\title{
Experimental Results From \\ Pressure Testing A 1:6-Scale \\ Nuclear Power Plant \\ Containment
}

Manuscript Completed: November 1991

Date Published: January 1992

Prepared by

D. S. Horschel

Sandia National Laboratories

Albuquerque, NM 87185-5800

Prepared for

Division of Engineering

Office of Nuclear Regulatory Research

U.S. Nuclear Regulatory Commission

Washington, DC 20555

NRC FIN A1249 


\begin{abstract}
This report discusses the testing of a 1:6-scale, reinforced-concrete containment building at Sandia National Laboratories, in Albuquerque, New Mexico. The scale-model, Light Water Reactor (LWR) containment building was designed and built to the American Society of Mechanical Engineers (ASME) code by United Engineers and Constructors, Inc., and was instrumented with over 1200 transducers to prepare for the test.

The containment model was tested to failure to determine its response to static internal overpressurization. As part of the U.S. Nuclear Regulatory Commission's program on containment integrity, the test results will be used to assess the capability of analytical methods to predict the performance of containments under severe-accident loads.

The scaled dimensions of the cylindrical wall and hemispherical dome were typical of a full-size containment. Other typical features included in the heavily reinforced model were equipment hatches, personnel air locks, several small piping penetrations, and a thin steel liner that was attached to the concrete by headed studs.

In addition to the transducers attached to the model, an acoustic detection system and several video and still cameras were used during testing to gather data and to aid in the conduct of the test.

The model and its instrumentation are briefly discussed, and is followed by the testing procedures and measured response of the containment model. A summary discussion is included to aid in understanding the significance of the test as it applies to real world reinforced concrete containment structures. The data gathered during SIT and overpressure testing are included as an appendix.
\end{abstract}




\section{TABLE OF CONTENTS}

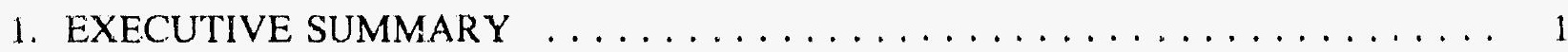

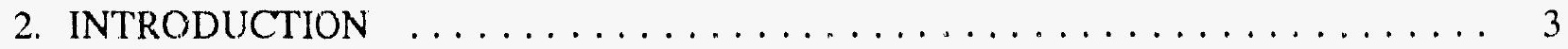

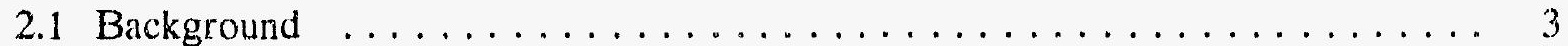

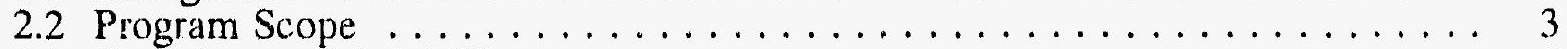

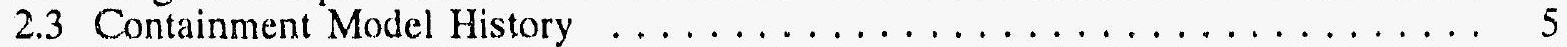

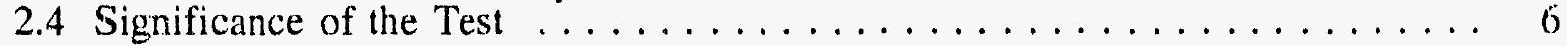

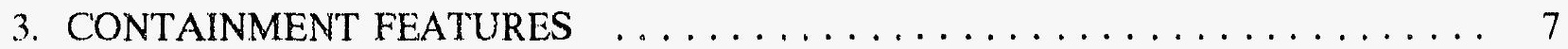

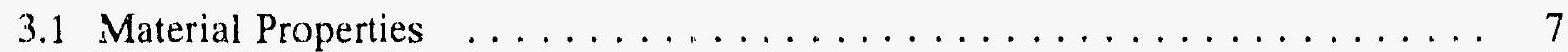

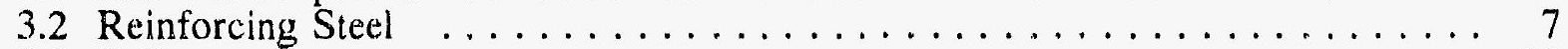

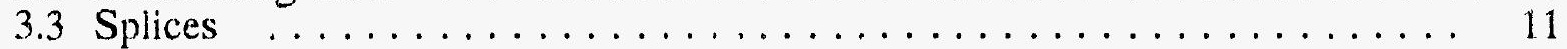

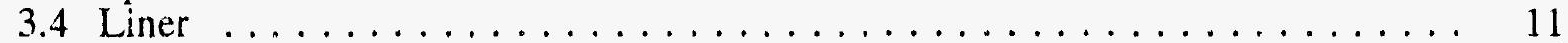

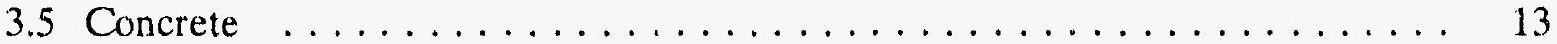

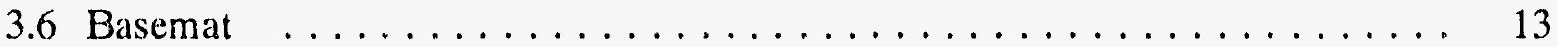

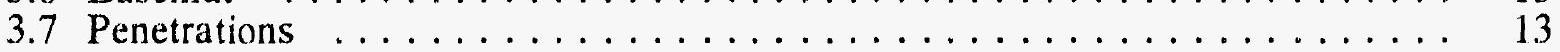

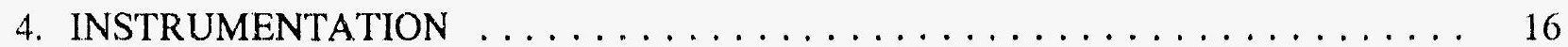

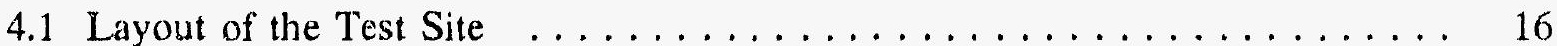

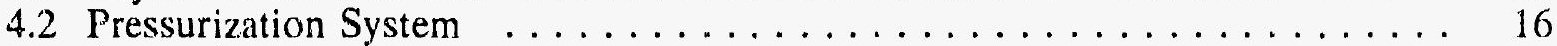

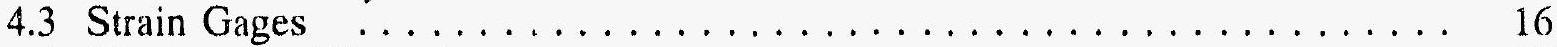

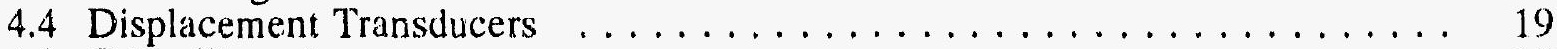

4.5 Other Transducers $\ldots \ldots \ldots \ldots \ldots \ldots$

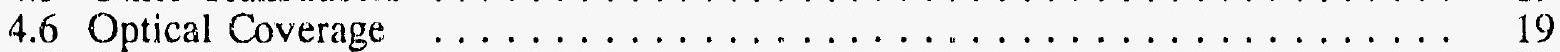

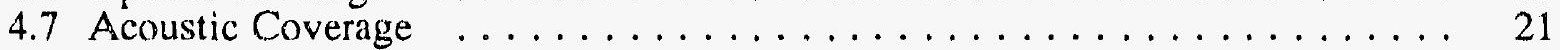

5. LOW-PRESSURE TESTING OF THE CONTAINMENT $\ldots \ldots \ldots \ldots \ldots$

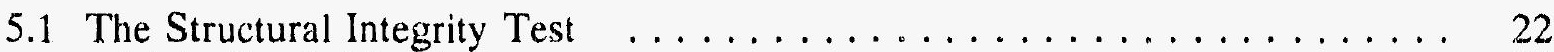

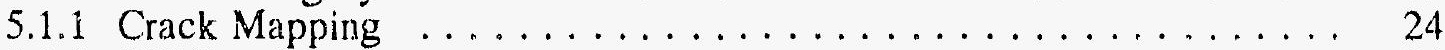

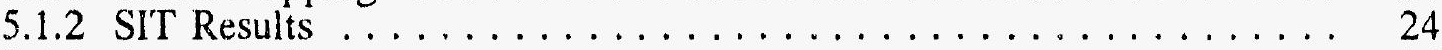

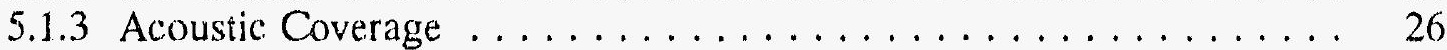

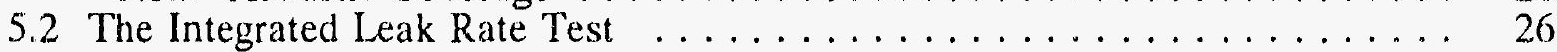

6. CONDUCT OF THE HIGH-PRESSURE TEST $\ldots \ldots \ldots \ldots \ldots \ldots$

6.1 Model Pressurization .............................. 29

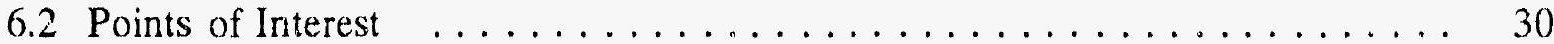

6.3 Events Leading to Test $\mathrm{Termination} \ldots \ldots \ldots \ldots \ldots \ldots \ldots \ldots \ldots$

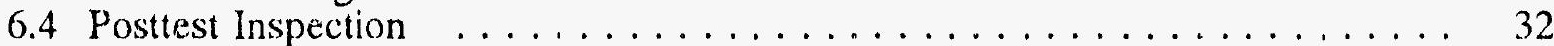

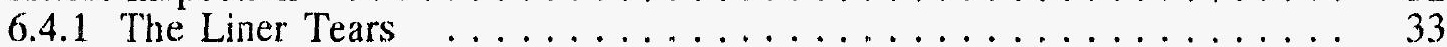

6.4 .2 Leakage Through the Small Tears ................ 36

6.4 .4 Ovalization of the Equipment Hatch Sleeves . . . . . . . . . . . 37

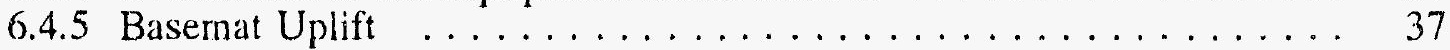




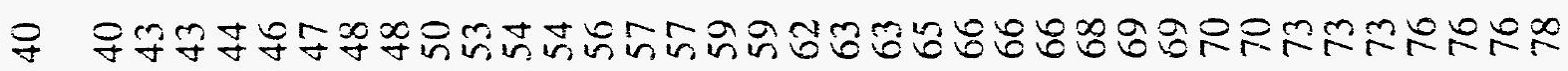

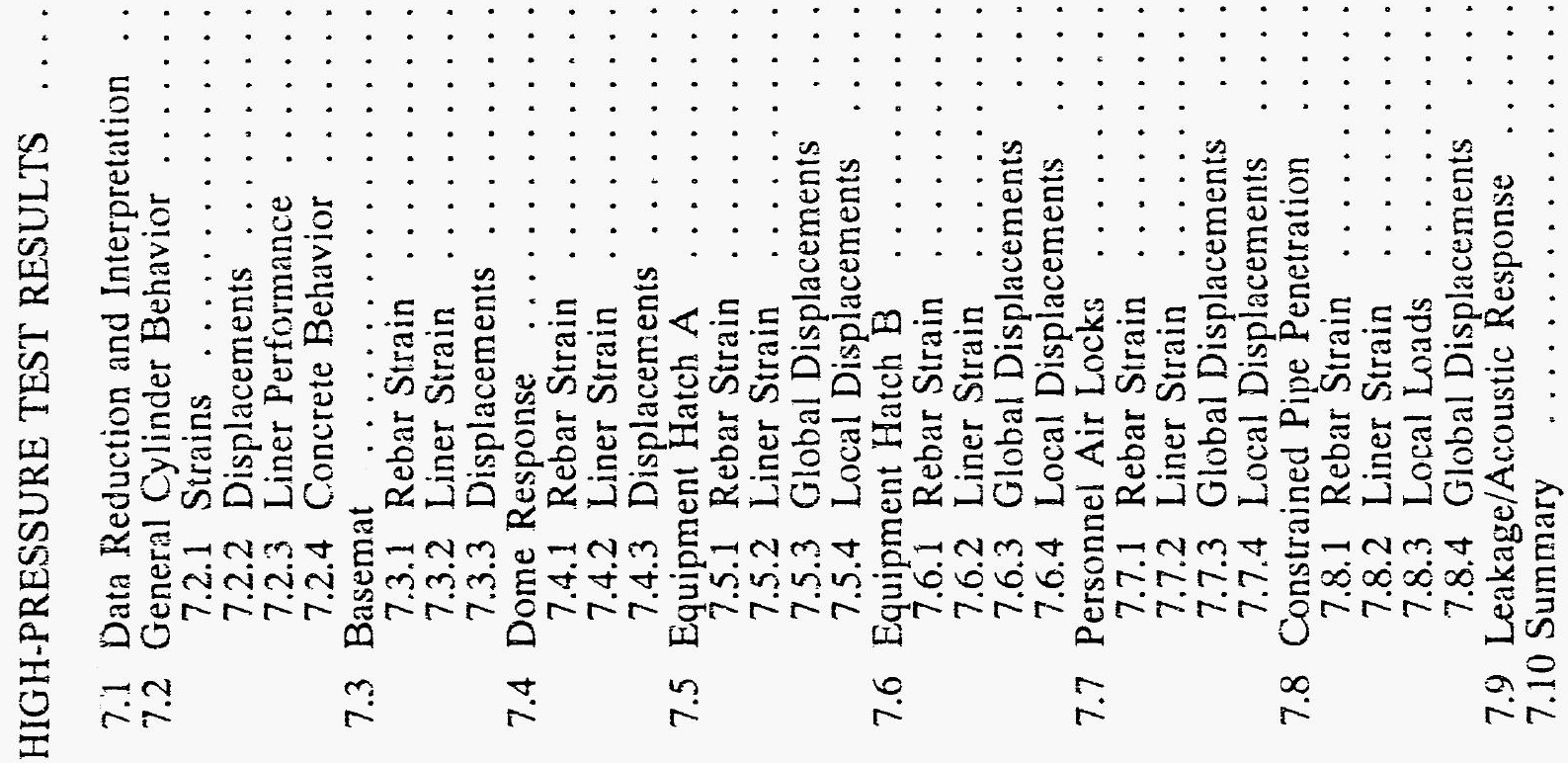




\section{TABLE OF CONTENTS CONTINUED}

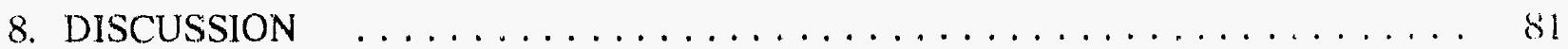

8.1 Repeatability of the Test and Other Failure Modes $\ldots \ldots \ldots \ldots \ldots \ldots$

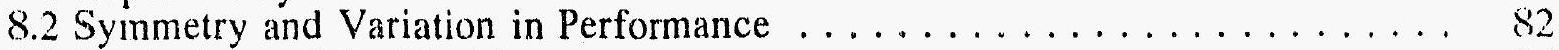

8.3 Limitations of the Model $\ldots \ldots \ldots \ldots \ldots \ldots \ldots \ldots \ldots \ldots \ldots \ldots$

8.3.1 Workmanship ............................ 84

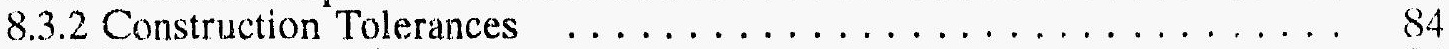

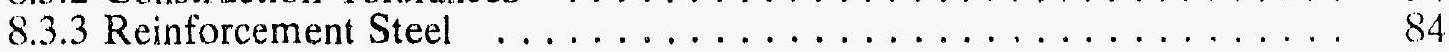

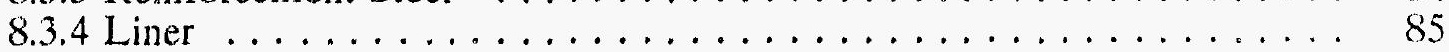

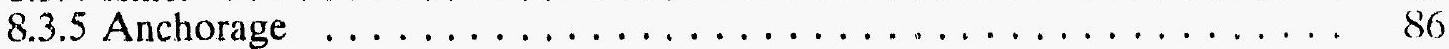

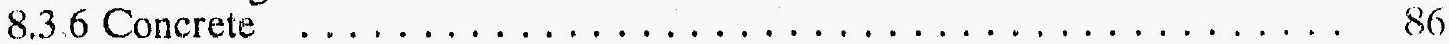

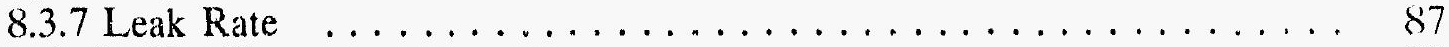

8.4 Trends In The Data . . . . . . . . . . . . . . . . . . . . 87

8.5 Test Determined Strain Concentration Factors $\ldots \ldots \ldots \ldots \ldots \ldots$

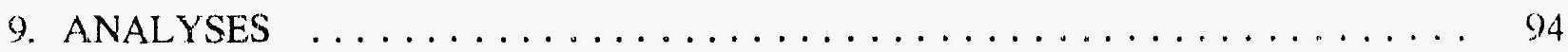

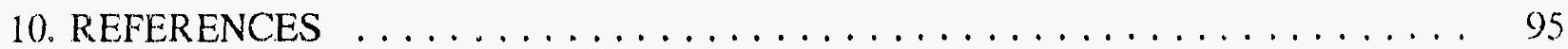

APPENDIX A: Crack Mapping Results From The Structural Integrity Test . . . . . A-1

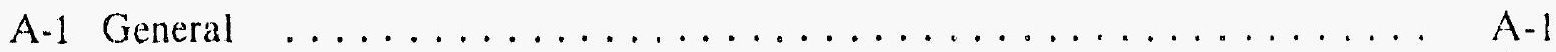

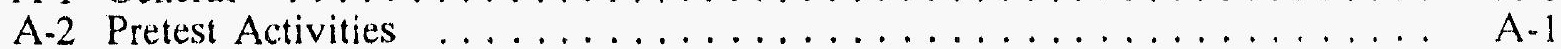

A-3 Crack Mapping Chronology $\ldots \ldots \ldots \ldots \ldots \ldots \ldots \ldots \ldots \ldots \ldots$

APPENDIX B: Results From The Track Transducers $\ldots \ldots \ldots \ldots \ldots \ldots$. . . . .

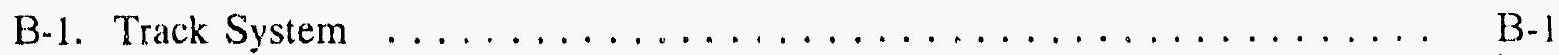

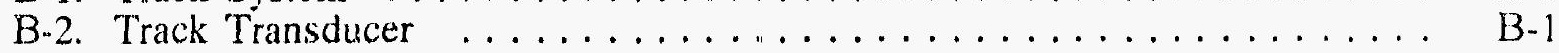

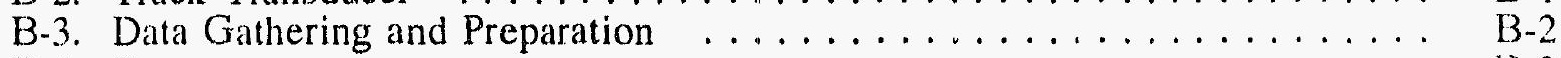

B-4. Data .............................

APPENDIX C: Test Results From The SIT and High-Pressure Test

(Appendix $C$ has been photoreduced into microfiche and inserted into a pocket in the back cover of this report. The following page numbers refer to the original printed pages.)

C-1 Introduction $\ldots \ldots \ldots \ldots \ldots \ldots \ldots \ldots \ldots \ldots \ldots \ldots \ldots \ldots \ldots$

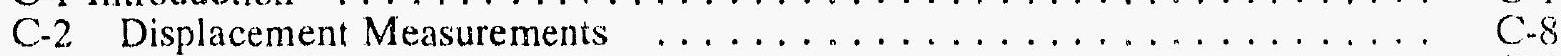

C-2.1 Potentiometers . . . . . . . . . . . . . . . . . C-8

C-2.2 Linear Variable Displacement Transducers . . . . . . . . . C-138

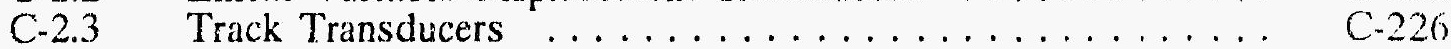

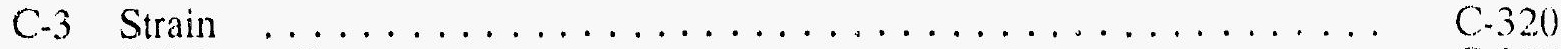

C-3.1 Rosettes ..................... C-320)

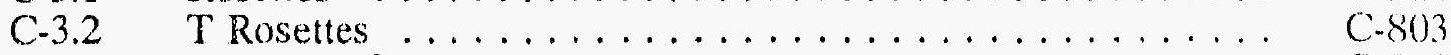

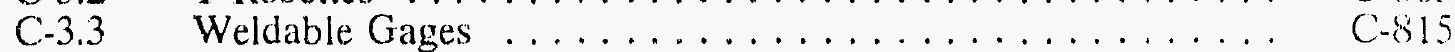

C -3.4 Strip Gages ....................... C.1.398

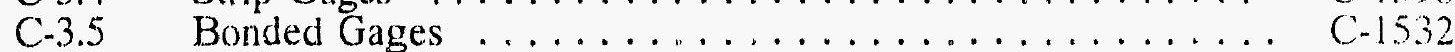

C-3.6 Singles ....................... C 1.580

C-4 Thermocouples ......................... C -1668

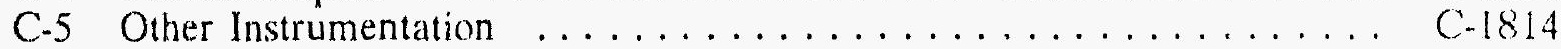




\section{LIST OF FIGURES}

1. Photograph of the Containment Model . . . . . . . . . . . . . . . . . . 4

2. Typical Section Through 1:6-Scale LWR Reinforced-Concrete Containment Model . 8

3. Typical Section Through the Basemat of the Containment Model . . . . . . . . . . 9

4. Typical Section Through the Cylinder Wall of the Containment Model . . . . . . 9

5. Typical Section Through the Dome of the Containment Model $\ldots \ldots \ldots \ldots$

6. Liner Stretchout Showing Containment Features $\ldots \ldots \ldots \ldots \ldots \ldots \ldots \ldots$

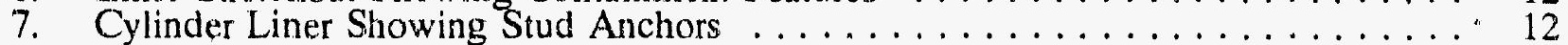

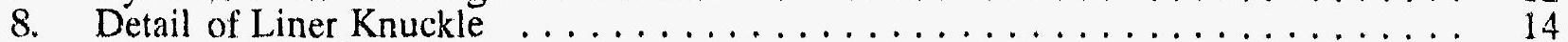

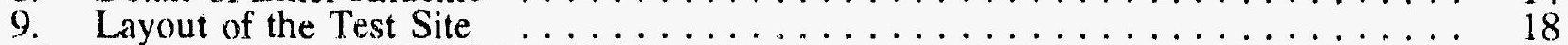

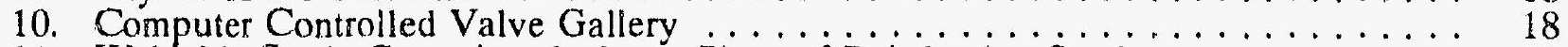

11. Weldable Strain Gage Attached to a Piece of Reinforcing Steel . . . . . . . . . 18

12. A Track Transducer System for Measuring Displacements of Containment Model 20

13. Liner Strains in the Hoop Direction Near Penetration R-2 . . . . . . . . . . . 25

14. Assessment of Leakage Rate Versus Time . . . . . . . . . . . . . . . . . . . . 27

15. Leakage Plot Using a 0.070 -In.-Diameter Orifice $\ldots \ldots \ldots \ldots \ldots \ldots \ldots$

16. Leakage Rate as a Function of Internal Pressure . . . . . . . . . . . . . . . 32

17. Stretchout of Containment Cylinder Showing Tears and Severely Distressed

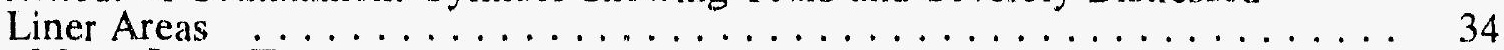

18. The Major Liner Tear $\ldots \ldots \ldots \ldots \ldots \ldots \ldots \ldots \ldots \ldots \ldots \ldots \ldots \ldots$

19. The Four Distressed Areas Around Equipment Hatch $\mathrm{A} \ldots \ldots \ldots \ldots \ldots$

20. Tear Next to Personnel Air Lock $A$. . . . . . . . . . . . . . . . . . . . 35

21. Holes in Liner Adjacent to Equipment Hatch B . . . . . . . . . . . . 36

22. Exposed Seal at Equipment Hatch $\mathrm{A}$ Because of Sleeve Ovalization $\ldots \ldots \ldots . . .38$

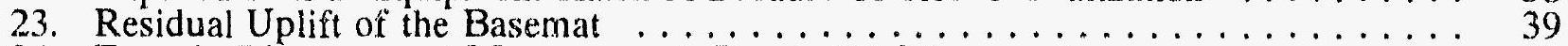

24. Error in Displacement Measurements Due to 2D Movement . . . . . . . . . 41

25. Hoop Strain in the Reinforcing Steel and Liner at Cylinder Midheight at the Final Pressure Level . . . . . . . . . . . . . . . . . . . . . . . . 44

26. Radial Displacement of Cylinder Wall Midheight $\ldots \ldots \ldots \ldots \ldots \ldots \ldots$

27. Hoop Strain in Liner Near an Assumed Intersection with a Crack in the Concrete at 7 Pressure Levels . . . . . . . . . . . . . . . . . . . . 46

28. Hoo Strain in Concrete Between Two Vertical Cracks in the Cylinder Wall .... 47

29 Strains in the Top Layer of Basemat Reinforcing Steel . . . . . . . . . . . . . 49

30. Strains in the Bottom Layer of Basemat Reinforcing Steel . . . . . . . . . . . . . 49

31. Meridional Strain vs Pressure for Strip Gage 1 in Liner Floor/Cylinder Knuckle . . 51

32. Meridional Strain vs Pressure for Strip Gage 4 in Liner Cylinder . . . . . . . . . . 51

33. Meridional Strain Field at Liner/Wall Junction at Seven Pressure Levels $\ldots . . . .52$

34. Inside and Outside Liner Strains Near the Knuckle $\ldots \ldots \ldots \ldots \ldots \ldots \ldots \ldots$

35. Uplift of the Basemat at Its Outer Edge $\ldots \ldots \ldots \ldots \ldots \ldots \ldots \ldots \ldots \ldots$

36. Strain Comparison Between the Inside and Outside Layei of Meridional Reinforcing Steel Above the Dome Springline . . . . . . . . . . . . . 55

37. Strain Comparison Between the Inside and Outside Layer of Hoop Reinforcing Steel Above the Dome Springline . . . . . . . . . . . . . . 56

38. Dome Liner Strains (Principal Maximum and Minimum) 67 from the Horizontal 57

39. Vertical Displacement of Dome Apex Including Meridional Shell Stretching . . . . 58

40. Concrete Embedment Gages Near the Boss of Equipment Hatch A . . . . . . . . . 59

41. Variation in Hoop Strain in the Reinforcing Bar Around the Slceve of Equipment Hatch A .......................... 60

42. Liner Strains Near the Insert Plate of Equipment Hatch A . . . . . . . . . . . . 61

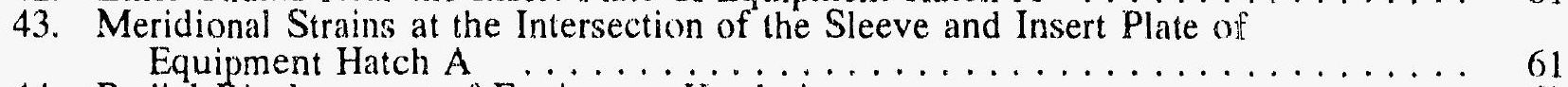

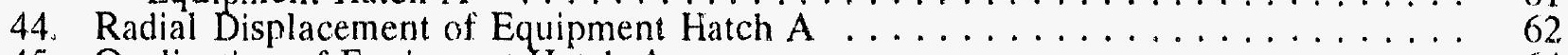

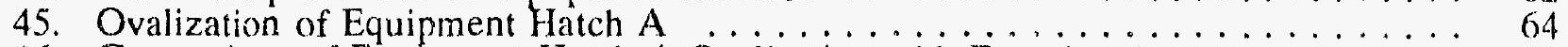

46. Comparison of Equipment Hatch A Ovalization with Equation $1 \ldots \ldots \ldots$

47. Plan View of Hatch B Showing Deformation of Liner at Boss Junction $\ldots \ldots$. . . 65

48. Variation in Hoop Strain in the Reinforcing Bar Around the Sleeve of

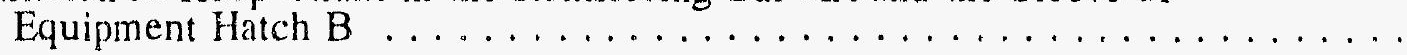




\section{LIST OF FIGURES CONTINUED}

49. Liner Hoop Strains Adjacent to the Conical Liner Section at Equipment Hatch B 67

50. Displacement of Equipment Hatch $B \ldots \ldots \ldots \ldots \ldots$

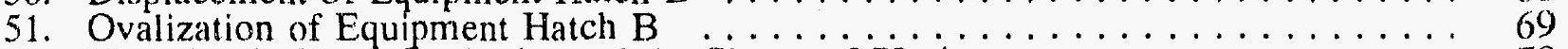

52. Variation in Hoop Strain Around the Sleeve of PL.$A \ldots \ldots \ldots$

53. Liner Strains Adjacent to PL-A Insert Plate and a Liner Tear . . . . . . . . . . 71

54. Dishing of the Center of the Bulkhead at PL-A $\ldots \ldots \ldots \ldots \ldots \ldots \ldots$

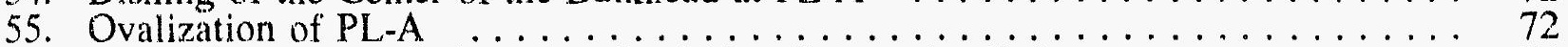

56. Hoop Strain in the Reinforcing Steel Near Penetration R-2 . . . . . . . . . 74

57. Meridional Strain in the Reinforcing Steel Near Penetration R-2 . . . . . . . . 74

58. Hoop Strain Near Penetration R-2 at Seven Pressure Levels . . . . . . . . . . . . 75

59. Radial Displacements at Elevation $20.125 \mathrm{ft}$ at Six Pressure Levels . . . . . . . . . 77

60. Mean Signal Level for an AE Sensor at Equipment Hatch B . . . . . . . . . . . 77

61. Ringdown Counts for an AE Sensor at Equipment Hatch A . . . . . . . . . . . 78

62. Histogram of Strain Ranges . Data Step $37 \ldots \ldots \ldots \ldots \ldots \ldots$

63. Group Histogram of Strain Kanges for Data Steps 26 to $36 \ldots \ldots \ldots$. . . . . 91

\section{APPENDIX A FIGURES}

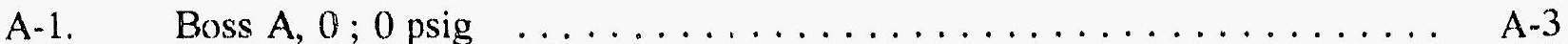

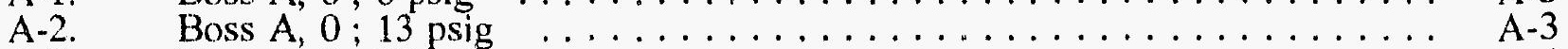

A-3. Boss A, $0 ; 22$ psig $\ldots \ldots \ldots \ldots \ldots \ldots \ldots \ldots \ldots \ldots \ldots \ldots \ldots$

A-4. Boss A, $0 ; 34$ psig $\ldots \ldots \ldots \ldots \ldots \ldots \ldots \ldots \ldots \ldots \ldots$ A-4

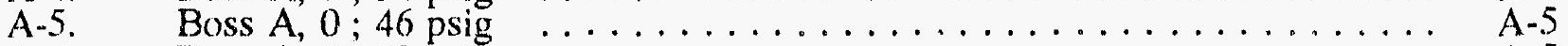

A-6. Boss A, $0 ; 53$ psig $\ldots \ldots \ldots \ldots \ldots \ldots \ldots \ldots \ldots \ldots \ldots \ldots$

A-7. Boss A, $0 ; 46$ psig $\ldots \ldots \ldots \ldots \ldots \ldots \ldots \ldots \ldots \ldots \ldots \ldots \ldots \ldots$ A $6 \ldots \ldots$

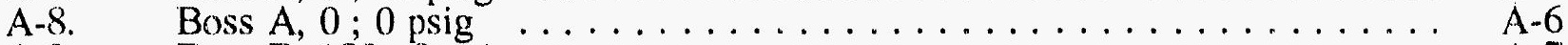

A-9. Boss B, $180 ; 0$ psig $\ldots \ldots \ldots \ldots \ldots \ldots \ldots \ldots \ldots \ldots \ldots \ldots$

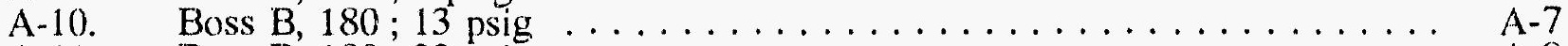

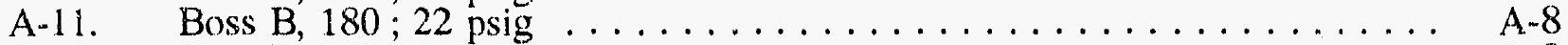

A-12. Boss B, $180 ; 34$ psig $\ldots \ldots \ldots \ldots \ldots \ldots \ldots \ldots \ldots \ldots \ldots \ldots \ldots \ldots$

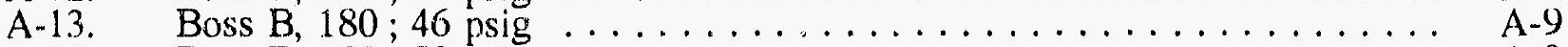

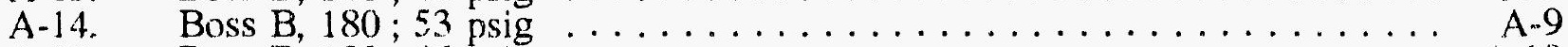

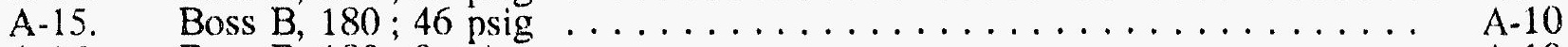

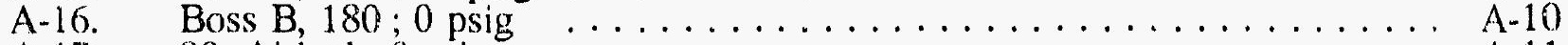

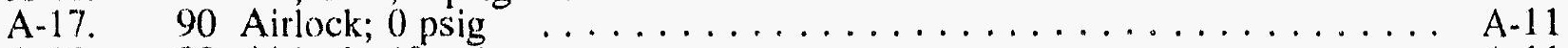

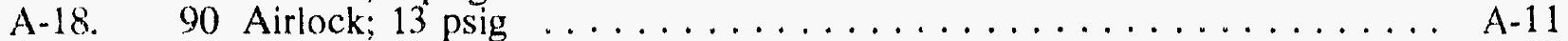

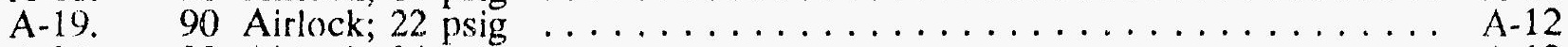

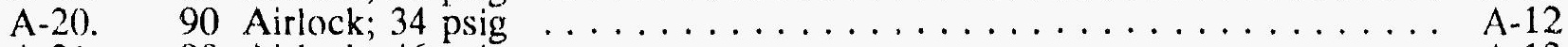

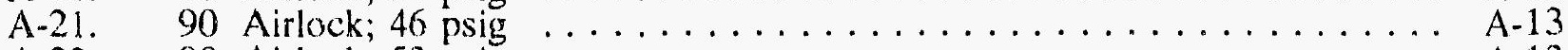

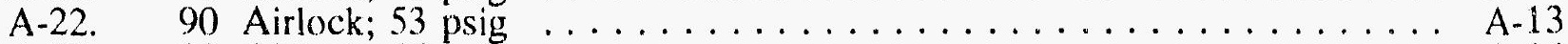

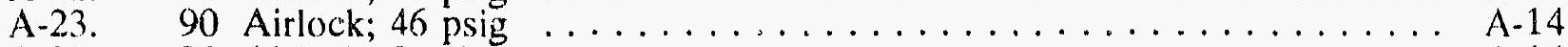

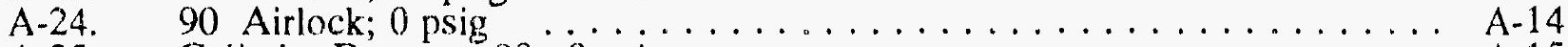

A-25. Cylinder Basemat, $90 ; 0$ psig $\ldots \ldots \ldots \ldots \ldots \ldots \ldots \ldots \ldots \ldots \ldots \ldots$

A-26. Cylinder Basemat, $90 ; 13$ psig $\ldots \ldots \ldots \ldots \ldots \ldots \ldots \ldots \ldots \ldots \ldots \ldots$

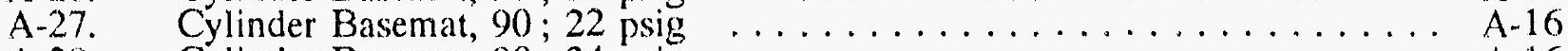

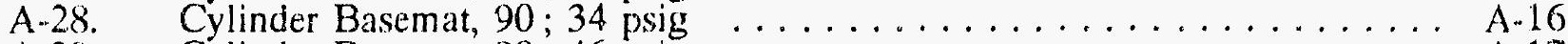

A-29. Cylinder Basemat, $90 ; 46$ psig $\ldots \ldots \ldots \ldots \ldots \ldots \ldots \ldots \ldots \ldots \ldots \ldots \ldots$

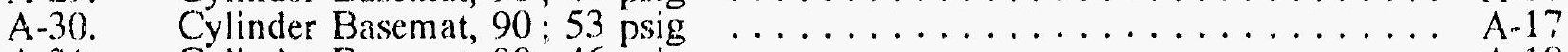

A-31. Cylinder Basemat, $90 ; 46$ psig $\ldots \ldots \ldots \ldots \ldots \ldots \ldots \ldots \ldots \ldots \ldots \ldots$

A-32. Cylinder Basemat, $90 ; 0$ psig $\ldots \ldots \ldots \ldots \ldots \ldots \ldots \ldots \ldots \ldots$ 


\section{LIST OF FIGURES CONTINUED}

APPENDIX A FIGURES

A-33.135@13;0 psig .................................. A-19

A-34.135@13;13 psig ................................ A-19

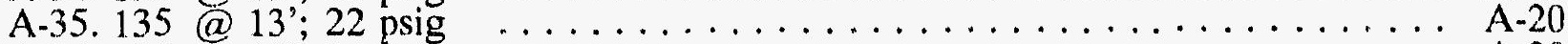

A-36.135@13;34 psig .............................. A-20

A-37.135@13;46 psig .............................. A-21

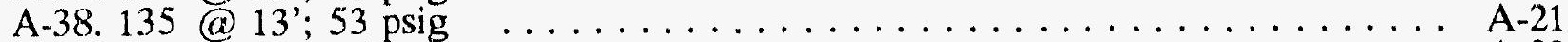

A-39.135@13; 46 psig .............................. A-22

A-40.135@13;0 psig .................................. A-22

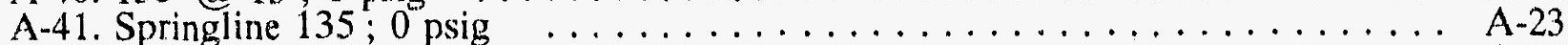

A-42. Springline $135 ; 13$ psig $\ldots \ldots \ldots \ldots \ldots \ldots \ldots \ldots \ldots \ldots \ldots \ldots \ldots \ldots \ldots \ldots \ldots$

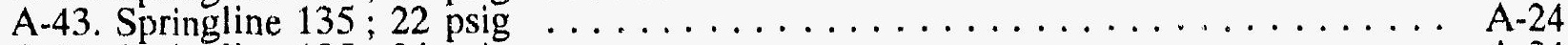

A-44. Springline $135 ; 34$ psig $\ldots \ldots \ldots \ldots \ldots \ldots \ldots \ldots \ldots \ldots \ldots \ldots \ldots \ldots$ A-24

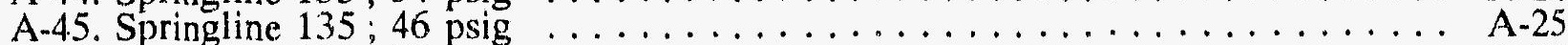

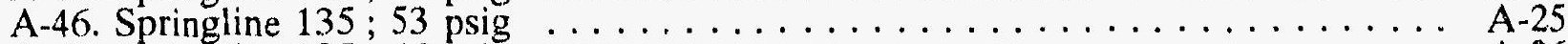

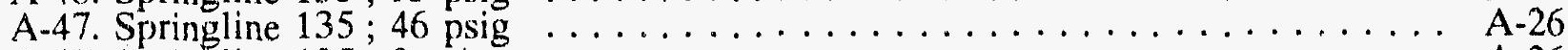

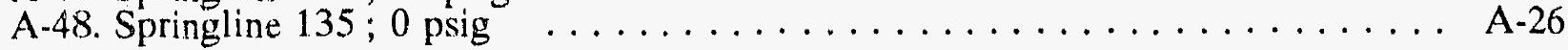

\section{APPENDIX B FIGURES}

B-1. Track 1, Interior Track Transducer Below EQ. A-1 $\ldots \ldots \ldots \ldots \ldots \ldots$ B-5

B-2. Track 2, Interior Track Transducer Near PL-A $\ldots \ldots \ldots \ldots \ldots \ldots \ldots \ldots$ B-5

B-3. Track 3, Interior Track Transducer Below EO.-B $\ldots \ldots \ldots \ldots \ldots \ldots \ldots \ldots$ B-6

B-4. Track 4, Interior Track Transducer Near PL-B . . . . . . . . . . . . . B B-6

B-5. Track 5, Exterior Track Transducer Below EQ.-A $\ldots \ldots \ldots \ldots \ldots \ldots \ldots$ B.

B-6. Track 6, Exterior Track Transducer Near PL-A . . . . . . . . . . . . .

B-7. Track 7, Exterior Track Transducer Below EQ -B . . . . . . . . . . . . . B B-8

B-8. Track 8, Exterior Track Transducer Near PL-B . . . . . . . . . . . . . . . B-8

B-9. Track 9, Basemat Track Transducer $\ldots \ldots \ldots \ldots \ldots \ldots \ldots \ldots \ldots$ B-9 


\section{LIST OF TABLES}

1. Still and Video Cameras Used During Containment Testing $\ldots \ldots \ldots \ldots \ldots$

2. Loading and Data Retrieval Intervals for the SIT $\ldots \ldots \ldots \ldots \ldots \ldots \ldots \ldots \ldots$

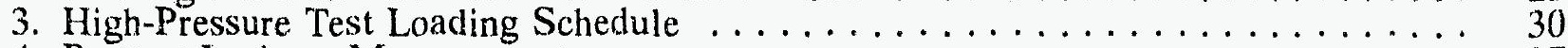

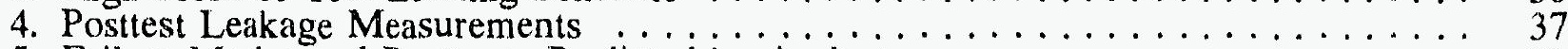

5. Failure Modes and Pressures Predicted by Analysts $\ldots \ldots \ldots \ldots \ldots \ldots \ldots$

6. Potential Differences Between the Model and a Prototype Containment $\ldots \ldots \ldots . . .83$

7. Strain Levels From The High Pressure Test $\ldots \ldots \ldots \ldots \ldots \ldots \ldots \ldots \ldots$

8. Experimentally Measured Strain Concentration Factors for the Containment Liner $\ldots$.

B-1. Location and Length of the Track Systems $\ldots \ldots \ldots \ldots \ldots \ldots \ldots \ldots$ B-1

B-2. Pressure Levels at Which Data Were Gathered During High Pressure Test .... . B-3

C-1. Instrumentation Listed by Channel Number $\ldots \ldots \ldots \ldots \ldots \ldots \ldots \ldots \ldots$ C.2 


\section{ACKNOWLEDGMENTS}

The testing of the containment model involved many people who aided in the success of the program.

David Clauss and Randy Weatherby helped review data and selected the interesting areas to monitor during the test. Their scrutinizing of the data following the test helped to select points of interest for this report. Their sharing of insights has made this report much more useful and interesting.

Marty Jinzo helped to prepare this report. He produced the entire set of plots found in Appendix $\mathrm{C}$ and wrote the report on the track transducers in Appendix B. His conscientious efforts helped to minimize errors in this report and in NUREG/CR-5083.

I also thank the Sandia and K-Tech personnel, many of whom have been instrumenting containment models since 1982. Dwight Lambert has led the efforts and orchestrated the many activities at the site. His understanding of the program, his multifaceted background, and his ability to work with the many people and crews are in a large way responsible for the success of the containment testing. His performance has been traly meritorious. The efforts of the following people in instrumenting the model--Jim Westmoreland, Marty Jinzo, Bob Eyers, Barry Spletzer, Pat Drozda, Rob Woodard, George Dibisceglie, Gary Loughrin, Frank Horine, Mike Turner, Mike Ramirez, and Bob Padilla--are gratefully acknowledged.

Thanks are due to Walt von Riesemann for his help and support throughout the entire containment testing effort.

Several groups at Sandia also deserve special recognition for their dedication and performance. The groups are Still Photography, Video Services, and the Nondestructive Technology Division, particularly Rob Smith, Joe Llamas, Bob Gardner, Ed Sisneros, Alan Beattie, Dan Costley, and Jean Sena.

Doug Brosseau and Doug Brinson from ERC, Inc., helped during the testing of the containment. Their help is gratefully acknowledged. Mr. Brosseau not only helped during testing, but also assisted during the construction of the containment model. He aided immensely in the crack mapping during the Structural Integrity Test and wrote the introduction to Appendix A. Mr. Brinson led the posttest effort to assess the leakage through the small liner tears.

Special thanks are due to the NRC and, in particular, to James Costello for their support of the containment integrity program. They provided a well-defined problem and the freedom to solve it in a proper manner.

Finally, thanks are due to the peer review group. Their many suggestions for this test and throughout the entire containment testing program helped to ensure that we were always looking at the right problem. They genuinely improved the testing efforts by their time, ideas, and expertise. The peer review group consists of Thomas J. Ahl, Wilfred E. Baker, William C. Black, Ted M. Brown, Richard Denning, Asadour H. Hadjian, Theodore E. Johnson, Mete A. Sozen, John D. Stevenson, H. T. Tang, Joseph J. Ucciierro, and Richard N. White. 


\section{EXECUTIVE SUMMARY}

Sandia National Laboratories completed testing of a 1:6-scale containment building for a Light Water Reactor (LWR) in July 1987. The containment building was made of reinforced concrete and had many of the features of full-size containments. The test program consisted of a Structural Integrity Test, an Integrated Leak Rate Test, and a concluding overpressurization test of the structure. The testing of the containment model and the results obtained are the subject of this report.

The containment model was tested to learn more about the behavior of containment structures subjected to pressure loadings beyond their design basis. The test results will aid in benchmarking analytical tools for assessing the performance of containment buildings subjected to a wide variety of accident scenarios.

The containment model was built by United Engineers and Constructors to the American Society of Mechanical Engineers (ASME) code standards with a design pressure of 46 psig. The 37foot-tall model had an inside diameter of $22 \mathrm{ft}$ and many typical features, including

- 2 personnel air locks,

- 2 equipment hatches and operable covers,

- 3 piping clusters,

- 2 steam lines,

- 8 layers of primary reinforcing in the cylinder, and

- a thin steel liner anchored to the concrete by headed studs.

The overall geometry of the containment model was a right-circular cylinder attached to the flat basemat and capped by a hemispherical dome.

The containment model had over 1200 discrete sensors, most of which were strain gages and displacement transducers. About 300 of these sensors were embedded in the concrete wall of the containment model. The remainder of the instrumentation was attached to the concrete or liner surfaces.

Low-pressure testing, which consisted of a Structural Integrity Test (SIT) and an Integrated Leak Rate Test (ILRT), was completed in early July 1987. These tests involved pressurization to 1.15 times design pressure for the SIT and to design pressure for the ILRT. The resultant cracks in the concrete were uniform in the cylinder with an average spacing of about $41 / 2$ in.; very few of the cracks exceeded a width of 0.010 in. Very few cracks were detected in the dome region, and those present had widths well below 0.010 in.

The ILRT was conducted at $46 \mathrm{psig}$, with the final leak rate measured as $0.14 \%$ mass/day. An orifice, which had a hole diameter of $0.070 \mathrm{in}$., was later used to simulate a leak in the model. The measured leak rate using this orifice was $11.5 \%$ at about $46 \mathrm{psig}$. Although this leak rate was somewhat large for normal ILRT testing, our goal was to determine typical leak sizes that might be encountered and measured during overpressurization of the containment model as well as typical times required for measuring them. Following the low-pressure tests, some additional instrumentation was added to the containment model and high-pressure testing began.

High-pressure testing of the containment model was conducted in July 1987 by pressurizing the containment with nitrogen gas until leakage exceeded the capacity of the pressurization system. During high-pressure testing, the load was increased in steps ranging in size from 2 to $10 \mathrm{psi}$. After the containment was held at each increased pressure level for about half an hour, all 
transducers were remotely scanned, and the results were stored on a data acquisition computer. Before the pressure was increased to the next level, photographs were taken via computer control from nine fixed camera positions, and portions of the model were videotaped by 12 video cameras, many of which were remotely controlled.

High-pressure testing ended after 32 hours. The containment model was pressurized to over three times its design pressure of $46 \mathrm{psig}$. At the peak pressure level of $145 \mathrm{psig}$, leakage exceeded $4000 \mathrm{scfm}$, which was the limit of the pressure source. This necessitated ending the test.

The cracks in the concrete that had developed during low-pressure testing became much wider (many were about $1 / 8$ in. wide), but few new cracks developed. Radial displacements at the cylinder midheight were roughly $11 / 2$ to $2 \mathrm{in}$., while typical strains in the hoop direction were between $11 / 2$ to $2 \%$. The peak liner strain recorded exceeded $8 \%$. One of the equipment hatch sleeves was deformed into an oval, which exposed one of the two gum-drop seals for a portion of its length. A dishing of the basemat was observed, which resulted in an average uplift of the outer edge of the basemat of about $3 / 8$ in.

Leakage from the containment model was quite small for most of the test; however, during the final few pressure steps, leakage grew rapidly. At 135 psig, a leak rate of about $8 \mathrm{scfm}$ was measured. This grew to $50 \mathrm{scfm}$ ( $62 \%$ mass/day) at the pressure level of $143 \mathrm{psig}$, and by the next pressure level, 145 psig, leakage grew to over $4000 \mathrm{scfm}$ ( $>5000 \%$ mass/day). The major source of leakage was a 22 -in.-long tear in the liner. Upon further investigation after the test, many other highly distressed areas were found in the liner; in five of these areas, tears existed that were about $1 / 2$ to $1 / 8$ in. long. Each of these tears, including the major tear, was adjac ini to a stud that attached the liner to the concrete.

Posttest evaluation revealed large strain concentrations near the penetration insert plates that were strongly influenced by the studs that anchored the liner to the rcinforced concrete wall. In addition to the six tears, there were many areas that had visible signs of distress.

The penetrations, in general, were stiffer than the non-perturbed wall: the cylinder wall moved radially farther outward than the penetrations. At the peak pressure level of $145 \mathrm{psig}$, equipment hatch $\mathrm{A}$ and $\mathrm{B}$ moved out radially 1.19 and 1.15 in. respectively, while the average radial displacement at mid-cylinder was $1.60 \mathrm{in}$. away from the large penetrations.

The perimeter of the basernat lifted off the work mat about $3 / 8$ in. at the peak pressure; however, only a small amount of uplift remained after the internal pressure was released. Very little plastic response was observed in the steel in the basemat or the dome of the containment.

The response at the junction of the dome and cylinder was measured by the transducers located at the springline. The meridional bending moment at the springline was evident from the strain measurements taken. The only plastic response measured in the dome was the small plastic strains in the reinforcing steel located near the springline.

The results collected during the test are presented with the insights gained during from this experiment. Included in the appendices is a complete tabulation and plotting of the all the transducer responses recorded during the test. 


\section{INTRODUCTION}

The U.S. Nuclear Regulatory Commission (NRC) is investigating the performance of Light Water Reactor (LWR) containments subjected to severe accidents. This work is being performed by the Containment Technology Division at Sandia National Laboratories (Sandia) in Albuquerque, New Mexico. The research effort described in this report involved testing a 1:6-scale, reinforced-concrete containment model. The containment model, which was designed and constructed by United Engineers and Constri:) [1-6], was the largest and most complex model of its kind (Figure 1).

The objective of the containment model tests was to generate data that could be used to qualify methods for reliably predicting the response of LWR containment buildings to severeaccident pressure loads. The test data recorded strains and deformations of the containment shell, as well as the leakage of the containment.

\subsection{Background}

Research into the behavior of LWR containment buildings subject to hypothesized severe accidents has been ongoing since the accident at Three Mile Island. The containment building, whether it is made of steel, reinforced concrete, or prestressed concrete, is the final engineered barrier to prevent the release of fission products and radioactive gases generated during reactor operations. The containment is designed to withstand accident conditions such as a Loss-of-Coolant Accident and environmental loading such as in earthquakes, and it relies not only on its structural capability, but also on its leak tightness.

After Three Mile Island, attention focused on the capability of containment buildings to withstand loadings beyond their design basis. To address containment performance beyond design capabilities, the NRC is sponsoring a testing and analytical program at Sandia. Sandia has conducted static overpressurization tests on five scale models of steel containment buildings $[7-14]$ and recently completed testing the reinforced-concrete containment model, that is the subjeat of this report.

\subsection{Program Scope}

The testing of the containment models is only one of the programs sponsored by the NRC to address containment integrity during severe accidents. Other programs sponsored by the NRC that deal with the performance of the containment system are also described below.

Tests and analyses are being conducted on mechanical penetrations of containment buildings, such as personnel air locks and bellows [15-20]. Some of these tests have included or will include both temperature and pressure as testing parameters. Structural data on equipment 


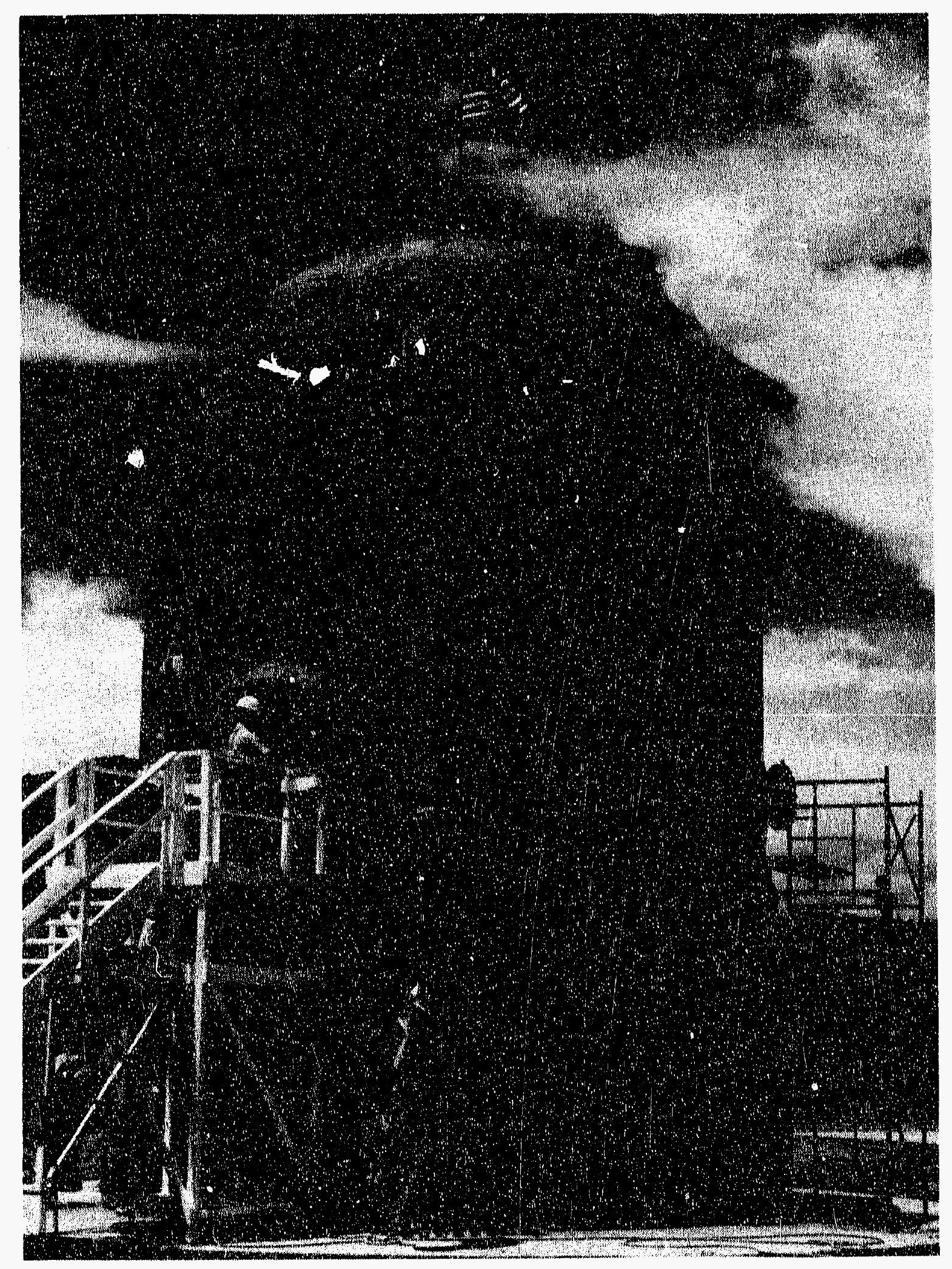

Figure 1. Photograph of the Containment Model. 
hatches, personnel air locks, and other mechanical penetrations are gathered from the containment tests and are used to augment additional tests on these penetrations when they are deemed necessary.

Three tests have been completed on the electrical penetration assemblies used in containments [21-23] and have included temperature and pressure profiles.

Extensive seal and gasket testing has also been conducted for the NRC. The elastomers used in sealing the mechanical penetrations are subject to severe accident environments that may include radiation exposure [24-26]. These tests determine the sealing capabilities of the elastomers when exposed to severe accident environments.

Tests on bellows, used to compensate for expansion and contraction in containment piping systems, are scheduled. Since bellows exist in many containments and they serve as part of the containment boundary, their integrity is important. Tests and analyses are being pursued, but no testing has been completed as of this writing.

When the information gained through these programs is considered, the bounds of behavior for a containment building system subjected to a severe accident can be deduced. These programs provide a basis for evaluating the performance of existing plants and may be used to improve the severe-accident performance of future plants.

\subsection{Containment Model History}

Several tests on scale-model containment buildings were conducted at Sandia from December 1982 to the latest test in July 1987. Four 1:32-scale steel containment models were tested from December 1982 to December 1983. These tests were followed by a test of a 1:8-scale steel containment model in November 1984. All tests were static, used nitrogen gas for pressurization, and continued until the containment model failed. The steel containment tests were followed by the test of the first reinforced-concrete containment model, described in this report.

The construction of the reinforced-concrete containment was much more complex than that of the steel containments. This necessitated more careful planning and design. The design of the containment model began in January 1985 and was completed in June 1985 . To ensure construction of a high-quality model, its design and a series of preconstruction tests was conducted in parallel. Because of the containment model's size, and in consideration of schedule constraints, particular attention was paid to liner welding, splices for the reinforcing steel, concrete placement, and other construction and sequencing details. Reference 27 is a report on the preconstruction tests performed during the design of the containment model.

Construction of the containment model began in August 1985 after comments and suggestions made by a peer review panel were incorporated into the design. The basemat concrete was placed in December 1985. Sections of the liner began arriving on site in January 1986, and the liner work was completed in February. The first concrete was placed in the cylinder wall in March, and concreting of the model concluded in May 1986. Model construction was completed in June 1986. Attaching, wiring, and checking over 1200 channels of instrumentation and other preparations for testing took several months. Low-pressure testing began in early July 1987; high-pressure testing followed in late July. 


\subsection{Significance of the 'Test}

Little information was available before this test on the ultimate performance of reinforcedconcrete pressure vessels, certainly nothing in sufficient detail to allow for validation of analytical tools. Further, there was only speculation about the failure mode and associated phenomena of a reinforced-concrete containment subjected to overpressurization.

The nonlinear behavior of a reinforced-concrete containment pressurized to failure is extremely difficult to model mathematically. Some simplifications are required, given the limitations of current computers and algorithms. This test has provided the engineering community with some indication of the critical items that should be included in a proper analysis of this type of structure. By having some assurance of what should be include in the analysis, the complexity and number of analyses can be greatly reduced.

With the completion of this test, an extremeiy large set of data was generated that can be used to validate computer analyses. Much was learned about the complex interaction between the reinforcing steel, the concrete, and the liner anchorage system in this large composite structure. The test provided insights into ultimate containment response, but also very importantly, it indicated where the analytical tools may need to be improved.

Certainly this single test has not answered all the questions--there is still need for additional research--but the test represents a significant milestone in our understanding of containment behavior and the first steps of validating the ability of analytical tools to predict reinforcedconcrete containment response in a severe accident environment. 


\section{CONTAINMENT FEATURES}

The 1:6-scale reinforced-concrete containment was designed in accordance with the American Society of Mechanical Engineers (ASME) code and had a design pressure of 46 psig. The inside diameter of the model was $22 \mathrm{ft}$ with a total height of $37 \mathrm{ft}$. The cylinder wall was 9 $3 / 4$ in. thick, and the dome wall was 7 in. thick. This model used $\# 4^{*}$ reinforcing bar for the primary reinforcing. The cylinder wall contained two layers of meridional, four layers of circumferential, and two layers of diagonal reinforcing steel. As these layers approached the apex of the dome, some layers were reduced in response to changes in the geometry and loading. The containment also had a steel liner on the inside surface that was $1 / 16$ in. thick along the base and cylinder wall and $1 / 12$ in. thick along the dome wall. The liner was attached to the concrete by $\approx 25000$ headed studs. Two equipment hatches, two air locks, constrained penetrations, and several piping penetrations were also included in the containment model. All construction materials had structural characteristics the same or nearly the same as those of actual containment buildings. A schematic of the containment is shown in Figure 2. Several reports that describe its design, construction, features, and instrumentation are available [1-6].

\subsection{Material Properties}

One crucial link between the containment model and the finite elemer $t$ analyses was the material properties. In addition to the many materials tests during the design and construction phases of the containment model, Sandia conducted additional material testing $[1,28]$. Since the material properties are important, more material samples were taken than were needed for the testing, and they may be used for further material testing.

\subsection{Reinforcing Steel}

The complex reinforcing patterns used in the containment model reflected those in actual containments. The primary reinforcing material was \#4 reinforcing steel (1/2 in. diameter), but some \#5 bars were used at the basemat/cylinder junction to resist the meridional moment, and \#5 and \#6 reinforcing steel was used in the basemat. Shear reinforcing called for \#2 and \#3 bars; however, \#2 bars were not readily available. Consequently, deformed 6-mm reinforcing bars of foreign manufacture were purchased, which had similar chemical and physical properties to the other reinforcing steel. All other reinforcing was A615 Grade 60.

Some typical reinforcement patterns in the model are shown in Figures 3 - 5. The reinforcing in the basemat and the bottom of the cylinder wall is shown in Figure 3. Figure 4 shows a typical section through the containment wall away from penetrations and the basemat. $A$ photograph of the dome and some of its unique features are shown in Figure 5.

\footnotetext{
*The reinforcing steel number indicates the diameter in eighths of an in., for example, a \#4 reinforcing bar has a 1/2-in. (4/8-in.) nominal diameter.
} 


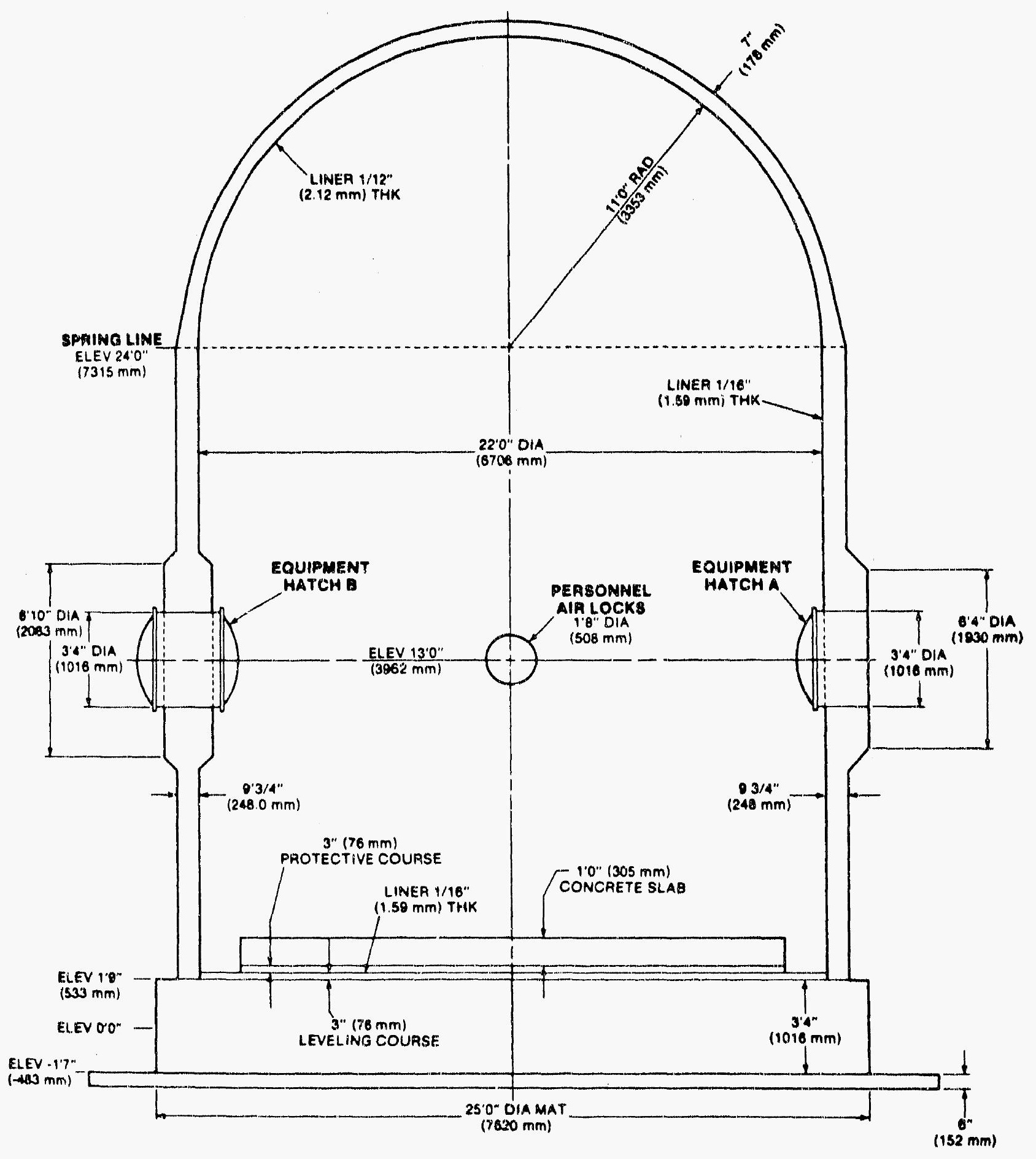

Figure 2. Typical Section Through 1:5-Scale LWR Reinforced-Concrete Containment Model. 


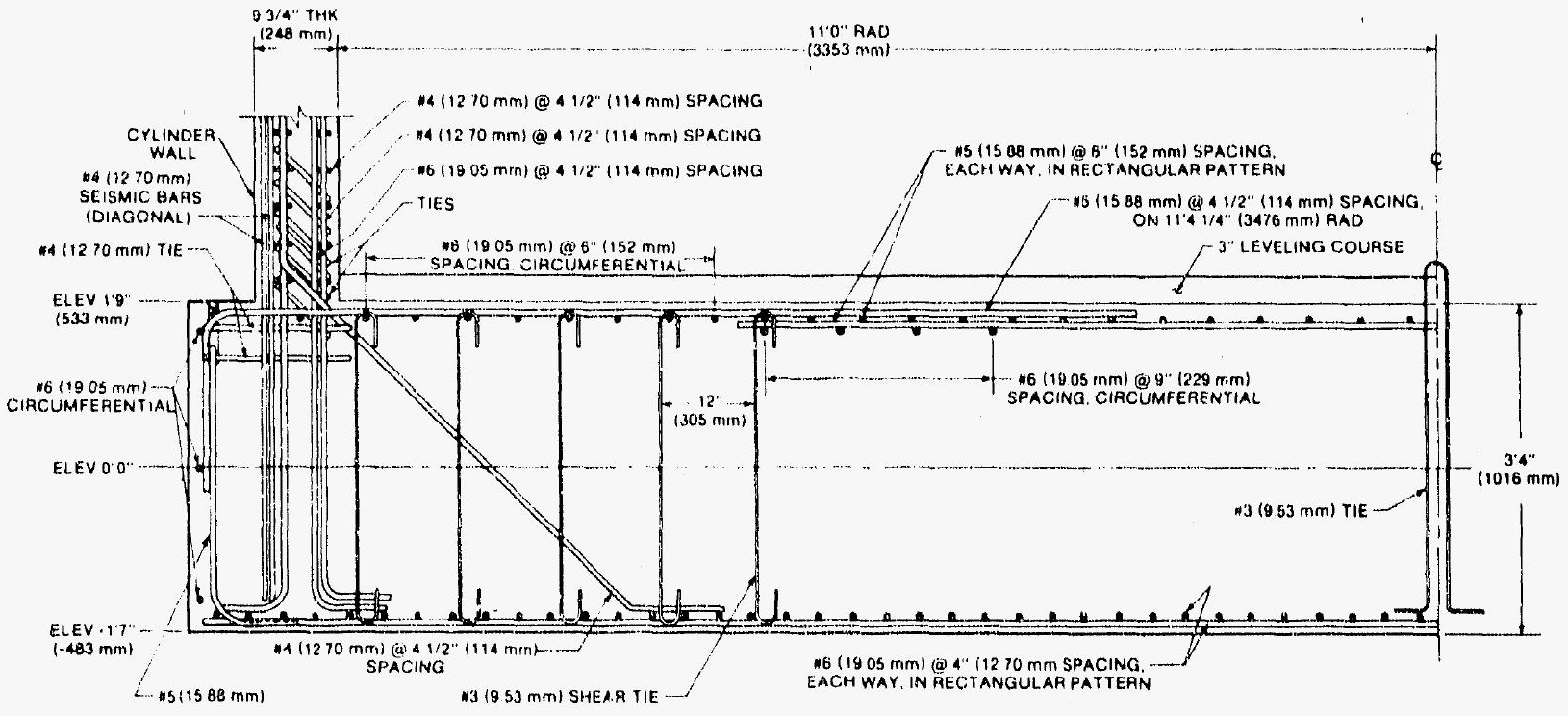

Figure 3. Typical Section Through the Basemat of the Containment Model.

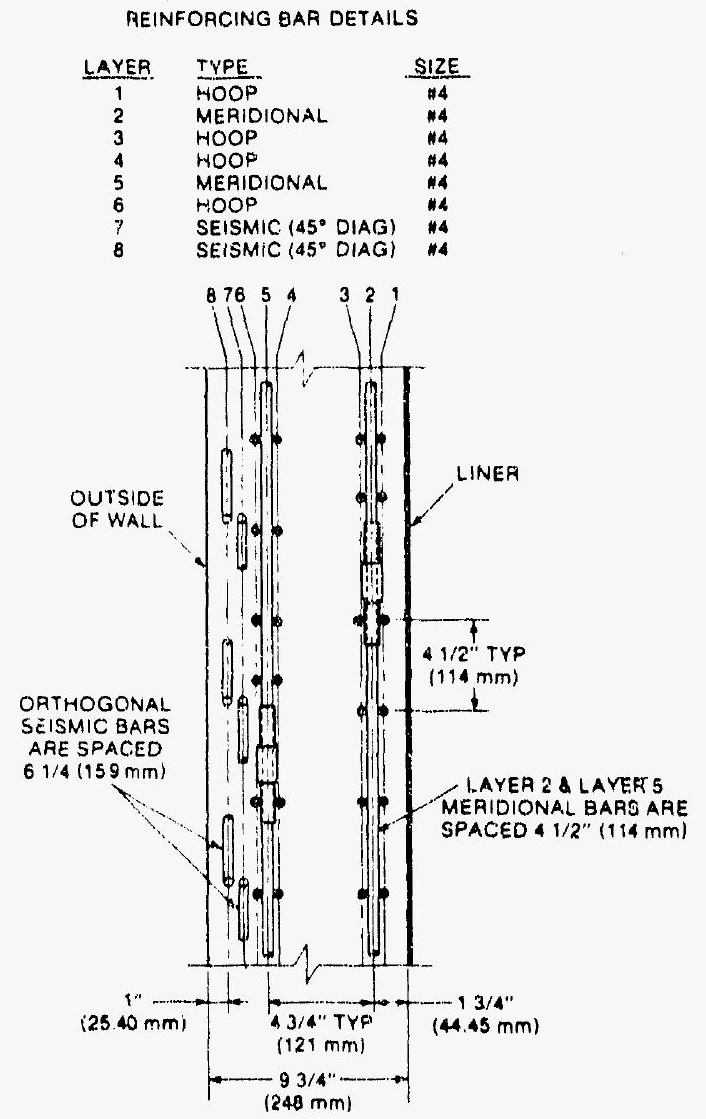

Figure 4. Typical Section Through the Cylinder Wall of the Containment Model. 


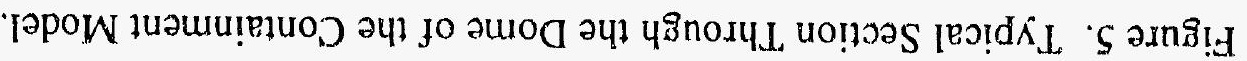

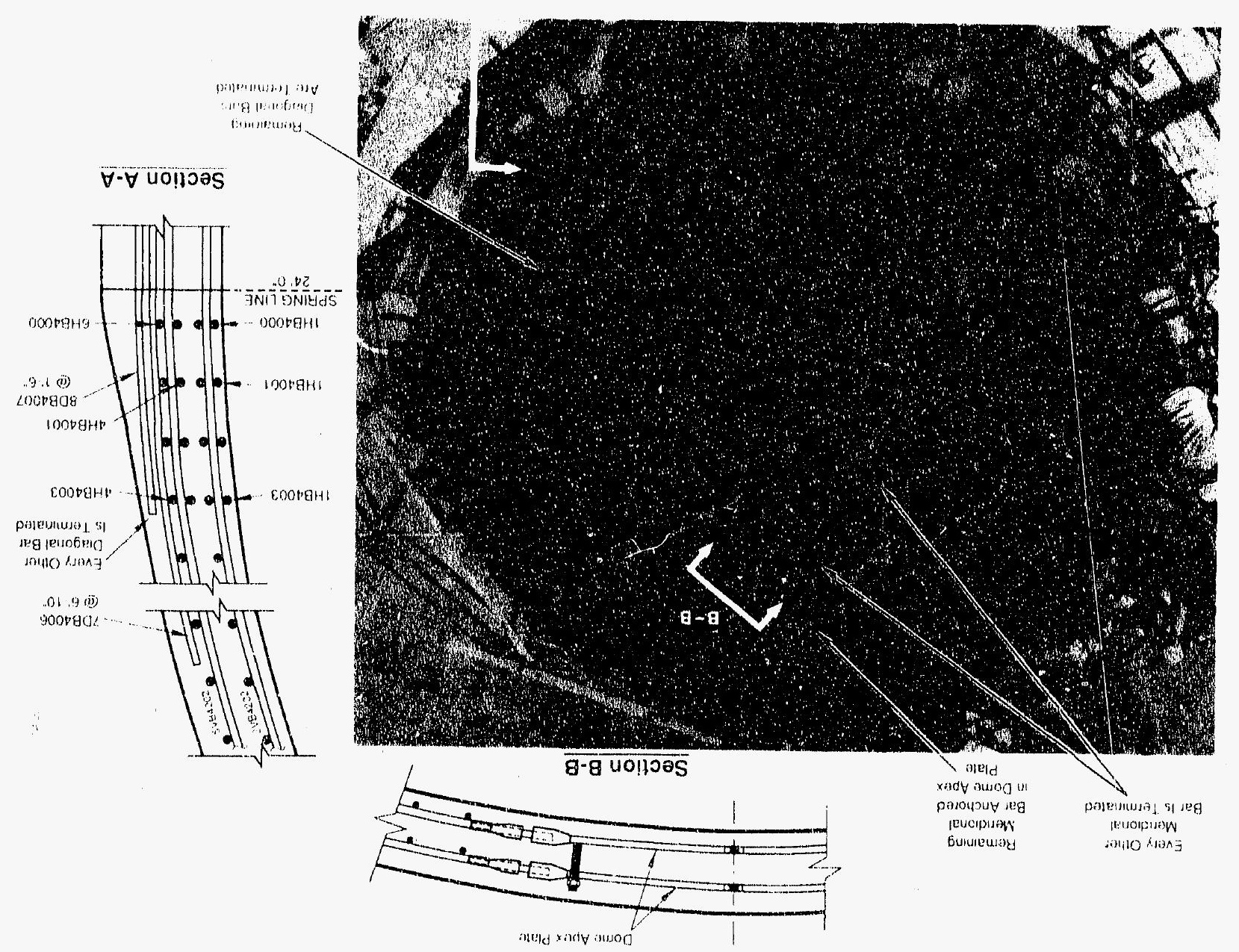


Large and complex reinforcing sections not requiring in-place installation were prefabricated to meet the critical placing schedule and to balance the construction workload. Entire sections of inner and outer layers of boss reinforcement were bent and assembled with the use of templates at the site. This permitted concurrent fabrication of several areas with an accuracy that could not be achieved by normal means but was required to maintain tolerances acceptable in a 1:6-scale model. The two dome reinforcement layers were also assembled using a template and each layer was fabricated as a single hemisphere.

\subsection{Splices}

About 5000 mechanically swaged connectors were used in splicing the reinforcing steel, most being installed on the $\# 4$ reinforcing steel. These splices allowed for flexible construction procedures--they could be installed either at the site using a bench-mounted press or as the bar was being installed in the model using a hand-held press. All main reinforcings bars in the mat and shell were spliced with a standard swaged or a swaged threaded coupler. Two phases of testing ensured that splices would meet all the design requirements, including the condition that the average of all break tests show results equal to or greater than the minimum certified ultimate strength of the reinforcement material. The first phase qualified the strength and nonslip characteristics of each connector type for each anticipated field condition, such as wet or frozen bars and/or coupling sieeves, and missing deformations caused by mill markings. The second phase qualified the splicers and instituted a continual sampling program io ensure the acceptability of the field work. The results of the construction tests and splice tests are in Reference 1.

\subsection{Liner}

Chicago Bridge and Iron (CB\&I) Company built the liner and the steel sections of the penetrations. The shell liner in the 1:6-scale containment was nominally $1 / 16 \mathrm{in}$. thick in the basemat and cylinder wall, stepping in the dome region of the containment to $1 / 12$ in. thick. Thickened sections of the liner around penetrations, referred to as insert plates, were 3/16 in. thick. Liners in U.S. containments are generally fabricated of SA516 Grade 60 steel. Because this material was not readily available in the small quantities needed in the $1 / 16$-in. and $1 / 12$ in. thicknesses, SA516 Grade 60 was used only for the construction of the insert plates and the penetrations. A suitable substitute was selected, SA414 Grade D, for the 1/12-in,-thick and 1/16-in.-thick sections of the liner, after a study by CB\&I. A stretchout of the liner cylinder is shown in Figure 6 where the thickened insert plates are indicated by crosshatching.

The liner was constructed in sections that included an internal frame system that supported the liner during transportation, construction, and placement of the concrete. All penetrations were added to the liner at the construction site. Over 25,000 headed studs were attached to the outside of the liner and eventually embedded in concrete. Studs with shank lengths of $1 / 2$ and $3 / 4$ in. were attached to the model by capacitive-discharge welding. The $1 / 2$-in.-long studs were used in the liner below elevation $6 \mathrm{ft} 3 \mathrm{in}$., while the $3 / 4$-in.-Iong studs were used at all other locations. The spacing of the studs varied from $2 \times 2$ in. in discontinuity regions to a maximum spacing of $8 \times 8$ in. in the dome. A construction photograph of the lower section of the cylinder liner and the studs is shown in Figure 7. 


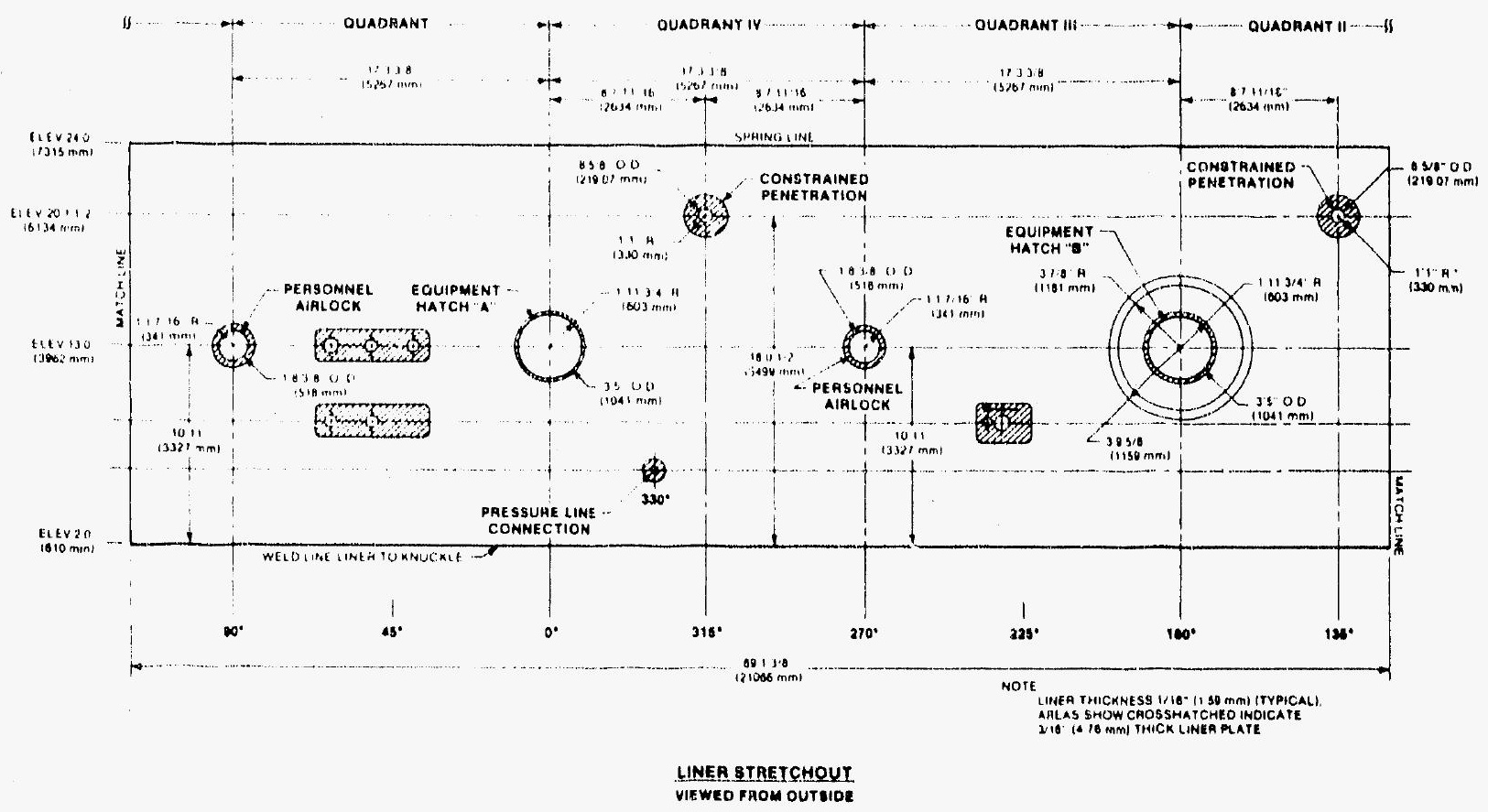

Figure 6. Liner Stretchout Showing Containment Features.

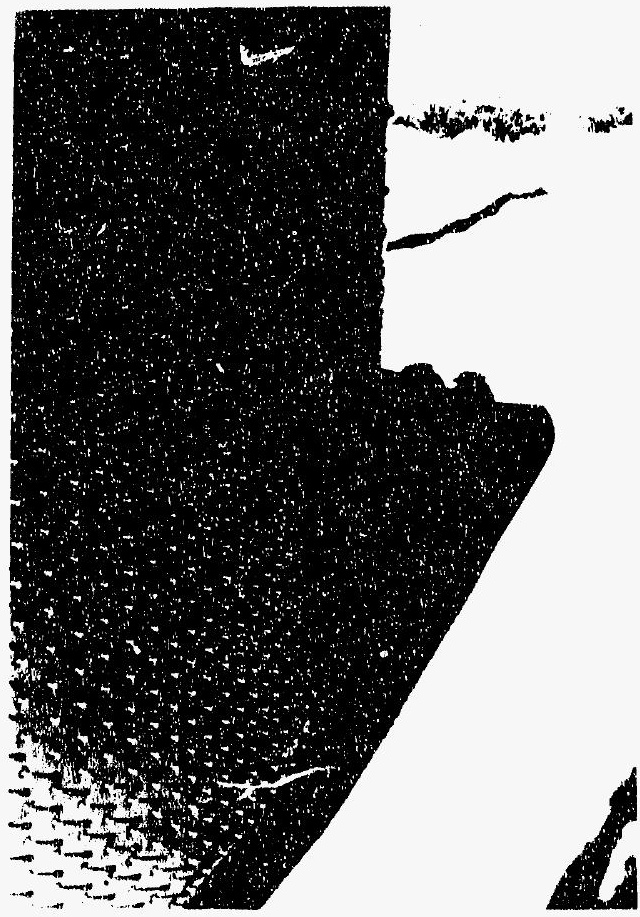

Figure 7. Cylinder Liner Showing Stud Anchors. 


\subsection{Concrete}

Several trial batches of concrete were made before the proportions of the final mix were decided upon. A well-rounded coarse aggregate with a nominal maximum size of $3 / 8$ in. was used. High-range water reducers (superplasticizers) were used to limit the water/cement ratio to the code-specified value of 0.53 . The slump required for the concrete lifts was about 8.5 in., except in the basemat and dome apex where the slump was reduced. To be properly placed in the containmeit model, the concrete had to be pumpable, flow well during vibration but not segregate, and consolidate well. To achieve these goals, several trial batches of concrete were tested. Trial batches were 4 to 5 cubic yards and were centrally batched and hauled to the site where they were pumped into a mock-up section of the containment wall. Through a plexiglass panel in the mock-up form system, the flow and consolidation characteristics of each trial mix were observed. While the concrete was still plastic, the mock-up was opened to seek out any lack of consolidation.

All concrete used for the model was centrally batched and hauled in trucks to the construction site, where it was pumped into the forms. Travel time from the central mixing plant to the construction site was about 45 minutes, necessitating the use of extended-life superplasticizers for the concrete that allowed a placing life of about three hours after batching. The compressive strength of the concrete at 28 days was about 5300 psi. Both direct tension and split-cylinder tests were performed [1]. The results of the concrete testing are in Reference 1.

\subsection{Basemat}

The 40-in.-thick basemat (43-in.-thick interior to the cylinder walls) in the 1:6-scale containment is somewhat thicker than would be linearly scaled from most full-size containments and does not include a reactor cavity, or other discontinuities. Because the dead weight of the model does not scale linearly from a full-size counterpart and because no internal structures of a containment were modeled, calculated moments at the base/wall intersection and uplift from bulging of the bottom of a linearly scaled basemat during pressurization were much higher than would be realized in a full-size containment. After scaling the moments at the basemat/cylinder wall intersection with the use of analyses, a 40-in. basemat thickness was selected.

Inside the cylinder wall, a backup bar for the welding of the liner was cast into a 3-in.-thick leveling course making the basemat $43 \mathrm{in}$. thick in that region. A schematic of this area is shown in Figure 8.

\subsection{Penetrations}

Both functional and representational penetrations were incorporated into the containment model. Most were scaled from typical penetrations in full-size containment buildings; however, several other penetrations were included for Sandia testing.

Two 40-in.-diameter equipment hatches, located at the same elevation, were scaled from typical equipment hatches in U.S. containment buildings. The major difference between the two equipment hatches was that the boss area of one projected entirely outward from the cylinder wall (equipment hatch $\mathrm{A}$ ), whereas the other boss was centered in the wall so that it projected both inward and outward from the cylinder wall (equipment hatch B; see Figure 2). The 
primary reinforcement around each of the equipment hatches was the same; however, their local reinforcement was different. Both equipment hatches had an inside pressure-seating cover; $B$ had a second pressure-unseating cover on the outside.

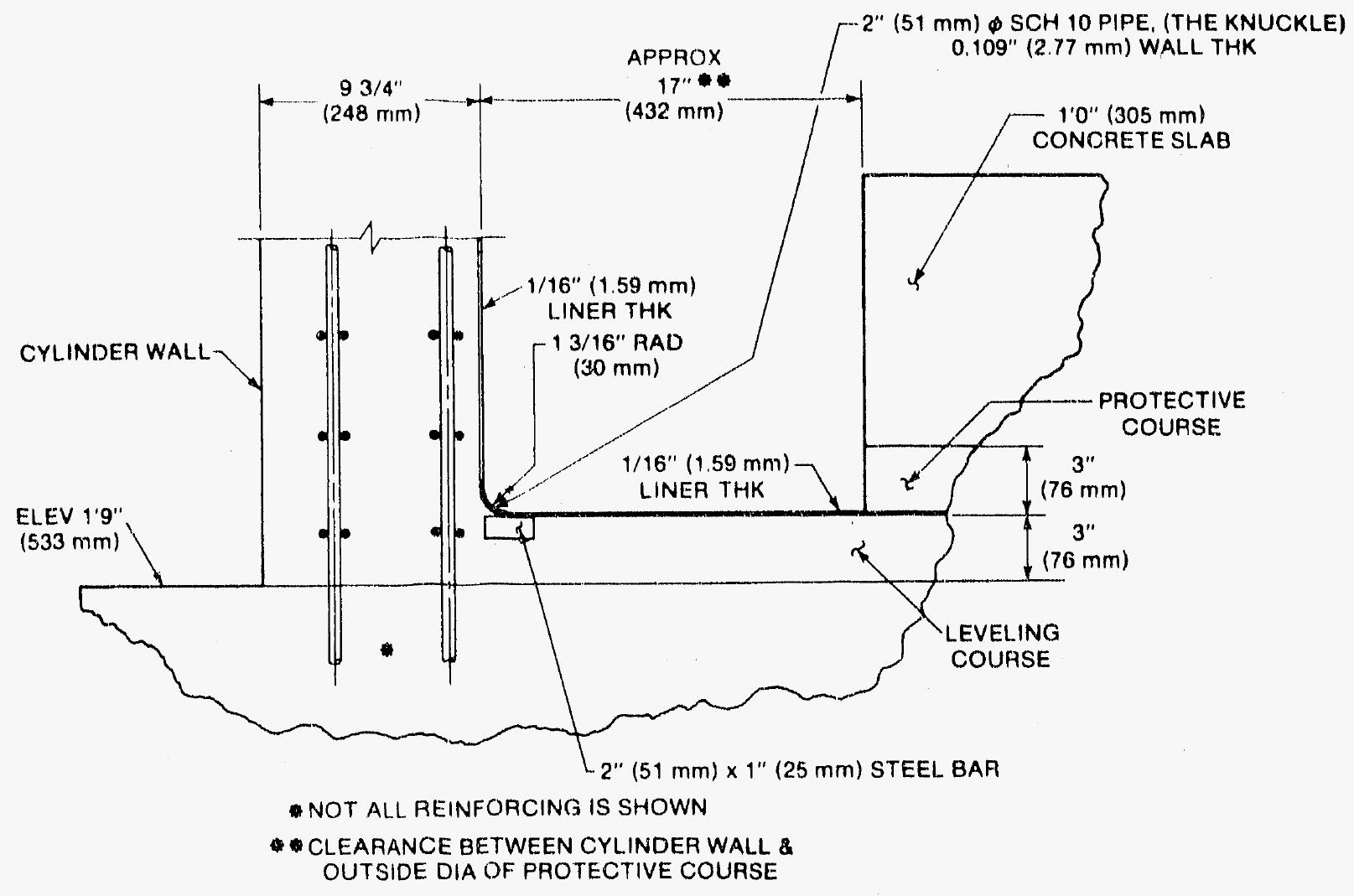

Figure 8. Detail of Liner Knuckle.

All three equipment hatch covers had typical scaled sealing arrangements: the two pressureseating equipment hatches had double gum-drop seals, and the pressure-unseating cover had a double tongue-and-groove seal. To gain some additional leakage information during the highpressure test, the seals in the pressure-unseating equipment hatch were thermally aged (no radiation aging) prior to the test so that the seals would have about a $95 \%$ compression set retention; however, only 50\% compression set retention was achieved.

This compression set retention was based on typical values when the seals were exposed to the Institute of Electrical and Electronic Engineers (IEEE) specifications:

- Thermal aging, $168 \mathrm{~h}$ at $300^{\circ} \mathrm{F}$

- Radiation aging, $200 \mathrm{Mrad}$ at a dose rate of less than $1 \mathrm{Mrad} / \mathrm{h}$.

Silicone seals exhibit $75 \%$ and $99 \%$ compression set retention for the IEEE thermal and radiation aging, respectively. As discussed in section 6.2, some leakage may have come from the pressure unseating equipment hatch at a pressure as low as 125 psig.

Two personnel air lock representations were included. The sleeve diameter in both air locks was about 20 in.; other sleeve dimensions were scaled from full-size personnel air locks. The reinforcing pattern around the air locks was identical, but the air locks themselves were different. One air lock, PL-B, used a thick flat plate as a pressure-unseating door so that it 
could be used as a "last-man-out" hatch. The other personnel air lock, PL-A, had scaled bulkheads and stiffener patterns typical of a full-size air lock, but no doors or sealing surfaces were included.

There were two 8-in.-diameter penetrations (designated R-1 and R-2) at the same elevation $180^{\circ}$ apart on the cylinder wall. They were connected with a smail rod, designed with a scaled stiffness, to simulate large-bore piping constraints on the growth of the shell.

Several other piping penetrations were also included in the containment model. One piping cluster included penetration diameters of 8 in. (1); 4 in. (1); 2 in. (2); and 1 in. (1). Other penetrations were provided to facilitate testing of the containment. These penetrations included five 6-in.-diameter penetrations that had 32 electrical couplings each for instrumentation lines, and a 4-in.-diameter penetration was used to connect the pressurization line to the model. The details of these "testing penetrations," such as liner and reinforcing steel details, were faithfully scaled from typical full-size containment penetrations with similar scaled diameters. 


\section{INSTRUMENTATION}

\subsection{Layout of the Test Site}

The containment model was instrumented to assess its structural behavior, which included large strains and displacements as well as leakage characteristics during testing. The transducers were connected to a data acquisition and control unit in the signal conditioning bunker, adjacent to the reinforced-concrete containment model (RCM) (Figure 9). Data scans were transmitted to the data acquisition computer in the command bunker about 1500 feet away from the containment model.

Most transducers on the containment were strain gages and displacement transducers. Over 300 strain gages were attached to the reinforcing steel during construction, and oyer 160 strain rosettes (480 gages) and 160 single and strip gages were applied to the steel liner. Also embedded in the concrete wall were about 40 thermocouples and 20 embedment gages. In addition to this instrumentation, several video and still cameras were used during testing to provide both qualitative and quantitative data. A complete description of all instrumentation used during testing is in Reference 1. A brief discussion of the types of test instrumentation follows.

\subsection{Pressurization System}

A computer-controlled valve gallery controls gas flow into/out of the containment model. The valve system has a maximum flow rate of $6 \mathrm{lb} / \mathrm{s}$, allowing a $2-3$ psi pressure step to be applied in seconds. Compressed air from a tube trailer was used for all low-pressure testing, while liquid nitrogen that was gasified as it entered the pressurization lines was used for the high-pressure test. The valve gallery is shown in Figure 10.

\subsection{Strain Gages}

Over 1200 transducers were attached to the containment model to measure its response during pressure testing. Encapsulated-weldable gages of a single-element design were predominantly used on the reinforcing steel. Their small size allowed them to be attached with minimal disturbance to the bar by spot welding. Figure 11 shows a gage attached to a $\# 4$ reinforcing bar. Although the gages were quite durable, they were coated with a silicone rubber and potted in epoxy to protect them during installation and placing of the concrete. The weldable gages were capable of measuring 6-7\% strain as determined by tests conducted at Sandia (manufacturer's claims are to $2 \%$ strain). Most of the gages on the liner were three-arm $45^{\circ}$ rosettes, made of annealed constantan with a polyimide backing. The liner was carefully prepared and the Arrnstrong A12 epoxy used to attach the rosettes to the containment model was heat cured for several hours, enabling the rosettes to measure strains up to 20\% [29]. Single gages and strip gages with the same properties as the rosettes were also used at various places on the containment liner. 


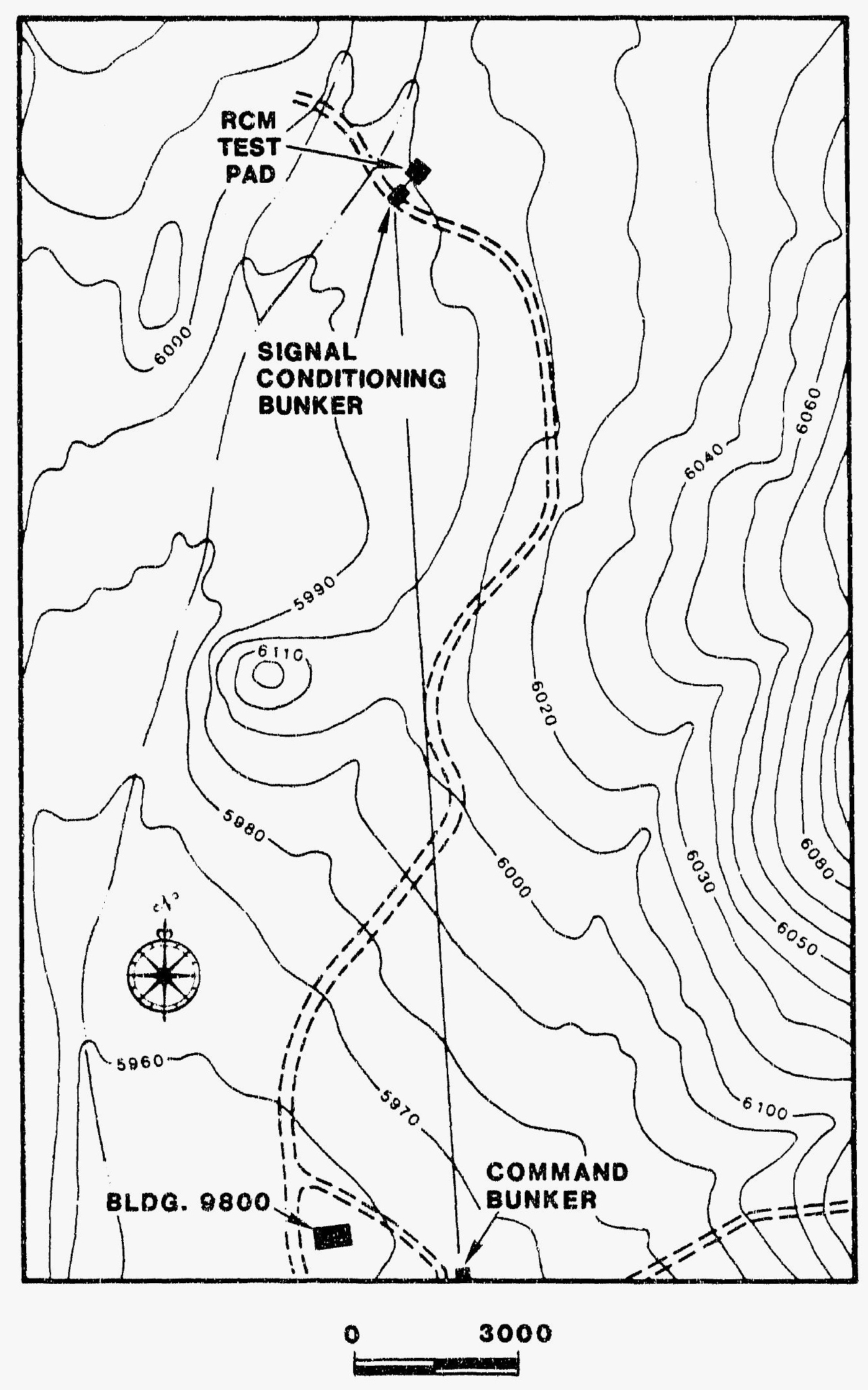

Figure 9. Layout of the Test Site. 


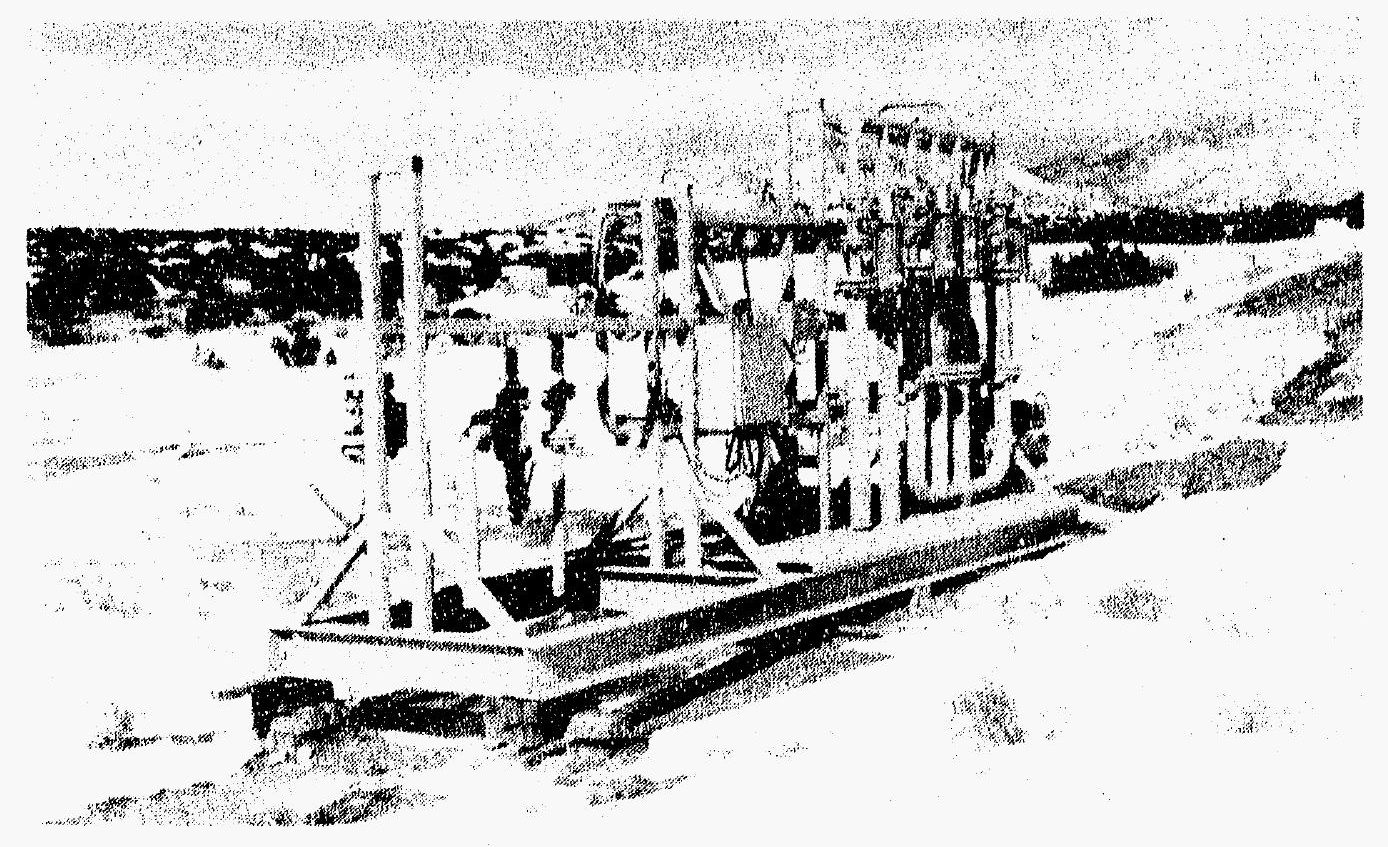

Figure 10. Computer Controlled Valve Gallery.

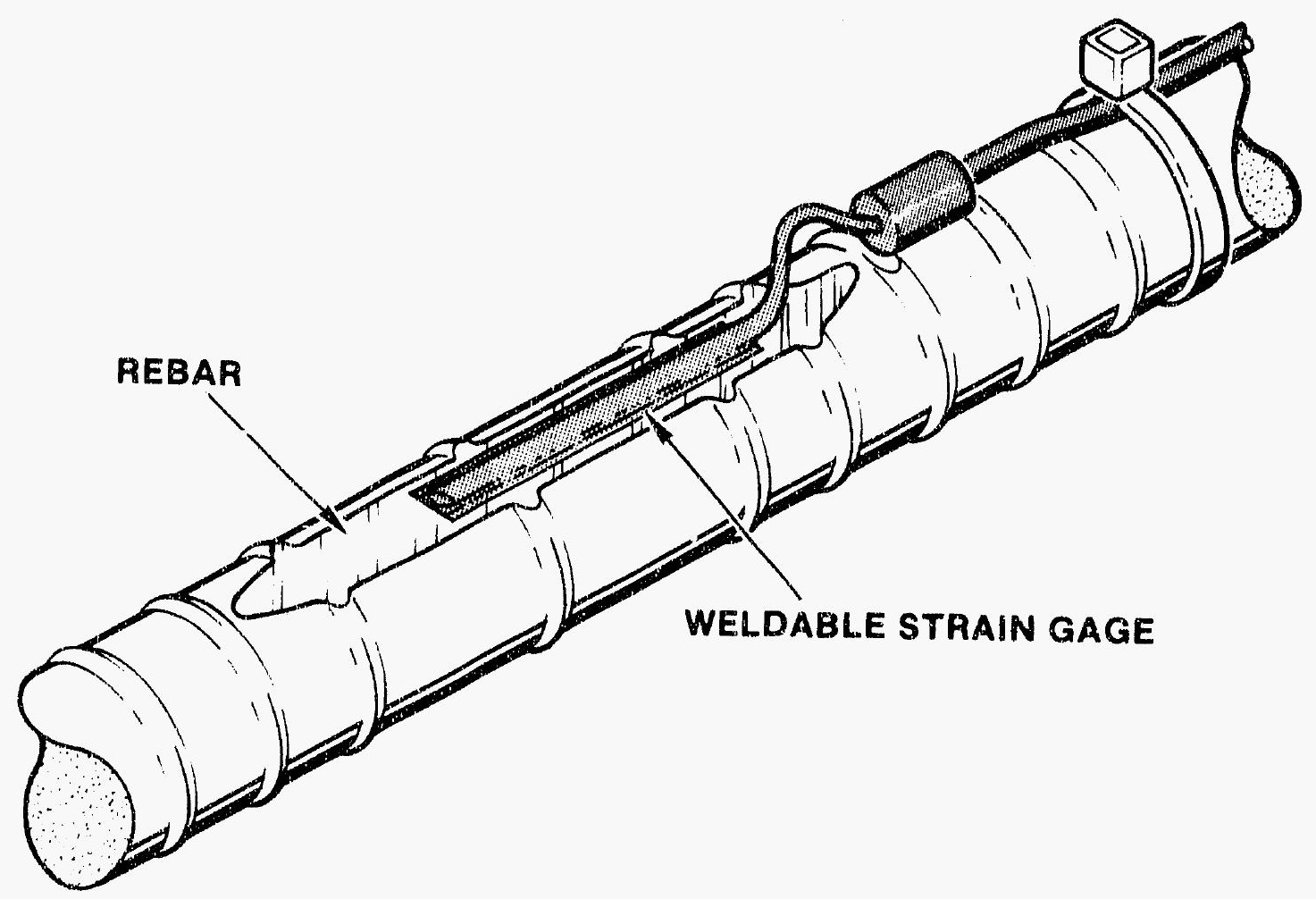

Figure 11. Weldable Strain Gage Attached to a Piece of Reinforcing Steel. 


\subsection{Displacement Transducers}

Nearly 150 displacement transducers were used on the containment model. Linear potentiometers and linear variable differential transformers (LVDTs) measured displacements of $4 \mathrm{in}$. or less. They were predominantly used to measure local deformations of the penetrations and for monitoring crack widths during testing. Cable potentiometers, with maximum ranges of 5 to 300 in., were used to measure displacements of the model. Several displacement transducers were attached at discrete locations to measure global deformations of the containment, and to augment them, nine-track transducers were designed and built. The track transducers were, as their name implies, mounted on tracks and powered by a gear motor, as shown in Figure 12. The motor pulled the transducer along a track, while an arm followed the contour of the containment. Each track system had two transducers-one measured the location along the track, while the second measured the movement of the track-arm perpendicular to the track. Since the track transducers were controlled separately from the other transducers, they were not scanned at every pressure level. After the test, the data obtained from the track transducers were plotted, giving a displacement profile of nine locations about the containment model. These plots are included in Appendix B.

\subsection{Other Transducers}

Over 40 thermocouples were embedded in the containment wall and 33 on the inside of the containment liner. The thermal data were used in the correction of the apparent strain read by the strain gages. Also in the containment wall were several embedment gages. Although they were similar to a strain gage and measure strain, they were not attached to the reinforcing steel, but placed directly in the concrete. Other instrumentation used for the containment testing included eight inclinometers (to measure rotation at selected locations and basemat uplift), three weather channels, five pressure channels, twelve resistance temperature detectors, two flow meters, and a load link.

\subsection{Optical Coverage}

Both the still camera and video camera stations changed from the low-pressure testing to the high-pressure test to optimize the information recorded. The cameras used during testing are listed in Table 1. The low-pressure test served as a "dry run" for determining the photographic needs during the high-pressure test.

Images from the video cameras were used during high-pressure testing to aid in selecting pressure step size, length of hold periods, and specific transducers to monitor areas that exhibited interesting behavior. 


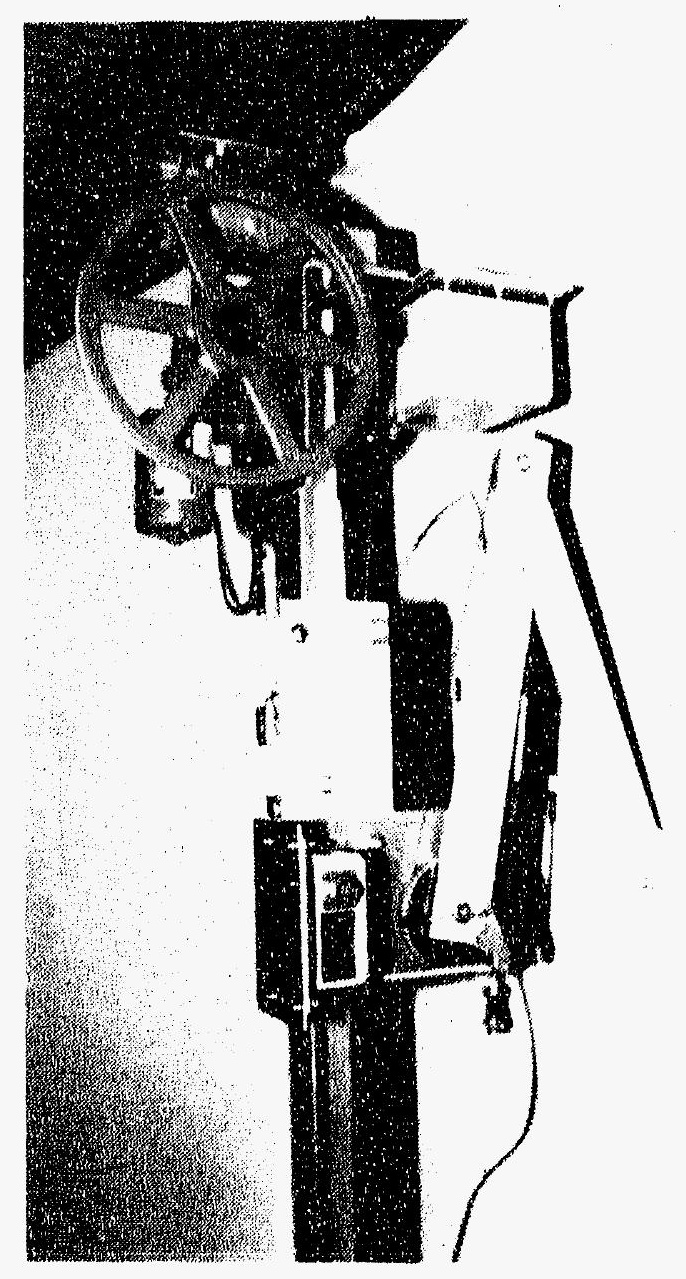

Figure 12. A Track Transducer System for Measuring Displacements of Containment Model.

Table 1.

Still and Video Cameras Used During Containment Testing

Low-Pressure
Test $\quad \begin{gathered}\text { High-Pressure } \\ \text { Test }\end{gathered}$

Close Range Cameras
Overview Cameras

Total Still Cameras

Video Cameras
10

5

15

$10^{*}$
18

12

6

$12^{*}$

"One video camera was located inside the containment model. 


\subsection{Acoustic Coverage}

The monitoring of acoustic emissions in a complex reinforced concrete structure is not a welldefined technology. In fact, few experiments on acoustic monitoring of reinforced concrete have been conducted. The primary purpose of the acoustic system was to determine if a leak was present in the containment model and to give its vicinity, but the system was not intended to be able to pinpoint the location of the leak nor be able to measure the leak rate.

The placement of six (seven for the high-pressure test) low-frequency acoustic sensors in the containment model was determined by the areas where leakage was most likely. From the pretest analyses, it was judged that the areas of potential leakage were near the major penetrations and near the basemat-cylinder wall junction.

The acoustic system was based on an extensively modified Acoustic Emission Technology (AET) Model 4900." The system was also modified for use with a MicroVax II computer. The sensors chosen had a maximum response between 30 and $100 \mathrm{kHz}$. The sensors had an internal amplifier that boosted the signal by $40 \mathrm{~dB}$ that was again amplified another $40 \mathrm{~dB}$ at the AET 4900.

The data from the sensors were processed for both burst noise and steady-state noise. Burst noise measurements indicated potential cracking either in the concrete or the steel, while the steady-state noise measurements were a strong indication of potential containment leakage. Although it is not possible to separate totally the two types of noise with any system, it is estimated that more than $90 \%$ separation was achieved in the containment experiment.

As leaks or increases in leakage were detected with the acoustic system, the small leak rates were determined by conducting mass measurements of the gas in the containment over time. The final large leak rate measurement was made by flowmeters in the gas supply system.

\footnotetext{
*Internal Report, A. G. Beattie, "Acoustic Monitoring of the One Sixth Scale Model of a Nuciear Reactor Containment Building," Nondestructive 'rest Report, Sandia National L.aboratories, Albuquerque, New Mexico, 1987.
} 


\section{LOW-PRESSURE TESTING OF THE CONTAINMENT'}

The model was tested much as a full-size prototypical containment is tested; that is, a Structural Integrity Test (SIT) and an Integrated Leak Rate Test (ILRT) were conducted at low pressure. These tests were followed by the high-pressure test. Highlights of the low-pressure testing are presented in the next two subsections.

\subsection{The Structural Integrity Test}

About 1200 transducers were used during the SIT [1], making this model the most heavily instrumented SIT ever conducted. Except for the track transducers, a few displacement transducers, and strain gages that were added after the low-pressure test, the same set of transducers was used for high-pressure testing. Although photographic and video coverage of the containment model was extensive for both tests, there were slight changes in the amount and location of the coverage. (There were 15 still cameras and 10 video cameras used during the SIT.)

The results from the instrumentation were compared to analysis results during the test at a minimum of 21 locations at each pressure step, which not only assured that the model behaved as expected but helped ensure that the containment model was safe to approach for crack mapping. (The analyses are discussed in References 30-33.)

The SIT was conducted in accordance with the ASME code, Section III, Division 2, CC 6000, and was pressurized using dry, compressed air, which also eliminated the need to measure the relative humidity in the vessel. Ten steps were required by the ASME code for increasing and decreasing the pressure in the containment during the SIT. In addition to these, intermediate steps were taken, and several additional data scans were performed. As pressure increased in the containment model, a data scan was taken at an intermediate step with no hold period. The pressure was then increased to the next SIT step, where a data scan was immediately initiated. After a one-hour hold at the required SIT pressure level, another data scan was performed, and crack mapping was conducted as required. If crack mapping was conducted, a third data scan was taken. Table 2 shows the loading schedule and data scans during the SIT. Standard tolerances for the pressure steps were about \pm 1 psi for all steps except peak pressure, which had a tolerance band of $+1 / 2$ psi to 0 psi.

One additional crack mapping was conducted that was not required by $\mathrm{CC} 6000$, at $46 \mathrm{psig}$, step 6. Since the previous two crack mappings were done under artificial light, a check was done the next morning in natural light, and no significant differences were found in the crack patterns.

On July 6, 1987, the first crack mapping was performed, with first pressurization occurring the next day. The last crack mapping was conducted four days later. The specific activities of the SIT follow and a chronology of the SIT may be found in Appendix A. 
Table 2.

Loading and Data Retrieval Intervals for the SIT

\begin{tabular}{|c|c|c|c|}
\hline Step & $\begin{array}{l}\text { Number of } \\
\text { Data Scans }\end{array}$ & $\begin{array}{l}\text { Pressure } \\
\text { (psig) }\end{array}$ & $\begin{array}{l}\text { Crack Mapping } \\
\text { Conducted }\end{array}$ \\
\hline 0 & 1 & 0 & yes \\
\hline 1 & $\begin{array}{l}1 \\
3\end{array}$ & $\begin{array}{l}5 \\
12\end{array}$ & $\begin{array}{l}\text { no } \\
\text { yes }\end{array}$ \\
\hline 2 & $\begin{array}{l}1 \\
3\end{array}$ & $\begin{array}{l}17 \\
22\end{array}$ & $\begin{array}{l}\text { no } \\
\text { yes }\end{array}$ \\
\hline 3 & $\begin{array}{l}1 \\
3\end{array}$ & $\begin{array}{l}27 \\
23\end{array}$ & $\begin{array}{l}\text { no } \\
\text { yes }\end{array}$ \\
\hline 4 & $\begin{array}{l}1 \\
3\end{array}$ & $\begin{array}{l}40 \\
46\end{array}$ & $\begin{array}{l}\text { no } \\
\text { yes }\end{array}$ \\
\hline 5 & $\begin{array}{l}1 \\
3\end{array}$ & $\begin{array}{l}50 \\
53\end{array}$ & $\begin{array}{l}\text { no } \\
\text { yes }\end{array}$ \\
\hline 6 & $\begin{array}{l}1 \\
2\end{array}$ & $\begin{array}{l}50 \\
46\end{array}$ & $\begin{array}{l}\text { no } \\
\text { yes }\end{array}$ \\
\hline 7 & $\begin{array}{l}1 \\
2\end{array}$ & $\begin{array}{l}40 \\
34\end{array}$ & $\begin{array}{l}\text { no } \\
\text { no }\end{array}$ \\
\hline 8 & $\begin{array}{l}1 \\
2\end{array}$ & $\begin{array}{l}27 \\
22\end{array}$ & $\begin{array}{l}\text { no } \\
\text { no }\end{array}$ \\
\hline 9 & $\begin{array}{l}1 \\
2\end{array}$ & $\begin{array}{l}17 \\
12\end{array}$ & $\begin{array}{l}\text { no } \\
\text { no }\end{array}$ \\
\hline 10 & $\begin{array}{l}1 \\
5\end{array}$ & $\begin{array}{l}5 \\
0\end{array}$ & $\begin{array}{l}\text { no } \\
\text { yes }\end{array}$ \\
\hline
\end{tabular}

As pressure was brought up to $12 \mathrm{psig}$, some troubles with the data scanner were encountered. The test was terminated, and the pressure released from the model until the problem could be rectified. Pressurization of the containment model began again the following day, and all transducer scans were taken again. (The crack mapping performed at 12 psig on the first day of pressurization was not repeated.)

Further testing proceeded with no notable problems through the peak pressure and down to the it psig pressure step (step 6). Some leakage, however, was noted from the containment model through the $\mathrm{AC}$ power feed throughs. Step 10 was completed in the evening of the fourth day of testily As can be seen in Table 2, additional data scans and crack mapping were done at 0 psig. 


\subsubsection{Crack Mapping}

Mapping of cracks was done at six locations about the containment model:

- equipment hatch $\mathrm{A}$ at $0^{\circ}$,

- equipment hatch $\mathrm{B}$ at $180^{\circ}$,

- personnel air lock at $90^{\circ}$,

- basemat/cylinder wall junction at $90^{\circ}$,

- free field at $135^{\circ}$, and

- springline at $135^{\circ}$.

Crack widths were not recorded unless they exceeded 0.010 in. Each mapped area was gridded with a 6" $\times 6$ " grid on the untreated concrete (that is, no whitewash or other coatings were used on the concrete). Crack orientation and spacing were fairly uniform on the model and tended to follow the reinforcing patterns. Cracks in the cylinder wall were in the hoop and meridional direction that appeared to follow the 4-1/2-in. pattern of reinforcing steel, although in some areas the cracks followed the outer layers of reinforcing steel oriented at $45^{\circ}$. In addition to these cracks, well-defined cracks developed around each of the major penetration bosses where they intersected the cylinder wall. Very few cracks on the model were more than 0.010 in. wide. A complete set of these crack maps is included in Appendix A.

\subsubsection{SI'T Results}

As with the crack widths, strains and displacements remained small throughout the SIT. Typical radial displacements measured at the peak pressure were in the range of 0.050 to 0.125 in. Typical strains varied by a like amount at 53 psig typical hoop strains in the free field at midheight of the cylinder were 0.06 to $0.10 \%$. (A complete set of results from the SIT is plotted and tabulated concurrently with the results from the high-pressure test in Appendix. C.) Some strains, however, deviated substantially from these typical ranges.

In the liner area adjacent to the insert plate for penetration R-2-a penetration that represented a steam line located at an elevation of $20 \mathrm{ft} 11$ in. at azimuth $315^{\circ}$-liner yielding was observed and local strains exceeded $0.55 \%$ at the peak pressure level of 53 psig. Figure 13 graphically shows the strain concentration near penetration R-2 at five pressure levels. Note that results from pressure level 46 and 53 psig overlay each other at some locations.

The first measured location, shown in Figure 13 as arc distance increases from the center of the penetration, is where a rosette was located on the insert plate. The insert plate intersected the nominal thickness liner material at an arc distance of 13.44 in. Adjacent to this was another rosette at a location of about 14 in. Beyond the second rosette was a series of seven gages on a common backing (a strip gage, $11 / 2$ in. long) that intersected the attachment of a liner stud to the liner. The final gage response plotted in Figure 13 is another rosette located slightly over 16 in. from the center of penetration R-2, which recorded unusually low strain levels throughout this test."

\footnotetext{
* The strain levels recorded by this rosette do not match the readings made during the high-pressure test at coinciding pressure levels. The reason for this discrepancy is not known.
} 


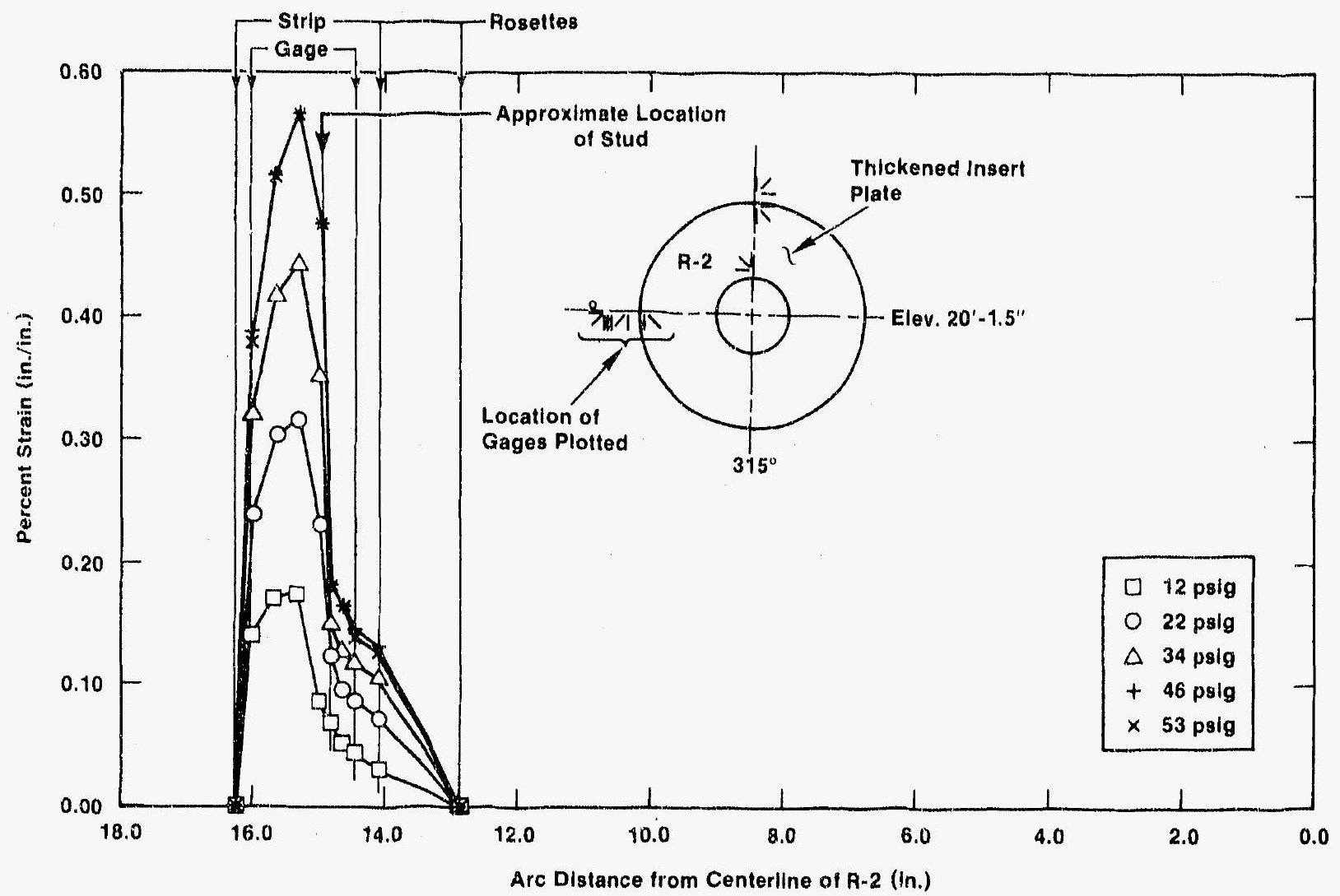

Figure 13. Liner Strains in the Hoop Direction Near Penetration R-2.

Figure 13 shows th it as pressure increased, strains remained small in the thicker insert plate. Strains in the neighboring nominal thickness liner showed a steady increase with increasing pressure, as well as a strain concentration that did not correlate with the presence of the stud. (During the high-pressure test, there was a strong correlation between the presence of the studs and the strain readings.) The plotted values of strain were connected by a line in Figure 13; caution should be used in interpreting values between those measured. A higher strain concentration possibly existed between gages but was not measured due to the discrete (and relatively large) size of the strain gage when compared to the relatively short distance over which the strain gradient likely occurred.

Compare the response of this area during the SIT with that of the high-pressure test in Figure 58. 'The concentration of the strains changed dramatically as the internal pressure increased. 


\subsubsection{Acoustic Coverage}

Use of the acoustic system during the SIT was more a final check of the system than an effort to gain acoustic data since leakage was not expected during this test. The SIT provided a vehicle to test the acoustic system's performance during pressurization of the containment model and in the presence of the other systems used for the containment model testing. Crosstalk from other systems and transducers could have prevented the acoustic system from gathering useful data, but the acoustic system functioned adequately during this containment test.

The acoustic system was able to sense the emissions from the model and performed without incident. "Background" noise from the model and noise generated from a pressurization step were measured and recorded. Some areas in the containment model emitted more noise than others, but nothing unusual in these areas was detected in this test or the high-pressure test. During the SIT, six acoustic sensors were used; one more was added at the midheight elevation of the cylinder for the high-pressure test for a total of seven acoustic sensors.

\subsection{The Integrated Leak Rate Test}

The standards in ANSI 56.8 [34] were followed during the ILRT, with some exceptions. The most notable exception was the accuracy of the pressure transducers. The standard required $\pm 0.001 \mathrm{psi}$ accuracy, and the two pressure transducers used during this ILRT were limited by the accuracy of the data scanner (approximately 5-1/2 digits). To compensate for this exception, the length of the test was extended, lasting nearly 50 hours instead of the 24-hour minimum required. Two methods--total time and mass-point-leak-rate--were used during leakage testing. As part of the ILRT, an orifice was placed in the containment model (at the valve gallery), and leakage was again measured. Details of this testing are presented below.

Following the leak repair of the AC feed throughs during the SIT, the ILRT began. The containment pressure was increased to $46 \mathrm{psig}$, the pressure source was effectively disconnected, and an isolation valve was closed that acted as a pressure boundary between the containment and ambient pressure. This allowed the air to leak from the containment model and at the same time prevented the pressure source from leaking into the containment.

Some leakage was detected going through the isolation valve, however. The first ILRT was conduct $:$ over approximately 48 hours. The leakage during this test was approximately $0.14 \%$ mass/duy at 46 psig. Because of the magnitude of this measurement and the notable leak at the isolation valve, most of the leakage measured was likely from this source. In any case, the leakage was within normal acceptable limits for an operational LWR containment building, which typically has maximum acceptable leakage rates of 0.1 to $0.5 \%$ mass/day at their design pressure. A plot of the leakage assessment versus time is shown in Figure 14.

Following the ILRT, an orifice was placed in the valve gallery to simulate leakage from the model. The measured leak rate from the 0.070 -in. diameter orifice was $11.5 \%$ at about 46 psig. Although this was somewhat large by industry standards, the goal was to use a typical leak size that might have been encountered and measured during overpressurization of the containment model as well as establishing the typical times necessary to measure leaks of these sizes.

The orifice used for this testing had a 0.070 -inch-diameter hole. The final result of this test yielded a leak rate of $11.5 \%$ mass/day and was determined after 21.8 hours. The pressure in the containment model changed noticeably during the test because of the leak. Testing began 
at a pressure of 57.1 psia and concluded with a pressure of 51.4 psia, while the temperature of the gas was relatively constant. The containment model's leakage history with the 0.070-in.diameter orifice in place is displayed in Figure 15.

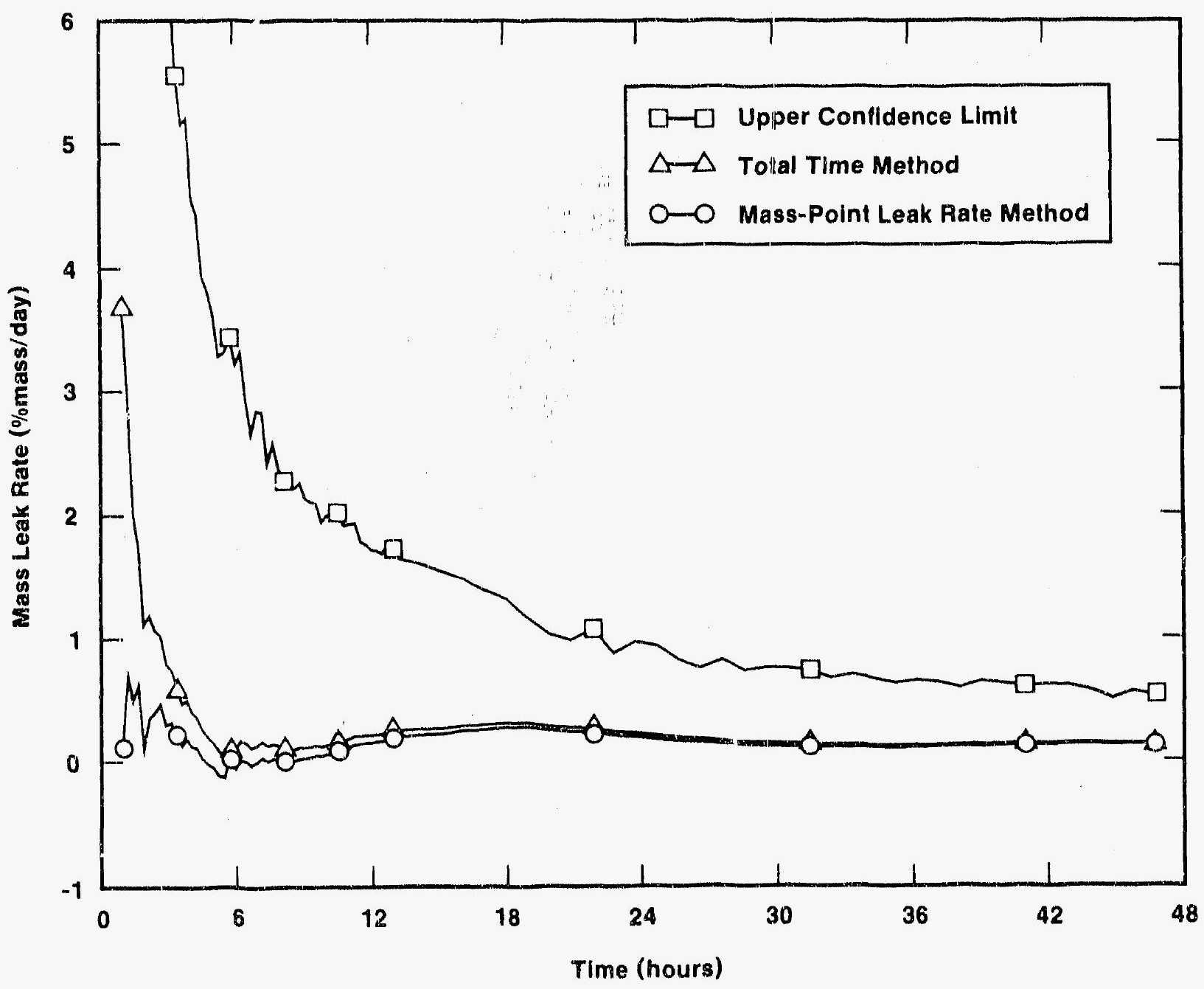

Figure 14. Assessment of Leakage Rate Versus Time. 


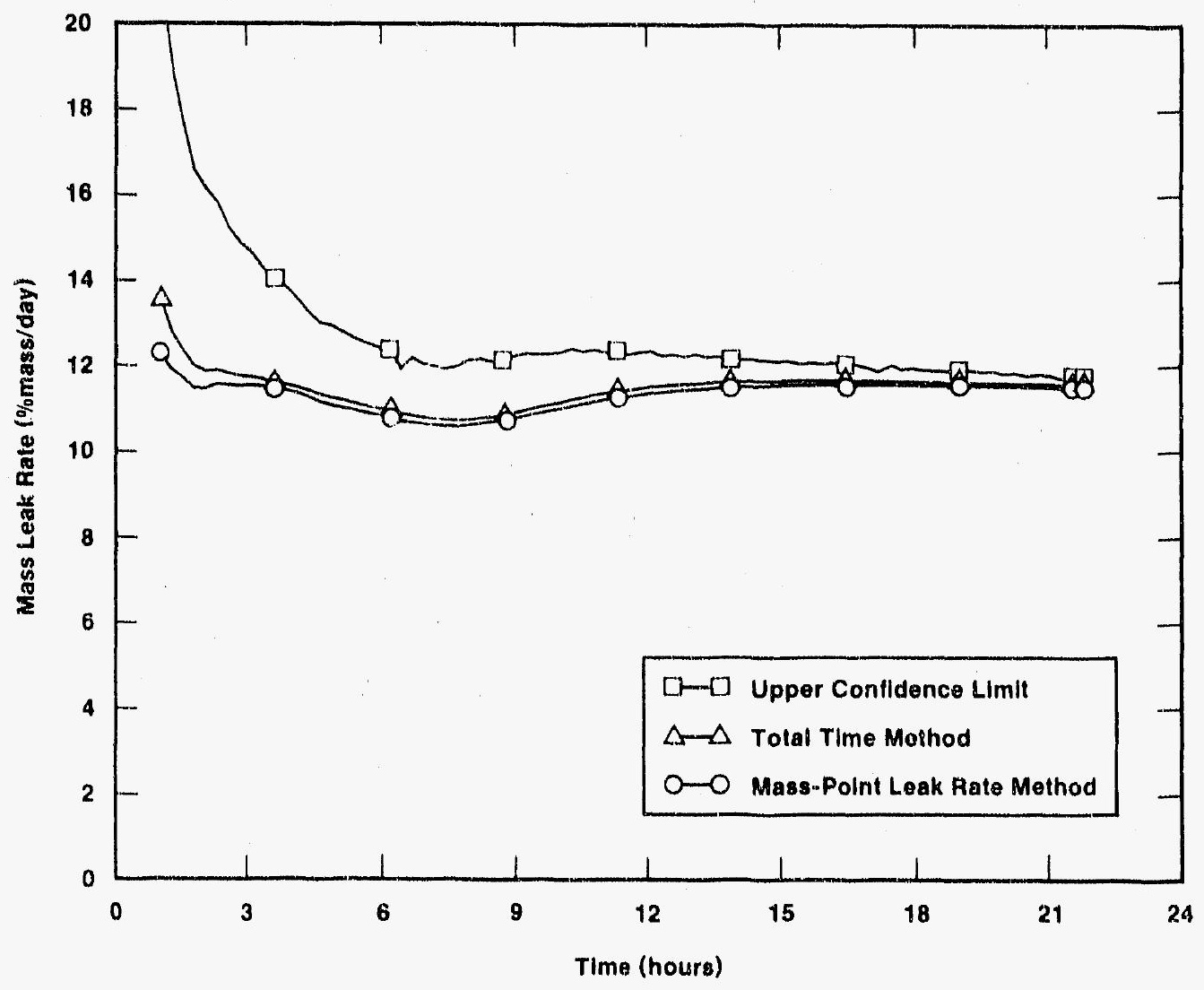

Figure 15. Leakage Plot Using a 0.070-In.-Diameter Orifice. 


\section{CONDUCT OF THE WIGH-PRESSURE TEST}

High-pressure testing was conducted continuously and was fully staffed around the clock. Nitrogen to pressurize the containment for high-pressure testing was trucked to the site in liquid form and gasified as it entered the pressure lines. Pressure-step size was based on both the predicted and the measured response of the model. Video information was also used to determine pressure-step size.

After the pressure was increased, several gages were monitored to determine the amount of creeping in the containment at the applied load. As anticipated, the model continued to creep slowly at the higher pressure levels; nevertheless, a full scan of all instrumentation was completed. (The track transducers were triggered independently and were not used at every pressure step.) If the model was at a pressure level for long, a second scan was usually conducted. At least one set of pictures from the 18 still cameras was taken at each pressure level.

The acoustic system was used primarily to determine whether the containment was leaking. The acoustic system could not pinpoint leakage, but a general location could be deduced.

Many predicted phenomena occurred during the test: cracks in the concrete became much wider, there was a noticeable cvaling of the sleeve of equipment hatch $\mathrm{A}$, and the outer edge of the basemat lifted off the work mat, to name a few. The test was terminated because of excessive leakage that began somewhat suddenly, going from $8 \mathrm{scfm}$ at 135 psig to over $4000 \mathrm{scfm}$ at 145 psig. Highlights of the testing are presented in the following subsections.

\subsection{Model Pressurization}

Pressurization of the containment began with 10-psi steps on the morning of July 28,1987 . As the maximum pressure reached during the SIT was exceeded, step size was reduced to 5-psi steps and finally to 2- to 3-psi steps as more signs of damage were evident. The loading schedule is shown in Table 3.

The pressure listed in Table 3 is the set pressure that the computer controlled valve gallery was attempting to maintain in the containment model. The actual pressures recorded at these steps varied slightly from those listed in the table. 
Table 3.

High-Pressure Test Loading Schedule

July 28

July 29

\begin{tabular}{cccccc} 
STEP & TIME & PRESSURE & STEP & TIME & PRESSURE \\
\cline { 3 - 4 } 1 & $11: 18 \mathrm{AM}$ & $10 \mathrm{psig}$ & 15 & $12: 34 \mathrm{AM}$ & 100 \\
2 & $11: 50$ & 20 & 16 & $2: 03$ & 102 \\
3 & $12: 18 \mathrm{PM}$ & 30 & 17 & $2: 55$ & 105 \\
4 & $12: 41$ & 40 & 18 & $3: 19$ & 107 \\
5 & $1: 29$ & 50 & 19 & $4: 10$ & 110 \\
6 & $2: 00$ & 55 & 20 & $4: 37$ & 112 \\
7 & $2: 31$ & 60 & 21 & $5: 20$ & 117 \\
8 & $3: 08$ & 65 & 22 & $6: 08$ & 120 \\
9 & $3: 39$ & 70 & 23 & $7: 44$ & 122 \\
10 & $4: 15$ & 75 & 24 & $8: 49$ & 125 \\
11 & $7: 26$ & 80 & 25 & $10: 37$ & 128 \\
12 & $8: 35$ & 85 & 26 & $11: 48$ & 130 \\
13 & $9: 44$ & 87 & 27 & $12: 24 \mathrm{PM}$ & 133 \\
14 & $11: 20$ & 95 & 28 & $1: 38$ & 135 \\
& & & 29 & $3: 38$ & 138 \\
& & & 30 & $4: 20$ & 140 \\
& & & 31 & $5: 20$ & 143 \\
& & & 32 & $6: 06$ & 145 \\
& & & & $7: 23$ & End of Test
\end{tabular}

\subsection{Points of Interest}

As the containment was pressurized, many phenomena were observed. At 125 psig some leakage from the vicinity of equipment hatch $B$ was sensed by the acoustic emission (AE) system. The AE system could not pinpoint the leakage; however, since the rate of leakage was small, it was most likely because of the unseating of the equipment hatch cover. With the accelerated aging of the seals (see section 3.7), very little separation would have caused some leakage." Leakage noise was continually monitored and increased at higher pressures. ${ }^{* *}$ The first leakage that was large enough to measure during testing was recorded at 135 psig and had a value of $11 \%$ mass/day $(8 \mathrm{scfm})$. L.eakage was noticed coming from the vicinity of equipment hatch $\mathrm{A}$ at $138 \mathrm{psig}$, and the ovalization of the sleeve of equipment hatch $\mathrm{A}$ was very

\footnotetext{
*Internal memorandurn, D.B. Clauss, "Comparison of Experiment and Analysis for the Behavior of Penetrations in the 1:6Scale Reinforced Concrete Containment Model," May 2, 1983.

** During the posttest inspections, two small holes in the liner near equipment hatch B were found, and leak lests were performed on these tears. It was concluded that the leakage through the liner from these liner tears would have been much greater than that occurring during the test at these pressure levels. This further supports the hypothesis that the leakage was due to the unseating of the equipment hatch.
} 
noticeable during the later stages of the test. The ovalization of equipment hatch $A$ was unlikely to be a source of leakage because metil-to-metal contact was maintained and one gasket was still intact. Displacement measurernents indicated nearly $1 / 2$-in. increase in the horizontal diameter.

During the final stages of pressurizatior, the uplift of the basemat from the concrete work mat was very noticeable. The amount of uplift averaged at three locations was $3 / 8 \mathrm{in}$. at the peak pressure of 145 psig.

During high-pressure testing, few nelv cracks developed in the model; however, cracks that were visible from the low-pressure tesing became much wider, some increasing to more than $1 / 8$ in. wide. The cracks followed seismic bars (angled at $45^{\circ}$ ) as well as the hoop and meridional directions. In the lower sections of the containment, few cracks were obvious. Although there was some cracking below elevation $6 \mathrm{ft} 3 \mathrm{in}$., the crack widths were very small during both low- and high-pressure testing. The lack of cracking is attributed to the constraint on radial $1:$ vement placed on the cylinder wall by the relatively stiff basemat and the additional reinforcing required in this region because of the additional forces (bending and shear) at the cylinder/basemat junction. A schematic of the reinforcing pattern in this area may be found in Appendix B of Reference 1.

\subsection{Events Leading to Test Termination}

Since some leakage was believed to come from both equipment hatches $\mathrm{A}$ and $\mathrm{B}$, a leakage test was conducted at 140 psig. 'The result of this leakage check was about $13 \%$ mass/day (10 $\mathrm{scfm})$. As the pressure was increased to $143 \mathrm{psig}$, even more noise was sensed from near equipment hatch $A$. The leakage check at this pressure level gave a result of $62 \%$ mass/day ( 50 scfm). Finally, at $145 \mathrm{psig}$, an attempt was made to determine how much leakage was coming from each hatch. By changing remotely controlled valves on the two covers at equipment hatch $B$, leakage from $B$ could be eliminated, assuming leakage was through the pressure unseating sealing surface.

The first attempt at measuring leakage was from the total containment, and a value of $234 \%$ mass/day (185 scfm) was obtained. Then, by changing the valves at equipment hatch $\mathrm{B}$ to eliminate leakage from it, a second reading was made. The leakage value was $352 \%$ mass/day $(275 \mathrm{scfm})$ and was completed at 6:57 PM. It was then apparent that leakage near equipment hatch $\mathrm{A}$ was slowly increasing. This was confirmed by the acoustic emissions in that area. By 7:05 pm, just 8 minutes later, the leakage rate had increased to $4000 \mathrm{scfm}$ (about $5000 \%$ mass/day), and the pressure in the containment was decreasing slightly even though nitrogen was being purnped into the containment n:odel at the capacity of the pressure system.

It should also be noted that $4000 \mathrm{scfm}$ is only a rough estimate of the flow rate because the rate was changing rapidly and significantly as the fill valves were opening and closing in the valve gallery, and there was a general increase of the leak rate with time. With this large leak, flow rates varied from about $3000 \mathrm{scfm}$ to about $4500 \mathrm{scfm}$.

A data scan was then initiated (actual model pressure decreased to about 142.5 psig at this time) and completed before the decision to discontinue testing was made. A plot of the increase in the leakage rate is shown in Figure 16.

Note that the pressure begins at 100 psig and that the last leakage value of over $4000 \mathrm{scfm}$ is not plotted in Figure 16. Figure 16 shows that the increase in leakage from the containment model grew quite rapidly over a few psi change in internal pressure. 


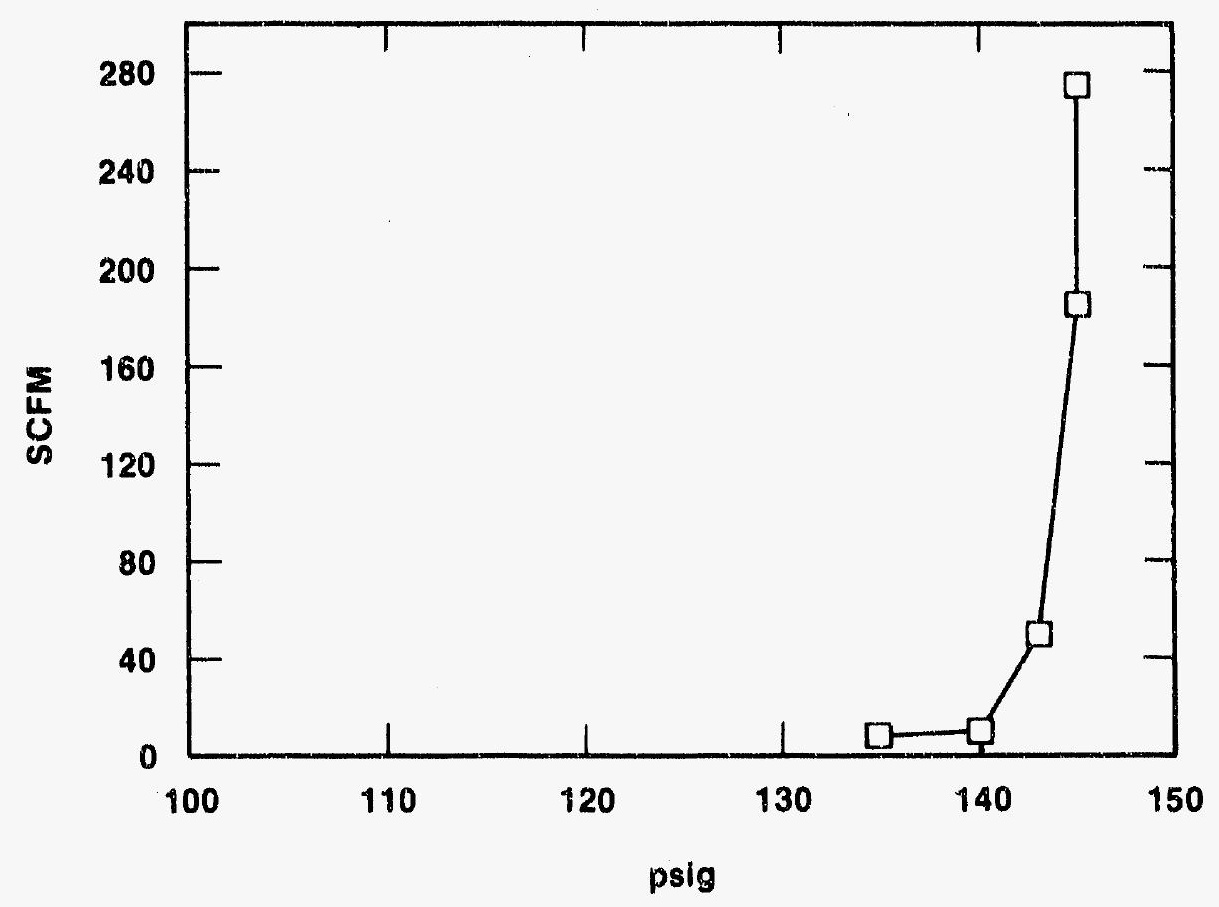

Figure 16. Leakage Rate as a Function of Internal Pressure.

These results should be tempered with an understanding of the effects testing procedure may have had on them. As pressure is increased in the containment model, the structure slowly responds--anelastically straining at a given pressure level. If pressure steps were taken at a faster or slower pace, leakage could arguably have occurred at a slightly higher or lower pressure. This can best be understood by estimating the amount of anelastic strain during a pressure step.

Using the creep data generated for the \#4 reinforcing steel [28] and a hoop stress of $75 \mathrm{ksi}$ (the lowest level that material tests were conducted) the amount of anelastic strain can be estimated. The strain in the reinforcing steel from these calculations at a time of 1 second is $1.24 \%$. The strain increases with time: holding the load constant for $3000 \mathrm{~s}$ (50 minutes) yields a strain of $1.45 \%$. The stress of $75 \mathrm{ksi}$ is close to the strain level in the hoop reinforcing at mid-height at an internal containment pressure of about $135 \mathrm{psi}$. The strains measured at the 134.42 psi pressure level at two mid-height hoop locations at $135^{\circ}$ was $1.04 \%$ and $1.24 \%$ for Channels 1104 and 1107 respectively. The variation in strain at these two locations is about the same level of variation of strain that one would expect to be present from anelastic straining of the material for a load that was sustained for 50 minutes.

\subsection{Posttest Inspection}

The model was approached after the internal pressure had been reduced to about $20 \mathrm{psig}$. The major leakage was from around, but not through, an instrumentation port. That is the nitrogen was flowing between the outside of the penetration sleeve and the concrete that surrounded it. The concrete in the wall was still intact but noticeably cooler from the large 
rush of nitrogen. The wall itself seemed somewhat delaminated because knocking on this area produced a noticeably different sound than that of neighboring areas of concrete.

\subsubsection{The Liner Tears}

Inside the containment, a 22 -in. tear by the insert plate was obvious-running in a vertical direction about 1 in. from the insert plate edge. Upon closer inspection, many small tears or areas of distress were found on the liner near other insert plates. Some of these areas of distress were from (apparently) the liner reaching its ultimate strain, while other areas of distress were because of the studs tearing a hole in the liner. Figure 17 shows a stretchout of the liner with the known cracks and tears indicated. As can be seen in Figure 17, all tears and distressed areas occurred near the mid-cylinder region of the containment, which is where the hoop strains were greatest.

In addition, every tear in the liner was associated with a stud that anchored the liner to the concrete, and the tears were also close to an insert plate, with the only exception being the two tears adjacent to equipment hatch B. (The tears have been categorized into two groups by the mechanism that caused the tear. The tearing associated with the insert plates is referred to as a shear tear while the two tears near equipinent hatch $B$ are referred to as a pull-out tear.) The maximum distance away from the closest insert plate was about $1.2 \mathrm{in}$. (The insert plates had a nominal thickness of $3 / 16$ in. and were butt-welded to the liner material, which was nominally $1 / 16$ in. thick.) It is clear that the combination of the penetration, its insert plate and the studs used to attach the liner to the concrete had a pronounced affect on the liner tearing.

Figure 18 shows the major liner tear next to the insert plate for a piping cluster used for the pass through of instrumentation wires. Note the buckling around the edge of the insert plate that occurred during unloading of the containment model. Close observation of the tear reveals shadows from the studs that anchor the liner to the concrete. Posttest analyses shed much light on the reasons and mechanisms for the tearing in this region $[33,36]$.

The four tears around equipment hatch $A$ are shown in Figure 19. The upper two tears were through the liner and exhibited leakage as discussed in the following section; the lower two areas were evidently not through the liner, since no measurable leakage was detected in measurements made after the test. Again note the proximity of the insert plates as well as the indentation in the liner because of the anchorage forces of the liner studs.

A distressed area occurred next to each of the insert plates of the two personnel air locks: one was a tear through the liner, and the other was severely distressed from the forces of the anchorage stud. The through liner tear adjacent to personnel air lock $\mathrm{A}$ is shown in Figure 20. The liner on the opposite side of each of the air locks had no visual signis of distress. Symmetry would suggest that some sign of distress would exist on each side of the air lock. Some thoughts on the air locks' lack of symmetry are presented in section 8 .

Note the similarity in the location and mode of liner tearing in personnel airlock $A$ versus equipment hatch $\dot{\mathrm{A}}$. Although the tearing was similar to the liner tearing around equipment hatch $\mathrm{A}$ (shear mode) there was only the one distressed area rather than the four, and further, the tear at equipment hatch $\mathrm{A}$ occurred on $45^{\circ}$ diagonals as opposed to the horizontal diameter for the personnel air locks. Further analysis may reveal a reason, but speculation is that the diameter of the penetration and the ability of the larger penetration to deform (to become oval in shape) more easily may have strongly influenced the location and number of the distressed areas. 


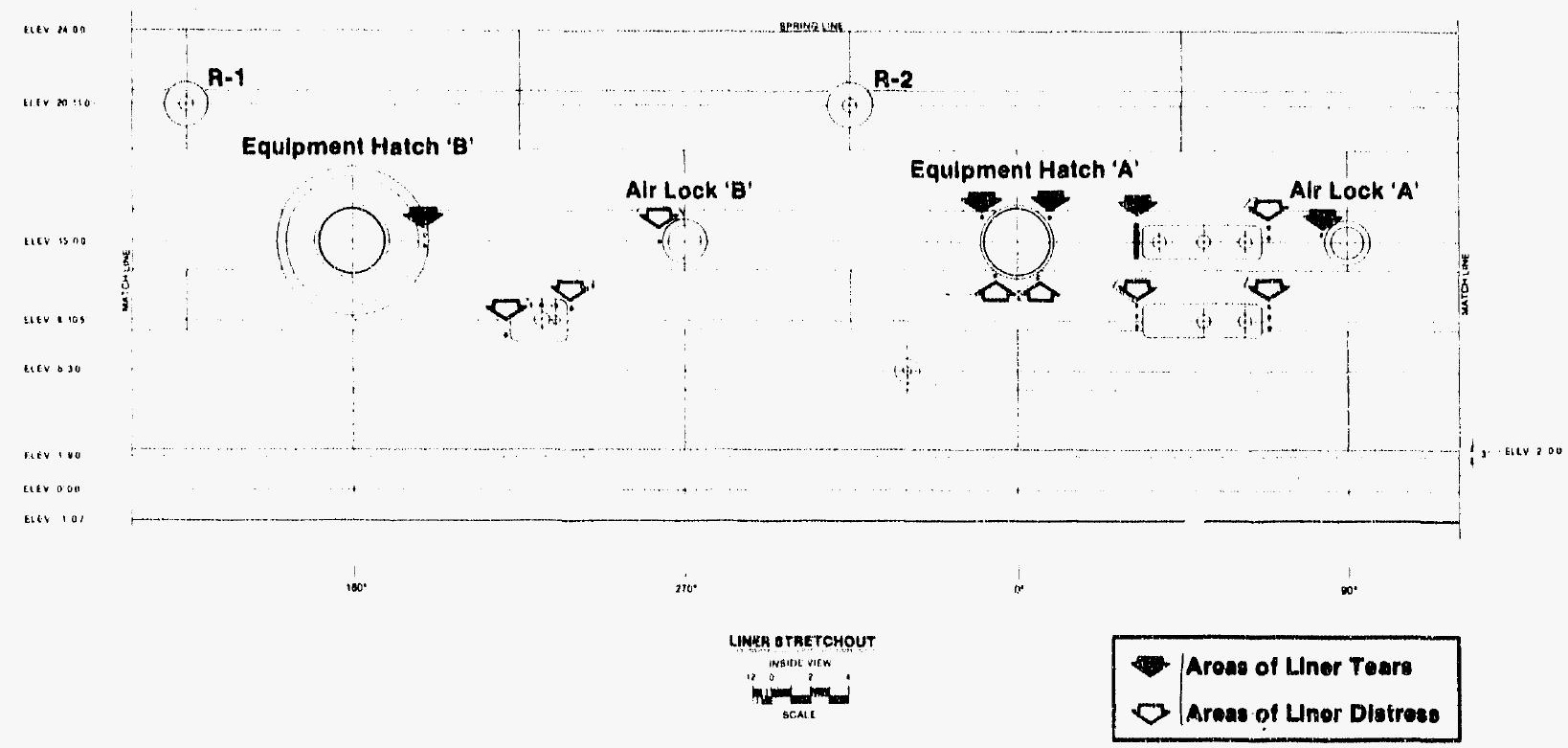

Figure 17. Stretchout of Containment Cylinder Showing Tears and Severely Distressed Liner Areas.

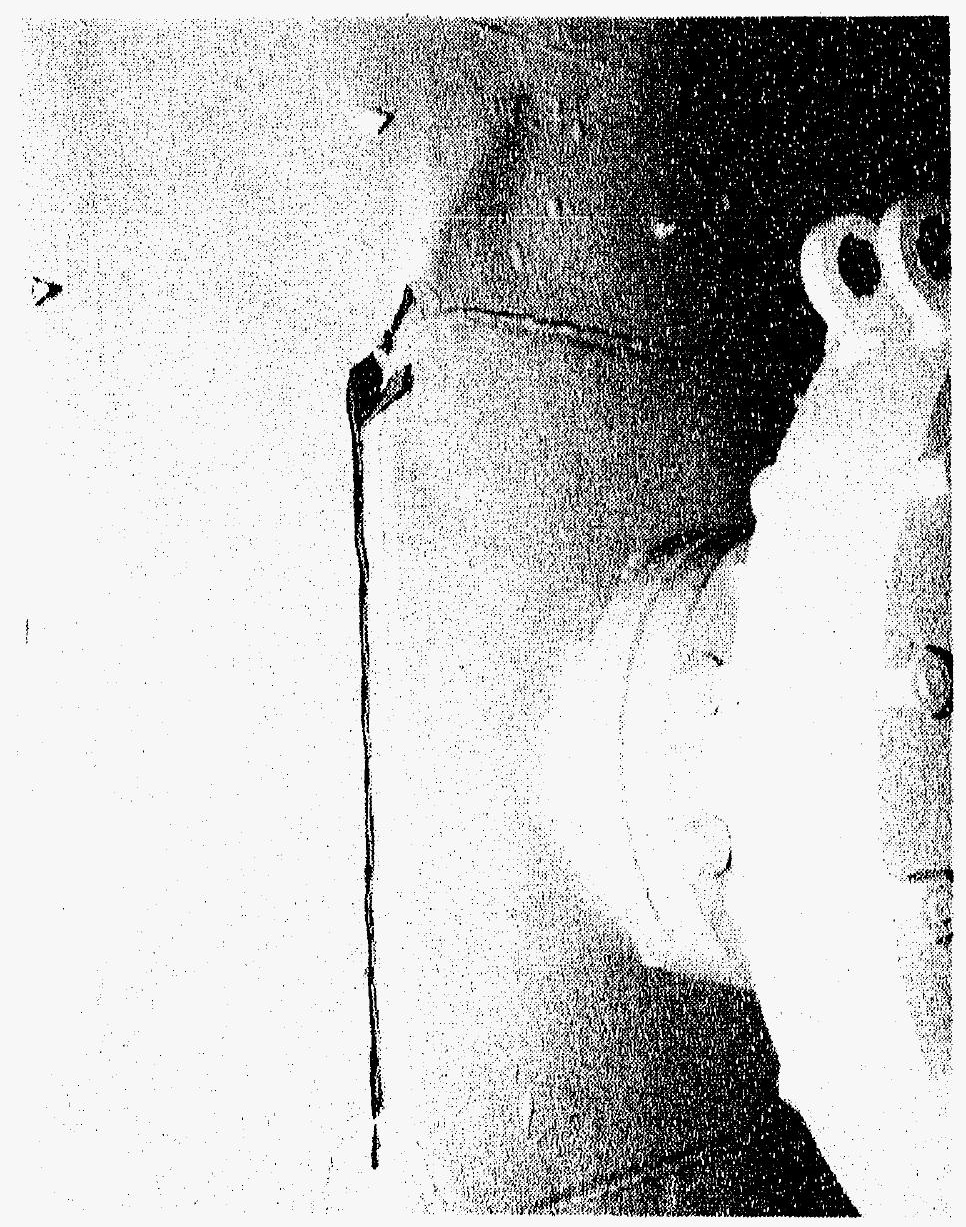

Figure 18. The Major Liner Tear. 


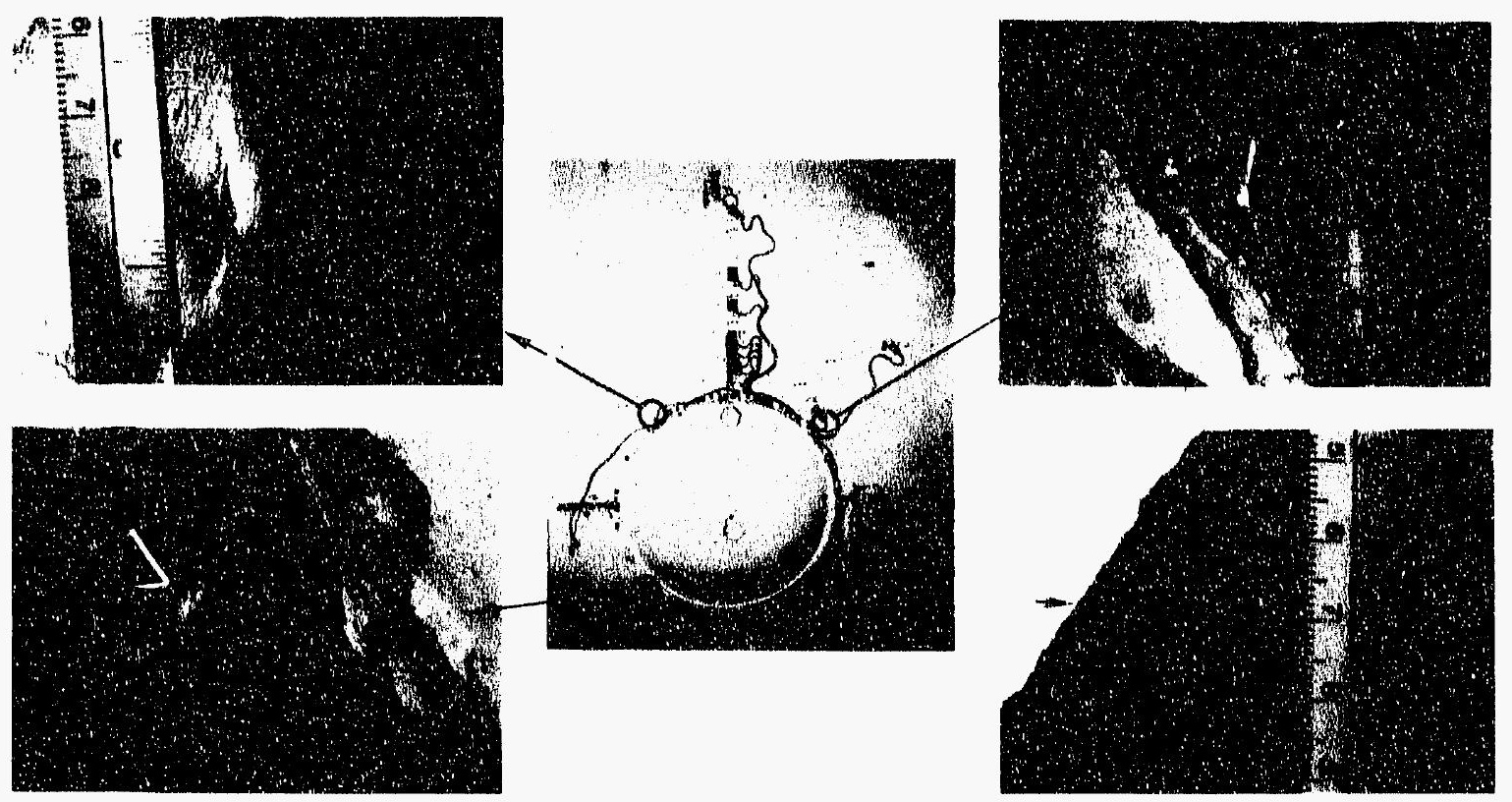

Figure 19. The Four Distressed Areas Around Equipment Hatch A.

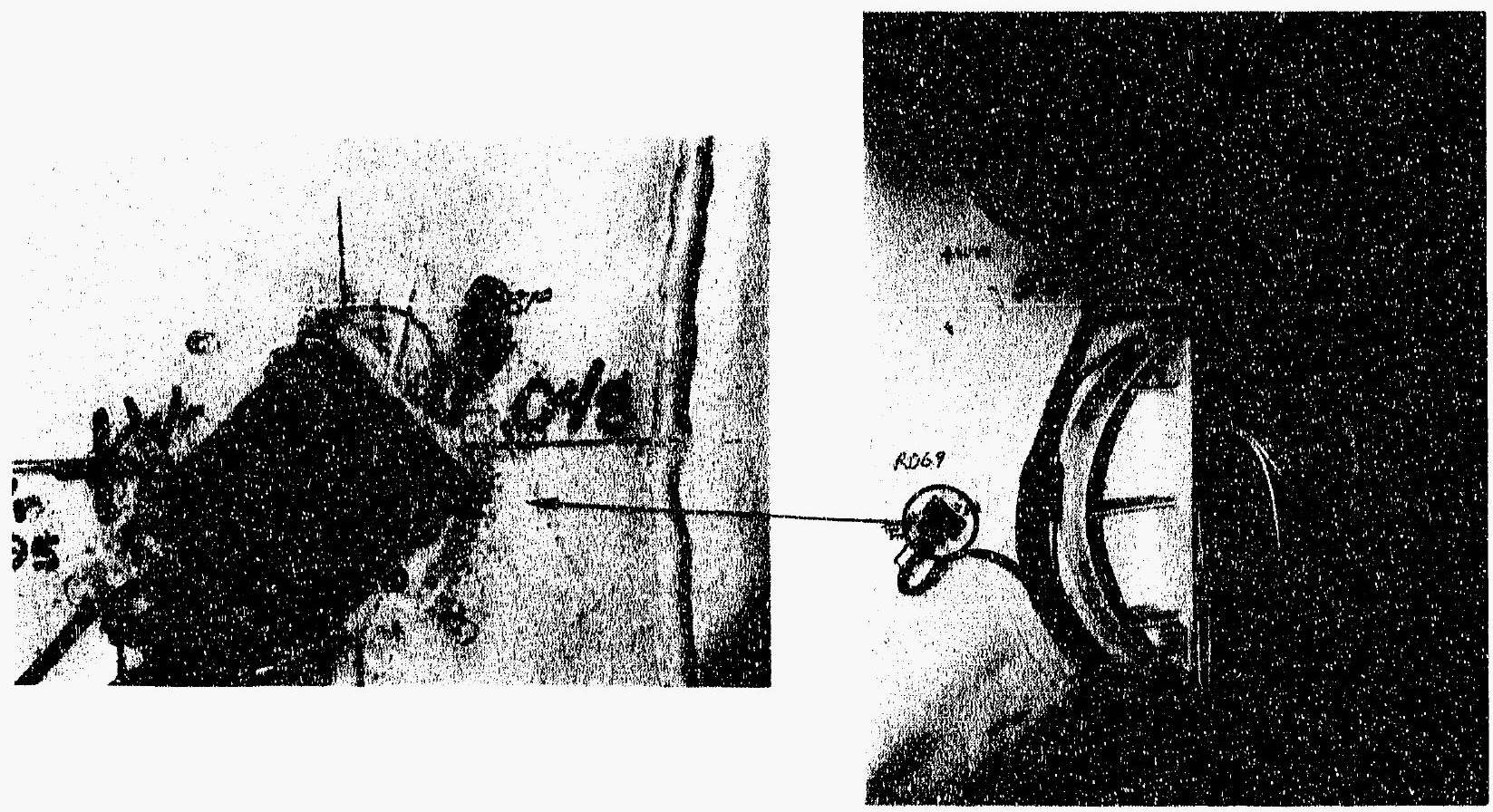

Figure 20. Tear Next to Personnel Air Lock A.

Two tears also occurred in the liner adjacent to equipment hatch $\mathrm{B}$ that were different from those previously discussed-a pull-out mode as opposed to a shear mode. The liner tears near equipment hatch $\mathrm{B}$ were associated with the local liner details that accommodated the inward projection of the equipment hatch boss. Because of this inward projection, there was a sharp discontinuity in the liner-an intersection of the cylinder wall with a truncated conical section-that was also attached to the concrete with studs. As the cylinder wall expanded under pressure, this angular junction tended to straighten out, thus loading the studs with tension. As the cylinder wall deformed, the studs tore holes in two locations on the truncated 
conical section of liner. These two tears can be seen in Figure 21. A more detailed discussion as well as strain measurements at this junction are presented in section 7.6.

Note the difference in the shape of these holes as opposed to the others in the model: here the base of the stud tore a circular hole and is no longer attached to the liner, while in other locations, the stud was still attached to the liner and adjacent to a linear tear. Additional insight into the liner tear is presented in section 7.6.

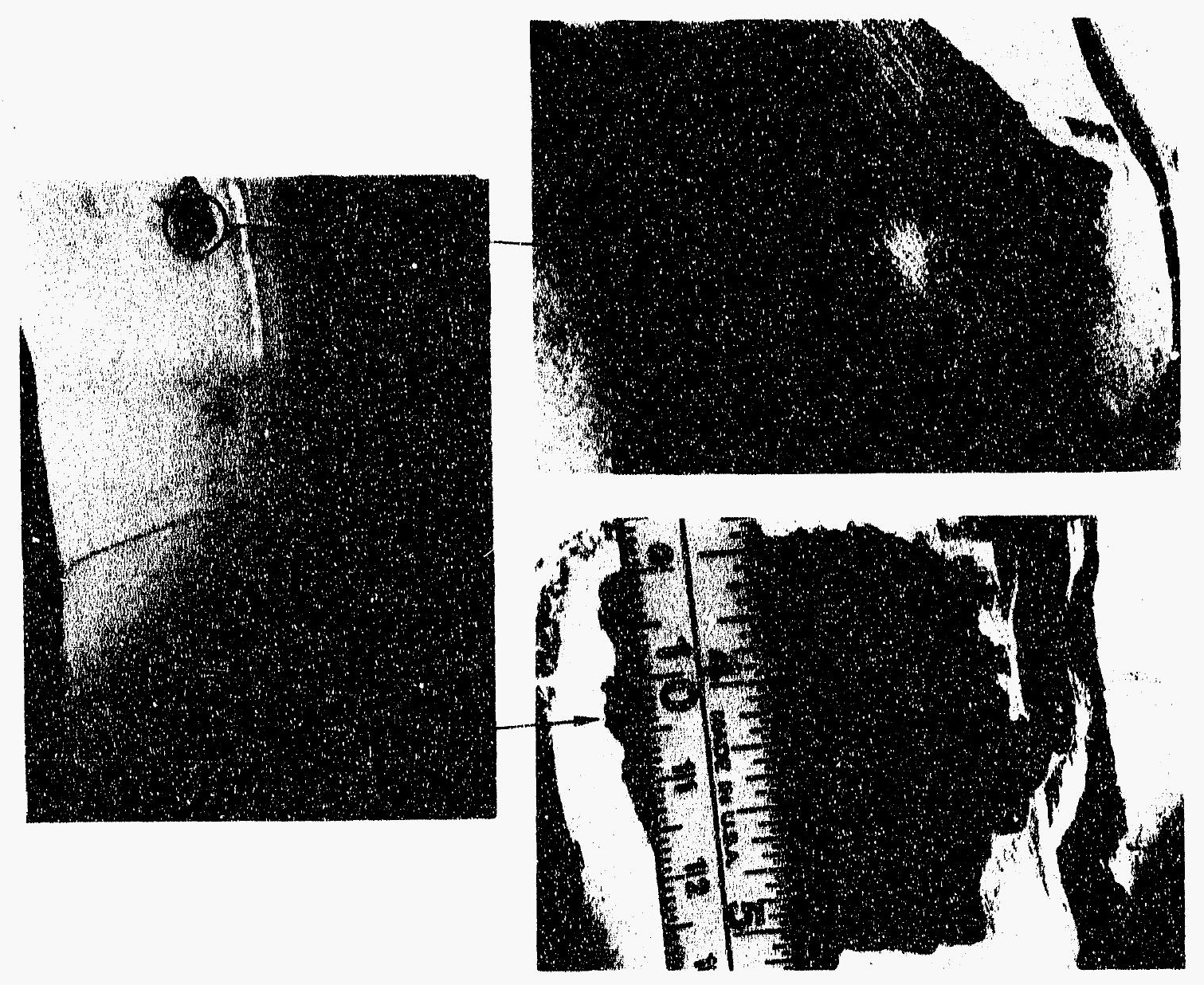

Figure 21. Holes in Liner Adjacent to Equipment Hatch B.

\subsubsection{Leakage Through the Small Tears}

A leakage assessment of each of the smaller tears and distressed areas shown in Figure 17 was made." Five small tears had measurable leakage, and a specially developed apparatus was made to measure the leakage through the small tears. The results from this testing are shown in Table 4.

The location presented in Table 4 is as viewed from the inside of the containment model. Other locations in Figure 17, but not included in Table 4, had no measurable leakage.

*Internal Report, D. Brinson, Leakage Flow Through the Small Breaches in the 1:6 Scale Reinforced Concrete Containment Model, ERC. International, April 1988. 
Table 4.

Posttest Leakage Measurements

\begin{tabular}{|c|c|c|c|c|}
\hline & \multicolumn{2}{|c|}{$\begin{array}{c}\text { Flow Rate } \\
\text { (scfm) }\end{array}$} & \multicolumn{2}{|c|}{$\begin{array}{l}\text { Leakage }^{2} \\
\text { (\%mass/day) }\end{array}$} \\
\hline Location & $125 \mathrm{psi}$ & $145 \mathrm{psi}$ & $125 \mathrm{psi}$ & $145 \mathrm{psi}$ \\
\hline $\begin{array}{l}90^{\circ} \text { Personnel Air Lock } \\
\text { Equipment Hatch A }\end{array}$ & 8 & 8 & 10.9 & 10.3 \\
\hline $\begin{array}{l}\text { Upper right } \\
\text { Upper left }\end{array}$ & $\begin{array}{c}8 \\
10\end{array}$ & $\begin{array}{c}9 \\
12\end{array}$ & $\begin{array}{l}10.9 \\
14.8\end{array}$ & $\begin{array}{l}11.6 \\
15.2\end{array}$ \\
\hline $\begin{array}{l}\text { Equipment Hatch B } \\
\text { Upper } \\
\text { Lower }\end{array}$ & $\begin{array}{c}27 \\
9\end{array}$ & $\begin{array}{l}30 \\
10\end{array}$ & $\begin{array}{l}38.0 \\
13.2\end{array}$ & $\begin{array}{l}37.6 \\
12.6\end{array}$ \\
\hline
\end{tabular}

1 Resolution of the flow meter is approximately $\pm 10 \%$.

2 Leakage rates are calculated from the flow rates measured and the volume of the containment model $\left(10890 \mathrm{ft}^{3}\right)$.

\subsubsection{Vacuum Box Testing}

Since the liner knuckle, the joint between the cylinder wall and basemat, was considered to be a likely place for liner tearing that could be caused by very small displacements, a thorough visual examination was conducted. No obvious tears or cracks were found. As an additional check of this area, a vacuum box test was conducted after all the paint was removed from the weld seams. Again, no signs of leakage through the weld seams were observed.

\subsubsection{Ovalization of the Equipment Hatch Sleeves}

Both during the test and the posttest inspection, significant ovalization at equipment hatch $\mathrm{A}$ was observed. This contrasts with a minimal amount of ovalization at equipment hatch $B$. The ovalization at equipment hatch $\mathrm{A}$ was sufficient to expose a portion of the one gum-drop seal at the left and right edge of the sleeve, as can be seen in Figure 22.

The detailed measurements and other data on the equipment hatches are presented in sections 7.5 and 7.6 .

\subsubsection{Basemat Uplift}

The basemat uplift was measured in three ways: use of displacement transducers attached to the basemat and working mat, use of a rail and tilt sensor, and use of an internally mounted track transducer cantilevered from the center column. All the displacement transducers were 
damaged during testing due to radial expansion of the basemat, but the tilt sensors and track transducers were able to measure the basemat uplift.

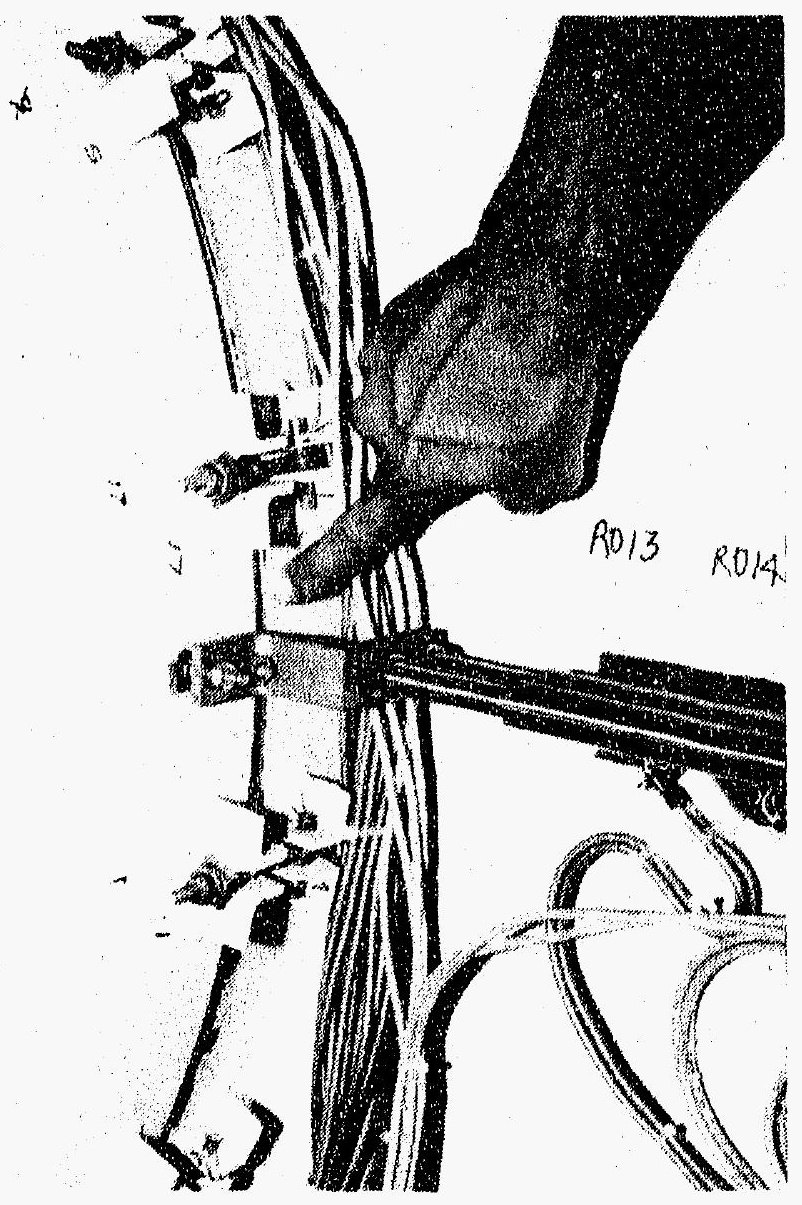

Figure 22. Exposed Seal at Equipment Hatch A Because of Sleeve Ovalization.

Three displacement transducers were attached to the outside edge of the basemat, and the working end of the transducer was attached to the top of the working mat. As pressure was increased in the containment model, there was also sufficient radial motion of the basemat to damage all three of these transducers. They were designed to allow for some small radial expansion, about 0.070 in., but this was obviously not enough.

The rail and tilt sensor method of measuring uplift used a rail that rested on the top edge of the basemat and continued radially to a pivot point about $10 \mathrm{ft}$ from the basemat edge (see Reference 1). A tilt sensor was on top of this rail; thus, as the basemat moved upward, a change of tilt was recorded that could then be converted to uplift using trigonometric functions. The average uplift of the basemat as measured by this means was $3 / 8 \mathrm{in}$. at the peak pressure level.

A picture of the residual uplift after depressurization of the containment model is shown in Figure 23. Since the uplift of the basemat was largely from elastic strains, only about $1 / 16$ in. of residual uplift existed after the model was depressurized. Section 7.3.3 describes the basemat displacements measured during the overpressurization of the model. 
The third method for measuring the uplift and also the profile of the basemat was with a track transducer (section 4.4) cantilevered from the internal column. In addition to the track transducer, a fixed displacement transducer was attached to the extreme end of the track. The track transducer performed well, but was able only to obtain the basemat profile for $\mathrm{R}=1 \mathrm{ft}$ $11.8 \mathrm{in}$. to $\mathrm{R}=9 \mathrm{ft} 0.9 \mathrm{in}$.

The chronology of the overpressurization test events have been qualitatively described in this section. Selected measurements made during the test as well as observations and conclusions from these measurements are discussed in the next section.

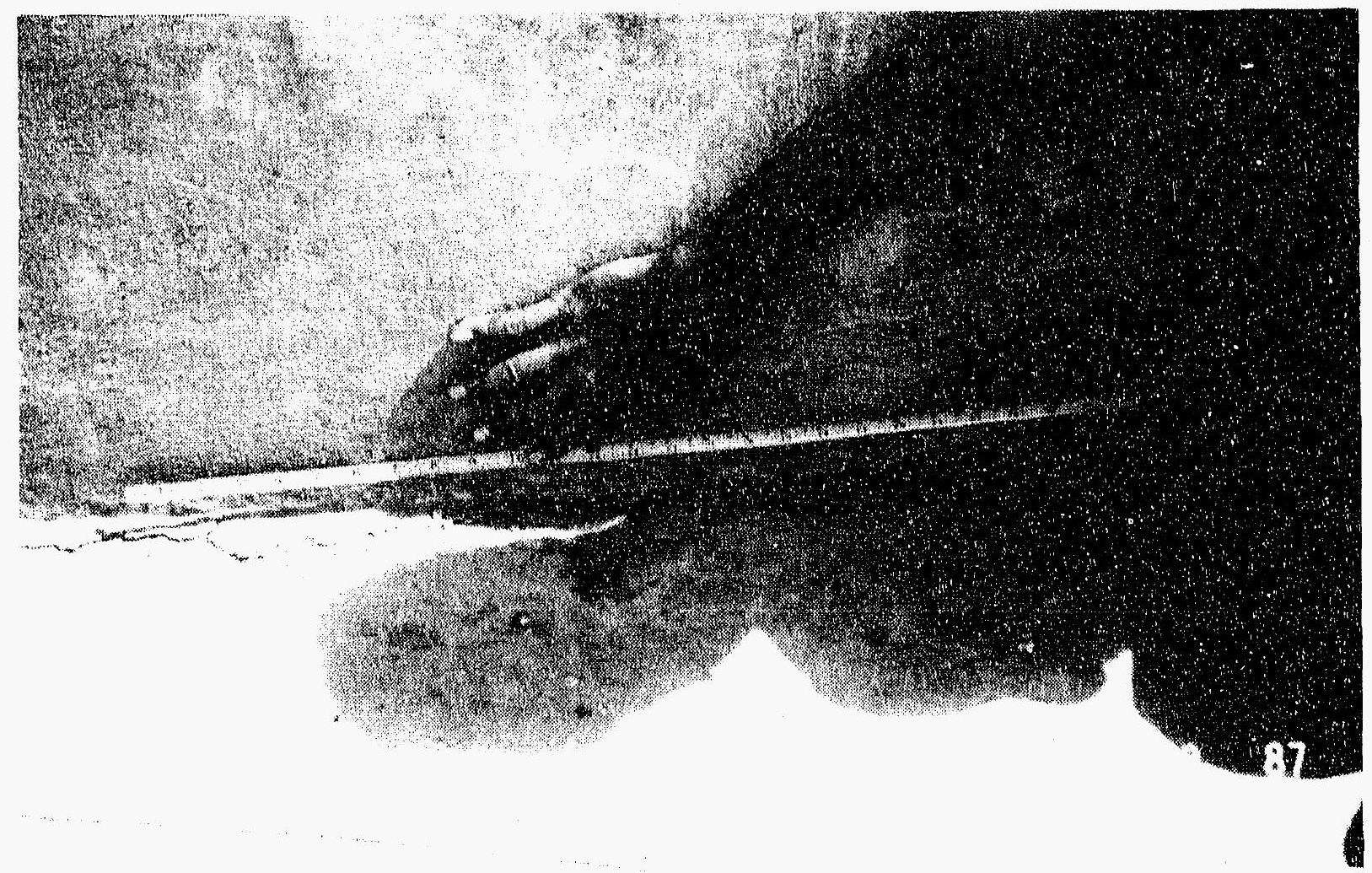

Figure 23. Residual Uplift of the Basemat. 


\section{HIGH-PRESSURE TEST RESULTS}

The results from the high-pressure test are presented in this section. Following a discussion on the accuracy of the measurements, the section is divided into general features of the containment, which include response of the feature. This is then followed by a summary of the results.

The instrumentation and its location and channel number may be found in Reference 1-they are not repeated here. Only a sampling of results is shown, but the measurements from all transducers are graphed and tabulated in Appendix $\mathrm{C}$, which also has a table relating channel numbers to sensor type and its alternate name, if applicable.

\subsection{Data Reduction and Interpretation}

A few important notes are necessary to understand the following sections fully. First, the model, as recorded by most of the transducers, experienced a permanent set after the completion of the Structural Integrity Test (SIT). All transducers were zeroed before the start of the high-pressure test. No attempt was made to correct the high-pressure test results based on the results of the SIT. Since other events associated with construction also influenced the elements-the reinforcing steel, for example, was plastically deformed during construction-the strain history for any element is not fully known. Similar examples are the amount of cold working of the materials from other construction sequences such as welding of the liner material. Since a systematic method for correcting the high-pressure data based on the results from the SIT may not be appropriate for all uses of the data, a correction was not applied to the high-pressure test data. However, the data for the SIT and high pressure tests are both plotted and listed in Appendix C, allowing users of the data to make the corrections appropriate for their application.

Second, global displacements are particularly difficult to quantify in this type of test-no fixed reference frame existed, but one needs to be assumed. For global measurements, this was the center column inside the containment. Where possible, the global measurements were made directly from this column; however, this was not possible in all cases. Measurements in the dome, for example, were made from the upper platform, which could move relative to the center column. This was recognized before the test and measurements were made to determine the amount of relative motion between the column and the platform. Since these relative motions were very small, no corrections were made to the global measurements of transducers attached to the platforms, most of which measured displacements in the dome.

Third, many of the displacement transducers were attached directly to the wall. As the containment was pressurized, the wall could move in three-dimensional space, measuring radial displacement, meridional displacement, and any tangential displacement of the wall. (Admittedly, tangential displacement would have been small and was not measured at any point in the containment model, but is mentioned for completeness.) To decrease the sensitivity of the transducer to other directions of movement, the length from the attachment point to the transducer in the direction of interest was kept as long as possible (Figure 24). 


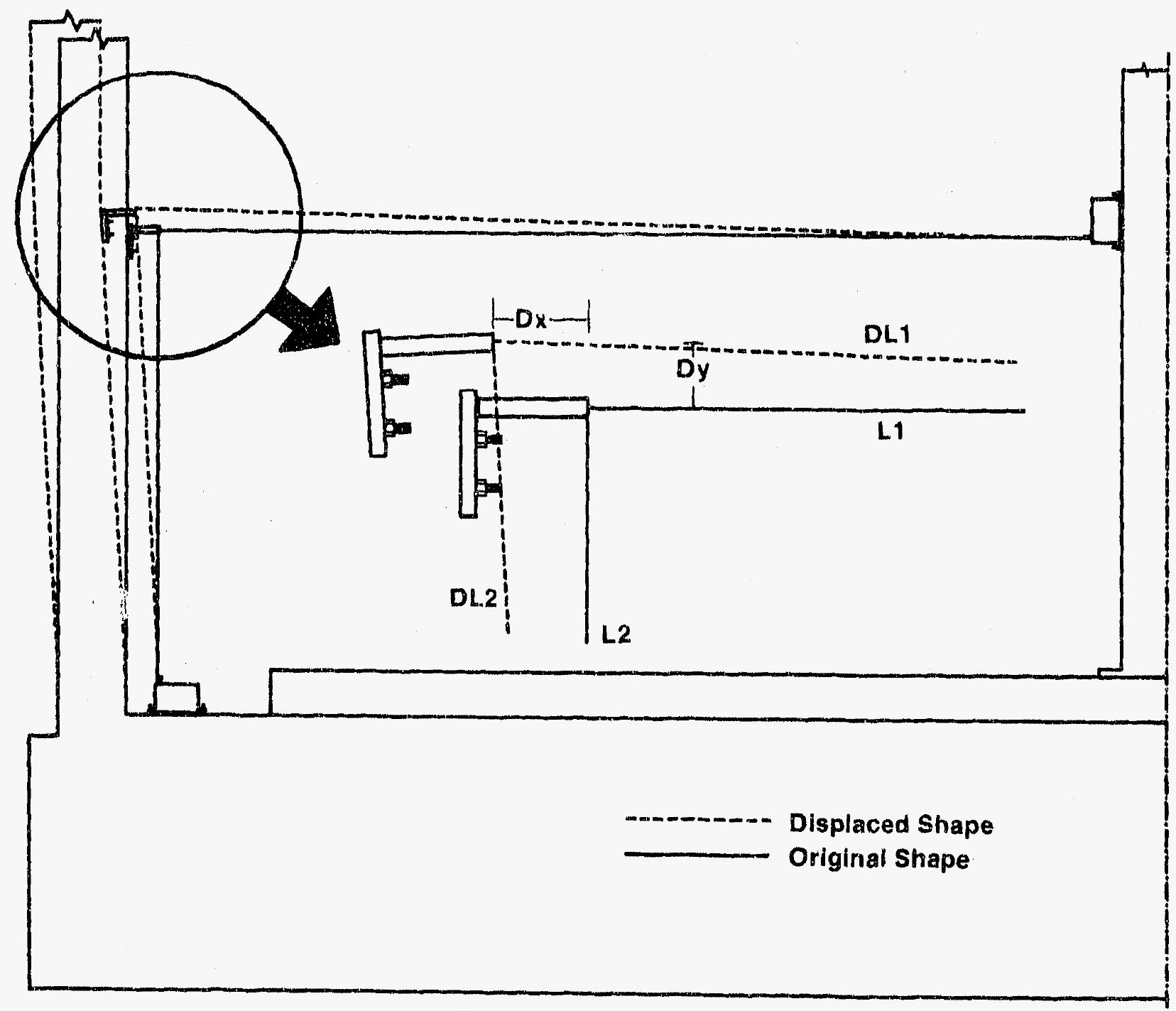

Figure 24. Error in Displacement Measurements Due to 2D Movement (Torsional Movement is Ignored).

However, since radial displacement dominated in many areas, the vertical displacements recorded for the cylinder wall were assessed to determine if corrections should be applied. The following equations illustrate this concept for channels 217 and 218 , which are located (approximately) at an azimuth of $228^{\circ}$ and an elevation of $11 \mathrm{ft}$. Channel 217 measured meridional deformation, while 218 measured radial displacement. The gages are assumed to be attached at the same point, and any tangential displacement is neglected.

From Figure 24, and by using the Pythagorean theorem, two equations with two unknowns can be writien:

$$
\begin{aligned}
& D L 1^{2}=D Y^{2}+(L 1+D X)^{2}, \text { and } \\
& D L 2^{2}=D X^{2}+(L 2+D Y)^{2}
\end{aligned}
$$

From the measurements report (Reference 1, Appendix A), the original lengths are obtained: 


$$
\begin{aligned}
& \mathrm{L} 1=126.81 \mathrm{in} . \\
& \mathrm{L} 2=107.72 \mathrm{in} .
\end{aligned}
$$

The deformed lengths are obtained by adding the recorded displacement to the original length:

$$
\begin{aligned}
& \text { DL1 }=128.76 \text { in. } \\
& \text { DL2 }=108.01 \text { in., }
\end{aligned}
$$

which were taken at the final pressure level.

Solving these equations yields the "independent" radial, DX, and meridional, DY, displacements.

Using the results of Channels 217 and 218 at a pressure of 143 psig, the corrected radial displacement is $1.9522 \mathrm{in}$. and the corrected meridional displacement is 0.2697 in., with respective errors of $0.0003 \mathrm{in}$. and $0.0176 \mathrm{in}$. Since $0.018 \mathrm{in}$. was the largest correction for this effect on any of the transducers at any pressure level, no corrections were applied. The displacement transducers that were assessed for this correction measured global meridional displacements in the cylinder wall: $0^{\circ}$ - D54 (Ch 173), D55 (Ch 174), D57 (Ch 176), D60 (Ch 179); $180^{\circ}$ - D88 (Ch 207), D89 (Ch 208), D91 (Ch 210), D93 (Ch 212); 225 - D95 (Ch 214), D98 (Ch 217), D104 (Ch 223), D105 (Ch 224).

Fourth, these same gages were also susceptible to another form of error. As shown in Figure 24 , some of these gages were attached directly to the floor liner; however, the liner was not necessarily in direct contact with the concrete below it. As a result, when pressure was applied in the containment model, the liner was displaced until it contacted the concrete below it. Where this error was evident, the recorded displacements were corrected by using a straight line extrapolation from the readings taken at 20 and 10 psig for the high pressure test and 5 or 10 psig for the SIT. The largest correction applied was slightly more than $1 / 8 \mathrm{in}$. The amount of correction is listed in the tabulated data.

Fifth, the materials of the containment model creeped at an applied load, making the test results somewhat time dependent. Note that this phenomenon of the steel materials used in fabrication of the containment model was investigated and reported in Reference 30 . No account for this phenomenon is applied to the data. Section 6.3 offers a discussion and example of the magnitude of variance that could be present in the data due to material creep.

Finally, there are the ever-present errors from the limitations of the transducers and the sensitivity of the digital voltage meter used to take measurements during the test, as well as the digital accuracy of the computer and its math routines to convert the voltage readings to engineering units. These errors are generally much smaller than those from the other sources mentioned above and have not been accounted for in any of the results presented here. 


\subsection{General Cylinder Behavior}

In this report, free-field response is defined as the response of the containment away from penetrations that disrupt its axisymmetric behavior. Since the region of influence of the four major penetrations particularly can be quite large, some perturbation in free-field response from the penetrations is present in the entire cylinder of the containment. This should be kept in mind when viewing the results in this section.

Significant containment building responses were associated with the cylinder wall. The largest strains and the largest displacements, as well as the liner failure that led to the termination of the test, occurred in the cylinder. Some of these responses, however, were more closely related to the penetrations and are covered in other sections.

As expected, the containment model response was not perfectly axisymmetric. There was noticeable variation in strains and displacements about the cylinder wall at a given elevation. In some cases, the variation could be attributed to features of the structure, while nonuniformity in material properties also had an effect.

\subsubsection{Strains}

Variations of hoop strain in the reinforcing steel and the liner near cylinder midheight are presented in a bar graph in Figure 25. The results in this figure were taken at the final pressure level, $143 \mathrm{psig}$, after the pressure decreased from the maximum level of $145 \mathrm{psig}$. The average value of strain recorded by the six gages plotted in Figure 25 is $1.69 \%$.

In Figure 25, the lowest value of hoop strain in the reinforcing steel is at an azimuth of $46^{\circ}$, which lies behind a thickened section in the liner. It is certainly logical to assume that some of the circumferential load was carried by the thickened liner, reducing the strains in this region of the wall. The strain reduction, however, was not nearly as large as expected if a perfect bond existed between the insert plate and the reinforced concrete wall. There was thus some flexibility of the anchorage system, which allowed the thickened insert plate to slip relative to the concrete wall.

Variation in the hoop-direction liner strains is more difficult to explain. The liner gages in Figure 25 were over four feet away from the insert plates of the penetrations, which was as far away as practicable, considering the spacing of the penetrations. The variation in the liner strain can be attributed to the liner anchorage system. Anchoring the liner to the cylinder wall allowed the liner to bridge cracks developing in the concrete. When a crack was located over a liner strain-gage position, the anchorage system (in this case headed studs) transferred this strain discontinuity into the liner, which created a higher strain reading even in the free field. Conversely, when liner strain gages were located on top studs, the strains were much smaller than the free-field strains. Variation of material properties is likely to have had an equally significant influence.

There is also the fact that the liner was not at all locations in intimate contact with the concrete. Even though the liner was used as the internal form during the placing of the concrete, when the liner's internal support frame was removed the liner pulled away from the concrete at some locations. (Particularly at field welds that ran in the hoop direction.) A record was kept on whether or not the liner near a strain gage was in solid contact with the concrete. This record is found in the Appendix A of Reference 1. 


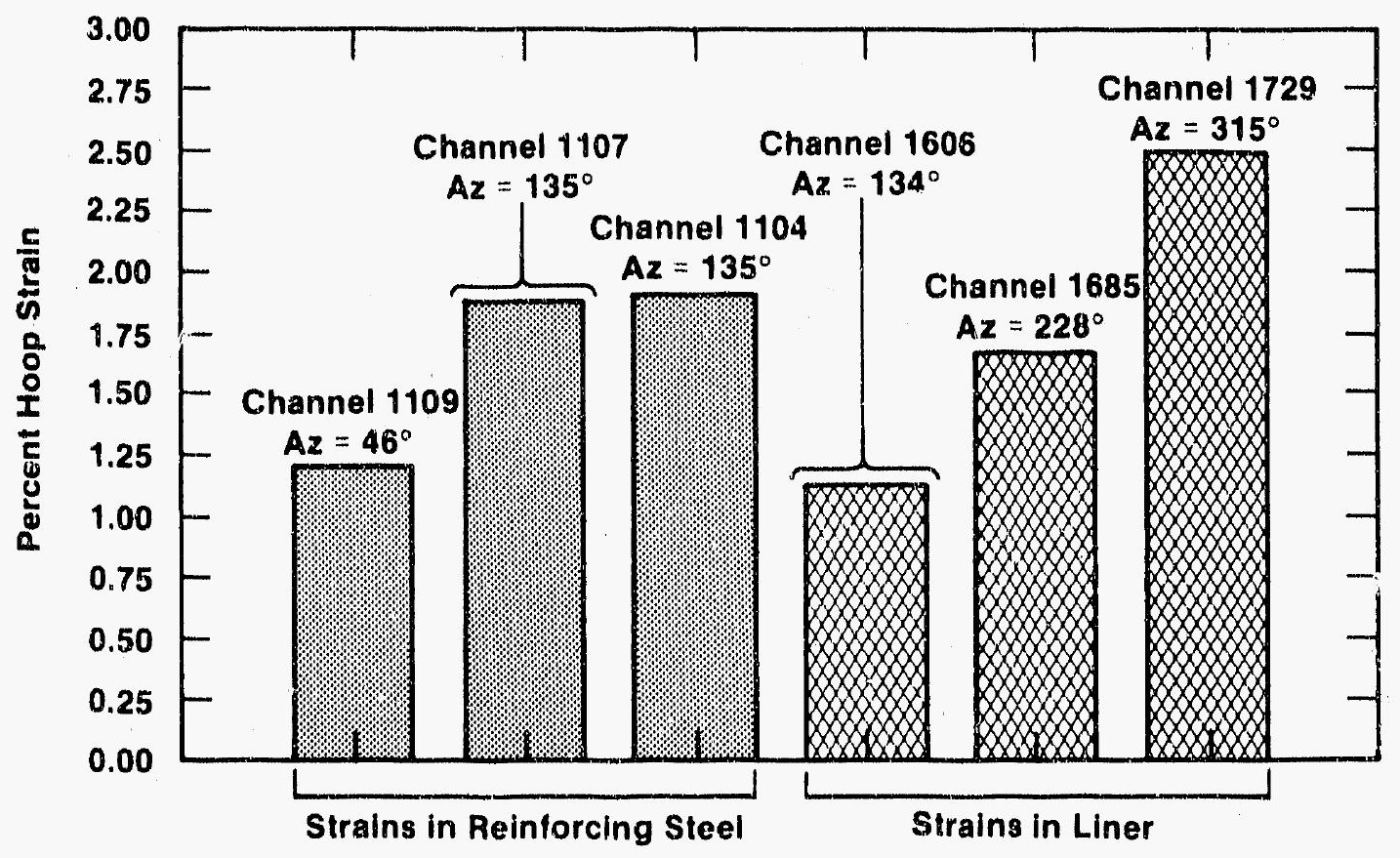

Figure 25. Hoop Strain in the Reinforcing Steel and Liner at Cylinder Midheight at the Final Pressure Level.

Finally, the effect of material variation is a constant source of variation in liner strain readings. Whether variation in the stress-strain properties or the variation in thickness of the liner itself, some statistical scatter in the strain readings is expected.

\subsubsection{Displacements}

Similar variations in the radial measurements were also observed in the containment model. Figure 26 shows six radial displacements: two are located on the two equipment hatches, one is on the personnel air lock, and the remaining three are "free-field" displacements. All of these plotted displacements are at cylinder midheight near elevation 13 feet. 
The largest radial displacements presented in Figure 26 were recorded in free-field areas. Shading indicates the range of free-field displacements, which indicate about a $1 / 2$-inch difference at the final pressure step plotted. In general, the displacements associated with the major penetrations were smaller than these free-field displacements. Furthermore, the two largest penetrations, equipment hatches $\mathrm{A}$ and $\mathrm{B}$, had the smallest displacements at the highest pressure readings. The smaller penetration, personnel air lock A (PL-A) located at $90^{\circ}$, had a radial displacement in the lower range of the free-field displacements at the later st $t$ ges of the high-pressure test, but also note that among the displacements measured for this group, PL-A showed the least displacement for pressures below 125 psig.

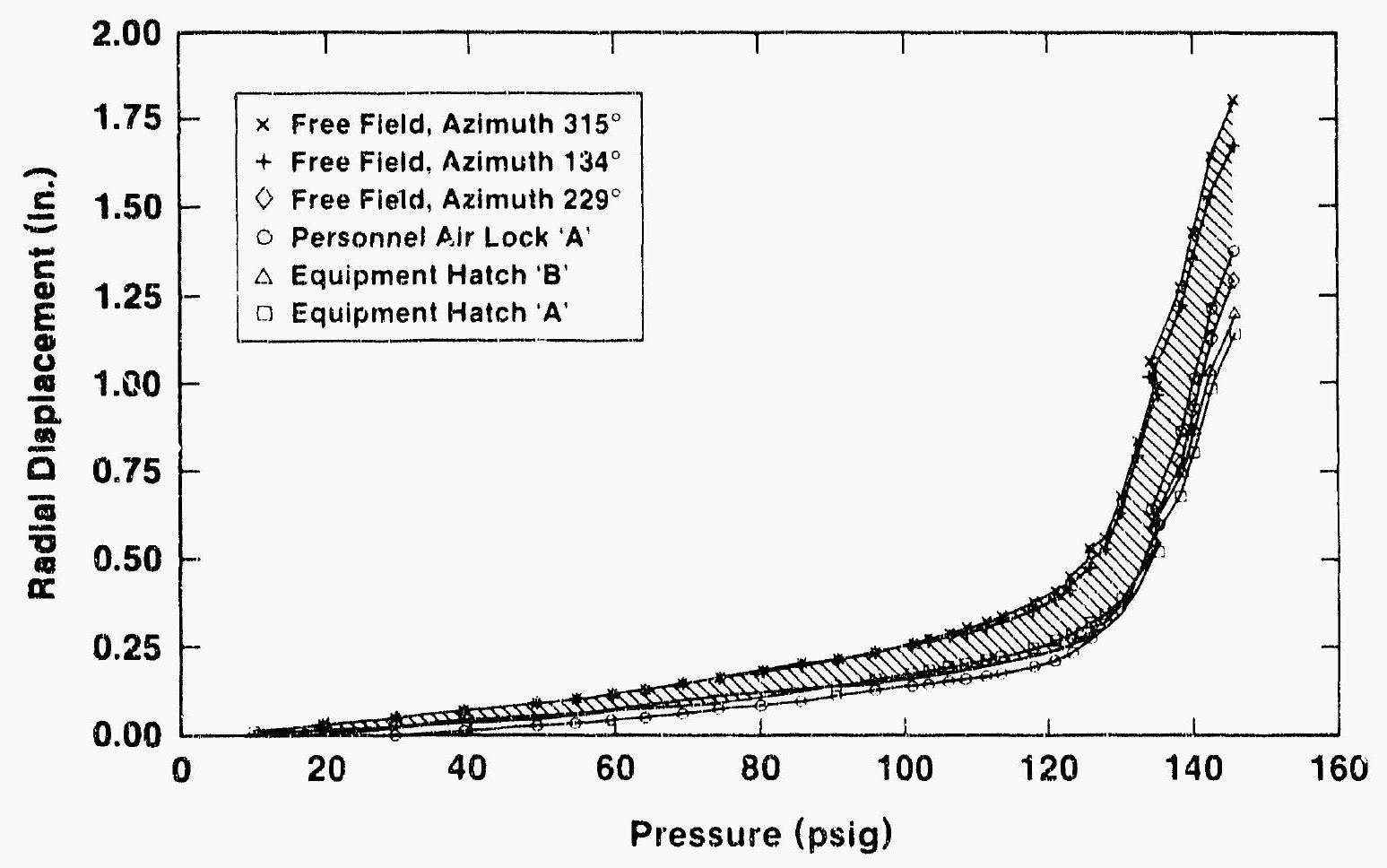

Figure 26. Radial Displacement of Cylinder Wall Midheight.

The decrease in radial displacements at the penetrations clearly reflected the relative increase in the cylinder wall stiffness from the penetration bosses; that is, the thickened boss and the increased reinforcing in this area stiffened the cylinder section and reduced the outward displacement.

\footnotetext{
*Berausa of the dicenntinuity of the nenctration, the wall har sthstantially more reinforcing steel and was also thiskened from its nominal 9-3/4 inch thickness to $1^{\prime} 9^{\prime \prime}, I^{\prime} 6-3 / 4^{\prime \prime}$, and ' $^{\prime} 3-3 / 4^{\prime \prime}$ for cquipment hatches $B$ and $A$, and PL-A, respectively [1].
} 


\subsubsection{Liner Performance}

Liner performance is discussed in the following sections with penetration behavior, but even away from the penetrations, some interesting liner responses were recorded during the highpressure test.

An attempt was made to measure the effect of a crack in the concrete on the behavior of the liner. Well-defined cracks developed on the outside face of the concrete from the low-pressure testing of the containment model. Two vertical cracks formed from the hoop stresses in the concrete were assumed to be through the wall thickness and intersect the liner. A strip gage was attached to the liner at this assurned intersection of the crack to measure the crack's effect on liner behavior. One of the strip gages (Strip 15) showed little unusual behavior, while the second strip gage (Strip 14, channel numbers 2364-2384) recorded a perturbation from a typical free-field response. The results from Strip 14 are shown in Figure 27.

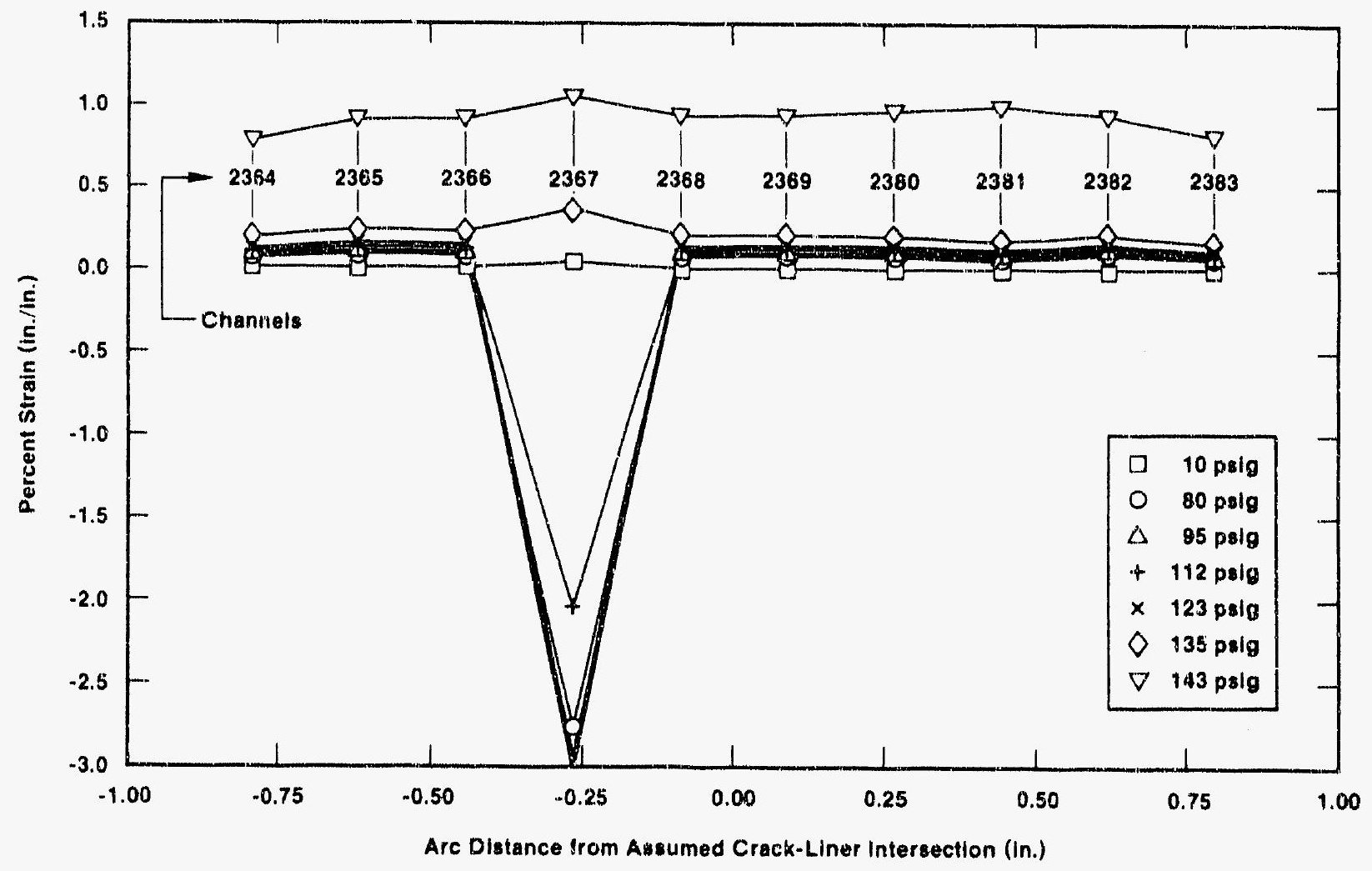

Figure 27. Hoop Strain in Liner Near an Assumed Intersection with a Crack in the Concrete at 7 Pressure Levels.

As the pressure in the containmen was increased, one gage on the strip showed an increase in compressive strains. As pressure was increased, the strain direction changed until at the final pressure ieveis it showed a tensiie strain siightiy greater than that of the neighboring gages on 
the strip. This trend could certainly have been from a crack, but the magnitude of the compressive strain at this one region was surprisingly high and could have indicated something other than a crack in the concrete (a small void, for example).

Certainly this evidence is not conclusive, but a thorough investigation of this region is planned. Removing the liner in this section should support or refute whether this surprising liner behavior was from a crack in the concrete or some other feature.

\subsubsection{Concrete Behavior}

Strain gages were placed on the concrete surface after the low-pressure testing was completed in an attempt to measure the straining of the concrete during high-pressure testing between the cracks that had developed. Specifically, four tee rosettes (channel numbers 2440 - 2447) were placed between azimuth $90.27^{\circ}$ and $91.94^{\circ}$ at an elevation of 22 feet, $2-1 / 2$ in. The recorded strain readings do not show a clear pattern for straining between the cracks. Lesser strains were anticipated near the cracks with increased tensile strains away from the crack edges. As can be seen in Figure 28, the strains were not always tensile, and in fact, as the internal pressure was increased, the strains dramatically decreased and in some cases became compressive for the last two pressure levels plotted. The reason is not fully understood, but the meridional bending in the cylinder wall from the constraint of the dome probably influenced the strain readings at this location. The heterogeneity of the concrete may also explain the strain readings. Whether a strain gage is located directly over a large piece of aggregate, or mortar can certainly influence the recorded strain gage response.

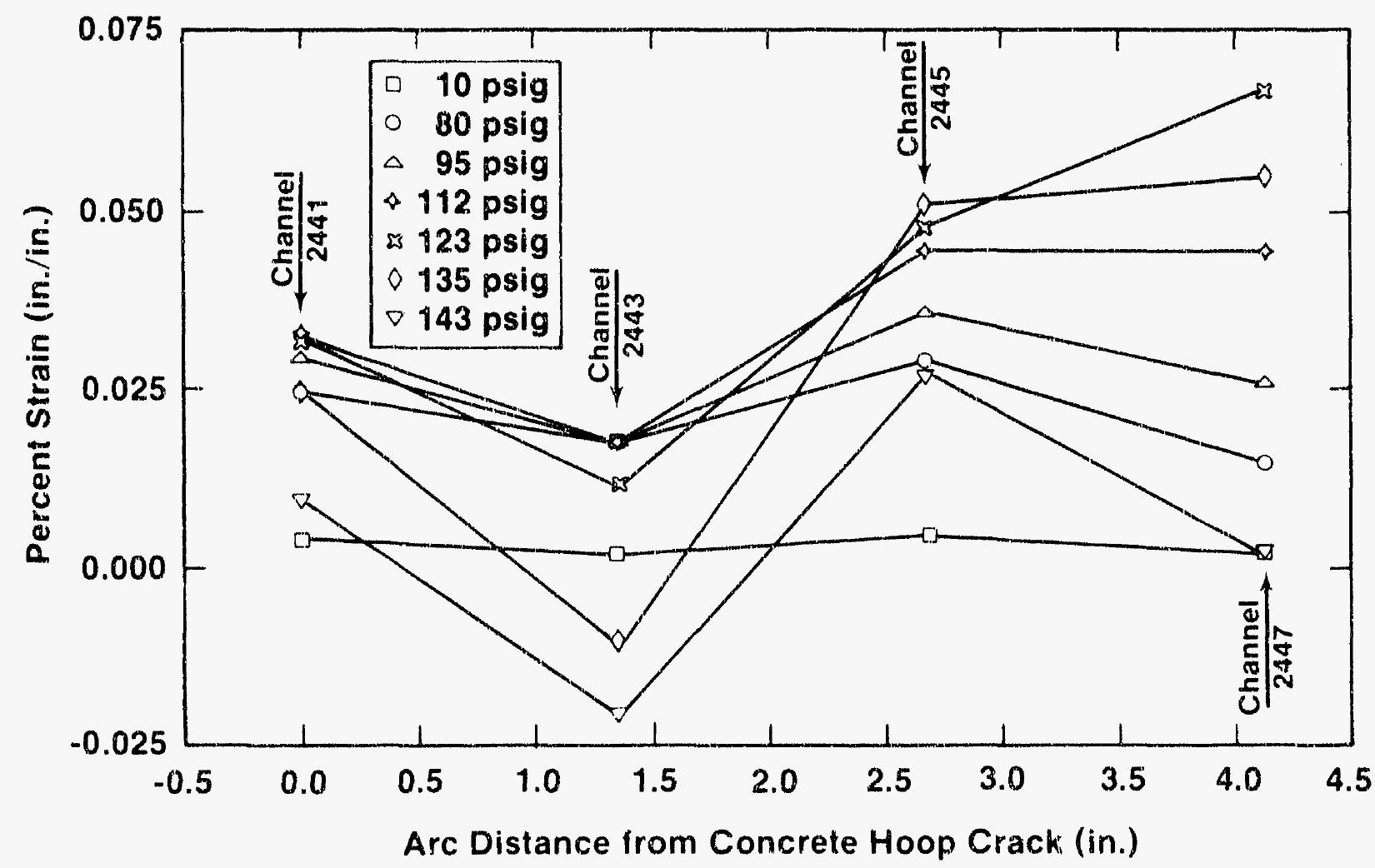

Figure 28. Honn Strain in Concrete Between Two Vertical Cracks in the Cylinder Wall. 


\subsection{Basemat}

The basemat of the containment model dished slightly from the loading of the internal pressure, and the concrete and the steel in the basemat showed slight nonlinear response. As a testimony to the magnitude of the forces involved in dishing the basemat, the meridional force applied to the rebar totaled 7.9 million lbs. at the peak test pressure of $145 \mathrm{psig}$. The following sections present some of the data on the rebar and liner strains and the displacements associated with the basemat.

\subsubsection{Rebar Strain}

The reinforcing steel in the basemat was instrumented with weldable strain gages. One of the difficulties in instrumenting the basemat was the change in reinforcing patterns the bottom reinforcing mat was arranged in an orthogonal pattern, while most of the top mat was arranged in a radial/circumferential pattern. To correlate more easily the bending/membrane response of the basemat, one of the orthogonal bars that passed through the center of the basemat (axis of revolution) was instrumented, since its performance should have been essentially the same as that of a radial bar. The radial bar directly above the orthogonal bar was also instrumented.

Figure 29 shows the measured response of the gages on the top basemat bar along its length at seven different pressure levels. Note that the strains towards the center on the basemat remained small throughout the test, but the strains in this radial bar near the intersection of the basemat with the cylinder clearly showed the tension associated with the bending moment due to the cylinder-basemat interaction. Also note that the fill slab that sat on top of the basemat extendec 115 in. from the basemat centerline and might have influenced the strains (and displacements see section 7.3.3) plotted in Figure 29.

This response contrasted with that recorded in the lower layer of basemat reinforcing, as can be seen in Figure 30. At the outer edge of the basemat, the outer bars experienced compression for most of the pressure history because of the bending moment introduced by the cylinder, but a large change in strain was measured at the third strain gage from the edge of the basemat, which measured the highest tensile strains in this region.

The drastic change in strain, from slight compression at 80 psig to $0.2 \%$ strain at 145 psig may be largely explained by the reinforcing pattern in this area. One of the meridional reinforcing bars (11VB4001, as designated in Figure 29) in the cylinder was bent at $45^{\circ}$ as it intersected the basemat and continued at this angle until it intersected the lower reinforcing mat at this location (96 in. from the basemat centerline) [1]. Some additional speculation or further analyses are needed to explain this response-the large change in strain may have been from the redistribution of the meridional forces in this bar or the angled meridional bar may have acted as a crack initiator and thus caused high strains in the area from the presence of a crack. Either or both of these factors may have influenced the strain in the reinforcing steel at this location in the basemat. 


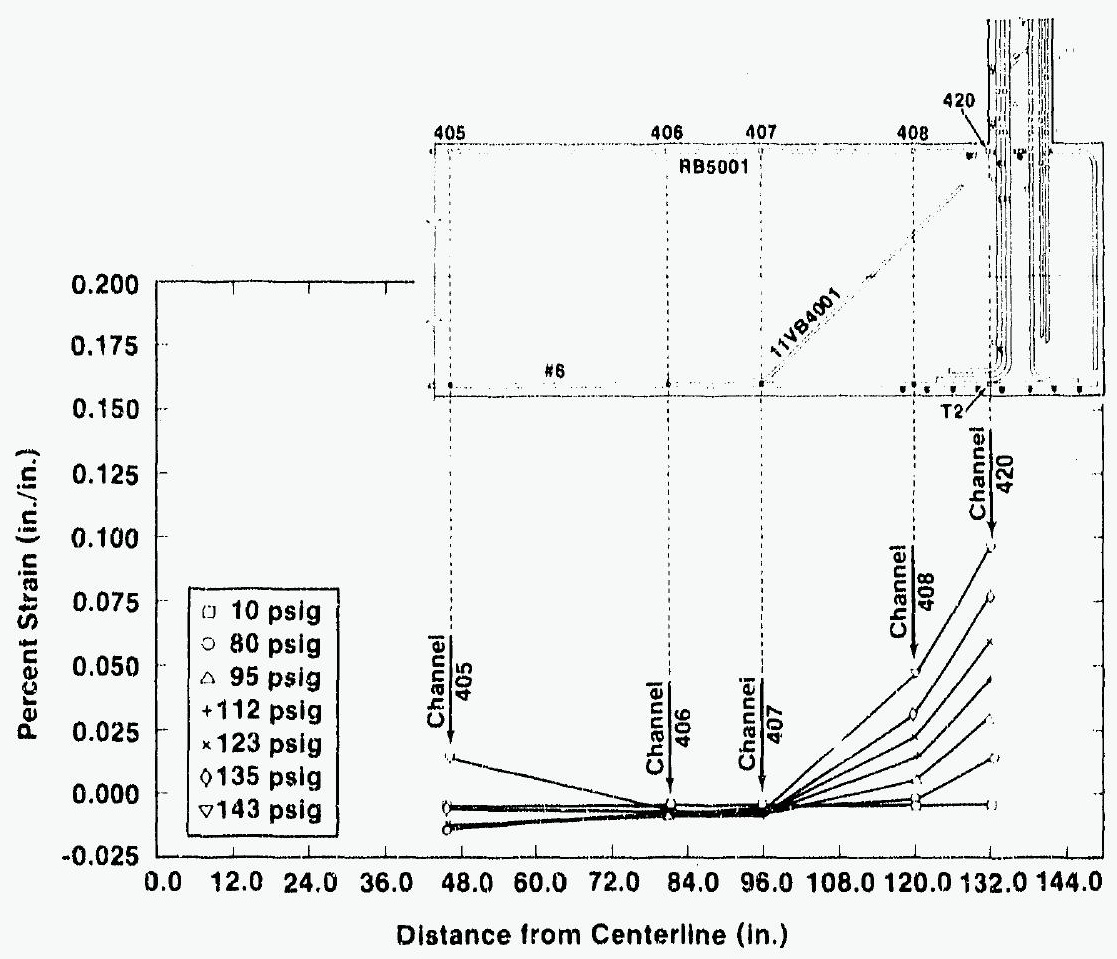

Figure 29 Strains in the Top Layer of Basemat Reinforcing Steel.

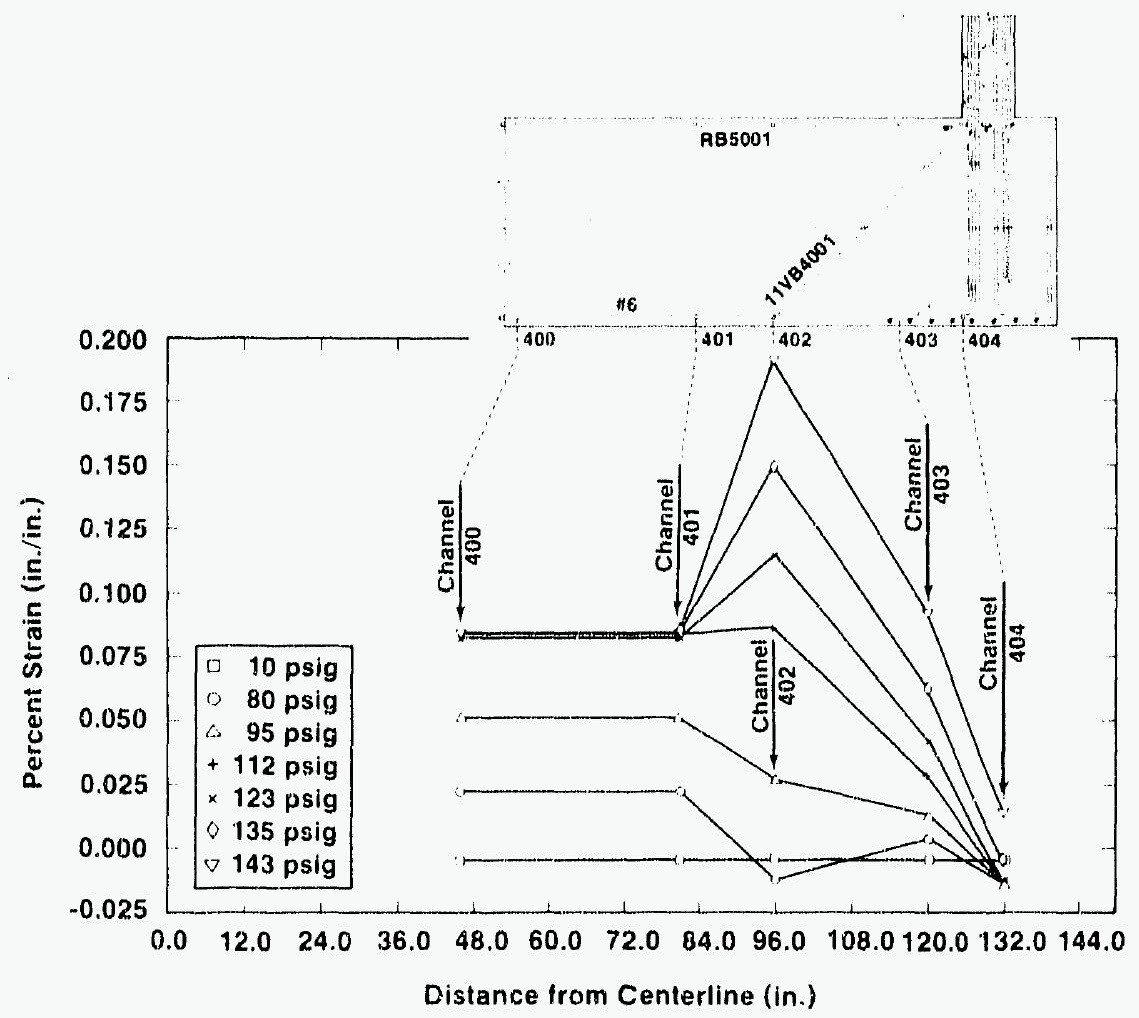

Figure 30. Strains in the Bottom Layer of Basemat Reinforcing Steel. 


\subsubsection{Liner Strain}

The intersection of the floor liner and the cylinder liner had several strain gages, including rosettes and strips attached to it. Because of the bending strains of the cylinder wall and the transition of the liner itself from the floor to the cylinder, some large strain gradients were recorded.

The knuckle was fabricated from a section of pipe that had a nominal wall thickness of 0.154 in. and was tapered to the 1/16-in. thickness as it abutted the liner material. A strip gage, Strip 1 (channel numbers 260 - 264), was placed on this knuckle section. By the final pressure steps, a very noticeable spread in recorded strain occurred between the five gages on this strip. The strains nearer the floor seam remained elastic, while the strains steadily increased as they approached the cylinder liner, having a maximum meridional strain of about $0.425 \%$. A similar but less significant change in strains was recorded with a strip gage about $130^{\circ}$ from Strip 1. The results of strip gage Strip 1 are shown graphically in Figure 31 .

Above this strip gage was strip 4 (channels 283 - 287), which was located entirely on the cylinder liner but adjacent to the knuckle-cylinder weld seam. While all the gages recorded elastic or nearly elastic strains, there were some differences in the results. Four gages showed very small increases in strain throughout the test and even some small compressive strains, while the fifth gage the one closest to the knuckle weld seam recorded a noticeably higher strain of $0.22 \%$ at the peak pressure. A plot of the results from this strip gage is shown in Figure 32.

Two comments should be made about measuring strain concentrations that occur over a very small (relative to the gage length) length as is the case here and in other liner strain measurements in this report. First, each strain gage on a strip averaged the strain over the area where the single gage was applied [35]. Second, the strain concentration in this area may have occurred over a small distance very near the weld seam (see page 161 of Reference 30). Because of the physical constraints in placing the gages adjacent to the weld seam, even this first gage may have missed the actual peak strain, and the peak strain at this location was probably not measured.

Combining the results from these two strip gages and plotting the results as a function of distance (at 7 pressure levels) gives the strain field depicted in Figure 33.

\footnotetext{
"One of the gages on this strip showed a substantial increase in strain as the model was depressurized, changing from very nciv zero strain at the peak pressure to about $2 \%$ strain after depressurization.
} 


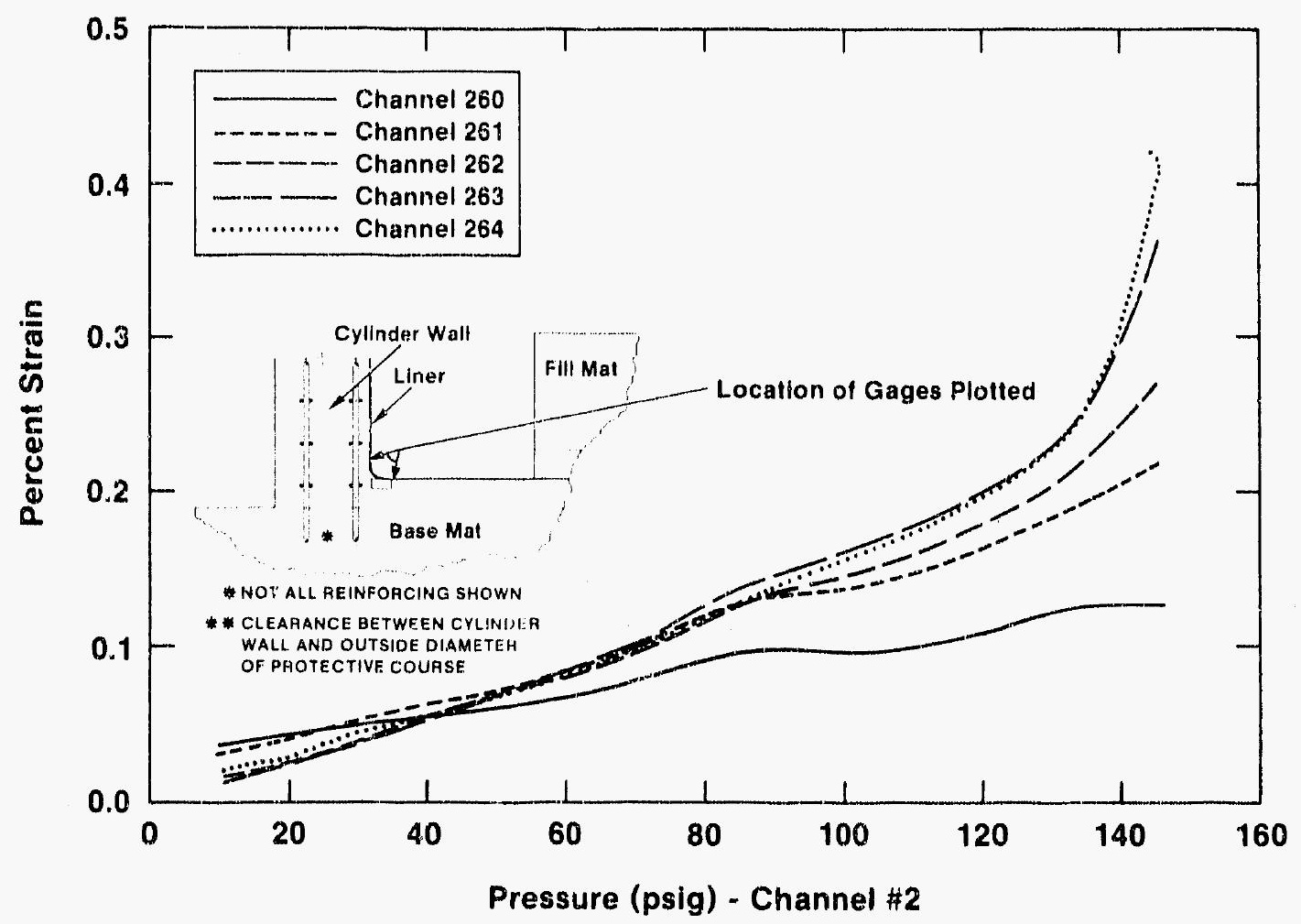

Figure 31. Meridional Strain vs Pressure for Strip Gage 1 in Liner Floor/Cylinder Knuckle.

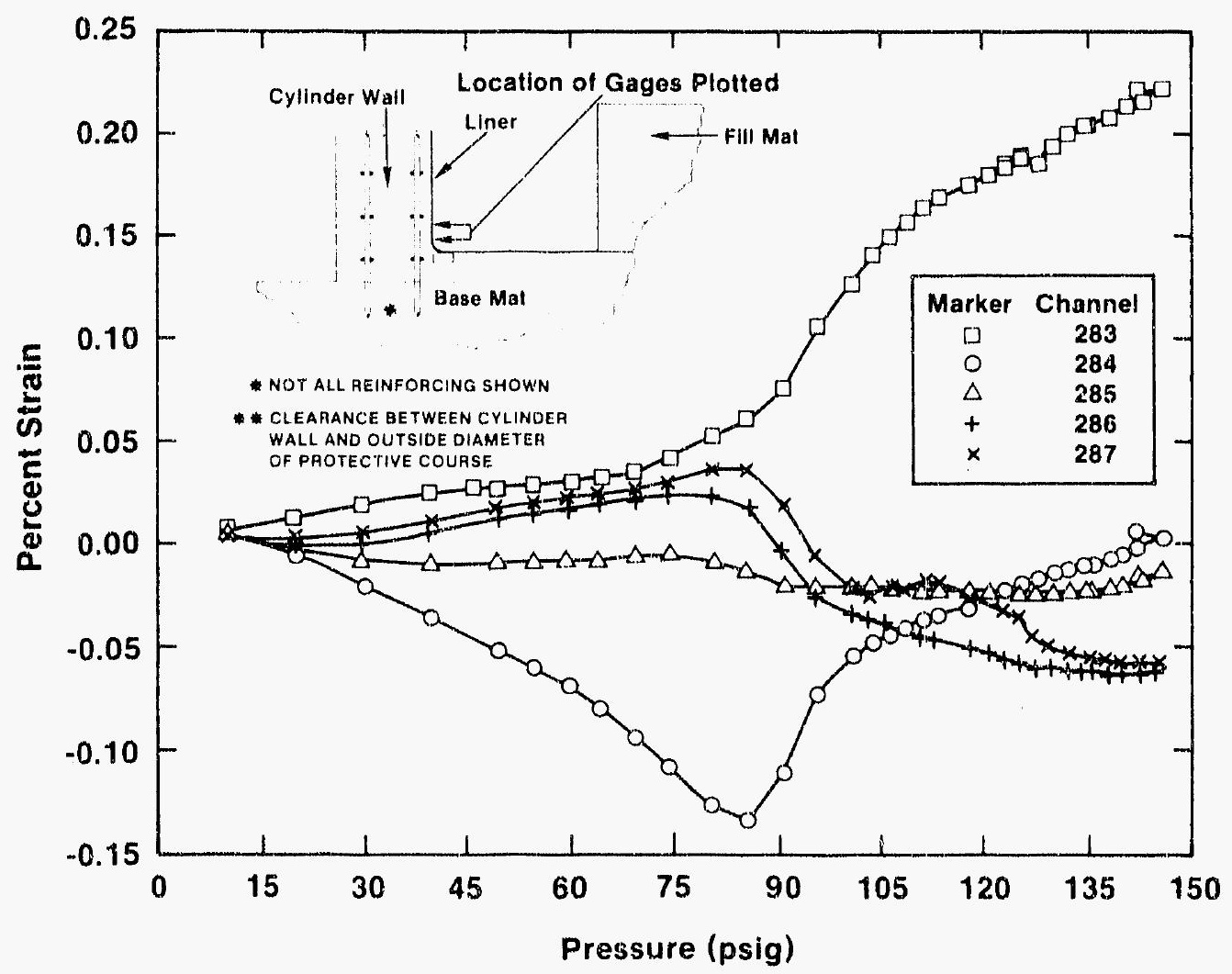

Figure 32. Meridional Strain vs Pressure for Strip Gage 4 in Liner Cylinder. 


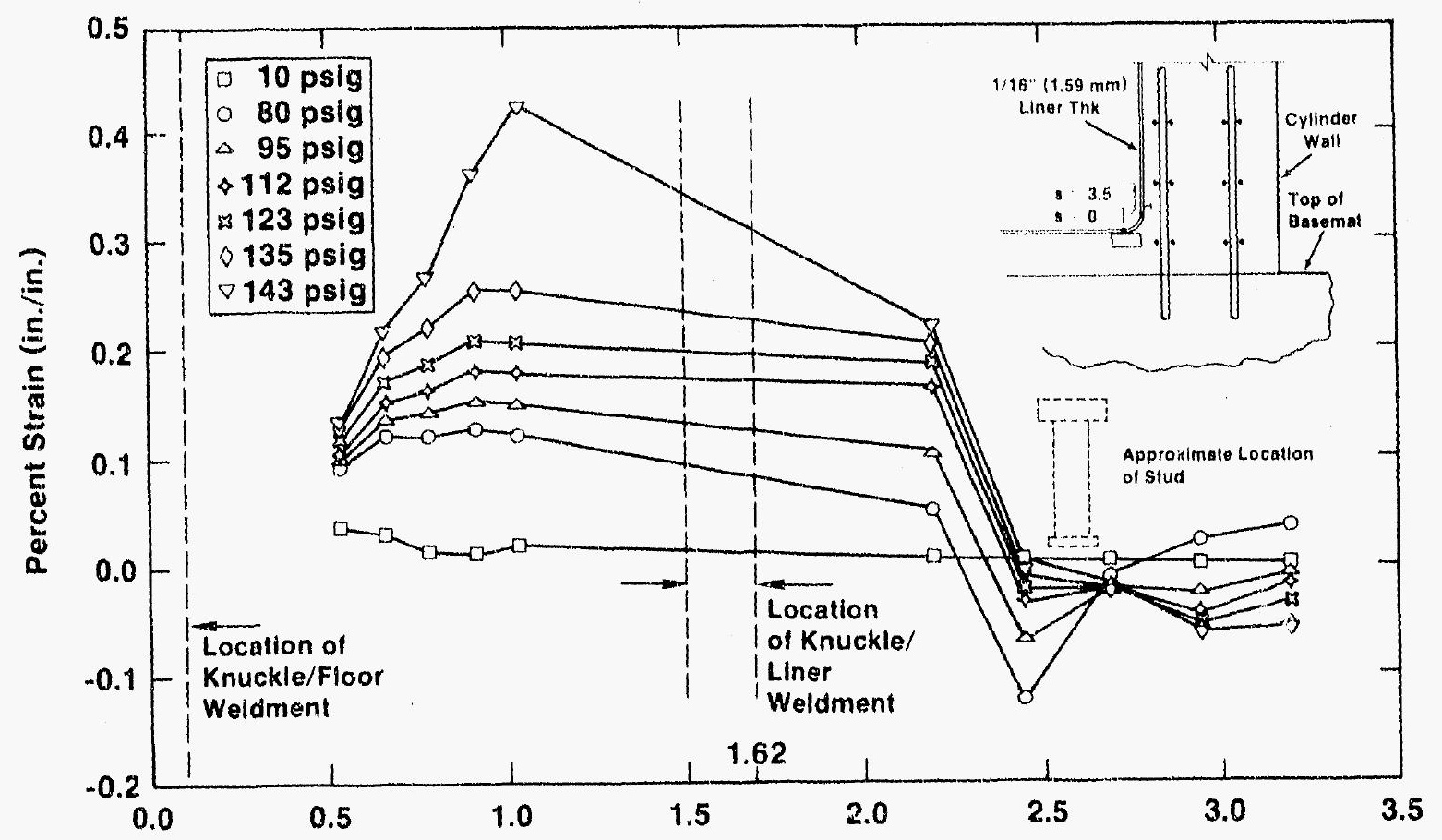

Arc Distance from Floor-Knuckle Junction (in.)

Figure 33. Meridional Strain Field at Liner/Wall Junction at Seven Pressure Levels.

The strains measured in the knuckle showed a steady increase in strain at each pressure level plotted. ${ }^{*}$ The results from the strip gage on the cylinder wall are more complex. The first gage (closest to the weld seam) also showed a steady increase in strain at each pressure level. The remaining four gages measured compressive strains for most of the pressure levels plotted, indicating bending in the liner from the movement of the wall and the reaction of the stud anchor. The influence of the stud, whose approximate location is shown in Figure 33, on these liner strains is quite obvious. Although the strains recorded are connected by a straight line in Figure 33, the strain field was probably more complex, and the actual peak strain was most. likely not measured. Since the strains measured inside the liner were compressive and were probably from local bending of the liner, it should be noted that the strain on the concrete side would be higher because the bending strains would be superimposed on the membrane strains. A look at the local liner strains in this area help support this hypothesis.

The local bending of the liner knuckle was measured in a few locations by placing weldable strain gages on both the concrete side and at matching locations on the inside of the liner. At azimuth $90^{\circ}$, six such gages existed, and their results are graphically shown in Figure 34 . It should be noted that the gage length of the weldable strain gages is about 0.61 in. with a physical length that is 1.09 in. This larger gage will average the strains in the liner over its gage length, thus obscuring the peaks in the strain that were measured by the shorter strip gages plotted above. That is, a direct comparison of the strains should not be made, but the weldable gages plotted in Figure 34 show the local variation in strain from the inside to the outside of the liner-there was some local bending of the liner in this area. The four gages

*Both of these gages measured meridional liner strains, contrary to what was reported in the Appendix of Reference 1. 
nearest the knuckle measured tension on the inside of the liner and compression on the concrete side of the liner for the entire duration of the test. The remaining two gages, located 4.5 in. above the containment floor, followed this same trend until 95 psig when both gages were measuring tensile strains. By 118 psig the tensile strain measured by the inner strain gage, channel 2189 , began to decrease. By 140 psig the strain gage on the concrete side of the liner, channel 886, was recording higher tensile strains than the inner strain gage. It is clear from this data that local bending of the liner was occurring-the liner was not acting as a membrane attached to the reinforced concrete wall.

\subsubsection{Displacements}

The uplift calculated from the measurements made with the inclinometers is presented in Figure 35. A sudden increase in the uplift of the basemat edge was recorded by two inclinometers as the internal pressure exceeded 75 psig, in contrast to the third inclinometer, which recorded a gradual increase in basemat uplift as the pressure increased. The sudden increase in uplift at the two measured locations was probably from either the breaking of the bond between the working mat and the basemat and/or the cracking of the basemat concrete. Note that the residual uplift was substantially less than the maximum uplift (see Figure 23), indicating that the uplift was largely from elastic strains in the basemat.

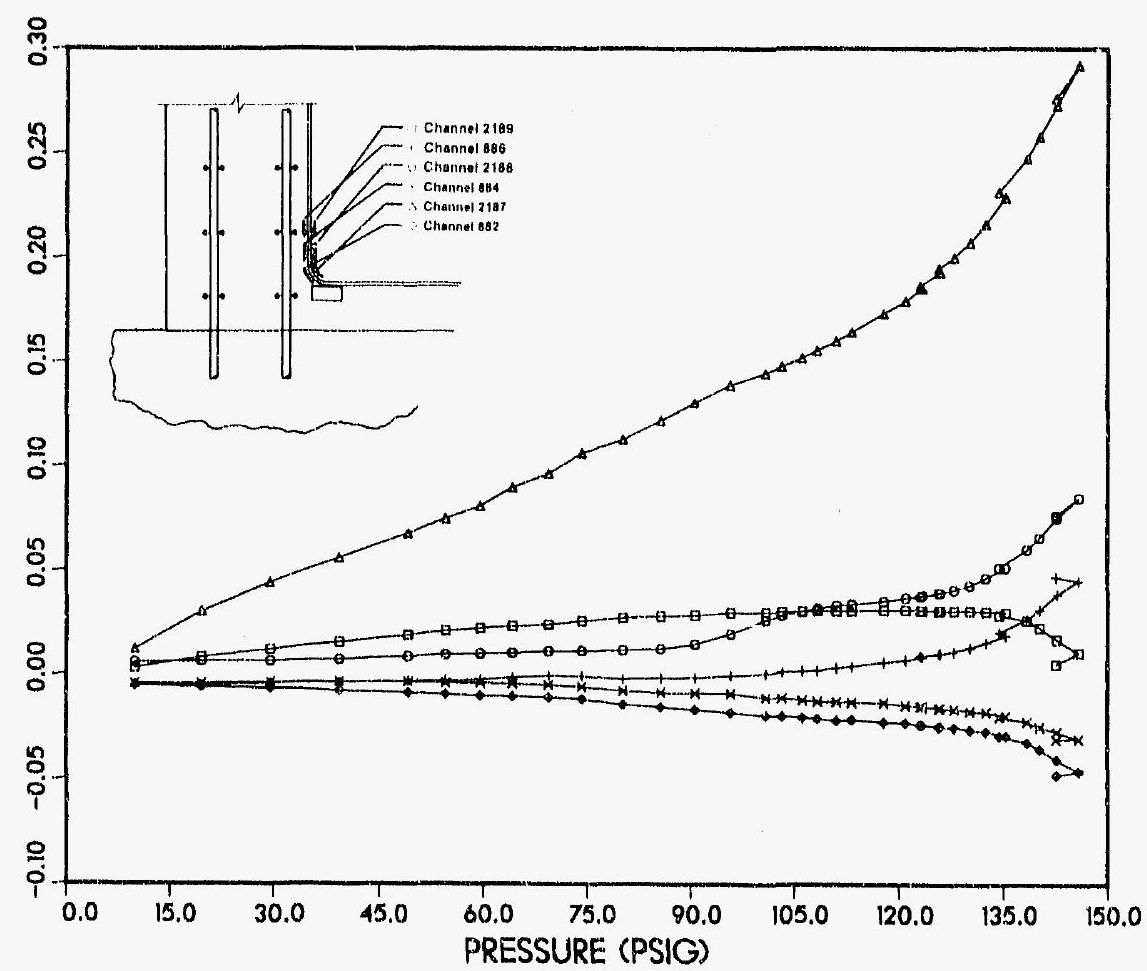

Figure 34. Inside and Outside Liner Strains Near the Knuckle. 


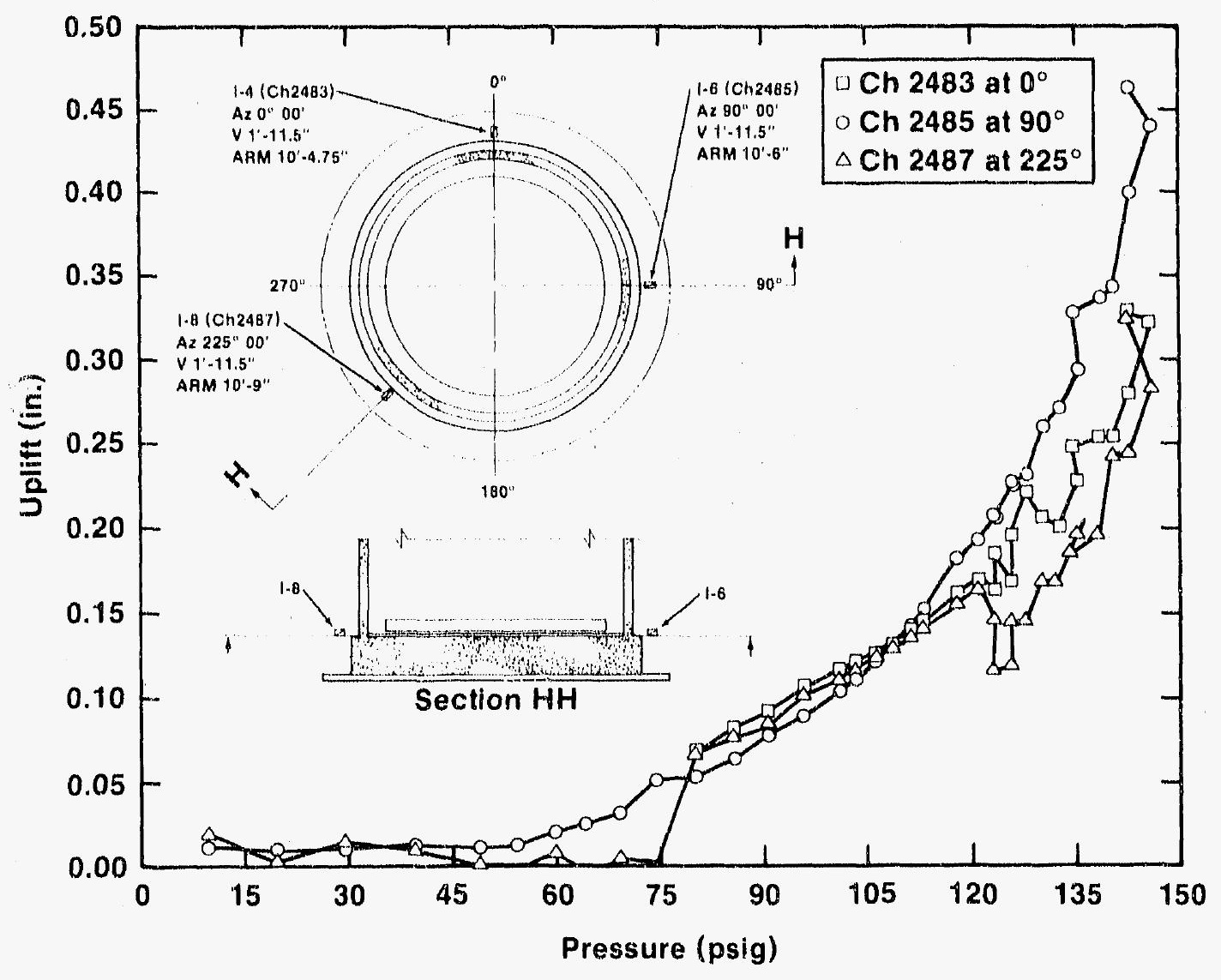

Figure 35. Uplift of the Basemat at Its Outer Edge.

\subsection{Dome Response}

The response of the liner and the reinforcing steel in the dome was mostly elastic throughout testing: cracks in the concrete and any plastic strains remained small. The termination of the meridional reinforcing bars as they approached the dome apex and the termination of the seismic and hoop reinforcing near the springline did not show sudden changes in strain in the neighboring reinforcing bars or in the liner. There was noticeable influence of the bending moment at the springline as recorded by the instruments in the region.

\subsubsection{Rebar Strain}

As was expected, the bending moment at the springline from the constraint of the dome on the cylinder radial displacement, noticeably affected the strains measured in the reinforcing steel. From the first pressure step to the last, the strains in the inside layer of meridional reinforcing were higher than those in the outside layer in the vicinity of the springline. This is illustrated by eight strain gages on the meridional reinforcing bars located in the dome above the springline: the strain as measured at the final pressure level (143 psig) is shown in a bar graph (Figure 36). 


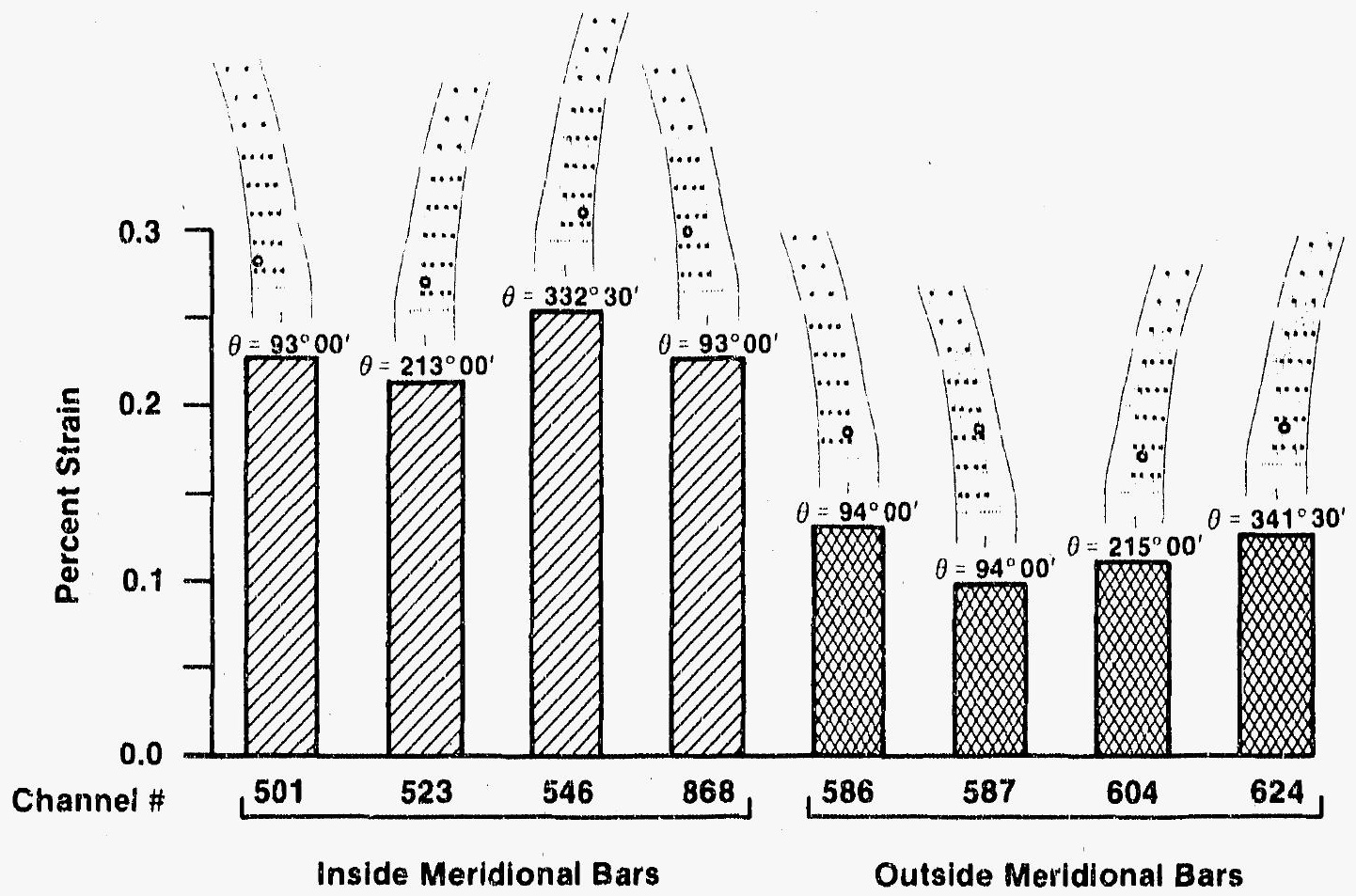

Figure 36. Strain Comparison Between the Inside and Outside Layer of Meridional Reinforcing Steel Above the Dome Springline.

As the reinforcing steel approached the dome apex, the difference between the outside layer and inside layer strains was smaller than observed at the springline, and a reversal of moment was also observed. At about $40^{\circ}$ from the springline, the strain in the outside layer of reinforcing $(0.14 \%)$ was slightly higher than inside $(0.13 \%)$ at the final pressure level. Again as the reinforcing bars approached the apex, the strain increased and the moment reversed. At an angle of about $79^{\circ}$, the inner layer of meridional reinforcing steel had a strain of $0.19 \%$, and the outer layer had a strain of $0.16 \%$ at the final pressure level.

The strain in the hoop-reinforcing steel near the springline also showed significant variation from the inside layer to the outside, with the inside layer of hoop exhibiting noticeably larger strains at three circumferential locations.

The variation in the hoop strains from the inner to the outer layer and the little evidence of circumferential bending may seem difficult to explain unless the performance of the seismic bars and the concrete is considered. As the meridional moment increases, the seismic bars aided in resisting this moment; however, since they were angled at $45^{\circ}$, they were also compressing the outer hoop bars because a hoop strain as well as a meridional strain was induced. In addition, the information gathered from these hoop bars during the test suggests that the inner hoop bars had little interaction with the concrete (the meridional moment that had certainly cracked the concrete on the inner surface), whereas the outer hoop bars apparently still resisted the hoop forces with the aid of the surrounding concrete. Figure 37 is a bar graph of the inner and outer hoop strains circumferentially about the springline as recorded at the last pressure level.

\footnotetext{
*One gage, W42 (channel 481), gave questionable results and is omitted from the figures and discussion.
} 


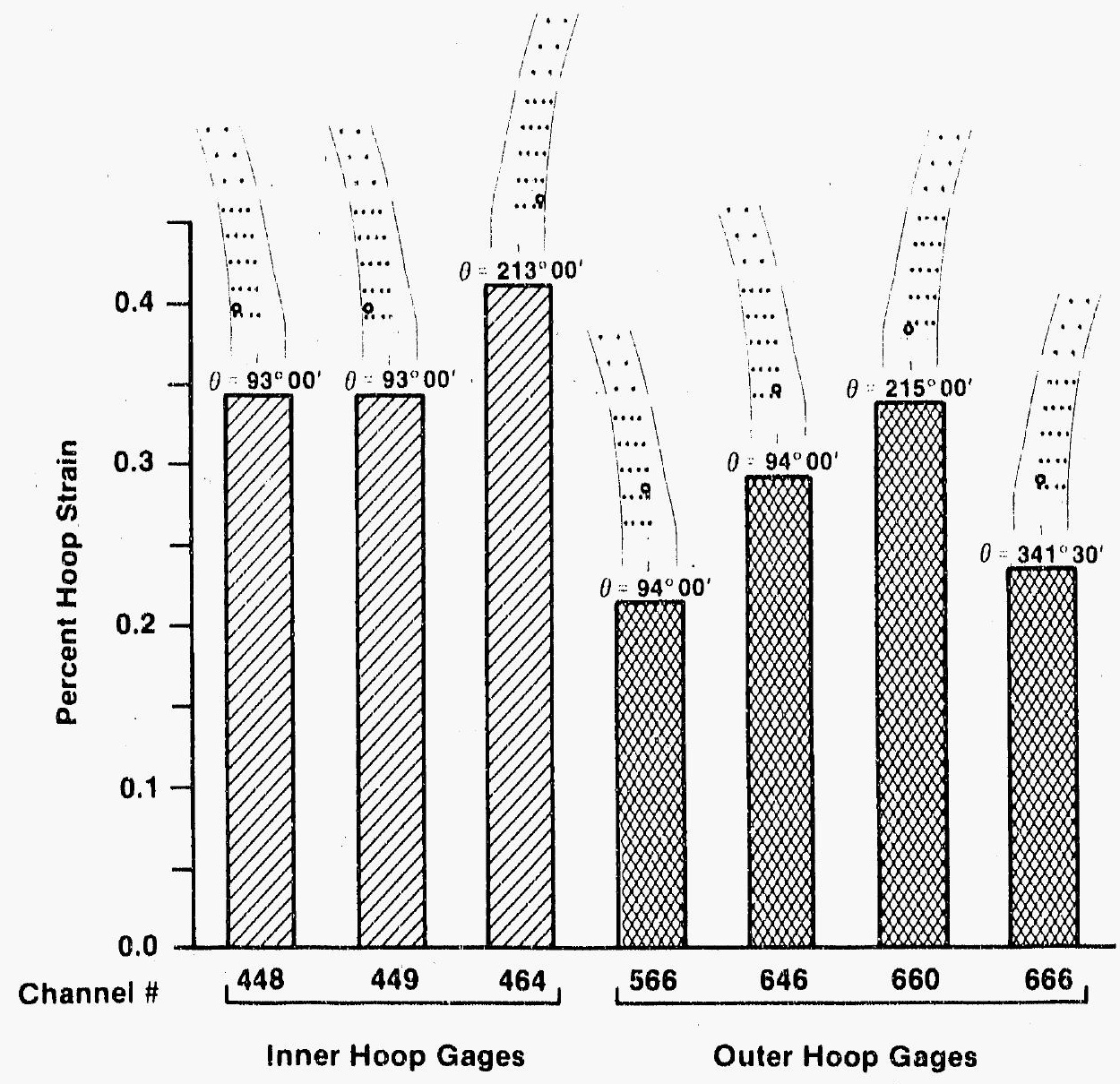

Figure 37. Strain Comparison Between the Inside and Outside Layer of Hoop Reinforcing Steel Above the Dome Springline.

\subsubsection{Liner Strain}

The liner strains varied much as the reinforcing strains did: strains were larger near the springline and reduced in magnitude as they approached the dome apex. The maximum and minimum principal strains calculated above the termination of the seismic bars were similar but not equal in magnitude (Figure 38).

As can be seen in Figure 38, and as was typical for other rosettes in the dome, the difference between the principal maximum and minimum strains increased as the pressure increased. In general, at locations above the termination of the seismic bars, the principal strains were in the hoop direction, but the strains nearly equaled the strains in the meridional direction at the later pressure levels. This was in contrast to the dome sections with the seismic bars where the meridional strains were about twice those of the hoop strains. 


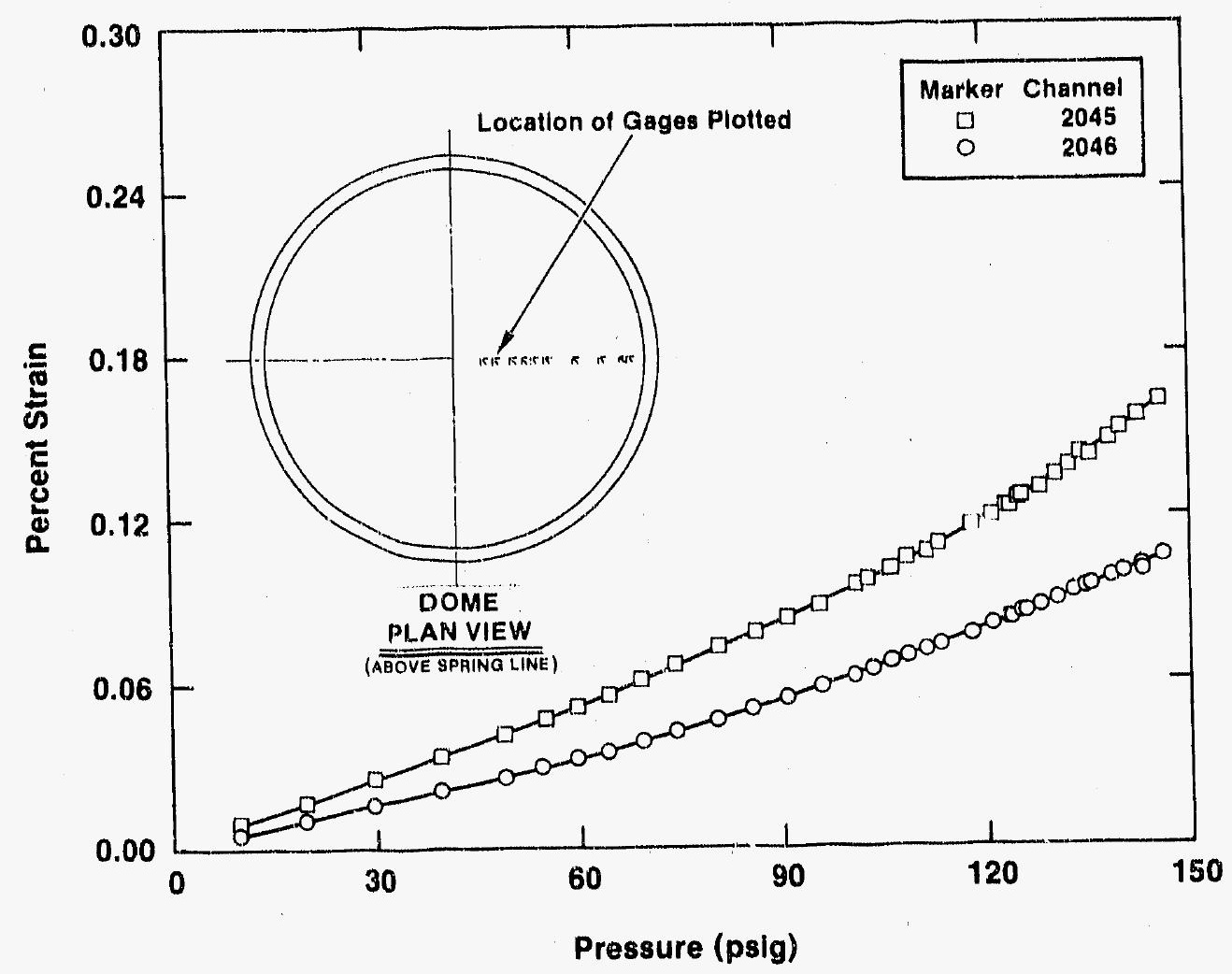

Figure 38. Dome Liner Strains (Principal Maximum and Minimum) $67^{\circ}$ from the Horizontal.

\subsubsection{Displacements}

The displacements in the dome were coupled with the meridional displacements of the cylinder, which averaged $0.55 \mathrm{in}$. The dome apex, including meridional displacement of the cylinder, rose over 1 in. during pressurization (Figure 39).

\subsection{Equipment Hatch A}

As with the other three major penetrations, a major crack formed between the boss and its intersection with the cylinder wall during the SIT and its width gradually increased during the high-pressure test.

Strains in the rebar that formed the thickened boss remained small (elastic or slightly plastic) during the high-pressure test. Primary reinforcing, present in the nonperturbed cylinder and merely bent around the penetration, accumulated less strain and displacement when compared to the free-field response of the reinforcing steel. 


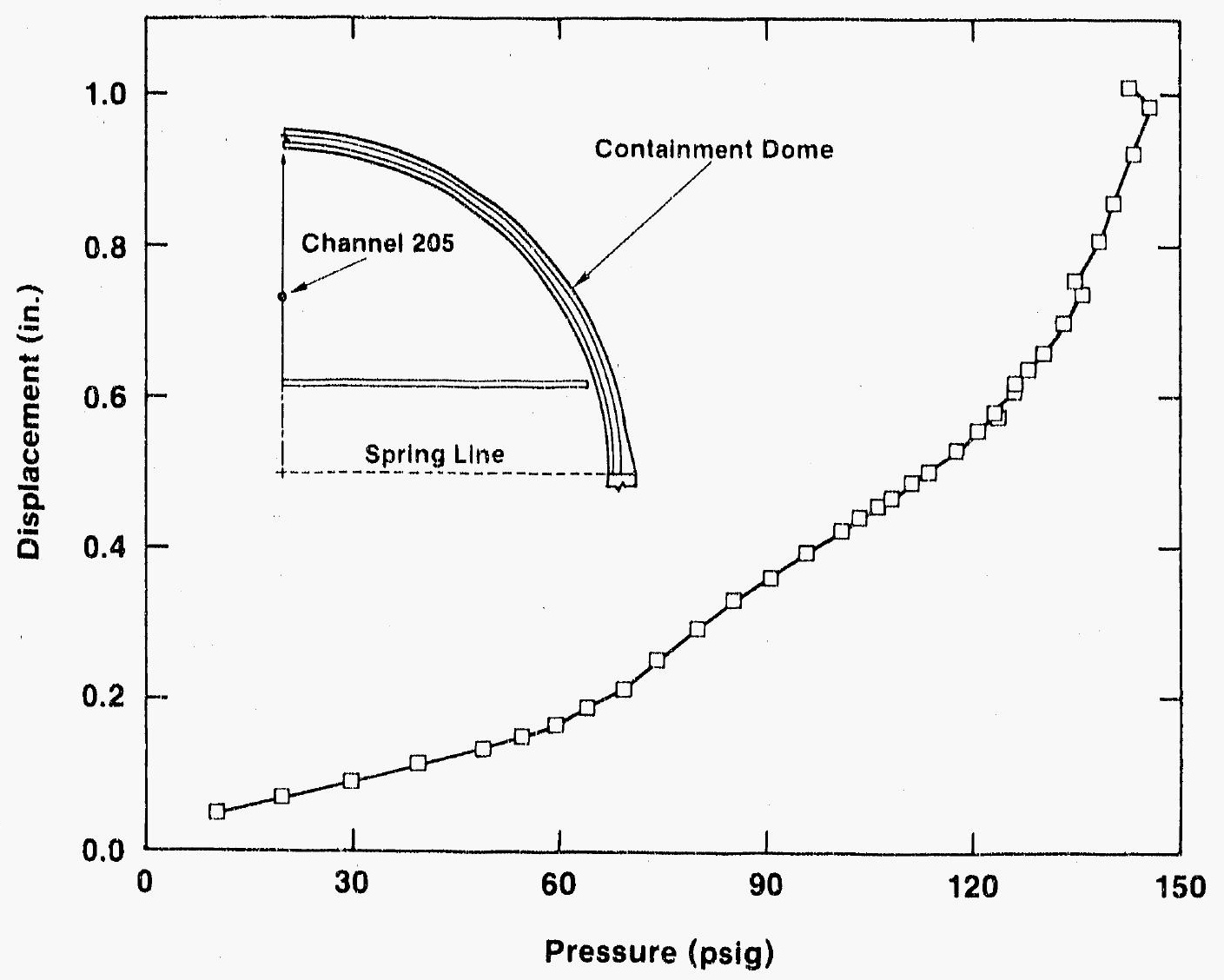

Figure 39. Vertical Displacement of Dome Apex Including Meridional Shell Stretching.

Global displacements of equipment hatch A were slightly smaller than those of the free-field counterparts. Displacement histories of the hatch and its cover demonstrated that the rotation of the sealing surface remained small throughout the high-pressure test, but translational motion from the hatch sleeve becoming oval was quite significant. One of the two gum-drop seals was exposed over a portion of its length because of the ovalization of the sleeve (see section 6.4.4).

Two embedment gages were located around the boss of equipment hatch $A$ at 12 o'clock and 9 $o^{\prime}$ clock* $^{*}$ where the thickened boss intersected the nominal wall thickness. The results of these two gages are contrasted in Figure 40.

As can be seen, the strain in the horizontal (hoop) direction was substantially greater than that in the meridional direction of the boss. The strains were substantially lower, however, than those measured in the free-field regions of the liner. The difference between the strains measured by the embedment gages and the free-field liner strains were probably from the bending induced by the discontinuity of the boss and the additional stiffness of the boss from the local reinforcing, some of which extended beyond the thickened boss region. (See Appendix B, page B-37, and Reference 1, page 38, for reinforcing details.)

*All clock faces in this report are as viewed from inside the containment model. 


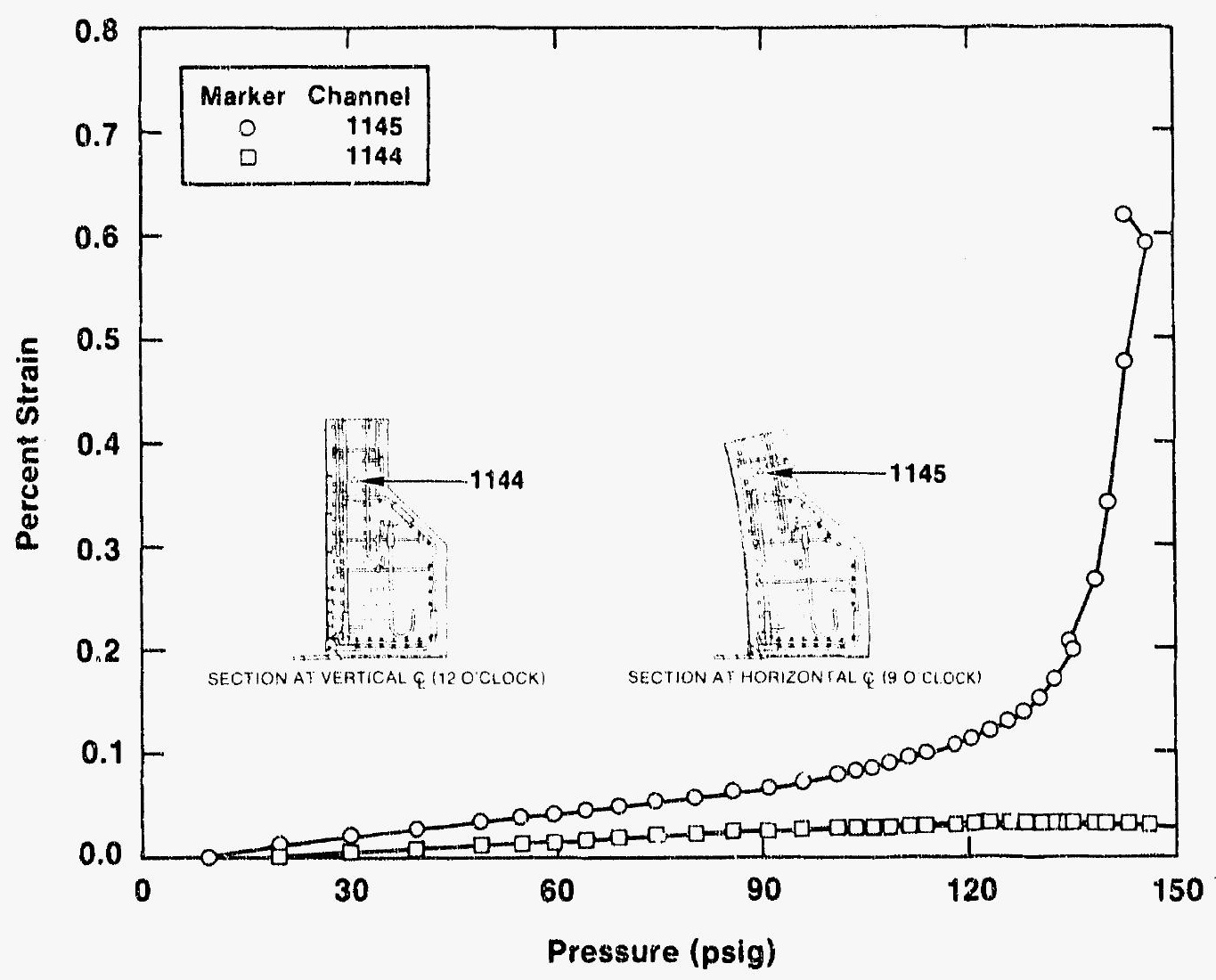

Figure 40. Concrete Embedment Gages Near the Boss of Equipment Hatch A.

Details of the strains and displacements of this equipment hatch are presented in the following sections.

\subsubsection{Rebar Strain}

The local circumferential bars that encircled the equipment hatch sleeve (bar 19CB4502; see page B-38 of Reference 1) varied in the strain measurements along a single bar. The strains were higher at the $120^{\prime}$ 'clock position than at the 9 o'clock position (Figure 41).

\subsubsection{Liner Strain}

Several strain gages were located about equipment hatch $\mathrm{A}$ to measure any bending in the liner. Six strain rosettes on a horizontal line at $3 o^{\prime}$ 'clock and six on a vertical line at 12 o'clock were each backed on the concrete side of the liner with a matching strain rosette (a total of 24 rosettes). Using a rosette on both the inside and concrete side of the liner allowed a measurement of local liner bending. 


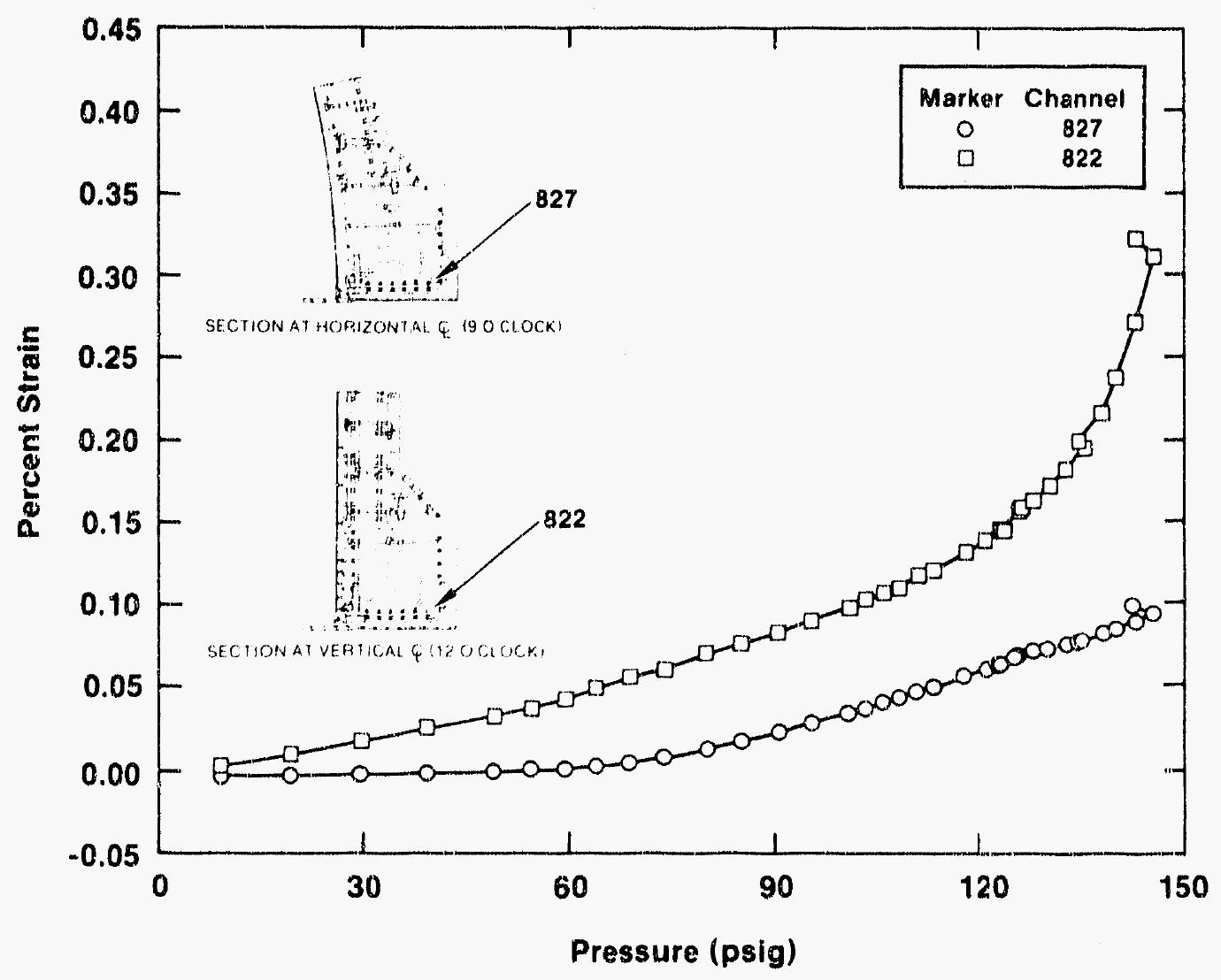

Figure 41. Variation in Hoop Strain in the Reinforcing Bar Around the Sleeve of Equipment Hatch A.

The largest strains recorded in the entire containment model were measured by a rosette near the insert plate of equipment hatch $\mathrm{A}$ at about 2 o'clock. This was very near where the liner developed a small tear during testing. The results from this strain rosette, after being converted to principal strains, are shown in Figure 42. Other rosettes were placed around the azimuth of equipment hatch A. There was a pair of rosettes at 3 o'clock and another pair at $12 \mathrm{o}^{\prime}$ 'clock. The pair consisted of a rosette on the inside of the liner and a rosette on the concrete side of the liner. Rosettes 1 and 13 were at the 3 o'clock position just beyond the insert plate. The maximum strains read at this location were $2.24 \%$ and $2.10 \%$, respectively. Rosettes 7 and 13 were at the corresponding 12 o'clock position. The respective maximum stains read at this position by these rosettes were $1.35 \%$ and $1.16 \%$. The average of tiese four strains, $1.71 \%$, is the same as the average hoop strains that were recorded at containment midheight $(1.69 \%)$ as discussed in section 7.2.1. The maximum strains that were measured at the 2 o'clock position, as can be seen in Figure 42, was $8.22 \%$-nearly five times greater than these other locations.

The intersection of the liner insert plate with the equipment hatch sleeve was instrumented with some strip gages that measured the meridional strains of the sleeve. At the 3 o'clock position of the sleeve, there was a strong influence on the meridional strain from the interaction with the insert plate. The variation in strain near the intersection of the insert plate with the liner is shown in Figure 43. The zero on the abscissa indicates the center (1/2. the plate thickness) of the insert plate; however, there was also a $3 / 8$-in.-thick collar behind the incert nlate that was designed to withstand radial forces from the equipment hatch (see page B-65 of Reference 1). 


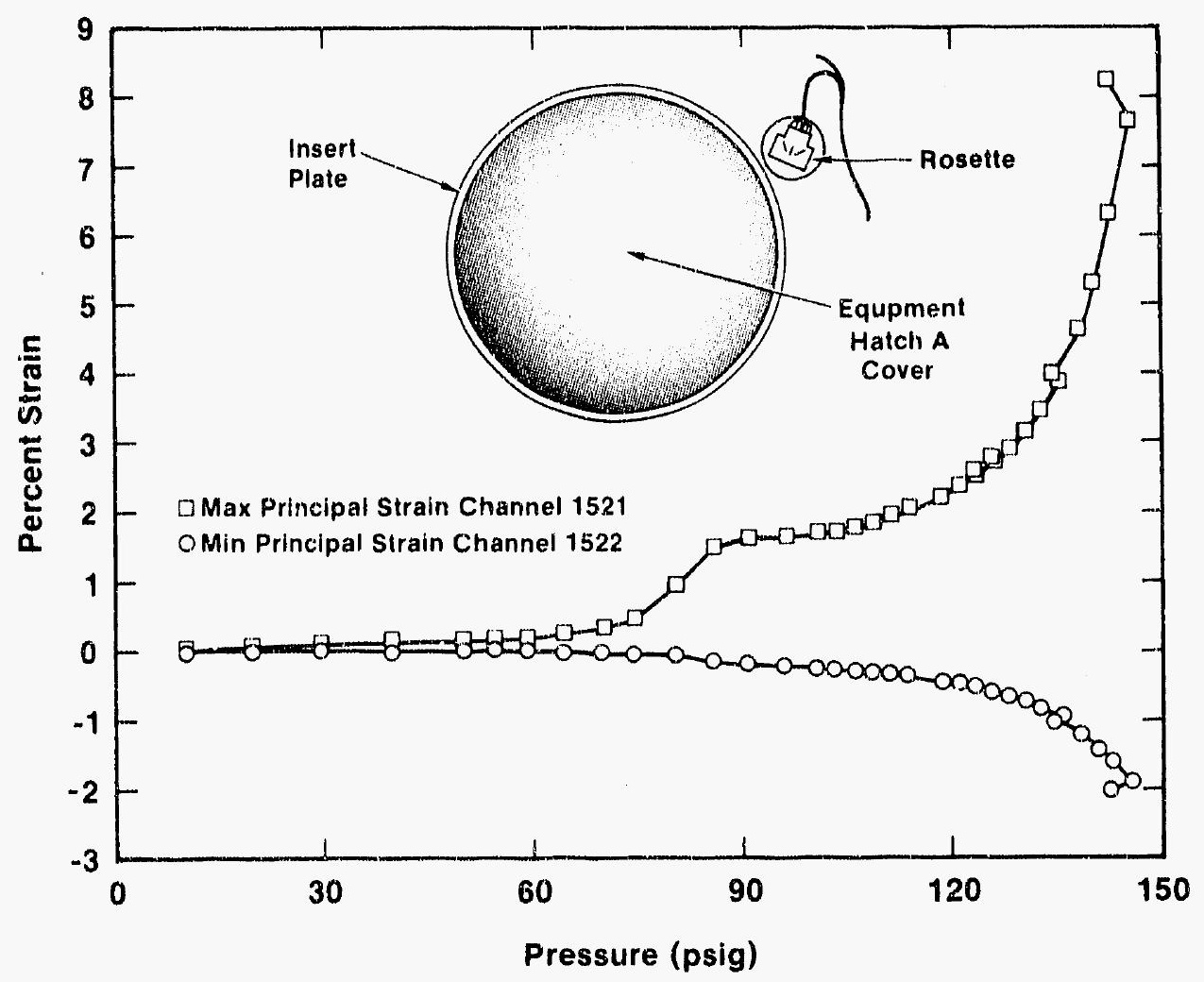

Figure 42. Liner Strains Near the Insert Plate of Equipment Hatch A.

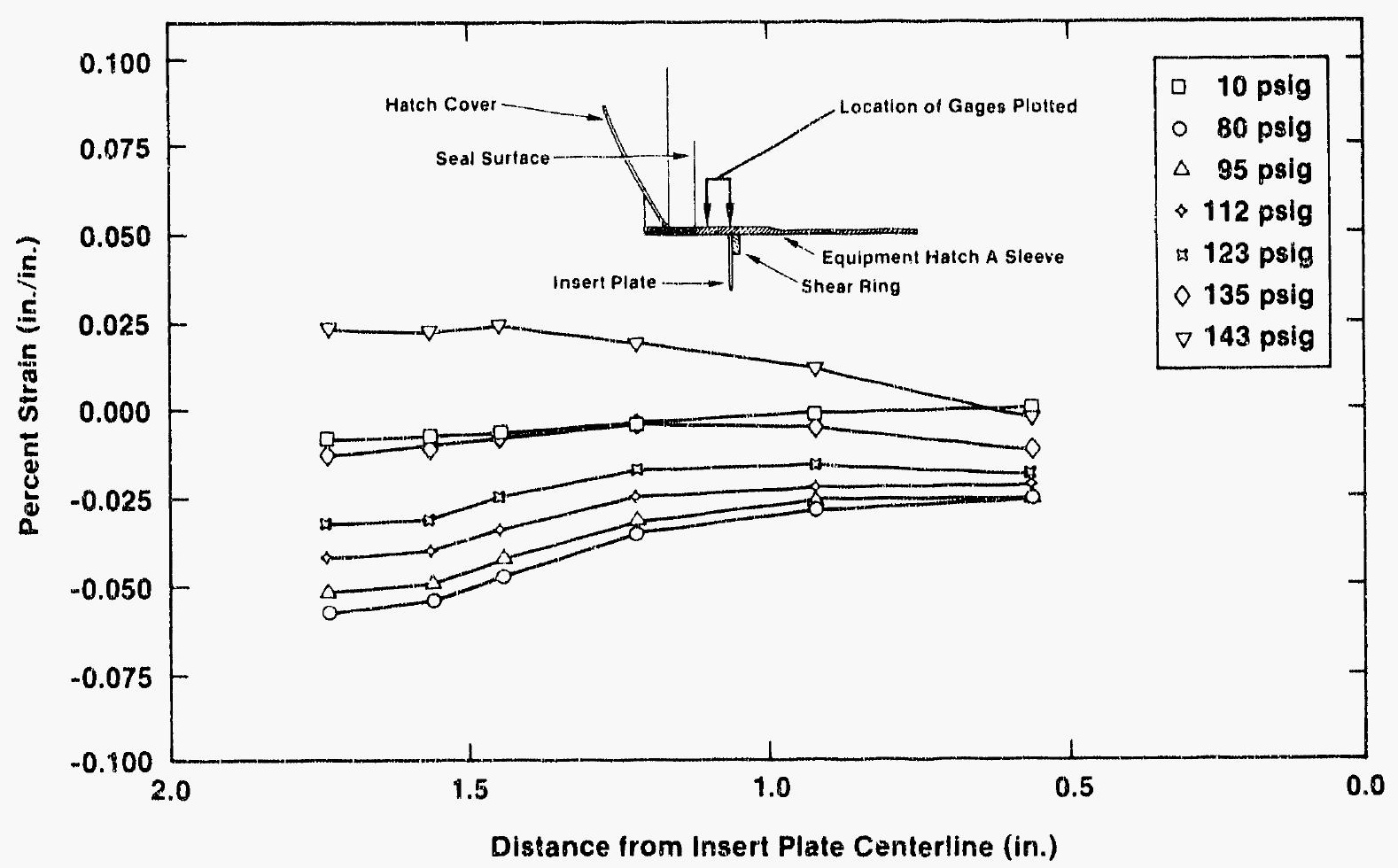

Figure 43. Meridional Strains at the Intersection of the Sleeve and İnsert Piate of Equipment Ḧatch Á. 
The strains became slightly negative at $10 \mathrm{psig}$ and, by $80 \mathrm{psig}$, had reached the maximum negative strain plotted in Figure 43. For the remaining pressure levels plotted, the strains became less negative as the internal pressure increased, with five of the six gages recording a tensile strain by the final pressure level. The insert plate clearly caused meridional bending in the equipment hatch sleeve, but the magnitude of the strains remained elastic throughout the test.

\subsubsection{Global Displacements}

As presented in section 7.2, the displacement of equipment hatch $\mathrm{A}$ was less than that of the cylinder away from penetrations at the same elevation. A plot of the displacement versus pressure is shown in Figure 44 for a displacement gage attached to the center of the hatch door and one attached to the hatch sleeve.

The two displacement measurements were expected to be very similar, but if the hatch door buckled, this sudden change in displacement would have been detected by comparing these two displacement transducers.

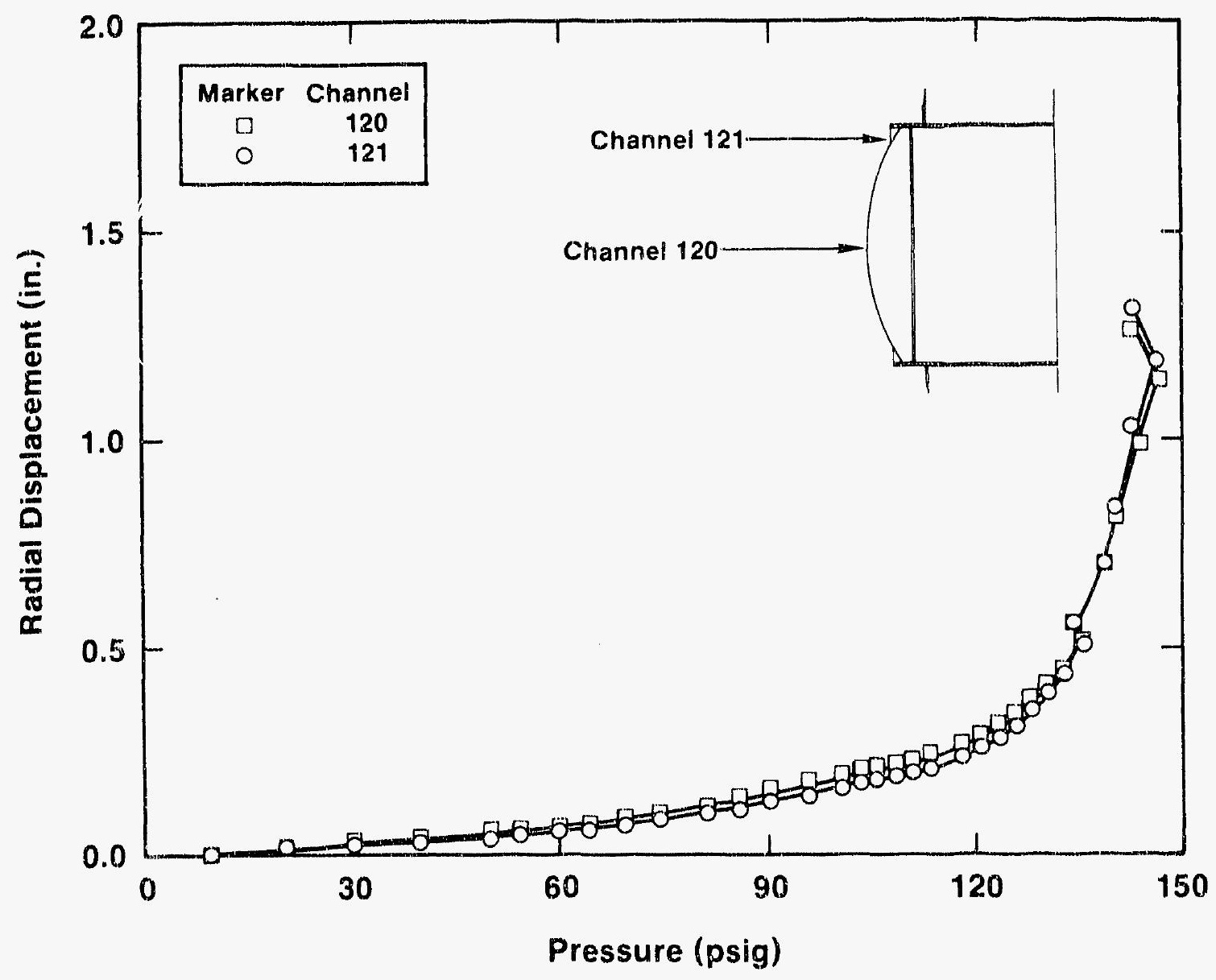

Figure 44. Radial Displacement of Equipment Hatch A. 


\subsubsection{Local Displacements}

A few highlights of the penetration response are presented in this section. In equipment hatch A, the thickened boss region projected entirely outward from the containment; thus, the liner had a constant radius as it approached the equipment hatch sleeve. As a consequence, the liner force was transmitted directly into the equipment hatch sleeve, causing it to deform into an oval. The change in the horizontal and vertical diameters is shown in Figure 45.

The amount of ovalization can be directly related to the amount of hoop strain at the elevation of the penetration as in

$$
\delta=\varepsilon_{\text {ff }} d_{s}
$$

where $\delta$ is the maximum change in sleeve diameter, $\varepsilon_{\mathrm{ff}}$ is the free-field hoop strain of the cylinder wall at the elevation of the penetration, and $\mathrm{d}_{\mathrm{s}}$ is the outside diameter of the penetration sleeve. This equation was developed and previously reported by Clauss [14], and captures the phenomenology amazingly well, considering its simplicity. The correlation between Eq. (1) and the experimental results is shown in Figure 46.

Note that the absolute value of the amount of ovalization was plotted in Figure 46. The "1" marker represents the sum of two transducers that were measuring the change in radius of the hatch and were diametrically opposed. The "2" and " 3 " markers represent measurements taken by transducers that spanned the diameter of the hatch in the direction noted on the figure. The decrease in the vertical diameter at a point sufficiently far from the intersection of the sleeve and liner is about equal in magnitude to the change in the horizontal diameter, but opposite in direction to it. The solid line is based upon Eq. (1), which uses the results (values of free-field strain) from an axisymmetric analysis of the containment model [31].

\subsection{Equipment Hatch B}

Equipment hatch B had two covers: one on the inside and one on the outside of the penetration sleeve. The gaskets of the pressure-unseating equipment hatch were thermally aged prior to testing. The aging caused the gaskets to retain a permanent set; thus, as the bolts that held the hatch cover in place elongated and the cover unseated, less resiliency of the gaskets caused the tongue to lose contact with the gasket at less deformation. This results in leakage at lower pressures. The gaskets were aged to about a 50\% compression set retention (see section 3.7). A very small leak from the vicinity of equipment hatch B was first detected by the acoustic emissions system at a pressure of 125 psig. Enough separation of the sealing surfaces seems to have occurred to allow leakage past the seal surface. This conclusion is based on the slow and steady increase in the acoustic noise in this area and the fact that the liner tears discovered after the termination of the test (see Figure 21) would have resulted in a sudden increase in leakage and a much higher rate of leakage (see Table 4).

The boss for equipment hatch B projected outward and inward, which caused a junction in the liner. As the containment was pressurized and the hoop strain began to increaste, this junction was loaded and caused the studs holding the liner to the concrete eventually to tear the liner. The result was two small circular tears that had a diameter equivalent to the base of the stud. The angle of the liner at this intersection was sharp at the initiation of the test but had rounded during high-pressure testing as shown in Figure 47, moving away from the concrete that backed it. 


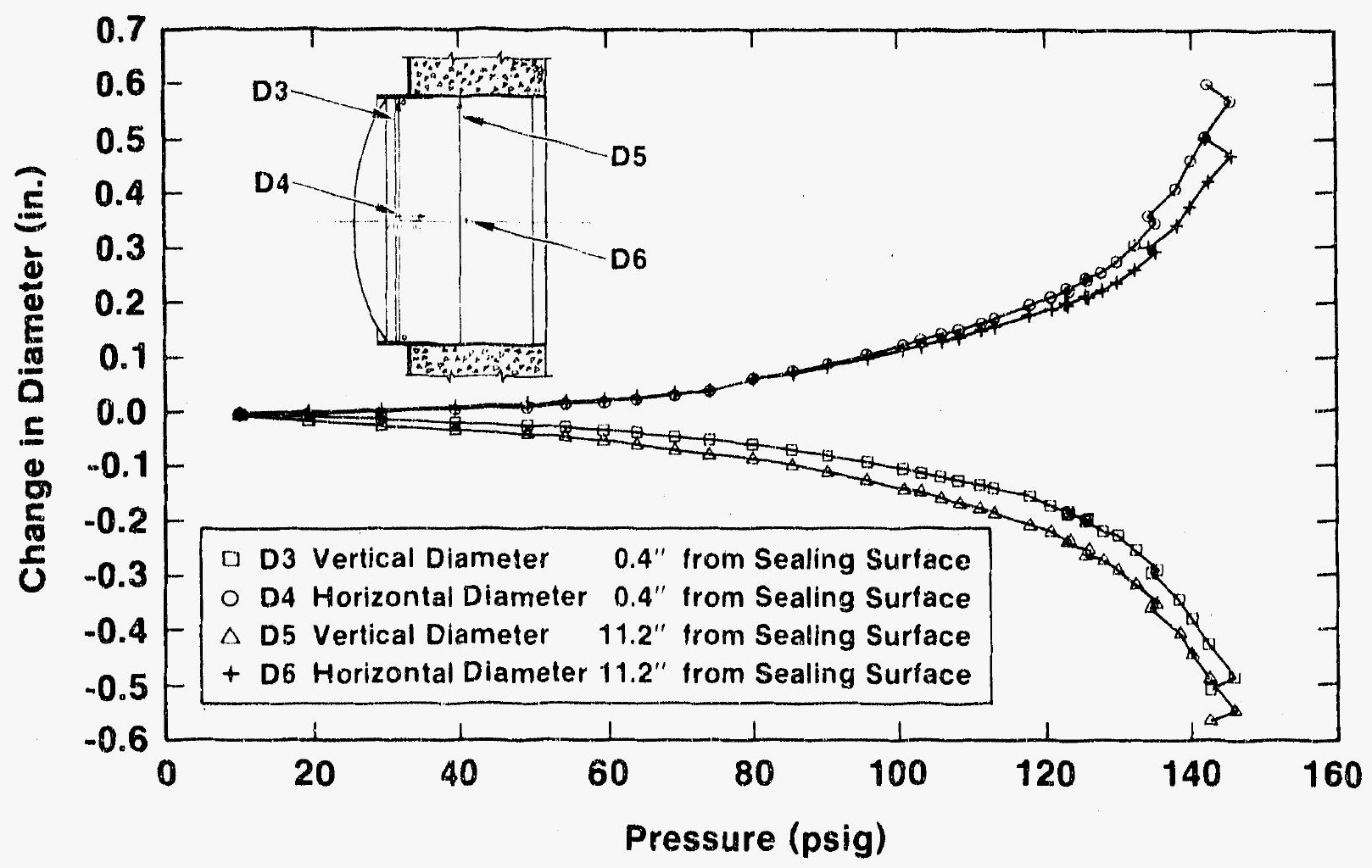

Figure 45. Ovalization of Equipment Hatch A.

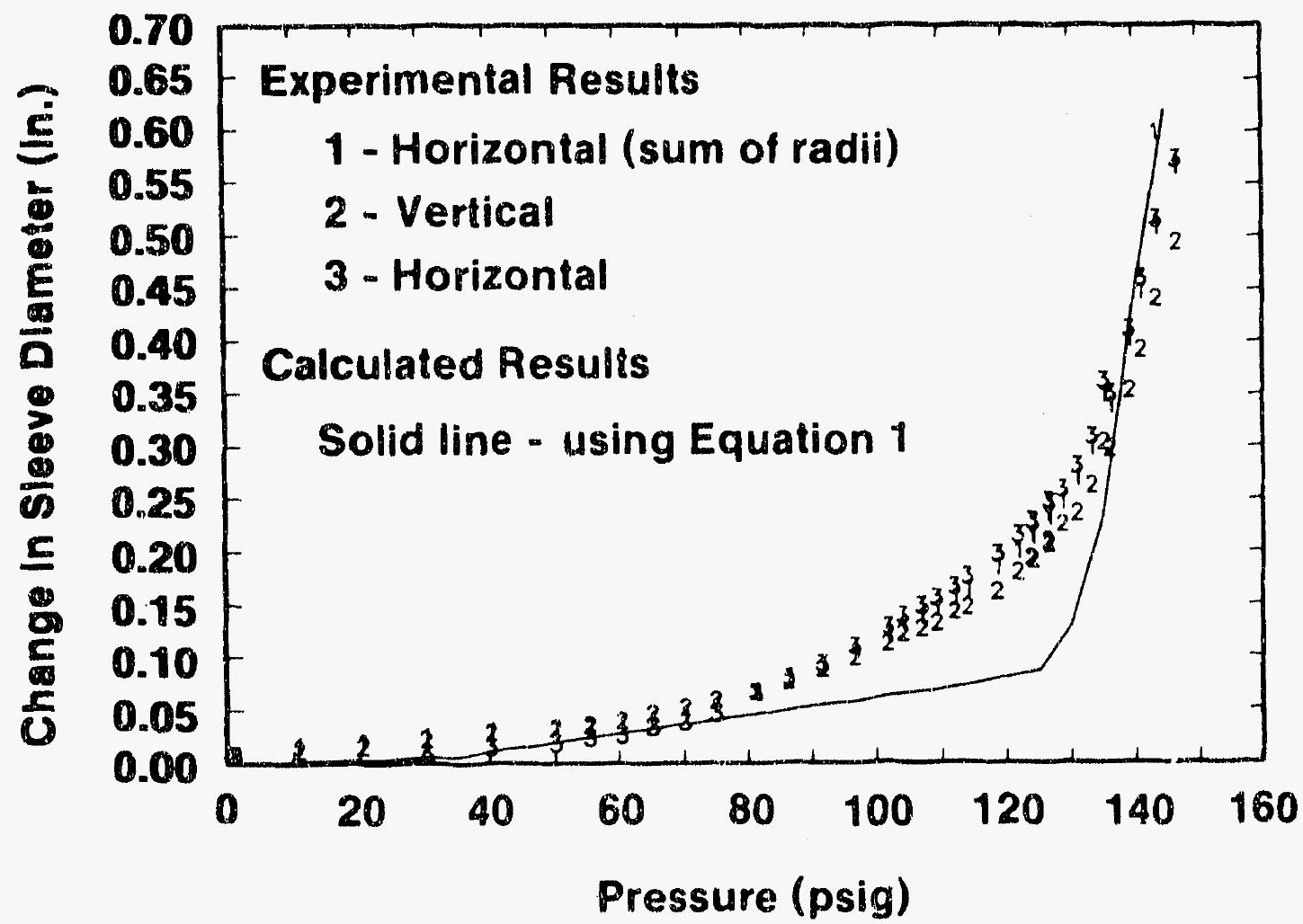

Figure 46. Comparison of Equipment Hatch A Ovalization with Equation 1. 

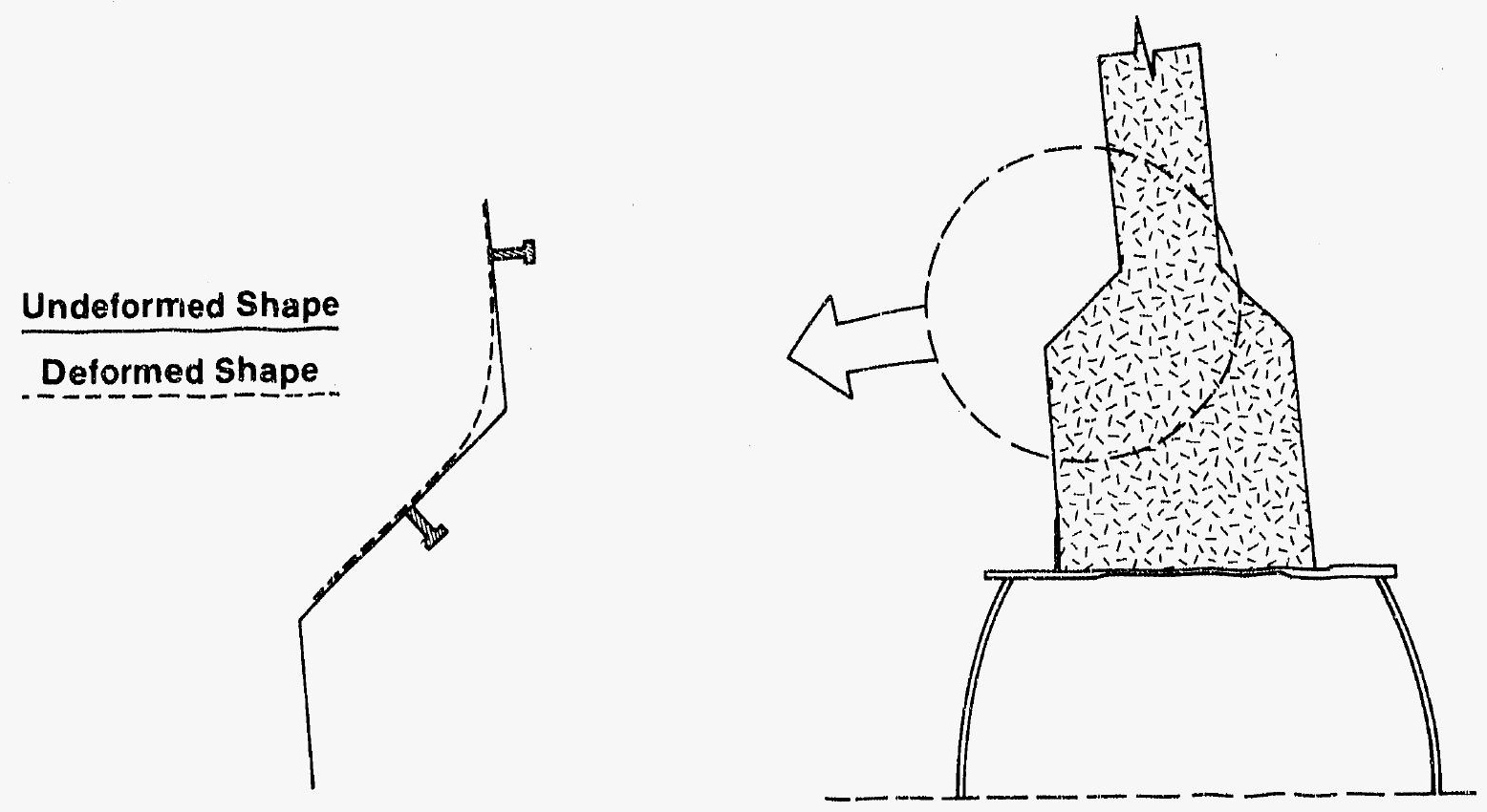

Figure 47. Plan View of Hatch B Showing Deformation of Liner at Boss Junction.

This liner discontinuity and the resultant flexibility of the liner at this point are probably the key factors in the lack of ovalization in this equipment hatch sleeve.

\subsubsection{Rebar Strain}

As in equipment hatch $A$, the circumferential bars that surrounded the sleeve of equipment hatch B were instrumented with weldable strain gages. Although a similar difference in strain was observed between that at the 9 o'clock versus the 12 o'clock position on this reinforcing bar, the strains plotted in Figure 48 were higher than those observed in equipment hatch A (see Figure 41.) 


\subsubsection{Liner Strain}

Since the boss for B projected inward, the liner had an abrupt change in radius. This effectively softened the liner and greatly reduced the force transmitted to the sleeve of equipment hatch $\mathrm{B}$, resulting in little ovalization of the sleeve. Other differences from equipment hatch $\mathrm{A}$ were also observed.

A truncated cone was used to transition the liner from its larger nominal radius to the face of the boss and the intersection of the liner with the equipment hatch sleeve. As the liner began to strain and deform during the test, an increasing amount of force was applied to this truncated conical section of liner. This had two obvious consequences determined after the test: this initially angular junction deformed, tending to round out the junction (Figure 47), and pull-out forces were placed on the studs anchoring the liner to the concrete.

In two cases, the studs on the conical liner section tore holes in the liner. These were not the only studs that aided in distributing this force. The studs adjacent to the conical liner section in the nominal wall radius held the liner in place, causing it to bend at the attachment of this stud to the liner. Figure 49 shows the strain distribution in the liner near the boss. The strain rosette at position 0.0 was adjacent to the weld seam between the conical liner section and the 132-in.-radius liner. About 1.25 in. to the left of this weld was a stud that had seven strain gages located about its attachment point. The third gage plotted was another strain rosette about 4.25 in. to the left of the first rosette.

As the pressure was increased in the containment model, the two rosettes measured a corresponding increase in liner hoop strain. This was in contrast to the gages located immediately adjacent to the stud, which measured a consistent increase in compressive strains in the liner. This was clearly because of bending caused by the discontinuity of the truncated conical liner section. It followed that the tensile strains where the stud was attached to the concrete face of the liner were much larger, since the bending tensile strains would be superimposed over the tensile membrane strains, but these strains were not measurable during the test.

\subsubsection{Global Displacements}

The radial displacement of equipment hatch $B$ was similar in response to those measured for equipment hatch $\mathrm{A}$ as can be seen in Figure 26. Radial response was measured at two locations on this hatch, but one of the displacement transducers gave some erratic response at lower pressures, though both correlated well at higher pressures. The response from the "better" sensor is plotted in Figure 50.

\subsubsection{Local Displacements}

The ovalization of the sleeve of equipment hatch B remained small relative to equipment hatch A throughout the high-pressure test, probably from the liner discontinuity as mentioned previously. A plot of the ovalization at the same scale as in Figure 46 is shown in Figure 51. Three pairs of transducers were positioned along the length of the sleeve to measure its change in diameter: a pair of transducers, one measuring the change in diameter in the horizontal direction and the other in the vertical direction, was attached near the inboard edge, mid-way, and near the outboard edge of the sleeve. 


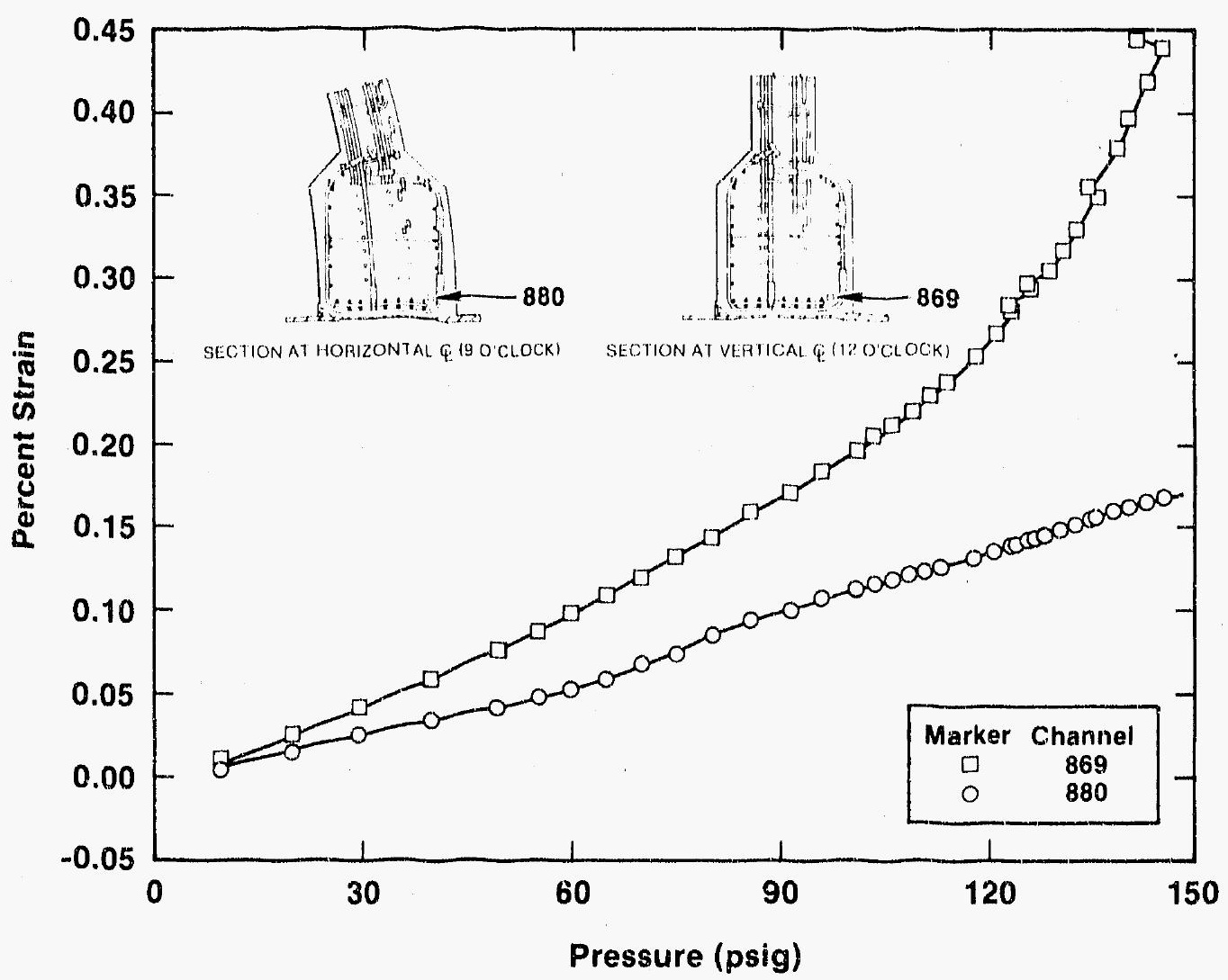

Figure 48. Variation in Hoop Strain in the Reinforcing Bar Around the Sleeve of Equipment Hatch B.

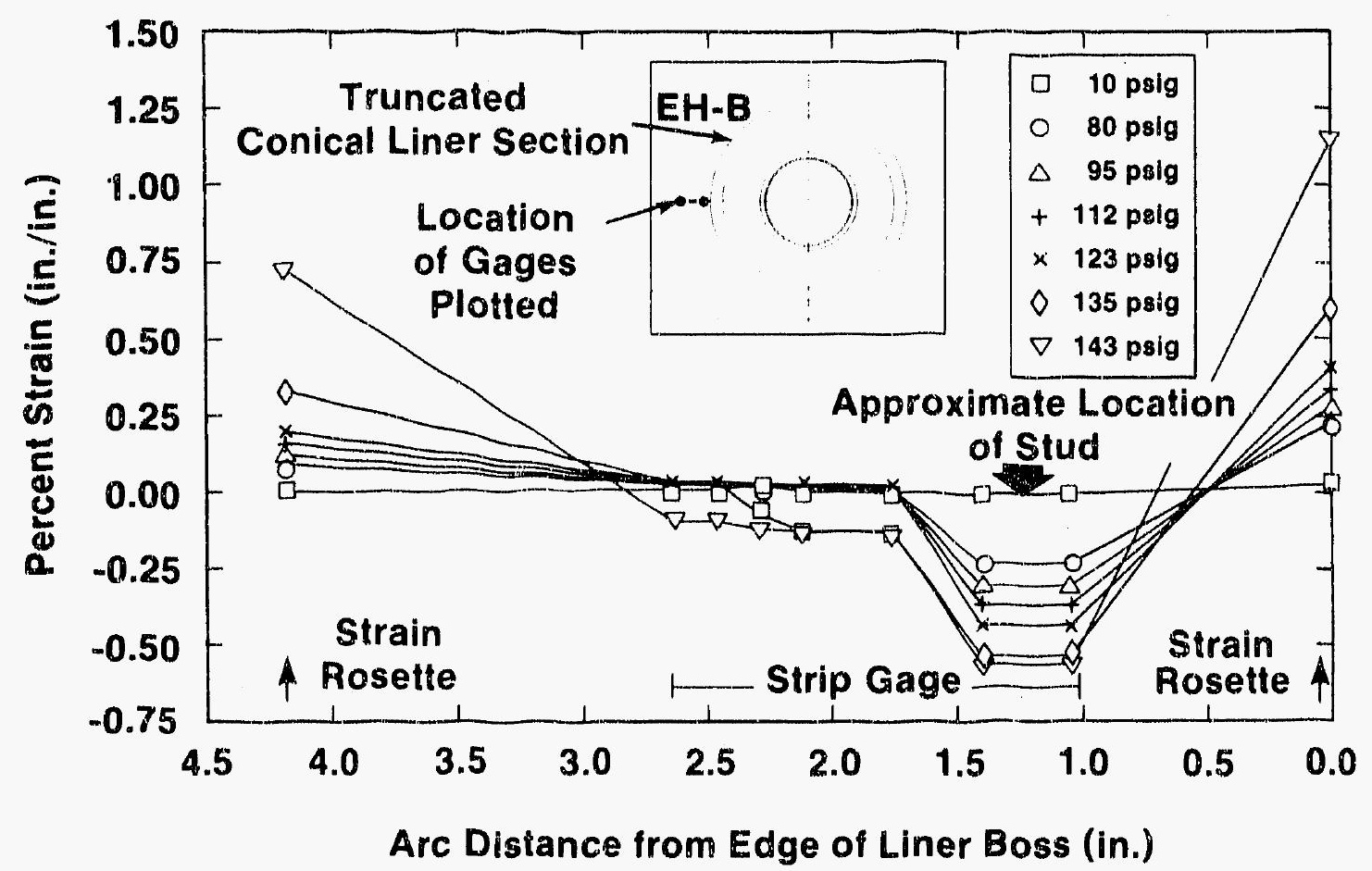

Figure 49. Liner Hoop Strains Adjacent to the Conical Liner Section at Equipment Hatch B. 


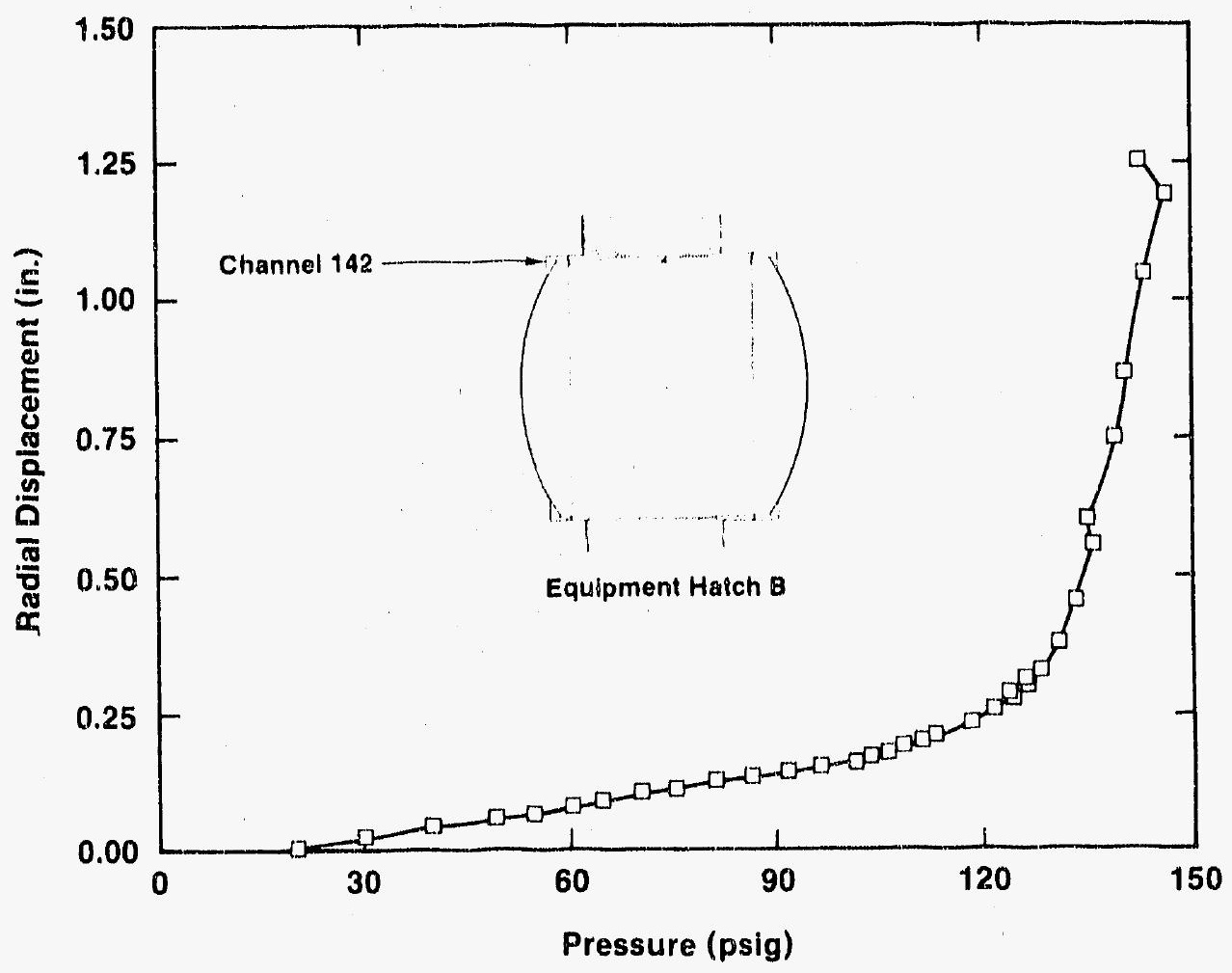

Figure 50. Radial Displacement of Equipment Hatch B.

The separation and rotation of the pressure-unseating equipment hatch cover and the equipment hatch sleeve were measured with eight displacement transducers, but in posttest interpretation of the data, some inconsistencies were found. To use these data to draw conclusions about these local deformations would be to base the conclusions more on speculation than sound measurements.

One example of this is the strain measurements recorded on the hatch swing bolts, which used an eye rather than a head. Although good strain measurements were made on the reduced section of the bolt, they did not correlate with the deformation of the bolt. This inconsistency led to a more detailed posttest inspection of the bolt and its overall deformation. The major finding of this inspection was that the eye of the bolt distorted significantly under the loads encountered during testing. Thus this additional flexibility in the bolt made correlation between the strain and the deformation impossible using the information gathered.

It should be noted that more testing of this equipment hatch is planned and will include both pressure and temperature loadings.

\subsection{Personnel Air Locks}

Since one of the air locks was used as a last man out hatch (PL-B), its cover was not typical of a full-size air lock, and it could not be as readily instrumented as the other, more typical air lock. The other air lock, PL-A, had a typical stiffener pattern on the inner and outer bulkheads but no operating doors or sealing surfaces because it was assembled entirely by weldments. 


\subsubsection{Rebar Strain}

As with the equipment hatches, a local reinforcing bar that encircled the personnel air lock sleeve was instrumented at the 9 o'clock and 12 o'clock positions with two gages at each location. The local strains showed a similar variation between the 9 o'clock and 12 o'clock positions, but the magnitude of the strains was much less than the analogous equipment hatch strains. The data from these gages have been plotted in Figure 52.

\subsubsection{Liner Strain}

The liner was either torn or distressed adjacent to each air lock insert plate (see Figure 20). In the case of PL-A, the liner tear occurred about 1-1/4 in. from a strain rosette. Although this was not the largest liner strain measured (see section 7.5.2), the strain measured at this location was substantially above the free-field strains. A plot of the response of this gage is shown in Figure 53.

As with other similar liner details, the strain concentration from the insert plate and the loading of the studs was very localized. A more highly instrumented area was monitored during the test and is presented in section 7.8.2, which shows the very local nature of the strain concentration adjacent to an insert plate.

The strains measured in the bulkhead of the personnel air lock remained small throughout the test, showing no signs of yielding.

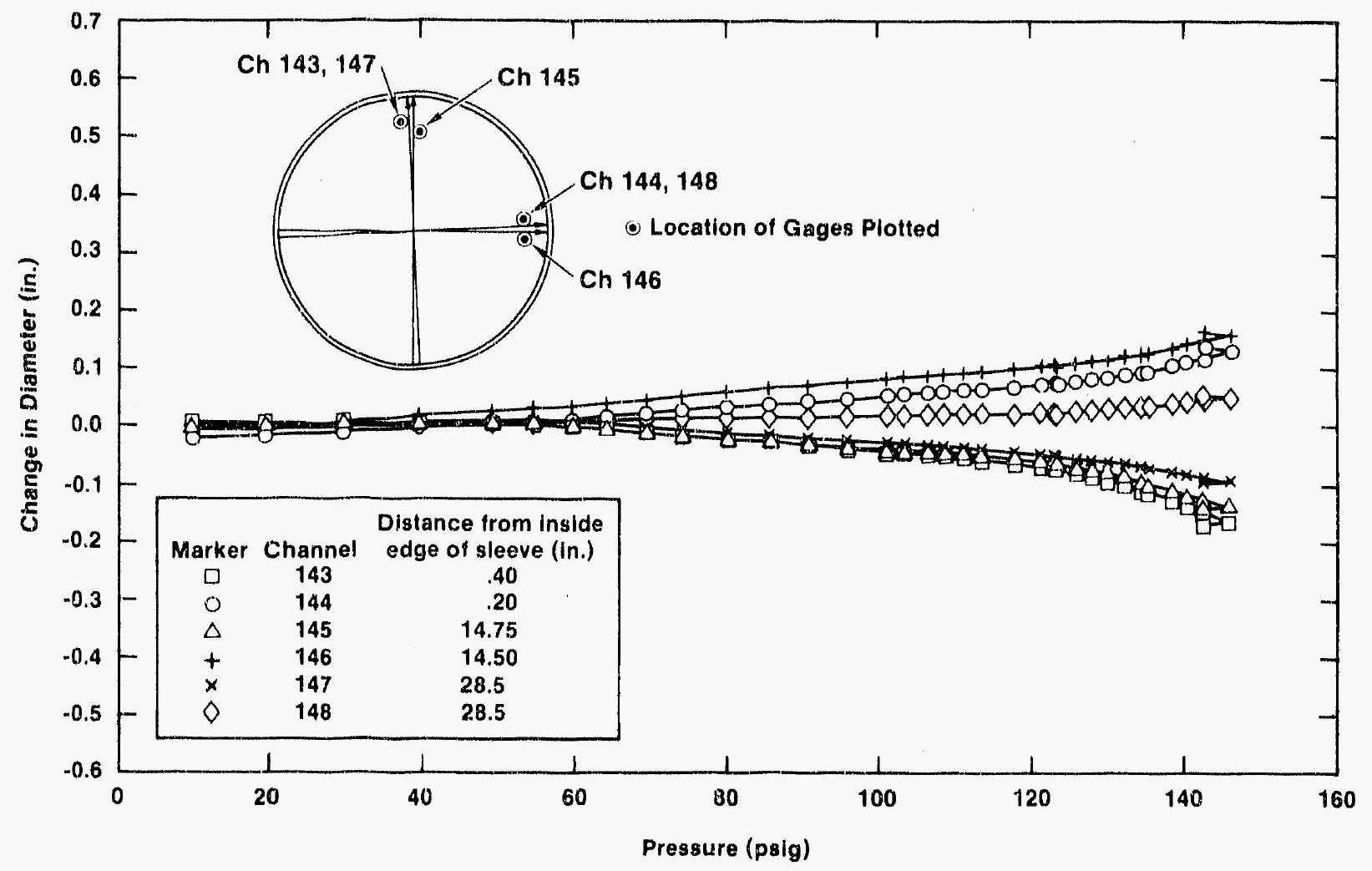

Figure 51. Ovalization of Equipment Hatch B. 


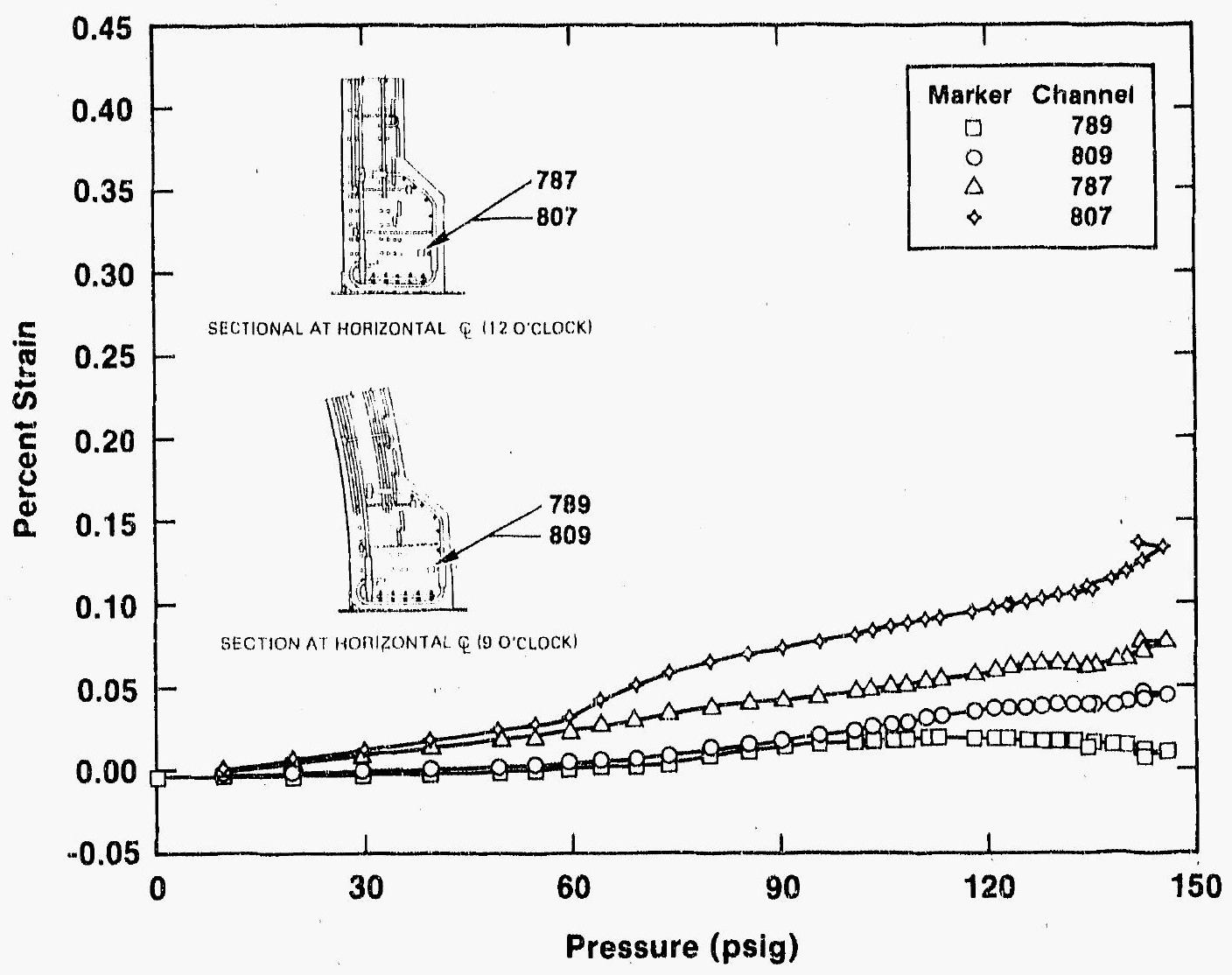

Figure 52. Variation in Hoop Strain Around the Sleeve of PL-A.

\subsubsection{Global Displacements}

The radial displacements of PL-A were presented in section 7.2. Of the free-field displacements measured and the two equipment hatches at this same elevation, PL-A showed the smallest radial displacement to a pressure of about 125 psig. Above this pressure, its radial displacement increased at a faster rate than that of the other areas. At the conclusion of the test, the radial displacement of PL-A was greater than that of the other two major penetrations and was even greater than some of the free-field displacements at this cylinder elevation.

\subsubsection{Local Displacements}

Several displacement transducers measured the dishing of the bulkhead, which was measured relative to the end of the sleeve. (The location of these gages may be found in Reference 1.) There were also displacement transducers in the air lock sleeve near the intersection of the sleeve and the cylinder liner. When compared to pretest predictions of the dishing of the 
bulkhead, the experimental results showed less bulging than expected by the analytical results. One logical explanation is that the stiffening of the bulkhead was from the tensile forces induced by the cylinder liner, which was not accounted for in the analyses, much like the tightening of a drum head

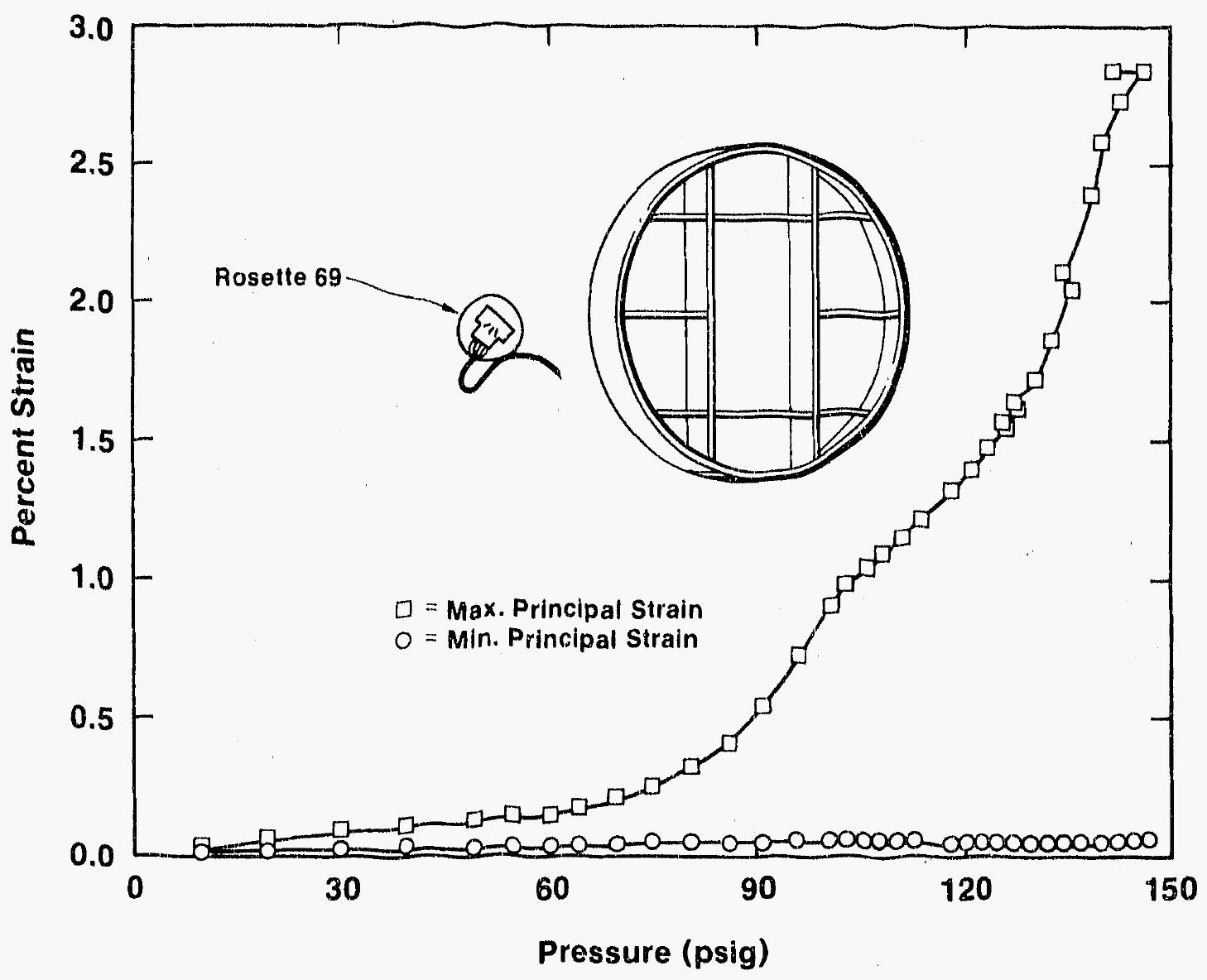

Figure 53. Liner Strains Adjacent to PL-A Insert Plate and a Liner Tear.

The measured deformation of the center of the bulkhead normal to its plane is shown in Figure 54. There were other displacement transducers on the bulkhead that measured similarly small deflections. Note that the larger the negative value of displacement, the greater the dishing of the bulkhead.

The sleeve of PL-A did not oval significantly, and there was no liner discontinuity as was the case with equipment hatch B. There is, however, one other consideration in applying Eq. (1): the sleeve must be free to deform inextensionally. In the case of PL-A, the bulkhead was welded to the sleeve and prevented this movernent. In contrast, there were only the frictional forces between the cover and the sleeve at equipment hatch $\mathrm{A}$. A plot of the sleeve deformation is shown in Figure 55, which is drawn at the same scale as Figures 46 and 52.

\footnotetext{
*Internal memorandum,D.B.Clauss, "Comparison of Experiment and Analysis for the Behaviorof Penetrations in the 1:6-Scale Reinforced Concrete Containment Model," May 2, 1988.
} 


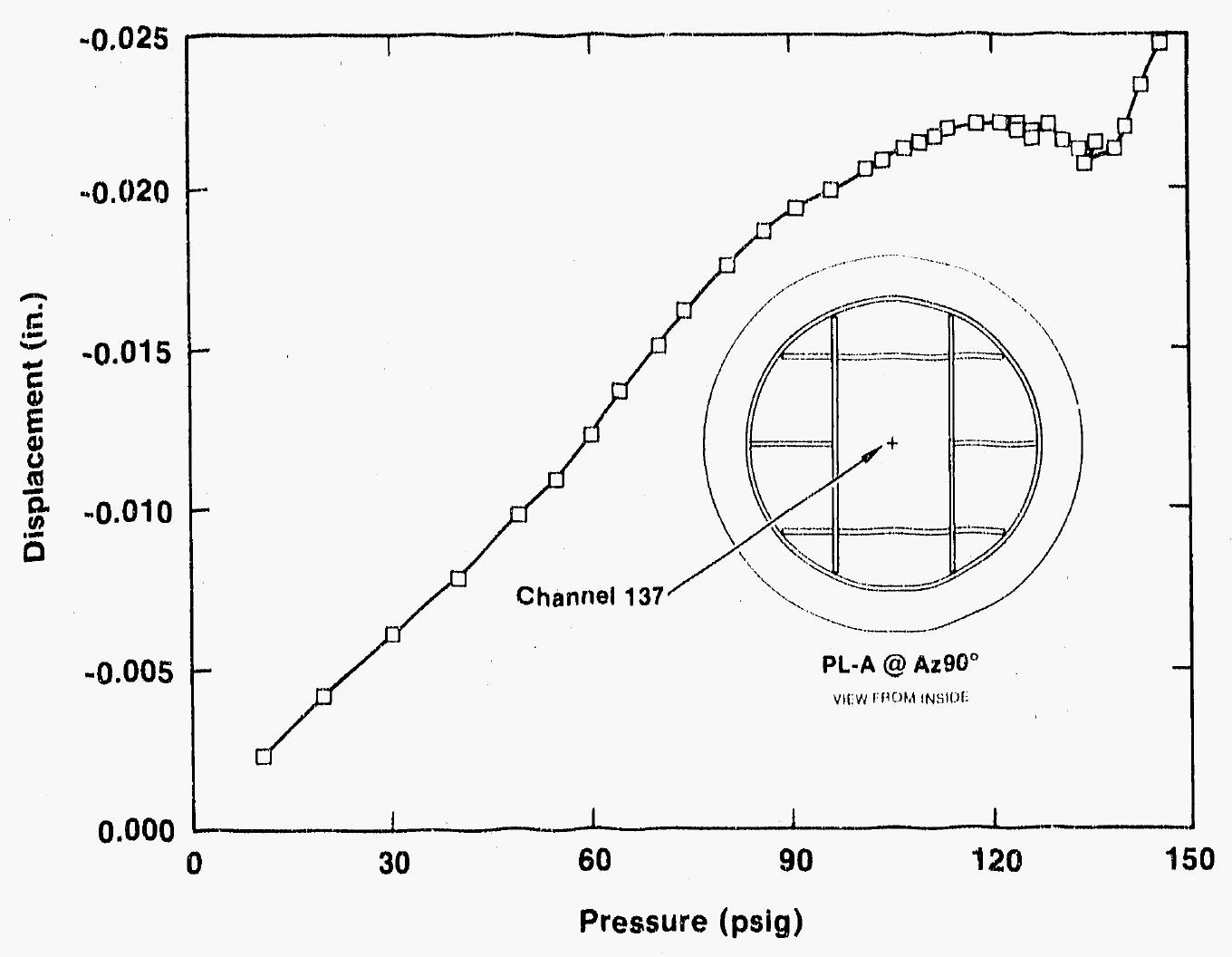

Figure 54. Dishing of the Center of the Bulkhead at PL-A.

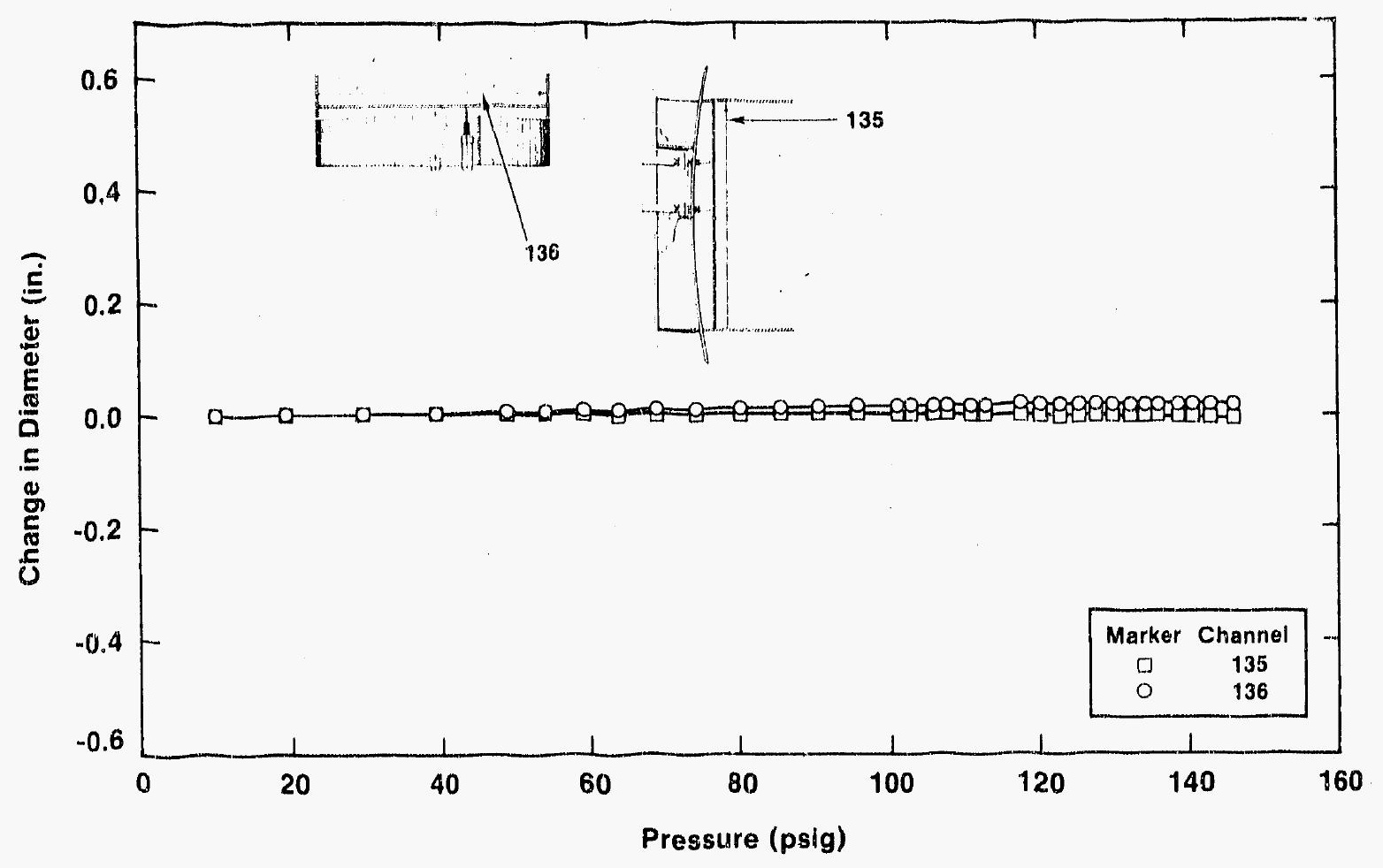

Figure 55. Ovalization of PL-A. 


\subsection{Constrained Pipe Penetration}

R-1 and R-2 were two 8-in.-diameter penetrations that were located at an elevation of 21.125 $\mathrm{ft}, 180^{\circ}$ apart at $135^{\circ}$ and $315^{\circ}$ on the cylinder wall. (See Figure 17.) They represented a major steam line penetrating the model. As a containment expands because of overpressurization during a severe accident, the steam line might have loaded the containment because of its inability to move freely. This loading, or constraint, was modeled in the containment by connecting the two penetrations by a small $(6-\mathrm{mm}$ diameter) reinforcing bar. The degree of constraint was selected by analyzing three piping systems of two full-size containment buildings during the design of the containment model. Although the degree of constraint varied significantly, a "typical" scaled stiffness was selected to represent this constraint in the containment model.

\subsubsection{Rebar Strain}

The reinforcing strains around this penetration were somewhat difficult to interpret, since it was difficult to determine whether the effect was from the constraint on the penetration, the constraint from the dome, or just typical variation of the strains in the reinforcing steel.

The hoop strains in a reinforcing bar that ran about a foot below the constrained penetration, R-2 averaged strains of $1.1 \%$ at the final pressure level, with the four locations on the bar being at azimuths $0^{\circ}, 46^{\circ}, 180^{\circ}$, and $242^{\circ}$. There was another hoop reinforcing bar 9.3 in. above the penetration. At a location of azimuth $44.5^{\circ}$, the strain was $.47 \%$ at the final pressure level. The strains in the reinforcing bar adjacent to the penetration had noticeably lower strain readings, as shown in Figure 56. One of the gages was on the innermost layer of hoop reinforcing steel, while the other two were located on the outermost layer of reinforcing. The response of the three gages is relatively consistent.

The strains in the meridional direction also showed variation because of the constrained penetration. For the outer layer of meridional reinforcing, the strains measured showed much less variation from the presence of R-2. In three separate bars, two near R-2 and one in the free field, the peak strains were $.23 \%, .19 \%$, and $.26 \%$ at locations of $312^{\circ}, 313^{\circ}$, and $47^{\circ}$, respectively. The strains measured on the inner layer of meridional reinforcing steel at these locations are plotted in Figure 57. The strains near the penetration were much lower than the free-field strains and also lower than those measured in the outer layer of meridional reinforcing steel near R-2. Although the loading of the reinforcing and the concrete in the vicinity of R-2 is more complex then in the surrounding free field, all indications from the measurements taken in this area indicate that the constraint placed on the containment shell through the fixity of the steam line reduced the strains and displacements in this area.

\subsubsection{Liner Strain}

Some interesting liner strains were also measured at penetration R-2. It should be noted that at the location of each tear discovered in the liner after the high-pressure test (with the exception of the tears near equipment hatch B), the liner geometry was similar to that at this location because a thickened liner section (3/16 in. thick) was welded to the nominal thickness liiner mäteriala (1/16 in. thick). This discontinuity and the liner anchorage system (the studs) caused very noticeable increase in the liner strain immediately adjacent to the insert plate. The response of the region adjacent to R-2 at seven pressure levels is shown in Figure 58. 


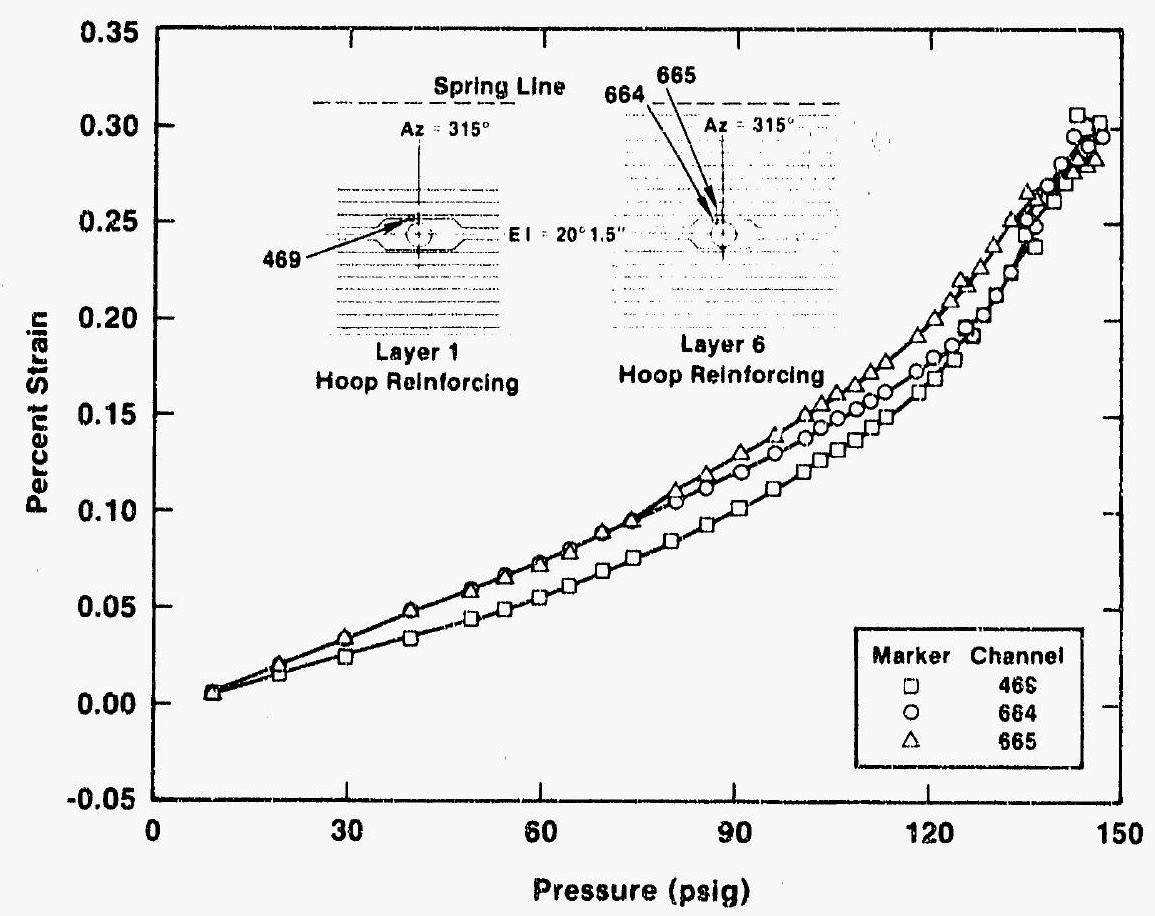

Figure 56. Hoop Strain in the Reinforcing Steel Near Penetration R-2.

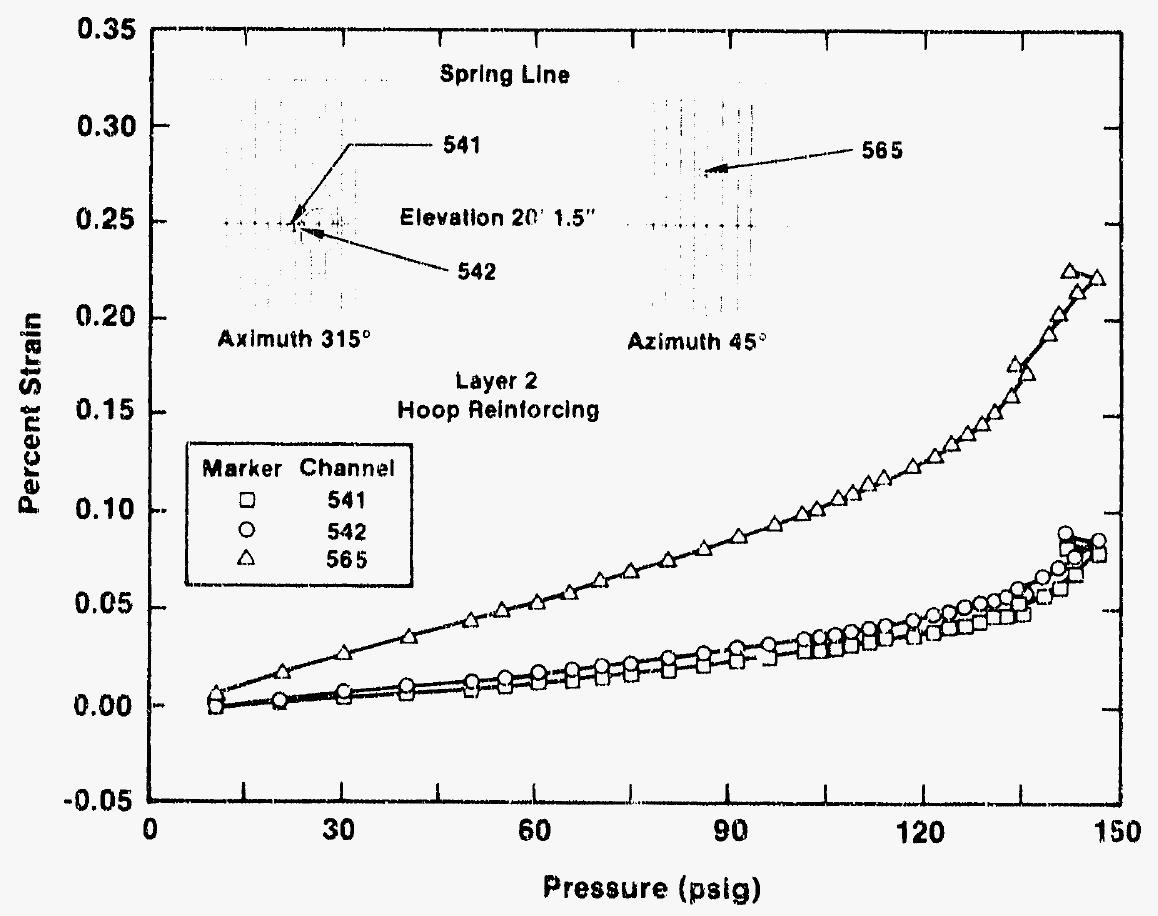

Figure 57. Meridional Strain in the Reinforcing Steel Near Penetration R-2. 


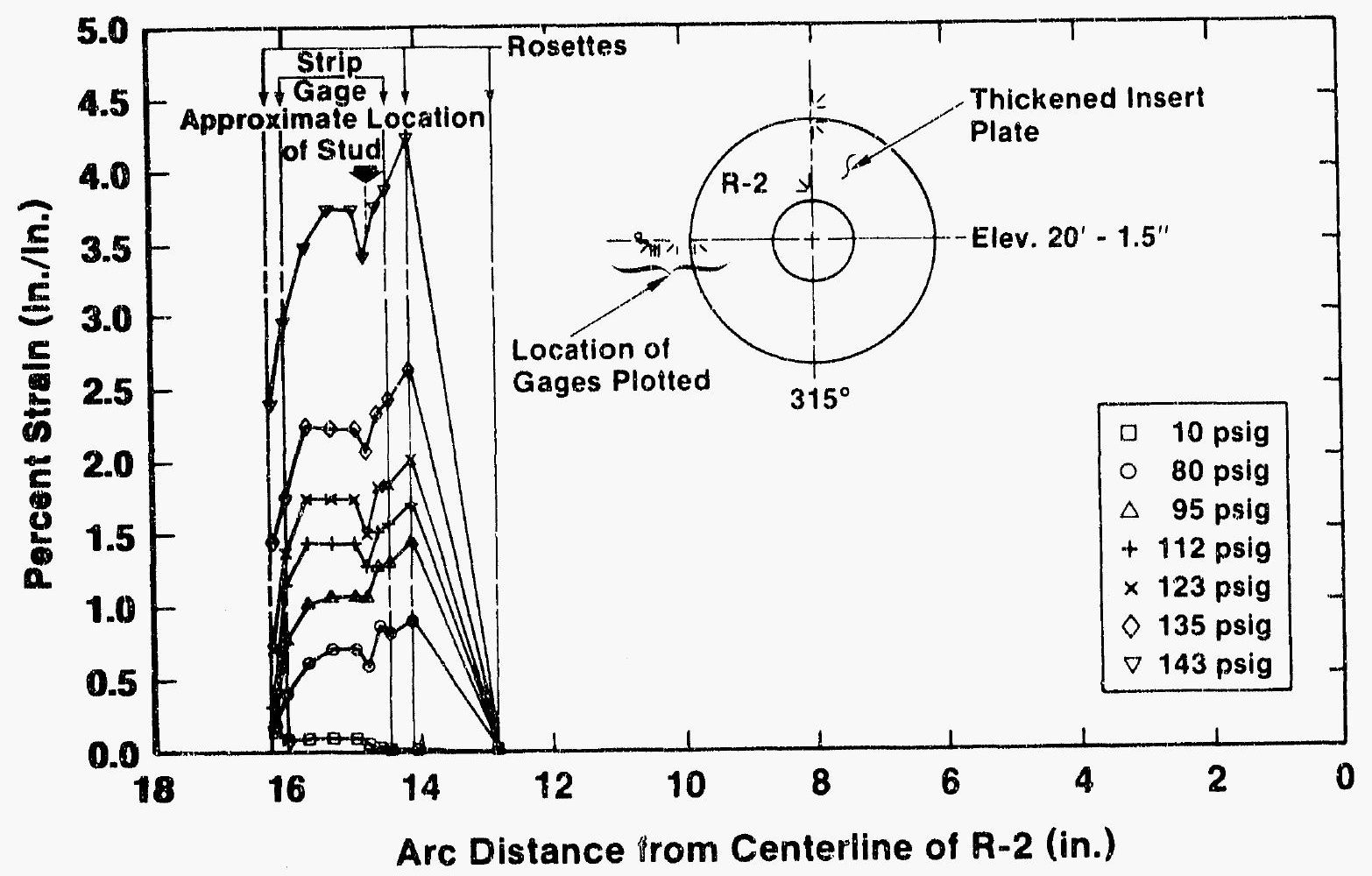

Figure 58. Hoop Strain Near Penetration R-2 at Seven Pressure Levels.

The first measured location, shown in Figure 58 as arc distance from the center of the penetration, is where a rosette was located on the insert plate. The insert plate intersecied the nominal thickness liner material at an arc distance of 13.44 in. Adjacent to this was another rosette at a location of about $14 \mathrm{in}$. Beyond the second rosette was a series of seven gages on a common backing (a strip gage) that intersected the attachment of a stud to the liner. The length of the strip gage was about $11 / 2 \mathrm{in}$. The final gage response plotted in Figure 58 is another rosette located slightly over 16 in. from the center of penetration R-2.

Figure 58 shows that as the pressure was increased, strains remained small in the thickened insert plate. Strains in the neighboring nominal thickness liner steadily increased with increasing pressure, as well as a strain concentration from this geometry and the presence of the stud. There was a relatively minor reduction in hoop strain at the point of stud attachment. The measured values of strain are connected by a line; caution should be used in interpreting values between those measured. It is entirely possible, if not probable, that a higher strain concentration exists at a point between the insert plate and the first measured location on the 1/16-in.-thick Iiner material.

These measurements and the tears observed at particular locations in the liner after the highpressure test present a strong argument for why the liner tore. Since this penetration, R-2, was in the cylinder near the intersection with the dome, the free-field strain was smaller than that recorded at the cylinder midheight (and no tear was present). It is easy to conclude that a similar response was present in other similar areas of the liner, but the magnitide of the maximum local strain was higher, since the strain concentration factor applies to higher values of free-field strain (resulting in tearing of the liner). 


\subsubsection{Local Loads}

A load cell was placed in series with the bar that connected penetrations R-1 and R-2 so that the load could be measured during pressurization. The yield load and maximum load for the bar that connected the two penetrations were $3340 \mathrm{lbs}$. and $4330 \mathrm{lbs}$. respectively. The load cell measurements proved to be unreliable; however, there was evidence that the bar connecting the two penetrations yielded during testing.

\subsubsection{Global Displacements}

If the constraint between the two steam-line penetrations was significant, the radial displacements near these penetrations would be less than in the neighboring areas (causing the cylinder to become oval in shape with the minor axis being at this constraint and the major axis being $90^{\circ}$ from it). The constraint imposed on this penetration can be seen in Figure 59 , as well as the influence of the constraint on the displacements. At the final pressure level, the lowest radial displacement recorded at the elevation of R-2 and R-1 was recorded at a location above equipment hatch $B$. This suggests that the other features of the containment, such as the major penetrations, had more influence on the radial displacements than did the constraint placed between R-1 and R-2.

\subsection{Leakage/Acoustic Response}

The acoustic monitoring system described in section 4.7 provided additional insight into the leakage of the containment model.

At pressures above 125 psig, a small amount of leakage was suspected from near equipment hatch B." In the data generated (see Figure 60), some indications appear of increased acoustic emissions (AE) at 122 psig. The large peaks in this figure were caused by the pressurization of the containment and then decreased to a baseline value. The increase in this baseline AE at pressures above 120 psig gave strong evidence for some small amounts of leakage, attributed to the unseating of the equipment hatch.

At a pressure of $135 \mathrm{psig}$, a dramatic increase in the ringdown counts was noted from a sensor at equipment hatch $\mathrm{A}$, and is graphically shown in (Figure 61). After the dramatic increase, the rate quickly reduced to a rate that was nearly the same before this event. This may indicate a tear, and then after a short time, the crack growth stopped.

It was somewhat surprising that none of the other sensors detected these AE signals, but the other sensors were possibly shielded by physical features of the model (the penetrations) and the natural attenuation from the large distances to other sensors. Even more surprising is that there was no immediate indication of leakage noise by the AE system. An integrated leak rate test measured a small $(8 \mathrm{scfm})$ leak in the model.

\footnotetext{
*Internal Report. A. G. Bealtie "Acnustir Monitoring of the One Sixth Scale Model of a Nuclear Reactor Containment Building," Nondestructive Test Report, Sandia National Laboratories, Albuquerque, New Mexico 1987.
} 


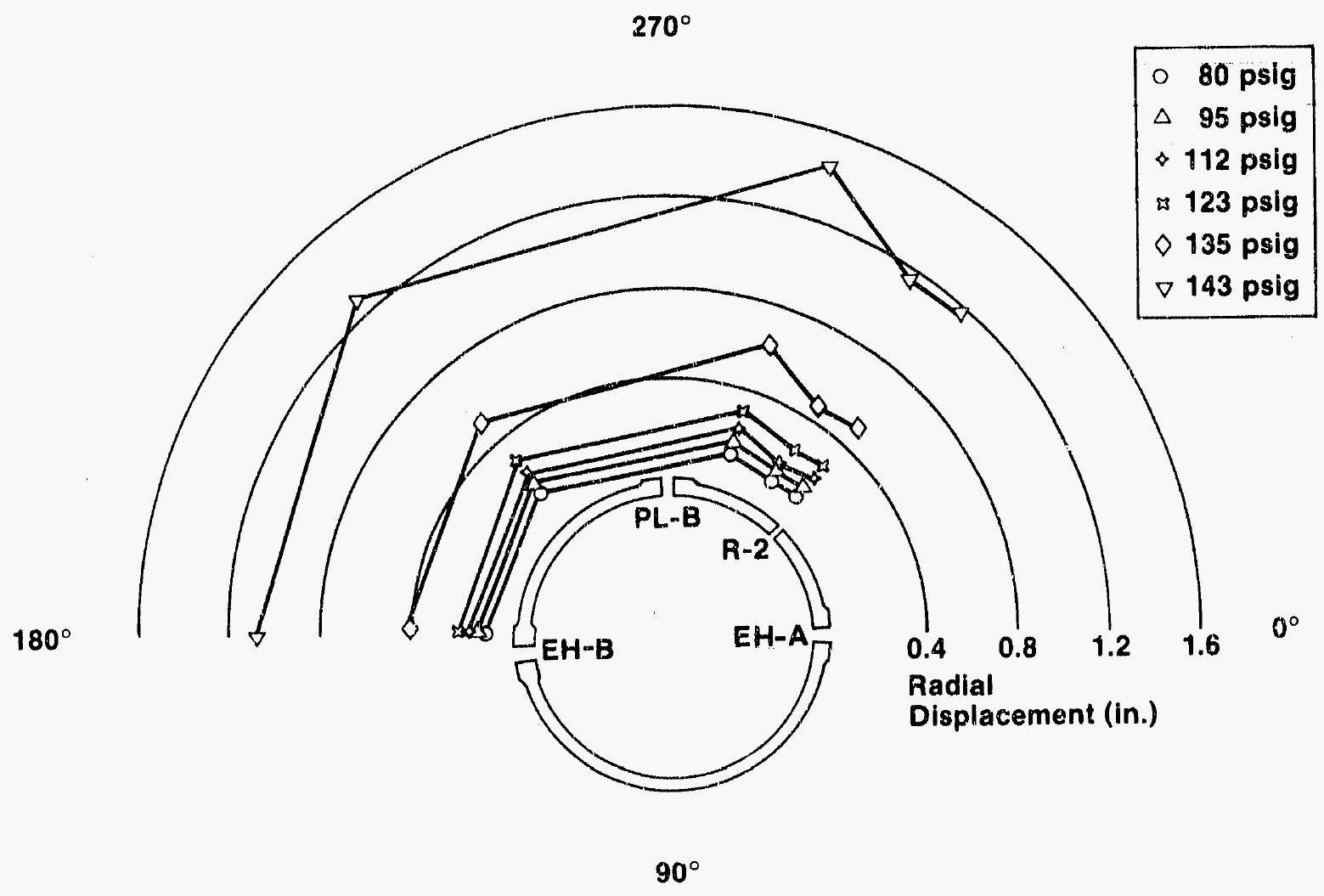

Figure 59. Radial Displacements at Elevation $20.125 \mathrm{ft}$ at Six Pressure Levels.

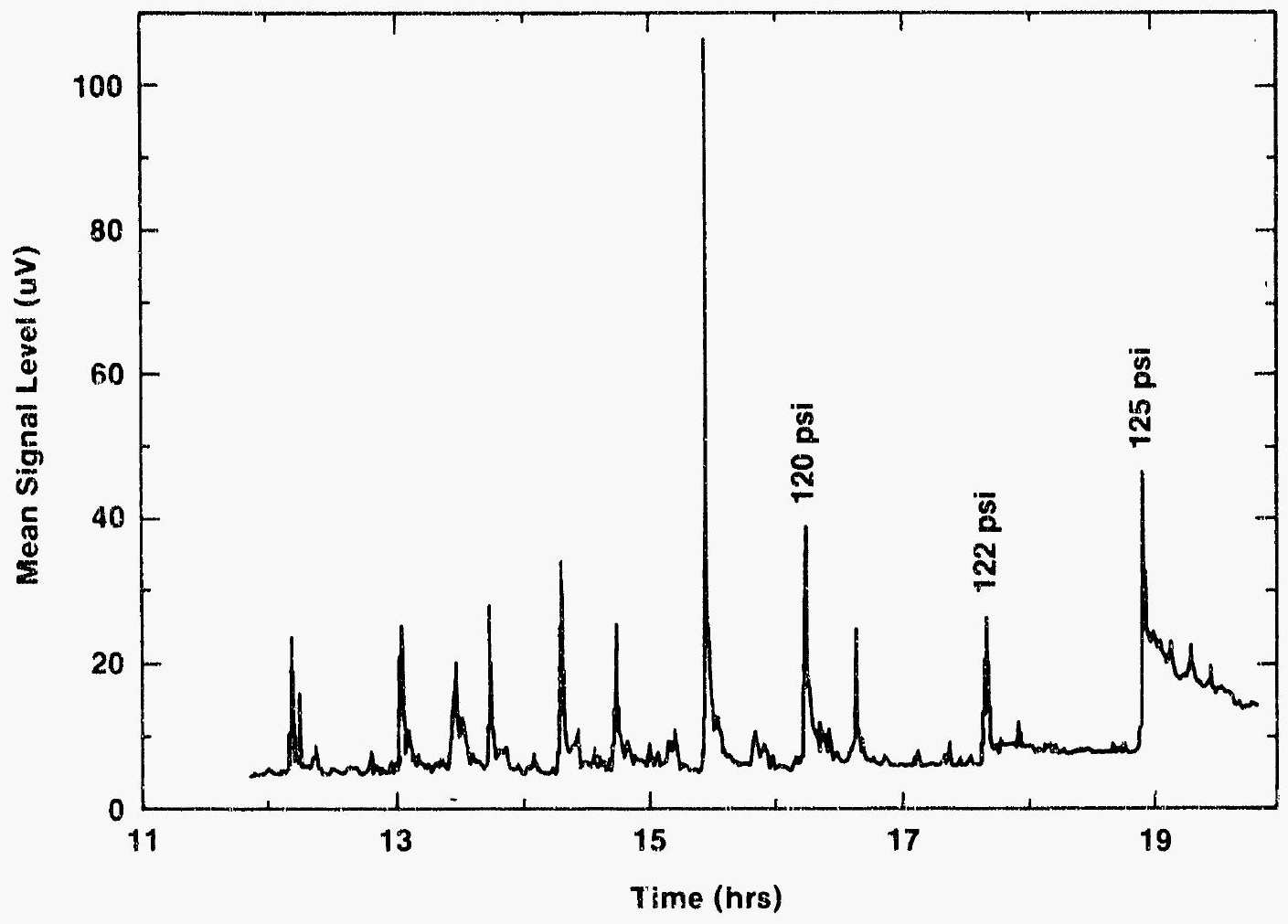

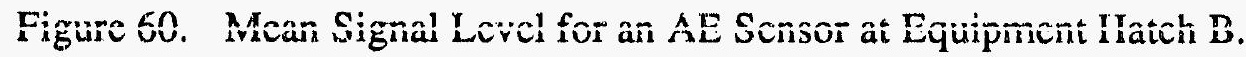




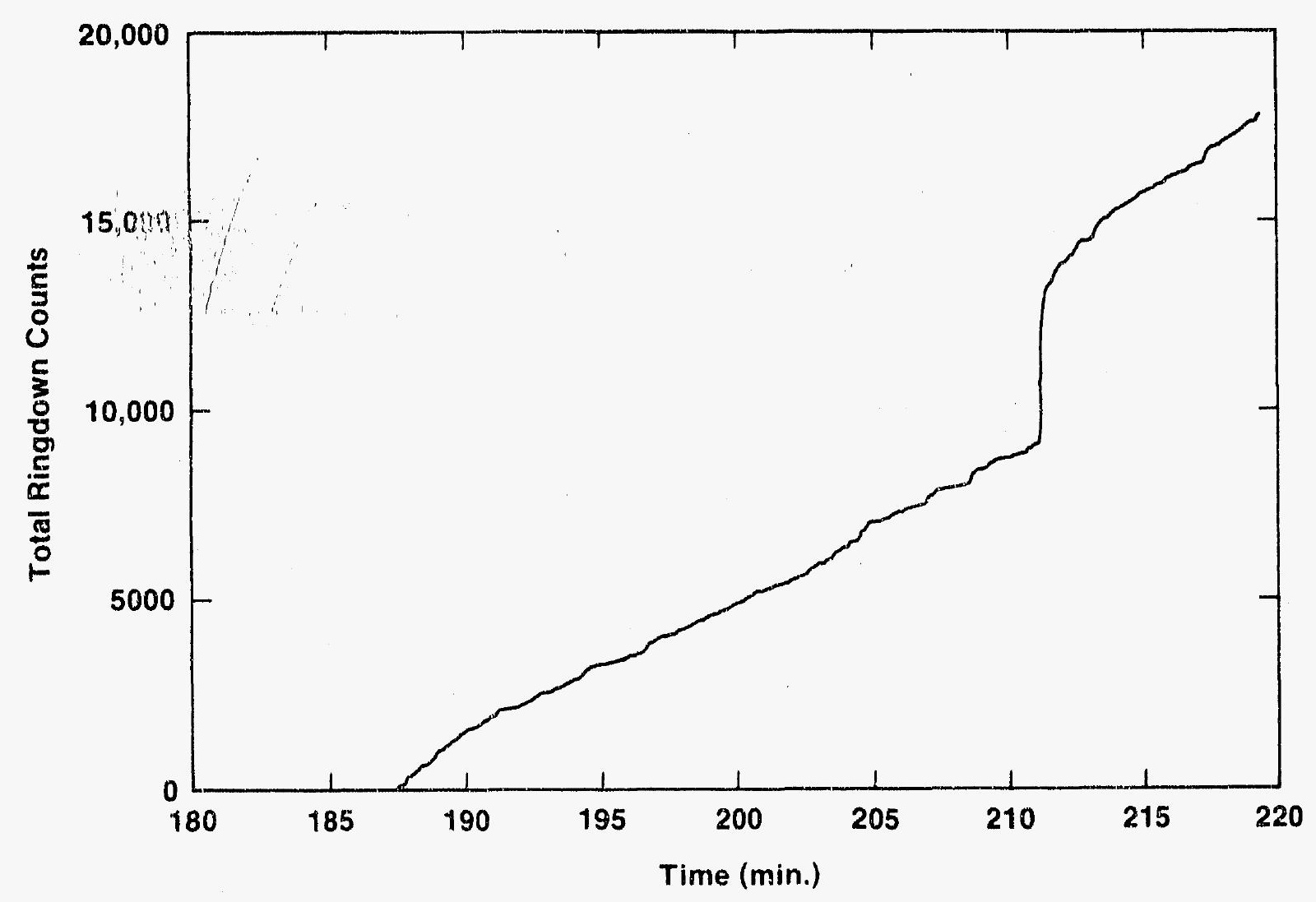

Figure 61. Ringdown Counts for an AE Sensor at Equipment Hatch A.

Ten minutes after the containment had been pressurized to the next pressure step, $138 \mathrm{psig}$, the sensor near equipment hatch $\mathrm{A}$ registered a large leak. The $\mathrm{AE}$ increased as the model was pressurized to $140 \mathrm{psig}$, and the signal was saturated as the pressure was further increased to $143 \mathrm{psig}$. Other sensors were able to detect the $\mathrm{AE}$ coming from this vicinity also. It was apparent that leakage was rapidly increasing. Although this leakage was large, the pressurization system had sufficient capacity to increase the internal pressure; where additional leakage measurements were taken (see sections 6.2 and 6.3).

Burst noise was also monitored during testing. Although this is not an exact science, it did provide some interesting qualitative results. All sensors showed very low activity up to about $50 \mathrm{psig}$. This is most likely because the model had been pressurized up to $53 \mathrm{psig}$ during the structural integrity test. At a pressure range of 50 to 70 psig, an increased AE rate was noted at all the sensors. The sensor near equipment hatch $A$ registered a small increase in crack rate at $110 \mathrm{psig}$ and stayed steady until the end of the test. Two other sensors at mid height of the cylinder recorded a significant increase in cracking at about 112 psig; however, this rate abruptly decreased at 135 and 138 psig for these sensors.

\subsection{Summary}

Data were generated at a total of 37 pressure levels for over 1200 sensors during the testing of a 1:6-scale reinforced concrete containment. The data and observations during testing have helned to forus ? ?n!ytyical efforts on the features important to containment nerformance. The 
data also have benchmarked the analytical predictions and increased understanding of the behavior of containments subjected to beyond design-basis loads.

The amount of data generated during this experiment and the many subtleties inherent in the data make it difficult to prove some of the hypotheses. As the interpretation of the data improves and posttest analyses continue, confidence in the accurate prediction of reinforcedconcrete containments will also increase.

Some of the conclusions and hypotheses are listed below. Admittedly, some of the following hypotheses are better substantiated than others:

- Insert plates can reduce the strains in the reinforcing steel.

- Insert plates cause a strain concentration in the liner.

- The studs near an insert plate contribute to a large strain variation that appears to have influenced the tears in the liner.

- Cracks in the concrete can cause a strain perturbation in the liner.

- Studs cause a strain gradient in the free-field liner.

- There was a strain gradient in the concrete between ccincrete cracks.

- The insert plate can cause noticeable bending strains in a penetration sleeve. (The resulting strain, however, remained small for this containment model.)

- The cylinder wall bending moment influences the strain in the reinforcing steel in the basemat.

- Penetrations tended to be stiffer than the free-field cylinder sections, resulting in smaller radial displacements than in the free field.

- Steam-line rigidity does not significantly constrain the cylinder.

- The liner junction between the floor and bottom of the cylinder causes a very local strain concentration.

- In the dome region, the seismic bars influence the response of other reinforcing members.

- Ovalization of equipment hatch $\mathrm{A}$ was related to the free-field hoop strain.

- Ovalization of equipment hatch $\mathrm{B}$ was prevented by the angular junction in the liner at the inward projection of the boss.

- The bulkhead of PL-A prevented its sleeve from becoming oval.

- Early leakage during the test was probably because of the unseating of the cover of equipment hatch $B$.

- Most of the liner tears occurred at a pressure level of $138-145 \mathrm{psig}$.

- Leakage can be detected by AE monitoring. 
Some of these conclusions are not at all surprising, while there are a few conclusions and hypotheses that were not anticipated. It is these new findings that necessitate more structural testing for areas that remain in question. 


\section{DISCUSSION}

This document describes a test that may have significant impact on future testing as well as in several areas of the nuclear industry. The author's thoughts on some frequent questions about the test may provide some additional points not brought out or fully obvious from the presentation of the data. Whether the reader embraces or rejects them, they should provide some further understanding of the test.

\subsection{Repeatability of the Test and Other Failure Modes}

A question from testing only one model is whether the outcome would be the same if the test were repeated. Although there is some reason for doubt, the answer is most likely to be that the same failure mode would be realized if another (identically designed) containment model were to be built.

A digression to the failure predictions may further explain this position. Ten organizations reported their failure predictions in Reference 30, which showed a relatively wide range of failure pressures and four general failure modes. Some of the organizations predicted equally likely failure mechanisms, which led to 14 predictions of failure mode and pressure as shown in Table 5.

Table 5.

Failure Modes and Pressures Predicted by Analysts

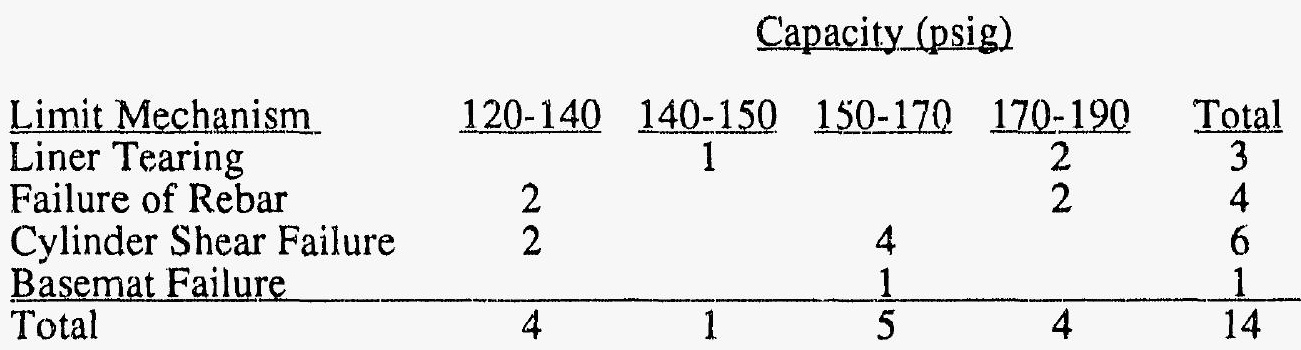

No organization flagged the failure location as a high priority. This in some cases may be attributed to the time allowed to conduct the analyses, which was constrained by the testing schedule, and funding limitations for the analyses within the organizations. Only a few of the organizations looked at the penetrations in detail, and those that analyzed penetrations chose the larger ones (equipment hat hes and personnel air locks) or the penetration that was constrained, R-2. No organization performed a pretest analysis of piping cluster 25-A.

With these analysus and some engineering judgment, the instrumentation plan evolved. In hindsight, it is clear that not only liner tearing but, more specifically, liner tearing next to insert plate $25-\mathrm{A}$ is very likely, as opposed to a one-time quirk of this test. This large insert plate, $6 \mathrm{ft} 3 \mathrm{in}$. in length, was located between two stiff and large penetrations, equipment hatch $\mathrm{A}$ and PL-A. Since these penetrations were stiffer (see Figure 26 and the associated discussion in section 7.2.2) than the neighboring shell, they forced more straining to occur between them. This in turn caused more load to be dumped into the insert plate 25-A. 
This plate has another characteristic that makes it a likely failure location: the three piping sleeves that penetrate it also penetrate the concrete wall. These penetrations also act as substantial anchors that resist slippage between the concrete and the liner. Another feature of insert plate $25-\mathrm{A}$ is its straight leading and trailing edge in the hoop direction, making the likelihood of a tear to propagate higher than if it were a semicircular shape. These features combined, create not only a high strain concentration, but one that had the ability to propagate over a relatively large length." Other tears in the liner may have occurred at a lower pressure, but because of their geometry, they were evidently less likely to propagate.

It must be stressed that other reinforced-concrete containments can have other failure modes, such as cylinder wall shear failure at its intersection with the basemat and general failure of the hoop reinforcing. This single test gives some insight into other failure modes. A study on the possibility of failure and other behavior of the cylinder wall at its intersection with the basemat is ongoing. Preliminary results indicate that a shear failure in this location was more likely than the visual evidence (lack of cracking and movement) would indicate [36]. The strains and forces at this location greatly exceeded code allowables.

Findings like these lead to caution when answering whether another containment model built and tested in a similar manner would fail in an identical fashion. A very good possibility of repeating this failure mode in another identically designed containment model exists; however, it is not known how near the containment model was to reaching another failure mode, which might change the outcome of a similar test.

\subsection{Symmetry and Variation in Performance}

There were many areas of the model that had a "twin" elsewhere in the model that had a somewhat different response. These differences may be because of construction, lay-out of the containment features, nonuniformity of materials, or other sources. In any case, some nonuniformity in response was evident in the containment model. A few examples of this follow.

- At the failure location near insert plate 25-A, the tear was on the left side (as viewed from inside the model) of the insert plate. While the right side showed some distress, no tears were found. This same pattern was repeated on the insert plate directly below $25-\mathrm{B},{ }^{* *}$ which is identical in size to $25-\mathrm{A}$; the left side showed severe necking of the steel (no tears, however), while the right edge exhibited much less distress.

- Both personnel air locks had one location of distress to the left of the penetration, while no such evidence was found for the right edge.

- For equipment hatch $\mathrm{B}$, the right edge of the truncated conical section of liner had two stud tears, while the left edge (the instrumented side, unfortunately) had no stud tears and showed less distortion from testing.

\footnotetext{
"Strain concentrations at other locations, for example, near the truncated conical section of liner adjacent to equipment hatch B, may have been higher, but once the liner tore locally, the mechanism to propagate the tear seems to have been missing. Analyses of this and other areas are needed to gain more insight into these areas.

** As in this case and the folloving example, there is uniformity from feature to feature but not around the feature itself.
} 
- A final example of nonuniform behavior was observed in the cylinder wall when comparing wall quadrant to wall quadrant. From $180^{\circ}$ to $270^{\circ}$, the displacements and crack widths were noticeably larger than in the wall sections from $90^{\circ}$ to $180^{\circ}$ or $270^{\circ}$ to $0^{\circ}$, which are all quite similar. The influence of the constrained penetration may be part of the explanation for these differences, but does not, in the author's mind, fully explain the measurements and observations.

Although there was variation in the performance of the model, none of the variances seem to be attributed to any anomalies associated with the model containment. That is, the same variances would be expected in a full-size containment and there is no evidence that the variances are smaller or larger in the containment model. Whether the variation was caused by construction quality, material properties, or penetration layout, or some other reason, it is not thought that the variance influenced the failure mode, failure pressure, or failure mechanism.

\subsection{Limitations of the Model}

A concern over the validation of computer analyses using scale model tests versus a full-size prototype is always present, and certain questions concerning this must be addressed before the methodology for predicting full-size containment performance is fully accepted. Table 6 lists some of the differences between the model and a full-size containment. Each of these attributes is discussed further in the following paragraphs.

Table 6.

Potential Differences Between the Model and a Prototype Containment

- Workmanship

- Construction tolerances

- Reinforcement Number of splices

Number of bars Ductility Bond

- Liner

Number/length of welds

Scaling of welds

Ductility at failure

- Anchorage

Stud Dimensions

Welding

- Concrete

Crack distribution/width

- Scaling of leak rate 


\subsubsection{Workmanship}

A few words on the effects of workmanship on containment failure should also be stated. Although there are differences in response recorded and also visually noted about the model, it does not seem that the workmanship played a significant role in failure of the containment.

The welds in the liner near where it tore were obviously of much interest after the test. Upon inspection by Sandia's metallurgists, it was conclusively determined that the liner failures were not related to the welding process, in fact all the tears were well away from the heat affected zone. All welds in the liner remained intact, with no signs or indications of deterioration. All tears occurred in association with the stud anchors, but pretest experiments on the stud-liner behavior $[1,27,28]$ show that there was not a perceivable degradation in liner performance due to the attachment of the studs.

Workmanship on this model was of high quality and there was no attempt at building flaws into this containment model that might duplicate poor quality in a full-size containment." The consequences of a full-size containment constructed to either higher or lower standards are subjective and would need to be determined on a case-by-case basis-no statements generalizing the effects of workmanship can be definitively made.

\subsubsection{Construction Tolerances}

In many cases, the tolerances assigned during normal full-size containment construction were scaled, but not in every case. In most instances the tolerances have little effect on the overall performance of the containment model; however, in some cases these tolerances can significantly effect the variance of actual model performance and anticipated performance. Take the simple example of the placement of the reinforcing bars in the containment wall. In the majority of the wall, which is responding as a membrane, the exact placement of the bars through the thickness of the wall makes little difference in the calculated strength of the shell wall. In contrast, placement of the reinforcing steel through the wall thickness can have a notable effect on moment resistance in areas of high bending, such as at the basemat-cylinder wall junction. Again, no generalized statements can be made about the effect of construction tolerances on the outcome of this test; however, in many cases construction variances can be assumed and its influence can be quantified and reasonably bounded by use of simple calculations.

\subsubsection{Reinforcement Steel}

The reinforcing steel presents several differences between the containment model and a full-size containment. Fewer bars were used for the construction of the containment model, since the steel area was scaled rather than scaling each bar's steel area. This resulted in a reduction to the number of reinforced bars and in a reduction of the bar splices needed. The consequences of this may be determined statistically, but no dramatic changes in performance in either case is apparent. Both have a statistically large population of splices as well as a significant number of redundant load carrying elements.

\footnotetext{
* There were both concrete repairs and liner repairs during the construction of the model. These are fully discussed in Reference 1.
} 
The ductility and the ultimate strength of the reinforcing steel increases with a decrease in bar diameter. For example, reinforcing bar made from A615 billet steel has a code specified minimum elongation of $9 \%$ and $7 \%$ in an 8 in. gage length for a \#4 and \#18 bar, respectively, with a minimum $60 \mathrm{ksi}$ yield and $90 \mathrm{ksi}$ tensile strength for all bar sizes [37]. Using actual material properties generally shows an even more dramatic difference. Certainly, if the failure of the model were related to reinforcing failure, this would be an important consideration; however, since the limits of the reinforcing bars were not encountered, they have no impact on the results of this experiment. Caution however, should be exercised when applying the results from this test to full-size containments. Since the larger reinforcing bars may have less ductility and plastic strains in the bars may be large due to bending of the bars during construction, a reinforcing bar failure on a full-size containment may occur at lower strain levels than were expected from results based on this containment model test. ${ }^{*}$

The bond between the reinforcing bar and the concrete also changes with bar size. A major factor influencing bond is that the cross-sectional area changes as the diameter squared, while the surface area changes as the diameter. As would be expected, the bond length required to develop the full strength of the bar increases in relation to the area of the bar rather than the diameter. Again, in a full-size containment a bond failure is more likely than this experimental data may suggest, but does not appear to be a problem based on experiments that used specimens constructed using full size bars and were loaded to high strain levels [38]. It should also be noted that the majority' of the primary reinforcing bars are made continuous through the use of mechanical connectors (Cadwelds) or are terminated in areas of lower stress (such as the meridional bars strength being developed within the basemat and dome.)

One additional point should be made concerning the bond of the concrete to the reinforcing steel. The bond can affect the crack spacing and thus it will directly affect the crack width size. If the cracks in the concrete are presumed to influence the tears in the liner, then the effects of bond on the crack spacing and size should be investigated moie thoroughly when failure modes are a part of the study."

\subsubsection{Liner}

There are several differences between the liner of the containment model and a full-size liner. Each sheet of liner material in the model covered a proportionally larger area of the containment shell, which has the consequence of reducing the number and length of welds in the liner. Also, the type of welding on the liner was different than that used in a full-size liner (single-pass, short-circuiting arc welding as opposed to multi-pass arc welding.) The result of this is that the number and the chance of having severe flaws will likely be different.

The SA-414 Grade D has properties that are similar to those of typical prototype liner material, SA-516 Grade 60, but there are notable differences. The SA-516 material and its thickness used for insert plates in the containment model are the same as typically used for liner

\footnotetext{
* Tests conducted at Construction Technology Laboratories, Inc. for the Electrical Power Research Institute used largediameter reinforcing steel $(\# 18)$ in specimens that were tested to simulate structural containment elements subjected to overpressurization [38]. Failure of some of the reinforcing bars in the specimens occurred at loads lower than expected and was thought by the researchers to be due to the plastic deformation experienced by the reinforcing bars during specimen fabrication.

** Current analyses at Sandia [36] indicate that the tearing of the liner is attributable to the anchorage system and that the concrete cracking has only a secondary (or tertiary) effect.
} 
material in a full-size containment liner, which allows a comparison of full scale liner material to that used in the containment model. The most significant difference between the two materials is the strain at failure. The strain at failure for the 0.2 in. thick SA-516 Grade 60 material used in the containment model is $45 \%$, while the $1 / 16$ in. thick SA-414 Grade D had a strain at failure of $26 \%$, both strains were measured in the rolling direction [28].

Since the model liner failure resulted in termination of the test, one may question what difference the performance of SA-516 Grade 60 would have had on the outcome of the test. Analyses [33] indicated that the strain in the liner increased very rapidly with increase in-the internal pressure. Although the SA-516 steel may have strained further before it tore, the change in pressure to get this change in strain would likely be very small. Thus, it is speculated that virtually no change in the outcome of the test would have occurred.

\subsubsection{Anchorage}

The studs used in the fabrication of the containment model are necessarily of a distorted scale. In addition to being shorter than the scaled length of a full-size stud, the diameter is larger than the typical diameter of a stud used for full-size containment construction (although it does scale to a larger diameter stud that is allowed by code). The dimensions of the stud diameter were intentionally distorted in an attempt to make a balance between the shear area (cross section area) and the bending resistance (moment of inertia), since it was not known whether bending or shear would be the dominate parameter in the performance of the studs. Whether the scaling of the studs struck this balance is still not known; however, separate effects tests that are designed to investigate the stud/liner performance will determine if this goal was achieved.

The process for welding the studs to the liner is also different for the model as compared to a full-size containment. The studs used for the containment model were welded to the liner using capacitive discharge techniques, which resulted in fusion of the entire stud base to the liner. A full-size stud is attached to the liner by fillet welding around the base of the stud, which does not fuse the stud base to the liner. It is speculated that failure modes associated with the liner and the anchorage system-whether bending, pull-out, or shear-may be different between the model and full-size containment. The separate effects tests planned at Sandia will address this issue.

\subsubsection{Concrete}

Judging by the number of published articles and the vast differences in findings, it is difficult to scale concrete and have all of the salient material properties unaffected; the quantification of the changes of the material properties are the subjects of these many articles. There is no doubt that the properties of concrete do change with changes in the size of the aggregate. Two material properties that are of concern on this test and are generally agreed to change with scale are its tensile and shear strength. In a study that used the data from the containment model test, some of the scaling issues of material properties are presented. ${ }^{*}$ It was summarized in this work that the shear resistance of the concrete may be as much as $10 \%$

\footnotetext{
*H. P. Walther, Evaluation of Behavior and the Radial Shear Strength of a Reinforced Concrete Containment Structure, NUREG/CR-5674, SAND91-7058, Sandia National Laboratories, Albuquerque, New Mexico (to be published).
} 
lower in a full-size containment structure, but consideration should also be given to the greater dead load of the full-size structure that would tend to enhance the aggregate interlock and thus the shear capacity of a section.

The quantification of the effects of scaled concrete in a scaled structure is difficult to parametrize and the associated debates are not reiterated here. The intent of raising the issue here is to make the reader aware of the gamut of potential differences between the response of a scale model versus a full-size containment. But, as can be seen from this brief introduction to the concerns of concrete scaling, there are (many) competing factors.

\subsubsection{Leak Rate}

The leak rates measured during the testing of the containment model are difficult to scale/extrapolate to full size leakage behavior. The parameters that may affect leakage are difficult to parametrize in the context of extrapolating "scaled" leak rate phenomenon to "fullsize" leak rates. Some of the differences between the containment model and a full-size containment that have already been mentioned will also have an affect on containment leakage. The bond between the concrete and the reinforcing steel and the number of reinforcing bars can change the frequency of concrete cracks, their spacing, and thus the crack width. The surface roughness of the crack is influenced by the tensile strength and aggregate size of the concrete. The surface roughness of the crack path will affect the aerodynamic drag and thus the leak rate due to crack paths roughness and tortuosity. These basic changes, when taken into account with large dimensional changes in the model versus full-size containment (such as the length of the tear and the through wall thickness) have a dramatic affect on leakage rates. The scaling of these parameters and their cumulative quantitative affect on leak rate are not well understood.

\subsection{Trends In The Data}

With the many channels of strain data to sort through, a group of strain histograms were plotted to aid in understanding the trends in the data gathered during model testing. The tabulated results from the generation of the histograms are presented in Table 7. About $800^{*}$ strain readings were categorized into the strain levels (in percent strain) listed below. Obviously bad data were not included in the table or the subsequent histograms.

Rosette 61 (Channels 1521-1523), which was located near equipment hatch $\mathrm{A}$ and near where the liner eventually tore, had the smallest strain (Channel 1522) for the last 9 pressure steps and the high strain (Channel 1521) for the last four steps. The largest strains for 33 of 37 steps were recorded by two strain gages: from steps 4 to 10 and steps 15 to 33 by channel 784 , and steps 12 to 14 and steps 34 to step 38 by channel 1521 .

The number of strain gages with readings in the 1 to $3 \%$ strain range increased significantly in the final few pressure steps. At pressure step 30 there were 23 strain readings in this range, by step 36 there were 75 gages with readings in this range.

The trends for the smaller strains on the containment model are more difficult to generalize. As pressure was increased, there was a general increase in the number of gages reading negative strains in the -0.3 to $-5.0 \%$ strain ranges while the strain range of 0.0 to $-0.1 \%$

\footnotetext{
*Note that the strain reading from the three-arm rosettes were converted to principal strains and an angle. Therefore for the 480 channels used for the strain rosettes, only 320 strains sre reported.
} 
showed a steady decrease in the number of gages recording these values. Of the more negative ranges, -0.1 to $-5.0 \%$, a general increase in the number of gages was observed until a plateau of gages (15-17) was reached at 90 psig. Very few additional gages were added to these ranges until pressure step 21. The most significant increases in negative strains occurred in the -0.3 to $-0.5 \%$ strain range where at step 21,12 gages recorded strains in this range, while at step 36,25 gages were recording strains in this range.

A histogram of the data taken at step 37 is shown in Figure 62. The same strain ranges used in Table 7 are used in plotting Figure 62; although not noted on the abscissa a -0.1 and 0.1 strain levels exist. The height of the bars reflects the number of gages in the range that is represented by the bar width. Since this histogram reflects the strains in the model after depressurization the number of gages in the range of -0.1 to 0.0 and 0.0 to 0.1 have increased significantly from the preceding step.

A group of histograms is presented in Figure 63 that use the same ranges as shown in Table 7 and Figure 62 and includes steps 26 to 36 with step 26 being in the foreground. Although it is difficult to determine the exact number of strain readings in a range, this grouped histogram clearly shows the trends of the strains. The number of strain readings is dramatically decreasing in the 0.1 to $0.3 \%$ range while the ranges that span from 0.3 to $3 \%$ strain are showing significant increases in the number of gages recording values of this magnitude. 
Table 7.

Strain Levels From The High Pressure Test

Strain Ranges $(\%)$

\begin{tabular}{llllllllllllllll}
\hline-5.0 & -3.0 & -1.0 & -0.7 & -0.5 & -0.3 & -0.1 & 0.0 & 0.1 & 0.3 & 0.5 & 0.7 & 1.0 & 3.0 & 5.0
\end{tabular}

Pressure to to to to to to to to to to to to to to to

Highest/ Lowest/

\begin{tabular}{|c|c|c|c|c|c|c|c|c|c|c|c|c|c|c|c|c|c|c|c|}
\hline Step & (psig 2 & -3.0 & -1.0 & $=0.7$ & -0.5 & $=0.3$ & 0.1 & 0.0 & 0.1 & 0.3 & 0.5 & 0.7 & 1.0 & 3.0 & 5,0 & 11.0 & Channel \# & Channel \# & Average \\
\hline 37 & 0.22 & 0 & 1 & 0 & 4 & 1 & 14 & 180 & 310 & 101 & 58 & 36 & 41. & 39 & 9 & 2 & $7.038 / 1521$ & $-1.880 / 1522$ & 0.2463 \\
\hline 36 & 142.52 & 0 & 1 & 0 & 4 & 3 & 25 & 93 & 191 & 223 & 83 & 41 & 45 & 75 & 10 & 2 & $8.219 / 152.1$ & $-2.061 / 1522$ & 0.3644 \\
\hline 35 & 145.78 & 0 & 1 & 0 & 4 & 2. & 2.5 & 97 & 186 & 233 & 80 & 42 & 41 & 73 & 10 & 2 & $7.708 / 1521$ & $-1.927 / 1522$ & 0.3555 \\
\hline 34 & 142.63 & 0 & 1 & 0 & 4 & 1 & 25 & 101 & 192 & 250 & 72 & 44 & 44. & 51 & 9 & 2 & $6.295 / 1521$ & $-1.587 / 1522$ & 0.3149 \\
\hline 33 & 140.16 & 0 & 1 & 0 & 4 & 1 & 25 & 105 & 197 & 269 & 80 & 31 & 34 & 42 & 5 & 2 & $6.213 / 784$ & $-1.320 / 1522$ & 0.2714 \\
\hline 32 & 138.35 & 0 & 1 & 0 & 4 & 1 & 24 & 110 & 200 & 283 & 80 & 30 & 26 & 31 & 4 & 1 & $6.006 / 784$ & $-1.165 / 1522$ & 0.2417 \\
\hline 31 & 134.42 & 0 & 0 & 2 & 3 & 1 & 17 & 115 & 211 & 320 & 55 & 25 & 20 & 25 & 1 & 1 & $5.907 / 784$ & $-0.985 / 1522$ & 0.2077 \\
\hline 30 & $135 n^{n}$ & 0 & 0 & 2 & 3 & 1 & 17 & 116 & 209 & 326 & 53 & 26 & 18 & 23 & 1 & 1 & $5.750 / 784$ & $-0.957 / 1522$ & 0.2026 \\
\hline 29 & 132.4 & 0 & 0 & 2 & 1 & 3 & 16 & 115 & 222 & 342 & 39 & 23 & 11 & 20 & 1 & 1 & $5.041 / 784$ & $-0.816 / 1522$ & 0.1783 \\
\hline 28 & 130.19 & 0 & 0 & 2 & 1 & 3 & 16 & 111 & 239 & 343 & 45 & 9 & 8 & 17 & 1 & 1 & $5.067 / 784$ & $-0.819 / 1083$ & 0.162 .4 \\
\hline 27 & 127.84 & 0 & 0 & 1 & 3 & 3 & 15 & 115 & 245 & 343 & 40 & 5 & 10 & 15 & 1 & 0 & $4.950 / 784$ & $-0.852 / 1083$ & 0.1503 \\
\hline 26 & 125.60 & 0 & 1 & 1 & 2 & 3 & 14 & 115 & 256 & 336 & 37 & 7 & 9 & 14 & 1 & 0 & $4.950 / 784$ & $-2.111 / 2367$ & 0.1433 \\
\hline 25 & 125.82 & 1 & 0 & 1 & 2 & 2. & 15 & 116 & 258 & 336 & 35 & 6 & 10 & 13 & 1 & 0 & $3.907 / 784$ & $-4.508 / 2367$ & 0.1368 \\
\hline 24 & 122.97 & 0 & 1 & 1 & 2 & 3 & 14 & 115 & 269 & 334 & 30 & 8 & 5 & 13 & 1 & 0 & $3.586 / 784$ & $-1.062 / 2367$ & 0.1325 \\
\hline 23 & 123.28 & 0 & 1 & 1 & 2 & 3 & 14 & 115 & 269 & 334 & 30 & 9 & 4 & 13 & 1 & 0 & 3.1927784 & $-2.924 / 2367$ & 0.1283 \\
\hline 22 & 120.92 & 0 & 1 & 1 & 1 & 4 & 13 & 119 & 276 & 335 & 24 & 8 & 2 & 13 & 1 & 0 & $3.027 / 784$ & $-1.995 / 2367$ & 0.1237 \\
\hline 21 & 117.83 & 0 & 1 & 1 & 1 & 4 & 12 & 119 & 290 & 327 & 24 & 3 & 2 & 14 & 0 & 0 & $2.808 / 784$ & $-1.850 / 2367$ & 0.1164 \\
\hline 20 & 113.24 & 1 & 0 & 1 & 1 & 4 & 10 & 122 & 312 & 310 & 20 & 3 & 1 & 13 & 0 & 0 & $2.479 / 784$ & $-3.217 / 2367$ & 0.1050 \\
\hline 19 & 111.08 & u & 1 & 1 & 1 & 4 & 10 & 126 & 319 & 304 & 15 & 3 & 1 & 13 & 0 & 0 & $2.028 / 784$ & $-2.043 / 2367$ & 0.1021 \\
\hline 18 & 108.31 & 0 & 1 & 1 & 1 & 4 & 9 & 128 & 340 & 287 & 10 & 3 & 1 & 13 & 0 & 0 & $1.967 / 784$ & $-2.668 / 2367$ & 0.0965 \\
\hline 17 & 106.11 & 1 & 0 & 1 & 1 & 4 & 9 & 126 & 359 & 271. & 10 & 2 & 1 & 13 & 0 & 0 & $1.897 / 784$ & $-3.077 / 2367$ & 0.0927 \\
\hline 16 & 103.25 & 0 & 1 & 1 & 1 & 3 & 10 & 129 & 367 & 263 & 7 & 2 & 3 & 11 & 0 & 0 & $1.805 / 784$ & $-2.325 / 2367$ & 0.0894 \\
\hline 1.5 & 100.9 .2 & 0 & 1 & 1 & 1 & 3 & 10 & 128 & 391 & 240 & 9 & 1 & 2 & 11 & 0 & 0 & $1.732 / 784$ & $-2.361 / 2.367$ & 0.0857 \\
\hline 14 & 95.69 & 1 & 0 & 2 & 0 & 3 & 9 & 130 & 415 & 219 & 6 & 0 & 4 & 9 & 0 & 0 & $1.639 / 1521$ & $1-3.022 / 2367$ & 0.0766 \\
\hline 13 & 90.58 & 1 & 0 & 2 & 0 & 1 & 12 & 130 & 448 & 186 & 5 & 2 & 6 & 5 & 0 & 0 & $1.629 / 1521$ & $1-3.219 / 2367$ & 0.0688 \\
\hline 12 & 85.61 & 0 & 1 & 2 & 0 & 1 & 11 & 130 & 486 & 151 & 4 & 2 & 6 & 4 & 0 & 0 & $1.493 / 1521$ & $1-2.741 / 2367$ & 0.0623 \\
\hline 11 & 80.16 & 0 & 1 & 2 & 0 & J & 8 & 130 & 517 & 124 & 5 & 3 & 7 & 0 & 0 & 0 & $0.914 / 329$ & $-2.757 / 2367$ & 0.0544 \\
\hline 10 & 74.16 & 0 & 0 & 2 & 0 & 1 & 6 & 130 & 552 & 94 & 6 & 5 & 2 & 0 & 0 & 0 & $0.874 / 784$ & $-0.806 / 1083$ & 0.0508 \\
\hline 9 & 69.32 & 0 & 0 & 2. & 0 & 1 & 5 & 132 & 588 & 57 & 8 & 4 & 1 & 0 & 0 & 0 & $0.776 / 784$ & $-0.833 / 1083$ & 0.0449 \\
\hline 8 & 64.20 & 0 & 0 & 2 & 0 & 1 & 3 & 133 & 612 & 40 & 6 & 1 & 0 & 0 & 0 & 0 & $0.642 / 784$ & $-0.837 / 1083$ & 0.0396 \\
\hline 7 & 59.57 & 0 & 0 & 1 & 0 & 1 & 3 & 137 & 623 & 29 & 3 & 1 & 0 & 0 & 0 & 0 & $0.578 / 784$ & $-0.737 / 2062$ & 0.0357 \\
\hline 6 & 54.50 & 0 & 0 & 1 & 0 & 1 & 3 & 138 & 632 & 19 & 4 & 0 & 0 & 0 & 0 & 0 & $0.465 / 784$ & $-0.746 / 2062$ & 0.0313 \\
\hline 5 & 49.16 & 0 & 0 & 1 & 0 & 1 & 3 & 146 & 628 & 18 & 1 & 0 & 0 & 0 & 0 & 0 & $0.438 / 784$ & $-0.747 / 2062$ & 0.0277 \\
\hline 4 & 39.42 & 0 & 1 & 1 & 0 & 1 & 2 & 153 & 625 & 15 & 0 & 0 & 0 & 0 & 0 & 0 & $0.288 / 784$ & $-1.161 / 1123$ & 0.0204 \\
\hline 3 & 29.57 & 0 & 0 & 1 & 0 & 1 & 2 & 163 & 620 & 11 & 0 & 0 & 0 & 0 & 0 & 0 & $0.202 / 325$ & $-0.736 / 2062$ & 0.0154 \\
\hline 2 & 19.55 & 0 & 0 & 1 & 0 & 1 & 2 & 173 & 614 & 7 & 0 & 0 & 0 & 0 & 0 & 0 & $0.162 / 323$ & $-0.769 / 2062$ & 0.0094 \\
\hline 1 & 9.89 & 0 & 0 & 1 & 0 & 1 & 3 & 207 & 583 & 3 & 0 & 0 & 0 & 0 & 0 & 0 & $0.152 / 1865$ & $5-0.778 / 2062$ & 0.0031 \\
\hline
\end{tabular}




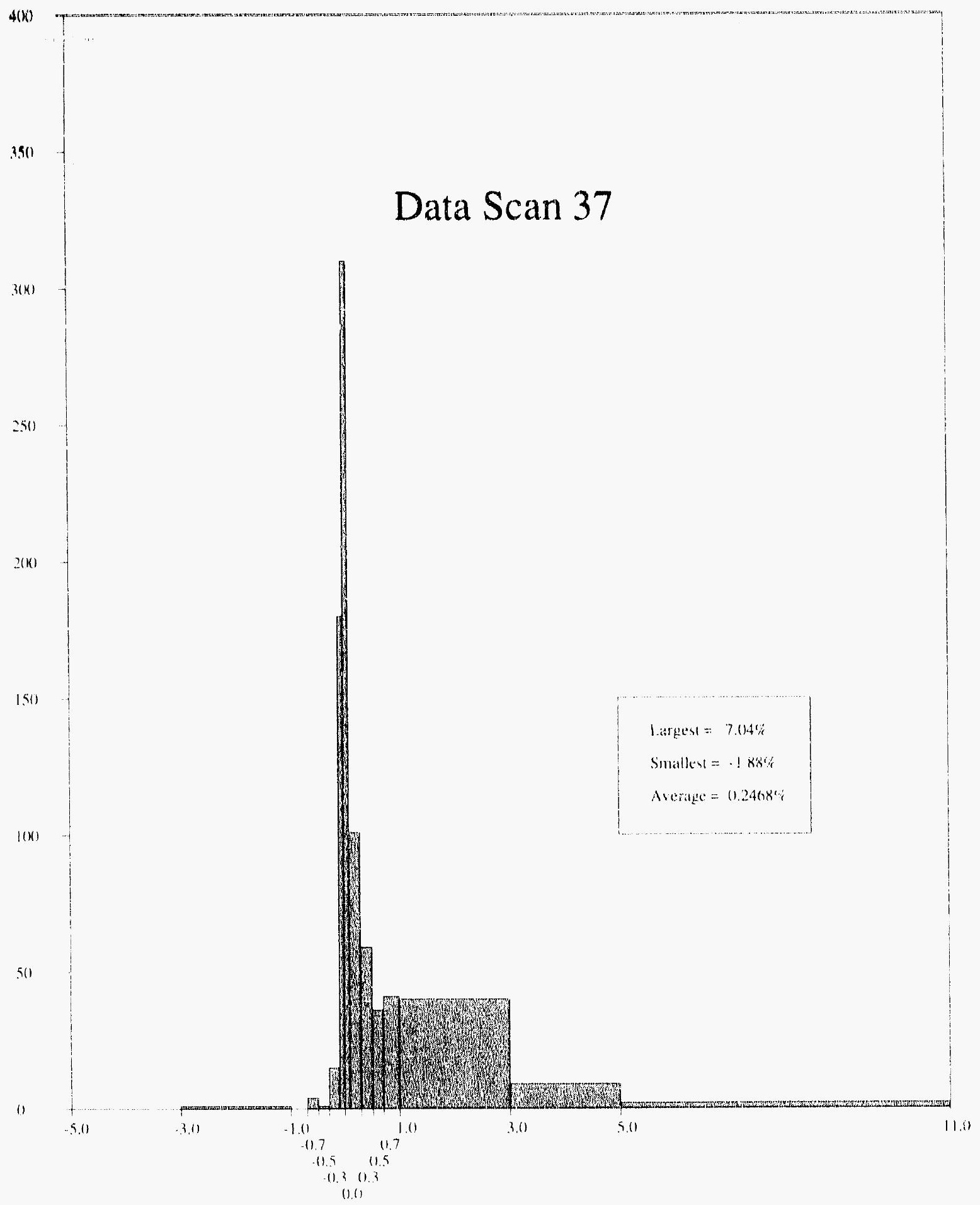

Figure 62. Histogram of Strain Ranges for Data Step 37. 


$$
1
$$




\subsection{Test Determined Strain Concentration Factors}

As was mentioned in section 7 , there was significant variation of the strains measured about the containment model especially in the liner. To explore this further, a brief comparison of the strains about the midheight or elevation $20.125 \mathrm{ft}$ of the model are compared to the freefield liner strains in Table 8. In interpreting the experimentally derived strain concentration factors the reader is cautioned to the limitations of the strain gage technique as introduced in section 7.8 .2 since there are physical limitations of the gages ability to measure peak strains. Six strain gages were used to determine an average free-field strain at cylinder mid-height (channels 1104, 1107, 1109, 1606, 1685, and 1729), and six gages were used to determine the average free-field response at elevation $20.125 \mathrm{ft}$ (channels 174, 1748, 1780, 1821, 1827, and 1849).

Table 8.

Experimentally Measured Strain Concentration Factors for the Containment Liner.

\begin{tabular}{|c|c|c|c|}
\hline & Channel & Strain at & (Avg FF $1.69 \%)$ \\
\hline Location & Number & $142.52 \mathrm{psig}$ & Concentration Factor \\
\hline \multirow{2}{*}{ Equipment hatch $\mathrm{A}$} & 1521 & 8.219 & 4.87 \\
\hline & 1162 & 2.236 & 1.32 \\
\hline Equipment hatch B & 1149 & 1.842 & 1.09 \\
\hline \multirow[t]{2}{*}{ Personnel Air Lock A } & 1565 & 2.833 & 1.68 \\
\hline & 1146 & 1.495 & 0.89 \\
\hline \multirow[t]{2}{*}{ Personnel Air Lock B } & 1707 & 4.725 & 2.80 \\
\hline & 1704 & 1.649 & 0.98 \\
\hline Piping Cluster & 1423 & 4.875 & 2.89 \\
\hline Instrumentation Feed ? & 784 & 5.799 & 3.43 \\
\hline
\end{tabular}

\begin{tabular}{llll}
\hline & Channel & Strain at & (Avg FF 0.63\%) \\
Location & Number & 142.52 psig & Concentration Factor \\
Penetration R-2 & 329 & 4.246 & 6.74 \\
& 1805 & 4.153 & 6.59 \\
& 328 & 3.894 & 6.18 \\
& 327 & 3.776 & 5.99 \\
& 325 & 3.746 & 5.94 \\
& 324 & 3.746 & 5.94 \\
& 323 & 3.485 & 5.53 \\
& 326 & 3.404 & 5.40 \\
& 322 & 2.961 & 4.70 \\
Penetration R-1 & 1865 & 2.392 & 3.79 \\
& 1805 & 4.153 & 6.59
\end{tabular}




$\begin{array}{llll} & \begin{array}{l}\text { Channel } \\ \text { Lumber }\end{array} & \begin{array}{l}\text { Strain at } \\ 127.84 \text { psig }\end{array} & \begin{array}{l}\text { (Avg FF 0.41\%) } \\ \text { Concentration Factor }\end{array} \\ \text { Location } & 1521 & 2.853 & 7.01 \\ \text { Equipment hatch A } & 1162 & 0.893 & 2.19 \\ & 11.49 & 0.701 & 1.72 \\ \text { Equipment hatch B } & 1565 & 1.607 & 3.95 \\ \text { Personnel Air Lock A } & 1146 & & \\ & 1707 & 1.968 & 4.83 \\ \text { Personnel Air Lock B } & 1704 & & \\ \text { Piping Cluster } & 1423 & 1.343 & 3.30 \\ \text { Instrumentation Feed Through } 784 & 4.950 & 12.16\end{array}$

\begin{tabular}{llll}
\hline & Channel & Strain at & (Avg FF 0.23\%) \\
Location & Number & $\frac{127.84}{\text { psig }}$ & Concentration Factor \\
\cline { 2 - 4 } Penetration R-2 & 329 & 2.219 & 9.81 \\
& 328 & 2.024 & 8.95 \\
& 327 & 2.004 & 8.86 \\
& 323 & 1.929 & 8.53 \\
& 325 & 1.906 & 8.43 \\
& 324 & 1.905 & 8.43 \\
& 326 & 1.654 & 7.31 \\
Penetration R-1 & 322 & 1.607 & 3.95 \\
& 1865 & 1.496 & 6.62 \\
& 1805 & 1.664 & 7.36
\end{tabular}

Some judgement should be exercised in using these experimentally measured strain concentrations due to the limits of the strain gage sensors in measuring peak strains and their direct dependence on the averaging of six gages to obtain the "free-field" response. To develop Table 8, the top forty strains were extracted from the strain data at each pressure step, which were then divided by the average strain at the appropriate elevation. At the first pressure step the top forty strains were much larger than the free field strains; resulting in large strain concentration factors: near penetration R-2, 5 gages had strain concentration factors in the 6 to 11 range. At the next pressure step the largest strain concentration factor was 5.8. The peak strain concentration factors continued to increase until many gages reached a peak at a pressure of 128 psig at pressure step 26, after which the gages that recorded the highest strains also recorded a decreasing strain concentration factor.

The average free-field strains used to calculate the strain concentration factors were nearly identical (1:1) up to step 14,95 psig; at subsequent pressure steps the mid-height free-field strain increased at a faster rate than the free-field strains at $20.125 \mathrm{ft}$ due to yielding of the cylinder. At pressure step 36,142 psig, the ratio of the average free-field strains at elevation $20.125 \mathrm{ft}$ to mid-height were $1: 2.7$.

Note that strain gage 784 recorded high strains for several of the pressure steps (see Table 7) and likewise showed a large strain concentration factor. Strain gage 784 was located on a seismic reinforcing bar near where the major liner tear occurred that caused the termination of the containment model test. The peak strain concentration factor recorded anywhere in the model was measured at pressure step 26,125 psig, by channel 784 , which was a factor of 12.98 . 


\section{ANALYSES}

Performing an adequate analysis of a containment model subjected to overpressurization was the motivation behind this testing program. This experiment is seen as a challenge to the analyst and the analyst's tools. It has always been viewed as impractical in terms of cost and time to test every containment design to every accident scenario. The intent of the program has been to generate data that can be used to quaiify analytical tools. These analytical tools can then be used to extrapolate reasonably to full-size containment buildings subject to hypothesized severe accidents. To this end, Sandia as well as several other organizations in the U.S. and Europe conducted pretest analysis of the containment building. The groups that participated or contributed to this round-robin exercise published in the report on this activity [30] are

- Sandia National Laboratories (USA)

- Argonne National Laboratory (USA)

- Electric Power Research Institute (USA)

- Commissariat a L'Energie Atomique (France)

- HM Nuclear Installations Inspectorate (UK)

- Comitato Nazionale per la ricerca e per lo sviluppo dell'Energia Nucleare e delle Energie Alternative, ENEA (Italy)

- U.K. Atomic Energy Authority, Safety and Reliability Directorate (UK)

- Gesellschaft fuer Reaktorsicherheit, GRS (FRG)

- Brookhaven National Laboratory (USA)

- Central Electricity Generating Board (UK)

Each group performing an analysis was given detailed construction drawings and specifications and actual material properties of the construction materials as they became available. Each organization worked independently using an analytical method of their choosing. The results of these pretest analyses are presented in Reference 30. Posttest evaluations and comparisons between experimental and analytical results can be found in Reference 36. Further analyses that were performed by Sandia can be found in References 30-33.

The presentation of the analyses and the subsequent comparisons to the experimentally derived data serve to further the understanding of the response of the containment model subjected to overpressurization.

\footnotetext{
* In addition to these ten, Taiwan Electric Power Company and Japan Atomic Energy Research Institute have reached agreements with the NRC that call for information exchange on the containment integrity programs. However they did not submit results to Sandia prior to test and were, therefore, nct included in the round-robin report.
} 


\section{REFERENCES}

[1] D. S. Horschel, Design, Construction, and Instrumentation of a 1:6-Scale Reinforced-Concrete Containment Model, NUREG/CR-5083, SAND88-0030, Sandia National Laboratories, Albuquerque, New Mexico (August 1988).

[2] D. S. Horschel, "Construction of a 1:6-Scale Concrete Containment," Nuclear Engineering and Design, Volume 104, pp. 341-347 (1987).

[3] J. J. Ucciferro, A. J. Hulshizer, and G. E. Gray, "Design and Construction of a 1:6 Scale LWR Reinforced Concrete Containment Model," in Proceedings of the Americal Power Conference, Chicago, Illinois (April 1986).

[4] D. S. Horschel, "The Testing of a 1:6-Scale Reinforced-Concrete Containment Model," in Proceedings of the American Power Conference, Volume 49, Chicago, Illinois (April 1987).

[5] J. J. Ucciferro and D. S. Horschel, "Design and Construction of a Large Reinforced-Concrete Containment Building to be Tested to Failure," in Transactions of the $9^{\text {th }}$ International Conference on Structural Mechanics in Reactor Technology, Volume J, pp. 209-214, Lausanne, Switzerland (August 1987).

[6] D. S. Horschel and L. D. Lambert, "Plans and Preparation for the Destructive Testing of a Reinforced-Concrete Containment Model," in Transactions of the $9^{\text {th }}$ International Conference on Structural Mechanics in Reactor Technology, Volume J, pp. 203-208, Lausanne, Switzerland (August 1987).

[7] D. S. Horschel and T. E. Blejwas, "An Analytical Investigation of the Response of Steel Containment Models to Internal Pressurization," in Transactions of the $7^{\text {th }}$ International Conference on Structural Mechanics in Reactor Technology, Volume J6/4, Chicago, Illinois (August 1983).

[8] D. S. Horschel and D. B. Clauss, "The Response of Steel Containment Models to Internal Pressurization," in Structural Engineering in Nuclear Facilities, J. J. Ucciferro, Ed., Raleigh, North Carolina (September 1984).

[9] T. E. Blejwas and D. S. Horschel, Containment Integrity Program Progress Report April 1983 to December 1984, NUREG/CR-3412/Vol. 2, SAND83-1482, Sandia National Laboratories, Albuquerque, New Mexico (December 1986).

[10] R. T. Reese and D. S. Horschel, Design and Fabrication of a 1/8-Scale Steel Contairment Model, NUREG/CR-3647, SAND84-0048, Sandia National Laboratories, Albuquerque, New Mexico (February 1985).

[11] D. B. Clauss, Pretest Predictions for the Response of a 1:8-Scale Steel LWR Containment Building Model to Static Overpressurization, NUREG/CR-4137, SAND85-0175, Sandia National Laboratories, Albuquerque, New Mexico (June 1985).

[12] D. B. Clauss, Comparison of Analytical Predictions and Experimental Results for a 1:8-Scale Steel Containment Model Pressurized to Failure, NUREG/CR-4209, SAND85-0679, Sandia National Laboratories, Albuquerque, New Mexico (July 1985). 
[13] L. N. Koenig, Experimental Results for a 1:8-Scale Steel Model Nuclear Power Plant Containment Pressurized to Failure, NUREG/CR-4216, SAND85-0790, Sandia National Laboratories, Albuquerque, New Mexico (December 1986).

[14] D. B. Clauss, D. S. Horschel, and T. E. Blejwas, "Insights into the Behavior of LWR Steel Containment Buildings During Severe Accidents," Nuclear Engineering and Design, Volume 100, pages 189-204 (1987).

[15] W. Wassef, L. Greimann, F. Fanous, M. B. Parks, "Analysis of Bellows Expansion Joints in Containment Buildings," in Transactions of the $11^{\text {th }}$ International Conference on Structural Mechanics in Reactor Technology, Volume F, pp. 79-84, Tokyo, Japan (August 1991).

[16] D. B. Clauss, "An Evaluation of the Leakage Potential of a Personnel Airlock Subject to Severe Accident Loads," in Transactions of the $9^{\text {th }}$ International Conference on Structural Mechanics in Reactor Technology, Volume J, pp. 147-152, Lusarne, Switzerland (August 1987).

[17] C. V. Subramanian, Integrity of Containment Penetrations Under Severe Accident Conditions FY84 Annual Report, NUREG/CR-4119, SAND85-0016, Sandia National Laboratories, Albuquerque, New Mexico (June 1985).

[18] R. F. Kulak, B. J. Hsieh, J. E. Ash, J. M. Kennedy, G. A. McLennan, and Y. C. Pan, Structural Response of Large Penetrations and Closures for Containment Vessels Subjected to Loadings Beyond Design Basis, NUREG/CR-4064, SAND847177, Sandia National Laboratories, Albuquerque, New Mexico (February 1985).

[19] T. R. Bump, R. W. Seidensticker, M. A. Shackelford, V. K. Gambhir, and G. L. McLennan, Characterization of Nuclear Reactor Containment Penetrations Preliminary Report, NUREG/CR-3855, SAND84-7139, Sandia National Laboratories, Albuquerque, New Mexico (June 1984).

[20] M. A. Shackelford, T. R. Bump, and R. W. Seidensticker, Characterization of Nuclear Reactor Containment Penetrations-Final Report, NUREG/CR-3855, SAND84-7180, Sandia National Laboratories, Albuquerque, New Mexico (February 1985).

[21] W. Sebrell, The Potential for Containment Leak Paths Through Electrical Penetration Assemblies Under Severe Accident Conditions, NUREG/CR-3234, SAND83-0538, Sandia National Laboratories, Albuquerque, New Mexico (July 1983).

[22] D. B. Clauss, Severe Accident Testing of Electrical Penetration Assemblies, NUREG/CR-5334, SAND89-0327, Sandia National Laboratories, Albuquerque, New Mexico (November 1989).

[23] J. D. Keck and F. V. Thome, "Leak Behavior Through EPAs Under Severe Accident Conditions," in Proceedings of the Third Workshop on Containment Integrity, T. Molina and R. Cochrell, Eds. NUREG/CP-0076, SAND86-0618, Sandia National Laboratories, Albuquerque, New Mexico (August 1986).

124] T. L. Bridges, Containment Penetration Elastomer Seal Leak Rate Tests, NUREG/CR-4944, SAND87-7118, Sandia National Laboratories, Albuquerque, New Mexico (July 1987). 
[25] D. A. Brinson and G. H. Graves, Evaluation of Seals for Mechanical Penetrations of Containment Buildings, NUREG/CR-5096, SAND88-7016, Sandia National Laboratories, Albuquerque, New Mexico (August 1988).

[26] L. N. Koenig, "Leakage Potential Through Mechanical Penetrations in a Severe Accident Environment," in Proceedings of the Third Workshop on Containment Integrity, T. Molina and R. Cochrell, Eds. NUREG/CP-0076, SAND86-0618, Sandia National Laboratories, Albuquerque, New Mexico (August 1986).

[27] D. F. Meinheit, S. R. Burns, J. Stecich, R. J. Isberner, and J. R. Janney, Support Tests for Sandia National Laboratories Design of LWR Reinforced Concrete Containment Model, WJE No. 841211, Wiss, Janney, Elstner Associates, Inc., Littleton, Colorado (July 1985).

[28] G. A. Knorovsky, P. W. Hatch, and M. R. Gutierrez, Evaluation of Materials of Construction for the Reinforced Concrete Reactor Containment Model, NUREG/CR5099, SAND88-0052, Sandia National Laboratories, Albuquerque, New Mexico (September 1988).

[29] R. P. Toth, The Search for a High Elongation Strain Gage System, NUREG/CR3222, SAND83-0549, Sandia National Laboratories, Albuquerque, New Mexico (September 1983).

[30] D. B. Clauss, Ed., Round-Robin Pretest Analyses of a 1:6-Scale Reinforced Concrete Containment Model Subject to Static Internal Pressurization, NUREG/CR4913, SAND87-0891, Sandia National Laboratories, Albuquerque, New Mexico (May 1987).

131] J. R. Weatherby, Axisymmetric Analysis of a 1:6-Scale Reinforced Concrete Containment Building Using a Distributed Cracking Model for the Concrete, NUREG/CR-4969, SAND87-1670, Sandia National Laboratories, Albuquerque, New Mexico (September 1987).

132] J. R. Weatherby and D. B. Clauss, "Analytical Predictions for the Performance of the Reinforced Concrete Containment Model Subject to Overpressurization," In Transactions of the $9^{\text {th }}$ International Conference on Structural Mechanics in Reactor Technology, Volume J, pp. 215-220, Lusanne Switzerland (August 1987).

[33] J. R. Weatherby, "Posttest Analysis of a Piping Penetration in a 1:6-Scale Model of a Reinforced Concrete Containment Building," in Proceedings of the Fourth Workshop on Containment integrity, Eds. R. Cochrell and T. Molina, NUREG/CP0095, SAND88-1836, Sandia National Laboratories, Albuquerque, NM (November 1988).

[34] ANSI/ANS 56.8-1981, "Containment System Leakage Testing Requirements," published by the American Nuclear Society through American National Standards Institute (1981).

[35] Strain Gage Selection Criteria, Procedures, Recommendations, Tech Note TN-505, Micromeasurements Group, Inc., Raleigh, NC, page 6 (1983).

[36] D. B. Clauss, Ed., Round-Robin Pretest Analyses of a 1:6-Scale Reinforced Concrete Containment Model Pressurized to Failure: Posttest Evaluations, NUREG/CR-5341, SAND89-0349, Sandia National Laboratories, Albuquerque, New Mexico (1989). 
[37] L. A. Lutz et al., "Steel Reinforcement Properties and Availability," in ACI Manual of Concrete Practice 1979. Part 2, ACI 439.2R-77, American Concrete Institute, Detroit, Michigan (1979).

[38] N. W. Hanson, J.J. Roller, D. M. Schultz, A. Azizinamini, "Concrete Containment Tests, Phase 3: Structural Elements With Penetration Sleeves," NP-6259-M Research Project 2172-2, Project Manager: H.T. Tang, Electric Power Research Institute, Palo Alto, CA, (March 1989). 


\section{APPENDIX A \\ CRACK MAPPING RESULTS \\ FROM THE \\ STRUCTURAL INTEGRITY TEST}

by

Doug Brosseau 


\title{
STRUCTURAL INTEGRITY TEST' (SIT)
}

\author{
Crack Mapping Activities
}

\section{A-1 General}

All procedures and personnel assignments for SIT testing were outlined before testing in the Test Plan (Rev. 2) and Safe Operating Procedure - SOP (Rev. 1) established for all containment model testing activities. Additional detailed instructions and schedules were issued before testing to ensure that enough personnel were available and last-minute details were clear to all involved. A detailed test prerequisite checklist was reviewed to ensure that all pretest activities were satisfactorily completed. Data sheets to document cracks on the model were dev'loped to match crack mapping grid patterns marked on selected areas of the containment model. These sheets included provisions to record the mapper, date, time, containment pres' ire, and area mapped.

\section{A.2 Pretest Activities}

On Monday, July 6, 1987, a short crack mapping orientation and training session was held at the test site. Ted Brown, of Wiss, Janney, Elstner, Associates, briefly explained the objectives and methods involved as are typically used in industry in accordance with ASME Section III, Division 2, CC 6000, specifications. Use of the optical comparators was explained and demonstrated. Logistical details to minimize the time to map cracks at the model while under pressure were discussed. Finally, the initial set of baseline cracks at 0 psig was mapped by the personnel who would act as mappers during the actual SIT. The following figures are copies of the actual field crack map data sheets. Any cracks 10 mils or greater in width are indicated on the maps.

An attempt was made to use the same mappers in each gridded area for consistency and familiarity with the progressive development of crack patterns. However, because of the week-long, multiple-shift srhedule and the use of personnel who also filled other testing roles, it was not always possible to have the same people map a given area through the entire pressure range of the SIT. Doug Brosseau reviewed the mapping books at each pressure level to ensure that all information was consistently acquired and to compensate for any lack of consistency from shifting personnel assignments.

\section{A-3 Crack Mapping Chronology}

The following is a brief description of the sequence of SIT activities with an emphasis on the crack mapping phases. Throughout the SIT, each set of maps was copied so that the next set at the higher pressure level would include those cracks noted at the previous pressure level. As crack widths changed, appropriate notes were made. Where cracks changed at 0 psig, this was noted. 
Tuesday, $7 / 7 / 87$

8:30 a.m. Model at 5 psig

9:20 a.m. Model at 13 psig; strain gage problems noted

11:15 a.m. Crack mapping initiated. All cracks were mapped.

12:15 p.m. Mapping at 13 psig completed

3.55 p.m. Testing was terminated; eventually, a bad cable connector on the data extender was located and repaired.

Wednesday, $7 / 8 / 87$

11:00 a.m. New 0 psig data scan was taken

12:00 p.m. Data scan at 5 psig

12:30 p.m. Mapping at 13 psig; map cracking was not repeated

3.35 p.m. Crack mapping at 22 psig was authorized.

4:10 p.m. Crack mapping complete

$6: 25 \mathrm{p} . \mathrm{m} . \quad$ Mapping at 34 psig initiated.

7:00 p.m. Mapping complete

8:54 p.m. Mapping at $46 \mathrm{psig}$ (design pressure) was initiated. Mapping at all pressures occurred only after the model had been allowed to stabilize at pressure for an hour.

9:45 p.m. Mapping complete

11:28 p.m. At 53 psig (1.15 times design pressure), crack mapping commenced

Thursday, 7/9/87

12:20 a.m. 53 psig crack mapping complete.

11:00 a.m. After an overnight hold at 46 psig, another set of crack maps was taken at a slightly reduced pressure of approximately $45 \mathrm{psig}$.

8:50 p.m. Pressure reduced to 0 psig

Friday, 7/10/87

1:30 p.m. A final set of maps of model crack status following the SIT was documented. This last set of maps represents the as-left condition of the gridded areas of the model following completion of the SIT test. 


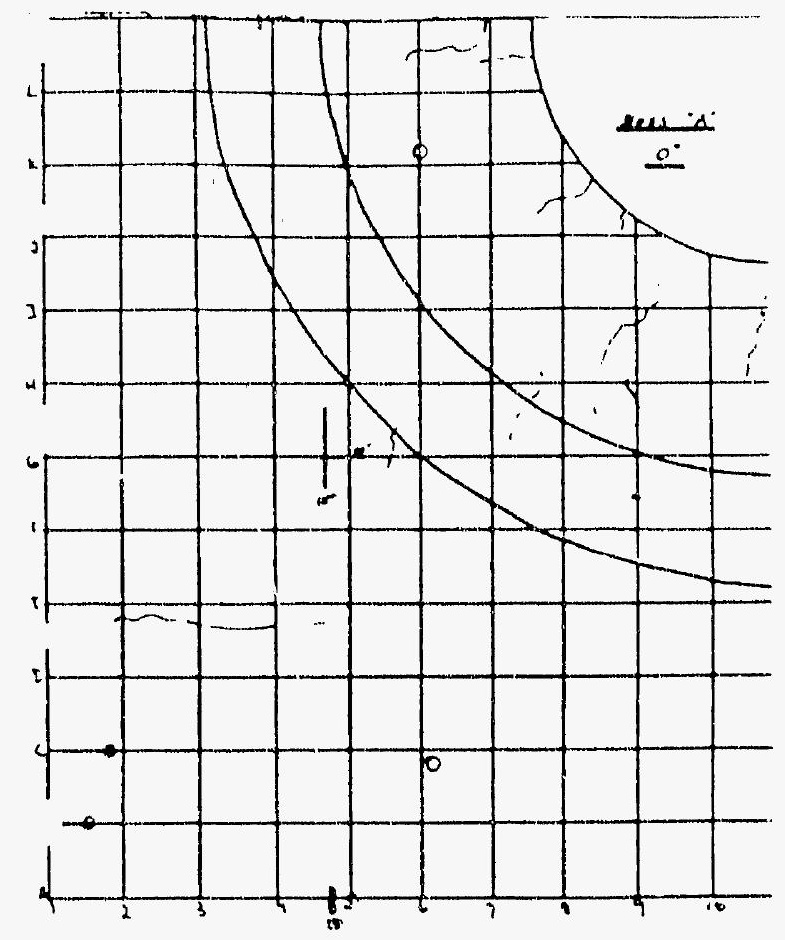

Figure A-1. Boss A, $0^{\circ}$; 0 psig; Date-6 July 1987; Time-1635; Mapper-Eyers.

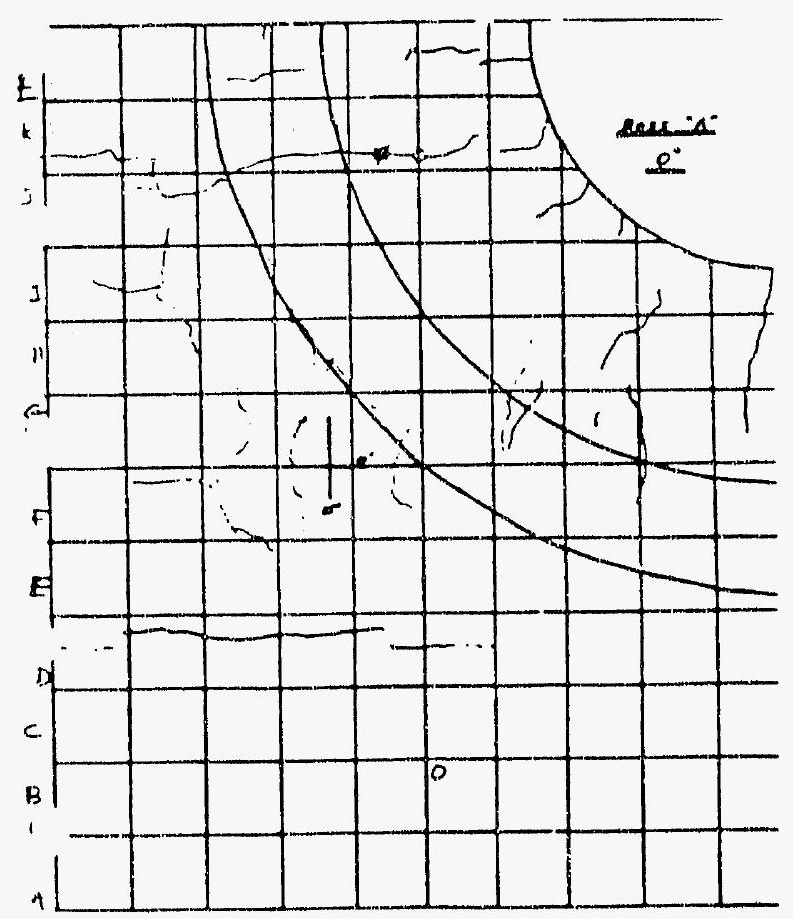

Figure A-2. Boss A, $0^{\circ}$; 13 psig; Date-7 July 1987; Time-1135; Mapper-Eyers. 


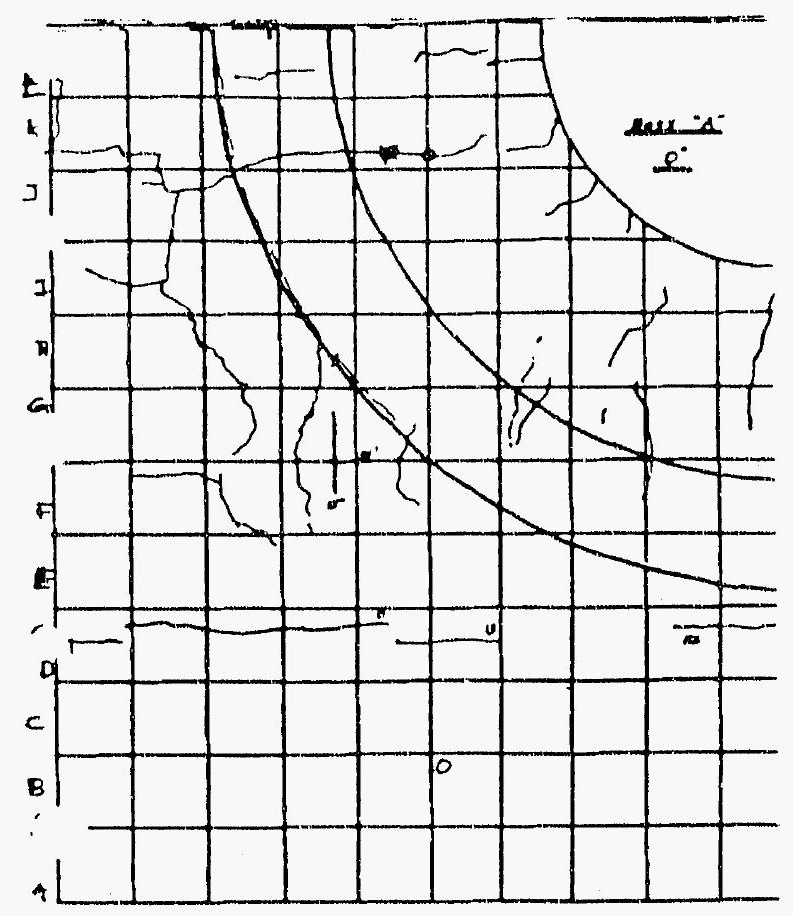

Figure A-3. Boss A, $0^{\circ} ; 22$ psig; Date-8 July 1987; Time-1550; Mapper-Eyers.

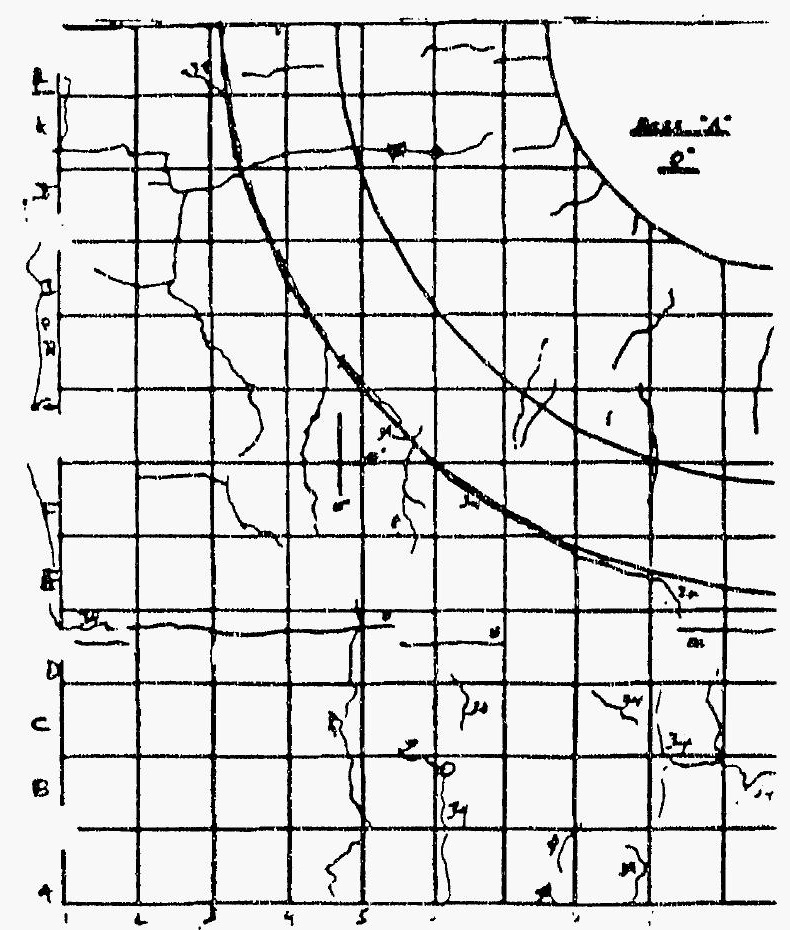

Figure A-4. Boss A, $0^{\circ}$; 34 psig; Date-8 July 1987; Time-0630; Mapper-Eyers. 


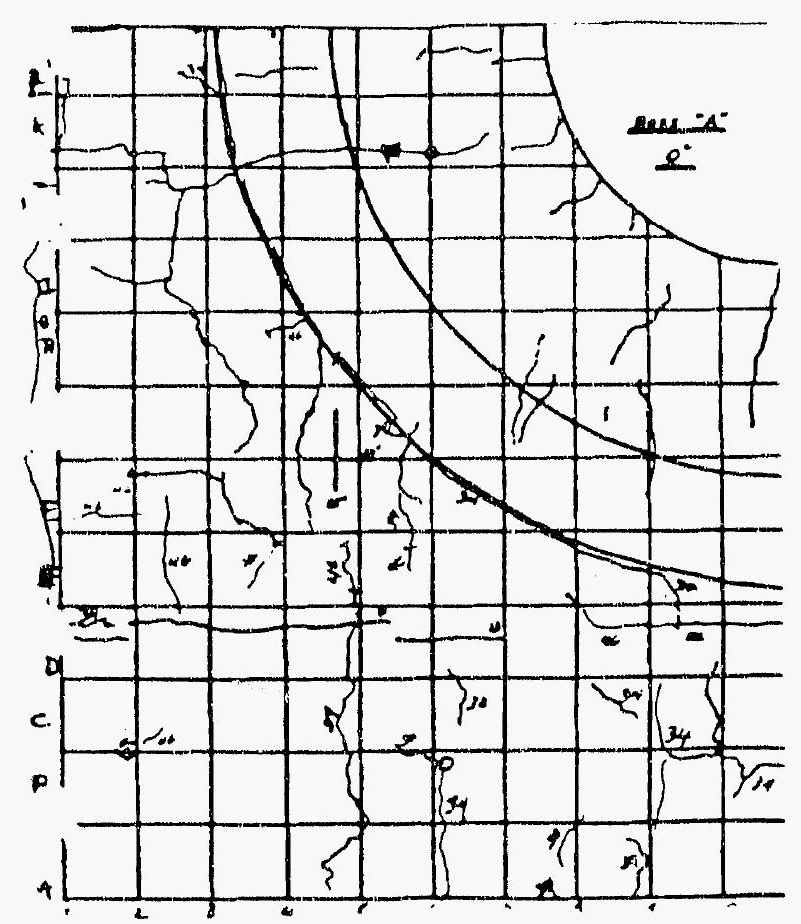

Figure A-5. Boss A, $0^{\circ}$; 46 psig; Date-8 July 1987; Time-1110; Mapper-Eyers.

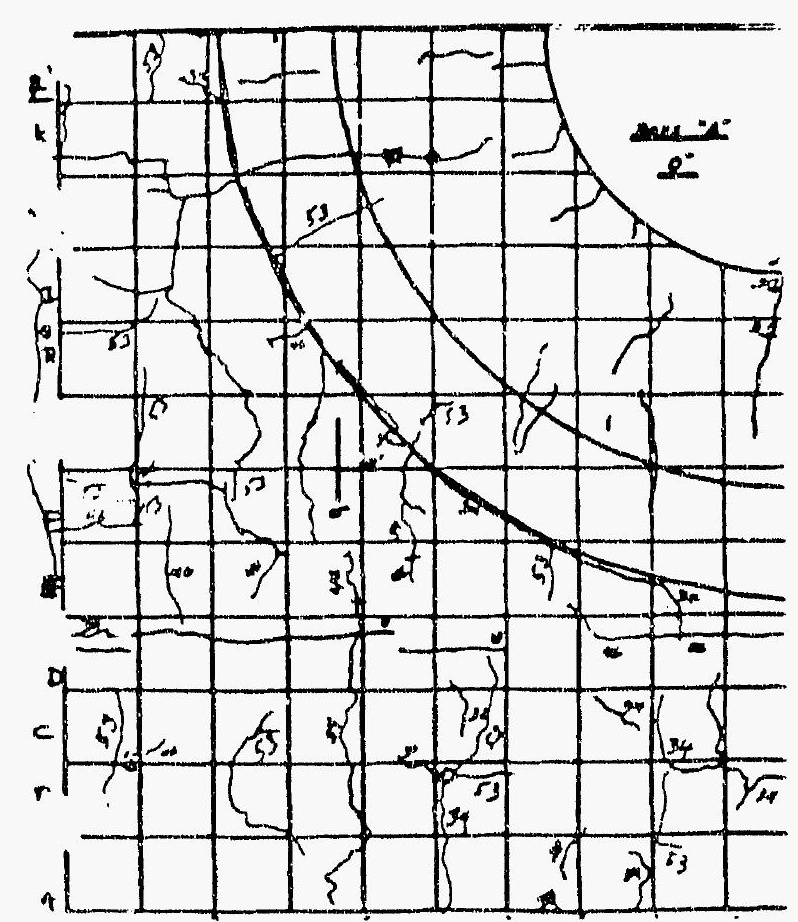

Figure A-6. Boss A, 0; 53 psig; Date-8 July 1987; Time-1145; Mapper-Eyers. 


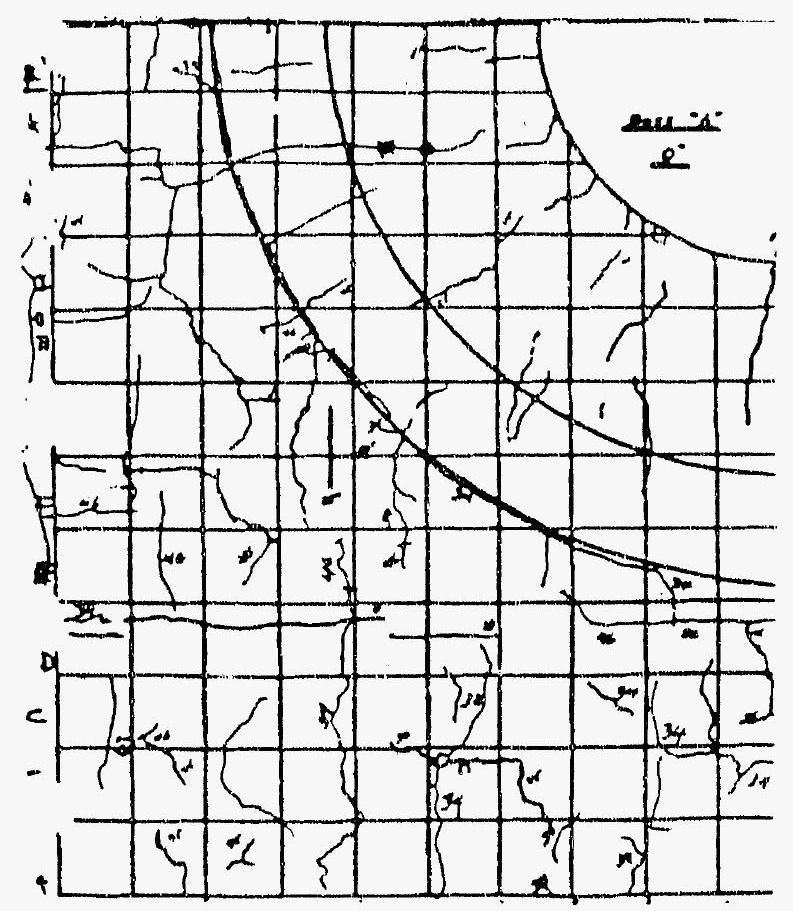

Figure A-7. Boss A, $0^{\circ}$; 46 psig; Date-9 July 1987; Time-1110; Mapper-Eyers/Jinzo.

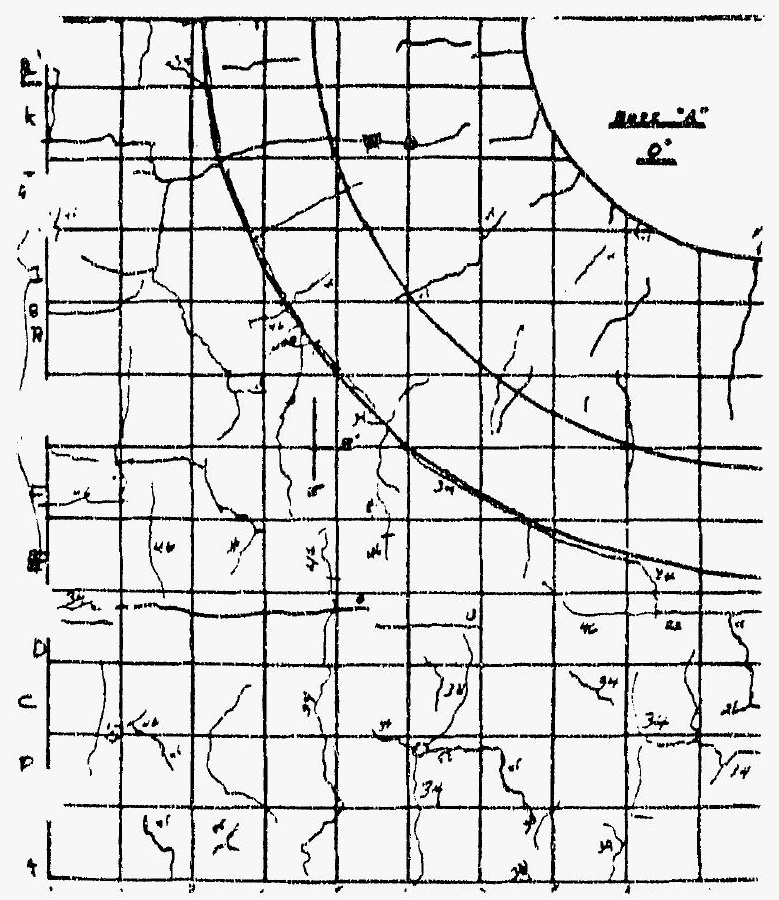

Figure A-8. Boss A, $0^{\circ}$; 0 psig; Date-10 July 1987; Time-1300; Mapper-Eyers. 


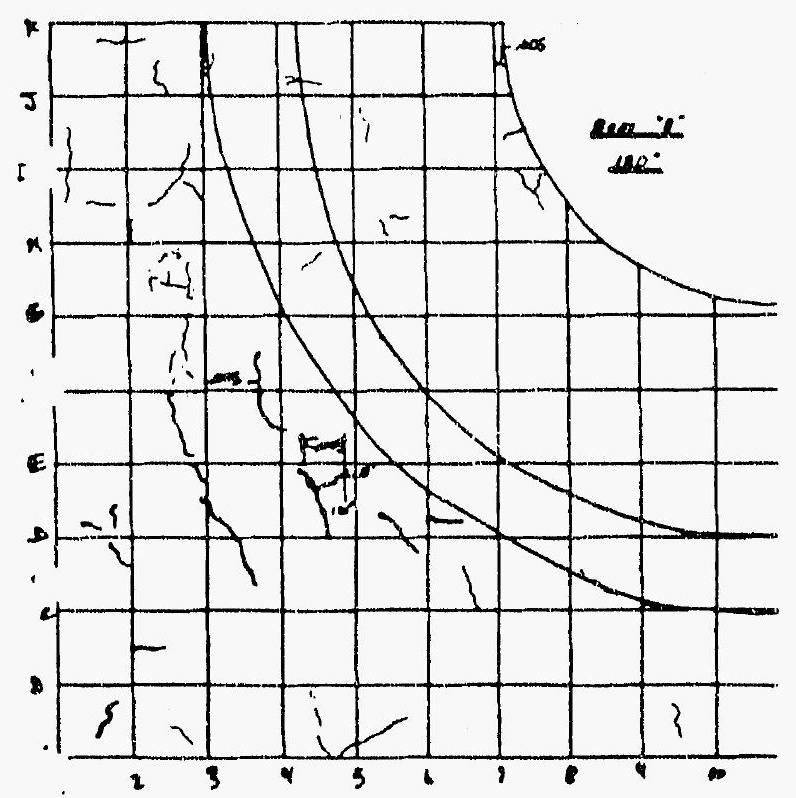

Figure A-9. Boss B, $180^{\circ}$; 0 psig; Date-6 July 1987; Time-1600; Mapper-Weatherby and Clauss.

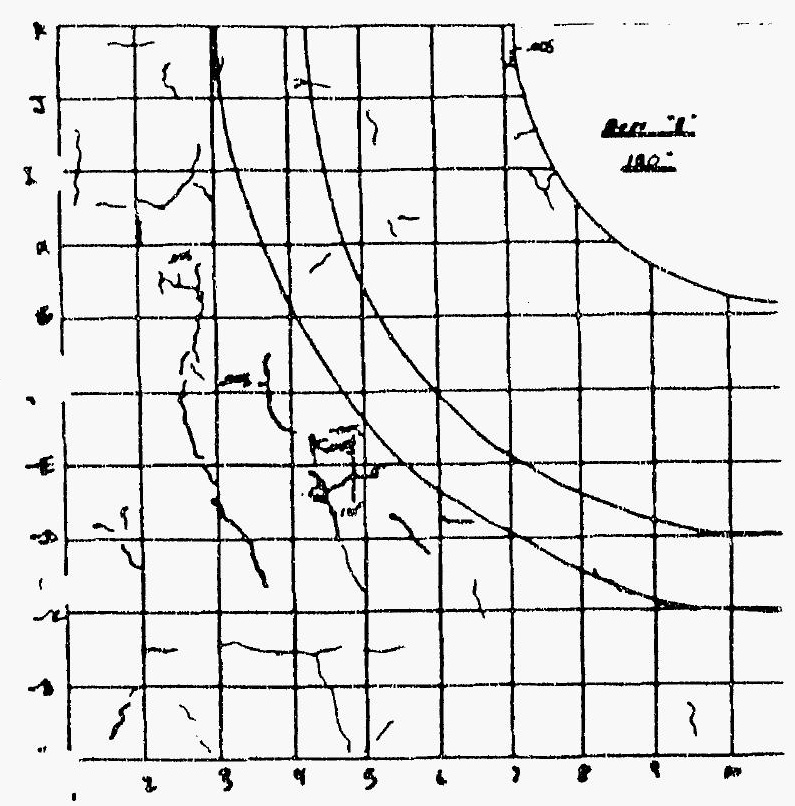

Figure A-10. Boss B, 180 13 psig; Date-7 July 1987; Time-1120; Mapper-Weatherby. 


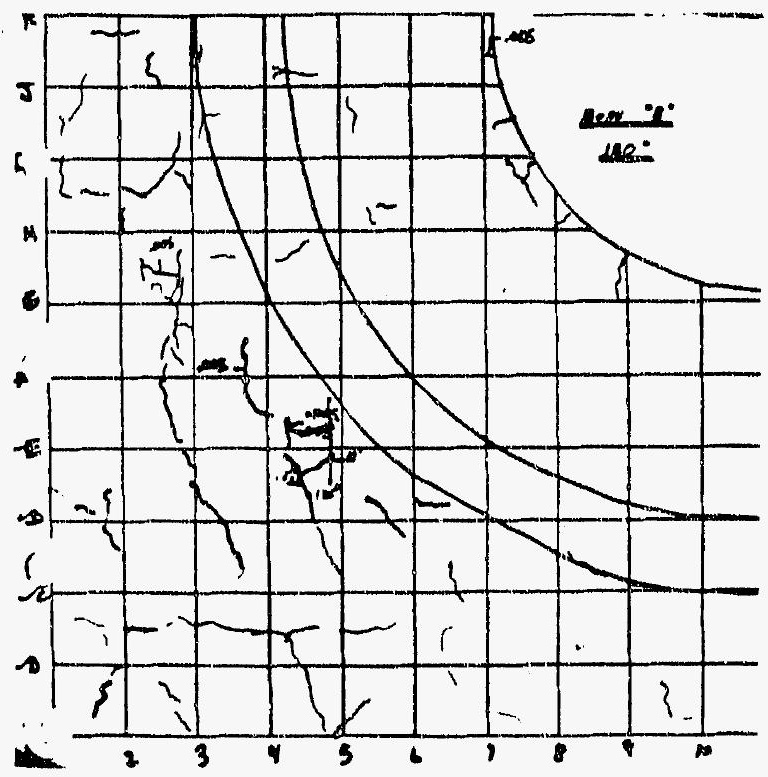

Figure A-11. Boss B, 180; 22 psig; Date-8 July 1987; Time-1545; Mapper-Clauss.

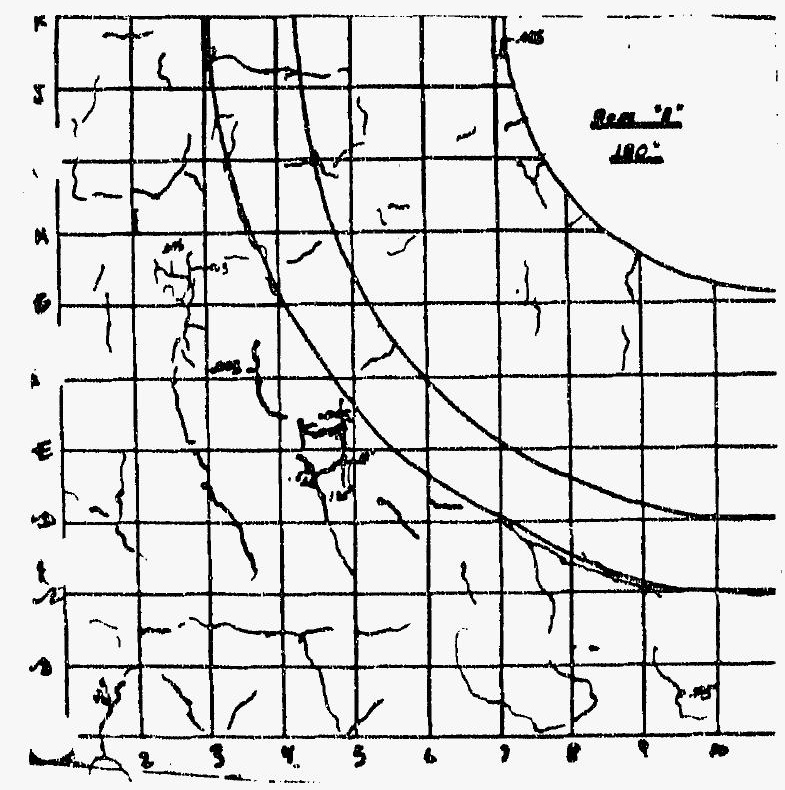

Figure A-12. Boss B, 180³ 34 psig; Date-8 July 1987; Time-1830; Mapper-Lambert. 


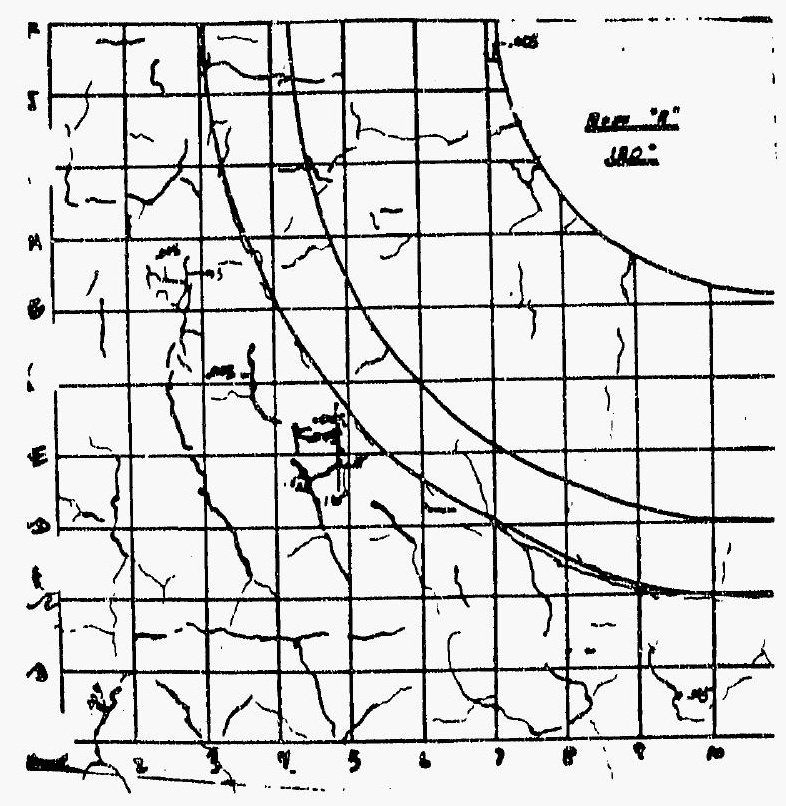

Figure A-13. Boss B, 180; 46 psig; Date-8 July 1987; Time-2012; Mapper-Lambert.

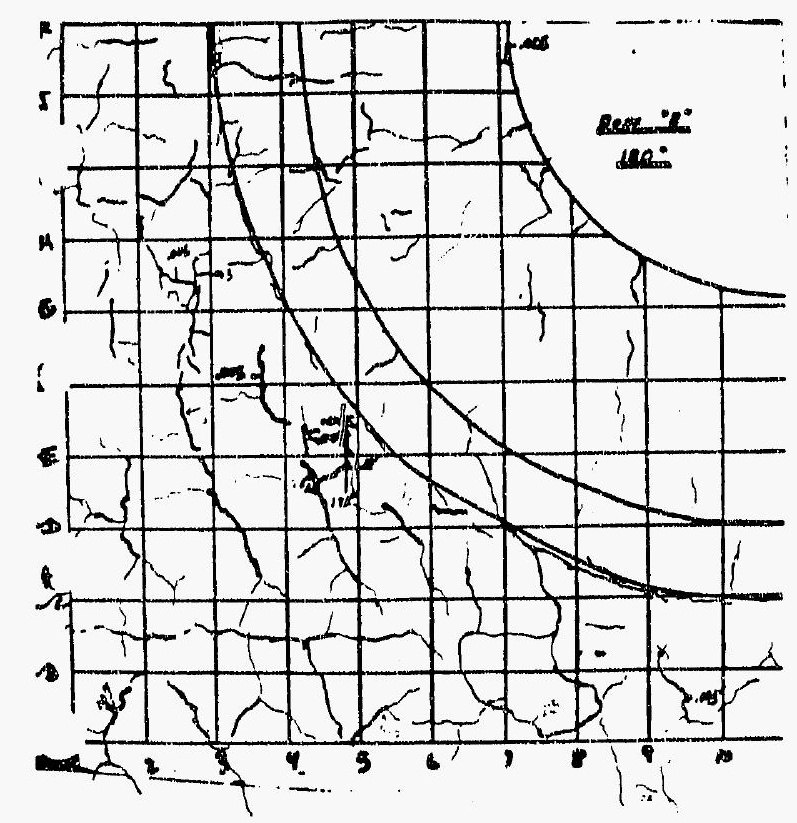

Figure A-14. Boss B, $180^{\circ}$; 53 psig; Date-8 July 1987; Time-2349; Mapper-Lambert. 


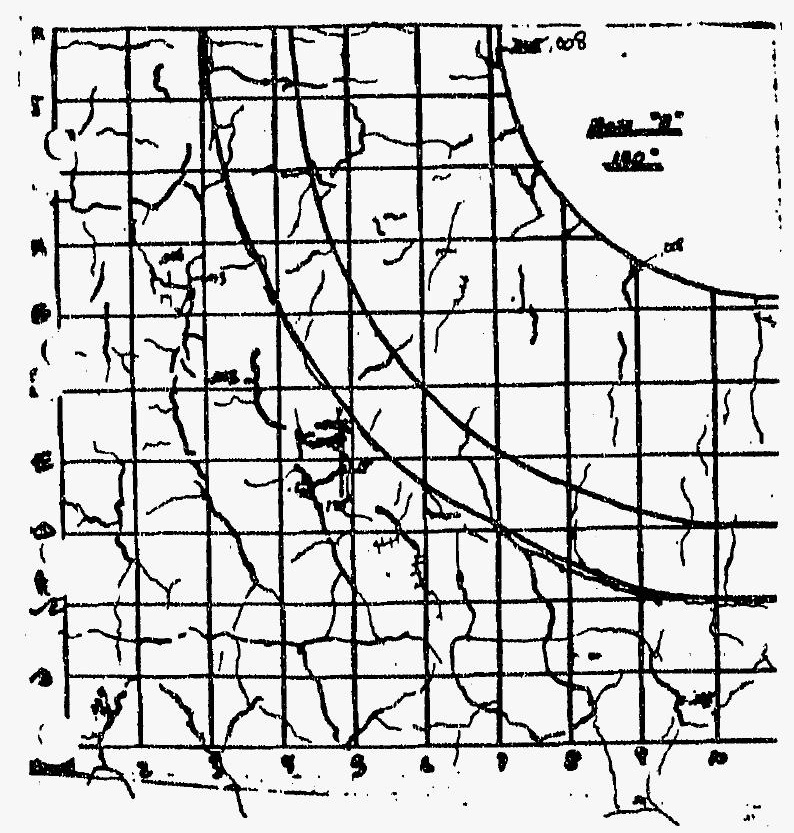

Figure A-15. Boss B, $180^{\circ}$; 46 psig; Date-9 July 1987; Time-1111; Mapper-Lambert.

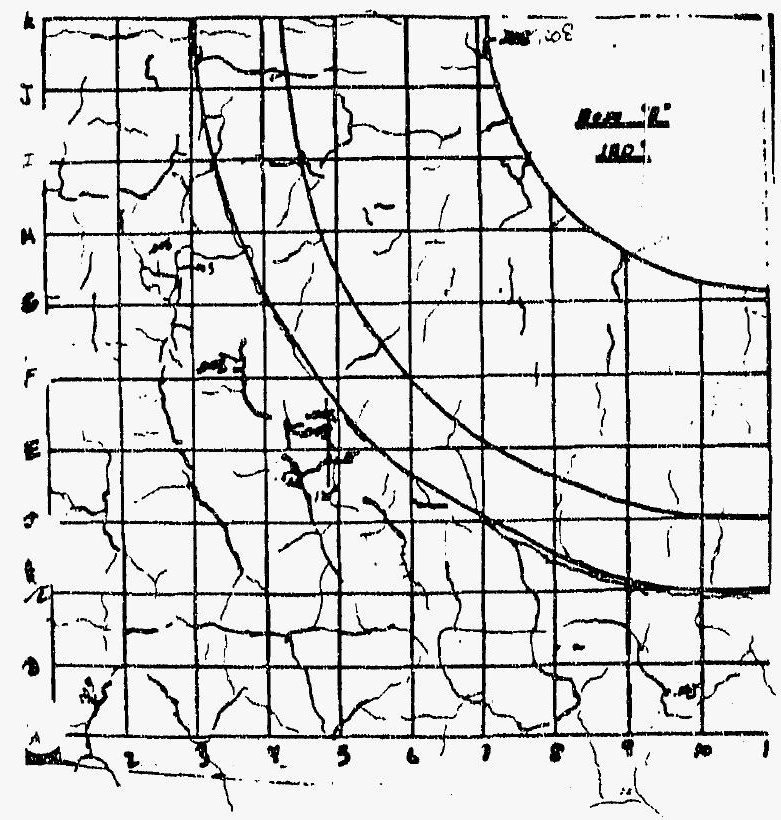

Figure A-16. Boss B, $180^{\circ}$; 0 psig; Date-10 July 1987; Time-1252; Mapper-Jinzo. 


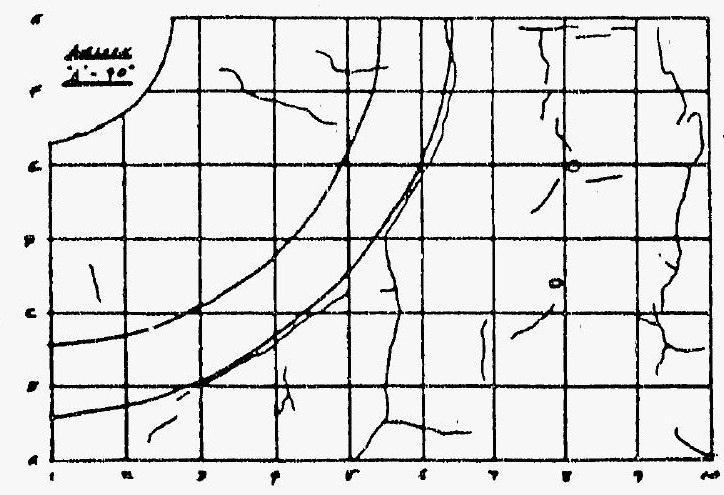

Figure A-17. $\quad 70^{\circ}$ Airlock; 0 psig; Date-7 July 1987; Time-0700; MapperBrosseau/Westmoreland.

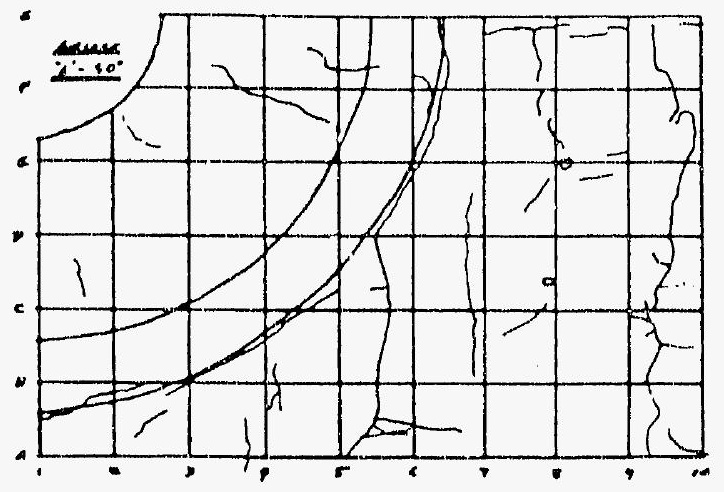

Figure A-18. 90 Airlock; 13 psig; Date-7 July 1987; Time-1145; Mapper-Brosseau/Jinzo. 


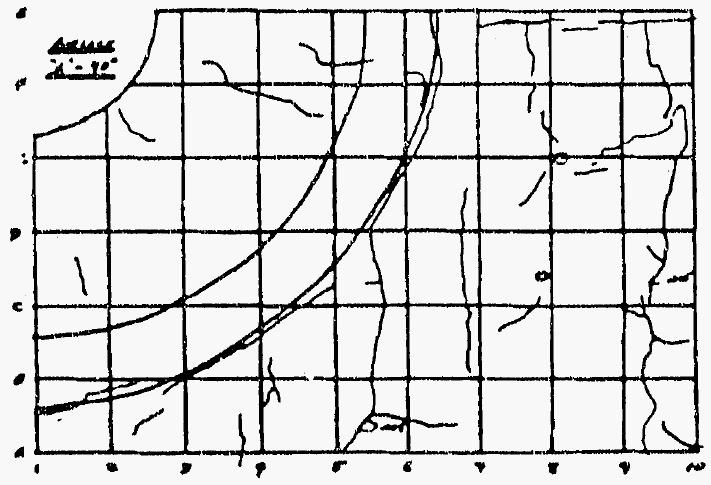

Figure A-19. $90^{\circ}$ Airlock; 22 psig; Date-8 July 1987; Time-1545; MapperWestmoreland/Brosseau.

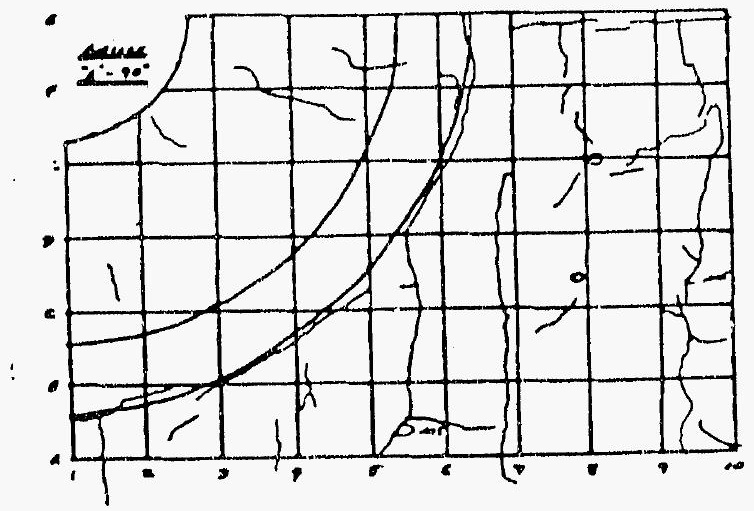

Figure A-20. $90^{\circ}$ Airlock; 34 psig; Date-8 July 1987; Time-1830; Mapper-Brosseau/Jinzo. 


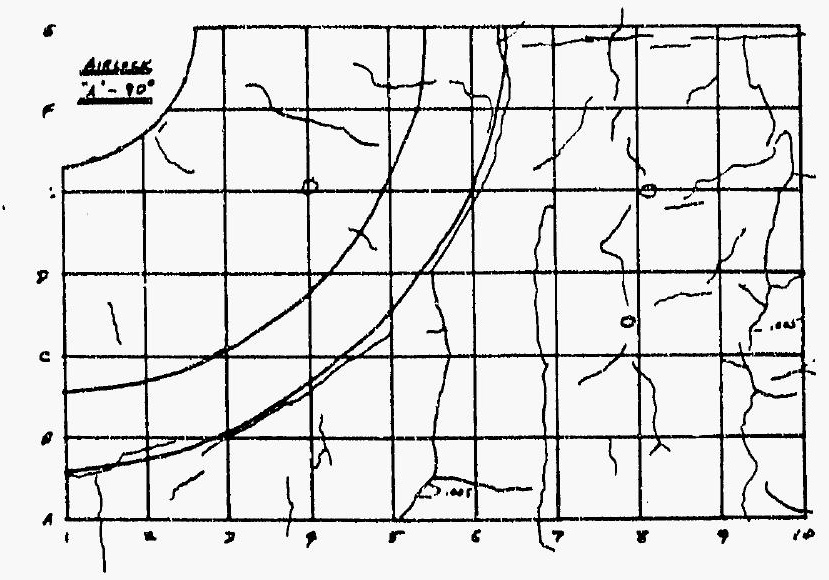

Figure A-21. 90 Airlock; 46 psig; Date-8 July 1987; Time-2105; MapperBrosseau/Westmoreland.

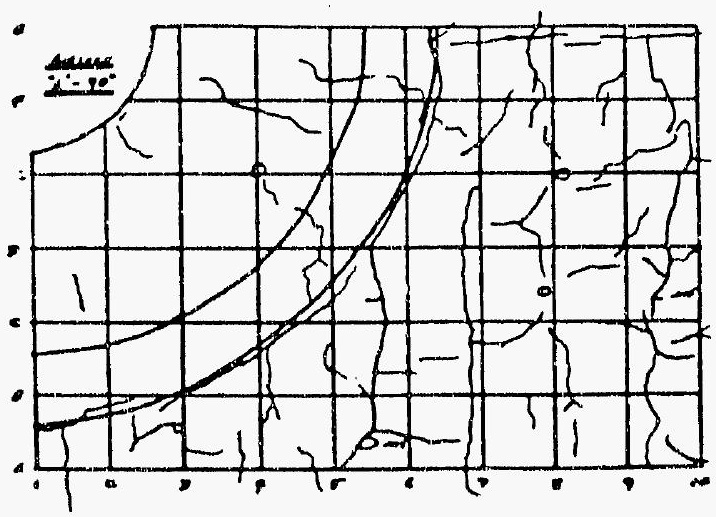

Figure A-22. $90^{\circ}$ Airlock; 53 psig; Date-8 July 1987; Time-2340; Mapper-Brosseau/Jinzo. 


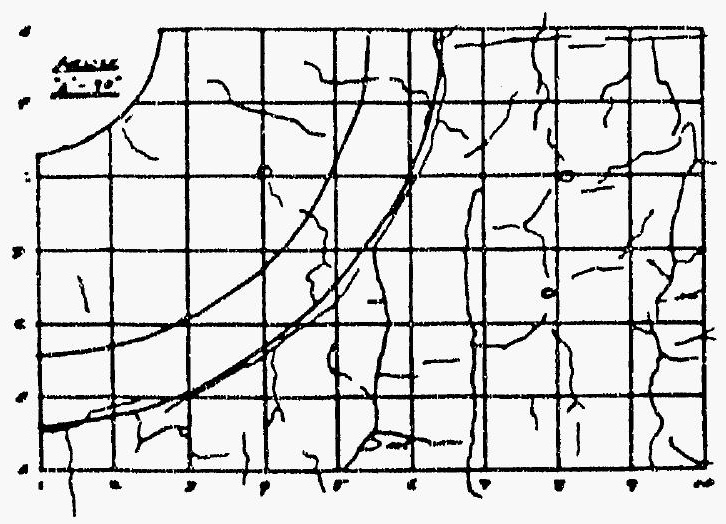

Figure A-23. $90^{\circ}$ Airlock; 46 psig; Date-9 July 1987; Time-1110; MapperWestmoreland/Brown.

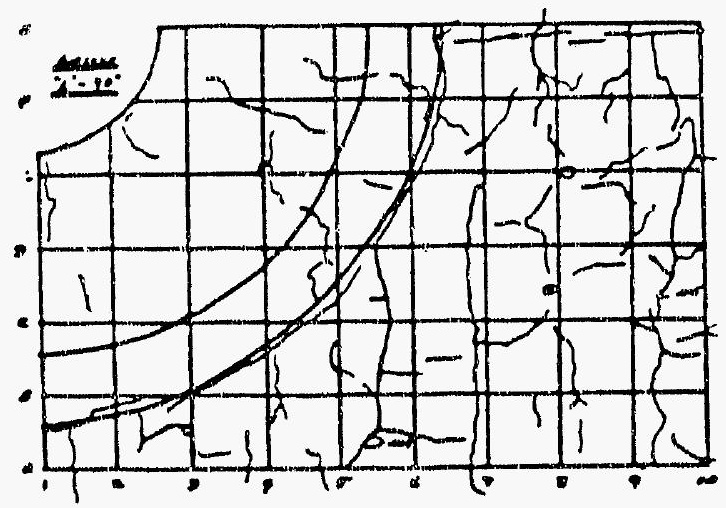

Figure A-24. $90^{\circ}$ Airlock: 0 psig: Date-10 July 1987; Time-1400; Mapper-Brosseau/Jinzo. 


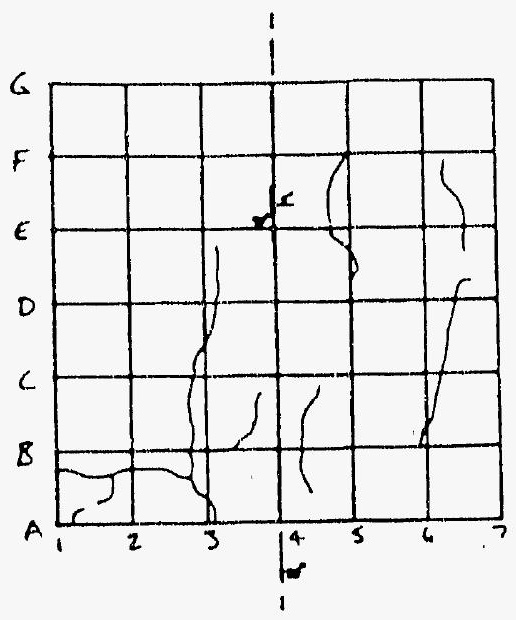

Figure A-25. Cylinder Basemat, 90% 0 psig; Date-6 July 1987; Time-1615; Mapper-Parks.

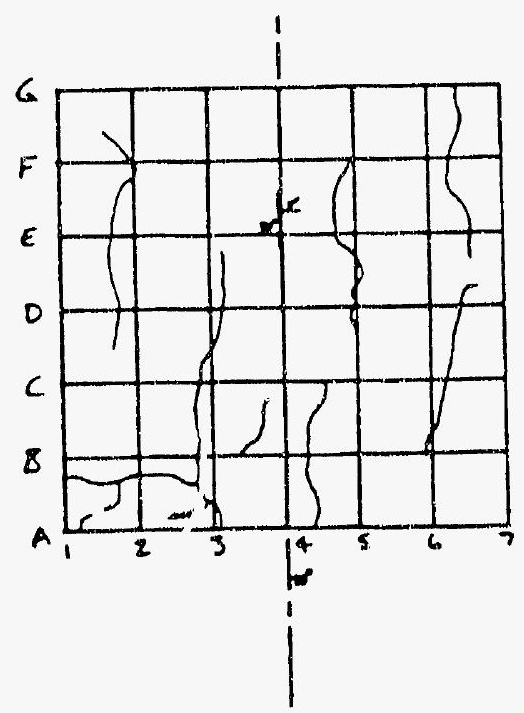

Figure A-26. Cylinder Basemat, 90; 13 psig; Date-7 July 1987; Time-1127; Mapper-Parks. 


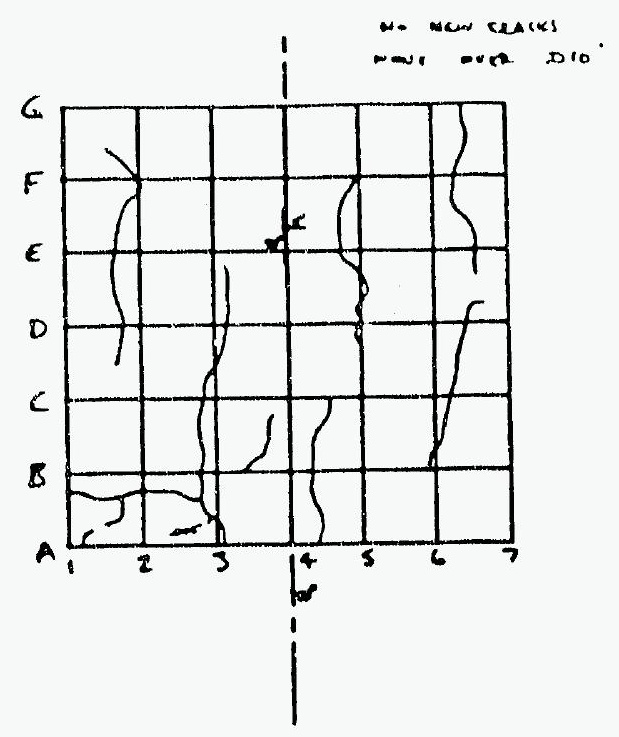

Figure A-27. Cylinder Basemat, 90 22 psig; Date-8 July 1987; Time-1545; MapperParks/Von Riesemann.

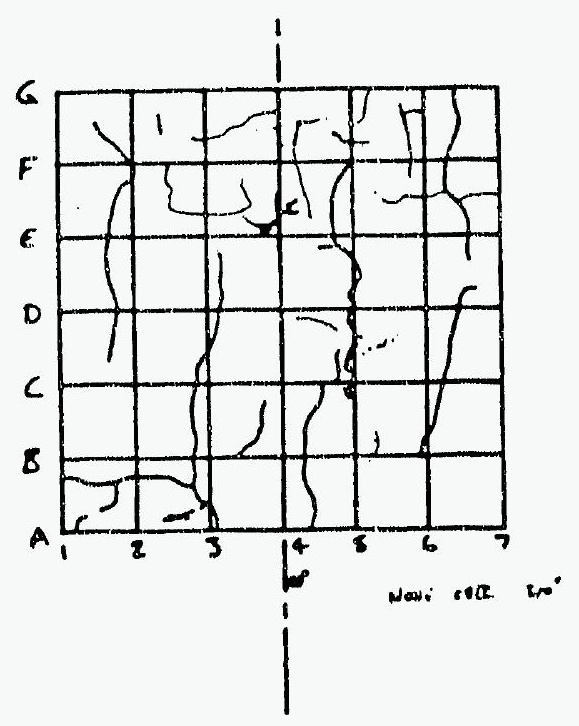

Figure A-28. Cylinder Basemat, $90^{\circ} ; 34$ psig; Date-8 July 1987; Time-1628; MapperParks/Von Riesernann. 


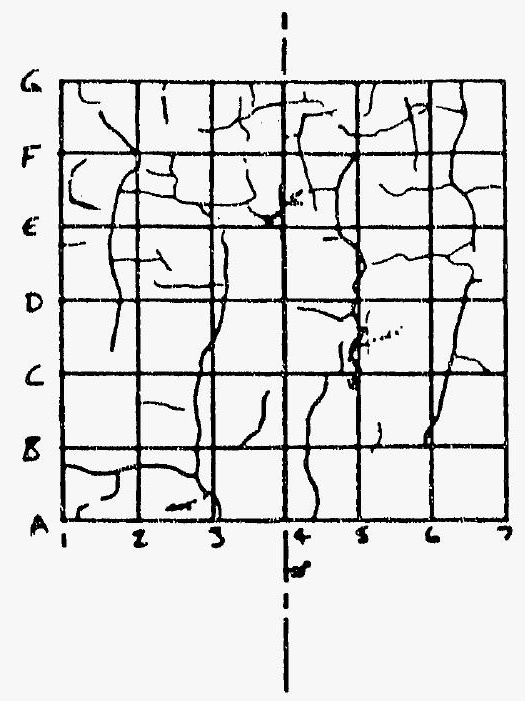

Figure A-29. Cylinder Basemat, $90^{\circ}$; 46 psig; Date-8 July 1987; Time-2100; MapperParks/Von Riesermann.

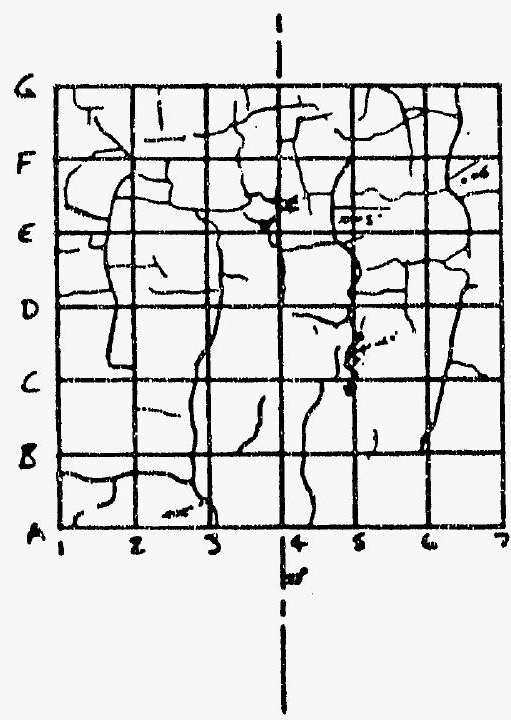

Figure A-30. Cylinder Basemat, 90 $; 53$ psig; Date-8 July 1987; Time-2335; MapperParks/Von Riesemann. 


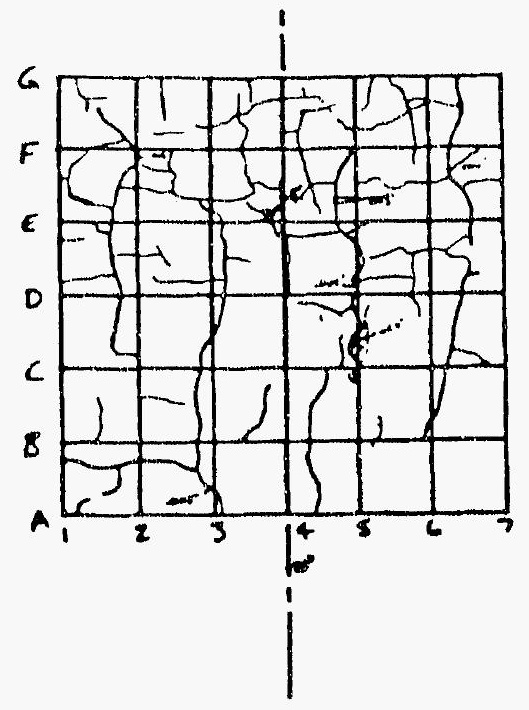

Figure A-31. Cylinder Basemat, 90 46 psig; Date-9 July 1987; Time-1110; Mapper-Parks.

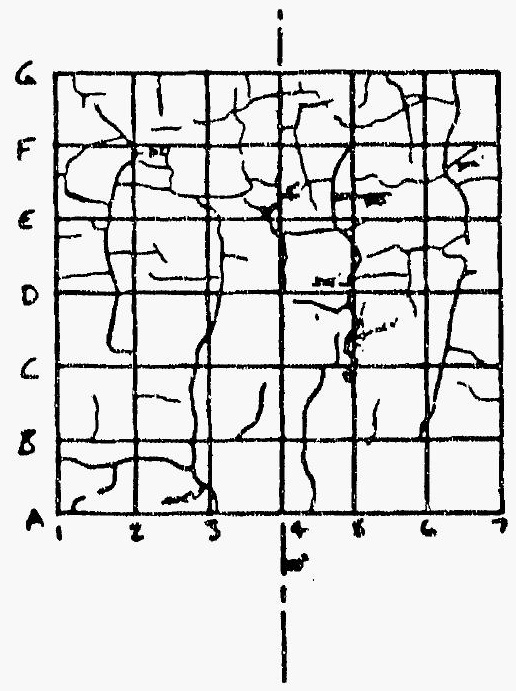

Figure A-32. Cylinder Basemat, 90 0 psig; Date-10 July 1987; Time-2450; Mapper-Parks. 


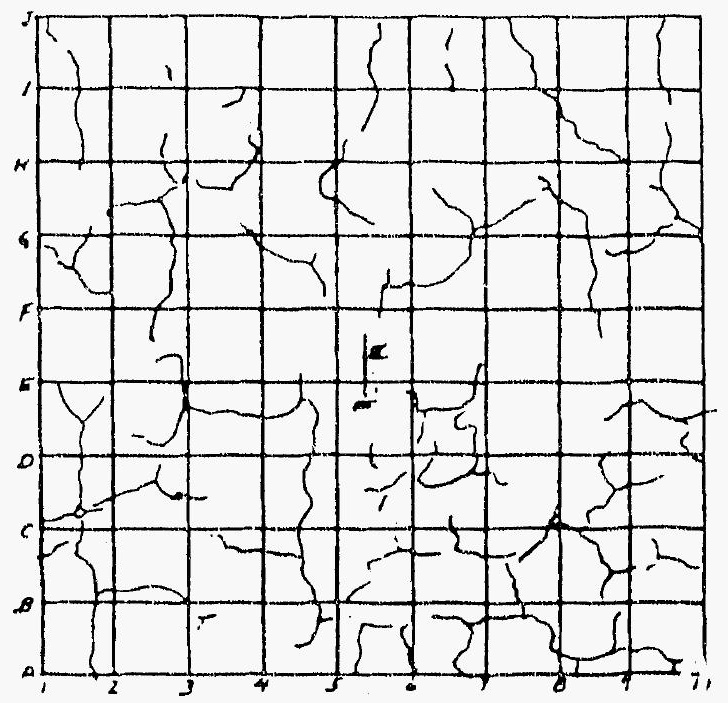

Figure A-33. 135 @ 13’; 0 psig; Date-7 July 1987; Time-1124; Mapper-Von Riesemann.

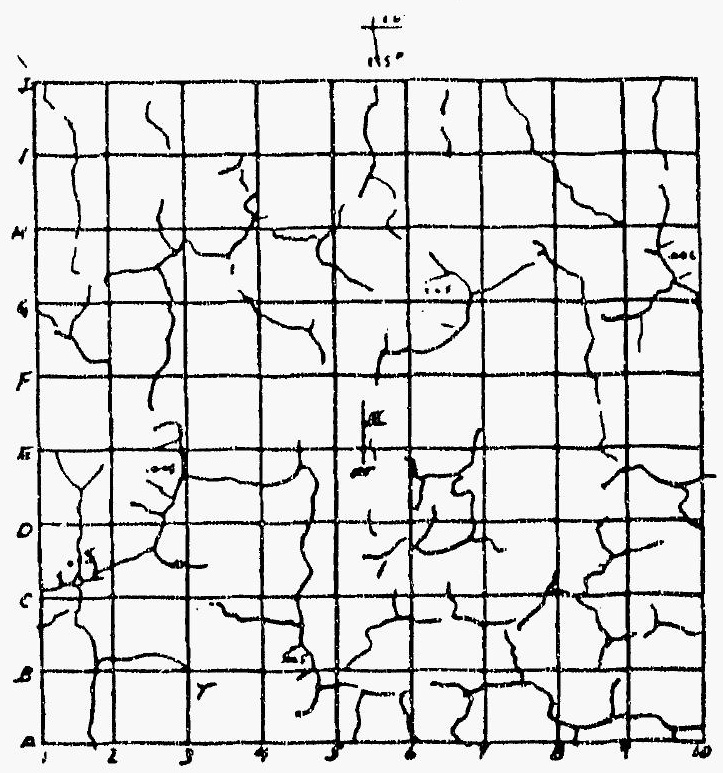

Figure A-34.135 @13'; 13 psig; Date-7-July 1987; Time-1124; Mapper-Von Riesemann. 


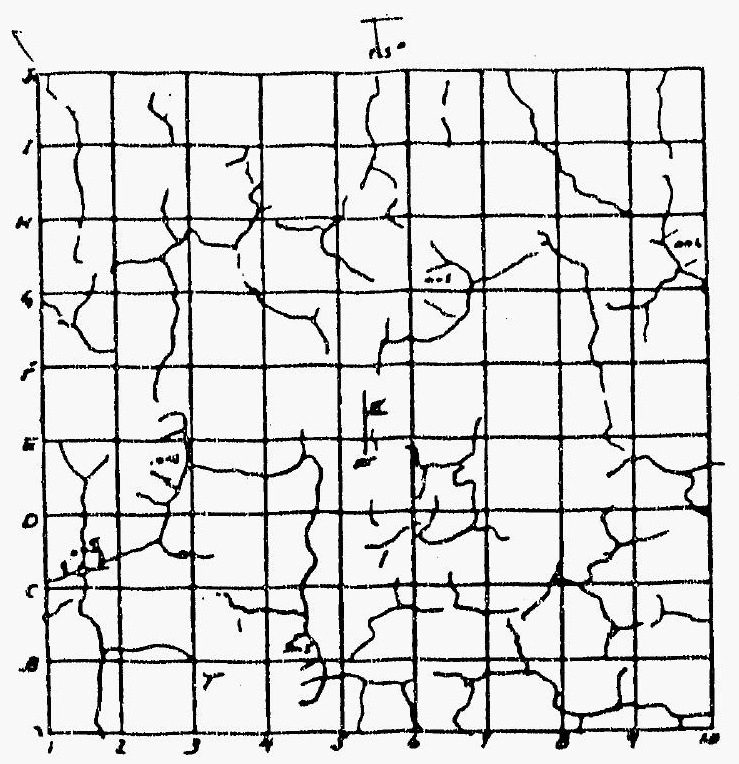

Figure A-35. 135 @ 13'; 22 psig; Date-8 July 1987; Time-1545; Mapper-Von Riesemann/Parks.

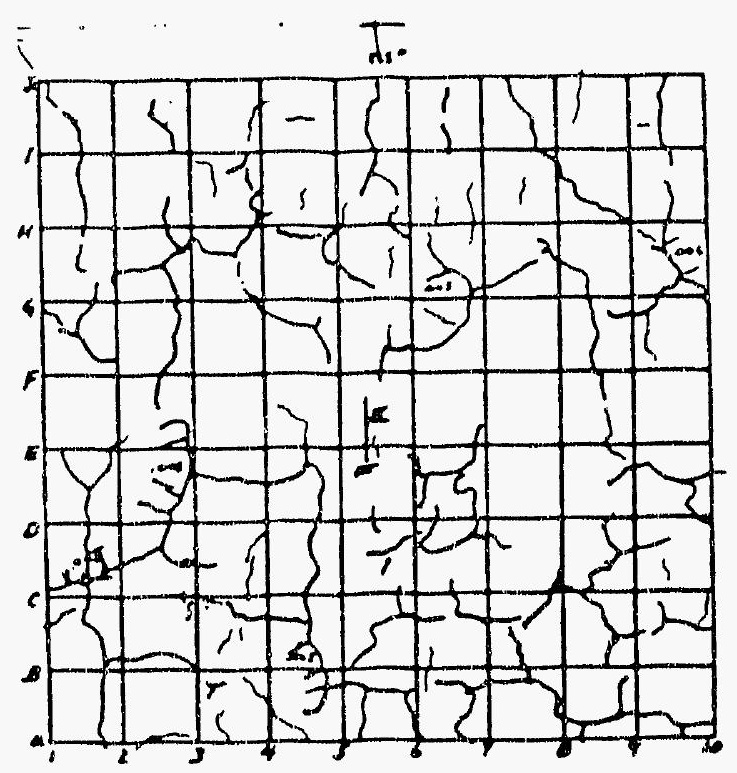

Figure A-36. 135 $@ 15^{\circ}$; 34 psig; Date-8 July 1987; Time-1947; Mapper-Von Riesemann/Parks. 


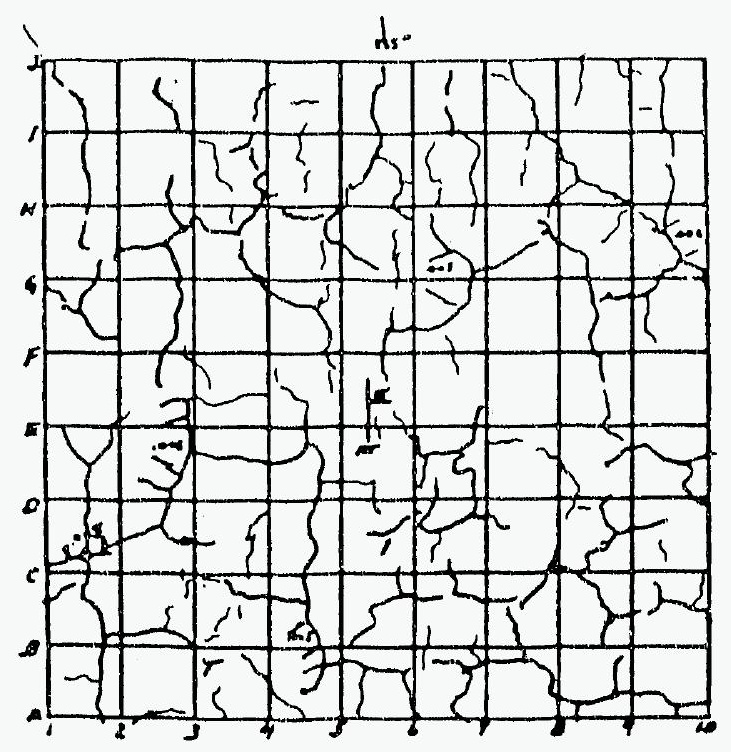

Figure A-37. $135^{\circ} @ 13^{\prime} ; 46$ psig; Date-8 July 1987; Time-2115; Mapper-Von Riesemann/Parks.

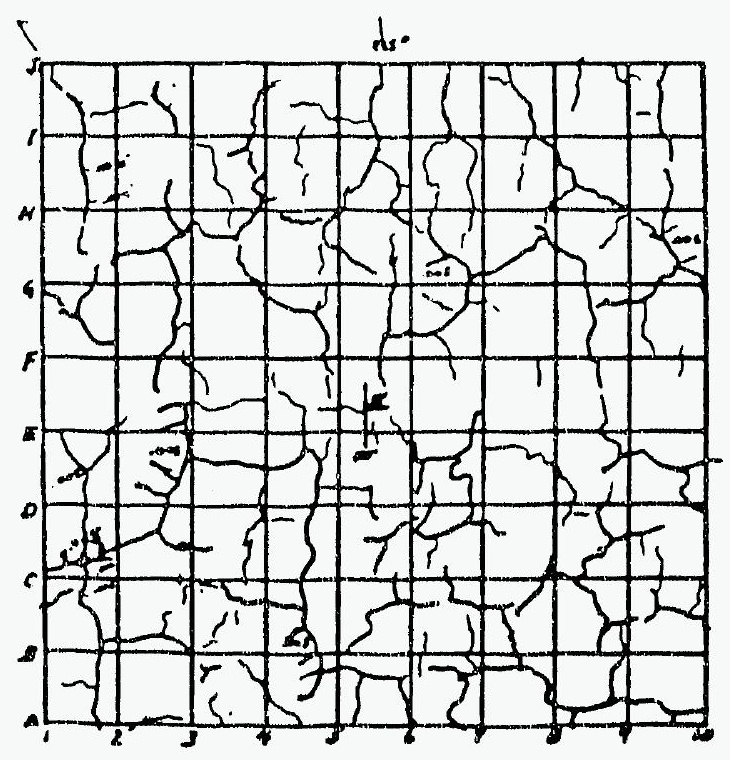

Figure A-38. 135 @ 13’; 53 psig; Date-8 July 1987; Time-2345; Mapper-Von Riesemann. 


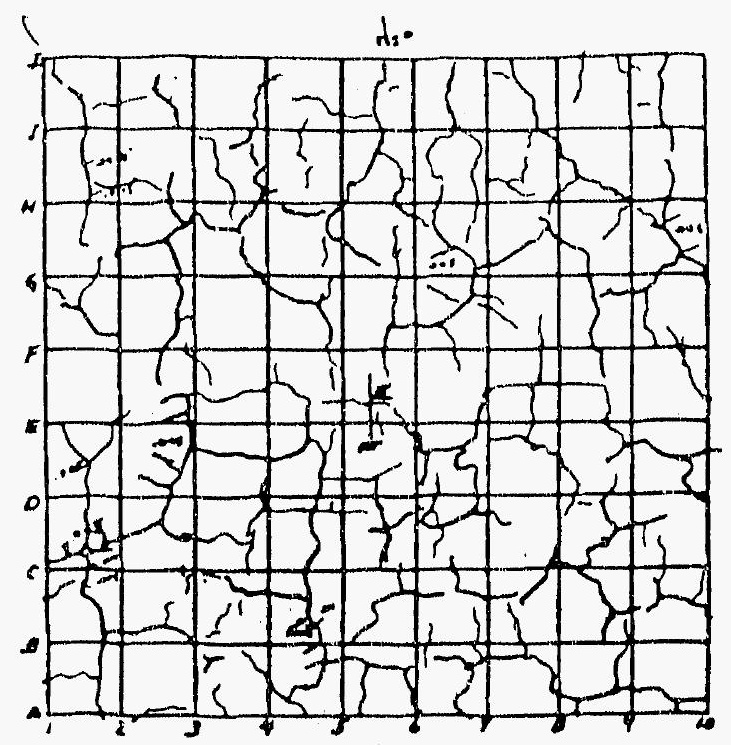

Figure A-39. 135 @ 13’; 46 psig; Date-9 July 1987; Time-1129; Mapper-Horschel.

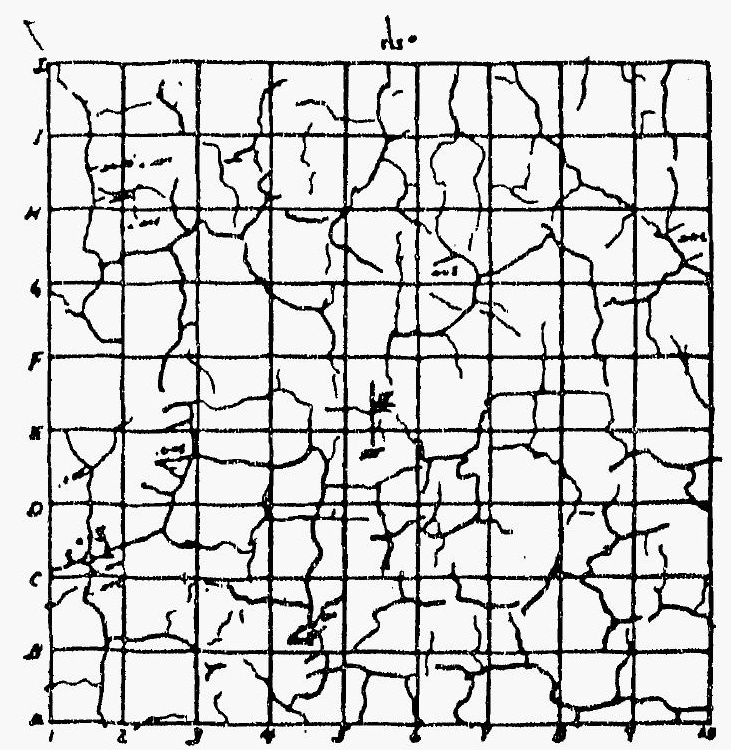

Figure A-40.135@ 13’; 0 psig; Date-10 July 1987; Time-1300; Mapper-Brosseau. 


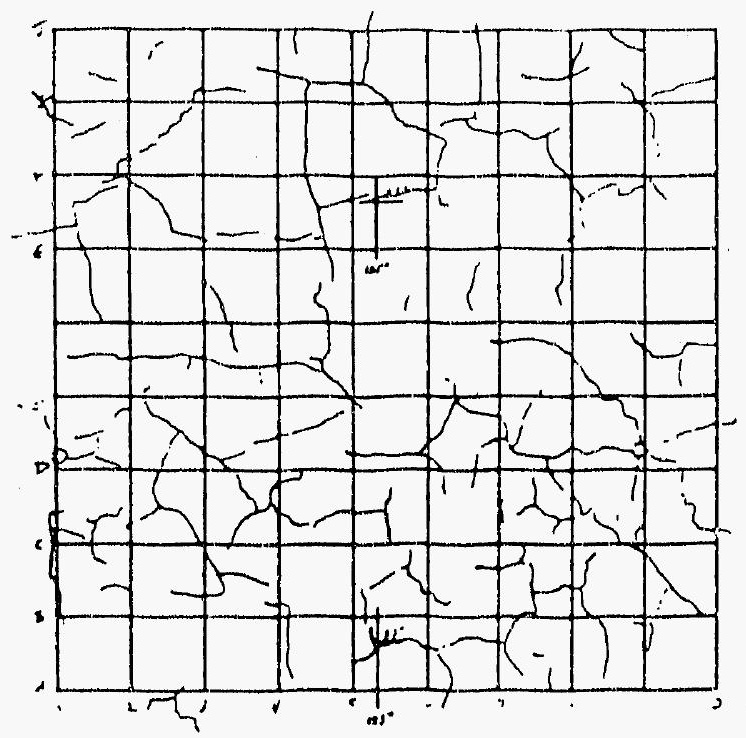

Figure A-41. Springline $135^{\circ} ; 0$ psig; Date-6 July 1987; Time-1700; MapperBrosseau/Westmoreland.

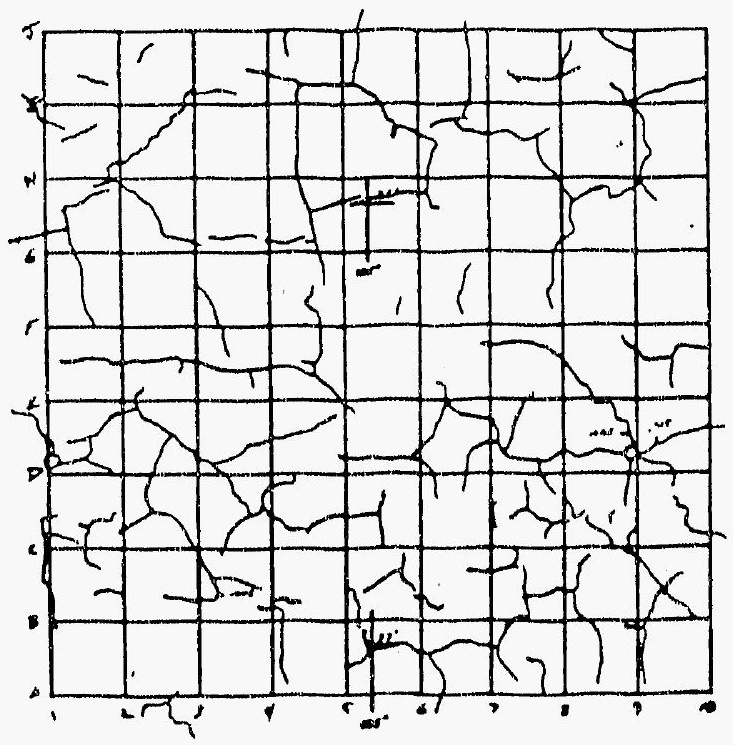

Figure A-42. Springline 135 ; 13 psig; Date-7 July 1987; Time-1130; MapperBrosseau/Jinzo. 


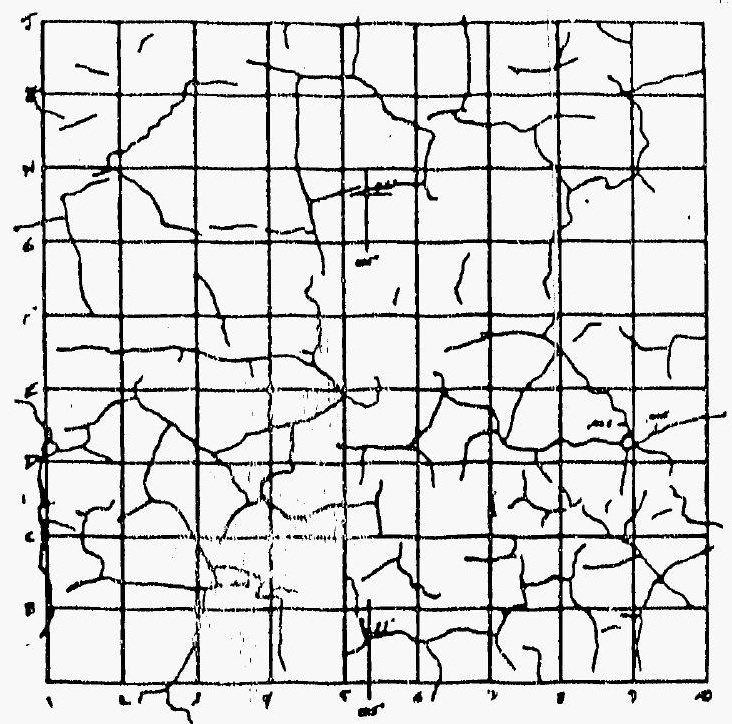

Figure A-43. Springline $135^{\circ} ; 22$ psig; Date-8 July 1987; Time-1600; MapperWestmoreland/Brosseau.

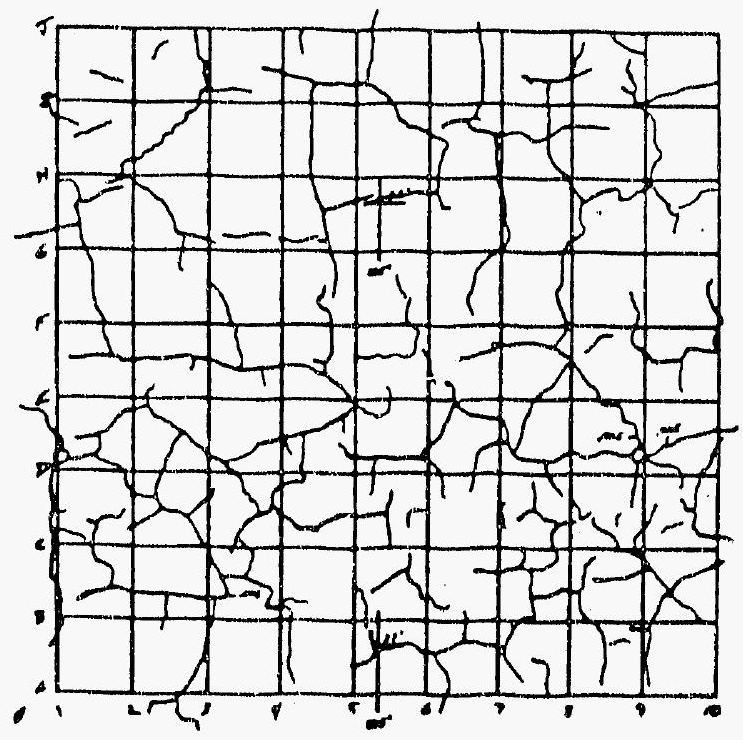

Figure A-44. Springline $135^{\circ} ; 34$ psig; Date-8 July 1987; Time-1845; MapperBrosseau/Jinzo. 


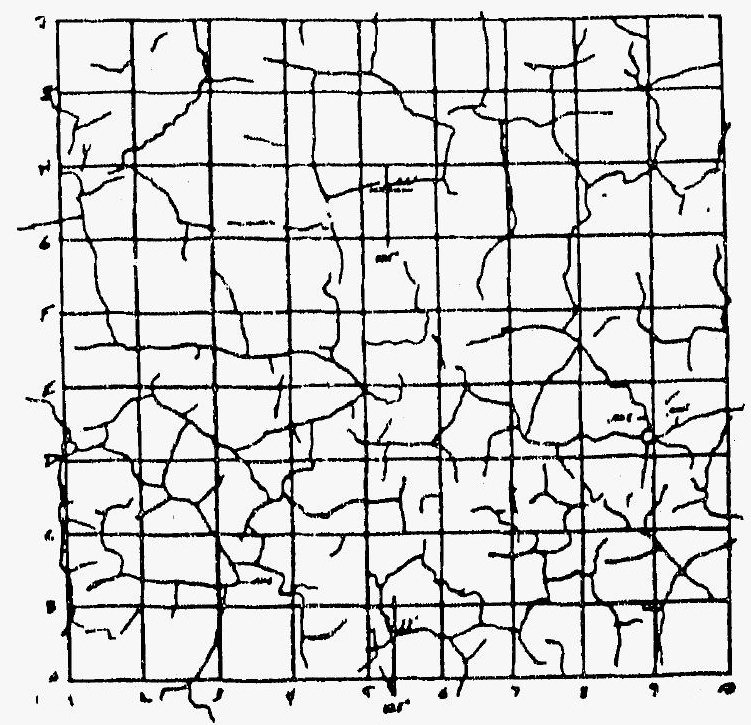

Figure A-45. Springline $135^{\circ} ; 46$ psig; Date-8 July 1987; Time-2120; MapperWestmoreland/Brosseau.

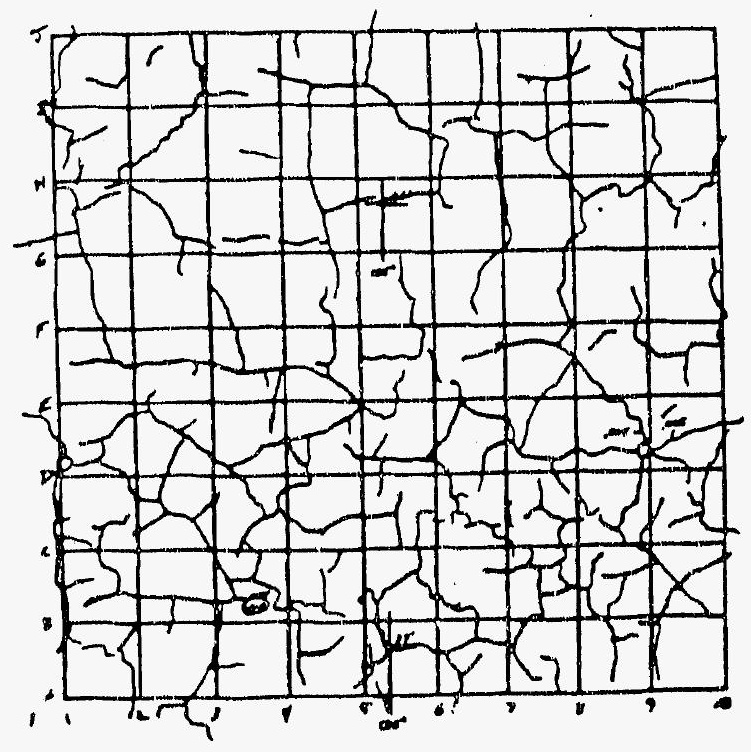

Figure A-46. Springline $135^{\circ} ; 53$ psig; Date-8 July 1987; Time-2350; MapperBrosseau/Jinzo. 


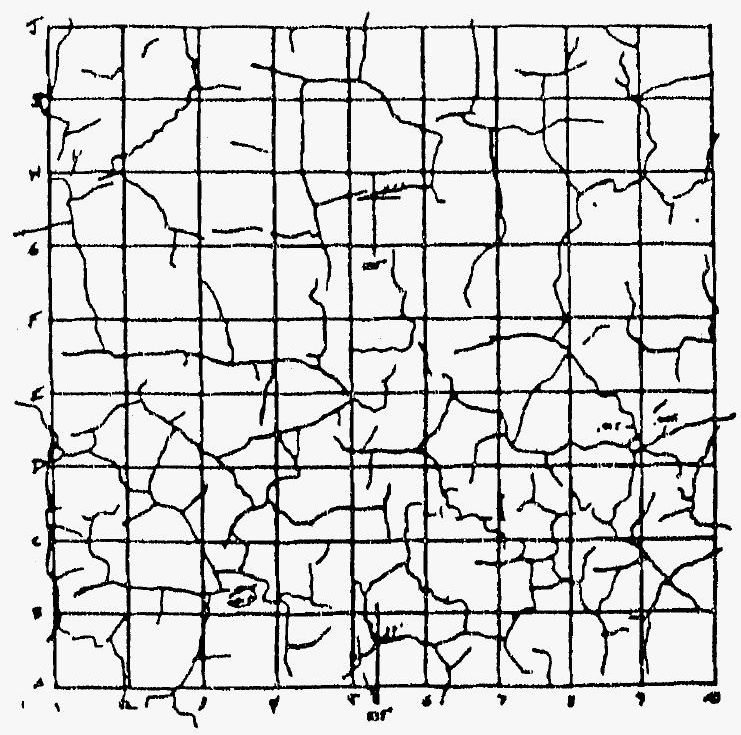

Figure A-47. Springline $135^{\circ} ; 46$ psig; Date-9 July 1987; Time-1100; MapperWestmoreland/Brosseau.

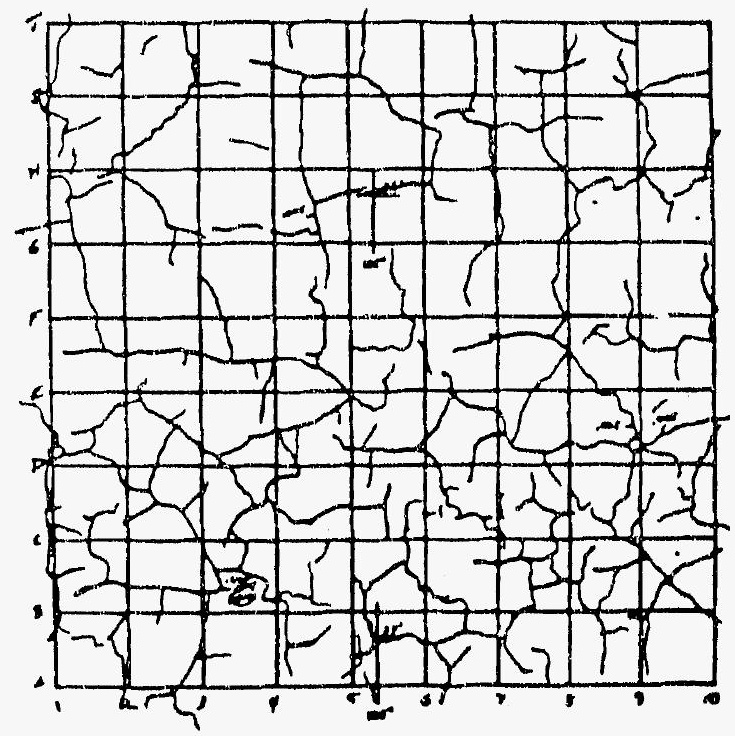

Figure A-48. Springline $135^{\circ}$; 0 psig; Date-10 July 1987; Time-1330; MapperBrosseau/Jinzo. 
APPENDIX B

RESULTS FROM THE TRACK TRANSDUCERS

by

M. G. Jinzo 


\section{B-1. Track System}

The track transducers measured deflection of the containment shell. The track system was designed and erected by Sandia personnel specifically for the containment test. The track transducers were, as their name suggests, mounted on tracks and powered by a gear motor as shown in Figure 12 in the body of this report. The motor pulled the transducer along a track, while an arm followed the contour of the model. Each track system had two transducers mounted on it one measured the location along the track, while the second measured the movement of the track arm perpendicular to the track.

\section{B-2. Track Transducc..}

Nine track systems were used on the model during testing. Potentiometric displacement transducers were selected ard sized to be most useful after the model had significantly deformed. The track systems could measure displacements up to $21.0 \mathrm{in}$. One track was in a horizontal position and measured the "dishing" of the basemat. The remaining eight systems measured the radial displacement of the cylinder wall. Table B-1 indicates the location and the approximate length for each track system. The computer that gathered the data was the same as for the other sensors on the model: an HP 1000 linked to an HP 3497 scanner and HP 3498 extenders that was operating custom-written software. The nine track transducers, however, were separately controlled by the software.

\section{TABLE B-1.}

\section{Location and Length of the Track Systems}

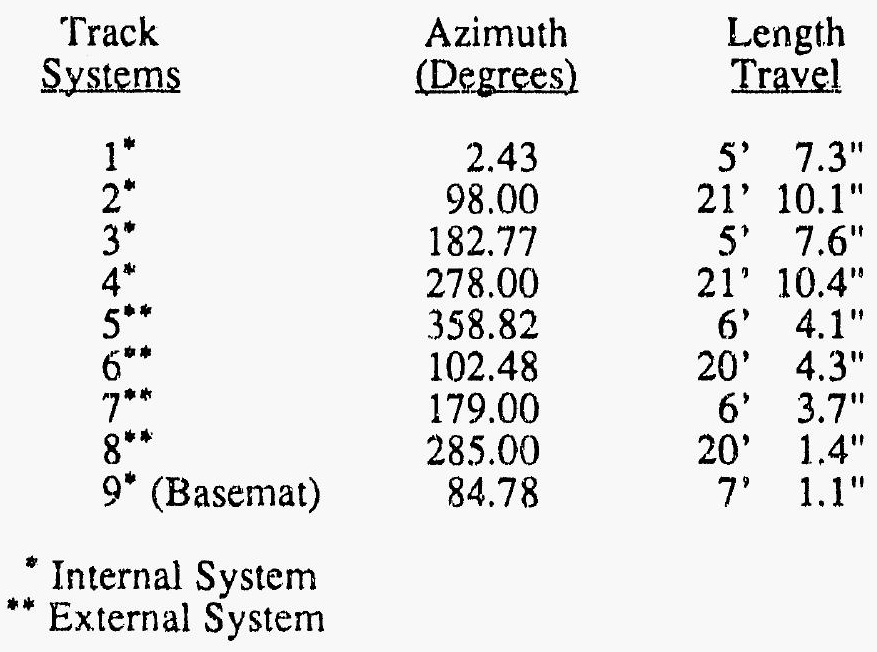

Since the track transducers were selected and sized to gather large deflection data (approximately $1 \mathrm{in}$. or more) and were designed to have a resolution of approximately $\pm 1 / 8$ in., track data were gathered at more frequent pressure steps as the radial displacement of the model exceeded 1 in. at its midheight. (Table B-2 shows all pressure leveis where data were taken.) 


\section{B-3. Data Gathering and Preparation}

The eight track systems used in the cylinder were designed so that data were gathered on both the up stroke and down stroke of the system. While the ninth track was designed to take data along the basemat from the outer edge of the fill slab towards the center column and back. The data taken in either direction of travel were more or less consistent with one another, but this report uses only the "up stroke" data, which were selected because it was felt that on the up stroke the gear motor had a steadier speed and applied a more consistent force on the track arm. (The down stroke of the vertically mounted track transducers relied on gravity to return the track mounted sensor to its bottom position. It was felt that any frictional forces could cause more racking of the transducers.)

The nine track systems were not scanned concurrently. The four inside tracks were started at the same time. When the scan on the shorter tracks finished, the two remaining longer tracks continued to scan, but because there were only four transducers left (2 transducers per track), the data were gathered at a faster rate. This process was repeated for the outside tracks, and finally, the basemat track was scanned separately.

Deflection data for the shorter tracks were taken at about 2.3-inch intervals along the cylinder. On the longer tracks; data were taken at 2.3-in. intervals for the lower onethird and at 0.55 -in. intervals for the remaining length of track.

To measure displacement with the tracks, a reading was taken at the start of the test (zero psig). This reading was subtracted from the subsequent track scans to obtain the deflection. Since the elevation at which the data were taken varied, as well as the number of data pairs (elevation, displacement) gathered at each track for each pressure level (Table B-2), straight line interpolation and extrapolation were used to subtract the "zero" readings from subsequent track scans.

\section{B-4. Data}

Plots of the data taken from the inside and outside track systems are shown in Figures B-2 through B-10. The plots show that after a pressure level of 125 psi was reached, an increase in pressure of approximately $10 \mathrm{psi}$, the model bowed considerably, with moderate but constant changes measured at pressures below 113 psi. It should also be noted that after a closer examination of the data and the containment model, it was determined that the major glitches in the data originated from the weld seam and construction joints on the model. The weld seams started at an elevation of approximately 60 in. and were repeated approximately every 38 in. ending at the springline for the internal vertically mounted track transducers. For the external track transducers, the construction joints started at an elevation of approximately 80 in. and were repeated again at approximately 156 in. Figures B-3 and B-7 provide more detailed information concerning the weld seams and construction joints. The symbols on the lines identify the curve and pressure level at which the data were taken. 
TABLE B-2

Pressure Levels at Which

Data Were Gathered During High Pressure Test

\begin{tabular}{|c|c|c|c|}
\hline Data & $\begin{array}{c}\text { Pressure } \\
\text { psig } \\
\text { (Channel \#2) } \\
\end{array}$ & $\begin{array}{l}\text { Track Data } \\
\text { Gathered }\end{array}$ & $\begin{array}{c}\text { Data Plotted } \\
\text { (Figs. B-3 - B-10 }\end{array}$ \\
\hline $1^{* *}$ & 9.9 & & \\
\hline 2 & 19.6 & & \\
\hline 3 & 29.6 & & \\
\hline 4 & 39.4 & & \\
\hline 5 & 49.2 & $\mathrm{X}$ & $X$ \\
\hline 6 & $\begin{array}{l}54.5 \\
596\end{array}$ & $X$ & \\
\hline $\begin{array}{l}7 \\
8\end{array}$ & $\begin{array}{l}59.6 \\
64.2\end{array}$ & & \\
\hline 9 & 69.3 & $X$ & \\
\hline 10 & 74.2 & & \\
\hline 11 & 80.2 & $X$ & $X$ \\
\hline 12 & 85.6 & & \\
\hline 13 & 90.6 & $X$ & \\
\hline 14 & 95.7 & $X$ & $X$ \\
\hline 15 & 100.9 & $X$ & \\
\hline 16 & 103.3 & & \\
\hline 17 & 106.1 & & \\
\hline 18 & 108.3 & $X$ & \\
\hline 19 & 111.1 & & \\
\hline 20 & 113.2 & $X$ & $X$ \\
\hline 21 & 117.8 & $X$ & \\
\hline 22 & 120.9 & $\mathrm{X}$ & \\
\hline $\begin{array}{l}23 \\
24\end{array}$ & 123.3 & & \\
\hline 25 & 125.8 & $X$ & $X$ \\
\hline 26 & 125.6 & & \\
\hline 27 & 127.8 & & \\
\hline 28 & 130.2 & $X$ & \\
\hline 29 & 132.5 & & \\
\hline 30 & 135.3 & $X$ & $X$ \\
\hline 31 & 134.4 & & \\
\hline 32 & 138.4 & & \\
\hline 33 & 140.2 & $\mathrm{X}$ & \\
\hline 34 & 142.6 & & \\
\hline 35 & 145.8 & $X$ & $\mathrm{X}$ \\
\hline 36 & 142.5 & & \\
\hline 37 & 0.2 & $X$ & \\
\hline
\end{tabular}

*Data were taken during high-pressure test.

** Data and track scans were taken at zero psig tc be used for zeroing subsequent readings. 


\section{Acknowledgements}

I wish to thank the following Sandia and K-tech personnel involved in the design, construction and installment of the track systems: Barry Spletzer, Pat Drozda, Frank Horine, and Gary Loughrin for their efforts in making the track systems a success. 


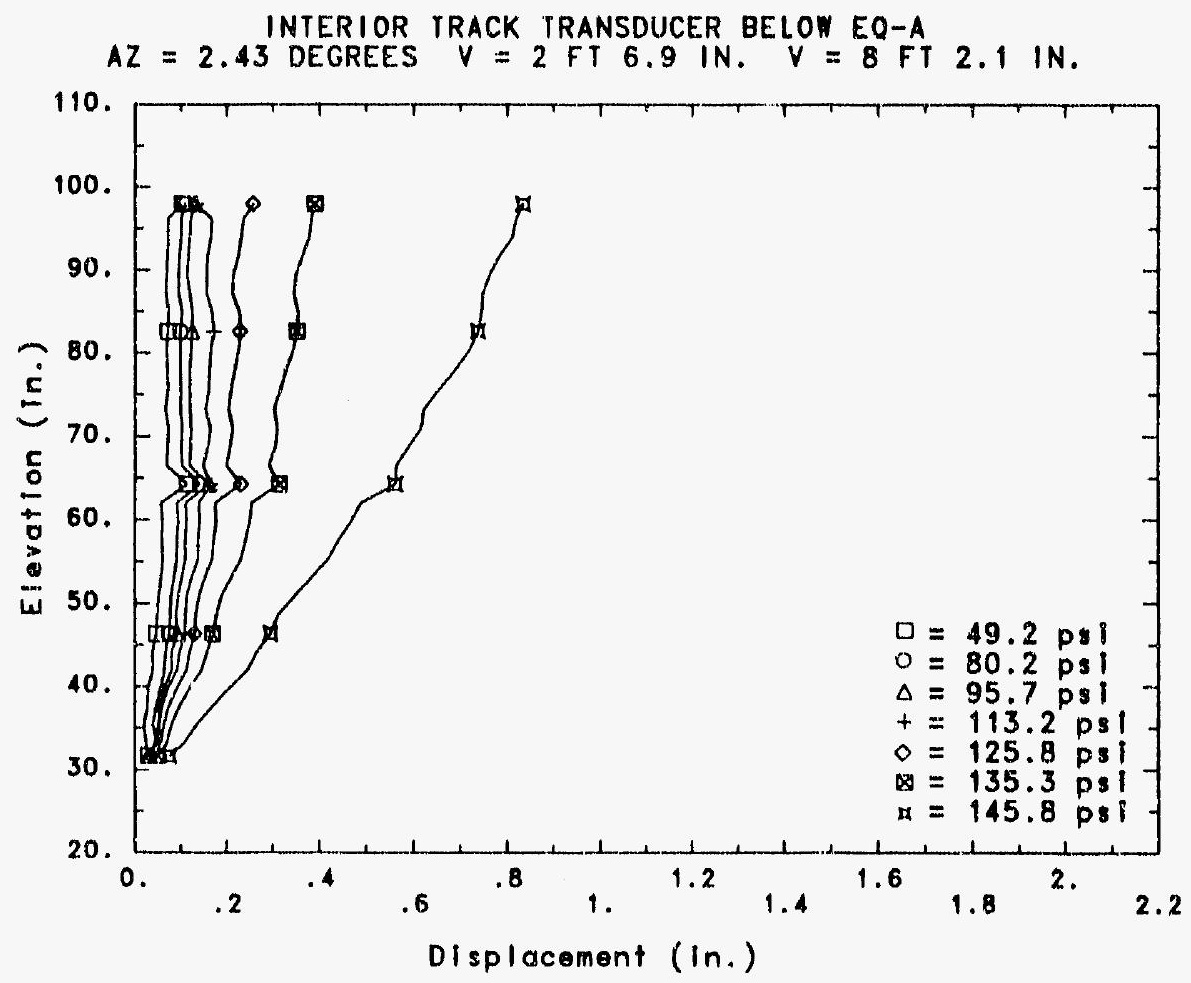

Figure B-1. Track 1, Interior Track Transducer Below EQ. A-1, Az=2.45 Degrees, V=2 Ft. 6.9 In., $V=8$ Ft. 2.1 In.

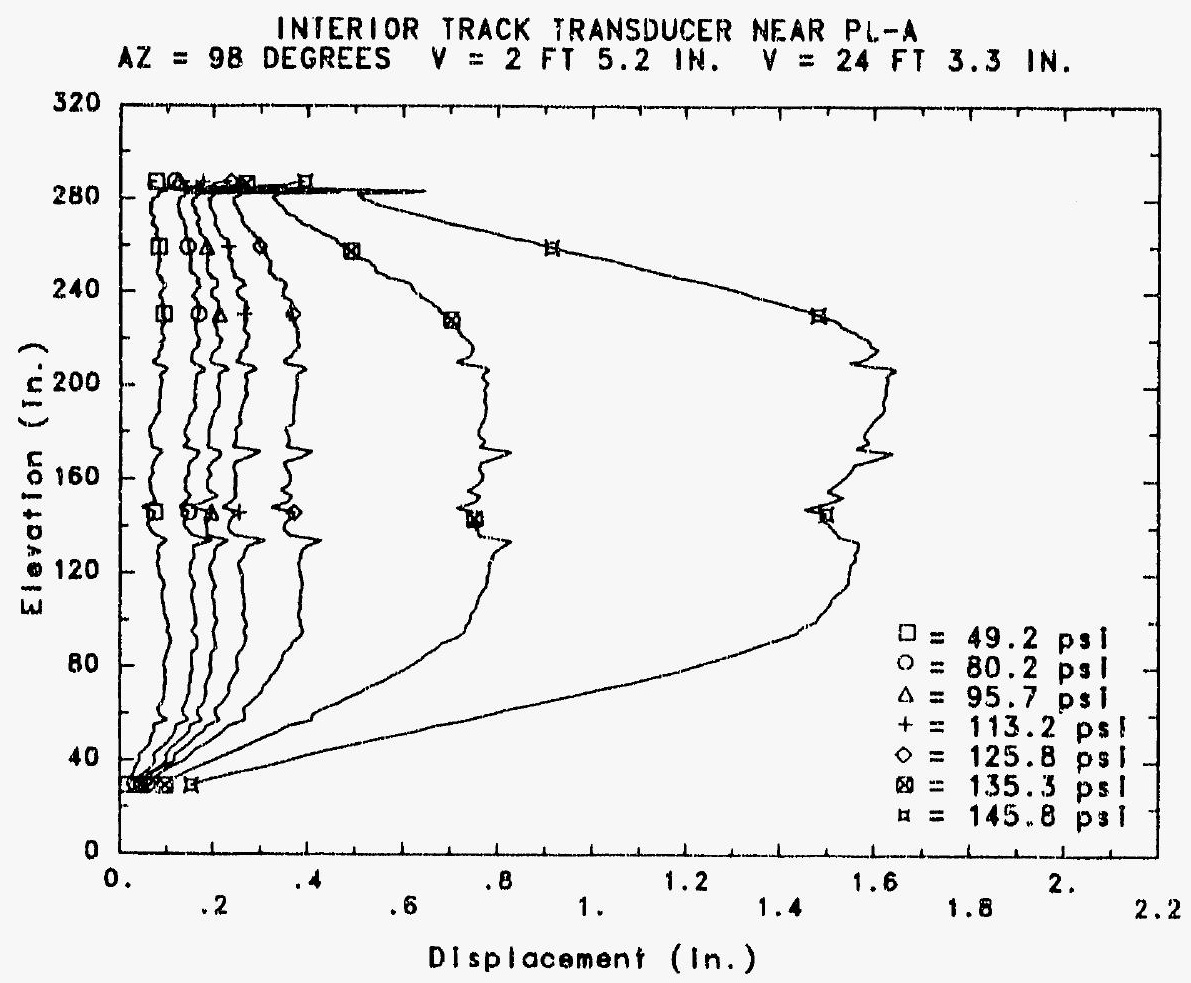

Figure B-2. Track 2, Interior Track Transducer Near PL-A; Az=98 Degrees, V=2 Ft. 5.2 In., $V=24$ Ft. 3.3 In. 


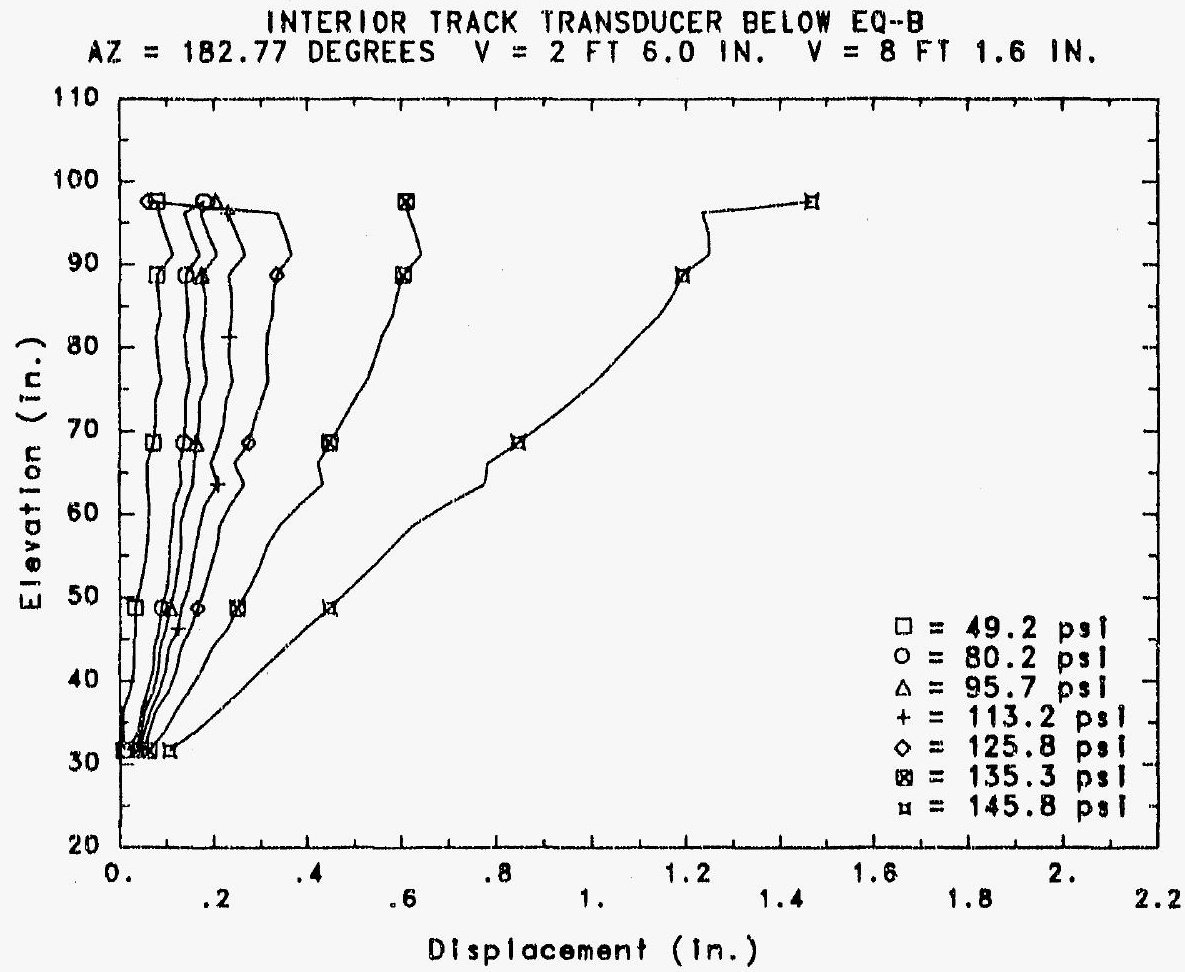

Figure B-3. Track 3, Interior Track Transducer Below EQ.-B, $A z=182.77$ Degrees; V=2 Ft. $6.0 \mathrm{In}$., V=8 Ft. $1.6 \mathrm{In}$.

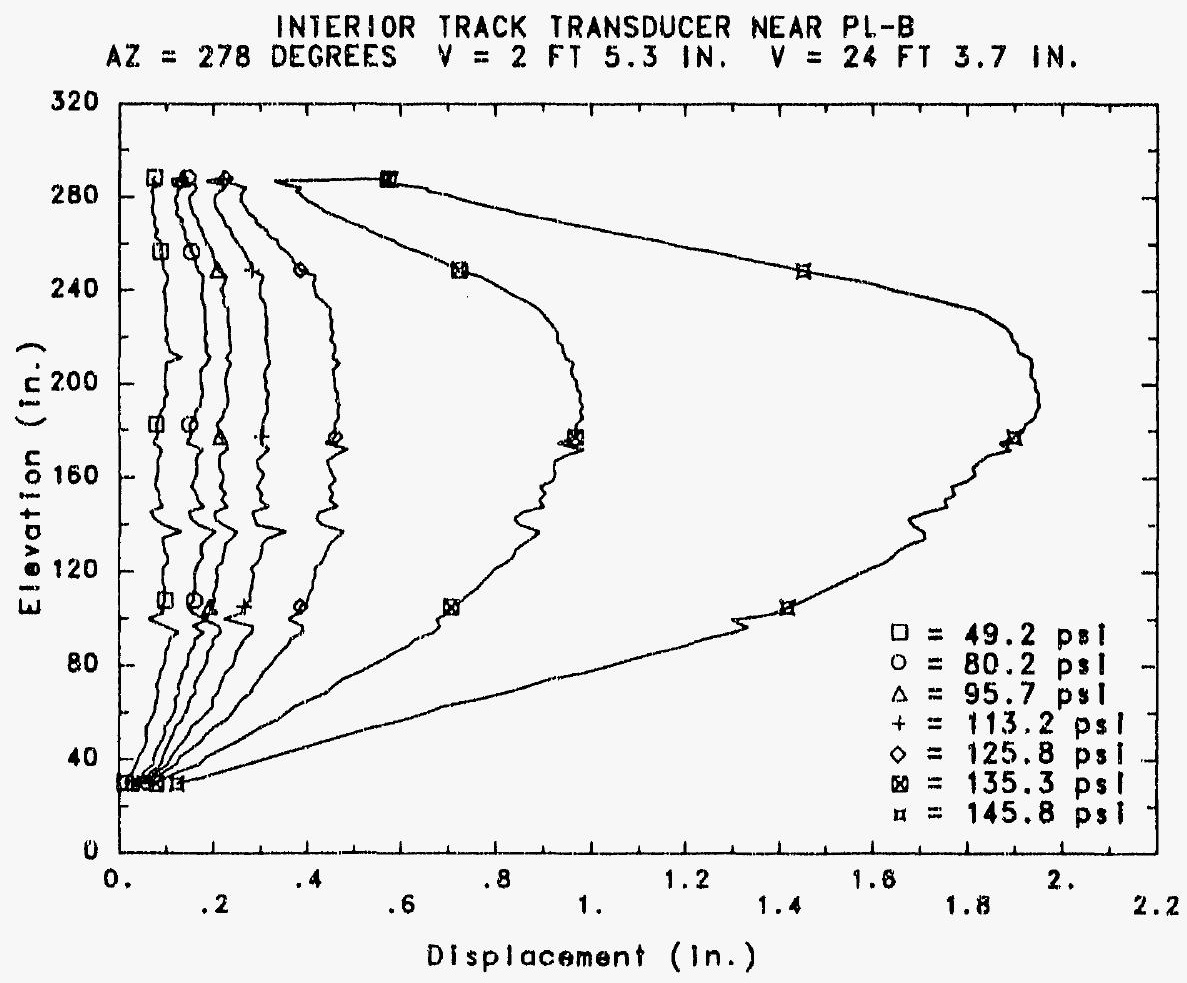

Figure B-4. Track 4, Interior Track Transducer Near PL-B; $A z=278$ Degrees, V=2 Ft. 5.3 In., V=24 Ft. 3.7 In. 


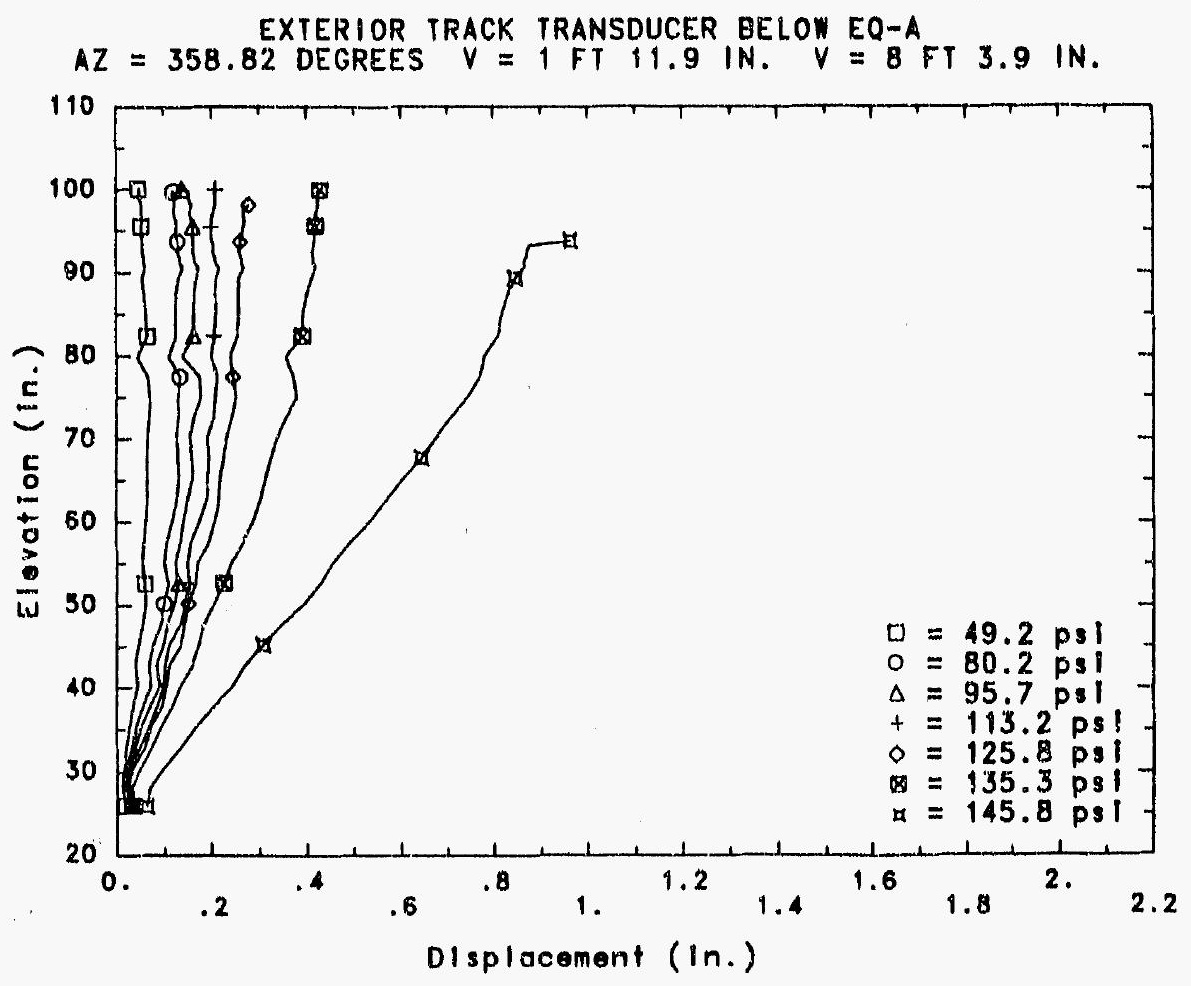

Figure B-5. Track 5, Exterior Track Transducer Below EQ.-A, Az=358.82 Degrees; V=1 Ft. 11.9 In., $\mathrm{V}=8 \mathrm{Ft}$. $3.9 \mathrm{In}$.

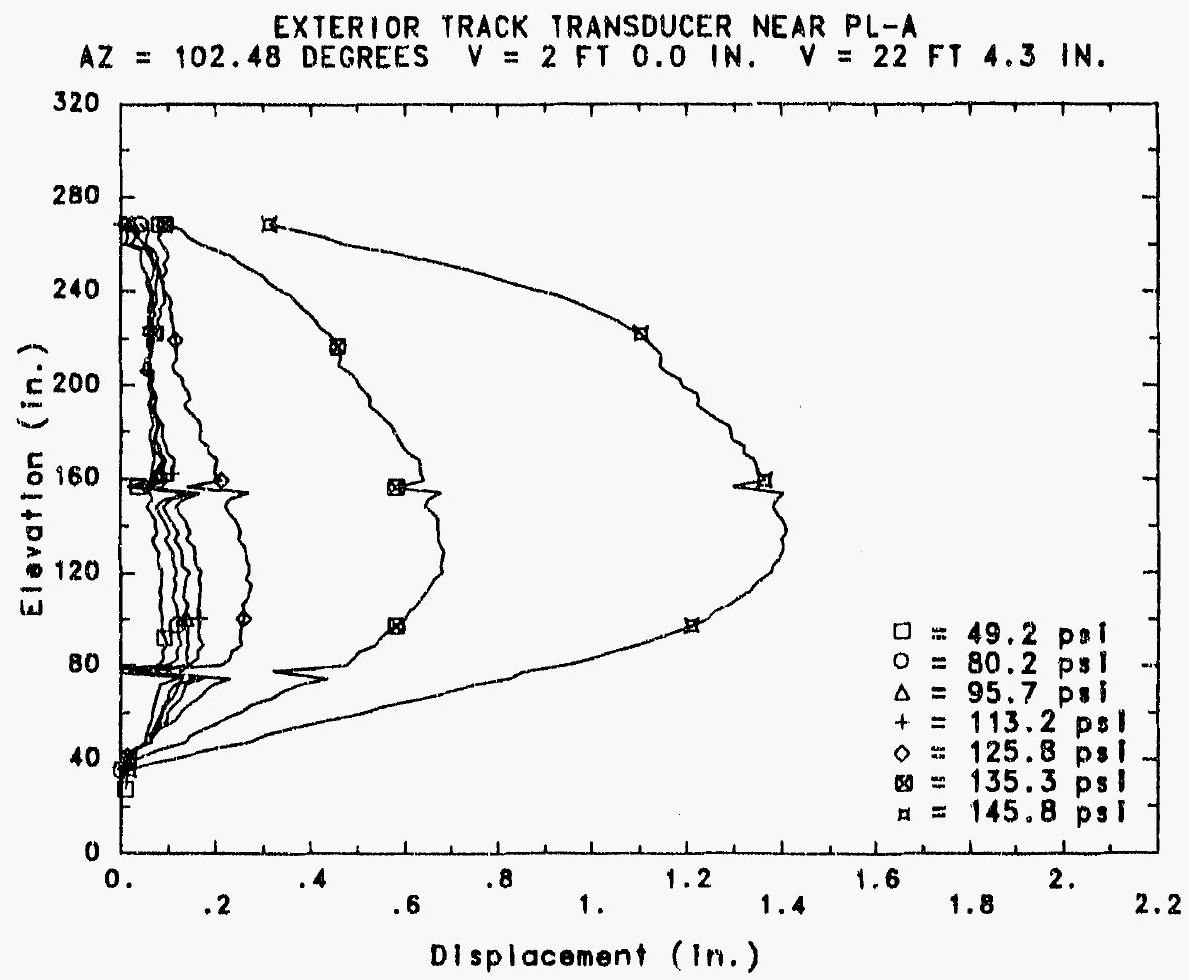

Figure B-6. Track 6, Exterior Track Transducer Near PL-A; Az=102.48 Degrees, $V=2 \mathrm{Ft}$. $0.0 \mathrm{In} ., \mathrm{V}=22 \mathrm{Ft} .4 .3 \mathrm{In}$. 


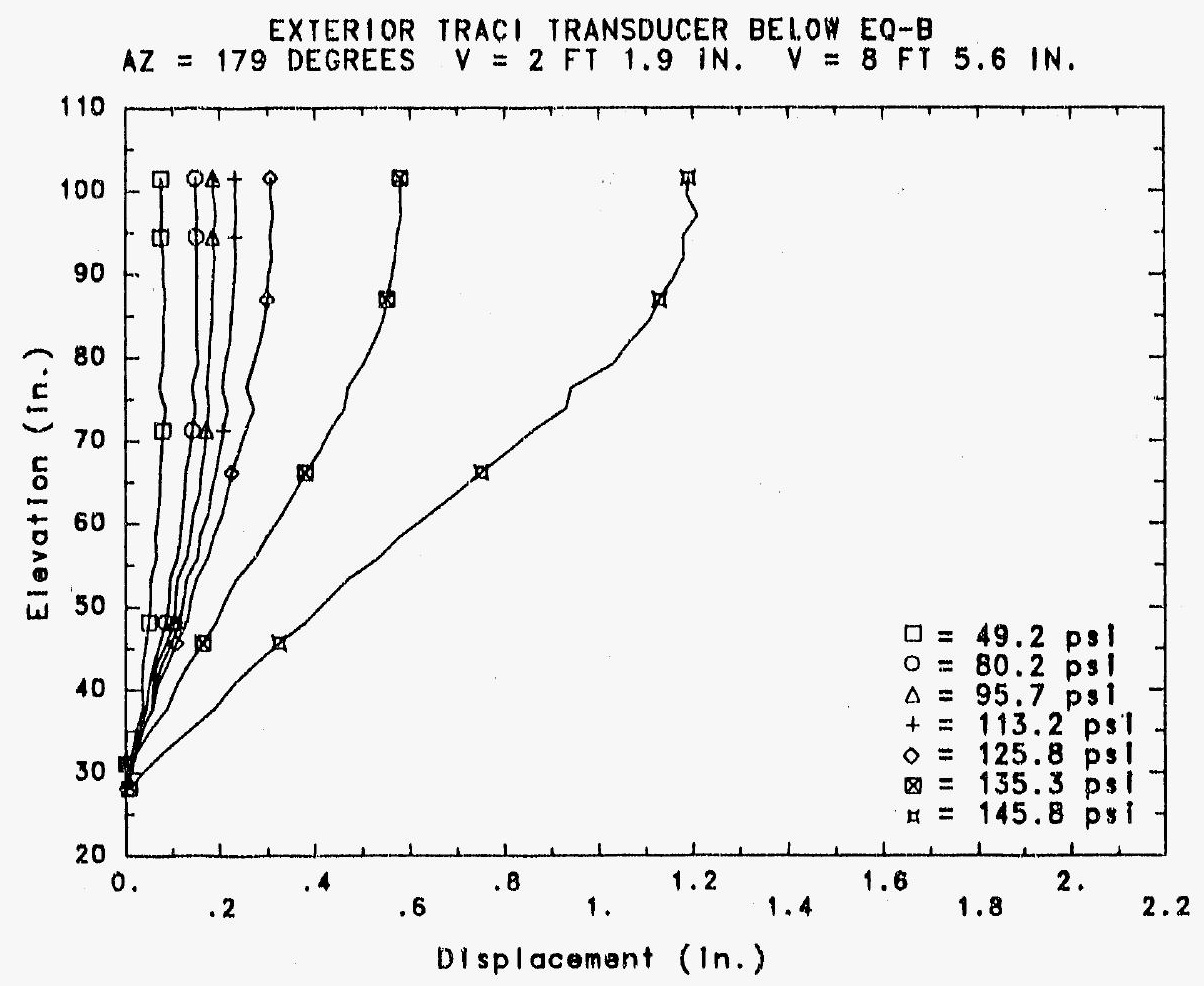

Figure B-7. Track 7, Exterior Track Transducer Below EQ.-B, Az=179 Degrees; V=2 Ft. 1.9 In., V=8 Ft. 5.6 In.

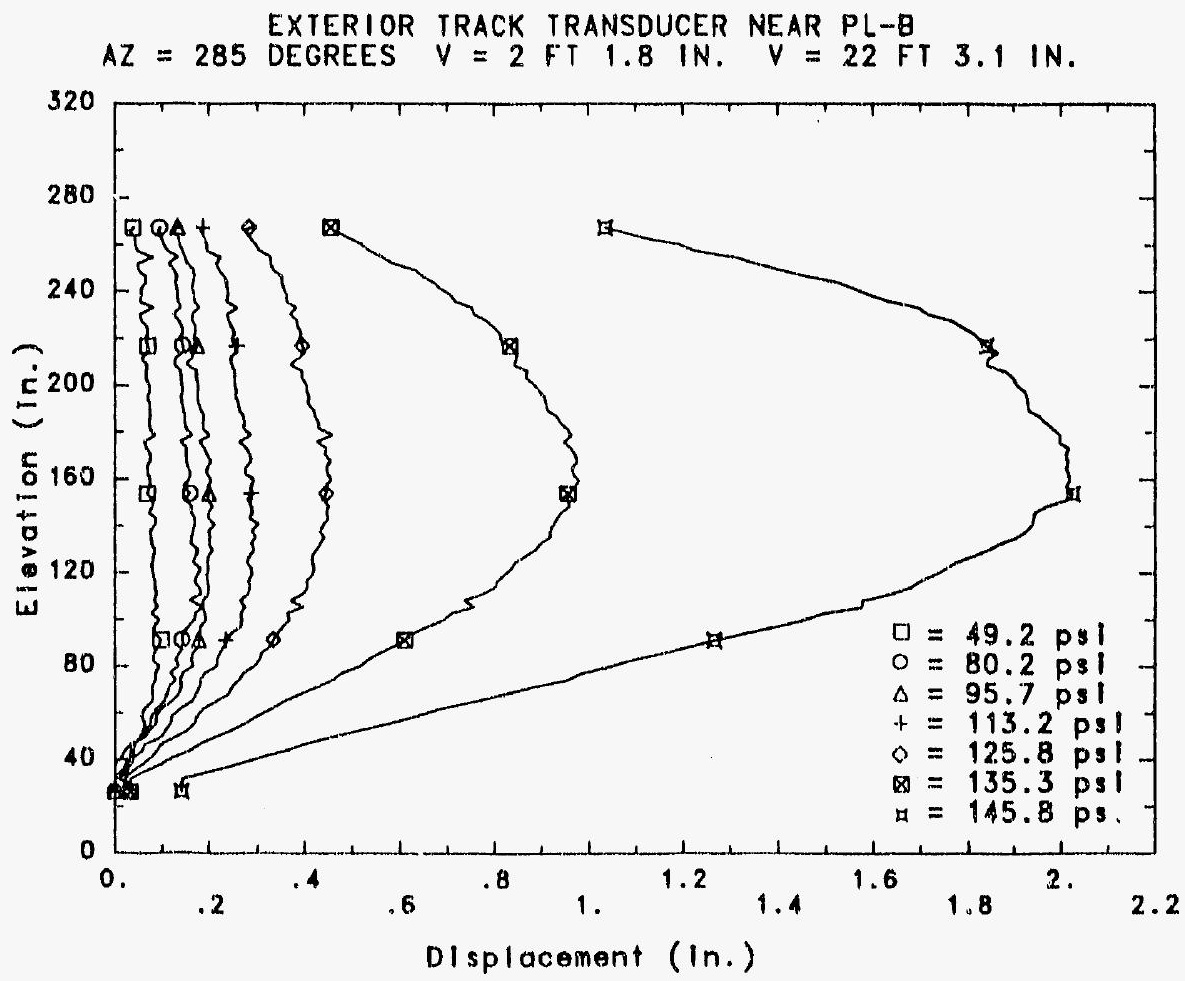

Figure B-8. Track 8, Exterior Track Transducer Near PL-B; Az=285 Degrees, V=2 Ft. 1.8 In., $V=22$ Ft. 3.1 In. 


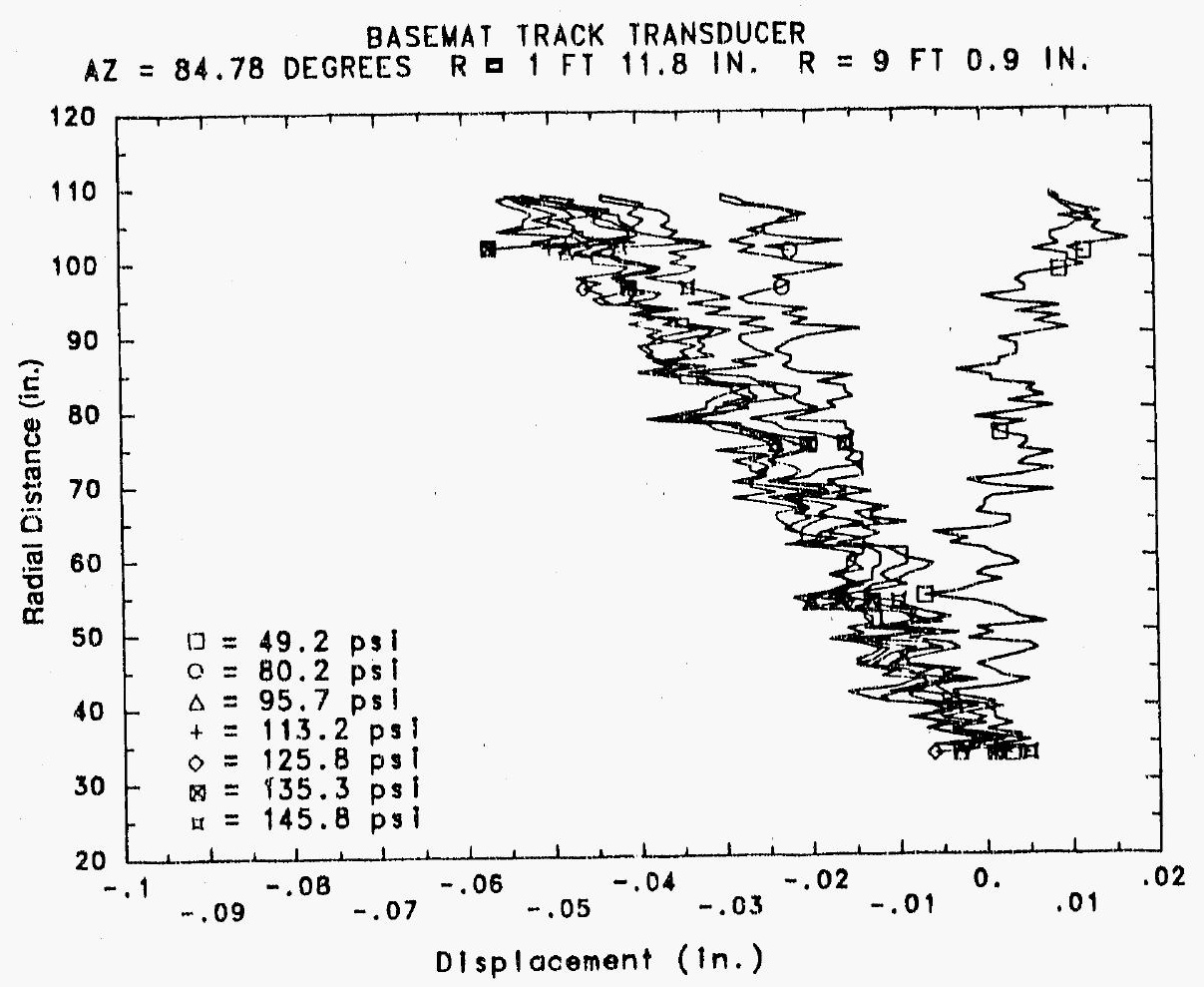

Figure B-9. Track 9, Basemat Track Transducer; $A z=84.78$ Degrees; $R=1$ Ft. 11.8 In., $R=9$ Ft. 0.9 In. 
Distribution:

J. F. Costello (20 Copies)

Structura1 \& Selsmic Engr. Branch

Mail Stop NL/S-217A

USNRC/RES

Washington, D. C. 20555

H. L. Graves, III

Structural \& Selsmic Engr. Branch

Mail Stop NL/S-217A

USNRC/RES

Washington, D.C. 20555

US Department of Energy

Office of Nuclear Energy

Attn: D. Giessing

W. Pasedag (2 coples)

Mail Stop B-107

NE -540

Washington, D.C. 20545

ABB Impe11 Corporation

Attn: Tim Lu

27401 Los Altos, Suite 480

Mission Viejo, CA 92691

ANATECH Research Corp.

Attn: Y. R, Rashid

P. O. Box 9165

LaJo11a, CA 92038

Argonne National Laboratory

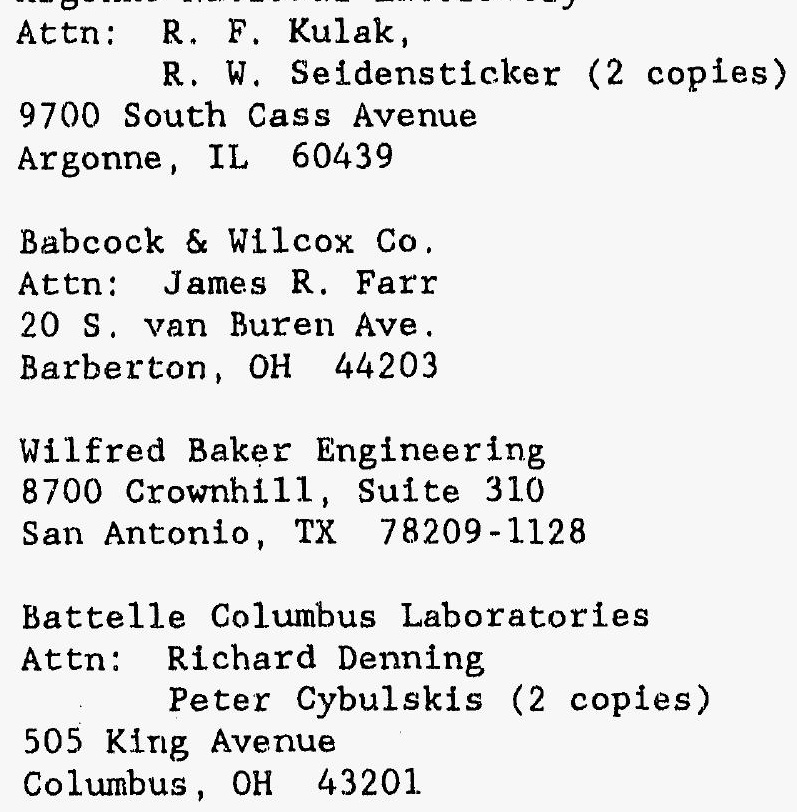

Bechtel Power Corporation

Attn: Asadour H. Hadjian Bertold W. Pfeifer (2 copies)

12440 E. Imperial Highway

Norwalk, CA 90650

Bechtel Power Corp.

Attn: Subir Sen

K. Y. Lee (2 copies)

15740 Shady Grove Rd.

Gaithersburg, MD 20877

Bechtel. Savannah River, Inc.

Attn: T. E. Johnson

802 E. Martintown Road

North Augusta, SC 29841

Brookhaven National Laboratory

Attn: C. Hofmayer, T. Pratt,

Building 130

M. Reich (3 copies)

Upton, NY 11973

Brookhaven National Laboratory

Attn: Ted Ginsberg

Building 820M

Upton, NY 11973

CBI NaCon, Inc.

Attn: Thomas J. Ah1

800 Jorie Boulevard

Oak Brook, IL 60521

City College of New York

Dept. of Civil Engineering

Attn: C. Costantino

140 Street and Convent Ave.

New York, NY 10031

Cornell University

Attn: Professor Richard N. White

School of Civil \& Environ. Engr.

Hollister Hall

Ithaca, NY 14853

EBASCO Services, Inc.

Attn: Robert C. Iotti

Two World Trade Center

New York, NY 10048 
EG\&G Idaho

Attn: B. Barnes, T. L. Bridges

(2 copies)

Willow Creek Bldg. W-3

PO Box 1625

Idaho Falls, ID 83415

Electrical Power Research Institute

Attn: H. T. Tang, Y. K. Tang ( 4 coples) Raf Sehga1, J. J. Taylor

3412 Hillview Avenue

PO Box 10412

Palo Alto, CA 94304

EQE Inc.

Attn: M. K. Ravindra

3300 Irvine Avenue

Suite 345

Newport Beach, CA 92660

General Electric Company

Attn: E. O. Swain, D. K. Henrie,

R. Gou, H. Townsend (4 copies)

175 Curtner Ave.

San Jose, CA 95125

Iowa State University

Department of Civil Englneering

Attn: L. Greimann

420 Town Engineering Bldg.

Ames, IA 50011

Los Alamos National Laboratorles

Attn: C. Anderson

PO Box 1663

Mail S top $N 576$

Los Alamos, NM 87545

Northern Illinois University

Mechanical Engineering Dept.

Attn: A. Marchertas

DeKalb, IL 60115

NUTECH Engineers, Inc.

Attn: John Clauss

1111 Pasquinelli Drive, Suite 100

Westmont, IL 60559

Oak Ridge National Laboratory

Attn: Steve Hodge

PO Box X

Oak Ridge, TN 37830
Quadrex Corporation

Attn: Quazi A. Hossain

1700 De11 Ave.

Campbe11, CA 95008

Sargent \& Lundy Engineers

Attn: B. A. Erler

P. K. Agrawal (2 copies)

55 E Monroe St.

Chicago, IL 60603

Stevenson \& Associates

Attn: John D. Stevenson

9217 Midwest Ave.

Cleveland, $\mathrm{OH} \quad 44125$

Tennessee Valley Authority

Attn: Nathaniel Foster

400 Summit Hill Rd.

W9D24C-K

Knoxville, TN 37902

TVA

Attn: D. Denton, W9A18

400 Commerce Ave.

Knoxville, TN 37902

United Engineers \& Constructors, Inc.

Attn: Joseph J. Ucciferro

$30 \mathrm{~S} .17$ th St.

Philadelphia, PA 19101

University of California at Santa Barbara

Dept. of Chemical \& Nuclear Engineering

Attn: T, G. Theofanous

Santa Barbara, CA 93106

University of Illinois

Attn: C. Siess

Dept. of Civil Engineering

Urbana, IL 61801

University of Illinois

1245 Newmark CE Lab

Attn: Prof. Mete A. Sozen

208 N. Romine

MC - 250

Urbana, IL 61801

University of Wisconsin

Nuclear Engineering Dept.

Attn: Prof. Michael Corradini Madison, WI 53706 
Westinghouse Electric Corp.

Attn: Vijay K. Sazawal

Waltz Mill Site

Box $1: 58$

Madison, PA 15663

Institut fuer Mechanik

Universitaet Innsbruck

Attn: Prof. G. I. Schueller

Technlkerstr. 13

A-6020 Innsbruck

AUSTRIA

Nuclear Studies \& Safety Dept.

Ontario Hydro

Attn: W. J. Penn

700 University Avenue

Toronto, Ontario

M5G 1 X 6

CANADA

University of Alberta

Dept. of Clvil Engineering

Attn: Prof. D. W. Murray

Edmonton, Alberta

T6G 2 G7

CANADA

Commissariat a l'Energle Atomique

Centre d'Etudes Nucleaires de Saclay

Attn: M. Livolant, P. Jamet (2 copies)

F-91191 Gif-sur-Yvette Cedex

FRANCE

Commissariat a l'Energie Atomique

Institut de Protection et de

Surete Nucleaire

Attn: M. Barbe

F-92660 Fontenay-aux-Roses

FRANCE

OECD Nuclear Energy Agency

Attn: K. Stadie

Deputy Director, Safety \& Regulation

38, Boulevard Suchet

F-75016 Paris

FRANCE

Kernforschungszentrum Karlsruhe GmbH

Attn: R. Krieg, P. Gast (2 copies)

Postfach 3640

D-7500 Karlsruhe

GERMANY
Technische Universitaet Muenchen

Lehrstuhl fuer Reakordynamik und Reaktorsicherheit

Attn: Prof. H. Karwat

D-8046 Garching

GERMANY

Staatliche Materialpruefungsanstalt (MPA)

University of Stuttgart

Attn: Prof. K. F. Kussmaul

Pfaffenwaldring 32

D-7000 Stuttgart 80 (Vaihingen)

GERMANY

Gesellschaft fuer Reaktorsicherheit

Attn: H. Schulz, A. Hoefler,

F. Schleifer ( 3 copies)

Schwertnergasse 1

D-5000 Koeln 1

GERMANY

Kraftwerk Union AG

Attn: M. Hintergraber

Hammerbacherstr. 12-14

D-8520 Erlangen

GERMANY

ISMES

Attn: A. Peano

Viale Giulio Cesare 29

I - 24100 Bergamo

ITALY

ENEA

Attn: Raffaele Di Sapia

Via Le Regina Margherita, 125

I-00198 Roma

ITALY

ENEA

Dipartimento Reattori Innovativi

Attn: Paolo Corticelli

Via Dell'Arcoveggio, 56/22-56/23

I-4012.9 Bologna

ITALY

ENEA-DISP

Attn: Giuseppe Pino

Gianni Petrangeli (2 copies)

Via Vitaliano Brancati, 48

I-00144 Roma

ITALY 
Nuclear Equipment Design Dept.

Hitachi Works, Hitachi, Ltd.

Attn: 0 . Oyamada

3-1-1 Saiwa1-Cho

Hitachi-Shi.

Ibaraki-ken

JAPAN

Division of Technical. Information

Japan Atomlic Energy Research Institute

Attn: Jun-ichi Shimokawa

2-2, Uchisaiwal-cho 2-chome

Chilyoda, Tokyo 100

JAPAN

University of Tokyo

Institute of Industrial Science

Attn: Prof. H. Shibata

22-1, Roppongi 7

Minatu-ku, Tokyo

JAPAN

Civil Engineering Laboratory

Central. Research Institute of

Electric Power Industry

Attn: Yuk1o Aoyagi

1646 Abiko Abiko-Shi Chiba

JAPAN

Kajima Corporation

Attn: $T$. Sugano, $K$. Umeda

KI Building

$$
\text { H. Tsubota (3 copies) }
$$

5-30, Akasada 6-chome

Minato-ku

Tokyo 107

JAPAN

Mitsubishi Heavy Industries, Ltd.

Attn: Kaoru Nagata

Manager, Nuclear Containment Vessel

Designing Section

Steel Structure Department

Kobe Shipyard \& Machinery Works

1-1, Wadasaki-cho, 1-Chome, Hyogo-ku,

Kobe 652

JAPAN
NUPEC

Attn: K. Takumi, A. Nonaka (2 coples)

Equipment and Components Dept.

Shuwa Kamiyacho Building

3-13, 4-Chome

Toranomon, Minato-ku

Tokyo 105

JAPAN

Japan Atomic Energy Research Inst.

Attn: Kunihisa Soda

Toshikuni Isozaki (2 coples)

Toka1-Mura, Ibaraki-Ken 319-11

JAPAN

Obayashi Corporation

Technical Research Institute

Attn: Toshikazu Takeda

Matsutaro Seki

4-640, Shimokiyoto, Xiyose-shi

T'okyo 204

JAPAN

Shimizu Coxporation

Attn: T. Kuroda, Y. Takeuchi (2 coples)

vuclear Power Division

Seavans South, No. 2-3, Shibaura

1.-chome, Minato-ku

Tokyo 105-07

JAPAN

Universidad Politecnica

Escuela Tecnica Superior

de Ingenieros Industriales

Attn: Agustin Alonso

Madrid

SPAIN

Unidad Electrica S.A.

Attn: Jose Puga

UNESA

ES-28020 Madrid

SPAIN

Principia Espana, SA

Attn: Joaquin Marti

Orense, 36-2

28020 Madrid

SPAIN 
Servicio Licenciamiento

Central Nuclear de Asco

Attn: Sr. D. Joaquin Sanchez Baptista

Tres Torres, 7

ES - 08017 Barcelona

SPAIN

Central Nuclear de Almaraz

Attn: Sr. D. Jose Maria Zamarron

Subdirector Tecnico

Claudio Coe11o, 123

ES - 28006 Madrid

SPAIN

Nuclenor, S.A.

Attn: Sr. D. Federico del Pozo Obeso Director General

Hernan Cortes, 26

ES-39003 Santander

SPAIN

UNESA

Attn: Sr. D. Jose Puga Fernandez

Francisco Gervas, 3

ES - 28020 Madrid

SPAIN

Studsvik Energiteknik $A B$

Attn: Kjell 0 . Johansson

S-611 82 Nykoping

SWEDEN

Swedish State Power Board

Nuclear Reactor Safety

Attn: Hans Cederberg

Per-Eric Ahlstrom

Ralf Espefaelt ( 3 copies)

S-162 87 Vallingby

SWEDEN

Swiss Federal Institute of Technology

Institute of Structural Engineering

Attn: W. Ammann

ETH-Hoenggerberg, HIL

CH-8093 Zurich

SWITZERLAND

Colenco Led.

Attn: K. Gahler, A. Huber

J. Jemielewski

A. Schopfer (4 copies)

Mellingerstrasse 207

CH-5405 Baden

SWITZERLAND
Paul Scherrer Institut

Attn: P. Hosemann

CH-5232 Villigen PSI

SWITZERLAND

Swiss Federal Nuclear Safety Inspectorate Federal Office of Energy

Attn: S. Chakraborty

CH-5303 Wuerenlingen

SWITZERLAND

Swiss Federal Institute of Technology

for Building Materials

Attn: Prof. F. H. Wittmann

ETH-Honggerberg

CH-8093 Zurich

SWITZERLAND

Elektrowatt Ingenieur"internehmung AG

Attn: John $\mathrm{P}$. Wolf

Bellerivestr. 36

CH-8022 Zurich

SWITZERLAND

Atomic Energy Authority

Safety and Reliability Directorate

Attn: D. W. Phtllips

Wigshaw Lane

Culcheth

Warrington WA3 $4 \mathrm{NE}$

UNITED KINGDOM

Atomic Energy Establishment

Attn: Peter Barr

Winfrith

Dorchester Dorset

DT2 8DH

UNITED KINGDOM

HM Nuclear Installations Inspectorate

Attn: R. Bye, R. J. Stubbs (2 copies)

St. Peter's House

Balliol Road

Bootle, Mereyside L2.0 3LZ.

UNITED KINGDOM

Nuclear Electric

Attn: J. Irving

Booths Hall

Chelford Road

Knutsford

Cheshire WA $168 Q \mathrm{QG}$

UNITED KINGDOM 
Taylor Woodrow Construction Limited

Attn: Carl C. Fleischer

Richard Crowder (2 copies)

345 Ruislip Road

Southall, Midalesex

UBI 2QX

UNITED KINGDOM

3141 S. A. Landenberger (5)

3151 G. C. Claycomb

6400 D. J. McCloskey

6460 J. V. Walker

6470 D. J. McCloskey

6473 W. A. von Rlesemann (31)

6473 D. S. Horsche1

6473 L. D. Lambert

6473 J. S. Ludwigsen

6473 D. W. Pace

6473 M. B. Parks

6473 B. L. Speltzer

6473 J. J. Westmoreland

6473 H. P. Walther

6473 R. A. Watson

8523-2 Central Technical files 


\begin{tabular}{|c|c|}
\hline $\begin{array}{l}\text { U.S. NUCLEAR REGULATORY COMMISSION } \\
\text { BIBLIOGRAPHIC DATA SHEET } \\
\text { ISee instructions on the reverse) }\end{array}$ & \multirow{2}{*}{ 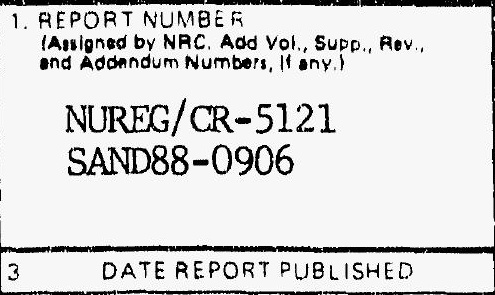 } \\
\hline \multirow{4}{*}{$\begin{array}{l}\text { 2. TITLE AND SUBTITLE } \\
\text { Experimental Results from Pressure Testing a 1:6-Scale Nuclear } \\
\text { Power Plant Containment }\end{array}$} & $\begin{array}{l}\text { NUREG/CR-5121 } \\
\text { SAND88-0906 }\end{array}$ \\
\hline & \\
\hline & \begin{tabular}{|l|l|} 
MONTM & $1992^{\text {TEAF }}$ \\
January & 199
\end{tabular} \\
\hline & $\begin{array}{l}\text { January } 1992 \\
\text { 4. FINOR GRANT NUMBER } \\
\text { FIN A1249 } \\
\end{array}$ \\
\hline $\begin{array}{l}\text { E. AUTHORISI } \\
\text { D. S. Horschel }\end{array}$ & \\
\hline \multicolumn{2}{|l|}{$\begin{array}{l}\text { Sandia National Laboratories } \\
\text { Albuquerque, NM } 87185\end{array}$} \\
\hline \multicolumn{2}{|c|}{ 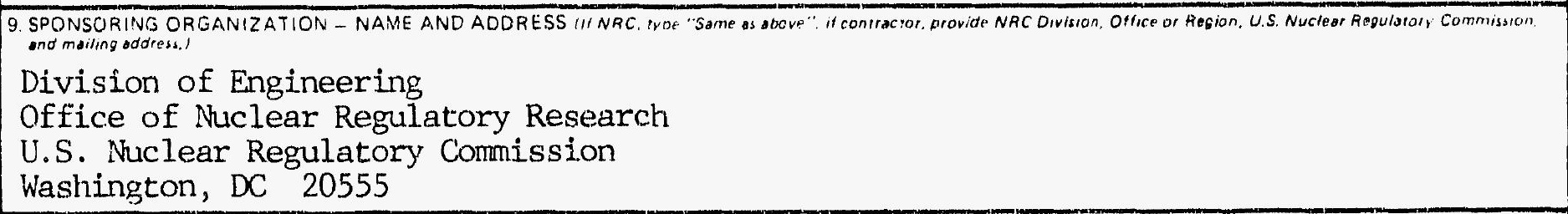 } \\
\hline \multicolumn{2}{|c|}{ 10. SUPPLEMENTARY NOTES } \\
\hline \multicolumn{2}{|c|}{ 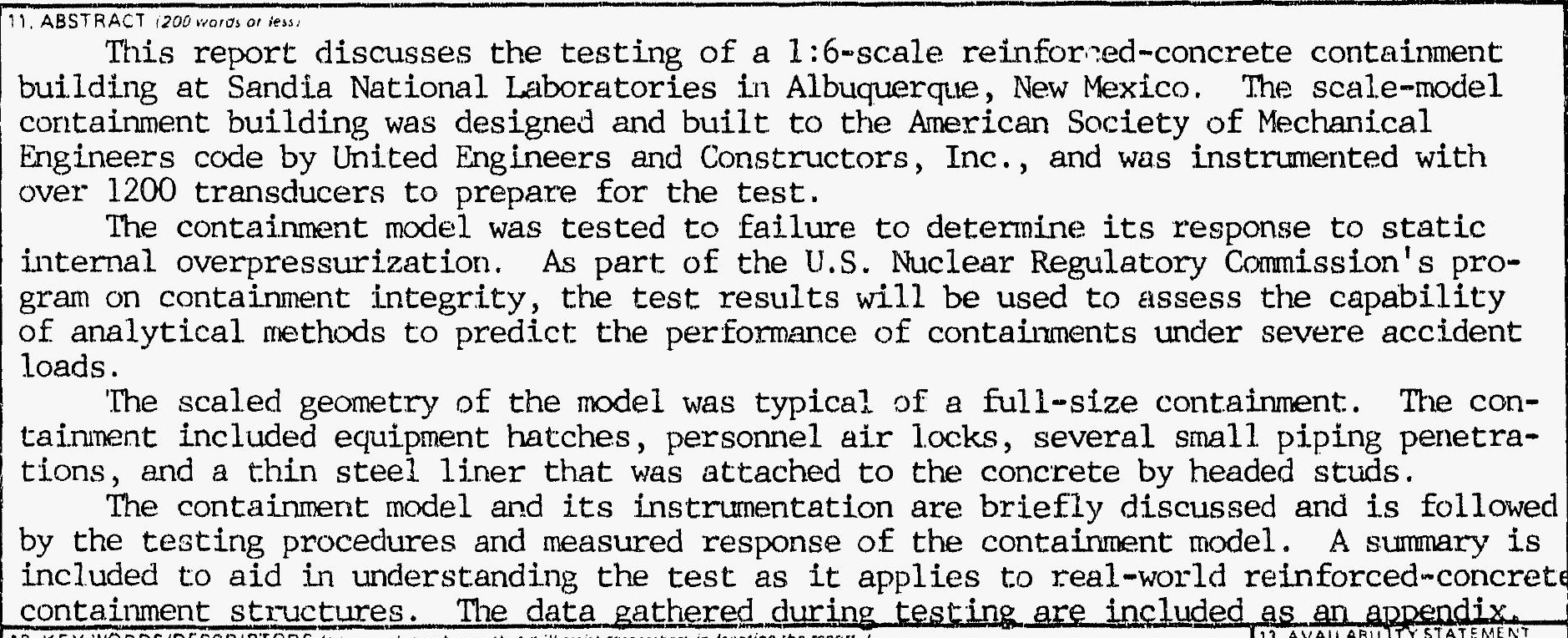 } \\
\hline \multirow[t]{2}{*}{ 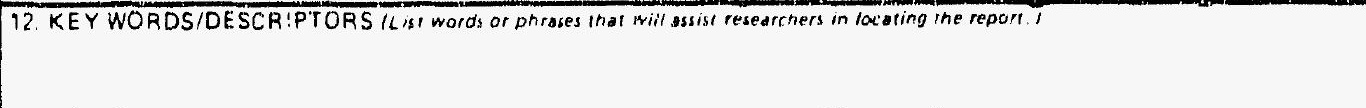 } & \\
\hline & 19. SECUAITYGLASSTFICATION \\
\hline \multirow{4}{*}{$\begin{array}{l}\text { Reinforced-Concrete } \\
\text { Nuclear Power } \\
\text { Testing } \\
\text { Containnent } \\
\text { Structures } \\
\text { Models } \\
\text { Failire }\end{array}$} & \\
\hline & \\
\hline & 15 NUMBER OF PAGES \\
\hline & ic price \\
\hline
\end{tabular}



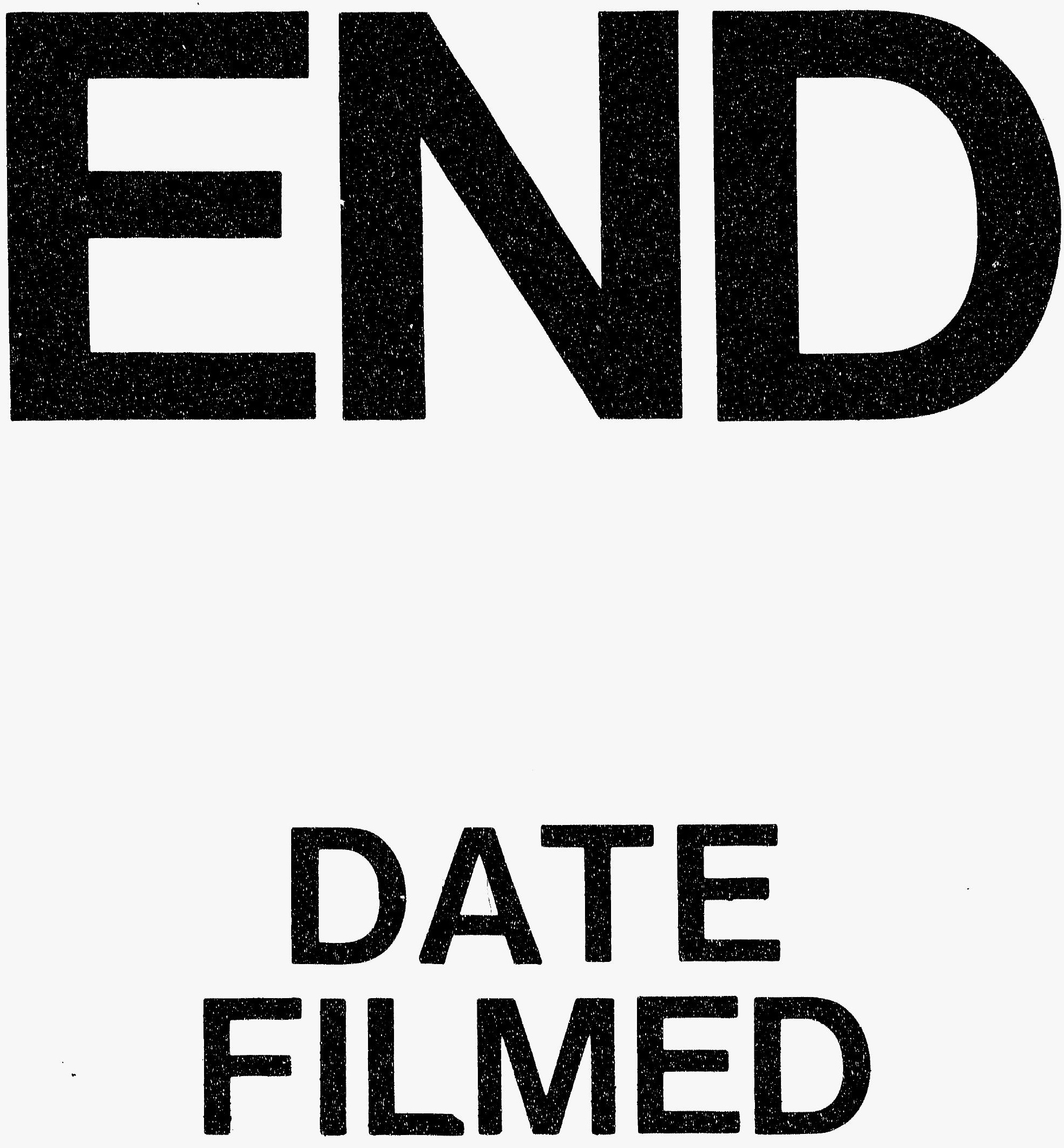

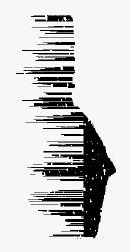

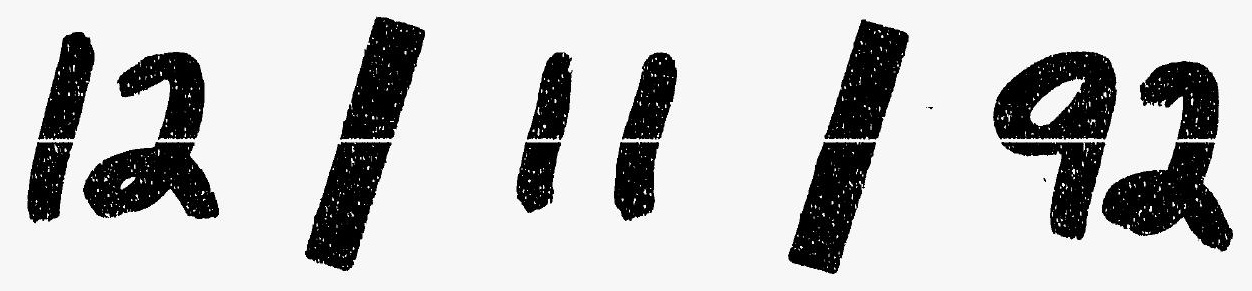





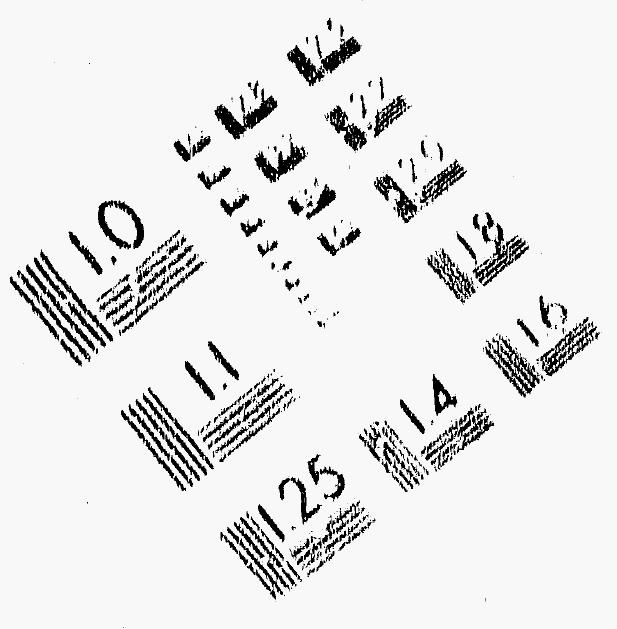

IMAGE EVALUATION

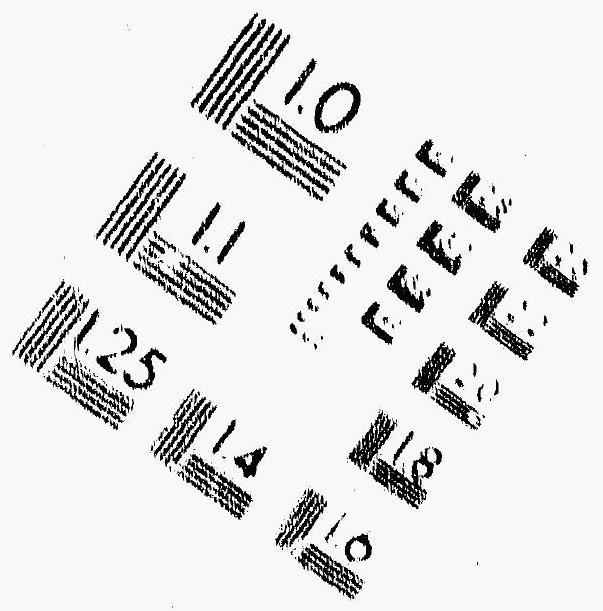

TEST TARGET (MT-3)
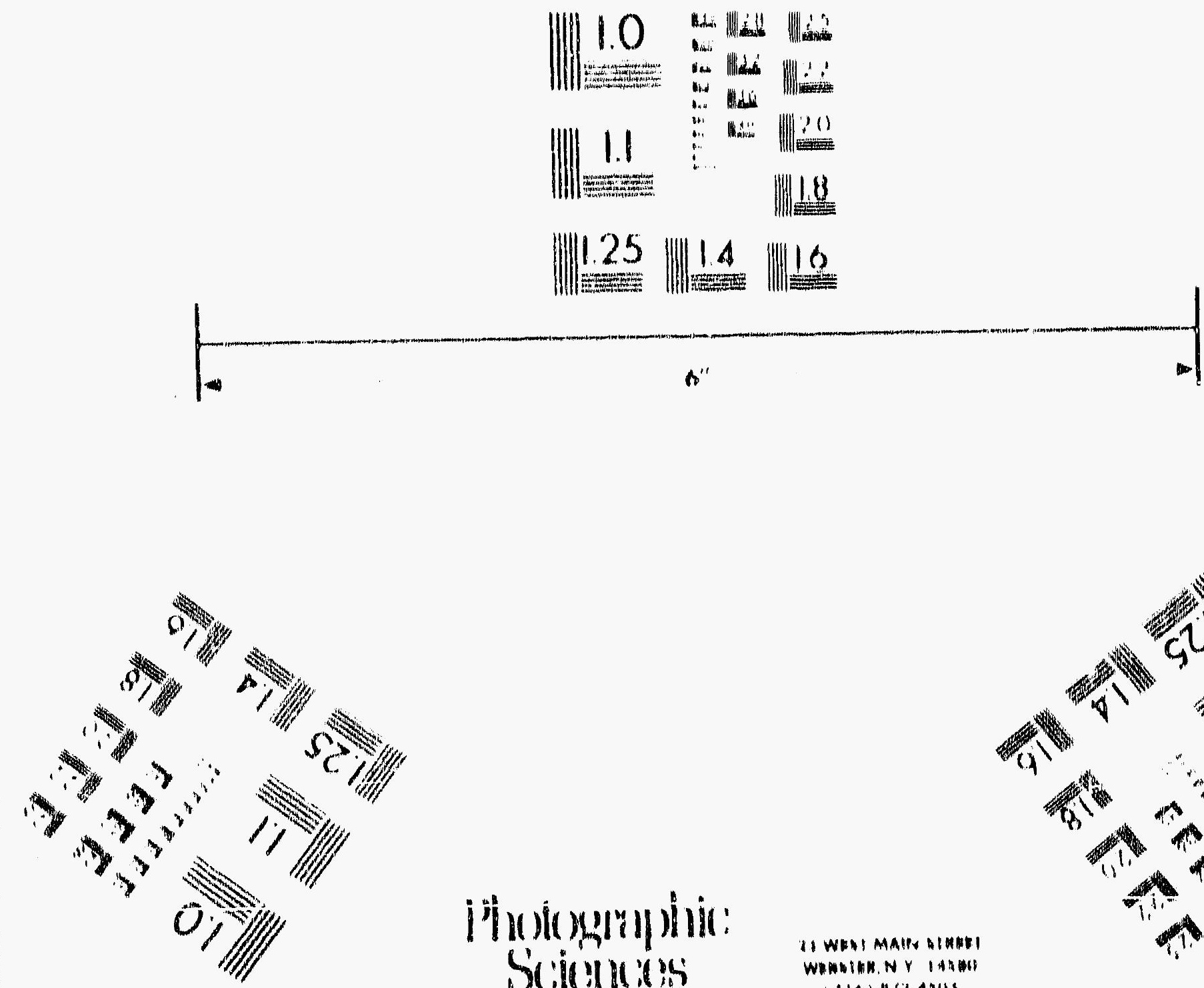

Ithofogmphis: Siciencess

(ciliperistion
II WEMI MAlit AlGMEI

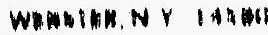

(I) hel alles

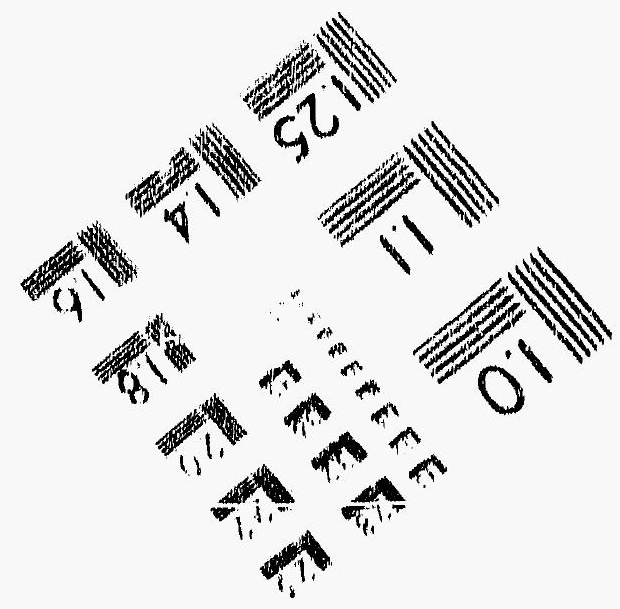




\section{SECURITY CLASSIFICATION}
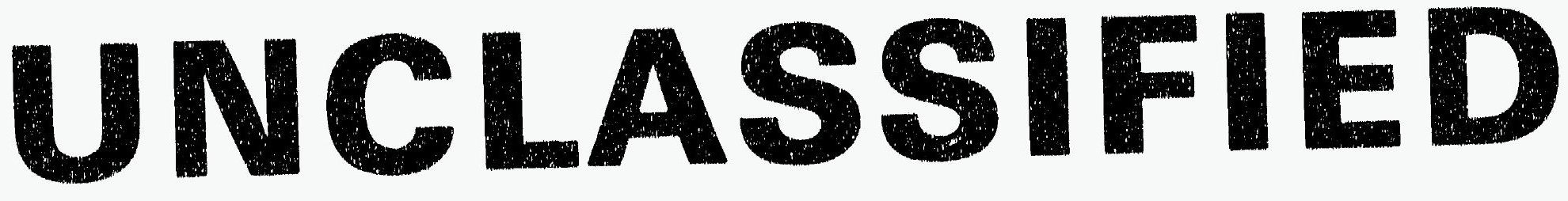

\section{DATE OF MICROFILMING $1-6-92$}

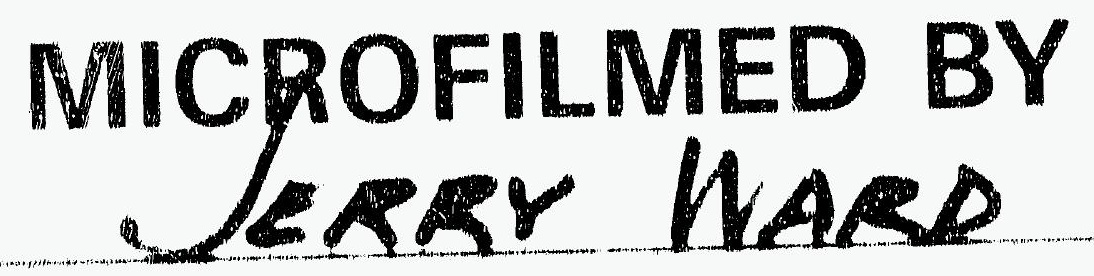

SANDIA NATIONAL LABS 
THIS DOCUMENT FILMED

AT

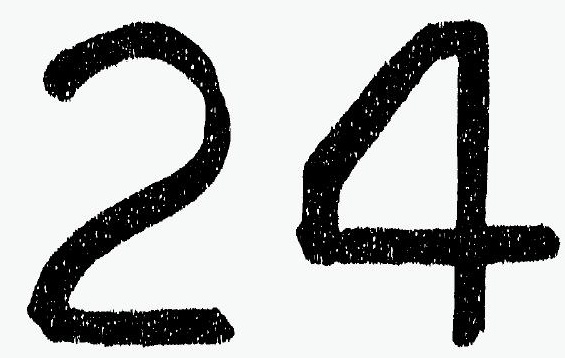

REDUCTION 
AMINIINI

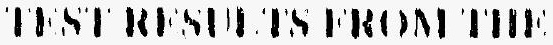

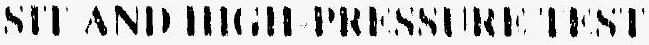




\section{('I INIKIIIIII IIIIN}

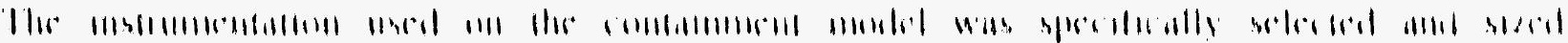

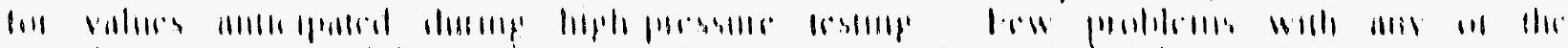

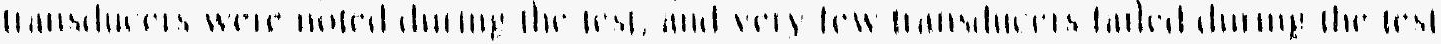

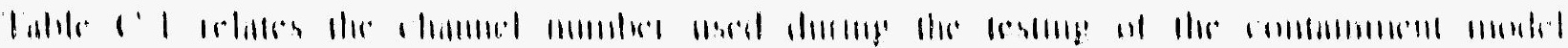

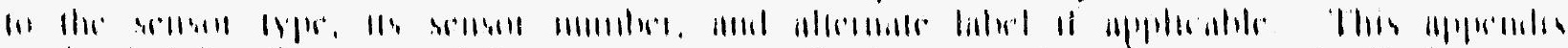

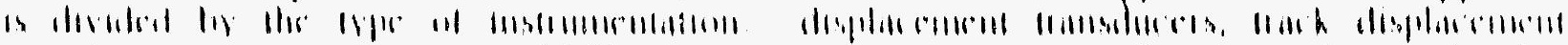

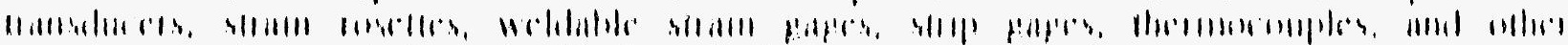

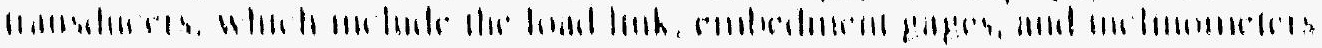

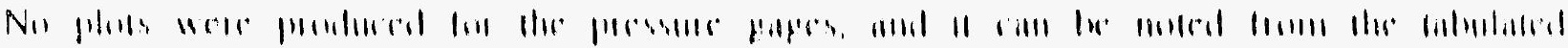

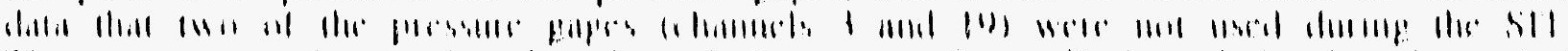

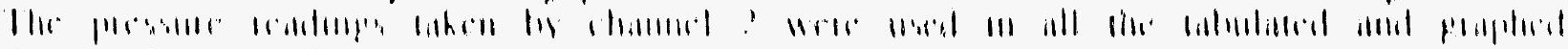

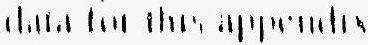

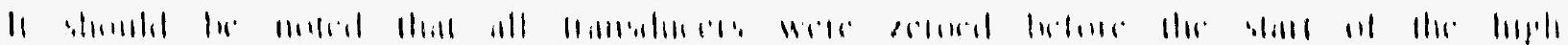

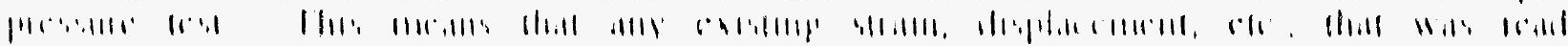

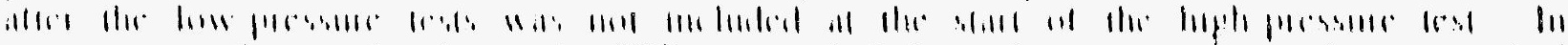

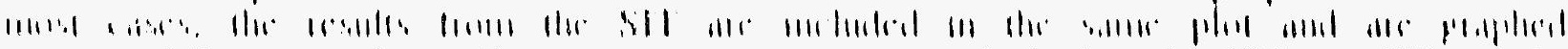

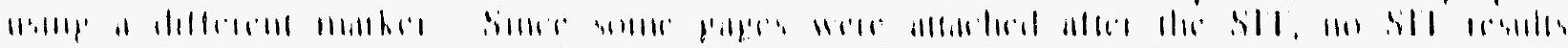

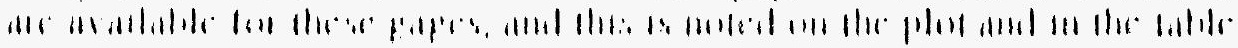

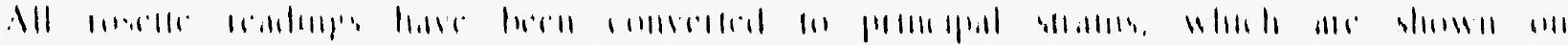

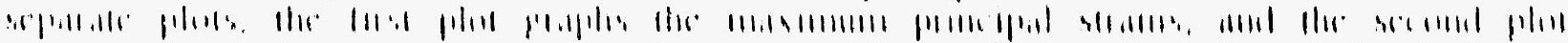

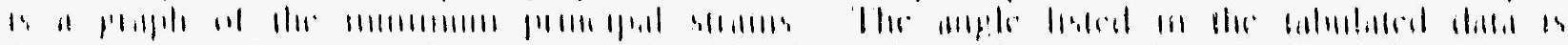

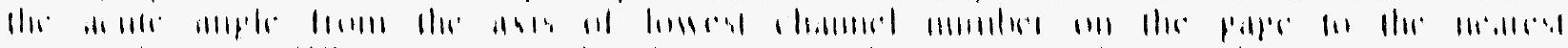

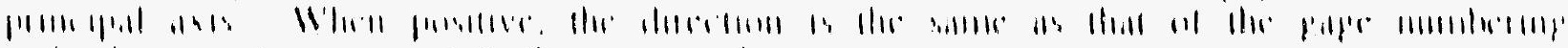
. 
linkile (' 1

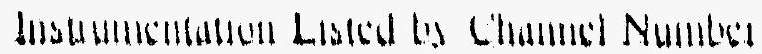

\begin{tabular}{|c|c|c|}
\hline $\begin{array}{c}\text { I hallinal } \\
\text { MHe }\end{array}$ & $\begin{array}{c}\text { Iinllaill } \\
\text { libu }\end{array}$ & $\begin{array}{c}\text { Allaniliala } \\
\text { Lutuel }\end{array}$ \\
\hline 1 & I'wantwi & \\
\hline$!$ & 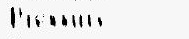 & \\
\hline 1 & I110:-111: & \\
\hline 1 & 1. fin & \\
\hline । & l/1,11 & \\
\hline n & I'וn, & \\
\hline IH & | noul | ind & \\
\hline 14 & l'wn+11. & \\
\hline (1) & 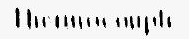 & \\
\hline 1.1 & 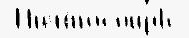 & \\
\hline $0 ! !$ & 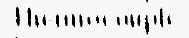 & \\
\hline 111 & 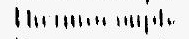 & \\
\hline 1,1 & 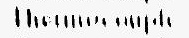 & \\
\hline$(1)$ & 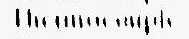 & \\
\hline nen & 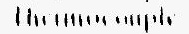 & \\
\hline n: & 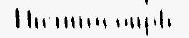 & \\
\hline rill & 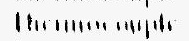 & \\
\hline$\therefore " 1$ & 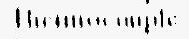 & \\
\hline 11 & 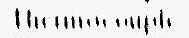 & \\
\hline 11 & 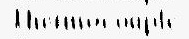 & \\
\hline$\because$ & 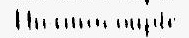 & \\
\hline 11 & 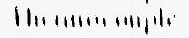 & \\
\hline 1.1 & 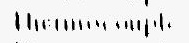 & \\
\hline 19 & 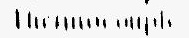 & \\
\hline ' 11 & 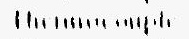 & \\
\hline$\therefore$ & 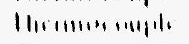 & \\
\hline In & 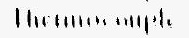 & \\
\hline 10 & 11 11н... & \\
\hline iil & 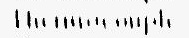 & \\
\hline ii & $|1,+1+\ldots, \ldots+1| \|_{1}$ & \\
\hline H.' & 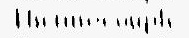 & \\
\hline ni & 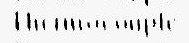 & \\
\hline $\mathrm{NI}$ & 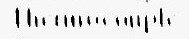 & \\
\hline hi & 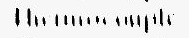 & \\
\hline $\mathrm{kli}$ & 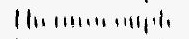 & \\
\hline$n ! !$ & | H. & \\
\hline प1 & 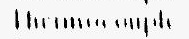 & \\
\hline 91 & 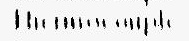 & \\
\hline $11:$ & 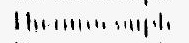 & \\
\hline 11 & 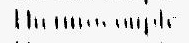 & \\
\hline 4 & 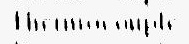 & \\
\hline 119 & 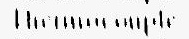 & \\
\hline $1 / 1_{1}$ & 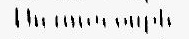 & \\
\hline$y^{\prime}$ & IIn 1116... & \\
\hline Uhi & \|\|$_{1}, \ldots+\ldots \ldots+1 \|_{1}$ & \\
\hline 0.1 & 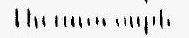 & \\
\hline 11111 & 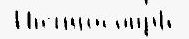 & \\
\hline $\mid 111$ & 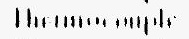 & \\
\hline $111:$ & Hor & \\
\hline 1111 & 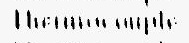 & \\
\hline 1111.1 & 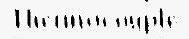 & \\
\hline 1114 & 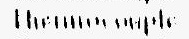 & \\
\hline 1116 & 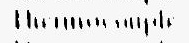 & \\
\hline$|i|$ & 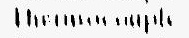 & \\
\hline $111 \mathrm{~h}$ & 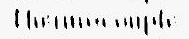 & \\
\hline 1114 & 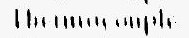 & \\
\hline 1111 & 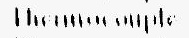 & \\
\hline 111 & 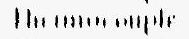 & \\
\hline 11: & 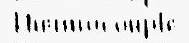 & \\
\hline 111 & 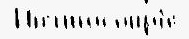 & \\
\hline 111 & 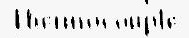 & \\
\hline 111 & 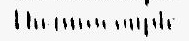 & \\
\hline 1101 & 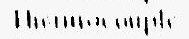 & \\
\hline 111 & 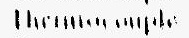 & \\
\hline $11 h$ & 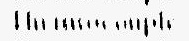 & \\
\hline 110 & 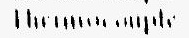 & \\
\hline
\end{tabular}

\begin{tabular}{|c|c|c|}
\hline $\begin{array}{c}\text { Ifaminal } \\
\text { No. }\end{array}$ & 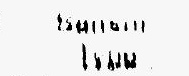 & $\begin{array}{c}\text { Alturunla } \\
\text { Labul }\end{array}$ \\
\hline 111 & $|1+1|+|1|+1+1+1,||$, & 111 \\
\hline $1: 1$ & 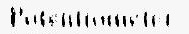 & $11:$ \\
\hline $1 \therefore !$ & 11111 & 111 \\
\hline $1: 1$ & 11111 & 111 \\
\hline $1: 1$ & 11111 & 111 \\
\hline 1.1 & $|110|$ & 1111 \\
\hline $1 ! 11$ & 11111 & 111 \\
\hline $1: 1$ & 11111 & $11 k$ \\
\hline $1 . \mathrm{H}$ & 11111 & 111 \\
\hline 1.11 & 11111 & 1111 \\
\hline 1111 & 11111 & 1111 \\
\hline 111 & 11111 & $111 !$ \\
\hline $11 !$ & 11111 & 1111 \\
\hline 111 & 11111 & 1111 \\
\hline 111 & |'w1, 11, & 1111 \\
\hline 114 & $\mid 1 / 11$ & 1111 \\
\hline 116 & 11111 & $|1| \mid$ \\
\hline 111 & $1111 \mid$ & $\| 1 \mid K$ \\
\hline 1111 & 11111 & 1114 \\
\hline 110 & 15111 & 11,11 \\
\hline 1111 & $|v| 1 \mid$ & $11: 1$ \\
\hline 111 & 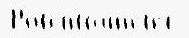 & $11 \because$ \\
\hline 11. & 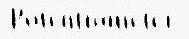 & $11: 1$ \\
\hline 111 & 11111 & $11: 1$ \\
\hline 111 & $|1| 1 \mid$ & $11: 1$ \\
\hline 111 & 11111 & $11 ! 11$ \\
\hline 116 & 11111 & $11: 1$ \\
\hline 111 & $|1| 1 \mid$ & $\|1.1\|$ \\
\hline $1 \mid h$ & 11111 & $11: 1\}$ \\
\hline $11+1$ & 11111 & 11111 \\
\hline 1111 & $1111 \mid$ & 1111 \\
\hline $1: 1$ & $1111 \mid$ & $111:$ \\
\hline $11 !$ & 11111 & 1111 \\
\hline 191 & 11111 & $1: 11$ \\
\hline 111 & 11111 & 1111 \\
\hline 19 & 11111 & 1111 \\
\hline 19 & 11111 & 111 \\
\hline 11 & 11111 & 1111 \\
\hline $\mathrm{I} / \mathrm{H}$ & 11111 & 1111 \\
\hline 1411 & 11111 & 11111 \\
\hline 16,11 & 11111 & $11 \|$ \\
\hline $10+1$ & $1111:$ & 1111 \\
\hline $16:$ & 1,111 & $11+1$ \\
\hline 161 & 11111 & 1111 \\
\hline $\mid 1,1$ & 11111 & 1119 \\
\hline 101 & 11111 & 11111 \\
\hline forr & 11111 & $111:$ \\
\hline 161 & $|1| 1 \mid$ & $\| \pi$ \\
\hline $16+1 \mathrm{i}$ & $|111|$ & $|1| 11$ \\
\hline $1(0,0)$ & 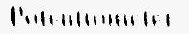 & 11911 \\
\hline $1 / 11$ & 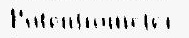 & $\mid 111$ \\
\hline 111 & 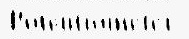 & $119 !$ \\
\hline $11 !$ & 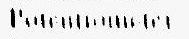 & 1191 \\
\hline 111 & 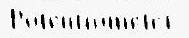 & 191 \\
\hline 111 & 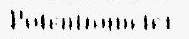 & 1191 \\
\hline $1: 1$ & 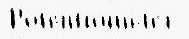 & 11911 \\
\hline $1 / 6$ & 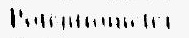 & 1191 \\
\hline $1: 1$ & 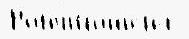 & $\operatorname{lin}$ \\
\hline $1: 4$ & 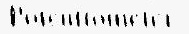 & 1110 \\
\hline 116 & 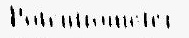 & $\|(x, \mid$ \\
\hline IUN & 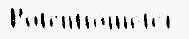 & $11: 1$ \\
\hline 1009 & 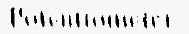 & IIHII \\
\hline$\therefore(111$ & 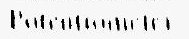 & ||$k \mid$ \\
\hline$\therefore(11$ & 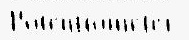 & lik: \\
\hline$\therefore 11$ & lentrithenterles & 1111 \\
\hline$\therefore 11$ & 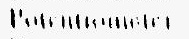 & $\| H \mid$ \\
\hline$! 11$ & 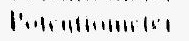 & ||$k \mid$ \\
\hline
\end{tabular}

\begin{tabular}{|c|c|c|}
\hline $\begin{array}{c}\text { ¿lasiliul } \\
\text { Nw. }\end{array}$ & $\begin{array}{l}\text { Tin11का" } \\
\text { |Xus }\end{array}$ & $\begin{array}{l}\text { Allenilialu } \\
\text { Lalusul }\end{array}$ \\
\hline ?114 & InII Illumblet & liks \\
\hline ! 114 & 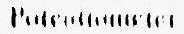 & lin! \\
\hline $161 !$ & 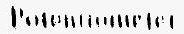 & ISHK \\
\hline$\therefore$ & 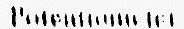 & $\|16\|$ \\
\hline$\therefore(111)$ & 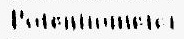 & $\| 1011$ \\
\hline$\because 11$ & 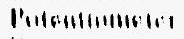 & $|\cdots|$ \\
\hline !1! & 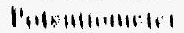 & $1110 !$ \\
\hline$: 1 !$ & P'wlemllullor|t & 1101 \\
\hline 111 & 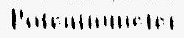 & 110.1 \\
\hline 111 & 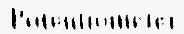 & 1109 \\
\hline$\therefore 1$ & I'silenthentwels & 11114 \\
\hline$\therefore 16$ & 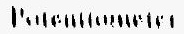 & $11: 1 !$ \\
\hline$\therefore 1 !$ & P'olsonltomenests & l10N \\
\hline$\therefore 11$ & 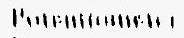 & liny \\
\hline .111 & 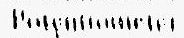 & 111111 \\
\hline$\therefore 11$ & 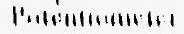 & 111111 \\
\hline$\therefore 1$ & l'mombnems & 11111. \\
\hline$\therefore !$ & 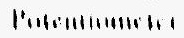 & 11111 \\
\hline$\therefore 1$ & Penchallomentet & $|1| 11 \mid$ \\
\hline$\therefore 1$ & 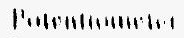 & 111119 \\
\hline$\because 1$ & 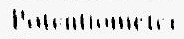 & lillk: \\
\hline$\therefore 61$ & 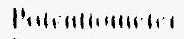 & 11111 \\
\hline$\because 1$ & 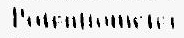 & 111111 \\
\hline$\because K$ & 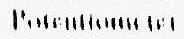 & 111114 \\
\hline$\therefore 11$ & 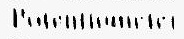 & 11111 \\
\hline 2111 & 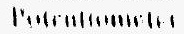 & 11111 \\
\hline$\therefore 11$ & 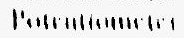 & $1118:$ \\
\hline$\therefore 1$. & 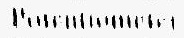 & 11111 \\
\hline$: 11$ & 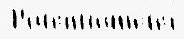 & 11111 \\
\hline .11 & 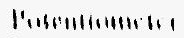 & 1111 \\
\hline$\therefore 11$ & 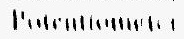 & 11110 \\
\hline$\therefore 16$ & 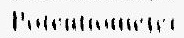 & $111 !$ \\
\hline$\therefore 11$ & 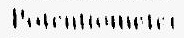 & $\| 11 k$ \\
\hline$\therefore H$ & 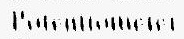 & 11111 \\
\hline .111 & 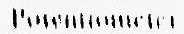 & 111.11 \\
\hline$\therefore 111$ & 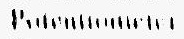 & $111: 1$ \\
\hline$\therefore 11$ & 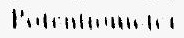 & $111 \therefore$ \\
\hline $11:$ & 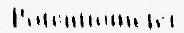 & $111: 1$ \\
\hline$\therefore 11$ & 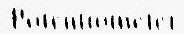 & $111 ! 1$ \\
\hline 11.1 & l'nlethlumentis & $111: 1$ \\
\hline$\because 1$ & Pandenthunetes & $\| 111$ \\
\hline$\therefore H$ & 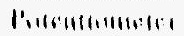 & $\| 1111$ \\
\hline
\end{tabular}




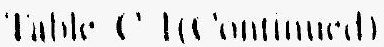

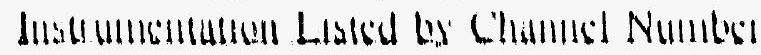

\begin{tabular}{|c|c|c|}
\hline $\begin{array}{l}\text { 1:||a|,|lu| } \\
\text { Ny. }\end{array}$ & 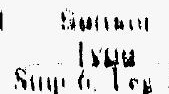 & \\
\hline 1111 & หIII \& I I & $i$ \\
\hline IIIH & bIII (1, I & 1 \\
\hline (1111) & bाता का I & \\
\hline 1.11 & 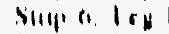 & H \\
\hline 1.1 & Siiif 11 & 111 \\
\hline $1: !$ & SIIII! I F & \\
\hline $1: 1$ & Viliय' I, I FN & ! \\
\hline $1: 1$ & \$1110 1,10 & 1 \\
\hline $1: 1$ & Мा1џ. I. 1. & \\
\hline $1 \because n$ & जIIf] 1.1 : & \\
\hline 1,11 & Min. 1.104 & H \\
\hline $1: H$ & Milf I $1 \mathrm{ch}$ & 111 \\
\hline 1.4 & Minrlle I:I. & I I B \\
\hline 1.111 & Knndl: $1: 1$. & lif! \\
\hline 1.11 & Kunclis I:I. & $1 \mathrm{rk}$ \\
\hline $11 !$ & Knowlls 1:1! & 1101 \\
\hline 1.11 & Kansils 1:1. & $1 \mathrm{ch}:$ \\
\hline 1.11 & Kunritr 1!! & 1101 \\
\hline 1.11 & Kinclli 19. & $|c|$ \\
\hline 1161 & Kundli I11. & 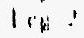 \\
\hline $18^{1}$ & Homlli 19, & 161 \\
\hline $1 / 1$ & Kunclle: ISr. & $1 .: 1$ \\
\hline 1.11 & Kundlle I Mri, & 110. \\
\hline 1011 & Kunlle I Mr & 1,41 \\
\hline $1 ; 1$ & Kintll! |Y! & $11 \mathrm{k}$ \\
\hline ln.: & Kindill 19! & $10 \mathrm{k}:$ \\
\hline 1,1 & Kinill: 19 ? & $10 k 1$ \\
\hline 141 & Kundl I9k & 161 \\
\hline (n) & 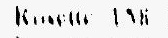 & $1.0:$ \\
\hline trint & Kindur Isk. & $1 \mathrm{CE} 1$ \\
\hline Irt' & Kanclli 1919 & $\mid 1+1$ \\
\hline $11,1 K$ & Nowith IVI & $11 k:$ \\
\hline 11,4 & Kandll |1" & $\log 1$ \\
\hline Itill & Kandur In's & 1,1 \\
\hline$|n|$ & Kusrlle Irol & 1.8 : \\
\hline lit.' & Kindle Intle. & $1+\mathrm{k}: 1$ \\
\hline$|k|$ & Hondull $\left|n_{1}\right|$ & 1.11 \\
\hline int. & Kanslle |rit. & $118:$ \\
\hline 4114 & Kunnll: ||$_{1} \mid$ & 1.41 \\
\hline IHor & 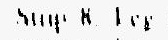 & 1 \\
\hline $1 x !$ & MIII' H l I B & $\therefore$ \\
\hline ink & Mili, I & 1 \\
\hline IRU & SIII) $\times$ IDE & 1 \\
\hline M11 & IIrilinils I & \\
\hline HII & Noldalm: & \\
\hline .111.' & Willalili 1 & \\
\hline HII & Wistiblils 1 & \\
\hline 111.8 & Wililalile a & \\
\hline 1119 & Melinalik o & \\
\hline HIr & Wirlialile! & \\
\hline .1111 & Wrlinhlir N & \\
\hline HIK & Wrlibalili N & \\
\hline .1110 & Wililulils III & \\
\hline 1.11 & Willalile 11 & \\
\hline $1: 1$ & Wililalili I: & \\
\hline $1 ! !$ & Willalile II & \\
\hline $1: 1$ & Wulilialile 1.1 & \\
\hline $1: 1$ & Hotiluble is & \\
\hline 1.9 & Wislinglili la & \\
\hline A's & Wrlibalile I: & \\
\hline$f: 1$ & Wrlilalils in & \\
\hline $1: x$ & II Llibalilis IU & \\
\hline $1: 10$ & Nolithlis ! & \\
\hline 1111 & Molilalile :I & \\
\hline 111 & Wrillalile $\therefore$ '. & \\
\hline 11: & Nolilulili :1 & \\
\hline
\end{tabular}

\begin{tabular}{|c|c|c|}
\hline 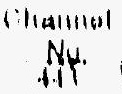 & 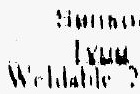 & \\
\hline 11.1 & Wrlilalll: : & $\therefore$ \\
\hline 119 & Nolilalile: & $\therefore 0$ \\
\hline $1+11$ & Wollitalite: & $\because 1$ \\
\hline +.1' & Wrilululs : & !K \\
\hline f.1K & Weldoblik: & $\therefore 0$ \\
\hline 4.14 & Woldutill I & 111 \\
\hline$t(1) \mid$ & Wrblaliw I & 11 \\
\hline$n+1$ & Wrildulite I & 1: \\
\hline 101! & Wrlilulili I & 11 \\
\hline $\mid 101$ & Wrlilulili I & 11 \\
\hline$\| n+1$ & Wrlablele I & 19 \\
\hline$(n+)$ & Wrlllulll: I & Mn \\
\hline Ithen & Wolilalilu 1 & 11 \\
\hline $4(1)$ & Wrlilulul: I & IN \\
\hline Hrik & Wrlinilile & IV \\
\hline$(1,1)$ & Wrallatilie & 111 \\
\hline InII & W'rlinalile: I & 11 \\
\hline$|h|$ & Wilinlile I & 1! \\
\hline In!' & 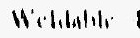 & 11 \\
\hline$|k|$ & Willalils & 11 \\
\hline$|t h|$ & Wrliballie & 11 \\
\hline ent & Nililalilit & fii \\
\hline Itiin & 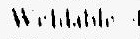 & 11 \\
\hline In' & Willialile & I \\
\hline lik & Wrl.1.11.1. & 11) \\
\hline$\| \mathrm{kil}$ & Nil l, al, & 111 \\
\hline 91111 & II. l,1,lils & 11 \\
\hline $11+1$ & $11,1,1,1,1$, & 1. \\
\hline $11:$ & Willullw & \\
\hline 9111 & Wuldalus & 11 \\
\hline 1111 & Wililallis & 19 \\
\hline 1119 & Whlililili & m \\
\hline $911 x_{1}$ & Wrillalil. & $1:$ \\
\hline 111 & Ninl,1,1,1: & iti \\
\hline Inth & Willlalule & (1) \\
\hline $1(114)$ & Willalile o & (i) \\
\hline 1.11 & Wullalili a & (1) \\
\hline $1: 1$ & Wrillillin a & $1:$ \\
\hline$\because \because !$ & Wrlulinlite & 81 \\
\hline $1 ! 1$ & Wirlablitie & (1) \\
\hline $1 ! 1$ & Wrillallir & (i) \\
\hline 1.9 & W'llalliter & riri \\
\hline 1,11 & Wralulike & (1: \\
\hline $9 ! !$ & W'allinlile & SH \\
\hline $1, k$ & Wratalue 1 & (11) \\
\hline 1,0 & Wrblublu & (1) \\
\hline 1111 & Writhalile & 11 \\
\hline 9.11 & Writulidi & $! !$ \\
\hline 111 & W'rlitalil & 11 \\
\hline 1.11 & Wrlilulili & 11 \\
\hline 1.11 & Wringlit. & 19 \\
\hline 1.11 & Welilatili & 101 \\
\hline 9.610 & Wrlilatily & 11 \\
\hline 141 & Mrlifalili & In \\
\hline 4.16 & Nolinalith & in \\
\hline 9.10 & Nilll.al.li & Bil \\
\hline lnel & Winlinlali & BI \\
\hline lin! & 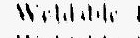 & N.' \\
\hline Yri: & $11 \cdot 1,1.111$ & Ni \\
\hline 181 & Willalls & ni \\
\hline 911 & Willialils & $\mathrm{NS}$ \\
\hline$x, 1$ & Willahlo & $\mathrm{Hn}$ \\
\hline rent. & Itrlibaluth & in \\
\hline$r n^{\prime}$ & Wildalli & HK \\
\hline YMH & Wrildulils & fiv \\
\hline y,is & Wholatele & \\
\hline
\end{tabular}

\begin{tabular}{|c|c|c|}
\hline $\begin{array}{l}\text { (lanllinwl } \\
\text { Nan. }\end{array}$ & 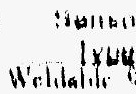 & \\
\hline$|K|$ & Wrlilalilis & U1 \\
\hline IK! & W'rlilalise & 10.1 \\
\hline$|k|$ & H'clilalite & U1 \\
\hline IN. & Helilalile & $u_{1}$ \\
\hline INI & H'rlilutile & 1) \\
\hline INsi & W'rlilatilir & UK \\
\hline IN! & IIrlilalile & (4I) \\
\hline INH & Wulilalils & 1,1 \\
\hline 180 & Welilalilis & 1111 \\
\hline :H川 & W' lalilo & 111. \\
\hline (H) & Wrilatili & 1111 \\
\hline nill! & W'rlilalilis & 114 \\
\hline (w) & Wrililalils & 1111 \\
\hline 6114 & Wrilalilu: & $1(x)$ \\
\hline (1)19 & Wolilaliks & $111 !$ \\
\hline ench & W'olidalile: & $111 \mathrm{~K}$ \\
\hline (1) (1) & Wolilalids & $\|(\omega)$ \\
\hline (il) & Wolifalils & 1111 \\
\hline (1)(11) & Wrifialus & 111 \\
\hline$n ! 11$ & Wrlilalili & 11. \\
\hline 0.1 & Wolilalils & 111 \\
\hline$(1,1:$ & Wrilatili & 11.1 \\
\hline$(1,1)$ & Hrlilalils: & 111 \\
\hline$(1.1$ & Wrlilaliler & 116 \\
\hline $1 . ! 1$ & Wrelilalils & 111 \\
\hline 1.61 & Wrhlablir & $11 \mathrm{H}$ \\
\hline 11.'! & Wirlilalilo & 110 \\
\hline 0.18 & Wrelilastide & 1.11 \\
\hline$(1.14)$ & Wrlilalils & $1: 1$ \\
\hline$(1.11)$ & Wolliahlis & $1 \because !$ \\
\hline 1.11 & Wrililulili & 1.1 \\
\hline (1.1.' & W'Rlilahis & 1.1 \\
\hline 1.11 & Wralishe & 1.9 \\
\hline 0.11 & Welinialis & 1.61 \\
\hline 6.19 & Withlafils & 1.1 \\
\hline$n 10$ & Walibulile & $1 . K$ \\
\hline n!! & Wrelilulils & 1.40 \\
\hline $1.61 \mathrm{H}$ & Willialilis & 1111 \\
\hline$(1.10)$ & Wolilatilis & 111 \\
\hline ensoll & W'dilatili & 11. \\
\hline$\{N, 1$ & Wilidafili & 111 \\
\hline phi.' & Wrhlahilr & 111 \\
\hline in: 1 & Wrelilutile. & 111 \\
\hline (n.) & Wrolilulile & 116 \\
\hline$(6)$ & Whliblike & 111 \\
\hline more & Wolilabili & $11 \mathrm{~N}$ \\
\hline (n)! & Wolikulilo: & I (I) \\
\hline mikin & Wolilalilo & 1.111 \\
\hline$(n, 1)$ & Wolilalilo & 1.11 \\
\hline nkikil & Welilaslile & 1.1. \\
\hline (IK) & W'slitalili & 111 \\
\hline ak: & Wridalile & 1.14 \\
\hline (IN) & Norlilatils & 111 \\
\hline nitil & Wriliblili & I. 11 \\
\hline lik & Winlilalik & 111 \\
\hline rithis & Wrililalilis & 1.18 \\
\hline (1)! & Wrshlalile & 110 \\
\hline nikst & W'Slilahils. & 41 \\
\hline (in's & Mrlibuliks & 11 \\
\hline 1111 & Wrilualiks & 19. \\
\hline 1111 & Wrlibalili & 1 \\
\hline 101 & Writholile: & 148 \\
\hline$! 11$ & W'studalilis & 119 \\
\hline 111.1 & Wralialilis & 10 \\
\hline 1119 & Wrlibulari & $19 !$ \\
\hline$!(1)$ & Wrlabilas & 116 \\
\hline
\end{tabular}




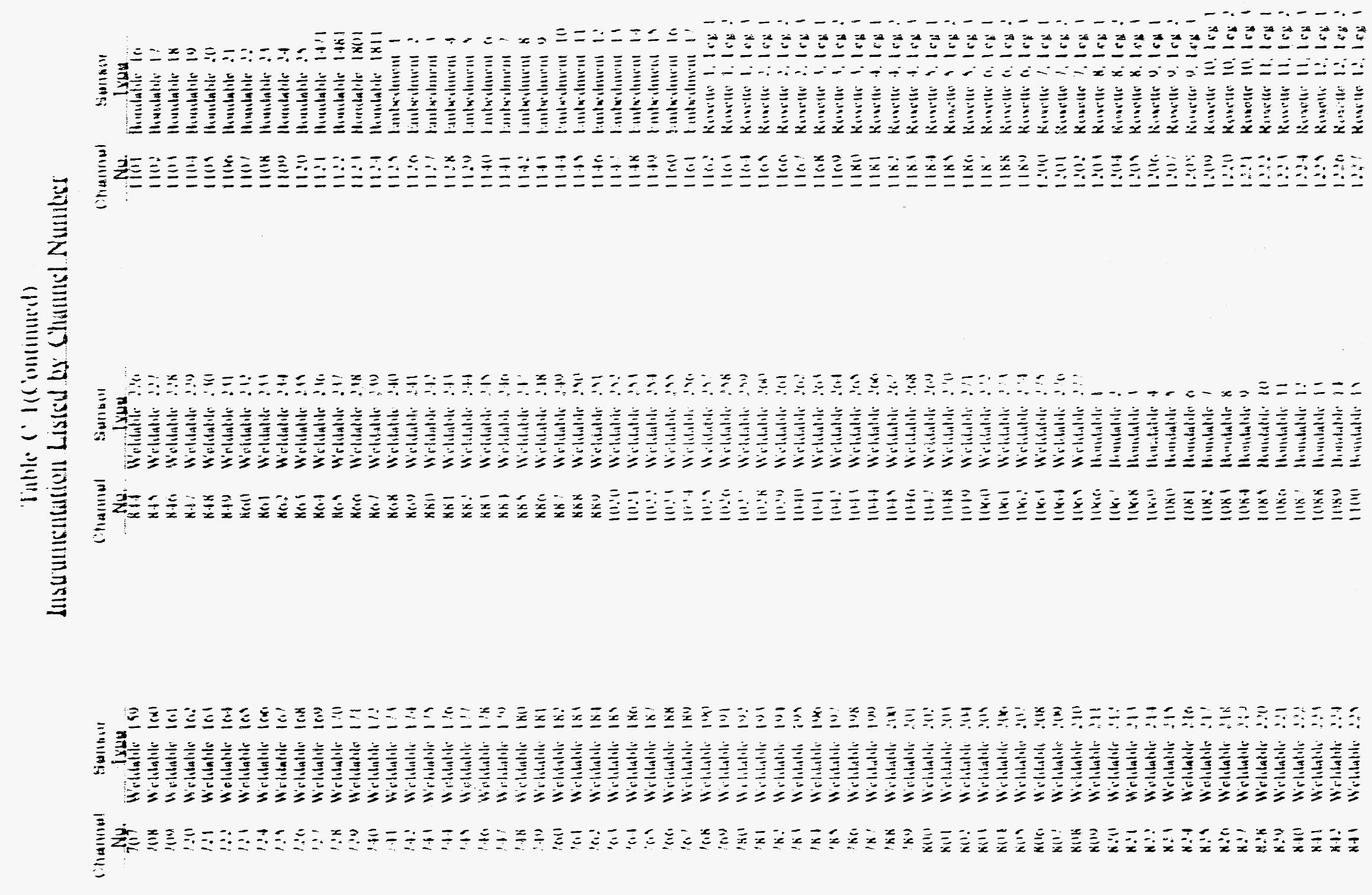

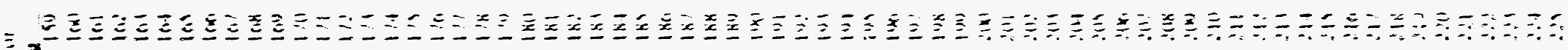

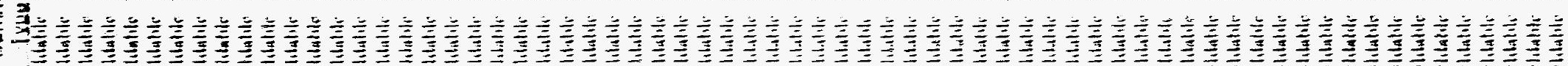

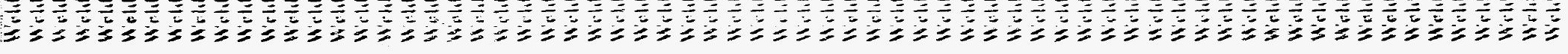

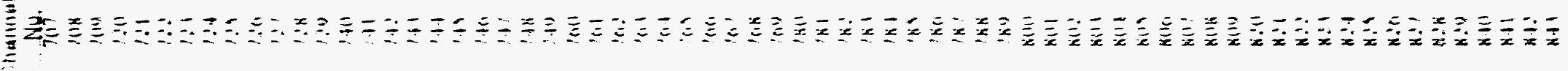


Tinble (' I(combinated)

\section{descrumsentation Lisked by Chamed Numbor}

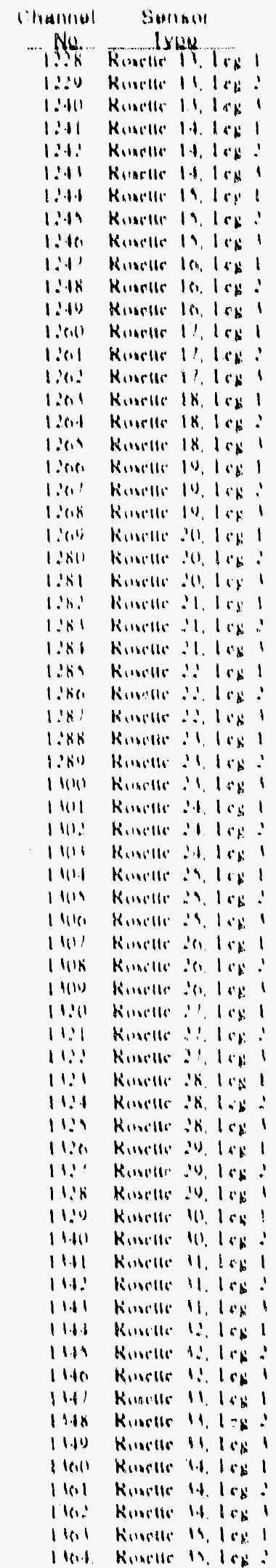

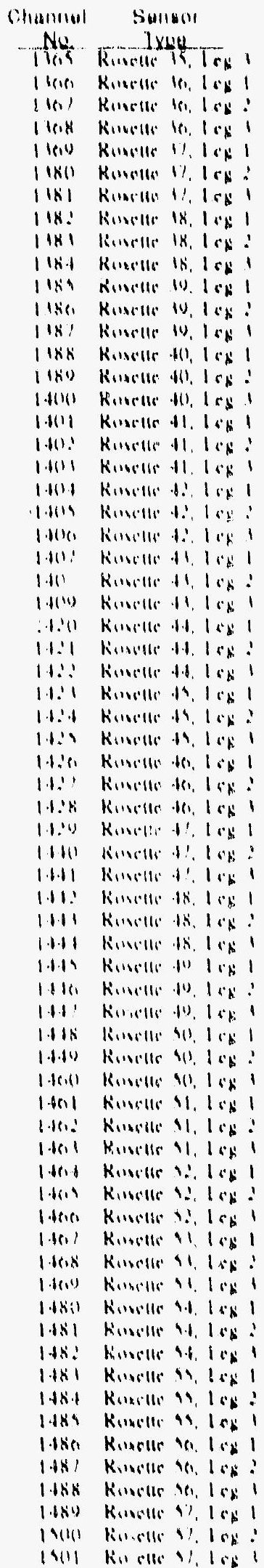

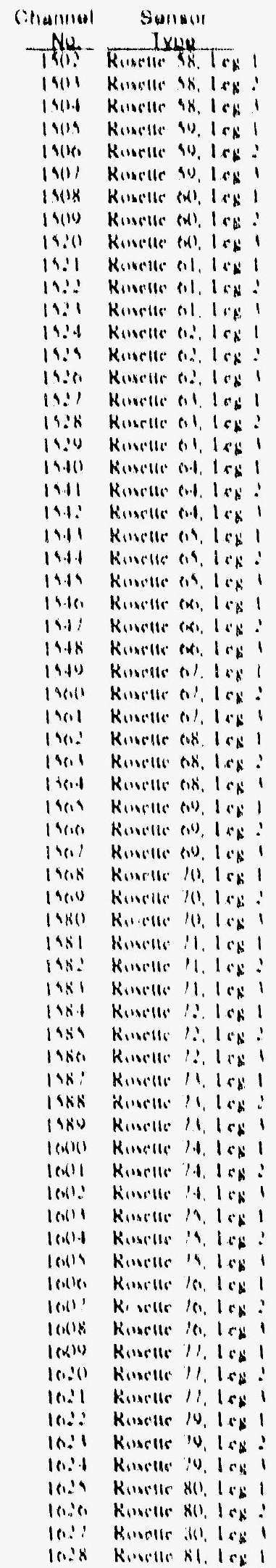


Table (' I(c)mbinmed)

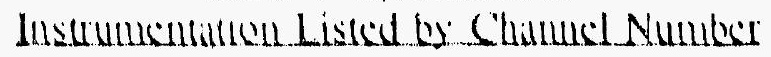

\begin{tabular}{|c|c|}
\hline $\begin{array}{c}C \text { hanmosl } \\
\mathrm{No}\end{array}$ & $\begin{array}{c}\text { Sullsil } \\
\text { Dyul }\end{array}$ \\
\hline $10=0$ & 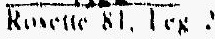 \\
\hline$|(m \mid)|$ & Kanclle $81,1 \times x \mid$ \\
\hline 10.11 & Humelle $8 ., 18 \%$ I \\
\hline 10.1: & Kumerlle K: I es: \\
\hline 10.11 & Rurlle si. Irs I \\
\hline 10.14 & Kunclle s'! I ss I \\
\hline 16.49 & Kunde K! I Is: \\
\hline 16.60 & Kusche KI, Ira I \\
\hline $10.1:$ & Finrlte Kil. IRA I \\
\hline 10.18 & Runelle K.I. I es: \\
\hline (16.f1) & 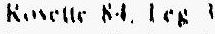 \\
\hline l(x)ll) & 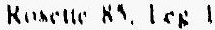 \\
\hline$|(x)|$ & Kurlle NG, I En : \\
\hline Iexi: & Kinclle Kh, I CA: I \\
\hline $\mid(x, \mid)$ & 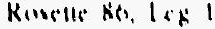 \\
\hline$|(x)+|$ & Kinbello Ke, I Ca: \\
\hline $\operatorname{lox}(x)$ & Kunsefle the las l \\
\hline lenses & 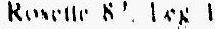 \\
\hline lene' & Kandli s' Ita : \\
\hline I $x, 1)$ & 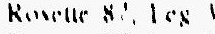 \\
\hline $1(x, 1)$ & Kuselle sk, I I 1 \\
\hline I & Kunlle si. I tof ! \\
\hline $\mid$ |nN| & Kunde sis Iry \\
\hline 101: & Kuselle sin, I rs I \\
\hline $\mid$ |ns $\mid$ & Kinslle fin. I cos : \\
\hline lRH. & Kundle \$4, I+म \\
\hline lnit & 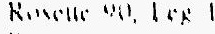 \\
\hline Insice & 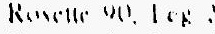 \\
\hline Inst & Kunder U(? l les \\
\hline Insist & Kumblle \\
\hline lnst) & 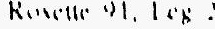 \\
\hline $1 ;(4)$ & 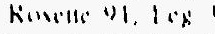 \\
\hline 1111 & 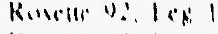 \\
\hline $1: 11:$ & 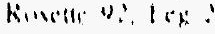 \\
\hline 1211 & Kundlk " $!$ I ag! I \\
\hline 111.1 & Kinclit 194,1 \& 1 \\
\hline 1119 & Kundl $11,1 \mathrm{sg}:$ \\
\hline $1: 110$ & 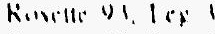 \\
\hline $1:(1)$ & Kuslu Us. Irs 1 \\
\hline 1 : & Kustll YI, I ck: \\
\hline 1 '(11) & Kundll ") I I \\
\hline $1 \because 1$ & 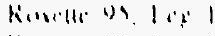 \\
\hline $1: 1$ & Kmolle o4, lof : \\
\hline $1 \because 2$ & Kщ, \\
\hline $1 \because 1$ & Kunctle ste las I \\
\hline $1 \div 1$ & K世, \\
\hline $1 \because 1$ & Kuncllic Jse, los \\
\hline $1 \because 11$ & Kunde y: 1 to 1 \\
\hline $1 ! 1$ & 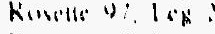 \\
\hline $1 \because x$ & Kunlle y: I on \\
\hline $1 \because 1)$ & 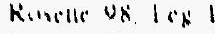 \\
\hline $1 \cdot 111$ & 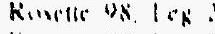 \\
\hline $1 \cdot 11$ & Knolle US, IEN \\
\hline $11:$ & Kunella (4), I rat \\
\hline $1: 11$ & Kwn \\
\hline 11.1 .1 & Kandle we I I 1 \\
\hline $1: 11$ & Kuslle lox I I sh \\
\hline $1: 41$ & Kinstle I(x), I ren \\
\hline 114 & Kondte IIK) I ch \\
\hline $1 \therefore$ & Kindll $1111.1 \mathrm{rk}$ \\
\hline 19.10 & Kustle I(I) I $\mathrm{rb}^{-}$ \\
\hline $1 '(1)$ & Kunlls IOII, I $\mathrm{ch}$ \\
\hline $1:(1)$ & Kundlle /11\%, I $\mathrm{cb}$ \\
\hline $1: n:$ & Runstle l11: I rg \\
\hline $1 ! 1$ & Kunele 119.9 I re! \\
\hline 101 & Kinells I10! I Is \\
\hline 114 & Kinelle I111, I is \\
\hline
\end{tabular}

\begin{tabular}{|c|c|}
\hline $\begin{array}{l}\text { Chammal } \\
\text { No. }\end{array}$ & $\begin{array}{c}\text { Sollsor } \\
\text { Ixwe } \\
\end{array}$ \\
\hline$?($ (B). & 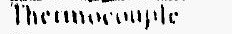 \\
\hline$\therefore(x) 4$ & Mormmisingle \\
\hline$\therefore(x))_{1}$ & Themomsenple \\
\hline$:(x))$ & Iheminconiflete \\
\hline$\therefore(x) s$ & Ihemencingles \\
\hline$\because(x \mid 1)$ & 'Thermiss \\
\hline $2(111)$ & 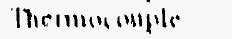 \\
\hline $2(1) 1$ & 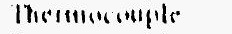 \\
\hline :(!): & 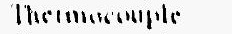 \\
\hline $2(111$ & 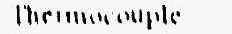 \\
\hline$\therefore(11.1$ & 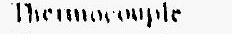 \\
\hline 21119 & Moreminsmiple \\
\hline$\therefore(1: 1)$ & Kisurte $1: 7,1$ es \\
\hline $20: 1$ & Kuncile 1:!, las \\
\hline$\therefore(1):$ & Kunctle 1:7, len \\
\hline $20 \div 1$ & Kurlls I2k, Ird \\
\hline $201: 4$ & 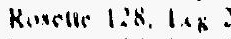 \\
\hline $2(1: 14$ & Kunstle l2k. I es \\
\hline$\therefore(1) \div$ & Kusells l:") I as I \\
\hline$\therefore(1: ?$ & Kurlls 1:6, I in \\
\hline$\therefore(1,8$ & Kusstle ILU, I fs \\
\hline$?(1) 113$ & Kosedle II(), I of I \\
\hline$\therefore(1.51)$ & Kunelle I I(I, I en. \\
\hline $2(1.1)$ & KMnlle I U), I on \\
\hline$\therefore(1.1$. & Kunclls $|11,| \mathrm{cs} \mid$ \\
\hline$\therefore(1.11$ & Kanclle $\mid 11,1 \% \mathrm{k}$. \\
\hline$\therefore(1.1 .1$ & Kmelle I11. I rg \\
\hline$\therefore(1.14$ & Kunclle $11 \therefore$ I as \\
\hline$\therefore(40)$ & 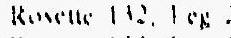 \\
\hline$\therefore(1.4)$ & Kuselfe 1 Ю I re \\
\hline$\therefore 1.18$ & Konelle 111.1 les \\
\hline$\therefore(1.10)$ & Kuselts 111,1 es \\
\hline$\therefore(k, 1)$ & Kunclli 111,1 is \\
\hline$\therefore\left|x_{1}\right|$ & Ronelle | $1.1,1$ es \\
\hline$\therefore\left(k_{1}:\right.$ & Ronctir $11.8,1$ es \\
\hline$\therefore(k, 1$ & Kunselle | 1, I es \\
\hline$?\left(k_{1} \cdot\right)$ & Kusstle 119, I ss \\
\hline$\therefore(x, 1)$ & Kunste $119,1 \mathrm{ch}$ \\
\hline$\therefore(x, s)$ & Kustlo I15, I, \\
\hline$\therefore\left(k_{1}\right)$ & RMelle I ls, I cos \\
\hline$\therefore k, n$ & Kundle I ka, I eg \\
\hline$\therefore(x, 1)$ & 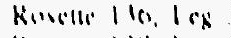 \\
\hline$\therefore(1)$ & Kinelle |l: I es \\
\hline$\geq(1, i)$ & Kmelle | $1: 1$ rg \\
\hline :11, : & Kurlu $11: 1$ es \\
\hline$\therefore 11$ & Kuncluc I Is, Iex \\
\hline 2115.1 & Knelle IIS, Irs \\
\hline : & Kmolle I Is, Ises \\
\hline ?IRE & Kunctio I I0, I cs \\
\hline$\therefore(1) !$ & Kunelle $\mid 10,1$ en \\
\hline OINK & Kowrte I to. I of \\
\hline$\therefore(1) \mathrm{s}$ & Kuselle Ith 1 es \\
\hline$\therefore 11118$ & Kunclla Ifll I If \\
\hline$\therefore 111\}$ & Kureile Id) I Es \\
\hline$\therefore 11 !$ & Kuselle $1+1$, I os \\
\hline$\therefore 1(1)$ & Kundte I.HI I rs \\
\hline$\therefore 111.1$ & Kurlle IHI I ef \\
\hline$: 1114$ & Kundle 14: I rs \\
\hline 21610 & Kmelle 1.42. I s"s \\
\hline$\because 1611$ & Kunclle It2, I en \\
\hline$\therefore 1118$ & Kaneste $111,1 \mathrm{CD}$ \\
\hline$\therefore 1110$ & Kaw!l $1+4,1 \mathrm{~g}$ \\
\hline$: 1: 11$ & Kundls 1.11, I 58 \\
\hline$: 1: 1$ & Konolle 1.4. I on \\
\hline$\because 1: 1$ & Kustle I.H. I is \\
\hline$: 1: 1$ & 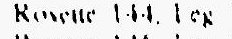 \\
\hline$\therefore 1: 1$ & Kasclls 1.19. I rg' \\
\hline
\end{tabular}


Table C-1(Continued)

\section{lenstrunsentation Listod by Chamed Number}

\begin{tabular}{|c|c|}
\hline $\begin{array}{l}\text { Channol } \\
\text { No. }\end{array}$ & $\begin{array}{l}\text { Sonsor } \\
\text { Irou } \\
\end{array}$ \\
\hline 1129 & Rosclle 1+5, Tes : \\
\hline$: 1: 0$ & Kusrue 1.\$4, Irg I \\
\hline $11: 9$ & Kunctle Its, I rs I \\
\hline $21: 8$ & Kanelle Ito, las: \\
\hline$\therefore 1: 4$ & Kuscll: Ito, Iss ! \\
\hline$: 140$ & Kusclue 147, l.es 1 \\
\hline 2141 & Kuselle 14?, I.ch : \\
\hline$: 14:$ & Kusclte 1\$7, Ins I \\
\hline 2141 & Kuselle IAs, I.en I \\
\hline 2144 & Kusctle 14k, Ies: \\
\hline$\therefore 145$ & Kosmile ItK, los I \\
\hline I4t & Kuselle 140. Iog I \\
\hline $214 ?$ & Kaselle 140. I rs: \\
\hline 21.48 & Konetle 140 I.en I \\
\hline 2140 & Kustlle IS(1, I ra \\
\hline$\therefore 1+1)$ & Kunctle 1.9(1. Ies \\
\hline $2|n|$ & Kunde 1.90, led \\
\hline$\because 10:$ & Kusctic IS1, Ies \\
\hline$\because 161$ & Kon:lls 191. I es \\
\hline$\because 16,1$ & Kusctse 191, I es \\
\hline$\because 101$ & 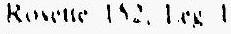 \\
\hline $2 ! 00$ & Koncts 19: I des. \\
\hline 2101 & Kustle 19:. lof \\
\hline $216 x$ & Kustos 191, leg l \\
\hline$\therefore(0,1)$ & Kwolls 191, I en. \\
\hline$: 1811$ & Kusclle ISI, I in \\
\hline ?|R่ & Kunelle 194, I ra \\
\hline :IN: & Kusclle 19.1, I co \\
\hline Iำ & Kunclls 19.4 I es \\
\hline 215.4 & Wald, : : is \\
\hline 218.9 & Mohdahle :Iu \\
\hline$\because \mathrm{lno}$ & Wrabalus 280 \\
\hline 218 & Whatus : 211 \\
\hline $215 x$ & 'Whldahic :S: \\
\hline :ISin & Moldille 251 \\
\hline$\because(1)$ & Woldallle $: 2.1$ \\
\hline$\because \because(1)$ & Wollatile 2is \\
\hline$\therefore(1)$ & Weldathe :ke \\
\hline$\because(1)$ & Wishuk :N! \\
\hline$\therefore(1) 1$ & Woldatio :As \\
\hline$\therefore(14$ & Wrltathe :SH \\
\hline$\because \because 10$ & Woldallite :ner \\
\hline$\therefore(1)$ & Wrldahls 201 \\
\hline$\because(1)$ & Wolihilile :"!: \\
\hline$\therefore(10$ & Mrlibils : 201 \\
\hline$\therefore \because()$ & Wolduhlse :20.4 \\
\hline $2 \because \because 1$ & Woldillk :wa \\
\hline$\because \because 2$ & Kundls II I I es I \\
\hline$\because 24(1)$ & Sแ1ए \&. los! \\
\hline$\therefore 21$ & SIIU स., Iss 10 \\
\hline$\because 4 !$ & Sinp" I CA 1 \\
\hline$\because \because 4$ & NIII, U. I Es: : \\
\hline$\therefore 24$ & Simf 4. I 1 \\
\hline$\because 14$ & 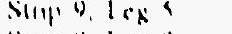 \\
\hline$\therefore+41$ & SHP U. ICA ! \\
\hline 24 & SIII 4. I RA 111 \\
\hline$\because+k$ & Sulp 11$),|E x|$ \\
\hline $23+4$ & NHII 111. I6N: \\
\hline$\because 201$ & SI1丩 10.15n! \\
\hline $2: 01$ & SIIIf 111, IER \\
\hline 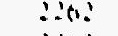 & Siny 10, I $\mathrm{Es}$ ? \\
\hline$\therefore+1$ & Siny 10. I.rk 1() \\
\hline$\therefore 604$ & $S t 1411,101$ \\
\hline$\therefore 01$ & Silly 11. IEx: \\
\hline$\because 00$ & Sแ川 |I. |Fू । \\
\hline$\therefore 20 !$ & SIIM II. Iens \\
\hline$\therefore 68$ & Siny 11. 108 : \\
\hline
\end{tabular}

\begin{tabular}{|c|c|c|}
\hline $\begin{array}{c}\text { Chamnal } \\
\text { Ne }\end{array}$ & $\begin{array}{c}\text { Sonsor } \\
\text { Ixose } \\
\end{array}$ & \\
\hline 220.0 & S(nI) 11, I. & 11 \\
\hline$\therefore x(1$ & Stmp 1: les 1 & \\
\hline ख̃र। & 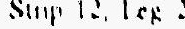 & ? \\
\hline$\therefore N !$ & Sump 12, 1 ext & 1 \\
\hline $2: K 1$ & Suy 12, lox & \\
\hline$\therefore \therefore 4$ & Sunp 12, 1es & \\
\hline$\because 18$ & Sinp 1: Ies 1 & 11 \\
\hline Into & 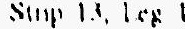 & 1 \\
\hline$\because 28$ & Sinf II, les & \\
\hline$\therefore A S$ & Sinp $1,1,150$ & 1 \\
\hline 2.86 & Siny 11! lsa! & 9 \\
\hline $21(16)$ & Sul $11, \mid<\mu^{\prime}$ & 9 \\
\hline 21111 & SIII 1.1. I.en & 16 \\
\hline $2 ! 1):$ & Sllu11 & \\
\hline ?:11)! & Suнuи & \\
\hline 211.4 & Strum & \\
\hline$: 1119$ & SHanII & \\
\hline 2106 & Sil:a11 & \\
\hline $21(1)$ & Sil.1111 & \\
\hline$? 1118$ & StI:I111 & \\
\hline$\because 11119$ & Sittin & \\
\hline$\because 1 ! 11$ & Sill,111 & \\
\hline $11: 1$ & Silau1 & \\
\hline 2121 & Silat11 & \\
\hline$\because 1: 1$ & S11:111 & \\
\hline$\therefore 1.1$ & Silain & \\
\hline 2124 & Silıu11 & \\
\hline 2121 & $S 11: 1111$ & \\
\hline 212 & Silıui & \\
\hline$\because 12$ & Sil.wn & \\
\hline $21: 0$ & Silıill1 & \\
\hline$\therefore .111$ & $S 11.111$ & \\
\hline 21.11 & Sil:al1 & \\
\hline$\therefore 1.1:$ & S11:111 & \\
\hline$: 1.11$ & Sil..111 & \\
\hline 21.4 & Sil:un & \\
\hline 24.45 & S11:1111 & \\
\hline$\therefore 1.10$ & $\$ 11,1411$ & \\
\hline$\therefore 1.1$ & Sil, & \\
\hline$? 1.15$ & Sitl,at1 & \\
\hline$\because 116$ & Sil:a111 & \\
\hline $210(1)$ & Sillan & \\
\hline$\because 10.1$ & Sil1:A111 & \\
\hline : In... & Silanil & \\
\hline 210,1 & Sillat11 & \\
\hline$\because !(1.8$ & Stip $14.10 \%$ & 1 \\
\hline$\because 10,5$ & Sinp H. Is & : \\
\hline$\because 10,0$ & Slllp 1.4, I En & 1 \\
\hline$\therefore 10 !$ & Suा 1.1. 1en & 4 \\
\hline 2105 & Sily 1.t. 10 & 4 \\
\hline$\because 10,0$ & Sill 1. IE & (1 \\
\hline$=\ln (1)$ & Sill 14. I ck & $?$ \\
\hline : |ล| & Sul 1.4. I ce & $s$ \\
\hline$\because 16:$ & Sunp 1.t. 1 es & 4 \\
\hline $2 \mid 81$ & 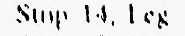 & 1 \\
\hline ? ISA & Smy is. I rs & 1 \\
\hline$? 18.9$ & Siny 15, !cs & : \\
\hline :Isn & Siup 19, 155 & 1 \\
\hline 2187 & SHap 19, les & 4 \\
\hline 2188 & Stup 19, $1 \mathrm{eg}$ & 9 \\
\hline 2160 & Sulp 15, 16A & o \\
\hline $2+1(x)$ & Suा̨ 19, $18 s$ & ? \\
\hline$\therefore d(11)$ & Stup R. IAd & s \\
\hline$\therefore+1):$ & Sup 15, Icg & U) \\
\hline$\therefore 4(14$ & Sแ1p 19, l cs & 11 \\
\hline $24: 4$ & Sil.u11 & \\
\hline$\therefore 1: 1$ & SHI:III & \\
\hline
\end{tabular}

\begin{tabular}{|c|c|}
\hline $\begin{array}{c}\text { Channal } \\
\text { No }\end{array}$ & $\begin{array}{l}\text { Swissor } \\
\text { Trute }\end{array}$ \\
\hline 2420 & St1ali \\
\hline $24: 1$ & Slıни) \\
\hline $24: 8$ & Silrail \\
\hline 2.120 & Sllaill \\
\hline 244() & T Ruscule 1, les \\
\hline $2+41$ & 'T Konsette 1, Ieg \\
\hline 2442 & T Kusetie 2. I $\mathrm{eg}$ \\
\hline 2443 & 1 Kuselte 2 I $\mathrm{eg}$ \\
\hline 24.4 & I Kusclle I Ies \\
\hline 2445 & I Kussule 1 les \\
\hline 24.40 & T Kusetie 4, log \\
\hline $24+4$ & IT Rousetle d, I $\mathrm{es}$ \\
\hline 2406 & Silain \\
\hline $2+41$ & Slunin \\
\hline $24+6$ & Siıыи \\
\hline $24 N(1)$ & finclinomente" \\
\hline 2451 & Inclinchusescl \\
\hline ZHE: & Inslinsunetel \\
\hline$: \sqrt{ } \mid 1$ & linslumsuneted \\
\hline $2.18+4$ & lnolinimenetel \\
\hline 2455 & Inclin, whetelel \\
\hline 2ASE & lnslingmelet \\
\hline$\therefore 18$ & lnsflumbulelst \\
\hline
\end{tabular}



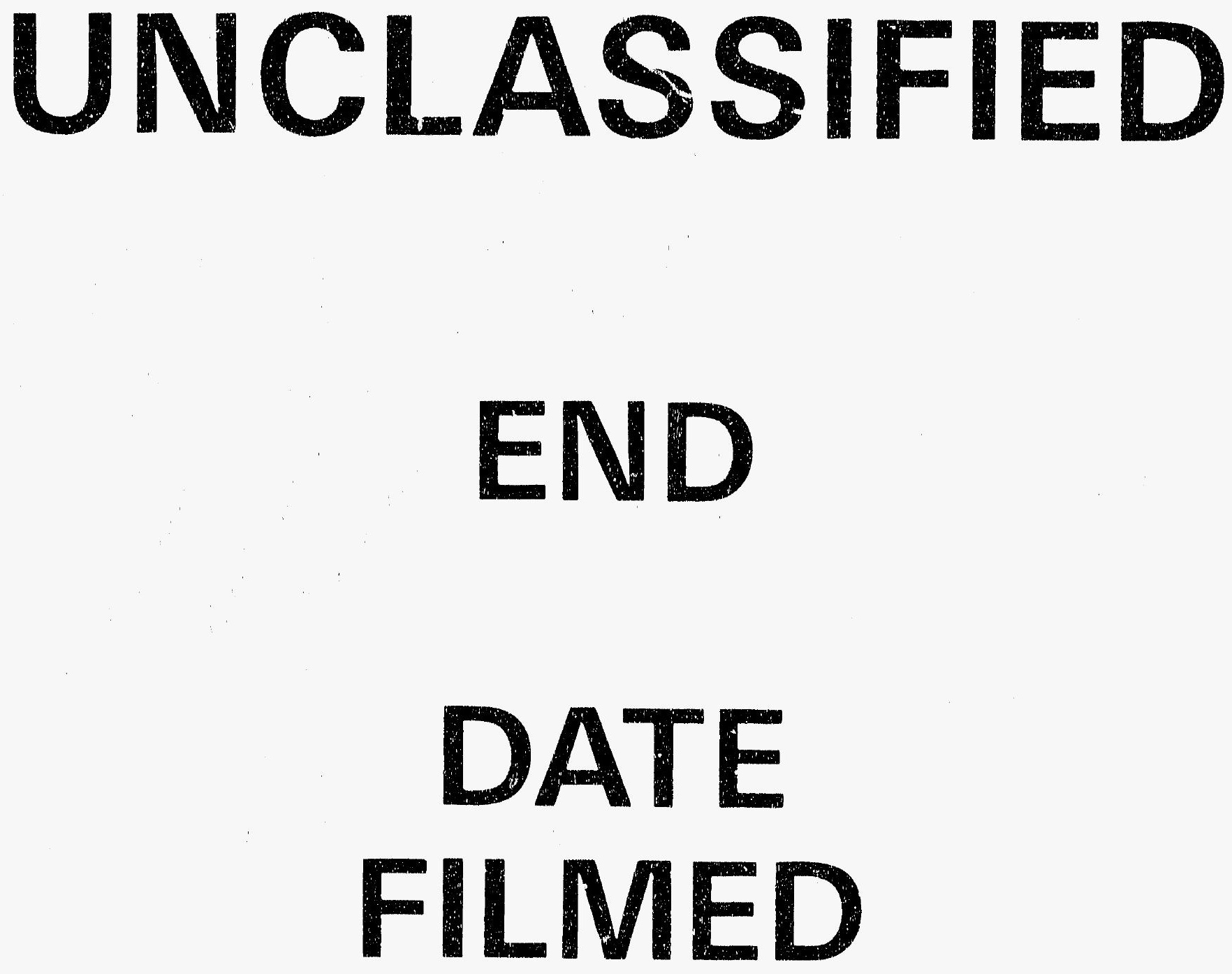

$$
1-6-92
$$

SANDIA NATIONAL LABS 


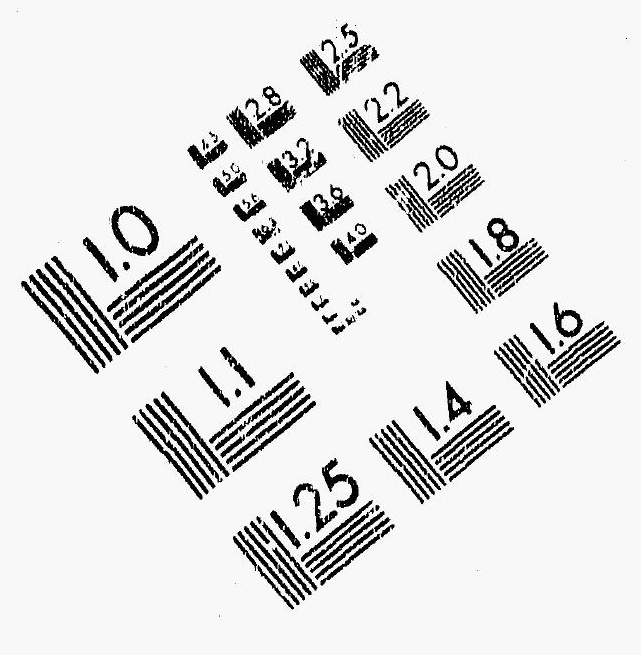

IMAGE EVALUATION

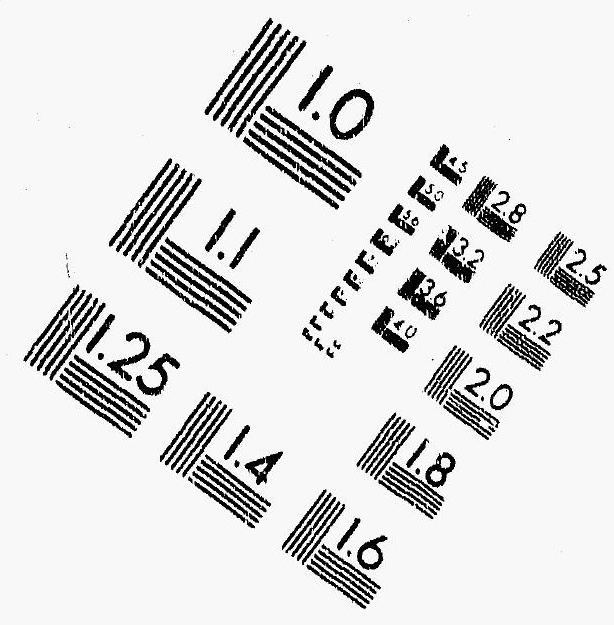

TEST TARGET (MT-3)
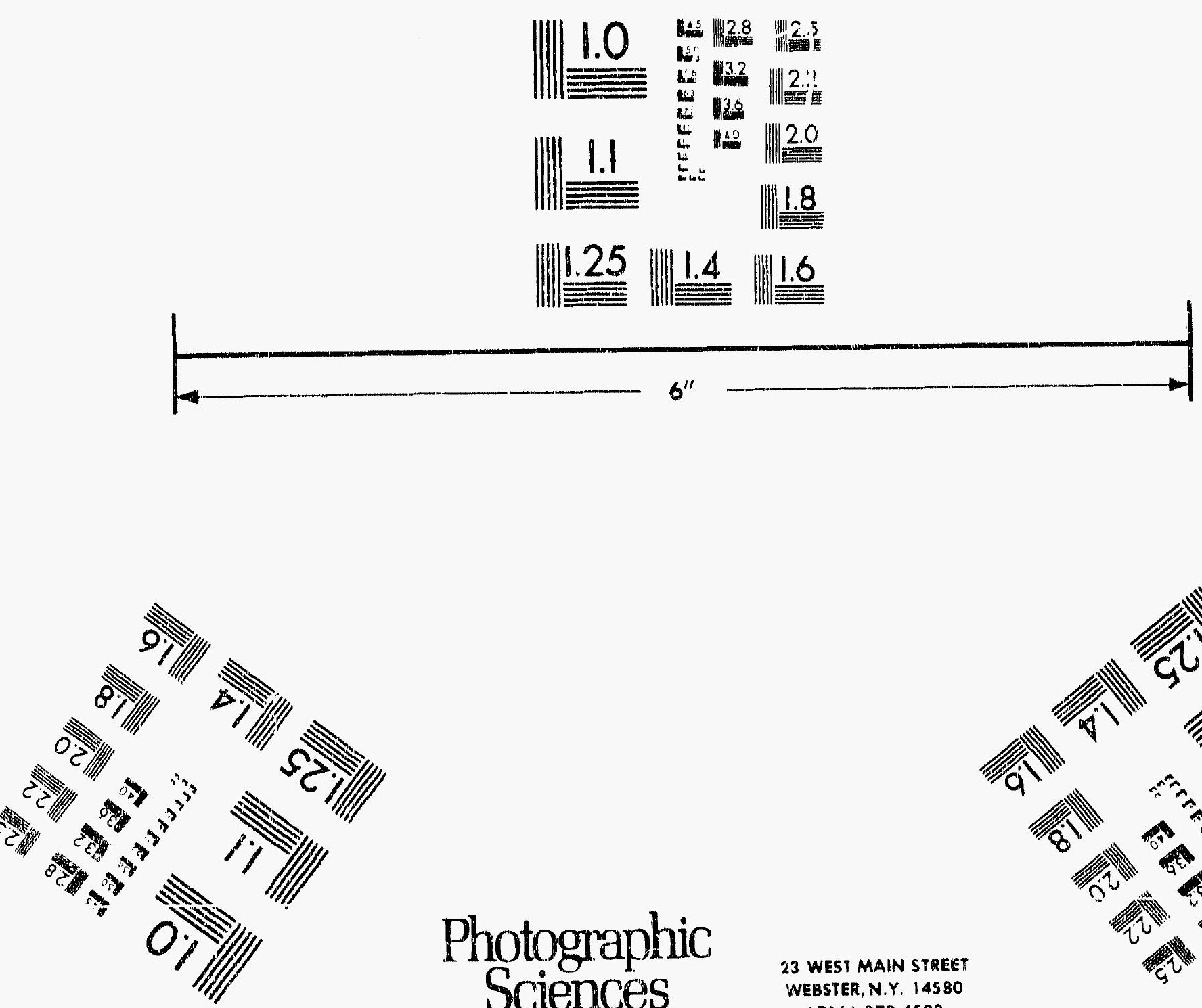

Photographic Sciences Corporation

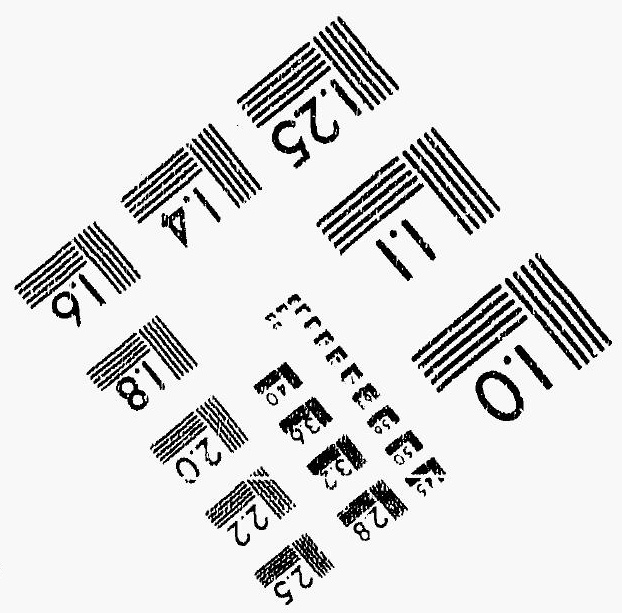




\section{SECURITY CLASSIFICATION}
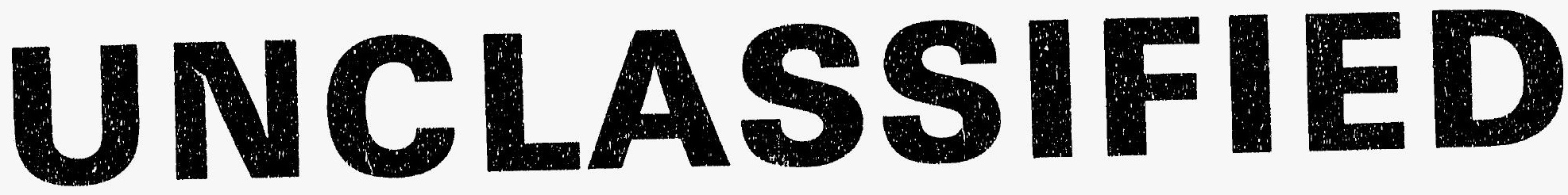

\section{DATE OF MICROFILMING $1-6-92$}

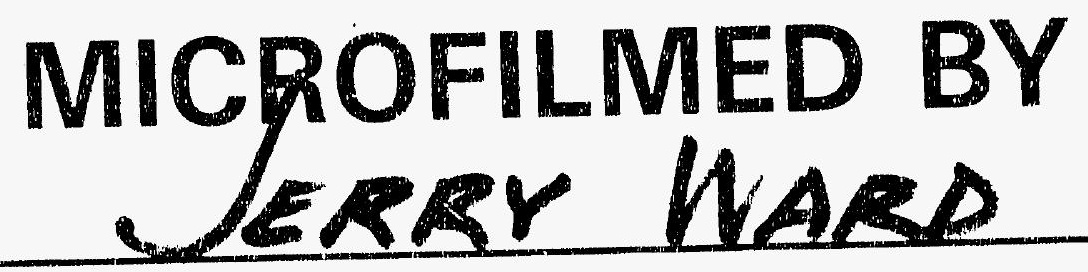

SANDIA NATIONAL LABS 
THIS DOCUMENT FILMED

AT

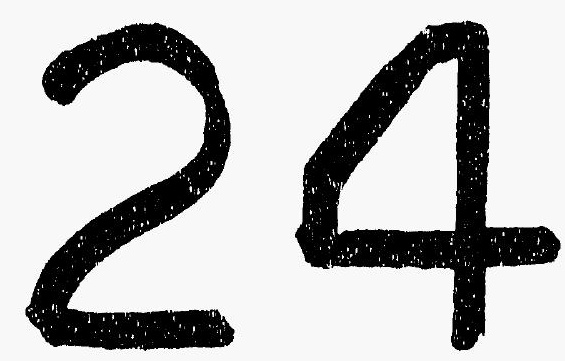

REDUCTION 


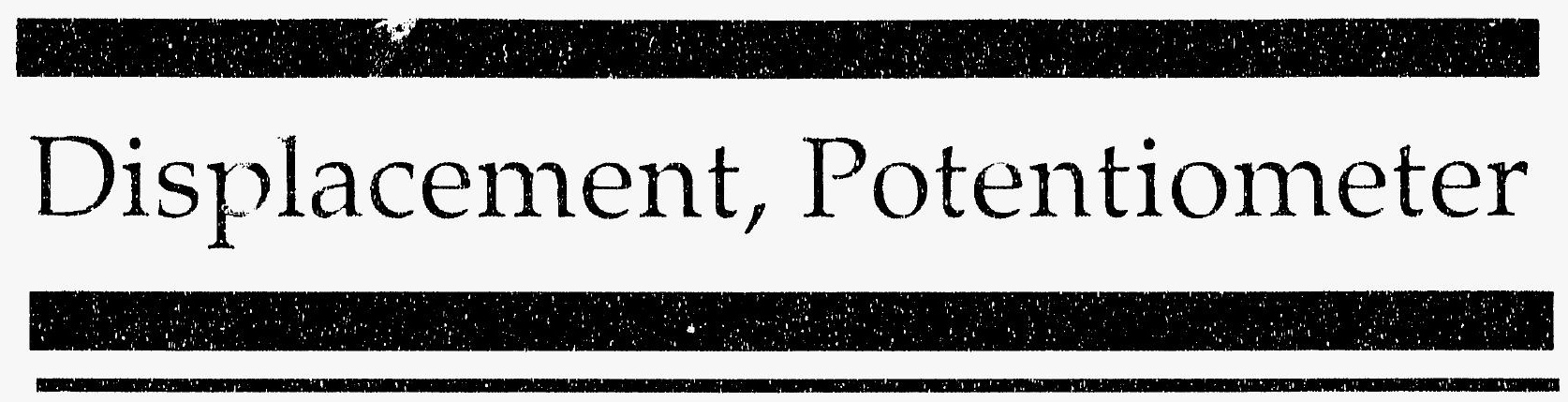




\section{Reinforced Concrete Test Data}

Potentiometer Channel 120

STRUCTURAL INTEGRITY TEST

\begin{tabular}{|c|c|c|c|}
\hline $\begin{array}{l}\text { Pressure } \\
(\text { psig) } \\
-0.05 \\
5.33 \\
12.31 \\
16.44 \\
20.51 \\
20.46 \\
20.47 \\
27.05 \\
34.69 \\
34.53 \\
34.53 \\
39.70 \\
45.90 \\
45.65 \\
45.62 \\
50.10 \\
53.47 \\
53.29 \\
53.21 \\
50.49 \\
46.14 \\
46.13 \\
44.97 \\
38.98 \\
33.99 \\
33.96 \\
26.83 \\
21.88 \\
21.90 \\
16.69 \\
11.74 \\
11.77 \\
3.05 \\
0.02 \\
-0.04 \\
-0.02 \\
-0.02 \\
0.02\end{array}$ & $\begin{array}{c}\text { Displacement } \\
\text { (inches) } \\
0.0000 \\
-0.0001 \\
0.0021 \\
0.0057 \\
0.0104 \\
0.0128 \\
0.0137 \\
0.0238 \\
0.0351 \\
0.0392 \\
0.0403 \\
0.0482 \\
0.0602 \\
0.0626 \\
0.0638 \\
0.0685 \\
0.0752 \\
0.0771 \\
0.0777 \\
0.0809 \\
0.0809 \\
0.0809 \\
0.0810 \\
0.0781 \\
0.0742 \\
0.0744 \\
0.0676 \\
0.0639 \\
0.0641 \\
0.0567 \\
0.0505 \\
0.0503 \\
0.0405 \\
0.0351 \\
0.0304 \\
0.0305 \\
0.0379 \\
0.0319 \\
0.04\end{array}$ & $\begin{array}{c}\text { Pressure } \\
(\text { ps } 1 \mathrm{~g}) \\
9.89 \\
19.55 \\
29.57 \\
39.42 \\
49.16 \\
54.50 \\
59.57 \\
64.20 \\
69.32 \\
74.16 \\
80.16 \\
25.61 \\
90.58 \\
95.69 \\
100.92 \\
103.25 \\
106.11 \\
108.31 \\
111.08 \\
113.24 \\
117.83 \\
120.92 \\
123.28 \\
122.97 \\
125.82 \\
125.60 \\
127.84 \\
130.19 \\
132.53 \\
135.33 \\
134.42 \\
138.35 \\
140.16 \\
142.63 \\
145.78 \\
142.52 \\
0.22 \\
\end{array}$ & $\begin{array}{c}\text { Displacement } \\
\text { (inches) } \\
0.0020 \\
0.0119 \\
0.0253 \\
0.0360 \\
0.0473 \\
0.0555 \\
0.0654 \\
0.0741 \\
0.0814 \\
0.0924 \\
0.1104 \\
0.1254 \\
0.1426 \\
0.1596 \\
0.1786 \\
0.1883 \\
0.1968 \\
0.2038 \\
0.2163 \\
0.2247 \\
0.2482 \\
0.2698 \\
0.2885 \\
0.2944 \\
0.3194 \\
0.3280 \\
0.3460 \\
0.3860 \\
0.4438 \\
0.5280 \\
0.5548 \\
0.6831 \\
0.8042 \\
0.9875 \\
1.1495 \\
1.2694 \\
0.9196 \\
0.96\end{array}$ \\
\hline
\end{tabular}

High Pressure test 


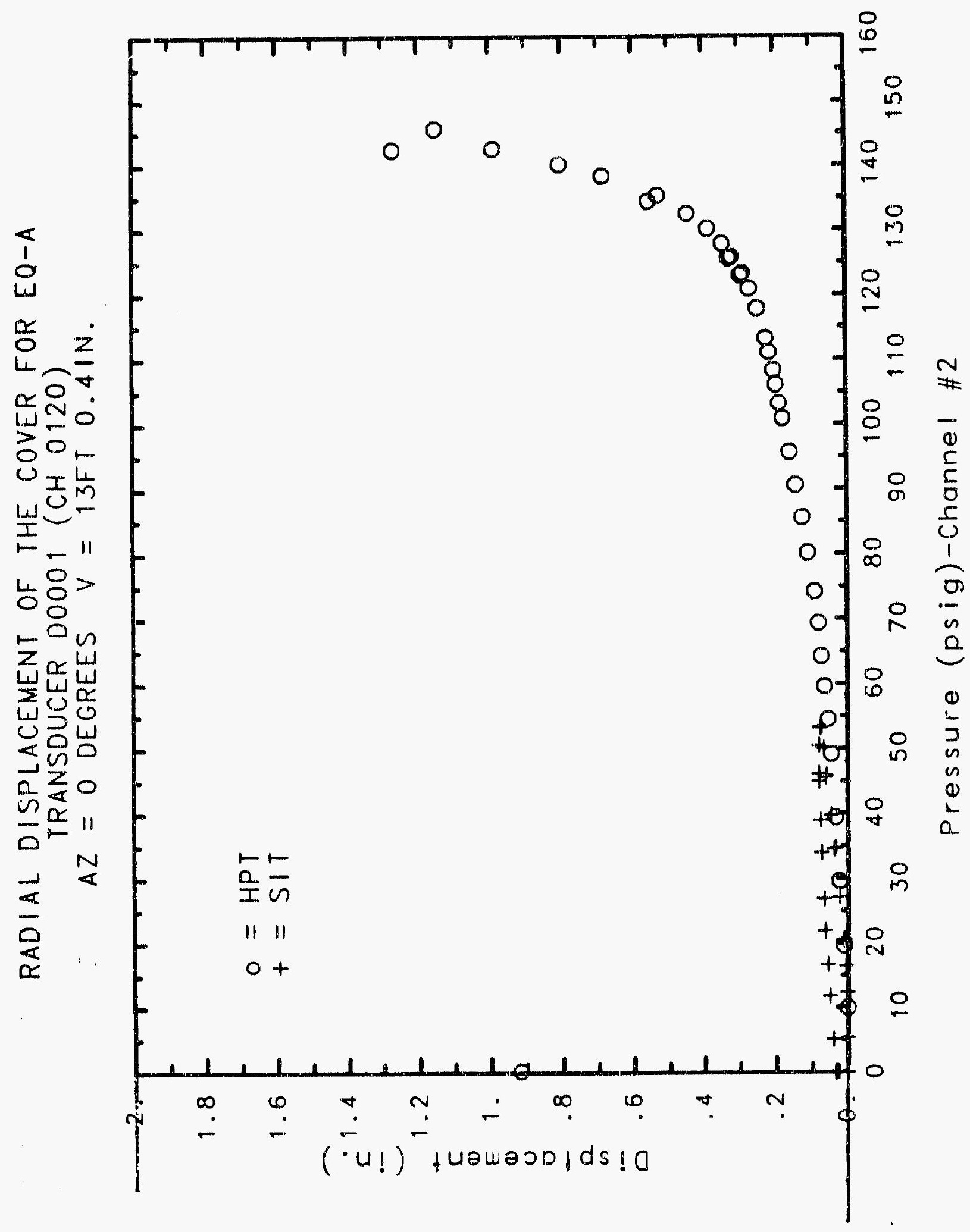

C-9 


\section{Reinforced Concrete Test Data}

Potentiometer Channel 121

STRUCTURAL INTEGRITY TEST

High Pressure Test

\begin{tabular}{|c|c|c|c|}
\hline $\begin{array}{l}\text { Pressure } \\
\text { (psig) } \\
-0.05 \\
5.33 \\
12.31 \\
16.44 \\
20.51 \\
20.46 \\
20.47 \\
27.05 \\
34.69 \\
34.53 \\
34.53 \\
39.70 \\
45.90 \\
45.65 \\
45.62 \\
50.10 \\
53.47 \\
53.29 \\
53.21 \\
50.49 \\
46.14 \\
46.13 \\
44.97 \\
38.98 \\
33.99 \\
33.96 \\
26.83 \\
21.88 \\
21.90 \\
16.69 \\
11.74 \\
11.77 \\
5.05 \\
0.02 \\
-0.04 \\
-0.02 \\
-0.02 \\
0.02 \\
\end{array}$ & $\begin{array}{c}\text { Displacement } \\
\text { (inches) } \\
0.0002 \\
0.0000 \\
0.0022 \\
0.0047 \\
0.0082 \\
0.0108 \\
0.0115 \\
0.0199 \\
0.0315 \\
0.0352 \\
0.0359 \\
0.0424 \\
0.0533 \\
0.0559 \\
0.0561 \\
0.0615 \\
0.0669 \\
0.0682 \\
0.0688 \\
0.0717 \\
0.0718 \\
0.0719 \\
0.0720 \\
0.0721 \\
0.0695 \\
0.0695 \\
0.0643 \\
0.0609 \\
0.0611 \\
0.0553 \\
0.0511 \\
0.0509 \\
0.0426 \\
0.0387 \\
0.0332 \\
0.0333 \\
0.0487 \\
0.0405 \\
0.05\end{array}$ & $\begin{array}{c}\text { Pressure } \\
\text { (ps1g) } \\
9.89 \\
19.55 \\
29.57 \\
39.42 \\
49.16 \\
54.50 \\
59.57 \\
64.20 \\
69.32 \\
74.16 \\
80.16 \\
85.61 \\
90.58 \\
95.69 \\
100.92 \\
103.25 \\
106.11 \\
108.31 \\
111.08 \\
113.24 \\
117.83 \\
120.92 \\
123.2 .8 \\
122.97 \\
125.82 \\
125.60 \\
127.84 \\
130.19 \\
132.53 \\
135.33 \\
134.42 \\
138.35 \\
14.0 .16 \\
142.63 \\
145.78 \\
142.52 \\
0.22 \\
\end{array}$ & $\begin{array}{c}\text { Displacement } \\
\text { (inches) } \\
0.0034 \\
0.0146 \\
0.0246 \\
0.0332 \\
0.0423 \\
0.0489 \\
0.0573 \\
0.0647 \\
0.0753 \\
0.0816 \\
0.0997 \\
0.1131 \\
0.1285 \\
0.1448 \\
0.1624 \\
0.1709 \\
0.1806 \\
0.1854 \\
0.1990 \\
0.2064 \\
0.2300 \\
0.2521 \\
0.2696 \\
0.2744 \\
0.3018 \\
0.3095 \\
0.3277 \\
0.3695 \\
0.4279 \\
0.5203 \\
0.5486 \\
0.6868 \\
0.8194 \\
1.0134 \\
1.1843 \\
1.3080 \\
0.9742\end{array}$ \\
\hline
\end{tabular}




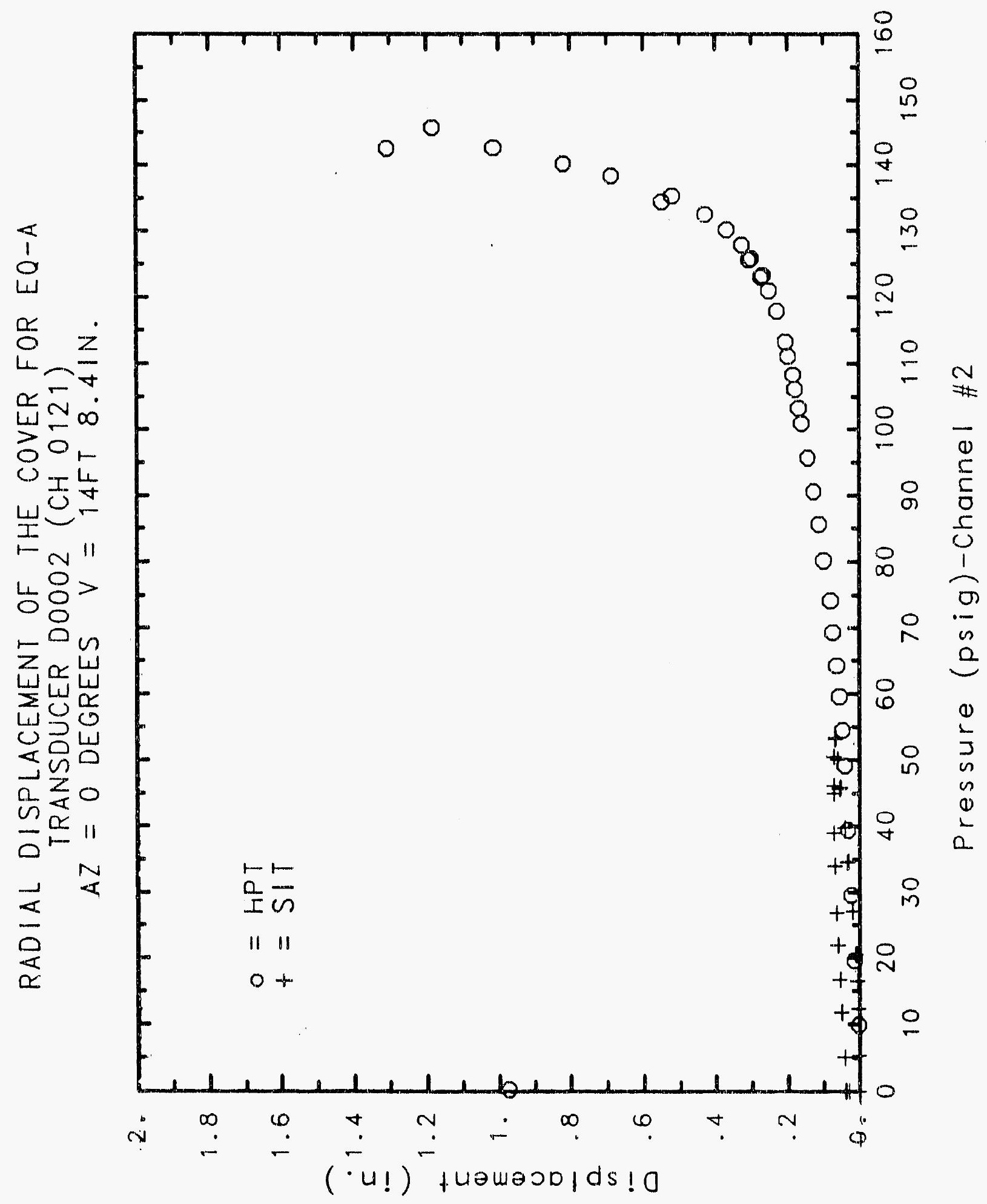




\section{Reinforced Concrete Test Data}

Potentiometer Channel 134

Structural Integrity Test

\begin{tabular}{cccc}
\hline & & & \\
Pressure & Displacement & Pressure & Displacement \\
(psig) & (inches) & (psig) & (inches) \\
-0.05 & 0.0000 & 9.89 & -0.0081 \\
5.33 & 0.0000 & 19.55 & -0.0082 \\
12.31 & 0.0001 & 29.57 & 0.0032 \\
16.44 & 0.0002 & 39.42 & 0.0170 \\
20.51 & 0.0002 & 49.16 & 0.0291 \\
20.46 & 0.0003 & 54.50 & 0.0374 \\
20.47 & 0.0004 & 59.57 & 0.0455 \\
27.05 & 0.0072 & 64.20 & 0.0540 \\
34.69 & 0.0214 & 69.32 & 0.0652 \\
34.53 & 0.0237 & 74.16 & 0.0776 \\
34.53 & 0.0239 & 80.16 & 0.0887 \\
39.70 & 0.0310 & 85.61 & 0.1000 \\
45.90 & 0.0421 & 90.58 & 0.1176 \\
45.65 & 0.0441 & 95.69 & 0.1316 \\
45.62 & 0.0444 & 100.92 & 0.1443 \\
50.10 & 0.0511 & 103.25 & 0.1512 \\
53.47 & 0.0575 & 106.11 & 0.1581 \\
53.29 & 0.0583 & 108.31 & 0.1634 \\
53.21 & 0.0583 & 111.08 & 0.1711 \\
50.49 & 0.0584 & 113.24 & 0.1787 \\
46.14 & 0.0584 & 117.83 & 0.1979 \\
46.13 & 0.0583 & 120.92 & 0.2148 \\
44.97 & 0.0587 & 123.28 & 0.2344 \\
38.98 & 0.0587 & 122.97 & 0.2407 \\
33.99 & 0.0588 & 125.82 & 0.2725 \\
33.96 & 0.0590 & 125.60 & 0.2825 \\
26.83 & 0.0590 & 127.84 & 0.3039 \\
21.88 & 0.0590 & 130.19 & 0.3656 \\
21.90 & 0.0591 & 132.53 & 0.4630 \\
16.69 & 0.0590 & 135.33 & 0.6077 \\
11.74 & 0.0562 & 134.42 & 0.8463 \\
11.77 & 0.0560 & 138.35 & 1.0068 \\
5.05 & 0.0425 & 140.16 & 1.2176 \\
0.02 & 0.0418 & 142.63 & 1.4480 \\
-0.04 & 0.0399 & 145.78 & \\
-0.02 & 0.0398 & 0.22 & \\
-0.02 & 0.0381 & & \\
0.02 & 0.0340 & & \\
\hline & & & \\
\hline
\end{tabular}

Higit Pressure Test 


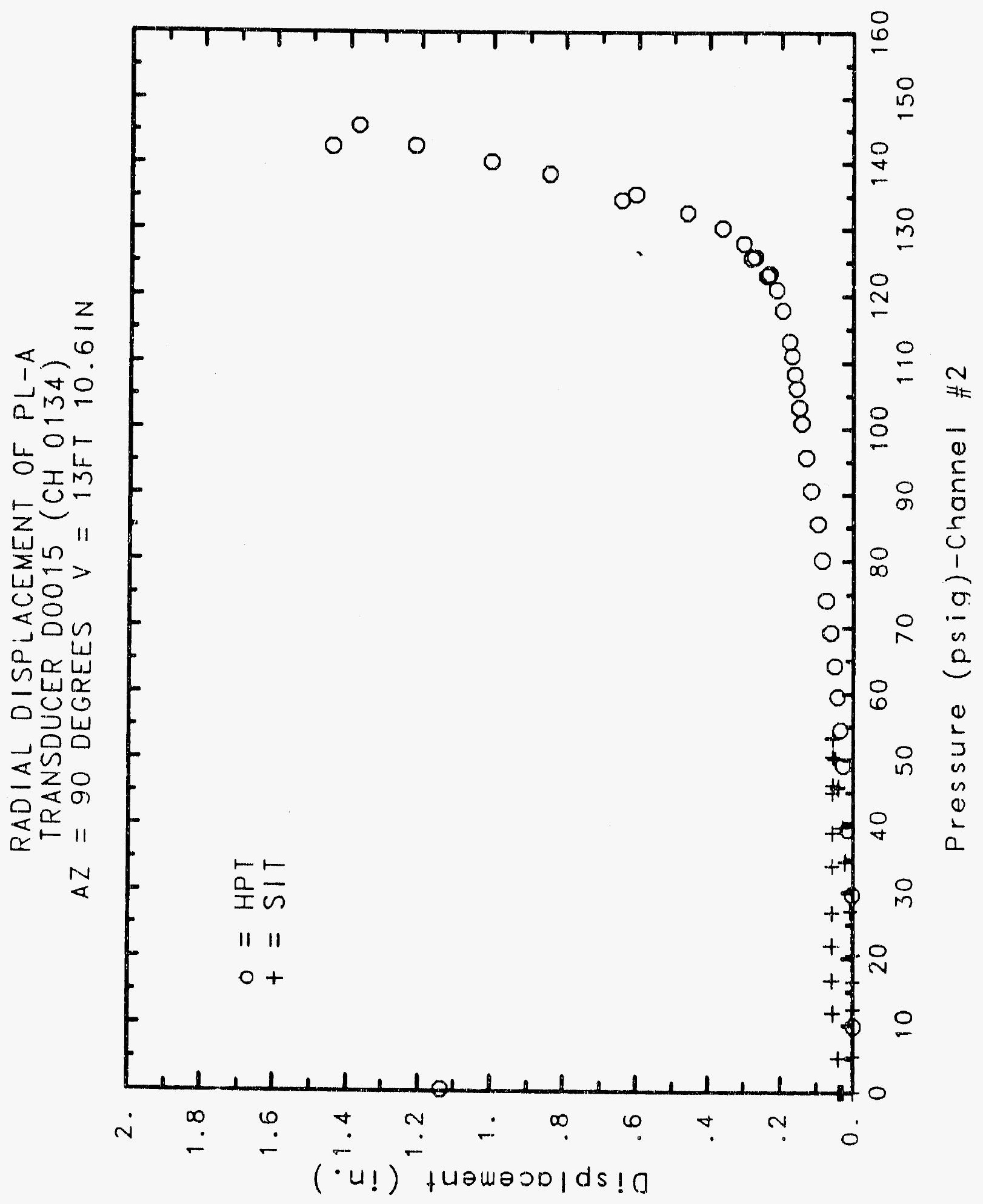




\section{Reinforced Concrete Test Data}

Potentiometer Channel 141

Structural INTEGRITY TEST

\begin{tabular}{|c|c|c|c|}
\hline $\begin{array}{l}\text { Pressure } \\
\text { (psig) } \\
-0.05 \\
5.33 \\
12.31 \\
16.44 \\
20.51 \\
20.46 \\
20.47 \\
27.05 \\
34.69 \\
34.53 \\
34.53 \\
39.70 \\
45.90 \\
45.65 \\
45.62 \\
50.10 \\
53.47 \\
53.29 \\
53.21 \\
50.49 \\
46.14 \\
46.13 \\
44.97 \\
38.98 \\
33.99 \\
33.96 \\
26.83 \\
21.88 \\
21.90 \\
16.69 \\
11.74 \\
11.77 \\
5.05 \\
0.07 \\
-0.06 \\
-0.02 \\
-0.02 \\
0.02 \\
\end{array}$ & $\begin{array}{c}\text { Displacement } \\
\text { (inches) } \\
-0.0001 \\
-0.0011 \\
-0.0010 \\
0.0011 \\
0.0087 \\
0.0113 \\
0.0124 \\
0.0269 \\
0.0457 \\
0.0494 \\
0.0503 \\
0.0621 \\
0.0805 \\
0.0838 \\
0.0839 \\
0.0914 \\
0.0995 \\
0.1004 \\
0.1006 \\
0.1020 \\
0.1022 \\
0.1022 \\
0.1024 \\
0.1026 \\
0.0984 \\
0.0986 \\
0.0879 \\
0.0819 \\
0.0817 \\
0.0694 \\
0.0605 \\
0.0603 \\
0.0458 \\
0.0436 \\
0.0380 \\
0.0382 \\
0.0202 \\
0.0272 \\
0.05\end{array}$ & $\begin{array}{c}\text { Pressure } \\
\text { (psig) } \\
9.89 \\
19.55 \\
29.57 \\
39.42 \\
49.16 \\
54.50 \\
59.57 \\
64.20 \\
69.32 \\
74.16 \\
80.16 \\
85.61 \\
90.58 \\
95.69 \\
100.92 \\
103.25 \\
106.11 \\
108.31 \\
111.08 \\
113.24 \\
117.83 \\
120.92 \\
123.28 \\
122.97 \\
125.82 \\
125.50 \\
127.84 \\
130.19 \\
132.53 \\
135.33 \\
134.42 \\
138.35 \\
140.16 \\
142.63 \\
145.78 \\
142.52 \\
0.22\end{array}$ & $\begin{array}{c}\text { Displacement } \\
\text { (inches) } \\
-0.0977 \\
-0.1427 \\
0.0971 \\
0.0965 \\
0.3564 \\
0.1021 \\
-0.4175 \\
-0.2232 \\
-0.2699 \\
-0.1471 \\
0.0430 \\
0.0550 \\
0.0639 \\
0.0765 \\
0.0919 \\
0.0997 \\
0.1082 \\
0.1153 \\
0.1266 \\
0.1366 \\
0.1614 \\
0.1800 \\
0.0514 \\
0.2037 \\
0.2319 \\
0.2427 \\
0.2616 \\
0.3156 \\
0.4010 \\
0.5167 \\
0.5574 \\
0.6940 \\
0.8199 \\
0.9971 \\
1.1413 \\
1.2035 \\
0.8073\end{array}$ \\
\hline
\end{tabular}

High Pressure test

Pressure

9.89

19.55

59.57

64.20

80.16

85.61

90.58

95.69

100.92

103.25

106.11

113.24

117.83

120.92

123.28

122.97

125.82

125.60

127.84

130.19

135.33

134.42

138.35

140.16

142.63

145.78

42.52

0.22 splacement

0.1427

0.0971

0.0965

0.3564

0.1021

$-0.4175$

0.2232

0.0430

0.0550

0.0639

0.0765

0.0919

0.0997

ก. 1266

0.1366

0.1614

0.18 .00

0.0514

0.2037

0.2319

0.3156

0.4010

0.5167

0.5574

0.6940

1.1413

1.2035

0.8073 


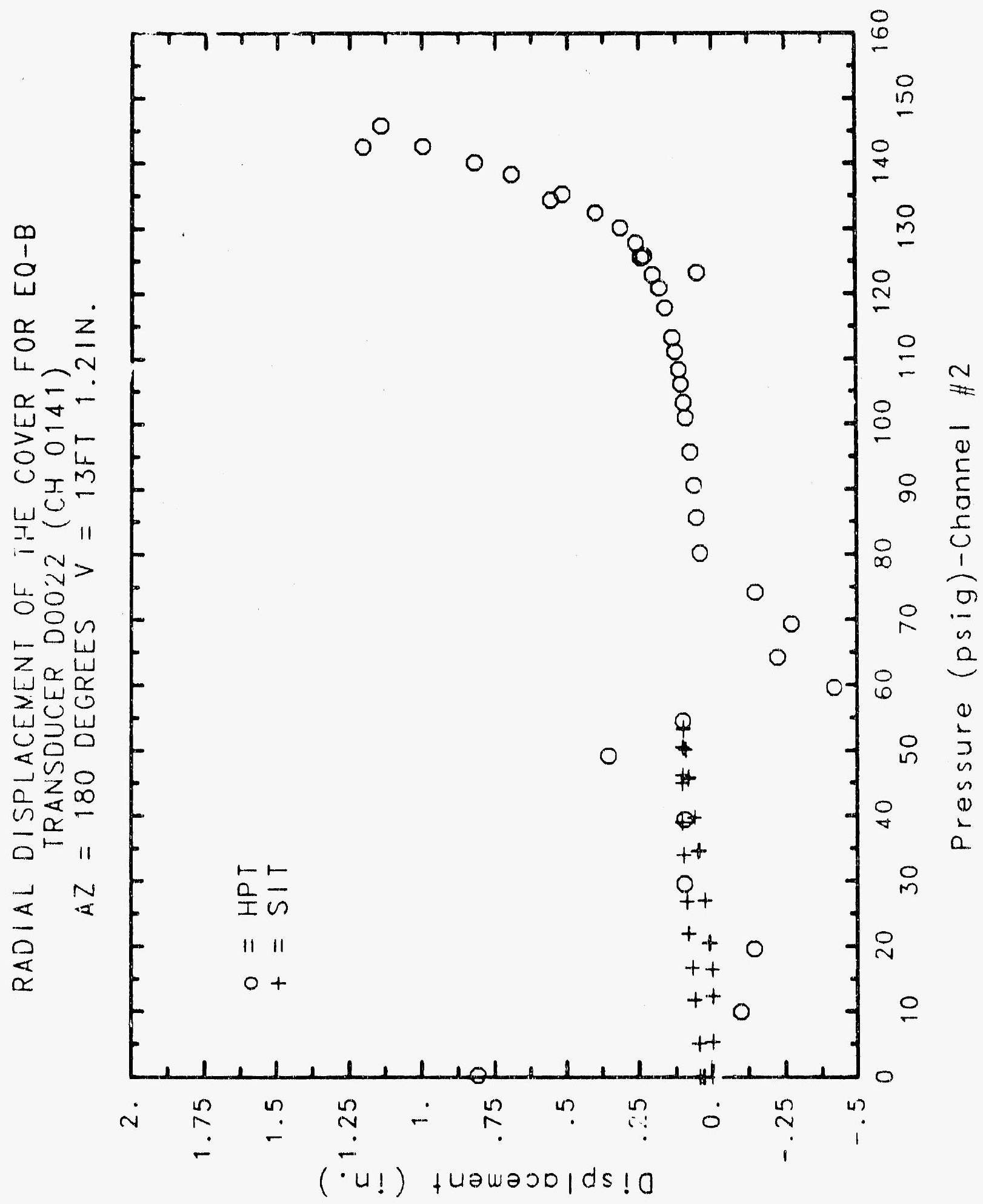




\title{
Reinforced Concrete Test Data
}

\author{
Potentiometer Channel 142
}

Structural INTEgRITY TEST

\begin{tabular}{|c|c|c|c|}
\hline $\begin{array}{c}\text { Pressure } \\
\text { (psig) } \\
-0.05 \\
5.33 \\
12.31 \\
16.44 \\
20.51 \\
20.46 \\
20.47 \\
27.05 \\
34.69 \\
34.53 \\
34.53 \\
39.70 \\
45.90 \\
45.65 \\
45.62 \\
50.10 \\
53.47 \\
53.29 \\
53.21 \\
50.49 \\
46.14 \\
46.13 \\
44.97 \\
38.98 \\
33.99 \\
33.96 \\
26.83 \\
21.88 \\
21.90 \\
16.69 \\
11.74 \\
11.77 \\
5.05 \\
0.02 \\
-0.04 \\
-0.02 \\
-0.02 \\
0.02\end{array}$ & $\begin{array}{c}\text { Displacement } \\
\text { (inches) } \\
0.0000 \\
-0.0031 \\
-0.0037 \\
-0.0010 \\
0.0074 \\
0.0114 \\
0.0117 \\
0.0216 \\
0.0403 \\
0.0442 \\
0.0447 \\
0.0542 \\
0.0688 \\
0.0714 \\
0.0717 \\
0.0779 \\
0.0845 \\
0.0855 \\
0.0858 \\
0.0874 \\
0.0875 \\
0.0875 \\
0.0877 \\
0.0880 \\
0.0849 \\
0.0846 \\
0.0757 \\
0.0712 \\
0.0710 \\
0.0612 \\
0.0532 \\
0.0530 \\
0.0403 \\
0.0398 \\
0.0327 \\
0.0327 \\
0.0138 \\
0.0174\end{array}$ & $\begin{array}{c}\text { Pressure } \\
(\text { psig) } \\
9.89 \\
19.55 \\
29.57 \\
39.42 \\
49.16 \\
54.50 \\
59.57 \\
64.20 \\
69.32 \\
74.16 \\
80.16 \\
85.61 \\
90.58 \\
95.69 \\
100.92 \\
103.25 \\
106.11 \\
108.31 \\
111.08 \\
113.24 \\
117.83 \\
120.92 \\
123.28 \\
122.97 \\
125.82 \\
125.60 \\
127.84 \\
130.19 \\
132.53 \\
135.33 \\
134.42 \\
138.35 \\
140.16 \\
142.63 \\
145.78 \\
142.52 \\
0.22\end{array}$ & $\begin{array}{c}\text { Displacement } \\
\text { (inches) } \\
-0.0029 \\
0.0077 \\
0.0235 \\
0.0432 \\
0.0566 \\
0.0658 \\
0.0757 \\
0.0894 \\
0.1020 \\
0.1142 \\
0.1250 \\
0.1346 \\
0.1405 \\
0.1503 \\
0.1660 \\
0.1714 \\
0.1787 \\
0.1859 \\
0.1978 \\
0.2057 \\
0.2296 \\
0.2468 \\
0.2645 \\
0.2693 \\
0.2952 \\
0.3061 \\
0.3227 \\
0.3720 \\
0.4520 \\
0.5619 \\
0.5944 \\
0.7412 \\
0.8651 \\
1.0415 \\
1.1921 \\
1.2513 \\
0.8678\end{array}$ \\
\hline
\end{tabular}

High Pressure test

Pressure

9.89

19.55

2.9 .57

39.42

59.57

64.20

69.32

85.61

95.69

106.11

108.31

113.24

117.83

122.97

125.82

125.60

127.84

135.33

134.42

138.35

140.16

142.63

45.78

42.52

1.2513

0.0138

0.0174 


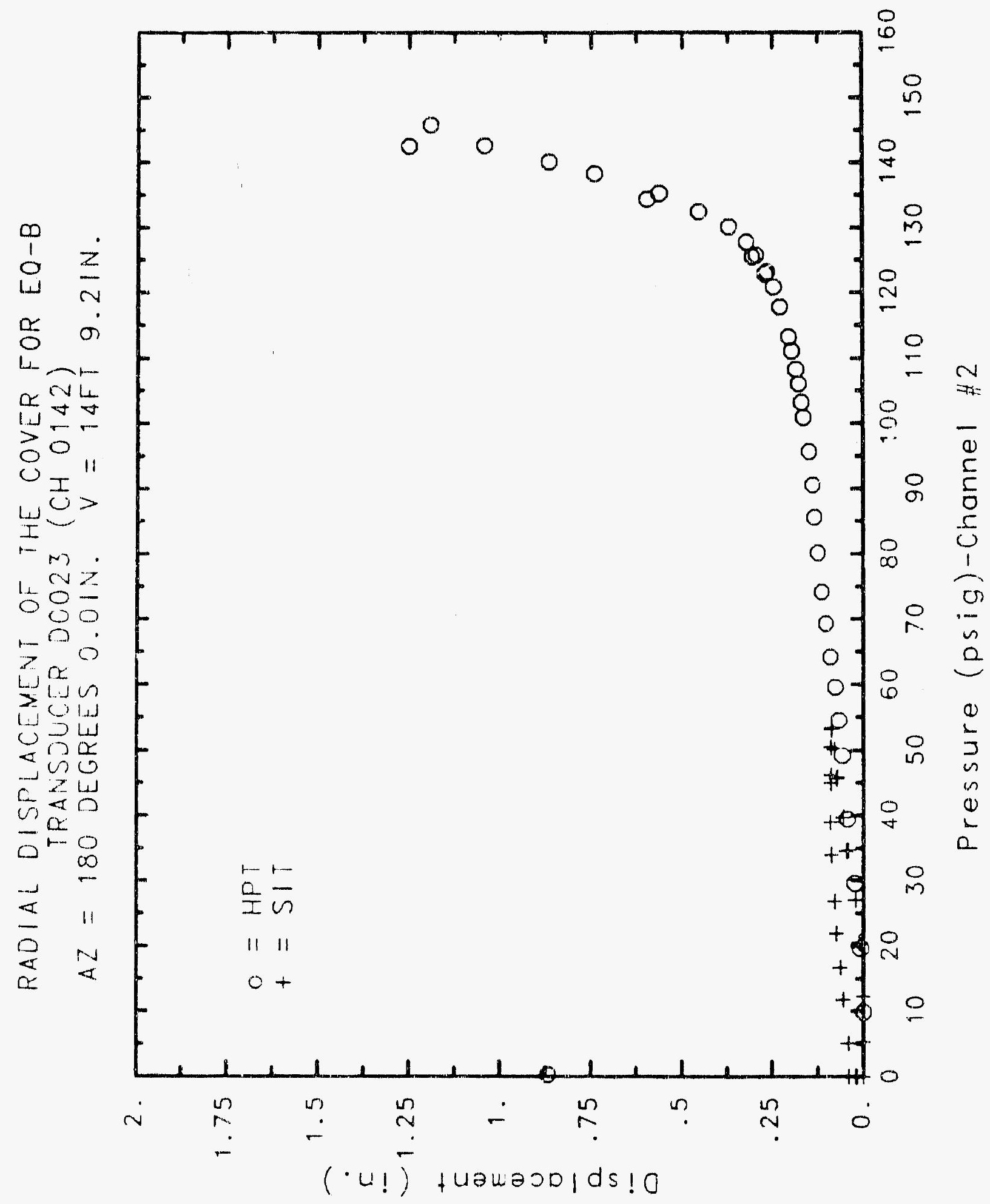




\section{Reinforced Concrete Test Data}

\section{Potentiometer Channel 169}

Structural InTEgrity TEST

\begin{tabular}{|c|c|c|c|}
\hline $\begin{array}{c}\text { Pressure } \\
\text { (psig) } \\
-0.05 \\
5.33 \\
12.31 \\
16.44 \\
20.51 \\
20.46 \\
20.47 \\
27.05 \\
34.69 \\
34.53 \\
34.53 \\
39.70 \\
45.90 \\
45.65 \\
45.62 \\
50.10 \\
53.47 \\
53.29 \\
53.21 \\
50.49 \\
46.14 \\
40.13 \\
44.97 \\
38.98 \\
33.99 \\
33.96 \\
26.83 \\
21.88 \\
21.90 \\
16.69 \\
11.74 \\
11.77 \\
5.05 \\
0.02 \\
-0.04 \\
-0.02 \\
-0.02 \\
0.02\end{array}$ & $\begin{array}{c}\text { Disp.acement } \\
\text { (inches) } \\
0.0127 \\
0.0126 \\
0.0171 \\
0.0100 \\
0.0108 \\
0.0168 \\
0.0121 \\
0.0171 \\
0.0267 \\
0.0339 \\
0.0262 \\
0.0209 \\
0.0251 \\
0.0347 \\
0.0296 \\
0.0245 \\
0.0290 \\
0.0329 \\
0.0304 \\
0.0255 \\
0.0218 \\
0.0196 \\
0.0091 \\
0.0084 \\
0.0122 \\
0.0198 \\
0.0096 \\
0.0138 \\
0.0197 \\
0.0168 \\
0.0132 \\
0.0214 \\
0.0093 \\
0.0093 \\
0.0027 \\
0.0022 \\
0.0065 \\
-0.0104 \\
0.04\end{array}$ & $\begin{array}{c}\text { Pressure } \\
(\text { psig) } \\
9.89 \\
19.55 \\
29.57 \\
39.42 \\
49.16 \\
54.50 \\
59.57 \\
64.20 \\
69.32 \\
74.16 \\
80.16 \\
85.61 \\
90.58 \\
95.69 \\
100.92 \\
103.25 \\
106.11 \\
108.31 \\
111.08 \\
113.24 \\
117.83 \\
120.92 \\
123.28 \\
122.97 \\
125.82 \\
125.60 \\
127.84 \\
130.19 \\
132.53 \\
135.33 \\
134.42 \\
138.35 \\
140.16 \\
142.63 \\
145.78 \\
142.52 \\
0.22\end{array}$ & $\begin{array}{c}\text { Displacement } \\
\text { (inches) } \\
0.0338 \\
0.0323 \\
0.0318 \\
0.0263 \\
0.0230 \\
0.0203 \\
0.0172 \\
0.0139 \\
0.0099 \\
-0.0092 \\
-0.0540 \\
-0.0759 \\
-0.0906 \\
-0.1018 \\
-0.1090 \\
-0.1159 \\
-0.1207 \\
-0.1208 \\
-0.1271 \\
-0.1287 \\
-0.1390 \\
-0.1426 \\
-0.1495 \\
-0.1496 \\
-0.1576 \\
-0.1611 \\
-0.1663 \\
-0.1702 \\
-0.1750 \\
-0.1847 \\
-0.1873 \\
-0.1925 \\
-0.1978 \\
-0.2073 \\
-0.2161 \\
-0.2298 \\
-0.2549\end{array}$ \\
\hline
\end{tabular}

\section{High Pressure Test}

splacement

0.0318

0.0263

0.0230

0.0203

0.0172

0.0139

0.0099

$-0.0092$

$-0.0906$

$-0.1018$

$-0.1090$

$-0.1159$

$-0.1207$

$-0.1208$

$-0.1390$

0.1426

$-0.1496$

$-0.1576$

$-0.1611$

$-0.1663$

$-0.1847$

$-0.1873$

$-0.1925$

$-0.1978$

$-0.2073$

$-0.2161$

$-0.2298$

$-0.2549$ 


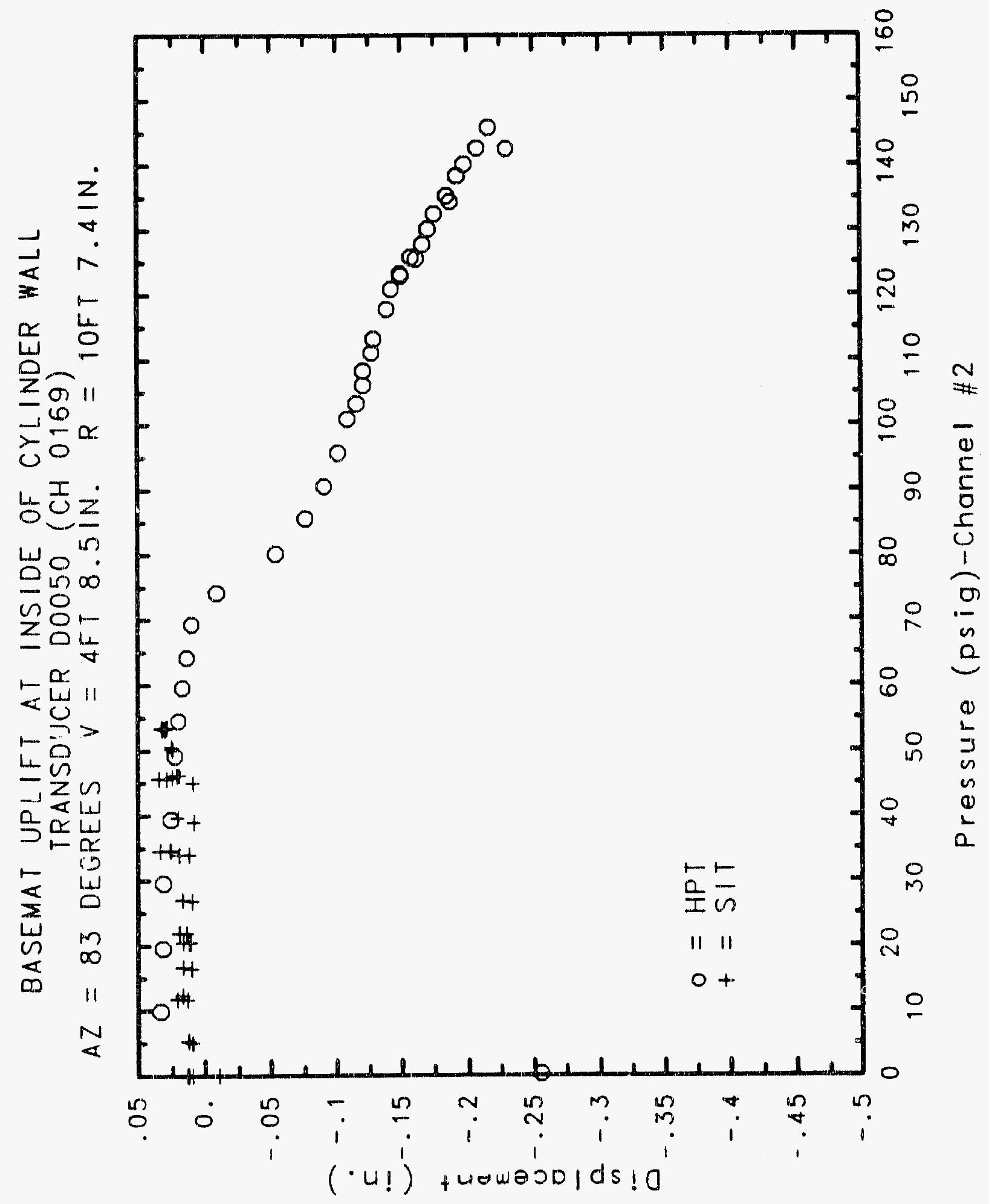




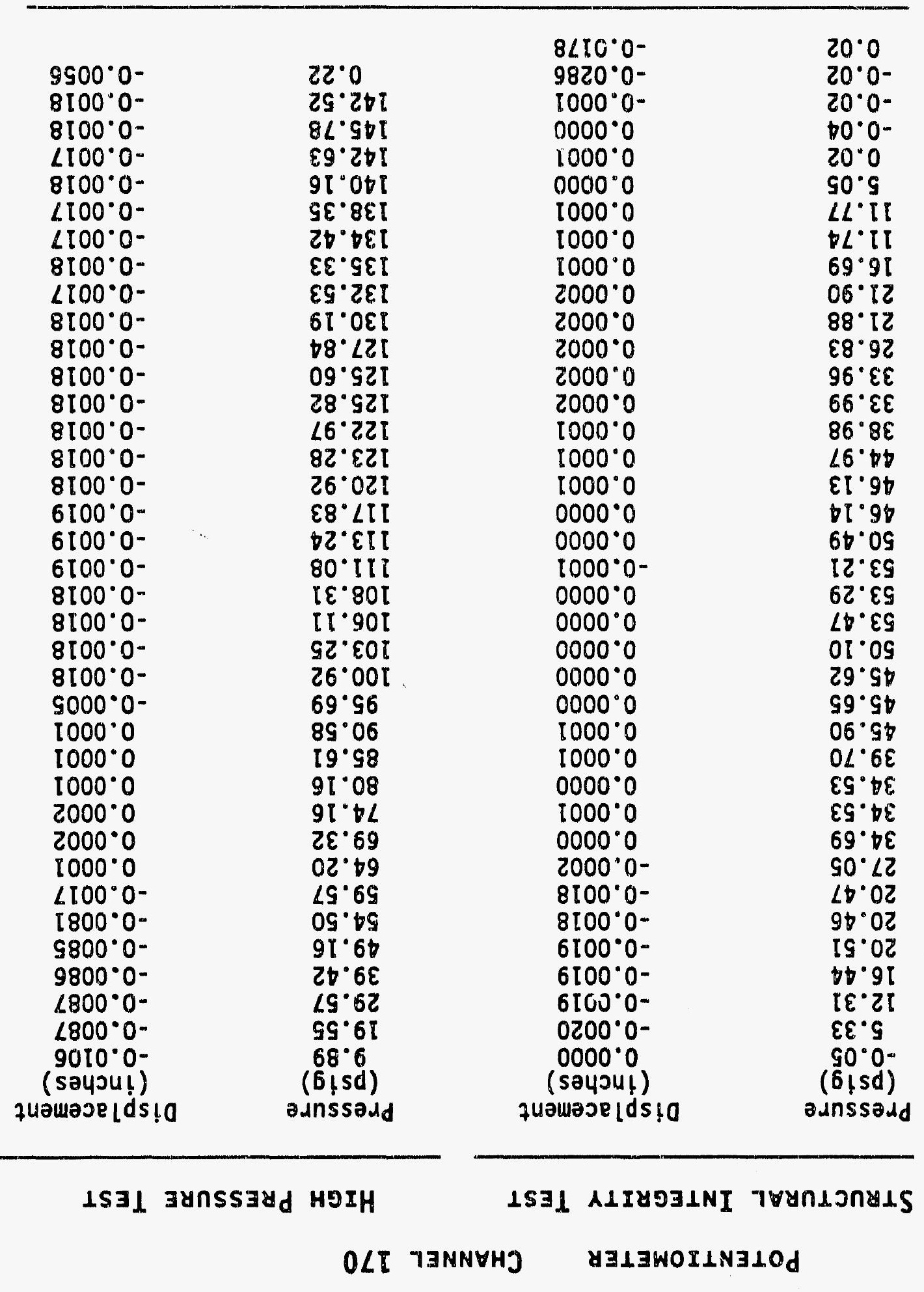

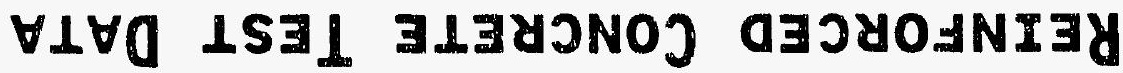




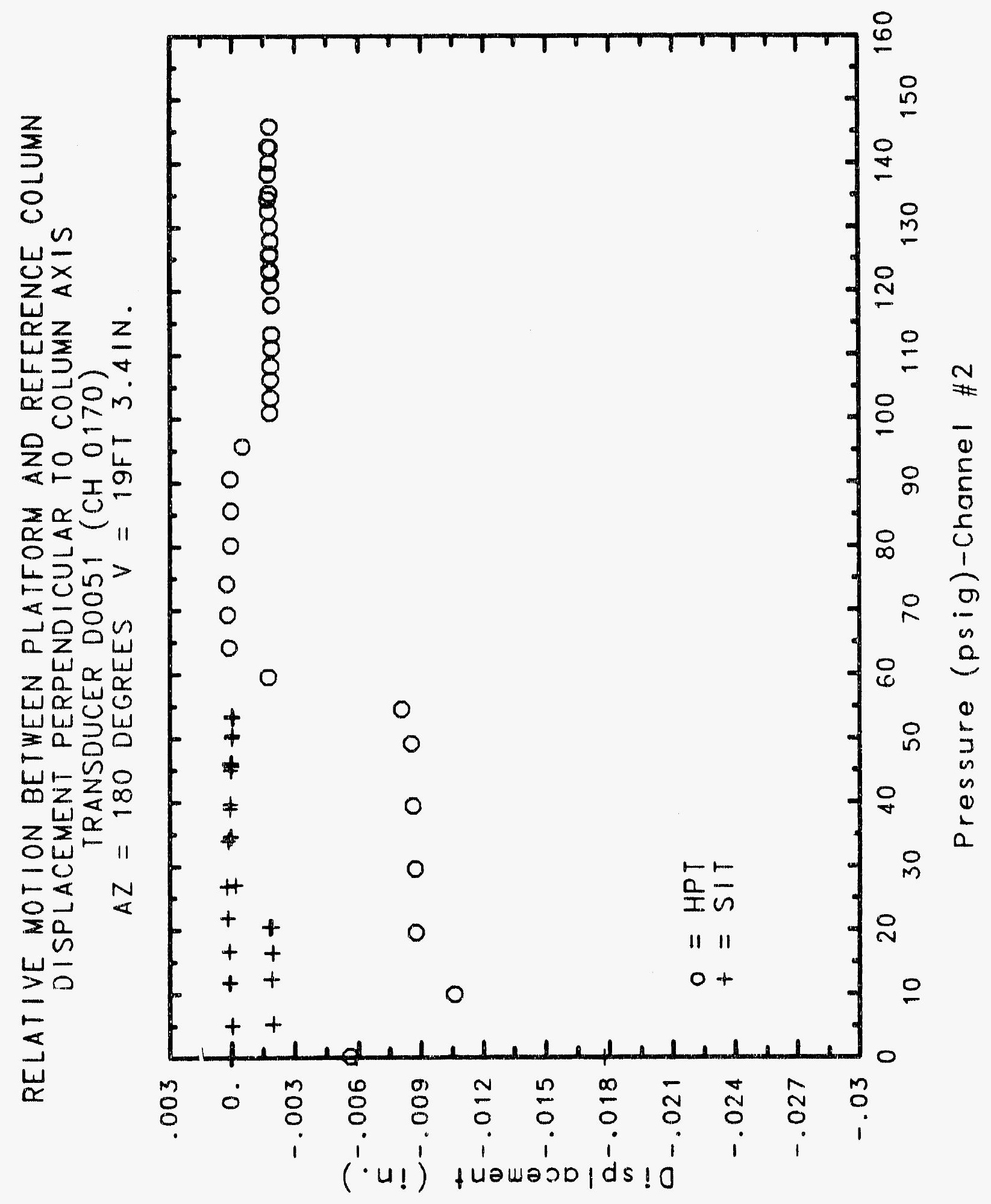




\section{Reinforced Concrete Test Data}

\section{Potentiometer Channel 171}

Structural Integrity Test

\begin{tabular}{|c|c|c|c|}
\hline $\begin{array}{l}\text { Pressure } \\
\text { (psig) } \\
-0.05 \\
5.33 \\
12.31 \\
16.44 \\
20.51 \\
20.46 \\
20.47 \\
27.05 \\
34.69 \\
34.53 \\
34.53 \\
39.70 \\
45.90 \\
45.65 \\
45.62 \\
50.10 \\
53.47 \\
53.29 \\
53.21 \\
50.49 \\
46.14 \\
46.13 \\
44.97 \\
38.98 \\
33.99 \\
33.96 \\
26.83 \\
21.88 \\
21.90 \\
16.59 \\
11.74 \\
11.77 \\
5.05 \\
0.02 \\
-0.04 \\
-0.02 \\
-0.02 \\
0.02 \\
\end{array}$ & $\begin{array}{c}\text { Displacement } \\
\text { (inches) } \\
0.0004 \\
0.0014 \\
0.0013 \\
0.0014 \\
0.0014 \\
0.0014 \\
0.0014 \\
0.0015 \\
0.0015 \\
0.0014 \\
0.0014 \\
0.0015 \\
0.0014 \\
0.0014 \\
0.0014 \\
0.0014 \\
0.0014 \\
0.0013 \\
0.0014 \\
0.0014 \\
0.0013 \\
0.0013 \\
0.0014 \\
0.0013 \\
0.0014 \\
0.0014 \\
0.0015 \\
0.0015 \\
0.0014 \\
0.0015 \\
0.0015 \\
0.0015 \\
0.0014 \\
0.0014 \\
0.0013 \\
0.0013 \\
-0.0098 \\
-0.0078\end{array}$ & $\begin{array}{c}\text { Pressure } \\
(\text { psig) } \\
9.89 \\
19.55 \\
29.57 \\
39.42 \\
49.16 \\
54.50 \\
59.57 \\
64.20 \\
69.32 \\
74.16 \\
80.1 \varepsilon \\
85.61 \\
90.58 \\
95.69 \\
100.92 \\
103.25 \\
106.11 \\
108.31 \\
111.08 \\
113.24 \\
117.83 \\
120.92 \\
123.28 \\
122.97 \\
125.82 \\
125.60 \\
127.84 \\
130.19 \\
132.53 \\
135.33 \\
134.42 \\
138.35 \\
140.16 \\
142.63 \\
145.78 \\
142.52 \\
0.22\end{array}$ & $\begin{array}{c}\text { Displacement } \\
\text { (inches) } \\
-0.0073 \\
-0.0073 \\
-0.0074 \\
-0.0074 \\
-0.0073 \\
-0.0073 \\
-0.0073 \\
-0.0073 \\
-0.0073 \\
-0.0072 \\
-0.0074 \\
-0.0074 \\
-0.0092 \\
-0.0093 \\
-0.0093 \\
-0.0093 \\
-0.0093 \\
-0.0093 \\
-0.0093 \\
-0.0093 \\
-0.0093 \\
-0.0093 \\
-0.0092 \\
-0.0092 \\
-0.0092 \\
-0.0093 \\
-0.0091 \\
-0.0092 \\
-0.0092 \\
-0.0092 \\
-0.0090 \\
-0.0091 \\
-0.0091 \\
-0.0091 \\
-0.0091 \\
-0.0092 \\
-0.0076 \\
-0.00\end{array}$ \\
\hline
\end{tabular}

High Pressure Test

(isplacement

$-0.0073$

$-0.0074$

$-0.0074$

$-0.0073$

$-0.0073$

$-0.0073$

$-0.0072$

$-0.0074$

$-0.0092$

0.0093

$-0.0093$

$-0.0093$

$-0.0093$

$-0.0093$

0.0092

0.0093

$-0.0092$

$-0.0092$

$-0.0092$

$-0.0091$

$-0.0091$

$-0.0091$

$-0.0092$

$-0.0076$ 


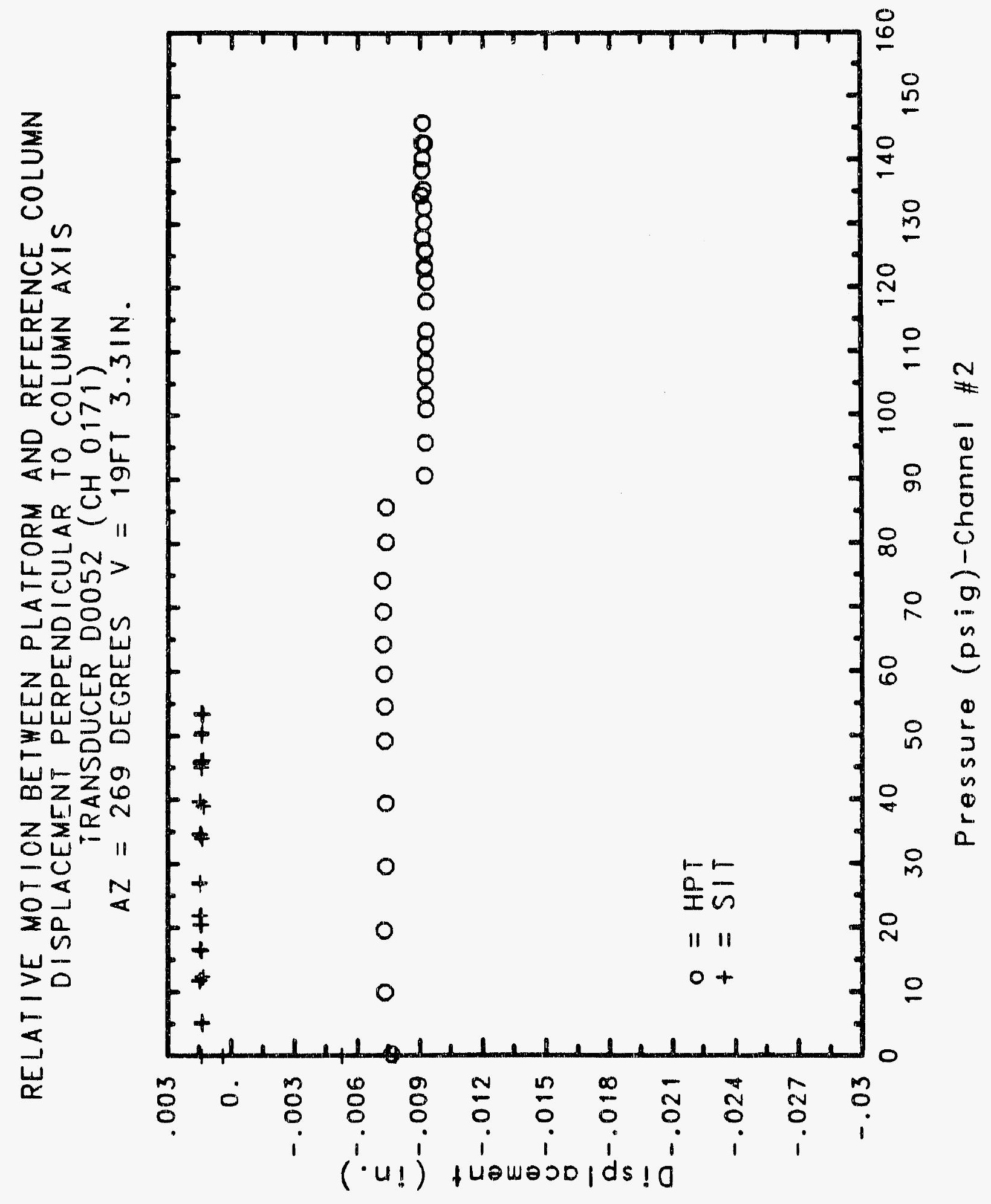




\section{Reinforced Concrete Test Data}

Potentiometer Channel 172

Structural Integrity Test

\begin{tabular}{|c|c|c|c|}
\hline $\begin{array}{l}\text { Pressura } \\
\text { (psig) } \\
-0.05 \\
5.33 \\
12.31 \\
16.44 \\
20.51 \\
20.46 \\
20.47 \\
27.05 \\
34.69 \\
34.53 \\
34.53 \\
39.70 \\
45.90 \\
45.65 \\
45.62 \\
50.10 \\
53.47 \\
53.29 \\
53.21 \\
50.49 \\
46.14 \\
46.13 \\
44.97 \\
38.98 \\
33.99 \\
33.96 \\
26.83 \\
21.88 \\
21.90 \\
16.69 \\
11.74 \\
11.77 \\
5.05 \\
0.02 \\
-0.04 \\
-0.02 \\
-0.02 \\
0.02\end{array}$ & $\begin{array}{c}\text { Displacement } \\
\text { (inches) } \\
-0.0008 \\
0.0025 \\
0.0038 \\
0.0042 \\
0.0037 \\
0.0048 \\
0.0047 \\
0.0022 \\
0.0000 \\
0.0019 \\
0.0022 \\
0.0011 \\
-0.001 \\
0.0003 \\
0.0006 \\
-0.0006 \\
-0.0010 \\
0.0000 \\
0.0002 \\
0.0017 \\
0.0030 \\
0.0021 \\
0.0014 \\
0.0023 \\
0.0026 \\
0.0024 \\
0.0033 \\
0.0035 \\
0.0028 \\
0.0034 \\
0.0041 \\
0.0030 \\
0.0045 \\
0.0055 \\
0.0060 \\
0.0051 \\
0.0085 \\
0.0073 \\
0.07\end{array}$ & $\begin{array}{l}\text { Pressure } \\
(\text { psig) } \\
9.89 \\
19.55 \\
29.57 \\
39.42 \\
49.16 \\
54.50 \\
59.57 \\
64.20 \\
69.32 \\
74.16 \\
80.16 \\
85.61 \\
90.58 \\
95.69 \\
100.92 \\
103.25 \\
106.11 \\
108.31 \\
111.08 \\
113.24 \\
117.83 \\
120.92 \\
123.28 \\
122.97 \\
125.82 \\
125.60 \\
127.84 \\
130.19 \\
132.53 \\
135.33 \\
134.42 \\
138.35 \\
140.16 \\
142.63 \\
145.78 \\
142.52 \\
0.22\end{array}$ & $\begin{array}{c}\text { Displacement } \\
\text { (inches) } \\
-0.0045 \\
-0.0057 \\
-0.0072 \\
-0.0077 \\
-0.0081 \\
-0.0155 \\
-0.0149 \\
-0.0140 \\
-0.0167 \\
-0.0224 \\
-0.0325 \\
-0.0408 \\
-0.0461 \\
-0.0462 \\
-0.0438 \\
-0.0421 \\
-0.0416 \\
-0.0414 \\
-0.0400 \\
-0.0394 \\
-0.0385 \\
-0.0368 \\
-0.0355 \\
-0.0349 \\
-0.0352 \\
-0.0348 \\
-0.0354 \\
-0.0358 \\
-0.0361 \\
-0.0362 \\
-0.0356 \\
-0.0370 \\
-0.0370 \\
-0.0381 \\
-0.0389 \\
-0.0390 \\
-0.0094\end{array}$ \\
\hline
\end{tabular}

High Pressure Test

splacement

(inches)

$-0.0045$

$-0.0057$

$-0.0072$

$-0.0155$

$-0.0149$

$-0.0140$

$-0.0167$

$-0.0224$

$-0.0461$

$-0.0462$

$-0.0438$

$-0.0421$

$-0.0416$

$-0.0394$

$-0.0385$

$-0.0368$

$-0.0355$

$-0.0349$

$-0.0352$

0.0348

$-0.0361$

$-0.0370$

$-0.0370$

0.0381

.0389

.0390

0.0094 


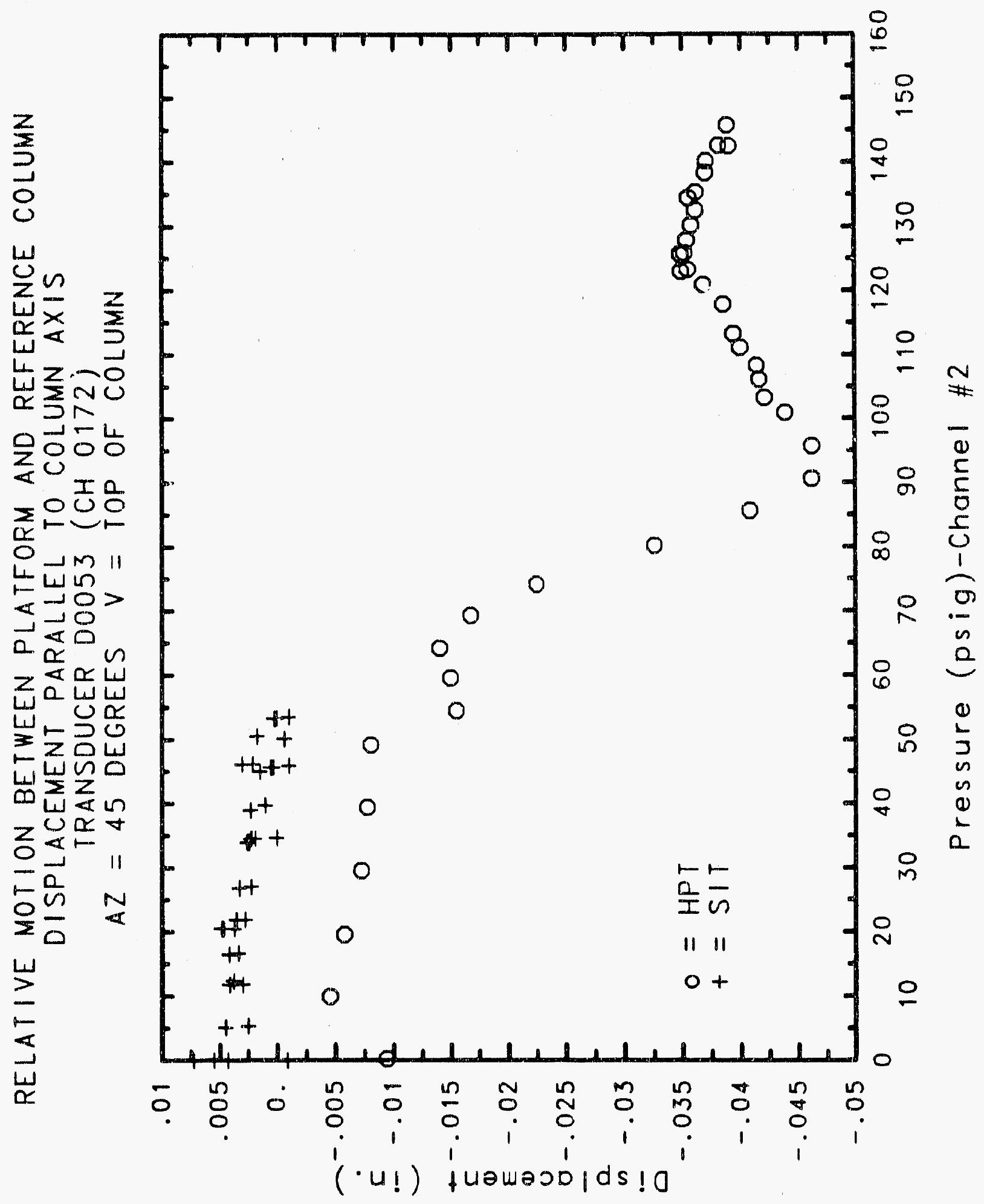




\section{Reinforced Concrete Test Data}

Potentiometer Channel 173

Structural Integrity Test

A correction of -0.0144 in. has been applied to each reading

Pressure

(psig)

$-0.05$

5.33

12.31

16.44

20.51

20.46

20.47

27.05

34.69

34.53

34.53

39.70

45.90

45.65

45.62

50.10

53.47

53.29

53.21

50.49

46.14

46.13

44.98

38.98

33.99

33.96

26.83

21.88

21.90

16.69

11.74

11.77

5.05

0.02

$-0.04$

$-0.02$

$-0.02$

0.02

0.02
Displacement

(inches)

$-0.0145$

0.0033

0.0076

0.0092

0.0111

0.0115

0.0118

0.0144

0.0192

0.0199

0.0201

0.0219

0.0249

0.0249

0.0263

0.0279

0.0281

0.0281

0.0291

0.0293

0.0293

0.0293

0.0280

0.0267

0.0269

0.0243

0.0232

0.0233

0.0205

0.0193

0.0193

0.0132

0.0041

0.0001

0.0003

0.0003

.0005

$-0.0081$
0.0245

\section{High Pressure Test}

A correction of -0.0152 in. has been applied to each reading.

Pressure

(psig)

9.89

19.55

29.57

39.42

49.16

54.50

59.57

64.20

69.32

74.16

80.16

85.61

90.58

95.69

100.92

103.25

106.11

108.31

111.08

113.24

117.83

120.92

123.28

122.97

125.82

125.60

127.84

130.19

132.53

135.33

134.42

138.35

140.16

142.63

145.78

142.52

0.22
Displacement (inches)

0.0038

0.0074

0.0114

0.0148

0.0179

0.0204

0.0231

0.0264

0.0294

0.0335

0.0376

0.0411

0.0450

0.0498

0.0547

0.0575

0.0602

0.0622

0.0664

0.0698

0.0745

0.0798

0.0844

0.0853

0.0901

0.0923

0.0962

0.1029

0.1101

0.1228

0.1256

0.1414

0.1546

0.1743

0.1917

0.2034

0.0866 


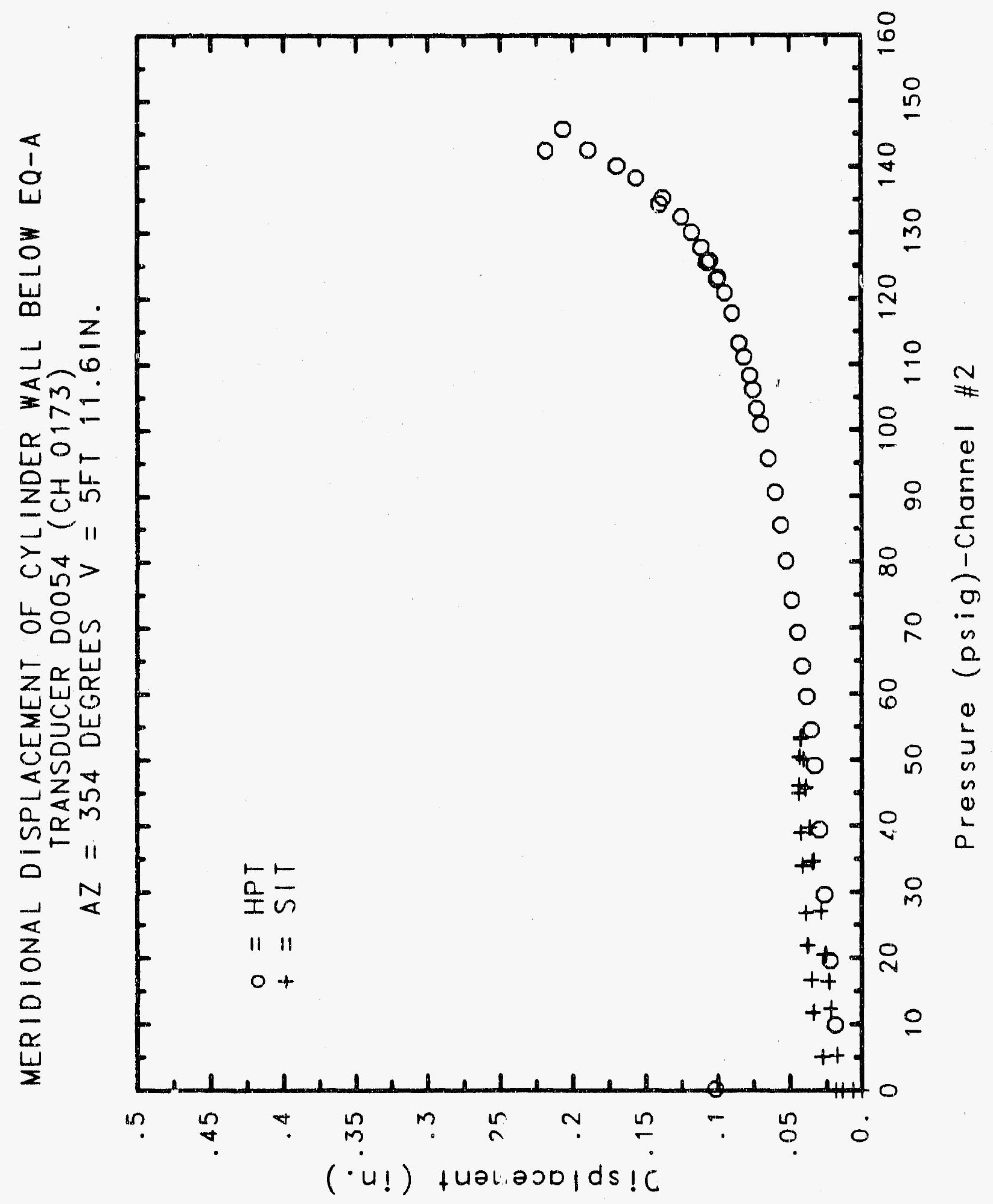




\section{Reinforced Concrete Test D.ita}

Potentiometer Channel 174

Structural. IntEgrity Test

A correction of -0.0121 in. has been applied to each reading

Pressure

(psig)

$-0.05$

5.33

12.31

16.44

20.51

20.46

20.47

27.05

34.69

34.53

34.53

39.70

45.90

45.65

45.62

50.10

53.47

53.29

53.21

50.49

46.14

46.13

44.98

38.98

33.99

33.96

26.83

21.88

21.90

16.69

11.74

11.77

5.05

0.02

$-0.04$

$-0.02$

$-0.02$

0.02

0.02
Displacement

(inches)

$-0.0118$

0.0030

0.0070

0.0089

0.0115

0.0124

0.0130

0.0183

0.0279

0.0299

0.0300

0.0342

0.0413

0.0422

0.0423

0.0461

0.0500

0.0506

0.0506

0.0534

0.0535

0.0536

$0.052 \%$

0.0509

0.0486

0.0487

0.0436

0.0408

0.0408

0.0358

0.0309

0.0307

0.0235

0.0196

0.0166

0.0166

0.0166

0.0165

0.0041
High Pressure test

A correction of -0.0110 in. has been applied to each reading.

Pressure

(psig)

9.89

19.55

29.57

39.42

49.16

54.50

59.57

64.20

69.32

74.16

80.16

85.61

90.58

95.69

100.92

103.25

106.11

108.31

111.08

113.24

117.83

120.92

123.28

122.97

125.82

125.60

127.84

130.19

132.53

135.33

134.42

138.35

140.16

142.63

145.78

142.52

0.22
Displ acement

(inches)

0.0071

0.0139

0.0211

0.0284

0.0355

0.0405

0.0455

0.0515

0.0579

0.0653

0.0734

0.0809

0.0890

0.0993

0.1108

0.1169

0.1227

0.1270

0.1343

0.1403

0.1524

0.1622

0.1710

0.1734

0.1837

0.1873

0.1937

0.2055

0.2191

0.2407

0.2457

0.2735

0.2946

0.3268

0.3584

0.3753

0.1794 


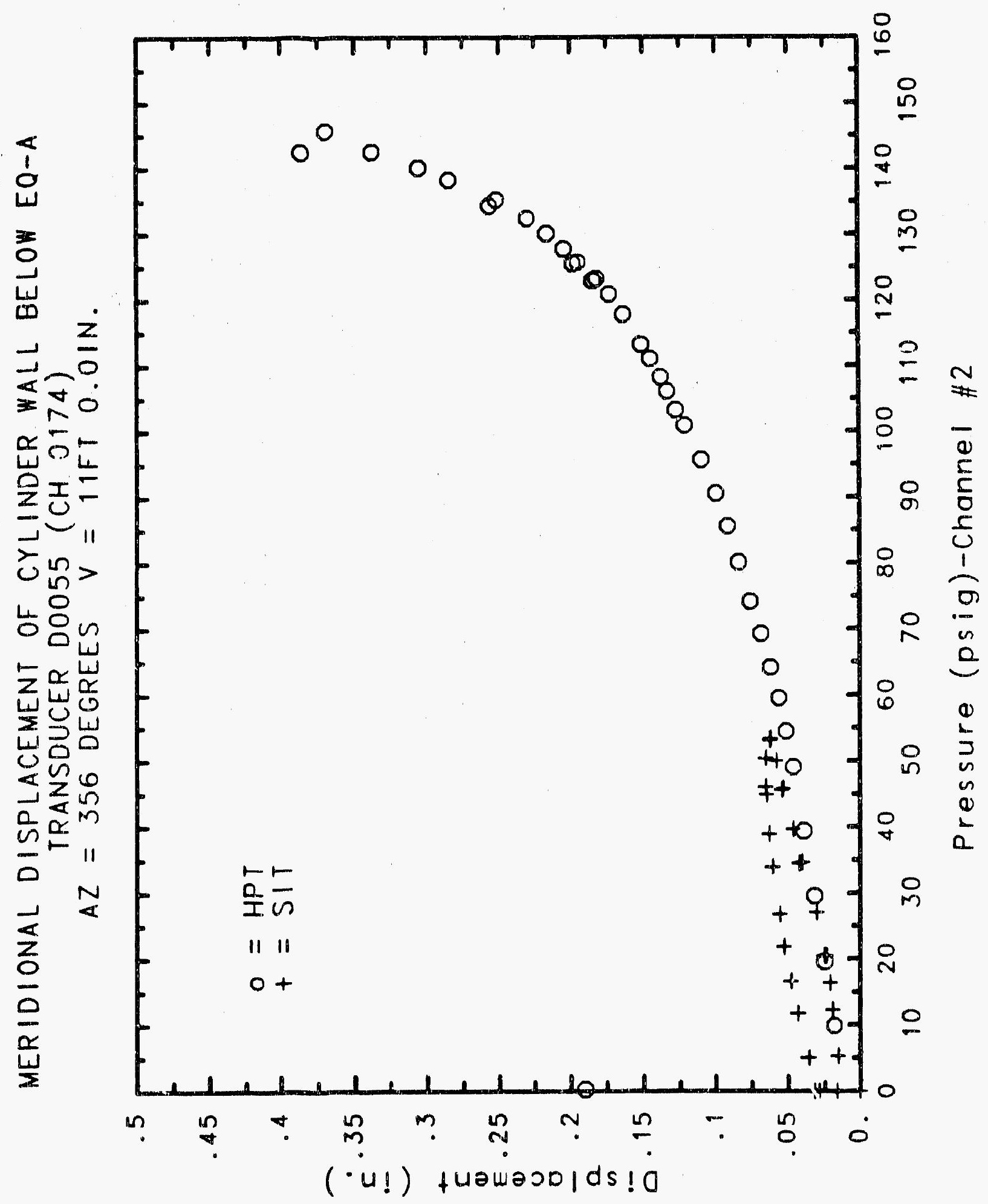

C-29 


\section{Reinforced Concrete Test Data}

Potentiometer Channel 175

Structural Integrity Test

\begin{tabular}{|c|c|}
\hline $\begin{array}{c}\text { Pressure } \\
\text { (psig) } \\
-0.05 \\
5.33 \\
12.31 \\
16.44 \\
20.51 \\
20.46 \\
20.47 \\
27.05 \\
34.69 \\
34.53 \\
34.53 \\
39.70 \\
45.90 \\
45.65 \\
45.62 \\
50.10 \\
53.47 \\
53.29 \\
53.21 \\
50.49 \\
46.14 \\
46.13 \\
44.97 \\
38.98 \\
33.99 \\
33.96 \\
26.83 \\
21.88 \\
21.90 \\
16.69 \\
11.74 \\
11.77 \\
5.05 \\
0.02 \\
-0.04 \\
-0.02 \\
-0.02 \\
0.02\end{array}$ & $\begin{array}{c}\text { Displacement } \\
\text { (inches) } \\
0.0001 \\
-0.0021 \\
-0.0022 \\
-0.0004 \\
0.0034 \\
0.0057 \\
0.0064 \\
0.0132 \\
0.0254 \\
0.0281 \\
0.0288 \\
0.0344 \\
0.0446 \\
0.0469 \\
0.0471 \\
0.0522 \\
0.0579 \\
0.0595 \\
0.0599 \\
0.0639 \\
0.0639 \\
0.0638 \\
0.0642 \\
0.0648 \\
0.0631 \\
0.0632 \\
0.0572 \\
0.0540 \\
0.0541 \\
0.0488 \\
0.0450 \\
0.0450 \\
0.0379 \\
0.0378 \\
0.0339 \\
0.0341 \\
0.0432 \\
0.0389\end{array}$ \\
\hline
\end{tabular}

high Pressure Test

Pressure

(psig)

9.89

19.55

29.57

39.42

49.16

54.50

59.57

64.20

69.32

74.16

80.16

85.81

90.58

95.69

100.92

103.25

106.11

108.31

111.08

113.24

117.83

120.92

123.28

122.97

125.82

125.60

127.84

$\$ 30.19$

132.53

135.33

134.42

138.35

140.16

142.63

145.78

142.52

0.22
Displacement

(inches)

$-0.0018$

0.0069

0.0158

0.0237

0.0330

0.0388

0.0459

0.0552

0.0629

0.0699

0.0858

0.0990

0.1159

0.1307

0.1473

0.1563

0.1649

0.1711

0.1817

0.1903

0.2118

0.2324

0.2510

0.2567

0.2824

0.2905

0.3064

0.3482

0.4067

0.4964

0.5241

0.6573

0.7901

0.9866

$1 . \lcm{559}$

1.2763

0.9538 
$[\varepsilon-]$

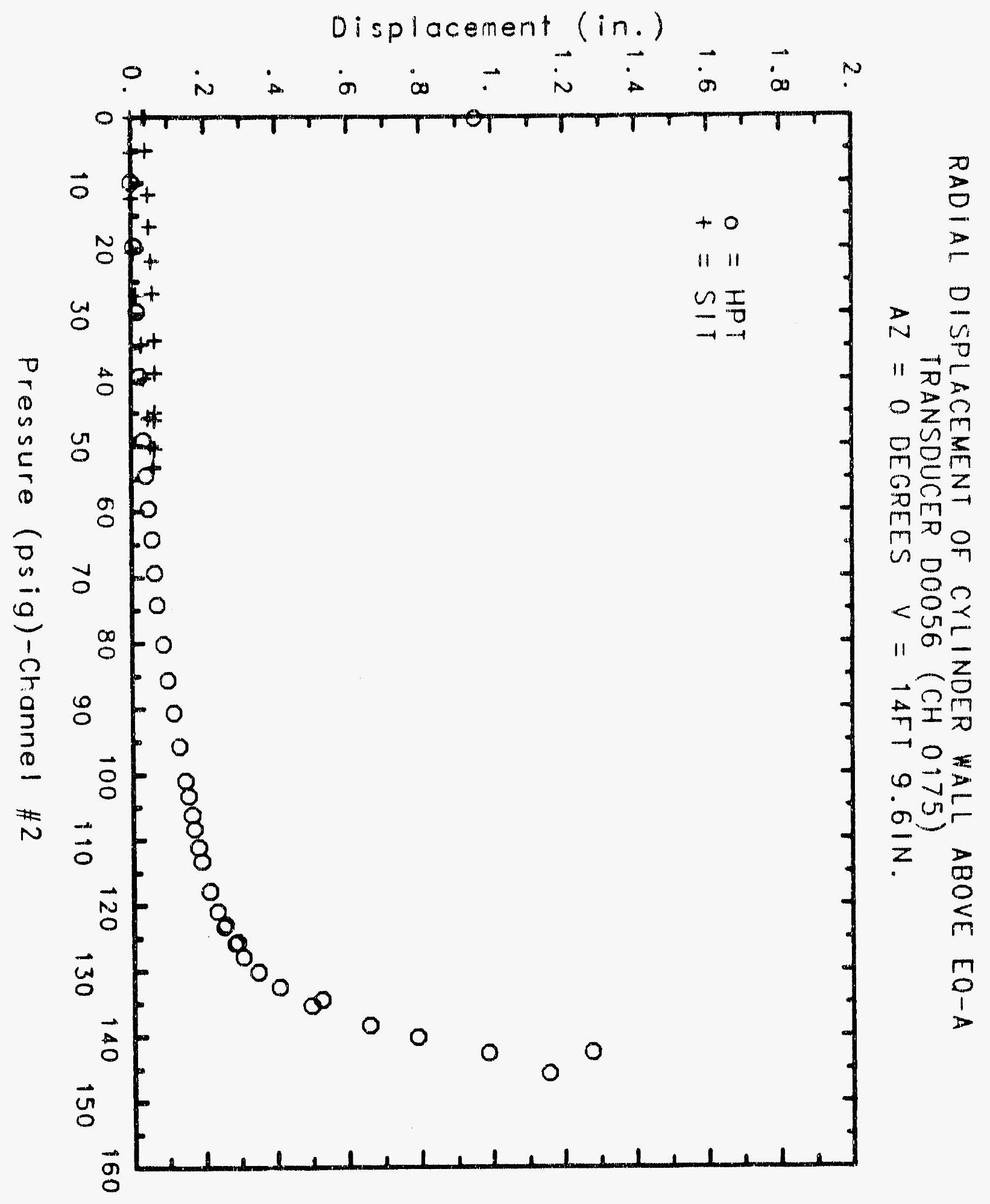




\section{Reinforced Concrete Test Data}

Potentiometer

Structural Integrity Test

A correction of $-0.1746 \mathrm{in}$. has been applied to each reading

Pressure

(psig)

$-0.05$

5.33

12.31

16.44

20.51

20.46

20.47

27.05

34.69

34.53

34.53

39.70

45.90

45.65

45.62

50.10

53.47

53.20

b3.21

50.49

46.14

46.13

44.98

38.98

33.99

33.96

26.83

21.88

21.90

16.69

11.74

11.77

5.05

0.02

$-0.04$

$-0.02$

$-0.0 \overline{2}$

0.02

0.02
Displacement

(inches)

$-0.1744$

0.0101

0.0233

0.0268

0.0346

0.0365

0.0384

0.0520

0.0665

0.0720

0.0720

0.0825

0.0938

0.0943

0.0943

0.1005

0.1070

0.1070

0.1070

0.1159

0.1161

0.1159

0.1156

0.1152

0.1138

0.1139

0.1098

0.1125

0.1125

0.1055

0.0983

0.0983

0.0780

$-0.0892$

$-0.1156$

$-0.1155$

$-0.1154$

$-0.1170$

$-0.1440$
Channel 176

High Pressure test

A correction of -0.1388 in. has been applied to each reading.

Pressure

(psig)

9.89

19.55

29.57

39.42

49.16

54.50

59.57

64.20

69.32

74.16

80.16

85.61

90.58

95.69

100.92

103.25

106.11

108.31

111.08

113.24

117.83

120.92

123.28

122.97

125.82

125.60

127.84

130.19

132.53

135.33

134.42

138.35

140.16

142.63

145.78

142.52

Uิ. $\bar{z}$
Displacement (inches)

0.0199

0.0393

0.0590

0.0740

0.0848

0.0900

0.0961

0.1090

0.1182

0.1334

0.1481

0.1585

0.1739

0.1840

0.1939

0.2109

0.2190

0.2249

0.2336

0.2452

0.2608

0.2711

0.2853

0.2891

0.2996

0.3031

0.3093

0.3280

0.3383

0.3621

0.3647

0.3960

0.4223

0.4632

0.5028

0.5165

0.0523 


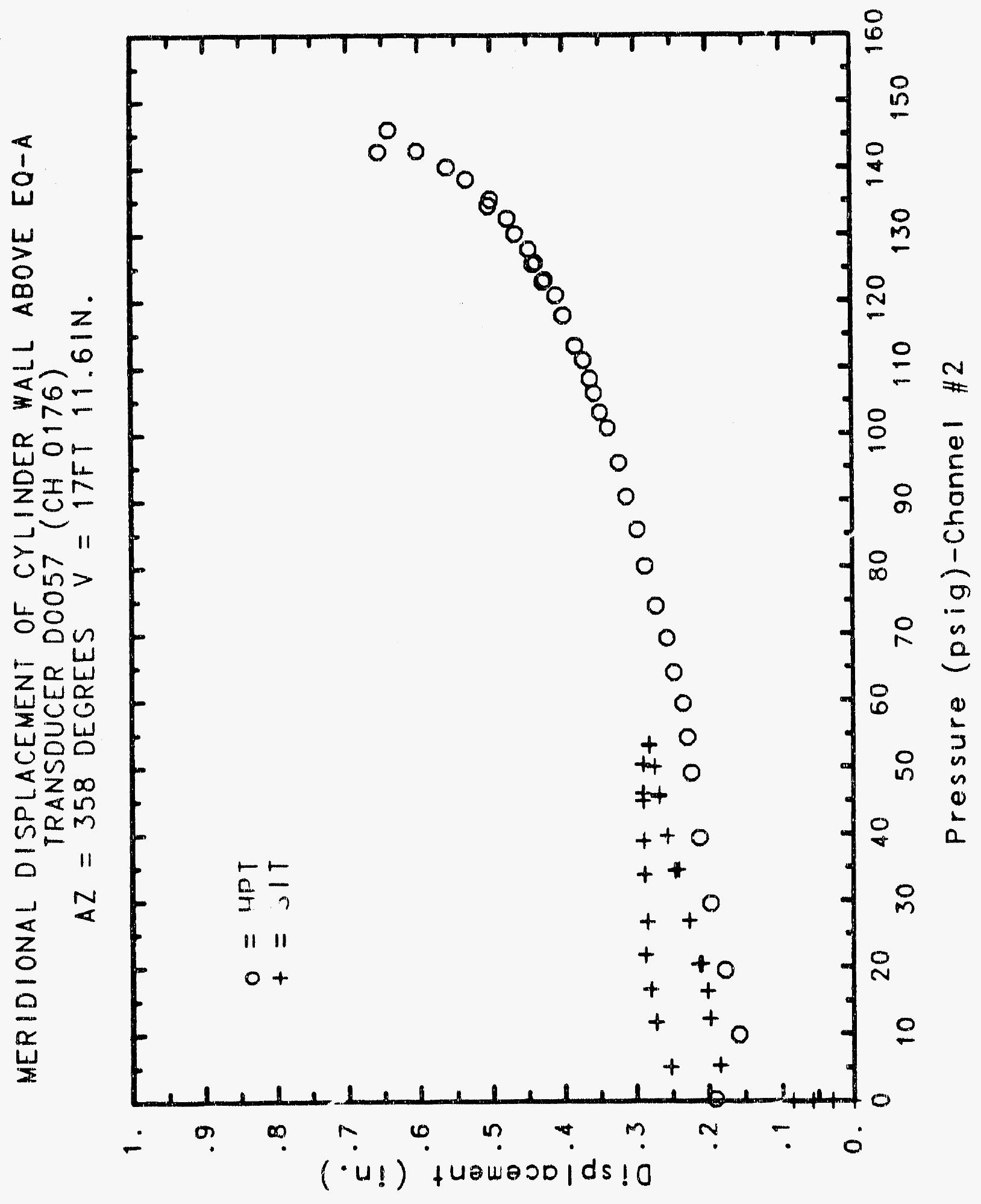




\section{Reinforced Concrete Test Data}

Potentiometer Channel 177

STRUCTURAL IntEgrity TEST

\begin{tabular}{|c|c|c|c|}
\hline $\begin{array}{c}\text { Pressure } \\
\text { (psig) } \\
-0.05 \\
5.33 \\
12.31 \\
16.44 \\
20.51 \\
20.46 \\
20.47 \\
27.05 \\
34.69 \\
34.53 \\
34.53 \\
39.70 \\
45.90 \\
45.65 \\
45.62 \\
50.10 \\
53.47 \\
53.29 \\
53.21 \\
50.49 \\
46.14 \\
46.13 \\
44.97 \\
38.98 \\
33.99 \\
33.96 \\
26.83 \\
21.88 \\
21.90 \\
16.69 \\
11.74 \\
11.77 \\
5.05 \\
0.02 \\
-0.04 \\
-0.02 \\
-0.02 \\
0.02\end{array}$ & $\begin{array}{c}\text { Displacement } \\
\text { (inches) } \\
0.0000 \\
-0.0042 \\
-0.0037 \\
-0.0005 \\
0.0050 \\
0.0081 \\
0.0090 \\
0.0193 \\
0.0376 \\
0.0413 \\
0.0425 \\
0.0494 \\
0.0634 \\
0.0658 \\
0.0661 \\
0.0733 \\
0.0805 \\
0.0824 \\
0.0826 \\
0.0858 \\
0.0859 \\
0.0858 \\
0.0862 \\
0.0827 \\
0.0779 \\
0.0780 \\
0.0692 \\
0.0650 \\
0.0650 \\
0.0576 \\
0.0520 \\
0.0519 \\
0.0423 \\
0.0417 \\
0.0373 \\
0.0366 \\
0.0544 \\
0.0423\end{array}$ & $\begin{array}{c}\text { Pressure } \\
(\text { psig) } \\
9.89 \\
19.55 \\
29.57 \\
39.42 \\
49.16 \\
54.50 \\
59.57 \\
64.20 \\
69.32 \\
74.16 \\
80.16 \\
85.61 \\
90.58 \\
95.69 \\
100.92 \\
103.25 \\
106.11 \\
108.31 \\
111.08 \\
113.24 \\
117.83 \\
120.92 \\
123.28 \\
122.97 \\
125.82 \\
125.60 \\
127.84 \\
130.19 \\
132.53 \\
135.33 \\
134.42 \\
138.35 \\
140.16 \\
142.63 \\
145.78 \\
142.52 \\
0.22\end{array}$ & $\begin{array}{c}\text { Displacement } \\
\text { (inches) } \\
0.0058 \\
0.0172 \\
0.0294 \\
0.0404 \\
0.0543 \\
0.0614 \\
0.0693 \\
0.0788 \\
0.0864 \\
0.0933 \\
0.1118 \\
0.1257 \\
0.1428 \\
0.1581 \\
0.1742 \\
0.1825 \\
0.1913 \\
0.1971 \\
0.2070 \\
0.2154 \\
0.2360 \\
0.2557 \\
0.2740 \\
0.2790 \\
0.3038 \\
0.3107 \\
0.3272 \\
0.3687 \\
0.4294 \\
0.5269 \\
0.5568 \\
0.7054 \\
0.8525 \\
1.0666 \\
1.2559 \\
1.3762 \\
1.0158\end{array}$ \\
\hline
\end{tabular}

\section{High Pressure Test}

Pressure

9.89

29.57

39.42

49.16

54.50

59.57

64.20

74.16

80.16

85.61

90.58

95.69

103.25

106.11

108.31

111.08

113.24

20.92

123.28

122.97

125.82

125.60

127.84

130.19

135.33

134.42

138.35

140.16

142.63

145.78

0.52 i splacement

(inches)

0.0058

0.0294

0.0404

0.0543

0.0614

0.0693

0.0864

0.0933

0.1118

0.1257

0.1428

0.1581

0.1825

0.1913

0.1971

0.2070

0.2557

0.2740

0.2790

0.3038

0.3107

0.3687

0.4294

0.5269

0.5568

0.7054

0.8525

1.0666

3762

1.0158 


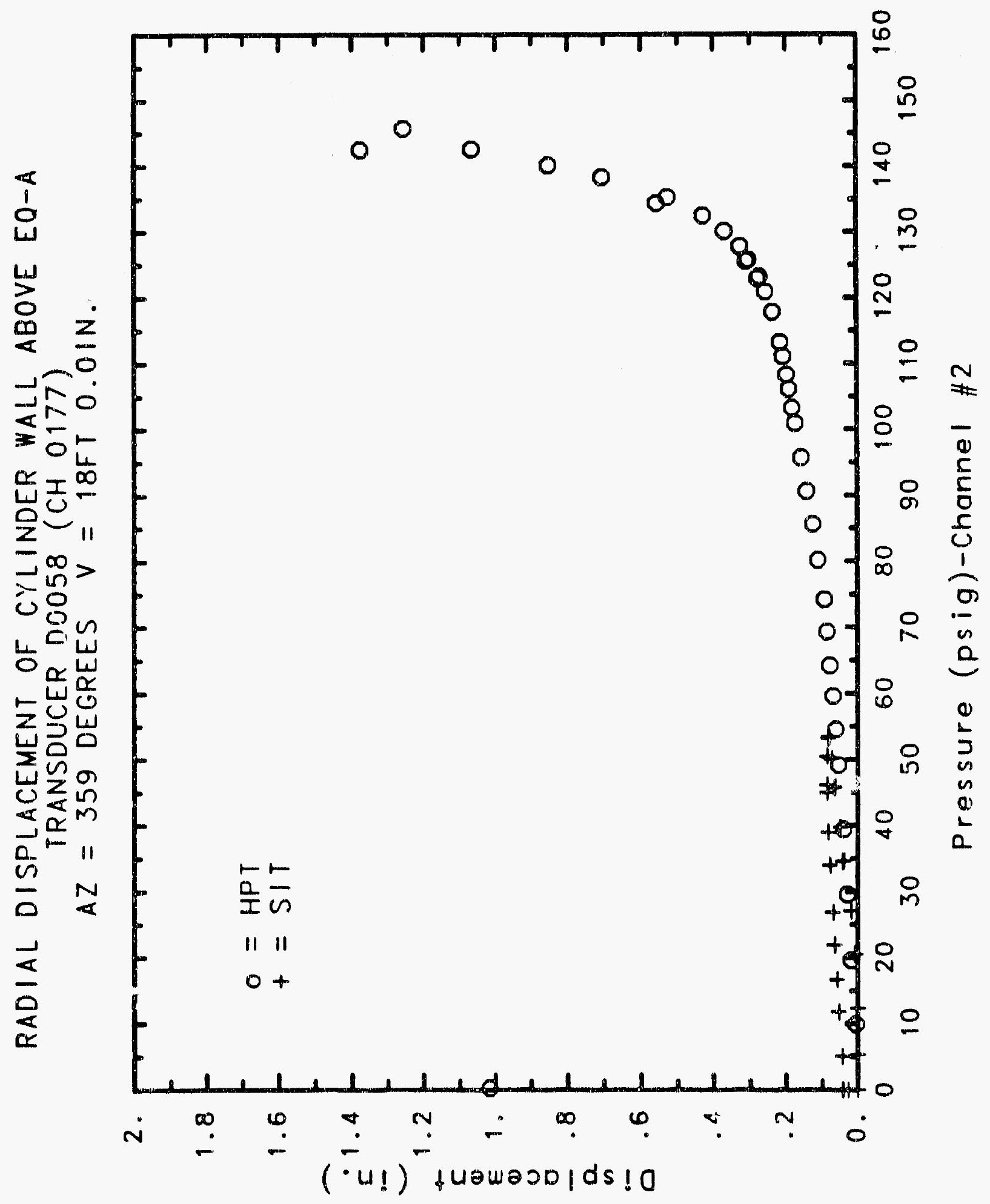




\section{Reinforced Concrete Test Data}

Potentiometer Chanael 178

STRUCTURAR IntEgrity TEST

High Pressure test

\begin{tabular}{|c|c|c|c|}
\hline $\begin{array}{c}\text { Pressure } \\
(\text { psig) } \\
-0.05 \\
5.33 \\
12.31 \\
16.44 \\
20.51 \\
20.46 \\
20.47 \\
27.05 \\
34.69 \\
34.53 \\
34.53 \\
39.70 \\
45.90 \\
45.65 \\
45.62 \\
50.10 \\
53.47 \\
53.29 \\
53.21 \\
50.49 \\
46.14 \\
46.13 \\
44.97 \\
38.98 \\
33.99 \\
33.96 \\
26.83 \\
21.88 \\
21.90 \\
16.69 \\
11.74 \\
11.77 \\
5.05 \\
0.02 \\
-0.04 \\
-0.02 \\
-0.02 \\
0.02\end{array}$ & $\begin{array}{c}\text { Displacement } \\
\text { (inches) } \\
0.0001 \\
-0.0021 \\
0.0006 \\
0.0037 \\
0.0101 \\
0.0136 \\
0.0144 \\
0.0273 \\
0.0496 \\
0.0547 \\
0.0560 \\
0.0637 \\
0.0782 \\
0.0818 \\
0.0821 \\
0.0892 \\
0.0965 \\
0.0985 \\
0.0991 \\
0.1014 \\
0.1018 \\
0.1017 \\
0.1001 \\
0.0950 \\
0.0898 \\
0.0898 \\
0.0797 \\
0.0747 \\
0.0750 \\
0.0661 \\
0.0601 \\
0.0600 \\
0.0483 \\
0.0480 \\
0.0417 \\
0.0403 \\
0.0656 \\
0.0490\end{array}$ & $\begin{array}{c}\text { Pressure } \\
(\text { psig) } \\
9.89 \\
19.55 \\
29.57 \\
39.42 \\
49.16 \\
54.50 \\
59.57 \\
64.20 \\
69.32 \\
74.16 \\
80.16 \\
85.61 \\
90.58 \\
95.69 \\
100.92 \\
103.25 \\
106.11 \\
108.31 \\
111.08 \\
113.24 \\
117.83 \\
120.92 \\
123.28 \\
122.97 \\
125.82 \\
125.60 \\
127.84 \\
130.19 \\
132.53 \\
135.33 \\
134.42 \\
138.35 \\
140.16 \\
142.63 \\
145.78 \\
142.52 \\
0.22\end{array}$ & $\begin{array}{c}\text { Displacement } \\
\text { (inches) } \\
0.0090 \\
0.0226 \\
0.0360 \\
0.0484 \\
0.0635 \\
0.0716 \\
0.0796 \\
0.0883 \\
0.0976 \\
0.1049 \\
0.1245 \\
0.1390 \\
0.1576 \\
0.1729 \\
0.1896 \\
0.1986 \\
0.2084 \\
0.2150 \\
0.2246 \\
0.2327 \\
0.2531 \\
0.2717 \\
0.2881 \\
0.2917 \\
0.3141 \\
0.3196 \\
0.3337 \\
0.3709 \\
0.4236 \\
0.5134 \\
0.5376 \\
0.0786 \\
0.8176 \\
1.0284 \\
1.2168 \\
1.3254 \\
0.9218 \\
0.04\end{array}$ \\
\hline
\end{tabular}




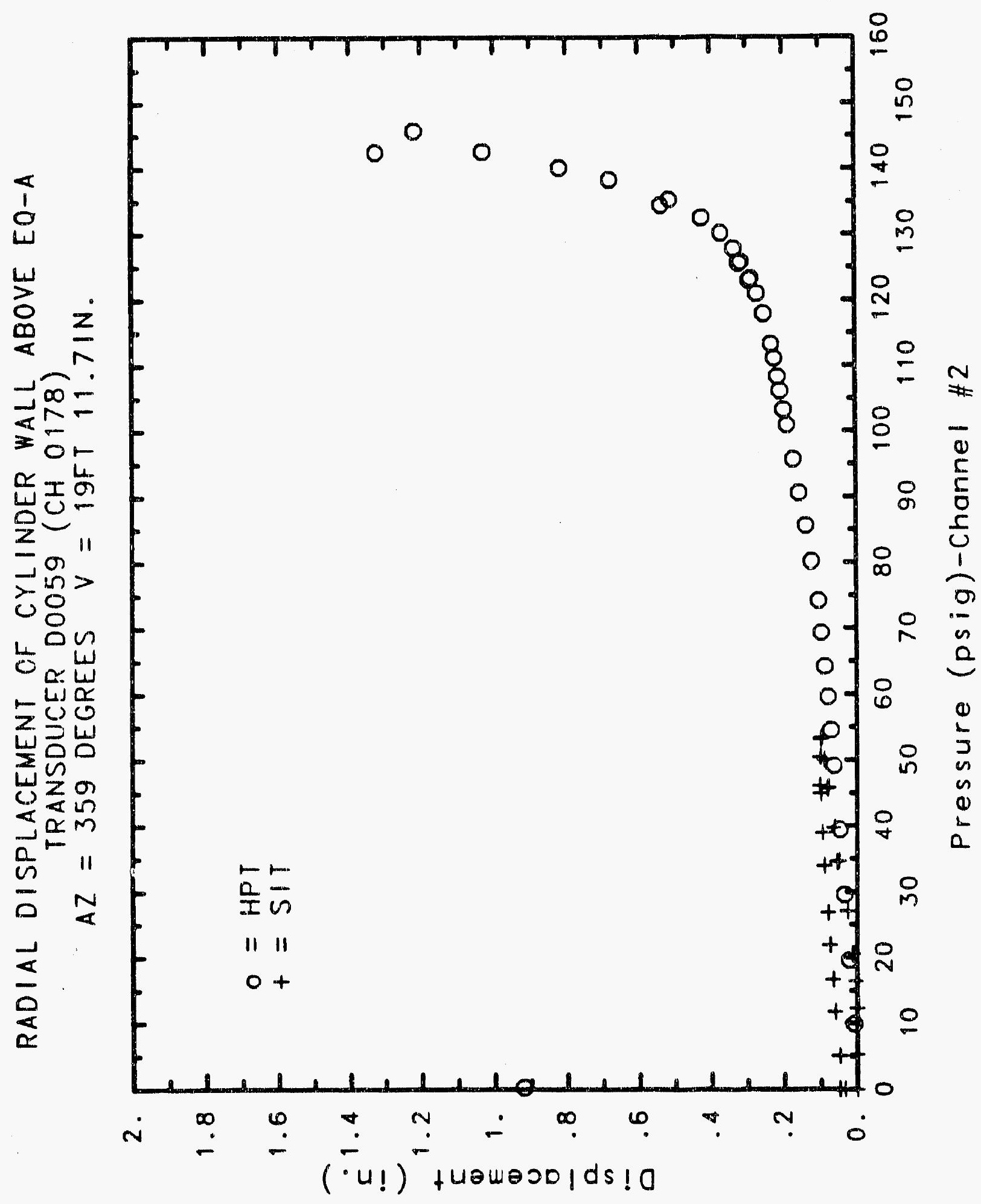




\section{Reinforced Concrete Test Data}

Potentiometer Channel 179

\section{Structural IntEgrity TEST}

A correction of -0.1087 in. has been applied to each reading

\begin{tabular}{|c|c|}
\hline $\begin{array}{c}\text { Pressure } \\
\text { (psig) } \\
-0.05 \\
5.33 \\
12.31 \\
16.44 \\
20.51 \\
20.46 \\
20.47 \\
27.05 \\
34.69 \\
34.53 \\
34.53 \\
39.70 \\
45.90 \\
45.65 \\
45.62 \\
50.10 \\
53.47 \\
53.29 \\
53.21 \\
50.49 \\
46.14 \\
46.13 \\
44.98 \\
38.98 \\
33.99 \\
33.96 \\
26.83 \\
21.88 \\
21.90 \\
16.69 \\
11.74 \\
11.77 \\
5.05 \\
0.02 \\
-0.04 \\
-0.02 \\
-0.02 \\
0.02 \\
0.02\end{array}$ & $\begin{array}{c}\text { Displacement } \\
\text { (inches) } \\
-0.1090 \\
0.0038 \\
0.0087 \\
0.0132 \\
0.0187 \\
0.0215 \\
0.0226 \\
0.0328 \\
0.0489 \\
0.0536 \\
0.0548 \\
0.0646 \\
0.0785 \\
0.0809 \\
0.0809 \\
0.0875 \\
0.0949 \\
0.0962 \\
0.0964 \\
0.1056 \\
0.1059 \\
0.1059 \\
0.1065 \\
0.1115 \\
0.1097 \\
0.1100 \\
0.0996 \\
0.0975 \\
0.0980 \\
0.0896 \\
0.0796 \\
0.0793 \\
0.0666 \\
-0.0455 \\
-0.0769 \\
-0.0766 \\
-0.0764 \\
-0.0768 \\
-0.1049\end{array}$ \\
\hline
\end{tabular}

High Pressure test

A correction of -0.1148 in. has been applied to each reading.

Pressure Displacement (psig) (inches)

9.89

19.55

29.57

39.42

49.16

54.50

59.57

64.20

69.32

74.16

80.16

85.61

90.58

95.69

100.92

103.25

106.11

108.31

111.08

113.24

117.83

120.92

123.28

122.97

125.82

125.60

127.84

130.19

132.53

135.33

134.42

138.35

140.16

142.63

145.78

142.52

0.22
0.0156

0.0309

0.0436

0.0594

0.0735

0.0814

0.0910

0.1052

0.1143

0.1283

0.1439

0.1536

0.1675

0.1817

0.1942

0.2035

0.2125

0.2168

0.2225

0.2268

0.2353

0.2431

0.2498

0.2516

0.2552

0.2571

0.2607

0.2673

0.2748

0.2865

0.2881

0.2981

0.3052

0.3143

0.3223

0.3274

$-0.0696$ 


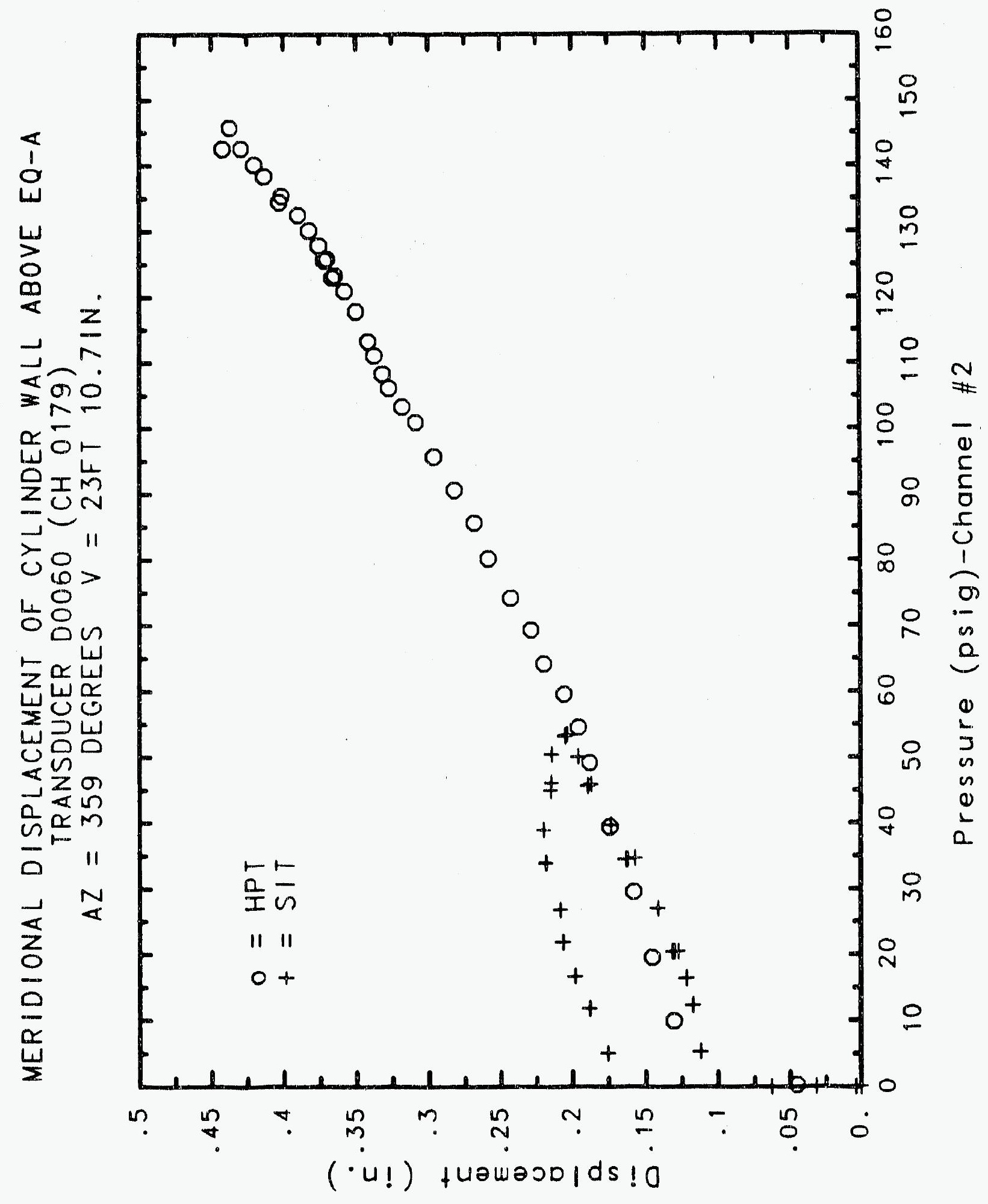




\section{Reinforced Concrete Test Data}

Potentrometer Channel 198

Structural Integrity Test

\begin{tabular}{|c|c|c|c|}
\hline $\begin{array}{c}\text { Pressure } \\
\text { (psig) } \\
-0.05 \\
5.33 \\
12.31 \\
16.44 \\
20.51 \\
20.46 \\
20.47 \\
27.05 \\
34.69 \\
34.53 \\
34.53 \\
39.70 \\
45.90 \\
45.65 \\
45.62 \\
50.10 \\
53.47 \\
53.29 \\
53.21 \\
50.49 \\
46.14 \\
46.13 \\
14.97 \\
38.98 \\
33.99 \\
33.96 \\
26.83 \\
21.88 \\
21.90 \\
16.69 \\
11.74 \\
11.77 \\
5.05 \\
0.02 \\
-0.04 \\
-0.02 \\
-0.02 \\
0.02\end{array}$ & $\begin{array}{c}\text { Displacement } \\
\text { (inches) } \\
0.0000 \\
0.0001 \\
0.0039 \\
0.0082 \\
0.0126 \\
0.0143 \\
0.0150 \\
0.0215 \\
0.0368 \\
0.0390 \\
0.0400 \\
0.0455 \\
0.0554 \\
0.0596 \\
0.0606 \\
0.0668 \\
0.0728 \\
0.0745 \\
0.0750 \\
0.0759 \\
0.0761 \\
0.0761 \\
0.0752 \\
0.0754 \\
0.0734 \\
0.0721 \\
0.0646 \\
0.0578 \\
0.0554 \\
0.0501 \\
0.0444 \\
0.0432 \\
0.0359 \\
0.0317 \\
0.0249 \\
0.0266 \\
0.0289 \\
0.0287\end{array}$ & $\begin{array}{c}\text { Pressure } \\
(\text { psig) } \\
9.89 \\
19.55 \\
29.57 \\
39.42 \\
49.16 \\
54.50 \\
59.57 \\
64.20 \\
69.32 \\
74.16 \\
80.16 \\
85.61 \\
90.58 \\
95.69 \\
100.92 \\
103.25 \\
106.11 \\
108.31 \\
111.08 \\
113.24 \\
117.83 \\
120.92 \\
123.28 \\
122.97 \\
125.82 \\
125.60 \\
127.84 \\
130.19 \\
132.53 \\
135.33 \\
134.42 \\
138.35 \\
140.16 \\
142.63 \\
145.78 \\
142.52 \\
0.22\end{array}$ & $\begin{array}{c}\text { Displacement } \\
\text { (inches) } \\
0.0076 \\
0.0174 \\
0.0272 \\
0.0392 \\
0.0500 \\
0.0583 \\
0.0658 \\
0.0745 \\
0.0871 \\
0.1049 \\
0.1149 \\
0.1249 \\
0.1390 \\
0.1541 \\
0.1650 \\
0.1708 \\
0.1764 \\
0.1800 \\
0.1862 \\
0.1916 \\
0.2012 \\
0.2083 \\
0.2184 \\
0.2221 \\
0.2342 \\
0.2399 \\
0.2472 \\
0.2568 \\
0.2667 \\
0.2782 \\
0.2804 \\
0.2908 \\
0.2987 \\
0.3078 \\
0.3185 \\
0.3243 \\
0.1087\end{array}$ \\
\hline
\end{tabular}

High Pressure test

splacement

(inches)

0.0076

0.0174

0.0272

0.0658

0.0745

0.0871

0.1249

0.1541

0.1650

0.1708

0.1862

0.1916

0.2012

0.2083

0.2184

0.2399

0.2568

0.2667

0.2782

0.2804

0.2908

0.3078

0.3185

0.3243

0.1087 


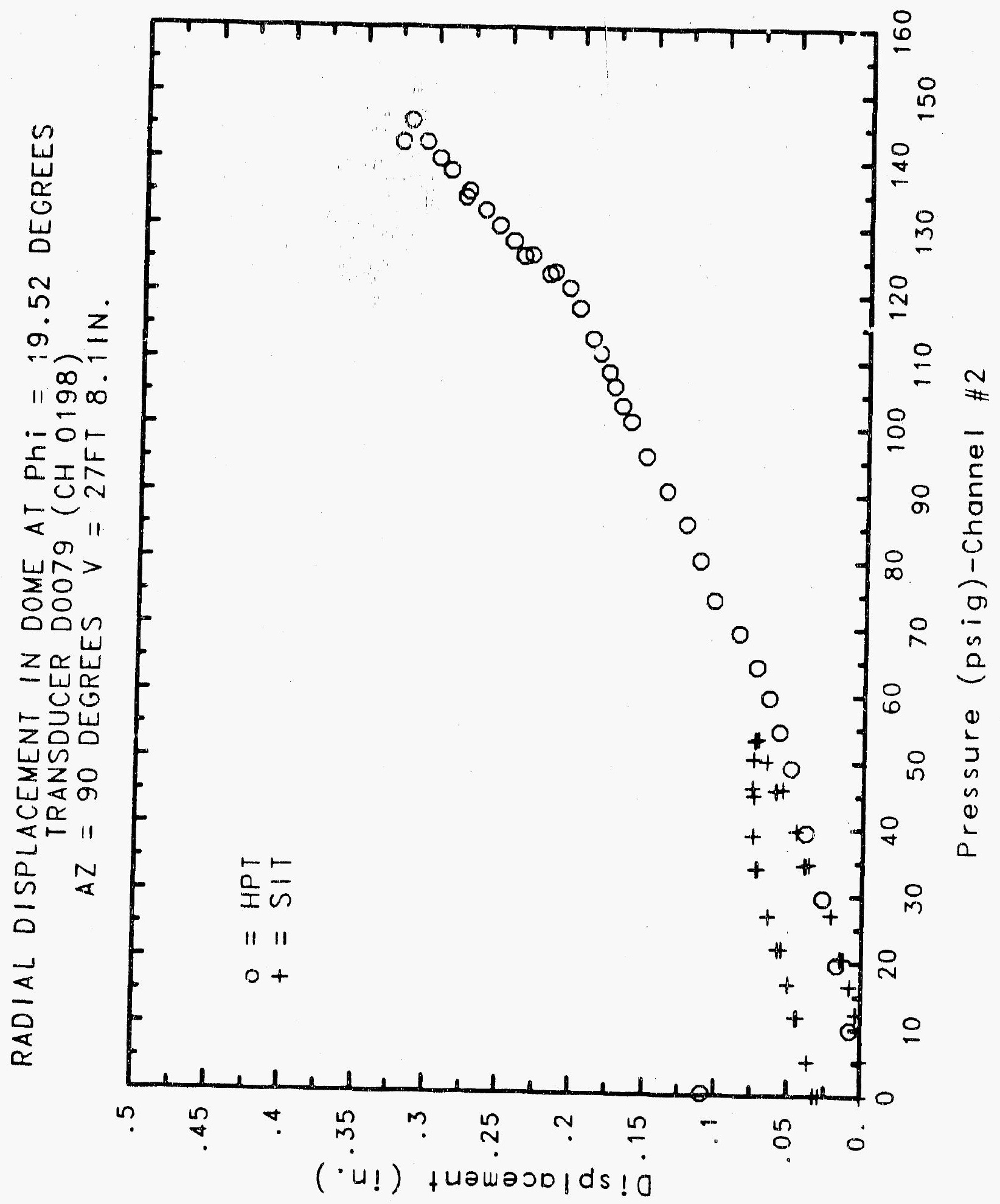




\section{Reinforced Concrete Test Data}

Potentiometer Channel 199

Structural Integrity Test

\begin{tabular}{|c|c|c|c|}
\hline $\begin{array}{c}\text { Pressure } \\
\text { (psig) } \\
-0.05 \\
5.33 \\
12.31 \\
16.44 \\
20.51 \\
20.46 \\
20.47 \\
27.05 \\
34.69 \\
34.53 \\
34.53 \\
39.70 \\
45.90 \\
45.65 \\
45.62 \\
50.10 \\
53.47 \\
53.29 \\
53.21 \\
50.49 \\
46.14 \\
46.13 \\
44.97 \\
38.98 \\
33.99 \\
33.96 \\
26.83 \\
21.88 \\
21.90 \\
16.69 \\
11.74 \\
11.77 \\
5.05 \\
0.02 \\
-0.04 \\
-0.02 \\
-0.02 \\
0.02\end{array}$ & $\begin{array}{c}\text { Displacement } \\
\text { (inches) } \\
0.0001 \\
-0.0020 \\
0.0066 \\
0.0129 \\
0.0229 \\
0.0272 \\
0.0288 \\
0.0441 \\
0.0699 \\
0.0768 \\
0.0782 \\
0.0890 \\
0.1104 \\
0.1155 \\
0.1163 \\
0.1267 \\
0.1395 \\
0.1419 \\
0.1425 \\
0.1435 \\
0.1435 \\
0.1435 \\
0.1402 \\
0.1371 \\
0.1286 \\
0.1278 \\
0.1150 \\
0.1049 \\
0.1029 \\
0.0937 \\
0.0815 \\
0.0803 \\
0.0662 \\
0.0573 \\
0.0445 \\
0.0442 \\
0.0444 \\
0.0449 \\
0.030\end{array}$ & $\begin{array}{c}\text { Pressure } \\
(\text { psig) } \\
9.89 \\
19.55 \\
29.57 \\
39.42 \\
49.16 \\
54.50 \\
59.57 \\
64.20 \\
69.32 \\
74.16 \\
80.16 \\
85.61 \\
90.58 \\
95.69 \\
100.92 \\
103.25 \\
106.11 \\
108.31 \\
111.08 \\
113.24 \\
117.83 \\
120.92 \\
123.28 \\
122.97 \\
125.82 \\
125.60 \\
127.84 \\
130.19 \\
132.53 \\
135.33 \\
134.42 \\
138.35 \\
140.16 \\
142.63 \\
145.78 \\
142.52 \\
0.22\end{array}$ & $\begin{array}{c}\text { Displacement } \\
\text { (inches) } \\
0.0097 \\
0.0290 \\
0.0506 \\
0.0714 \\
0.0947 \\
0.1089 \\
0.1252 \\
0.1444 \\
0.1689 \\
0.2043 \\
0.2374 \\
0.2640 \\
0.2948 \\
0.3208 \\
0.3479 \\
0.3623 \\
0.3756 \\
0.3865 \\
0.4002 \\
0.4113 \\
0.4368 \\
0.4558 \\
0.4775 \\
0.4819 \\
0.5060 \\
0.5132 \\
0.5287 \\
0.5498 \\
0.5770 \\
0.6128 \\
0.6200 \\
0.6604 \\
0.6940 \\
0.7408 \\
0.7816 \\
0.8020 \\
0.3948\end{array}$ \\
\hline
\end{tabular}

High Pressure test

inches)

0.0097

0.0290

0.0947

0.1252

0.1444

0.1689

0.2043

0.2374

0.3208

0.3479

0.3623

0.3756

0.3865

0.4002

0.4558

0.4775

0.4819

0.5060

0.5498

0.5128

0.6200

0.6604

0.6940

0.7408

0.7816

0.8020

0.3948 


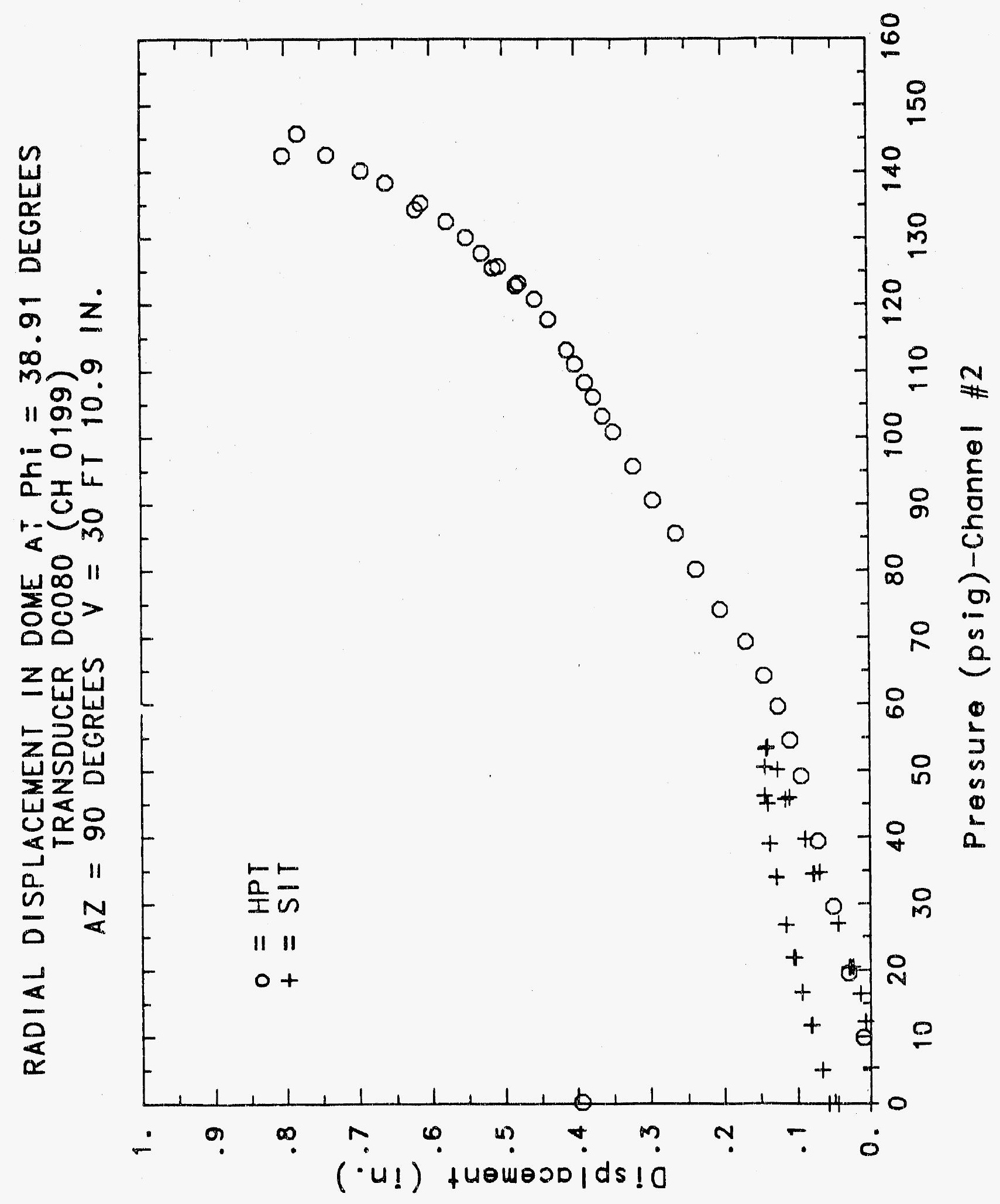




\section{Reinforced Concrete Test Data}

Potentrometer Channel. 200

STRUCTURAL INTEGRITY TEST

\begin{tabular}{|c|c|c|c|}
\hline $\begin{array}{l}\text { Pressure } \\
\text { (psig) } \\
-0.05 \\
5.33 \\
12.31 \\
16.44 \\
20.51 \\
20.46 \\
20.47 \\
27.05 \\
34.69 \\
34.53 \\
34.53 \\
39.70 \\
45.90 \\
45.65 \\
45.62 \\
50.10 \\
53.47 \\
53.29 \\
53.21 \\
50.49 \\
46.14 \\
46.13 \\
44.97 \\
38.98 \\
33.99 \\
33.96 \\
26.83 \\
21.88 \\
21.90 \\
16.69 \\
11.74 \\
11.77 \\
5.05 \\
0.02 \\
-0.04 \\
-0.02 \\
-0.02 \\
0.02 \\
\end{array}$ & $\begin{array}{c}\text { Displacement } \\
\text { (inches) } \\
0.0002 \\
-0.0003 \\
-0.0012 \\
0.0024 \\
0.0069 \\
0.0078 \\
0.0083 \\
0.0154 \\
0.0290 \\
0.0307 \\
0.0306 \\
0.0363 \\
0.0454 \\
0.0481 \\
0.0487 \\
0.0538 \\
0.0597 \\
0.0603 \\
0.0603 \\
0.0628 \\
0.0626 \\
0.0628 \\
0.0627 \\
0.0632 \\
0.0632 \\
0.0633 \\
0.0574 \\
0.0530 \\
0.0530 \\
0.0443 \\
0.0384 \\
0.0384 \\
0.0299 \\
0.0288 \\
0.0245 \\
0.0248 \\
0.0249 \\
0.0256 \\
0.05\end{array}$ & $\begin{array}{c}\text { Pressure } \\
(\text { psig) } \\
9.89 \\
19.55 \\
29.57 \\
39.42 \\
49.16 \\
54.50 \\
59.57 \\
64.20 \\
69.32 \\
74.16 \\
80.16 \\
85.61 \\
90.58 \\
95.69 \\
100.92 \\
103.25 \\
106.11 \\
108.31 \\
111.08 \\
113.24 \\
117.83 \\
120.92 \\
123.28 \\
122.97 \\
125.82 \\
125.60 \\
127.84 \\
130.19 \\
132.53 \\
135.33 \\
134.42 \\
138.35 \\
140.16 \\
142.63 \\
145.78 \\
142.52 \\
0.22\end{array}$ & $\begin{array}{c}\text { Displacement } \\
\text { (inches) } \\
-0.0021 \\
0.0091 \\
0.0193 \\
0.0309 \\
0.0420 \\
0.0494 \\
0.0563 \\
0.0639 \\
0.0735 \\
0.0870 \\
0.0916 \\
0.0991 \\
0.1095 \\
0.1189 \\
0.1270 \\
0.1309 \\
0.1348 \\
0.1379 \\
0.1418 \\
0.1453 \\
0.1545 \\
0.1609 \\
0.1690 \\
0.1721 \\
0.1845 \\
0.1884 \\
0.1967 \\
0.2097 \\
0.2273 \\
0.2545 \\
0.2593 \\
0.3007 \\
0.3301 \\
0.3718 \\
0.4085 \\
0.4216 \\
0.2127\end{array}$ \\
\hline
\end{tabular}

High Pressure Test

(inches)

0.0091

0.0193

0.0309

0.0420

0.0639

0.0735

0.0870

0.0916

0.0991

0.1095

0.1309

0.1348

0.1379

0.1418

0.1453

0.1545

0.1721

0.1845

0.1884

0.1967

0.2097

0.2593

0.3007

0.3301

0.3718

0.4085

0.4216

0.2127 


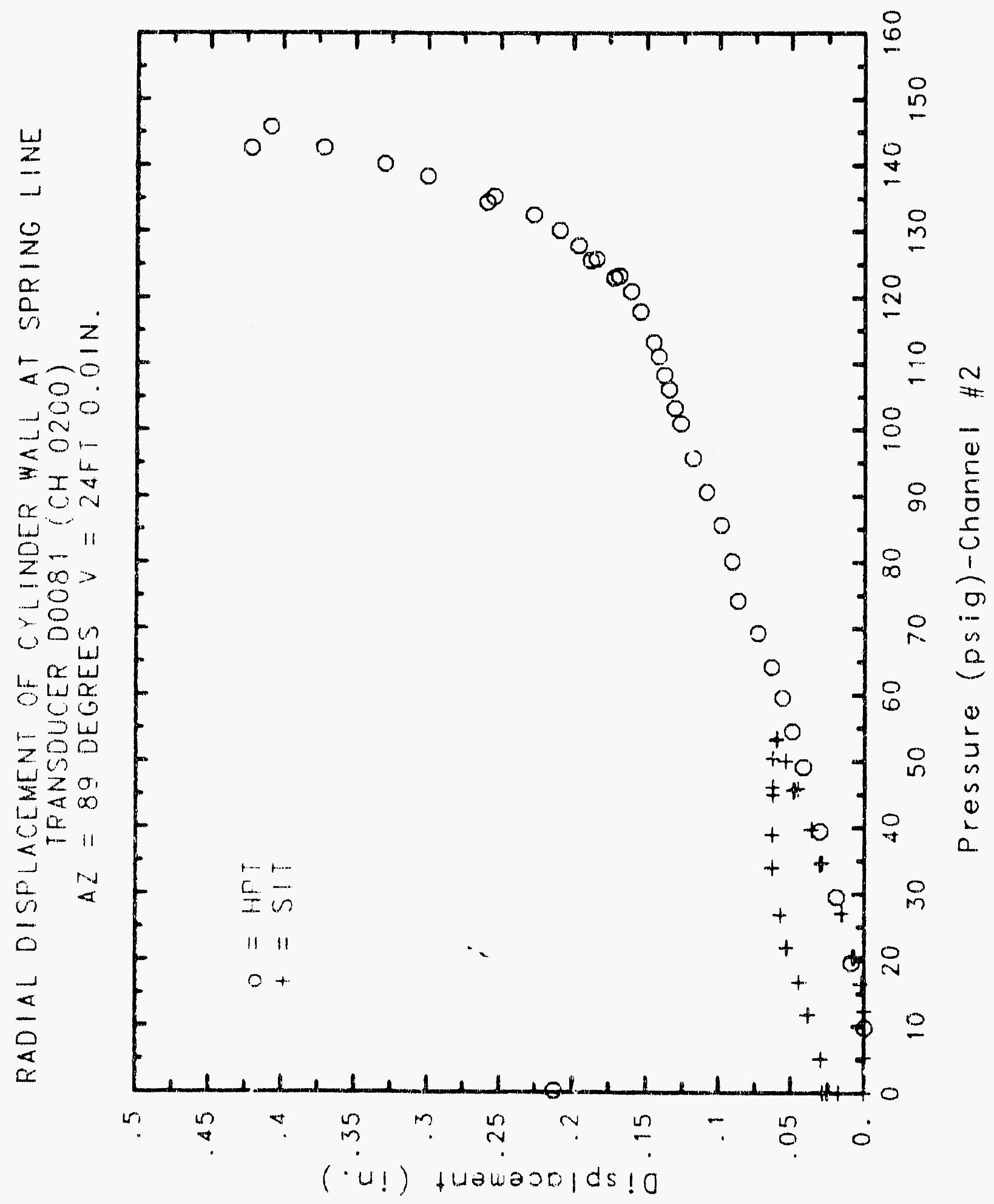




\section{Reinforced Concrete Test Data}

Potentiometer Channel 201

Structural Integraty TEST

\begin{tabular}{|c|c|c|c|}
\hline $\begin{array}{l}\text { Pressure } \\
\text { (psig) } \\
-0.05 \\
5.33 \\
12.31 \\
16.44 \\
20.51 \\
20.46 \\
20.47 \\
27.05 \\
34.69 \\
34.53 \\
34.53 \\
39.70 \\
45.90 \\
45.65 \\
45.62 \\
50.10 \\
53.47 \\
53.29 \\
53.21 \\
50.49 \\
46.14 \\
46.13 \\
44.97 \\
38.98 \\
33.99 \\
33.96 \\
26.83 \\
21.88 \\
21.90 \\
16.69 \\
11.74 \\
11.77 \\
5.05 \\
0.02 \\
-0.04 \\
-0.02 \\
-0.02 \\
0.02 \\
\end{array}$ & $\begin{array}{c}\text { Displacement } \\
\text { (inches) } \\
0.0000 \\
0.0217 \\
0.0429 \\
0.0529 \\
0.0625 \\
0.0645 \\
0.0651 \\
0.0824 \\
0.1026 \\
0.1047 \\
0.1054 \\
0.1145 \\
0.1291 \\
0.1329 \\
0.1340 \\
0.1425 \\
0.1499 \\
0.1518 \\
0.1522 \\
0.1537 \\
0.1540 \\
0.1540 \\
0.1540 \\
0.1512 \\
0.1440 \\
0.1438 \\
0.1284 \\
0.1167 \\
0.1151 \\
0.1000 \\
0.0832 \\
0.0831 \\
0.0527 \\
0.0238 \\
0.0170 \\
0.0168 \\
0.0167 \\
0.0171 \\
0.070\end{array}$ & $\begin{array}{c}\text { Pressure } \\
(p s 1 g) \\
9.89 \\
19.55 \\
29.57 \\
39.42 \\
49.16 \\
54.50 \\
59.57 \\
64.20 \\
69.32 \\
74.16 \\
80.16 \\
85.61 \\
90.58 \\
95.69 \\
100.92 \\
103.25 \\
106.11 \\
108.31 \\
111.08 \\
113.24 \\
117.83 \\
120.92 \\
123.28 \\
122.97 \\
125.82 \\
125.60 \\
127.84 \\
130.19 \\
132.53 \\
135.33 \\
134.42 \\
138.35 \\
140.16 \\
142.63 \\
145.78 \\
142.52 \\
0.22\end{array}$ & $\begin{array}{c}\text { Displacement } \\
\text { (inches) } \\
0.0424 \\
0.0731 \\
0.0981 \\
0.1182 \\
0.1354 \\
0.1452 \\
0.1554 \\
0.1568 \\
0.1820 \\
0.2029 \\
0.2188 \\
0.2336 \\
0.2505 \\
0.2673 \\
0.2822 \\
0.2901 \\
0.2966 \\
0.3021 \\
0.3099 \\
0.3158 \\
0.3298 \\
0.3399 \\
0.3517 \\
0.3558 \\
0.3705 \\
0.3764 \\
0.3863 \\
0.3993 \\
0.4141 \\
0.4345 \\
0.4377 \\
0.4614 \\
0.4796 \\
0.5034 \\
0.5273 \\
0.5373 \\
0.2571\end{array}$ \\
\hline
\end{tabular}

htgh Pressure test

splacement

0.0731

0.0981

0.1182

0.1354

0.1568

0.1820

0.2029

0.2188

0.2673

0.2822

0.2901

0.2966

0.3099

0.3158

0.3399

0.3517

0.3558

0.3764

0.3863

0.4141

0.4345

0.4377

0.4614

0.4796

0.5034

0.5373

0.257 


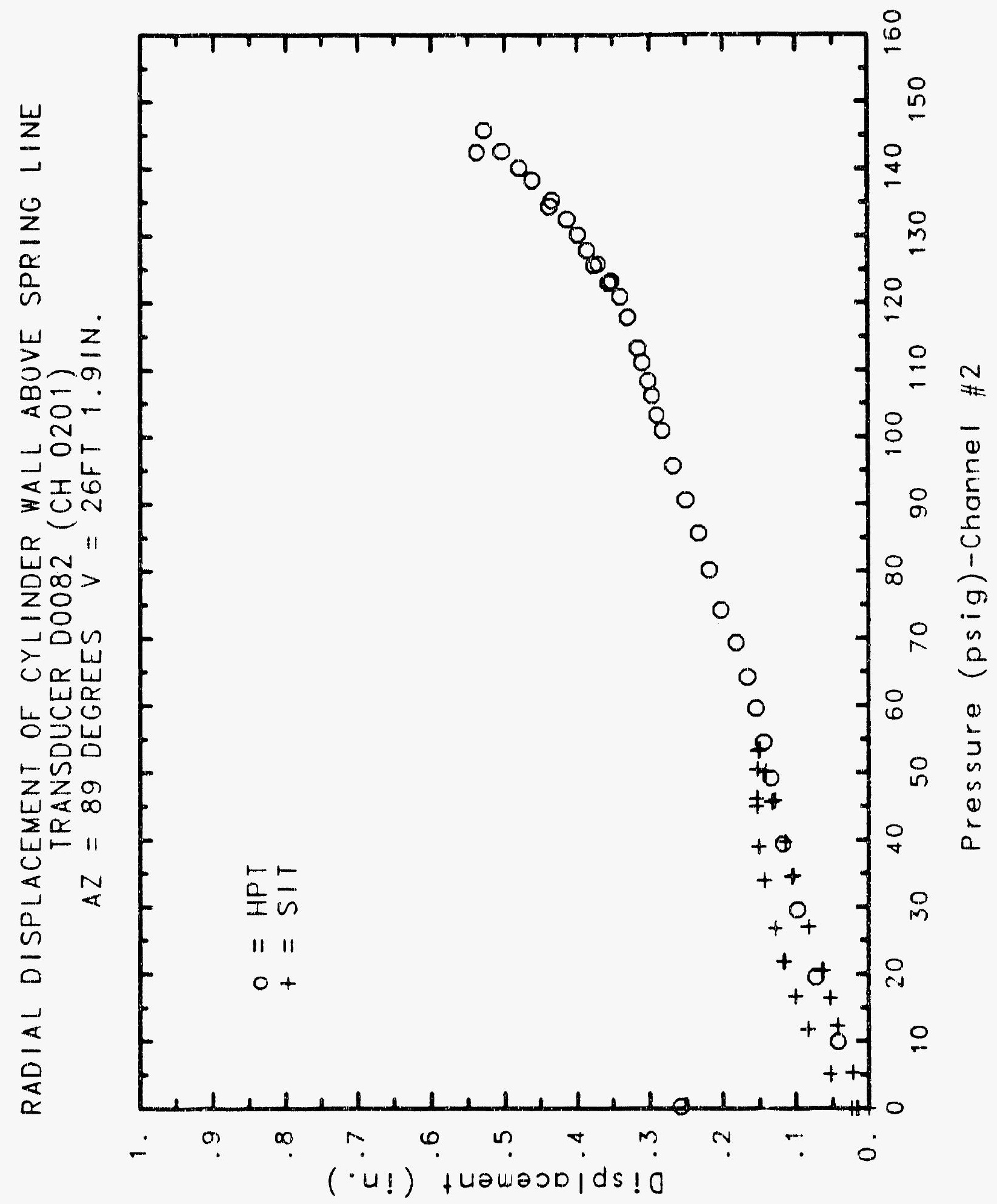




\section{Reinforced Concrete Test Data}

Potentiometer Channel 202

StRUCTURAL INTEgRITY TEST

\begin{tabular}{|c|c|c|c|}
\hline $\begin{array}{c}\text { Pressure } \\
\text { (psig) } \\
-0.05 \\
5.33 \\
12.31 \\
16.44 \\
20.51 \\
20.46 \\
20.47 \\
27.05 \\
34.69 \\
34.53 \\
34.53 \\
39.70 \\
45.90 \\
45.65 \\
45.62 \\
50.10 \\
53.47 \\
53.29 \\
53.21 \\
50.49 \\
46.14 \\
46.13 \\
44.97 \\
38.98 \\
33.99 \\
33.98 \\
26.83 \\
21.88 \\
21.90 \\
16.69 \\
11.74 \\
11.77 \\
5.05 \\
0.02 \\
-0.04 \\
-0.02 \\
-0.02 \\
0.02\end{array}$ & $\begin{array}{c}\text { Displacement } \\
\text { (inches) } \\
0.0000 \\
-0.0022 \\
0.0028 \\
0.0087 \\
0.0164 \\
0.0203 \\
0.0216 \\
0.0355 \\
0.0592 \\
0.0655 \\
0.0675 \\
0.0783 \\
0.0970 \\
0.1023 \\
0.1031 \\
0.1114 \\
0.1215 \\
0.1243 \\
0.1250 \\
0.1264 \\
0.1267 \\
0.1265 \\
0.1236 \\
0.1224 \\
0.1155 \\
0.1150 \\
0.1036 \\
0.0945 \\
0.0924 \\
0.0826 \\
0.0733 \\
0.0720 \\
0.0576 \\
0.0503 \\
0.0387 \\
0.0387 \\
0.0393 \\
0.0396 \\
0.076\end{array}$ & $\begin{array}{c}\text { Pressure } \\
(\text { psig) } \\
9.89 \\
19.55 \\
29.57 \\
39.42 \\
49.16 \\
54.50 \\
59.57 \\
64.20 \\
69.32 \\
74.16 \\
80.16 \\
85.61 \\
90.58 \\
95.69 \\
100.92 \\
103.25 \\
106.11 \\
108.31 \\
111.08 \\
113.24 \\
117.83 \\
120.92 \\
123.28 \\
122.97 \\
125.82 \\
125.60 \\
127.84 \\
130.19 \\
132.53 \\
135.33 \\
134.42 \\
138.35 \\
140.16 \\
142.63 \\
145.78 \\
142.52 \\
0.22\end{array}$ & $\begin{array}{c}\text { Displacement } \\
\text { (inches) } \\
0.0079 \\
0.0252 \\
0.0431 \\
0.0631 \\
0.0828 \\
0.0961 \\
0.1106 \\
0.1278 \\
0.1502 \\
0.1822 \\
0.2100 \\
0.2336 \\
0.2591 \\
0.2838 \\
0.3060 \\
0.3188 \\
0.3308 \\
0.3391 \\
0.3528 \\
0.3627 \\
0.3848 \\
0.4028 \\
0.4199 \\
0.4259 \\
0.4461 \\
0.4555 \\
0.4691 \\
0.4883 \\
0.5115 \\
0.5418 \\
0.5477 \\
0.5810 \\
0.6091 \\
0.6442 \\
0.6792 \\
0.6964 \\
0.3265\end{array}$ \\
\hline
\end{tabular}

\section{High Pressure Test}

placement

0.0079

0.0431

0.0631

0.0828

0.0961

0.1106

0.1278

0.1502

0.1822

-1
0

0.2591

0.2838

0.3060

0.3391

0.3528

0.3627

0.3848

0.4028

0.4199

0.4259

0.4691

0.4883

0.5115

0.5418

0.5477

0.5810

0.6792

0.6964

0.3265 


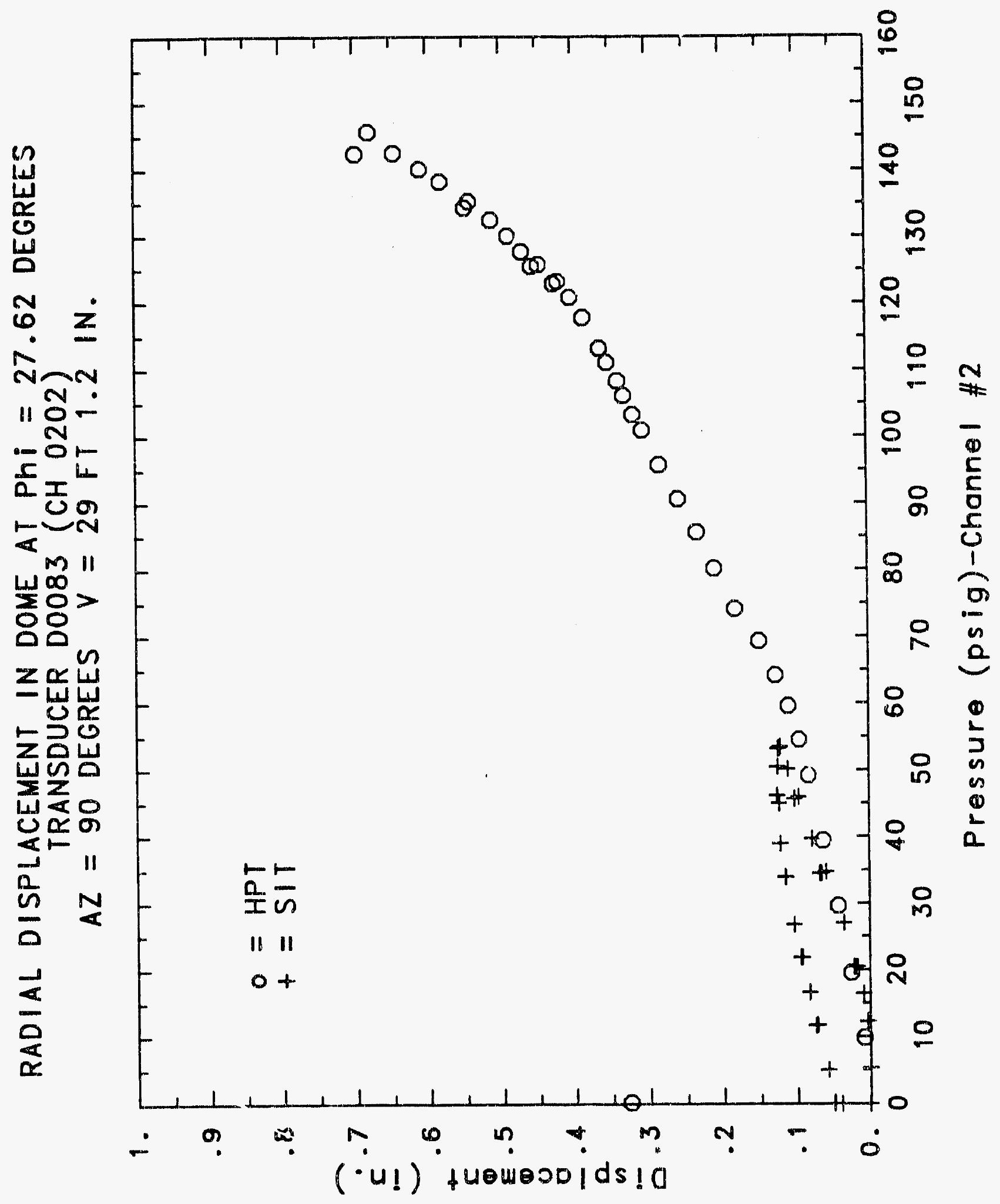




\section{Reinforced Concrete Test Data}

Potentrometer Channel 203

Structural Integrity Test

\begin{tabular}{|c|c|c|c|}
\hline $\begin{array}{c}\text { Pressure } \\
\text { (psig) } \\
-0.05 \\
5.33 \\
12.31 \\
16.44 \\
20.51 \\
20.46 \\
20.47 \\
27.05 \\
34.69 \\
34.53 \\
34.53 \\
39.70 \\
45.90 \\
45.65 \\
45.62 \\
50.10 \\
53.47 \\
53.29 \\
53.21 \\
50.49 \\
46.14 \\
46.13 \\
44.97 \\
38.98 \\
33.99 \\
33.96 \\
26.83 \\
21.88 \\
21.90 \\
16.69 \\
11.74 \\
11.77 \\
5.05 \\
0.02 \\
-0.04 \\
-0.02 \\
-0.02 \\
0.02\end{array}$ & $\begin{array}{c}\text { Displacement } \\
\text { (inches) } \\
0.0000 \\
0.0002 \\
0.0117 \\
0.0198 \\
0.0308 \\
0.0358 \\
0.0380 \\
0.0553 \\
0.0823 \\
0.0901 \\
0.0917 \\
0.1043 \\
0.1265 \\
0.1334 \\
0.1348 \\
0.1451 \\
0.1558 \\
0.1597 \\
0.1607 \\
0.1626 \\
0.1618 \\
0.1595 \\
0.1552 \\
0.1500 \\
0.1424 \\
0.1416 \\
0.1269 \\
0.1158 \\
0.1134 \\
0.1012 \\
0.0874 \\
0.0856 \\
0.0691 \\
0.0592 \\
0.0441 \\
0.0440 \\
0.0441 \\
0.0445\end{array}$ & $\begin{array}{c}\text { Pressure } \\
(\text { psig) } \\
9.89 \\
19.55 \\
29.57 \\
39.42 \\
49.16 \\
54.50 \\
59.57 \\
64.20 \\
69.32 \\
74.16 \\
80.16 \\
85.61 \\
90.58 \\
95.69 \\
100.92 \\
103.25 \\
106.11 \\
108.31 \\
111.08 \\
113.24 \\
117.83 \\
120.92 \\
123.28 \\
122.97 \\
125.82 \\
125.60 \\
127.84 \\
130.19 \\
132.53 \\
135.33 \\
134.42 \\
138.35 \\
140.16 \\
142.63 \\
145.78 \\
142.52 \\
0.22\end{array}$ & $\begin{array}{c}\text { Displacement } \\
\text { (inches) } \\
0.0120 \\
0.0339 \\
0.0579 \\
0.0844 \\
0.1077 \\
0.1241 \\
0.1412 \\
0.1622 \\
0.1866 \\
0.2252 \\
0.2630 \\
0.2942 \\
0.3259 \\
0.3562 \\
0.3847 \\
0.4009 \\
0.4147 \\
0.4269 \\
0.4429 \\
0.4564 \\
0.4843 \\
0.5064 \\
0.5295 \\
0.5363 \\
0.5597 \\
0.5700 \\
0.5845 \\
0.6111 \\
0.6406 \\
0.6818 \\
0.6905 \\
0.7374 \\
0.7772 \\
0.8298 \\
0.8799 \\
0.9012 \\
0.4433\end{array}$ \\
\hline
\end{tabular}

High Pressure test

$$
\begin{aligned}
& \text { (inches) } \\
& 0.0120 \\
& 0.0339 \\
& 0.0579 \\
& 0.0844 \\
& 0.1077 \\
& 0.1241 \\
& 0.1412 \\
& 0.1622 \\
& 0.1866 \\
& 0.2252 \\
& 0.2630 \\
& 0.2942 \\
& 0.3259 \\
& 0.3562 \\
& 0.3847 \\
& 0.4009 \\
& 0.4147 \\
& 0.4269 \\
& 0.4429 \\
& 0.4564 \\
& 0.4843 \\
& 0.5064 \\
& 0.5295 \\
& 0.5363 \\
& 0.5597 \\
& 0.5700 \\
& 0.5845 \\
& 0.6111 \\
& 0.6406 \\
& 0.6818 \\
& 0.6905 \\
& 0.7374 \\
& 0.7772 \\
& 0.8298 \\
& 0.8799 \\
& 0.9012 \\
& 0.4433
\end{aligned}
$$




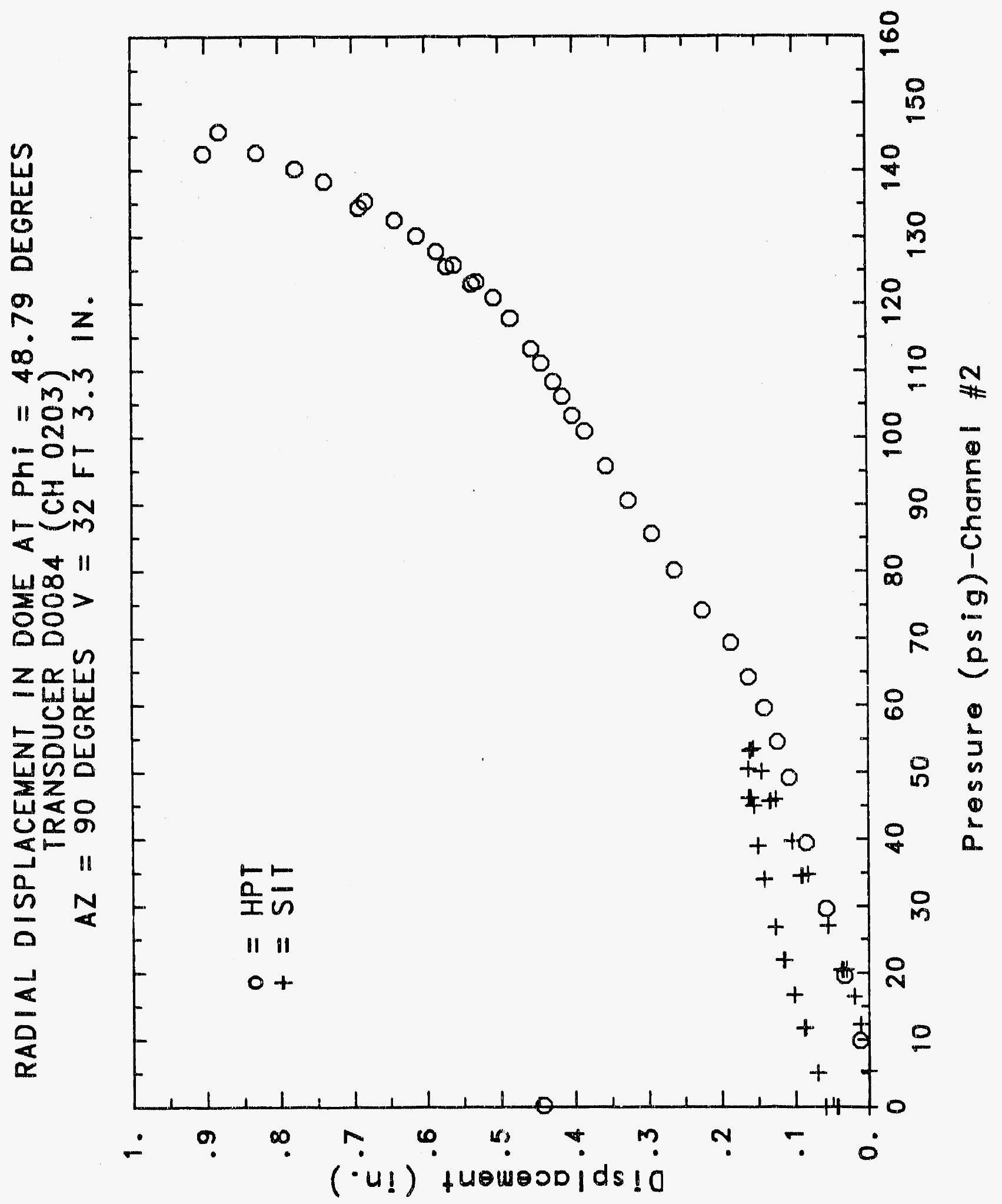

C-51 


\section{Reinforced Concrete Test Data}

Potentiometer Channel 204

Structural Integrity Test

\begin{tabular}{|c|c|c|c|}
\hline $\begin{array}{c}\text { Pressure } \\
\text { (psig) } \\
-0.05 \\
5.33 \\
12.31 \\
16.44 \\
20.51 \\
20.46 \\
20.47 \\
27.05 \\
34.69 \\
34.53 \\
34.53 \\
39.70 \\
45.90 \\
45.65 \\
45.62 \\
50.10 \\
53.47 \\
53.29 \\
53.21 \\
50.49 \\
46.14 \\
46.13 \\
44.97 \\
38.98 \\
33.99 \\
33.96 \\
26.83 \\
21.88 \\
21.90 \\
16.69 \\
11.74 \\
11.77 \\
5.05 \\
0.02 \\
-0.04 \\
-0.02 \\
-0.02 \\
0.02 \\
\end{array}$ & $\begin{array}{c}\text { Disp'acement } \\
\text { (ilches) } \\
0.0000 \\
-0.0034 \\
0.0101 \\
0.0191 \\
0.0309 \\
0.0357 \\
0.0382 \\
0.0551 \\
0.0830 \\
0.0923 \\
0.0942 \\
0.1075 \\
0.1293 \\
0.1353 \\
0.1360 \\
0.1471 \\
0.1588 \\
0.1624 \\
0.1632 \\
0.1650 \\
0.1656 \\
0.1653 \\
0.1632 \\
0.1555 \\
0.1478 \\
0.1477 \\
0.1334 \\
0.1227 \\
0.1223 \\
0.1092 \\
0.0955 \\
0.0941 \\
0.0760 \\
0.0653 \\
0.0495 \\
0.0497 \\
0.0496 \\
0.0499 \\
0.075\end{array}$ & $\begin{array}{c}\text { Pressure } \\
(\text { psig) } \\
9.89 \\
19.55 \\
29.57 \\
39.42 \\
49.16 \\
54.50 \\
59.57 \\
64.20 \\
69.32 \\
74.16 \\
80.16 \\
85.61 \\
90.58 \\
95.59 \\
100.92 \\
103.25 \\
106.11 \\
108.31 \\
111.08 \\
113.24 \\
117.83 \\
120.92 \\
123.28 \\
122.97 \\
125.82 \\
125.60 \\
127.84 \\
130.19 \\
132.53 \\
135.33 \\
134.42 \\
138.35 \\
140.16 \\
142.63 \\
145.78 \\
142.52 \\
0.22\end{array}$ & $\begin{array}{c}\text { Displacement } \\
\text { (inches) } \\
0.0066 \\
0.0301 \\
0.0556 \\
0.0822 \\
0.1074 \\
0.1237 \\
0.1434 \\
0.1653 \\
0.1917 \\
0.2354 \\
0.2804 \\
0.3179 \\
0.3528 \\
0.3851 \\
0.4163 \\
0.4348 \\
0.4517 \\
0.4639 \\
0.4846 \\
0.4987 \\
0.5333 \\
0.5576 \\
0.5827 \\
0.5890 \\
0.6130 \\
0.6249 \\
0.6401 \\
0.6681 \\
0.7020 \\
0.7514 \\
0.7637 \\
0.8225 \\
0.8681 \\
0.9342 \\
0.9934 \\
1.0194 \\
0.5363\end{array}$ \\
\hline
\end{tabular}

High Pressure test

splacement

inches)

0.0066

0.0301

0.1074

0.1237

0.1434

0.1653

0.1917

0.3179

0.3528

0.3851

0.4163

0.4348

0.4517

0.4639

0.4987

0.5333

0.5576

0.5827

0.5890

0.6249

0.6401

0.6681

0.7020

0.8225

0.8681

0.9342

0.9934

1.0194

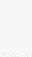




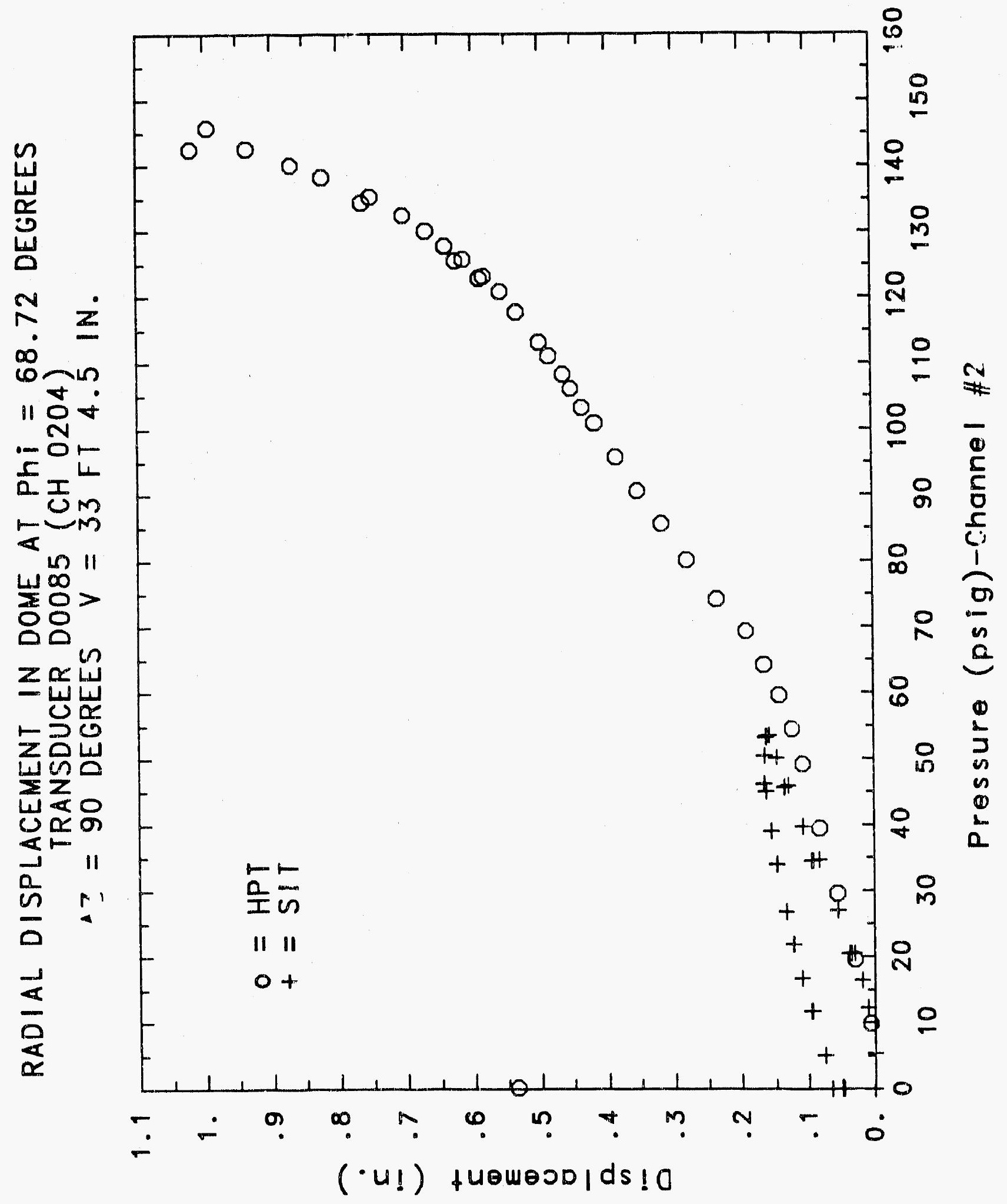




\section{Reinforced Concrete Test Data}

\begin{tabular}{|c|c|c|c|}
\hline \multirow[b]{2}{*}{ STRUCTURAL } & \multicolumn{3}{|c|}{ Channel 205} \\
\hline & INTEGRITY TEST & HIGH $P_{F}$ & RE TEST \\
\hline $\begin{array}{l}\text { Pressure } \\
\text { (psig) } \\
-0.05 \\
5.33 \\
12.31 \\
16.44 \\
20.51 \\
20.46 \\
20.47 \\
27.05 \\
34.69 \\
34.53 \\
34.53 \\
39.70 \\
45.90 \\
45.65 \\
45.62 \\
50.10 \\
53.47 \\
53.29 \\
53.21 \\
50.49 \\
46.14 \\
46.13 \\
44.97 \\
38.98 \\
33.99 \\
33.96 \\
26.83 \\
21.88 \\
21.90 \\
16.69 \\
11.74 \\
11.77 \\
5.05 \\
0.02 \\
-0.04 \\
-0.02 \\
-0.02 \\
0.02 \\
\end{array}$ & $\begin{array}{c}\text { Displacement } \\
\text { (inches) } \\
0.0000 \\
0.0200 \\
0.0471 \\
0.0597 \\
0.0755 \\
0.0820 \\
0.0852 \\
0.1038 \\
0.1321 \\
0.1433 \\
0.1454 \\
0.1563 \\
0.1771 \\
0.1847 \\
0.1857 \\
0.1940 \\
0.2067 \\
0.2106 \\
0.2114 \\
0.2126 \\
0.2133 \\
0.2126 \\
0.2106 \\
0.2047 \\
0.1974 \\
0.1974 \\
0.1863 \\
0.1784 \\
0.1783 \\
0.1666 \\
0.1541 \\
0.1527 \\
0.1321 \\
0.0874 \\
0.0676 \\
0.0672 \\
0.0672 \\
0.0676 \\
0.076\end{array}$ & $\begin{array}{c}\text { Pressure } \\
(\text { psig) } \\
9.89 \\
19.55 \\
29.57 \\
39.42 \\
49.16 \\
54.50 \\
59.57 \\
64.20 \\
69.32 \\
74.16 \\
80.16 \\
85.61 \\
90.58 \\
95.69 \\
100.92 \\
103.25 \\
106.11 \\
108.31 \\
111.08 \\
113.24 \\
117.83 \\
120.92 \\
123.28 \\
122.97 \\
125.82 \\
125.60 \\
127.84 \\
130.19 \\
132.53 \\
135.33 \\
134.42 \\
138.35 \\
140.16 \\
142.63 \\
145.78 \\
142.52 \\
0.22 \\
\end{array}$ & $\begin{array}{c}\text { Displacement } \\
\text { (inches) } \\
0.0459 \\
0.0689 \\
0.0898 \\
0.1132 \\
0.1355 \\
0.1506 \\
0.1662 \\
0.1884 \\
0.2128 \\
0.2519 \\
0.2964 \\
0.3300 \\
0.3618 \\
0.3933 \\
0.4214 \\
0.4397 \\
0.4555 \\
0.4666 \\
0.4892 \\
0.5026 \\
0.5323 \\
0.5566 \\
0.5810 \\
0.5877 \\
0.6094 \\
0.6216 \\
0.6364 \\
0.6634 \\
0.6978 \\
0.7441 \\
0.7569 \\
0.8118 \\
0.8584 \\
0.9253 \\
0.9854 \\
1.0119 \\
0.5827\end{array}$ \\
\hline
\end{tabular}




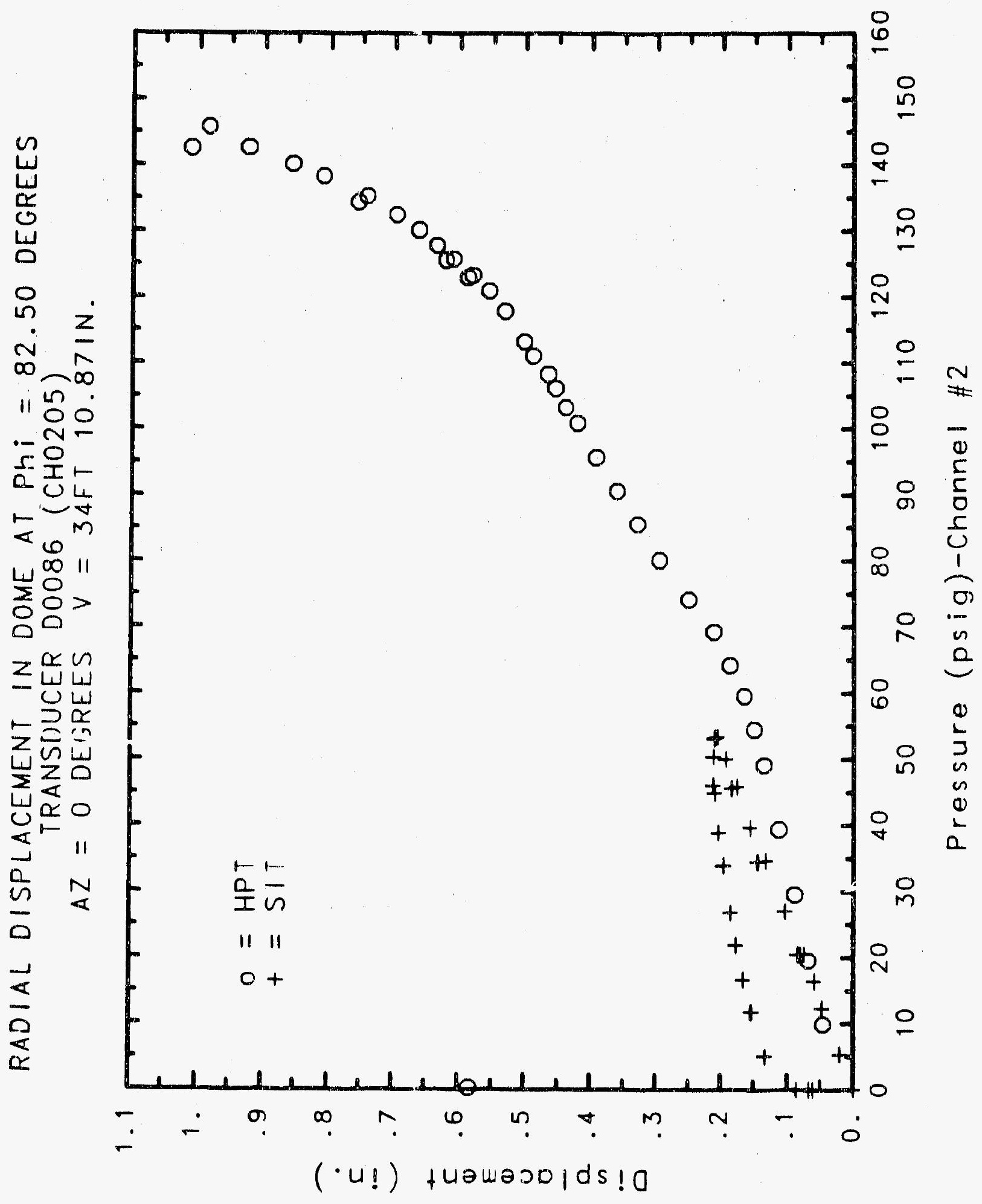




\section{Reinforced Concrete Test Data}

\begin{tabular}{|c|c|c|c|}
\hline \multirow{2}{*}{\multicolumn{2}{|c|}{ STRUCTURAL INTEGRITY TEST }} & \multicolumn{2}{|c|}{ Channel 206} \\
\hline & & HIGH P & RE TEST \\
\hline $\begin{array}{c}\text { Pressure } \\
\text { (psig) } \\
-0.05 \\
5.33 \\
12.31 \\
16.44 \\
20.51 \\
20.46 \\
20.47 \\
27.05 \\
34.69 \\
34.53 \\
34.53 \\
39.70 \\
45.90 \\
45.65 \\
45.62 \\
50.10 \\
53.47 \\
53.29 \\
53.21 \\
50.49 \\
46.14 \\
46.13 \\
44.97 \\
38.98 \\
33.99 \\
33.96 \\
26.83 \\
21.88 \\
21.90 \\
16.69 \\
11.74 \\
11.77 \\
5.05 \\
0.02 \\
-0.04 \\
-0.02 \\
-0.02 \\
0.07\end{array}$ & $\begin{array}{c}\text { Displacement } \\
\text { (inches) } \\
-0.0001 \\
-0.0004 \\
0.0087 \\
0.0136 \\
0.0211 \\
0.0236 \\
0.0244 \\
0.0411 \\
0.0659 \\
0.0728 \\
0.0747 \\
0.0873 \\
0.1054 \\
0.1067 \\
0.1080 \\
0.1161 \\
0.1243 \\
0.1249 \\
0.1255 \\
0.1263 \\
0.1265 \\
0.1264 \\
0.1211 \\
0.1126 \\
0.1059 \\
0.1060 \\
0.0918 \\
0.0842 \\
0.0846 \\
0.0749 \\
0.0655 \\
0.0654 \\
0.0480 \\
0.0419 \\
0.0336 \\
0.0337 \\
0.0270 \\
0.0267\end{array}$ & $\begin{array}{c}\text { Pressure } \\
\text { (psig) } \\
9.89 \\
19.55 \\
29.57 \\
39.42 \\
49.16 \\
54.50 \\
59.57 \\
64.20 \\
69.32 \\
74.16 \\
80.16 \\
85.61 \\
90.58 \\
95.69 \\
100.92 \\
103.25 \\
106.11 \\
108.31 \\
111.08 \\
113.24 \\
117.83 \\
120.92 \\
123.28 \\
122.97 \\
125.82 \\
125.60 \\
127.84 \\
130.19 \\
132.53 \\
135.33 \\
134.42 \\
138.35 \\
140.16 \\
142.63 \\
145.78 \\
142.52 \\
0.22\end{array}$ & $\begin{array}{c}\text { Displacement } \\
\text { (inches) } \\
0.0110 \\
0.0301 \\
0.0522 \\
0.0743 \\
0.0933 \\
0.1061 \\
0.1179 \\
0.1327 \\
0.1499 \\
0.1690 \\
0.1829 \\
0.1980 \\
0.2160 \\
0.2365 \\
0.2596 \\
0.2694 \\
0.2816 \\
0.2920 \\
0.3079 \\
0.3217 \\
0.3589 \\
0.3886 \\
0.4209 \\
0.4287 \\
0.4803 \\
0.4944 \\
0.5305 \\
0.6340 \\
0.7932 \\
0.9785 \\
1.0195 \\
1.2219 \\
1.3652 \\
1.5469 \\
1.6852 \\
1.7283 \\
1.0883\end{array}$ \\
\hline
\end{tabular}




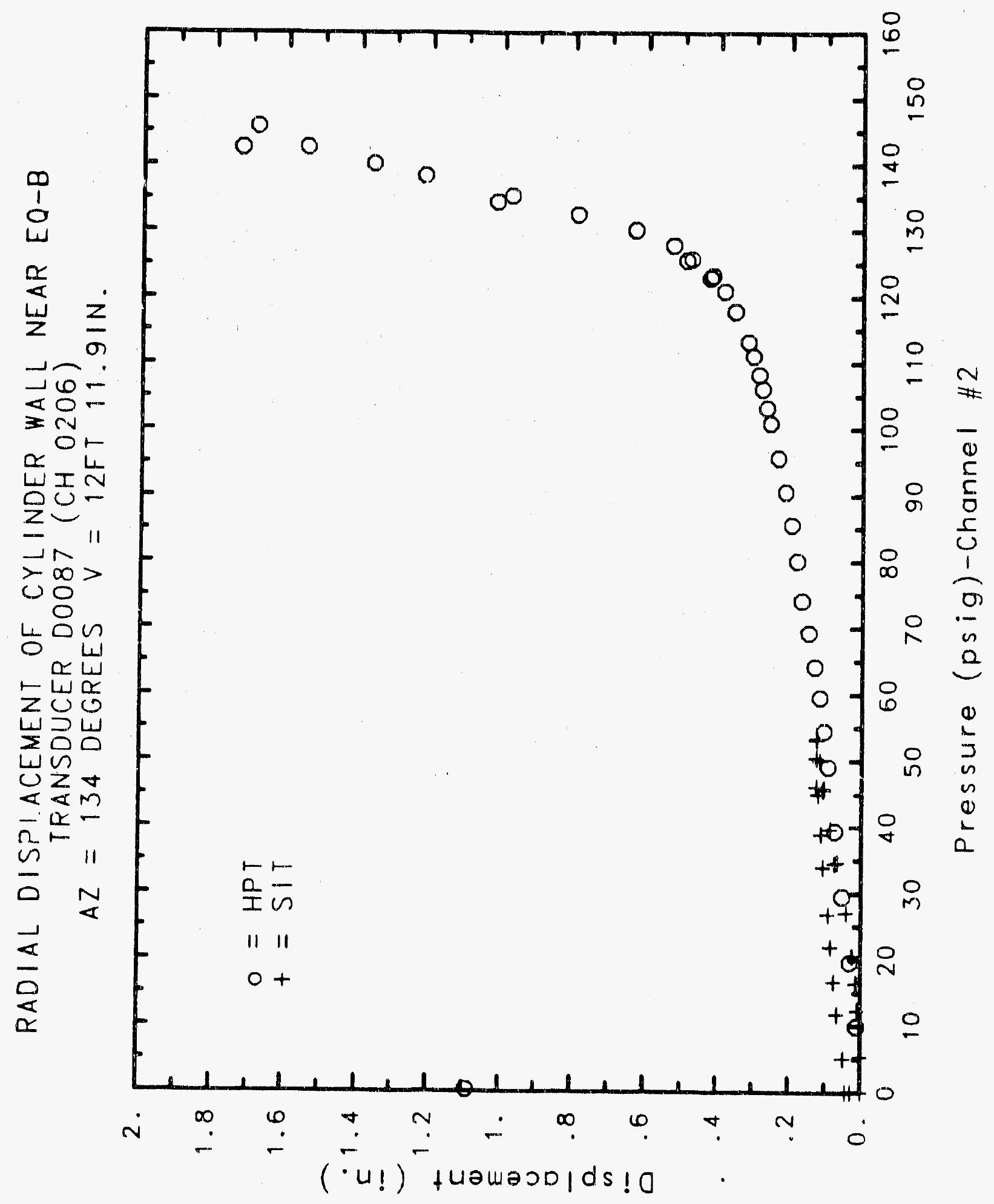




\section{Reinforced Concrete Test Data}

PotentTOMETER

Structural Integrity Test

A correction of -0.0123 in. has been applied to each reading

Pressure

(psig)

$-0.05$

5.33

12.31

16.44

20.51

20.46

20.47

27.05

34.69

34.53

34.53

39.70

45.90

45.65

45.62

50.10

53.47

53.29

53.21

50.49

46.14

46.13

44.98

38.98

33.99

33.96

26.83

21.88

21.90

16.69

11.74

11.77

5.05

0.02

$-0.04$

$-0.02$

$-0.02$

0.02

0.02
Displacement

(inches)

$-0.0123$

0.0071

0.0164

0.0205

0.0253

0.0255

0.0265

0.0356

0.0432

0.0446

0.0447

0.0493

0.0565

0.0639

0.0639

0.0665

0.0691

0.0693

0.0692

0.0699

0.0701

0.0701

0.0628

0.0672

0.0649

0.0650

0.0606

0.0573

0.0574

0.0509

0.0378

0.0442

0.0290

0.0045

0.0010

0.0011

$-0.0006$

$-0.0064$

$-0.0160$
Channel 207

High Pressure test

A correction of $-0.0205 \mathrm{in}$. has been applied to each reading.

Pressure

(psig)

9.89

Displacement

19.55

29.57

39.42

49.16

54.50

59.57

64.20

69.32

74.16

80.16

85.61

$90 \quad 58$

$9^{8} .69$

110.92

103.25

106.11

108.31

111.08

113.24

117.83

120.92

123.28

122.97

125.82

125.60

127.84

130.19

132.53

135.33

134.42

138.35

140.16

142.63

145.78

142.52

0.22 (inches)

0.0144

0.0285

0.0372

0.0467

0.0526

0.0557

0.0586

0.0656

0.0713

0.0781

0.0848

0.0939

0.0999

0.1057

0.1154

0.1184

0.1219

0.1253

0.1300

0.1336

0.1437

0.1517

0.1576

0.1598

0.1684

0.1714

0.1756

0.1900

0.2073

0.2298

0.2341

0.2626

0.2859

0.3184

0.3543

0.3671

0.1693 


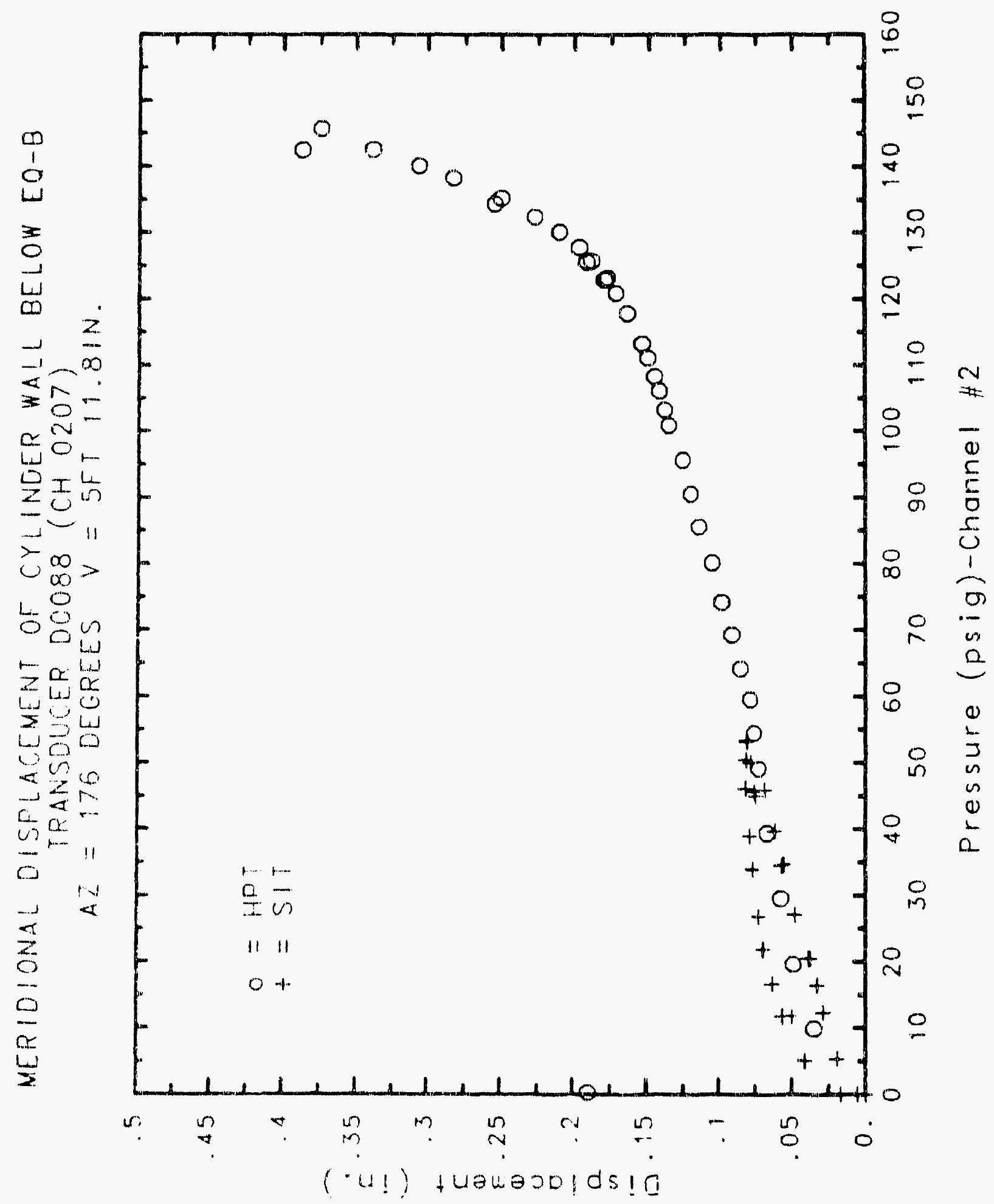




\section{Reinforced Concrete Test Data}

Potentiometer Channel 208

StRUCTURAL InTEgRITY TEST

A correction of -0.0548 in. has been applied to each reading

\begin{tabular}{cc} 
Pressure & Displacement \\
(psig) & $($ inches) \\
-0.05 & -0.0547 \\
5.33 & 0.0078 \\
12.31 & 0.0180 \\
16.44 & 0.0217 \\
20.51 & 0.0261 \\
20.46 & 0.0284 \\
20.47 & 0.0294 \\
27.05 & 0.0359 \\
34.69 & 0.0501 \\
34.53 & 0.0533 \\
34.53 & 0.0542 \\
39.70 & 0.0614 \\
45.90 & 0.0734 \\
45.65 & 0.0757 \\
45.62 & 0.0758 \\
50.10 & 0.0805 \\
53.47 & 0.0868 \\
53.29 & 0.0876 \\
53.21 & 0.0879 \\
50.49 & 0.0898 \\
46.14 & 0.0900 \\
46.13 & 0.0900 \\
44.98 & 0.0858 \\
38.98 & 0.0316 \\
33.99 & 0.0735 \\
33.96 & 0.0786 \\
26.83 & 0.0717 \\
21.88 & 0.0687 \\
21.90 & 0.0688 \\
16.69 & 0.0629 \\
11.74 & 0.0569 \\
11.77 & 0.0568 \\
5.05 & 0.0414 \\
0.02 & -0.0106 \\
-0.04 & -0.0210 \\
-0.02 & -0.0208 \\
-0.02 & -0.0219 \\
0.02 & -0.0216 \\
0.02 & -0.0352 \\
& \\
\hline 109 &
\end{tabular}

High Pressure Test

A correction of -0.0572 in. has been applied to each reading.

Pressure Displacement

(psig) (inches)

9.89

19.55

29.57

0.0130

0.0257

0.0371

39.42

0.0488

49.16

0.0591

54.50

59.57

0.0652

64.20

69.32

0.0728

0.0803

0.0929

74.16

0.1070

80.16

0.1225

85.61

90.58

95.69

100.92

0.1355

0.1506

0.1636

0.1780

103.25

106.11

0.1872

108.31

0.1969

111.08

0.2026

0.2113

113.24

0.2185

117.83

0.2348

120.92

0.2464

123.28

0.2587

122.97

0.2614

125.82

125.60

0.2741

0.2789

0.2861

0.3051

130.19

0.3281

132.53

0.3587

0.3679

0.4059

0.4362

0.4818

0.5255

0. 5434

0.1790 


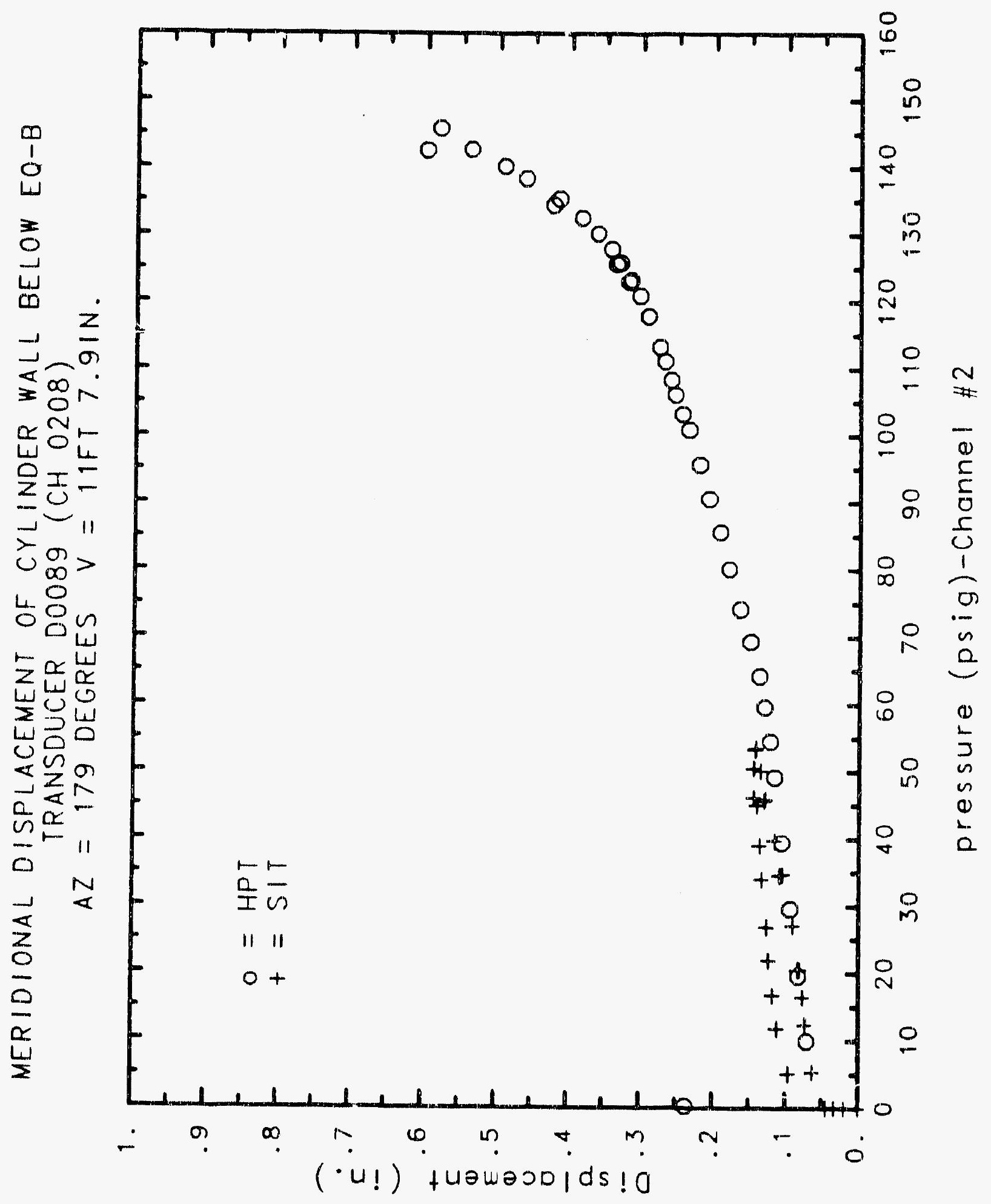




\section{Reinforced Concrete Test Data}

Potentiometer Channel 209

Structural Integrity Test

\begin{tabular}{|c|c|c|c|}
\hline $\begin{array}{c}\text { Pressure } \\
\text { (psig) } \\
-0.05 \\
5.33 \\
12.31 \\
16.44 \\
20.51 \\
20.46 \\
20.47 \\
27.05 \\
34.69 \\
34.53 \\
34.53 \\
39.70 \\
45.90 \\
45.65 \\
45.62 \\
50.10 \\
53.47 \\
53.29 \\
53.21 \\
50.49 \\
46.14 \\
46.13 \\
44.97 \\
38.98 \\
33.99 \\
33.96 \\
26.83 \\
21.88 \\
21.90 \\
16.69 \\
11.74 \\
11.77 \\
5.05 \\
0.02 \\
-0.04 \\
-0.02 \\
-0.02 \\
0.02\end{array}$ & $\begin{array}{c}\text { Displacement } \\
\text { (inches) } \\
-0.0002 \\
-0.0052 \\
-0.0027 \\
-0.0002 \\
0.0051 \\
0.0070 \\
0.0077 \\
0.0190 \\
0.0357 \\
0.0378 \\
0.0383 \\
0.0458 \\
0.0588 \\
0.0593 \\
0.0594 \\
0.0652 \\
0.0715 \\
0.0723 \\
0.0724 \\
0.0753 \\
0.0755 \\
0.0754 \\
0.0758 \\
0.0737 \\
0.0729 \\
0.0729 \\
0.0641 \\
0.0597 \\
0.0599 \\
0.0502 \\
0.0430 \\
0.0428 \\
0.0311 \\
0.0298 \\
0.0293 \\
0.0294 \\
-0.0014 \\
0.0037\end{array}$ & $\begin{array}{c}\text { Pressure } \\
(p s i g) \\
9.89 \\
19.55 \\
29.57 \\
39.42 \\
49.16 \\
54.50 \\
59.57 \\
64.20 \\
69.32 \\
74.16 \\
80.16 \\
85.61 \\
90.58 \\
95.69 \\
100.92 \\
103.25 \\
106.11 \\
108.31 \\
111.08 \\
113.24 \\
117.83 \\
120.92 \\
123.28 \\
122.97 \\
125.82 \\
125.60 \\
127.84 \\
130.19 \\
132.53 \\
135.33 \\
134.42 \\
138.35 \\
140.16 \\
142.63 \\
145.78 \\
142.52 \\
0.22\end{array}$ & $\begin{array}{c}\text { Displacement } \\
\text { (inches) } \\
\text {-0.0078 } \\
0.0029 \\
0.0171 \\
0.0313 \\
0.0437 \\
0.0528 \\
0.0609 \\
0.0694 \\
0.0803 \\
0.0899 \\
0.0918 \\
0.0989 \\
0.0999 \\
0.1056 \\
0.1162 \\
0.1196 \\
0.1239 \\
0.1275 \\
0.1362 \\
0.1455 \\
0.1604 \\
0.1769 \\
0.1894 \\
0.1921 \\
0.2151 \\
0.2213 \\
0.2391 \\
0.2814 \\
0.3499 \\
0.4513 \\
0.4814 \\
0.6327 \\
0.7598 \\
0.9414 \\
1.1067 \\
1.1662 \\
0.8607\end{array}$ \\
\hline
\end{tabular}

High Pressure test

splacement

(inches)

$-0.0078$

0.0029

0.0437

0.0609

0.0694

0.0803

0.0899

0.0999

0.1056

0.1162

0.1196

0.1239

0.1362

0.1455

0.1604

0.1769

0.1894

0.2213

0.2391

0.2814

0.3499

0.6327

7598

.1067

1.1662 


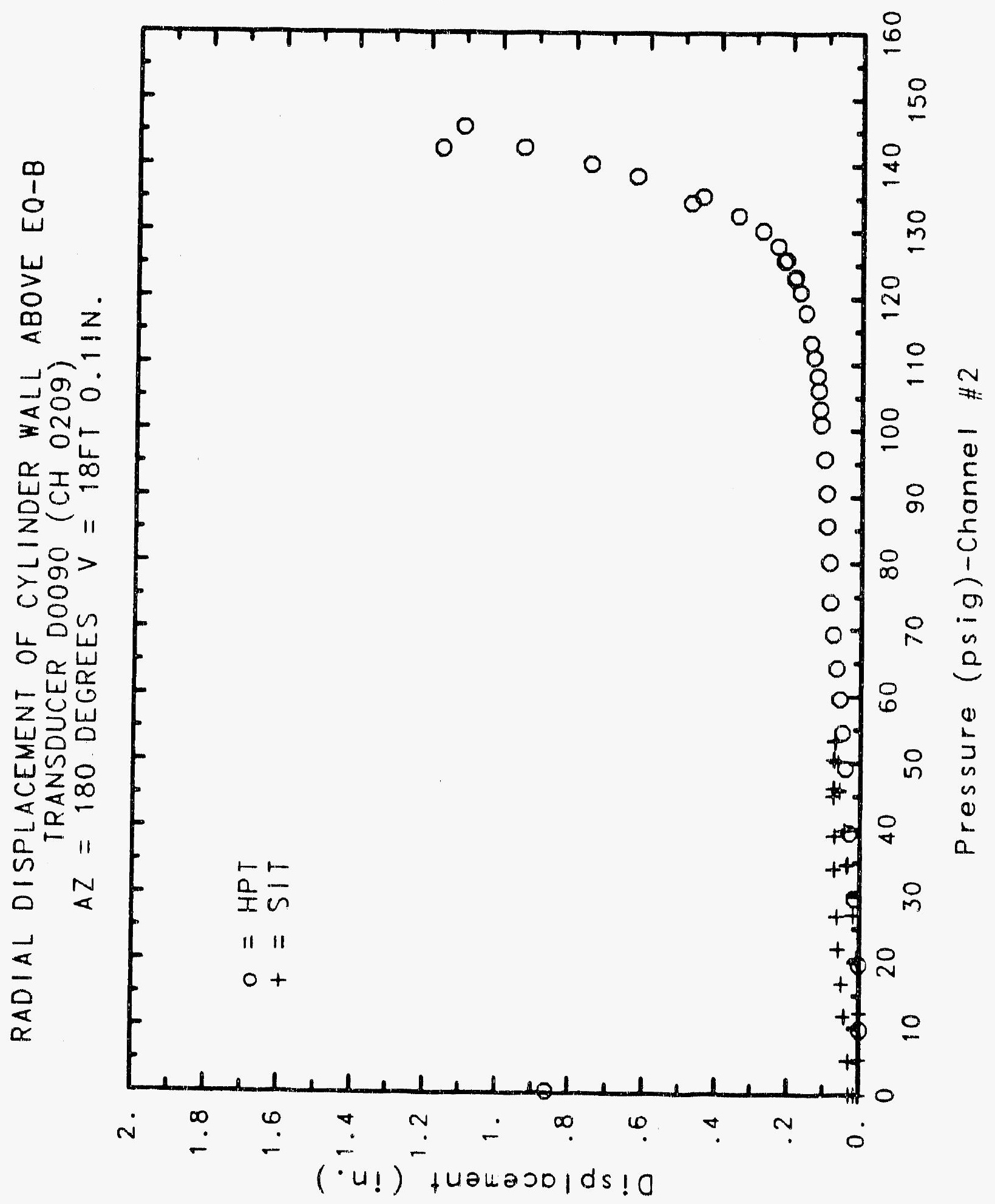




\section{Reinforced Concrete Test Data}

Potentiometer Channel 210

Structural Integrity Test

\begin{tabular}{|c|c|c|c|}
\hline $\begin{array}{c}\text { Pressure } \\
(p \text { ig) } \\
-0.05 \\
5.33 \\
12.31 \\
16.44 \\
20.51 \\
20.46 \\
20.47 \\
27.05 \\
34.69 \\
34.53 \\
34.53 \\
39.70 \\
45.90 \\
45.65 \\
45.62 \\
50.10 \\
53.47 \\
53.29 \\
53.21 \\
50.49 \\
46.14 \\
46.13 \\
44.97 \\
38.98 \\
33.99 \\
33.96 \\
26.83 \\
21.88 \\
21.90 \\
16.69 \\
11.74 \\
11.77 \\
5.05 \\
0.02 \\
-0.04 \\
-0.02 \\
-0.02 \\
0.02\end{array}$ & $\begin{array}{c}\text { Displacement } \\
\text { (inches) } \\
0.0001 \\
0.0000 \\
0.0001 \\
0.0001 \\
0.0003 \\
0.0004 \\
0.0004 \\
0.0006 \\
0.0006 \\
0.0006 \\
0.0007 \\
0.0007 \\
0.0006 \\
0.0004 \\
0.0004 \\
0.0004 \\
0.0004 \\
0.0001 \\
0.0000 \\
0.0000 \\
0.0001 \\
-0.0001 \\
0.0001 \\
0.0003 \\
0.0004 \\
0.0006 \\
0.0006 \\
0.0004 \\
0.0007 \\
0.0006 \\
0.0006 \\
0.0006 \\
0.0003 \\
0.0001 \\
-0.0004 \\
-0.0004 \\
-0.0003 \\
0.0000 \\
0.00\end{array}$ & $\begin{array}{c}\text { Pressure } \\
(\text { psig) } \\
9.89 \\
19.55 \\
29.57 \\
39.42 \\
49.16 \\
54.50 \\
59.57 \\
64.20 \\
69.32 \\
74.16 \\
80.16 \\
85.61 \\
90.58 \\
95.69 \\
100.92 \\
103.25 \\
106.11 \\
108.31 \\
111.08 \\
113.24 \\
117.83 \\
120.92 \\
123.28 \\
122.97 \\
125.82 \\
125.60 \\
127.84 \\
130.19 \\
132.53 \\
135.33 \\
134.42 \\
138.35 \\
140.16 \\
142.63 \\
145.78 \\
142.52 \\
0.22\end{array}$ & $\begin{array}{c}\text { Displacement } \\
(\text { inches) } \\
-0.0142 \\
-0.0139 \\
-0.0139 \\
-0.0138 \\
-0.0136 \\
-0.0138 \\
-0.0136 \\
-0.0135 \\
-0.0121 \\
0.0036 \\
0.0393 \\
0.0631 \\
0.0878 \\
0.1169 \\
0.1501 \\
0.1645 \\
0.1791 \\
0.1855 \\
0.2072 \\
0.2153 \\
0.2424 \\
0.2699 \\
0.2812 \\
0.2878 \\
0.3032 \\
0.3077 \\
0.3119 \\
0.3419 \\
0.3605 \\
0.3947 \\
0.4126 \\
0.4538 \\
0.5112 \\
0.5859 \\
0.6781 \\
0.7199 \\
0.5150\end{array}$ \\
\hline
\end{tabular}

High Pressure test

splacement

(inches)

$-0.0142$

$-0.0139$

$-0.0139$

$-0.0138$

$-0.0136$

$-0.0135$

0.0121

0.0036

0.0393

0.1169

0.1501

0.1645

0.1791

0.1855

0.2072

0.2699

0.3032

0.3077

0.3119

0.3419

0.3605

0.4538

.5859

0.6781

0.7199

0.5150 


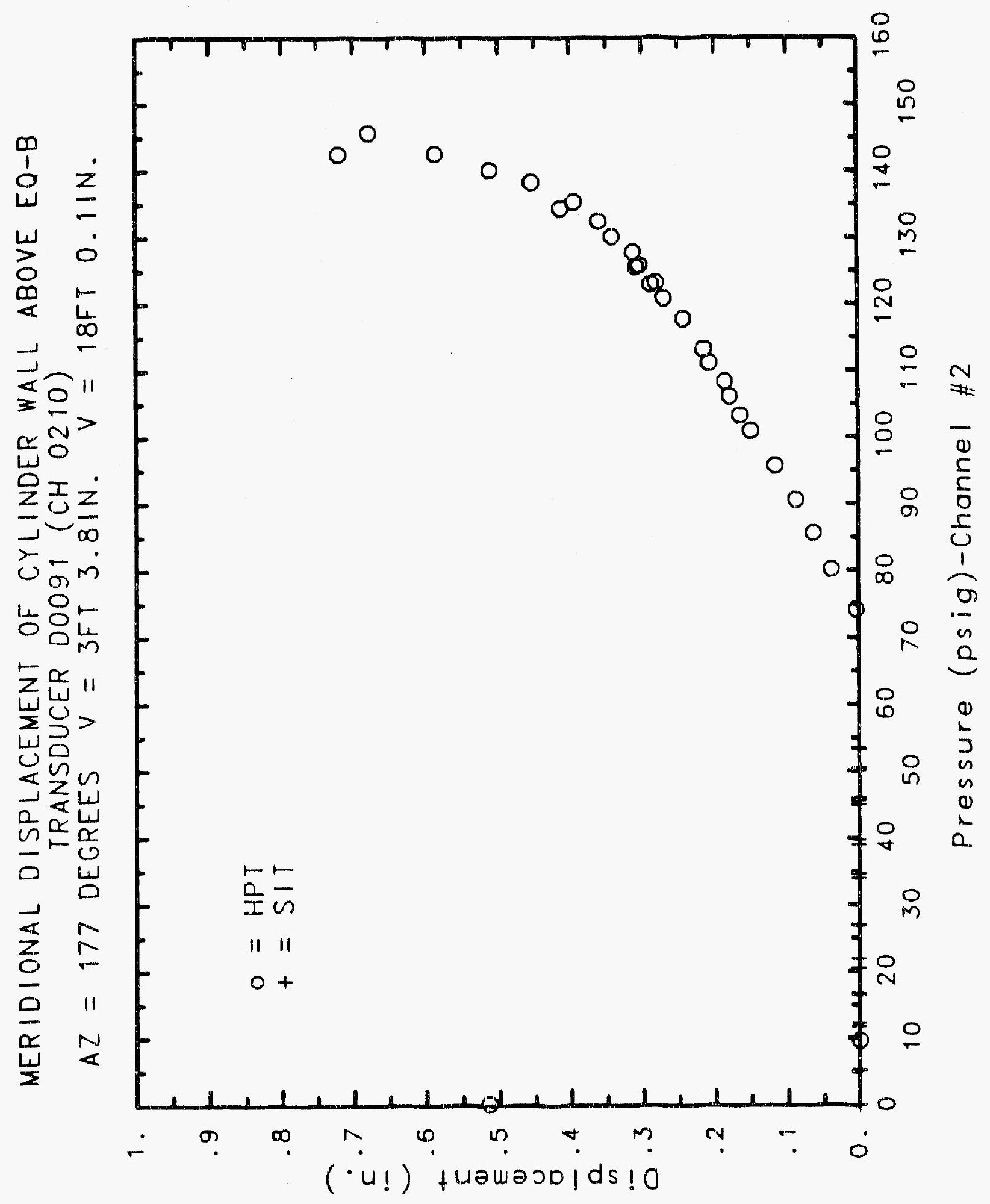




\section{Reinforced Concrete Test Data}

\section{Potentiometer Channel 211}

Structural Integrity Test

\begin{tabular}{|c|c|c|c|}
\hline $\begin{array}{c}\text { Pressure } \\
\text { (psig) } \\
-0.05 \\
5.33 \\
12.31 \\
16.44 \\
20.51 \\
20.46 \\
20.47 \\
27.05 \\
34.69 \\
34.53 \\
34.53 \\
39.70 \\
45.90 \\
45.65 \\
45.62 \\
50.10 \\
53.47 \\
53.29 \\
53.21 \\
50.49 \\
46.14 \\
46.13 \\
44.97 \\
38.98 \\
33.99 \\
33.96 \\
26.83 \\
21.88 \\
21.90 \\
16.69 \\
11.74 \\
11.77 \\
5.05 \\
0.02 \\
-0.04 \\
-0.02 \\
-0.02 \\
0.02\end{array}$ & $\begin{array}{c}\text { Displacement } \\
\text { (Inches) } \\
-0.0001 \\
-0.0019 \\
0.0028 \\
0.0064 \\
0.0146 \\
0.0165 \\
0.0174 \\
0.0330 \\
0.0452 \\
0.0476 \\
0.0483 \\
0.0576 \\
0.0684 \\
0.0697 \\
0.0698 \\
0.0752 \\
0.0814 \\
0.0823 \\
0.0823 \\
0.0844 \\
0.0846 \\
0.0847 \\
0.0848 \\
0.0792 \\
0.0747 \\
0.0748 \\
0.0661 \\
0.0610 \\
0.0613 \\
0.0495 \\
0.0431 \\
0.0431 \\
0.0334 \\
0.0334 \\
0.0322 \\
0.0322 \\
-0.0051 \\
0.0067\end{array}$ & $\begin{array}{c}\text { Pressure } \\
(\text { psig) } \\
9.89 \\
19.55 \\
29.57 \\
39.42 \\
49.16 \\
54.50 \\
59.57 \\
64.20 \\
69.32 \\
74.16 \\
80.16 \\
85.61 \\
90.58 \\
95.69 \\
100.92 \\
103.25 \\
106.11 \\
108.31 \\
111.08 \\
113.24 \\
117.83 \\
120.92 \\
123.28 \\
122.97 \\
125.82 \\
125.60 \\
127.84 \\
130.19 \\
132.53 \\
135.33 \\
134.42 \\
138.35 \\
140.16 \\
142.63 \\
145.78 \\
142.52 \\
0.22\end{array}$ & $\begin{array}{c}\text { Displacement } \\
\text { (inches) } \\
0.0040 \\
0.0177 \\
0.0283 \\
0.0467 \\
0.0596 \\
0.0703 \\
0.0791 \\
0.0916 \\
0.0993 \\
0.1107 \\
0.1152 \\
0.1209 \\
0.1221 \\
0.1305 \\
0.1385 \\
0.1438 \\
0.1489 \\
0.1535 \\
0.1637 \\
0.1693 \\
0.1816 \\
0.1959 \\
0.2093 \\
0.2104 \\
0.2289 \\
0.2371 \\
0.2458 \\
0.2818 \\
0.3307 \\
0.4157 \\
0.4418 \\
0.5712 \\
0.6920 \\
0.8761 \\
1.0319 \\
1.0902 \\
0.7670\end{array}$ \\
\hline
\end{tabular}

High Pressure test

(inclacement

0.0177

0.0283

0.0467

0.0596

0703

0.0916

0.0993

0.1107

0.1152

0.1209

0.1305

0.1385

0.1438

0.1489

0.1535

0.1693

0.1816

0.1959

0.2093

0.2371

0.2458

0.2818

0.3307

0.4157

0.6920

0.8761

1.0319

1.0902

0.7670 


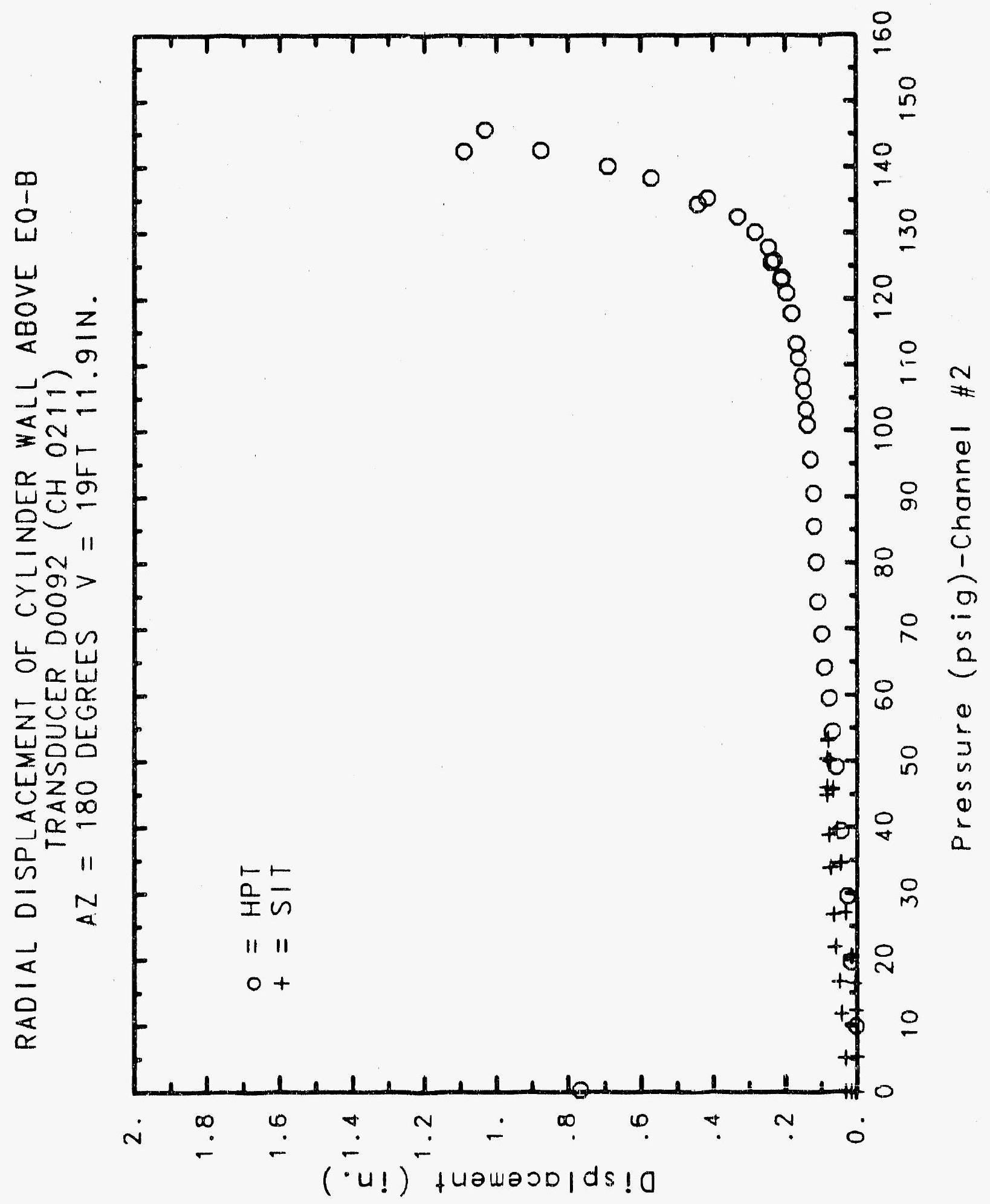




\section{Reinforced Concrete Test Data}

Potentiometer Channel 212

Structural Integrity Test

\begin{tabular}{|c|c|c|c|}
\hline $\begin{array}{l}\text { Pressure } \\
\text { (psig) } \\
-0.05 \\
5.33 \\
12.31 \\
16.44 \\
20.51 \\
20.46 \\
20.47 \\
27.05 \\
34.69 \\
34.53 \\
34.53 \\
39.70 \\
45.90 \\
45.65 \\
45.62 \\
50.10 \\
53.47 \\
53.29 \\
53.21 \\
50.49 \\
46.14 \\
46.13 \\
44.97 \\
38.98 \\
33.99 \\
33.96 \\
26.83 \\
21.88 \\
21.90 \\
16.69 \\
11.74 \\
11.77 \\
5.05 \\
0.02 \\
-0.04 \\
-0.02 \\
-0.02 \\
0.02 \\
\end{array}$ & $\begin{array}{c}\text { Displacement } \\
\text { (inches) } \\
-0.0001 \\
-0.0018 \\
0.0053 \\
0.0191 \\
0.0193 \\
0.0195 \\
0.0197 \\
0.0432 \\
0.0512 \\
0.0511 \\
0.0512 \\
0.0801 \\
0.0837 \\
0.0833 \\
0.0833 \\
0.0834 \\
0.1053 \\
0.1051 \\
0.1050 \\
0.1047 \\
0.1049 \\
0.1048 \\
0.1051 \\
0.1052 \\
0.1054 \\
0.1057 \\
0.1057 \\
0.1058 \\
0.1063 \\
0.0882 \\
0.0552 \\
0.0551 \\
0.0008 \\
-0.0230 \\
-0.0243 \\
-0.0240 \\
-0.0514 \\
-0.0513 \\
0.05\end{array}$ & $\begin{array}{c}\text { Pressure } \\
(\text { psig) } \\
9.89 \\
19.55 \\
29.57 \\
39.42 \\
49.16 \\
54.50 \\
59.57 \\
64.20 \\
69.32 \\
74.16 \\
80.16 \\
85.61 \\
90.58 \\
95.69 \\
100.92 \\
103.25 \\
106.11 \\
108.31 \\
111.08 \\
113.24 \\
117.83 \\
120.92 \\
123.28 \\
122.97 \\
125.82 \\
125.60 \\
127.84 \\
130.19 \\
132.53 \\
135.33 \\
134.42 \\
138.35 \\
140.16 \\
142.63 \\
145.78 \\
142.52 \\
0.22 \\
\end{array}$ & $\begin{array}{c}\text { Displacement } \\
\text { (inches) } \\
0.0002 \\
0.0283 \\
0.0785 \\
0.1045 \\
0.1371 \\
0.1455 \\
0.1455 \\
0.1461 \\
0.1765 \\
0.1816 \\
0.2078 \\
0.2200 \\
0.2329 \\
0.2444 \\
0.2682 \\
0.2922 \\
0.3017 \\
0.3076 \\
0.3192 \\
0.3337 \\
0.3506 \\
0.3733 \\
0.3864 \\
0.3865 \\
0.4170 \\
0.4171 \\
0.4273 \\
0.4418 \\
0.4633 \\
0.4904 \\
0.4911 \\
0.5211 \\
0.5410 \\
0.5512 \\
0.5578 \\
0.5836 \\
0.3057\end{array}$ \\
\hline
\end{tabular}

High Pressure test 


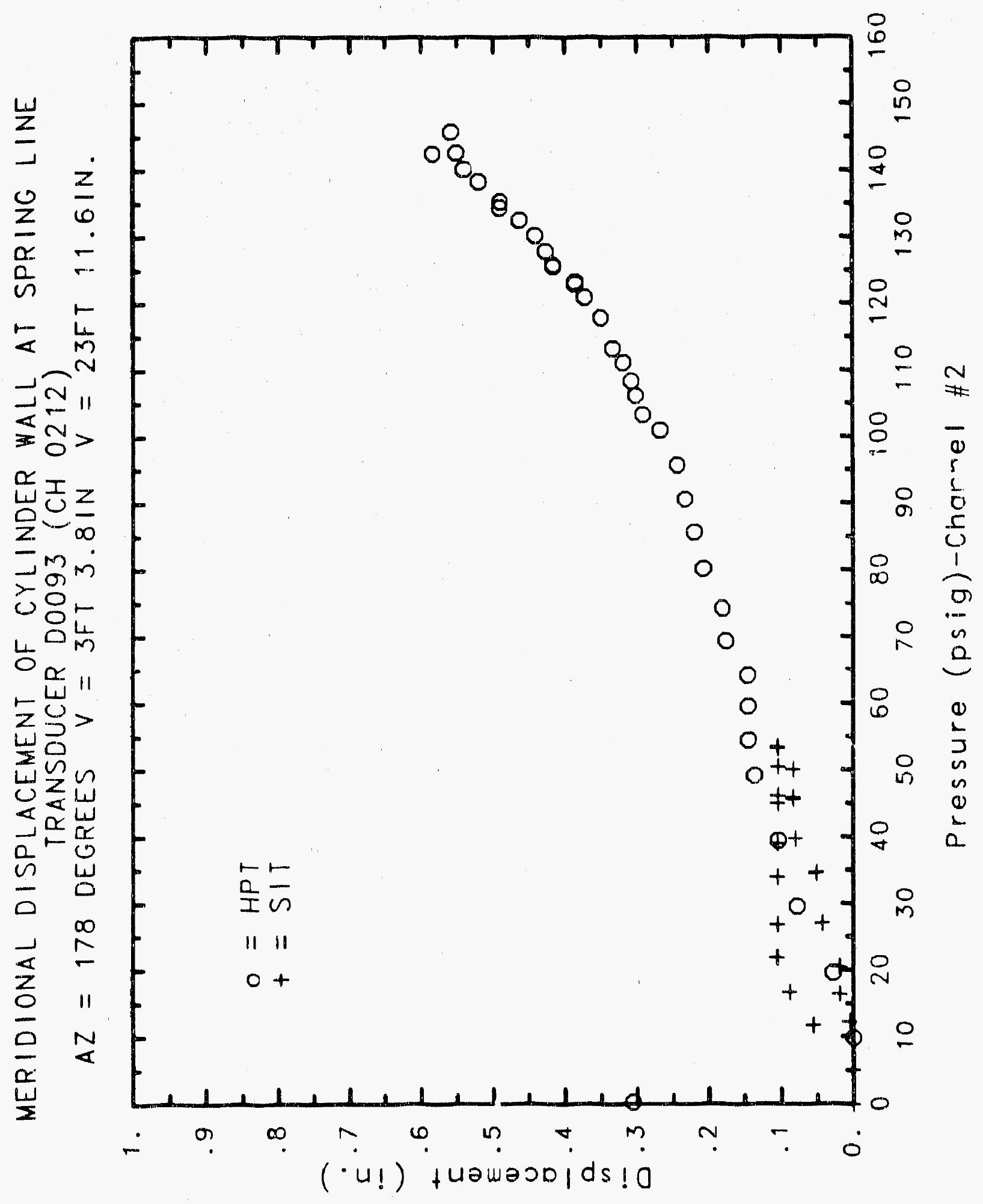




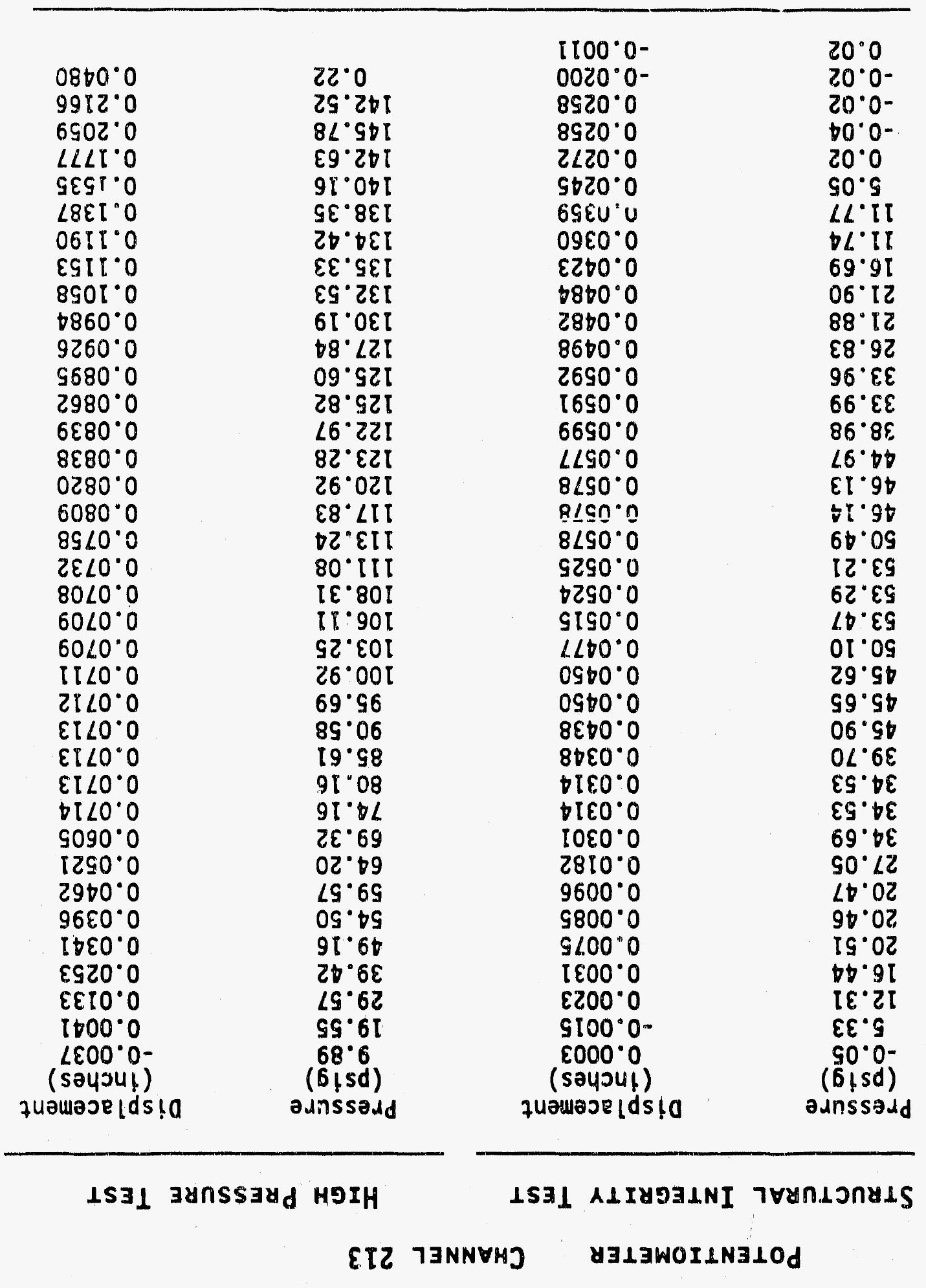

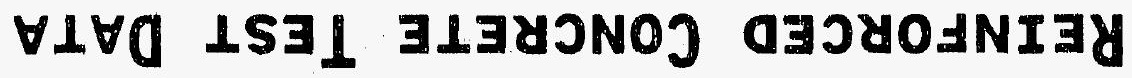




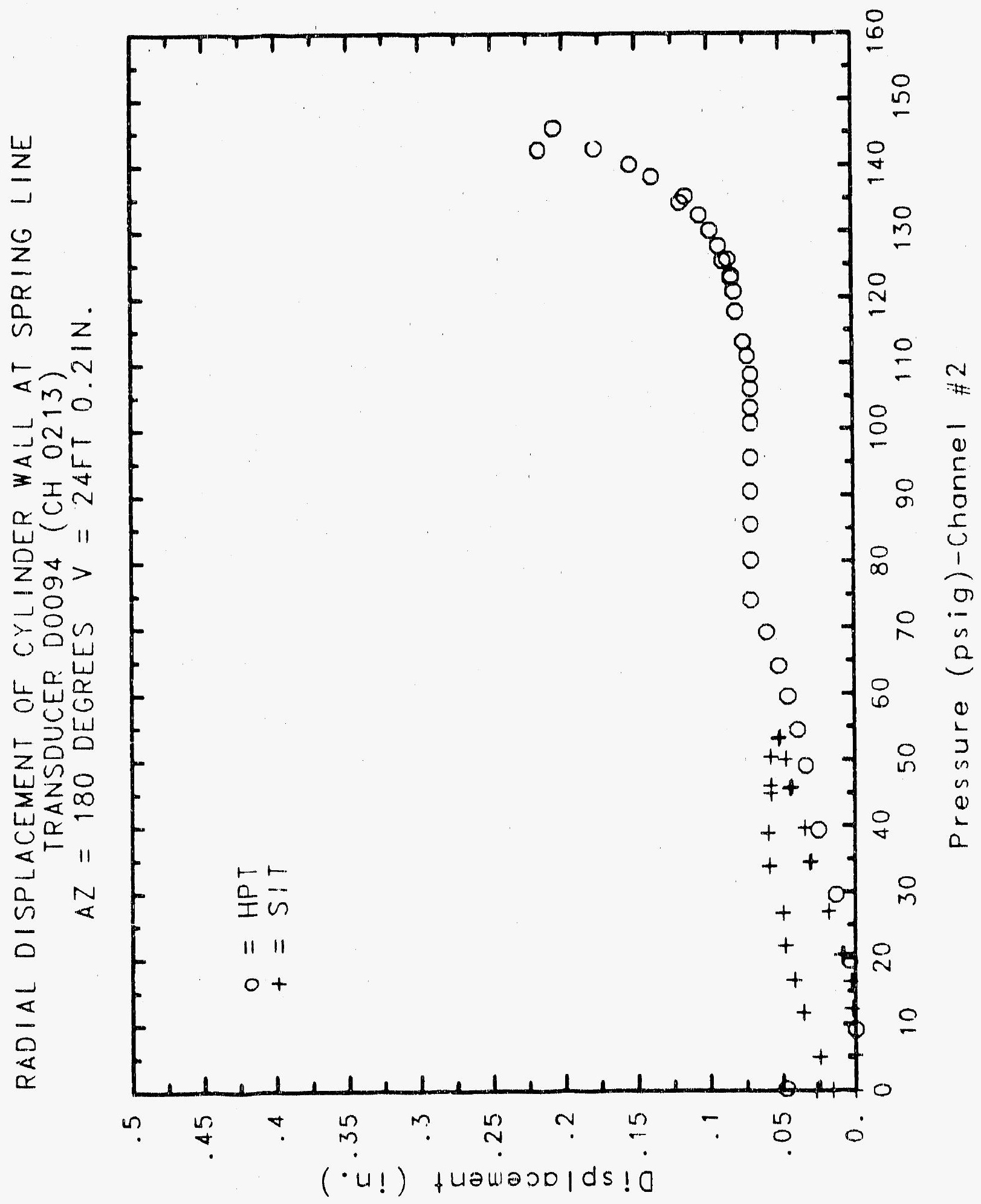




\section{Reinforced Concrete Test Data}

Potentiometer Channel 214

Structural Integrity Test

A correction of 0.0006 in. has been applied to each reading

\section{Pressure}

(psig)

$-0.05$

5.33

12.31

16.44

20.51

20.46

20.47

27.05

34.69

34.53

34.53

39.70

45.90

45.65

45.62

50.10

53.47

53.29

53.21

50.49

46.14

46.13

44.98

38.98

33.99

33.96

26.83

21.88

21.90

16.59

11.74

11.77

5.05

0.02

$-0.04$

$-0.02$

$-0.02$

0.02

0.02
Displacement

(inches)

0.0006

0.0013

0.0029

0.0039

0.0055

0.0062

0.0064

0.0099

0.0158

0.0168

0.0169

0.0192

0.0225

0.0229

0.0229

0.0247

0.0263

0.0263

0.0264

0.0272

0.0272

0.0272

0.0270

0.0271

0.0255

0.0255

0.0231

0.0218

0.0219

0.0194

0.0175

0.0174

0.0125

0.0123

0.0121

0.0122

0.0122

0.0123

0.0067 high Pressure test

A correction of $0.0036 \mathrm{in.} \mathrm{has}$ been applied to each reading.

Pressure

(psig)

9.89

19.55

29.57

39.42

49.16

54.50

59.57

64.20

69.32

74.16

80.16

85.61

90.58

95.69

100.92

103.25

106.11

108.31

111.08

113.24

117.83

120.92

123.28

122.97

125.82

125.60

127.84

130.19

132.53

135.33

134.42

138.35

140.16

142.63

145.78

142.52

0.22
Displacement

(inches)

0.0041

0.0082

0.0122

0.0156

0.0192

0.0217

0.0241

0.0273

0.0308

0.0351

0.0397

0.0439

0.0489

0.0544

0.0604

0.0632

0.0661

0.0687

0.0726

0.0801

0.0843

0.0902

0.0957

0.0974

0.1066

0.1092

0.1144

0.1257

0.1405

0.1597

0.1633

0.1864

0.2048

0.2312

0.2537

0.2612

0.1688 


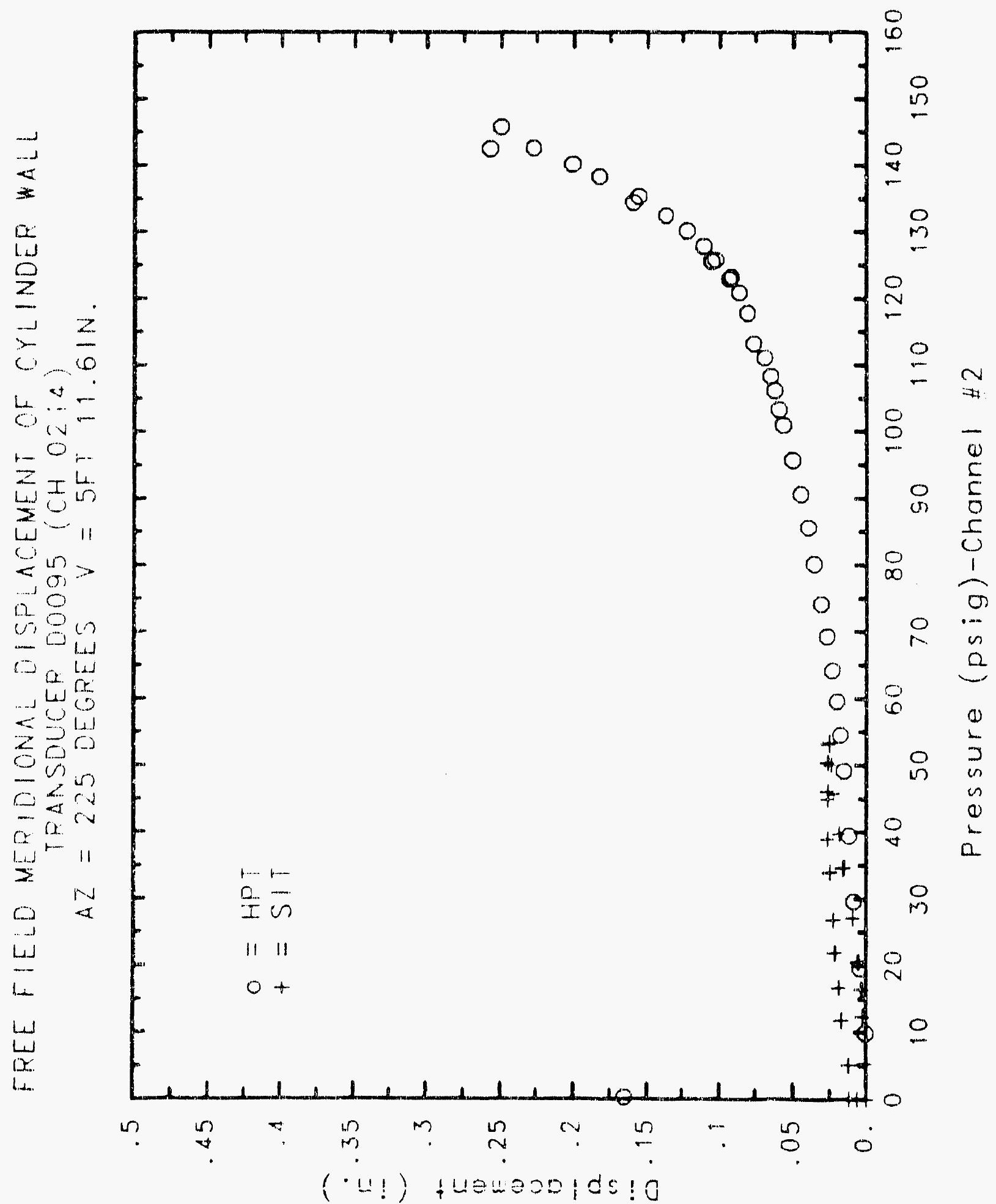

C.7? 


\section{Reinforced Concrete Test Data}

Potentiometer Channel 215

StRuctural InTEgRITY TEST

\begin{tabular}{|c|c|c|c|}
\hline $\begin{array}{l}\text { Pressure } \\
\text { (psig) } \\
-0.05 \\
5.33 \\
12.31 \\
16.44 \\
20.51 \\
20.46 \\
20.47 \\
27.05 \\
34.69 \\
34.53 \\
34.53 \\
39.70 \\
45.90 \\
45.65 \\
45.62 \\
50.10 \\
53.47 \\
53.29 \\
53.21 \\
50.49 \\
46.14 \\
46.13 \\
44.97 \\
38.98 \\
33.99 \\
33.96 \\
26.83 \\
21.88 \\
21.90 \\
16.69 \\
11.74 \\
11.77 \\
5.05 \\
0.02 \\
-0.04 \\
-0.02 \\
-0.02 \\
0.02\end{array}$ & $\begin{array}{c}\text { Displacement } \\
\text { (inches) } \\
0.0001 \\
0.0037 \\
0.0117 \\
0.0152 \\
0.0240 \\
0.0239 \\
0.0239 \\
0.0328 \\
0.0539 \\
0.0549 \\
0.0552 \\
0.0697 \\
0.0785 \\
0.0804 \\
0.0819 \\
0.0996 \\
0.0993 \\
0.1000 \\
0.1001 \\
0.1052 \\
0.1055 \\
0.1057 \\
0.1051 \\
0.0984 \\
0.0934 \\
0.0936 \\
0.0799 \\
0.0713 \\
0.0717 \\
0.0614 \\
0.0518 \\
0.0515 \\
0.0356 \\
0.0336 \\
0.0306 \\
0.0306 \\
-1.0309 \\
-1.0306\end{array}$ & $\begin{array}{c}\text { Pressure } \\
\text { (psig) } \\
9.89 \\
19.55 \\
29.57 \\
39.42 \\
49.16 \\
54.50 \\
59.57 \\
64.20 \\
69.32 \\
74.16 \\
80.16 \\
85.61 \\
90.58 \\
95.69 \\
100.92 \\
103.25 \\
106.11 \\
108.31 \\
111.08 \\
113.24 \\
117.83 \\
120.92 \\
123.28 \\
122.97 \\
125.82 \\
125.60 \\
127.84 \\
130.19 \\
132.53 \\
135.33 \\
134.42 \\
138.35 \\
140.16 \\
142.63 \\
145.78 \\
142.52 \\
0.22\end{array}$ & $\begin{array}{c}\text { Displacement } \\
\text { (inches) } \\
0.0065 \\
0.0263 \\
0.0476 \\
0.0646 \\
0.0328 \\
0.0936 \\
0.1073 \\
0.1151 \\
0.1312 \\
0.1426 \\
0.1568 \\
0.1725 \\
0.1844 \\
0.1950 \\
0.2110 \\
0.2241 \\
0.2351 \\
0.2364 \\
0.2518 \\
0.2631 \\
0.2798 \\
0.2971 \\
0.3162 \\
0.3226 \\
0.3535 \\
0.3633 \\
0.3826 \\
0.4296 \\
0.4918 \\
0.5736 \\
0.5945 \\
0.6991 \\
0.7828 \\
0.9000 \\
1.0008 \\
1.0499 \\
0.7066\end{array}$ \\
\hline
\end{tabular}

High Pressure test

(inches)

0.0065

0.0263

0.0936

0.1073

0.1151

0.1312

0.1725

0.1844

0.22 .41

0.2518

0.2631

0.2798

0.2971

0.3162

0.3226

0.3633

0.3826

0.4296

0.4918

0.6991

0.7828

0.9000

1.0008

1.0499

0.7066 


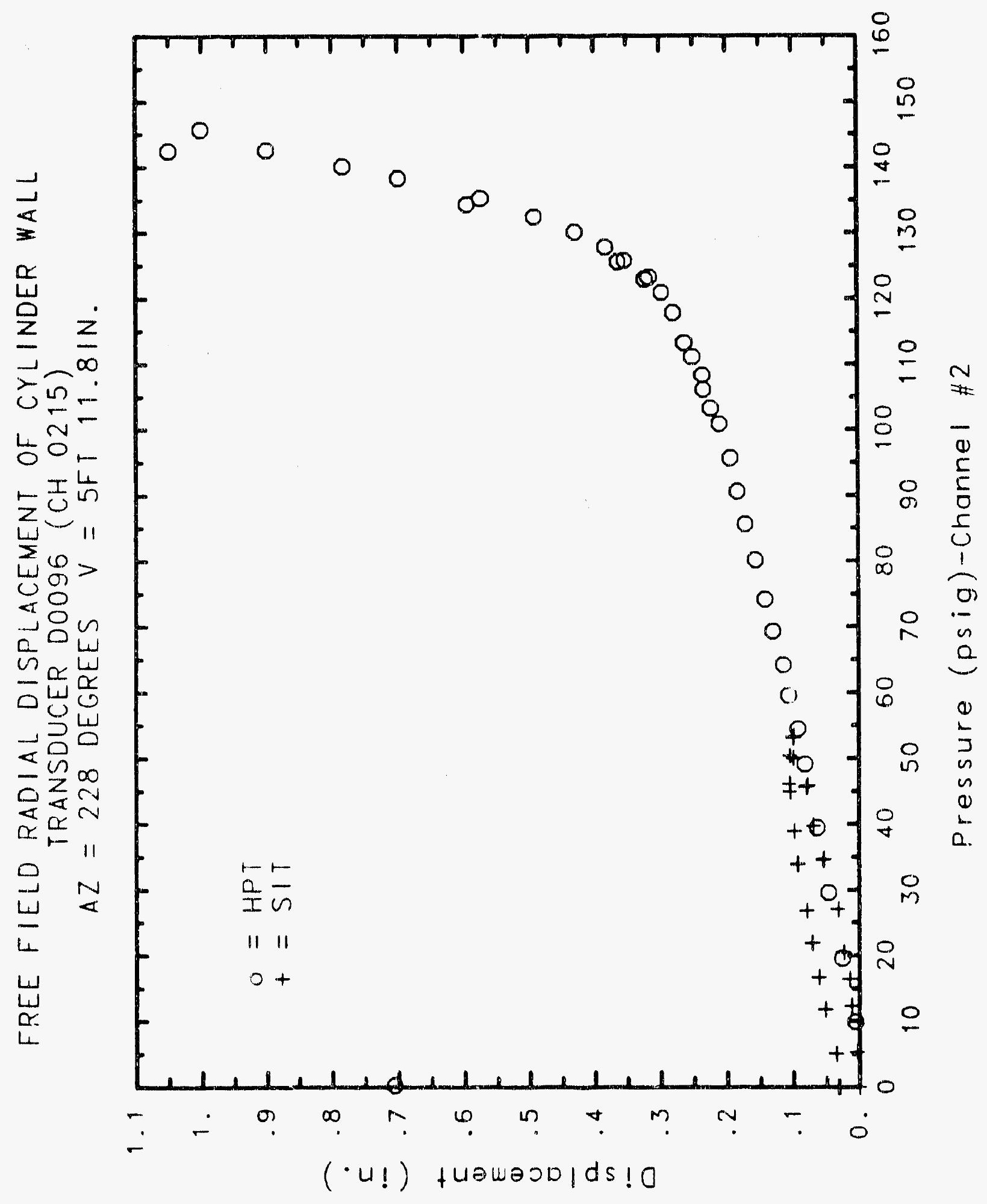




\section{Reinforced Concrete Test Data}

\begin{tabular}{|c|c|c|c|}
\hline \multirow[b]{2}{*}{ STRUCTURAL } & \multicolumn{2}{|c|}{ Channel 216} & \multirow[b]{2}{*}{ RE TEST } \\
\hline & INTEGRITY TEST & High Pf & \\
\hline $\begin{array}{l}\text { Pressure } \\
\text { (psig) } \\
-0.05 \\
5.33 \\
12.31 \\
16.44 \\
20.51 \\
20.46 \\
20.47 \\
27.05 \\
34.69 \\
34.53 \\
34.53 \\
39.70 \\
45.90 \\
45.65 \\
45.62 \\
50.10 \\
53.47 \\
53.29 \\
53.21 \\
50.49 \\
46.14 \\
46.13 \\
44.97 \\
38.98 \\
33.99 \\
33.96 \\
26.83 \\
21.88 \\
21.90 \\
16.69 \\
11.74 \\
11.77 \\
5.05 \\
0.02 \\
-0.04 \\
-0.02 \\
-0.02 \\
0.02\end{array}$ & $\begin{array}{c}\text { Displacement } \\
\text { (inches) } \\
-0.0001 \\
-0.0012 \\
0.0050 \\
0.0098 \\
0.0193 \\
0.0227 \\
0.0237 \\
0.0441 \\
0.0731 \\
0.0771 \\
0.0776 \\
0.0903 \\
0.1112 \\
0.1143 \\
0.1146 \\
0.1255 \\
0.1364 \\
0.1381 \\
0.1385 \\
0.1408 \\
0.1410 \\
0.1410 \\
0.1388 \\
0.1300 \\
0.1218 \\
0.1218 \\
0.1050 \\
0.0963 \\
0.0962 \\
0.0813 \\
0.0687 \\
0.0685 \\
0.0497 \\
0.0455 \\
0.0414 \\
0.0415 \\
-1.0206 \\
-1.0202 \\
0\end{array}$ & $\begin{array}{c}\text { Pressure } \\
\text { (psig) } \\
9.89 \\
19.55 \\
29.57 \\
39.42 \\
49.16 \\
54.50 \\
59.57 \\
64.20 \\
69.32 \\
74.16 \\
80.16 \\
85.61 \\
90.58 \\
95.69 \\
100.92 \\
103.25 \\
106.11 \\
108.31 \\
111.08 \\
113.24 \\
117.83 \\
120.92 \\
123.28 \\
122.97 \\
125.82 \\
125.60 \\
127.84 \\
130.19 \\
132.53 \\
135.33 \\
134.42 \\
138.35 \\
140.16 \\
142.63 \\
145.78 \\
142.52 \\
0.22 \\
\end{array}$ & $\begin{array}{c}\text { Displacement } \\
\text { (inches) } \\
0.0072 \\
0.0305 \\
0.0553 \\
0.0795 \\
0.0999 \\
0.1127 \\
0.1284 \\
0.1431 \\
0.1604 \\
0.1758 \\
0.1988 \\
0.2189 \\
0.2361 \\
0.2577 \\
0.2836 \\
0.2967 \\
0.3121 \\
0.3249 \\
0.3447 \\
0.3617 \\
0.4047 \\
0.4415 \\
0.4795 \\
0.4895 \\
0.5495 \\
0.5666 \\
0.6040 \\
0.6991 \\
0.8279 \\
0.9840 \\
1.0248 \\
1.2163 \\
1.3666 \\
1.5680 \\
1.7376 \\
1.8056 \\
1.3050 \\
0.04\end{array}$ \\
\hline
\end{tabular}




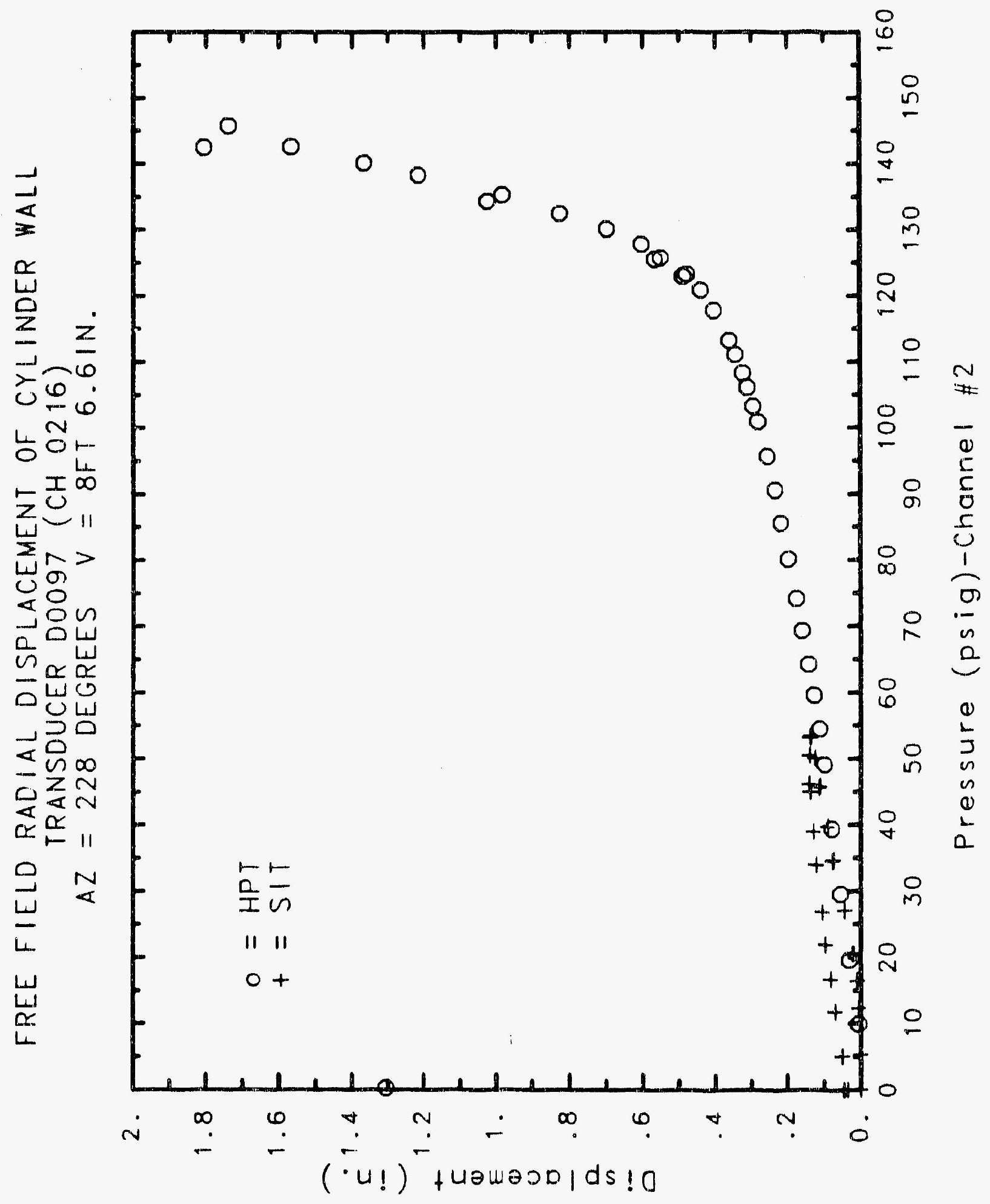




\section{Reinforced Concrete Test Data}

Potentiometer Channel 217

STRUCTURAL INTEGRITY TEST

A correction of 0.0089 in. has been applied to each reading

Pressure

(psig)

$-0.05$

5.33

12.31

16.44

20.51

20.46

20.47

27.05

34.69

34.53

34.53

39.70

45.90

45.65

45.62

50.10

53.47

53.29

53.21

50.49

46.14

46.13

44.98

38.98

33.99

33.96

26.83

21.88

21.90

16.69

11.74

11.77

5.05

0.02

$-0.04$

$-0.02$

$-0.02$

0.02

0.02
Displacement

(inches)

0.0011

0.0034

0.0077

0.0098

0.0126

0.0137

0.0142

0.0194

0.0283

0.0311

0.0312

0.0347

0.0408

0.0420

0.0420

0.0450

0.0484

0.0489

0.0489

0.0513

0.0515

0.0515

0.0496

0.0481

0.0455

0.0453

0.0411

0.0388

0.0391

0.0343

0.0298

0.0297

0.0221

0.0252

0.0245

0.0245

0.0248

0.0252

0.0225 hitgh Pressure test

A correction of 0.0016 in. has been applied to each reading.

Pressure Displacement

(psig) (inches)

$9.89 \quad 0.0073$

$19.55 \quad 0.0145$

$29.57 \quad 0.0215$

$39.42 \quad 0.0280$

$49.16 \quad 0.0348$

$\begin{array}{ll}54.50 & 0.0384\end{array}$

$59.57 \quad 0.0425$

$64.20 \quad 0.0475$

$69.32 \quad 0.0529$

$74.16 \quad 0.0599$

$80.16 \quad 0.0680$

$85.61 \quad 0.0750$

$90.58 \quad 0.0826$

$95.69 \quad 0.0908$

$100.92 \quad 0.0990$

$103.25 \quad 0.1034$

$106.11 \quad 0.1086$

$108.31 \quad 0.1119$

$111.08 \quad 0.1164$

$113.24 \quad 0.1230$

$117.83 \quad 0.1301$

$120.92 \quad 0.1376$

$123.28 \quad 0.1435$

$122.97 \quad 0.1457$

$125.82 \quad 0.1545$

$125.60 \quad 0.1576$

$127.84 \quad 0.1630$

$130.19 \quad 0.1739$

$132.53 \quad 0.1906$

$135.33 \quad 0.2065$

$134.42 \quad 0.2100$

$138.35 \quad 0.2319$

$140.16 \quad 0.2481$

$142.63 \quad 0.2678$

$145.78 \quad 0.2845$

$\$ 42.52 \quad 0.2889$

$\begin{array}{ll}0.22 & 0.1681\end{array}$ 


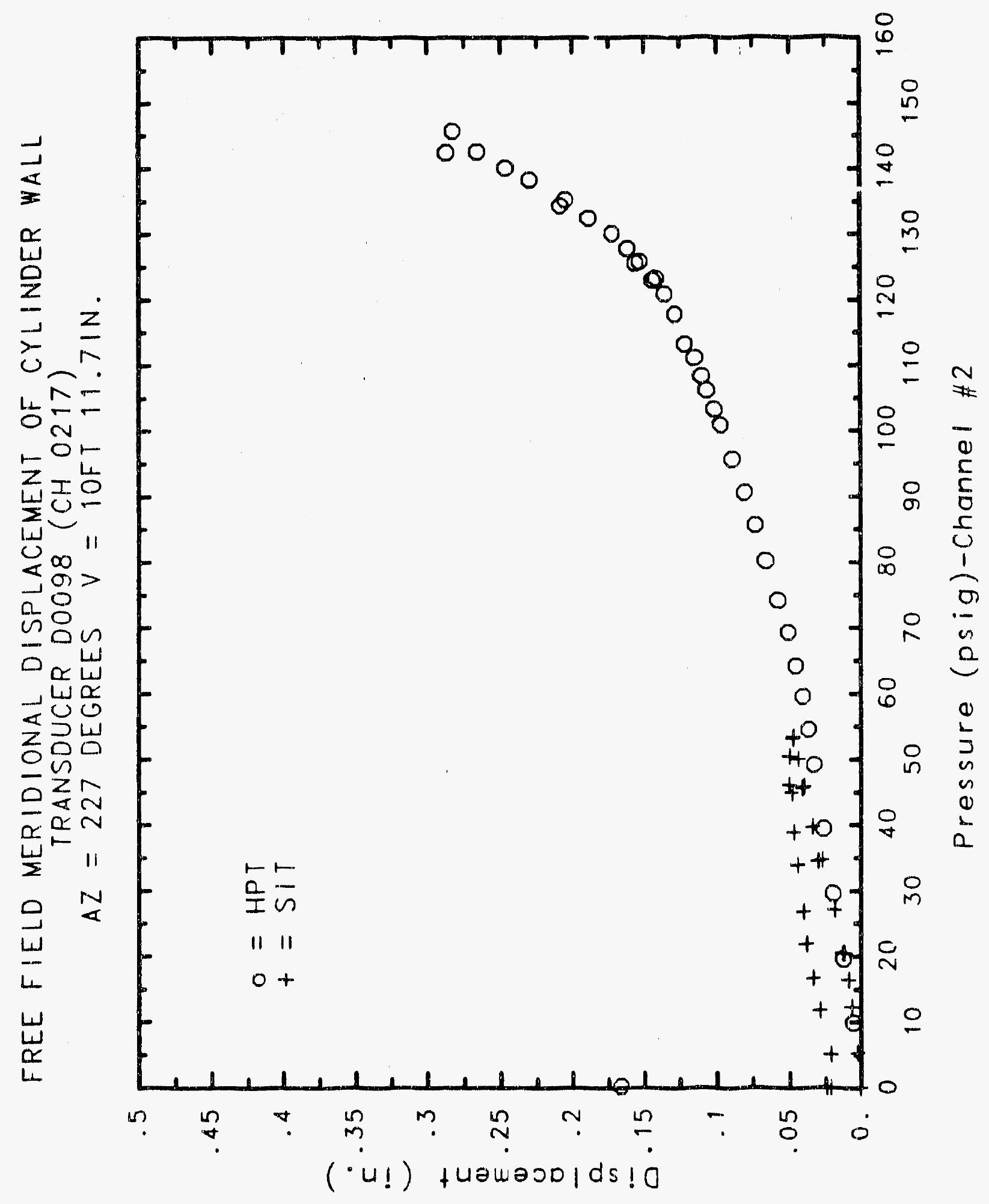




\section{Reinforced Concrete Test Data}

Potentrometer Channel 218

Structural Integrity test

\begin{tabular}{|c|c|c|c|}
\hline $\begin{array}{c}\text { Pressure } \\
\text { (psig) } \\
-0.05 \\
5.33 \\
12.31 \\
16.44 \\
20.51 \\
20.46 \\
20.47 \\
27.05 \\
34.69 \\
34.53 \\
34.53 \\
39.70 \\
45.90 \\
45.65 \\
45.62 \\
50.10 \\
53.47 \\
53.29 \\
53.21 \\
50.49 \\
46.14 \\
46.13 \\
44.97 \\
38.98 \\
33.99 \\
33.96 \\
26.83 \\
21.88 \\
21.90 \\
16.69 \\
11.74 \\
11.77 \\
5.05 \\
0.02 \\
-0.04 \\
-0.02 \\
-0.02 \\
0.02\end{array}$ & $\begin{array}{c}\text { Displacement } \\
\text { (inches) } \\
-0.0001 \\
-0.0001 \\
0.0075 \\
0.0122 \\
0.0207 \\
0.0241 \\
0.0255 \\
0.0449 \\
0.0728 \\
0.0768 \\
0.0770 \\
0.0902 \\
0.1107 \\
0.1142 \\
0.1146 \\
0.1260 \\
0.1361 \\
0.1386 \\
0.1394 \\
0.1420 \\
0.1420 \\
0.1420 \\
0.1369 \\
0.1288 \\
0.1206 \\
0.1207 \\
0.1053 \\
0.0977 \\
0.0973 \\
0.0835 \\
0.0713 \\
0.0711 \\
0.0544 \\
0.0501 \\
0.0460 \\
0.0461 \\
0.0337 \\
0.0284\end{array}$ & $\begin{array}{c}\text { Pressure } \\
\text { (psig) } \\
9.89 \\
19.55 \\
29.57 \\
39.42 \\
49.16 \\
54.50 \\
59.57 \\
64.20 \\
69.32 \\
74.16 \\
80.16 \\
85.61 \\
90.58 \\
95.69 \\
100.92 \\
103.25 \\
106.11 \\
108.31 \\
111.08 \\
113.24 \\
117.83 \\
120.92 \\
123.28 \\
122.97 \\
125.82 \\
125.60 \\
127.84 \\
130.19 \\
132.53 \\
135.33 \\
134.42 \\
138.35 \\
140.16 \\
142.63 \\
145.78 \\
142.52 \\
0.22\end{array}$ & $\begin{array}{c}\text { Displacement } \\
\text { (inches) } \\
0.0128 \\
0.0343 \\
0.0575 \\
0.0817 \\
0.1030 \\
0.1164 \\
0.1295 \\
0.1470 \\
0.1626 \\
0.1793 \\
0.2012 \\
0.2229 \\
0.2361 \\
0.2589 \\
0.2837 \\
0.2980 \\
0.3128 \\
0.3252 \\
0.3453 \\
0.3619 \\
0.4050 \\
0.4428 \\
0.4820 \\
0.4933 \\
0.5571 \\
0.5773 \\
0.6189 \\
0.7321 \\
0.8899 \\
1.0784 \\
1.1253 \\
1.3434 \\
1.5078 \\
1.7183 \\
1.8875 \\
1.9525 \\
1.4761\end{array}$ \\
\hline
\end{tabular}

High Pressure test

(inches)

0.0128

0.0817

0.1164

0.1295

0.1470

0.2229

0.2361

0.2589

0.2837

0.3252

0.3453

0.3619

0.4050

0.4428

0.4820

0.5773

0.6189

0.7321

0.8899

1.0784

.5078

.7183

1.8875

1.9525

1.4761 


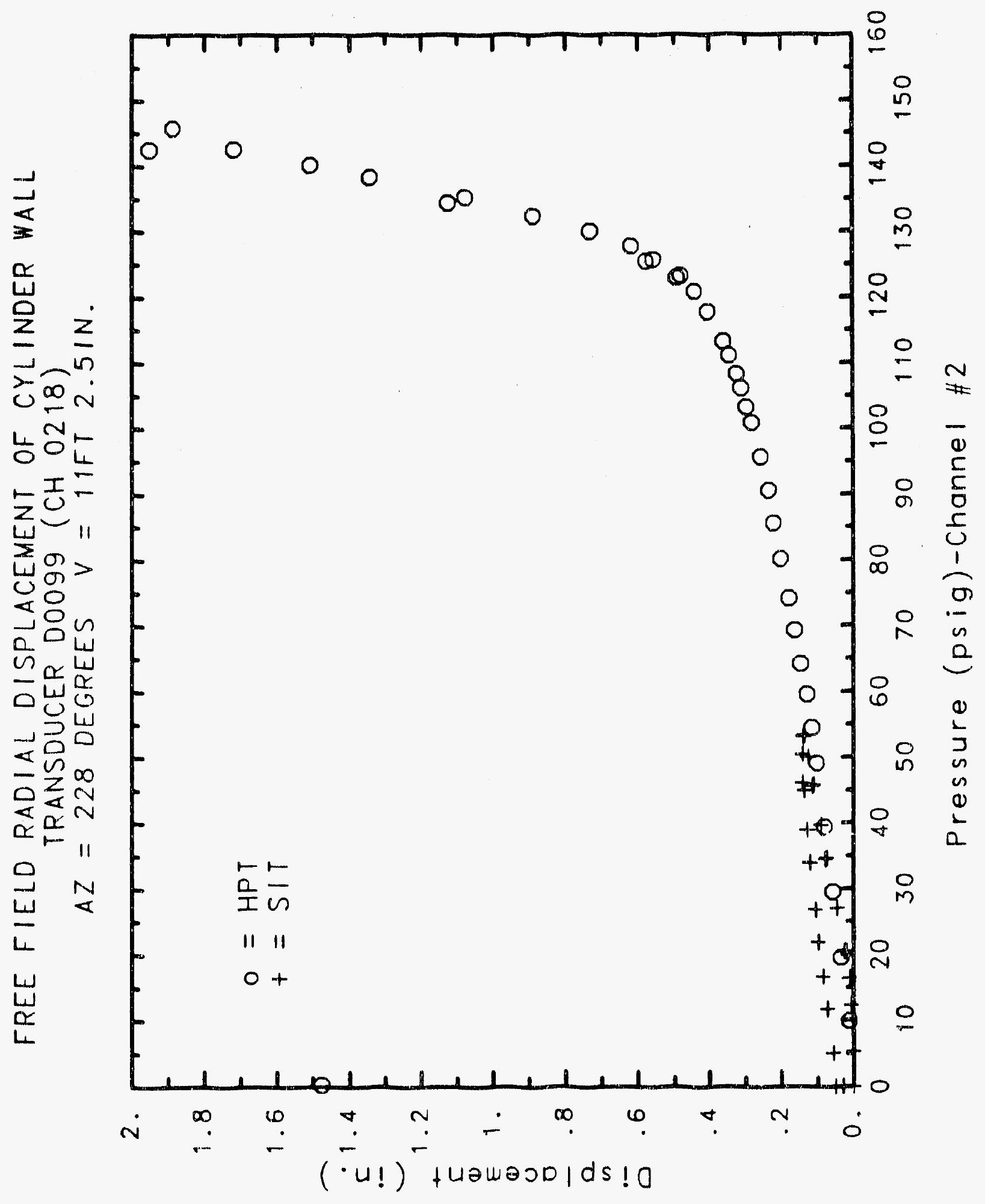




\section{Reinforced Concrete Test Data}

Potentiometer Channel 219

Structural InTEgrity TEST

\begin{tabular}{c} 
Pressure \\
(psig) \\
-0.05 \\
5.33 \\
12.31 \\
16.44 \\
20.51 \\
20.46 \\
20.47 \\
27.05 \\
34.69 \\
34.53 \\
34.53 \\
39.70 \\
45.90 \\
45.65 \\
45.62 \\
50.10 \\
53.47 \\
53.29 \\
53.21 \\
50.49 \\
46.14 \\
46.13 \\
44.97 \\
38.98 \\
33.99 \\
33.96 \\
26.83 \\
21.88 \\
21.90 \\
16.69 \\
11.74 \\
11.77 \\
5.05 \\
0.02 \\
-0.04 \\
-0.02 \\
-0.02 \\
0.02 \\
\\
\hline
\end{tabular}

Displacement

(inches)

0.0000

$-0.0018$

0.0026

0.0052

0.0153

0.0182

0.0192

0.0375

0.0669

0.0701

0.0703

0.0836

0.1052

0.1084

0.1089

0.1197

0.1310

0.1330

0.1336

0.1363

0.1371

0.1372

0.1345

0.1261

0.1190

0.1191

0.1050

0.0982

0.0980

0.0829

0.0720

0.0720

0.0553

0.0507

0.0475

0.0476

0.0281

0.0301
High Pressure test

Pressure

(psig)

9.89

19.55

29.57

39.42

49.16

54.50

59.57

54.20

69.32

74.16

80.16

85.61

90.58

95.69

100.92

103.25

106.11

108.31

111.08

113.24

117.83

120.92

123.28

122.97

125.82

125.60

127.84

130.19

132.53

135.33

134.42

138.35

140.16

142.63

145.78

142.52

0.22
Displacement (inches)

0.0066

0.0253

0.0458

0.0707

0.0921

0.1060

0.1193

0.1342

0.1506

0.1668

0.1865

0.2051

0.2177

0.2392

0.2628

0.2761

0.2897

0.3038

0.3209

0.3370

0.3773

0.4101

0.4468

0.4554

0.5125

0.5280

0.5680

0.6732

0.8247

1.0161

1.0650

1.2811

1.4445

1.6552

1.8145

1.8750

1.4324 


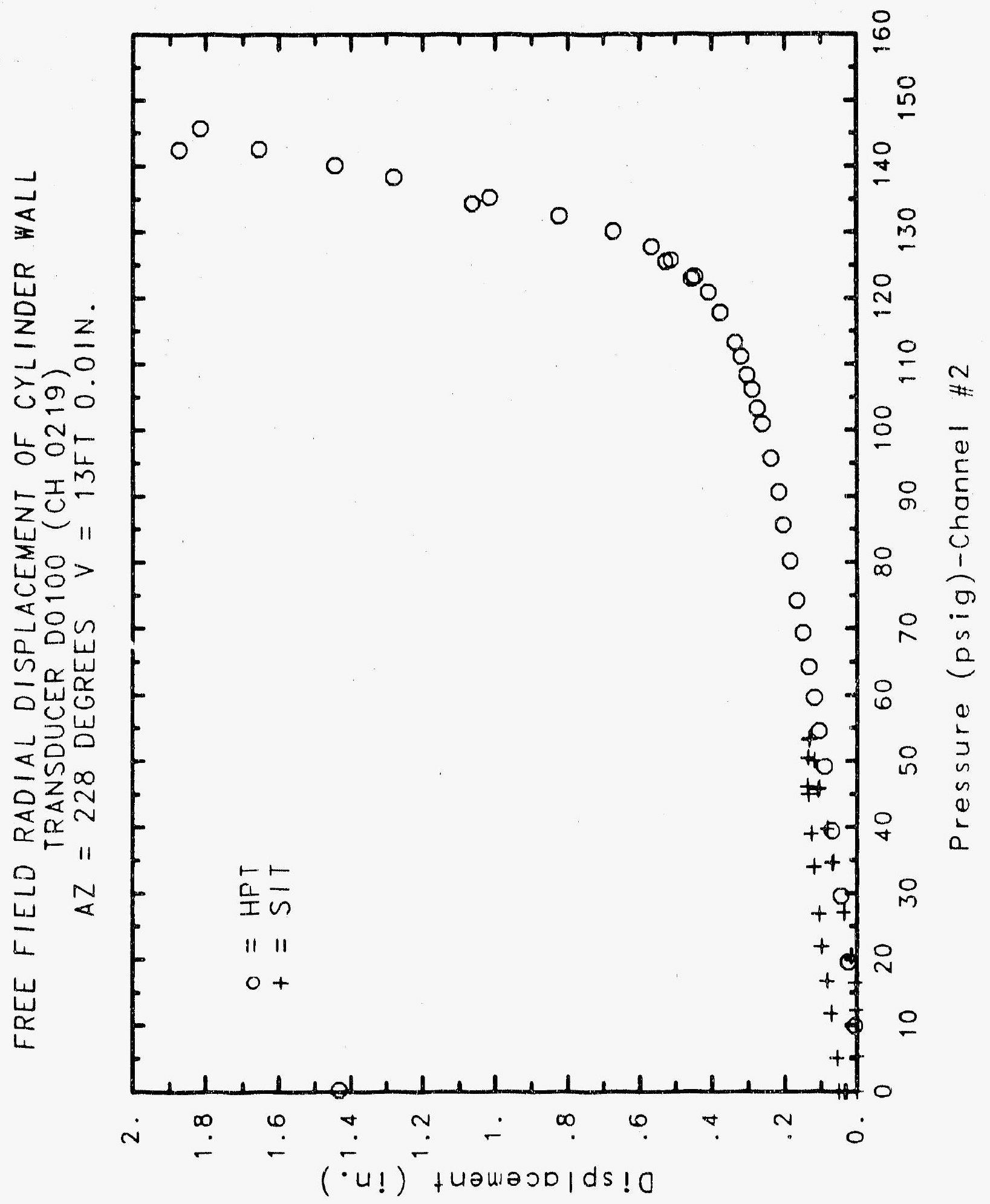




\section{Reinforced Concrete Test Data}

Potentiometer Channel 220

STRUCTURAL. INTEGRITY TEST

\begin{tabular}{|c|c|c|c|}
\hline $\begin{array}{l}\text { Pressure } \\
\text { (psig) } \\
-0.05 \\
5.33 \\
12.31 \\
16.44 \\
20.51 \\
20.46 \\
20.47 \\
27.05 \\
34.69 \\
34.53 \\
34.53 \\
39.70 \\
45.90 \\
45.65 \\
45.62 \\
50.10 \\
53.47 \\
53.29 \\
53.21 \\
50.49 \\
46.14 \\
46.13 \\
44.97 \\
38.98 \\
33.99 \\
33.96 \\
26.83 \\
21.88 \\
21.90 \\
16.69 \\
11.74 \\
11.77 \\
5.05 \\
0.02 \\
-0.04 \\
-0.02 \\
-0.02 \\
0.02\end{array}$ & $\begin{array}{c}\text { Displacement } \\
\text { (inches) } \\
0.0001 \\
-0.0020 \\
0.0020 \\
0.0050 \\
0.0144 \\
0.0208 \\
0.0227 \\
0.0394 \\
0.0663 \\
0.0701 \\
0.0704 \\
0.0814 \\
0.1021 \\
0.1044 \\
0.1048 \\
0.1163 \\
0.1267 \\
0.1289 \\
0.1296 \\
0.1325 \\
0.1321 \\
0.1319 \\
0.1320 \\
0.1240 \\
0.1174 \\
0.1176 \\
0.1030 \\
0.0981 \\
0.0980 \\
0.0817 \\
0.0714 \\
0.0713 \\
0.0578 \\
0.0549 \\
0.0520 \\
0.0523 \\
0.0259 \\
0.0287\end{array}$ & $\begin{array}{c}\text { Pressure } \\
(\text { psig) } \\
9.89 \\
19.55 \\
29.57 \\
39.42 \\
49.16 \\
54.50 \\
59.57 \\
64.20 \\
69.32 \\
74.16 \\
80.16 \\
85.61 \\
90.58 \\
95.69 \\
100.92 \\
103.45 \\
106.11 \\
108.31 \\
111.08 \\
113.24 \\
117.83 \\
120.92 \\
123.28 \\
122.97 \\
125.82 \\
125.60 \\
127.84 \\
130.19 \\
132.53 \\
135.33 \\
134.42 \\
138.35 \\
140.16 \\
142.63 \\
145.78 \\
142.52 \\
0.22\end{array}$ & $\begin{array}{c}\text { Displacement } \\
\text { (inches) } \\
0.0074 \\
0.0229 \\
0.0431 \\
0.0662 \\
0.0872 \\
0.0993 \\
0.1124 \\
0.1270 \\
0.1411 \\
0.1539 \\
0.1735 \\
0.1892 \\
0.1982 \\
0.2146 \\
0.2363 \\
0.2475 \\
0.2611 \\
0.2707 \\
0.2877 \\
0.3013 \\
0.3335 \\
0.3604 \\
0.3898 \\
0.3967 \\
0.4398 \\
0.4527 \\
0.4831 \\
0.5699 \\
0.7066 \\
0.8840 \\
0.9298 \\
1.1467 \\
1.3090 \\
1.5153 \\
1.6744 \\
1.7320 \\
1.3198\end{array}$ \\
\hline
\end{tabular}

High Pressure test 


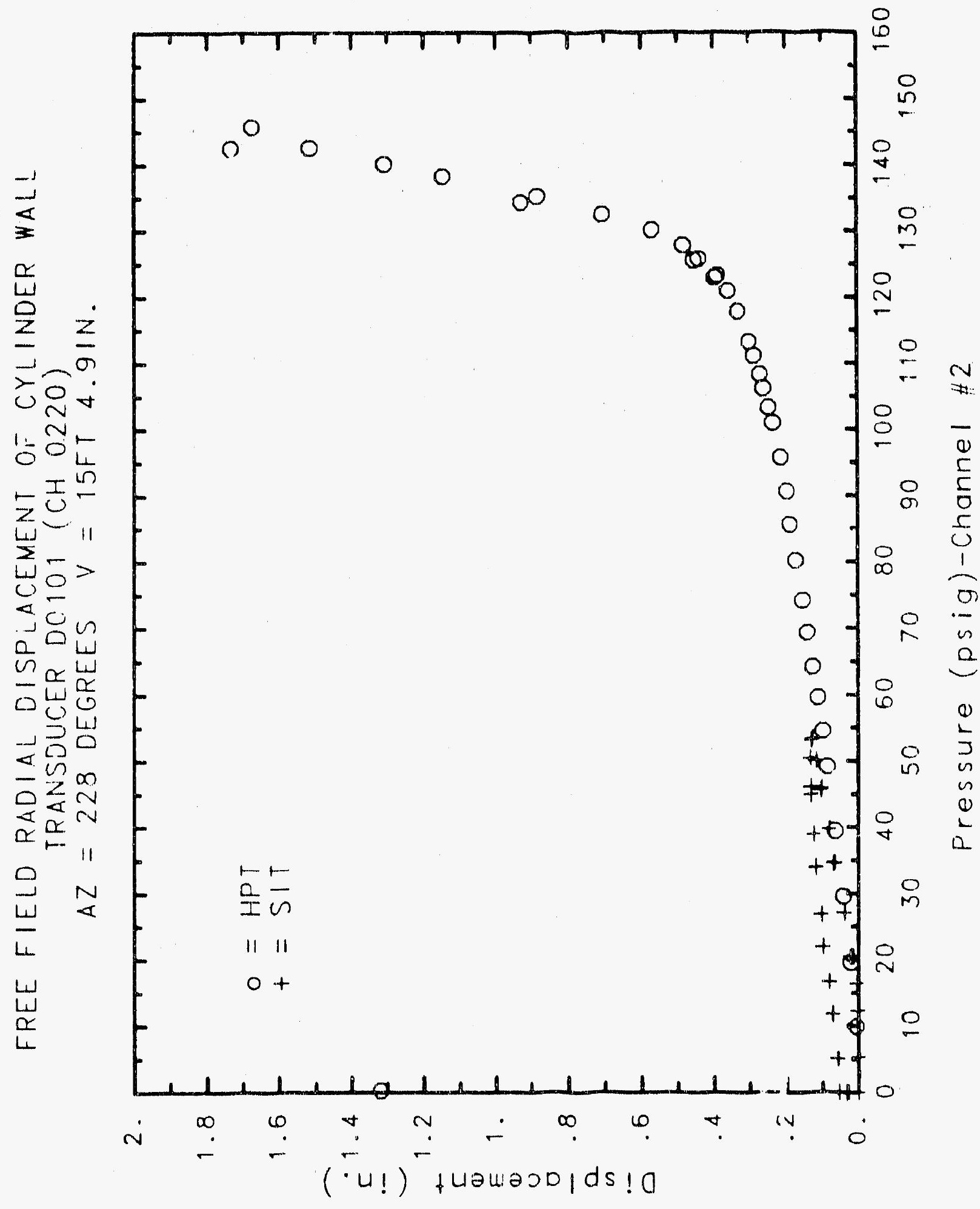




\section{Reinforced Concrete Test Data}

Potentiometer Channel 221

Structural Integrity Test

\begin{tabular}{|c|c|c|c|}
\hline $\begin{array}{l}\text { Pressure } \\
\text { (psig) } \\
-0.05 \\
5.33 \\
12.31 \\
18.44 \\
20.51 \\
20.46 \\
20.47 \\
27.05 \\
34.69 \\
34.53 \\
34.53 \\
39.70 \\
45.90 \\
15.65 \\
45.62 \\
50.10 \\
53.47 \\
53.29 \\
53.21 \\
50.49 \\
46.14 \\
46.13 \\
44.97 \\
38.98 \\
33.99 \\
33.96 \\
26.83 \\
21.88 \\
21.90 \\
16.69 \\
11.74 \\
11.77 \\
5.05 \\
0.02 \\
-0.04 \\
-0.02 \\
-0.02 \\
0.02\end{array}$ & $\begin{array}{c}\text { Displacement } \\
\text { (inches) } \\
-0.0001 \\
0.0035 \\
0.0160 \\
0.0204 \\
0.0347 \\
0.0388 \\
0.0405 \\
0.0588 \\
0.0861 \\
0.0893 \\
0.0896 \\
0.0980 \\
0.1176 \\
0.1198 \\
0.1201 \\
0.1298 \\
0.1392 \\
0.1404 \\
0.1405 \\
0.1424 \\
0.1426 \\
0.1425 \\
0.1427 \\
0.1370 \\
0.1309 \\
0.1310 \\
0.1188 \\
0.1122 \\
0.1121 \\
0.0972 \\
0.0873 \\
0.0872 \\
0.0679 \\
0.0554 \\
0.0510 \\
0.0510 \\
0.0175 \\
0.0339\end{array}$ & $\begin{array}{c}\text { Pressure } \\
(p s 1 g) \\
9.89 \\
19.55 \\
29.57 \\
39.42 \\
49.16 \\
54.50 \\
59.57 \\
64.20 \\
69.32 \\
74.16 \\
80.16 \\
85.61 \\
90.58 \\
95.69 \\
100.92 \\
103.25 \\
106.11 \\
108.31 \\
111.08 \\
113.24 \\
117.83 \\
120.52 \\
123.28 \\
122.97 \\
125.82 \\
125.60 \\
127.84 \\
130.19 \\
132.53 \\
135.33 \\
134.42 \\
138.35 \\
140.16 \\
142.63 \\
145.78 \\
142.52 \\
0.22\end{array}$ & $\begin{array}{c}\text { Displacement } \\
\text { (inches) } \\
0.0091 \\
0.0314 \\
0.0492 \\
0.0718 \\
0.0901 \\
0.0997 \\
0.1123 \\
0.1258 \\
0.1407 \\
0.1517 \\
0.1664 \\
0.1802 \\
0.1838 \\
0.1999 \\
0.2171 \\
0.2253 \\
0.2358 \\
0.2444 \\
0.2607 \\
0.2695 \\
0.2956 \\
0.3179 \\
0.3366 \\
0.3422 \\
0.3723 \\
0.3808 \\
0.3996 \\
0.4557 \\
0.5519 \\
0.6944 \\
0.7317 \\
0.9222 \\
1.0812 \\
1.2975 \\
1.4674 \\
1.5318 \\
1.0972\end{array}$ \\
\hline
\end{tabular}

\section{High Pressure test}

splacement

0.0314

0.0492

0.0718

0.0901

0.0997

0.1123

0.1407

0.1517

0.1664

0.1802

0.1838

0.217

0.2253

0.2358

0.2695

0.3366

0.3422

0.3996

0.4557

0.5519

0.6944

0.7317

0.9222

1.0812

1.2975

1.4674

1.5318

1.0972 
$(.8$.

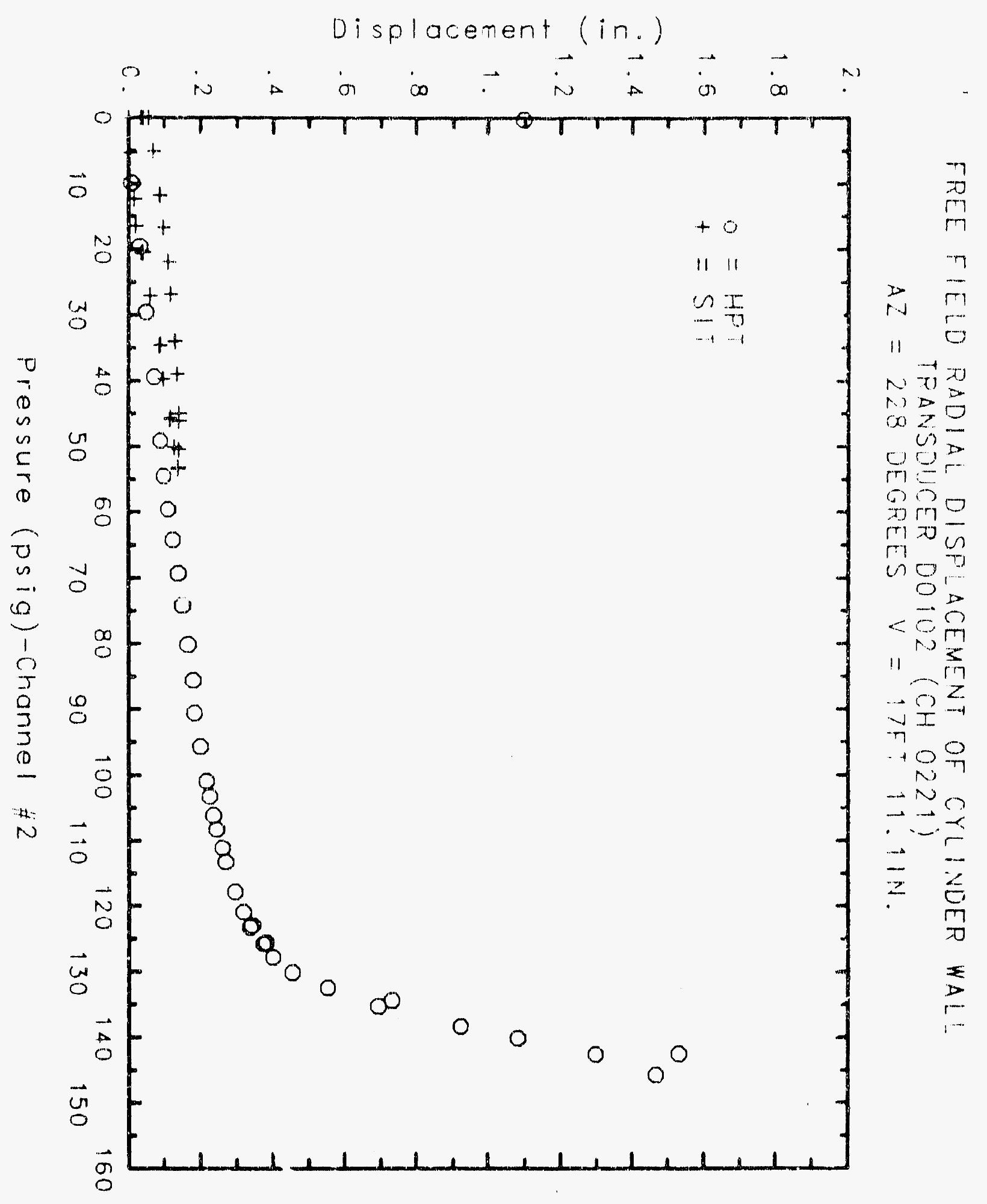




\section{Reinforced Concrete Test Data}

\section{Potentiometer Channel 222}

\section{STRUCTURAL InTEgRITY TEST}

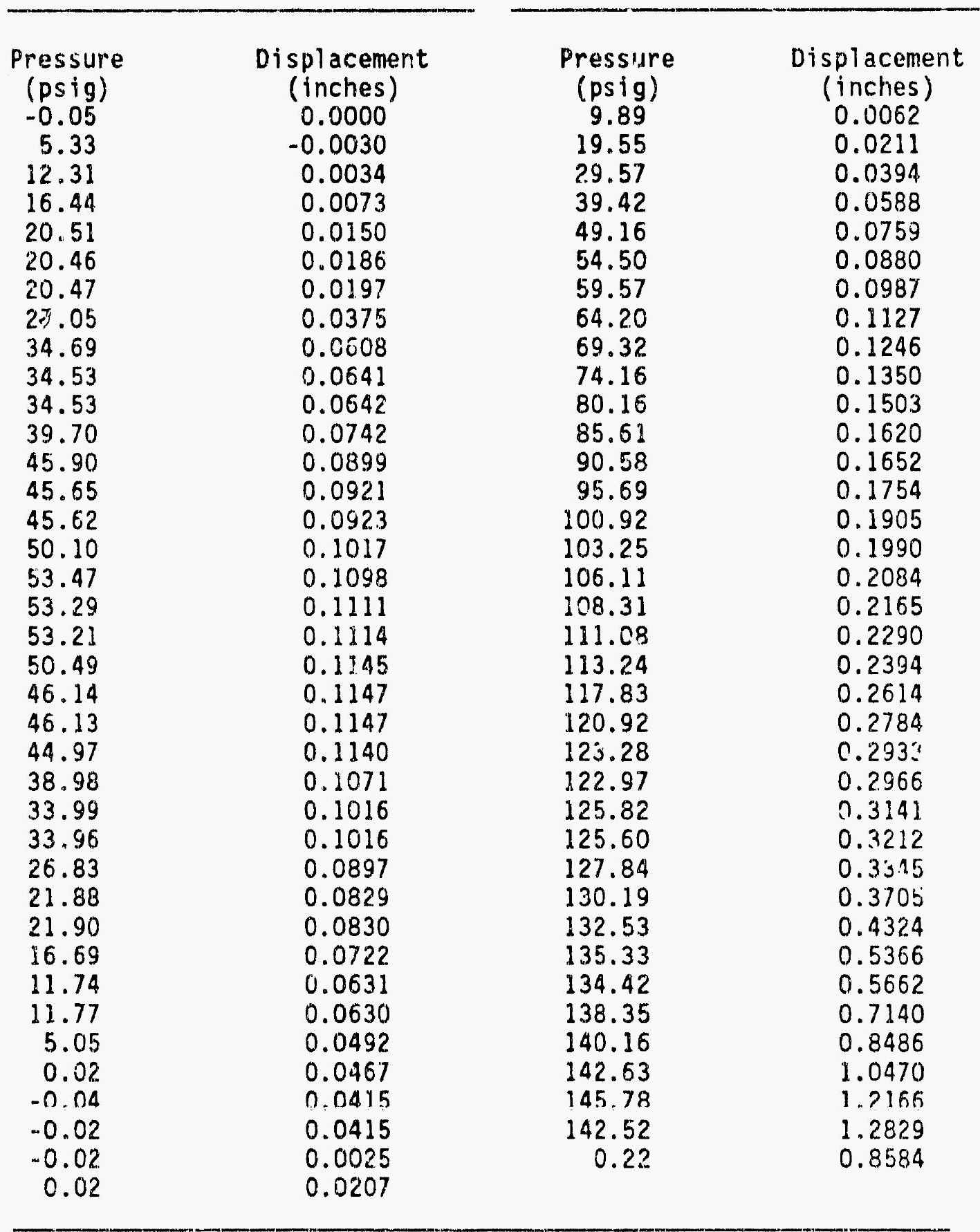

\section{High Pressure test}

placement

0.0211

0.0759

0.0987

0.1350

1620

0.1652

0.1754

2084

0.2394

0.2614

0.2784

.3141

$0.3,115$

0.3705

0.4324

0.5366

0.5662

0.7140

2166

1.2829

0.8584 


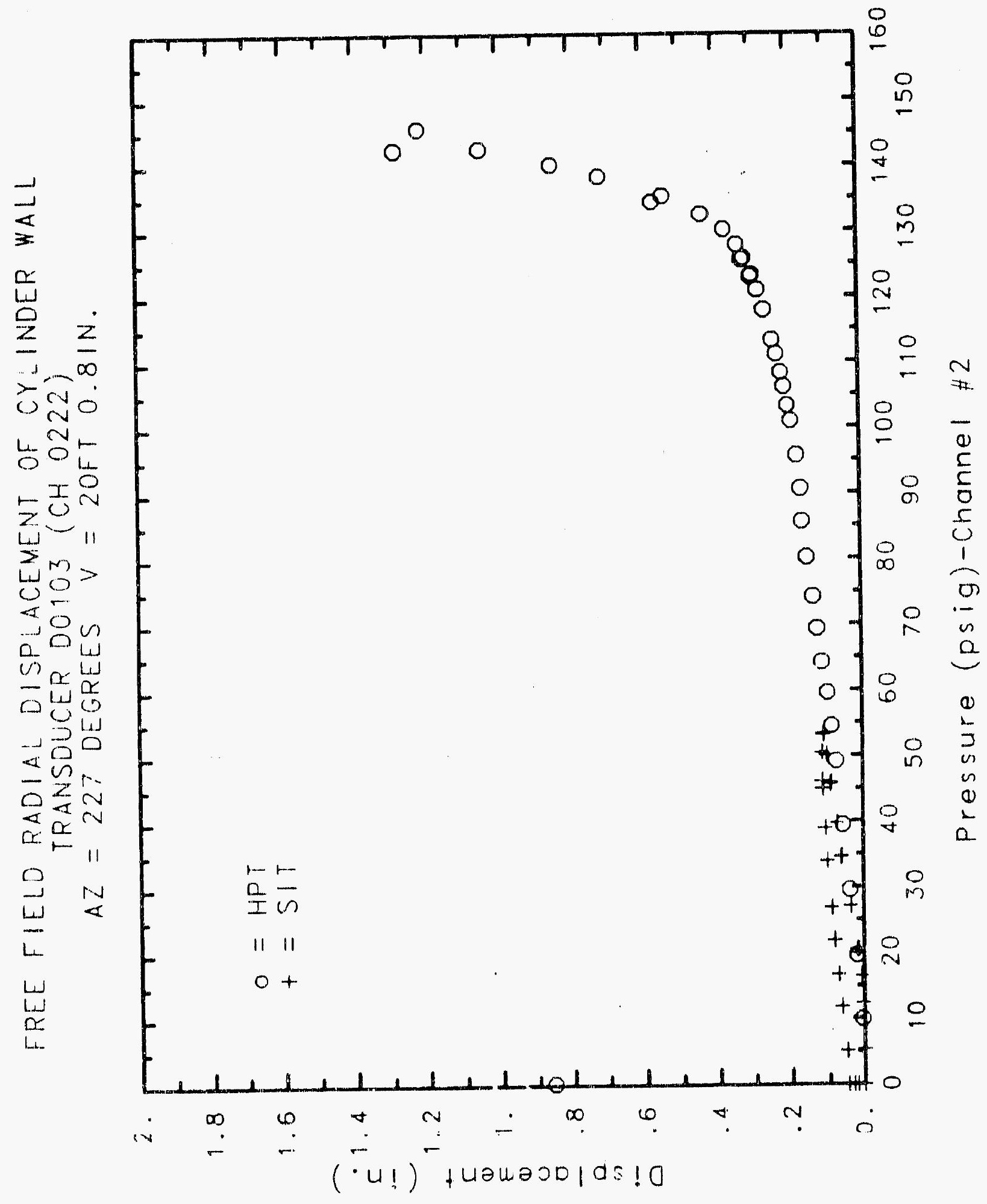




\section{Reinforced Concrete Test Data}

POTENTIOMETER

Structural IntEgraty Test

A correction of -0.0510 in. has been applied to each reading

Pressure

(psig)

$-0.05$

5.33

12.31

16.44

20.51

20.46

20.47

27.05

34.69

34.53

34.53

39.70

45.90

45.65

45.62

50.10

53.47

53.29

53.21

50.49

46.14

46.13

44.98

38.98

33.99

33.96

26.83

21.88

21.90

16.69

11.74

11.77

5.05

0.02

$-0.04$

$-0.02$

$-0.02$

0.02

0.02
Displacement

(inches)

$-0.0513$

0.0404

0.0933

0.0987

0.1040

0.1058

0.1058

0.1123

0.1262

0.1316

0.1317

0.1366

0.1475

0.1523

0.1523

0.1577

0.1638

0.1658

0.1658

0.1719

0.1719

0.1719

0.1722

0.1717

0.1674

0.1676

0.1590

0.1544

0.1546

0.1469

0.1451

0.1449

0.1275

0.0534

0.0385

0.0386

0.0227

0.0272

0.0119
Channel 223

High Pressure test

A correction of -0.0839 in. has been applied to each reading.

Pressure

(psig)

9.89

19.55

29.57

39.42

49.16

54.50

59.57

64.20

69.32

74.16

80.16

85.61

90.58

95.69

100.92

103.25

106.11

108.31

111.08

113.24

117.83

120.92

123.28

122.97

$125.8 \hat{c}$

125.60

127.84

130.19

132.53

135.33

134.42

138.35

140.16

142.63

145.78

142.52

0.22
Displacement

(inches)

0.0039

0.0077

0.0216

0.0354

0.0490

0.0602

0.0673

0.0787

0.0915

0.1067

0.1263

0.1443

0.1606

0.1786

0.1964

0.2066

0.2171

0.2243

0.2344

0.2436

0.2606

0.2738

0.2877

0.2921

0.3046

0.3080

0.3154

0.3267

0.3378

0.3582

0.3623

0.3953

0.4253

0.4711

0.5146

0.5320

$-0.0491$ 


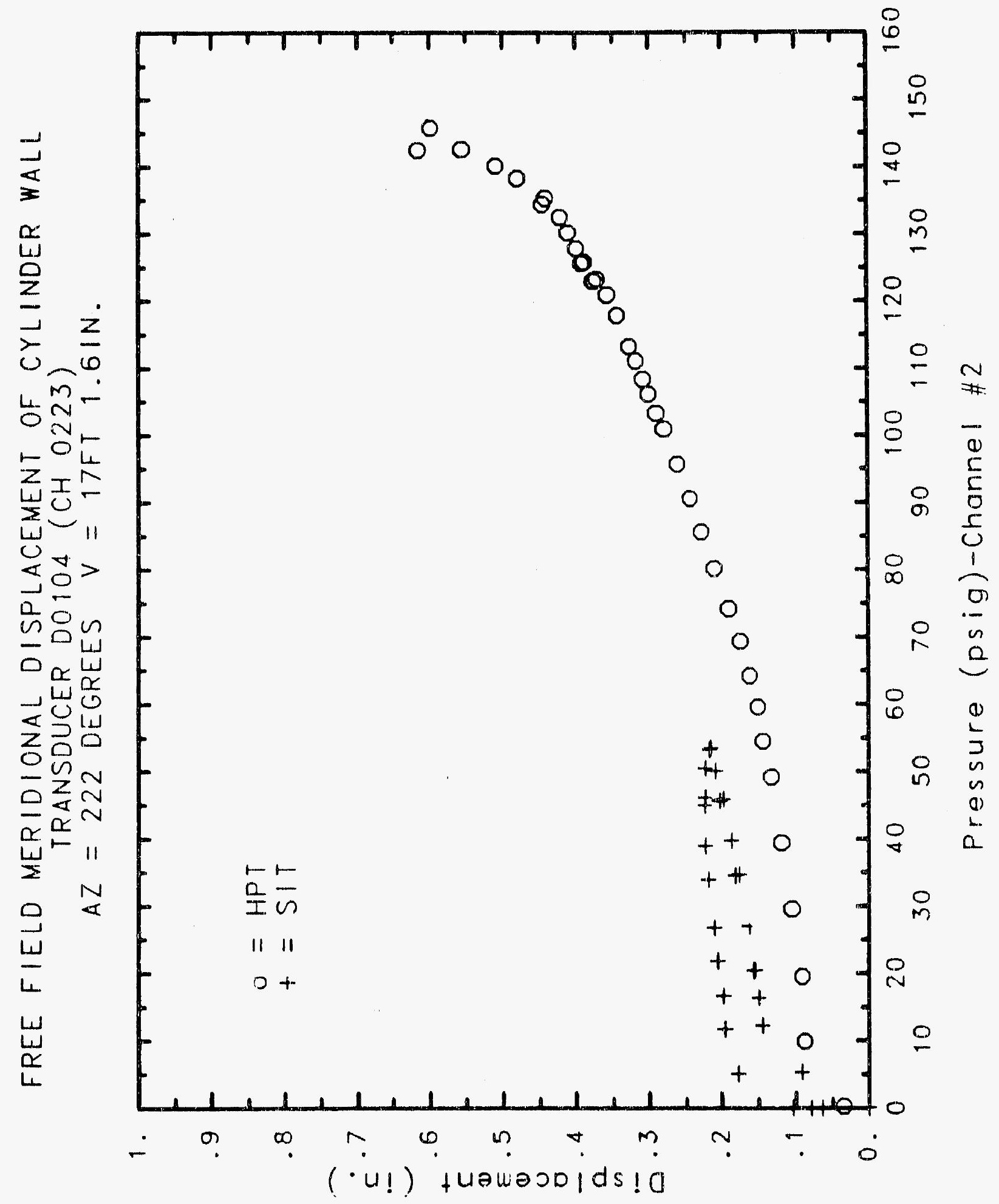




\section{Reinforced Concrete Test Data}

Potentiometer Channel. 224

StRUCTURAL INTEgRITY TEST

High Pressure test

A correction of -0.0035 in. has been applied to each reading

\begin{tabular}{|c|c|c|c|}
\hline $\begin{array}{l}\text { Pressure } \\
\text { (psig) } \\
-0.05 \\
5.33 \\
12.31 \\
16.44 \\
20.51 \\
20.46 \\
20.47 \\
27.05 \\
34.69 \\
34.53 \\
34.53 \\
39.70 \\
45.90 \\
45.65 \\
45.62 \\
50.10 \\
53.47 \\
53.29 \\
53.21 \\
50.49 \\
46.14 \\
46.13 \\
44.98 \\
38.98 \\
33.99 \\
33.96 \\
26.83 \\
21.88 \\
21.90 \\
16.69 \\
11.74 \\
11.77 \\
5.05 \\
0.02 \\
-0.04 \\
-0.72 \\
-0.02 \\
0.02 \\
0.02 \\
\end{array}$ & $\begin{array}{c}\text { Displacement } \\
\text { (inches) } \\
\text {-0.0035 } \\
0.0044 \\
0.0101 \\
0.0137 \\
0.0212 \\
0.0255 \\
0.0263 \\
0.0370 \\
0.0569 \\
0.0626 \\
0.0637 \\
0.0714 \\
0.0829 \\
0.0859 \\
0.0859 \\
0.0972 \\
0.1049 \\
0.1058 \\
0.1058 \\
0.1143 \\
0.1144 \\
0.1143 \\
0.1094 \\
0.1060 \\
0.1011 \\
0.1011 \\
0.0891 \\
0.08: 5 \\
0.0811 \\
0.0753 \\
0.0673 \\
0.0675 \\
0.0436 \\
0.0487 \\
0.0379 \\
0.0379 \\
0.0383 \\
0.0391 \\
0.0211\end{array}$ & $\begin{array}{c}\text { Pressure } \\
(p s i g) \\
9.89 \\
19.55 \\
29.57 \\
39.42 \\
49.16 \\
54.50 \\
59.57 \\
64.20 \\
69.32 \\
74.16 \\
80.16 \\
85.61 \\
90.58 \\
95.69 \\
100.92 \\
103.25 \\
106.11 \\
108.31 \\
111.08 \\
113.24 \\
117.83 \\
120.92 \\
123.28 \\
122.97 \\
125.82 \\
125.60 \\
127.84 \\
130.19 \\
132.53 \\
135.33 \\
134.42 \\
138.35 \\
140.16 \\
142.63 \\
145.79 \\
142.52 \\
0.22\end{array}$ & $\begin{array}{c}\text { Displacement } \\
\text { (inches) } \\
0.0201 \\
0.0398 \\
0.0532 \\
0.0716 \\
0.0851 \\
0.0968 \\
0.1041 \\
0.1146 \\
0.1314 \\
0.1500 \\
0.1656 \\
0.1849 \\
0.2092 \\
0.2293 \\
0.2530 \\
0.2633 \\
0.2714 \\
0.2832 \\
0.2996 \\
0.3077 \\
0.3323 \\
0.3488 \\
0.3671 \\
0.3753 \\
0.3958 \\
0.4018 \\
0.4146 \\
0.4319 \\
0.4562 \\
0.4849 \\
0.4896 \\
0.5199 \\
0.5476 \\
0.5859 \\
0.6241 \\
0.6365 \\
0.3016\end{array}$ \\
\hline
\end{tabular}

A correction of 0.0044 in. has been applied to each reading.

Pressure

9.89

(inches)

inche

0.0035

0.0212

0.0255

0.0569

0.0626

0.0859

0.0972

0.1049

0.1144

0.1143

0.1011

0.0891

0.08 ،

19.55

0.0398

0.0532

0.0851

0.0968

0.1500

0.1656

0.1849

0.2530

0.2633

0.2714

0.3323

0.3488

0.3671

0.4018

0.4146

0.4319

0.4562

0.4896

0.5199

0.0487

0.6241

0.0379

0.0391

0.6365

0.0211 


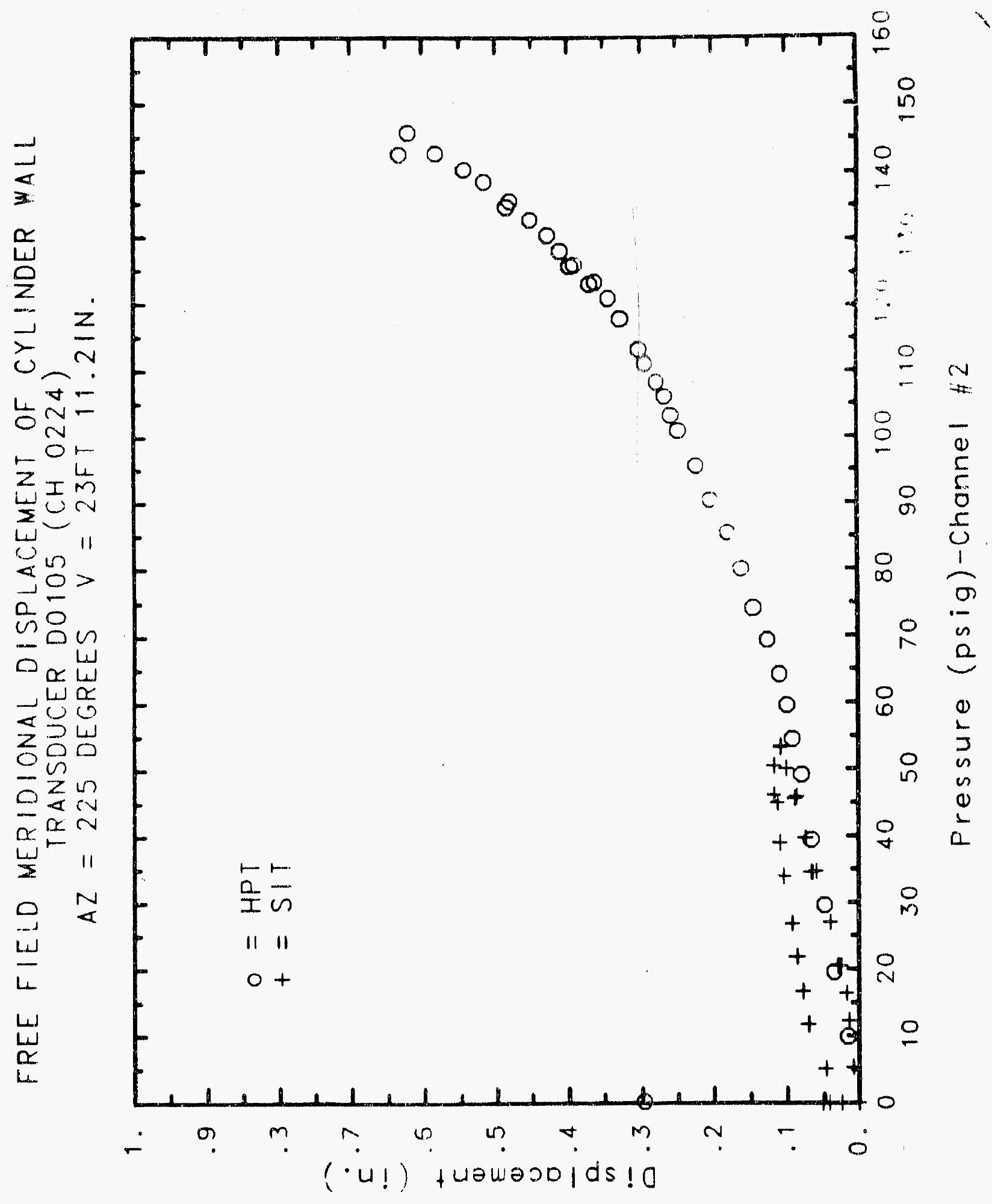




\section{Reinforced Concrete Test Data}

Pu: NTIOMETER Channel 225

StRUCTURAL IntEgrITy TEST

\begin{tabular}{|c|c|c|c|}
\hline $\begin{array}{l}\text { (psigure } \\
\text { (psig) } \\
-0.05 \\
5.33 \\
12.31 \\
16.44 \\
20.51 \\
20.46 \\
20.47 \\
27.05 \\
34.69 \\
34.53 \\
34.53 \\
39.70 \\
45.90 \\
45.65 \\
45.62 \\
50.10 \\
53.47 \\
53.29 \\
53.21 \\
50.49 \\
46.14 \\
46.13 \\
44.97 \\
38.98 \\
33.99 \\
33.96 \\
26.83 \\
21.88 \\
21.90 \\
16.69 \\
11.74 \\
11.77 \\
5.05 \\
0.02 \\
-0.04 \\
-0.02 \\
-0.02 \\
0.02\end{array}$ & $\begin{array}{c}\text { Displacement } \\
\text { (inches) } \\
0.0001 \\
-0.0001 \\
0.0054 \\
0.0095 \\
0.0165 \\
0.0194 \\
0.0206 \\
0.0351 \\
0.0551 \\
0.0602 \\
0.0612 \\
0.0701 \\
0.0835 \\
0.0846 \\
0.0845 \\
0.0919 \\
0.0992 \\
0.1001 \\
0.1001 \\
0.1026 \\
0.1025 \\
0.1025 \\
0.1005 \\
0.0935 \\
0.0875 \\
0.0877 \\
0.0768 \\
0.0706 \\
0.0709 \\
0.0621 \\
0.0544 \\
0.0541 \\
0.0404 \\
0.0387 \\
0.0296 \\
0.0287 \\
0.0128 \\
0.0168\end{array}$ & $\begin{array}{c}\text { Pressure } \\
(p 519) \\
9.89 \\
19.55 \\
29.57 \\
39.42 \\
49.16 \\
54.50 \\
59.57 \\
64.20 \\
69.32 \\
74.16 \\
80.16 \\
85.61 \\
90.58 \\
95.69 \\
100.92 \\
103.25 \\
105.11 \\
108.31 \\
111.08 \\
113.24 \\
117.83 \\
120.92 \\
123.28 \\
122.97 \\
125.82 \\
125.60 \\
127.84 \\
130.19 \\
132.53 \\
135.33 \\
134.42 \\
138.35 \\
140.16 \\
142.63 \\
145.78 \\
142.52 \\
0.22\end{array}$ & $\begin{array}{c}\text { Displacement } \\
\text { (inches) } \\
0.0077 \\
0.0229 \\
0.0400 \\
0.0561 \\
0.0728 \\
0.0825 \\
0.0917 \\
0.1032 \\
0.1118 \\
0.1196 \\
0.1371 \\
0.1532 \\
0.1602 \\
0.1756 \\
0.1945 \\
0.2038 \\
0.2139 \\
0.2226 \\
0.2366 \\
0.2464 \\
0.2704 \\
0.2925 \\
0.3105 \\
0.3149 \\
0.3405 \\
0.3487 \\
0.3667 \\
0.4137 \\
0.4919 \\
0.6237 \\
0.6607 \\
0.8458 \\
0.9998 \\
1.2081 \\
1.3812 \\
1.4424 \\
0.9648\end{array}$ \\
\hline
\end{tabular}

High Pressuke Tle:

placement

0.0077

0.0400

0.0561

0.0728

0.0825

0.1118

0.1196

0.1371

0.1756

0.1945

0.2038

0.2139

0.2464

0.2704

0.2925

0.3105

0.3487

0.3667

0.4137

0.4919

0.8458

0.9998

1.2081

1.3812

1.4424 
ऽ6-J

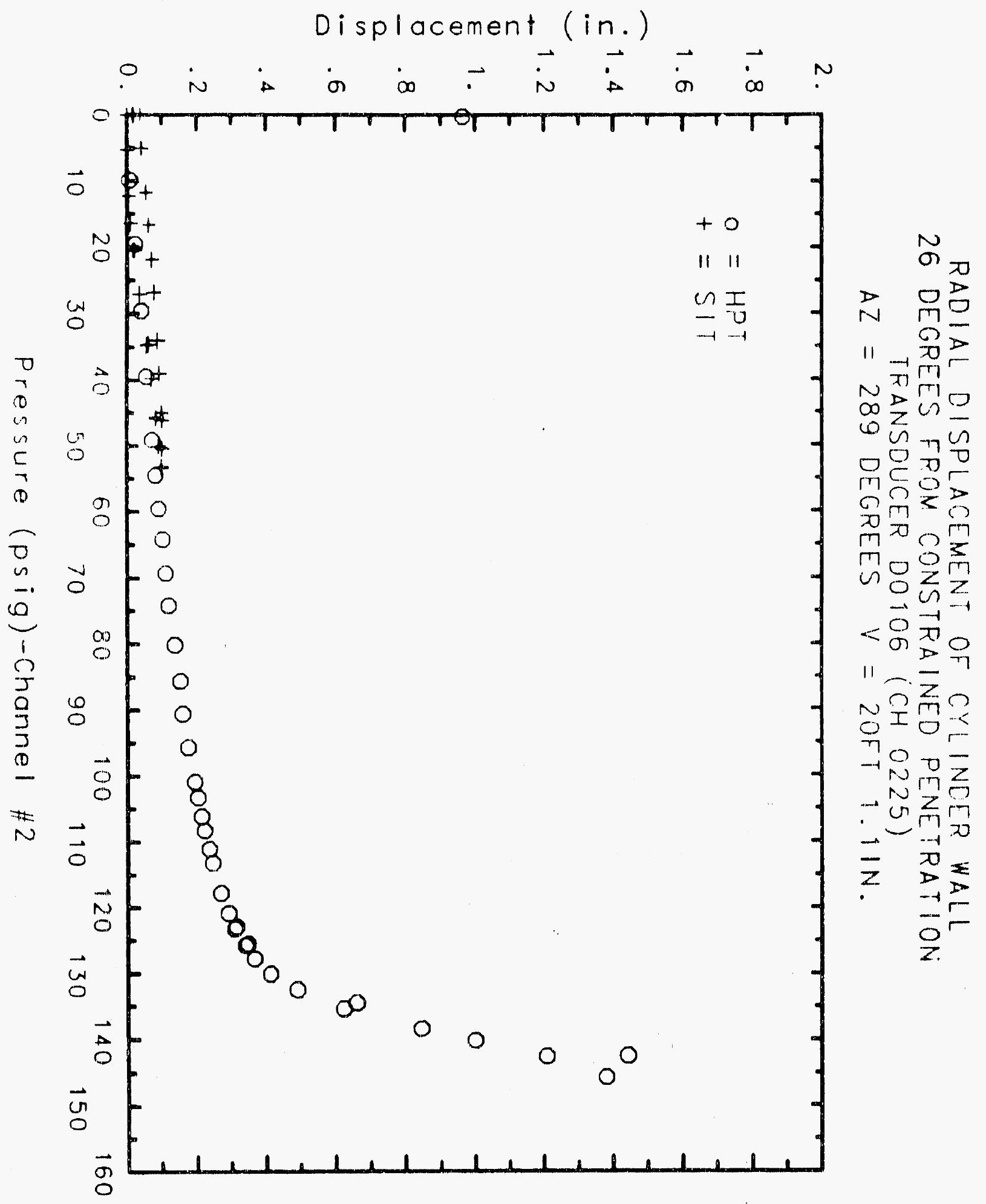




\section{Reinforced Concrete Test Data}

\section{Potentiometer Channel 226}

Structural INTEgrity Test

\begin{tabular}{|c|c|c|c|}
\hline $\begin{array}{c}\text { Pressure } \\
\text { (psig) } \\
-0.05 \\
5.33 \\
12.31 \\
16.44 \\
20.51 \\
20.46 \\
20.47 \\
27.05 \\
34.69 \\
34.53 \\
34.53 \\
39.70 \\
45.90 \\
45.65 \\
45.62 \\
50.10 \\
53.47 \\
53.29 \\
53.21 \\
50.49 \\
46.14 \\
46.13 \\
44.9 ! \\
38.98 \\
33.99 \\
33.96 \\
26.83 \\
21.88 \\
21.90 \\
16.69 \\
11.74 \\
11.77 \\
5.05 \\
0.02 \\
-0.04 \\
-0.02 \\
-0.02 \\
0.02 \\
\end{array}$ & $\begin{array}{c}\text { Displacement } \\
\text { (inches) } \\
-0.0001 \\
0.0025 \\
0.0108 \\
0.0138 \\
0.0190 \\
0.0226 \\
0.0241 \\
0.0379 \\
0.0545 \\
0.0581 \\
0.0593 \\
0.0721 \\
0.0859 \\
0.0870 \\
0.0868 \\
0.0912 \\
0.0970 \\
0.0977 \\
0.0976 \\
0.1002 \\
0.1004 \\
0.1002 \\
0.0984 \\
0.0924 \\
0.0876 \\
0.0877 \\
0.0760 \\
0.0715 \\
0.0718 \\
0.0594 \\
0.0541 \\
0.0538 \\
0.0420 \\
0.0403 \\
0.0319 \\
0.0318 \\
0.0250 \\
0.0232 \\
0.030\end{array}$ & $\begin{array}{c}\text { Pressure } \\
(p s i g) \\
9.89 \\
19.55 \\
29.57 \\
39.42 \\
49.16 \\
54.50 \\
59.57 \\
64.20 \\
69.32 \\
74.16 \\
80.16 \\
85.61 \\
90.58 \\
95.69 \\
100.92 \\
103.25 \\
106.11 \\
108.31 \\
111.08 \\
113.24 \\
117.82 \\
120.92 \\
123.28 \\
122.97 \\
125.82 \\
125.60 \\
127.84 \\
130.19 \\
132.53 \\
135.33 \\
134.42 \\
138.35 \\
140.16 \\
142.63 \\
145.78 \\
142.52 \\
0.22\end{array}$ & $\begin{array}{c}\text { Displacement } \\
\text { (inches) } \\
0.0106 \\
0.0236 \\
0.0356 \\
0.0523 \\
0.0688 \\
0.0760 \\
0.0852 \\
0.0953 \\
0.1021 \\
0.1068 \\
0.1250 \\
0.1383 \\
0.1464 \\
0.1596 \\
0.1735 \\
0.1807 \\
0.1882 \\
0.1975 \\
0.2072 \\
0.2138 \\
0.2303 \\
0.2452 \\
0.2564 \\
0.2595 \\
0.2776 \\
0.2845 \\
0.2951 \\
0.3227 \\
0.3730 \\
0.4620 \\
0.4912 \\
0.6435 \\
0.7701 \\
0.9453 \\
1.1068 \\
1.1687 \\
0.5954\end{array}$ \\
\hline
\end{tabular}

\section{High Pressure test}

splacement

0.0236

0.0356

0.0523

0.0688

0.0760

0.1021

0.1068

0.1250

0.1383

0.1464

0.1735

0.1807

0.1882

0.1975

0.2072

0.2138

0.2303

0.2564

0.2595

0.2776

0.2845

0.2951

0.3227

0.4620

0.4912

0.6435

0.7701

0.9453

1.1068

1.1687

0.5954 


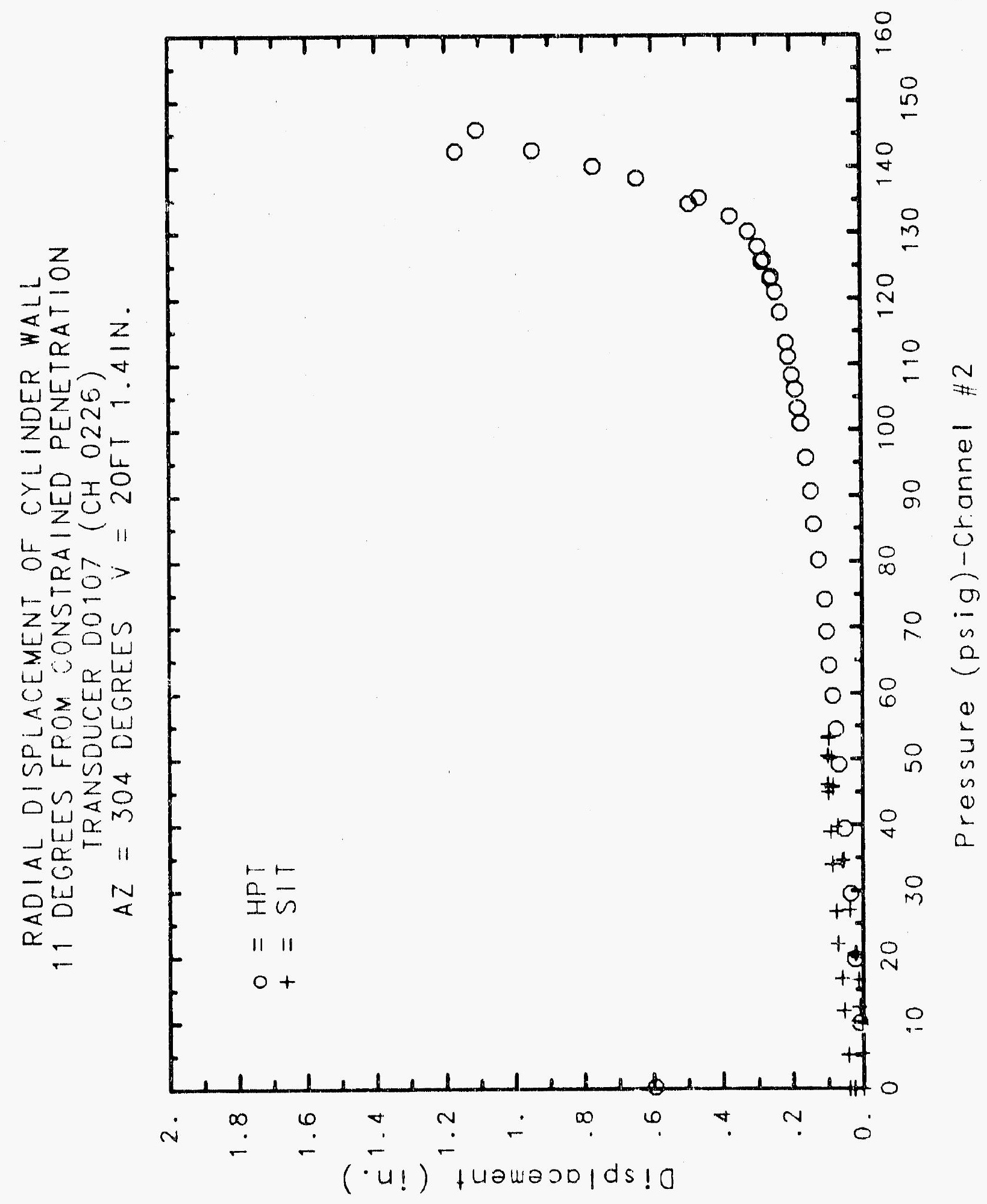




\section{Reinforced Concrete Test Data}

Potentiometer Chiannel 227

STRUCTURAL INTEGRITY TEST

\begin{tabular}{c}
\hline \\
Pressure \\
(psig) \\
-0.05 \\
5.33 \\
12.31 \\
16.44 \\
20.51 \\
20.46 \\
20.47 \\
27.05 \\
34.69 \\
34.53 \\
34.53 \\
39.70 \\
45.90 \\
45.65 \\
45.62 \\
50.10 \\
53.47 \\
53.29 \\
53.21 \\
50.49 \\
46.14 \\
46.13 \\
44.97 \\
38.98 \\
33.99 \\
33.96 \\
26.83 \\
21.88 \\
21.90 \\
16.69 \\
11.74 \\
11.77 \\
5.05 \\
0.02 \\
-0.04 \\
-0.02 \\
-0.02 \\
0.02 \\
\\
\hline
\end{tabular}

Displacement

(inches)

0.0001

$-0.0012$

0.0061

0.0096

0.0151

0.0179

0.0190

0.0348

0.0515

0.0567

0.0579

0.0665

0.0803

0.0816

0.0815

0.0880

0.0940

0.0944

0.0944

0.0961

0.0963

0.0964

0.0957

0.0907

0.0844

0.0845

0.0734

0.0672

0.0674

0.0586

0.0512

0.0510

0.0398

0.0383

0.0268

0.0271

0.0229

0.0215

\section{High Pressure test}

Pressure

(psig)

9.89

19.55

29.57

39.42

49.16

54.50

59.57

64.20

69.32

.4 .16

bu่. 16

85.61

90.58

95.69

100.92

103.25

106.11

108.31

111.08

113.24

117.83

120.92

123.28

122.97

125.82

125.60

127.84

130.19

132.53

135.33

134.42

138.35

140.16

142.63

145.78

142.52

0.22
Displacement

(inches)

0.0072

0.0229

0.0361

0.0508

0.0679

0.0765

0.0827

0.0947

0.1016

0.1070

0.1246

0.1404

0.1508

0.1637

0.1817

0.1903

0.1979

0.2048

0.2174

0.2230

0.2407

0.2586

0.2708

0.2748

0.2920

0.3022

0.3110

0.3414

0.3880

0.4786

0.5098

0.6522

0.7751

0.955 ?

1.1179

1.1848

0.8572 


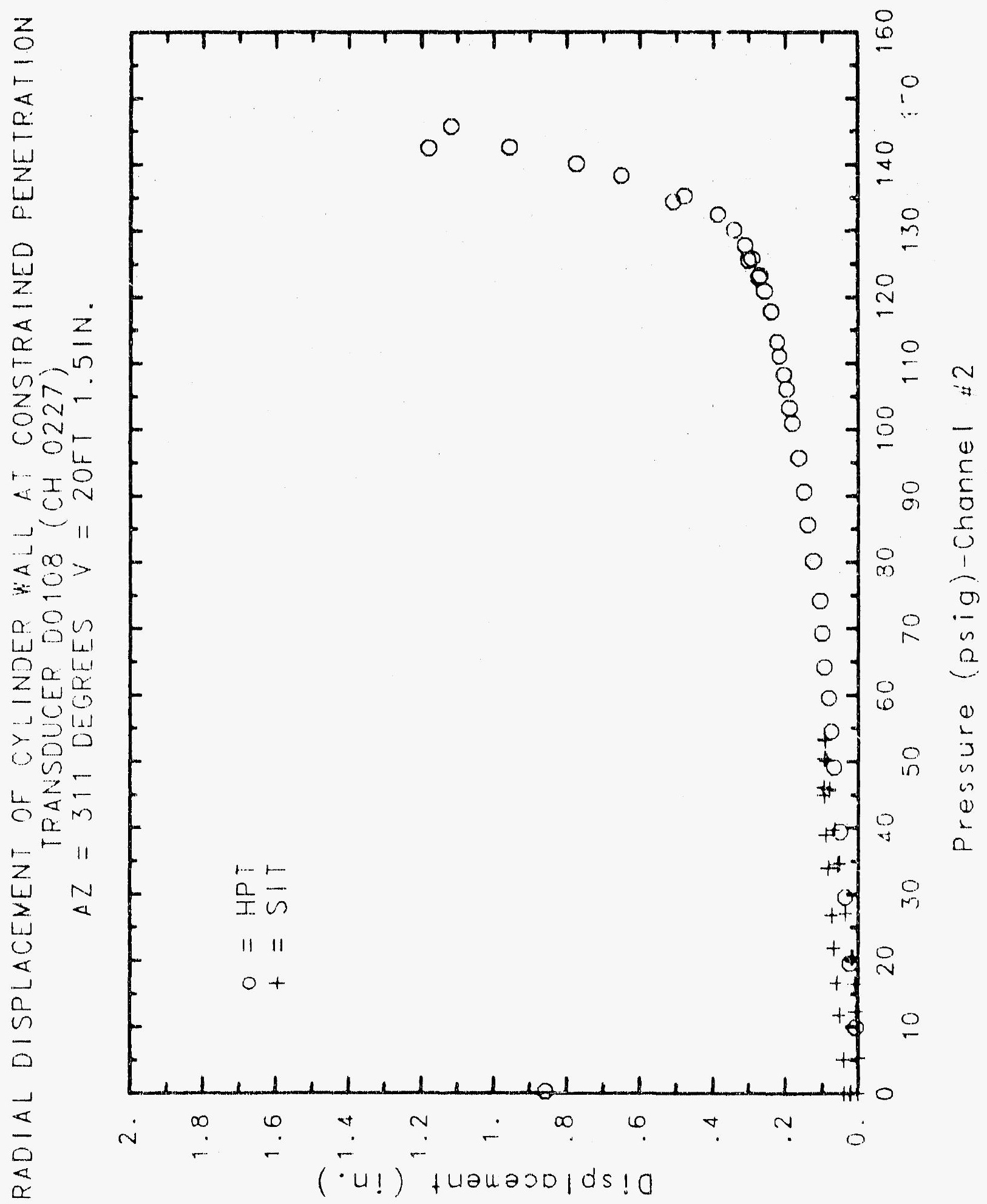




\section{Reinforced Concrete Test Data}

Potentiometer Channel 228

Structural Integrity Test

\begin{tabular}{cc}
\hline & \\
Pressure & Displacement \\
(psig) & (inches) \\
-0.05 & -0.0007 \\
5.33 & 0.0021 \\
12.31 & 0.0049 \\
16.44 & 0.0098 \\
20.51 & 0.0165 \\
20.46 & 0.0188 \\
20.47 & 0.0197 \\
27.05 & 0.0342 \\
34.69 & 0.0520 \\
34.53 & 0.0556 \\
34.53 & 0.0566 \\
39.70 & 0.0659 \\
45.90 & 0.0776 \\
45.65 & 0.0783 \\
45.62 & 0.0783 \\
50.10 & 0.0858 \\
53.47 & 0.0917 \\
53.29 & 0.0919 \\
53.21 & 0.0918 \\
50.49 & 0.0939 \\
46.14 & 0.0940 \\
46.13 & 0.0940 \\
44.97 & 0.0950 \\
38.98 & 0.0921 \\
33.99 & 0.0881 \\
33.96 & 0.0880 \\
26.83 & 0.0772 \\
21.88 & 0.0716 \\
21.90 & 0.0719 \\
16.69 & 0.0619 \\
11.74 & 0.0539 \\
11.79 & 0.0537 \\
5.05 & 0.0407 \\
0.02 & 0.0391 \\
-0.04 & 0.0326 \\
-0.02 & 0.0330 \\
-0.02 & 0.0340 \\
0.02 & 0.0314 \\
& \\
\hline &
\end{tabular}

High Pressure test

\begin{tabular}{|c|c|}
\hline $\begin{array}{c}\text { Pressure } \\
\text { (psig) } \\
9.89 \\
19.55 \\
29.57 \\
39.42 \\
49.16 \\
54.50 \\
59.57 \\
64.20 \\
69.32 \\
74.16 \\
80.16 \\
85.61 \\
90.58 \\
95.69 \\
100.92 \\
103.25 \\
116.11 \\
108.31 \\
111.08 \\
11 . .24 \\
111.83 \\
120.92 \\
12328 \\
122.97 \\
125.32 \\
125.60 \\
127.81 \\
130.10 \\
132.52 \\
135.33 \\
134.42 \\
138.35 \\
140.16 \\
142.63 \\
145.78 \\
142.52 \\
0.22\end{array}$ & $\begin{array}{c}\text { Displacement } \\
\text { (inches) } \\
-0.0003 \\
0.0128 \\
0.0273 \\
0.0430 \\
0.0585 \\
0.0584 \\
0.0676 \\
0.0807 \\
0.0890 \\
0.0978 \\
0.1139 \\
0.1267 \\
0.1409 \\
0.1553 \\
0.1729 \\
0.1793 \\
0.1373 \\
0.1939 \\
0.2039 \\
0.2099 \\
0.2254 \\
0.2424 \\
0.2569 \\
0.2600 \\
0.2816 \\
0.2900 \\
0.3075 \\
0.3471 \\
0.4176 \\
0.5498 \\
0.5875 \\
0.7774 \\
0.9256 \\
1.1316 \\
1.2988 \\
1.3529 \\
0.9855\end{array}$ \\
\hline
\end{tabular}




\section{IMAGE EVALUATION \\ TEST TARGET (MT-3)}
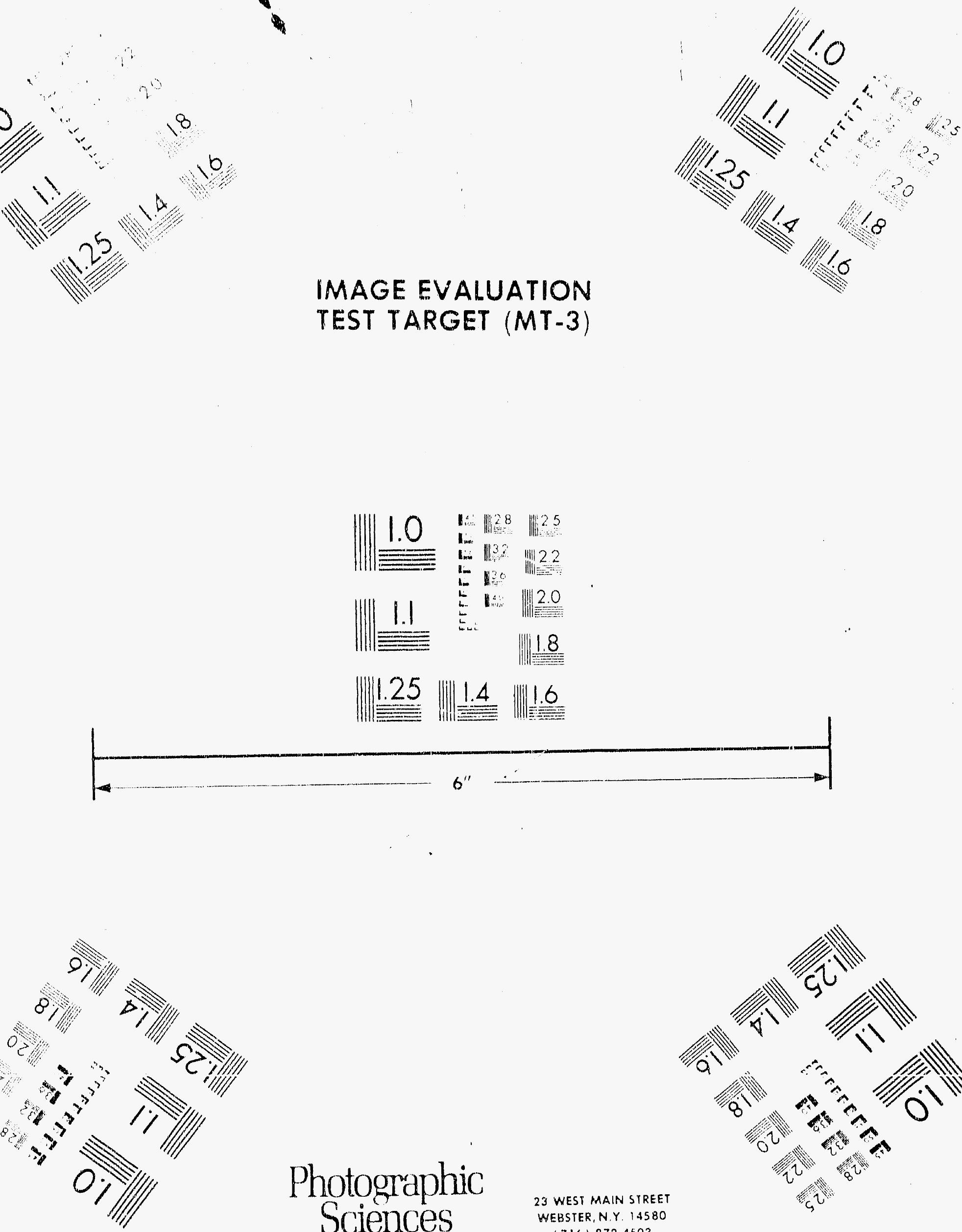

Photographic Sciences Corporation
23 WEST MAIN STREET WEBSTER, N.Y, 14580 (716) 872.4503

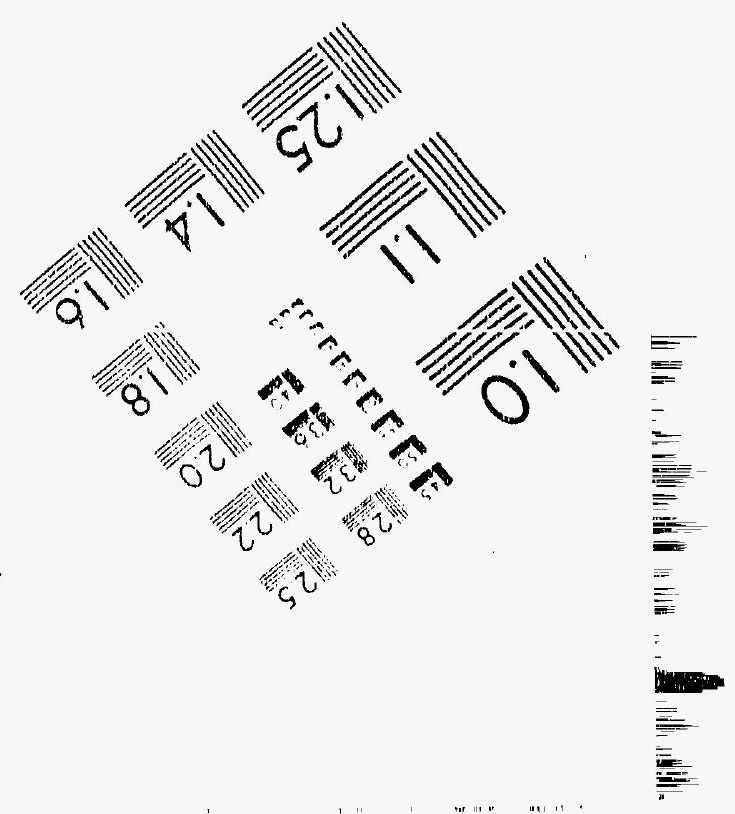




\section{SECURITY CLASSIFICATION}

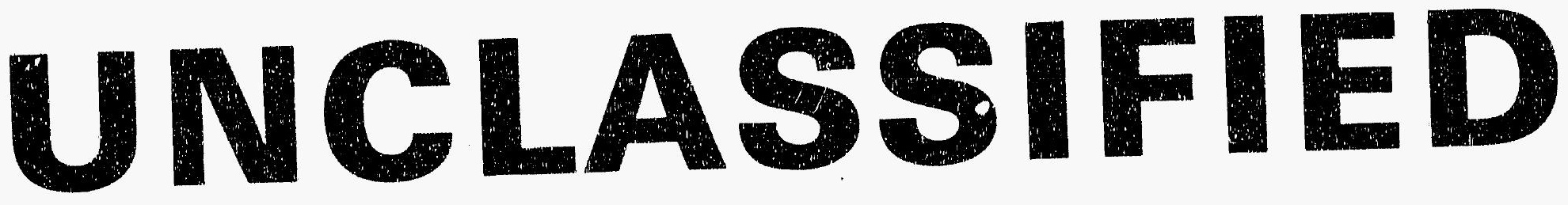

\section{DATE OF MICROFILMING $1-6-92$}

\section{MICROFILMED BY - lerer llard}

SANDIA NATIONAL LABS 


\section{THIS DOCUMENT FILMED}

AT

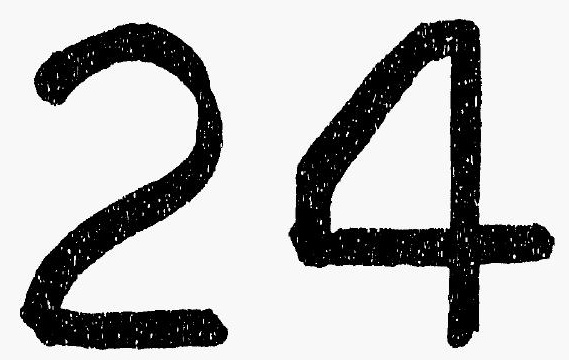

REDUCTION 


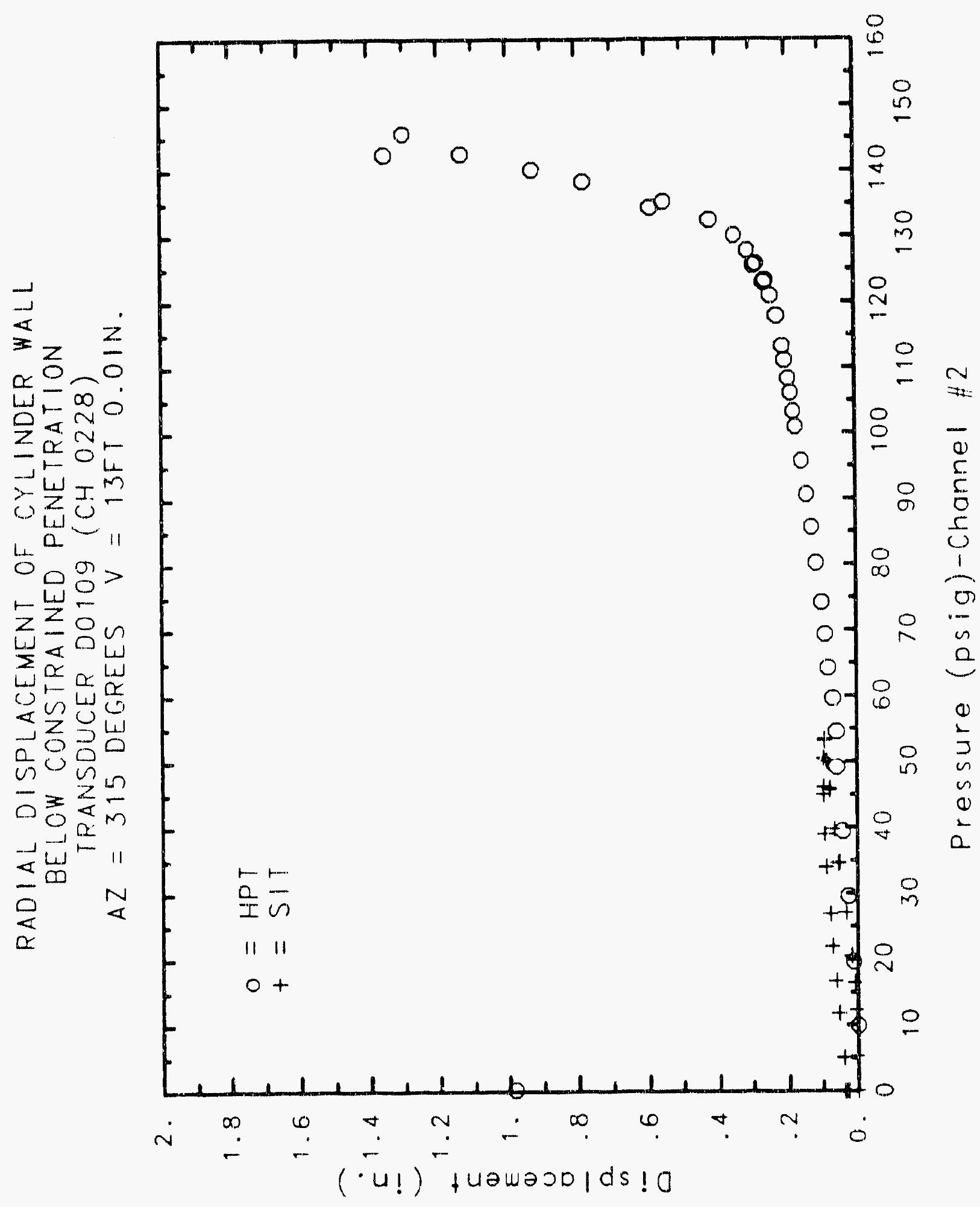

C- 101 


\section{Reinforced Concrete Test Data}

Potentiometer Channel 229

Structural INTEgRITY TEST

\begin{tabular}{|c|c|c|c|}
\hline $\begin{array}{c}\text { Pressure } \\
\text { (psig) } \\
-0.05 \\
5.33 \\
12.31 \\
16.44 \\
20.51 \\
20.46 \\
20.47 \\
27.05 \\
34.69 \\
34.53 \\
34.53 \\
39.70 \\
45.90 \\
45.65 \\
45.62 \\
50.10 \\
53.47 \\
53.29 \\
53.21 \\
50.49 \\
46.14 \\
46.13 \\
44.97 \\
38.98 \\
33.39 \\
33.96 \\
26.83 \\
21.88 \\
21.90 \\
16.69 \\
11.74 \\
11.77 \\
5.05 \\
0.02 \\
-0.04 \\
-0.02 \\
-0.02 \\
0.02\end{array}$ & $\begin{array}{c}\text { Displacement } \\
\text { (inches) } \\
0.0022 \\
0.0061 \\
0.0081 \\
0.0127 \\
0.0200 \\
0.0222 \\
0.0234 \\
0.0378 \\
0.0548 \\
0.0590 \\
0.0600 \\
0.0686 \\
0.0804 \\
0.0815 \\
0.0815 \\
0.0883 \\
0.0941 \\
0.0945 \\
0.0950 \\
0.0965 \\
0.0965 \\
0.0964 \\
0.0969 \\
0.0962 \\
0.0911 \\
0.0912 \\
0.0790 \\
0.0750 \\
0.0754 \\
0.0656 \\
0.0591 \\
0.0589 \\
0.0466 \\
0.0444 \\
0.0366 \\
0.0369 \\
0.0305 \\
0.0333\end{array}$ & $\begin{array}{c}\text { Pressure } \\
(\text { psig) } \\
9.89 \\
19.55 \\
29.57 \\
39.42 \\
49.16 \\
54.50 \\
59.57 \\
64.20 \\
69.32 \\
74.16 \\
80.16 \\
85.61 \\
90.58 \\
95.69 \\
100.92 \\
103.25 \\
106.11 \\
108.31 \\
111.08 \\
113.24 \\
117.83 \\
120.92 \\
123.28 \\
122.97 \\
125.82 \\
125.60 \\
127.84 \\
130.19 \\
132.53 \\
135.33 \\
134.42 \\
138.35 \\
140.16 \\
142.63 \\
145.78 \\
142.52 \\
0.22\end{array}$ & $\begin{array}{c}\text { Displacement } \\
\text { (inches) } \\
\text { o.0061 } \\
0.0108 \\
0.0264 \\
0.0429 \\
0.0584 \\
0.0600 \\
0.0712 \\
0.0802 \\
0.0900 \\
0.0970 \\
0.1014 \\
0.1150 \\
0.1264 \\
0.1471 \\
0.1642 \\
0.1724 \\
0.1800 \\
0.1857 \\
0.1973 \\
0.2035 \\
0.2204 \\
0.2359 \\
0.2481 \\
0.2537 \\
0.2544 \\
0.2570 \\
0.2782 \\
0.3170 \\
0.3731 \\
0.4920 \\
0.5312 \\
0.6815 \\
0.8322 \\
1.0152 \\
1.1946 \\
1.2654 \\
0.9011\end{array}$ \\
\hline
\end{tabular}

\section{High Pressure test}

placement

(inches)

0.0108

0.0264

0.0429

0.0584

0.0802

0.0900

0.0970

0.1014

0.1642

0.1724

0.1800

0.2035

0.2204

0.2359

0.2481

0.2570

0.2782

0.3170

0.3731

0.4920

0.5312

0.8322

1.0152

1.1946

1.2654

0.9011 


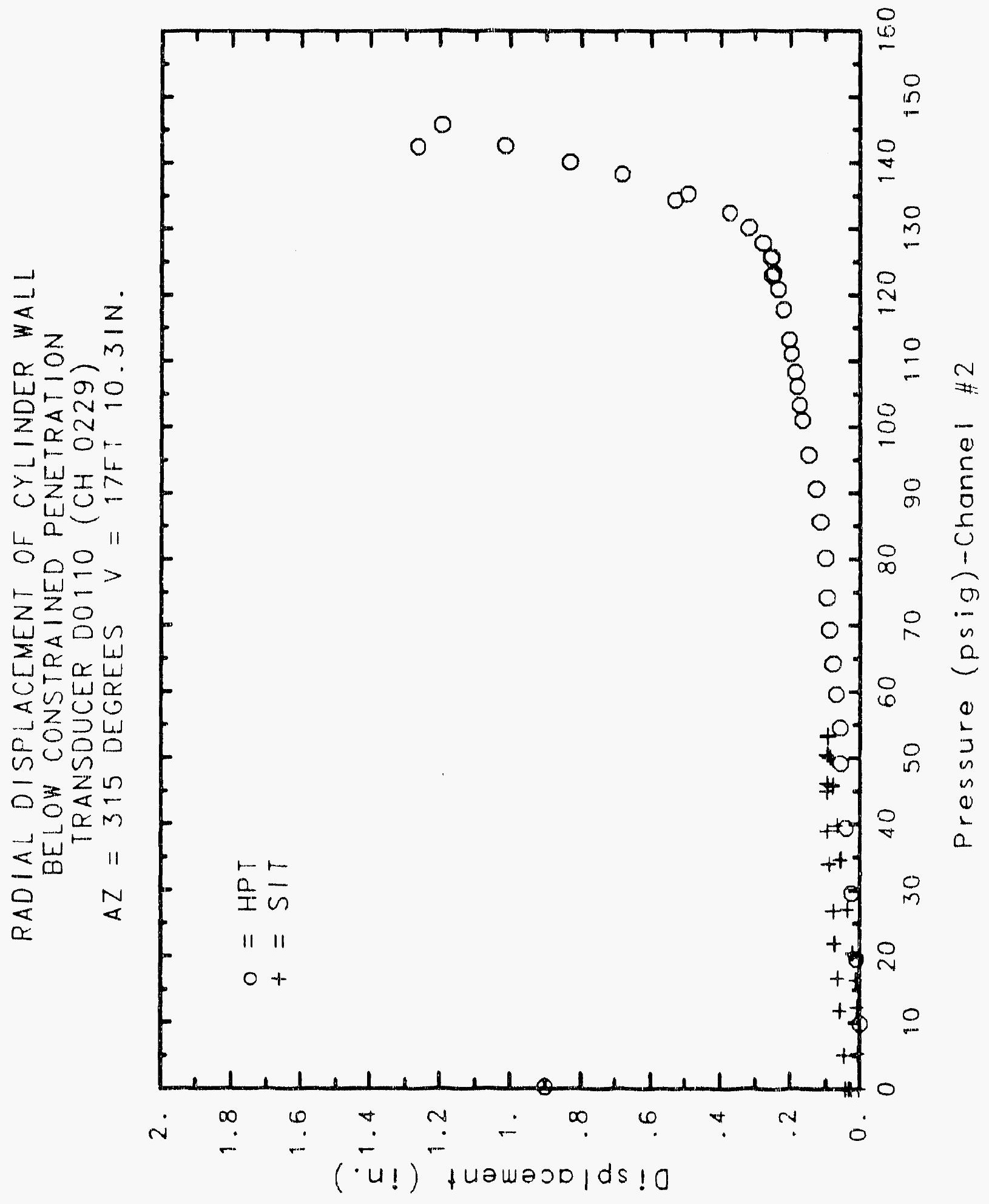




\section{Reinforced Concrete Test Data}

Potentiometer Channel 230

Structural IntEgrity TEST

\begin{tabular}{|c|c|c|c|}
\hline $\begin{array}{l}\text { Pressure } \\
\text { (psig) } \\
-0.05 \\
5.33 \\
12.31 \\
16.44 \\
20.51 \\
20.46 \\
20.47 \\
27.05 \\
34.69 \\
34.53 \\
34.53 \\
39.70 \\
45.90 \\
45.65 \\
45.62 \\
50.10 \\
53.47 \\
53.29 \\
53.21 \\
50.49 \\
46.14 \\
46.13 \\
44.97 \\
38.98 \\
33.99 \\
33.96 \\
26.83 \\
21.88 \\
21.90 \\
16.69 \\
11.74 \\
11.77 \\
5.05 \\
0.02 \\
-0.04 \\
-0.02 \\
-0.02 \\
0.02\end{array}$ & $\begin{array}{c}\text { Displacement } \\
\text { (inches) } \\
-0.0006 \\
0.0006 \\
0.0016 \\
0.0017 \\
0.0014 \\
0.0011 \\
0.0011 \\
0.0014 \\
0.0011 \\
0.0012 \\
0.0014 \\
0.0016 \\
0.0014 \\
0.0015 \\
0.0016 \\
0.0017 \\
0.0018 \\
0.0020 \\
0.0020 \\
0.0021 \\
0.0020 \\
0.0020 \\
0.0011 \\
0.0011 \\
0.0011 \\
0.0008 \\
0.0007 \\
0.0007 \\
0.0007 \\
0.0007 \\
0.0008 \\
0.0010 \\
0.0012 \\
0.0015 \\
0.0013 \\
-0.0003 \\
0.0002 \\
0.0012 \\
0.0\end{array}$ & $\begin{array}{c}\text { Pressure } \\
\text { (psig) } \\
9.89 \\
19.55 \\
29.57 \\
39.42 \\
49.16 \\
54.50 \\
59.57 \\
64.20 \\
69.32 \\
74.16 \\
80.16 \\
85.61 \\
90.58 \\
95.69 \\
100.92 \\
103.25 \\
106.11 \\
108.31 \\
111.08 \\
113.24 \\
117.83 \\
120.92 \\
123.28 \\
122.97 \\
125.82 \\
125.60 \\
127.84 \\
130.19 \\
132.53 \\
135.33 \\
134.42 \\
138.35 \\
140.16 \\
142.63 \\
145.78 \\
142.52 \\
0.22\end{array}$ & $\begin{array}{c}\text { Displacement } \\
\text { (inches) } \\
-0.0013 \\
-0.0012 \\
-0.0011 \\
-0.0011 \\
-0.0009 \\
-0.0044 \\
-0.0040 \\
-0.0030 \\
-0.00<8 \\
-0.0017 \\
-0.0020 \\
-0.0020 \\
-0.0019 \\
-0.0016 \\
-0.0013 \\
-0.0013 \\
-0.0012 \\
-0.0011 \\
-0.0012 \\
-0.0011 \\
-0.0010 \\
-0.0008 \\
-0.0005 \\
-0.0003 \\
-0.0001 \\
-0.0004 \\
-0.0003 \\
-0.0003 \\
-0.0001 \\
0.0000 \\
0.0000 \\
0.0002 \\
0.0001 \\
-0.0001 \\
-0.0002 \\
-0.0007 \\
-0.0012\end{array}$ \\
\hline
\end{tabular}

\section{High Pressure test}

placement

$-0.0012$

$-0.0009$

$-0.0044$

$-0.0040$

$-0.0030$

0.0019

$-0.0016$

0.0013

0.0011

$-0.0010$

$-0.0008$

$-0.0005$

$-0.0004$

$-0.0003$

$-0.0003$

$-0.0001$

0.0000

0.0002

$-0.0002$

$-0.0007$

$-0.0012$ 


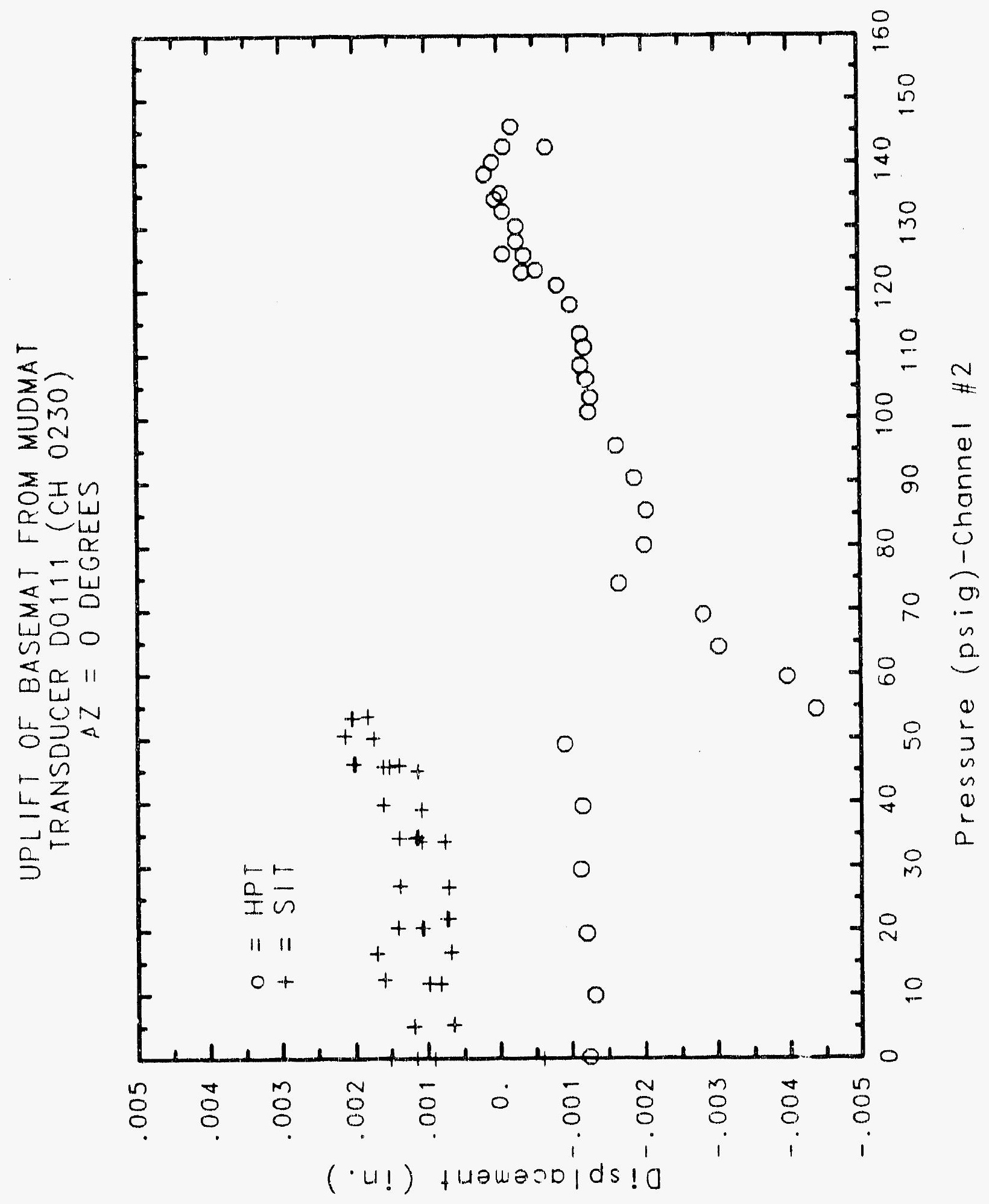




\section{Reinforced Concrete Test Data}

Potentiometer Channel 231

StRuctural IntEgrity TEST

\begin{tabular}{|c|c|c|c|}
\hline $\begin{array}{c}\text { Pressure } \\
\text { (psig) } \\
-0.05 \\
5.33 \\
12.31 \\
16.44 \\
20.51 \\
20.46 \\
20.47 \\
27.05 \\
34.69 \\
34.53 \\
34.53 \\
39.70 \\
45.90 \\
45.65 \\
45.62 \\
50.10 \\
53.47 \\
53.29 \\
53.21 \\
50.49 \\
46.14 \\
46.13 \\
44.97 \\
38.98 \\
33.99 \\
33.96 \\
26.83 \\
21.88 \\
21.90 \\
16.69 \\
11.74 \\
11.77 \\
5.05 \\
0.02 \\
-0.04 \\
-0.02 \\
-0.02 \\
0.02\end{array}$ & $\begin{array}{c}\text { Displacement } \\
\text { (inches) } \\
0.0011 \\
0.0028 \\
0.0038 \\
0.0038 \\
0.0032 \\
0.0028 \\
0.0013 \\
0.0021 \\
0.0025 \\
0.0022 \\
0.0019 \\
0.0034 \\
0.0041 \\
0.0048 \\
0.0050 \\
0.0053 \\
0.0056 \\
0.0061 \\
0.0065 \\
0.0066 \\
0.0065 \\
0.0065 \\
0.0062 \\
0.0059 \\
0.0046 \\
0.0036 \\
0.0025 \\
0.0022 \\
0.0019 \\
0.0030 \\
0.0024 \\
0.0037 \\
0.0041 \\
0.0044 \\
0.0055 \\
0.0052 \\
0.0049 \\
0.0037\end{array}$ & $\begin{array}{c}\text { Pressure } \\
\text { (psig) } \\
9.89 \\
19.55 \\
29.57 \\
39.42 \\
49.16 \\
54.50 \\
59.57 \\
64.20 \\
69.32 \\
74.16 \\
80.16 \\
85.61 \\
90.58 \\
95.69 \\
100.92 \\
103.25 \\
106.11 \\
108.31 \\
111.08 \\
113.24 \\
117.83 \\
120.92 \\
123.28 \\
122.91 \\
125.82 \\
125.60 \\
127.84 \\
130.19 \\
132.53 \\
135.33 \\
134.42 \\
138.35 \\
140.16 \\
142.63 \\
145.78 \\
142.52 \\
0.22\end{array}$ & $\begin{array}{c}\text { Displacement } \\
\text { (inches) } \\
-0.0033 \\
-0.0021 \\
-0.0010 \\
0.0005 \\
0.0008 \\
-0.0030 \\
-0.0007 \\
-0.0010 \\
0.0010 \\
0.0028 \\
-0.0022 \\
-0.0022 \\
-0.0010 \\
0.0018 \\
0.0031 \\
0.0033 \\
0.0034 \\
0.0034 \\
0.0038 \\
0.0040 \\
0.0167 \\
0.0205 \\
0.0206 \\
0.0210 \\
0.0132 \\
0.0112 \\
0.0142 \\
0.0172 \\
0.0231 \\
0.0292 \\
0.0310 \\
0.0225 \\
0.0301 \\
0.0477 \\
0.0774 \\
0.0859 \\
0.0845\end{array}$ \\
\hline
\end{tabular}

High pressure test

splacement

$-0.0033$

$-0.0021$

$-0.0010$

0.0005

0.0008

$-0.0010$

0.0010

0.0028

$-0.0022$

0.0018

0.0031

0.0033

0.0034

0.0034

0.0040

0.0167

0.0205

0.0206

0.0210

0.0112

0.0142

0.0172

0.0231

0.0292

0.0310

0.0301

0.0477

0.0774

0.0859

0.0845 


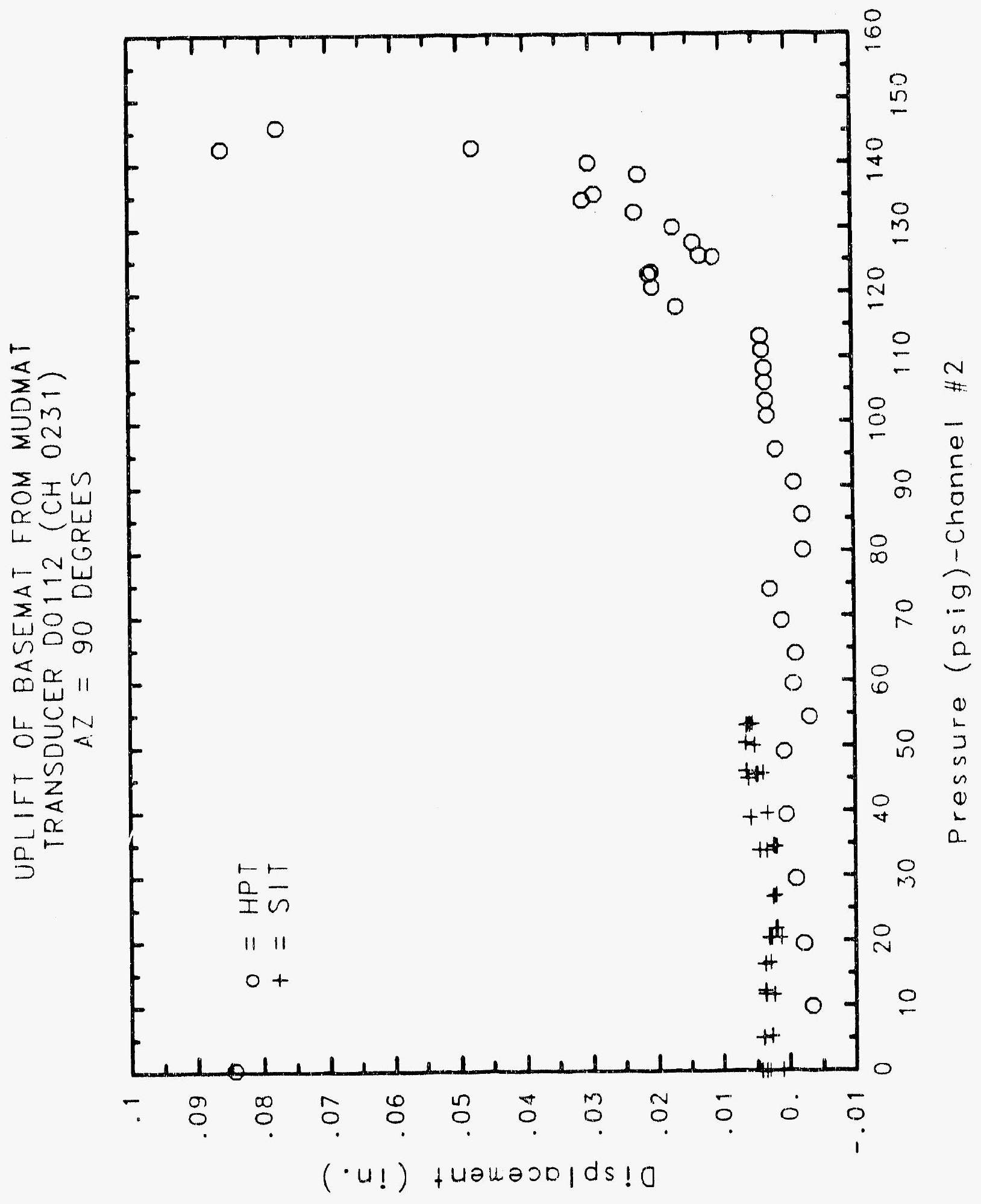




\section{Reinforced Concrete Test Data}

Potentiometer Channel 232

Structural Integrity Test

\begin{tabular}{|c|c|c|c|}
\hline $\begin{array}{c}\text { Pressure } \\
\text { (psig) } \\
-0.05 \\
5.33 \\
12.31 \\
16.44 \\
20.51 \\
20.46 \\
20.47 \\
27.05 \\
34.69 \\
34.53 \\
34.53 \\
39.70 \\
45.90 \\
45.65 \\
45.62 \\
50.10 \\
53.47 \\
53.29 \\
53.21 \\
50.49 \\
46.14 \\
46.13 \\
44.97 \\
38.98 \\
33.99 \\
33.96 \\
26.83 \\
21.88 \\
21.90 \\
16.69 \\
11.74 \\
11.77 \\
5.05 \\
0.02 \\
-0.04 \\
-0.02 \\
-0.02 \\
0.02\end{array}$ & $\begin{array}{c}\text { Displacement } \\
\text { (inches) } \\
-0.0002 \\
0.0009 \\
0.0011 \\
0.0013 \\
0.0011 \\
0.0010 \\
0.0010 \\
0.0011 \\
0.0011 \\
0.0011 \\
0.0011 \\
0.0016 \\
0.0020 \\
0.0025 \\
0.0026 \\
0.0029 \\
0.0031 \\
0.0036 \\
0.0037 \\
0.0038 \\
0.0037 \\
0.0037 \\
0.0023 \\
0.0023 \\
0.0022 \\
0.0021 \\
0.0017 \\
0.0014 \\
0.0012 \\
0.0011 \\
0.0010 \\
0.0011 \\
0.0011 \\
0.0013 \\
0.0061 \\
0.0007 \\
0.0008 \\
0.0009\end{array}$ & $\begin{array}{c}\text { Pressure } \\
\text { (psig) } \\
9.89 \\
19.55 \\
29.57 \\
39.42 \\
49.16 \\
54.50 \\
59.57 \\
64.20 \\
69.32 \\
74.16 \\
80.16 \\
85.61 \\
90.58 \\
95.69 \\
100.92 \\
103.25 \\
106.11 \\
108.31 \\
111.08 \\
113.24 \\
117.83 \\
120.92 \\
123.28 \\
122.97 \\
125.82 \\
125.60 \\
127.84 \\
130.19 \\
132.53 \\
135.33 \\
134.42 \\
138.35 \\
140.16 \\
142.63 \\
145.78 \\
142.52 \\
0.22\end{array}$ & $\begin{array}{c}\text { Displacement } \\
\text { (inches) } \\
-0.0006 \\
-0.0007 \\
-0.0004 \\
-0.0003 \\
-0.0001 \\
-0.0029 \\
-0.0024 \\
-0.0011 \\
-0.0001 \\
0.0014 \\
0.0011 \\
0.0011 \\
0.0011 \\
0.0012 \\
0.0015 \\
0.0015 \\
0.0015 \\
0.0016 \\
0.0015 \\
0.0015 \\
0.0016 \\
0.0017 \\
0.0021 \\
0.0023 \\
0.0025 \\
0.0023 \\
0.0023 \\
0.0022 \\
0.0023 \\
0.0023 \\
0.0023 \\
0.0025 \\
0.0025 \\
0.0023 \\
0.0022 \\
0.0018 \\
0.0008\end{array}$ \\
\hline
\end{tabular}

High Pressure Test

Pressure

(inches) $-0.0007$ $-0.0004$ $-0.0003$ $-0.0001$ $-0.0011$ 0.0014 0.0011 0.0012 0.0015 0.0015 0.0015 0.0016 0.0015 0.0017 0.0021 0.0023 0.0023 0.0023 0.0023 0.0025 0.0025 $\begin{array}{rl}42.52 & 0.0018 \\ 0.22 & 0.0008\end{array}$ 


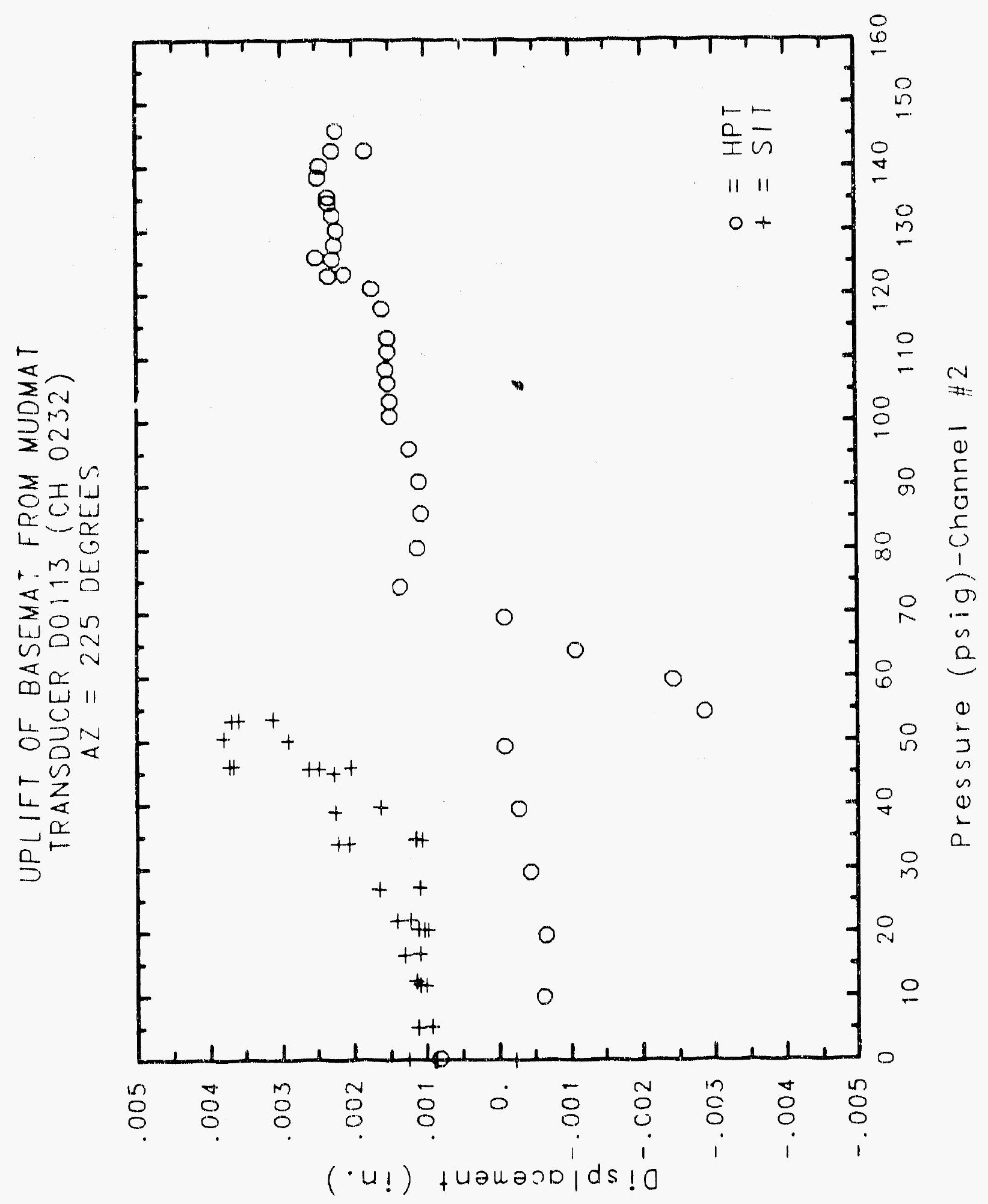




\section{Reinforced Concrete Test Data}

Potentiometer Channel. 233

Structural Integrity test

\begin{tabular}{|c|c|c|c|}
\hline $\begin{array}{c}\text { Pressure } \\
\text { (psig) } \\
-0.05 \\
5.33 \\
12.31 \\
16.44 \\
20.51 \\
20.46 \\
20.47 \\
27.05 \\
34.69 \\
34.53 \\
34.53 \\
39.70 \\
45.90 \\
45.65 \\
45.62 \\
50.10 \\
53.47 \\
53.29 \\
53.21 \\
50.49 \\
46.14 \\
46.13 \\
44.97 \\
38.98 \\
33.99 \\
33.96 \\
26.83 \\
21.88 \\
21.90 \\
16.69 \\
11.74 \\
11.77 \\
5.05 \\
0.02 \\
-0.04 \\
-0.02 \\
-0.02 \\
0.02\end{array}$ & $\begin{array}{c}\text { Displacement } \\
\text { (inches) } \\
0.0304 \\
0.0784 \\
0.1095 \\
0.1155 \\
0.1236 \\
0.1270 \\
0.1297 \\
0.1439 \\
0.1784 \\
0.1824 \\
0.1872 \\
0.2041 \\
0.2432 \\
0.2547 \\
0.2581 \\
0.3074 \\
0.3318 \\
0.3462 \\
0.3581 \\
0.3658 \\
0.3671 \\
0.3658 \\
0.6074 \\
0.6095 \\
0.6007 \\
0.6014 \\
0.5779 \\
0.5597 \\
0.5568 \\
0.5365 \\
0.5081 \\
0.5063 \\
0.4894 \\
0.4624 \\
0.4475 \\
0.4446 \\
0.4428 \\
0.4189 \\
0.45\end{array}$ & $\begin{array}{c}\text { Pressure } \\
(p s i g) \\
9.89 \\
19.55 \\
29.57 \\
39.42 \\
49.16 \\
54.50 \\
59.57 \\
64.20 \\
69.32 \\
74.16 \\
80.16 \\
85.61 \\
90.58 \\
95.69 \\
100.92 \\
103.25 \\
106.11 \\
108.31 \\
111.08 \\
113.24 \\
117.83 \\
120.92 \\
123.28 \\
122.97 \\
125.82 \\
125.60 \\
127.84 \\
130.19 \\
132.53 \\
135.33 \\
134.42 \\
139.35 \\
140.16 \\
142.63 \\
145.78 \\
142.52 \\
0.22\end{array}$ & $\begin{array}{c}\text { Displacement } \\
\text { (inches) } \\
-0.0892 \\
-0.0459 \\
0.0203 \\
0.1034 \\
0.1428 \\
0.0550 \\
0.1496 \\
0.1739 \\
0.2401 \\
0.3171 \\
0.2507 \\
0.3178 \\
0.4023 \\
0.5151 \\
0.6158 \\
0.6448 \\
0.6793 \\
0.7198 \\
0.7928 \\
0.8415 \\
0.9732 \\
1.0820 \\
1.1631 \\
1.2084 \\
1.1354 \\
1.1273 \\
1.2969 \\
1.5563 \\
1.9692 \\
2.5644 \\
2.7692 \\
3.2439 \\
4.0345 \\
4.8236 \\
5.7392 \\
6.1986 \\
4.9257\end{array}$ \\
\hline
\end{tabular}

High Pressure test

splacement

$-0.0459$

0.1034

0.1496

0.1739

0.2401

3178

0.4023

0.6448

0.6793

$(8415$

0.9732

1.2084

1.1354

1.1273

1.2969

2.5644

2.7692

3.2439

5.7392

6.1986

4.9257 


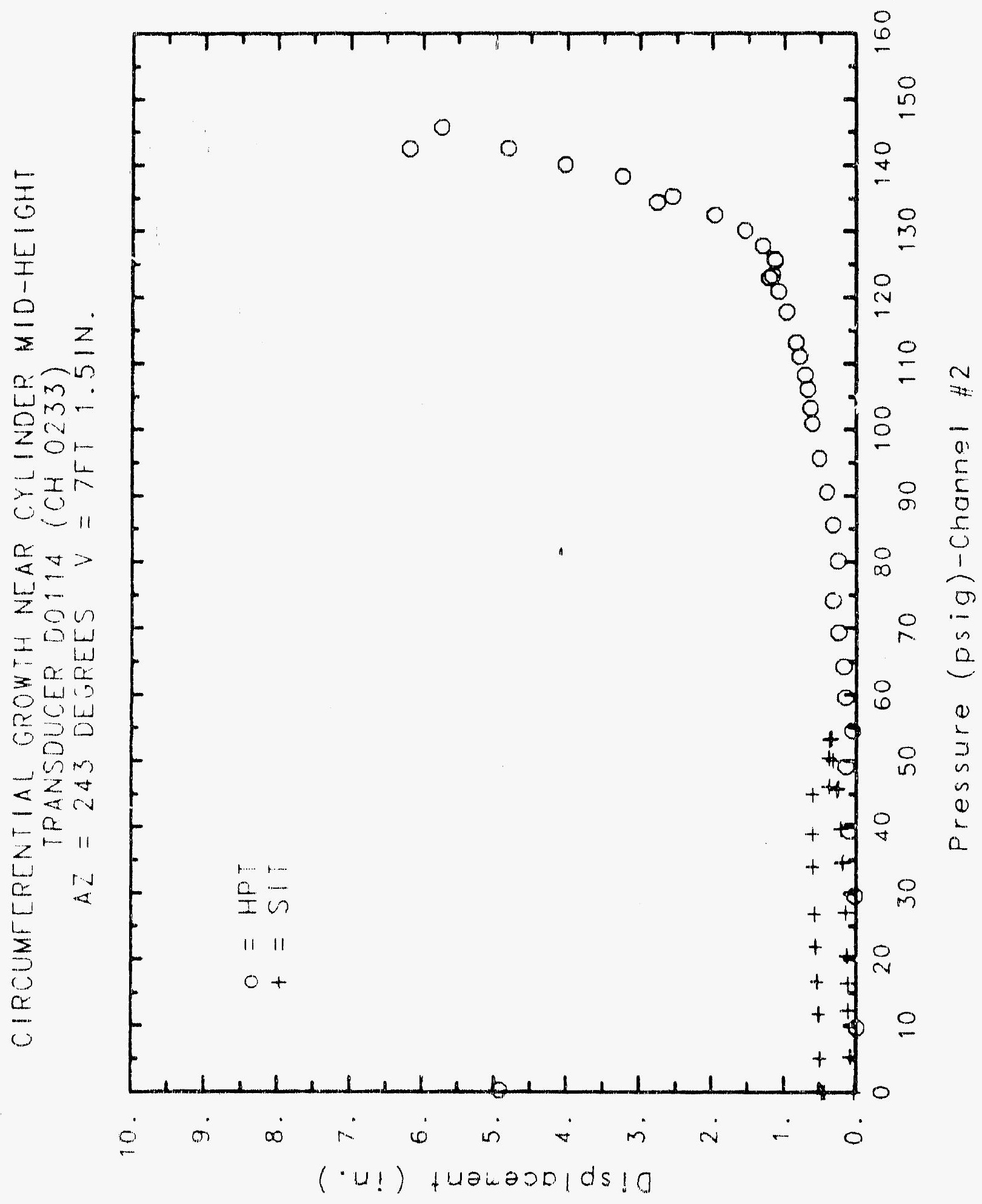




\section{Reinforced Concrete Test Data}

\begin{tabular}{|c|c|c|c|}
\hline \multirow[b]{2}{*}{ STRUCTURAL } & \multicolumn{2}{|c|}{ Channel 234} & \multirow[b]{2}{*}{ E TEST } \\
\hline & EGRITY TEST & HIGH PF & \\
\hline $\begin{array}{l}\text { Pressure } \\
\text { (psig) } \\
-0.05 \\
5.33 \\
12.31 \\
16.44 \\
20.51 \\
20.46 \\
20.47 \\
27.05 \\
34.69 \\
34.53 \\
34.53 \\
39.70 \\
45.90 \\
45.65 \\
45.62 \\
50.10 \\
53.47 \\
53.29 \\
53.21 \\
50.49 \\
46.14 \\
46.13 \\
44.97 \\
38.98 \\
33.99 \\
33.96 \\
26.83 \\
21.88 \\
21.90 \\
16.69 \\
11.74 \\
11.77 \\
5.05 \\
0.02 \\
-0.04 \\
-0.06 \\
-0.02 \\
0.02\end{array}$ & $\begin{array}{c}\text { Cisplacement } \\
\text { (inches) } \\
-0.0115 \\
0.0338 \\
0.0330 \\
0.0384 \\
0.0404 \\
0.0397 \\
0.0384 \\
0.0532 \\
0.0769 \\
0.0816 \\
0.0842 \\
0.1071 \\
0.1456 \\
0.1544 \\
0.1625 \\
0.1956 \\
0.2200 \\
0.2294 \\
0.2342 \\
0.2384 \\
0.2416 \\
0.2377 \\
0.2532 \\
0.2519 \\
0.2526 \\
0.2499 \\
0.2472 \\
0.2377 \\
0.2382 \\
0.2242 \\
0.2139 \\
0.2125 \\
0.2024 \\
0.1909 \\
0.1708 \\
0.1715 \\
0.1681 \\
0.1681\end{array}$ & $\begin{array}{c}\text { Pressure } \\
\text { (psig) } \\
9.89 \\
19.55 \\
29.57 \\
39.42 \\
49.16 \\
54.50 \\
59.57 \\
64.20 \\
69.32 \\
74.16 \\
80.16 \\
85.61 \\
90.58 \\
95.69 \\
100.92 \\
103.25 \\
106.11 \\
108.31 \\
111.08 \\
113.24 \\
117.83 \\
120.92 \\
123.28 \\
122.97 \\
125.82 \\
125.60 \\
127.84 \\
130.19 \\
132.53 \\
135.33 \\
134.42 \\
138.35 \\
140.16 \\
142.63 \\
145.78 \\
142.52 \\
0.22 \\
\\
\end{array}$ & $\begin{array}{c}\text { Displacement } \\
\text { (inches) } \\
-0.0319 \\
-0.0333 \\
-0.0123 \\
0.0181 \\
0.0492 \\
-0.0508 \\
-0.0130 \\
0.0546 \\
0.0931 \\
0.1526 \\
0.2424 \\
0.2850 \\
0.3404 \\
0.4147 \\
0.4965 \\
0.5336 \\
0.5749 \\
0.6059 \\
0.6728 \\
0.7323 \\
0.8465 \\
0.9370 \\
1.0478 \\
1.1242 \\
1.2499 \\
1.3005 \\
1.4046 \\
1.7363 \\
2.3404 \\
3.1708 \\
3.3904 \\
4.5984 \\
5.6240 \\
6.8876 \\
8.0936 \\
8.5800 \\
7.2975 \\
\end{array}$ \\
\hline
\end{tabular}




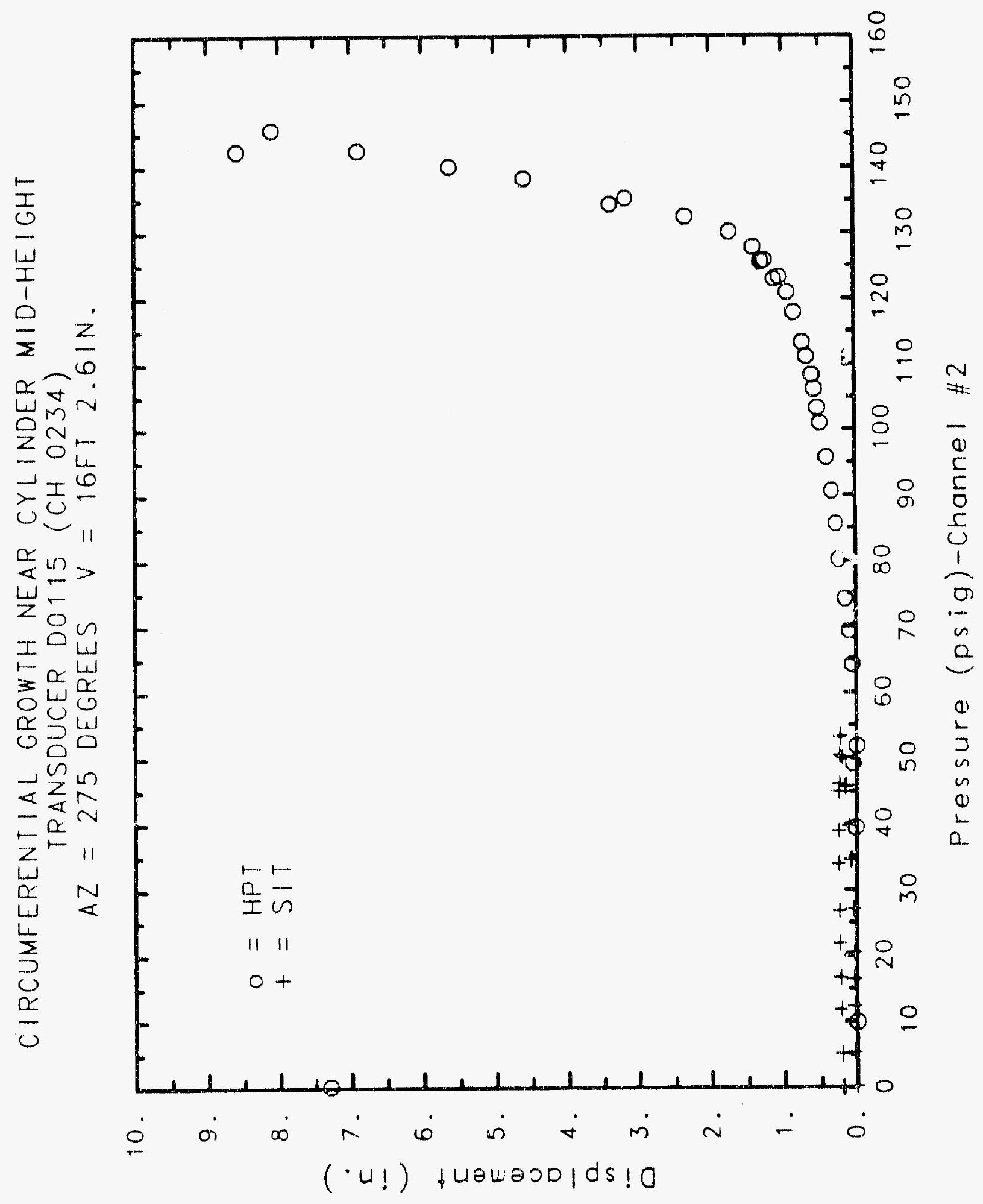

C. -11.3 


\section{Reinforcen Concrete Test Data}

Potentiometer Channel 235

Structural Integrity Test

\begin{tabular}{|c|c|c|c|}
\hline $\begin{array}{l}\text { Pressure } \\
\text { (psig) } \\
-0.05 \\
5.33 \\
12.31 \\
16.44 \\
20.51 \\
20.46 \\
20.47 \\
27.05 \\
34.69 \\
34.53 \\
34.53 \\
39.70 \\
45.90 \\
45.65 \\
45.62 \\
50.10 \\
53.47 \\
53.29 \\
53.21 \\
50.49 \\
46.14 \\
46.13 \\
44.97 \\
38.98 \\
33.99 \\
33.96 \\
26.83 \\
21.88 \\
21.90 \\
16.69 \\
11.74 \\
11.77 \\
5.05 \\
0.02 \\
-0.04 \\
-0.02 \\
-0.02 \\
0.02 \\
\end{array}$ & $\begin{array}{c}\text { Displacement } \\
\text { (inches) } \\
0.0000 \\
0.0002 \\
0.0005 \\
0.0009 \\
0.0010 \\
0.0010 \\
0.0012 \\
0.0011 \\
0.0010 \\
0.0010 \\
0.0011 \\
0.0009 \\
0.0007 \\
0.0004 \\
0.0004 \\
0.0002 \\
0.0001 \\
-0.0001 \\
-0.0001 \\
0.0005 \\
0.0008 \\
0.0008 \\
0.0017 \\
0.0038 \\
0.0036 \\
0.0038 \\
0.0053 \\
0.0051 \\
0.0051 \\
0.0056 \\
0.0048 \\
0.0046 \\
0.0022 \\
0.0021 \\
0.0028 \\
0.0030 \\
-0.0133 \\
-0.0054\end{array}$ & $\begin{array}{c}\text { Pressure } \\
\text { (psig) } \\
9.89 \\
19.55 \\
29.57 \\
39.42 \\
49.16 \\
54.50 \\
59.57 \\
64.20 \\
69.32 \\
74.16 \\
80.16 \\
85.61 \\
90.58 \\
95.69 \\
100.92 \\
103.25 \\
106.11 \\
108.31 \\
111.08 \\
113.24 \\
117.83 \\
120.92 \\
123.28 \\
122.97 \\
125.82 \\
125.60 \\
127.84 \\
130.19 \\
132.53 \\
135.33 \\
134.42 \\
138.35 \\
140.16 \\
142.63 \\
145.78 \\
142.52 \\
0.22\end{array}$ & $\begin{array}{l}\text { Displacement } \\
\text { (inches) } \\
-0.0130 \\
-0.0132 \\
-0.0130 \\
-0.0128 \\
-0.0127 \\
-0.0127 \\
-0.0126 \\
-0.0121 \\
-0.0107 \\
-0.0085 \\
-0.0090 \\
-0.0091 \\
-0.0090 \\
-0.0091 \\
-0.0090 \\
-0.0091 \\
-0.0091 \\
-0.0091 \\
-0.0091 \\
-0.0091 \\
-0.0091 \\
-0.0091 \\
-0.0090 \\
-0.0090 \\
-0.0083 \\
-0.0083 \\
-0.0082 \\
-0.0082 \\
-0.0081 \\
-0.0080 \\
-0.0079 \\
-0.0078 \\
-0.0079 \\
-0.0080 \\
-0.0081 \\
-0.0083 \\
-0.0057\end{array}$ \\
\hline
\end{tabular}

high Pressure test

placement

$-0.0132$

$-0.0128$

$-0.0127$

$-0.0127$

$-0.0107$

$-0.0085$

$-0.0090$

$-0.0091$

$-0.0090$

$-0.0091$

0.0091

$-0.0091$

0.009

$-0.0091$

$-0.0091$

$-0.0090$

$-0.0083$

$-0.0082$

0.0082

$-0.0081$

$-0.0078$

$-0.0079$

$-0.0080$

$-0.0081$

0.0083 


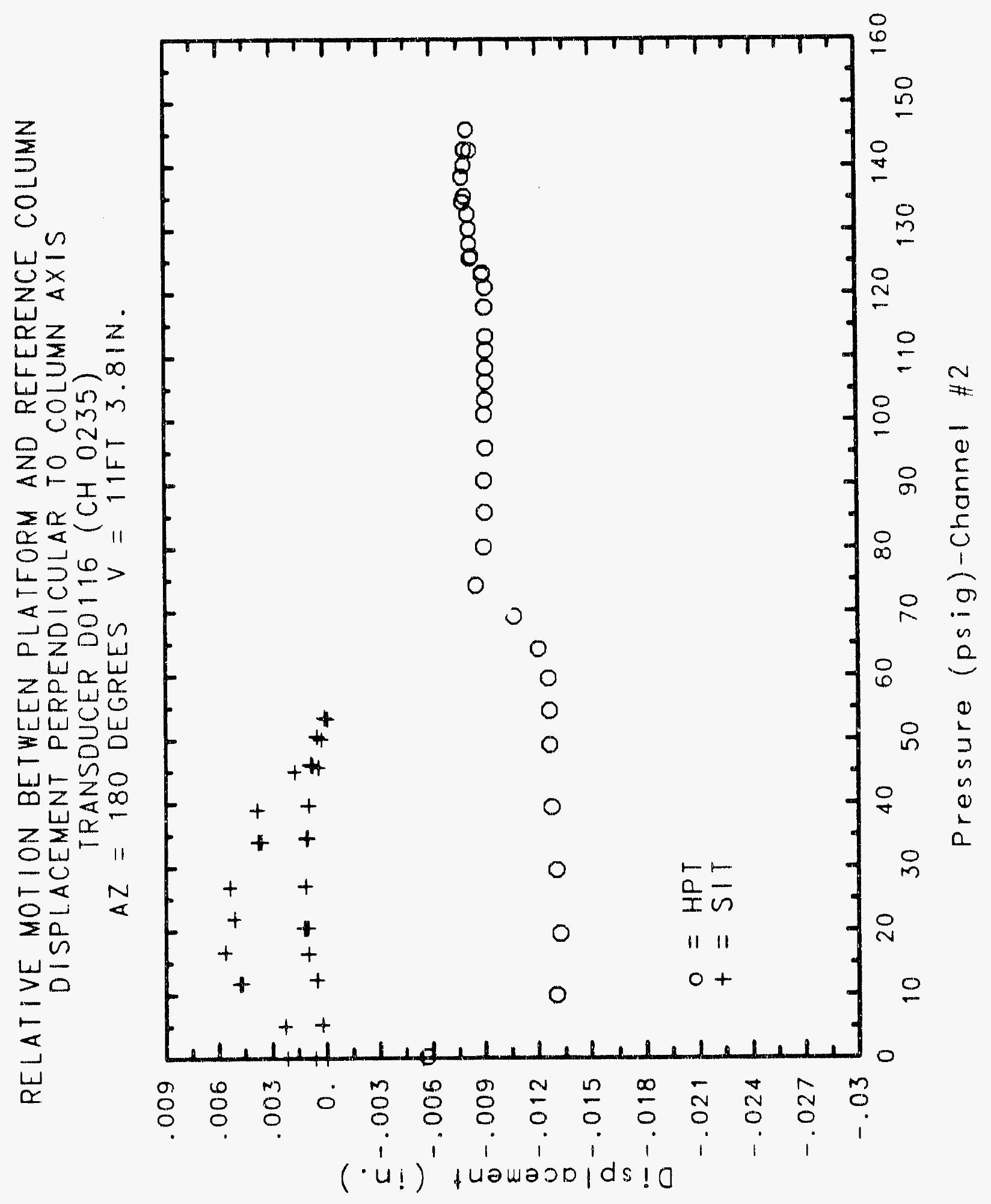




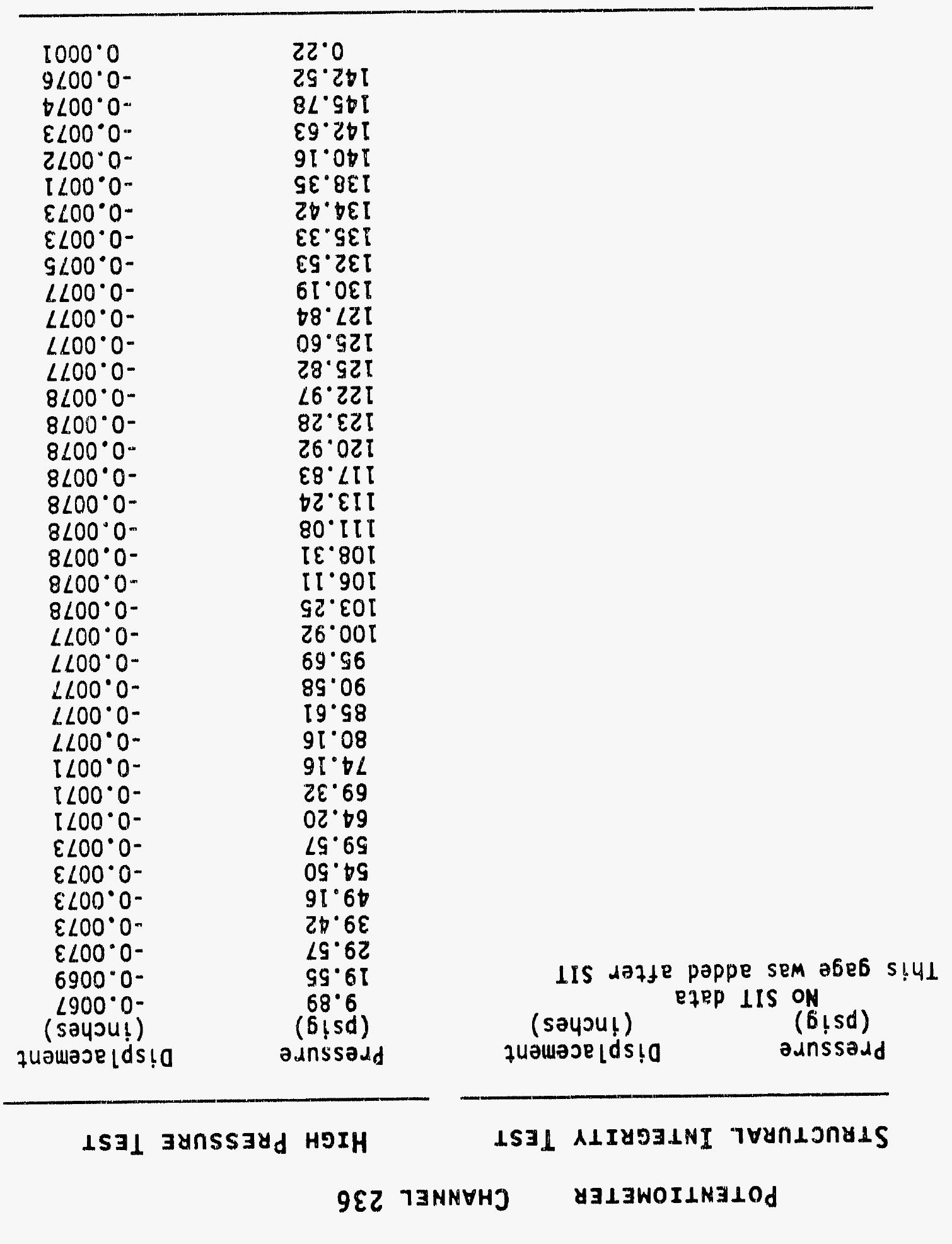

\section{$\forall \perp \forall O \perp S 3 \perp$ BLIVJNOJ 0JJYOANIJY}




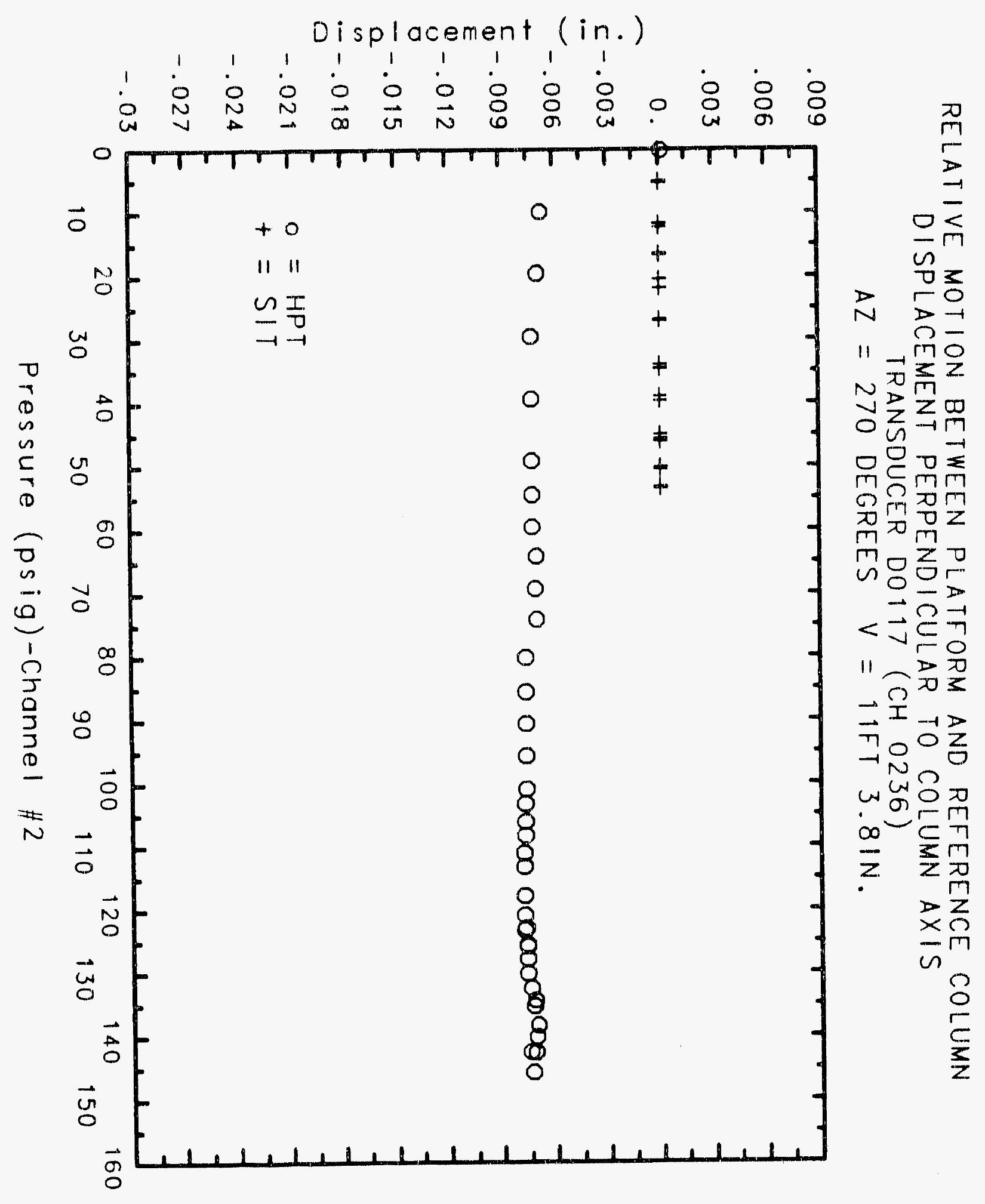




\section{Reinforced Concrete Test Data}

\begin{tabular}{l} 
Potentiometer \\
Structural Integrity Test \\
\hline $\begin{array}{c}\text { Pressure } \\
\text { (psig) } \\
\text { No SIT data (inches) }\end{array}$ \\
$\begin{array}{c}\text { gisplacement } \\
\text { gage was added after SIT }\end{array}$
\end{tabular}

This gage was added after SIT

High Pressure test

\begin{tabular}{|c|c|}
\hline $\begin{array}{c}\text { Pressure } \\
\text { (psig) } \\
9.89 \\
19.55 \\
29.57 \\
39.42 \\
49.16 \\
54.50 \\
59.57 \\
64.20 \\
69.32 \\
74.16 \\
80.16 \\
85.61 \\
90.58 \\
95.69 \\
100.92 \\
103.25 \\
106.11 \\
108.31 \\
111.08 \\
113.24 \\
117.83 \\
120.92 \\
123.28 \\
122.97 \\
125.82 \\
125.60 \\
127.84 \\
130.19 \\
132.53 \\
135.33 \\
134.42 \\
138.35 \\
140.16 \\
142.63 \\
145.78 \\
142.52 \\
0.22\end{array}$ & $\begin{array}{c}\text { Displacement } \\
\text { (inches) } \\
0.0011 \\
0.0037 \\
0.0058 \\
0.0078 \\
0.0098 \\
0.0113 \\
0.0131 \\
0.0147 \\
0.0165 \\
0.0184 \\
0.0226 \\
0.0248 \\
0.0270 \\
0.0298 \\
0.0324 \\
0.0340 \\
0.0355 \\
0.0367 \\
0.0383 \\
0.0397 \\
0.0425 \\
0.0446 \\
0.0463 \\
0.0462 \\
0.0478 \\
0.0484 \\
0.0498 \\
0.0523 \\
0.0546 \\
0.0587 \\
0.0599 \\
0.0654 \\
0.0718 \\
0.0806 \\
0.0911 \\
0.0945 \\
0.0429\end{array}$ \\
\hline
\end{tabular}




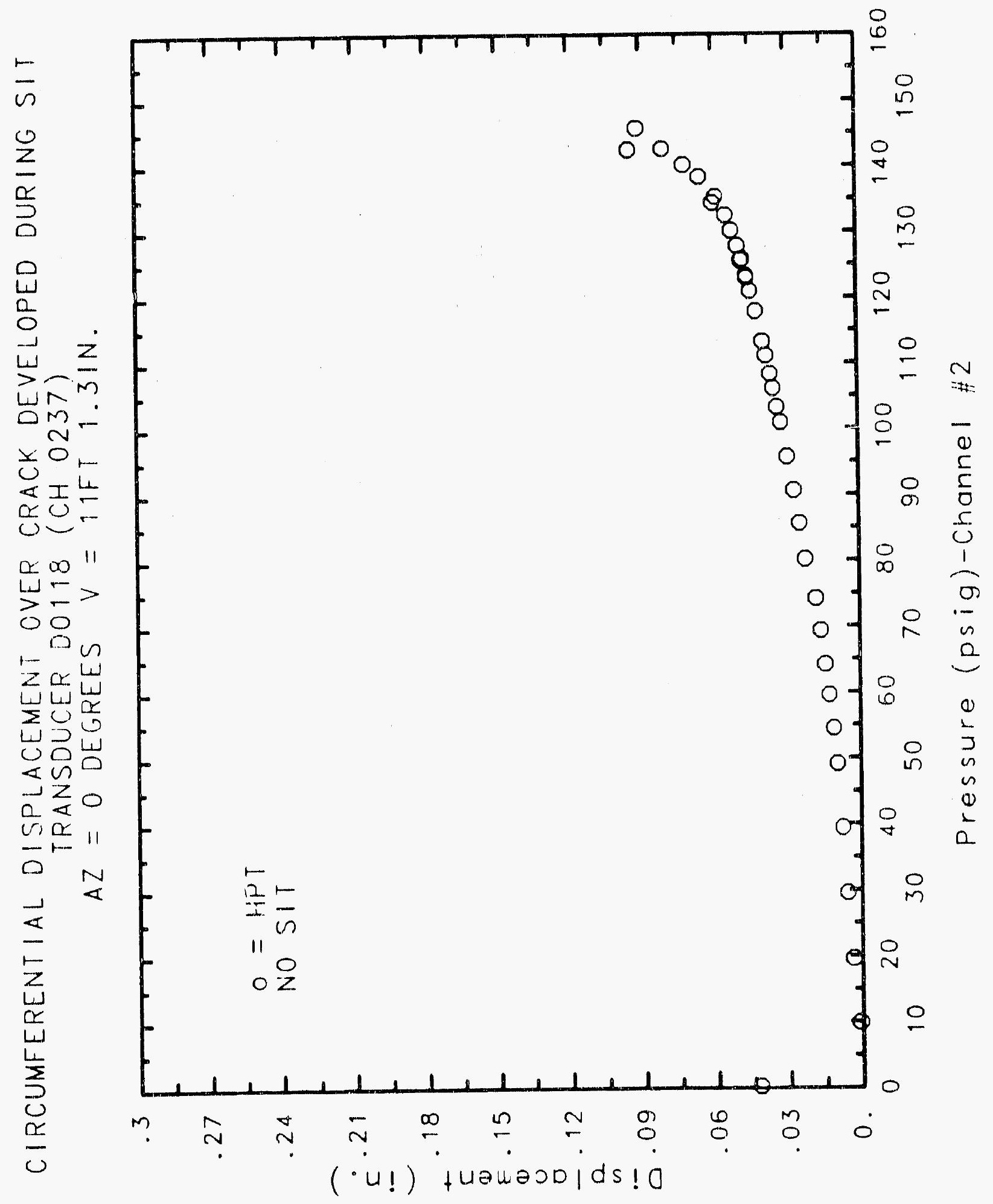




\section{Reinforced Concrete Test Data}

\section{Potentiometer Channel 238}

\section{Structural Integrity TEST}

$\begin{array}{cc}\text { Pressure } & \text { Displacement } \\ \text { (psig) } & \text { (inches) }\end{array}$

This gage was added after SIT

\section{High Pressure test}

Pressure

(psig)

9.89

19.55

29.57

39.42

49.16

54.50

59.57

64.20

69.32

74.16

80.16

85.61

90.58

95.69

100.92

103.25

106.11

108.31

111.08

113.24

117.83

120.92

123.28

122.97

125.82

125.60

127.84

130.19

132.53

135.33

134.42

138.35

140.16

142.63

145.78

142.52

0.22
Displacement

(inches)

0.0007

0.0018

0.0028

0.0036

0.0044

0.0047

0.0052

0.0056

0.0000

0.0065

0.0084

0.0093

0.0101

0.0111

0.0123

0.0129

0.0135

0.0140

0.0148

0.0154

0.0170

0.0182

0.0192

0.0193

0.0206

0.0207

0.0215

0.0232

0.0253

0.0291

0.0305

0.0430

0.0622

0.0949

0.1164

0.1229

0.0875 


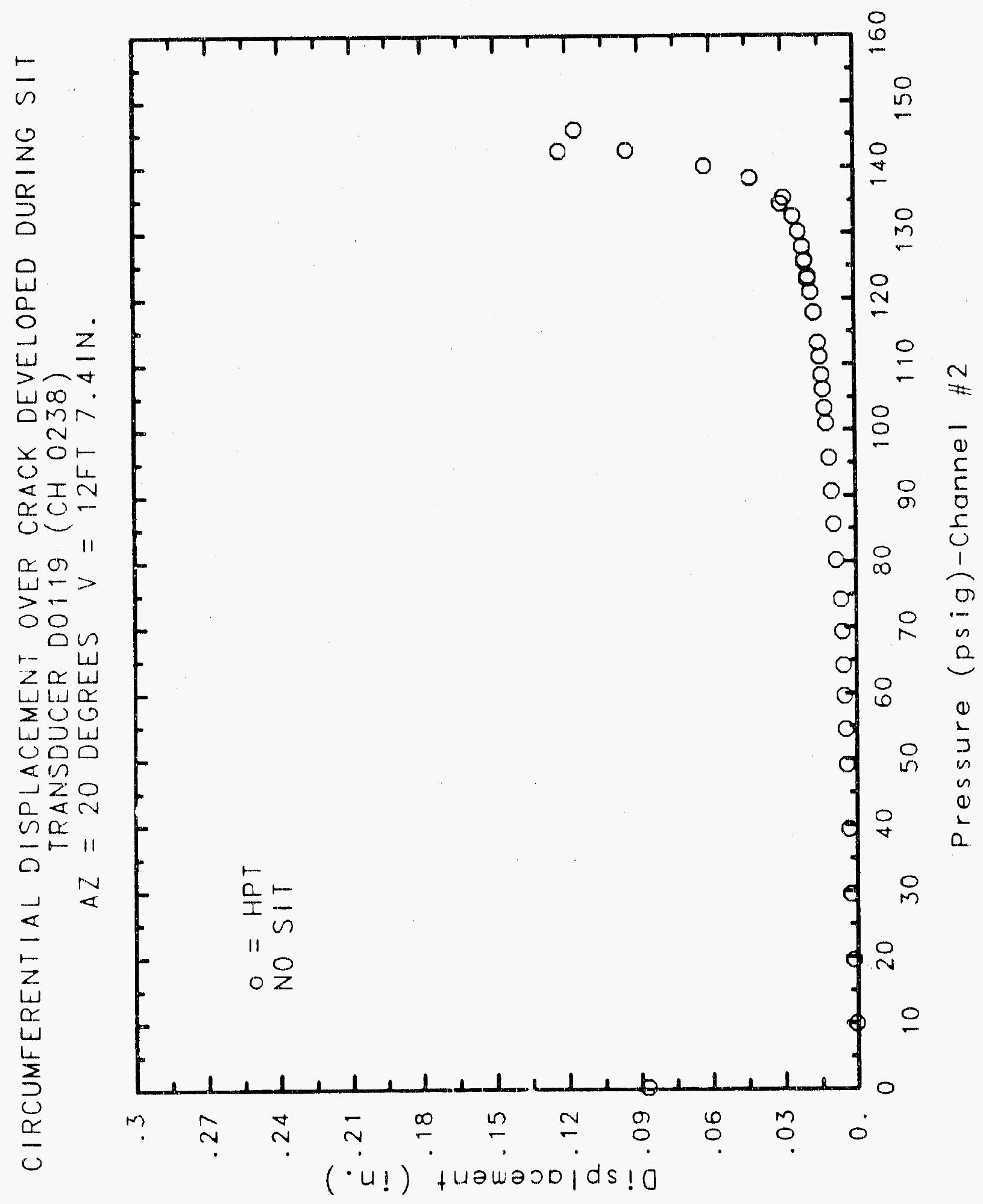




\section{Reinforced Concrete Test Data}

Potenttcmeter Channel 239

Structural Integrity test

Pressure

(psig)

No SIT data

This gage was added after SIT (inches)
Displacement

\section{High Pressure test}

\begin{tabular}{|c|c|}
\hline $\begin{array}{c}\text { Pressure } \\
\text { (psig) } \\
9.89 \\
19.55 \\
29.57 \\
39.42 \\
49.16 \\
54.50 \\
59.57 \\
64.20 \\
69.32 \\
74.16 \\
80.16 \\
85.61 \\
90.58 \\
95.69 \\
100.92 \\
103.25 \\
106.11 \\
108.31 \\
111.08 \\
113.24 \\
117.83 \\
120.92 \\
123.28 \\
122.97 \\
125.82 \\
125.60 \\
127.84 \\
130.19 \\
132.53 \\
135.33 \\
134.42 \\
138.35 \\
140.16 \\
142.63 \\
145.78 \\
142.52 \\
0.22\end{array}$ & $\begin{array}{c}\text { Displacement } \\
(\text { inches) } \\
0.0005 \\
0.0012 \\
0.0021 \\
0.0033 \\
0.0043 \\
0.0049 \\
0.0057 \\
0.0058 \\
0.0068 \\
0.0072 \\
0.0100 \\
0.0108 \\
0.0114 \\
0.0122 \\
0.0131 \\
0.0135 \\
0.0139 \\
0.0142 \\
0.0147 \\
0.0151 \\
0.0159 \\
0.0166 \\
0.0169 \\
0.0168 \\
0.0174 \\
0.0174 \\
0.0182 \\
0.0203 \\
0.0259 \\
0.0367 \\
0.0385 \\
0.0456 \\
0.0505 \\
0.0569 \\
0.0619 \\
0.0633 \\
0.0468\end{array}$ \\
\hline
\end{tabular}




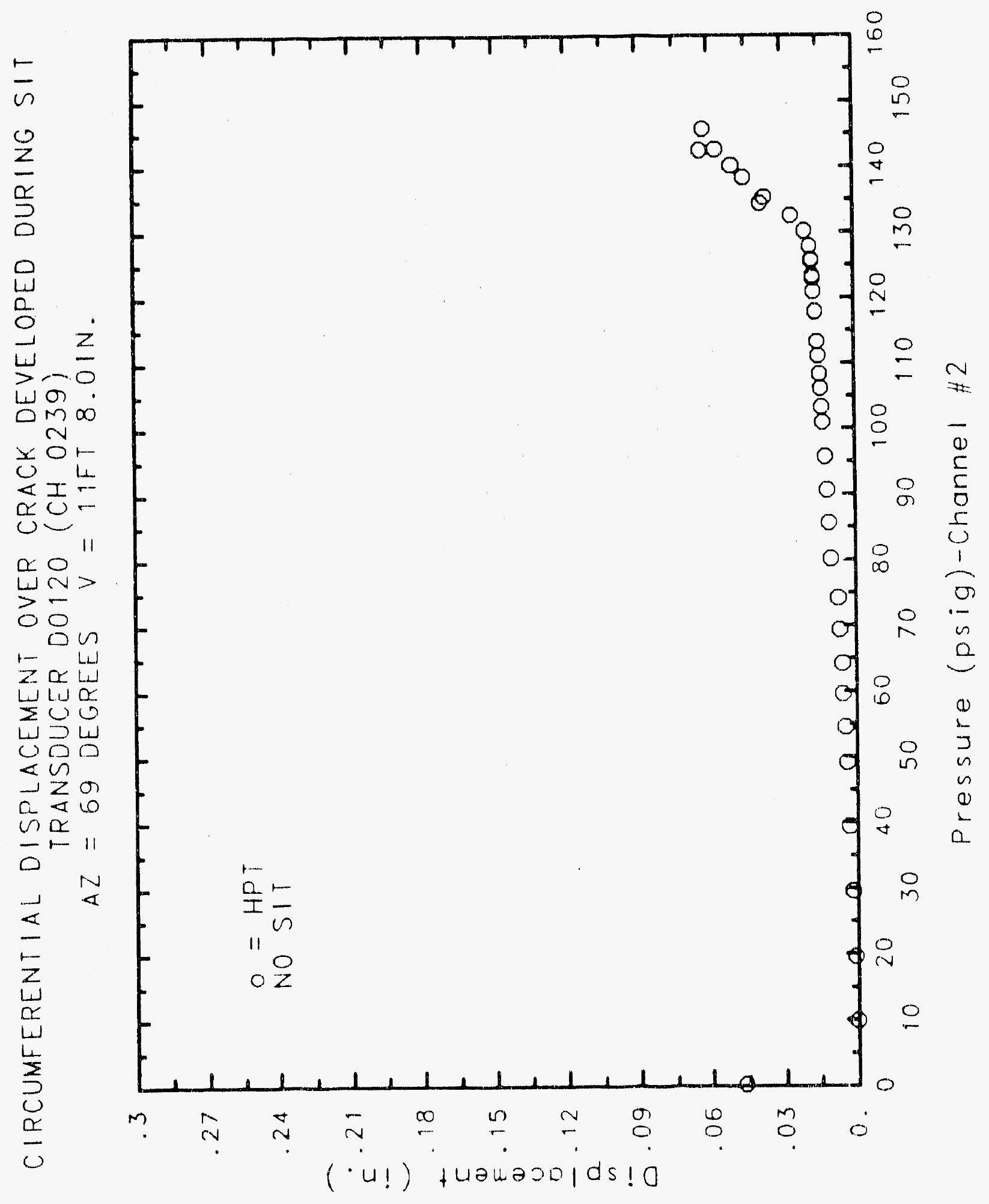




\section{Reinforced Concrete Test Data}

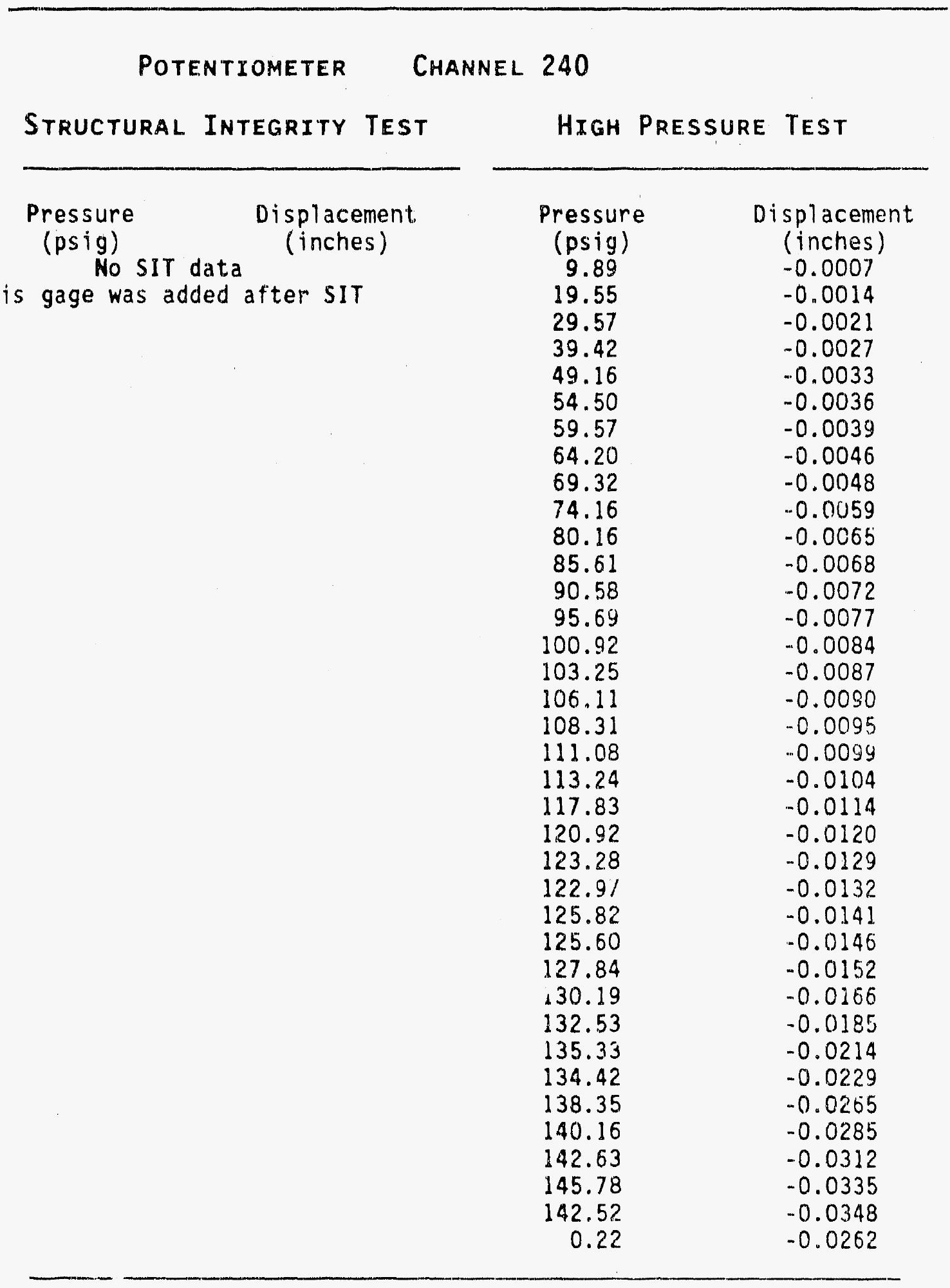




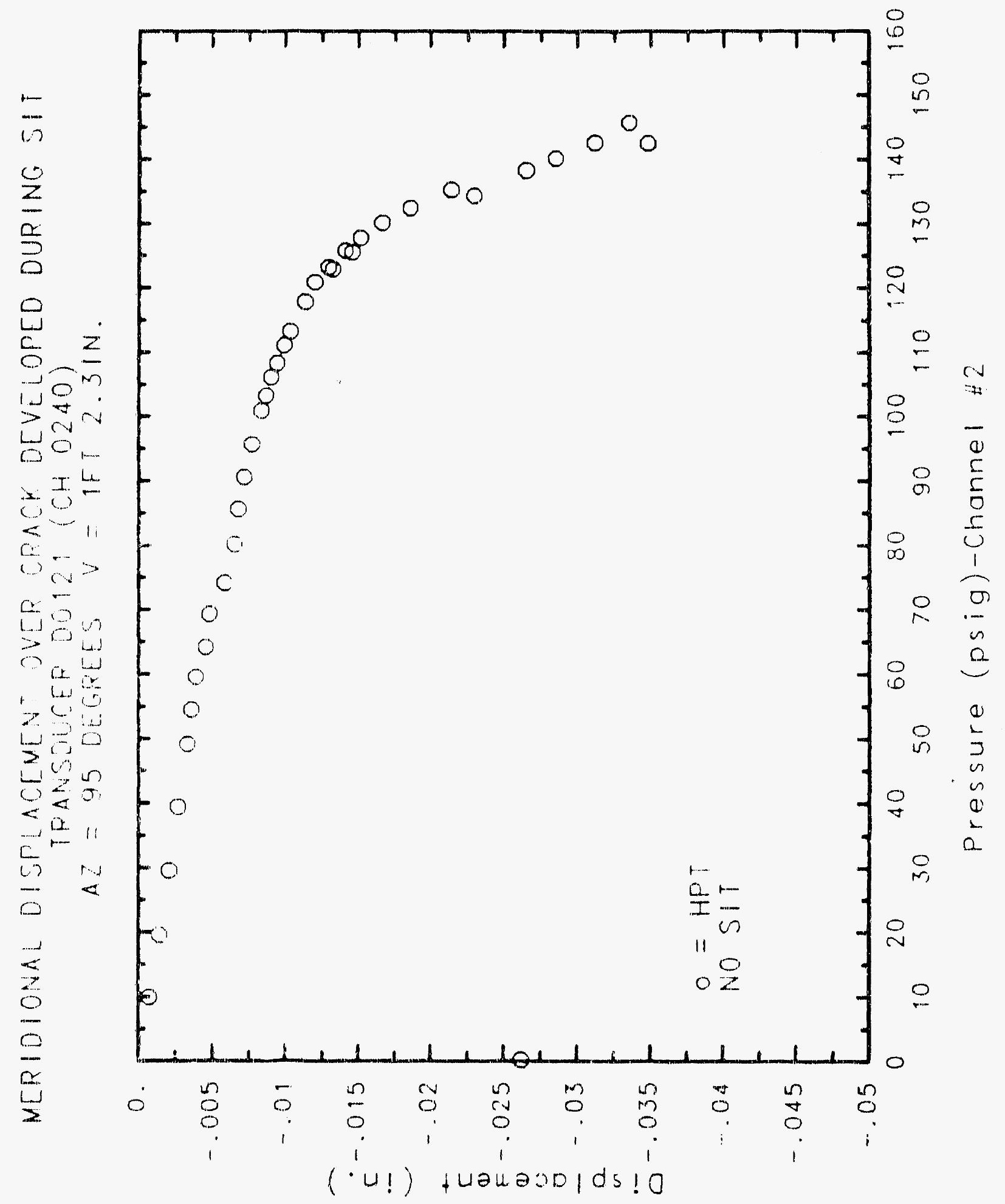

C. -125 


\section{Reinforced Concrete Test Data}

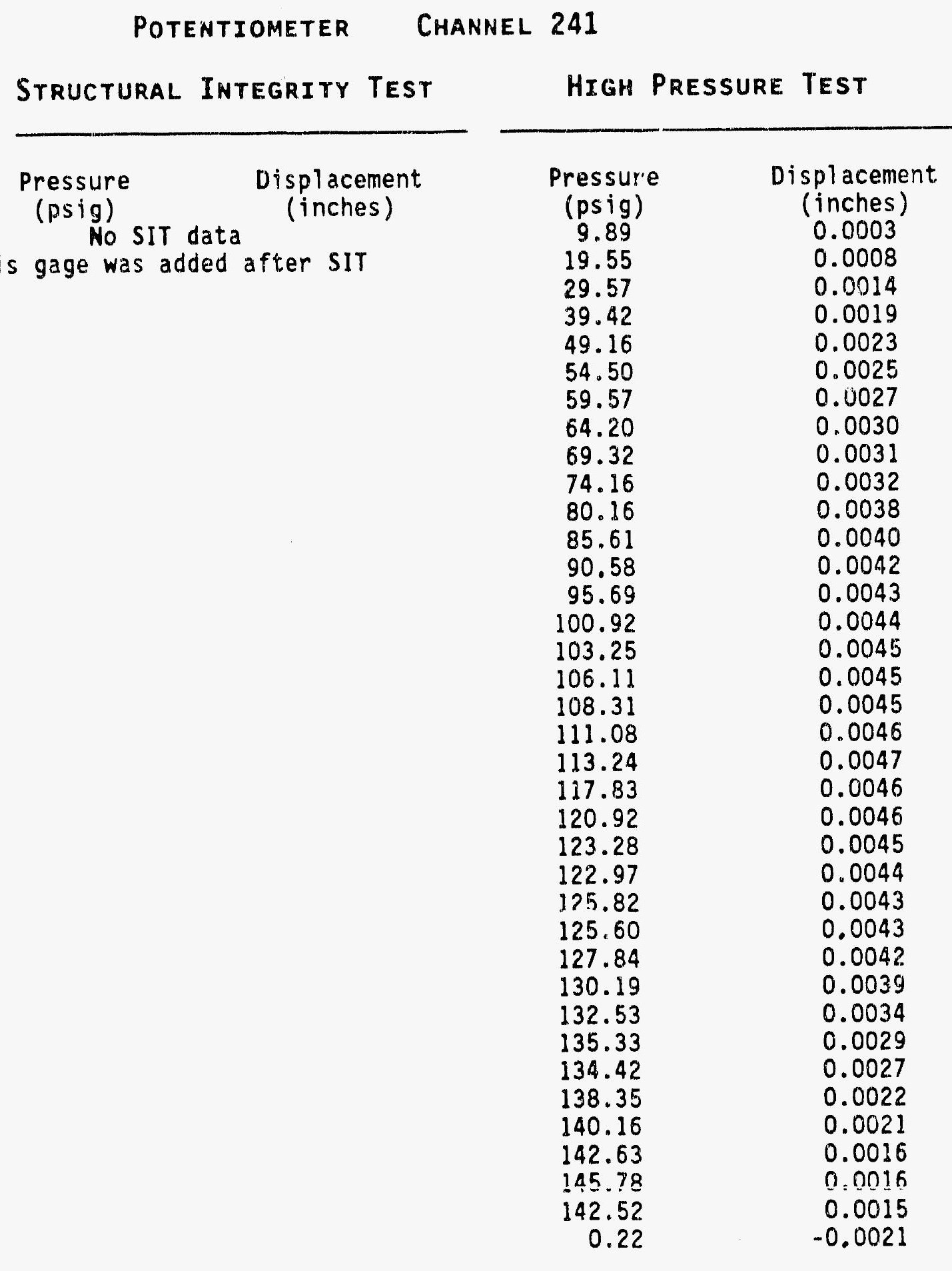




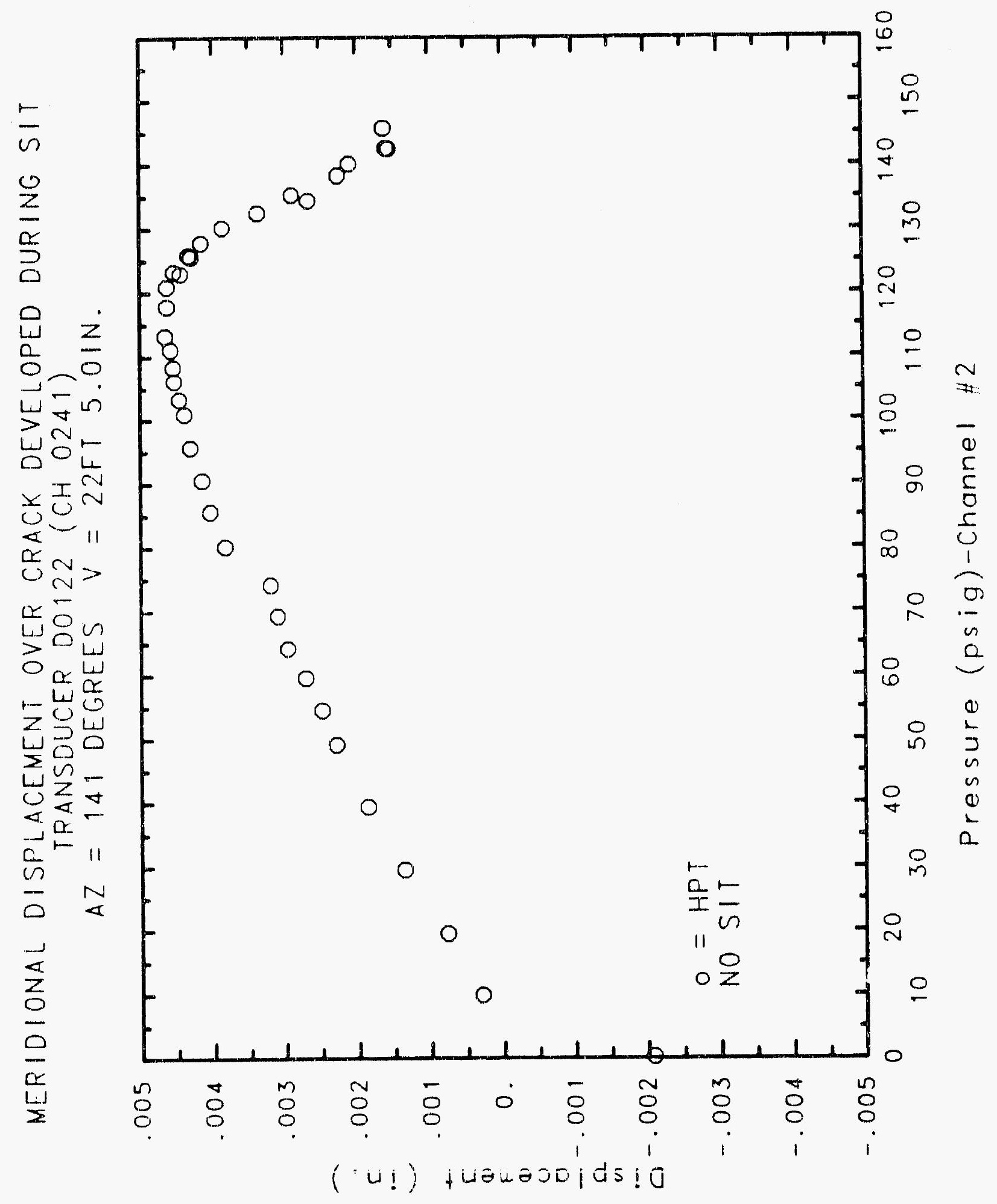




\section{Reinforced Concrete Test Data}

Potentiometer Channel 242

Structural Integrity TEST

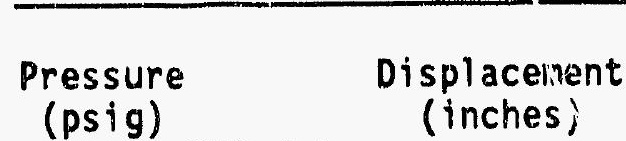

Mo SIT data

This gage was added after SIT

\section{High Pressure Test}

Pressure

(psig)

9.89

19.55

29.57

39.42

49.16

54.50

59.57

64.20

69.32

74.16

80.16

85.61

90.58

95.69

100.92

103.25

106.11

108.31

111.08

113.24

117.83

120.92

123.28

122.97

125.82

125.60

127.84

130.19

132.53

135.33

134.42

138.35

140.16

142.63

145.78

142.52

U..$\hat{c}$
Displacement

(inches)

0.0012

0.0026

0.0040

0.0054

0.0066

0.0073

0.0081

0.0089

0.0098

0.0104

0.0123

0.0133

0.0143

0.0156

0.0168

0.0176

0.0184

0.0191

0.0203

0.0213

0.0242

0.0268

0.0300

0.0306

0.0375

0.0393

0.0444

0.0538

0.0633

0.0732

0.0747

0.0822

0.0874

0.0943

0.1003

0.1019

0.0800 


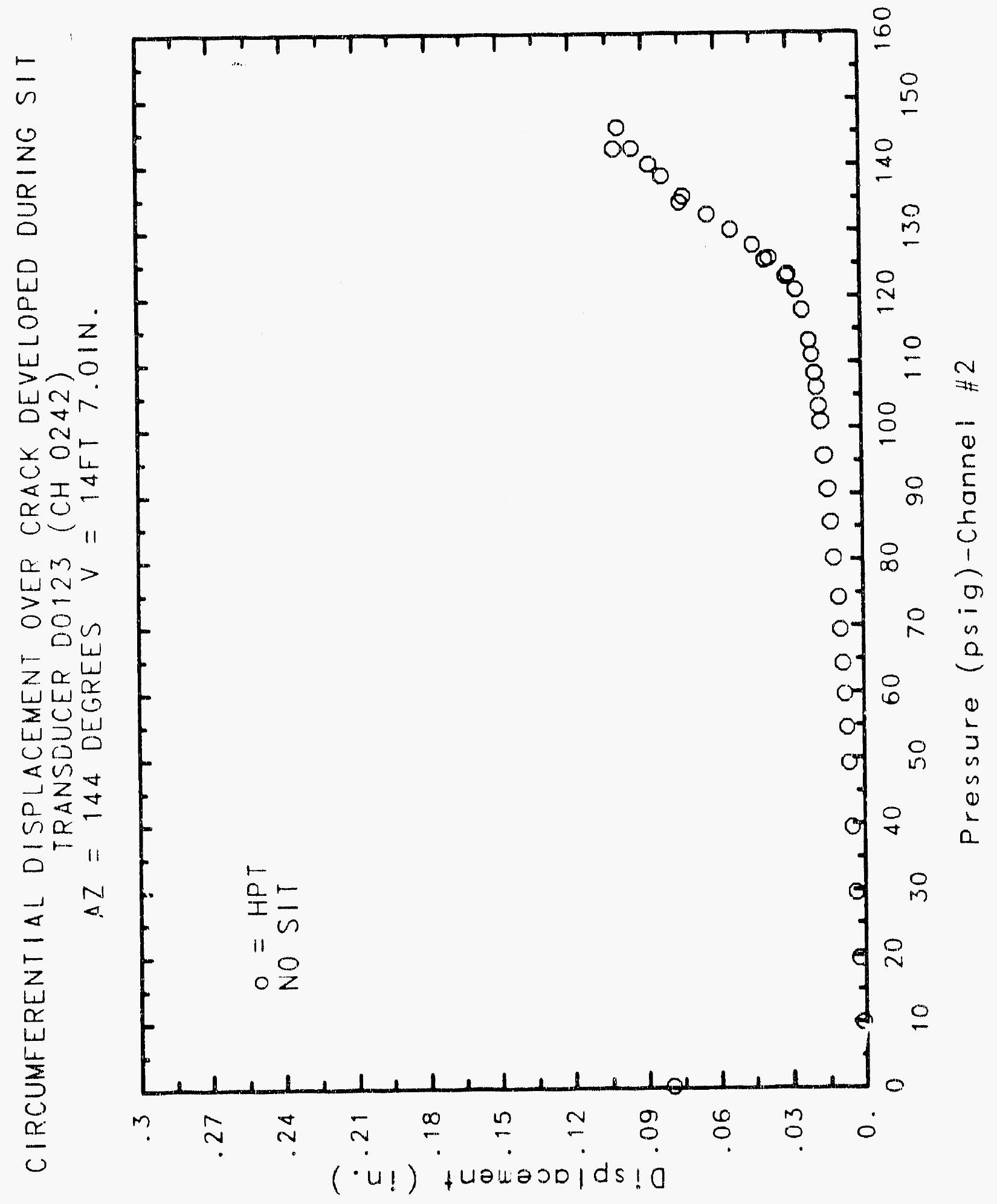




\section{Reinforced Concrete Test Data}

\section{Potentiometer Channel 243}

Structural INTEgRity TEST

$\begin{array}{cc}\begin{array}{c}\text { Pressure } \\ \text { (psig) }\end{array} & \text { Dispiacement } \\ \text { (inches) }\end{array}$

This gage was added after SIT
High Pressure Test

\begin{tabular}{|c|c|}
\hline $\begin{array}{c}\text { Pressure } \\
\text { (psig) } \\
9.89 \\
19.55 \\
29.57 \\
39.42 \\
49.16 \\
54.50 \\
59.57 \\
64.20 \\
69.32 \\
74.16 \\
80.16 \\
85.61 \\
90.58 \\
95.69 \\
100.92 \\
103.25 \\
106.11 \\
108.31 \\
111.08 \\
113.24 \\
117.83 \\
120.92 \\
123.28 \\
122.97 \\
125.82 \\
125.60 \\
127.84 \\
130.19 \\
132.53 \\
135.33 \\
134.42 \\
138.35 \\
140.16 \\
142.63 \\
145.78 \\
142.52 \\
0.22\end{array}$ & $\begin{array}{c}\text { Displacement } \\
\text { (inches) } \\
0.0008 \\
0.0023 \\
0.0038 \\
0.0053 \\
0.0066 \\
0.0073 \\
0.0081 \\
0.0090 \\
0.0098 \\
0.0107 \\
0.0127 \\
0.0139 \\
0.0151 \\
0.0167 \\
0.0181 \\
0.0188 \\
0.0195 \\
0.0200 \\
0.0208 \\
0.0213 \\
0.0224 \\
0.0233 \\
0.0239 \\
0.0240 \\
0.0247 \\
0.0249 \\
0.0253 \\
0.0260 \\
0.0267 \\
0.0272 \\
0.0271 \\
0.0273 \\
0.0275 \\
0.0278 \\
0.0283 \\
0.0280 \\
0.0084\end{array}$ \\
\hline
\end{tabular}




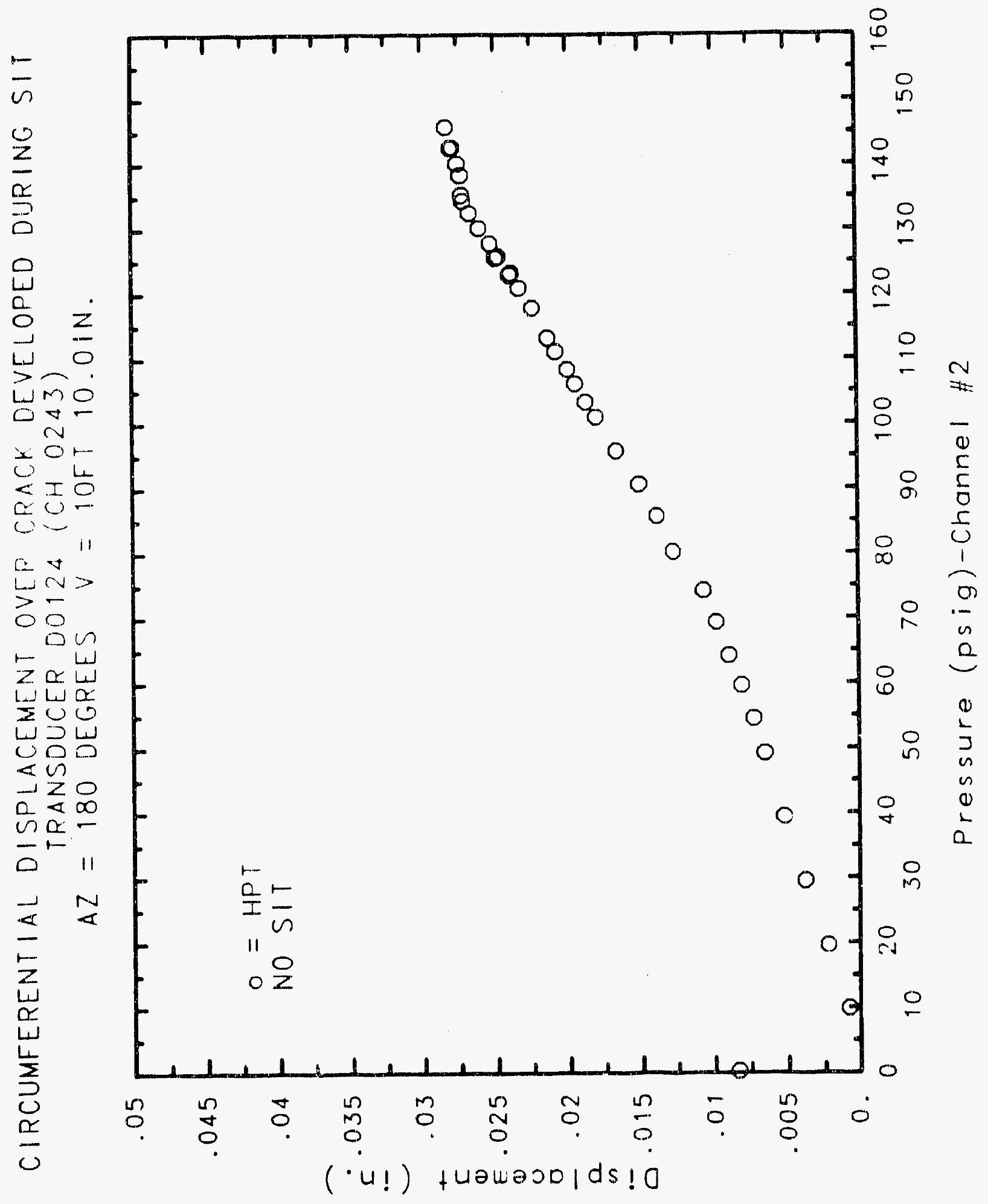




\section{Reinforced Concrete Test Data}

\begin{tabular}{|c|c|c|}
\hline \multicolumn{3}{|c|}{ Potentiometer Channel 244} \\
\hline $\begin{array}{l}\text { Pressure } \quad \text { Displacement } \\
\text { (psig) (inches) } \\
\text { No SIT data gage was added after SIT }\end{array}$ & $\begin{array}{c}\text { Pressure } \\
(\text { psig) } \\
9.89 \\
19.55 \\
29.57 \\
39.42 \\
49.16 \\
54.50 \\
59.57 \\
64.20 \\
69.32 \\
74.16 \\
80.16 \\
85.61 \\
90.58 \\
95.69 \\
100.92 \\
103.25 \\
106.11 \\
108.31 \\
111.08 \\
113.24 \\
117.83 \\
120.92 \\
123.28 \\
122.97 \\
125.82 \\
125.60 \\
127.84 \\
130.19 \\
132.53 \\
135.33 \\
134.42 \\
138.35 \\
140.16 \\
142.63 \\
145.78 \\
142.52 \\
0.22\end{array}$ & $\begin{array}{c}\text { Displacement } \\
\text { (inches) } \\
-0.0002 \\
-0.0004 \\
-0.0006 \\
-0.0008 \\
-0.0011 \\
-0.0012 \\
-0.0013 \\
-0.0014 \\
-0.0016 \\
-0.0019 \\
-0.0018 \\
-0.0021 \\
-0.0020 \\
-0.0023 \\
-0.0027 \\
-0.0027 \\
-0.0030 \\
-0.0032 \\
-0.0034 \\
-0.0037 \\
-0.0044 \\
-0.0049 \\
-0.0061 \\
-0.0065 \\
-0.0073 \\
-0.0075 \\
-0.0080 \\
-0.0082 \\
-0.0092 \\
-0.0107 \\
-0.0102 \\
-0.0132 \\
-0.0163 \\
-0.0194 \\
-0.0212 \\
-0.0220 \\
-0.0160\end{array}$ \\
\hline
\end{tabular}




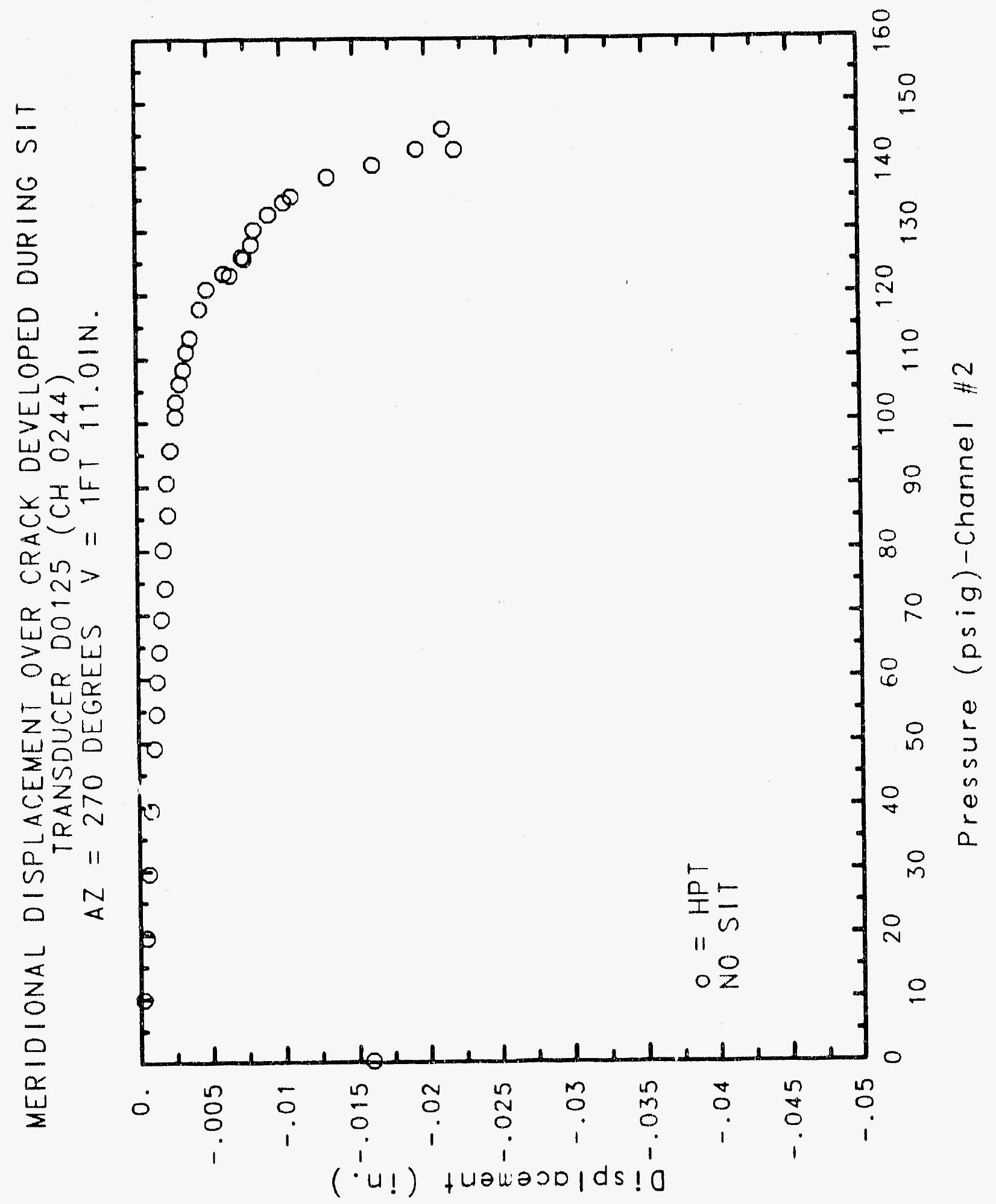




\section{Reinforced Concrete Test Data}

Potentiometer Channel 257

Structural Integrity Test

Pressure $\quad$ Displacement
(psig)
No SIT data (inches)

This gage was added after SIT
High Pressure test

$\begin{array}{cc}\text { Pressure } & \text { Displacement } \\ \text { (psig) } & \text { (inches) } \\ 9.89 & -0.0025 \\ 19.55 & 0.0245 \\ 29.57 & 0.0525 \\ 39.42 & 0.0810 \\ 49.16 & 0.1088 \\ 54.50 & 0.1125 \\ 59.57 & 0.1371 \\ 64.20 & 0.1621 \\ 69.32 & 0.1936 \\ 74.16 & 0.2415 \\ 80.16 & 0.2606 \\ 85.61 & 0.2929 \\ 90.58 & 0.3333 \\ 95.69 & 0.3745 \\ 100.92 & 0.4091 \\ 103.25 & 0.4281 \\ 106.11 & 0.4432 \\ 108.31 & 0.4558 \\ 111.08 & 0.4737 \\ 113.24 & 0.4860 \\ 117.83 & 0.5136 \\ 120.92 & 0.5385 \\ 123.28 & 0.5633 \\ 122.97 & 0.5713 \\ 125.82 & 0.5620 \\ 125.60 & 0.5659 \\ 127.84 & 0.5960 \\ 130.19 & 0.6309 \\ 132.53 & 0.6607 \\ 135.33 & 0.7170 \\ 134.42 & 0.7379 \\ 138.35 & 0.7515 \\ 140.16 & 0.8045 \\ 142.63 & 0.8437 \\ 145.78 & 0.9082 \\ 142.52 & 0.9436 \\ 0.22 & 0.4626 \\ & \\ & \end{array}$




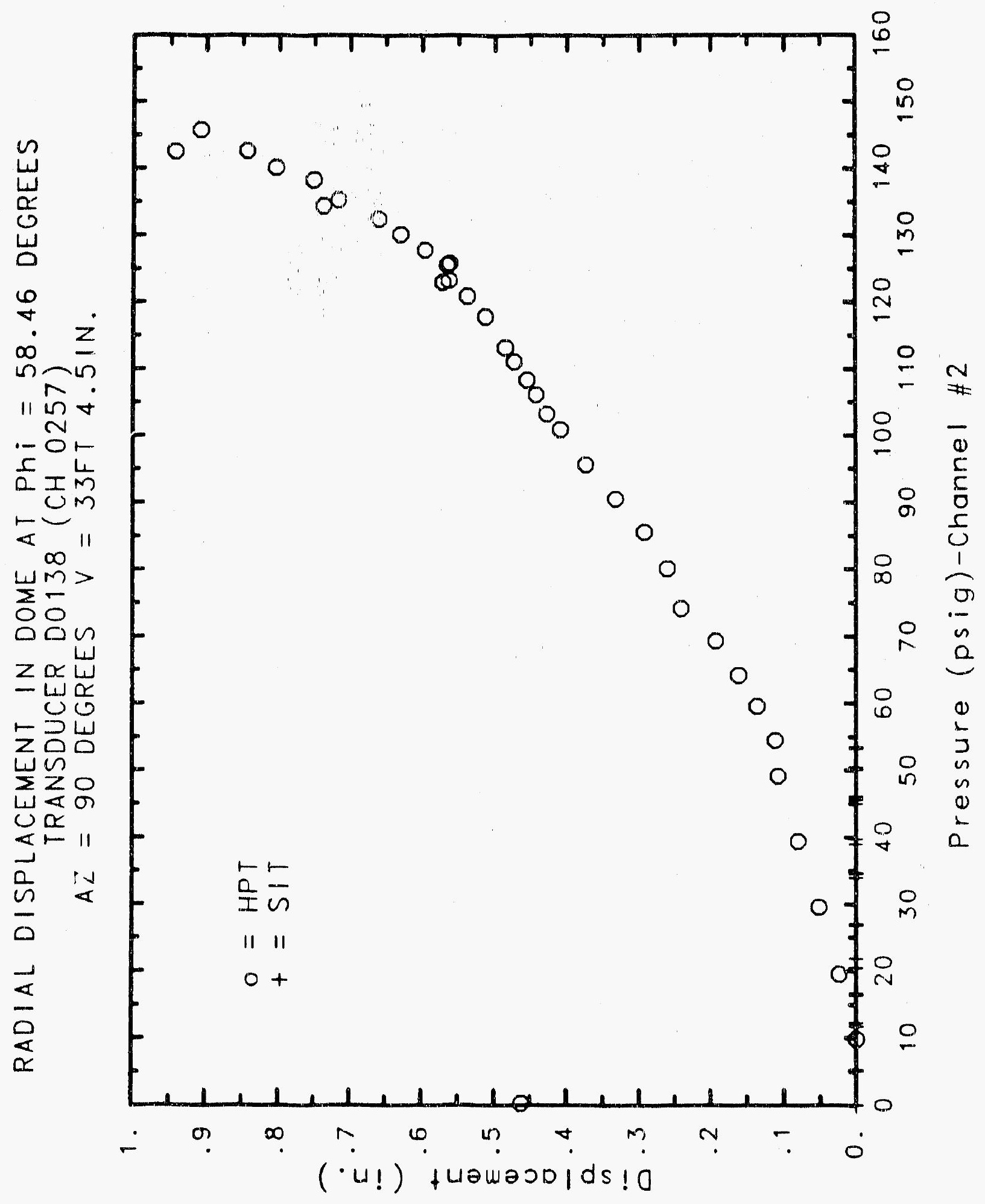

C. 135 


\section{Reinforced Concrete Test Data}

Potentiometer Channel 258

Structural Integrity test

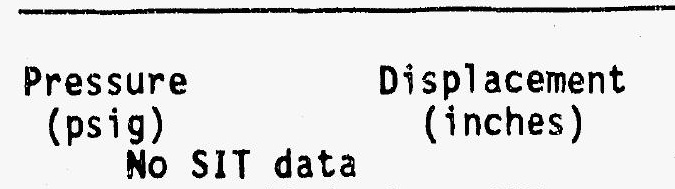

This gage was added after SIT
High Pressure Test

\begin{tabular}{cc}
\hline Pressure & Displacement \\
(psig) & (inches) \\
9.89 & -0.0046 \\
19.55 & 0.0169 \\
29.57 & 0.0421 \\
39.42 & 0.0706 \\
49.16 & 0.0931 \\
54.50 & 0.0955 \\
59.57 & 0.1202 \\
64.20 & 0.1410 \\
69.32 & 0.1708 \\
74.16 & 0.2174 \\
80.16 & 0.2416 \\
85.61 & 0.2763 \\
90.58 & 0.3142 \\
95.69 & 0.3544 \\
100.92 & 0.3893 \\
103.25 & 0.4094 \\
106.11 & 0.4236 \\
108.31 & 0.4351 \\
111.08 & 0.4544 \\
113.24 & 0.4685 \\
117.83 & 0.4988 \\
120.92 & 0.5213 \\
123.28 & 0.5447 \\
122.97 & 0.5530 \\
125.82 & 0.5410 \\
125.60 & 0.5448 \\
127.84 & 0.5714 \\
130.19 & 0.6042 \\
132.53 & 0.6370 \\
135.33 & 0.6959 \\
134.42 & 0.7170 \\
138.35 & 0.7339 \\
140.16 & 0.7902 \\
142.63 & 0.8359 \\
145.78 & 0.9030 \\
142.52 & 0.9371 \\
0.22 & 0.4946 \\
& \\
&
\end{tabular}




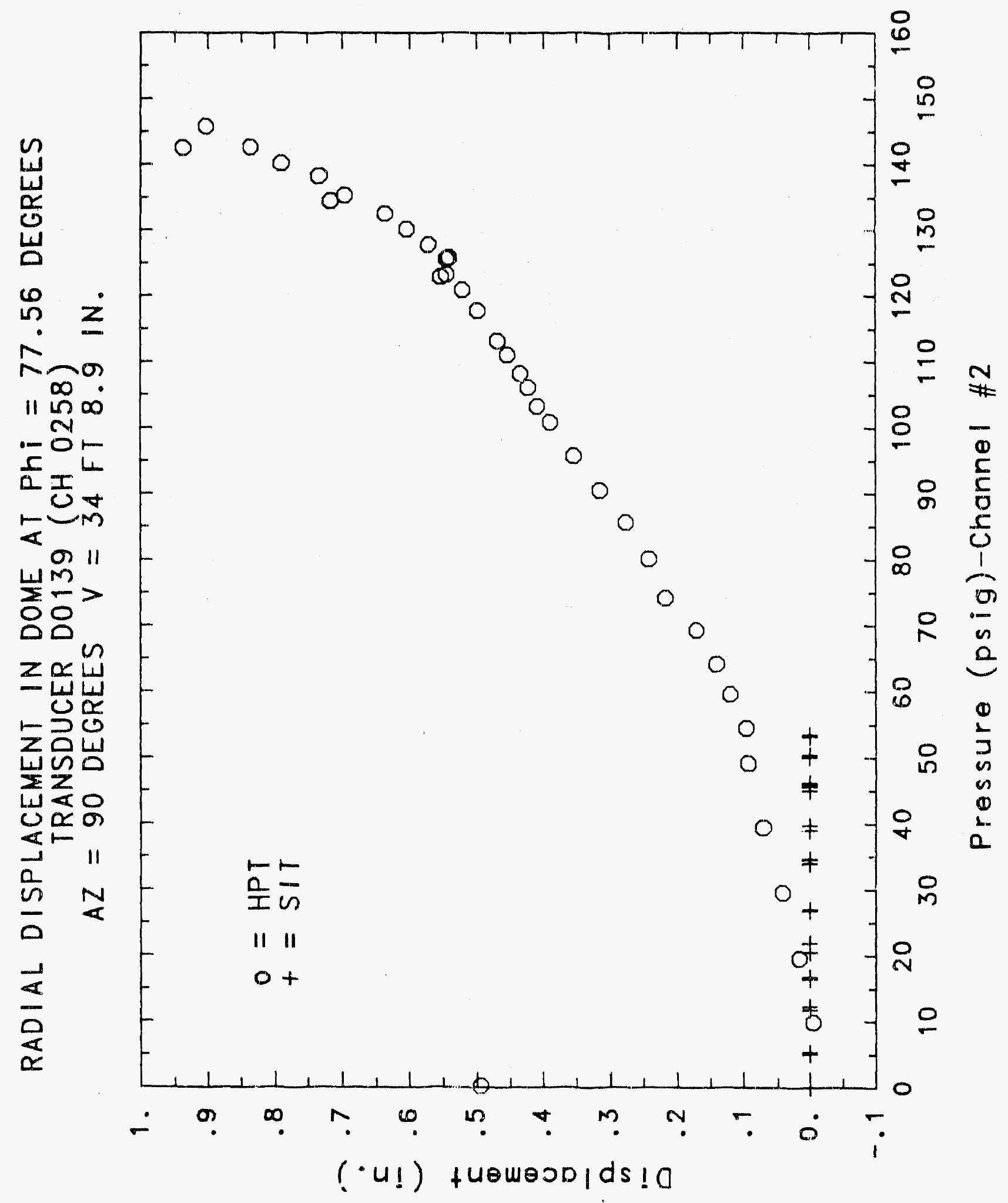



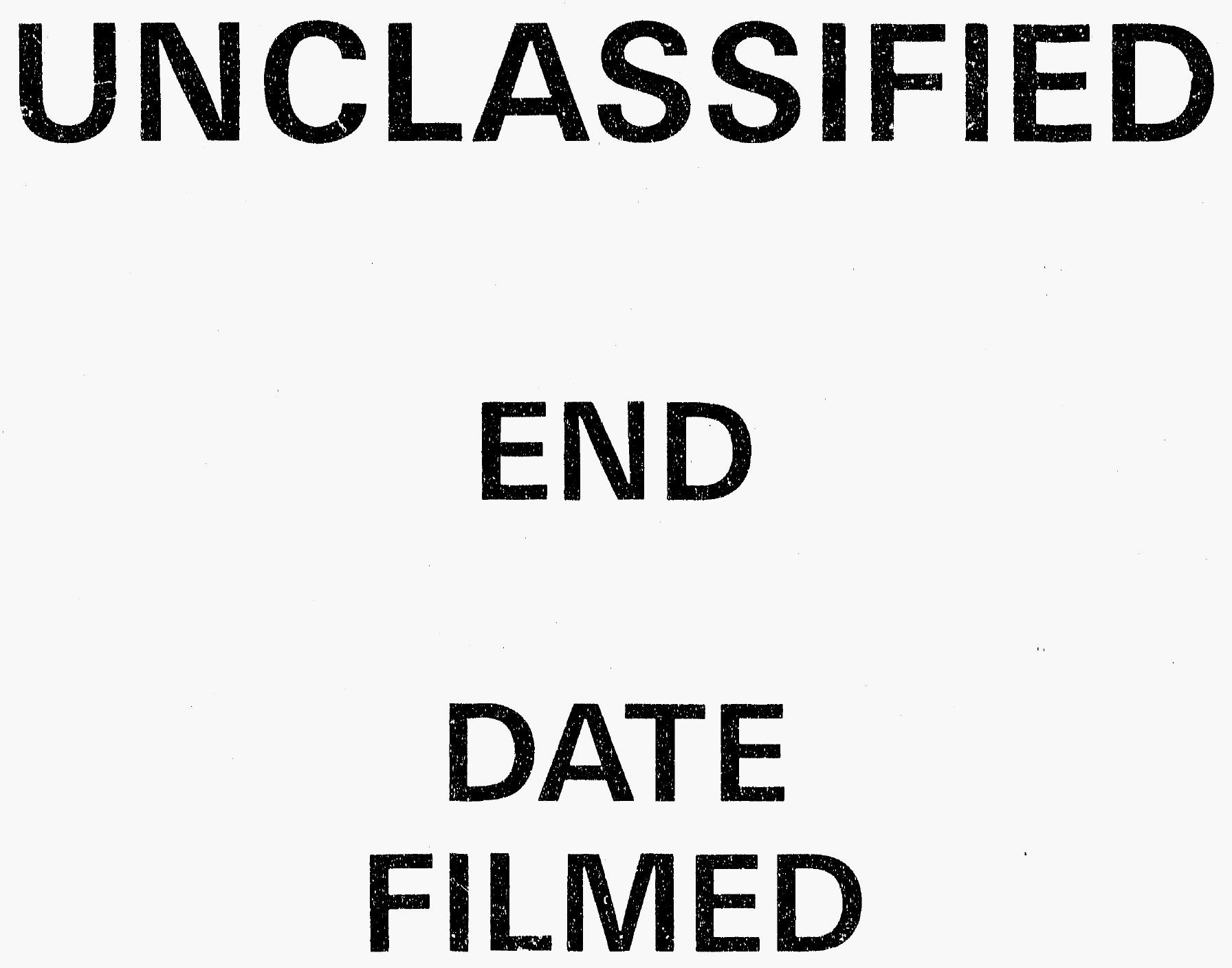

$$
1-6-92
$$

SANDIA NATIONAL LABS 


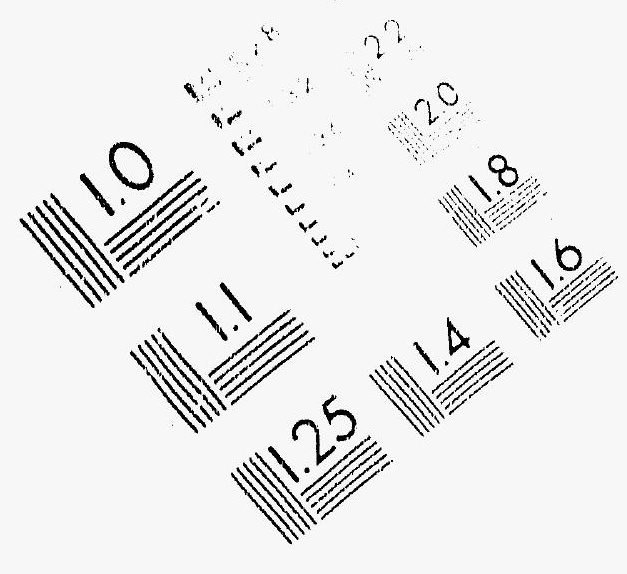

\section{IMAGE EVALUATION \\ TEST TARGET (MT-3)}
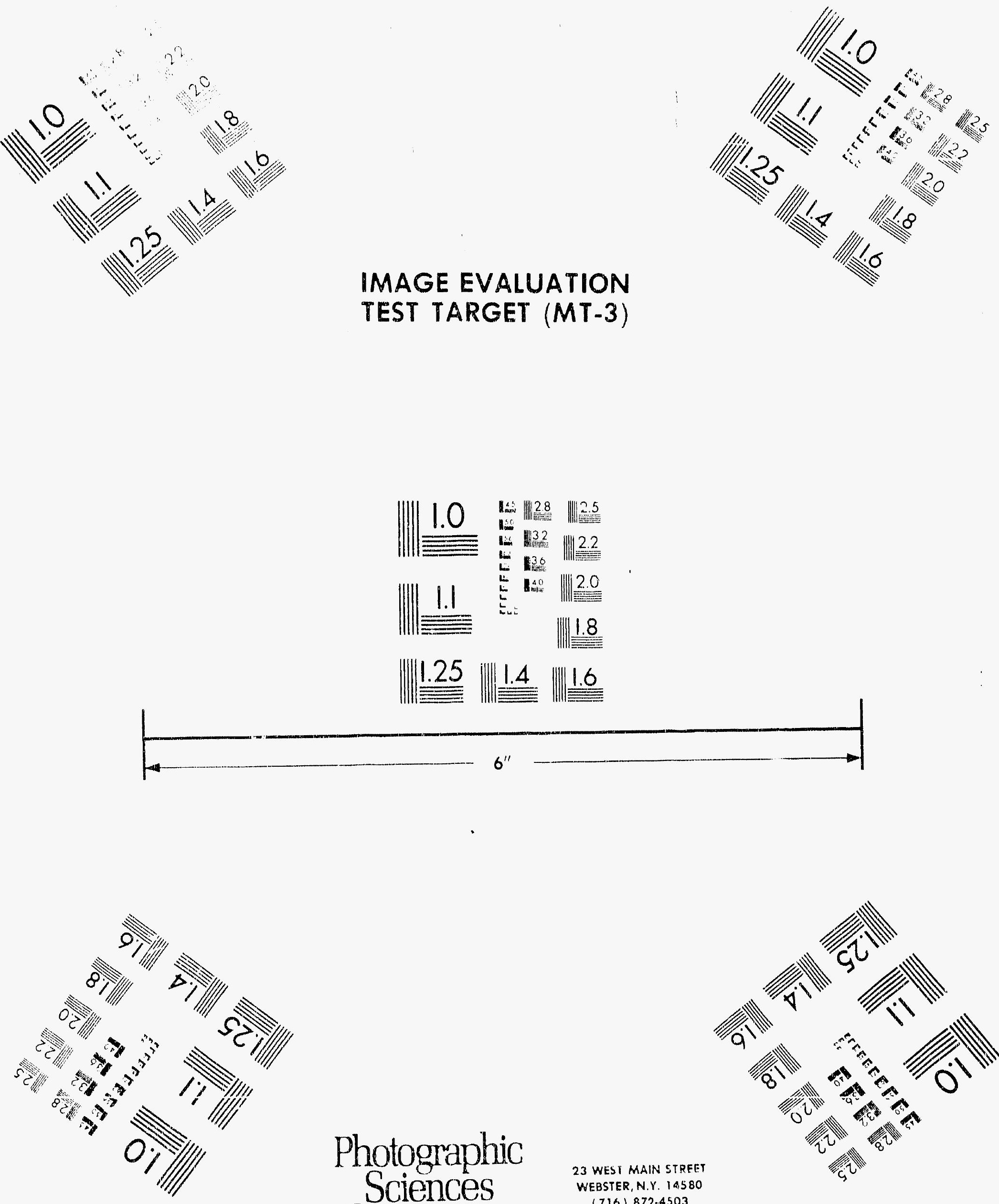

Photographic Corporation

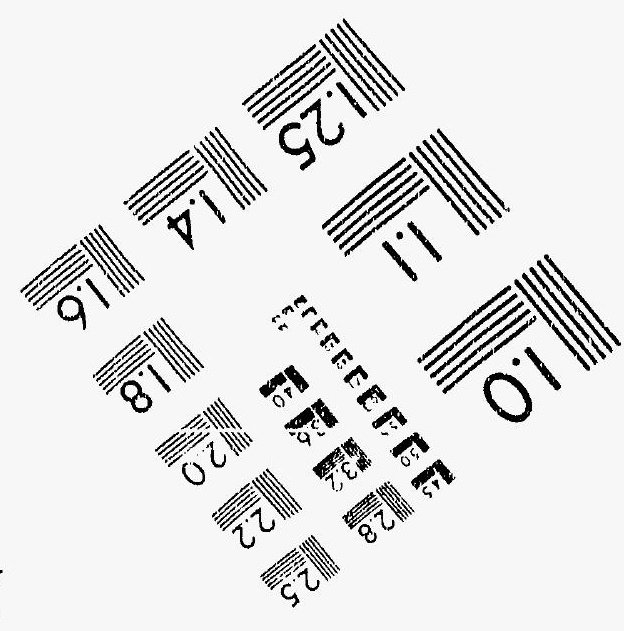




\section{SECURITY CLASSIFICATION}
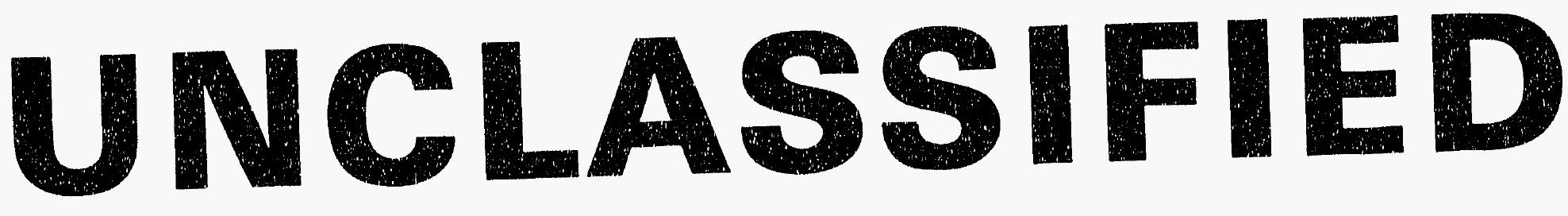

\section{DATE OF MICROFILMING $1-6-92$}

\section{MICROFILMED BY - erar hard}

SANDIA NATIONAL LABS 


\section{THIS DOCUMENT FILMED}

AT

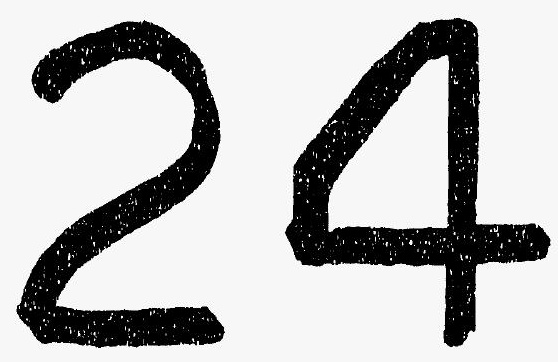

REDUCTION 


\section{Displacement, LVDT}

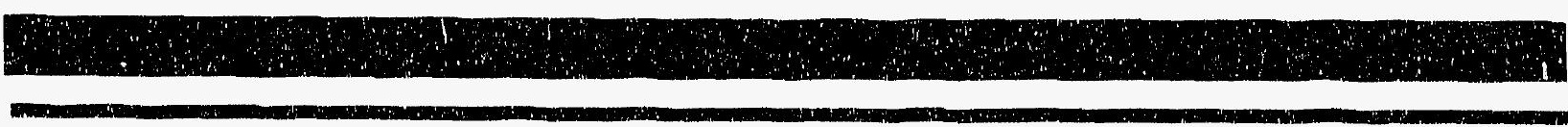




\section{Reinforced Concrete Test Data}

LVDT Channel 122

STRUCTURAL INTEGRITY TEST

\begin{tabular}{|c|c|c|c|}
\hline $\begin{array}{c}\text { Pressure } \\
\text { (psig) } \\
-0.05 \\
5.33 \\
12.31 \\
16.44 \\
20.51 \\
20.46 \\
20.47 \\
27.05 \\
34.69 \\
34.53 \\
34.53 \\
39.70 \\
45.90 \\
45.65 \\
45.62 \\
50.10 \\
53.47 \\
53.29 \\
53.21 \\
50.49 \\
46.14 \\
46.13 \\
44.97 \\
38.98 \\
33.99 \\
33.96 \\
26.83 \\
21.88 \\
21.90 \\
16.09 \\
11.74 \\
11.77 \\
5.05 \\
0.02 \\
-0.04 \\
-0.02 \\
-0.02 \\
0.02\end{array}$ & $\begin{array}{c}\text { Displacement } \\
\text { (inches) } \\
0.0003 \\
0.0010 \\
0.0018 \\
0.0034 \\
0.0042 \\
0.0048 \\
0.0057 \\
0.0094 \\
0.0156 \\
0.0165 \\
0.0165 \\
0.0191 \\
0.0235 \\
0.0235 \\
0.0235 \\
0.0258 \\
0.0305 \\
0.0308 \\
0.0308 \\
0.0320 \\
0.0328 \\
0.0330 \\
0.0355 \\
0.0356 \\
0.0349 \\
0.0350 \\
0.0340 \\
0.0311 \\
0.0313 \\
0.0268 \\
0.0225 \\
0.0216 \\
0.0151 \\
0.0105 \\
0.0058 \\
0.0075 \\
0.0081 \\
0.0079\end{array}$ & $\begin{array}{c}\text { Pressure } \\
(\text { psig) } \\
9.89 \\
9.55 \\
29.57 \\
39.42 \\
49.16 \\
54.50 \\
59.57 \\
64.20 \\
69.32 \\
74.16 \\
80.16 \\
85.61 \\
90.58 \\
95.69 \\
100.92 \\
103.25 \\
106.11 \\
108.31 \\
111.08 \\
113.24 \\
117.83 \\
120.92 \\
123.28 \\
122.97 \\
125.82 \\
125.60 \\
127.84 \\
130.19 \\
132.53 \\
135.33 \\
134.42 \\
138.35 \\
140.16 \\
142.63 \\
145.78 \\
142.52 \\
0.22\end{array}$ & $\begin{array}{c}\text { Displacement } \\
\text { (inches) } \\
0.0038 \\
0.0074 \\
0.0135 \\
0.0202 \\
0.0249 \\
0.0277 \\
0.0323 \\
0.0383 \\
0.0449 \\
0.0512 \\
0.0593 \\
0.0696 \\
0.0805 \\
0.0920 \\
0.1046 \\
0.1148 \\
0.1194 \\
0.1245 \\
0.1353 \\
0.1401 \\
0.1541 \\
0.1727 \\
0.1847 \\
0.1867 \\
0.1994 \\
0.2027 \\
0.2186 \\
0.2289 \\
0.2551 \\
0.2908 \\
0.2973 \\
0.3465 \\
0.3850 \\
0.4338 \\
0.4866 \\
0.5088 \\
0.3680\end{array}$ \\
\hline
\end{tabular}




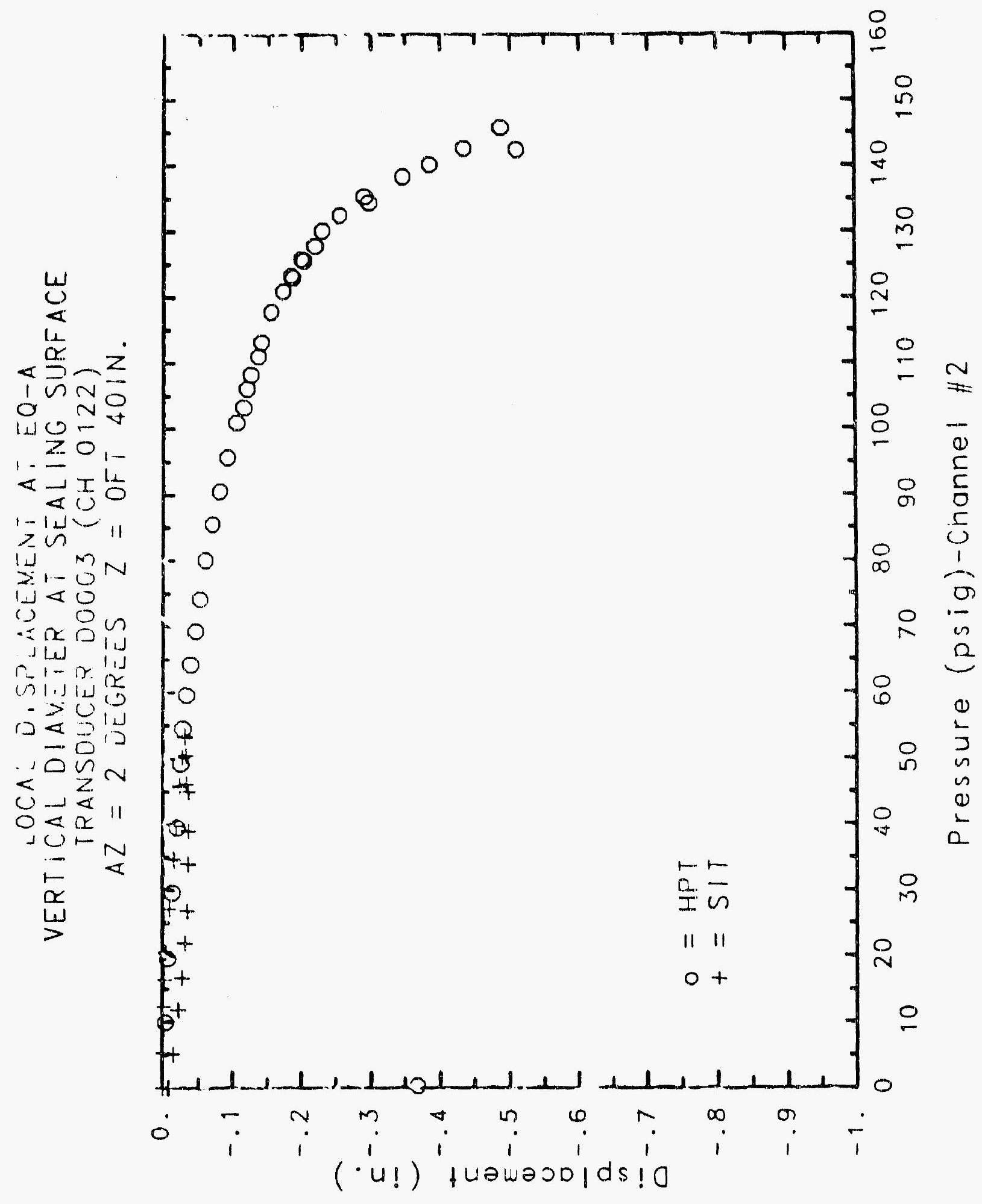




\section{Reinforced Concrete Test Data}

\section{LVDT Channel 123}

STRUCTURAL INTEGRITY TEST

\begin{tabular}{|c|c|c|c|}
\hline $\begin{array}{l}\text { Pressure } \\
\text { (psig) } \\
-0.05 \\
5.33 \\
12.31 \\
16.44 \\
20.51 \\
20.46 \\
20.47 \\
27.05 \\
34.69 \\
34.53 \\
34.53 \\
39.70 \\
45.90 \\
45.65 \\
45.62 \\
50.10 \\
53.47 \\
53.29 \\
53.21 \\
50.49 \\
46.14 \\
46.13 \\
44.97 \\
38.98 \\
33.99 \\
33.96 \\
26.83 \\
21.88 \\
21.90 \\
16.69 \\
11.74 \\
11.77 \\
5.05 \\
0.02 \\
-0.04 \\
-0.02 \\
-0.02 \\
0.02\end{array}$ & $\begin{array}{c}\text { Displacement } \\
\text { (inches) } \\
0.0001 \\
0.0004 \\
0.0009 \\
0.0008 \\
0.0011 \\
0.0011 \\
0.0011 \\
0.0012 \\
-0.0063 \\
-0.0062 \\
-0.0063 \\
-0.0120 \\
-0.0179 \\
-0.0178 \\
-0.0179 \\
-0.0230 \\
-0.0275 \\
-0.0300 \\
-0.0300 \\
-0.0342 \\
-0.0343 \\
-0.0342 \\
-0.0346 \\
-0.0346 \\
-0.0348 \\
-0.0347 \\
-0.0347 \\
-0.0349 \\
-0.0345 \\
-0.0347 \\
-0.0345 \\
-0.0345 \\
-0.0332 \\
-0.0290 \\
-0.0281 \\
-0.0281 \\
-0.0277 \\
-0.0244 \\
0\end{array}$ & $\begin{array}{c}\text { Pressure } \\
\text { (psig) } \\
9.89 \\
19.55 \\
29.57 \\
39.42 \\
49.16 \\
54.50 \\
59.57 \\
64.20 \\
69.32 \\
74.16 \\
80.16 \\
85.61 \\
90.58 \\
95.69 \\
100.92 \\
103.25 \\
106.11 \\
108.31 \\
111.08 \\
113.24 \\
117.83 \\
120.92 \\
123.28 \\
122.97 \\
125.82 \\
125.60 \\
127.84 \\
130.19 \\
132.53 \\
135.33 \\
134.42 \\
138.35 \\
140.16 \\
142.63 \\
145.78 \\
142.52 \\
0.22\end{array}$ & $\begin{array}{c}\text { Displacement } \\
\text { (inches) } \\
0.0005 \\
0.0007 \\
-0.0002 \\
-0.0060 \\
-0.0102 \\
-0.0153 \\
-0.0183 \\
-0.0255 \\
-0.0302 \\
-0.0388 \\
-0.0593 \\
-0.0726 \\
-0.0858 \\
-0.1028 \\
-0.1210 \\
-0.1311 \\
-0.1409 \\
-0.1482 \\
-0.1589 \\
-0.1685 \\
-0.1902 \\
-0.2081 \\
-0.2199 \\
-0.2215 \\
-0.2383 \\
-0.2411 \\
-0.2518 \\
-0.2736 \\
-0.3017 \\
-0.3429 \\
-0.3552 \\
-0.4044 \\
-0.4527 \\
-0.5086 \\
-0.5659 \\
-0.5969 \\
-0.4520\end{array}$ \\
\hline
\end{tabular}

high Pressure test 


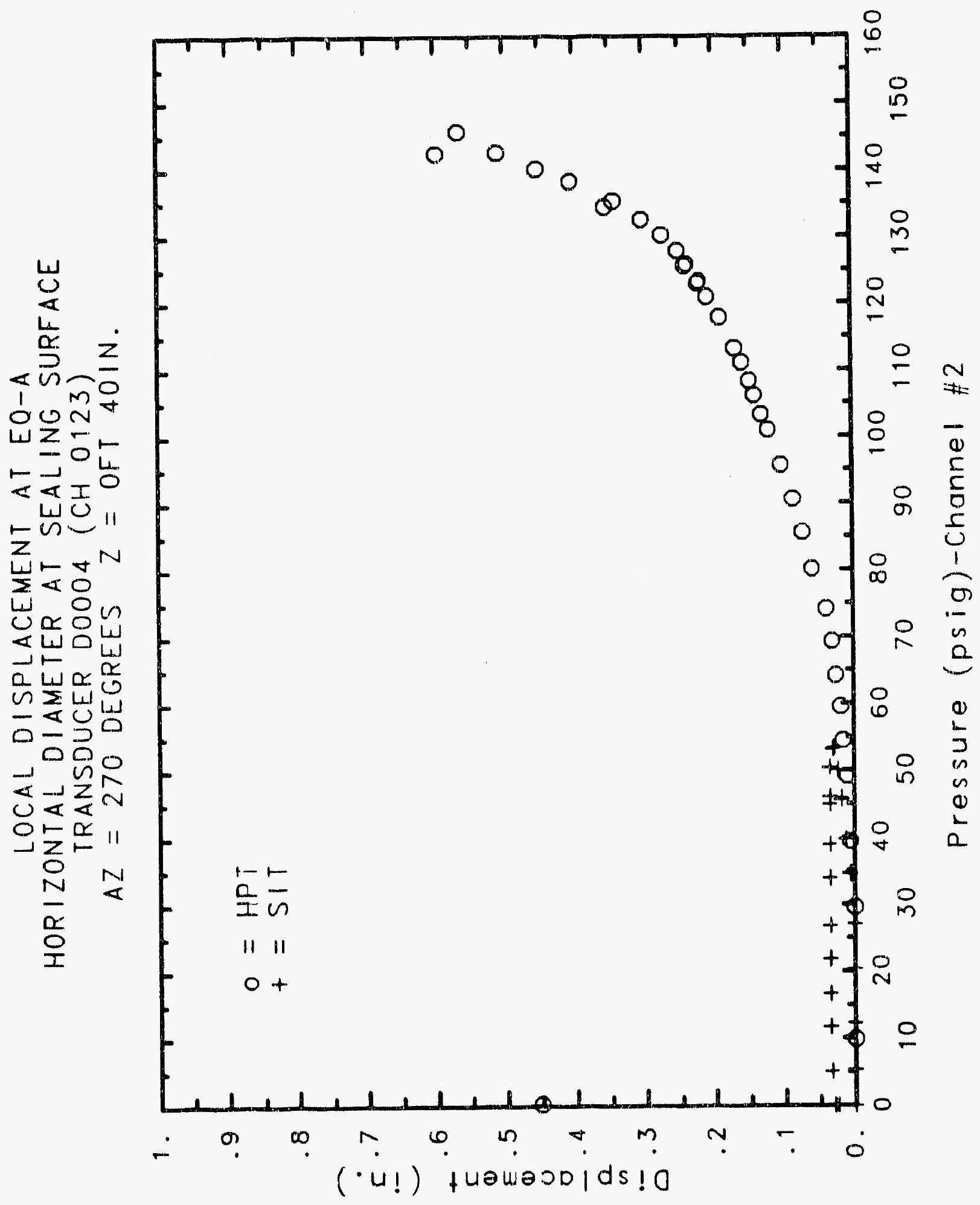




\title{
Reinforced Concrete Test Data
}

\author{
LVDT Channel 124
}

Structural INTEgRITY TEST

\begin{tabular}{|c|c|c|c|}
\hline $\begin{array}{l}\text { Pressure } \\
\text { (psig) } \\
-0.05 \\
5.33 \\
12.31 \\
16.44 \\
20.51 \\
20.46 \\
20.47 \\
27.05 \\
34.69 \\
34.53 \\
34.53 \\
39.70 \\
45.90 \\
45.65 \\
45.62 \\
50.10 \\
53.47 \\
53.29 \\
53.21 \\
50.49 \\
46.14 \\
46.13 \\
44.97 \\
38.98 \\
33.99 \\
33.96 \\
26.83 \\
21.88 \\
21.90 \\
16.69 \\
11.74 \\
11.77 \\
5.05 \\
0.02 \\
-0.04 \\
-0.02 \\
-0.02 \\
0.02\end{array}$ & $\begin{array}{c}\text { Displacement } \\
\text { (inches) } \\
-0.0037 \\
0.0075 \\
0.0105 \\
0.0020 \\
0.0042 \\
-0.0140 \\
-0.0110 \\
0.0055 \\
0.0013 \\
-0.0133 \\
-0.0098 \\
0.0025 \\
0.0012 \\
-0.0108 \\
-0.0086 \\
0.0018 \\
0.0041 \\
-0.0082 \\
-0.0078 \\
-0.0033 \\
0.0022 \\
-0.0015 \\
-0.0006 \\
0.0061 \\
0.0053 \\
-0.0091 \\
0.0067 \\
0.0080 \\
-0.0090 \\
0.0031 \\
0.0048 \\
-0.0086 \\
0.0048 \\
0.0067 \\
0.0258 \\
0.0095 \\
0.0134 \\
0.0108\end{array}$ & $\begin{array}{c}\text { Pressure } \\
(p s i g) \\
9.89 \\
19.55 \\
29.57 \\
39.42 \\
49.16 \\
54.50 \\
59.57 \\
64.20 \\
69.32 \\
74.16 \\
80.16 \\
85.61 \\
90.58 \\
95.69 \\
100.92 \\
103.25 \\
106.11 \\
108.31 \\
111.08 \\
113.24 \\
117.83 \\
120.92 \\
123.28 \\
122.97 \\
125.82 \\
125.60 \\
127.84 \\
130.19 \\
132.53 \\
135.33 \\
134.42 \\
138.35 \\
140.16 \\
142.63 \\
145.78 \\
142.52 \\
0.22 \\
\end{array}$ & $\begin{array}{c}\text { Displacement } \\
\text { (inches) } \\
0.0078 \\
0.0160 \\
0.0250 \\
0.0330 \\
0.0406 \\
0.0452 \\
0.0510 \\
0.0595 \\
0.0679 \\
0.0771 \\
0.0849 \\
0.0975 \\
0.1110 \\
0.1248 \\
0.1417 \\
0.1501 \\
0.1587 \\
0.1656 \\
0.1774 \\
0.1854 \\
0.2064 \\
0.2224 \\
0.2376 \\
0.2413 \\
0.2553 \\
0.2611 \\
0.2727 \\
0.2912 \\
0.3176 \\
0.3523 \\
0.3618 \\
0.4071 \\
0.4439 \\
0.4982 \\
0.5472 \\
0.5646 \\
0.3121\end{array}$ \\
\hline
\end{tabular}

High Pressure test
(inches)
0.0078
0.0160
0.0406
0.0510
0.0595
0.0679
0.0849
0.1248
0.1417
0.1656
0.1774
0.1854
0.2064
0.2413
2611
0.2727
0.2912
0.3176
0.4071
0.4439
0.4982
0.5472
0.5646
0.3121 


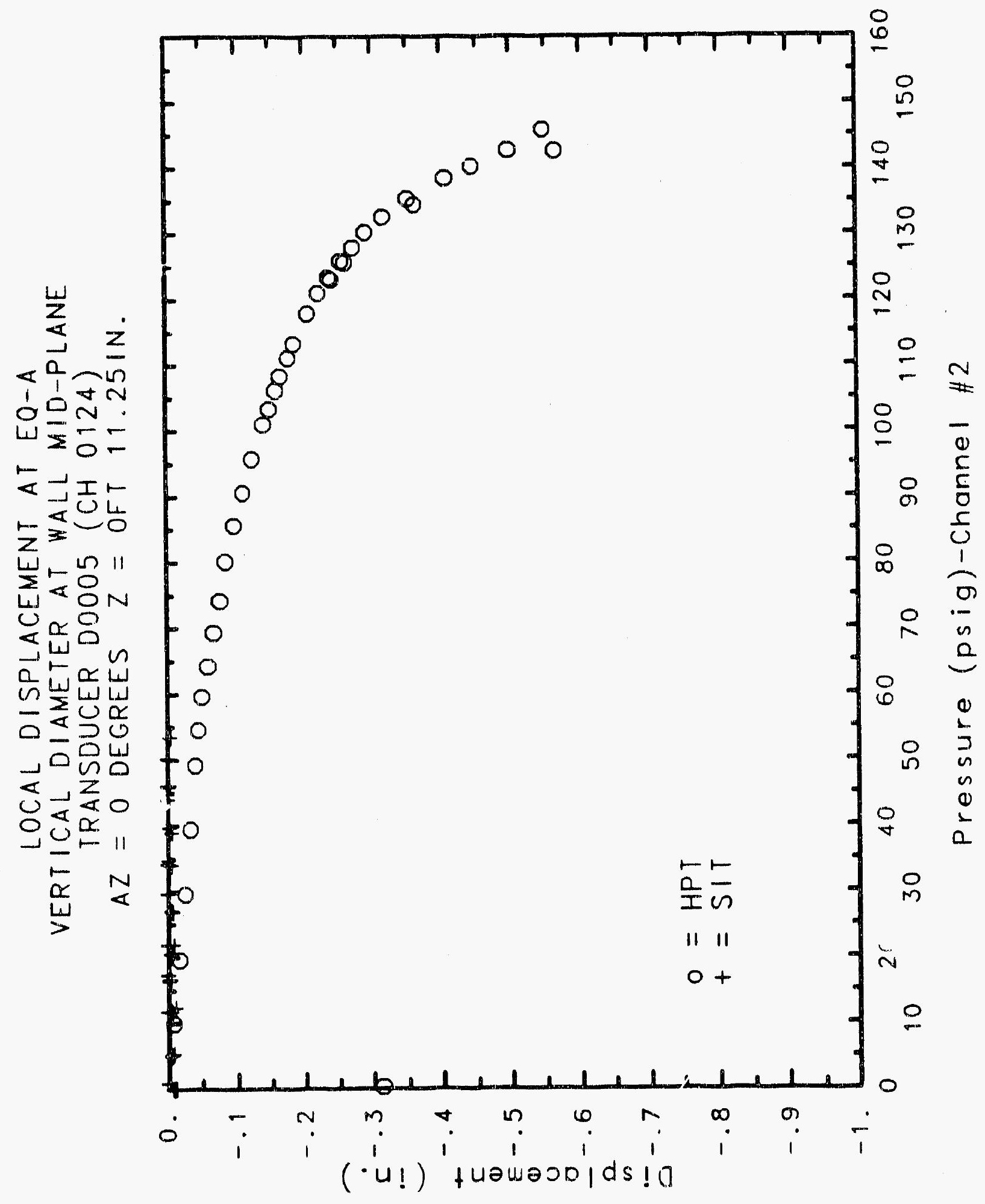




\title{
Reinforced Concrete Test Data
}

\author{
LVDT Channel 125
}

\section{STRUCTURAL INTEgRITY TEST}

\begin{tabular}{|c|c|c|c|}
\hline $\begin{array}{l}\text { Pressure } \\
\text { (psig) } \\
-0.05 \\
5.33 \\
12.31 \\
16.44 \\
20.51 \\
20.46 \\
20.47 \\
27.05 \\
34.69 \\
34.53 \\
34.53 \\
39.70 \\
45.90 \\
45.65 \\
45.62 \\
50.10 \\
53.47 \\
53.29 \\
53.21 \\
50.49 \\
46.14 \\
46.13 \\
44.97 \\
38.98 \\
\end{array}$ & $\begin{array}{c}\text { Displacement } \\
\text { (Inches) } \\
0.0000 \\
0.0001 \\
0.0002 \\
0.0004 \\
0.0007 \\
0.0009 \\
0.0010 \\
0.0006 \\
-0.0053 \\
-0.0052 \\
-0.0056 \\
-0.0099 \\
-0.0170 \\
-0.0186 \\
-0.0188 \\
-0.0220 \\
-0.0253 \\
-0.0280 \\
-0.0287 \\
-0.0291 \\
-0.0292 \\
-0.0292 \\
-0.0294 \\
-0.0295 \\
-0.0298 \\
-0.0297 \\
-0.0298 \\
-0.0299 \\
-0.0299 \\
-0.0299 \\
-0.0302 \\
-0.0305 \\
-0.0307 \\
-0.0308 \\
-0.0310 \\
-0.0306 \\
-0.0309 \\
-0.0310 \\
-0.0\end{array}$ & $\begin{array}{c}\text { Pressure } \\
(p s 1 g) \\
9.89 \\
19.55 \\
29.57 \\
39.42 \\
49.16 \\
54.50 \\
59.57 \\
64.20 \\
69.32 \\
74.18 \\
80.16 \\
85.61 \\
90.58 \\
95.69 \\
100.92 \\
10.25 \\
106.11 \\
108.31 \\
111.08 \\
113.24 \\
117.83 \\
120.92 \\
123.28 \\
122.97 \\
125.82 \\
125.60 \\
127.84 \\
130.19 \\
132.53 \\
135.33 \\
134.42 \\
138.35 \\
140.16 \\
142.63 \\
145.78 \\
142.52 \\
0.22\end{array}$ & $\begin{array}{c}\text { Displacement } \\
\text { (inches) } \\
0.0002 \\
-0.0011 \\
-0.0049 \\
-0.0099 \\
-0.0158 \\
-0.0185 \\
-0.0225 \\
-0.0273 \\
-0.0331 \\
-0.0401 \\
-0.0586 \\
-0.0699 \\
-0.0819 \\
-0.0970 \\
-0.1126 \\
-0.1209 \\
-0.1288 \\
-0.1350 \\
-0.1453 \\
-0.1537 \\
-0.1723 \\
-0.1861 \\
-0.1950 \\
-0.1957 \\
-0.2088 \\
-0.2121 \\
-0.2199 \\
-0.2370 \\
-0.2584 \\
-0.2905 \\
-0.2991 \\
-0.3414 \\
-0.3764 \\
-0.4235 \\
-0.4701 \\
-0.4975 \\
-0.3264\end{array}$ \\
\hline
\end{tabular}

High Pressure test

splacement

0.0002

$-0.0011$

$-0.0099$

$-0.0158$

$-0.0185$

$-0,0225$

$-0.0273$

$-0.0586$

$-0.0699$

$-0.0819$

$-0.0970$

$-0.1126$

$-0.1350$

$-0.1453$

$-0.1537$

$-0.1723$

$-0.1861$

$-0.1950$

$-0.2121$

$-0.2199$

$-0.2370$

$-0.2584$

$-0.2905$

$-0.3764$

$-0.4235$

$-0.4975$

$-0.3264$ 


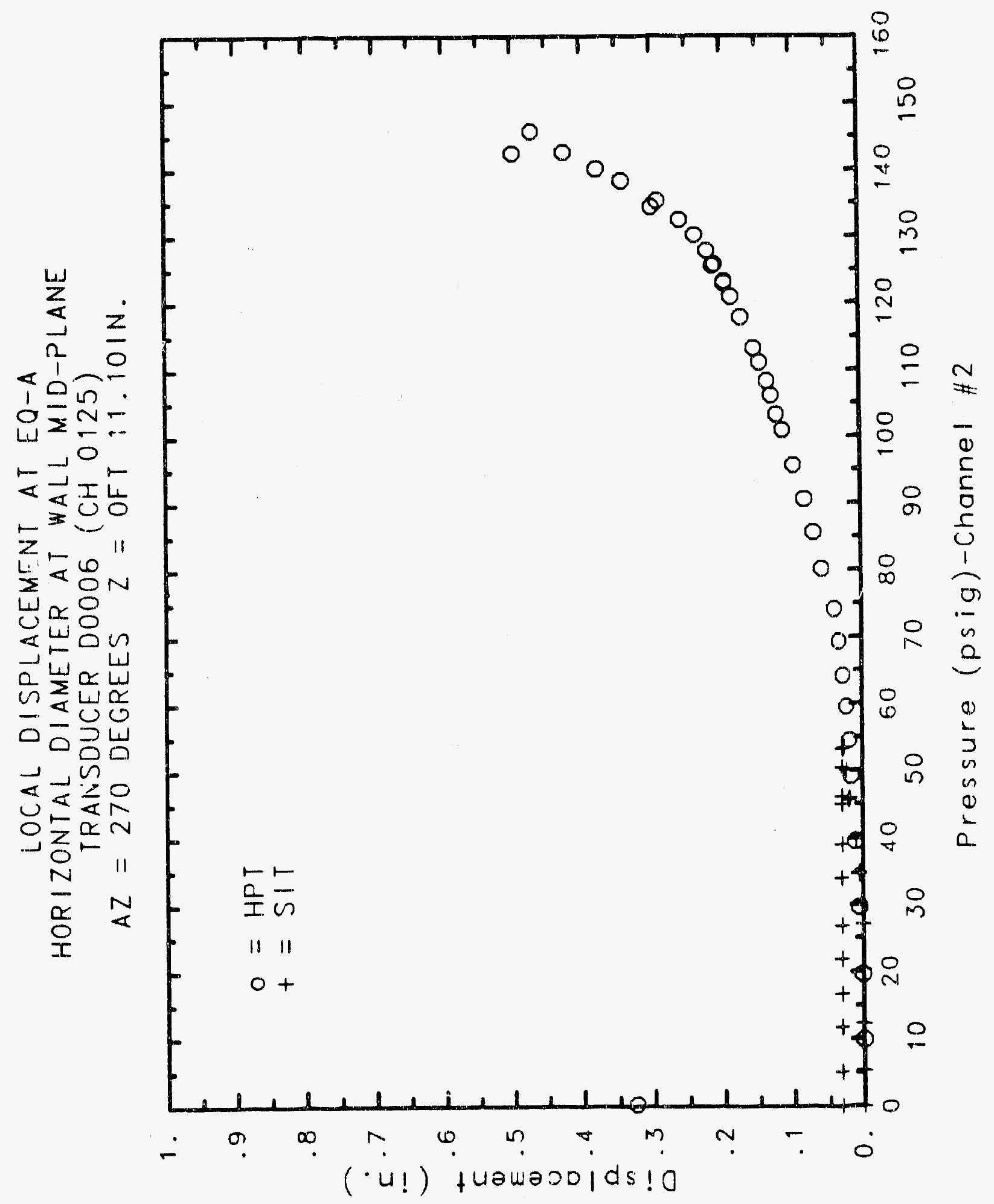




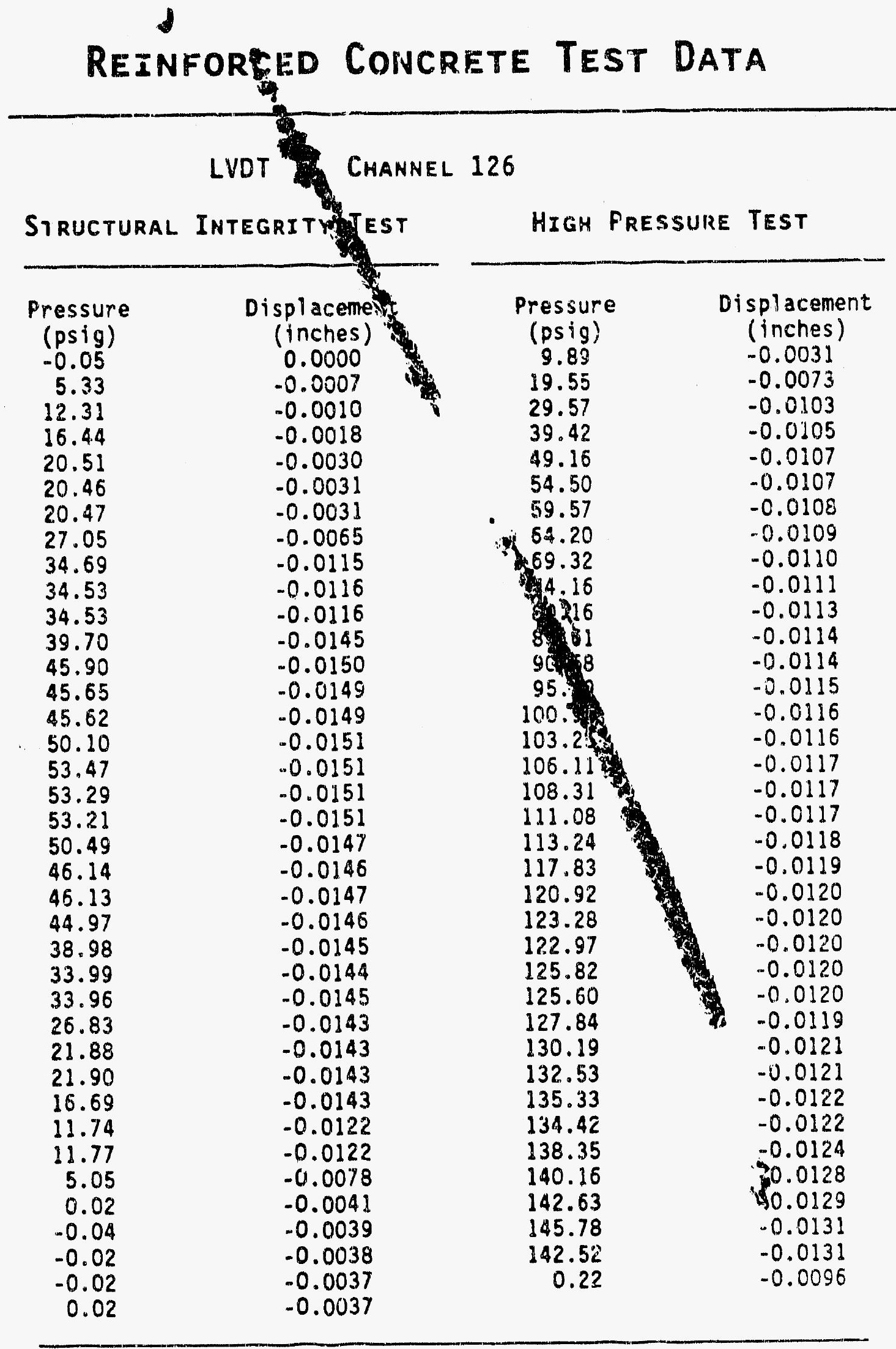




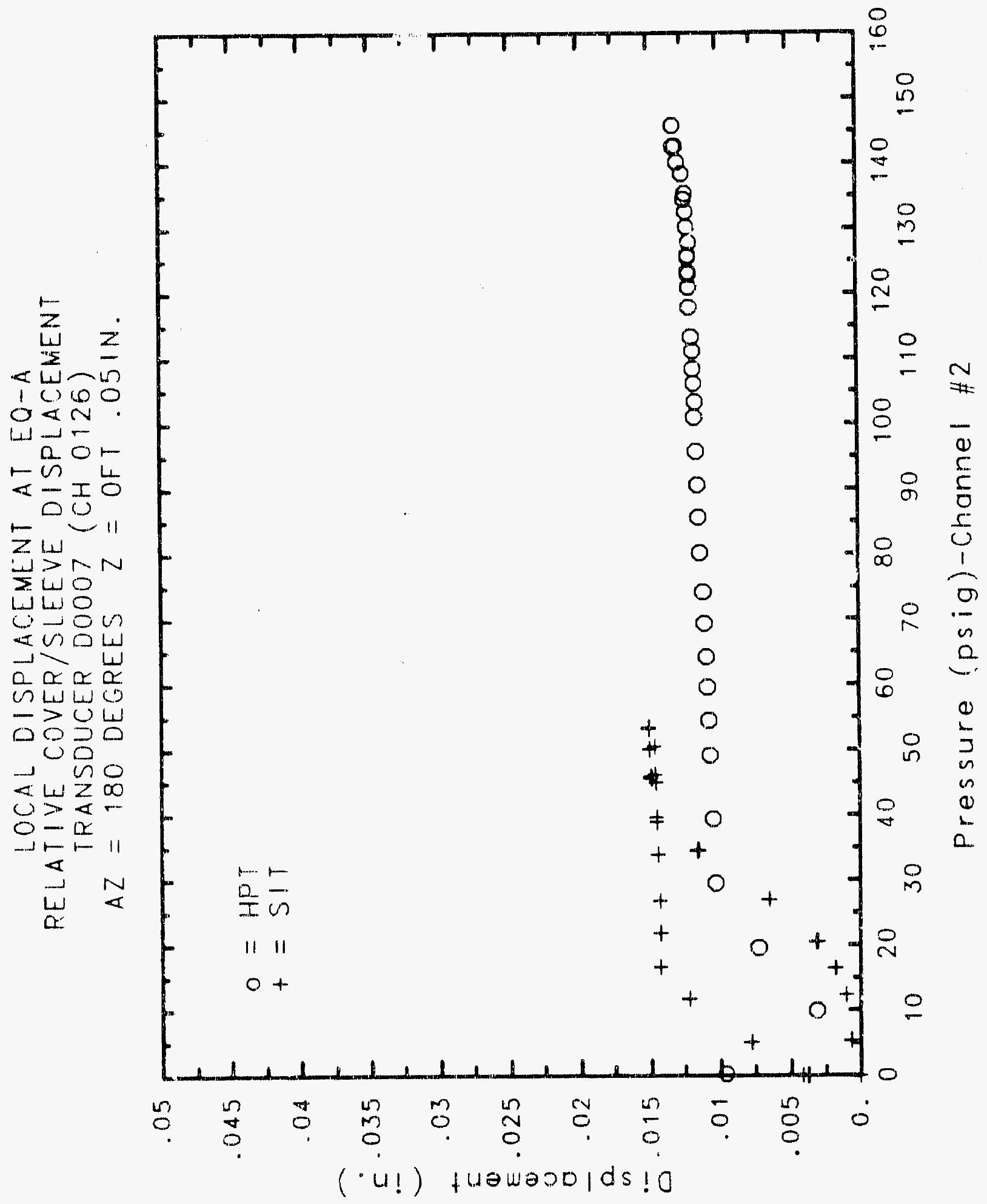




\section{Reinforced Concrete Test Data}

lvdi Channel 127

Structural Tntegrity Test

\begin{tabular}{|c|c|c|c|}
\hline $\begin{array}{l}\text { Pressure } \\
\text { (psig) } \\
-0.05 \\
5.33 \\
12.31 \\
16.44 \\
20.51 \\
20.46 \\
20.47 \\
27.05 \\
34.69 \\
34.53 \\
34.53 \\
39.70 \\
45.90 \\
45.65 \\
45.62 \\
50.10 \\
53.47 \\
53.29 \\
53.21 \\
50.49 \\
46.14 \\
46.13 \\
44.97 \\
38.98 \\
33.99 \\
33.96 \\
26.83 \\
21.88 \\
21.90 \\
16.69 \\
11.74 \\
11.77 \\
5.05 \\
0.02 \\
-0.04 \\
-0.02 \\
-0.02 \\
0.02\end{array}$ & $\begin{array}{c}\text { Displacement } \\
\text { (inches) } \\
0.0000 \\
-0.0011 \\
-0.0015 \\
-0.0021 \\
-0.0031 \\
-0.0031 \\
-0.0031 \\
-0.0059 \\
-0.0102 \\
-0.0103 \\
-0.0103 \\
-0.0129 \\
-0.0162 \\
-0.0164 \\
-0.0164 \\
-0.0188 \\
-0.0207 \\
-0.0207 \\
-0.0207 \\
-0.0214 \\
-0.0215 \\
-0.0214 \\
-0.0215 \\
-0.0217 \\
-0.0219 \\
-0.0219 \\
-0.0214 \\
-0.0189 \\
-0.0189 \\
-0.0155 \\
-0.0121 \\
-0.0121 \\
-0.0081 \\
-0.0044 \\
-0.0039 \\
-0.0037 \\
-0.0037 \\
-0.0036 \\
-0.00\end{array}$ & $\begin{array}{c}\text { Pressure } \\
(\text { psig) } \\
9.89 \\
19.55 \\
29.57 \\
39.42 \\
49.16 \\
54.50 \\
59.57 \\
64.20 \\
69.32 \\
74.16 \\
80.16 \\
85.61 \\
90.58 \\
95.69 \\
100.92 \\
103.25 \\
106.11 \\
108.31 \\
111.08 \\
113.24 \\
117.83 \\
120.92 \\
123.28 \\
122.97 \\
125.82 \\
125.60 \\
127.84 \\
130.19 \\
132.53 \\
135.33 \\
134.42 \\
138.35 \\
140.16 \\
142.63 \\
145.78 \\
142.52 \\
0.22 \\
\end{array}$ & $\begin{array}{c}\text { Displacement } \\
\text { (inches) } \\
-0.0032 \\
-0.0068 \\
-0.0105 \\
-0.0145 \\
-0.0174 \\
-0.0191 \\
-0.0212 \\
-0.0238 \\
-0.0268 \\
-0.0303 \\
-0.0365 \\
-0.0418 \\
-0.0471 \\
-0.0531 \\
-0.0591 \\
-0.0647 \\
-0.0661 \\
-0.0680 \\
-0.0743 \\
-0.0754 \\
-0.0804 \\
-0.0907 \\
-0.0937 \\
-0.0938 \\
-0.1012 \\
-0.1013 \\
-0.1090 \\
-0.1119 \\
-0.1229 \\
-0.1378 \\
-0.1386 \\
-0.1639 \\
-0.1860 \\
-0.2040 \\
-0.2254 \\
-0.2371 \\
-0.1939\end{array}$ \\
\hline
\end{tabular}

high Pressure test

splacement

$-0.0032$

$-0.0145$

0.0212

$-0.0303$

$-0.0418$

$-0.0471$

$-0.0647$

$-0.0661$

$-0.0680$

$-0.0743$

0.0907

$-0.0938$

$-0.1012$

0.1013

0.1090

0.1378

$-0.1386$

$-0.1639$

$-0.1860$

0.2040

(3)

$-0.1939$ (inches) 


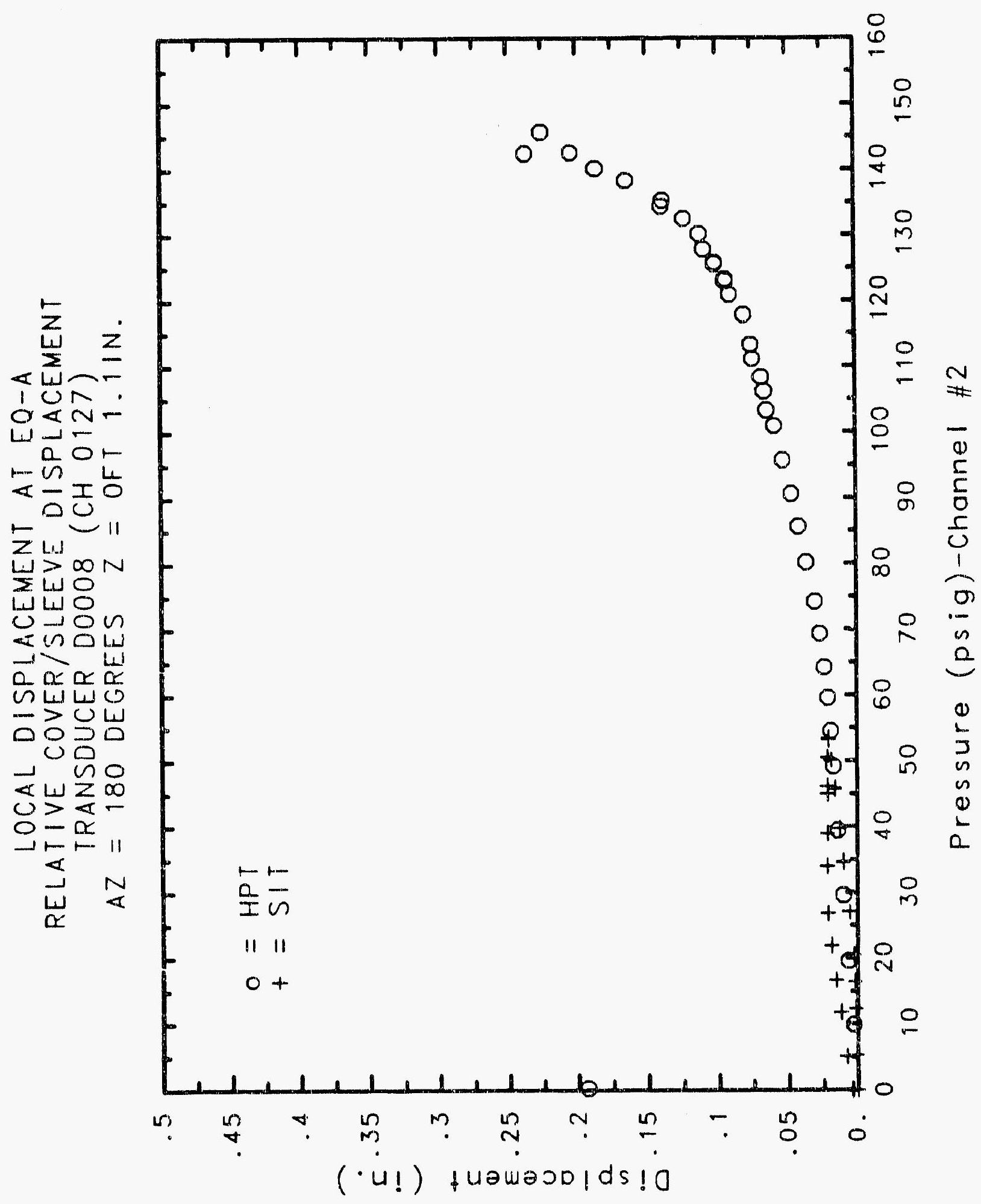




\title{
Reinforced Concrete Test Data
}

\author{
lvot Channel 128
}

\begin{tabular}{|c|c|c|c|}
\hline \multicolumn{2}{|c|}{ STRUCTURAL INTEGRITY TEST } & \multicolumn{2}{|c|}{ High Pressure Test } \\
\hline $\begin{array}{l}\text { Pressure } \\
\text { (psig) } \\
-0.05 \\
5.33 \\
12.31 \\
16.44 \\
20.51 \\
20.46 \\
20.47 \\
27.05 \\
34.69 \\
34.53 \\
34.53 \\
39.70 \\
45.90 \\
45.65 \\
45.62 \\
50.10 \\
53.47 \\
53.29 \\
53.21 \\
50.49 \\
46.14 \\
46.13 \\
44.97 \\
38.98 \\
33.99 \\
33.96 \\
26.83 \\
21.88 \\
21.90 \\
16.69 \\
11.74 \\
11.77 \\
5.05 \\
0.02 \\
-0.04 \\
-0.02 \\
-0.02 \\
0.02 \\
\end{array}$ & $\begin{array}{c}\text { Displacement } \\
\text { (inches) } \\
\text {-0.0001 } \\
0.0016 \\
0.0026 \\
0.0029 \\
0.0035 \\
0.0035 \\
0.0036 \\
0.0052 \\
0.0078 \\
0.0080 \\
0.0081 \\
0.0093 \\
0.0112 \\
0.0114 \\
0.0114 \\
0.0129 \\
0.0141 \\
0.0142 \\
0.0143 \\
0.0148 \\
0.0148 \\
0.0149 \\
0.0148 \\
0.0147 \\
0.0148 \\
0.0147 \\
0.0143 \\
0.0133 \\
0.0134 \\
0.0114 \\
0.0092 \\
0.0092 \\
0.0062 \\
0.0031 \\
0.0027 \\
0.0023 \\
0.0023 \\
0.0023 \\
0.04\end{array}$ & $\begin{array}{c}\text { Pressure } \\
\text { (psig) } \\
9.89 \\
19.55 \\
29.57 \\
39.42 \\
49.16 \\
54.50 \\
59.57 \\
64.20 \\
69.32 \\
74.16 \\
80.16 \\
85.61 \\
90.58 \\
95.69 \\
100.92 \\
103.25 \\
106.11 \\
108.31 \\
111.08 \\
113.24 \\
117.83 \\
120.92 \\
123.28 \\
122.97 \\
125.82 \\
125.60 \\
127.84 \\
130.19 \\
132.53 \\
135.33 \\
134.42 \\
138.35 \\
140.16 \\
142.63 \\
145.78 \\
142.52 \\
0.22 \\
\end{array}$ & $\begin{array}{c}\text { Displacement } \\
\text { (inches) } \\
0.0050 \\
0.0077 \\
0.0099 \\
0.0117 \\
0.0133 \\
0.0142 \\
0.0158 \\
0.0178 \\
0.0206 \\
0.0244 \\
0.0306 \\
0.0368 \\
0.0431 \\
0.0504 \\
0.0586 \\
0.0629 \\
0.0672 \\
0.0707 \\
0.0761 \\
0.0803 \\
0.0904 \\
0.0981 \\
0.1051 \\
0.1062 \\
0.1150 \\
0.1168 \\
0.1227 \\
0.1340 \\
0.1486 \\
0.1683 \\
0.1731 \\
0.2007 \\
0.2235 \\
0.2550 \\
0.2843 \\
0.3011 \\
0.2216\end{array}$ \\
\hline
\end{tabular}




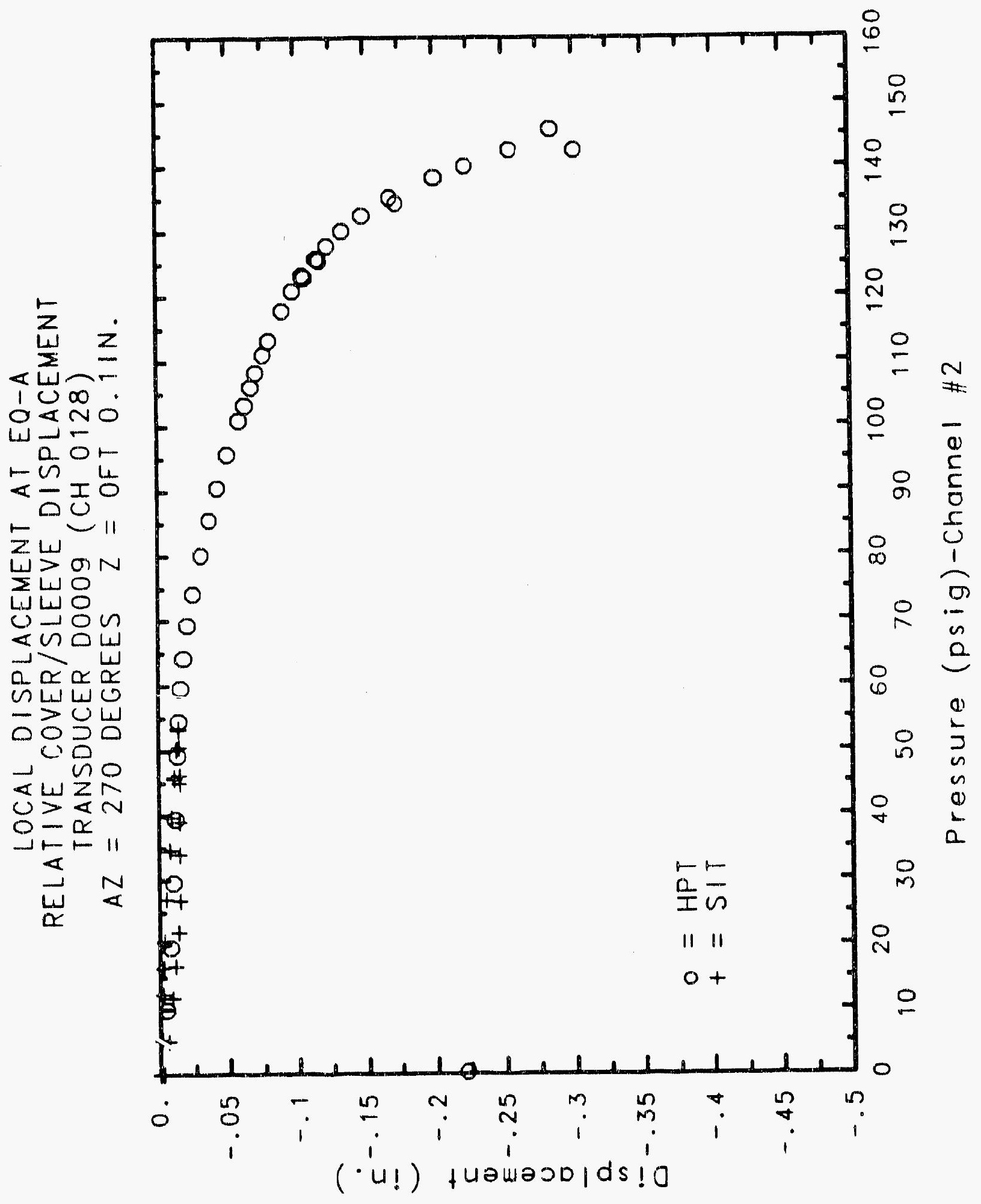




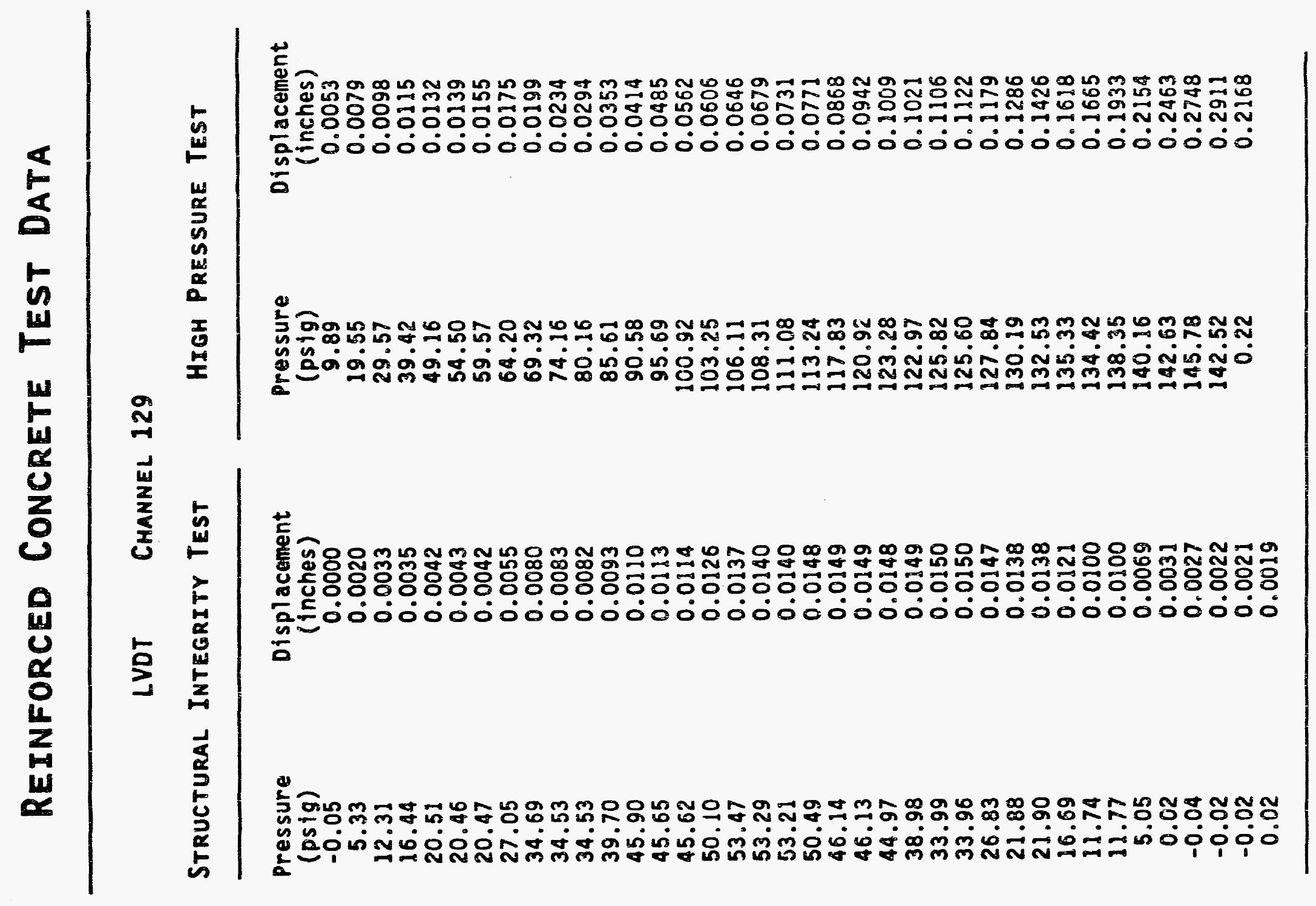




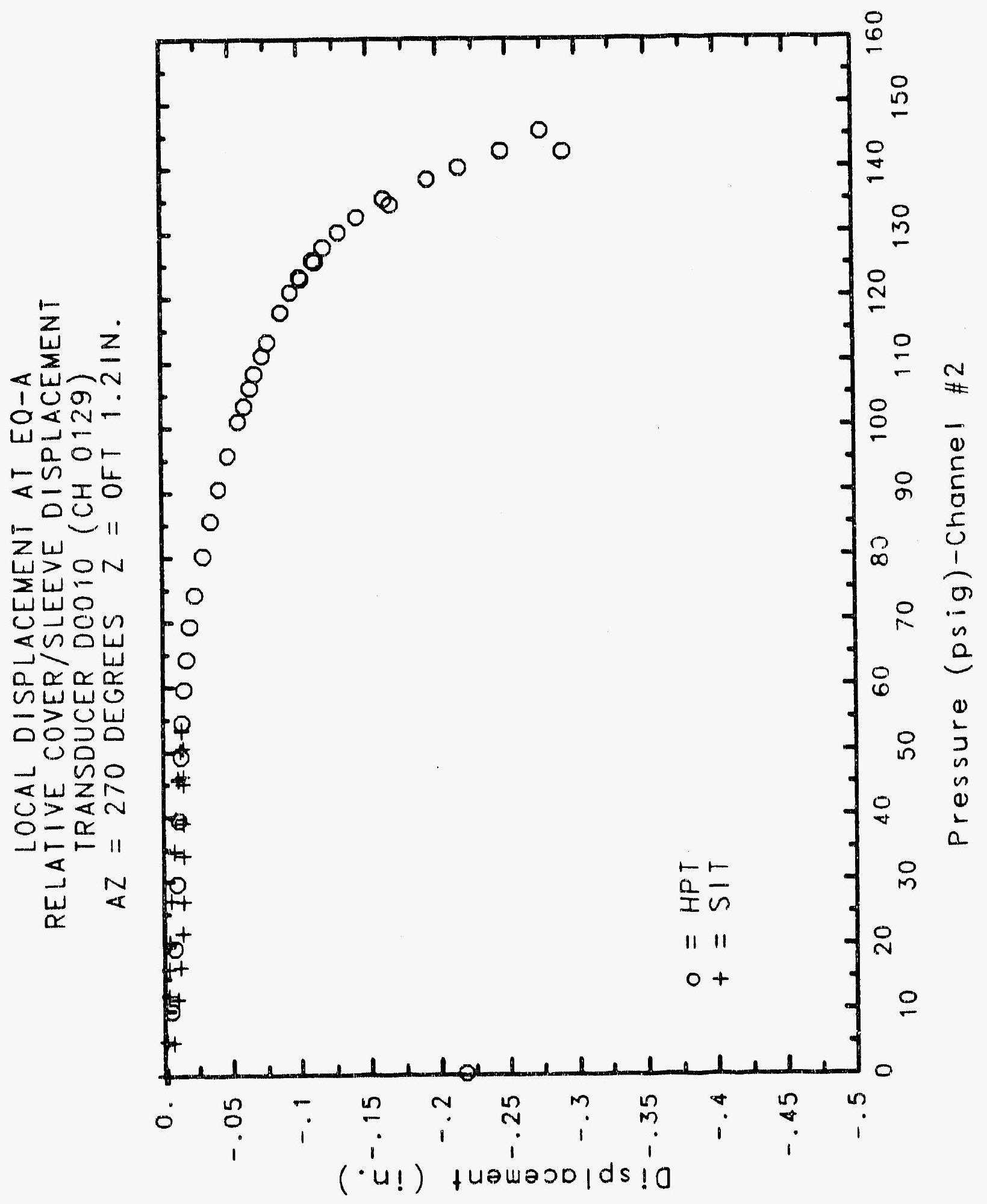




\section{Reinforced Concrete Test Data}

LVDt Channel 130

Structural Integrity Test

\begin{tabular}{|c|c|c|c|}
\hline $\begin{array}{c}\text { Pressure } \\
(p s i g) \\
-0.05 \\
5.33 \\
12.31 \\
16.44 \\
20.51 \\
20.46 \\
20.47 \\
27.05 \\
34.69 \\
34.53 \\
34.53 \\
39.70 \\
45.90 \\
45.65 \\
45.62 \\
50.10 \\
53.47 \\
53.29 \\
53.21 \\
50.49 \\
46.14 \\
46.13 \\
44.97 \\
38.98 \\
33.99 \\
33.96 \\
26.83 \\
21.88 \\
21.90 \\
16.69 \\
11.74 \\
11.77 \\
5.05 \\
0.02 \\
-0.04 \\
-0.02 \\
-0.02 \\
0.02 \\
\end{array}$ & $\begin{array}{c}\text { Displacement } \\
\text { (inches) } \\
0.0000 \\
-0.0028 \\
-0.0036 \\
-0.0039 \\
-0.0049 \\
-0.0049 \\
-0.0048 \\
-0.0086 \\
-0.0136 \\
-0.0135 \\
-0.0136 \\
-0.0164 \\
-0.0201 \\
-0.0701 \\
-0.0 .70 \\
-0.0228 \\
-0.0249 \\
-0.0250 \\
-0.0250 \\
-0.0260 \\
-0.0261 \\
-0.0261 \\
-0.0261 \\
-0.0260 \\
-0.0259 \\
-0.0259 \\
-0.0254 \\
-0.0235 \\
-0.0235 \\
-0.0198 \\
-0.0157 \\
-0.0156 \\
-0.0086 \\
-0.0018 \\
-0.0016 \\
-0.0015 \\
-0.0014 \\
-0.0013 \\
-0.00\end{array}$ & $\begin{array}{l}\text { Pressure } \\
(\text { psig) } \\
9.89 \\
19.55 \\
29.57 \\
39.42 \\
49.16 \\
54.50 \\
59.57 \\
64.20 \\
69.32 \\
74.16 \\
80.16 \\
85.61 \\
90.58 \\
95.69 \\
100.92 \\
103.25 \\
106.11 \\
108.31 \\
111.08 \\
113.24 \\
117.83 \\
120.92 \\
123.28 \\
122.97 \\
125.82 \\
125.60 \\
127.84 \\
130.19 \\
132.53 \\
135.33 \\
134.42 \\
138.35 \\
140.16 \\
142.63 \\
145.78 \\
142.52 \\
0.22\end{array}$ & $\begin{array}{c}\text { Displacement } \\
\text { (inches) } \\
-0.0042 \\
-0.0057 \\
-0.0103 \\
-0.0147 \\
-0.0179 \\
-0.0188 \\
-0.0221 \\
-0.0253 \\
-0.0297 \\
-0.0317 \\
-0.0375 \\
-0.0434 \\
-0.0492 \\
-0.0558 \\
-0.0624 \\
-0.0692 \\
-0.0713 \\
-0.0736 \\
-0.0791 \\
-0.0817 \\
-0.0884 \\
-0.0989 \\
-0.1052 \\
-0.1053 \\
-0.1110 \\
-0.1110 \\
-0.1222 \\
-0.1271 \\
-0.1407 \\
-0.1600 \\
-0.1613 \\
-0.1840 \\
-0.1981 \\
-0.2201 \\
-0.2518 \\
-0.2629 \\
-0.1856\end{array}$ \\
\hline
\end{tabular}

High Pressure test

9.89

19.55

39.42

49.16

54.50

59.57

74.16

80.16

85.61

100.92

113.24

117.83

120.92

122.97

125.82

125.60

127.84

130.19

132.53

134.42

138.35

140.16

42.52

0.22 splacement

$-0.0057$

$-0.0103$

$-0.0147$

$-0.0179$

$-0.0188$

$-0.0297$

$-0.0317$

$-0.0375$

$-0.0434$

$-0.0492$

0.0558

$-0.0692$

$-0.0713$

$-0.0736$

$-0.0791$

$-0.0989$

$-0.1052$

$-0.1053$

$-0.1110$

$-0.1110$

$-0.1407$

$-0.1600$

$-0.1613$

$-0.1840$

$-0.1981$

$-0.2629$

$-0.1856$ 


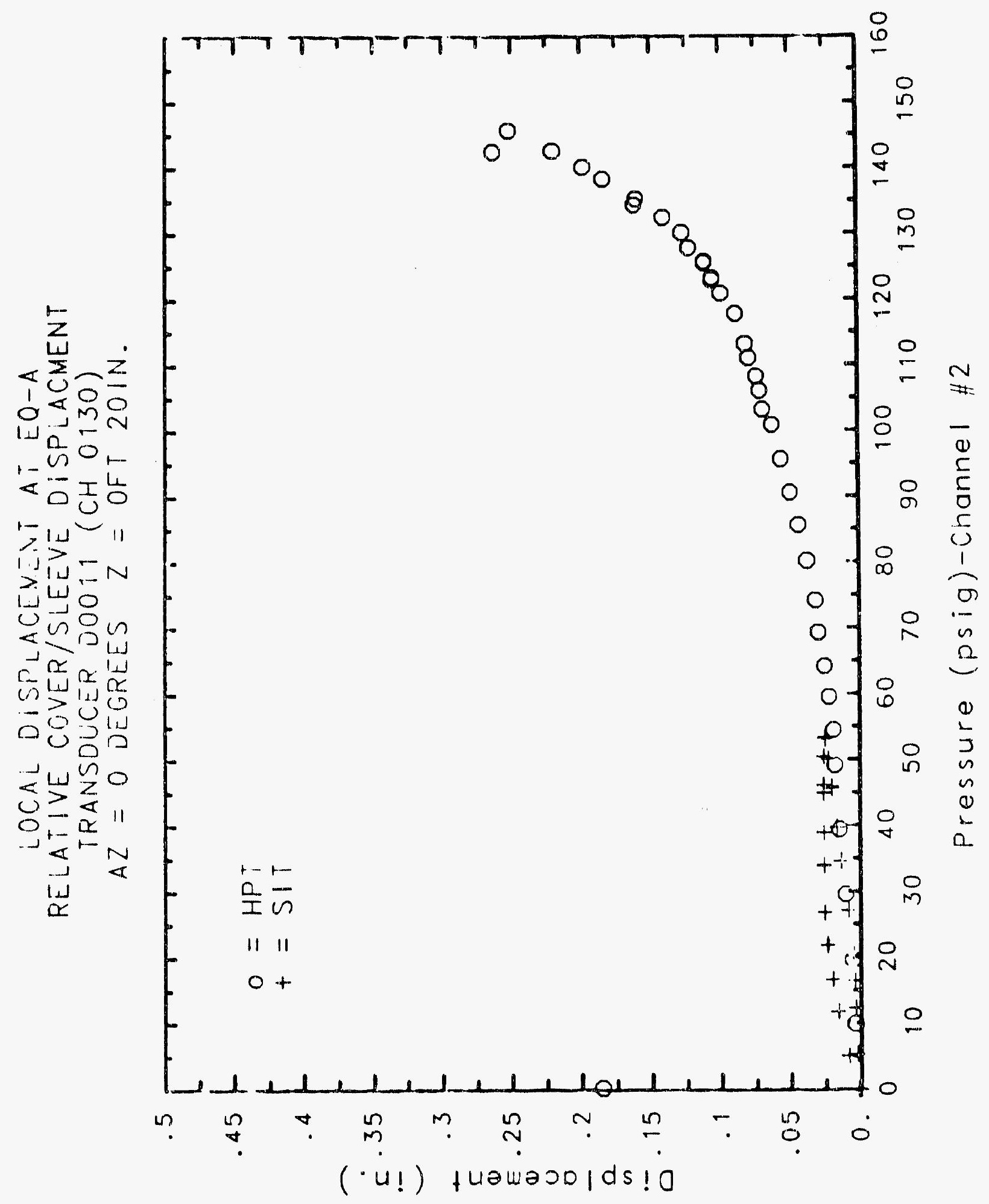




\section{Retnforced Concrete Test Data}

lvot Channel 131

Structural Integrity Test

\begin{tabular}{|c|c|c|c|}
\hline $\begin{array}{c}\text { Pressure } \\
\text { (psig) } \\
-0.05 \\
5.33 \\
12.31 \\
16.44 \\
20.51 \\
20.46 \\
20.47 \\
27.05 \\
34.69 \\
34.53 \\
34.53 \\
39.70 \\
45.90 \\
45.65 \\
45.62 \\
50.10 \\
53.47 \\
53.29 \\
53.21 \\
50.49 \\
46.14 \\
46.13 \\
44.97 \\
38.98 \\
33.99 \\
33.96 \\
26.83 \\
21.88 \\
21.90 \\
16.69 \\
11.74 \\
11.77 \\
5.05 \\
0.02 \\
-0.04 \\
-0.02 \\
-0.02 \\
0.02 \\
\end{array}$ & $\begin{array}{c}\text { Displacement } \\
\text { (inches) } \\
-0.0001 \\
-0.0029 \\
-0.0041 \\
-0.0044 \\
-0.0052 \\
-0.0052 \\
-0.0051 \\
-0.0084 \\
-0.0129 \\
-0.0129 \\
-0.0129 \\
-0.0154 \\
-0.0187 \\
-0.0187 \\
-0.0188 \\
-0.0212 \\
-0.0231 \\
-0.0232 \\
-0.0232 \\
-0.0244 \\
-0.0244 \\
-0.0244 \\
-0.0246 \\
-0.0248 \\
-0.0249 \\
-0.0249 \\
-0.0244 \\
-0.0225 \\
-0.0226 \\
-0.0191 \\
-0.0152 \\
-0.0152 \\
-0.0084 \\
-0.0016 \\
-0.0013 \\
-0.0014 \\
-0.0013 \\
-0.0011\end{array}$ & $\begin{array}{c}\text { Pressure } \\
(\text { psig) } \\
9.89 \\
19.55 \\
29.57 \\
39.42 \\
49.16 \\
54.50 \\
59.57 \\
64.20 \\
69.32 \\
74.16 \\
80.16 \\
85.61 \\
90.58 \\
95.69 \\
100.92 \\
103.25 \\
106.11 \\
108.31 \\
111.08 \\
113.24 \\
117.83 \\
120.92 \\
123.28 \\
122.97 \\
125.82 \\
125.60 \\
127.84 \\
130.19 \\
132.53 \\
135.33 \\
134.42 \\
138.35 \\
140.16 \\
142.63 \\
145.78 \\
142.52 \\
0.22\end{array}$ & $\begin{array}{c}\text { Displacement } \\
\text { (inches) } \\
-0.0045 \\
-0.0058 \\
-0.0100 \\
-0.0141 \\
-0.0167 \\
-0.0174 \\
-0.0204 \\
-0.0234 \\
-0.0275 \\
-0.0294 \\
-0.0351 \\
-0.0411 \\
-0.0467 \\
-0.0533 \\
-0.0598 \\
-0.0665 \\
-0.0687 \\
-0.0711 \\
-0.0766 \\
-0.0791 \\
-0.0860 \\
-0.0964 \\
-0.1026 \\
-0.1028 \\
-0.1083 \\
-0.1085 \\
-0.1196 \\
-0.1243 \\
-0.1380 \\
-0.1570 \\
-0.1582 \\
-0.1803 \\
-0.1937 \\
-0.2151 \\
-0.2456 \\
-0.2559 \\
-0.1860\end{array}$ \\
\hline
\end{tabular}

High Pressure test 


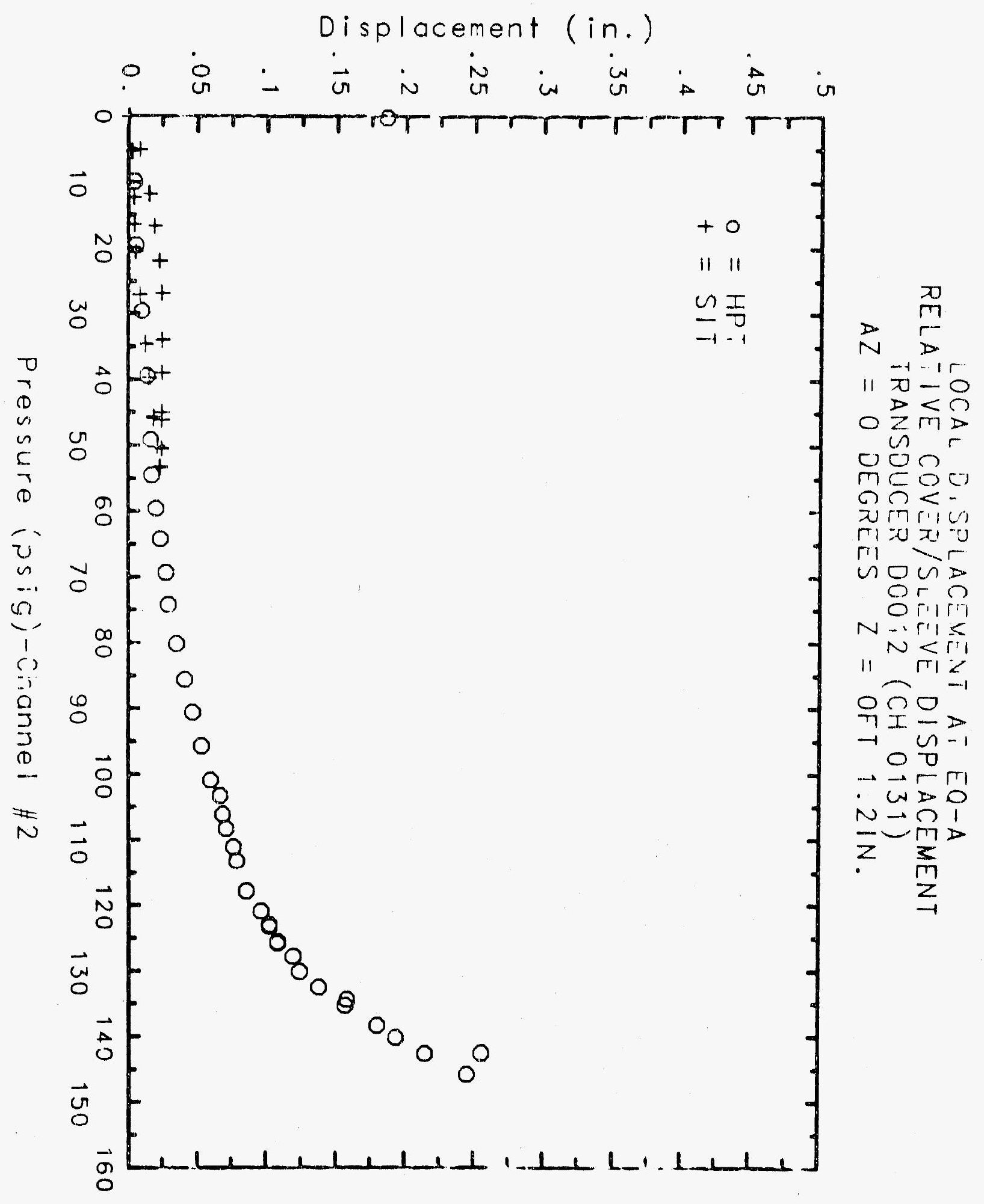




\section{Reinforced Concrete Test Data}

LVDt Channel 132

STRUCTURAL INTEGRITY TEST

\begin{tabular}{|c|c|c|c|}
\hline $\begin{array}{c}\text { Pressure } \\
\text { (psig) } \\
-0.05 \\
5.33 \\
12.31 \\
16.44 \\
20.51 \\
20.46 \\
20.47 \\
27.05 \\
34.69 \\
34.53 \\
34.53 \\
39.70 \\
45.90 \\
45.65 \\
45.62 \\
50.10 \\
53.47 \\
53.29 \\
53.21 \\
50.49 \\
46.14 \\
46.13 \\
44.97 \\
38.98 \\
33.99 \\
33.96 \\
26.83 \\
21.88 \\
21.90 \\
16.69 \\
11.74 \\
11.77 \\
5.05 \\
0.02 \\
-0.04 \\
-0.02 \\
-0.02 \\
0.02 \\
\end{array}$ & $\begin{array}{c}\text { Displacement } \\
\text { (1nches) } \\
0.0000 \\
0.0014 \\
0.0017 \\
0.0017 \\
0.0018 \\
0.0019 \\
0.0019 \\
0.0031 \\
0.0059 \\
0.0063 \\
0.0064 \\
0.0080 \\
0.0106 \\
0.0110 \\
0.0110 \\
0.0123 \\
0.0138 \\
0.0141 \\
0.0142 \\
0.0149 \\
0.0149 \\
0.0149 \\
0.0149 \\
0.0148 \\
0.0148 \\
0.0148 \\
0.0147 \\
0.0141 \\
0.0140 \\
0.0122 \\
0.0098 \\
0.0097 \\
0.0066 \\
0.0043 \\
0.0038 \\
0.0034 \\
0.0031 \\
0.0029 \\
0.03\end{array}$ & $\begin{array}{c}\text { Pressure } \\
\text { (ps g) } \\
9.89 \\
19.55 \\
29.57 \\
39.42 \\
49.16 \\
54.50 \\
59.57 \\
64.20 \\
69.32 \\
74.16 \\
80.16 \\
85.61 \\
90.58 \\
95.69 \\
100.92 \\
103.25 \\
106.11 \\
108.31 \\
111.08 \\
113.24 \\
117.83 \\
120.92 \\
123.28 \\
122.97 \\
125.82 \\
125.60 \\
127.84 \\
130.19 \\
132.53 \\
135.33 \\
134.42 \\
138.35 \\
140.16 \\
142.63 \\
145.78 \\
142.52 \\
0.22 \\
\end{array}$ & $\begin{array}{c}\text { Displacement } \\
\text { (inches) } \\
0.0032 \\
0.0051 \\
0.0075 \\
0.0095 \\
0.0112 \\
0.0121 \\
0.0138 \\
0.0159 \\
0.0188 \\
0.0222 \\
0.0284 \\
0.0342 \\
0.0404 \\
0.0478 \\
0.0558 \\
0.0598 \\
0.0639 \\
0.0672 \\
0.0726 \\
0.0765 \\
0.0865 \\
0.0944 \\
0.1008 \\
0.1022 \\
0.1098 \\
0.1117 \\
0.1176 \\
0.1280 \\
0.1426 \\
0.1635 \\
0.1689 \\
0.1947 \\
0.2161 \\
0.2464 \\
0.2748 \\
0.2901 \\
0.2181\end{array}$ \\
\hline
\end{tabular}

high Pressure test

$$
\begin{aligned}
& \text { splacement } \\
& \text { (inches) } \\
& 0.0032 \\
& 0.0051 \\
& 0.0075 \\
& 0.0095 \\
& 0.0112 \\
& 0.0121 \\
& 0.0138 \\
& 0.0159 \\
& 0.0188 \\
& 0.0222 \\
& 0.0284 \\
& 0.0342 \\
& 0.0404 \\
& 0.0478 \\
& 0.0558 \\
& 0.0598 \\
& 0.0639 \\
& 0.0672 \\
& 0.0726 \\
& 0.0765 \\
& 0.0865 \\
& 0.0944 \\
& 0.1008 \\
& 0.1022 \\
& 0.1098 \\
& 0.1117 \\
& 0.1176 \\
& 0.1280 \\
& 0.1426 \\
& 0.1635 \\
& 0.1689 \\
& 0.1947 \\
& 0.2161 \\
& 0.2464 \\
& 0.2748 \\
& 0.2901 \\
& 0.2181
\end{aligned}
$$




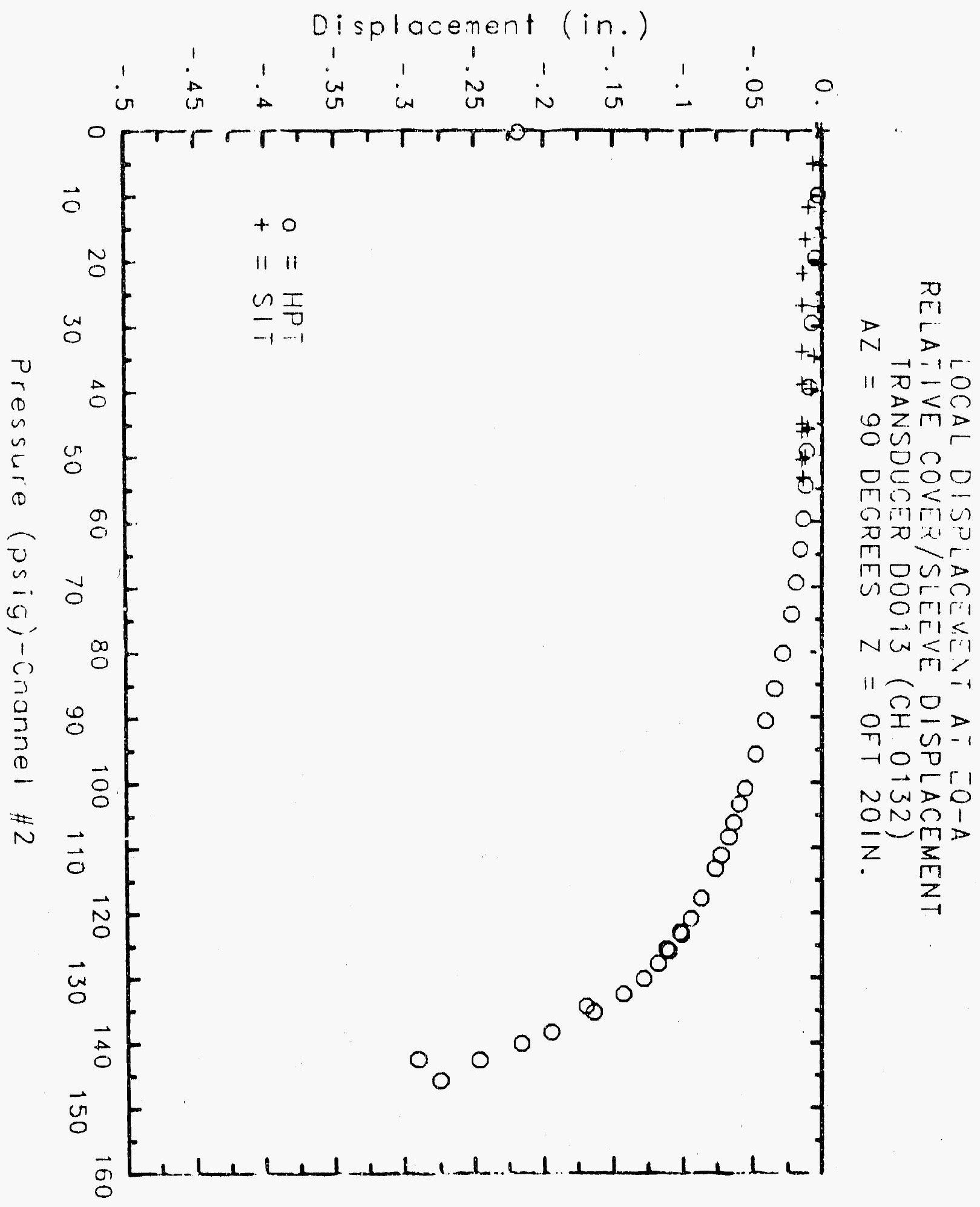




\title{
Reinforced Concrete Test Data
}

\author{
LVDt Channel 133
}

STRUCTURAL INTEgRITY TEST

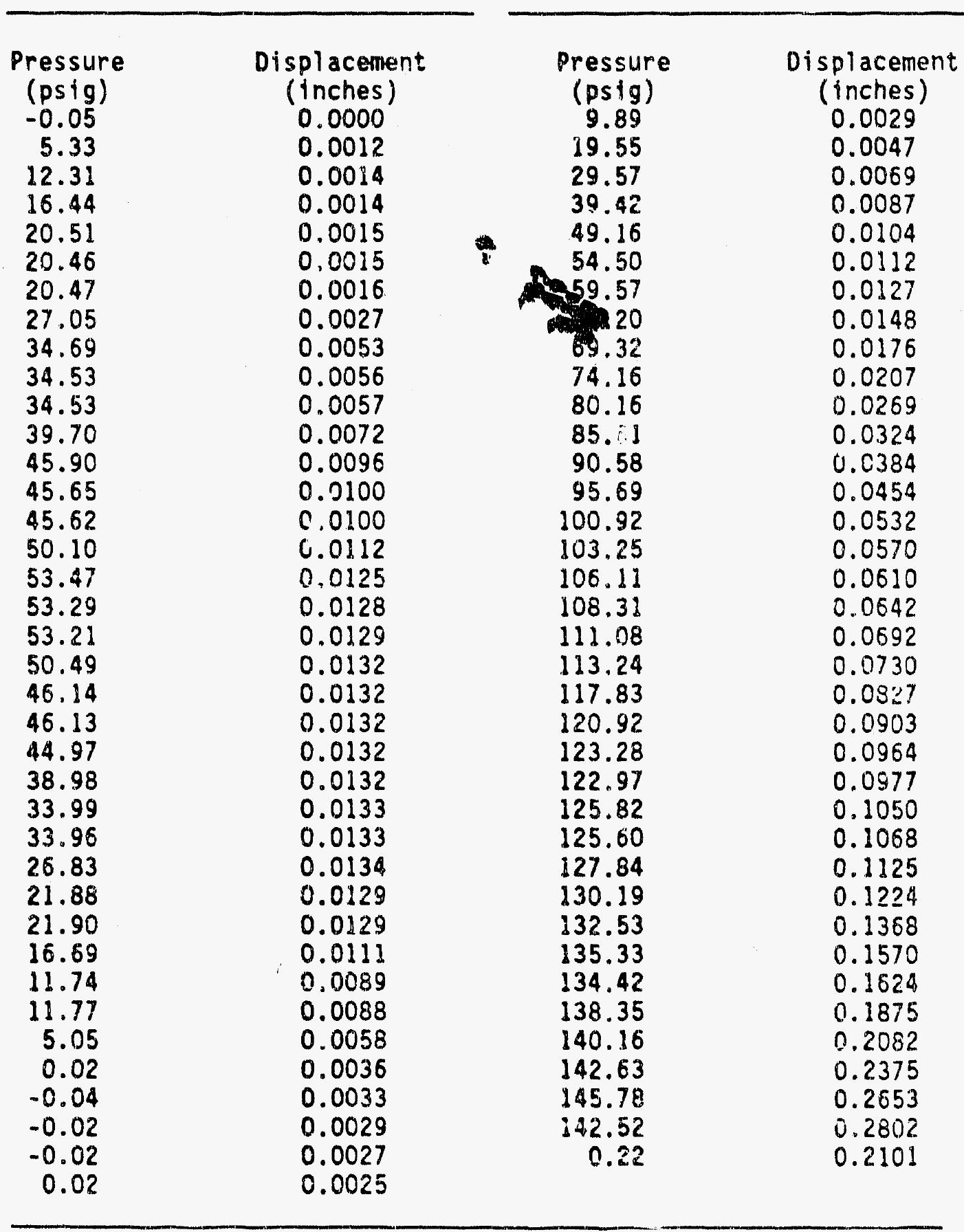

High pressure test

placement

0.0047

0.0069

0.0087

0.0104

0.0148

0.0176

0.0384

0.0454

0.0532

0.0642

0.0692

0.0730

$0.08 \% 7$

0.0977

0.1050

0.1068

0.1368

0.1570

0.1624

0.1875

0.2082

.2375

0.2802 


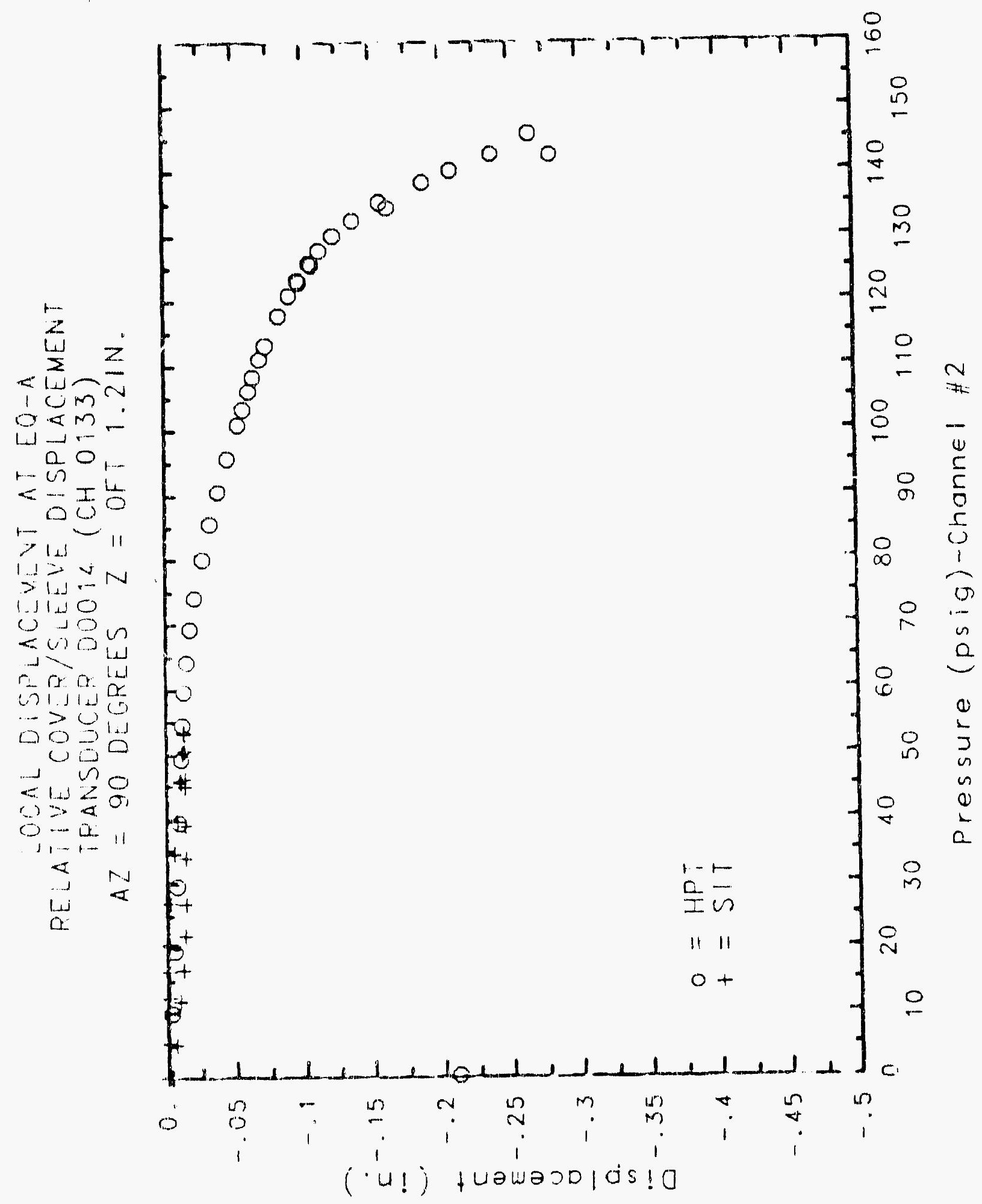




\section{Reinforced Concrete Test Data}

\section{LVDT CHANNEL 135}

StRuctural InTEgRITY TEST

\begin{tabular}{|c|c|c|c|}
\hline $\begin{array}{l}\text { Pressure } \\
\text { (psig) } \\
-0.05 \\
5.33 \\
12.31 \\
16.44 \\
20.51 \\
20.46 \\
20.47 \\
27.05 \\
34.69 \\
34.53 \\
34.53 \\
39.70 \\
45.90 \\
45.65 \\
45.62 \\
50.10 \\
53.47 \\
53.29 \\
53.21 \\
50.49 \\
46.14 \\
46.13 \\
44.97 \\
38.98 \\
33.99 \\
33.96 \\
26.83 \\
21.88 \\
21.90 \\
16.89 \\
11.74 \\
11.77 \\
5.05 \\
0.02 \\
-0.04 \\
-0.02 \\
=0.02 \\
0.02\end{array}$ & $\begin{array}{c}\text { Displacement } \\
\text { (inches) } \\
0.0002 \\
0.0008 \\
0.0008 \\
0.0009 \\
0.0010 \\
0.0011 \\
0.0011 \\
0.0014 \\
0.0013 \\
0.0014 \\
0.0014 \\
0.0013 \\
0.0006 \\
0.0000 \\
-0.0001 \\
-0.0004 \\
-0.0004 \\
-0.0008 \\
-0.0011 \\
-0.0011 \\
-0.0010 \\
-0.0011 \\
-0.0002 \\
0.0004 \\
0.0007 \\
0.0009 \\
0.0017 \\
0.0021 \\
0.0022 \\
0.0024 \\
0.0023 \\
0.0021 \\
0.0020 \\
0.0019 \\
-0.0002 \\
0.0010 \\
0.0013 \\
0.0017\end{array}$ & $\begin{array}{c}\text { Pressure } \\
(\text { psig) } \\
9.89 \\
19.55 \\
29.57 \\
39.42 \\
49.16 \\
54.50 \\
59.57 \\
64.20 \\
69.32 \\
74.16 \\
80.16 \\
85.61 \\
90.58 \\
95.69 \\
100.92 \\
103.25 \\
106.11 \\
108.31 \\
111.08 \\
113.24 \\
117.83 \\
120.92 \\
123.28 \\
122.97 \\
125.82 \\
125.60 \\
127.84 \\
130.19 \\
132.53 \\
135.33 \\
134.42 \\
138.35 \\
140.16 \\
142.63 \\
145.78 \\
142.52 \\
0.22\end{array}$ & $\begin{array}{c}\text { Displacement } \\
(\text { Inches) } \\
0.0004 \\
0.0005 \\
0.0004 \\
0.0002 \\
-0.0001 \\
-0.0002 \\
-0.0001 \\
-0.0002 \\
-0.0002 \\
0.0003 \\
-0.0021 \\
-0.0019 \\
-0.0016 \\
-0.0012 \\
-0.0008 \\
-0.0005 \\
-0.0003 \\
-0.0001 \\
0.0004 \\
0.0006 \\
0.0014 \\
0.0020 \\
0.0030 \\
0.0035 \\
0.0040 \\
0.0046 \\
0.0050 \\
0.0055 \\
0.0060 \\
0.0071 \\
0.0076 \\
0.0087 \\
0.0090 \\
0.0095 \\
0.0096 \\
0.0095 \\
0.0078\end{array}$ \\
\hline
\end{tabular}

High Pressure test

Pressure

0.89

0.0004

0.0005

0.0004

0.0002

0.0001

$-0.0001$

$-0.0002$

$-0.0021$

$-0.0019$

$-0.0016$

0.0012

$-0.0003$

0.0001

0.0014

0.0020

0.0030

0.0035

0.0050

0.0060

0.0087

0.0096

0.0095 


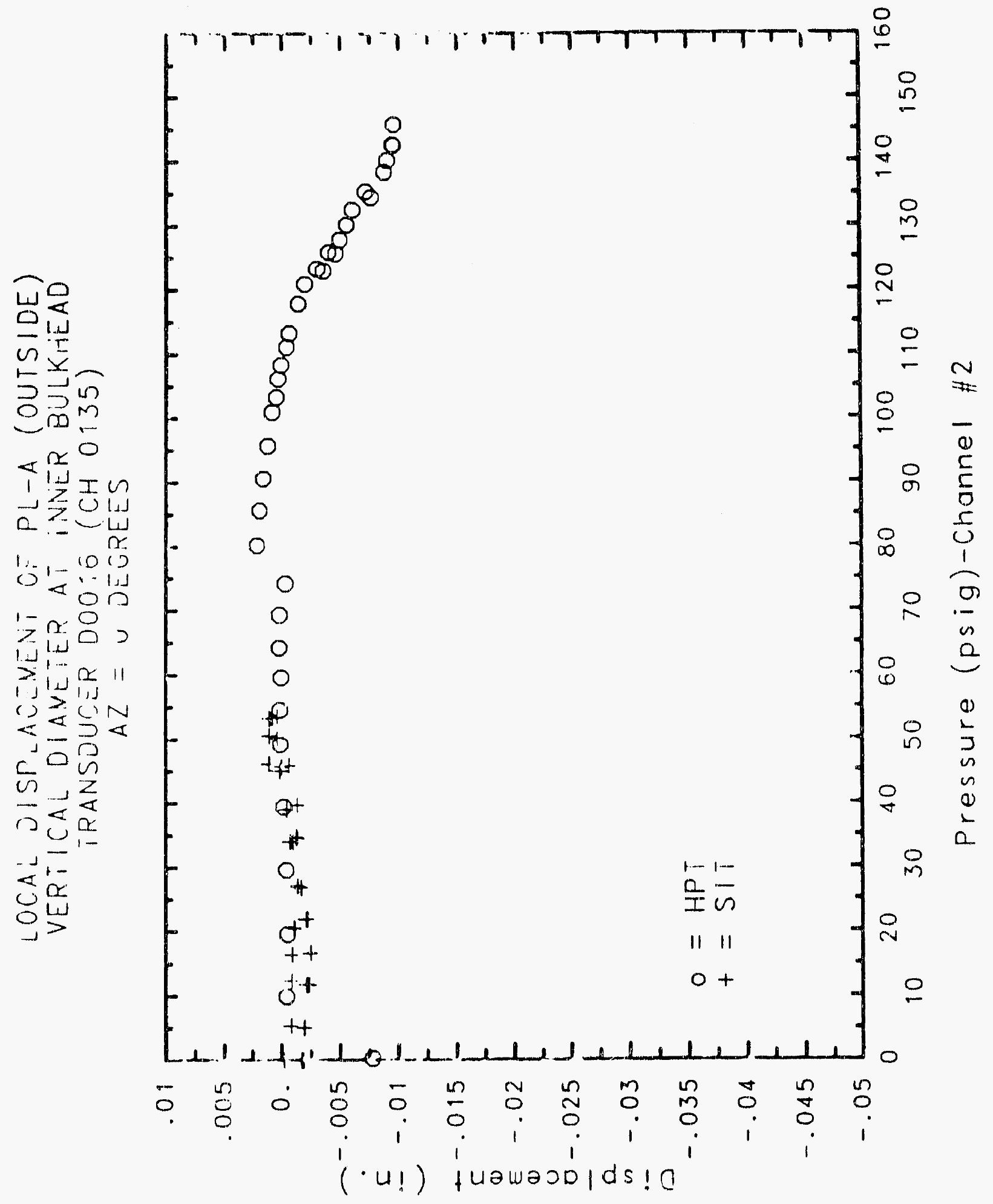

C- 163 


\section{Reinforced Concrete Test Data}

\section{LVDT Channel 136}

STRUCTURAL INTEGRITY TEST

\begin{tabular}{|c|c|c|c|}
\hline $\begin{array}{l}\text { Pressure } \\
\text { (psig) } \\
-0.05 \\
5.33 \\
12.31 \\
16.44 \\
20.51 \\
20.46 \\
20.47 \\
27.05 \\
34.69 \\
34.53 \\
34.53 \\
39.70 \\
45.90 \\
45.65 \\
45.62 \\
50.10 \\
53.47 \\
53.29 \\
53.21 \\
50.49 \\
46.14 \\
46.13 \\
44.97 \\
38.98 \\
33.99 \\
33.96 \\
26.83 \\
21.88 \\
21.90 \\
16.69 \\
11.74 \\
11.77 \\
5.05 \\
0.02 \\
-0.04 \\
-0.02 \\
-0.02 \\
0.02 \\
\end{array}$ & $\begin{array}{c}\text { Displacement } \\
\text { (Inches) } \\
0.0000 \\
0.0001 \\
0.0004 \\
0.0003 \\
0.0003 \\
0.0004 \\
0.0001 \\
0.0000 \\
-0.0011 \\
-0.0016 \\
-0.0016 \\
-0.0021 \\
-0.0037 \\
-0.0042 \\
-0.0047 \\
-0.0051 \\
-0.0058 \\
-0.0060 \\
-0.0067 \\
-0.0075 \\
-0.0072 \\
-0.0076 \\
-0.0070 \\
-0.0058 \\
-0.0047 \\
-0.0043 \\
-0.0033 \\
-0.0020 \\
-0.0018 \\
-0.0011 \\
-0.0008 \\
-0.0006 \\
-0.0003 \\
-0.0003 \\
0.0001 \\
0.0004 \\
0.0005 \\
0.0006\end{array}$ & $\begin{array}{c}\text { Pressuree } \\
(p s 1 g) \\
9.89 \\
19.55 \\
29.57 \\
39.42 \\
49.16 \\
54.50 \\
59.57 \\
64.20 \\
69.32 \\
74.16 \\
80.16 \\
85.61 \\
90.58 \\
95.69 \\
100.92 \\
103.25 \\
106.11 \\
108.31 \\
111.08 \\
113.24 \\
117.83 \\
120.92 \\
123.28 \\
122.97 \\
125.82 \\
125.60 \\
127.84 \\
130.19 \\
132.53 \\
135.33 \\
134.42 \\
138.35 \\
140.16 \\
142.63 \\
145.73 \\
142.51 \\
0.22 \\
\end{array}$ & $\begin{array}{c}\text { Displacement } \\
\text { (iriches) } \\
-0.0003 \\
-0.0010 \\
-0.0025 \\
-0.0042 \\
-0.0064 \\
-0.0066 \\
-0.0074 \\
-0.0075 \\
-0.0079 \\
-0.0075 \\
-0.0100 \\
-0.0104 \\
-0.0108 \\
-0.0115 \\
-0.0119 \\
-0.0128 \\
-0.0132 \\
-0.0134 \\
-0.0136 \\
-0.0138 \\
-0.0146 \\
-0.0147 \\
-0.0150 \\
-0.0148 \\
-0.0146 \\
-0.0144 \\
-0.0146 \\
-0.0146 \\
-0.0149 \\
-0.0153 \\
-0.0152 \\
-0.0149 \\
-0.0153 \\
-0.0158 \\
-0.0162 \\
-0.0170 \\
-0.0011\end{array}$ \\
\hline
\end{tabular}

high Pressure test

splacement

(iriches)

$-0.0003$

$-0.0010$

$-0.0042$

$-0.0064$

0.0075

$-0.0100$

$-0.0104$

$-0.0119$

0.0134

$-0.0138$

$-0.0146$

$-0.0147$

0.0150

$-0.0144$

$-0.0146$

0.0146

$-0.0152$

$-0.0149$

$-0.0153$

0.0158 


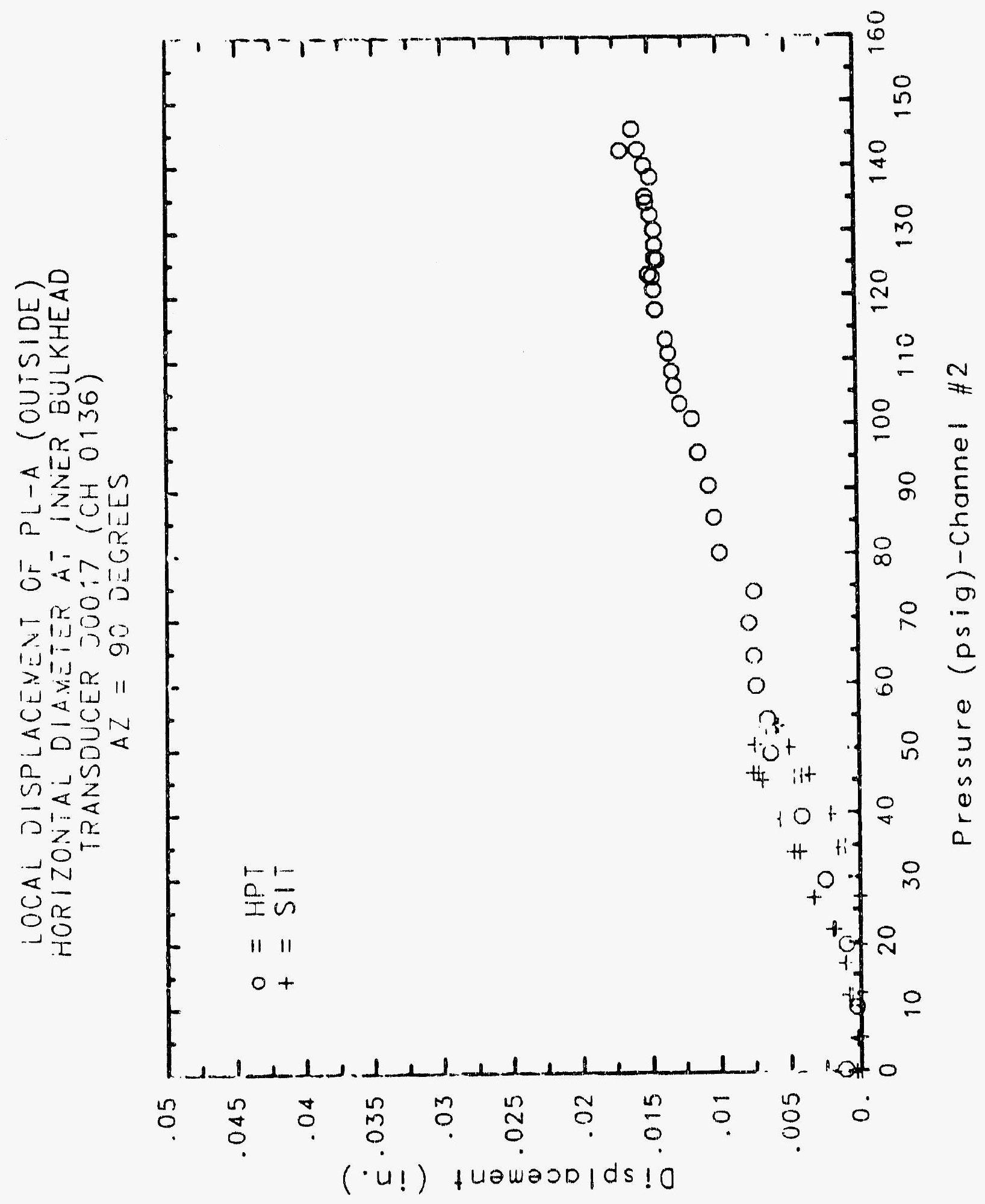




\section{Reinforced Concrete Test Data}

\section{LVDt Channel 137}

Structural IntEgrity Test

\begin{tabular}{|c|c|c|c|}
\hline $\begin{array}{l}\text { Pressure } \\
\text { (psig) } \\
-0.05 \\
5.33 \\
12.31 \\
16.44 \\
20.51 \\
20.46 \\
20.47 \\
27.05 \\
34.69 \\
34.53 \\
34.53 \\
39.70 \\
45.90 \\
45.65 \\
45.62 \\
50.10 \\
53.47 \\
53.29 \\
53.21 \\
50.49 \\
46.14 \\
46.13 \\
44.97 \\
38.98 \\
33.99 \\
33.96 \\
26.83 \\
21.88 \\
21.90 \\
16.69 \\
11.74 \\
11.77 \\
5.05 \\
0.02 \\
-0.04 \\
-0.02 \\
-0.02 \\
0.02\end{array}$ & $\begin{array}{c}\text { Displacement } \\
(\text { Inches) } \\
0.0000 \\
-0.0017 \\
-0.0040 \\
-0.0053 \\
-0.0064 \\
-0.0065 \\
-0.0065 \\
-0.0075 \\
-0.0086 \\
-0.0086 \\
-0.0087 \\
-0.0098 \\
-0.0107 \\
-0.0106 \\
-0.0106 \\
-0.0114 \\
-0.0119 \\
-0.0119 \\
-0.0119 \\
-0.0114 \\
-0.0108 \\
-0.0107 \\
-0.0103 \\
-0.0093 \\
-0.0083 \\
-0.0084 \\
-0.0069 \\
-0.0059 \\
-0.0060 \\
-0.0051 \\
-0.0040 \\
-0.0039 \\
-0.0023 \\
-0.0009 \\
-0.0003 \\
-0.0002 \\
-0.0002 \\
-0.0003 \\
-0.00\end{array}$ & $\begin{array}{c}\text { Pressure } \\
(\text { psig) } \\
9.89 \\
19.55 \\
29.57 \\
39.42 \\
49.16 \\
54.50 \\
59.57 \\
64.20 \\
69.32 \\
74.16 \\
80.16 \\
85.61 \\
90.58 \\
95.69 \\
100.92 \\
103.25 \\
106.11 \\
108.31 \\
111.08 \\
113.24 \\
117.83 \\
120.92 \\
123.28 \\
122.97 \\
125.82 \\
125.60 \\
127.84 \\
130.19 \\
132.53 \\
135.33 \\
134.42 \\
138.35 \\
140.16 \\
142.63 \\
145.78 \\
142.52 \\
0.22 \\
\end{array}$ & $\begin{array}{l}\text { Displacement } \\
(\text { inches) } \\
-0.0022 \\
-0.0042 \\
-0.0061 \\
-0.0080 \\
-0.0099 \\
-0.0110 \\
-0.0124 \\
-0.0138 \\
-0.0151 \\
-0.0162 \\
-0.0177 \\
-0.0188 \\
-0.0195 \\
-0.0200 \\
-0.0206 \\
-0.0209 \\
-0.0212 \\
-0.0215 \\
-0.0217 \\
-0.0219 \\
-0.0220 \\
-0.0221 \\
-0.0221 \\
-0.0220 \\
-0.0220 \\
-0.0218 \\
-0.0220 \\
-0.0217 \\
-0.0213 \\
-0.0213 \\
-0.0210 \\
-0.0212 \\
-0.0218 \\
-0.0233 \\
-0.0246 \\
-0.0234 \\
0.0055\end{array}$ \\
\hline
\end{tabular}

High Pressure test

splacement

(inches)

$-0.0022$

$-0.0061$

$-0.0080$

$-0.0099$

$-0.0110$

$-0.0124$

$-0.0138$

$-0.0151$

0.0177

$-0.0188$

$-0.0195$

$-0.0200$

$-0.0206$

$-0.0215$

0.0217

$-0.0219$

$-0.0220$

0.0221

$-0.0221$

$-0.0218$

$-0.0220$

$-0.0217$

$-0.0213$

$-0.0213$

$-0.0210$

$-0.0212$

0.0218

0.0234

0.0055 


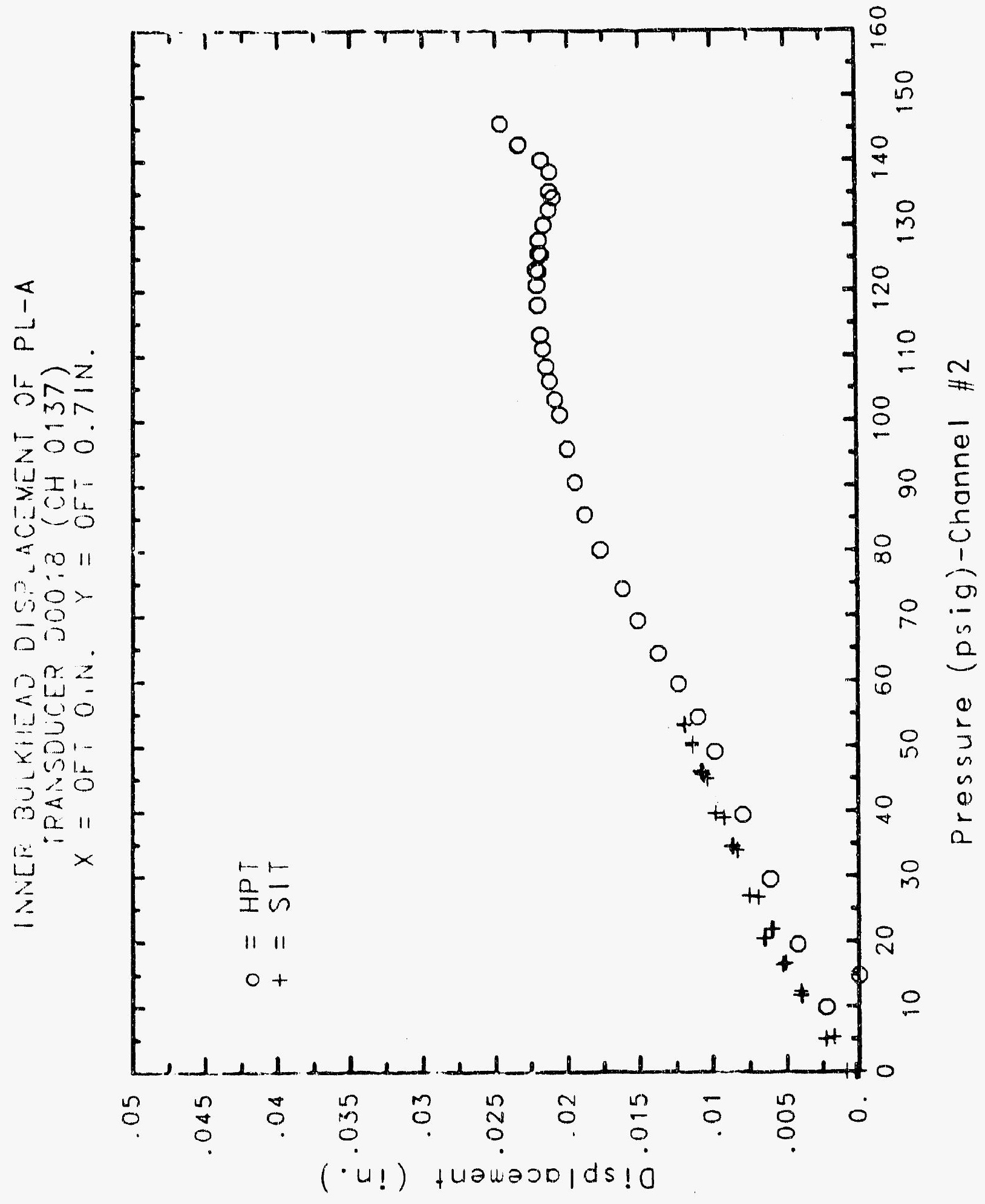




\section{Reinforced Concrete Test Data}

LVDT Channel 138

STRUCTURAL InTEGRITY TEST

\begin{tabular}{|c|c|}
\hline $\begin{array}{l}\text { Pressure } \\
(\text { psig) } \\
-0.05 \\
5.33 \\
12.31 \\
15.44 \\
20.51 \\
20.46 \\
20.47 \\
27.05 \\
34.69 \\
34.53 \\
34.53 \\
39.70 \\
45.90 \\
45.65 \\
45.62 \\
50.10 \\
53.47 \\
53.29 \\
53.21 \\
50.49 \\
46.14 \\
46.13 \\
44.97 \\
38.98 \\
33.99 \\
33.96 \\
26.83 \\
21.88 \\
21.90 \\
16.69 \\
11.74 \\
11.77 \\
5.05 \\
0.02 \\
-0.04 \\
-0.02 \\
-0.02 \\
0.02\end{array}$ & $\begin{array}{c}\text { Displacement } \\
(\text { Inches) } \\
0.0000 \\
-0.0002 \\
-0.0008 \\
-0.0009 \\
-0.0011 \\
-0.0012 \\
-0.0012 \\
-0.0012 \\
-0.0013 \\
-0.0013 \\
-0.0013 \\
-0.0014 \\
-0.0014 \\
-0.0014 \\
-0.0014 \\
-0.0014 \\
-0.0015 \\
-0.0015 \\
-0.0015 \\
-0.0015 \\
-0.0015 \\
-0.0015 \\
-0.0014 \\
-0.0013 \\
-0.0012 \\
-0.0013 \\
-0.0011 \\
-0.0011 \\
-0.0011 \\
-0.0010 \\
-0.0009 \\
-0.0008 \\
-0.0006 \\
-0.0005 \\
-0.0002 \\
-0.0001 \\
-0.0001 \\
-0.0001\end{array}$ \\
\hline
\end{tabular}

High Pressure Test

\begin{tabular}{|c|c|}
\hline $\begin{array}{c}\text { Pressure } \\
\text { (psig) } \\
9.89 \\
19.55 \\
29.57 \\
39.42 \\
49.16 \\
54.50 \\
59.57 \\
64.20 \\
69.32 \\
74.16 \\
80.16 \\
85.61 \\
90.58 \\
95.69 \\
100.92 \\
103.25 \\
106.11 \\
108.31 \\
111.08 \\
113.24 \\
117.83 \\
120.92 \\
123.28 \\
122.97 \\
125.82 \\
125.60 \\
127.84 \\
130.19 \\
132.53 \\
135.33 \\
134.42 \\
138.35 \\
140.16 \\
142.63 \\
145.78 \\
142.52 \\
0.22\end{array}$ & $\begin{array}{l}\text { Displacement } \\
\text { (inches) } \\
-0.0003 \\
-0.0004 \\
-0.0006 \\
-0.0009 \\
-0.0011 \\
-0.0013 \\
-0.0015 \\
-0.0017 \\
-0.0019 \\
-0.0021 \\
-0.0022 \\
-0.0024 \\
-0.0025 \\
-0.0026 \\
-0.0026 \\
-0.0026 \\
-0.0027 \\
-0.0027 \\
-0.0026 \\
-0.0026 \\
-0.0026 \\
-0.0025 \\
-0.0025 \\
-0.0025 \\
-0.0025 \\
-0.0025 \\
-0.0025 \\
-0.0023 \\
-0.0022 \\
-0.0022 \\
-0.0022 \\
-0.0022 \\
-0.0023 \\
-0.0025 \\
-0.0027 \\
-0.0026 \\
-0.0005\end{array}$ \\
\hline
\end{tabular}




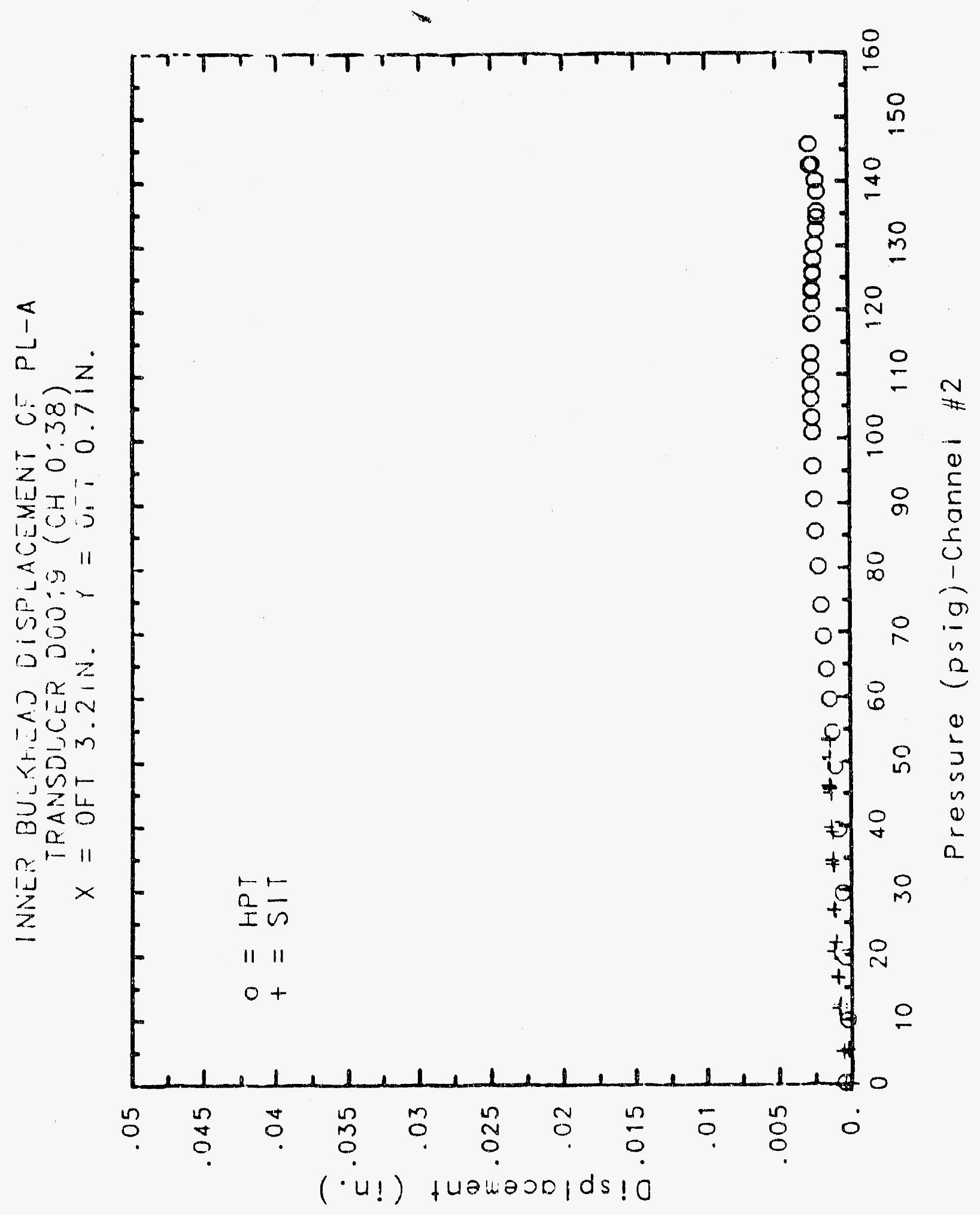




\section{Reinforced Concrete Test Data}

LVDt Channel 139

STRUCTURAL IN'TEGRITY TEST

\begin{tabular}{|c|c|c|c|}
\hline $\begin{array}{c}\text { Pressure } \\
\text { (psig) } \\
-0.05 \\
5.33 \\
12.31 \\
16.44 \\
20.51 \\
20.46 \\
20.47 \\
27.05 \\
34.69 \\
34.53 \\
34.53 \\
39.70 \\
45.90 \\
45.65 \\
45.62 \\
50.10 \\
53.47 \\
53.29 \\
53.21 \\
50.49 \\
46.14 \\
46.13 \\
44.97 \\
38.98 \\
33.99 \\
33.96 \\
26.83 \\
21.88 \\
21.90 \\
16.69 \\
11.74 \\
11.77 \\
5.05 \\
0.02 \\
-0.04 \\
-0.02 \\
-0.02 \\
0.02\end{array}$ & $\begin{array}{c}\text { Displacement } \\
\text { (inches) } \\
0.0000 \\
-0.0007 \\
-0.0017 \\
-0.0023 \\
-0.0028 \\
-0.0029 \\
-0.0029 \\
-0.0030 \\
-0.0032 \\
-0.0033 \\
-0.0034 \\
-0.0037 \\
-0.0038 \\
-0.0038 \\
-0.0038 \\
-0.0039 \\
-0.0040 \\
-0.0041 \\
-0.0040 \\
-0.0040 \\
-0.0040 \\
-0.0038 \\
-0.0037 \\
-0.0034 \\
-0.0031 \\
-0.0032 \\
-0.0027 \\
-0.0024 \\
-0.0026 \\
-0.0023 \\
-0.0020 \\
-0.0019 \\
-0.0014 \\
-0.0009 \\
-0.0003 \\
-0.0003 \\
-0.0003 \\
-0.0003\end{array}$ & $\begin{array}{c}\text { Pressure } \\
\text { (psig) } \\
9.89 \\
19.55 \\
29.57 \\
39.42 \\
49.16 \\
54.50 \\
59.57 \\
64.20 \\
69.32 \\
74.16 \\
80.16 \\
85.61 \\
90.58 \\
95.69 \\
100.92 \\
103.25 \\
106.11 \\
108.31 \\
111.08 \\
113.24 \\
117.83 \\
120.92 \\
123.28 \\
122.97 \\
125.82 \\
125.60 \\
127.84 \\
130.19 \\
132.53 \\
135.33 \\
134.42 \\
138.35 \\
140.16 \\
142.63 \\
145.78 \\
142.52 \\
0.22\end{array}$ & $\begin{array}{c}\text { Displacement } \\
(\text { inches) } \\
-0.0006 \\
-0.0011 \\
-0.0016 \\
-0.0022 \\
-0.0027 \\
-0.0031 \\
-0.0037 \\
-0.0043 \\
-0.0048 \\
-0.0051 \\
-0.0056 \\
-0.0058 \\
-0.0059 \\
-0.0057 \\
-0.0056 \\
-0.0056 \\
-0.0056 \\
-0.0056 \\
-0.0054 \\
-0.0053 \\
-0.0050 \\
-0.0047 \\
-0.0045 \\
-0.0044 \\
-0.0041 \\
-0.0041 \\
-0.0040 \\
-0.0035 \\
-0.0030 \\
-0.0028 \\
-0.0027 \\
-0.0025 \\
-0.0029 \\
-0.0039 \\
-0.0047 \\
-0.0038 \\
0.0042\end{array}$ \\
\hline
\end{tabular}

High Pressure test

splacement

$-0.0016$

$-0.0022$

$-0.0027$

$-0.0031$

$-0.0037$

$-0.0051$

$-0.0056$

$-0.0058$

$-0.0059$

$-0.0056$

$-0.0056$

$-0.0056$

$-0.0054$

$-0.0053$

$-0.0045$

$-0.0044$

$-0.0041$

$-0.0041$

$-0.0040$

$-0.0028$

$-0.0027$

$-0.0025$

$-0.0029$

$-0.0039$

0.0038 


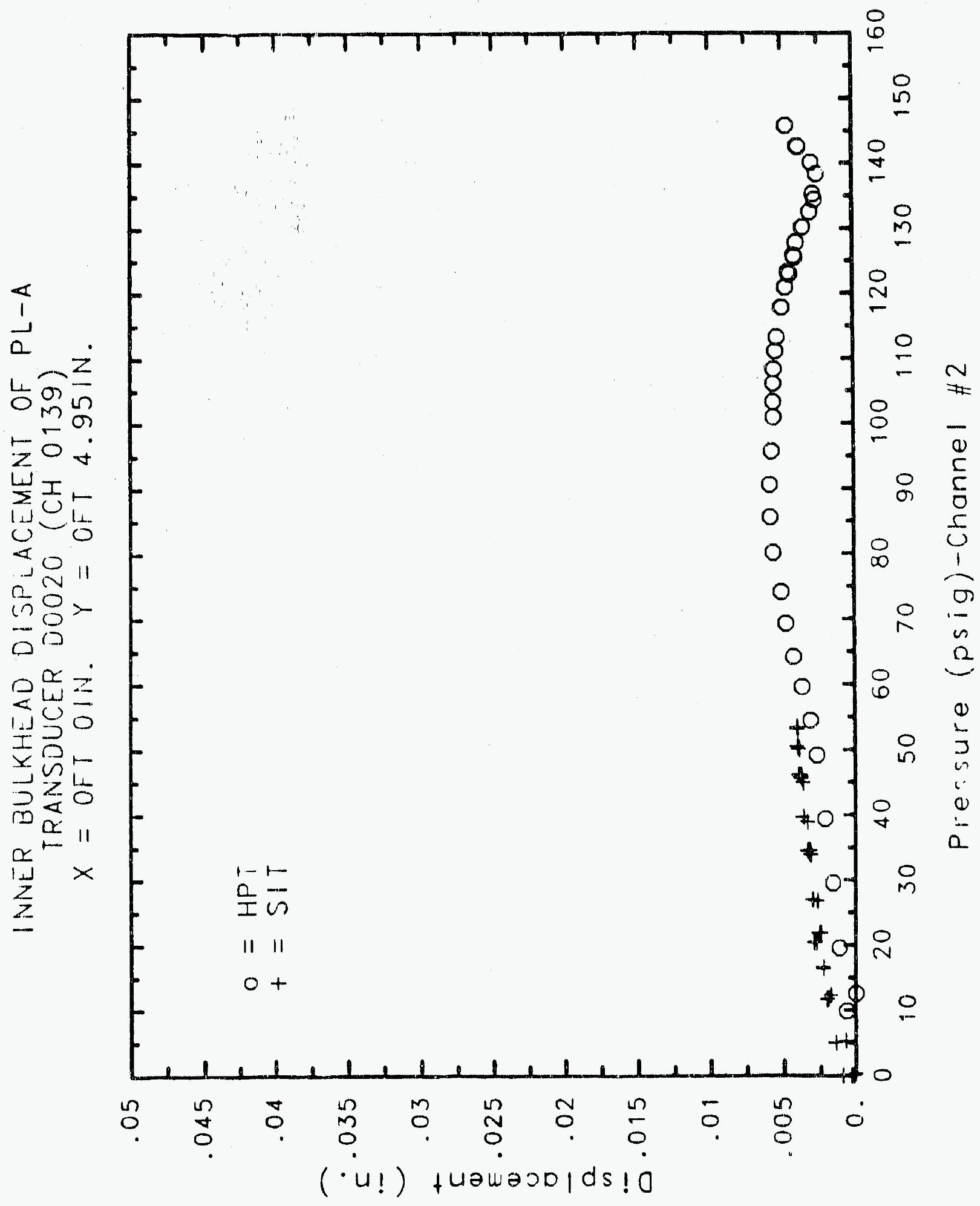

C. -171 


\section{Reinforced Concrete Test Data}

Lvd Chanmel 140

Structural InTEgrity TEST

\begin{tabular}{|c|c|c|c|}
\hline $\begin{array}{l}\text { Pressure } \\
(p s 1 g) \\
-0.05 \\
5.33 \\
12.31 \\
16.44 \\
20.51 \\
20.46 \\
20.47 \\
27.05 \\
34.69 \\
34.53 \\
34.53 \\
39.70 \\
45.90 \\
45.65 \\
45.62 \\
50.10 \\
53.47 \\
53.29 \\
53.21 \\
50.49 \\
46.14 \\
46.13 \\
44.97 \\
38.98 \\
33.99 \\
33.96 \\
26.83 \\
21.83 \\
21.90 \\
16.69 \\
11.74 \\
11.77 \\
5.05 \\
0.02 \\
-0.04 \\
-0.02 \\
-0.02 \\
0.02\end{array}$ & $\begin{array}{c}\text { Displacement } \\
\text { (inches) } \\
0.0000 \\
-0.0001 \\
-0.0005 \\
-0.0005 \\
-0.0006 \\
-0.0007 \\
-0.0007 \\
-0.0006 \\
-0.0006 \\
-0.0007 \\
-0.0007 \\
-0.0007 \\
-0.0007 \\
-0.0007 \\
-0.0007 \\
-0.0007 \\
-0.0007 \\
-0.0007 \\
-0.0007 \\
-0.0007 \\
-0.0008 \\
-0.0007 \\
-0.0007 \\
-0.0006 \\
-0.0006 \\
-0.0006 \\
-0.0006 \\
-0.0006 \\
-0.0006 \\
-0.0005 \\
-0.0005 \\
-0.0004 \\
-0.0004 \\
-0.0003 \\
-0.0001 \\
-0.0001 \\
-0.0001 \\
-0.0001\end{array}$ & $\begin{array}{c}\text { Pressure } \\
\text { (ps1g) } \\
9.89 \\
19.55 \\
29.57 \\
39.42 \\
49.16 \\
54.50 \\
59.57 \\
64.20 \\
69.32 \\
74.16 \\
80.16 \\
85.61 \\
90.58 \\
95.69 \\
100.92 \\
103.25 \\
106.11 \\
108.31 \\
111.08 \\
113.24 \\
117.83 \\
120.92 \\
123.28 \\
122.97 \\
125.82 \\
125.60 \\
127.84 \\
130.19 \\
132.53 \\
135.33 \\
134.42 \\
138.35 \\
140.16 \\
142.63 \\
145.78 \\
142.52 \\
0.22\end{array}$ & $\begin{array}{l}\text { Displacement } \\
\text { (inches) } \\
-0.0001 \\
-0.0001 \\
-0.0002 \\
-0.0003 \\
-0.0004 \\
-0.0005 \\
-0.0006 \\
-0.0007 \\
-0.0008 \\
-0.0009 \\
-0.0009 \\
-0.0009 \\
-0.0009 \\
-0.0009 \\
-0.0008 \\
-0.0008 \\
-0.0008 \\
-0.0008 \\
-0.0008 \\
-0.0008 \\
-0.0006 \\
-0.0006 \\
-0.0006 \\
-0.0006 \\
-0.0005 \\
-0.0005 \\
-0.0005 \\
-0.0004 \\
-0.0003 \\
-0.0002 \\
-0.0002 \\
-0.0002 \\
-0.0002 \\
-0.0003 \\
-0.0004 \\
-0.0004 \\
-0.0004\end{array}$ \\
\hline
\end{tabular}

High Pressure test

spiacement

$-0.0001$

$-0.0001$

$-0.0002$

$-0.0003$

$-0.0004$

$-0.0005$

$-0.0008$

$-0.0009$

$-0.0009$

$-0.0009$

0.0009

$-0.0008$

$-0.0008$

$-0.0008$

$-0.0008$

$-0.0008$

$-0.0006$

$-0.0006$

$-0.0006$

$-0.0006$

$-0.0005$

$-0.0005$

$-0.0004$

$-0.0003$

$-0.0002$

$-0.0002$

$-0.0002$

$-0.0003$

$-0.0004$

$-0.0004$

$-0.0004$ 


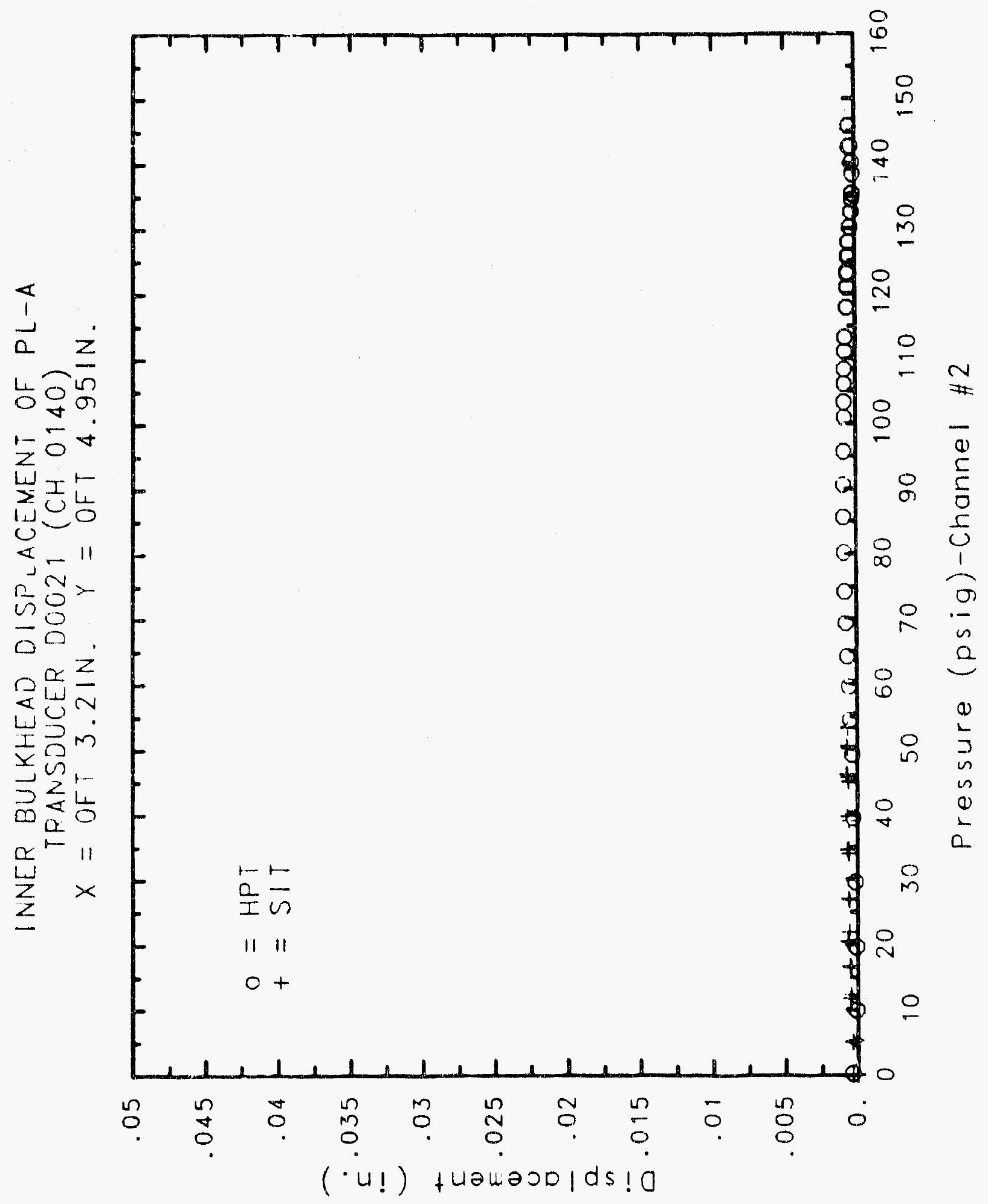




\section{Reinforced Concrete Test Data}

LVd Channel 143

\section{STRUCTURAL IATEGRITY TEST}

\begin{tabular}{c} 
Pressure \\
(psig) \\
-0.05 \\
5.33 \\
12.31 \\
16.44 \\
20.51 \\
20.46 \\
20.47 \\
27.05 \\
34.69 \\
34.53 \\
34.53 \\
39.70 \\
45.90 \\
45.65 \\
45.62 \\
50.10 \\
53.47 \\
53.29 \\
53.21 \\
50.49 \\
46.14 \\
46.13 \\
44.97 \\
38.98 \\
33.99 \\
33.96 \\
25.83 \\
21.88 \\
21.90 \\
16.69 \\
11.74 \\
11.77 \\
5.05 \\
0.02 \\
-0.04 \\
-0.02 \\
-0.02 \\
0.02 \\
\\
\hline
\end{tabular}

Displacement

(inches)

0.0000

0.0000

0.0003

$-0.0002$

$-0.0002$

$-0.0003$

$-0.0007$

0.0043

0.0050

0.0052

0.0090

0.0148

0.0148

0.0146

0.0167

0.0190

0.0197

0.0196

0.0197

0.0195

0.0196

0.0199

0.0199

0.0199

0.0200

0.0200

0.2200

0.0202

0.0201

0.0203

0.0201

0.0200

0.0182

0.0150

0.0151

0.0150

0.0151
0.0002
High Pressure test

\begin{tabular}{|c|c|}
\hline $\begin{array}{c}\text { Pressure } \\
(\text { psig) } \\
9.89 \\
19.55 \\
29.57 \\
39.42 \\
49.15 \\
54.50 \\
59.57 \\
64.20 \\
69.32 \\
74.16 \\
80.16 \\
85.61 \\
90.58 \\
95.69 \\
100.92 \\
103.25 \\
106.11 \\
108.31 \\
111.08 \\
113.24 \\
117.83 \\
120.92 \\
123.28 \\
122.97 \\
125.82 \\
125.60 \\
127.84 \\
130.19 \\
132.53 \\
135.33 \\
134.42 \\
138.35 \\
140.16 \\
142.63 \\
145.78 \\
142.52 \\
0.22\end{array}$ & $\begin{array}{c}\text { Displacement } \\
\text { (inches) } \\
-0.0071 \\
-0.0053 \\
-0.0052 \\
-0.0039 \\
-0.0018 \\
-0.0004 \\
0.0018 \\
0.0060 \\
0.0112 \\
0.0167 \\
0.0225 \\
0.0274 \\
0.0316 \\
0.0383 \\
0.0445 \\
0.0478 \\
0.0500 \\
0.0518 \\
0.0553 \\
0.0574 \\
0.0631 \\
0.0671 \\
0.0721 \\
0.0738 \\
0.0793 \\
0.0806 \\
0.0841 \\
0.0901 \\
0.0978 \\
0.1078 \\
0.1130 \\
0.1251 \\
0.1344 \\
0.1479 \\
0.1599 \\
0.1669 \\
0.0696\end{array}$ \\
\hline
\end{tabular}




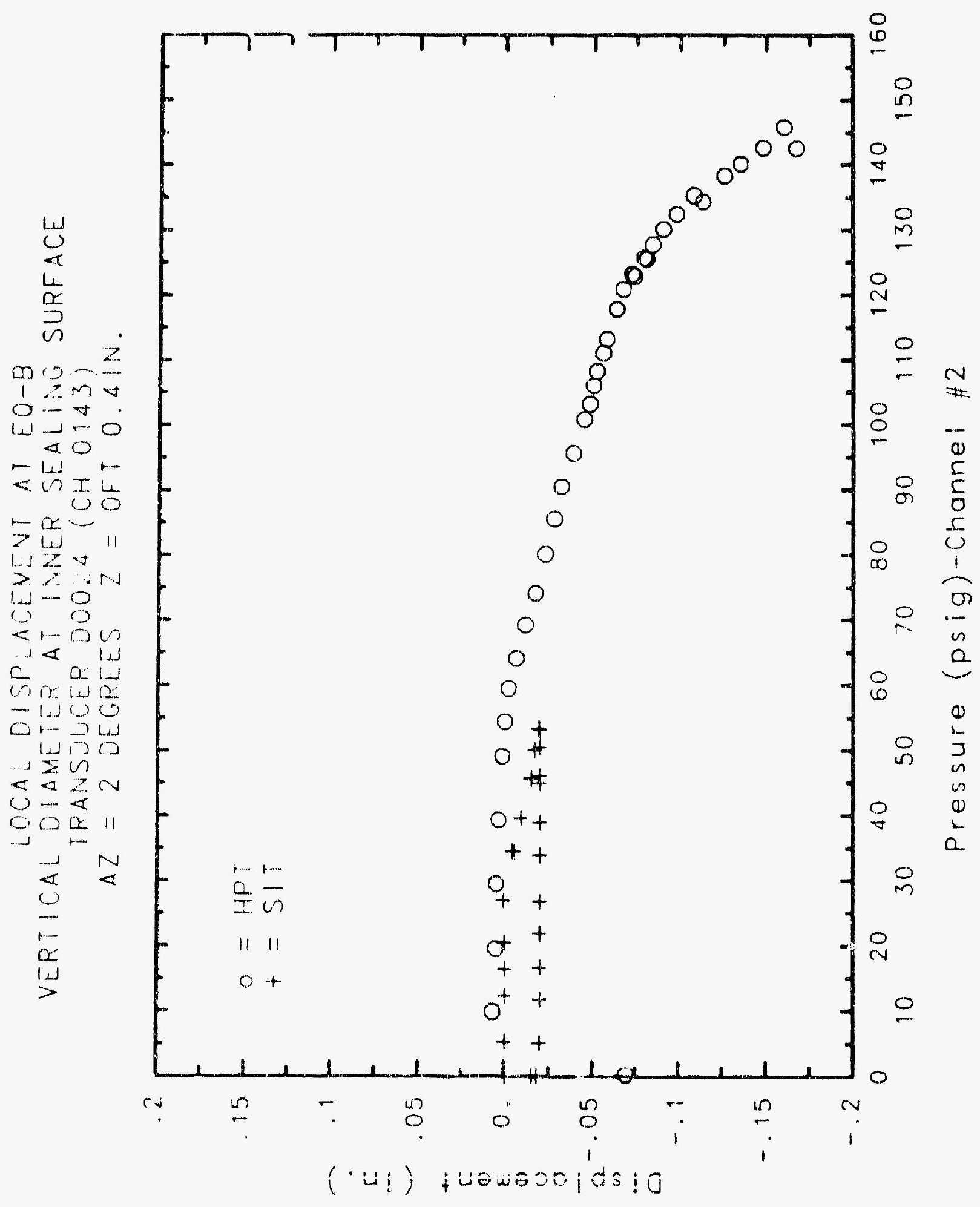




\section{Reinforced Concrete Test Data}

LVDt Channel 144

\begin{tabular}{|c|c|c|c|}
\hline $\begin{array}{c}\text { Pressure } \\
\text { (psig) } \\
-0.05 \\
5.33 \\
12.31 \\
16.44 \\
20.51 \\
20.46 \\
20.47 \\
27.05 \\
34.69 \\
34.53 \\
34.53 \\
39.70 \\
45.90 \\
45.65 \\
45.62 \\
50.10 \\
53.47 \\
53.29 \\
53.21 \\
50.49 \\
46.14 \\
46.13 \\
44.97 \\
38.98 \\
33.99 \\
33.96 \\
26.83 \\
21.88 \\
21.90 \\
16.69 \\
11.74 \\
11.77 \\
5.05 \\
0.02 \\
-0.04 \\
-0.02 \\
-0.02 \\
0.02\end{array}$ & $\begin{array}{c}\text { Displacement } \\
\text { (Inches) } \\
0.0001 \\
0.0002 \\
0.0003 \\
0.0003 \\
0.0007 \\
0.0009 \\
0.0009 \\
0.0024 \\
0.0048 \\
0.0054 \\
0.0054 \\
0.0066 \\
0.0085 \\
0.0060 \\
0.0051 \\
0.0022 \\
0.0015 \\
0.0011 \\
0.0008 \\
-0.0026 \\
-0.0027 \\
-0.0028 \\
-0.0023 \\
-0.0023 \\
-0.0021 \\
-0.0020 \\
-0.0021 \\
-0.0020 \\
-0.0016 \\
-0.0015 \\
-0.0015 \\
-0.0018 \\
-0.0019 \\
-0.0020 \\
-0.0013 \\
-0.0015 \\
-0.0012 \\
-0.0009\end{array}$ & $\begin{array}{c}\text { Pressure } \\
\text { (psig) } \\
9.89 \\
19.55 \\
29.57 \\
39.42 \\
49.16 \\
54.50 \\
59.57 \\
64.20 \\
69.32 \\
74.16 \\
80.16 \\
85.61 \\
90.58 \\
95.69 \\
100.92 \\
103.25 \\
106.11 \\
108.31 \\
111.08 \\
113.24 \\
117.83 \\
120.92 \\
123.28 \\
122.97 \\
125.82 \\
125.60 \\
127.84 \\
130.19 \\
132.53 \\
135.33 \\
134.42 \\
138.35 \\
140.16 \\
142.63 \\
145.78 \\
142.52 \\
0.22\end{array}$ & $\begin{array}{c}\text { Displacement } \\
\text { (inches) } \\
0.0192 \\
0.0169 \\
0.0103 \\
0.0027 \\
-0.0022 \\
-0.0076 \\
-0.0114 \\
-0.0166 \\
-0.0219 \\
-0.0276 \\
-0.0344 \\
-0.0395 \\
-0.0433 \\
-0.0485 \\
-0.0538 \\
-0.0578 \\
-0.0607 \\
-0.0624 \\
-0.0650 \\
-0.0681 \\
-0.0714 \\
-0.0738 \\
-0.0772 \\
-0.0770 \\
-0.0804 \\
-0.0805 \\
-0.0832 \\
-0.0887 \\
-0.0941 \\
-0.0999 \\
-0.0999 \\
-0.1069 \\
-0.1137 \\
-0.1224 \\
-0.1340 \\
-0.1392 \\
-0.0782\end{array}$ \\
\hline
\end{tabular}

High Pressure test

placement

0.0169

0.0103

0.0027

$-0.0022$

$-0.0166$

$-0.0219$

$-0.0276$

$-0.0344$

$-0.0395$

$-0.0485$

$-0.0538$

$-0.0578$

$-0.0607$

0.0650

$-0.0681$

$-0.0714$

$-0.0738$

$-0.0772$

$-0.0805$

$-0.0832$

$-0.0887$

$-0.0941$

$-0.1069$

$-0.1137$

$-0.1224$

$-0.1340$

$-0.1392$
Structural Integrity test 


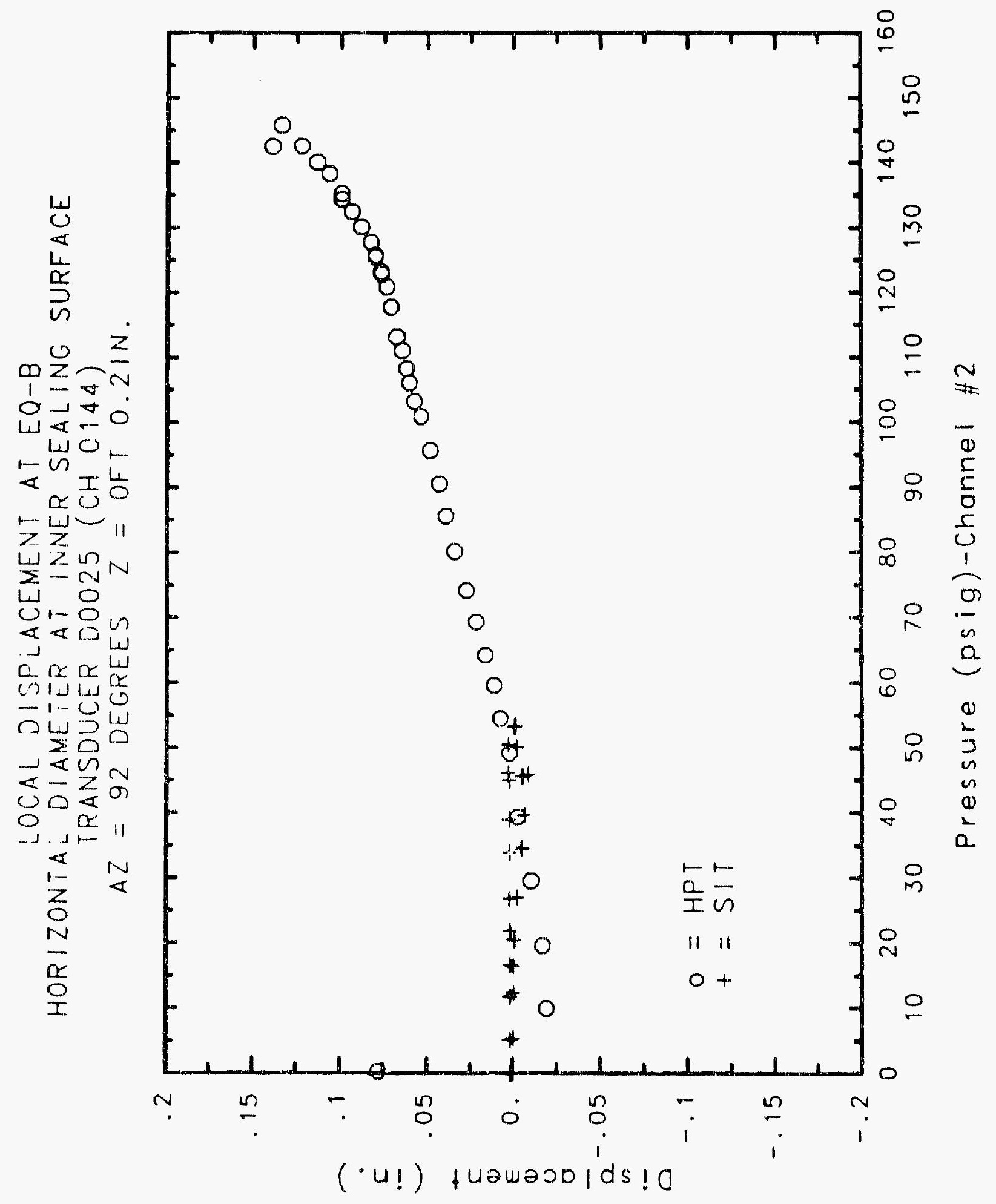




\section{Reinforced Concrete Test Data}

\section{LVDT Channel 145}

Structural Integrity TEST

\begin{tabular}{|c|c|c|c|}
\hline $\begin{array}{l}\text { Pressure } \\
\text { (psig) } \\
-0.05 \\
5.33 \\
12.31 \\
16.44 \\
20.51 \\
20.46 \\
20.47 \\
27.05 \\
34.69 \\
34.53 \\
34.53 \\
39.70 \\
45.90 \\
45.65 \\
45.62 \\
50.10 \\
53.47 \\
53.29 \\
53.21 \\
50.49 \\
46.14 \\
46.13 \\
44.97 \\
38.98 \\
33.99 \\
33.96 \\
26.83 \\
21.88 \\
21.90 \\
16.69 \\
11.74 \\
11.77 \\
5.05 \\
0.02 \\
-0.04 \\
-0.02 \\
-0.02 \\
0.02\end{array}$ & $\begin{array}{c}\text { Displacement } \\
\text { (inches) } \\
0.0002 \\
0.0004 \\
0.0009 \\
0.0017 \\
0.0028 \\
0.0037 \\
0.0039 \\
0.0068 \\
0.0096 \\
0.0111 \\
0.0114 \\
0.0139 \\
0.0187 \\
0.0184 \\
0.0177 \\
0.0179 \\
0.0203 \\
0.0206 \\
0.0201 \\
0.0197 \\
0.0193 \\
0.0189 \\
0.0204 \\
0.0200 \\
0.0189 \\
0.0188 \\
0.0174 \\
0.0165 \\
0.0171 \\
0.0164 \\
0.0149 \\
0.0139 \\
0.0114 \\
0.0090 \\
0.0060 \\
0.0068 \\
0.0072 \\
0.0071\end{array}$ & $\begin{array}{c}\text { Pressure } \\
(\text { psig) } \\
9.89 \\
19.55 \\
29.57 \\
39.42 \\
49.16 \\
54.50 \\
59.57 \\
64.20 \\
69.32 \\
74.16 \\
80.16 \\
85.61 \\
90.58 \\
95.69 \\
100.92 \\
103.25 \\
106.11 \\
108.31 \\
111.08 \\
113.24 \\
117.83 \\
120.92 \\
123.28 \\
122.97 \\
125.82 \\
125.60 \\
127.84 \\
130.19 \\
132.53 \\
135.33 \\
134.42 \\
138.35 \\
140.16 \\
142.63 \\
145.78 \\
142.52 \\
0.22\end{array}$ & $\begin{array}{c}\text { Displacement } \\
\text { (inches) } \\
0.0021 \\
0.0029 \\
0.0021 \\
0.0013 \\
0.0015 \\
0.0024 \\
0.0041 \\
0.0068 \\
0.0119 \\
0.0169 \\
0.0200 \\
0.0244 \\
0.0283 \\
0.0331 \\
0.0385 \\
0.0408 \\
0.0427 \\
0.0444 \\
0.0476 \\
0.0501 \\
0.0552 \\
0.0600 \\
0.0650 \\
0.0669 \\
0.0722 \\
0.0737 \\
0.0762 \\
0.0819 \\
0.0881 \\
0.0960 \\
0.0985 \\
0.1080 \\
0.1148 \\
0.1256 \\
0.1348 \\
0.1404 \\
0.0746\end{array}$ \\
\hline
\end{tabular}

High Pressure test

Pressure

0.89

49.16

59.57

64.20

80.16

85.61

90.58

103.25

106.11

108.31

123.28

122.97

130.19

132.5

142.63

145.78

42.52

0.22 inches

0.0021

0.0013

0.0041

0.0068

0.0200

0.0283

0.0408

0.0476

0.0600

0.0669

0.0819

0.0881

0.1080

0.1348

0.1404 


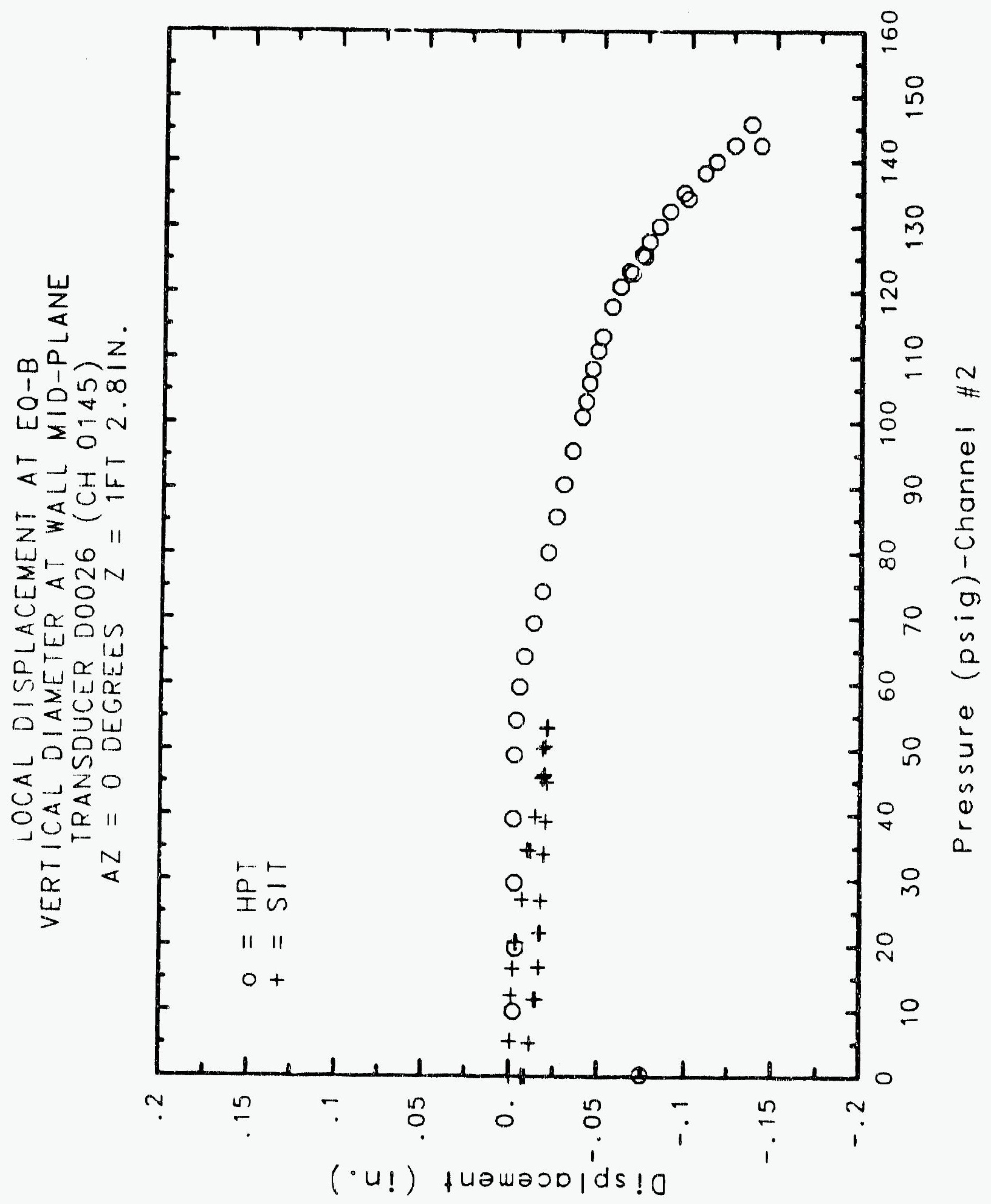

C- 179 


\title{
Reinforced Conr.rete Test Data
}

\author{
LVDT Channel 146
}

Structural INTEGRITY TEST

\begin{tabular}{|c|c|c|c|}
\hline $\begin{array}{l}\text { Pressure } \\
\text { (psig) } \\
-0.05 \\
5.33 \\
12.31 \\
16.44 \\
20.51 \\
20.46 \\
20.47 \\
27.05 \\
34.69 \\
34.53 \\
34.53 \\
39.70 \\
45.90 \\
45.65 \\
45.62 \\
50.10 \\
53.47 \\
53.29 \\
53.21 \\
50.49 \\
46.14 \\
46.13 \\
44.97 \\
38.98 \\
33.99 \\
33.96 \\
26.83 \\
21.89 \\
21.90 \\
16.69 \\
11.74 \\
11.77 \\
5.05 \\
0.02 \\
-0.04 \\
-0.02 \\
-0.02 \\
0.02 \\
\end{array}$ & $\begin{array}{c}\text { Displacement } \\
\text { (inches) } \\
0.0003 \\
0.0001 \\
0.0000 \\
0.0000 \\
0.0004 \\
0.0003 \\
0.0005 \\
-0.0015 \\
-0.0074 \\
-0.0072 \\
-0.0071 \\
-0.0086 \\
-0.0118 \\
-0.0136 \\
-0.0146 \\
-0.0164 \\
-0.0181 \\
-0.0187 \\
-0.0191 \\
-0.0193 \\
-0.0196 \\
-0.0192 \\
-0.0196 \\
-0.0196 \\
-0.0195 \\
-0.0194 \\
-0.0196 \\
-0.0195 \\
-0.0193 \\
-0.0193 \\
-0.0192 \\
-0.0194 \\
-0.0193 \\
-0.0192 \\
-0.0195 \\
-0.0197 \\
-0.0196 \\
-0.0195\end{array}$ & $\begin{array}{c}\text { Pressure } \\
(p s 1 g) \\
9.89 \\
19.55 \\
29.57 \\
39.42 \\
49.16 \\
54.50 \\
59.57 \\
64.20 \\
69.32 \\
74.16 \\
80.16 \\
85.61 \\
90.58 \\
95.69 \\
100.92 \\
103.25 \\
106.11 \\
108.31 \\
111.08 \\
113.24 \\
117.83 \\
120.92 \\
123.28 \\
122.97 \\
125.82 \\
125.60 \\
127.84 \\
130.19 \\
132.53 \\
135.33 \\
134.42 \\
138.35 \\
140.16 \\
142.63 \\
145.78 \\
142.52 \\
0.22\end{array}$ & $\begin{array}{c}\text { Displacement } \\
\text { (inches) } \\
-0.0007 \\
-0.0030 \\
-0.0110 \\
-0.0195 \\
-0.0277 \\
-0.0312 \\
-0.0357 \\
-0.0402 \\
-0.0453 \\
-0.0514 \\
-0.0613 \\
-0.0666 \\
-0.0707 \\
-0.0777 \\
-0.0830 \\
-0.0860 \\
-0.0893 \\
-0.0907 \\
-0.0935 \\
-0.0964 \\
-0.0999 \\
-0.1040 \\
-0.1080 \\
-0.1079 \\
-0.1109 \\
-0.1118 \\
-0.1150 \\
-0.1187 \\
-0.1239 \\
-0.1301 \\
-0.1313 \\
-0.1390 \\
-0.1448 \\
-0.1528 \\
-0.1617 \\
-0.1658 \\
-0.0811\end{array}$ \\
\hline
\end{tabular}

High Pressije test

splacement

$-0.0030$

$-0.0110$

$-0.0195$

$-0.0277$

0.0312

$-0.0453$

$-0.0514$

$-0.0613$

$-0.0666$

$-0.0777$

$-0.0830$

$-0.0860$

$-0.0893$

$-0.0964$

$-0.0999$

$-0.1040$

$-0.1080$

$-0.1118$

$-0.1150$

$-0.1187$

$-0.1239$

0.1390

$-0.1448$

$-0.1528$

$-0.1617$

$-0.1658$

0.0811 


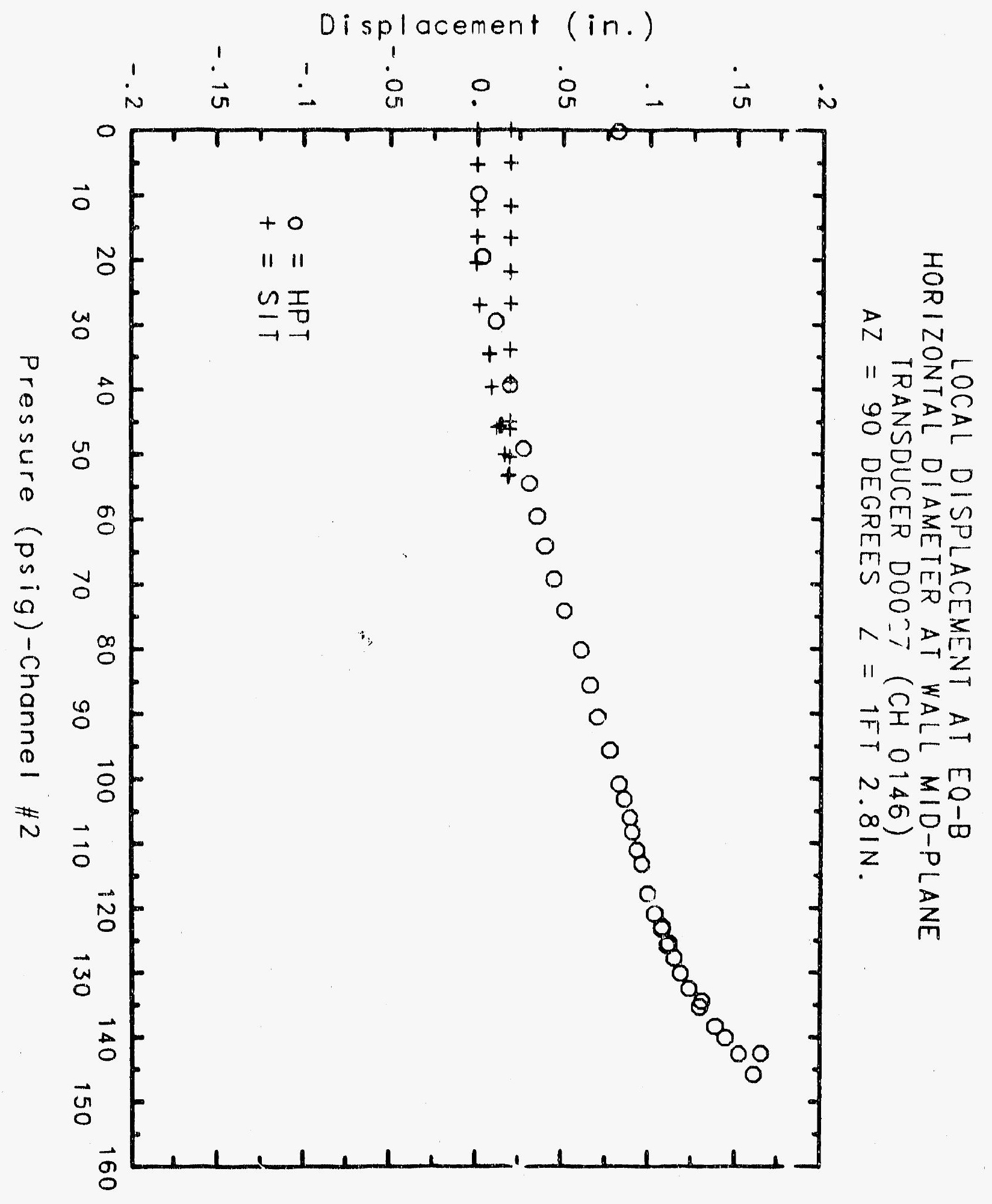




\section{Reinforced Concrete Test Data}

LVDT Channel 147

Structural IntEgrity TEst

\begin{tabular}{|c|c|c|c|}
\hline $\begin{array}{l}\text { Pressure } \\
(p s i g) \\
-0.05 \\
5.33 \\
12.31 \\
16.44 \\
20.51 \\
20.46 \\
20.47 \\
27.05 \\
34.69 \\
34.53 \\
34.53 \\
39.70 \\
45.90 \\
45.65 \\
45.62 \\
50.10 \\
53.47 \\
53.29 \\
53.21 \\
50.49 \\
46.14 \\
46.13 \\
44.97 \\
38.98 \\
33.99 \\
33.96 \\
26.83 \\
21.88 \\
21.90 \\
16.69 \\
11.74 \\
11.77 \\
5.05 \\
0.02 \\
-0.04 \\
-0.02 \\
-0.02 \\
0.02\end{array}$ & $\begin{array}{c}\text { Displacement } \\
\text { (Inches) } \\
0.0001 \\
0.0008 \\
0.0013 \\
0.0009 \\
0.0018 \\
0.0024 \\
0.0020 \\
0.0027 \\
0.0027 \\
0.0027 \\
0.0035 \\
0.0039 \\
0.0061 \\
0.0074 \\
0.0070 \\
0.0077 \\
0.0085 \\
0.0090 \\
0.0088 \\
0.0066 \\
0.0055 \\
0.0063 \\
0.0062 \\
0.0056 \\
0.0054 \\
0.0054 \\
0.0053 \\
0.0043 \\
0.0046 \\
0.0042 \\
0.0038 \\
0.0045 \\
0.0037 \\
0.0025 \\
0.0021 \\
0.0021 \\
0.0025 \\
0.0030\end{array}$ & $\begin{array}{c}\text { Pressure } \\
(p s 1 g) \\
9.89 \\
19.55 \\
29.57 \\
39.42 \\
49.16 \\
54.50 \\
59.57 \\
64.20 \\
69.32 \\
74.16 \\
80.16 \\
85.61 \\
90.58 \\
95.69 \\
100.92 \\
103.25 \\
106.11 \\
108.31 \\
111.08 \\
113.24 \\
117.83 \\
120.92 \\
123.28 \\
122.97 \\
125.82 \\
125.60 \\
127.84 \\
130.19 \\
132.53 \\
135.33 \\
134.42 \\
138.35 \\
140.16 \\
142.63 \\
145.78 \\
142.52 \\
0.22\end{array}$ & $\begin{array}{c}\text { Displacement } \\
(\text { inches) } \\
0.0007 \\
0.0001 \\
-0.0038 \\
-0.0056 \\
-0.0064 \\
-0.0068 \\
-0.0065 \\
-0.0026 \\
0.0026 \\
0.0064 \\
0.0112 \\
0.0143 \\
0.0182 \\
0.0224 \\
0.0272 \\
0.0292 \\
0.0311 \\
0.0330 \\
0.0351 \\
0.0372 \\
0.0414 \\
0.0452 \\
0.0486 \\
0.0496 \\
0.0535 \\
0.0546 \\
0.0562 \\
0.0600 \\
0.0635 \\
0.0682 \\
0.0698 \\
0.0753 \\
0.0800 \\
0.0859 \\
0.0907 \\
0.0938 \\
0.0612\end{array}$ \\
\hline
\end{tabular}

High Pressure test

$$
\begin{aligned}
& \text { splacement } \\
& \text { (inches) } \\
& 0.0007 \\
& 0.0001 \\
& -0.0038 \\
& -0.0056 \\
& -0.0064 \\
& -0.0068 \\
& -0.0065 \\
& -0.0026 \\
& 0.0026 \\
& 0.0064 \\
& 0.0112 \\
& 0.0143 \\
& 0.0182 \\
& 0.0224 \\
& 0.0272 \\
& 0.0292 \\
& 0.0311 \\
& 0.0330 \\
& 0.0351 \\
& 0.0372 \\
& 0.0414 \\
& 0.0452 \\
& 0.0486 \\
& 0.0496 \\
& 0.0535 \\
& 0.0546 \\
& 0.0562 \\
& 0.0600 \\
& 0.0635 \\
& 0.0682 \\
& 0.0698 \\
& 0.0753 \\
& 0.0800 \\
& 0.0859 \\
& 0.0907 \\
& 0.0938 \\
& 0.0612 \\
&
\end{aligned}
$$




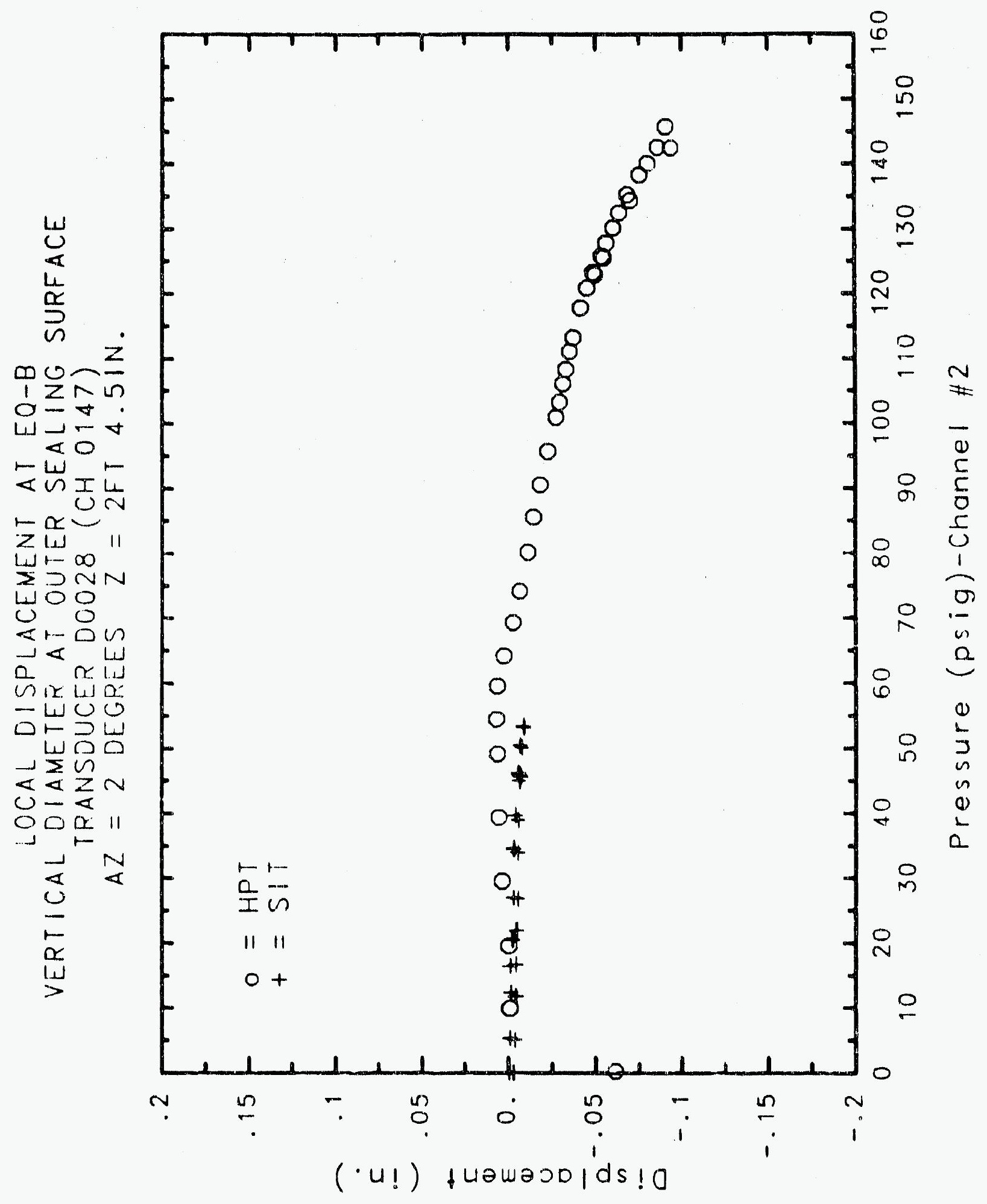

C. 183 


\section{Reinforced Concrete Test Data}

LVDt Channel 148

Structural IntegrIty TEST

\begin{tabular}{|c|c|c|c|}
\hline $\begin{array}{l}\text { Pressure } \\
\text { (psig) } \\
-0.05 \\
5.33 \\
12.31 \\
16.44 \\
20.51 \\
20.46 \\
20.47 \\
27.05 \\
34.69 \\
34.53 \\
34.53 \\
39.70 \\
45.90 \\
45.65 \\
45.62 \\
50.10 \\
53.47 \\
53.29 \\
53.21 \\
50.49 \\
46.14 \\
46.13 \\
44.97 \\
38.98 \\
33.99 \\
33.96 \\
26.83 \\
21.88 \\
21.90 \\
16.69 \\
11.74 \\
11.77 \\
5.05 \\
0.02 \\
-0.04 \\
-0.02 \\
-0.02 \\
0.02\end{array}$ & $\begin{array}{c}\text { Displacement } \\
\text { (inches) } \\
0.0000 \\
0.0003 \\
-0.0001 \\
0.0002 \\
0.0002 \\
0.0005 \\
0.0004 \\
0.0005 \\
0.0010 \\
0.0016 \\
0.0016 \\
0.0019 \\
0.0021 \\
0.0020 \\
0.0016 \\
0.0018 \\
0.0018 \\
0.0020 \\
0.0016 \\
0.0017 \\
0.0016 \\
0.0020 \\
0.0020 \\
0.0020 \\
0.0021 \\
0.0023 \\
0.0020 \\
0.0024 \\
0.0025 \\
0.0024 \\
0.0025 \\
0.0023 \\
0.0023 \\
0.0023 \\
0.0017 \\
0.0017 \\
0.0015 \\
0.0013\end{array}$ & $\begin{array}{c}\text { Pressure } \\
(\text { psig) } \\
9.89 \\
19.55 \\
29.57 \\
39.42 \\
49.16 \\
54.50 \\
59.57 \\
64.20 \\
69.32 \\
74.16 \\
80.16 \\
85.61 \\
90.58 \\
95.69 \\
100.92 \\
103.25 \\
106.11 \\
108.31 \\
111.08 \\
113.24 \\
117.83 \\
120.92 \\
123.28 \\
122.97 \\
125.82 \\
125.60 \\
127.84 \\
130.19 \\
132.53 \\
135.33 \\
134.42 \\
138.35 \\
140.16 \\
142.63 \\
145.78 \\
142.52 \\
0.22 \\
\end{array}$ & $\begin{array}{c}\text { Dispiacement } \\
\text { (inches) } \\
-0.0001 \\
-0.0004 \\
-0.0004 \\
-0.0022 \\
-0.0049 \\
-0.0066 \\
-0.0082 \\
-0.0102 \\
-0.0114 \\
-0.0131 \\
-0.0153 \\
-0.0164 \\
-0.0176 \\
-0.0193 \\
-0.0208 \\
-0.0213 \\
-0.0221 \\
-0.0224 \\
-0.0231 \\
-0.0235 \\
-0.0245 \\
-0.0260 \\
-0.0274 \\
-0.0284 \\
-0.0287 \\
-0.0287 \\
-0.0292 \\
-0.0321 \\
-0.0329 \\
-0.0355 \\
-0.0365 \\
-0.0383 \\
-0.0430 \\
-0.0462 \\
-0.0501 \\
-0.0540 \\
-0.0583\end{array}$ \\
\hline
\end{tabular}

High Pressure test

spiacement

(inches)

$-0.0004$

$-0.0004$

$-0.0022$

$-0.0049$

$-0.0066$

0.0082

$-0.0114$

$-0.0131$

$-0.0153$

$-0.0164$

$-0.0208$

$-0.0213$

$-0.0221$

$-0.0224$

$-0.0235$

$-0.0245$

$-0.0260$

$-0.0274$

$-0.0287$

$-0.0292$

$-0.0321$

$-0.0329$

$-0.0383$

$-0.0430$

$-0.0462$

$-0.0501$

$-0.0540$

$-0.0583$ 


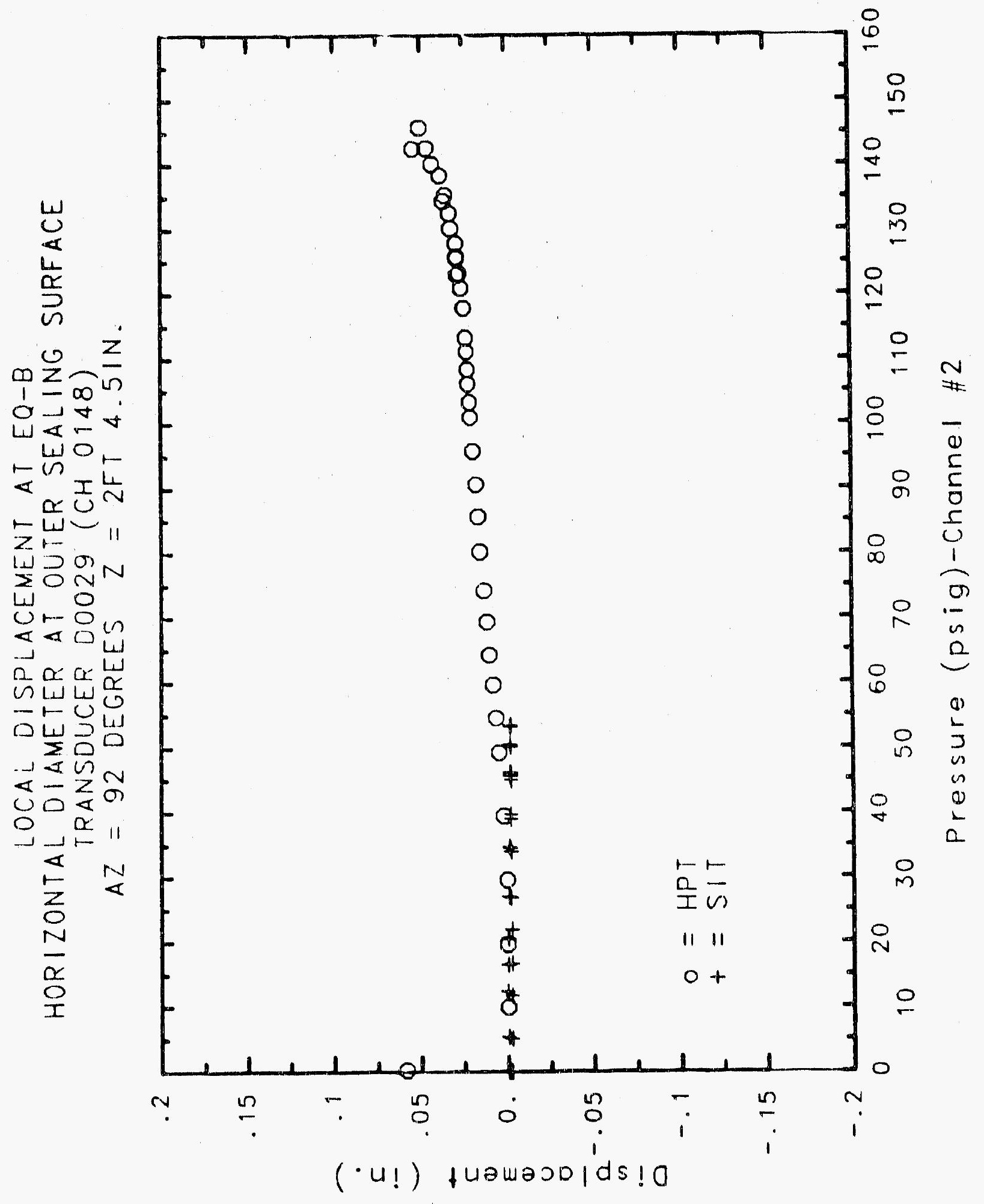

C- 185 


\section{Reinforced Concrete Test Data}

\section{LVDT Channel 149}

Structural Integrity Test

\begin{tabular}{|c|c|c|c|}
\hline $\begin{array}{c}\text { Pressure } \\
\text { (psig) } \\
-0.05 \\
5.33 \\
12.31 \\
16.44 \\
20.51 \\
20.46 \\
20.47 \\
27.05 \\
34.69 \\
34.53 \\
34.53 \\
39.70 \\
45.90 \\
45.65 \\
45.62 \\
50.10 \\
53.47 \\
53.29 \\
53.21 \\
50.49 \\
46.14 \\
46.13 \\
44.97 \\
38.98 \\
33.99 \\
33.96 \\
26.83 \\
21.88 \\
21.90 \\
16.69 \\
11.74 \\
11.77 \\
5.05 \\
0.02 \\
-0.04 \\
-0.02 \\
-0.02 \\
0.02\end{array}$ & $\begin{array}{c}\text { Displacement } \\
(\text { inches) } \\
0.0002 \\
0.0006 \\
0.0006 \\
0.0001 \\
0.0002 \\
0.0001 \\
-0.0001 \\
-0.0002 \\
-0.0028 \\
-0.0034 \\
-0.0030 \\
-0.0052 \\
-0.0079 \\
-0.0085 \\
-0.0084 \\
-0.0107 \\
-0.0122 \\
-0.0122 \\
-0.0123 \\
-0.0126 \\
-0.0121 \\
-0.0122 \\
-0.0123 \\
-0.0123 \\
-0.0119 \\
-0.0123 \\
-0.0118 \\
-0.0117 \\
-0.0120 \\
-0.0119 \\
-0.0119 \\
-0.0119 \\
-0.0112 \\
-0.0094 \\
-0.0090 \\
-0.0087 \\
-0.0085 \\
-0.0090 \\
-0.0\end{array}$ & $\begin{array}{c}\text { Pressure } \\
(\text { ps 1g) } \\
9.89 \\
19.55 \\
29.57 \\
39.42 \\
49.16 \\
54.50 \\
59.57 \\
64.20 \\
69.32 \\
74.16 \\
80.16 \\
85.61 \\
90.58 \\
95.69 \\
100.92 \\
103.25 \\
106.11 \\
108.31 \\
111.08 \\
113.24 \\
117.83 \\
120.92 \\
123.28 \\
122.97 \\
125.82 \\
125.60 \\
127.84 \\
130.19 \\
132.53 \\
135.33 \\
134.42 \\
138.35 \\
140.16 \\
142.63 \\
145.78 \\
142.52 \\
0.22\end{array}$ & $\begin{array}{c}\text { Displacement } \\
\text { (inches) } \\
-0.0003 \\
-0.0002 \\
-0.0009 \\
-0.0037 \\
-0.0068 \\
-0.0088 \\
-0.0104 \\
-0.0127 \\
-0.0156 \\
-0.0184 \\
-0.0221 \\
-0.0255 \\
-0.0288 \\
-0.0320 \\
-0.0349 \\
-0.0371 \\
-0.0386 \\
-0.0395 \\
-0.0420 \\
-0.0428 \\
-0.0467 \\
-0.0491 \\
-0.0510 \\
-0.0516 \\
-0.0546 \\
-0.0552 \\
-0.0573 \\
-0.0610 \\
-0.0647 \\
-0.0702 \\
-0.0708 \\
-0.0772 \\
-0.0814 \\
-0.0881 \\
-0.0937 \\
-0.0974 \\
-0.0329\end{array}$ \\
\hline
\end{tabular}

High Pressure Test

(inches)

0.0003

$-0.0002$

$-0.0009$

0.0037

0.0088

$-0.0104$

$-0.0127$

$-0.0156$

0.0221

$-0.0255$

$-0.0288$

$-0.0320$

$-0.0386$

$-0.0395$

$-0.0420$

$-0.0491$

$-0.0510$

$-0.0516$

$-0.0573$

$-0.0610$

$-0.0647$

$-0.0702$

$-0.0814$

$-0.0881$

$-0.0937$

$-0.0974$

0.0329 


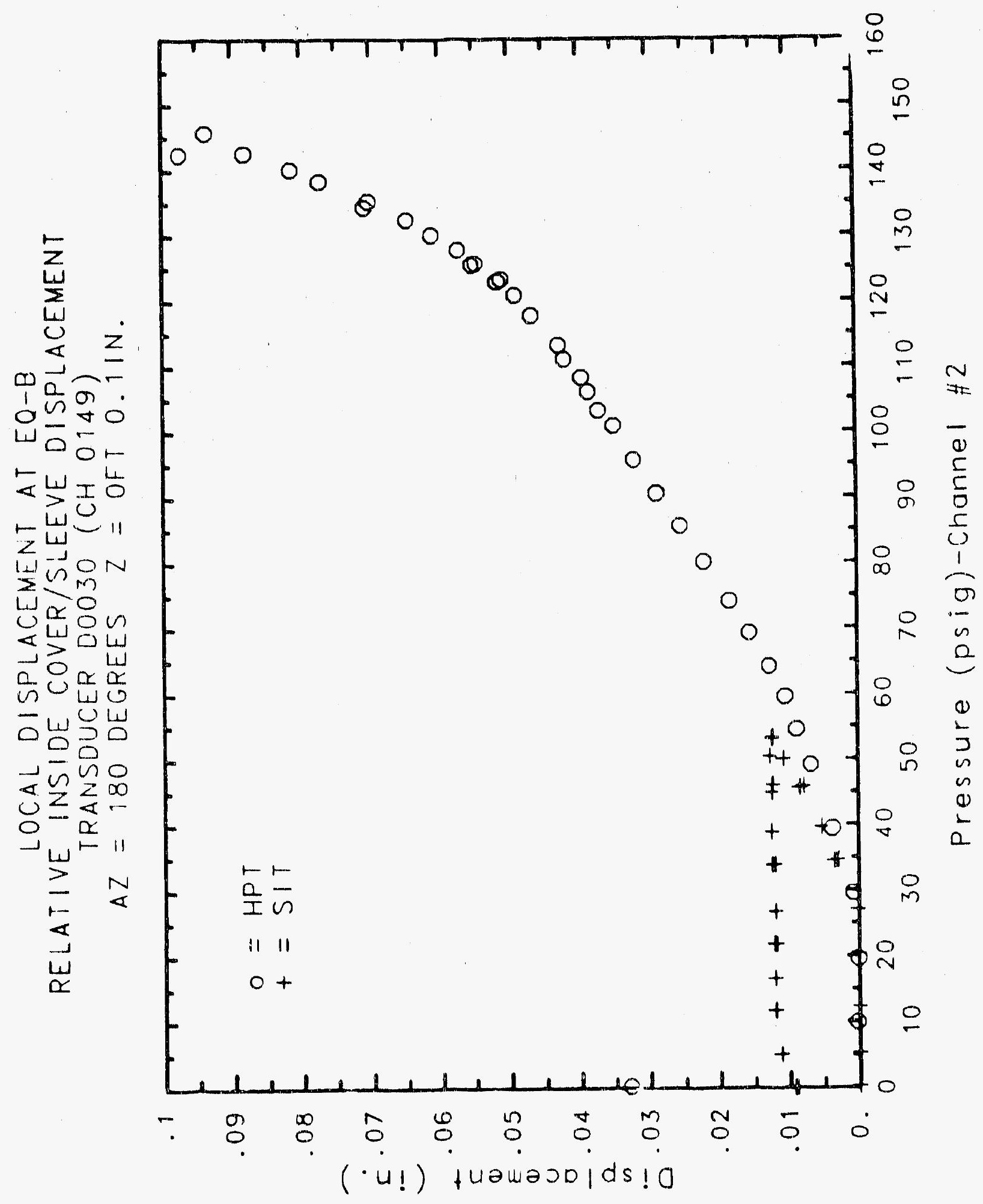

C. 187 


\title{
Reinforced Concrete Test Data
}

\author{
LVDT Channel 150
}

Structural Integrity Test

\begin{tabular}{|c|c|c|c|}
\hline $\begin{array}{c}\text { Pressure } \\
\text { (psig) } \\
-0.05 \\
5.33 \\
12.31 \\
16.44 \\
20.51 \\
20.46 \\
20.47 \\
27.05 \\
34.69 \\
34.53 \\
34.53 \\
39.70 \\
45.90 \\
45.65 \\
45.62 \\
50.10 \\
53.47 \\
53.29 \\
53.21 \\
50.49 \\
46.14 \\
46.13 \\
44.97 \\
38.98 \\
33.99 \\
33.96 \\
26.83 \\
21.88 \\
21.90 \\
16.69 \\
11.74 \\
11.77 \\
5.05 \\
0.02 \\
-0.04 \\
-0.02 \\
-0.02 \\
0.02\end{array}$ & $\begin{array}{c}\text { Displacement } \\
\text { (inches) } \\
0.0011 \\
0.0011 \\
0.0010 \\
0.0000 \\
0.0008 \\
0.0009 \\
-0.0001 \\
0.0000 \\
-0.0020 \\
-0.0031 \\
-0.0026 \\
-0.0043 \\
-0.0073 \\
-0.0075 \\
-0.0083 \\
-0.0095 \\
-0.0119 \\
-0.0114 \\
-0.0123 \\
-0.0118 \\
-0.0119 \\
-0.0110 \\
-0.0116 \\
-0.0118 \\
-0.0115 \\
-0.0117 \\
-0.0117 \\
-0.0109 \\
-0.0112 \\
-0.0108 \\
-0.0113 \\
-0.0105 \\
-0.0108 \\
-0.0084 \\
-0.0082 \\
-0.0077 \\
-0.0078 \\
-0.0078\end{array}$ & $\begin{array}{c}\text { Pressure } \\
\text { (psig) } \\
9.89 \\
19.55 \\
29.57 \\
39.42 \\
49.16 \\
54.50 \\
59.57 \\
64.20 \\
69.32 \\
74.16 \\
80.16 \\
85.61 \\
90.58 \\
95.69 \\
100.92 \\
103.25 \\
106.11 \\
108.31 \\
111.08 \\
113.24 \\
117.83 \\
120.92 \\
123.28 \\
122.97 \\
125.82 \\
125.60 \\
127.84 \\
130.19 \\
132.53 \\
135.33 \\
134.42 \\
138.35 \\
140.16 \\
142.63 \\
145.78 \\
142.52 \\
0.22\end{array}$ & $\begin{array}{c}\text { Displacement } \\
\text { (inches) } \\
0.0010 \\
0.0003 \\
-0.0004 \\
-0.0030 \\
-0.0059 \\
-0.0066 \\
-0.0078 \\
-0.0104 \\
-0.0134 \\
-0.0155 \\
-0.0199 \\
-0.0228 \\
-0.0252 \\
-0.0281 \\
-0.0318 \\
-0.0329 \\
-0.0347 \\
-0.0364 \\
-0.0379 \\
-0.0391 \\
-0.0422 \\
-0.0445 \\
-0.0471 \\
-0.0472 \\
-0.0506 \\
-0.0505 \\
-0.0527 \\
-0.0561 \\
-0.0602 \\
-0.0648 \\
-0.0665 \\
-0.0716 \\
-0.0767 \\
-0.0820 \\
-0.0884 \\
-0.0916 \\
-0.0321\end{array}$ \\
\hline
\end{tabular}

High Pressure test

splacement

0.0003

$-0.0004$

$-0.0030$

$-0.0059$

$-0.0204$

$-0.0134$

$-0.0155$

$-0.0199$

$-0.0281$

$-0.0318$

0.0329

0.0347

$-0.0391$

$-0.0422$

$-0.0445$

$-0.0471$

$-0.0472$

$-0.0527$

$-0.0561$

$-0.0602$

$-0.0648$

$-0.0767$

.

$-0.0916$

$-0.0321$ 


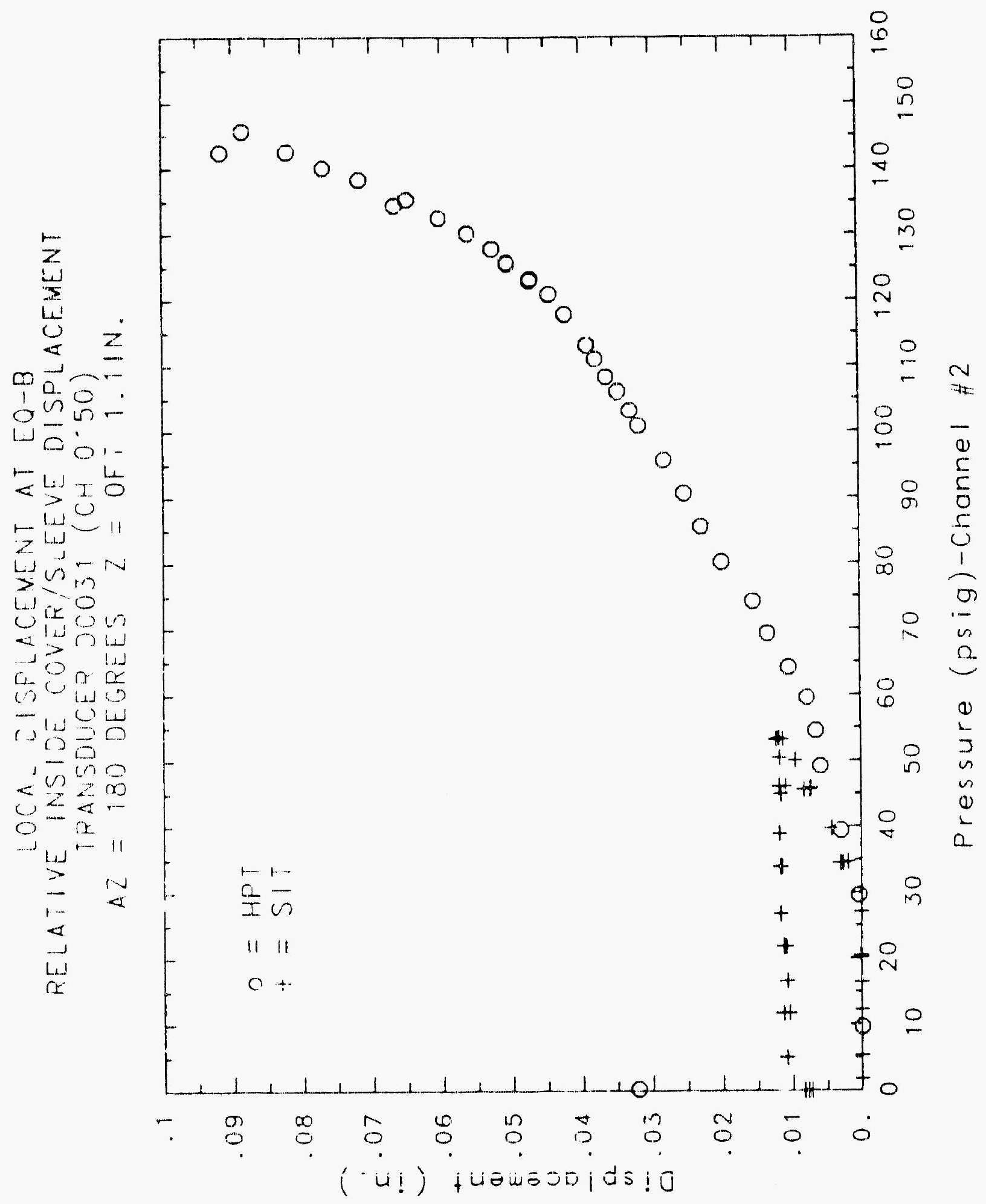

C. 189 


\section{Reinforced Concrete Test Data}

LVDT Channel 151

Structural Integrity Test

\begin{tabular}{|c|c|c|c|}
\hline $\begin{array}{l}\text { Pressure } \\
\text { (psig) } \\
-0.05 \\
5.33 \\
12.31 \\
16.44 \\
20.51 \\
20.46 \\
20.47 \\
27.05 \\
34.69 \\
34.53 \\
34.53 \\
39.70 \\
45.90 \\
45.65 \\
45.62 \\
50.10 \\
53.47 \\
53.29 \\
53.21 \\
50.49 \\
46.14 \\
46.13 \\
44.97 \\
38.98 \\
33.99 \\
33.96 \\
26.83 \\
21.88 \\
21.90 \\
16.69 \\
11.74 \\
11.77 \\
5.05 \\
0.02 \\
-0.04 \\
-0.02 \\
-0.02 \\
0.02\end{array}$ & $\begin{array}{c}\text { Displacement } \\
\text { (inches) } \\
-0.0002 \\
-0.0003 \\
-0.0003 \\
-0.0003 \\
-0.0004 \\
-0.0003 \\
-0.0002 \\
-0.0005 \\
-0.0003 \\
-0.0004 \\
-0.0002 \\
-0.0002 \\
0.0000 \\
-0.0001 \\
0.0001 \\
0.0002 \\
0.0003 \\
0.0004 \\
0.0003 \\
0.0006 \\
0.0007 \\
0.0005 \\
0.0007 \\
0.0009 \\
0.0009 \\
0.0009 \\
0.0015 \\
0.0019 \\
0.0021 \\
0.0026 \\
0.0030 \\
0.0029 \\
0.0032 \\
0.0036 \\
0.0036 \\
0.0037 \\
0.0036 \\
0.0037\end{array}$ & $\begin{array}{c}\text { Pressure } \\
(\text { psig) } \\
9.89 \\
19.55 \\
29.57 \\
39.42 \\
49.16 \\
54.50 \\
59.57 \\
64.20 \\
69.32 \\
71.16 \\
80.16 \\
85.61 \\
90.58 \\
95.69 \\
100.92 \\
103.25 \\
106.11 \\
108.31 \\
111.08 \\
113.24 \\
117.83 \\
120.92 \\
123.28 \\
122.97 \\
125.82 \\
125.60 \\
127.84 \\
130.19 \\
132.53 \\
135.33 \\
134.42 \\
138.35 \\
140.16 \\
142.63 \\
145.78 \\
142.52 \\
0.22\end{array}$ & $\begin{array}{c}\text { Displacement } \\
\text { (inches) } \\
0.0002 \\
0.0008 \\
0.0017 \\
0.0028 \\
0.0046 \\
0.0060 \\
0.0074 \\
0.0094 \\
0.0112 \\
0.0136 \\
0.0166 \\
0.0190 \\
0.0213 \\
0.0235 \\
0.0260 \\
0.0273 \\
0.0284 \\
0.0293 \\
0.0307 \\
0.0317 \\
0.0340 \\
0.0358 \\
0.0374 \\
0.0375 \\
0.0394 \\
0.0398 \\
0.0411 \\
0.0434 \\
0.0460 \\
0.0491 \\
0.0497 \\
0.0532 \\
0.0559 \\
0.0599 \\
0.0639 \\
0.0661 \\
0.0679\end{array}$ \\
\hline
\end{tabular}

high Pressure test

placement

0.0008

0.0060

0.0112

0.0136

0.0235

0.0260

0.0307

0.0317

0.0340

0.0375

0.0394

0.0398

0.0411

0491

0.0497

0.0532

0.0639

0.0661

0. 


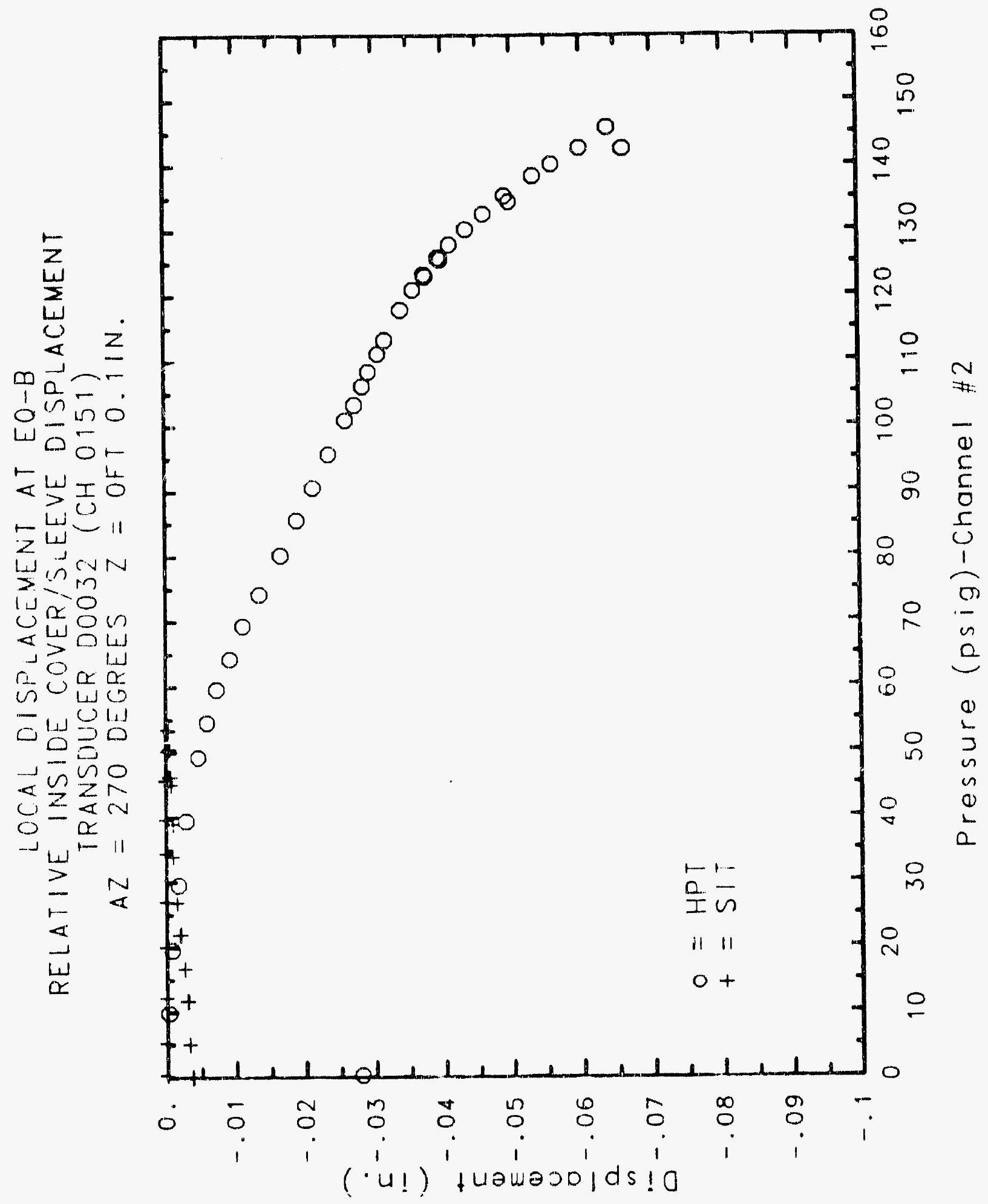




\section{Reinforced Concrete Test Data}

LVDT Channel 152

STRUCTURAL INTEGRITY TEST

\begin{tabular}{|c|c|c|c|}
\hline $\begin{array}{l}\text { Pressure } \\
\text { (psig) } \\
-0.05 \\
5.33 \\
12.31 \\
16.44 \\
20.51 \\
20.46 \\
20.47 \\
27.05 \\
34.69 \\
34.53 \\
34.53 \\
39.70 \\
45.90 \\
45.65 \\
45.62 \\
50.10 \\
53.47 \\
53.29 \\
53.21 \\
50.49 \\
46.14 \\
46.13 \\
44.97 \\
38.98 \\
33.99 \\
33.96 \\
26.83 \\
21.88 \\
21.90 \\
16.69 \\
11.74 \\
11.77 \\
5.05 \\
0.02 \\
-0.04 \\
-0.02 \\
-0.02 \\
0.02\end{array}$ & $\begin{array}{c}\text { Displacement } \\
\text { (inches) } \\
0.0001 \\
0.0000 \\
-0.0001 \\
0.0000 \\
-0.0003 \\
-0.0001 \\
0.0000 \\
-0.0003 \\
-0.0005 \\
-0.0004 \\
-0.0001 \\
-0.0004 \\
-0.0004 \\
-0.0002 \\
0.0000 \\
-0.0002 \\
0.0000 \\
-0.0002 \\
-0.0001 \\
0.0002 \\
0.0001 \\
0.0003 \\
0.0001 \\
0.0006 \\
0.0007 \\
0.0004 \\
0.0008 \\
0.0015 \\
0.0016 \\
0.0024 \\
0.0027 \\
0.0026 \\
0.0034 \\
0.0036 \\
0.0037 \\
0.0039 \\
0.0037 \\
0.0037 \\
0.00\end{array}$ & $\begin{array}{c}\text { Pressure } \\
(p s i g) \\
9.89 \\
19.55 \\
29.57 \\
39.42 \\
49.16 \\
54.50 \\
59.57 \\
64.20 \\
69.32 \\
74.16 \\
80.16 \\
85.61 \\
90.58 \\
95.69 \\
100.92 \\
103.25 \\
106.11 \\
108.31 \\
111.08 \\
113.24 \\
117.83 \\
120.92 \\
123.28 \\
122.97 \\
125.82 \\
125.60 \\
127.84 \\
130.19 \\
132.53 \\
135.33 \\
134.42 \\
138.35 \\
140.16 \\
142.63 \\
145.78 \\
142.52 \\
0.22\end{array}$ & $\begin{array}{c}\text { Displacement } \\
\text { (inches) } \\
0.0002 \\
0.0007 \\
0.0012 \\
0.0023 \\
0.0044 \\
0.0053 \\
0.0067 \\
0.0086 \\
0.0109 \\
0.0129 \\
0.0163 \\
0.0184 \\
0.0208 \\
0.0229 \\
0.0255 \\
0.0267 \\
0.0280 \\
0.0288 \\
0.0302 \\
0.0313 \\
0.0336 \\
0.0352 \\
0.0371 \\
0.0371 \\
0.0391 \\
0.0394 \\
0.0407 \\
0.0431 \\
0.0460 \\
0.0490 \\
0.0497 \\
0.0533 \\
0.0563 \\
0.0604 \\
0.0646 \\
0.0670 \\
0.0287\end{array}$ \\
\hline
\end{tabular}

High Pressure test

splacement

0.0012

0.0023

0.0044

0.0053

0.0109

0.0129

0.0163

0.0184

0.0208

0.0267

0.0313

0.0371

0.0371

0.039

0.0394

0.0407

0.0490

0.0563

0.0604

0.0670

0.0287 


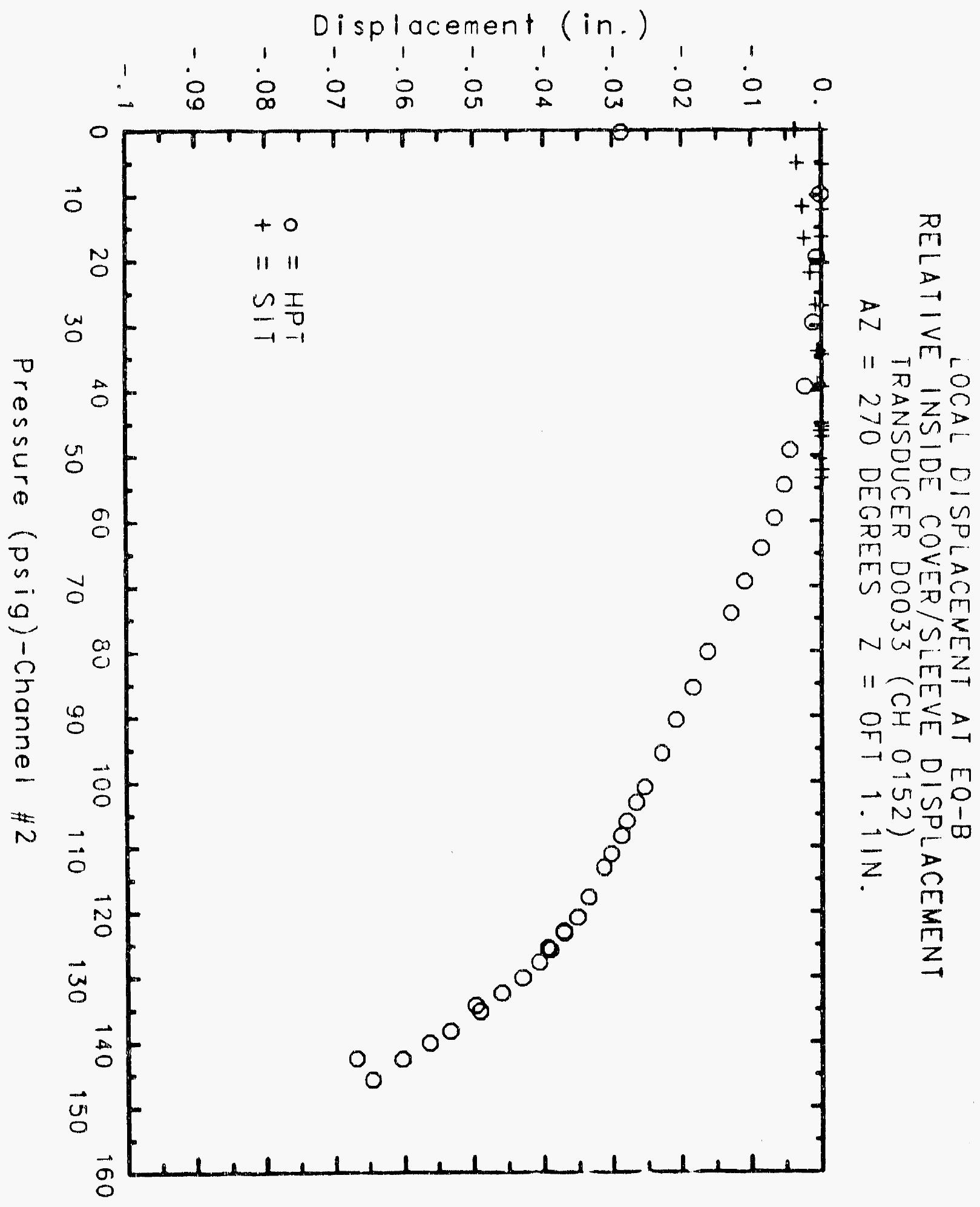




\section{Reinforced Concrete Test Data}

LVDT Channel 153

STRUCTURAL INTEGRITY TEST

\begin{tabular}{|c|c|c|c|}
\hline $\begin{array}{c}\text { Pressure } \\
\text { (psig) } \\
-0.05 \\
5.33 \\
12.31 \\
16.44 \\
20.51 \\
20.46 \\
20.47 \\
27.05 \\
34.69 \\
34.53 \\
34.53 \\
39.70 \\
45.90 \\
45.65 \\
45.62 \\
50.10 \\
53.47 \\
53.29 \\
53.21 \\
50.49 \\
46.14 \\
46.13 \\
44.97 \\
38.98 \\
33.99 \\
33.96 \\
26.83 \\
21.88 \\
21.90 \\
16.69 \\
11.74 \\
11.77 \\
5.05 \\
0.02 \\
-0.04 \\
-0.02 \\
-0.02 \\
0.02\end{array}$ & $\begin{array}{c}\text { Displacement } \\
\text { (Inches) } \\
0.0000 \\
0.0000 \\
-0.0001 \\
-0.0001 \\
-0.0002 \\
-0.0002 \\
-0.0002 \\
-0.0005 \\
-0.0022 \\
-0.0025 \\
-0.0026 \\
-0.0053 \\
-0.0098 \\
-0.0102 \\
-0.0103 \\
-0.0124 \\
-0.0140 \\
-0.0142 \\
-0.0142 \\
-0.0139 \\
-0.0138 \\
-0.0138 \\
-0.0138 \\
-0.0137 \\
-0.0137 \\
-0.0137 \\
-0.0137 \\
-0.0136 \\
-0.0136 \\
-0.0136 \\
-0.0134 \\
-0.0135 \\
-0.0127 \\
-0.0102 \\
-0.0100 \\
-0.0099 \\
-0.0100 \\
-0.0101\end{array}$ & $\begin{array}{c}\text { Pressure } \\
(\text { psig) } \\
9.89 \\
19.55 \\
29.57 \\
39.42 \\
49.16 \\
54.50 \\
59.57 \\
64.20 \\
69.32 \\
74.16 \\
80.16 \\
85.61 \\
90.58 \\
95.69 \\
100.92 \\
103.25 \\
106.11 \\
108.31 \\
111.08 \\
113.24 \\
117.83 \\
120.92 \\
123.28 \\
122.97 \\
125.82 \\
125.60 \\
127.84 \\
130.19 \\
132.53 \\
135.33 \\
134.42 \\
138.35 \\
140.16 \\
142.63 \\
145.78 \\
142.52 \\
0.22\end{array}$ & $\begin{array}{l}\text { Displacement } \\
\text { (inches) } \\
-0.0001 \\
-0.0002 \\
-0.0010 \\
-0.0035 \\
-0.0065 \\
-0.0082 \\
-0.0101 \\
-0.0128 \\
-0.0157 \\
-0.0186 \\
-0.0226 \\
-0.0258 \\
-0.0285 \\
-0.0317 \\
-0.0349 \\
-0.0366 \\
-0.0382 \\
-0.0394 \\
-0.0412 \\
-0.0425 \\
-0.0455 \\
-0.0477 \\
-0.0498 \\
-0.0499 \\
-0.0523 \\
-0.0527 \\
-0.0547 \\
-0.0580 \\
-0.0618 \\
-0.0664 \\
-0.0672 \\
-0.0727 \\
-0.0770 \\
-0.0833 \\
-0.0882 \\
-0.0890 \\
-0.0331\end{array}$ \\
\hline
\end{tabular}

High Pressure test 


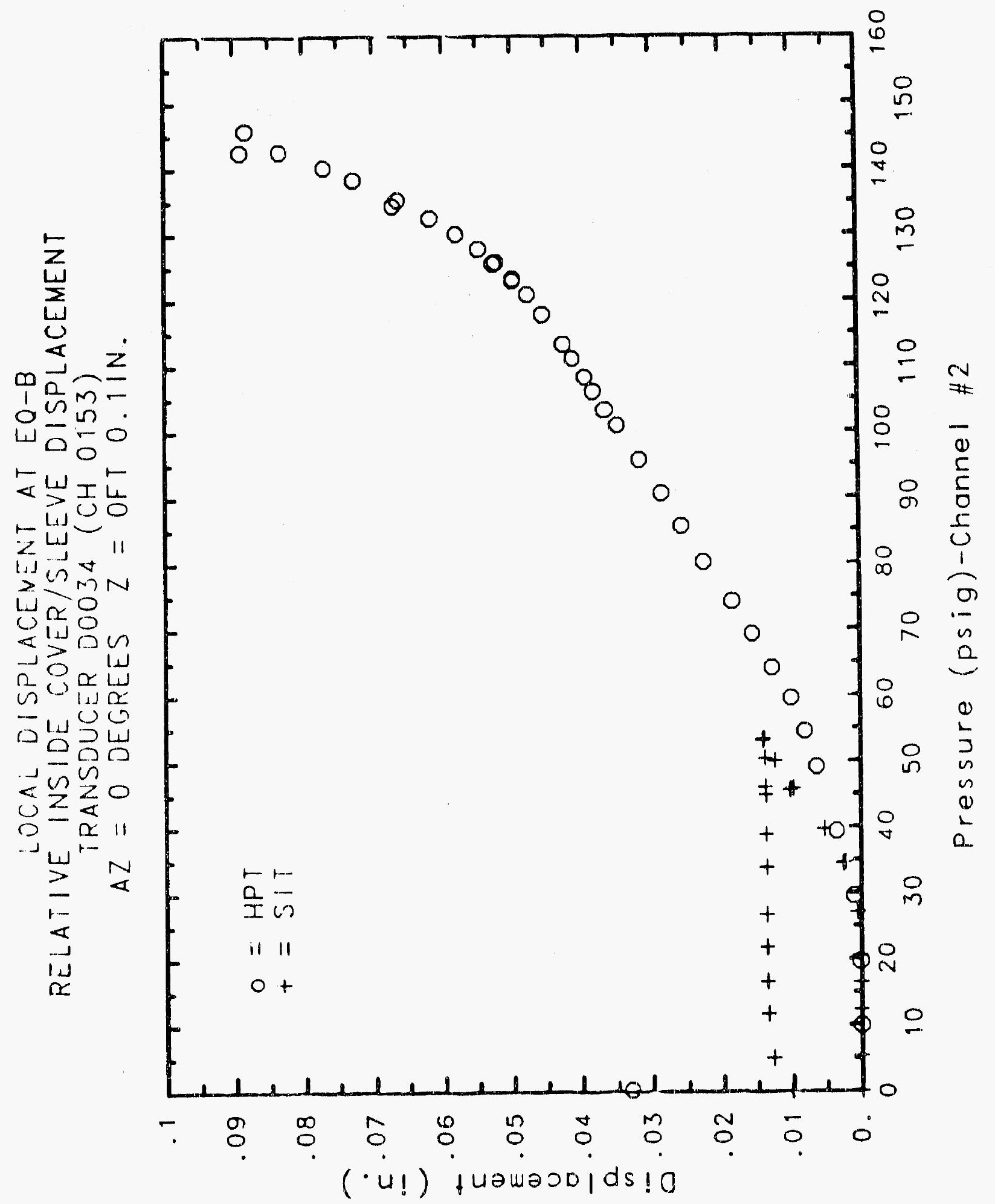

C-195 


\section{Reinforced Concrete Test Data}

LyDT Channel 154

StruCtURAL INTEGRITY TEST

\begin{tabular}{|c|c|c|c|}
\hline $\begin{array}{l}\text { Pressure } \\
\text { (psig) } \\
-0.05 \\
5.33 \\
12.31 \\
16.44 \\
20.51 \\
20.46 \\
20.47 \\
27.05 \\
34.69 \\
34.53 \\
34.53 \\
39.70 \\
45.90 \\
45.65 \\
45.62 \\
50.10 \\
53.47 \\
53.29 \\
53.21 \\
50.49 \\
46.14 \\
46.13 \\
44.97 \\
38.98 \\
33.99 \\
33.96 \\
26.83 \\
21.88 \\
21.90 \\
16.69 \\
11.74 \\
11.77 \\
5.05 \\
0.02 \\
-0.04 \\
-0.02 \\
-0.02 \\
0.02\end{array}$ & $\begin{array}{c}\text { Displacement } \\
\text { (inches) } \\
0.0000 \\
0.0000 \\
-0.0001 \\
-0.0001 \\
-0.0001 \\
-0.0001 \\
-0.0001 \\
-0.0002 \\
-0.0019 \\
-0.0021 \\
-0.0022 \\
-0.0048 \\
-0.0091 \\
-0.0095 \\
-0.0095 \\
-0.0116 \\
-0.0131 \\
-0.0133 \\
-0.0133 \\
-0.0133 \\
-0.0133 \\
-0.0133 \\
-0.0133 \\
-0.0133 \\
-0.0132 \\
-0.0132 \\
-0.0132 \\
-0.0132 \\
-0.0132 \\
-0.0132 \\
-0.0131 \\
-0.0131 \\
-0.0124 \\
-0.0100 \\
-0.0096 \\
-0.0096 \\
-0.0096 \\
-0.0096 \\
-0.0\end{array}$ & $\begin{array}{c}\text { Pressure } \\
\text { (psig) } \\
9.89 \\
19.55 \\
29.57 \\
39.42 \\
49.16 \\
54.50 \\
59.57 \\
64.20 \\
69.32 \\
74.16 \\
80.16 \\
85.61 \\
90.58 \\
95.69 \\
100.92 \\
103.25 \\
106.11 \\
108.31 \\
111.08 \\
113.24 \\
117.83 \\
120.92 \\
123.28 \\
122.97 \\
125.82 \\
125.60 \\
127.84 \\
130.19 \\
132.53 \\
135.33 \\
134.42 \\
138.35 \\
140.16 \\
142.63 \\
145.78 \\
142.52 \\
0.22\end{array}$ & $\begin{array}{c}\text { Displacement } \\
\text { (inches) } \\
0.0000 \\
0.0001 \\
-0.0005 \\
-0.0027 \\
-0.0053 \\
-0.0068 \\
-0.0085 \\
-0.0109 \\
-0.0136 \\
-0.0163 \\
-0.0200 \\
-0.0230 \\
-0.0255 \\
-0.0284 \\
-0.0315 \\
-0.0331 \\
-0.0346 \\
-0.0357 \\
-0.0375 \\
-0.0387 \\
-0.0416 \\
-0.0434 \\
-0.0454 \\
-0.0456 \\
-0.0478 \\
-0.0482 \\
-0.0501 \\
-0.0531 \\
-0.0567 \\
-0.0611 \\
-0.0619 \\
-0.0671 \\
-0.0711 \\
-0.0771 \\
-0.0831 \\
-0.0863 \\
-0.0325\end{array}$ \\
\hline
\end{tabular}

High Pressure test

splacement

(inches)

0.0000

0.0001

$-0.0005$

0.0027

$-0.0085$

$-0.0109$

$-0.0136$

$-0.0163$

$-0.0255$

$-0.0284$

$-0.0315$

$-0.0331$

$-0.0375$

$-0.0454$

$-0.0482$

$-0.0501$

$-0.0531$

$-0.0567$

$-0.0611$

$-0.0619$

$-0.0771$

$-0.0831$

$-0.0863$ 


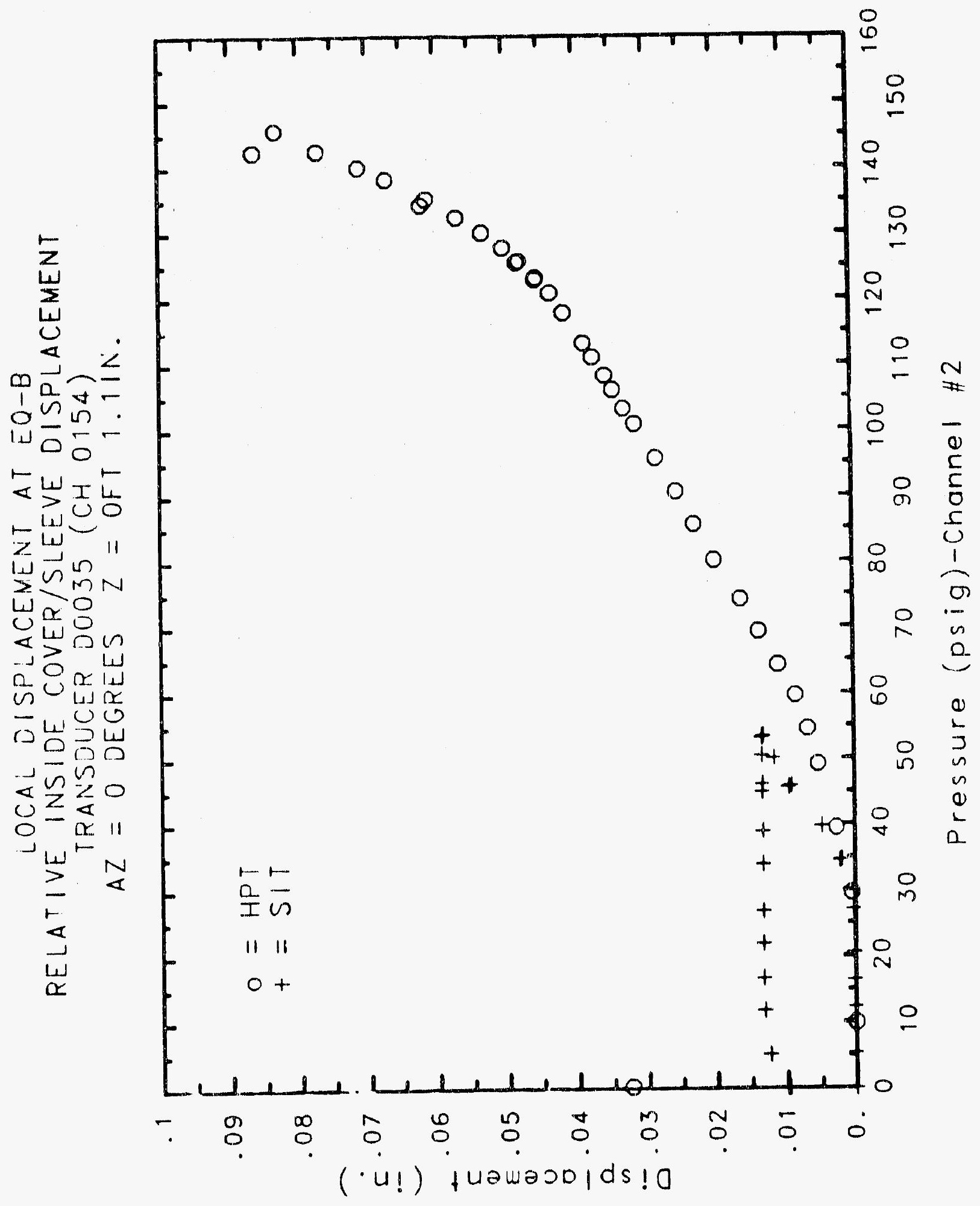




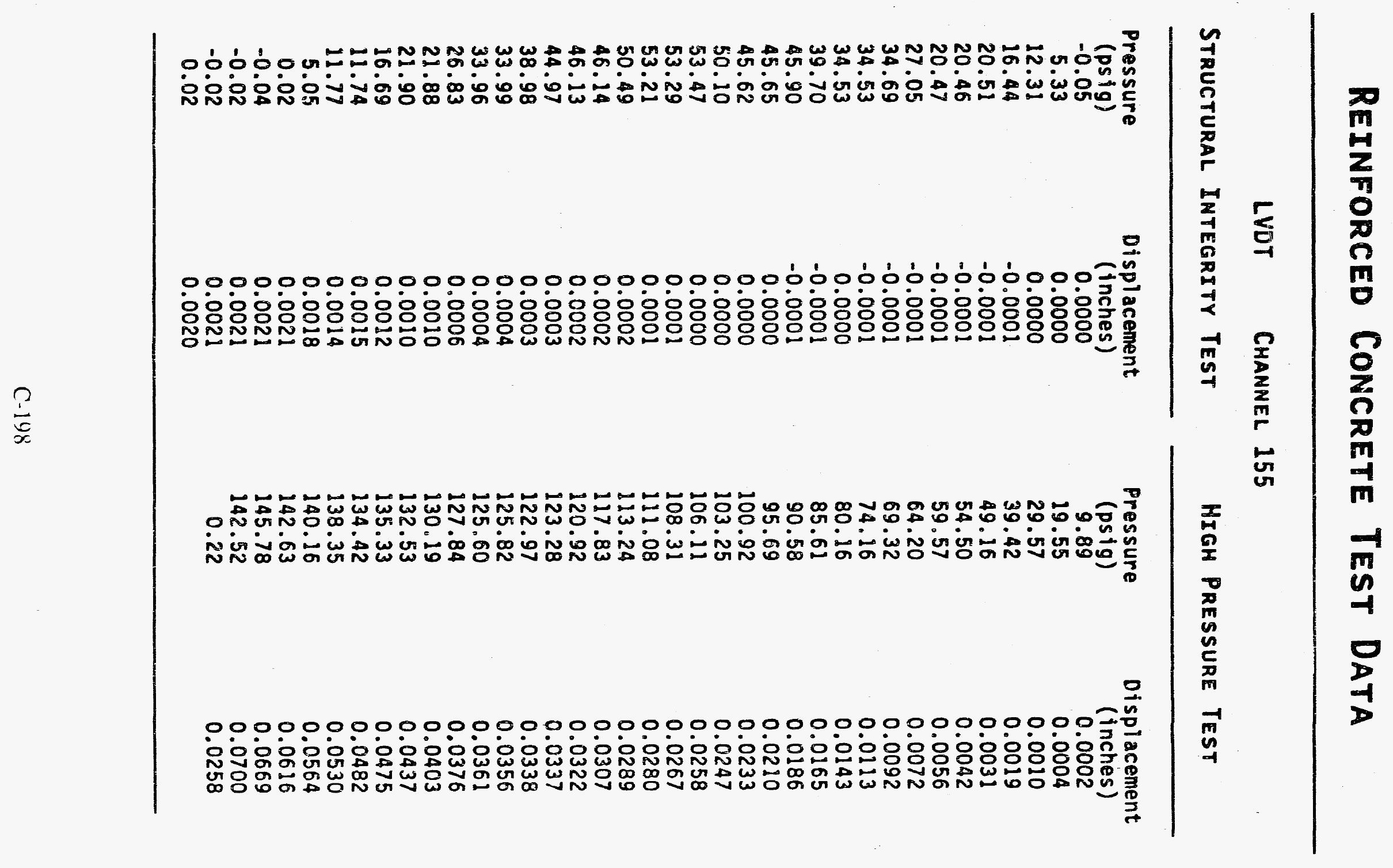




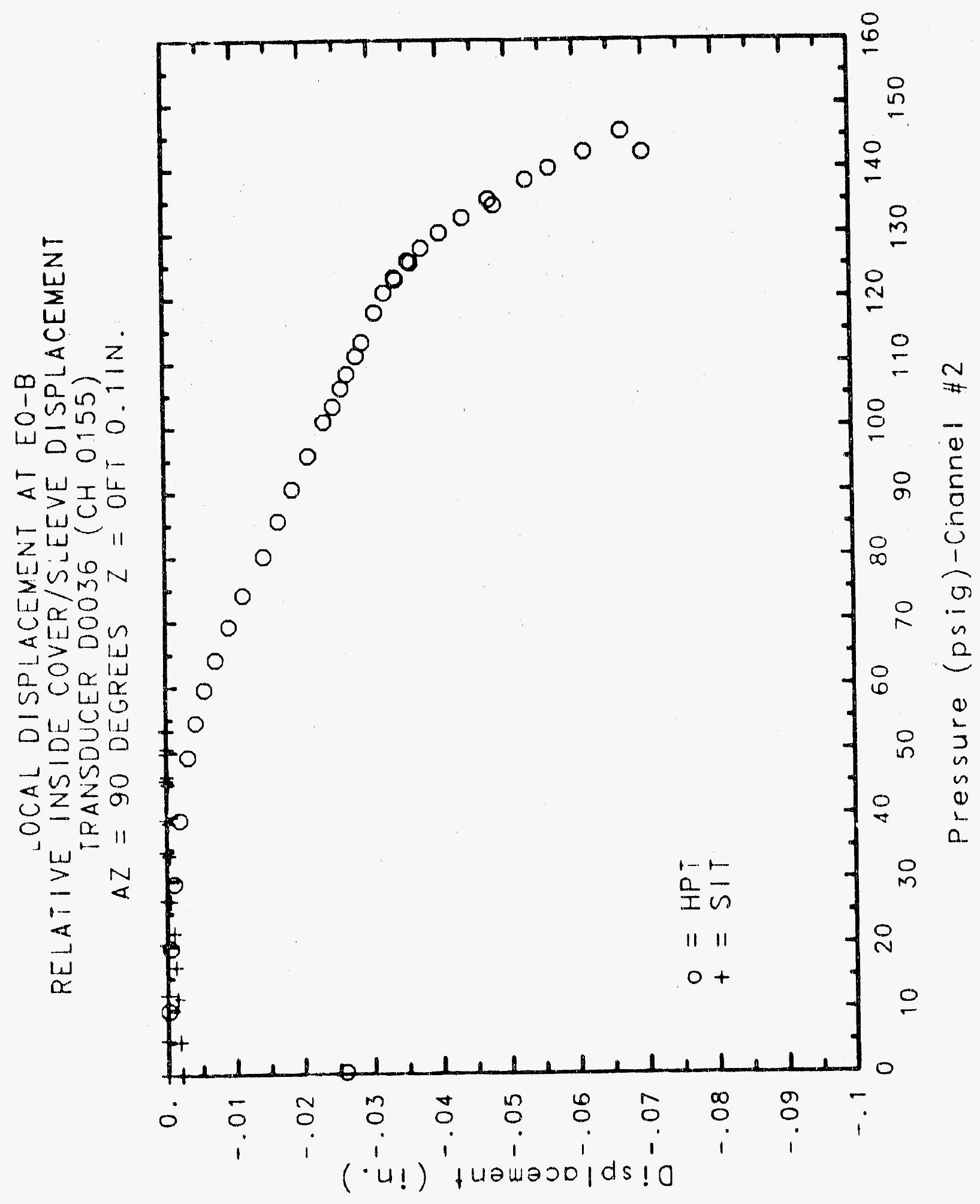




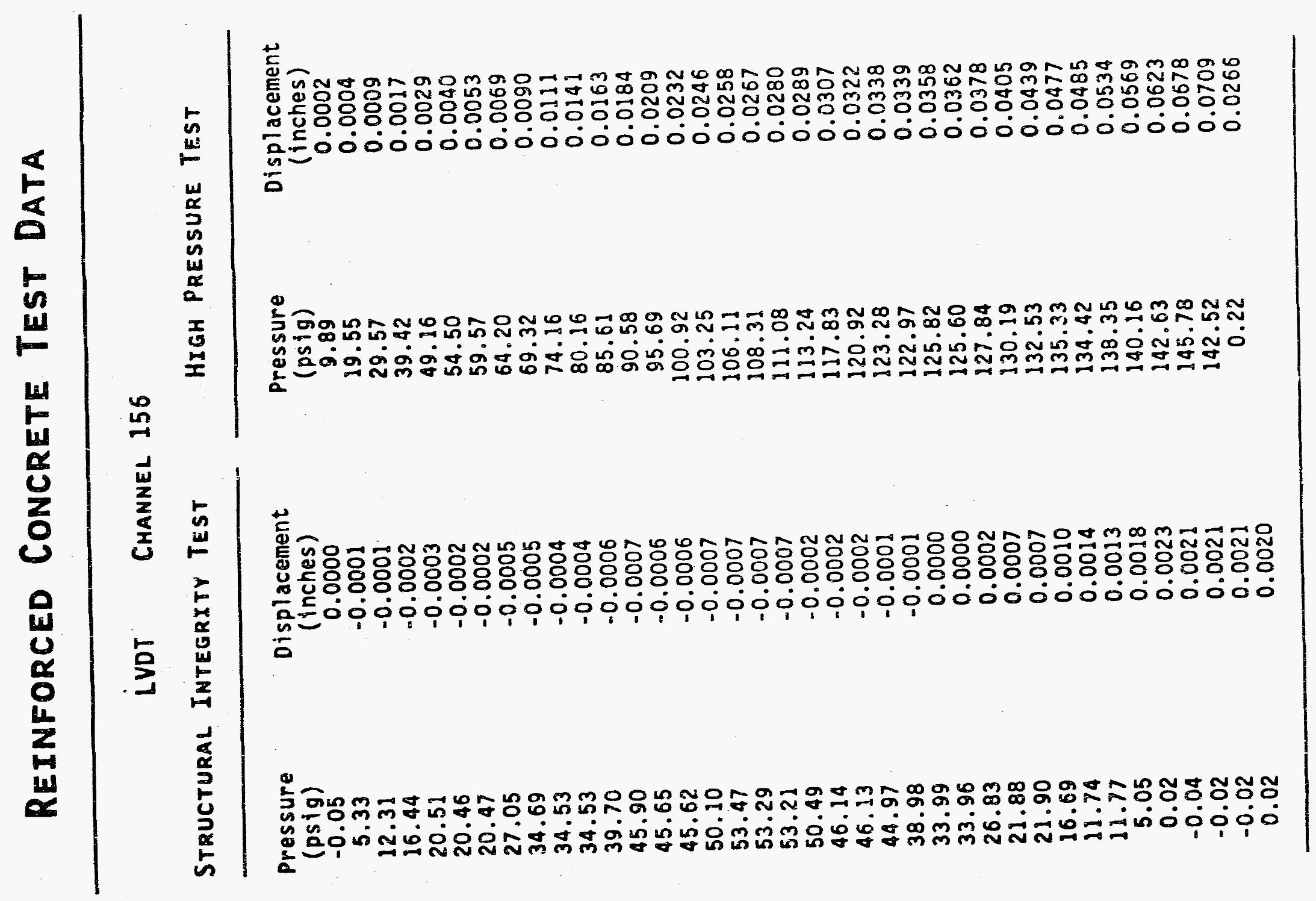




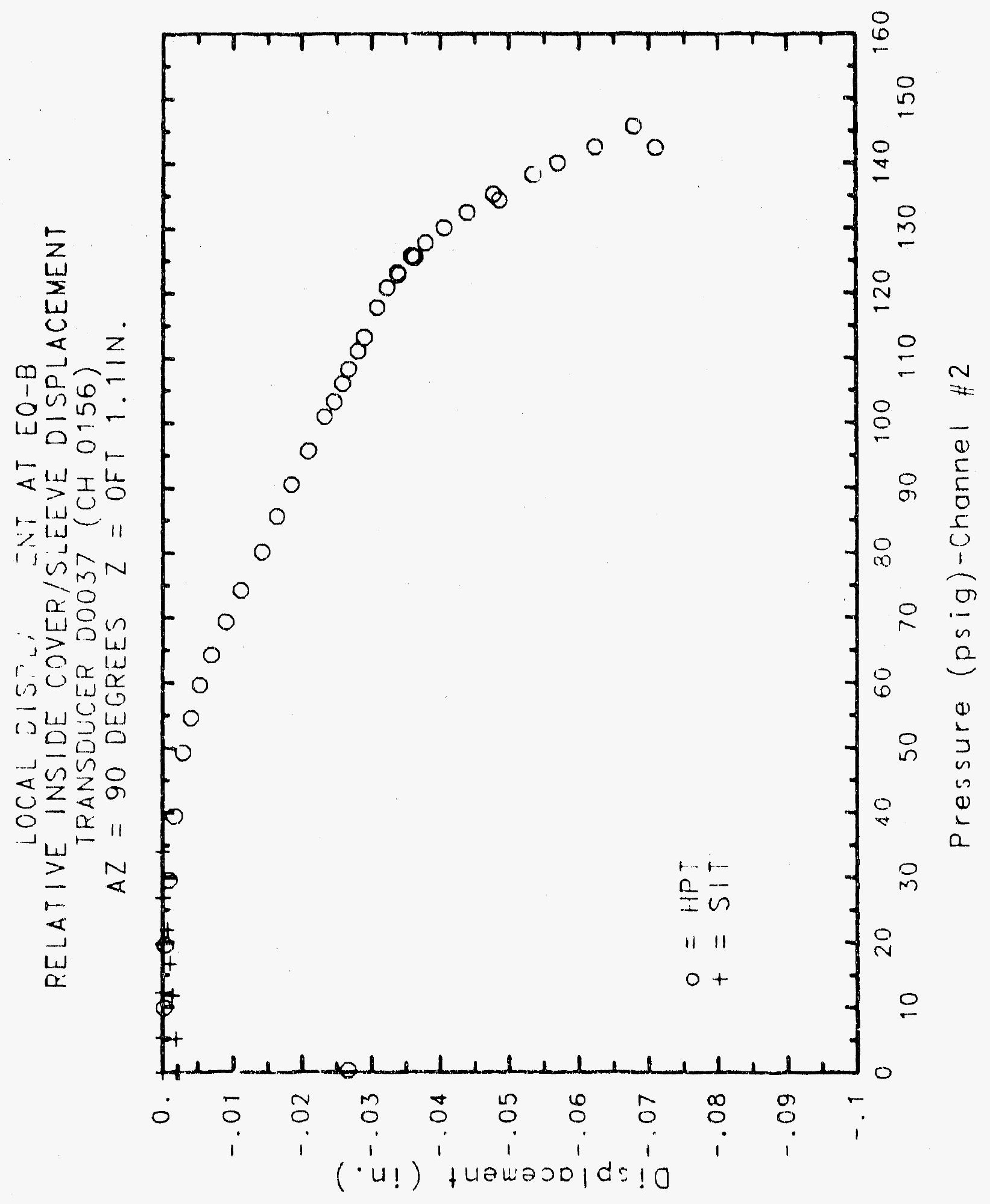




\section{Reinforced Concrete Test Data}

Lvd Channel 157

Structural Integrity test

\begin{tabular}{|c|c|c|c|}
\hline $\begin{array}{l}\text { Pressure } \\
\text { (psig) } \\
-0.05 \\
5.33 \\
12.31 \\
16.44 \\
20.51 \\
20.46 \\
20.47 \\
27.05 \\
34.69 \\
34.53 \\
34.53 \\
39.70 \\
45.90 \\
45.65 \\
45.62 \\
50.10 \\
53.47 \\
53.29 \\
53.21 \\
50.49 \\
46.14 \\
46.13 \\
44.97 \\
38.98 \\
33.99 \\
33.96 \\
26.83 \\
21.88 \\
21.90 \\
16.69 \\
11.74 \\
11.77 \\
5.05 \\
0.02 \\
-0.04 \\
-0.02 \\
-0.02 \\
0.02\end{array}$ & $\begin{array}{c}\text { Displacement } \\
\text { (Inches) } \\
0.0000 \\
0.0000 \\
0.0000 \\
0.0000 \\
0.0000 \\
-0.0001 \\
-0.0001 \\
-0.0001 \\
-0.0001 \\
-0.0006 \\
-0.0006 \\
-0.0005 \\
-0.0006 \\
-0.0005 \\
-0.0005 \\
-0.0006 \\
-0.0009 \\
-0.0009 \\
-0.0009 \\
-0.0009 \\
-0.0009 \\
-0.0009 \\
-0.0011 \\
-0.0013 \\
-0.0012 \\
-0.0013 \\
-0.0013 \\
-0.0013 \\
-0.0015 \\
-0.0016 \\
-0.0016 \\
-0.0013 \\
-0.0012 \\
-0.0011 \\
-0.0010 \\
-0.0011 \\
-0.0010 \\
-0.0011\end{array}$ & $\begin{array}{c}\text { Pressure } \\
\text { (psig) } \\
9.89 \\
19.55 \\
29.57 \\
39.42 \\
49.16 \\
54.50 \\
59.57 \\
64.20 \\
69.32 \\
74.16 \\
80.16 \\
85.61 \\
90.55 \\
95.69 \\
100.92 \\
103.25 \\
106.11 \\
108.31 \\
111.08 \\
113.24 \\
117.83 \\
120.92 \\
123.28 \\
122.97 \\
125.82 \\
125.60 \\
127.84 \\
130.19 \\
132.53 \\
135.33 \\
134.42 \\
138.35 \\
140.16 \\
142.63 \\
145.78 \\
142.52 \\
0.22\end{array}$ & $\begin{array}{c}\text { Displacement } \\
\text { (inches) } \\
0.0000 \\
0.0001 \\
0.0003 \\
0.0005 \\
0.0006 \\
0.0005 \\
-0.0001 \\
-0.0017 \\
-0.0042 \\
-0.0059 \\
-0.0060 \\
-0.0060 \\
-0.0073 \\
-0.0081 \\
0.0033 \\
-0.0091 \\
-0.0094 \\
-0.0097 \\
-0.0102 \\
-0.0105 \\
-0.0115 \\
-0.0122 \\
-0.0134 \\
-0.0138 \\
-0.0143 \\
-0.0146 \\
-0.0148 \\
-0.0152 \\
-0.0157 \\
-0.0164 \\
-0.0169 \\
-0.0172 \\
-0.0175 \\
-0.0180 \\
-0.0182 \\
-0.0185 \\
-0.0269\end{array}$ \\
\hline
\end{tabular}

High Pressure test

placement

0.0001

0.0003

0.0005

0.0006

0.0005

$-0.0017$

$-0.0042$

$-0.0059$

$-0.0073$

$-0.0081$

0.053

$-0.0097$

$-0.0102$

$-0.0105$

$-0.0134$

$-0.0138$

$-0.0143$

$-0.0146$

$-0.0148$

$-0.0164$

$-0.0169$

$-0.0172$

$-0.0175$

$-0.0182$

$-0.0185$

$-0.0269$ 


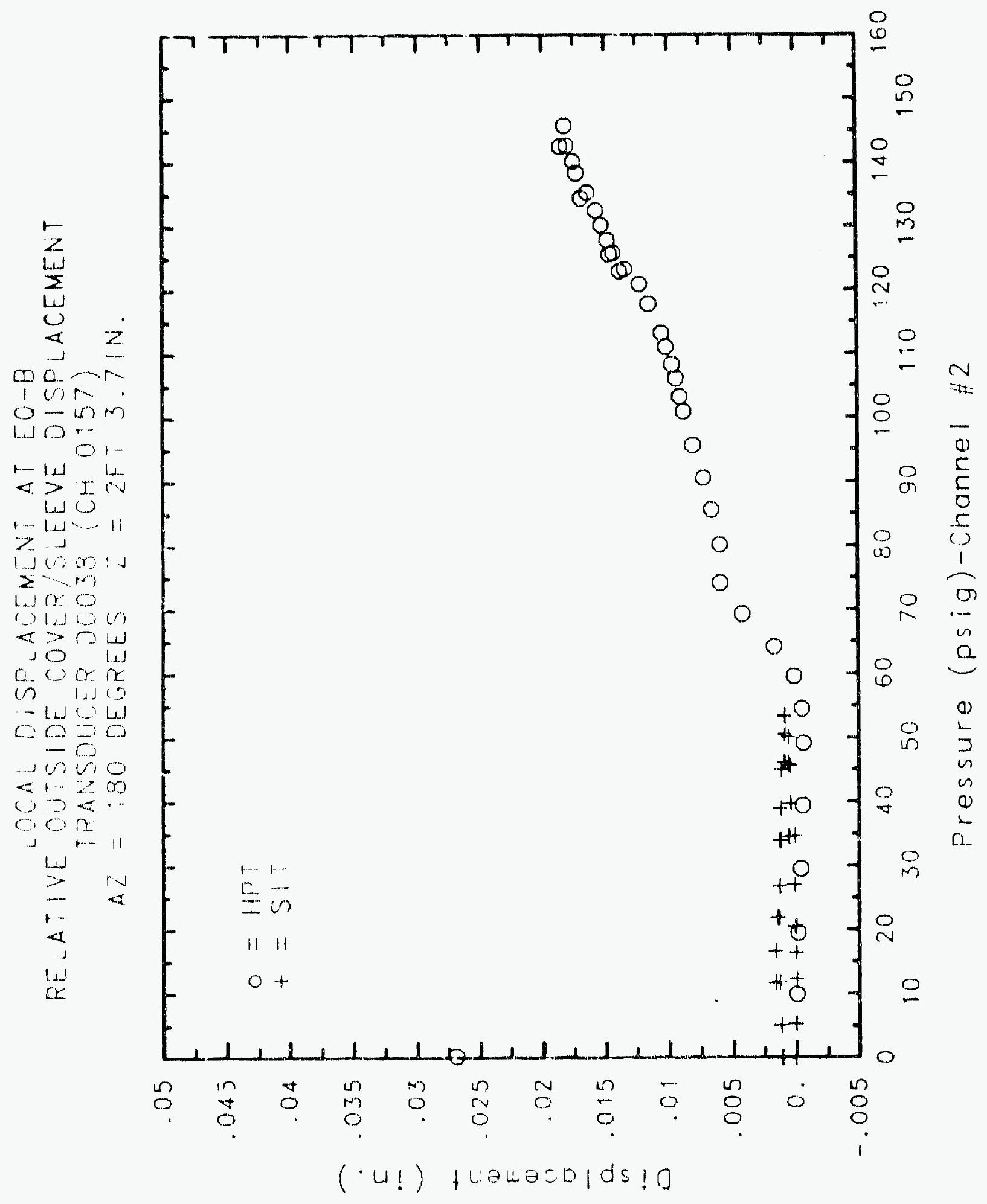




\section{Reinforced Concrete Test Data}

LVDT Channel 158

Structural InTEgrity TEST

\begin{tabular}{|c|c|c|c|}
\hline $\begin{array}{c}\text { Pressure } \\
\text { (psig) } \\
-0.05 \\
5.33 \\
12.31 \\
16.44 \\
20.51 \\
20.46 \\
20.47 \\
27.05 \\
34.69 \\
34.53 \\
34.53 \\
39.70 \\
45.90 \\
45.65 \\
45.62 \\
50.10 \\
53.47 \\
53.29 \\
53.21 \\
50.49 \\
46.14 \\
46.13 \\
44.97 \\
38.98 \\
33.99 \\
33.96 \\
26.83 \\
21.88 \\
21.90 \\
16.69 \\
11.74 \\
11.77 \\
5.05 \\
0.02 \\
-0.04 \\
-0.02 \\
-0.02 \\
0.02\end{array}$ & $\begin{array}{c}\text { Displacement } \\
\text { (1nches) } \\
0.0000 \\
-0.0001 \\
-0.0002 \\
-0.0003 \\
-0.0004 \\
-0.0005 \\
-0.0005 \\
-0.0006 \\
-0.0007 \\
-0.0011 \\
-0.0011 \\
-0.0010 \\
-0.0010 \\
-0.0008 \\
-0.0008 \\
-0.0010 \\
-0.0013 \\
-0.0012 \\
-0.0013 \\
-0.0013 \\
-0.0014 \\
-0.0013 \\
-0.0016 \\
-0.0017 \\
-0.0016 \\
-0.0017 \\
-0.0018 \\
-0.0018 \\
-0.0020 \\
-0.0020 \\
-0.0021 \\
-0.0016 \\
-0.0015 \\
-0.0013 \\
-0.0013 \\
-0.0013 \\
-0.0013 \\
-0.0015 \\
-0.0\end{array}$ & $\begin{array}{c}\text { Pressure } \\
\text { (psig) } \\
9.89 \\
19.55 \\
29.57 \\
39.42 \\
49.16 \\
54.50 \\
59.57 \\
64.20 \\
69.32 \\
74.16 \\
80.16 \\
85.61 \\
90.58 \\
95.69 \\
100.92 \\
103.25 \\
106.11 \\
108.31 \\
111.08 \\
113.24 \\
117.83 \\
120.92 \\
123.28 \\
122.97 \\
125.82 \\
125.60 \\
127.84 \\
130.19 \\
132.53 \\
135.33 \\
134.42 \\
138.35 \\
140.16 \\
142.63 \\
145.78 \\
142.52 \\
0.22 \\
\end{array}$ & $\begin{array}{c}\text { Displacement } \\
\text { (inches) } \\
0.0000 \\
0.0000 \\
0.0001 \\
0.0001 \\
-0.0001 \\
-0.0004 \\
-0.0012 \\
-0.0030 \\
-0.0057 \\
-0.0079 \\
-0.0086 \\
-0.0101 \\
-0.0116 \\
-0.0132 \\
-0.0148 \\
-0.0155 \\
-0.0162 \\
-0.0169 \\
-0.0178 \\
-0.0185 \\
-0.0202 \\
-0.0214 \\
-0.0233 \\
-0.0237 \\
-0.0246 \\
-0.0249 \\
-0.0253 \\
-0.0262 \\
-0.0269 \\
-0.0280 \\
-0.0286 \\
-0.0294 \\
-0.0297 \\
-0.0305 \\
-0.0308 \\
-0.0311 \\
-0.0307\end{array}$ \\
\hline
\end{tabular}

High Pressure test

placement

(inches)

0.0000

0.0000

0.0001

$-0.0004$

$-0.0030$

$-0.0057$

$-0.0079$

$-0.0116$

$-0.0155$

$-0.0214$

$-0.0233$

$-0.0237$

$-0.0246$

$-0.0262$

$-0.0286$

$-0.0294$

$-0.0297$

$-0.0311$ 


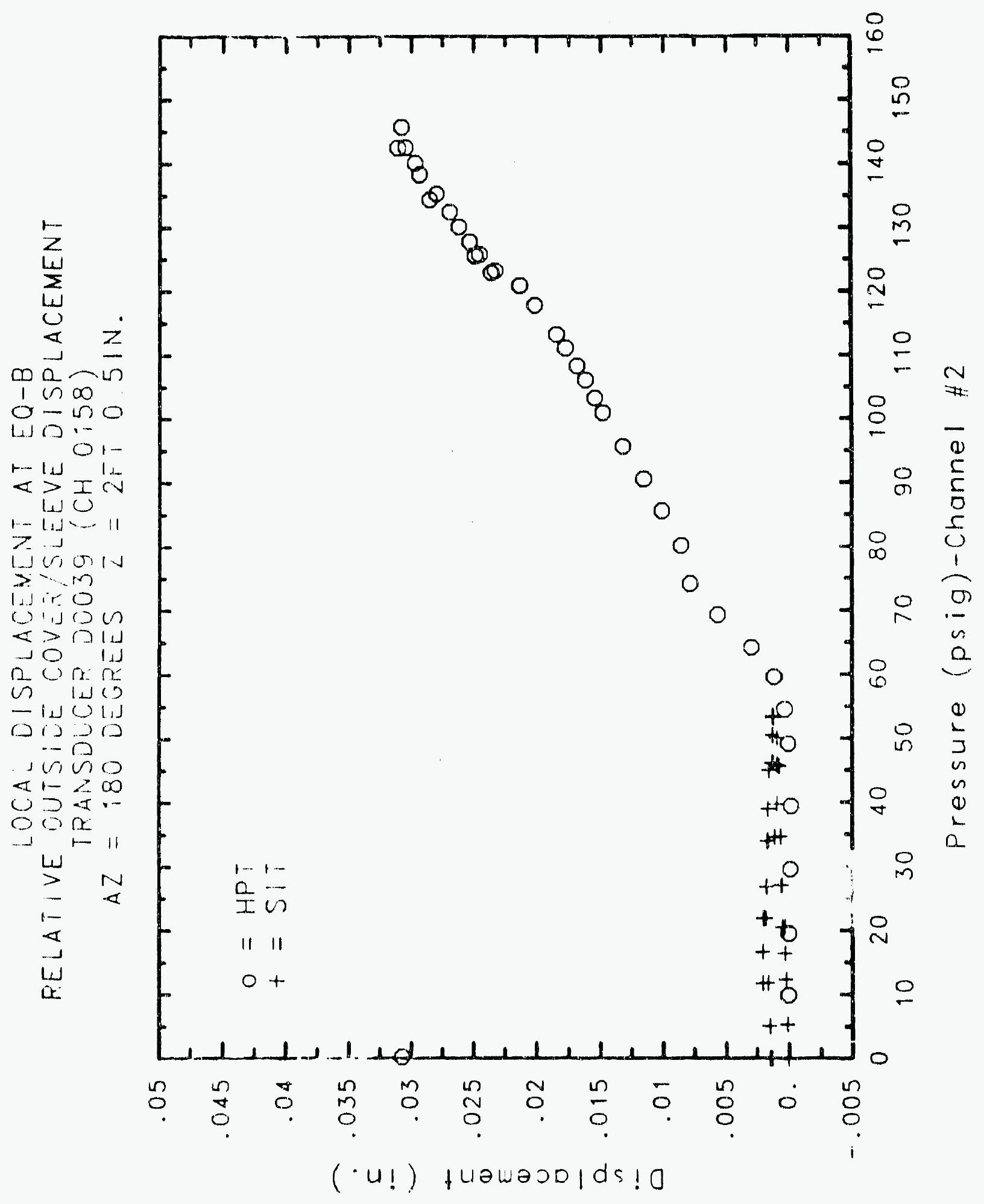




\title{
Reinforced Concrete Test Data
}

\author{
LVDT Channel 159
}

Structural InTEgrity Test

\begin{tabular}{|c|c|c|c|}
\hline $\begin{array}{l}\text { Pressure } \\
\text { (psig) } \\
-0.05 \\
5.33 \\
12.31 \\
16.44 \\
20.51 \\
20.46 \\
20.47 \\
27.05 \\
34.69 \\
34.53 \\
34.53 \\
39.70 \\
45.90 \\
45.65 \\
45.62 \\
50.10 \\
53.47 \\
53.29 \\
53.21 \\
50.49 \\
46.14 \\
46.13 \\
44.97 \\
38.98 \\
33.99 \\
33.96 \\
26.83 \\
21.88 \\
21.90 \\
16.69 \\
11.74 \\
11.77 \\
5.05 \\
0.02 \\
-0.04 \\
-0.02 \\
-0.02 \\
0.02\end{array}$ & $\begin{array}{c}\text { Displacement } \\
\text { (inches) } \\
0.0000 \\
0.0002 \\
0.0003 \\
0.0004 \\
0.0005 \\
0.0005 \\
0.0006 \\
0.0006 \\
0.0007 \\
0.0008 \\
0.0008 \\
0.0008 \\
0.0005 \\
0.0004 \\
0.0005 \\
0.0005 \\
0.0006 \\
0.0005 \\
0.0005 \\
0.0004 \\
0.0004 \\
0.0003 \\
0.0004 \\
0.0005 \\
0.0004 \\
0.0005 \\
0.0005 \\
0.0005 \\
0.0005 \\
0.0005 \\
0.0005 \\
0.0003 \\
0.0002 \\
0.0002 \\
0.0003 \\
0.0002 \\
0.0003 \\
0.0003\end{array}$ & $\begin{array}{c}\text { Pressure } \\
\text { (psig) } \\
9.89 \\
19.55 \\
29.57 \\
39.42 \\
49.16 \\
54.50 \\
59.57 \\
64.20 \\
69.32 \\
74.16 \\
80.16 \\
85.61 \\
90.58 \\
95.69 \\
100.92 \\
103.25 \\
106.11 \\
108.31 \\
111.08 \\
113.24 \\
117.83 \\
120.92 \\
123.28 \\
122.97 \\
125.82 \\
125.60 \\
127.84 \\
130.19 \\
132.53 \\
135.33 \\
134.42 \\
138.35 \\
140.16 \\
142.63 \\
145.78 \\
142.52 \\
0.22 \\
\end{array}$ & $\begin{array}{c}\text { Displacement } \\
\text { (inches) } \\
0.0001 \\
0.0002 \\
0.0003 \\
0.0004 \\
0.0007 \\
0.0009 \\
0.0011 \\
0.0013 \\
0.0014 \\
0.0011 \\
0.0003 \\
-0.0003 \\
-0.0009 \\
-0.0015 \\
-0.0022 \\
-0.0025 \\
-0.0028 \\
-0.0030 \\
-0.0033 \\
-0.0035 \\
-0.0038 \\
-0.0041 \\
-0.0041 \\
-0.0040 \\
-0.0041 \\
-0.0040 \\
-0.0041 \\
-0.0040 \\
-0.0040 \\
-0.0039 \\
-0.0037 \\
-0.0036 \\
-0.0035 \\
-0.0032 \\
-0.0029 \\
-0.0026 \\
0.0018\end{array}$ \\
\hline
\end{tabular}

\section{High Pressure Test}

0.0002

0.0003

0.0009

0.0011

0.0013

0.0014

$-0.0003$

$-0.0009$

$-0.0015$

$-0.0028$

$-0.0030$

$-0.0038$

0.0040

$-0.0040$

$-0.0039$

$-0.0035$

$-0.0032$

0.0029

$-0.0026$

0.0018 splacement 


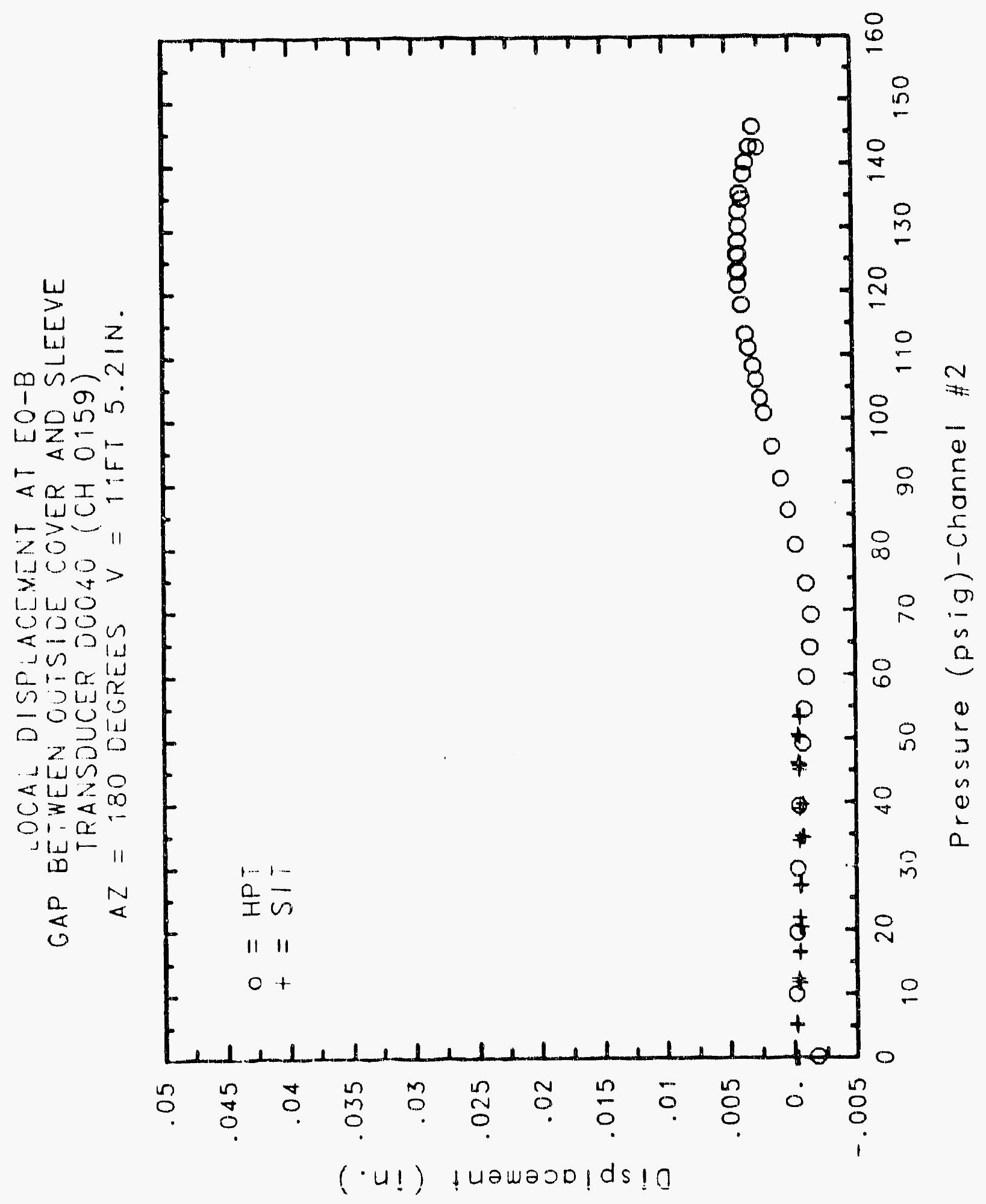




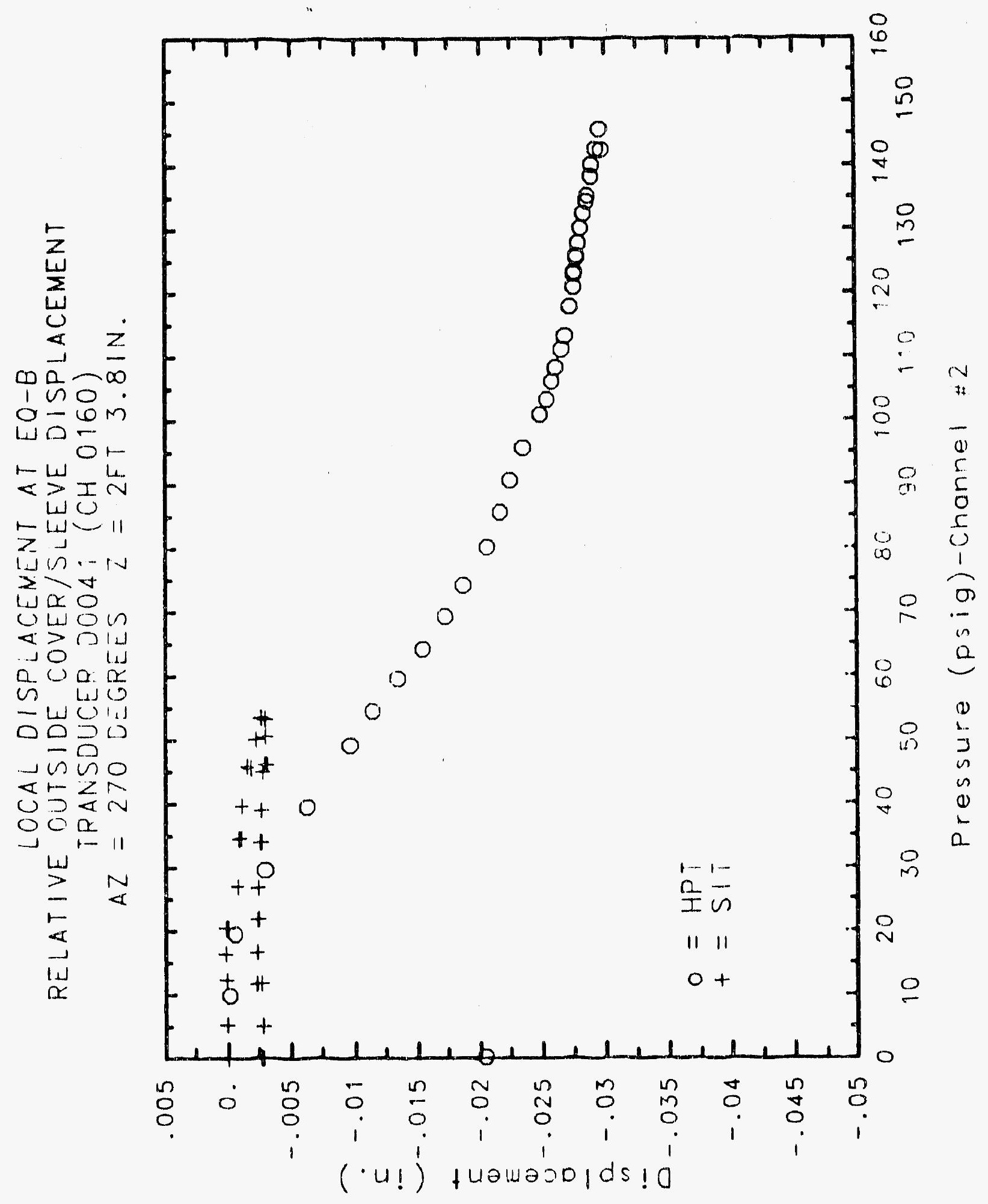




\section{Reinforced Concrete Test Data}

lvdt Channel 161

STRUCTURAL INTEGRITY TEST

\begin{tabular}{|c|c|c|c|}
\hline $\begin{array}{l}\text { Pressure } \\
\text { (psig) } \\
-0.05 \\
5.33 \\
12.31 \\
16.44 \\
20.51 \\
20.46 \\
20.47 \\
27.05 \\
34.69 \\
34.53 \\
34.53 \\
39.70 \\
45.90 \\
45.65 \\
45.62 \\
50.10 \\
53.47 \\
53.29 \\
53.21 \\
50.49 \\
46.14 \\
46.13 \\
44.97 \\
38.98 \\
33.99 \\
33.96 \\
26.83 \\
21.88 \\
21.90 \\
16.69 \\
11.74 \\
11.77 \\
5.05 \\
0.02 \\
-0.04 \\
-0.02 \\
-0.02 \\
0.02 \\
\end{array}$ & $\begin{array}{c}\text { Displacement } \\
\text { (inches) } \\
-0.0001 \\
-0.0002 \\
-0.0004 \\
-0.0006 \\
-0.0005 \\
-0.0005 \\
-0.0010 \\
0.0001 \\
0.0005 \\
-0.0002 \\
-0.0001 \\
0.0003 \\
0.0011 \\
0.0016 \\
0.0017 \\
0.0020 \\
0.0024 \\
0.0030 \\
0.0030 \\
0.0030 \\
0.0028 \\
0.0031 \\
0.0024 \\
0.0023 \\
0.0023 \\
0.0021 \\
0.0017 \\
0.0018 \\
0.0016 \\
0.0015 \\
0.0015 \\
0.0022 \\
0.0024 \\
0.0026 \\
0.0029 \\
0.0027 \\
0.0026 \\
0.0024\end{array}$ & $\begin{array}{c}\text { Pressure } \\
(\text { psig) } \\
9.89 \\
19.55 \\
29.57 \\
39.42 \\
49.16 \\
54.50 \\
59.57 \\
64.20 \\
69.32 \\
74.16 \\
80.16 \\
85.61 \\
90.58 \\
95.69 \\
100.92 \\
103.25 \\
106.11 \\
108.31 \\
111.08 \\
113.24 \\
117.83 \\
120.92 \\
123.28 \\
122.97 \\
125.82 \\
125.60 \\
127.84 \\
130.19 \\
132.53 \\
135.33 \\
134.42 \\
138.35 \\
140.16 \\
142.63 \\
145.78 \\
142.52 \\
0.22\end{array}$ & $\begin{array}{c}\text { Displacement } \\
\text { (inches) } \\
0.0000 \\
0.0004 \\
0.0029 \\
0.0061 \\
0.0093 \\
0.0110 \\
0.0129 \\
0.0146 \\
0.0163 \\
0.0174 \\
0.0194 \\
0.0201 \\
0.0206 \\
0.0213 \\
0.0224 \\
0.0228 \\
0.0232 \\
0.0234 \\
0.0238 \\
0.0240 \\
0.0243 \\
0.0245 \\
0.0243 \\
0.0242 \\
0.0243 \\
0.0243 \\
0.0244 \\
0.0244 \\
0.0245 \\
0.0247 \\
0.0246 \\
0.0248 \\
0.0249 \\
0.0252 \\
0.0257 \\
0.0259 \\
0.0221\end{array}$ \\
\hline
\end{tabular}

High Pressure Test

placement

(inches)

0.0000

0.006

0.0093

0.0110

0.0163

0.0174

0.0194

0.0201

0.0206

0.0224

0.0228

0.0238

0.0240

0.0243

0.0245

0.0243

0.0243

0.0244

0.0247

0.0246

0.0248

0.0249

0.0252

0.0257

.0259

0.0221 


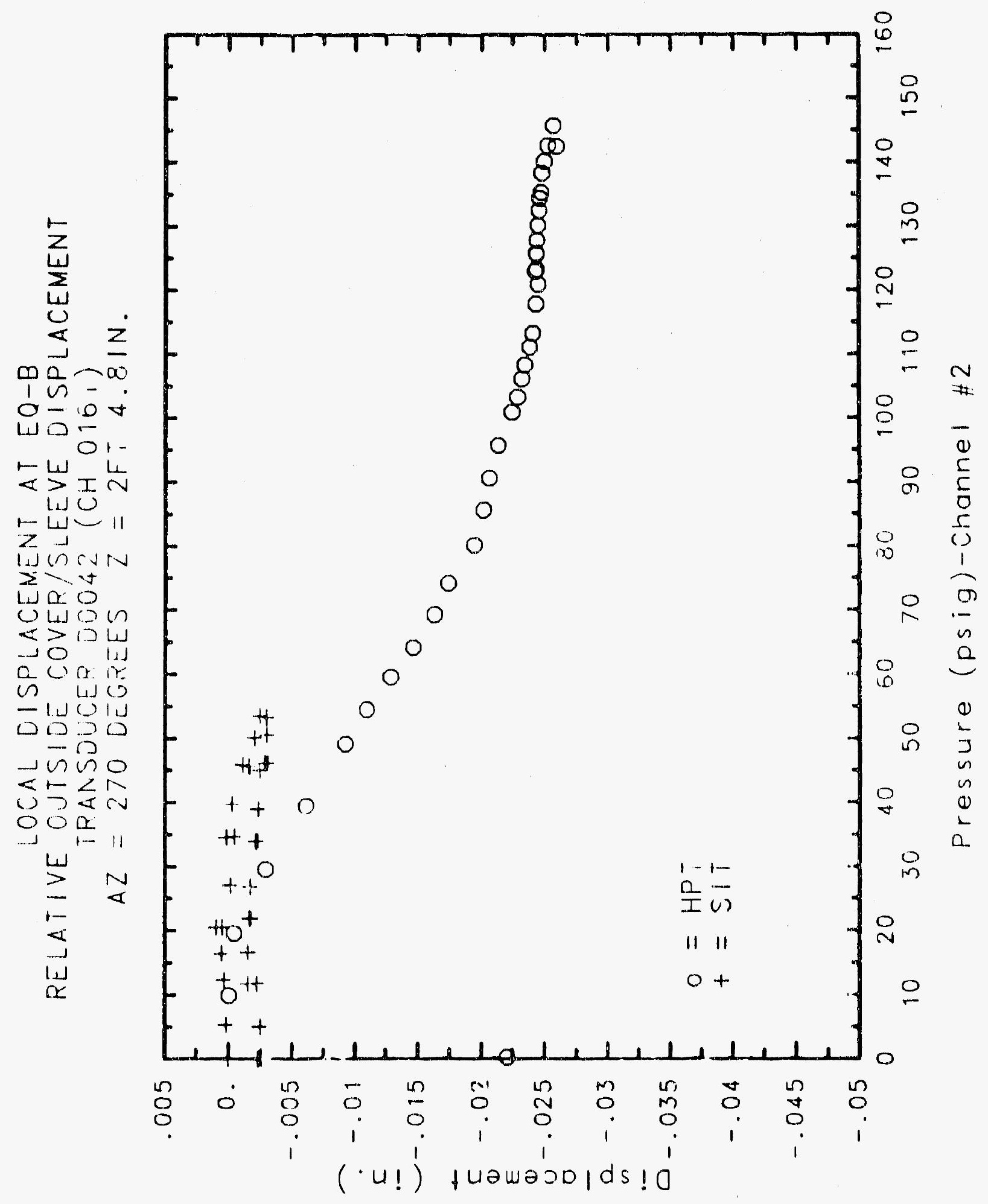




\section{Reinforced Concrete Test Data}

LVDT Channel 162

Structural IntEgrity TEST

\begin{tabular}{|c|c|c|c|}
\hline $\begin{array}{c}\text { Pressure } \\
\text { (psig) } \\
-0.05 \\
5.33 \\
12.31 \\
16.44 \\
20.51 \\
20.46 \\
20.47 \\
27.05 \\
34.69 \\
34.53 \\
34.53 \\
39.70 \\
45.90 \\
45.65 \\
45.62 \\
50.10 \\
53.47 \\
53.29 \\
53.21 \\
50.49 \\
46.14 \\
46.13 \\
44.97 \\
38.98 \\
33.99 \\
33.96 \\
26.83 \\
21.88 \\
21.90 \\
16.69 \\
11.74 \\
11.77 \\
5.05 \\
0.02 \\
-0.04 \\
-0.02 \\
-0.02 \\
0.02\end{array}$ & $\begin{array}{c}\text { Displacenent } \\
\text { (Inches) } \\
0.0000 \\
0.0000 \\
0.0001 \\
0.0001 \\
0.0000 \\
0.0000 \\
0.0001 \\
0.0000 \\
0.0000 \\
0.0001 \\
0.0001 \\
0.0000 \\
-0.0003 \\
-0.0002 \\
-0.0002 \\
-0.0003 \\
-0.0003 \\
-0.0004 \\
-0.0004 \\
-0.0005 \\
-0.0004 \\
-0.0005 \\
-0.0004 \\
-0.0003 \\
-0.0003 \\
-0.0003 \\
-0.0002 \\
-0.0003 \\
-0.0003 \\
-0.0002 \\
-0.0002 \\
-0.0003 \\
-0.0003 \\
-0.0003 \\
-0.0001 \\
-0.0002 \\
-0.0001 \\
-0.0001\end{array}$ & $\begin{array}{c}\text { Pressure } \\
(\text { ps 1g) } \\
9.89 \\
19.55 \\
29.57 \\
39.42 \\
49.16 \\
54.50 \\
59.57 \\
64.20 \\
69.32 \\
74.16 \\
80.16 \\
85.61 \\
90.58 \\
95.69 \\
100.92 \\
103.25 \\
106.11 \\
108.31 \\
111.08 \\
113.24 \\
117.83 \\
120.92 \\
123.28 \\
122.97 \\
125.82 \\
125.60 \\
127.84 \\
130.19 \\
132.53 \\
135.33 \\
134.42 \\
138.35 \\
140.16 \\
142.63 \\
145.78 \\
142.52 \\
0.22\end{array}$ & $\begin{array}{c}\text { Displacem nt } \\
\text { (inches) } \\
0.0000 \\
-0.0001 \\
-0.0002 \\
-0.0003 \\
-0.0002 \\
-0.0003 \\
-0.0005 \\
-0.0007 \\
-0.0009 \\
-0.0011 \\
-0.0020 \\
-0.0028 \\
-0.0037 \\
-0.0048 \\
-0.0060 \\
-0.0066 \\
-0.0072 \\
-0.0077 \\
-0.0084 \\
-0.0089 \\
-0.0100 \\
-0.0109 \\
-0.0115 \\
-0.0117 \\
-0.0124 \\
-0.0126 \\
-0.0130 \\
-0.0137 \\
-0.0145 \\
-0.0156 \\
-0.0159 \\
-0.0170 \\
-0.0180 \\
-0.0196 \\
-0.0213 \\
-0.0222 \\
-0.0060\end{array}$ \\
\hline
\end{tabular}

high Pressure test

splacement

(aces)

0.0000

$-0.0001$

$-0.0002$

$-0.0003$

$-0.0005$

$-0.0007$

$-0.0020$

$-0.0028$

$-0.0037$

$-0.0048$

$-0.0060$

$-0.0077$

$-0.0084$

$-0.0089$

$-0.0100$

$-0.0109$

$-0.0124$

0.0137

$-0.0159$

.0170

$-0.0196$

$-0.0213$

$-0.0222$

$-0.0060$ 


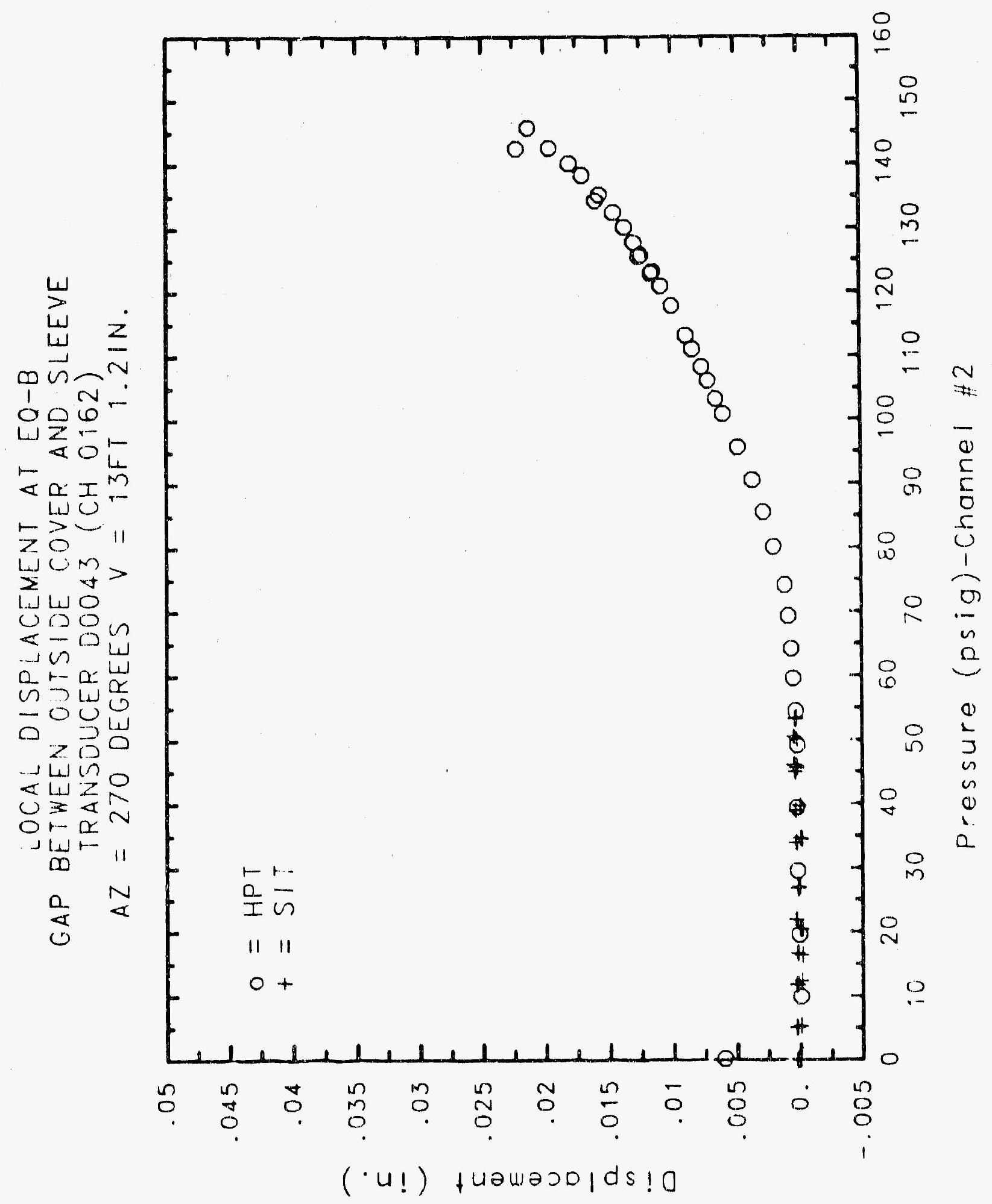




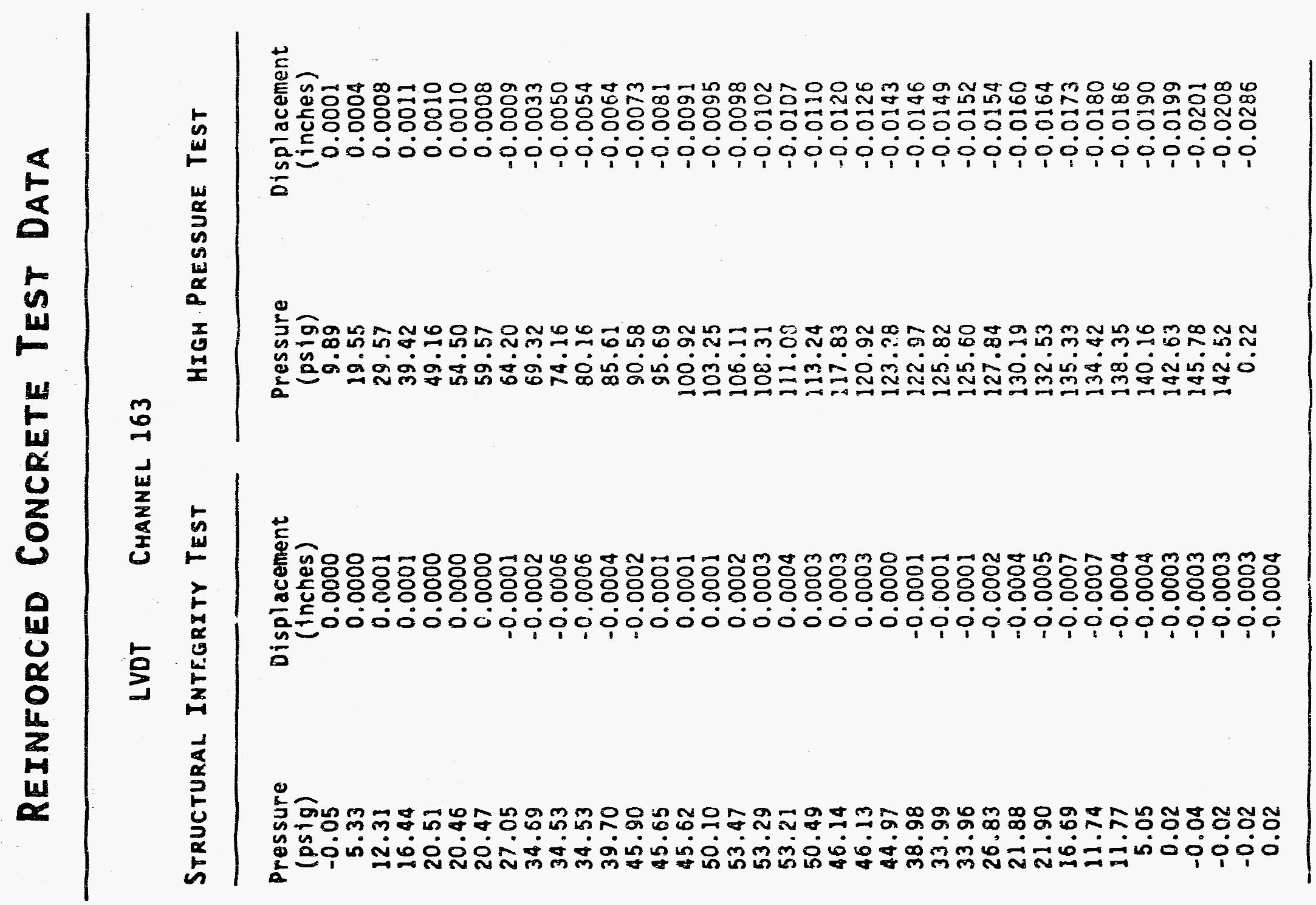




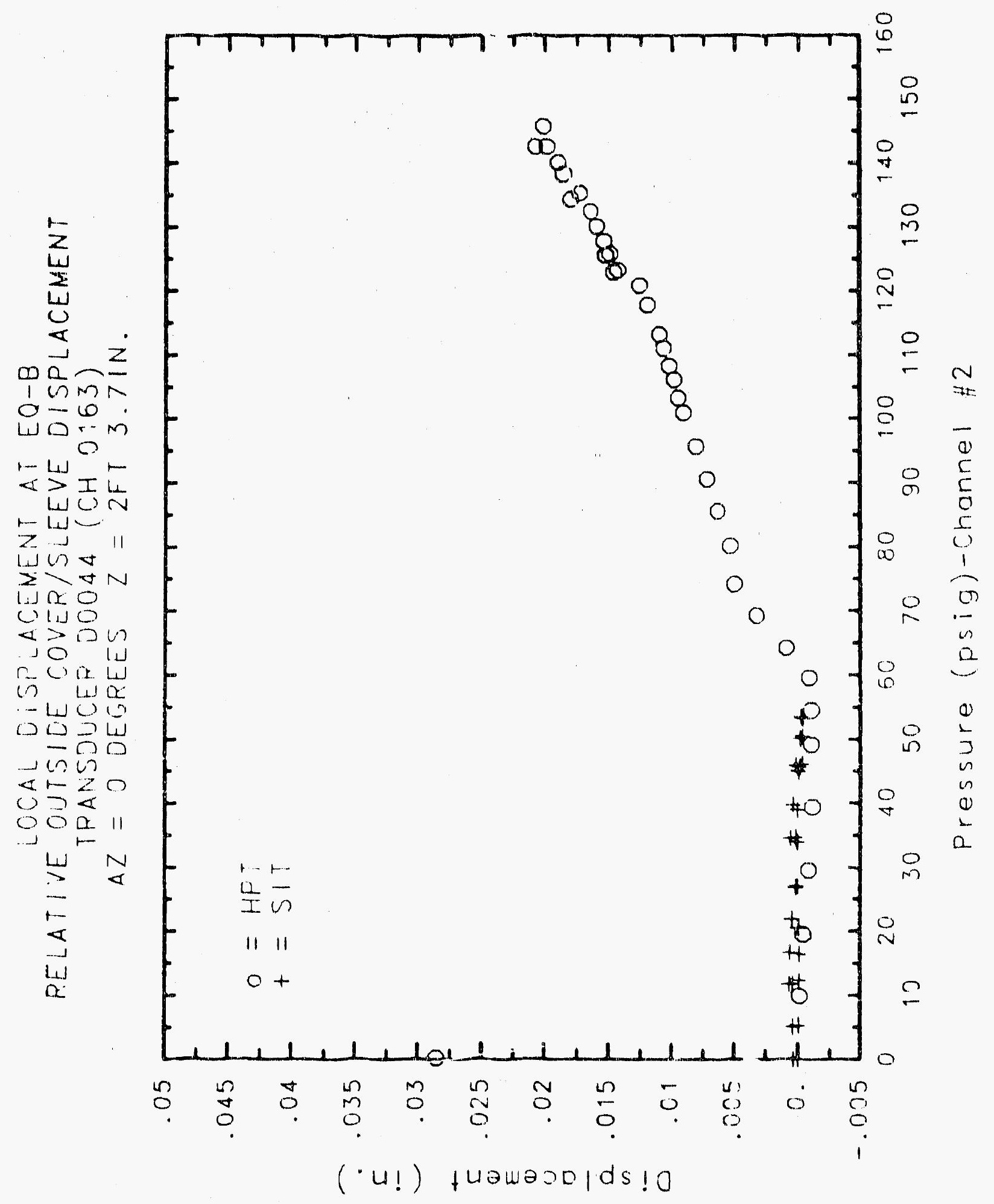




\section{Reinforced Concrete Test Data}

LVDT ChANNEL 164

STRUCTURAL INTEGRITY TEST

\begin{tabular}{cccc}
\hline & & & \\
Pressure & Displacement & Pressure & Displacement \\
(psig) & $($ inches) & (psig) & (inchts) \\
-0.05 & 0.0000 & 9.89 & -0.0002 \\
5.33 & -0.0003 & 19.55 & 0.0000 \\
12.31 & -0.0005 & 29.57 & 0.0005 \\
16.44 & -0.0007 & 39.42 & 0.0006 \\
20.51 & -0.0008 & 49.16 & 0.0002 \\
20.46 & -0.0010 & 54.50 & 0.0002 \\
20.47 & -0.0010 & 59.57 & -0.0001 \\
27.05 & -0.0012 & 64.20 & -0.0020 \\
34.69 & -0.0014 & 69.32 & -0.0045 \\
34.53 & -0.0023 & 74.16 & -0.0069 \\
34.53 & -0.0023 & 80.16 & -0.0071 \\
39.70 & -0.0017 & 85.61 & -0.0088 \\
45.90 & -0.0011 & 90.58 & 0.0101 \\
45.65 & -0.0004 & 95.69 & -0.0113 \\
45.62 & -0.0004 & 100.92 & -0.0127 \\
50.10 & -0.0004 & 103.25 & -0.0131 \\
53.47 & -0.0004 & 106.11 & -0.0137 \\
53.29 & 0.0001 & 108.31 & -0.0143 \\
53.21 & -0.0001 & 111.08 & -0.0149 \\
50.49 & -0.0001 & 113.24 & -0.0154 \\
46.14 & -0.0003 & 117.83 & -0.0169 \\
46.13 & -0.0001 & 120.92 & -0.0178 \\
44.97 & -0.0007 & 123.28 & -0.0206 \\
38.98 & -0.0009 & 122.97 & -0.0212 \\
33.99 & 0.0009 & 125.32 & -0.0212 \\
33.96 & -0.0010 & 125.60 & -0.0214 \\
26.83 & -0.0011 & 127.84 & -0.0216 \\
21.88 & -0.0015 & 130.19 & -0.0226 \\
21.90 & -0.0017 & 132.53 & -0.0233 \\
16.69 & -0.0020 & 135.33 & -0.0245 \\
11.74 & -0.0020 & 134.42 & -0.0255 \\
11.77 & -0.0010 & 138.35 & -0.0256 \\
5.05 & -0.0006 & 140.16 & -0.0270 \\
0.02 & -0.0003 & 142.63 & -0.0283 \\
-0.04 & -0.0008 & 145.78 & -0.0287 \\
-0.02 & -0.0005 & 142.52 & -0.0293 \\
-0.02 & -0.0005 & 0.22 & -0.0284 \\
0.02 & -0.0008 & & \\
& & & \\
\hline
\end{tabular}

High Pressure test

(inchus)

$-0.0002$

0.0000

0.0005

0.0006

0.0002

0.0001

$-0.0020$

$-0.0045$

$-0.0069$

$-0.0071$

.0 .0101

$-0.0113$

$-0.0127$

$-0.0131$

$-0.0137$

$-0.0149$

$-0.0154$

$-0.0169$

$-0.0178$

$-0.0206$

$-0.0214$

$-0.0216$

$-0.0226$

$-0.0233$

$-0.0245$

$-0.0258$

$-0.0270$

$-0.0283$

$-0.0287$

$-0.0293$

$-0.0284$ 


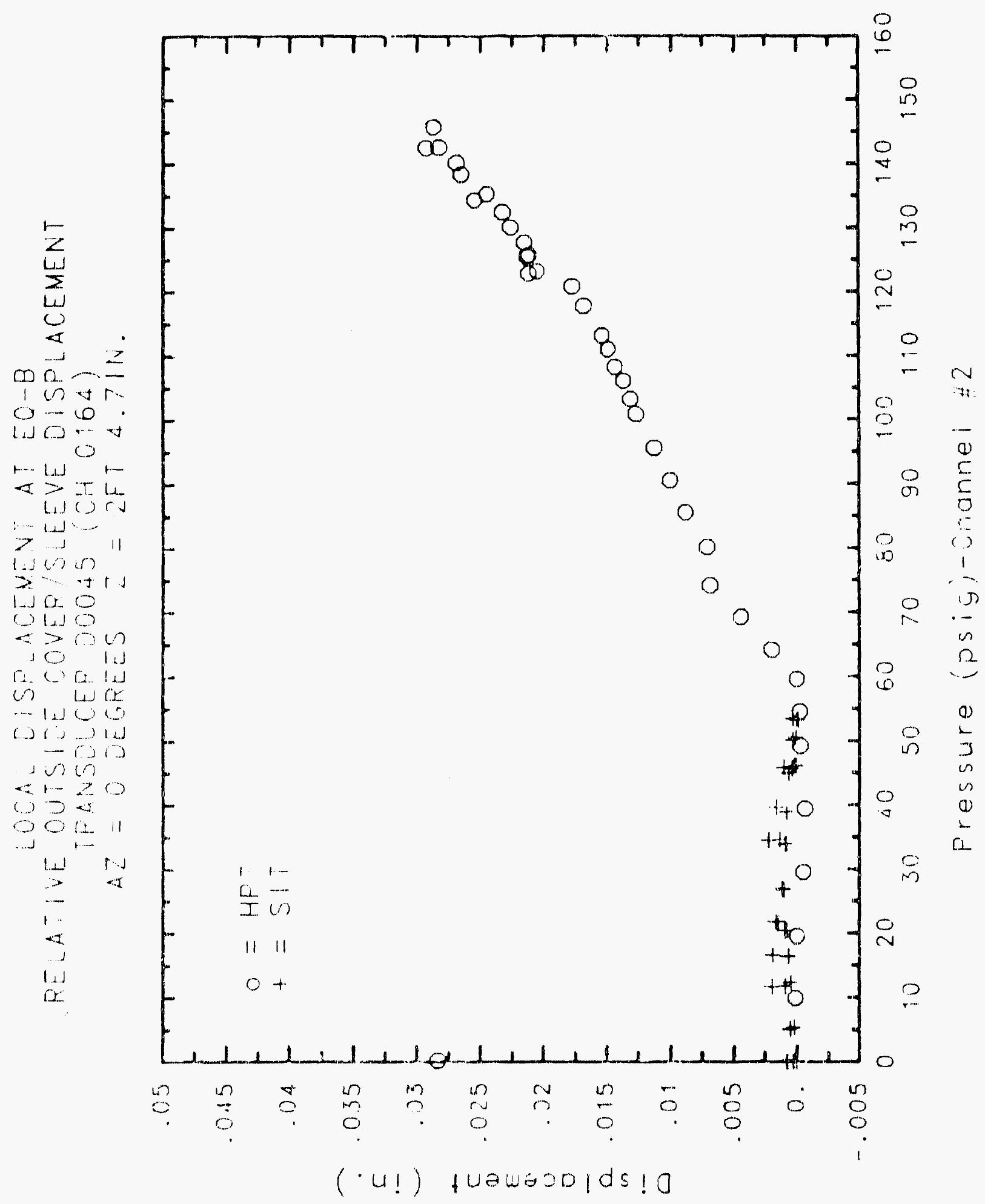

C.217 


\section{Reinforced Concrete Test Data}

LVDT Channel 165

Structural IntEgrity Test

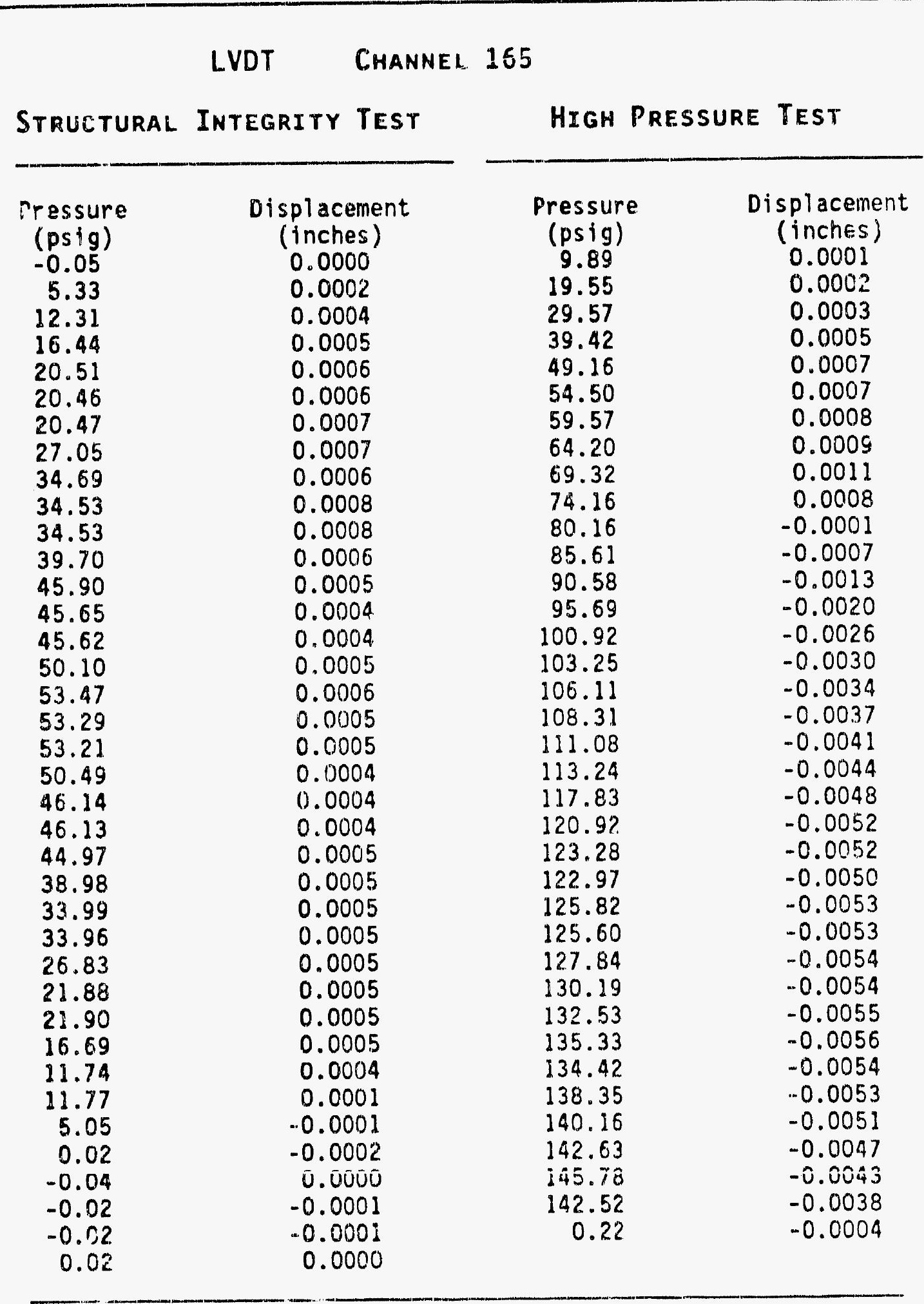

High pressure test

Pr 


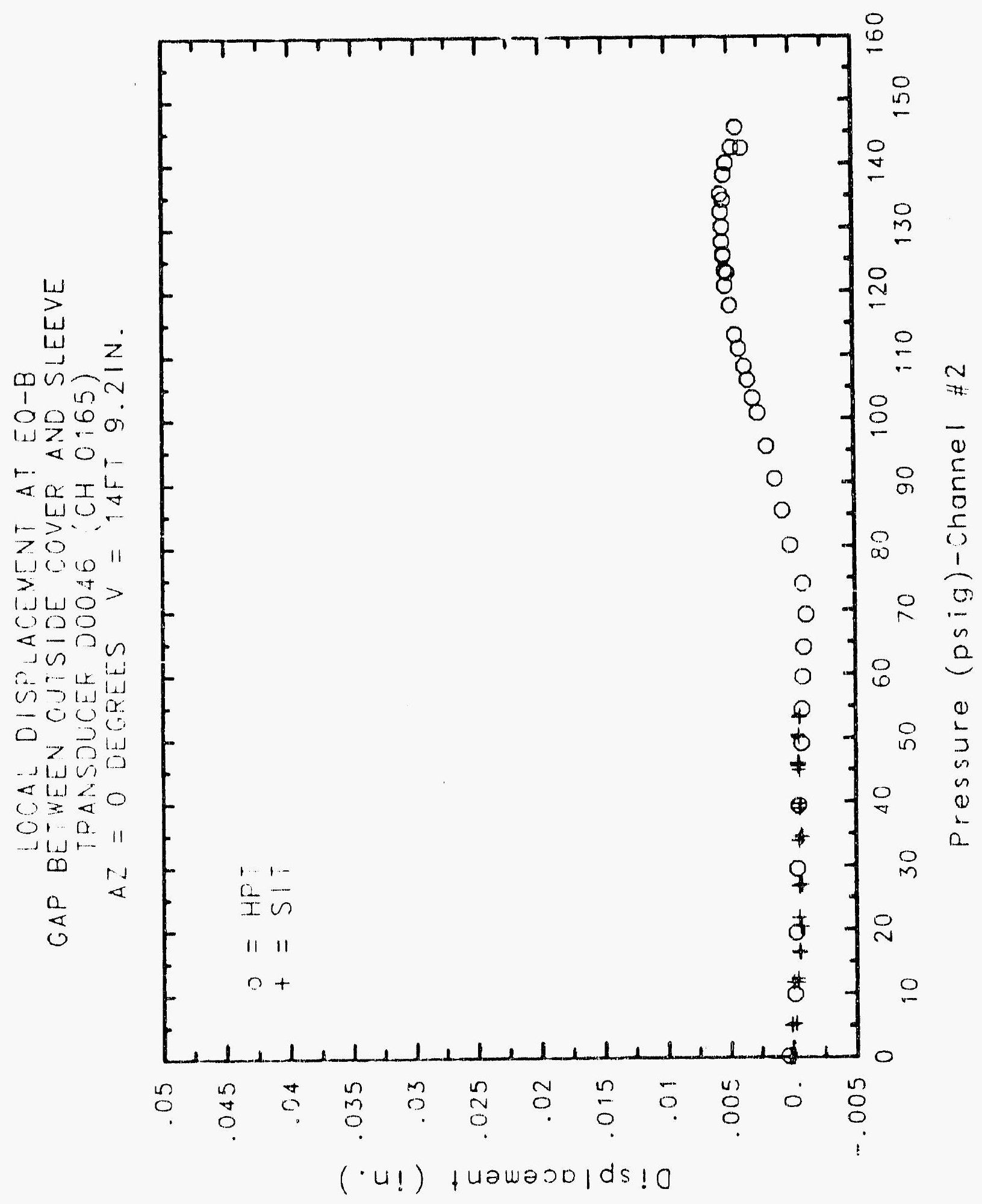




\title{
Reinforced Concrete Test Data
}

\author{
LVDT Channel 166
}

STRUCTURAL INTEGRITY TEST

\begin{tabular}{|c|c|c|c|}
\hline $\begin{array}{c}\text { Pressure } \\
\text { (psig) } \\
-0.05 \\
5.33 \\
12.31 \\
16.44 \\
20.51 \\
20.46 \\
20.47 \\
27.05 \\
34.69 \\
34.53 \\
34.53 \\
39.70 \\
45.90 \\
45.65 \\
45.62 \\
50.10 \\
53.47 \\
53.29 \\
53.21 \\
50.49 \\
46.14 \\
46.13 \\
44.97 \\
38.98 \\
33.99 \\
33.96 \\
26.83 \\
21.88 \\
21.90 \\
16.69 \\
11.74 \\
11.77 \\
5.05 \\
0.02 \\
-0.04 \\
-0.02 \\
-0.02 \\
0.02\end{array}$ & $\begin{array}{c}\text { Displacement } \\
\text { (inches) } \\
0.0000 \\
-0.0001 \\
\text {-0.0001 } \\
-0.0002 \\
-0.0001 \\
0.0000 \\
0.0000 \\
0.0006 \\
0.0007 \\
0.0006 \\
0.0006 \\
0.0007 \\
0.0008 \\
0.0009 \\
0.0009 \\
0.0009 \\
0.0009 \\
0.0010 \\
0.0010 \\
0.0010 \\
0.0009 \\
0.0010 \\
0.0007 \\
0.0007 \\
0.0007 \\
0.0006 \\
0.0006 \\
0.0006 \\
0.0006 \\
0.0006 \\
0.0006 \\
0.0007 \\
0.0007 \\
0.0008 \\
0.0005 \\
0.0007 \\
0.0007 \\
0.0007\end{array}$ & $\begin{array}{c}\text { Pressure } \\
\text { (psig) } \\
9.89 \\
19.55 \\
29.57 \\
39.42 \\
49.16 \\
54.50 \\
59.57 \\
64.20 \\
69.32 \\
74.16 \\
80.16 \\
85.61 \\
90.58 \\
95.69 \\
100.92 \\
103.25 \\
106.11 \\
108.31 \\
111.08 \\
113.24 \\
117.83 \\
120.92 \\
123.28 \\
122.97 \\
125.82 \\
125.60 \\
127.84 \\
130.19 \\
132.53 \\
135.33 \\
134.42 \\
138.35 \\
140.16 \\
142.63 \\
145.78 \\
142.52 \\
0.22\end{array}$ & $\begin{array}{c}\text { Displacement } \\
\text { (inches) } \\
0.0001 \\
0.0025 \\
0.0055 \\
0.0082 \\
0.0103 \\
0.0114 \\
0.0126 \\
0.0139 \\
0.0152 \\
0.0163 \\
0.0185 \\
0.0199 \\
0.0211 \\
0.0224 \\
0.0234 \\
0.0240 \\
0.0244 \\
0.0246 \\
0.0249 \\
0.0251 \\
0.0254 \\
0.0257 \\
0.0257 \\
0.0255 \\
0.0258 \\
0.0259 \\
0.0261 \\
0.0263 \\
0.0265 \\
0.0268 \\
0.0268 \\
0.0272 \\
0.0279 \\
0.0285 \\
0.0290 \\
0.0291 \\
0.0201\end{array}$ \\
\hline
\end{tabular}

High Pressure test

placement

0.0025

0.0055

0.0082

0.0103

0.0126

0.0139

0.0152

0.0199

0.0224

0.0234

0.0246

0.0257

0.0258

0.0259

0.0261

0.0263

0.0265

0.0268

0.0285

0.0290

0.0291 


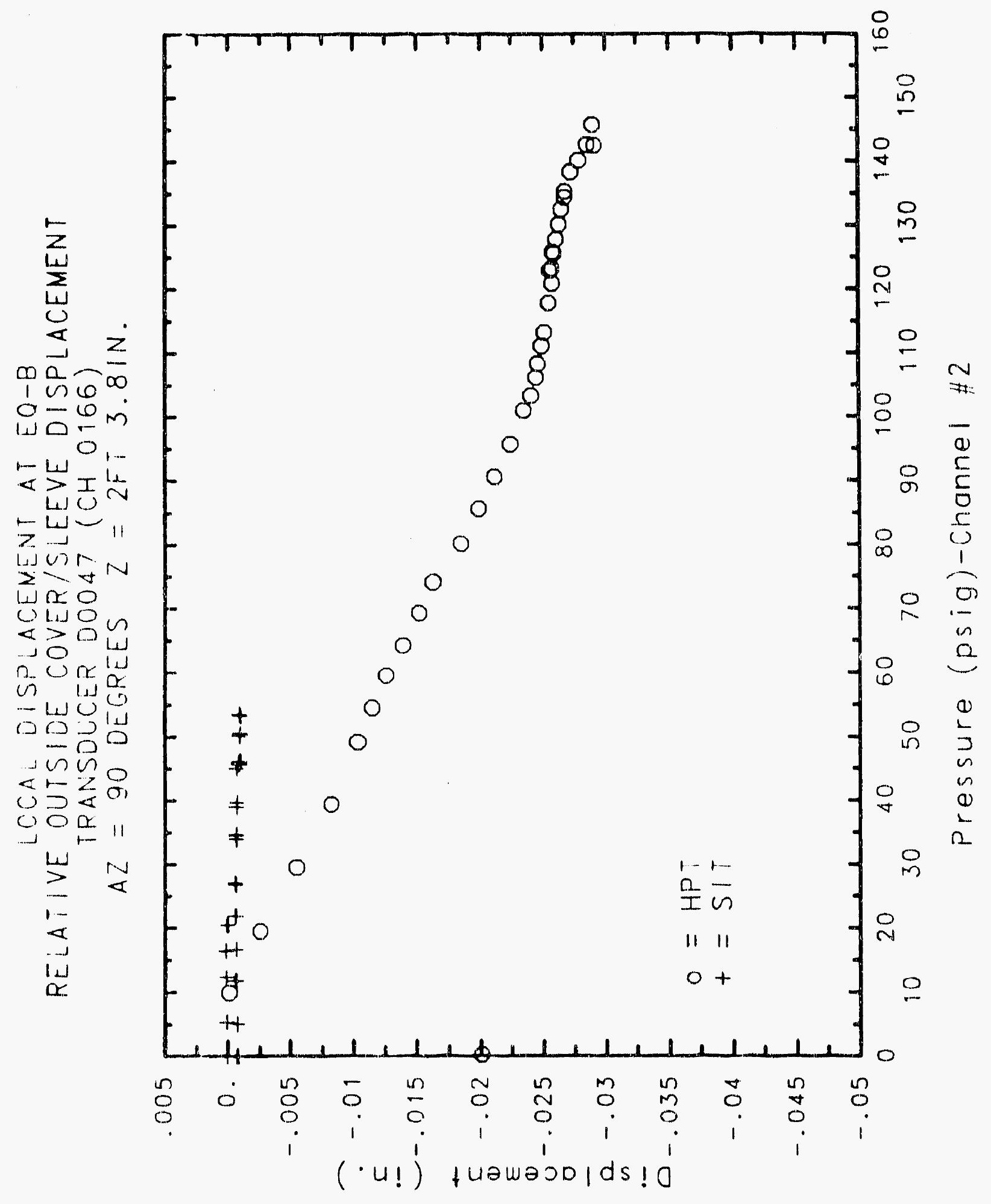




\section{Reinforced Concrete Test Data}

\section{LVDT Channel. 167}

StRUCTURAL InTEgRITY TEST

\begin{tabular}{|c|c|c|c|}
\hline $\begin{array}{l}\text { Pressure } \\
\text { (psig) } \\
-0.05 \\
5.33 \\
12.31 \\
16.44 \\
20.51 \\
20.46 \\
20.47 \\
27.05 \\
34.69 \\
34.53 \\
34.53 \\
39.70 \\
45.90 \\
45.65 \\
45.62 \\
50.10 \\
53.47 \\
53.29 \\
53.21 \\
50.49 \\
46.14 \\
46.13 \\
44.97 \\
38.98 \\
33.99 \\
33.96 \\
26.83 \\
21.88 \\
21.90 \\
16.69 \\
11.74 \\
11.77 \\
5.05 \\
0.02 \\
-0.04 \\
-0.02 \\
-0.02 \\
0.02\end{array}$ & $\begin{array}{c}\text { Displacement } \\
\text { (inches) } \\
0.0000 \\
-0.0001 \\
-0.0002 \\
-0.0003 \\
-0.0002 \\
-0.0002 \\
-0.0002 \\
0.0003 \\
0.0003 \\
0.0001 \\
0.0002 \\
0.0003 \\
0.0005 \\
0.0006 \\
0.0006 \\
0.0006 \\
0.0006 \\
0.0008 \\
0.0008 \\
0.0007 \\
0.0007 \\
0.0008 \\
0.0005 \\
0.0004 \\
0.0004 \\
0.0004 \\
0.0003 \\
0.0003 \\
0.0003 \\
0.0002 \\
0.0002 \\
0.0004 \\
0.0005 \\
0.0006 \\
0.0004 \\
0.0005 \\
0.0005 \\
0.0004\end{array}$ & $\begin{array}{c}\text { Pressure } \\
(\text { psig) } \\
9.89 \\
19.55 \\
29.57 \\
39.42 \\
49.16 \\
54.50 \\
59.57 \\
64.20 \\
69.32 \\
74.16 \\
80.16 \\
85.61 \\
90.58 \\
95.69 \\
100.92 \\
103.25 \\
106.11 \\
108.31 \\
111.08 \\
113.24 \\
117.83 \\
120.92 \\
123.28 \\
122.97 \\
125.82 \\
125.60 \\
12784 \\
130.19 \\
132.53 \\
135.33 \\
134.42 \\
138.35 \\
140.16 \\
142.63 \\
145.78 \\
142.52 \\
0.22\end{array}$ & $\begin{array}{c}\text { Displacement } \\
\text { (inches) } \\
0.0001 \\
0.0024 \\
0.0054 \\
0.0080 \\
0.0100 \\
0.0111 \\
0.0121 \\
0.0134 \\
0.0146 \\
0.0153 \\
0.0175 \\
0.0187 \\
0.0196 \\
0.0205 \\
0.0210 \\
0.0214 \\
0.0216 \\
0.0216 \\
0.0217 \\
0.0217 \\
0.0218 \\
0.0219 \\
0.0216 \\
0.0214 \\
0.0215 \\
0.0216 \\
0.0216 \\
0.0216 \\
0.0217 \\
0.0218 \\
0.0218 \\
0.0220 \\
0.0226 \\
0.0231 \\
0.0235 \\
0.0237 \\
0.0220 \\
0.0\end{array}$ \\
\hline
\end{tabular}

High Pressure test (inches) 0.0001 0.0024 0.0054 0.0080 0.0111 0.0121 0.0134 0.0146 0.0153 0.0216 0.0216 0.0217 0.0217 0.0218 0.0219 0.0216 0.0214 0.0215 0.0216 0.0216 0.0216 0.0217 0.0218 0.0220 0.0226 0.0231 


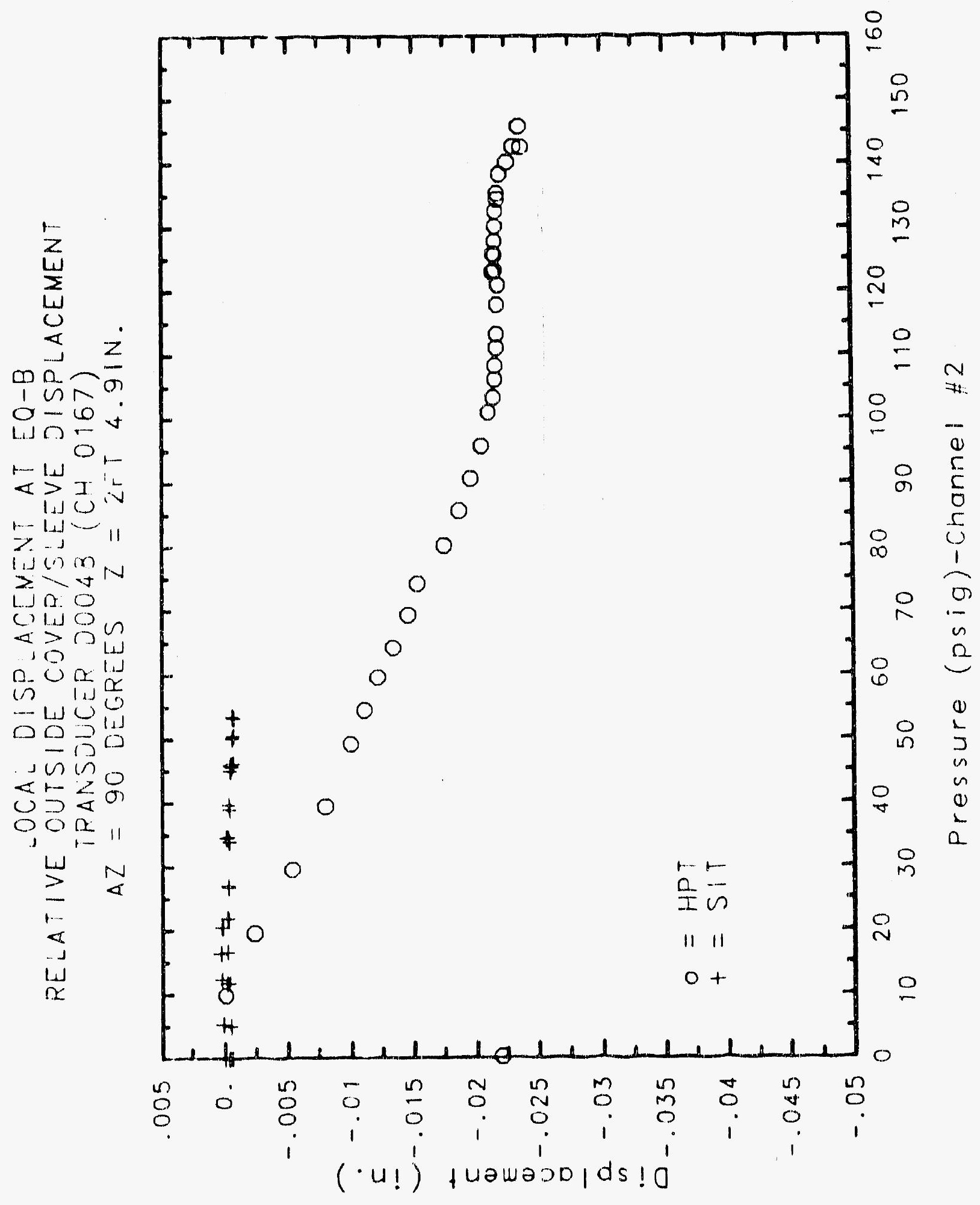




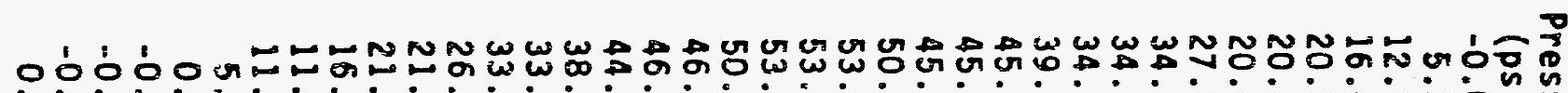

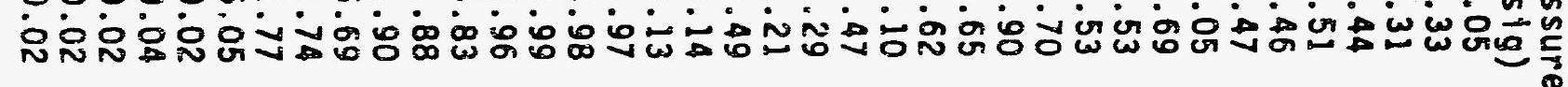

$00000000000000000000000000000000000000 \frac{1}{3}$

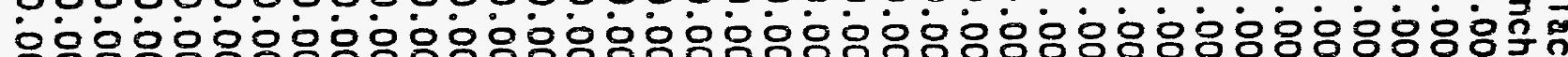

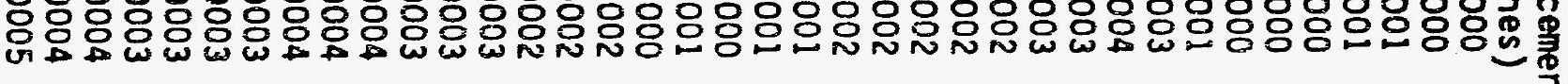

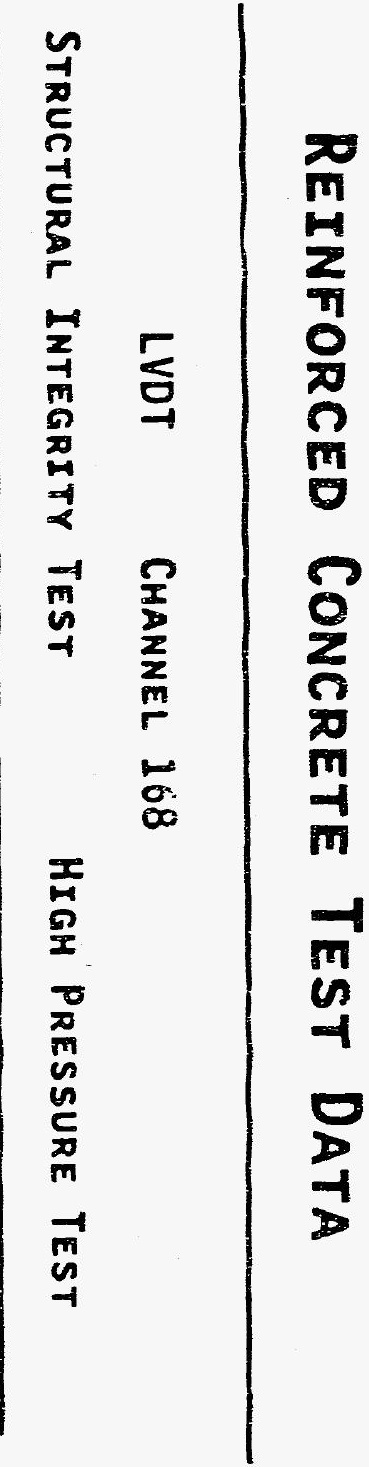

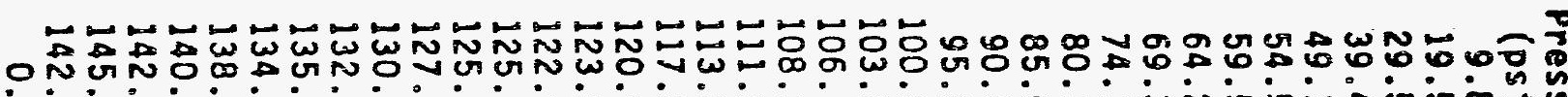

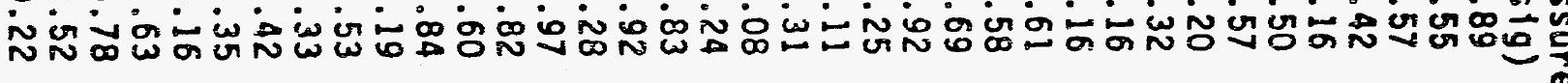

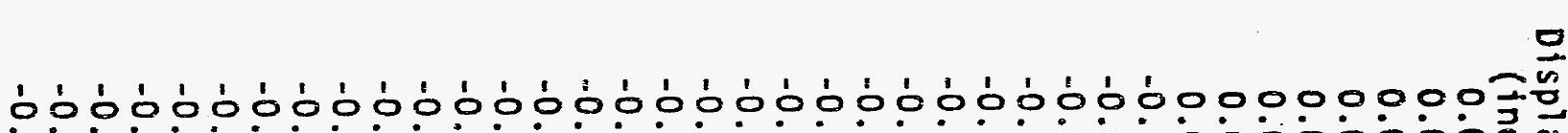

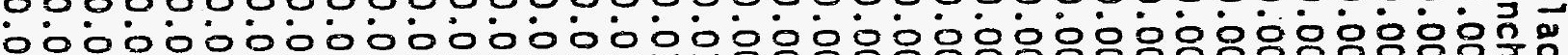

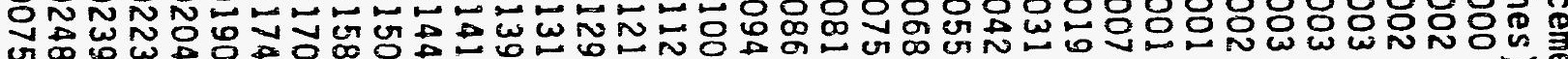




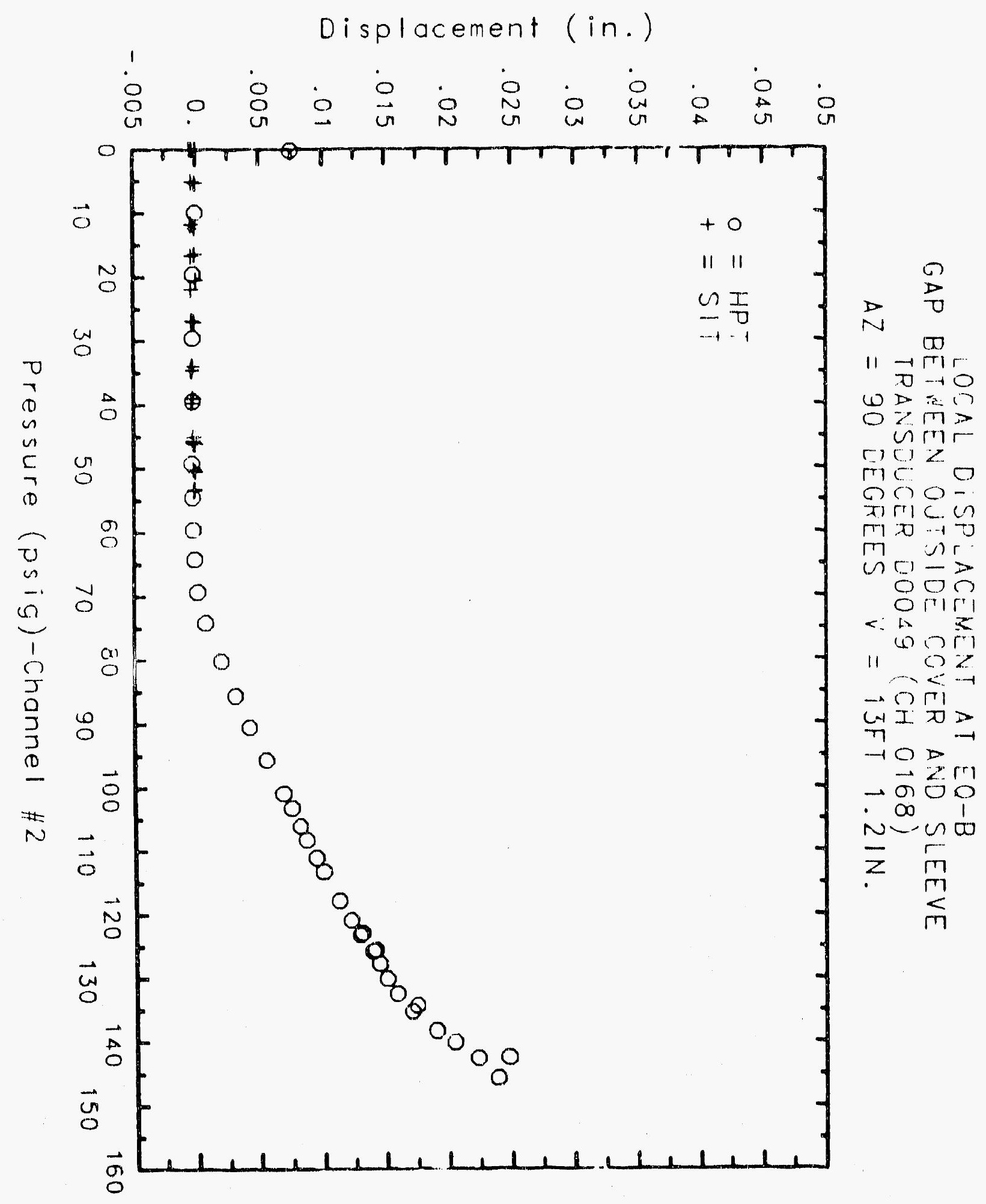



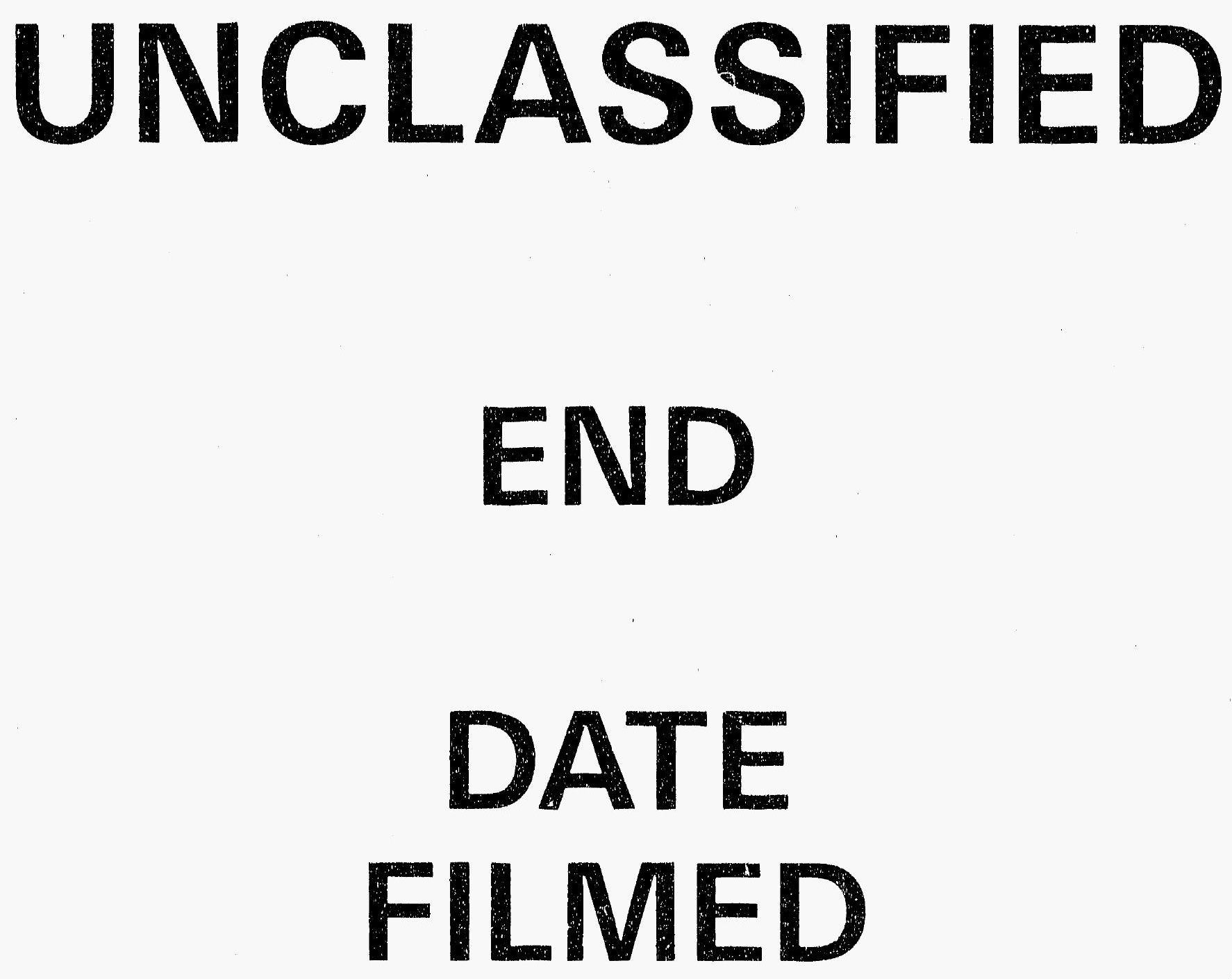

$$
1-6-92
$$

SANDIA NATIONAL LABS 


\section{uog̣erodiog

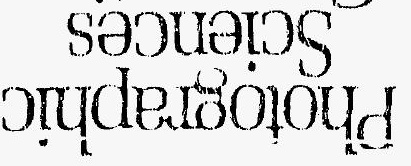
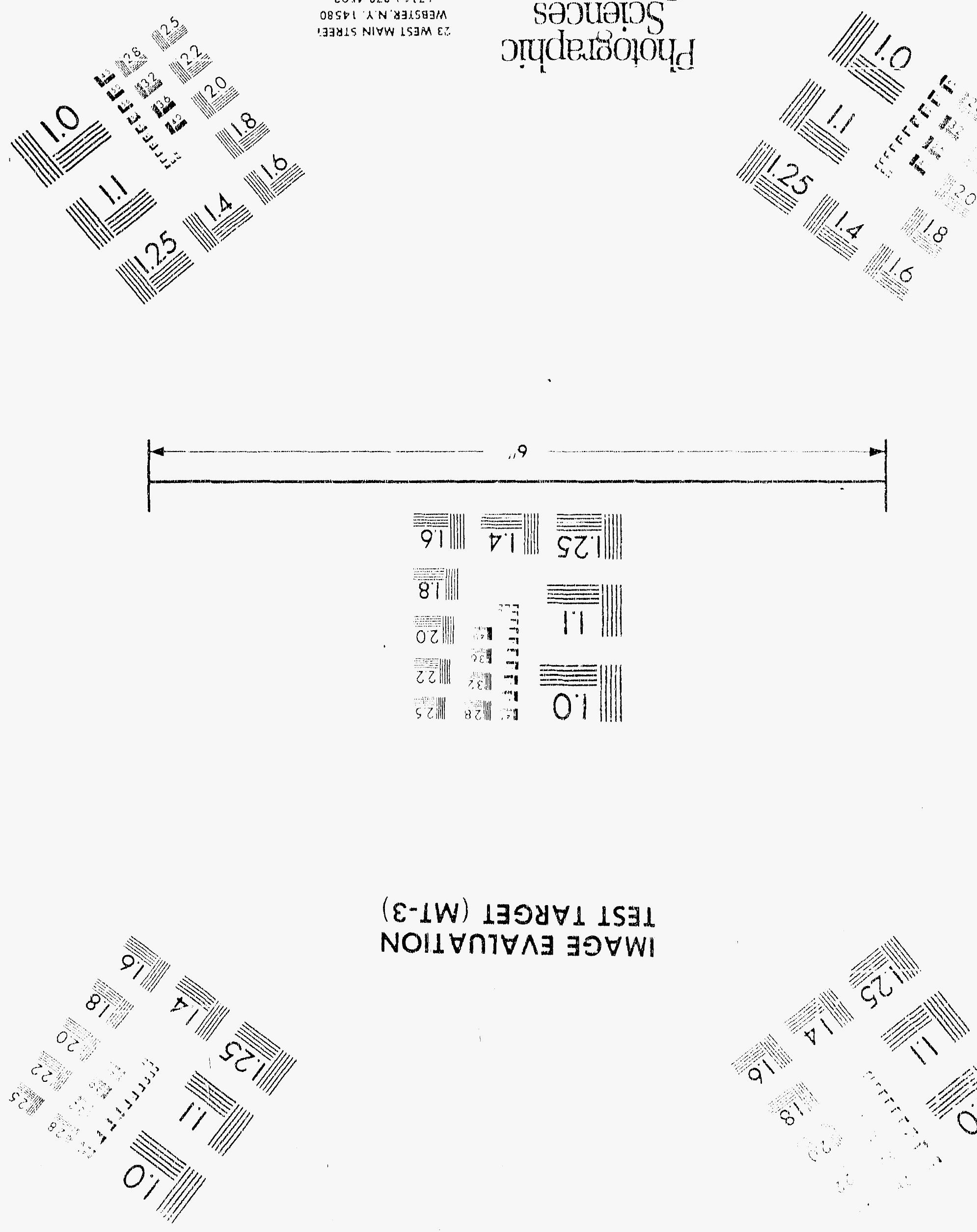


\section{SECURITY CLASSIFICATION}
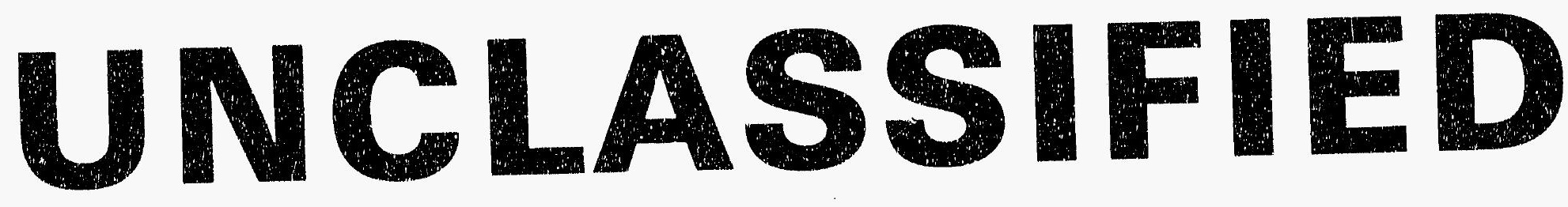

\section{DATE OF MICROFILMING $1-6-92$}

\section{MICROFILMED BY - Lerer hara}

SANDIA NATIONAL LABS 


\section{THIS DOCUMENT FILMED}

AT

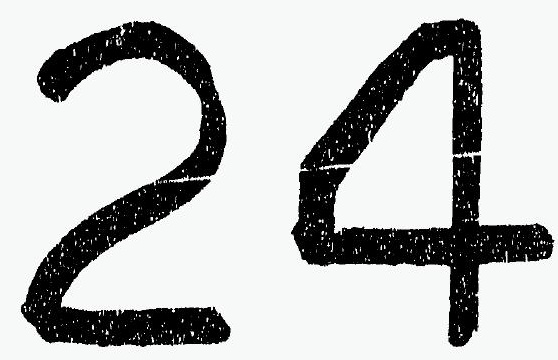

REDUCTION 


\section{Track Transducers}

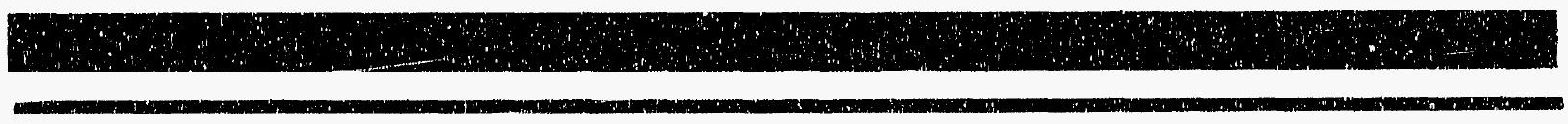




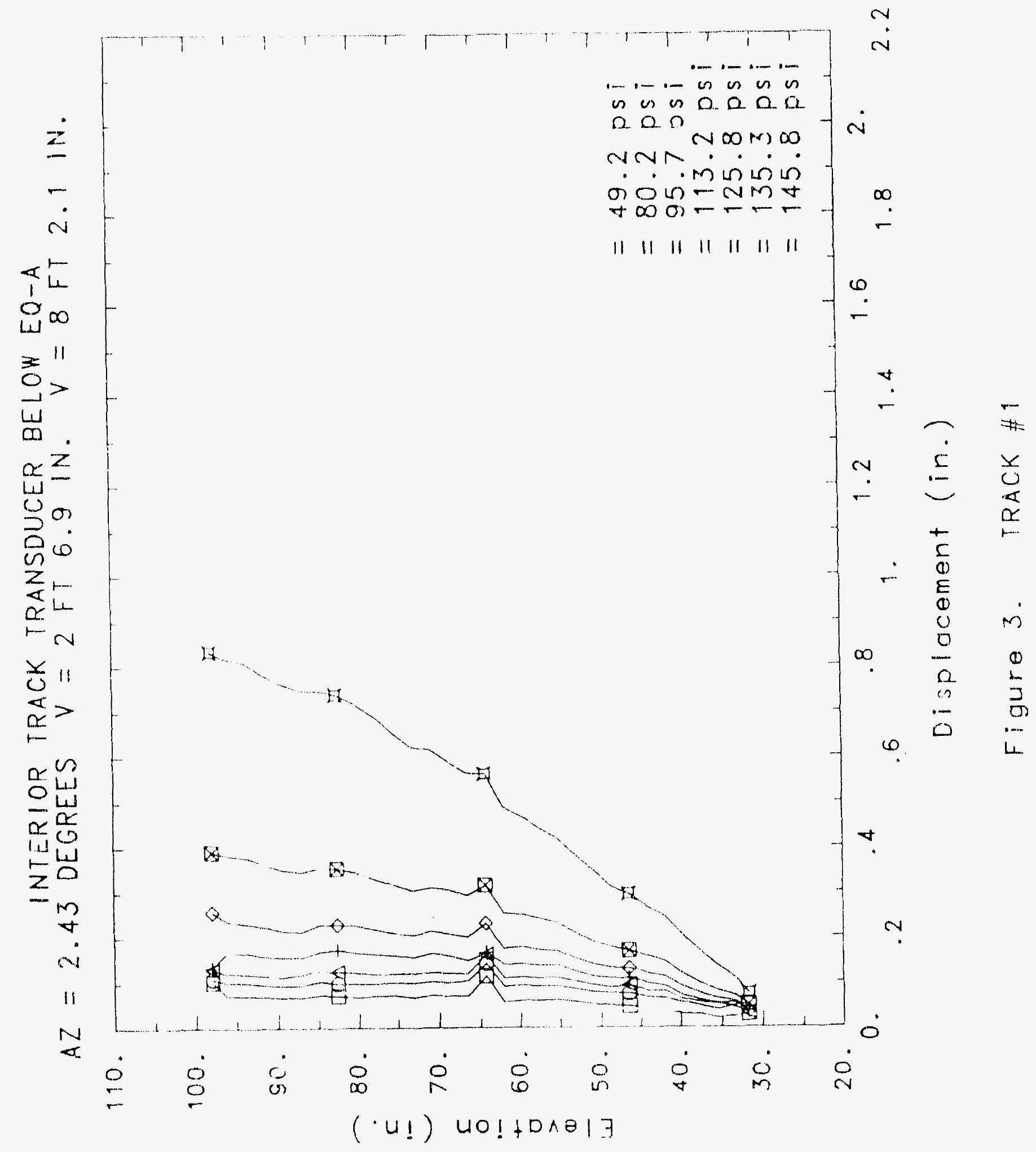

C. 226 


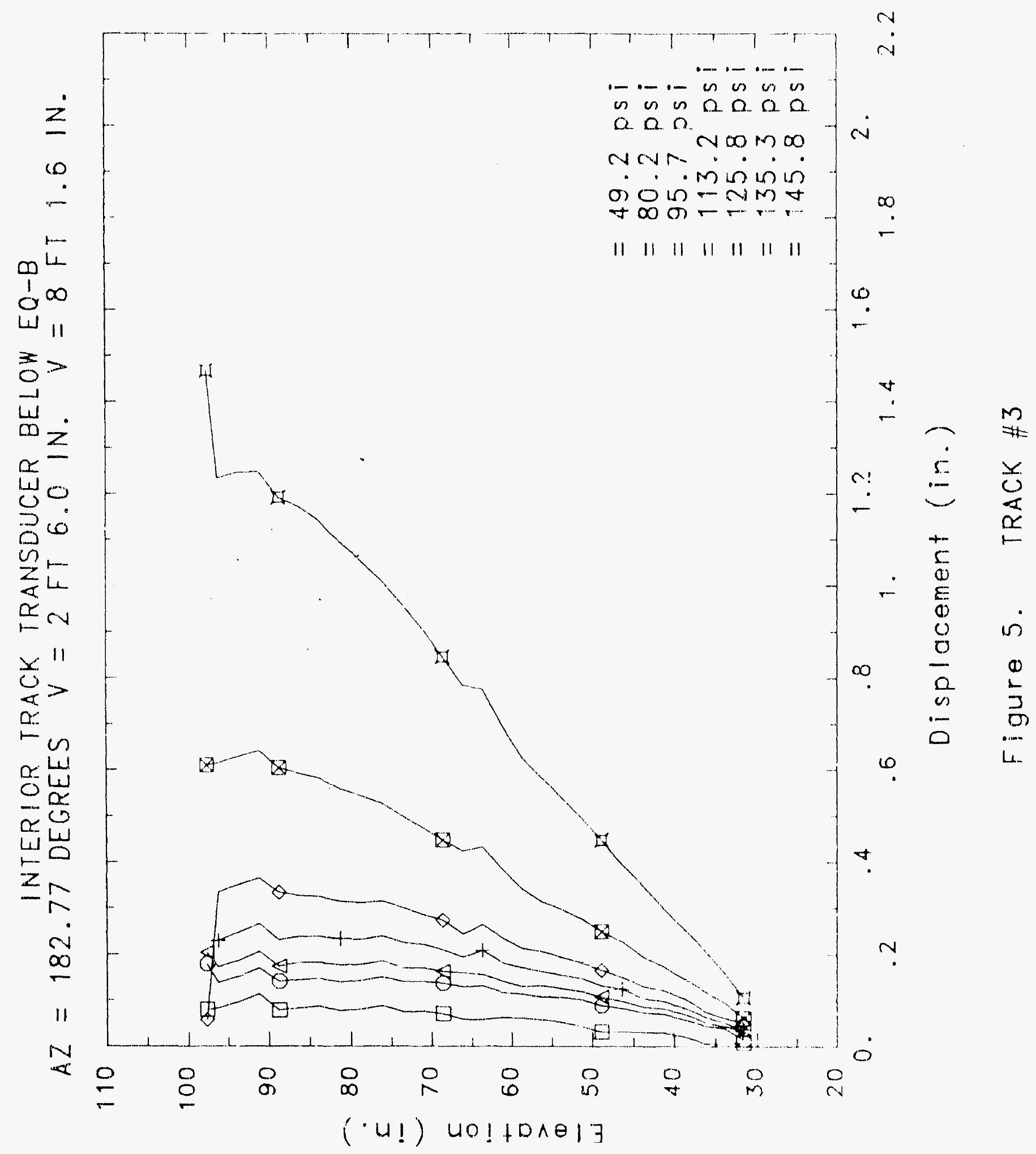




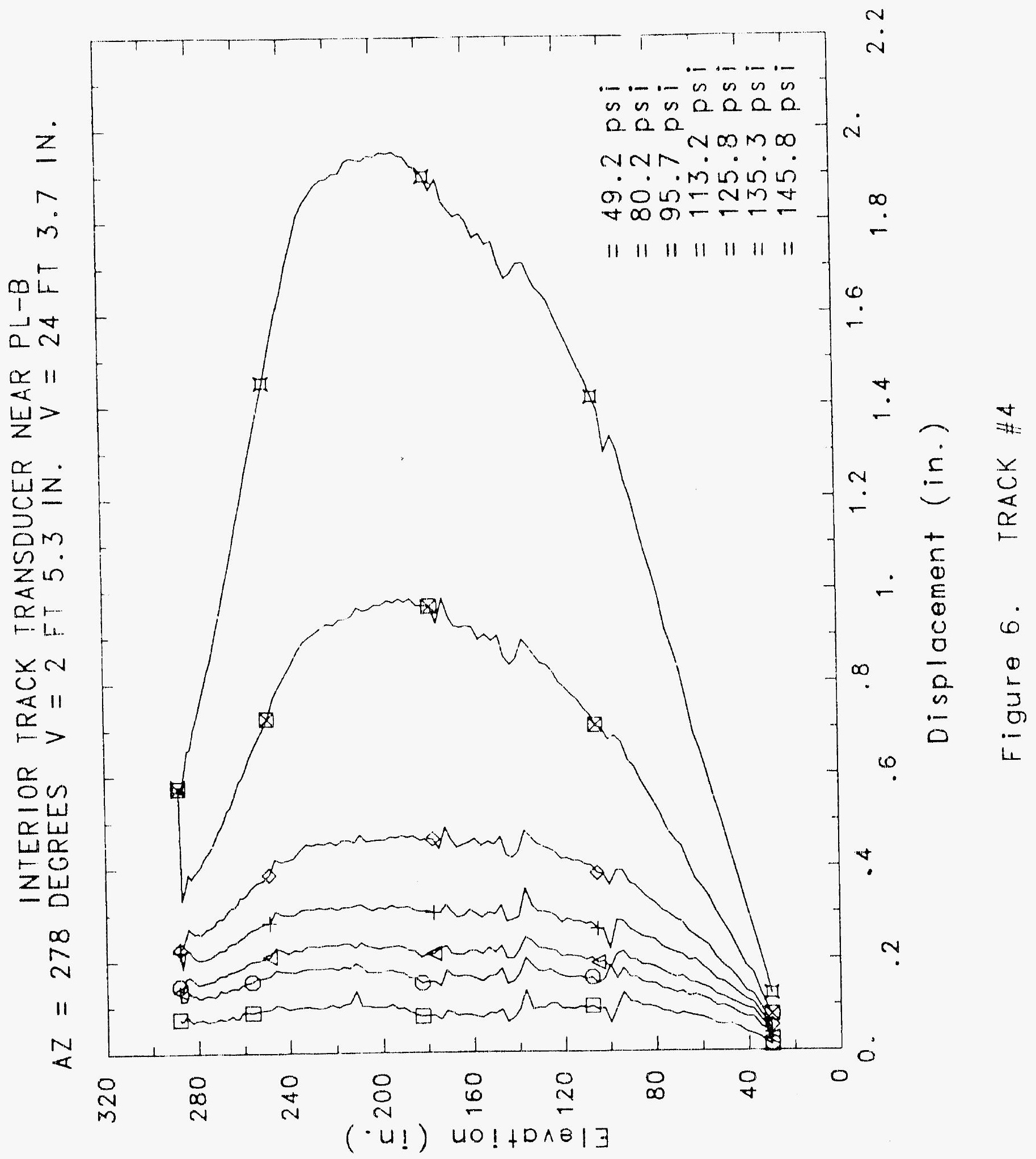




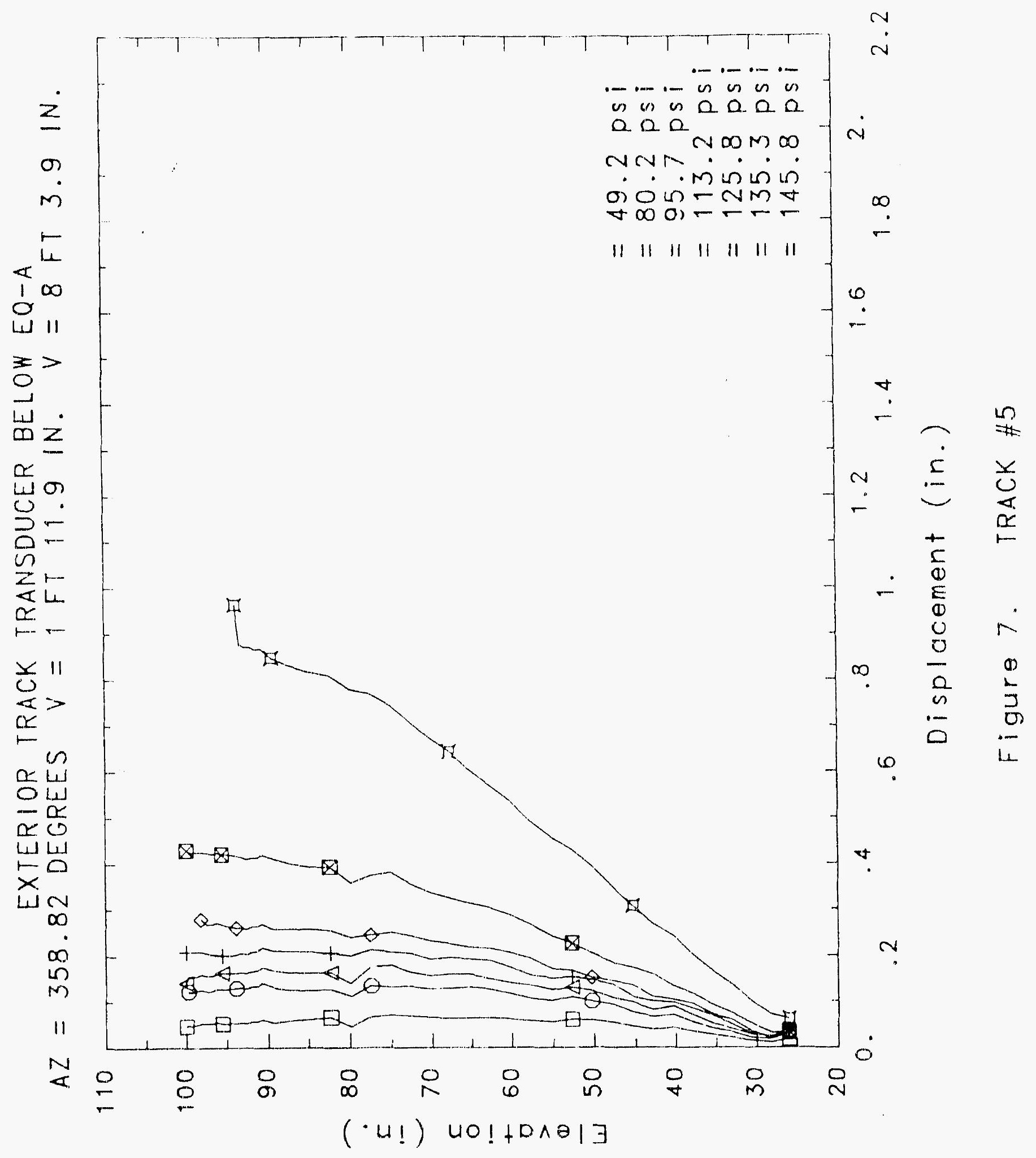

C. 230 


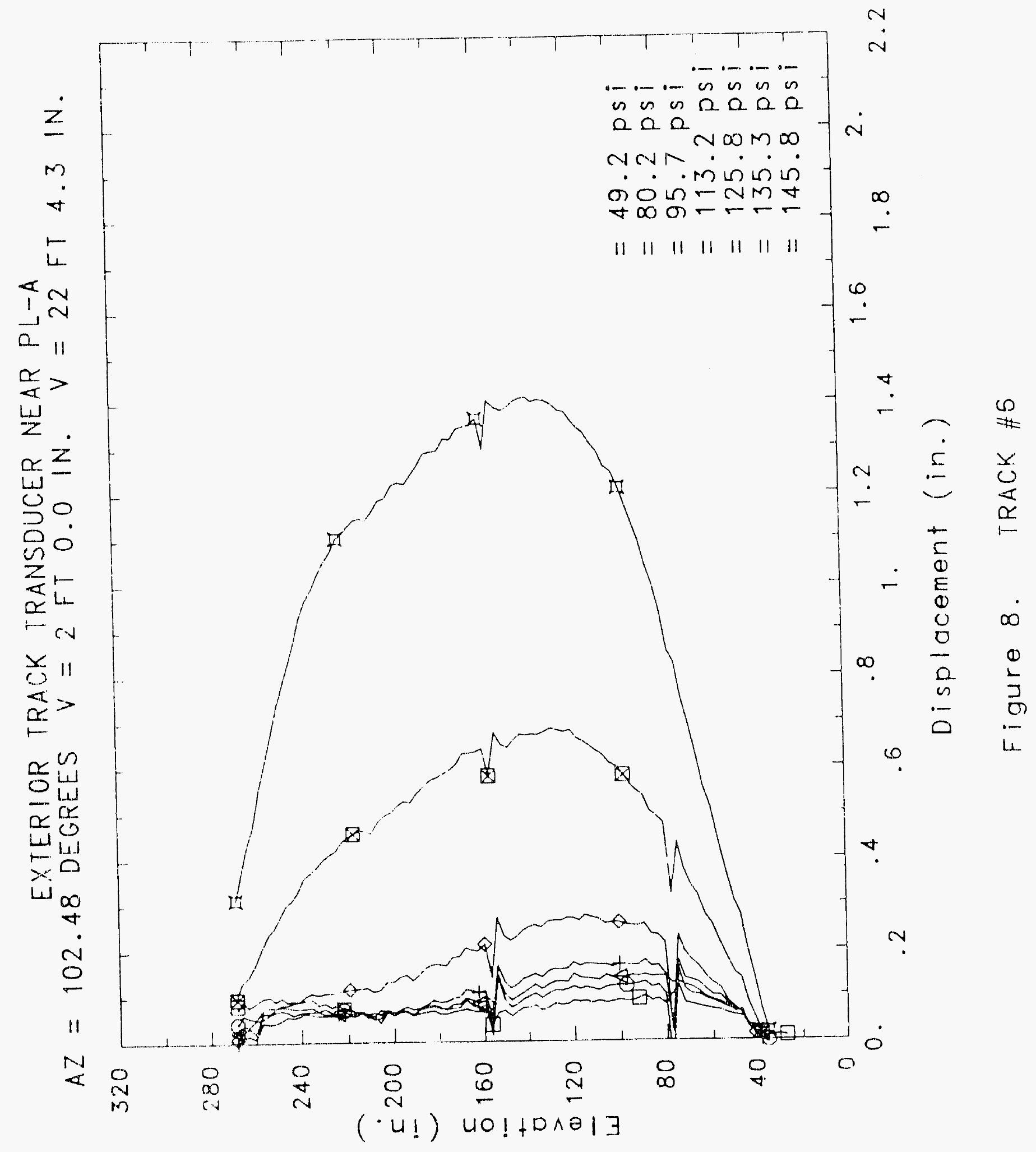




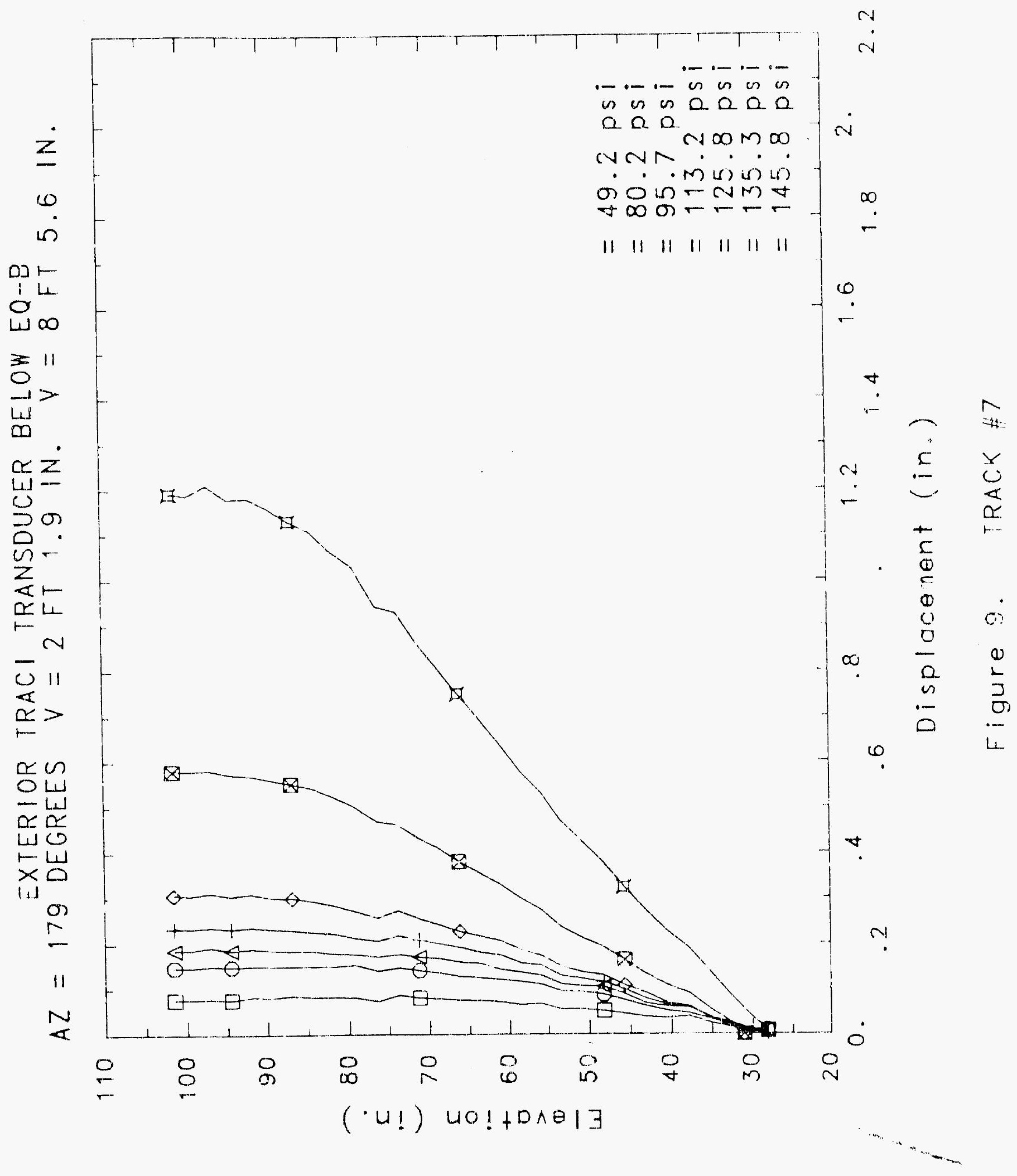




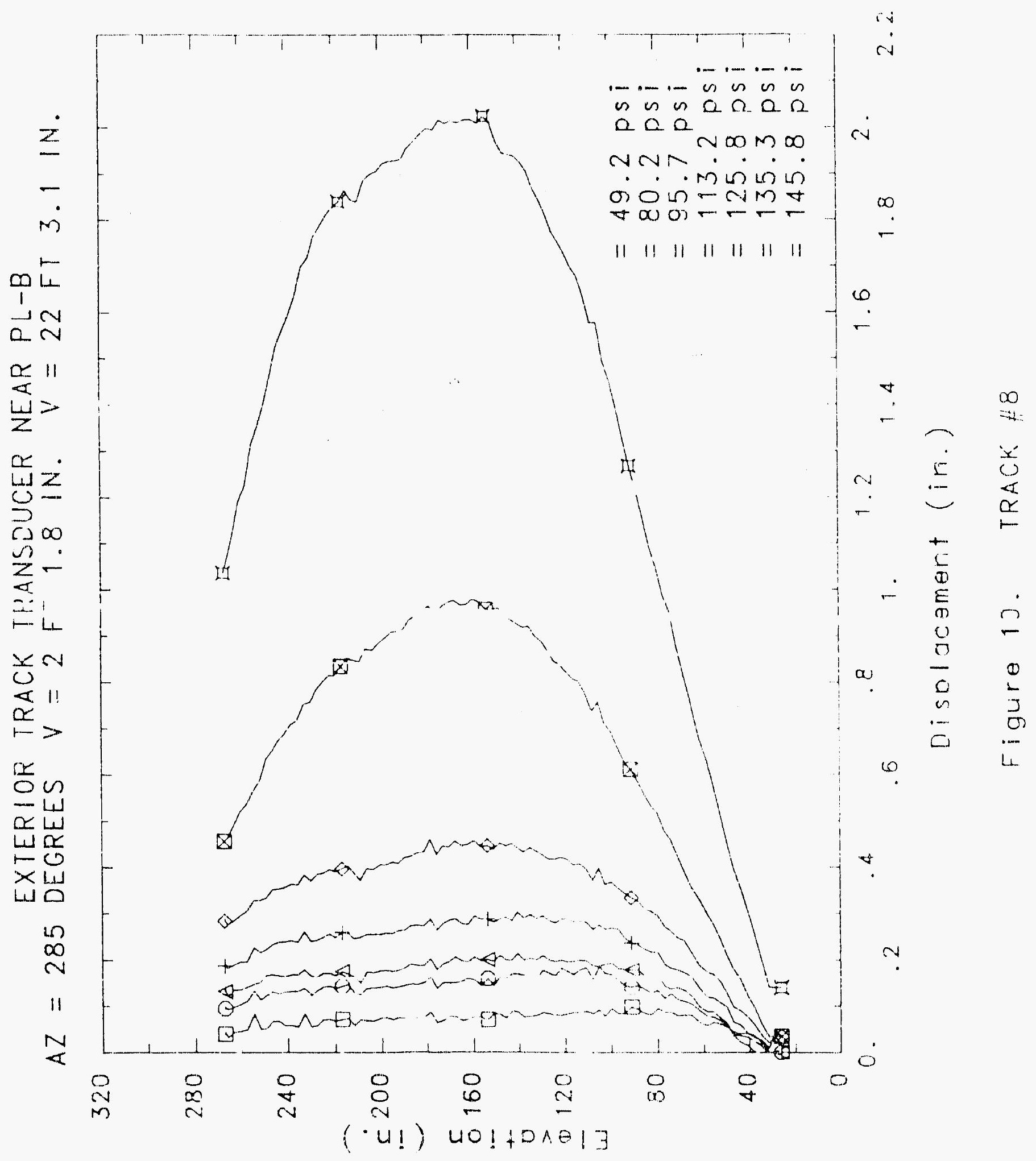




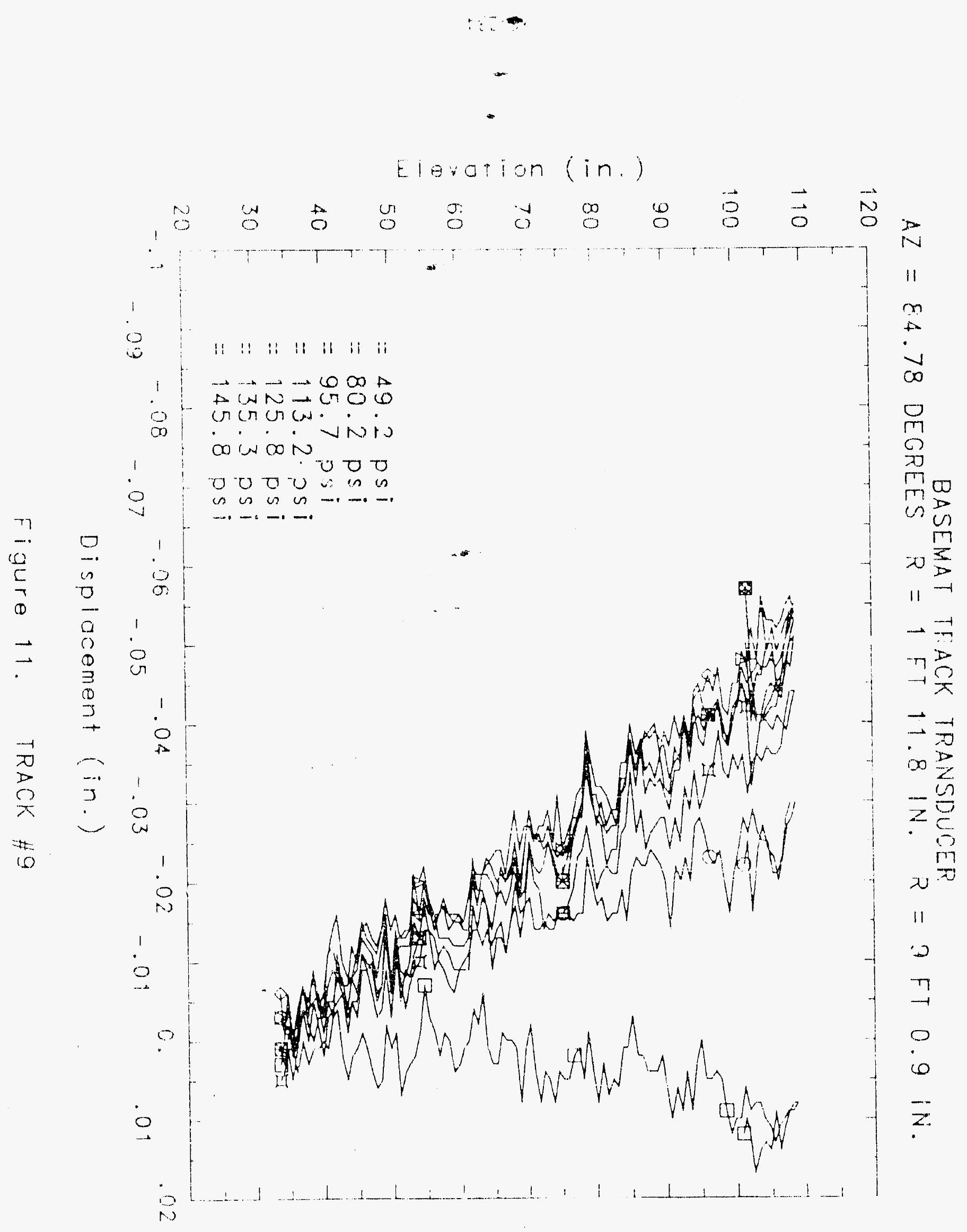




\section{Reinforced Concrete Test Data}

\section{Track 1 - High Pressure Test}

\begin{tabular}{|c|c|c|c|c|c|c|c|}
\hline \multicolumn{2}{|c|}{ 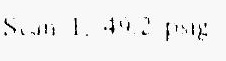 } & $\begin{array}{lllll}8 & 1 & 1 \\
0 & 0 & 1 & 1\end{array}$ & $\begin{array}{l}016,15 \\
011329\end{array}$ & $6,5+11$ & $0(150)$ & $\begin{array}{lll}1 & 1 \\
1 & 1\end{array}$ & $\begin{array}{l}1115012 \\
1111280\end{array}$ \\
\hline & & $810.1 \% 1$ & $(1) 18: 5$ & $(x)(1) \leq 11$ & olosili & 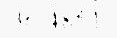 & 111,25 \\
\hline $0,12(x)$ & 111962 & $\delta(1, q 1)])$ & 01130 & 57.40121 & $\|(1)\| 1)$ & $\therefore \cdots$ & $11+1, \cdots+1$ \\
\hline $4 \therefore 1 \geq(x)$ & $111^{\prime \prime} \div ;$ & $73 x+1$ & $(1)()^{\prime}\{t)$ & $54.50 \%$ & 11005 & $\therefore 1 \ldots{ }_{i}$ & 111021 \\
\hline $4 " 144$ & $1: 110: 1$ & $\because 41111$ & $1111 \% 2$ & $522 \times 1$ & $18 x+x$ & $\cdots 1 ;: !$ & $(1) 216)$ \\
\hline $45<112$ & $\left(1,11^{\prime \prime}+1\right)$ & 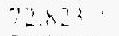 & 11914 & 4i) $2: 41$ & $1 ! 0,1.4$ & $\because 4: 61$ & $(112.16)$ \\
\hline $121311:$ & $\therefore i x<1+$ & $-1128+1$ & $(16, \cdots, 1$ & $4.4(4) 1$ & (1in:) & $\therefore \quad|\cdots|$ & (1,ing, \\
\hline $401 \times 1=9$ & 1 Hon & $0^{7}>2 \times 1$ & 0.0411 & 44.91121 & $(1015)$ & $x+21$ & $(1) i, 1,1$ \\
\hline $8 \because 12 \cdots 1$ & $(1,16,1) \cdot\}$ & $\left(1^{5} 1.4\right)$ & $(1,1,5) x$ & 41281 & () $11.1 \%$ & 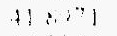 & inkis \\
\hline$\therefore \leq 5,1$ & 11818,6 & $(\therefore<i)$ & $11110 x$ & 34.1311 & (1): : & $\therefore: 1: 11$ & li (k,in) \\
\hline $5 \div 14<1$ & $(1)(1+x)$ & $|n: 11 ;| 1$ & (1):11: & $36.4 \times<1$ & $: 01,4.4$ & At $:\{1\}$ & 1111'is \\
\hline$k(1+46] i$ & $1+\{k, 1\}$ & $4 \div+12 !$ & $\{1(k, 1)\}$ & $3 x-8\}(1)$ & 110.21 & $A B<\ldots 11$ & $11.61 \%$ \\
\hline$\because \quad 1 \leq<1$ & 0,145 & 98121 & $(114: 21)$ & 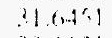 & $(1.1): 4$ & $\therefore \cdots$ & 1111,13 \\
\hline$\because 1111$ & $11)(15.18$ & $\therefore 221$ & $0(x)$ (x): & 311131 & $(1,1): x$ & $\leftrightarrow i .1$ & nitisi \\
\hline$\because \therefore x^{\prime} 1$ & $(1, x, i$ & $40(1,41$ & $(1)(1.41)$ & $3<2<7$ & $011: 21$ & $\because \therefore \because 1$ & $(1)(0) 10)$. \\
\hline $112 \times 14$ & $0.17 x \ldots$ & an, misti! & $111 \ldots 1$ & $27 . f(1)]$ & $(1,11: 1)^{\prime}$ & $\therefore \quad ; \quad 1$ & f: 19414 \\
\hline 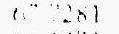 & (1) Whi: & $4+111$ & $11641 k$ & 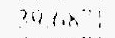 & $11,14:$ & $4, \quad: \quad 1$ & $(1(1)+1)$ \\
\hline $1 * 11 \% 1$ & 11,13 & $\therefore 11,1$ & $11119: 8$ & $4151 ? !$ & 11,1614 & $\therefore 1 " 1$ & ()$(\mu) ! 5$ \\
\hline$t_{1} .<<!$ & $(1+\cdots$ & $\therefore+1+1$ & $1: 11.01$ & $+41<1$ & $1105:$ & $1.1 \div .11$ & 111421 \\
\hline$\cdots \cdots !$ & $\therefore 1: 411$ & $26,14<1$ & 11021 & H. $2: 111$ & $0(1), 1$ & $\{t \cdot 1: 1\}$ & i) 1112,4 \\
\hline$\therefore \quad 4.2$ & $(101,4.6$ & $\{: x i f$ & $1191 \% 2$ & 45 50011 & $11(x, i)$ & $1 \therefore \quad 1$ & $11.1114: 8$ \\
\hline$\therefore \div \div 1$ & $(1,11.1))$ & $: 11.161$ & $11+i, 1)$ & $511 d_{1}$ & $(11)^{\prime}+2 ?$ & $\because 11$ & (1) $11 \because " 1$ \\
\hline$\because \ldots n$ & $11(1.4:$ & $\therefore \quad 111$ & 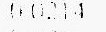 & $53(1): 1$ & $\log (1,4)$ & $\because \therefore 1$ & $(i)(x), x$. \\
\hline$A \ldots+1+1$ & 11,12 & $\therefore \quad 154$ & $1,1, \ldots$ & $5<2: 11$ & $(1+1+1, x$ & $4 \quad: 11:$ & $11(k)+1)=$ \\
\hline 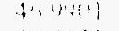 & Hilis & $: 4+3+1$ & $(1)(1414)$ & $524 x i$ & 11165 & 41 & $11(x):-2$ \\
\hline$+4+1: 1$ & $1:, 1, d_{1} \cdot 1$ & $41, k \mid$ & $(11,+)^{\prime}$ & $\langle 1,7+1\}$ & $11(14: 1$ & $0 \therefore 1|x|$ & $(1, k) \ldots)$ \\
\hline$\$ 11$ & 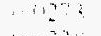 & $1 ! * ; 1$ & (1)(1.f) & (1) $\cdot x_{1}<1$ & $1111 k: 2$ & $\therefore \therefore: 11$ & $11(x+6)$ \\
\hline$\because 1:: 1$ & $11,-3$ & $4+1 \cdot 4$ & $\{1: 3.141$ & $(x+2) 11)$ & $11 \mid 1, \ldots:$ & $=1 \times-1$ & $111: 21$ \\
\hline 24,165 & $1: 11: \therefore 1$ & $46,: 1: 1$ & itite? & $(x \cdot 1241$ & $0(1,4)$ & $k=1 k+1$ & $11(k)$ \\
\hline$\because B i b 1$ & 11 fifm & $1: 5 i$ & (1)kx! & 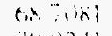 & $115 x<1$ & $\therefore 4 \ldots 1$ & $(1)(x) \mid 1$ \\
\hline "1 $1 .+51$ & $(111)$ & $41, \because 11$ & $11\left(x_{1}, 4\right)$ & $\because 192 \ldots 1$ & \|\|$N N_{1}$ & $18 \div$ & $1 \because(n) ; 0)$ \\
\hline$: 21 ! 2 !$ & $111: 1$ & 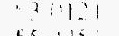 & (i.ln $n+x$ & $7(18)$ & (1) $1,4$. & $111: 4,5$ & (1) 11114 \\
\hline is $15: 1$ & $1 ! i: 3|1|$ & \& $\therefore 16$ & (1) & $75+111$ & 11011 & $m: 11 !$ & $\because 1111:$ \\
\hline$\because 426$ & $(11)=1$ & $\{1+1$ & $(111: \vdots$ & 9061 & 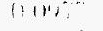 & $4+119$ & $(1 \mid 11): 1$ \\
\hline 41,4$\}$ & (1) 1312 & (1) 4111 & $(1+1) \cdot 1$ & (ni) 2101 & $11(1+2.4$ & & \\
\hline 41941 & $11: 1312$ & $(1), 4,1$ & $(1,1,1$ & $x_{2}=2 x y$ & (B) & & \\
\hline+2121 & 11112,2 & $\left.\left\{+\frac{1}{4}=1\right]\right\}$ & (i) $114.4^{2}$ & $84 \times 3$ & 1111121 & $x+14<4$ & $161=1 \%$ \\
\hline$x(1: 21: 1$ & $1)+i k n$ & $\ln 12+4$ & $(3118 ? ! ?$ & $8 \div(1)+1$ & $(i)(k, t)$ & & \\
\hline $1:<8,1$ & 110.114 & 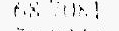 & dolste: & $84, i t, 2.1$ & (:!n!)! & $12 k 13(11)$ & (1) 1211 \\
\hline $9: 1+1$ & $11191^{6}$ & $3 \cdot \ldots+41$ & $11 \ldots$ & $4 x^{2}=27$ & (1) & $\times 12(11:$ & $\because 211$ \\
\hline $54112 !$ & $11015 n$ & $1|x|$ & 116.8 & 42 is 6 & $\left(1: t^{\prime} m\right)$ & ia lok & 01211 \\
\hline$\therefore 2: 51$ & $\left.(1)\left(m_{1}\right)\right)$ & $\because 1.4 i$ & $|+m=|$ & (x) If(i) & \|\|$\mu k$ & $14<002 ?$ & 111121 \\
\hline$\because 4 \mathrm{Si}$ & 11,1914 & $t \times 1$ & $(01: 5$, & 17.814 .4 & $(1,11,2$ & $\because 21,100$ & 11,1102 \\
\hline$a_{i},+11 i$ & litex) & $x+1=1 x .1$ & $11: m, 1$ & & & $\because 0 t x: 4$ & 01.1111 \\
\hline (1) $\cdots, 4$ & $11(15) !$ & $\therefore=6 n, 1$ & $110, t_{1}$ & & & 4.0 .21 & (1) 111.:! \\
\hline$\therefore 11$ & 11113 & $x .4+31$ & (1) (1) & 8.211 .4 & $\therefore \quad 1: 11$ & 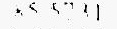 & 1111.46 \\
\hline$n+4: 41$ & (1'kn) & $x^{\prime} \quad k 1$ & $1101,1 \mathrm{~s}$ & & & $k: 111,51$ & ('If(x) \\
\hline 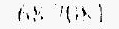 & $(10,19$ & $x=4,34$ & $(118)$ & Uस: & (1) Hal & $x+4+11$ & $(1) 11.4$ \\
\hline $792: 941$ & $\because 19=$ & $4: 5 ?$ & 111126 & $4(2)(\pi)$ & (1) list & $1 \because 41$ & (f) $(k)$ ) \\
\hline$\because 18 \geqslant 1$ & $(1), 4,4 ;$ & $y=x_{1} x^{2}$ & (1) llakn & of $12 x$ & (1) 1 kid & $\because 9.9111$ & $0(k)+1$ \\
\hline$=41.71$ & 191172. & He: lik!! & (1) 116R & y5<4:? & $111 \%$ & $\because 231$ & $0.101, \mathrm{~s}$ \\
\hline$=\cos$ " & 110,12 & $\because 42$ & $(1)+48$ & $(1) 12(x)$ & $11: \cdots+d$ & $42 \times 11$ & (1) $1: 1^{6}$ \\
\hline 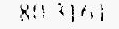 & $1,(x+x)$ & & & $4+\{x+1\}$ & $6\{r+4$ & 1. $\quad \therefore 1$ & $0 ! k+1, \ldots$ \\
\hline $\sin <\div 1$ & $110 \%$ & & & $n+1: 1$ & $111+.12$ & 16171 & 1016 \\
\hline $8 .+2.1$ & $1100^{-12} 2$ & $\therefore$ an? & $\because p \times f$ & 86074 & $\theta\left(r+t_{4}\right.$ & $12<21$ & dif $18 x$ \\
\hline $8: 101:$ & $4 k$, & & & $0^{2}(1.45)$ & (1) 6m, & $1.4+1411$ & $(1)(x)+1)$ \\
\hline$\therefore 26,2.1$ & $11(4 ;)^{5}$ & $46: 26:$ & (1) $(19)$ & $x(1+7+1)$ & $(1 ! n+1)$ & $47(1.1)$ & $(1 \leq x+x)$ \\
\hline $115 ?$ & $(1,1)^{\prime \prime}$ & th $12 r_{1}$ & $1111 \div+1$ & $\because 1,\langle 61$ & $11+\ldots+$ & $445(12)$ & $(1618)$ \\
\hline$" x<3$, & 1192 & $411 \%$ & 1113.84 & 72111 & $111 \% 11$ & $\because \therefore B 1$ & (1) \\
\hline ur $\mid 1 k !$ & $i 1, j !$ & w口 4,1, & $\{11: 2\}$ & $72 \therefore 21$ & $11, \ldots . i$ & $1,0,34$ & $116 \%$ \\
\hline $8+1.4$ & " ! :" & $4: 120$ & $1: i m \varepsilon^{\prime}$ & $3 ! 1511$ & $(1) 11)$ & $\{\therefore$ rind & $111^{25}$ \\
\hline & & $901+16$ & (1)"18.2) & c)? & 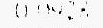 & $11+11,: 1$ & Hix, he \\
\hline & & $3 \times 121$ & $0(1+146$ & $a^{6} ; 4$ & $1+n \cdot 2$ & $41 \% 71$ & $11.6=$ \\
\hline$\therefore$ and $\quad \therefore$ & . 1. 1.it & $x \cdot c \cdot 1$ & 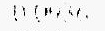 & 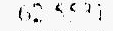 & $11, n^{14}$ & $2131: 31$ & $(1,16) 1$ \\
\hline & & $x^{2}+1.4^{6}+1$ & $1: \ln n+x^{*}$ & (201:21) & $1: \ldots$ & if $+x<1$ & $(1: 10 \cdots$ \\
\hline 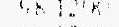 & $117 \mathrm{tat}$ & $(1+411)$ & $111,2$. & $54+12$ & $\because \cdots r^{2}$ & $\because\{\therefore<1$ & "11+1). \\
\hline th $12 \%$ & $(m+\ldots$ & $\because 45 \times 1$ & $\because \because r+\ldots$ & 54811 & 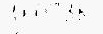 & $.128: 51$ & (1) $\left.11^{2}: 1\right)$ \\
\hline it 10 & $\left\{n_{1},:\right.$ & $3611: 1$ & (1) 118011 & $\therefore \therefore n 1$ & $(1+x) \cdot 1)$ & $\because 1141$ & (1; i.... \\
\hline 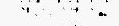 & $\therefore \cdots$ & 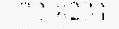 & $i,^{\prime \prime} i$ & $4 \div \therefore i$ & 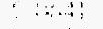 & 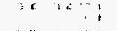 & $\because: \therefore \%:$ \\
\hline $4: 12 x$ & $(1+a) \times$ & $\because \cdots i$ & $1: k_{i}, "$ & He 94 ? & $11 \times x$ & $\because 24$ & $11+\because$ \\
\hline $1116 x<2$ & wink! & $f, \cdots " \therefore:$ & $\mid,\{\ldots+1$ & $21911: 1$ & $1: 4:$ & $4: 1,1 !$ & $111 \times \ldots$ \\
\hline
\end{tabular}


Reinforced Concrete Test Data

Track 1 - High Pressure Test

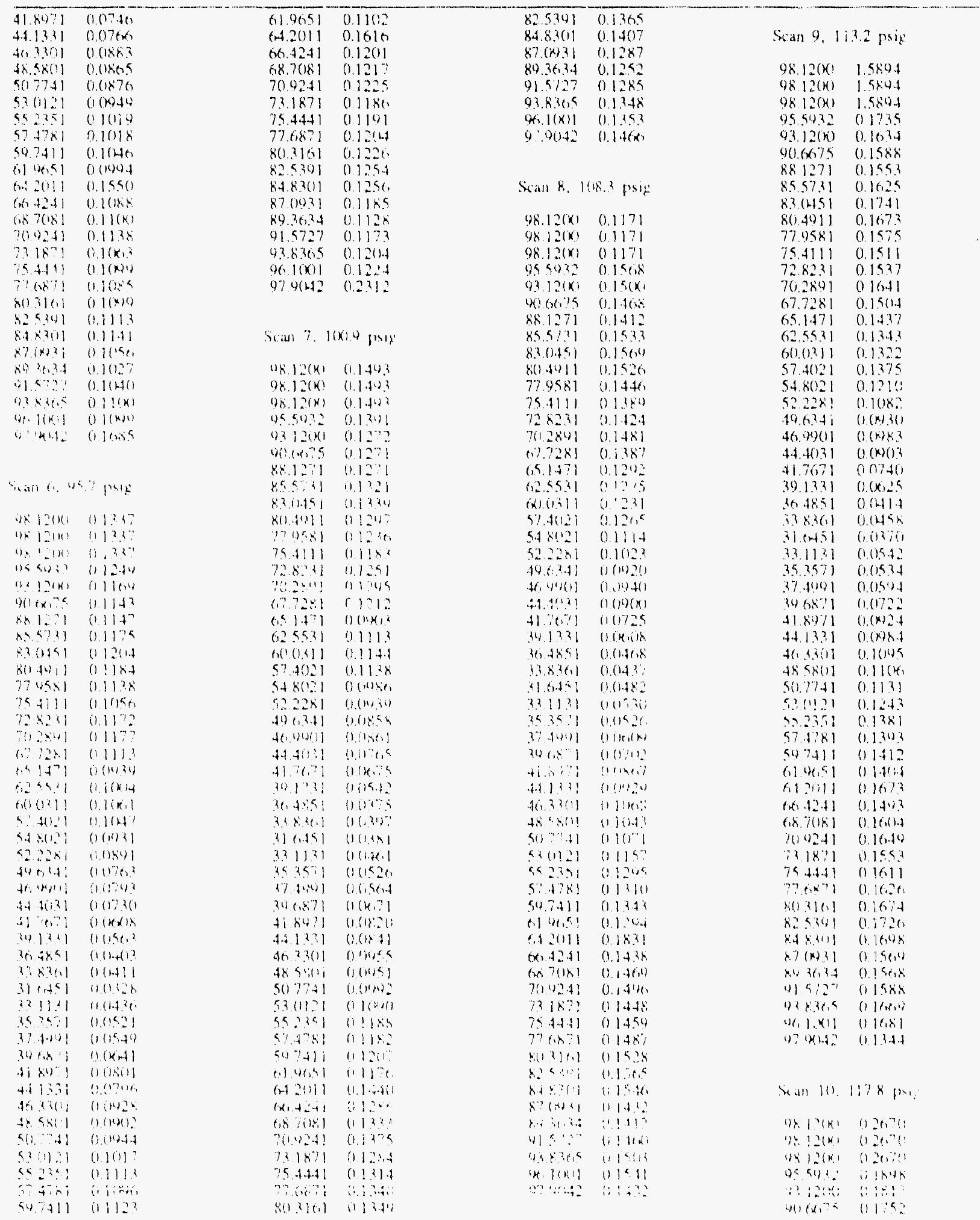


Reinforced Concrete Test Data

Track 1 - High Pressure Test

\begin{tabular}{|c|c|c|c|c|c|c|c|}
\hline 88.1271 & 0.1689 & 70.2891 & 0.1867 & $46.99(11$ & 0.1188 & 37.4991 & 0.0827 \\
\hline 85.5731 & 0.178 .3 & 67.7281 & 0.1759 & 44.40131 & 0.1128 & $39.68 \% 1$ & 0.0971 \\
\hline $83(45)$ & 0.1878 & 65.1471 & 0.1686 & $41.76 \%$ & 0.0450 & 418971 & 0.123 .3 \\
\hline $8(1.4911$ & $0.18+2$ & 62.5531 & 0.1574 & 39.1331 & 0.0741 & 44.1331 & 0.1334 \\
\hline 77.9 .81 & 0.1724 & 6000311 & 0.1487 & 36.4851 & 0.0576 & 46.3 .301 & $0.1+19$ \\
\hline 75.4111 & $0.16+4$ & $57.4(121$ & 0.1536 & 33.8361 & 0.05 .41 & 485801 & 0.1451 \\
\hline 72.8231 & $0.16 \cos 4$ & 54.8021 & 0.1360 & $31 .(4.51$ & $0.03\left(x^{\prime}\right)$ & 507741 & 0.1534 \\
\hline $7(0.2801$ & 0.1774 & 52.2281 & 0.1226 & 33.1131 & 0.0973 & 53.0121 & 0.17015 \\
\hline 6.7281 & 0.163 .5 & 49.6 .391 & 0.1082 & 35.3571 & 0.0 tits & 55.2351 & 0.1879 \\
\hline 6.51471 & 0.1548 & $46.99(1)$ & 0.1074 & 37.4001 & 0.07 .37 & 57.4781 & 01971 \\
\hline 62.5531 & 0.1400 & 44.40131 & 0.1018 & 39.6871 & $O(\mu)(x)$ & 50.7411 & 0.1973 \\
\hline$f(1)(13) 11$ & 0.1186 & 41.7671 & 0.08 .18 & 41.8971 & 0.1121 & $01 \% .51$ & 0.198 .5 \\
\hline 57.4121 & 0.14 .50 & 39.1331 & 0.06 .43 & 4.1331 & 0.1100 & $64.2(1) 11$ & 0.26 .38 \\
\hline 54.8021 & 0.127 & 30.48 .51 & $0.04 .5 i$ & 46.3301 & 0.1310 & 66.4241 & 0.2288 \\
\hline 52.2281 & (1.1121: & $3,3.8,36,1$ & 0.04 .54 & 48.5801 & 0.1327 & 68.081 & 0.2345 \\
\hline $44,0.71$ & 0.10111 & 31.6451 & 0.042 .5 & $5(1) 7741$ & U. I 4012 & 70.9241 & $0.2466)$ \\
\hline $460(1)$ & 0.1012 & 33.11 .31 & 0.01536 & 53.0121 & 0.193 .3 & 73.1871 & 023.50 \\
\hline $44.4(1) ? 1$ & 0.01075 & 35.3571 & 0.0565 & 55.2351 & 0.1068 & 75.4421 & 0.2411 \\
\hline $41.7(17)$ & 001812 & $37.4(29)$ & $0.06,76$ & 57.4781 & 0.1731 & $77.6 \$ 71$ & 0.2504 \\
\hline 30.13 .31 & $0(x, \div)$ & 306871 & 0.01822 & 59.7411 & 0.1788 & 80.3161 & 0.254 .3 \\
\hline 36.48 .51 & 0.0426 & 41.8471 & 0.0485 & 61.96 .51 & 0.1750 & 82.5391 & (0. 26609 \\
\hline 3.38 .361 & 0.1470 & 44.1331 & $0.1(k, 1$ & $6+.20111$ & 0.2313 & 8.483011 & $0.26+6$ \\
\hline 31,451 & 0.01277 & $46.23(1)$ & 0.1158 & 66.42 .41 & $0.21 K 18$ & $87(x) ? 1$ & $0.25(1)$ \\
\hline 32.1121 & $0.0<5 ?$ & 18.58011 & (1) $1140 ?$ & $6 \times 7(18)$ & 0.2080 & $89.36,19$ & 0.2538 \\
\hline 35.2571 & $0,0,53$ & $50 \div 741$ & 0.120 .5 & 70.92 .41 & 0.21 .37 & $91.5 \% 27$ & 0.26 .48 \\
\hline $37.4(17)$ & ()$_{1}\left(M_{2}\right)$. & $5.1(1) 21$ & 0.1397 & $73.18 \div 1$ & 0.205 .3 & 93.8 .305 & 0.2747 \\
\hline $96(x) !$ & $0 .(175)$ & 5.5 .2351 & 0.1510 & $75.4+1.1$ & $(1.2(19) 8$ & $96.1(k) 1$ & 0.2818 \\
\hline 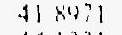 & $(1.60 \% 4$ & 57.4781 & $0.15 \times 3$ & 776871 & $0.218 x$ & $97.9(1.42$ & 0.28 .35 \\
\hline 441231 & (1) 11.4 & 54.7 .411 & $0.15 i 5$ & $8(1.3) 61$ & 0.2267 & & \\
\hline $4(1,3,3(1)$ & (1.11+1 & 60051 & 0.157 .3 & 82.5391 & 0.2217 & & \\
\hline $485 x(1)$ & $(1,120$ & $6.4 .2(1) 1$ & 0.1013 & 8.4 .8301 & 0.2282 & \multirow{2}{*}{\multicolumn{2}{|c|}{ Scan 14, 1.35.3 psig: }} \\
\hline$\{(17: 41$ & 0.1167 & 6,6211 & i) $11 \times, 8$ & $87 .(1931$ & $(1.21 .34$ & & \\
\hline $4:(11: 1$ & 0.1 .12 & 6x.? & $0|k S|$ & $89.36,3.4$ & 0.2148 & 98.1201 & 0.3472 \\
\hline $5<2351$ & $014: 4$ & 70.9241 & 0.1887 & $91.5 \% 27$ & 0.2250 & 48.1200 & 0.3972 \\
\hline 574781 & (1) 1.46010 & 73.1871 & 01746 & 0.3 .8 .26 .5 & $(1.23) 8$ & $98.12(x)$ & 0.3472 \\
\hline (1).7411 & 0.1511 & 75.4 .4 .41 & 0.1816 & $46.10(\mathrm{il}$ & $0.236,7$ & 95.5932 & 0.3883 \\
\hline $6 \mid(x, 5)$ & $(1.1 .509$ & $77.6 \times 71$ & 0.1875 & $07.9(14.2$ & 0.2579 & $43.12(x)$ & $0.1 / 0.8$ \\
\hline+2.20111 & 01.1721 & $80.316 \mathrm{i}$ & 0.14 .22 & & & $9(1.6 x)$ is & (1).361: \\
\hline $6 t_{1}: 241$ & i). $1(x, 5$ & 82.5 .301 & $0.2(x) 2$ & & & 88.1271 & 0.346 .5 \\
\hline $68.7(181$ & 0.1714 & 84.8 .301 & 0.1980 & $\sin 13$. & 1.30 .2 psiz & 85.5731 & 0.3471 \\
\hline 704241 & $0.17 k 0$ & 87.0431 & $0.18 .4 i_{1}$ & & & 8.1451 & 0.3578 \\
\hline $73.187 !$ & 0.10 .58 & $89 . .36,3.4$ & 0.1826 & $98.12(x)$ & 0.2887 & 80.41911 & 0.35 .47 \\
\hline 75.44 .1 & 01723 & 91.5727 & (1) 188. & $9812(0)$ & 0.2887 & 77.9581 & 0.3301 .4 \\
\hline 77.5871 & $(1.170)$ & 938365 & 0.1484 & $98.12(x)$ & 0.2887 & 75.4111 & 0.30 .43 \\
\hline $8\left(12^{2} 16\right)$ & $0.1 \times 25$ & $66.1\left(x^{\prime}\right) 1$ & $0.2(1) 4$ & 95.5912 & 0.2826 & 72.8231 & $0.302\}$ \\
\hline $82\{31) 1$ & $0.1 \times(16)$ & $97.9(14.2$ & 0.17111 & $0.3 .12(x)$ & 11.2587? & $702 \times 91$ & $(13080$ \\
\hline$\left.\therefore 4 k^{\prime}\right\}(! 1$ & $(1.16+1$ & & & $40.66,75$ & 0.2572 & 67.7281 & 0.29 .30 \\
\hline 87.011 & 11.i7:! & & & 88.1271 & $0.253 k^{\prime}$ & 6.5 .1471 & 0.1344 \\
\hline $80.30,37$ & $0.1 \%(14$ & $S_{c}$ (a) 12, & 1258 priz: & 8.5 .57 .31 & $0.25(x)$ & 62.5531 & 0.25 .94 \\
\hline 41527 & 01755 & & & 83.01451 & 0.2609 & 600.0311 & 0.24 .73 \\
\hline $43 \times 365$ & 0.1198 & $98.12(n)$ & 02.4 .01 & 80.4411 & 0.2673 & 57.4011 & 0.2348 \\
\hline $96.1(x) 1$ & 0.1871 & $98.12(x)$ & 0.2 .1501 & 77.9581 & 0.2503 & 54.8021 & 0.2124 \\
\hline \multirow[t]{5}{*}{$979 \times 112$} & 0.16 .10 & $98.12(x)$ & 0.2 .450 & $79+i i i$ & 0.2 .356 & $52.278 !$ & 0.1896 \\
\hline & & 95.59 .32 & $0.2 \cdot \$ 0.3$ & 72.82 .31 & 0.2 .141 & 496341 & 0.1684 \\
\hline & & $43.12(x)$ & 0.2264 & 70.2841 & $0.24+1$ & 460901 & 0.1578 \\
\hline & & 90.06175 & $0.22(k)$ & 67.7281 & 0.235. & 44.4141 & 0.1535 \\
\hline & & 881271 & 0.2146 & 6.5 .1471 & 0.213 .4 & 41.7671 & 0.1209 \\
\hline \multirow{2}{*}{\multicolumn{2}{|c|}{ Saan 11, 120.4 pon: }} & 85.57 .31 & 0.219 .4 & 63.5531 & $0.20(0) ?$ & 39.13 .31 & 0.1019 \\
\hline & & 83.(4.51 & 0.23 .52 & $6 \times 10311$ & 0.1864 & 36.48 .51 & 0.0784 \\
\hline $98.121 k !$ & $(1.2127$ & 80.4911 & 0.2289 & 57.40 .21 & 0.1917 & 33.836 .1 & (1) (k).4t \\
\hline $08.12(k)$ & 11.2127 & $77.05 \times 1$ & $0.21 .4 k$ & 548021 & 0.1722 & $3 ! \cdot 6+51$ & 0.0512 \\
\hline$O K 12 C$ & 0.2127 & $75.411 i$ & 0.211 .2 & 52.2261 & 0.1545 & .3 .3 .1131 & $0.07(1) ?$ \\
\hline $05503 ?$ & 0.21157 & 72.8231 & 0.2010 & $44.6 .3+1$ & 0.1 .371 & $35.35 \% 1$ & $0.08(1)$ \\
\hline $03.12(x)$ & 01440 & 702891 & 0.2121 & $40.490 ! !$ & 0.1323 & 37.4091 & 0.0966 \\
\hline $90.6 x=5$ & 018,11 & 67.7281 & $0.190(1)$ & .14 .40 .31 & $0.12+11$ & 39.6871 & $0.110 \mathrm{~s}$ \\
\hline 881271 & 0.18 .57 & 651471 & 01875 & 41.7671 & 0.1122 & 41.8971 & 0.1462 \\
\hline 185.5721 & 0.180 .3 & 6. $553 !$ & $0 ! 721$ & $30.1 \% 11$ & 0.0957 & 44.1211 & $(1.158 .4$ \\
\hline 831691 & A2iiil & 60.0 .311 & $0.16+19$ & $3(1.4855$ & $(1,(10,30)$ & $46, .3301$ & 0.1715 \\
\hline 80.4911 & $0.1(x)()$ & 57.4021 & 0.17211 & 33.8361 & 0.05 .36 & 48.58011 & 0.1794 \\
\hline 71.4581 & 0.180 .7 & 54.8021 & 0.15 .32 & 31.6451 & o.otion & 50.7741 & $0.1 \times 91$ \\
\hline 75.4111 & 0.1754 & $\{22 \pi x\}$ & $0.13 k 4$ & 3.1131 & $0 .(x, 72$ & $53 .(1) 21$ & 0.2101 \\
\hline 128211 & $(1.1 / 1 / x$ & $49,0.41$ & 0.1211 & $39.19 / 1$ & $(1)(x, 14)$ & 59.2351 & U.2. 10 \\
\hline
\end{tabular}




\section{Reinforced Concrete Test Data}

Track 1 - High Pressure Test

\begin{tabular}{|c|c|c|c|c|c|}
\hline $574 \times 1$ & $(2+1:$ & 77.6871 & 0.47 .36 & 47.9012 & 0.8 .339 \\
\hline $507+11$ & $11: 4.4$ & 80.3161 & 0.4878 & & \\
\hline 0140,51 & $112<c_{1}$ & 82.5391 & $0.5(x) 2$ & \multicolumn{2}{|c|}{ san 17.0 psig (jxisttest) } \\
\hline $\operatorname{tin} 2(1) 11$ & (1) 1911 & 84.8301 & 0.5055 & & \\
\hline $6 x+2.41$ & 112.014 & $87 .(x) 31$ & 0.4488 & 98.1200 & $0.7 \times 39$ \\
\hline $687(16)$ & $1131 \times 2)$ & 89.36 .34 & 0.5088 & $98.12(x)$ & 0.586 .3 \\
\hline $7\left(i 4_{2}^{2}: 1\right.$ & 12111 & $91 \leq 12 \%$ & 0.3 .1 .5 & 97.7 .348 & 0.5869 \\
\hline 77 ini & $1141: ?$ & 93.8365 & 0.5456 & 95.5932 & 0.5820 \\
\hline $7544 i$ & 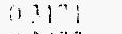 & $90 .|(x)|$ & 0.5507 & 93.1200 & 0.56 .29 \\
\hline$P+\cdots+1$ & $1: \because \cdots$ & 07.0042 & 0.5723 & 90.6675 & 0.5400 \\
\hline $801 ?-1+1$ & $1: 4.4$ & & & 88.1271 & 0.5142 \\
\hline$\ddot{n}=+1$ & 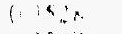 & \multirow{3}{*}{\multicolumn{2}{|c|}{ Scan 10, 1.958 psig }} & 85.57 .31 & 0.5026 \\
\hline 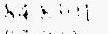 & 1.19 .4 & & & 83.0451 & 0.50111 \\
\hline$x^{*}$ ind: & $\therefore:|x| 1$ & & & $80.4 ? 11$ & 0.400 .3 \\
\hline$s i$ is. : & 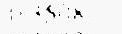 & $98.12(x)$ & 0.8 .309 & 77.95811 & 0.45 .15 \\
\hline $41 \div$ & "1+.2: & $98.12(x)$ & $0.83(19)$ & 75.4111 & 0.412 .3 \\
\hline $4: 406$ & 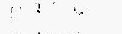 & $98.12(x)$ & $0 . R\}\langle k\rangle$ & 72.8231 & 0.391 .4 \\
\hline$\because 1 \times 1$ & $\because \therefore \cdots$ & 95.5932 & 0.8214 & 70.7891 & 0.38 .15 \\
\hline+1 & $24:=$ & $\begin{array}{l}93.12150 \\
90.6675\end{array}$ & $\begin{array}{l}0.81129 \\
0.7 \% 62\end{array}$ & 67.7281 & 0.3576 \\
\hline & & $\begin{array}{l}90.6675 \\
88.1271\end{array}$ & $\begin{array}{l}0.7762 \\
0.7531\end{array}$ & $\begin{array}{l}6.5 .1471 \\
62.5531\end{array}$ & $\begin{array}{l}0.3288 \\
0.2474\end{array}$ \\
\hline$\because \ldots t \quad 1^{k}$ & 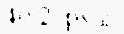 & 85.5731 & 0.7427 & $6(1.0 .311$ & 0.2741 \\
\hline & & 8.0 .451 & 0.7393 & $57.4(12)$ & 0.2611 \\
\hline$\because+1$. & $11<e n$ & 80.4911 & 0.728 .3 & $5.4 .8(1) 1$ & $0.2,3,30$ \\
\hline 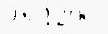 & $.41, \ldots$ & 77.9581 & 0.686 .5 & 52.2281 & $(0.1902$ \\
\hline 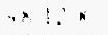 & $=n+n$ & 75.4111 & 0.6401 & 49.6 .371 & 0.1758 \\
\hline 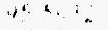 & $1145 \%$ & 72.8231 & 0.6161 & $46.99(1)$ & $0.16 .5 !$ \\
\hline w $12 *$ & $(153), 1$. & 70.2891 & 0.6039 & $4.4 .4(1)+1$ & 0.1512 \\
\hline$\because i, 1, \cdots$ & $1,9$. & 67.7281 & 0.572 .4 & $41.76 ? 1$ & 0.1207 \\
\hline b. $61: 1$ & {$[: 4+1)$} & 65.1 .171 & 0.5221 & 39.1331 & 0.0001 \\
\hline$x^{2}+4$ & $\because 41+9$ & 6.2 .5531 & 0.4059 & 36.4851 & $0.06,49$ \\
\hline$x: 451$ & $(1<$ injiti & $60(1311$ & (i. $1 \in \times)^{-1}$ & 33.8361 & 0.0572 \\
\hline$n: 11 !$ & $11+14$ & $57.4(121$ & 0.48 si. & $316-151$ & 0.0536 \\
\hline$\cdots+15$ & $11.1: 1$ & $54.8(12)$ & 0.3941 & 31.1131 & $0.16,37$ \\
\hline$\because<1111$ & $(1+111)$ & $52.2 \times 1$ & 0.2551 & 35.3577 & 0.0730 \\
\hline$\therefore \because 1$ & $(1.1251)$ & 40.6 .341 & 0.311 .3 & $37.4(1) 19$ & $\left(1.0 x^{3} y\right)$ \\
\hline$\therefore \therefore \infty^{\prime}$ & $11+2.40$ & $46.90(1) 1$ & 0.2816 & $3068=1$ & 0.1131 \\
\hline A, $\cdots !$ & 114139 & 44.40 .11 & 0.2598 & 118471 & 0.1423 \\
\hline$+\left\{14^{-1}\right\}$ & $027 \% 7$ & 41.7671 & 0.2226 & $44.132 !$ & $0.15(k)$ \\
\hline$f_{2} \leq<\div$ & $(1+4,60)$ & 39.1331 & 0.176 .8 & 46.3301 & $0.166 \mathrm{~m}$ \\
\hline $4 x: 1: 11$ & (1) 3..8 & 36.4851 & 0.1327 & 48.58011 & 4.1771 \\
\hline $57 \div 1$ & 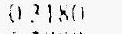 & 3.3 .8 .361 & 0.10151 & 50.7741 & (1)19is \\
\hline $4+x i) \geq 1$ & 0.2sing & 31.6451 & $0.0^{-1} ; 61$ & 5.101..1 & 0.2216 \\
\hline $52: 2 \times 1$ & 0.2510 & 33.1131 & 0.1080 & 55.2 .351 & 0.2477 \\
\hline 406211 & $(1 \div 101$ & $35.35 ? !$ & 0.1 .153 & $\left.57.478^{\prime}\right]$ & 0.2629 \\
\hline $4(, 4)(1)$ & $(12(1) x$ & 37.4991 & 0.1676 & 59.7411 & 0.2765 \\
\hline $4 \div \mid(1) 1$ & (1) 1437 & 39.6871 & 0.20 .50 & 61.9651 & 0.2907 \\
\hline \pm 101 & id $10+5$ & $41 \times 271$ & 0.24 .54 & $6+.2011$ & 0.346 .3 \\
\hline 201123 & (1) 1.325 & $44.13,31$ & 0.265 .5 & $(x+42+1)$ & 0.3 .4 .36 \\
\hline$x_{1}+\mathrm{p}^{9} \mathrm{i}$ & 011115 & $46.3 .3(1)$ & 0.29 .40 & 68.7081 & 0.9677 \\
\hline$\because\{3(1)$ & $0(1 \times x+1$ & 48.58011 & 0.213 .3 & $70.42+41$ & 0.3805 \\
\hline 31451 & 018.2 & $5(0.77 .41$ & $0.3 .40 k)$ & 73.1571 & 0.390 .1 \\
\hline 321111 & $0(1 \times 7)$ & $53(112)$ & $0.3 \times 17$ & 75.4 .41 & $(1.2225$ \\
\hline 353571 & 0.111201 & 55.2351 & 0.4194 & 77.68871 & 0.4493 \\
\hline $37+4191$ & $0.126 \%$ & $\$ 7.4781$ & 0.44012 & 80.3161 & $0.48(1)$ \\
\hline $30+2.7$ & (1) 1554 & 59.7411 & 0.46770 & 82.5391 & 0.494 .3 \\
\hline $41.80 \div 1$ & $0.15 \varsigma 0$ & 61.9651 & $0.48 \times 7$ & $x 4: ? \div !$ & $0 . .40 x$ \\
\hline 4.81 .3 .11 & $0.2(x) 7$ & 64.2011 & $056 \sin$ & 87.01? & 0.5111 \\
\hline $40,33(1)$ & 0.21 .50 & $(0.42 .11$ & 0.5614 & 80.36 .34 & 0.5246 \\
\hline $42<00:$ & (1.). & $68.7(18.1$ & 0.5801 & 91.5727 & 0.5152 \\
\hline 511.7741 & 0.2486 & 709241 & 0.6161 & 93.8 .365 & $0.56+3$ \\
\hline $53(1) 21$ & 0.270 .8 & 731871 & 0.6221 & $96.10(1)$ & 0.5777 \\
\hline $5 \% 224 i$ & $0.3(k, 8$ & 75.4411 & 0.6519 & 97.9042 & 0.59 .57 \\
\hline $57 \times 1 \times 1$ & 0.124 & 77.6871 & (1) 6156 & $98 .(17) 27$ & $\left(0.546_{1}\right.$ \\
\hline 54,711 & 0.3330 & $8(1.3161$ & 0.718 .5 & 98.0793 & (0.5ysi) \\
\hline $5 ! \times<1$ & $(1.3425$ & 82.5341 & 0.73 .8 & & \\
\hline thd $2(1) 1$ & 0.4157 & 8.48 .3018 & 0.7454 & & \\
\hline$(x, 424)$ & 0.3040 & 87.0931 & 0.7 .18 ? & & \\
\hline tit. $7(1) 1$ & 0.4145 & 83.363 .4 & 0.70 .30 & & \\
\hline $7(1) 192.11$ & 0.1305 & $0: 572 \%$ & 0.78 .311 & & \\
\hline 73.1871 & $(1.4121$ & 93.8 .365 & $0.8111)$ & & \\
\hline 75.441 & 0.4488 & $96.100(1)$ & $0 \times 192$ & & \\
\hline
\end{tabular}




\section{Reinforced Concrete Test Data}

Track 2 - High Pressure Test

\begin{tabular}{|c|c|c|c|c|c|c|c|}
\hline \multicolumn{2}{|c|}{ Sean 1,44.2 psig } & 118.8904 & 0.0752 & 114.42214 & 0.0947 & 2363204 & 0.0914 \\
\hline 287.2670 & 00594 & $\begin{array}{l}116.6004 \\
114.2204\end{array}$ & $\begin{array}{l}0.0825 \\
0.0918\end{array}$ & $\begin{array}{l}116.8504 \\
119.404\end{array}$ & $\begin{array}{l}0.0917 \\
0.08 .10\end{array}$ & $\begin{array}{r}236.8914 \\
2375004\end{array}$ & 0.0887 \\
\hline 287.2 .128 & $0.0<01$ & 111.6904 & 0.08 .37 & $121.6(x) 4$ & 0.0826 & 238.0754 & 0.0821 \\
\hline 286.7603 & 0.0528 & 109.2604 & 0.0857 & 12.401014 & 0.0785 & 238.6464 & $0.08 .4 ?$ \\
\hline 284.3547 & $0.096 x^{\prime}$ & 106.930 .4 & 0.0804 & $126.44(14$ & 0.0838 & 239.2594 & 0.0847 \\
\hline 281.4904 & 0.07166 & 104.7404 & 0.0899 & 128.7304 & (1) 0.801 & 239.5444 & 0.08618 \\
\hline 278.6584 & 0.0739 & 102.1504 & 0.0910 & 131.1904 & 0.0833 & 240.4724 & $0.091,3$ \\
\hline $276.30 \mathrm{kH}$ & $0,06,32$ & 99.7004 & 0.0954 & $133.75(1.4$ & $0.10 \times 2$ & 241.0544 & 0.0913 \\
\hline 272.7274 & 0.05 .32 & $97.50 \times 14$ & 0.1069 & 136.1704 & 0.0768 & 241.5854 & 0.0045 \\
\hline 271.2704 & 0.0620 & 94.9704 & 0.0989 & 138.6704 & 0.0650 & 242.1464 & $0 .(4) 59$ \\
\hline 268.7494 & 0.0686 & $92.62(04$ & 0.0913 & 140.9104 & 0.0584 & 242.789 .4 & 0.0974 \\
\hline 266.2594 & $0.0 \times 384$ & 90.2 .314 & 0.0968 & 143.4004 & 0.0709 & 243.4024 & $0 .(19+40$ \\
\hline $263.83 \mathrm{~cm}$ & 0.0738 & 87.9104 & 0.1092 & 145.8304 & 0.0751 & 244.0134 & 0.01899 \\
\hline 261.2794 & 0.0698 & 85.6004 & 0.0995 & 148.0504 & $0.04688^{\circ}$ & 244.6254 & 0.08901 \\
\hline 258.768 .4 & 0.0793 & 83.2004 & 0.0920 & 150.5804 & 0.0759 & 245.1554 & 0.0856 \\
\hline 256.3614 & 0.0810 & 80.7304 & 0.0922 & 152.8904 & 0.0699 & 245.768 .4 & 0.0896 \\
\hline 25395524 & 0.0887 & 78.14014 & 0.0956 & 155.380 .4 & $0.06+5$ & 246.2994 & $0.08+1$ \\
\hline 251.4014 & 0.08 .31 & 75.6204 & 0.09013 & 157.5604 & 0.0647 & 246789.4 & 0.0826 \\
\hline 2489524 & 0.0725 & 73.09094 & 0.0870 & 159.8704 & 0.0618 & 247.3814 & 00797 \\
\hline 246.5 .93 .4 & 0.0830 & $70.52(14$ & 0.0871 & $162.1(x) 4$ & 0.0702 & 247.9734 & 0.0780 \\
\hline $2+4.1154$ & $0 \cap 924$ & 67.9704 & 0.0815 & 164.5204 & 0.06801 & 248.5854 & 0.0770 \\
\hline 241.58 .54 & 0.040 .3 & $6:+604$ & 0.09012 & 167.0104 & 0.0719 & 249.1774 & $0.08(k)$ \\
\hline 238.902 .4 & 0.08115 & 624504 & 0.0829 & $169.46(1.4$ & 0.0795 & 249.7 .184 & 0.0788 \\
\hline $236.7(184$ & 0.0827 & $6(1) .50(14$ & 0.1798 & 171.8304 & 0.0916 & $250.4 ?(144$ & 0.0801 \\
\hline 234.2044 & 0.0int? & $57.40(1) 4$ & 0.0893 & 174.010 .4 & 0.06 .58 & 250.9324 & 0.0820 \\
\hline 231.83024 & 0.01598 & 55.6104 & $0.06 \times 2$ & $170.5+14$ & 0.0681 & 251.5244 & 0.08201 \\
\hline$\because 92614$ & $0 .(1),(13$ & 53.0904 & 0.06194 & $179.16(4)$ & 0.0625 & 252.1164 & 0.0815 \\
\hline 226.9524 & 0.086 .1 & $5(1.2404$ & 0.0627 & 181.5204 & 0.0622 & 252.7274 & 0.018 .11 \\
\hline 2344834 & 0.0894 & 47.7804 & 0.0587 & 183.91114 & $0.07(1)$ & 253.3194 & 0.0867 \\
\hline 2220.344 & 0.08 .56 & $44.93(14$ & 0.05 .22 & 186.381 .4 & 00751 & 25391114 & 0.0823 \\
\hline 2196.244 & 0.188 .4 & $42.27(14$ & 0.0 .346 & 188.931 .4 & 0.08 .28 & 254.5224 & $0.0 \% ! 4$ \\
\hline 216.911 .4 & 0.086 .5 & 39.8 .304 & 0.04 .35 & 191.3384 & 0.0817 & 255.1304 & 0.08 .32 \\
\hline 21.15644 & 0.080 .1 & 37.38014 & 0.0 .365 & 193.8684 & 0.09 .34 & 255.7284 & 0.0835 \\
\hline 212.19744 & 0.0727 & 35.1304 & 0,0319 & 146.3614 & $0.08+3$ & 256.3404 & 0.01856 \\
\hline $2(x) .828 .4$ & 0.0689 & $32.6(x) \leqslant$ & 0.0111 & 198.1544 & 0.0841 & $256.87(1.4$ & $0.08 \cdot 45$ \\
\hline 207.4634 & $0 .(14) ?$ & 30.2404 & 0.02015 & 199.9324 & 0.0873 & 251.5854 & 0.08 .30 \\
\hline 205.115 .4 & 0.1876 & $29.42(14$ & 0.0157 & 201105.34 & 0.0876 & 258.1 .354 & 0.0828 \\
\hline $202 \operatorname{sen}(x)$ & 0.01533 & $31.87(14$ & 0.02013 & 202.1754 & $0.09 \times 10$ & 258.6654 & 0.0818 \\
\hline $2(0) .3194$ & 0.0864 & .345264 & 0.0247 & $203.34(14$ & 0.0853 & 259.2594 & 0.0829 \\
\hline 197.4714 & 0.0755 & 369704 & 0.0 .3 .32 & 204.521 .4 & 0.08 .46 & 259.8714 & 0.1829 \\
\hline 195.5654 & 0.0780 & 39.6304 & 0.01 .22 & 205.6674 & $0.09+2$ & $260.5+44$ & 0.0829 \\
\hline $193 .(1524$ & $0.0 \% 100$ & 42,1704 & 0.0413 & $206.800) .4$ & 0.1011 & 261.1574 & 0.0840 \\
\hline $19(16074$ & $0.0180 ?$ & 44.7304 & 0.0561 & 2017.9724 & 0.0927 & 261.7084 & 0.0839 \\
\hline 188.2794 & 0.0770 & 47.180 .4 & $0 .(666) 0$ & 209.01754 & 0.0701 & 262.36014 & 0.0828 \\
\hline $185.77 \mathrm{CH}$ & 0.0708 & 49.8304 & 0.0656 & 210.2174 & $0.06,95$ & 262.9534 & 0.0798 \\
\hline $183.36,64$ & $0.06+16$ & 52.2804 & 0.0744 & 211.3384 & 0.0792 & 263.5234 & 0.0775 \\
\hline 180.9304 & 0.0586 & 54.8704 & 0.0722 & 212.4424 & 0.0832 & 264.1364 & 0.0769 \\
\hline 178.4 .404 & 0.06 .24 & $5722(14$ & 0.1019 & 213.6854 & 0.0808 & 264.7284 & 0.0751 \\
\hline 175.6704 & 0.0550 & 39.62614 & 0.0840 & 214.8104 & $0.08+0$ & 265.340 .4 & 0.0786 \\
\hline 173.20004 & 0.0548 & $61.97(14$ & 00812 & 215.9114 & 0.0891 & 365.8914 & 0.0797 \\
\hline 170.9104 & 0.0739 & 64.3504 & 0.0886 & 217.0344 & 0.0902 & 266.4424 & 00797 \\
\hline 167.8504 & 0.0624 & 66.7104 & 0.0938 & 218.1554 & 0.08 .38 & 266.9724 & 0.0795 \\
\hline $165.420 \mathrm{~s}$ & $0.060+1$ & $69.1: 04$ & 0.03251 & 219.48 .34 & $0.09+1$ & 267.5854 & 0.076 .5 \\
\hline $162.87 \mathrm{ks}$ & 0.0623 & 71.4204 & 0.01945 & 220.6044 & 0.0944 & 208.1364 & 0.0738 \\
\hline $160.40(4)$ & 0.0654 & 7.3 .8504 & 0.0980 & 221.8714 & 0.0880 & 268.728 .4 & $0.06 \mathrm{hit} 4$ \\
\hline $1.57 .990 \mathrm{M}$ & 0.0544 & 10.160 .1 & 0.159 .49 & $223(114.4$ & 0.0868 & 269.4014 & 0.0670 \\
\hline 155.7504 & $0.0 \times 122$ & 78.5204 & 0.0977 & 224.1974 & 0.0875 & 270.0754 & Ontwiti \\
\hline $15309(14$ & $0.08 \%$ & $80.80 \times 4$ & 0.092 .3 & 225.5854 & 0.0914 & 270.6874 & 0.0710 \\
\hline $1506,90 !$ & 0.0574 & 83.2804 & 0.096 .5 & 226.9114 & 0.0942 & 271.3004 & $0071 \%$ \\
\hline $1.48 .10 \times 4$ & 01.014 .34 & $85.60 \times 14$ & 0.01996 & 228.0744 & 0.0977 & 271.8304 & 0.0742 \\
\hline 145.8504 & $0.074 y$ & 87.0404 & 01055 & 229.2594 & 0.09018 & 272.4424 & 0.0710 \\
\hline 14.3 .3204 & $0(x) 5 \theta$ & 90.3104 & $0.110 ; 3$ & 230.5244 & 0.19426 & 272.953 .4 & 0.06286 \\
\hline 140.770 .4 & $0(x+16)$ & 92.7504 & $0.10: 3$ & 231.1164 & 0.0924 & 273.5444 & $0.06 x+6$ \\
\hline 1.38 .3414 & $0.0 x, 5 x$ & 95.0904 & $0.10 i 1$ & 231.7084 & 0.0895 & 274.1564 & $0.06+3$ \\
\hline 135.85614 & 0.076 .9 & 97.6704 & 01040 & 232.2504 & 0.0926 & $27 \$ 7084$ & 0.0645 \\
\hline $133.76(4$ & O.okiki & 408704 & 0.10111 & 232.5144 & 0.0926 & 275.3 .40 .4 & 0.0612 \\
\hline 131.0704 & 10728 & 10123804 & $0(x) 54$ & 233.462 .4 & 0.0865 & 275.9524 & 0.06701 \\
\hline $1.8 .50 \times 14$ & $0.07(x)$ & 104.89014 & $\left.0 .(x) x_{12}\right)$ & 234.0554 & 0.0846 & 2766264 & 0.0675 \\
\hline $126.26 \times 19$ & $0.081 x$ & 107.0004 & $0,(x) \geq 24$ & $2346 \times 64$ & 0.0886 & $.277 .2 .3(44$ & $0 .(x \leq 5)$ \\
\hline $123.99(44$ & 0.0715 & 109.540 .4 & $0 .(x) 05$ & 2352584 & $0 .(k) 11$ & 277.8814 & $0.04+45$ \\
\hline $121.120 \mathrm{M}$ & 0.0176 .3 & 111.800 .4 & 00895 & 2.58 .8074 & ก. (100) 5 & 278.4509 & 0.06 .77 \\
\hline
\end{tabular}




\section{Reinforced Concrete Test Data}

Track 2 - High Pressure Test

\begin{tabular}{|c|c|c|c|c|c|c|c|}
\hline 279.1427 & $0.07(x)$ & $1(x)+11 \times 4$ & 0.0814 & $\because \because 4$ & 111162 & 221.8714 & 0.1109 \\
\hline 279.7590 & 0.0677 & $15: 4 \times 14$ & (1) 0803 & $70 \mid 1 \times w$ & 0112 & 223.0144 & 0.1084 \\
\hline 280.3917 & 0.0752 & 1557504 & 0.0840 & $78<24$ & 0118.3 & 224.1974 & 0.1077 \\
\hline 281.0345 & 0.0819 & $153(x)(14$ & 0.1012 .3 & का मानाम & (1) 11 th & 225.5854 & 0.1109 \\
\hline 281.6305 & 0.0801 & 15060004 & 0.0706 & $83: 914$ & 01153 & 226.9114 & 0.1157 \\
\hline 282.2366 & 0.0747 & $1+8.16 \times 4$ & 0.0741 & $856 x+4$ & 0.1180 & 228.0744 & 0.118 .8 \\
\hline 282.8631 & 0.15 .37 & 1458504 & 0.0974 & $x^{\prime \prime} y 514$ & $(1) \times 177$ & 229.2594 & 0.1141 \\
\hline 283.53 .46 & 00089 & 14.3 .2144 & 0.0807 & $4(1) ? 114$ & (1) 1208 & 230.5244 & 0.1180 \\
\hline 284.2896 & $0.0 \times 18.5$ & 141.7704 & 0.013 .37 & $42-5(H$ & 0.12 .77 & 231.1164 & 0.1155 \\
\hline 285.0120 & $0.06-17$ & 1.383 .404 & 0.0844 & of rovit: & 0.1216 & 231.7084 & 0.1115 \\
\hline 285.6183 & 0.0588 & 1358.514 & 0.0956 & yit.iul & 01207 & 232.2594 & 0.1145 \\
\hline 285.2792 & 0.06 .32 & $133.36(4)$ & 0.1168 & $(4) \times x^{\prime \prime}(\mu)$ & 01258 & 232.8504 & 0.1159 \\
\hline 286.8976 & 0.0632 & $13161 \%$ & 0.09 .41 & $11: 22814.4$ & 01104 & 233.4624 & 0.1149 \\
\hline 287.1877 & 0.0656 & $120.5(k) 4$ & 0.0960 & $1(4) \times x)$ & (1)11kt & 234.0554 & 0.1176 \\
\hline 287.2046 & O.iii(1) & $126.20(1-4$ & 0.10 .49 & I07.112kit & $(1.1)(x)$ & 234.66664 & 0.1170 \\
\hline \multirow[t]{2}{*}{2.87 .1425} & 0.07111 & $12 ? .74014$ & $0(x)(1 . ?$ & $f(1)<40.4$ & (1) 1121 & 235.2584 & 0.11 .57 \\
\hline & & 1214214 & $0 .(x) .35$ & $1114+14$ & (i $i(x))$ & 235.8044 & 0.1140 \\
\hline \multirow{2}{*}{\multicolumn{2}{|c|}{ Scan 2, 51.6 psig }} & $118 \times 90(1.4$ & $0 .(x) \geq 1$ & $11+4211$ & (i. 1131$)$ & 236.320 .4 & 0.1125 \\
\hline & & $116+x(x) .4$ & 0.1014 & 110.55114 & $01(x, 7$ & 236.8914 & 0.1104 \\
\hline 287.2670 & 0.0605 & 1142204 & 0.10190 & $1191+14$ & () $1(x) 7$ & 237.5024 & 0.1077 \\
\hline 287.2428 & $0.06(x)$ & $1116,1)(1.4$ & 0.10 .42 & $1216 x+4$ & 0.10137 & 238.0754 & 0.1036 \\
\hline $286.76,93$ & $0 .(x ; 1)$ & $10 \mathrm{irin}$ & 0106.5 & 124011914 & $0.16 r)$ & 238.6464 & 0.1050 \\
\hline 284.3547 & 0.1254 & $11(x, 33112$ & 0.1052 & $1.2+4+11$ & $011 x_{1} ?$ & 239.259 .4 & 0.1070 \\
\hline 281.4999 & 0.0892 & 10.7404 & 0.1110 & $128 \div 3114$ & 018116 & 2.39 .8494 & $(1.1079$ \\
\hline 278.8584 & $0.08(x)$ & !(1: $) 5012$ & 0.1157 & $! 1 !:(x) !$ & $1111 \% 2$ & 240.4424 & 0.1116 \\
\hline $276.3(\mathrm{kH}$ & 0.0791 & $047(x+4$ & 0.1130 & $1: 37904$ & $0.1 .47 \%$ & 2410.544 & 0.1136 \\
\hline 273.7274 & 0.0748 & $07.5 \mathrm{ins}$ & 0.1253 & 136,1704 & $0 .(1+x) .3$ & 241.5854 & 0.1141 \\
\hline 271.279 .4 & 0.0840 & $94.47(14$ & 0.1173 & $19 \times 670.4$ & 0.01876 & 242.1964 & 0.115 .1 \\
\hline 26397494 & 0.0894 & $926.2(1.4$ & 0.11 .58 & 14010114 & (1) 17765 & 242.789 .4 & 0.1207 \\
\hline 266.2504 & 0.11900 & 90.2304 & 0.11 हून & $143.41 \times 1.4$ & 01952 & 243.4024 & 0.1128 \\
\hline $263.831(14$ & $0.0 \times 8 \times 7$ & 87.4101 .4 & 0.1316 & $1.4^{4} \times 301.4$ & 0.11007 & 244.0134 & 0.1119 \\
\hline 201.2794 & $0.0 y(x)$ & $85 n(x) 4$ & 0.1234 & $1.4 .018(1.4)$ & $(1076,7$ & 244.6254 & 0.11 .37 \\
\hline 258.7684 & $0.10(15$ & $8^{2}=2 x 14$ & $(1.1) \mid 81$ & $1: 215 \times 14$ & 0.0575 & 245.1554 & 0.11 .37 \\
\hline 256.3614 & 0.10117 & $80.7 ? 11.9$ & 0.1122 & 142 s. H. & 0.1049 & 245.7684 & $0.10 k 2$ \\
\hline 253.9524 & 0.1110 & 78.1111 .8 & 0.1153 & $155.3 \times 114$ & (1)(18) & 246.2994 & 0.1041 \\
\hline 251.4014 & $0.101 .3 k$ & 750.2014 & 0.116 .3 & $15 \operatorname{sen} 4$ & $(1071) 0$ & 2.46 .7894 & 0.10137 \\
\hline 248.4524 & $0 .(14.34)$ & 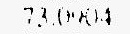 & 0.11 .43 & $15.08 \times 114$ & 0,01743 & 247.3814 & 0.1011 \\
\hline $246.5+34$ & $(1) 11(x)$ & $70.52(1.4$ & $0.107 \%$ & $\ln : 1(x) 4$ & $0.018+6$ & 247.97 .34 & 0.0998 \\
\hline $2+1.115 .4$ & $0.10 \times 4$ & 6707014 & $(1.0459)$ & $10+52(14$ & 0.08442 & 248.5854 & 0.01972 \\
\hline $2+1.585 .4$ & $0.10(4)$ & $65+1014$ & $01.10 x)^{2}$ & 167.01104 & 0.0878 & 249.1774 & 0.0980 \\
\hline 2389924 & 0.1020 & $0 \geq 0511.1$ & 0.0907 & $10,4)+(x) .4$ & $0.1(k) ?$ & 249.748 .4 & $0.10 \times 12$ \\
\hline 23671184 & 0.1019 & $6015.5(x+4$ & 0.0996 & $171.83(1.2$ & 0.11 .50 & 250.3204 & 0.1015 \\
\hline 231.209 .4 & 0.1102 & $57.40 \times 1.4$ & 0.1011 & 1241110.4 & 0.0818 & 250.9324 & 0.103 .4 \\
\hline 231.8364 & (1) $1(x-1$ & 3501014 & 0.0917 & $17+4+x) .1$ & 0.0394 & 251.5244 & 0.1034 \\
\hline 229.3614 & $0.111 ?$ & $5104(1.4$ & 0,0815 & $1.4|+x| .4$ & 0.0829 & 252.1164 & 0.1015 \\
\hline 2769524 & $0.11\left(k^{\prime}\right.$ & 11.2414 & 0.0701 & $3 \therefore \leq ? \div 14$ & $00 \times 15$ & 252.7274 & 0.1029 \\
\hline 224.40 .24 & 0.1074 & 41.780 .4 & $(107.49$ & $18=01(1.4$ & $(101899$ & 253,3194 & $0.107 t$ \\
\hline 222.03 .4$. & $0.10 \% 3$ & 41430.4 & 0.0627 & $15+38(1.4$ & $0(x) 15$ & 253.9114 & 0.10 .44 \\
\hline 219.6254 & (1) $11(1)$ & $42.2^{-1}(14$ & 0.0 .16 ? & $\operatorname{lin} 4214$ & 0.10 .27 & 254.5224 & 0.1001 \\
\hline 216.9114 & 0.1044 & 3083014 & 0.0502 & 141334 & 0.1040 & 255.1364 & 0.10114 \\
\hline 214.56 .41 & 0.10687 & 3738004 & $(0.614 \times 4)$ & lat sint & $(1,1024$ & 255.728 .4 & 0.1028 \\
\hline 212.0744 & $0 .(5), 37$ & 3513144 & $0(0) 12$ & $140,2011+4$ & $0.1(k, 8$ & 256.3404 & 0.1017 \\
\hline 204.8284 & $0.09(\mathbf{k})$ & $326(x) 4$ & 0.0197 & $10 x$ is 544 & $0.10 x+8$ & 256.8704 & 0.1011 \\
\hline 207.46 .84 & $(1.121)$ & 30.240 .4 & $0.02(1)$ & fow ylat & $0.11 \times 18$ & 257.5854 & 0.0998 \\
\hline 205.1154 & 0.1114 & $29+2(14$ & 0.0208 & 201101534 & 0.10182 & 258.1354 & 0.00997 \\
\hline $202.666 \mathrm{~m}$ & 0.1026 & 318714 & 11.0 .267 & $30: 734$ & (1. 10181 & 258.6654 & $0.0 \times 997$ \\
\hline 200.2194 & 0.10 .22 & $3+452114$ & 0.0359 & 203.2404 & 0.10170 & 259.2594 & 0.0971 \\
\hline 197.9714 & 0.0938 & 3697044 & 0.0428 & 214.5214 & 0.1056 & 259.8714 & 0.09887 \\
\hline 195.5654 & 0.0482 & 39.6304 & 0.0545 & $2(15.6(x) 4$ & 0.1151 & 260.5444 & 0.09997 \\
\hline 193.0524 & $0.10 \times(9)$ & 42.07014 & $0.0 .5(6)$ & $2(x, \sin () 4$ & 0.1242 & 261.1574 & $0.10 \times 6$ \\
\hline 190.6674 & $0.10 \mathrm{nn}$ & 44.7364 & 0.0709 & 2194924 & 0.1195 & 261.7684 & 0.1012 \\
\hline 188.279 .4 & $0.04 \times 1$ & 47.18144 & 0.0788 & 20090754 & $0.1000(1)$ & 262.3604 & 0.1006 \\
\hline 185.77124 & 0.0863 & 498.304 & 0.0766 & $211121: 4$ & 0.0995 & 262.9534 & 0.0964 \\
\hline 183.3604 & 0.0837 & 52.28614 & 0.0913 & 211338.4 & $0(1473$ & 263.5234 & $0.09 .4 i$ \\
\hline 180.9304 & 0.0829 & $54.87(14$ & 0.04 .41 & 2124424 & 0.1028 & 264.1 .364 & $0.09 ? 6$ \\
\hline 1784404 & 0.0892 & 57.2204 & (1) 1167 & 21.3054 & 0.1034 & 264.7284 & 0.0912 \\
\hline 175.6704 & 0.0867 & $59+2.14$ & 0.1120 & $21+\times 1114$ & $0.16 \mathrm{H})$ & 265.3 .104 & 0.0932 \\
\hline $173.2(x) 4$ & C. (N) 19 & 61.97111 & $0.1(x) 3$ & $215: 111$ & 0.1110 & 265.8914 & 0.0955 \\
\hline $170.91(4$ & (1.09k1) & 6435,14 & (1.)114.5 & $21-1124.8$ & $(1.111)$ & $26 \times 6.442 .4$ & $0 .(19) 60$ \\
\hline $167.850 \mathrm{k}$ & 0.0768 & $\left(x_{1}>1014\right.$ & 0.1110 & 2 areg & $(1) 11,5$ & 266.9724 & 0.0090 \\
\hline 165.420 .4 & 0.0846 & 64) 1104 & 0.1124 & $: 14+x: 1$ & $1111^{20}$ & 267.5854 & $0 .(14925$ \\
\hline $162.87(\mathrm{H}$ & 0.0919 & 71.9204 & $0.1: 34$ & $221: 0141$ & 011167 & $268.136 \mathrm{~A}$ & 0.0904 \\
\hline
\end{tabular}




\section{Reinforced Concrete Test Data}

Track 2 - High Pressure Test

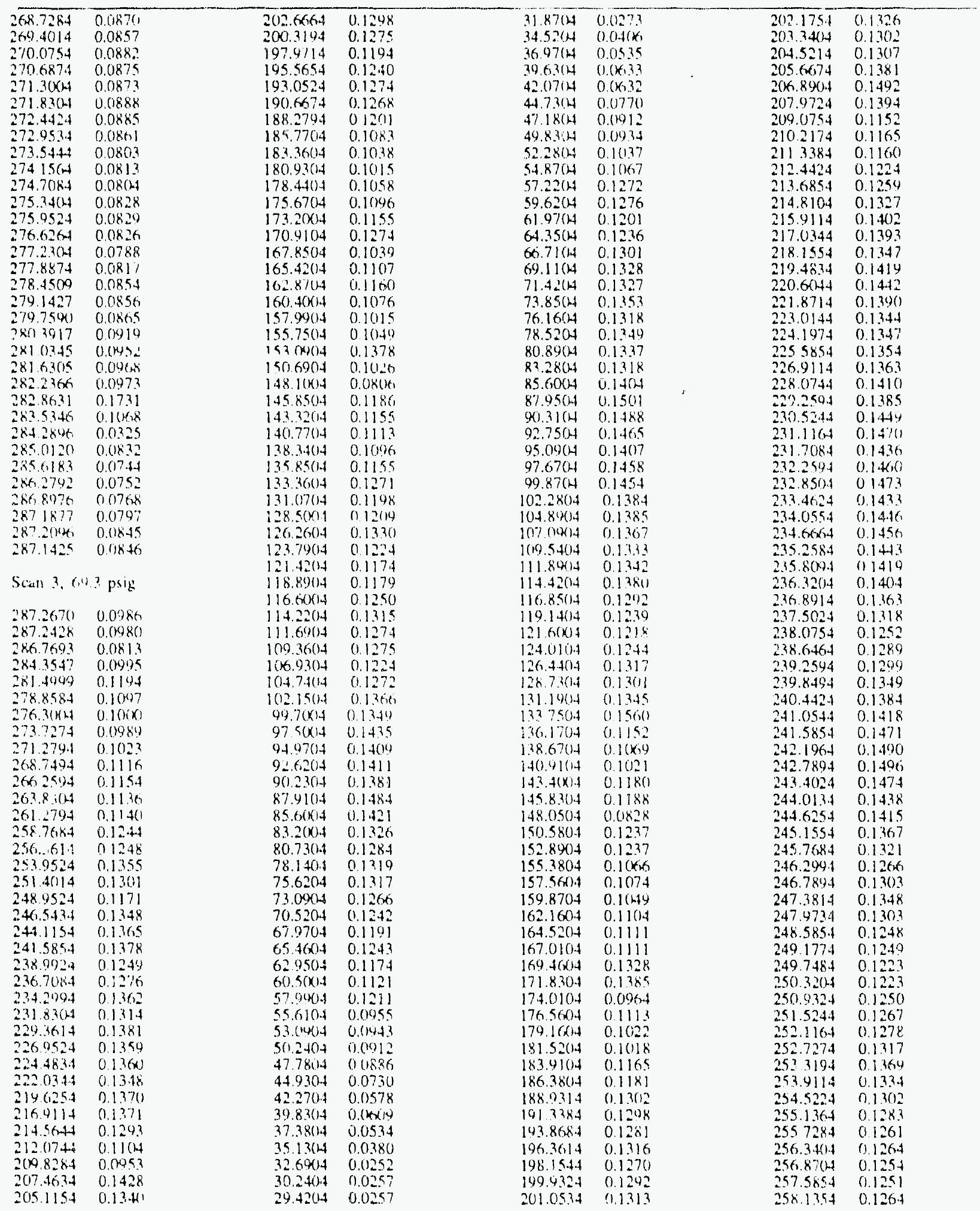




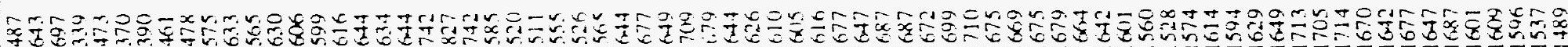

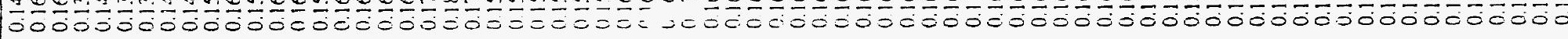

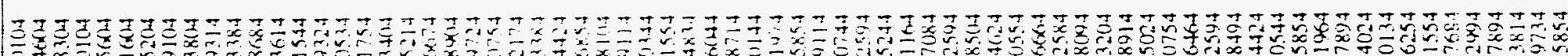

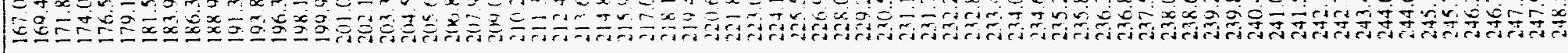

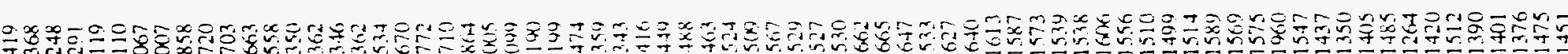
00000000000000000000000000000000000000000000000000000000000030000

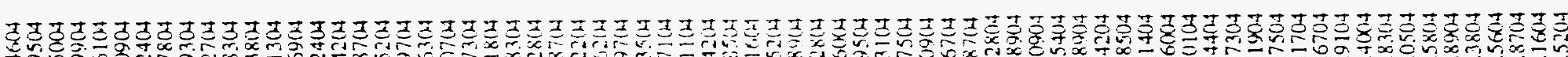

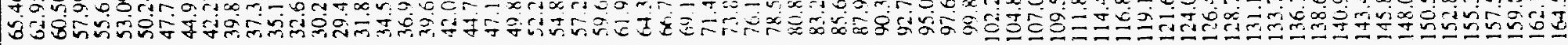

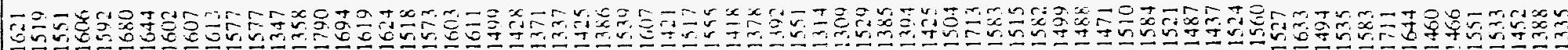
00000000000000000000000000005000000000000000000000000000000000000000000

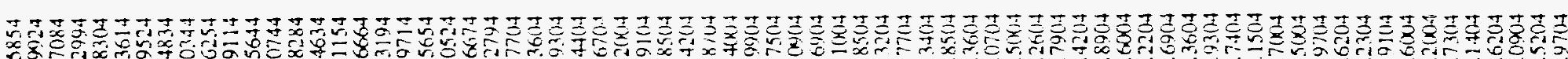

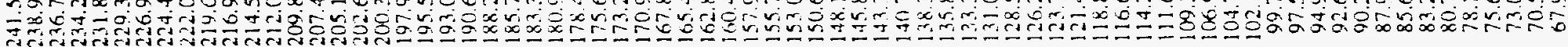

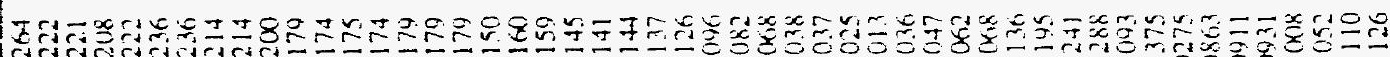

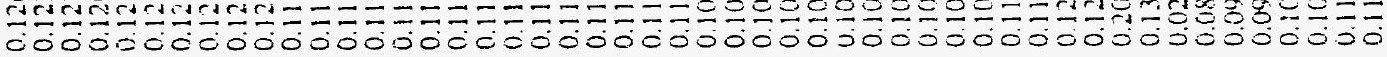

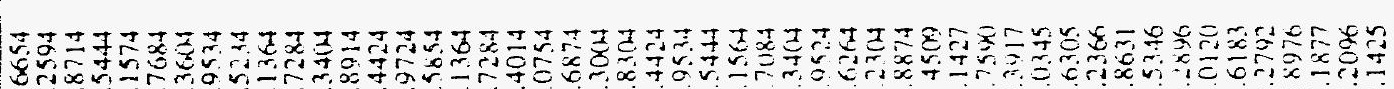

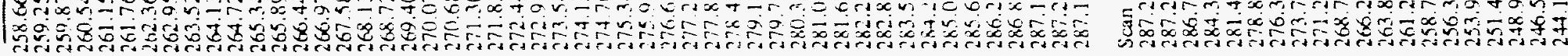

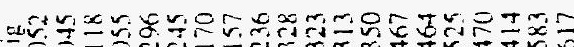
-

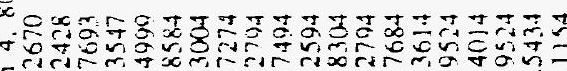




\section{Reinforced Concrete Test Data}

Track 2 - High Pressure Test

\begin{tabular}{|c|c|c|c|c|c|c|c|}
\hline 249.1774 & 0.1488 & 284.3547 & 0.1172 & 106.9304 & 0.1717 & 1264404 & 0.1862 \\
\hline 249.7484 & 0.1475 & 281.4999 & 0.1515 & 104.7404 & 0.1781 & 128.7 .304 & 0.1922 \\
\hline 250,3204 & 0.1489 & 278.8 .584 & 0.14 .20 & 102.1504 & 0.1841 & 131.1904 & 0.1941 \\
\hline 2509324 & 0.1514 & 276.3004 & 0.1345 & 99.7004 & 0.1841 & 133.7504 & 0.2338 \\
\hline 251.5244 & 0.1502 & 273.7274 & 0.1379 & $97.50 \times 14$ & 0.1910 & 136.1704 & 0.1724 \\
\hline 252.1164 & 0.1475 & 271.2794 & 0.14 (il & 94.9704 & 0.1813 & 138.6704 & 0.1608 \\
\hline 252.7274 & 0.1495 & 268.749 .4 & 0.1553 & $92.620 \mathrm{H4}$ & 0.1784 & 140.910 .4 & 0.1661 \\
\hline 253.3194 & 0.1542 & 266.2594 & 0.1554 & 90.2304 & 0.186 .3 & $143.4(024$ & 0.16 .25 \\
\hline 253.9114 & 0.1513 & 263.8304 & 0.1 .509 & 87.9104 & 0.1985 & 145.83014 & 0.18 .52 \\
\hline 254.5224 & 0.1406 & 261.2794 & 0.1552 & 85.6004 & 0.1842 & 148.0504 & 0.1573 \\
\hline 255.1364 & $0.14 \div 6$ & 258.7684 & 0.1709 & 8.3 .2004 & 0.1729 & 150.580 .4 & 0.1525 \\
\hline 255.7284 & 0.1448 & 256.3614 & 0.1726 & 80.7304 & 0.1759 & 152.8904 & 0.1978 \\
\hline 256,3404 & 0.1510 & 253.952 .4 & 0.18 .31 & 78.1401 & 0.1779 & 155.3804 & 0.1123 \\
\hline $2.56 .87 \mathrm{~cm}$ & 0.1446 & 251.4014 & 0.1675 & $75.62(14$ & 0.1798 & $157.56(04)$ & 0.1732 \\
\hline 257.5854 & 0.1489 & 248.9524 & 0.1631 & 73.0904 & 0.1601 & $159.87(44$ & 0.1714 \\
\hline 258.1354 & 0.1464 & 246.5434 & 0.1720 & $70.52(14$ & 0.1610 & 162.1604 & 0.1787 \\
\hline 258.66 .54 & 0.1482 & 244.1154 & 0.1911 & 67.9704 & 0.1573 & 164.5204 & 0.1750 \\
\hline 250.250 .4 & 0.14 .48 & 241.5854 & 0.1870 & 0.5 .40 .124 & 0.1621 & 167.01104 & 0.1872 \\
\hline 254.8714 & 0.1 .450 & 238.9024 & 0.1744 & 62.95014 & 0.1566 & $16,9.46(14$ & $0.1 \% 64$ \\
\hline 260154.44 & 0.1447 & 236.708 .4 & 0.17 .31 & $60.50(1)+$ & 0.1516 & 171.8304 & 0.2279 \\
\hline 2611574 & 014.59 & 234.2994 & 0.1853 & 57.99141 & 0.1580 & 174.0104 & 0.1657 \\
\hline 261.768 .4 & 0.14 .50 & 231.8 .304 & 0.1653 & 55.6114 & 0.1331 & 176.560 .4 & 0.1753 \\
\hline 262.3604 & $0.1+47$ & 229.3614 & $0.19(14$ & 53.0904 & 0.1284 & $179.1(0) .1$ & 0.1741 \\
\hline 28,20534 & 0.1419 & 226.9524 & 0.1850 & 50.2414 & 0.1274 & 181.5211 .4 & 0.1712 \\
\hline 20.2 .5234 & $0.1+10$ & 224.48 .34 & 0.1865 & 47.7804 & 0.1188 & 183.9104 & 0.1703 \\
\hline $26+13104$ & 0.13014 & 222.0344 & 0.188 .5 & 44.93114 & 0.0992 & 180.380 .4 & 0.174 .3 \\
\hline 264.7284 & $0.1 .7(x)$ & 219.6 .54 & 0.19 .48 & 42.2704 & 0.08 .56 & 188.9314 & $0.186 x$ \\
\hline $265.34(4)$ & 011384 & 216.9114 & 0.19119 & $39.83(\mathrm{kd}$ & (0)618? & 101.3384 & $0.195 ?$ \\
\hline $265 \times 914$ & $(1.1 .2(x)$ & 214.564 .4 & 0.1871 & $37.380 \mathrm{H}$ & 0.0741 & $193.66 \times 1$ & 0.14 .11 \\
\hline 266.4424 & $(1.13126)$ & 212.0744 & 0.1752 & 35.1304 & $0.0 \times 17$ & 190.3614 & 0.1081 \\
\hline $2(x, 4) 724$ & 0.1404 & 2019828.4 & 0.1721 & 326004 & 0.01 .44 & 198.194 .1 & $0.1 \times(x)$ \\
\hline 161.5854 & (1. 1.344 & $2(17,46.34$ & $(0.2 ! 00)$ & 30.240 .4 & 0.1496 & 149.4 .124 & 0.1910 \\
\hline hax $136 \mathrm{~d}$ & 0.1340 & 2015.1154 & 0.2013 .3 & $29+234$ & $0.047 k$ & $2(11.010 .3 .1$ & 0.1047 \\
\hline $26872 \times 4$ & $0.1 .35 i$ & 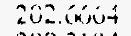 & ‥1800 & $31.87(4$ & $0.05(1)$ & $2(12.1754$ & $010 ? 1$ \\
\hline $2(19) 4014$ & 0.1354 & 200.3104 & $0.184 !$ & 34.5214 & 0.0612 & $203.3+14$ & 0.1057 \\
\hline 270.0754 & $(0.1350$ & 197.9714 & 0.18 .59 & 36,9714 & $0.08(x)$ & $2(1) 4.9214$ & o:1:! \\
\hline 270.6874 & 0.136 .2 & 195.56 .54 & 0.1809 & 30.6304 & $0 .(19)(x)$ & $205 .(x, 14$ & $0.2018 ?$ \\
\hline $271.31 \times 44$ & 0.1305 & 193.0524 & 0.1931 & 42.0704 & 0.087 .5 & $2(16.89) 4$ & 0.2120 \\
\hline 271.8304 & 0.1295 & $190.6 \times 74$ & 0.1590 & 44.7304 & 0.10 .31 & 207.9724 & $0.20 \div 1$ \\
\hline 2724424 & $0.12(4)$ & 188.2794 & 0.1839 & 47.18124 & 0.11 .5 .3 & 209.07 .54 & 0.1800 \\
\hline 272453.4 & 0.1277 & 18.5 .7704 & 0.1729 & 49.8314 & 0.1250 & 210.2174 & 0.1816 \\
\hline 273.5441 & $0.12+6$ & 18.3 .3604 & 0.17 .38 & 52.2804 & 0.1380 & 211.3384 & 0.1801 \\
\hline $274.156 \mathrm{~m}$ & 0.1 .270 & 180.9304 & $0.17(11$ & $54.870 \mathrm{H}$ & 0.1387 & 212.4 .424 & $01 \times 33$ \\
\hline 2747084 & 0.1264 & 178.4104 & 0.1787 & 47.2214 & 0.1657 & $213.6 \times 54$ & 0.1820 \\
\hline $275.34(44$ & 0.1252 & $175.6 \% 14$ & 0.17 .33 & 59.6204 & 0.1501 & $214 \times 10.4$ & 01862 \\
\hline 275.952 .4 & 0.12 .36 & $173.2(1044$ & 0.1815 & 61.9704 & 0.1520 & 215.911 .4 & 014.5 \\
\hline 276.6264 & 0.124 .3 & 170.9104 & 0.2020 & 64.3504 & 0.16 .25 & 217.034 .4 & 0.1904 \\
\hline 277.2314 & 0.12018 & 167.8504 & 0.178 .4 & 66.7114 & $0.17(x)$ & 218.1554 & 0.1400 \\
\hline 277.8874 & (0.12.34 & 16.5 .4204 & 0.1885 & 69.1104 & 0.1708 & 219.48 .34 & 0.1987 \\
\hline $278.45(19)$ & $0.1 \div 67$ & 162.8704 & 0.1852 & 71.4204 & $0.16 x y$ & 220.6014 .4 & 0.10 .58 \\
\hline $279.142 \%$ & 0.1259 & $160.4(x) 4$ & 0.1730 & 73.8504 & 0.1753 & 221.871 .4 & 0.19 .37 \\
\hline 279.7590 & 0.12 .35 & 157.9904 & 0.1703 & 76.160 .1 & 0.1737 & 223.11144 & 0.1941 \\
\hline 280.3917 & 0.1324 & 155.7504 & 0.1775 & 78.5204 & 0.1770 & 224.1974 & 0.1897 \\
\hline 28101345 & 0.1 .773 & 153.0904 & 0.1939 & 80.8904 & 0.1773 & 225.5854 & 0.18 .4 \\
\hline 281.6305 & 0.1369 & 150.6904 & 0.1619 & 83.2804 & 0.17 .40 & 220.9114 & 0.1808 \\
\hline 287.2316 & 0.1359 & 148.1004 & 0.1482 & 85.6004 & 0.1747 & 228.0744 & 0.1898 \\
\hline 282.86 .11 & $0.18(11$ & 145.850 .4 & 0.18 .24 & 87.950 .4 & $0.18 \times 6$ & 229.2594 & 0.1911 \\
\hline 283.5346 & 0.1397 & 143.32144 & 0.1739 & 90.310 .1 & 0.1902 & 2.30 .5244 & $0.15 x+2$ \\
\hline 284.2896 & $(110,38$ & 140.7704 & 0.1768 & 92.7504 & 0.1898 & 2.31 .116 .4 & $0.1 \% 0$ \\
\hline 285.0120 & 0.1172 & 138.3404 & 0.1766 & 95.09014 & 0.1874 & 2.31 .7084 & 0.1928 \\
\hline $285.61 \times 3$ & 0.1105 & 135.850 .4 & 0.1766 & 97.670 .4 & 0.1903 & 232.2544 & 0.1936 \\
\hline 286.2792 & 0.1110 & $1,33,3604$ & 0.19 .28 & 99.8704 & 0.1896 & 232.8 .504 & 0.1959 \\
\hline 286.8976 & 0.11 .39 & $131.07(1.4$ & 0.18 .39 & 102.280 .4 & 0.18 .88 & 233.4624 & 0.1936 \\
\hline 207.1877 & $0 ! 183$ & 128.5004 & 0.1848 & 104.890 .4 & $0.18 \mathrm{sin}$ & 2.34 .0554 & 0.1896 \\
\hline \multirow[t]{3}{*}{287.3241} & 0.116 .3 & $126.260-1$ & 0.1424 & $107 \log (0) 4$ & 0.1828 & $234.64 \times 64$ & 0.1870 \\
\hline & & 123.7904 & 0.1820 & 109.5404 & 0.1821 & $235 ? 584$ & 0.1902 \\
\hline & & 121.4204 & $0.17(17$ & 111.8004 & 0.1774 & 235.8094 & $0.1 \times x i 3$ \\
\hline \multirow{2}{*}{\multicolumn{2}{|c|}{ Scan 5. 90.6 psig }} & 118.890 .1 & 0.1724 & 114.4204 & 0.1875 & 236.3204 & 0.1875 \\
\hline & & $116.6(k 14$ & 0.1786 & 116.8504 & 0.18 .40 & 236.8914 & 0.1830 \\
\hline 287.20711 & $0.128 \%$ & $114.22(14$ & 0.186 .3 & 119.1404 & 0.1740 & 237.5024 & 0.1782 \\
\hline 287.2428 & 0.1279 & 111.60014 & 0.1804 & $121.66 \times 14$ & 0.1784 & 238.0754 & 0.1738 \\
\hline 286.7603 & 0.12499 & 109.3604 & 0.1742 & 12.4 .0104 & 0.1758 & $238.6+64$ & 0.1754 \\
\hline
\end{tabular}




\section{Reinforced Concrete Test Data}

Track 2 - High Pressure Test

\begin{tabular}{|c|c|c|c|c|c|c|c|}
\hline 239,2504 & 0.17901 & 282.2366 & 0.1583 & $145.850 \mathrm{H}$ & 0.1969 & 87.9504 & 0.2067 \\
\hline 239.8494 & 0.1811 & 282.8631 & 0.2088 & 143.32014 & 0.1946 & 90.3104 & 0.2058 \\
\hline 240.4424 & 0.1841 & 283.5346 & 0.1619 & 140.7714 & 0.1892 & 92.7504 & 0.2022 \\
\hline 241.0544 & 0.1854 & 284.2896 & 0.0776 & $138.3+14$ & 0.1841 & 95.0904 & 0.1986 \\
\hline 241.5854 & 0.1904 & 285.0120 & 0.1339 & $135.850 \mathrm{~d}$ & 0.1971 & 07.6704 & 0.2052 \\
\hline 242.1964 & 0.1960 & 285.6183 & 0.1246 & 1.33 .3614 & 0.2309 & 99.8704 & 0.2097 \\
\hline 242.7894 & 0.1970 & 286.2792 & 0.1295 & 131.0704 & 0.20 .30 & 102.2804 & 0.2018 \\
\hline 243.4024 & 0.1948 & 286.8976 & 0.1331 & 128.5004 & 0.2043 & 104.8904 & 0.2036 \\
\hline 244.013 .4 & 0.1929 & 287.1877 & 0.1367 & $126.26(04$ & 0.207 .3 & 107.0904 & $(1.202 ?$ \\
\hline 244.6254 & 0.1910 & 287.3244 & 0.1511 & 123.7014 & 0.1911 & 109.5404 & $0.2(0) 3$ \\
\hline 245.1554 & 0.1902 & & & 121.4204 & 0.1966 & 111.8904 & 0.1975 \\
\hline 245.7684 & 0.1883 & $\operatorname{Scan} 6,9$. & 7 psig & 118.8904 & 0.1927 & 114.4204 & 0.2046 \\
\hline 246.2994 & 0.1786 & & & $116.60 \times 14$ & 0.1980 & $116.850-4$ & 0.2005 \\
\hline 246.789 .4 & 0.1809 & 287.2670 & 0.1459 & 114.2204 & 0.2041 & 119.1404 & 0.1971 \\
\hline 2473814 & 0.1803 & 287.2428 & 0.1450 & 111.6904 & 0.1958 & 121.6004 & 0.1921 \\
\hline 247.9734 & 0.1762 & 286.7693 & 0.1428 & 109.360 .4 & 0.1897 & 124.0104 & 0.1947 \\
\hline 248.5854 & 0.1714 & 284.3547 & 0.1358 & 1069304 & 0.1915 & 126.4404 & 0.2011 \\
\hline 249.1774 & 0.1711 & 281.4999 & 0.16 .34 & 104.7404 & 0.20018 & 178.730 .4 & 0.1025 \\
\hline 249.7484 & 0.1677 & 278.8584 & 0.1529 & 102.1504 & 0.20116 & 131.1904 & 0.2084 \\
\hline 250.3204 & 0.1684 & $2763(40) 4$ & 0.1474 & $99.7(104$ & 0.1972 & 133.7504 & 0.2317 \\
\hline 2.90 .9324 & 0.1710 & $273.72 \% 4$ & 0.1533 & $97.5(x) 4$ & 0.2047 & 136.1704 & 0.1862 \\
\hline 251.5244 & 0.1741 & 211.2794 & 0.1617 & $44.9 \%(04$ & 0.1978 & 138.6704 & 0.1871 \\
\hline 252.1164 & 0.1722 & 268.7494 & 0.1714 & $92.6 ? 04$ & 0.1984 & 140.9104 & 0.1831 \\
\hline 252.7274 & 0.1736 & 266.259 .4 & 0.1681 & 90.2304 & 0.2058 & $143.4(0) 4$ & 0.1932 \\
\hline 253.3194 & 0.1770 & 26.3 .8 .114 & $0.16+1$ & 87.9104 & 0.2133 & 145.8304 & 0.1959 \\
\hline 2539114 & 0.1770 & 261.2794 & 0.1718 & $85.60 \times 4$ & 0.20 .36 & 148.0504 & 0.1552 \\
\hline 254.5224 & 0.1726 & 258.7684 & 0.1876 & $8.20 \times 4$ & 0.1900 & 1.50 .5804 & 0.1833 \\
\hline 255.1364 & 0.1741 & 256.3614 & 0.1908 & 80.7304 & 0.1919 & 192.890 .4 & $0.2(\times 19$ \\
\hline 255.7284 & 0.1737 & 253.9524 & 0.1881 & 78.1404 & 0.1969 & 155.38024 & 0.1888 \\
\hline $2.56, .34014$ & 0.1777 & $251+4114$ & 0.18 .9 .6 & 75.6204 & 0.1927 & 157.5604 & 0.1871 \\
\hline $256.87 \mathrm{CH}$ & 0.1770 & $24 \times 9524$ & $0.179:$ & $73.100(4)$ & 0.1770 & 159.8704 & 0.1867 \\
\hline 257.5854 & 0.17 .59 & 246.5434 & 0.1960 & 70.5214 & 0.1757 & $162.16(14$ & 0.1958 \\
\hline 2581354 & 0.1714 & 244.1154 & 02052 & 67.9714 & 0.174 .3 & 164.5204 & 0.1881 \\
\hline 258.6654 & 0.1702 & 241.585 .4 & 0.20 .36 & $65+1014$ & 0.1826 & 167.0104 & 0.1960 \\
\hline 250.2544 & 0.16 .54 & 238.0924 & 0.1862 & 02.9514 & $0.17(x)$ & $169.46 \times 14$ & 0.2136 \\
\hline 250.8714 & 0.1657 & 236.7084 & 0.1014 & 60.5 (k) & 0.1588 & 171.8304 & 0.216 .3 \\
\hline $260.54+4$ & $0.16(x)$ & 234.2994 & 0.20171 & $57.990(44$ & 0.1566 & 174.01144 & 0.1858 \\
\hline 261.1574 & 0.1689 & 231.8304 & 0.20 .501 & 55.6104 & 0.1 .488 & $1 / 6.5604$ & 0.1910 \\
\hline 261.7684 & 0.1713 & 229.3614 & 0.2071 & 53.0904 & (1) 1.130 & 179.1604 & 0.1897 \\
\hline 2tastring & $\cong . ! 7 \cap 3$ & 226.9524 & $0.20(10$ & 50.2 .404 & 0.1380 & 181.5204 & 0.1901 \\
\hline 262.9534 & 0.16 .51 & $224 .+634$ & 0.1981 & $97.7 \mathrm{not}$ & 0.1225 & 183.9114 & 0.195 .1 \\
\hline 263.5234 & 0.1623 & 222.0344 & 0.2078 & 41.9304 & 0.1071 & 186.3804 & 0.1977 \\
\hline 264.1364 & 0.1591 & 219.6254 & 0.2125 & 42.2704 & 0.0981 & 188.9 .314 & 0.2031 \\
\hline 264.7284 & 0.1554 & 216.9114 & $0.2(19 !$ & 39.8304 & 0.0953 & 191.3384 & 0.2116 \\
\hline 265.34144 & 0.1554 & 214.5644 & 0.2043 & $37.380 \mathrm{Ar}$ & 0.0829 & 193.8684 & 0.21 .31 \\
\hline 265.8914 & 0.1559 & 2120744 & 0.1903 & $35.130 \mathrm{~cm}$ & 0.0596 & 196.3614 & 0.21 .411 \\
\hline $2(x, 4+24$ & $0.16(x)$ & 209.8284 & 0.1885 & 32.60014 & $0.0460)$ & 198.1544 & 0.2102 \\
\hline 266.9724 & $0.162^{7}$ & 207.46 .34 & 0.2265 & 30.2404 & $0.05(1)$ & 199.9324 & $0.2(x) 3$ \\
\hline 267.5854 & 0.1615 & 205.1154 & 0.2189 & 29.1204 & 0.0401 & 201.0534 & 0.2118 \\
\hline 268.1304 & 0.1615 & 202.626 .4 & $0.19 \% 8$ & 31.8704 & 0.01506 & 202.1754 & 0.2140 \\
\hline 268.7284 & 0.1514 & $? 00,3194$ & 0.20 .27 & 34.5204 & 0.0655 & 203.3404 & 0.213 .3 \\
\hline 269.40114 & 0.1502 & 197.4714 & 0.2010 & 36.9704 & 0.08 .54 & 204.5214 & 0.2107 \\
\hline 270.0754 & 0.1514 & 195.56 .54 & 0.2053 & 306314 & 0.0908 & 205.6674 & 0.218 .3 \\
\hline 270.6874 & 0.151 .3 & 193.0524 & $0.208 x$ & 42.07021 & 0.048 .3 & 206.8904 & 0.23 .32 \\
\hline $271.30 \times 4$ & 0.1523 & 190.6674 & 0.2 (Kt) & 44.7 .304 & 0.1087 & 207.9724 & 0.2274 \\
\hline 271.8 .90 .4 & 0.1546 & 188.2794 & 0.1974 & 47.1804 & 0.1219 & 209.0754 & 0.2018 \\
\hline 3724424 & 0.1522 & 185.7704 & 0.1900 & 49.8304 & 0.1363 & 210.2174 & 0.19 .40 \\
\hline 272.9534 & 0.1509 & 183.3604 & 0.1940 & 52.2804 & 0.1489 & 211.3384 & 0.1920 \\
\hline 273.5444 & 01466 & 180.9304 & 0.1884 & 54.8704 & 0.1502 & 212.4424 & 0.1967 \\
\hline 274.15 int & 0.1451 & 178.4404 & 0.1961 & 57.2204 & 0.1774 & 213.6854 & 0.1980 \\
\hline 274.7084 & 0.1461 & 175.6704 & 0.1875 & 59.6204 & 0.166 .5 & 214.8104 & 0.2033 \\
\hline 275.3404 & 0.1448 & 173.20044 & 0.20 .35 & 61.9704 & 0.1681 & 215.9114 & 0.2120 \\
\hline 275.9524 & $0.1+46$ & 170.9104 & 0.2168 & 64.3504 & 0.1798 & 217.03 .44 & 0.2145 \\
\hline 276.6264 & 0.1 .71 & 167.8504 & 0.1945 & 66.7114 & 0.18 .30 & 218.1554 & 0.2144 \\
\hline 277.2304 & 0.14 .79 & 165.4204 & 0.196 .3 & $69.11(4$ & 0.1860 & 219.48 .34 & 0.2202 \\
\hline 277.8874 & 0.1418 & 162.8704 & 0.190 .4 & 71.4214 & 0.1828 & 220.6044 & 0.2152 \\
\hline $278.45(x)$ & 0.1442 & $160.4(0) 4$ & 0.19 .3 .3 & 73.8504 & 0.1882 & 221.8714 & 0.2075 \\
\hline 279.1427 & 0.1460 & $157.090(14$ & 0.1867 & 76.1604 & 0.1928 & 223.0144 & 0.2088 \\
\hline 279.7500 & 01427 & 155.7504 & 0.1926 & 78.5214 & 0.1071 & 224.1974 & 0.2085 \\
\hline 280.3917 & 0.1441 & $153.00(14$ & 0.2168 & $80.89(24$ & 0.1934 & 225.5854 & 0.2038 \\
\hline 281.0345 & (1) 1541 & 150.64014 & $0.176 ?$ & 83.2804 & 0.1956 & 226.9114 & $0.20 \times 1$ \\
\hline 2810.3015 & 0.1584 & 148.1004 & 0.1575 & 85.6004 & 0.1951 & 228.074 .4 & $0.2(x) 6$ \\
\hline
\end{tabular}




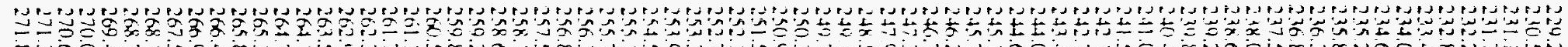

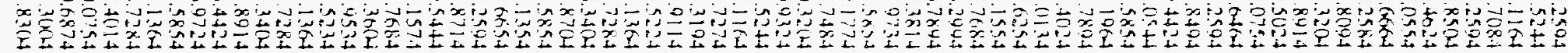

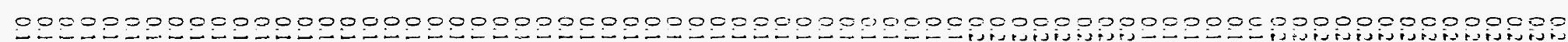

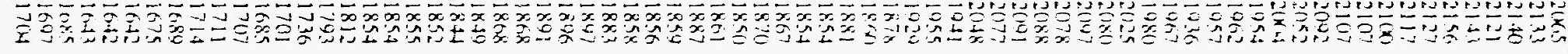

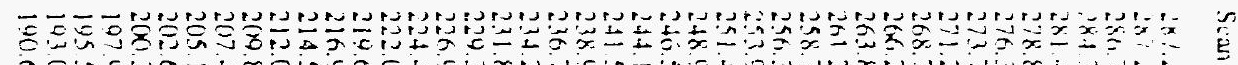

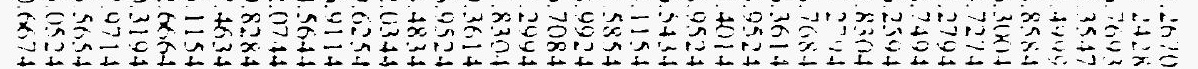

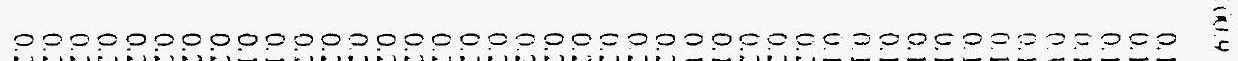

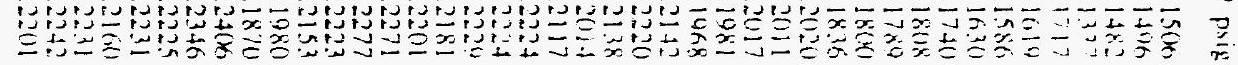

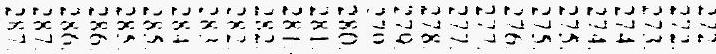

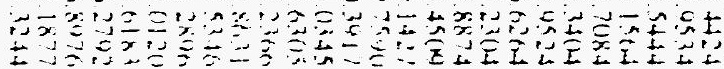

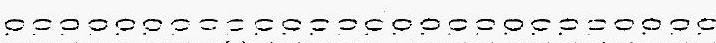

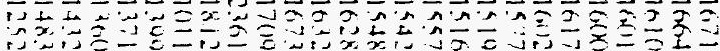

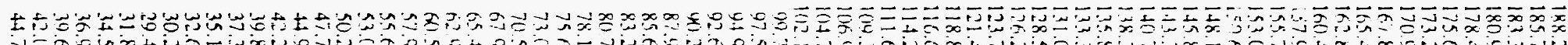

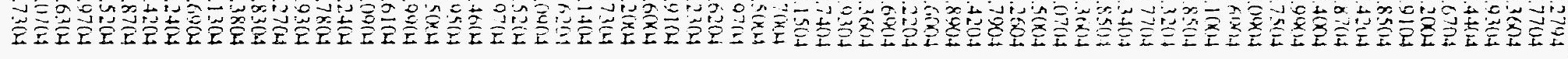

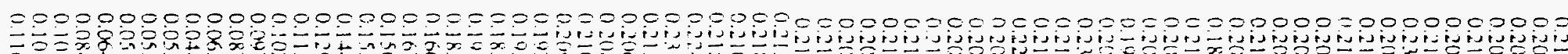

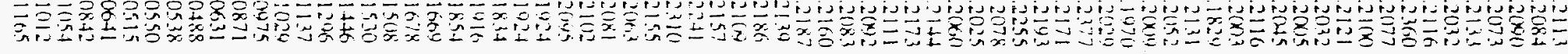

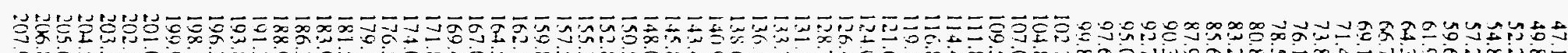

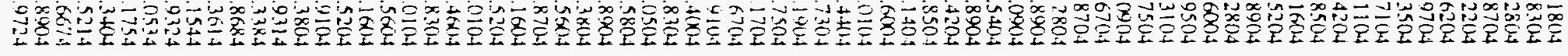
$00000000000000000000000000000000000000000000000000000=00000000000000000$

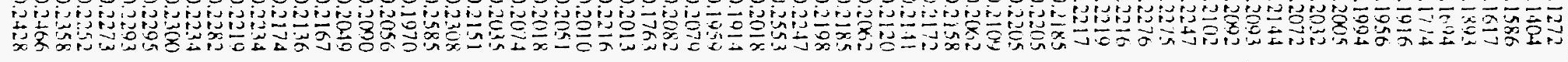


Reinforced Concrete Test Data

Track 2 - Migh Pressure Test

\begin{tabular}{|c|c|c|c|c|c|c|c|}
\hline $2(x) .17554$ & 0.2183 & $26236(1) 4$ & 0.11224 & 229.3614 & 0.2487 & $53 .(x)(H)$ & 0.1674 \\
\hline 210.2174 & 0.2031 & 2629534 & 0.1864 & 226.9524 & 0.24 .36 & 50.2404 & 0.1551 \\
\hline 2113384 & $0.2(1998$ & 263.5244 & 0.185 .3 & 224.4834 & 0.2420 & 47.7804 & 0.1403 \\
\hline 212.4424 & 0.2140 & $264.136+4$ & 0.1825 & 222.0344 & 0.2512 & 44.9304 & 0.1243 \\
\hline 213.6854 & 0.2134 & 264.7284 & 0.1817 & 219.6254 & 0.2520 & 42.2704 & 0.1115 \\
\hline 214.8104 & 0.2168 & 265.3404 & 0.1820 & 216.9114 & 0.2488 & 39.8304 & 0.1091 \\
\hline 215.9114 & $0.22 \times 6$ & $265.89: 4$ & 0.18 .32 & $214.56+4$ & $0.2+0.2$ & 37.3804 & $0.09(4)$ \\
\hline 217.01344 & 0.2317 & 266.4424 & 0.18 .31 & 212.0744 & 0.2190 & 35.1304 & 00702 \\
\hline 218.1554 & 0.2276 & 266.9724 & 0.1831 & 200.8284 & 0.2143 & 32.6904 & 0.05509 \\
\hline 219.48 .34 & 0.2320 & 267.5854 & 0.1826 & $207.46,34$ & 0.2600 & $30.24(14$ & 0.0572 \\
\hline 220.00144 & 0.2308 & 268.1364 & 0.1826 & 205.1154 & 0.2545 & 29.4204 & 0.0557 \\
\hline 221.8714 & 0.2246 & 268.7284 & 0.1760 & $202.666+4$ & 0.2376 & 31.8704 & 0.1526 \\
\hline 223.014 & 0.2236 & $269+(1) 14$ & 0.178 .3 & 200.3194 & 0.2422 & 34.5204 & 0.0717 \\
\hline 2241974 & 0.2244 & 270.079 .4 & 0.178 .4 & 197.971 .4 & 0.24610 & 36.9704 & $0.090(0)$ \\
\hline 225.585 .54 & 0.22 .1 & 270.6874 & 0.1795 & 195.5654 & 0.2492 & 39.63144 & $0.1(x) 0$ \\
\hline 220.9114 & 0.2226 & $271.30 \times 4$ & 0.798 & 193.0524 & 0.2478 & 42.0704 & 0.1163 \\
\hline $228.07+4$ & 0.2264 & 271.8304 & 0.1822 & 190.6074 & 0.2388 & $44.73,4$ & 0.1271 \\
\hline 220.2544 & 0.2238 & $27-424$ & 0.1780 & 188.2794 & 0.2350 & 47.1804 & 0.14115 \\
\hline 2301.5244 & 0.2264 & 272.4534 & 0.1702 & 185.7714 & 0.2249 & 49.8304 & 0.15 .34 \\
\hline $231.116 \mathrm{H}$ & 0.2275 & $273.54+4$ & $0.1 \%$ & $183.3(x)-4$ & 0.2343 & 52280.4 & 0.1686 \\
\hline 2.41 .7084 & 0.2275 & $27+1.504$ & $0.17(1)$ & 180.9304 & $0.2 .3(1.1$ & 54.8704 & 0.1719 \\
\hline 232.259 .4 & 0.2317 & $27.470 \mathrm{st}$ & 01719 & $17 k+404$ & 0.2346 & 57.2204 & 0.20 .60 \\
\hline 2328504 & $0.23+1$ & 275.3404 & 0.171 .3 & 1756704 & 0.2313 & 59.6204 & 0.1840 \\
\hline $233+4624$ & 0.236 & 2754524 & $0.17\left(x_{1}\right.$ & $17.32(x) \cdot 1$ & 0.2362 & 61.0704 & 0.1882 \\
\hline 2340.554 & 0.2245 & 276.6264 & 0.16 .5 & 170.01014 & 0.2513 & for. 35014 & 0.2015 \\
\hline $274.6 x+604$ & 0.2277 & 277.23614 & $0.16,5 ?$ & 167.8501 .4 & 0.2284 & 66.7104 & 0.213 .5 \\
\hline $215.25 \mathrm{kH}$ & 0.226 .8 & $277.8 \times 74$ & 0.1667 & $165+2.14$ & 0.2307 & 89.1104 & 0.2150 \\
\hline 235800.4 & 0.2253 & $278.45(19$ & $0.16+12$ & 162.870 .4 & $0.23=7$ & $71+42(1.4$ & 0.2178 \\
\hline $236.32(4)$ & 0.2228 & 279.1427 & 0.161 .3 & 160.404 & 0.2388 & 73.8544 & 0.2230 \\
\hline 2368914 & 0.2170 & $: 79.7590$ & 0.16 .52 & .57 .9904 & 0.2247 & 76.1604 & 0.2254 \\
\hline $23 \div .5024$ & 0.2141 & 280.7917 & 0.1644 & $155.74(1.1$ & 0.2314 & 78.5214 & 0.2337 \\
\hline 2.88 .01754 & 0.2107 & 281.0345 & $0.172 x$ & $153.01 \times 14$ & $0.248 ?$ & $80.89(14$ & 0.2290 \\
\hline 238.6464 & 0.2122 & $281.6,305$ & 0.1755 & 1501.6914 & 0.2292 & 832804 & 0.22144 \\
\hline 239.2504 & 0.2126 & 282.2316 & $0.179, ?$ & $148.1(0) 4$ & 0.2013 & $85.60(0) 4$ & 0.2307 \\
\hline 230.849 .4 & 0.2113 & 282.8531 & 0.2 .111 & 1458514 & 0.2278 & 87.4504 & 0.24 .32 \\
\hline $2+104+2=4$ & 0.21 .46 & $283.53+h$ & $0.19(1,3$ & 14.3 .320 .4 & 0.22192 & 90.3104 & $0.248 \mathrm{H}$ \\
\hline $2+1.015+1$ & 0.2151 & 284.2896 & 0.0479 & $14077(14$ & 0.2231 & 92.7504 & 0.2482 \\
\hline $2+1.58 .54$ & 0.2188 & 285.0120 & 0.1535 & $138.7+4.4$ & 0.2164 & 950004 & 0.2 .473 \\
\hline $242.196 \mathrm{~A}$ & 0.2218 & 285.618 .3 & 0.14 .32 & 135.8504 & 0.23 .37 & 97.67014 & 0.2501 \\
\hline 242.7894 & 0.2252 & 286.2792 & 0.1452 & 133.3601 .4 & $0.2 x+9$ & 098701.1 & 0.2178 \\
\hline $2434(124$ & 0.2241 & 286.8976 & 0.1546 & 131.070 .4 & $(1.2517$ & 102.2804 & 0.2415 \\
\hline 24.0134 & 0.2214 & $287.187 ?$ & 0.1565 & $128.5(x)-4$ & 0.2432 & 10.18344 & 0.24 .36 \\
\hline 24.625 .4 & $0.21 \mathrm{so}$ & 287.3244 & 0.1526 & $126.26(1.4$ & 0.2499 & $107 .(4 \times 104$ & 0.2442 \\
\hline 245.1554 & 0.217 .4 & & & $123.7 \times 14.4$ & 0.2335 & 109.5404 & $0.240 x$ \\
\hline 245.7684 & $0.21(x)$ & \multirow{3}{*}{\multicolumn{2}{|c|}{$\mathrm{Sc}$ an 8, 108.3 psig }} & 121.4204 & 0.2373 & $111.89(14$ & 0.2372 \\
\hline $2+6.2994$ & 0.2077 & & & 118.840 .4 & 0.2137 & 114.42014 & $0.2+40$ \\
\hline 246.7894 & 0.2104 & & & $116.60 \times 4$ & 0.2390 & 116.8 .504 & 0.2428 \\
\hline 247.3814 & 0.2087 & 287.2670 & 0.1702 & 114.2204 & 0.2430 & $119.1+14$ & $0 \geq 272$ \\
\hline 247.973 .4 & $0.2(145$ & 287.2670 & 0.1702 & $111.6 \times 4$ & 0.2359 & 121.6004 & 0.235 .4 \\
\hline $2.48 .5 \times 5.4$ & 0.2023 & 286.7693 & 0.1655 & $109.38 \times 14$ & 0.2331 & 124.0104 & 0.2316 \\
\hline 249.1774 & 0.2032 & 284.35477 & 0.1 .114 & $1(k, 1) 704$ & $0.234 k^{\prime}$ & 126.4 .404 & 0.24 .33 \\
\hline 244.7 .484 & 0.2010 & 281.4999 & 0.1888 & 104.7404 & 0.2431 & $128.730 \mathrm{~s}$ & 0.241 .3 \\
\hline $250.32(44$ & $0.2(118$ & 278.898 .4 & 0.1770 & 102.1504 & $0.2+6.5$ & 131.1904 & 0.2481 \\
\hline 250.9324 & 0.2023 & $276.30 \times 4$ & 0.1758 & $99 . ?(x) 4$ & 0.2369 & 133.75014 & 0.2825 \\
\hline $251.52+4$ & $0.2(123$ & 273.7274 & 0.1795 & $07.50 \times 4$ & 0.246 .5 & 136.1704 & 0.2280 \\
\hline 252.1164 & $0.2(k)$ & $271.2 \% 94$ & 0.1884 & $94.97(\mathrm{~N}$ & 0.24 .35 & $138.670 \mathrm{H}$ & 0.2200 \\
\hline 252.7274 & 0.1966 & 268.1474 & 0.1450 & 02,6124 & 0.2453 & 140.9104 & 0.2210 \\
\hline 253.3194 & $0.2(x) 18$ & 266.2594 & 0.1925 & $90.23(4-4$ & $11243 \%$ & 143.4004 & $0.23\left(k_{1}\right.$ \\
\hline 253.9114 & 0.1970 & $263.83(44$ & 0.1978 & 81.9104 & 0.2530 & $1+5.8 .3(44$ & 0.2247 \\
\hline 254.5224 & 0.1950 & 261.2794 & 0.20 .32 & $8561 k 4$ & 0.2413 & 148.0504 & 0.1935 \\
\hline 255.1364 & $0.19 \% 1$ & 2587684 & 0.2196 & 8.2644 & 0.2252 & 150.5804 & 0.2231 \\
\hline 235.7204 & 0.1992 & 256,3614 & 0.2145 & 80.73014 & (1) $22 \mathrm{rm}$ & 1528004 & 0.2416 \\
\hline $256.3+604$ & 0.2013 & 2539524 & 0.22 .38 & 78.1404 & 02338 & 155.3804 & 0.2243 \\
\hline 256.8704 & 0.2012 & 251.4014 & 0.22 .14 & $15.62(4)$ & 0.2215 & $157.56 \times 4$ & 0.2205 \\
\hline 257.5854 & $(1.2031$ & 248.9524 & 0.2186 & $73 .(6)(4)$ & 0.2170 & 150.8704 & 0.2282 \\
\hline $25 \times .1394$ & 0.2009 & $246: 96$ & 0.2354 & 70.52121 & 0.211. & $162.16(x)$ & 0.2302 \\
\hline 258.6654 & 0.1900 & 244.1154 & 0.24 .34 & 67.9704 & $0.2(k, 3$ & 164.5204 & 0.2293 \\
\hline 259.2594 & 0.1948 & 241.585 .4 & 0.2347 & $65.46(4)$ & $0.21+4$ & $167.01(2.4$ & 0.2314 \\
\hline 259.871 .4 & 0.19 .41 & 238.9424 & 0.2277 & 62.9514 & $0.2(1.48$ & $16946 \times 4$ & 0.2514 \\
\hline 260.544 & 0.1963 & 236.7084 & 0.233 .2 & $601.5 \mathrm{~km}$ & 0.1839 & 171.8304 & 0.2724 \\
\hline $261.15 \%$ & 0.1941 & 234.2944 & 0.249 .3 & 57,90014 & 0.1821 & 174.010 .4 & 0.2229 \\
\hline $261.76,84$ & 0.1938 & 231.8304 & 02470 & 55.6104 & 0.1715 & $176.56(x) 4$ & 0.2333 \\
\hline
\end{tabular}




\section{Reinforced Concrete Test Data}

Track 2. High Pressure Test

\begin{tabular}{|c|c|}
\hline $1: 41644$ & $(1: 210$ \\
\hline$|x| 52134$ & $11 \ldots n$ \\
\hline 1839114 & $11: \div$ \\
\hline $18 \times, 3 \operatorname{lins}$ & $11: 214$ \\
\hline 188.1114 & $(1 \div \div 11$ \\
\hline 1013354 & (1)24: \\
\hline 193.8684 & 12511 \\
\hline 106.3014 & $(129)$ \\
\hline 10815.44 & $0: 201$ \\
\hline 1009.0324 & $0.25(x)$ \\
\hline 2010534 & $11.24:$ \\
\hline $20: 175.4$ & $112.4 \times 8$ \\
\hline $20,3.3464$ & 11.2482 \\
\hline 204.5214 & 0.120 \\
\hline $2015.6 \times 77$ & 0.2570 \\
\hline $206.89(4$ & $0.2(19)$ \\
\hline 207.9724 & 0.26 .20 \\
\hline $2(x) 0754$ & 0.240 .3 \\
\hline 210.2174 & 0.2332 \\
\hline 211.3384 & $(1.2 .214$ \\
\hline $212+42+$ & 112373 \\
\hline $213.6, k \leq 4$ & 0.232 \\
\hline $21+5164$ & 0.27 .45 \\
\hline $21<0114$ & $(1.2+2)$ \\
\hline $2103+4$ & 11.25 (15 \\
\hline $21 \times 1554$ & 0.2 .46 .3 \\
\hline 210.4814 & 0.2577 \\
\hline $22(0)+x+1$ & 0.2582 \\
\hline 221.8714 & 0.243 \\
\hline $223019+4$ & $0.2+47$ \\
\hline $22+1401$ & 0 ith \\
\hline $225 \leq 854$ & $0 .+16=$ \\
\hline 220114 & $18 x(x)$ \\
\hline $2 x-1,+1$ & 0.316 \\
\hline 234.254 & $0 .-x)$ \\
\hline 231150 & 0 \\
\hline और & $02+4$ \\
\hline 23225134 & $0.25+4$ \\
\hline $232.85(4)$ & (1.25, \\
\hline $233+6.24$ & 0.2502 \\
\hline 244.1159 .4 & (1. $250 x$ \\
\hline $2346 \times 6+1$ & $0.2 \leq 33$ \\
\hline 235.2584 & 0.2534 \\
\hline $2.398(14.4$ & 0.2513 \\
\hline 2.36 .3254 & $0.2+7 t$ \\
\hline 236.6014 & $0: 2+2$ \\
\hline 237.9124 & $0.2+12$ \\
\hline 238.0754 & (1) $22(51)$ \\
\hline $238 \cdot 6+46-4$ & 0.2354 \\
\hline 239.2594 & 0.2352 \\
\hline 239.8 .194 & 1) $2+1 ?$ \\
\hline $2+1) \cdot 14=4$ & $0.2 .3(x)$ \\
\hline $2+1.015+1$ & $0.237(1$ \\
\hline $2415 \times 54$ & $0.2+13$ \\
\hline 242.1964 & 0.24 .47 \\
\hline 2427894 & 0.2485 \\
\hline 243.4024 & $0.24(x)$ \\
\hline 244.0134 & 0.2445 \\
\hline 246625.4 & 0.2453 \\
\hline 2451594 & $0.2+(x)$ \\
\hline 245.7684 & 0.2442 \\
\hline 246.2994 & 0.2738 \\
\hline 3467804 & 0.2330 \\
\hline 2473814 & 0.2 .104 \\
\hline 247.973 .4 & $0.22(x)$ \\
\hline 348.5854 & $(1.221$ \\
\hline 2441174 & 0.2224 \\
\hline $249.7+84$ & $0.22(x)$ \\
\hline 250.3204 & 0.2219 \\
\hline $25(1) 4324$ & 0.2210 \\
\hline $251.52+4$ & 9.2218 \\
\hline
\end{tabular}

\begin{tabular}{|c|c|c|c|c|}
\hline$\therefore \because: 1+1$ & $1 \because \therefore 1 s$ & & $\therefore \cdots+4$ & $\because \because:$ \\
\hline$\because \because \because 4$ & $\because \because 44$ & & 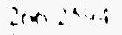 & $12:$ \\
\hline$\because \div: \because 1$ & 112.94 & & $211: 2 \times 4.4$ & $11 \therefore A$ \\
\hline$\because 5+4: 4$ & $(1: 1 \%)$ & & $2+1: \geq+i$ & 11.1. \\
\hline$\because \because \because \because 4$ & $11: 1 \% 1$ & & $249^{\circ}$ ins: & $\therefore: 141$ \\
\hline$\{:<+.4$ & $1121 \%$ & & 2 se : : : : & $11: 211^{\circ}$ \\
\hline$\because \because 1$ & 4218. & & $26,19: 4$ & $\because 24$ \\
\hline $6,4: 4$ & 112215 & & $251+1+$ & $11: 3 a$ \\
\hline - \& 14 & 1122119 & & $24 x: 2,-1$ & $(12741)$ \\
\hline$\because \because 4$ & (1:2:1) & & $\therefore 10 \div 1: 1$ & $\therefore 2 \div \times$ \\
\hline 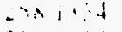 & $\therefore::^{\circ}$ & & 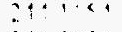 & 11251 \\
\hline $2 x+204$ & 1:2014 & & $2+1.994$ & $1: \div \div 5$ \\
\hline 29425,1 & $\therefore ?: 1,11$ & & $23 \times 4+1$ & $\because 24 i n$ \\
\hline 25,314 & 1121.15 & & In '....4 & $11: 1+1$ \\
\hline $21 x^{2}<4+4$ & $11: 115$ & & $\because 1422 \times 1$ & $112+40$ \\
\hline$\therefore 1: 4$ & $(121.40$ & & $211 \times 4.4$ & $1.1,14$ \\
\hline$\therefore 1+A 1$ & $(121+8$ & & 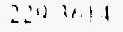 & 12212 \\
\hline 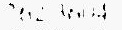 & $(12110$ & & $\therefore a y^{2}+1$ & 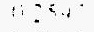 \\
\hline$\therefore: 34$ & (12 Elex & & $\therefore 4+4 i$ & $1 \therefore$ \\
\hline 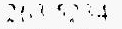 & 112.140 & & $\therefore: 11: 4: 4$ & s...? \\
\hline$\therefore+11<6.4$ & $11:-1.41\}$ & & $\therefore+\cdots$ & $\because c$, \\
\hline$\therefore+4 .+1$ & 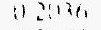 & & :Dariat & $2 \ldots$ \\
\hline$\therefore 18 \times 14$ & i) 23415 & & $\because 1+2+1$ & $\therefore$ \\
\hline $211=2114$ & $(1140$ & & $21: \quad 11$ & $\therefore \cdots$ \\
\hline $2+4+4+21$ & $(\because 14 ? .1$ & & $2 !+4: \because 1$ & $21+4$ \\
\hline $2+x, y=1$ & $111 \%=$ & & $\because 11 !: 4$ & $\therefore 1$ \\
\hline $2 x=84$ & 119.10 & & $2: 1:<1$ & $\because \because=$ \\
\hline $2 t a: 16,1$ & (1) 1080 & & $2-x+1$ & 1: ar: : \\
\hline$\therefore \cdots \cdots 1$ & 111583 & & $\therefore x+1,1$ & 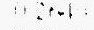 \\
\hline$\therefore+1+11$ & $(! \ln (i)$ & & $1, \cdots::$ & $\therefore \therefore$ \\
\hline $2: 1: 1<4$ & (1) $194 ?$ & & $1+4,1$ & $\because \ldots 1$ \\
\hline $2 \cdots+24$ & $(192011)$ & & $1.1 \because:$ & $\alpha \alpha$ \\
\hline$=1 \quad 1, \ldots$ & $1: 1,19$ & & $12 \cdots+$ & $11:$ \\
\hline $2 \times 1$ & 119.14 & - & $1 \cdots: 4$ & $\because \because \because$ \\
\hline$\therefore 11: 1$ & i $14, \ldots$ & & $\cdots+$ & \\
\hline$\therefore \because y 1$ & $11 ! 1 ! !$ & & $1 \cdots \cdots$ & $\therefore$ \\
\hline$\therefore a 4 b 4$ & $+19=$ & & 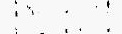 & $\therefore$ \\
\hline$=1 ! \cdots 1$ & $11: 94$ & . & $\therefore 2:$ & $\therefore$ \\
\hline$\div 4$ & $\because 1 \cdots !$ & & $\because \vdots$ & $\because 2.1$ \\
\hline$\therefore$ a d & $\because 4$ & & $1 \div 1$ & $\therefore$ \\
\hline$\therefore \sin$ & $\because \cdots$ & $\cdots$ & $1 ; \vdots \vdots$ & $\overline{7}:$ \\
\hline 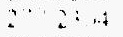 & $\because: \cdots$ & & $164^{5}+4$ & 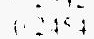 \\
\hline$\because \cdots !$ & $\because 1: \therefore$ & & $11: 2 \times 11$ & $1+\angle 2$. \\
\hline $27 * 161$ & $\because i \therefore . ?$ & & 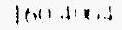 & $11.5 \%$ \\
\hline 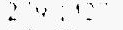 & $1.1 \cdots$ & & $15 \% \cdots \mathrm{MA}$ & 112400 \\
\hline 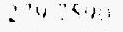 & 111412 & & 1557514 & (1) $24 x)$ \\
\hline$\therefore+\cdots \mid$ & $\because: 2: i$ & & $153.1,0,1$. & i1 $\because 1 \% 5$ \\
\hline $2 x: 1, i 4^{6}$ & (1) $1 \ldots, ?$ & & $1506,0.44$ & 1124.4 \\
\hline $2 \times 1+1,19$ & $\left.\|i\|\right|^{\varsigma}$ & & $148.11 \times 4$ & (1):25: \\
\hline$\therefore x:=210$ & (1) 1943 & & $145 \times 594$ & 0.2911 \\
\hline $2 x-\operatorname{sen} 31$ & 12312 & & 14.3 .3214 & $01.2+164$ \\
\hline 28.15340 & 0.1410 & & 1407704 & 0.233 \\
\hline 264 ander & () (1sis) & & $1383+144$ & 0.2320 \\
\hline 28501120 & $0 . \ln 34$ & & 1.3585014 & $0.2+12+2$ \\
\hline 2856153 & 0.1591 & & $133.3(24$ & $0.2 \times 15$ \\
\hline 286.2912 & $(1.1905$ & & $1.3107(144$ & 01.2714 \\
\hline $2 S 680 ?$ & $0.16,80$ & & $12850 \times 4$ & 0.2627 \\
\hline $287.187 ?$ & 0.1415 & & $126.26 \times 4$ & 0.2080 \\
\hline & & & $12.7 .7 \times 14$ & 02408 \\
\hline & & & $121.121 \mathrm{~m}$ & 0.2502 \\
\hline \multirow{2}{*}{\multicolumn{3}{|c|}{ Stan 7.11 .32 psig }} & $118 \mathrm{sin}(4)$ & $0.257 x$ \\
\hline & & & $116 \cdot 0(x) 4$ & $0.2017 ?$ \\
\hline 87.2क? & $(1.1798$ & & $11+.22(4.4$ & $0.2 n^{2} t_{1}$ \\
\hline $287.26 ?$ & (1.17.4 & & 111064 & 0.2550 \\
\hline $280,7 f, 1) ?$ & 0.17 .4 & & $10030 \times 4$ & $(1.540$ \\
\hline $3 x+15+7$ & (0.) $(x) 3$ & & $1(k, 4) 301$ & 0.20 .64 \\
\hline $281+40(1) 0$ & $0.14+4$ & & 104.114 & (1) $26 \ldots$ \\
\hline $78 \times 58.4$ & 0.1 sinte & & 102.15144 & 02604 \\
\hline $276.3(614$ & (1.18.84 & & (94). $7 \mathrm{kH}$ & $012511 ?$ \\
\hline $273.2=4$ & (1) 1011 & & $07.5 \ln 4$ & 112729 \\
\hline 2712704 & (1).2112] & & $1947(1)+1$ & 0.2051 \\
\hline
\end{tabular}

\begin{tabular}{|c|c|}
\hline 1) 2. 62 ( & 0.26 .51 \\
\hline 90.2301 .4 & (1.268? \\
\hline 87.9104 & 0.276 .3 \\
\hline $85.6 \times(4)$ & $0.26(13$ \\
\hline $83.26 \times 4$ & 0.2450 \\
\hline 80.1314 & $0.5(14)$ \\
\hline 18.14114 & 0.2540 \\
\hline 75.6204 & 0.2454 \\
\hline 1.5.0x) (u) & (1.2.97: \\
\hline $7(1.520 .4$ & 0.2275 \\
\hline $1.707(1.1$ & 02323 \\
\hline 65.46014 & 0.2338 \\
\hline $62.99(1.4$ & 0.2176 \\
\hline $60.5(x) 4$ & 0.20134 \\
\hline $57000(14$ & 0.1985 \\
\hline 55.6104 & 0.1881 \\
\hline $57 .(49)$ & 0.1820 \\
\hline 5012.404 & () $16 \leqslant, 0$ \\
\hline 47.78014 & $0.1 .5(14)$ \\
\hline 4.40301 .4 & 0.13 .70 \\
\hline 42.27144 & 0.1140 \\
\hline 34.8304 & 0.1124 \\
\hline 37.3804 & 0.04 .4 .4 \\
\hline 35.13114 & 01.0725 \\
\hline 32.64014 & $0(1+10)$ \\
\hline 30.2404 & 0.0518 \\
\hline 24.42114 & 0.0534 \\
\hline $31 \times 79(4$ & $0 .(k \times)$ \\
\hline 345214 & 0.070 \\
\hline $3(1,97(4)$ & $0 .(9) 12$ \\
\hline 306304 & 0.1198 \\
\hline $4: 11711.1$ & $0.11: 5$ \\
\hline 44 731:4 & 0.1348 \\
\hline $4^{7} 18(1)+4$ & 0.1514 \\
\hline (40) 8101.4 & (1. $160(1)$ \\
\hline 52280.4 & 0.170 .4 \\
\hline 54874 & 0.1879 \\
\hline $5 \div .22(1.4$ & 0.21 .54 \\
\hline (5) tian) & $(12045$ \\
\hline 0.1 .47194 & 0.2054 \\
\hline 64.3504 & 0.2186 \\
\hline 66.7104 & 0.2285 \\
\hline 69.1164 & 02245 \\
\hline $71+2114$ & 0.2334 \\
\hline 73.5514 & 0.23136 \\
\hline 70.1614 & $(0.2 .452$ \\
\hline 78.5204 & 0.2545 \\
\hline 80.8404 & 0.2525 \\
\hline 83.2504 & $0.2+440$ \\
\hline $85.6(x) 4$ & 0.2537 \\
\hline $87.05(4.4$ & 0.20 .45 \\
\hline $4071: 114$ & (1).2675 \\
\hline 427504 & 0.27118 \\
\hline $0.5(x)(4)$ & 0.26 .75 \\
\hline 97.6714 & 0.2731 \\
\hline $0987(4)$ & 0.2674 \\
\hline 10228804 & 0.263 .4 \\
\hline 104.8904 & $0.26,70$ \\
\hline $1017(\times \times 14$ & $0.2(x+1)$ \\
\hline $1(x) .5+(4-4$ & $0.20+4$ \\
\hline 111 soxh & $0.262:$ \\
\hline $114+214$ & $0.2+21$ \\
\hline 1168514 & $0.26,32$ \\
\hline $1190.1+124$ & 0.201 ? \\
\hline $1216 \times 14$ & $(1.2 .56 x$ \\
\hline $12 \div 0164$ & 0.2594 \\
\hline $126+44$ & $(1.249)$ \\
\hline $12 \times ? 314$ & $02(x)$ \\
\hline 1211604 & 0.273 \\
\hline $13 \times .7504$ & (1) $3(x)$ \\
\hline $17,170.4$ & $0.2+4$ \\
\hline 1366796 & 0.2110 \\
\hline
\end{tabular}




\section{照: \\ Reinforced Concrete Test Data}

Track 2 - High Pressure Test

\begin{tabular}{|c|c|c|c|c|c|c|c|}
\hline 1.101 .4104 & 0.2 .343 & 212.7801 & $02,2=$ & $\therefore x c_{1} \ldots$ & $11.10+4+4$ & 1.34.0\%? & $0.2 x x$ \\
\hline it? fints & 0.2304 & 24.401 .24 & $(12)(n)$ & $\therefore 6,340$ & $111: 4 h$ & $1285(x 4)$ & 1) 2xixt \\
\hline $1.45 \times 1.24$ & 0.2562 & $2440 ! 34$ & 0.20 .34 & $\therefore=18$ & i) $19 ?$ & $12628(x) 4$ & 0.2840 \\
\hline 1480.514 & 0.2203 & 244.6254 & 0.20 .26 & & & $123.7 \times 4$ & $0272 !$ \\
\hline $1501.580 \mathrm{H}$ & $0.23(x)$ & 245.1554 & $0.25 \times 1$ & & & $1: 1+214$ & 0.284 .1 \\
\hline 152.8904 & 0.2748 & 245.7654 & $0.25+5$ & \multirow{2}{*}{\multicolumn{2}{|c|}{ Sxan $10.11^{-1} \dot{x}$ por }} & 118.8444 & $0.28+4)$ \\
\hline 155.3804 & 0.24 .66 & 246.2094 & $0.2+28$ & & & $116.0 \times 4$ & $0.2 \times 15$ \\
\hline $157.50 \mathrm{OH}$ & $0.24 \times 7$ & 246.7804 & 0.2 .157 & $28 \div 2510$ & (1) |xलmi & $114.22 ! 1.4$ & n? $?(x) ?$ \\
\hline 180.8714 & 0.2472 & 247.3814 & $0.24 \%$ & $2 n 7.2: 0^{-101}$ & $0.1 \times 4+4$ & $111 .(6,4) .4$ & 0.2827 \\
\hline $161<x+1$ & u. 0.4 & $2: 2,0724$ & 0.2460 & 72h Tr.w: & $018 ?$ & $1(x, 3+14$ & 0.28 .36 \\
\hline 164.5204 & $0.2+110$ & 24858.54 & $0.241 ?$ & 2043.47 & 01586 & 115.4 .414 & $\because \therefore x \times 1$ \\
\hline 167.0164 & 0.2179 & $\therefore 401774$ & $n ?+i T$ & $201+440$ & (1.2). 219 & $104-201$ & 0.20 .11 \\
\hline $160.46(4$ & 0.2701 & $249.7+84$ & 0.2749 & $2 \sin 86 t$ & 0.1400 & 10215154 & $0.29 ? 6$ \\
\hline 171.8314 & $0.3(x) 1$ & 2503204 & 0.23104 & $276.4 \times 14$ & 01201 & 9) 9 (x) & (1. 2867 \\
\hline 174.0114 & $02 f(x)$ & 250.9324 & $0.2 \times 14$ & $27 \quad 214$ & (1) $2(x+x-1$ & 4) $7.5(x) 1$ & 0.2432 \\
\hline $176.50 \mathrm{~m}$ & (1) $2+4)$ & $251.52+4$ & 0.2414 & 2712704 & $0.21+0$ & 0.40714 & $(12 \times 2) 1$ \\
\hline $179.160 \mathrm{~N}$ & 0.2408 & $252.11 \mathrm{th}$ & $0.23 \times 4$ & $26 \times>744$ & $0 \geq 1 \times 3$ & $9262(14$ & 0.2424 \\
\hline 1815214 & 0.244 & 252:2: it & $0.236 t$ & 260.2504 & $(1: 21)$ & 90.2304 & $0.204+1$ \\
\hline 1830164 & 0.594 & 2533194 & $112+14$ & $20 . \leqslant \leq 1.4$ & 1) 2285 & 87.910 .4 & $0.2(0), 0$ \\
\hline $18 \times 3514$ & $0: 5+4$ & 2519114 & $(1.234)$ & $2012^{204}$ & 0.2757 & $85(x \times)+$ & 0.2814 \\
\hline $18 \times 9314$ & 0.2586 & 2445224 & $0.201 x$ & $25 \times-7$ tist & 0.2519 & 83.20034 & $0.272 \%$ \\
\hline (4) 3364 & 0.2018 & $255,1^{2} 04$ & 0.23115 & $25(1,3 f, 14$ & $0.24 / 6$ & 80.7304 & $0.27+4$ \\
\hline 19:3ntint & (1.2tutn & 255.7284 & $(1.2 \div 21$ & 2574524 & 0.2555 & 78.14014 & (0.7.7. \\
\hline $10,27,1.4$ & 0.2720 & isn itim & 02.356 & $251.10 ! 1$ & 0.2568 & $75+204$ & $026>0$ \\
\hline $14815+4$ & 0.2679 & 256.8004 & 0.2352 & 2484524 & 02543 & 1.10 .14 & 0.2552 \\
\hline 1100424 & 0.20150 & 25.5854 & 0.23 .13 & 240.5434 & $(1.26) 6$ & $70.52(14$ & 0.2512 \\
\hline 2010534 & $0.27(n)$ & 258.1354 & 0.2336 & 244.1154 & 112745 & $67.07 \%(44$ & $0.2+21$ \\
\hline $21121: 94$ & 0.2725 & $2586 \times 54$ & 03258 & 241.5854 & 0.2711 & 6,5 whot & (1) 24 the \\
\hline 213,3414 & $0.20,0 i$ & 259.250 .4 & 0.2324 & 238.0424 & $0.2+523$ & 6205014 & 41.2114 \\
\hline $2(445214$ & $0.26,1)$ & $250.8^{-11}$ & 0.2316 & 236.7024 & 1) 2724 & $605(x) 4$ & 0.2157 \\
\hline 2015 t $x, 1]$ & $0.27 \times 0$ & 260.5444 & 0.2104 & $2312(x) 4$ & 02870 & $57.40(1.4$ & 0.2078 \\
\hline (K) S.74 & $[1.21)] 0$ & 2611574 & $0.23(1)$ & $231.83(14$ & 0.2820 & 55.6114 & 02014 \\
\hline $2(1), 4: 24$ & 0.2854 & 201.7664 & 0.230 & $270.3(164$ & 0.28 .411 & $53(\mu)(1.4$ & 0.1913 \\
\hline-301194 & 11206-t & 36.23 tiond & (1 220$) 6$ & 2204524 & $(1,25.60)$ & 50.704 & (1.1753 \\
\hline 210214 & (1) $2+6 n$ & $21.25<34$ & 0.2234 & $22+454$ & (1) 281. & $4^{7} 78014$ & $015: 1)$ \\
\hline $21133 n+$ & 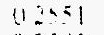 & 2035544 & 1).216. & $22: 01: 144$ & $(1.24561$ & $44.1) 3(14$ & $(1.1 .139$ \\
\hline 2124.124 & 1) $: 9 x^{2}:$ & $26+1+10 t$ & 2611.5 & 2190254 & $(1200)+$ & $4.2=74$ & 0.12811 \\
\hline $213 \cos 54$ & 0.2500 & 20, ?2B1 & $0.21+4$ & 216,0114 & U.25int & $30.8 \div(14$ & $11.11: 0$ \\
\hline 2148114 & 1). 2617 & 265.3404 & $0 . ? 13$ & 2145044 & (1).25:3 & $37.3 \times 14$ & $0 .(x) \leq 3$ \\
\hline 215.9114 & $0.2(1)$ & 20.58014 & 0.21110 & $212.117+4$ & (1).21 147 & $35130 \mathrm{it}$ & 0.01771 \\
\hline 2110344 & $(1.27) 7$ & $2+6+124$ & $0.2(k)$ & $2(10 \times 2 \times 54$ & 0.2536 & $3 \ldots 60(14$ & 0.058 .0 \\
\hline 2181554 & 02580 & $2 t(x, 4)=4$ & $0.2(14) 4$ & 2017.4034 & $0.3 ! 16$ & 30.2454 & $0.0 \times(12$ \\
\hline $214+8.34$ & 0.2753 & $20.79 \times 94$ & 0.2077 & 2051154 & 0.2051 & 29.4214 & $0.06: 4$ \\
\hline $2 \therefore(1)+1)$ & 0.275 .4 & $208.13 \mathrm{mt}$ & $0.2(x))$ & $20 ?(x)+1$ & $0.2 \mathrm{kndl}$ & 31.8714 & $0 .(x, 1)$ \\
\hline $22 ! \therefore 14$ & 0.2739 & $268 x^{\prime}>284$ & (1) 20125 & 200.3104 & $(1,2,6 ; 0)$ & 34.520 .4 & 0.1706 \\
\hline 2230114 & (1) 262 & $260 .+(1)+$ & 0.2013 .5 & 107.0714 & $(128 \times 4$ & $36.97(\mathrm{mt}$ & nomen \\
\hline $2241 \cdots+$ & $0.20,51$ & $\therefore$ Rivolst & (1) $214+2$ & $105.5+54$ & 01.245011 & $34.63 ; 4$ & 0.1234 \\
\hline 225.565 .4 & $0.26,56$ & $276 \mathrm{~m}^{2}+4$ & $0201+1$ & 10301524 & 0.25 .2 & 4307114 & 0.1280 \\
\hline $2264 ! 1.4$ & $(1) \operatorname{Ln}(x)$ & $213 \times 14$ & $02014)$ & $1001.66: 4$ & 0270 & 4473114 & $0.1+42$ \\
\hline $2230 H$ & 0.2716 & 271.8311 & $0.20 \times 4$ & $185 ? 294$ & 0.2783 & 47.1804 & 0.1558 \\
\hline 214.2504 & 0.76 .5 & $272+124$ & (1.) 2018 & 185.7114 & (1.29.23 & 44.8314 & 0.1712 \\
\hline$\therefore 3(1<24$ & $0.20+3$ & $272454 t$ & $0: 2(126$ & $18336 \times 4$ & 0.27301 & 52.2804 & 0,1897 \\
\hline$\therefore 311101$ & (1.2t $2+5)$ & 2795444 & (1.2!x:? & 1804304 & $0.20 \% 1$ & 54.8004 & 0. $2(1) 6$ \\
\hline 23191144 & $1,26,55$ & $27+1504$ & $(1 . ! 01) !$ & $178+404$ & $0.2^{2} \div 4$ & 57.220 .1 & 0.2313 \\
\hline 722411 & 0.2711 & - 74 cind & $(111) \cdot 3$ & 175.604 & 0.2734 & 54.5214 & 0.2188 \\
\hline 2123514 & (1.2:4t & $275 \quad 2.101$ & 0.1197 & $1: 3.2014$ & 0.2714 & $61.47(1.4$ & 0.218 \\
\hline $213+40-4$ & 0.27 .25 & $=54524$ & (1) int & 170,0104 & $0.2 x+2$ & 6.4 .3504 & $0.232:$ \\
\hline 2310554 & $(i \geq 2)$ & $27 t: 6,2 t-1$ & $11) 4 ! 6$ & 1678504 & 0.2701 & 66.7104 & (1.24t22 \\
\hline $23 ! \cos d x$ & (1) 2f,is & $=7: 23(14$ & 01546 & 165.4204 & 0.2006 & 64.1104 & 0.2514 \\
\hline 235251 & $0 . ?(x)$ & $27 \mathrm{sin}+4$ & $11 v_{0}^{2}$ & 1628704 & 0.2816 & i). +20.4 & 0.2520 \\
\hline 234864 & (1) 26.84 & $2 x+40$ & $0.17+0$ & $10(x) 4(x) 4$ & 0.2713 & 7985144 & 0.2545 \\
\hline$\because 403: 24$ & (1) $20+x$ & $\because 4142$ & (1) 1420 & $157000 \mathrm{~d}$ & 0.2710 & 96.1044 & $0.26+n$ \\
\hline$\because 70804$ & 0.26io & $=10-5,1$ & Uthrid & 155.7504 & 0.2684 & 78.5204 & 0.2730 \\
\hline $278<1.4$ & 0.251 & $260.80 !^{\circ}$ & $(1) 1^{\prime}$ & $15306 \times 4$ & (1) $2 x x_{2}$ & $80.84(1.4$ & 0.274. \\
\hline 21804 & $\because 12 \div 1$ & $2 \times 16134$ & $614 \%$ & $150100 \times 3.1$ & $02 \leq 24$ & $82.2 \times(1.4$ & 0.2742 \\
\hline$\therefore \therefore n 1-4$ & 0.252 & $2 \times 14.31$ & 119012 & 148.164 & 0.2370 & $85(x) 4$ & 0 1) \\
\hline$\therefore " w \geq \cos$ & 12511 & $\therefore \because \therefore ?$ & $11211 \times$ & $1+5<504$ & 02615 & 8,05104 & 112855 \\
\hline$\because 1.4 \times+44$ & (1) $24 \times 1$ & $2 \therefore \cdots+i$ & " & 143.214 & 0.24 & य, $7 \mid 14$ & (1).2110x \\
\hline$\because 4.423$ & (1) 214 & $\because \therefore<t$ & $(12) 1^{\circ}$ & $1+11-7 ?(1.4$ & $11.2 t 1 x$ & 927904 & $(1), 4$ \\
\hline$\because \because \quad 1^{\prime \prime} \div$ & $(25, x)$ & $\therefore t \cdots$ & $11: 4$ & $148+44$ & $(1.2(x) 2$ & 95 ixont & (i. 2.28 \\
\hline$\because+19 x+4$ & $\begin{array}{l}112488 \\
17+240\end{array}$ & 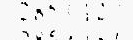 & $11 ;$ & 1758564 & (1) $-x+n$ & $\because 6114$ & $1,29+1$ \\
\hline$-1:: \because n$ & i1.2tit? & & $\therefore \ldots !$ & $194.3(x) 4$ & $(2.2) 618$ & 96 in i)t & 1.20 .70 \\
\hline
\end{tabular}




\section{Reinforced Concrete Test Data}

Track 2 - High Pressure Tesi

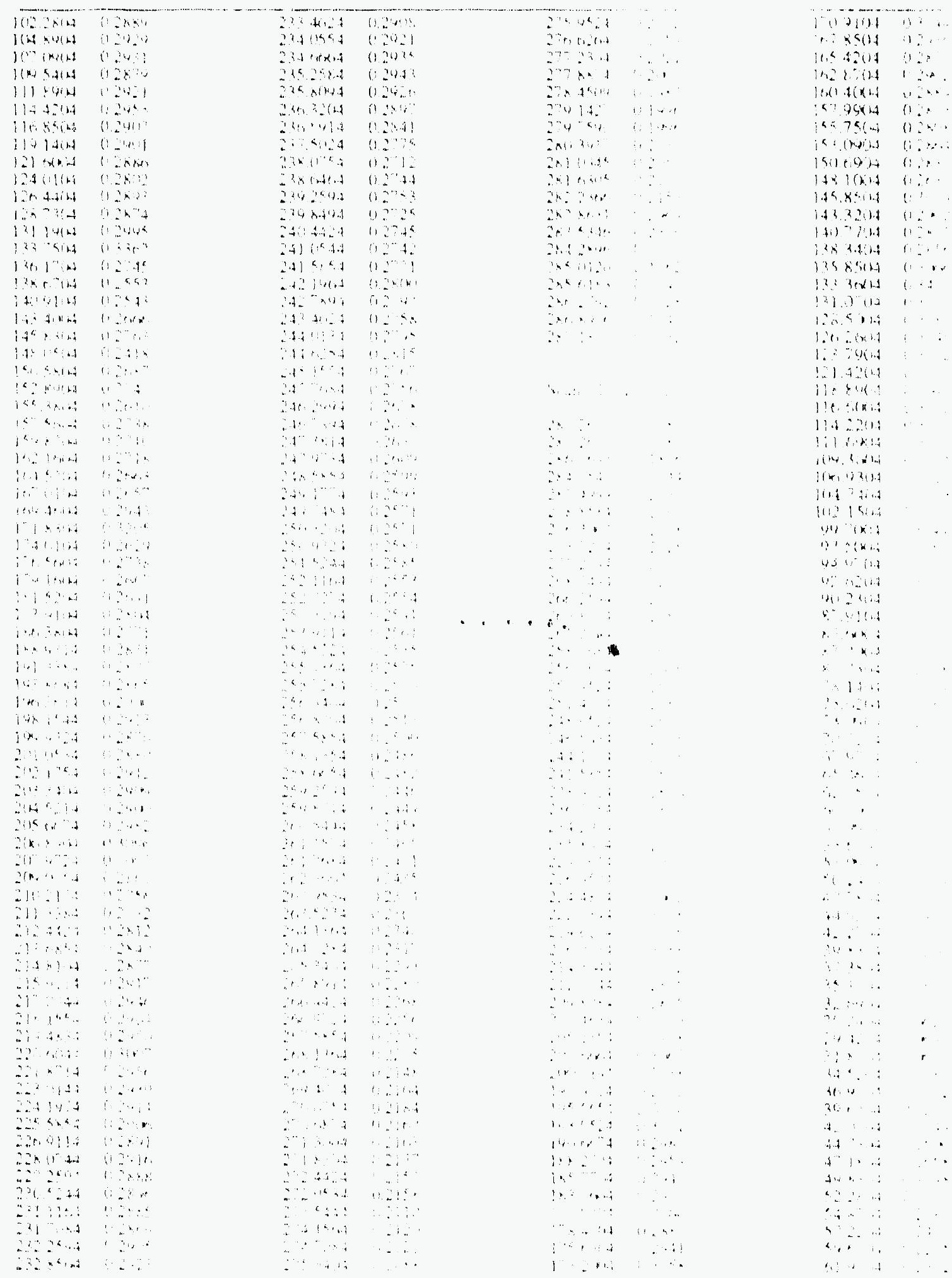




\section{Reinforced Concrete Test Data}

Track 2 - High Pressure Test

\begin{tabular}{|c|c|c|c|c|c|c|c|}
\hline ind 35ib & 02430 & 217.013 .44 & 0.3127 & $I(x), 442.4$ & 0.2375 & 207.46 .34 & 0.39013 \\
\hline 66.710 .4 & 0.2555 & $218.1^{5} 4$ & $C_{1} 3\left(\times_{2} 0\right)$ & 2669724 & 0.2360 & 2015.1154 & 0.3811 \\
\hline 69.111 .4 & 0.2654 & 219.48 .34 & 0.3141 & 267.5854 & 0.2433 & $202.66 x+4$ & 0.3666 \\
\hline 71.420 .4 & (1) $2(x) ?$ & 220.60144 & 0.3167 & 268.1 .364 & 0.2318 & 200.110 .4 & 0.3777 \\
\hline $73 \mathrm{sin}$ & 0.27604 & 221.8714 & 0.3130 & 268.7284 & 0.2275 & 197.9714 & 0.3727 \\
\hline $70.16 x-4$ & $6279 x^{\circ}$ & $\therefore 23.0144$ & 03123 & 269.4014 & 0.2281 & 105.5654 & 0.3750 \\
\hline $78.52(14$ & $0.2 \times \times 1$ & 224.1074 & 0.3128 & 270.0754 & 0.2270 & 193.0524 & 0.3715 \\
\hline $80.89(14)$ & 0.2943 & 225.5854 & 0.36184 & 270.6874 & 0.22 .43 & 190.6674 & 0.3692 \\
\hline 83.2804 & $0.204 !$ & 220.9114 & $0.3(k, y)$ & $271.30 \times 14$ & 0.2236 & 188.2794 & 0.3688 \\
\hline ES coloth & 0.20 .47 & 228.0744 & 0.3080 & 271.8301 .4 & 0.2240 & $18.5 .77(1) 4$ & 0.36 .4 \\
\hline 87.9514 & 0.30150 & 224.254 .4 & $0.30 \% 1$ & 272.4 .124 & 0.22 .47 & 183.360 .4 & $0.36 \times, 7$ \\
\hline 9031144 & (1) $33 x=7$ & 230.53 .44 & 0.3110 & 2729534 & $(1.2237$ & 180.9304 & 0.3577 \\
\hline $92.740 \mathrm{Li}$ & $(1.31 \times .5$ & 231.1164 & $0.30 \times 2$ & 273.5414 & 0.2224 & 178.41814 & $0.36+1.5$ \\
\hline $45(r)(4)$ & 0.31111 & $231.7(18.4$ & 0.30127 & 274.1564 & 0.22018 & 175.6704 & 0.3648 \\
\hline $97.6 ? 1.4$ & (i) Xlith & 232254.4 & 0.3170 & 274.7084 & 0.2199 & $173.2(0) 4$ & 0.3745 \\
\hline 908714 & 0.9210 & 222.850 .4 & 0.310 .4 & $275.34(1) 4$ & $0.218^{2}$ & 170.9104 & $0.3 \% 62$ \\
\hline $10 \%: 2 \times 14$ & $(1,2) 21 !$ & $233+4.24$ & $0.3(109)$ & 2749524 & 0.216 .5 & 167.85014 & $(0.3+0.05$ \\
\hline $1(4.8004$ & $(1.2) .9$ & 32289654 & 0.2110 & $276: 6264$ & 0.21 .40 & 165.42014 & 0.36 tit \\
\hline $10 \div .1914$ & $\left(1 . . i^{*} \because\right.$ & $244.6 x i 1.4$ & 11.4111 & 277.2304 & $0.2(x) 4$ & $162 \times 704$ & $0.36 .9 ?$ \\
\hline $10 \times 58114$ & $0.417 ?$ & 235.2484 & $6 ? 100$ & 277.8814 & u.zive & $160.4(x) 4$ & 0.3643 \\
\hline $111.31+4)$ & 0.318 & $2158(10) 1$ & (1) $4(1)$ & 278.4504 & (1.20) & 130.564 & 0.3572 \\
\hline $114+214$ & 0.3270 & 37622114 & (5.3) & $27414: 1$ & Bizitin & 15.5750 .4 & 0.35 .46 \\
\hline $136 \leqslant 512$ & $(1,3) 16$ & $236: 514$ & 11.201195 & 214.75001 & $0.2(k) 5$ & 153.00014 & 0.3822 \\
\hline 110 iftal & $0.31+4$ & 23750124 & (1).2128 & $280 \cdot 917$ & 0.2110 & $150.00 \times 14$ & $0.36 x+0$ \\
\hline $1: 1,604.4$ & (1) illwen & 238.1154 & 102872 & $2 \times 1.1345$ & 0.2177 & $148.11 \times 1.4$ & $0.33(x)$ \\
\hline $12+1: 1(H$ & $0.3(1.711$ & $2 \& B$ GHCH & U. 240 & 2616.3015 & 0.2213 & 145.851 .4 & $0 \tan 7$ \\
\hline $12 t .1 \times 11.1$ & $(1.3154$ & 239.2504 & 02015 & $28: 230,0$ & 0.2211 & $143.32(1.4$ & $0.36 \times 6$ \\
\hline 128,1124 & $(1,114$ & $2 \therefore \because 814$ & $0.26(19$ & $2 x_{2}=k 6,31$ & 0.2324 & 140.7764 & 0.3585 \\
\hline 13110124 & $11.17 \mathrm{th}$ & 240424 & 0.254 .1 & 2625340 & 0.2218 & $138.1+114$ & $0.2^{2} 54$ \\
\hline $1325(\mathrm{H})$ & 0.30 .21 & 241.01544 & $11.2411 ?$ & $2 x+2500$ & (1. $(19.58$ & 125.850 .4 & $0.374 \pi$ \\
\hline $136,1: 14$ & 0.21111 & $241.5 \times 44$ & 0.2434 & 28501201 & $0.110: 5$ & $1.39 .3(x) .4$ & $0.414 ;$ \\
\hline $1.386 ? 0 \mathrm{nd}$ & 0.2742 & 242164.4 & $(1.075$ & $2 \times 561 \times 3$ & 0.1767 & 131.0714 & $0.30 \times 5$ \\
\hline $1+(0,9)(4$ & 0.2 .20 & 242.184 .4 & $(1.2) / 51$ & $28(1.271) ?$ & 0.1790 & $1285(1 \times 1.4$ & () 3841 \\
\hline$: 14.4(k) .4$ & $(12,8 x$ & $213.4(124$ & 0.24 .49 & 286,8406 & 0.1919 & $126.26(4)$ & 0.39 .38 \\
\hline 1.758 .465 & 0.2452 & $24.4(1) 3.4$ & 0.21421 & 287.1877 & 0.11880 & $123.7 \times 1.4$ & 0.3817 \\
\hline $1.181151 \mathrm{H}$ & 112944 & 2446.254 & 0.2924 & \multirow{3}{*}{\multicolumn{2}{|c|}{ Soan 12. 1258 poip }} & 121.42014 & 0.3814 \\
\hline $19011.5 \times 124$ & 02840 & 2.15 .1564 & $(1,2) 1^{2}$ & & & $118.86(1) 4$ & 0.3015 \\
\hline $152 \sin x(4$ & 0.2873 & 215768.1 & $0.2(1.31)$ & & & $116.6(x) 4$ & 0.38 .45 \\
\hline 155,28511 & 02781 & 2462344.4 & 0.2851 & 287.2670 & 0.2266 & 1142204 & $0.3(x) ?$ \\
\hline 157.50014 & $0.20(x)$ & 246.7804 & $0.28 . \therefore$ & 287.2428 & 0.2141 & 111.60014 & 0.3867 \\
\hline $150 . x^{-1}(1+1$ & 91.20142 & 2.47 .3814 & 0.2142 & 24670,45 & $(1.2) 117$ & $1(k) .2604$ & $0.38 \times 11$ \\
\hline $16.216,14$ & $(1.24 .213$ & 24.973 .4 & $(127 \% 1$ & $20,0<4$ & $0.1-60$ & $1(x, 9) 3014$ & $0.386,4$ \\
\hline $1+.4 .5 \geq(1.4$ & $0.2 \times+1$ & $248 \times 154$ & 0.2740 & $281+4004$ & $(1.2)$ & $1(1-1.7+46)$ & $0.30(4)$ \\
\hline 1610114 & $(: 2 \div 34$ & 2411774 & 0.27 .46 & $278 \times 481$ & $0.2+(x)$ & $11121.10 \mathrm{~m}$ & 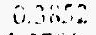 \\
\hline 160.46 .64 & 0.2120 & 249.7464 & 0.2726 & $276.3(x) 4$ & 1.2435 & 90.7164 & 0.3790 \\
\hline $17 ! R .414$ & (1) $42(x)$ & 2403204 & 0.2725 & 273.727 .4 & $(1.24,37$ & $07.5(x):$ & 0.39011 \\
\hline 1740104 & $0.18+8$ & 250.4324 & 11276 & 271.2794 & 0.2520 & $9407(14$ & 0.3645 \\
\hline 17056014 & 029.41 & 251.5244 & 0.2725 & 268.7404 & (). $2 \operatorname{trta}$ & 92.61214 & $(0.1700$ \\
\hline 179.16612 & 0.384 & 2521164 & 11.2704 & 266.2564 & $0.2+192$ & $902.2(1.4$ & 0.3750 \\
\hline 181.5214 & $0.2 \Delta t t^{4}$ & 252.727 .4 & 02722 & $26383(4)$ & $0.28(1)$ & $87.4)(1.4$ & 0.378 .5 \\
\hline 1839114 & 0.2970 & 2533194 & 0.2718 & 261.2744 & 0.285 & $85,4 \times 14$ & $0.76,4.3$ \\
\hline 180.3804 & $0.205 x$ & 2529114 & $0.26,51$ & 258.7064 & 0.2000 & 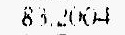 & 0.3941 \\
\hline 188.9314 & 0.31615 & 254.5224 & $(1)+6-10$ & 240.3614 & $0 . .1127$ & 801730.4 & 0.3473 \\
\hline $19 ! 3384$ & 6.2005 & 255.1364 & $(1.264)$ & 2539524 & 0.3120 & 78.1404 & 0.3421 \\
\hline 193 kfthis & 0.311013 & 259.7281 & $0,26,52$ & $2<1.4014$ & 0.3716 & 7562012 & 0.3288 \\
\hline 10630.1 .4 & 60.801 & 2562404 & 0.20 .50 & 248.4524 & (1).31761 & $7.3 .(x)(1) 4$ & 0.2179 \\
\hline 198.154 & $0.307:$ & 256.87014 & $0.20 ? 5$ & 2.46 .5 .47 .4 & (). .270 & $70 \leq 201.4$ & () 31400 \\
\hline 1046224 & $0.361 \times 4$ & $2.9 .5 \times 54$ & $(1.20 .30$ & 244.1154 & 03419 & 67.0764 & 0.3012 \\
\hline$\therefore(1), 1424$ & 0.30701 & $25: 1354$ & 0.26 .39 & 2415854 & 0.3446 & $65.46(14$ & 0.208 .4 \\
\hline 201754 & 0.3112 & $258+54$ & 02632 & 238.492 .4 & 01.3 .3 .50 & 6245014 & 0.2800 \\
\hline $2(133.414$ & 0.3150 & 3592514 & 0.2572 & 236.7034 & 0.3456 & $6(x) .50 \times 4$ & 0.2553 \\
\hline $2(4,52) 14$ & $0.30 x_{2}$ & 254,3714 & $024 ? 0$ & 2.34 .2904 & 0.3618 & 57.9604 & 0.2579 \\
\hline $2016(x, 7)$ & 0.3151 & Zncirit11 & 0.570 & 231.83014 & 0.3554 & 55.6104 & 0.2168 \\
\hline $2(6,8)(2-8$ & 0.3252 & 261.1571 & 0.2584 & ans.abit & 0.36 .27 & $53.190(1) 4$ & 0.2241 \\
\hline 2179724 & 0.318 .9 & 261.7684 & 0.2501 & 2264524 & $0.36 .2 ?$ & 50.2404 & 030159 \\
\hline 214.1194 & $(1,2,7$ & 2623601 & 0256 & $224+48.4$ & $0,3+42$ & 47.7814 & (1). 1 k1th \\
\hline 210.2174 & $(124) 4$ & 262.9534 & 0.25011 & 22201344 & 0.3740 & $4.493(1.1$ & 01723 \\
\hline 211.2384 & 0.2931 & 26.35 .234 & $0.2 .4+4$ & 219.6 .754 & $0.981 k 1$ & $42.27(1.4$ & 0.3474 \\
\hline 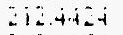 & üinin & anitiona & $0: \because\}$ & $2161: 1: 4$ & 8.7? & $30 \mathrm{~g} 3 \mathrm{rL}$ & $0 ! 317$ \\
\hline 21326854 & 0.3026 & 264.7284 & 0.2416 & 214.56 .4 .4 & 0.3741 & $37.380 \mathrm{~A}$ & 0.1101 \\
\hline$\because 12.8104$ & $0.3(1.4 .5$ & 205.3404 & $(1.2401)$ & 21207.4 & 0.3527 & 321504 & $0.6 \times 129$ \\
\hline 2150114 & 0.3127 & $? 68914$ & 0.2315 & $20982 \times 4$ & 0.33887 & 32.6004 & $0.0 \times 185$ \\
\hline
\end{tabular}




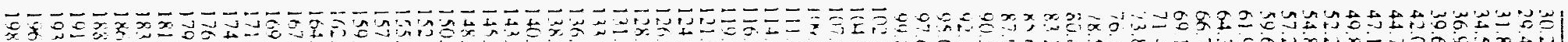

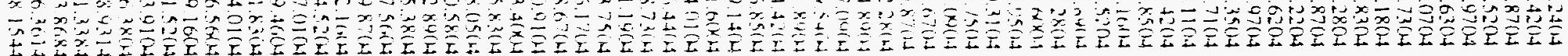
$c 0=00=0=00000=0000000000000000000000000=0000000000000000000000000000$

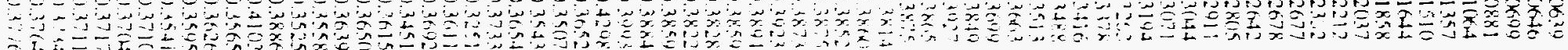

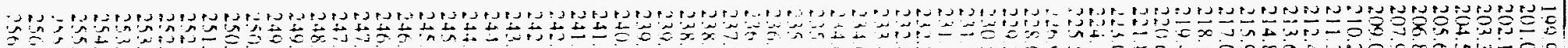

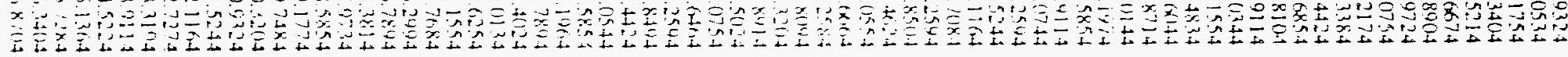

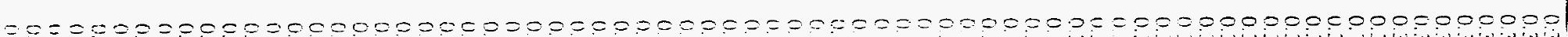
等

$?$

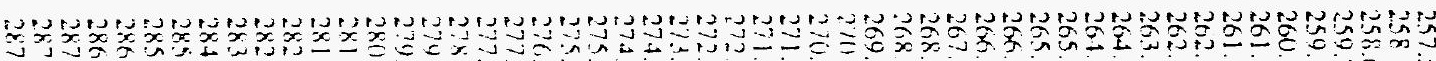

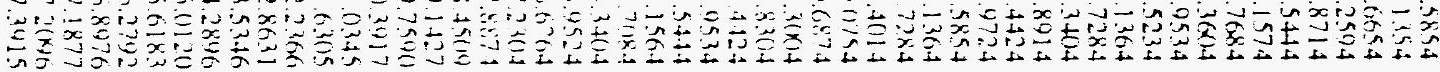

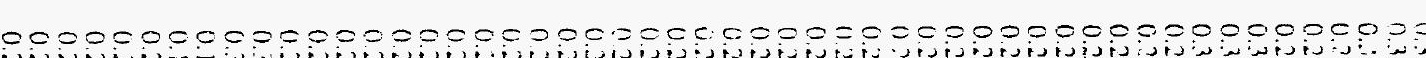

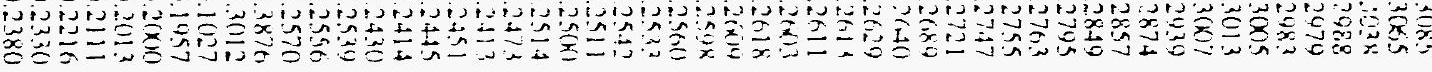

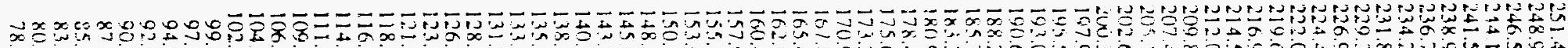

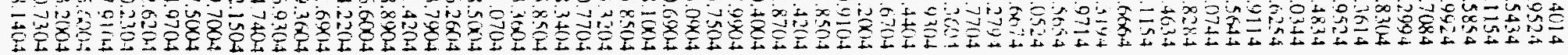
000000000000000000000000000000000000000000000000000000000000000000000000

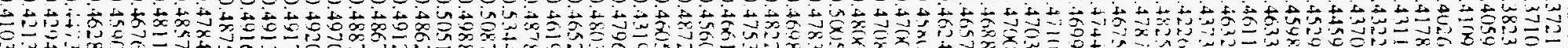




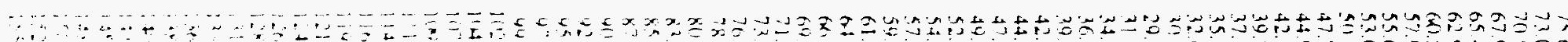

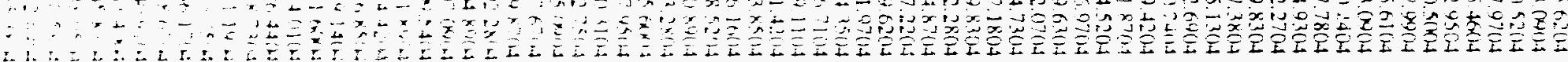

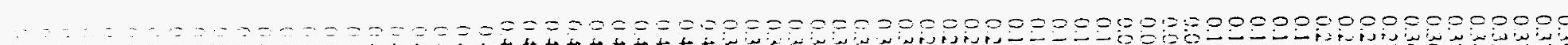

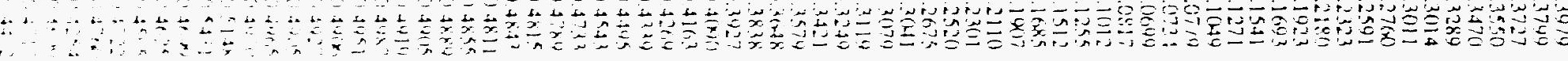

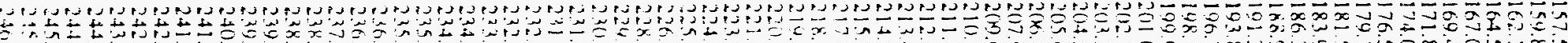
W

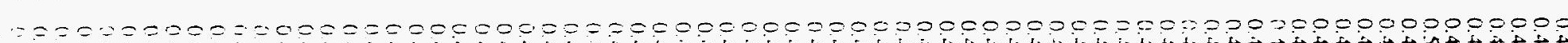

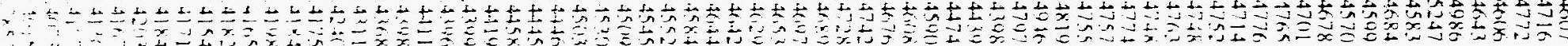

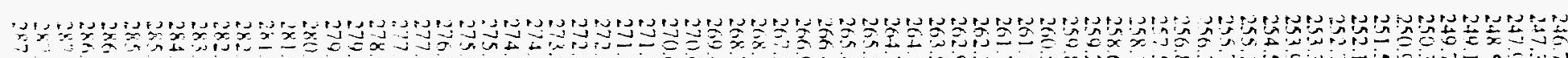

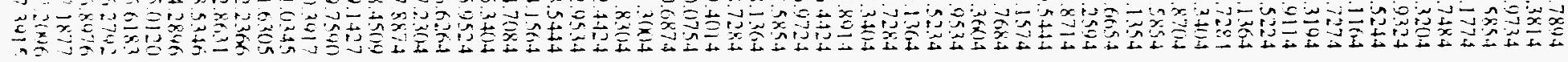

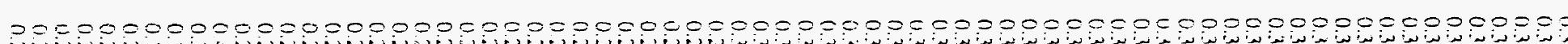

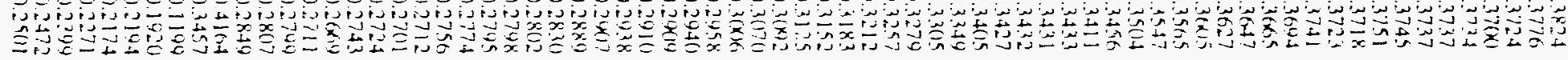

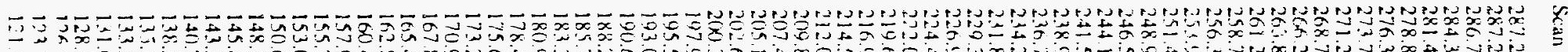

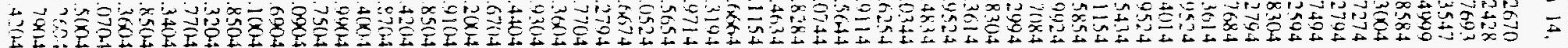

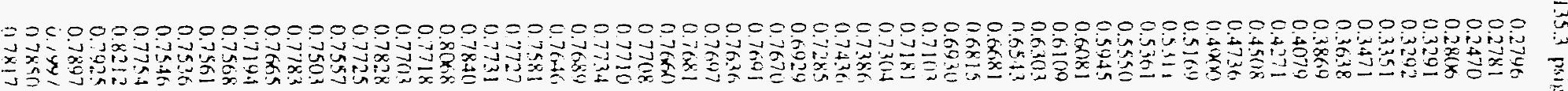




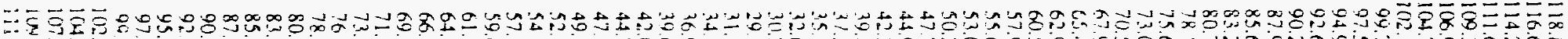

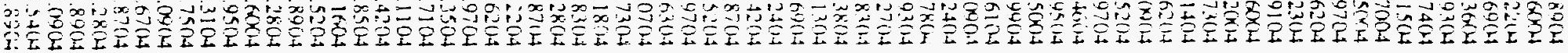

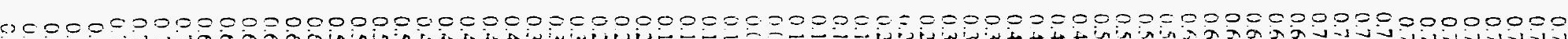

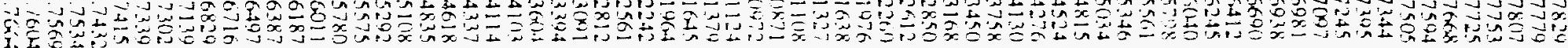

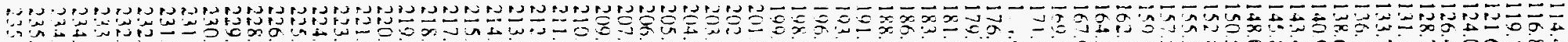

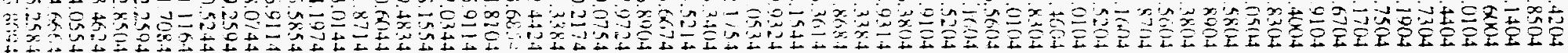
$=000000=00000000000000000000=0000=000=0000000000=00=00=00000000000000000$

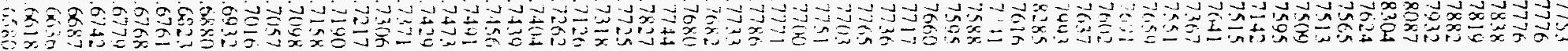

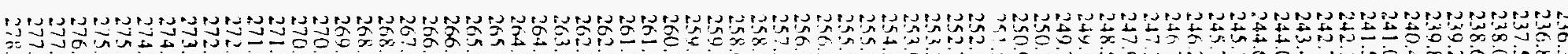

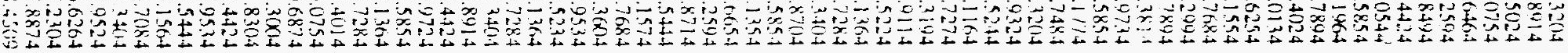
000000000000000000000000000000000000000000000000000000000000000000

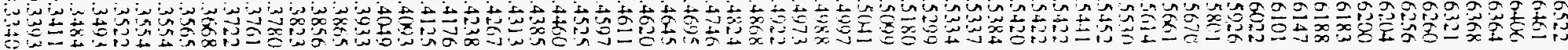

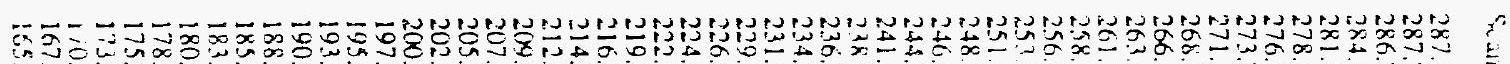

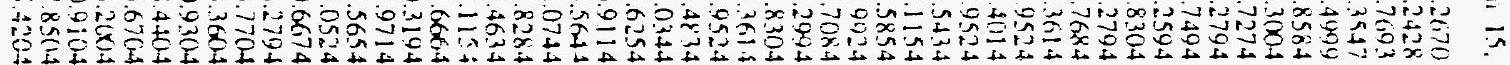

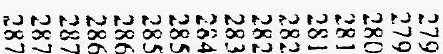

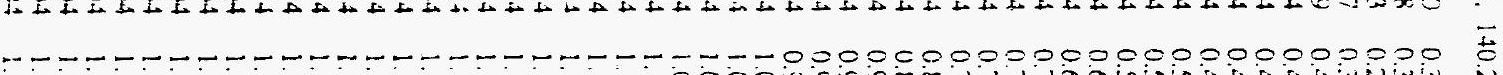

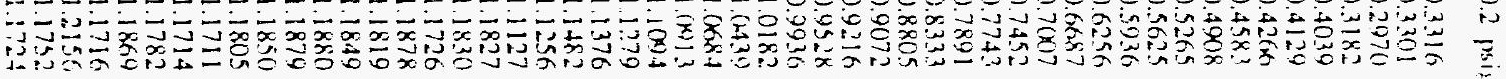

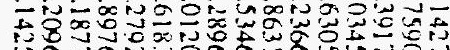
0000000000000000

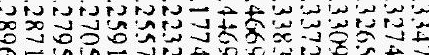




\section{Reinforced Concrete Test Data}

Track 2 - High Pressure Test

\begin{tabular}{|c|c|c|c|c|c|c|c|}
\hline $162.876 \mathrm{~m}$ & $1.18(1) ?$ & 71.4204 & 0.7569 & 2211 end & 1.1316 & $26,8.1364$ & 0.5451 \\
\hline $160.40 \times 4$ & $(\mid f \infty) ?$ & 73.8504 & 0.7947 & $21 \times: 14$ & 11227 & $26872 \times 4$ & 11.5327 \\
\hline $157 . \overline{9} 4(14$ & 1.1300 & 76.1614 & 0.8277 & $\therefore 30114$ & 1.1140 & 269.4014 & 0.5227 \\
\hline 155.7504 & 11315 & $7852(44$ & $0.8(x) 4$ & $22+104$ & 11140 & 270.0754 & 0.5193 \\
\hline 157.19004 & 1 I $68 k$ & $80 k 1 \times 1.4$ & 0.8882 & $\therefore \operatorname{ses} 4$ & $100 s 0$ & 270.6874 & 0.5144 \\
\hline 150.6904 & $1.136 x$ & 83.3804 & 0.9222 & $22+6 ! 14$ & 1.0852 & 271.3004 & 0.5072 \\
\hline $148.1 \mathrm{ckm}$ & $1 .(14)(x)$ & searsh & 0.9465 & $\therefore \times 1: 44$ & 10774 & 271.8304 & 0.5024 \\
\hline 149.8504 & 1.1540 & $\delta^{-0}$ us, od & $(1,9) 8 \mathrm{~s} ?$ & $\therefore 4: 84$ & 10590 & 272.4424 & 0.4926 \\
\hline $143.32 \mathrm{~cm}$ & $1.12 \%$ & w : lint & (1.)4K, & $\therefore 41 \div+4$ & $i 05(k)$ & 272.9534 & 0.483 .4 \\
\hline 140.7704 & $1.132=$ & $4 ?, 25,1$, & 10314 & $z: 1|1| x .4$ & i. (45) & 273.5144 & 0.4738 \\
\hline 138.3404 & $1133 \mathrm{~m}$ & is inn if. & 1.61841 & 231 ind & $1013 \times 1$ & 274.1564 & 0.4683 \\
\hline $135.85(\mathrm{lt}$ & 11544 & $1+11.4$ & 1.0708 & $22: 2001$ & 101277 & 274.7084 & 0.4626 \\
\hline 133.3604 & $118: 4$ & $44 \times: 4$ & l.6xt-4 & $\because \therefore<14$ & 101347 & 275.3404 & 0.4566 \\
\hline $131.07 \mathrm{ht}$ & $1.173+$ & 1122814 & 1.09 .36 & $\therefore: 12+4$ & 1020. & 275.9524 & 0.4510 \\
\hline $128.50(0) 4$ & 1.1002 & 1148014 & 1.1112 & $234+1<8:$ & $1111 \times 4$ & 276.6264 & 0.4455 \\
\hline 126.2604 & $11 ? 16$ & $\therefore:$ indis & 1.1120 & $24+2 x+4$ & $1(x, x, 3$ & 277.2304 & 0.4331 \\
\hline $123.74(4$ & 11602 & lint sitint & 1211 & $\therefore \therefore: 4 a t$ & (1) ocisis & 277.8874 & 0.4284 \\
\hline 121.4204 & 1.16 .4 .4 & iil suld & $1.179 ?$ & $\therefore<A+1$ & (i) () () 44 & 278.4509 & 0.4273 \\
\hline $118 \times 794$ & 1.15317 & $11.4+2(1.4$ & 1.14 .30 & $24, \cdot 11.4$ & inits & 279.1427 & 0.4218 \\
\hline $116.60 \mathrm{KH}$ & .1524 & $1: 0.561 .4$ & 1.1 .415 & $\because \cdots, \times+1+$ & (1) 1$) " 16.1$ & 279.7590 & 0.4129 \\
\hline 114.2204 & 1.19 .2 & ild j.11.4 & 1.1525 & $\therefore 4.24$ & (1) $90 \mathrm{k} x \mathrm{x}$ & 280.3917 & 0.4127 \\
\hline $111.600 \mathrm{H}$ & $1.1 .7+1$ & $3: 1 \times \times 4$ & 1.1582 & $\therefore \therefore \cdots 4$ & $(196,10)$ & 281.0345 & 0.4110 \\
\hline $16 x .3614$ & 1.1326 & 12111114 & 1.1547 & $\because \because 1,1 / .1$ & 114579 & 281.6305 & (0.4086 \\
\hline $1(6.0314$ & 11172 & $\because+1+111$ & 1 leist & $2 \times 2<4$ & $(105) 4$ & 282.2366 & 0.41 .39 \\
\hline 104.7404 & 1110 & $12 x-31.4$ & $1.16 .7=$ & $2: 51+1$ & $\left(1199_{2}\right)$ & 282.8631 & $0.540^{\circ}$ \\
\hline $1012.15(\mathrm{~L})$ & $\mid$ |xis & 121014 & 1.1821 & $\because 41: 1$ & $(10.384$ & 283.5346 & 0.5374 \\
\hline 99.7004 & $1.174^{-1}$ & $1: 2: 6114$ & 1.2683 & $\therefore 4: 44$ & $09 \div 21$ & 284.2896 & 0.24 .30 \\
\hline 97.5004 & 1.0733 & $130)^{-1}(14$ & 1.910 & $\therefore 4<\because 4$ & 114263 & 28.5 .01201 & 0.2442 \\
\hline 94.97014 & $1.050,3$ & 1350204 & 1.1368 & $272: 21$ & $(10178$ & 285.6183 & 0.2976 \\
\hline $92.62(1.4$ & 1.0242 & $1+1410104$ & 1.1250 & $\because 42-x+1$ & $(4,4):$ & 286.2792 & 0.3012 \\
\hline 90.2 .301 & $1.0 \times 12 ?$ & $1.2311 \times 14$ & 1.1155 & $\therefore 4+24$ & $(191 k+1$ & 286.8976 & 0.3132 \\
\hline 87.910 .4 & 0.9408 & $186 x^{2} 14$ & 1.1284 & $24+144$ & $(1 \times 1): 1$ & 287.1877 & 0.32 .44 \\
\hline $85.6(x) 4$ & 0.96 .70 & $14 \pi 01511.4$ & 1.0820 & $24+1.254$ & $1+8 B(13$ & 287.2096 & 0.3 .340 \\
\hline $83.20 \times 14$ & 00914 & 1501 sidet & 1.1126 & $\therefore<1<x$ & $08: 10$ & 287.1425 & 0.3371 \\
\hline 80.73014 & OBnis & $1 \because x(8) 14$ & 1.1 .116 & $24 \cos 4$ & $f x x_{1} x^{\prime}$ & & \\
\hline 78.1404 & $0.85 \times 11$ & $1553 \times 14$ & $111 \%$ & 246. $2(x) 1$ & $1) \times 426$ & & \\
\hline 75.6204 & 0.8223 & $15 ? \operatorname{sen} 14$ & 11.3 .42 & $2+40-84$ & $(1.836,2$ & Scan 16, 1 & 45.8 psizz \\
\hline 73.090 .4 & $0.78: 2$ & 1548014 & 1.1 .5196 & $24: 9 \times 14$ & O $k ? \%$ & & \\
\hline 70.5204 & (0) $7 \mathrm{ki}^{3}$ & $10: 1+0(14$ & $1: 16,25$ & $25: 474$ & $(18177$ & 287.2670 & 0.3920 \\
\hline 67.9704 & $0 . n^{\prime}(\mu) h$ & $10.4 \div 201.4$ & 11001 & $2465 x+4$ & 0.8104 & 287.2428 & $0.390 \times 1$ \\
\hline 65.460 .4 & $0.6,22$ & 10.09104 & 0.1712 & $\therefore 41 \% 4$ & $(180187$ & 286.7693 & 0.3730 \\
\hline 62.9504 & 06.311 & $10.1)$ tix) & 1.2167 & 24.741 & $(18014$ & 284.3547 & 0.3820 \\
\hline $60.50(1) 4$ & 0.582 .5 & $1:: 33(14$ & $1.23,31$ & .9142911 .4 & $(1)-7007$ & 281.4999 & 0.503 .5 \\
\hline 57.90014 & 0.56111 & $10+1914$ & 1.1736 & $2: 1 \div 1$ & 07021 & 278.8584 & 0.5245 \\
\hline 55.610 .4 & $0.5(r) 1)$ & $170.5(104$ & 1.1807 & $2<15344$ & 0.78 .41 & $2 \% 6.3(014$ & 0.5527 \\
\hline 53.01904 & $0 . t \tan A$ & 1016,114 & 1.1737 & $\therefore: 1 i+n d$ & (i)7.36 & 273.7274 & 0.5946 \\
\hline $502+0.4$ & $0431 ?$ & $1 \mathrm{H} ! 3214$ & 1.1110 & 2394 & 11.7 .73 & 271.2794 & 0.6424 \\
\hline 47.78114 & $0.34: 7$ & $\left.1 x^{2}+1\right) 14$ & 118015 & $2<2 \times 14.4$ & $(1,70.25$ & 268.7494 & 0.6981 \\
\hline 44.4304 & 0.1476 & Ixt, in: & 1.1857 & $2 \times 21114$ & $11.74 x^{\prime}:$ & 266.2594 & 0.7511 \\
\hline 42.2704 & 0.246 .1 & 1884314 & 1.1051 & $24 \times 24$ & 017.164 & 26.8 .8304 & 0.7928 \\
\hline 39.83014 & $0.24 \times 3$ & 1413284 & 1.1473 & $2 \ll<134$ & (1)7284 & 261.2794 & 0.8468 \\
\hline $37.38(44$ & 0.2254 & 14 xeint & $1.19(11)$ & $295: 2 \times 4$ & 07210 & 258.7684 & 0.9115 \\
\hline 35.1304 & $0 . \mid 8.1$ & 1403014 & 1.1974 & 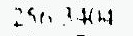 & (0).7126 & 256.3614 & 0.9635 \\
\hline $32.69(14$ & $0.144 x$ & 1175.1544 & 1.193 .4 & $29+\times 7(14$ & $07(x+1)$ & 253.9524 & 1.0232 \\
\hline 30.2404 & 0.1256 & 144022.4 & 1.1962 & $20: 584$ & $(1)(1, x)()$ & 251.4014 & 1.0691 \\
\hline $25.420-8$ & 0.1150 & (1) 1614 & 1.1971 & $2 \times 1 \times 4$ & $(1), 1) 11$ & 248.9524 & 1.0936 \\
\hline $31.87(14$ & 0.1517 & $\because \because 1754$ & 1.1990 & $2 x+x<4$ & $(i t) \times 36$ & 246.5434 & 1.1510 \\
\hline 34.5204 & 0.1852 & $2(1: 3414$ & 110.40 & $\because k ., 2<i, 1$ & 0.6704 & 244.1154 & 1.2300 \\
\hline 36.9704 & 0.2210 & $2+4<: 1+$ & 1.1603 & $2<+14$ & $\theta(x) 18$ & 241.5854 & 1.2698 \\
\hline 39.6304 & $0.2(1) x$ & $21,4,4$ & 11944 & 2010444 & 06534 & 238.9924 & 1.2971 \\
\hline 42.0704 & 0.2480 & $\therefore x \times 14$ & 1.2115 & $20: 1<+4$ & $0(m+1$ & 236.7084 & 1.3420 \\
\hline $44.730 \mathrm{At}$ & 0.3474 & $31^{*}+24$ & $118 \times 3$ & $\therefore i^{-1}+1$ & (1) tod & 234.2094 & 1.3990 \\
\hline $47.1804^{\circ}$ & $0.3 \times 19$ & $2+4<1$ & 1.1728 & $\therefore \therefore \quad 24$ & 00,46 & 231.8304 & $1.4 .36 i$ \\
\hline 49.8304 & $0.4 \div 1 ?$ & $\therefore \mathrm{Zi}: \mathrm{i}^{-}+$ & 11145 & $\therefore \therefore 44$ & 06.38 & 229.3614 & 1.4737 \\
\hline 52.280 .4 & $0.40^{\circ}=$ & $211: 3 \times 4$ & 1.1304 & $\therefore: 2<: 34$ & (1) (1) 40 & 226.9524 & 1.5010 \\
\hline 54.8704 & $0.49 \pi, 1 \mathrm{~m}$ & $\therefore 12+124$ & $1.194 x^{\prime}$ & $\therefore+41\}+4$ & $0.6 \times 151$ & 224.4834 & 1.5234 \\
\hline $57.22(\mathrm{H}$ & 0.5544 & $\because 12 \cos 4$ & $11+(01$ & $21.4 \quad 2 \times 1$ & $(18074$ & 222.0344 & 1.5493 \\
\hline 59.6204 & $0.571 ?$ & $314 \times 1014$ & 1. Isis & $2+<3+11.4$ & 0.5845 & 219.6254 & 1.5694 \\
\hline 61.9704 & 11.60174 & $215+114$ & 1.1534 & $2 t<\operatorname{sidj}$ & 119827 & 210.9114 & 1.5820 \\
\hline 64.3504 & 0.6536 & 2111244 & $15: ?$ & ine $44: 4$ & 015746 & 214.5644 & 1.5860 \\
\hline $66.71 \mathrm{in}$ & Uneils & :in 1994 & 11400 & $2(x, 1) \geq 4$ & (1) $\left\{(x)_{2}\right)$ & 212.0744 & 1.5673 \\
\hline 69.1104 & 0.7212 & $2104+134$ & $114.4 ?$ & $2678 \times 94$ & 0.5553 & 209.8284 & 1.5331 \\
\hline
\end{tabular}




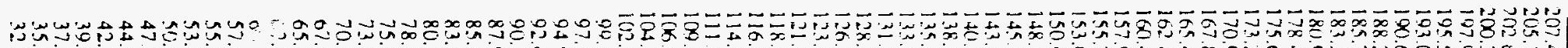

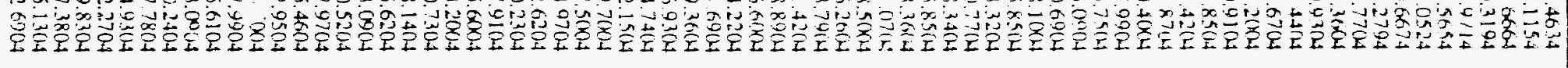
copocoon 0000000

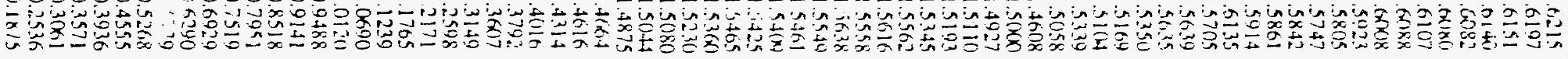

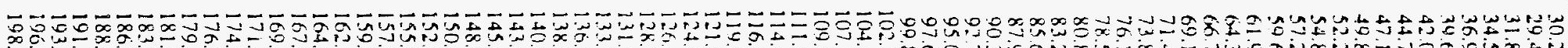

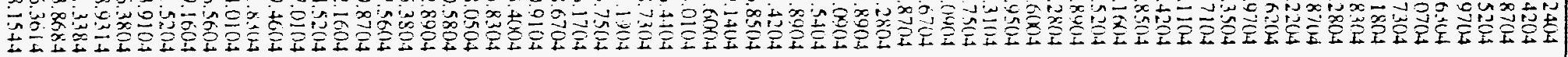
更

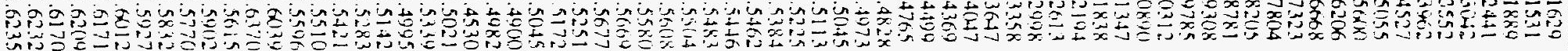

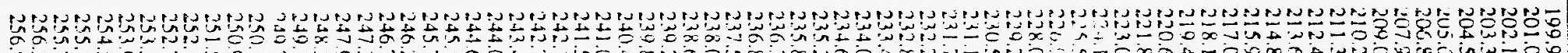

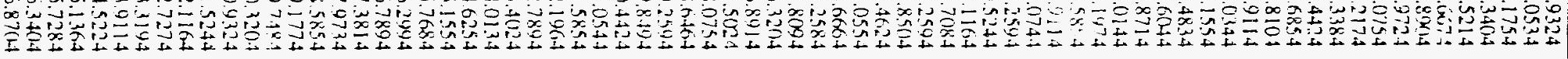

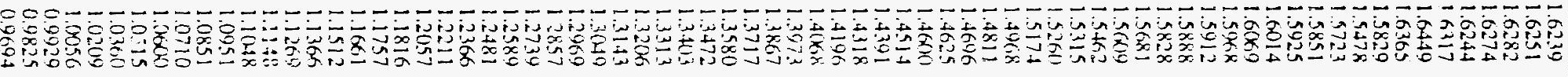

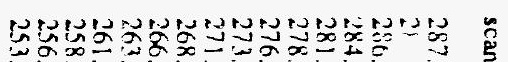

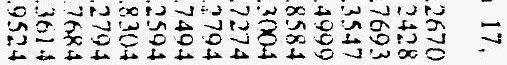
$000000000000=00, \bar{m}$

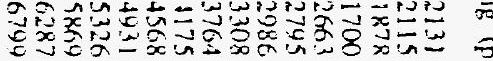

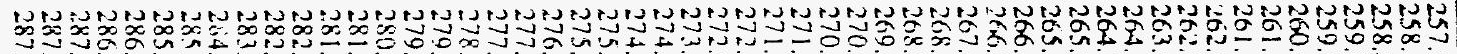

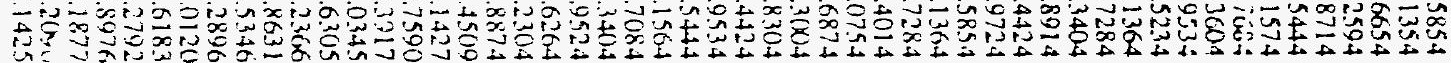

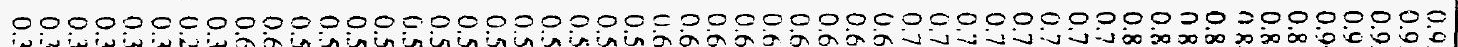

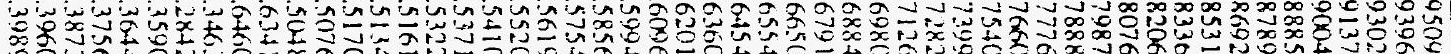




\section{Reinforced Concrete Test Data}

Track 2 - High Pressure Test

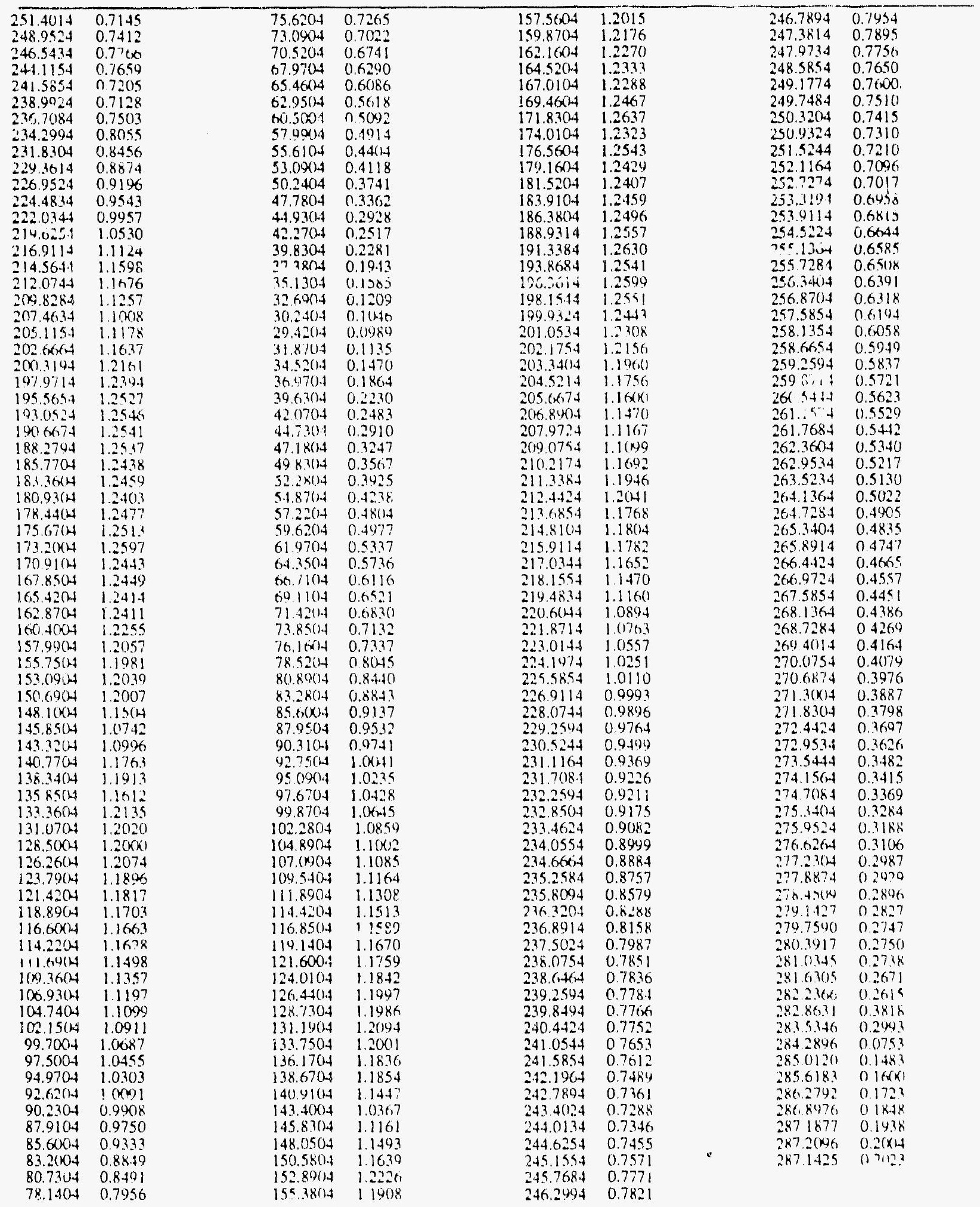


Track 3 - High Pressure Test

\begin{tabular}{|c|c|c|c|c|c|c|c|}
\hline \multicolumn{2}{|c|}{ Scan 1, 49.2 psig } & \multirow{2}{*}{$\begin{array}{l}80.69 .38 \\
78.328 .8 \\
75.9578\end{array}$} & \multirow{2}{*}{$\begin{array}{l}0.09588 \\
0.10688 \\
0.1009\end{array}$} & \multirow{2}{*}{$\begin{array}{l}54.4918 \\
52.0718 \\
49.6268\end{array}$} & \multirow{2}{*}{$\begin{array}{l}0.0811 \\
0.0800 \\
0.0697\end{array}$} & \multirow{2}{*}{$\begin{array}{l}36.3248 \\
38.7948 \\
41.3228\end{array}$} & \multirow{2}{*}{$\begin{array}{l}1.0446 \\
0.0581\end{array}$} \\
\hline 97.6000 & 0.0729 & & & & & & \\
\hline 97.5931 & $0.078 \%$ & 73.5928 & $0.10 \times 18$ & 47.25 .38 & 0.0646 & 43.8428 & 0.074 .3 \\
\hline 97.2149 & 0.0755 & 71.1538 & 0.0999 & 44.7828 & 0.0509 & 46.3158 & 0.0825 \\
\hline 95.0729 & 0.0801 & 68.8018 & 0.0890 & 42.4108 & 0.0538 & 48.7748 & 0.0889 \\
\hline 92.7148 & 0.1123 & 66.4 .368 & 0.0865 & 39.9178 & 0.0492 & 51.2488 & 0.1007 \\
\hline 90.2487 & 0.1119 & 04.0378 & 0.1419 & 37.4978 & 0.0365 & 5.3 .7548 & 0.1061 \\
\hline 87.8428 & 0.08017 & 61.6538 & 0.0816 & 35.0658 & 0.0280 & 56.2348 & 0.1078 \\
\hline 85.44 .38 & 0.0816 & 59.2828 & 0.0731 & 32.5788 & 0.0203 & 58.6798 & 0.1140 \\
\hline 83.0658 & 0.086 .3 & 56.8558 & 0.0768 & 31.6388 & 0.0216 & 61.1268 & 0.1159 \\
\hline 80.69 .38 & 0.07 .39 & 54.4918 & $0.0 \% 22$ & 33.8758 & 0.0241 & 63.6198 & 0.1320 \\
\hline 78.3288 & 0.0843 & 52.0718 & $0.0 \times 88$ & 36.3298 & 0.0250 & 66.1068 & 0.1289 \\
\hline 75.9578 & 0.0779 & 49.6268 & 0.0630 & .38 .7948 & $0.04(18$ & 68.5728 & 0.1366 \\
\hline 73.5928 & 0.07 .45 & 47.25 .38 & 0.0541 & 41.3228 & 0.0512 & $71.0(148$ & 0.1396 \\
\hline 71.1538 & $0.077 ?$ & 44.7828 & 0.0457 & $43.842 x$ & 0.01535 & 73.5118 & 0.1398 \\
\hline 68.8018 & $\operatorname{lick>0}$ & 42.4108 & $0.04+4$ & 46.3158 & 0.06615 & 76.0118 & 0.1499 \\
\hline $\operatorname{tac} .4368$ & 0.0006 & $34017 k$ & 0.0 .580 & 48.7718 & $0 .(x+4$ & $78.559 \mathrm{~g}$ & 0.1427 \\
\hline 64.0378 & 0.1650 & $3 \% .4978$ & 0.0240 & $51.24 k x$ & 0.0771 & $81.295 \mathrm{k}$ & 0.1393 \\
\hline 61.6538 & $0.0 \times 17$ & 35.0658 & 0.1211 & 537548 & 0.0879 & 8.7806 & 0.1174 \\
\hline 59.2828 & 0.0597 & 32.5788 & 0.01155 & $56 ? 348$ & 0.0891 & 86.2548 & 0.1450 \\
\hline 56.8558 & 0.0549 & 31.6388 & $0.00 \times 0)$ & 58.6798 & 0.0893 & $8 \times 6945$ & 0.1418 \\
\hline$\$ 4.4918$ & 0.0540 & 33.8758 & 0.018 .4 & 61.1268 & 0.0948 & $01153 k$ & 0.1705 \\
\hline 52.0718 & $0.0 .49 ?$ & 36.3208 & 0.020 .3 & 63.6198 & 0.1209 & $93.6 \times 608$ & 0.1524 \\
\hline 49.6268 & 0.0417 & 38.7948 & $0.03(14$ & $66.10 \times, 8$ & 0.1052 & 96.1674 & 0.1375 \\
\hline 47.2538 & 0.0407 & 41.3228 & $0.64 \leqslant 9$ & 68.5728 & $0.10+6$ & 97.56602 & 0.1787 \\
\hline $44.7 k 2 k$ & 0.0347 & $4 ? 8.4 ? 8$ & 0.0473 .3 & 71.0048 & 0.1121 & & \\
\hline 42.4108 & 0.0337 & 46.3158 & (). (1.493 & 73.5118 & 0.1123 & & \\
\hline 39.9178 & 0.0271 & 48.7748 & 0.0546 & 76.0118 & 0.1222 & \multirow{2}{*}{\multicolumn{2}{|c|}{ Sran $5,90,6$ psig }} \\
\hline 37.4078 & 0.0181 & 51.2488 & 0.06 .77 & 78.5598 & 0.1152 & & \\
\hline $350 x, 58$ & 0.0184 & 53.7548 & $0.075 \%$ & $81.205 x$ & 0.117. & $97.60 \times 00$ & 0.2163 \\
\hline 32.5788 & 0.6447 & 50.2348 & $0 .(17917)$ & 33.7888 & $0.12(x$ & $97.6(0) 0)$ & 0.2163 \\
\hline 31.6388 & 0.1007 & 58.6708 & 0.08015 & 86.2548 & 0.1175 & 97.2149 & 0.1514 \\
\hline 33.8758 & $0.6 \times 75$ & 61.1268 & $00 \times 34$ & $88.67+45$ & $0.11+6$ & 95.0720 & 0.1609 \\
\hline 36.3248 & $0.00 \times 1.5$ & 63.6198 & $0.1(477$ & 91.1538 & 0.146 .3 & 92.7148 & 0.1912 \\
\hline $38.79+8$ & 0.02 .07 & $66.1(k) k$ & 0.0821 & 93.6608 & 0.1274 & 90.2487 & 0.1927 \\
\hline 41.3228 & 0.0301 & 68.5728 & $0 .(19)(17$ & 96.1674 & 0.1107 & 87.8428 & 0.1619 \\
\hline $43.8+28$ & $0.031 ?$ & $71.0(148$ & $(1.09065$ & 97.5662 & $0.134(1$ & 85.4438 & 0.1614 \\
\hline 46.3158 & 0.0317 & 73.5118 & $0.1(x) 6$ & & & 83.0658 & 0.1653 \\
\hline 48.7748 & 0.0317 & 76.0118 & 0.1115 & Scan 4.80 & $1.2 \mathrm{psig}$ & 80.6938 & 0.1528 \\
\hline 51.2488 & 0.0427 & $78.559 \mathrm{~K}$ & 0.01993 & 97.60100 & 0.1266 & 78.3288 & 0.1613 \\
\hline 53.7548 & 0.0515 & 81.2958 & 0.1005 & $97.60 \times 0$ & 0.1266 & 15.9578 & 0.1578 \\
\hline 56.2318 & 0.0583 & 83.7888 & 0.1075 & 97.2149 & 0.1291 & $73.592 \mathrm{k}$ & 0.1537 \\
\hline 58.6798 & 0.0610 & 86.25 .48 & 0.1055 & 95.0729 & 0.1392 & 71.1538 & 0.1540 \\
\hline 01.1268 & 0.0630 & 88.0075 & 0.1035 & 92.7148 & 0.17 .37 & 68.8018 & 0.1462 \\
\hline 636198 & 0.0576 & 911538 & 0.1318 & 90.2487 & 0.1710 & 66.4368 & $0.13 k$ \\
\hline $66.10 \times 18$ & 0.0591 & $936(6) 8$ & 0.1179 & 87.8428 & 0.1397 & 64.0378 & 0.1419 \\
\hline 68.5728 & $0 .(6,08$ & 96.1674 & 0.1028 & 85.4438 & 0.1425 & 61.6538 & 0.1270 \\
\hline 71.0048 & 0.0754 & 97.5662 & 0.1472 & 83.0658 & 0.1452 & 59.2828 & 0.1172 \\
\hline 73.5118 & 0.0751 & & & 80.6938 & 0.1337 & 56.8558 & 0.1175 \\
\hline 76.0118 & 0.0880 & Scan 3. 69 & 3 psig & 78.3288 & 0.1403 & 54.4918 & 0.1131 \\
\hline 78.5598 & 0.0812 & & & 75.9578 & 0.1366 & $52.071 \mathrm{~K}$ & 0.1057 \\
\hline 81.2458 & 0.0768 & $97.6000)$ & 0.09017 & 73.5928 & 0.1336 & 49.6268 & 0.1000 \\
\hline 83.7888 & 0.086 .9 & $97.60(6)$ & $\sin (x 17$ & 71.1538 & 0.1342 & 47.2538 & 0.0878 \\
\hline 86.2548 & 0.08 .33 & 97.2149 & 0.111 .4 & 68.8018 & 0.1295 & 44.7828 & 0.0787 \\
\hline 88.6945 & 0.0785 & 95.0729 & 0.1134 & 66.4368 & 0.1097 & 42.4108 & 0.0747 \\
\hline 91.1538 & 0.11 .16 & 92.7148 & 0.1463 & 64.0378 & 0.1549 & 39.9178 & 0.0709 \\
\hline $93.66,08$ & 0.090 & 90.248 .7 & 0.1470 & 61.6538 & 0.1152 & 37.4978 & 0.0562 \\
\hline 96.1674 & 0.0828 & 87.8428 & 0.1130 & 59.2828 & 0.1660 & 35.0658 & 0.0419 \\
\hline \multirow[t]{2}{*}{97.5662} & 0.0801 & 89.44 .38 & 0.1150 & 56.8558 & 01015 & 32.5788 & 0.0330 \\
\hline & & 83.0658 & 0.1213 & 54.4918 & $0 .(19.39$ & 31.6388 & 0.0314 \\
\hline \multirow{2}{*}{\multicolumn{2}{|c|}{ Scan 2, 59.6 psig }} & 80.69 .38 & 0.1072 & 52.0718 & 0.09 .39 & 33.8758 & 0.0123 \\
\hline & & 78.3288 & 0.1164 & 49.6268 & 0.08 .34 & 36.3298 & 0.0192 \\
\hline 97.6000 & 0.0956 & $75.957 x$ & $0.11 \div 7$ & 47.2538 & 0.0780 & 38.7948 & 0.0682 \\
\hline 97.6000 & 0.0956 & 73.5928 & 0.1110 & 47626 & 0.0712 & 41.3228 & 0.0788 \\
\hline $97.2 ! 49$ & 0.0490 & 71.1538 & 0.1128 & 42.41108 & $0 .(k i 15$ & 43.8428 & 0.0812 \\
\hline 95.0729 & 0.10012 & $6 \times .8(1) 18$ & 0.114 .11 & $39.917 \mathrm{~K}$ & 0.05126 & 46.3158 & $0 .(19.39$ \\
\hline 92.7118 & 0.1 .90 & 66.4368 & (1)(k)13 & $37.497 k$ & 0.0476 & 48.7748 & 0.1015 \\
\hline 90.2487 & 0.1350 & 0.40378 & ก) 1.45 ! & $35(k, 58$ & 0.013 .13 & 51.2488 & 0.1125 \\
\hline 87.8428 & 0.102 .4 & $61.6,538$ & 0.0454 & $32.578 x$ & 0.0271 & 53.75 .48 & 0.1172 \\
\hline 8.5 .4138 & $0.1^{1} 059$ & $=4.2 \times 26$ & $0.0 \%(0) 1$ & 31.6388 & 010151 & 56.23 .48 & 0.1222 \\
\hline 83.0658 & $0.1(1 \times 1$ & 56.8558 & $0.4 \times 8 \div 1$ & 13.8758 & 0.0388 & 58.6798 & 0.1226 \\
\hline
\end{tabular}




\section{Reinforced Concrete Test Data}

Track 3 - High Pressure Test

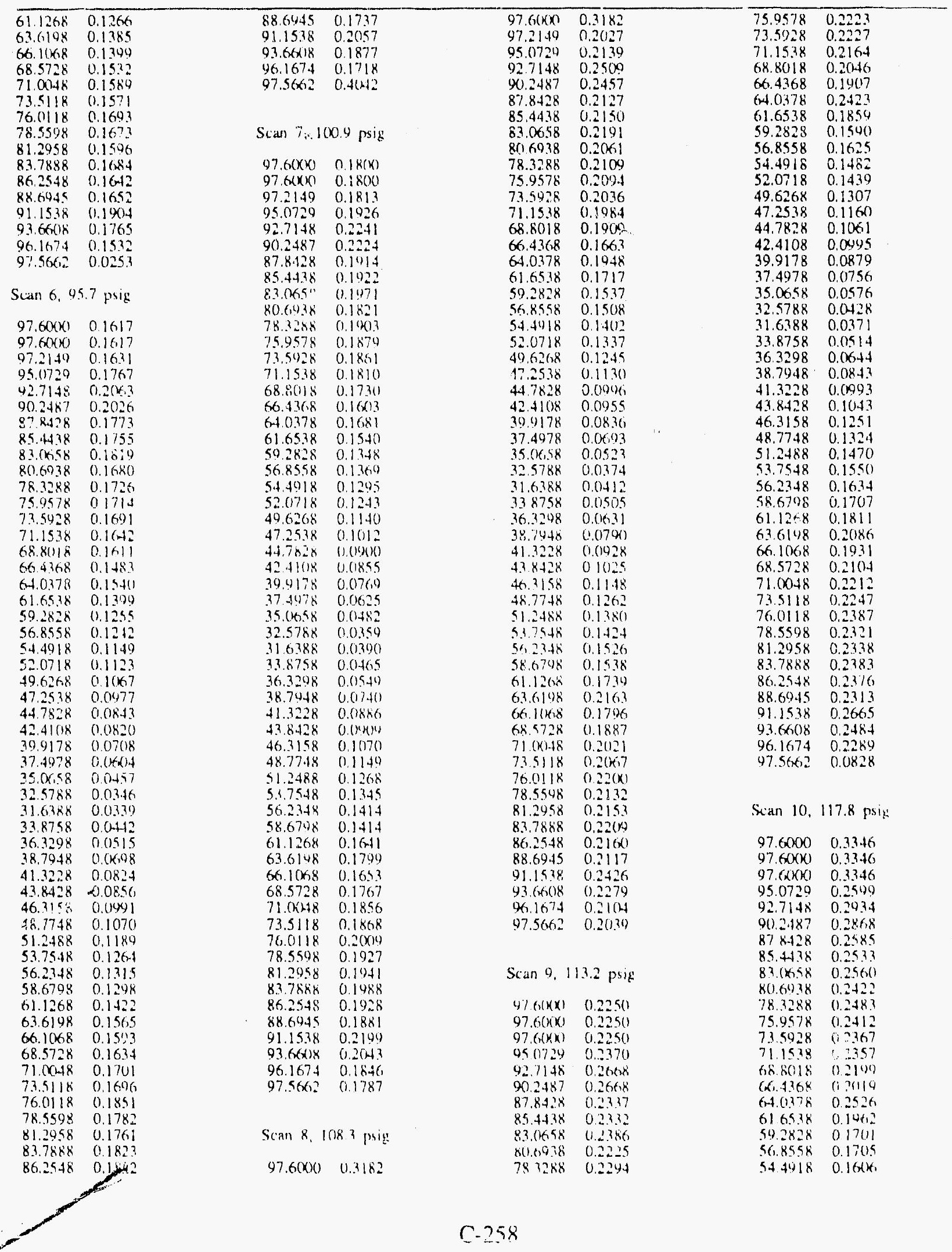




\section{Reinforced Concrete Test Data}

Track 3 - High Pressure Test

\begin{tabular}{|c|c|c|}
\hline $52018 x$ & 0.1495 & \\
\hline 4) & 0.1381 & \\
\hline $4724+8$ & (1.1257 & \\
\hline $4.18: k$ & $(1 . 1 1 \longdiv { 2 3 }$ & \\
\hline $42+116$ & 01147 & \\
\hline 349170 & $0,(\mu): 1$ & \\
\hline 3.4078 & 0.080 & \\
\hline 39169 & 0.0605 & \\
\hline 329788 & 0.0419 & \\
\hline 36388 & 0.0401 & \\
\hline 338758 & (0.0533 & \\
\hline 363208 & $0.06,54$ & \\
\hline 38.7448 & 0.086 .7 & \\
\hline 41.3228 & $0.105 ?$ & \\
\hline 438.25 & $01118 ?$ & \\
\hline th 31.58 & 0.1288 & \\
\hline 48774 & $0.14(1)$ & \\
\hline $51248 \pi$ & 0.1536 & \\
\hline $53744 k$ & (1) 1608 & $\ldots$ \\
\hline 50,246 & 0.1124 & \\
\hline $5 x \pi^{-2} 4 x$ & 0.182 .3 & \\
\hline (1) 1.1268 & 0.180 .1 & \\
\hline 630148 & $(1.2738$ & \\
\hline$(x+1),(x, k)$ & $0 ?(x) 4$ & \\
\hline (6) 4728 & 0.226 .8 & \\
\hline $710 \times 148$ & 0.2368 & \\
\hline 735118 & 0.2424 & \\
\hline $7+0118$ & (1).25.45 & $\sim$ ata \\
\hline $78<54$ & $(12526$ & \\
\hline 812058 & $0251 ?$ & \\
\hline 83.885 & (1.2578 & \\
\hline$k(1) .2948$ & (i) $255 \%$ & \\
\hline sis f.0.45 & (1) 25.4 .4 & \\
\hline $41153 x$ & $0.28 \div 7$ & \\
\hline प? Rols & 0221 & \\
\hline ine $10-1$ & 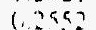 & \\
\hline$y-5+k=2$ & (1) $2+400$ & \\
\hline
\end{tabular}

Scan 11. 1209 prig

\begin{tabular}{|c|c|}
\hline $97.6 \times(x)$ & 0.2714 \\
\hline $47.6 \times 1 \times()$ & 0.2714 \\
\hline $97 \cdot(\alpha)(x)$ & 0.2714 \\
\hline $9511 \% 20$ & 0.28 .31 \\
\hline$y \geq 7148$ & 03111 \\
\hline 00.2487 & $0.1(m) ?$ \\
\hline$x=k+2 k$ & 0.2757 \\
\hline$x^{x}+41 ;$ & 112725 \\
\hline $8.1 \times 58$ & (1) २७६ ? \\
\hline ה! & 112482 \\
\hline 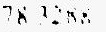 & (1) $\therefore+1,1$ \\
\hline 75450 & $11 .<\div$ \\
\hline $93<2 x$ & $12\{4,1$ \\
\hline 711548 & (1) : Xes) \\
\hline tor $3(11)$ & $11: 14$ \\
\hline (xiting & 112145 \\
\hline tod $1110 x$ & $\because 2601\}$ \\
\hline $0 ! 6536$ & 02116 \\
\hline $54.252 k$ & $(1|k| k \mid$ \\
\hline 56855 & $9 ! 1$ \\
\hline $5+4018$ & 110,54 \\
\hline 52.0718 & (1.1576 \\
\hline 40.6268 & $0.14 k x$ \\
\hline 47.2538 & 01301 \\
\hline $44.782 k$ & 01183 \\
\hline 42.4118 & $(1.111 .5$ \\
\hline 99.9178 & $0.1(x) 5$ \\
\hline 37.4478 & $(1.61828$ \\
\hline $35 . \ln 8$ & $0.0 K S .8$, \\
\hline 325788 & \\
\hline $31.6318 x$ & \\
\hline
\end{tabular}

\begin{tabular}{|c|c|}
\hline & \\
\hline 33879 & $0(1960)$ \\
\hline 36.208 & (1). $(x, 7 x$ \\
\hline $3 k-4.18$ & $0(\mathrm{~m}) ? 7$ \\
\hline $412: 2 K$ & $(1)(x) 2$ \\
\hline $43 \times 4: 8$ & 01140 \\
\hline 46.2158 & $(1.1359$ \\
\hline $4 \pi .778$ & 0.147 .3 \\
\hline $52+3 x$ & 01612 \\
\hline $5379+8$ & 0.1725 \\
\hline $5+134 \pi$ & 0.1817 \\
\hline $5 x<14 x$ & 0.180 .4 \\
\hline $6112+1 x$ & 0.21158 \\
\hline $63+6148$ & 0.743 \\
\hline for $1(x) x^{\prime \prime}$ & 0.2212 \\
\hline 68528 & 0.24 .14 \\
\hline $711 x+18$ & $0.25(17$ \\
\hline 735118 & 0.35801 \\
\hline $70(1) 13$ & $0.27 \%$ \\
\hline $7 x-554 x$ & $(1.22, \cdots 7$ \\
\hline 812456 & $0.21 \times 1=$ \\
\hline $8.7 .7858^{2}$ & 027.1 \\
\hline $86.25+k$ & 0.280 \\
\hline 880.1445 & 0.2312 \\
\hline 411538 & 0.3154 \\
\hline 9.3. $(x \times 18$ & $(1.24=1$ \\
\hline Q6i $16: 4$ & $(1.2754$ \\
\hline $97.56(x) 2$ & 0.3 .44 \\
\hline$S c+4 ; 12$. & $1 . . .4 \mathrm{~s}$ \\
\hline $070(x)$ & (1).327? \\
\hline $0 !(x) x)$ & (1) 3 \\
\hline $4 ? 010 \times 4)$ & $(132 \%=1$ \\
\hline 45,242 & $1: x+4 x$ \\
\hline 927148 & $013(x, y)$ \\
\hline $0\left(3,243^{\circ}\right.$ & $(1,3 t, t)$ \\
\hline$x>8+2 x$ & $(1,3214 \%$ \\
\hline $85+43 k$ & (1) $.3+6$ \\
\hline $87,(k, 48$ & (1) $32+8$ \\
\hline $8(1,61) 38$ & $01.314 x$ \\
\hline $7 x .22 x$ & (1) $3(n), 3$ \\
\hline $75.45^{\circ} 8$ & 0.305 \\
\hline 7.35028 & 11.21452 \\
\hline 71.1538 & 01.254 .4 \\
\hline $6 x m=15$ & 11223 \\
\hline (xw tiens & (1.24.8 \\
\hline 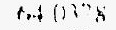 & 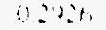 \\
\hline (i) 1.540 & $(1 \geq 1, i$ \\
\hline 502528 & $11 ? 11 \%$ \\
\hline s. & $11: \times 14$ \\
\hline $4+4.15$ & 41424 \\
\hline $5211^{\circ} 18$ & 1.1744 \\
\hline 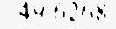 & $1: 11 x, 6$ \\
\hline$x^{-2}=18$ & $11+.1$ \\
\hline 4.45 & 11194 \\
\hline $42+10$ & 111228 \\
\hline $3+417 x$ & $(1 \mid 12+4$ \\
\hline 3740 & $01\left(x, x_{i}+\right.$ \\
\hline 3514,5 & $110: 12$ \\
\hline באים & 11 (sith \\
\hline $116,18 \mathrm{~B}$ & $\left(10166^{2}\right)$ \\
\hline 33.8598 & $11\left(x_{4}\right) i$ \\
\hline $36.320 x$ & (1. (c) $1 \mathrm{mt}$ \\
\hline 38.794 .48 & 0.10330 \\
\hline 41.7228 & (1) 1214 \\
\hline $438+20$ & $(1.1 .312$ \\
\hline 46.3158 & 015115 \\
\hline 487748 & $(1).(x) \mid$ \\
\hline 51.248 .8 & $(1) \sin 110$ \\
\hline $5.754 x$ & (1) 1930 \\
\hline $96.2 \ldots+4$ & $\left(1,2: x_{1}+1\right.$ \\
\hline 58.071$)$ & $0.212 x$ \\
\hline
\end{tabular}

$\begin{array}{ll}61.1268 & 0.2348 \\ 63.6198 & 0.2649 \\ 66.1068 & 0.2437 \\ 68.5728 & 0.277 .47 \\ 71.0048 & 0.2855 \\ 73.5118 & 0.2940 \\ 76.0118 & 0.3153 \\ 78.5598 & 0.3119 \\ 81.29 .58 & 0.3141 \\ 83.7888 & 0.3262 \\ 86.2948 & 0.3266 \\ 88.6945 & 0.3340 \\ 91.1538 & 0.3648 \\ 93.6608 & 0.3520 \\ 96.1674 & 0.3341 \\ 07.56612 & 0.0583\end{array}$

Scan 13. 130.2 psiz

$97.6(k) \quad 0.4(15)$

$97.60 \mathrm{kn} 0.105$

$97.600(0) \quad 0.4051$

$95.0729 \quad 0.4201$

$02.7148 \quad 0.4479$

$90.2487 \quad 0.44(44$

$87.8428 \quad 0.406 .5$

$85.4438 \quad 0.3054$

83.016.58 0.3037

$80.6038 \quad 0.3731$

$\begin{array}{ll}78.3288 & 0.3707\end{array}$

$750578 \quad 0.3620$

7356280.3514

$71.1538 \quad 0.3415$

$68.8018 \quad 0.3201$

$66.4368 \quad 0.21729$

$6.0378 \quad 03353$

$61.6538 \quad 0.2825$

$59.2828 \quad 0.2471$

$56.8558 \quad 0.2281$

$54.4918 \quad 0.2266$

$52.0718 \quad 0.2077$

$49.6268 \quad 0.1928$

$47.25 .38 \quad 0.1691$

$4.7828 \quad 0.1526$

$424108 \quad 0.1307$

$369178 \quad 0.1258$

$37.4978 \quad 0.1095$

$350658 \quad 0.1810$

3.57880 .0500

$31.6389 \quad 0.0581$

$33.8758 \quad 0.0724$

36.32080 .0932

$38.7048 \quad 0.1167$

41.3228 0.1384

$438+28 \quad 0.1505$

$46.3158 \quad 0.1724$

$48.7748 \quad 0.1808$

$51.2488 \quad 0.2(193$

$53.7548 \quad 0.2221$

$56.73 .48 \quad 0.2357$

$58.6798 \quad 0.2516$

$61.1268 \quad 0.2717$

$03.6198 \quad 0.2977$

$66.1068 \quad 0.2901$

$68.5728 \quad 0.3240$

$71.00748 \quad 0.3425$

$735118 \quad 0.3548$

$76.0118 \quad 0.3749$

785508003753

$81.2058 \quad 0.3845$

$83.7888 \quad 0.3982$

$\begin{array}{ll}86.2548 & 0.4026 \\ 88.6945 & 0.4049 \\ 91.1538 & 0.4436 \\ 93.6608 & 0.4306 \\ 96.1674 & 0.4153 \\ 97.5662 & 0.6722\end{array}$

Scan 14, 135.3 psiz

\begin{tabular}{|c|c|}
\hline (x) & \\
\hline .60001 & \\
\hline $.6(x)(x)$ & \\
\hline 0729 & \\
\hline & \\
\hline & \\
\hline 7.8428 & ـ \\
\hline 44.38 & \\
\hline .0658 & \\
\hline & \\
\hline & \\
\hline & \\
\hline & \\
\hline 1.538 & \\
\hline 018 & \\
\hline & \\
\hline 178 & \\
\hline & \\
\hline & \\
\hline & \\
\hline & \\
\hline 52.017 & \\
\hline & \\
\hline & \\
\hline & \\
\hline & \\
\hline & \\
\hline 7.4978 & \\
\hline .0558 & \\
\hline 2.5 & \\
\hline t & \\
\hline 1.8 & \\
\hline 36 & \\
\hline & \\
\hline & \\
\hline & \\
\hline & \\
\hline & \\
\hline & \\
\hline .7 & \\
\hline 5.236 & \\
\hline & \\
\hline 1 & \\
\hline 6 & \\
\hline & \\
\hline & \\
\hline & \\
\hline & \\
\hline & \\
\hline & \\
\hline & \\
\hline & \\
\hline & \\
\hline & \\
\hline & \\
\hline & \\
\hline & \\
\hline & \\
\hline
\end{tabular}

Scan 15. 140.2 psiz 
() $9[-3$

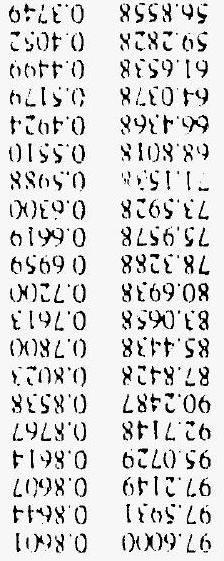

$(15 a)$ sond) sisd 0 "il unos

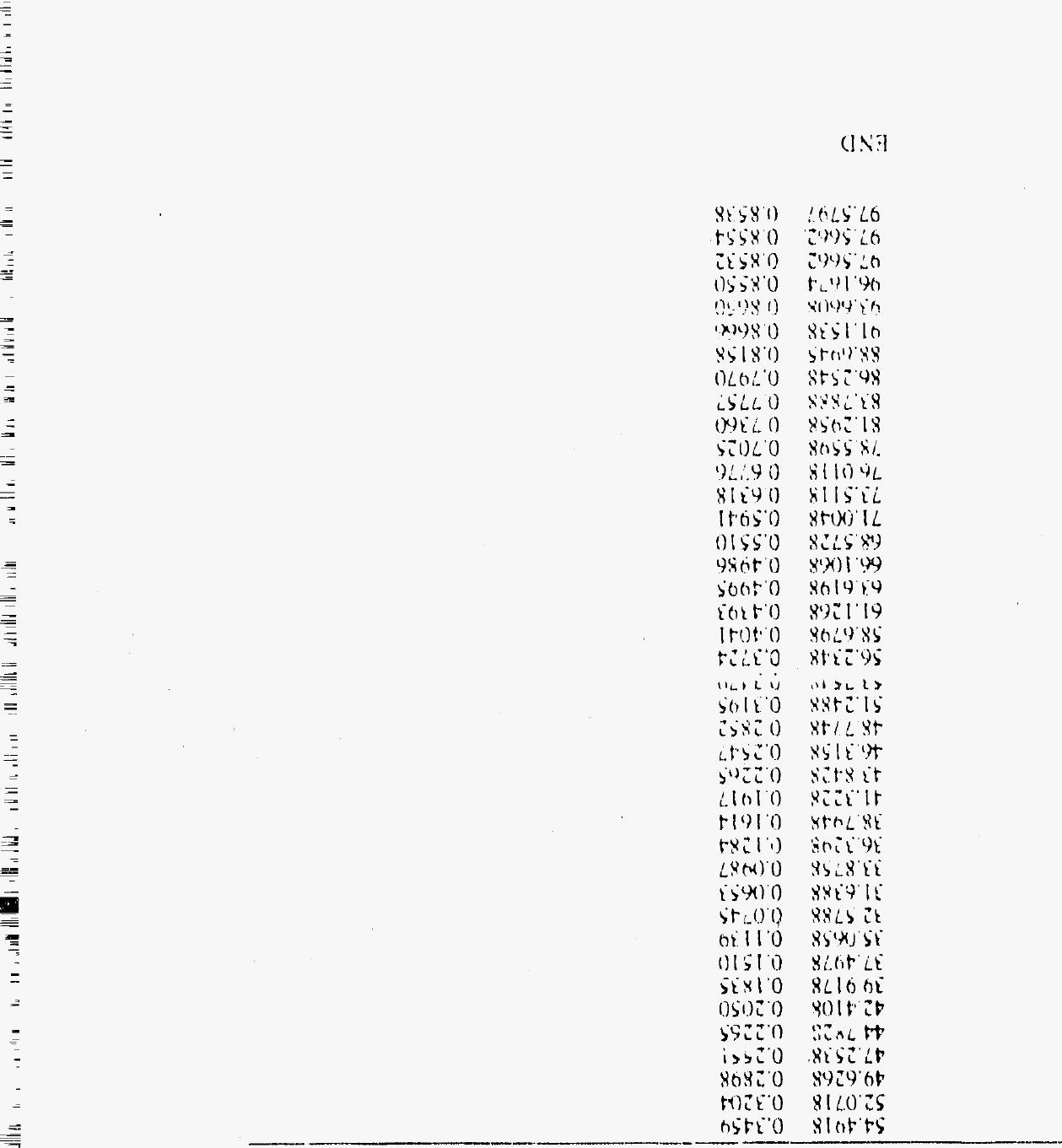

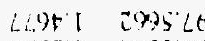

जelil 1.9190

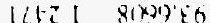

oitel xisllo

olol. 550988

हE1 815298

It1 $888<28$

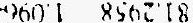

1) 15018058

S(x)il 811096

95500 RIISEL

9 anto $8(4)) 1 i$

(1558.0 820589

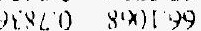

1.200

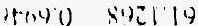

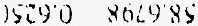

ivino stroys

kitso stsics

Linto 8ALIIS

91504851685

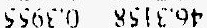

5iseo 8it8it

E(x)t0 8ERElt

$5550^{\circ} 0$ 8troL 82

ESUEO $80 E E^{\circ} 9 E^{\circ}$

$5(x) 10 \quad 8518.6$

85010 88.9.

(2)

1910 85स्त

antio seotic

$1+820801600$

sitio $8011 \mathrm{z}$

S19.") 828L5t

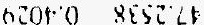

055t0 84096

tioso 812025

$510+50$ 810t5s

55850 8558.95

850

$880<0$ 8is?

$158<0$ 8LiOtO

01LLO 84Lt

$00+88^{\circ} 810889$

rratio 8is!ll

90100 8itisl

08860 8LSOSL

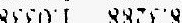

8ELO 820908

SЕII 8590ह8

8051 8itt 58

8LLII 8DF?L8

تEE $\angle 85 T 0$

885i $8+12: 0$

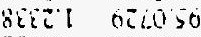

EEITI (N)OYlLA

ZiEZ! (1009'L6

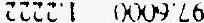

Bisd ystr 91 uens

E98LOO $2995 \%$

$\therefore 880 \quad 5 \angle 9196$

5000 8049\%6

$E(T) 0^{\circ} 0 \quad 8 E 5110$

() $8580 \quad 550988$

$0158^{\circ} 0$ 8552.98

HFE 880 88L

SOKLO 85 की 18

i) $55 \% 0$ 8RT5\%8L

ISELO 811096

56090 8115\%

$81990 \quad 3+(x) 12$

11290 YE 1589

$05 \angle 50$ 8x 81140

SLLSO 801929

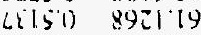

Existo 802985

LEF

lioto 8t5i.

icion 8stils

SSELO XWLLO

98600 851805

I1)

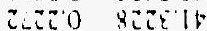

Otolo stolse

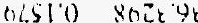

(i)

(x) 800 8sic

sit(x) $891.5 \mathrm{C}$

Eसा

4981 ij $8106 t 2$

SSIZ0 8iloot

itrio 8015 it

XSOEO BESLIt

$\left.1(x) \sigma^{\circ}\right)$ 8ंड

Ditro 89:06

HLEU 81:0

intin vinmit

1)rito 8558.95

5(x)t 820765

58150 रहकेष

10550 8(20)59

brysu gyitiga

Otت9' 810889

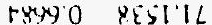

7) 1690 8ITSEL

IELLO 8LSOSL

$915 L^{\circ}$ 88त8 $8 \mathrm{~L}$

i.iLO $8: 0908$

1i.18.0 8590.

Fin 88158

80580 रu18.8

$1988.0 \quad \angle 850^{\circ} 00$

$9516^{\circ} 0$ 8\$1L 26

(x) $\$ 8.0$ OELOSO

rin) 1 (x) (x)

ilix) 1 (x)

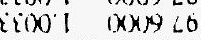




\section{Reinforced Concrete Test Data}

Track 4 - High Pressure Test

\begin{tabular}{|c|c|c|c|c|c|c|c|}
\hline \multicolumn{2}{|c|}{ Scan $1,40.2$ psig } & 97.4425 & $0.01 \times(x)$ & $16+1.2725$ & $n 01814$ & 267.5545 & $0 .(\mu) \times 15$ \\
\hline 288.23211 & 0.1356 & 050429 & $f .111(x)$ & 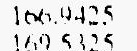 & $0.08(1,3\}$ & $\begin{array}{l}204.7115 \\
2010825\end{array}$ & $0(x) 42$ \\
\hline 288,2320 & 0.1356 & $\begin{array}{l}92.2225 \\
89.4529\end{array}$ & $\begin{array}{l}0.11(x) \\
0.1\left(x_{1}\right) ?\end{array}$ & $\begin{array}{l}1698325 \\
172.0825\end{array}$ & $\begin{array}{l}0.018 \mathrm{k} \\
0 .(1805\end{array}$ & $\begin{array}{l}2010825 \\
290.2279\end{array}$ & $\begin{array}{l}0.1(12) \\
0(x)\end{array}$ \\
\hline 287.5156 & 0.0544 & $\begin{array}{l}89.4525 \\
867529\end{array}$ & $\begin{array}{l}0.1\left(x_{1}\right) \\
0.1(124\end{array}$ & 174.8019 & 0.0724 & $256+796$ & $0.1(x, 4)$ \\
\hline 284.9207 & $0(k) !:$ & 83.4625 & $(1.0(1)+40$ & 177.4725 & o.osik, & 253.61155 & 0.1620 \\
\hline $281.99 \div 2$ & $0 .(x+4)$ & 81.0625 & b. (k)*14 & 180.16 .25 & 0.08001 & $250 . n+x) 5$ & $0.115 \%$ \\
\hline 278.8891 & $0.1 k+45$ & 78.3125 & 0 (1) (H) () & 1827525 & 0.0785 & 248.1255 & 0.1117 \\
\hline 275.9215 & $0.0 x+1,3$ & 75.3725 & $0018 \mathrm{sin}$ & 185.5425 & $0.06+1$ & $2+5.4115$ & 0.1268 \\
\hline $27304+5$ & 0.0715 & 72.4325 & 0.01045 & 185.1125 & $0 .(180)\}$ & 2425445 & 011144 \\
\hline 270.2685 & 0.0710 & 69.6725 & 0.0812 & 140.7179 & 0.4075 & 2319.0015 & $0113^{7}$ \\
\hline 267.5545 & $0 .(1 \%)$ & 60.4025 & 0.074 & 103.2275 & $0(1)+1$ & 237.1615 & 0.1214 \\
\hline 264.7175 & 007593 & 64.1425 & $0.017 .4 ?$ & 145.8Bicis & $0.10 .36 \mathrm{~B}$ & $23432 \times 5$ & 0.1170 \\
\hline 261.9825 & (1) 0821 & 01.2225 & (1) $11(N 2$ & 198.4605 & 0.0981 & 2316359 & 0.1172 \\
\hline 259.2275 & 0.076 .8 & 58.4325 & $0 ! k \times 1$ & 201.1255 & $0 .(k) N^{7}$ & 28840 & 0.1106 \\
\hline $256+725$ & $0 .(k)(1)$ & 55.282 .5 & 00.97 .4 & 2036355 & $0.00 \times 1$ & 226.1255 & $0.111 \%$ \\
\hline 2536159 & 0.0825 & 52.2229 & ootent? & $2(k, 2485$ & $(1.10111$ & $223+115$ & 0.12 .10 \\
\hline $250 \times(015$ & 0.0882 & 49.3625 & $0 .(15) . ?$ & 208.7585 & 0.1011 & 220.670 .5 & 01115 \\
\hline $2+8.1259$ & $0.0 \times 14$ & +0.9129 & 0.0530 & 211.3245 & 0.13 .77 & 217.0425 & $0.12(\alpha)$ \\
\hline 245.4115 & $0 .(k+t), 4$ & 43.4429 & $0(145)$ & 21301115 & 0.108 .3 & 215.1465 & $0.122 ?$ \\
\hline 2425045 & 0.0042 & 10.5025 & nondis & 210.5735 & 0.1042 & 212.3405 & 0.1259 \\
\hline $230.9(115$ & $0.095 x^{\circ}$ & 37.5325 & $0.03: 8$ & 219.1455 & (1.114:4 & 2094725 & $0.130 !$ \\
\hline 2371655 & 0.1012 & 346725 & $0.111 \times 1$ & 221.7986 & 0.1612 & $2(6.770 .5$ & $0.121 . ?$ \\
\hline 234.285 & $0 .(9)+2$ & 31.0125 & 0.0111 & 22430015 & const & 204.1225 & 0.111 \\
\hline $231.6,345$ & 0.04822 & $24.7 \div 5$ & 00127 & $227.04+15$ & 0.04 .1 & 20128855 & (1.116: \\
\hline 2288405 & 0.11935 & 121029 & $0.018 ?$ & 2206745 & (1. $(1487$ & 198.5335 & (1)114? \\
\hline $22+1255$ & 0.1427 & 346725 & $\left(0.02 x^{1}\right)$ & 232160,5 & $0.1(x) 4$ & 1058105 & $011 \%$ \\
\hline $22+115$ & $0(1) 1$. & 37.9425 & (1) 10304 & 2368105 & $0.60 ; 0$ & 1029615 & 0.1181 \\
\hline $220.676^{6}$ & 0.0 .223 & 41003 & 0,0112 & 2376145 & 0.01905 & 1002885 & 0.1188 \\
\hline 217.0425 & 0.0 .27 & H. 20.25 & 011514 & $2+10.34105$ & $0+1930$ & 187.5 .25 & 1).1117 \\
\hline 2151 the & $0.014:-1$ & 472325 & 01105 & 2432675 & 0.11122 & 18.186 .25 & $(\because 161 x$ \\
\hline $21 ? 3119$ & $0.63+11$ & 508025 & (1) (15?? & $24600+15$ & () 101 & $1 \times 20 k 25$ & 0.04066 \\
\hline $2(x) .4725$ & $0(19)(1)$ & $53 \times 10.5$ & 0115 ino & $? 488105$ & 0.000 .3 & 170.31225 & $01(x) ?$ \\
\hline $2 x+70$ & $0 .(19)(-1)$ & 50,6425 & (1) (1) (tis) & 251.0305 & $0 .(1), 33$ & 170.6125 & $0.144: 2$ \\
\hline $2(40) 225$ & (1) 0095 & 50.9825 & 0.61726 & 254.4425 & (i)ivi? & 1738425 & 0.180 \\
\hline $201.2 \times 55$ & $0 .(m)<\}$ & 0280125 & $0 \leqslant 58$ & 2.50 .5535 & $000+1$ & $17(1.0 .125$ & $0.1611 \%$ \\
\hline 1485.396 & $0 .(x) \geq k$ & $65(6) \geq 5$ & 0.0703 & $258-15$ & 0.0840 & 10.81 .25 & Ch klows \\
\hline 1058105 & 0.01904 & $6 \times 54 \geq 5$ & 0.18330 & 260)(knts & 0.0901 .3 & 165,3325 & $0.11 \times(x)$ \\
\hline 192.9815 & (i. $(10+45$ & $314+25$ & 0.679 & $201.3(n) 5$ & 0.0850 & $1 f_{2}+125$ & $0(\mu)+B$ \\
\hline 150.2855 & 00005 & 74.3225 & (1) 184 ? & $2 f_{1} 2, t 155$ & 0.0750 & $19474=5$ & $(1.1(x)=$ \\
\hline 187.5325 & 0.0823 & 77.1025 & $0 .(187 ? 3$ & 2630825 & 0.6106 & 156.725 & alliats \\
\hline 18486125 & $(1.0)=0$ & $802(1) 5$ & $0(x)+1$ & $2052 k 85$ & $01.0 \times 34$ & $153+425$ & 01.1642 \\
\hline 1220825 & 0.0735 & 820425 & 00077 & 260.5055 & $0.0771 \%$ & 150 nons & 100032 \\
\hline 174.4925 & $0.078 . ?$ & 858125 & $0(1)+x d$ & $2(17 x) \cdot(x) 5$ & 0.07 .36 & $11: 4025$ & (1) lil. \\
\hline 176.6125 & un?: & 880525 & (1) $110 \times 4$ & 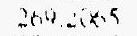 & $0.0^{-1} 31$ & $1: 5.1825$ & 11.1:2: \\
\hline 1718.82 & 010,23 & $4 ! 31125$ & $9110 \mathrm{~s}$ & 2014425 & $\cong 1170.4$ & $1+24125$ & olwst \\
\hline 170.4425 & $00:-71$ & $4+1425$ & (1.1.2: & 271.7785 & 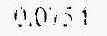 & 134.725 & $(1,11: 1$ \\
\hline 168.1825 & 0.0122 & 90,4325 & $0(11972$ & $2720 \leq 29$ & $(107)$ & $130 \div 925$ & $\left(11^{-1}: \cdots 11\right.$ \\
\hline 165.332 .5 & 0.0550 & 40.6425 & $(1.16 \times 10)$ & 2741265 & $0 !(x, y)$ & 131725 & $(11120$ \\
\hline 162.4125 & 0.0830 & 11124125 & $0(1)+1)$ & 275.6765 & ii. $(x, x)$ & 13101425 & $1111: 2$ \\
\hline 159.7425 & 0.0805 & 105.1225 & $n(x) 11$ & 278.6135 & 0.0201 & 128.225 & (i) 1135 \\
\hline 1567225 & $(101.31$ & 107825 & $0 . \cos \mathrm{se}$ & $278.3(\mathrm{NS}$ & $0 .(17.32$ & 1255525 & (1) 1 L 2.1 \\
\hline $153+425$ & $0.08+11$ & 1104.395 & $0 x+1$ & 270.7317 & 0.0723 & 122.9525 & 0.1109 \\
\hline 150.8025 & 0.0777 & 113.0825 & (1. $(x)(x)$ & 281.0807 & 0.1719 & 120.2724 & 0.104 \\
\hline 1479625 & $0.08: ?$ & 1158025 & (0): 2 & 282.809 & $0 \times k^{2}=$ & $1174<25$ & $0.111 x$ \\
\hline 145.1839 & (i.). $18(2)$ & 118.4925 & $0.094 ?$ & 283.7474 & 0.0735 & 1140735 & $01.11(x)$ \\
\hline $142+125$ & $0.07(x)$ & 121.1425 & 11.091 .3 .3 & 285.2650 & $06 \pi+4$ & 1118029 & (1) $11+1$ \\
\hline 1.19 .7125 & 0.6910 & 121.7325 & 0.1034 & 286.7339 & 0.0716 & $|\{n\}| 1325$ & () $\int(x, 5$ \\
\hline 136.7025 & $01+12$ & 126.2625 & 0.104 .4 & 287.8052 & $0 .(1)(x)$ & $1(x, 4325$ & 0114.3 \\
\hline 133.7725 & $n(M) 12$ & 124.425 & $0 .(k)(6.3$ & $288.119 x$ & 0.0759 & 103505 & () $1 ! 111$ \\
\hline 131.0425 & $6.160(12$ & 131.5725 & $0(n) \leqslant 1$ & & & $1(x)-25$ & (1) $(\mathrm{m})=$ \\
\hline 128.3225 & $0.09180^{\circ}$ & 134.2325 & $O(x)=t$ & Scan 2,5 & 6 psig & 978425 & $(1118$ \\
\hline 125.5525 & $0114+1$ & $137(1829$ & 0.1320 & & & $45(14 \div 5$ & 01305 \\
\hline 1224525 & (1.(k):? & 13480525 & $O(x) 1^{7}$ & $28 \times 230$ & 0.1212 & 92.2225 & 0.1314 \\
\hline 120.2725 & $0 .(10) 11$ & 1425125 & 0.1754 & $2 \times 52201$ & $0.12: 2$ & 8uts is & 0.1201 \\
\hline 117.4525 & $0.08^{-2}=$ & 1452225 & $u(x \leq s$ & 201.5150 & (1) onit & 86.525 & $0.121,4$ \\
\hline 114025 & $0.11 \times 1 \times 4$ & 147.8225 & $(1)(x) 24$ & $3 x+4247$ & 0.074 & $83.4,25$ & $(1) 121 \mathrm{x}$ \\
\hline 1118025 & 0.0882 & 15115525 & 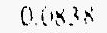 & $281.94 \%$ & 0.6137 & xilk:s & 0.1134 \\
\hline $01010-3$ & (1.06as & 1532015 & $0.08+6$ & 278.8841 & $0118: 1$ & 78.3125 & 01124 \\
\hline $106+325$ & 0.0124 & 1550825 & 0.0756 & 275.11215 & 0.065 & 753725 & (1) 1035 \\
\hline 107.57 .2 & 0.085 & 158.825 & 0.01795 & $27.311+4.5$ & 0.6412 & $72+325$ & 0.9015 \\
\hline $1(x) 77.25$ & $0.019=0$ & 161.4025 & 0.0810 & $270 ? 6(x) 5$ & $0(\mu+1)$ & $0,067: 5$ & 01041 \\
\hline
\end{tabular}




\section{Reinforced Concrete Test Data}

Track 4 - High Pressure Test

\begin{tabular}{|c|c|c|c|c|c|c|c|}
\hline$(x, \infty) 125$ & 11 lowsto & 191225 & 0.1167 & $2.77 .16,55$ & $0.1+.58$ & 340725 & 0.113 .36 \\
\hline $0+1425$ & $\theta(N) \mid x$ & 195.3805 & (1.1223 & 2343265 & 0.1421 & 31.0174 & 0.1963 \\
\hline 61 2235 & (1) $12 \ldots$ & $10 \times+(10)$ & 0.12117 & 231.6355 & 0.1465 & $2977 ? 5$ & 0.025 \\
\hline $58+325$ & $0.018+i i$ & 2011255 & 0.1218 & 228.8 .415 & $0.1+10$ & 3201225 & 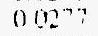 \\
\hline 55.2825 & $0\left(1 k^{\prime}\right) s^{\prime}$ & 203.5355 & $012(x)$ & 226.1259 & $0.1+11$ & $3+0725$ & $0.037 x$ \\
\hline 5272.25 & ()$(k, 1)()$ & $20 \times .2485$ & 0.1216 & 221.4115 & 11.149? & 37.9425 & $0.05(1)$ \\
\hline 40,3625 & $0(k-)$ & $2(18.7589$ & 0.1270 & 220.6765 & 01415 & $41.10 ! 25$ & l) \\
\hline $46.51: 9$ & $0 .(k)=$ & 211.3245 & 0.1250 & 2174425 & $0.1+51$ & $4+\ldots 105$ & (). (x.4. \\
\hline 434125 & (1.155? & $213.2 \times 115$ & 0.120 .1 & 2151145 & $0.1+6.7$ & 47.7325 & $0 .(1 k 1)$ \\
\hline 40.51$): 5$ & 110510 & 2105735 & $0.13(1)$ & 212.3409 & $0.1 \% 5$ & $50.80: 5$ & $0.0 \times 20$ \\
\hline $37.53: 5$ & (1) (M) & 214.1455 & (i lik? & 209.4725 & (1.1420 & $53 \dot{M x}=5$ & $0.18: 8$ \\
\hline 390116 & (1):1:54 & 221.7485 & 0.121 .0 & 2060.770 .5 & 0.1 .425 & 56.0 .425 & (1) \\
\hline 310125 & 0.01225 & $224.49(19$ & (1) $11 k$. & 204.0225 & (1) 13 (ind & 54.4825 & 0.11 .25 \\
\hline 24.7725 & (1) (11) & $22 \%(4+45$ & 0.1218 & 2011.2655 & (1.134? & 6280125 & (1) 1108 \\
\hline 32.0225 & 00183 & 229.5745 & 0.12018 & $19 \times 53.55$ & 0.1 .10 & 6.5 .6425 & 0.1082 \\
\hline 34675 & 0.0314 & $23: 10015$ & 11.1238 & 1958199 & 1). 1.54 & $6 \times 50: 5$ & 01100 \\
\hline 37.12425 & (1)intte & $214 \times 1+9$ & 0.1244 & 1924815 & $0 !+23$ & $71+425$ & 0.1195 \\
\hline $41(x)=5$ & (1) $115 / \mathrm{h}$ & 2376145 & 0.116 & 1902885 & 0.1 .401 & $743: 25$ & $0.120 \%$ \\
\hline $44.2+.25$ & $0.05^{2}+4$ & $240.3+05$ & $0.12: 11$ & $18^{7} 7.5325$ & (1).12?3 & 77.3025 & (1) 1230 \\
\hline 477325 & $(1) k x+1$ & 2432175 & 0.12 .40 & 181.8625 & 0.1233 & 81205 & (1.13.11 \\
\hline 501005 & $(3002)$ & $2+0 ! k+4$ & $0.13(10$ & 18201825 & $\{1.117 .3$ & $8314: 5$ & $0.133 \mathrm{i}$ \\
\hline 5130.5 & 01971. & 2488145 & $0.11 \mathrm{~K}^{7}$ & $179.34 \times 5$ & $0123:$ & 858125 & 01.56 \\
\hline $5+19425$ & $0.082 ?$ & $251.6,365$ & (1.115? & 176.6125 & 0.1214 & $8 x \in \cos$ & $(1)+1)$ \\
\hline 54 4 8.5 & $(3)(k) 18$ & $25+412: 5$ & 0.1115 & $178 x 425$ & 0.14018 & $01.34: 5$ & $0.1+6$ \\
\hline $6 \geq x(1 \geq 5$ & Onilpive & 250.95 .15 & 0.195 & 170.12225 & 0.1287 & 441429 & $010 \div 1$ \\
\hline $05.60 \div 5$ & 0 (11) & $25594: 5$ & $0.1(15)$ & 168.1825 & $0.1 ! 81$ & $90,9 \div 25$ & (1) 1.355 \\
\hline $08.54 \geq 5$ & (i) 10.35 & $260(x-45$ & 0. Jont & 165.334 & 0.1290 & $0(9,61) \geq 5$ & 1117119 \\
\hline 31.44 .9 & $11(11 \cdots)$ & $201.4 m=$ & (1) 16: & $102+125$ & 0.1290 & $112+125$ & $(1.1252$ \\
\hline $74: 325$ & 111041 & 260155 & $(1,(\mu) \leq 3$ & 154.7475 & 0.1330 & $10 \% .225$ & 0.1 .1118 \\
\hline 77.1115 & $(1|1| n) ?$ & 20,2085 & $(1 .(x) \leq 0)$ & $15 n 7215$ & 01212 & 107.8224 & $0.112 ?$ \\
\hline 80210.5 & $(1.11+1)$ & $2652 n \times 5$ & (1.10)14 & 153.4725 & 0.1285 & $110+125$ & (1.) 13.40 \\
\hline 814225 & $(1.11+1$ & 200,5055 & $0 .(x) 51$ & 150.3125 & 01205 & $113 .(1825$ & (1.1. $40 \%$ \\
\hline $85+125$ & 11140 & 260 antis & 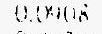 & $14 \div 90_{2} 5$ & $(1.1 .17 \%$ & $115 \times 125$ & 11.134 \\
\hline 88.0529 & (1) $1 \geq 5$ & Znd $20 x-5$ & $0.1 \times 2-11$ & 1451825 & $011+x$ & 118.4025 & 0.1 .30 .1 \\
\hline 013012 & 111275 & 20104025 & $0(n)=1$ & 142.4125 & 0.1184 & 1211.125 & 0.141 \\
\hline 44.1425 & (1.) $1+4$ & 27.758 & $(1,(x))=$ & 1.347125 & 0.1356 & 1237225 & 0.1432 \\
\hline 404125 & 0.1280 & 20yi:s & 0.0125 & 130.7425 & $0.1 ? 5 y$ & 126.26 .25 & 01450 \\
\hline $4(1)(5)=5$ & 0.1555 & $=i+3205$ & (口) 1870 & 133.7725 & (1) 1.68 & $12 y .1425$ & 0.1 .107 \\
\hline 102.4125 & $0.117 \mathrm{~s}$ & 2750685 & $0.015 ? ?$ & 1.3104 .55 & 0.1358 & 131.5725 & 0.1301 \\
\hline 1651225 & $0.1(x) ?$ & 277.90195 & Oowis & 128.7225 & 0.1 .30 .5 & $13+2.25$ & 0.1504 \\
\hline $1617 \times 225$ & $0.12(x)$ & 278 Sins & $(1)(18 \div 6$ & 125.5525 & 0.1516 & 137.0825 & $(1 !)(1)$ \\
\hline $1111+226$ & 0.11 .18 & 2701117 & (1) 1858 & 122.4525 & $0.1 .31 \%$ & 1.74 .3625 & 01.6 .3 \\
\hline 11211825 & 0.1186 & $28161 \mathrm{O}$ & $0.080 \div$ & 1202725 & 0.1345 & 142.5125 & $0.11 \mathrm{n.4}$ \\
\hline 11580125 & $01110 \mathrm{x}$ & $282+7(1)$. & 01958 & 117.4525 & (0.1.21 & $145.242:$ & ( 11220 \\
\hline 118.4425 & 0.11 .1 & 2037474 & $(;(x)) \times$ & $11.6,725$ & (1.1.114 & 147.8225 & $0.120 \%$ \\
\hline $1211+25$ & 0.1112 & 2852550 & $0.10 ! !$ & $111.8: 25$ & $(1.1 .310)$ & 1501.5525 & 0.1225 \\
\hline 12373.5 & $012: 1$ & $3 \times(, .733)$ & $1195+2$ & 104.1825 & 0.13 .2 & 1532025 & $(1) 13.90$ \\
\hline $120.2+25$ & (i) $1: 01$ & 257.805 & 1)(15is & $101,4.25$ & 0.15 .7 & 155.4825 & $(1.12(n)$ \\
\hline 1201425 & $0.112 n$ & Inst1148 & ii. $12(1)$. & 103598 & 0. 1308 & $158.8+25$ & $(1,1214$ \\
\hline $1 \div 1.57: 5$ & $0.110 ?$ & & & $1607: 29$ & 0.140 & $161+425$ & $0.125 !$ \\
\hline 134.2325 & 01182 & Scan 30,0 & 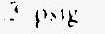 & 97.9425 & (i) $1.3(x)$ & it-1.2725 & 0.1252 \\
\hline $13 ?, 1825$ & $0.1+40$ & & & y5 010.125 & ก.148. & 106.0425 & 012511 \\
\hline 17986.5 & 0.1121 & 2852220 & $11(\%: 2$ & 92.2235 & 0.1504 & 1645325 & 0.1283 \\
\hline 1425125 & $(1)(x) 3 !$ & $28 x^{2} \leq 3261$ & (1) $(x+2)$ & 84.4525 & 0.1 .158 & 172.1125 & 0.1 .24 \\
\hline $1452: 29$ & $0 .(18.30$ & $2 \times 7.5150$ & $O(x) 1 ?$ & 86.7525 & 0.14 .31 & 174.8025 & (1.13): \\
\hline $147 \times 225$ & $0.1 i 2 t$ & $28+420 ?$ & 01.1365 & 8.39625 & 0.1 .373 & 177.4725 & $0.12(x)$ \\
\hline $15155 \times 29$ & 0.101 .1 & 281.4472 & 0010 & 81.1625 & 0.1 .22 & 180.1625 & $01.122=$ \\
\hline 153.2020 & 0.1082 & 2788801 & $0.0 \times 4.45$ & 28.30 .25 & $0.12 ? 1$ & 1827525 & 0.1161 \\
\hline 155.9825 & 0 (iv) 50 & 2754215 & 01045 & $75 . .1725$ & 0.1230 & 185.5925 & 01210 \\
\hline $15 x \times 12.9$ & $0 .(x) 8)$ & 27.2445 & $0.106 ?$ & 724.325 & 0.1218 & 188.1025 & (1) 13324 \\
\hline 10140.5 & 0.1038 & 270 .465 & 0 linted & 69.6725 & $0.117 \%$ & 140.7175 & (1.1211 \\
\hline 104.2725 & 0.1094 & $267 \leq 45$ & 011106 & 6041125 & $0.16)$ & 1012275 & (1) 1.133 \\
\hline $160.4+25$ & (I) (M) Bis & 204.7175 & 01124 & $6+1425$ & (i) 1047 & 145.8505 & $0.1+6.4$ \\
\hline 1605325 & (1,iiild & 2614525 & 0.1227 & 41279 & () 11211 & 198.4695 & (1.142? \\
\hline 17201825 & (1) llix & $250: 275$ & 0.12115 & $58+325$ & $O(x) S ?$ & 2111.1254 & (1.1.4. \\
\hline 17805 & $0.115 x$ & $250+4 \geq 5$ & $0.13(1)$ & 35.2825 & $0 .(11) \div 6$ & 2013.6 .55 & $0.14^{\circ} 6$ \\
\hline 177.124 & $0.1(k) 1$ & 2536155 & (1) $1: 5.1$ & 52.2235 & $005: ?$ & $2(x, 24 \times 5$ & O.i-10.\} \\
\hline $18(1) x_{1} 25$ & $(i,(M) ? !$ & 250.80115 & $(1.1341$ & fiv. 20.25 & (1) (1)it & 2018.758 .9 & (i) 1sur) \\
\hline 1827526 & $U(x) 56$ & $\because 16.1255$ & $(1.13 ! 1$ & $\operatorname{tn} .5125$ & $0(1)(i)$ & 2113245 & 0.1534 \\
\hline 1855025 & 0.1018 & $245+115$ & 0.1 .445 & $43+4.25$ & $0.1 k: 2 k$ & 213.0015 & 0.1570 \\
\hline 186.1025 & 0.10145 & 2425445 & $01,3,6$ & $2(154)=$ & $(1,1020$ & $210.5 ? 35$ & (1) 15.4? \\
\hline $11 \times 1.11=5$ & 01142 & $2.34 *(115$ & (0.1.36) & 37.5325 & 0.61517 & 219.1459 & 0.1505 \\
\hline
\end{tabular}




\section{Reinforced Concrete Test Data}

Track 4 - High Pressure Test

\begin{tabular}{|c|c|c|c|c|c|c|c|}
\hline $22174 x^{6}$ & (i) 1529 & 211235 & fistats & $6 x^{\prime}(1: 5$ & 1.1 .15 & $256 \$ 515$ & 0155.4 \\
\hline 22430015 & $01.4 \%$ & jur $\leq$ as & $0 i(w j$ & $6: 0425$ & 0.1110 & 258.934 & 0.1518 \\
\hline $217(14)^{4}$ & $01 \%$ & $126 \times 116$ & $11 \div 4$ & (1. 5925 & $0.1 \geq 8$ & $2 f(0)\left(k+e^{k}\right.$ & 0.14 .1 \\
\hline 22654 & 1014 & [4: $\left.4 x_{1}\right]=$ & $1: 1 " \cdots$ & 11.4428 & $0.1+1+1$ & 261 rema & (1) 1.482 \\
\hline$\therefore 210 x^{5}$ & $115 \mathrm{i}$ & $19112 x=$ & $\because 1^{17-2}$ & $7422=4$ & $0.12 \div 1$ & 26206155 & (1) 140 \\
\hline-348116 & $01+40$ & $1 k^{\prime}=2$ & (1) 611 ! & 77.21128 & 013244 & $26 ?$ ouge & (i) $1+x+$ \\
\hline $227+.1 .4$ & $(1) 140$ & iktsens & (1) 1 sren & k11 211 & 01456 & 26,52865 & (1) 1114 \\
\hline $2+11: 4 \%$ & 014 & $1 x^{2}+46$ & $\| 1 f_{2}$ & 820.4 .4 & (1) itst & 27655655 & (1) 3181 \\
\hline $21+110$ & $1114)^{\prime}$ & $1.4 \ldots$ & 1115 & $85 \times 1=6$ & $1115 i 11$ & $20,7 \times x+15$ & () $13 \%$ \\
\hline $2 f(x, k, 1$ & (') \& & $11 \cdots 1 . \alpha$ & (i) 10 & $8 x+\ldots$ & a i : ! ! & (1) 2) & 01220 \\
\hline 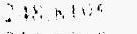 & $i: 1+11^{1}$ & $1: 2 \times 1: 8$ & $1: 1: 1$ & 4) $7+23$ & (1) / (cin) & Mifit?25 & $(13604$ \\
\hline $2<10$ & $1::^{2} 4$ & $16 \ldots, \ldots$ & $111 c^{6}$ & 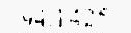 & 111,114 & 271.775 & $012 k 2$ \\
\hline$x+7+26$ & !' : ' & $14.2 \div$ & $(i) \therefore i$ & $\left(x_{1}, 1,3\right)$ & (1.1 S r. & $2724 \times 24$ & $(51230$ \\
\hline $2<5<5$ & $(1)$ & $10^{2}+20$ & $11+\cdots$ & $0 x_{1} \theta_{2}$ & $018 \mathrm{~s}, \mathrm{c}$ & 774205 & 0.1247 \\
\hline $1 \leq 2: 0$ & $0112 \%$ & $11,11 \ldots$ & intit & 1012916 & (1) 1 sto & atos & 0.1212 \\
\hline $2+x)+k+16$ & $1: 2 x$ & $16+7=$ & (1) $1: 0$ & $104 ! 28$ & (1.). 14 & $277(k)<s$ & 0.1227 \\
\hline$\therefore 1$ lind & $: 126$ & 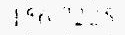 & $: 15$ & $161 " 216$ & (1) it: 14 & $2 \because x .4(x) 5$ & 01212 \\
\hline $2+21.1<6$ & $1111: \cdots$ & 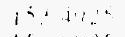 & $\because 1 \quad \because$ & $116+215$ & $|1|<7 \mid$ & $\therefore \quad ; i i ?$ & $0 ! 2 ! n$ \\
\hline$\therefore 0^{x}+0^{2}$ & $011^{4}$ & 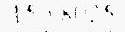 & $1: 11: 2$ & $11 \% 11.24$ & $(1: 6, \ldots)$ & $2 x+1517$ & 0.1 .7 \\
\hline 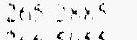 & $11 \%$ & $14^{\circ} " 4$ & $011^{\cdots}$ & $114 \times 12<$ & $0: 4,1.4$ & $2 n \times 4)(14$ & $(1) 251$ \\
\hline$\Rightarrow+\infty<1,64$ & 0314 & 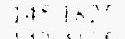 & $\because, \cdots, 1$ & $11 k+202$ & (1) loit & $2 x^{7} \quad 44$ & 0.1932 \\
\hline It $x_{1}$ & \begin{tabular}{l|ll}
$1 !$ & $1 !$ \\
$1111 \%$
\end{tabular} & & $\begin{array}{l}31^{6} \\
(1)^{\prime \prime}\end{array}$ & 121.1126 & (1) 16 th & $2852 \times 41$ & $111<1$ \\
\hline-4 & (1) 110. & $1+4,4$ & 1121 & 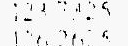 & 0170 & $25,7<20$ & 0.11 .411 \\
\hline$\therefore 1 \quad \%$ & 11 & $\therefore=$ & $11: 111$ & anidices & (1) 1110 & $2546=$ & $0.111 ?$ \\
\hline$\therefore \quad]_{1}$ & $1+1 \cdot x=$ & $1: 4 \ldots$ & $\therefore 11$. & $121<24$ & $11 \ldots$ & & \\
\hline $2 \cdots+3$ & $\because \ldots$ & $1 \therefore \therefore$ & $111, \cdots$ & $121 ? 24$ & 11 lire? & & \\
\hline$\because \leqslant 6$ & $1 i:$ & 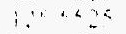 & $11:-$ & 1402 & 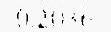 & \multirow{2}{*}{\multicolumn{2}{|c|}{ 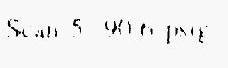 }} \\
\hline$\therefore \cdots+:$ & 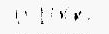 & $\therefore 1 \therefore=$ & $\because \cdots \cdots$ & $1+10$ sit..'s & bivi & & \\
\hline$\therefore n \because \cdots$ & $\vdots 1: \cdots$ & $1+\cdots$ & 1 it is & $1+\alpha_{2}^{+}: 12$ & $1, i, i$ & $2 \sin 220$ & $(1), 27$ \\
\hline $2 \cdots * 1$ & a,$\ldots$ & $1: t^{4}: 3$ & $1, i \cdots$ & $1: 232$ & $0, \cdots$ & 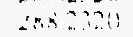 & $41 ?$ \\
\hline$\therefore \therefore: \cdots$ & $\because: \ldots \phi^{\circ}$ & ! t. & $11: 40$ & $1.17 \times 2$ & a i:?: & 24.73150 & 0.1241 \\
\hline 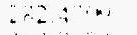 & $\| i n k$ & $i 1 i+16$ & 11 i & 196556 & $\| 1|x|$, & $26+1990$ & (1) 1005 \\
\hline$\therefore x 2 \cdots+4$ & 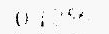 & $\because \cdots+\infty<$ & $(i)$ & $192 ? 129$ & $11,1,:$ & $280.7 \%=$ & 01.78 \\
\hline $2652 \times 4$ & $(113.4$ & $110+\ldots x$ & $11<i$ & 1554826 & $015 \%$ & $\therefore$ Acenis & $6: 277$ \\
\hline 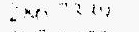 & $\because \ldots+4)$ & $1 i+60$ & $\| 1+2$ & $15 n \times i 25$ & $(1)(x):$ & $=41.14$ & 0.1415 \\
\hline$\therefore \quad k a^{6}=$ & $1,13 m$ & 140 & $\begin{array}{lllll}1 & i & \vdots\end{array}$ & $1111 . \div$ & (i) 18 & $2 \therefore(1+4)$ & 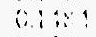 \\
\hline $5 \sin 4 \times$ & 2 J'a & 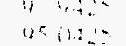 & 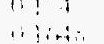 & It $1: 2$ & $\begin{array}{l}0165 \\
01164 .\end{array}$ & 20205 & $(1.19) 1 \%$ \\
\hline $24 \times 221$ & $\because 11$, & $4: 235$ & (1) it:il & $\begin{array}{l}106,4.92 \\
16,502\end{array}$ & U.tixi: & $\begin{array}{l}10 \\
21.1 \\
21\end{array}$ & $010 ! 9$ \\
\hline 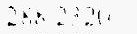 & $0: 1 n:$ & g(i) $14 \div 2$ & $1, \ldots$ & 172182 & (1) : & 1,19825 & 11328 \\
\hline $3+\cdots$ & 1.115 & 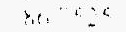 & i): & $1+n-x$ & "isis? & $\therefore 4397$ & (1) \\
\hline$\therefore \cdots 4,4$ & 111 & $x: 1:<$ & $111 \therefore=$ & 17.48 & 1115.711 & $2<14$ & $0.15<3$ \\
\hline$\therefore+1+8$ & $(1) 1 r^{5}$ & $A: D: c$ & $11: 1:$ & listive is & (i) $1: 2$ & Sinol.5 & $0.1+8$ \\
\hline$\Rightarrow x<4$ & $1111: 4$ & $\cdots 4 \quad \therefore<$ & $11: 4=$ & $1 \therefore 2 \cdots r$ & $1: 111$ & (5) a(x) & (1) Ksis \\
\hline $38+216$ & $i_{1} 112 \ldots$ & 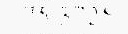 & $\therefore: \because 2$ & $18<5,1$ & $i 1: 26$ & $24+1255$ & $(1.11,4)$ \\
\hline$Z^{7}: 1144^{6}$ & $1 i ! \cdots$ & $3+4$ & 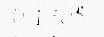 & Jk: : & lis.' & 2451115 & $0.20: 27$ \\
\hline$\therefore \quad 1$, & $\cdots: n 1$ & til $=$ & " & $\left.|+|\right|^{-1} 1<$ & $1^{\prime}:{ }^{\prime \prime},{ }^{\prime \prime} 4$ & $21: 54.45$ & (1) 1955 \\
\hline $31-19$ & $\| 1 \%$ & $(H) \quad \because B$ & in $1 \mathrm{in}$ & $110 \therefore$ & $1: 1 \%$ & (2) (1)(1) & (1) 167 \\
\hline $21+21^{-1}$ & $1+11+\ldots$ & $\therefore: \quad \dagger$ & $1+1 i 1$ & $\because 1^{6} \times 12$ & (i. $1^{-1}: \vdots$ & $\because 21645$ & $0.26 \mathrm{k} .1$ \\
\hline$\therefore 190$ & 111.4 & $\therefore \therefore$ & $11 ! ! 4$ & 11541,15 & $1: 1: 1$ & $\therefore 4.286$ & $0.201 !$ \\
\hline $2 x+2=$ & $(1) 11,2$ & $\Leftrightarrow 42$ & (1) j:kit & 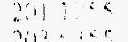 & $111^{\cdots}$ & 2316395 & $0.14 \pi$ \\
\hline$\therefore c_{1}+\alpha$ & 61,1 & 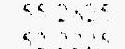 & $\therefore$ and & $31^{2}=4$ & $01 \%$ & $22 \times 8105$ & (1) $11 \cdots$ \\
\hline $7 c \cdot 01<\div$ & 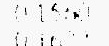 & $\begin{array}{l}6 \\
a\end{array}$ & $\because 19$ & $\begin{array}{l}310-10 k \\
310\end{array}$ & $111^{13}$ & 201045 & 01205 \\
\hline 25 atec & in it a... & 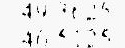 & $1+11 \%$ & $\begin{array}{llc}118 & 6 \\
3 ! 1 & 4\end{array}$ & $11 \mid \ldots+1$ & $22+115$ & 0.2118 \\
\hline$\therefore+1206$ & $\begin{array}{l}1161: \\
111\end{array}$ & $\begin{array}{lll}4 i< & 36\end{array}$ & $1: 1=$ & $\begin{array}{ll}2 ! 1 \\
31+410\end{array}$ & $1,1,1$ & $201+105$ & $0 ? 2127$ \\
\hline $2^{a c} 11 k$ & $\begin{array}{l}\text { (1) } 1.4 \\
\text { (i) } 1+4.4\end{array}$ & $\begin{array}{l}41286 \\
21626\end{array}$ & is $k$ ret & 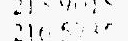 & $11 \times n$ & 212.4 .125 & $0.2 \%$ \\
\hline $24: 416.86$ & $\begin{array}{l}\text { (i) } 1+A+2 \\
1: N\end{array}$ & 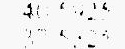 & $11: x+1$ & 2165 & $1: 1,1$ & $215.1+65$ & $02(x) \mid$ \\
\hline$\therefore 4+16$ & $\operatorname{lin}$ & $i_{1}<$ & $11: 1.9$ & & $41172 \%$ & 2123106 & 0.2122 \\
\hline$y^{2} / a^{\prime}$ & 110 & $21 t=$ & $\therefore \quad \therefore x$ & 21948 & $11 \times 4:$ & $2(k)+725$ & 0213.4 \\
\hline $2 \div 426$ & $11 \% 4$. & $\because 1+4$ & $1=$ & $\therefore 1 \therefore 1^{6}$ & $11, \ldots$ & Iik 7,es & $1 . ?(x) 5$ \\
\hline 231,169 & 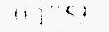 & $\because 4 \quad=$ & $\therefore$ & $21+1 \div 4$ & $(i \mid h)^{\prime}$ & $20(1+11225$ & $0.2(k 17)$ \\
\hline$\therefore \therefore \quad \therefore .4$ & if $i t+b$ & $\because \quad \therefore$ & & $24^{6} 84$ & $11: \div 1$ & 211,206 & $0,1 \times 15$ \\
\hline$\therefore+205$ & $\because \because$ & $\therefore, \cdots$ & $(\cdots$, & $=2 i t n^{\circ}$ & $11: 21$ & ith $5 \square$ & 02011 \\
\hline$\therefore 2+11^{\alpha}$ & $111 \cdots$ & 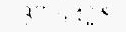 & $\because \quad \therefore$ & $24+12$ & $01 \% 1$ & 1058145 & $0.20 \%$ \\
\hline$=111, c^{2}$ & $i ; " \ldots$ & $\therefore: \cdots$ & $\vdots: \cdots$ & $2:+1,:=$ & $(1) \div$ & $11=0$ & $0.105:$ \\
\hline 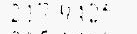 & $1: 2$ & $a+\ldots$ & $\quad \cdots$ & $\therefore 11<4,6$ & $11\} \cdots$ & 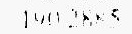 & $1 \because 801$ \\
\hline $215: 1 \%$ & $11^{11}$ & $\therefore \cdots$ & 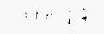 & $\therefore 1 \ldots$ & $\because 1+1$ & $10^{-15}: 25$ & 119457 \\
\hline$\therefore 3^{2}=i^{c}$ & 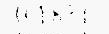 & $\because+\because$ & $A n$ & $2 t+i x_{n}$ & $11 \times 1,1$ & $18.7+25$ & (1) 19.2? \\
\hline $2(x+1=6$ & $(1) \therefore$ & $4 \quad \cdots=$ & $i, n \ldots$ & 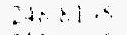 & $\{110 \%$ & Honsto & $11, \therefore$ \\
\hline $2(x, \cdots$ & 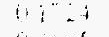 & $\because \quad 4$ & 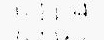 & $21 \ldots 5$ & $\| 1 \cdot \because$ & $1 \%, 2 y_{0}^{\prime} 2$ & $111 \%$ \\
\hline $214+26$ & $a x+1,4$ & 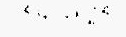 & i: : & $26+2=$ & $1111: "$ & $|x+1| 2<$ & 0118 \\
\hline
\end{tabular}




\section{Reinforced Concrete Test Data}

Track 4 - High Pressure Test

\begin{tabular}{|c|c|c|c|}
\hline 173.445 & 0.2 .56 & 91.3926 & $0.18 . ? 2$ \\
\hline 170.98 .25 & $(1.2(170)$ & 94.1425 & 0.2155 \\
\hline 160.1825 & 0.1412 & 96.0224 & $0.176 \%$ \\
\hline $10^{5}, 3,25$ & 0.21118 & 9060929 & $0.19 x-4$ \\
\hline 162.4125 & $0.2 ! \times ! ?$ & 102.4125 & 0.1760 .4 \\
\hline 1.94 .7 .425 & 0.21112 & 105.1295 & 0.1770 \\
\hline 156.7225 & $014 \%$ & 1117.8226 & 0.18600 \\
\hline $15: 4075$ & $(1.26174$ & $110.4: 25$ & (1) 1841 \\
\hline 1.50 .801 .25 & $0.16(x)$ & 11.308 .25 & (1) 1808 \\
\hline 147.96 .26 & 0.2117 & 115.6025 & (1.) $140(14$ \\
\hline 145.18 .9 & 0.1254 & 118.5925 & $018 \mathrm{~s}$ \\
\hline $142+125$ & $0.18 \pi$ & 121.1429 & 01896 \\
\hline 134.115 & 0.20137 & $1237+29$ & 0.100011 \\
\hline 1.36 .7926 & U. $2 .(x) .5$ & 126.20 .25 & $0.2(x, 1)$ \\
\hline 1.3777 .5 & 0.2027 & $127(1.425$ & $0.92, \mathrm{i}$ \\
\hline $131.14+25$ & $0.20,25$ & 131.5725 & 0.2022 \\
\hline $128 \quad 3225$ & $0.201 ! 4$ & 144295 & 0.2121 \\
\hline $125<5.25$ & $0.21+2$ & 13761025 & $02 \cdot 4 ?$ \\
\hline 1224525 & 0.1904 & $134.80=5$ & 9 गयाद \\
\hline 19112725 & 0.1918 & 142.5125 & (1) 166 ? \\
\hline $11745 \ldots 5$ & $018: 2$ & 1159125 & $0.11 /(1)$ \\
\hline $1140^{725}$ & $018 \mathrm{~s}$ & 1478225 & 0.2144 \\
\hline 111.8725 & $0.1 \times 15$ & $1561<525$ & $(1.2111)$ \\
\hline lint $15=5$ & $(1) 17 \%$ & 1532125 & $0.21 k 14$ \\
\hline$i\left(x_{1}+2,25\right.$ & 019.1 & 1594025 & 0.19501 \\
\hline $11145: 2:$ & $0.1^{-12}=$ & 1964825 & 0201,1 \\
\hline 1601793 & (1) $1.7,8$ & $101.42=5$ & $02: 12$ \\
\hline $4 \div .425$ & (1) $17(1)$. & 16.42975 & $(1.211 .4 t$ \\
\hline 461915 & $91 \times 1.4$ & 166.492 .5 & 11.14011 \\
\hline (1) 2.236 & (1) 18.4. & 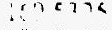 & 1) 2.11 .4 .8 \\
\hline E4. 4625 & $11.1 \times+1$, & 17201825 & (1) 210 \\
\hline 80.25 & $0.175 x$ & 174.805 & $0.1 \times 12$ \\
\hline $\bar{x} 3.9 t .25$ & $\left.(1)^{\prime}\right)$ & 1774.9 & 01316 \\
\hline $811 \times .5$ & (1) ic: & Isitik, 25 & $0.1 .9 \%$ \\
\hline $783(125$ & (1): $16 . \%$ & 10025 & $01: \ldots$ \\
\hline 35.3725 & $(0.15: 5$ & 1850,25 & $(12)(H)$ \\
\hline 791.5 & $0.1 .+6.1$ & 15811125 & (1). 142,1 \\
\hline 64.6725 & $0: 44$ & $!(10171>4$ & (1) $2(1,14$ \\
\hline 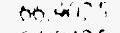 & 0.1231 & 1192379 & 0,2123 \\
\hline 5.401 .425 & () 121,10 & $115=x+16$ & $621(n)$ \\
\hline 012325 & 1). $1 .+2+1$ & $145+406$ & lin \\
\hline $56+425$ & (1) $12: 1 ?$ & 20111.24 & $(12,18$, \\
\hline 552826 & 111111 & $26121,2+4$ & $0: 11 i$ \\
\hline 53925 & $010 i ?$ & 216.2165 & $1121 \cdots$ \\
\hline $4 y+34.25$ & (likiss & $260^{2} 756$ & 1212 \\
\hline 40.5125 & $0(15)$ & 2119246 & 112.84 \\
\hline 42.6425 & $111 k<1 !$ & $2 ! 3(21)^{5}$ & (1).21 $\mathrm{ir}$ \\
\hline 2051125 & $(191), 4)$ & $216,9 ? 25$ & 0.21 .41 \\
\hline 37.9426 & $0\left(1^{-1}+1\right)$ & 21171455 & (1) $2116 \%$ \\
\hline $3.4+528$ & 010500 & $\because 17455$ & $0.21+1$ \\
\hline 316125 & 1). (1.46.? & 22434015 & $(1.21) ! 1$ \\
\hline 29.1726 & (j) $(1+2)$ & $227.6+45$ & 9.2110 \\
\hline $32.1=8$ & bisto & 209.5745 & $0.216 i$ \\
\hline $346^{-2} 25$ & !'ख! ? & 23210,05 & 02113 \\
\hline 374.425 & $0 .(1812$ & andoin & $\because y$ 化 \\
\hline 4$)(0.25$ & $(1)(1 k 6)$ & 230.145 & 0.2017 \\
\hline $44: 6.25$ & $(1)(H) 5, t$ & 2410.345 & $0.196 x$ \\
\hline 47.7325 & $0.11: 7$ & 2422015 & $0.2(1.8)$ \\
\hline 56.5125 & $(1) 1132$ & 240,6045 & 0.2123 \\
\hline 53 bit.24 & 01111 & $2408: 95$ & 10.1922 \\
\hline 90.9425 & 0.1252 & 2510265 & (1) $18: \pi$ \\
\hline 59 th:3 & (1) 1 रा1 & $24+91795$ & (i) $18+1$ \\
\hline $6.2 \times 112$ & $014.4^{7}$ & 250,545 & O 1sits? \\
\hline 65,0125 & 01252 & 258.7275 & $(1) 1315$ \\
\hline$(1, k+51), 5$ & 0.12 .7 & $2 f_{1}(1)(x+1=$ & 0192 \\
\hline $71+125$ & $0151 \mathrm{~A}$ & $26,1\} 3(1+15$ & $\because 170.2$ \\
\hline $7+3225$ & $(1)+1+1)$ & $20,20: 4$ & $0.10(\mathrm{~s})$ \\
\hline 7736 & 015.5 & 20,2005 & 111128 \\
\hline (xit) 210 & $i i^{2} ?$ & 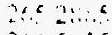 & $\therefore 1: 12$ \\
\hline $82142=6$ & (1) linh & $20 x+5, \leq 5$ & (1) $\mid+1.4$ \\
\hline $8<+126$ & $(1.177 .4$ & $26:$ ston & (1. ! stik \\
\hline s.t. 1.4 & (1) 180 & $2,0,4)(k, 2$ & (1) $15,4,1)$ \\
\hline
\end{tabular}

\begin{tabular}{|c|c|c|c|}
\hline 270405 & 0.1551 & 130.7125 & $0.218 k$ \\
\hline 271778.5 & $(1.14174$ & 1367925 & 0.2518 \\
\hline 272.1825 & 0.1415 & 133.7725 & 0.2222 \\
\hline 2741245 & 0.1137 & 131.0425 & 0.2198 \\
\hline $275.6 / 0.5$ & 0.1428 & 128.3225 & 0.2179 \\
\hline $277 .(1)<3$ & 0.1401 & 125.5575 & 0.22111 \\
\hline $278.31 \times 15$ & 0.136 .4 & 122.9525 & $0.210 \hat{~}$ \\
\hline $2 \% 7317$ & $0.1 .34 k$ & 120.2725 & 0.2078 \\
\hline 28101807 & 0.1424 & 117.4525 & $0.2(114$ \\
\hline $2 k_{2}^{2}+7(\mu)$ & 0.1474 & 114.6725 & $0.2(122$ \\
\hline $28,74 \%$ & 11.1522 & 111.8725 & 0.1972 \\
\hline 285.2850 & $(1.1 .145$ & $10+1825$ & $0.1(2,13$ \\
\hline $280 / 39 y$ & 0.1270 & 106.425 & 0.1950 \\
\hline $287.805 ?$ & 0.1 .46 & 103.5725 & $0.167 ?$ \\
\hline & & 100.7725 & $0.16 \times 18$ \\
\hline Scan $f$. U. & "źn & 97.9 .425 & 0.2190 \\
\hline & & $95 .(425$ & $0.2(1)^{5} 1$ \\
\hline 280.2320 & 010154 & $9 ? 2225$ & $0.203 k^{\circ}$ \\
\hline 2862520 & 0.1018 & 89.4525 & 0.2019 \\
\hline $207<156$ & $(1.131 ?$ & 86.7525 & 0.1924 \\
\hline $28+4247$ & $01.1 \times 10.9$ & 83,2625 & 0.186 .5 \\
\hline $2 x \mid 1897$ & 0.1 .410 & $81.0 \times 1,5$ & $0.1 k ! 6$ \\
\hline $27 \times x \times 11$ & (1).14.6) & $78.3(125$ & 0.1762 \\
\hline $2 ? 54215$ & 0.1511 & 75.3775 & (0.16.2 \\
\hline$? 7 ? 014.45$ & 0.15197 & 7.4225 & $0.10 .5 !$ \\
\hline $21121 \times 5$ & $0.10,5 !$ & $0,9,6725$ & $0.16 t^{2}$ \\
\hline $20,55+5$ & $01.17 \mathrm{tm}$ & 60.20125 & 0.1520 \\
\hline $20+77: 3 ?$ & 0.17 .41 & 6.4 .14255 & 0.1 .12 .1 \\
\hline $26.14 \times 25$ & $(1.184010$ & 61.2225 & $(1.16(x, 5)$ \\
\hline 25112315 & $(1.1821)$ & 56.4225 & $(11328$ \\
\hline 256.17 .5 & (1.1141.4 & 54.01255 & 0.1270 \\
\hline 2546,159 & $0.1 \cdot 1 \cdot 4+4$ & 522225 & 0.115 .3 \\
\hline 250 solkis & 0.21121 & 40.3625 & $0.116,8$ \\
\hline 2461255 & (1)11,35 & 46.5125 & $(i,(x) 70$ \\
\hline $24 \div 1115$ & $023 \%$ & 43.4425 & $(1 .(k)(1) 7$ \\
\hline$\therefore 4.5445$ & $0.21 \cdots:$ & 80.5925 & 50881 \\
\hline 73011$)^{2}$ & (1.2173 & 37.5325 & $0(11.94$ \\
\hline $2710^{6} \cdot 5$ & 0.2215 & 3.46725 & 0.0562 \\
\hline 23,3269 & 0.2207 & 310125 & 0.0500 \\
\hline 2316,755 & (1) 2.2Ku & 29.7715 & 0.0267 \\
\hline $225611=5$ & $0.2211 !$ & 32.1225 & 0.15481 \\
\hline 2261255 & 0.2244 & $3+6725$ & of́nin." \\
\hline $223+115$ & $(1.210$ & 17.9425 & 0.01695 \\
\hline ?hon:s, & $0.220 \div 4$ & $41(x) 25$ & $0.0(0,3)$ \\
\hline 2170.925 & $11.2+2=$ & $4+20: 5$ & $0 !\left(n^{\prime}\right) !$ \\
\hline $2 ! 514+5$ & 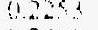 & $4 \% 7325$ & 0.16192 \\
\hline $21 \geq 2.945$ & (1.2.64 & 501801.25 & (1.116, \\
\hline 244.2725 & $(1+2) !)$ & 53.8125 & (1.1221 \\
\hline $2(k, 7), 0,5$ & 0.2257 & 56.4425 & 0.136 .1 \\
\hline $2(1401) 225$ & (1.2.24t & 59.9325 & 0.1422 \\
\hline 201.2585 & 0.21144 & 6280125 & (1).1.4\% \\
\hline $148<3<9$ & 0.2210 & $65.6,925$ & 0.1508 \\
\hline $195.8: 45$ & 0.2260 & 68.5925 & 0.16 .25 \\
\hline $1929 \times 1 \%$ & 6. án & $71 .+25$ & 0.1598 \\
\hline 1911.2835 & $(1.226)$ & 74.3225 & 0.1657 \\
\hline $10, \quad \therefore 25$ & 11,915 & 77.3020. & 01744 \\
\hline $18+86.25$ & 0.2145 & 80.2025 & 0.1824 \\
\hline $182(1825$ & $(1.2015 .2$ & $83 .(1425$ & 0.1870 \\
\hline 174.7625 & 0.21 .35 & $85 \times 1.25$ & $0.187 ?$ \\
\hline $1 ?(.6,125$ & $(120127$ & $8 \times 6,625$ & 0.1482 \\
\hline $173 \times 425$ & (1):xys & 91.3425 & 0.1953 \\
\hline $1: 110425$ & 11.22 .43 & 44.1425 & 0.2106 \\
\hline 11.8 .1525 & (1):124 & 46.925 & 92114 \\
\hline 16.53 .325 & $0.216,8$ & $94(1925$ & $9.18 \mathrm{mg}$ \\
\hline 1024125 & 0.2143 & 102.4125 & 0.105 .3 \\
\hline 1597425 & 0.2235 & 1015.1225 & 0.1920 \\
\hline 150.7225 & $(1.2) 10.5$ & 107.8225 & (1.2) $2(4)$ \\
\hline 1534125 & 0.2286 & 110.4 .325 & $0.19 k_{2}^{2}$ \\
\hline $151: 56126$ & $(121<1$ & $1 ! 311825$ & 0.2155 \\
\hline 127.4025 & (1)2.24 & 11586325 & $0.2(x)$ \\
\hline $1.15,196$ & $\because 767 \mathrm{th}$ & $118+425$ & $(1.2(k) 0$ \\
\hline $142+125$ & $2(x, 4$ & i.:1.2425 & $0.21 \times 1$ \\
\hline
\end{tabular}




\section{Reinforced Concrete Test Data}

Track 4 - High Pressure Test

\begin{tabular}{|c|c|c|c|c|c|c|c|}
\hline 123.7325 & 0.2100 & 2867344 & 0.1378 & $1(x) .1825$ & 0.2152 & 153.2025 & 0.2448 \\
\hline 126.2625 & $0.22(\mathrm{~N})$ & $287.015 \%$ & 0.1350 & 106,4325 & 0.2145 & 155.9825 & 02358 \\
\hline 129.1425 & 0.2243 & & & 103.5725 & $0.2(x)(1)$ & 158.8425 & 0.2456 \\
\hline 131.5725 & 0.2244 & & & $1(x) 7725$ & 0.2121 & 161.4925 & 0.2457 \\
\hline 134.2325 & 0.2341 & $S_{c a}, 7, i c$ & 0.9 posiz & 97.9425 & 0.2149 & 164.2725 & 0.2395 \\
\hline 1.370825 & $(1.2492$ & & & $95 .(1425$ & 0.2260 & 166.9425 & 0.2 .34 .3 \\
\hline 1.398625 & $0 . .22(x)$ & 288.2320 & $0.317 x$ & 92.2225 & 0.2222 & 169.5325 & 0.2405 \\
\hline 14291.25 & 0.2021 & 288.2370 & $0.317 x$ & 89.4525 & $0.22(18$ & 172.0825 & 0.2598 \\
\hline 1452425 & $0.2(11 \%$ & 287.5156 & 0.1384 & 86.75 .25 & (1).2088 & 174.8025 & 0.2249 \\
\hline 147.8225 & $0.29(x)$ & 284.9207 & 0.1910 & 83.963 & 0.21155 & 177.477 .5 & 0.2311 \\
\hline 1505525 & 0.2104 & 2810972 & 0.1500 & $81(x, 25$ & 0.1952 & 1800625 & 0.2377 \\
\hline 1532025 & 0.2254 & 278.8981 & $0.154 ?$ & 76.3025 & 0.194 .4 & 182.7525 & 0.228 .5 \\
\hline 1554825 & 0.2111 & 275.9215 & 0.1610 & 75.2795 & $018<3$ & 18.5 .592 .5 & 012427 \\
\hline 158.8.4: & $0 ? ?(x)$ & 273.1445 & $0.1 \% 4 x$ & 72.4325 & 0.176 .3 & 188.1025 & 0.2455 \\
\hline$i 01.4925$ & 0.2147 & 270.2685 & 0.18013 & 69.6725 & 0.17 .37 & 190.7175 & $(1.2492$ \\
\hline 16.4 .272 .5 & 0.111 & 267.55 .15 & $0.106 ?$ & $6 x 01925$ & 0.1 tuth & 193.2275 & 0.250 .3 \\
\hline $10 \mathrm{kn}, 942.5$ & 0.2115 & 264.7175 & 0.19017 & $6+0.425$ & 0.1542 & 195.8805 & 0.2489 \\
\hline $169.5 \times 25$ & 012195 & $96.198 ? 5$ & $0 ?(x) 0$ & (6) 2225 & $0.166,3$ & $100: 095$ & 1.2445 \\
\hline $172082 x$ & $0.2 .2(x)$ & 2642275 & 0.1980 & 584225 & 0.1411 & 201.1255 & 024.34 \\
\hline 174.805 & 0.2250 & 256.4725 & 0.2124 & $55.2 \times 25$ & 0.1331 & 2036355 & 0.2434 \\
\hline 177.47 .95 & $(12)+3$ & 2336155 & 0.2101 & 52.2225 & $0.130 !$ & $20 \times .248 .5$ & $0.25(x)$ \\
\hline $180(x, 25$ & 0.21 .31 & $250.800(15$ & $(0.22)\left(k_{1}\right.$ & 49.76 .29 & 0.11 .38 & 208.7585 & 0.2570 \\
\hline 182.7925 & $0.2(171$ & 248.1255 & $(0.2158$ & +6.5125 & 0.10 .36 & 211.3295 & 0.2626 \\
\hline 1855025 & 0.2175 & $245+115$ & 0.2477 & 43.4425 & $0.017+18$ & 2139015 & 0.2538 \\
\hline $18 \times 1025$ & 0.2223 & .42 .54 .45 & 023.44 & 40.54725 & $0(19)$ is & 216.5735 & 0.2588 \\
\hline $1(x) 71115$ & $(0.2041$ & $239.9(115$ & 0.2350 & $\$ 7.5325$ & 0.0786 & 219.1455 & 0.2508 \\
\hline 1432274 & 012320 & 2271695 & $(0.2 .430)$ & 34.6725 & 0.0573 & 221.7985 & 0.2535 \\
\hline $195.8 \times 15$ & 0.220. & 22.13254 & $(121 . ?)$ & $31 \mathrm{nl}: 5$ & $0(1526$ & 224.3905 & $0.2\{?\}$ \\
\hline 148.7405 & $0.23 \%$ & 2310.255 & $(1.2+1)$ & 29.7725 & $0.052(1$ & 2270.115 & $0.25(17$ \\
\hline 201.1255 & $(1.2251$ & 228.84015 & (1.2.78iv) & 32.0225 & $0.05(x)$ & $229.57+5$ & 0.2475 \\
\hline 20136,255 & 012276 & 226.1255 & ():440 & 34.6725 & $0.06,8,4$ & 232.1665 & $0.250,7$ \\
\hline $26 \times .2+84$ & $0.211 x_{1}$ & 2234115 & 0.2513 & 37.9425 & 0.114 .45 & 1.44 .8145 & 0.2 .427 \\
\hline 2018.7565 & 0.2277 & 220.6765 & 0.2127 & $41 .(k) 25$ & 0104.57 & 237.6145 & 0.2402 \\
\hline 211.3795 & 11226.5 & 217.4425 & $0.2+45$ & 4.1 .26 .25 & 0.1073 & 240.3495 & 0.2365 \\
\hline 213,91115 & $0 \div 23,3$ & $2151+1.5$ & (1).24601 & 47.7325 & $0.11 / 1$ & 243.2075 & $0.24(k)$ \\
\hline $216,57.35$ & 012258 & 2123409 & $62+10$ & 5018025 & 0.1257 & $2.46 .(k \times-15$ & 0.2491 \\
\hline 219.1455 & 0.2 .332 & $2(k)\{725$ & $\{1,2517$ & 33805 & 0.1308 & 2488195 & 0.2292 \\
\hline 221.9085 & 0.23 .42 & 2007765 & (1).2481 & 56.9425 & $0.1+(x)$ & 251.6365 & 0.2243 \\
\hline 22439115 & 0.2 .31 & 2040275 & 0.2428 & 59.9825 & 0.1548 & 254.4925 & $0.219 t_{1}$ \\
\hline 2270.4 .49 & $\because 2 x ! 12$ & 20: In:se & $0.2 .9 ?$ & 628025 & 0.150 .2 & 2565525 & 0.2152 \\
\hline 239.5745 & $0.23 \%$ & ix & (1) $24+1$ & 656025 & $0.1 \mathrm{xiH}$ & 258.7375 & 0.207 .4 \\
\hline $23: 1665$ & 0.280 & 1058105 & 0.2455 & 685.525 & 0.17 .22 & 260.0645 & 0.2013 .3 \\
\hline 2448105 & 0.2 .15 & 1920815 & $0.2 .4^{7}(1$ & 714425 & $0.171,3$ & $261.30 \times 15$ & 0.2015 \\
\hline $237+145$ & $(1.229$ & 100.2805 & 0.24115 & 74.3215 & 0.1773 & 262.6155 & 0.19 .56 \\
\hline 2413405 & 0.236 & 167.625 & 0.2124 & 77.30125 & $0.1 \times 6$ & 263.9825 & $0.1 \% 2$ \\
\hline 24320175 & 0.226 & $184 x+25$ & 0.2375 & $8(0.20 .25$ & 0.106 .4 & 265.2885 & 0.1935 \\
\hline $2+46,\left(x_{1}-1\right)$ & 0123.4 & 1821325 & 012200 & 8.3 .0425 & $0.2(\because) 5$ & 266.5955 & 0.1857 \\
\hline $2.2 \times 8195$ & $0.211 \times 1$ & 179.2195 & 0.2375 & 85.8125 & 0.26119 & 267.8605 & 0.1810 \\
\hline 2510,105 & 0.20130 & 176,6125 & 0.2215 & 88.6525 & 0.2134 & 269.206 .5 & 0.1836 \\
\hline 24.4425 & $0.2(x)$ & $1738+25$ & 0.267 & 91.3925 & 0.216 .3 & 270.4425 & 0.18 .41 \\
\hline 25605535 & 0.1975 & 17619.925 & (1. 244.4 & 94.1425 & 0.2322 & 271.7785 & 0.1771 \\
\hline$? 9873: 5$ & $0192 \mathrm{~h}$ & 1681525 & 0.2306 & 46.9325 & 0.2172 & 272.9825 & 0.1689 \\
\hline $26 x)(x+4)$ & $0 . \mid g(k)$ & 105.7325 & 0.22 .46 & 99.6925 & 0.1866 & 274.3295 & 0.166 .3 \\
\hline $2(1) 3(x) 5$ & $0 \mid \& 11$ & 162.4125 & 0.2300 & 102.4125 & 0.2106 & 2756765 & $0.16 \times 0$ \\
\hline $2+2001 \leq 5$ & $0.18 k$ & 154.7425 & 0124.34 & 105.1225 & 0.21 .21 & $27 \% .003 .35$ & 0.1661 \\
\hline 2609805 & $0.1 / k 8$ & 156.72 .25 & (0) 2.349 & 107.8225 & 0.2241 & $278.3(\mu) 5$ & 0.1620 \\
\hline 265 AM & (2.:!'! & $152+925$ & () 2.142 & 110.1325 & 0.22117 & $279.731^{7}$ & $0.164 . ?$ \\
\hline Thtsyse & 0179 & 19580125 & $0 ? 2,1$ & 113.08 .25 & 0.2250 & 281.0807 & $0 .|\tan |$ \\
\hline 26786015 & 0.17111 & 147.4025 & $0.252 \div$ & 115.80 .5 & 0.2256 & 282.4709 & 0.1737 \\
\hline $20,20 x, 5$ & $0.1+\sin$ & 1451825 & $(12200$ & $118+925$ & $0.228:$ & 283.7974 & 0.1790 \\
\hline 2704025 & $(1) 68.1$ & 1424125 & 0.2367 & 121.1425 & 0.2295 & $285.2 \times 50$ & 0.1 .491 \\
\hline 271.7785 & 0.10 .501 & 12971.5 & 02236 & 123.7325 & 0.2 .376 & 28673.39 & 0.1489 \\
\hline 2724825 & $016 R_{2}$ & $1367: 25$ & (1).214 & $126,26,25$ & $0.2+105$ & 287.8052 & 0.1419 \\
\hline 274.2344 & $0.15 ?$ & 1.2 .77 .25 & 6.2424 & 124.1425 & $0.2+14$ & & \\
\hline 2756709 & $0.152 \%$ & 131.1424 & $(1.2+21$ & 1.31 .5725 & 0.2417 & \multirow{3}{*}{\multicolumn{2}{|c|}{ F́an 8, 108.3 psiz }} \\
\hline $277\left(a^{\prime}\right) .25$ & 0.1518 & 128.3225 & $(12.4(1)$ & 134.2325 & 0.2530 & & \\
\hline $27 x 3(105$ & $(1.1512$ & 1255524 & $0.24 \%$ & 1.370825 & 112721 & & \\
\hline 279,217 & $0.14 \times 0$ & 1224525 & 0.2348 & $13986: 5$ & 0.2362 & 256.2320 & 0.5311 \\
\hline 29101807 & $0.154 i$ & 1201225 & 0.2 .47 & 1425125 & $(1.221$ & 288.2320 & 05211 \\
\hline $15: 2914$ & (1) lon & 137.424 & 02280 & 145.2425 & 0.236 .2 & 2875150 & 0.1585 \\
\hline $28: 709:$ & $0.16 \div$ & 1146126 & $(19221)$ & 147.8225 & 0.2552 & $28+4.4 ?$ & 01.2179 \\
\hline 2652850 & 0.1615 & 1118725 & 128164 & 1.50 .5525 & 0.283 & 281 W4i2 & 01820 \\
\hline
\end{tabular}




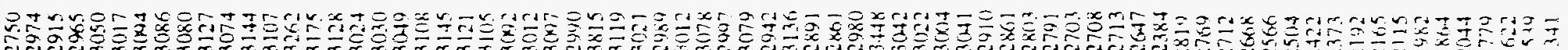

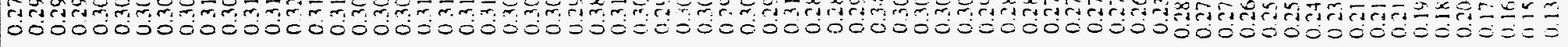

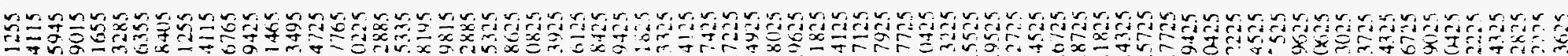

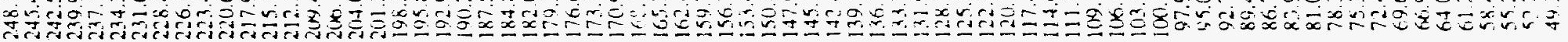

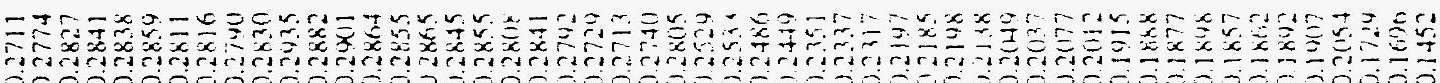
00000000000000020000000000000000000.0000000000000000

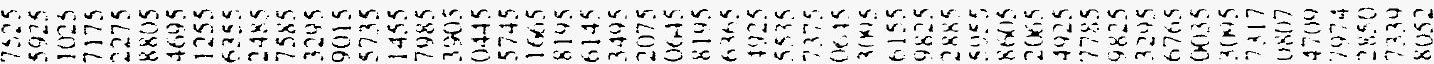

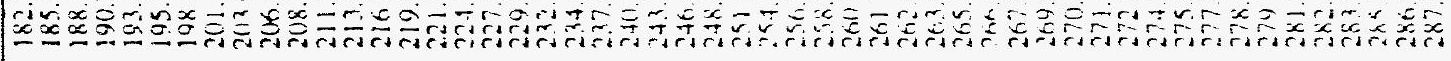

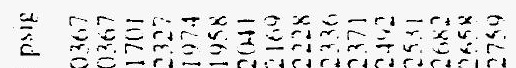

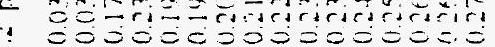
$=$ or S

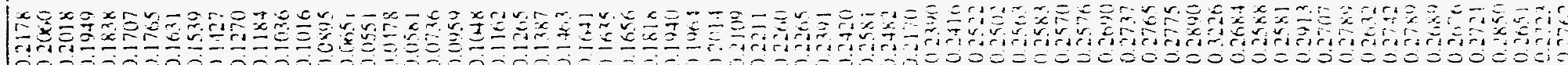

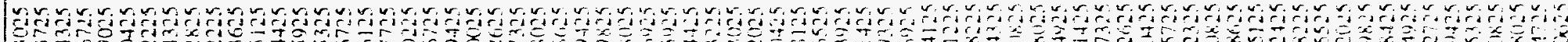

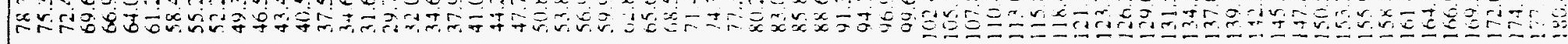

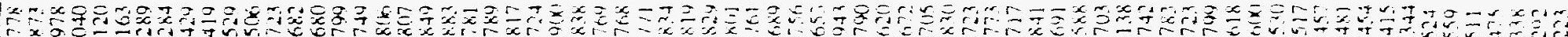

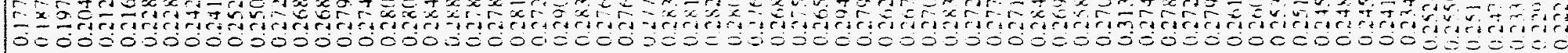

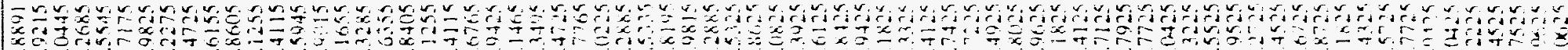

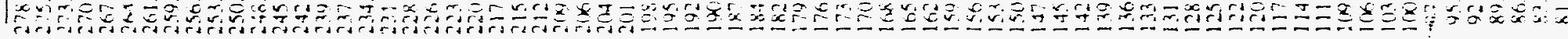




\section{Reinforced Concrete Test Data}

Track 4 - High Pressure Test

\begin{tabular}{|c|c|c|c|c|c|c|c|}
\hline 46.5125 & 0.1273 & 211.3295 & 0.3177 & 217.9425 & 0.3466 & $44.26,25$ & 0.1290 \\
\hline 43.4425 & 0.1131 & 213.9015 & 0.3150 & 215.1465 & U.:177 & 47.7325 & 0.14 .31 \\
\hline 40.5925 & 0.1094 & 216.5735 & 0.3145 & 212.3495 & 0.3217 & 50.8025 & 0.1553 \\
\hline 37.5325 & 0.088 .3 & 219.1455 & 0.3102 & 209.4725 & 0.7575 & 53.8625 & 0.1695 \\
\hline 34.6725 & $0.06 \times 2 ?$ & 221.7985 & 0.3149 & 206.776 .5 & 0.3474 & 56.9425 & 0.1871 \\
\hline 31.6125 & 0.0582 & 224.3905 & 0.3115 & 204.0225 & U. 3.369 & 59.9825 & $0.20 \times 15$ \\
\hline 29.7725 & 00391 & 227.0445 & 0.3146 & 201.2885 & 0.3377 & 628025 & 0.2070 \\
\hline 32.0225 & 0.1655 & 229.5745 & 0.3108 & 198.5335 & 0.3418 & 65.6025 & 0.211 .3 \\
\hline 34.6725 & 0.08012 & 232.160 .5 & 0.3121 & 195.8195 & 013485 & 68.5025 & 0.2277 \\
\hline 37.9 .425 & 0.105 .3 & 234.8145 & $0.311 k 6$ & 192.9815 & 0.3501 & 71.4425 & 0.2284 \\
\hline $41.0 \times 125$ & 0.1119 & $21 \% 6145$ & $0.700 ?$ & 190.2885 & 0.3488 & 74.3225 & 0.2393 \\
\hline 44.2015 & 0.1229 & 240.3495 & 0.2995 & 187.5325 & $0.346(1$ & 77.30 .25 & 0.2447 \\
\hline 47.712 .5 & 0.1377 & 243.207 .5 & $0.30 \times 17$ & 184.8625 & 0.3446 & 80.2025 & 0.259 .3 \\
\hline 50.8025 & 0.1491 & 246.06 .45 & 0.3077 & 182.0825 & 0.3374 & 83.0425 & 0.2651 \\
\hline 53.86725 & 0.1547 & 2.48 .8195 & 0.28 .34 & 179.3925 & 0.3397 & 85.8125 & 0.2706 \\
\hline 56.9425 & 0.1729 & 251.6365 & 0.2780 & 176.6125 & 03302 & 88.6525 & 0.2831 \\
\hline 59.9825 & $0.18 \times 44$ & 2544025 & 0.2714 & 173.8425 & (1).37(14 & 91.3925 & 0.2871 \\
\hline 62.80125 & 0.1080 & 256.5539 & 0.266 .8 & $17(1.9425$ & 0.3460 & 94.1425 & 0.3166 \\
\hline 65.6025 & 0.197 .3 & 258.7375 & (0.25193 & 168.1825 & 0.32 .58 & 96.9 .325 & 0.2981 \\
\hline 08.5025 & $0.2(k) 4$ & $260(k-1)$ & 0.2522 & 165.3329 & 0.3287 & 99.6925 & 0.3240 \\
\hline 71.4125 & 0.2127 & $261.3(145$ & 0.2498 & 162.4125 & 0.33 .54 & 102.4125 & 0.2052 \\
\hline 74.3225 & 0.2187 & 26261595 & 0.24 .66 & 159.7425 & 0.74 .54 & 105.1225 & 0.2926 \\
\hline $77.3(1: 5$ & 0.2254 & 26.39825 & 0.2302 & 156.7225 & 0.3368 & 107.8225 & $0 . .1(1) 81$ \\
\hline $8(1.20) 25$ & $O+n i$ & 2652885 & ().2Xk3 & 153.4925 & $0.3+10)$ & 1104325 & $0.30 \mathrm{no}$ \\
\hline 8.31425 & $0.2+41$ & $2(x-54) 55$ & 0.2440 & 150.80105 & 0.3 .307 & 113.4525 & 0.3107 \\
\hline 858125 & $112\{4\}$ & $207.86,15$ & 02201 & 147.9625 & 0.3417 & 115.8015 & 0.311 .5 \\
\hline $88.0^{<} 25$ & $0 ? 570$ & $264.2(105)$ & $\left(1.2 .2 p_{1}\right.$ & 145.1825 & () 2225 & 118.4925 & 0.318 .3 \\
\hline 41.3925 & (1). $.0,5\}$ & $27(14025$ & (1) 2234 & 142.4125 & 0.3190 & 121.1425 & 0.314 .4 \\
\hline 941.125 & $1928+1$ & $271.77 \times 5$ & 0.2188 & 1.39 .7125 & 0.32618 & 123.7325 & 0.3262 \\
\hline 90.0325 & $0.2 \times+46$ & 272.9825 & 021.27 & 1.36 .7425 & 0.3731 & 126.26 .05 & 0.3 .327 \\
\hline $94.619=5$ & $0.2 .2+4$ & 274.3245 & $0120 \% 3$ & 133.7725 & 0.3356 & 129.0425 & 0.33 .318 \\
\hline 1012.4125 & $11.26,23$ & 279.670 .5 & $0.2(150$ & 131.1425 & 0.33 .54 & 131.5725 & 0.3 .365 \\
\hline 1015.1225 & 0.20 .58 & $277 .(x) 25$ & $0.2(1+2)$ & 128.3225 & 0.3270 & 1.34 .2325 & 0.35136 \\
\hline 107.6225 & 11274.1 & 2753 (105 & $(1.201 .4 .4$ & 125.5525 & 0.3367 & 137.0825 & $0 . .35 k x$ \\
\hline 1104.25 & 0.2762 & 279.7117 & (1.2(1) 7 & 122.9525 & 0.3215 & 1398625 & 0.3247 \\
\hline 1130825 & $O 2 R^{\prime} I K$ & 2Eloke? & $0.2(17) 2$ & 120.2725 & 0.3148 & 142.5125 & 0.313 .4 \\
\hline 1158025 & $0.2 \times 21)$ & $282+7(14$ & 0.2142 & 117.452 .5 & $0.3(x) 6$ & 145.2425 & 0.3251 \\
\hline 118.4026 & $\left(12 x / x^{\prime}\right.$ & $2 \times 37974$ & 0.2234 & 114.672 .5 & 0.3087 & 147.8225 & $0.3+11$ \\
\hline 121.1425 & $0.28(4)$ & 2852850 & 0.2210 & 111.8725 & 0.30 .30 & 150.5525 & 0.3339 \\
\hline 123.7325 & 0.2444 & 286.7334 & $0.1 x+4$ & 104.1825 & $0.3(1) 2$ & 153.2025 & 0.3 .391 \\
\hline $12+2+26$ & $0,3 x_{1}$ & 287.81952 & $(1.22(k)$ & 106.1225 & $0.3(x) 3$ & 1550825 & 0.1301 \\
\hline $129(1.425$ & 0130137 & & & $10125: 15$ & $0.28 \% 8$ & 1588.125 & 0.3417 \\
\hline 131.675 & $(1.311) 2$ & & & 100.7725 & azuñ & 161.4025 & $0.37,86$ \\
\hline 1.342325 & 0.2172 & Scan 10.1 & 17.8 puy & 91.4425 & 0.3117 & 164.2725 & 0.1341 \\
\hline 177.4825 & $1136: 2$ & & & 450.0425 & 0.2984 & 166.9425 & $0.32(x)$ \\
\hline 134.5025 & 112032 & $7 \times 82: 211$ & $0.1 \times 18$ & 92.2225 & 0.208 .3 & 169.5325 & $0.3 .3 \times 5$ \\
\hline 142.5125 & 0.2871 & $2 \times \times 2320$ & (1) $18: 8$ & 84.1525 & 0.24015 & 172.0825 & 0.36 .38 \\
\hline 1.15 .2425 & U.282? & 287.5150 & (1.) 18.20 & 86.752 .5 & $(1.2810$ & 174.8025 & 0.3 .597 \\
\hline 1.478225 & $(13) \mathrm{kZ}$ & $2 k+4290 ?$ & (1.24114) & 83.90 .25 & 0.27 .30 & 177.4725 & $0.34(\mu)$ \\
\hline 150.5525 & $(1.294) ?$ & 2810072 & $0.2\left(1 x_{2} 2\right.$ & 81 (16?29 & 0.26 .36 & $180 .(k) 25$ & 0.3417 \\
\hline 15321125 & 0.3020 & $278.86 \times 1$ & $0.2(1)) !$ & 78.31125 & 0.2984 & 182.7 .525 & 0.3412 \\
\hline 1559825 & 01.2072 & 275.9215 & (1.2226 & 75.3725 & 0.2382 & 185.5925 & 0.3464 \\
\hline 158.5425 & 0.3012 & $27 ? .14 .45$ & 0.2334 & 724325 & 0.2 .3 .7 & 188.1025 & 0.3407 \\
\hline iti1 4025 & $0.3(1) \times 6$ & 270.2685 & $0.2+20$ & 69.6725 & 0.2298 & 190.7175 & $0.351 \mathrm{~K}$ \\
\hline 164.2725 & $0.24(6.2$ & 2675545 & 0.2517 & 66.9025 & 0.2 .52 & 193.2275 & 0.3480 \\
\hline 16019425 & 0.29 .34 & 304.7175 & 0.2571 & 64.0425 & 0.20 .35 & 195.8805 & 0.3528 \\
\hline 164.5125 & 0,3051 & 261.9825 & $0.27) 6$ & 612225 & $0.14(x)$ & 198.4695 & $0.344 ?$ \\
\hline 17201825 & 0.3140 & $250 \quad 2275$ & 0.2724 & 58.4325 & $0.184 \times 1$ & 201.1255 & 0.3 .465 \\
\hline 17.48025 & $0.3(1.34$ & 296.4725 & $0.21) !(1$ & 55.2825 & 0.1780 & 2013.6355 & $0.3+40$ \\
\hline 177.4725 & (1) 31132 & 2536155 & $0.29(k)$ & 52.2225 & 0.1613 & 206.2485 & 0.1416 \\
\hline $180(k, 25$ & 0.3072 & 250180,115 & 0.30 .31 & 49.3625 & 0.1 .153 & 208.7585 & $0.358:$ \\
\hline 182.7525 & 03153 & 248.1255 & $0.2(1.4$ & 46.5125 & 0.1 .158 & 211.3295 & 0.34 .56 \\
\hline 185.5425 & $(1.3(x) !$ & $245+1115$ & $(1.2247$ & 43.4425 & (1) 1211) & 213.9015 & 0.3409 \\
\hline 188.1105 & $(1,3)(x)$ & $24: 50.45$ & (1) 3220 & $4(154) 25$ & 0.1114 & 216.5735 & 0.3419 \\
\hline 160.7175 & 0.31501 & $230 x 015$ & $(1.320 .5$ & 37.53 .5 & (1)(19) & 219.1455 & 0.3440 \\
\hline 1932275 & 091.36 & 23716,55 & $(1.3313$ & 3.46725 & 00.46 & 221.7985 & $0.3+600$ \\
\hline 14588615 & $(1,314 \%$ & $23432 \times 5$ & 0.3350 & 31.0125 & () $\left(k_{1}\right) k$ & $224.34 \times 5$ & 0.35140 \\
\hline 198.4705 & $(1,3)(x)$ & 2310259 & 03.4114 & 207225 & $0.05(x)$ & 227014.45 & 0.2440 \\
\hline $2(11.1245$ & $(1.3) 3 \%$ & $2285+4115$ & 033100 & 32.11215 & $\left(1,1 k, 2 k^{\prime}\right.$ & 229.5745 & $0.2 \div 1 ?\}$ \\
\hline $2(1), 1.355$ & $(1.3018)$ & 220.1255 & 02426 & 346725 & $0 .(182 ?$ & 232.166 .5 & $0 .{ }^{3} 43 x$ \\
\hline $2(x, 24) 5$ & $0.3 ; 35$ & $223+115$ & 0.246 .5 & $37.4+25$ & $0.1\left(x_{1}\right)$ & 234.8195 & 0.3368 \\
\hline $2(18.7565$ & 0.3208 & 22001010 & 0.3341 & $41 .(10) 25$ & 0.1152 & $23 / 6145$ & (i..128..) \\
\hline
\end{tabular}




\section{Reinforced Concrete Test Data}

Track 4 - High Pressure Test

\begin{tabular}{|c|c|c|c|c|c|c|c|}
\hline 240.3495 & 0.3287 & 187.5325 & 0.37 .48 & 77.3025 & 0.2621 & 263.9825 & 0.2725 \\
\hline 24.3 .2075 & 0.37 .74 & 184.8625 & 0.3772 & 80.20125 & 0.27 .33 & $265.28 \times 5$ & 0.2760 \\
\hline $246 .(1645$ & 0.3373 & 182.0825 & 0.370 .4 & 8.3 .0425 & 0.2700 & 266.5955 & 0.2700 \\
\hline 248.8195 & $0.3(1999$ & 179.3925 & 0.3691 & 85.8125 & 0.2886 & 267.8605 & $0.260 x^{\prime}$ \\
\hline 251.6365 & 0.3042 & 176.6125 & 0.36 .11 & 88.6525 & 0.3023 & $269.2(\times 55$ & $0.25^{2} 2$ \\
\hline 2544925 & 0.2934 & 173.8425 & 0.4449 & 91.3925 & 0.3058 & 270.4925 & 0.2558 \\
\hline 256.553 .35 & 0.2875 & 170.9425 & $0.3760)$ & 941425 & 0.33015 & 271.7785 & 0.2518 \\
\hline 258.7375 & 0.2811 & 168.1825 & 0.3596 & 96.9325 & 0.329 .3 & 272.9825 & 0.2440 \\
\hline $2(60)(x+45$ & 0.2749 & 165.3325 & (0.3615 & 99.6025 & 0.2770 & 274.3295 & 0.2 .377 \\
\hline $26130 \times 5$ & 0.2731 & 1624125 & 0.3641 & 102.4125 & 0.3144 & 275.676 .5 & 0.23 .39 \\
\hline 202.6155 & 0.2627 & 159.7425 & 0.3675 & 105.1225 & 0.3181 & $277.0 \times 13.5$ & 0.2345 \\
\hline 263.9825 & 0.2574 & 156.7225 & 0.3547 & 107.822 .5 & 0.3318 & 278.3095 & 0.2314 \\
\hline 269.2885 & 0.2585 & 153.4925 & 0.3645 & 110.4325 & 0.3273 & 279.7317 & 0.2341 \\
\hline 266.59 .55 & 0.2552 & 150.8025 & 0.3575 & 113.0825 & 0.3372 & 281.0807 & 0.2307 \\
\hline 267.8605 & 0.2453 & 147.9625 & $0.37+2$ & 115.8025 & 0.3365 & 282.4709 & 0.2357 \\
\hline 269.2065 & 0.2398 & 145.1825 & $0.35(19$ & 118.4025 & 0.3 .39 .3 & 283.7974 & 0.2516 \\
\hline 270.4925 & 0.2405 & 142.4125 & 0.3476 & 121.1425 & 0.3305 & 2852850 & 0.2486 \\
\hline 271.7785 & 0.2368 & 139.7125 & 0.3671 & 173.7325 & $(1.3 .19)(0)$ & 286.7339 & 0.2085 \\
\hline $272.4 \times 25$ & 02270 & 1.6 .7025 & 0.:Bi) & 126.2625 & 0.357 .7 & 287.8052 & $0.18+1$ \\
\hline 214.3296 & 0.2224 & 132.7725 & $(1.3627$ & $120(1425$ & 0.3611 & \multirow{3}{*}{\multicolumn{2}{|c|}{ Scan 12. 125.8 psiz }} \\
\hline 275.6765 & 0.2107 & 121.0125 & (1).36.22 & 131.5725 & 0.36 .5 .7 & & \\
\hline $277.0(1.35$ & 0.2201 & 128.3225 & 0.3571 & 134.2325 & 0.376 .7 & & \\
\hline 278.3095 & $0.216 x$ & 125.5525 & 0.362 .3 & 137.0825 & 0.41 .31 & 288.2320 & 0.1965 \\
\hline 279.7317 & 0.21 .56 & 122.9525 & 0.3425 & 139.86 .25 & 0.3512 & 288.2320 & 0.1465 \\
\hline 281.0807 & 0.2194 & 120.2725 & $0.34 i k)$ & 142.5125 & $0.3 .4(0.3$ & 287.51 .56 & 0.2166 \\
\hline $282.47(99$ & $0.22+4$ & 117.4525 & $0,3,341)$ & 145.2425 & 0.342 .5 & 284.9207 & $0.289:$ \\
\hline 283.7974 & 0.2374 & 114.6725 & 0.3316 & 147.8225 & 0.3817 & 281.9972 & 0.2487 \\
\hline 285.2850 & 0.2 (a)ia & $11 \mathrm{i} 8724$ & 0.326 .5 & 150.55 .25 & 0.3022 & 278.8891 & 0.2514 \\
\hline 286.73 .34 & 0.2011 & 109.1825 & 0.310 .3 & 1522025 & 0.36135 & 275.9215 & 0.2657 \\
\hline \multirow[t]{3}{*}{287.8052} & 0.1879 & $106,4,325$ & 0.32 .45 & 15.5 .982 .5 & 0.3526 & $273.0+1.15$ & 0.2813 \\
\hline & & 10.3 .5725 & 0.3104 & 156.8 .225 & 0.3674 & 270.2685 & 0.2908 \\
\hline & & $1(k) .7725$ & 0.911 .5 & 161.4425 & 0.36 tit & 267.5545 & 0.304 .3 \\
\hline \multirow{2}{*}{\multicolumn{2}{|c|}{ Scan 11.1200 psig }} & 97.4 .225 & $9.32+2$ & 164.2725 & 0.3586 & 264.7175 & 0.313 .4 \\
\hline & & 9.5 .19 .429 & $0.3^{3} \cdot 3$ & $16,9.9 .5$ & 0.3530 & 261.9825 & 0.33 .3 .4 \\
\hline 288.2 .20 & 0.2845 & 92.2215 & 0.316 .3 & $1,60.5325$ & $0.3(x)(x)$ & 259.2275 & 0.3 .363 \\
\hline $7692:=0$ & 0.2845 & .7 .7525 & (). 31.1 & $172.11 \times 25$ & (1)34)13 & 256.4725 & $0.358 R$ \\
\hline 287.5150 & 0.1402 & 80.1525 & 0.2482 & 174.60125 & 0.36 .39 & 253.6155 & 0.3617 \\
\hline 284.9297 & $0.258 . ?$ & 83.41 .25 & $(02.015$ & 177.4725 & $0.3(x) .5$ & $250.8(0) 5$ & 0.380 .2 \\
\hline 281.9472 & 0.22 .32 & $81.06,25$ & 0.28 .11 & $18(1.161,25$ & 0.3745 & 248.12 .55 & 0.386 .3 \\
\hline 278.8891 & 0.2201 & 76.3025 & $0.2721)$ & 162.7525 & 0.3686 & 245.4115 & 0.41 .31 \\
\hline 275.9215 & 02364 & 75.3725 & 0.2567 & 185.5925 & 0.3774 & 242.54 .45 & $0.4(x) 5$ \\
\hline 273.0445 & 0.2488 & 72.4325 & 0.2525 & 188.1025 & 0.1760 & .239 .90115 & 0.1168 \\
\hline 270.2685 & 0.2538 & $0,9.6725$ & 0.2 .41 & 100.7175 & 0,3769 & 2.37 .1655 & 0.4270 \\
\hline 267.5545 & 0.266 .5 & 66.90125 & $0.2 .3(k)$ & 193.2275 & 0.3772 & 234.328 .5 & 0.4313 \\
\hline $2+4.7175$ & n 2723 & 6.4 .1425 & 0.2131 & 149.88019 & 0.3760 & 271.6355 & $0.4+11$ \\
\hline 261.9825 & $0.285 x$ & 61.2225 & 0.2355 & $19 R+6,45$ & 0.3727 & $228.84(15$ & 0.4 .151 \\
\hline 259.2275 & 0.2915 & 58.4375 & 01082 & 2111.1255 & 0.1723 & 226.1255 & 0.4519 \\
\hline 256.4725 & 0.30107 & 55.2825 & $0.18+7$ & 2033.6359 & 0.370 .3 & 223.4115 & 0.4532 \\
\hline 253.61155 & 0.31 .35 & 52.2225 & 0.1774 & $2(16.248 .5$ & 0.3753 & 220.6755 & 0.4461 \\
\hline 25086015 & 0.2217 & 49.2625 & 0.1510 & 208.7585 & $0.38+3$ & $2 \mathrm{i} 7.9425$ & 0.4448 \\
\hline 248.1255 & 0.3247 & 46.5125 & 0.1404 & 211.3295 & 0.365 .5 & $215.1+0.5$ & 0.4538 \\
\hline 245.1115 & 0.3511 & 4.3 .442 .5 & $0.1252 ?$ & 213.9015 & 0.3771 & 212.3495 & 0.459 .3 \\
\hline 242.5945 & $0.34(x)$ & 40.54 .5 & 0.116 .3 & 216.573 .5 & 0.3792 & $2(19.4725$ & 0.4704 \\
\hline 239.9015 & $0.34 t .7$ & 17.5375 & 0.0998 & 2101455 & 0.36490 & 206.7765 & 0.454() \\
\hline 2.17 .1655 & 01.3591 & 346725 & 0.0760 & 221.7985 & 0.3778 & 204.0225 & 0.45 .37 \\
\hline 234.3285 & 0.36 .23 & 31.6125 & 0.16125 & $224,3(x) 5$ & 0.3703 & 201.2885 & 0.4558 \\
\hline 231.6255 & 0.3693 & 29.7725 & 110.402 & $227 .(1+45$ & 0.3767 & 198.53 .35 & 0.4561 .2 \\
\hline 228.8 .905 & 0.3667 & 320225 & $0.064 i 0$ & 224.5745 & 0.3690 & 195.8195 & 0.466 .5 \\
\hline 226.1255 & 0.36103 & 3.16725 & $01.00(1)$ & $232.16 \times 15$ & 0.3767 & 192.4815 & 0.4655 \\
\hline 223.411 .5 & 0.3720 & 37.9425 & 0.1112 & 23.4 .8195 & $0.3(x) ?$ & 190.288 .5 & 0.4702 \\
\hline 220.6765 & $0.36+5$ & $41.0 \times 25$ & 0.1216 & 237.6145 & 0.1552 & $18 \% .5 .25$ & 0.467 .3 \\
\hline 217.94 .5 & 0.3699 & 44.2625 & 0.13 .44 & 240.3495 & 0.3497 & 184.8625 & $0.46 k 0$ \\
\hline 215.1465 & 0.3713 & 47.732 .5 & 0.1527 & 243.2075 & 0.3474 & $182.08 \pm 5$ & 0.4577 \\
\hline 232.3495 & $0.376 x$ & 50.80125 & $0.16,31$ & $216.0 \times \mathrm{m}+5$ & $0.3(x) 2$ & 179.3925 & 0.460 .3 \\
\hline $2(x) .4725$ & 0.3829 & 5.380625 & 0.17 .30 & 248.8195 & 0.3287 & 176.6125 & 0.4561 \\
\hline 206.776 .5 & $03 ? 17$ & 56.9425 & 0.9948 & 2.51 .0 .365 & 0.3212 & 173.8425 & $0.53(1) 8$ \\
\hline 244.0225 & $0.36 \times 12$ & 59.9825 & 0.2110 & 254.4925 & 0.3149 & 170.9425 & 0.47 .48 \\
\hline 201.2885 & 0.368 .2 & 62.8025 & 0.22 .35 & 25655535 & $0.3(180$ & 168.1825 & 0.44 .56 \\
\hline 198.4335 & $0.3(1 \times) \cdot$ & $65.6(1) 25$ & 0.2240 & 258.7 .375 & 0.2456 & 165.3325 & 0.4519 \\
\hline 195.8195 & 0.375 .3 & 68.5925 & 0.2 .40 .4 & $260.0 \mathrm{~K}+\mathrm{S}$ & 0.2925 & 102.4125 & $0.4 .5(12$ \\
\hline 192.9815 & 0.3701 & 71.4425 & 0.2437 & $261.3(k) 5$ & 0.2412 & 159.742 .5 & 0.4640 \\
\hline 190.2885 & $0.37 \mathrm{dAt}$ & 74.322 .5 & 0.7532 & 2626155 & $0.27 \times 5$ & 156.7225 & 0.4 .536 \\
\hline
\end{tabular}




\section{Reinforced Concrete Test Data}

Track 4 - High Pressure Test

\begin{tabular}{|c|c|c|c|c|c|c|c|}
\hline 153.4925 & 0.4550 & 110.4325 & 0.4054 & 279.7317 & 0.2607 & 122.952 .5 & 0.53001 \\
\hline 150.8025 & 0.4490 & 113.0825 & 0.4127 & $281.080^{\circ}$ & 0.2586 & 120.2725 & $0.52(6)$ \\
\hline 147.9625 & 0.4606 & 115.8025 & 0.4131 & 282.4709 & $0.2+40$ & 117.4525 & 0.5137 \\
\hline 145.1825 & 0.4456 & 118.4925 & $0.419 ?$ & 283.7974 & 0.2714 & 114.6725 & 0.5005 \\
\hline 142.4125 & 0.4307 & 121.1425 & $0.417:$ & 3.85 .2850 & $0.25(x)$ & 111.8725 & 0.4990 \\
\hline 139.7125 & $0.44(0)$ & 123.7325 & 0.4317 & 286,7339 & 0.2298 & 109.1825 & 0.4911 \\
\hline 136.7925 & 0.4695 & 126.2625 & 0.4388 & 287.805. & 0.2261 & 106.432 .5 & 0.4862 \\
\hline 133.7725 & 0.4504 & 129.14425 & 0.4446 & & & 103.5725 & 0.4685 \\
\hline 131.0425 & 0.448 .3 & 131.5725 & 0.450 .3 & & & 100.772 .5 & 0.4338 \\
\hline 128.3225 & 0.4366 & 134.2325 & 0.46 .36 & Scan 13,1 & 30.2 psig & 97.9425 & 0.4674 \\
\hline 125.5525 & 0.4470 & 137.0825 & 0.4782 & & & 95.0425 & 0.466 .5 \\
\hline 122.9525 & $0.429:$ & 139.8625 & 0.4340 & 288.2320 & 0.1606 & 92.2275 & 0.4588 \\
\hline 120.2725 & 0.4240 & 142.5125 & 0.42012 & 288.2320 & $0.16 \times 16$ & 89.4525 & 0.442 .3 \\
\hline 117.4525 & 0.4151 & 145.2425 & 0.4262 & 287.5156 & 0.2391 & 86.7525 & 0.4289 \\
\hline 114.6725 & 0.4089 & 147.8225 & 0.46 .59 & 284.9297 & 0.3165 & 83.9625 & 0.4116 \\
\hline 111.8725 & 0.4072 & 150.5525 & 0.4405 & 281.9072 & 0.2848 & 81.0625 & 0.3982 \\
\hline 109.1825 & 0.3987 & 153.2025 & 0.4561 & 278.8801 & 0.2860 & 78.3025 & 0.387 .3 \\
\hline 106.4325 & 0.3968 & 155.9825 & 0.4468 & 275.9215 & 0.3013 & 75.3725 & 0.3568 \\
\hline 103.5725 & 0.3811 & 158.8425 & 0.78 .20 & 273.0445 & 0.3194 & 72.4325 & 0.3441 \\
\hline 100.7725 & 0.3517 & 101.4925 & 0.45313 & 270.2685 & 0.331 .3 & 60.6725 & 0.3 .306 \\
\hline 97.9425 & 0.4019 & 164.2725 & 0.4492 & 267.5545 & 0.3510 & 66.9025 & 0.3694 \\
\hline $95 .(1425$ & 0.3894 & 166.9425 & 0.4410 & 204.7175 & 0.3631 & 64.0425 & 0.2847 \\
\hline 92.2275 & 0.3795 & 169.5325 & 0.4618 & 261.9825 & 0.3862 & 61.2225 & 0.2946 \\
\hline 89.452 .5 & 0.3667 & 172.0825 & 0.487 .5 & 259.2275 & 0.3970 & 58.4325 & 0.2643 \\
\hline 86.7525 & 0.3572 & 174.81125 & 0.4113 & 256.4725 & 0.4240 & 55.282 .5 & 0.2444 \\
\hline $83.96,25$ & 0.3475 & 177.4725 & $0.14 ? .4$ & 253.6155 & 0.4318 & 52.2225 & 0.22 .52 \\
\hline $31.0 \times 25$ & 0.3 .162 & 180.1625 & 0.46 .37 & $250.81 \times 15$ & 0.4513 & 49.3625 & 0.2010 \\
\hline $76.3(125$ & 0.3277 & 182.752 .5 & 0.4594 & 2481255 & 0.4572 & 46.5125 & 0.1777 \\
\hline 75.3725 & 0.30175 & 185.5925 & 0.4701 & 245.4115 & 0.498 .3 & 41.4425 & 0.1616 \\
\hline 72.4 .325 & 0.29 .32 & $18 \times .1025$ & 0.46 .58 & 242.5045 & 0.4975 & 40.5925 & 0.1451 \\
\hline 69.6725 & 0.2827 & 190.7175 & 0.4710 & 23990115 & 0.5064 & 37.5325 & 0.1233 \\
\hline 66.9025 & $0.26 \div 5$ & 193.2275 & $0 .+6,55$ & 237.16 .55 & 0.5274 & 34.6725 & 0.0959 \\
\hline 640425 & 0.251 .3 & 19588115 & 0.4677 & 234.3285 & 0.5340 & 31.6125 & 0.0749 \\
\hline 61.222 .5 & 0.2724 & $190 .+695$ & 0.46115 & 231.6355 & 0.54 .57 & 24.7725 & 0.0583 \\
\hline 58.4325 & 0.2 .306 & $2(11.1255$ & 0.1612 & $228.8 .4(15$ & $0.5566(1$ & 32.0225 & 0.0829 \\
\hline 55.282 .5 & $02(x)^{2}$ & 203.6355 & $0.151) 2$ & 2261255 & 0.56250 & 34.6725 & 0.10 .33 \\
\hline 52.2225 & 0.1902 & $20(1,2+65$ & $0.4\langle 8\}$ & 223.4115 & 0.56011 & 37.9425 & 0.1360 \\
\hline 49.3625 & 0.1762 & $208.75 \times 5$ & 04727 & 220.676 .5 & 0.5647 & 41,0025 & 0.146 .5 \\
\hline 46.512 .5 & $0.151)(1)$ & 211.3205 & 0.4551 & 217.9425 & 0.56195 & 44.262 .5 & $0.16 \times 9$ \\
\hline 43.4425 & 0.1416 & 2139015 & 0 frik) & 215.1465 & 0.5738 & 47.7325 & 0.1941 \\
\hline 40.5925 & 0.1316 & 216.5735 & 0.46 .13 & 212.3445 & $0.58+5$ & 50.8025 & 0.21 .74 \\
\hline 27.5325 & 0.1144 & 219.1455 & 0.4501 & 200.4725 & 0.5869 & 53.8625 & 0.2305 \\
\hline 3.6795 & 0.0850 & 221.7985 & $0.456 k^{\circ}$ & 206.7765 & 0.5778 & 56.4425 & $0.255 R$ \\
\hline $31.01: 9$ & $0 .(k, 9) 7$ & 224.3905 & 0.4511 & 201.0225 & 0.5150 & 59.9825 & 0.2728 \\
\hline 29.7725 & 0.0549 & 227.1445 & 0.4578 & 2011.2885 & 0.5760 & 62.8025 & 0.2828 \\
\hline 32.0225 & 0.0745 & 229.574 .5 & 0.4488 & 198.5335 & 0.5787 & 6.5.6425 & 02991 \\
\hline 34.6725 & $0.019+6$ & 232.1665 & 0.4523 & 145.8105 & 0.5803 & 68.5925 & 0.325 .3 \\
\hline $37.17+25$ & $\cap 12.11$ & 234.8105 & 0.1 .367 & $14>0915$ & 0.5898 & 71.442 .5 & 0.33 .36 \\
\hline $41 .(k) 25$ & 0.1302 & $237.61+5$ & 0.4210 & 190.2885 & $(1.5040$ & 74.3225 & 0.3469 \\
\hline 44.2625 & 0.1521 & $? 4013405$ & 0.1177 & 187.5325 & 0.5910 & 77.3025 & 0.3648 \\
\hline 47.7325 & 0.1742 & 2432015 & $0.41+5$ & 184.8625 & 0.51765 & 80.2025 & 0.3826 \\
\hline $50 \times 0175$ & 0.1851 & $246(x)+5$ & 0.4214 & 18201825 & 0.5880 & 83.0425 & 0.3065 \\
\hline $53.86,25$ & 0.203 .4 & 248.8195 & 0.3858 & 179.3925 & 0.5921 & 85.8125 & 0.4115 \\
\hline 56,9425 & $0.2+41$ & 251.6365 & 0.1731 & 176.6125 & 0.5870 & 88.6525 & 0.4318 \\
\hline $59.98 \div 5$ & 0.2399 & 254.4925 & 0.367 .3 & 173.8425 & 0.6287 & 91.3925 & 0.43 .54 \\
\hline $62.8(125$ & 0.2488 & 256.5535 & 0.3574 & 170.9425 & $0 . f_{1} 115$ & 94.1425 & 0.4681 \\
\hline 65.6025 & 0.2508 & 258.7375 & 0.3421 & $16 x .1825$ & $0.5 x^{\circ}(x)$ & 96.9325 & 0.4716 \\
\hline 68.5925 & 0.2802 & $2600,0 \times+5$ & 0.31775 & 165.3325 & 0.5810 & 99.6925 & 0.4106 \\
\hline 71.4429 & 0.2856 & 261.3095 & 0.3372 & 162.4125 & 0.5802 & 102.4125 & 0.4684 \\
\hline 74.3225 & $0.298 . ?$ & 262.6155 & 0.3235 & 159.7425 & 0.5662 & 105.1225 & 0.4702 \\
\hline 77.301 .5 & $0.30 \times 00$ & 263.9825 & 03162 & 156.7225 & 0.5728 & 107.8225 & 0.4898 \\
\hline 80.2025 & 0.3207 & 265.2885 & 0.3164 & 15.3 .4925 & 0.5760 & 110.4325 & 0.4972 \\
\hline 83. 0425 & 0.3313 & 266.5955 & $0.3(11) 4$ & 150.8025 & 0.56 .53 & 113.0825 & $0.5(x) 6$ \\
\hline 858125 & $0.3+11$ & 267.8605 & 0.2440 & 147.962 .5 & 0.5814 & 115.8025 & $0.51(x)$ \\
\hline 88.6525 & 0.3594 & $2(19), 2(k, 5$ & 0.2911 & 145.1825 & 0.5502 & 118.4025 & 0.5190 \\
\hline 91.3925 & 0.3665 & 2704925 & (1) 2017 & $1+2.4125$ & $0.5+22$ & 121.1425 & 0.519 .3 \\
\hline 94.1425 & $0.39 ? 0$ & 271.778 .5 & 0.2871 & 139.7125 & 0.5530 & 123.7325 & 0.5364 \\
\hline 96.4325 & 0.3921 & 272.9825 & (1.2774 & 136.7925 & 0.0235 & 126.2625 & 0.5440 \\
\hline 9961925 & $0.3(x) 1$ & 27.4 .3205 & (1).2721 & 133.7725 & $0.961 ?$ & 129.0425 & 0.5101 \\
\hline 102.4125 & 0.38 .33 & 275.6765 & 0.2688 & 131.0425 & 0.5542 & 1.31 .5725 & 0.556 .3 \\
\hline 105.1225 & 0.3856 & $277 .(x) 35$ & 0.2642 & 128.3225 & 0.5440 & 134.2325 & 0.5736 \\
\hline 107.8225 & 0.40 .27 & 278.30195 & 0.2576 & 1255525 & 0.5517 & 137.0825 & 0.5956 \\
\hline
\end{tabular}


Track 4 - High Pressure Test

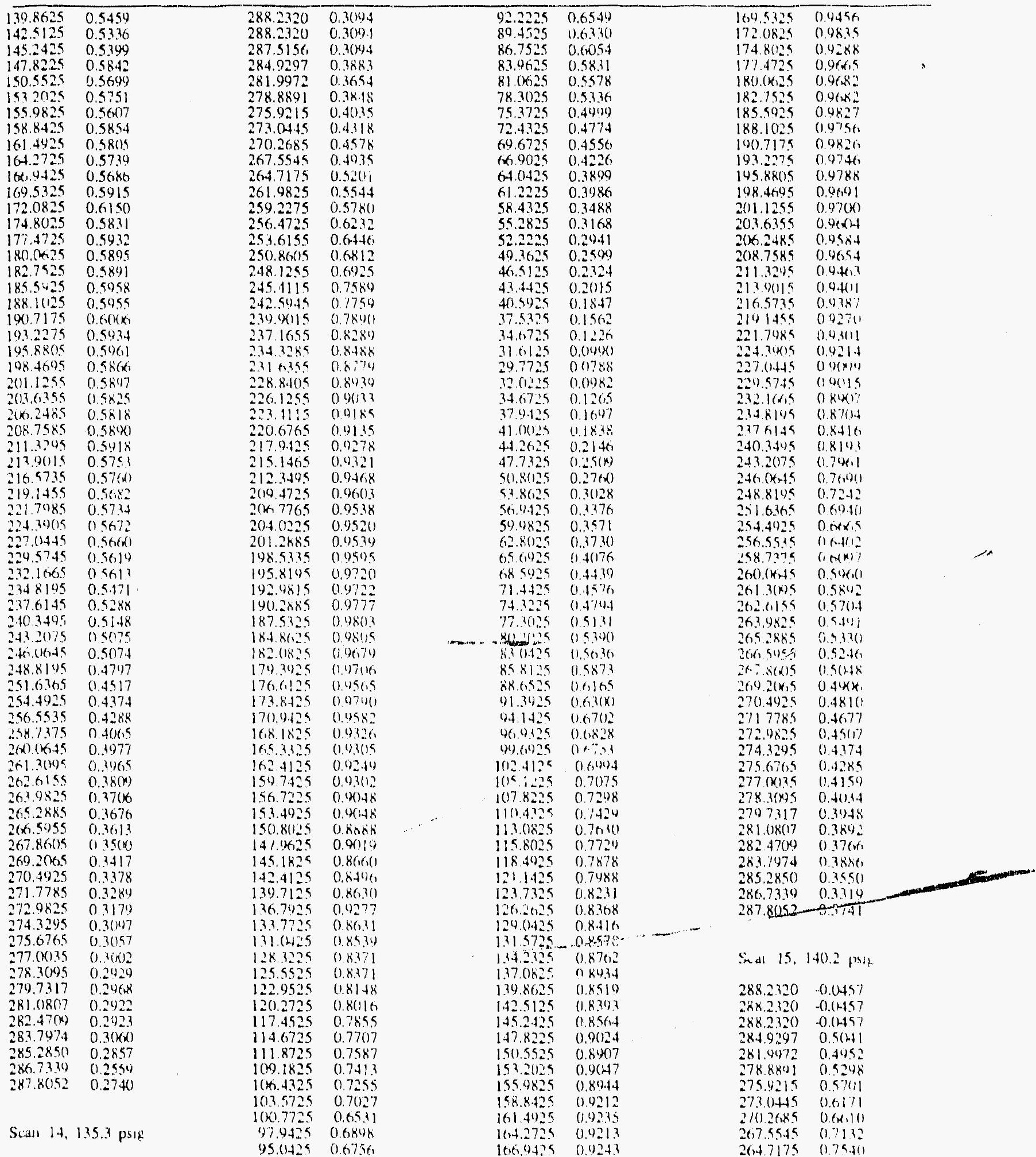




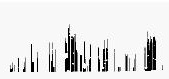

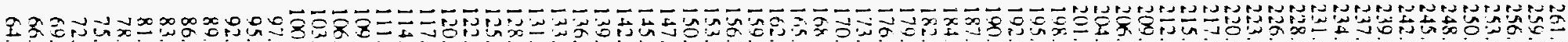
Fo

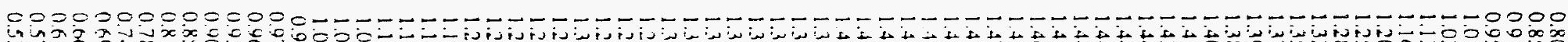

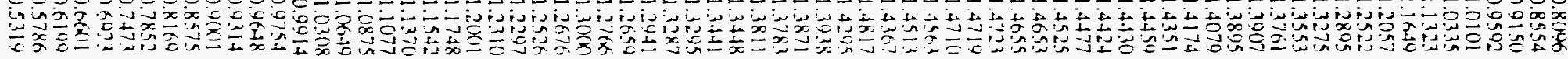

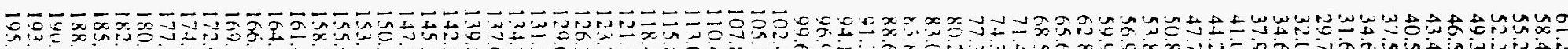

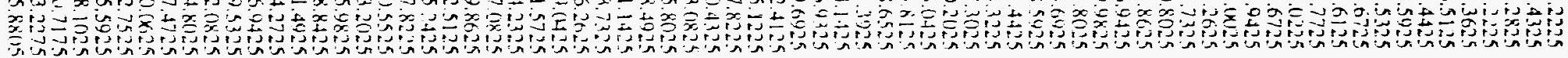
-

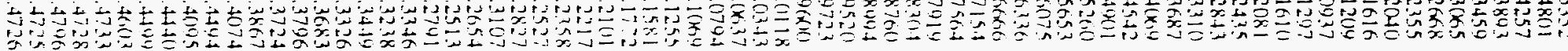

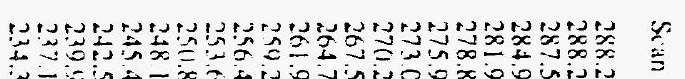

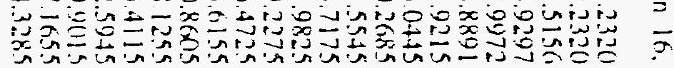

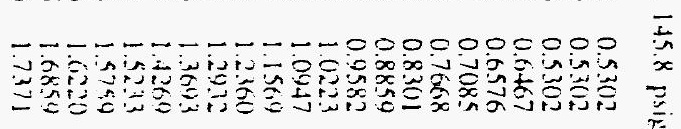

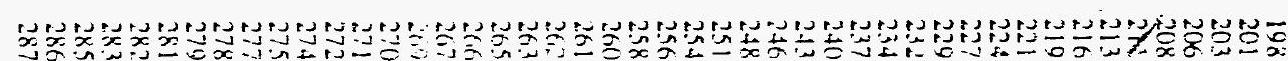

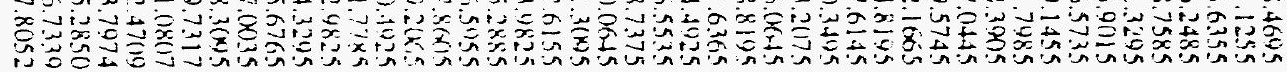

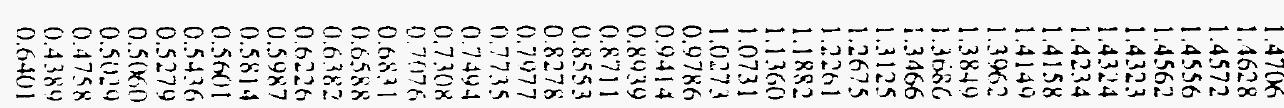

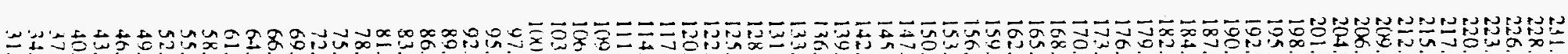
O

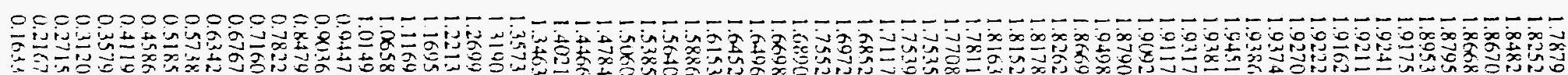




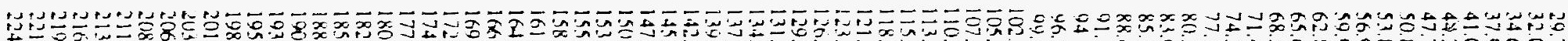

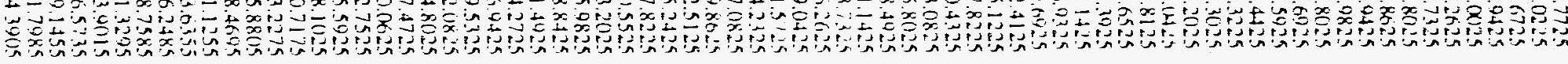

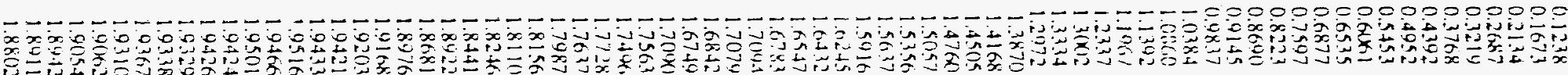

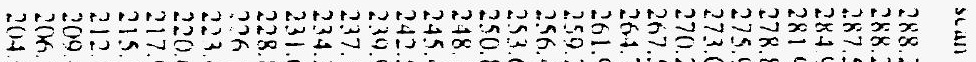

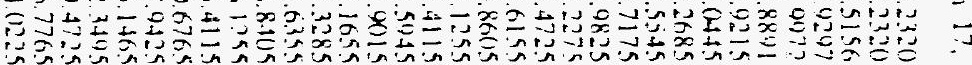

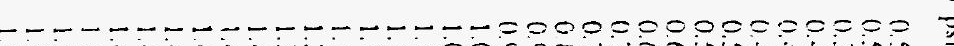

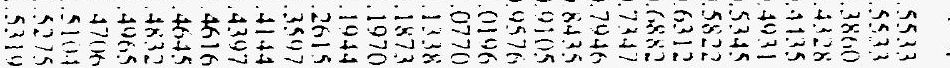

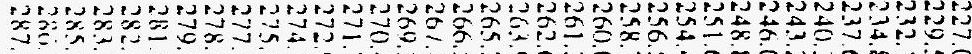

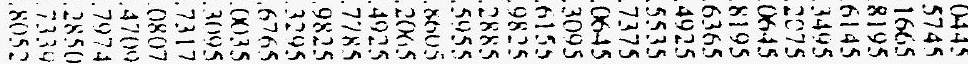

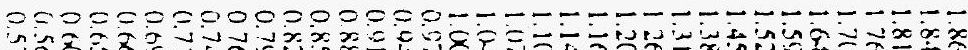

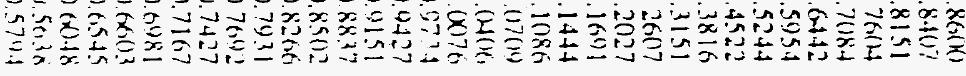

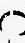

is

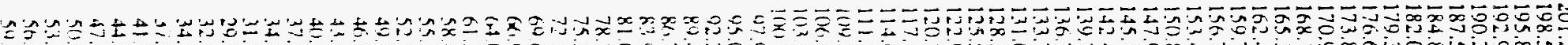

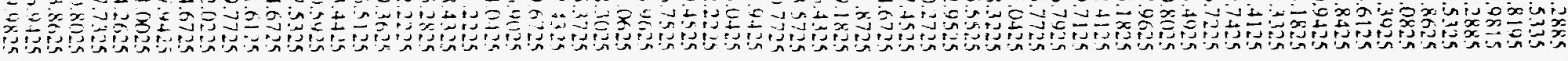

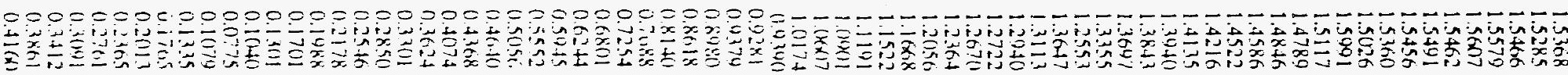

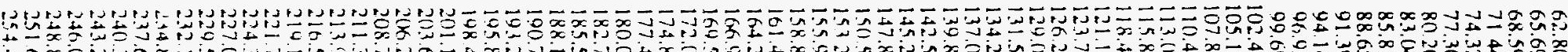

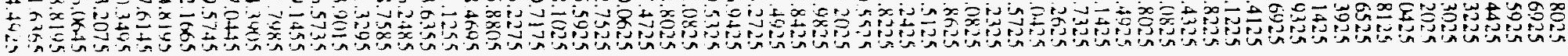

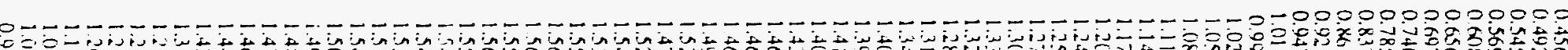

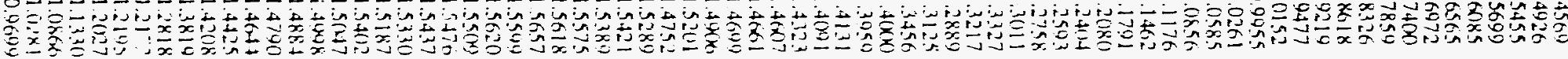

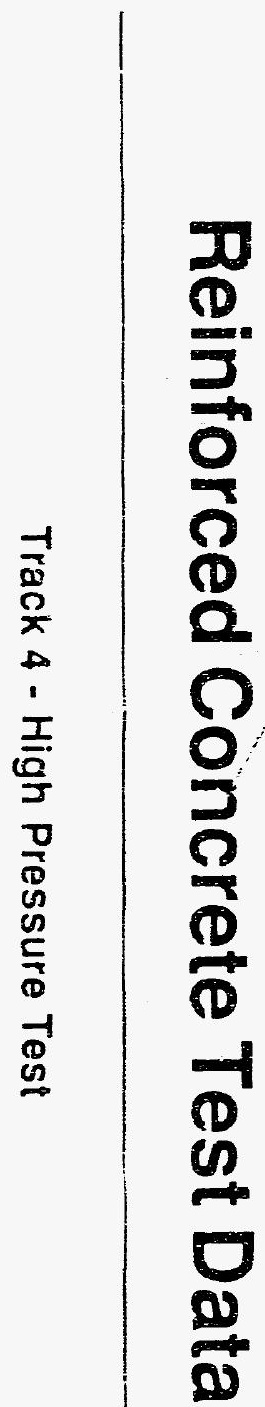




\section{Reinforced Concrete Test Data}

Track 4 - High Pressure Test

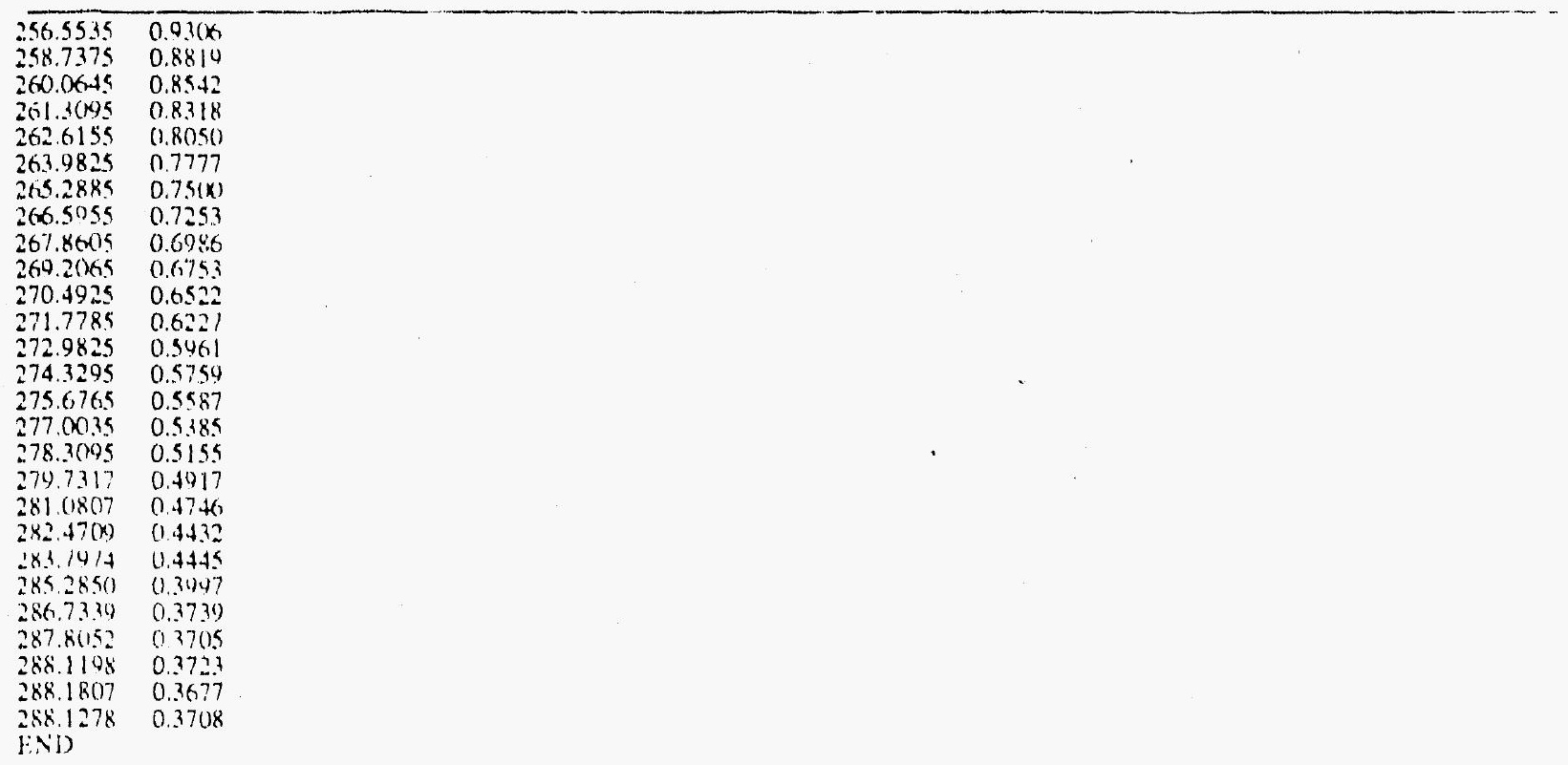




\section{Reinforced Concrete Test Data}

Track 5 - High Pressure Test

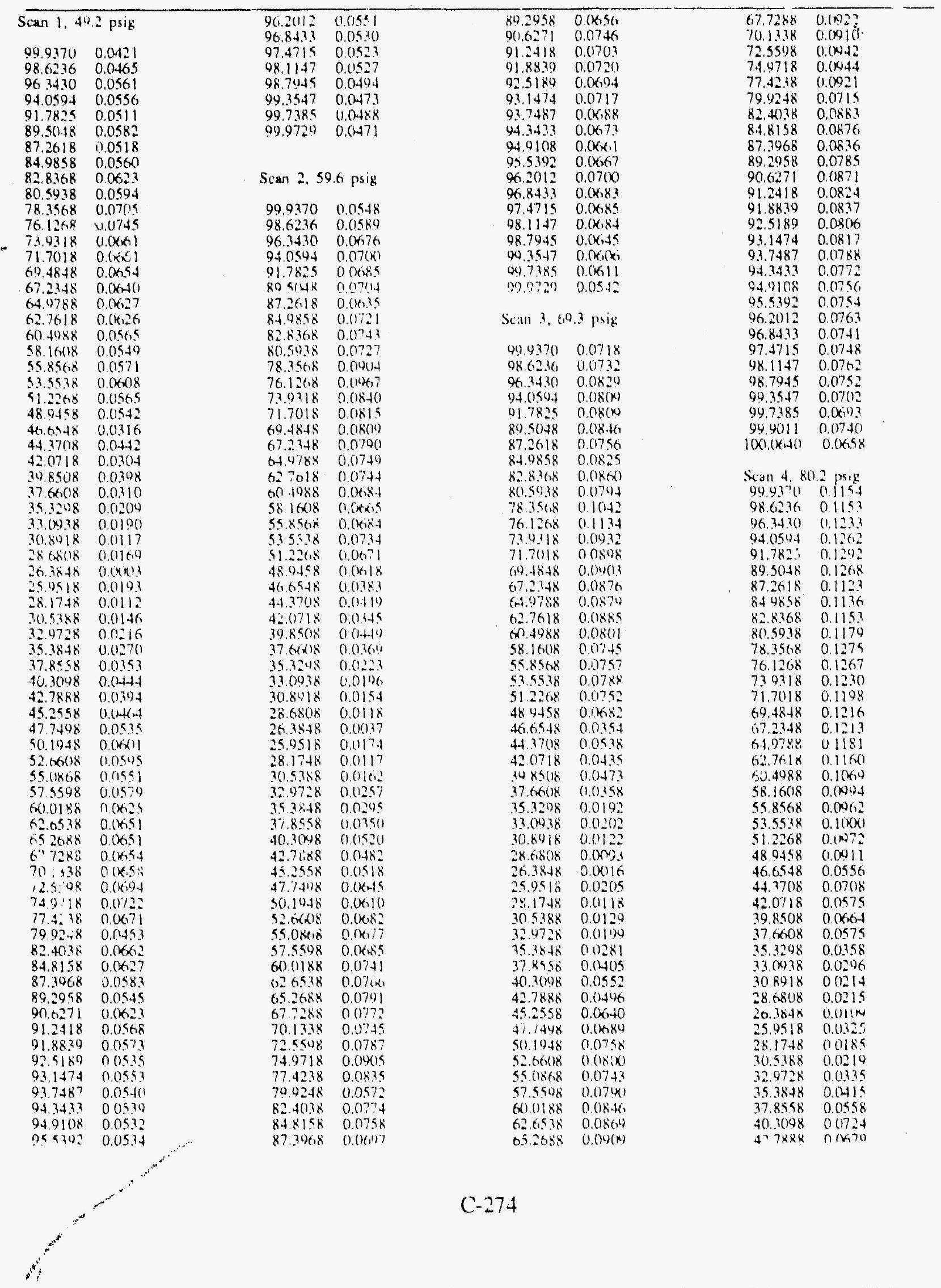




\section{Reinforced Concrete Test Data}

Track 5 - High Pressure Test

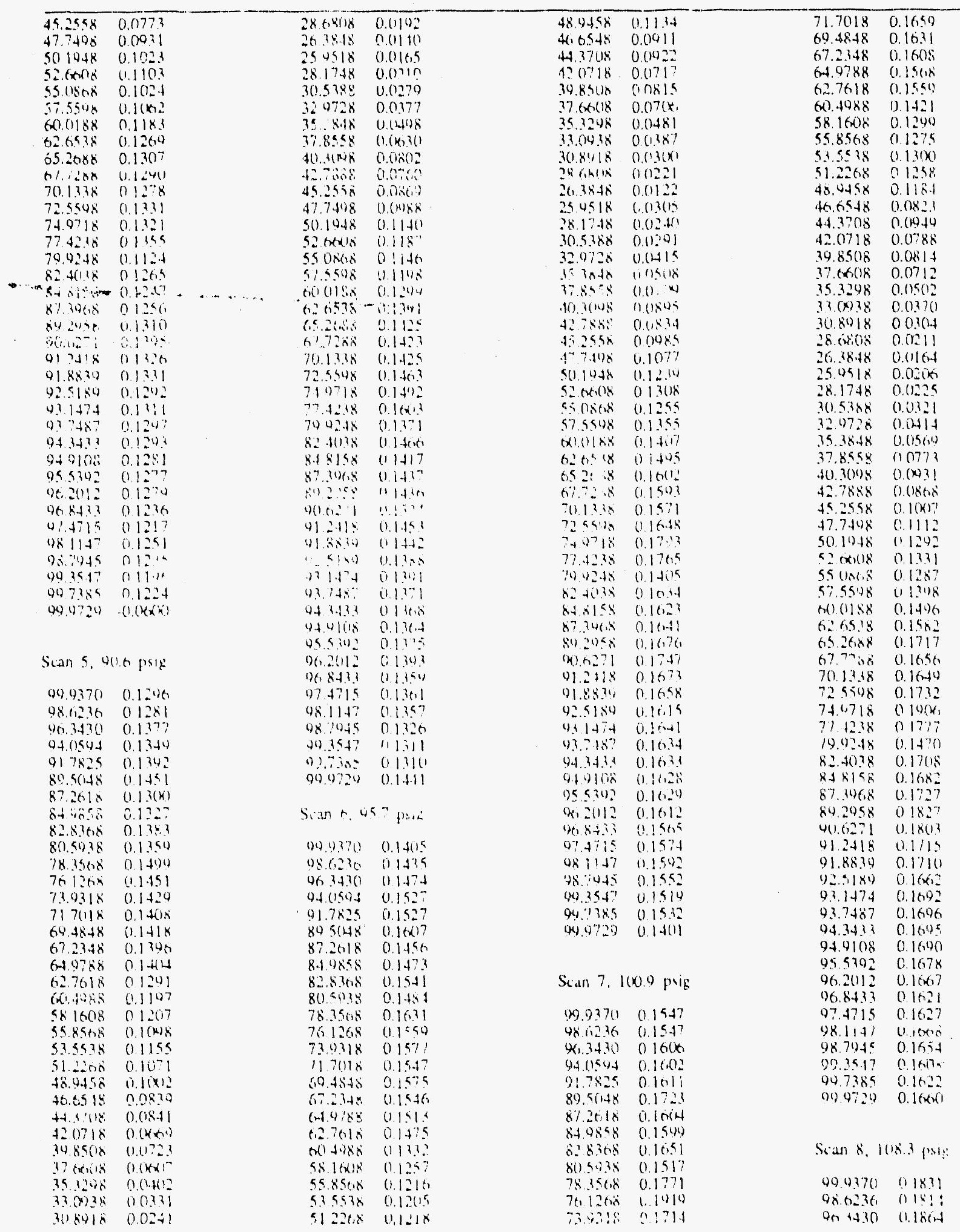




\section{Reinforced Concrete Test Data}

Track 5 - High Pressure Test

\begin{tabular}{|c|c|c|c|c|c|c|c|}
\hline $44(15124$ & 0.187 & 99.2547 & $0.18(x)$ & $92.14: 4$ & $(1.2(1) \$$ & 77.9238 & $(12341$ \\
\hline 01.7825 & 0.1808 & 09.7385 & 0.1814 & 0.7487 & 0.21125 & $7902+10$ & 0.2011 \\
\hline 845048 & 0.1918 & 99.97 .24 & $0.2\left(x_{1}\right)$ & $94.34: 1$ & $0.2(1) 2$ & $82+(0.38$ & $0.226 n$ \\
\hline 87.2618 & $017 \% 4$ & & & 944108 & $0.2(x)$ & 848158 & 0.2242 \\
\hline $84.985 x$ & 0.18 .2 & & & 95.9392 & $0.2(1) 3$ & 87.3468 & (1.2611? \\
\hline $8=538$ & 0.1818 & \multirow{2}{*}{\multicolumn{2}{|c|}{ SuAn 9.11.3.2 psig }} & 94.2012 & $0.2(1.3)$ & 84.2658 & $0.231 \%$ \\
\hline 80.54 .88 & $0.1 / 41$ & & & $9+5+3.3$ & $0.2(110)$ & 006.71 & 0.238 .3 \\
\hline 78.1508 & 0.1854 & $99.9+70$ & 0.2082 & 97.4715 & (1).211.5 & 01.2418 & 0.224 .4 \\
\hline $7+1268$ & $(1.20119$ & 58.6276 & 0.2152 & $98.11+7$ & 0.2074 & 918830 & 0.2284 \\
\hline 730.118 & 0.1885 & 96.14 .30 & $0.2(2))$ & 9870.15 & (). $20 k 1$ & 925150 & $0.2 ? 30$ \\
\hline 71.018 & (1) $8: k)$ & 94.11504 & $0.2 \operatorname{li}(x)$ & 90.3547 & 0.2078 & $9 \times 147.4$ & (1).2154 \\
\hline 60.45 .48 & 0.1705 & 91.7825 & 0.2020 & 007365 & 0.2015 & $9.7+87$ & (1).252 \\
\hline (i) 5 ifi & $(1.17 .40)$ & (8) $5(2) 8$ & $0 ? 08 \mathrm{i}$ & 90.9724 & 0.21082 & 44.34 .3 & $0.22+x$ \\
\hline$(4+1)=88$ & 0.1712 & $97.24,1 n$ & $0.14 t^{3}$ & & & 44.4118 & $(1.22) x$ \\
\hline$\therefore=7018$ & $01: 27$ & $8+450$ & $0.140+1$ & & & 95.5942 & $0.22 \times 5$ \\
\hline$(2)+40 \pi$ & $0 \mathrm{i}$ in? & 82.83rig & (1) $1148^{-1}$ & Scini $11 \%$ & 1?? psiz & $06.2(112$ & 0.220 .4 \\
\hline 58.16016 & (i)! +6! & $k^{\prime}(1.54 .18$ & $(1.180) 2$ & & & 46.84 .3 & 0.223 \\
\hline 55856.8 & $(1.130)$ & $\because 8.3564$ & $11.2(150)$ & 94.1370 & 0.2203 & 074119 & 6.2301 \\
\hline 535538 & 0.1350 & 16.1208 & 02217 & 98.6236 & 0227 & $98114 ?$ & 11.2320 \\
\hline 51.2264 & (1.) 16.5 & 730318 & 0.21158 & $60.3+30$ & $0.22(x)$ & 48.1045 & 0.2356 \\
\hline 45958 & (1) 126.4 & 717018 & $0.14 k, 16$ & $44(1504$ & $0123=$ & (1) $754 ?$ & $0.27+4$ \\
\hline $1+.49 .16$ & $01(x)$ & $60,46+18$ & 0.1912 & 91.7825 & 0.2711 & 307185 & 0.2432 \\
\hline $4+20$ & 0.1154 & 07.2348 & (1.1818. & $80.5\left(1.4 x^{\circ}\right.$ & $(1.2252$ & (31).19?217 & 0.2011 \\
\hline 42018 & 116180 & 64. $78 x$ & $0.18 .1^{-7}$ & $87 ? 018$ & 0.2916 & & \\
\hline $30 \times 618$ & O) $1+n ! ?$ & $6: 0,18$ & (1) 1201 & 81.085 & 0.214 & & \\
\hline $1 \because(x, 1) s$ & $(1.11 \% ?$ & (6) +1) & $\because 170$ & 82.8304 & $0.210:$ & \multirow{2}{*}{\multicolumn{2}{|c|}{ San 1!. 120.4 p }} \\
\hline 353218 & 01040,5 & $5818 x+4$ & 0.1568 & $80 \leq 074$ & $0.2(1) n$ & & \\
\hline$\{i \mid x): i x$ & $(1: 114)$ & ธS\$5स & 0.158 & is 3506 & 0.2207 & (U) & $0 .+112$ \\
\hline$\{1+x+1=$ & $11(1 ; i)$ & $5: 6438$ & (1) I fo.? & $?(1.120,3$ & $0.2+23$ & 48620 & (1.2.1n: \\
\hline Ix chits & $1111: 1, \cdots$ & 51220 & $\because 14: 1)$ & $\because 298$ & (1).S(1) & $00^{2}+40$ & 02420 \\
\hline$\therefore+\cdots+i$ & $11111+10$ & tisustis & $(1,1+1)$ & $\because 171 !$ & 1.2193 & 114 lisid & (1) $2.41 \%$ \\
\hline$\therefore=5 / 1$ & $(1,12,2)$ & for $69+4$ & $11,16 \div 1$ & (1) $+x+8$ & (1)!Mn) & 417525 & 112320 \\
\hline $231^{-1}+4$ & $1111+41$ & $41+3$ & 111146 & $0 ?: 240$ & $(1.211=?$ & $50501.4 \therefore$ & $(1.2+1)^{2}$ \\
\hline $41: 28 n$ & $1: \vdots: ?$ & 1211. & $(11) 12 !)$ & (w) & $0107=$ & $\because 2+18$ & $(123) 6$ \\
\hline$\therefore 4$ & (1) (1.te) & 30.56 in & $0(x) 1$ & 62.7618 & (1) lyil & $\therefore .6848$ & (1) 2 ii \\
\hline 35 indis & $0(x, 1)$ & 3 texis & 116184 & $(x)(11)$ sis & (1) 185 & $8 \geq 8\}$ & $(1,2 \ldots)$ \\
\hline 1" & $\{1(1,1)$ & 35.3914 & $(1) \mid 0)$ & 58 Itwis & (1) I rint & 8(i) 5ios: & $11219 k$ \\
\hline 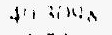 & $11, N_{i-i}$ & $\{1,16 ; 18$ & (1) $14.4 \div$ & 55848 & $(1: \leq: \vdots)$ & 78.7568 & $0228:$ \\
\hline $4: 7 x \times$ & $116 \mathrm{mon}, "$ & $\because(184) x$ & $\left(12 b^{6}\right.$ & 535538 & 0.1542 & in. 1200 & $1.2+1.1$ \\
\hline $4: 24$ & $(1): 1.1$ & $2861, \ldots 14$ & (1) (1) $2+2$ & $51226 x$ & 0.1513 & 734118 & $0.13(60)$ \\
\hline 4.45 & $1112: 11$ & $20+1 \times 15$ & 0111120 & $4 x y 48$ & $011+i d$ & 71.7118 & $0.24=$ \\
\hline $51104 \pi$ & $\{1.11\}$ & $2505 i s$ & $(1) 11: ?$ & $40,0.5+x$ & $(1) 12(4)$ & $6(4)+6.18$ & 11.2169 \\
\hline$\because x^{2}+4$ & (1) is.d & $\therefore 10+1$ & $11(1), 1$ & It 3 (ix & $01.11^{7} \mathrm{n}$ & $07.23+0$ & 0.2114 \\
\hline S Int & (1.14ix & $104 \times 8$ & $11113 \mathrm{~A}$ & 42018 & 0.0803 & $6+0^{-1} \times S$ & $(1.2(x)-13$ \\
\hline$\Leftrightarrow 596$, & 114 & $\because 40 \therefore$ & $1111, "$ & $3015 \mathrm{in}$ & $(i(M) \geq\}$ & 62068 & $(12)(14 x$ \\
\hline rat:ilan & $(1+1 \times$ & 15154 & $(1, x, \ldots$ & $\because$ chtin & $01,1,1)$ & $\{w\}+x$ & 1) 1472 \\
\hline$A: 1,<: 2$ & 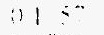 & 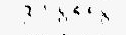 & $111:$ & \& $\because \cdots$ & 1110,101 & $4010 x+5$ & $(11$ lt, \\
\hline $65 \geq 2, x$ & $11 / 0$ & 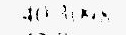 & $\because \because 1$ & $J:$ : H. i i & $(1: 16: 1)$ & 59 5. & $0.1(x, 1)$ \\
\hline$r:-9 k$ & 119. & $4: \cdots$ & $1 ! 1: 1$ & $\cdots, \ldots i n$ & $(1,11) ?$ & 515 . & (i) $\mid(x+1)$ \\
\hline$\because 1114$ & $11 \times 11$ & $4 \div \therefore$ & $\because ! \vdots " *$ & $\therefore+\ldots$ & 111128 & 51220 & 01584 \\
\hline$\because y^{\prime}$ & $\because$ Ixw & +4 & $\because: \vdots A^{\prime}$ & $=2010$ & $11(1) \div$ & $4 x \cdot 16 x$ & 11151.4 \\
\hline$\therefore 4+1 x$ & $\because 11)$, & $5 ; 14$ & $11=$ & $\because a^{*}$ is & $(1116)^{-}$ & $46,+5+x$ & 0.12111 \\
\hline$\Rightarrow+\ldots h$ & $11-11-4$ & $\therefore \ln a:$ & $\therefore$ & $\therefore$ : is & $11(12+, 14$ & $442: 13$ & $011 \%$ \\
\hline$\because,-240$ & $01 \cdots 1$ & $\because \therefore a$ & $11 ;$ & $: \quad \because 4,:$ & 111120 & $42(1,18$ & $0(k+1,)^{-7}$ \\
\hline$B=4 i n$ & $(1) 10.64$ & , sian & i1 184 & $\because+\because 9$ & 11115.15 & $14.851 / 4$ & (bik) \\
\hline $345 ! 58$ & i: Inx.' & 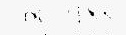 & $1: i^{-} \vdots 1$ & "W ix & 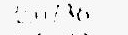 & Pixus & $0\left(1 x^{2}+2\right.$ \\
\hline 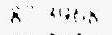 & 11104 & $\therefore: ; A$ & $! \quad ! \cdots !$ & $;^{\prime \prime}, \cdots$ & ii $z^{\prime \prime \prime}$ & 15.3248 & (3) (kns) \\
\hline$n+2 x<$ & 11,4 & $\cdots=\cdots$ & 1 1 iv: & $4 \ldots$ & jiils & 11048 & 0.0930 \\
\hline$w_{11} \ldots$ & (i) $\mid \cdots, 1$ & t, $\because \cdots$ & $\because 11+d t$ & $4_{2} \cdots$ & $\| 11 K_{1}=$ & $3(1.8918$ & (1) $11 ! 5 ?$ \\
\hline 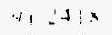 &,$\ldots$ & $\because 11 ; n$ & i i : & $4=\because a$ & $\because \quad 11=$ & $2 x \operatorname{ton}(1)$ & $01 w^{5}$ \\
\hline $11 \ldots 1$ & 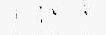 & $\because 4$ & $\because \because n$ & \pm & 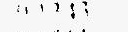 & $26.3 x+8$ & 0.013 \\
\hline w<1 & $1: 1$ & $741 x$ & $\because \because \therefore n 1$ & $\zeta_{1}: \therefore$ & $\because 1 \div: 1$ & $254^{\circ}: 8$ & $0.1117 t$ \\
\hline$\because \cdots:$ & $: \quad \because$ & " l" & $11 ! 5$ & $\Omega$ ser. & $.1<4$ & $2 k 17+6$ & 0.6253 \\
\hline $4: \quad:$ & $\because \cdots$ & $\because \because \because \div$ & $111 w$ & $\therefore \quad$ & $\because \vdots$ & 30 द3⿻ & 00.6 .7 \\
\hline 4. 1.t 1: & $\therefore 1: 11$ & $511: 2$ & $\because \ldots 0^{\circ}$ & 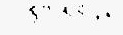 & $\| 1 \ldots$ & $\{y \therefore n$ & 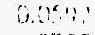 \\
\hline$\vdots \cdots 11$, & $"$ “ $\therefore$ ' & A.tSifs, & 111125 & w..... & $\because: \cdots$ & $1532: 9$ & 11075 \\
\hline 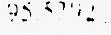 & 0.1314 & $\because \because \ldots \ldots$ & 11121 & $n_{4} n^{\prime}$ & $: 1: 2$ & ?7 & $0 .(x) 1: ?$ \\
\hline$\therefore, \cdots$ & (1) 1 $1801 ?$ & (i) $205 x$ & $(1 \leq 1) !$ & $\cdots: \cdots$ & 1. $\therefore 4$ & $\therefore 18148$ & 1) $16: 4 !$ \\
\hline we $x-4:$ : & $\because 1 "$, & wat an & $0 ? 141$ & A $\quad \because \cdots$ & $\because \therefore 11=$ & 42.7568 & (1) $112 \%$ \\
\hline$w^{-1}+16$ & 1101 & $41=-3: 3$ & (1).21?!! & $7 ! 1: \cdots$ & 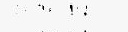 & 4.52454 & (1) 1200 \\
\hline $4: 11$. & $.111 \mathrm{nn}$ & $0 ! 8 x i n$ & 11.2084 & 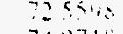 & $\because 1 \cdot 1$ & t7.74a & 0.147 \\
\hline 120.16 & $111: 1$ & 9! 5120 & $02(4)$ & $7+17+15$ & : & $5(11048$ & U. ISS \\
\hline
\end{tabular}




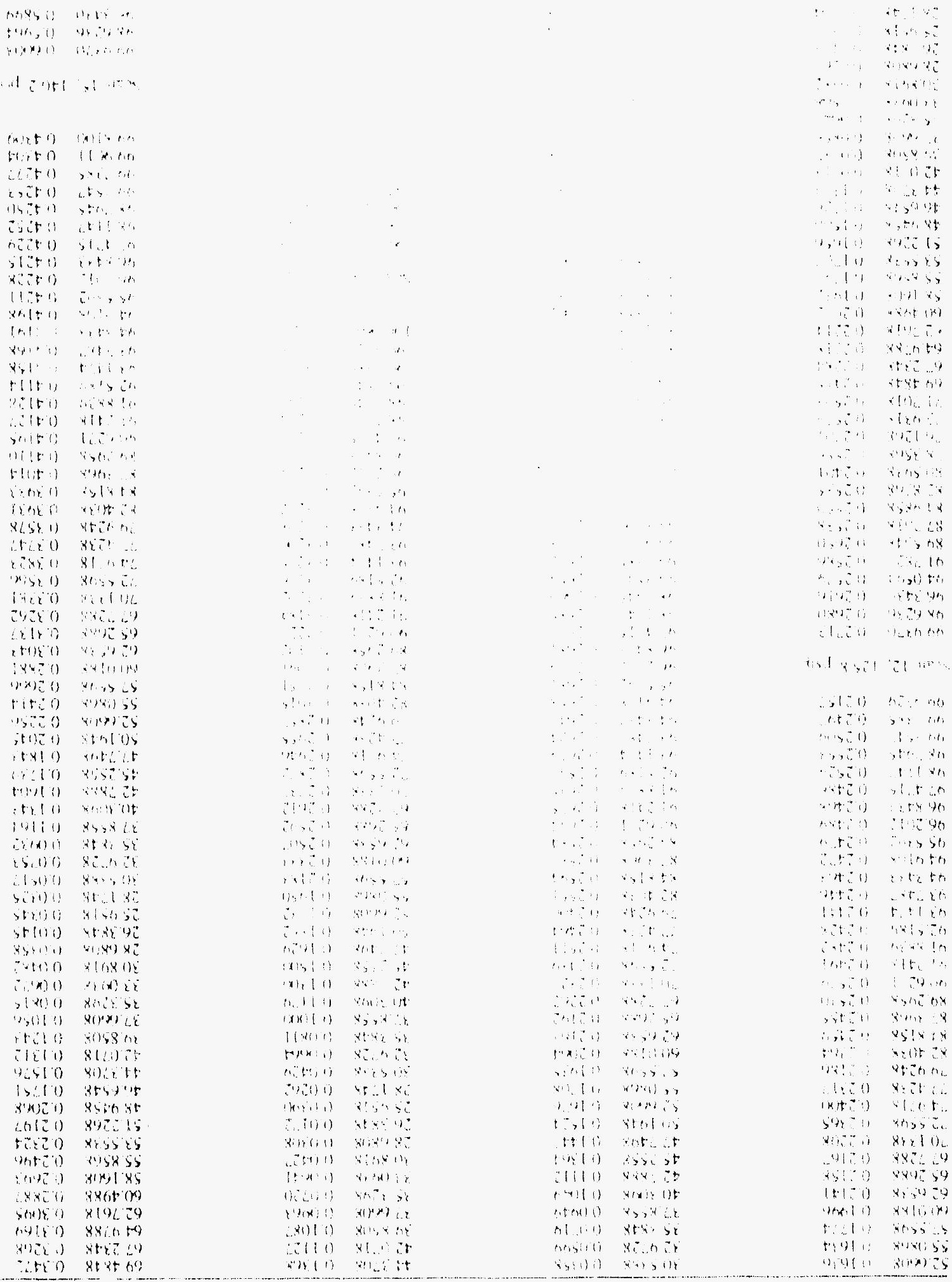

ISa1 anssald 4D!H - SYOEd1.

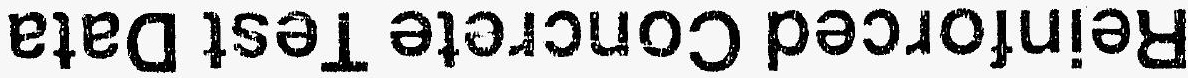




\section{Reinforced Concrete Test Data}

Track 5 - High Pressure Test

\begin{tabular}{|c|c|c|c|c|c|c|c|}
\hline 0.4 .1504 & 0.5838 & 49.75 .47 & $(1,51)(;)$ & 92.5180 & 0.8714 & 72.5598 & 0.438 .8 \\
\hline 41.7425 & (1) $5 \times 1.5$ & 49.7885 & 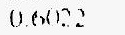 & 93.1474 & $0.877 k$ & 74.9718 & 0.4081 \\
\hline $895(148$ & $(1.56,30$ & $09: 1)(1: 1$ & $0.0,0152$ & 93.7487 & 0.8819 .7 & 77.42 .38 & 0.50 .42 \\
\hline 872618 & $(15.453$ & $49811 k$ & 0.0058 & 943.413 & 0.8640 & 74.4248 & 0.504 .1 \\
\hline 8.49858 & (j) 5.926 & & & 94.9168 & $0.8 \times 74$ & 82.4038 & 0.5296 \\
\hline $8: 8308$ & 0.9 .121 & \multirow{3}{*}{\multicolumn{2}{|c|}{ Sian 16. 145.8 psip }} & 95.5302 & 08913 & 84.8158 & 0.5490 \\
\hline 80.5038 & $0.51 ? ?$ & & & 96.2012 & 0.8 .956 & 87.346 .8 & $0.55,44$ \\
\hline 18.394 .8 & 0.5274 & & & 468.73 .3 & $0.856 \times 8$ & 89.2958 & 0.5719 \\
\hline 16.126 .8 & 0.5272 & $90.4 \times 70$ & 000132 & 97.4715 & 0.9020 & 90.6271 & 0.54 .48 \\
\hline 734318 & $0.40 \times 8$ & 980.2 .46 & $0.9(1.30)$ & 98.1147 & $0.9(159$ & $91.241 K$ & $0.594 ?$ \\
\hline $7 ! .7018$ & $0.4: 7 k$ & 96.34 .30 & $0.88,619$ & $98 \div 4.45$ & 0.90 .54 & 91.8834 & 0.5980 \\
\hline $69.48+3$ & 0.40 .11 & 4.4054 .4 & 0.8721 & 99.3547 & 0.9054 & 92.5189 & 0.5960 \\
\hline 67.2345 & $(14.3)$ & 91.78 .5 & 0.8574 & (4) 7385 & $0.4(18.3$ & 93.1474 & $0.6(x) 14$ \\
\hline of $978 x$ & $0.4 ! 31$ & 84.5148 & $0.8+1)$ & 0.4 .10011 & 09110 & 93.7487 & $0.6 x) 38$ \\
\hline 027016 & $0.40)^{2}$ & 87.2616 & $0.8 .2(n)$ & 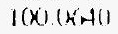 & 0.4164 & 94.3433 & 0.60174 \\
\hline $60+406$ & 0.3742 & $8.4 .985 \times$ & $0.8 .12=$ & & & $94910 x$ & 0.6117 \\
\hline So $16 .(1)$ & $0 \times 5.40$ & $82.811,4$ & $0.7 \cdot 16$ & & & 95.5392 & $0615 k$ \\
\hline $55 x^{2} 6.8$ & $0.23(x)$ & $x i 543 k$ & $(1, \because 6612$ & \multicolumn{2}{|c|}{ 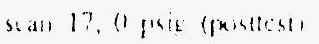 } & $9+2,2(1) 2$ & 0.6190 \\
\hline 534528 & $0.3(k, t)$ & $78,35 r, x$ & (1) 79.1 & & & $90 x+33$ & $06,(19)$ \\
\hline 5122618 & $(1.25 .47$ & $7613 f, k$ & (1) $7.4+1.1$ & (j) 1$) \times ? 0$ & 0.0 .352 & 07.471 .5 & 6.626 .3 \\
\hline 480.958 & 0.26 .12 & 7213118 & 0.713. & Uh $(1,2\}$ & $0.6,297$ & 48.11 .47 & 0.6 .3 .41 \\
\hline $41104 x$ & 0.21 .4 & $717(1) x$ & $\left(16, x^{2}+3\right.$ & 90,340 & 116165 & 9871345 & 0.6375 \\
\hline $442^{2}(16$ & (1) $2\left(x_{10}\right)$ & (51) $48+8$ & $0.6,2)$ & 9.461504 & 1). susth & 99.3547 & $0.63+40$ \\
\hline $42017 \mathrm{~h}$ & $0.17,1$ & 6723.46 & 0.1 .2 .95 & 41.78 .5 & ().5821 & $0,725.5$ & 0. 63.33 \\
\hline 39.5501 & 010.32 & (it 1$)^{2}$ kik & $(1.51+1)$ & 8. $5(1.4 k$ & (1).57615 & $94(x) 11$ & (). 6.356 \\
\hline $37+x+16$ & $01^{2<7}$ & $\therefore 7(1) s$ & $11.9620 ?$ & 872618 & (). $5 f(0.4$ & $99 . k i(k)$ & $0.621) 1$ \\
\hline 49.3245 & 0.10 .4 & (1) (1) & 0.53101 & 844855 & 05147 & & \\
\hline $31 .(x) 3 x^{\prime}$ & $0(18)+$ & $5 x /(x) 8$ & $\left(0,4 x_{1}\right)$ & $x=8.60$ & 0143113 & & \\
\hline 308016 & $(1)(k,+))$ & 558568 & 0.91 .23 & $k(1<1) 38$ & $(1501) 2$ & $1: \therefore 1)$ & \\
\hline 280 resth & i) $01511^{6}$ & 535508 & 0.4214 & 78 र & 119117 & & \\
\hline In 3 inth & $0 .(1240)$ & $51221, k$ & $(1,4,2) ?$ & 7012016 & (1) 4.5 & & \\
\hline 259518 & $(1)(142)$ & $4 \times 4458$ & 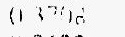 & 73.4215 & litidis & & \\
\hline $2 \times 1746$ & 00046,4 & $4+.65$ & $4: 192$ & $71701 \%$ & $1+3 i k$ & & \\
\hline$\because: \because n$ & $61 x_{x} \cdot x$ & 443011 & $113 \times 1$ & (i) $4 x+x$ & $(1+1) .21)$ & & \\
\hline$\because 4: 26$ & $0(k) \cdot 2$ & $4: 11718$ & (1) $245 i$ & $\theta^{-} \geq 3.4 k$ & $113 \times 11^{5}$ & & \\
\hline $252 x+8$ & (1) 1218 & $306<18$ & 11214 & $(x+1) / 8 x$ & (1) $30,1+1$ & & \\
\hline $37 \times 5 i$ & (1) $\mathrm{knh}$ & 37 cordit: & $(1) 111,4$ & $\therefore=0,18$ & 01845 & & \\
\hline $4(12)(x)$ & $(1) 74$ & 342918 & $111<9 ?$ & tst posk & $(12914)$ & & \\
\hline $42 x+2$ & (1) i se. & $? 3114$ is & $0: 121$ & $5 x$ itris & $(1.24,27$ & & \\
\hline $442<58$ & $(1) \ldots$ & $31+x+41 x$ & $\left.(111)^{\prime}\right)$ & 64856 & $(127,1)$ & & \\
\hline $47-749 x$ & $11:$ & In walls & $1111^{-3} i k$ & 545538 & 6.254 & & \\
\hline $514+6$ & $(1) \quad 1$ & $2 f, 4 x+3$ & 01114.44 & 51.2210 & $0 . ?$ ? & & \\
\hline$\Leftrightarrow$ exrlik & $\left(\begin{array}{lll}1 & 21\end{array}\right)$ & $2545 \mid x$ & $(1)(x, 2)$ & $464.15 x$ & $0.2(x)$ & & \\
\hline $\begin{array}{l}5 \% 5 x+k \\
5 \% 550 k\end{array}$ & (1) $12 x$ & $2819+4$ & (i) & te 69.80 & () $/ k(x)$ & & \\
\hline (1) & $(12 \cdots+4$ & 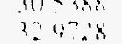 & 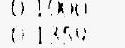 & $\begin{array}{l}63701 \\
420718\end{array}$ & $\begin{array}{l}(1.1622 \\
1.1 .97\end{array}$ & & \\
\hline $120<58$ & $11+1: 3$ & 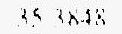 & $(1) 10,50$ & .19. 85,18 & 0.1371 & & \\
\hline tis 2 ansh & $01.21 ?$ & $978<0 x$ & $(1,2 ! 1: 1$ & 37 thilik & 0.1151 & & \\
\hline (1? $7: 8, \alpha$ & 0.13112 & $4(1) 20+k$ & 11.2 .416 & $35.321+8$ & $\left.\left(1,(1)^{\prime}\right)\right)$ & & \\
\hline$\because\left(11^{2} 3\right.$ & $1145(14)$ & 4: ?kisin & $(1271)$ & $3300 x+4$ & $0 .(i) .34$ & & \\
\hline$? 2<426$ & 0.4642 & $44.2<58$ & $(1): 178$ & $3(1.84)$ & $(1)(15+4 x$ & & \\
\hline $30 ? 1 x$ & (1)<促, & $47 \because 48$ & $02 \$ 1,6$ & 28.68018 & $0.0 .1(t)$ & & \\
\hline 7428 & $0511 k$ & $501.14+1$ & $(0,2 m)$ & 26 indente & 01.04111 .4 & & \\
\hline$\because 4 y^{\prime} 1 x$ & i) & $\because(A x) S$ & $11+10)$ & 259518 & $003 k 5$ & & \\
\hline र. मारू & (1) 5.tur. & $r(1), k$ & $(1.2453$ & $=8.1748$ & $0 .(1+4) 2$ & & \\
\hline 34 nis & $(1<419)$ & $c \%<48$ & (i) $24 \times 15$ & $3(1.5\} \times 6 t^{\prime}$ & $0 .\left(x_{1}(1)\right.$ & & \\
\hline h, 36,6 & $y=s, t$ & $001118 x$ & 015215 & 324728 & $0(18.30)$ & & \\
\hline 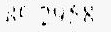 & $0,50,5$ & $\theta_{2} \mathrm{C}^{k}$ is & (14-1) & 359645 & $0(104)$ & & \\
\hline$v\left(1,0_{2} \geq\right\}$ & 1552,2 & $0,520,25$ & $0.05 k$ & 5.8558 & $(1.12) 9$ & & \\
\hline $412.11 \%$ & 1,4752 & 077258 & $1 ; 6.14 ?$ & 4(1) $+(4) \mathrm{s}$ & 01442 & & \\
\hline $91 \mathrm{sen} \%$ & $0.57 \pi^{6}$ & 70,1328 & $0.6 \div 42$ & $42.7 \sin 8$ & $0.15(x)$ & & \\
\hline 425160 & (1) $x>74$ & 72 ic14k & $0.7(k)_{2}^{3}$ & 452558 & 01702 & & \\
\hline 431474 & $(15 x ! 1)$ & 749716 & $1174 x$ & 47.1408 & 0.140 .7 & & \\
\hline 14. 74.7 & $0.5 \times 21$ & 77.428 & $11760 \%$ & $5(1) 4.48$. & $0.225 k$ & & \\
\hline 94.3423 & 05857 & $70021.2 x$ & $(177,4)$ & Sב Exklk & 11.417 & & \\
\hline $44 y 16 x$ & $\left.0.58^{\circ}\right)$ & $8.191 \%$ & (j) xind & $\ll 11$ ses & $0.2<y$ & & \\
\hline $0454 \% ?$ & $04011)$ & $8+819 y$ & (1) & $5 \pi<511)$ & (1)284) & & \\
\hline 46.2112 & $0.54+11$ & $5 ? 3+41$, & 11522 & $(x)(1 ! 68$ & $(0.3155$ & & \\
\hline $9+k+1\}$ & $(1,51,24$ & $x, 1458$ & $114 x+2$ & 62059 & 11.1414 & & \\
\hline $974: 5$ & $(1,9, j=?$ & $(x)+1,1$ & $1 \times 1, \%$ & 652068 & 03.379 & & \\
\hline 461117 & (1) $5.18<$ & $412.41 \%$ & $(, n, x, 3$ & 677268 & 02010 & & \\
\hline $9,74.4$ & $05 \mathrm{~ms}$ & 17) $8 \times 14$ & $19+12$ & $7013: 8$ & (1). 41175 & & \\
\hline
\end{tabular}




\section{Reinforced Concrete Test Data}

Track 6 - High Pressure Test

\begin{tabular}{|c|c|c|c|c|c|c|c|}
\hline \multicolumn{2}{|c|}{ Sianl 1.442 posk } & 79.2951 & 0.01800 & $174.70 x_{1} 1$ & $0.0 \times 17$ & 176.2771 & $0(19: 4$ \\
\hline $2(x) 3(k x)$ & 0.01382 & 73.7251 & $\begin{array}{l}0.06110 \\
0.13886\end{array}$ & $\begin{array}{l}182.6011 \\
185.5401\end{array}$ & $\begin{array}{l}0.0687 \\
01 K \times 00\end{array}$ & $\begin{array}{l}173.6621 \\
17049(1)\end{array}$ & 0.075 \\
\hline $2(x)(n+1)$ & $0.06+!$ & 70.4061 & gonotio & 186.3791 & $0.06 .3(1$ & $16820<1$ & $0\left(\mathrm{Ni}_{i}\right)$ \\
\hline 203.7113 & $0.056 n t$ & $68.0 \times 1$ & 0.11802 & 191.2451 & 0.0588 & $165(x) 51$ & $0\left(k_{i}\right)$ \\
\hline $2(x) .70,14$ & $0(k, 10$ & 0.5 .21 .51 & 0.0746 & 144.1341 & $0 .(k x) 4$ & $|62405|$ & (1) \\
\hline $257.74(1)$ & 0.01544 & $6334<1$ & $0.0 \div 4$ & $1008+41$ & 0.07018 & 100020991 & $0(x) \div 5$ \\
\hline 254.7231 & $0 .(k, 14$ & $54(605)$ & $0(x+1)$ & $199 \times(-9)$ & O. (kitit & $157.705 j$ & $0(x+1)$ \\
\hline $252(14 t)$ & $0(k, 7)$ & 50.8751 & $0(k \times) 2$ & $2(1280(1))$ & $0.07(19$ & 155.1551 & (1.1 $11_{12}$ \\
\hline .2402791 & $0 .(1 \% 105$ & 54.1151 & $0,(x, 1)$ & $?(1500,5)$ & 0.0545 & 1524351 & 0.122 \\
\hline$=26.56 .31$ & $0(k, 50)$ & 51.2751 & 0.01574 & $208+42(1)$ & $0.16 ? 88$ & 1.447451 & (1) IKA \\
\hline 2438251 & $0(1771)$ & 48.5451 & $0 .(152) 1$ & 211.1341 & $0 .(x+1) 9$ & 1470151 & 0.coste: \\
\hline 2411141 & 1) 67.45 & 4.5 .7251 & 6.14 .36 & 213.8281 & $0 .(657 ?$ & 144.3551 & $0.08 i=$ \\
\hline $248.44 i 11$ & $011 \%$ & 429051 & $0(1342$ & 210.5011 & $0 .(10.5 ?$ & $1+1.6 .251$ & $(1)(N), 3)$ \\
\hline $234(x, 4)$ & () $\mid[n \|$ & 40.1 .351 & $0 .(1,70$ & $219.2 \times 101$ & 0.0764 & 138.8 .451 & $0 .(k+1)$ \\
\hline 231119 & 1111117 & $37 ? 59 !$ & $003 i 5$ & 221.0501 & $(1.0744$ & 13.9 .9051 & 0.1025 \\
\hline $23(1,2581$ & (1) (rupid & 343551 & $(1)(1) 10$ & 274.6851 & (1) (1k20) & 132.9051 & $0 .(\mu)$ \\
\hline$\because \quad 0, \$ 1$ & $0.1(x)$ & 31.4851 & 0.11125 & 227.3781 & $0.017(x)$ & 130.2751 & $0 .(19 k 5$ \\
\hline 22.40 .11 & $0.0 k 18)$ & $2 \times 6.451$ & $(3)(k \times)$ & $2.30 .1 ! 41$ & 0.015 .57 & 127.5351 & 0.1027 \\
\hline $22211 \times 1$ & $0.1(k, 1)$ & 27.2491 & ()$(K)+1)$ & 232.8281 & 0.0821 & 124.9251 & $O(\mu) 7 k$ \\
\hline 213583 & () (r)ent & $208+8 \leq 1$ & $0(1) 117$ & 235.5221 & 0.04 .36 & 122.2351 & 0.0797 \\
\hline$\therefore 16,01(11$ & $01(k+1$ & $3268<1$ & $(1111) 14$ & 238.1551 & 0.0916 & 119.4 .351 & 0.1028 \\
\hline $21+1+41$ & $01112 x$ & 255751 & 0.0125 & 240.80181 & () likity & 116.7451 & 0.1048 \\
\hline $211<(11)$ & (1) (ridis & $38.5(15)$ & $0 \log 50$ & 243.4421 & 0.0014 & 114.1351 & $0(3972$ \\
\hline $2(1 x+x)$ & $0.0 \times<1$ & $414:<1$ & $0(1): 4$ & $2+4+2101$ & (1) (1)8xi? & 111.4 .451 & 0.1012 \\
\hline$\because \times, 1)^{-2} \geq$ & $(1,4)^{*} ?$ & $4.22<1$ & $(1)(1.3 .40$ & 288.0711 & $0.010 \times()$ & 108.7051 & $0.1(x) 1$ \\
\hline $21134(k, 1)$ & onsu? & $4 \div 3051$ & $1105 i^{7}$ ? & $251 .(1.16)\}$ & $0.0 x^{\prime} y k$ & 104.1451 & 0.1053 \\
\hline $2(x)(x) 1)$ & "I lix!) & $5(14) 51$ & $(1.018 .4$ & $25+4(k) 1$ & $(1 .(12) 7)$ & 1013.3951 & 0.1018 \\
\hline $10.45(11]$ & (1 $1811_{2}^{\prime}$ & 524551 & $(1)(151) 4$ & $2572(x) 1$ & 1).(1wers & 100.765 .51 & 0.10135 \\
\hline $14<2471$ & $11(1,4: ?$ & 50.7851 & $(0 .(16)+1)$ & 2001571 & (0.08kens & 97.9851 & $(1,(x) \times-1$ \\
\hline $142<5+1$ & $(1.16 .1 .4$ & 5.74 .451 & $(:)$ was? & $26,2(1)(1 \times)$ & $(1\langle 17\rangle)^{\prime}$ & 45.3751 & $0.1(k)$ \\
\hline leir nils. & $016 \times 8$ & 624251 & $(1)(x, 1) k$ & $26<6347$ & $0 \log (\operatorname{sis}$ & 92.7451 & 0.1024 \\
\hline 1811121 & $0(1) 4:$ & 0,54591 & 0117.15 & 26711776 & $017+42$ & $Q(i .1151$ & 0.1054 \\
\hline $\ln 19050$ & fliwat, & $\{1 \times x+4\}$ & $0(1) 78 ?$ & $20 x-5 y$ & (1) Gini: & $8 \% 4251$ & 0.1047 \\
\hline$(x)(1+4)$ & $(1,1)^{\circ} \mathrm{K}$ & $\because 1$ xisi & (1)(15?: & $20 k 20 \% 3$ & (1)(182) & $84.1 \times 51$ & $0.1(k)=$ \\
\hline 17898 & $1111: 46$ & 740.151 & 012017 & & & $82 .(x 15)$ & 0.10110 \\
\hline 170.2711 & $0(1,551$ & 7.9 .191 & $-(1)(1,2) 3$ & Sian :. So & o psit! & 79.2951 & 0.0035 \\
\hline $173(x, 2)$ & $(1)(150,1)$ & 804251 & $(1)(3, y)$ & & & 76.3951 & $0.0 .0 \times 1$ \\
\hline $17(145,1)$ & $\left(10^{-1}\right) 11$ & $8 ? 2751$ & $0(k) 11$ & $26,8 ?(x, 6)$ & $0.0)\left(x_{1}\right.$ & 7.3 .7251 & 0.1024 \\
\hline $10 \times 2.2451$ & () () kntix & 86,1751 & $0.18 ? ?$ & Ixt.cotity & 0.101 .5 & 70.90151 & $0.09+1$ \\
\hline $105(n) 15)$ & 001124 & 8901151 & $\left(i .(k+)_{2}\right)$ & 203.7713 & $0 .(19+42$ & $0,8(0) \leq 1$ & $0 .(x) 73$ \\
\hline $16: 4051$ & O) & 41.9451 & $0 .(1) \geq 21$ & $26(1.7624)$ & $0.1643^{2}$ & $65.2 \mathrm{i} 51$ & 0.0889 \\
\hline $1(x)=491$ & 0.97 .40 & $44 \times 1151$ & $0 .(18 \% 2$ & $257.74(11$ & 0.10125 & 6.3 .3951 & 0.01821 \\
\hline $157^{\circ}, 1151$ & ofinis & $0,1,5(5,5)$ & $0.05 ? 2$ & 254.72111 & $0.11(x, 4)$ & $59(x) 51$ & 0.0810 \\
\hline $1 \leq 5.11<41$ & 0.1107 & $10\left(x+44^{\circ} 1\right.$ & $0 .(1+2) 8$ & $252(1.46,1$ & 0.1150 t & 56.8151 & $0(x, 1)$ \\
\hline $152+44$ & (1) 1010 & 103.1351 & $0 .(185.4$ & 2.44 .2771 & $0.10 \times 4$ & $54011<1$ & Ensii \\
\hline 194.7851 & $(111 ; 9 k$ & $1\left(k, 2, k_{1}\right)$ & 01.08 .45 & $240,56,31$ & 0.10196 & 51.27 .51 & $0.1 \times 1,58$ \\
\hline $14 \%(105)$ & $0 .(k, 70)$ & $1(19)(15.5)$ & U(1hs) & $=1 \cdots \cdots-c 1$ & 01.1130 & 48.54 .51 & 0.0 .5 .36 \\
\hline 1442551 & (1) (Ki, it) & $111 \mathrm{ket}<1$ & $\therefore$ …‥ & 241.1141 & 0.1121 & 457251 & $\theta(\mu 1 *)$ \\
\hline $1+1.62<1$ & 1111755 & 1146.251 & (1) $(1 \times 1 \times)$ & $23 \times .4+4(1)$ & 0.1378 & 420051 & 0.0392 \\
\hline $14 x+451$ & 0.073. & 117.3751 & 01086.4 & 23.500 .51 & 0.11 .34 & 40.1351 & 0.0333 \\
\hline $1.350(15)$ & $0.0 \times 2 !$ & 12011251 & $0.618 \times 10$ & 234.11 .41 & $0.1(1+4$ & 37.2551 & $0.03(x)$ \\
\hline 1320051 & $(1061)$ & $12: 24551$ & $(1)(1)$ : & 230.3981 & $0.10<7$ & 34.3551 & 0.0212 \\
\hline 1.30 .2751 & 0.070 .0 & $125 \%(151$ & 0.0708 & $227.6(1,5)$ & 0.1058 & 31.4651 & 0.0071 \\
\hline 127.5351 & coustis & 128.541 & $0.0 \times 23$ & 2240711 & $(1.1111 .7$ & $28.6+51$ & $-0.0 \times 125$ \\
\hline 124.9251 & $0.074 ?$ & 1.11 .3191 & (1)(17): & 222.3181 & 0.11 .33 & 27.2951 & $.0 .0 !(x)$ \\
\hline 132.2351 & (1). $(x,+1)$ & 1.34 .1351 & $(1,9,74$ & $219.588^{2}$ & $0.11(x)$ & 29.8451 & $0 .(x) 19$ \\
\hline $119+351$ & $0 .(M)(L)$ & $13(0,4: 5)$ & 0.675 & 2164101 & 0.1110 & 32.0451 & $0 .(x) 1 x$ \\
\hline 116.7491 & $(114) 7$ & 1347051 & 04740 & $\therefore \mid 4.194\}$ & $0.1(\pi) 7$ & 255751 & $0.0 \times 175$ \\
\hline 114.1751 & 0.0186 & 1425251 & (1)194? & 211.50111 & 0.1111 & 38.5051 & 0.0148 \\
\hline 111.4451 & 0.11818 & 1453351 & $0.16,5 x$ & $2(18 \times 48)$ & (1)(11)? ? & 41.4551 & $0 .(1,313$ \\
\hline $1018.76,51$ & $0(1)+1 i$ & 148.154 & $(1)(15+1)$ & $2(16,1173)$ & $0.1(x) .1$ & 44.3751 & 0.03 .30 \\
\hline $\mid(x, 1.45)$ & (i.cisic: & 151.1151 & $0\left\{x_{1} ? ?\right.$ & $20134(x) 1)$ & $0.11 \times 15$ & 47.3941 & $0.0 .55 \%$ \\
\hline$!(12345)$ & 0.0874 & 1534751 & 0.11 .23 & 2 (k) fors) & $0.10+11$ & 50.4151 & $0.05+x$ \\
\hline $1(x)=0,1$ & $0(1882$ & $15 n 7104$ & $(1,1,274$ & 107.95011 & (U. $1(x) 1$ & 53.4551 & $0.0 x_{1}(x)$ \\
\hline 97.451 & (1018.9? & $1\left\{4\left(9.5^{5}\right\}\right.$ & $0(x, x, x$ & 1452071 & $0.1(x, 3$ & 56.7851 & $0(x, 0) 4$ \\
\hline $45 \div \div 51$ & $0(154 x$ & 16.2 .4261 & OIx:Bi & |142\{k,3] & 0.11 .411 & 50.4451 & $(1.0714$ \\
\hline 92.7 .451 & olks? & $10.52,291$ & $(1), ?]$ & $1808: 151$ & $0(x) 74$ & 62.9251 & $(1,17) \times 4$ \\
\hline Gii.iisi & iifinis? & inis:isi & 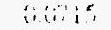 & 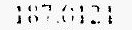 & 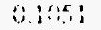 & $650 \mathrm{ces}$ & 60? \\
\hline 80.4251 & 60014 & 171.1621 & $(1)(x, 1) !$ & 184.2051 & $0.11 \times+4$ & $68 \times 451$ & 0.0816 \\
\hline 847851 & $0.167 \%$ & 1734,301 & $01,1 \times 1,57$ & $1816+41$ & (1).(1):? & 71.8041 & (1) (1) \\
\hline $82(x) 151$ & $0.0 \times 1.4$ & 1768001 & (1) (x):-t & 178.3501 & $0 .(14.363$ & 746491 & U. $1(x) 2$ \\
\hline
\end{tabular}




\section{Reinforced Concrete Test Data}

Track 6 - High Pressure Test

\begin{tabular}{|c|c|c|c|c|c|c|c|}
\hline 77.5451 & $0 . \mathrm{KS}_{2} \mathrm{O}$ & \multirow{2}{*}{\multicolumn{2}{|c|}{ Scan 3.603 psip }} & 79.2451 & 0119.4 & $179.7(x, 1)$ & $0.09 ? 23$ \\
\hline 80.4251 & $0.1(x) 7$ & & & 76.3951 & $0 .(14)\left(x^{4}\right)$ & $182.6(1) 11$ & $0 .(k+k) ?$ \\
\hline $83 . .3351$ & 0.10111 & $26,8.3(x .6)$ & $0.17+60$ & 73.7251 & 0.1215 & $18.5540(1)$ & $0 .(4) 7 t$ \\
\hline 86.1751 & O. (Kyst & $26 x \cdot 6+1 k 4$ & $0.6 k_{2} \mathrm{~B}$ & 70.9051 & 0.10155 & 18.8 .3791 & 0.0055 \\
\hline 89.0151 & 0.1077 & 263.7713 & $0 .(180117$ & $68(k) 151$ & $0.11 \mathrm{ks}$ & 191.2951 & $0.0888^{\prime}$ \\
\hline 01.4 .451 & $0.10(x)$ & 260.7620 & $0.00(15$ & 05.21 .51 & 0.0999 & 19) 4.1341 & 0.1031 \\
\hline 94.8051 & 0.10122 & 257.7401 & $0,(x) \mid i$ & $(2.37) 51$ & 0.09 .75 & 196.8441 & 0.10 .51 \\
\hline $97.5(1.51$ & $0 .(4997$ & 24.47201 & U. (14.40) & 50.6051 & 0.088 .5 & $190.8(, 9) 1$ & 0.1016 \\
\hline 100.4451 & $0.1(1.4 !$ & $252(46)$ & $0 .(x) 78$ & $56 . \times 451$ & $0.0 \times 1.8$ & $2(1) 28(10)$ & $0.1(x) ?$ \\
\hline $10 ., 3.351$ & 0.10124 & 2402771 & $0: 10 k ?$ & 54.01151 & 0.0776 & 20.60 .51 & $0.0 \%(0)$ \\
\hline 106.23 .51 & 0.10 .30 & 246.56 .31 & $(1,(x))^{2} ?$ & 51.2751 & 0070.3 & 208.42011 & 0.0944 \\
\hline 1090551 & 0.0972 & 243.8281 & $0.1(x) 8$ & 48.54 .51 & 0.0617 & 211.1341 & 0.1059 \\
\hline 111.8651 & 0.1045 & 241.1141 & 0.107 .3 & 45.7251 & 0.04 .1 .3 & 213.8281 & $0.11 \times 8.8$ \\
\hline 114.6251 & $0.1(k 17)$ & 238.4401 & 0.1486 & 42.9051 & 0.0 .372 & 216.51111 & $0.11 \times, 5$ \\
\hline 117.3751 & 0.0995 & $235.6 \times 551$ & 01282 & 40.13 .51 & 0.013 .54 & 219.2461 & 0.1196 \\
\hline $\mid 2(1) 1351$ & 0.1124 & 233.1141 & $0.127 \%$ & 37.2591. & 0.11212 & $221.95(11$ & 0.1157 \\
\hline 122.4551 & $0 .(k)+4$ & $23(1.3581$ & 0.1242 & 3.13541 & $(1)(1) 47$ & 224.6851 & (1) $12+2$ \\
\hline $125.7(15.1$ & $0.09 \div 4$ & $227.6 \times 151$ & 0.121 .5 & $3148 \leq 1$ & $0 .(K K, S$ & 227.3781 & 0.1162 \\
\hline 128.5251 & f) $(\mu) \xi^{2}$ & 2240711 & $0.12(x)$ & $28(0.451$ & $.0(x) .13$ & 230.1141 & 0.1174 \\
\hline 1213151 & (1)16.33 & $\therefore 23 \mid \times 1$ & 0.1240 & 27.2451 & $0(x) 77$ & 232.8281 & 0.1202 \\
\hline 1341291 & $(3,1) 812$ & $2145 k 31$ & () $120 x$ & $24 x+51$ & () $\{1,1.1\}$ & 235.5221 & 0.1330 \\
\hline 130,00151 & $0 .(M) 4$ & $2|(1.1)|(1)$ & $11.12+1$ & 320,591 & $(1) x \times x)$ & 238.1551 & 0.134 .3 \\
\hline $1 ? 9181$ & 0.0420 & 214.14 .11 & (1.1.5.4 & $35.57 \$ 1$ & $(1)\left(k x_{1}\right)$ & $241) \times(181$ & 0.1204 \\
\hline $14: 5251$ & $0.18 \times 6$ & $2115(111$ & $012 \pi$ & $38.5(1) 51$ & $0.02+1$ & 24.9 .5421 & (1.1284 \\
\hline 1456251 & $0 .(18) 1)$ & $2(18.8+k 1$ & 0.113 .4 & $11+5 \leqslant 1$ & 0.113 .43 & 246.2161 & 0.1322 \\
\hline 1481551 & $0.11 \% 26$ & $2(k, 1) ? 3)$ & 0.12112 & 4.43751 & $0 .(1.4(1)$ & 248.9711 & $0.1+112$ \\
\hline 1511151 & Otsisis & $2(1) 3.4(x) 1$ & $0.1(11) 7$ & 47.31151 & $0 .(1 ; 44$ & $251.60+611$ & 0.1 .411 \\
\hline 1534751 & 0.14 .2 & $2(x)(x .51$ & 0.110 .11 & 501.4151 & $0 .(5,30$ & $254.4(x) 1$ & 0.1426 \\
\hline $150.7(151$ & 0.12018 & 107.45011 & 0.1070 & 53.15 .51 & 0.67 .36 & $257.2(x) 1$ & 0.13 .31 \\
\hline 1949451 & 0.0871 & 105.2071 & $\ldots 1(1) 1)$ & $56,7 \times \leq 1$ & $0.118(x)$ & 260.1571 & 0.1182 \\
\hline 162.4351 & 11.0705 & [4! $58.1 !$ & $\therefore 11(11)$ & $51,9+51$ & $\cos (\sin x)$ & $263 .(4) 181$ & 0.1221 \\
\hline 10.520 .51 & $(1)(18<0)$ & 1848195 & ()$(x), x)$ & 6.24251 & 011858 & 265.8347 & 0.1284 \\
\hline $10,8.2191$ & $(1 .(1) 4 \times 5$ & 187.0121 & (1) 1082 & $65,1,551$ & $(1,11) \div 5$ & 26,0175 & 01122 \\
\hline $171<1621$ & (1.). & $18+? y^{5} 1$ & $(1.1124$ & $6 \times 8.1<1$ & 0.1023 & 268.2950 & 0.1260 \\
\hline 17293011 & (j) $\left(1 k^{\prime} 3.1\right.$ & 181.64 .81 & (1) $11: 15$ & 71.80151 & 0.16147 & & \\
\hline $170 x(18)$ & 0.117 .4 & 1784801 & $0.10(x)$ & $7.1+1.451$ & $11.1^{21,5}$ & Scan 4,8 & $I$ pipk \\
\hline $174.7(k)$. & 0 or tret & 178.271 & likisin & 775.951 & i) $(1 \mathrm{~s}) \mathrm{s}$ & $20,81(x)$ & 0.0128 \\
\hline$\left|x_{2} .(x) 1\right|$ & $0.18+7$ & $1718 \times 1.1$ & $0(\ln (1)$ & $x(1+2,51$ & (1) $\mid 1181$ & $2 f(x)\left(-4 x^{\prime}\right)$ & (1).(x)14 \\
\hline 185.54018 & $0 .(18+x$ & $176(; 50) !$ & $(1,(x)+1.4$ & $83 \div 351$ & (1) $12\left(k_{1}\right.$ & 20.3 .771 .3 & $0.1 \times+18$ \\
\hline $1 \times 8.3741$ & 0.18218 & $\mid(k-2) \leq 1$ & (1) lltit) & 80.1751 & $(111: 3$ & $20,1.76207$ & $0.01+2$ \\
\hline 101.2051 & $0.172 ?$ & $16.5+151$ & $31 x, x=$ & 8901151 & $(1) i 1 i$ & 257.7401 & $0 .(01855$ \\
\hline$[1.413 .41$ & 0,0182 & $|6 . !(x) \leq|$ & $(0,1 \times 1) ?$ & $0 ! 10.64$ & (1) 1..5in & 254.7201 & $0.0(x, 1)$ \\
\hline$|4+n+4|$ & Gowat & 16012041 & $\because(x) B(;$ & $(94: 11<1$ & (1) 1 '.4 & 2520.4611 & $0 .(12(x)$ \\
\hline $194 . x(3,4)$ & 061850 & 157.2151 & $\left(1, k, k^{2} 2\right.$ & 9754 & 0.121 .1 & 244.2771 & $0.0 .2 k$ \\
\hline $201 . x+41$ & $0(1) R^{2}=$ & $155(1051$ & $\because ! 194$ & $i(n)+1,5\}$ & 0.1243 & $2.46 .56,31$ & $0.02 \%$ \\
\hline $2015 \cos 51$ & $0(14) 18$ & $152 .+351$ & (1) 1181 & 1113.235 & ii.:?: & 243.8281 & $0.0+4.0$ \\
\hline $2(18.42(1)$ & 0.1713 & 149.7 .451 & 0.10 .311 & $1(k .235)$ & $0: 11)(1$ & 21111.11 & 0.0 .387 \\
\hline 211.1341 & $(0,(1)(x)$ & $14:(11: 1$ & $(1)(x) 2 ?$ & $104(155)$ & $0.12(10)$ & $2 ? 4 \times 1 \times 1$ & U.unii \\
\hline$\because 13 k 201$ & 0.62300 & 144.2553 & (1)(16:7\% & 111 Xfisl & $0.12(1)$ & $2.15,6(0,5)$ & 0.01 .94 \\
\hline 210.50111 & $0.11 \times \leq 1$ & $1+1.101$ & $(1)(x) \leq 1)$ & $114.6129 !$ & $(0.1197$ & $233.11+1$ & $0.080 ?$ \\
\hline 2192361 & $0,118<2$ & 138.8251 & $(i, i n i)()$ & $111.3 \% 1$ & 0.12018 & 230.3 .581 & $0(176.9$ \\
\hline 2219501 & $(10878$ & $135(414)$ & () 10.3 .4 & 120.1 .351 & 0.126 .5 & $227(6,5)$ & 0.0831 \\
\hline $2246 k<1$ & $0.08 \times 103$ & 13.21051 & 010184 & $12: 19 \leq 51$ & 0.1114 & 2249711 & $0 .(x, 0 ; 7$ \\
\hline 227.3781 & $0.08,05$ & $130 \div 31$ & $(1.104 .2$ & $125 \%(15)$ & $0.116 x$ & 2223181 & $0.08+10$ \\
\hline $23(1.1141$ & 0.0018 & 127.5351 & (1)11.45 & $128 \leq 251$ & 0.116 .8 & 219.58 .31 & 0.0818 \\
\hline $23282 \times 1$ & 0.0917 & 1240251 & $(1,111 \div 0$ & 131.3151 & $(1.1118$ & 216.9101 & 0.0875 \\
\hline 2355221 & $(0.194 .42$ & $122 \therefore 34$ & () (1) 5 & 13.4 .3 .51 & 011178 & 214.1441 & $0 .(k) 13$ \\
\hline 2.361591 & 010114 & $114+251$ & 01131 & 120,4051 & (1) 1110.4 & $211.5(1) 1$ & 0.01877 \\
\hline $2108(18)$ & $0.114^{-1} 4$ & $11+7.451$ & (1) 1216 & 1.34 .71151 & $(1,(1) x)_{2}$ & $2(18.8+8)$ & 0.073 .3 \\
\hline 24.5 .5421 & $0(1) 72$ & 11.1 .351 & $1110 \cdot 14$ & 1.12 .5241 & $01(1)+5$ & $2(x,(173)$ & $0.0 \times 30$ \\
\hline$?+16.2161$ & $0.119 \times 5$ & $111.4 \div 1$ & $0: 1.11$ & 145.3351 & 0.11126 & $2(13.4(x))$ & $0 .(1841$ \\
\hline 248.17711 & 0.10 .2 & 10070.59 & $0.116 ?$ & $19 k 15$ & $0.14)$ & $2(k) \cdot(x), 1$ & $0.082 \pi$ \\
\hline $251,6.461$ & (1) $(0,1) 4$ & $1(x, 1,1 \times 1$ & 01140 & 1511151 & $(1.10187$ & $197.95(11$ & 0.18119 \\
\hline $1944(k)$ & $01(x)$ & $1037 y \leq 1$ & $(1.110 .3$ & 1539751 & $0.161 k ?$ & 105.2071 & 0.0815 \\
\hline 257.2 Kkil & (0.:1i: & $l(k) 7 f, 5)$ & (1.i1?? & 156,7651 & $0(18: 20$ & {$[92.58 .1$} & 0.013 .30 \\
\hline $2601.57 !$ & 0.083010 & 97.9851 & 011.19 & 150.5 .751 & 0. r.ws & $189.8(151$ & 0.07 .28 \\
\hline $26 ?(x), 1$ & $U(\mu) .11$ & 95.9751 & $(1,11, \%$ & $10: 4251$ & $11(x) 2)$ & 18701121 & (1). $1 k 71$ \\
\hline $205 \times 21 ?$ & $U(H) Z_{2}$ & 927451 & 0.1202 & $16,5.2151$ & (1) $111: 2$ & 1842051 & 0.0890 \\
\hline $20,7.9775$ & 0.08 .12 & $4(: ; 15)$ & $(1): 18$ & 168.2150 & 0.1122 & $181.6+41$ & $0.11 x+8$ \\
\hline 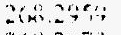 & 6.iongo & 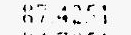 & i.i:i i & "Yi ${ }^{2} \geq$ & 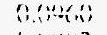 & 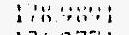 & $6.647 ?$ \\
\hline $206 \pi^{\prime} .20^{-2}$ & 00855 & $x+7851$ & $012: 411$ & $1 i\{1,311 i$ & $(11432$ & 176.2711 & (1) $(k+14$ \\
\hline & & $82.1 \times k)$ & 11119.9 & $\left|\because 6 x^{\prime}\right| x \mid$ & (1)orisit & $173(x, 2)$ & (1). $(k+2+2$ \\
\hline
\end{tabular}




\section{Track 6 - High Pressure Test}

\begin{tabular}{|c|c|c|c|c|c|c|c|}
\hline 170.9501 & $0.07(1)$ & 8.3 .3351 & $0.120 \%$ & \multicolumn{2}{|c|}{ Scan 5. (x).6 psig } & 79.2951 & 0.12 .56 \\
\hline 16202051 & 0.0851 & 86.1751 & 0.1161 & & & 16.39 .1 & 0.1136 \\
\hline $165(6) 51$ & $0.0 \times 6-4$ & 89.0151 & 0.1286 & $268.3(00)$ & $(1.0274$ & 73.7251 & 0.1273 \\
\hline 16.2 .0951 & 0.0775 & 91.44 .51 & 0.1246 & $266(-480)$ & 0.0318 & 70 भisi & 0.1168 \\
\hline$i(x) 2051$ & 0.0872 & 94.80151 & 0.1234 & 26.7 .7713 & 0.03 .56 & 68.0051 & 0.1208 \\
\hline 157.7051 & $0,(x, 3)$ & 97.5651 & 0.1210 & 200.7024 & 0.0479 & 65.2151 & 0.1116 \\
\hline 1550551 & 0.1181 & 100.4151 & 0.1212 & $257.74(1)$ & 0.06 .17 & 62.3951 & 0.1016 \\
\hline 152.4351 & $0.13(x)$ & 10333.51 & $0.12(19)$ & 254.7201 & $0 .(1.1499$ & 59.6051 & 0.0975 \\
\hline 149.7451 & $0(10) \times 8$ & 106.2351 & 0.1178 & 252.0461 & 0.056 .5 & 56.8451 & $0.08: 22$ \\
\hline 147.0151 & (1).(1803 & 1090551 & 0.1008 & 240.2771 & 0.0575 & 54.0151 & 0.0783 \\
\hline 144.3541 & 0.0780 & 111.86551 & 0.11 .54 & $246,56,31$ & 0.0574 & 51.2751 & 0.07 .17 \\
\hline 141.6 .251 & $0 .(x)(x)$ & 114.6 .251 & 0.1180 & 24.3 .8281 & $0 .\left(k+h^{\prime} 4\right.$ & 46.5 .5 .51 & $0.0,16$ \\
\hline 178.8451 & $0 .(x) 41$ & 117.3751 & 0.11 .39 & 241.1141 & $0.06,22$ & 45.7251 & 0.0478 \\
\hline 13590151 & $01(18)$ & 120.1351 & 0.12112 & $2.38 .4+11$ & 0.101 & $42 .(x) 51$ & 0.0 .321 \\
\hline 1320451 & $0.10 \times, 7$ & 122.9551 & 0.11 .37 & 23.5 .60 .51 & 0.0716 & 40.1351 & 0.0254 \\
\hline 1.30 .2751 & $(1)(x)$ & 125.7051 & 0.111 .4 & 233.1141 & $(1)(1) ; 1)$ & 37.2551 & 0.02 .32 \\
\hline 127.5151 & $(1.1170$ & 128.5251 & 0.1112 & $23\left(1.358^{\prime} 1\right.$ & (1.0795 & 34.3551 & 0.0015 .5 \\
\hline 1249211 & 0.113 .3 & 1313151 & 0.16148 & $227(x+5)$ & $11\left(1 k^{\prime}\right) 3$ & 31.4851 & $-(0 .(x) \times 0$ \\
\hline 1222351 & (1) (1) us & 134.1351 & () $(x)+1 ?$ & 2240711 & $(1.1722$ & 28.6451 & .0 .0175 \\
\hline $110+251$ & 0.116 is & $136(x) 5)$ & (1). 118.40 & $2223 \mid 81$ & $(1.118 .14$ & 27.2451 & -0.0176 \\
\hline 1107451 & 0.1226 & 1707651 & (i.f(x)?t. & $210.5 \times 21$ & $(1)(18.34)$ & 24.8 .151 & -0.01171 \\
\hline $11+131$ & U. $I \mid(1)$ & 1425251 & $(1.1 \times 121$ & $216,()\}(11)$ & U. $1 \times 70$ & $320 \times 31$ &.$(1)(x) 09$ \\
\hline 1114451 & (1) 1228 & 145.33451 & $(1)(1) 2(1)$ & $.3 .1+14.11$ & 0.0874 & $35<141$ & $-0 .(x) \geq 0$ \\
\hline $1(18.70 .5)$ & 0.1126 & $\left|4 x^{\prime}\right| 5 \mid$ & 0.17 .45 & 211.5011 & 0.08 .876 & 38.5051 & 0.0142 \\
\hline $1(x, 145 ;$ & (i.|2x| & 1511151 & $11,\left(x_{i}\right) 5$ & $2018 \times+4 \times 1$ & 0.077 .3 & 41.4551 & $0(0) ?(1) 7$ \\
\hline 11133951 & 0.1211 & $15.90^{-5} 51$ & $011+11$ & $2(x, 6) ? .11$ & $0.07(x)$ & 44.3751 & (). (1).194 \\
\hline $1(x), 76<1$ & 0.1222 & $150115 !$ & (i) $111^{5} 2$ & $2(13.4(x))$ & 0.17170 & 47.3951 & $0 . x \times 2\}$ \\
\hline $474 \times 51$ & $(1.125()$ & $154,5.451$ & () (1)ill. & $2(x)(x), 5)$ & 0.16125 & $504+151$ & (). $\{x, 8\}$ \\
\hline 95.3751 & $(1.110 x$ & $|(12)+45|$ & $(1,19)(4)$ & $11) ; 05(1)$ & 0.1824 & 314551 & 110755 \\
\hline 42.7451 & $(1.1254$ & 105.2451 & (1.0) 112 & 1652071 & (1) IIs.t, & Se: $78: 1$ & (1) 1858 \\
\hline $9(1.1151$ & () 1 t & $\mid 682151$ & $0(x)(18$ & 192.5631 & ")in:s & 549.451 & $(1)(x+2)$ \\
\hline 874251 & 0.1123 & 17111521 & $(0.1) \div(1)$ & 1808051 & $(1)(1 k) s$ & $0: 4 \geq 51$ & $(1)(x) 5 x$ \\
\hline$x+7891$ & 0.1103 & $173(1) 3(1)$ & $(1)(1)+1)$ & $1 \times 7.1121$ & (1) (nit.) & ถ⿻ 4551 & $(1) 106.5$ \\
\hline $82(x) ; 1)$ & 0.1158 & 176.8081 & $0,(k, i)()$ & $18.1206]$ & $0(14)$, & ox xitis & $(1) 11 k$ \\
\hline 742451 & 0.1194 & 17470101 & () (1): it & $1 \div 1(1.4 .11$ & $(0.08 .6$ & $71 \times 1151$ & 01253 \\
\hline 70.2051 & 0.1189 & $18: 2 x^{\prime} ! 1$ & $\left.0.11^{-1}\right) ! 1$ & lis 4 nith & (1)(1) 17 & $74+451$ & (i.1.537 \\
\hline 737251 & (1.1274 & $18 \leq 54(11$ & G(1):? & 176.2771 & $0(x, 5)$ & 77.5 .451 & $0 .(1) 7(1) .4$ \\
\hline $70.6 \times 151$ & () $101 / 4$ & $|\sin 374|$ & $0(1711 \%$ & $1736 x_{2}=1$ & $\{(x, n)$ & $811+251$ & 0.12114 \\
\hline $6 K^{\prime}(x): 1$ & () $f(k) \varphi$ & 191.2451 & $0(15 k)$ & $17 ! 104011$ & $0.076)_{2}$ & $8: 1351$ & 0.1 .351 \\
\hline$(1.5 .2151$ & 0 litl & 194.1341 & $0\langle x, \delta\rangle$ & $\mid 08.2051$ & vonis & $k 0.1751$ & (i. 1240 \\
\hline 623451 & 0.119 .36 & 190.8491 & $0,(x) i f(1)$ & 16.5 .60051 & 011872 & wo 11151 & 01452 \\
\hline $59 .(x) 51$ & (1) $(1 \times 1) 3$ & $197.86,4)$ & $0,(x, 1 ?$ & $\mid 6.9495]$ & $(1)(x) x^{3}$ & 91.0451 & 1). 1910 \\
\hline $50 \times 45 !$ & $0 .(17 t, 7$ & $2(1) 8(20)$ & $(3,(x, 1), 5$ & $10,0.2451$ & $0\left(M^{\urcorner}\right)$ & 94.80 .51 & 0.1 .47 \\
\hline 54.11151 & $0 .(x, 0) 1$ & $2015 .(x, 5)$ & $0 .(x, 4)$ & $157.7(15)$ & $0(14) 2$ & 07.56 .51 & 0.1 .220 \\
\hline 51.2751 & $0,0)(-13$ & $2(2 x \cdot 1201$ & $0 .(15.50)$ & 155.01551 & $0.0(x)=$ & $1(x)+4.51$ & 0.1134 \\
\hline 485451 & $0.0156,4$ & 211.1741 & $0 .(x, 1)$ & $152+351$ & 01150 & 1114.2341 & 0.1320 \\
\hline 15.7251 & $0(1.10)$ & 21.7 .8 .81 & 0.06 .8 & 149,7451 & 0.11157 & 116.2351 & 0.1311 \\
\hline $12 \%(5)$ & 061201 & $216.5(111$ & $0 .(x, i)$ & $197(1191$ & 0.101 .5 & 109.0551 & $0.13(14)$ \\
\hline 40.1351 & $0 .(126.4$ & $2 \mid 9, ?\{6 \mid$ & $0.17(14)$ & $1+4.3551$ & (1) 10.1 & 111.8601 & 0.13 .32 \\
\hline 37.2551 & 0.11210 & $221.95(11$ & $0 .(1 ; 0) 3$ & $1+41,0,251$ & 010112 & 114.651 & 0.1324 \\
\hline 34.3551 & 0.01115 & $22+6 x^{2}=1$ & 0.1724 & $13 k .8 .451$ & 0.11112 & 117.3751 & 0.1275 \\
\hline $3 i .90<1$ & $-1)(x), 5$ & 227.3781 & $0, x_{10}+2$ & 1359051 & 01193 & $120135 !$ & 0.1310 \\
\hline 26.6451 & -0.0168 & 2301.11 .11 & 0.07 .4 & 132.7451 & 0.1178 & 1224551 & (1).1235 \\
\hline 27.2951 & -0.01177 & $29=026 !$ & (1) (xiy) & 130.2751 & 111170 & 125.7051 & (1).1239 \\
\hline 24.8151 & 0.0107 & 214 $322 !$ & 0.01014 & $12 \% \leqslant 351$ & 0.126 .5 & 128.5251 & 0.1240 \\
\hline 32.6851 & $.0 .0 \times 153$ & 238.1551 & 00.5 .94 & $12: 1225 i$ & 0.1242 & 131.1151 & $0.11 \% 4$ \\
\hline 3.5 .57 .51 & $0.0(x) 1.4$ & $24(1.8(18)$ & 0.0736 & 122.2351 & 0.1619 & 134.1 .351 & $0.1(x) y$ \\
\hline 36.5051 & $0.02(x)$ & $24.35+21$ & 0.0743 & $11^{0}+3.51$ & 0.1255 & $1360(151$ & $0.1(k) k$ \\
\hline 41.4581 & $0.024 n$ & 240.2101 & 0.0777 & 110.74 .51 & 01.355 & 130.7051 & u.iw2 \\
\hline 44.3751 & 0.01323 & 248.0711 & 0.0742 & 114.1 .351 & (1) 1235 & 142.5251 & $0.0 \% 8$ \\
\hline 473951 & $0(k, 3)$ & 25160401 & $0 .(1750)$ & $111+451$ & 0.1276 & 145.3351 & $0 .(k) \times) 1$ \\
\hline 50.4151 & $0 .(1+K) 2$ & $254+4 k ! !$ & 110779 & 168.76 .51 & 0.1343 & 148.1551 & 0.0442 \\
\hline 5.14551 & $(1.0171) 4$ & $257.2(x) 1$ & $(1.07(1)$ & $11 \times, 1151$ & 0113.40 & 151.1151 & $0.11 \times 4$ \\
\hline$\{6.785\}$ & $(1.6744$ & $260(1.157 !$ & $0.15 ? 1$ & 103.3951 & 0.1 .353 & 153.0751 & 0111111 \\
\hline 540.551 & 0.0 sint & $263(x) 81$ & 0.0540 & $1(0), 76,51$ & $0.136 \mathrm{mt}$ & 156.7051 & 0.166 .38 \\
\hline 624251 & (). $(k x+k)$ & $26,5.83 .47$ & $0 .(15118$ & 979851 & 0.1 .320 & 159.5451 & $0.0 \times 47$ \\
\hline 659551 & $0.1(x)$ & 261.077 .5 & $(1)(1+1)$ & 95.2751 & (1.1.3\%2 & 162,4351 & 0.01801 .4 \\
\hline 68.8451 & $11) 1125$ & 268.2050 & $0 .(4 i)$ & 92.7451 & 0.1413 & 165.2951 & $0.0 \times 05$ \\
\hline$? 18(19)$ & (1.1130 & $2618 . ? 6: 3$ & $(1 .(4.3)$ & (x) 11.51 & 0.13118 & $10 k .2151$ & $U(\mu) 7)$ \\
\hline 74.451 & 0.1572 & 268.2456 & (). (H) & 87.4251 & 0.1247 & $171.115 ? \mid$ & 0.0765 \\
\hline $7 \% .451$ & 00480 & & & 84.78 .51 & $0.131(1)$ & $113.93(11$ & (1) 1186.1 \\
\hline kii.4251 & (1) 1114 & & & $k ?\{x, 5\}$ & 0.1240 & 176.8081 & () $1176(1)$ \\
\hline
\end{tabular}


Track 6 - High Pressure Test

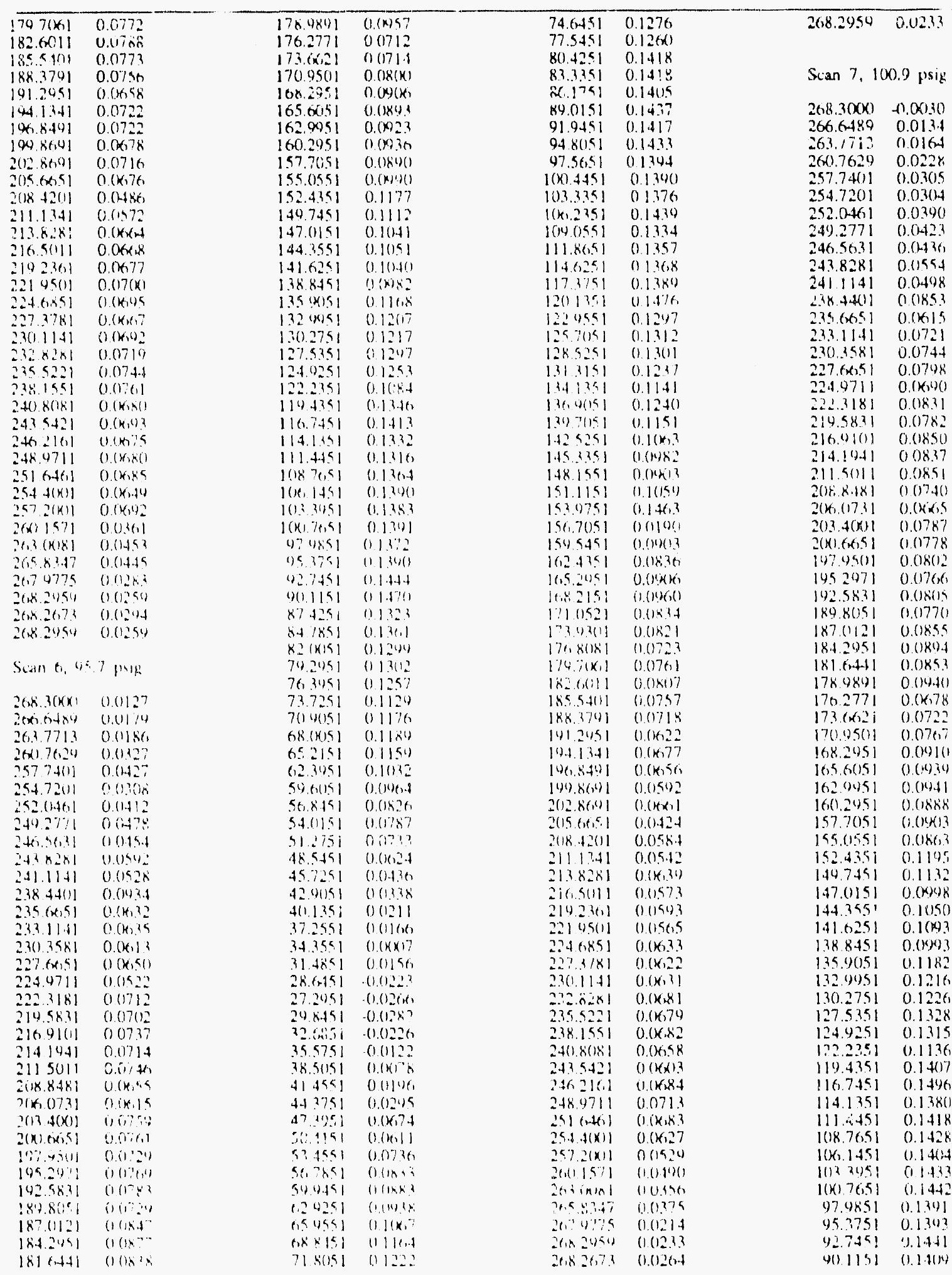




\section{Reinforced Concrete Test Data}

Track 6 - High Pressure Test

\begin{tabular}{|c|c|c|c|c|c|c|c|}
\hline 87.4251 & 0.1396 & 171.01521 & 0.018012 & 180.8051 & 0.08 .31 & 62.9251 & 0.1032 \\
\hline 84.78 .51 & $0.1+38$ & $173.93(11$ & $0.08 \times 47$ & 187.0121 & $0 .(H)(1) 2$ & 65.9551 & 0.1182 \\
\hline $82 .(10151$ & 0.136 .5 & 176.8081 & 0.0758 & 184.2451 & 0.00093 & 68.4451 & 0.1277 \\
\hline $74.295 i$ & 0.1341 & $179.7(661$ & 0.0774 & $181.64+1$ & $0 .(10) 21$ & 71.8051 & 0.134 .5 \\
\hline 76.3951 & 0.1216 & 182.6011 & $0.0 \times 17$ & 178.9801 & 0.1024 & 74.6451 & 0.1397 \\
\hline 73.7251 & 0.1256 & 185.5401 & 0.0767 & 176.2771 & 0.01727 & 77.5451 & 0.1053 \\
\hline 70.0051 & 0.1262 & 188.3791 & $0 .(x, 1)(1)$ & $173 .(x+2) 1$ & 0.0740 & 80.4251 & 0.1669 \\
\hline $68 .(x) 51$ & 0.12 .19 & 191.2051 & $0,(x, 1)$ & $170.95(11$ & 0.0870 & 83.3351 & 0.1549 \\
\hline 65.21 .51 & 0.1187 & 194.1341 & 0.07 .3 .3 & 168.2451 & 0.0888 & 86.1751 & 0.15 .34 \\
\hline 62.3951 & 0.1089 & 196.8401 & o.ixis? & 10.560151 & $0 .(194)$ & 89.0151 & 0.1649 \\
\hline $59 .(x) 51$ & 0.112 .4 & $109.86,91$ & $(1)(x, 2)$ & 162.4451 & 0.095 .5 & 91.9453 & 0.1615 \\
\hline 56.8 .451 & 0.08 .35 & 202.86901 & $0 .(x, 5)$ & 160.2951 & $0.10 \times 4$ & 94.8051 & 0.1608 \\
\hline 54.0151 & 0.0817 & $205(x, 5)$ & 0.0157 .3 & 157.7051 & $0.11 \times 5$ & $97.565 i$ & 0.1567 \\
\hline 51.2751 & 0.0773 & 208.4201 & 0.0413 & 155.0551 & 0.0939 & 100.4451 & 0.1570 \\
\hline $4 \times .745$ ! & 0.06 .29 & 211.1341 & 0.053 .5 & 152.4351 & 0.1311 & 103.3351 & $0.15(x)$ \\
\hline 4.5 .7251 & $0.04: x$ & 213,8281 & $0,(x, 1+1$ & 149.7451 & 0.1221 & 106.2351 & 0.1618 \\
\hline 42.9051 & $0.02 \times 5$ & Ziúson!! & 0.0948 & 147.11151 & (1) 1150 & $1,00.0551$ & 0.1522 \\
\hline $4(1.1 .351$ & $0.01 \%$ & 214.2361 & $(1.051) 8$ & $1+4.3551$ & 0.11 .50 & 111.8651 & 0.1540 \\
\hline .37 .2551 & 0.011 .48 & $221.95(11$ & $(1)(x, 1.5$ & 1410251 & $\because 1180$ & 114.6251 & 0.1528 \\
\hline 34.3551 & $-0 .(x)_{2}^{2}-1$ & 224.68 .51 & $0 .\left(x_{1}\right) 4$ & 138.8451 & 0.1148 & 117.3751 & 0.1517 \\
\hline 31.4851 & -0.0188 & $22 \% .3781$ & $0 .(x) 14$ & 135.4051 & 0.131 .4 & 120.1341 & 0.16 .12 \\
\hline $28 .(145)$ & .0 .0267 & $23(1.11+1$ & $0 .(x+1.5$ & $1.82(3051$ & 0.13411 & 122.9551 & 0.1446 \\
\hline 27.2051 & $-0.02 \times .5$ & 2328281 & $(1)(x, 20)$ & 1.012751 & 0.13 .38 & $125.7(151$ & 0.1 .462 \\
\hline 29.8451 & 0.0257 & 2.15 .5221 & () $(x, 78$ & 127.5351 & 0.14 .22 & 128.57 .51 & $0.14+11$ \\
\hline 726851 & 0.01171 & $23 \times .1551$ & $0 .(x, 4)$ & 124.9251 & 0.1 .110 & 1.11 .3151 & 0.1 .374 \\
\hline 35.5751 & (i) (x) & $27(1.8(18)$ & $0 .(15)(1)$ & 122.2351 & 0.1296 & 134.1351 & (1.125. \\
\hline 38.50551 & $0 .(x(x))$ & 2475421 & $0.0506)$ & 119.4 .351 & 0.151 .3 & 1.36 .9051 & 0.1448 \\
\hline 41.45 .51 & (i) (1) 18 & $24(1,2161$ & $(105 \div 4$ & 110.1 .851 & 0.1 .748 & 130.7051 & $(1.1202$ \\
\hline 44.3751 & (1).1134) & $24 x^{\circ} 0711$ & () $(k, 1)$ & 11.41 .351 & 0140.4 & 142.5251 & $0.11 \mathrm{ke}$ \\
\hline 47.3051 & 0.0725 & $251 .(2+0)$ & $(1.1570$ & 111.4451 & $0.19(x)$ & $1,45,3,1,51$ & 0.1174 \\
\hline 50.41 .51 & (1) $(x, y)$ ? & $25.4 .41(1)$ & 0.15018 & $1(18: 0,51$ & $(1.15 \% 2$ & 1.48 .1551 & (1. $116(1)$ \\
\hline 534551 & 0.1751 & $257.2(1111$ & $(1015861)$ & $10,1.451$ & 0.15 .97 & 151.11 .51 & 0.1231 \\
\hline 547851 & $0(1670)$ & $2 f(0,1571$ & (1.01687 & 1013.3051 & $(11544$ & 15.3 .9751 & 0.1 .8 .47 \\
\hline 50.4 .51 & $0 .(\mathrm{M}) \mathrm{B})$ & $26,3(x)(8)$ & $(1,1) \geq 0$ & $1(k) .7(1.5)$ & 0.1571 & 156.70 .51 & 0.0577 \\
\hline $624+41$ & (1). 1050 & 2658.747 & $(0.01311)$ & 47.4851 & () $\left.1.5 t_{1}\right)$ & 1.54 .54 .51 & $0.1(x) .4$ \\
\hline 650551 & 0.1182 & $26 ? .1775$ & (1) (i) 14$)$ & 45,3751 & $0.157 \%$ & $1(1,243.51$ & $0 .(19) .27$ \\
\hline 68.8451 & (1).12(18 & $2\left(1 x^{2} .954\right)$ & $(1.0113$ & 92.7451 & $0.15(, 0)$ & 16.5 .2051 & 01091 \\
\hline 71.8051 & 0.13113 & 26826,73 & 0.011 .16 & $(x) .1151$ & $0.15 k 8$ & $\mid 0,8.2151$ & $0.1(128$ \\
\hline 74.6451 & $0.17(1)$ & $2(18.2054$ & $0 .(111.4$ & 87.4251 & $(0.151) 2$ & 171.0521 & 0.0182 .5 \\
\hline 77.5451 & -0.01437 & & & K. 7591 & 0.16 .48 & $17.3 .93(11$ & 0.0812 \\
\hline 80.4251 & 0.1 .302 & & & $82 .(5) .5)$ & 0.1542 & 176.8081 & $0.077+4$ \\
\hline 8.3 .3 .351 & (1.1.128 & $S(a), x, 10$ & B..3 poin & 74.215 .51 & (1) $15+1$ & $170.70 k, 1$ & 0.0708 \\
\hline 86.1751 & 0.1410 & & & 70.3051 & (1) 1203 & $182.6(1) 1]$ & $0.0187 \times$ \\
\hline 89.0151 & 0.158 .5 & $20,8.3(x)(x)$ & $0(x) 1) ?$ & 73.7251 & 0.13 .44 & $18.5 .5+(1) 1$ & 0.0775 \\
\hline 91.0451 & 0.1474 & Itete $\operatorname{tat} x y$ & $(1)(x) \times 5$ & $70.4(151$ & 0.14 .4 & $188 . .1701$ & 0.0713 \\
\hline $17.4 .8(1.51$ & 0.1 .949 & 20.3 .771 .3 & (1.)(x)7? & $6 x(x) 51$ & $01) 3(k)$ & 101.2051 & $0 .(k x) 1$ \\
\hline 07.50 .51 & 0.14 .3 & $2611.7(126)$ & (). () $16, B^{\prime}$ & 0.52151 & $(1.12 i)$ & $14+13+1$ & $0 .\left(x_{1}()_{2}\right)$ \\
\hline $1(x)+451$ & 0.14 .41 & $257.7 .4(11$ & $0(1) 14$. & $(12.3451$ & 0.1117 & 146.84131 & $0.160+5$ \\
\hline 1013.93 .51 & 0.1414 & 25.472011 & $0(12112$ & $51) .6(151$ & $(111140$ & 199.8041 & $0.0590 ?$ \\
\hline $1(k, 2351$ & 0.1 .1 .13 & $252(1+46)$ & 0.013101 & $50.8+51$ & $0.118(x)$ & $202.86,91$ & 0.0592 \\
\hline $1(x), 0551$ & $0.14 t x)$ & 249.2771 & 0.14015 & $54 .(1) 51$ & 0.01815 & 20.5 .6651 & 0.0538 \\
\hline 111.8651 & $0.1+x)$ & 246.5631 & $0 .(40) ?$ & 51.2751 & 0.07775 & $208 .+201$ & $0.0 .40(0)$ \\
\hline 114.6251 & $0.1+56$ & 213.8281 & 0.05 .37 & 48.54 .51 & (1). 158.5 & 211.1341 & 0.0522 \\
\hline 117.3751 & 0.1410 & 241.1141 & $0 .(14 \times 77$ & 4.5 .7251 & 0.0 .398 & 213.8281 & 0.0591 \\
\hline 120.1351 & $0.14 .5 x$ & $2.38 .44(1)$ & $0 .(K) 38$ & 420051 & $0.026 x$ & 216.50111 & 0.0585 \\
\hline 122.9551 & $0.13: \times 1$ & $2,35 .(x, 5)$ & 0.07 .56 & 40.1 .351 & 0.0181 & 219.2361 & 0.0599 \\
\hline 125.7051 & 0.1342 & 233.1141 & 0.018 .40 & 37.2551 & $0 .(x) 86$ & 221.9501 & 0.0510 \\
\hline 128.5251 & 0.136 .3 & $230.358 !$ & 0.0798 & 34.3551 & $-0 .(x) .56$ & 234.6851 & 0.0574 \\
\hline 131.3151 & 0.1310 & $227 .(x .5)$ & $0.0 \times 36$ & 31.4851 & $-0(1272$ & $2 ? 7.3781$ & $0.0 .5 \times 6$ \\
\hline 1.34 .1 .351 & 0.1246 & 224.9711 & 0.01737 & 28.6 .451 & $-0.0 .+(0) 1$ & 2.50 .1141 & 0.060 .5 \\
\hline 1.360051 & (1) 1162 & 222.3181 & $(1)(x)(k)$ & 27.2951 & $0.0+10$ & 232.8281 & 0.0593 \\
\hline 139.7051 & 0.11 .55 & 214.5831 & $0,018,7$ & $? 4.84 .51$ & -0.0 .342 & 235.5221 & $0 .(16+4$ \\
\hline 1.12 .5251 & 018170 & 210.9101 & (i) (k)ifif & $32 \cos 51$ & $-0.11 \geq 71$ & $238155 !$ & $0.0 x+12$ \\
\hline 145.1 .51 & 0.1078 & 214.1941 & $0(x) \mid ?$ & .35 .5751 & -(1)1)KS & 240.8081 & 0.0526 \\
\hline 148.1551 & 0.10 .41 & $2115(111$ & $(1 .(x) 50$ & 38.5051 & $0 .(x) 2 y$ & 243.5421 & 0.01522 \\
\hline 1511151 & 0.1127 & $2\left(1 x^{\prime} x+x\right)$ & $(1)(8,17$ & $41+5.51$ & 0.0116 .8 & 246.2161 & 0.0535 \\
\hline 1534751 & 0.1210 & $2(10.0171)$ & $0,(x, 1]$ & 4.4 .3751 & ().11:8.5 & 2.48 .9711 & 0.0159 .3 \\
\hline $1.56 .7(1.5)$ & $0,(x,+x)$ & $2(13.4 \times 1)$ & $(0.0770$ & 47.3451 & $0 .(17.30)$ & $251.6+(1]$ & $0 .(14) 8$ \\
\hline 159.54 .51 & $(1)(x) 50)$ & $2(x)(x, 5)$ & $0 .(1749$ & 50.4151 & $0.06,53$ & $254.4(x) 1$ & $0.1+46 x$ \\
\hline 102.43 .51 & 0.0893 & 197.95118 & $0 .(1825$ & 53.4551 & $(101) 10$ & $257.2(x) 1$ & 0.01418 \\
\hline 165.2951 & 0.1450 & $195.20 \% 1$ & $0.118+48$ & 56.78 .5 & ómis & $2(x) .1571$ & 0.010 .5 \\
\hline 16022151 & 0.0963 & 192.5811 & 0.01807 & 54.9451 & 0.0979 & $263.0016:$ & 0.0167 \\
\hline
\end{tabular}


Track 6 - High Pressure Test

\begin{tabular}{|c|c|c|c|c|c|c|c|}
\hline 265.83 .47 & $0.01(x)$ & 97.9851 & $0.16+45$ & 159.5451 & 0.1121 & $2(x),(x, 5)$ & 0.0818 \\
\hline 267.9775 & 0.0052 & 95.3751 & 0.168. & 162.4 .351 & 0.1075 & $147.195(11$ & 0.0876 \\
\hline 268.2959 & 0.0049 & 92.7451 & 0.1641 & 16.5 .2451 & 0.1119 & 195.2071 & 0.0954 \\
\hline 268.267 .3 & 0.00182 & 90.1151 & 0.1756 & 168.2151 & 0.1151 & 102.5831 & 0.1012 \\
\hline \multirow[t]{3}{*}{268.2959} & 0.0049 & 87.4251 & 0.1773 & 171.0521 & $0.14) 86$ & 184.8051 & $0.0 \times 980$ \\
\hline & & 84.7851 & 0.1787 & $173.93(1) !$ & 0.00 .17 & 187.0121 & 0.1045 \\
\hline & & $82 .(x) 51$ & 0.1682 & 176.8081 & 0.0861 & 184.2951 & $0.1(x) 7$ \\
\hline \multirow{2}{*}{\multicolumn{2}{|c|}{ Scan 9.11.3.2 pisig }} & 79.2951 & 0.1679 & $179.7(x) 1$ & $0.087(1$ & $181.64+1$ & $0.11(x)$ \\
\hline & & 76.3951 & 0.158 .5 & 182.6011 & 0.0010 & 178.9891 & $0.1(x, 4)$ \\
\hline 268.30000 & -0.0139 & 73.7251 & 0.1594 & 185.54011 & 0.0811 & 176.2771 & 0.0896 \\
\hline 2666.6480 & -0.002 .1 & 70.90151 & 0.1407 & 188.3791 & 0.0724 & 173.66521 & 0.0971 \\
\hline 26.3 .7713 & 0.00 .39 & 68.0051 & 0.13189 & 191.2951 & 0.072 .3 & $17(0.45(1)$ & $0.110 \%$ \\
\hline $2(x) .7629$ & 0.0244 & 65.2151 & 0.1275 & 194.1 .341 & 0.0777 & 16.8 .2451 & 0.1223 \\
\hline $257.74(11$ & 0.0234 & 62.3951 & 0.11 .55 & 196.8441 & 0.0721 & $16.5 .6 \times 1.51$ & $0.129(1$ \\
\hline 254.7201 & 0.02 .3 .3 & 54.6051 & $0.11 x i 5$ & 190.8601 & $0 .(K) 2(1)$ & 162.9951 & 0.128 .3 \\
\hline 252.04611 & 0.0308 & $56.8+51$ & $0 .(1452$ & $2(1) .86(1) 1$ & $0(x, 74$ & $|(n) .245|$ & 0.1 .37 .3 \\
\hline 249.2771 & $0.0 .278^{\circ}$ & 54.0151 & (1.)(18.54) & $2015.6(15)$ & 0.053 .32 & $157.7(151$ & $0.1(46$ \\
\hline 246.56 .31 & $0.038, \mathrm{~K}$ & 51.2751 & $0 .(17) 7$ & $208.42(11$ & $0.015(1)$ & 155.0551 & 0.1422 \\
\hline 24.82881 & 0.0529 & $48.545 !$ & $0 .(1)\left(x_{1}()\right.$ & 211.1 .341 & $0 .(1502$ & $152+3.51$ & 0.1672 \\
\hline 241.1141 & $0 .(1)+40)$ & .15 .7251 & $0.01 .6 \mathrm{k})$ & 213.8281 & $0,(x \times 1)$ & 147.7451 & 0.159() \\
\hline $2.38 .44(11$ & $0 .(6)-48$ & $42.3(151$ & $0 .(1) \times 4$ & 216.5011 & $0(16,28)$ & 147.0151 & 0.1 .501 \\
\hline 23.5 aci.51 & $007(x)$ & 40.13 .51 & (1).(1).5() & 219.2361 & $0 .(K, 3()$ & 144.3551 & 0.1428 \\
\hline 233.1141 & 0.0871 & 37.2551 & $0 .(k 1.44)$ & $221.45(11$ & $\left(1 .\left(6,78^{\prime}\right.\right.$ & 141.6251 & 0.14 .56 \\
\hline $23(1.3581$ & 0.08 .35 & .3 .4 .35 .51 & $.0 .(1)+46$ & $22+.6851$ & 0.0 .10 & $1.38 . x+51$ & 0.1474 \\
\hline 227.6051 & (0.08' & 31.4851 & -0.1258 & 227.3781 & $(1 .(15 \times 6)$ & $1.35 .0(551$ & $0.16,5.5$ \\
\hline 224.4111 & 0.07 Wh & 28.6451 & -0.0136 .5 & 230.11 .41 & 0.058 .3 & 1.32 .4951 & 0.166 .5 \\
\hline 222.3181 & $0.09+6$ & 27.2951 & $0 .(1.4(12)$ & 2328281 & 0.0512 .1 & 130.2751 & 0.16194 \\
\hline 219.5831 & $0.196(1)$ & $29 x+51$ & 0.618 .1 & 23.5 .5221 & $0.0 K_{1} \geq 4$ & 127.5351 & 0.1781 \\
\hline 210.4101 & 0.0952 & 326.851 & $-0 .(1.20 .1$ & 2.38 .1551 & $0.0 \times 1.54$ & 124.9251 & 01773 \\
\hline 214.1941 & $0.09(18$ & 35551 &.$(101180)$ & 240.81101 & 0.1574 & 122.2351 & 0.10 .51 \\
\hline $211.5(1) 11$ & $0.096,1$ & $38.5(15)$ & $01.0(1) 2$ & 241.5421 & 0.05 .40 & 119.43 .51 & $0.18+8$ \\
\hline $2(0) \times+(k)$ & (1) & 41.4551 & 190112 & 240.2161 & 0.0157 .3 & 116.74 .1 & 0.190 \\
\hline $2(x,() 731$ & 0.0787 & 443751 & 110.117 & 248.4711 & $(0 .(1.41)(1)$ & 114.13 .51 & 0.1807 \\
\hline $2019.4(x) 1$ & 0.01770 & .97 .31951 & ()elents & $25 ! 6(1+61)$ & 0.114 .35 & $111.4+51$ & 0.18 .98 \\
\hline $2(x) .6(1,5)$ & 0.01875 & $5(1.4151$ & (j.ikntu & $25.4 .4(x) 1)$ & (). $(1.41 .4$ & $10 \times .76 .51$ & $0.18(1,2)$ \\
\hline$\lfloor 47.95(1)$ & $0,(14,41$ & $5.3+4<1$ & $(1.17 \%)$ & $257.2(x) 1$ & (0.0.1.42 & $\mid(k, 145 \mid$ & 0.1863 \\
\hline 195.2971 & 0,18179 & $50,785)$ & 0.11174 & $26(1.15171$ & (1). ()1(1).3 & 1013,3951 & 0.1881 \\
\hline $192.5 \times 31$ & $0 .(1070$ & 54.4 .451 & $0.110 \% 0$ & $26,3,(x) k 1$ & 0.016 .5 & $1(x) .76 .51$ & 0.1646 \\
\hline $189.8(151$ & 1).(19)2? & $62.4 \geq 51$ & 0.1110 & 26.5 .8 .3 .47 & 0.0112 & 070851 & 0.1881 \\
\hline 187.0121 & 19.11112 & 65.4551 & $(1.121,8$ & 267.0775 & 0.00004 & 45.7751 & 0.1875 \\
\hline 184.2451 & 0.1045 & 68 8.451 & $0.1 .31 ;$ & 268.2950 & $0.0(x) 77$ & 02.7451 & $0.186,8$ \\
\hline $181.61+1$ & $0.111+0$ & 71.8051 & 01374 & 26.8 .2673 & $0.0(1.39)$ & 90.1151 & 0.1923 \\
\hline 178.4891 & $0.1(150)$ & 746451 & 0.1752 & $268.2451)$ & $0.06 \times 17$ & 87.4251 & 0.1846 \\
\hline 176.2771 & $(1.0870)$ & 77.5451 & $(10.819$ & & & 8478.51 & 0.1010 \\
\hline $173 .(x+21$ & $0.177+1$ & $8(0,4251$ & $0.1117 ?$ & & & $82(x) 51$ & (0.1817 \\
\hline $170.45(1)$ & 0.0878 & 83.3351 & $0.16+6$ & Sran 10 , & 117.8 psig & 79.2951 & 0.1859 \\
\hline 168.2451 & $0.1(x)$ & 86.1751 & 0.163 .4 & & & 76,7951 & 0.1548 \\
\hline 10.5 .60151 & 0.1047 & 89.0151 & 0.17 .54 & $2688.3(x)(1)$ & $-(1.01 / 6$ & 7.3 .7251 & 0.1676 \\
\hline 162.0451 & 0.1021 & 91.04 .51 & 0.16 .50 & zenting & - (1). $(1) 47$ & $70.9 \times 151$ & 0.1507 \\
\hline 1600.2051 & 0.1131 & $94.8(15)$ & $(0.16,50)$ & $\therefore(1,3,771.3$ & $0 .(x) .35$ & $68 .(x) 51$ & $0.1488^{\circ}$ \\
\hline $157.7(151$ & $0 .(17(x)$ & 97.56 .51 & 0.1058 & $2610.76 .29)$ & 0.0257 & 6.52151 & 0.1388 \\
\hline $155.0 \leq 51$ & $0.13(4)$ & $100.445 !$ & (i.16.8.4 & $257.74(1)$ & $0.02(13.3$ & 62.3951 & $0.124 ?$ \\
\hline 152.4351 & 0.148 .3 & 103.4351 & $(1,16,30)$ & 254.2011 & 0.0218 & $54 .(x) 51$ & 0.1178 \\
\hline 1.14 .1751 & 0.1 .1 .22 & $10 x, 2351$ & $0.10,18$ & $252 .(1+6+1$ & 0.0280 & 50.0 .451 & $0 .(x) 89$ \\
\hline 147.01 .51 & 0.1311 .5 & 109.05 .51 & 0.10 .70 & 249.2771 & 0.041 .3 & 54.111 .51 & 0.0867 \\
\hline 144.35951 & $0.12+1$ & 111.86 .51 & $0.160(1$ & 246.56 .31 & $0 .(1428$ & 51.2751 & 0.08100 \\
\hline 1410.251 & 0.1290 & 114.6251 & $0.16,80$ & 24.3 .8281 & 0.0556 & 48.5451 & $0 .(x .39$ \\
\hline $138.8+51$ & $0.13(x)$ & 117.3751 & 0.16 .51 & 2.11 .1141 & 0.0522 & 45.7251 & 0.0 tiny \\
\hline 135.9051 & $0.15(11$ & $12(1.1351$ & 0.1717 & $2.38 .44(11$ & $0.1(x)$ & 42.40151 & 0.0255 \\
\hline 132.9451 & $0.15(1) 2$ & 1220551 & (1.158\% & 2.35 .66 .51 & 0.08 .2 .3 & 40.1351 & 0.0150 \\
\hline 130.2751 & 0.1518 & $125.7(151$ & $0.150,3$ & 233.1141 & 0.11856 & 37.2551 & $0 .(x+4 y$ \\
\hline 127.5351 & $0.16\left(x_{1}\right)$ & $128<251$ & 0.1584 & 230.7581 & 0.088 .5 & 34.7551 & -0.017 .1 \\
\hline 12.4 .9251 & 0.1577 & 131.3151 & 0.15 .17 & 227.66 .51 & 0.09 .30 & 31.4851 & -0.0 .325 \\
\hline 122.2351 & 0.1397 & 1.34 .1 .351 & 0.1301 & 224.9711 & 0.0804 & 28.6451 & $-0 .(142)$ \\
\hline 119.4 .351 & 0.16 .22 & 136.6051 & 0.1475 & 222.3181 & 0.10 .31 & 27.2951 & -0.0527 \\
\hline 116.7 .4 .51 & 0.1648 & 139.70151 & 01718 & 210.58 .31 & 0.0906 & 29.8451 & $0 .(1+27$ \\
\hline 114.1351 & 0.1631 & 142.5251 & $0.13 \% 2$ & $2 ! 6,(2)(1)$ & 0.16157 & 326851 & $(0 .(1) 3+3$ \\
\hline 111.4451 & 0.16 .32 & 145,3351 & 0.1251 & $214.14+1$ & 0.1140 & 3.5 .575 .51 &.$(1,1221$ \\
\hline 108.765$)$ & 0.1718 & 148.1551 & 0.1171 & $211.5(0) 1$ & $0.1(x, 6)$ & $.38<9(151$ & $(0 .(x) 14$ \\
\hline 106.1451 & 0.1684 & 151.1151 & 0.1 .312 & $2(18.8+81$ & 0.0972 & 41.4551 & (1).(1) 10 \\
\hline 10.4 14:1 & (1.) 1501 & 153.9751 & $0.160 ?$ & 206.07 .31 & $0 .(18)(1)$ & 14.1751 & 0.0124 \\
\hline 100.76 .51 & (1.16.58 & $15677(151$ & 0.046 .7 & $20.3 .4(x)]$ & 0.urit & $47.36,51$ & $0.0 x, 21$ \\
\hline
\end{tabular}




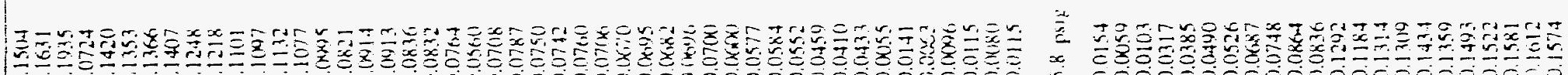

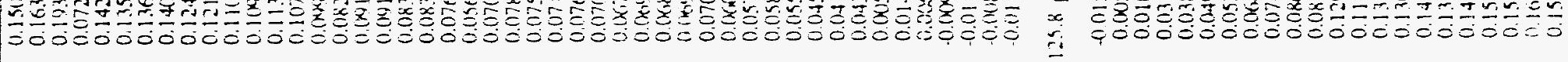

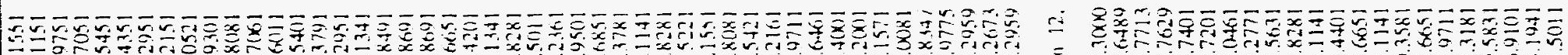

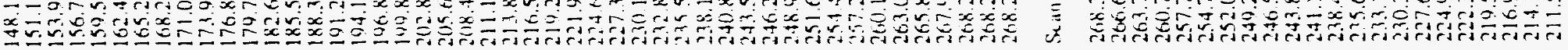

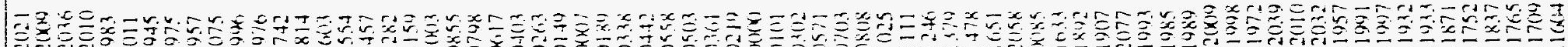

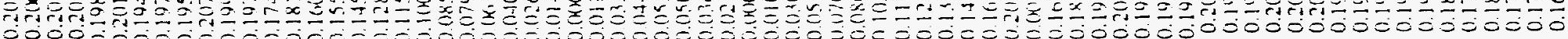

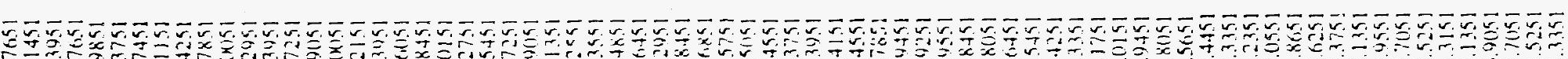

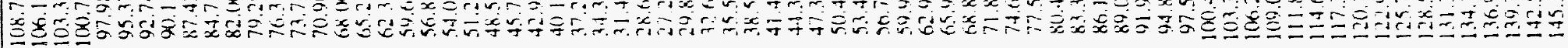

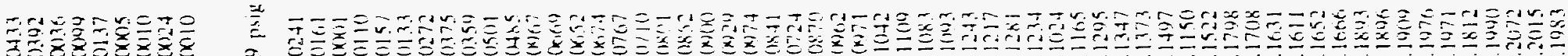

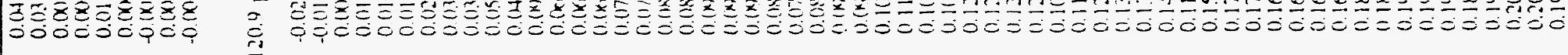

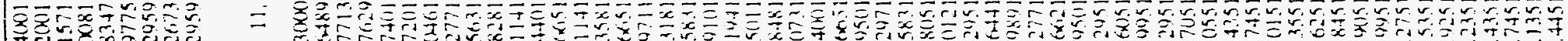

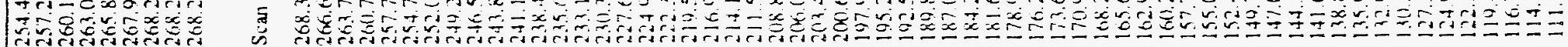

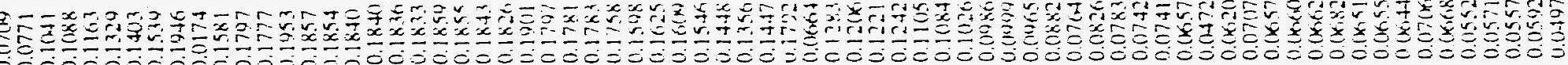

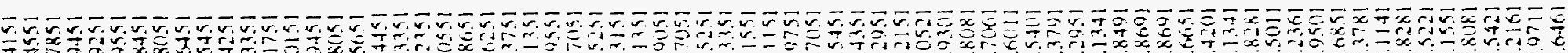

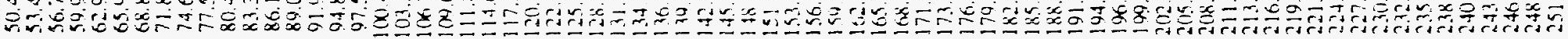

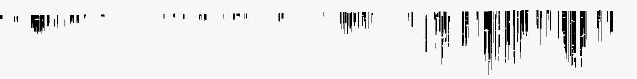




\section{Reinforced Concrete Test Data}

Track 6 - High Pressure Test

\begin{tabular}{|c|c|c|c|c|c|c|c|}
\hline $2(18 \times x+8)$ & $0.1+112$ & 41.4551 & (1). 11$)(12$ & 246.2161 & $0.0 \times 11$ & 110.7451 & 0.1892 \\
\hline $2(x .07 .31$ & 0.14 .51 & 44.3751 & 0.0258 & 248.9711 & 0.08 .30 & 114.1 .351 & 0.377 .3 \\
\hline $203,4(x) 1)$ & $0.15(1)$ & 47.3051 & 0050.3 & $251.6+611$ & $0.076(1)$ & 111.4451 & $0.37+1$ \\
\hline $2(x),(x, 5)$ & 0.14 .7 & 50.4151 & $0.117(1)$. & $254.4(x) 1$ & $O(K, 3 B$ & $108.70 .5)$ & $0.78(1)$ \\
\hline 197.9501 & 0.1534 & 53.4551 & 0.18 .10 & $257.2(x) !$ & $0.16,18$ & $10 x, 1451$ & $0.3(x) .3$ \\
\hline 195.2071 & 0.1580 & 56.78 .51 & 0.1145 & 200.1571 & $0.02(x)$ & 1013.3051 & (1) 36xit \\
\hline 192.58 .11 & $(1.15(1)$ & 54.4 .451 & $0.12(.4)$ & 203 (K) & $0 .(1), 10$ & 100.76 .51 & 0.3628 \\
\hline 184.80 .51 & $0.14(x)$ & 62.9251 & $0.14(1)$ & 26.5 .83 .47 & (1)(1).44 & 97.98 .51 & 0.34 .51 \\
\hline 187.0121 & 0.1544 & 0.54551 & $(1.1020$ & 267.9775 & $0 .(X Y) ?$ & 95.3751 & $0.34(44$ \\
\hline 184.2451 & 0.16 .33 & 68.8451 & 0.1755 & 208.21754 & (1) (xisis & 92.7451 & 0.336 .7 \\
\hline $181.6+41$ & 0.1738 & 71.8051 & 0.1452 & 20.8 .267 .3 & (0)(1):1 & $9(0.1151$ & (1).3320 \\
\hline 178.9801 & $0.18(11$ & 74.6451 & 0.2316 & 266.2059 & $0.0 x \sin$ & 87.4251 & 0.3230 \\
\hline 176.27 .71 & 0.1780 & 77.54 .51 & $(1.0120)$ & & & 84.78 .51 & 0.3217 \\
\hline $173.6(6.21$ & 0.1807 & 801.42 .51 & 01.21 .37 & & & $82 .(0) 51$ & $0.31 x_{1} 2$ \\
\hline$i 70.45(1)$ & $0.10(18$ & 8.3 .3351 & 0.2112 & \multirow{2}{*}{\multicolumn{2}{|c|}{ Scan 13. 13(1).2 psig }} & 79.2951 & $0.28+(1)$ \\
\hline 168.2051 & 0.2021 & 80.1751 & 0.2321 & & & 76.3951 & 0.250 .7 \\
\hline $105.6(15)$ & $0.2(18)$ & 80.11591 & 0.2558 & $268.2(x)(x)$ & $0 .(1111$ & 73.7251 & 0.2382 \\
\hline$|0.2005|$ & 01074 & 91.9151 & (1) 1523 & $26+20.480$ & 0.01250 & 70.90151 & 0.2312 \\
\hline$i(x) .2051$ & $0.2(1) 70$ & 04.8051 & 0.25 .24 & $26,3.7713$ & $(1.0 .414$ & $68(x) 51$ & 0.2210 \\
\hline 157.7051 & 0.2151 & $07.56,51$ & 0.2547 & 260.7624 & $0 .(151) 5$ & 6.5 .2151 & $0.2(x)$ \\
\hline 1550.551 & 0.2018 .4 & $1(x)+151$ & $(1.2\} 1(1)$ & 257.74011 & $\left.(1)(1,1,)_{2}\right)$ & 62.3451 & 0.1826 \\
\hline 152.4751 & 0.2480 & $3(13.3351$ & $\left(12 l_{1}(1)\right.$ & 254.7201 & $0 .(1807$ & $59 .(x) 5 i$ & $010 \% 3$ \\
\hline 140.7451 & $0.245:$ & $1(x, 2,2 \times 1$ & 0.2573 & 252.0 .10 .1 & $0 .(14) 1.1$ & 568451 & 0.140 .3 \\
\hline $147 .(1151$ & 0.2374 & $1(14)(155)$ & $01.20,77$ & 249.2771 & 0.108 .5 & 54.0151 & 0.1252 \\
\hline $1+4 . .3551$ & 0.2132 & 111.86 .51 & 0.271 .9 & 246.96 .31 & 0.1101 & $\$ 1.2751$ & 0.1087 \\
\hline 141.6251 & $112+42$ & $11+624$ & 01275.2 & $2+38281$ & 0.132 .4 & -48.5451 & $0.0 \times 129$ \\
\hline $1.38 .8+51$ & 0.2518 & 117.3751 & 0.20145 & $2+111+1$ & 0.1 .10 .5 & 45.7251 & (1). 15.33 \\
\hline $135(9) 151$ & 0.2587 & $12(1,13.51$ & 0.2700 & $238.4+111$ & 0.1742 & $42 .(x \times 151$ & $(0,0) 3(x)$ \\
\hline 132.9051 & $0.2 f(x)$ & 1220551 & 0.2703 & $2.15 \cdot(x, 5)$ & 0.15 .34 & 40.1 .551 & 0.01 .18 \\
\hline 1.30 .2751 & 0.2675 & 125.7051 & $0.210+5$ & $23311+1$ & 0.1567 & 37.2551 & $0 .(x) \geq 0$ \\
\hline 127.5351 & $0.2 \% 4.3$ & $12 x \leq 5251$ & $0.2(6.10)$ & $2.31,3.581$ & $(1, \mid 6,1)$ & $3.1,35,51$ & $0 .(1284 t$ \\
\hline 124.9251 & 0.2722 & 131.3151 & $(1.2017$ & $227.60,51$ & 0.1757 & 31.4851 & $-0.0 .5(17$ \\
\hline 1222351 & 0.2561 & 134.1351 & $(1.2415 .5$ & 224.8711 & $0.174 k^{\prime}$ & $280+51$ & -0.0.15, \\
\hline 114.4351 & 0.2741 & $136.2 \times 151$ & (1) 2540 & 2223181 & 0.1435 & 27.24 .51 & $0 .(x, 77$ \\
\hline 1107451 & 0.2702 & $1.30 .7(5.5)$ & $0.25(1)$ & 21458.11 & 0.1401 & $24.8+51$ & -0.0506 \\
\hline 114.1351 & $0.26 .50)$ & 1425251 & 0.2122 & 216.9101 & $(1.20198$ & 32.6851 & .0.0.54 \\
\hline $111 .+451$ & 0.2714 & 1.45 .3351 & $(123.20)$ & 2141041 & $0.21 ?(1)$ & 35.5751 & -0.0 .3014 \\
\hline 105.76 .51 & 0.2720 & 148.1551 & (1.221. & $211.5(11)$ & $0.21+1$ & $38.5(151$ & $.0 .(x) 53$ \\
\hline $10 x .1451$ & $\left.(1) 20^{1}\right) 6$ & 151.1191 & 0.2315 & $2\left(1 x^{\prime} x+101\right.$ & $11.2(x) .5$ & 41.4551 & 0.0185 \\
\hline 103.3451 & $0.2(2+4)$ & 1539751 & 0.2717 & $20(1), 1$ & 021.1 & 4.4 .3751 & 0.0370 \\
\hline $1(n) 70,5)$ & 0.2610 & 1.56 .70151 & $(1)+25$ & $20.34(k) 1$ & 0.2165 & 47.3951 & 0.0733 \\
\hline 07.4591 & 0.2543 & $150.5+51$ & $(1.21+4)$ & Znim(m, $(x)$ & $0.2 \geq 9 ?$ & $5(1.41 .51$ & ONAL'S \\
\hline 45.3751 & () 25? 3 & 102.4251 & 11.1040( & $197.95(11$ & $(0.2340)$ & 53.45 .51 & $0.1(k, 7)$ \\
\hline 92.7451 & 0.2531 & 105.2451 & $02(1) 4$ & 1952071 & $0.2+(1,0)$ & 56.78 .51 & 0.1380 \\
\hline 00.11 .51 & (1. 2516 & 161.2151 & (1) 2015u & $192.5 \times 31$ & 0.2524 & 50.4451 & 0.1570 \\
\hline 87.4251 & 0.2453 & 171.1521 & (1) 1810.5 & 180.8091 & $(1.2+1)(0)$ & 629251 & 0.17 .57 \\
\hline 84.851 & Q. 215.1 & 17341018 & (1) 1548 & $187(11: 1$ & $11.2(x)$. & 650551 & $0.2(k)$ \\
\hline $8:(x) 51$ & 0.2332 & I?ts.tik) & 0.161. & 184.2051 & $02: 53$ & 68.84 .51 & $(0.2151$ \\
\hline 70.2051 & 0.2 .115 & $174.7(16.1$ & $0.1(, 0)$ & $18 !+.1 .11$ & (0.2768 & 71.80 .51 & 0.2380 \\
\hline $7 t, 345.51$ & 0.1872 & $182(x) 11$ & $0.17(15$ & $178.98(1)$ & 0.2956 & 71.6191 & 0.2821 \\
\hline 73.7251 & $0.2(1,30)$ & $185.54(1)$ & 0.1617 & $176.27 \%$ & 0.2kiti? & 77.5451 & 0.073 .1 \\
\hline $70.5 \times 51$ & $0.19 ! 2$ & 188.3741 & $0 . \mid 481$ & 173.60121 & 0.2724 & 80.4251 & 0.27 .32 \\
\hline$G K .(x) S 1$ & 0.1767 & 1912051 & (1). 1347 & 171.9501 & 0.28 .10 & 83.3351 & $0.3(x) 2$ \\
\hline 0.5 .2151 & 0.1613 & 104.1341 & 0.147 .3 & 1682051 & 01.30113 & 86.1751 & $(1.3(k), 3$ \\
\hline 62.3951 & 0.144 .3 & $10(, 8.401$ & 0.1432 & 165 o 1151 & (). $.3(1.57$ & 80.0151 & 0.3297 \\
\hline $59.6(15)$ & 0.1 .387 & $100.8(1,0)$ & 0.1321 & 1620491 & $0.3(4.3$ & 91.4451 & 0.326 .5 \\
\hline 50.8451 & $011(x)$ & $2(1) .8601$ & 0.1324 & liil? & 0.1219 & $0.48(1 \times 1$ & 0.3127 \\
\hline 54.1151 & 0.9081 & $205(x, 4)$ & 0.113 .32 & 157.7015 .1 & 0.2 keroi & 97.56 .51 & 0.3415 \\
\hline 512751 & $0 .(1850)$ & $208.42(1)$ & $0.11(11)$ & 155.0551 & 0.34 .32 & $1(x)+4.51$ & 0.3443 \\
\hline 48.5 .451 & o (ksis & 2111341 & 0.1142 & 152.4 .351 & $0.350 ?$ & 103.3 .3 .351 & $0.35(15$ \\
\hline 45.7251 & $0 .(2+11$ & 2138281 & 0.118 .4 & 1.9075) & 0.7525 & $1(x), 2351$ & 0.350 .2 \\
\hline $42(x) 51$ & $(0,(124)$ & $210.5(1) 11$ & 0.1198 & $14 \div 0151$ & 0.3478 & 109.0551 & 0.36 .56 \\
\hline $4(0.1 .351$ & $(1) 011 k$ & 210.23111 & $(1) 11(, 1)$ & 14.41951 & 0.110 .2 & 111.80 .51 & 0.37501 \\
\hline 37.2551 & $(3.0(0) \geq 0$ & $221.45(1)$ & (1) 1125 & 141.6251 & (1) 3530 & 114.6251 & 0.3761 \\
\hline 34.3551 & 0.01972 & 2946851 & 0.111 .5 & 1388451 & 0.3574 & 1173751 & 0.3726 \\
\hline 31.4851 & $-0.04+32$ & 287.1781 & (1) 11178 & $135(20) 51$ & 0.3752 & 120.1 .351 & $0.38(1)$ \\
\hline 28.6451 & 0.0551 & $2.3(1.11+1$ & $(1.113 .31)$ & 13280051 & 0.3786 & 1220551 & 0.3702 \\
\hline 272051 & $-0 .\left(k_{1}\left(k_{1}\right)\right.$ & 2328.81 & 0.10112 & 1.191791 & $(1.3772$ & 125.7051 & 0.3781 \\
\hline 29.84 .51 & -0.11534 & $215,5 \geq 21$ & $0.11 k i n$ & 127.5 .351 & $0.382 ?$ & 128.5251 & $0.37 \times 4$ \\
\hline 32.6851 & 0.01412 & 2.38 .1551 & 0.111161 & $12+11251$ & 0.3627 & 1313151 & 0.376 .7 \\
\hline 35.5751 & 0.0290 & $2408(18)$ & $0 .(x)(x)$ & 122.2351 & $(1.35(1,1)$ & 1.34 .1351 & 0.36 .2 \\
\hline 36.5051 & $-(1)(214.3$ & $2+3.5+21$ & $0113: 1$ & $|10+15|$ & $0.3 \% 90$ & 136.0051 & $0.3\left(x_{1}()\right.$ \\
\hline
\end{tabular}




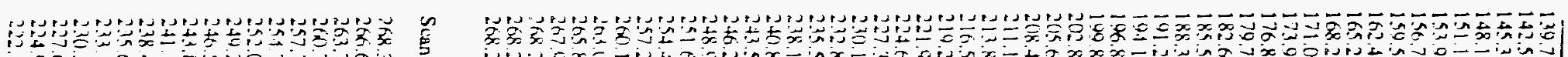

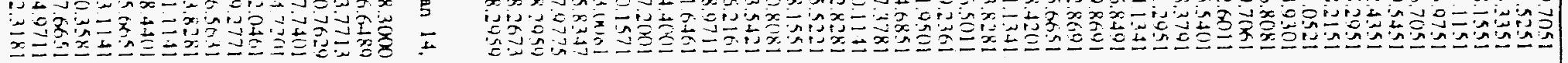

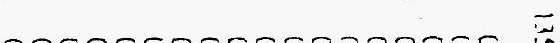

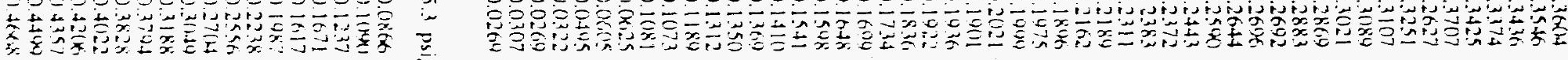

obounc000000000000c00000000000000000000000000000000

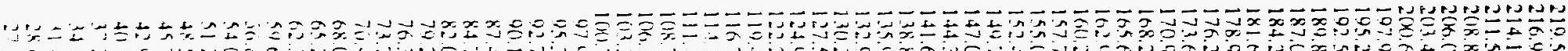

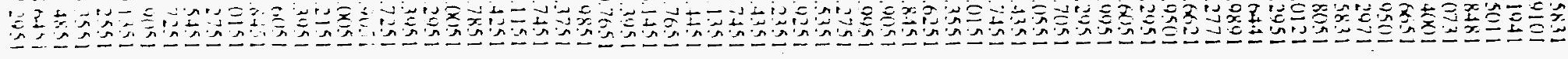

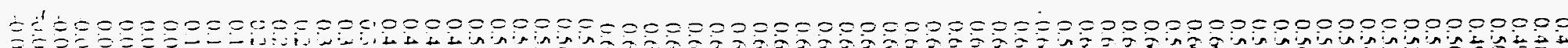

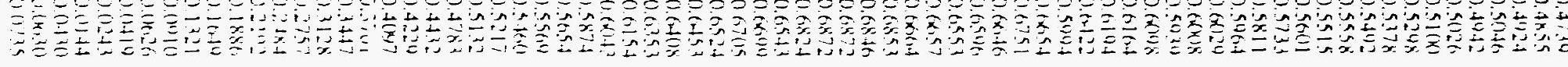

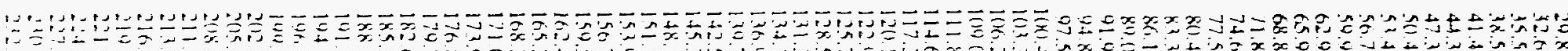

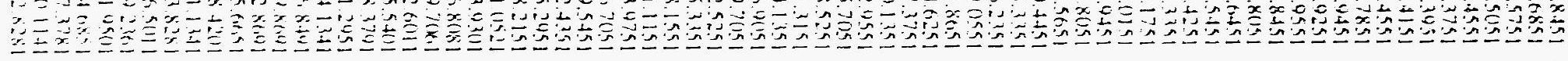

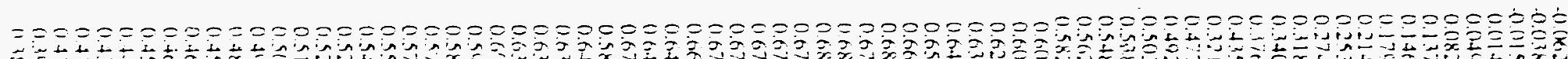

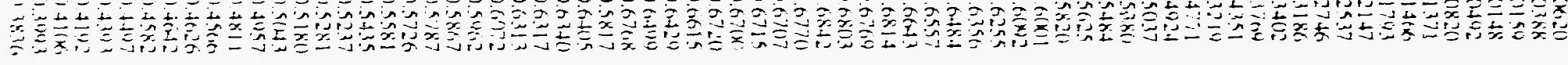

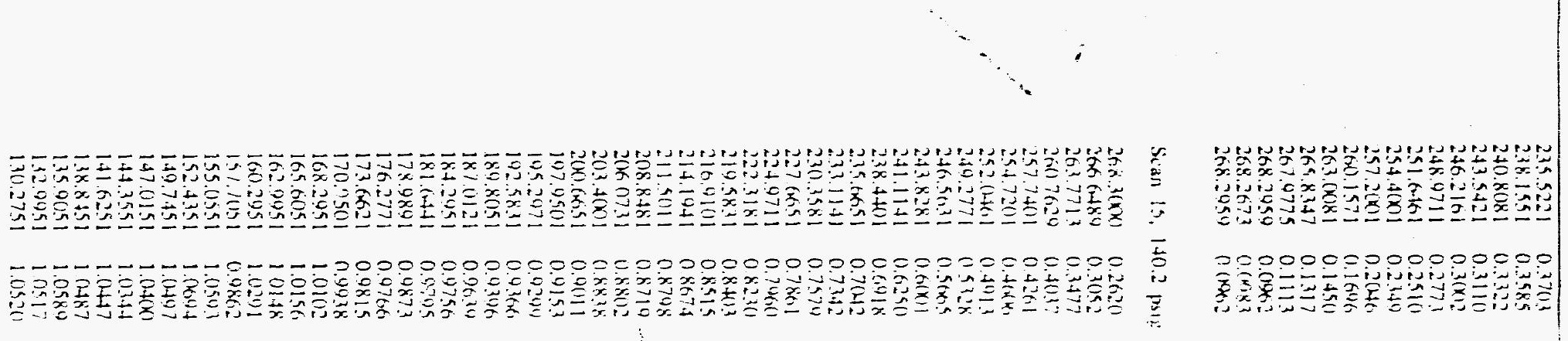




\section{Reinforced Concrete Test Data}

Track 6 - High Pressure Test

\begin{tabular}{|c|c|c|c|c|c|c|c|}
\hline 127.5351 & 1.0912 & 128.5251 & 1.1478 & 224.10111 & $1(1) .4 ?$ & 28.0 .51 & $-0.0,13$ \\
\hline $124.025 i$ & 1.1448 & 131.3151 & $1 .(x+2.5$ & $222.3|8|$ & 1.126 .3 & 27.2951 & (1). $18(x)$ \\
\hline 122.2191 & 1.0251 & 1.341 .351 & 1.0572 & 210.9811 & $1.14 k$ & $298+51$ & (1) (1564) \\
\hline 119.4351 & 1.0276 & $1360(151$ & $1,(x, 14)$ & $216.41(11$ & $1.15(1)$ & $32+x 51$ & 0.0180 \\
\hline 116.7451 & 10260 & $1307(19)$ & $1.156,7$ & 21410.11 & 1.1111 & 35.5751 & (1). (1) 80 \\
\hline 114.1 .351 & $1 .(x+1$ & 1425251 & 1.1501 & $2115(1 \mathrm{i})$ & 1.178. & .38 .5051 & $0(x)$ \\
\hline 111.4451 & 0.4451 & 1453151 & 1. (4.55) & 2(18. & $1.16,50$ & 41.45 .51 & 0.1350 \\
\hline 108.7651 & 0.9770 & $1.98 \quad 1551$ & 1. (I.Si\} & $2(x, 0) 3)$ & 1.1724 & 44.3751 & $0.196 . ?$ \\
\hline $1(x) .1+51$ & 040.57 & 1511151 & 1. (4. 16 & $2(1) 1,4(x))$ & 1.1502 & 47.3951 & $0.27(\mathrm{~m})$ \\
\hline 103.3051 & 0.9380 & 1.53 .4751 & $1 .(1786)$ & $2(x)(x, 5)$ & 1.2121 & 50.4151 & $0.3(k) .1$ \\
\hline $1(x), 7051$ & 0.0160 & 1.50 .7051 & $(0.4440$ & 197.4501 & 12251 & 53.4551 & 0.30 .50 \\
\hline 07.9851 & 0.8023 & 1505451 & 1.0318 & 105.2071 & 1.2158 & 56.78 .51 & 0.4 .44 \\
\hline 95.3751 & $0 . \mathrm{H}(\times)$ & $\mid 02.4 .351$ & 1. (1) 55 & 102.58 .11 & $1.2+44$ & 504451 & 0.5110 \\
\hline 02.7451 & 0.8 .433 & $10,5.2051$ & $1(114)$ & 180.8051 & 1.2476 & 62.9251 & 0.5557 \\
\hline $9(1.1151$ & 0.82311 & 108.2151 & 10105 & 187.0121 & 1.2727 & 659551 & $0.0 \times 13$ \\
\hline 87.4251 & 0.7773 & 171.0621 & $(1,0)(1) 8$ & $\mid 8.4241$ & $12 x(11$ & .68 .8451 & $0.64 ? 0$ \\
\hline 84.7851 & $(1.7548$ & $17.343(11$ & $\left.(1.41)^{\circ}\right) 8$ & 181.0 .441 & $1.20: 1$ & 71.80151 & $0.75(17$ \\
\hline $82 .(x) 45)$ & 0.7180 & I ThSISI & (1) 14$)(1$ & 178.4801 & $1.3(1\} 0)$ & $?+4.91$ & $(1.820, ?$ \\
\hline 79.2051 & (1.676) & $174.7(k)$. & $0.4 \%, 47$ & 176.2771 & $1.3(1): 2$ & 77.5451 & 0.8575 \\
\hline 76.3051 & $0.611)(1)$ & $18 \geq(x+1)$ & $(14) 12$ & $|=3 x+2|$ & 1.3171 & 80.4251 & $(1.4146$, \\
\hline 73.7251 & $0.54 \div 7$ & 1855.4111 & (1) 1$) 5(1)$ & $170.05(1)$ & $1.3 ?(x)$ & 83.1351 & 0.4042 \\
\hline $70 .(x) 51$ & $0.5+(1)$ & 185.1741 & (1) $11.4(11)$ & $|618.205|$ & 1.320 & 80.1751 & 1. $(4(k)$ \\
\hline GR. $(x) 15)$ & 0.5014 & 1012191 & (1) 1910 & $\mid 0,5$ tous & 1.3200 & $80(1151$ & $1 .(x)+4$ \\
\hline 6.5 .21 .51 & 0.4580 & 14.4 .1 .41 & $(1,4): 27$ & $|02.405|$ & 1.3353 & 41.4 .151 & 1.13 .38 \\
\hline 62.3951 & $(1+144$ & $10+8.8+1) \mid$ & (1) $(2)(x)$ & $i(1) 204 i$ & $1.39 \% 9$ & $0+80151$ & 1.1729 \\
\hline $59 .(x) 51$ & $13.37+3$ & $|(x) x(x)|$ & (1.8sint & 157.7051 & 1.116 .4 & $075(1.51$ & $1.2(k) 6$ \\
\hline $50 \times 451$ & 0.3338 & $202 x(1,1)$ & $(1.8150$ & 145.1551 & $1.3(x)+1$ & $1(x) .4451$ & 1.2 $H_{1}(1)$ \\
\hline $54(1) 51$ & 0.2880 & $2(1),(0,5)$ & 0.8559 & $152+351$ & 1.3015 & $1(13.3351$ & 1.2581 \\
\hline 51.245 & 0.25011 & $2(1)+2(1)$ & 0.8201 & $1+10.7+51$ & 1.1870 & $1(x) .2351$ & $1.29 x_{1}$ \\
\hline 485.551 & $0.2(x) .8$ & 2111.3 .11 & (1) sill & 1.47 .1195 & 1.3820 & 100,0551 & 1. $3(187)$ \\
\hline 457251 & 0.1 .71 & 213.8281 & $(1): 311$ & 1443551 & 1.3780 & 111.86151 & 1.331 .4 \\
\hline $42(x) \leq 1$ & 0.11 .0 & $216.9(1) 1$ & $0 \times 151$ & $1410,25 !$ & 1. $3 k^{\prime} 05$ & $11+6251$ & 1.3 .132 \\
\hline $4(1.1 .351$ & 0.01795 & 2102361 & $(1.80)=8$ & 136.8551 & 1.31440 & 111.3751 & $1.35 \%$ \\
\hline 37.551 & $0 .(1.40)$ & 22119501 & 0.70115 & $1: 5$ (u) & $1+1 \times 0$ & $12(1.1 .351$ & 1.2485 \\
\hline 34.3551 & $\left(0,\left(k k_{1}\right)\right.$ & 2246154 & (i) $"(1,1)]$ & 1320458 & $1.4(x)-7$ & 1220551 & $38(1) ?$ \\
\hline $11+651$ &.$(1.11 .1)$ & 227.371 & 07453 & 13112751 & $1+11) 1$ & 125.7051 & 1.344 .5 \\
\hline 28.6451 & $-(1)(k x)^{-1}$ & $2.31111+1$ & $117 \geq 93$ & $1 \because \div<391$ & $1.4(1) 0)$ & $\mid 2 k 5251$ & $1401 ?$ \\
\hline 27.2051 & (1):1?: ? & 2328281 & $07(1) 8$ & $12+6.251$ & $1.30 \times 11$ & 191.1151 & 1.4143 \\
\hline $20.8 .+51$ & $0.115 .+5$ & 235,521 & $(1)(8) 10$ & 1222351 & 130.15 & 134.1 .351 & $1.8(2)(\mathrm{v}$ \\
\hline$\therefore 20591$ & $-(1)(12.81)$ & 2581591 & (1) $(x, 15$ & $111.1 .35 \mid$ & 11751 & $136.9(15)$ & 1. $f(x)(y$ \\
\hline 155751 & $0(x) \times s$ & $2 f(1) 8(1,8)$ & $(10,12$ & 110.2451 & 1.375 .5 & 1.34 .70 .51 & $1.4(1 \% 74$ \\
\hline $38.5(15)$ & $(10.12010$ & 2435421 & $(15 \%) 8$ & $11+1.351$ & 1.350 .4 & $1+2.5251$ & $1401 \%=$ \\
\hline 41.4551 & $(1)$ & 270.210 .1 & (1) 511 & 111.4 .451 & 13324 & 1.45 .3151 & 1.3848 \\
\hline $44.3:$ & 01.1 .36 & $2450 \div 11$ & $(1.546 ?$ & 1018.7651 & 1.316 .8 & $148.15: 1$ & 1.38211 \\
\hline 47.7951 & $0.2(1) 1$ & $251.64(1)$ & (1) $+4+1$ & $116,1.151$ & 1.2483 & 1.51 .1151 & 1. knot \\
\hline 50.41 .51 & $0.21 \%$ & $254+(k) 1$ & $01+2 \div 10$ & 101120151 & 1.2505 & 15.0751 & $1.4(130)$ \\
\hline $53+541$ & $11270^{-9}$ & $257.2(k) 1)$ & 0.43 .14 & $l(k)>0.1$ & 1.24it & 1.50 .10 .1 & $1.24 ! 1$ \\
\hline 50.7851 & $(13224$ & 20111571 & 0.3075 & 074851 & 12 (1) & 154.5451 & 1.30 .30 \\
\hline 50.0451 & (1).3(6)1) & $203(x) 181$ & () 14()$\times$ & $053 \div 1$ & $1.1 \%(x)$ & 162.1151 & 1.3512 \\
\hline 0202.51 & 04178 & $20,58.37 ?$ & $0.3(k) 2$ & $192+51$ & $1.1+5.1$ & 16.5 .2451 & 1.2873 \\
\hline 0,50551 & $(1.10 .27$ & $26.07: 5$ & 0.7011 & $1 x: 1151$ & 1.1152 & 168.2151 & $1.3+15$ \\
\hline $0 \times 8451$ & (1) 914$\}$ & 208.20517 & 0.25.42 & $8: 4291$ & $f(k, 1)$ & 1710521 & 1.2148 \\
\hline 71.8051 & $(1.55 .58$ & & & $84.7 \times 51$ & 1.11287 & 173.0301 & 1.3205 \\
\hline 74.6451 & 0.0 .85 & & & $8 ?|1015|$ & $04)(12 \div$ & 170.8081 & 1.24.4t \\
\hline 77.5451 & (1.54tit & Siaul 16, & $458^{\prime}$ p's! & 702051 & $(1)(x)\left(x_{1}\right)$ & $170.71 \times 1$ & 1.243 .5 \\
\hline $80+251$ & 0.6056 & & & 76.3051 & $0.8+422$ & $1826(111$ & 1.2882 \\
\hline 833351 & $0.74(x)$ & $2063(1)$ & $(1.2577$ & 737251 & () 81125 & $18.5 .5+111$ & $1.20 .2(1)$ \\
\hline 86.1751 & 0.7712 & $\left.2 \operatorname{trn}(x+1)^{\prime}\right)$ & (1) 245 & $7(1)(15)$ & 0.7253 & 188.3741 & 1.2419 \\
\hline $89 .(115 i$ & $(1,1214$ & $2037 \% 13$ & (1) 76,33 & of $(x) \leq i$ & 0.67 .2 & 101.2051 & 1.2217 \\
\hline 91.9451 & 0.8380 & $20(1)(12 x)$ & $114+4 ?$ & 652151 & $(1.01 .41)$ & $104.17+1$ & $1.22 t .2$ \\
\hline 48051 & 086.22 & $\therefore 790$ & 0.48 .11 & 62.3451 & $(1 \leq\{, 1\}$ & $|9(, x+4)|$ & 1.2108 \\
\hline $97.5(1.5)$ & 0.8023 & $25+72010$ & $0.50,25$ & $540(195)$ & 114078 & $17968(19) 1$ & 1.1425 \\
\hline $1(x)+151$ & $040 ! 1):$ & $\therefore 52(46)$ & 0.6211 & $508+51$ & $0+38 n$ & $2028(9,9)$ & 1. $|\mathrm{Bn}|$ \\
\hline 109335 & 0.0172 & 244.2771 & $0(1,6) \geq 8$ & 540151 & $(1,4)(x)$ & $2015+x(1.5)$ & $1.1(x)$. \\
\hline $10 x, 2341$ & 0.9585 & 2.46 .90 .31 & $07.11 ?$ & 512751 & 0.1332 & $208+401]$ & 1.1427 \\
\hline$I(x) .(155)$ & 0.075 .4 & 2438281 & (1.50) & 485451 & (1).27(x) & $2 \mid 1.13 .4 !$ & 11.170 \\
\hline 111.8051 & $(1,1)(1) 561$ & 241.1141 & $\left(1,851 x_{1}\right.$ & 457251 & 0.2111 & 21.8281 & 11454 \\
\hline 114.61251 & $1(x)(1) 1)$ & $235+101$ & 0.4 .420 & $4: 0(151$ & 0.10015 & 210.51111 & 1.1 .311 \\
\hline 117.37 .51 & 10170 & $245(x, 5)$ & (1) $)$ incis & $+(1) 1.351$ & (1) 1116 & 2114.2101 & 1.1153 \\
\hline $120,13,51$ & 1.0255 & $233.1 i+1$ & $1(x) 50)$ & .37 .2551 & $0.0(x) 3\}$ & $22144(11$ & $1.111 \%$ \\
\hline 17215551 & 1.14tis & $23(1.3581$ & $1.03(15$ & 34.1551 & $(1.01171$ & 2246859 & 1.1130 \\
\hline 12570.51 & 1.0 .4 .45 & $227(x, 5)$ & 101706 & $31+851$ & $(101) 301)$ & 227.3781 & $1015 ? 26$ \\
\hline
\end{tabular}




\section{Reinforced Concrete Test Da}

Track 6 - High Prescure Test

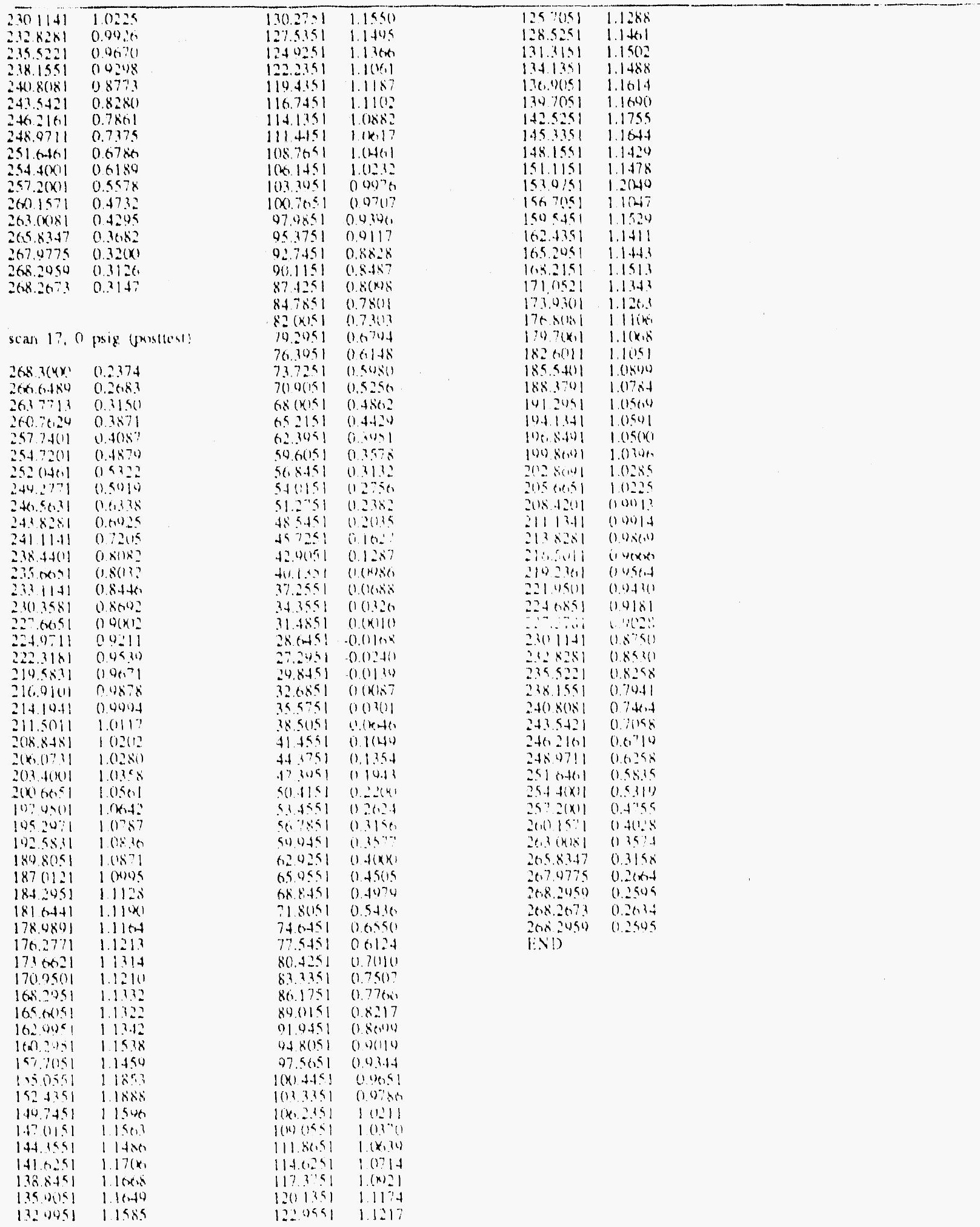




\section{Reinforced Concrete Test Data}

Track 7 - High Pressure Tect

\begin{tabular}{|c|c|c|c|}
\hline \multicolumn{2}{|c|}{$\therefore$ sall 1, 1:? pous } & $x: 11 x: 2$ & (1) (kot.pu \\
\hline 1016.250 & 01734 & $\begin{array}{l}81+4420 \\
81 \text { is }\end{array}$ & $\begin{array}{l}(11114 \\
(1,12)\end{array}$ \\
\hline If(x)(1)24 & (1) $117,7(1$ & $70 ?+?$ & (I) (ive)? \\
\hline $47.61: ?$ & 0.0100 & ? & $011 \times 11^{7}$ \\
\hline us (xil) & 011754 & $7+1792$ & 11100 \\
\hline 02.300 .4 & (1)iilen & ? +151: & $0 .(1) ?(1)$ \\
\hline 80 (1)? & (1) (ISto & 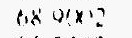 & $0\left(1 x^{-1}\right)$ \\
\hline $87.182: 2$ & $(1) B:$ & $6(1,711)$ & 0 (rixks \\
\hline $8+44+2$ & 0104 10 & $4,1741^{\prime}$ & $0 ! 1 x: 1$ \\
\hline 818502 & $(1,1,1)$ & $61: 1: 2$ & (nlitent \\
\hline 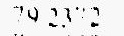 & $0(1)+1,4$ & SR'HW? & 11010 \\
\hline $10.0+12.2$ & $0\left(1 x^{2}+\right.$ & 56.146. & $(101,3\}$ \\
\hline 74.052 & $0010 \%$ & $5+48: 2$ & $\|(1)$ \\
\hline $71.4+12$ & 0.0701 & $50105 ?$ & $(1)(x+1)$ \\
\hline tor wain & $11,1): 1$ & $4: 3^{*}=2$ & $\left(1111^{4} 10\right.$ \\
\hline$(x+3.302$ & $10.0 \div 53$ & $45-1,22$ & (111.41.) \\
\hline 6.37012 & 00124 & $4+24+4$ & $0(1.6+1 .-1)$ \\
\hline $01: 172$ & $1)(x, ?$ & (1) $: 4$ ? & (1) (1) 14 \\
\hline$\because \because 4107$ & nollik, & in Ilks? & $\left(111: 1^{\circ}\right.$ \\
\hline$S 6(4) R$ ? & $(1117 \mathrm{i} 0)$ & $\therefore i n$ & $6: 2,2 !$ \\
\hline 514372 & (1) $(1554)$ & $3: 10 \div 2$ & $110 \mid+1$ \\
\hline 5114532 & $11(x)=$ & $304 \times 2=$ & $0: x \times n$ \\
\hline 485722 & 0.043 .4 & 281052 & $(1,(x)=)$ \\
\hline 1570,2 & $(1)(1,1,1)$ & 3110195 & $116111 ?$ \\
\hline 432442 & $(1,1,1,0)$ & 35,2 & $\left.(111)^{7}\right)$ \\
\hline 411014 & $11019-$ & $3515: 2$ & 0.01 .74$. \\
\hline $181 \cdots 2$ & 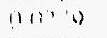 & $37811=$ & (16): \\
\hline $35464 ?$ & (1) : id & $411+4 \div$ & $1 \cdots \cdots+1$ \\
\hline$\because 28: 4$ & $(1+11:=$ & $4 ?(13)$ & $\{1.1 .1)$ \\
\hline ib fik: & 110128 & $f \div 0,2: 2$ & 100512 \\
\hline $2 \times 16 \div 2$ & bientert & Is lnis & 111 arde. \\
\hline $3111^{-2}:$ & $(1) \times x, 3$ & $50{ }^{4}$ & 1114,11 \\
\hline$\because 503$ & ficis & $\therefore ?$ & "ikntis \\
\hline 181222 & $1112: 16$ & $5411 \because$ & 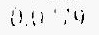 \\
\hline $3 ? \times 112$ & $11113 \mathrm{~mm}$ & 544149 & in (n) 2 ) \\
\hline $4111^{1}=$ & $0 ! 1759$ & (1) 1012 & $1.11: n+4$ \\
\hline $4102 x=$ & $11: 3,5:$ & $f(x)(x+2)$ & 1: $m 11$ \\
\hline$+5+23$ & $11 ! 1+4$ ki & int: 1 t 1 & $11+w_{n}-1 \mathrm{~s}$ \\
\hline $4 \times 10(1)$ & $11(19: 3$ & 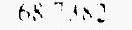 & $1161 \%=$ \\
\hline (6. ") & 0.1550 & 1243 & $(1018)$ \\
\hline$(3) \div ?$ & $005 \mathrm{ts}$ & 395 & $\because 1: x " 1$ \\
\hline $540 \geq 1 ?$ & $(1)+k \cdot 1)$ & 70,282 & $(1(n) \cdots)$ \\
\hline $5: 515 ?$ & $(1)\left(k, c_{1}\right.$ & 701702 & (1) (1) : \\
\hline 01.1122 & $(16 \because \because$ & $81816=$ & $(1 / 1)$ \\
\hline$(13,(x) 1)=$ & (1) $1: 44$ & $A 4: \therefore$ & 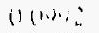 \\
\hline $0,110$. & (1) 1140 ? & S! ssil! & $111: x: 4$ \\
\hline $6 x^{7}+1,2=$ & (1)!..1 & $8+412$ & $(1 / 1 \times 18$ \\
\hline$\because 12 ! " \div$ & $(1 ! n): 1$ & (1) 1.5411 & (1:13,n \\
\hline ? & $1111 \cdots: 4$ & $94+111$ & $(1(11) \cdots$ \\
\hline$\theta_{1} x^{2}=5$ & $(1)(1,2)$ & $97,1,57$ & 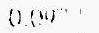 \\
\hline (1). 172 & $(101810$ & $9(x+f(x)=$ & (1). (m)+t. \\
\hline $81815=$ & $0(152)$ & $1111 \because 1$ & $n(m): 0$ \\
\hline $8+192$ & $1:(18: 2=$ & | & $(:(x)+2)$ \\
\hline sit, sins: & 01556 & $1015: 111$ & (1) $(x+4:$ \\
\hline (4) $413:$ & $0.62=$ & \multirow{2}{*}{\multicolumn{2}{|c|}{ 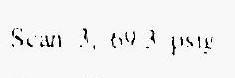 }} \\
\hline 41.45 .611 & $0.0182 ?$ & & \\
\hline 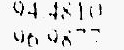 & $\begin{array}{l}01100 \\
\left.110^{-1}+10\right)\end{array}$ & 1011.62501 & (1) $1 ! k \cdot x$ \\
\hline wo de,1! & $110 \times 2$ & $\left.1(x) \mid N_{2}\right) t$ & 0.105 \\
\hline $16113: 1$ & $1111 \mathrm{r}$. & if $(11: 3$ & $(111 \times .5$ \\
\hline 11119351 & $\left(11^{-1}\right)(2)^{\prime}$ & DS $(k 1)$ & (1) 114): \\
\hline$|1| \leq 210$ & i) $11: 10^{-7}$ & $y=3 k t+4$ & 0140 \\
\hline \multirow{3}{*}{\multicolumn{2}{|c|}{ Sian 2 sito prog }} & $80: 129$ & $611 ; \%$ \\
\hline & & 871822 & $011 x+5$ \\
\hline & & $8+4 i 12$ & 011143 \\
\hline $1+1 \mid 6,24$ & "1) & 818512 & $(1.1126$ \\
\hline $11 x+1 x=4$ & 01,200 & $79.23 \% 2$ & (1)11:6 \\
\hline$u^{2} .2 \times 1=$ & (1im) & $70(1.12)$ & (1) $111 \%$ \\
\hline US $(x) 1)$ ? & (f(1):-1) & $7+1192$ & $1111+1$, \\
\hline$\because 3 \mathrm{scm}$ & $(1) x+4$ & $\because 1$ 15... & $(1) 1011$ \\
\hline xis 714 ! & $0.11 x)^{2}$ & $(\therefore)(x)$ & ii $1.12 i$ \\
\hline
\end{tabular}

\begin{tabular}{|c|c|}
\hline 60.1342 & (1) $1611 ?$ \\
\hline 0.17412 & $0(n)>1$ \\
\hline 01.2172 & $0 .(N) 12$ \\
\hline Sitotil? & $(0,(n) ! 4$ \\
\hline SCIUIS: & $O(x)(1)$ \\
\hline 524872 & $0.08(x)$ \\
\hline 50.0512 & 0.08612 \\
\hline 48.372 & $i)(x, 1)$ \\
\hline 457053 & (1) $05(x)$ \\
\hline $11 ?+4 ?$ & 00522 \\
\hline 40.012 & 00.83 .1 \\
\hline 1810182 & $0.11: 2$, \\
\hline is SORS? & $0,(1:+4)$ \\
\hline 3207.42 & 0.0169 \\
\hline $304+2=$ & $0\left(x^{\prime}\right)(y)$ \\
\hline 281155 & $11(x)<x$ \\
\hline 31045 & $0(x),(x)$ \\
\hline$\because 4+2$. & 1811170 \\
\hline 391821 & $0102 a_{2}$ \\
\hline 178112 & 011.450 \\
\hline $4(1+4) 2$ & $1114: 2$ \\
\hline $43112 \times 2$ & $111+471$ \\
\hline $440: 2$ & $(1)(x)$ \\
\hline An lises & $0(x) \cdot s)$ \\
\hline $5(1 ?) \div$ & $11111 \mathrm{H}$ \\
\hline $5311: 2$ & 1111014 \\
\hline 59192112 & (1) (11i) \\
\hline $58<1<$ & (itovi: \\
\hline (1) 102 & (1)(ivilite) \\
\hline$t .26(i)=$ & $(1) \mid x(x)$ \\
\hline$t x, 1 \cdots=?$ & (.111': \\
\hline$k+4: a$ & 0115.1 \\
\hline$\because 1: 1 \%$ & (i low \\
\hline - 152 & 111194 \\
\hline $363 \div$ & 0102 \\
\hline$\because 417 !=$ & 1111111 \\
\hline (4) sile? & $1111<?$ \\
\hline$n+1: 22$ & $11115 "$ \\
\hline ses sise: & 01120 \\
\hline (i) $+1,32$ & 01121 \\
\hline (1) 1354 & $1111: 4$ \\
\hline $445 ! !$ & (1) likx) \\
\hline $4 x+4 x^{-12}$ & (i) 1054 \\
\hline (3) $+(1,1)$ & 111198 \\
\hline $111 \mid 1: 1$ & $011,5 \mid$ \\
\hline 1011.5351 & $(\because|1| x, \mid$ \\
\hline \multirow{2}{*}{\multicolumn{2}{|c|}{ 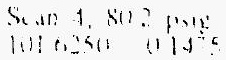 }} \\
\hline & \\
\hline Ifkilla? & $1115 \div 4$ \\
\hline wite? & $(1.15) 0$ \\
\hline$\because c\left(H_{11}\right)^{\prime}$ & $(1.1 .5)^{7}$ \\
\hline 1) ב...BSt & $0.1+1.1$ \\
\hline sid ? ? (1). & (1.) thise \\
\hline $8 ? .182$ & 01.661 \\
\hline $8+4.42$ & $0.1+85$ \\
\hline $8 \mid 8 \div 0)$ & $111+38$ \\
\hline $7421:$ & 0.1530 \\
\hline $706+22$ & $(11+35$ \\
\hline-11175 & $0.15 \%(1$ \\
\hline $71+212$ & (1)118is \\
\hline (sis) & $0.131 k 1$ \\
\hline$(x+3)=$ & 111.4 \\
\hline $6 ?-7,2$ & $(11: 12$ \\
\hline $0121: 2$ & (1.118.t \\
\hline $53(6+4) \div$ & 011.2 \\
\hline $50(1.45)$ & $\left(11110^{\circ}\right.$ \\
\hline $48+4$ & 1) (S: \\
\hline $5(+1) 532$ & (19) \\
\hline 46322 & 01814 \\
\hline 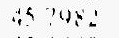 & 11018 \\
\hline $4324+2$ & $(1,(k, \ldots)$ \\
\hline
\end{tabular}

\begin{tabular}{|c|c|}
\hline $167(4)=$ & (1.k1: \\
\hline 36 108' & 0.14 .40 \\
\hline 3456182 & 0.11121 \\
\hline $12474 !$ & (1) 1224 \\
\hline $.10+1\left(k_{1}=2\right.$ & (ivil)i \\
\hline 280552 & or (xis) \\
\hline $30 .(1,: 4$ & $\because(x+1+$ \\
\hline $12.50, \cdots$ & 119211 \\
\hline 35.182 .2 & $01112 \mathrm{~h}$ \\
\hline 378112 & 0.461 .1 \\
\hline $4(1+4: 2$ & 0.11914 \\
\hline t?.0:4? & $(1)(x, 1)$ \\
\hline 496122 & $011 \% 5 ?$ \\
\hline בR. & $0110^{\circ} 2$ \\
\hline 2) & $(1)(x) . ?$ \\
\hline 53.3172 & $(1)\left(x_{1}\right) n^{\prime}$ \\
\hline $5542(12$ & 0.1111 .8 \\
\hline 585152 & $0.11 \div 4$ \\
\hline 0111022 & $01.1: 11$ \\
\hline$(x+2)(1)=$ & (1) 1254 ) \\
\hline$\left.(* 6,)^{-1}\right)$ & (1) $12 \times 1$ \\
\hline $6 x-82$ & $(1) 110$ \\
\hline $112: 2$ & (:) $1+1 k$, \\
\hline 71 is & (1) $40^{-1}$ \\
\hline גים & $\begin{array}{l}(1.9+4 x) \\
(11543\end{array}$ \\
\hline BI Kis? & 11.1516 \\
\hline $8+1922$ & (1.) 1.98 \\
\hline stiosto: & $0.15,1$ \\
\hline 841122 & $11 ! 51$ : \\
\hline (1) 105419 & $11151: 4$ \\
\hline $44+310$ & (।小!1 \\
\hline $4 f_{1}+x_{2}=$ & $(1) 5,3$ \\
\hline 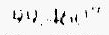 & (1) BAs? \\
\hline $\mid 11: 31$ & $111 \ldots=$ \\
\hline 11115151 & $014 \%$ \\
\hline 1015910 & (1) 1.1 \\
\hline
\end{tabular}

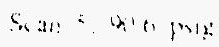

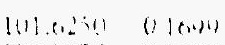

l(x)iset oles.

1): $2 x)^{-1}$ (1) $1 ? 3$

o. 3ind $1110 \%$

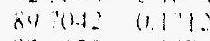

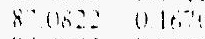

$8+40.42$ i 1605

it $24=2$ intot

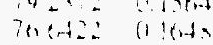

$7+10520160$

7145120160

bet $(x)=0.1: 43$

(x) 3.34: () 1.44)

6. 74120111

(1) $21^{\circ}=0.11 \mathrm{~B}$

skithe 0110

St, (bts.? $0,12 \div$

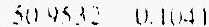

to. $172 \mathrm{O}$ (IINA

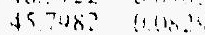

4:2412 $0(10: 18$

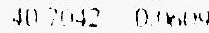

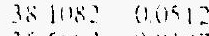

$34 \%+2 \quad 1162+3$

$3(1+4)=(1611) !$

solis? $11(x)$ 


\section{Reinforced Concrete Test Data}

Track 7 - Hot Pressue Test

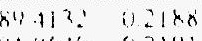

(1) $144,4(1) \quad(1219)$

13.4 ikl11 0218.8

wisin 0.2201

(19.6) 0.217

(11) 153 19.2102

111) 5.111 (1217\%

$\operatorname{xan} 9.114: 94$

$1919.240 \quad 01.2211$

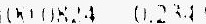

y (2) 1220

uर $1 \times 11200.23 x$

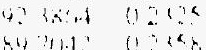

कiv $19: 11$

bit 1040 (1) 22:

$81+112 \cdots 1$

$14+2 \quad 028$

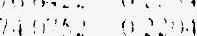

3. $281: 4214$

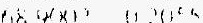

tin 31142 (i) 1112

(1) 14.1113 .4

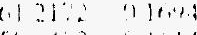

So.tin? (1) 161.4

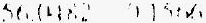

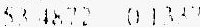

(1):3 01814

$4 \times 12 \times 11122^{2}$

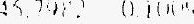

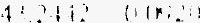

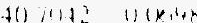

in latr? (1)

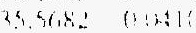

3942 (16)

(i) Hx (1)

2in

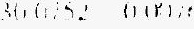

$\therefore$ a 12 ? $1101 \%$

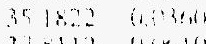

(1) $x: 1$

$4(1)+17=0 \mathrm{c}$

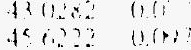

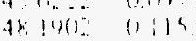

का अ(1): (i.1.24

4137100119

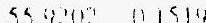

$58.514=015 \%$

(1) 110200171

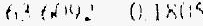

(x) 171.2 0.14)

(4) 320000101

i1 $237 \%$ (1.2!)

$7,7 \times 9011.174$

16.325 (1) 131

141 112 oा 212

8) $811^{2} \quad(122)$

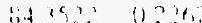

sichen 1120

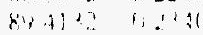

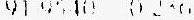

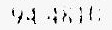

'to 46 .

(w) knth on

bis 12010 
Reinforced Concrete Test Data

Track 7 . High Pressure Test

\begin{tabular}{|c|c|c|c|c|c|c|c|}
\hline \multirow[t]{3}{*}{10153161} & 02310 & $976(1) \div$ & $0.27 .4 x$ & $76 .(.12)$ & $0.26 .2 \mathrm{y}$ & 58.6492 & 0.2327 \\
\hline & & $95 .(x) ! 2$ & 0.2752 & $74 .(1) \div 52$ & $0.24(11$ & $56(1482$ & 0.2157 \\
\hline & & 92140.9 & 0.27119 & $71.4 \times 12$ & 0.2492 & $53,4 ! ! ?$ & 0.1410 \\
\hline \multirow{2}{*}{ Sant 10.1} & 117.8 psig & $89: 10.12$ & $0.27 \%$ & $(x+9)(x) 2$ & $0.246 ?$ & 50,4532 & 0.1845 \\
\hline & & $870 \times 22$ & $0.260^{2}$ & $(x, 339)$ & $0.23(x)$ & $4 \times .3722$ & 0.14801 \\
\hline 1011250 & (1).2408 & 84.9042 & 0.2621 & 6.17412 & 0.21314 & 45.7082 & 0.1348 \\
\hline lix (18:2t & $0.25,44$ & $81.854 \%$ & 0.2512 & (i) $.7 \%$ & 0.2119 & 43.24 .42 & 0.1215 \\
\hline $47(x)=$ & 0.2551 & $70.2 .1 \% ?$ & $(1) 25.88$ & 580.742 & $0.14 .9 x$ & $4(1) 7(1+2)$ & 0.0853 \\
\hline $45(x) 1 ?$ & $0.25 \%$ & $76,(1.222$ & 0.2 .4801 & So 10.18 ? & $0.18+5$ & 38.1082 & $0\left(x, x^{\prime}\right)$ \\
\hline $9=386$ & $(1.25) 4$ & 14.6752 & 0.25 .41 & $53+4872$ & (1) $1(1,2)$ & $35.56,82$ & 0.1455 \\
\hline$\left.x^{\prime}\right) 7(42$. & $(1.25(1) ?$ & 71.4812 & $(1.231)($. & 50.9512 & (1) $16, k$ & 32.4742 & 0.0240 \\
\hline $8 .(1 \times 22$ & 0.24 int & $6.89(x)_{2}^{3}$ & 0.2290 & $1 \times 3722$ & $0.12 \times 3$ & $\left.30.4 k_{12}\right)$ & $0 .(x) \geqslant 1$ \\
\hline$\therefore 41042$ & $(1,2.40 .7$ & $66 . .3392$ & 0.2153 & 45.794 .2 & 0.1137 & $2 k .0552$ & -0.1214 \\
\hline (i) $n<4:$ & $0215 \%$ & $60^{2} 7912$ & $0.20 \% 5$ & $43.24 .1 \%$ & $(1 .(1) .58$ & $30.075:$ & $0(x) 4 ?$ \\
\hline$\because 4 \therefore \%$ & 022016 & 612172 & (1) 11) 10 & $40.7(1.42$ & () & 32.56 .22 & (1).0164 \\
\hline $7(1,(x)) 2$ & (1).241 & $58 .(.-19)_{+}$ & (1) $) \wedge(1)$ & 38.1018 & 0.01545 & 35.1822 & 0.016 .5 \\
\hline$\because 4019: 3$ & $11 .+11$ & $56(1.18 .22$ & 111740 & 35.56182 & 011.14 & 31.8112 & 0.0726 \\
\hline 714912 & (1) 2.3(1) & $534 \times 2$ & $1114: 11$ & $32474 ?$ & (i) (1) 6 i & 40.4172 & $0.082 !$ \\
\hline in $1 \times n$ : & 19.215 .4 & $(116)^{6}: 2$ & 11.1911 .4 & $3(1.4(16) ?$ & $(1,(K))$ & 4.11282 & (1) (x) 8.4 \\
\hline$(6,2(1))$ & $(12)\left(x_{1}\right)$ & $48 \times 2 \%$ & 01.125 & $2 \times 1<4 ?$ & $(1 / H 14) 3$ & 45.1122 & $(11178$ \\
\hline 617012 & $\because$ : ime & 4871152 & $(1))\left(x_{1} \cdot\right)$ & 401175 & $0(10) 1$ & .481002 & 0.1 .457 \\
\hline$+1=1 \vdots$ & $111 \times 2$ & 422492 & $(1)(14) \subseteq)$ & $\because 4622$ & 010170 & $5(1) 4(1) ?$ & (1) $r_{1}() .1$ \\
\hline SAtris & $1: 17(x)$ & $417(1,2$ & $1101: 21$ & $\because 1 x^{\prime}$ & $(1)(1<1,5$ & 52,1172 & 0.17 .34 \\
\hline$\left\{6,1+1 k_{n}\right.$ & $(1) 10$. & $3 k 11 k \ldots$ & $(1(1) \alpha) ?$ & $37 \therefore 112$ & $0(1) 5 i, 5$ & $5.5192(12$ & (1. 20770 \\
\hline 54 is & (1) $1=115$ & 3556 & $(1)(1.1 .6)$ & $4\left(1.44^{12} 2\right.$ & $(1)(k, 1)()$ & $5 \times 5152$ & $(1.22 .47$ \\
\hline $4(1)<! 2$ & $0.1 \% 11$ & $3211: 12$ & $(1.11 .411)$ & $4 ?(1.212$ & $0(18) 18$ & 0.1 .1022 & (1) 2.41 .4 \\
\hline $48: 372$ & 111240 & $3019 x=$ & $(1,11) .35$ & 45022 & 0.101 .4 & $6.3(x) 2$ & $0.26,17$ \\
\hline 4594.2 & $0.11+4 ! 1$ & $26 k<4$ & $.(1): 14 \times 18$ & 46.1402 & $(1) 3(x)$ & $(x, 1)(0)$ & 0.276)$. \\
\hline 4.2412 & $(1118 \times 1)$ & $31,(1) \div 2$ & (1) $(1)(1)$ & $50.740 \%$ & 11.96 .0 & 687382 & (0.21,54 \\
\hline 457012 & $(1\{k, m\}$ & 12862 & 110918 & 531172 & $0.1+87$ & 71.2372 & $(1.31(1)$ \\
\hline${ }^{2}<116$ & $0.141)$ & $35.16: 2$ & $(1) 1181) 2$ & is प:21: & 01773 & 7.37852 & 0.3374 \\
\hline \& $96 \ldots ?$ & $11(12: 2)$ & $4: 8: 12$ & $(1,2 x+7)$ & 584152 & $11.18 \div 1$ & 76.3252 & 0.3145 \\
\hline $1: 4: 12$ & $0(1) \leq(1)$, & 411.142 & $(1 !) \vdots 11)$ & 6111,28 & $02(k 6$ & $701 \% / 62$ & 0.3514 \\
\hline$x_{1}+\mu_{n}$ & $0,(1), 4$ & 42119 & $\| 1 \ldots \ldots 1$ & $6,3(1)=$ & $0.21(x)$ & 81.80152 & (3. 75.26 \\
\hline $2 l_{1}, c_{1}<x_{2}$ & (1) & $4, \quad 2, \ldots$ & (1) 110 & at.17ta! & 01272 & 8.9522 & (1).36, 82 \\
\hline $21,1,4$ & ()$(x) !$ & $98110 ! !$ & 111.11 & $\cos 7262$ & $(1.2+17)$ & 80.866 .2 & $(1.17(\mathrm{~d})$ \\
\hline 22562 & 0.01254 & $4(17)$ & $(1)+46$ & 71.2372 & 0.2558 & 89.1122 & $(1.3816$ \\
\hline 19132 & $0(1) 30,1$ & $41\left\{1^{\cdots}\right\}$ & 111.42. & 137552 & (1) $2 \%\{0\}$ & (4). 45.10 & 0.3870 \\
\hline 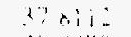 & $0(x, 2)$ & $\$ 54.21 \%$ & 11 itif & ${ }^{7}(1,95 ?$ & $0.25 \%$ & 94.4810 & $0.186,5$ \\
\hline $4 i 1+1 \%$ & $0 ! x \in$ & $58-1<?$ & 011.90 & 10110.0 & $(1.2 \% 21$ & $969 n^{\circ} 17$ & 0.19 .27 \\
\hline$d 1_{1}^{\prime} x^{\prime}$ & $1117 \mathrm{BH}$ & 61.11123 & $(1) 111)$ & 4181152 & $0.2 \times 5.1$ & (3) $4 x(17$ & $0.34(k)$ \\
\hline $94<, \ldots 2$ & i) 11112 & $\beta_{1}, x_{1}, \cdots$ & 1121112 & 615522 & $(1.21) 30$ & $101 \ldots 3111$ & $(0.34)(1$ \\
\hline $4: 14:$ & $(1) 1, n$ & $\because i, 1, \ldots$ & 0.2111 & sonkit? & $(1,4 k)$. & 10115351 & 0.3815 \\
\hline s,i ग) & (1) $12 \times 1$ & $t, t: \ldots$ & i) 2.161 & $50+12$ & (1) $3(1) \cdot 4$ & 1011.5310 & $0.38 \% 5$ \\
\hline $5341 \div$ & 1114.411 & $71, \because \because$ & $11 .{ }^{\prime} \cdot$ & 1114540 & 0.3111 & & \\
\hline$\{<1\} ?$ & (1) $18 \times 1$ & $146=$ & $(1: \div 11)$ & $0.4+4 \times 10$ & $(1,21) 51$ & & \\
\hline $54<15 ?$ & $0,1,6 \%$ & $76, \ldots ?$ & $112 \ldots, 1$ & $4(1,48) ?$ & 0.1114 & San 14. & 15? psia: \\
\hline $6,111 \ldots$ & (1) Bl & 74 1: & $0<1$ & $14.161(1)$ & 0306 & & \\
\hline$t ; t\left(m_{2}\right)$ & (1) 1. pin & xislez & $(1) \div<1$ & 1011.2271 & $(131 k 4$ & $1011.250)$ & $0.5 \% 60$ \\
\hline$(x, 1)$ & $(12) 11.4$ & Bd $2 \div$ & (1 $21, ? 1$ & $111 \mid<251$ & $0.3(x, y)$ & $10 \times 1.1024$ & 0.576 .4 \\
\hline l.s an. & 0.2111 & stosen: & $112 t 14$ & $1011 \leq 410$ & $(1.3(x, 1)$ & $97 .(6) 27$ & $(0.5 \% 24)$ \\
\hline 71.24. & $11: 221\}$ & $\left.\left.x^{\prime}\right)+1\right\}$ & $02: 14$ & & & $95 .(x) 12$ & $0.57 .3 \%$ \\
\hline 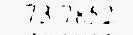 & (1) 1201 & $4105: 11$ & $(12, \cdots 1)$ & & & $0.386 t .4$ & () $50, k 1$ \\
\hline it 1242 & 0.221 & $44+131$ & $0 ? 2 \div 14$ & Sianl? & 1302 plose: & 847042 & $\left(1.56 x^{\prime}\right) \cdot t$ \\
\hline 79.180 & (1) ב & $(y, 1,1,1,7)$ & $6 \therefore 7$ & & & 87.01523 & 0.5 .46 .3 \\
\hline $818019 .$. & $0 \therefore 4 k 1$ & $(y)+f(x) ?$ & $112-411$ & 11116,2511 & (1) $2 k i d$ & 8444.2 & $1153 \%$ \\
\hline $4+4522$ & (1) $\therefore+1+\ldots$ & 101314 & (1):? & $11 x)(1824$ & 0 the & 81.8542 & $(1.5) 4)$ \\
\hline $81_{1}+x x_{1}=$ & $\therefore 44$ & $101<24 !$ & 1.2 .18 & $97+1127$ & $\{1,3, i f\}$, & 79.2372 & $(1.5) f_{2}$ \\
\hline sid $11 ? 2$ & $1 \quad 22$ & 101153111 & $6.274 x$ & $45(x) 1)$ & $0.38 \mathrm{~kg}$ & $76,(-122$ & $0.475 \%$ \\
\hline 91 (4. & 11.2564 & & & 42386 & 1: $3 k(x)$ & 7.407 .52 & $0.4 x+1$ \\
\hline 4.4 .36111 & 0.28 .14 & Sual !a. & $125 x p$ & git.7(1.4. & $113 \times 21$ & $71.48: 12$ & $0.4-421$ \\
\hline 96.967 & $(1: 57)$ & & & $87 .(16,2)$ & $(1.27 .12$ & $(18, x), 2$ & 0.4125 \\
\hline $34.41,11 ?$ & $(1.25 \%$ & 1111.6280 & 1) $31 \% 4$ & $8.9+9.82$ & $0.36,7.4$ & 66.3392 & 1).3.8.4. \\
\hline $1(1) 137$ & $1: 2574$ & $1(k)(1,24$ & $0 \quad 2(1), 4$ & $81.859=$ & $(1,35) k$ & 6.7 .7912 & $(1.3(n) .4$ \\
\hline $101<191$ & $(1,15)$, & $47+6(127$ & $\left.(1)^{2}\right)(x)$ & $74.277 ?$ & 0.3 .260 & 61.2172 & 0.1173 \\
\hline 11153$\} 1$ & $02 x$ & $4(k))$ & (1) 2(ik? & $?+1+\cdots+2 ?$ & (1).32.4 & $5 x \cdot(+1) 2$ & (1) $3(1 .+4$ \\
\hline & & is 3 sild & (1) 311011 & $-4115 ?$ & 11.116 & $\sin (148) 2$ & 1128.14 \\
\hline & & $84.71+2$ & 031145 & $11+4.1=$ & $1111+4$ & 53.4872 & 0.2412 \\
\hline$S_{\text {citll }} 11$. & 121100 pis & $y_{1}^{\prime \prime}\left(1 x_{2}\right)$ & $02 x+4$ & $\left(x_{1}^{\prime}(1) 1\right)$ & 11.21854 & $5(1) 1812$ & 0.2 .115 \\
\hline & & $8.9+172$ & (1) 24) & $(x)$ (1) & (12)is & $48 \div 22$ & 0.14 .4 .4 \\
\hline 1010.141 & 11.27 & $818<12$ & $112 \div 46$ & $0.941=$ & (1) ? $1,1 ?$ & 157482 & $01(x)$ \\
\hline $1(x, 0), 2$ & $11: \cdots$ & $4,2,19$ & (1) $\therefore \therefore$; & (3) & $(1 . . .+41)$ & 412.42 & (1.14?3 \\
\hline
\end{tabular}




\section{Reinforced Concrete Test Data}

Track 7 - High Pressure Test

\begin{tabular}{|c|c|c|c|c|c|c|c|}
\hline $40 . / 1142$ & 0.1081 & 32.5622 & $0(1), 1)$ & 50.7002 & $0.42(x)$ & $68.7382 ?$ & $(1.551 ?$ \\
\hline 38.1082 & 0.18 .32 & 35.1822 & 0.18607 & 43.3172 & $0.47(4$ & 71.2372 & (1.980R \\
\hline 35.568 .9 & $0 .(15.50)$ & 378112 & 0.1200 & 55.9202 & 0.5347 & 717852 & $(0.6,579$ \\
\hline 320742 & 0.11275 & 40.4 .172 & 0.1 .501 & 585152 & $0.5 \times 12$ & 76,3252 & 0.6715 \\
\hline $30.4 \times 2$ & $0(x+4)$ & 43982 & $0.14 \times 13$ & 61.1022 & $0.6+11$ & 74176.2 & 07118 \\
\hline 280552 & (1) (1) 24 & 456222 & 0.2282 & $6.3 .6(k) 2$ & 06055 & 818052 & $0.76(1)$ \\
\hline 30.61752 & $\theta(x) x)$ & $48.10(1)$ & $0.2(8)$ & 66.1762 & 0.7496 & 8.4 .3527 & 0.7857 \\
\hline .32 .40 .2 & 11.0257 & \&i) $7 \times 12$ & 0.311 & 60.7382 & $0.8(16)$ & sicisticis & $0.8(1) ?$ \\
\hline 35.1822 & 0.0552 & 3.3 .3172 & $(1.3 .44)$ & 71.2 .172 & (Ifini? & $80+1132$ & $0.8 .\{1 \times$. \\
\hline .77 .8112 & (1). (one) & $55.12(1)$ & $0.3 k .11$ & 73.1653 & 0.9714 & $9 i 4<10$ & $0 x+4 t i x$ \\
\hline $4(1+4) 2$ & $0.1(x, x)$ & $5 \times 5152$ & 0.4211 & 76.3252 & 0.9 .432 & $4+4 \times 10$ & (1) sit?) \\
\hline $43028:$ & (i) 1310 & 0.1 .10127 & 0.46 .113 & 70.1762 & $1.03(15$ & $40,46 ? 7$ & $11858 \mathrm{~K}$ \\
\hline $45.622 ?$ & (1) $1+x+1)$ & $f_{1} x_{x}(x)$ & $0.408 i f$, & 81.8052 & $1,(k, 5)$ & Q(1) $4(x) 7$ & (1) $85 \times 1$ \\
\hline $48.19(1)$ & 0.1118 & $0 x+1102$ & $0<\{3,0)$ & 84.3522 & 1.iciss & 1011327 & (1) $8(151)$ \\
\hline $5(1740) 2$ & (1)2124 & 187382 & 0.50 & $86 \times 8862$ & $\therefore 126 x$ & 101.9151 & (1) $x \in ?\}$ \\
\hline 533172 & (1) 2.256 & $71.22 \%$ & 0.6 .14 .4 & 84.4132 & $1.15(7)$ & 10115.10 & $0.86,+3$ \\
\hline 5502112 & $13.274 \%$ & 197812 & $(1) 6,(1)$ & $91.95 .4(1$ & $1.1 \times 1.77$ & & \\
\hline 585152 & $0.3(x,)^{r}$ & 703252 & ()finc? & (1) 4.4610 & $1.1 / 42$ & & \\
\hline 61.11122 & $0321 \%$, & 79.1762 & 07.310 & $96.987 ?$ & $1.2(16) x$ & $i \cdot(1)$ & \\
\hline $63(x) 192$ & $11.351,5$ & है। & $0.7<51$ & (x) $4(1,1) 7$ & 1.1862 & & \\
\hline 601.1762 & $0.3 / 41$ & $x+14.21$ & 0.7818 & 101.3371 & 1.1922 & & \\
\hline $6 \times .73 \times 2$ & 0.1117 & $x\left(1, x \times 1 n_{n}^{\prime}\right.$ & 0 ait) & 1011.351 & 1.1910 & & \\
\hline 71.2372 & 0.4728 & $80+112$ & $(1, k): 0)$ & $101.5310)$ & $1.14(4)$ & & \\
\hline 73785 & $(1,+1,1)$ & 1) 45. & $(18+20)$ & & & & \\
\hline $7(3.3259$ & $0 .+4(i) t)$ & $(1.4 .4 x) 11$ & 0832 & & & & \\
\hline $74.176:$ & $0.4(1) 4$ & $90.487 ?$ & 1).m. & s'inl $1 \%$ & I psiz! (gxinlten) & & \\
\hline BI Bits? & 0.5238 & (9) fext: & $0.8+2)$ & & & & \\
\hline$x+4.152: "$ & $(1,5.44)$ & $101121: 1$ & $(184.41$ & 1011.6250 & $0 \times x(1) 6$ & & \\
\hline sitikt.? & $(1)<40$ & $1011<351$ & $(1+131)$ & $10(x)(182.4$ & $(1 \times 0,12$ & & \\
\hline $84.41 \%:$ & 0.56 .22 & 10115.10 & $(1 \times 1+i)$ & $07 .(1) 27$ & $(1.852 .4$ & & \\
\hline 1)! (15.11 & $(15 t, 4)$ & \multirow{4}{*}{\multicolumn{2}{|c|}{ Scath l:. 1 15. prip }} & $95,(4,12$ & $(1.8+14)$ & & \\
\hline $19+4 \times 111$ & $(1,5 \% 1)$ & & & $1) \leq 1 \times(r-1$ & 0.839 & & \\
\hline 1$)(1,7)$ & $115 \times 14$ & & & $84.7(1.12$ & $(1.8247$ & & \\
\hline 1). $4(1)$ & 0.58014 & & & 870822 & 11.8125 & & \\
\hline 1113321 & $0 \sin 10$ & 10110.250 & $1.1 \times 3 h$ & $8+11.12$ & $\begin{array}{l}(17650 \\
(1) .8(1) 0\end{array}$ & & \\
\hline $\begin{array}{l}1011<\div<1 \\
1011<3101\end{array}$ & $11.9,81,1$ & $1(x) .13 .2 .4$ & $1.18 \times 0$ & 尚然, & $\begin{array}{l}0.79(1) \\
07.72\end{array}$ & & \\
\hline $1(1): 311)$ & $04801 \%$ & $\begin{array}{l}97(1) 107 \\
05(1) ! 2\end{array}$ & 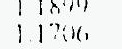 & $76,1.22$ & 0.6855 & & \\
\hline & & $92.1601 t$ & 1.162 & 14.1752 & $(1)(1584)$ & & \\
\hline \multirow{2}{*}{\multicolumn{2}{|c|}{ Sian 15, 140:2 meg }} & 80.7142 & $1.1 .5(1)$ & $7\} .451:$ & $0.51) 2 k$ & & \\
\hline & & ritis..? & 11218 & $68 x(x) ?$ & 0.547 .3 & & \\
\hline 1111.62501 & $0.8 .131)$ & $8.4+41.42$ & 1.1082 & $(x, 3,3,1) 2$ & $0.9(150)$ & & \\
\hline $1(x)(1): 24$ & $(1.8 .124$ & (i) $\times\{19:$ & 1.05.40 & $(0,3.7912$ & $0.41 .8 K$ & & \\
\hline 47.61 .7 & $0 \times 316$ & $792 \div ! 1$ & 1.0182 & $61.21 \div 2$ & $(0.4205$ & & \\
\hline $0<\{x \mid 12$ & $(18.4 .4$ & $760.42:$ & $\langle 19(1\rangle$, & 586.442 & $(1.31) \geq 4$ & & \\
\hline $42,36,6,1$ & 0.82 .5 & 7101752 & $(11) 337$ & S(i.).1.1.] & $0.2(, 0)\}$ & & \\
\hline $80.7(1.42$ & (1) 81$) ?$ & 714512 & 118.853 & 53.487 .2 & $0,3\left(x_{1}, 5\right.$ & & \\
\hline $87 .\left(1 k_{1} 23\right.$ & $0.81) \%$ & $(3 \times 4)(x) 2$ & $(1) B(k)$ & 50,19532 & 0.2484 & & \\
\hline 844142 & $0.78,(14)$ & $606230 ?$ & $0.74 \mathrm{k})$ & 482722 & 0.2554 & & \\
\hline bi) $8<1, ?$ & $0.7+76$ & $6,3.7412$ & $(1,6,0) 7(1)$ & $45, \because) 82$ & 0.22 .27 & & \\
\hline 7112172 & $(1.7 .3 .4 .4$ & 01.2172 & $116,301$. & 43.24 .42 & (1.1924 & & \\
\hline $70(+1))$ & $(1) 6,1)(13$ & $58.6942=$ & $(1.5 i k) 1$ & 411.711 .42 & (1) $16(1) 1$ & & \\
\hline $7411: 5$ & $0,6,0,4)$ & $56,0,162$ & $0.9+14$ & 38.10182 & 0.1344 & & \\
\hline 114512 & 06180 & $\{24 x\}$ & () $4 k_{2} 7$ & $35.561 \times 2$ & $0.11,31$ & & \\
\hline $68(x \times 12$ & (1).57ik & $511,45{ }^{\prime}$ & $0.45(14)$ & .32 .974 .2 & 010755 & & \\
\hline 60,3342 & 11.5318 & 48372 & $0.3 \times(1)$ & $3(1.4(4) 2$ & 0.0 .527 & & \\
\hline 617912 & $(0.4477$ & 45.7462 & 0.3277 & $2 \times(15.52$ & $0.021) 5$ & & \\
\hline 61.2172 & 11.4576 & $4.3 .2+4.2$ & $(1.27,7.4$ & 3011752 & 0.0421 & & \\
\hline $58.6-19 ?$ & 1142201 & $407(1+2$ & $0.214 x$ & 325012 & 0.07 .34 & & \\
\hline $56(4.8:$ & $0.2 k, 0,1)$ & 1811182 & 0.1621 & 351822 & 0.10117 & & \\
\hline $5148 \% ?$ & $\div-1+2: ?$ & $35.56, k 12$ & (1).135? & 3.8112 & $0.1+301$ & & \\
\hline 511.9632 & 11.2236 & 324742 & $0(14) 13$ & 411.4572 & 0.1617 & & \\
\hline $4 \times 3722$ & (1.2) & $30.4(x) ?$ & () $(1<1) .4$ & 4.11 .82 & $0.14 ! 0$ & & \\
\hline $\begin{array}{l}45.7982 \\
43244 ?\end{array}$ & $\begin{array}{l}0.26 k \\
(11) ? 9\end{array}$ & $\begin{array}{l}214011562 \\
11107<2\end{array}$ & $\begin{array}{l}\text { i) (nint } \\
\text { (1) risis? }\end{array}$ & $\begin{array}{l}456122 \\
440(1)\end{array}$ & $\begin{array}{l}0.2241 \\
0.2567\end{array}$ & & \\
\hline 40.70 .72 & (1.) 5,1 & $32 x$ & (1) 141 ? & 50 $74(1)$ & $0.28 \%$ & & \\
\hline $38.1015: 2$ & 01221 & 35.16 .2 & $(1) 3 f_{1} c$ & $<\&\{1: 2$ & $11+11^{2}:$ & & \\
\hline $34 s_{1} b^{\prime}$ & $111: 51.2$ & $\{: \times 1:\rangle$ & $\|11\| 1.1$ & 45097 & i) $35 x x$ & & \\
\hline $324 \div 42$ & 1) $115 ! 4$ & $4014 \% ?$ & $(12)(1)$ & $3 x 515$ & (1) 11011 & & \\
\hline $81.4(x)_{2}^{2}$ & 0.11225 & $43168:$ & $(1.2 \%, 41$ & $(1) 1012]$ & 0.142 & & \\
\hline In 155. & (1)(K).2 & 4.562. & $1123 n$ & $4,3614$. & $41.4) 4$ & & \\
\hline 3010752 & $(0.1(k) 7 \mathrm{~A}$ & $48.1(x) 2$ & 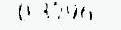 & $(x):,()_{\infty}^{\prime}$ & 0.50161 & & \\
\hline
\end{tabular}




\section{Reinforced Concrete Test Data}

Track 8 - High Pressure Test

\begin{tabular}{|c|c|c|c|c|c|c|c|}
\hline \multicolumn{2}{|c|}{ Sian 1, 44.2 pisig } & 70.5335 & 0.01746 & $184,(6,55$ & 0.07 .37 & 169.982 .5 & $0.0(x)+.4$ \\
\hline $267.10 \times()$ & 0.0448 & 64.67 .35 & $0 .(k, \times 0)$ & $\begin{array}{l}180.7615 \\
180.5755\end{array}$ & $\begin{array}{l}0.07)(1, \\
0.0753\end{array}$ & $\begin{array}{l}167.20335 \\
164.3735\end{array}$ & $\begin{array}{l}0.1044 \\
0.0484\end{array}$ \\
\hline 205.3715 & 0.0510 & 62.1835 & $(0 .(K+1)$ & 192.2805 & 0.0754 & $161.6,53.5$ & 0.087 .1 \\
\hline 262.5386 & 0.0614 & 54.34 .15 & $0 .(K, 5:$ & $105 .(0.46 .5$ & $(1)(1) 70 \%$ & $15 k .8 .43 .5$ & 0.1882 \\
\hline 254.3919 & $0 .(6,16)$ & 56.57 .15 & $0 .(k, 7)$ & 1078225 & 0.17764 & $15 t, 0 \times, 35$ & (). $(1440$ \\
\hline 256.1125 & $0 .(15(x)$ & 53.7 .135 & $0 .(x \times)(1)$ & $2(x) .4755$ & $0.0 \% 142$ & 1.53 .18 .35 & $0 .(k) 10$ \\
\hline 2520825 & $0(151 ?$ & 50.9245 & 0.0 .522 & 203.475 .5 & $0.0 \times 1,77$ & 150.3335 & $0 .||+i \mid$ \\
\hline 249.8425 & 0.0 .505 & 48.110 .5 & 0 ritul & $2(x+10) / 5$ & U.iking & 147.43 .35 & 0.1027 \\
\hline 246.12245 & 0.0574 & 45.321 .5 & 0.011 .25 & $20 \times 88.5$ & $0.070) ?$ & 144.55 .35 & 0.10110 \\
\hline 2441085 & $0 .\left(K_{1},(), 4\right.$ & 47.55 .35 & $0 .(14)$ & 211.14 .95 & 0.0575 & 141.6935 & 0.1010 \\
\hline$? 41.2505$ & $\left.0 .\left(K_{1}()\right)\right]$ & 30.7435 & 00.118 & 214.23015 & 0.013 .1 & 138.803 .5 & $0 .(x)>7$ \\
\hline 238.45 .45 & $0 .(19.9)$ & 3711239 & 0.0 .300 & 210.9235 & 0.0721 & 1250235 & $0 .(k x) y$ \\
\hline $2.35 .6,595$ & 0.01550 & 34.16 .35 & $0.01)(.2$ & 219.6505 & $0.0 \sin 4$ & 1.32983 .5 & 0.08 sit \\
\hline 212.8015 & $0(k, 1) 2$ & 31.3235 & 0.0124 & 222.3125 & $(0.0 \div 201$ & 129.70135 & $0.080 ?$ \\
\hline $230.1 \times 6.5$ & $(1)(k, 70)$ & $7 \times 2735$ & $0.0 .390)$ & $215.0 \times 155$ & 0.0710 & 126.7435 & (1. (x) kn? \\
\hline 227.2715 & (). $1 x, 56$ : & 20.2235 & niti,i) & 222.61045 & $0.058 \%$ & $12 \times 8215$ & 0.0095 \\
\hline 2.4570 .5 & o.trivi & 28.6895 & (1)(1.119) & 2307925 & $11(149 i$ & 121010139 & 011174 \\
\hline 2218.425 & (). $(k, 1)]$ & 31.5115 & $(1)(1)(1)$ & $23,3.180 .5$ & 0.11838 & 118.1435 & $0098+$ \\
\hline 214.1275 & $0.16(1 .+7)$ & $3.4(10) 3$ & ().121. & 2358.425 & 0.11592 & 115.45 .45 & $0.1(x) 2$ \\
\hline $216.3<25$ & olokfor & 37.80139 & $(10<27$ & $23 k 5,50,5$ & $01,\{150) 1$ & 112.533 .5 & 0.1031 \\
\hline$? 136$ ins & 010727 & 10.6415 & (1)6.110 & $241: 1115$ & (o.cositi & $1(11) 68.75$ & 0.1020 \\
\hline 210.88 .25 & $(j,(x, 1 x)$ & $4.1,1,15$ & $(1) 11111$ & $244 \times 215$ & $0,(x, k: 2$ & $1(k, 0) 235$ & $0.1(184)$ \\
\hline $2(18.1664$ & Oofretis & $41,5.45$ & $(161) 6.42$ & 2.86 .4055 & $0(x) 8$, & 114.1215 & 0.1014 .4 \\
\hline 20155355 & 0.0815 & 411.91945 & $005+1$ & $2+40.11065$ & (1). 11526 & 1111.3735 & $01(150$ \\
\hline 2012.81 .35 & $0.16<3$ & 52.4335 & $116 x \cdot 13$ & $251.84,15$ & $(1.050)$ & 98.5715 & 0.1214 \\
\hline $2(x, 2,4) 5$ & 100127 & 55.1135 & $0,16,58$ & 25.45475 & $0 .(18.3 .4$ & 9.76 .15 & 0.1085 \\
\hline 197.9455 & 1107.48 & $5 x<525$ & $00 \operatorname{lsin} 1)$ & $25: 4+6,5$ & $0 .(1)+4.4$ & 92.4 .935 & 0.114 .5 \\
\hline $144.621=$ & $(1.1)] 1+4$ & 6idias & $6.26)^{2}$ & $7+11 ? 79.1$ & 011.44 .4 & 9()$,(x, 35$ & $0 .(x) \leq 0$ \\
\hline 19211155 & $11.1749 ?$ & $(1 \therefore 4,2,5$ & (1) $10 \mathrm{mon}$ & $20,3(0 .+44)$ & () $(1.4 .38$ & 87.31 .35 & $(1, y)=1$ \\
\hline $1 \times 4.3724$ & $01112(1$ & fixpluis & $0.1 \times 11$ & $2058+40$ & (1). 11.13 & $8: 51.15$ & $0 .(k) .13$ \\
\hline $1201+1215$ & $(1.61+4)$ & 71.0219 & (1)(18) 11. & $207(1022$ & $(1.0) .136$ & 81.8215 & $\{1(x) 7 ?$ \\
\hline 163.1215 & $(1) 11 ! 628$ & 7.411 .435 & 11116 & $267)(16,52$ & $0.61+12$ & 78.96 .15 & ()$(x) c)$ \\
\hline 181.11 .45 & $(1,(1), 24$ & $77(101,35$ & $(1)(18)(1)$ & $2 \therefore \pi,(1), 34$ & $0(1) 114 k$ & 76.2139 & $0(1 \times) 7$ \\
\hline 178.1525 & $0(1712$ & $710 \leqslant 10^{16}$ & (ifiagy & & & 733335 & $(1(k)()\}$ \\
\hline 175 6544 & $(1)(1)(\mu)$ & 82.2015 & 111901 & Bxan 2, 51 & 6 pखp & 70.53 .39 & $(1,(x) \geq y)$ \\
\hline 17.7795 & 1) 11768 & die r.i.s & ockistt & & & $0,7.73 .3 .5$ & (1) osite \\
\hline $16(1)(4) \times 25$ & $0 .(18.23$ & 88.1535 & $0(16) 20)$ & $267 x \ln (x)$ & $(1.11 .30) !$ & 64.8735 & $0 .(x+10)$ \\
\hline 167.21134 & O.0Bfi:- & (7) 1.2225 & $(1.1147)$ & $2(1,5,1 / 15$ & 0.11 .2 .4 .4 & $6.1 \times 35$ & 0.01127 \\
\hline 164.3725 & undet) & $0.9(k): 5$ & $(0,1) \mathrm{B}+111$ & 26,25386 & $(1)(1514$ & $50,2+15$ & 0.11703 \\
\hline in. 105.5 & U) $(4,1, x)$ & 96.12129 & $1)(1 \times .12$ & 2519.31114 & $(1.11 .46,6$ & 505715 & 0.0770 \\
\hline 158.84 .5 & $(1.1786)$ & 40.79 .15 & $(1,(1) 14,2$ & 256.11 .5 & 0.01.90\%: & 53.7435 & 0.617 .28 \\
\hline $1.5 n,()+35$ & $0.07 .41)$ & 1025.35 & $1111 \% 4$ & 2528615 & $0.0(6,70$ & 50.4795 & $0 .\left(x_{1}\right)(x)$ \\
\hline 153.18 .35 & 1111221 & $1052.26,45$ & $(0 .(4) 2)$ & $24 \% 8.25$ & $0.0 .5 \times 3$ & 48.103 .5 & 0.0540 \\
\hline 1503335 & () (18ki) & $16 \times .1+1.5$ & $0.018+1$ & $240.4+5$ & 0.06185 & 4.5 .32 .35 & (1).0554 \\
\hline $14 \% 4335$ & $0(1) ? 11$ & 110.7735 & $(1,08)$ & $34.411 k 5$ & 0.0750 & 42.55 .35 & 0.0484 \\
\hline $1+4.5535$ & $(1.17721$ & 113.13 is & U1, IS $(1, ?$ & 241.25015 & $(0)(36) 5$ & 39.714 .15 & (1) $(1,4)(1)$ \\
\hline $1+1.6935$ & $0(17.11$ & 116.1615 & 0.0861 .1 & 236.454 .5 & $(1.0718$ & 37.123 .35 & 0.0375 \\
\hline 138.0765 & (i) $\left(3 n^{\prime}+1\right.$ & 118.98 .35 & 0.183 .31 & 235.61505 & $0.076,1$ & 34.16 .44 & (1). (1):21) \\
\hline $1.3542: 39$ & 0.194 .15 & 121.51835 & $(1,19,9 k$ & $232 k(1) 5$ & $(1,1) 76.4$ & $31 . .3235$ & $0 .(K K M)$ \\
\hline 1.32 .9835 & (1) $11 / 18$ & 1242435 & (0.18) 117 & $23 i(k)(x, 5$ & $0.0 \times 77$ & 282737 & $(1 .(x) \times)$ \\
\hline 124.70 .35 & $0 .\left(x_{1}\right)(1)$ & $12.6+4.434$ & $0 .\left(k x^{\prime} 2 k\right.$ & 227.2715 & 0.11812 & 26.2275 & $0 .(x)(x)$ \\
\hline 126.74 .5 & $(10 ; i+1)$ & $12 y,(x, 13)$ & (1) (1) & $22+.5165$ & $0 ! 1152$ & $28(x \times 1)$ & $U .(x \times x)$ \\
\hline 123823.5 & 0.1635 & 1323125 & 016904 & 2218125 & 0.07 .57 & 31.53 .35 & -()$.(x) .54$ \\
\hline 121.04325 & $0,6155 !$ & $12511 \times 25$ & $11018 ! 1$ & 214.1275 & $0.11^{\circ} 7(x)$ & 34.6435 & $0 .(1) 2.5$ \\
\hline 118.1729 & $0.0160+1$ & 13776,35 & $0.017+5$ & 216.349 & U.unit & 37.8035 & 0.0214 \\
\hline 115.45 .35 & $0(y)(x)$ & $1.4091 ? 5$ & 0.1870 & 213.6745 & 0.07801 & 40.60 .35 & $0.02 .40)$ \\
\hline 1125435 & $(1010 ; 55$ & 1.431235 & (1)(1tixy) & $210.8 \times 15$ & ().08(14) & $4.3 .6,3.35$ & 0.0 .276 \\
\hline $1(x), f(x)+5$ & $0.16 k^{2}+4$ & 1458235 & (1) $(1742$ & 208.1885 & 0.0708 & 46.53 .35 & 0.0 .350 \\
\hline$f(x, 9) \geq 35$ & $0.0 n+1$ & 140.4135 & 1) $\left(10^{2}\right\}$ & 2015.5 .555 & Ooket & 49.44 .35 & 0.)(1-1) \\
\hline 1114.124 & 0.0198 & 151.17 .95 & $(1.110 .5$ & $2(1) k 0,4$ & $(1)(07,35$ & 52.4335 & $0 .(1+7)$ \\
\hline 1011.37 .15 & $(0 .(18 ! x)$ & 153.8 .4 .35 & 0.01712 & $3(4) .25(15$ & 0.018 .301 & 55.41 .35 & 0.01577 \\
\hline 98.573 .5 & $004 y 5$ & 150.681 .45 & 0.0753 & 197.4955 & $0.08+4$ & 58.553 .5 & 0.0487 \\
\hline 95.76 .35 & 0.014 .48 & 150.40 .15 & $(1 .(1)) / 4$ & 1448215 & $0.08(1) 1$ & 61.613 .35 & 0.0550 \\
\hline 92.9 .4 .5 & $(1 .(k)())$ & 16.210145 & 110116.4 & 1921055 & o.t)ken & $64.40,35$ & (1.0758 \\
\hline $40(x, 15$ & 0.0623 & 16.478 .3 .5 & 0. (x, site & 1803725 & 0.11818 & $6, x(x, 3,5$ & Onofits \\
\hline 873139 & () $18 \mathrm{~s}(\mathrm{k})$ & $16,7<36.5$ & $0117(, 1)$ & 186.83 .15 & 0.018 .50 & 71.0215 & 0.11850 \\
\hline $84.51+5$ & 0.1793 & $17(12285$ & 00780 & 10.3 .72615 & $0 .(16.2)$ & 74.1439 & 0.11807 \\
\hline 81.8235 & 1107.46 & 1721464 & $0,(x, 1,0)$ & $1 k 1.11 .45$ & $0 .(14,1)$ & $(1 .(x) 1)$ & $(1.01 / 0.7$ \\
\hline $7 k, 14.35$ & 0.18ifine & 17580,35 & $(1)(x, 1)$ & $17 \times .3525$ & 0.118 .31 & 79.80 .35 & () $(x) 31$ \\
\hline 76.2139 & $01,17) 51$ & 1760375 & lisinge) & 175.5555 & (1.0k.34 & 82.7175 & $0.183 x^{\prime}$ \\
\hline 73.3235 & 0.95 .17 & 181.3945 & $(1,4 i), 7$ & 1727745 & $0.06 x+1)$ & 85.51 .15 & Quintl \\
\hline
\end{tabular}




\section{Reinforced Concrete Test Data}

Track 8 - High Pressure Test

\begin{tabular}{|c|c|c|c|c|c|c|c|}
\hline 88.3535 & $0.09 \%$ & $2671(x)$ & $0 .(3,1) 2$ & 64.87 .35 & $(0 .(x)+10)$ & 189.5755 & 0.09496 \\
\hline 91.2235 & 0.0445 & 70.5 .3715 & 0.0774 & $6 \because 16$ is & (o.cwe.t & 192.289 .5 & $0 . i$ ink \\
\hline $94 .(k, 3)$ & $0 .(\mu)>7$ & 2625860 & 01164 ? & $59,34.35$ & $0.0 \times 50(1)$ & 105.040 .5 & $0.0 \%, 1$ \\
\hline 90.9235 & $0 .(\mu) .31$ & 259.31910 & () 118.34 & 56.57 .35 & 0.0970 & 197.8225 & $0.1(x)$ \\
\hline $99.74+5$ & $0 .(k) 78$ & 256.1125 & $(3)(x)) 4$ & .5 .7429 & $0 .(1808$ & $2(x) .4755$ & $0.0 x+x)$ \\
\hline 1112.5735 & $0 .(1)(x)$ & 2528835 & $0.016,1\}$ & 50.92 .35 & $0 .(x, 4)$ & 203.4755 & 0.04913 \\
\hline 101526.35 & 0.1101 & 2408425 & $\left(1,\left(x_{1}\right)\right)$ & 48.1035 & $0(k, 26$ & $2(x) .1675$ & 0.09 .30 \\
\hline 1018604.5 & 0.0 isis & 246.9245 & $0(1680)$ & 45.32 .35 & 0.0527 & $2018.8 \times 35$ & $0 .(9326$ \\
\hline 110.7735 & $0 .(k) 0)$ & 244.1018 .5 & ()$(n)(1)$ & 42.553 .5 & 0.0585 & 211.4045 & 0.0853 \\
\hline 113.5335 & $0 .(k)(0) 7$ & 241.25015 & $(1.0847$ & 39.7935 & 0.0476 & 2142305 & $0 .(6924$ \\
\hline 116.1835 & $0 .(10)(1.3$ & $238.45+5$ & 0.0888 & $37 .(12.15$ & 0.0 .387 & 216.9235 & $0 .(x+14$ \\
\hline 118.98 .35 & $0(\mu)(X)$ & 235.6545 & (o.ckus & 34.163 .5 & 0.011 .16 & 219.6505 & 0.0887 \\
\hline 121.5435 & U.tr)tol & 2328015 & $(1 .(x) 6.3$ & 313235 & $-0(x) 18$. & 222.3125 & $0 .(4) 29$ \\
\hline 124.24 .35 & 0.0874 & 230.000 .5 & 0.10121 & 28.27 .35 & $0 .(x)(x)$ & 2250055 & 0.0898 \\
\hline 126.4439 & $0.0 \times 75$ & 227.2715 & 0.10121 & 26.2215 & $0.0 \times(x)$ & 297.6905 & 0.0896 \\
\hline 121) $\times \times, 35$ & $(0)(k / k)$ & $224.9 / 0.5$ & 01120 & 28.68335 & $0 .(x)(x)$ & 230.3935 & 0.08 .36 \\
\hline 1323139 & O(Givi) & 271.8 .125 & $0.110 k$, & 3153335 & $(1)(x \times x)$ & 233.0805 & $0.111 ?$ \\
\hline 135.1839 & 0.10115 & $214127 \mathrm{~s}$ & $0.110(1)$ & $3.46(1) 35$ & 111114.5 & 235.8425 & $0.096(1)$ \\
\hline 197.7615 & orosici? & 210.15 .4 & (1).1258 & 37.86194 & $0.02+10$ & 238.456 .5 & $0.0 \times 62$ \\
\hline $1+101.51 .35$ & $0(1)+x)$ & 2136,705 & 0.1150 & $4(1,4,1) \div 5$ & 001372 & 241.2105 & 0.0782 \\
\hline 1.93 .133 .34 & $0.04) 57$ & $210.88^{25}$ & 1) II in & $43.0,335$ & 0.01286 & 2438215 & $0 .(18.47$ \\
\hline 1458245 & (1)(11)) & 208.1664 & 01.107 .5 & 40,5315 & $0.04+8$ & 246.4455 & 0.01821, \\
\hline 1484215 & (1)(M)Sil & 2115.5255 & (1.12.4.4 & 44.81935 & (0.0.5.4? & 249.11185 & $0 .(k, 7)$ \\
\hline $1511: 26$ & Sinnil! & 201281.25 & (1) 10i: i & 52.4315 & $0(1)(120)$ & $251.86,3.5$ & (1) $(k, 5,3$ \\
\hline i.518.175 & (i)(K)( ? ? & 20025015 & $(11.11)$ & $55+135$ & $00^{-}(x)$ & 2.5 .4 .5177 .5 & (1) $1(x, 5$ \\
\hline 15000125 & $0(14) 10$ & 167.8055 & (1) 124 & 56.5535 & $1)\left(K_{1}(x)\right.$ & $25: 4+6.5$ & $0 .(17) 8$ \\
\hline 159.41125 & $(101456$ & $11+8 x_{2} 15$ & (1) 1254 & 0.10 .135 & $(0.1) 724)$ & 200.2754 & (1).054! \\
\hline 16211135 & (1) 1105 & 11) 2.1045 & (1) $) 17(1$ & $(+4)(1): 5$ & $0 .\left(1 x^{\prime} 54\right)$ & 36.301449 & $0.0147 \%$ \\
\hline 100.7835 & $(i)(K)(x)$ & 184.3725 & $(1.11 .41)$ & $1.80(1) .5$ & (1)(14) 57 & 26.5 .84440 & 0.01 .18 \\
\hline $10,7.5360^{2}$ & (1) (ixiser & 180143.45 & (1) 118.t & 71.0325 & $0 .(10) 70$ & $267 . i(178$ & 0.0 .325 \\
\hline$\because 7(1):{ }^{\prime}$ & $0(1)\left(x_{1}\right.$ & $1 \times 17 ? 115$ & 012017 & 74.11 .4 .5 & $(1.18 \times 53$ & & \\
\hline $173^{2} 1 .+13^{5}$ & $0(181) 5$ & 181014.45 & $(1.12115$ & $77(10.55$ & $(0.111) 21$ & $\operatorname{Sian} 4.80$ & 2 psik \\
\hline 1758625 & (1) (1).10 & $17 \times 15: 4$ & $11.11: 2$ & 74.8 .6 .35 & $(1.101 .4 .4$ & $26.7 .1(x)$ & $(1) i(x, 1)$ \\
\hline $1 / 80.75$ & liti:5 & $175 \leqslant 64$ & 0.12012 & $8: 1939$ & (1) $110(1)$ & 265.3715 & 0.1112 \\
\hline 1813035 & (1)(1i)(1)) & 172.7745 & (0.1280 & $65 \$ 135$ & 0.10 .1 & 262.5386 & $0.12 \%$ \\
\hline 181.055 & $0.0 \mathrm{isth}$ & $16,4.0 x_{2}: 5$ & 0.13011 & 863535 & 0.11018 & 250.3919 & 0.1153 \\
\hline Inci.7(1). & $0.04)(3$ & 16791135 & 111318 & 41.2235 & $(1.10 .37$ & 256.1125 & 0.1147 \\
\hline 180.5755 & $(1)(1) ? 2$ & 16.1 .37 .15 & 0.1316 & 0.1110 .15 & 0.1075 & 252.88 .15 & 0.122 .1 \\
\hline 102.28 .95 & $0(14+10)$ & 1610,535 & 01111 & $(y, 1): 19$ & 6.10 .55 & 2.19 .8425 & 0.1102 \\
\hline $195(1.465$ & $0.114(1)$ & 158.8135 & $(1.1201$ & 447675 & 0.1140 & 246.924 .5 & 0.1136 \\
\hline 107.8225 & $(1 . \operatorname{lin}(x)$ & 156.16 .15 & 0.13014 & 1012.5719 & $(i .1(x) !$ & 244.1085 & $(1.1250$ \\
\hline $2(x) .4755$ & $0 .(14) 1.7$ & 152.1535 & $(1.1 .31)($, & $1015.21,15$ & 011.42 & 291.2505 & 0.1228 \\
\hline $2(1.3 .4755$ & (1). (1) he - & 1.902185 & (1) 15.12 & 101801.2 .5 & $0.101 \times 0$ & $23 x+15+5$ & 0.116 .5 \\
\hline $2(x), 1075$ & () $(1, \times) !$ & 147.1235 & (1) 12kes & 110.9739 & $(1.1112$ & 235.6545 & $0.1(x) y)$ \\
\hline $201 \times$ pin is & $0.010,8$ & 144.55 .55 & $(1.1311)$ & 113.5335 & 01115 & 232.8015 & 0.12 .48 \\
\hline 2114445 & (1.08:20) & 141.61935 & $(1.12 \%)$ & 116.1815 & $(1.115)$ & $230 .(k x, 5$ & 0.1260 \\
\hline 214.23015 & $0.10 \mathrm{~km}$ & $138 \times 1175$ & () 124 & $1: 8.9 x+5$ & 0.1210 & 227.2715 & 0.1 .127 \\
\hline $216,42,14$ & ócisis & 135.92 .45 & $(1.1 .11)$ & $121.51 \% 35$ & $(1.1 \div 1.5$ & 224.576 .5 & 0.1337 \\
\hline $21905 \%$ & $0 .(18801)$ & 1320849 & $0.111 \%$ & 124.293 .5 & () 11158 & 2218.425 & 0.13 .42 \\
\hline $2: 2.9125$ & 0.0962 & 12671125 & (1) 1(ifly & 126.9445 & 0.1118 & 219.1275 & $0.1 .39 \mathrm{~s}$ \\
\hline $225 .(\times 15.5$ & $0(14) 14$ & 126.7 .435 & $0.12(1)$ & 12466.35 & 0.1155 & 216.1525 & 0.1525 \\
\hline $227.6,045$ & 0.18 .30 & 123.823 .5 & 0.124 .9 & 132.333 .5 & $0.1(x, 5$ & 213.6745 & 0.1 .970 \\
\hline $23(1.35 .35$ & $0(1) 702$ & $121 .(x) .35$ & 0.1181 & 135.0835 & 0.1144 & 210.8875 & 0.1377 \\
\hline 231.0865 & $0(14.12$ & 118.1935 & $0.12(13$ & 137.76 .35 & 0.1112 & 208.1885 & $0.13(1)$ \\
\hline $215 \times 8425$ & $(1.0789)$ & 115.45 .15 & 0.132 .3 & $14(1.51 .45$ & 0.1115 & 205.5 .355 & 0.1476 \\
\hline 238.5565 & (). 01830 & 112.5335 & 0.1111 & 1433.1335 & 0.1102 & 202.8635 & 0.1 .390 \\
\hline 241.1116 & $0\left(1 x^{2} 24\right)$ & 10968.35 & $(0.120)$ & 145.8235 & $0,(x)(0)$ & $2(x) .2505$ & 0.1424 \\
\hline 2438215 & 0.618013 & $10 \times 1.92 \times 5$ & 0.1178 & 148.43 .15 & 0.1057 & 197.4955 & 0.1 .406 \\
\hline 2.40 .4055 & o.cisis & 1014.123 .5 & 0.1200 & 151.17 .15 & 0.0970 & (1)4.8215 & $0.13 \%$ \\
\hline 24010184 & 0.17301 & $1011.3 \% 35$ & $0.111) 2$ & 153.8435 & $0(1) \geqslant 1+$ & 192.1055 & $0.1+19$ \\
\hline 2518635 & $0,(k, ?)$ & $9 \times .5715$ & $(0.1211$ & 15e.unsus & (i) (w) 26 & 189.3725 & 0.1 .36 .2 \\
\hline 354 suts & $0016: 3$ & 95.76 .25 & $0.12 \times 5$ & 159.4935 & $0.1(1 / 3$ & 186.4345 & 0.1453 \\
\hline 257.4 .46 .9 & 0.015017 & 920.435 & $0.11 \%$ & 16.210 .35 & $01(x) s$ & 18.3 .7205 & $0 . i \cdot 26.2$ \\
\hline $2(x) .2754$ & $0 .(x, 50)$ & $9(i, 14,4.5$ & 0.1191 & 16.4 .78 .35 & (0.0.1925 & 181.0445 & 0.1543 \\
\hline $203 .(14.49$ & $0 .(k) 3$ & 87.9135 & $0.1(N)(1)$ & 167.530 .9 & 0.10 .1 .1 & 178.3525 & $0.130, k$ \\
\hline 26584.50 & (i) 1148.3 & 84.5135 & 0.1142 & 170.7285 & 0.10150 & 175.55 .15 & 0.1451 \\
\hline 20111422 & $0 .\left(k_{1}\right)+4$ & 81.8235 & 0.1040 & 17.3 .0 .40 .5 & $(1)(14) 4$ & 1727745 & () 1.380 \\
\hline $267.16,52$ & i) $1 v_{1}, 2 k$ & 78.96 .35 & 0.1087 & $175,86.35$ & $(1.6) 86.3$ & 1090825 & 0.1474 \\
\hline $267 \cdot(\mu) 35$ & 0.0501 & 76.21 .15 & $0.1(1.2)$ & 178.6 .375 & 0.1156 & 10.720 .25 & 0.1553 \\
\hline \multirow{3}{*}{\multicolumn{2}{|c|}{ Scall 3, (1) a paigh }} & 1.1 .1415 & U.!Mx: & inisivi= & (i) jiin & icmisis: & שים \\
\hline & & 70.533 .5 & 0.1012 .4 & 1840655 & 0.1146 & 16.16 .535 & 0.14 .32 \\
\hline & & 0.7 .7335 & 0.1035 & $180.76,15$ & 0.1024 & 158.8435 & 0.1557 \\
\hline
\end{tabular}




\section{Reinforced Concrete Test Data}

Track 8 - High Pressure Test

\begin{tabular}{|c|c|c|c|c|c|c|c|}
\hline $15 x: 4$ is & $11 \mid+\ldots 1$ & $10257 ?$ & 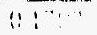 & $\therefore 9011.14$ & $(1) 1021$ & 53.7435 & 0.1090 \\
\hline $111 \times 19$ & 1114 & 105.2634 & $(1,14,0)$ & $252 \times 815$ & $0.128 .1)$ & 50.9235 & 0.0880 \\
\hline 1981125 & $\| 11:$ & $1018(4) 15$ & $(1) \times$ & 240 6425 & $0.120 ?$ & 48.1035 & $0.07 \times 9$ \\
\hline 14.418 & $t: 1+18$ & 110.7739 & (1) In: & 2460245 & 0.13017 & 45.3235 & 0.07 .11 \\
\hline $14<<15$ & 11.11 & 113.5135 & $111^{n}$ & $24410 \times 5$ & (1) 1.9012 & 42.5535 & $0.0 \times 477$ \\
\hline $1.1 !+1016$ & $11: 41:$ & 116.18 .19 & $1: t_{1}$ & $241: 5(15$ & $0.14(1)$ & 39.7935 & $0.0 \times(K)$ \\
\hline $13 x+1:=$ & $11<\because$ & $118 \mathrm{cs}$ is & $11 !<$ & Znstiss & 0.1370 & 370235 & 0.0171 \\
\hline 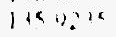 & $1 . i$ & 121.41134 & $11): i$ & 2350,549 & $(1.1474$ & $.14 .16,3.5$ & $0.0(1) 10$ \\
\hline 11240 & $i: 1: \cdots$ & 1242114 & $111 \%$ & $232 x+15$ & () 1 (1) & 31,3235 & $0 .(x \times x)$ \\
\hline 120,116 & 1114 & $126,4+4$ is & $11):$ & $23(1110 \times 15$ & (1) Iexix & 28.2115 & $0 .(x \times x)$ \\
\hline $1.07,6$ & 1114 & $1211 .(x, 15$ & 1111 & $\therefore 7 ? 15$ & 0.1541 & 26.2235 & $0 .(x)(x)$ \\
\hline $122 x: 16$ & $(114 m)$ & 132146 & $\left(11^{\prime}:\right.$ & $21+5: 0,5$ & (1) 1.536 & $28.0 \times 35$ & $0.0(x)(x)$ \\
\hline $1: 1(n) \cdot a$ & (1) 1106 & 135018.19 & $\because 1 \cdot$, & $211 \times+2 \div 5$ & (1) 14,1 & 31.53 .35 & $0 .(x) 76$ \\
\hline 1181414 & $(11.142$ & $1.37 \% 1,14$ & $11 \ldots$ & 2141275 & (1.) 1415.5 & 34.64335 & 0.0117 \\
\hline $1151<16$ & 111410 & $14051+5$ & $1: \ldots$, & $216,24.5$ & $(1.1613$ & 37.8035 & 0.026 .5 \\
\hline $13: 4,25$ & (1) itent & 1431118 & $\because \mid \cdots 1$ & $\therefore 10,045$ & 0.1558 & 40,6015 & 0.0310 \\
\hline line tim is & $11<13$ & $1.458: 16$ & (1) : 1, : & 211182815 & (1) 1521 & $43,6,3,35$ & $0 .(1,3.51)$ \\
\hline$l_{1} \pi, y_{2}$ is & $1115: 2$ & $14 \times 14$ & $111 \ldots$ & $20 \times 1585$ & 0.1316 & $46.53,35$ & 0. (45) \\
\hline 11b 1): & $111<4$ & $1511: 16$ & is : : : & 21155355 & $(1.1506$ & 49.4035 & $(0,(x, 2)$ \\
\hline $1011 \%$ & $11) 4 i 11$ & 1538415 & $1: 1:$ & 211 Notis & (1). 1.107 & 52.4335 & $0.076(1)$ \\
\hline $4 x<\cdots x$ & (1) $1+x^{4}$ & 156,106 & $1+112$ & $2(x) \geq 5(15$ & $(1) 16133$ & 5.5 .413 .5 & $0(x) 10$ \\
\hline ya 1,14 & 0140.4 & $151) .1019$ & $1: 1 \cdot 1$ & $14: 3455$ & (i.) 11 ) & 58.5535 & o. okik. \\
\hline $12+14$ & 110.11 & $1021111 \mathrm{c}$ & $(1 ;<, i$ & 14. 8219 & $0.156 x$ & 6101135 & ()$(k) .4()$ \\
\hline$\left(y_{1}\right) 1 \times, 16$ & 111.92 .1 & $16.1 ? \mathrm{a}<\mathrm{c}$ & $1,1: 3$ & WU Kk5 & 01.157 .1 & $(04.96 .35$ & (1) 11.5 .3 \\
\hline$x: 11<$ & (1) $10, ?$ & $\mid \theta_{1}=\left\{p_{1}<\right.$ & $111 \%$ & 1801725 & 0.1518 & $0,8,(x), 1,5$ & $(1.1+42)$ \\
\hline$x+1 \times 1=5$ & $111<11$. & 1712385 & $1: 1: 1$ & $181,41.15$ & 0.1612 & 71.0235 & (1) 1.3 .14 \\
\hline $8: \therefore 16$ & $0.13(1)\}$ & $173: 411.6$ & $111^{<}$ & $1817: 04$ & (1. 16,28 & $74.14 \div 5$ & 0.1376 \\
\hline $7 x(x, 16$ & 0118 & 174616 & 1111 & $|k| 01.4 .15$ & (1) 10.14 & $77(x) 35$ & (). 13.12 \\
\hline 1631 & (1) 14in & $\operatorname{lin} x \cdot 4$ & $111+11$ & 17825 & (1) 1.556 & $79.80,35$ & 0.156 .1 \\
\hline 712216 & 1112.4 & $|x| 161 c$ & 11111 & $175<559$ & 0.150 .1 & $82.7(1) 35$ & $0.14 k \mathrm{~K}$ \\
\hline$\because 14<1<$ & (1) 1124 & $18.10<4$ & $11 ; 10$ & $172.7,015$ & $0.1(x) 1$ & 85.5135 & (1). 1520 \\
\hline$\iota^{\prime \prime}: 1$, & $\because 1, i k$ & $181,1,1^{5}$ & $1111 . \ldots$ & (1,1) & 0.1720 & 88.3535 & 0.1623 \\
\hline $0.4 \times:<6$ & (1) & inis in & (1) 110 & $161: 3114$ & (1) inilu & $1) 1.2235$ & 0.1610 \\
\hline taleis & $111121 k$ & jii: 2 Ans & $11 i 11 \mathrm{k}$ & 16.13785 & $(0.17 .11)$ & $04.1 \times 1.5$ & 0.16 .50 \\
\hline $4.4: 4$ & $\left.(1) \mid 1 x_{1}\right)$ & jus (1) & $111 \%$ & $1010,0<5$ & (1.15.12 & 90.9235 & ().17.14 \\
\hline $41,<14$ & 11161, & $107 \times 23$ & $111+11$ & $158 \times 145$ & 0.1732 & 00.70 .35 & (). 18.38 \\
\hline $41: 124$ & 11 (k) & $2(H) 1: 4$ & $111 \because$ & $150,14,35$ & $0.10,50$ & $1012.5 \% 15$ & $0.1 \times 12$ \\
\hline 8110216 & 11119.4 & $211+4: 4$ & $(11,1)$ & 152.1639 & 11.581 & 10526,39 & $0.2(x)$ \\
\hline an 11,25 & $1111 \div 1$ & $2(x) i x^{\prime}$ & $(1):$ & $1502 \div 3$ & () $\mid(6 \times 3$ & 10180435 & $0.16 x i 7$ \\
\hline de 216 & (1) (k- $+4:)$ & zolithis & ii i.ri & 197.4715 & (1) 15015 & 110.77 .35 & 0.10 .47 \\
\hline $42<8<5$ & $0(k \alpha k)$ & $211.1111^{6}$ & $111 . \mathrm{in}$ & 1445835 & (1) 1.5 .80 & 113.5335 & 0.1217 \\
\hline$(1)^{2}+x_{1}$ & 018972 & $21.42: 16$ & $1: 114$ & $111.0,4,35$ & (1). i(x). & $116.1 \times 35$ & 01847 \\
\hline 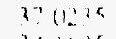 & (1) fistid & 21011215 & 1111 & $136 \therefore(1) .5$ & () $|k| 1$ & 118.96 .35 & 0.1840 \\
\hline 3.110 .25 & 1111211 & $\because 14,1,0$ & $(1) \cdots 1$ & 125.17235 & $(1.1771$ & 121.5935 & 0.1931 \\
\hline 11912 & $(1, \mid K K K)$ & $\because \because 214$ & 111.111 & 1324845 & $0.172 k$ & 124.21735 & 0.1825 \\
\hline $24: \because 16$ & $111 x \times n)$ & 226 1rias & $1111 ! 1$ & 124.1135 & O.lis: & 1260435 & 0.18 .53 \\
\hline 202315 & (INABH: & $22)(4,4$ & $111 \vdots$ & $12+7.415$ & $0.16 \times 37$ & $120(x, 3,35$ & 0.1020 \\
\hline $28,15: 5$ & $11(k) x)$ & 2361570 & $111.2 n$ & $1218 \div<5$ & (1) 1721 & 132.3335 & 0.1707 \\
\hline $11<3<$ & $(1)(k, 5)$ & $22310 \times 5$ & $111 i_{s}:$ & $121 .(k), 15$ & 0.176 .3 & 125.10835 & $\left.0.1 \sin ^{\prime}\right)$ \\
\hline 140,1425 & (1)(1)14? & 215,642 & $111: 11$ & 118.1435 & (1) 1718 & 137.70 .35 & 0.1850 \\
\hline $1 / x+14$ & $(1111.9)$ & $238<545$ & 01328 & 115.4535 & $0.1 \% 0$ & 1.10 .51 .15 & 0.1812 \\
\hline 4010,14 & $0(1224)$ & 2412116 & $(: 1: 16)$ & 112.5335 & 0.16 .51 & 143.13 .35 & 0.18 .11 \\
\hline 420144 & $(1)(i) k, 4$ & 2438215 & (1) $12 x, 1)$ & $1(k) .6835$ & 0.1816 & 145.8235 & 0.1808 \\
\hline $40,41: 5$ & $(1)(1,11) ?$ & 216 174s & 01310 & $11(x, 0) 235$ & (). 17.50 & 148.4335 & 0.1823 \\
\hline (1) 14 is & (I) istis & $2.4431 \mathrm{~h}^{6}$ & $(1123)$ & $1(1.1 .1235$ & 0.1777 & 15117.45 & 01658 \\
\hline 521115 & $(1(x, 1))$ & 25181,24 & 111120 & 1011.3735 & 0.1680 & $153 \times 135$ & 0.1768 \\
\hline [4] & $11 \%(1)$ & 25.45014 & $(1) 120$ & $9 \times .573 .35$ & $(0.171) 4$ & 156.08 .35 & $0.16+1$. \\
\hline sis 454 & $(i 11 ?)(1)$ & $25^{1}+11^{6}$ & 111111 & 95.26 .35 & 0.1720 & 150.403 .5 & $0.1 \times 4 ?$ \\
\hline (.) 0.134 & (1)(xis) & $2 x, 11: 4.1$ & $41: 111$ & 929.835 & 0.1801 & 162.1035 & 0.1787 \\
\hline $6 \cdot 2 \times k_{1}+;$ & (i: $:\{: 1$ & $\therefore(14(2+.41)$ & 11 ind & $x) 16, ? 5$ & $0.16+10$ & 1647835 & $01 \%$ \\
\hline $68 i x, 35$ & $(1) 101$ & $\therefore(1+4+4)$ & $(1+\cdots) !$ & 87.31 .35 & 0.1588 & $16,7.536 .5$ & 01780 \\
\hline $71112+4$ & 111151 & $? t, ?\left(x_{i}\right) \ldots$ & (1) Min: & 015135 & (1) ISch & 170.228 .5 & 0.1730 \\
\hline $7+4+1: 9$ & $1: 12 i e_{1}$ & $2(1) 7$ ov & (1) 1,1 & 81.8 .19 & 0.148 .5 & 173.01 .46 .5 & 0.1716 \\
\hline $77(x) 15$ & $1 ; 11: 4$ & $26,7(n) 25$ & 111161 & $78.46,35$ & (1.) 15.41 & $175.86,35$ & (0.1.580) \\
\hline 74 stis & i. 1 i. . & \multirow{4}{*}{\multicolumn{2}{|c|}{ 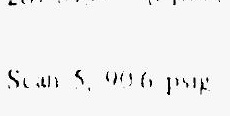 }} & 76.21 .35 & (). 1.180 & 178.6375 & $0.19+40$ \\
\hline $8: 70<4$ & 11285 & & & 1.13335 & (1.1280 & 181.3935 & 0.1761 \\
\hline $4<51<9$ & (1) 1350 & & & 7055336 & 0.1 .735 & $184 .(\times 155$ & $0.1(x)$, \\
\hline 881539 & 01450 & & & 67.7135 & $0.131 \mathrm{H}$ & 186.015 & 0.167 .9 \\
\hline 412286 & (1) 1.111 & $2(1 \div 10 k)$ & 1111110 & (r) 8735 & 0.1327 & 189.5753 & 0.16 His \\
\hline if $(x, 15$ & $111.4 \mathrm{~h}$ & 21,5115 & Hinger & 0.218 .15 & 0.1175 & 142.2895 & 0.1548 \\
\hline 901.9275 & () 1.50 & $262 \leq s+x i$ & 111111 & 34.4415 & $0.12(19)$ & $145(1+4) 5$ & (1.15in \\
\hline 09.7635 & $610 x ?$ & 2543010 & 111114 & 56.57 .35 & (1.1215 & 197.8225 & 0.1576 \\
\hline
\end{tabular}




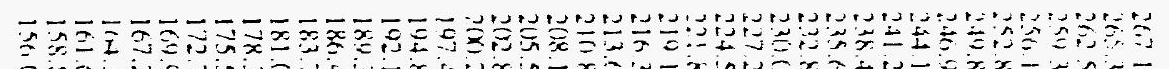

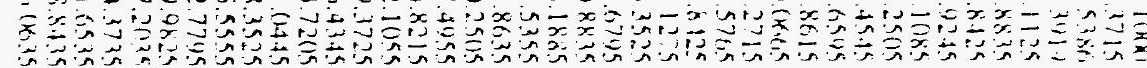

lo $=0=0000000=0000000000=0=0=0=0=0=0=0$

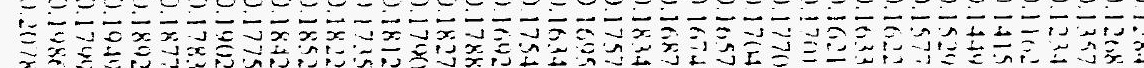

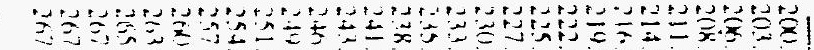

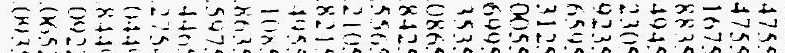

$\mathrm{U}=0=0=000=0=0=0=0000000=0=0$

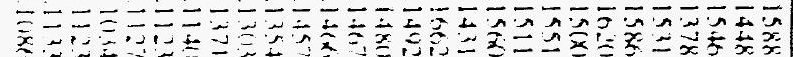

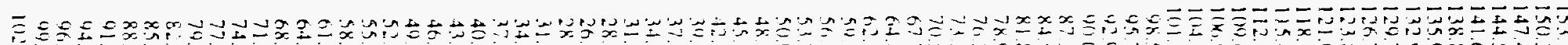
y

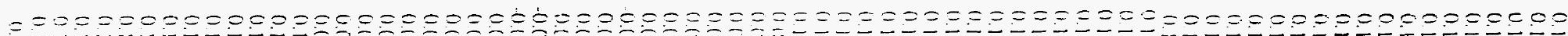

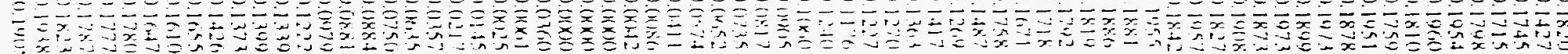

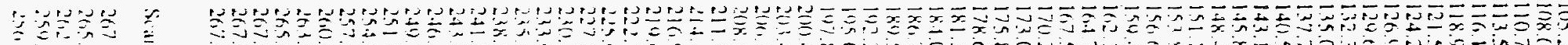

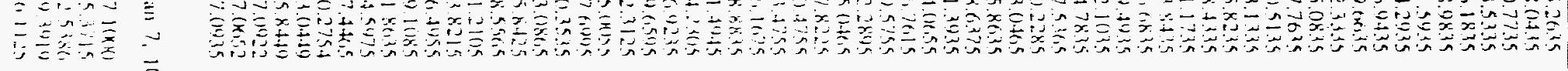

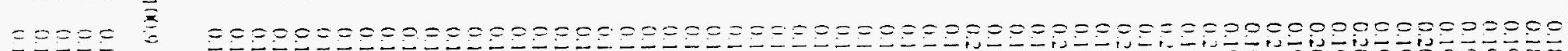

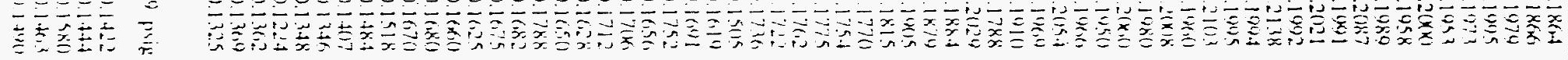

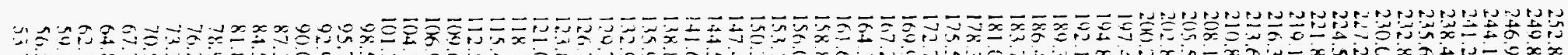

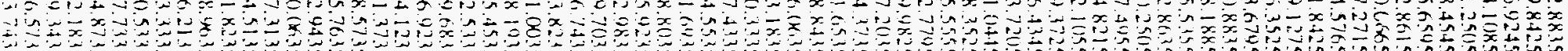
然

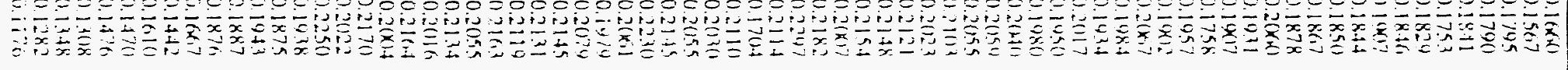




\section{Reinforced Concrete Test Data}

Track 8 - High Pressure Test

\begin{tabular}{|c|c|c|c|c|c|c|c|}
\hline 50.9235 & $0 .(1) \times x$ & 203.4755 & (1) 11334 & 153.18 .3 .5 & 0.2407 & 105.26 .15 & 0.2 .172 \\
\hline 48.107 .5 & 0.0665 & $2(x .16 .75$ & 0.19015 & 150.3335 & 0.20 .45 & 1080439 & 0.23 .39 \\
\hline 45.32 .45 & 0.0784 & 208.88 .35 & 0.1786 & 147.4335 & 0.2272 & 110.7775 & 02.443 \\
\hline 42.5535 & 0.070 & 211.4045 & 0.184 .4 & 144.553 .5 & 0.2320 & 11.5 .375 & 0.24 .54 \\
\hline 39,7935 & $0.1 \times 7)$ & 214.2305 & 0.20135 & 141.6935 & 0.2424 & 116.18 .35 & $0.24: 1$ \\
\hline .37 .0235 & 0.0445 & 210.4235 & 0.1450 & 1.38 .80 .15 & $0.2+18.5$ & $11 \times 08.14$ & 0.2468 \\
\hline 34.16 .35 & 0.01140 & 219.6545 & (1. 18.58 & 135.923 .4 & 0.2401 & 121.5035 & 0.2464 \\
\hline 31.3235 & $0.0(0) 28$ & 222.3125 & 0.1912 & 132.9835 & 0.2342 & 124.2435 & $0.24,7$ \\
\hline 28.27 .35 & $0 .(X) \times(x)$ & 225.0155 & 0.1800 & 129.7035 & 0.22110 & 126.9475 & $0.250(1$ \\
\hline 26.22 .35 & $0.0 \times(x)$ & 227.6995 & 0.18 .52 & $126.7+15$ & $0.2+17$ & $129.6 \times 135$ & $0.258:$ \\
\hline 28.68 .35 & $0.0(x)$ & 230.35 .35 & $0.182(1$ & 123.8235 & $0.24(k)$ & 132.333 .35 & $0.257 \%$ \\
\hline 31.533 .5 & $0.0 \times 100$ & 233.0865 & (1) ?(1) & 121.0019 & 0.2444 & 175.08 .35 & 0.257 .3 \\
\hline 34.69 .35 & $0 .(x) .3$ & 2.358 .125 & 0.18 .317 & 118.1935 & 0.2394 & 137.76 .15 & $0.2+111$ \\
\hline 37.80 .15 & 0.11267 & 2.38 .5565 & 0.182 .3 & 115.45 .35 & 0.2458 & 140.51 .35 & 0.2595 \\
\hline 40.603 .5 & $0.0 .3 \times 1$ & 241.2105 & 0.1741 & 112.53335 & 0.2318 & 143.1335 & 0.2580 \\
\hline 43.6335 & $(1 .(1+1)$ & 243.8215 & $0.1 \times .10$ & $1(N) .683 .5$ & 0.2783 & 145.82 .35 & 0.2505 \\
\hline 46.53 .3 .5 & $0,(k, 5)$ & $246.41) \leq 5$ & $0.1 \% 85$ & $1(k, 4) 2.35$ & 0.236 .8 & 148.4334 & $0.25 \% 2$ \\
\hline 40.49 .35 & 0.11778 & 249.10185 & $(0.16,1) 7$ & $1(14.1235$ & $0.23(10)$ & 191.1735 & 0.2314 \\
\hline 52.43 .35 & $0.086(1.4$ & 251.863 .5 & $(1,150)$ & 1011.3735 & 0.2 .317 & 153.84 .35 & $0.25(14)$ \\
\hline 5.5 .41 .35 & 0.1014 & 254.5075 & () $18(10$ & 98.57 .35 & 0.2430 & 1506,835 & (1.25+61 \\
\hline 58.5535 & 0.1126 & 257.440 .5 & 0.1318 & 05.76 .15 & $0.18 \sin$ & 154.44 .75 & $0.25(10)$ \\
\hline 61.613 .5 & 0.114 .3 & $20(1.2754$ & 0.1533 & 92.94 .35 & 029171 & 162.100 .15 & $(1.2514$ \\
\hline $6-4,4,35$ & () 1.74 & $26.3(4.44)$ & 0.1 .450 & $0(1)(x, 35$ & $0.15 \mathrm{KK}$ & 164.78 .35 & (1) 25.5 .4 \\
\hline $68.1 \times 1 \times 5$ & 0.1 .876 & $265.84+40$ & 0.1322 & $87.3 ! 3.1$ & $0.184 \pi$ & $16,7536,5$ & 0.2406 \\
\hline 71.11235 & $0.150(1$ & $267(x) \geq 2$ & $(1.1450$ & 84.51 .15 & 0.1841 & $17(1.22 \times 1.5$ & () 2.561. \\
\hline 74.14 .39 & $(0.15 .10$ & $267\left(x_{1} 92\right.$ & (1) 1.18 (1) & 81.82 .35 & 0.1745 & 173046.5 & $0.2+26$ \\
\hline $7 \%(x) 35$ & $0.15(, 0)$ & 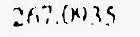 & $0.1+4.2$ & 78.100 .15 & $(11880$ & 175860.35 & 02.387 \\
\hline $70.85,35$ & 0.1786 & & & 76.21 .35 & 0.1811 & $178.61,5$ & 0.2574 \\
\hline 82.70135 & $(1.1700)$ & & & $73 . .3335$ & 0.16111 & 1813415 & $02.47 \mathrm{~K}$ \\
\hline 85.5135 & $0185 ?$ & Sis and $x^{\prime}, 10$ & 8.2 psip. & $7(1,5335$ & $0.1 ? 0\}$. & $184.16,55$ & 0.2 .446 \\
\hline 88.3535 & 0.11050 & & & 60.7315 & 0.17018 & $1 \times 6.76119$ & $11.2+21)$ \\
\hline 41.2239 & 0.14 .40 & $2671(x)(1$ & $(1.150) !$ & $6+18795$ & () issits & 184.5755 & (1).2351 \\
\hline $44 . x 35$ & $\left(1,2\left(x_{1}\right)\right.$ & $20,5.3715$ & (1. 1 Sk.t & 621815 & (0.1.164 & 1922805 & 0.2 .41 \\
\hline $9(1.0235$ & 0.21 .98 & $202<380$ & 0.17411 & (5) 7415 & (1.) 14.16$)$ & 1050.0 .40 .5 & 012325 \\
\hline 49.79 .35 & 0.21 .55 & $25(1) .31910$ & (). 16.32 & $5(1,5) 35$ & $(1.14+12$ & 197.8225 & 0.2371 \\
\hline 102.573 .5 & 0.2168 & 250.1125 & $0.155 \%$ & 5.17 .4 .15 & 0.1 .3 .31 & $2(x), 4755$ & 0.22014 \\
\hline 105.2635 & $0.21 \mathrm{kit}$ & 2528635 & 0.1 Sitot: & 501.42 .3 .5 & 0.1101 & 201.1 .4755 & 0.2147 \\
\hline 108.0435 & 0.2173 & 2458.125 & (). $1 k^{\prime}((1)$ & 48.10135 & (1)(11) (1) & $2(x, 16) 5$ & 0.2148 \\
\hline 110.77 .35 & $0.2 ? t x t$ & $240.02+5$ & $(0.14): 8$ & 45.12 .15 & 0.0876 & $2(18.80 .35$ & $0.2(1) 0)$ \\
\hline 11.1 .5335 & 0.2265 & 244.1065 & (1) $2(1)$ & 42.553 .5 & $0 .(1) \div 1$ & 211.40 .15 & $0.2 ?(17$ \\
\hline 116.18 .15 & 0.22 .66 & 2.41 .25015 & $(0.2(11))$ & $31) .7915$ & 0()$(x) 1$ & 214.2305 & 0.22 .36 \\
\hline 1180835 & 0.2 .3 .31 & 238.4545 & $0.2(k) ?$ & 17.11235 & $0.01 .5(.8)$ & 210.9245 & 0.2157 \\
\hline 121.5435 & $(1.2315$ & 235.0505 & $0.2(1) .4$ & 34.16 .15 & $0 .(x) \geq 1$ & 219.65195 & 0.2218 \\
\hline 1.4424 .15 & 0.2105 & 7228015 & 0.2170 & 7.32 .5 & -0.01 .1 .50 & 222.3125 & 0.219 .3 \\
\hline 126.44 .35 & 0.2210 & $23(1)\left(x_{1}, 5\right.$ & (). $22(x)$ & 28.273 .5 & $0.0135(1$ & $225.1 \times 155$ & 0.2177 \\
\hline 124$)(6,35$ & 0.2310 & 227.2715 & 0.2230 & 96,2235 & .0 .01750 & $227 .(1965$ & $(1.2144$ \\
\hline 132.3135 & $0.22\left(x_{1}\right)$ & $224.5 \% 615$ & 0.2133 & 28.6335 & $.010,150$ & 2311.3539 & (1 2$\} 11$ \\
\hline 1.35 .08 .35 & 0.2311 & $2218+25$ & 0.2172 & 31.335 & 0.013 .50 & 233.08015 & 0.2285 \\
\hline 137.70 .15 & 0.2220 & 2101215 & (0.2193 & 346035 & $-0 .(x)(x) 7$ & 2358.425 & $0 ?(x) 2$ \\
\hline $1.4(1.51 .15$ & $(1.2 .320$ & 216.3525 & 01.23017 & $.37 .8(13.5$ & $(0.011) 6$ & 238.5505 & 0.218 .4 \\
\hline 143.133 .5 & $(12210$ & 21367745 & 0.2212 & 40.603 .5 & $0.02+4$ & 241.2105 & $0.2(x)(1$ \\
\hline 145.82 .15 & 0.2270 & 210.88 .35 & (0. $2 \mid\langle 1\}$ & $43.6,335$ & 0.01450 & 243.8215 & 0.2101 .4 \\
\hline 148.43 .35 & 01.2213 & 208.1885 & $(1.2(1) .3$ & 40.533 .35 & 0.04 .54 & 246.4455 & $0.210 K_{1}$ \\
\hline 151.17 .34 & 01.21 .36 & 2015.5355 & 0.2250 & 40.4935 & 0.0757 & 240.108 .5 & $0.19 ? 6$ \\
\hline 153.84 .35 & 0.2101 & 202.80 .35 & $(1.2212$ & 524335 & $0 .(1927$ & 251.86 .35 & $0.18(.4)$ \\
\hline 156.68 .35 & 0.21 .19 & $20(1.25015$ & 0.2112 & 55.41 .35 & 0.1050 & 25.1 .507 .5 & 0.2101 \\
\hline 159.43 .35 & 0.22 .30 & 197.4955 & 0.23 .40 & 58.5535 & 0.10 .36 & 257.4 .46 .5 & 0.16 .94 \\
\hline 16.1035 & 0.2126 & 194.8215 & $(1.2203$ & 61.013 .5 & 0.1100 & 260.2754 & $(0.16(0))$ \\
\hline 164.78 .35 & 0.2171 & 1921055 & 0.230 .1 & $6+.90,35$ & 0.1454 & $263 .(14.40)$ & 0.16 .54 \\
\hline 167.536 .5 & $0 ? 21 k$ & 1803725 & 0.2365 & $68.0 \times 3.35$ & $0.16 i 11$ & 26.5 .8 .4 .49 & $0.157 i$ \\
\hline 170228.5 & 0.218 .4 & 186.43 .45 & 0.23 .45 & 71.012 .35 & 0.1676 & $267,(k) 22$ & (1) 17017 \\
\hline $173(1.4015$ & $0.2(184)$ & 183.7205 & (1) 2.3 .47 & 74.0 .835 & $0.16,72$ & $267)(k, 52$ & 0.1713 \\
\hline 175.86 .15 & (0.1010) & $181(4.45$ & 0.2 .4511 & $77 .(x) 3.5$ & 0.1721 & $2(17 .(k) 3.5$ & $\left(1.16\left(1^{0}\right)\right.$ \\
\hline $178.6,375$ & 0.2235 & 178.3525 & 0.2350 & $79.86,35$ & 0.197 .5 & & \\
\hline 181.3935 & $0.211 \% 4$ & 175.5555 & 012175 & 827013.5 & $(0.11)+2$ & & \\
\hline 18406.55 & $0.2(1) *$ & 172.7705 & $(1.2 .350$ & 8.5 .51 .35 & $0.19 \times 9$ & \multirow{2}{*}{\multicolumn{2}{|c|}{$\operatorname{Scan} 9.1112$ psip }} \\
\hline $186.76,1.5$ & (1).2(18: & $1(1,1) 8325$ & 0.2 .422 & $88 . .3535$ & 0.2125 & & \\
\hline 180.5755 & $(1.19 .9) .1$ & 107.2113 .3 & $(1.2 .401$ & 41.2235 & 0.21 .57 & $267.16 \times(x)$ & $0.180(1)$ \\
\hline 192.2805 & 0.14 .42 & 10.4 .27 .35 & 0.2542 & 0.4 .616 .35 & $(1.216)$ & $20,5,3715$ & 0.187 .5 \\
\hline 195.046 .5 & $0.19 \div 29$ & $16,1.6,5.35$ & $0.2 .47 \mathrm{~S}^{\circ}$ & 96.9235 & 1).?18.7 & 262.5386 & $0.2(113$ \\
\hline 107.8225 & Q16in? & 158.84 .35 & (1).2581 & 49.793 .35 & 0.2 .337 & 2503010 & () 18 in \\
\hline$?(x) .4755$ & 0.1005 & $196.1 x, 15$ & $0.26 . .61$ & 102.5725 & (1).2.3(16) & 256.1125 & 0.1781 \\
\hline
\end{tabular}




\section{Reinforced Concrete Test Data}

Track 8 - High Pressure Test

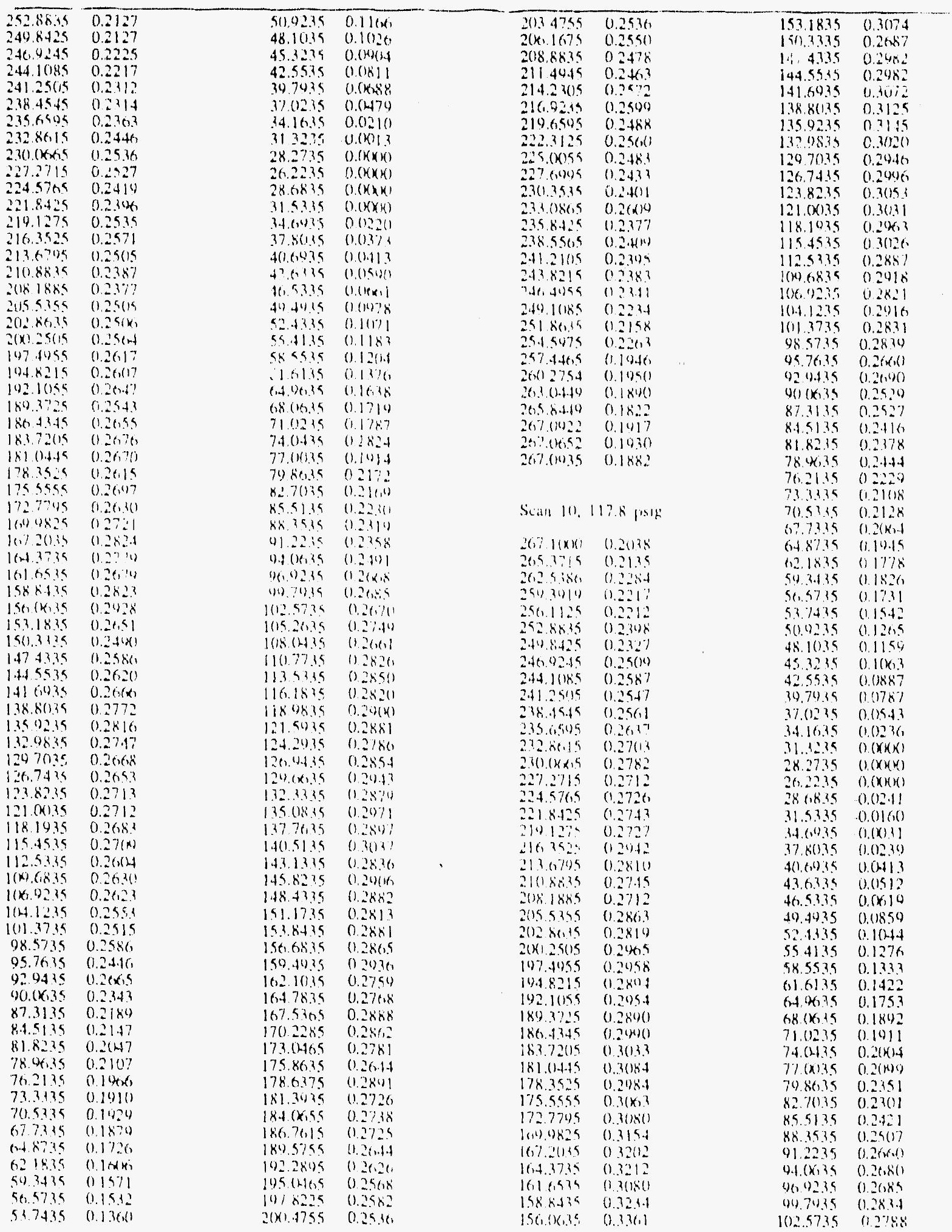


Reinforced Concrete Test Data

Track 8 - High Pressure Test

\begin{tabular}{|c|c|c|c|c|c|c|c|}
\hline 105.20 .35 & $0.3(1.48$ & 25286835 & 11.20 .51 & $5(1.02 .15$ & 0.1370 & $201 .+1755$ & $0.311^{6}$ \\
\hline 108.04 .35 & 0.276 .1 & 249.8425 & $0.25 \%)$ & 48.10 .15 & 0.1227 & $2(x, 16,15$ & (1) $f(x):$ \\
\hline 1111.77 .35 & (1) $3(x) 1$ & $246.92+5$ & $0.27+42$ & 45.32 .15 & $0.11(x)$ & 208.883 .5 & (1) $f(x)-1$ \\
\hline 113.5339 & $0.3(k-1$ & 24411185 & 0.270 .4 & 42.553 .5 & $0 .(\omega)(1) 1$ & 2114115 & $0.2(k) .1$ \\
\hline 116.18 .35 & 0.3027 & 241.25115 & 0.2885 & 39.7935 & 0.0783 & 214.21115 & 0.3180 \\
\hline 118.983 .35 & 0.3118 & 2.38 .454 .5 & $0.2 x+1$ & 37.0235 & 0.0526 & 216.0235 & $0.3 ! 14$ \\
\hline 121.5939 & 0.3124 & 215.6505 & $(0.2929$ & 34.16 .35 & 0.0280 & 219.65195 & 0.1190 \\
\hline 124.2039 & 0.3054 & 2323615 & $0.29)(04$ & 31.3235 & $0 . \cos (x)$ & 222.3125 & $0.3111 x^{\prime}$ \\
\hline 126.44 .39 & 0.3116 & $23(1,(k 6,5$ & $0.2(39) .3$ & 28.27 .35 & $0.1 \times(x) 1$ & $225(k) 55$ & $0.7($ N1) \\
\hline $124.6 x 35$ & 0.1225 & 227.2715 & $0.26,54$ & 26.2235 & $0 . \cos (x)$ & $227.6(0) 6$ & 0.208 .4 \\
\hline 132.3335 & 0.3246 & 224.9765 & $0.3(1) 8$ & 28.6835 & (1). $($ KKKX) & 230.3539 & $0.2 \times 6.1$ \\
\hline 135.188 .35 & $0.32(x)$ & 221.8425 & 0.20193 & 31.5335 & $-0 .(1)+8$ & 233.08695 & $0.31 \% 4$ \\
\hline $137.26,35$ & 0.320 .3 & 210.1275 & $0.3(k+1$ & 34.6935 & 0.1248 & 2358426 & $028+5$ \\
\hline $1.4(15135$ & 013.340 & 216.3525 & 0.316 .7 & 37.803 .5 & $0 .(1.1 .14$ & 236.5565 & () $? k+1$ \\
\hline 143.13315 & 0.3258 & 213.67995 & 0.3150 & 40.619 .35 & 0.0527 & 241.2105 & $0: 1.4 .4$ \\
\hline 1458235 & $0.328 x$ & 2108835 & (1.3(154) & 436,35 & (). $(k+155$ & 2478215 & 0.2900 \\
\hline $1+4 k+135$ & 0.3242 & 2181585 & $(1.2(1) 2.3$ & $+6,53 ? 5$ & $(1)(1)(x)$ & 2.46 .9455 & $0.2+14.4$ \\
\hline 151.1735 & $0.3(1 ! 2$ & 20155.155 & $(1,31201$ & 40.4915 & 0.1058 & 240.1185 & (1.26n:2 \\
\hline 1528.835 & 0.3242 & 201280,35 & (1.34156) & 52.1335 & $0.12 \%$ & $251.86,35$ & $(1.25 .10$ \\
\hline 150.01835 & $0.3 ! 70$ & $2(0) .2515$ & 0.3244 & $55+1135$ & 0.1793 & 25.45979 & (i) 25.tu \\
\hline 154.49 .15 & 0.3270 & 197.4755 & (1.3.12, & 589519 & $(1.1+32$ & $25 / 4+49$ & (1).2.175 \\
\hline 102.1035 & 0.32111 & 1948215 & $0.316 . ?$ & 0.61 .35 & 0.158 .4 & $26(0) .2754$ & $(1.2394$ \\
\hline 1647835 & 0.3181 & 1021055 & 0.3180 & (n) 190.15 & 0.1748 & 26.304 .40 & $0.2 \times 1$ \\
\hline 167.536 .5 & 0.3254 & 154.3725 & 0.31 .55 & 6.8 .16 .15 & $0.2(k x)$ & $20,5.8 .4 .49$ & 021.14 \\
\hline $170.22 \mathrm{~K}^{6}$ & 0.3140 & 180.4345 & 0.320 .5 & 71.1239 & $(1.21 .30$ & $267.692 !$ & $01 \therefore 9$ \\
\hline 173.0486 .5 & 0.3102 & 183.72115 & (1) $328 \% ?$ & 74.1415 & (1 22238 & $26)(k, 5)$ & $0.223^{\prime}$ \\
\hline $175.80,3.5$ & $0.3(k(x)$ & 181.0445 & (0) 31.22 & $77 .(00.15$ & $0.2 !(x)$ & 267.00935 & $0.21 \div 1$ \\
\hline 178.6379 & 0.3 .365 & 178.3525 & $(0.329 .1$ & 70.80 .35 & $0.24 k k$ & & \\
\hline 181.3035 & 0.3132 & 17555559 & 0.3 .461 & 82.7035 & 0.25077 & Scan 12, & 25.8 p" \\
\hline $18+<k, 55$ & 0.301 .98 & $172.7,15$ & (1)3154 & 85.5135 & 0.2587 & & \\
\hline $180,10.15$ & 0.31139 & $1(x, 4), 4,25$ & $0.346 \%$ & 88.15 .15 & $(1.272 .3$ & $26 \% .1(x)(x)$ & 0.2744 \\
\hline 180.595 & $0.3111 ?$ & 16.7201 .35 & $(1.39 .11$ & 41.2235 & $0.28(1.9$ & 265.3715 & 0.2767 \\
\hline $175034+465$ & 0.2018 & $\mid 0,10,535$ & 0.2120 & $\{x, 11: 15$ & $(1.3(19.1)$ & 251931918 & 0.32011 \\
\hline 14.8225 & $11.21 \times 12$ & 1588115 & (1) $15: 5$ & $(4) 71715$ & 0.31101 & 250.1125 & $0.196)$ \\
\hline $2(101.454$ & $0.25+1$ & $1.56,1 \times 1,35$ & $(1,3,1,2)$ & 101.5915 & $(191114$ & $252 \times 515$ & $0.133 .4 x^{\circ}$ \\
\hline 2014759 & $0.2 \geqslant(x)$ & 153.1535 & (1) 216.4 & 10520.95 & 0.3457 & 249.8425 & $0.3 .150)$ \\
\hline $2(x) 1675$ & (1).2815 & 150,3345 & (1).311.36) & $108 .(1+1.15$ & $0.3(x, 3$ & 246.9245 & 0.33142 \\
\hline $208 \times 815$ & 0.2515 & 147.4315 & $(1.32101$ & $110.7 \% 35$ & 01.3335 & 2.44 .1185 & $0.358 \mathrm{t}$ \\
\hline $211+14.5$ & $(126004$ & 1.4 .553 .5 & (1) $1212 ?$ & 113.5335 & $0.3+11$ & $2+1.250 .5$ & 0.3551 \\
\hline 214.23115 & 0.27719 & 141.61939 & 0.33 .36 & 116.1835 & $0.33 \% 1$ & 2384.445 & $0.37 \times 0)$ \\
\hline 21619234 & $(1.282 .4$ & 1.38 .811 .35 & $(1.7 .761 .4$ & 118385 & 0.3450 & 215.0 .595 & $0,367 \%$ \\
\hline 21900114 & 0.271 & 135.92 .15 & 0.34319 & 121.51145 & 0.3522 & 232.8015 & $0.1866 ?$ \\
\hline 2223125 & $0.2 k(x)$ & 13298.35 & 0.3285 & 124.2435 & 0.3475 & $230(x \times 1.5$ & $(0.7 x) ?$ \\
\hline $2.2 .(k 155$ & 0.2722 & 121.7045 & 0.324 .4 & 126.64 .435 & $(1.34 \%)$ & 227.271 .5 & 0.384 .1 \\
\hline 227.610175 & $01.2 \pi(k)$ & $126.7+35$ & 0.3314 & 129.60 .35 & 0.3507 & 2245765 & $(0,364$. \\
\hline .30 .1535 & 0.2616 & $123 \times 235$ & 0.3344 & 132.3335 & 0.34143 & 2218429 & 0.3901 \\
\hline 233.01605 & 0.285 .4 & 121.14135 & $0.3267 i$ & 135.11835 & $0.35 \% 2$ & 219.1275 & 0.4077 \\
\hline $2358+25$ & $0.26(x)$ & 1181935 & $0.12 \times 0$ & 13776,35 & 0.3576 & 216,3525 & (1).4(1)2 \\
\hline 238.55015 & 0.2582 & 115.4515 & (1) 3111? & 1.40 .5135 & (1.3tilo & 21367745 & $0.34 \times 1$ \\
\hline 241.21165 & 0.2525 & 112.5935 & (1. 31.42 & 143.1335 & $0.36,58$ & 21118015 & 0.39 .38 \\
\hline $2438 \geq 15$ & $0.2(1.34$ & 1010685 & 0.3151 & 1.15 .8235 & 0.3572 & 208.188 .5 & $0.3 \times 54$ \\
\hline$\therefore$ Ho sysas & 0.2510 & 116,0235 & 0.20071 & $1+5+335$ & (1). 36,57 & 2015.9159 & 0.408 .3 \\
\hline 240.10185 & $0.24+13$ & 104.1235 & $0.31 ! k$ & 191.1735 & 0.35993 & $2012.86,3.5$ & 0.40132 \\
\hline 251.86 .35 & $0.22 \% 1$ & 101.3335 & $(1.24711$ & $153.8+35$ & 0.3529 & $2(k) .25015$ & 0.4129 \\
\hline 254.5079 & 0.2517 & 48.5735 & $(1.3128$ & 15611815 & 0.3567 & 107.4055 & 0.4237 \\
\hline $257+400^{4}$ & 0.2122 & 9970.35 & 0.2522 & 150.193 .5 & $(0.36,91$ & 194.8219 & $0.4 ! 33$ \\
\hline $2(x) .27 .54$ & 0.2274 & 920419 & $0.2 \times 70$ & 162.10135 & 0.353 .3 & 102.1059 & 0.4221 \\
\hline $263 .(1.4+4)$ & $0.22(11$ & $9(1.1 k, 15$ & $0.2(x, 1)$ & 164.7839 & 0.1423 & 189.3725 & $0.41+8$ \\
\hline $205 \times 4+49$ & $0.2(x) 7$ & 87.313 .5 & $0.2 \operatorname{2n}(1)$ & 167.536 .5 & $0.3517(1)$ & 186.434 .5 & 0.4275 \\
\hline 267.0122 & 0.210 .3 & 84.9115 & 0.2576 & 170.2285 & 0.3586 & 183.7205 & $043(12$ \\
\hline $267.06,52$ & 0.2112 & 81.8235 & 0.2512 & $173.61+46.5$ & 0.34 .53 & 181.0145 & 0.4 .419 \\
\hline \multirow[t]{3}{*}{$267 .(19) .35$} & $02(k \times 1$ & 78.96 .3 .5 & 0.2514 & 175.86 .35 & 0.3271 & $1 / 8.3525$ & 0.4240 \\
\hline & & 16.21 .35 & 112381 & 178.6 .37 .5 & 0.35 .38 & 175.5555 & 0.4 .111 \\
\hline \multirow{3}{*}{\multicolumn{2}{|c|}{ Som $11,120.4$ prigk }} & 73.333 .5 & 02319 & 181.7935 & 0.1424 & 1727749 & 0.4176 \\
\hline & & 70.533 .5 & 0.2244 & $184.1 k+5,5$ & $0.33 \% 6$ & 3609829 & 0.4 .472 \\
\hline & & $6.7,15$ & 1.2101 & 180.76119 & $0.335 \%$ & $10112(1) 35$ & $0.46,34$ \\
\hline 207.10000 & 0.2170 & 0.48735 & 0.2128 & 1005555 & $(0.3261$ & 16.4 .37 .35 & 0.401 .4 \\
\hline 26153719 & 0.2214 .4 & 0.183 .5 & 0.1881 & (1)? 28105 & $0.4 \div 11$ & 101,01535 & 0.4537 \\
\hline $2612.51 \mathrm{sin}$ & 0.2162 & 514.3419 & 01801 & $1145 .(1.461 .5$ & $(1.32(1)$ & 198.8479 & $(0.459()$ \\
\hline 254.3914 & 0.2 .1018 & 56.57 .34 & 0.1816 & 1078225 & 0.3221 & 150.06 .35 & 0.4701 \\
\hline 250,1125 & 0.230 .1 & 5.64435 & 0\}.(x, 1)$ & $2(51 .+155$ & 0.3230 & 153.18 .15 & $0.46 .5 \mathrm{k}$ \\
\hline
\end{tabular}




\section{Reinforced Concrete Test Data}

Track 8 - High Pressure Test

\begin{tabular}{|c|c|}
\hline 150.3375 & 0.1224 \\
\hline $14 ?+315$ & (1. $4+4501$ \\
\hline 14.5535 & () 4.111 \\
\hline $1+1,(, 0) 35$ & (1) 4.4 .88 \\
\hline $13: 80139$ & $(1+1(x)$ \\
\hline 1354215 & $0.44+41$ \\
\hline $1.12 .4 \times 19$ & $04 \times 30$ \\
\hline 129.71144 & 0.4187 \\
\hline 120.7 .434 & $0+24 ?$ \\
\hline 1218235 & $(1+1 k 2$ \\
\hline $1: 1 .(x) 15$ & 0.4224 \\
\hline 118.1034 & $(1,81,31$ \\
\hline 1154546 & $041+1$ \\
\hline 112.5335 & 0.3974 \\
\hline$I(x)(68,35$ & 0.3451 \\
\hline $1(k, 0215$ & 0.31927 \\
\hline $1141: 15$ & (1) $18 k(1)$ \\
\hline 101137.5 & $\left(11^{3}\right) 10$ \\
\hline 90515 & 0.1520 \\
\hline 9570,35 & $(1,1+x)$ \\
\hline 420415 & 0.3743 \\
\hline $9(1)(x, 15$ & (1) 3.40 .1 \\
\hline 87.1135 & (1).3.315 \\
\hline 84.5135 & $(1 \times 240$ \\
\hline $818 \geq 35$ & (1) 3188 \\
\hline $76.40,15$ & (1) $4(14.4$ \\
\hline 76.21 .45 & (1) 26,53 \\
\hline $\begin{array}{r}73.3335 \\
705335\end{array}$ & 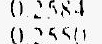 \\
\hline 67.7335 & (1) $2+4(1)$ \\
\hline 6ot $x=15$ & $0121 \% 1$ \\
\hline $621 \times 19$ & (1) $21 \% 14$ \\
\hline $5(1) .3+4.5$ & 0.2145 \\
\hline$\{0,579$ & (1) $14 ? 01$ \\
\hline 537434 & (1) $1^{-1} 1,11$ \\
\hline 5014$) 39$ & 0.1554 \\
\hline 48.11135 & 0.1 .382 \\
\hline 45,3245 & 0.1258 \\
\hline 425535 & ().1154 \\
\hline $30.74,35$ & $0(1)+1, y$ \\
\hline 37.0139 & 0.1750 \\
\hline $3 .+16.15$ & 0.11378 \\
\hline 31.3234 & $(1,0)](k)$ \\
\hline 282735 & $0 .(13501)$ \\
\hline $\begin{array}{l}26.22 .35 \\
28.6275\end{array}$ & $\begin{array}{l}0 .(1.350 \\
010350\end{array}$ \\
\hline $\begin{array}{l}28.6184 \\
315335\end{array}$ & (1) (1) 211 \\
\hline 34 (3) 35 & 0.0 .131 \\
\hline 77.80 .15 & $0 .(151 / 8$ \\
\hline $4(0.6,0.35$ & $0 .(17460$ \\
\hline $43,6,335$ & () (ISi) \\
\hline 40.5335 & 0.0080 \\
\hline 49.40 .35 & $0.11 / 8$ \\
\hline 52.4335 & $(1.15 .10)$ \\
\hline 55.4135 & ()) $16 k \pi$ \\
\hline 58.5515 & 0.17 .319 \\
\hline 61.01 .35 & 0.180 .8 \\
\hline 64.176 .35 & (1. 2161 \\
\hline $6.8 .1 x, 35$ & $0.24(k)$ \\
\hline $11.12 \div 5$ & $(0.2478$ \\
\hline 710415 & $0.26,10$ \\
\hline $77 .(x) .35$ & 0.2718 \\
\hline 70.80 .35 & 0.20 .50 \\
\hline 82.70115 & (1) 11210 \\
\hline 85.51 .35 & (1) $3(x)]$ \\
\hline 88.1515 & $032 \% 1$ \\
\hline 91.2235 & (1) 3...1 \\
\hline $9.4 .16,35$ & (1) $7+4.5$ \\
\hline 969735 & $(1,39.4 i$ \\
\hline 00.7475 & (1) $\{(x) \mid$ \\
\hline 102573.5 & (1) $7(1,2$ ? \\
\hline 10.5 .26 .35 & $(0.348)$ \\
\hline
\end{tabular}

\begin{tabular}{|c|c|}
\hline 11814145 & $017(19)$ \\
\hline 1111.15 & (1. 308.5 \\
\hline 111583.5 & $0.4(16.5$ \\
\hline 116,1875 & () $4\left(1488^{\circ}\right.$ \\
\hline 11848.35 & $(1.41 \%$ \\
\hline 1215415 & 0.4222 \\
\hline 124.2935 & 0.4182 \\
\hline 126,4435 & 0,4167 \\
\hline $129 .(x) .5$ & 0.425 .5 \\
\hline 132.33 .35 & $0.43 .4 i$ \\
\hline 135.08 .35 & $0.4+18$ \\
\hline 1.37 .76 .35 & 0.4328 \\
\hline 140.51 .35 & $(1.4422$ \\
\hline 143.133 .35 & $(1.4511)$ \\
\hline 145.8215 & 0.416 .1 \\
\hline 148.43 .35 & 0.4525 \\
\hline 1.51 .1735 & $0.43^{* 1} 6$ \\
\hline 153.8 .1 .15 & (1).477) \\
\hline 1.56 cisis 15 & $(1 .+4+12$ \\
\hline 1519.4075 & $(1+5 \div ?$ \\
\hline 162.111 .5 & $04+145$ \\
\hline 164.7835 & 0.4 .4518 \\
\hline $16 \% .5365$ & 0.4558 \\
\hline 170.2285 & 1) $4+4.11$ \\
\hline 173.046 .5 & $(1.4 .151)$ \\
\hline $175.80,15$ & $(1+2=4$ \\
\hline 1786.6375 & $0 .+(1+1)$ \\
\hline$|8| .(1)\} 5$ & $(1.4223$ \\
\hline $184 .(x, 55$ & $\left(1.4 ?\left(k_{1}\right)\right.$ \\
\hline 180.7015 & (1. 1.244 \\
\hline 1805755 & (1.4:15 \\
\hline 142.25145 & $(1) 4(1): 3$ \\
\hline $10,0 \times 1316,5$ & $11 .+1115$ \\
\hline 1478225 & 11.101 .7 \\
\hline $2(x) .4755$ & 0.404 \\
\hline $20.3 .1 \div 55$ & (1) 197.411 \\
\hline $2\left(k_{1}, 10,5\right.$ & (1). (1) 14 \\
\hline $2(18.8815$ & $(1,3711)$ \\
\hline $211 .+1745$ & 0. 3 ind \\
\hline $21+.21115$ & 040.30 \\
\hline 216.02335 & $030 \% 1$ \\
\hline $210.0,505$ & $(9.311) 1.4$ \\
\hline 222.3125 & () 391.58 \\
\hline $225 .(x) 55$ & 0.3874 \\
\hline $227 .(6,0) 5$ & () 3855 \\
\hline 230,3515 & $0.36(1)(1$ \\
\hline $2330 \sin (15$ & 0.1820 \\
\hline 235.8 .425 & 11.3616 \\
\hline $2.88556,5$ & $(1,30,31$ \\
\hline 241.21115 & (1 15555 \\
\hline 243.8215 & $(0.35 .31$ \\
\hline 2.46 .4055 & 0.3525 \\
\hline 296.1185 & (1) 3.3 .4 \\
\hline 251.861 .15 & $0.1261)$ \\
\hline 25.4 .5675 & (0. $32 \times 1$ \\
\hline 257.446 .5 & (1).3(1.+8 \\
\hline $26(0.2754$ & $0.2 ! 58$ \\
\hline $26.3 .(444)$ & 0.286 .3 \\
\hline $20.5 .8+4.69$ & $0.27+4 k$ \\
\hline $267 .(x) 22$ & 0.28861 \\
\hline $26 \%(x, 52)$ & $0.288 \%$ \\
\hline $26,7 .(x) 35$ & 0.28 .43 \\
\hline \multicolumn{2}{|c|}{ Soan 13, 130.2 prigk } \\
\hline $207.10(1)(1)$ & 0.3118 \\
\hline $20,5,3715$ & $(1) 116.1$ \\
\hline $20.25+46$ & $(1,211)$ \\
\hline $29(9.31110$ & (1).3142 \\
\hline 250.1125 & (i 1.494 \\
\hline 25285.75 & (1.377.4 \\
\hline
\end{tabular}

\begin{tabular}{|c|c|c|c|}
\hline $2+13 \times 124$ & $0.3 \times 40$ & 48.103 .5 & 0.1730 \\
\hline $24(.1) 249$ & (1) $4(x) .4$ & 45.32 .15 & 0.1551 \\
\hline $2.4 .111 k^{4}$ & 0.4251 & 42.553 .5 & 0.1336 \\
\hline $2+125115$ & $(1.4244$ & 39.793 .5 & 0.121 .1 \\
\hline$? 48+549$ & $(1.4 .1(x)$ & 37.0235 & 0.100 .3 \\
\hline 2150.505 & 0.4 .4 .16 & 34.16 .35 & $0.06 x+1)$ \\
\hline$\therefore \because k t_{1}$ & 0.4624 & $31.32,35$ & 0.0354 \\
\hline $3.311(k \times, 5$ & 0.4750 & 18.2735 & 0.0350 \\
\hline 227.2715 & $(1+48+7$ & 26.2235 & 0.0350 \\
\hline $23+.5765$ & $0.48(17$ & 28.683 .5 & $(0: 0,350$ \\
\hline $2218+29$ & (0.485i3 & 31.5315 & 0.0350 \\
\hline 210.1275 & 0.491 .5 & 34.60 .35 & $0.0 .5 \%$ \\
\hline 216.35 .5 & $0.5(1) ?$ & 37.80135 & $0.074(1)$ \\
\hline 2130745 & $0.9(40)$ & 40.69 .35 & 0.(1885 \\
\hline 21086.5 & $(0.493 .9$ & 4.3 .6 .3 .3 .5 & 0.1126 \\
\hline $2(1810 \% 5$ & $0.5(1) 18$ & 46.53 .35 & 0.1 .368 \\
\hline 20194355 & 0.5108 & 49.493 .5 & 0.1702 \\
\hline $2(1) \leq 86,14$ & $(1.51 .57$ & 52.43 .35 & 0.181 .4 \\
\hline $3(x) 25(15$ & 0.5 .104 & 55.41 .75 & 0.2050 \\
\hline $117 \div+1955$ & 0.53 .51 & 58.553 .35 & 0.2164 \\
\hline 10.18215 & 0.5 .350 & $(1.613 .5$ & 0.2350 \\
\hline 102.1155 & $(0.53 .41$ & 64.96 .35 & 0.2584 \\
\hline 180.3725 & 0.53 .38 & $68 .(x, 3.5$ & $0.278-1$ \\
\hline 180.44 .45 & $0.5+5 \%$ & 71.023 .5 & 0.304 .3 \\
\hline 18.1 .72015 & $(1.5+406$ & $74 .(4.3 .5$ & 0.318 .3 \\
\hline $181 .(14.45$ & 0.5713 & 77.00 .35 & $(1.1200)$ \\
\hline 1783525 & $0.56 \times 14$ & 74.86 .3 .5 & 0.36 .35 \\
\hline 175,4555 & 0.5679 & 82.703 .5 & 0.30 .75 \\
\hline 172704 & 0.56 .36 & 85.5135 & 0.38 .30 \\
\hline $\mid(x), 4825$ & 0.9817 & 88.153 .15 & (1) 4026 \\
\hline 16122175 & $(1.5475$ & 91.2235 & $0 . \$ 130$ \\
\hline 16.11715 & (). $(x,(x, 1)$ & $94 .(x, 3,5$ & 0.4270 \\
\hline $10.10,5.19$ & $0.58+1$ & 96.0215 & 0.44 .34 \\
\hline $158+195$ & $(0.519 .12$ & 0917.7039 & (1). $+6 .+11$ \\
\hline $156,60,15$ & $(1,6(1) 2$ & 1012.573 .5 & $0.4(x)$ \\
\hline 15.1 .18 .15 & (I.Sא.? & 105.263 .35 & $0.4 \div 1.9$ \\
\hline 150.3335 & 0.5058 & $108 .(04.35$ & $0.48(17$ \\
\hline 1.424 .35 & 0.5785 & 1101.773 .5 & 0.5120 \\
\hline 144.5535 & 0.57 .51 & 113.533 .5 & 0.5151 \\
\hline $141(6,1)+5$ & 0.5716 & 116.18 .35 & 0522.1 \\
\hline 13.8 .51135 & 0.5710 & 118.0835 & $0.52(1)$ \\
\hline 1.35 .12 .35 & 0.56 the & 121.5435 & 0.5328 \\
\hline 132188.35 & 0.562010 & 1242935 & 0.5353 \\
\hline 124.70135 & $0.5+27$ & 126.4 .135 & $0.5+47$ \\
\hline 136.7 .135 & $(0.5+4(10)$ & 124.00 .15 & 0.5620 \\
\hline 123.8235 & $0.54+317$ & 132.3335 & $0.5(x) 8$ \\
\hline $121 .(k) 35$ & 0.5440 & 135.08 .35 & 0.5680 \\
\hline 118.1735 & $(1.5328$ & 137.76 .35 & $(0.570) 1$ \\
\hline 1154535 & 0.52 .11 & 1.40 .51 .35 & $0.55(x)$ \\
\hline 112.53 .35 & $0.5(x) 1$ & 143.133 .5 & 0.5750 \\
\hline $1(19), 618.15$ & 0.4970 & 145.82 .15 & 0.5752 \\
\hline $1(40,1) 235$ & 0.48 .14 & $1.48+3335$ & $0.58(1)$ \\
\hline $1(1.4 .12 .35$ & $(0 .+1) 14$ & 151.1135 & $0.586,2$ \\
\hline $101.3 \% 3.5$ & 0.4750 & $153.8+39$ & $(0.5911$ \\
\hline 48.5735 & $(0.48+3$ & 156.68 .35 & 0.517 .18 \\
\hline 0.9 .76 .15 & $(1.4616)$ & 1.59 .49 .35 & 0.5482 \\
\hline (9) 20.435 & 0.45 .32 & 162.10 .35 & 0.580 .2 \\
\hline$\varphi(1),(16,3.5$ & 0.420 .5 & 16.4 .7835 & 0.58 .10 \\
\hline 87.11 .15 & 0.413 .3 & 167.536 .5 & 0.5010 \\
\hline 84.51 .3 .5 & $(0.34 \geq 8$ & 170.2285 & 0.5874 \\
\hline 81.8235 & $0,38 \times 3$. & 173.040 .5 & 0.5771 \\
\hline $78.9(1.15$ & $(0.37 .40)$ & 17.5 .86 .35 & 0.55027 \\
\hline 70.21 .5 & $0.35(x)$ & 178.6 .175 & 0.58551 \\
\hline 71.13 .35 & $0.3(1)(1)$ & 181.39 .35 & $0.5(2+1)$ \\
\hline 70.53 .3 .5 & $(1,3,(x)$ & $184 .(5,5.5$ & $0.5(4.4$ \\
\hline 617135 & 0.30152 & 180.70 .1 .5 & $0.550,7$ \\
\hline $6+18735$ & (1.28s': & 180.5755 & $0.5+44$ \\
\hline $621 \times 35$ & 0.261015 & $10 ? .8095$ & $0.53 \mathrm{cos}$ \\
\hline $54,3.435$ & 0.25 .41 & 105.01405 & $0.55+31$ \\
\hline $56,57.35$ & $0.2+10$ & 1078229 & 0.53 .301 \\
\hline 5474.15 & (1.2142 & $2(x) 4: 55$ & $(1.5 \leq+1$ \\
\hline $5(1.423 .5$ & $(1.190) 2$ & 2034755 & $(1.512)$ \\
\hline
\end{tabular}




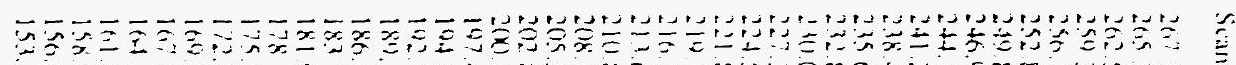
等

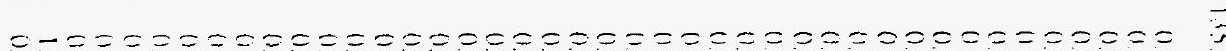

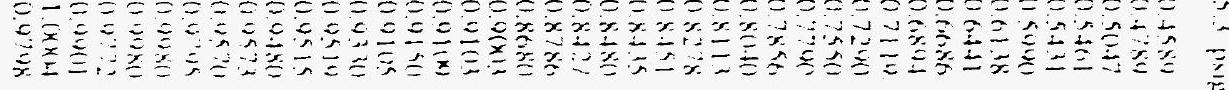

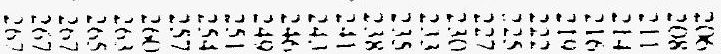

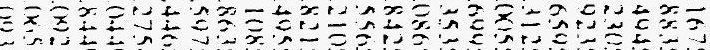

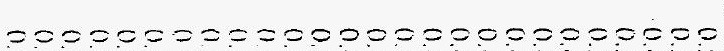

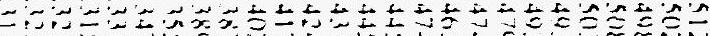

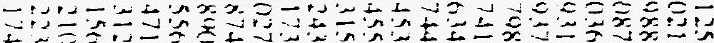

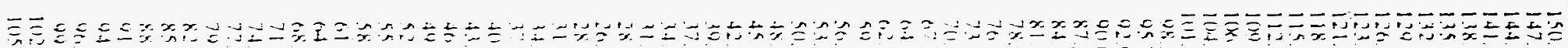
然 =о三

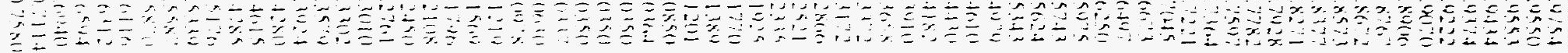

ב"

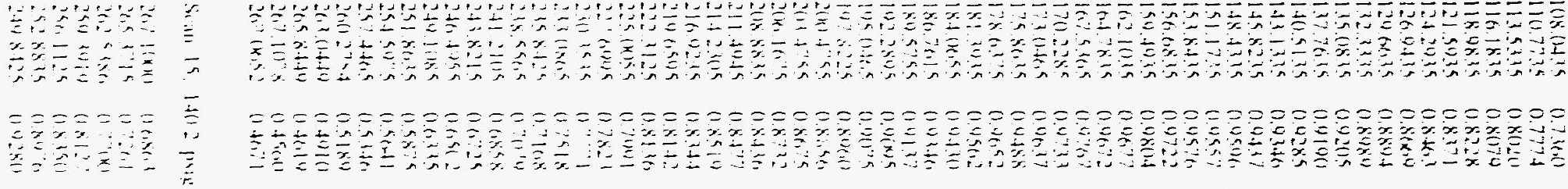

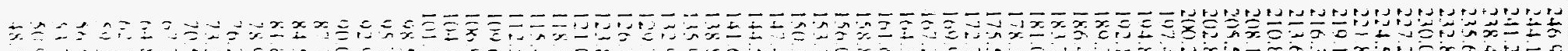

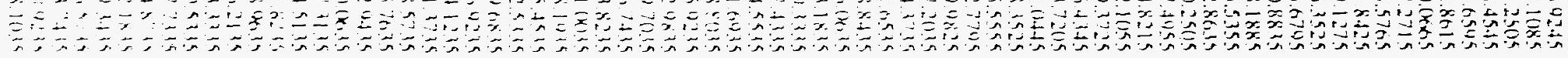

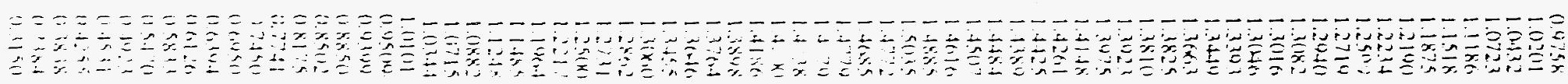




\section{Reinforced Concrete Test Data}

Track 8 - High Pressure Test

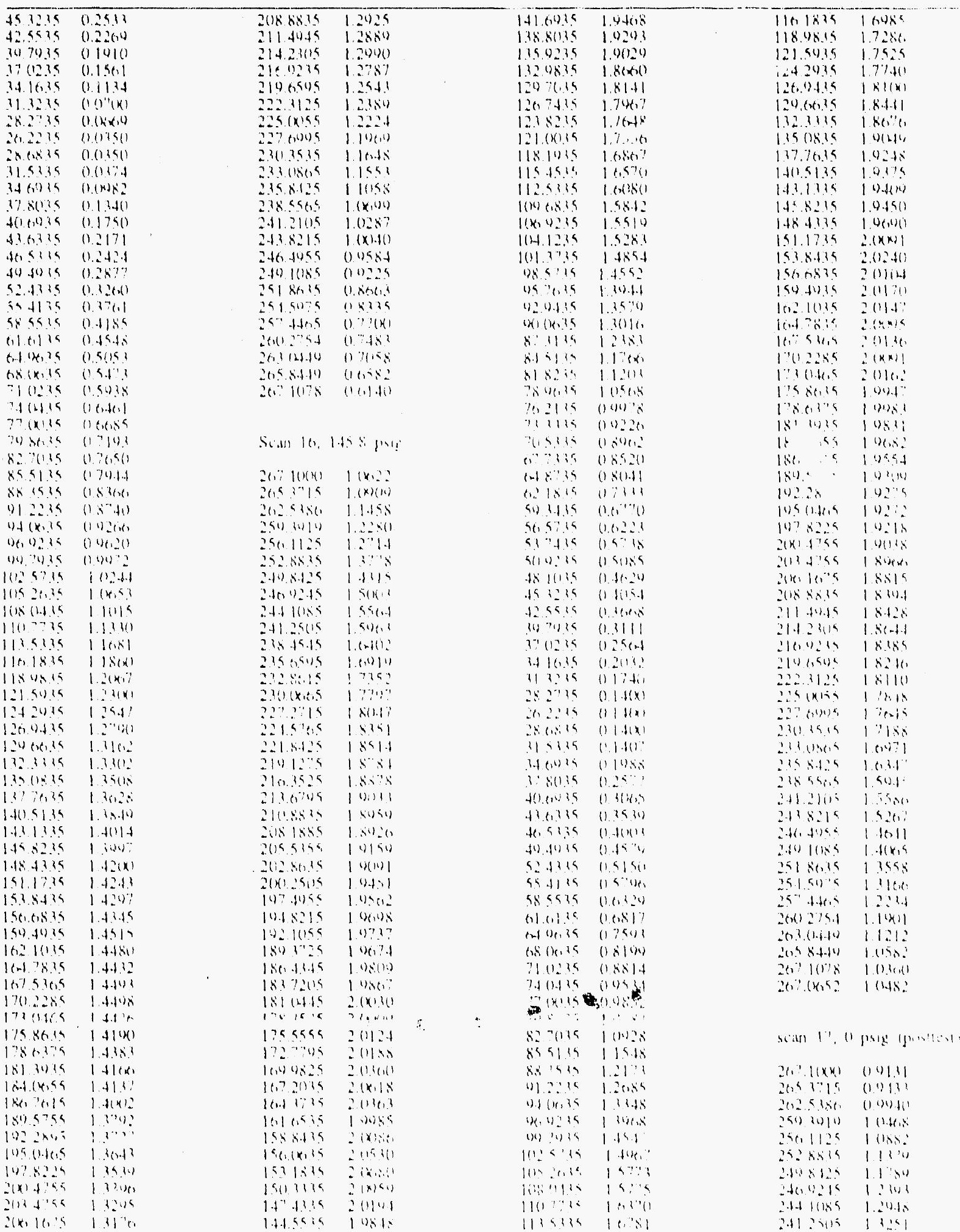




\section{Reinforced Concrete Test Data}

Track 8 - High Pressisre Test

\begin{tabular}{|c|c|c|c|c|c|c|}
\hline$\therefore 354549$ & $1,2 t, 5.2$ & 1711219 & $1 \because 114$ & 21610215 & 1.5410 & 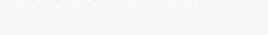 \\
\hline$=160.54 \%$ & 14115 & 34.16 .15 & 111712 & I14.6.50) & $1.5 \% \cdot 1$ & \\
\hline$\because 28 \times 19$ & 1402.4 & $\because 1215$ & (") HW" & 223125 & 1.5503 & \\
\hline ? $3111 \times x 1^{5}$ & $140=$ & 28.2715 & 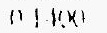 & 22506155 & $1.578=$ & \\
\hline$\because 2: 2^{7} 15$ & $151 \%$ & 262215 & () $\mid H(K)$ & $22 \therefore 0(0) 9$ & $1.5(x) 5$ & \\
\hline $23+40$ & 15401 & 286615 & $(1.1+(x)$ & $210.14,35$ & $1 . f(x, 7$ & \\
\hline 2218425 & $15 \%$ & .11 .53 .15 & $0.1 f(k)$ & 2330180,5 & $1.4(x, 1)$ & \\
\hline 210125 & isks: & $1.1(10)$ is & 0.1710 & 2358425 & 14110 & \\
\hline$\therefore 10.3524$ & $1,111: 2$ & 19.801 .5 & 1). $.1 \times 1.4$ & $238.550,4$ & 13610 & \\
\hline 21.26 .08 & $|t| 1)^{\prime}$ & 40.61918 & $(1.2)^{\circ}$ & 241.21015 & 1.1386 & \\
\hline 2108515 & $10 \ldots \%$ & 410.135 & (1) $: 58 . ?$ & 2438215 & 1.2414 & \\
\hline ? & 16284 & 40.51 .14 & 0278 & $246+4055$ & $1.26 \% 2$ & \\
\hline 2015.5155 & $10,50$. & 41).4195 & $(1.316 .1$ & 2411.1185 & $1.2(171)$ & \\
\hline $2018<16$ & $1(1, \therefore)$ & $52+135$ & 11.354 & 251.80 .15 & 1.1901 & \\
\hline $2(x): 5: 15$ & $1+x, 1$ & 5.511 .15 & $(1.41 \% 4)$ & $25+5005$ & 11200 & \\
\hline 107.1055 & $16 \%$ & sis & (1) $+4+$ & $25 ?+405$ & 105.2 & \\
\hline 1445215 & $|(0,0)| 1 \mid$ & 0.10 .119 & 11 thim & $\therefore x(1) 2754$ & $1.11 \% 7$ & \\
\hline 1111110 & $16 \times 2.4$ & 6.190 .15 & $16519 !$ & $260(1 .+10)$ & $(10) 18$ & \\
\hline $18013: 5$ & $16 \times 16$ & (1):ke., & $(15 x)$ & It. 5 s.t.tu & (1) (1) & \\
\hline $180+145$ & $|(3)<|$ & 111235 & $116: 4$ & $26 !(11) 22$ & $0014 ?$ & \\
\hline $19 ! \therefore k$ & $111 \%$ & 7111.135 & $\| 1, \ldots$ & $2 n^{-1}(k, 5)$ & $(1) \cdot 111) \div .1$ & \\
\hline 151.11 .49 & 1716.4 & $7.1 \times 135$ & $11 \because 210$ & $2(1, ;(k)+s$ & 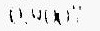 & \\
\hline $1: 19: 4$ & $1 " 1.40$ & 30 sols & $11: 3)$ & $1 \vee 11$ & & \\
\hline 1 \& \$4 & $1 \because n$ & $B 29115$ & $(1 x: 1$ & & & \\
\hline $1 \because-1,8$ & $i " 1, x$ & $85<515$ & $11 \because 11$ & & & \\
\hline $16.40 x=$ & 1."1 & Bis 1915 & $(11)=110$ & & & \\
\hline 10.21144 & $1 \cdots 4$ & $111 \ldots 16$ & $(1, \sin x)$ & & & \\
\hline $10+193$ & $i \cdots$ & 1) 1910.18 & $111 \cdots 5$ & & & \\
\hline $10.16: 16$ & 111 & $1701: 4$ & $10(1,1)$ & & & \\
\hline $15 \times 110$ & $1 \because \therefore$ & $(y 1)^{\prime} \cdot 116$ & 1.111 & & & \\
\hline $140: k, 15$ & $1: 111$ & $10: 946$ & ||$\cdots \mid$ & & & \\
\hline 1411315 & $1 j i$ & $119 \therefore 15$ & 124 & & & \\
\hline $14,12: 19$ & $110 x$ & $1: 1,1: 4$ & 1281 & & & \\
\hline $11^{\prime \prime}+130$ & 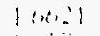 & $1: 11 \cdots i$ & 1 ifil & & & \\
\hline $1.145: 5$ & $i t: \cdots$ & 1115315 & 11115 & & & \\
\hline 1.118 .216 & $11 \cdots$ & 110,1315 & 1411 & & & \\
\hline 116.10 & in: : 1 & $11 \times 4516$ & 1 k1/e..: & & & \\
\hline $140: 46$ & 192 & $1.15: 1:=$ & 1.425 & & & \\
\hline 1121815 & $1<5$ & 124214 & $1+1.7$ & & & \\
\hline $120 \div 118$ & $19 \mid(\cdot)$ & 1261115 & 11,51 & & & \\
\hline 126.743 & 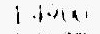 & $12 x(x, \cdots$ & $1 \because 1 x$ & & & \\
\hline $123: 3: 5$ & $116, \cdots$ & $11+11$ & 1.54t: & & & \\
\hline $12114: 5$ & $1412=$ & $1: 50136$ & $1<" 11$ & & & \\
\hline 1118.1415 & $1: \| 1$ & $1: 70: 5$ & 15,1 & & & \\
\hline 115153 & $1 \cdots x$ & $14151: 5$ & 1. $(x+1)(x)$ & & & \\
\hline $11: 51: 5$ & $1: 41$ & 1.11115 & 10.235 & & & \\
\hline$f(x)$ (in is & $12 ! " A$ & $1.15: 14$ & $11+158$ & & & \\
\hline $1(x, \because 2]^{6}$ & $121 \%$ & 1111125 & $1: a^{\prime}$ & & & \\
\hline 1141219 & 1111 & 141114 & $1+x \ldots 1$ & & & \\
\hline 1191.1916 & $115: 1$ & $1918+18$ & I (12)/ & & & \\
\hline 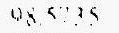 & 11131 & 150 en is & | (4) & & & \\
\hline 1) 5 th & $1(1)$ & $1514+1135$ & I (i)is: & & & \\
\hline $9: 19.15$ & $164^{4} .1$ & $10: 11035$ & 1. "1) & & & \\
\hline 40$)(x, 19$ & $(1) \div 21$ & $10.17 \times 15$ & $1 \div 1.1 ?$ & & & \\
\hline $8 \div 11.15$ & (1) 1 ) $3(4)$ & $16 \therefore 510^{5}$ & $1 \geq 14$ & & & \\
\hline 845115 & 11 singt & 1710228 & $122+1$ & & & \\
\hline 8112.15 & (18.1.8 & $1: 1(4), 3$ & 111.1 & & & \\
\hline 7514,15 & $0 k(1)$ & 1758615 & 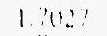 & & & \\
\hline 76.21 .15 & $11 \%: 1$ & 178.1829 & 1. 1018 & & & \\
\hline 33.3335 & 0.6711 & 181.3115 & $\mid(t, 1),(1)$ & & & \\
\hline$\because 4: 6$ & $\cdots \cdots$ & $1: 1 ; \%$ & 1, & & & \\
\hline 67.336 & 0 cils.t & $\mid 86,7615$ & $10 \times 10^{7}$ & & & \\
\hline $6.4 \times 15$ & (1) $5+4+6$ & 180575 & istas & & & \\
\hline OAisis & $11 \therefore 11$ & 1422865 & $f(i)(\mu)$ & & & \\
\hline (1) 3.115 & $1,152)$ & $10<146,5$ & $10 ; 111$ & & & \\
\hline$\left.S_{1}, \varsigma^{\circ}\right), 5$ & (1) 150 & $11: x: 2=$ & $1\left(x_{1}\right) !$ & & & \\
\hline $5:-1: 5$ & $11.11 \times$ & $214+4<5$ & $10+1$ & & & \\
\hline 4011234 & $\because ! \cdots$ & $\therefore 11+3$ & $1(x))^{2}$ & & & \\
\hline 181115 & $11: 411$ & $\therefore m \mid n^{-1}=$ & $1(-1) 16$ & & & \\
\hline 453244 & $\| 11: x_{1}:$ & $\because 12 \therefore 8: 6$ & I (x) & & & \\
\hline 42546 & $(1: \cdots)$ & $211+1.45$ & $1(x \div x)$ & & & \\
\hline (1) :10:5 & $11 \therefore 4 \div 1$ & $21.4: 109$ & 10.1111 & & & \\
\hline
\end{tabular}




\section{Reinforced Concrete Test Data}

Track 9 - High Pressure Test

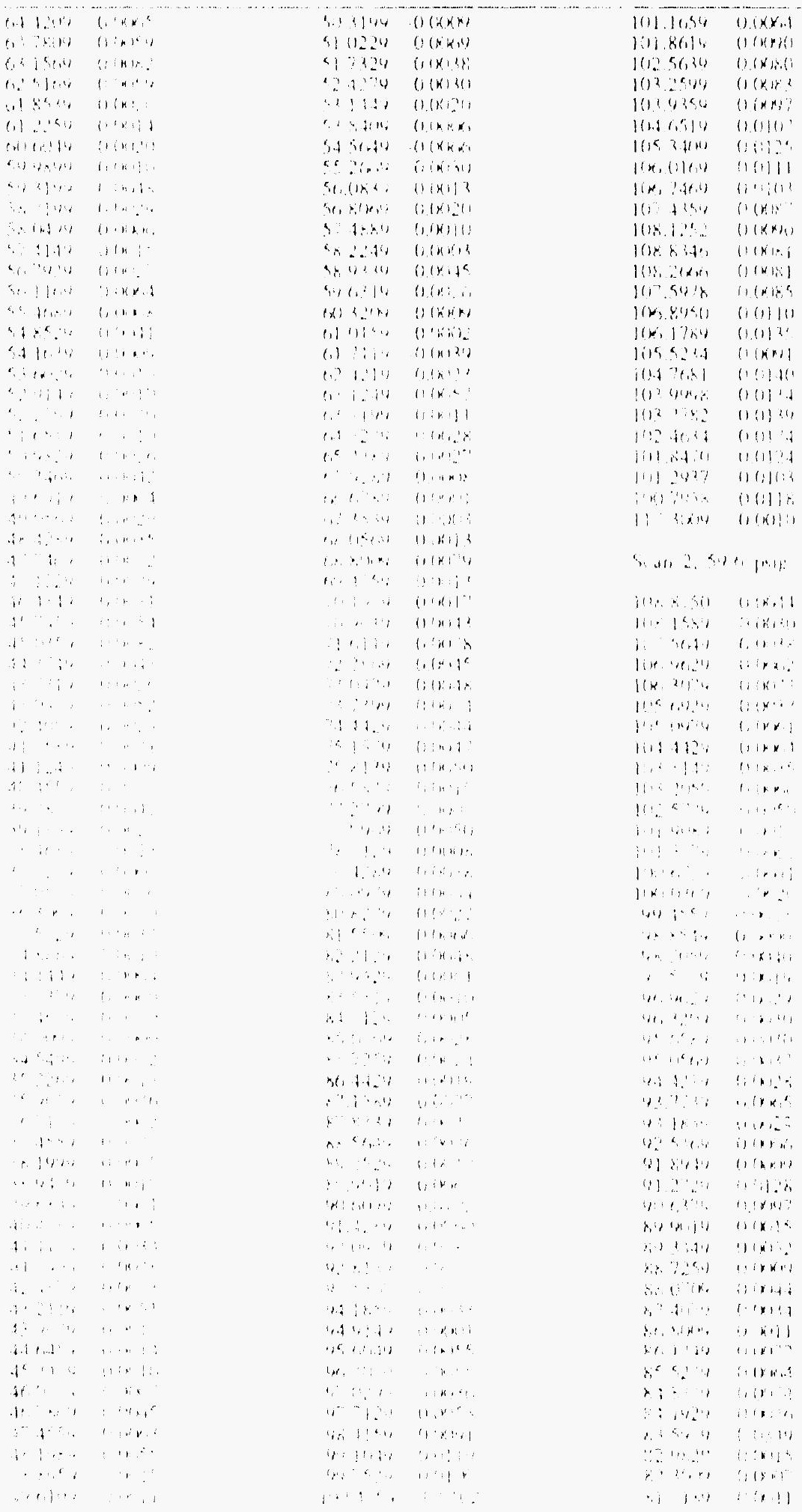




\section{Reinforced Concrete Test Data}

Track 9 - High Pressure Test

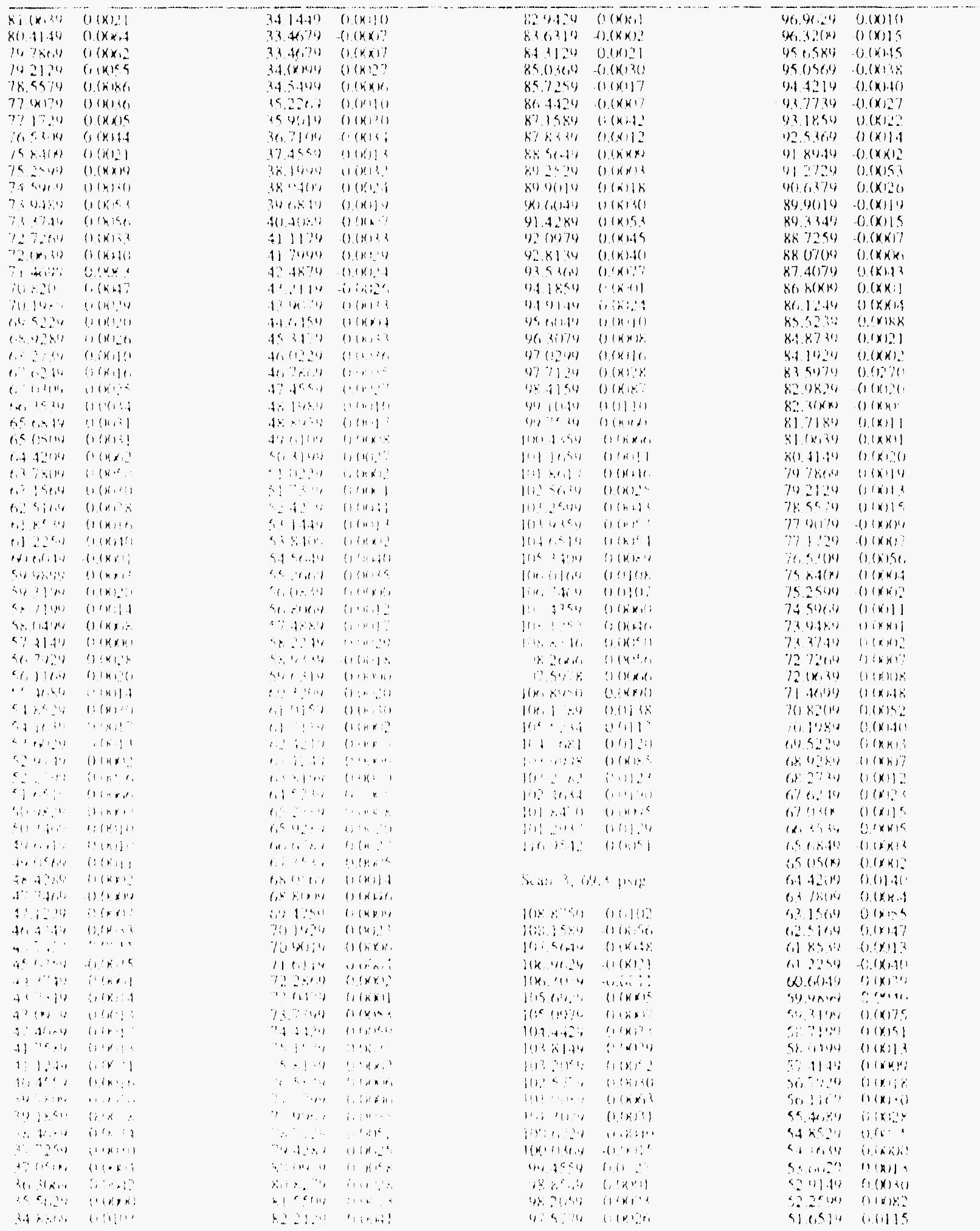




\section{Reinforced Concrete Test Data}

Track 9 - High Pressure Test

\begin{tabular}{|c|c|c|c|c|c|c|}
\hline$s(1.082)$. & $(1)(x) \geq 1$ & 652260 & $(1)(x) !: !$ & 101.8170 & 67.6249 & .0 .0110 \\
\hline 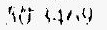 & (). (k)1)? & $05,4=84$ & (1) $10 s_{0}$ & $101: 303$ & $67.013(\mathrm{k})$ & (1) $(x) k x)$ \\
\hline $40(1,1)$ & $(1)(x) \div)$ & $(x, c), x y)$ & $(1)(x) 1 \div$ & $116,95.42$ & $(x, .3530$ &.$(1.0120)$ \\
\hline du $(1)^{2}(1,4)$ & $(! 1 \times 417)$ & $(1) .1539$ & $(1)(x) 4$ & & $65.6 \times 19$ &.$(0 .(x) \mu) 7$ \\
\hline $4 \times 4: 2: 8$ & (i) $\left.(x)_{a}\right)$ & $\left(x_{i}(1) \cdot(x)\right.$ & ()$(x \times)_{2}^{\prime}$ & & $6,5(0,5(x)$ & (1).(1)(14) \\
\hline $1 \% 1,11$ & $\Leftrightarrow(x)=x$ & $6 x^{2} \sin (x)$ & $(1)(k 113$ & \multirow[t]{2}{*}{ 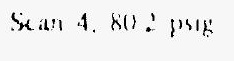 } & $6+42(k)$ & $0 .(x)(x)$ \\
\hline 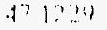 & $0 .(k) 14$ & $(14+1.54)$ & $0(5 \times 15$ & & $6.3 .78(k)$ & $-(1 .(x), 4$ \\
\hline $4(1,+1,40)$ & $0(x+1)$ & $7(1.10 \geq 1)$ & $.0(x), 4$ & 1018.8960 & 62.1569 & fo $(x)\{i$ \\
\hline 45.74517 & (1) $(k+1)$ & $7(1(x)) 14$ & $-(1)(x, k)$ & lok.1584 & $62<16,4$ & $0 .(x) 20$ \\
\hline 450154 & $(1)(4 x, d)$ & 710.114 & $0.0(1), 6$ & $10786+10$ & (1) .5539 & (). (x)>x \\
\hline 4187417 & (1) $(x) 1\}$ & $72 x(1,1)$ & $06 \times 120$ & $16 x, 10,04$ & 0,12250 & (1) 111128 \\
\hline 437111 & $(1: x) 11)$ & $71.1+1.14$ & $0(4))^{2}$ & $11(x, 101)$ & $(x) \cdot(10)+4)$ & .0 .1130 \\
\hline $43(x) ? 4$ & $(1)(x) \leq n$ & 7372114 & ().(H) & $10.50,0,14$ & sy todigy & $-10 .(1120$ \\
\hline $42+\sin 4$ & $(1,6)$ & 74.4214 & $0.01 \times 1:$ & $1(151127 / 4)$ & $59.3 !(k)$ & $0 .(1)+0$ \\
\hline $4 i 19 x^{\circ}$ & $(1)(x+4)$ & $3<154$ & $(1 \leq x)<1$ & $10.4+1.20$ & $58.71(x)$ & $0.011 \%$ \\
\hline 41.1214 & $(1.1 \times 1.01)$ & 75.81311 & $11(101)^{2}$ & $\left.1(1)^{2} \lambda 1.14\right)$ & $5 k .(1.1(x)$ & $(1)(x) \div 1$ \\
\hline $40,4<54$ & $0(k+1)$ & $\left.\eta_{0}<x<1\right\}$ & Ufí:.') & 10123154 & 57.41 .44 & $0(x)<(1)$ \\
\hline 147 7ins & $111 k 11:$ & $7127(89$ & $(1(x)) !$ & $1125 \% 24$ & $.5(1,79) 20$ & () (1)ils \\
\hline $9+1 ; 0.11$ & $11+\infty), 1)$ & $77(40,0)$ & $0101: 4$ & $1(1) 18(16)$ & $5(;, 1] 1,1)$ & $0 .(x) \geq 2$ \\
\hline $3 x+1+x)$ & $0(x): ?$ & $7 \mathrm{~h}: 1124$ & $11(w r)$ & $\operatorname{lil}\left\{\left(1^{-1}\right)\right.$ & $55.16: 2)$ & $91 .(x) .94$ \\
\hline 19.94 & $\therefore$ an & $1142 \times 1$ & $|1+n| 1 \mid$ & H!H & 5.485 .24 & (1). $(x) 1.4$ \\
\hline $1,11, i \cdots 1$ & lifrke & 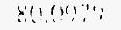 & finne: & $1(k)(1,3,4)$ & $5.416,3.4$ & $0 .(x) \div 1$ \\
\hline if $41,10,1\}$ & 11 KN1t & $80182 \%$ & $(1)(x) ?$ & $13+95517$ & $531,(120)$ & (1) (x) 10 \\
\hline $344_{1}, \ldots !$ & (1) & $3.1<4(14)$ & $(110)(1)$ & 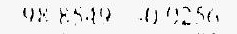 & 5.51 .40 & (1) $(x: 7)$ \\
\hline S.4 Rentit & $11: k+14$ & $x: ? 1 .: 1$ & $(1)(11,1)$ & (16. $: 140$ & $32 S(y)$ & U. $(x))^{2}$ ? \\
\hline $141+11$ & $(1+1 k)^{\prime}$ & $x_{*}: 1,(2,1)$ & $(1)(x+1)$ & $19 \div$ in & 51.514 & locion: \\
\hline $3: 41,71$ & ()$(x)) 1$ & $\left.b^{2}+x^{2} \mid 1\right)$ & $(1114: 4$ & $(x, 1), 2,4$ & $5(1,462)$ & $0(x) i 19$ \\
\hline$\therefore<41,4\}$ & $1: 1 h 1) 11$ & $x+11: 2$ & $(1) 11,1)$ & $11,2,2110$ & $8(1) 3.40,1)$ & $(1(x) \cdots 0)$ \\
\hline $31(k)$, & 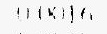 & $8511^{\prime}, 1$, & $1111 x<$ & 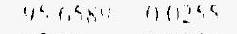 & $(40(1): 1)$ &.$(1)(x) 55$ \\
\hline$x+4<13.4$ & $11: n+4$ & $x<9,1411$ & $1 .|x| x+1 \mid$ & $18: 11^{t} \cdot 1 \cdot:$ & $(19)(15(, 1)$ & (1)(11:25 \\
\hline is $\therefore 1$ ' & $1,1 \mathrm{H} H \mathrm{H}$ & s.t. 1.1." & : : nit & $\because 1 i: 1+1$ & qu tan & $(1)(k) .1 ?$ \\
\hline (4) $x(1)$ ) & $(i d, i)$ & $\left.h: 1^{\prime} \because \cdot\right)$ & $(1: M) \cdot$ & $1,1,7+4,1$ & $41,2,4(1)$ & $0(x)<1$ \\
\hline is. 'Ilx; & $n+n+$ & $x^{\prime}: x^{\prime}: 11$ & $(1+1+1)$ & 1141801 & 171224 & $(11 x) 18$ \\
\hline$\because 4(x)$ & $\| \ln x$ & $\sin (x+1)$ & (1) $1: 4$ & $11): 4,1,4$ & 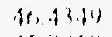 & $(1)(x))^{\prime}$ \\
\hline 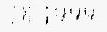 & :1 $1 \times x \times$ & 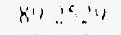 & $\mid 1+11.11$ & $11 ! \therefore, 13$ & $15: 140$ & $(1 . ; x+\cdots)$ \\
\hline 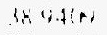 & $1+(k+1)$ & xusping & 1:161!61 & (1! :11 & 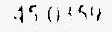 & $0\left(x^{\prime}\right)+1$ \\
\hline $301+x$ & (bix':) & 1)(16,10) & $\left.(1, t)^{1}\right\}$ & $(11,12,4)$ & $4.17 .4 \%$ & $0(1) \times k)$ \\
\hline $41+1,21$ & $1: \ln x: 1$ & $111+2 \times 1$ & (ilni): & $x+1519=$ & 427.10 & $0 .(x) 18 \%$ \\
\hline$\$ 1114$ & $(1)\left(k_{i}\right)$ & 1? 3111 & $\{(k, 1)$ & $x 18=1.11$ & $4\{(n+3)$ & (1)(x)ix \\
\hline 414311 & 1) 1 (n) $1:$ & 128.111 & (1)!11:"? & 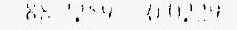 & $424(x)$ & $(1, \times 12)$ \\
\hline 4. AR 4 & $(1 \mid x): 1$ & 101631, & 1,1114 & $B_{1} 11^{\circ}(\mathrm{m})$ & $4: .5611$ & $(1 .(x) .19)$ \\
\hline $12: 1118$ & (1) $11 !: !$ & $1112=1$ & $(1111)^{r}$ & $a_{1}: 11+4$ & 41.1214 & (1)(x):? \\
\hline 42 (x)क & $\because \because: ?:$ & $14.91191)$ & 1140,10 & he Sol & $4(1,9<44$ & $(1)\{x)\{8$ \\
\hline$f\{1, f(1)$ & $(1,1):$ & $1,601,14$ & $(1,1) \times 4$, & nri 1:11n & $347 \times(k)$ &.$(1.1 \times 1.711)$ \\
\hline $\left.4^{\prime} \quad 44^{-1}\right)$ & $(: 1,1:, 1)$ & $m, a_{i}$, & 111111,11 & $3,5<24$ & $41) .15(1)$ & of (x)itu \\
\hline He:112:11 & 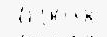 & $1: 11 . "$, & $(1), 11 \cdots, 1$ & $\therefore 4 \div$ & $3 k+k, k l$ & $\{1,6,2\}$ \\
\hline$k_{1} \quad x+\cdots$ & $f(x)^{2}$ & "1: ? & illit. & $x+112$ & $1,7\{, 1,1)$ & $(t)(x) ! 1)$ \\
\hline 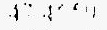 & "1:HI. & $12+11$ & $(1,1 ! \cdots)$ & $n+1, n$ & $\{"|r| x \mid$ & $\{1(x) 1$ ? \\
\hline 901.19 & (1) & $1 ": ! 114$ & $(16), \cdots$ & $\therefore 114$ & $16.8(x, 4)$ & 0101211 \\
\hline $4 \therefore \therefore 1$ & $11\{0,1\}$ & $i, 1 ;, 4, i, i$ & lnil & $\therefore \quad$ in & $44(0 ., 1)$ & $0,(x):=$ \\
\hline $4+1, ! 314$ & OIKKK! & $101+1101$, & (:) 1 r $2:$ & 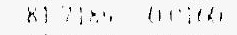 & $.4 \times(6,1)$ & (1) $(x) /(1)$ \\
\hline $411 \cdot 101$ & ipton & :i1: $\mid 1 \cdot 0 \cdot 1$ & $(10 x)$ & $k \mid 1, k, k 1$, & 4.1 .4 .11 & $01 x+15$ \\
\hline 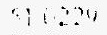 & $(1+4)$ & $11,1 \times 1.1=$ & 11:1:1 & $8+1+4=$ & 3158194 & $0(x) 10$ \\
\hline 5172515 & $\langle 1\}|1\rangle)$ & $111=x, \cdots$ & i)(x)i: & (3) & 33.96 .74 & (1) $1 x) 14$ \\
\hline $5: 1211$ & $1, k+114$ & $101:-61,1\}$ & $(:\{1: 1)$ & 14.21 .11 & $1.4(k)(m)$ & $f(x) \mid 11$ \\
\hline 51,140 & 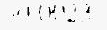 & 1921901 & (1) $x: y$ & $7: \ldots 64$ & 345.424 & (1)(x!!? \\
\hline as pi:ite. & (Hoki & $10\{1, \ldots 1\}$ & $\left(11 k_{1}\right)$. & 728019 & 45.2014 & $(1: 141$, \\
\hline $4: 4,64$ & $11+111^{6}$ & $16, \ldots 140$ & $(1)(x), 0)$ & 77.1124 & 35,191918 & $?(x)(1) ?$ \\
\hline$\because ?$ & (n) $x$ & $\mid 1 k: 11\|x\|)$, & $1+12: ! 4$ & 10.1 .112 & $\mid t, \eta \| r)$ & $A(k+i)$ \\
\hline 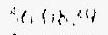 & $(1) k \mid:)$ & lok, Then & 1610.11 & $74 h-164$ & $3 ? 155,4$ & $(1.1 \times) 10$ \\
\hline 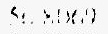 & $(\cdots) \mid$ & 110.1054 & $(1)(x) \mid)$ & $7 a: x(1,4)$ & is lon & (1) (x) \\
\hline \&ans & Ninits & $19: 8.1 .2^{12}$ & frílth.? & 745014 & $3 \alpha \log \mid(\alpha)$ & $.11(x)+k$ \\
\hline $5 x^{2} 22+11$ & $1)(\mu: 4$, & $1 ! ; r, c 1 p$ & $1+1 \times 1,11$ & $721 / 1: 1$, & $317+3.94$ & $(1) x k: 1$ \\
\hline $36+2319$ & l) $(x)+1)$ & 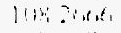 & 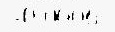 & 111111 & 414180 & unisita \\
\hline 51$)(619$ & link & $\left\{(\therefore 64)^{\prime}\right\}$ & $(1,\{r):$ & $98+1$ & $\$ 111 ;$ & 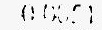 \\
\hline (W) $32 \mathrm{WH}$ & $1114 x: 3$ & $\left.1\left(k, b_{1}\right)<1\right)$ & $0(x) .2^{\prime}$ & $\because 2(x, 1)$ & 41.740 & $11(k \div)$ \\
\hline $0,111 ! 4$ & $111 n_{1} \cdot 1$ & $\left|1 w, l^{\prime} i k\right|$. & 11 a & $31+1+48$ & $12+480$ & (1) $\left(x_{1}\right)<$ \\
\hline $6 ! 7: 14$ & ino. & $1 / 1 \times<2: 1$ & $\therefore 1015$ & $m, 11 m$ & 212111 & $11(x) 2)$ \\
\hline $62+2118$ & $1(x) 1$ & 111.17 .31 & (1) ix).?.! & 70110101 & $x \mid 1)\left(1^{9} ; i\right.$ & $0: x+28$ \\
\hline$(1,1) \ldots$ & 119,12 & 11 1 म幺: & $O(x)$ & (1) 8.21 & H. r. ? & inists \\
\hline$t,\{k \mid n$ & $\left(1+()^{\prime}\right) i$ & $160^{2}: y^{\prime}$ & $11(1) \cdot 1_{c}$ & (xit) & $1534: 4$ & $01.1)(1$, \\
\hline $1.1 \% 23$ & and: & $: 12 \because 4, \because 4$ & $\therefore i n \because \cdots$ & $\therefore: 17$ & $H_{1}(1) 24$ & $(111: 61)$ \\
\hline
\end{tabular}




\section{Reinforced Concrete Test Data}

Track 9 - High Pressure Test

\begin{tabular}{|c|c|c|c|}
\hline .607869 & $(1)(x) 8.5$ & 977124 & .0 .112 .88 \\
\hline 47.4550 & -f) $1 \times(4+1)$ & 48.41 .59 & 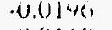 \\
\hline 481980 & $(1)(x) / 4$ & 99.10 .19 & $0 .(1)(8)$ \\
\hline 48.8450 & 0.01127 & 94,194 & $0 .(12) 10$ \\
\hline (1) $610(x)$ & $-(1)(x)(x)$ & $1(x)+4.54)$ & (1) 612.40 \\
\hline 5()$>11(x)$ & 0.01120 & $1011.16,50)$ & 0.112801 \\
\hline 51.0120 & (0) (1015.4 & $10 ! 1.8014$ & (1)(1)?(1) \\
\hline 9171218 & $(1)(k k))$ & $1023 x+17$ & (1) सं $8 \mathrm{sic}$ \\
\hline $5 \div 12 \% 0$ & $11.0 \times 178$ & 101325134 & (1) $(1 ?, !$ \\
\hline 5.31 .4 .810 & $0.01)\left(1 x^{\prime}\right.$ & 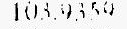 & .1102501 \\
\hline $34 x \cdot(1 x)$ & (1).01.30 & $1(1.40410)$ & $(1.112 .811$ \\
\hline 5.956 .94 & $11(1171$ & 0053.4149 & $(1,11,1(1)$ \\
\hline (4) ? 1,4 & $(1)(1) 30$ & $10,1,110,1)$ & $110.11 \%$ \\
\hline st, (18.31) & aina? & $10,7.40,4$ & $-(1.112,10)$ \\
\hline iosikn & (1).19112 & 167.4759 & -1111.28 .4 \\
\hline s. $18 x+4$ & $(1,0111)$ & $308,125 ?$ & $(1,1)(1)$ \\
\hline \&1 22,44 & $(1)(1) 11)$ & 1188.46 & $(1,(1) 14)$ \\
\hline $98 \cdot 1\langle\langle 4$ & $(1)(k k))$ & litk itrue & $11(1,14$ \\
\hline 30,110 & (1)Kr! 1 & $11) !<1)+x_{i}$ & $\left(1, l_{0} t^{\prime} x_{1}\right.$ \\
\hline$(x) !(x)$ & (1)(11)! & $10 x, 8,0901$ & 111124 \\
\hline (1) 11$)$ S4 & (1) $1.11: n$ & $l\left(k_{1},\left.\right|^{\top} \mathrm{kir}\right.$ & $(;\{1,\{1)$ \\
\hline$(.17114$ & $(1.11)+1)$ & $1015 \leq 284$ & $(1,1 \ldots 1,1$ \\
\hline$(1.4211)$ & 011119 & 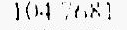 & $(1.112,26)$ \\
\hline$(1,12.14)$ & .1111110 & 1612 crats & $11112: 3$ \\
\hline 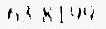 & 11111.18 & $10 \times 2 \%$ & $-(1,11278$ \\
\hline$(2)<214$ & $11+11 ?$ & $101.81,2 !$ & $(1)(1)(1)$, \\
\hline $11^{6}:\langle 3,1\rangle$ & $(1)(1) 14$ & $111 ! n+16$ & $(11: 11)$ \\
\hline$B \times 1 ", A 1$ & $11 \mathrm{iith}$ & $1111.214\}$ & (1):? \\
\hline 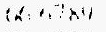 & 1111168 & $136,46$. & $(11:), 16$ \\
\hline (i) $15: 14$ & oij)" & \multirow{4}{*}{\multicolumn{2}{|c|}{ 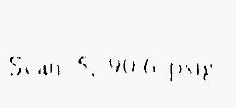 }} \\
\hline the licisen & $11111: \therefore$ & & \\
\hline 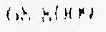 & $(1.11): 1$ & & \\
\hline$(14.4 \div 91)$ & $(1)(1) ?]$ & & \\
\hline $7(1) 10.34$ & $0(1) " 1)$ & lots b. & (i) (1.111? \\
\hline (1) 1)!1!? & $(1)(1) .92$ & 1061584 & 110.6010 .4 \\
\hline$! 1(1) 11)$ & $(1.11218)$ & $1019.9(.+4)$ & $11(4+1,5$ \\
\hline 7228,48 & (\}$(1) \mid 1)$ & $1\left(x, x_{1}, 4\right.$ & fincts \\
\hline 7101,21$)$ & $(1)(1) 36$ & $10(x, 411,1)$ & 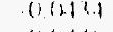 \\
\hline $747: 4$ & $(1)(1) 17$ & $1(15,(1) 21)$ & $(1)(1+1)+k$ \\
\hline 7.4 .9 .16 & $(111) 5,4)$ & $1015(x) 70)$ & $\{()|x|$ \\
\hline$i \leqslant 1 \leqslant 3$ & $(1)(1) 51)$ & 111.411 .4 & $(1111.4)$ \\
\hline $75 \times 1.96$ & (1)(1) $1 \ldots .1$ & $111 ? 81.41)$ & $(11+2.1)$ \\
\hline 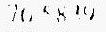 & $1111)^{4} 11$ & 1112.0164 & $(1114,81)$ \\
\hline $7: 710$ & $(1) \mid 1,1$ & $101<? ; 1)$ & $(1) 1,1,4)$ \\
\hline$\because 7()(1), 4$ & $(1) 11)_{1}(1)$ & $1(1) 1,161)$ & $(1114, \vdots)$ \\
\hline $787 i^{\prime \prime \prime}$ & 1101,25 & $14 ; 1+11: 1$ & $(1)(1) 11)$ \\
\hline 7442814 & $01(1) 1 \%$ & 11011,7201 & $.0(14)$ \\
\hline 8010140 & $(1)(1)(1,0)$ & $(x+1)^{\prime}(1, y)$ & 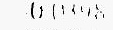 \\
\hline $8(1+\cdots)$ & (j) 11,1$]$ & 174.15 & $11(11) \div$ \\
\hline$\therefore 1(4+n)$ & $11111+1$ & 48564 & ()(i1k: \\
\hline$x: 21.4$ & (1)ii]6.1 & 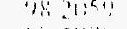 & 111912 \\
\hline $820.4 \%$ & $(101) 6,5$ & औव? & $0 !(1) 11 !$ \\
\hline$x ?+1,10$ & $11111 \mathrm{Ag}$ & $4 t, 16,24$ & $11\left(12 x^{\prime}\right)$ \\
\hline$x+1, \cdots$ & \{1) & $(6 t: 4(x)$ & $1111.41 ?$ \\
\hline$u<\cdots$, & 0.1196 & 45.650 .4 & (1)(1), \\
\hline $85.12<.11$ & (1)1.2? & us $(1,56,4)$ & $1 ! 1), 11$ \\
\hline kn $4.1="$ & $-(1) 11 ! \times 1$ & $42.921\}$ & $1)(11 \%$ \\
\hline $8: 1450$ & $(111): 1 x$ & 417,713 & flolis \\
\hline$k^{\prime} x: 24$ & $1111: 15$ & $4 \div 18-4$ & 011160 \\
\hline 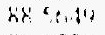 & ii 11:'? & $y \geq\{x\}$ & (1)i.1:) \\
\hline niv .:3" & .111228 & $4(x-1)$ & $(1614,45$ \\
\hline$x+4010$ & $(1(1) \cdot 11)$ & H: :थ" & 1112,1 \\
\hline (x) $(x+1)$ & $116,1: 9$ & $4(1), 2,9)$ & 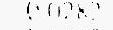 \\
\hline$\therefore\{2 k 4$ & $(111,21.2$ & 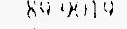 & $01611)$ \\
\hline $4: 14011$ & $4169) 191$ & 543.3 .94 & $11 ! 1911.4$ \\
\hline 421814 & $11,19: 5$ & $r k i 2 k, 1$ & 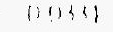 \\
\hline 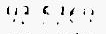 & $\therefore \cdots-1 !$ & $5 \times 1111112$ & แ!い: \\
\hline $4.913: 511$ & $(1: 1, \ldots, 1,4$ & 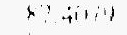 & $1101.1 \%$ \\
\hline 4.941 .41 & i) $\left(1 !^{\prime \prime 11}\right.$ & Pre a's(l) & $(1112.1$ \\
\hline 1$)^{6}(1,1.14)$ & $(1)(1+2$ & $\therefore t, \quad \therefore d u$ & $(11,2,1)$ \\
\hline 14,$111 ;$ & $(1)(1) i_{1}$ & $85<\leq 30$ & $011+1.2$ \\
\hline 0701010 & 1111.18 & $5.45,739$ & 1161.2 \\
\hline
\end{tabular}

\begin{tabular}{|c|c|c|c|}
\hline & & & \\
\hline $8+11020$ & (1)0 12.55 & $37, .250$ & (1) $(x)(1)$ \\
\hline $8 x ; 079$ & $0 .(k) .77$ & $37.0 .5(x)$ & $(1)(x) 3)$ \\
\hline $8: 0824$ & -0.01267 & $36.316 .4)$ & - (1) $(x)_{2}^{2}(1$ \\
\hline $8 \ldots .3(x \times 11)$ & $.0 .0 .10 k$ & 35.5629 & ก. $(15 ?$ \\
\hline 81.7189 & (6.1)29? & $34.886,0)$ & (c) $\left(x^{\prime}\right) 94$ \\
\hline $81.8 x, 49$ & $(0.02(2)$ & .34 .14 .84 & $-0 .(x) .47$ \\
\hline $8(3.41 .44)$ & (1).(1) 58 & 33.4679 & - (). $(x)(x, 4)$ \\
\hline 70.7860 & $(1,0) \div 50$ & $33.46 \% 9$ & $f(x)(x, 0)$ \\
\hline 19.2120 & $0.012(x)$ & $34 .(k)(x)$ & $(1)(x))(0)$ \\
\hline $78<5 \% 4$ & $(1), 14$ & 34.5400 & $(0.1 \times 170$ \\
\hline $719(179$ & $0112=$ & $35.226,4)$ & ()$.(x)(x) 1$ \\
\hline 771720 & $0.011 \% 6$ & 35.10140 & $-(1) .(x) 27$ \\
\hline $70.53(1)$ & 01118.4 & $36.11(x)$ & $(x)(x) 7()$ \\
\hline 75 s.14.4 & 0.02 .45 & 37.4554 & $13(x+41)$ \\
\hline 75.2549 & $(1,0211)$ & $38.14(x)$ & $0 .(x) 30$ \\
\hline $74.54()$. & $-0.1122 t$ & $38.94(k)$ & $-0 .(x) .36$ \\
\hline $\left.7.3 .4 .9 x^{\prime \prime}\right)$ & $(0.1) 223$ & $39.6 n+4 ;$ & $O(x) ? 0$ \\
\hline 793740 & $-(1,12) 11$ & $+(j .+10(4)$ & (). $(0) \div 8$ \\
\hline $12.72(19)$ & (1).1131. & 41.1174 & $(I)(x M) \cdot 1$ \\
\hline $7 ?(x, 3)$ & $(1)(121)$ & 41.70040 & $-(1) .(1) \div ?$ \\
\hline if $1(1,21)$ & $(1)(1) 11)$ & .42 .9871$. & $-(1,(x)) / 4$ \\
\hline $1(18.2(x)$ & $(1)(1)+4$ & 472110 & $0 .(x: 55$ \\
\hline 70.1486 & (1)(1) 1.9 .4 & $4.17(171)$ & (o.(x)s \\
\hline$(1,4)\{2 . .14$ & $(1)(1) .14$ & 14.6 .150 & (1).0110! \\
\hline is ugat & $(1,1,2)^{r}$ & 1.4. 1.914 & .0111 .47 \\
\hline$t, x: ?\langle 1\rangle$ & $\left.(1.1)^{2}\right) 10$ & $4 x, 11: 21)$ & (1) $(x) / 1)$ \\
\hline 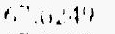 & $1)(1) 101$ & $40,7 \times(1,1)$ & (1) intik \\
\hline$(1 ; 1)(10)$ & $(191) 1]$ & $4 / 4550$ & $0(x+2)$ \\
\hline $0,+5 \cdot 0$ & $(101)(1)$. & is 14ky & $11(1) 11)$ \\
\hline (is (in) & $(1,1) 1+1$ & 46.5050 & (1) 11174 \\
\hline 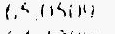 & $(1)(1) \% 1$ & fingl(x) & $01111 k$ \\
\hline 0.1 .1814 & $(1)(1.4)$ & $5(1,11: x)$ & 11111.15 \\
\hline$(1.378(14)$ & $0.111: 1$ & $\{162$ & $-0(1) 16$ \\
\hline $0,315(1,2)$ & $(111) \cdot 1)$ & $5,12,19$ & 01117 \\
\hline (2. $\{1,1\}$ & (1)(1) 1 & $\{3+12,4$ & $11(1)+1)$ \\
\hline$(1) \times 8<10$ & 011185 & $\{11.140$ & (1). (1) 157 \\
\hline 612254 & (1)(1) 118 & $\{3(x)(x)$ & $(1.1115 .9$ \\
\hline 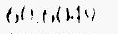 & $(1) 1 ; 1)$ & $51.5(1.40)$ & $(0.01) 00$ \\
\hline \{1) $1 / 204$ & 1801201 & $54.2(x, 4)$ & (1.1)lin \\
\hline $41, .3140$ & 1101145 & 50.118310 &.$(1 .(1) \cdot 81)$ \\
\hline Sx? I(1) & $(1,11) 52$ & $\{i, K(K, U)$ & (1)(1) \\
\hline$S B(1) .404$ & (1)1141 & $57.18 \times 4$ & $.11017 \%$ \\
\hline 571114 & (1)(1) $\therefore 4$ & $\{x, 2,4\}$ & $(i .11) 78$ \\
\hline$\left.S t_{1}, 11\right) 11$ & $0(1) .2$ & $5 x^{\prime} .9 ; 315$ & $0,(1)+(1)$ \\
\hline$\{f,|1| f, ?$ & $(1) 11] 115$ & $50(1,210$ & 110110.2 \\
\hline $55.7\left(\begin{array}{ll}2 \\
5\end{array}\right.$ & 011114 & $(x) ? \because(x)$ & (0)(1151) \\
\hline $5.4 k: 4$ & $(i)|1| x \mid$ & $(1)(1)$ (i) & $(1) 11) 1(1)$ \\
\hline$\{1,16,31\}$ & $01,1) \ldots 1$ & $\therefore 1 ? 11.1$ & (1.011)8 \\
\hline $51: \ldots 1.4$ & $11611: !$ & $(\because 4.11)$ &.$(1) 180$ \\
\hline 524140 & $11(1111$ & 6.12 .44 & $-(1)(1) 2 t_{1}$ \\
\hline (4) ? $\{x \mid y$ & (1)(1) i.t. & $(1,+1,+1)$ & (1).11:211 \\
\hline $310<16$ & $00 ! 16$ & (21 5274 & $111172 \%$ \\
\hline$: 110824$ & $-(1)(0118$ & or.t.guv & 0.112 .24 \\
\hline$\$ 11<.16,0$ & $(0,(k, 1) !$ & $(15.07 \times 9)$ &.$(10) 2.24$ \\
\hline$\left.x^{4}(1)+1\right)$ & i) (k'u & $(x), 618)$ & 0.620 .5 \\
\hline$(1)(1,0,0)$ & $11(1) 42$ & (i) $3\{34$ & (1) 11292 \\
\hline $48-428$ & $11(1) 16$ & $(x)(1)(x)$ & $0 .(1.64 .4$ \\
\hline $9: 7.4(14)$ & $(1)(1)\} 1$ & $\left(x^{k} k(K k)\right.$ & $0(1)(116$ \\
\hline 47129 & (1) (1).1! & (i) $1 ; 15,4)$ & $11(12.4)$ \\
\hline $96+4164$ & $(1)(H)(x)$ & 7111.4 & $01(1,1) / 1$ \\
\hline .15 .7 .46 .4 & $(1,61) 11)$ & $7(1,1) 119$ & $(111,211)$ \\
\hline $45(148)$ & $(1: 1) 13$ & 716114 &.$(1)(1.4)$ \\
\hline $11: 110$ & $(1)(x) 3.1$ & $72: B(1)$ & (1) (1. 1? \\
\hline 92314 & $11.5 \times 51$ & 7.30 .424 & 0.11277 \\
\hline 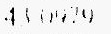 & $\| 1(K)+\rangle$ & $717(10)$ & $(10.40$ \\
\hline $9 .+184$ & ()$, k) ?\}$ & 7.14 .920 & $40 \% !$ \\
\hline $9 !(5 \times 1)$ & $111 \times 172$ & $7515 \%$ & $611 \% 95$ \\
\hline - i i 44 & i) in 2 & 75801911 & $0.0125,5$ \\
\hline $7(1.15 .50)$ & (1): $\left.\mathrm{H}_{\mathrm{H}}\right]$ & $76,58 \cdots$ & (1) $10 \times 2$ \\
\hline $30: 5114$ & $(1,1,15)$ & $77: 104$ & $61127 t)$ \\
\hline 31618,4 & Uikil1 & $77.906+4$ & (1) Tnt. \\
\hline 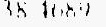 & 811145 & 18.1126 & (1) $414(1)$ \\
\hline
\end{tabular}




\section{Reinforced Concrete Test Data}

Track 9 - High Pressure Test

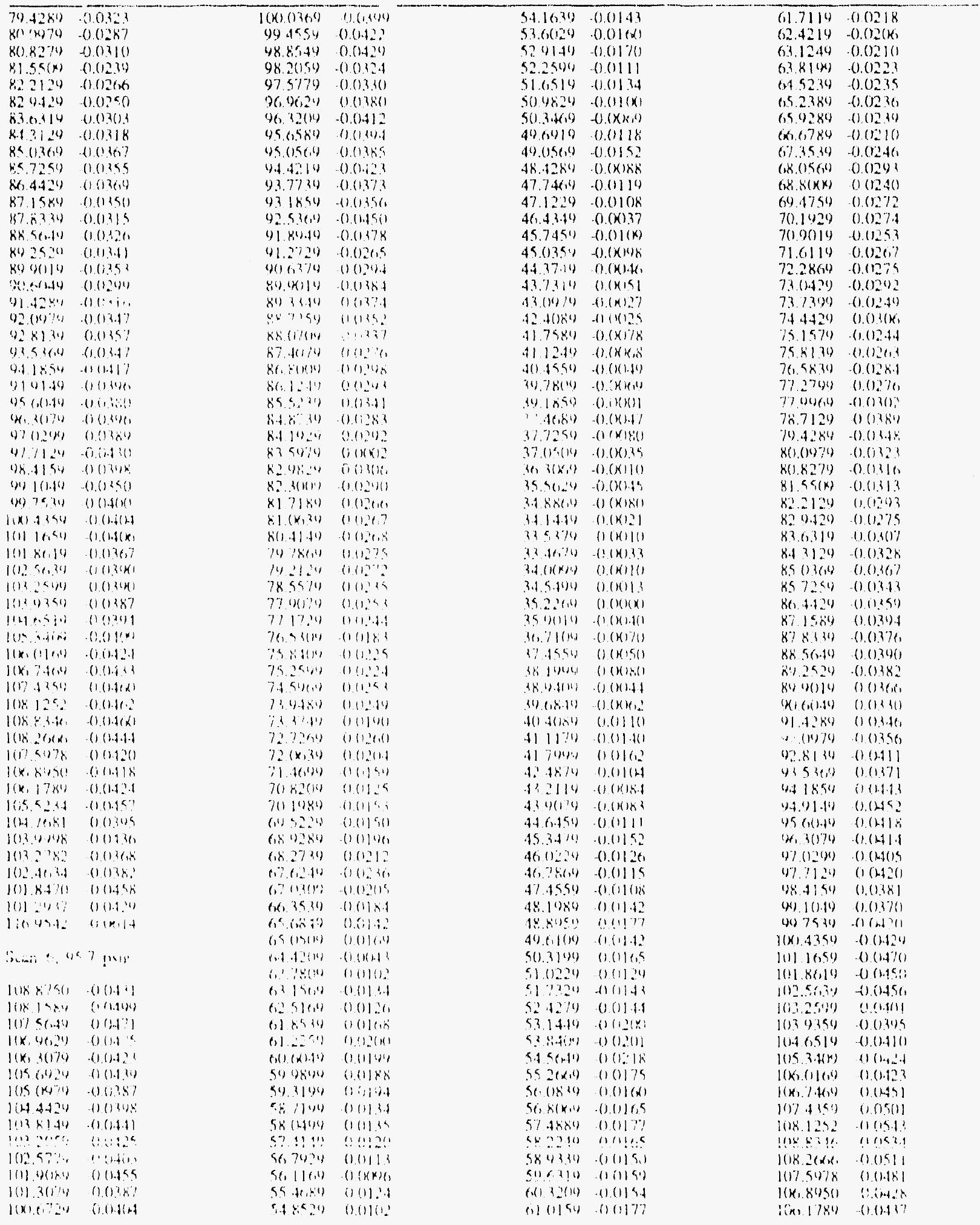


Track 9 - High Pressure Test

\begin{tabular}{|c|c|c|c|c|c|c|c|}
\hline 1019.9234 & $-(0.115(1) 3$ & $7(1.82(1)$ & (1)(1) & 43.2114 & .0 .0102 & 0.1 .18 .50 & $.0 .(4)()$ \\
\hline $1(4.768)$ & (1)(1.20) & $7(1.118 \times 1)$ & $-(1)(11)$ & $4.3 .17(174$ & $-(1,0) 108$ & 94.9149 & 0.01438 \\
\hline 10130008 & $0 .(1.46,1)$ & (1). 9234 & $(0,12 \%$ & $4.4 \cdot(.450)$ & -0.0110 & $9 \sec (x) 1 !$ & (1.1422 \\
\hline 1013.2782 & (1) $(14(x)$ & (1K.).ร1) & $(1 .(1: 40)$ & 4.5 .3 .974 & -0.01110 & 96.3079 & $-0 .(1+1) 4$ \\
\hline 102.46 .34 & (1) 0.125 & $68.2 \%$ & $(1,112.14)$ & $4(1.10120)$ & $0 .(x) x)$ & $97.02(x)$ & $0 .(131) 8$ \\
\hline 1018.671 & $(1) .(1.877$ & (4). (12.4) & (1).(12:3) & $46.786(10)$ & $0(1) 10$ & 97.7120 & 0.01 .31 \\
\hline 101.29 .37 & $(1.1 .480)$ & (6) (1).1(m) & $0(1) 6 . ;$ & 47.4554 & .0 .0112 & 98.4159 & -0.038 .3 \\
\hline 110.45 .42 & $(1)(1.46 .1$ & $6(x), 35: 0$ & $.11(1) 11 . ?$ & 481080 & (1) $(1126$ & $(2) .10 .40$ & -0.010162 \\
\hline & & 6,5 (2)+f. & 0.01740 & $48.80,50$ & (1)(1)111 & (29.75.79 & -1)(0.184 \\
\hline & & $(0,5 .(1) 5(1))$ & $0 .(1) \%$ & 40.61014 & - (1). (x)k.t & $\gamma(x) .4359$ & (0.0.387 \\
\hline \multirow{2}{*}{\multicolumn{2}{|c|}{ Scan i. lik) lo psiz }} & $(1+4 .+2(1)$ & $(1)(x))(1)$ & $5(1.3 ! 40)$ & .0 .01601 & 101.10 .50 & (). $(1 .+50)$ \\
\hline & & 6.3 .78016 &.$(1.1115 i)$ & $51(122)$ & 0.01173 & 10118619 & $(1)(1.417)$ \\
\hline 1018.8750 & (1) (1.44), & $0,3.150,4)$ & (1)(1) 50 & 51.7 .327 & $41,(1120)$ & $102,56.70$ & (1) (1).4(1). \\
\hline $1(181586)$ & $0.14 \times 7$ & $(12.510,0)$ & (1)(1) 15 & 52.4274 & .0 .0122 & 10.3 .2509 & $0.0 .1 .4(1.4$ \\
\hline $1075(x+1)$ & (1). (1.48,t. & 01.85314 & $(1) 11) ;$ & 5.3 .1 .4 .49 & (0.01).95 & 10.1 .0 .359 & -0.041616 \\
\hline $1(x, 4), 24)$ & $0.17 \%$ & $(61.22<11$ & $(1,1) 81)$ & 438.416 & $-(1) .01 .4(1$ & $10.40,514$ & $0(1 .+17)$ \\
\hline $1(x, 1(1) 70$ & (1) $0.4 .5(1)$ & $(11)(x 1.81)$ & (1)111,) & 5.451 .40 & $.0 .(1) i) k$ & 105.14010 & (1)10382 \\
\hline $1(19,1,1), 4)$ & $.01 .11 .150 !$ & $5,1)(18121)$ & $(1,1)<.1$ & $55 \quad 2(x, 1)$ & $11019 !$ & $|(x, 1)|(0)$, & (1) 11.143 \\
\hline $1115(1474)$ & $-0.0138 \mathrm{sil}$ & (1) 2104 & $(1)(1) ! 1)$ & $5(1,1) 30$ & $(101152$ & $11(x, 7,4(1)$ & (1).0.41.? \\
\hline $1(4.1420)$ & $.0 .1137 k$ & 58.71011 & $0111) 11$ & $5 \theta s(x, 1)$ & $(111) \%$ & $1(17,415:)$ & $0 .(1) \cdot 4 .(1)$ \\
\hline 10181.814 & $\left(1,1.4 .1 \mathrm{R}^{\prime}\right.$ & SE $14+20)$ & (1)(1)1,.. & $5 \% 1864$ & $(1)(1): 5$ & 1618.1252 & $(0.115)_{2}(1$ \\
\hline 101201540 & $(1)(1.114$ & $51.11 .1 \%$ & (1)(1) (1) & $56: 23.49$ & $(1.11)(x)$ & 108.8 .346 & (1).(15.15 \\
\hline $10257 \%$ & 011.120 & 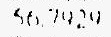 & $1111) 40$ & 454330 & () 111311 & $108.2(k)$, & $0.615 \%$ \\
\hline $10110(180)$ & () $(1.18)^{2}$ & $5(1,1)(1,0)$ & $(1)(1) K$ & 5110,114 & $0111\{11$ & $107.56 \% 8$ & (1) (1) 1516 \\
\hline 1011.30100 & $0(1.12)$ & $55+434$ & (1)11) 1? & $(x, 12,114)$ & $(1.11) \%$ & $1(k, 80) 50)$ & (1)(1) 5.401 \\
\hline$f(x), f, 7, y$ & $-(0.11 .12 ?$ & 5165316 & ()$(H) B\}$ & (1) (i) 4 (u &.$(1)(1) 62$ & $l i k, 1)$ & (1) 119211 \\
\hline $1(x, 1), 3,6)$ & (1) (1.4.2 & $54.11,31)$ & $(111): 1$ & 617114 & $(1)(1,46)$ & $1015 \$ 234$ & -0.0512 \\
\hline (2) $+15(1)$ & $(1.01,34)$ & $\{3,(x, 2)\}$ & $.(1) 11131)$ & $6: 1: 14$ & $(: 1) 16$ & 191.1681 & 0.0511 \\
\hline Uns 54.11 & $(1)(1)(1)()$ & $5: 1 / 4.4$ & 11111.2 & 0141.2 .14 & $(1,1) 25$ & 1111.89098 & .0101532 \\
\hline $4 x: 2(1<1)$ & (1).112.12 & 522514 & $(1 ; 1)$ in & tos side & $(101) 11$ & $1012 \% 162$ & $-(0,0), 3\}$ \\
\hline 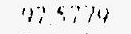 & $(1), ?$ & if $6 \mathrm{i}$. & -11111.111 & $(1,5)(1)$ & $(16) \div 4)$ & 102.463 .4 & $.010 .15,1$ \\
\hline$\left(x_{1}, x_{1} x_{2}\right)$ & $111: 21)$, & $5(14 x .1)$ & 1111111 & $0.5: 46,1\}$ & (1)11:2!) & 1011.8400 & .0 .09 .3 .1 \\
\hline$(x, 2 Z(x)$ & $11(1) 111$ & $5018(1,0)$ & 11164. & 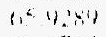 & $(1,11)(x)$ & 101.2017 & (l) (U) I \\
\hline (1) $5(1 \leq x+1)$ & $(1)(1.4) ;\}$ & $41)(11) 11$ & $11(10)$ & $\left(x, x_{1}, x\right)$ & $(1 ; 1)(1)$ & 110.4512 & (I)KHI \\
\hline $149115(1)$ & $(1) 11\}<1$ & $f(1)(1,2,4)$ & $(1,1) 9(1)$ & 671514 & (1)(1)(1) & & \\
\hline (1.) 1214; & $116 .+2 ;$ & $484 x^{\prime}$ & $(1) 11)(11)$ & $(1, x)(1,4)$ & (1) (1:31 & \multirow{3}{*}{\multicolumn{2}{|c|}{ 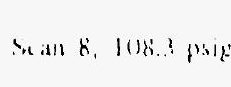 }} \\
\hline $11+7)(11)$ & $(1)(1) 1)$ & $47 \div+1,1)$ & (1.14kis & $6 x i(x)$ & $(11) ?(18$ & & \\
\hline 41854 & $0(1): 1)$ & $41 \ldots$ & $\left.(1) k x_{1}\right)$ & $0,4.475,4$ & (i.11.24.4 & & \\
\hline $0 ?<3(, 1)$ & $(10.116$ & 46.41 .14 & $(1+k) ?)$ & 711924 & (1) (1) $x x^{\prime}$ & $1018 \times 750$ &.$(1)(1159)$ \\
\hline (1) 8.4. & $(1)(1.810)$ & 457.1511 & $11111.11:$ & $7(1.1)(016)$ & $(1)(1,280)$ & 108.1580 & $0(1555$ \\
\hline 41.214 & .0101125 & $4.5(1) 154$ & $(i 11 ! 6)$ & 71,1114 & 0.11256 & $101.5 \operatorname{tr} 10)$ & .0 .01514 \\
\hline$(x),\left(1, Y^{\circ}, 0\right)$ & (1)(1)3.14 & $442 ?+4$ & $11 x+1)$ & 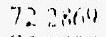 & $(101240$ & $1(k) .9620$ & 0.11511 \\
\hline$x^{\prime}(1)(1)$ & $0.61,3.4$ & 43.7 .14 & $(1+k)^{2}$ & $3.3(1.24$ & $1101: 4$ & $1(x, .30)$ & $(1,(1.476)$ \\
\hline $69 \leq 199$ & 06257 & $4<(x), \div$ & $\because \because n \cdot a$, & $? 23114$ & $0.01 \ldots$ & $10 \times(0) 29$ & $0(1,4: 0$ \\
\hline 88.7251 & $0,11<101$ & 424184 & $1 / 1 k k, 5$ & $71.1 .11)$ & $(1011) 10$ & $1(15.61979)$ & $(1 .(1.4)$ \\
\hline 点(1) & $(1)(1,270)$ & 41.7564 & (1) (1) & 741514 & $(1) ?$ & $1(1.4+42)$ & (1.)(1.47? \\
\hline $87.4(174)$ & $(11) 113$ & 4112.44 & $-(1), x)$ & 75814 & $(1,1,1,4)$ & 1018.81 .64 & (1).(1.14) \\
\hline$x_{1} \sin \left(x x_{1}\right.$ & $(1,1132 ?$ & $4(1) .1550$ & $1111(x) !$ & $\because 1.58113$ & $(1),(3,2) !$ & $1013.2(154)$ & 1). $(1)+k n$ \\
\hline $81.12+41$ & 1101124 & $31) 7 x(31)$ & $(2,1):)$ & 792749 & (1) $114 k$, & $10125 \% 74$ & $0,11.450$ \\
\hline $855 \therefore(1)$ & $00<?$ & 301856 & (1) $11 \times())$ & $\because y+40$ & 11014 & $10119(16 \mathrm{~d}$ & $.11(1.174)$ \\
\hline $848: 48$ & $(1)(1,2) .1$ & $3 \& 4 x+4$ & $.(1)(k, 1)\}$ & 1871.14 & $(1 \sin \theta)$ & $10113(1) 4$ & $0 .(1.120)$ \\
\hline 8.4 .1124 & $(101)+i$ & 1772017 & (1)KH) & 76.1284 &.$(111,147$ & $l\left(x_{1}, \ldots\right)$ & (1). (1).116 \\
\hline$x,\{, 94: 0)$ & lini? & ?7.65ind & $(1) \mid 1+1)$ & $4 !+(1,1) 4$ & (1)?? & $i(x) \quad\{(1)$ & $1161.4 \ldots$ \\
\hline Bung y & $(1)(1) \leq 56$ & $2 x, 3(x, 1)$ & $0,1, \cdots: ?$ & $n(1, \ldots)$ & $(111), 11.1$ & $(x)+4550$ & f) 14.41 \\
\hline$x: 4(x)$ & $(1) 280$ & $35<(.21)$ & - & 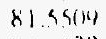 & $(1)(1)(1))$ & $98 \times 5.80$ & 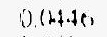 \\
\hline $81: 180$ & 8.6277 & 34 singen & olnkil) & nE II:" & $\because 1171$ & $96 . ?(150)$ & (0.0.4.1. \\
\hline $81(k, 3\}$ & $(1,1) 214$ & 3.11 .4 .44 & $(1) x)\}$ & $x=(6120)$ & (1) (1)'A) & $37 \ldots 9010$ & nlat 30 \\
\hline $8(1 .+1.41)$ & $(11126,1$ & $23.5,3014$ & $11(x)+1)$ & $\times 3.6,140$ & (1) $114 \pi$ & $\left(K_{1}, 9(1,1)\right.$ & $0 .(1+1)$ \\
\hline $79.78(x)$ & $(1)(1) \cdot f_{1}$ & $13+114$ & $(1)(k)) ?$ & 5.12124 & 0114.14 & $(4.32(,)$. & 0.01341 \\
\hline 79.2124 & .011205 & $3.4 k 11)$ & $1, \ldots 1011$ & $k \leq(1), 0,0$ & (1)1110\%, & ys $(1,5 \mathrm{x}$ & (0)(1)+11) \\
\hline 78.5570 & $(1)(125)$ & 3.45 .800 & $(1)(x)<x$ & 85.7254 & $(1.111 .4 .1$ & $95.05(.1)$ & $0 .(1+1)$ \\
\hline $77.9(1 ; 1)$ & (1) 11.2 .1 & $.35,22(16)$ & $11(111) 5$ & $80.412=$ & $(1,1915,4)$ & 14.4214 & $.11(4.5)$ \\
\hline 771720 & (1) 112.90 & $35(k 1) 17$ & $\left(1,\left(M_{i}\right)\right)$ & 87.158 .4 & 010313 & 93.77 .34 & $(1)(1.1 .11)$ \\
\hline $76.530 k$ & $(1)(1) 41$ & $.16 .711 M$ & $0\left(x x_{i, 1}\right.$ & $878<14$ & (1) (1) 1.41$)$ & $4.1 \times 89$ & (1). $14+12$ \\
\hline $79 \times 4(\mathrm{k})$ & 010244 & 37.45 .511 & () $(K) !(1$ & $\therefore x^{\prime} 5(6.10$ & $.0 .0113,3$ & $92.53(6)$ & $0.64+4.4$ \\
\hline $75.25(x)$ & $0(1)+2$ & 281804 & $(1 ! k) \leq S$ & K(1) 25.14 & 010.458 & $9180+4$ & $0 .(1+20)$ \\
\hline $1+.34(, 4)$ & $(111 !), 1)$ & 38.4 .4164 & $(1)(1,1)$. & (3) $14(1) 4$ & .110 .1 .15 & 91.2729 & $.0 .13(x)$ \\
\hline 139489 & $\{1,(1 \leq+2)$ & $34(564)$ & (i)ikis? & $4(1)(1+1)$ & $(1.01+21$ & $x(1,1,7)$ & $(1.013 .1 .1$ \\
\hline 73.37 .1$. & $.11,11237$ & 4010184 & $(1 ! 1) 21$ & $y_{1} \ldots \ldots$ & $1114: 4$ & xy.d119 & $(1)(1+1)=$ \\
\hline 7972 tid & $(1,1,()$, & 411170 & ()(1)il) & $42(19) 9)$ & 0.11234 & 33210 & .1111152 \\
\hline$\because \therefore(K, i)$ & (i.ioive & 41.7404 & $(111)(11)$ & 428111 & 0,01977 & 88.72511 & (1)(13KK \\
\hline 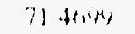 & 1111153 & 424574 & $(1)(1): 0)$ & 0.153 .64 & (1) (1) 1.5k & $k s(r)(x)$ & $0(1,13)$ \\
\hline
\end{tabular}




\section{Reinforced Concrete Test Data}

Track 9 - High Pressure Test

\begin{tabular}{|c|c|c|c|c|c|c|}
\hline 87.4070 & .0 .0 .353 & 41.12 .41 & $-(3.011) 5$ & $758130 \quad(1.192 .44$ & $1013.2(154)$ & (1). $(14 ? 11$ \\
\hline $8(x) \delta(x)$ & 0.0 .11 .4 & 40.4550 & -()$\cdot(k x)+1)$ & 76.5816 & $1012.5 \%)$ & $-(1) .(14.3 .4$ \\
\hline 86.12 .40 & .0 .01321 & $30.75(14)$ & $-(1.1 k x, 1)$ & 77.271040 .0288 & $101.9(180)$ & $.0 .(0.41) 2$ \\
\hline 85.52317 &.$(1)(1,3) 2$ & 30.18 .51 & $0 .(1)(x) 7$ & 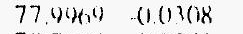 & 10133794 & (1). $(1.228$ \\
\hline 84.87 .30 & $-(1.0274$ & .86 .40801 & $0 .(K n)()$ & 78.712 .400 .181 & $10(1) 6720$ & $-(1,(143)$ \\
\hline 84.1020 & -0.013 .40 & 37.7259 & $0(x+140$ & $744280 \quad-0.0132 .1$ & l(k) citt : & $-(1)(4+4)$ \\
\hline $8.3 .5074:$ & (1).(1)(4) & $37.0 .5(16)$ & (1). (x) & $8(10(6) 71)+0.0288$ & $(x) .4594$ & $-0(1+24$ \\
\hline 82.4824 & $\$ 1.0 .345$ & $3(1, ?(x, y)$ & $0(k x)()$ & $8(1.827) \quad 0 .(13) 4$ & $08 \times 544$ & $-(1,(4) 10)$ \\
\hline $82,3(x)$ & $(1)(0.346$ & $355(120)$ &.$(1)(190)$ & $81.55(14 \quad 0.01277$ & $48.2(150)$ & $0112 ! 13$ \\
\hline 81.7164 & (). $(13.53$ & $3488(10$ & (1) $(x),()$ & $82.2124 \quad 0 .(12.48$ & 07.5779 & $-(0.01915$ \\
\hline $81 .(k, 34)$ & $0 .(1315$ & 34.14 .44 & ()$(x) 15$ & $82.4 .424 \quad-0.01267$ & $(x, 1)(1,21)$ & -0.018 .3 \\
\hline $8(1) .4141)$ & $-0 .(1281)$ & 3.3 .5 .970 & $0 .(x) 10)$ & $83 .(1319$ & $(x) .32(x)$ & $-\langle 1 .(40)\}$ \\
\hline $79.78(1)$ & $0(1) \geq(1,0)$ & $13.40,70$ & $0 .(x) 15$ & $4.13120 \quad(0 .(19.47$ & 05.6580 & (1) $(144(k)$ \\
\hline 74.2120 & 000289 & $34 .(x)(1) 0$ & $0.11(x) 1$ & $x 5(0) 3(1) \quad(1,0)\} 860$ & 95.0 .569 & (1) 1420 \\
\hline 78.5570 & $-(1)(1241$ & 34.5 .400 & $(1)(k) \geq 0)$ & $85.7254 \quad 0.013 .32$ & 44.4219 & (1) $(142 k$ \\
\hline $77.9(171)$ & $0 .(12)(x)$ & $35.22(, 1)$ & $(1)(16 \times)$ & $8(1.4 .4 .1)$ & 0.7739 & (1) $0.36,9$ \\
\hline $77.17 \%$ & $-0.1131 k$ & $35,(x, 11)$ & $11(K)_{2}^{\prime}()$ & 87.1584 & 0) 3.1850 & -()$(1)(1))$ \\
\hline$\therefore(1.541 x)$ & $.0 .(1 \geq 11$ & 36.71114 &.$(1)(1+1)^{-1} ?$ & 87.8141 & $17253(1,0$ & ()(4) \\
\hline $758.4(x)$ & $(1)(1,21$ & $37 .+5513$ & $-1)(11)(1)$ & $8.5 .5(x+1)$ & 41.80 .41 & $(1013,7)$ \\
\hline 752540 & 0.1126 .1 & $38.10(1)$ & $0(x): 8$ & $512<20$ & 412720 & $(101)(1 / 8)$ \\
\hline $74.996,14$ & .1111212 & 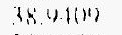 & $(1)(1) 119$ & $840(114$ & $(x) 63 \%$ & (1)(1.2) \\
\hline $710.18^{\prime \prime}$ & () $(: 327$ & $34(x) 14$ & $0(x) 11)$ & $0(1, t, 1+1)$ & $89.9(110$ & $(1.0 .328$ \\
\hline 73.37 .918 & $.(1,1) \geq 4.4$ & $41: .4(1,1)$ & $(1)(1) 11)$ & $41 .+284$ & 84.33417 & $-(1.0718$ \\
\hline $7272(16)$ & $(1)(1) \geq 4$ & $+111 \% 10$ & $(1)(1) 13$ & $9 \div(111)$ & 88.7251 & 0.01172 \\
\hline $2(k, 1 i)$ & $(1025 i)$ & $41.3(1)$ & $(111110$ & 428134 & $88.07(x)$ & $(1)(1,1,1)$ \\
\hline $71+(1,1)(x)$ & $.0102(16)$ & $42+40$ & $(1) ! k x, 1$ & $4=.58(0.0)-(1.0 .37()$ & $8 ? .4(179)$ & .0 .0 .11010 \\
\hline $7\left(1 x_{n}^{\prime}(x)\right.$ & $(1.0) 10(x)$ & 1321111 & $(1)(1)$ & $4 .+1 \times 54$ & $86.8(x(x)$ & $-(1,1,3)$ \\
\hline $71 . j 124$ & $(1,11) 1 !$ & $43(x+1)$ & (1) handi & $(2.4(1) .41) \quad 00(1.437$ & $8(1.12 .91)$ & $0.11921)$ \\
\hline (1) 19.520 & $-111 \div 21$ & $.4 .4(+4) 13$ & $(1: 11 k) !$ & $45(1,1) .10)$ & $85.5: 14$ & $(1.1132)$ \\
\hline (k) 4254 & 011.21 & $45,31: 18$ & $1,11<1$ & $1)(1,1(1,1)$ & 8.18 .320 & $0.1121,11$ \\
\hline$(B 2273)$ & 01.62 .411 & $f 1,(1: \ldots)$ & $(1: 1) 11)$ & $0 ? 11240$ & 8.4 .1724 & $(1) 112$ \\
\hline 0.1210 & $1:(1) 2(1,1$ & de incer & $111111 ! 2$ & 1): :1:4 & $83.90 \% 10$ & $0(x), 1)$ \\
\hline$(n) \| s(p)$ & $11(121 ?$ & $4 ! 9(41)$ & $(1) 1 k)$ & $46+154$ & 8248.20 & $0(1) 204$ \\
\hline$(x, 1014$ & $(1,13: 1 k$ & fis foxid & (1) $11^{1 !}$ & (5) $1(1.80)$ & $x \geq 1(k *)$ & $0.114 / 8$ \\
\hline $0.5(1, i)$ & $(1)(1) 11)$ & 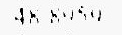 & $(1+1) \because$ & $(9117511$ & 81.7184 & $0 .(1) 207$ \\
\hline $0,5(15(m)$ & $11012: 2$ & $f(1)(1,1)$ & $(111) \div$ & $1(k)+7551)$ & $81(x, 10)$ & $10.04(4)$ \\
\hline$(m 8 .+2(m)$ & $(1) 11) 111$ & $5(121411$ & $(111)<1$ & $1111164)$ & ki1 $+1+11$ & (1)(1)5.1 \\
\hline $027 K(\mathrm{~K})$ & $.1)(11.80$ & $\$ 1(1,21)$ & $111111^{\prime}$ & $1(11.81,1)$ & $79 \div 8(1)$, & $-0.05 \times 10$ \\
\hline $0.115(0,1)$ & (1) (1) 4.1 & 817121 & $(111) 1 !)$ & $10: 2.56,30 \quad-01(1.45 x$ & 74.2124 & -11.11250 \\
\hline$(1,2 \leq\{\mid(, 1)$ & $11(1)<1$ & $52+111$ & 1111189 & $101.25(4) \quad-(10.11 .411)$ & $7 x<570$ &.$(1)(1214)$ \\
\hline$(1) 8<11)$ & $(1)(120)$ & 531.111 & 11111311 & $1113415(1)$ & $779(1) 4$ & 0.01252 \\
\hline$(1) .2 \leq 54$ & $(1)(1: 23)$ & Tisif(1:) & $0113)^{\circ}$ & $1(14.0 .517)$ & 77.1720 & $.0 .02 \% 1$ \\
\hline (1). $1 .(1.14)$ &.$(1)(12.211$ & $5.45(1.1)$ & 111911 & 1115.3 .4114 & $76 \leqslant 3(x)$ & $(1.0216$ \\
\hline $50(1) 800$ & $(1)(1)\{1)$ & $.952(1,4)$ & $(1) 11 \%$ & $1(1,1,(1) 6,1)$ & $75.64(x)$ & -11.11281 \\
\hline $50 x \mid 00$ & 1101240 & $50,18<1)$ & 1111151 & $106, ? \cdot 40,4$ & $7525(40$ & $0.0126)$ \\
\hline$\{x: 1101$ & $11(1211:$ & $S t, \therefore(k, 6)$ & $(1)(1) \% 1$ & $107.43(51)$ & 7.4 .5060 & .0 .012018 \\
\hline $5 x(1.4(2)$ & $(1.0170)$ & 57.4880 & $.(1)(1): 5$ & $108125:$ & $\left.73.4 .9 x^{\prime}\right)$ & $-(1 .(1 \geq 3)$ \\
\hline $57+1+11$ & 1111152 & 58.240 & $13(1) .14$ & $1018 \times 240$ & 73.37 .49 & (1)(1)14) \\
\hline $50,79.11$ & $(1) 115)$ & $5 x(4)(11)$ & $0 .(1)^{5}, 1:$ & $1(18.21 x+6) \quad-1105019$ & $72.72(, 0)$ & 1) 11225 \\
\hline$x(1.116,1)$ & $(1)(1) 10)$ & 540214 & 0111411 & 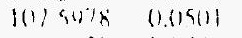 & $72,(x, 30)$ & $-1)(1224$ \\
\hline sa 90.64 & vinis" & (11) $12(11)$ & 01111.411 & $1 ! 16,8050$ & $11.46,201$ & -0.11880 \\
\hline $5.4 \times 5.46$ & 11111.12 & $011(1150)$ & $11(1) 111$ & $10 k .18 \mathrm{sin}$ & $7(18.2(K)$ & -(1.)(1) 81 \\
\hline$(-1.10,21)$ & $1101) a n$ & 0117110 & $(1)(1) \times 2$ & 1055234 & $7(1.11810$ & $0 .(1) 118$ \\
\hline $53(6) ! 17$ & (1) 11$) 11$ & $(1.2+111)$ & $0(1) 5 ?$ & $101.10 \mathrm{kn} 1$ & (3) 5210 & $0.02 \div \%$ \\
\hline$\{2(1)|1\rangle$ & $11(1) 11$ & 0.112 .44 &.$(14120)$ & 101191196 & $(38,9284$ & (0). 12.40 \\
\hline $52.25(x)$ & (1)(1).2) & $(0.1 .8104$ & $(1,0) 2117$ & $1013.2 \%$ & $\left(\mathrm{~s}^{2}: 2136\right.$ & $0(0210$ \\
\hline 51.6510 & 911135 & 6.45230 & $-(1)(12) !$ & $10 ?+1014$ & $67 .(12+4)$ & $\left.(1)(1) s^{\prime}\right)$ \\
\hline $5(1)(1826)$ & $(1)(11) k$ & $0,5.2380$ & $(10)] 13)$ & $1(1) 8.4 \%$ & 67.010 .10 & (1.01189 \\
\hline$S(1,3.4(, 1)$ & $0 .(k \mid k)$ & $(6,5) \geq 84$ & 211218 & $110.450,1$ & $(6,1,3534)$ & $0 .(1) 9(1)$ \\
\hline$(19): 1910$ & $0(x) y 3$ & $(6)(17 \times)$ & $(1) ! 10)$ & & 65.656 .46 & $0 .(11) 97$ \\
\hline$f(1)(1) 5(, 1)$ & $(0.01301$ & 67.15 .30 & $-(1.02(3)$ & & $0.5 .(1) 5 / 10)$ & (1).(1)! (1) \\
\hline $4 k_{i}+2 \sin ^{2}$ & $.0(1114$ & 68.11904 & 0,01210 & 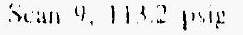 & $(x+42(x)$ & $0.01)(1.4$ \\
\hline $47.7 .40,0$ & (1) insici) & 088.8014 & $-(1.021 \%$ & & $0.3 .7 k(k)$ & (1.01.36 \\
\hline $47.122 !$ & $0 .(11112$ & 604150 &.$(1) 11232$ & $1(18.8950$ & $0.3 .15(10)$ & 0.011 .31 \\
\hline $16,43.94$ & (). $(x) 5 x$ & $7(1.1420$ & $0.0 .2(1)$ & 108.1584 & $(2) .5 \mid(, 1)$ & (0).(1115 \\
\hline 45.7 .454 & (1)0115? & $?(1)(1)$ & $(1,1,2.43$ & 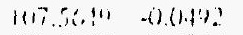 & 01.8534 & \{\}(\}$! 51)$ \\
\hline $45,0,35.6$ & (10)15: & 1101,14 & $(1.112 .28$ & $1(16,9)(.24)$ & 01.2250 & 0.01170 \\
\hline $44.1 / 44$ & $f(x), ?\}$ & 722206 & $-(1,1) 267$ & $1\left(x_{1}, 3(1) / 4\right)$ & $(x+1,(1)+4)$ & $0(1) 80$ \\
\hline 43.7314 & (1)11111.4 & $19(2.12)$ & -11.1254 & $1(1<(, 1))^{2} 4$ & $504 \sin 4$ & (1)(1)18' \\
\hline $43(1070)$ & $(1) \times 100$ & $73 . / 3131)$ & $.(1,1), 144$ & $119.113 / 4$ & $M: 1 M$ & (1) 11215 \\
\hline 92.9183 .8 & $11(x)\}$ & $74+129$ & $-11.12 \cdot 11$ & $1(14.4421)$ & $s k^{-2} \mid x !$ & -(1).(1) B. \\
\hline$+19: 104$ & $11(k)^{*}+1$ & $75.15 \%$ & $(1) ! 12(1)$ & $1(1: 81.41)$ & $58(1.41 \%)$ &.$(1 .(1) 7)$ \\
\hline
\end{tabular}




\section{Reinforced Concrete Test Data}

Track 9 - High Pressure Test

\begin{tabular}{|c|c|c|c|c|c|c|}
\hline 57.4149 & $-1)(1) 6(1)$ & 58.22 .49 & (1).011.10 & $108^{\prime} 8140$ & 73.1794 & (1.).1.240 \\
\hline 56.7929 & -0.0140 & 58.9330 & $.0 .(1150$ & $108.2(x x)$ & 72.7269 & (1). 0.22 \\
\hline $5(1.1160$ & 0.0110 & 59.6119 &.$(1)(115)$ & 107.5978 & 72.06 .30 & $-(1.01) 76$ \\
\hline 55.4684 & $-0.010(x)$ & $(0.132(1) 0$ & $-(1)(1) 140$ & 106.80501 & 71.46189 & .011131 \\
\hline 548520 & $0.0 \times 178$ & $61 .(1150)$ & $-(0.111 .38$ & $1(6.1780)$ & $70.82(x)$ & $.0 .013 k$ \\
\hline $54.16,30$ & $-(0.0133$ & 01.7111 & $(1 .(1) 2(x)$ & 105.5234 & 70.1984 & $0.0116(x)$ \\
\hline 53.60120 & $-(1.011 .38$ & 62.4214 & $.0(1167$ & 104.7631 & (i) 5224 & $(1.1221$ \\
\hline 52.9144 & .0 .01152 & $63.12+17$ &.$(1.112017$ & 103.13908 & $6 k^{\prime}, 9 \geq 80$ & (0.0) $2+40$ \\
\hline $52.25(x)$ & $0 .(115.1$ & 63.8140 & $(1.0216)$ & 10.3 .2782 & 68.2730 & $0.1921 \%$ \\
\hline 51.6514 & $0.0113 \%$ & 6.4 .5234 &.$(1.112(1)$ & 1012.46 .34 & $67 .(12+1)$ & .11 .0111 \\
\hline $5(1.982 .14$ & fl. $(k x)(1)$ & 05.2389 & $.(1)(1), 81$ & 101.8 .470 & $67 .(1) 3(x)$ & -0.01170 \\
\hline $50,3.404$ & $-0 .(K) S t$ & $(65.9284$ & -0.12211 & 116.4 .56 .3 & $(x .3530$ & .0 .0166 .1 \\
\hline $49 .(1914)$ & -()$(x) 7)(1$ & $(x, 6,750)$ & -0.01145 & & 6.5 .68 .56 & $-(1.01178$ \\
\hline 41$)(15(, 0)$ & 0.111 .4 & 07.3539 & 0.01215 & & $6.5 \cup S(x)$ &.$(1.0177$ \\
\hline 48.4280 & () $(x) !(1$ & $0,8,(1,5(, 1)$ & $0.01 \div 1.4$ & \multirow[t]{2}{*}{ Scanl 10. 117.8 pisig } & $(n+42(x)$ & $0 .(x)(x, 1)$ \\
\hline $47.74(10)$ & c). $(x+44$ & $68.81(3)$ & $(i,(1))$ & & $6.3 .78(x)$ & $(1)(1) 42$ \\
\hline 47.1224 & $(1 .(x+70)$ & (11). 4754 & $1)(1): 11$ & $108.5 \% 50$ & $03.15(, 0)$ & (1.01.1\%) \\
\hline $46,43.40$ & $0 .(k+1)$ & $?(1)(4) .11$ & () (1.25 & 1081580 & $62.51(10)$ & (1).(1) $: 4$ \\
\hline $45.745,9$ & 00131 & $7(1.9(1) 11)$ & $(1.11,4.4$ & $101,56+10)$ & 61.85 .34 & Q.015i \\
\hline $45 .(1) 154$ & $.0 .(1) 2(1)$ & $11 .(1114$ & 0.025 & $1\left(x_{1} \cdot\right)_{1}(2)$ & 01.22513 & $-(1 .(1) 6,16$ \\
\hline 4.137 .49 & $11(K 2) 7$ & $\because 228(1)$ & (1) (1).44 & $1(k, 1) ; 0$ & $(x),(1) \cdot f(1)$ & 00150 \\
\hline 417314 & $-(1.61777$ & $\because 3(421)$ & $0.1124 ?$ & $105+(4), 1)$ & $5048(1)$ & $(1,())(x)$ \\
\hline $41(107)$ & $(1)(K) \div 4$ & $73 \% 3+24$ & () $012: 5$ & $105(1070)$ & 54.31407 & 0.0187 \\
\hline $42.4(184)$ & (1). (kL) & 7.4 .4 .421 & $-(1.1) \geq 13$ & $194+424$ & 58.7100 & $(1)(1) 4$ \\
\hline 41.75810 & $0 .(k) 58$ & 75.15 .4 &.$(1)(12(1)$ & $103.81+10$ & $5 k .0 .7110)$ & $(0.010 .4$ \\
\hline 41.12 .49 & $-(1)(10155$ & 7.551 .34 & (1)(1):26, & $10.1 .2(181)$ & $5 i+11+17$ & $-(1.11)(1)$ \\
\hline $.1(1.4554)$ & $(1)(x))_{2}^{7}$ & $70,5 \times 30$ & $(1) 11)^{<} \because$ & $10297 \%$ & $56.7424)$ & $-(1)(1): 1$ \\
\hline .76) $78(x)$ & $1 /(k) s_{0}$ & 771700 & $11(1,28)$ & 101141818 & $50.110,0$ & (1)(1) I \\
\hline $34.15<4$ & $0(k),(1)$ & $7^{-1}$ (n) $(1)$ & 110.18 .4 & 1019.10114 & $55+6180$ & $-11(k M) S$ \\
\hline$\{k \cdot A\langle, i u$ & $(1)(1)(x) !$ & $78-120$ & $011 ! 1$ & $1141612)$ & $5.4 \times 5=11$ & $-(1)(x)(x, 5$ \\
\hline 3.72519 & (1)(KIS) & $90+2\langle 4\rangle$ & $11(12) 1.1$ & $1(x+1)(x, 1)$ & 5.1 .10 .10 & $.0(1) 127$ \\
\hline$\because(15(x)$ & $(1)(k) 1.4$ & $\left.x_{1}()(x) ; 0\right)$ & $(11 ;: 11$ & (1) $.55(4)$ & $53+1,(129$ & (1)(1) +1! \\
\hline (f) $3(x, 1)$ & f.tuxi. & nill $2: 11$ & $(111) \cdot \cdots$ & 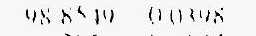 & $\{2,4 \mid+4)$ & $0(114)$ \\
\hline 1549.24 & () $(x+1.1)$ & $81<5,14$ & (1) $(1,1,1)$ & $48: 2(150)$ & $52.54 \%$ & $(111) 18$ \\
\hline $54 \times 4(1)$ & $(1)(k)\{1)$ & $8: \therefore 1$. & 1111211 & $4: 5 \% 14$ & $51(1514)$ & (). (1) $16:$ \\
\hline 34.144$)$ & lif (1) & $k: 4: 2$, & $\because 1126$ & $(x, 1)(1,2)$ & (ilun:y) & $-(1)(x)(1)$ \\
\hline $33+460$ & $(1 \mid k)) \mid()$ & $\lambda^{\prime}(1,1)$ & $11(1)+11$ & $4(1,3(14)$ & $5(1), 4(0,4)$ & (1). (x) \\
\hline $12+61,4$ & $\|(k)\| \|$ & $0.1112,1)$ & $11111 \ldots$ & $4)(1,5,11)$ & $(1)(1)(1)$ & (1) $(x)>(1)$ \\
\hline $3+8(x+1)(1)$ & $(1)(k)<)$ & t' 6 in 1,11$)$ & G() isi & $1),(15,0,4)$ & $4(1)(1<(, 1)$ & $11.111)$ \\
\hline $3.4+1,10)$ & $\|(x)\}$ & $84 \quad 1211$ & $11+4.11$ & $14+1211$ & thing & $(1)(x) 15()$ \\
\hline $35: 26,4$ & (1) (x) & $x(1,+1.1)$, & (1) 11$\}^{-6}$ & $117^{-1}(1)$ & $47 \% 1(1,1)$ & $0 .(x): 8$ \\
\hline $35.19(1) 10$ & $(1)(x+11)$ & $8: 1941$ & $(1,1) ! 3$ & $4315(1)$ & 11.1224 & $(1,(x), 1$ \\
\hline $3(1,1)(k)$ & $(1(x) !)$ & 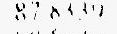 & $(1918.61)$ & $4 \ldots<1,1) \quad(1,1, x$ & 16,4140 & $01(x) 114$ \\
\hline $17+(551$ & (1) (hint) & $h, x^{\prime}<x+14$ & $411 \cdots: 1$ & 11.84 .11 & 15.74 .56 & (1). (1) 16 \\
\hline 3814130 & diңLlis & 5125216 & (1) $1131:=$ & (1) $\therefore 2 y$ & $15(1,150)$ &.$(1.1111$ \\
\hline$\therefore(x), f(x)$ & (). $(H \times x, i)$ & $80(x+11)$ & $(111,2)$ & $9(: 0,17)$ & 4131.11 & $0(x) i .4$ \\
\hline "16 (18) & $(1)(k) .:)$ & $111)\left(x^{1} .11\right)$ & $(161<1)$ & for $40190 \quad 010318$ & 41.7311 & $(1,(K K)$, \\
\hline $4(1+118)$ & $0(1)(1)$ & 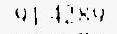 & $(1,1) ?$ & (8) $33.140 \quad 0.11318$ & $41(x) \div 1)$ & 1114151 \\
\hline 41114 & $(1)(x) 1$ & $y \div 1119 y$ & 010141 & $8 k: 24$ & $42+1864$ & $(1)(x) s)$ \\
\hline $11: 040$ & $1)(k+1)$ & 42313 & $(1) .1119$ & 850101001034 & 41.1580 & $(1)(x)(x, 1)$ \\
\hline $4 \div 4571$ & ()(K) & $y)<\{(1,1)$ & $1111 ! 11$ & $87.4012 \quad 0.10) 314$ & 2112.14 & (). $(x)+x$ \\
\hline 4121111 & (1)(K) & $0.818<11$ & $(1,11114)$ & $8(1, k(x) 1) \quad 11.1140) 7$ & $.4(1.45 .54$ & $(1)(x) y:$ \\
\hline $430(9)$ & Oinds: & 14.181 .41 & 10.11 .61 & $86,1290 \quad 001016$ & $30.78(k)$ & $0.1 \times 170$ \\
\hline $4.9 \cdot(1.451)$ & (1) (kis) & $1) 5(x) .14$ & $-1,(1,1) 1$ & $85.5230 \quad(11) .85$ & 34.1850 & $(0,(x), 31$ \\
\hline 15.3 .717 & $-(1.61120$ & $1)(1,3174$ & $(10.16)$ & $84.8730)-(1)(1) 33$ & 18.1680 & $0.1(x) .1(1)$ \\
\hline $40,112.27$ & $(1.1) 11 \mathrm{n}$ & 47.11200 & $-(1)(1.41) .2$ & $84.1924 \quad(10+1$ & 37.7250 & $0 .(x) 57$ \\
\hline $46.78(1)$ & 0.011 .2 & 9711217 & $(1.01 .4 ?$ & $83.50)-(1.14 k) \geq$ & $37 .(1) 5(x)$ & $0 .(\times 1.15$ \\
\hline 47.4590 & (1). $(418) ?$ & $48.41(1)$ & $-(1 .(1) .4(1)$ & $829820 \quad-1(10211$ & $36.3(5,4)$ & $0 .(x) 18$ \\
\hline 48.1782 & (1).11118 & $(p) ! 1(1.91)$ & $.0(1) 7.1$ & $423(x)(1) \quad-(1.1) 2 x+4$ & $35.50,29$ & (1). (4) 4$)$ \\
\hline $4 x+5(1)$ & (1).(1).5.? & 04.7536 & $0 .(1.4(k)$ & $81.71840-(1.01 .84$ & $34.858(1)$ & $-(1.1 \times \times 1.4$ \\
\hline $40.01(k)$ & $0.1 \times 18\}$ & $1(k) .1 .150$ & $.0(413)$ & $81 .(4,30-0.01270$ & $.941+14$ & (1). $(x) 1.1$ \\
\hline $5(1,194$ & $(1.11111$ & $1(11.1(1,56)$ & $-(1.1459$ & $8(1.4144) \quad(1.01 .34$ & 33.5371 & $(1 .(x) 30$ \\
\hline $51(1: 21\}$ & 0.01121 & 10118010 & $0 .(1.4 .30$ & $70.78600 \quad-(1.0270$ & $33.40,74$ & $(0 .(x) 16$ \\
\hline 537210 & $(1.1) 11.5$ & $10) .50,30$ & $(1)(4)^{1)}$ & $79.2124 \quad(10.12+8$ & $34 .(x+10)$ & $0 .(x) 17$ \\
\hline $52+9270$ & $0 .(1) .20$ & 1032.3100 & $-1)(H)(K)$ & $78.5574 \quad(1.120 .5$ & $34.54(10)$ & $0 .(x)+1)$ \\
\hline 5.1 .440 & $.(1)(1), 80)$ & 103.93 .500 & $(1)(4) 1)_{2}^{2}$ & $77.9(170 \quad-0.112(x)$ & $3 \div 22(1,1)$ & $0 .(x), 3(1$ \\
\hline 53 kialn: & $0: 1154$ & $10+6,519$ & (1)(1.92) & $77.1720 \quad(101190)$ & $359(119$ & $-(1,(x) 21)$ \\
\hline $5.1 .5(1 .+4)$ & (1) $(1: 1 / 4$ & $1114.3 .4(16)$ & 11.1434 & $76.5 .1(14) \quad-(1) .01701)$ & $3(1,1)(x)$ & $-(1 .(x) 18 !)$ \\
\hline $552(x, 4)$ &.$(1)(1) 10.8$ & $\mid\left(x_{1}(1) \mid(1)\right.$ & 01.142 & $18.8+140 \quad 010.44$ & 17.45517 & $(1)(x) 3)$ \\
\hline Sor. 118.34 & $(1,0) 1.4 ?$ & $1(x: ;, 4(, 1)$ & 0.14 .13 & $7525(10)$ & $38 \mid(3)$ & $.0 .1 \times k .1$ \\
\hline$. x_{1}, k\left(x_{1},\right)$ & $(1),(11) 44$ & $111:+294$ & $.11+3(1) \mid$ & $1+5 \sin$ & ingoing & dicing \\
\hline$\because 4 x^{\prime \prime}$ & $0.11](x)$ & 11181252 & $0(4) 18$ & $134.484 \quad-10(12.85$ & 30.68 .44 &.$(1)(x) 111$ \\
\hline
\end{tabular}




\section{Reinforced Concrete Test Data}

Track 9 - High Pressure Test

\begin{tabular}{|c|c|c|c|c|c|c|c|}
\hline 40.41180 & $0.0 x \times i)$ & 91.4284 & $.0 .(1.30 .1$ & 80.1 .40 & $-0 .(1.120)$ & $4.1 .(5970$ & $0(x)+1$ \\
\hline 41.1170 & $0 .(x) 78$ & $92 .(x) 70$ & $(9 .(1) 71) 8$ & 88.7250 & $-(1) .(1) 42$ & 424080 & $-0.0 \times 1.52$ \\
\hline 41.7000 & $-0.0(1) 70$ & 92.8139 & $-(1.0 .3 .14$ & $88.07(19)$ & 0.01371 & 41.7580 & $0.01(x)$ \\
\hline 42.4879 & -0.10188 & $9.5 .3(19)$ & $-0.0 .3,36$ & 87.1070 & $.0 .02(0.4$ & 41.1244 & $.0 .0 \times 147$ \\
\hline 43.110 & $-0.0(0) 79$ & 94.18 .50 & $-(1.0411 .5$ & $86.8(x) 14)$ & $-(1.0 .320$ & 40.45 .50 & $-0 .(0)(x)$ \\
\hline 43.9079 & $-(0.0) 7(1)$ & 94.9144 & -0.01 .42 .2 & 86.12 .40 & 0.0251 & $39.78(x)$ & $-0,(0) .18$ \\
\hline 44.6450 & $\left.0 .(K)^{\prime}\right) 2$ & $95 .(x) .19$ & $-(1.0 .382$ & $85.5731)$ & -0.026 .4 & 30.18 .59 & $-(1,0(1), 1$ \\
\hline 45.3470 & -0.011 .31 & $96.307 \%$ & 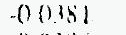 & $84.8 \% 30$ & -0.02 .31 & 38.4684 & .0. (x) 25 \\
\hline 46.0220 & 0.01153 & $41 .(12)(2)$ & $-0)(13 k 6$ & 8.4 .1920 & 1101225 & 37.7250 & $.(1)(x) \leq 4$ \\
\hline $4(1.78(1)$ & 0.0120 & 97.7129 & $-(1.01 .411$ & $8.25(470$ & $0 .(x) \| 1$ & $37.0 .5(x)$ & $.0(x+14)$ \\
\hline 47.9559 & -orkis' & 08.150 & $0.0 .14,7$ & 8.48 .9 & -0.028 .7 & $36.30 \times 19$ & $-0.0 y) .43$ \\
\hline $4 k^{\prime} .1\left(4 k^{\prime \prime \prime}\right.$ & .0 .0110 .4 & $40.1(1.46)$ & .0 .0 .350 & $82.7(x) 11)$ & $0 .(1280)$ & $35.5(1.7)$ & $-0 .(x) 3()$ \\
\hline 48.8050 & (1)(1)+1) & $49.75,70$ & $0.11 .41(1)$ & 81.7184 & .0 .0217 & $34.88(10)$ & (0). $(\times 145$ \\
\hline $40.61(x)$ & $-0 .(x) 76$ & $10(x)+354$ & (). (4)? & $81(x, 3 !)$ & -0.01281 & 34.1447 & $0 .(x) 10$ \\
\hline $5(1.31(8)$ & $-(1 .(1) 31$ & $101 \cdot 1(1,51)$ & -(1)(2)+1) & $8(1,1111)$ & 0.01247 & 15.4674 & $0 .(x) .3(1)$ \\
\hline 510224 & (1) Kust & $1(11.8010)$ & (1.).4. 11 & $31) 78(1,1)$ & $(1,1) 2$.10 & 13.40 .70 & $0 .(x) .31$ \\
\hline 51.7 .210 & 0.01103 & 10125010 & 0.0108 & 10.2124 & $(1)(1)(1)$ & $34 .(x)(1) 0$ & ()$(x) 51$ \\
\hline 52.4274 & .0 .0101 .4 & 1013.25040 & $(10113.4$ & $785571)$ & $(1)(1)(1)()$ & $34.5 .1(8)$ & $0 .(x) 201)$ \\
\hline 53.14 .14 & $-0 .(1) 152$ & $1(13.49,5.9)$ & $-0 .(1): 5$ & $77.9(1 \% 4)$ & $(1.1) !(x)$ & $35.226,0$ & $0.0(x) 10$ \\
\hline $53.8 .1(k)$ & $-(1)(1) 01$ & $1(1.4(1,5) 14$ & $-(0)(1+1)$ & $77.1: 20$ & $.11 .113(12)$ & $15.0(1) 10$ &.()$(x)(1)$ \\
\hline 54.90 .4 & $-(10.111911$ & $10.8 .31(10)$ & $-(0.121014)$ & $7(1.53(14)$ & (1).(1) $k_{2}$ & $36.71(x)$ & $(1)(x)(1)$ \\
\hline $55.26 x, 4$ & 0.0117 .3 & $!\left(k_{1},(1)(, 1)\right.$ & $-(0.01308$ & $75.64(114)$ & .0112944 & 37.4 .554 & $(0 .(x) 17$ \\
\hline $50,18.36$ & $(1.11193$ & $10(x, 7,+1,1)$ & (i) (14.3) & 75.251811 & $(1.112,29)$ & 38.1804 & $0 .(x)(k)$ \\
\hline it, $8(1), 13$ & (1)(1)/6 & $107.2351)$ & (1).14) 10 & $74.417(1)$. & 0,1913 & $38^{\circ} \cdot 4.4(8)$ & $-11,(k) 31)$ \\
\hline 57.4585 & $-(1)(1) 1.1)$ & 108.1252 & $-(1) .(15) 1(1$ & 73.484 & $.(1)(1) 11]$ & (1) fikitl) & $.0 .(x) .30$ \\
\hline $5 x-2+4$ & $0(1) 10)$ & $111 \times k 1.40$ & $-(1)(15())$ & 7.3 .37 .13 &.$(1) 1185$ & 40.4080 & (1)(x).iv) \\
\hline$\{1,()\{11\}$ & $0 .(1130$ & $1018206 x$ & $.(1) .(4.54$ & $12.72(1,4$ & $-0(1215$ & $1 ! .17 \%$ & $11(x) 1:$ \\
\hline 5401314 & $(1111)+2$ & $111 ! 911: 20$ & $-(1)(1) 11$, & 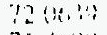 & -iifilims & $41 ;()(3)$ & $(1,(x) 17:$ \\
\hline$(x), x^{2}(x)$ & (1) 11117 & $1(x, 8) 513$ & (1) 141$) ?$ & $71.4(1,1)$ & $(1)(1)+4)$ & 42.4670 & $(1)(x) 50$ \\
\hline t.giisin & (1) $6115:$ & Iinglin & $(1,1+1.4)$ & $11182(x)$ & $(1111.10$ & 412114 & (1.ixkin \\
\hline $0171: 4$ & $(1,1) 225$ & $10<521.4$ &.$(1)(414)(1)$ & 701 lutin & $-(1)(11) 10)$ & $4.3 .0(1 ; 0)$ & $0.1 \times 17.3$ \\
\hline$(12.4214$ & (1) $11 ! 21$ & $1(1.4)(2,8)$ & $(1)(1+a)$ & 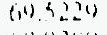 & (1) 0.1? & $+4 .(1.41)$ & () (x) \\
\hline $61+240$ & $0(12: 2$ & $1(18.64)(3)$ & $111+40(1)$ & $(181) .54$ & $(1,11: 10$ & $19.8 .4 \div 18$ & -0.010 .5 \\
\hline 0.381100 & $(10186 i$ & 111, Ys. & $0 .(1)<.4$ & $(1,2) / 31)$ & $(1)(11 \%)$ & $H(1): 20$ & $(1) x(1)$ \\
\hline$(-1,5210)$ & $(0)(1) 13)$ & $10 ! 1624$ & (1)if 188 & $\because \because 12+11$ & 11.11105 & $40,18(1)$ & $(1) 11117$ \\
\hline$(0,23,40)$ & (1)(1) $\mathrm{A}$ ? & 1119819 & (1). (1H.). & (1) $10.3(11)$ & (1).01) & 47.1580 & $f(x))(1$ \\
\hline $0.5192(1)$ & (i) (i)k' & $116.156,3$ & $(1)(1.1 .5$ & $(x, 1,1510)$ & $(1.0) ! 51$ & 481080 & (1). (x) 185 \\
\hline$(x, 6) x$ & (1) (1) 161 & & & of (rit) & (1) (1) $(0,1)$ & 48.86515 &.$(1)(11.4$ \\
\hline (7) 3530 & $.0(1) 14$ & & & 6119116 & $\left.(1.01)^{-17}\right)$ & $f(1)+\{(x)$ & $-(1)(x), 7$ \\
\hline$(18)(150,1)$ & $-0 .(1.944$ & Sicanl 11. & 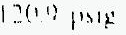 & $0.4+2(16)$ & $11)(k) 3$ & 50.3100 & -0.11128 \\
\hline $\cos x(k x)$ & $-(1)(1) 45$ & & & $(1,3,7 \mathrm{n}(14)$ & $11(1) 16$ & $\{1.022\}$ & $\left.-(1)(x) s^{\prime}\right]$ \\
\hline (1) $1 ! 54$ & $-(1)(129)$ & 10188,50 & $0,(1, k)$ & $0.3 .150,1)$ & $(1)(1) .35$ & 917.211 & $0(x) \geqslant 1)$ \\
\hline$(1.11121)$ & $(1.01278$ & 108.1584 & $(1.05) .4$ & (1): Silent) & (1). $(x) 185$ & $\$ 2.4271$ & $0 .(x) \div 8$ \\
\hline $7(1.9(1) 19$ & (1). (1):45 & $1(1 ;, 4(1.81)$ & $(1)(191) 1$ & $01189(1)$ & $(1.1) 13(1)$ & 53.1 .447 & $-(1.0121$ \\
\hline $71+116$ & $0 .(123)$ & $1(4,4), \ldots)$ & $(1)(1.1)$. & 61.2256 &.$(1.111401$ & $5.1 . R \cdot f(x)$ & $.0 .(11.1 .3$ \\
\hline$\therefore 2(8, n)$ & (1)(1) 19 & $1(1)(1,2(1), 1)$ & $(1)(21110)$ & $(1), 6(1)+4)$ & $(1.01)(1)$ & $5+2.3(1+4)$ & $-11111 / 11$ \\
\hline $73(1.4 .60$ & .1111211 & $11,561721)$ & 1111415 & Sw vous & 11.111 .11 & $55 \therefore t(x)$ & -i).,1! If \\
\hline$\because\{? \mid+(n)$ & 0.0 .10 & $1(15(k) \cdot 1)$ & $11(16 i-1$ & 513.39014 & $-(1)(1) 1 ; 1)$ & $5 n,(1), 30)$ & (1)(1) 15 \\
\hline is 14.4 & $.0(1211$. & $10.4 .4 .11)$ & flílk\} & 58.7114 & $-(1.111 .17$ & $S\left(x, g^{\prime}(x, y)\right.$ & $(1) 111)$ \\
\hline 1515,4 & .0 .01 .615 & 10181.14 & $1 ! 1+14$ & $58(9.911)$ & $(1.61), 36$ & $5 ? 140 .+1$ & 2.01141 \\
\hline 55813 & (1) 11,251 & $10132(151)$ & (1.14.) & $57+1411$ & $(1,(1)+16$ & $\therefore n .2244$ & 0.01100 \\
\hline 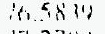 & $-4)(128\}$ & $1(1.25174$ & (1) (1.) 1.4 & $51,742)$ & $0,(1) 1\}$ & 58.93 .39 & f) (x) ( \\
\hline 17.2700 & $0 .(1251)$ & $1011.4(1,21)$ & (). (4):8 & $56.116,9$ & (towy & $411\{1,3\}$ & (1)(1) 20 \\
\hline $77(5)(1,4)$ & $-0.1) 2(18$ & $161136)$ & $(1)(1) \times()$ & $55.4+.81)$ & (1)(xin) & $(x), 12(x)$ & (1)(111? \\
\hline 78.71 .10 &.$(1)(1.3 .611$ & $1(x(1,6, \ldots)$ & $-(10)\{160$ & 348520 & ()$(n) ?(1)$ & $01(1154)$ & $(1.01125$ \\
\hline $79 .+286$ & $-1) 0.86$ & $i(x) .11 .10,4)$ & (1) $(1)(, 0)$ & 54.16 .14 & $\left.(1)(x)^{t}\right)(1)$ & 0.1 .1110 & $.0 .018 . ?$ \\
\hline $8(0.1) 974$ & .0 .11 .7 .3 & 40.4580 & $-1)(1,10)$ & $536(1) 24$ & $-(0)(1) ! 21$ & $(12+210$ & -110155 \\
\hline 8018279 & $-(0 .(1) \geq()(3)$ & $48 x+44$ & (1).1382 & 52191.40 & 0.01301 & 6.3 .12 .40 & $-(1) .02(1) .1$ \\
\hline $81.55(k)$ & $0 .(1270)$ & 98.20150 & -0101370 & 52.25601 & $-(1)(11) 5$ & (1).81以 & $.11 .11 / 1.1$ \\
\hline ‥2 2124 & 0.1277 & अ氵illy & $11(14 / 11$ & 51.6 .514 & $0 .(1)+1)$ & $(41.5231)$ & 0.011 .1 \\
\hline 82.4426 & (1)(1)272 & $1)(0,1)(1, \ldots)$ & $0.13,3$ & $5(1.9820$ & $-(0 .(K) \& 9)$ & $\left.0,5.23 x^{\prime}\right)$ & (1). (1) 161 \\
\hline 83.0 .314 & $-(1)(12 k 3.1$ & $9(3.2114)$ & $(1 .(1,11))$ & $50,3.7(6)$ & $(1)(1)(3)$ & 0.54250 & (1)(1)i(k) \\
\hline 8.1 .3120 & $(0,029)$ & प) 6950 & $-(1)(1)<(1)$ & $4(1)(1) \mid 0$, & (1). (k) & $(x, 1,6781)$ & $.0 .(11.31$ \\
\hline $850,30,0$ & $-(1.0 .3 .41$ & 1991180,1 & (1)(1) 3k & $10(1504)$ & $.0(1) .31$ & $07 . .15 .318$ & (0.0) 1811 \\
\hline 85,72518 & $-(i) 1,3015$ & 10.4 .4 .10 & $(1)(1)+10$ & 48.4284 & $(1(K) 1) 5$ & $0.805(14)$ & $-(1.021 .4$ \\
\hline 80.4126 & $(1)(1,35)$ & $14: 31\}$ & (1) 11451 & $47.7 \cdot(0,1)$ & $(1 .()(1) 19)$ & (stisikm) & $.0 .(1180)$ \\
\hline 87.1584 & $11.111 .1 n^{\circ}$ & $41 \mid 801)$ & $(1) 181$ & 471224 & -()$.(k) 5.4$ & $(0,+750$ & $(1.112 .30$ \\
\hline 87.5134 & $(1) 113.13$ & $(1)<4(, 1)$ & $(1: 1\{1) !$ & $4(1 .+1.14)$ & $(1)(1) 1201$ & $7(1.1920$ & $-(1) 1256$ \\
\hline $8 \therefore<(1, f)$ & $(1111)$ & "1! husu & $.1111\{t .8$ & .157 .450 & $-(1.11121$ & $7(10)(1)$ & $.0(12.3)$ \\
\hline 80.94 & $(1) 11344$ & (j) : : :v & $1: 112: 1$ & 49.1165 & $-0 . i i+i$ & 116114 & .0 .1122 .4 \\
\hline รูy $9(1)$ & $(1) 11) 16$ & $4(164)$ & 1111201 & $4.4 .2 \div+4$ & $(1.14136$ & $72.286(1)$ & -0.11 .118 \\
\hline$(x)(111)$ & $1111 \therefore .8$ & Bund & $i, i, i_{i}$ & : $: 3: 1 ;: 4$ & finitit? & $1.19 ! 2 ! ?$ & (40!)! \\
\hline
\end{tabular}




\section{Reinforced Concrete Test Data}

Track 9 - High Pressure Test

\begin{tabular}{|c|c|c|c|c|c|c|c|}
\hline $73.73(x)$ & -0.11207 & 104.4429 & $.0 .(45.1$ & 58.7100 & 0.011 .17 & 50.800 .4 & .0 .01612 \\
\hline 74.4420 & ().0247 & $1013.81+9$ & $-(1)(1) S(K)$ & $58(1) 4(7)$ & $-(1) .0140$ & 57.4889 & -0.017 .1 \\
\hline 75.1570 & 0.0201 & 103.2050 & (f) $(1+1, ?$ & 57.4140 &.$(1) 137$ & 58.2240 & $.0(1)(x)$ \\
\hline 75.81 .30 & (1). (1) & 102.5770 & (1).14.54 & 56.7924 & -0.0127 & 58.93 .39 & (1) (1) 610 \\
\hline 76.5830 & $-(1.027()$ & $101.9(180)$ & (1) $(40,1)$ & 56.1160 & .0 .0105 & 54.6 .310 & $-0 .(1)(1.5$ \\
\hline $77.27(x)$ & $.01(1) 40$ & $101,3(170$ & .0 .14 .15 & 5.5.tiky & .0 .0112 & $(x) .12(x)$ & $-0.01+3$ \\
\hline $77.49(1)$ & $0.02(1.5$ & $1(x) .6729$ & $-(n(1)+4)$ & 5.4 .8520 & $(0,0) \mid(x)$ & 6.1 .0154 & .0 .0138 \\
\hline 78.7120 & $-(1.0,3(1)$ & $1(k),(1) 26,0)$ & (1). (4) 28 & $54,16,318$ & $-0 .(1) 22$ & 61.7110 & $-0.0) 2(N)$ \\
\hline 79.42814 & .0 .01 .5 .3 & 40.4554 & $0.0 .1501)$ & $51,6(2)(4)$ & $0 .(1151$ & 62.4210 & $(0,0)(0,0)$ \\
\hline$x(1(N) ? !$ & $-11.02+13$ & 48.85 .47 &.$(1 .(1.851)$ & $4201.11)$ & $-0,01 \mid 01$ & 0.3 .1240 & $0 .(1210$ \\
\hline$x(1,8274$ &.$\left\langle\gamma_{1}()_{2}\right)(0)$ & 98.21150 & 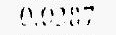 & $52.25(3)$ &.$(1,0) 127$ & $6.3 .81(x)$ & 0.0211 \\
\hline $81.55(x)$ & nne? & 97.5774 & $(1)(1.4)(1)$ & 51.0510 & $-0 .(1) 30$ & $(44.5230$ & .0 .0221 \\
\hline 82.2120 & (1). $(1,251)$ & $9(1.9(1,2)$ & -(1)(1).38 & $5(1.08 .21)$ & 0.0123 & 6.52180 & -0.01002 \\
\hline 824424 & .0 .0255 & $4(1) .3(14)$ & -0.11278 & $5(1.1 .1+10$ & $-(1 .(x)>8$ & $6.3 .9: 280$ & -0.0212 \\
\hline $8.3+(0.310$ & -0.12877 & 4.5 .0 .5840 &.$(3)(0) 18()$ & $.19 .(1011)$ & (1).0(185 & $(x), 6784$ & .0 .0108 \\
\hline 84.3120 & $(1 .(1) 295$ & $05(15(.0)$ & $(1)(13(5))$ & (1) $(1 \leq(, 0)$ & $-0.0) \leqslant(1$ & 67.3430 & $-(1) .02(1) \varepsilon_{1}$ \\
\hline $85 .(1) 3(.1)$ & .0 .0337 & 144.111 & 0.14 .52 & 481286 & . () (x) & (and $(1500)$ & 0.01250 \\
\hline $85, ?: 250$ & (1).(1)20? & 0.77 .11 & $(1)(1) .110)$ & 41710,0 & $-(1) 0 \mid\left(x_{1}\right)$ & $(\omega) s(k)$ & 0.0107 \\
\hline $8(1+42)$ & $-0 .(14.12$ & 0.3 .15511 & (1.)(1.4).. & 471230 & $(1.11) 115$ & 0.44790 & .0 .01 .10 \\
\hline 87.1580 & $-(0.0) 3(\mathrm{Ki}$ & $9):\{(0,1)$ & $(1.0 .4) .4$ & 46.1 .114 & $0 .(111 \%)$ & $7(111920)$ & -0.11 six \\
\hline $8 \div 8.816$ & $-(1.01 .38$ & 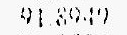 & 0.0115 & 45.7 .45 & $f(x))$ & $7(1,9(1] 1)$ & $0.11 \div 5 \%$ \\
\hline $88.5(1.19$ & .1103 .18 & (1) I'? & $(10)(1)$ & $4.5 .(1.551)$ &.$(1)(1) 17$ & 1101111 & $(0.11) 52$ \\
\hline (4) 15.21 & $0,01313.1$ & $9(1)(1 ; 0)$ & $(1.0118$ & $4.4 .3 \% 4 "$ & ()$,(x) S_{e}$ & 72.280 .10 & (1) (125? \\
\hline$\left.x^{\prime}\right), \dot{1}(1) \varphi$ & $0.01,20)$ & $80.0(114)$ & .010 .360 & 4.7316 & $0 .(x))(1)$ & $73(1.920)$ & $-(1,(1,27)$ \\
\hline$(x)(x(1 . f 1)$ & $0 .(1) 20.5$ & 817.3 .317 & $(1.1) 3.10)$ & $+13(11) 7(1)$ & $-(1)(1) 2()$ & 73.7 .490 & $-(1)(1274$ \\
\hline $41 .+256$ & 1111280 & 88.72517 & $.0(1) 360$ & $42.4(250)$ & $0(1) 12$ & 74.4420 & 0.020 \\
\hline $4 ?(10) \div 4$ & $(1,1) \cdot 4)$ & $8 \times 11 \div(\mu)$ & $.(1)(1 ; 1)\}$ & $41.75 \mathrm{k}^{\prime \prime}$ & $0(k) S)$ & 751570 & 0.01218 \\
\hline 1180180 & $-\left(1(1)^{2}\right) 8$ & $87.4(170)$ & $.(1) 18 \leq 15$ & 41.1 .416 & $(1)(n) !()$ & 75.81 .317 & $.0(1237)$ \\
\hline 4$)\{5\}(, 1)$ & (1).(14,1) & $\therefore(\forall) W H)$ & 110114 & $1(1.455(1)$ & (I) (K) is & $76,58,30$ & -(1).12kit \\
\hline $9418^{8}$ & $(1)(121)$ & $8(1.1211)$ & $0.614(1)$ & 39.78114 & $(1)(1,1), 6$ & 77.270. & 0.0 .3015 \\
\hline 0.1 .91 .10 & (i.011:5 & 859314 & $(1013) 0$ & (1) 1854 & 0.010150 & $77.94(1,9)$ & $-(1.01323$ \\
\hline $45(1) \cdot 12$ & .110371 & $8+8716$ & $(1) 1)_{2} \%(1$ & $38 \cdot(2, i)$ & 0.1415 .5 & 75.1120 & (1).11.8.4 \\
\hline $48,117,1)$ & $(1)(1,1)$ & $8.4 .11921)$ & $-(111.21 .4$ & 17,7254 & -()$(1)(x)$ & 70.4280 & $(1.03211$ \\
\hline$(y \div \ldots(12(x)$ & $-(1618)$ & $8 \geq 819 ; 0$ & $\left.(1)(1)(1)^{\prime}\right\}$ & $37 .(19(11)$ & $-(1)(0) 17 ?$ & $(i(1)(x) \geqslant y$ & (1)(1)\%() \\
\hline 47.71 .0 & $.010 .10(1)$ & 824520 & $0 ! 13\} 11$ & $3(1,3(x, 4)$ & $-(0)(x) 11$ & $8(1.8279$ & .010 .104 \\
\hline $4 x+150$ & (1) & $B=36(16)$ & 4111811 & $35 \$(10)$ & $0 .(k 195$ & $81.5 .5(x)$ & -(1)1) \\
\hline$(x) \mid 10.11)$ & $-(1)(13 .+1)$ & 81.7180 & $-(0,01,1) 1$ & $34 \sinh$ & .0 .111135 & 82.2124 & $-0,0,1: i$ \\
\hline (w) 7530 & (1)(1):4-4 & $81.1 k+10)$ & $\left.(1)(1)^{1}\right)=$ & $34.1+40$ & (). (K)ik & $82(9.124$ & $(1)(1 ! 1) 1$ \\
\hline $1(x)+150)$ & $0 .(1.417$ & $8(1.41 .14)$ & $-(1.11242$ & $33.40,74$ & $-0 .(k)(x)$ & 8.0 .0 .310 & $-(1,0.350$ \\
\hline $1111.1(1,51)$ & $-(1)(1.425$ & $7978(.9)$ & $01(1271$ & $31.4(17)$ & -()$(K)(x)$ & 8.1 .3124 & $0 .(1,354$ \\
\hline $1(1) \mathrm{st}, 10$ & $-1)(1.40)$ & 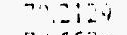 & $\cdot(1 .(1) 2(1))$ & $2.4 .(k x) y$ & $-(0 .(1) 10$ & $85 .(1)\{(x)$ & .0 .11348 \\
\hline 10.90 .10$)$ & $-1101 \cdots$ & ?iisory & $.11 .(1,251$ & 3.1 .5 .9170 & $.(1,1)(k) 1)$ & 8.5 .72 .54 & 011157 \\
\hline anduy & $-11 .(1): 1)$ & $\because 7(x) \geqslant 1)$ & (1) 11.9 .1 & $35.2 ?(i)$ & $(0)(x) 10$ & 86.4 .426 & $0.113 \alpha_{2}^{2}$ \\
\hline $1(1,3.1) 350$ & $-(1)(1.97)$ & 77.1724 & $(1)(1,1,4)$ & 35.19119 & $-0 .(811.35$ & $87.15 \mathrm{k0}$ & $(1,1374$ \\
\hline 104.6516 & 011245 & $76: 5:(14)$ & (1) 11232 & $\because: ., 1(10)$ & 1). $(x)(1)(1)$ & 87.8336 & 0.01380 \\
\hline $105.34(x)$ & 0.01352 & $75.4 \cdot 1$ & $(1,(1),(1,1)$ & 37.1550 & $-(0.0(1) i()$ & $88.5(0.47)$ & $-(1.01307$ \\
\hline$|(x, 1)| f, y)$ & $0(1,17)$ & 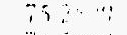 & $-11.11 \leq 64$ & 35.1406 & $0(x) \delta^{\prime} \varsigma$ & 80.5211 & $0.0361,5$ \\
\hline$\left(i x_{1} \cdot 7+(1)\right.$, & .0 .11 .111 & $7+4(1)(1)$ & 0191261 & $.38 .97(14)$ & $-0 .(x)(1.4)$ & $x 0,9(1) 0$ & $0,0,30(1)$ \\
\hline 197.19 .4 &.$(1) .1 .18(x)$ & $7\{4.65 y$ & $-(1) 1218$ & $30,618+44$ & $-0.1(1) .38$ & $(x)(6,1), 11)$ & $-0)(1.367$ \\
\hline $118125 ?$ & $0(1)(1.21) 6)$ & $11.1 \% 1 \%$ & 0.11212 & $4(0.4(150)$ & $-(0.01167$ & 41.4280 & (1)(4) 14 \\
\hline $108.83 .+0$ & $-0 .(1-1+x)$ & $72,72(14)$ & $0 .\left(1,<_{2}\right.$ & +1.1179 & 0.0116 & $02 .(1971)$ & 0.11385 \\
\hline $11+12=2(x)$ &.$(1)(0.12 \%$ & $7: 3(x, 40)$ & 0.02018 & $41.70(11)$ & $.0 .(1) 11) 5$ & 92.8130 & (1). (4) \\
\hline 1075078 & -0.0 .415 & 71.4000 & $.1111 / 4$ & $42+8 . ?$ & $-(1)(x) 171)$ & $0,1.53(6,0)$ & (1). (1)3? \\
\hline $1(x, 8050)$ & 0.134018 & $70.8 .2(14)$ & $(1.0) 106)$ & 43.2111 &.$(1.0(x), 0)$ & 04.18 .54 & 0.01 .12 \\
\hline $1(x, 1,8,1)$ & $(1) 1.113$ & $(0.1980$ & 0.0152 & $47,1)(170$ & 0.01110 & 9.401 .94 & $0 .(14.41$ \\
\hline 105.523 .4 & $(0.11 .427$ & $1,05521)$ & .0 .11150 & 44.6 .450 & 0.01018 & $65.6(1.14)$ & $11 .(4.137$ \\
\hline 10470,181 & $.0(1.412$ & $\therefore 80380$ & $0 .(1)(1)(0)$ & 45.3 .474 & $(1.011 .40)$ & $(x) .1(1 ; y)$ & $-(1)(46)$ \\
\hline $1(1,3.010())^{\prime}$ & $.0(1.155$ & $(18.2730$ & $(1.112018$ & 46.01 .26 & .0 .011 .77 & $41 .(12 \times)$ & (1). (4.1.1. \\
\hline 1013.2782 & 0.0187 & $6.7+2 .+40$ & -11.112 .2 & $40.780,0$ &.$(1.0) 1.3(1$ & 97.7120 & $(1)(2+7)(1)$ \\
\hline $102+40,3.4$ & 0.0 .42 .4 & 01.01014 & $-0.11: 11$ & 47.1550 & 0.01121 & 178.21 .54 & -0.1142 .2 \\
\hline $101847(1)$ & U.\{1.161) & (xí.35.19) & (1)(1)1)(1) & 48.1480 & $0(1) .18$ & $(x) .1(1.41)$ & $0 .(4 ! 5$ \\
\hline \multirow[t]{2}{*}{$110 .+50.1$} & $0 .(1.4 \%$ & 650.8 .14 & $-(i)(1) 58$ & 48.84513 & $.0(1150)$ & (96).75.30 & $-0.1+4+4.5$ \\
\hline & & $6.5 .(15)(M)$ & $-(1)(1))(16)$ & 40.61119 & () $\left(k k_{1}\right)$ & $1(x) .4 .159$ & $.0,045(1$ \\
\hline \multirow[t]{2}{*}{ Sian 12.} & $125 k$ psig & $64.42(14)$ & (I) $(K, K) \cdot$ & $.5(1.3100)$ & 0.0113 .3 & $1(11.165 .57$ & -0.0487 \\
\hline & & 0.378110 & $(1)(1)(k)$ & 91.0220 & $-(1.01113 .4$ & 101.80110 & 0.0 .17 .5 \\
\hline $108.815(1)$ & . () (1.18.'. & $0,3,1,5(0,0)$ & $.(1) 11) 13$ & 51.7 .320 & $-(0.1) 112$ & $1(1, \ldots 5(1,3)$ & $-(0.0 .51 .5$ \\
\hline 108.158. & $(1)(1.41) 7$ & $0,2.5 \mid(1,0)$ & (1)(11111) & 5242,4 & $0.0(1) .13$ & $1(1.1 .25014$ & 0.01 .881 \\
\hline $107.5(2.14)$ & -0.185401 & $(1) 8510$ & $.0 .(11 \% 0$ & $53.1+14$ & $-1)(1)(x)$ & 10184.1517 & (1) $(1.471$ \\
\hline$f(x, 9)(1,2)$ & 0.01526 & 012250 & (1)(1) & 5.5480 & 0.11150 & 101.10 .510 & $-(0.0 .471$ \\
\hline $11 x_{1} .10170$ & $0 .(1.917)$ & $(x)+(x)+4)$ & $0.119(1)$ & SA. $\{(x+1)$ & $-(0,010)(1)$ & 105.5 .400 & 0.01 .451 \\
\hline $1015(61) 20$ & $0 .(1.41) 2$ & 506800 & $-110113 i$ & $552(x, 0)$ & 1111178 & $l(k,(1) t, 1)$ & $(1 .(1.4)$ \\
\hline $105(1)>0)$ & (1). (1).13 & So $(1) \cdot)$ & $0(111)$ & $56,11,10)$ & (1).011.501 & $1(k, .7+k, 1)$ & (1)(1.183 \\
\hline
\end{tabular}




\section{Reinforced Concrete Test Data}

Track 9 - High Pressure Test

\begin{tabular}{|c|c|c|c|c|c|}
\hline $107+354$ & $.0 .050 \times 1$ & $74.54)(14$ &.$(1) 2501$ & $3 \mathrm{~s}^{\prime} 11.4 \mathrm{~kJ}$ & $\because \quad, \quad:$ \\
\hline 106.1252 & 0.05 .11 & 73.0486 & $.0(12,40$ & $(1)(19.4)$ & $1:$ \\
\hline $108.33+16$ & -0.0 .5 .11 & 73.3740 & $.0 .(1) 1.43$ & $f(1, \ldots)$ & . \\
\hline $1(x) .2 \tan (x)$ & .0 .0 .56 .3 & 72.726 .0 & .0 .118 .5 & $4: 1 ! \%$ & a $n$, \\
\hline $107.54 \% 8$ & -0.01547 & $72 .(x, 34)$ & -0.01804 & $41 \%$ & $\cdots$ \\
\hline $10 \times .80 .50$ & 0.05 .12 & $71.4(1,0)$ & $(1)(1) 11 !$ & $4 \therefore \therefore$ & $\cdots$ \\
\hline $1(* 1.1784)$ & (). $(151.5$ & $7(0.82(x)$ &.$(1) .11 .34$ & $41: 1: 2$ & $\cdots$ \\
\hline 105.9244 & -0.115 .14 & 70.1980 & .0 .0157 & $\$ 10$. & $\cdots$ \\
\hline 104.76 .81 & (1) $(15.47$ & $(10.5221)$ & $-(0.01157$ & $.4+1.96$ & . \\
\hline 101.40908 & 0.0950 & 68. & -0.0215 & $f^{<}: 1: 1$ & \\
\hline $103.27 \mathrm{kz}$ & $-0.0+181$ & $(2)^{\prime} .27 .39$ & $.0 .01 x_{1}$ & $\operatorname{sen} 12$. & \\
\hline 10.286 .3 .1 & (1).(4) & $(.7,(.2 .41)$ & -0.0170 & th $\quad \therefore$. & $\cdots:$ \\
\hline 101.8470 & $-(1.115(1,1)$ & 07.011119 & 0.01 .51 & $+1+2$ & $\cdots$ \\
\hline \multirow{3}{*}{116.450 .9} & $-0.058(1)$ & $(x, 35,39)$ & 0.011 .54 & $\$ \therefore \mid \ldots$, & 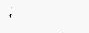 \\
\hline & & 65.6844 & 0.011 .10 & $4 x+x, 2$ & ‘ \\
\hline & & $6.5 .(15(1)$ & $-0,0151$ & $1+\ldots !$ & $\cdot$ \\
\hline \multirow{2}{*}{\multicolumn{2}{|c|}{ Scan 13,13() a prige }} & $(1.442(10)$ & $-(1)(k x, 1$ & $4 \quad i \vdots$ & . \\
\hline & & $6.376(14)$ & (1)(1)1/k & $41 \ldots$ & $\cdot$ \\
\hline 108.87511 & 010.41 & (i.) $15(6,0)$ & $\begin{array}{l}0(1) 11\} \\
-0(x) x)\}\end{array}$ & $\because \because$ & \\
\hline $\begin{array}{l}108.158 .4 \\
107 \text { sethly }\end{array}$ & $\begin{array}{l}-1)(05)(1 \\
1101977\end{array}$ & (1) $25 \mid(1,1)$ & $\begin{array}{l}-(1)(x+3) 3 \\
(1,0) ! 25\end{array}$ & $\because 1$ & . \\
\hline$!(x, 1)(2,3)$ & .011946 & 61.2250 & $(1,(1) 56)$ & c $\cdot \cdots$ & \\
\hline$f(x, 3(1), 0)$ & (1)(1.14) & (1)(1)(x) $\{1\}$ & $(1,(1)(0,0)$ & 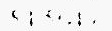 & \\
\hline $10<6(1) 21)$ & $0.01 .1^{\circ 4}$ & 810,9000 & $(1.01) 5.1$ & « . & \\
\hline $105(119)$ & $0 .(1.4: !$ & $5(1) .110()$ & $(1)(1) \times(1)$ & $\therefore \quad \cdots$ & . \\
\hline $1121 .+410$ &.$(1.1 .42)$ & 9891818 & .0 .01501 & $\varepsilon_{i} \cdot$ & \\
\hline $10: 8140$ & 0.01 .405 & $58 .(1+4)$ & $(1.01)(1)$ & $<\quad: \cdots$ & \\
\hline 10.3 .2050 & $-(1)(1.1 .+11)$ & 57.1149 & -0.011 .50 & $4 \ldots$ & \\
\hline 102.5774$)$ & 0.01 .51 & $5(1,7021)$ & $-(111) 28$ & $\Leftrightarrow \ldots$ & \\
\hline $1(1) .0(1) 50$ & $(i)(1) 5(4)$ & $S(1,11(, 0)$ & $11(1906$ & $4 \ldots$ & \\
\hline 101.31100 & 60140 & 55.10 .818 & $-(1)(k 18)-1$ & en $\because \cdots$ & \\
\hline$: 100,0.20$ & $-0 .(1.458$ & 54.85917 & $(1)(17)$ & $\therefore ; \cdot$, & \\
\hline$\ldots(1),(1,1)$ & $\cdot(1)(1.4(x)$ & 5.4 .16 .36 & $\theta(1)(x, 6)$ & $1:$ : : & \\
\hline $4(1) 45511$ & $-(1)(2+1))$ & $53(x)-3)$ & (1) (1)10? & 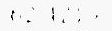 & \\
\hline (1) 585.11 & $-(1)(1)+14)$ & $52.41+40$ & $-(1)(11) .4$ & t: : : : & \\
\hline 06.2050 & $-(1)(1)(1) !$ & $5: 25014$ & 0.0138 & $\ldots$ & \\
\hline 07.5770 & $0.11 .30,1)$ & 51.6514 & $(1,01,30)$ & $\therefore: \because \cdots$ & . \\
\hline $9(1,7), 2)$ & $10.11 .41 k_{1}$ & $5(1.69 .24)$ & $-(1.0111)^{2}$ & $\cdots \cdots$ & \\
\hline$m, 32(k)$ & $(1)(1.124)$ & $50.1 .4(1)$, & $(1)(1) 19)$ & $1 \cdots \cdots$ & \\
\hline 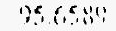 & 6.ant & $4(1)(1)(0)$ &.$(1.191 .1 \%$ & , . . . & \\
\hline $0.5 .05(.1)$ & .0 .11 .450 & $40.115(14)$ & $(1)(1): 1$ & $1 \quad \cdots$ & \\
\hline 04.4210 & $010+21$ & 48.4254 & $(1.61) 1$ & $\therefore$ & \\
\hline 93.77 .114 & $(1.01 .10) 1$ & $47.74(1,4)$ & 0.0113010 & $\therefore \cdots$ & \\
\hline 9.3 .18 .54 &.$(1(1,30) 1$ & 47.1290 & $\|(k),(1)$ & $\cdots:$ & \\
\hline $42.5 .3(14)$ & (1)(13). & $4(1,4.4 .4)$ &.$(1.1(x) 10$ & $\because \because$ & \\
\hline $91 . k^{(3.140}$ & -0.01 .410 & 4.7450 & 0.11167 & $\because \quad \because$ & \\
\hline 171.2721 & $-(1,(1)-8)$ & 45.0150 & $(1)(1) !+11$ & $\because \cdots$ & \\
\hline $90(1,17.9$ & $-(1.0 .316$ & 4487.47 & (1). $(1)+16)$ & $\because \therefore$ & \\
\hline$(81) 0(1) 0$ & 0.0 .374 & 427310 & $0(x) ! 1$ & $\because \ldots i$ & $\ldots$ \\
\hline 89.3 .3 .117 & 0.0 .321 & $45(x) 70$ & $(1)(x) 1)$ & $\because \quad:$ & $\because$ \\
\hline $88 \div ? 2511$ & 0.01324 & $42+18 \times$ & 0.11152 & $+1: 2$ & \\
\hline $88,07(k)$ & -0.0 .3 .44 & 41358 & .016188 & $"$ & t \\
\hline $87.4(174)$ & $.0 .113(1.4$ & 411211 & 1.1 188 & $\because \ldots$ & \\
\hline$K(1, S(x)$ & .0 .032 .4 & $4(1.4559$ &.$(1)(x)(14)$ & " & \\
\hline 86.12 .47 & $-(1)(1)+1 ?$ & (31). $78(10)$ & $.010(1.1 .1$ & $\cdots$ & \\
\hline 55.52317 & $0.0,3,1\}$ & 39.1856 & $-(1)(1) 28$ & $\cdots$ & \\
\hline$(818 \div .11)$ & $0(12,42$ & 38.46 .84 & - $1 .(1+10)$ & $\because \quad 1$ & \\
\hline 8.1090 & $0 .(1285$ & 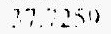 & .)(KH)! & $\because \ldots$ & \\
\hline $8.5(140)$ & (1) (k!!) & $37019(11)$ & $(1 .(1), 31)$ & $\therefore \quad$, & ' \\
\hline 120824 & 0.0128 .1 & $3(1,3(k, 1)$ & (1)(x)1S & $\cdots$ & \\
\hline $8 \div . ?(k \mu)$ & $0.0)^{(6)(1)}$ & $35.5(2.26)$ & (1).61) & $\therefore$ & : \\
\hline $81.71 \mathrm{~K}^{4}$ & -0.11286 & $34.850(1)$ & (). 1410 & $\because \ldots$ & \\
\hline $81,(x, 3)$ & $.0 .(1) 250$ & 3.4 .1448 & $0($ (H) $)(1)$ & $\therefore \quad \therefore$ & . \\
\hline $8(1) .41 .+4)$ & $-(0.02 ? \div 1$ & 315,5979 & $0 .(1) 11)$ & $\therefore: \cdots$ & ? \\
\hline 79.7860 & $0.01)_{2}^{2} i$ & $3\}+4(1)$ & () (k) 14$)$ & $\because: \cdots$ & . \\
\hline 79.2129 & (1.).1255 & $3 . f(x)(y)$ & $0114+1)$ & $n$ & \\
\hline 78.5570 & (1).(1) 2110 & 3.4 .5 .1908 & $.0 .(60) 5$ & $\therefore \because$ & \\
\hline $7 \div .9(176)$ & $.0 .112(1.4$ & $15,22(19)$ & $0 .(x) 27$ & $M:$ : & \\
\hline 77.1720 & (1).0? $2(k$, & $.35 .6(111)$ & $.11 .111: 3$ & $\therefore \quad \cdots$ & \\
\hline $76.53(x)$ & .0 .01177 & so $711(m)$ & $(1)\left(k x_{1}()\right.$ & $A$ & $\therefore$ \\
\hline $75.8 . f(x)$ & 0.02 .30 & $37+550$ &.$(1.61) .3(1)$ & $n \cdot \cdots$ & \\
\hline $75.25(x)$ & (1).112.t6 & 38.1307 & $11.14+1.4$ &.$\quad$. & \\
\hline
\end{tabular}




\section{Reinforced Concrete Test Data}

Track 9 - High Pressure Test 


\section{Reinforced Concrete Test Data}

Track 9 - High Pressure Test

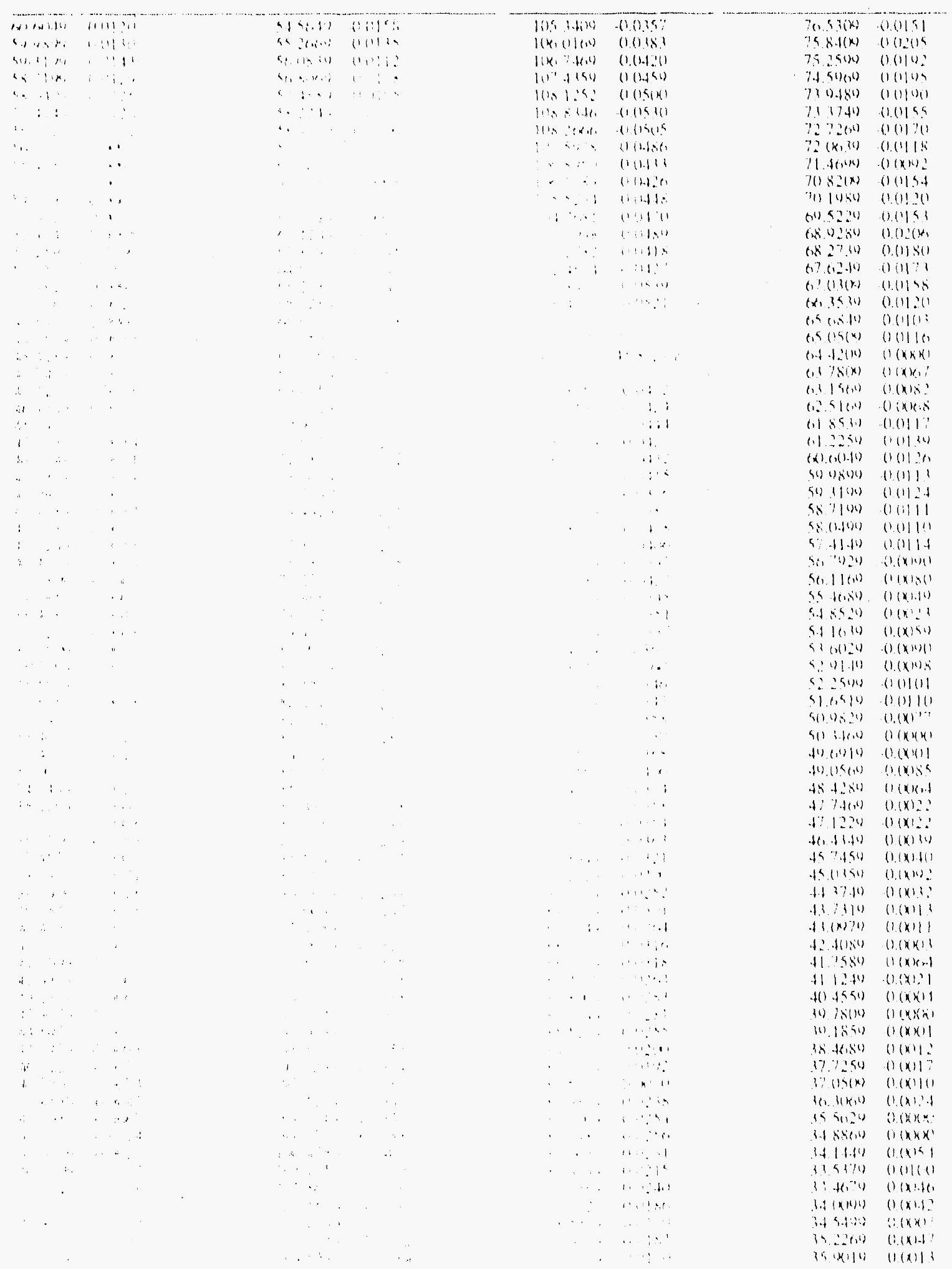




\section{Reinforced Concrete Test Data}

Track 9 - High Pressure Test

\begin{tabular}{|c|c|}
\hline $3(1 \cdot 1(n)$ & $(1)(x+8)$ \\
\hline $3+196.8$ & $(1.1 \times x \times)$ \\
\hline$|x|(3)(n)$ & $(1)(x+4)$ \\
\hline $\operatorname{lin}(1) .1(x)$ & $01(x) \div 1$ \\
\hline (1) $(4)+1$ & $0(x) 16$ \\
\hline$+(1) .4(1,6)$ & (1)(x)B) \\
\hline 111114 & $(1 .(4)(1)$ \\
\hline 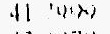 & $(1 / k)<)$ \\
\hline $42+5=0$ & $11(x)+1)$ \\
\hline $4: 211.1$ & $(1(x)))$ \\
\hline$+(1)(10)$ & !Cul? \\
\hline $4.4(.190)$ & (i) \\
\hline $16\left(1^{-10}\right)$ & $(1)(k x))$ \\
\hline$t(10,20)$ & (1) \\
\hline ter sis & $(1)(x+5)$ \\
\hline$d: f(4,4)$ & (1) H11: \\
\hline His 145 & $0(x+4)$ \\
\hline fiñ & $(1)(x), x$ \\
\hline$f(x)(1(x)$ & $(!|x|:)$ \\
\hline$\{(1) 1 / \cdots)$ & $(1,11111)$ \\
\hline $5112 \ldots$ & 1914181 \\
\hline $4,1,1$ & $11(x+1)$ \\
\hline$\therefore \therefore 4: 4$ & $16 \ln x \cdot 5$ \\
\hline$\$ 1114$ & $(1)(x))$ \\
\hline$\{\because B f(n)$ & $(191) \div ?$ \\
\hline $5.1(1.14)$ & $(11) \|\}$ \\
\hline$\Leftrightarrow 2 \cos (4)$ & $11+111:$ \\
\hline $4,\left(\begin{array}{lll}4 & 10\end{array}\right)$ & $(119)$ \\
\hline 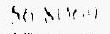 & $1: 1.111:$ \\
\hline$(\cdots, 1)$ & $i n n+1$ \\
\hline $4: \cdots$ & $\because:$ : ! ! ! \\
\hline$\because 210: i n$ & $1114 \times 1.1$ \\
\hline$\because 4,1,4$ & 1145 \\
\hline (1): ? $\because x)$ & $114 \ldots$ \\
\hline i.1.1!2, & $\because 1: 4 \times \ldots 1$ \\
\hline 11.110 & .111 .1 .1 \\
\hline (1)1:1: & 114. \\
\hline 611211 & $11: 1$ \\
\hline $018 \mid(x)$ & Hill : \\
\hline 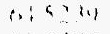 & $(1: n, \ldots)$ \\
\hline 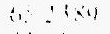 & $(1,11,1$ \\
\hline$(51) ? \times 1)$ & $1 \cdots \cdots !$ \\
\hline (11, $1,(x+1)$ & $(: 4 !(\cdots)$ \\
\hline$(1,4(1)$ & $11,111^{\circ}$ \\
\hline$($ ( & (1) $11 \ldots$. \\
\hline $\sin (\sin x)$ & $11 \mathrm{lin}$ \\
\hline$(n) \mid(1)$ & $, 141,1$, \\
\hline$\because 1 \div: 2$ & 11.11 \\
\hline$(1,16110$ & $\because \ldots$ \\
\hline 1.111 .1 & $1 \cdots: \ldots$ \\
\hline$\because \therefore(x)$ & $(1,: 1 \%$ \\
\hline $4.16 .4,9$ & $\because \because 11, \cdots$ \\
\hline$\therefore \quad \therefore(\mu)$ & $11: 1 \cdot 1$ \\
\hline$\therefore+1.9$ & $11 ! ! ! 1,$. \\
\hline 15150 & $11: 1: \%$ \\
\hline 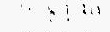 & 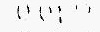 \\
\hline$" \cdots, "$, & $1 ; i 1 ; 1,1$ \\
\hline$\therefore M$ & $11 \cdots$ \\
\hline I'suls & 11.4 \\
\hline \& 1.0 & $\cdots$ \\
\hline$\because+$, & $1 \therefore$ \\
\hline 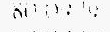 & $\therefore \quad 1 \cdots$ \\
\hline (1) $\because \because y$ & $\because \because \cdots$ \\
\hline (i) $\quad \mu_{i}$ & $(1,1,1)$ \\
\hline$\therefore \therefore 11$ & $1 \quad \because 1$ \\
\hline$\because 4.4$ & 1:1\%:": \\
\hline itsiti & $\because 1 \because "$ \\
\hline A.t in an & $1: \because 4$ \\
\hline$\alpha+\{+, d$ & 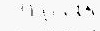 \\
\hline$\therefore \quad \therefore 11$ & $11, \cdots$ \\
\hline $\mathrm{sin}+1.4$ & $411 \therefore$ \\
\hline
\end{tabular}

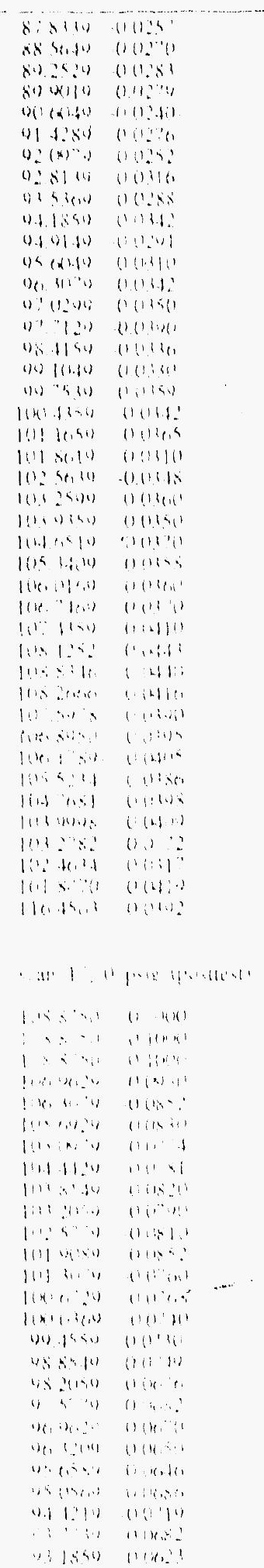

1): $51(1,4) \quad(1,1 k, 80)$

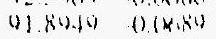

(1) $2 \% 210 \%$ (3) (19:9

$00<1 \% 0$ d)

(i) billu $0 .\left(x_{1}(x)\right.$

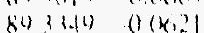

sis 250 (1) 015 (s)

Bis (1) (1)

8. 11120 001010

Sosing ons?

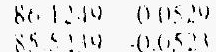

Bis 10 (1) (1)

8.1 19!. $0111 \%$

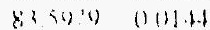

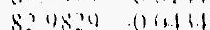

8. l(x) (i) (1)

si. 1.:4 011.45 ?

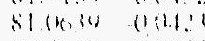

$80+110$ (1) 1102$)$

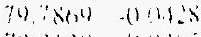

7).21:4 110169

sis 011112

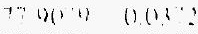

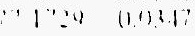

(0) 51(m) 11011111

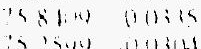

पद⿱

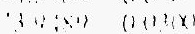

3 ind (111),

$\because 2 \therefore) \quad(1010)$

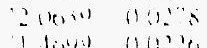

1)

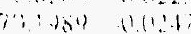

(1) 5204000

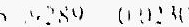

$\therefore \therefore 30 \quad(1,1) ! 1: 1)$

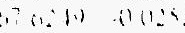

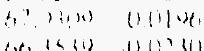

(1) की

(is (1) in (1) (1)

(i.) 1. (1) (1) (19):

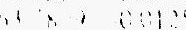

(1) $15, \mathrm{n}$ (1)

a ? ind

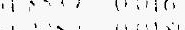

t11 4 (1) 10 (1)

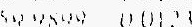

(4) (1) (1)

4. 910100111.

(3) (1) (1) (1):

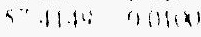

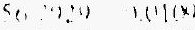

4. 116,0 10116

anse 11110

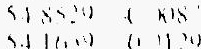

5:(11)!) (1)11

Sग) (11) (1)

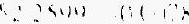

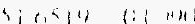

(11)

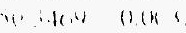

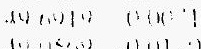

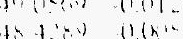

flat ante

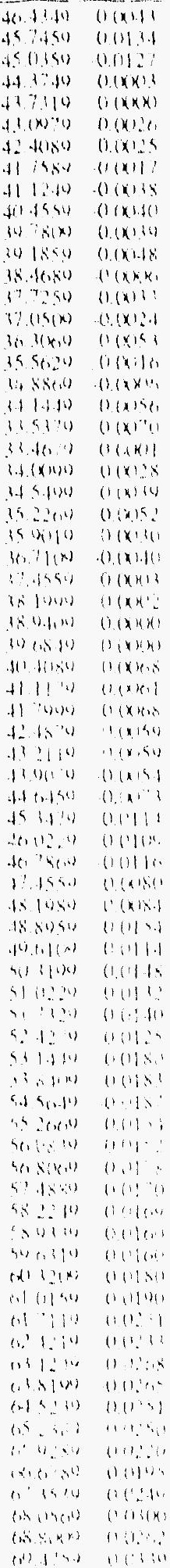




\section{IMAGE EVALUATION \\ TEST TARGET (MT-3)}
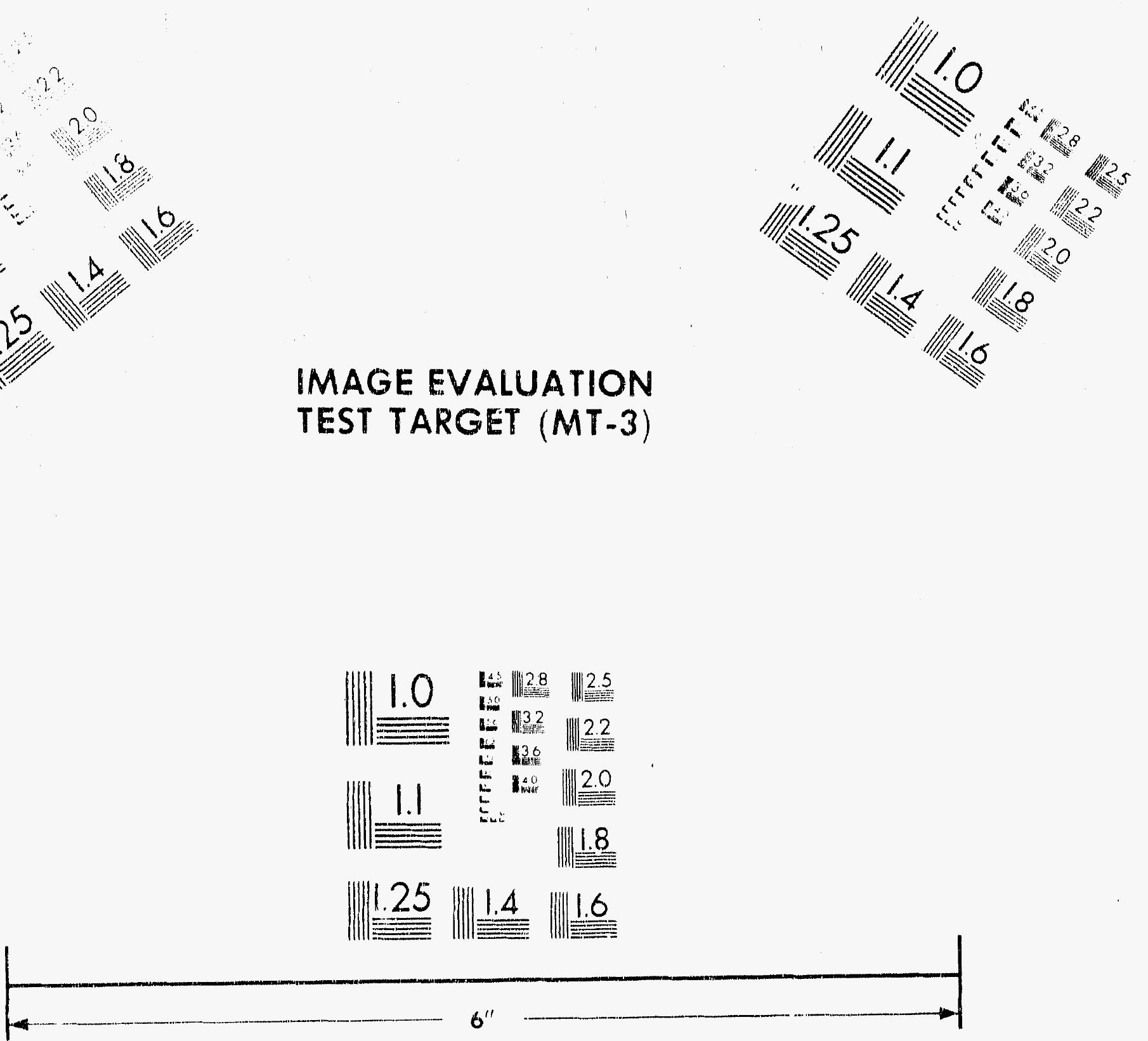

Photographic Sciences Corporation

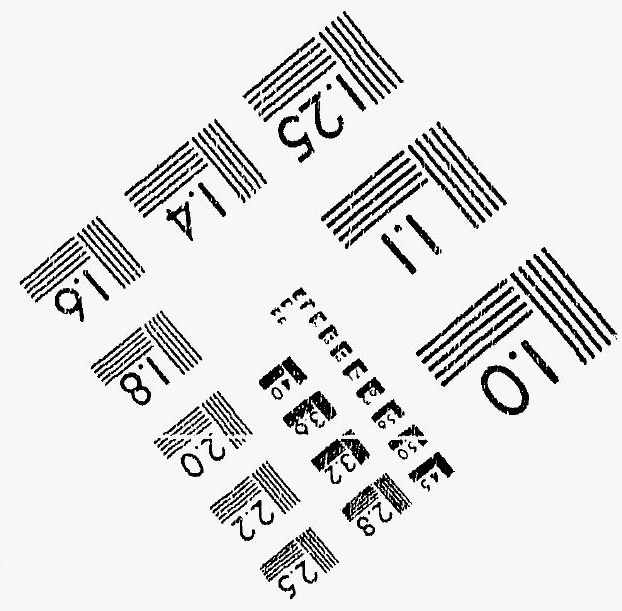

23 WEST MAIN STREET

WEBSTER, N.Y. 14580

(716) B72.4503 


\section{SECURITY CLASSIFICATION}
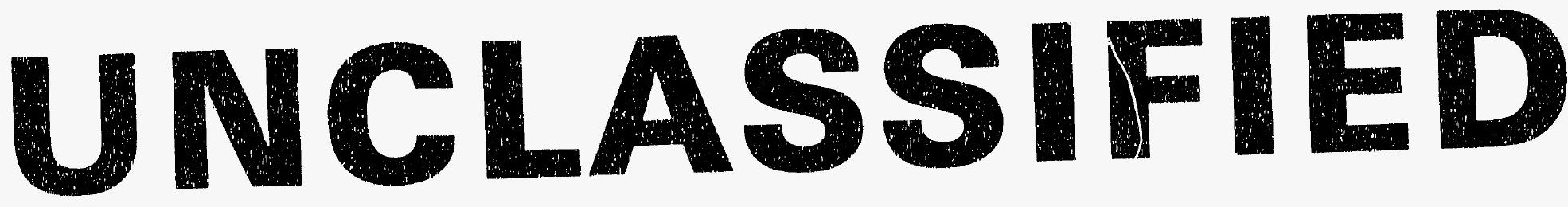

\section{DATE OF MICROFILIMING $1-6-92$}

\section{MICROFILMED BY ERRY WARD}

SANDIA NATIONAL LABS 


\section{THIS DOCUMENT FILMED}

AT

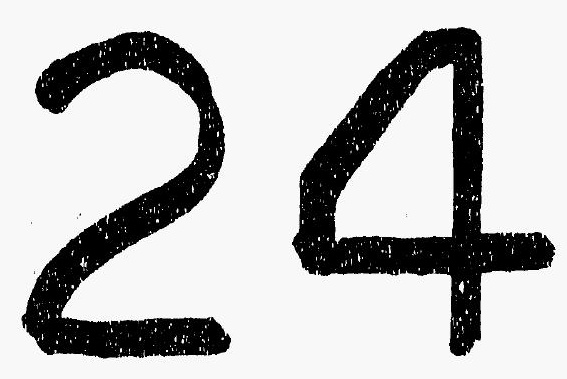

REDUCTION 
$615-5$

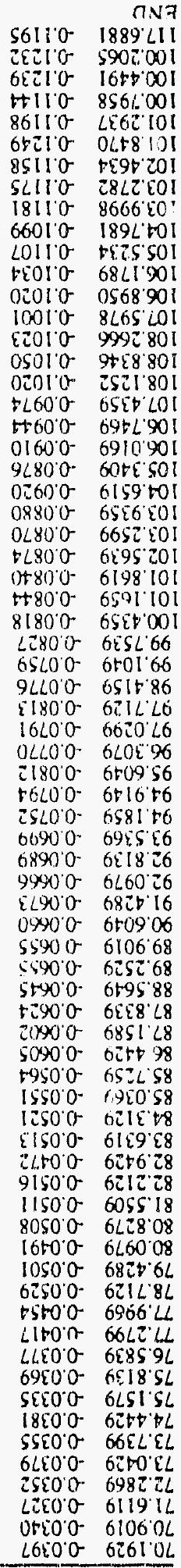

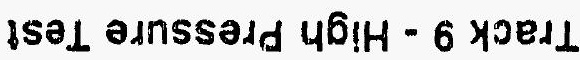

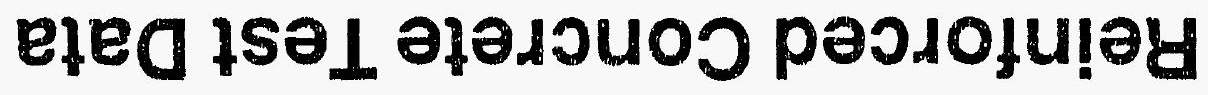



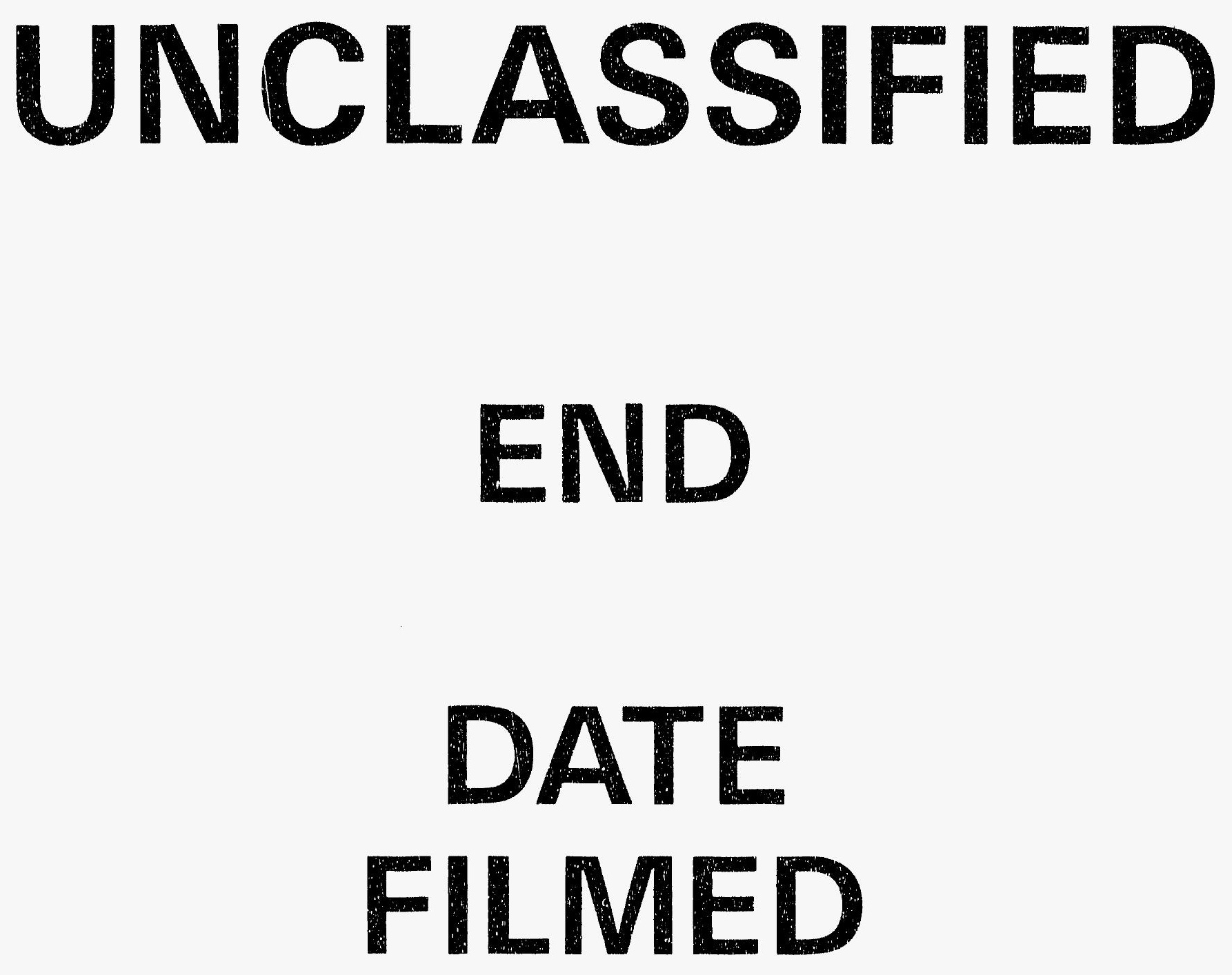

$$
1-6-92
$$

SANDIA NATIONAL LABS 
IMAGE EVALUATION

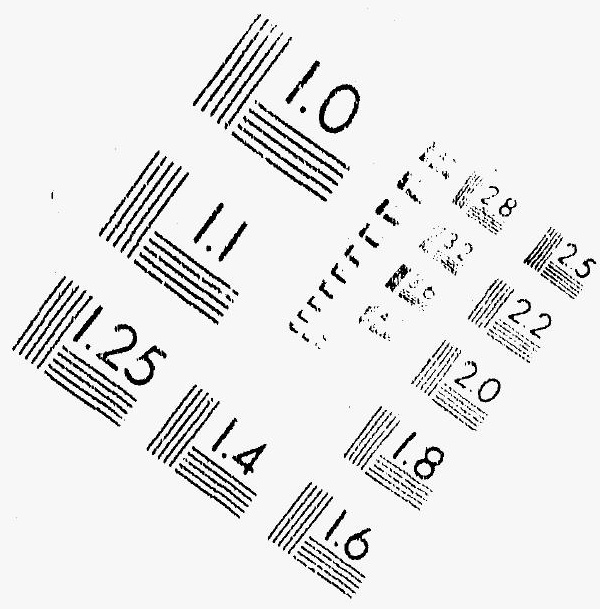

TEST TARGET (MT-3)
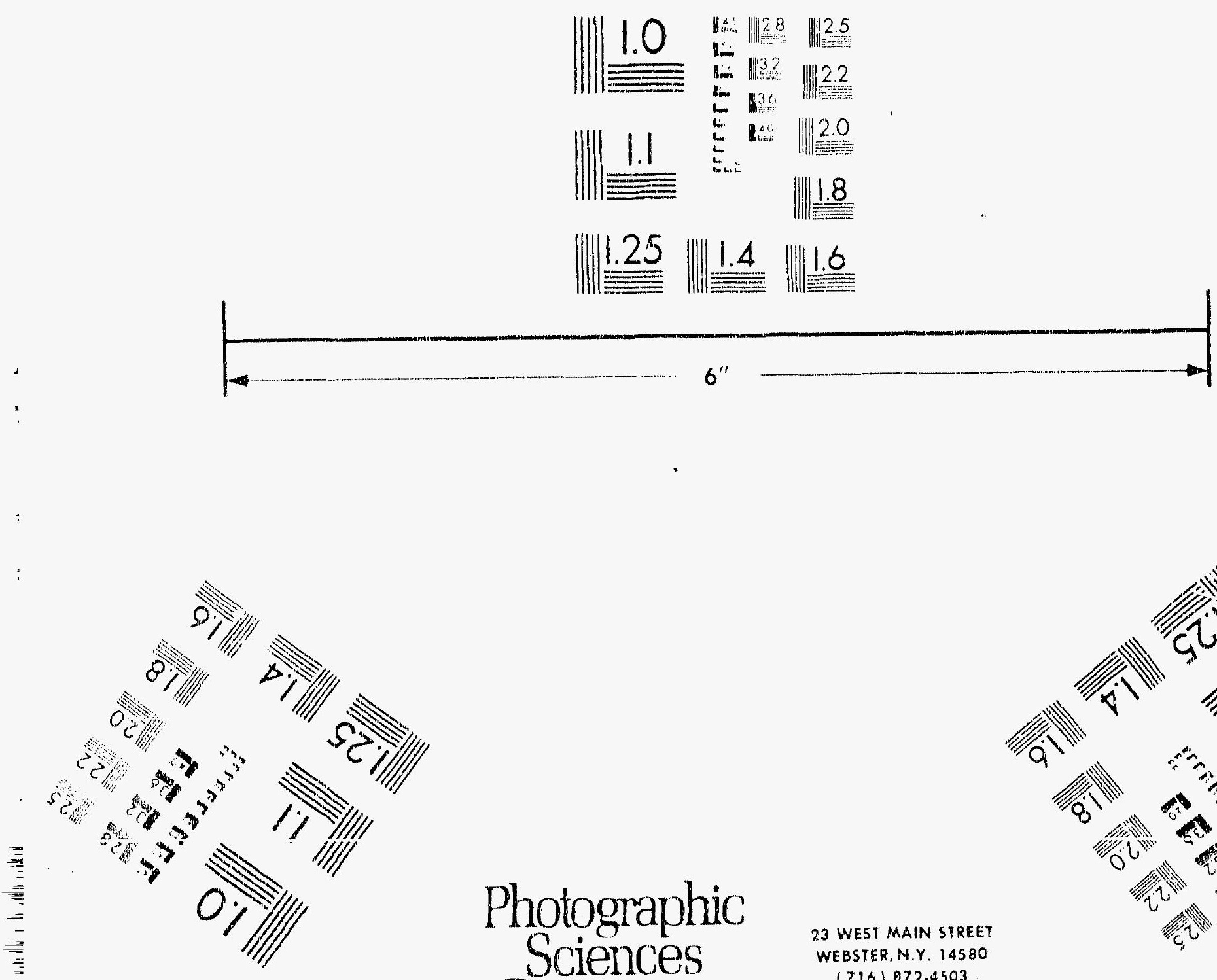

Photographic Sciences
Corporation

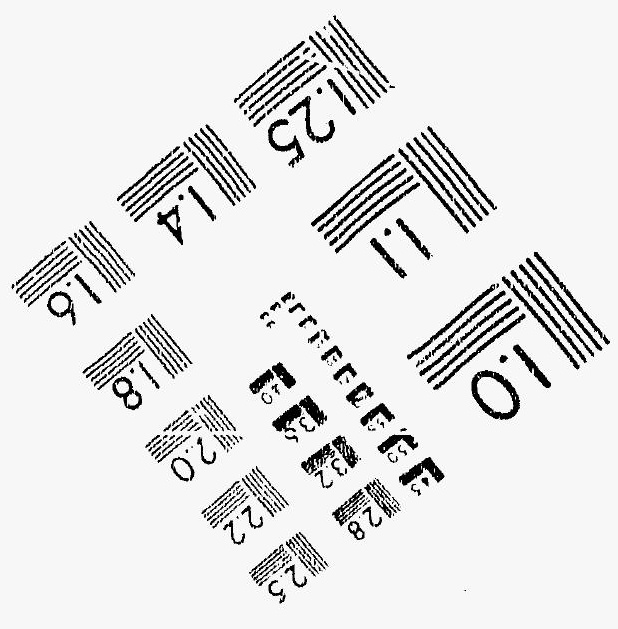




\section{SECURITY CLASSIFICATION}

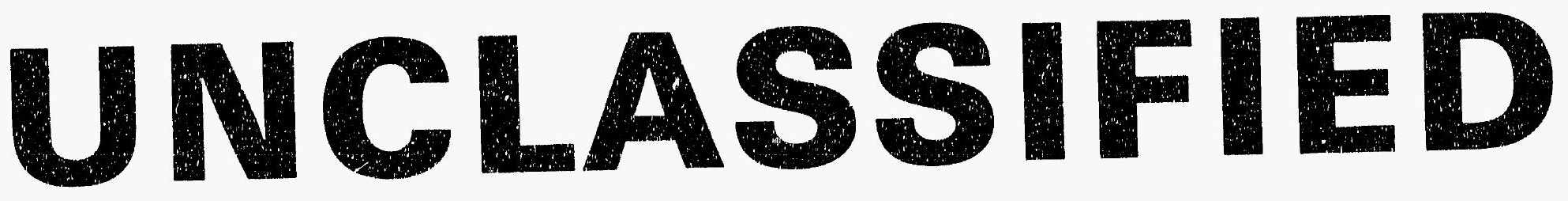

\section{DATE OF MICROFILMING $1-6-92$}

\section{MICROFILMED BY erar hard}




\section{THIS DOCUMENT FILMED}

AT

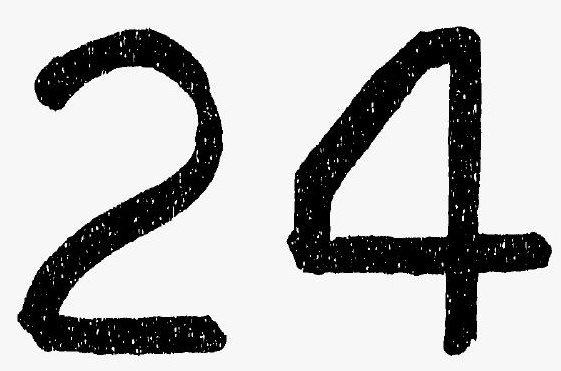

REDUCTION 


\section{Strain Gages, Rosettes}

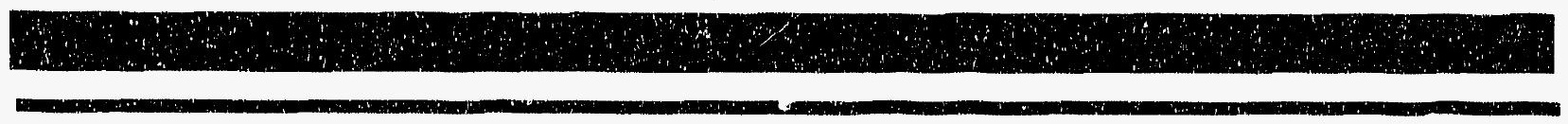





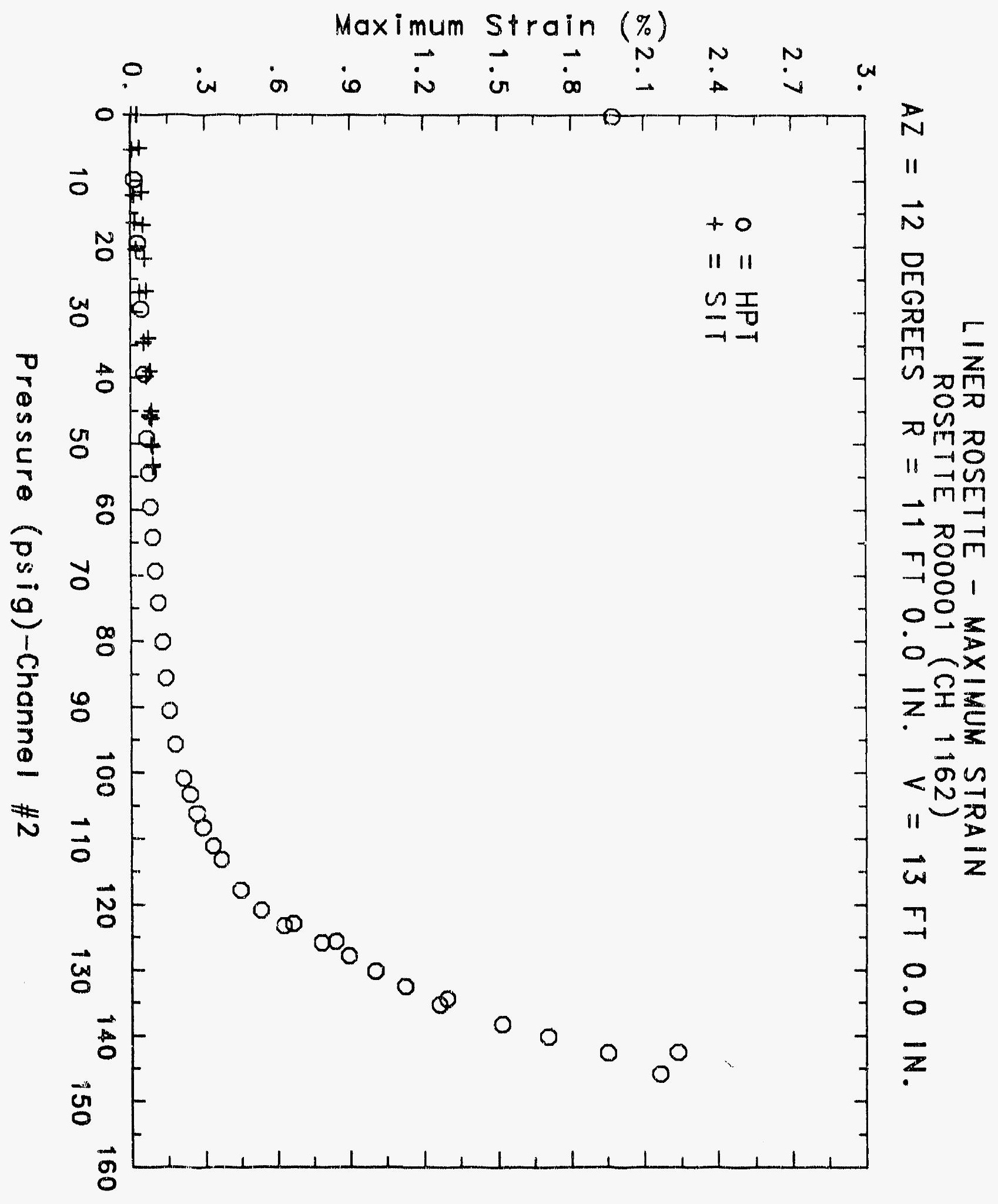




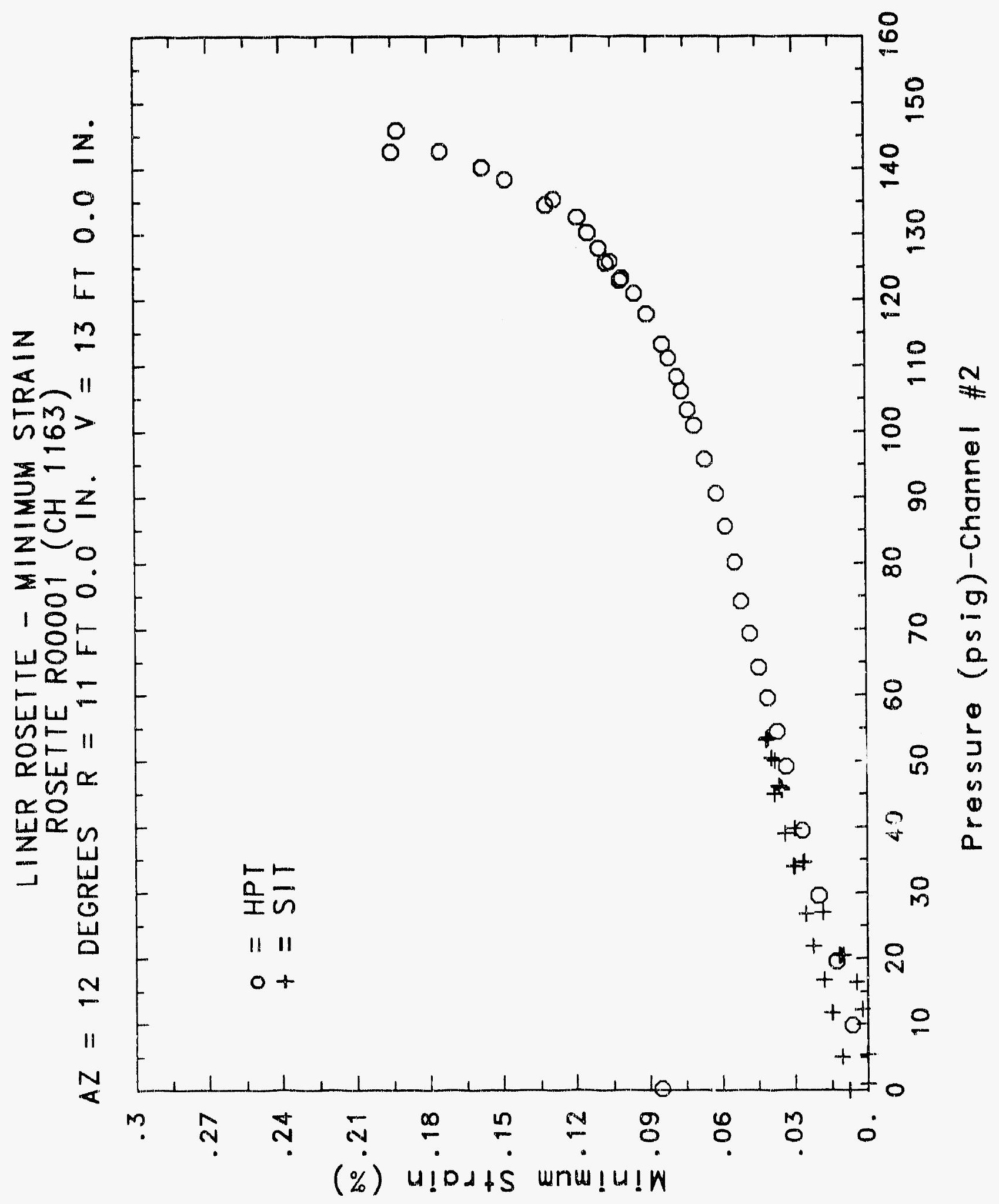




\section{Reinforced Concrete Test Data}

Rosette 2 Channels 11651166 116\%

STRUCTURAL INTEGRITY TEST

\begin{tabular}{|c|c|c|c|c|c|c|c|}
\hline $\begin{array}{c}\text { Pressure } \\
\text { Psig } \\
-0.05 \\
5.33 \\
12.31 \\
16.44 \\
20.51 \\
20.46 \\
20.47 \\
27.05 \\
34.69 \\
34.53 \\
34.53 \\
39.70 \\
45.90 \\
45.65 \\
45.62 \\
50.10 \\
53.47 \\
53.29 \\
53.21 \\
50.49 \\
46.14 \\
46.13 \\
44.97 \\
38.98 \\
33.99 \\
33.96 \\
26.83 \\
21.88 \\
21.90 \\
16.69 \\
11.74 \\
11.77 \\
5.05 \\
0.02 \\
-0.04 \\
-0.02 \\
-0.02 \\
0.02\end{array}$ & $\begin{array}{l}\text { Maximum } \\
\text { \%Strain } \\
0.0020 \\
0.0022 \\
0.0059 \\
0.0084 \\
0.0147 \\
0.0174 \\
0.0179 \\
0.0312 \\
0.0470 \\
0.0482 \\
0.0489 \\
0.0565 \\
0.0684 \\
0.0705 \\
0.0705 \\
0.0768 \\
0.0830 \\
0.0838 \\
0.0841 \\
0.0823 \\
0.0790 \\
0.0789 \\
0.0769 \\
0.0721 \\
0.0666 \\
0.0662 \\
0.0582 \\
0.0521 \\
0.0516 \\
0.0456 \\
0.0393 \\
0.0389 \\
0.0304 \\
0.0233 \\
0.0217 \\
0.0210 \\
0.0205 \\
0.0202\end{array}$ & $\begin{array}{l}\text { Minimum } \\
\text { \%Strain } \\
-0.0009 \\
0.0012 \\
0.0034 \\
0.0045 \\
0.0065 \\
0.0067 \\
0.0068 \\
0.0110 \\
0.0150 \\
0.0156 \\
0.0152 \\
0.0173 \\
0.0217 \\
0.0218 \\
0.0218 \\
0.0241 \\
0.0261 \\
0.0269 \\
0.0266 \\
0.0247 \\
0.0229 \\
0.0224 \\
0.0244 \\
0.0219 \\
0.0191 \\
0.0194 \\
0.0161 \\
0.0127 \\
0.0128 \\
0.0105 \\
0.0090 \\
0.0080 \\
0.0052 \\
0.0027 \\
0.0027 \\
0.0038 \\
0.0041 \\
0.0032\end{array}$ & $\begin{array}{c}\text { Angle } \\
\text { Degrees } \\
38.68 \\
-37.90 \\
-31.88 \\
-23.06 \\
-8.73 \\
-9.41 \\
-6.69 \\
0.13 \\
5.49 \\
6.16 \\
6.74 \\
7.85 \\
8.06 \\
7.51 \\
8.02 \\
8.01 \\
7.67 \\
7.24 \\
7.49 \\
8.32 \\
8.30 \\
8.61 \\
8.71 \\
8.04 \\
8.89 \\
8.82 \\
9.10 \\
10.08 \\
10.58 \\
11.09 \\
9.57 \\
11.03 \\
12.27 \\
10.85 \\
8.99 \\
7.10 \\
10.90 \\
12.34\end{array}$ & $\begin{array}{c}\text { Pressure } \\
\text { psig } \\
9.89 \\
19.55 \\
29.57 \\
39.42 \\
49.16 \\
54.50 \\
59.57 \\
64.20 \\
69.32 \\
74.16 \\
80.16 \\
85.61 \\
90.58 \\
95.69 \\
100.92 \\
103.25 \\
106.11 \\
108.31 \\
111.08 \\
113.24 \\
117.83 \\
120.92 \\
123.28 \\
122.97 \\
125.82 \\
125.60 \\
127.84 \\
130.19 \\
132.53 \\
135.33 \\
134.42 \\
138.35 \\
140.16 \\
142.63 \\
145.78 \\
142.52 \\
0.22\end{array}$ & $\begin{array}{l}\text { Maximum } \\
\text { xStrain } \\
0.0138 \\
0.0272 \\
0.0400 \\
0.0517 \\
0.0626 \\
0.0689 \\
0.0767 \\
0.0839 \\
0.0923 \\
0.1018 \\
0.1147 \\
0.1244 \\
0.1341 \\
0.1470 \\
0.1618 \\
0.1708 \\
0.1799 \\
0.1872 \\
0.1989 \\
0.2091 \\
0.2329 \\
0.2578 \\
0.2857 \\
0.2929 \\
0.3167 \\
0.3288 \\
0.3454 \\
0.3897 \\
0.4555 \\
0.5808 \\
0.6405 \\
0.8298 \\
0.9730 \\
1.1058 \\
1.2253 \\
1.2646 \\
0.9900\end{array}$ & $\begin{array}{l}\text { Minimum } \\
\text { \%Strain } \\
0.0047 \\
0.0096 \\
0.0141 \\
0.0186 \\
0.0232 \\
0.0262 \\
0.0293 \\
0.0319 \\
0.0348 \\
0.0380 \\
0.0402 \\
0.0433 \\
0.0468 \\
0.0508 \\
0.0552 \\
0.0574 \\
0.0601 \\
0.0621 \\
0.0654 \\
0.0682 \\
0.0747 \\
0.0804 \\
0.0856 \\
0.0868 \\
0.0916 \\
0.0940 \\
0.0968 \\
0.1022 \\
0.1085 \\
0.1182 \\
0.1195 \\
0.1339 \\
0.1472 \\
0.1675 \\
0.1867 \\
0.1898 \\
0.1074\end{array}$ & $\begin{array}{r}\text { Angle } \\
\text { Degrees } \\
6.16 \\
6.07 \\
6.56 \\
6.28 \\
6.51 \\
6.25 \\
5.28 \\
5.25 \\
4.72 \\
4.19 \\
4.01 \\
3.74 \\
3.03 \\
3.17 \\
3.05 \\
2.93 \\
2.91 \\
2.94 \\
3.08 \\
3.09 \\
3.53 \\
3.69 \\
3.62 \\
3.57 \\
3.69 \\
3.61 \\
3.75 \\
3.98 \\
3.97 \\
4.37 \\
4.45 \\
3.59 \\
2.45 \\
1.80 \\
1.63 \\
1.69 \\
0.09\end{array}$ \\
\hline
\end{tabular}

High pressure test

\subsection{6}

6.07

6.56

.28

5.28

.25

.72

4.19

4.01

3.74

.03

3.05

2.93

2.91

2.94

3.08

3.09

3.69

3.62

3.57

3.69

3.61

3.75

3.97

4.37

.45

3.59

2.45

1.80

.63

.69

0.09 


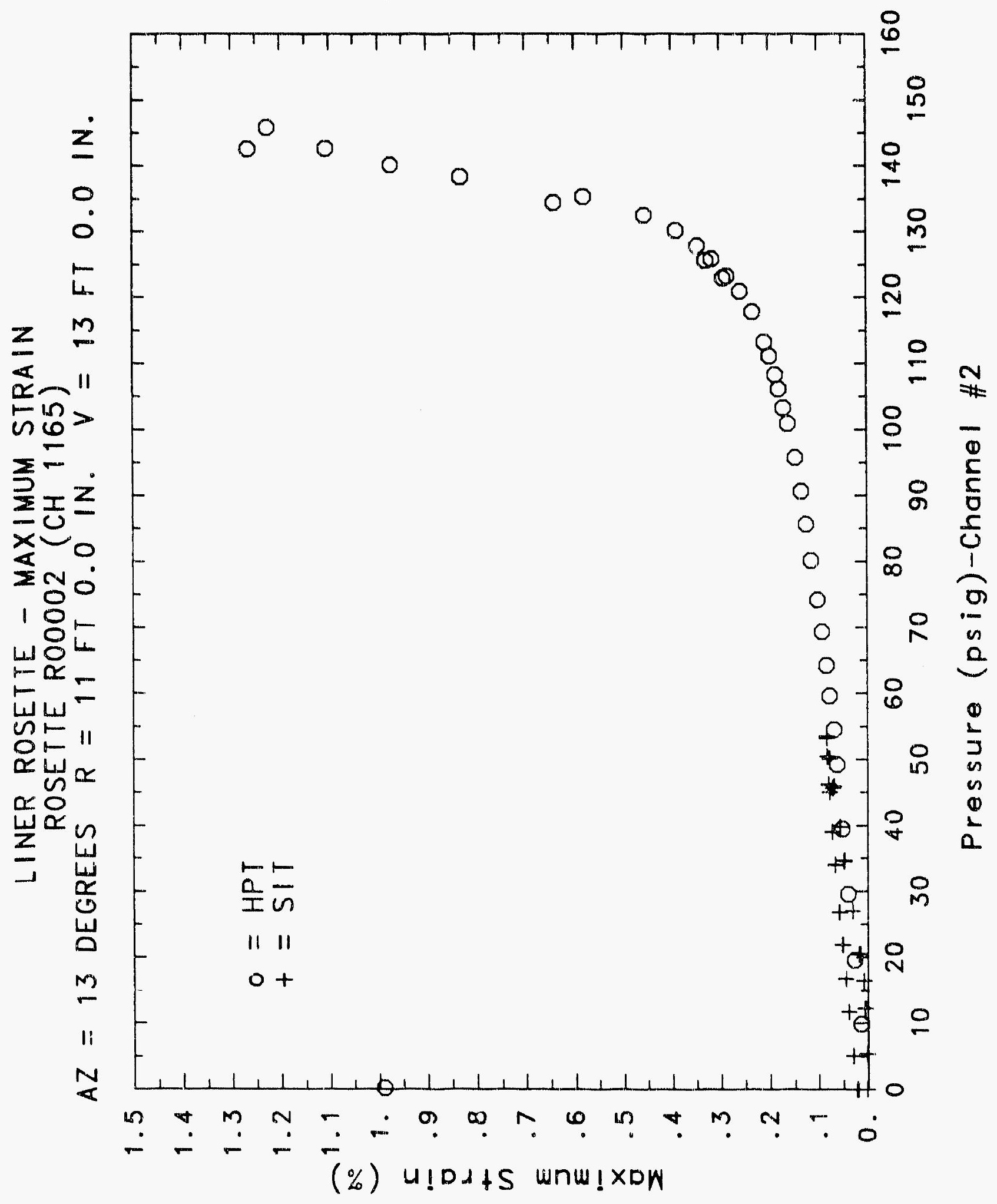




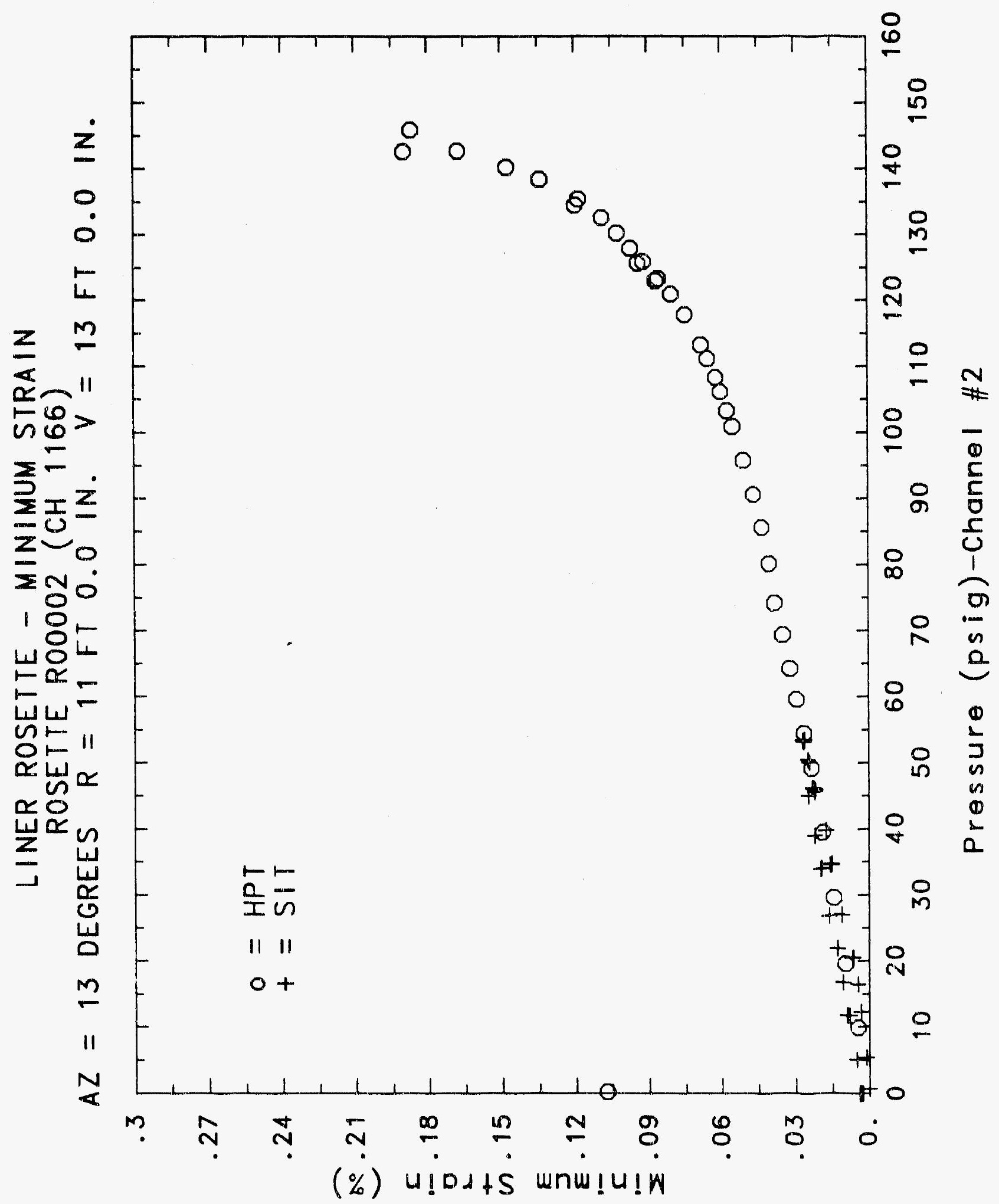

C. 325 


\title{
Reinforced Concrete Test Data
}

\author{
Rosette 3 Channels $1168 \quad 1169 \quad 1180$
}

\section{STRUCTURAL INTEgRITY TEST}

\begin{tabular}{|c|c|c|c|c|c|c|c|}
\hline $\begin{array}{c}\text { Pressure } \\
\text { psig } \\
-0.05 \\
5.33 \\
12.31 \\
16.44 \\
20.51 \\
20.46 \\
20.47 \\
27.05 \\
34.69 \\
34.53 \\
34.53 \\
39.70 \\
45.90 \\
45.65 \\
45.62 \\
50.10 \\
53.47 \\
53.29 \\
53.21 \\
50.49 \\
46.14 \\
46.13 \\
44.97 \\
38.98 \\
33.99 \\
33.96 \\
26.83 \\
21.88 \\
21.90 \\
16.69 \\
11.74 \\
11.77 \\
5.05 \\
0.02 \\
-0.04 \\
-0.02 \\
-0.02 \\
0.02\end{array}$ & $\begin{array}{l}\text { Maximum } \\
\text { \%Strain } \\
0.0005 \\
0.0031 \\
0.0070 \\
0.0096 \\
0.0156 \\
0.0180 \\
0.0189 \\
0.0342 \\
0.0523 \\
0.0536 \\
0.0542 \\
0.0627 \\
0.0758 \\
0.0776 \\
0.0782 \\
0.0850 \\
0.0915 \\
0.0927 \\
0.0932 \\
0.0912 \\
0.0870 \\
0.0867 \\
0.0856 \\
0.0798 \\
0.0736 \\
0.0734 \\
0.0643 \\
0.0577 \\
0.0574 \\
0.0509 \\
0.0441 \\
0.0434 \\
0.0344 \\
0.0268 \\
0.0247 \\
0.0243 \\
0.0238 \\
0.0234\end{array}$ & $\begin{array}{l}\text { Minimum } \\
\text { \%Strain } \\
-0.0002 \\
0.0006 \\
0.0020 \\
0.0029 \\
0.0031 \\
0.0038 \\
0.0040 \\
0.0074 \\
0.0118 \\
0.0118 \\
0.0115 \\
0.0132 \\
0.0154 \\
0.0154 \\
0.0151 \\
0.0163 \\
0.0179 \\
0.0181 \\
0.0172 \\
0.0179 \\
0.0171 \\
0.0171 \\
0.0205 \\
0.0196 \\
0.0180 \\
0.0179 \\
0.0156 \\
0.0141 \\
0.0139 \\
0.0124 \\
0.0105 \\
0.0096 \\
0.0074 \\
0.0063 \\
0.0063 \\
0.0074 \\
0.0076 \\
0.0074\end{array}$ & $\begin{array}{c}\text { Angle } \\
\text { Degrees } \\
34.81 \\
-22.51 \\
-5.75 \\
-0.04 \\
-0.35 \\
2.76 \\
2.20 \\
-1.33 \\
-2.57 \\
-3.17 \\
-3.14 \\
-3.14 \\
-3.56 \\
-2.84 \\
-3.31 \\
-3.58 \\
-3.00 \\
-2.92 \\
-2.94 \\
-2.72 \\
-2.61 \\
-2.99 \\
-2.65 \\
-1.79 \\
-2.19 \\
-1.95 \\
-2.11 \\
-2.14 \\
-2.00 \\
-1.49 \\
-1.74 \\
-2.42 \\
-2.59 \\
-0.84 \\
0.52 \\
2.51 \\
-0.88 \\
-0.26\end{array}$ & $\begin{array}{c}\text { Pressure } \\
\text { Psig } \\
9.89 \\
19.55 \\
29.57 \\
39.42 \\
49.16 \\
54.50 \\
59.57 \\
64.20 \\
69.32 \\
74.16 \\
80.16 \\
85.61 \\
90.58 \\
95.69 \\
100.92 \\
103.25 \\
106.11 \\
108.31 \\
111.08 \\
113.24 \\
117.83 \\
120.92 \\
123.28 \\
122.97 \\
125.82 \\
125.60 \\
127.84 \\
130.19 \\
132.53 \\
135.33 \\
134.42 \\
138.35 \\
140.16 \\
142.63 \\
145.78 \\
142.52 \\
0.22\end{array}$ & $\begin{array}{l}\text { Maximum } \\
\text { XStrain } \\
0.0149 \\
0.0294 \\
0.0436 \\
0.0568 \\
0.0691 \\
0.0764 \\
0.0842 \\
0.0921 \\
0.1010 \\
0.1100 \\
0.1229 \\
0.1316 \\
0.1404 \\
0.1514 \\
0.1628 \\
0.1698 \\
0.1765 \\
0.1818 \\
0.1907 \\
0.1978 \\
0.2139 \\
0.2273 \\
0.2422 \\
0.2456 \\
0.2595 \\
0.2654 \\
0.2746 \\
0.2945 \\
0.3184 \\
0.3583 \\
0.3736 \\
0.4342 \\
0.5096 \\
0.6471 \\
0.8001 \\
0.8547 \\
0.5629\end{array}$ & $\begin{array}{l}\text { Minimum } \\
\text { XStrain } \\
0.0039 \\
0.0075 \\
0.0112 \\
0.0141 \\
0.0165 \\
0.0179 \\
0.0192 \\
0.0208 \\
0.0223 \\
0.0241 \\
0.0246 \\
0.0263 \\
0.0283 \\
0.0308 \\
0.0339 \\
0.0357 \\
0.0373 \\
0.0389 \\
0.0414 \\
0.0435 \\
0.0480 \\
0.0520 \\
0.0554 \\
0.0569 \\
0.0602 \\
0.0622 \\
0.0639 \\
0.0691 \\
0.0738 \\
0.0824 \\
0.0849 \\
0.0958 \\
0.1049 \\
0.1175 \\
0.1315 \\
0.1345 \\
0.0824\end{array}$ & $\begin{array}{c}\text { Angle } \\
\text { Degrees } \\
-3.38 \\
-2.80 \\
-2.29 \\
-2.36 \\
-2.50 \\
-3.07 \\
-2.87 \\
-2.43 \\
-2.47 \\
-3.02 \\
-2.53 \\
-2.60 \\
-2.60 \\
-2.59 \\
-2.37 \\
-2.21 \\
-2.32 \\
-2.29 \\
-2.29 \\
-2.34 \\
-2.82 \\
-3.43 \\
-3.71 \\
-3.80 \\
-4.09 \\
-4.20 \\
-4.44 \\
-4.67 \\
-5.13 \\
-5\end{array}$ \\
\hline
\end{tabular}

\section{High Pressure test}




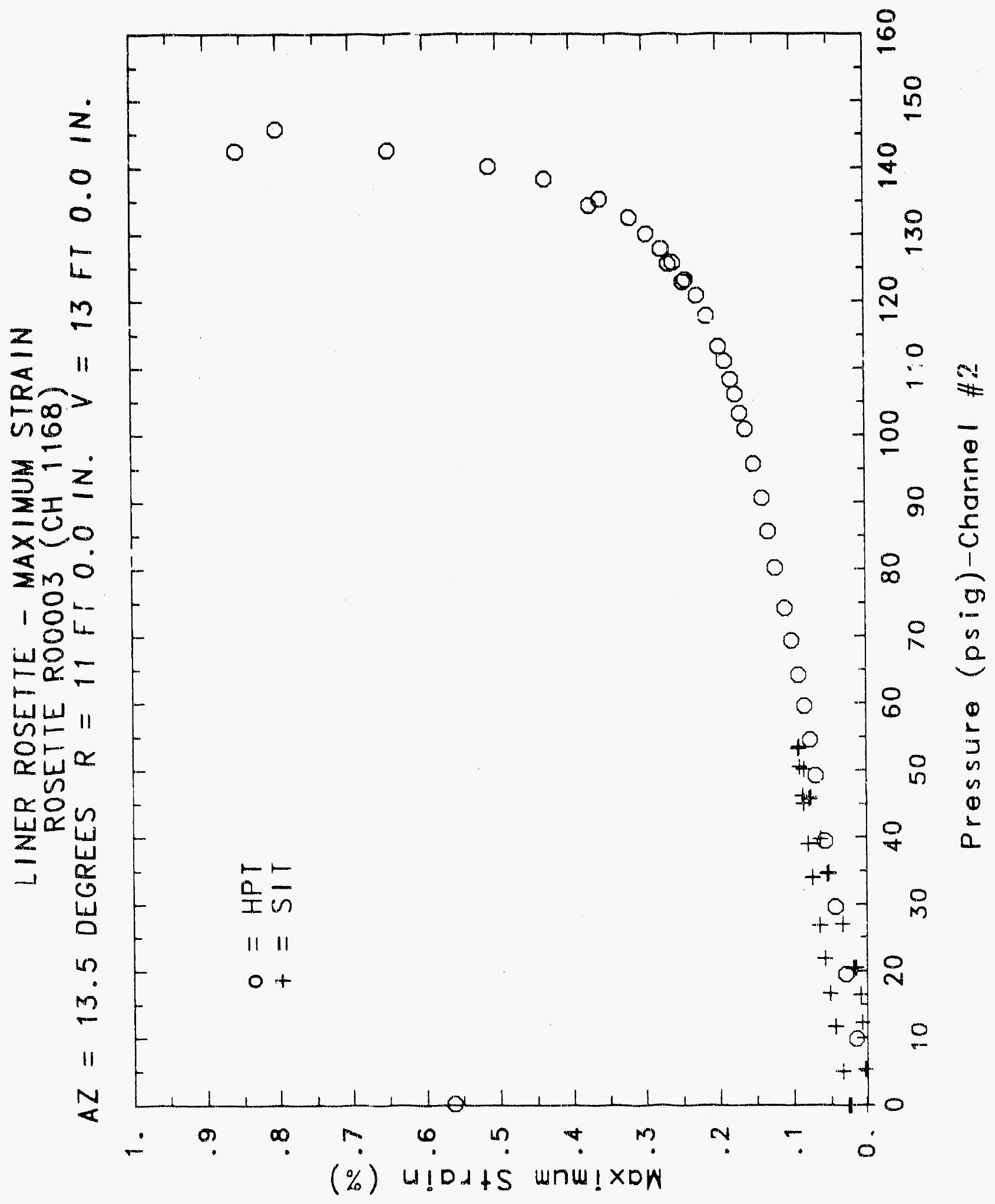




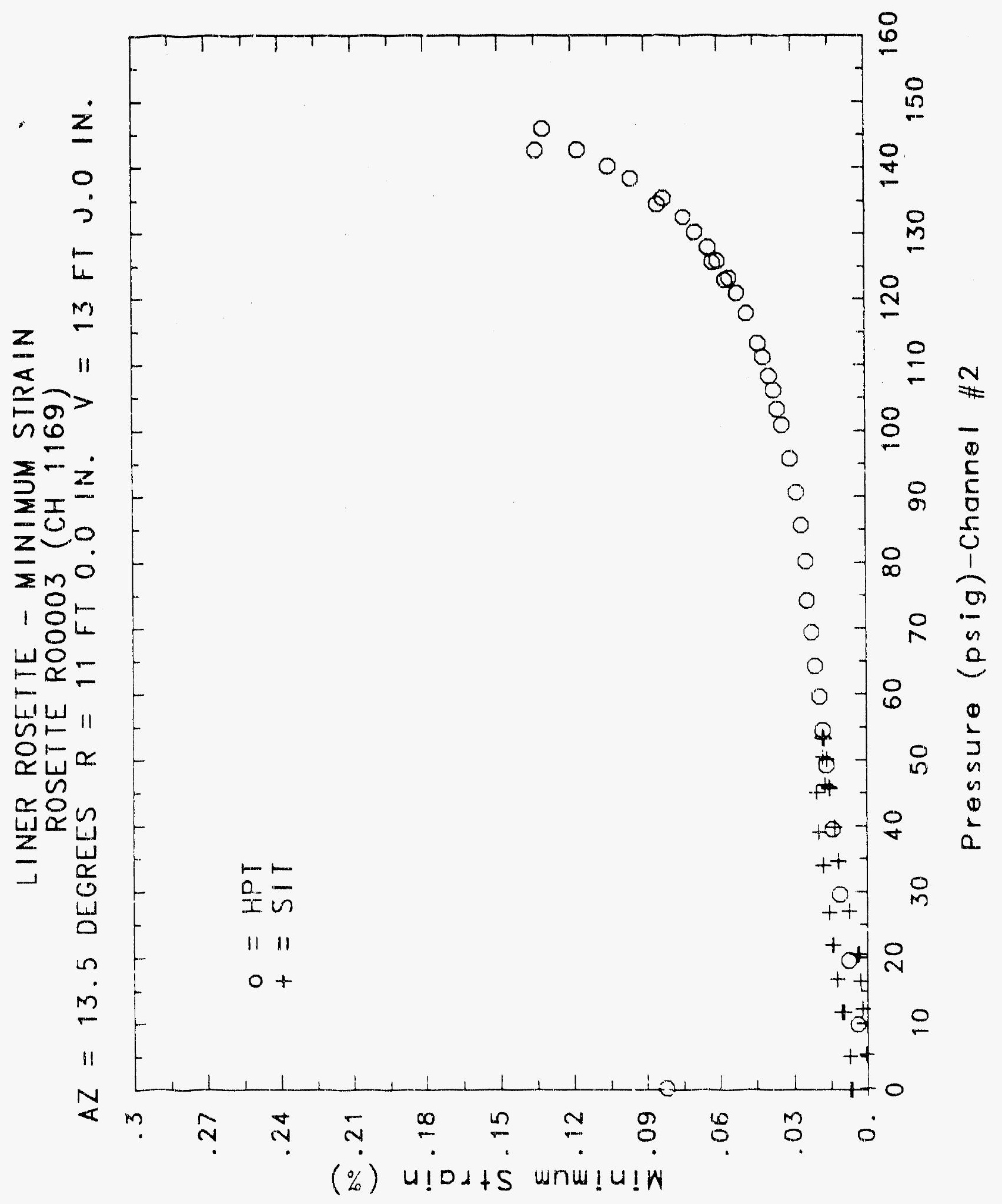




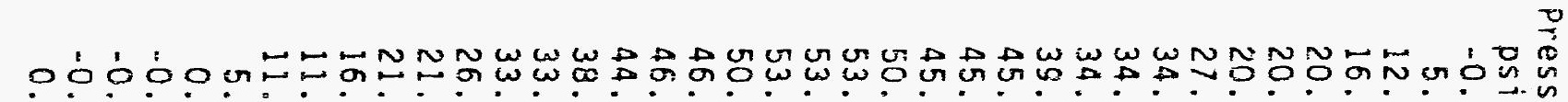

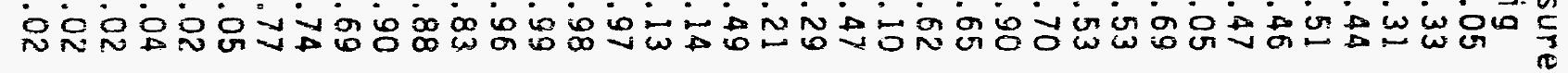
0000000000000000000000000000000000000003 00000000000000000000000000000

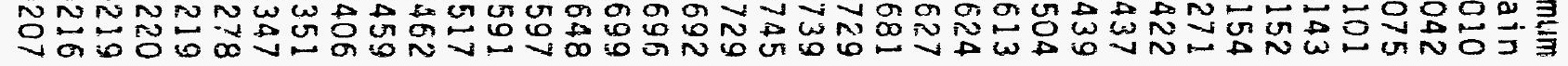

00000000000000000000000000000000000000 至

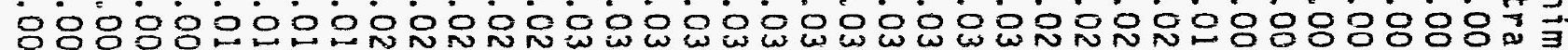

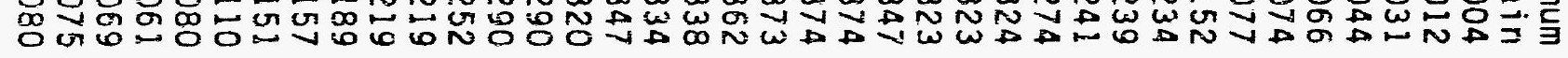

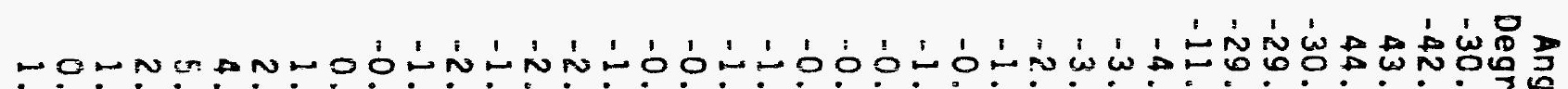

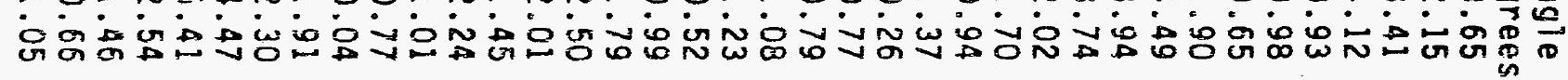

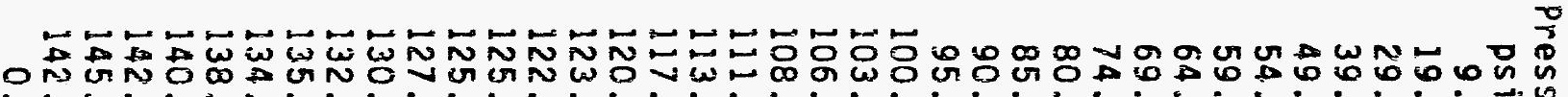

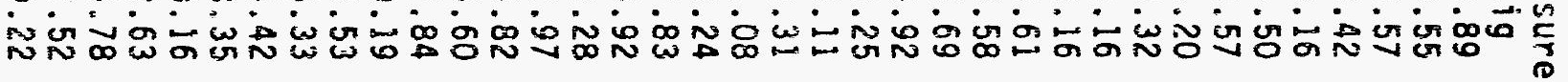
$0000000000000000000000000000000000000 \mathrm{H}$

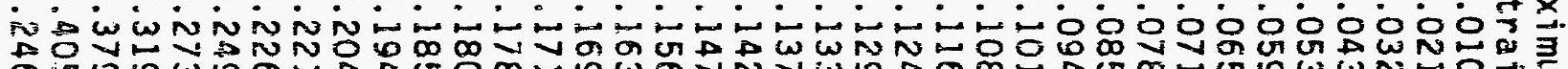

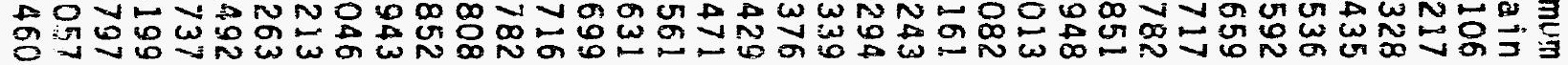

$0000000000000000000000000000000000000 \mathrm{H}^{3}$ సNN

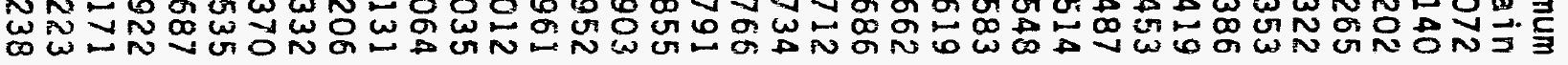

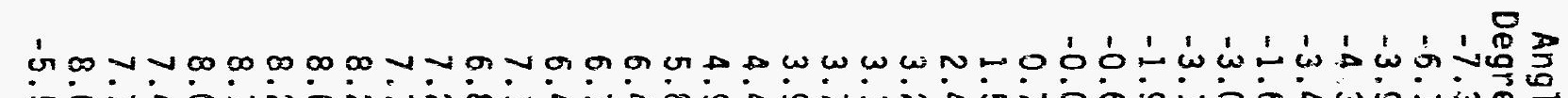

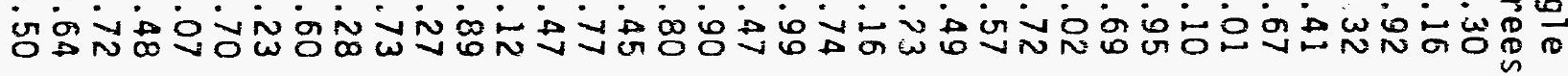




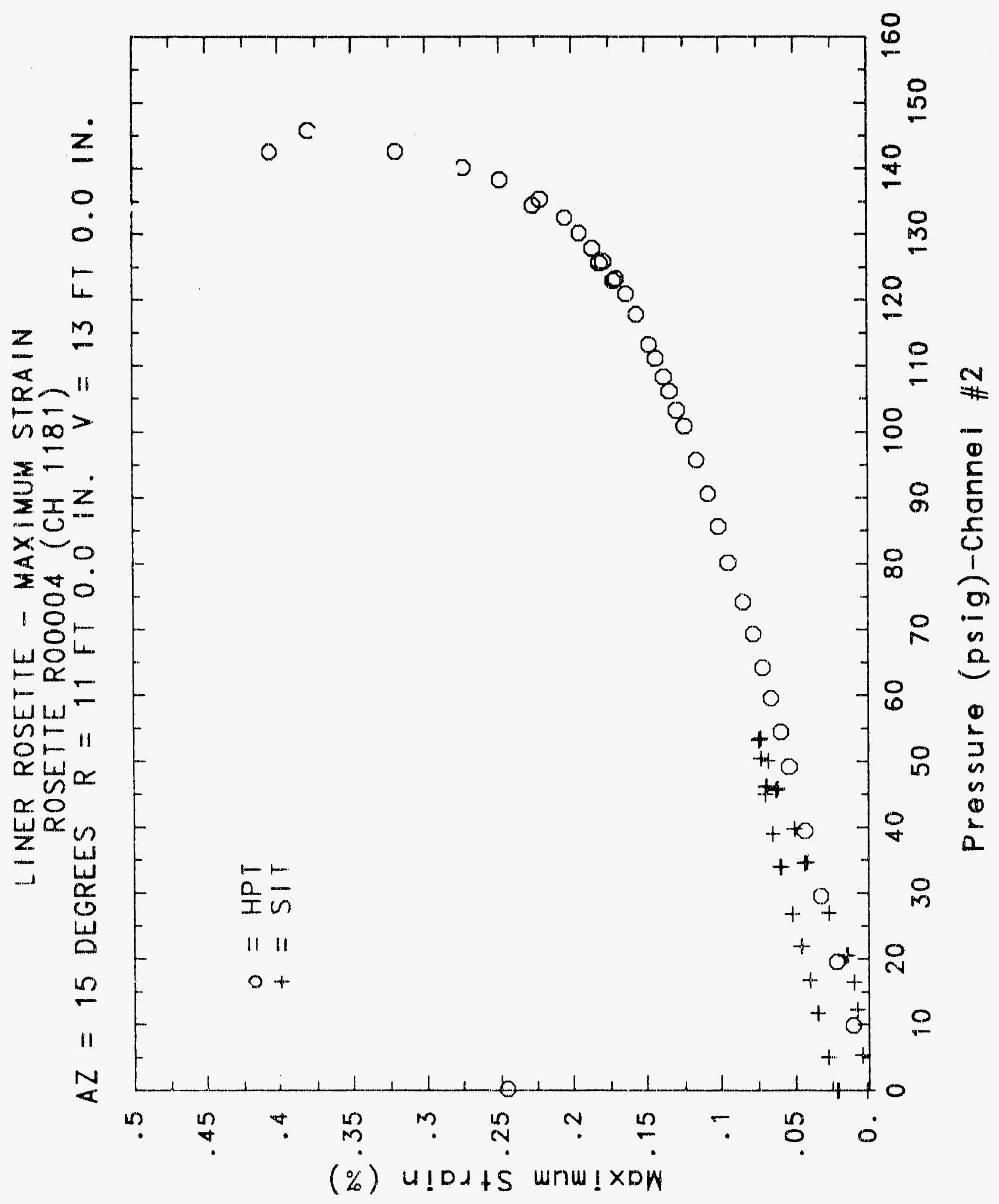




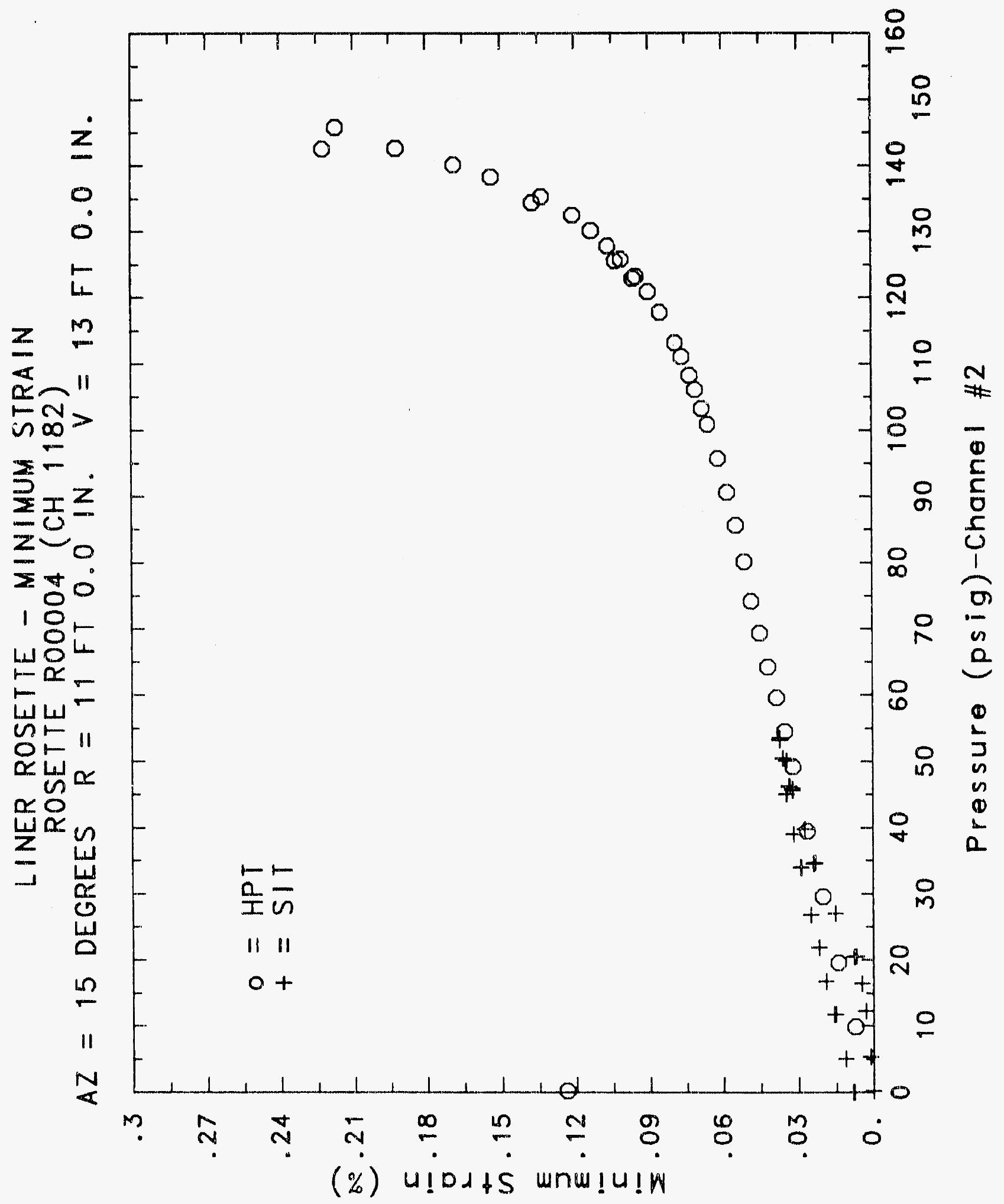




\title{
Reinforced Concrete Test Data
}

\author{
$\begin{array}{llllll}\text { Rosette } & 5 & \text { Channels } & 1184 & 1185 & 1186\end{array}$
}

\section{StRUCTURAL INTEgRITY TEst}

\begin{tabular}{|c|c|c|c|c|c|c|c|}
\hline $\begin{array}{c}\text { Pressure } \\
\text { psig } \\
-0.05 \\
5.33 \\
12.31 \\
16.44 \\
20.51 \\
20.46 \\
20.47 \\
27.05 \\
34.69 \\
34.53 \\
34.53 \\
39.70 \\
45.90 \\
45.65 \\
45.62 \\
50.10 \\
53.47 \\
53.29 \\
53.21 \\
50.49 \\
46.14 \\
46.13 \\
44.97 \\
38.98 \\
33.99 \\
33.96 \\
26.83 \\
21.88 \\
21.90 \\
16.69 \\
11.74 \\
11.77 \\
5.05 \\
0.02 \\
-0.04 \\
-0.02 \\
-0.02 \\
0.02\end{array}$ & $\begin{array}{c}\text { Maximum } \\
\% \text { Strain } \\
0.0002 \\
0.0079 \\
0.0242 \\
0.0353 \\
0.0486 \\
0.0495 \\
0.0500 \\
0.0629 \\
0.0740 \\
0.0748 \\
0.0746 \\
0.0810 \\
0.0908 \\
0.0912 \\
0.0913 \\
0.0979 \\
0.1029 \\
0.1033 \\
0.1032 \\
0.1009 \\
0.0967 \\
0.0966 \\
0.0955 \\
0.0896 \\
0.0845 \\
0.0844 \\
0.0763 \\
0.0711 \\
0.0709 \\
0.0651 \\
0.0563 \\
0.0559 \\
0.0372 \\
0.0225 \\
0.0218 \\
0.0222 \\
0.0221 \\
0.0215\end{array}$ & $\begin{array}{l}\text { Minimum } \\
\% \text { Strain } \\
-0.0008 \\
-0.0009 \\
-0.0066 \\
-0.0076 \\
-0.0053 \\
-0.0050 \\
-0.0048 \\
-0.0001 \\
0.0111 \\
0.0128 \\
0.0133 \\
0.0242 \\
0.0372 \\
0.0383 \\
0.0385 \\
0.0448 \\
0.0505 \\
0.0511 \\
0.0514 \\
0.0489 \\
0.0446 \\
0.0442 \\
0.0437 \\
0.0377 \\
0.0315 \\
0.0314 \\
0.0211 \\
0.0129 \\
0.0128 \\
0.0086 \\
0.0057 \\
0.0053 \\
0.0021 \\
0.0021 \\
0.0011 \\
0.0014 \\
0.0018 \\
0.0021\end{array}$ & $\begin{array}{c}\text { Angle } \\
\text { Degrees } \\
-2.58 \\
-4.19 \\
-1.14 \\
-1.04 \\
-2.19 \\
-1.98 \\
-1.93 \\
-3.39 \\
-5.11 \\
-5.64 \\
-5.43 \\
-9.50 \\
-14.58 \\
-15.26 \\
-15.37 \\
-18.97 \\
-21.04 \\
-21.31 \\
-21.27 \\
-20.24 \\
-18.43 \\
-18.29 \\
-16.52 \\
-14.52 \\
-11.95 \\
-12.07 \\
-7.59 \\
-4.45 \\
-4.21 \\
-2.86 \\
-3.01 \\
-3.30 \\
-5.95 \\
-12.37 \\
-14.71 \\
-13.19 \\
-12.50 \\
-11.85\end{array}$ & $\begin{array}{c}\text { Pressure } \\
\text { psig } \\
9.89 \\
19.55 \\
29.57 \\
39.42 \\
49.16 \\
54.50 \\
59.57 \\
64.20 \\
69.32 \\
74.16 \\
80.16 \\
85.61 \\
90.58 \\
95.69 \\
100.92 \\
103.25 \\
106.11 \\
108.31 \\
111.08 \\
113.24 \\
117.83 \\
120.92 \\
123.28 \\
122.97 \\
125.82 \\
125.60 \\
127.84 \\
130.19 \\
132.53 \\
135.33 \\
134.42 \\
138.35 \\
140.16 \\
142.63 \\
145.78 \\
142.52 \\
0.22\end{array}$ & $\begin{array}{c}\text { Maximum } \\
\text { \%Strain } \\
0.0283 \\
0.0491 \\
0.0599 \\
0.0707 \\
0.0823 \\
0.0890 \\
0.0970 \\
0.1048 \\
0.1141 \\
0.1216 \\
0.1316 \\
0.1399 \\
0.1484 \\
0.1585 \\
0.1693 \\
0.1736 \\
0.1801 \\
0.1849 \\
0.1918 \\
0.1975 \\
0.2098 \\
0.2192 \\
0.2254 \\
0.2267 \\
0.2360 \\
0.2371 \\
0.2431 \\
0.2540 \\
0.2675 \\
0.2852 \\
0.2885 \\
0.3161 \\
0.3508 \\
0.4335 \\
0.5420 \\
0.5703 \\
0.4343\end{array}$ & $\begin{array}{l}\text { Minimum } \\
\text { \%Strain } \\
0.0011 \\
0.0069 \\
0.0203 \\
0.0352 \\
0.0470 \\
0.0531 \\
0.0588 \\
0.0647 \\
0.0707 \\
0.0773 \\
0.0841 \\
0.0911 \\
0.0576 \\
0.1048 \\
0.1130 \\
0.1173 \\
0.1218 \\
0.1253 \\
0.1306 \\
0.1349 \\
0.1450 \\
0.1520 \\
0.1604 \\
0.1621 \\
0.1707 \\
0.1745 \\
0.1805 \\
0.1937 \\
0.2112 \\
0.2433 \\
0.2533 \\
0.2970 \\
0.3294 \\
0.3700 \\
0.4098 \\
0.4139 \\
0.2091\end{array}$ & $\begin{array}{c}\text { Angle } \\
\text { Degrees } \\
-1.74 \\
-2.08 \\
-6.54 \\
-14.42 \\
-21.67 \\
-25.33 \\
-29.30 \\
-32.43 \\
-35.14 \\
-36.82 \\
-39.05 \\
-41.87 \\
-43.84 \\
43.97 \\
42.54 \\
41.87 \\
41.00 \\
40.41 \\
39.86 \\
39.42 \\
37.77 \\
37.08 \\
36.39 \\
36.59 \\
36.08 \\
35.87 \\
35.47 \\
34.78 \\
34.25 \\
31.85 \\
30.07 \\
13.80 \\
-23.13 \\
40.75 \\
32.93 \\
34.33 \\
23.51\end{array}$ \\
\hline
\end{tabular}

\section{High Pressure Test}




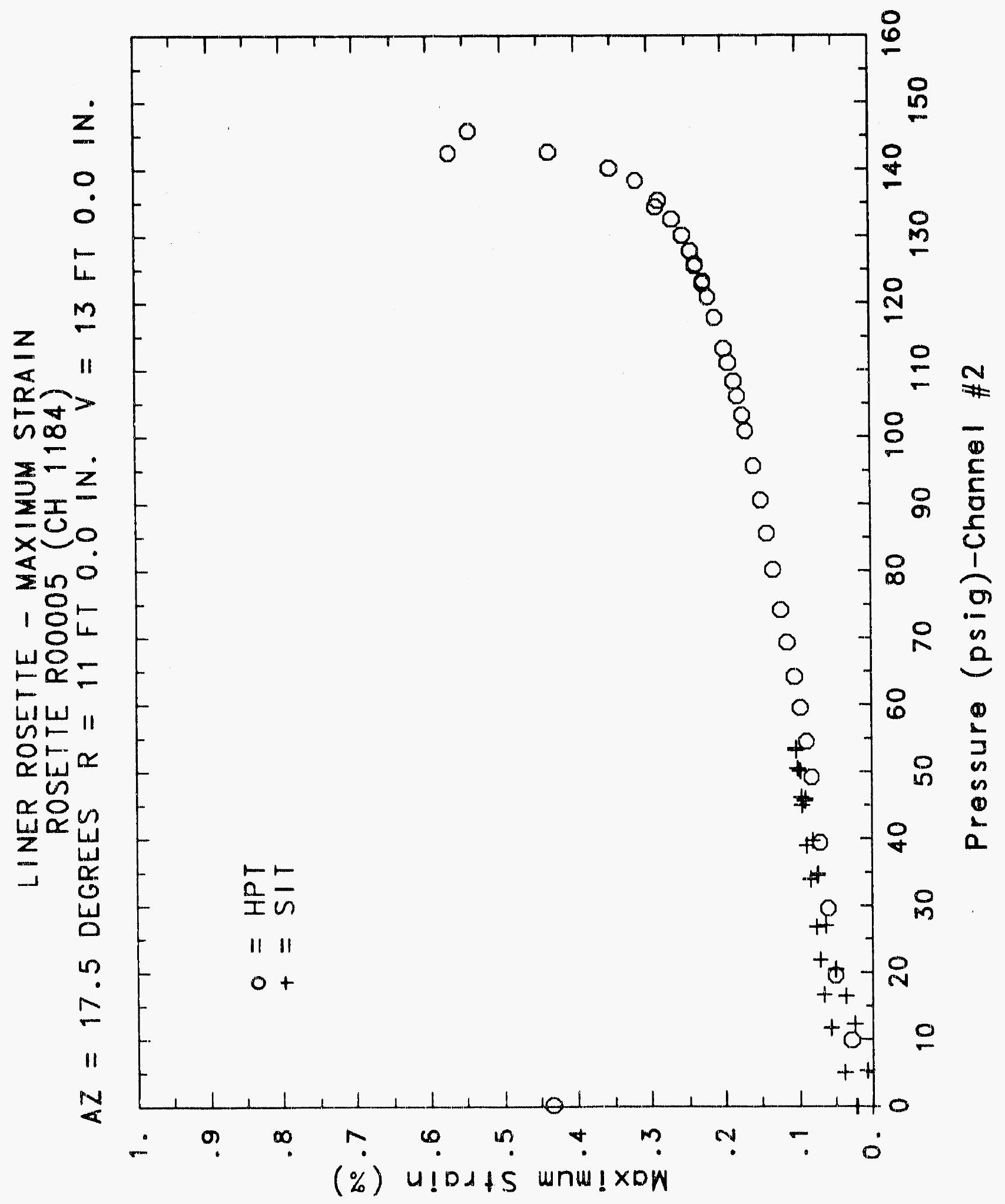




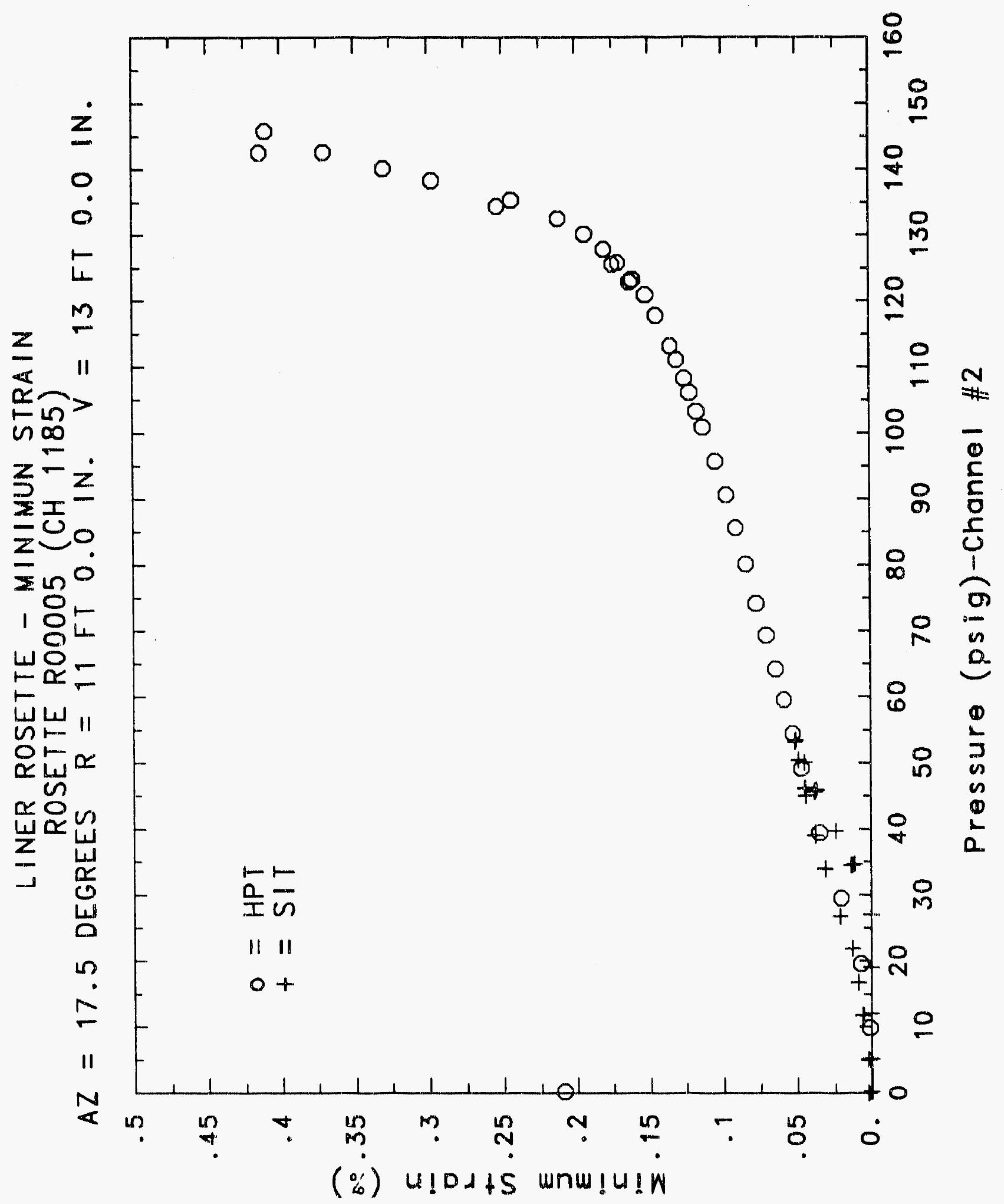




\title{
Reinforced Concrete Test Data
}

\author{
$\begin{array}{llllll}\text { Rosetre } & 6 & \text { Channel.s } & 1187 & 1188 & 1189\end{array}$
}

\section{STRUCTURAL. INTEGRITY TEST}

\begin{tabular}{|c|c|c|c|c|c|c|c|}
\hline 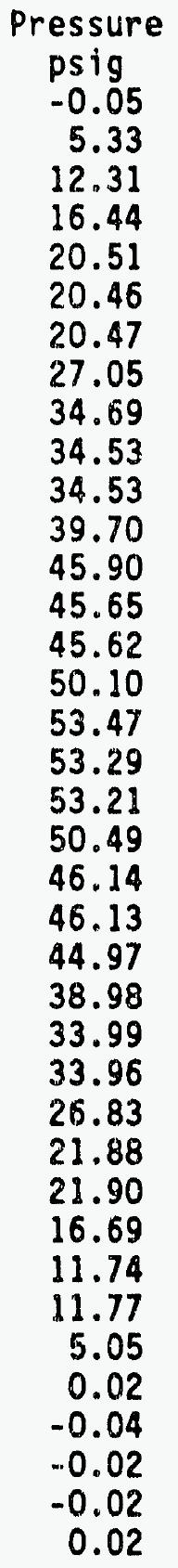 & $\begin{array}{l}\text { Maximum } \\
\text { XStrain } \\
0.0004 \\
0.0048 \\
0.0112 \\
0.0144 \\
0.0190 \\
0.0202 \\
0.0211 \\
0.0297 \\
0.0414 \\
0.0420 \\
0.0422 \\
0.0483 \\
0.0575 \\
0.0575 \\
0.0575 \\
0.0628 \\
0.0675 \\
0.0678 \\
0.0679 \\
0.0659 \\
0.0623 \\
0.0621 \\
0.0630 \\
0.0576 \\
0.0529 \\
0.0528 \\
0.0459 \\
0.0409 \\
0.0408 \\
0.0356 \\
0.0304 \\
0.0297 \\
0.0227 \\
0.0169 \\
0.0149 \\
0.0158 \\
0.0158 \\
0.0156\end{array}$ & $\begin{array}{l}\text { Minimum } \\
\text { \%Strain } \\
-0.0006 \\
-0.0015 \\
-0.0006 \\
0.0011 \\
0.0035 \\
0.0039 \\
0.0041 \\
0.0085 \\
0.0140 \\
0.0144 \\
0.0144 \\
0.0176 \\
0.0218 \\
0.0212 \\
0.0215 \\
0.0241 \\
0.0262 \\
0.0263 \\
0.0260 \\
0.0255 \\
0.0238 \\
0.0235 \\
0.0240 \\
0.0216 \\
0.0192 \\
0.0190 \\
0.0150 \\
0.0127 \\
0.0126 \\
0.0099 \\
0.0073 \\
0.0069 \\
0.0042 \\
0.0049 \\
0.0048 \\
0.0053 \\
0.0055 \\
0.0054\end{array}$ & $\begin{array}{c}\text { Angle } \\
\text { Degrees } \\
38.09 \\
-11.49 \\
-14.77 \\
-10.48 \\
-7.99 \\
-7.77 \\
-7.96 \\
-2.71 \\
1.27 \\
2.18 \\
2.25 \\
3.29 \\
5.44 \\
5.77 \\
5.79 \\
5.64 \\
6.68 \\
6.91 \\
6.96 \\
6.24 \\
5.27 \\
5.16 \\
5.84 \\
5\end{array}$ & $\begin{array}{c}\text { Pressure } \\
\text { psig } \\
9.89 \\
19.55 \\
29.57 \\
39.42 \\
49.16 \\
54.50 \\
59.57 \\
64.20 \\
69.32 \\
74.16 \\
80.16 \\
85.61 \\
90.58 \\
95.69 \\
100.92 \\
103.25 \\
106.11 \\
108.31 \\
111.08 \\
113.24 \\
117.83 \\
120.92 \\
123.28 \\
122.97 \\
125.82 \\
125.60 \\
127.84 \\
130.19 \\
132.53 \\
135.33 \\
134.42 \\
138.35 \\
140.16 \\
142.63 \\
145.78 \\
142.52 \\
0.22\end{array}$ & $\begin{array}{l}\text { Maximum } \\
\text { XStrain } \\
0.0130 \\
0.0239 \\
0.0349 \\
0.0453 \\
0.0553 \\
0.0606 \\
0.0669 \\
0.0734 \\
0.0803 \\
0.0874 \\
0.0941 \\
0.1016 \\
0.1090 \\
0.1173 \\
0.1267 \\
0.1316 \\
0.1372 \\
0.1413 \\
0.1477 \\
0.1525 \\
0.1636 \\
0.1717 \\
0.1796 \\
0.1813 \\
0.1899 \\
0.1930 \\
0.1992 \\
0.2125 \\
0.2307 \\
0.2644 \\
0.2747 \\
0.3407 \\
0.4233 \\
0.6267 \\
0.8195 \\
0.8389 \\
0.6735\end{array}$ & $\begin{array}{l}\text { Minimum } \\
\text { xStrain } \\
0.0011 \\
0.0068 \\
0.0126 \\
0.0179 \\
0.0228 \\
0.0256 \\
0.0290 \\
0.0317 \\
0.0350 \\
0.0386 \\
0.0414 \\
0.0449 \\
0.0484 \\
0.0517 \\
0.0556 \\
0.0574 \\
0.0597 \\
0.0614 \\
0.0637 \\
0.0655 \\
0.0696 \\
0.0722 \\
0.0744 \\
0.0746 \\
0.0778 \\
0.0782 \\
0.0803 \\
0.0835 \\
0.0874 \\
0.0942 \\
0.0953 \\
0.1061 \\
0.1164 \\
0.1386 \\
0.1558 \\
0.1572 \\
0.0809\end{array}$ & $\begin{array}{c}\text { Angle } \\
\text { Degrees } \\
-8.62 \\
-1.51 \\
2.37 \\
5.30 \\
7.81 \\
9.18 \\
8.88 \\
9.46 \\
10.12 \\
11.27 \\
12.22 \\
13.01 \\
13.90 \\
14.86 \\
15.72 \\
16.03 \\
16.37 \\
16.75 \\
17.14 \\
17.47 \\
18.41 \\
19.20 \\
19.76 \\
20.03 \\
20.46 \\
20.77 \\
21.01 \\
21.47 \\
22.01 \\
22.40 \\
22.42 \\
21.89 \\
20.48 \\
17.30 \\
15.30 \\
15.50 \\
17.14\end{array}$ \\
\hline
\end{tabular}

\section{High Pressure test}




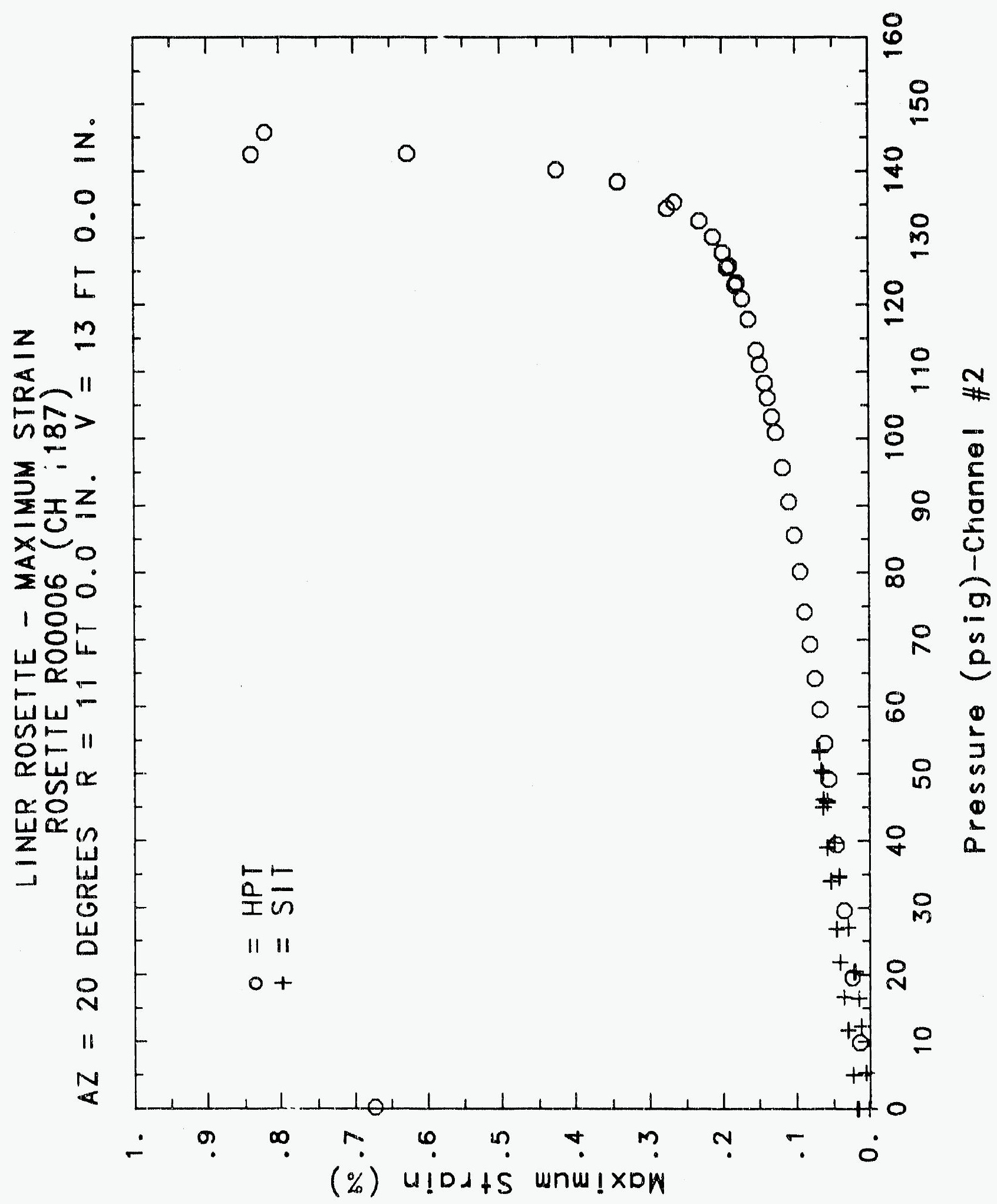




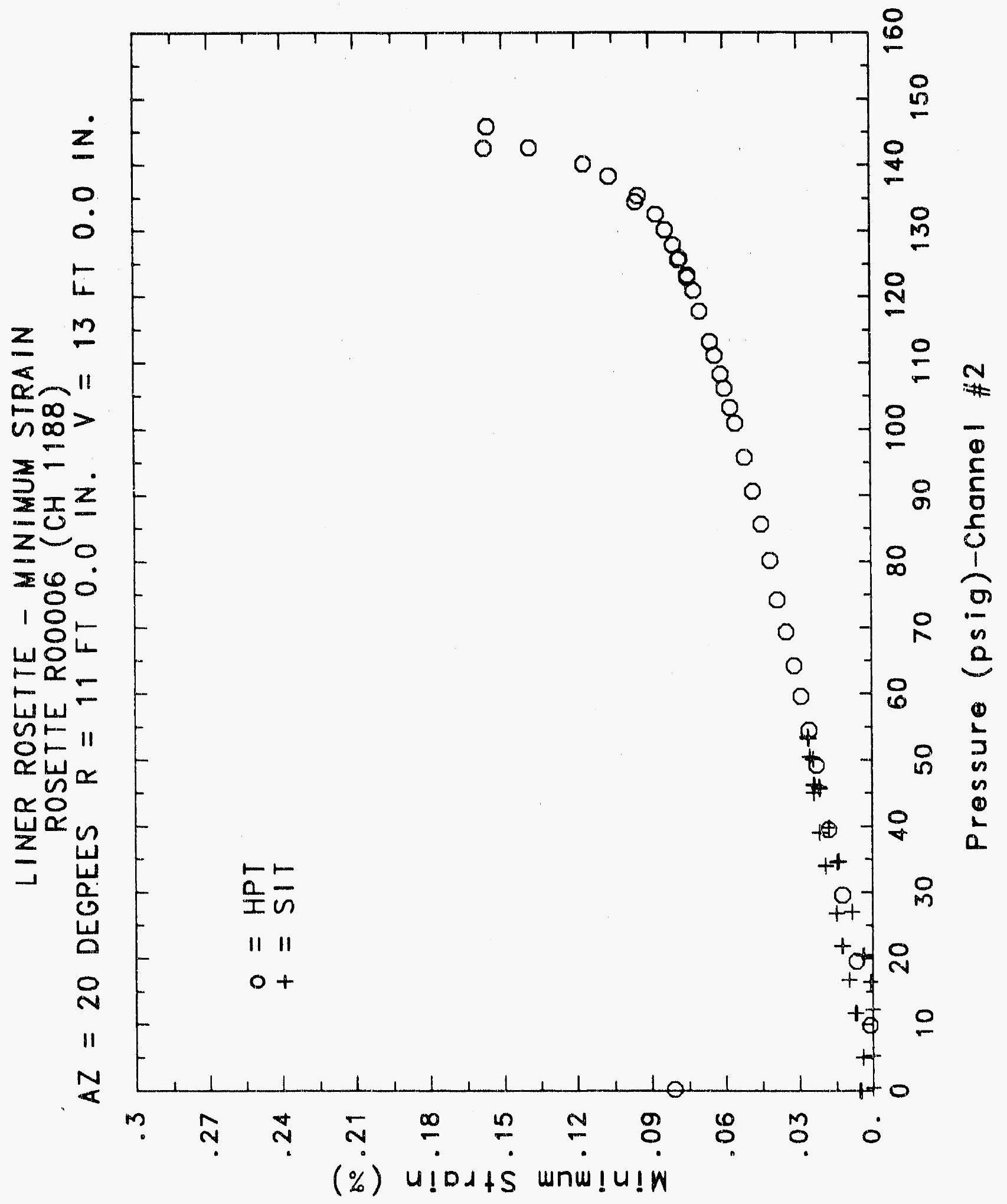




\title{
Reinforced Concrete Test Data
}

\author{
Rosette 7 Channels $12001201 \quad 1202$
}

STRUCTURAL INTEGRITY TEST

\begin{tabular}{|c|c|c|c|c|c|c|c|}
\hline $\begin{array}{c}\text { Pressure } \\
\text { psig } \\
-0.05 \\
5.33 \\
12.31 \\
16.44 \\
20.51 \\
20.46 \\
20.47 \\
27.05 \\
34.69 \\
34.53 \\
34.53 \\
39.70 \\
45.90 \\
45.65 \\
45.62 \\
50.10 \\
53.47 \\
53.29 \\
53.21 \\
50.49 \\
46.14 \\
46.13 \\
44.97 \\
38.98 \\
33.99 \\
33.96 \\
26.83 \\
21.88 \\
21.90 \\
16.69 \\
11.74 \\
11.77 \\
5.05 \\
0.02 \\
-0.04 \\
-0.02 \\
-0.02 \\
0.02\end{array}$ & $\begin{array}{c}\text { Maximum } \\
\text { xStrain } \\
0.0009 \\
0.0045 \\
0.0123 \\
0.0174 \\
0.0235 \\
0.0253 \\
0.0256 \\
0.0399 \\
0.0593 \\
0.0595 \\
0.0599 \\
0.0689 \\
0.0861 \\
0.0880 \\
0.0890 \\
0.0993 \\
0.1107 \\
0.1118 \\
0.1123 \\
0.1120 \\
0.1070 \\
0.1078 \\
0.1078 \\
0.1007 \\
0.0937 \\
0.0931 \\
0.0835 \\
0.0742 \\
0.0744 \\
0.0660 \\
0.0561 \\
0.0554 \\
0.0425 \\
0.0339 \\
0.0332 \\
0.0340 \\
0.0327 \\
0.0328\end{array}$ & $\begin{array}{l}\text { Minimum } \\
\text { XStrain } \\
-0.0009 \\
0.0032 \\
0.0078 \\
0.0110 \\
0.0161 \\
0.0167 \\
0.0171 \\
0.0304 \\
0.0455 \\
0.0470 \\
0.0470 \\
0.0542 \\
0.0632 \\
0.0646 \\
0.0647 \\
0.0706 \\
0.0759 \\
0.0775 \\
0.0781 \\
0.0763 \\
0.0728 \\
0.0723 \\
0.0708 \\
0.0651 \\
0.0597 \\
0.0595 \\
0.0505 \\
0.0445 \\
0.0444 \\
0.0367 \\
0.0299 \\
0.0296 \\
0.0199 \\
0.0122 \\
0.0109 \\
0.0097 \\
0.0097 \\
0.0093\end{array}$ & $\begin{array}{c}\text { Angle } \\
\text { Degrees } \\
-37.53 \\
-23.41 \\
-32.57 \\
-34.54 \\
-38.58 \\
-41.16 \\
-41.00 \\
29.86 \\
36.82 \\
36.24 \\
35.68 \\
40.53 \\
44.53 \\
43.29 \\
43.06 \\
44.08 \\
43.99 \\
43.41 \\
42.95 \\
42.53 \\
42.42 \\
42.27 \\
45.00 \\
44.66 \\
-44.93 \\
-44.46 \\
-43.94 \\
-42.68 \\
-42.86 \\
-41.73 \\
-40.45 \\
-41.20 \\
-39.89 \\
-38.93 \\
-38.07 \\
-36.04 \\
-35.24 \\
-35.14\end{array}$ & $\begin{array}{c}\text { Pressure } \\
\text { psig } \\
9.89 \\
19.55 \\
29.57 \\
39.42 \\
49.16 \\
54.50 \\
59.57 \\
64.20 \\
69.32 \\
74.16 \\
80.16 \\
85.61 \\
90.58 \\
95.69 \\
100.92 \\
103.25 \\
106.11 \\
108.31 \\
111.08 \\
113.24 \\
117.83 \\
120.92 \\
123.28 \\
122.97 \\
125.82 \\
125.60 \\
127.84 \\
130.19 \\
132.53 \\
135.33 \\
134.42 \\
138.35 \\
140.16 \\
142.63 \\
145.78 \\
142.52 \\
0.22\end{array}$ & $\begin{array}{l}\text { Maximum } \\
\text { xStrain } \\
0.0150 \\
0.0325 \\
0.0484 \\
0.0643 \\
0.0805 \\
0.0898 \\
0.1042 \\
0.1223 \\
0.1440 \\
0.1681 \\
0.2157 \\
0.2613 \\
0.3123 \\
0.3956 \\
0.4970 \\
0.5392 \\
0.5793 \\
0.6102 \\
0.6623 \\
0.6965 \\
0.7745 \\
0.8340 \\
0.8690 \\
0.8721 \\
0.9038 \\
0.9064 \\
0.9343 \\
0.9655 \\
1.0108 \\
1.0700 \\
1.0743 \\
1.1570 \\
1.2084 \\
1.2643 \\
1.3414 \\
1.3497 \\
1.0435\end{array}$ & $\begin{array}{l}\text { Minimum } \\
\text { xStrain } \\
0.0120 \\
0.0246 \\
0.0386 \\
0.0518 \\
0.0644 \\
0.0718 \\
0.0809 \\
0.0920 \\
0.1043 \\
0.1203 \\
0.1456 \\
0.1683 \\
0.1887 \\
0.207 \\
0.2260 \\
0.2336 \\
0.2425 \\
0.2496 \\
0.2600 \\
0.2677 \\
0.2854 \\
0.2994 \\
0.3078 \\
0.3085 \\
0.3193 \\
0.3200 \\
0.3293 \\
0.3413 \\
0.3589 \\
0.3728 \\
0.3721 \\
0.3867 \\
0.3947 \\
0.4047 \\
0.4141 \\
0.4143 \\
0.2993\end{array}$ & $\begin{array}{c}\text { Angle } \\
\text { Degrees } \\
-38.45 \\
-44.00 \\
37.64 \\
33.31 \\
29.65 \\
26.77 \\
28.50 \\
30.36 \\
31.35 \\
29.69 \\
23.35 \\
17.02 \\
11.37 \\
8.57 \\
8.12 \\
7.78 \\
7.97 \\
8.10 \\
8.23 \\
8.27 \\
8.33 \\
8.12 \\
7.91 \\
7.98 \\
7.67 \\
7.68 \\
7.23 \\
6.68 \\
5.73 \\
4.55 \\
4.49 \\
3.56 \\
3.00\end{array}$ \\
\hline
\end{tabular}

high Pressure Test 


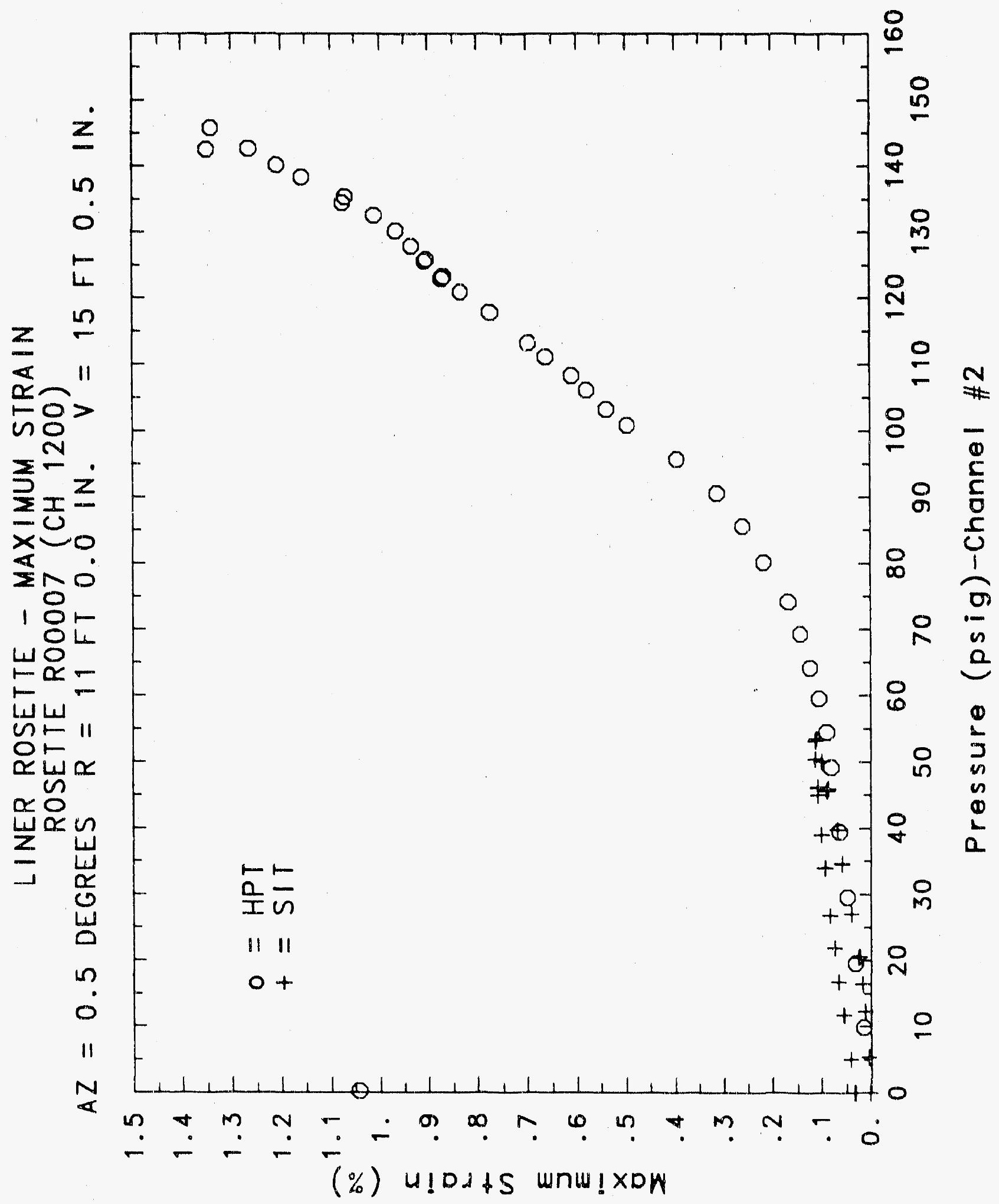




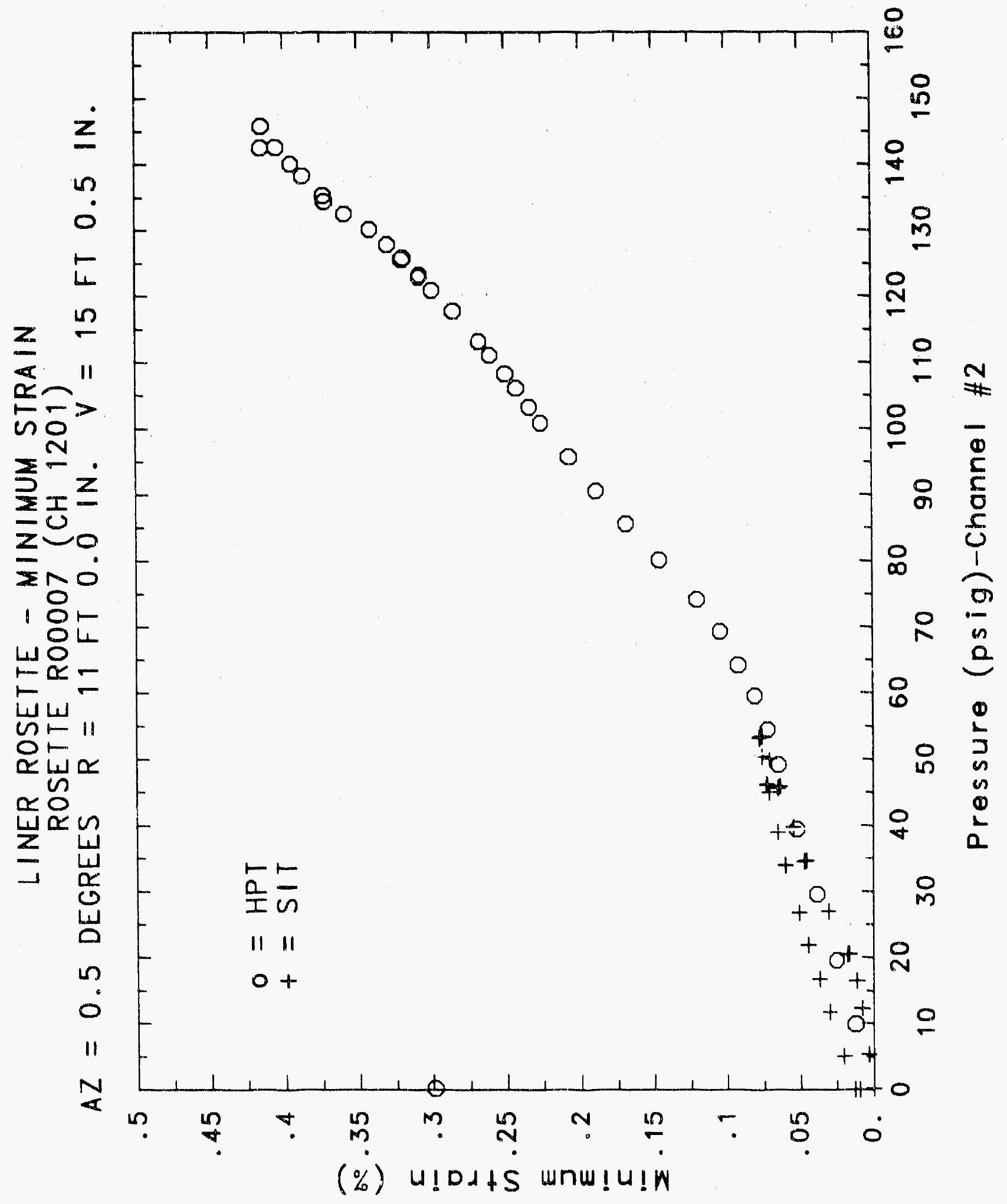




\title{
Reinforced Concrete Test Data
}

\author{
Rosette 8 Channels $1203 \quad 1204 \quad 1205$
}

\section{Structural InTEgrity TEST}

\begin{tabular}{|c|c|c|c|c|c|c|c|}
\hline $\begin{array}{c}\text { Pressure } \\
\text { psig } \\
-0.05 \\
5.33 \\
12.31 \\
16.44 \\
20.51 \\
20.46 \\
20.47 \\
27.05 \\
34.59 \\
34.53 \\
34.53 \\
39.70 \\
45.90 \\
45.65 \\
45.62 \\
50.10 \\
53.47 \\
53.29 \\
53.21 \\
50.49 \\
46.14 \\
46.13 \\
44.97 \\
38.98 \\
33.99 \\
33.96 \\
26.83 \\
21.88 \\
21.90 \\
16.69 \\
11.74 \\
11.77 \\
5.05 \\
0.02 \\
-0.04 \\
-0.02 \\
-0.02 \\
0.02\end{array}$ & $\begin{array}{l}\text { Maximum } \\
\text { \%Strain } \\
0.0005 \\
0.0042 \\
0.0147 \\
0.0305 \\
0.0508 \\
0.0535 \\
0.0455 \\
0.0614 \\
0.0883 \\
0.0900 \\
0.0904 \\
0.1015 \\
0.1130 \\
0.1021 \\
0.1027 \\
0.1159 \\
0.1248 \\
0.1387 \\
0.1385 \\
0.1352 \\
0.1282 \\
0.1280 \\
0.1272 \\
0.1233 \\
0.1158 \\
0.1130 \\
0.1017 \\
0.0934 \\
0.0940 \\
0.0851 \\
0.0776 \\
0.0677 \\
0.0552 \\
0.0490 \\
0.0538 \\
0.0533 \\
0.0530 \\
0.0549\end{array}$ & $\begin{array}{r}\text { Minimum } \\
\text { \%Strain } \\
-0.0004 \\
0.0011 \\
-0.0038 \\
-0.0141 \\
-0.0256 \\
-0.0265 \\
-0.0183 \\
-0.0118 \\
-0.0091 \\
-0.0071 \\
-0.0067 \\
-0.0014 \\
0.0122 \\
0.0259 \\
0.0266 \\
0.0279 \\
0.0336 \\
0.0224 \\
0.0229 \\
0.0234 \\
0.0241 \\
0.0245 \\
0.0276 \\
0.0191 \\
0.0166 \\
0.0200 \\
0.0147 \\
0.01121 \\
0.0115 \\
0.0071 \\
0.0015 \\
0.0114 \\
0.0077 \\
0.0047 \\
-0.0008 \\
0.0004 \\
-0.0002 \\
-0.0015\end{array}$ & $\begin{array}{c}\text { Angle } \\
\text { Degrees } \\
\text {-6.92 } \\
27.20 \\
39.53 \\
42.05 \\
43.42 \\
43.74 \\
43.51 \\
44.74 \\
44.92 \\
45.00 \\
-44.94 \\
45.00 \\
44.24 \\
44.17 \\
44.19 \\
43.79 \\
42.97 \\
43.41 \\
43.47 \\
43.76 \\
43.70 \\
43.80 \\
43.17 \\
43.51 \\
43.89 \\
43.72 \\
44.18 \\
44.27 \\
44.46 \\
44.55 \\
44.53 \\
44.81 \\
-44.79 \\
-44.73 \\
-43.94 \\
-44.47 \\
-44.86 \\
-44.75\end{array}$ & $\begin{array}{c}\text { Pressure } \\
\text { psig } \\
9.89 \\
19.55 \\
29.57 \\
39.42 \\
49.16 \\
54.50 \\
59.57 \\
64.20 \\
69.32 \\
74.16 \\
80.16 \\
85.61 \\
90.58 \\
95.69 \\
100.92 \\
103.25 \\
106.11 \\
108.31 \\
111.08 \\
113.24 \\
117.83 \\
120.92 \\
123.28 \\
122.97 \\
125.82 \\
125.60 \\
127.84 \\
130.19 \\
132.53 \\
135.33 \\
134.42 \\
138.35 \\
140.16 \\
142.63 \\
145.78 \\
142.52 \\
0.22\end{array}$ & $\begin{array}{l}\text { Maximum } \\
\text { xStrain } \\
0.0194 \\
0.0373 \\
0.0556 \\
0.0737 \\
0.0922 \\
0.1052 \\
0.1192 \\
0.1362 \\
0.1533 \\
0.1732 \\
0.1927 \\
0.2149 \\
0.2341 \\
0.2533 \\
0.2733 \\
0.2819 \\
0.2932 \\
0.3024 \\
0.3170 \\
0.3287 \\
0.3617 \\
0.3888 \\
0.4088 \\
0.4108 \\
0.4327 \\
0.4352 \\
0.4526 \\
0.4755 \\
0.5047 \\
0.5432 \\
0.5469 \\
0.5990 \\
0.6379 \\
0.6929 \\
0.7637 \\
0.7788 \\
0.5717\end{array}$ & $\begin{array}{l}\text { Minimum } \\
\text { XStrain } \\
0.0002 \\
0.0042 \\
0.0105 \\
0.0168 \\
0.0238 \\
0.0268 \\
0.0331 \\
0.0423 \\
0.0539 \\
0.0665 \\
0.0983 \\
0.1191 \\
0.1396 \\
0.1623 \\
0.1888 \\
0.2035 \\
0.2182 \\
0.2279 \\
0.2433 \\
0.2538 \\
0.2730 \\
0.2855 \\
0.2941 \\
0.2958 \\
0.3043 \\
0.3060 \\
0.3129 \\
0.3226 \\
0.3349 \\
0.3494 \\
0.3506 \\
0.3683 \\
0.3791 \\
0.3975 \\
0.4202 \\
0.4268 \\
0.1785\end{array}$ & $\begin{array}{c}\text { Angle } \\
\text { Degrees } \\
40.37 \\
41.02 \\
41.89 \\
42.60 \\
42.87 \\
43.00 \\
42.33 \\
41.32 \\
40.21 \\
39.15 \\
38.28 \\
39.08 \\
40.66 \\
43.06 \\
-43.00 \\
-40.12 \\
-36.13 \\
-33.40 \\
-27.25 \\
-23.08 \\
-14.67 \\
-9.45 \\
-6.18 \\
-4.72 \\
-3.79 \\
-2.40 \\
-2.48 \\
-1.54 \\
-0.72 \\
0.28 \\
0.06 \\
0.54 \\
1.17 \\
1.72 \\
2.20 \\
3.13 \\
21.45\end{array}$ \\
\hline
\end{tabular}

\author{
high Pressure test
}




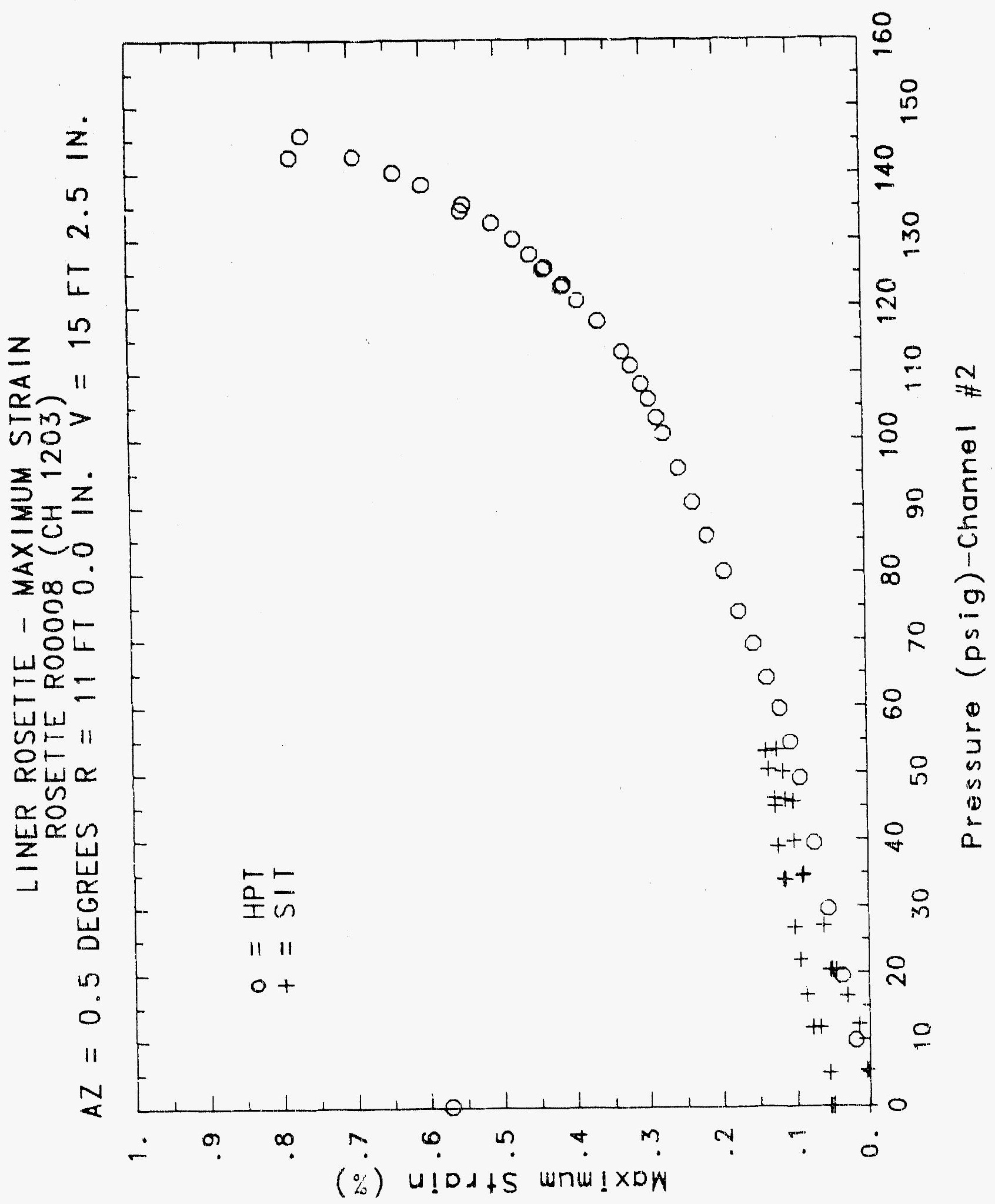




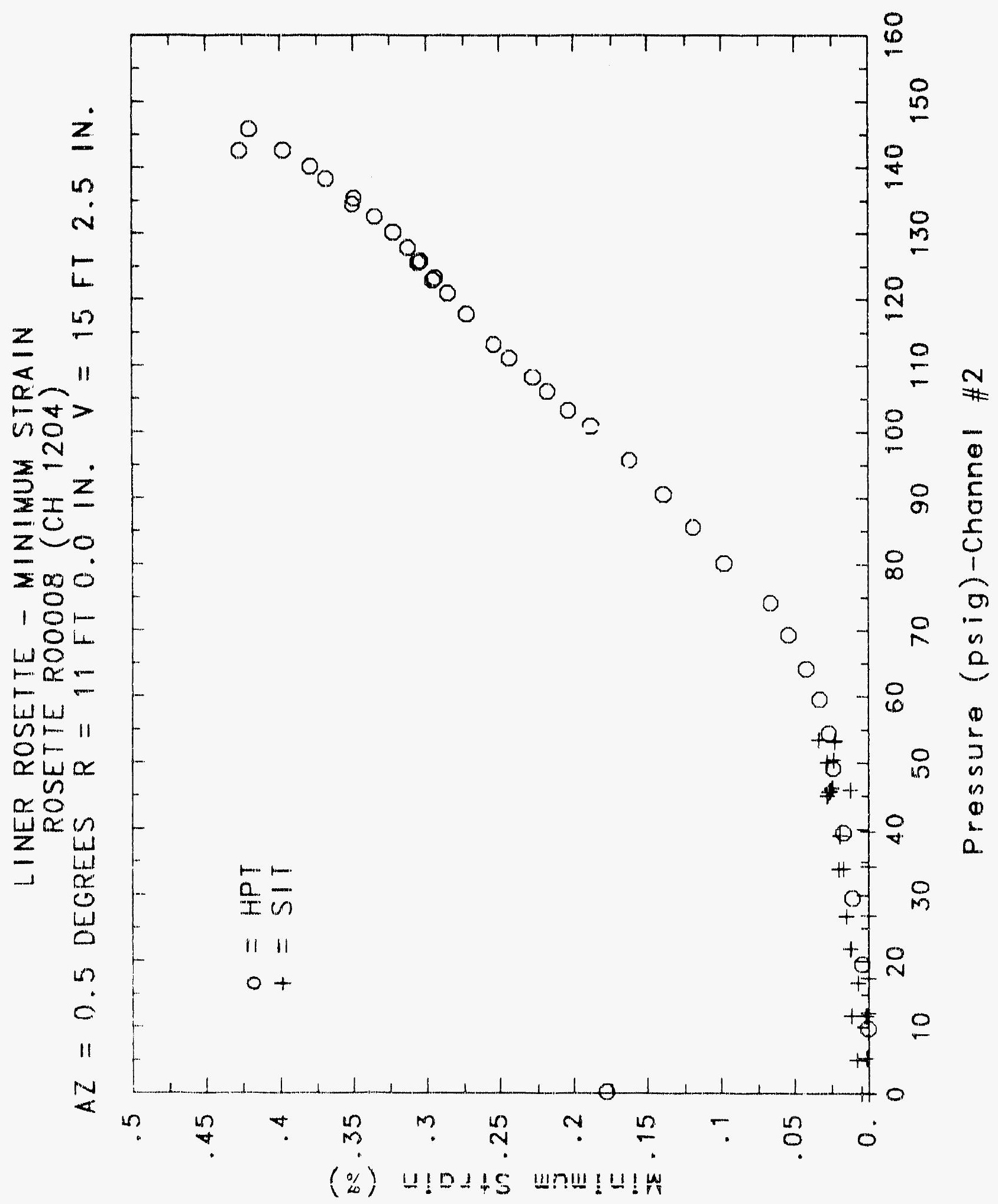




\title{
Reinforced Concrete Test Data
}

\author{
$\begin{array}{llllll}\text { Rosette } & 9 & \text { Channels } & 1206 & 1207 & 1208\end{array}$
}

STRUCTURAL INTEGRITY TEST

\begin{tabular}{|c|c|c|c|c|c|c|c|}
\hline $\begin{array}{c}\text { Pressure } \\
\text { psig } \\
-0.05 \\
5.33 \\
12.31 \\
16.44 \\
20.51 \\
20.46 \\
20.47 \\
27.05 \\
34.69 \\
34.53 \\
34.53 \\
39.70 \\
45.90 \\
45.65 \\
45.62 \\
50.10 \\
53.47 \\
53.29 \\
53.21 \\
50.49 \\
46.14 \\
46.13 \\
44.97 \\
38.98 \\
33.99 \\
33.96 \\
26.83 \\
21.88 \\
21.90 \\
16.69 \\
11.74 \\
11.77 \\
5.05 \\
0.02 \\
-0.04 \\
-0.02 \\
-0.02 \\
0.02\end{array}$ & $\begin{array}{c}\text { Maximum } \\
\text { \%Strain } \\
0.0003 \\
0.0031 \\
0.0067 \\
0.0084 \\
0.0129 \\
0.0128 \\
0.0124 \\
0.0221 \\
0.0326 \\
0.0333 \\
0.0334 \\
0.0387 \\
0.0472 \\
0.0492 \\
0.0493 \\
0.0558 \\
0.0601 \\
0.0623 \\
0.0620 \\
0.0618 \\
0.0600 \\
0.0597 \\
0.0636 \\
0.0604 \\
0.0568 \\
0.0569 \\
0.0521 \\
0.0477 \\
0.0481 \\
0.0443 \\
0.0406 \\
0.0400 \\
0.0329 \\
0.0279 \\
0.0263 \\
0.0280 \\
0.0283 \\
0.0272\end{array}$ & $\begin{array}{l}\text { Minimum } \\
\% \text { Strain } \\
-0.0004 \\
0.0016 \\
0.0040 \\
0.0061 \\
0.0085 \\
0.0094 \\
0.0101 \\
0.0174 \\
0.0278 \\
0.0290 \\
0.0291 \\
0.0353 \\
0.0459 \\
0.0476 \\
0.0487 \\
0.0526 \\
0.0583 \\
0.0594 \\
0.0594 \\
0.0583 \\
0.0561 \\
0.0563 \\
0.0543 \\
0.0502 \\
0.0467 \\
0.0465 \\
0.0404 \\
0.0360 \\
0.0358 \\
0.0306 \\
0.0258 \\
0.0258 \\
0.0189 \\
0.0139 \\
0.0132 \\
0.0119 \\
0.0115 \\
0.0114\end{array}$ & $\begin{array}{c}\text { Angle } \\
\text { Degrees } \\
-0.32 \\
-43.65 \\
-34.70 \\
-32.40 \\
-43.63 \\
-44.91 \\
-39.58 \\
26.85 \\
5.66 \\
4.85 \\
2.05 \\
-4.41 \\
41.37 \\
31.16 \\
26.22 \\
25.24 \\
-14.56 \\
-17.11 \\
2.69 \\
7.77 \\
3.06 \\
3.11 \\
0.20 \\
0.98 \\
0.00 \\
-2.07 \\
-3.38 \\
-3.69 \\
-5.12 \\
-5.27 \\
-8.17 \\
-8.80 \\
-7.55 \\
-2.44 \\
-9.13 \\
-8.99 \\
-9.77 \\
-7.06\end{array}$ & $\begin{array}{c}\text { Pressure } \\
\text { psig } \\
9.89 \\
19.55 \\
29.57 \\
39.42 \\
49.16 \\
54.50 \\
59.57 \\
64.20 \\
69.32 \\
74.16 \\
80.16 \\
85.61 \\
90.58 \\
95.69 \\
100.92 \\
103.25 \\
106.11 \\
108.31 \\
111.08 \\
113.24 \\
117.83 \\
120.92 \\
123.28 \\
122.97 \\
125.82 \\
125.60 \\
127.84 \\
130.19 \\
132.53 \\
135.33 \\
134.42 \\
138.35 \\
140.16 \\
142.63 \\
145.78 \\
142.52 \\
0.22\end{array}$ & $\begin{array}{l}\text { Maximum } \\
\text { xStrain } \\
0.0116 \\
0.0209 \\
0.0288 \\
0.0376 \\
0.0472 \\
0.0534 \\
0.0614 \\
0.0727 \\
0.0840 \\
0.0959 \\
0.1150 \\
0.1294 \\
0.1417 \\
0.1549 \\
0.1679 \\
0.1740 \\
0.1801 \\
0.1852 \\
0.1930 \\
0.1988 \\
0.2128 \\
0.2237 \\
0.2333 \\
0.2340 \\
0.2449 \\
0.2478 \\
0.2559 \\
0.2676 \\
0.2822 \\
0.3028 \\
0.3060 \\
0.3336 \\
0.3563 \\
0.3914 \\
0.4375 \\
0.4531 \\
0.3261\end{array}$ & $\begin{array}{l}\text { Minimum } \\
\text { \%Strain } \\
0.0068 \\
0.0156 \\
0.0258 \\
0.0344 \\
0.0412 \\
0.0452 \\
0.0510 \\
0.0597 \\
0.0697 \\
0.0808 \\
0.0962 \\
0.1079 \\
0.1186 \\
0.1300 \\
0.1436 \\
0.1508 \\
0.1574 \\
0.1624 \\
0.1704 \\
0.1755 \\
0.1867 \\
0.1947 \\
0.2010 \\
0.2024 \\
0.2084 \\
0.2096 \\
0.2147 \\
0.2224 \\
0.2322 \\
0.2454 \\
0.2474 \\
0.2647 \\
0.2787 \\
0.3003 \\
0.3300 \\
0.3335 \\
0.2063\end{array}$ & $\begin{array}{r}\text { Angle } \\
\text { Degrees } \\
-41.28 \\
-40.29 \\
40.38 \\
7.81 \\
-0.27 \\
-6.69 \\
-13.02 \\
-16.83 \\
-15.14 \\
-19.83 \\
-20.38 \\
-21.11 \\
-20.77 \\
-18.58 \\
-14.05 \\
-9.90 \\
-6.26 \\
-3.51 \\
1.94 \\
7.03 \\
14.12 \\
17.85 \\
21.15 \\
22.40 \\
25.65 \\
27.29 \\
27.88 \\
29.96 \\
31.92 \\
34.35 \\
35.46 \\
36.96 \\
38.16 \\
40.45 \\
42.07 \\
43.38 \\
-23.43\end{array}$ \\
\hline
\end{tabular}

High Pressure test 


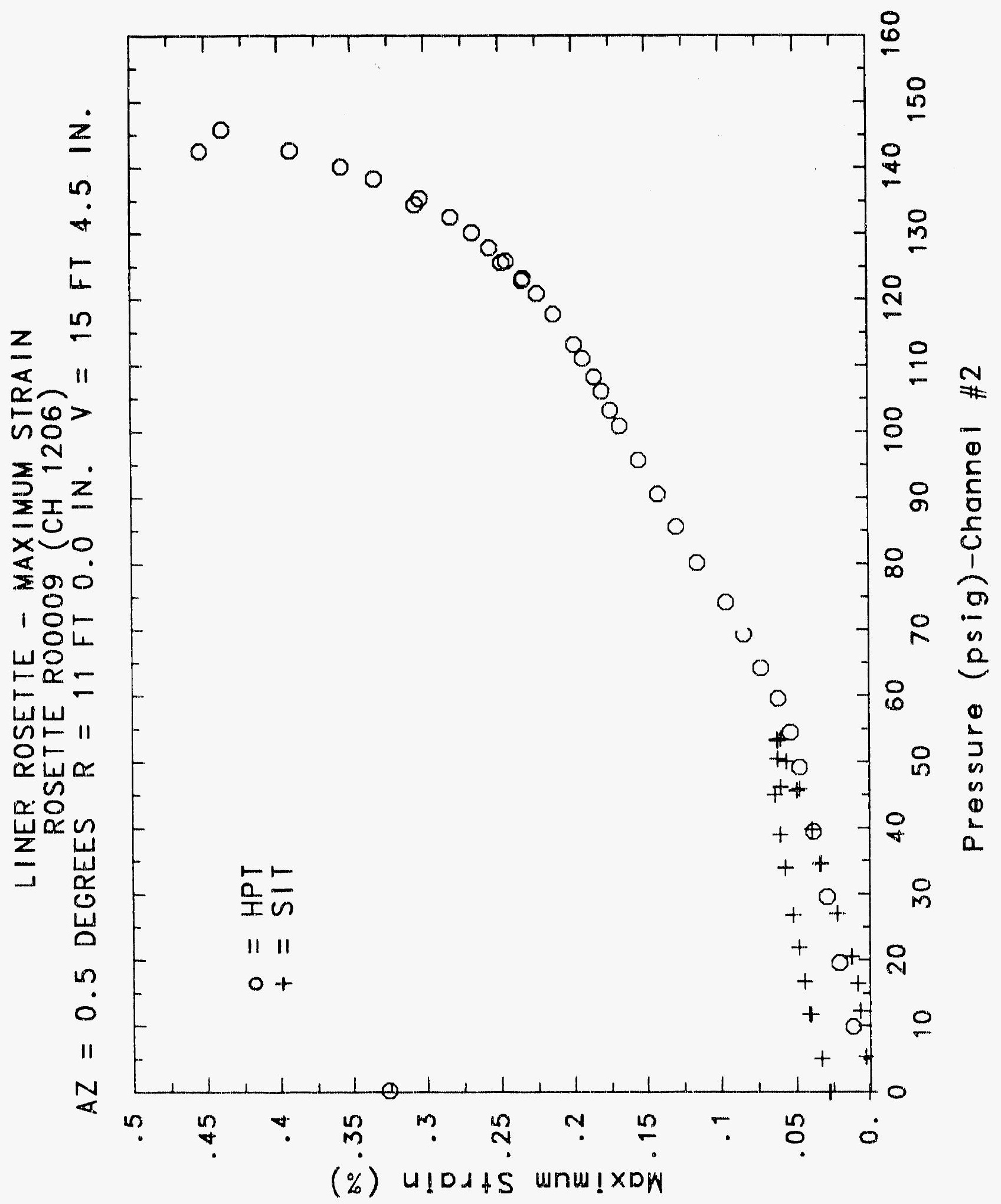




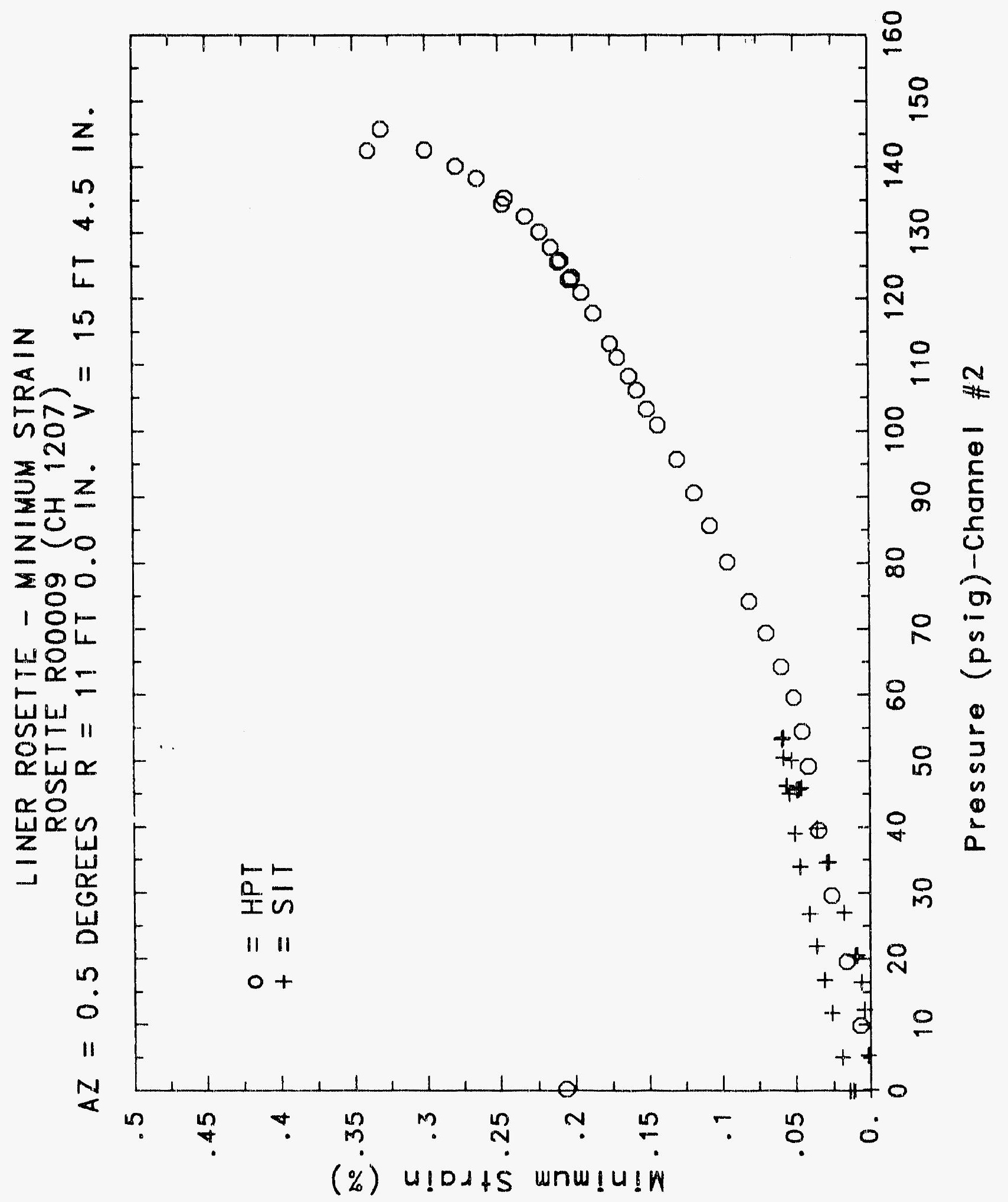




\section{Reinforced Concrete Test Data}

Rosette 10 Channels $1209 \quad 12201221$

STRUCTURAL INTEGRITY TEST

\begin{tabular}{|c|c|c|c|c|c|c|c|}
\hline $\begin{array}{c}\text { Pressure } \\
\text { psig } \\
-0.05 \\
5.33 \\
12.31 \\
16.44 \\
20.51 \\
20.46 \\
20.47 \\
27.05 \\
34.69 \\
34.53 \\
34.53 \\
39.70 \\
45.510 \\
45.65 \\
45.62 \\
50.10 \\
53.47 \\
53.29 \\
53.21 \\
50.49 \\
46.14 \\
46.13 \\
44.97 \\
38.98 \\
33.99 \\
33.96 \\
26.83 \\
21.88 \\
21.90 \\
16.69 \\
11.74 \\
11.77 \\
5.05 \\
0.02 \\
-0.04 \\
-0.02 \\
-0.02 \\
0.02\end{array}$ & $\begin{array}{l}\text { Maximum } \\
\text { xStrain } \\
0.0006 \\
0.0030 \\
0.0082 \\
0.0119 \\
0.0154 \\
0.0161 \\
0.0167 \\
0.0292 \\
0.0462 \\
0.0472 \\
0.0474 \\
0.0558 \\
0.0700 \\
0.0714 \\
0.0716 \\
0.0791 \\
0.0864 \\
0.0886 \\
0.0888 \\
0.0873 \\
0.0838 \\
0.0823 \\
0.0845 \\
0.0781 \\
0.0728 \\
0.0725 \\
0.0643 \\
0.0581 \\
0.0578 \\
0.0516 \\
0.0453 \\
0.0449 \\
0.0351 \\
0.0273 \\
0.0279 \\
0.0282 \\
0.0280 \\
0.0275\end{array}$ & $\begin{array}{l}\text { Minimum } \\
\text { \%Strain } \\
-0.0012 \\
0.0021 \\
0.0047 \\
0.0065 \\
0.0093 \\
0.0099 \\
0.0101 \\
0.0163 \\
0.0239 \\
0.0244 \\
0.0247 \\
0.0289 \\
0.0344 \\
0.0356 \\
0.0360 \\
0.0393 \\
0.0425 \\
0.0429 \\
0.0432 \\
0.0425 \\
0.0404 \\
0.0409 \\
0.0406 \\
0.0376 \\
0.0345 \\
0.0344 \\
0.0296 \\
0.0259 \\
0.0258 \\
0.0217 \\
0.0178 \\
0.0174 \\
0.0128 \\
0.0088 \\
0.0069 \\
0.0072 \\
0.0073 \\
0.0071\end{array}$ & $\begin{array}{c}\text { Angle } \\
\text { Degrees } \\
38.29 \\
3.59 \\
-19.81 \\
-23.19 \\
-28.02 \\
-29.62 \\
-32.02 \\
44.55 \\
44.86 \\
44.15 \\
43.82 \\
-44.67 \\
-42.18 \\
-42.22 \\
-42.33 \\
-40.81 \\
-39.77 \\
-39.46 \\
-39.08 \\
-39.44 \\
-39.04 \\
-38.98 \\
-37.06 \\
-36.62 \\
-36.53 \\
-36.30 \\
-35.95 \\
-35.14 \\
-34.96 \\
-34.37 \\
-33.65 \\
-33.52 \\
-31.32 \\
-26.42 \\
-30.13 \\
-26.41 \\
-25.66 \\
-25.35\end{array}$ & $\begin{array}{c}\text { Pressure } \\
\text { psig } \\
9.89 \\
19.55 \\
29.57 \\
39.42 \\
49.16 \\
54.50 \\
59.57 \\
64.20 \\
69.32 \\
74.16 \\
80.16 \\
85.61 \\
90.58 \\
95.69 \\
100.92 \\
103.25 \\
106.11 \\
108.31 \\
111.08 \\
113.24 \\
117.83 \\
120.92 \\
123.28 \\
122.97 \\
125.82 \\
125.60 \\
127.84 \\
130.19 \\
132.53 \\
135.33 \\
134.42 \\
138.35 \\
140.16 \\
142.63 \\
145.78 \\
142.52 \\
0.22\end{array}$ & $\begin{array}{c}\text { Maximum } \\
\text { xStrain } \\
0.0134 \\
0.0283 \\
0.0421 \\
0.0548 \\
0.0674 \\
0.0755 \\
0.0861 \\
0.0990 \\
0.1143 \\
0.1318 \\
0.1536 \\
0.1728 \\
0.1902 \\
0.2099 \\
0.2300 \\
0.2392 \\
0.2496 \\
0.2574 \\
0.2695 \\
0.2788 \\
0.3011 \\
0.3176 \\
0.3298 \\
0.3319 \\
0.3463 \\
0.3490 \\
0.3593 \\
0.3757 \\
0.3952 \\
0.4214 \\
0.4256 \\
0.4629 \\
0.4940 \\
0.5430 \\
0.6066 \\
0.6262 \\
0.4195\end{array}$ & $\begin{array}{l}\text { Minimum } \\
\text { XStrain } \\
0.0076 \\
0.0142 \\
0.0218 \\
0.0291 \\
0.0362 \\
0.0402 \\
0.0446 \\
0.0497 \\
0.0553 \\
0.0610 \\
0.0719 \\
0.0822 \\
0.0933 \\
0.1063 \\
0.1206 \\
0.1284 \\
0.1360 \\
0.1416 \\
0.1505 \\
0.1567 \\
0.1725 \\
0.1843 \\
0.1938 \\
0.1948 \\
0.2040 \\
0.2055 \\
0.2129 \\
0.2238 \\
0.2391 \\
0.2596 \\
0.2618 \\
0.2895 \\
0.3120 \\
0.3451 \\
0.3880 \\
0.4004 \\
0.2649\end{array}$ & $\begin{array}{c}\text { Angle } \\
\text { Degrees } \\
-38.08 \\
-39.29 \\
-40.33 \\
-40.80 \\
-41.60 \\
-41.70 \\
-40.17 \\
-37.55 \\
-35.20 \\
-32.20 \\
-30.31 \\
-28.62 \\
-27.67 \\
-27.45 \\
-27.84 \\
-28.23 \\
-28.53 \\
-28.76 \\
-29.09 \\
-29.45 \\
-30.01 \\
-30.48 \\
-30.93 \\
-30.95 \\
-31.17 \\
-31.32 \\
-31.34 \\
-31.57 \\
-31.20 \\
-31.02 \\
-31.02 \\
-30.72 \\
-30.66 \\
-30.03 \\
-29.09 \\
-28.16 \\
-14.73\end{array}$ \\
\hline
\end{tabular}

High Pressure test 


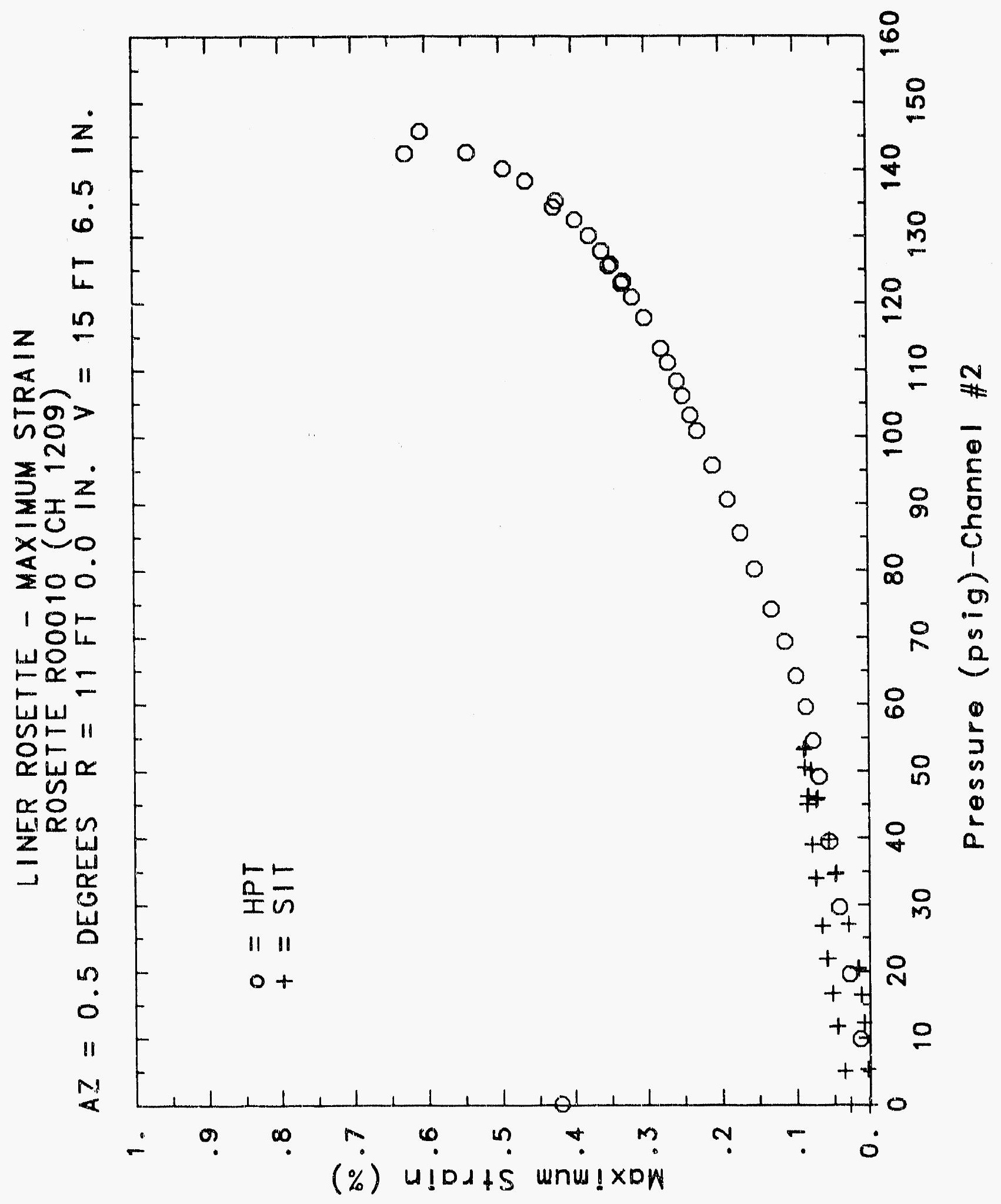




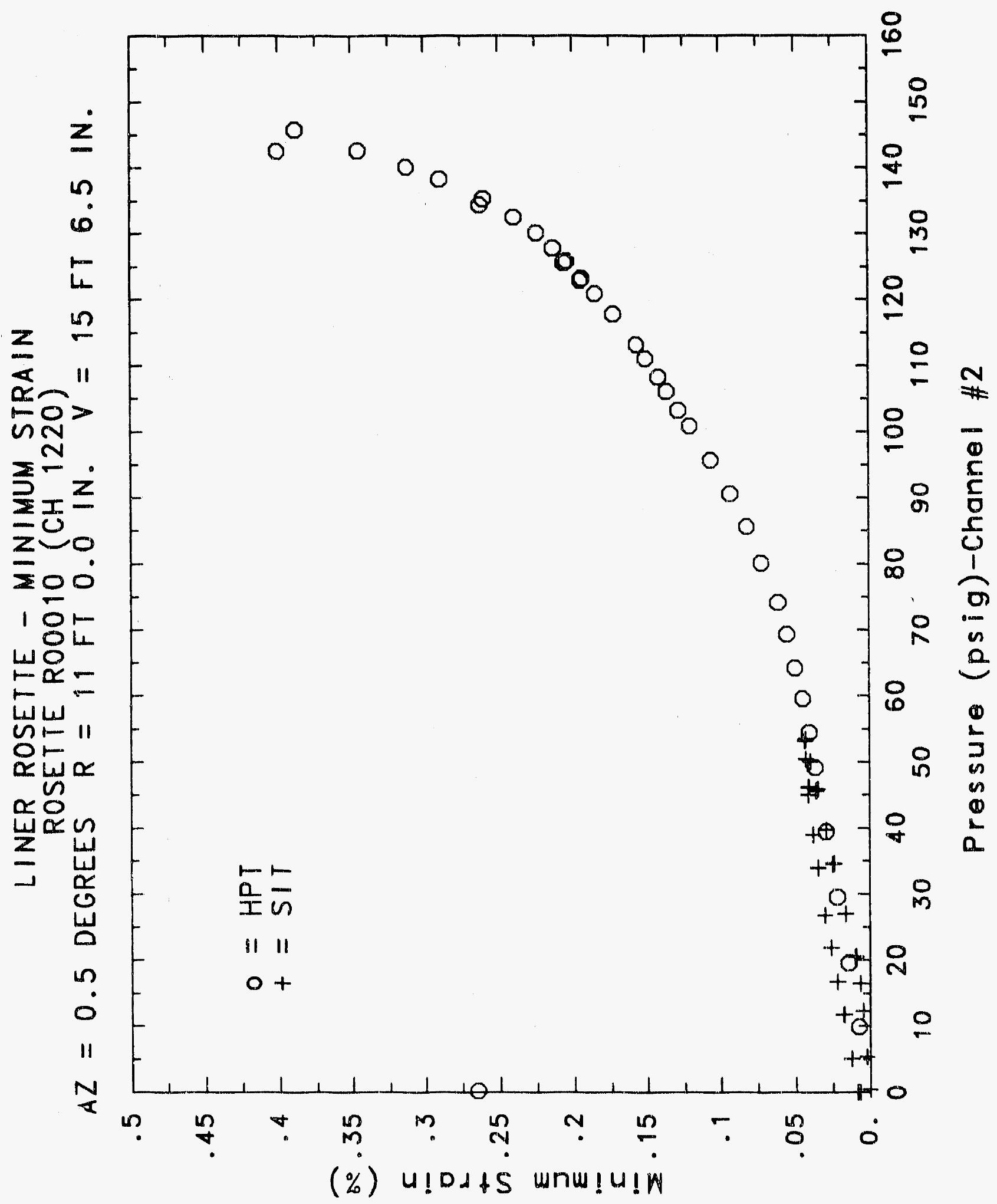




\section{Reinforced Concrete Test Data}

Rosette 11 Channels $1222 \quad 1223 \quad 1224$

Structural INTEGRITY TEST

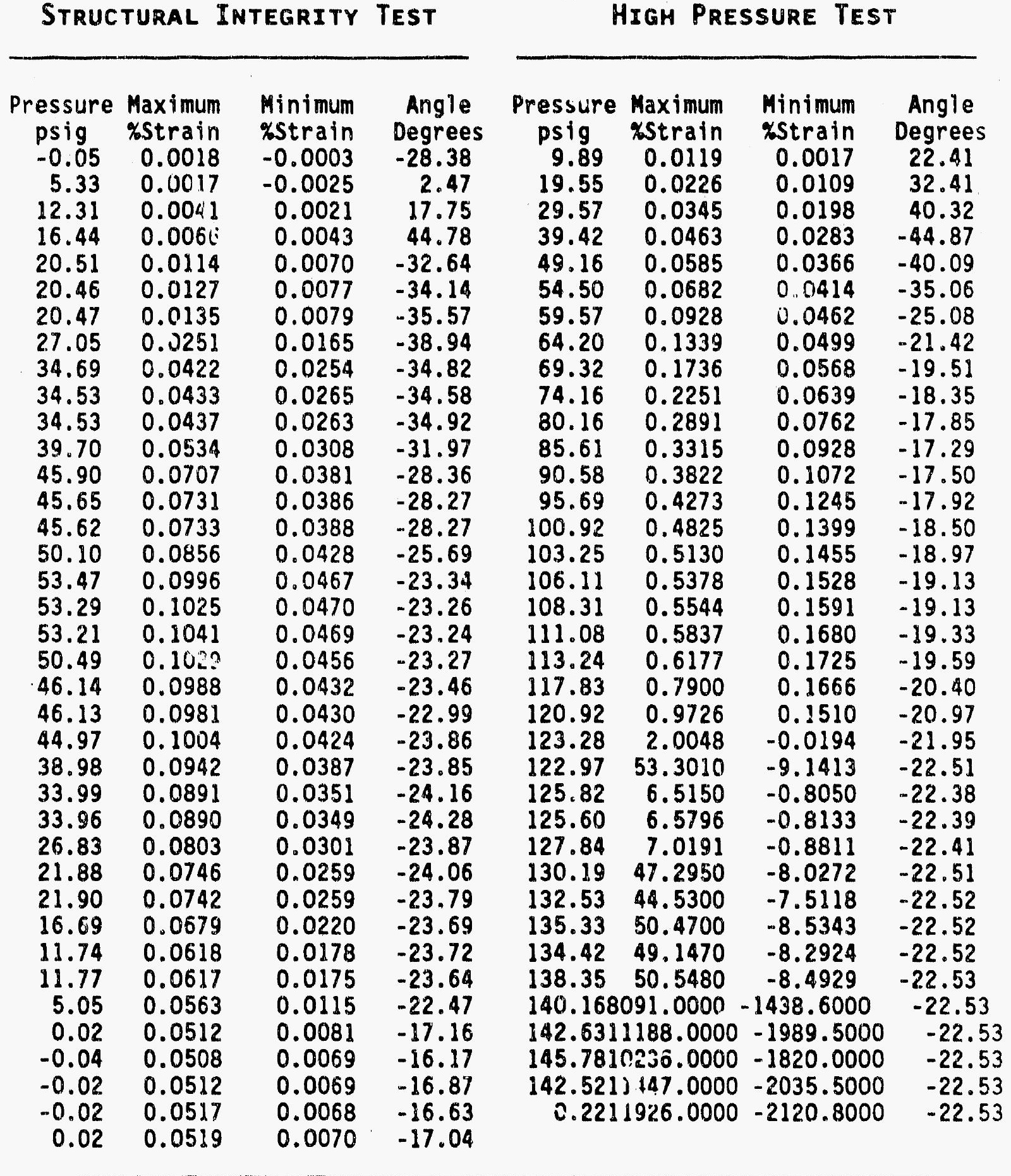

High Pressure test 


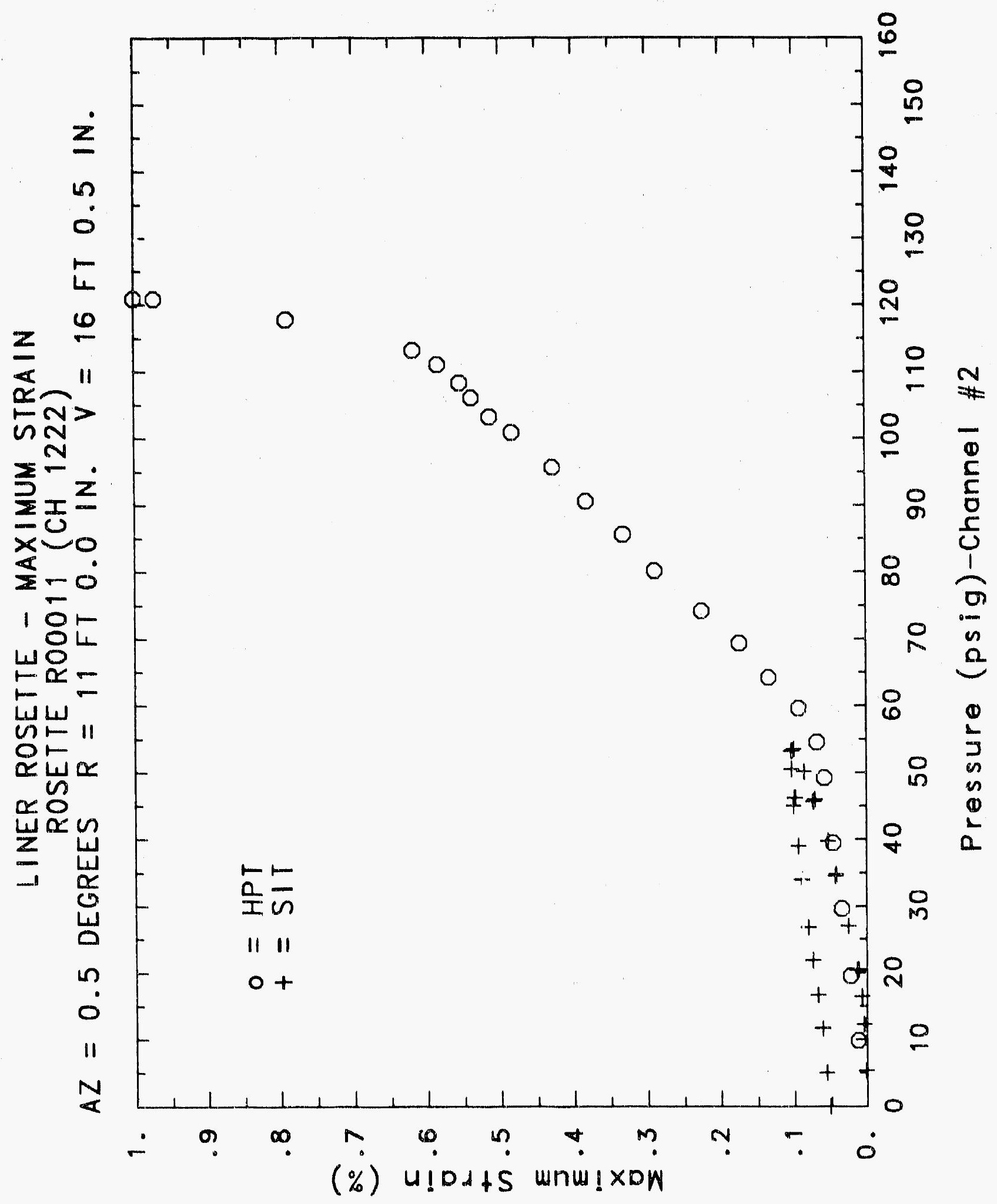




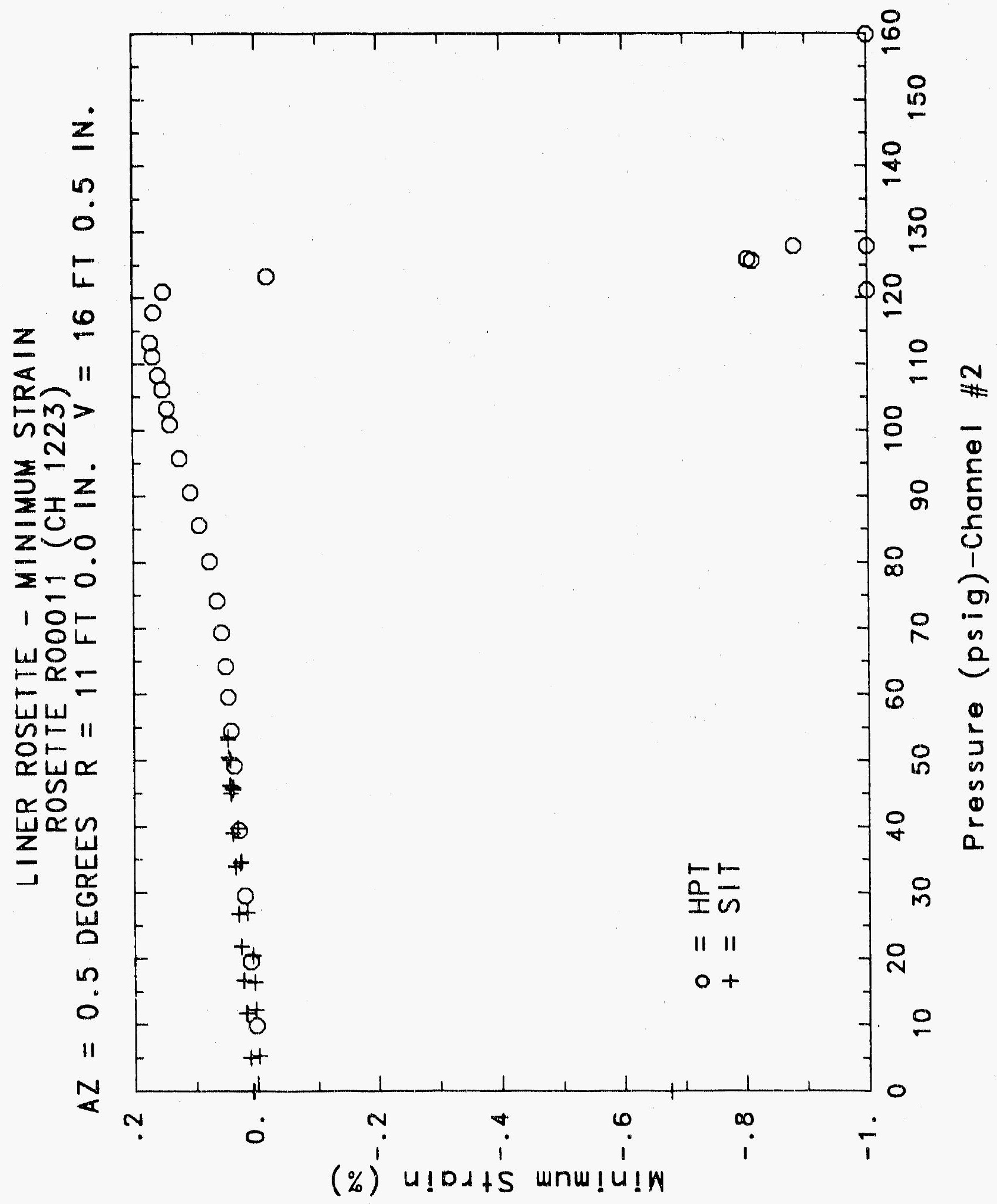




\section{Reinforced Concrete Test Data}

Rosette 12 Channels $1225 \quad 1226 \quad 1227$

\section{STRUCTURAL INTEGRITY TEST}

\begin{tabular}{|c|c|c|c|c|c|c|c|}
\hline $\begin{array}{c}\text { Pressure } \\
\text { psig } \\
-0.05 \\
5.33 \\
12.31 \\
16.44 \\
20.51 \\
20.46 \\
20.47 \\
27.05 \\
34.69 \\
34.53 \\
34.53 \\
39.70 \\
45.90 \\
45.65 \\
45.62 \\
50.10 \\
53.47 \\
53.29 \\
53.21 \\
50.49 \\
46.14 \\
46.13 \\
44.97 \\
38.98 \\
33.99 \\
33.96 \\
26.83 \\
21.88 \\
21.90 \\
16.69 \\
11.74 \\
11.77 \\
5.05 \\
0.02 \\
-0.04 \\
-0.02 \\
-0.02 \\
0.02\end{array}$ & $\begin{array}{c}\text { Maximum } \\
\text { xStrain } \\
0.0018 \\
0.0035 \\
0.0069 \\
0.0082 \\
0.0106 \\
0.0116 \\
0.0117 \\
0.0187 \\
0.0250 \\
0.0254 \\
0.0256 \\
0.0292 \\
0.0329 \\
0.0328 \\
0.0324 \\
0.0342 \\
0.0350 \\
0.0348 \\
0.0345 \\
0.0329 \\
0.0311 \\
0.0308 \\
0.0317 \\
0.0283 \\
0.0258 \\
0.0257 \\
0.0220 \\
0.0195 \\
0.0196 \\
0.0173 \\
0.0152 \\
0.0150 \\
0.0130 \\
0.0127 \\
0.0109 \\
0.0116 \\
0.0118 \\
0.0118\end{array}$ & $\begin{array}{r}\text { Minimum } \\
\text { XStrain } \\
-0.0009 \\
-0.0111 \\
-0.0128 \\
-0.0108 \\
-0.0084 \\
-0.0083 \\
-0.0083 \\
-0.0059 \\
0.0018 \\
0.0024 \\
0.0023 \\
0.0055 \\
0.0105 \\
0.0102 \\
0.0100 \\
0.0137 \\
0.0167 \\
0.0160 \\
0.0165 \\
0.0142 \\
0.0114 \\
0.0111 \\
0.0096 \\
0.0056 \\
0.0028 \\
0.0026 \\
-0.0014 \\
-0.0038 \\
-0.0038 \\
-0.0061 \\
-0.0075 \\
-0.0075 \\
-0.0076 \\
-0.0024 \\
-0.0017 \\
-0.0022 \\
-0.0019 \\
-0.0016\end{array}$ & $\begin{array}{c}\text { Angle } \\
\text { Degrees } \\
40.25 \\
19.95 \\
18.74 \\
18.28 \\
13.91 \\
12.19 \\
10.70 \\
-1.05 \\
-1.00 \\
-0.34 \\
0.11 \\
-1.25 \\
-2.33 \\
-2.09 \\
-3.03 \\
-2.97 \\
-4.51 \\
-5.02 \\
-3.39 \\
-6.24 \\
-5.05 \\
-5.46 \\
-3.98 \\
-5.61 \\
-4.53 \\
-4.83 \\
-4.89 \\
-4.62 \\
-5.08 \\
-5.87 \\
-6.25 \\
-5.79 \\
-8.73 \\
-22.52 \\
-21.54 \\
-22.73 \\
-23.59 \\
-22.24\end{array}$ & $\begin{array}{c}\text { Pressure } \\
\text { psig } \\
9.89 \\
19.55 \\
29.57 \\
39.42 \\
49.16 \\
54.50 \\
59.57 \\
64.20 \\
69.32 \\
74.16 \\
80.16 \\
85.61 \\
90.58 \\
95.69 \\
100.92 \\
103.25 \\
106.11 \\
108.31 \\
111.08 \\
113.24 \\
117.83 \\
120.92 \\
123.28 \\
122.97 \\
125.82 \\
125.60 \\
127.84 \\
130.19 \\
132.53 \\
135.33 \\
134.42 \\
138.35 \\
140.16 \\
142.63 \\
145.78 \\
142.52 \\
0.22\end{array}$ & $\begin{array}{l}\text { Maximum } \\
\text { XStrain } \\
0.0050 \\
0.0096 \\
0.0146 \\
0.0199 \\
0.0257 \\
0.0287 \\
0.0311 \\
0.0339 \\
0.0406 \\
0.0492 \\
0.0635 \\
0.0803 \\
0.0994 \\
0.1229 \\
0.1514 \\
0.1640 \\
0.1792 \\
0.1916 \\
0.2094 \\
0.2234 \\
0.2567 \\
0.2791 \\
0.2962 \\
0.2989 \\
0.3199 \\
0.3243 \\
0.3388 \\
0.3633 \\
0.3975 \\
0.4453 \\
0.4539 \\
0.5154 \\
0.5679 \\
0.6455 \\
0.7289 \\
0.7586 \\
0.7384\end{array}$ & $\begin{array}{l}\text { Minimum } \\
\text { KStrain } \\
-0.0066 \\
-0.0035 \\
0.0015 \\
0.0076 \\
0.0143 \\
0.0188 \\
0.0233 \\
0.0272 \\
0.0296 \\
0.0309 \\
0.0328 \\
0.0366 \\
0.0434 \\
0.0546 \\
0.0703 \\
0.0792 \\
0.0889 \\
0.0963 \\
0.1082 \\
0.1170 \\
0.1388 \\
0.1550 \\
0.1692 \\
0.1720 \\
0.1906 \\
0.1957 \\
0.2102 \\
0.2371 \\
0.2709 \\
0.3144 \\
0.3219 \\
0.3798 \\
0.4242 \\
0.4874 \\
0.5535 \\
0.5698 \\
0.3742\end{array}$ & $\begin{array}{c}\text { Angle } \\
\text { Degrees } \\
13.82 \\
13.76 \\
12.46 \\
12.33 \\
15.04 \\
14.69 \\
22.04 \\
41.41 \\
-28.83 \\
-19.35 \\
-15.16 \\
-12.70 \\
-11.89 \\
-11.06 \\
-10.99 \\
-11.70 \\
-12.08 \\
-12.46 \\
-13.27 \\
-13.91 \\
-15.18 \\
-16.56 \\
-17.94 \\
-18.48 \\
-20.39 \\
-21.31 \\
-23.00 \\
-25.88 \\
-29.27 \\
-32.76 \\
-32.89 \\
-34.63 \\
-34.78 \\
-33.39 \\
-31.73 \\
-30.07 \\
-2.07\end{array}$ \\
\hline
\end{tabular}

\section{high Pressure test}




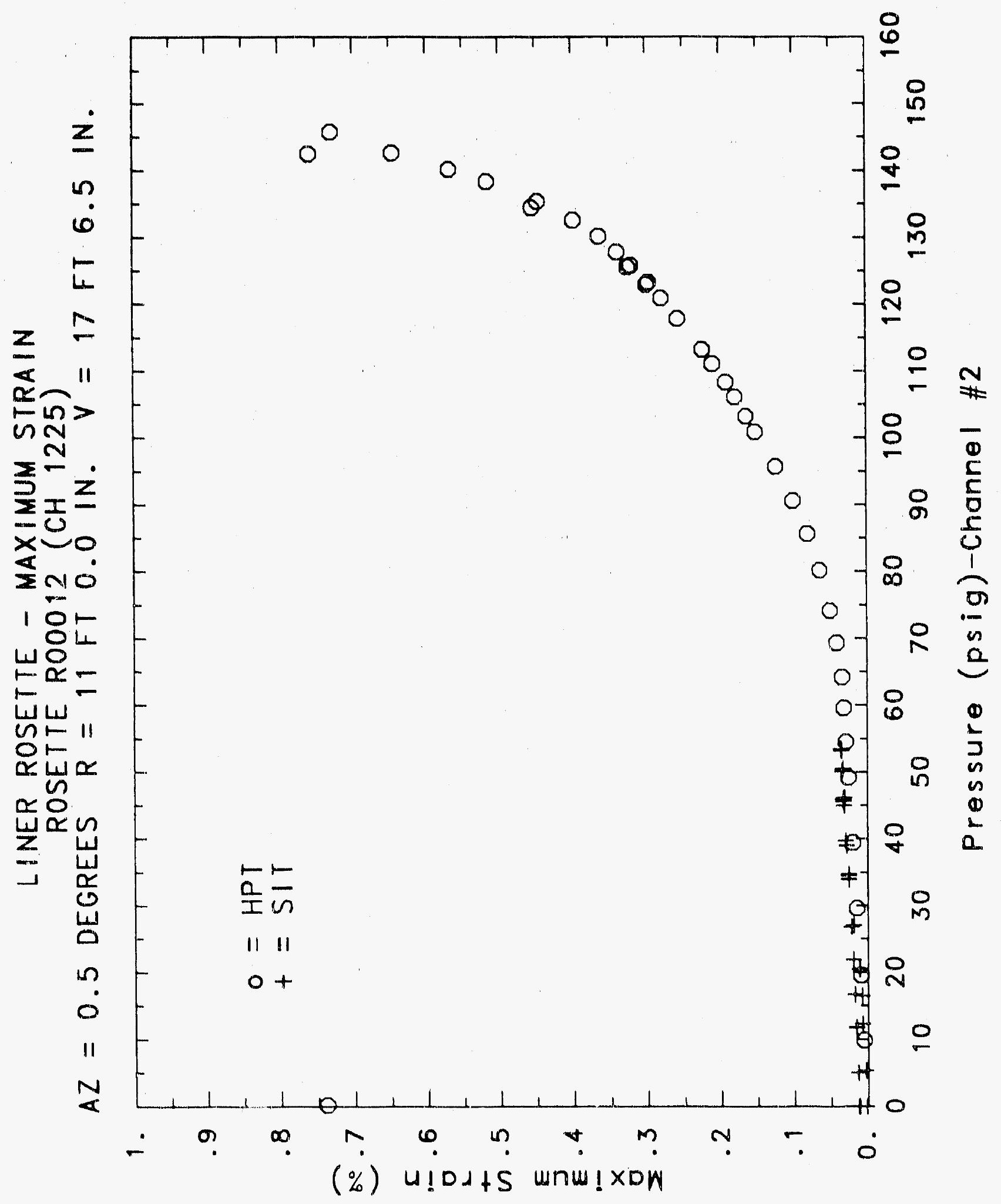




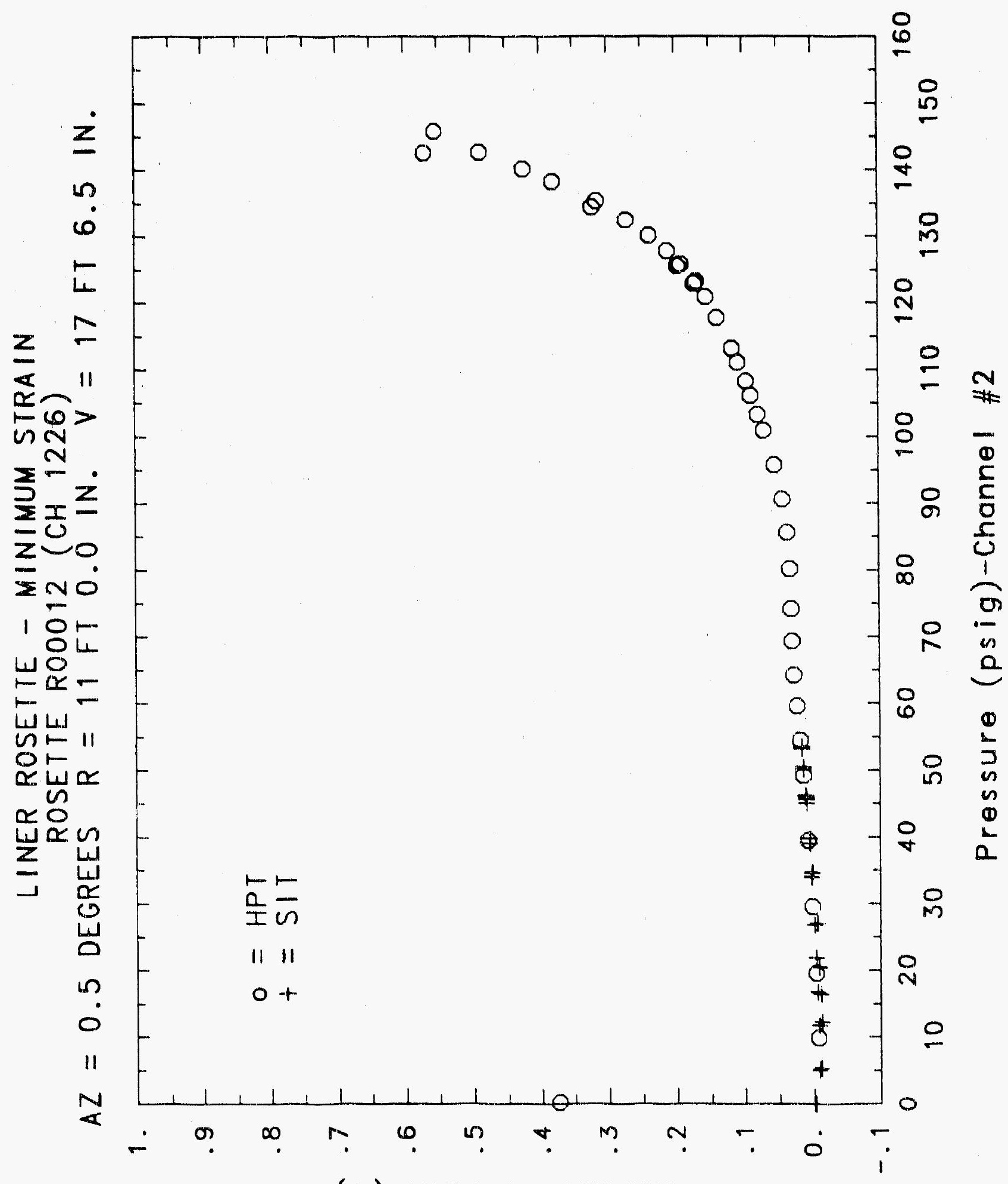

(\%) U!Dd+S mnw!U!w 


\section{Reinforced Concrete Test Data}

Rosette 13 Channels 122812291240

STRUCTURAL INTEGRITY TEST

\begin{tabular}{|c|c|c|c|c|c|c|c|}
\hline $\begin{array}{l}\text { Pressure } \\
\text { psig } \\
-0.05 \\
5.33 \\
12.31 \\
16.44 \\
20.51 \\
20.46 \\
20.47 \\
27.05 \\
34.69 \\
34.53 \\
34.53 \\
39.70 \\
45.90 \\
45.65 \\
45.62 \\
50.10 \\
53.47 \\
53.29 \\
53.21 \\
50.49 \\
46.14 \\
46.13 \\
44.97 \\
38.98 \\
33.99 \\
33.96 \\
26.83 \\
21.88 \\
21.90 \\
16.69 \\
11.74 \\
11.77 \\
5.05 \\
0.02 \\
-0.04 \\
-0.02 \\
-0.02 \\
0.02\end{array}$ & $\begin{array}{l}\text { Maximum } \\
\text { XStrain } \\
0.0002 \\
0.0015 \\
0.0041 \\
0.0062 \\
0.0141 \\
0.0161 \\
0.0169 \\
0.0308 \\
0.0467 \\
0.0483 \\
0.0493 \\
0.0572 \\
0.0697 \\
0.0718 \\
0.0723 \\
0.0790 \\
0.0852 \\
0.0860 \\
0.0867 \\
0.0851 \\
0.0818 \\
0.0815 \\
0.0779 \\
0.0731 \\
0.0684 \\
0.0681 \\
0.0603 \\
0.0542 \\
0.0545 \\
0.0486 \\
0.0427 \\
0.0428 \\
0.0350 \\
0.0283 \\
0.0258 \\
0.0249 \\
0.0245 \\
0.0240\end{array}$ & $\begin{array}{l}\text { Minimum } \\
\text { xstrain } \\
-0.0009 \\
0.0006 \\
0.0007 \\
0.0022 \\
0.0033 \\
0.0046 \\
0.0044 \\
0.0069 \\
0.0106 \\
0.0113 \\
0.0118 \\
0.0135 \\
0.0166 \\
0.0166 \\
0.0164 \\
0.0183 \\
0.0202 \\
0.0196 \\
0.0197 \\
0.0193 \\
0.0176 \\
0.0172 \\
0.0183 \\
0.0166 \\
0.0152 \\
0.0151 \\
0.0123 \\
0.0103 \\
0.0109 \\
0.0084 \\
0.0071 \\
0.0061 \\
0.0036 \\
0.0022 \\
0.0003 \\
0.0006 \\
0.0024 \\
0.0016\end{array}$ & $\begin{array}{c}\text { Angle } \\
\text { Degrees } \\
-27.37 \\
27.23 \\
32.80 \\
42.28 \\
-20.96 \\
-17.87 \\
-19.70 \\
-18.89 \\
-19.44 \\
-19.87 \\
-19.13 \\
-19.84 \\
-19.09 \\
-18.61 \\
-18.93 \\
-18.59 \\
-18.06 \\
-18.47 \\
-18.19 \\
-18.13 \\
-17.74 \\
-17.88 \\
-18.03 \\
-18.56 \\
-18.32 \\
-18.88 \\
-19.31 \\
-20.05 \\
-19.33 \\
-20.26 \\
-19.37 \\
-19.79 \\
-19.51 \\
-17.92 \\
-13.93 \\
-16.04 \\
-15.32 \\
-19.84\end{array}$ & $\begin{array}{c}\text { Pressure } \\
\text { psig } \\
9.89 \\
19.55 \\
29.57 \\
39.42 \\
49.16 \\
54.50 \\
59.57 \\
64.20 \\
69.32 \\
74.16 \\
80.16 \\
85.61 \\
90.58 \\
95.69 \\
100.92 \\
103.25 \\
106.11 \\
108.31 \\
111.08 \\
113.24 \\
117.83 \\
120.92 \\
123.28 \\
122.97 \\
125.82 \\
125.60 \\
127.84 \\
130.19 \\
132.53 \\
135.33 \\
134.42 \\
138.35 \\
140.16 \\
142.63 \\
145.78 \\
142.52 \\
0.22\end{array}$ & $\begin{array}{l}\text { Maximum } \\
\text { xStrain } \\
0.0136 \\
0.0272 \\
0.0402 \\
0.0515 \\
0.0624 \\
0.0688 \\
0.0764 \\
0.0851 \\
0.0946 \\
0.1059 \\
0.1216 \\
0.1338 \\
0.1466 \\
0.1666 \\
0.1955 \\
0.2177 \\
0.2417 \\
0.2620 \\
0.2982 \\
0.3<92 \\
0.4030 \\
0.4777 \\
0.5684 \\
0.6046 \\
0.7192 \\
0.7745 \\
0.8269 \\
0.9298 \\
1.0391 \\
1.1533 \\
1.1756 \\
1.3569 \\
1.5371 \\
1.7893 \\
2.0215 \\
2.1001 \\
1.7295\end{array}$ & $\begin{array}{l}\text { Minimum } \\
\text { zStrain } \\
0.0060 \\
0.0101 \\
0.0141 \\
0.0179 \\
0.0213 \\
0.0237 \\
0.0259 \\
0.0283 \\
0.0305 \\
0.0327 \\
0.0321 \\
0.0334 \\
0.0344 \\
0.0354 \\
0.0363 \\
0.0367 \\
0.0369 \\
0.0380 \\
0.0397 \\
0.0414 \\
0.0458 \\
0.0499 \\
0.0549 \\
0.0560 \\
0.0617 \\
0.0647 \\
0.0684 \\
0.0765 \\
0.0866 \\
0.1019 \\
0.1057 \\
0.1169 \\
0.1283 \\
0.1399 \\
0.1501 \\
0.1504 \\
0.0277\end{array}$ & $\begin{array}{r}\text { Angle } \\
\text { Degrees } \\
-22.16 \\
-19.63 \\
-19.62 \\
-19.63 \\
-19.84 \\
-19.34 \\
-18.21 \\
-17.39 \\
-16.49 \\
-15.99 \\
-14.83 \\
-14.82 \\
-15.00 \\
-14.65 \\
-13.82 \\
-13.25 \\
-12.67 \\
-12.12 \\
-11.26 \\
-10.62 \\
-9.54 \\
-8.62 \\
-7.52 \\
-7.04 \\
-5.45 \\
-5.45 \\
-5.11 \\
-4.54 \\
-3.94 \\
-3.34 \\
-3.22 \\
-2.21 \\
-1.58 \\
-0.87 \\
-0.46 \\
-0.40 \\
-0.62\end{array}$ \\
\hline
\end{tabular}

High Pressure TEST 


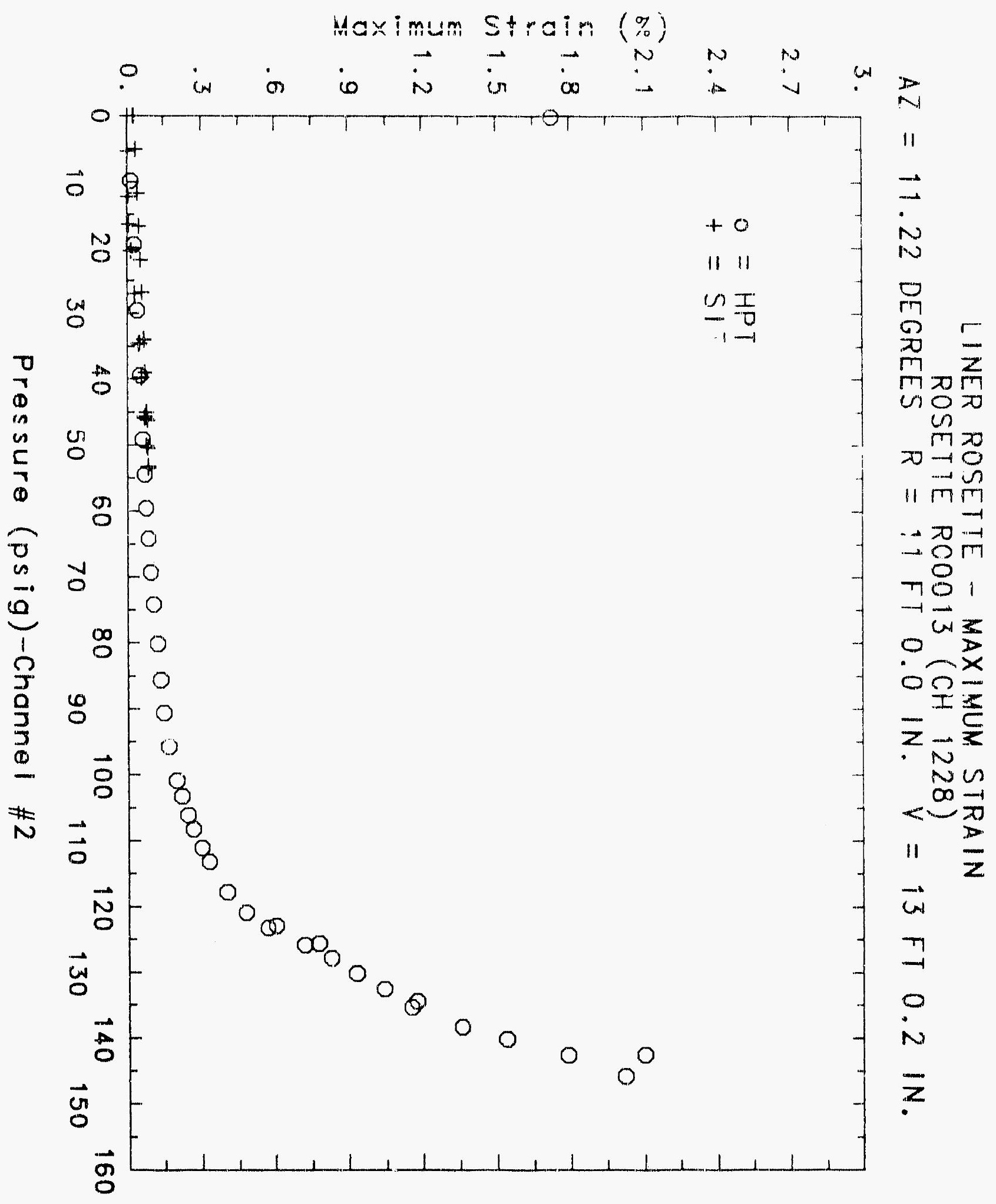




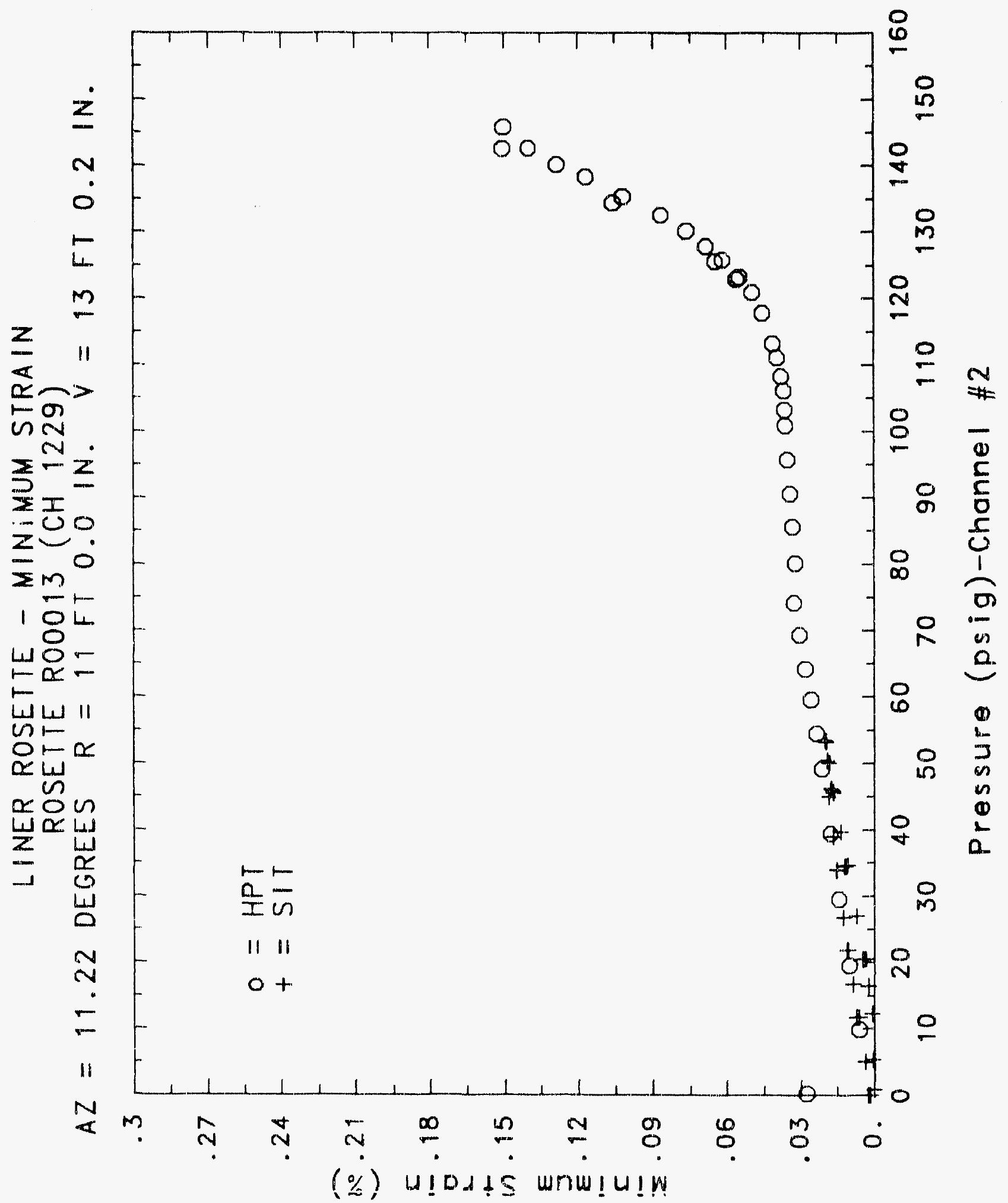




\section{Reinforced Concrete Test Data}

Rosette 14 Channels $1241 \quad 1242 \quad 1243$

Structural Integrity Test

\begin{tabular}{|c|c|c|c|c|c|c|c|}
\hline $\begin{array}{c}\text { ressure } \\
\text { psig } \\
-0.05 \\
5.33 \\
12.31 \\
16.44 \\
16 \\
20.51 \\
20.46 \\
20.47 \\
27.05 \\
34.69 \\
34.53 \\
34 \\
34.53 \\
39.70 \\
45.90 \\
45.65 \\
45.62 \\
50.10 \\
53.47 \\
53\end{array}$ & $\begin{array}{l}\text { Maximium } \\
\text { \%Strain } \\
0.0006 \\
0.0011 \\
0.0027 \\
0.0038 \\
0.0081 \\
0.0100 \\
0.0111 \\
0.0245 \\
0.0398 \\
0.0417 \\
0.0426 \\
0.0502 \\
0.0620 \\
0.0645 \\
0.0647 \\
0.0712 \\
0.0771 \\
0.0783 \\
0.0787 \\
0.0776 \\
0.0741 \\
0.0739 \\
0.0707 \\
0.0662 \\
0.0618 \\
0.0614 \\
0.0543 \\
0.0488 \\
0.0487 \\
0.0437 \\
0.0388 \\
0.0389 \\
0.0325 \\
0.0266 \\
0.0246 \\
0.0234 \\
0.0229 \\
0.0226\end{array}$ & $\begin{array}{l}\text { Minimum } \\
\text { \%Strain } \\
-0.0014 \\
-0.0016 \\
-0.0009 \\
0.0004 \\
0.0050 \\
0.0058 \\
0.0064 \\
0.0111 \\
0.0165 \\
0.0172 \\
0.0167 \\
0.0195 \\
0.0231 \\
0.0228 \\
0.0229 \\
0.0246 \\
0.0269 \\
0.0266 \\
0.0267 \\
0.0262 \\
0.0238 \\
0.0238 \\
0.0245 \\
0.0229 \\
0.0205 \\
0.0206 \\
0.0179 \\
0.0155 \\
0.0154 \\
0.0131 \\
0.0112 \\
0.0105 \\
0.0078 \\
0.0062 \\
0.0038 \\
0.0042 \\
0.0060 \\
0.0065\end{array}$ & $\begin{array}{c}\text { Angle } \\
\text { Degrees } \\
24.16 \\
32.74 \\
20.25 \\
17.57 \\
-21.53 \\
-15.87 \\
-11.21 \\
-6.69 \\
-9.29 \\
-7.55 \\
-9.45 \\
-8.74 \\
-9.02 \\
-8.50 \\
-8.77 \\
-9.00 \\
-8.01 \\
-8.34 \\
-7.96 \\
-6.94 \\
-7.39 \\
-6.94 \\
-7.72 \\
-6.92 \\
-6.26 \\
-6.20 \\
-4.73 \\
-6.18 \\
-6.71 \\
-6.73 \\
-5.93 \\
-6.97 \\
-8.33 \\
-6.73 \\
-7.56 \\
-0.49 \\
-8.14 \\
-8.96\end{array}$ & $\begin{array}{c}\text { Pressure } \\
\text { psig } \\
9.89 \\
19.55 \\
29.57 \\
39.42 \\
49.16 \\
54.50 \\
59.57 \\
64.20 \\
69.32 \\
74.16 \\
80.16 \\
85.61 \\
90.58 \\
95.69 \\
100.92 \\
103.25 \\
106.11 \\
108.31 \\
111.08 \\
113.24 \\
117.83 \\
120.92 \\
123.28 \\
122.97 \\
125.82 \\
125.60 \\
127.84 \\
130.19 \\
132.53 \\
135.33 \\
134.42 \\
138.35 \\
140.16 \\
142.63 \\
145.78 \\
142.52 \\
0.22\end{array}$ & $\begin{array}{l}\text { Maximum } \\
\text { \%Strain } \\
0.0103 \\
0.0222 \\
0.0335 \\
0.0439 \\
0.0538 \\
0.0599 \\
0.0670 \\
0.0744 \\
0.0824 \\
0.0913 \\
0.1043 \\
0.1132 \\
0.1221 \\
0.1338 \\
0.1468 \\
0.1547 \\
0.1626 \\
0.1691 \\
0.1799 \\
0.1893 \\
0.2121 \\
0.2361 \\
0.2634 \\
0.2702 \\
0.2941 \\
0.3051 \\
0.3237 \\
0.3710 \\
0.4403 \\
0.5774 \\
0.6452 \\
0.8515 \\
1.0044 \\
1.1438 \\
1.2676 \\
1.3071 \\
1.0017\end{array}$ & $\begin{array}{r}\text { Minimum } \\
\text { zStrain } \\
0.0045 \\
0.0090 \\
0.0137 \\
0.0179 \\
0.0217 \\
0.0243 \\
0.0267 \\
0.0293 \\
0.0319 \\
0.0340 \\
0.0344 \\
0.0367 \\
0.0387 \\
0.0399 \\
0.0415 \\
0.0410 \\
0.0426 \\
0.0433 \\
0.0440 \\
0.0448 \\
0.0471 \\
0.0486 \\
0.0493 \\
0.0498 \\
0.0515 \\
0.0526 \\
0.0537 \\
0.0555 \\
0.0566 \\
0.0577 \\
0.0561 \\
0.0604 \\
0.0647 \\
0.0748 \\
0.0870 \\
0.0852 \\
-0.0105\end{array}$ & $\begin{array}{c}\text { Angle } \\
\text { Degrees } \\
-8.69 \\
-6.03 \\
-5.28 \\
-6.62 \\
-7.87 \\
-7.88 \\
-7.52 \\
-6.62 \\
-6.37 \\
-6.78 \\
-6.73 \\
-6.85 \\
-6.88 \\
-7.45 \\
-7.94 \\
-8.39 \\
-8.57 \\
-8.78 \\
-9.25 \\
-9.33 \\
-9.82 \\
-9.94 \\
-9.87 \\
-9.65 \\
-9.77 \\
-9.70 \\
-9.67 \\
-9.38 \\
-8.82 \\
-8.21 \\
-7.79 \\
-6.11 \\
-4.95 \\
-4.62 \\
-4.54 \\
-4.66 \\
-4.86\end{array}$ \\
\hline
\end{tabular}

High Pressure test 


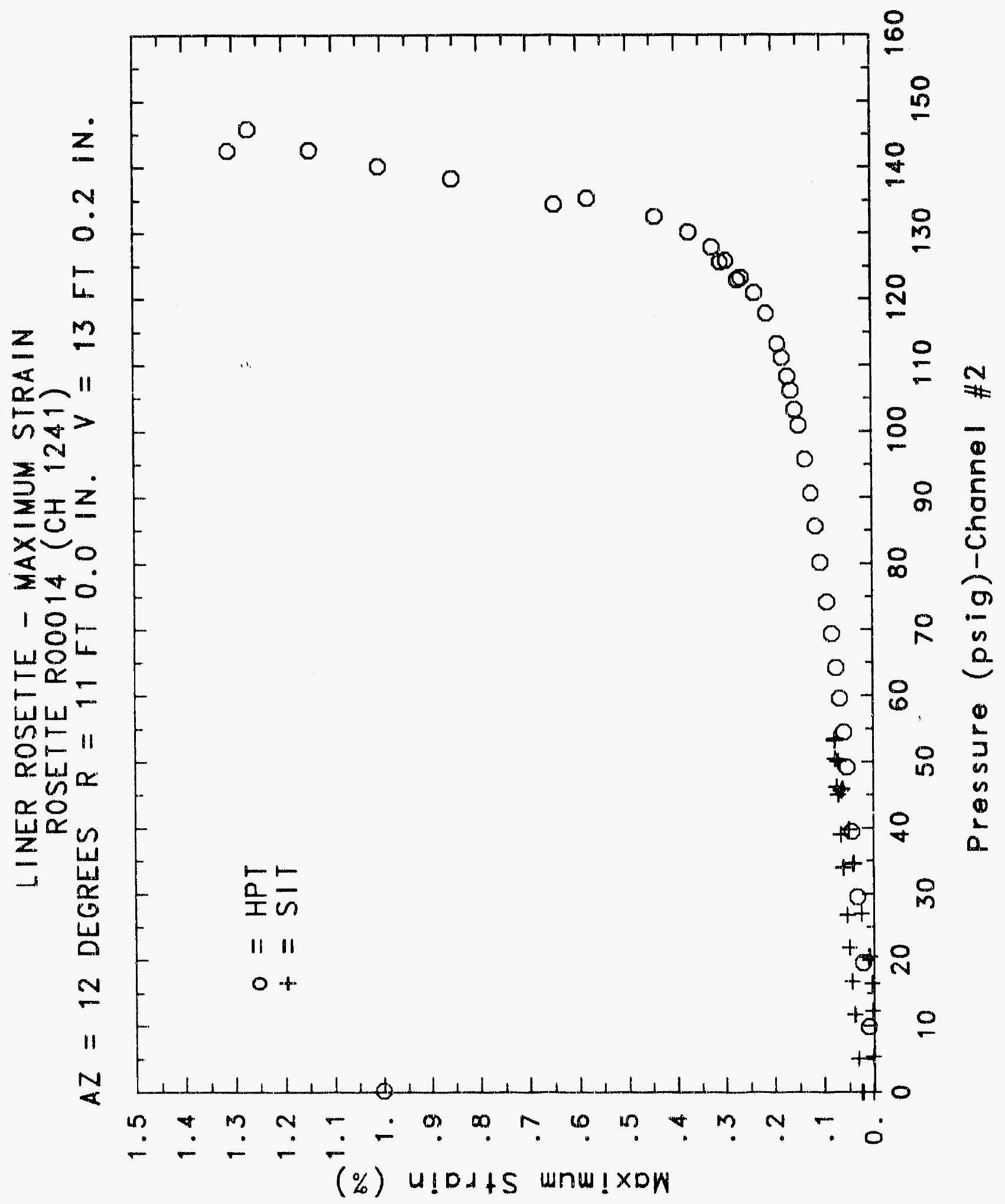

C. $36(1)$ 


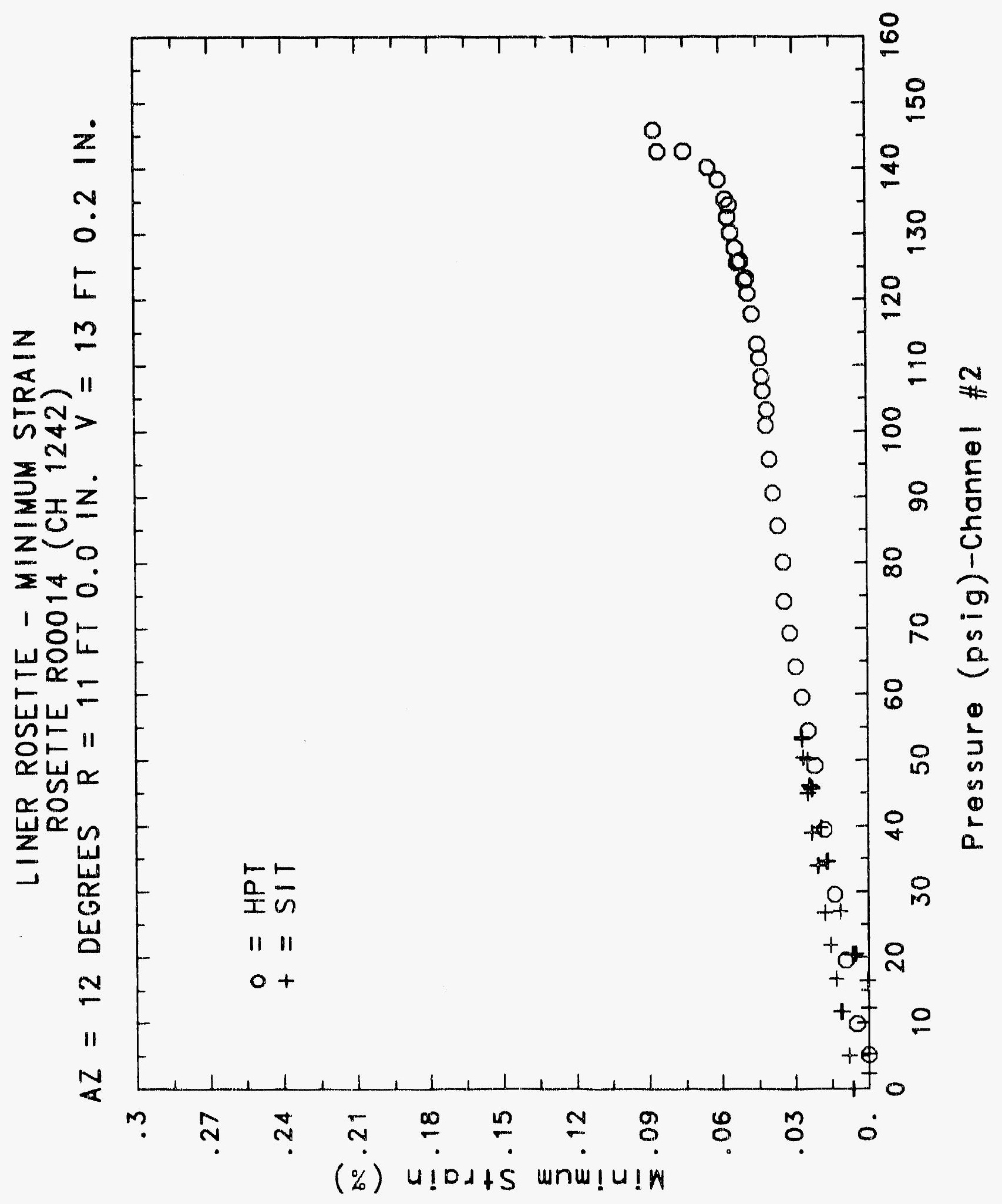




\title{
Reinforced Concrete Test Data
}

\author{
Rosette 15 Channels $1244 \quad 1245 \quad 1246$
}

Structural INTEGRITY TEST

\begin{tabular}{|c|c|c|c|c|c|c|c|}
\hline $\begin{array}{c}\text { Pressure } \\
\text { psig } \\
-0.05 \\
5.33 \\
12.31 \\
16.44 \\
20.51 \\
20.46 \\
20.47 \\
27.05 \\
34.69 \\
34.53 \\
34.53 \\
39.70 \\
45.90 \\
45.65 \\
45.62 \\
50.10 \\
53.47 \\
53.29 \\
53.21 \\
50.49 \\
46.14 \\
46.13 \\
44.97 \\
38.98 \\
33.99 \\
33.96 \\
26.83 \\
21.88 \\
21.90 \\
16.69 \\
11.74 \\
11.77 \\
5.05 \\
0.02 \\
-0.04 \\
-0.02 \\
-0.02 \\
0.02\end{array}$ & $\begin{array}{l}\text { Maximum } \\
\text { XStrain } \\
0.0009 \\
0.0005 \\
0.0005 \\
0.0004 \\
0.0042 \\
0.0058 \\
0.0066 \\
0.0178 \\
0.0322 \\
0.0341 \\
0.0344 \\
0.0411 \\
0.0515 \\
0.0534 \\
0.0536 \\
0.0590 \\
0.0637 \\
0.0640 \\
0.0646 \\
0.0631 \\
0.0597 \\
0.0596 \\
0.0562 \\
0.0517 \\
0.0480 \\
0.0475 \\
0.0411 \\
0.0368 \\
0.0368 \\
0.0326 \\
0.0287 \\
0.0287 \\
0.0236 \\
0.0189 \\
0.0180 \\
0.0172 \\
0.0167 \\
0.0161\end{array}$ & $\begin{array}{l}\text { Minimum } \\
\text { XStrain } \\
-0.0007 \\
-0.0010 \\
-0.0009 \\
-0.0004 \\
0.0003 \\
0.0007 \\
0.0013 \\
0.0035 \\
0.0063 \\
0.0066 \\
0.0063 \\
0.0068 \\
0.0097 \\
0.0091 \\
0.0098 \\
0.0105 \\
0.0113 \\
0.0114 \\
0.0111 \\
0.0109 \\
0.0103 \\
0.0100 \\
0.0104 \\
0.0102 \\
0.0084 \\
0.0093 \\
0.0070 \\
0.0071 \\
0.0069 \\
0.0061 \\
0.0047 \\
0.0047 \\
0.0037 \\
0.0033 \\
0.0017 \\
0.0022 \\
0.0026 \\
0.0036\end{array}$ & $\begin{array}{c}\text { Angle } \\
\text { Degrees } \\
-12.66 \\
22.57 \\
18.84 \\
33.84 \\
-1.10 \\
-5.00 \\
-2.25 \\
-9.71 \\
-12.39 \\
-13.34 \\
-13.42 \\
-13.32 \\
-12.03 \\
-12.53 \\
-11.67 \\
-11.69 \\
-11.86 \\
-11.56 \\
-11.36 \\
-11.16 \\
-11.15 \\
-10.80 \\
-12.18 \\
-11.43 \\
-12.78 \\
-12.90 \\
-13.79 \\
-13.21 \\
-13.59 \\
-14.33 \\
-15.53 \\
-15.06 \\
-15.85 \\
-16.24 \\
-12.25 \\
-15.53 \\
-17.69 \\
-16.80\end{array}$ & $\begin{array}{c}\text { Pressure } \\
\text { Psig } \\
9.89 \\
19.55 \\
29.57 \\
39.42 \\
49.16 \\
54.50 \\
59.57 \\
64.20 \\
69.32 \\
74.16 \\
80.16 \\
85.61 \\
90.58 \\
95.69 \\
100.92 \\
103.25 \\
106.11 \\
108.31 \\
111.08 \\
113.24 \\
117.83 \\
120.92 \\
123.28 \\
122.97 \\
125.82 \\
125.60 \\
127.84 \\
130.19 \\
132.53 \\
135.33 \\
134.42 \\
138.35 \\
140.16 \\
142.63 \\
145.78 \\
142.52 \\
0.22\end{array}$ & $\begin{array}{l}\text { Maximum } \\
\text { XStrain } \\
0.0086 \\
0.0180 \\
0.0275 \\
0.0363 \\
0.0451 \\
0.0502 \\
0.0555 \\
0.0609 \\
0.0672 \\
0.0737 \\
0.0830 \\
0.0890 \\
0.0949 \\
0.1024 \\
0.1104 \\
0.1150 \\
0.1199 \\
0.1238 \\
0.1303 \\
0.1357 \\
0.1489 \\
0.1605 \\
0.1720 \\
0.1738 \\
0.1857 \\
0.1899 \\
0.1978 \\
0.2138 \\
0.2342 \\
0.2693 \\
0.2827 \\
0.3386 \\
0.4078 \\
0.5392 \\
0.6876 \\
0.7383 \\
0.5174\end{array}$ & $\begin{array}{l}\text { Minimum } \\
\text { XStrain } \\
0.0020 \\
0.0040 \\
0.0059 \\
0.0078 \\
0.0099 \\
0.0116 \\
0.0129 \\
0.0147 \\
0.0163 \\
0.0179 \\
0.0176 \\
0.0185 \\
0.0194 \\
0.0204 \\
0.0213 \\
0.0216 \\
0.0220 \\
0.0232 \\
0.02 .39 \\
0.0251 \\
0.0274 \\
0.0290 \\
0.0306 \\
0.0304 \\
0.0325 \\
0.0336 \\
0.0343 \\
0.0378 \\
0.0409 \\
0.0467 \\
0.0478 \\
0.0580 \\
0.0661 \\
0.0828 \\
0.1060 \\
0.1087 \\
0.0046\end{array}$ & $\begin{array}{c}\text { Angle } \\
\text { Degrees } \\
-14.91 \\
-11.45 \\
-11.49 \\
-11.63 \\
-10.78 \\
-10.65 \\
-10.30 \\
-9.63 \\
-9.32 \\
-9.51 \\
-8.93 \\
-9.27 \\
-9.49 \\
-9.87 \\
-10.35 \\
-10.56 \\
-10.96 \\
-11.06 \\
-11.42 \\
-11.56 \\
-12.23 \\
-12.61 \\
-12.75 \\
-12.83 \\
-13.01 \\
-13.01 \\
-13.40 \\
-13.30 \\
-13.47 \\
-13.67 \\
-13.65 \\
-13.63 \\
-13.31 \\
-12.27 \\
-10.29 \\
-9.58 \\
-9.76\end{array}$ \\
\hline
\end{tabular}

High Pressure test 


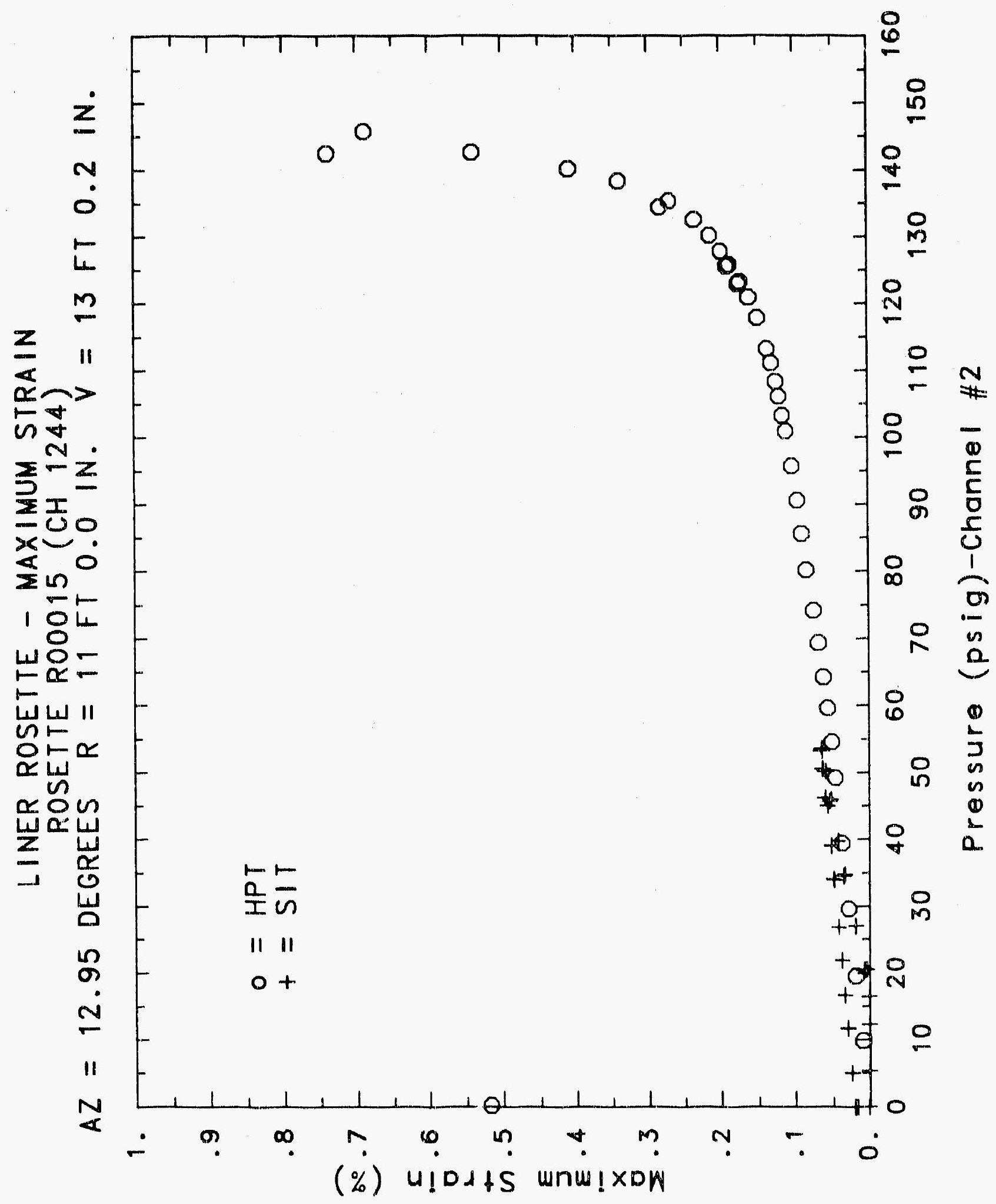




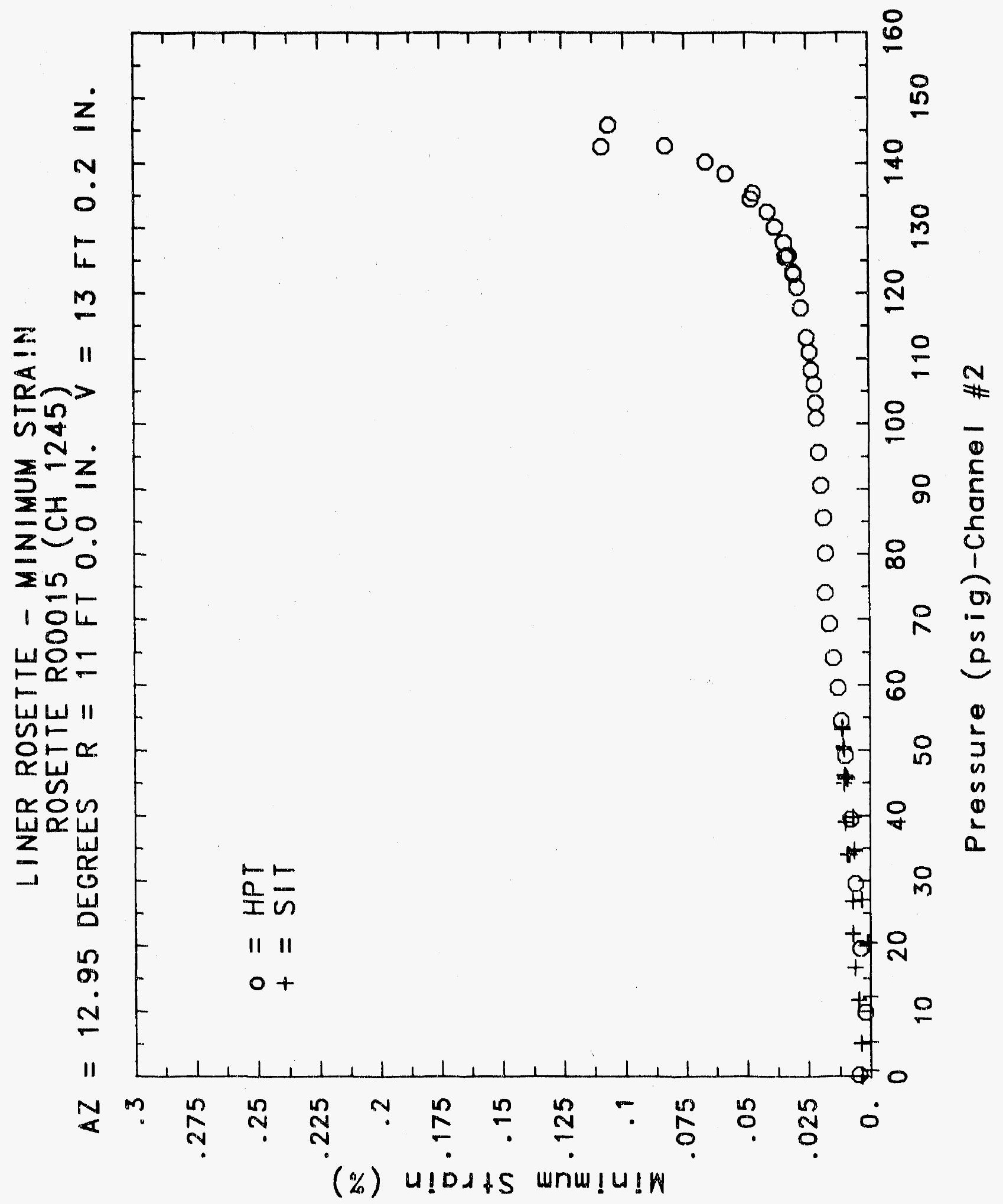




\section{Reinforced Concrete Test Data}

Rosette 16 Channels $1247 \quad 1248 \quad 1249$

Structural IntEgrity TEST

\begin{tabular}{|c|c|c|c|c|c|c|c|}
\hline $\begin{array}{c}\text { ressure } \\
\text { psig } \\
-0.05 \\
5.33 \\
12.31 \\
16.44 \\
20.51 \\
20.46 \\
20.47 \\
27.05 \\
34.69 \\
34.53 \\
34.53 \\
39.70 \\
45.90 \\
45.65 \\
45.62 \\
50.10 \\
53.47 \\
53.29 \\
53.21 \\
50.49 \\
46.14 \\
46.13 \\
44.97 \\
38.98 \\
33\end{array}$ & $\begin{array}{c}\text { Maximum } \\
\text { XStrain } \\
0.0024 \\
0.0029 \\
0.0053 \\
0.0064 \\
0.0096 \\
0.0107 \\
0.0114 \\
0.0230 \\
0.0376 \\
0.0393 \\
0.0396 \\
0.0457 \\
0.0552 \\
0.0566 \\
0.0567 \\
0.0614 \\
0.0658 \\
0.0664 \\
0.0667 \\
0.0651 \\
0.0622 \\
0.0619 \\
0.0597 \\
0.0554 \\
0.0518 \\
0.0514 \\
0.0453 \\
0.0408 \\
0.0409 \\
0.0366 \\
0.0322 \\
0.0322 \\
0.0266 \\
0.0209 \\
0.0197 \\
0.0191 \\
0.0186 \\
0.0183\end{array}$ & $\begin{array}{r}\text { Minimum } \\
\text { XStrain } \\
-0.0011 \\
0.0005 \\
-0.0011 \\
-0.0004 \\
0.0013 \\
0.0018 \\
0.0023 \\
0.0051 \\
0.0083 \\
0.0081 \\
0.0087 \\
0.0094 \\
0.0110 \\
0.0111 \\
0.0112 \\
0.0125 \\
0.0128 \\
0.0121 \\
0.0119 \\
0.0110 \\
0.0098 \\
0.0097 \\
0.0092 \\
0.0093 \\
0.0074 \\
0.0079 \\
0.0066 \\
0.0059 \\
0.0055 \\
0.0044 \\
0.0032 \\
0.0034 \\
0.0025 \\
0.0017 \\
-0.0012 \\
-0.0004 \\
0.0004 \\
0.0014\end{array}$ & $\begin{array}{c}\text { Angle } \\
\text { Degrees } \\
40.51 \\
40.47 \\
43.51 \\
44.26 \\
-27.34 \\
-25.81 \\
-23.44 \\
-21.67 \\
-22.15 \\
-21.88 \\
-20.94 \\
-21.13 \\
-20.89 \\
-19.65 \\
-19.62 \\
-19.41 \\
-19.37 \\
-19.07 \\
-18.90 \\
-18.74 \\
-18.30 \\
-17.70 \\
-19.77 \\
-18.08 \\
-18.31 \\
-18.12 \\
-17.53 \\
-16.62 \\
-16.90 \\
-15.90 \\
-15.50 \\
-14.11 \\
-11.71 \\
-8.07 \\
-7.76 \\
-9.21 \\
-10.64 \\
-9.87\end{array}$ & $\begin{array}{c}\text { Pressure } \\
\text { psig } \\
9.89 \\
19.55 \\
29.57 \\
39.42 \\
49.16 \\
54.50 \\
59.57 \\
64.20 \\
69.32 \\
74.16 \\
80.16 \\
85.61 \\
90.58 \\
95.69 \\
100.92 \\
103.25 \\
106.11 \\
108.31 \\
111.08 \\
113.24 \\
117.83 \\
120.92 \\
123.28 \\
122.97 \\
125.82 \\
125.60 \\
127.84 \\
130.19 \\
132.53 \\
135.33 \\
134.42 \\
138.35 \\
140.16 \\
142.63 \\
145.78 \\
142.52 \\
0.22\end{array}$ & $\begin{array}{c}\text { Maximum } \\
\text { xStrain } \\
0.0124 \\
0.0221 \\
0.0317 \\
0.0405 \\
0.0484 \\
0.0536 \\
0.0583 \\
0.0631 \\
0.0683 \\
0.0742 \\
0.0802 \\
0.0854 \\
0.0902 \\
0.0958 \\
0.1011 \\
0.1035 \\
0.1067 \\
0.1089 \\
0.1121 \\
0.1146 \\
0.1205 \\
0.1246 \\
0.1282 \\
0.1283 \\
0.1329 \\
0.1338 \\
0.1371 \\
0.1433 \\
0.1502 \\
0.1617 \\
0.1639 \\
0.1808 \\
0.1980 \\
0.2330 \\
0.2819 \\
0.3036 \\
0.1354\end{array}$ & $\begin{array}{l}\text { Minimum } \\
\text { XStrain } \\
0.0026 \\
0.0044 \\
0.0070 \\
0.0095 \\
0.0127 \\
0.0133 \\
0.0152 \\
0.0169 \\
0.0188 \\
0.0194 \\
0.0195 \\
0.0187 \\
0.0183 \\
0.0178 \\
0.0172 \\
0.0170 \\
0.0166 \\
0.0169 \\
0.0164 \\
0.0162 \\
0.0160 \\
0.0160 \\
0.0150 \\
0.0155 \\
0.0155 \\
0.0158 \\
0.0169 \\
0.0170 \\
0.0194 \\
0.0222 \\
0.0231 \\
0.0303 \\
0.0374 \\
0.0517 \\
0.0687 \\
0.0661 \\
-0.0524\end{array}$ & $\begin{array}{c}\text { Angle } \\
\text { Degrees } \\
-27.59 \\
-25.56 \\
-24.40 \\
-23.73 \\
-23.06 \\
-23.12 \\
-22.28 \\
-22.23 \\
-21.74 \\
-22.48 \\
-21.31 \\
-21.94 \\
-22.49 \\
-23.06 \\
-23.72 \\
-23.91 \\
-24.59 \\
-24.87 \\
-25.48 \\
-25.99 \\
-27.39 \\
-28.33 \\
-29.05 \\
-29.26 \\
-30.05 \\
-30.50 \\
-30.82 \\
-31.50 \\
-32.06 \\
-32.84 \\
-32.82 \\
-32.87 \\
-32.04 \\
-29.83 \\
-27.06 \\
-26.24 \\
-29.62\end{array}$ \\
\hline
\end{tabular}

high Pressure test 


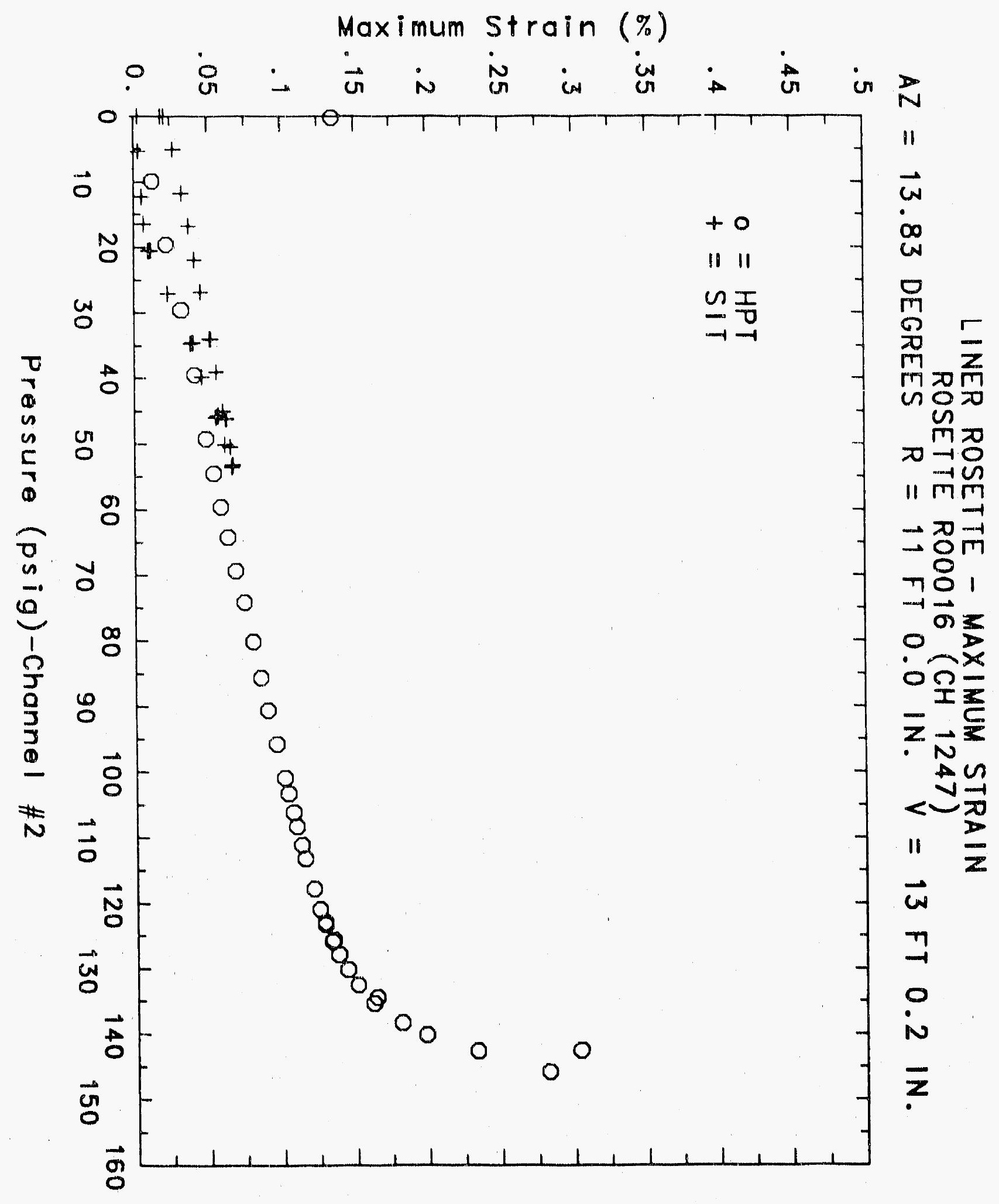




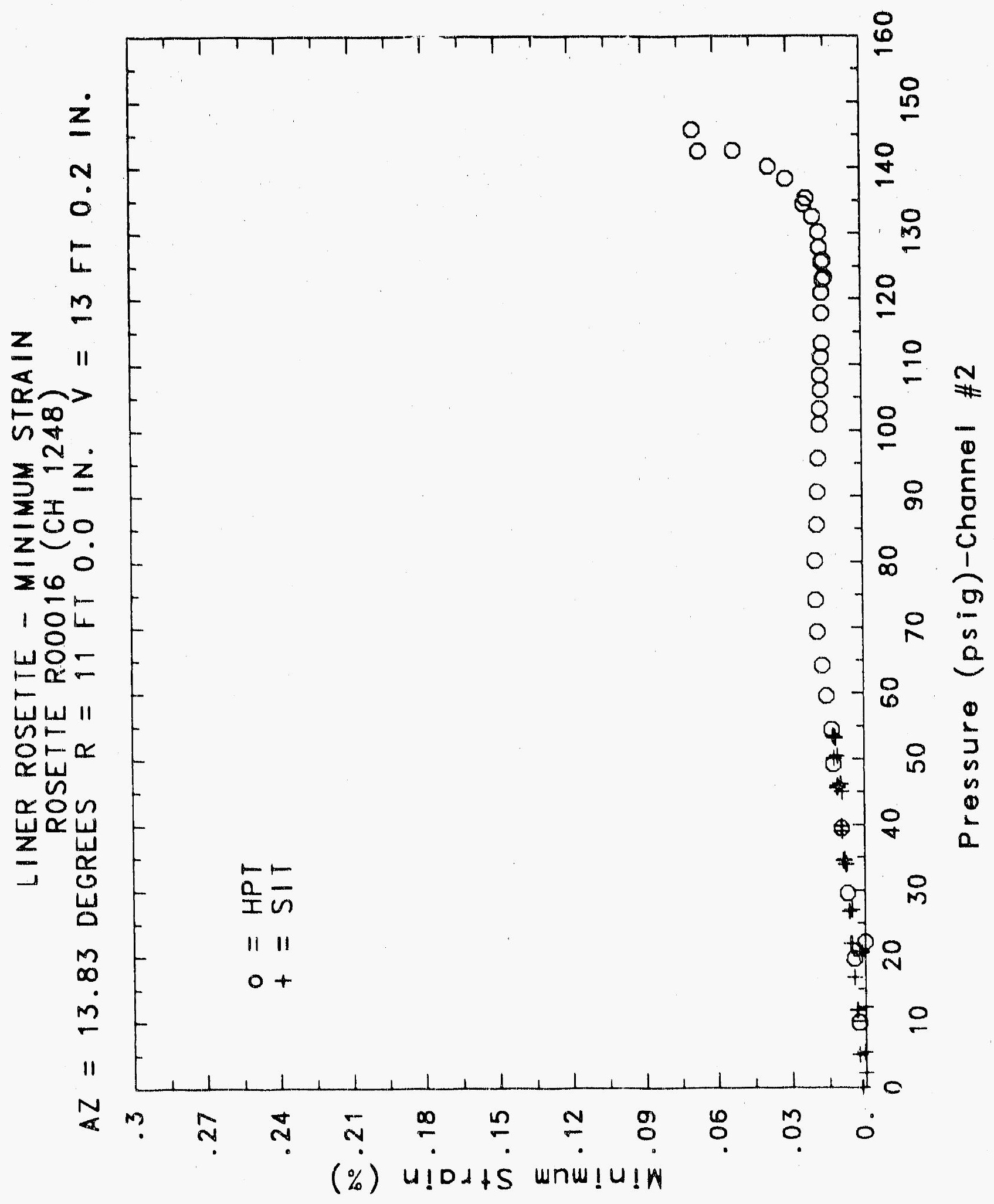




\section{Reinforced Concrete Test Data}

\section{Rosette 17 Channels $1260 \quad 1261 \quad 1262$}

Structural Integrity Test

\begin{tabular}{|c|c|c|c|c|c|c|c|}
\hline $\begin{array}{c}\text { ressure } \\
\text { psig } \\
-0.05 \\
5.33 \\
12.31 \\
16.44 \\
20.51 \\
20.46 \\
20.47 \\
27.05 \\
34.69 \\
34.53 \\
34.53 \\
39.70 \\
45.90 \\
45.65 \\
45.62 \\
50.10 \\
53.47 \\
53.29 \\
53.21 \\
50.49 \\
46.14 \\
46.13 \\
44.97 \\
38.98 \\
33.99 \\
33.96 \\
26.83 \\
21.88 \\
21.90 \\
16.69 \\
11.74 \\
11.77 \\
5\end{array}$ & $\begin{array}{r}\text { Maximum } \\
\text { XStrain } \\
0.0005 \\
-0.0058 \\
-0.0171 \\
-0.0238 \\
-0.0261 \\
-0.0251 \\
-0.0253 \\
-0.0208 \\
-0.0065 \\
-0.0052 \\
-0.0046 \\
0.0009 \\
0.0099 \\
0.0106 \\
0.0107 \\
0.0152 \\
0.0192 \\
0.0197 \\
0.0196 \\
0.0186 \\
0.0161 \\
0.0158 \\
0.0165 \\
0.0127 \\
0.0088 \\
0.0090 \\
0.0028 \\
-0.0018 \\
-0.0024 \\
-0.0040 \\
-0.0022 \\
-0.0033 \\
0.0042 \\
0.0125 \\
0.0126 \\
0.0123 \\
0.0124 \\
0.0112\end{array}$ & $\begin{array}{l}\text { Minimum } \\
\text { xStrain } \\
-0.0011 \\
-0.0197 \\
-0.0338 \\
-0.0407 \\
-0.0435 \\
-0.0422 \\
-0.0413 \\
-0.0388 \\
-0.0333 \\
-0.0324 \\
-0.0323 \\
-0.0294 \\
-0.0239 \\
-0.0238 \\
-0.0237 \\
-0.0208 \\
-0.0180 \\
-0.0177 \\
-0.0180 \\
-0.0191 \\
-0.0210 \\
-0.0216 \\
-0.0207 \\
-0.0230 \\
-0.0250 \\
-0.0248 \\
-0.0272 \\
-0.0286 \\
-0.0285 \\
-0.0299 \\
-0.0286 \\
-0.0292 \\
-0.0208 \\
-0.0095 \\
-0.0080 \\
-0.0085 \\
-0.0085 \\
-0.0080\end{array}$ & $\begin{array}{c}\text { Angle } \\
\text { Degrees } \\
-41.16 \\
14.21 \\
24.59 \\
26.93 \\
24.11 \\
23.01 \\
20.15 \\
9.73 \\
1.57 \\
0.58 \\
0.06 \\
-1.72 \\
-2.53 \\
-1.92 \\
-1.72 \\
-2.40 \\
-3.50 \\
-2.15 \\
-2.55 \\
-1.82 \\
-0.63 \\
0.21 \\
-1.27 \\
-0.92 \\
-0.32 \\
0.00 \\
2.09 \\
4.60 \\
3.47 \\
9.83 \\
13.98 \\
14.02 \\
12.83 \\
6.90 \\
12.01 \\
9.85 \\
10.80 \\
5.23\end{array}$ & $\begin{array}{c}\text { Pressure } \\
\text { psig } \\
9.89 \\
19.55 \\
29.57 \\
39.42 \\
49.16 \\
54.50 \\
59.57 \\
64.20 \\
69.32 \\
74.16 \\
80.16 \\
85.61 \\
90.58 \\
95.69 \\
100.92 \\
103.25 \\
106.11 \\
108.31 \\
111.08 \\
113.24 \\
117.83 \\
120.92 \\
123.28 \\
122.97 \\
125.82 \\
125.60 \\
127.84 \\
130.19 \\
132.53 \\
135.33 \\
134.42 \\
138.35 \\
140.16 \\
142.63 \\
145.78 \\
142.52 \\
0.22\end{array}$ & $\begin{array}{l}\text { Maximum } \\
\text { xStrain } \\
-0.0113 \\
-0.0147 \\
-0.0060 \\
0.0028 \\
0.0111 \\
0.0162 \\
0.0207 \\
0.0264 \\
0.0321 \\
0.0381 \\
0.0430 \\
0.0485 \\
0.0538 \\
0.0597 \\
0.0666 \\
0.0698 \\
0.0739 \\
0.0770 \\
0.0813 \\
0.0849 \\
0.0938 \\
0.1008 \\
0.1072 \\
0.1085 \\
0.1171 \\
0.1203 \\
0.1272 \\
0.1411 \\
0.1607 \\
0.1974 \\
0.2098 \\
0.2644 \\
0.3153 \\
0.4062 \\
0.5131 \\
0.5411 \\
0.3663\end{array}$ & $\begin{array}{l}\text { Minimum } \\
\text { XStrain } \\
-0.0220 \\
-0.0244 \\
-0.0209 \\
-0.0162 \\
-0.0103 \\
-0.0068 \\
-0.0030 \\
0.0006 \\
0.0046 \\
0.0088 \\
0.0121 \\
0.0158 \\
0.0192 \\
0.0223 \\
0.0258 \\
0.0270 \\
0.0287 \\
0.0303 \\
0.0323 \\
0.0333 \\
0.0364 \\
0.0374 \\
0.0390 \\
0.0390 \\
0.0413 \\
0.0412 \\
0.0420 \\
0.0442 \\
0.0466 \\
0.0502 \\
0.0484 \\
0.0588 \\
0.0704 \\
0.1020 \\
0.1491 \\
0.1480 \\
0.0242\end{array}$ & $\begin{array}{c}\text { Angle } \\
\text { Degrees } \\
21.12 \\
8.15 \\
-8.19 \\
-10.99 \\
-12.32 \\
-13.38 \\
-13.63 \\
-15.64 \\
-16.83 \\
-18.94 \\
-19.18 \\
-21.98 \\
-24.50 \\
-27.00 \\
-29.12 \\
-29.69 \\
-31.22 \\
-32.18 \\
-33.36 \\
-34.46 \\
-36.94 \\
-38.52 \\
-39.42 \\
-39.31 \\
-40.36 \\
-40.60 \\
-41.23 \\
-41.99 \\
-42.76 \\
-43.78 \\
-44.24 \\
44.58 \\
43.93 \\
-44.86 \\
-41.80 \\
-41.74 \\
44.05\end{array}$ \\
\hline
\end{tabular}

High Pressure test 


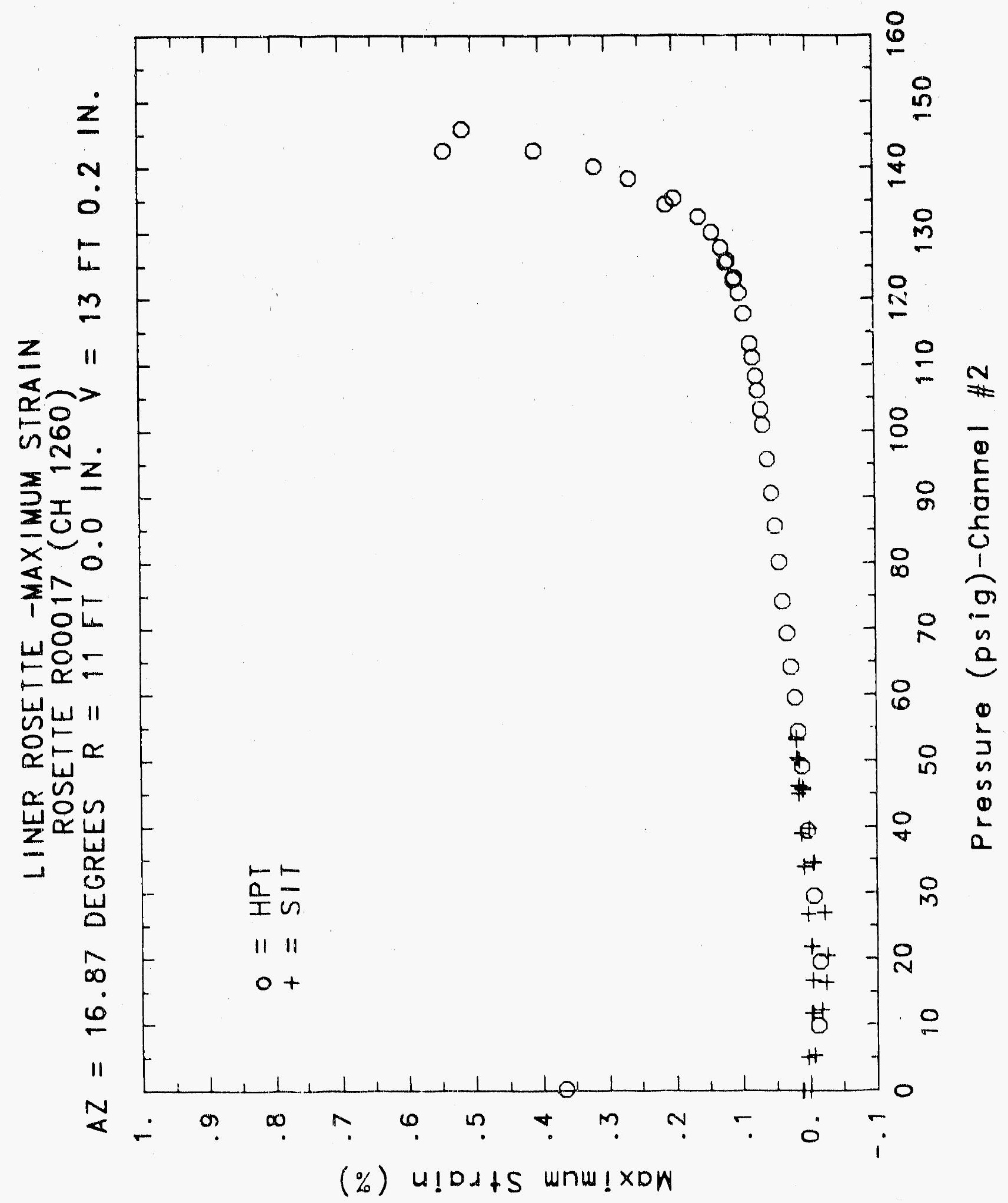




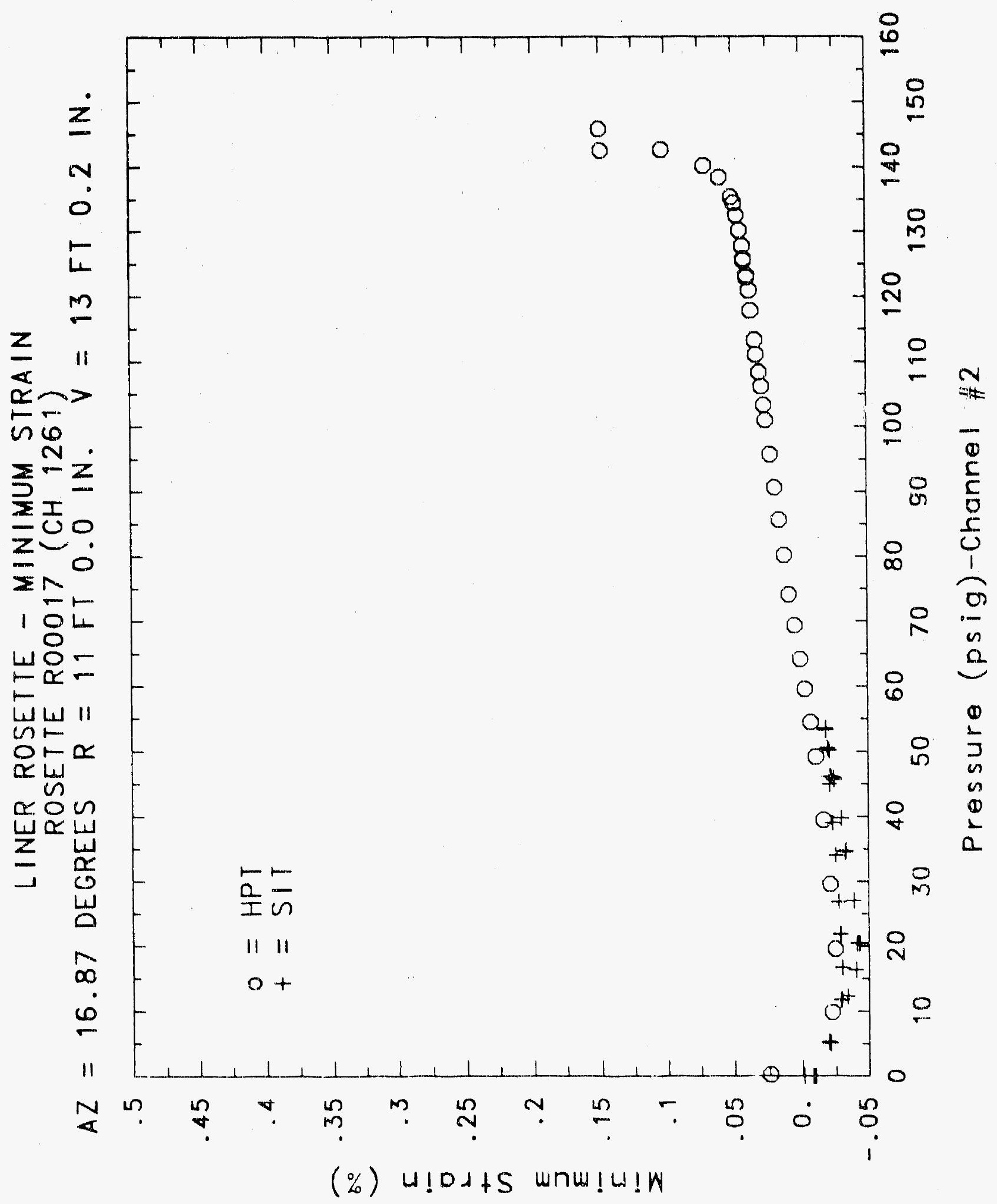




\title{
Reinforced Concrete Test Data
}

\author{
Rosette 18 Channels $1263 \quad 1264 \quad 1265$
}

STRUCTURAL INTEGRITY TEST

\begin{tabular}{|c|c|c|c|c|c|c|c|}
\hline Pressure & Maximum & Minimum & Angle & Pressure & Maxinum & Minimum & Angle \\
\hline psig & \%strain & \%strain & Degrees & psig & xstrain & xstrain & Degrees \\
\hline-0.05 & $0.001^{r}$ & $-0,0008$ & 34.43 & 9.89 & 0.0142 & 0.0001 & 5.30 \\
\hline 5.33 & 0.0037 & -0.0014 & 3.33 & 19.55 & 0.0249 & 0.0043 & 2.26 \\
\hline 12.31 & 0.0077 & -0.0030 & 2.32 & 29.57 & 0.0350 & 0.0089 & -1.16 \\
\hline 16.44 & 0.0102 & -0.0022 & 1.18 & 39.42 & 0.0451 & 0.0136 & -2.13 \\
\hline 20.51 & 0.0152 & -0.0006 & 0.71 & 49.16 & 0.0543 & 0.0177 & -3.59 \\
\hline 20.46 & 0.0166 & -0.0002 & 0.05 & 54.50 & 0.0599 & 0.0202 & -4.16 \\
\hline 20.47 & 0.0173 & 0.0003 & 1.04 & 59.57 & 0.0655 & 0.0229 & -4.79 \\
\hline 27.05 & 0.0273 & 0.0037 & -1.74 & 64.20 & 0.0710 & 0.0266 & -4.78 \\
\hline 34.69 & 0.0403 & 0.0092 & -2.24 & 69.32 & 0.0780 & 0.0293 & -5.18 \\
\hline 34.53 & 0.0420 & 0.0102 & -1.04 & 74.16 & 0.0848 & 0.0330 & .6 .11 \\
\hline 34.53 & 0.0421 & 0.0100 & -2.01 & 80.16 & 0.0897 & 0.0347 & -7.85 \\
\hline 39.70 & 0.0479 & 0.0126 & -2.35 & 85.61 & 0.0962 & 0.0378 & -8.86 \\
\hline 45.90 & 0.0568 & 0.0167 & -4.04 & 90.58 & 0.1025 & 0.0407 & -9.78 \\
\hline 45.65 & 0.0572 & 0.0169 & -3.82 & 95.69 & 0.1099 & 0.0435 & -10.86 \\
\hline 45.62 & 0.0572 & 0.0171 & -3.21 & 100.92 & 0.1182 & 0.0468 & -11.71 \\
\hline 50.10 & 0.0622 & 0.0189 & -4.45 & 103.25 & 0.1224 & 0.0480 & -12.27 \\
\hline 53.47 & 0.0669 & 0.0216 & -3.94 & 106.11 & 0.1271 & 0.0497 & -12.73 \\
\hline 53.29 & $0.067 ?$ & 0.0219 & -3.80 & 108.31 & 0.1308 & 0.0509 & -13.13 \\
\hline 53.21 & 0.0672 & 0.0220 & -4.04 & 111.08 & 0.1360 & 0.0525 & -13.59 \\
\hline 50.49 & 0.0658 & 0.0215 & -3.03 & 113.24 & 0.1401 & 0.0537 & -14.08 \\
\hline 46.14 & 0.0627 & 0.0201 & -2.27 & 117.83 & 0.1493 & 0.0568 & -14.96 \\
\hline 45.13 & 0.0625 & 0.0199 & -1.87 & 120.92 & 0.1558 & 0.0588 & -15.81 \\
\hline 44.97 & 0.0653 & 0.0217 & -1.08 & 123.28 & 0.1624 & 0.0598 & -16.62 \\
\hline 38.98 & 0.0607 & 0.0200 & 0.51 & 122.97 & 0.1639 & 0.0603 & -16.60 \\
\hline 33.99 & 0.0563 & 0.0177 & 1.33 & 125.82 & 0.1715 & 0.0624 & -17.26 \\
\hline 33.96 & 0.0560 & 0.0174 & 0.95 & 125.60 & 0.1742 & 0.0632 & -17.36 \\
\hline 26.83 & 0.0499 & 0.0148 & 3.10 & 127.84 & 0.1800 & 0.0646 & -17.69 \\
\hline 21.88 & 0.0453 & 0.0128 & 5.19 & 130.19 & 0.1913 & 0.0672 & -18.47 \\
\hline 21.90 & 0.0445 & 0.0126 & 4.85 & 132.53 & 0.2078 & 0.0697 & -19.07 \\
\hline 16.69 & 0.0394 & 0.0105 & 5.53 & 135.33 & 0.2384 & 0.0749 & -19.97 \\
\hline 11.74 & 0.0350 & 0.0083 & 8.69 & 134.2 & 0.2480 & 0.0746 & -20.34 \\
\hline 11.77 & 0.0336 & 0.0073 & 7.14 & 138.35 & 0.3110 & 0.0846 & -19.50 \\
\hline 5.05 & 0.0268 & 0.0056 & 11.48 & 140.16 & 0.3914 & 0.0940 & -18.73 \\
\hline 0.02 & 0.0203 & 0.0060 & 6.87 & 142.63 & $0.598 ?$ & 0.1088 & .15 .94 \\
\hline .0 .04 & 0.0184 & 0.0048 & y.js & 145.78 & 0.7945 & 0.1284 & $-14.6 \dot{y}$ \\
\hline-0.02 & 0.0195 & 0.0057 & 8.53 & 142.52 & 0.8096 & 0.1254 & -15.76 \\
\hline-0.02 & 0.0198 & 0.0059 & 10.12 & 0.22 & 0.5352 & 0.0286 & -17.34 \\
\hline 0.02 & 0.0196 & 0.0058 & 10.38 & & & & \\
\hline
\end{tabular}

High Pressure test 


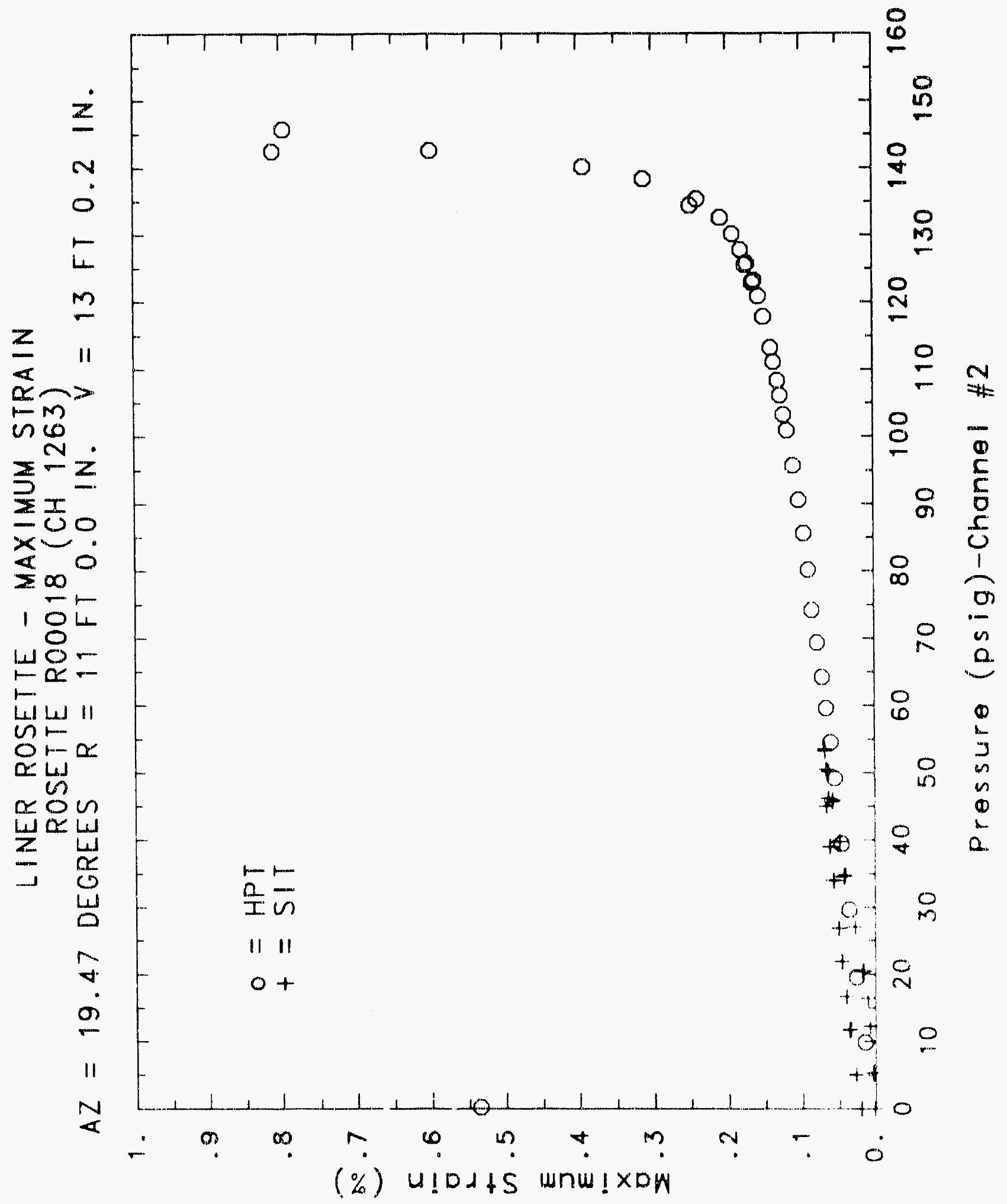

C. 372 


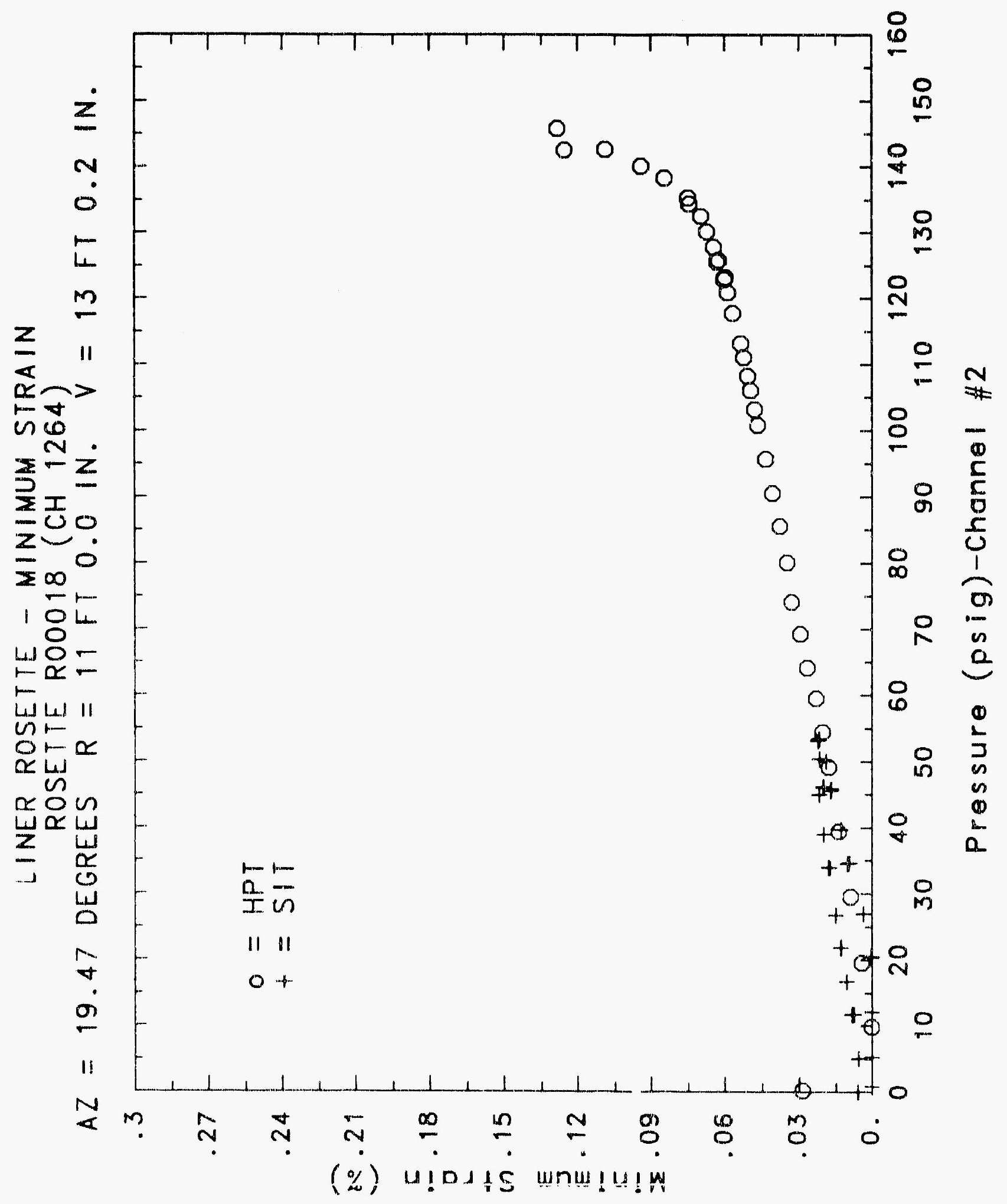




\title{
Reinforced Concrete Test Data
}

\author{
$\begin{array}{llllll}\text { Rosette } & 19 & \text { Chanmels } & 1266 & 1267 & 1268\end{array}$
}

StRUCTURAL INTEgRITY TEST

\begin{tabular}{|c|c|c|c|c|c|c|c|}
\hline $\begin{array}{c}\text { Pressure } \\
\text { psig } \\
-0.05 \\
5.33 \\
12.31 \\
16.44 \\
20.51 \\
20.46 \\
20.47 \\
27.05 \\
34.69 \\
34.53 \\
34.53 \\
39.70 \\
45.90 \\
45.65 \\
45.62 \\
50.10 \\
53.47 \\
53.29 \\
53.21 \\
50.49 \\
46.14 \\
46.13 \\
44.97 \\
38.98 \\
33.99 \\
33.96 \\
26.83 \\
21.88 \\
21.90 \\
16.69 \\
11.74 \\
11.77 \\
5.05 \\
0.02 \\
-0.04 \\
-6.002 \\
-0.02 \\
0.02\end{array}$ & $\begin{array}{c}\text { Maximum } \\
\text { yStrain } \\
0.0017 \\
0.0011 \\
0.0024 \\
0.0041 \\
0.0068 \\
0.0075 \\
0.0079 \\
0.0167 \\
0.0228 \\
0.0232 \\
0.0235 \\
0.0258 \\
0.0292 \\
0.0301 \\
0.0305 \\
0.0324 \\
0.0351 \\
0.0365 \\
0.0371 \\
0.0368 \\
0.0356 \\
0.0353 \\
0.0338 \\
0.0311 \\
0.0289 \\
0.0290 \\
0.0250 \\
0.0217 \\
0.0217 \\
0.0184 \\
0.0149 \\
0.0148 \\
0.0102 \\
0.0078 \\
0.0065 \\
0.0057 \\
0.0056 \\
0.0060\end{array}$ & $\begin{array}{l}\text { Minimum } \\
\text { XStrain } \\
-0.0012 \\
-0.0028 \\
-0.0068 \\
-0.0089 \\
-0.0120 \\
-0.0128 \\
-0.0128 \\
-0.0195 \\
-0.0252 \\
-0.0256 \\
-0.0259 \\
-0.0278 \\
-0.0302 \\
-0.0314 \\
-0.0315 \\
-0.0326 \\
-0.0342 \\
-0.0350 \\
-0.0353 \\
-0.0355 \\
-0.0356 \\
-0.0355 \\
-0.0348 \\
-0.0346 \\
-0.0340 \\
-0.0338 \\
-0.0328 \\
-0.0309 \\
-0.0308 \\
-0.0288 \\
-0.0260 \\
-0.0260 \\
-0.0217 \\
-0.0186 \\
-0.0178 \\
-0.0181 \\
-0.0181 \\
-0.0179\end{array}$ & $\begin{array}{c}\text { Angle } \\
\text { Degrees } \\
-42.92 \\
-4.39 \\
6.49 \\
6.11 \\
3.75 \\
4.50 \\
4.05 \\
2.91 \\
2.62 \\
2.19 \\
2.31 \\
0.85 \\
-1.83 \\
-2.06 \\
-2.80 \\
-3.30 \\
-4.72 \\
-5.46 \\
-5.47 \\
-5.81 \\
-6.23 \\
-6.32 \\
-7.41 \\
-8.03 \\
-8.70 \\
-8.52 \\
-9.57 \\
-10.85 \\
-10.81 \\
-12.41 \\
-14.98 \\
-14.40 \\
-19.89 \\
-27.84 \\
-30.18 \\
-31.54 \\
-32.32 \\
-32.31\end{array}$ & $\begin{array}{c}\text { Pressure } \\
\text { psig } \\
9.89 \\
19.55 \\
29.57 \\
39.42 \\
49.16 \\
54.50 \\
59.57 \\
64.20 \\
69.32 \\
74.16 \\
80.16 \\
85.61 \\
90.58 \\
95.69 \\
100.92 \\
103.25 \\
106.11 \\
108.31 \\
111.08 \\
113.24 \\
117.83 \\
120.92 \\
123.28 \\
122.97 \\
125.82 \\
125.60 \\
127.84 \\
130.19 \\
132.53 \\
135.33 \\
134.42 \\
138.35 \\
140.16 \\
142.63 \\
145.78 \\
142.52 \\
0.22\end{array}$ & $\begin{array}{l}\text { Maximum } \\
\text { zStrain } \\
0.0066 \\
0.0137 \\
0.0212 \\
0.0288 \\
0.0366 \\
0.0420 \\
0.0473 \\
0.0539 \\
0.0635 \\
0.0751 \\
0.1045 \\
0.1388 \\
0.1840 \\
0.2543 \\
0.3303 \\
0.3637 \\
0.3944 \\
0.4188 \\
0.4612 \\
0.4897 \\
0.5559 \\
0.6082 \\
0.6387 \\
0.6411 \\
0.6720 \\
0.6750 \\
0.7057 \\
0.7421 \\
0.7932 \\
0.8573 \\
0.8624 \\
0.9514 \\
1.0084 \\
1.0726 \\
1.1532 \\
1.1603 \\
0.8793\end{array}$ & $\begin{array}{l}\text { Minimum } \\
\text { \%Strain } \\
-0.0072 \\
-0.0125 \\
-0.0173 \\
-0.0212 \\
-0.0240 \\
-0.0253 \\
-0.0272 \\
-0.0303 \\
-0.0353 \\
-0.0414 \\
-0.0550 \\
-0.0668 \\
-0.0784 \\
-0.0928 \\
-0.1070 \\
-0.1129 \\
-0.1182 \\
-0.1221 \\
-0.1288 \\
-0.1335 \\
-0.1420 \\
-0.1490 \\
-0.1534 \\
-0.1543 \\
-0.1573 \\
-0.1580 \\
-0.1601 \\
-0.1638 \\
-0.1671 \\
-0.1723 \\
-0.1738 \\
-0.1800 \\
-0.1849 \\
-0.1888 \\
-0.1951 \\
-0.1384 \\
-0.1796\end{array}$ & $\begin{array}{c}\text { Angle } \\
\text { Degrees } \\
2.76 \\
2.12 \\
2.26 \\
2.53 \\
2.40 \\
1.85 \\
-0.08 \\
-3.04 \\
-5.45 \\
-6.98 \\
-8.50 \\
-8.63 \\
-8.82 \\
-9.42 \\
-9.81 \\
-9.98 \\
-10.14 \\
-10.26 \\
-10.48 \\
-10.61 \\
-10.83 \\
-10.87 \\
-10.86 \\
-10.86 \\
-10.85 \\
-10.87 \\
-10.86 \\
-10.90 \\
-10.78 \\
-10.53 \\
-10.51 \\
-10.40 \\
-10.27 \\
-10.16 \\
-9.91 \\
-3.88 \\
-10.84\end{array}$ \\
\hline
\end{tabular}

High Pressure test 


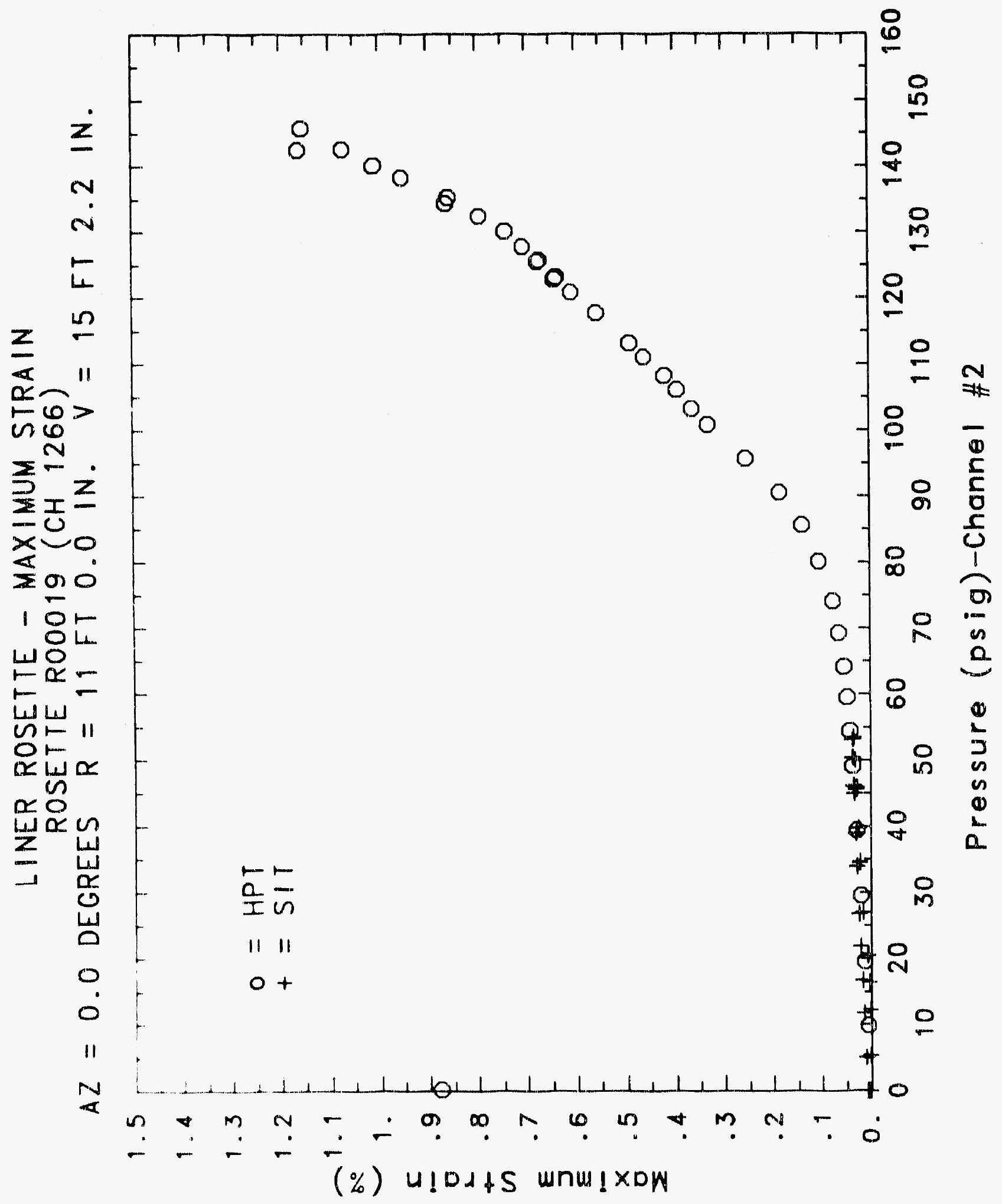

C -375 


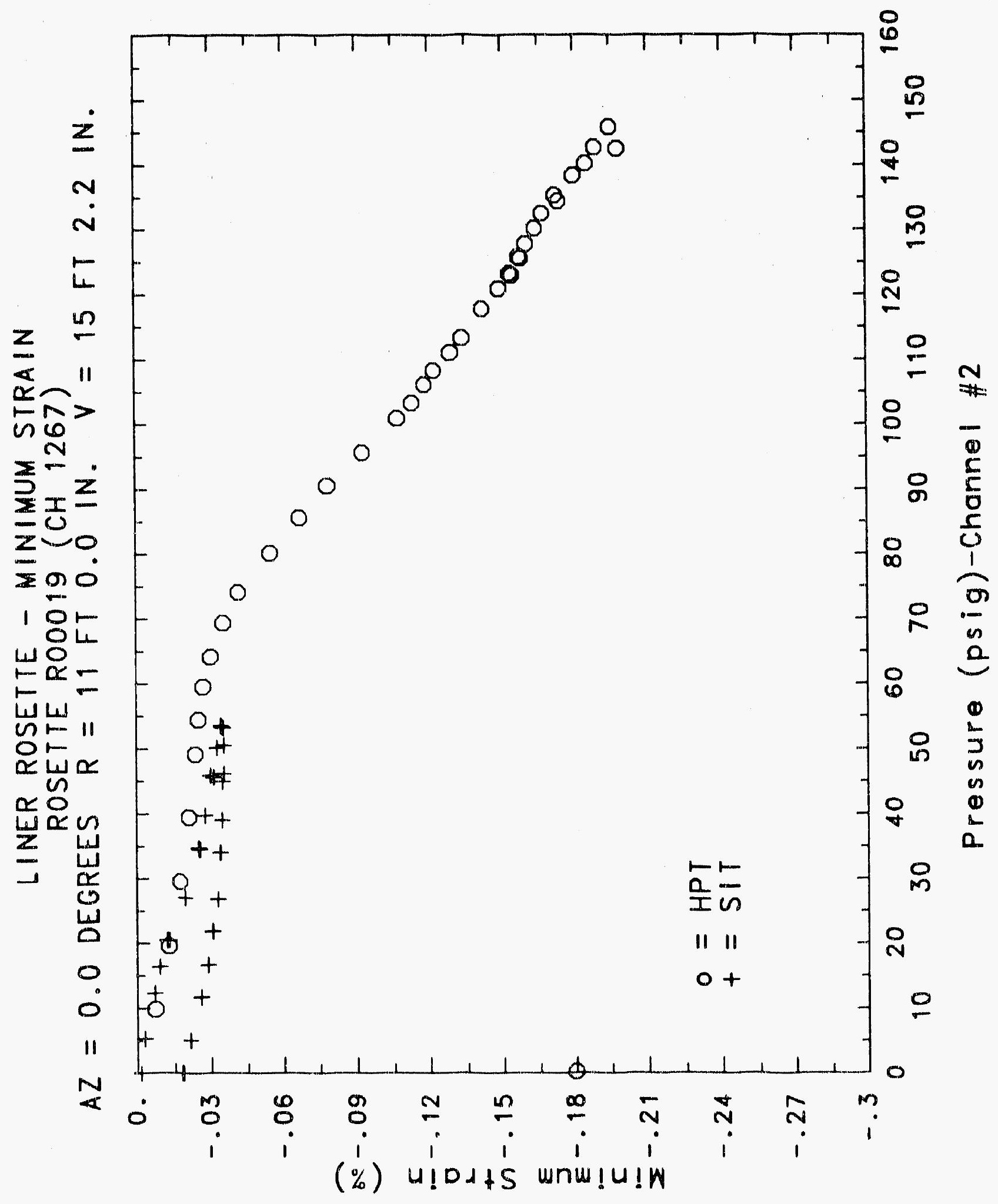




\title{
Reinforced Concrete Test Data
}

\author{
Rosette 20 Channels $1269 \quad 1280 \quad 1281$
}

\section{Structural Integrity Test}

\begin{tabular}{|c|c|c|c|c|c|c|c|}
\hline $\begin{array}{c}\text { Pressure } \\
\text { psig } \\
-0.05 \\
5.33 \\
12.31 \\
16.44 \\
20.51 \\
20.46 \\
20.47 \\
27.05 \\
34.69 \\
34.53 \\
34.53 \\
39.70 \\
45.90 \\
45.65 \\
45.62 \\
50.10 \\
53.47 \\
53.29 \\
53.21 \\
50.49 \\
46.14 \\
46.13 \\
44.97 \\
38.98 \\
33.99 \\
33.96 \\
26.83 \\
21.88 \\
21.90 \\
16.69 \\
11.74 \\
11.77 \\
5.05 \\
0.02 \\
-0.04 \\
-0.02 \\
-0.02 \\
0.02\end{array}$ & $\begin{array}{l}\text { Maximum } \\
\text { xStrain } \\
0.0020 \\
0.0018 \\
0.0041 \\
0.0058 \\
0.0093 \\
0.0106 \\
0.0111 \\
0.0222 \\
0.0329 \\
0.0341 \\
0.0344 \\
0.0388 \\
0.0460 \\
0.0484 \\
0.0496 \\
0.0537 \\
0.0590 \\
0.0616 \\
0.0625 \\
0.0625 \\
0.0612 \\
0.0609 \\
0.0586 \\
0.0559 \\
0.0529 \\
0.0525 \\
0.0473 \\
0.0430 \\
0.0427 \\
0.0382 \\
0.0324 \\
0.0328 \\
0.0255 \\
0.0201 \\
0.0184 \\
0.0177 \\
0.0181 \\
0.0166\end{array}$ & $\begin{array}{l}\text { Minimum } \\
\text { XStrain } \\
-0.0007 \\
0.0001 \\
-0.0006 \\
-0.0009 \\
-0.0016 \\
-0.0022 \\
-0.0025 \\
-0.0051 \\
-0.0061 \\
-0.0076 \\
-0.0079 \\
-0.0078 \\
-0.0073 \\
-0.0092 \\
-0.0092 \\
-0.0087 \\
-0.0089 \\
-0.0091 \\
-0.0100 \\
-0.0114 \\
-0.0126 \\
-0.0131 \\
-0.0148 \\
-0.0163 \\
-0.0171 \\
-0.0168 \\
-0.0177 \\
-0.0177 \\
-0.0173 \\
-0.0173 \\
-0.0166 \\
-0.0164 \\
-0.0153 \\
-0.0136 \\
-0.0120 \\
-0.0123 \\
-0.0125 \\
-0.0119\end{array}$ & $\begin{array}{c}\text { Angle } \\
\text { Degrees } \\
28.92 \\
-22.56 \\
-4.43 \\
-0.04 \\
-2.02 \\
-3.66 \\
-5.80 \\
-3.85 \\
-3.12 \\
-2.62 \\
-2.98 \\
-2.56 \\
-4.07 \\
-4.25 \\
-4.99 \\
-4.89 \\
-5.47 \\
-6.22 \\
-6.31 \\
-6.12 \\
-6.96 \\
-6.35 \\
-7.38 \\
-8.05 \\
-8.23 \\
-8.24 \\
-8.52 \\
-8.65 \\
-8.77 \\
-9.55 \\
-10.27 \\
-10.45 \\
-13.72 \\
-19.06 \\
-21.19 \\
-23.24 \\
-24.77 \\
-22.70\end{array}$ & $\begin{array}{c}\text { Pressure } \\
\text { psig } \\
9.89 \\
19.55 \\
29.57 \\
39.42 \\
49.16 \\
54.50 \\
59.57 \\
64.20 \\
69.32 \\
74.16 \\
80.16 \\
85.61 \\
90.58 \\
95.69 \\
100.92 \\
103.25 \\
106.11 \\
108.31 \\
111.08 \\
113.24 \\
117.83 \\
120.92 \\
123.28 \\
122.97 \\
125.82 \\
125.60 \\
127.84 \\
130.19 \\
132.53 \\
135.33 \\
134.42 \\
138.35 \\
140.16 \\
142.63 \\
145.78 \\
142.52 \\
0.22\end{array}$ & $\begin{array}{l}\text { Maximum } \\
\text { xStrain } \\
0.0097 \\
0.0189 \\
0.0280 \\
0.0371 \\
0.0462 \\
0.0526 \\
0.0609 \\
0.0731 \\
0.0845 \\
0.0965 \\
0.1218 \\
0.1430 \\
0.1667 \\
0.1936 \\
0.2257 \\
0.2447 \\
0.2645 \\
0.2795 \\
0.3039 \\
0.3201 \\
0.3574 \\
0.3851 \\
0.4040 \\
0.4057 \\
0.4242 \\
0.4263 \\
0.4415 \\
0.4605 \\
0.4849 \\
0.5203 \\
0.5243 \\
0.5736 \\
0.6123 \\
0.6653 \\
0.7366 \\
0.7508 \\
0.5185\end{array}$ & $\begin{array}{l}\text { Minimum } \\
\text { XStrain } \\
-0.0021 \\
-0.0042 \\
-0.0051 \\
-0.0048 \\
-0.0038 \\
-0.0031 \\
-0.0021 \\
-0.0007 \\
0.0015 \\
0.0046 \\
0.0057 \\
0.0102 \\
0.0139 \\
0.0179 \\
0.0234 \\
0.0244 \\
0.0277 \\
0.0307 \\
0.0349 \\
0.0384 \\
0.0489 \\
0.0564 \\
0.0608 \\
0.0603 \\
0.0683 \\
0.0681 \\
0.0750 \\
0.0839 \\
0.0972 \\
0.1130 \\
0.1133 \\
0.1353 \\
0.1501 \\
0.1729 \\
0.2020 \\
0.2075 \\
0.1528\end{array}$ & $\begin{array}{c}\text { Angle } \\
\text { Degrees } \\
7.55 \\
8.16 \\
4.57 \\
3.68 \\
2.61 \\
1.22 \\
0.04 \\
-2.14 \\
-3.59 \\
-5.14 \\
-7.70 \\
-9.15 \\
-9.95 \\
-10.47 \\
-10.70 \\
-10.75 \\
-10.93 \\
-11.04 \\
-11.23 \\
-11.25 \\
-11.24 \\
-11.17 \\
-11.08 \\
-11.08 \\
-11.04 \\
-11.06 \\
-10.91 \\
-10.66 \\
-10.33 \\
-9.95 \\
-9.90 \\
-9.44 \\
-9.19 \\
-8.77 \\
-8.48 \\
-8.38 \\
19.31\end{array}$ \\
\hline
\end{tabular}

\section{High Pressure Test}




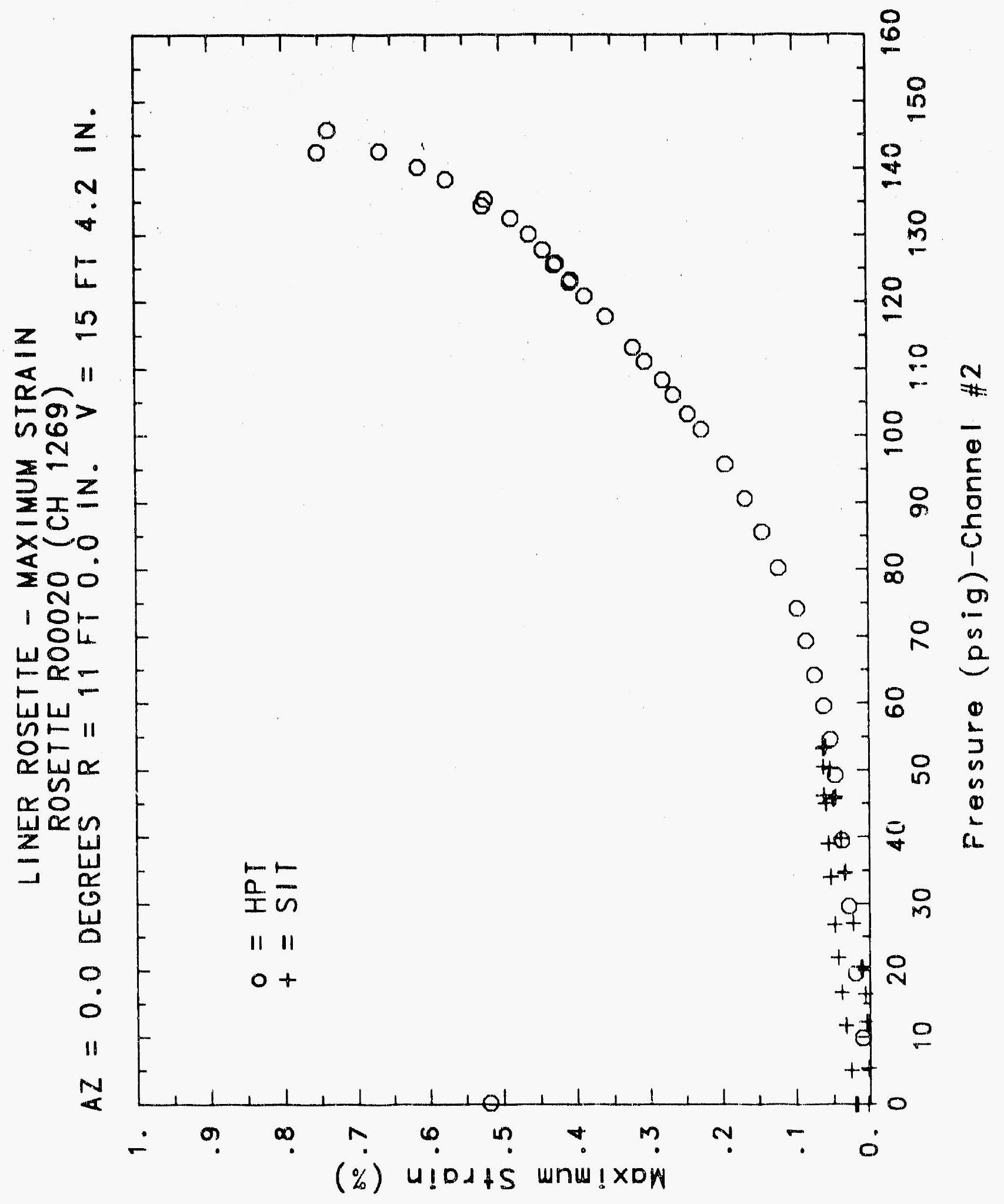




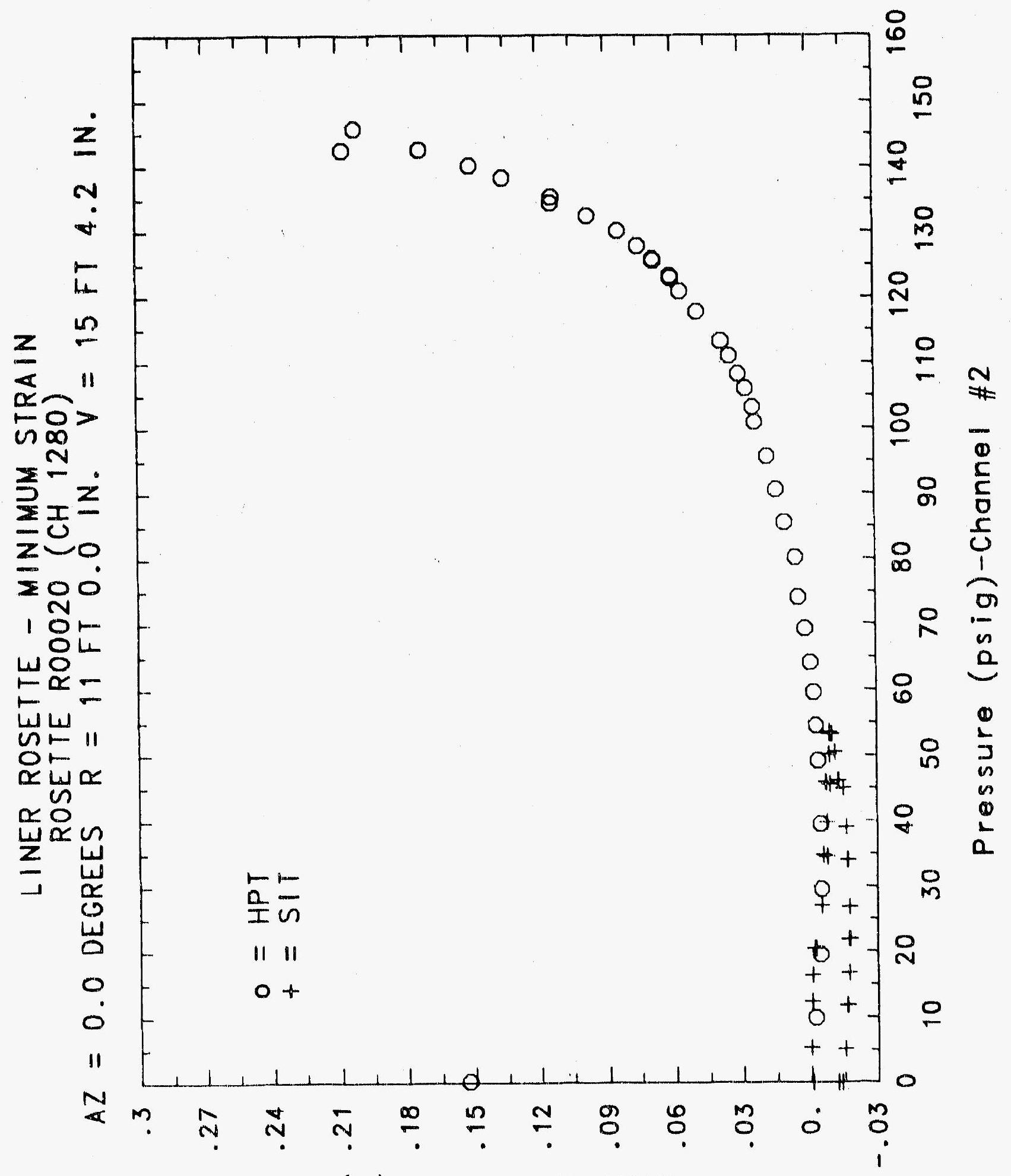

(\%) w!Ddts wnw!u!w 


\section{Reinforced Concrete Test Data}

\begin{tabular}{|c|c|c|c|c|c|c|c|}
\hline $\begin{array}{c}\text { Pressure } \\
\text { psig } \\
\text {-0.05 } \\
5.33 \\
12.31 \\
16.44 \\
20.51 \\
20.46 \\
20.47 \\
27.05 \\
34.69 \\
34.53 \\
34.53 \\
39.70 \\
45.90 \\
45.65 \\
45.62 \\
50.10 \\
53.47 \\
53.29 \\
53.21 \\
50.49 \\
46.14 \\
46.13 \\
44.97 \\
38.98 \\
33.99 \\
33.96 \\
26.83 \\
21.88 \\
21.90 \\
16.69 \\
11.74 \\
11.77 \\
5.05 \\
0.02 \\
-0.04 \\
-0.02 \\
-0.02 \\
0.02\end{array}$ & $\begin{array}{c}\text { Maximum } \\
\text { xStrain } \\
0.0007 \\
0.0017 \\
0.0045 \\
0.0069 \\
0.0103 \\
0.0107 \\
0.0109 \\
0.0192 \\
0.0287 \\
0.0297 \\
0.0298 \\
0.0343 \\
0.0397 \\
0.0410 \\
0.0410 \\
0.0444 \\
0.0471 \\
0.0473 \\
0.0476 \\
0.0472 \\
0.0455 \\
0.0457 \\
0.0432 \\
0.0401 \\
0.0373 \\
0.0370 \\
0.0325 \\
0.0288 \\
0.0286 \\
0.0249 \\
0.0212 \\
0.0208 \\
0.0140 \\
0.0085 \\
0.0076 \\
0.0065 \\
0.0061 \\
0.0059\end{array}$ & $\begin{array}{r}\text { Minimum } \\
\text { xStrain } \\
-0.0018 \\
-0.0003 \\
-0.0004 \\
0.0002 \\
-0.0001 \\
0.0005 \\
0.0000 \\
0.0038 \\
0.0074 \\
0.0069 \\
0.0071 \\
0.0078 \\
0.0080 \\
0.0072 \\
0.0070 \\
0.0078 \\
0.0077 \\
0.0077 \\
0.0079 \\
0.0066 \\
0.0044 \\
0.0043 \\
0.0054 \\
0.0032 \\
0.0007 \\
0.0002 \\
-0.0034 \\
-0.0057 \\
-0.0065 \\
-0.0096 \\
-0.0126 \\
-0.0121 \\
-0.0131 \\
-0.0134 \\
-0.0135 \\
-0.0128 \\
-0.0125 \\
-0.0129\end{array}$ & $\begin{array}{c}\text { Angle } \\
\text { Degrees } \\
-28.64 \\
-22.31 \\
-40.32 \\
-43.55 \\
39.66 \\
38.00 \\
38.30 \\
22.46 \\
14.02 \\
13.34 \\
12.95 \\
13.10 \\
16.73 \\
16.59 \\
16.86 \\
17.62 \\
19.05 \\
19.03 \\
18.34 \\
18.30 \\
18.81 \\
18.54 \\
17.96 \\
17.77 \\
18.46 \\
19.57 \\
20.85 \\
22.10 \\
23.32 \\
25.51 \\
28.31 \\
27.80 \\
31.17 \\
35.23 \\
36.92 \\
38.13 \\
39.36 \\
39.38\end{array}$ & $\begin{array}{c}\text { Pressure } \\
\text { psig } \\
9.89 \\
19.55 \\
29.57 \\
39.42 \\
49.16 \\
54.50 \\
59.57 \\
64.20 \\
69.32 \\
74.16 \\
80.16 \\
85.61 \\
90.58 \\
95.69 \\
100.92 \\
103.25 \\
106.11 \\
108.31 \\
111.08 \\
113.24 \\
117.83 \\
120.92 \\
123.28 \\
122.97 \\
125.82 \\
125.60 \\
127.84 \\
130.19 \\
132.53 \\
135.33 \\
134.42 \\
138.35 \\
140.16 \\
142.63 \\
145.78 \\
142.52 \\
0.22\end{array}$ & $\begin{array}{l}\text { Maximum } \\
\text { xStrain } \\
0.0144 \\
0.0205 \\
0.0299 \\
0.0381 \\
0.0473 \\
0.0532 \\
0.0580 \\
0.0625 \\
0.0669 \\
0.0722 \\
0.0815 \\
0.0886 \\
0.0951 \\
0.1020 \\
0.1082 \\
0.1122 \\
0.1151 \\
0.1175 \\
0.1213 \\
0.1244 \\
0.1309 \\
0.1371 \\
0.1429 \\
0.1429 \\
0.1488 \\
0.1498 \\
0.1554 \\
0.1621 \\
0.1705 \\
0.1832 \\
0.1844 \\
0.2033 \\
0.2185 \\
0.2439 \\
0.2831 \\
0.2920 \\
0.1632\end{array}$ & $\begin{array}{l}\text { Minimum } \\
\text { xStrain } \\
-0.0028 \\
0.0017 \\
0.0056 \\
0.0095 \\
0.0137 \\
0.0163 \\
0.0185 \\
0.0208 \\
0.0234 \\
0.0257 \\
0.0275 \\
0.0320 \\
0.0372 \\
0.0432 \\
0.0498 \\
0.0530 \\
0.0572 \\
0.0607 \\
0.0651 \\
0.0694 \\
0.0796 \\
0.0870 \\
0.0919 \\
0.0924 \\
0.0995 \\
0.0999 \\
0.1059 \\
0.1142 \\
0.1263 \\
0.1416 \\
0.1426 \\
0.1645 \\
0.1804 \\
0.2062 \\
0.2405 \\
0.2501 \\
0.0628\end{array}$ & $\begin{array}{c}\text { Angle } \\
\text { Degrees } \\
-35.92 \\
-24.43 \\
-21.13 \\
-18.89 \\
-17.79 \\
-16.07 \\
-12.61 \\
-8.54 \\
-5.40 \\
-2.10 \\
-0.71 \\
-0.74 \\
-1.61 \\
-2.38 \\
-2.50 \\
-2.96 \\
-3.32 \\
-3.44 \\
-3.48 \\
-3.83 \\
-4.13 \\
-4.15 \\
-4.83 \\
-4.63 \\
-5.64 \\
-6.25 \\
-6.59 \\
-7.62 \\
-8.21 \\
-8.90 \\
-8.74 \\
-11.33 \\
-12.08 \\
-17.20 \\
-24.92 \\
-26.85 \\
-12.12\end{array}$ \\
\hline
\end{tabular}

High Pressure test

\section{Rosette 21 Channels $1282 \quad 1283 \quad 1284$}

Structural InTEgRItY TEST 


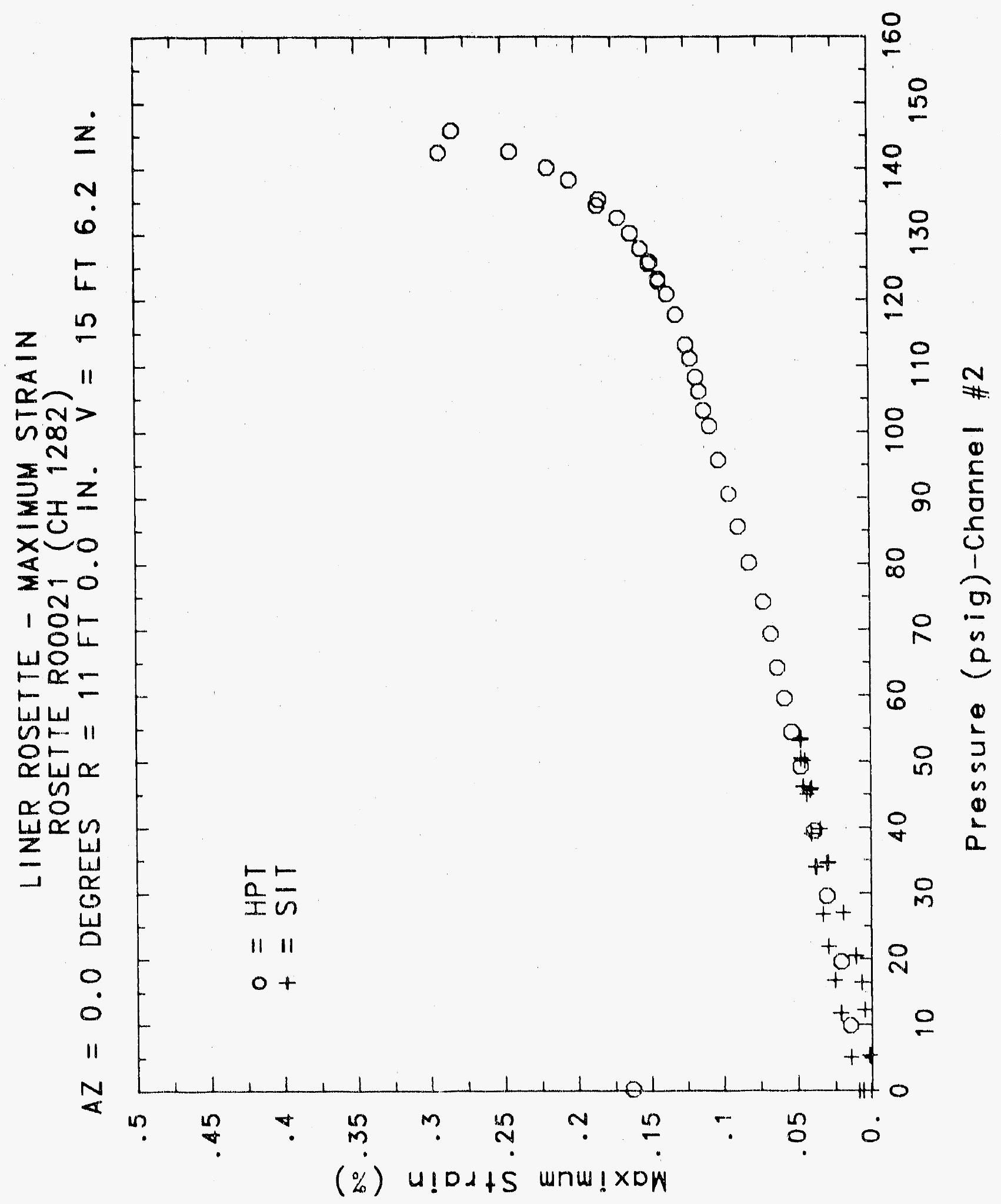




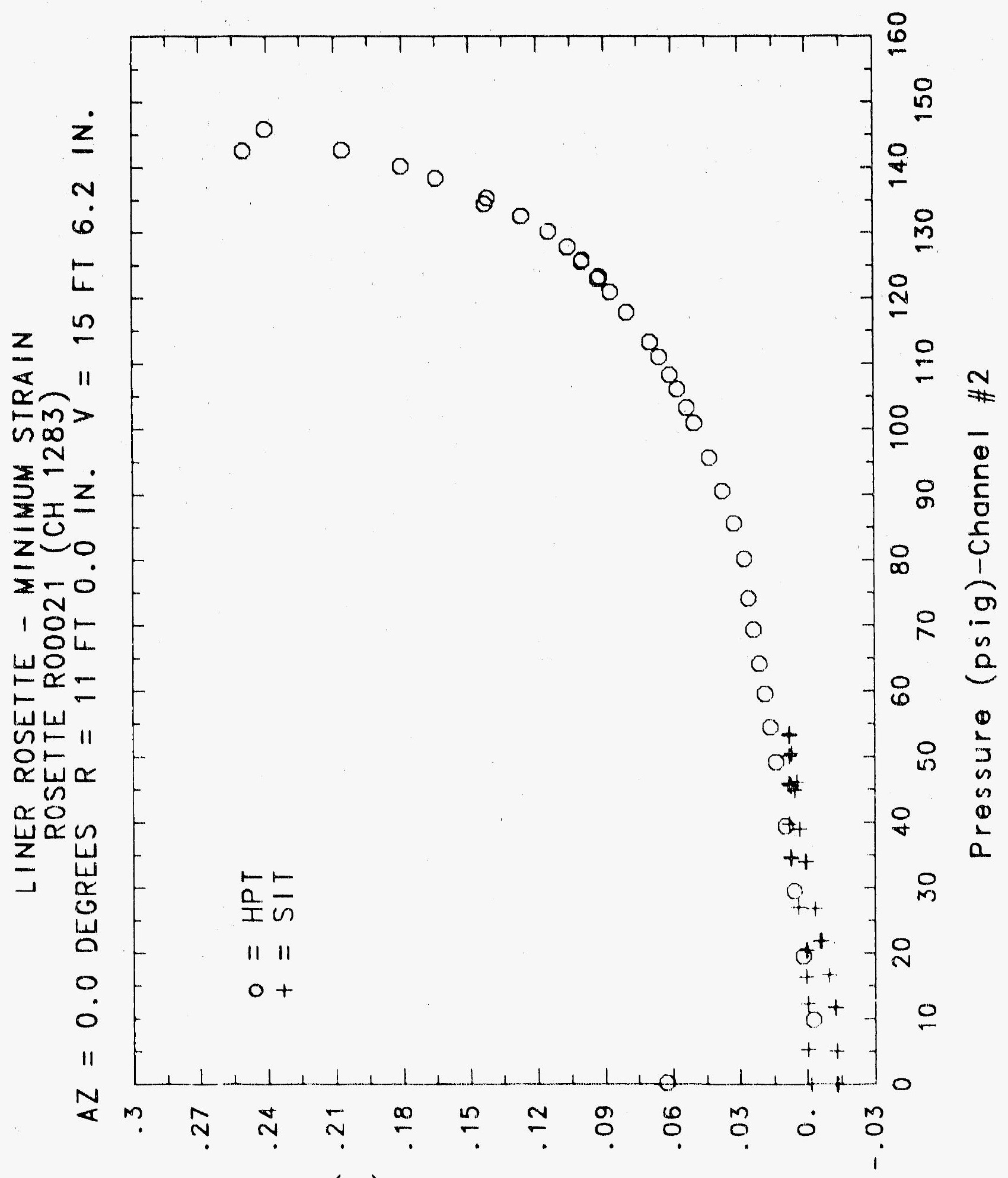

(\%) $u ! D \perp+S$ wnw! $u ! w$ 


\section{Reinforced Concrete Test Data}

\section{Rosette 22 Channels $1285 \quad 1286 \quad 1287$}

Structural Integrity Test

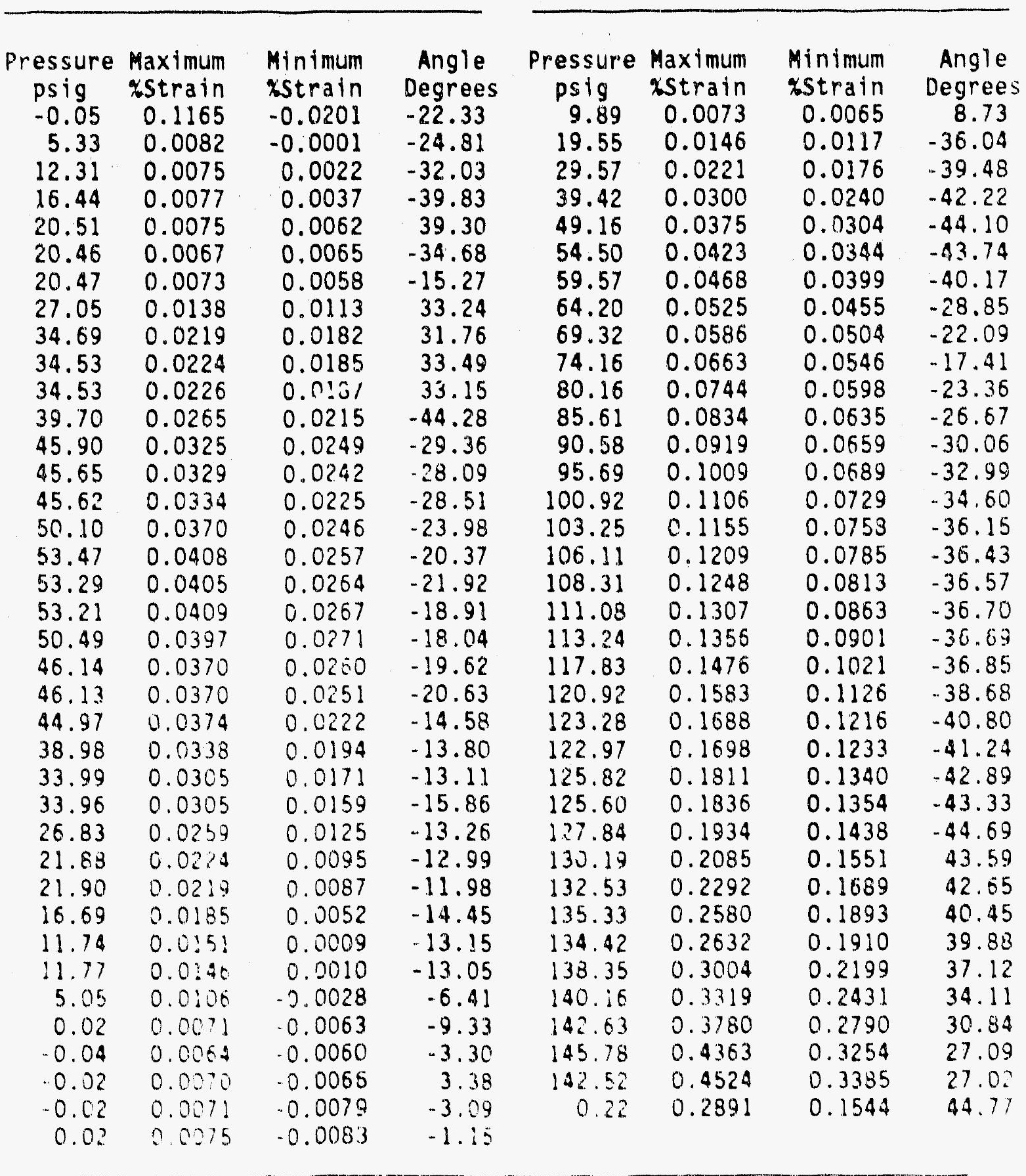

High Pressure test 


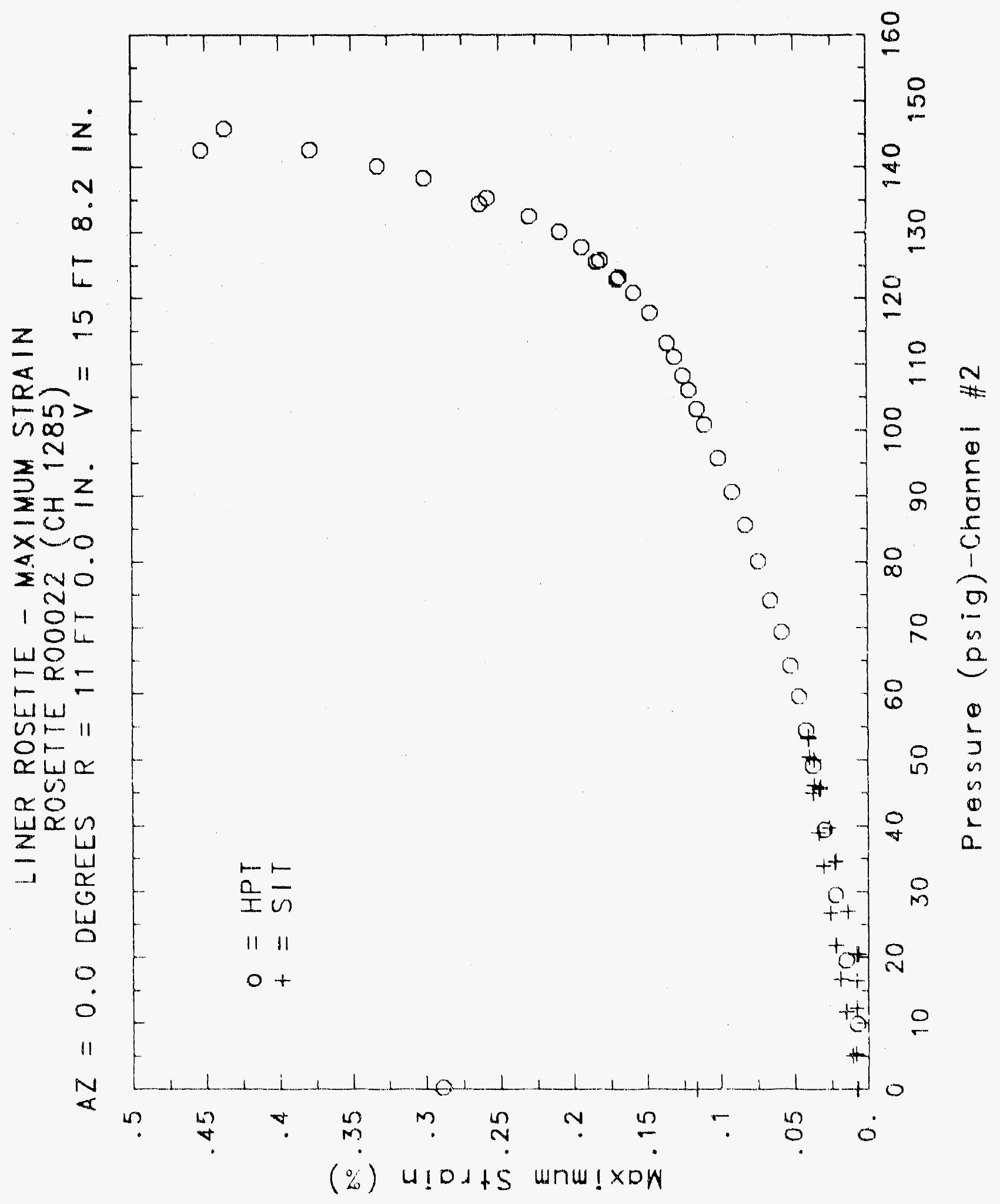




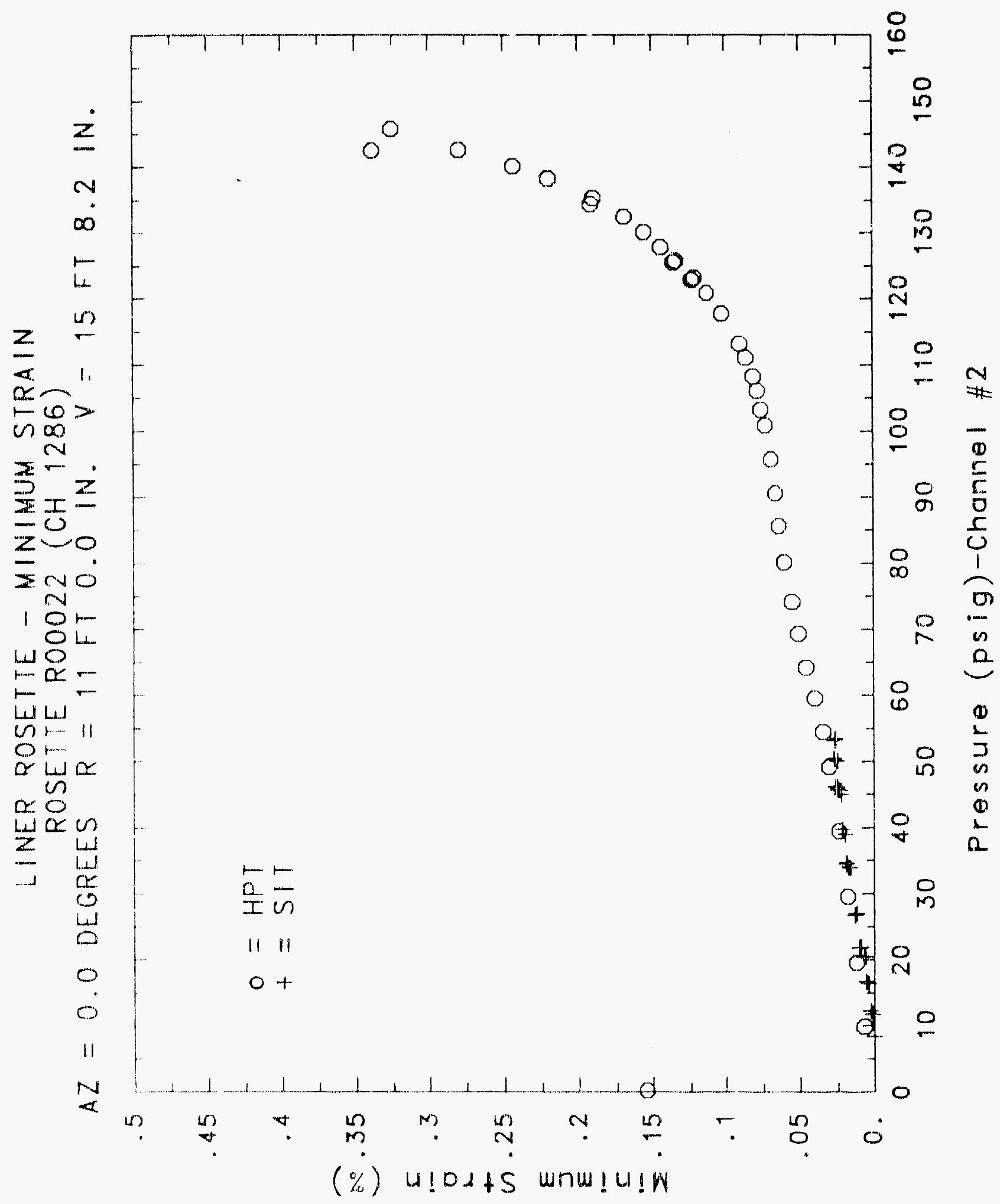


ㅇำ以

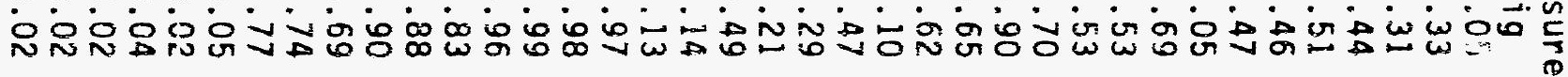

00000000000000000000000000000000000000 要

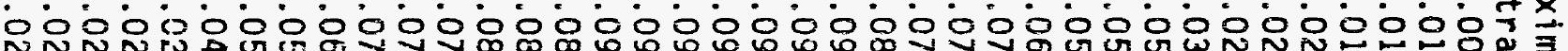

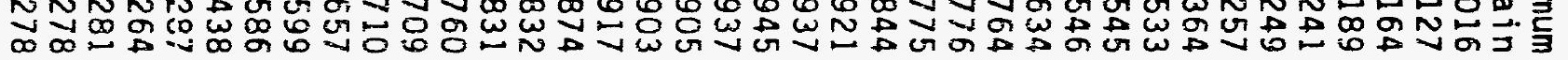

00000000000000000000000000000000000000 势

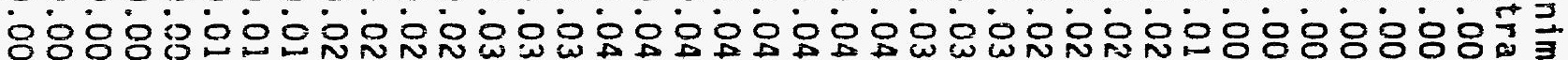

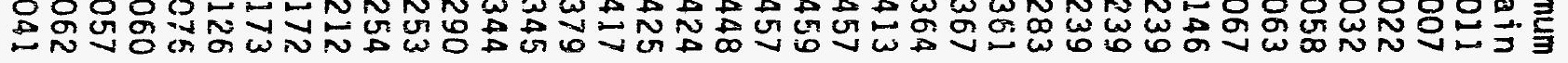

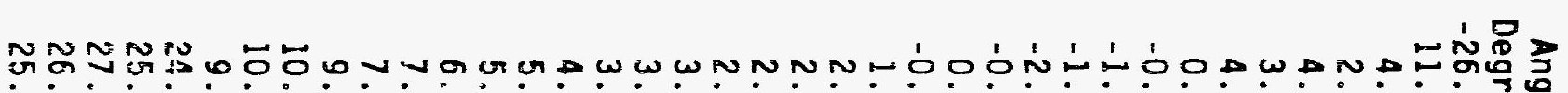

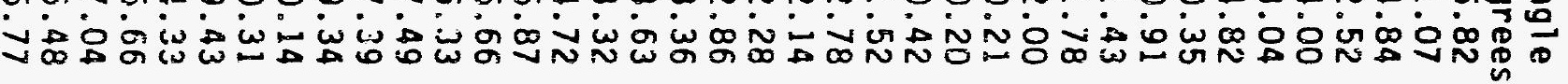

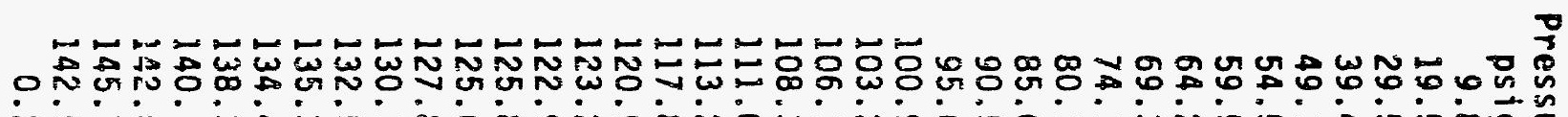

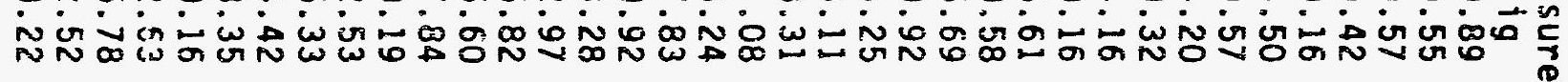
$0000000000000000000000000000000000000 \%$

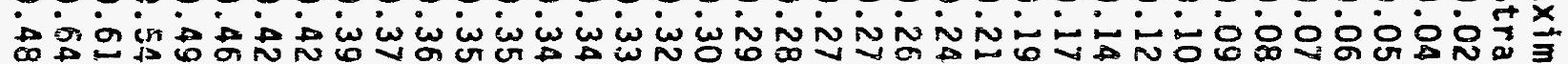

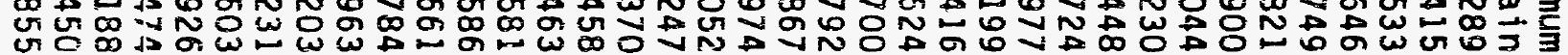

0000000000000000000000000000000000000 势

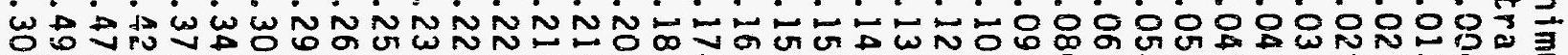

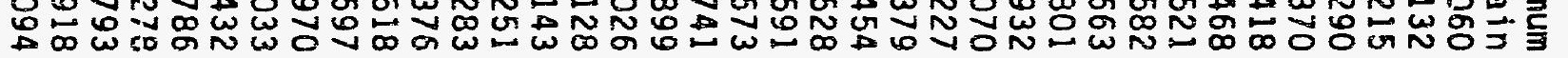

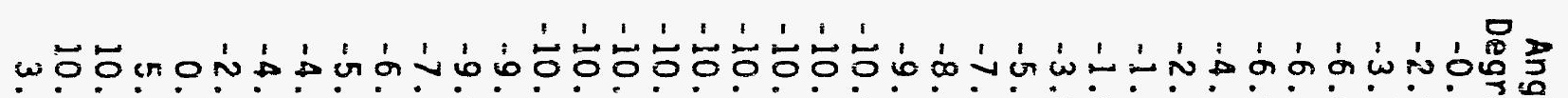

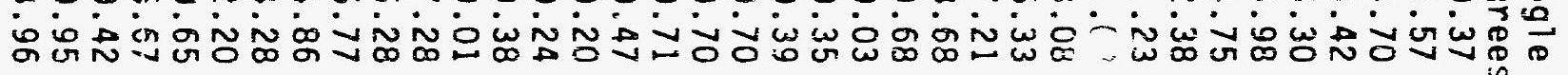




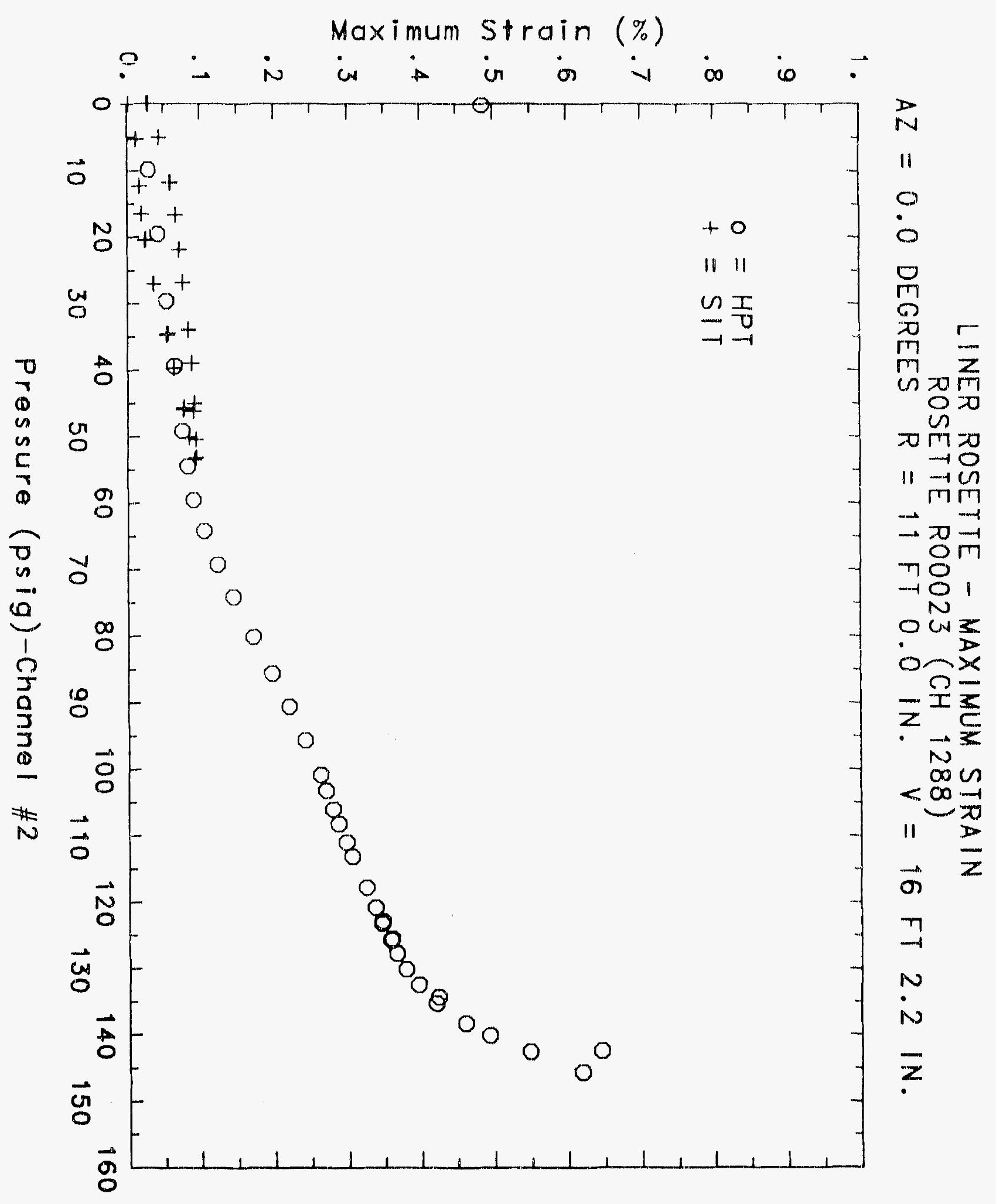


8880

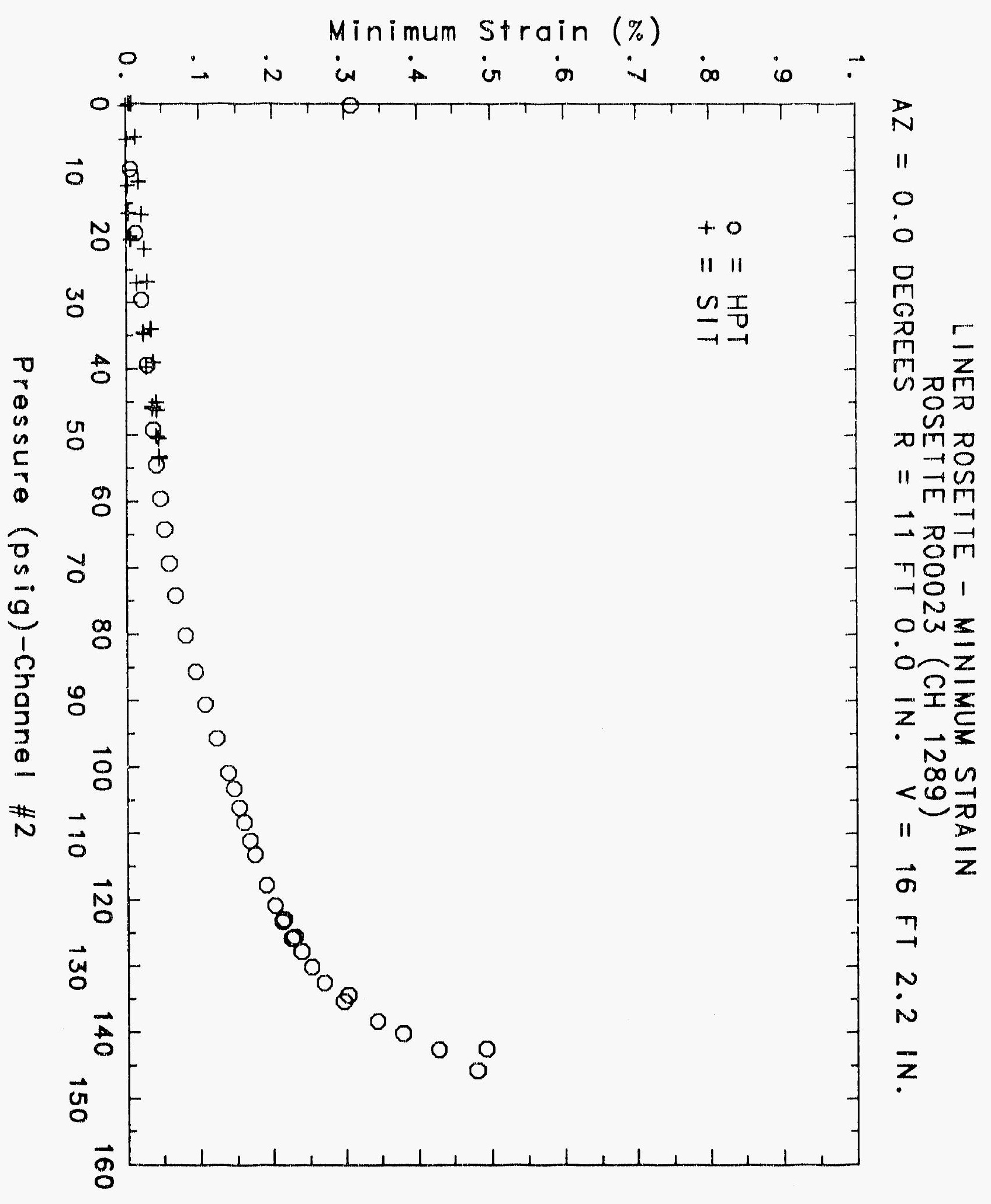




\section{Reinforced Concrete Test Data}

\section{Rosette 24 Channels $1301 \quad 1302 \quad 1303$}

StRUCTURAL INTEgRITY TEST

\begin{tabular}{|c|c|c|c|c|c|c|c|}
\hline $\begin{array}{c}\text { Pressure } \\
\text { psig } \\
-0.05 \\
5.33 \\
12.31 \\
16.44 \\
20.51 \\
20.46 \\
20.47 \\
27.05 \\
34.69 \\
34.53 \\
34.53 \\
39.70 \\
45.90 \\
45.65 \\
45.62 \\
50.10 \\
53.47 \\
53.29 \\
53.21 \\
50.49 \\
46.14 \\
46.13 \\
44.97 \\
38.98 \\
33.99 \\
33.96 \\
26.83 \\
21.88 \\
21.90 \\
16.69 \\
11.74 \\
11.77 \\
5.05 \\
0.02 \\
-0.04 \\
-0.02 \\
-0.02 \\
0.02\end{array}$ & $\begin{array}{l}\text { Maximum } \\
\text { XStrain } \\
0.0006 \\
0.0133 \\
0.0206 \\
0.0235 \\
0.0285 \\
0.0296 \\
0.0302 \\
0.0424 \\
0.0632 \\
0.0654 \\
0.0656 \\
0.0761 \\
0.0925 \\
0.0941 \\
0.0939 \\
0.1032 \\
0.1122 \\
0.1132 \\
0.1131 \\
0.1120 \\
0.1083 \\
0.1083 \\
0.1091 \\
0.1031 \\
0.0969 \\
0.0967 \\
0.0864 \\
0.0781 \\
0.0781 \\
0.0687 \\
0.0584 \\
0.0588 \\
0.0425 \\
0.0287 \\
0.0276 \\
0.0285 \\
0.0288 \\
0.0285\end{array}$ & $\begin{array}{l}\text { Minimum } \\
\text { \%Strain } \\
0.0005 \\
0.0024 \\
0.0046 \\
0.0074 \\
0.0133 \\
0.0148 \\
0.0157 \\
0.0270 \\
0.0394 \\
0.0399 \\
0.0400 \\
0.0449 \\
0.0518 \\
0.0517 \\
0.0517 \\
0.0554 \\
0.0588 \\
0.0588 \\
0.0586 \\
0.0571 \\
0.0539 \\
0.0537 \\
0.0541 \\
0.0494 \\
0.0452 \\
0.0452 \\
0.0388 \\
0.0344 \\
0.0346 \\
0.0298 \\
0.0249 \\
0.02488 \\
0.0183 \\
0.0117 \\
0.0109 \\
0.0111 \\
0.0108 \\
0.0096\end{array}$ & $\begin{array}{c}\text { Angle } \\
\text { Degrees } \\
-25.27 \\
-3.40 \\
-3.70 \\
-2.04 \\
-0.07 \\
1.65 \\
1.85 \\
5.14 \\
6.88 \\
7.55 \\
6.79 \\
7.92 \\
9.06 \\
9.78 \\
9.09 \\
9.56 \\
10.25 \\
10.10 \\
9.89 \\
10.53 \\
11.33 \\
11.53 \\
13.24 \\
14.03 \\
14.67 \\
13.92 \\
14.87 \\
15.18 \\
14.58 \\
15.52 \\
16.40 \\
16.02 \\
19.50 \\
33.53 \\
27.46 \\
31.77 \\
32.30 \\
32.12\end{array}$ & $\begin{array}{c}\text { Pressure } \\
\text { Ps1g } \\
9.89 \\
19.55 \\
29.57 \\
39.42 \\
49.16 \\
54.50 \\
59.57 \\
64.20 \\
69.32 \\
74.16 \\
80.16 \\
85.61 \\
90.58 \\
95.69 \\
100.92 \\
103.25 \\
106.11 \\
108.31 \\
111.08 \\
113.24 \\
117.83 \\
120.92 \\
123.28 \\
122.97 \\
125.82 \\
125.60 \\
127.84 \\
130.19 \\
132.53 \\
135.33 \\
134.42 \\
138.35 \\
140.16 \\
142.63 \\
145.78 \\
142.52 \\
0.22\end{array}$ & $\begin{array}{l}\text { Maximum } \\
\text { XStrain } \\
0.0272 \\
0.0460 \\
0.0628 \\
0.0768 \\
0.0891 \\
0.0967 \\
0.1065 \\
0.1189 \\
0.1324 \\
0.1484 \\
0.1695 \\
0.1903 \\
0.2106 \\
0.2319 \\
0.2532 \\
0.2607 \\
0.2700 \\
0.2779 \\
0.2884 \\
0.2964 \\
0.3169 \\
0.3291 \\
0.3389 \\
0.3405 \\
0.3551 \\
0.3584 \\
0.3718 \\
0.3994 \\
0.4428 \\
0.5056 \\
0.5173 \\
0.5921 \\
0.6495 \\
0.7273 \\
0.8091 \\
0.8327 \\
0.6403\end{array}$ & $\begin{array}{l}\text { Minimum } \\
\text { XStrain } \\
0.0094 \\
0.0182 \\
0.0277 \\
0.0369 \\
0.0456 \\
0.0502 \\
0.0547 \\
0.0592 \\
0.0650 \\
0.0717 \\
0.0819 \\
0.0940 \\
0.1085 \\
0.1275 \\
0.1511 \\
0.1633 \\
0.1763 \\
0.1864 \\
0.2027 \\
0.2147 \\
0.2446 \\
0.2659 \\
0.2847 \\
0.2885 \\
0.3115 \\
0.3173 \\
0.3325 \\
0.3558 \\
0.3806 \\
0.4125 \\
0.4184 \\
0.4673 \\
0.5103 \\
0.5772 \\
0.6503 \\
0.6756 \\
0.3615\end{array}$ & $\begin{array}{c}\text { Angle } \\
\text { Degrees } \\
2.70 \\
5.31 \\
6.36 \\
6.54 \\
6.20 \\
5.95 \\
6.71 \\
7.70 \\
8.42 \\
9.16 \\
10.06 \\
10.03 \\
9.35 \\
8.16 \\
7.26 \\
7.08 \\
6.88 \\
6.91 \\
7.19 \\
7.58 \\
9.39 \\
11.28 \\
14.65 \\
16.30 \\
24.13 \\
28.64 \\
38.47 \\
-35.12 \\
-24.74 \\
-18.71 \\
-17.82 \\
-16.44 \\
-17.40 \\
-20.04 \\
-23.53 \\
-26.21 \\
-14.42\end{array}$ \\
\hline
\end{tabular}

High Pressure test 


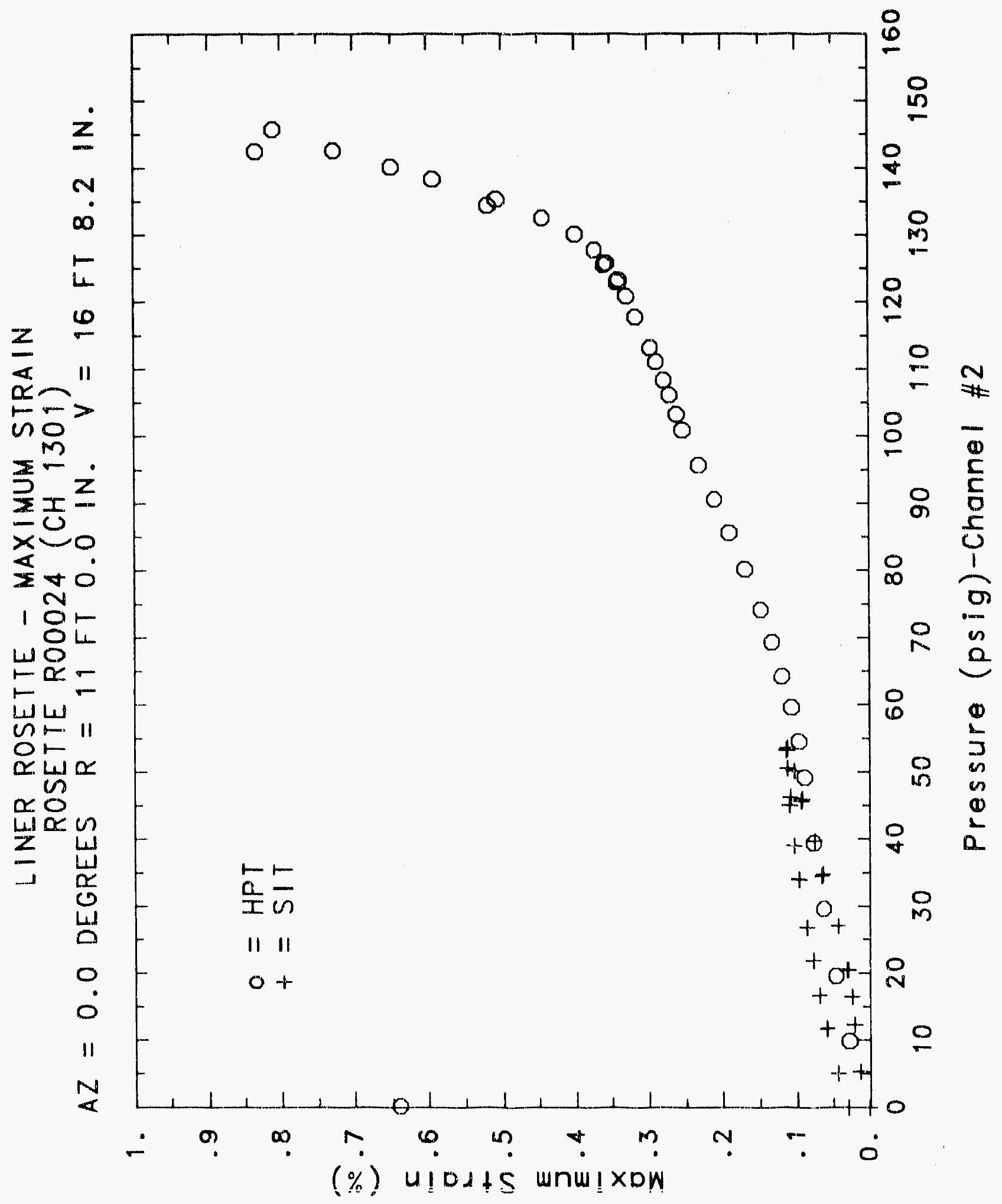




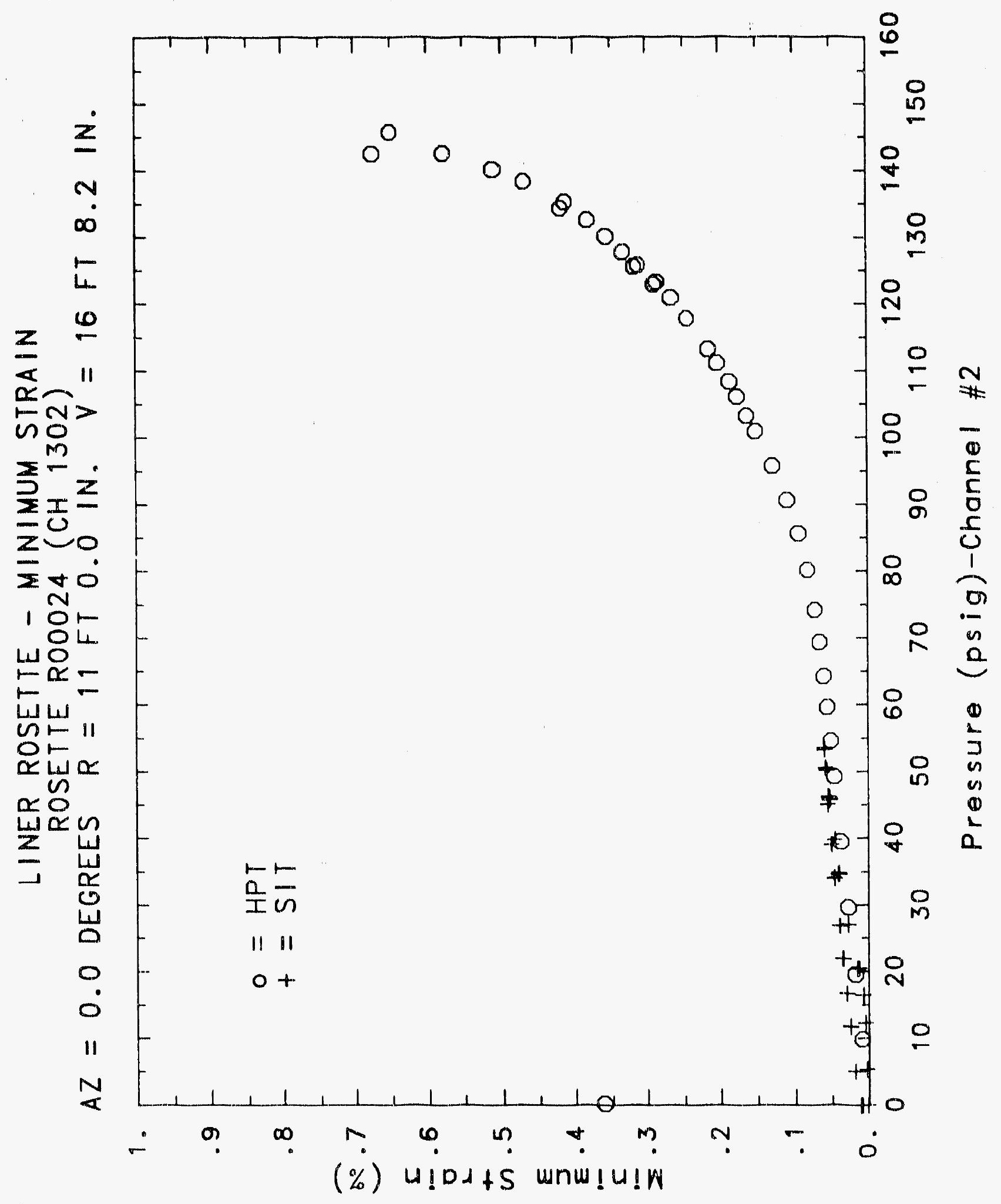




\title{
Reinforced Concrete Test Data
}

\author{
Rosette 25 Channels $1304 \quad 1305 \quad 1306$
}

\section{STRUCTURAL INTEgRITY TEST}

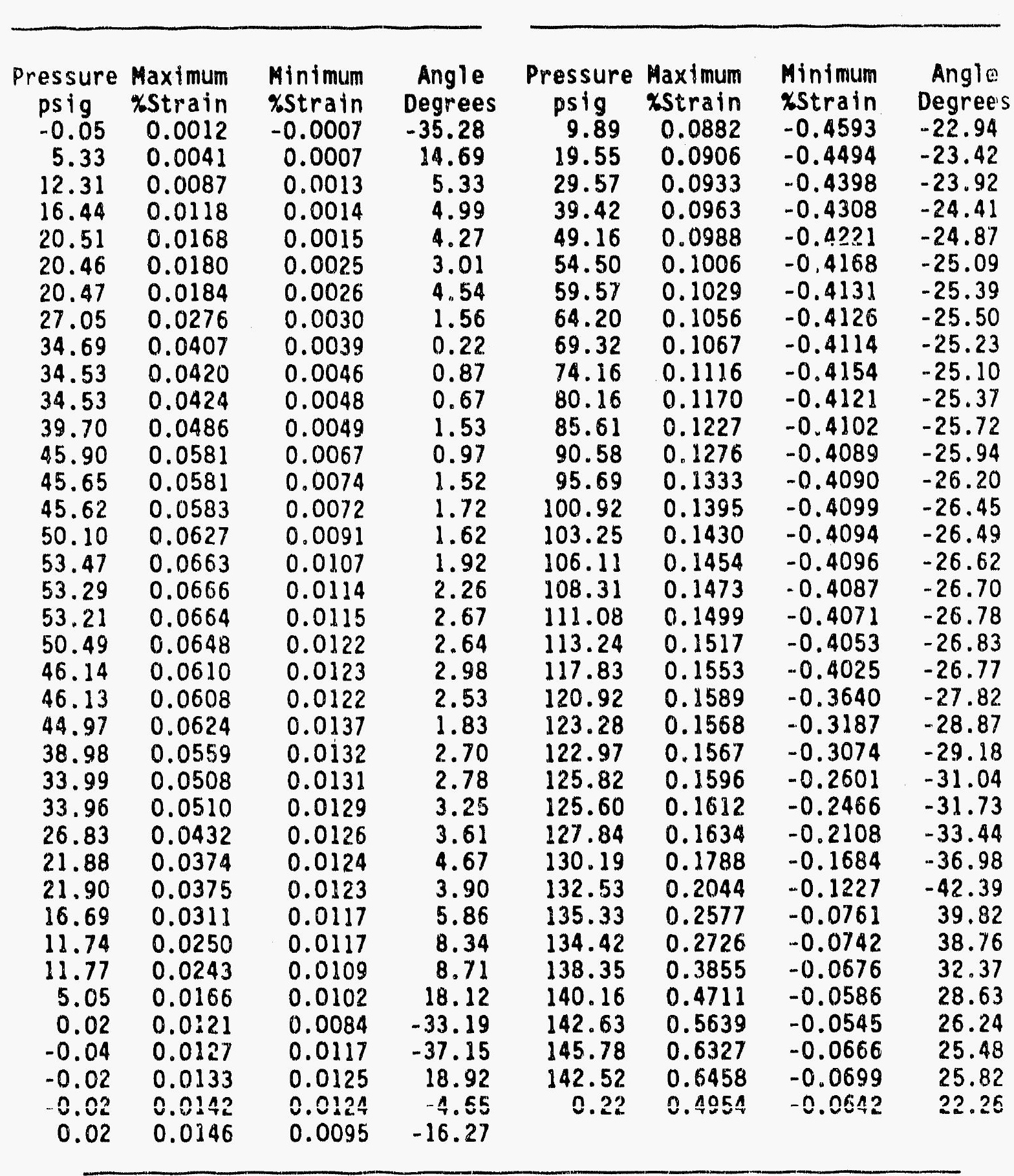

High Pressure test 


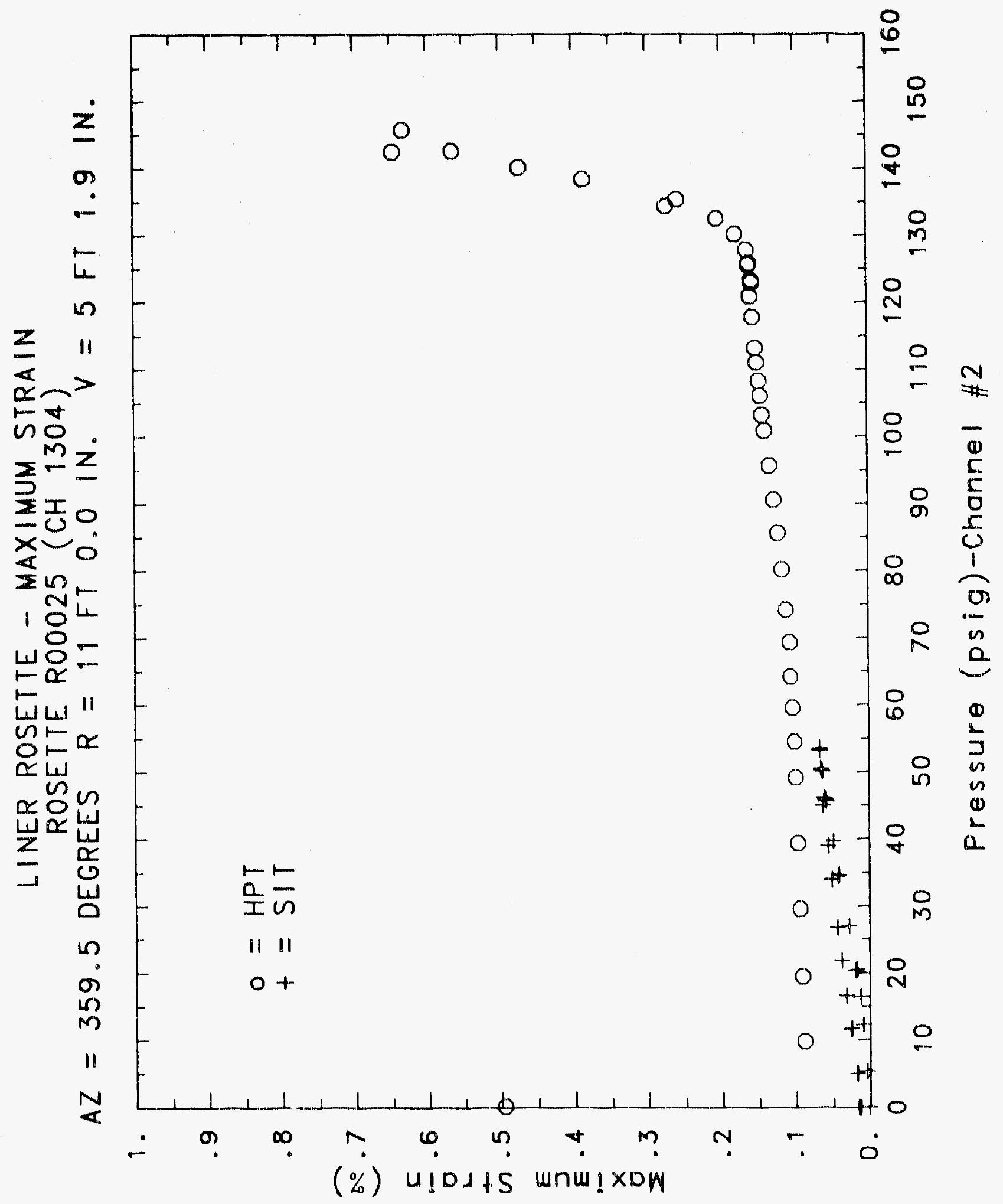




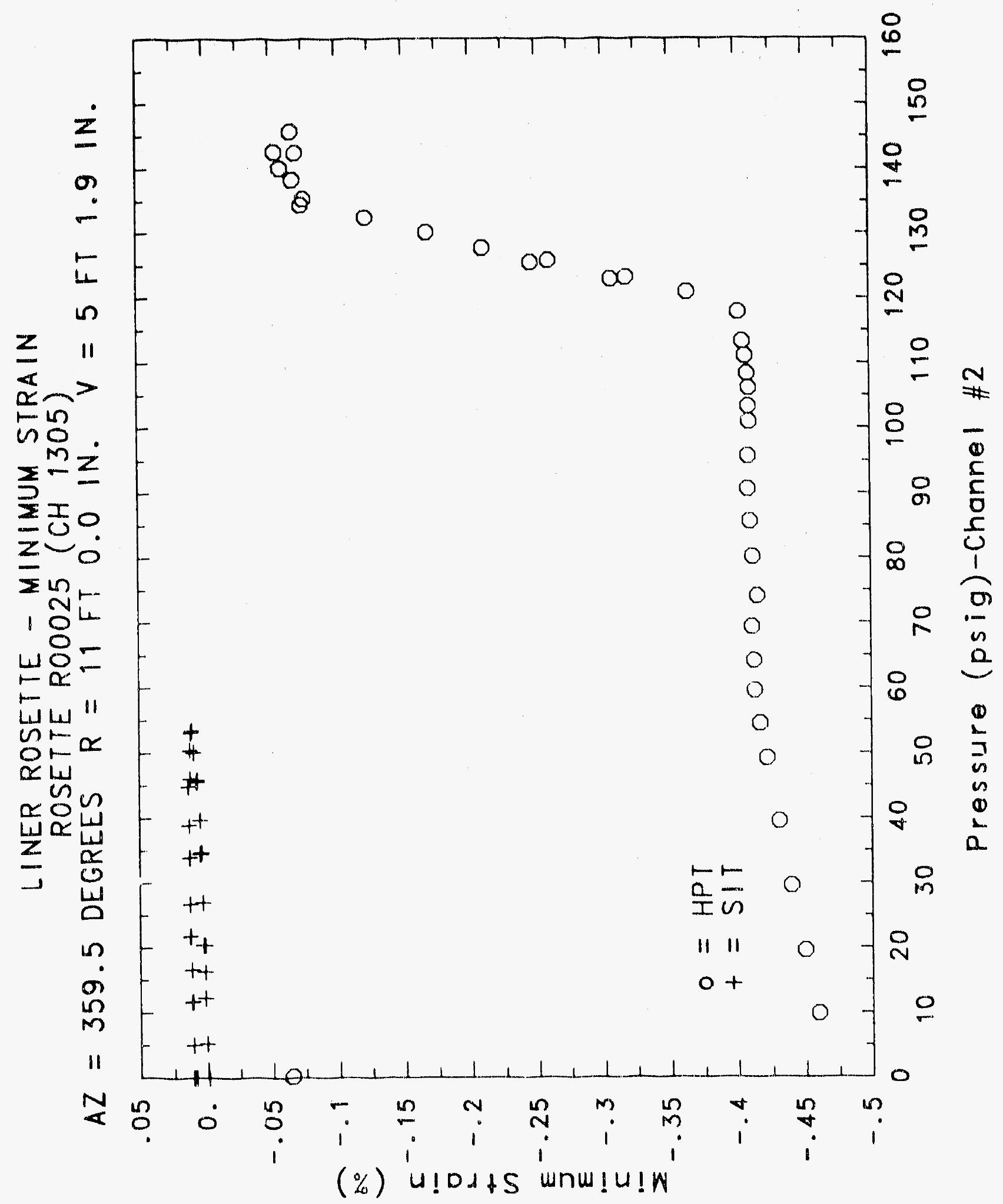




\section{Reinforced Concrete Test Data}

\section{Rosette 26 Channels $1307 \quad 1308 \quad 1309$}

$$
\text { Structural Integrity Test }
$$

\begin{tabular}{|c|c|c|c|c|c|c|c|}
\hline $\begin{array}{c}\text { Pressure } \\
\text { psig } \\
-0.05 \\
5.33 \\
12.31 \\
16.44 \\
20.51 \\
20.46 \\
20.47 \\
27.05 \\
34.69 \\
34.53 \\
34.53 \\
39.70 \\
45.90 \\
45.65 \\
45.62 \\
50.10 \\
53.47 \\
53.29 \\
53.21 \\
50.49 \\
46.14 \\
46.13 \\
44.97 \\
38.98 \\
33.99 \\
33.96 \\
26.83 \\
21.88 \\
21.90 \\
16.69 \\
11.74 \\
11.77 \\
5.05 \\
0.02 \\
-0.04 \\
-0.02 \\
-0.02 \\
0.02\end{array}$ & $\begin{array}{c}\text { Maximum } \\
\text { \%strain } \\
0.0012 \\
0.0143 \\
0.0182 \\
0.0210 \\
0.0253 \\
0.0264 \\
0.0267 \\
0.0344 \\
0.0472 \\
0.0479 \\
0.0481 \\
0.0557 \\
0.0657 \\
0.0657 \\
0.0658 \\
0.0712 \\
0.0751 \\
0.0752 \\
0.0749 \\
0.0722 \\
0.0674 \\
0.0671 \\
0.0659 \\
0.0591 \\
0.0543 \\
0.0544 \\
0.0485 \\
0.0448 \\
0.0441 \\
0.0405 \\
0.0367 \\
0.0363 \\
0.0313 \\
0.0229 \\
0.0226 \\
0.0243 \\
0.0245 \\
0.0231\end{array}$ & $\begin{array}{c}\text { Minimum } \\
\text { X.Strain } \\
-0.0014 \\
0.0008 \\
0.0039 \\
0.0065 \\
0.0102 \\
0.0111 \\
0.0119 \\
0.0194 \\
0.0313 \\
0.0326 \\
0.0331 \\
0.0375 \\
0.0447 \\
0.0442 \\
0.0445 \\
0.0476 \\
0.0503 \\
0.0505 \\
0.0503 \\
0.0499 \\
0.0478 \\
0.0476 \\
0.0486 \\
0.0451 \\
0.0410 \\
0.0409 \\
0.0333 \\
0.0278 \\
0.0285 \\
0.0222 \\
0.0173 \\
0.0170 \\
0.0101 \\
0.0041 \\
0.0055 \\
0.0060 \\
0.0050 \\
0.0054\end{array}$ & $\begin{array}{c}\text { Angle } \\
\text { Degrees } \\
-38.97 \\
43.13 \\
38.42 \\
32.92 \\
25.28 \\
25.87 \\
25.18 \\
20.59 \\
16.85 \\
15.07 \\
14.61 \\
9.90 \\
7.35 \\
8.36 \\
7.14 \\
6.71 \\
7.03 \\
5.72 \\
6.85 \\
5.75 \\
7.65 \\
7.46 \\
11.48 \\
18.50 \\
25.89 \\
25.06 \\
35.80 \\
40.76 \\
40.16 \\
43.38 \\
44.89 \\
44.08 \\
44.78 \\
38.67 \\
35.36 \\
41.02 \\
41.54 \\
38.82\end{array}$ & $\begin{array}{c}\text { Pressure } \\
\text { psig } \\
9.89 \\
19.55 \\
29.57 \\
39.42 \\
49.16 \\
54.50 \\
59.57 \\
64.20 \\
69.32 \\
74.16 \\
80.16 \\
85.61 \\
90.58 \\
95.69 \\
100.92 \\
103.25 \\
106.11 \\
108.31 \\
111.08 \\
113.24 \\
117.83 \\
120.92 \\
123.28 \\
122.97 \\
125.82 \\
125.60 \\
127.84 \\
130.19 \\
132.53 \\
135.33 \\
134.42 \\
138.35 \\
140.16 \\
142.63 \\
145.78 \\
142.52 \\
0.22\end{array}$ & $\begin{array}{l}\text { Maximum } \\
\text { XStrain } \\
0.0133 \\
0.0212 \\
0.0327 \\
0.0437 \\
0.0551 \\
0.0610 \\
0.0681 \\
0.0750 \\
0.0799 \\
0.0860 \\
0.0960 \\
0.1112 \\
0.1310 \\
0.1566 \\
0.1830 \\
0.1967 \\
0.2131 \\
0.2241 \\
0.2402 \\
0.2542 \\
0.2937 \\
0.3211 \\
0.3461 \\
0.3518 \\
0.3915 \\
0.4028 \\
0.4264 \\
0.4653 \\
0.5091 \\
0.6017 \\
0.6250 \\
0.7489 \\
0.8392 \\
0.9526 \\
1.0426 \\
1.0713 \\
0.8279\end{array}$ & $\begin{array}{l}\text { Minimum } \\
\text { XStrain } \\
0.0108 \\
0.0193 \\
0.0261 \\
0.0325 \\
0.0380 \\
0.0421 \\
0.0442 \\
0.0464 \\
0.0494 \\
0.0524 \\
0.0540 \\
0.0533 \\
0.0487 \\
0.0419 \\
0.0374 \\
0.0358 \\
0.0342 \\
0.0346 \\
0.0369 \\
0.0397 \\
0.0513 \\
0.0623 \\
0.0722 \\
0.0740 \\
0.0856 \\
0.0877 \\
0.0949 \\
0.1067 \\
0.1199 \\
0.1291 \\
0.1289 \\
0.1361 \\
0.1410 \\
0.1485 \\
0.1644 \\
0.1703 \\
0.1091\end{array}$ & $\begin{array}{c}\text { Angle } \\
\text { Degrees } \\
5.68 \\
-42.61 \\
-23.94 \\
-19.88 \\
-16.74 \\
-16.35 \\
-15.64 \\
-16.02 \\
-13.59 \\
-11.53 \\
-9.40 \\
-6.59 \\
-5.27 \\
-4.25 \\
-4.08 \\
-4.32 \\
-4.51 \\
-4.84 \\
-5.79 \\
-6.78 \\
-9.31 \\
-10.68 \\
-12.35 \\
-12.75 \\
-14.96 \\
-15.44 \\
-16.20 \\
-17.04 \\
-17.58 \\
-18.48 \\
-18.73 \\
-19.18 \\
-19.50 \\
-19.91 \\
-19.96 \\
-19.90 \\
-24.87\end{array}$ \\
\hline
\end{tabular}

High Pressure Test 
()(1).

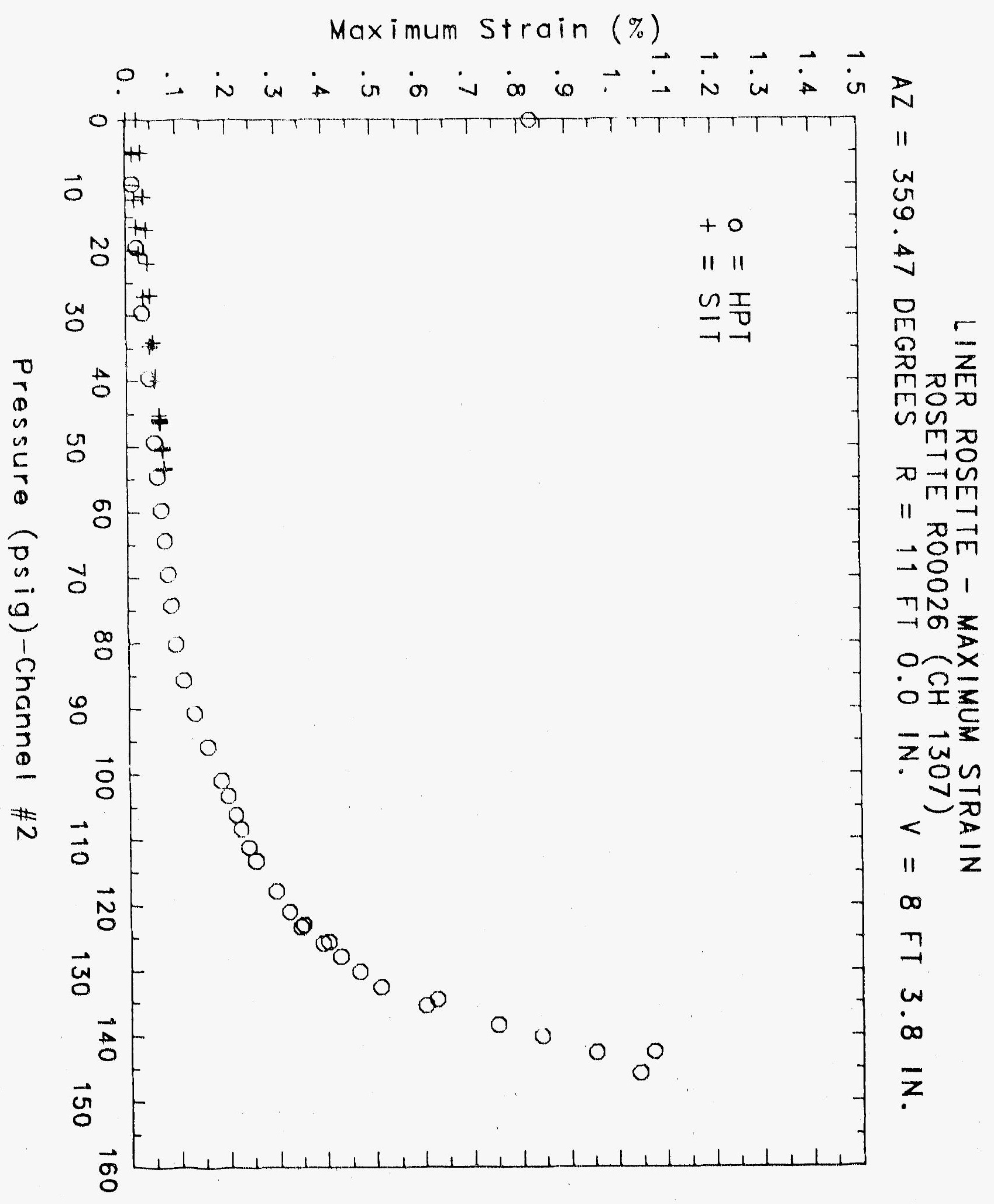




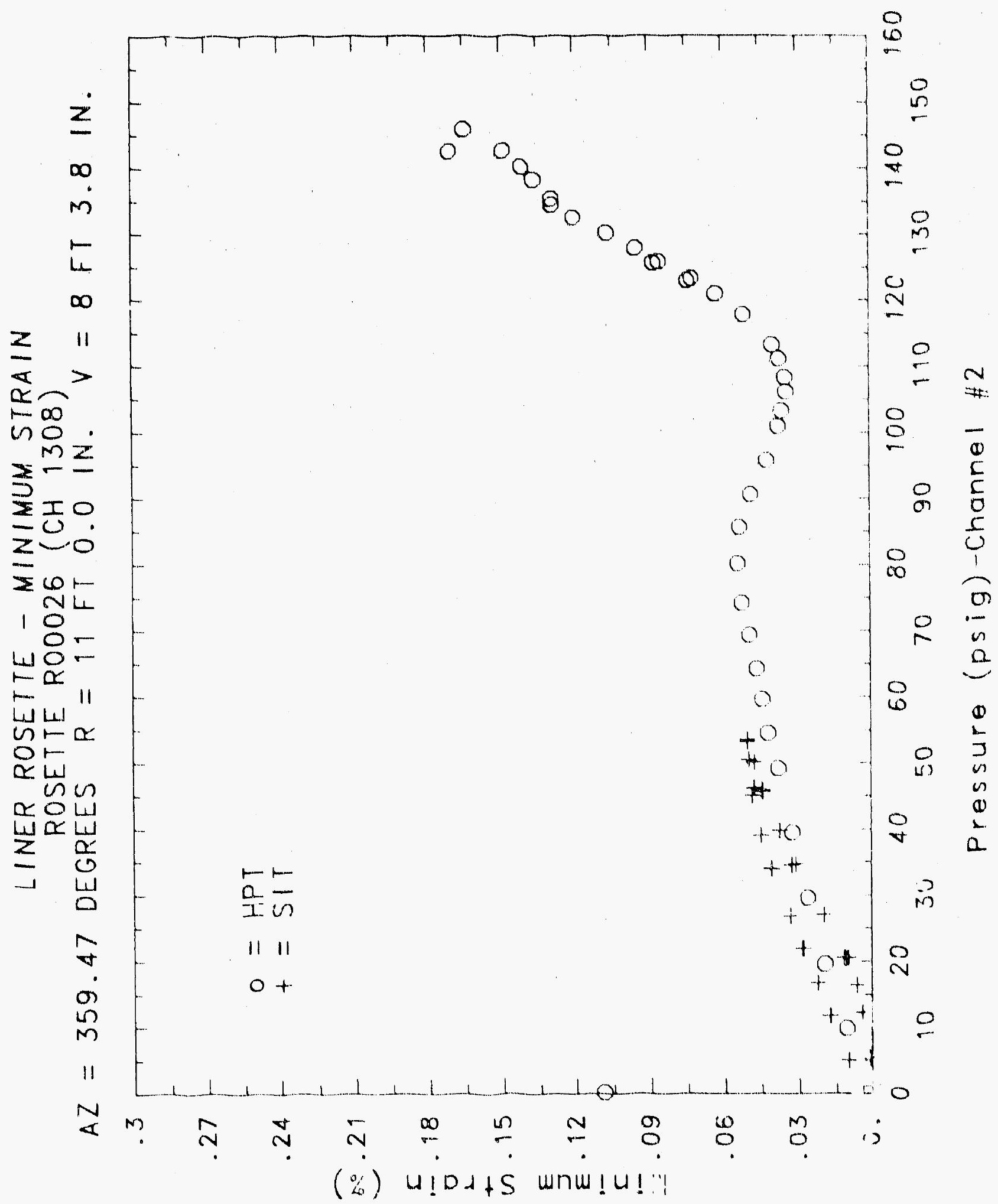




\section{Reinforced Concrete Test Data}

Rosette 27 Channels $1320 \quad 1321 \quad 1322$

STRUCTURAL INTEGRITY TEST

\begin{tabular}{|c|c|c|c|c|c|c|c|}
\hline Pressure & Maximum & Minimum & Angle & Pressure & Maximum & Minimum & Angle \\
\hline $\begin{array}{l}\text { psig } \\
-0.05\end{array}$ & $\begin{array}{r}\text { xstrain } \\
0.0001\end{array}$ & $\begin{array}{c}\text { \%strain } \\
0.0001\end{array}$ & $\begin{array}{c}\text { Degrees } \\
21.54\end{array}$ & $\begin{array}{l}\text { psig } \\
9.89\end{array}$ & $\begin{array}{c}\text { x Strain } \\
0.0041\end{array}$ & $\begin{array}{l}\text { Xstrain } \\
-0.0005\end{array}$ & $\begin{array}{l}\text { Degrees } \\
-19.87\end{array}$ \\
\hline 5.33 & 0.0024 & -0.0008 & 20.77 & 19.55 & 0.0027 & $-0.005\rfloor$ & 42.55 \\
\hline 12.31 & 0.0037 & -0.0012 & 18.51 & 29.57 & 0.0020 & -0.0132 & 30.35 \\
\hline 16.44 & 0.0029 & -0.0018 & 24.57 & 39.42 & 0.0018 & -0.0219 & 26.00 \\
\hline 20.51 & 0.0011 & -0.0032 & 40.26 & 49.16 & 0.0015 & -0.0303 & 23.25 \\
\hline 20.46 & 0.0004 & -0.0034 & -44.10 & 54.50 & 0.0012 & -0.0352 & 22.04 \\
\hline 20.47 & 0.0001 & -0.0037 & -42.25 & 59.57 & 0.0014 & -0.0404 & 21.47 \\
\hline 27.05 & -0.0026 & -0.0059 & -6.32 & 64.20 & 0.0011 & -0.0460 & 20.58 \\
\hline 34.69 & -0.0028 & -0.0190 & 22.14 & 69.32 & 0.0004 & -0.0509 & 19.79 \\
\hline 34.53 & -0.0025 & -0.01 .99 & 23.12 & 74.16 & 0.0007 & -0.0589 & 19.87 \\
\hline 34.53 & -0.0024 & -0.0200 & 23.59 & 80.16 & 0.0005 & -0.0656 & 19.31 \\
\hline 39.70 & -0.0021 & -0.0238 & 24.21 & 85.61 & -0.0006 & -0.0708 & 18.19 \\
\hline 45.90 & -0.0019 & -0.0294 & 24.23 & 90.58 & -0.0016 & -0.0738 & 10.31 \\
\hline 45.65 & -0.0016 & -0.0291 & 24.42 & 95.69 & -0.0024 & -0.0762 & 14.88 \\
\hline 45.62 & -0.0019 & -0.0287 & 24.15 & 100.92 & -0.0032 & -0.0797 & 13.73 \\
\hline 50.10 & -0.0019 & -0.0324 & 23.53 & 103.25 & -0.0035 & -0.0821 & 13.08 \\
\hline 53.47 & -0.0024 & -0.0361 & 21.64 & 106.11 & -0.0039 & -0.0842 & 12.56 \\
\hline 53.29 & .0 .0023 & -0.0361 & 21.34 & 108.31 & -0.0042 & -0.0864 & 12.15 \\
\hline 53.21 & -0.0023 & -0.0360 & 21.08 & 111.08 & -0.0047 & -0.0907 & 11.69 \\
\hline 50.49 & -0.0021 & -0.0343 & 21.56 & 113.24 & -0.0051 & -0.0940 & 11.28 \\
\hline 46.14 & -0.0017 & -0.0309 & 22.31 & 127.83 & -0.0062 & -0.1010 & 10.78 \\
\hline 46.13 & -0.0014 & -0.0305 & 22.56 & 120.92 & -0.0071 & -0.1073 & 10.54 \\
\hline 44.97 & -0.0012 & -0.0304 & 20.95 & 123.28 & -0.0074 & -0.1120 & 10.30 \\
\hline 38.98 & -0.0006 & -0.0258 & 22.83 & 122.97 & -0.0075 & -0.1127 & 10.26 \\
\hline 33.99 & -0.0004 & -0.0215 & 24.79 & 125.82 & -0.0083 & -0.1185 & 9.89 \\
\hline 33.96 & -0.0004 & $\ldots .0221$ & 24.88 & 125.60 & -0.0086 & -0.1209 & 9.86 \\
\hline 26.83 & 0.0001 & -0.0157 & 28.90 & 127.84 & -0.0094 & -0.1255 & 9.85 \\
\hline 21.88 & 0.0005 & -0.0113 & 34.04 & 130.19 & -0.0105 & .0 .1321 & 9.90 \\
\hline 21.90 & 0.0004 & -0.0114 & 34.90 & 132.53 & -0.0111 & -0.1386 & 9.95 \\
\hline 16.69 & 0.0008 & -0.0077 & 43.43 & 135.33 & -0.0118 & -0.1483 & 10.04 \\
\hline 11.74 & 0.0016 & -0.0046 & -32.53 & 134.42 & -0.0118 & -0.1505 & 10.07 \\
\hline 11.77 & 0.0019 & -0.0045 & -28.91 & 138.35 & -0.0120 & -0.1624 & 10.34 \\
\hline 5.05 & 0.0029 & $\cdots 0.0027$ & -15.81 & 140.16 & -0.0120 & -0.1698 & 10.53 \\
\hline 0.02 & 0.0032 & -0.0019 & -13.67 & 142.63 & -0.0116 & -0.1784 & 10.83 \\
\hline-0.04 & 0.0024 & 0.0001 & 2.02 & 145.78 & -0.0114 & -0.1885 & 11.07 \\
\hline-0.02 & 0.0025 & 0.0002 & 0.93 & 142.52 & -0.0091 & -0.1880 & 11.47 \\
\hline & 0.0025 & -0.0001 & 1.79 & 0.22 & 0.0655 & -0.0727 & 18.57 \\
\hline & 0.0024 & -0.0018 & 5.42 & & & & \\
\hline
\end{tabular}

High Pressure test 


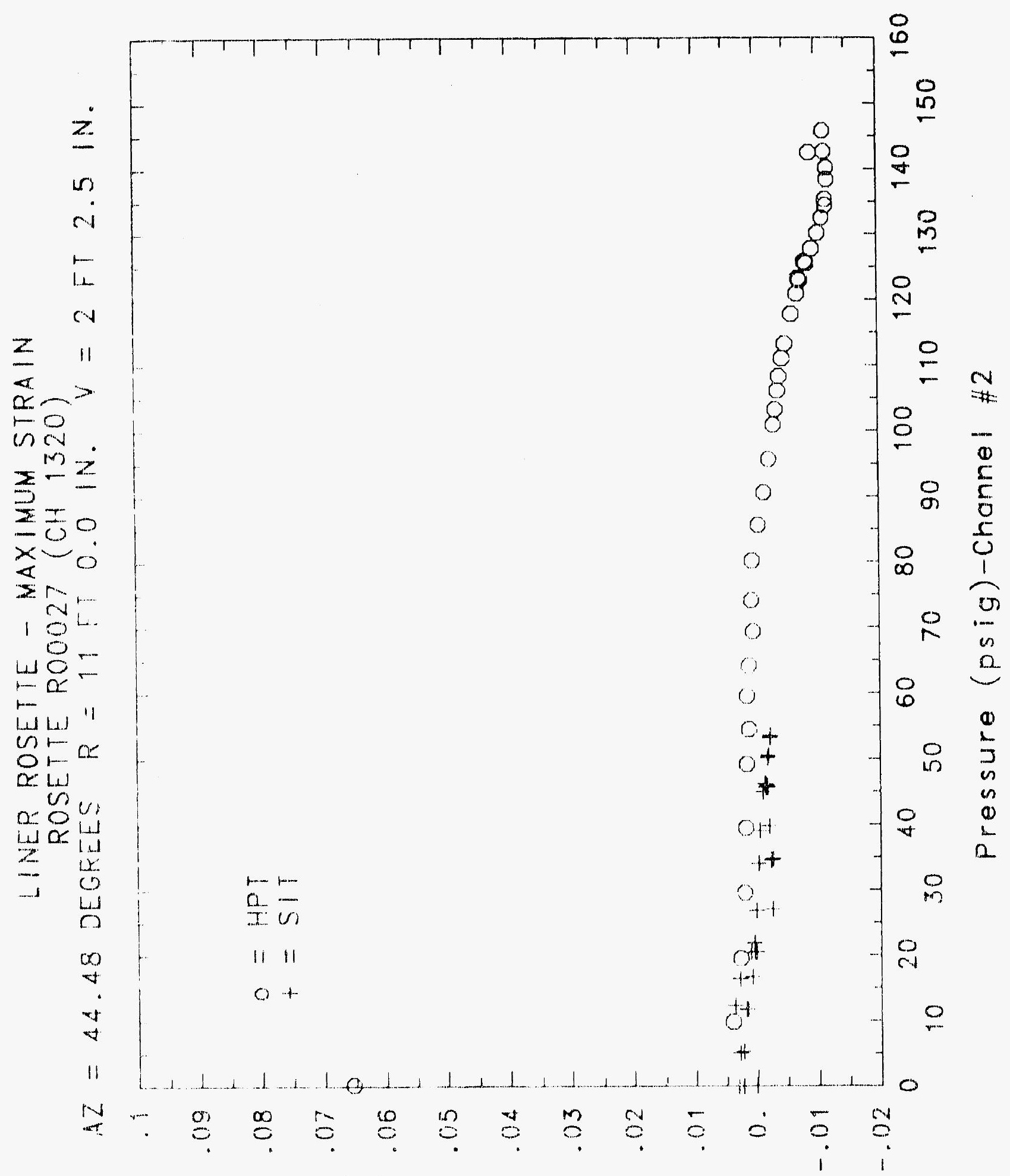

(\%) $4 ! D \perp+5$ wnw! XDW 


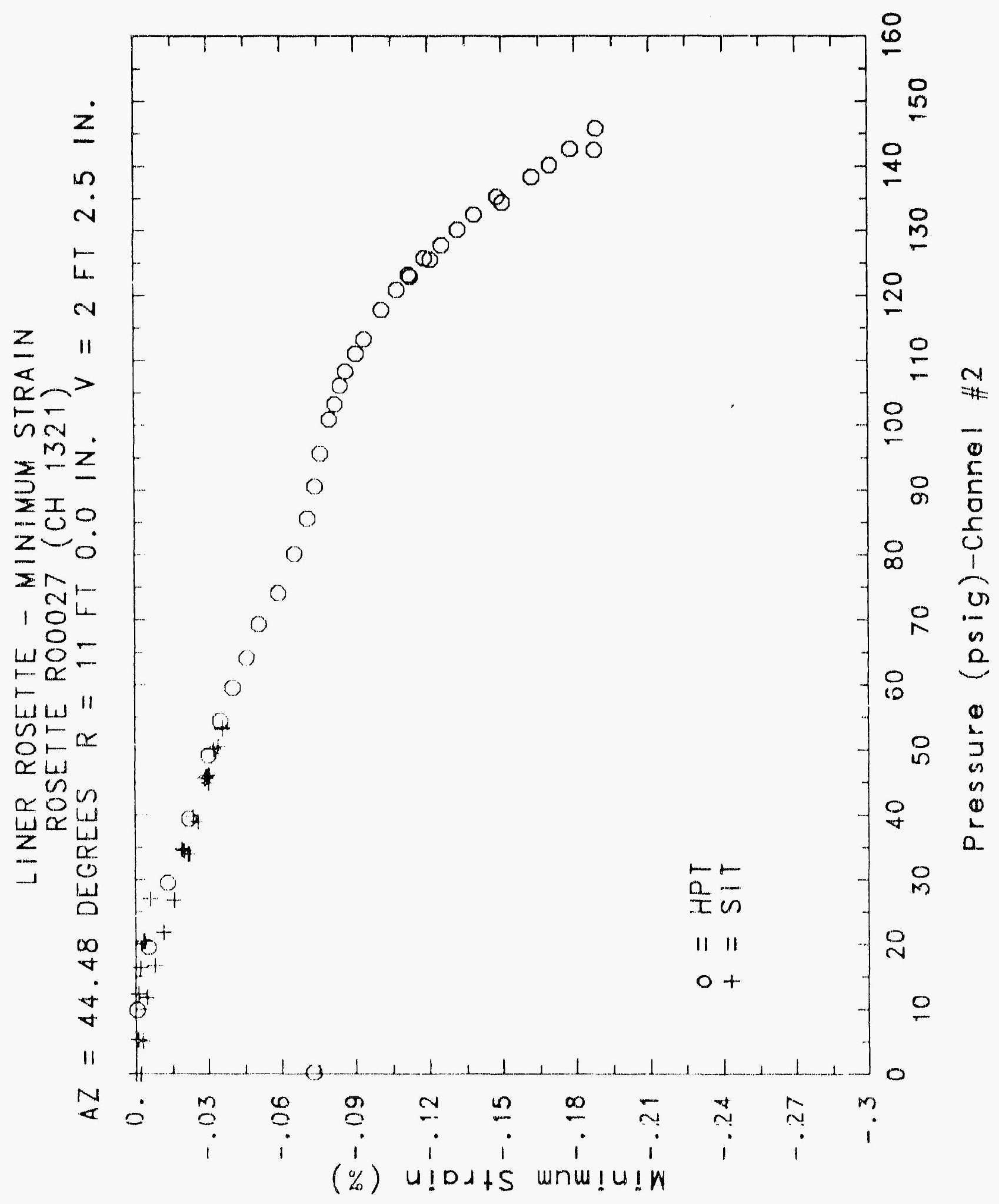




\title{
Reinforced Concrete Test Data
}

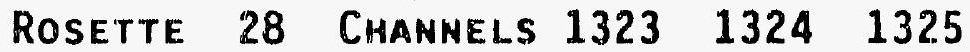

\section{Structural Integrity TEST}

\begin{tabular}{|c|c|c|c|c|c|c|c|}
\hline Pressure & Maximum & Minimum & Angle & Pressure & Maximum & Minimum & Angle \\
\hline psig & \%strain & \%strain & Degrees & psig & \%strain & *strain & Degrees \\
\hline-0.05 & 0.0022 & -0.0018 & -44.40 & 9.89 & 0.0221 & -0.0140 & 41.02 \\
\hline 5.33 & 0.0118 & -0.0092 & -42.94 & 19.55 & 0.02 .49 & -0.0102 & 36.86 \\
\hline 12.31 & 0.0234 & -0.0183 & -42.77 & 29.57 & 0.0304 & -0.0091 & 34.28 \\
\hline 16.44 & 0.0274 & -0.0205 & -42.45 & 39.42 & 0.0310 & -0.0037 & 28.78 \\
\hline 20.51 & 0.0306 & -0.0209 & -41.90 & 49.16 & 0.0335 & 0.0003 & 24.74 \\
\hline 20.46 & 0.0320 & -0.0214 & -41.86 & 54. & 0.0339 & 0.0038 & 19.68 \\
\hline 20.47 & 0.0331 & -0.0221 & -41.98 & 59.57 & 0.0373 & 0.0044 & 19.46 \\
\hline 27.05 & 0.0379 & -0.0218 & -40.87 & 64.20 & 0.0384 & 0.0079 & 13.32 \\
\hline 34.69 & 0.0441 & -0.0199 & -39.25 & 69.32 & 0.0407 & 0.0107 & 4.91 \\
\hline 34.53 & 0.0470 & -0.0219 & -39.58 & 74.16 & 0.0445 & 0.0128 & 10.55 \\
\hline 34.53 & 0.0485 & -0.0234 & -39.77 & 80.16 & 0.0507 & 0.0119 & 19.57 \\
\hline 39.70 & 0.0528 & -0.0241 & -39.24 & 85.61 & 0.0579 & 0.0102 & 24.24 \\
\hline 45.90 & 0.0629 & -0.0284 & -38.96 & 90.58 & 0.0630 & 0.0105 & 25.28 \\
\hline 45.65 & 0.0780 & -0.0434 & -40.38 & 95.69 & 0.0691 & 0.0113 & 26.50 \\
\hline 45.62 & 0.0821 & -0.0474 & -40.67 & 100.92 & 0.0731 & 0.0144 & 26.91 \\
\hline 50.10 & 0.0898 & -0.0519 & -40.61 & 103.25 & 0.0771 & $0 . C 145$ & 28.30 \\
\hline 53.47 & 0.1054 & -0.0643 & -40.92 & 106.11 & 0.0799 & 0.0154 & 28.82 \\
\hline 53.29 & 0.1244 & -0.0828 & -41.63 & 108.31 & 0.0834 & 0.0149 & 29.73 \\
\hline 53.21 & 0.1329 & -0.0912 & -41.84 & 111.08 & 0.0919 & 0.0109 & 32.21 \\
\hline 50.49 & 0.1471 & -0.1059 & -42.25 & 113.24 & 0.0953 & 0.0110 & 32.66 \\
\hline 46.14 & 0.1725 & -0.1332 & -42.82 & 117.83 & 0.1014 & 0.0133 & 33.14 \\
\hline 46.13 & 0.1886 & -0.1496 & -43.03 & 120.92 & 0.1060 & ن.0151 & 33.49 \\
\hline 44.97 & 0.2503 & -0.2096 & -43.55 & 123.28 & 0.1074 & 0.0197 & 32.93 \\
\hline 38.98 & 0.2804 & -0.2433 & -43.81 & 122.97 & 0.1077 & 0.0206 & 32.73 \\
\hline 33.99 & 0.2720 & -0.2378 & -43.87 & 125.82 & 0.1144 & 0.0204 & 33.75 \\
\hline 33.96 & 0.2833 & -0.2490 & -43.91 & 125.60 & 0.1171 & 0.0196 & 34.09 \\
\hline 26.83 & 0.2934 & -0.2637 & -44.09 & 127.84 & 0.1199 & 0.0208 & 34.09 \\
\hline 21.88 & 0.2969 & -0.2705 & -44.20 & 130.19 & 0.1272 & 0.0223 & 34.44 \\
\hline 21.90 & 0.2828 & $-r .2566$ & -44.18 & 132.53 & 0.1369 & 0.0249 & 34.69 \\
\hline 16.69 & 0.2744 & -0.2518 & -44.26 & 135.33 & 0.1520 & 0.0269 & 35.36 \\
\hline 11.74 & 0.2539 & -0.2347 & -44.34 & 134.42 & 0.1538 & 0.0283 & 35.28 \\
\hline 11.77 & 0.2563 & -0.2381 & -44.35 & 138.35 & 0.1680 & 0.0346 & 35.50 \\
\hline 5.05 & 0.2582 & -0.2444 & -44.54 & 140.16 & 0.1749 & 0.0413 & 35.32 \\
\hline 0.02 & 0.2530 & -0.2426 & -44.68 & 142.63 & 0.1849 & 0.0504 & 34.64 \\
\hline-0.04 & 0.4897 & -0.4774 & -44.86 & 145.78 & 0.1972 & 0.0591 & 33.56 \\
\hline-0.02 & 0.4104 & -0.3974 & -44.83 & 142.52 & 0.2029 & 0.0631 & 33.43 \\
\hline $\begin{array}{r}-0.02 \\
0.02\end{array}$ & 0.4189 & -0.4061 & -44.83 & 0.22 & 0.6152 & -0.5021 & 44.95 \\
\hline & & & & & & & \\
\hline
\end{tabular}

High Pressure test 


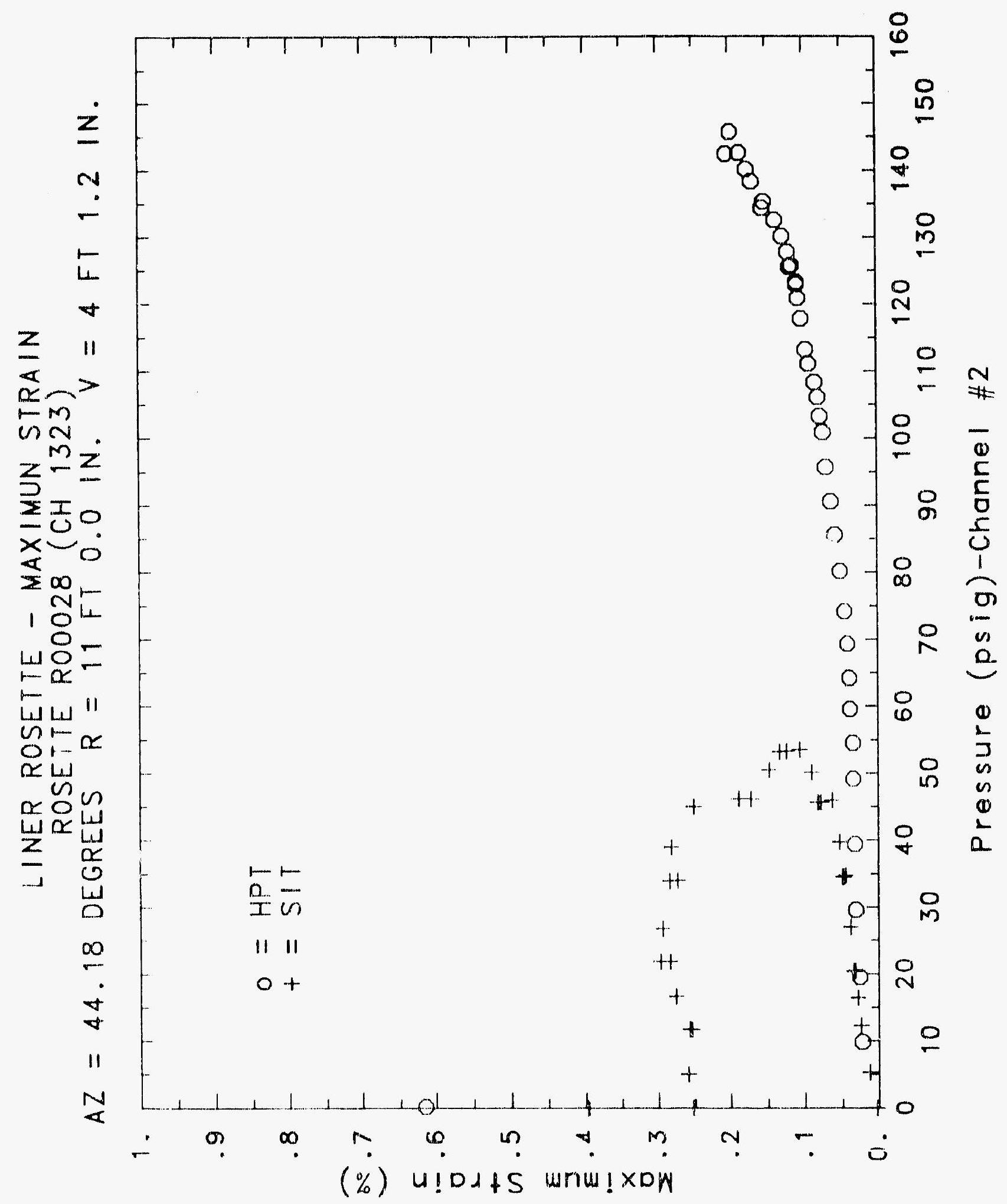




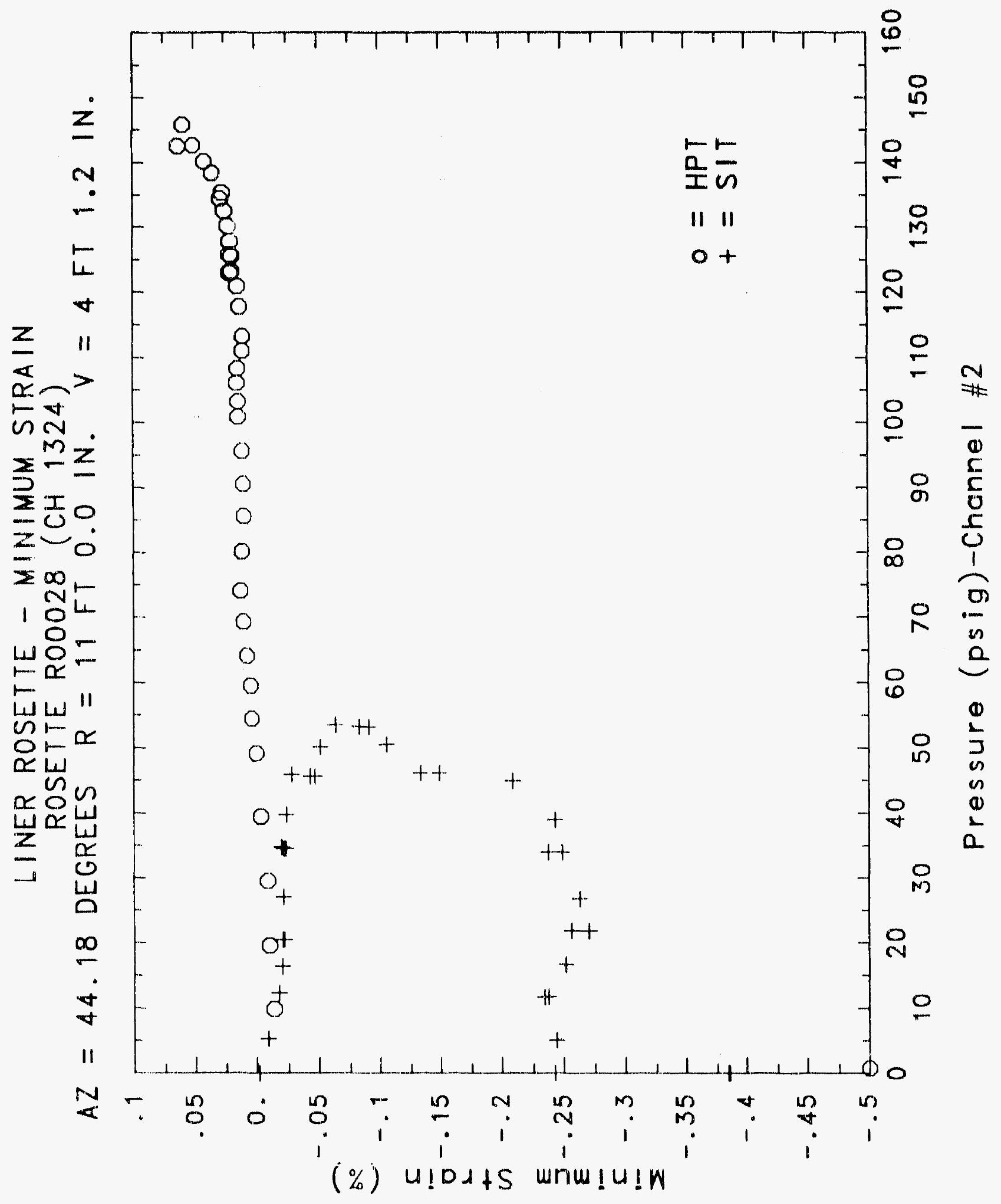




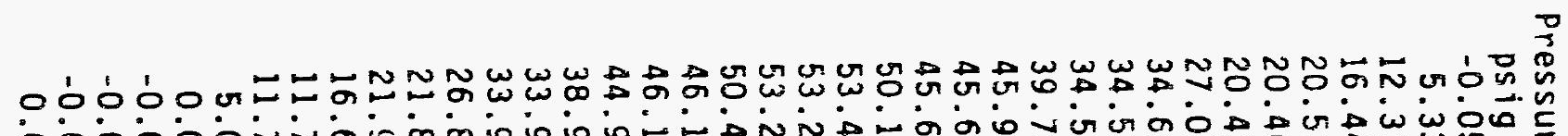

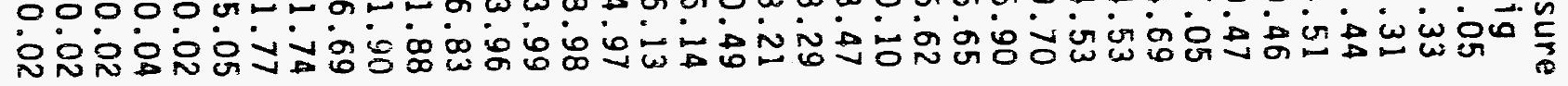
$000000000000000000000000000000000000000 \mathrm{w}^{2}$ - o o o o

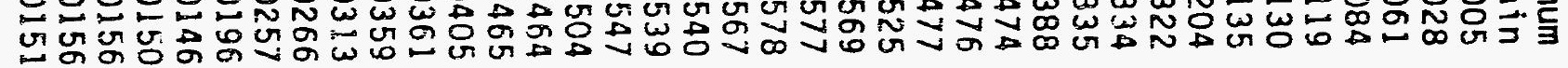

000000000000000000000000000000000000004 -

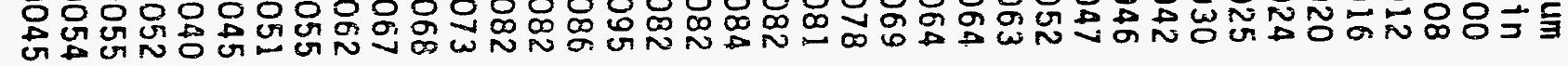

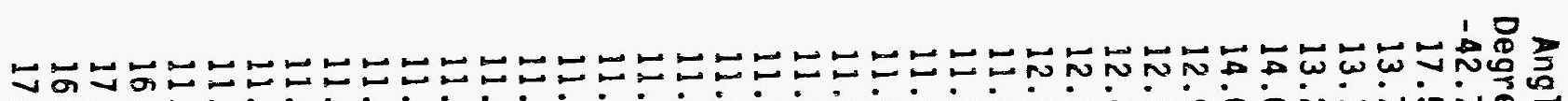

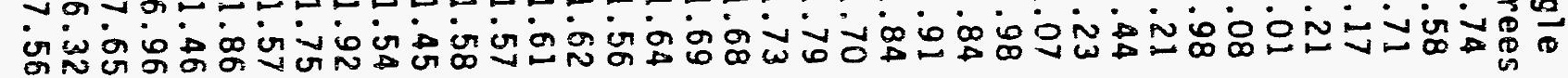

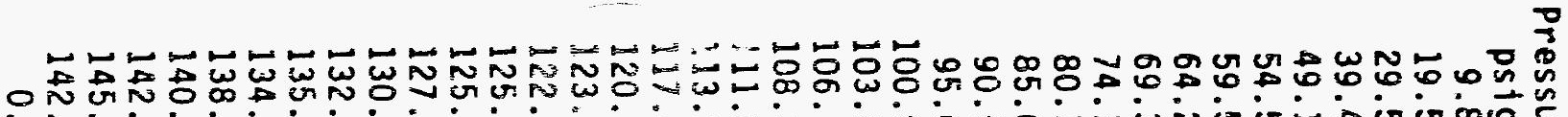
N

$00000000000000000000000000000000000000 \frac{\pi}{x}$

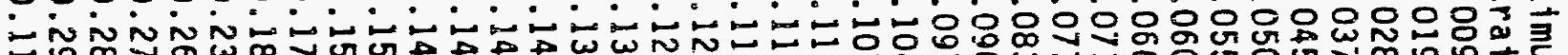

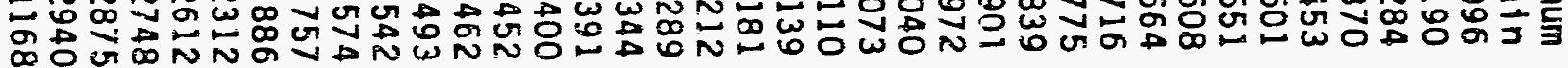

1 00000000000000000000000000000000000000

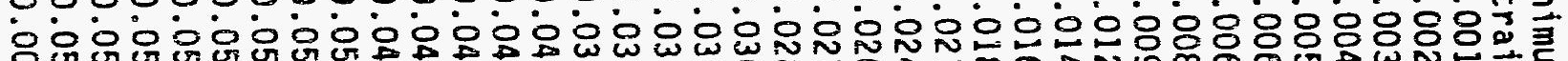

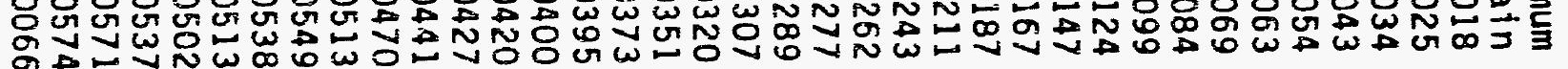

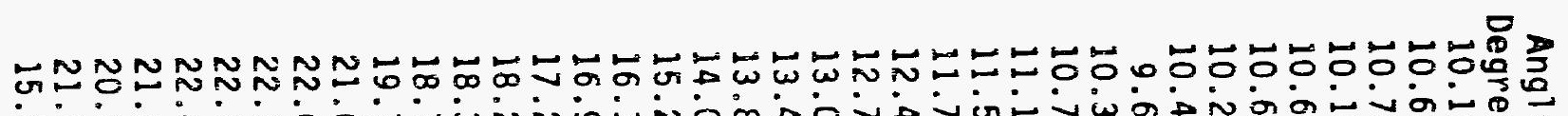

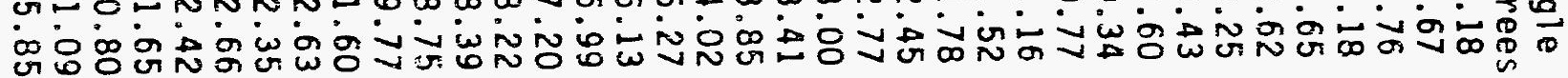




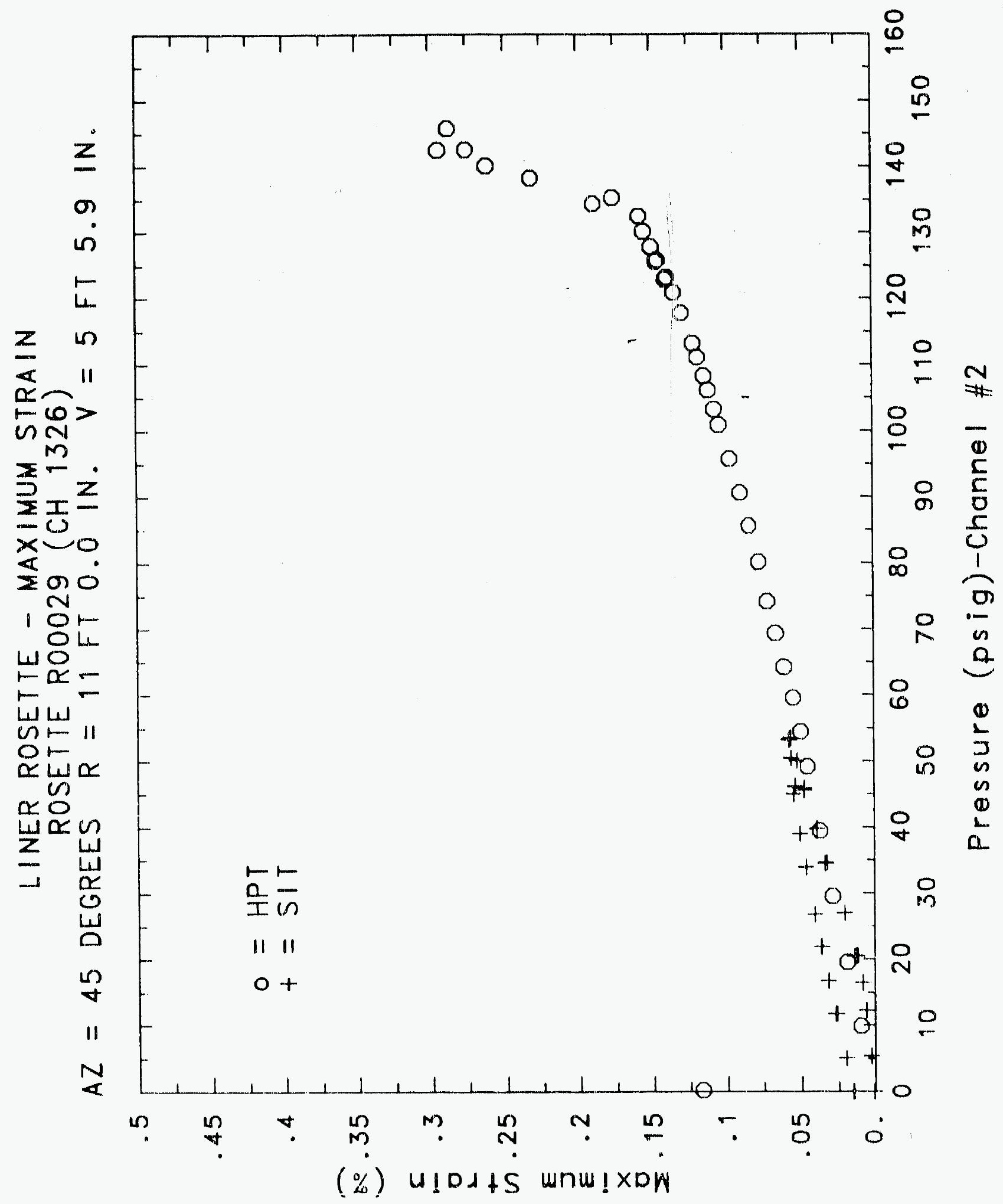




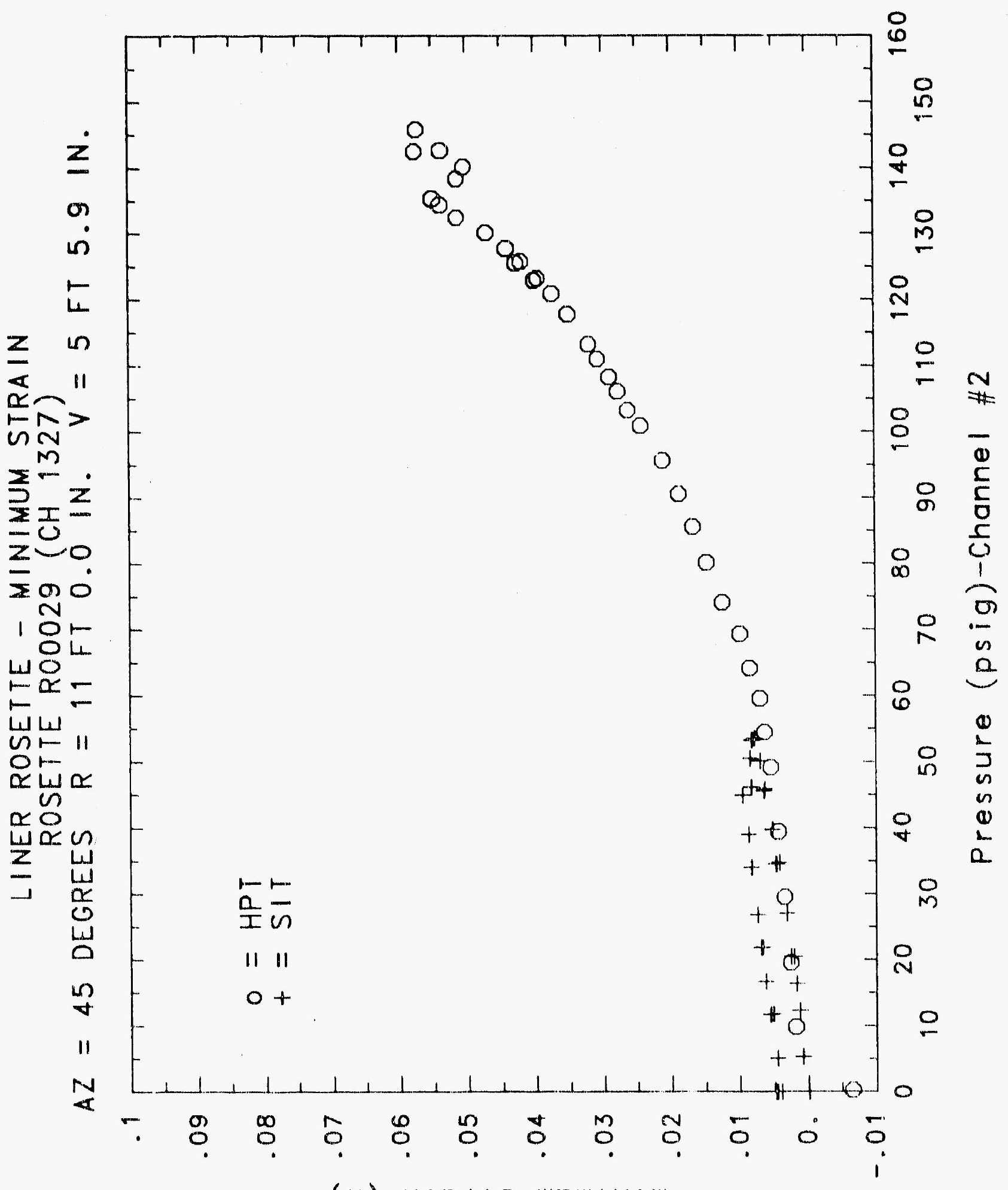

(\%) $u ! D \lambda+S$ unw! $u ! W$ 


\title{
Reinforced Concrete Test Data
}

\author{
Rosette 30 Channels $1329 \quad 1340 \quad 2222$
}

StRuCtural InTEgRity TEST

\begin{tabular}{|c|c|c|c|c|c|c|c|}
\hline $\begin{array}{c}\text { Pressure } \\
\text { psig } \\
-0.05 \\
5.33 \\
12.31 \\
16.44 \\
20.51 \\
20.46 \\
20.47 \\
27.05 \\
34.69 \\
34.53 \\
34.53 \\
39.70 \\
45.90 \\
45.65 \\
45.62 \\
50.10 \\
53.47 \\
53.29 \\
53.21 \\
50.49 \\
46.14 \\
46.13 \\
44.97 \\
38.98 \\
33.99 \\
33.96 \\
26.83 \\
21.813 \\
21.90 \\
16.69 \\
11.74 \\
11.77 \\
5.05 \\
0.02 \\
-0.04 \\
-0.02 \\
-0.02 \\
0.02\end{array}$ & $\begin{array}{c}\text { Maximum } \\
\text { xStrain } \\
0.0007 \\
0.0056 \\
0.0122 \\
0.0157 \\
0.0188 \\
0.0200 \\
0.0207 \\
0.0270 \\
0.0375 \\
0.0390 \\
0.0389 \\
0.0451 \\
0.0547 \\
0.0551 \\
0.0548 \\
0.0598 \\
0.0641 \\
0.0647 \\
0.0649 \\
0.0034 \\
0.0597 \\
0.0594 \\
0.0608 \\
0.0567 \\
0.0518 \\
0.0518 \\
0.0445 \\
0.0403 \\
0.0380 \\
0.0333 \\
0.0297 \\
0.0290 \\
0.0252 \\
0.0231 \\
0.0233 \\
0.0241 \\
0.0240 \\
0.0234\end{array}$ & $\begin{array}{l}\text { Minimum } \\
\text { xStrain } \\
0.0003 \\
0.0010 \\
0.0023 \\
0.0027 \\
0.0032 \\
0.0034 \\
0.0034 \\
0.0046 \\
0.0072 \\
0.0080 \\
0.0083 \\
0.0115 \\
0.0194 \\
0.0205 \\
0.0207 \\
0.0233 \\
0.0265 \\
0.0275 \\
0.0278 \\
0.0278 \\
0.0270 \\
0.0268 \\
0.0279 \\
0.0299 \\
0.0286 \\
0.0287 \\
0.0267 \\
0.0254 \\
0.0256 \\
0.0231 \\
0.0200 \\
0.0192 \\
0.0131 \\
0.0059 \\
0.0050 \\
0.0060 \\
0.0064 \\
0.0058\end{array}$ & $\begin{array}{c}\text { Angle } \\
\text { Degrees } \\
18.56 \\
10.93 \\
12.81 \\
11.73 \\
9.36 \\
8.52 \\
7.59 \\
6.88 \\
5.71 \\
6.11 \\
5.78 \\
6.94 \\
8.95 \\
9.13 \\
9.03 \\
8.96 \\
8.80 \\
9.22 \\
9.45 \\
9.71 \\
9.90 \\
10.05 \\
12.70 \\
20.41 \\
21.89 \\
21.00 \\
25.00 \\
31.53 \\
33.17 \\
41.36 \\
-34.53 \\
-31.52 \\
-18.23 \\
-10.75 \\
-12.49 \\
-14.29 \\
-14.77 \\
-12.38\end{array}$ & $\begin{array}{c}\text { Pressure } \\
\text { psig } \\
9.89 \\
19.55 \\
29.57 \\
39.42 \\
49.16 \\
54.50 \\
59.57 \\
64.20 \\
69.32 \\
74.16 \\
80.16 \\
85.61 \\
90.58 \\
95.69 \\
100.92 \\
103.25 \\
106.11 \\
108.31 \\
111.08 \\
113.24 \\
117.83 \\
120.92 \\
123.28 \\
122.97 \\
125.82 \\
125.60 \\
127.84 \\
130.19 \\
132.53 \\
135.33 \\
134.42 \\
138.35 \\
140.16 \\
142.63 \\
145.78 \\
142.52 \\
0.82\end{array}$ & $\begin{array}{l}\text { Maximul. } \\
\text { x.Strain } \\
0.0081 \\
0.0145 \\
0.0209 \\
0.0268 \\
0.0325 \\
0.0360 \\
0.0395 \\
0.0439 \\
0.0486 \\
0.0530 \\
0.0570 \\
0.0607 \\
0.0643 \\
0.0681 \\
0.0722 \\
0.0741 \\
0.0760 \\
0.0773 \\
0.0790 \\
0.0802 \\
0.0815 \\
0.0867 \\
0.0917 \\
0.0927 \\
0.0990 \\
0.1007 \\
0.1051 \\
0.1163 \\
0.1352 \\
0.1665 \\
0.1745 \\
0.2183 \\
0.2525 \\
0.2928 \\
0.3199 \\
0.3447 \\
0.3303\end{array}$ & $\begin{array}{l}\text { Minimum } \\
\text { xStrain } \\
0.0081 \\
0.0145 \\
0.0209 \\
0.0268 \\
0.0325 \\
0.0360 \\
0.0395 \\
0.0439 \\
0.0486 \\
0.0530 \\
0.0570 \\
0.0607 \\
0.0643 \\
0.0681 \\
0.0722 \\
0.0741 \\
0.0760 \\
0.0773 \\
0.0790 \\
0.0802 \\
0.0815 \\
0.0821 \\
0.0825 \\
0.0824 \\
0.0818 \\
0.0817 \\
0.0819 \\
0.0810 \\
0.0786 \\
0.0776 \\
0.0779 \\
0.0809 \\
0.0879 \\
0.1047 \\
0.1292 \\
0.1349 \\
0.0305\end{array}$ & $\begin{array}{c}\text { Angle } \\
\text { Degrees } \\
22.47 \\
23.68 \\
23.94 \\
24.66 \\
24.96 \\
24.98 \\
24.76 \\
24.21 \\
23.61 \\
23.38 \\
23.18 \\
22.93 \\
22.91 \\
22.97 \\
23.11 \\
23.16 \\
23.21 \\
23.23 \\
23.26 \\
23.29 \\
23.46 \\
23.57 \\
23.73 \\
23.81 \\
23.90 \\
23.88 \\
23.91 \\
24.08 \\
24.15 \\
24.25 \\
24.27 \\
24.28 \\
24.27 \\
24.11 \\
23.85 \\
23.64 \\
24.91\end{array}$ \\
\hline
\end{tabular}

High Pressure test 


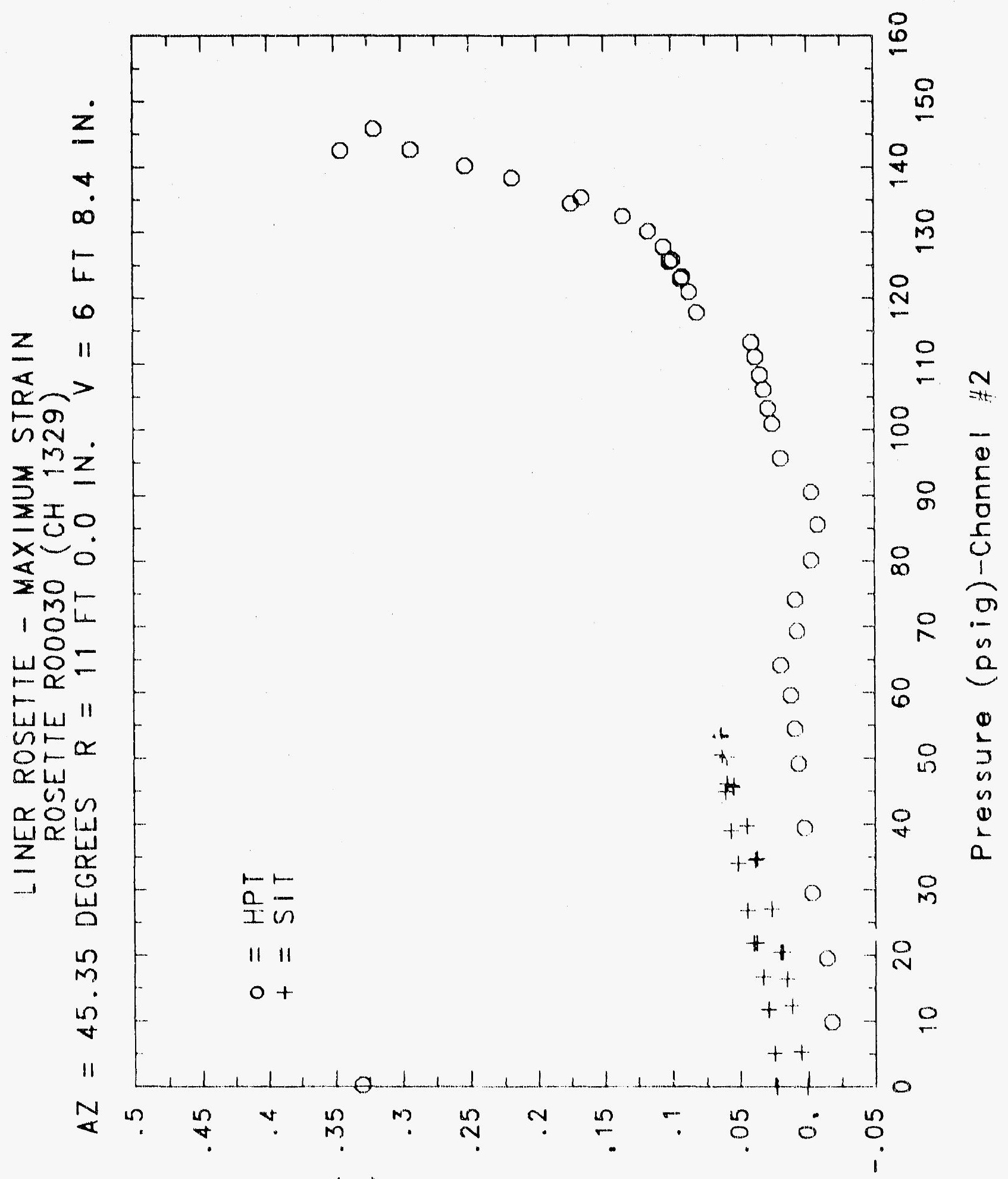

(\%) $u ! 0 \lambda+S$ wnw! $\times 0 w$ 


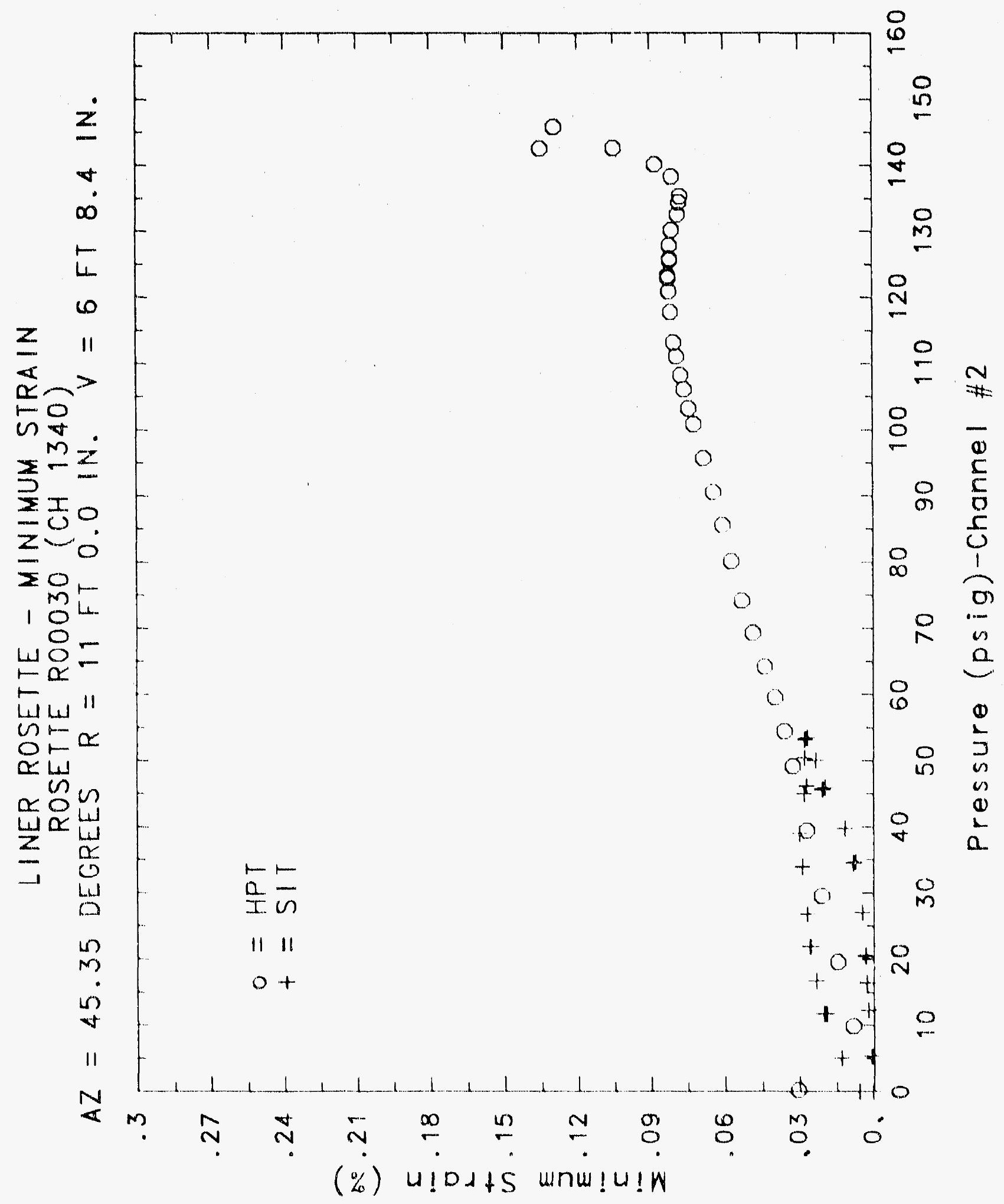




\section{Reinforced Concrete Test Data}

Rosette 31 Channels $1341 \quad 1342 \quad 1343$

Structural Integrity Test

\begin{tabular}{|c|c|c|c|c|c|c|c|}
\hline Pressure & Maximum & Minimum & Angle & Pressure & Maximum & Minimum & Angle \\
\hline psig & xstrain & \%train & Degrees & psig & xstrain & xstrain & Degrees \\
\hline-0.05 & 0.0003 & 0.0000 & 44.93 & 9.89 & 0.0188 & 0.0025 & 11.16 \\
\hline 5.33 & 0.0041 & 0.0019 & 11.50 & 19.55 & 0.0305 & 0.0041 & 7.27 \\
\hline 12.31 & 0.0090 & 0.0040 & 6.57 & 29.57 & 0.0424 & 0.0057 & 5.55 \\
\hline 16.44 & 0.0127 & 0.0051 & 5.78 & 39.42 & 0.0536 & 0.0074 & 4.83 \\
\hline 20.51 & 0.0186 & 0.0059 & 5.72 & 49.16 & 0.0642 & 0.0488 & 3.84 \\
\hline 20.46 & 0.0199 & 0.0061 & 5.06 & 54.50 & 0.0702 & 0.0097 & 3.51 \\
\hline 20.47 & 0.0212 & 0.0062 & 5.00 & 59.57 & 0.0765 & 0.0102 & 3.05 \\
\hline 27.05 & 0.0308 & 0.0064 & 4.05 & 64.20 & 0.0870 & 0.0106 & 3.75 \\
\hline 34.69 & 0.0441 & 0.0070 & 1.99 & 69.32 & 0.1046 & 0.0110 & 5.77 \\
\hline 34.53 & 0.0447 & 0.0071 & 1.59 & 74.16 & 0.0858 & 0.0135 & -1.84 \\
\hline 34.5 .3 & 0.0446 & 0.0071 & 1.27 & 80.16 & 0.0632 & 0.0049 & -23.60 \\
\hline 39.70 & 0.0513 & 0.0076 & 0.82 & 85.61 & 0.0680 & 0.0060 & .22 .55 \\
\hline 45.90 & 0.0619 & 0.0080 & 0.24 & 90.58 & 0.0723 & 0.0009 & -21.81 \\
\hline 45.65 & 0.0615 & 0.0077 & -0.05 & 95.69 & 0.0767 & 0.0071 & -21.36 \\
\hline 45.62 & 0.0614 & 0.0075 & -0.10 & 100.92 & 0.0806 & 0.0074 & -21.17 \\
\hline 50.10 & 0.0670 & 0.0082 & -0.23 & 103.25 & 0.0824 & 0.0073 & -21.05 \\
\hline 53.47 & 0.0720 & 0.0084 & -0.55 & 106.11 & 0.0852 & 0.0055 & -20.89 \\
\hline 53.29 & 0.0720 & 0.0084 & -0.61 & 108.31 & 0.0869 & 0.0052 & -20.85 \\
\hline 53.21 & 0.0719 & 0.0083 & -0.69 & 111.08 & 0.0893 & 0.0053 & -20.47 \\
\hline 50.49 & 0.0693 & 0.0080 & -1.00 & 113.24 & 0.0905 & 0.0053 & -20.42 \\
\hline 46.14 & 0.0648 & 0.0073 & -1.29 & 117.83 & 0.0915 & 0.0059 & -20.23 \\
\hline 46.13 & 0.0643 & 0.0073 & -1.33 & 120.92 & 0.0921 & 0.0060 & -20.02 \\
\hline 44.97 & 0.0649 & 0.0080 & -0.41 & 123.28 & 0.0920 & 0.0058 & -19.71 \\
\hline 38.98 & 0.0590 & 0.0070 & -0.54 & 122.97 & 0.0913 & 0.0052 & -19.99 \\
\hline 33.99 & 0.0538 & 0.0063 & -0.59 & 125.82 & 0.0912 & 0.0044 & -20.78 \\
\hline 33.96 & 0.0543 & 0.0063 & -0.91 & 125.60 & 0.0913 & 0.0042 & -20.64 \\
\hline 26.83 & 0.0464 & 0.0051 & -1.10 & 127.84 & 0.1135 & 0.0127 & .7 .95 \\
\hline 21.88 & 0.0407 & 0.0043 & -0.92 & 130.19 & 0.1295 & 0.0135 & -2.40 \\
\hline 21.90 & 0.0409 & 0.0043 & -1.24 & 132.53 & 0.1334 & 0.0128 & -1.49 \\
\hline 16.69 & 0.0349 & 0.0035 & -1.68 & 135.33 & 0.1328 & 0.0125 & -2.25 \\
\hline 11.74 & 0.0289 & 0.0024 & -2.37 & 134.42 & 0.0840 & -0.0107 & -30.24 \\
\hline 11.77 & 0.0284 & 0.0021 & -2.30 & 138.35 & 0.1861 & 0.0122 & 4.77 \\
\hline 5.05 & 0.0210 & 0.0006 & -3.40 & 140.16 & 0.1356 & 0.0145 & -9.23 \\
\hline 0.02 & 0.0153 & -0.0008 & -4.97 & 142.63 & 0.2198 & -0.0710 & -32.95 \\
\hline-0.04 & 0.0149 & -0.0002 & -3.90 & 145.78 & 0.5365 & -0.2238 & -33.66 \\
\hline-0.02 & 0.0158 & 0.0006 & -2.51 & 142.52 & 0.6114 & -0.1334 & -25.57 \\
\hline-0.02 & 0.0161 & 0.0008 & -2.67 & 0.22 & 0.4489 & -0.1881 & -31.93 \\
\hline 0.02 & 0.0164 & 0.0007 & -2.23 & & & & \\
\hline
\end{tabular}

High Pressure test 


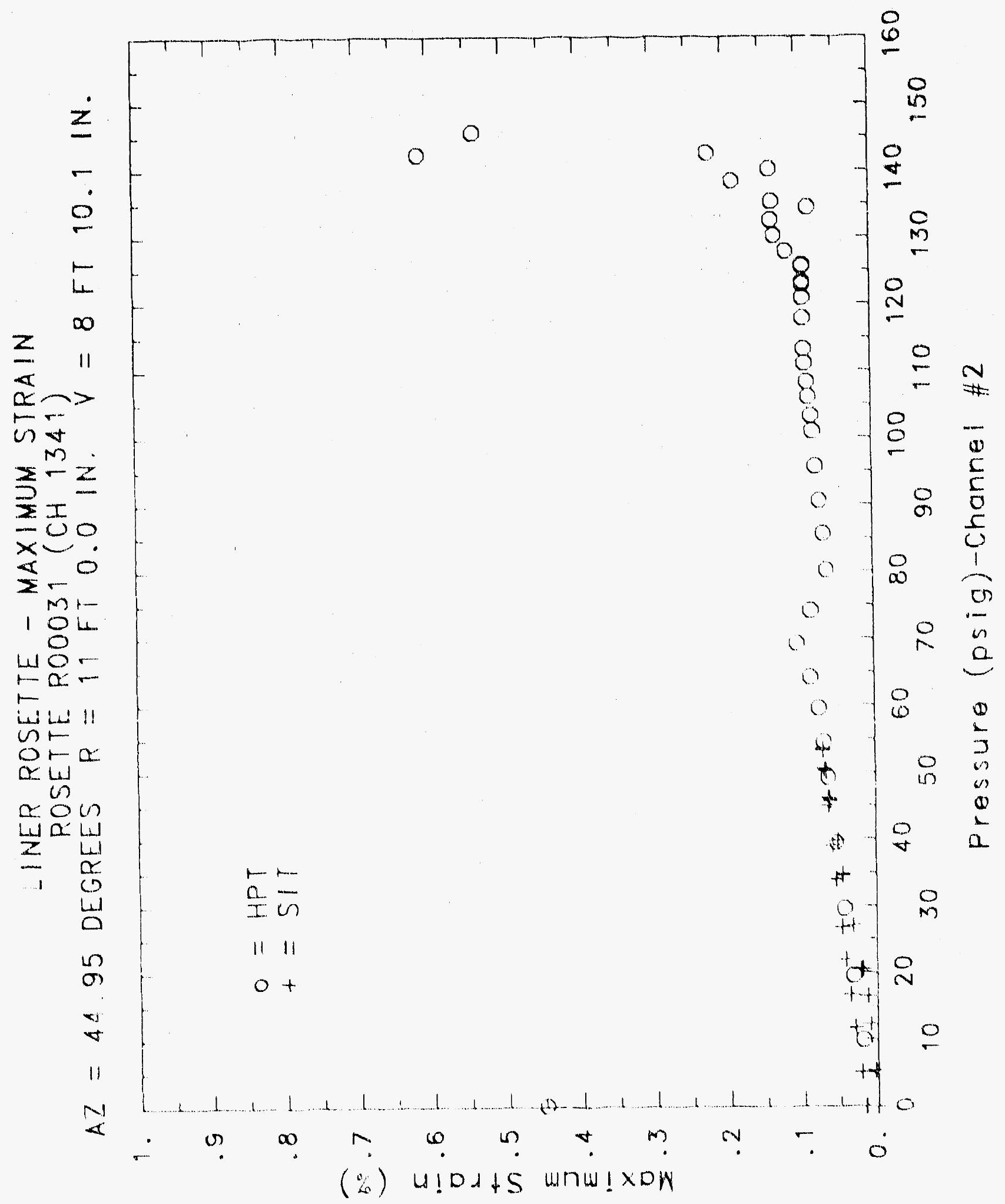




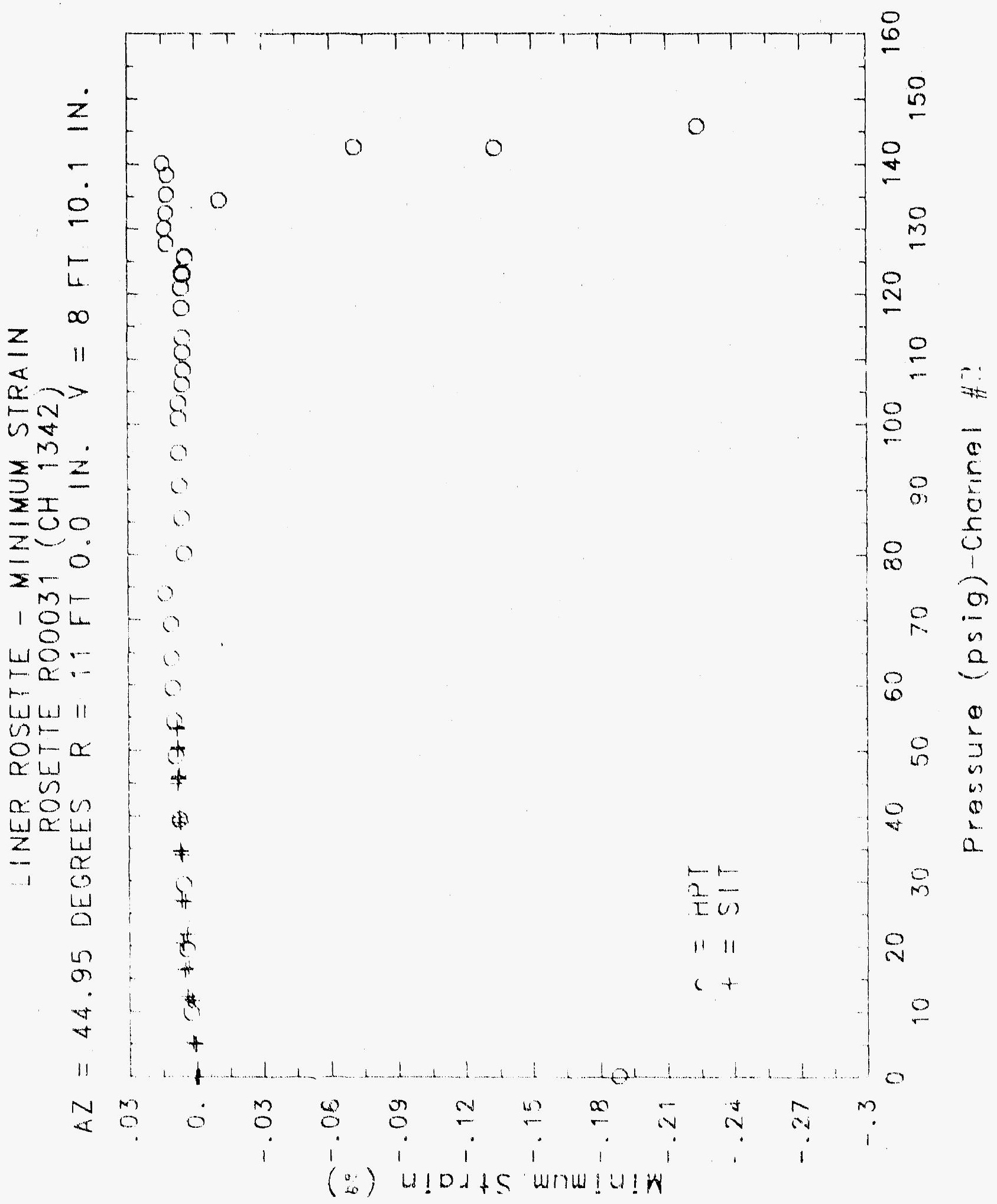




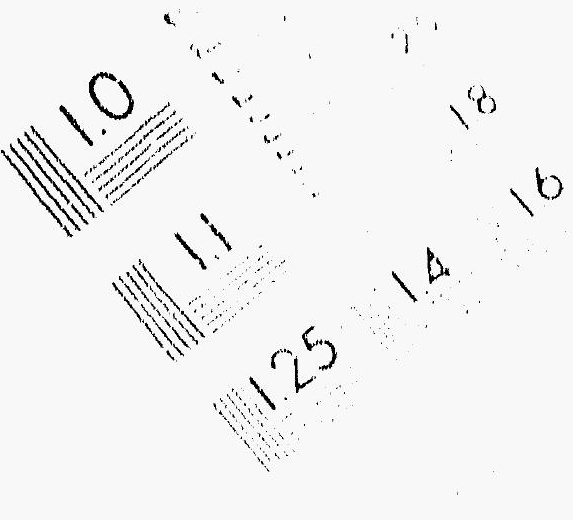

IMAGE EVALUATION

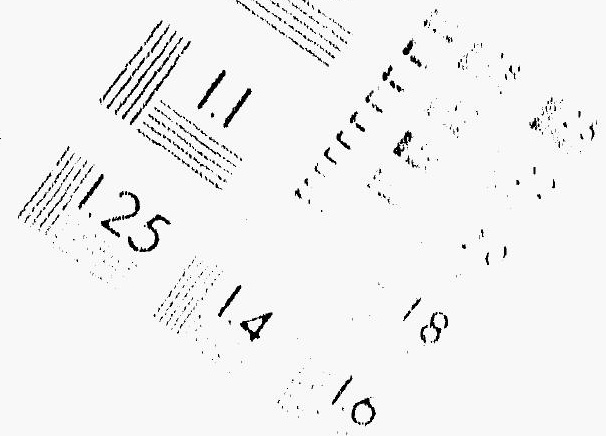

TEST TARGET (MT-3)
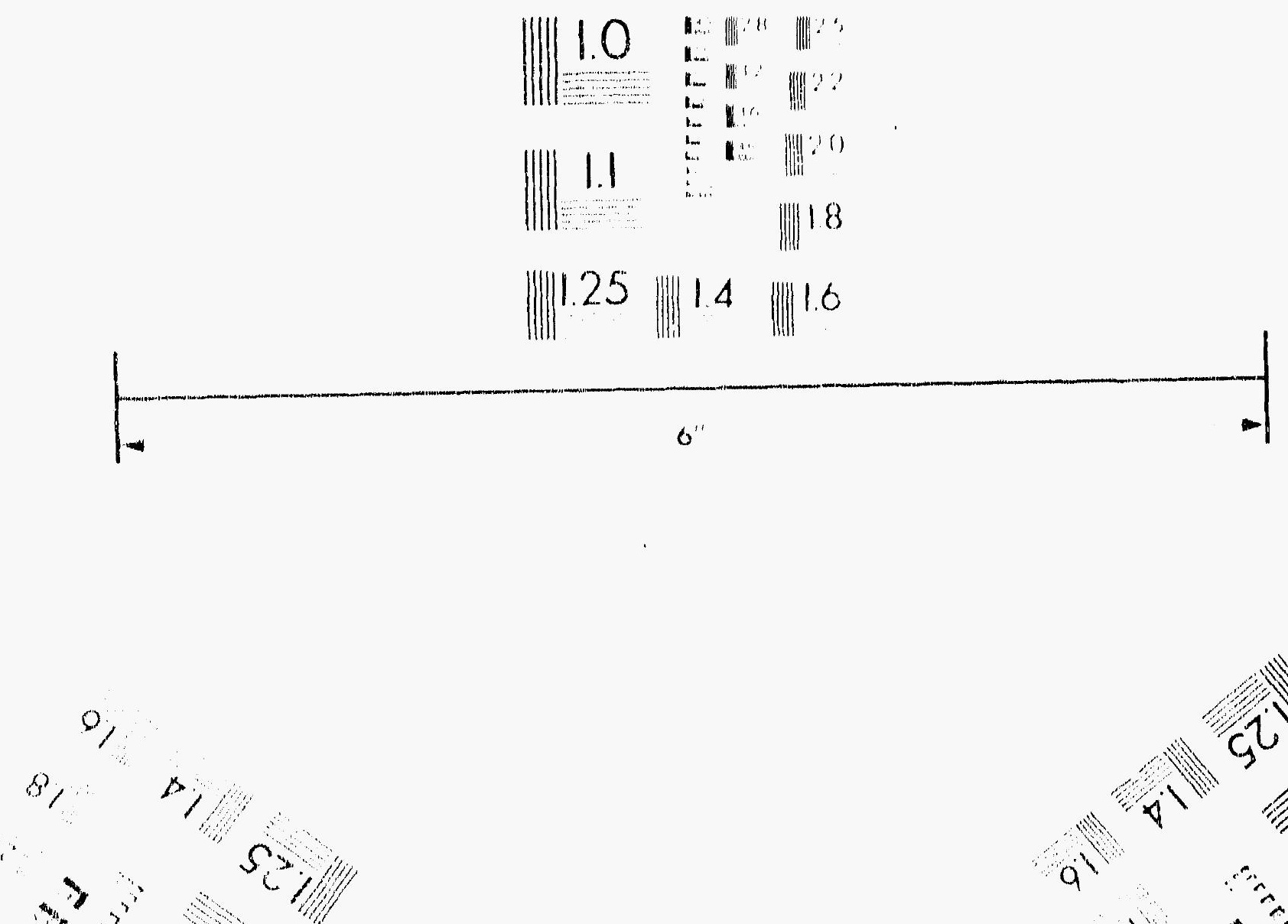

Phologluphic: Sciencess (oriporation

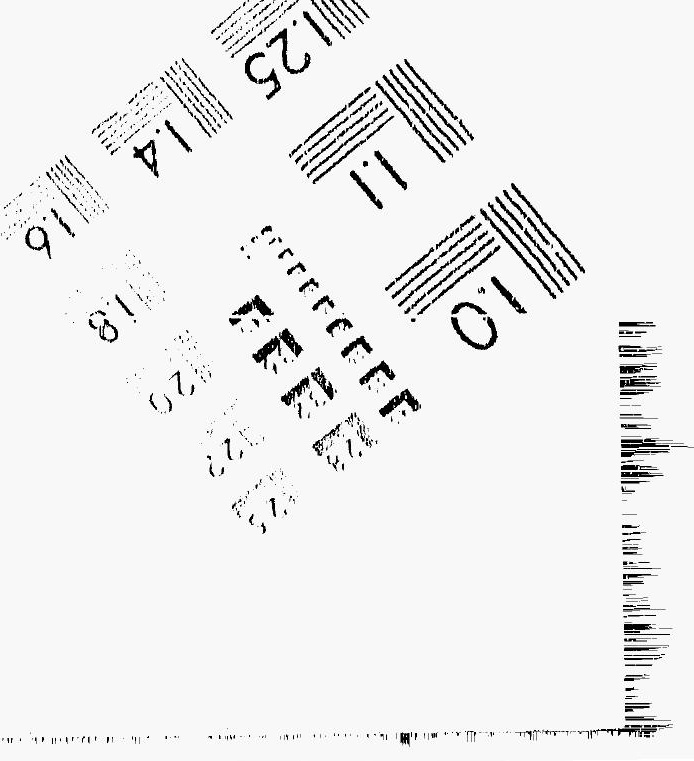




\section{SECURITY CLASSIFICATION}
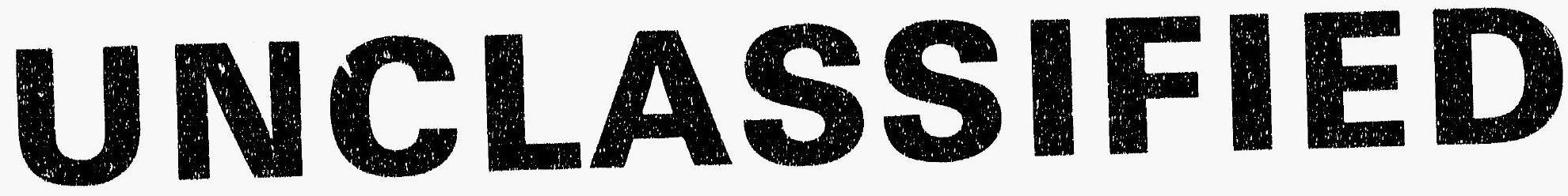

\section{DATE OF MICROFILMING $1-6-92$}

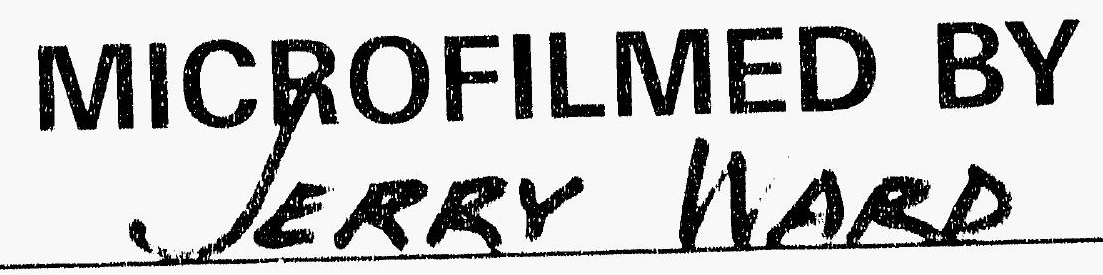

SANDIA NATIONAL LABS 


\section{THIS DOCUMENT FILMED}

AT

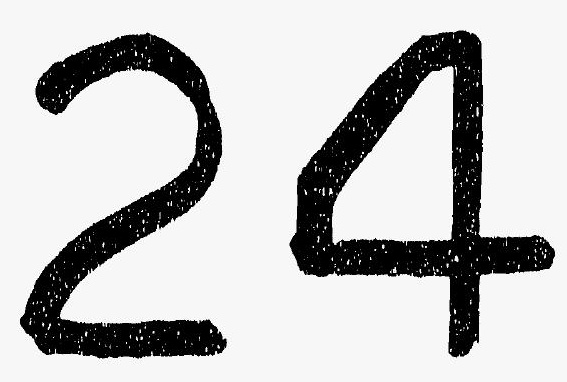

REDUCTION 


\title{
Reinforced Concrete Test Data
}

\author{
Rosette 32 Channels $1344 \quad 1345 \quad 1346$
}

Structural IntEgrity Test

\begin{tabular}{|c|c|c|c|c|c|c|c|}
\hline $\begin{array}{c}\text { Pressure } \\
\text { ps 1g } \\
-0.05 \\
5.33 \\
12.31 \\
16.44 \\
20.51 \\
20.46 \\
20.47 \\
27.05 \\
34.69 \\
34.53 \\
34.53 \\
39.70 \\
45.90 \\
45.65 \\
45.62 \\
30.10 \\
53.47 \\
53.29 \\
53.21 \\
50.49 \\
46.14 \\
46.13 \\
44.97 \\
38.98 \\
33.99 \\
33.96 \\
26.83 \\
21.88 \\
21.90 \\
16.69 \\
11.74 \\
11.77 \\
5.05 \\
0.02 \\
-0.04 \\
-0.02 \\
-0.02 \\
0.06\end{array}$ & $\begin{array}{c}\text { Maximum } \\
\text { x.Strain } \\
0.0003 \\
0.0043 \\
0.0084 \\
0.0109 \\
0.0146 \\
0.0157 \\
0.0164 \\
0.0251 \\
0.0385 \\
0.0395 \\
0.0397 \\
0.0457 \\
0.0552 \\
0.0547 \\
0.0544 \\
0.0594 \\
0.0641 \\
0.0641 \\
0.0642 \\
0.0624 \\
0.0590 \\
0.0588 \\
0.0581 \\
0.0533 \\
0.0490 \\
0.0493 \\
0.0427 \\
0.0381 \\
0.0385 \\
0.0336 \\
0.0284 \\
0.0277 \\
0.0210 \\
0.0155 \\
0.0140 \\
0.0148 \\
0.0154 \\
0.0154\end{array}$ & $\begin{array}{l}\text { Minimum } \\
\text { XStra1n } \\
0.0002 \\
0.0020 \\
0.0057 \\
0.0081 \\
0.0110 \\
0.0112 \\
0.0115 \\
0.0145 \\
0.0182 \\
0.0184 \\
0.0185 \\
0.0211 \\
0.0246 \\
0.0244 \\
0.0244 \\
0.0267 \\
0.0288 \\
0.0289 \\
0.0288 \\
0.0279 \\
0.0260 \\
0.0260 \\
0.0261 \\
0.0233 \\
0.0210 \\
0.0212 \\
0.0180 \\
0.0155 \\
0.0157 \\
0.0131 \\
0.0105 \\
0.0104 \\
0.0069 \\
0.0041 \\
0.0052 \\
0.0057 \\
0.0059 \\
0.0058\end{array}$ & $\begin{array}{c}\text { Angle } \\
\text { Degrees } \\
1.38 \\
-5.57 \\
4.69 \\
12.53 \\
22.22 \\
20.53 \\
20.05 \\
29.89 \\
30.53 \\
29.55 \\
29.50 \\
30.32 \\
31.05 \\
30.55 \\
30.51 \\
31.24 \\
31.62 \\
31.34 \\
31.35 \\
30.66 \\
29.80 \\
30.15 \\
28.83 \\
27.62 \\
26.64 \\
26.51 \\
24.88 \\
23.34 \\
23.49 \\
21.48 \\
19.11 \\
20.07 \\
15.98 \\
11.51 \\
12.07 \\
12.03 \\
12.63 \\
13.90\end{array}$ & $\begin{array}{c}\text { Pressure } \\
\text { ps1g } \\
9.89 \\
19.55 \\
29.57 \\
39.42 \\
49.16 \\
54.50 \\
59.57 \\
64.20 \\
69.32 \\
74.16 \\
80.16 \\
85.61 \\
90.58 \\
95.69 \\
100.92 \\
103.25 \\
106.11 \\
108.31 \\
111.08 \\
113.24 \\
117.83 \\
120.92 \\
123.28 \\
122.97 \\
125.82 \\
125.60 \\
127.84 \\
130.19 \\
132.53 \\
135.33 \\
134.42 \\
138.35 \\
140.16 \\
142.63 \\
145.78 \\
142.52 \\
0.22\end{array}$ & $\begin{array}{l}\text { Haximum } \\
\text { XStratn } \\
0.0111 \\
0.0218 \\
0.0312 \\
0.0410 \\
0.0500 \\
0.0541 \\
0.0605 \\
0.0669 \\
0.0737 \\
0.0810 \\
0.0875 \\
0.0952 \\
0.1022 \\
0.1090 \\
0.1169 \\
0.1208 \\
0.1247 \\
0.1277 \\
0.1305 \\
0.1319 \\
0.1354 \\
0.1390 \\
0.1415 \\
0.1420 \\
0.1462 \\
0.1466 \\
0.1502 \\
0.1529 \\
0.1575 \\
0.1652 \\
0.1672 \\
0.1937 \\
0.2436 \\
0.3453 \\
0.4618 \\
0.5037 \\
0.3569\end{array}$ & $\begin{array}{c}\text { Minimum } \\
\text { XStrain } \\
0.0049 \\
0.0091 \\
0.0120 \\
0.0166 \\
0.0212 \\
0.0238 \\
0.0267 \\
0.0295 \\
0.0319 \\
0.0349 \\
0.0372 \\
0.0401 \\
0.0424 \\
0.0451 \\
0.0471 \\
0.0483 \\
0.0495 \\
0.0509 \\
0.0518 \\
0.0526 \\
0.0540 \\
0.0552 \\
0.0564 \\
0.0565 \\
0.0578 \\
0.0579 \\
0.0586 \\
0.0585 \\
0.0578 \\
0.0562 \\
0.0558 \\
0.0535 \\
0.0493 \\
0.0464 \\
0.0537 \\
0.0660 \\
-0.0637\end{array}$ & $\begin{array}{r}\text { Angle } \\
\text { Degree } \\
12.87 \\
19.85 \\
17.56 \\
20.97 \\
23.14 \\
23.88 \\
25.05 \\
25.48 \\
25.67 \\
24.96 \\
25.69 \\
26.37 \\
26.91 \\
27.58 \\
28.66 \\
29.18 \\
30.05 \\
30.87 \\
32.54 \\
33.79 \\
35.83 \\
37.14 \\
37.86 \\
38.15 \\
39.96 \\
40.17 \\
41.21 \\
42.62 \\
44.30 \\
-43.69 \\
-43.08 \\
-38.71 \\
-34.34 \\
-31.62 \\
-30.11 \\
-27.28 \\
-18.36\end{array}$ \\
\hline
\end{tabular}

High Pressure Test 


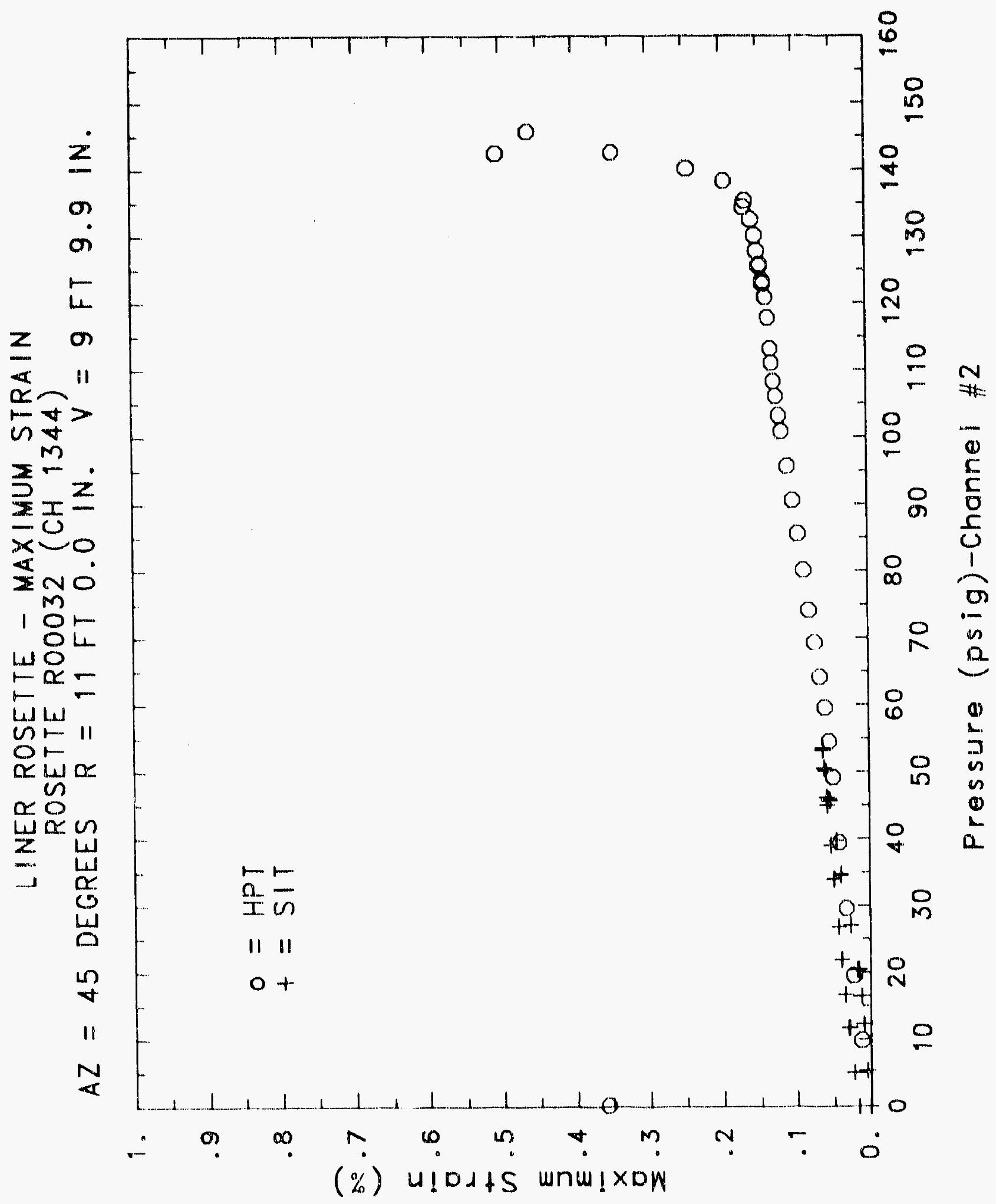




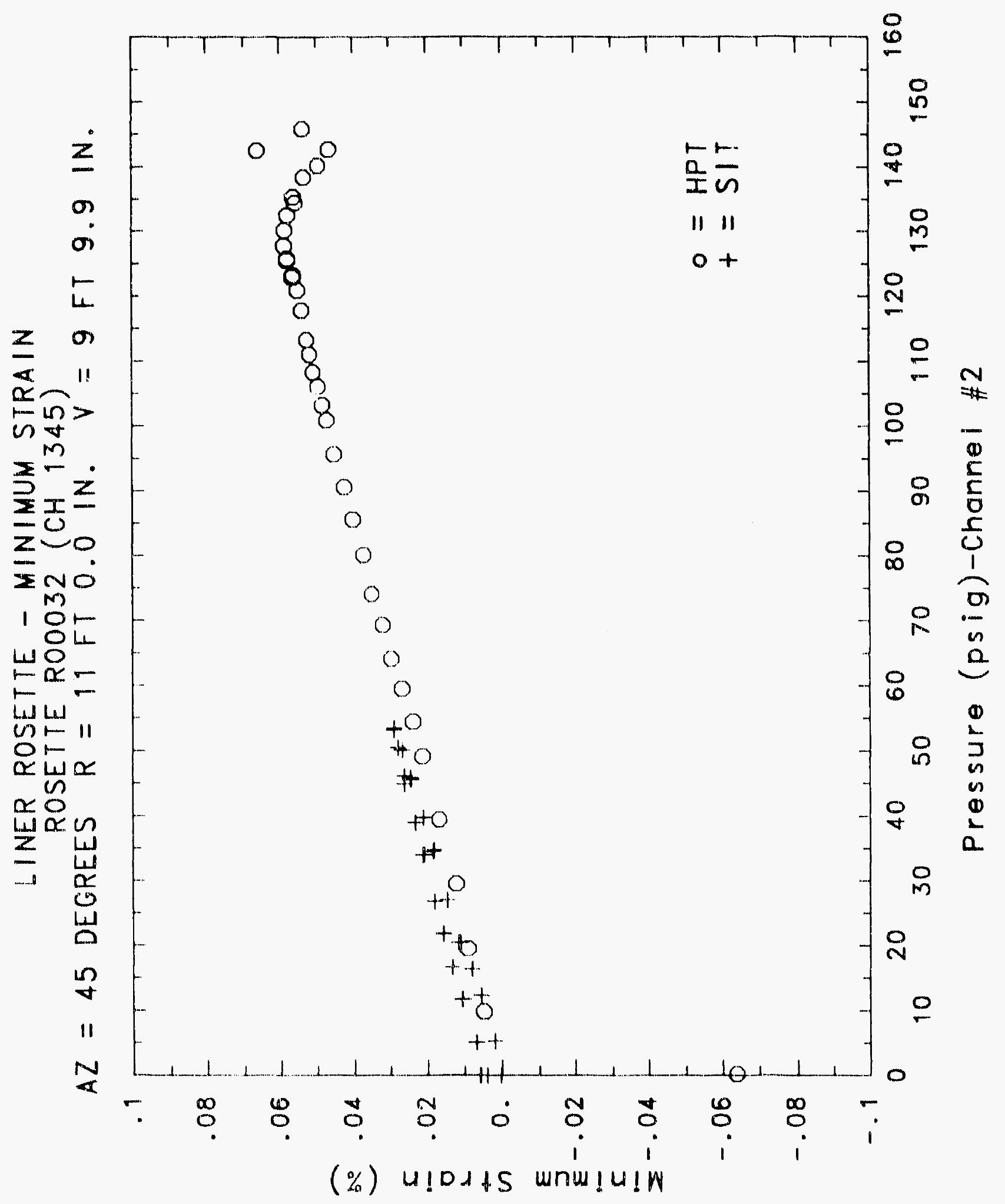




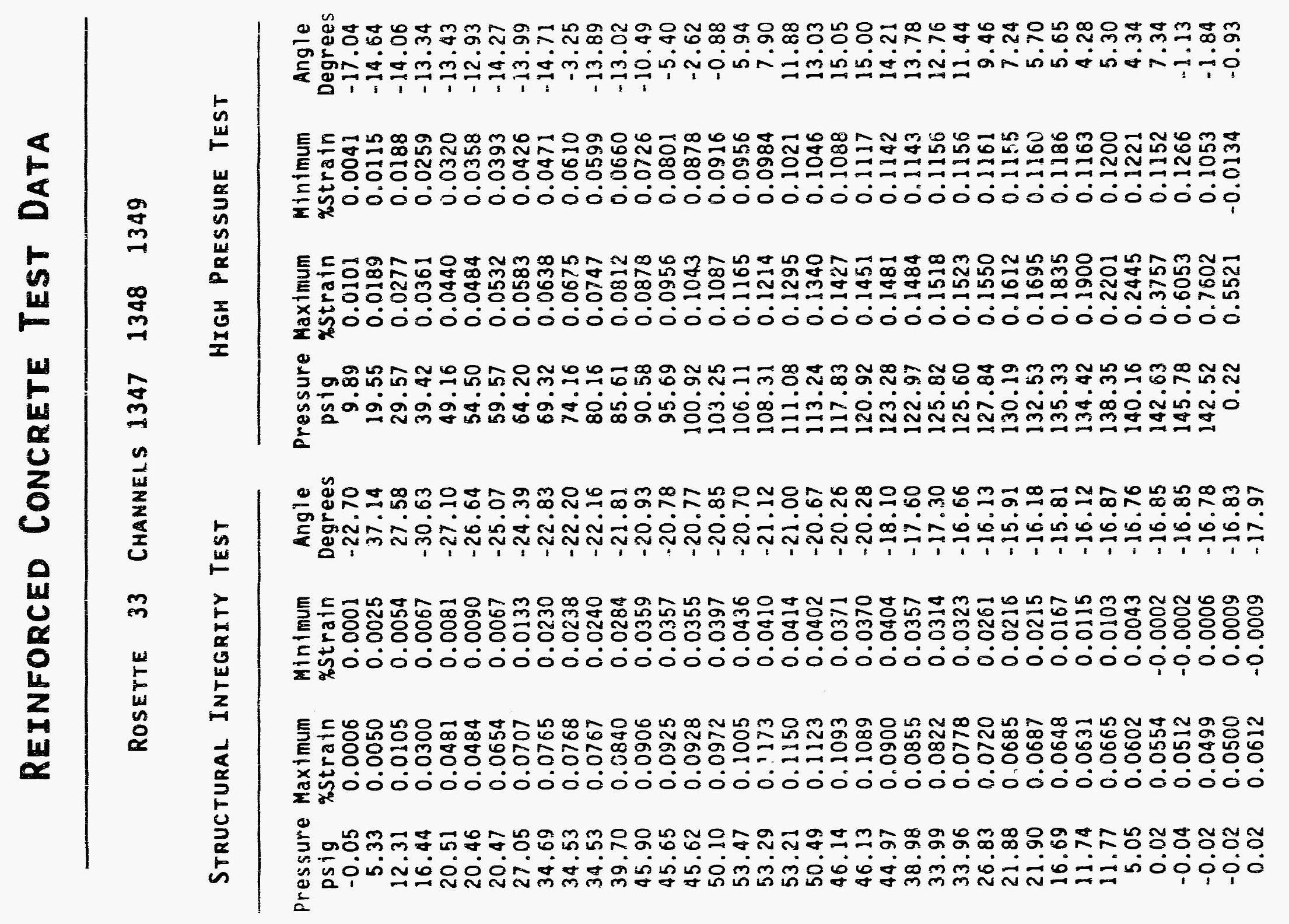




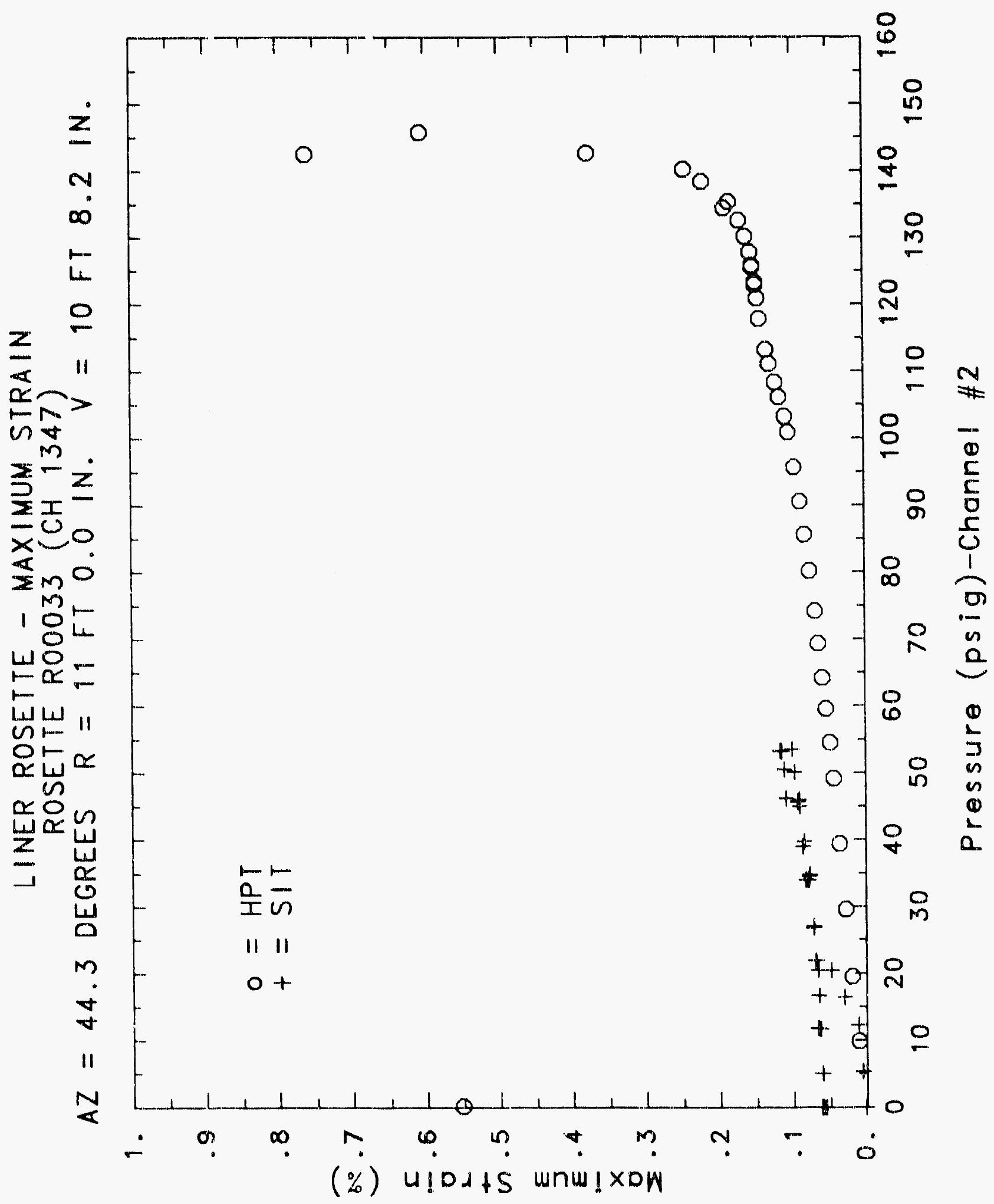




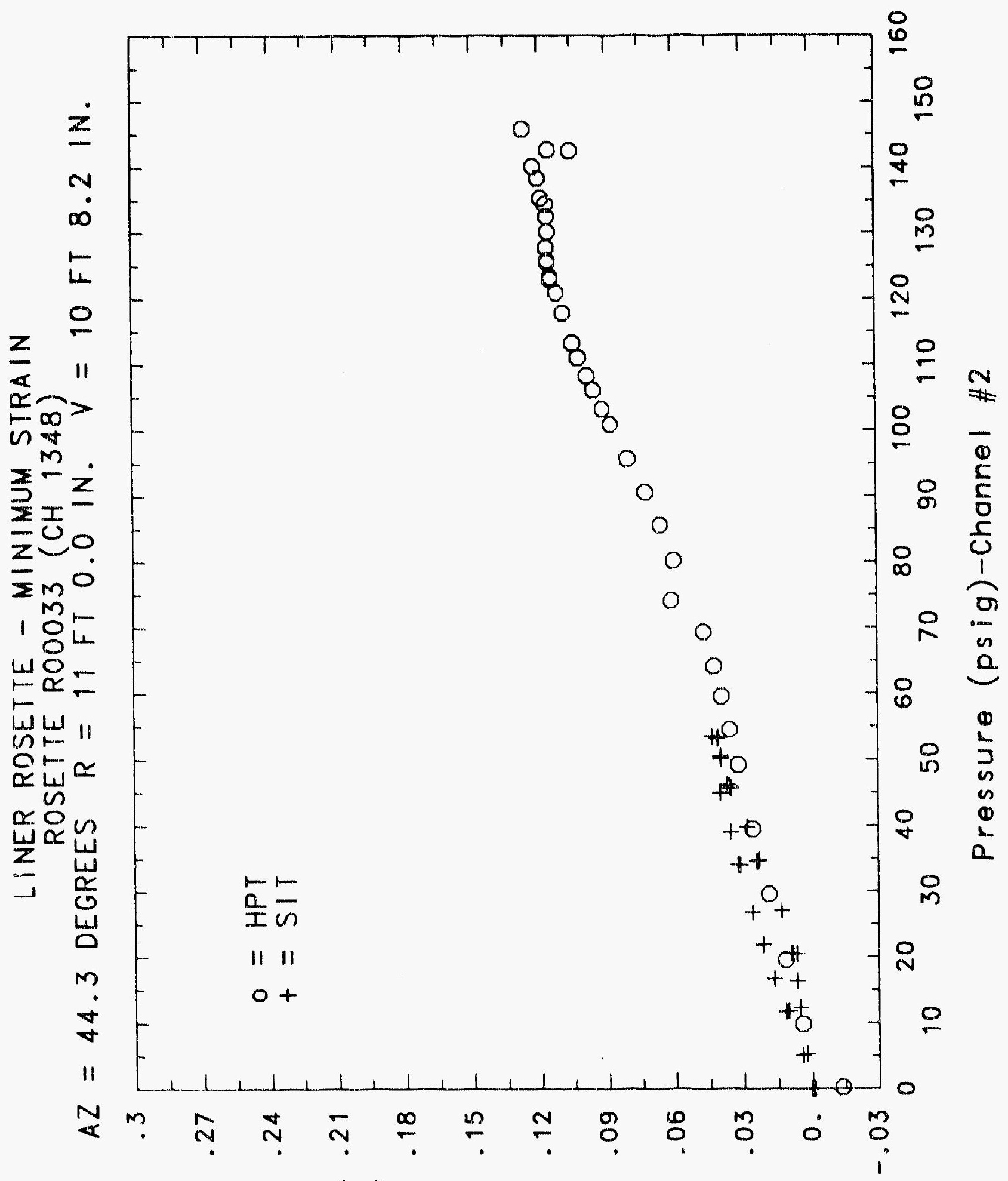

(\%) $u ! D d+S$ wnm!u!W 


\title{
Reinforced Concrete Test Data
}

\author{
Rosette 34 Channels $1360 \quad 1361 \quad 1362$
}

\section{STRUCTURAL INTEgRITY TEST}

\begin{tabular}{|c|c|c|c|c|c|c|c|}
\hline $\begin{array}{c}\text { Pressure } \\
\text { psig } \\
-0.05 \\
5.33 \\
12.31 \\
16.44 \\
20.51 \\
20.46 \\
20.47 \\
27.05 \\
34.69 \\
34.53 \\
34.53 \\
39.70 \\
45.90 \\
45.65 \\
45.62 \\
50.10 \\
53.47 \\
53.29 \\
53.21 \\
50.49 \\
46.14 \\
46.13 \\
44.97 \\
38.98 \\
33.99 \\
33.96 \\
23.83 \\
21.88 \\
21.90 \\
16.69 \\
11.74 \\
11.77 \\
5.05 \\
0.02 \\
-0.04 \\
-0.02 \\
-0.02 \\
0.02\end{array}$ & $\begin{array}{c}\text { Maximum } \\
\text { xStrain } \\
0.0003 \\
0.0030 \\
0.0060 \\
0.0079 \\
0.0106 \\
0.0120 \\
0.0125 \\
0.0179 \\
0.0248 \\
0.0260 \\
0.0261 \\
0.0297 \\
0.0336 \\
0.0334 \\
0.0334 \\
0.0355 \\
0.0375 \\
0.0374 \\
0.0373 \\
0.0359 \\
0.0339 \\
0.0334 \\
0.0327 \\
0.0299 \\
0.0277 \\
0.0285 \\
0.0249 \\
0.0229 \\
0.0230 \\
0.0202 \\
0.0175 \\
0.0170 \\
0.0134 \\
0.0110 \\
0.0093 \\
0.0095 \\
0.0092 \\
0.0083\end{array}$ & $\begin{array}{c}\text { Minimum } \\
\text { xStrain } \\
0.0001 \\
0.0003 \\
0.0009 \\
0.0012 \\
0.0016 \\
0.0018 \\
0.0019 \\
0.0026 \\
0.0036 \\
0.0035 \\
0.0035 \\
0.0038 \\
0.0047 \\
0.0047 \\
0.0047 \\
0.0055 \\
0.0063 \\
0.0066 \\
0.0068 \\
0.0069 \\
0.0067 \\
0.0068 \\
0.0073 \\
0.0064 \\
0.0057 \\
0.0060 \\
0.0051 \\
0.0045 \\
0.0044 \\
0.0035 \\
0.0030 \\
0.0027 \\
0.0020 \\
0.0014 \\
0.0026 \\
0.0024 \\
0.0020 \\
-0.0008\end{array}$ & $\begin{array}{c}\text { Angle } \\
\text { Degrees } \\
-5.39 \\
-27.47 \\
-22.04 \\
-20.84 \\
-19.88 \\
-19.36 \\
-19.48 \\
-17.54 \\
-17.77 \\
-17.73 \\
-17.28 \\
-17.54 \\
-17.93 \\
-17.73 \\
-17.76 \\
-17.91 \\
-18.09 \\
-18.43 \\
-18.32 \\
-18.47 \\
-18.71 \\
-18.91 \\
-18.88 \\
-18.96 \\
-18.89 \\
-18.49 \\
-17.95 \\
-17.99 \\
-17.08 \\
-17.08 \\
-17.13 \\
-16.32 \\
-15.07 \\
-13.81 \\
-13.60 \\
-13.93 \\
-11.69 \\
-6.21\end{array}$ & $\begin{array}{c}\text { Pressure } \\
\text { psig } \\
9.89 \\
19.55 \\
29.57 \\
39.42 \\
49.16 \\
54.50 \\
59.57 \\
64.20 \\
69.32 \\
74.16 \\
80.16 \\
85.61 \\
90.58 \\
95.69 \\
100.92 \\
103.25 \\
106.11 \\
108.31 \\
111.08 \\
113.24 \\
117.83 \\
120.92 \\
123.28 \\
122.97 \\
125.82 \\
125.60 \\
127.84 \\
130.19 \\
132.53 \\
135.33 \\
134.42 \\
138.35 \\
140.16 \\
142.63 \\
145.78 \\
142.52 \\
0.22\end{array}$ & $\begin{array}{l}\text { Maximum } \\
\text { XStrain } \\
0.0062 \\
0.0108 \\
0.0156 \\
0.0205 \\
0.0254 \\
0.0285 \\
0.0312 \\
0.0344 \\
0.0376 \\
0.0407 \\
0.0446 \\
0.0480 \\
0.0499 \\
0.0519 \\
0.0541 \\
0.0553 \\
0.0569 \\
0.0582 \\
0.0594 \\
0.0605 \\
0.0624 \\
0.0638 \\
0.0648 \\
0.0649 \\
0.0662 \\
0.0662 \\
0.0678 \\
0.0696 \\
0.0725 \\
0.0811 \\
0.0819 \\
0.0900 \\
0.0948 \\
0.1032 \\
0.1118 \\
0.1126 \\
0.0671\end{array}$ & $\begin{array}{c}\text { Minimum } \\
\text { xStrain } \\
0.0016 \\
0.0029 \\
0.0043 \\
0.0058 \\
0.0072 \\
0.0080 \\
0.0089 \\
0.0100 \\
0.0109 \\
0.0119 \\
0.0125 \\
0.0136 \\
0.0148 \\
0.0164 \\
0.0180 \\
0.0189 \\
0.0196 \\
0.0204 \\
0.0216 \\
0.0225 \\
0.0246 \\
0.0262 \\
0.0279 \\
0.0282 \\
0.0300 \\
0.0305 \\
0.0314 \\
0.0333 \\
0.0370 \\
0.0412 \\
0.0419 \\
0.0467 \\
0.0496 \\
0.0528 \\
0.0556 \\
0.0558 \\
-0.0007\end{array}$ & $\begin{array}{c}\text { Angle } \\
\text { Degrees } \\
-16.53 \\
-19.40 \\
-20.16 \\
-20.38 \\
-20.45 \\
-20.20 \\
-20.03 \\
-21.01 \\
-21.08 \\
-21.07 \\
-20.07 \\
-19.90 \\
-20.49 \\
-21.40 \\
-21.68 \\
-22.03 \\
-22.17 \\
-22.51 \\
-22.50 \\
-23.00 \\
-24.08 \\
-24.56 \\
25.62 \\
-15.87 \\
-26.60 \\
-26.98 \\
-27.67 \\
-29.56 \\
-33.61 \\
-35.63 \\
-37.08 \\
-41.94 \\
43.94 \\
37.22 \\
31.83 \\
28.39 \\
3.49\end{array}$ \\
\hline
\end{tabular}

High Pressure test 


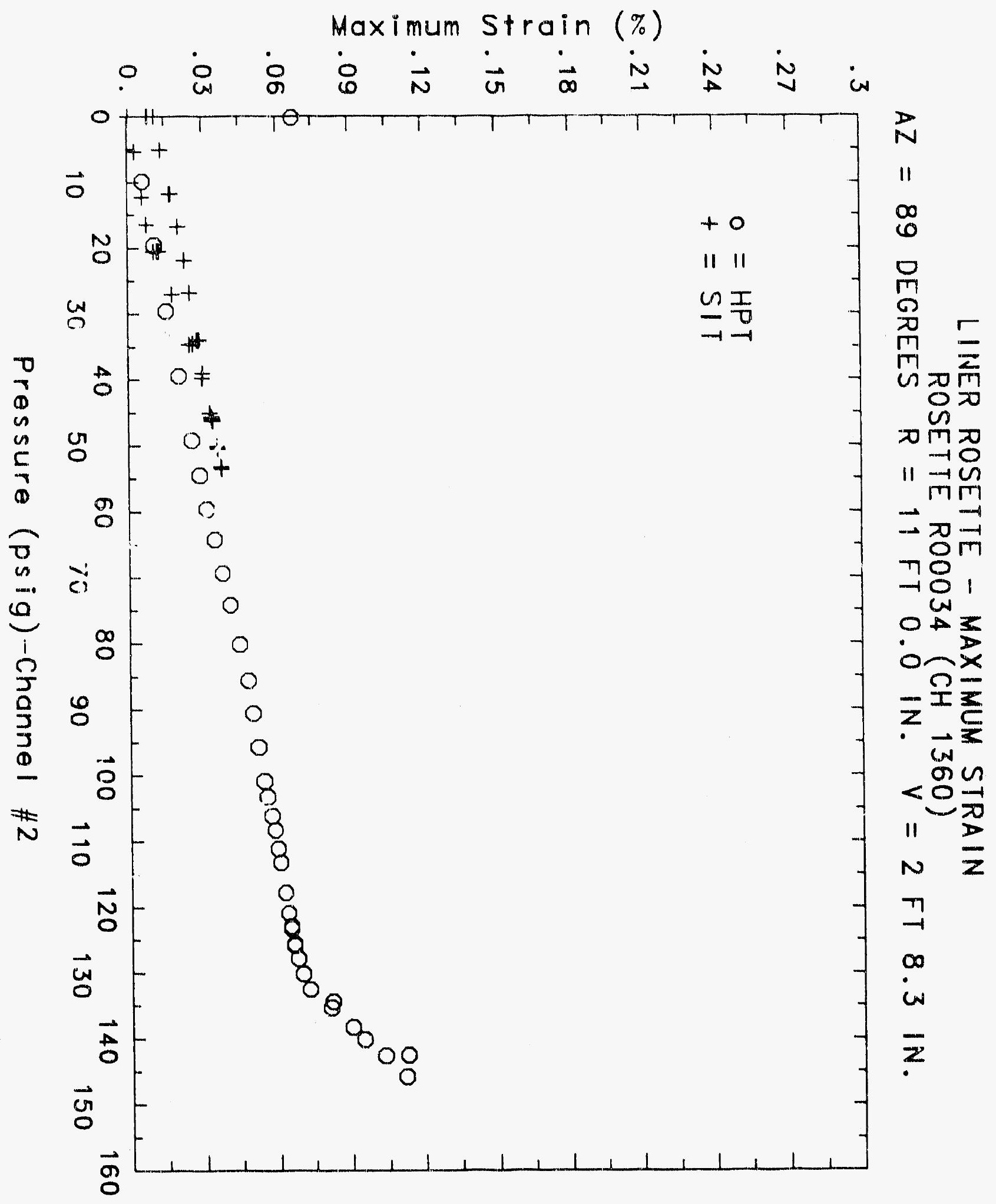




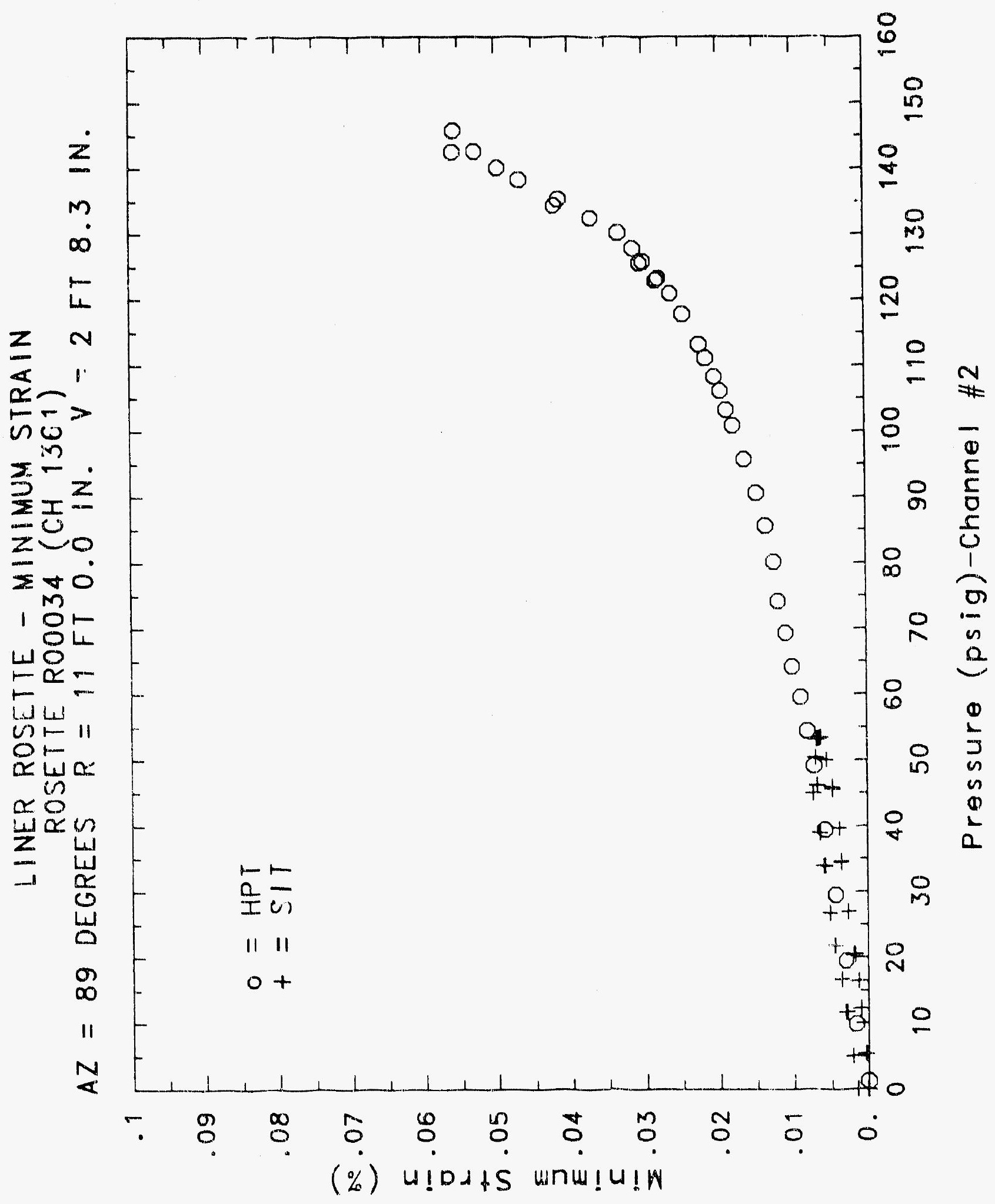




\section{Reinforced Concrete Test Data}

Rusetta 35 Channels $1363 \quad 1364 \quad 1365$

STRUCTURAL.. INTEGRITY TEST

\begin{tabular}{|c|c|c|c|c|c|c|c|}
\hline Pressure & Maxinum & Minimum & Angle & Pressure & Maximum & Minimum & Angle \\
\hline psig & \%strain & *strain & Degrees & psig & xstrain & xstrain & Degrees \\
\hline-0.05 & 0.0004 & 0.0000 & -7.11 & 9.89 & 0.0074 & 0.0042 & 9.84 \\
\hline 5.33 & 0.0019 & 0.0013 & 1.65 & 19.55 & 0.0134 & 0.0074 & 9.28 \\
\hline 12.31 & 0.0040 & 0.0026 & 0.59 & 29.57 & 0.0188 & 0.0110 & 12.22 \\
\hline 16.44 & 0.0054 & 0.0032 & -3.70 & 39.42 & 0.0234 & 0.0145 & 11.35 \\
\hline 20.51 & 0.0072 & 0.0046 & -4.01 & 49.16 & 0.0275 & 0.0183 & 11.31 \\
\hline 20.46 & 0.0077 & 0.0050 & -4.46 & 54.50 & 0.0301 & 0.0207 & 12.86 \\
\hline 20.47 & 0.0080 & 0.0051 & -8.37 & 59.57 & 0.0328 & 0.0230 & 13.03 \\
\hline 27.05 & 0.0113 & 0.0087 & -3.97 & 64.20 & 0.0359 & 0.0254 & 11.53 \\
\hline 34.69 & 0.0160 & 0.0149 & -11.12 & 69.32 & 0.0391 & 0.0278 & 10.60 \\
\hline 34.53 & 0.0163 & 0.0162 & 40.00 & 74.16 & 0.0421 & 0.0303 & 8.43 \\
\hline 34.53 & 0.0165 & 0.0163 & -10.65 & 80.16 & 0.0433 & 0.0349 & 12.47 \\
\hline 39.70 & 0.0194 & 0.0185 & -18.90 & 85.61 & 0.0468 & 0.0387 & 13.41 \\
\hline 45.90 & 0.0240 & 0.0223 & -30.40 & 90.58 & 0.0503 & 0.0415 & 12.54 \\
\hline 45.65 & 0.0239 & 0.0224 & -28.09 & 95.69 & 0.0547 & 0.0442 & 10.55 \\
\hline 45.62 & 0.0238 & 0.0224 & -26.22 & 100.92 & 0.0586 & 0.0473 & 8.72 \\
\hline 50.10 & 0.0262 & 0.0247 & -34.49 & 103.25 & 0.0607 & 0.0488 & 7.60 \\
\hline 53.47 & 0.0281 & 0.0269 & 37.48 & 106.11 & 0.0628 & 0.0507 & 7.04 \\
\hline 53.29 & 0.0284 & 0.0273 & 24.89 & 108.31 & 0.0644 & 0.0521 & 6.15 \\
\hline 53.21 & 0.0285 & 0.0274 & 13.82 & 111.08 & 0.0671 & 0.0541 & 4.74 \\
\hline 50.49 & 0.0283 & 0.0267 & 7.91 & 113.24 & 0.0691 & 0.0553 & 2.95 \\
\hline 46.14 & 0.0270 & 0.0252 & 3.63 & 117.83 & 0.0737 & 0.0584 & -0.36 \\
\hline 46.13 & 0.0271 & 0.0250 & 0.61 & 120.92 & 0.0774 & 0.0605 & -2.75 \\
\hline 44.97 & 0.0295 & 0.0247 & 0.35 & 123.28 & 0.0809 & 0.0616 & -5.32 \\
\hline 38.98 & 0.0269 & 0.0224 & .1 .68 & 122.97 & 0.0817 & 0.0613 & -6.64 \\
\hline 33.99 & 0.0247 & 0.0205 & -1.38 & 125.82 & 0.0855 & 0.0633 & -8.15 \\
\hline 33.96 & 0.0247 & 0.0206 & -6.09 & 125.60 & 0.0869 & 0.0627 & -8.65 \\
\hline 26.83 & 0.0213 & 0.0178 & -9.46 & 127.84 & 0.0896 & 0.0640 & -9.46 \\
\hline 21.88 & 0.0189 & 0.0154 & -15.14 & 130.19 & 0.0964 & 0.0667 & -10.34 \\
\hline 21.90 & 0.0181 & 0.0155 & -22.56 & 132.53 & 0.1086 & 0.06536 & -12.13 \\
\hline 16.69 & 0.0150 & 0.1129 & -31.67 & 135.33 & 0.1207 & 0.0102 & -14.42 \\
\hline 11.74 & 0.0123 & 0.0103 & -44.80 & 134.42 & 0.1246 & 0.0683 & -14.84 \\
\hline 11.77 & 0.0114 & 0.0094 & 32.61 & 138.35 & 0.1456 & 0.0699 & -15.77 \\
\hline 5.05 & 0.0088 & 0.0054 & 23.50 & 140.16 & 0.1600 & 0.0728 & -15.53 \\
\hline 0.02 & 0.0073 & 0.0022 & 18.22 & 142.63 & 0.1803 & 0.0777 & -14.36 \\
\hline 0.34 & 0.0092 & 0.0052 & 21.96 & 145.78 & 0.1976 & 0.0816 & -12.86 \\
\hline-0.02 & 0.0091 & 0.0055 & 27.34 & 142.52 & 0.2034 & 0.0791 & -13.05 \\
\hline-0.02 & 0.0092 & 0.0056 & 2.7 .45 & 0.22 & 0.0967 & 0.0278 & -25.74 \\
\hline 0.02 & 0.0082 & 0.0048 & 26.00 & & & & \\
\hline
\end{tabular}

High Pressure test 


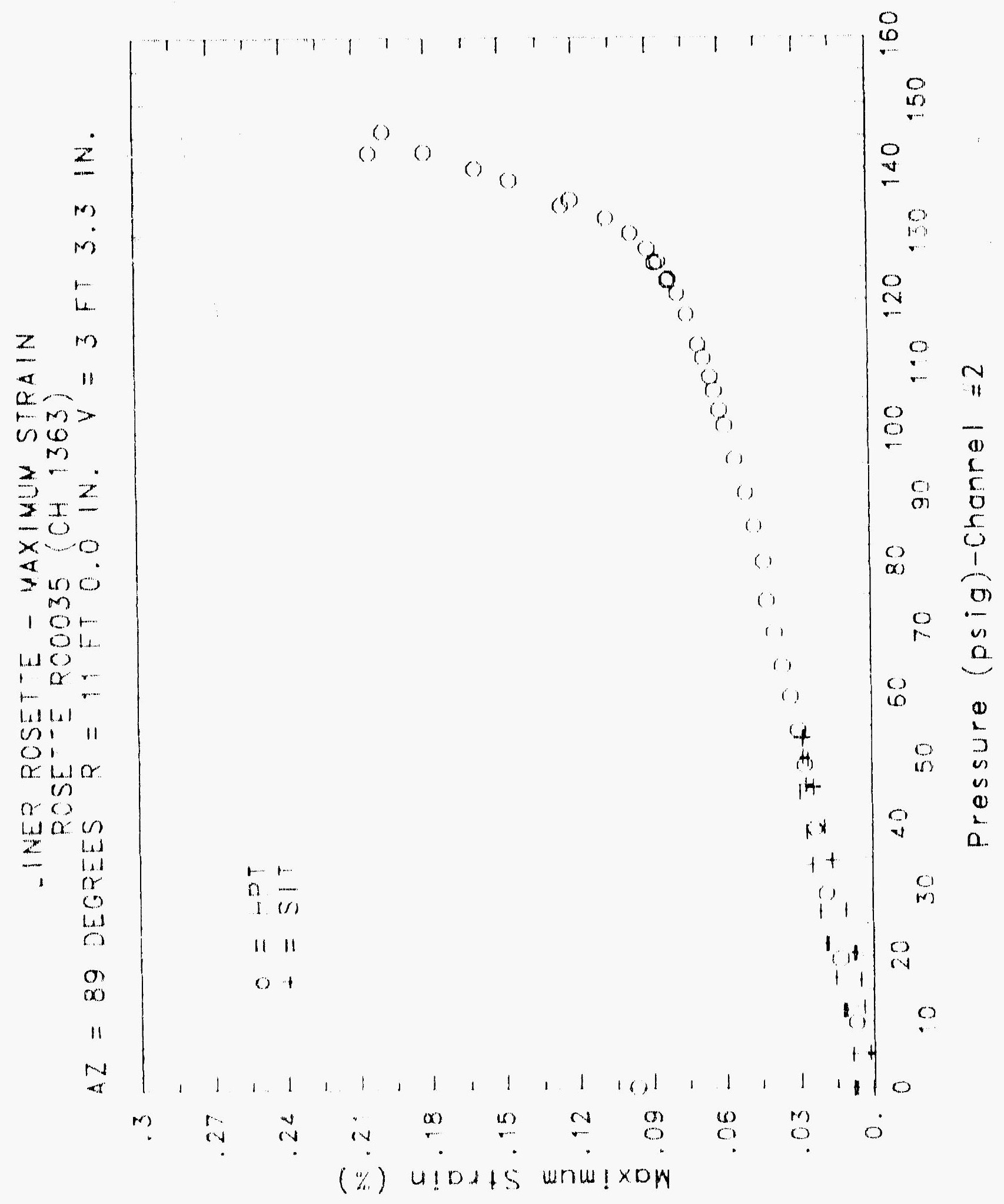

$(1.18$ 


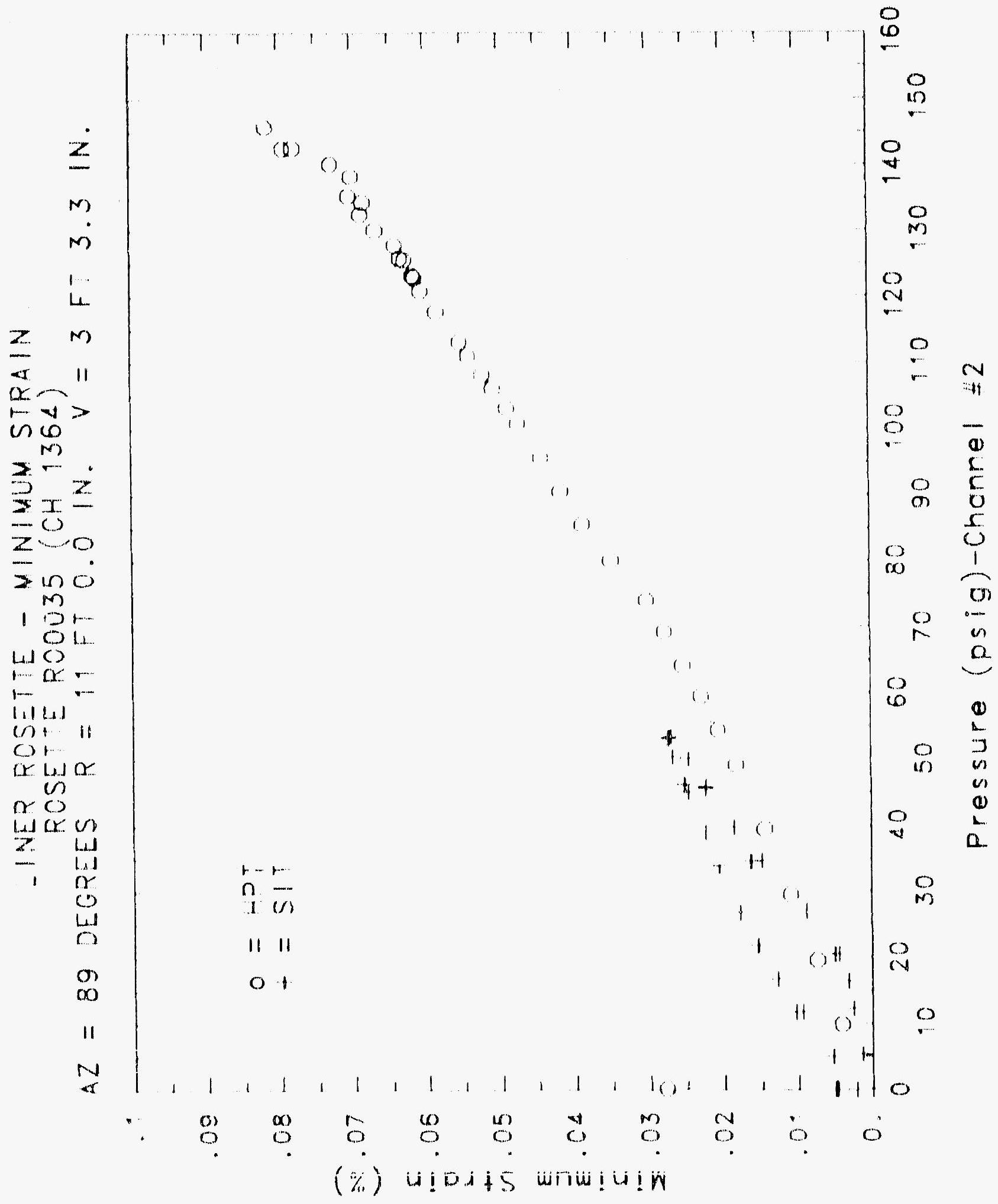




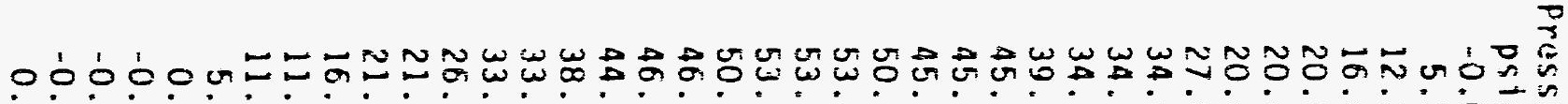

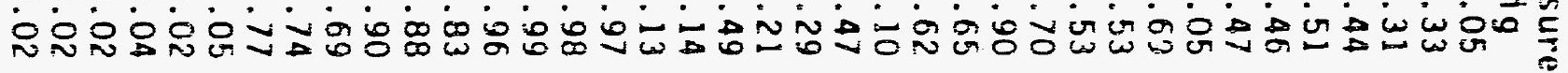
$00000000000000000000000000000000000000 \% \frac{1}{4}$

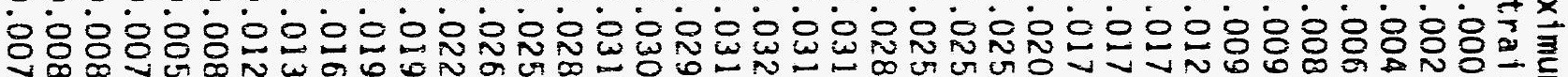

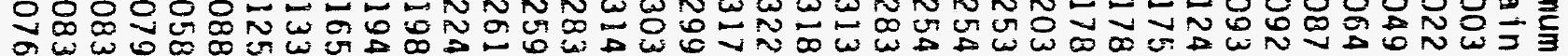

$00000000000000000000000000000000000000 \mathrm{~h}$

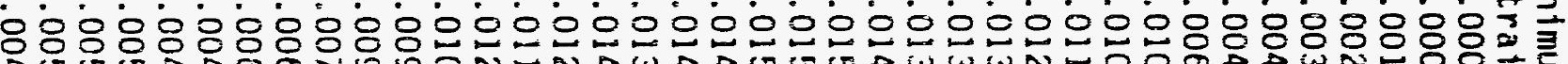

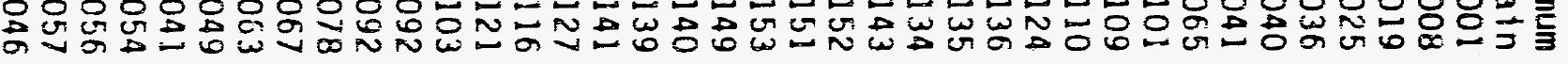

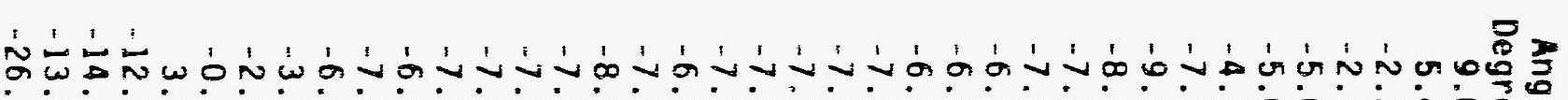

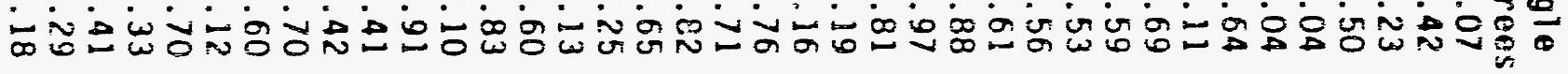

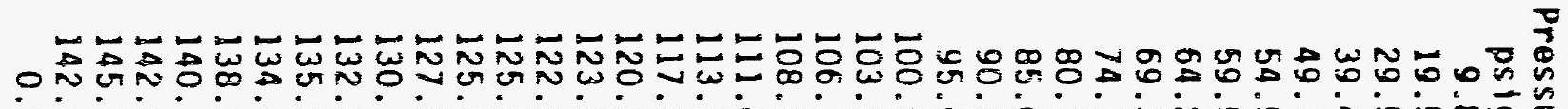

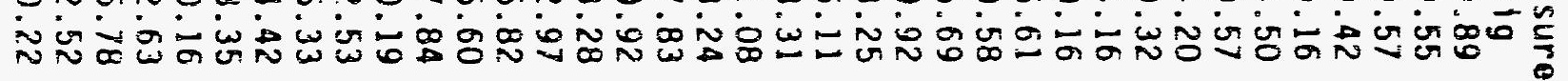
0000000000000000000000000000000000000贷

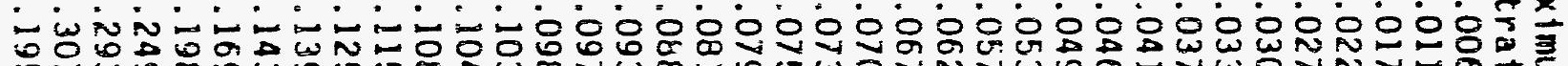

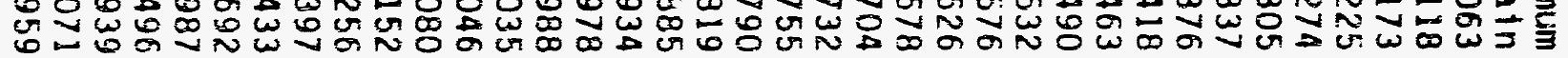

0000000000000000000000000000000000000永

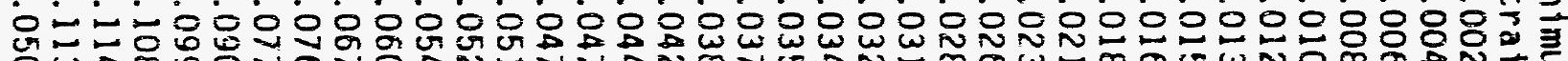

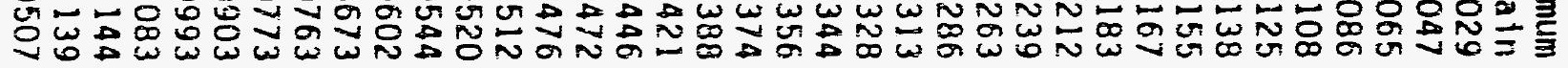

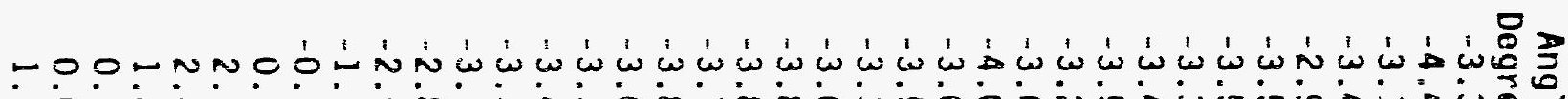

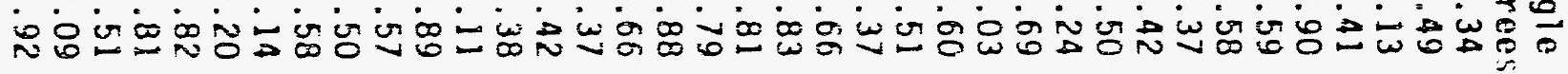




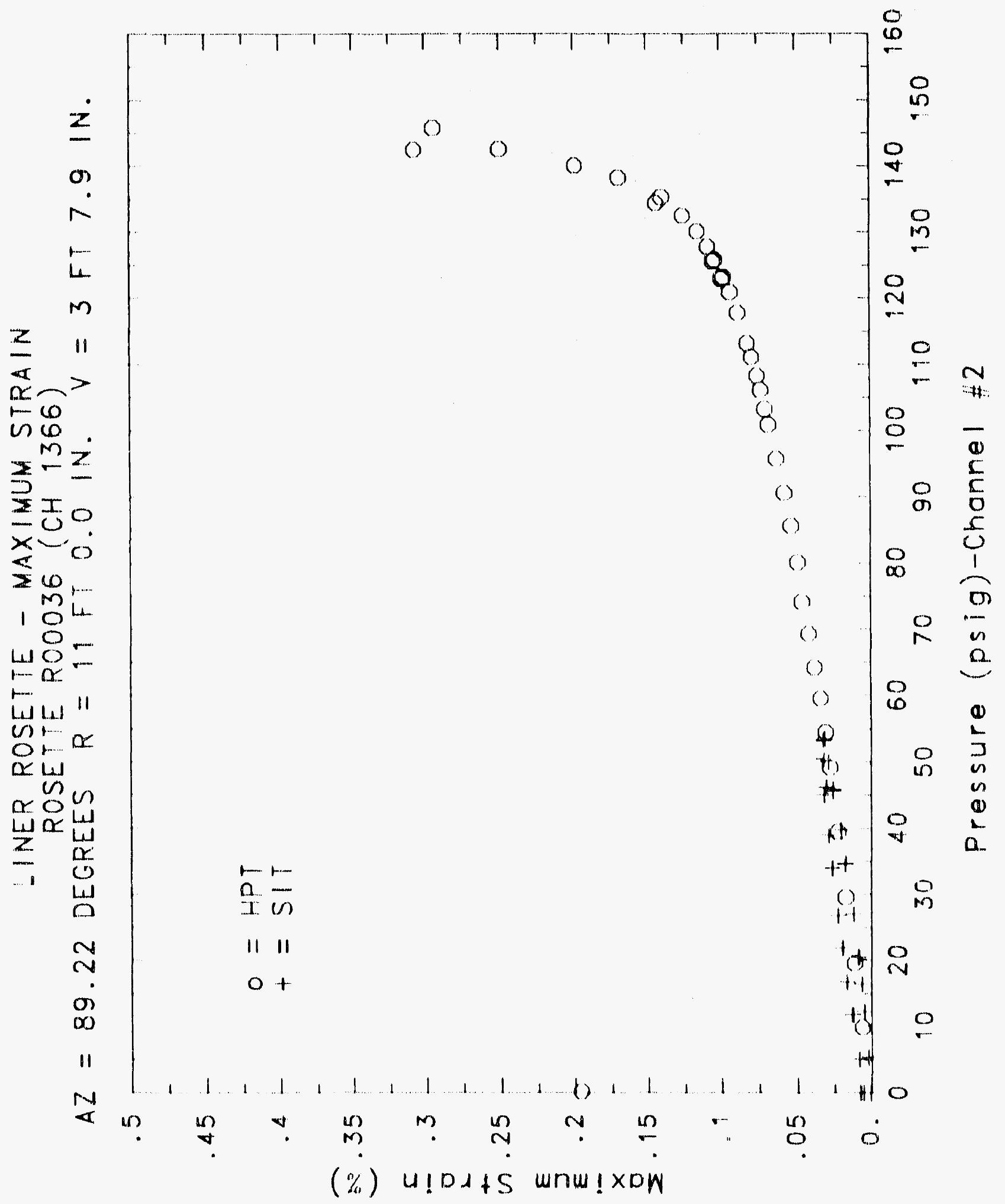




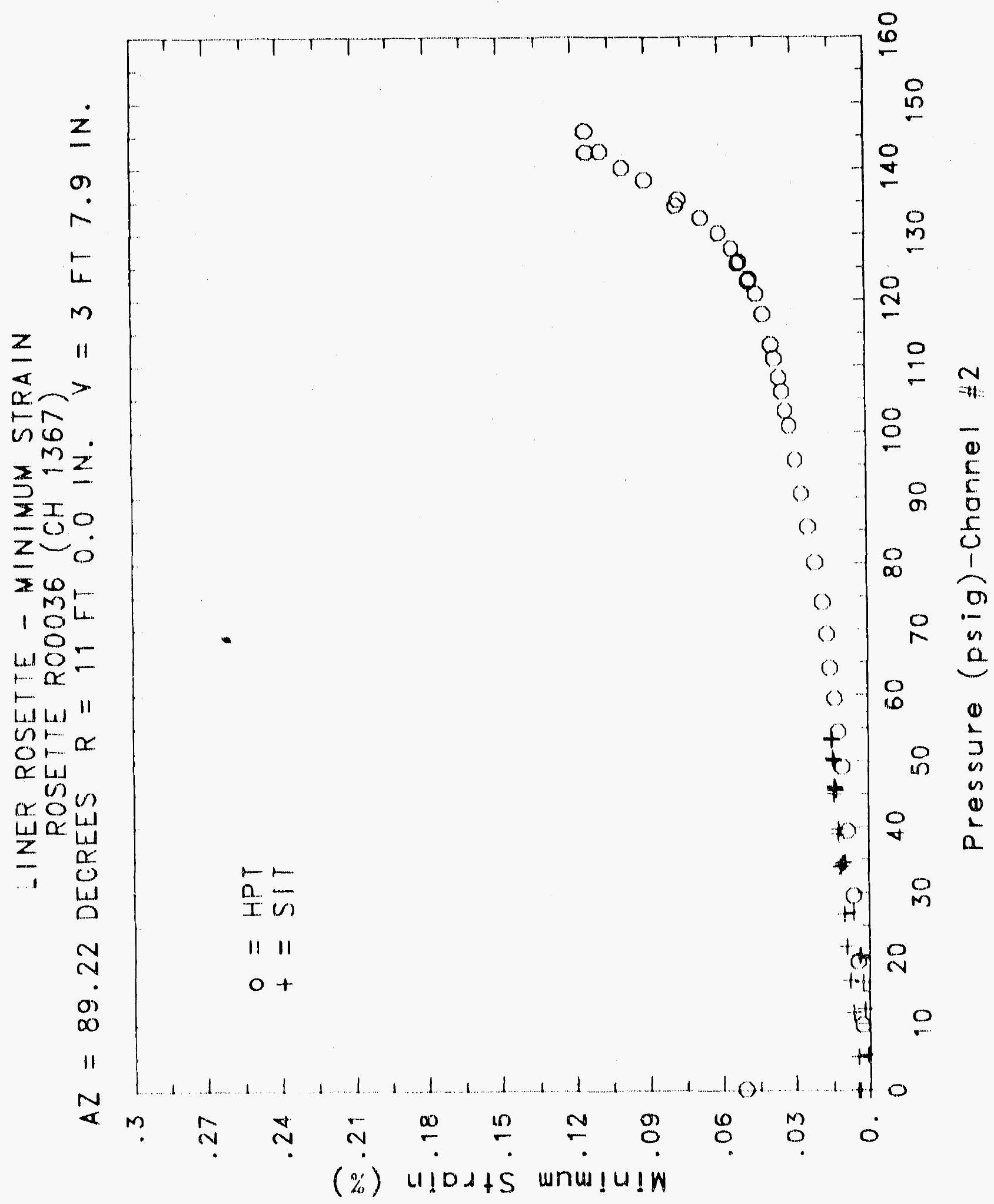




\section{Reinforced Concrete Test Data}

Rosette 37 Channels $1369 \quad 1380 \quad 1381$

StRUCTURAL INTEGRITY TEST

\begin{tabular}{|c|c|c|c|c|c|c|c|}
\hline $\begin{array}{c}\text { Pressure } \\
\text { psig } \\
-0.05 \\
5.33 \\
12.31 \\
16.44 \\
20.51 \\
20.46 \\
20.47 \\
27.05 \\
34.69 \\
34.53 \\
34.53 \\
39.70 \\
45.90 \\
45.65 \\
45.62 \\
50.10 \\
53.47 \\
53.29 \\
53.21 \\
50.49 \\
46.14 \\
46.13 \\
44.97 \\
38.98 \\
33.99 \\
33.96 \\
26.83 \\
21.88 \\
21.90 \\
16.69 \\
11.74 \\
11.77 \\
5.05 \\
0.02 \\
-0.04 \\
-0.02 \\
-0.02 \\
0.02\end{array}$ & $\begin{array}{c}\text { Maximum } \\
\text { \%Strain } \\
0.0007 \\
0.0033 \\
0.0071 \\
0.0096 \\
0.0130 \\
0.0141 \\
0.0147 \\
0.0218 \\
0.0351 \\
0.0363 \\
0.0366 \\
0.0412 \\
0.0485 \\
0.0483 \\
0.0483 \\
0.0524 \\
0.0566 \\
0.0567 \\
0.0570 \\
0.0559 \\
0.0531 \\
0.0529 \\
0.0534 \\
0.0486 \\
0.0446 \\
0.0450 \\
0.0392 \\
0.0350 \\
0.0346 \\
0.0296 \\
0.0248 \\
0.0230 \\
0.0166 \\
0.0118 \\
0.0124 \\
0.0131 \\
0.0134 \\
0.0125\end{array}$ & $\begin{array}{c}\text { Minimum } \\
\text { XStratn } \\
0.0000 \\
0.0008 \\
0.0016 \\
0.0021 \\
0.0032 \\
0.0037 \\
0.0039 \\
0.0052 \\
0.0076 \\
0.0081 \\
0.0082 \\
0.0088 \\
0.0104 \\
0.0104 \\
0.0103 \\
0.0111 \\
0.0119 \\
0.0121 \\
0.0122 \\
0.0121 \\
0.0116 \\
0.0115 \\
0.0128 \\
0.0119 \\
0.0113 \\
0.0116 \\
0.0103 \\
0.0096 \\
0.0093 \\
0.0082 \\
0.0072 \\
0.0064 \\
0.0056 \\
0.0055 \\
0.0078 \\
0.0080 \\
0.0082 \\
0.0073\end{array}$ & $\begin{array}{c}\text { Angle } \\
\text { Degrees } \\
44.97 \\
3.92 \\
2.07 \\
1.64 \\
-1.20 \\
-1.80 \\
-2.50 \\
-6.28 \\
-3.38 \\
-2.68 \\
-1.89 \\
-2.14 \\
-1.52 \\
-1.55 \\
-1.43 \\
-1.45 \\
-0.27 \\
-0.20 \\
-0.09 \\
-0.04 \\
0.12 \\
0.10 \\
0.21 \\
0.50 \\
0.91 \\
0.79 \\
1.16 \\
1.53 \\
1.42 \\
2.09 \\
3.95 \\
3.67 \\
5.70 \\
11.07 \\
8.00 \\
7.39 \\
8.94 \\
9.37\end{array}$ & $\begin{array}{c}\text { Pressure } \\
\text { psig } \\
9.89 \\
19.55 \\
29.57 \\
39.42 \\
49.16 \\
54.50 \\
59.57 \\
64.20 \\
69.32 \\
74.16 \\
80.16 \\
85.61 \\
90.58 \\
95.69 \\
100.92 \\
103.25 \\
106.11 \\
108.31 \\
111.08 \\
113.24 \\
117.83 \\
120.92 \\
123.28 \\
122.97 \\
125.82 \\
125.60 \\
127.84 \\
130.19 \\
132.53 \\
135.33 \\
134.42 \\
138.35 \\
140.16 \\
142.63 \\
145.78 \\
142.52 \\
0.22\end{array}$ & $\begin{array}{c}\text { Maximum } \\
\text { xStra1n } \\
0.0103 \\
0.0198 \\
0.0291 \\
0.0377 \\
0.0460 \\
0.0507 \\
0.0554 \\
0.0609 \\
0.0667 \\
0.0727 \\
0.0769 \\
0.0831 \\
0.0895 \\
0.0973 \\
0.1060 \\
0.1103 \\
0.1150 \\
0.1192 \\
0.1256 \\
0.1313 \\
0.1447 \\
0.1548 \\
0.1647 \\
0.1669 \\
0.1798 \\
0.1837 \\
0.1925 \\
0.2167 \\
0.2555 \\
0.3257 \\
0.3486 \\
0.5394 \\
0.6890 \\
0.8627 \\
0.9498 \\
0.9698 \\
0.7864\end{array}$ & $\begin{array}{l}\text { Minimum } \\
\text { zStrain } \\
0.0019 \\
0.0028 \\
0.0041 \\
0.0059 \\
0.0079 \\
0.0092 \\
0.0104 \\
0.0123 \\
0.0141 \\
0.0153 \\
0.0175 \\
0.0203 \\
0.0229 \\
0.0258 \\
0.0289 \\
0.0306 \\
0.0325 \\
0.0339 \\
0.0358 \\
0.0373 \\
0.0410 \\
0.0440 \\
0.0467 \\
0.0472 \\
0.0514 \\
0.0523 \\
0.0552 \\
0.0612 \\
0.0686 \\
0.0764 \\
0.0766 \\
0.0819 \\
0.0872 \\
0.0957 \\
0.1021 \\
0.1030 \\
0.0389\end{array}$ & $\begin{array}{c}\text { Angle } \\
\text { Degrees } \\
-1.48 \\
-1.47 \\
-1.51 \\
-1.62 \\
-1.15 \\
-1.92 \\
-1.07 \\
-0.31 \\
0.87 \\
0.70 \\
0.35 \\
0.20 \\
0.42 \\
0.84 \\
1.41 \\
1.66 \\
1.87 \\
2.14 \\
2.29 \\
2.65 \\
2.86 \\
2.80 \\
2.60 \\
2.50 \\
2.39 \\
2.37 \\
2.21 \\
1.47 \\
-0.25 \\
-3.04 \\
-3.34 \\
-3.88 \\
-2.80 \\
-0.28 \\
0.78 \\
0.84 \\
0.68\end{array}$ \\
\hline
\end{tabular}

high Pressure test 


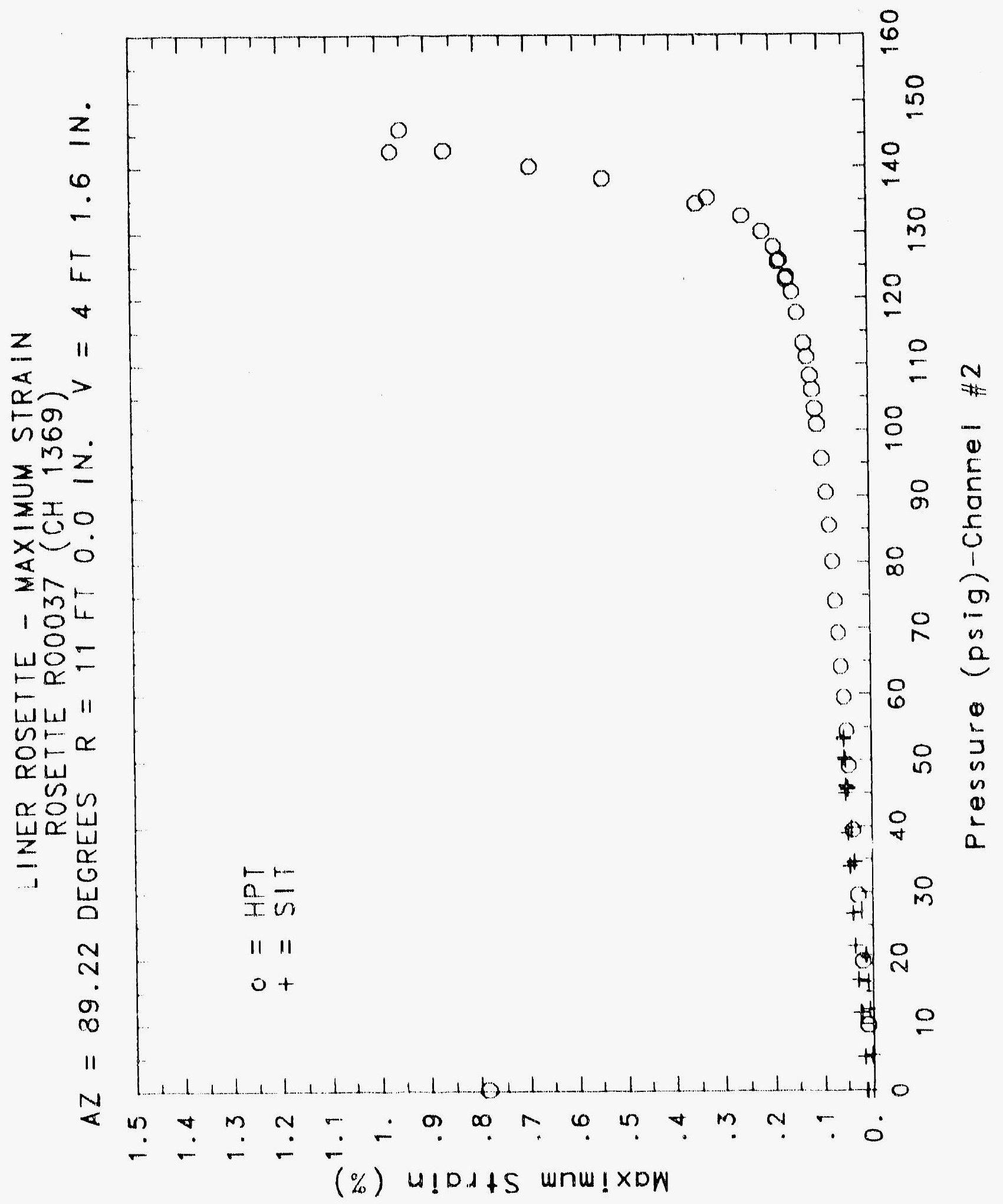




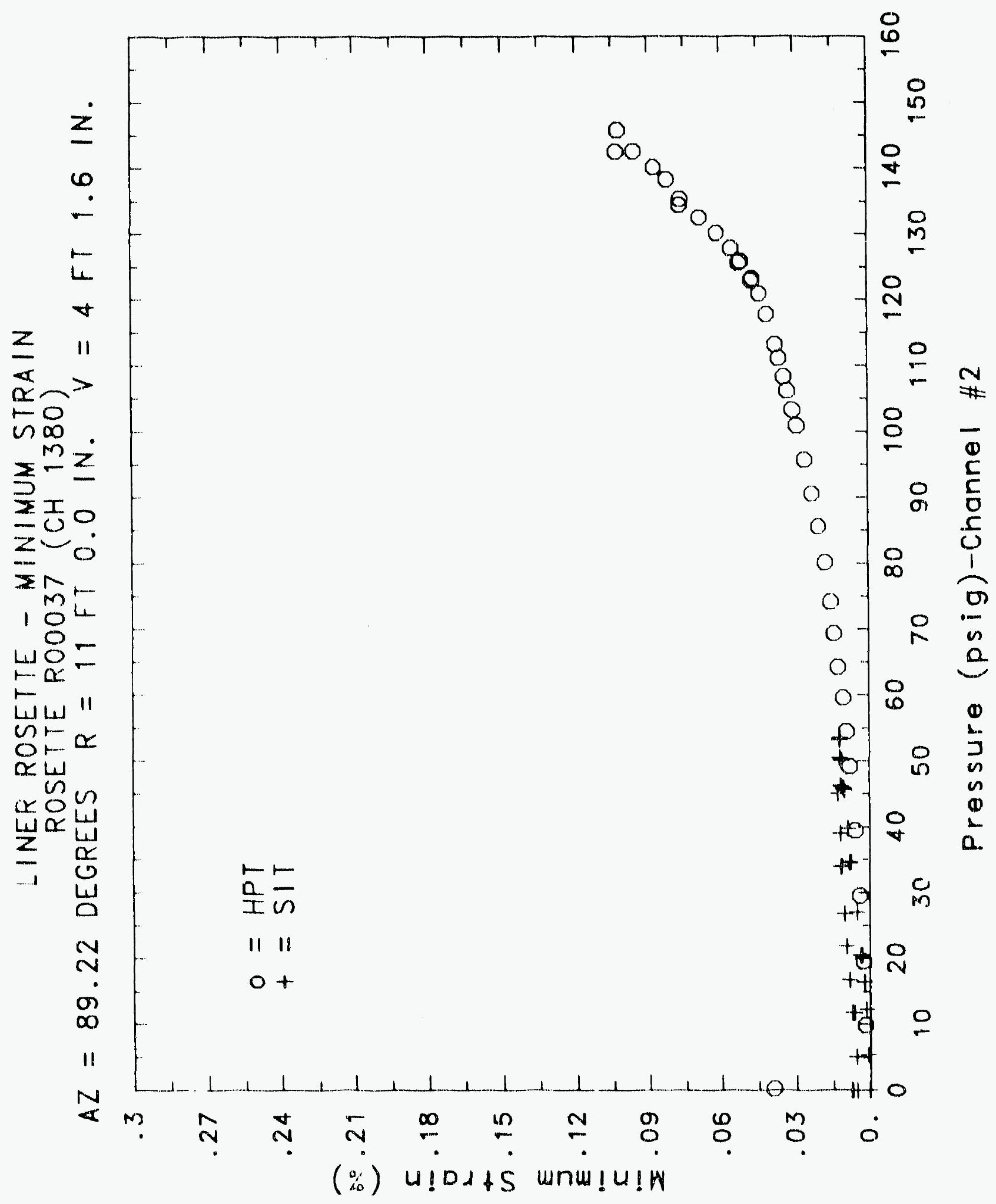




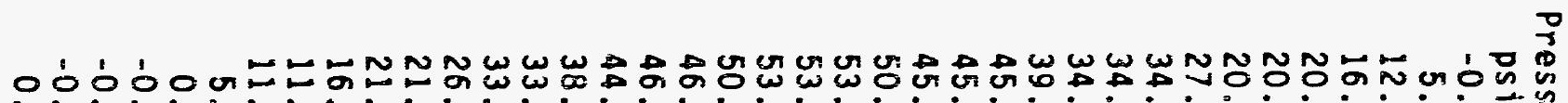

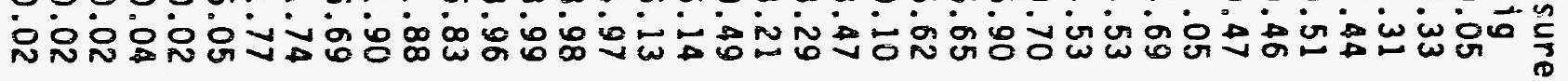
$00000000000000000000000000000000000000 \mathrm{H}$

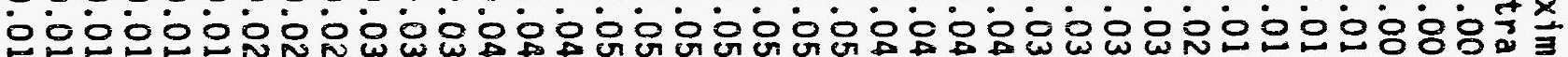

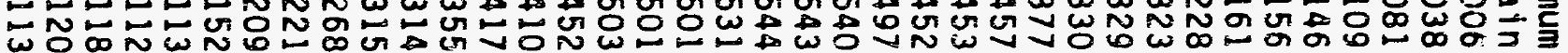

000000000000000000000000000000000000004

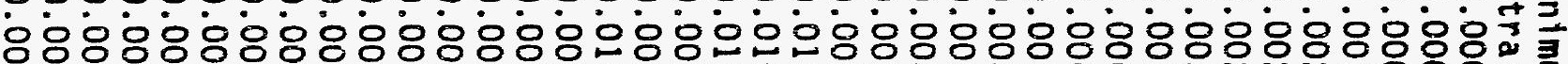
出出

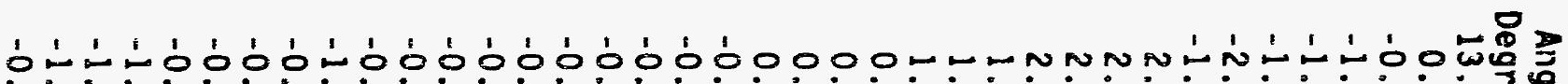

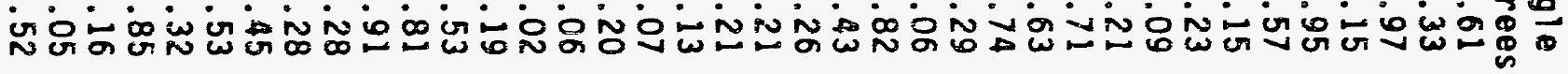

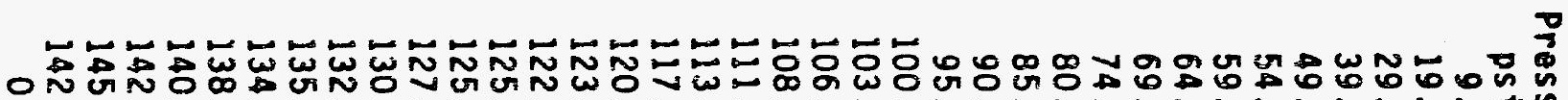
స 0- 0000000000000000000000000000000000 开

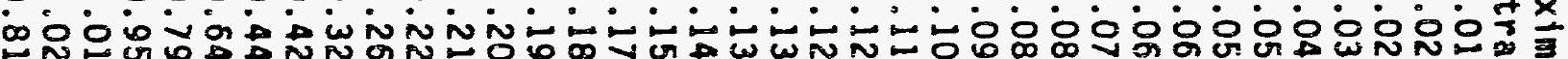

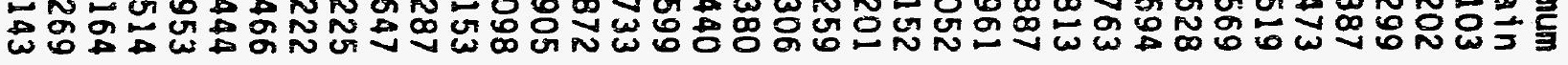

$0000000000000000000000000000000000000 \% \frac{1}{3}$

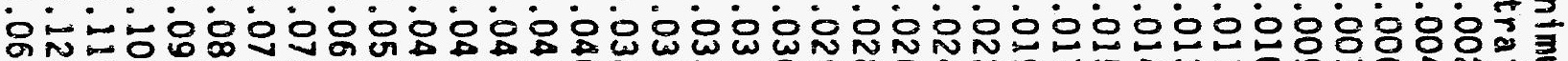

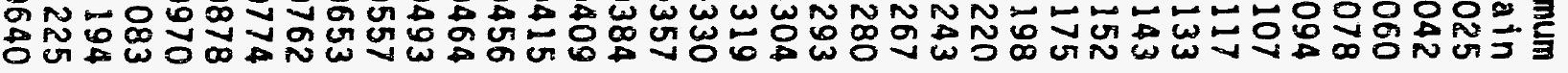

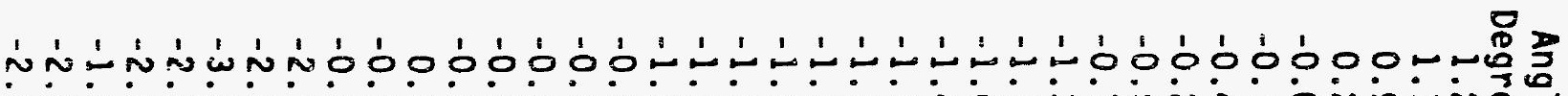

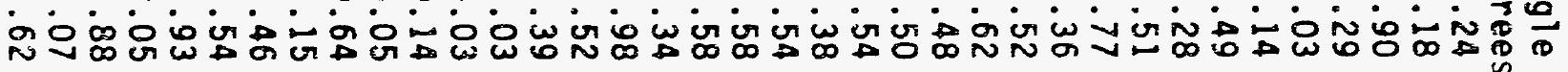




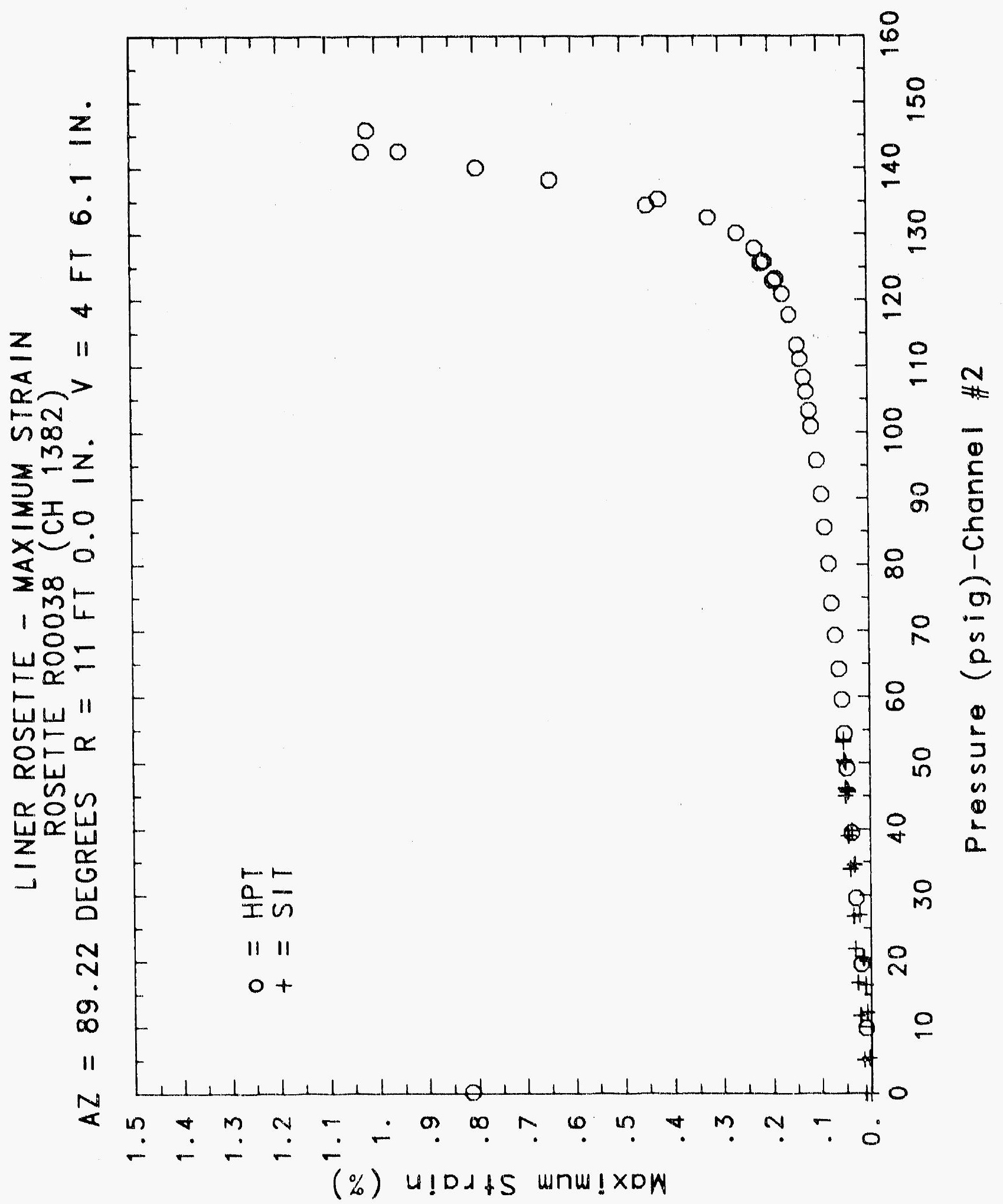




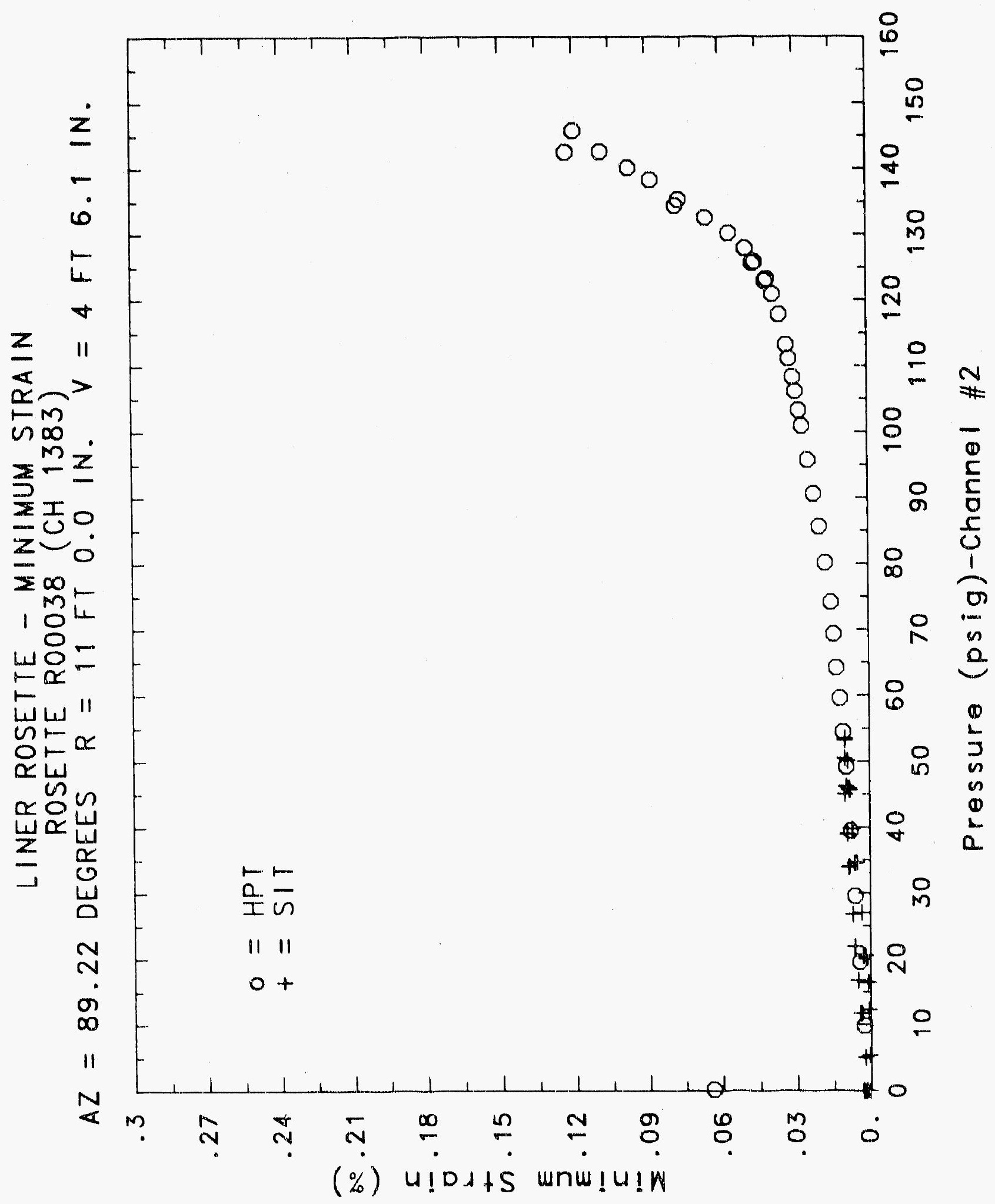




\title{
Reinforced Concrete Test Data
}

\author{
Rosette $39 \quad$ Channels $1385 \quad 1386 \quad 1387$
}

\section{StRuCtural INTEgRITY TEST}

\begin{tabular}{|c|c|c|c|c|c|c|c|}
\hline $\begin{array}{c}\text { Pressure } \\
\text { psig } \\
-0.05 \\
5.33 \\
12.31 \\
16.44 \\
20.51 \\
20.46 \\
20.47 \\
27.05 \\
34.69 \\
34.53 \\
34.53 \\
39.70 \\
45.90 \\
45.65 \\
45.62 \\
50.10 \\
53.47 \\
53.29 \\
53.21 \\
50.49 \\
46.14 \\
46.13 \\
44.97 \\
38.98 \\
33.99 \\
33.96 \\
26.83 \\
21.88 \\
21.90 \\
16.69 \\
11.74 \\
11.77 \\
5.05 \\
0.02 \\
-0.04 \\
-0.02 \\
-0.02 \\
0.02\end{array}$ & $\begin{array}{c}\text { Maximum } \\
\text { xStrain } \\
0.0000 \\
-0.0051 \\
-0.0072 \\
-0.0061 \\
-0.0018 \\
-0.0005 \\
0.0006 \\
0.0114 \\
0.0271 \\
0.0292 \\
0.0297 \\
0.0379 \\
0.0492 \\
0.0494 \\
0.0493 \\
0.0559 \\
0.0617 \\
0.0621 \\
0.0620 \\
0.0594 \\
0.0541 \\
0.0538 \\
0.0521 \\
0.0447 \\
0.0379 \\
0.0380 \\
0.0277 \\
0.0201 \\
0.0202 \\
0.0133 \\
0.0107 \\
0.0097 \\
0.0045 \\
0.0088 \\
0.0067 \\
0.0070 \\
0.0072 \\
0.0069\end{array}$ & $\begin{array}{l}\text { Minimum } \\
\text { KStrain } \\
-0.0003 \\
-0.0139 \\
-0.0132 \\
-0.0098 \\
-0.0072 \\
-0.0062 \\
-0.0057 \\
-0.0020 \\
0.0063 \\
0.0086 \\
0.0092 \\
0.0133 \\
0.0200 \\
0.0207 \\
0.0209 \\
0.0237 \\
0.0267 \\
0.0272 \\
0.0274 \\
0.0270 \\
0.0255 \\
0.0252 \\
0.0249 \\
0.0226 \\
0.0205 \\
0.0208 \\
0.0175 \\
0.0153 \\
0.0156 \\
0.0120 \\
0.0035 \\
0.0033 \\
-0.0110 \\
-0.0141 \\
-0.0123 \\
-0.0126 \\
-0.0126 \\
-0.0124\end{array}$ & $\begin{array}{c}\text { Angle } \\
\text { Degrees } \\
-38.01 \\
-19.22 \\
-25.30 \\
-41.31 \\
19.51 \\
16.35 \\
14.04 \\
2.19 \\
-3.00 \\
-3.00 \\
-3.26 \\
-3.72 \\
-3.59 \\
-3.56 \\
-3.76 \\
-4.46 \\
-4.77 \\
-4.77 \\
-4.83 \\
-4.27 \\
-3.29 \\
-3.29 \\
-2.71 \\
-1.19 \\
-0.90 \\
-1.49 \\
-0.32 \\
-1.95 \\
-1.78 \\
10.66 \\
5.44 \\
6.94 \\
17.12 \\
22.27 \\
24.33 \\
24.19 \\
23.91 \\
24.29\end{array}$ & $\begin{array}{c}\text { Pressure } \\
\text { psig } \\
9.89 \\
19.55 \\
29.57 \\
39.42 \\
49.16 \\
54.50 \\
59.57 \\
64.20 \\
69.32 \\
74.16 \\
80.16 \\
85.61 \\
90.58 \\
95.69 \\
100.92 \\
103.25 \\
106.11 \\
108.31 \\
111.08 \\
113.24 \\
117.83 \\
120.92 \\
123.28 \\
122.97 \\
125.82 \\
125.60 \\
127.84 \\
130.19 \\
132.53 \\
135.33 \\
134.42 \\
138.35 \\
140.16 \\
142.63 \\
145.78 \\
142.52 \\
0.22\end{array}$ & $\begin{array}{l}\text { Maximum } \\
\text { xStrain } \\
0.0050 \\
0.0217 \\
0.0359 \\
0.0497 \\
0.0629 \\
0.0706 \\
0.0789 \\
0.0878 \\
0.0977 \\
0.1089 \\
0.1225 \\
0.1382 \\
0.1560 \\
0.1781 \\
0.1993 \\
0.2071 \\
0.2145 \\
0.2195 \\
0.2264 \\
0.2311 \\
0.2415 \\
0.2505 \\
0.2596 \\
0.2613 \\
0.2710 \\
0.2741 \\
0.2813 \\
0.2967 \\
0.3198 \\
0.3628 \\
0.3755 \\
0.4444 \\
0.4909 \\
0.5805 \\
0.6886 \\
0.7235 \\
0.5633\end{array}$ & $\begin{array}{l}\text { Minimum } \\
\text { xStrain } \\
-0.0028 \\
0.0056 \\
0.0126 \\
0.0187 \\
0.0243 \\
0.0278 \\
0.0312 \\
0.0342 \\
0.0370 \\
0.0393 \\
0.0435 \\
0.0479 \\
0.0523 \\
0.0569 \\
0.0626 \\
0.0658 \\
0.0693 \\
0.0720 \\
0.0756 \\
0.0779 \\
0.0839 \\
0.0882 \\
0.0924 \\
0.0933 \\
0.0981 \\
0.0994 \\
0.1016 \\
0.1059 \\
0.1130 \\
0.1219 \\
0.1229 \\
0.1270 \\
0.1308 \\
0.1308 \\
0.1302 \\
0.1285 \\
0.0322\end{array}$ & $\begin{array}{c}\text { Angle } \\
\text { Degrees } \\
38.65 \\
24.56 \\
14.02 \\
7.90 \\
4.50 \\
3.16 \\
2.03 \\
1.63 \\
1.52 \\
0.31 \\
-1.19 \\
-2.92 \\
-4.68 \\
-6.57 \\
-8.02 \\
-8.52 \\
-9.08 \\
-9.49 \\
-10.09 \\
-10.59 \\
-12.15 \\
-13.31 \\
-14.16 \\
-14.33 \\
-14.84 \\
-14.88 \\
-14.81 \\
-14.00 \\
-12.32 \\
-11.61 \\
-11.87 \\
-13.42 \\
-14.26 \\
-15.06 \\
-15.26 \\
-15.73 \\
-15.48\end{array}$ \\
\hline
\end{tabular}

\section{HXgh Pressure test}




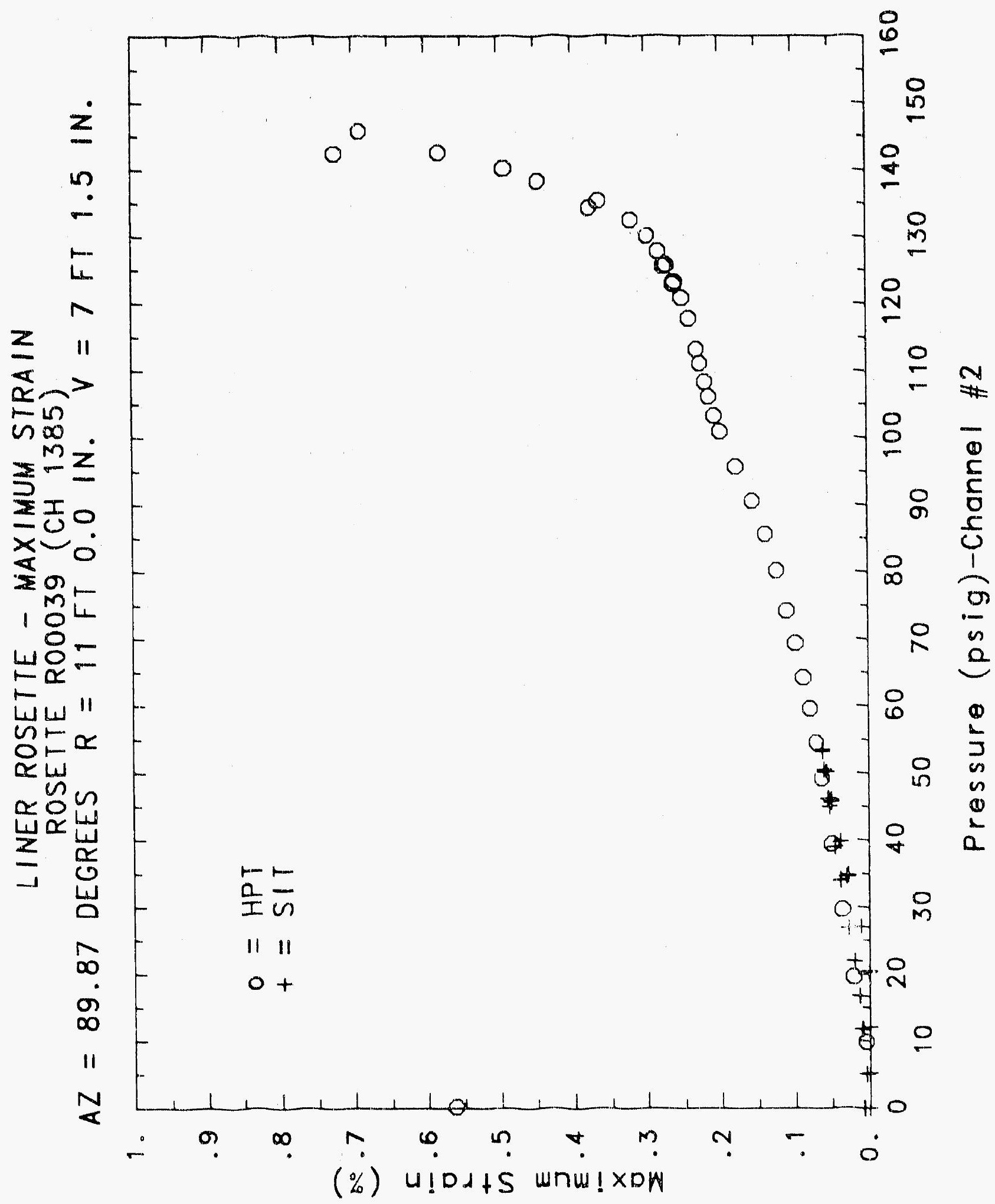




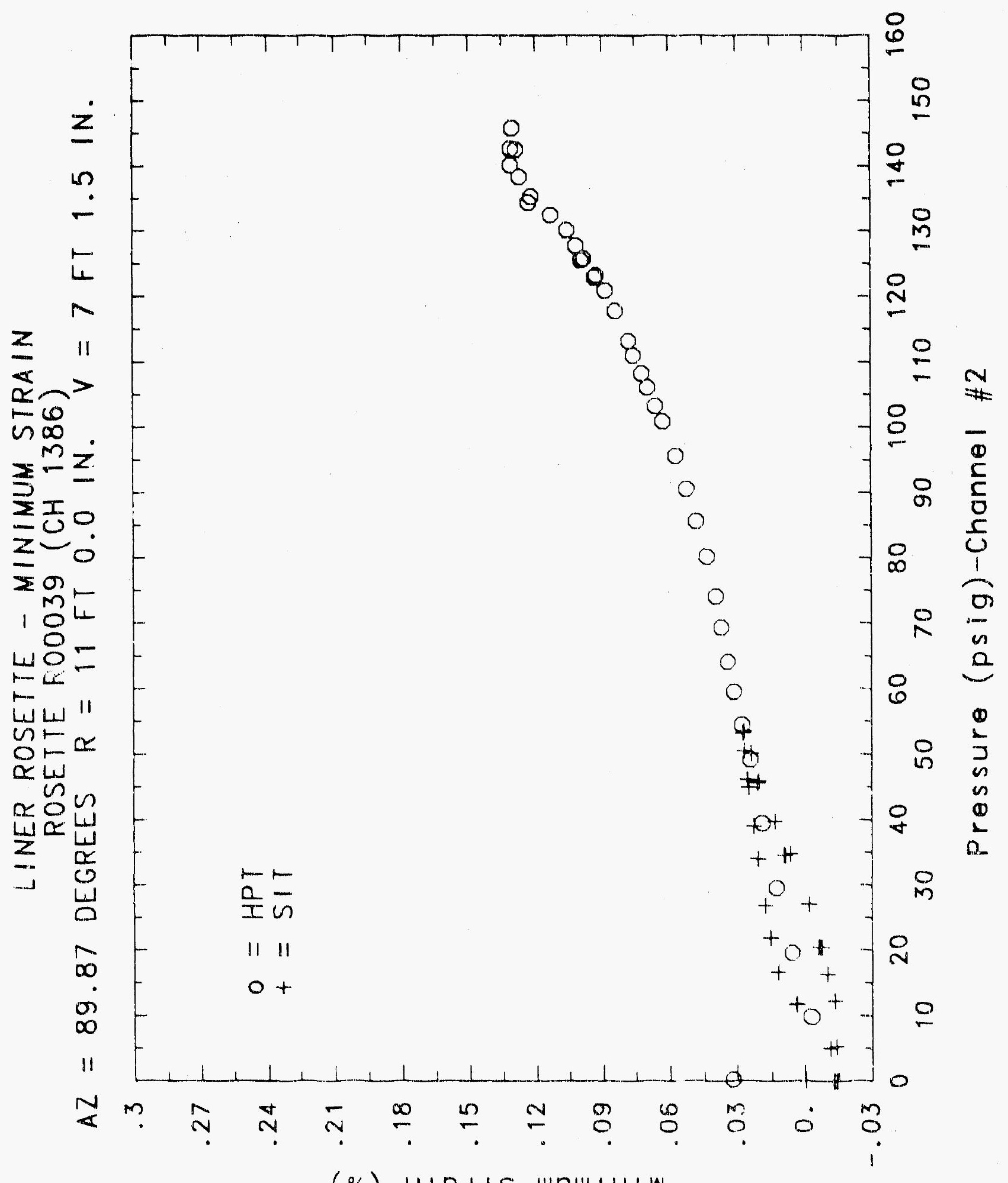

(\%) $u ! 0 d+S$ unw!u! 


\title{
Reinforced Concrete Test Data
}

\author{
Rosette 40 Channels $1388 \quad 1389 \quad 1400$
}

\section{Structukal Integrety TEST}

\begin{tabular}{|c|c|}
\hline Pressure & Max I mum \\
\hline psig & xstraln \\
\hline-0.05 & 0.0003 \\
\hline 5.33 & 0.0032 \\
\hline 12.31 & $0.00 / 13$ \\
\hline 16.44 & 0.0110 \\
\hline 20.51 & 0.0163 \\
\hline 20.46 & 0.0113 \\
\hline 20.41 & 0.0184 \\
\hline 27.05 & 0.0301 \\
\hline 34.69 & 0.0459 \\
\hline 34.53 & $0.0 .14 / 8$ \\
\hline 34.53 & 0.0482 \\
\hline 39.10 & 0.0565 \\
\hline 45.90 & 0.0669 \\
\hline 95.65 & 0.0613 \\
\hline $4 b .6 ?$ & $0.06,11$ \\
\hline 50.10 & 0.0118 \\
\hline 53.41 & 0.0791 \\
\hline 83.29 & 0.080 .3 \\
\hline 53.21 & 0.0803 \\
\hline 60.49 & 0.0784 \\
\hline 46.14 & 0.0141 \\
\hline 46.13 & 0.0738 \\
\hline 44.97 & 0.0131 \\
\hline 38.98 & $0.066,8$ \\
\hline 31.99 & 0.0613 \\
\hline 33.96 & 0.0618 \\
\hline 20.81 & 0.0512 \\
\hline 21.88 & 0.0414 \\
\hline 21.90 & $0.04 / 6$ \\
\hline 16.69 & 0.0413 \\
\hline 11.14 & $0.03 ! 0$ \\
\hline 11.11 & 0.0339 \\
\hline 5.05 & 0.0269 \\
\hline $0.0 ?$ & 0.0173 \\
\hline 0.04 & 0.0160 \\
\hline $0.0 ?$ & 0.0161 \\
\hline-0.02 & 0.0161 \\
\hline $0.0 \%$ & 0.0141 \\
\hline
\end{tabular}

Mintmuin

xStrain

0.0001

0.0016

0.0038

0.005 ?

0.007 ?

0.0080

0.0086

0.0131

$0.019 \%$

0.0208

0.0212

0.0248

0.0291

$0.024 ?$

0.0293

0.0338

0.0341

0.0340

0.0334

0.0 .114

0.0310

0.0312

0.0294

0.6215

0.0218

0.0250

0.0232

$0.0 \% 36$

0.0215

0.019 ?

0.0182

0.0151

0.0120

$0.0 n g$ ?

$0.010 \%$

0.0100

0.0096
0.0314
Angle Degraes

17.53

$-9.38$

$-8.06$

$-8.08$

$-8.00$

$-9.13$

10.03

$-8.77$

.7 .50

.7 .44

$-7.15$

..7. 10

6.90

.1 .11

.7 .38

.7 .21

7.45

7.34

7.40

7.39

7.54

1.41

7.32

7.47

7.60

7.88

8.55

$-9.24$

- 3.38

9.61

10.37

11.24

13.80

.8 .19

?. 5?

.7 .12

$-7.72$

$-6.39$
High Pressure test

\begin{tabular}{|c|c|c|c|}
\hline Pressure & Maximum & Minimum & Angle \\
\hline$p s+g$ & xstrain & xstrain & Degree. \\
\hline 9.89 & 0.0140 & 0.0061 & -15.64 \\
\hline 19.55 & 0.0263 & 0.0108 & -12.49 \\
\hline 29.57 & 0.0385 & 0.0156 & -11.88 \\
\hline 39.42 & 0.0504 & 0.0201 & .10 .42 \\
\hline 49.16 & 0.0619 & 0.0245 & -9.14 \\
\hline 54.50 & 0.0687 & 0.0271 & $-9.6 ?$ \\
\hline 59.57 & 0.0764 & 0.0300 & -9.36 \\
\hline 64.20 & 0.0854 & 0.0335 & .9 .04 \\
\hline 69.32 & $0.095 \%$ & 0.0372 & 8.46 \\
\hline 74.16 & 0.1054 & 0.0406 & 9.20 \\
\hline 80.16 & 0.1156 & 0.0441 & 8.13 \\
\hline 85.61 & 0.1236 & 0.0490 & .7 .28 \\
\hline 90.58 & 0.1258 & 0.0543 & 6.39 \\
\hline 95.69 & 0.1275 & 0.0591 & $6.1 \%$ \\
\hline $100.9 ?$ & 0.1290 & 0.0650 & 6.14 \\
\hline 103.25 & 0.1308 & $0.06,14$ & 0.24 \\
\hline 106.11 & 0.1323 & 0.0697 & 6.40 \\
\hline 108.31 & 0.1395 & 0.0718 & 1.54 \\
\hline 111.08 & 0.1353 & 0.0744 & 6.86 \\
\hline 113.24 & 0.1371 & 0.0763 & 0.82 \\
\hline 117.83 & 0.1409 & $0.080 ?$ & 0.11 \\
\hline $120.9 \%$ & 0.1455 & $0.08 \% \%$ & 6.81 \\
\hline 123.28 & 0.1509 & 0.0838 & 6.96 \\
\hline 122.91 & 0.1518 & 0.0833 & 0.46 \\
\hline $125.8 \%$ & 0.1861 & 0.0848 & 1.07 \\
\hline 125.60 & 0.1385 & 0.01847 & 1.20 \\
\hline 127.84 & 0.1623 & (1). .1954 & 1.23 \\
\hline 130.19 & 0.1691 & 1). 089.4 & $7.8 \%$ \\
\hline 132.53 & 0.1712 & 0.0846 & 7.11 \\
\hline 135.33 & $0.19 / 5$ & 0.08320 & 8.06 \\
\hline 134.42 & 0.20 .00 & 0.0810 & 8.4 .3 \\
\hline 138.35 & 0.2460 & 0.0719 & 11.24 \\
\hline 140.16 & 0.2994 & 0.0181 & 11.59 \\
\hline 142.03 & 0.5314 & 0.0893 & -3.04 \\
\hline 146.79 & $0.859 ?$ & (1.0945 & $3.5 !$ \\
\hline 142.52 & 0.9763 & 0.0976 & .4 .29 \\
\hline 0.22 & 0.7141 & 0.0403 & -4.03 \\
\hline
\end{tabular}




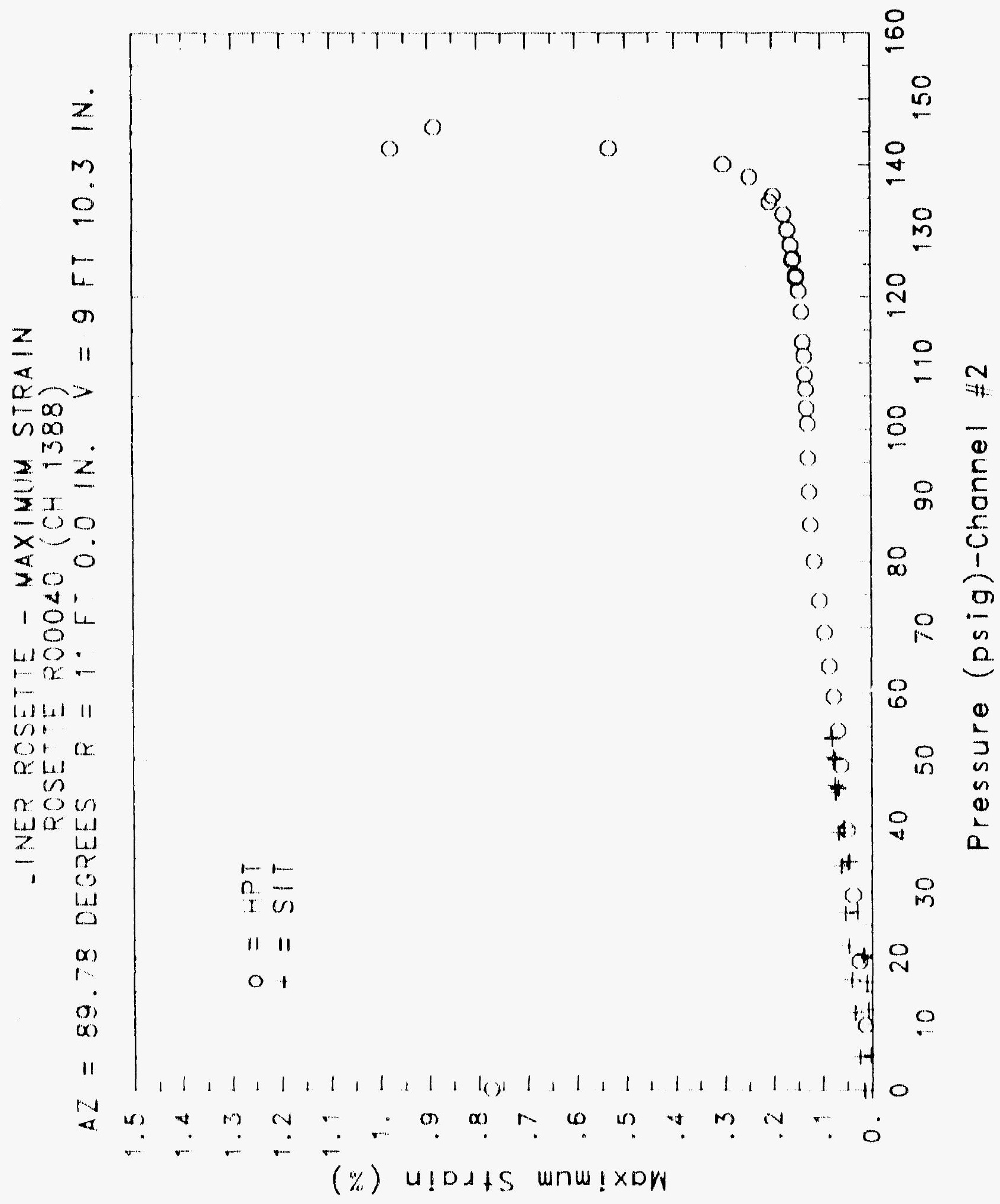




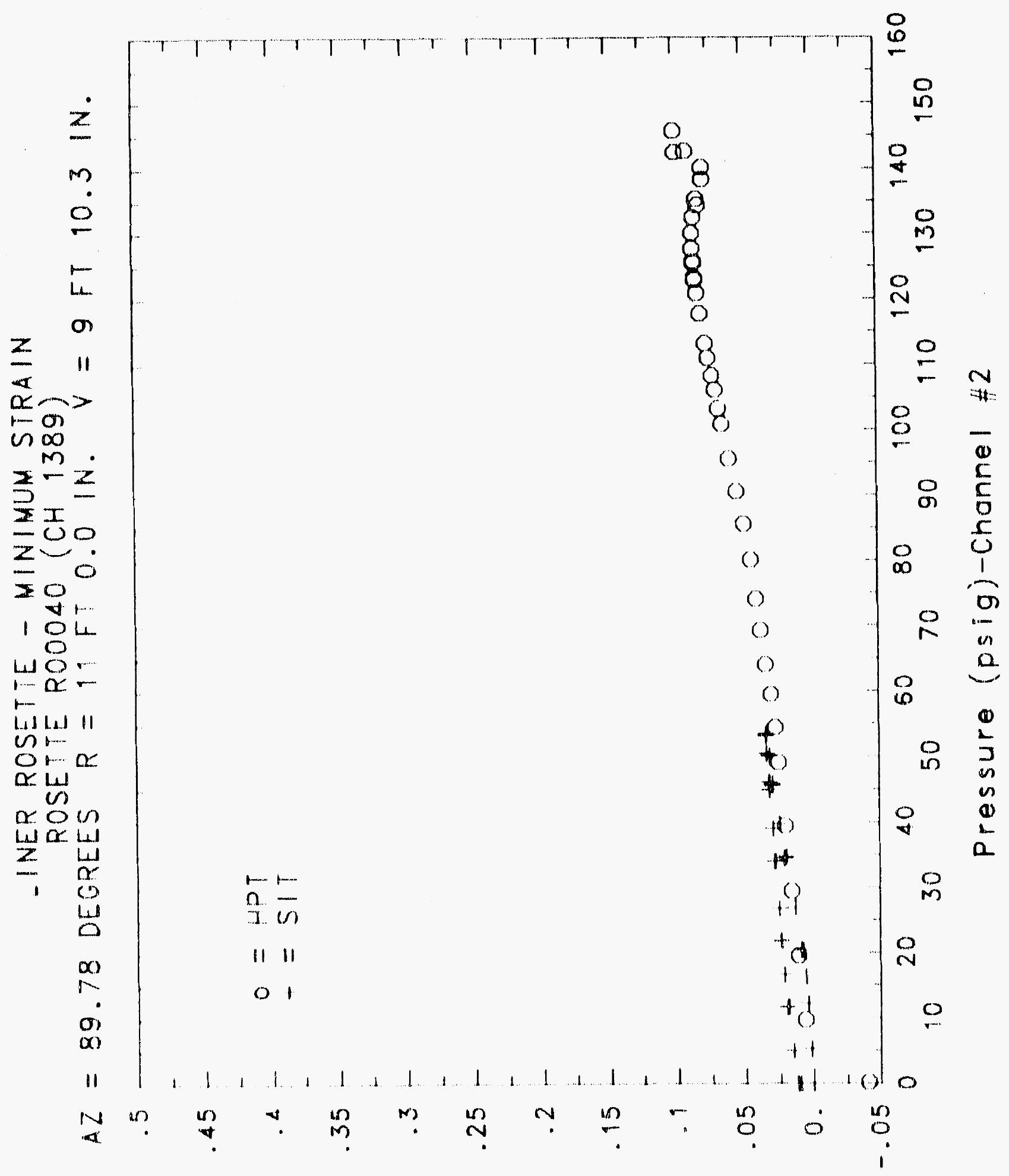

(\%) U!DdtS wnw!U!W 


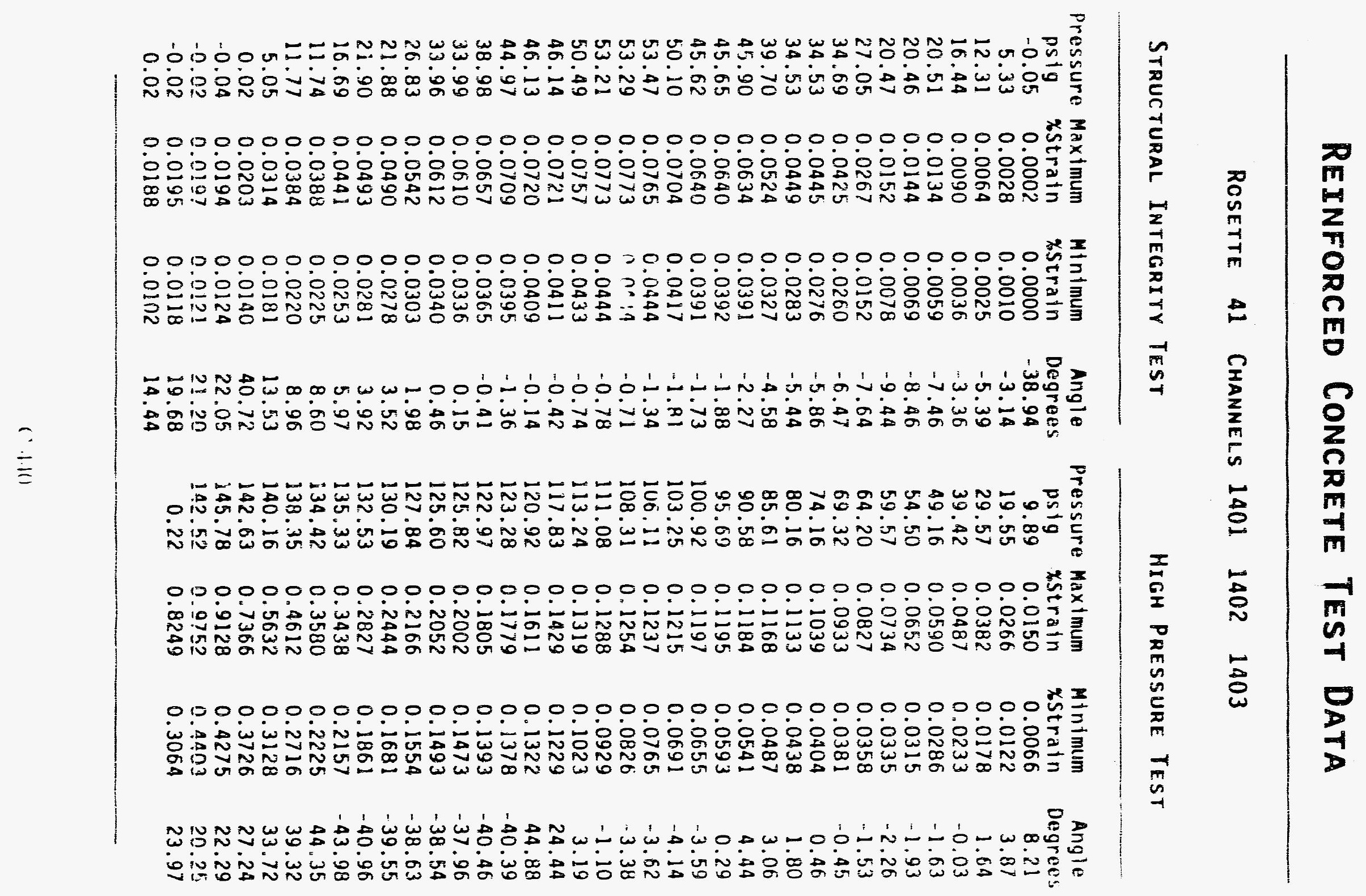




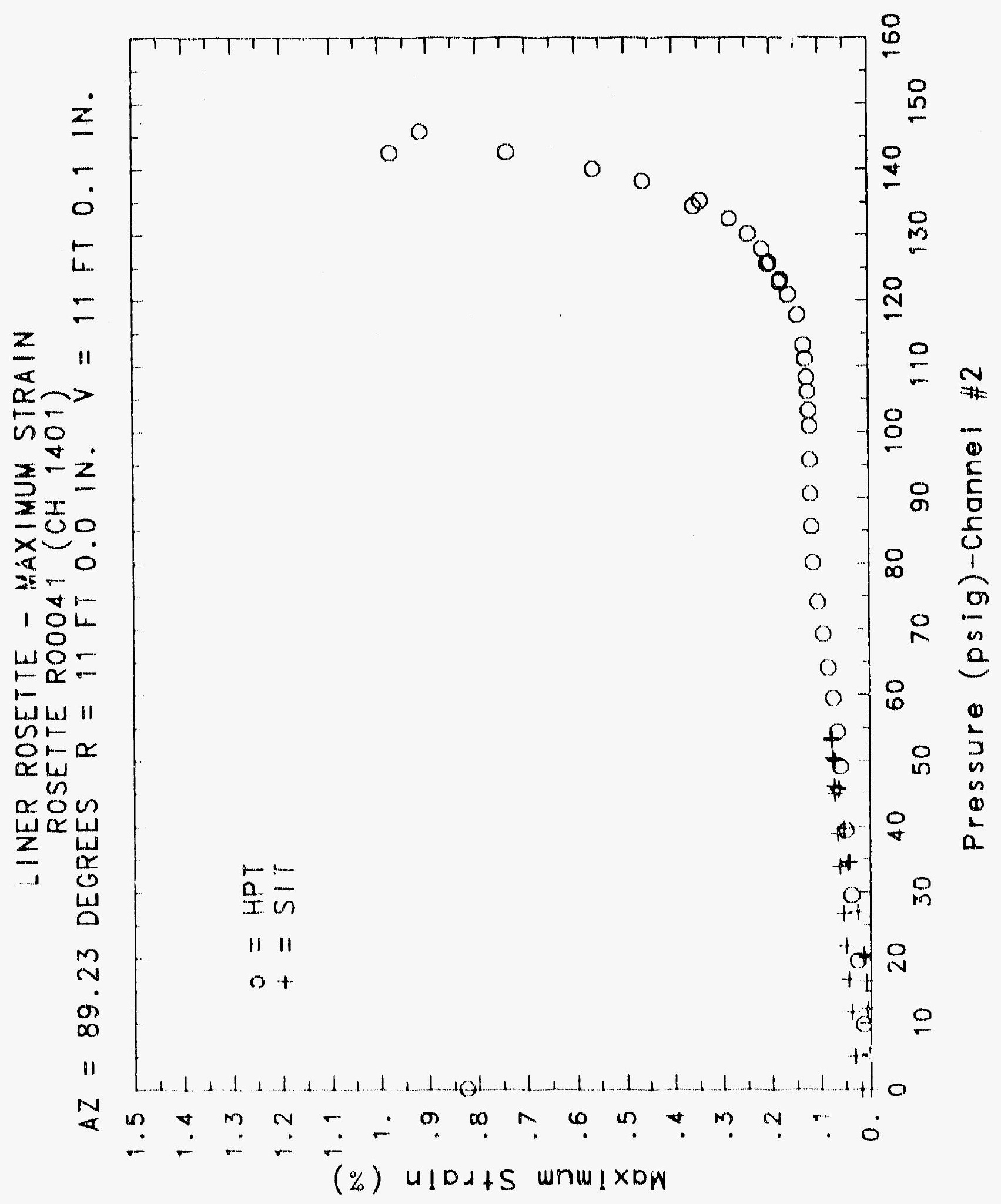




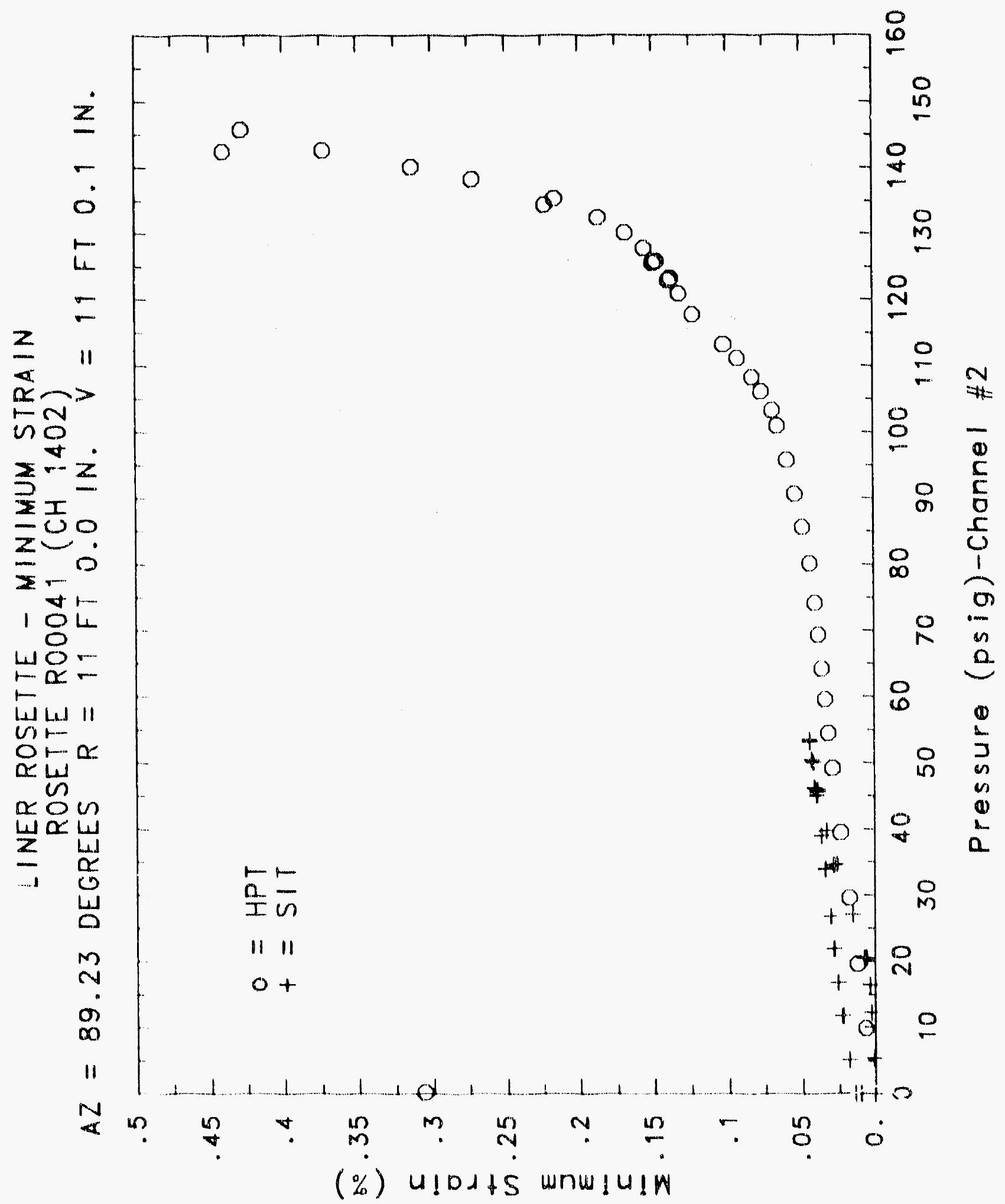

('4) 


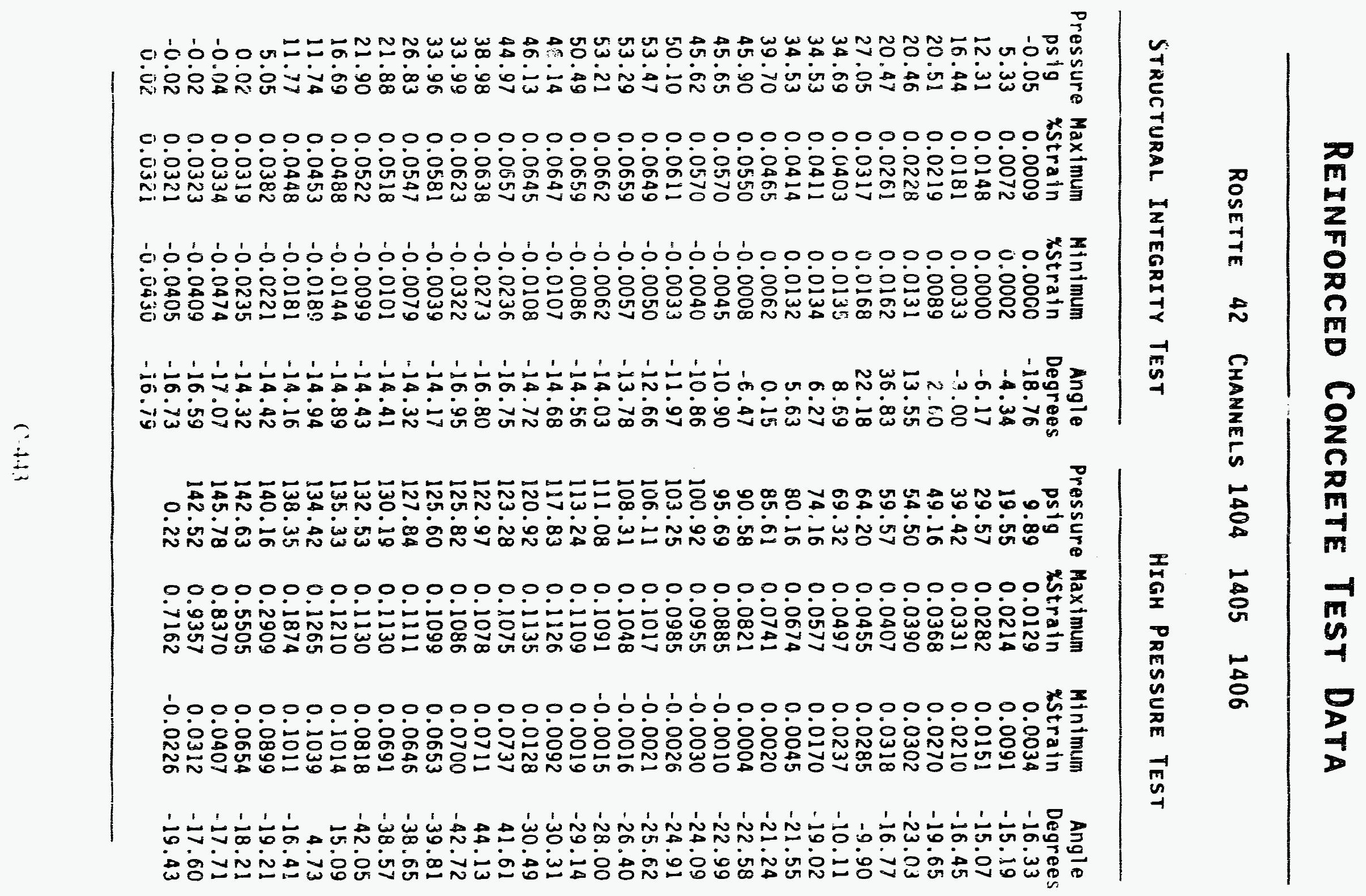




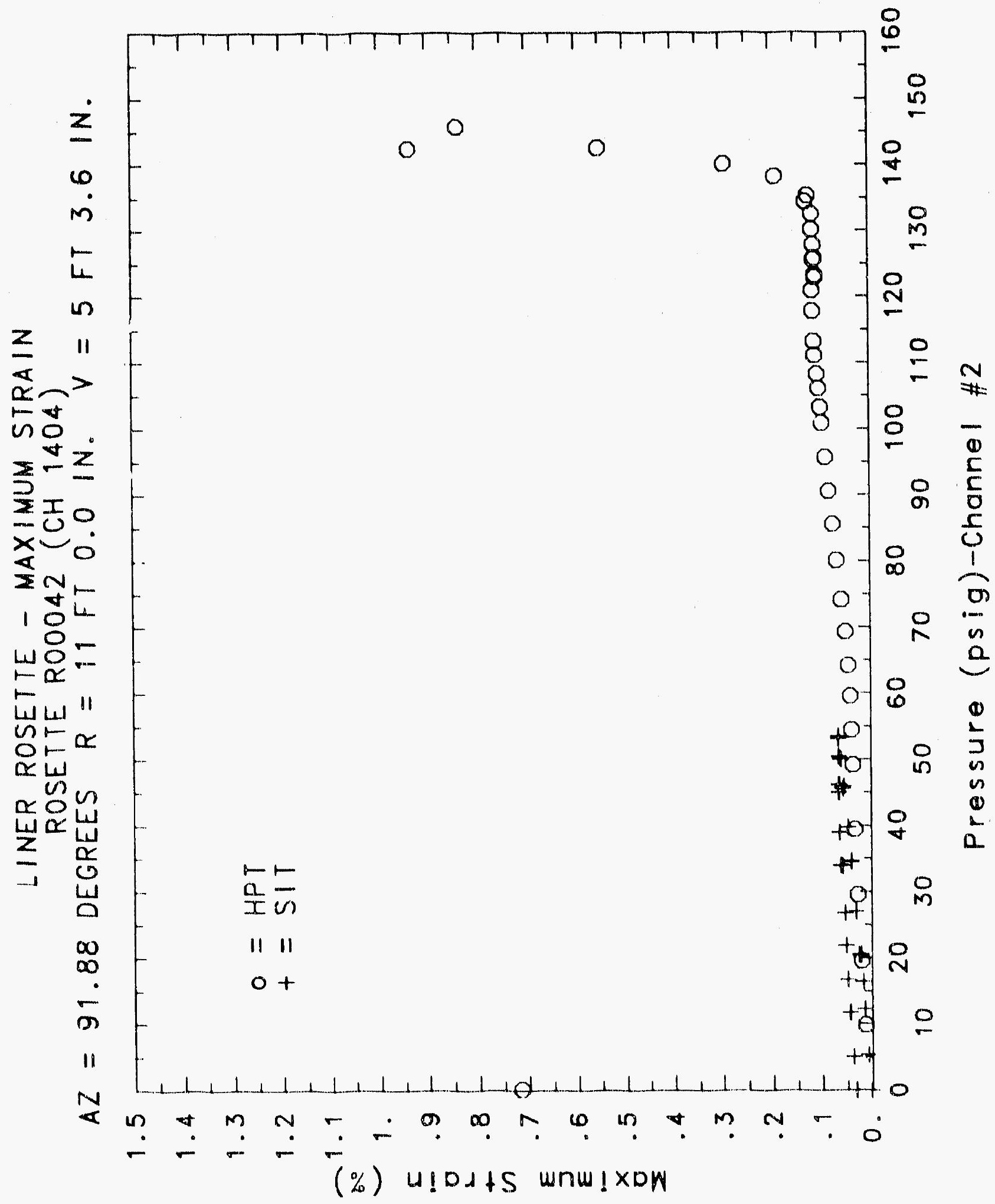




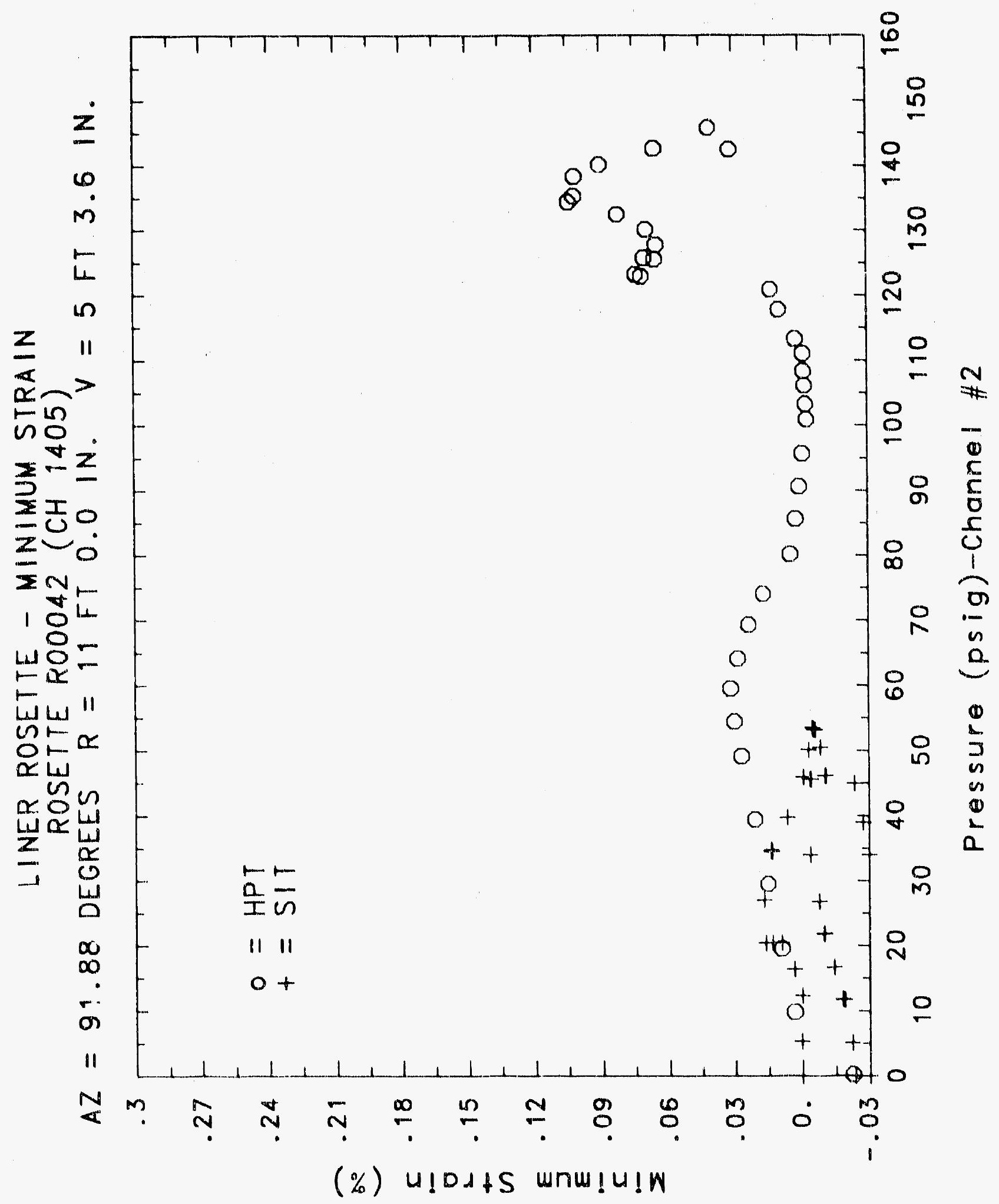




\section{Reinforced Concrete Test Data}

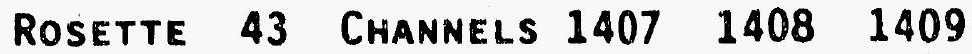

$$
\text { Structural Integrity Test }
$$

\begin{tabular}{|c|c|c|c|c|c|c|c|}
\hline $\begin{array}{c}\text { Pressure } \\
\text { psig } \\
-0.05 \\
5.33 \\
12.31 \\
16.44 \\
20.51 \\
20.46 \\
20.47 \\
27.05 \\
34.69 \\
34.53 \\
34.53 \\
39.70 \\
45.90 \\
45.65 \\
45.62 \\
50.10 \\
53.47 \\
53.29 \\
53.21 \\
50.49 \\
46.14 \\
46.13 \\
44.97 \\
38.98 \\
33.99 \\
33.96 \\
26.83 \\
21.88 \\
21.90 \\
16.69 \\
11.74 \\
11.77 \\
5.05 \\
0.02 \\
-0.04 \\
-0.02 \\
-0.02 \\
0.02\end{array}$ & $\begin{array}{c}\text { Maximum } \\
\text { x.Strain } \\
0.0001 \\
0.0016 \\
0.0046 \\
0.0070 \\
0.0107 \\
0.0116 \\
0.0120 \\
0.0201 \\
0.0310 \\
0.0320 \\
0.0320 \\
0.0443 \\
0.0656 \\
0.0669 \\
0.0669 \\
0.0741 \\
0.0817 \\
0.0830 \\
0.0836 \\
0.0827 \\
0.0797 \\
0.0795 \\
0.0814 \\
0.0762 \\
0.0713 \\
0.0712 \\
0.0632 \\
0.0572 \\
0.0569 \\
0.0503 \\
0.0429 \\
0.0410 \\
0.0305 \\
0.0218 \\
0.0196 \\
0.0201 \\
0.0199 \\
0.0189\end{array}$ & $\begin{array}{c}\text { Minimum } \\
\text { XStrain } \\
-0.0001 \\
0.0006 \\
0.0013 \\
0.0017 \\
0.0029 \\
0.0035 \\
0.0035 \\
0.0056 \\
0.0082 \\
0.0068 \\
0.0039 \\
0.0080 \\
0.0205 \\
0.0207 \\
0.0206 \\
0.0229 \\
0.0247 \\
0.0252 \\
0.0254 \\
0.0252 \\
0.0242 \\
0.0241 \\
0.0260 \\
0.0240 \\
0.0221 \\
0.0221 \\
0.0193 \\
0.0173 \\
0.0173 \\
0.0151 \\
0.0129 \\
0.0118 \\
0.0090 \\
0.0064 \\
0.0082 \\
0.0084 \\
0.0081 \\
0.0072\end{array}$ & $\begin{array}{c}\text { Angle } \\
\text { Degrees } \\
45.00 \\
-15.91 \\
-7.08 \\
-4.63 \\
-5.17 \\
-5.74 \\
-4.65 \\
-4.82 \\
-6.37 \\
-9.60 \\
-12.84 \\
-8.18 \\
-2.66 \\
-2.29 \\
-1.93 \\
-2.39 \\
-1.74 \\
-1.33 \\
-1.26 \\
-0.68 \\
-0.37 \\
-0.36 \\
0.14 \\
0.08 \\
0.14 \\
0.13 \\
0.06 \\
0.05 \\
-0.32 \\
-0.68 \\
-1.65 \\
-2.01 \\
-4.80 \\
-9.24 \\
1.83 \\
7.20 \\
9.05 \\
8.89\end{array}$ & $\begin{array}{c}\text { Pressure } \\
\text { psig } \\
9.89 \\
19.55 \\
29.57 \\
39.42 \\
49.16 \\
54.50 \\
59.57 \\
64.20 \\
69.32 \\
74.16 \\
80.16 \\
85.61 \\
90.58 \\
95.69 \\
100.92 \\
103.25 \\
106.11 \\
108.31 \\
111.08 \\
113.24 \\
117.83 \\
120.92 \\
123.28 \\
122.97 \\
125.82 \\
125.60 \\
127.84 \\
130.19 \\
132.53 \\
135.33 \\
134.42 \\
138.35 \\
140.16 \\
142.63 \\
145.78 \\
142.52 \\
0.22\end{array}$ & $\begin{array}{c}\text { Maximum } \\
\text { x.Strain } \\
0.0435 \\
0.0554 \\
0.0602 \\
0.0677 \\
0.0784 \\
0.0844 \\
0.0912 \\
0.0986 \\
0.1061 \\
0.1121 \\
0.1215 \\
0.1302 \\
0.1386 \\
0.1484 \\
0.1588 \\
0.1639 \\
0.1686 \\
0.1723 \\
0.1777 \\
0.1819 \\
0.1908 \\
0.1974 \\
0.2028 \\
0.2038 \\
0.2099 \\
0.2120 \\
0.2161 \\
0.2246 \\
0.2382 \\
0.2553 \\
0.2586 \\
0.2828 \\
0.3079 \\
0.3902 \\
0.5613 \\
0.6060 \\
0.5515\end{array}$ & $\begin{array}{l}\text { Minimum } \\
\text { XStrain } \\
-0.0243 \\
-0.0174 \\
-0.0038 \\
0.0057 \\
0.0109 \\
0.0152 \\
0.0191 \\
0.0239 \\
0.0284 \\
0.0340 \\
0.0393 \\
0.0448 \\
0.0499 \\
0.0557 \\
0.0612 \\
0.0640 \\
0.0668 \\
0.0689 \\
0.0723 \\
0.0745 \\
0.0806 \\
0.0853 \\
0.0898 \\
0.0910 \\
0.0964 \\
0.0974 \\
0.1006 \\
0.1075 \\
0.1158 \\
0.1270 \\
0.1284 \\
0.1444 \\
0.1534 \\
0.1494 \\
0.1584 \\
0.1610 \\
-0.1355\end{array}$ & $\begin{array}{c}\text { Angle } \\
\text { Degrees } \\
\text {-40.34 } \\
-36.11 \\
-29.73 \\
-24.31 \\
-22.11 \\
-20.06 \\
-18.19 \\
-16.42 \\
-14.61 \\
-13.26 \\
-11.40 \\
-9.63 \\
-7.75 \\
-5.87 \\
-4.21 \\
-3.42 \\
-2.70 \\
-2.19 \\
-1.24 \\
-0.44 \\
1.16 \\
2.53 \\
3.42 \\
3.44 \\
4.96 \\
5.97 \\
6.66 \\
8.93 \\
12.32 \\
15.90 \\
16.44 \\
18.46 \\
19.33 \\
21.48 \\
14.84 \\
13.30 \\
-34.84\end{array}$ \\
\hline
\end{tabular}

High fressure TEST

Structural Integrity Test 


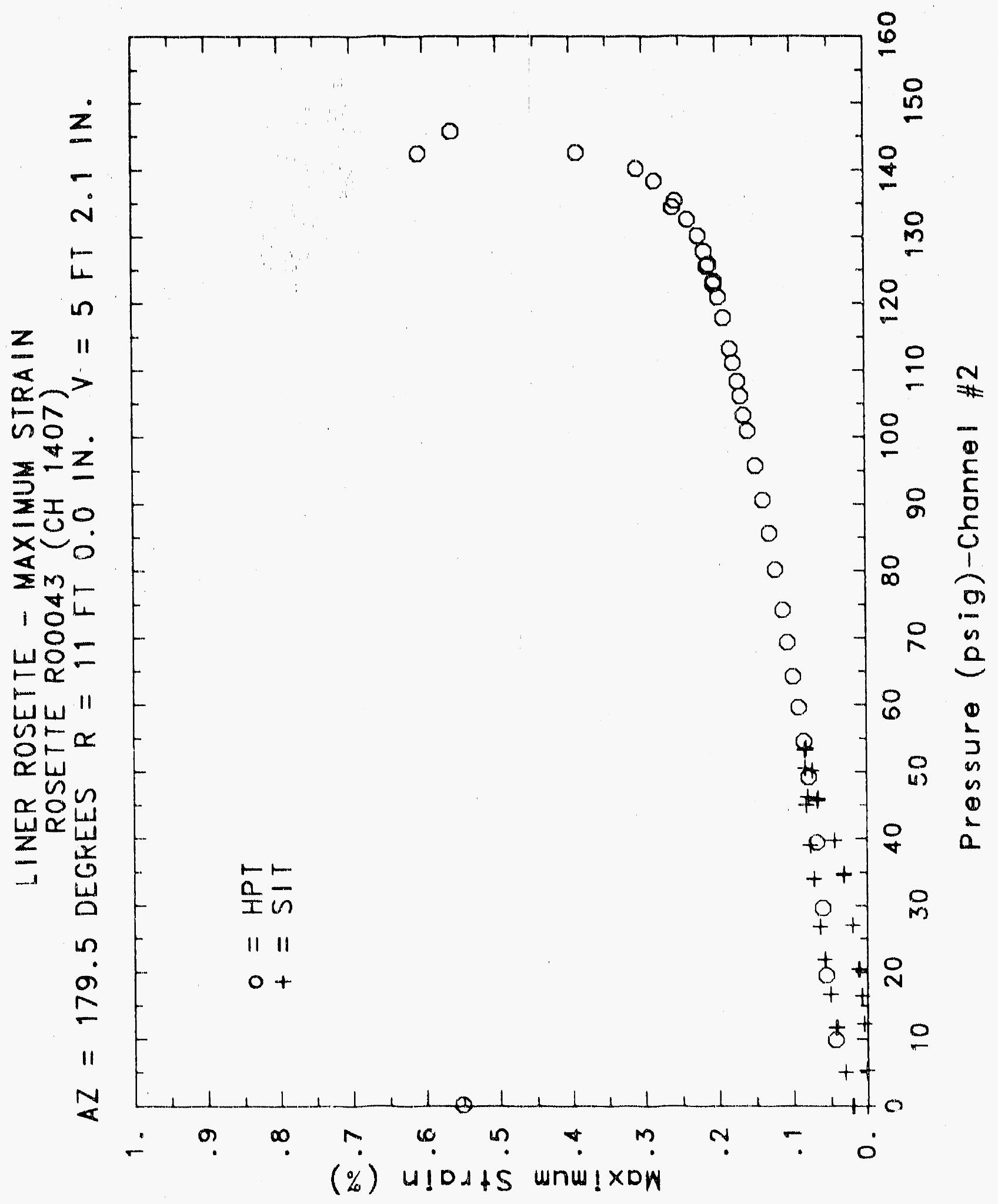




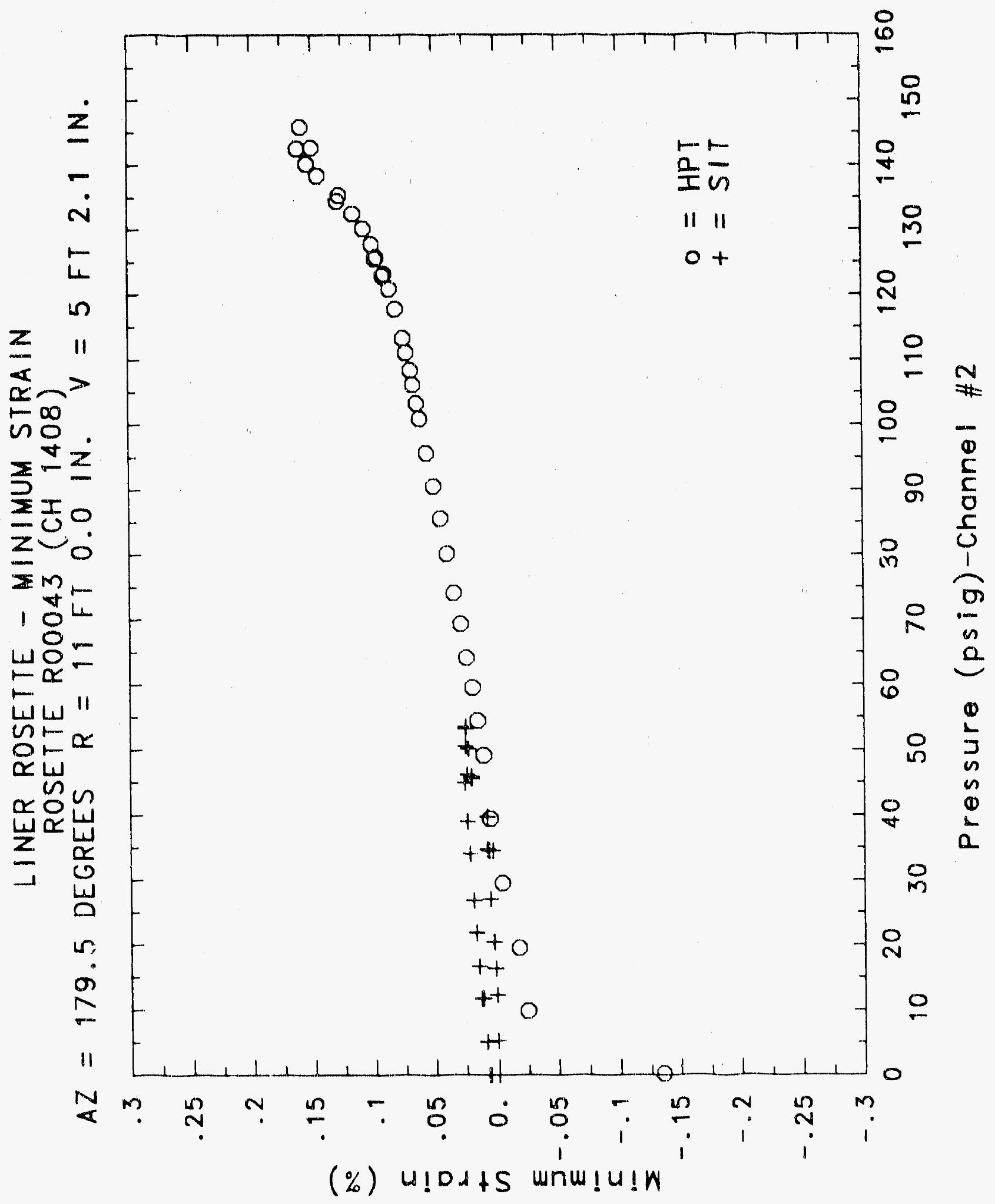




\title{
Reinforced Concrete Test Data
}

\author{
Rosette 44 Channels $14201421 \quad 1422$
}

StRUCTURAL IntEgrity TEST

\begin{tabular}{|c|c|c|c|c|c|c|c|}
\hline $\begin{array}{c}\text { Pressure } \\
\text { psig } \\
-0.05 \\
5.33 \\
12.31 \\
16.44 \\
20.51 \\
20.46 \\
20.47 \\
27.05 \\
34.69 \\
34.53 \\
34.53 \\
39.70 \\
45.90 \\
45.65 \\
45.62 \\
50.10 \\
53.47 \\
53.29 \\
53.21 \\
50.49 \\
46.14 \\
46.13 \\
44.97 \\
38.98 \\
33.99 \\
33.96 \\
26.83 \\
21.88 \\
21.90 \\
16.69 \\
11.74 \\
11.77 \\
5.05 \\
0.02 \\
-0.04 \\
-0.02 \\
-0.02 \\
0.02\end{array}$ & $\begin{array}{c}\text { Maximum } \\
\text { \%Strain } \\
0.0000 \\
0.0016 \\
0.0032 \\
0.0052 \\
0.0081 \\
0.0088 \\
0.0092 \\
0.0192 \\
0.0341 \\
0.0356 \\
0.0361 \\
0.0432 \\
0.0553 \\
0.0550 \\
0.0547 \\
0.0609 \\
0.0662 \\
0.0660 \\
0.0660 \\
0.0638 \\
0.0592 \\
0.0591 \\
0.0586 \\
0.0518 \\
0.0454 \\
0.0454 \\
0.0363 \\
0.0299 \\
0.0301 \\
0.0233 \\
0.0165 \\
0.0156 \\
0.0083 \\
0.0023 \\
0.0022 \\
0.0023 \\
0.0024 \\
0.0030\end{array}$ & $\begin{array}{l}\text { Minimum } \\
\text { xStrain } \\
-0.0002 \\
-0.0101 \\
-0.0135 \\
-0.0092 \\
-0.0037 \\
-0.0025 \\
-0.0018 \\
0.0067 \\
0.0146 \\
0.0156 \\
0.0158 \\
0.0187 \\
0.0175 \\
0.0169 \\
0.0167 \\
0.0173 \\
0.0168 \\
0.0165 \\
0.0164 \\
0.0153 \\
0.0134 \\
0.0132 \\
0.0143 \\
0.0115 \\
0.0088 \\
0.0090 \\
0.0051 \\
0.0027 \\
0.0029 \\
0.0004 \\
-0.0020 \\
-0.0025 \\
-0.0072 \\
-0.0124 \\
-0.0102 \\
-0.0102 \\
-0.0103 \\
-0.0105\end{array}$ & $\begin{array}{c}\text { Angle } \\
\text { Uegrees } \\
-0.39 \\
-33.36 \\
-39.06 \\
-37.96 \\
-38.40 \\
-39.93 \\
-39.45 \\
-24.18 \\
-13.87 \\
-14.20 \\
-14.43 \\
-10.23 \\
-9.12 \\
-9.18 \\
-9.12 \\
-7.46 \\
-5.02 \\
-4.83 \\
-4.96 \\
-5.54 \\
-6.66 \\
-6.67 \\
-6.75 \\
-8.86 \\
-10.32 \\
-10.12 \\
-12.65 \\
-14.53 \\
-14.92 \\
-17.85 \\
-21.97 \\
-22.24 \\
-35.64 \\
-42.87 \\
43.61 \\
42.52 \\
42.21 \\
43.47\end{array}$ & $\begin{array}{c}\text { Pressure } \\
\text { psig } \\
9.89 \\
19.55 \\
29.57 \\
39.42 \\
49.16 \\
54.50 \\
59.57 \\
64.20 \\
69.32 \\
74.16 \\
80.16 \\
85.61 \\
90.58 \\
95.69 \\
100.92 \\
103.25 \\
106.11 \\
108.31 \\
111.08 \\
113.24 \\
117.83 \\
120.92 \\
123.28 \\
122.97 \\
125.82 \\
125.60 \\
127.84 \\
130.19 \\
132.53 \\
135.33 \\
134.42 \\
138.35 \\
140.16 \\
142.63 \\
145.78 \\
142.52 \\
0.22\end{array}$ & $\begin{array}{l}\text { Maximum } \\
\text { xStrain } \\
0.0120 \\
0.0274 \\
0.0424 \\
0.0564 \\
0.0696 \\
0.0767 \\
0.0826 \\
0.0878 \\
0.0934 \\
0.0994 \\
0.1063 \\
0.1135 \\
0.1200 \\
0.1262 \\
0.1326 \\
0.1351 \\
0.1390 \\
0.1418 \\
0.1451 \\
0.1475 \\
0.1532 \\
0.1565 \\
0.1587 \\
0.1587 \\
0.1604 \\
0.1602 \\
0.1619 \\
0.1620 \\
0.1611 \\
0.1611 \\
0.1602 \\
0.1624 \\
0.1700 \\
0.2101 \\
0.364 ! \\
0.4201 \\
0.2641\end{array}$ & $\begin{array}{l}\text { Minimum } \\
\text { xStrain } \\
0.0069 \\
0.0097 \\
0.0114 \\
0.0138 \\
0.0168 \\
0.0182 \\
0.0192 \\
0.0194 \\
0.0194 \\
0.0189 \\
0.0178 \\
0.0187 \\
0.0193 \\
0.0204 \\
0.0215 \\
0.0217 \\
0.0226 \\
0.0233 \\
0.0240 \\
0.0246 \\
0.0259 \\
0.0266 \\
0.0272 \\
0.0272 \\
0.0276 \\
0.0276 \\
0.0278 \\
0.0280 \\
0.0279 \\
0.0270 \\
0.0269 \\
0.0281 \\
0.0288 \\
0.0264 \\
0.0211 \\
0.0196 \\
0.0078\end{array}$ & $\begin{array}{c}\text { Angle } \\
\text { Degrees } \\
8.22 \\
5.10 \\
3.11 \\
2.34 \\
2.40 \\
2.55 \\
3.14 \\
3.50 \\
3.49 \\
3.80 \\
2.36 \\
1.39 \\
0.08 \\
-1.13 \\
-2.69 \\
-3.07 \\
-3.25 \\
-3.72 \\
-3.83 \\
-3.85 \\
-4.68 \\
-4.69 \\
-4.68 \\
-4.73 \\
-4.32 \\
-3.93 \\
-3.49 \\
-2.95 \\
-2.17 \\
-1.48 \\
-1.40 \\
0.25 \\
2.51 \\
8.14 \\
7.89 \\
7.00 \\
7.45\end{array}$ \\
\hline
\end{tabular}

high Pressure test 


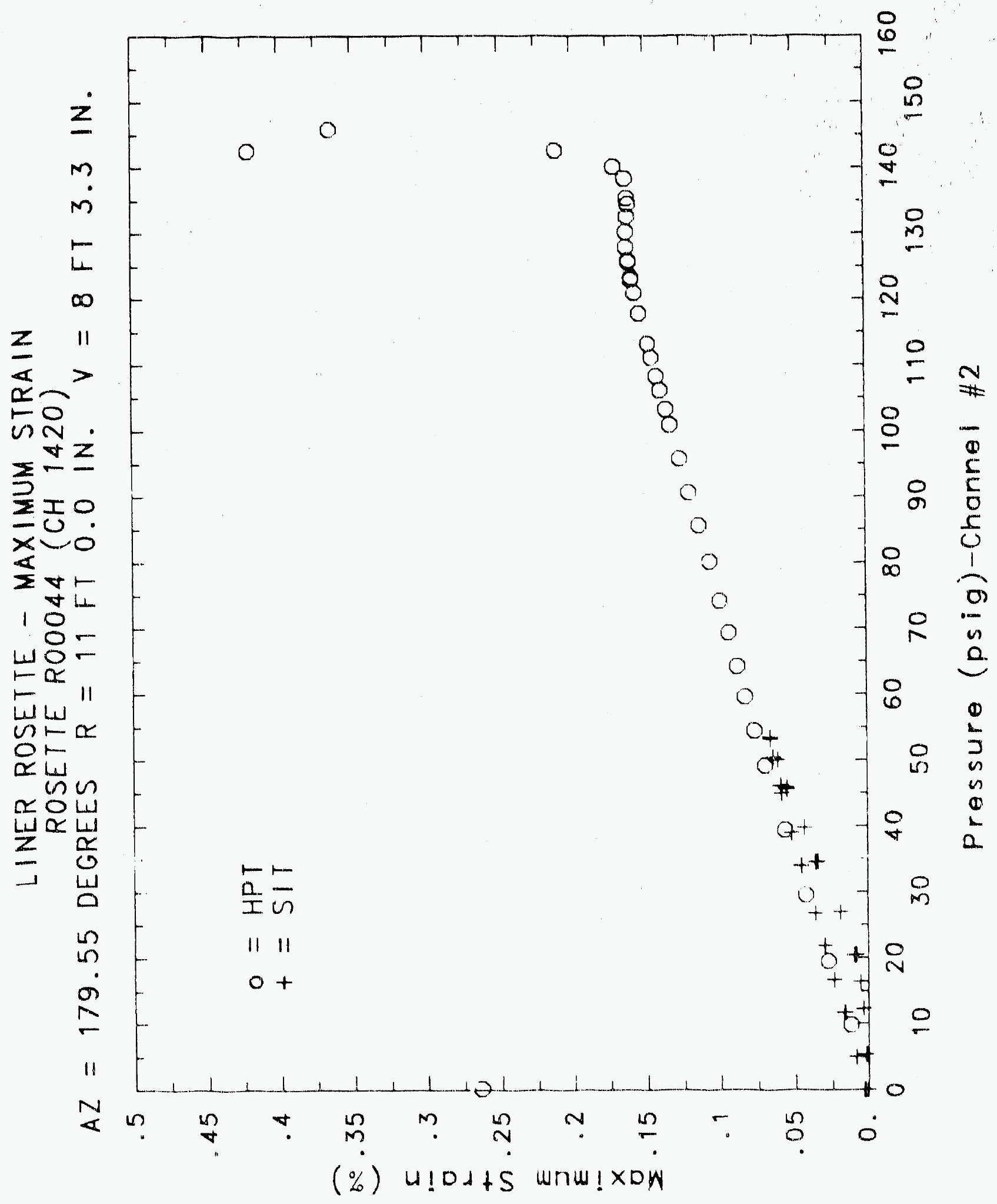




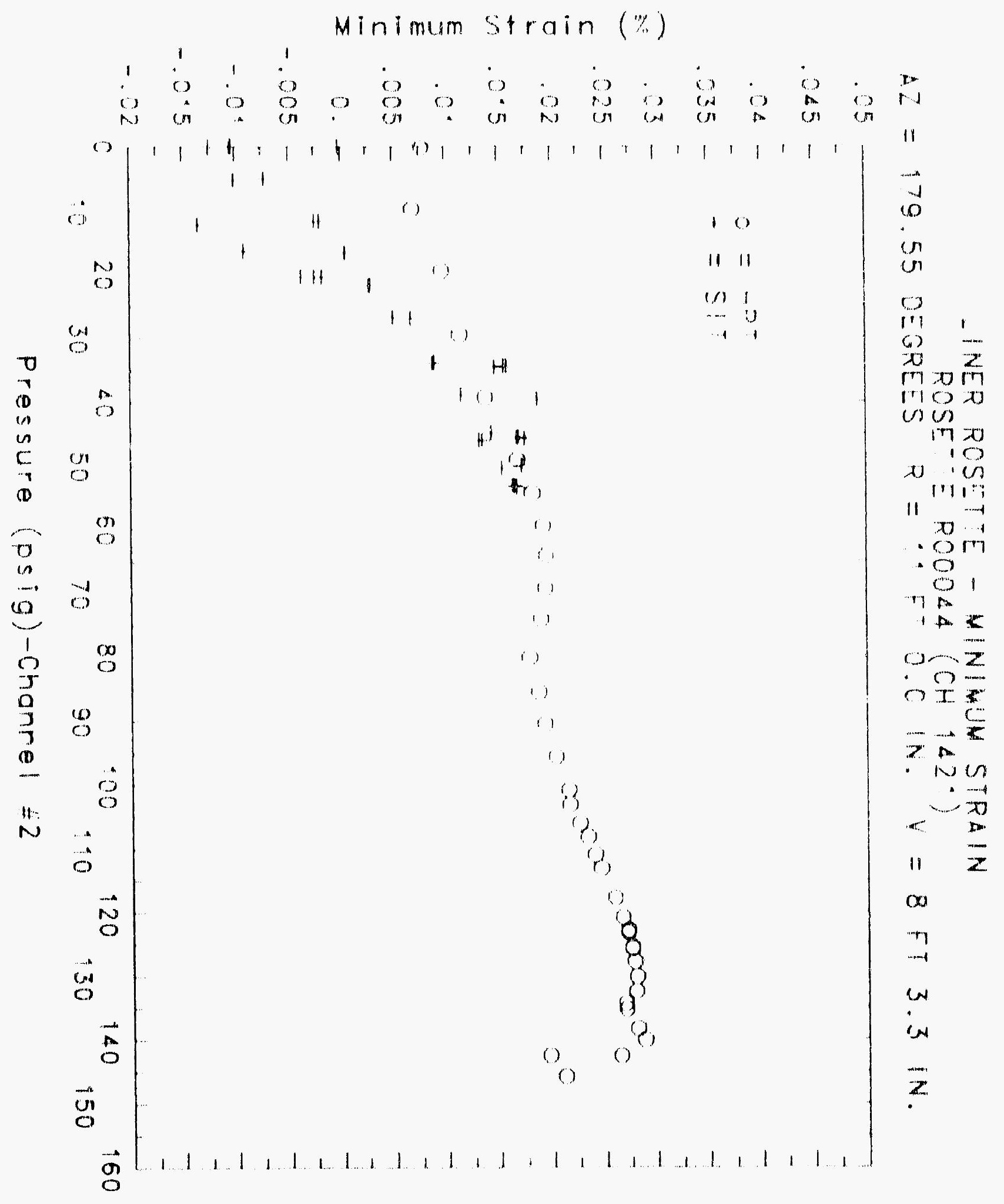




\title{
Reinforced Concrete Test Data
}

\author{
$\begin{array}{llllll}\text { Rosette } & 45 & \text { Channels } & 1423 & 1424 & 1425\end{array}$
}

\section{Structural Integrity Test}

\begin{tabular}{|c|c|c|c|}
\hline Pressure & Maximum & Miritmum & Angle \\
\hline psig & *strain & xstrain & Degre \\
\hline 0.05 & 0.0175 & -0.0174 & Aa 0 \\
\hline 5.33 & 0.1686 & -0.1612 & \\
\hline 12.31 & 0.1743 & -0.1561 & \\
\hline 16.14 & & & \\
\hline 20.51 & 0.1871 & $0.148 /$ & -43.0 \\
\hline 20.46 & 0.1892 & -0.1486 & \\
\hline 20.47 & 0.1898 & 0.1495 & -43.1 \\
\hline 27.05 & 0.2012 & 0.1423 & -42 \\
\hline 34.69 & 0.2173 & 0.1325 & .41 \\
\hline 34.53 & 0.2183 & -0. & \\
\hline 34.53 & 0.2187 & & \\
\hline 39.70 & 0.2262 & -0.1256 & -41. \\
\hline 45.90 & 0.2349 & 0.1160 & .41. \\
\hline 45.65 & 0.2348 & 0.1152 & -41. \\
\hline 45.62 & 0.2348 & -0.1152 & -41 \\
\hline 50.10 & 0.2404 & 0 & -41 \\
\hline 53.47 & & & \\
\hline 53.29 & 0.2455 & -0.1050 & -41 \\
\hline 53.21 & 0.2455 & -0.1047 & -41.2 \\
\hline 50.49 & 0.2431 & .0 .1064 & 41. \\
\hline 46.14 & 0.2381 & -0.1098 & -41. \\
\hline 46.13 & 0.2378 & 0 & -41 \\
\hline 44.97 & 0.2 & -0 . & \\
\hline 38.98 & 0. & & \\
\hline 33.99 & & & \\
\hline 33.96 & 0.2236 & -0.1202 & -42.6 \\
\hline 26.83 & 0.2137 & -0.1273 & -43.2 \\
\hline 21.188 & 0.2071 & -0.1324 & -43. \\
\hline 21.90 & 0.2070 & -0.1325 & -43. \\
\hline 16.69 & 0.2000 & 0.1376 & -44 \\
\hline 11.74 & 0.1931 & -0.1427 & -44 \\
\hline 11.71 & & & \\
\hline 5.05 & 0.1840 & 0.1501 & 44. \\
\hline $0.0 \%$ & 0.1772 & -0.1551 & 14. \\
\hline 10.04 & $0.1 / 18$ & 0.1558 & $4 \dot{4}$. \\
\hline $0.0 ?$ & 0.1119 & 0.1565 & 44. \\
\hline $0.0 \%$ & $0.1 / 69$ & 0.1571 & \\
\hline $0.0 ?$ & $0.1 / 60$ & 0.1577 & \\
\hline
\end{tabular}

High Pressure Test

\begin{tabular}{|c|c|c|c|}
\hline Pressure & Maximum & Minimum & Angle \\
\hline psig & *strain & xstrain & Degree \\
\hline 9.89 & 0.0163 & 0.0070 & -9.13 \\
\hline 19.55 & 0.0332 & 0.0136 & -8.14 \\
\hline 29.57 & 0.0506 & 0.0206 & .7 .09 \\
\hline 39.12 & 0.0677 & 0.0275 & $-6.8 ?$ \\
\hline 49.16 & 0.0847 & 0.0343 & -6.57 \\
\hline 54.50 & 0.0934 & 0.0386 & -6.41 \\
\hline 59.57 & 0.1005 & 0.0442 & -8.02 \\
\hline 64.20 & 0.1094 & .0462 & .5 .65 \\
\hline 69.32 & 0.1221 & .0487 & -2.82 \\
\hline 74.16 & 0.1346 & 0.0544 & 2.10 \\
\hline 80.16 & 0.1625 & 0.0588 & 11.10 \\
\hline 85.61 & 0.2336 & 0.0433 & 21.82 \\
\hline 90.58 & 0.3491 & 0.0158 & 24.98 \\
\hline 95.69 & 0.4957 & $.020 \%$ & 20.70 \\
\hline 100.92 & 0.6384 & .0458 & 14.32 \\
\hline 103.25 & 0.7075 & 0.0583 & 11.19 \\
\hline 106.11 & 0.7857 & 0.0681 & 8.26 \\
\hline 108.31 & 0.8404 & 0.0726 & 6.61 \\
\hline 111.08 & 0.9139 & 0.0766 & 4.75 \\
\hline 113.24 & 0.9591 & 0.0781 & 3.87 \\
\hline 117.83 & 1.0330 & 0.0778 & 3.15 \\
\hline 120.92 & 1.0740 & 0.0766 & 3.14 \\
\hline 123.28 & 1.1212 & 0.0754 & 3.42 \\
\hline 122.97 & 1.1335 & 0.0754 & 3.52 \\
\hline 125.82 & 1.2274 & 0.0735 & 4.24 \\
\hline 125.60 & 1.2561 & 0.0732 & 4. 34 \\
\hline 127.84 & 1.3428 & 0.0718 & 4.66 \\
\hline 130.19 & 1.5931 & 0.0673 & 5.21 \\
\hline 132.53 & 1.9912 & 0.0533 & 5.09 \\
\hline 135.33 & 2.5450 & 0.0536 & 4.47 \\
\hline 134.42 & 2.6375 & 0.0505 & 4.37 \\
\hline 138.35 & 3.1821 & 0.0339 & 4.02 \\
\hline 140.16 & 3.5940 & 0.0201 & 3.93 \\
\hline 142.63 & 4.1629 & $0.00 \% 1$ & 3.84 \\
\hline 195.78 & 4.7211 & -0.0130 & 3.72 \\
\hline 142.52 & 4.8751 & 0.0192 & 3.63 \\
\hline 0.22 & 4.5987 & -0.1544 & 9.28 \\
\hline
\end{tabular}




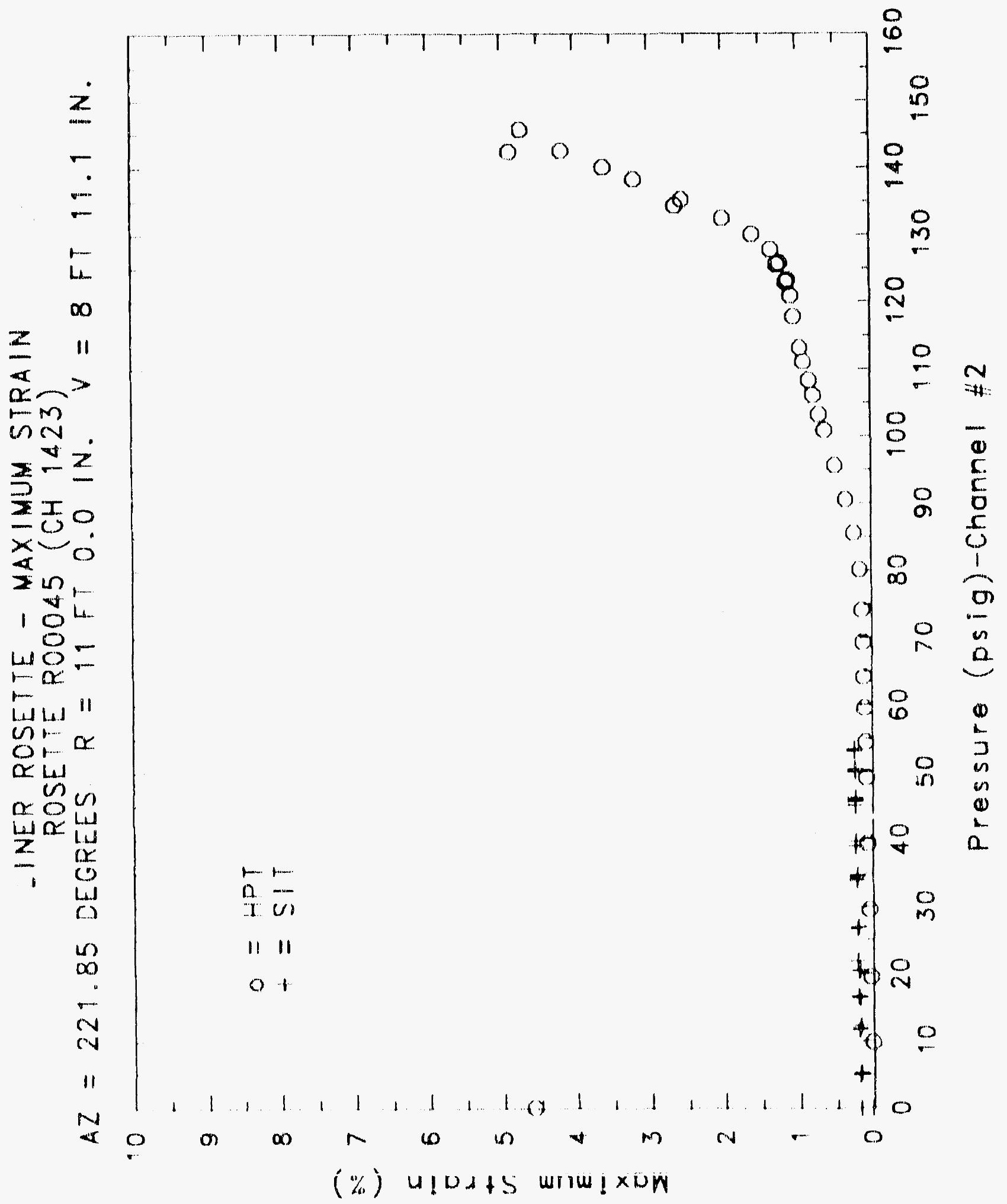




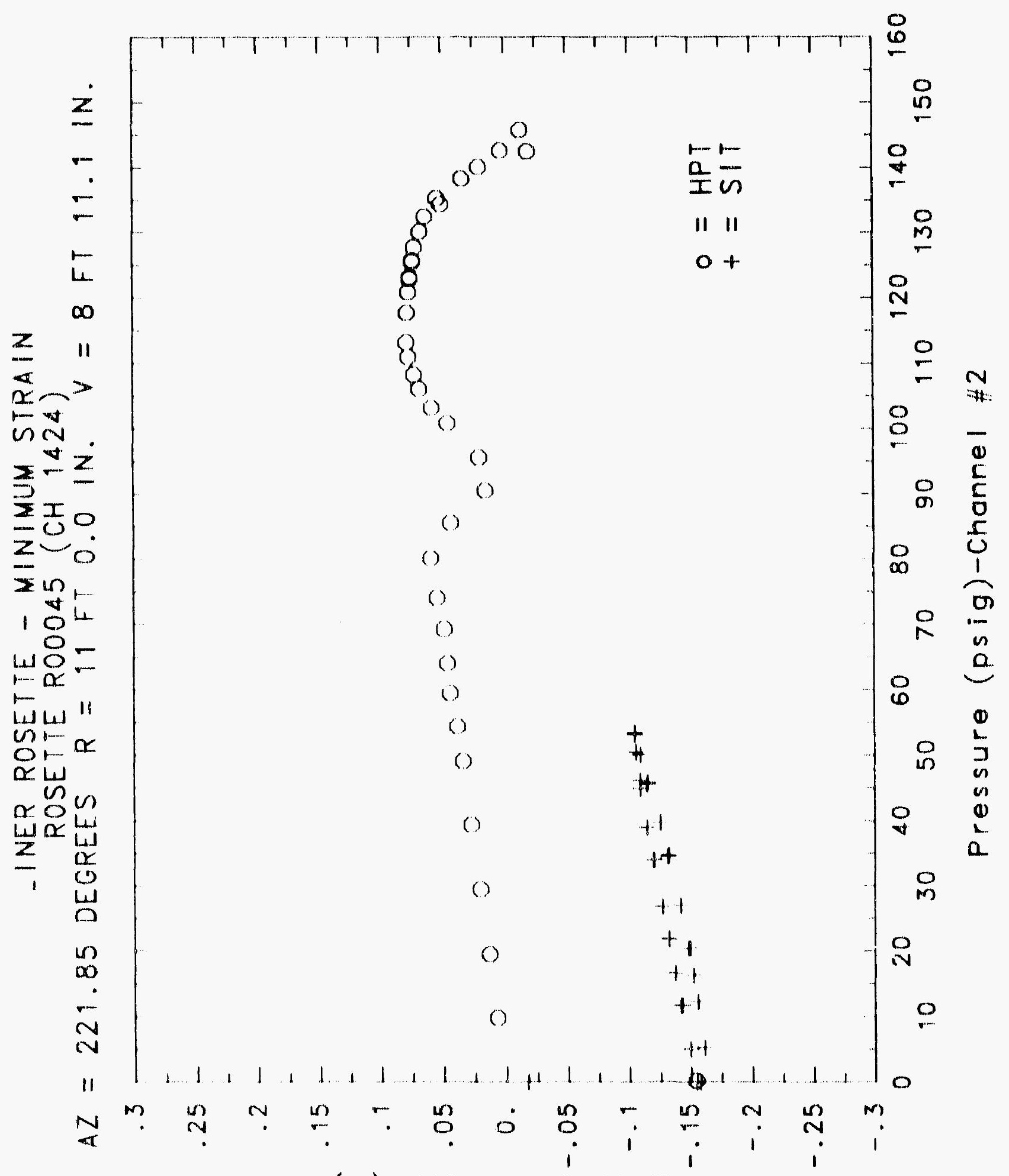

(\%) 


\section{Reinforced Concrete Test Data}

Rosette 46 Channels $1426 \quad 1427 \quad 1428$

Structural IHTEgRITY TEST

\begin{tabular}{|c|c|c|c|c|c|c|c|}
\hline $\begin{array}{c}\text { Pressure } \\
\text { psig } \\
-0.05 \\
5.33 \\
12.31 \\
16.44 \\
20.51 \\
20.46 \\
20.47 \\
27.05 \\
34.69 \\
34.53 \\
34.53 \\
39.70 \\
45.90 \\
45.65 \\
45.62 \\
50.10 \\
53.47 \\
53.29 \\
53.21 \\
50.49 \\
46.14 \\
46.13 \\
44.97 \\
38.98 \\
33.99 \\
33.96 \\
26.83 \\
21.88 \\
21.90 \\
16.69 \\
11.74 \\
11.77 \\
5.05 \\
0.02 \\
-0.04 \\
-0.02 \\
-0.02 \\
0.02\end{array}$ & $\begin{array}{c}\text { Maximum } \\
\text { xStrain } \\
0.0002 \\
0.0027 \\
0.0048 \\
0.0057 \\
0.0073 \\
0.0076 \\
0.0075 \\
0.0096 \\
0.0089 \\
0.0087 \\
0.0088 \\
0.0102 \\
0.0146 \\
0.0153 \\
0.0154 \\
0.0183 \\
0.0211 \\
0.0214 \\
0.0214 \\
0.0205 \\
0.0184 \\
0.0181 \\
0.0164 \\
0.0137 \\
0.0114 \\
0.0111 \\
0.0082 \\
0.0065 \\
0.0065 \\
0.0051 \\
0.0047 \\
0.0047 \\
0.0044 \\
0.0049 \\
0.0059 \\
0.0053 \\
0.0048 \\
0.0035\end{array}$ & $\begin{array}{r}\text { Minimum } \\
\text { XStra1n } \\
-0.0003 \\
-0.0004 \\
-0.0002 \\
0.0001 \\
0.0006 \\
0.0007 \\
0.0006 \\
0.0017 \\
0.0016 \\
0.0013 \\
0.0012 \\
-0.0003 \\
-0.0011 \\
-0.0009 \\
-0.0010 \\
-0.0018 \\
-0.0032 \\
-0.0032 \\
-0.0035 \\
-0.0033 \\
-0.0024 \\
-0.0024 \\
-0.0024 \\
-0.0010 \\
0.0002 \\
0.0001 \\
0.0021 \\
0.0031 \\
0.0031 \\
0.0041 \\
0.0043 \\
0.0041 \\
0.0037 \\
0.0020 \\
0.0017 \\
0.0027 \\
0.0027 \\
0.0020\end{array}$ & $\begin{array}{c}\text { Angle } \\
\text { Degrees } \\
22.41 \\
-20.81 \\
-21.43 \\
-22.71 \\
-24.98 \\
-24.34 \\
-24.38 \\
-28.08 \\
39.36 \\
36.09 \\
35.48 \\
26.23 \\
18.12 \\
17.32 \\
17.26 \\
16.17 \\
15.63 \\
16.08 \\
15.81 \\
15.39 \\
14.90 \\
14.83 \\
12.30 \\
11.66 \\
11.50 \\
10.91 \\
11.66 \\
9.98 \\
12.40 \\
24.72 \\
-20.71 \\
-8.36 \\
3.09 \\
-16.77 \\
-21.05 \\
-25.40 \\
-27.50 \\
-18.22\end{array}$ & $\begin{array}{c}\text { Pressure } \\
\text { ps } 1 \text { g } \\
9.89 \\
19.55 \\
29.57 \\
39.42 \\
49.16 \\
54.50 \\
59.57 \\
64.20 \\
69.32 \\
74.16 \\
80.16 \\
85.61 \\
90.58 \\
95.69 \\
100.92 \\
103.25 \\
106.11 \\
108.31 \\
111.08 \\
113.24 \\
117.83 \\
120.92 \\
123.28 \\
122.97 \\
125.82 \\
125.60 \\
127.84 \\
130.19 \\
132.53 \\
135.33 \\
134.42 \\
138.35 \\
140.16 \\
142.63 \\
145.78 \\
142.52 \\
0.22\end{array}$ & $\begin{array}{l}\text { Maximum } \\
\text { Xstrain } \\
0.0051 \\
0.0053 \\
0.0100 \\
0.0154 \\
0.0215 \\
0.0255 \\
0.0294 \\
0.0333 \\
0.0374 \\
0.0421 \\
0.0486 \\
0.0533 \\
0.0578 \\
0.0616 \\
0.0657 \\
0.0682 \\
0.0698 \\
0.0707 \\
0.0727 \\
0.0744 \\
0.0781 \\
0.0795 \\
0.0809 \\
0.0810 \\
0.0829 \\
0.0824 \\
0.0829 \\
0.0829 \\
0.0829 \\
0.0840 \\
0.0834 \\
0.0851 \\
0.0856 \\
0.0845 \\
0.0856 \\
0.0826 \\
0.0045\end{array}$ & $\begin{array}{r}\text { Minimum } \\
\text { XStrain } \\
0.0002 \\
0.0004 \\
-0.0024 \\
-0.0053 \\
-0.0074 \\
-0.0088 \\
-0.0105 \\
-0.0138 \\
-0.0174 \\
-0.0223 \\
-0.0268 \\
-0.0296 \\
-0.0319 \\
-0.0341 \\
-0.0353 \\
-0.0358 \\
-0.0369 \\
-0.0376 \\
-0.0381 \\
-0.0394 \\
-0.0418 \\
-0.0441 \\
-0.0465 \\
-0.0472 \\
-0.0495 \\
-0.0494 \\
-0.0500 \\
-0.0513 \\
-0.0529 \\
-0.0554 \\
-0.0549 \\
-0.0561 \\
-0.0569 \\
-0.0651 \\
-0.0548 \\
-0.0510 \\
-0.0075\end{array}$ & $\begin{array}{c}\text { Angle } \\
\text { Degrees } \\
-21.20 \\
25.72 \\
16.39 \\
14.34 \\
14.40 \\
14.69 \\
15.14 \\
15.01 \\
15.61 \\
17.00 \\
18.90 \\
20.17 \\
21.23 \\
21.71 \\
22.40 \\
22.70 \\
22.71 \\
22.63 \\
22.93 \\
23.08 \\
23.35 \\
23.09 \\
22.79 \\
22.60 \\
22.43 \\
22.33 \\
22.34 \\
22.14 \\
21.78 \\
22.16 \\
22.23 \\
22.94 \\
23.32 \\
22.33 \\
24.11 \\
24.27 \\
13.35\end{array}$ \\
\hline
\end{tabular}

High Pressure test 


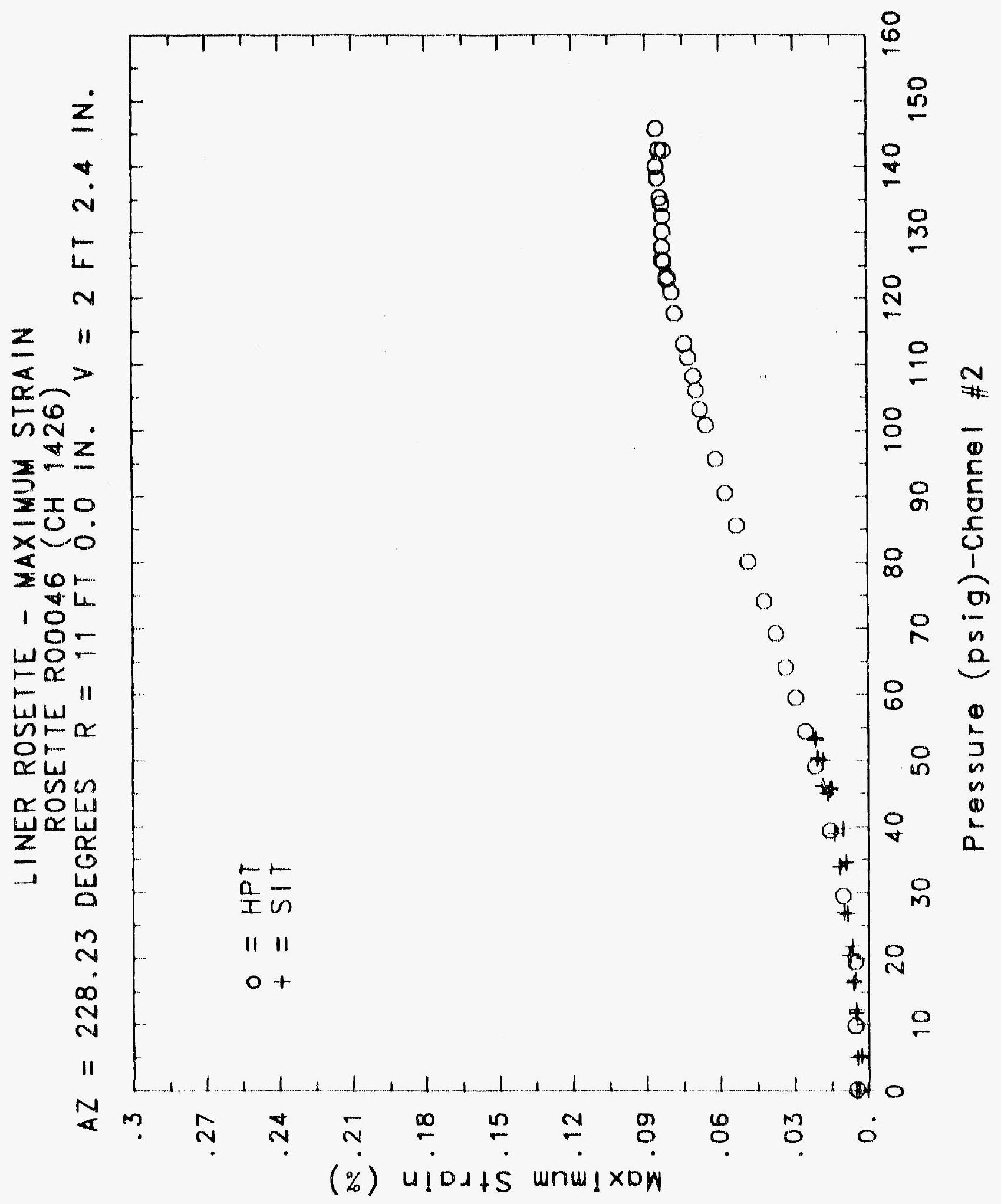




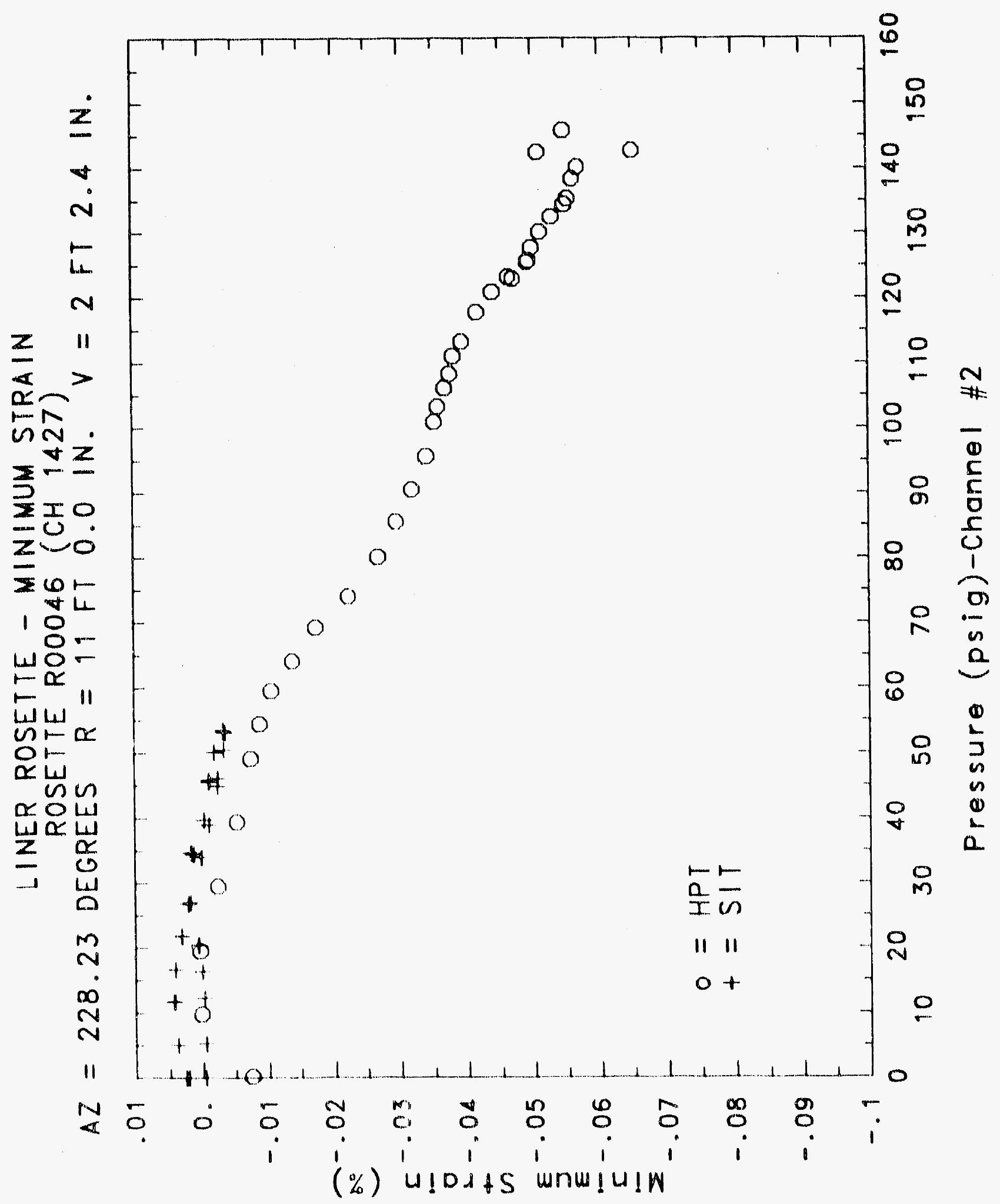




\section{Reinforced Concrete Test Data}

\section{Rosette 47 Channels $1429 \quad 1440 \quad 1441$}

Structural InTEgrity TEST

\begin{tabular}{|c|c|c|c|c|c|c|c|}
\hline $\begin{array}{c}\text { Pressure } \\
\text { Ps Ig } \\
-0.05 \\
5.33 \\
12.31 \\
16.44 \\
20.51 \\
20.46 \\
20.47 \\
27.05 \\
34.69 \\
34.53 \\
34.53 \\
39.70 \\
45.90 \\
45.65 \\
45.62 \\
50.10 \\
53.47 \\
53.29 \\
53.21 \\
50.49 \\
46.14 \\
46.13 \\
44.97 \\
38.98 \\
33.99 \\
33.96 \\
26.83 \\
21.88 \\
21.90 \\
16.69 \\
11.74 \\
11.77 \\
5.01 \\
0.02 \\
-0.04 \\
-0.02 \\
-0.02 \\
0.02\end{array}$ & $\begin{array}{l}\text { Maximum } \\
\text { \%Strain } \\
0.0004 \\
0.0025 \\
0.0058 \\
0.0085 \\
0.0127 \\
0.0136 \\
0.0140 \\
0.0219 \\
0.0341 \\
0.0358 \\
0.0361 \\
0.0424 \\
0.0537 \\
0.0555 \\
0.0564 \\
0.0643 \\
0.0736 \\
0.0755 \\
0.0761 \\
0.0750 \\
0.0719 \\
0.0718 \\
0.0722 \\
0.0676 \\
0.0633 \\
0.0633 \\
0.0566 \\
0.0518 \\
0.0517 \\
0.0465 \\
0.0415 \\
0.0410 \\
0.0341 \\
0.0289 \\
0.0328 \\
0.0378 \\
0.0385 \\
0.0366\end{array}$ & $\begin{array}{l}\text { Minimum } \\
\text { xStrain } \\
0.0002 \\
0.0010 \\
0.0014 \\
0.0020 \\
0.0027 \\
0.0035 \\
0.0036 \\
0.0055 \\
0.0083 \\
0.0093 \\
0.0094 \\
0.0107 \\
0.0133 \\
0.0137 \\
0.0138 \\
0.0148 \\
0.0159 \\
0.0161 \\
0.0161 \\
0.0161 \\
0.0156 \\
0.0154 \\
0.0159 \\
0.0149 \\
0.0141 \\
0.0143 \\
0.0130 \\
0.0120 \\
0.0121 \\
0.0112 \\
0.0104 \\
0.0100 \\
0.0087 \\
0.0079 \\
0.0081 \\
0.0073 \\
0.0065 \\
0.0054\end{array}$ & $\begin{array}{c}\text { Angle } \\
\text { Degrees } \\
29.11 \\
14.48 \\
13.88 \\
11.86 \\
12.72 \\
12.95 \\
13.33 \\
13.34 \\
11.74 \\
11.53 \\
11.80 \\
11.00 \\
8.41 \\
8.11 \\
7.61 \\
6.04 \\
4.17 \\
3.81 \\
3.75 \\
3.68 \\
3.54 \\
3.65 \\
2.78 \\
2.52 \\
2.23 \\
2.11 \\
1.51 \\
0.89 \\
0.96 \\
0.22 \\
-0.76 \\
-1.13 \\
-3.15 \\
-5.44 \\
-10.24 \\
-12.68 \\
-13.27 \\
-12.64\end{array}$ & $\begin{array}{c}\text { Pressure } \\
\text { Psig } \\
9.89 \\
19.55 \\
29.57 \\
39.42 \\
49.16 \\
54.50 \\
59.57 \\
64.20 \\
69.32 \\
74.16 \\
80.16 \\
85.61 \\
90.58 \\
95.69 \\
100.92 \\
103.25 \\
106.11 \\
108.31 \\
111.08 \\
113.24 \\
117.83 \\
120.92 \\
123.28 \\
122.97 \\
125.82 \\
125.60 \\
127.84 \\
130.19 \\
132.53 \\
135.33 \\
134.42 \\
138.35 \\
140.16 \\
142.63 \\
145.78 \\
142.52 \\
0.22\end{array}$ & $\begin{array}{c}\text { Maximum } \\
\text { xStrain } \\
0.0111 \\
0.0220 \\
0.0330 \\
0.0433 \\
0.0530 \\
0.0590 \\
0.0746 \\
0.0927 \\
0.1072 \\
0.1222 \\
0.1460 \\
0.1659 \\
0.1735 \\
0.1856 \\
0.2068 \\
0.2119 \\
0.2264 \\
0.2336 \\
0.2478 \\
0.2567 \\
0.2814 \\
0.3017 \\
0.3154 \\
0.3187 \\
0.3288 \\
0.3325 \\
0.3462 \\
0.3780 \\
0.4095 \\
0.4403 \\
0.4442 \\
0.4664 \\
0.5027 \\
0.5721 \\
0.6337 \\
0.6474 \\
0.4469\end{array}$ & $\begin{array}{l}\text { Minimum } \\
\text { XStrain } \\
0.0026 \\
0.0042 \\
0.0060 \\
0.0082 \\
0.0104 \\
0.0115 \\
0.0134 \\
0.0142 \\
0.0142 \\
0.0141 \\
0.0135 \\
0.0135 \\
0.0144 \\
0.0157 \\
0.0170 \\
0.0178 \\
0.0191 \\
0.0207 \\
0.0234 \\
0.0255 \\
0.0307 \\
0.0351 \\
0.0398 \\
0.0405 \\
0.0437 \\
0.0440 \\
0.0463 \\
0.0508 \\
0.0557 \\
0.0641 \\
0.0646 \\
0.0731 \\
0.0782 \\
0.0820 \\
0.0867 \\
0.0857 \\
0.0164\end{array}$ & $\begin{array}{c}\text { Angle } \\
\text { Degrees } \\
7.31 \\
7.40 \\
7.18 \\
7.28 \\
7.00 \\
7.06 \\
2.10 \\
-1.78 \\
-2.99 \\
-4.68 \\
-5.74 \\
-5.59 \\
-4.74 \\
-3.37 \\
-2.65 \\
-2.11 \\
-1.50 \\
-0.85 \\
-0.58 \\
-0.53 \\
-0.59 \\
-0.25 \\
0.01 \\
0.00 \\
0.69 \\
0.59 \\
0.43 \\
0.83 \\
0.61 \\
1.95 \\
2.34 \\
3.81 \\
4.20 \\
3.75 \\
3.94 \\
3.88 \\
0.96\end{array}$ \\
\hline
\end{tabular}

high Pressure test

grees

7.40

.18

7.28

2.10

.78

4.68

$-5.74$

$-5.59$

$-4.74$

37

$-2.11$

1.50

0.85

0.58

$-0.59$

0.25

0.00

.69

43

0.83

0.61

.81

4.20

.75

.94

3.82
0.96 


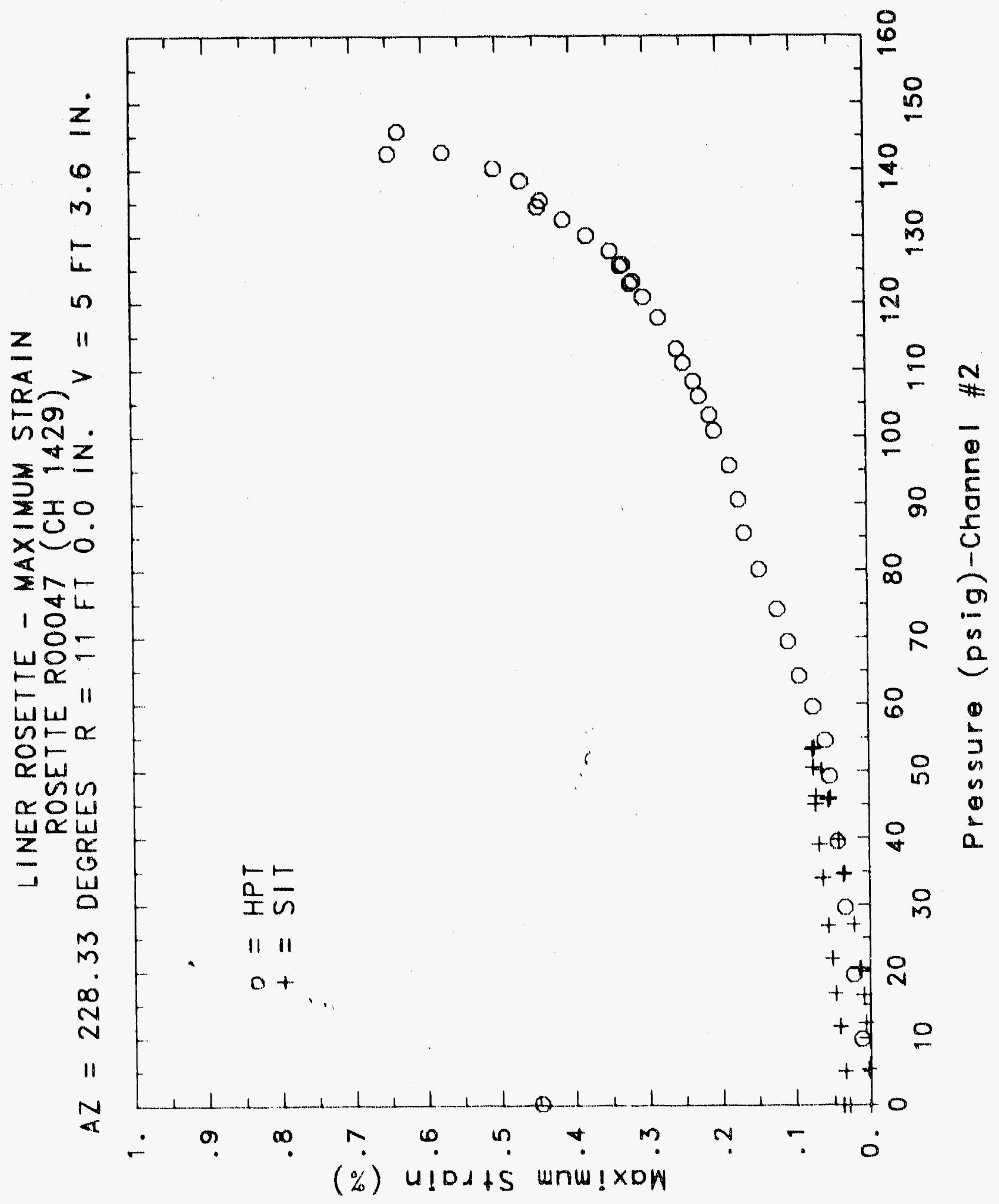




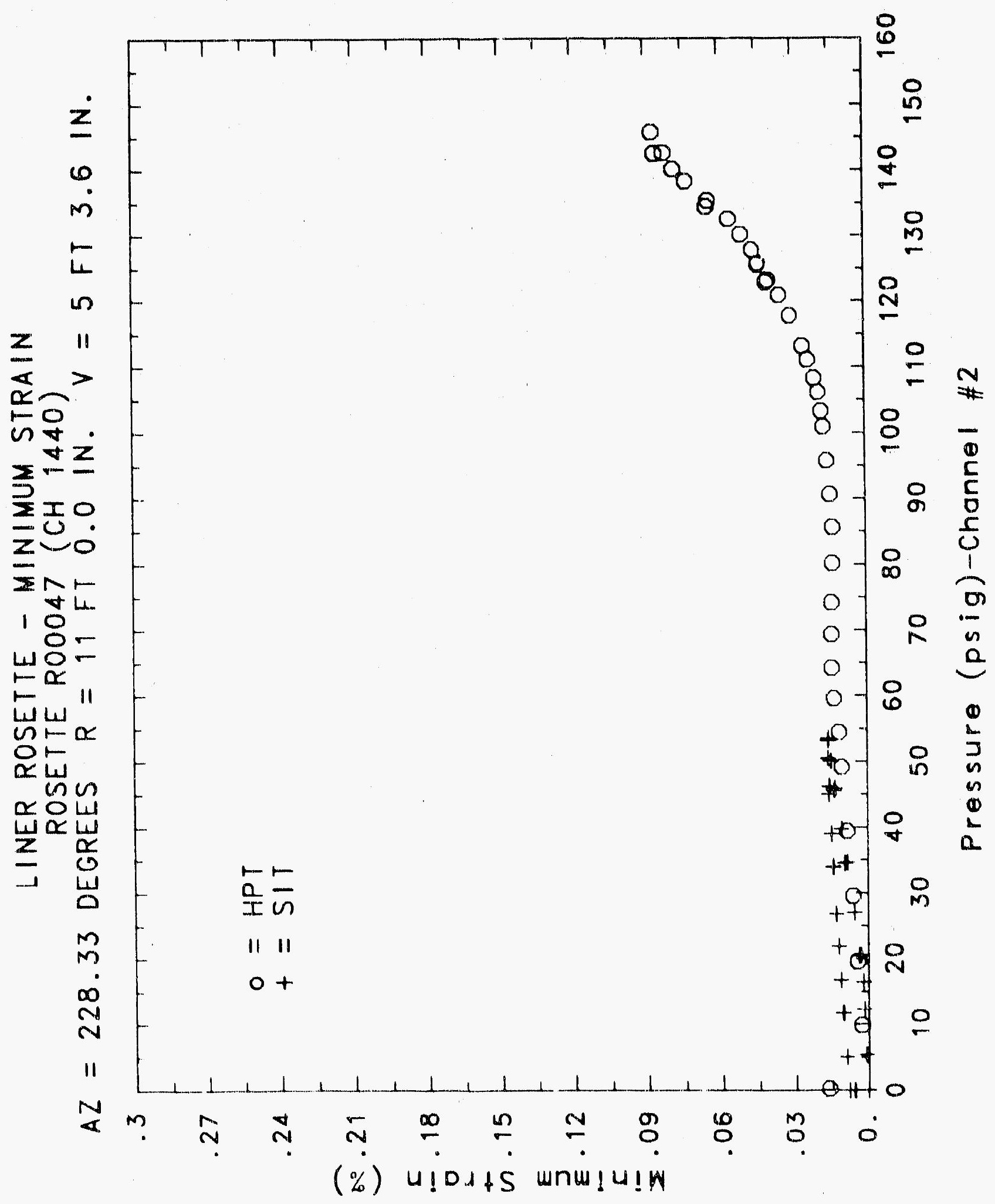




\section{Reinforced Concrete Test Data}

Rosette 48 Channels $1442 \quad 1443 \quad 1444$

STRUCTURAL INTEGRITY TEST

\begin{tabular}{|c|c|c|c|c|c|c|c|}
\hline $\begin{array}{c}\text { Pressure } \\
\text { psig } \\
-0.05 \\
5.33 \\
12.31 \\
16.44 \\
20.51 \\
20.46 \\
20.47 \\
27.05 \\
34.69 \\
34.53 \\
34.53 \\
39.70 \\
45.90 \\
45.65 \\
45.62 \\
50.10 \\
53.47 \\
53.29 \\
53.21 \\
50.49 \\
46.14 \\
46.13 \\
44.97 \\
38.98 \\
33.99 \\
33.96 \\
26.83 \\
21.88 \\
21.90 \\
16.69 \\
11.74 \\
11.77 \\
5.05 \\
0.02 \\
-0.04 \\
-0.02 \\
-0.02 \\
0.02\end{array}$ & $\begin{array}{c}\text { Maximum } \\
\text { xStrain } \\
0.0007 \\
0.0029 \\
0.0068 \\
0.0099 \\
0.0154 \\
0.0165 \\
0.0172 \\
0.0252 \\
0.0371 \\
0.0380 \\
0.0380 \\
0.0427 \\
0.0510 \\
0.0518 \\
0.0522 \\
0.0579 \\
0.0652 \\
0.0667 \\
0.0668 \\
0.0656 \\
0.0631 \\
0.0628 \\
0.0644 \\
0.0603 \\
0.0561 \\
0.0562 \\
0.0494 \\
0.0443 \\
0.0439 \\
0.0383 \\
0.0324 \\
0.0311 \\
0.0228 \\
0.0163 \\
0.0154 \\
0.0155 \\
0.0146 \\
0.0138\end{array}$ & $\begin{array}{l}\text { Minimum } \\
\text { xStrain } \\
0.0000 \\
0.0014 \\
0.0031 \\
0.0042 \\
0.0064 \\
0.0071 \\
0.0073 \\
0.0130 \\
0.0218 \\
0.0227 \\
0.0231 \\
0.0266 \\
0.0360 \\
0.0373 \\
0.0374 \\
0.0408 \\
0.0440 \\
0.0443 \\
0.0444 \\
0.0437 \\
0.0415 \\
0.0412 \\
0.0398 \\
0.0365 \\
0.0335 \\
0.0331 \\
0.0286 \\
0.0253 \\
0.0253 \\
0.0220 \\
0.0187 \\
0.0187 \\
0.0141 \\
0.0106 \\
0.0109 \\
0.0107 \\
0.0102 \\
0.0091\end{array}$ & $\begin{array}{c}\text { Angle } \\
\text { Degrees } \\
30.92 \\
28.13 \\
26.21 \\
25.47 \\
20.56 \\
20.68 \\
21.47 \\
19.45 \\
25.60 \\
26.72 \\
27.07 \\
29.27 \\
43.57 \\
-43.97 \\
-43.02 \\
-41.78 \\
-43.04 \\
-43.09 \\
-42.87 \\
-42.16 \\
-41.22 \\
-41.23 \\
-40.54 \\
-39.41 \\
-38.58 \\
-38.90 \\
-38.12 \\
-37.18 \\
-37.30 \\
-36.31 \\
-35.54 \\
-34.78 \\
-32.64 \\
-28.89 \\
-24.89 \\
-25.73 \\
-26.32 \\
-32.74\end{array}$ & $\begin{array}{c}\text { Pressule } \\
\text { psig } \\
9.89 \\
19.55 \\
29.57 \\
39.42 \\
49.16 \\
54.50 \\
59.57 \\
64.20 \\
69.32 \\
74.16 \\
80.16 \\
85.61 \\
90.58 \\
95.69 \\
100.92 \\
103.25 \\
106.11 \\
108.31 \\
111.08 \\
113.24 \\
117.83 \\
120.92 \\
123.28 \\
122.97 \\
125.82 \\
125.60 \\
127.84 \\
130.19 \\
132.53 \\
135.33 \\
134.42 \\
138.35 \\
140.16 \\
142.63 \\
145.78 \\
142.52 \\
0.22\end{array}$ & $\begin{array}{l}\text { Maximum } \\
\text { XStrain } \\
0.0126 \\
0.0253 \\
0.0388 \\
0.0506 \\
0.0606 \\
0.0671 \\
0.0722 \\
0.0786 \\
0.0846 \\
0.0919 \\
0.0994 \\
0.1048 \\
0.1471 \\
0.2213 \\
0.3031 \\
0.3315 \\
0.3590 \\
0.3794 \\
0.4074 \\
0.4276 \\
0.4628 \\
0.4865 \\
0.5039 \\
0.5091 \\
0.5221 \\
0.5286 \\
0.5339 \\
0.5423 \\
0.5571 \\
0.5798 \\
0.5854 \\
0.6115 \\
0.6349 \\
0.6528 \\
0.6628 \\
0.6647 \\
0.5085\end{array}$ & $\begin{array}{c}\text { Minimum } \\
\text { xStrain } \\
0.0069 \\
0.0136 \\
0.0201 \\
0.0265 \\
0.0327 \\
0.0362 \\
0.0393 \\
0.0438 \\
0.0482 \\
0.0529 \\
0.0628 \\
0.0749 \\
0.0833 \\
0.0814 \\
0.0773 \\
0.0768 \\
0.0756 \\
0.0753 \\
0.0745 \\
0.0742 \\
0.0743 \\
0.0741 \\
0.0737 \\
0.0733 \\
0.0724 \\
0.0703 \\
0.0701 \\
0.0689 \\
0.0677 \\
0.0672 \\
0.0664 \\
0.0666 \\
0.0664 \\
0.0684 \\
0.0731 \\
0.0708 \\
-0.0481\end{array}$ & $\begin{array}{c}\text { Angle } \\
\text { Degrees } \\
\text {-41.69 } \\
-40.76 \\
-39.25 \\
-39.39 \\
-40.16 \\
-40.66 \\
-39.14 \\
-40.11 \\
-40.01 \\
-42.00 \\
40.52 \\
40.25 \\
-22.82 \\
-18.43 \\
-16.62 \\
-15.93 \\
-15.43 \\
-15.08 \\
-14.38 \\
-13.90 \\
-12.88 \\
-12.22 \\
-11.85 \\
-11.73 \\
-11.37 \\
-11.75 \\
-11.66 \\
-11.43 \\
-11.27 \\
-11.08 \\
-11.11 \\
-11.03 \\
-10.85 \\
-10.88 \\
-10.73 \\
-10.76 \\
-5.41\end{array}$ \\
\hline
\end{tabular}

High Pressure test 


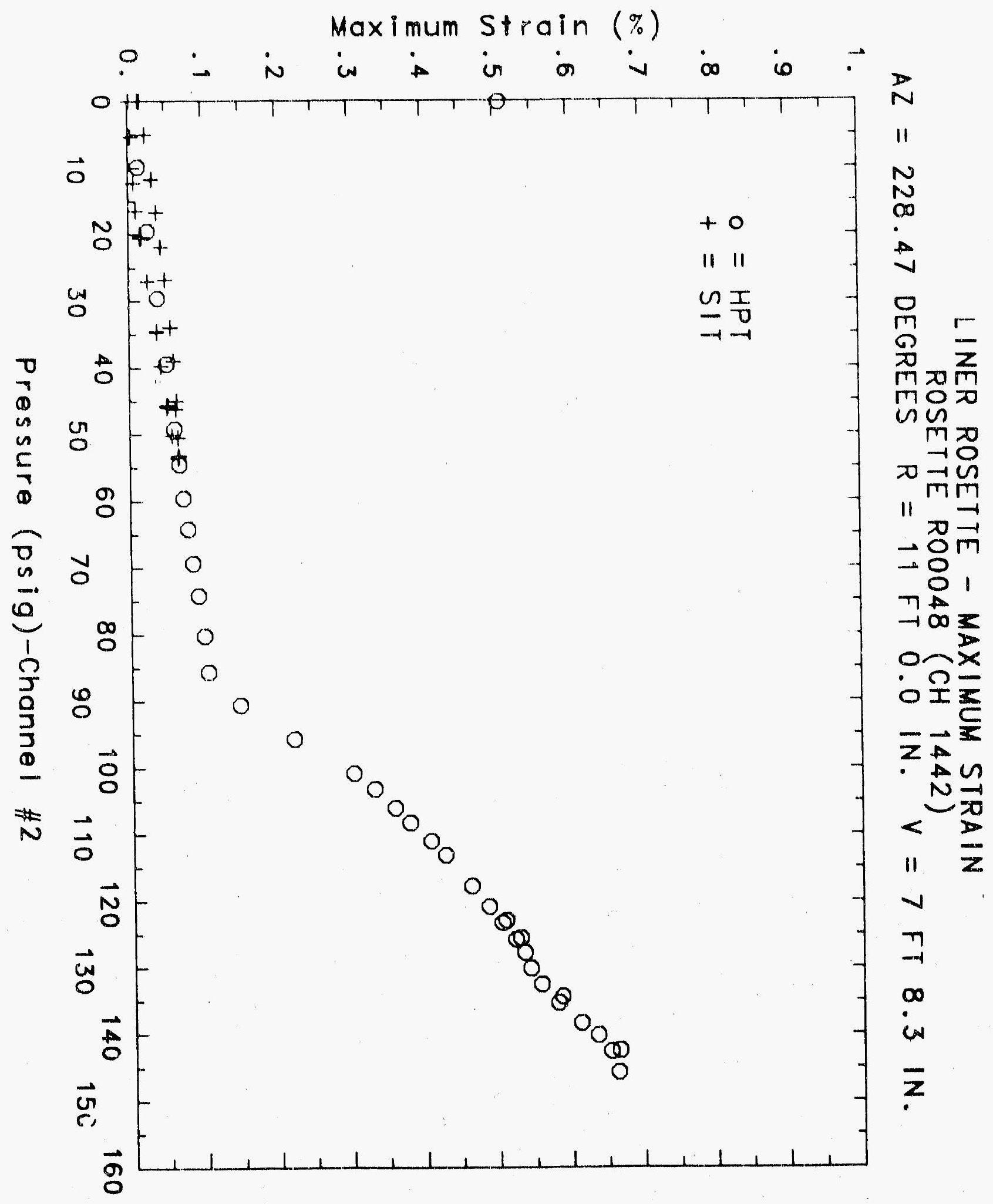




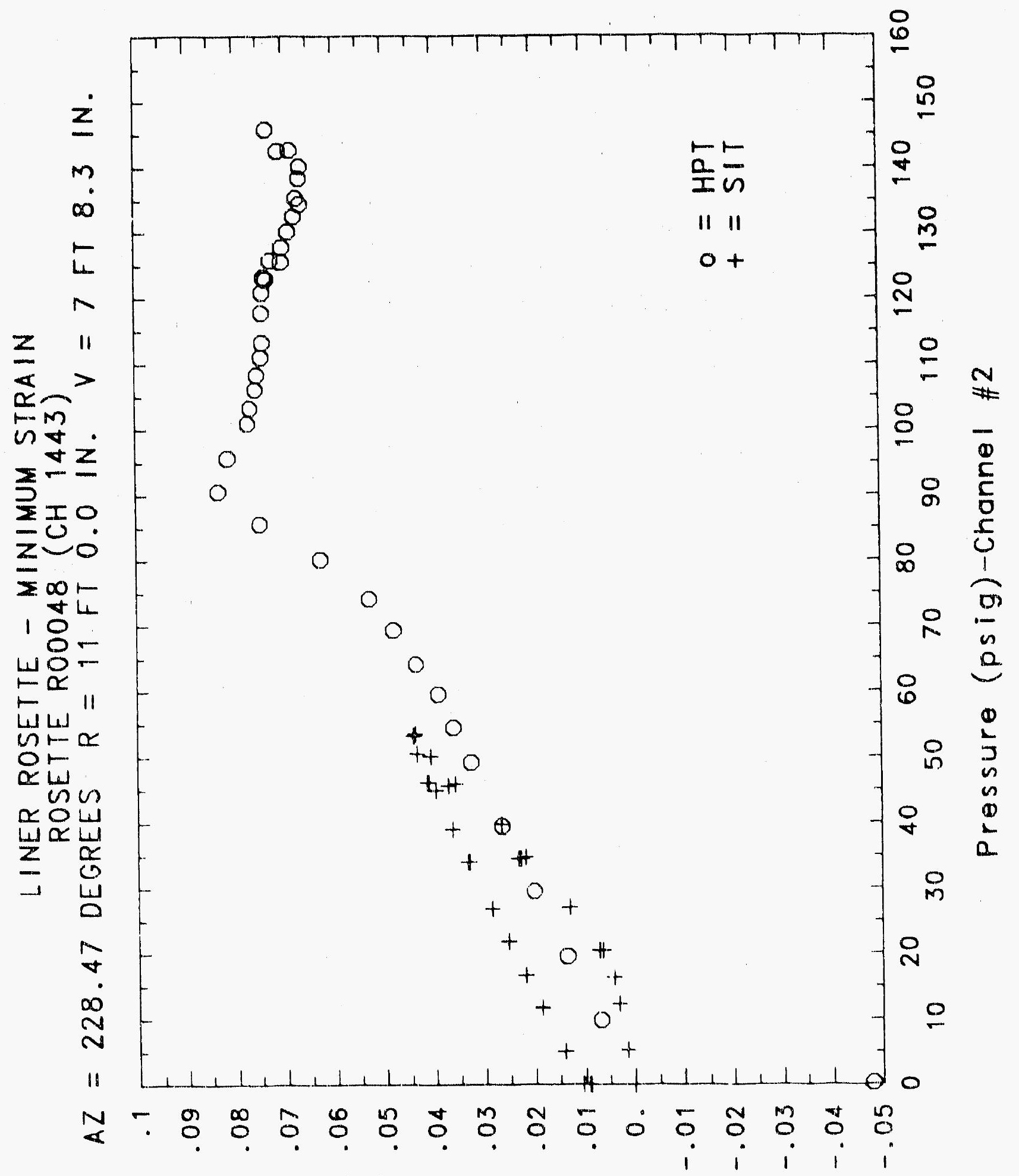

(\%) $4 ! D d+S$ WnW!U!W 


\section{Reinforced Concrete Test Data}

Rosette 49 Channels $1445 \quad 1446 \quad 1447$

STRUCTURAL INTEgRITY TEST

\begin{tabular}{|c|c|c|c|c|c|c|c|}
\hline Pressure & Maximum & Minimum & Angle & Pressure & Maximum & Minimum & Angle \\
\hline psig & xstrain & strain & Degrees & psig & xstrain & Strain & Degrees \\
\hline-0.05 & 0.0002 & -0.0003 & -15.18 & 9.89 & 0.0104 & 0.0041 & -1.64 \\
\hline 5.33 & 0.0024 & 0.0011 & 2.01 & 19.55 & 0.0200 & 0.0074 & -3.26 \\
\hline 12.31 & 0.0001 & 0.0026 & -0.01 & 29.57 & 0.0298 & 0.0104 & $-4.4 !$ \\
\hline .44 & 0.0090 & 0.0035 & -2.66 & 39.42 & 0.0388 & 0.0132 & -4.38 \\
\hline .51 & 0.0133 & 0.0046 & -5.05 & 49.16 & 0.0479 & 0.0158 & -4.92 \\
\hline .46 & 0.0140 & 0.0047 & -4.37 & 54.50 & 0.0531 & 0.0173 & -5.26 \\
\hline 20.47 & 0.0143 & 0.0046 & -3.23 & 59.57 & 0.0576 & 0.0186 & -4.97 \\
\hline & 222 & 0.0064 & -4.88 & 64.20 & 0.0623 & 0.0198 & -5.05 \\
\hline 34.69 & 324 & 093 & -5.52 & .32 & 671 & 0.0209 & -6.02 \\
\hline .53 & 0.0335 & 0.0096 & -5.63 & 74.16 & 0.0714 & 0.0219 & -6.97 \\
\hline .53 & 0.0336 & 0.0099 & -5.61 & 80.16 & 0.0756 & 0.0237 & -7.44 \\
\hline .70 & 0.0392 & 0.0114 & -5.37 & 85.61 & 0.0792 & 0.0249 & -8.09 \\
\hline .90 & 0.0471 & 0.0138 & -5.13 & 90.58 & 0.0823 & 0.0259 & -8.67 \\
\hline .65 & 0.0477 & 0.0140 & -5.07 & 95.69 & 0.0851 & 0.0268 & -9.24 \\
\hline .62 & 0.0477 & 0.0141 & -5.16 & 100.92 & 0. & 0.0274 & -9.97 \\
\hline .10 & 0.1 & 0.0153 & -5.1 & 103. & & & -10.23 \\
\hline 53.47 & 0.1 & 0.1 & -5.0 & & & 0.1 & -10.53 \\
\hline 53.29 & 0.6 & 0.0166 & -5.09 & 108.31 & 0.0909 & 0.0 & -10.72 \\
\hline .21 & 0.0565 & 168 & -5.51 & 111.08 & .0918 & 0. & -11.17 \\
\hline 50.49 & 0.0553 & 0.0162 & -5.3 & 113.24 & 0.0923 & 0.0 & -11.35 \\
\hline 46.14 & 0.0520 & 0.0154 & -5.19 & 117.83 & 0.0925 & 0.0288 & -12.25 \\
\hline 46.13 & 0.0519 & 0.0 & -5.46 & 120.92 & 0.0928 & 0.0289 & -13.00 \\
\hline 44.97 & 0.0514 & & -5. & 123.28 & 928 & 0.0292 & -13.44 \\
\hline 38.98 & 0.0468 & 0.0 & -5.61 & 122.97 & 923 & 0.0293 & -13.43 \\
\hline .99 & 0.0425 & 0.0 & -5.22 & 125.82 & 921 & 0.0295 & -13.90 \\
\hline 33.96 & 0.0425 & 0.0 & -5.06 & 125.60 & 0.0913 & 0.0 & -13.94 \\
\hline 26.83 & 0.0351 & 0.0105 & -4.86 & 127.84 & 0.0922 & 0.0295 & -14.02 \\
\hline 21.88 & 0.0315 & 0.0089 & -4.28 & 130.19 & 0.0923 & 0.0299 & -14.63 \\
\hline 21.90 & 0.0315 & 0.0089 & -4.4 & 132.53 & 0.0937 & 0.0309 & -14.34 \\
\hline 16.69 & 0.0267 & 0.0073 & -3.32 & 135.33 & 0.0974 & 0.0316 & -14.04 \\
\hline 11.74 & 0.0220 & 0.0058 & -2.76 & 134.42 & 0.0980 & 0.0309 & -13.82 \\
\hline 11.77 & 0.0214 & 0.0056 & -2.59 & 138.35 & 0.1046 & 0.0314 & -13.25 \\
\hline 5.05 & 0.0153 & 0.0034 & -2.61 & 140.16 & 0.1095 & 0.0312 & -12.95 \\
\hline 0.02 & 0.0106 & 0.0018 & -0.35 & 142.63 & 0.1153 & 0.0311 & -12.80 \\
\hline-0.04 & 0.0107 & 0.0028 & -4.67 & 145.78 & 0.1223 & 0.0309 & -12.72 \\
\hline-0.02 & 0.0107 & 0.0024 & -4.40 & 142.52 & 0.1204 & 0.0296 & -12.67 \\
\hline-0.02 & 0.0100 & 0.0020 & -4.30 & 0.22 & 0.0035 & -0.0194 & -32.41 \\
\hline 0.02 & & 0.0009 & -1.90 & & & & \\
\hline
\end{tabular}

High Pressuke Test 


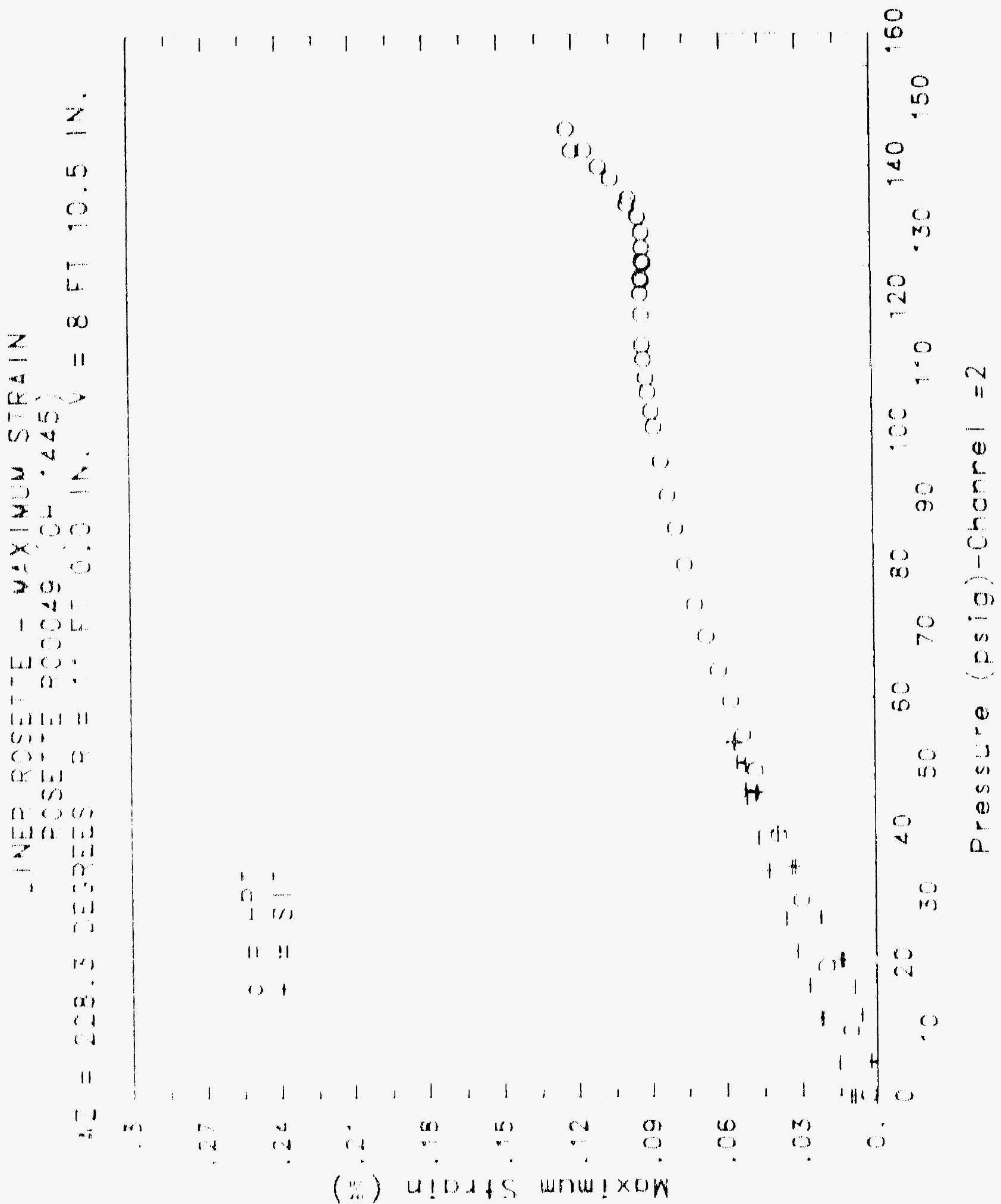




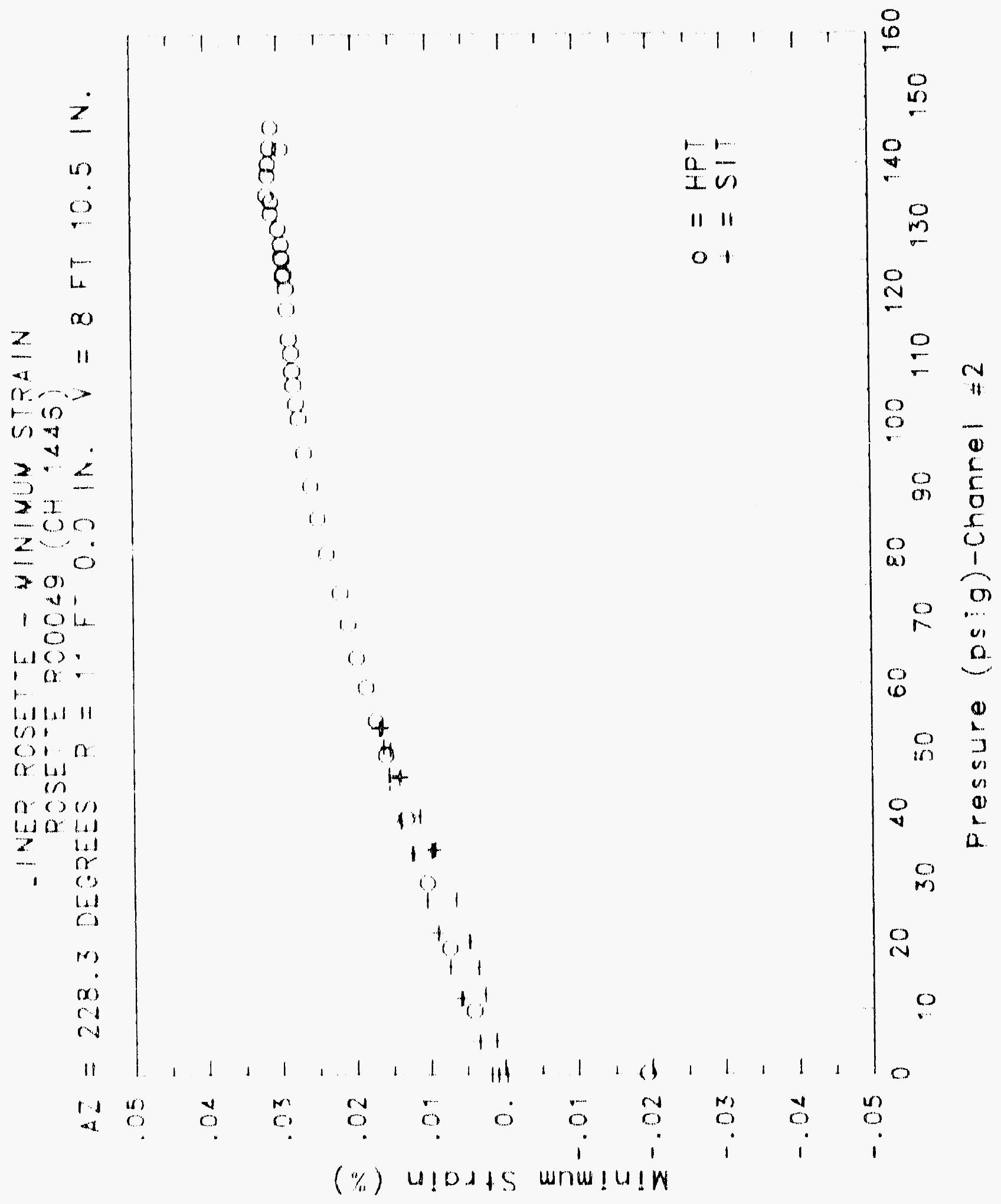




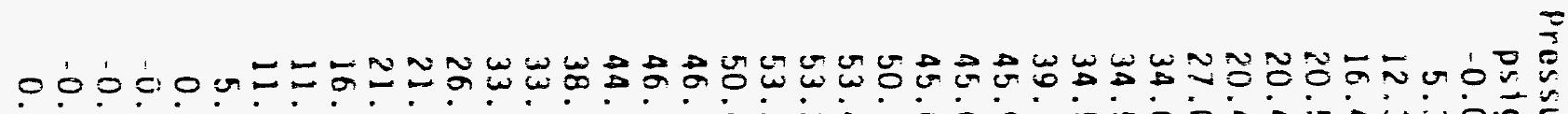

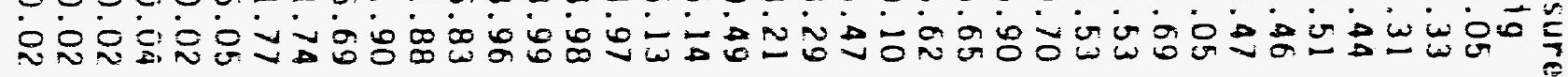

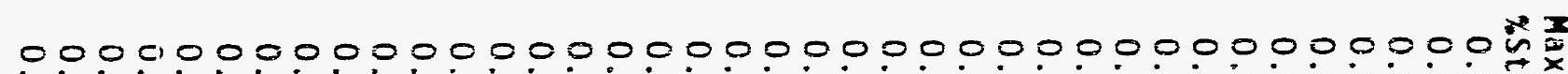

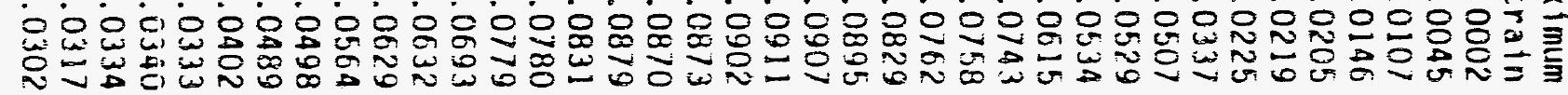

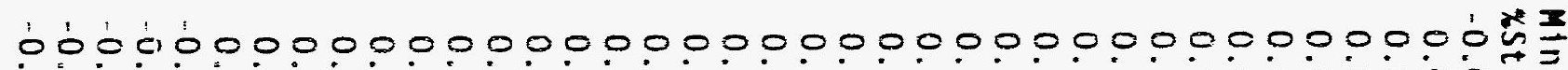

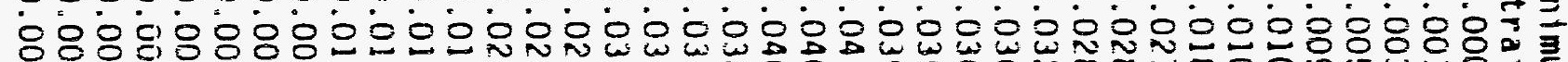

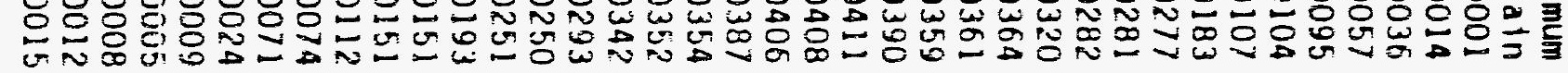

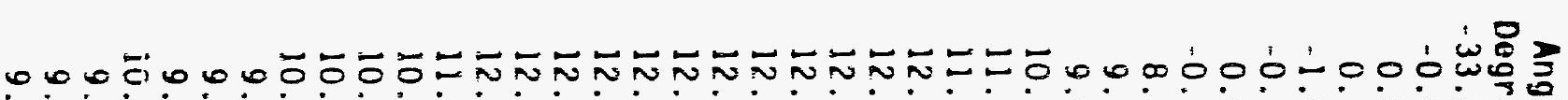

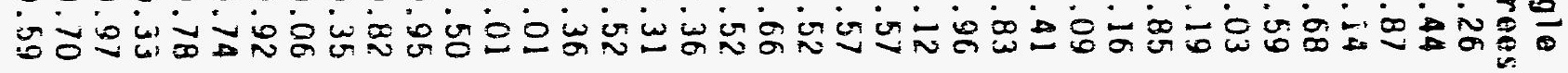

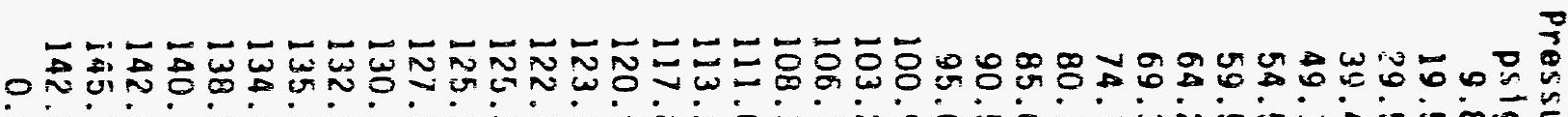

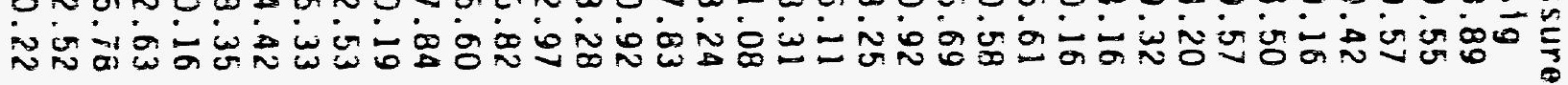
$00000000000000000000000000000000000000 \frac{1}{3}$

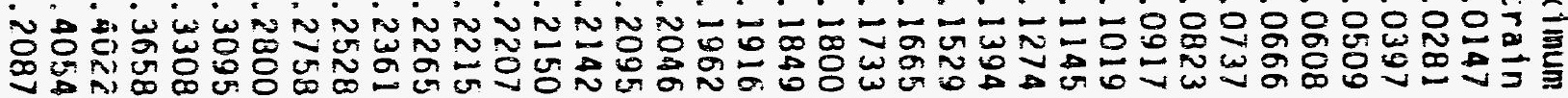

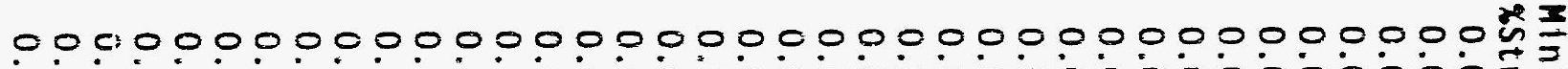

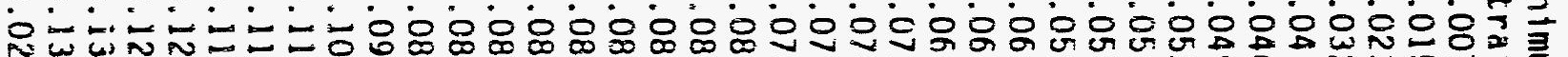

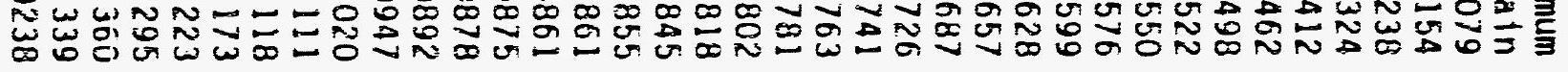

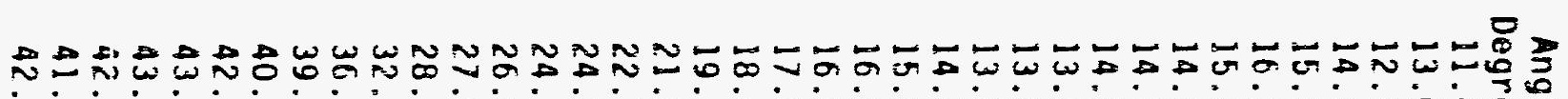

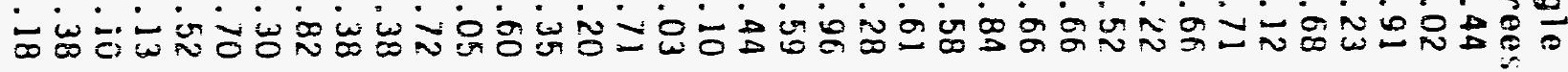




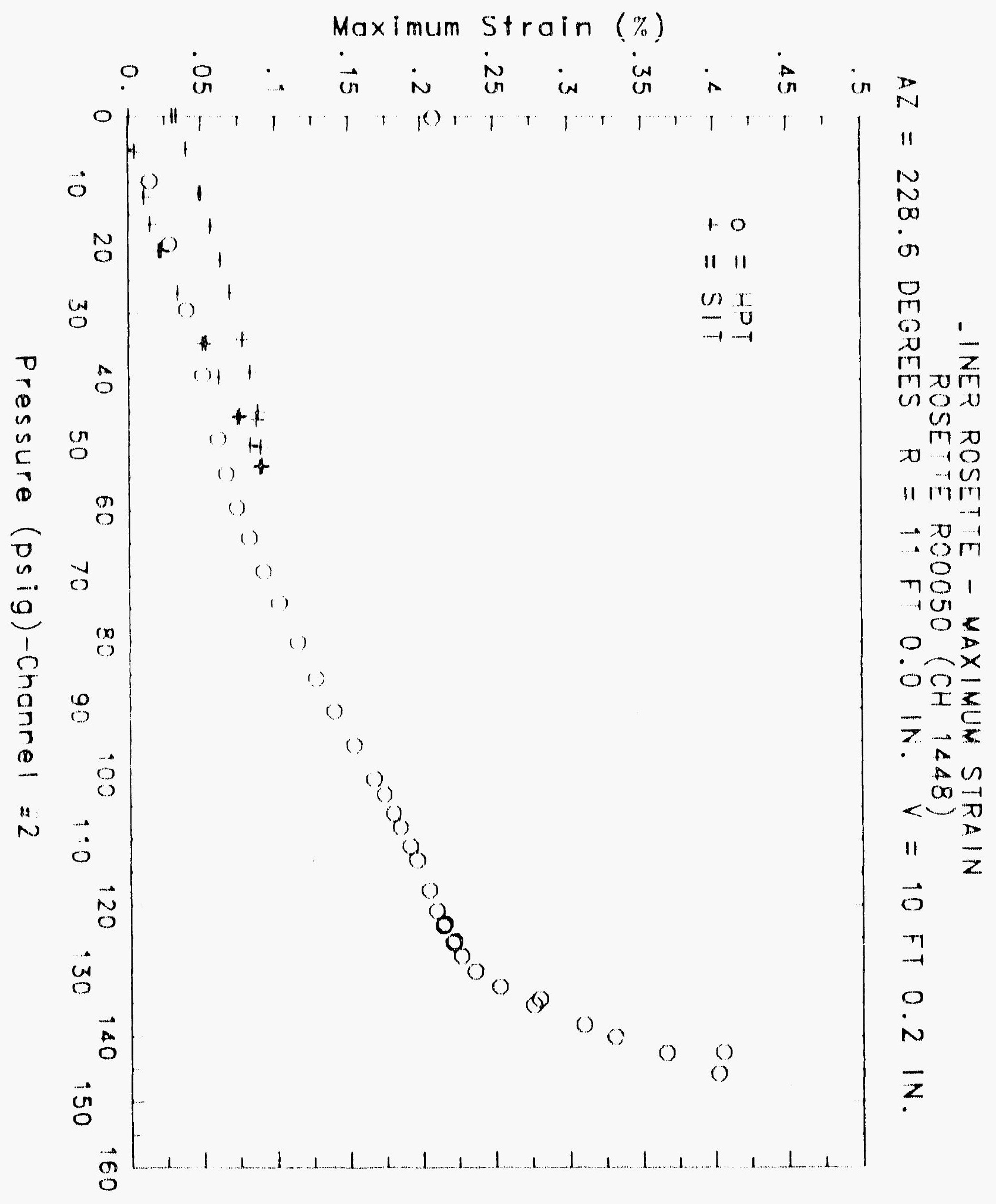




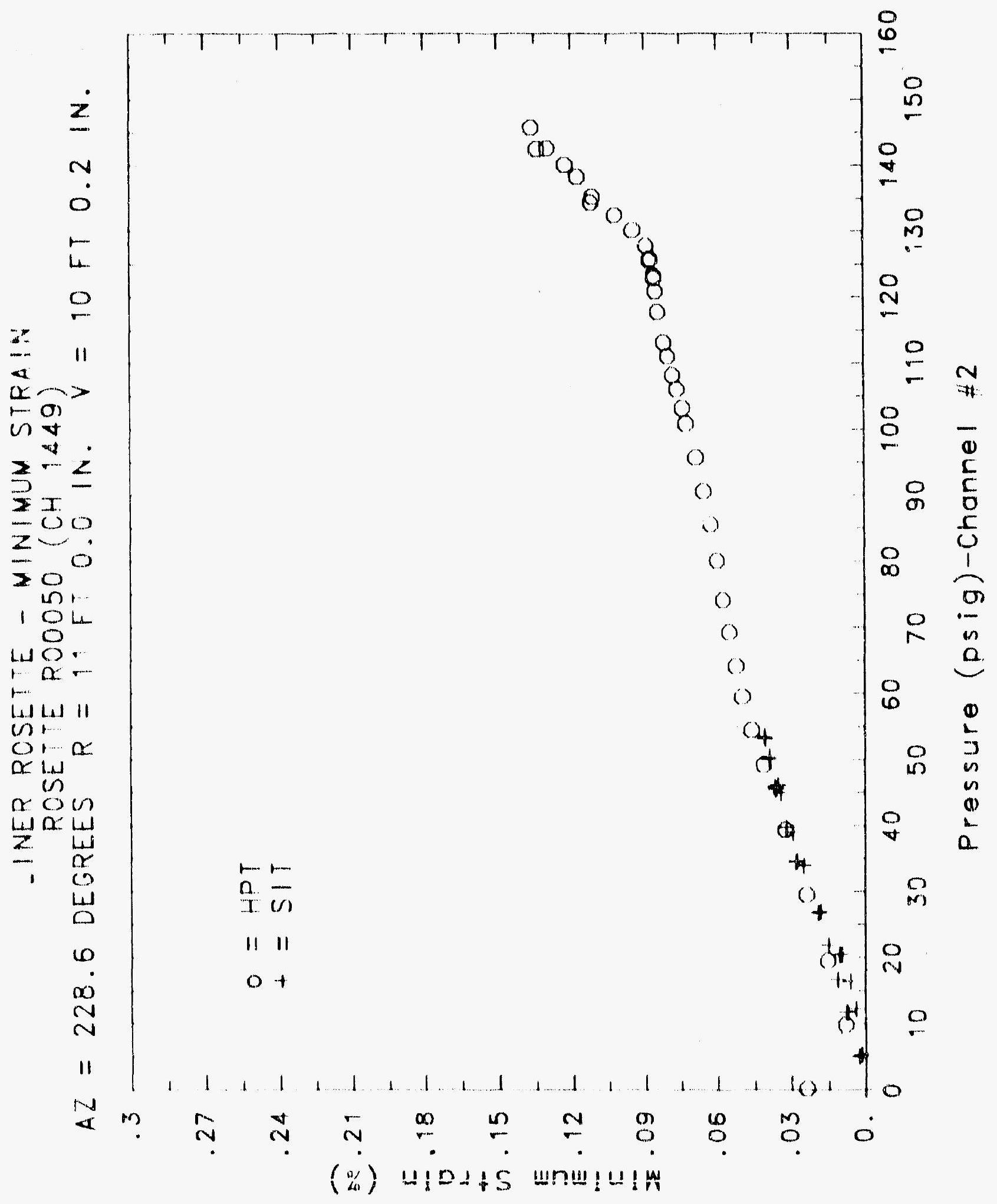




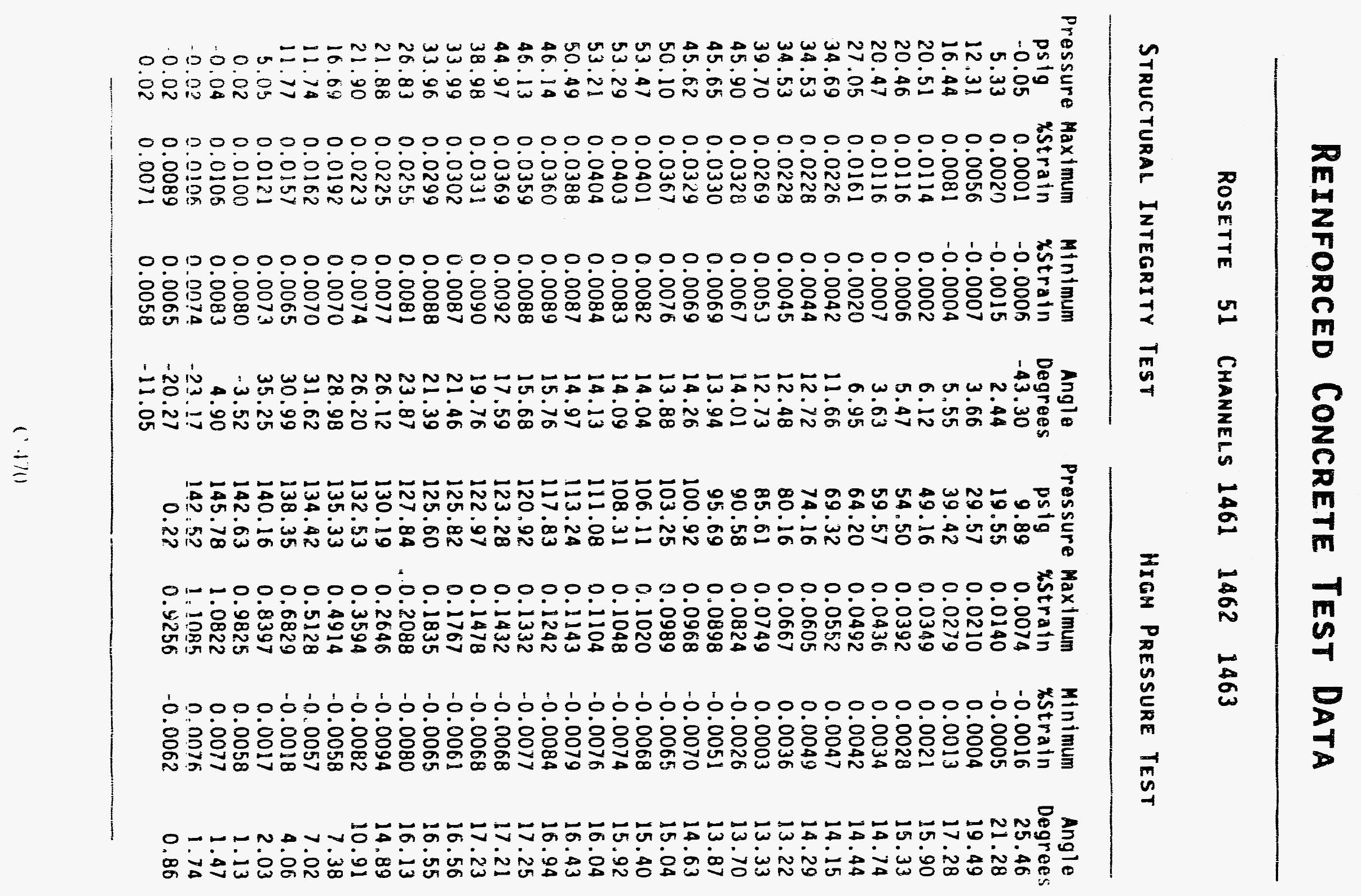




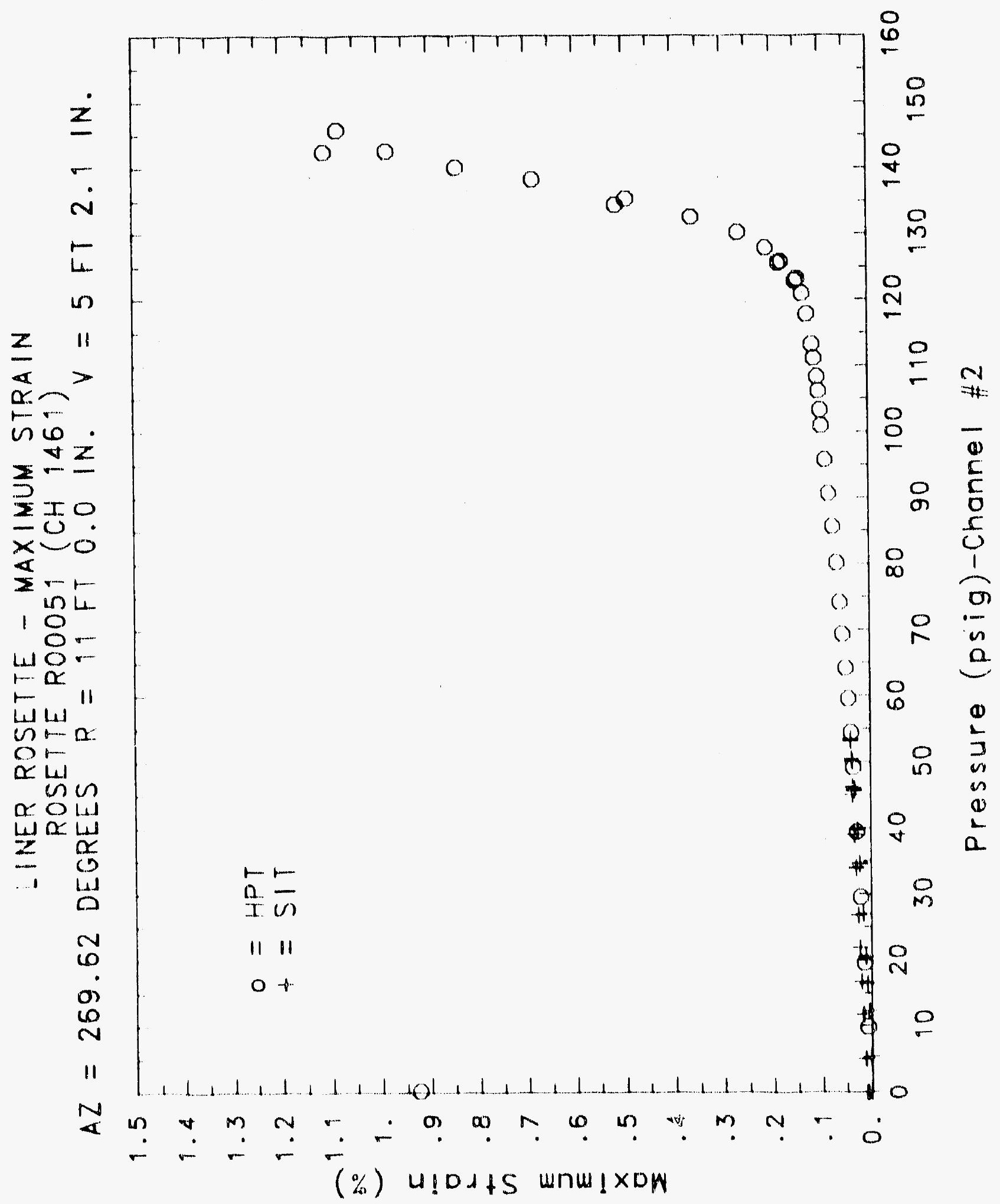




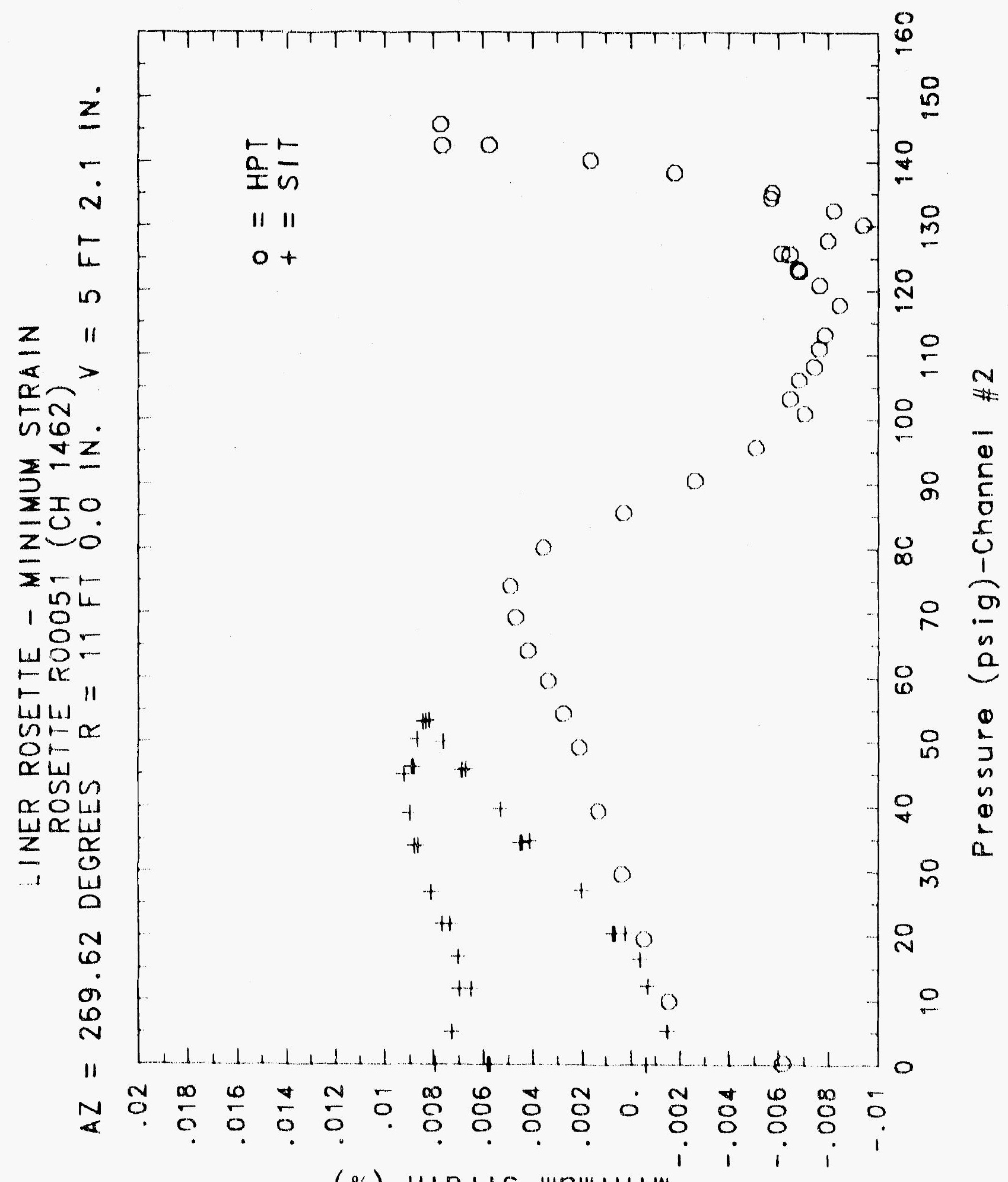

(\%) U!Dג WS wnw!U!W 


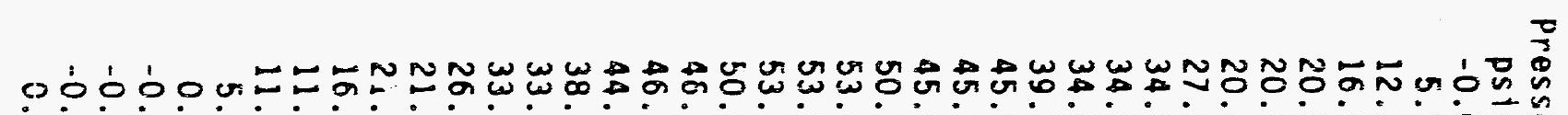

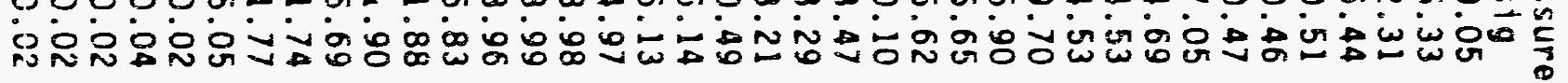
$00000000000000000000000000000000000000 \%$

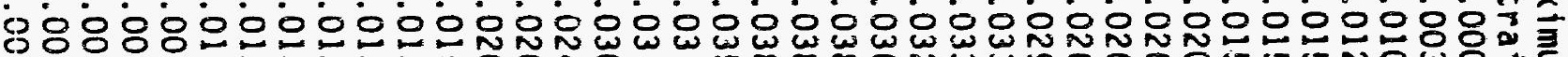

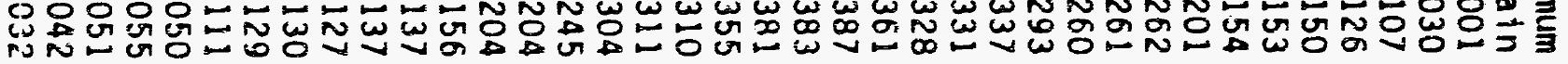

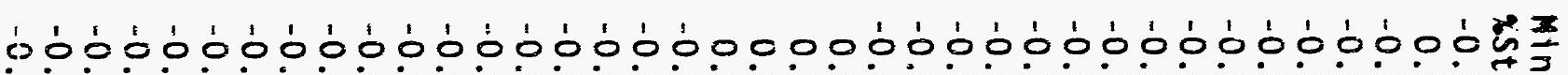

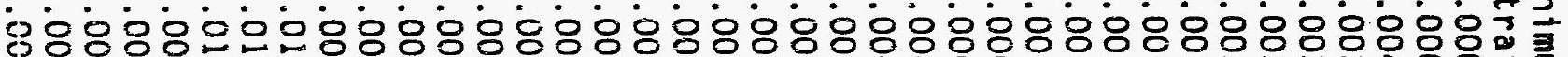

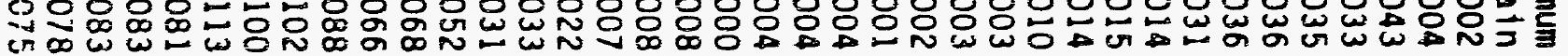

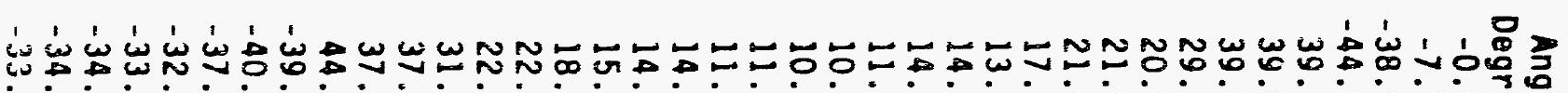

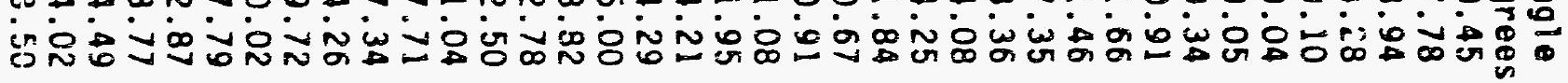

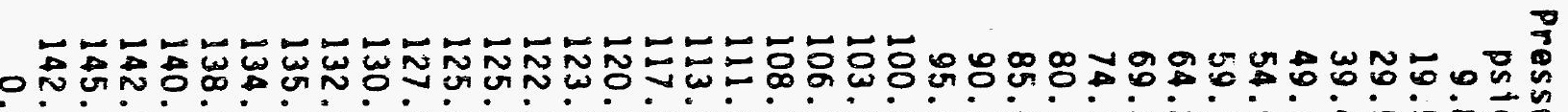

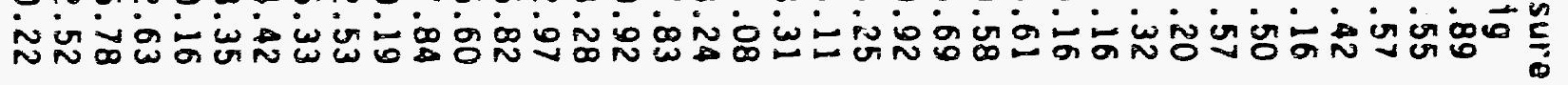

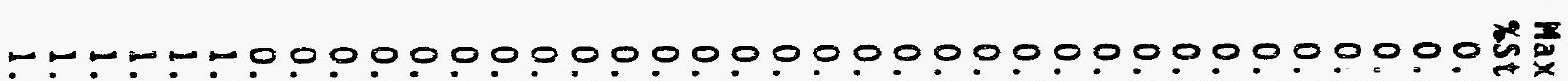

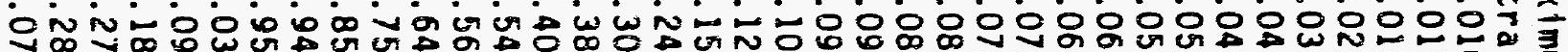

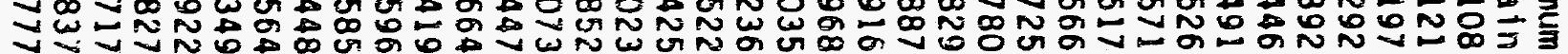

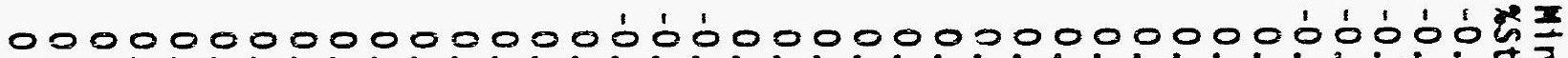
웅ㅇㅇㅇㅇㅇㅇㅇㅇㅇㅇㅇㅇㅇㅇㅇㅇㅇㅇㅇㅇㅇㅇㅇㅇㅇㅇㅇㅇㅇㅇㅇㅇㅇㅇㅇㅇㅇㅝ

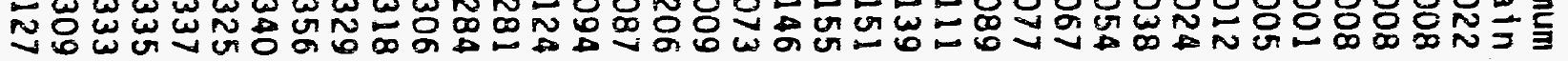

ஸ்ப்

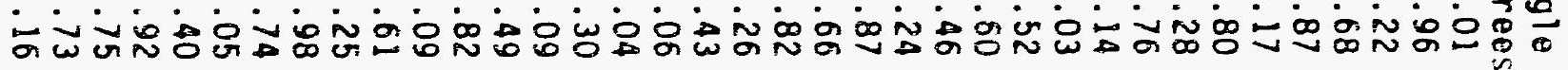




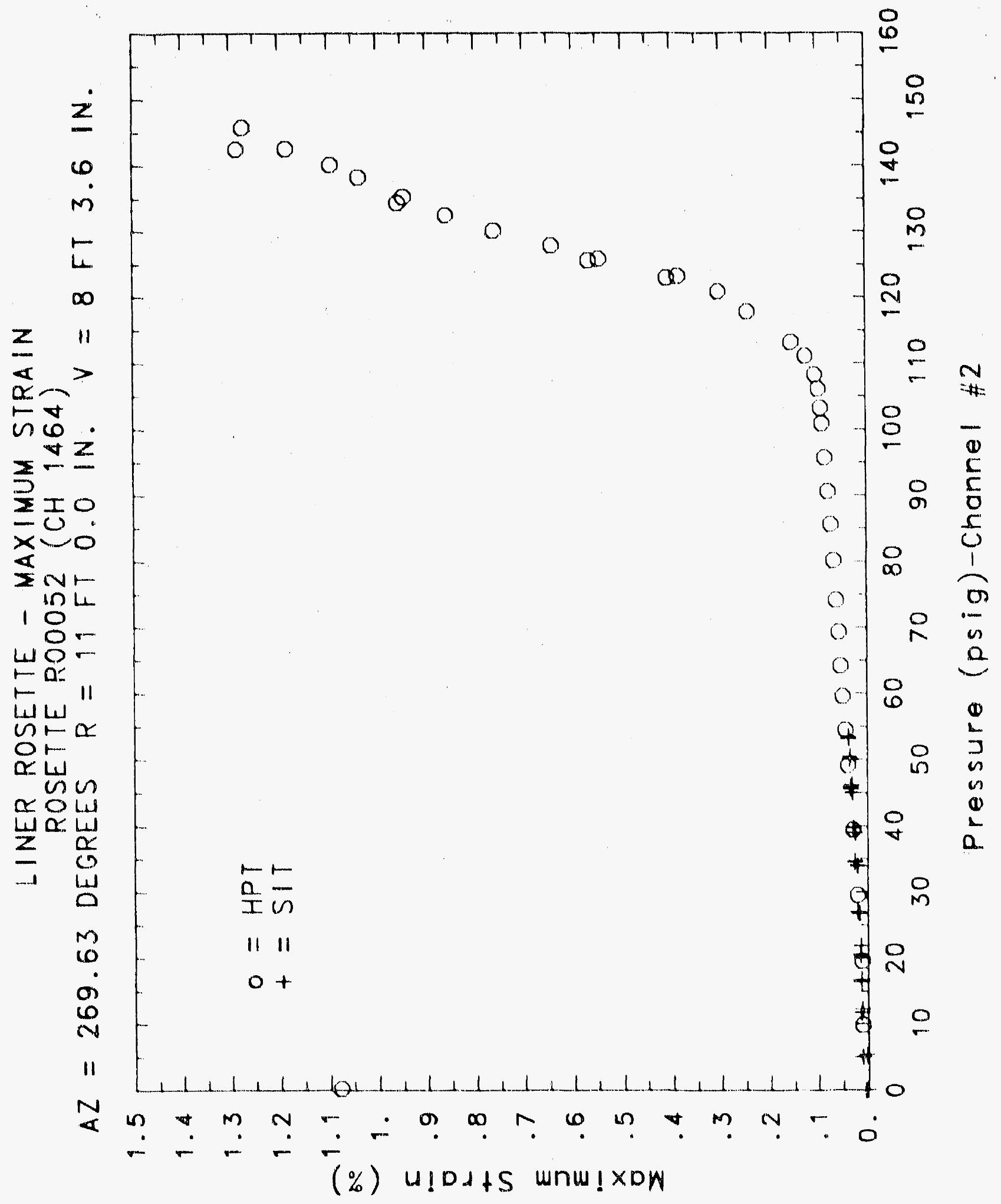




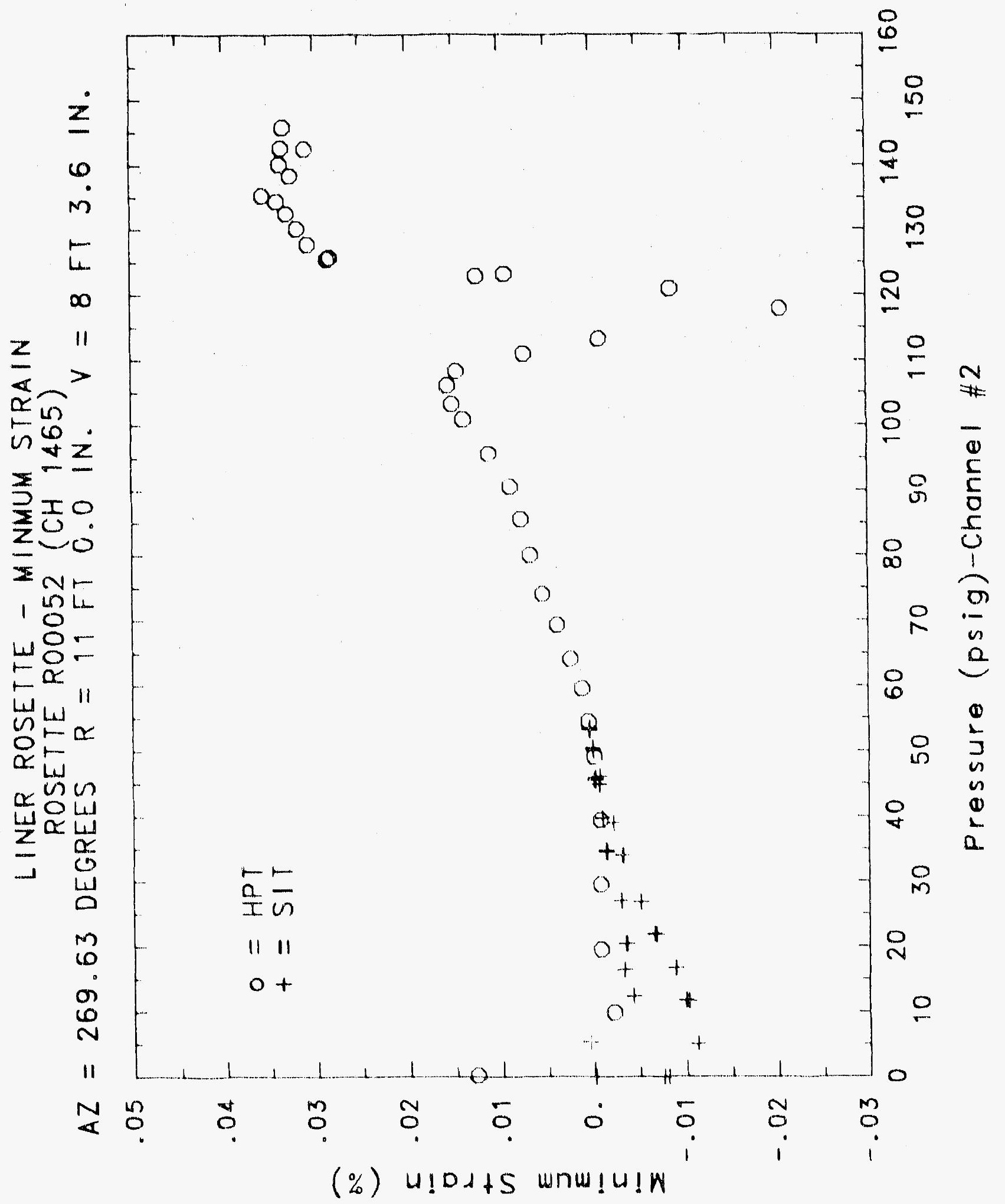




\section{Reinforced Concrete Test Data}

\section{Rosette 53 Channels $1467 \quad 1468 \quad 1469$}

Structural InTEgrity TEST

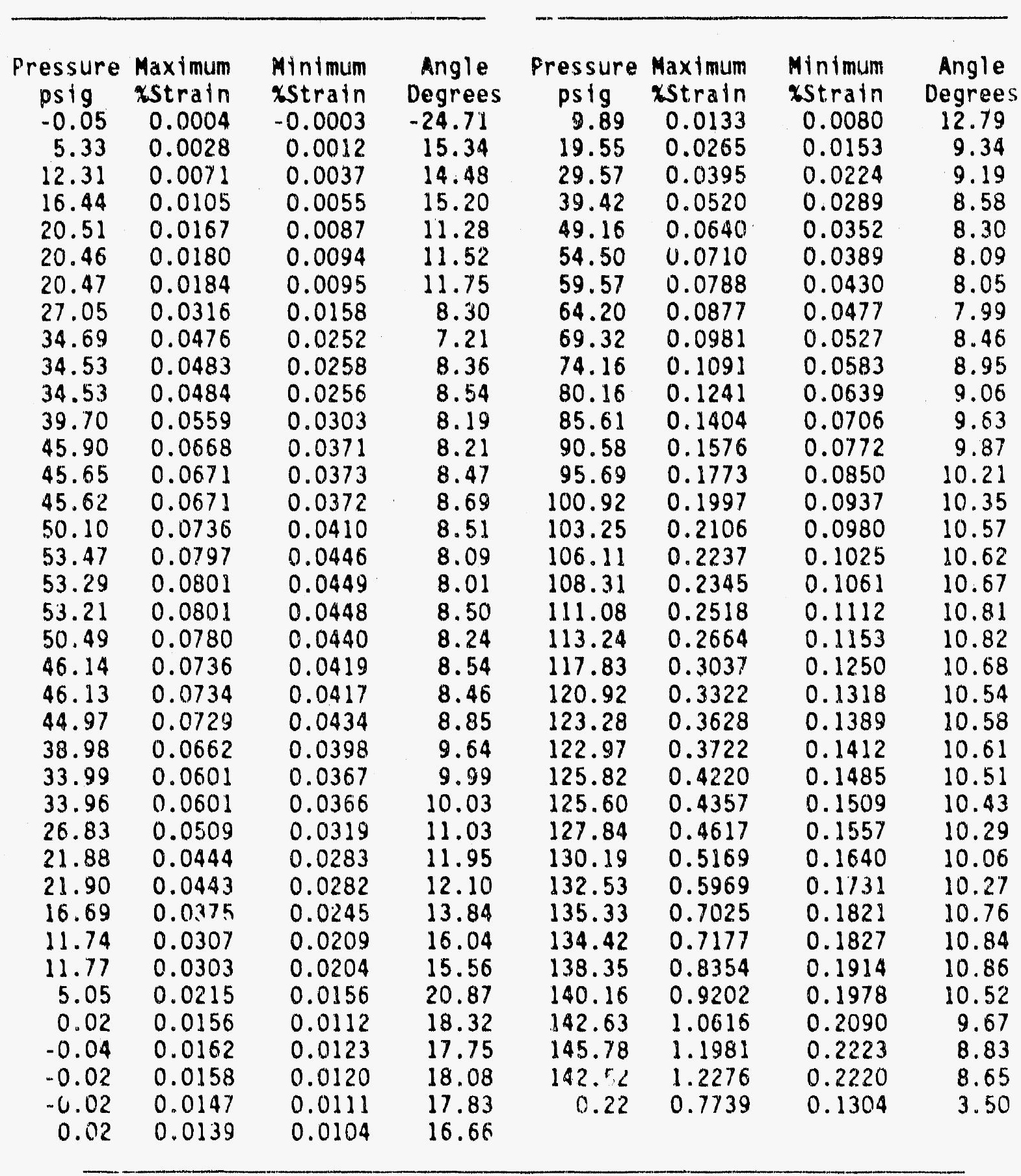

High Pressure test 


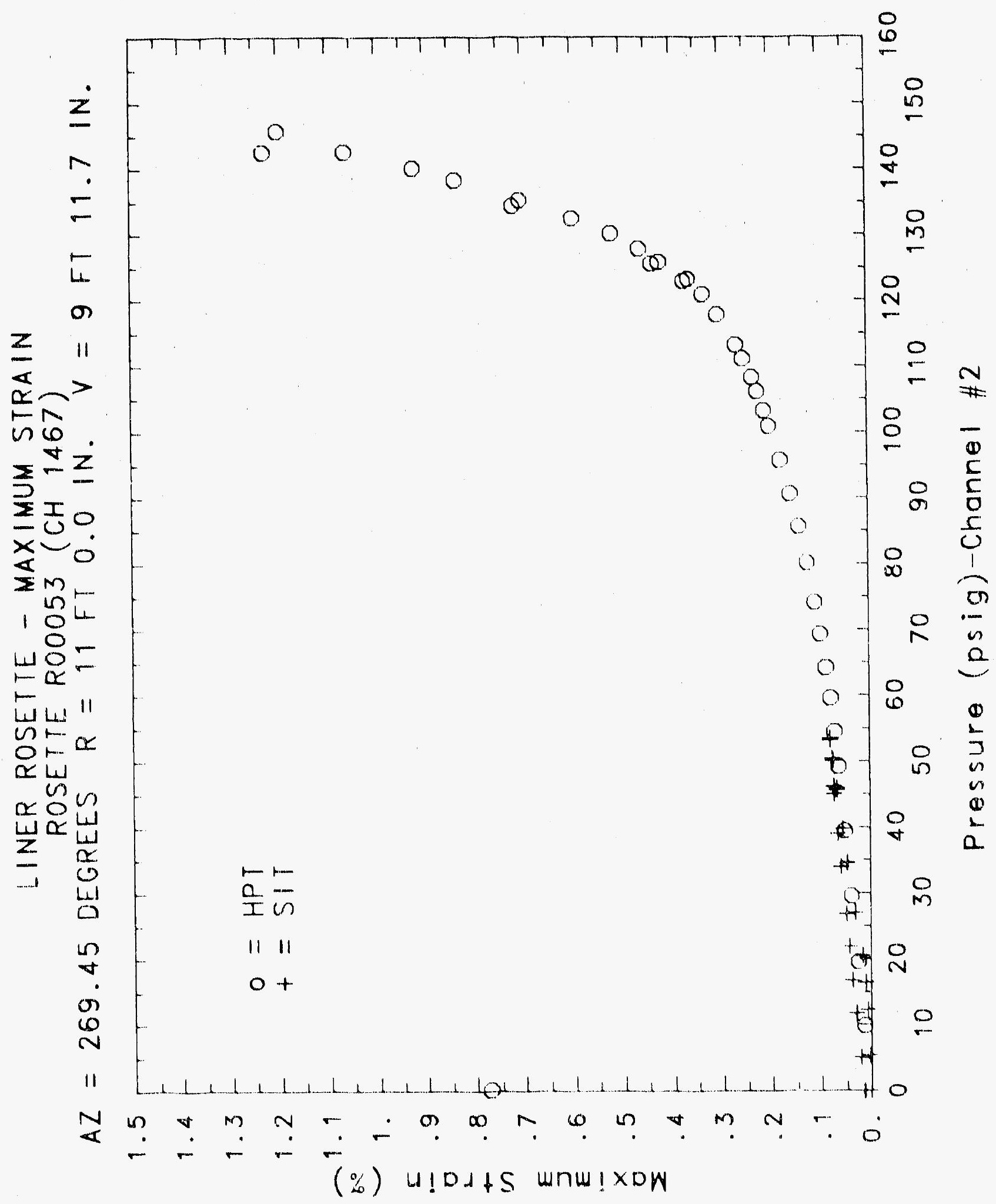




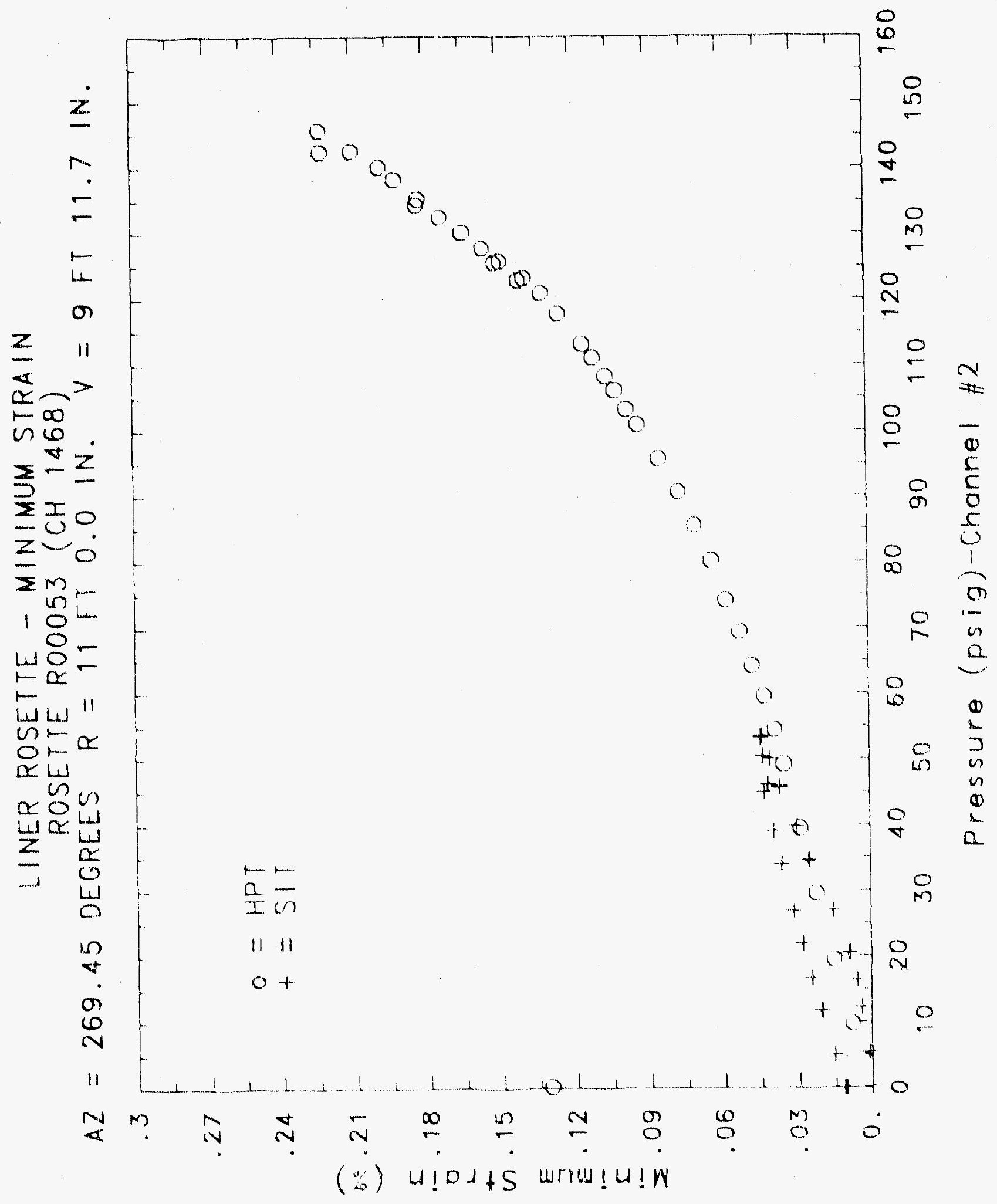

c. +3 


\title{
Reinforced Concrete Test Data
}

\author{
Roserte 54 Channels $1480 \quad 1481$ iAR?
}

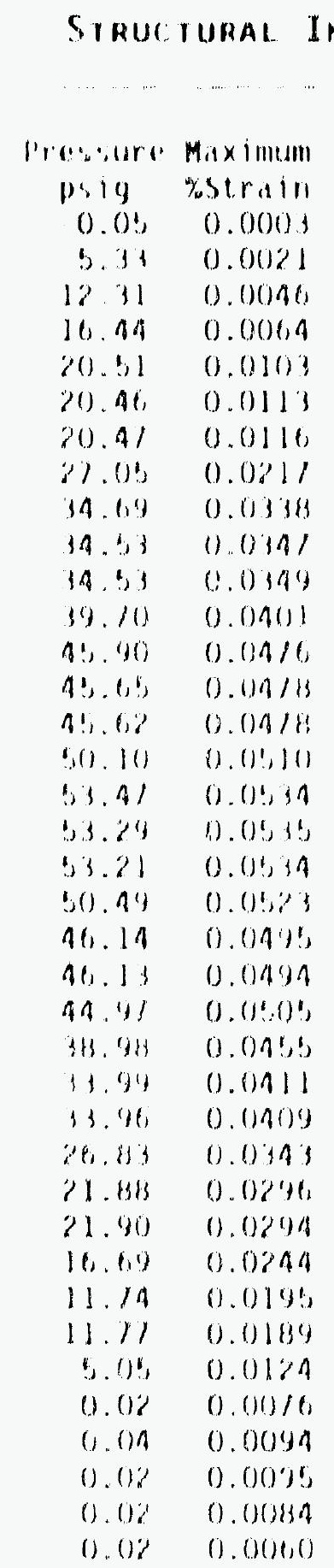

Minimum

*strain

0.0000

$0.000 \mathrm{~s}$

0.0001

$0.000 \%$

0.0006

0.0009

0.0011

0.0013

$0.00 \% 6$

0.0033

0.0034

0.0036

0. 0048

(1).001)!

$0.005 \%$

0.00199

$0.0(06,8)$

$0.0(1) / 0$

(). (1)()/1

$0.00 / \%$

$0.00 / 3$

$0.001 \%$

0.00834

0.0011

(). $00 / 3$

0.0014

$0.006,1$

0.0064

$0.006 \%$

$0.001,9$

$0.00 ? 6$

0.0053

$0.00 ! 0)$

0.0048

0.006 .8

0.0069

0.0014

0.).0041
TEST

Angle Pressure Miximum

Degrees:

33.28

1. 10

(). $5 !$

1.11

0.19

1.63

1.9?

0.18

0.23

0.24

0.35

0.60

0.2818

0.88

1.09

1.61

2.19

$2.6,4$

2. 18

7.61

?. 61

2.94

?. 35

2.09

1.91

1.12

1.1\%

1.03

1.29

1.29

2.11

2.41

1. 48

12.94

21.10

9.46

12. 31

0.32

\begin{tabular}{|c|c|}
\hline y & \\
\hline 9.89 & \\
\hline 19.56 & \\
\hline 29.57 & \\
\hline 39.42 & \\
\hline 49.16 & $.0 n$ \\
\hline 54.50 & 0 \\
\hline 59.51 & $0.0 !$ \\
\hline 0.7 .70 & \\
\hline 69.32 & 0. \\
\hline 14.16 & 0.01 \\
\hline 80.16 & \\
\hline 85.61 & 0.0 \\
\hline 90.58 & \\
\hline 94.69 & \\
\hline 100.97 & \\
\hline 03.25 & \\
\hline 00.11 & \\
\hline 1013.31 & 0.1 \\
\hline 111.018 & 0. \\
\hline & \\
\hline 117.87 & 0.0836 \\
\hline $120.9 \%$ & 0.09 \\
\hline 173.28 & 0.1 \\
\hline 127.97 & 0.096 \\
\hline 128.87 & 0.099 \\
\hline 125.00 & 0.101 \\
\hline 121.834 & 0.103 \\
\hline 130.19 & 0.106 \\
\hline 1.12 .53 & \\
\hline 135.33 & 0.119 \\
\hline 134.98 & \\
\hline 1313.39 & 0.14 \\
\hline 140.16 & 0.14 .9 \\
\hline 198.63 & 0.1800 \\
\hline 145.18 & 0.194 \\
\hline $148.5 \%$ & \\
\hline $0.2 \%$ & $0.0 \%$ \\
\hline
\end{tabular}

High Pressure Test
Minilimem

xstraln

0.0008

0.0010

0.0011

0.0019

0.0025

0.0030

0.0036

$0.1004 ?$

0.0060

$0.00 / 4$

0.0088

0.0103

0.0121

0.0155

0.0183

0.0199

$0.0 \% 14$

0.0278

$0.0 \% 49$

0.0263

0.0903

0.0335

0.0361

$0.03 / 3$

0.0408

(1. 0411

0.0436

0.0469

0.0519

0.0579

0.0 .884

0.0648

$0.06 / 6$

$0.0 / 383$

0.0191

0.0718

(). $01 / 8$
Angle:

Degreos.

0.13

$0.6 \%$

0.94

1.21

1.3!

$1.4 \%$

1.19

$0.83 !$

0.99

0.1 !

1.99

1.09

(). 19

0.01

0.54

0.91

0.06

(). 12

0.11

1.60

4.93

5. 91

1.61

1. 15

9. (1)

9.10

10.18

13.20

15.91

19.89

19.96

21.11

20.83

19.93

18.11

$1 / .68$

H. : 1 


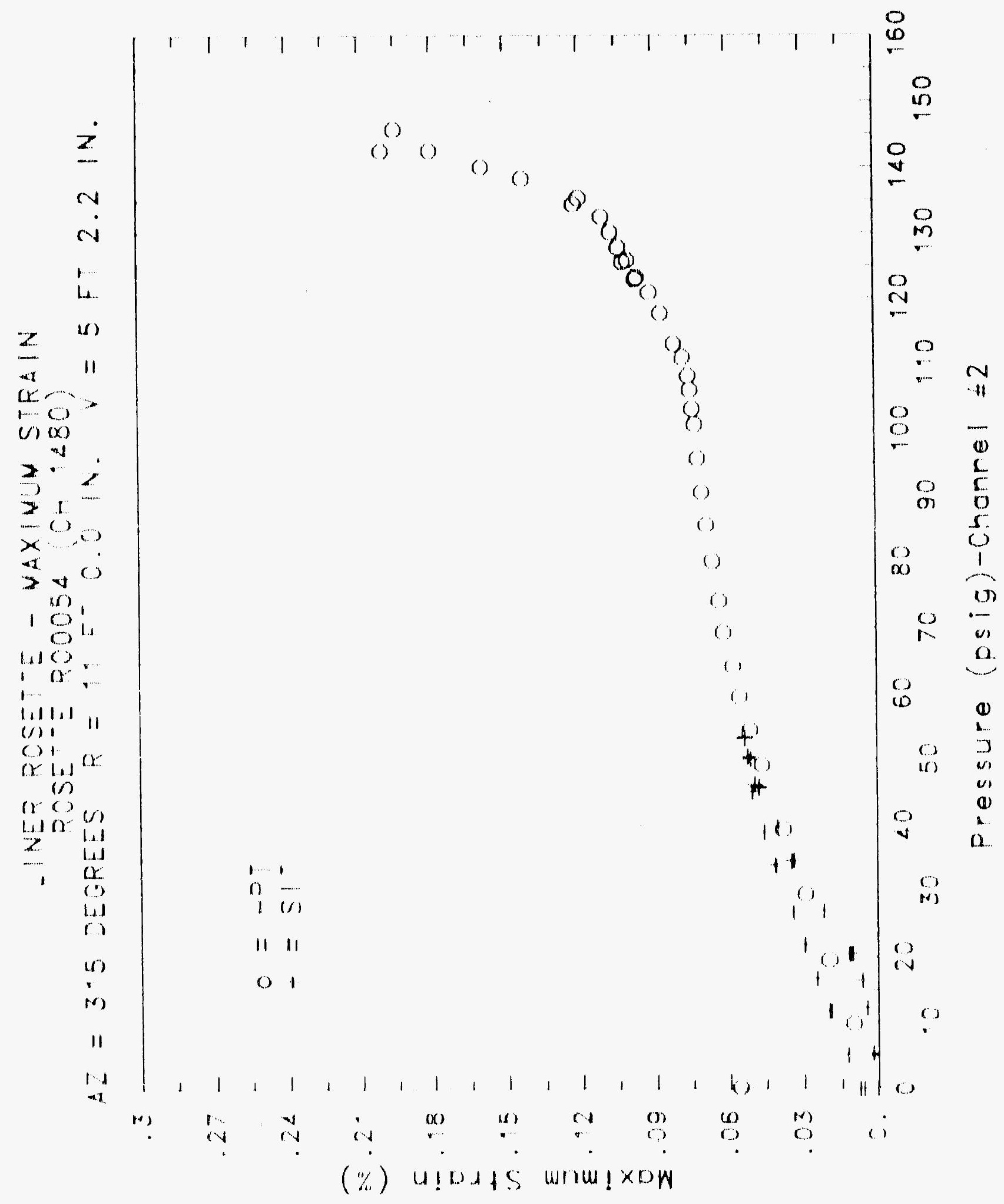




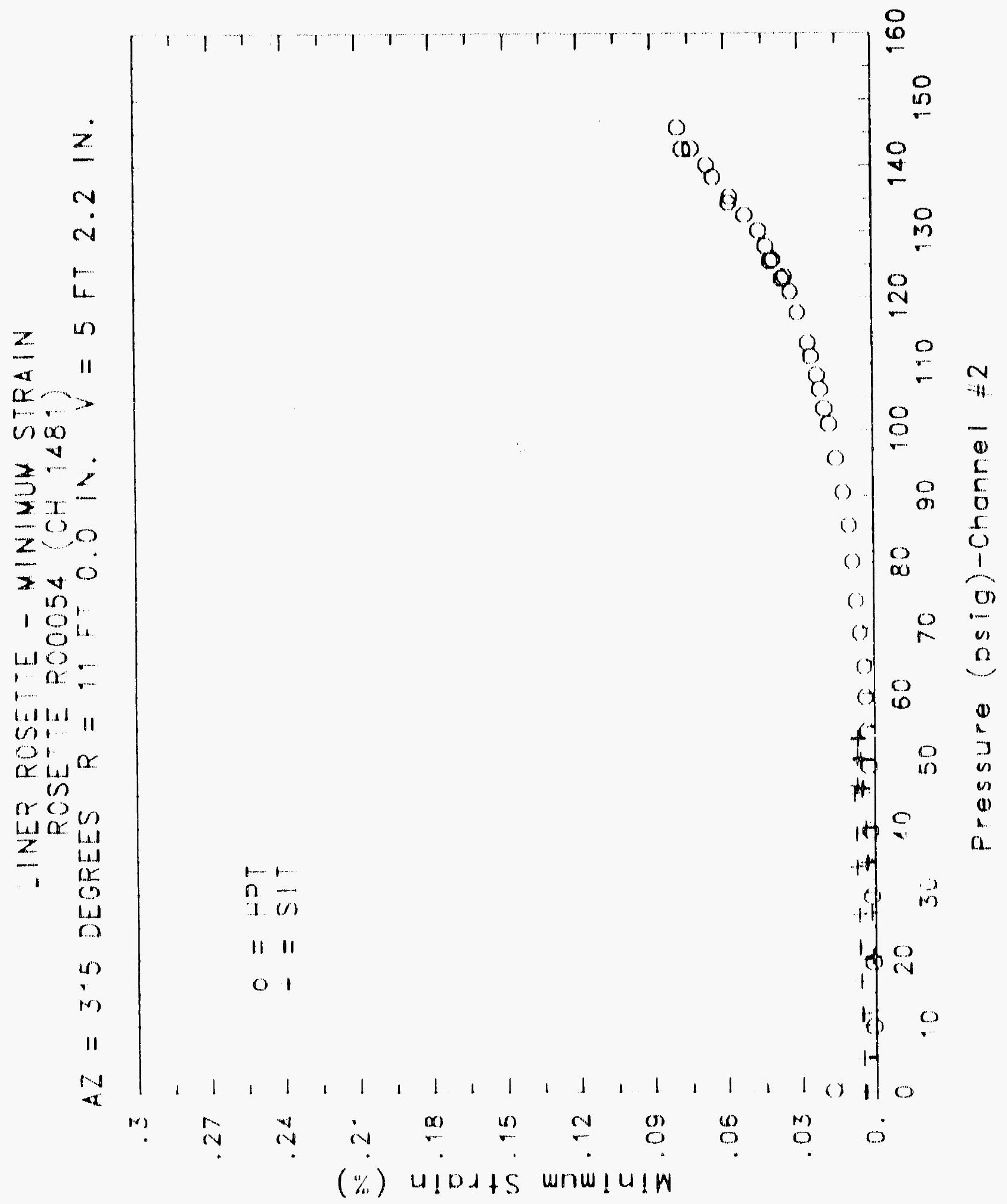




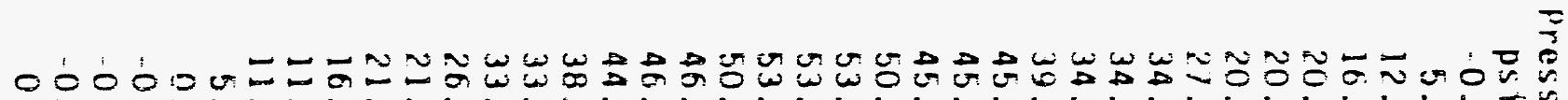

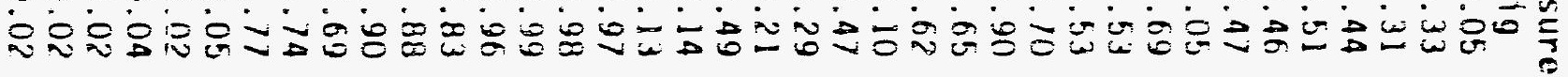

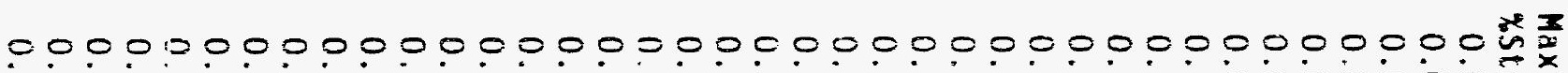

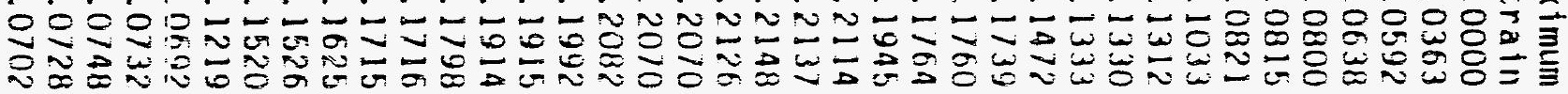

$00000000000000000000000000000000000000 \frac{1}{1}$

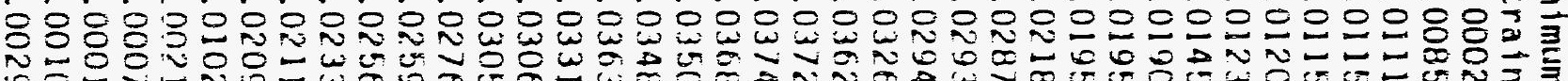

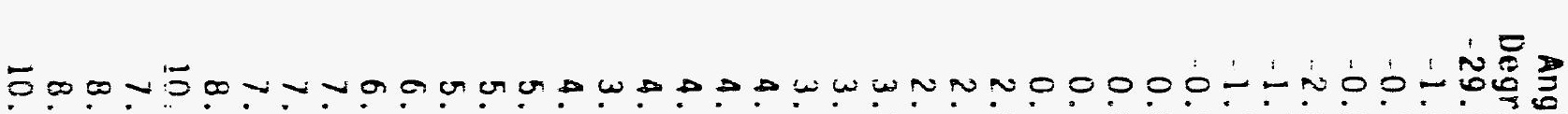

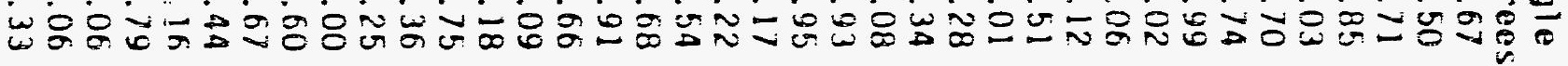

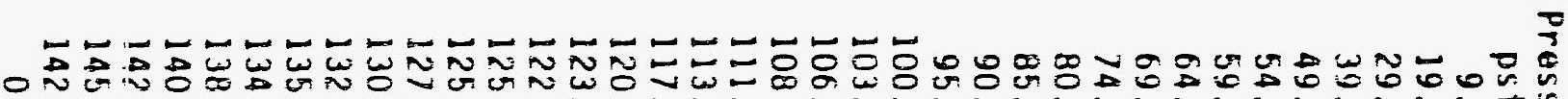

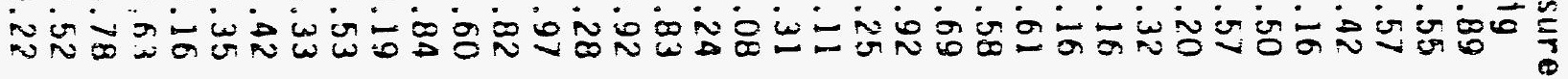
$0-1-0000000000000000000000000000000000$

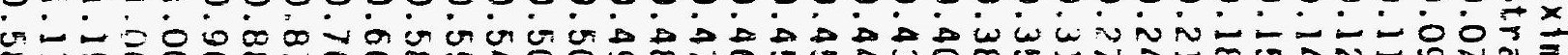

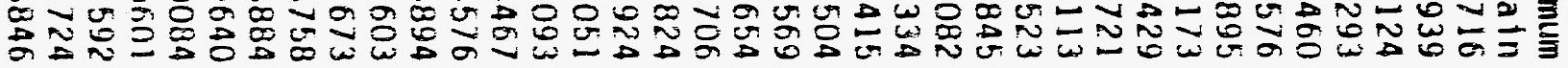

1 $0000000000000000000000000000000000000 \frac{1}{1}$

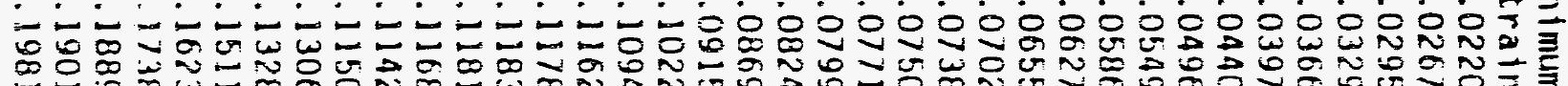

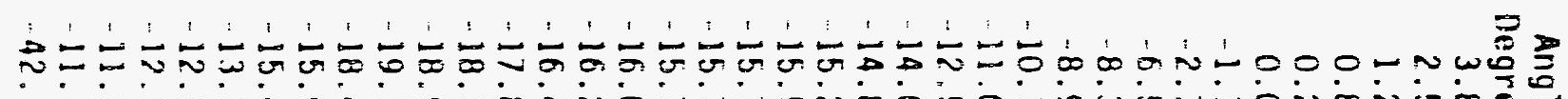
えजか。 


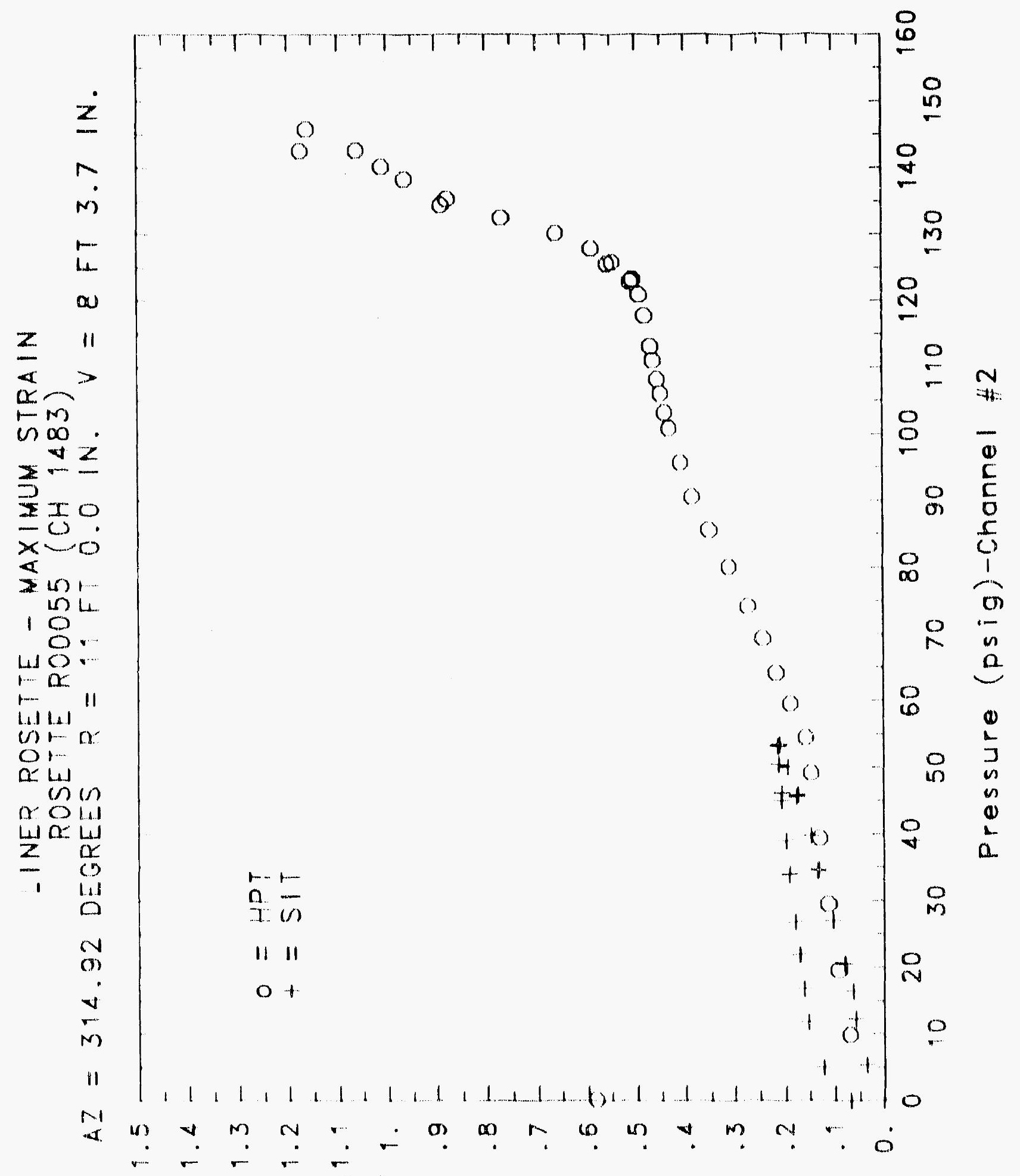

$(\%) \quad 4 ! 0 \lambda+5$ wnw! $\times$ DW 


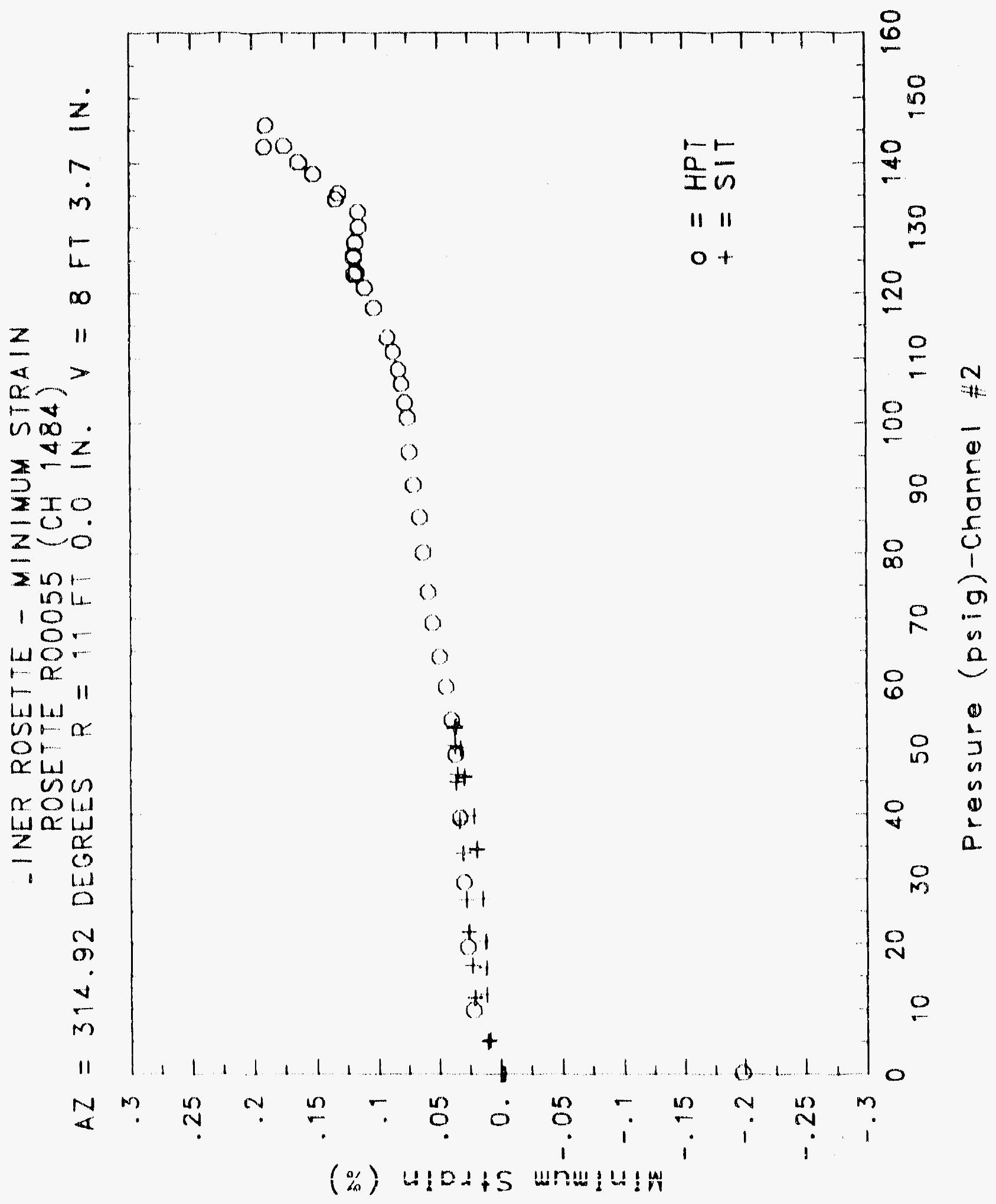




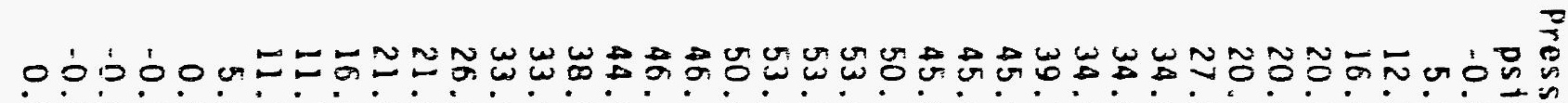

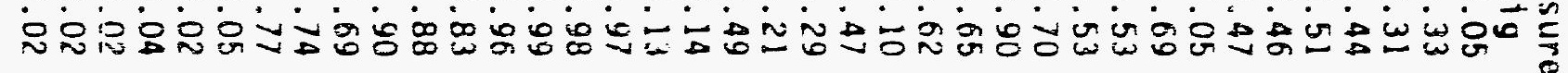
$000000000000000000000000000000000000004 \frac{1}{2}$

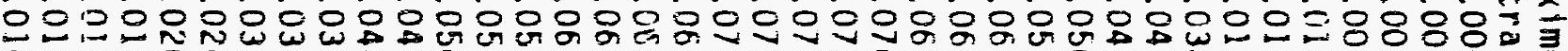

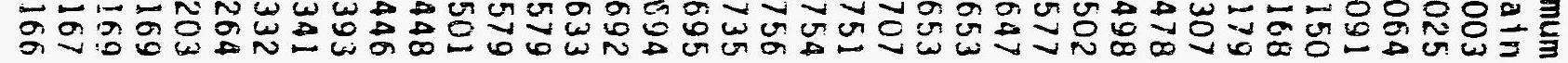

$0010000000000000000000000000000000000000 \frac{1}{3}$

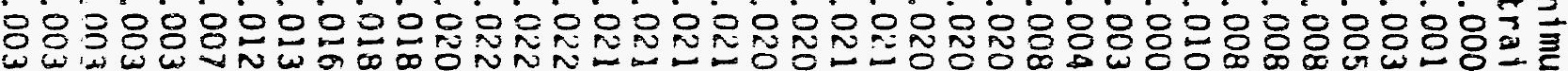

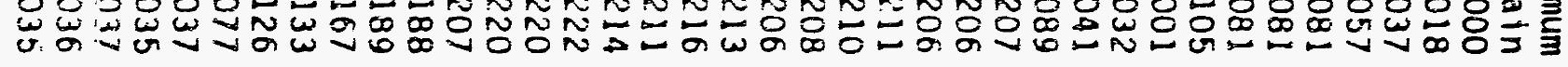

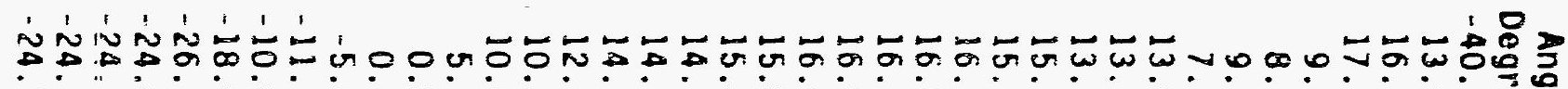

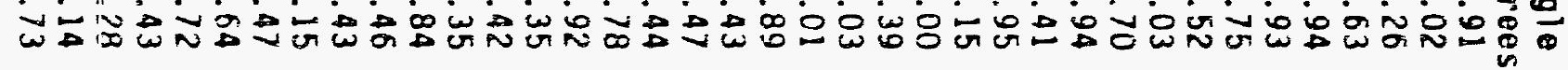

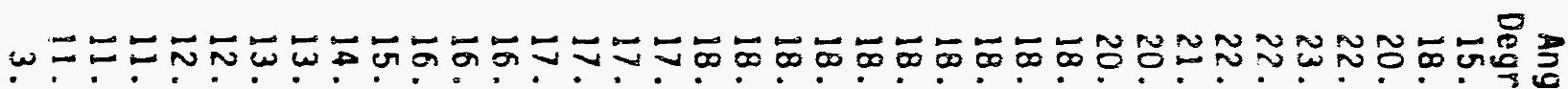

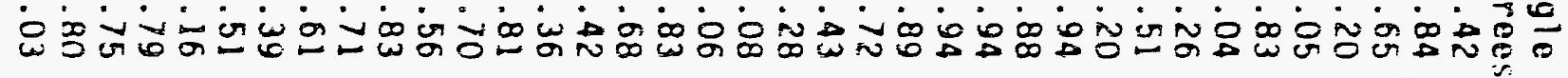

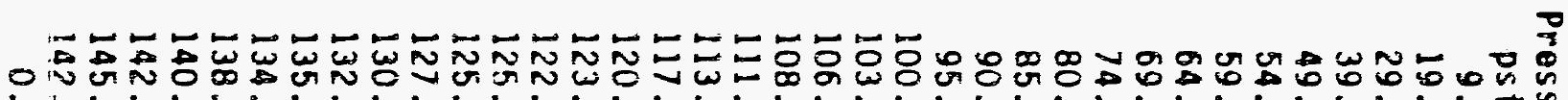

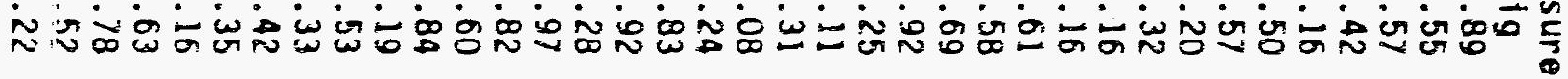

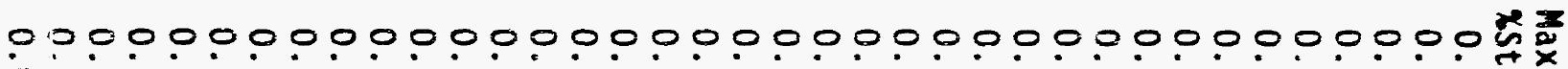

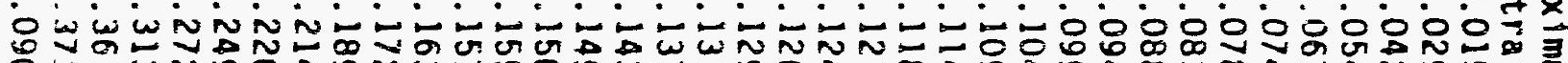

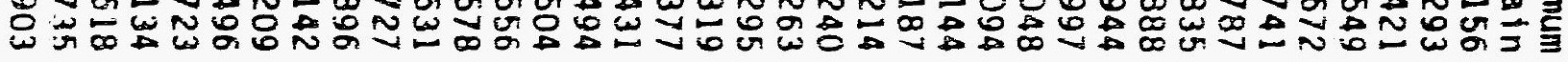

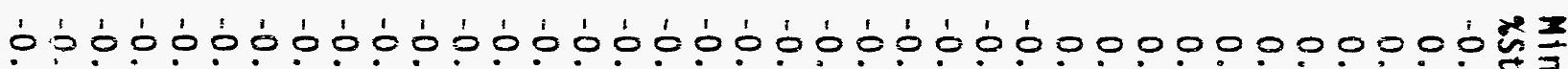
는

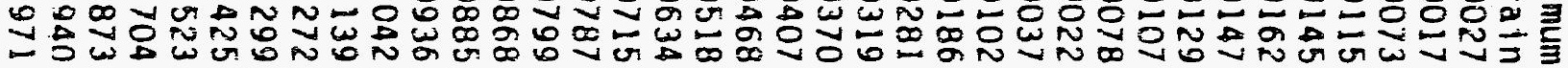




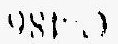

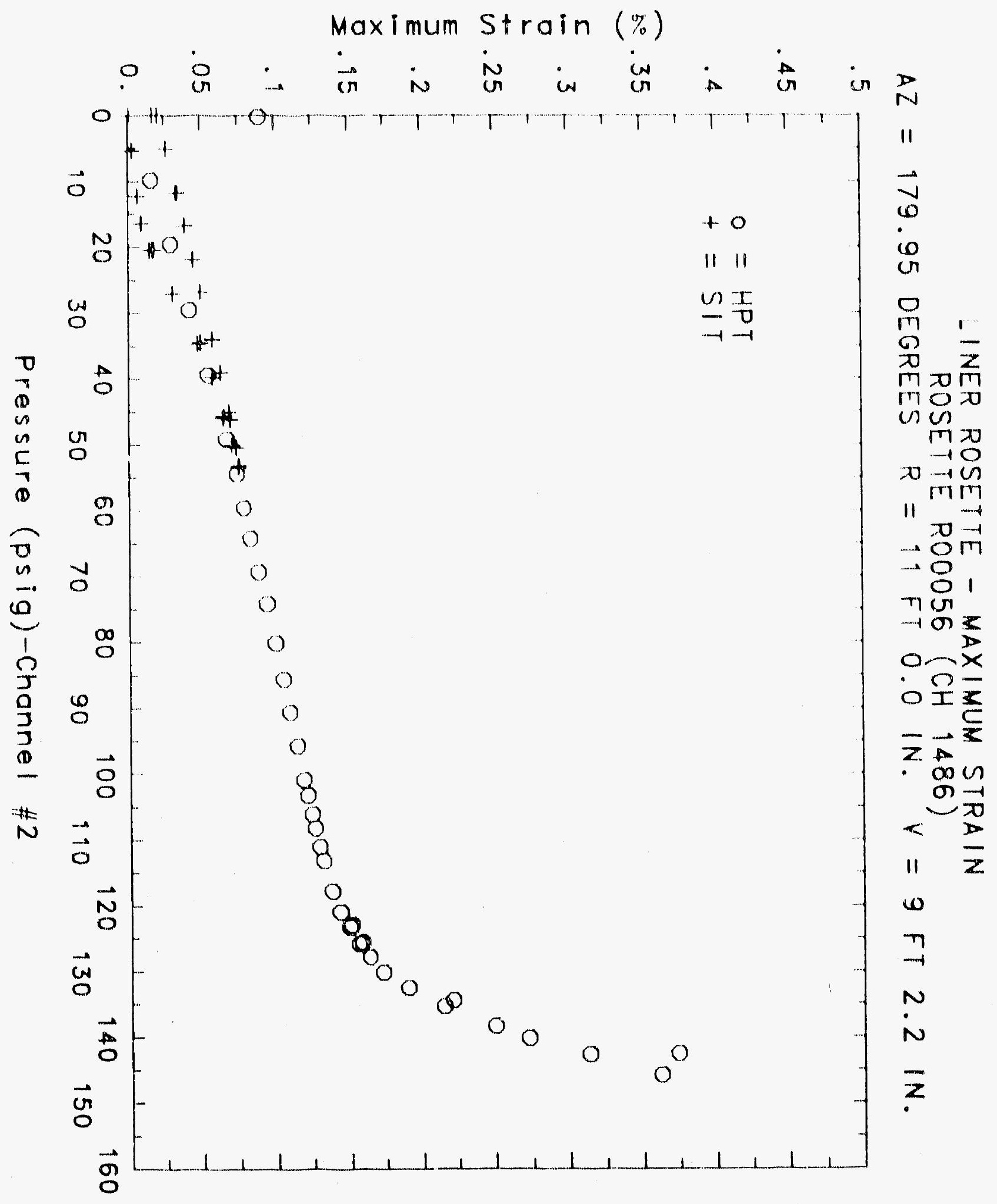




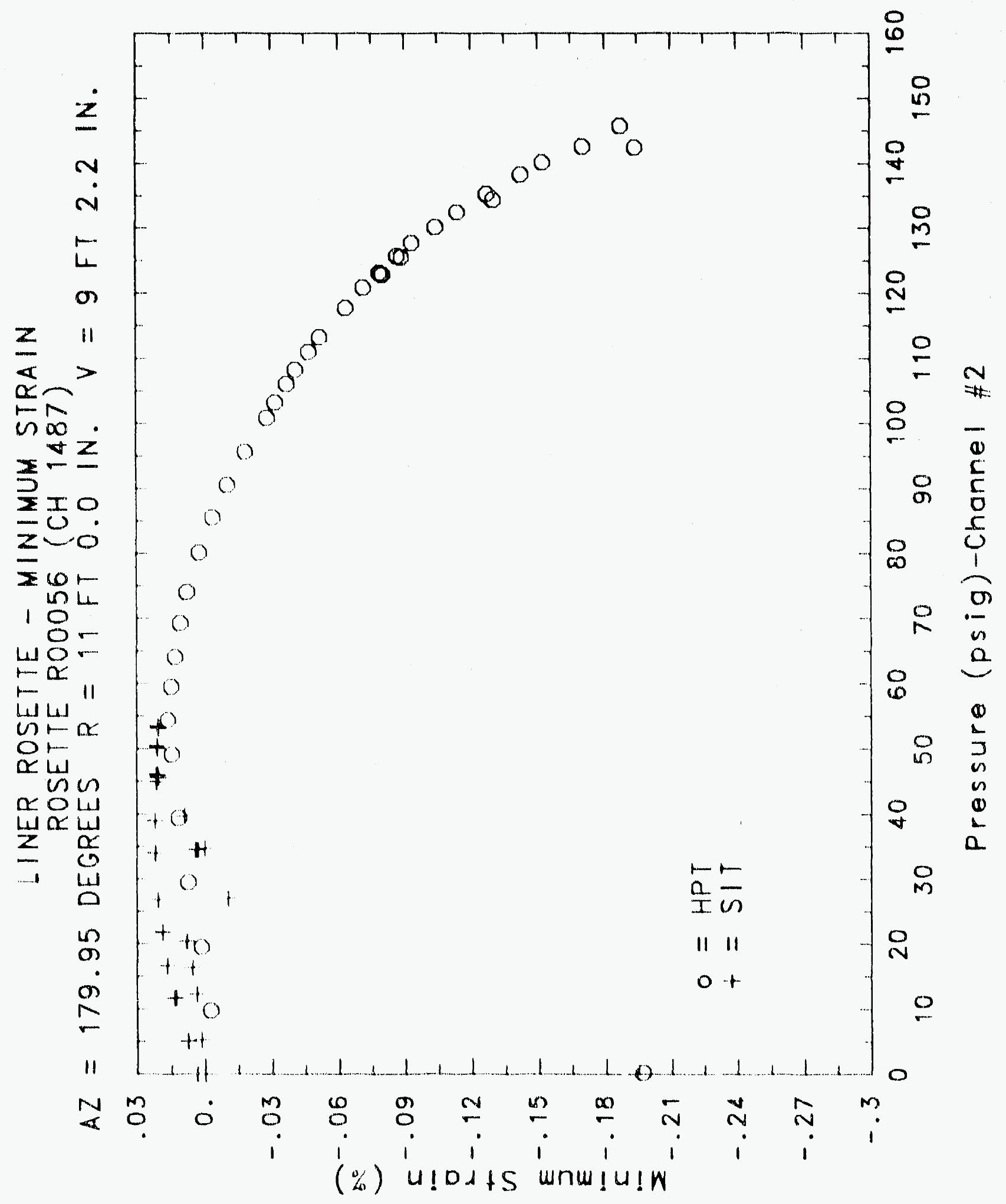




\title{
Reinforced Concrete Test Data
}

\author{
Rosette 57 Channels $1489 \quad 1500 \quad 1501$
}

\section{Structural InTEgRITY TEST}

\begin{tabular}{|c|c|c|c|}
\hline Pressure & Maximum & Minimum & Angle \\
\hline psig & xstrain & xstratn & Degrees \\
\hline-0.05 & 0.0003 & $\begin{array}{l}-0.0001 \\
-0.0070\end{array}$ & $\begin{array}{r}11.45 \\
-35.70\end{array}$ \\
\hline 5.33 & -0.0004 & -0.0070 & -35.79 \\
\hline 12.31 & -0.0022 & -0.0119 & 40.13 \\
\hline 16.44 & -0.0032 & -0.0141 & 33.56 \\
\hline 20.51 & -0.0041 & -0.0169 & 27.52 \\
\hline 20.46 & -0.0037 & -0.0176 & 27.39 \\
\hline 20.47 & -0.0036 & -0.0178 & 27.15 \\
\hline 27.05 & 0.0024 & -0.0216 & 16.64 \\
\hline 34.69 & 0.0043 & -0.0226 & 11.45 \\
\hline 34.53 & 0.0047 & -0.0223 & 10.84 \\
\hline 34.53 & 0.0048 & -0.0222 & 10.73 \\
\hline 39.70 & 0.0056 & -0.0224 & 8.93 \\
\hline 45.90 & 0.0068 & -0.0220 & 6.57 \\
\hline 45.65 & 0.0071 & -0.0215 & 6.44 \\
\hline 45.62 & 0.0072 & -0.0214 & 6.39 \\
\hline 50.10 & 0.0079 & -0.0210 & 5.59 \\
\hline 53.47 & 0.0096 & -0.0201 & 4.14 \\
\hline 53.29 & 0.0101 & -0.0196 & 4.05 \\
\hline 53.21 & 0.0103 & -0.0193 & 3.89 \\
\hline 50.49 & 0.0103 & -0.0191 & 4.08 \\
\hline 46.14 & 0.0100 & -0.0190 & 4.40 \\
\hline 46.13 & 0.0100 & -0.0187 & 4.29 \\
\hline 44.97 & 0.0103 & -0.0180 & 4.26 \\
\hline 38.98 & 0.0095 & -0.0182 & 5.06 \\
\hline 33.99 & 0.0089 & -0.0180 & 6.08 \\
\hline 33.96 & 0.0089 & -0.0181 & 6.26 \\
\hline 26.83 & 0.0079 & -0.0173 & 7.48 \\
\hline 21.88 & 0.0074 & -0.0162 & 7.81 \\
\hline 21.90 & 0.0073 & -0.0161 & 7.51 \\
\hline 16.69 & 0.0064 & -0.0141 & 6.42 \\
\hline 11.74 & 0.0054 & -0.0120 & 5.18 \\
\hline 11.77 & 0.0052 & -0.0121 & 4.80 \\
\hline 5.05 & 0.0037 & -0.0082 & 2.66 \\
\hline 0.02 & 0.0070 & -0.0051 & -8.85 \\
\hline-0.04 & 0.0076 & -0.0048 & .6 .49 \\
\hline-0.02 & 0.0077 & -0.0047 & -6.01 \\
\hline-0.02 & 0.0076 & -0.0048 & -5.59 \\
\hline $0.0 ?$ & 0.0070 & -0.0046 & -4.76 \\
\hline
\end{tabular}

\section{High Pressure Test}

\begin{tabular}{|c|c|c|c|}
\hline Pressure & Maximum & Minimum & Angle \\
\hline psig & xstrain & xStrain & Degrees \\
\hline 9.89 & 0.0005 & -0.0074 & 32.76 \\
\hline 19.55 & 0.0013 & -0.0113 & 21.04 \\
\hline 29.57 & 0.0024 & -0.0135 & 18.38 \\
\hline 39.42 & 0.0037 & -0.0131 & 15.01 \\
\hline 49.16 & 0.0054 & -0.0118 & 13.88 \\
\hline 54.50 & 0.0065 & -0.0109 & 13.45 \\
\hline 59.57 & 0.0077 & -0.0102 & 12.64 \\
\hline 64.20 & 0.0093 & -0.0093 & 11.85 \\
\hline 69.32 & 0.0107 & -0.0083 & 12.47 \\
\hline 74.16 & 0.0124 & -0.0073 & 12.50 \\
\hline 80.16 & 0.0133 & -0.0066 & 14.00 \\
\hline 85.61 & 0.0146 & -0.0059 & 15.43 \\
\hline 90.58 & 0.0157 & -0.0054 & 17.28 \\
\hline 95.69 & 0.0167 & -0.0052 & 19.94 \\
\hline 100.92 & 0.0175 & -0.0046 & 22.24 \\
\hline 103.25 & 0.0178 & -0.0045 & 23.15 \\
\hline 106.11 & 0.0181 & -0.0046 & 24.38 \\
\hline 108.31 & 0.0182 & -0.0044 & 25.30 \\
\hline 111.08 & 0.0184 & -0.0044 & 26.81 \\
\hline 113.24 & 0.0184 & -0.0043 & 27.88 \\
\hline 117.83 & 0.0189 & -0.0045 & 31.67 \\
\hline 120.92 & 0.0192 & -0.0048 & 34.14 \\
\hline 123.28 & 0.0199 & -0.0049 & 35.70 \\
\hline 122.97 & 0.0200 & -0.0046 & 36.15 \\
\hline 125.82 & 0.0199 & -0.0049 & 38.61 \\
\hline 125.60 & 0.0199 & -0.0046 & 39.53 \\
\hline 127.84 & 0.0202 & -0.0051 & 40.90 \\
\hline 130.19 & 0.0203 & -0.0051 & 43.90 \\
\hline 132.53 & 0.0210 & -0.0056 & -43.87 \\
\hline 135.33 & 0.0220 & -0.0063 & -41.56 \\
\hline 134.42 & 0.0225 & -0.0067 & -40.83 \\
\hline 138.35 & 0.0251 & -0.0073 & -40.57 \\
\hline 140.16 & 0.0271 & -0.0074 & -39.99 \\
\hline 142.63 & 0.0307 & -0.0074 & -39.25 \\
\hline 145.78 & 0.0346 & -0.0071 & -39.39 \\
\hline 142.52 & 0.0356 & -0.0071 & -38.22 \\
\hline 0.22 & 0.0046 & -0.0431 & -12.52 \\
\hline
\end{tabular}




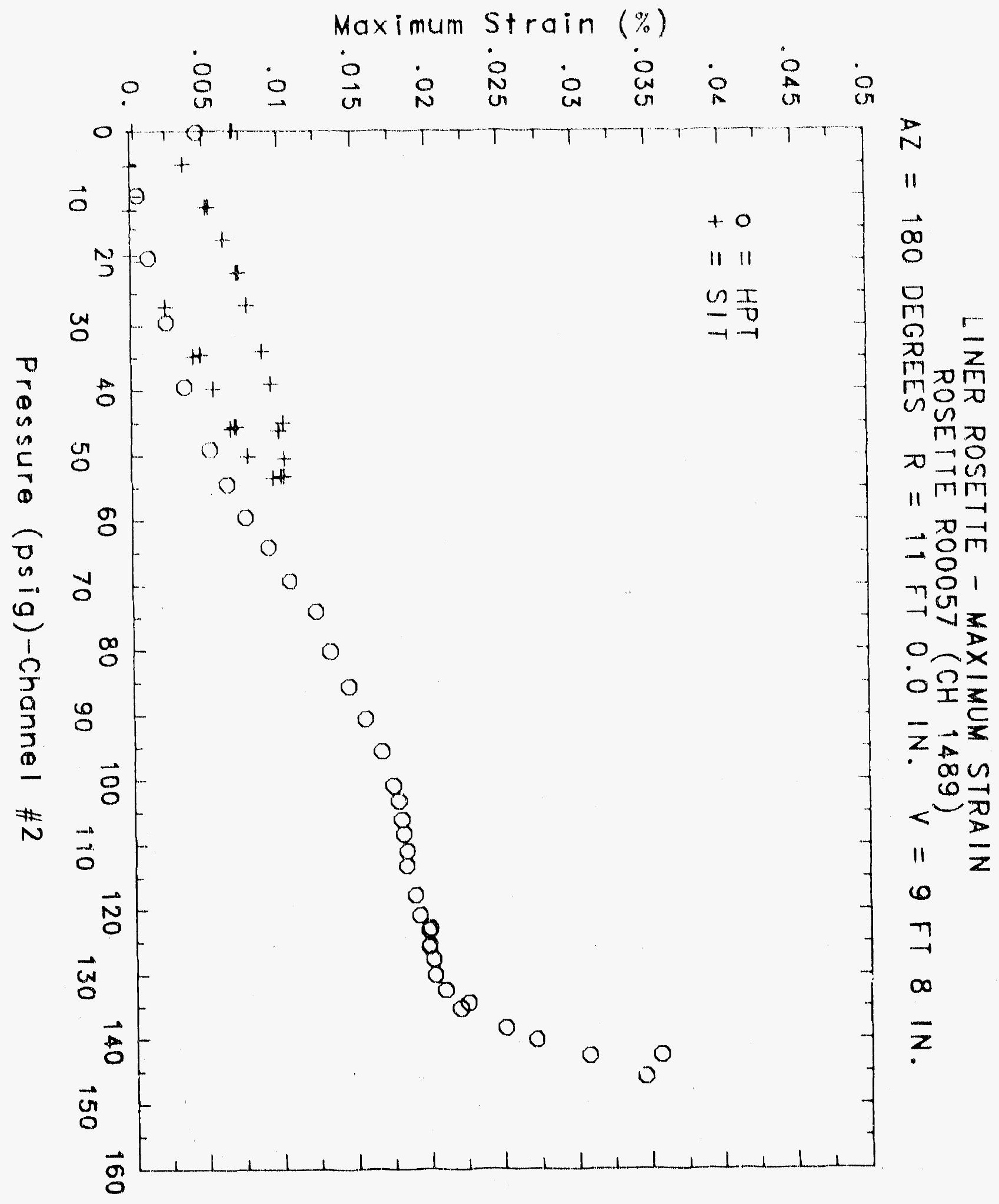




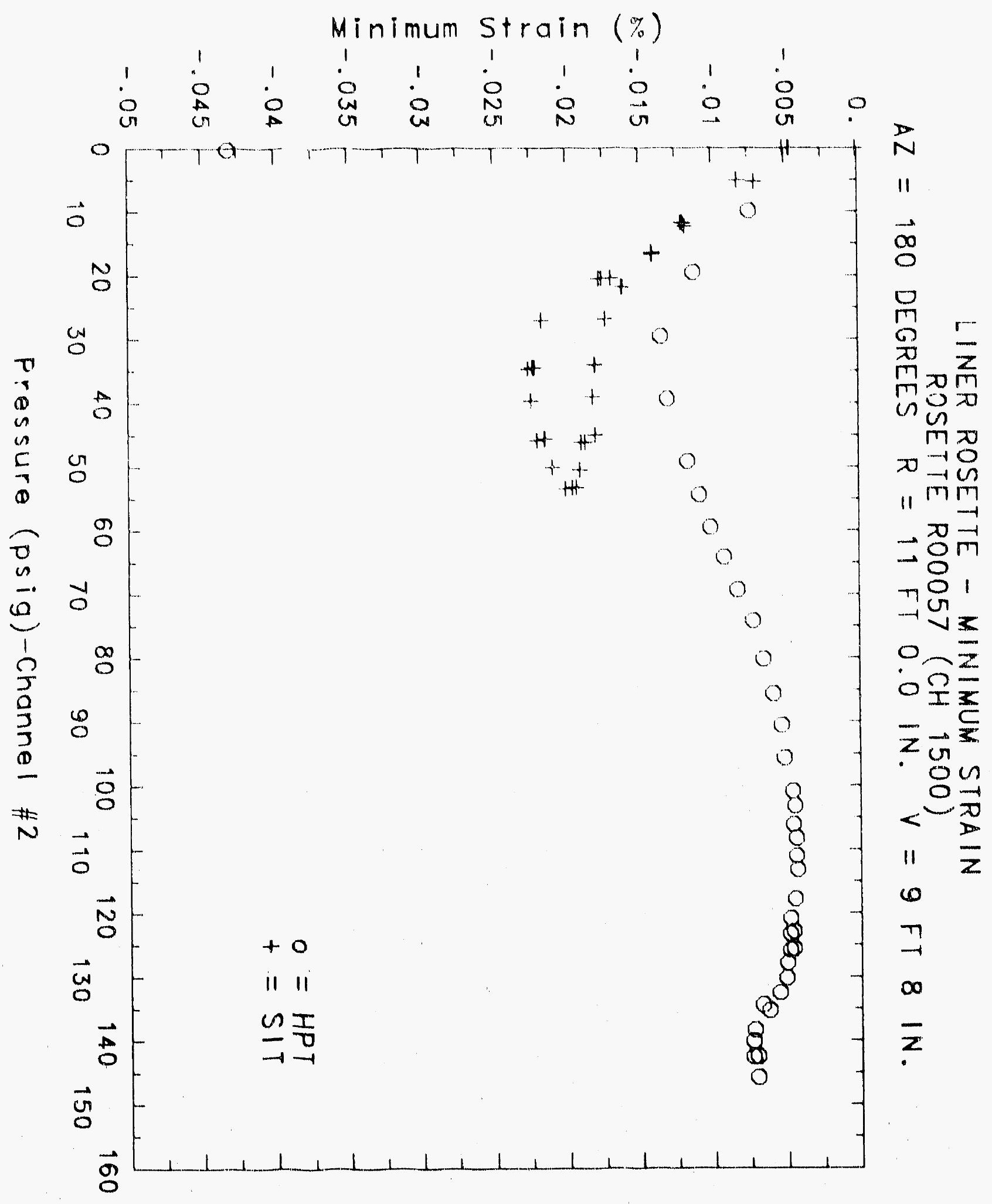




\section{Reinforced Concrete Test Data}

\section{Rosette 58 Channels $1502 \quad 1503 \quad 1504$}

Structural InTEgRity TEST

\begin{tabular}{|c|c|c|c|c|c|c|c|}
\hline Pressure & Maximum & Minimum & Angle & Pressure & Maximum & Minimum & Angle \\
\hline psig & \%Strain & xstrain & Degrees & psig & x Strain & xstrain & Degree \\
\hline-0.05 & 0.0004 & 0.0002 & -32.10 & 9.89 & 0.0032 & -0.0075 & -40.83 \\
\hline 5.33 & 0.0026 & -0.0055 & 44.08 & 19.55 & 0.0025 & -0.0081 & -29.68 \\
\hline 12.31 & 0.0006 & -0.0087 & -38.44 & 29.57 & 0.0034 & -0.0088 & -18.85 \\
\hline 16.44 & -0.0004 & -0.0097 & -32.34 & 39.42 & 0.0052 & -0.0092 & -10.17 \\
\hline 20.51 & -0.0019 & -0.0097 & -20.04 & 49. & 0.0078 & -0.0091 & -3.82 \\
\hline 20.46 & -0.0 & 092 & & & 0.0098 & & -0.84 \\
\hline 20.4 & -0.0 & 91 & -12 & & 0.0117 & 187 & 1.59 \\
\hline 27.05 & 0.0046 & -0.0115 & 2.73 & 64.20 & 0.0140 & -0.0083 & 3.79 \\
\hline 34.69 & 0.0067 & -0.0131 & 5.15 & 69.32 & 0.0163 & -0.0079 & 6.60 \\
\hline 34.53 & 0.0070 & -0.0126 & 4.44 & 74.16 & 0.0185 & -0.0073 & 9.26 \\
\hline 34.53 & 0.0069 & -0.0126 & 4.04 & 80.16 & 0.0205 & -0.0065 & 11.21 \\
\hline 39.70 & 0.0078 & -0.0133 & 4.19 & 85. & 0.0225 & -0 . & 12.97 \\
\hline & 0.0082 & & 6.47 & & & & \\
\hline 45.6 & 0.0084 & 25 & 6.65 & & 0.1 & & 65 \\
\hline 45.62 & 0.0084 & -0.0125 & 7.19 & 100.92 & 0.0269 & -0.0054 & 18.37 \\
\hline 50.10 & 0.0093 & -0.0130 & 8.17 & 103.25 & 0.0271 & -0.005. & 19.23 \\
\hline 53.47 & 0.0105 & -0.0122 & 6.92 & 106.11 & 0.0276 & -0.0054 & 20.08 \\
\hline 53.29 & 0.0106 & -0.0118 & 7.33 & 108.31 & 0.0280 & -0.0054 & 20.07 \\
\hline 53.21 & 0.0107 & -0.0117 & 7.62 & 111.08 & 0.0283 & -0 & 21.62 \\
\hline 50.49 & 0.0105 & -1 & 7.7 & & & & \\
\hline & & -0.4 & 6.9 & 3 & 290 & & 1.44 \\
\hline 46.13 & 0.0 & & 6. & & 294 & 54 & 5.91 \\
\hline 44.97 & 0.0092 & -0.0101 & 6.38 & 123 & 0.0297 & -0.0053 & 27.25 \\
\hline 38.98 & 0.0080 & -0.0097 & 5.41 & 122.97 & 0.0295 & -0.0052 & 27.52 \\
\hline 33.99 & 0.0070 & -0.0089 & 5.36 & 125.82 & 0.0294 & -0.0050 & 28.82 \\
\hline 33.96 & 0.0069 & -0.0090 & 4.69 & 125.60 & 0.0290 & -0.0047 & 29.44 \\
\hline 26.83 & 0.0055 & -0.0073 & 2.41 & 127.84 & 0.0292 & -0.0048 & 30.24 \\
\hline 21.88 & 0.0041 & -0.0059 & 1.52 & 130.19 & 0.0290 & -0 . & 32.14 \\
\hline 21.90 & 0.0041 & & 0. & & & & 33.82 \\
\hline 16.69 & & -0.0 & -1.59 & & 0.0217 & & \\
\hline 11.74 & 0.0025 & -0.0033 & -5.08 & 134.42 & $0.012+3$ & -0.0041 & 37.17 \\
\hline 11.77 & 0.0024 & -0.0034 & -3.55 & 138.35 & 0.1312 & -0.0035 & 37.86 \\
\hline 5.05 & 0.0025 & -0.0013 & -13.42 & 140.16 & $0 . \$ 26$ & -0.0029 & 39.11 \\
\hline 0.02 & 0.0075 & -0.0023 & 29.73 & 142.63 & 0.0651 & -0.0018 & 40.49 \\
\hline-0.04 & 0.0094 & -0.0036 & 29.01 & 145.78 & 0.0386 & -0.0007 & 41.06 \\
\hline-0.02 & 0.0094 & -0.0034 & 29.11 & 142.52 & $0: 183$ & 0.0000 & 42.94 \\
\hline-0.02 & 0.0092 & -0.0033 & 28.92 & 0.22 & 0.069 & -0.0463 & 30.41 \\
\hline 0.02 & 0.0082 & -0.0033 & 30.11 & & & & \\
\hline
\end{tabular}

High Pressure test 


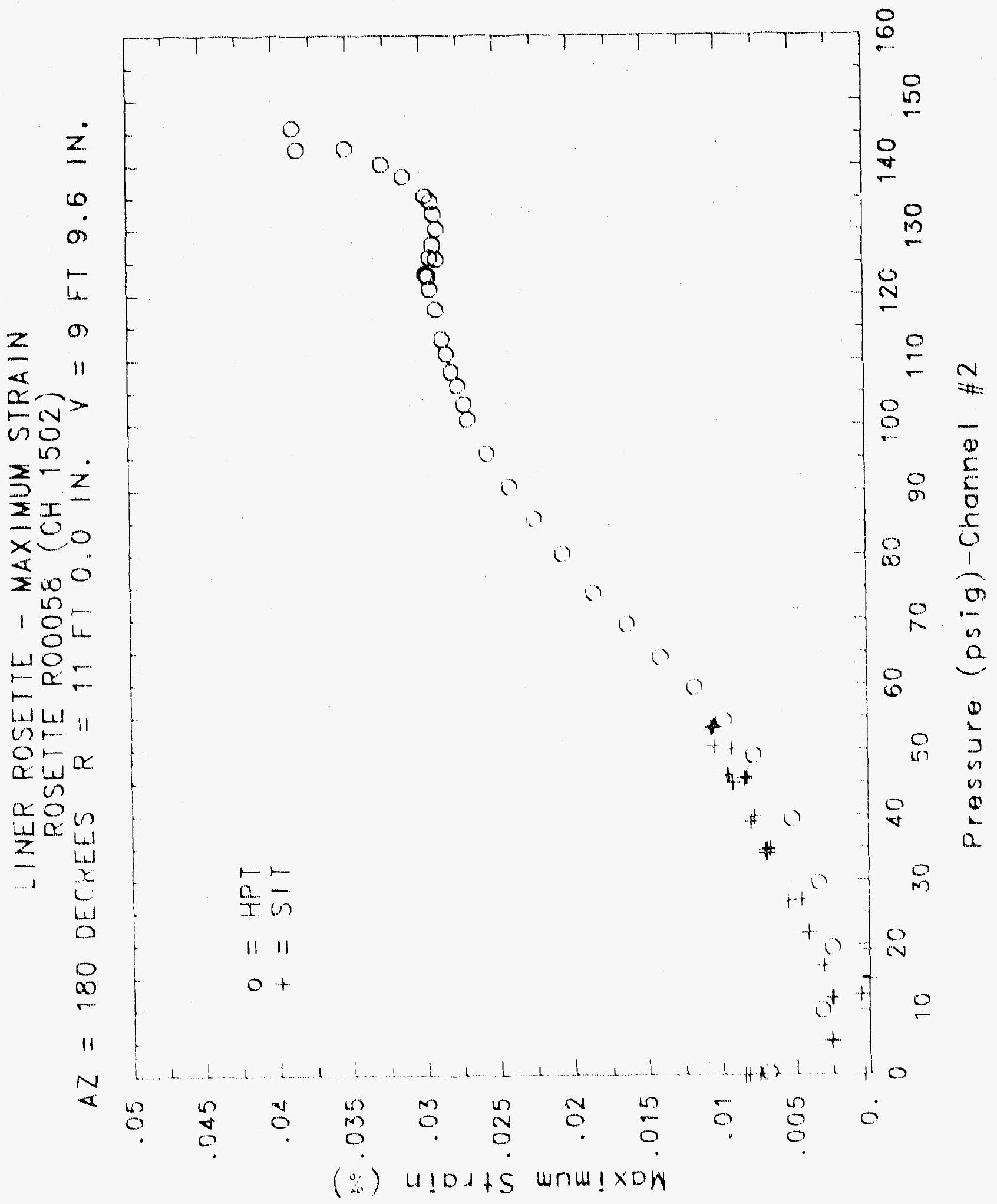

(') (1) : 


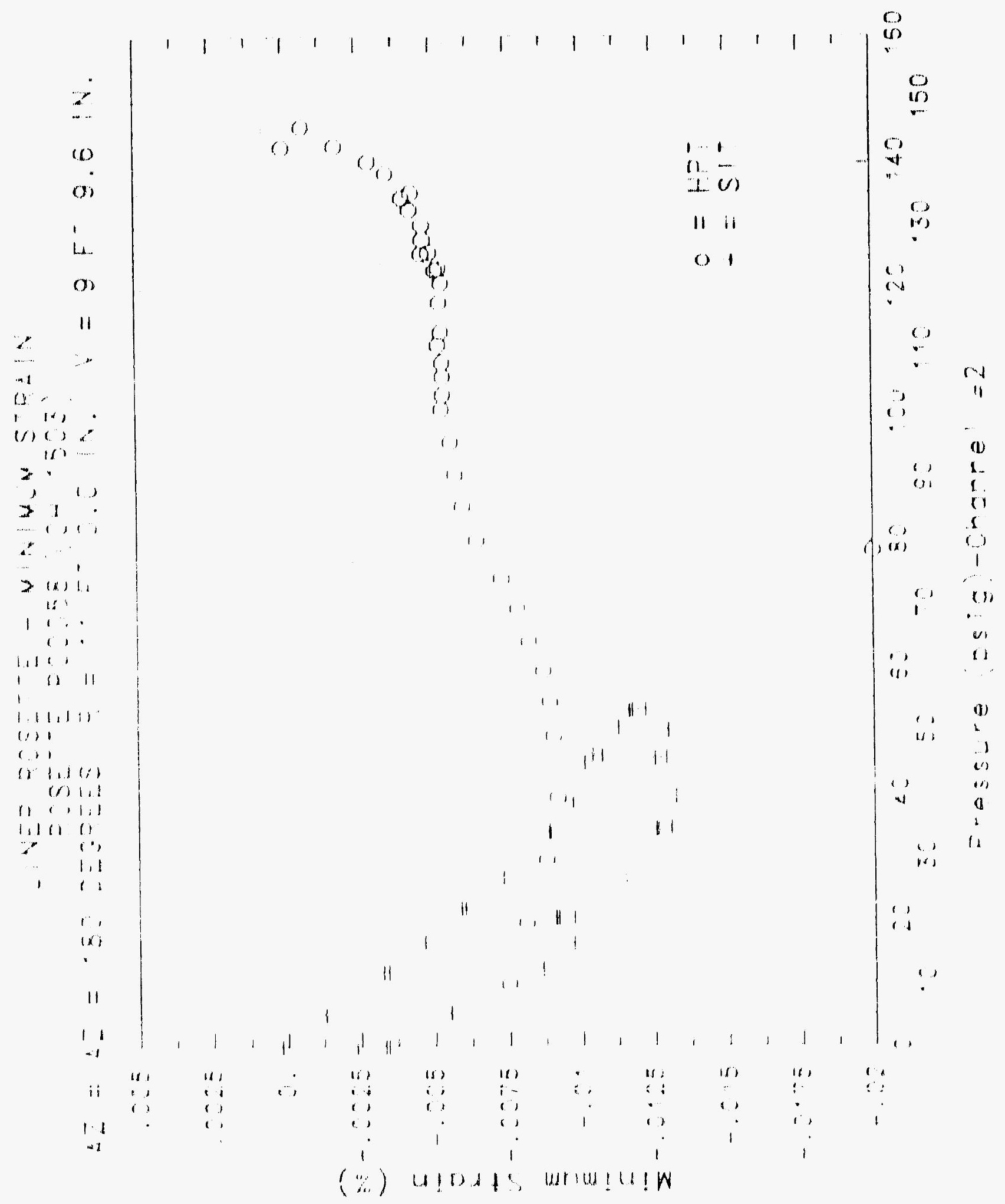




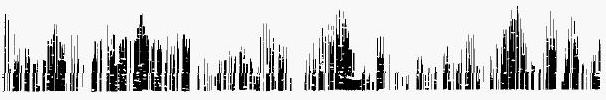

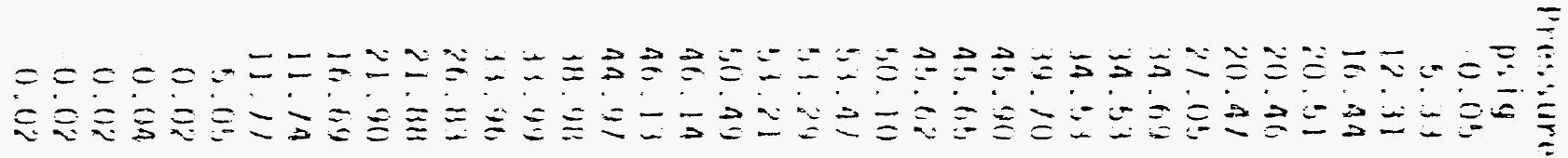

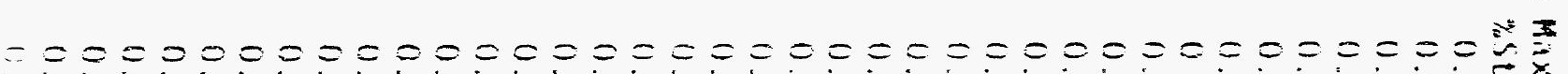

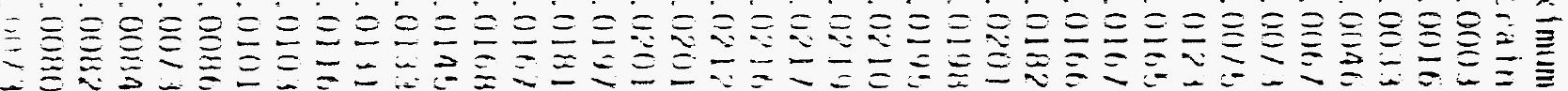

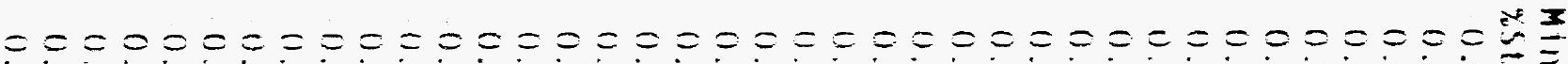

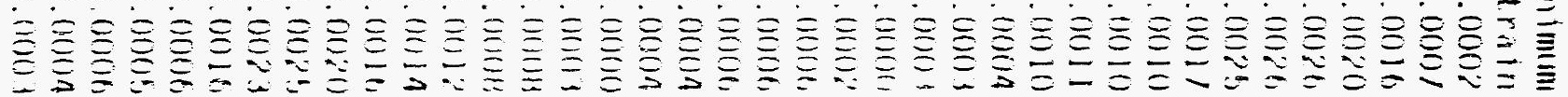

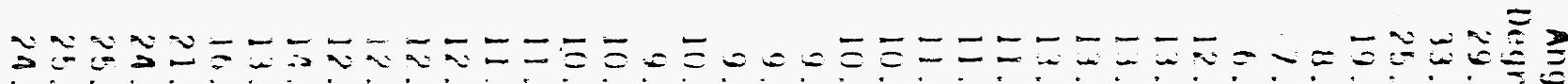

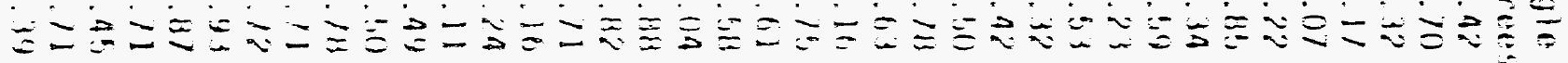

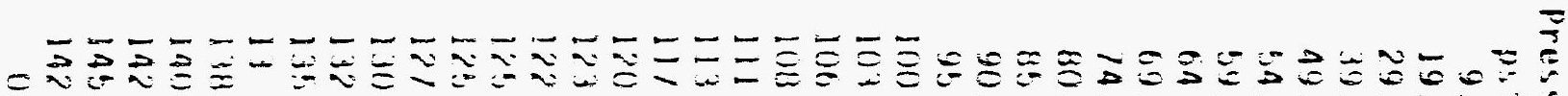

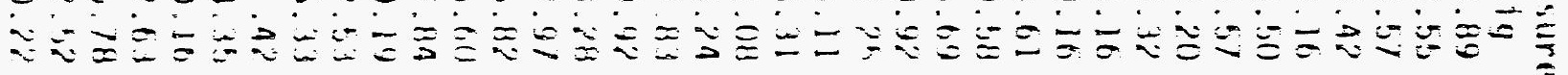

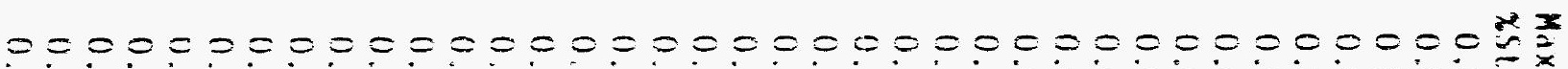

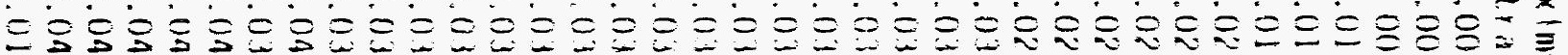

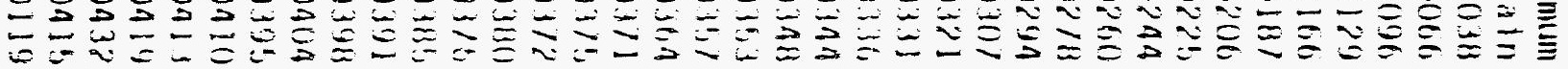

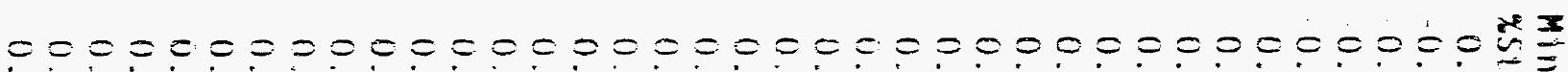

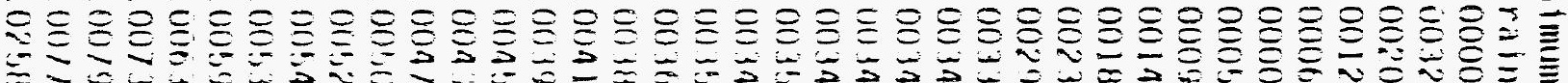

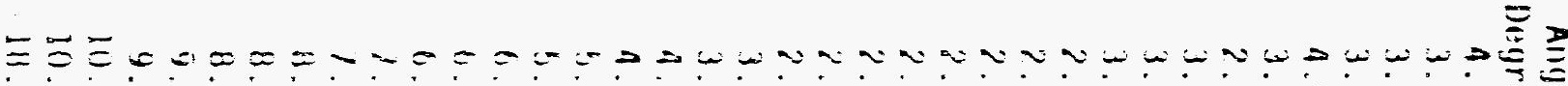

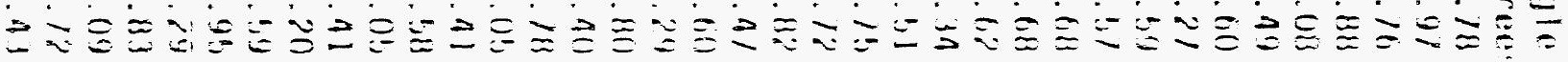




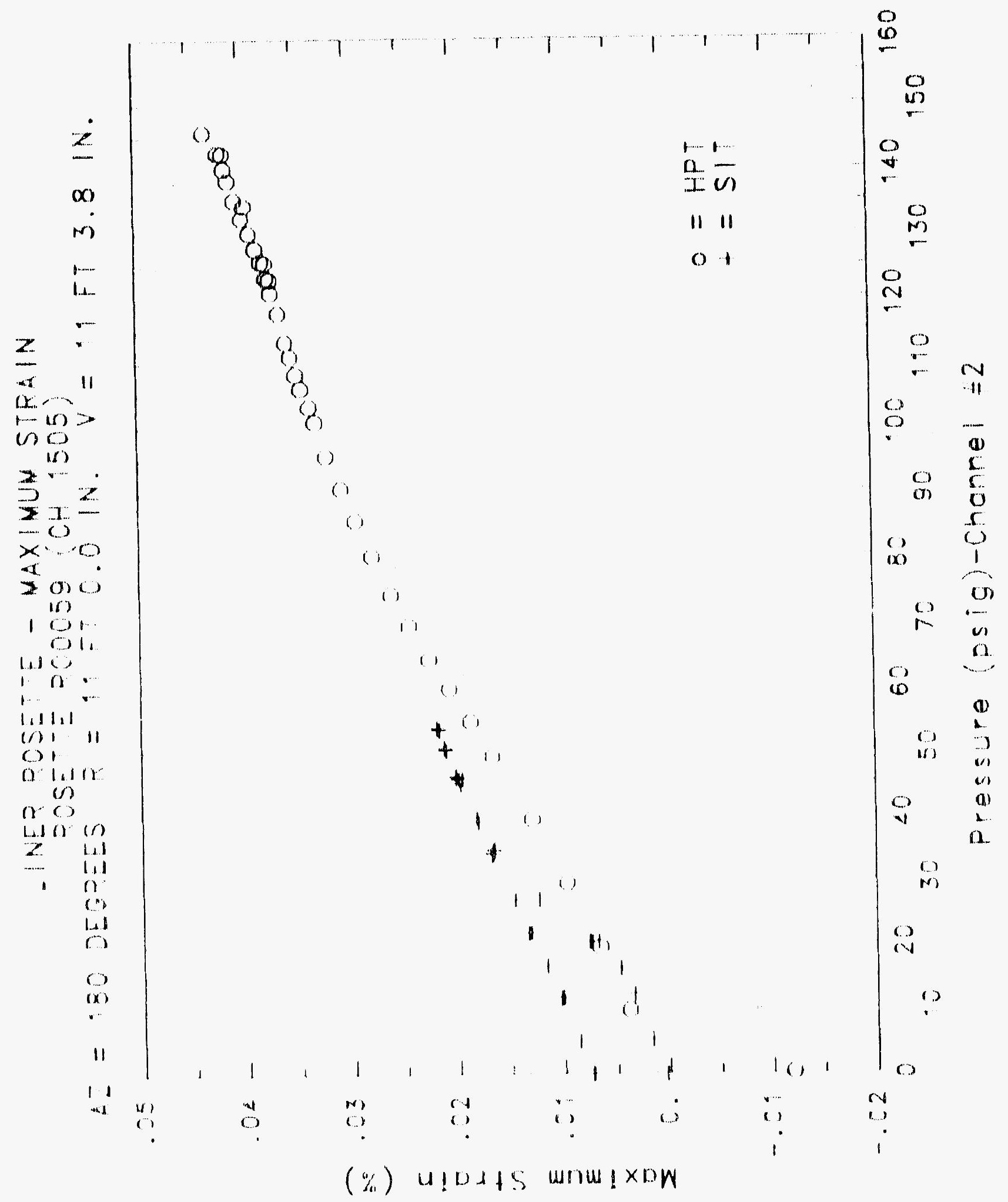




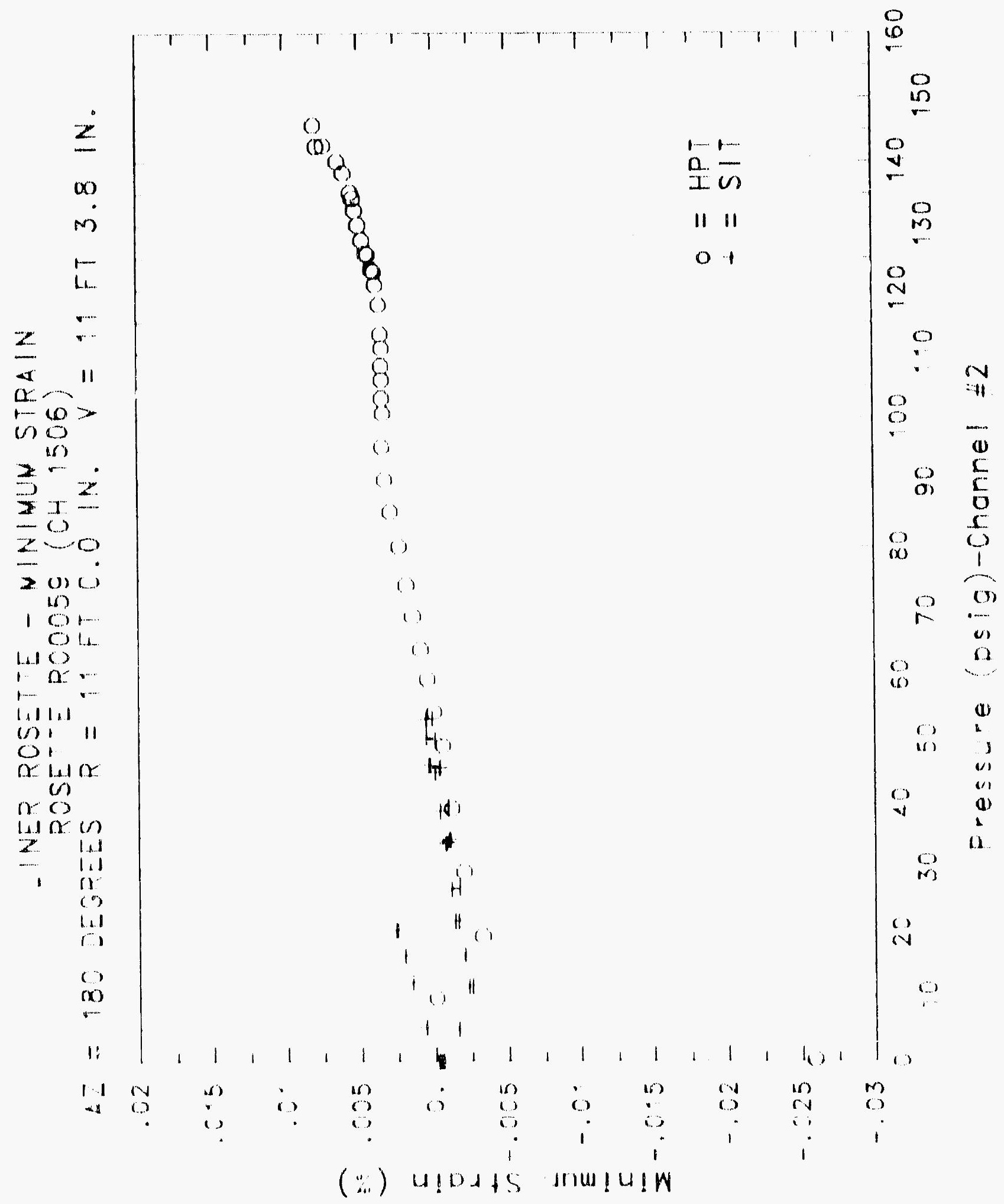




\title{
Reinforced Concrete Test Data
}

\author{
Rosette 60 Channels $1508 \quad 1509 \quad 1520$
}

STRUCTURAL INTEGRITY TEST

\begin{tabular}{|c|c|c|c|}
\hline Pressure & Maximum & Minimum & Angle \\
\hline psig & xstrain & xstrain & Degrees \\
\hline-0.05 & 0.0003 & 0.0000 & 41.90 \\
\hline 5.33 & 0.0019 & -0.0035 & 20.73 \\
\hline 12.31 & 0.0062 & -0.0063 & 21.25 \\
\hline 16.44 & 0.0122 & 0.0053 & 23.72 \\
\hline 30.51 & 0.0223 & 0.0023 & 25.18 \\
\hline 20.46 & 0.0242 & 0.0011 & 25.51 \\
\hline 20.47 & 0.0250 & -0.0006 & 25.68 \\
\hline 27.05 & 0.0397 & 0.0072 & 24.79 \\
\hline 34.69 & $0.05 / 1$ & 0.0174 & 21.85 \\
\hline 3.4 .53 & $0.05 / 3$ & 0.0178 & 21.85 \\
\hline 34.53 & 0.0574 & $0.01 / 9$ & 21.94 \\
\hline 39.10 & 0.0655 & 0.0217 & 21.11 \\
\hline 45.90 & 0.0803 & 0.0274 & 21.14 \\
\hline 45.65 & 0.0816 & 0.0270 & 22.21 \\
\hline 45.62 & 0.0817 & 0.0269 & 22.33 \\
\hline 50.10 & 0.0908 & 0.0294 & 22.31 \\
\hline 53.47 & 0.1000 & 0.0308 & 23.29 \\
\hline 53.29 & 0.1015 & 0.0309 & 23.90 \\
\hline 53.21 & 0.1016 & 0.0310 & 23.97 \\
\hline 50.49 & 0.1001 & 0.0296 & 25.05 \\
\hline 46.14 & 0.0961 & 0.0268 & 26.18 \\
\hline 46.13 & 0.0960 & 0.0266 & 26.09 \\
\hline 44.97 & $0.09 b 1$ & 0.0279 & 27.31 \\
\hline 38.98 & 0.0906 & 0.0235 & 28.88 \\
\hline 33.99 & 0.0862 & 0.0193 & 29.90 \\
\hline 33.96 & 0.0860 & 0.0192 & 29.78 \\
\hline 26.83 & 0.0794 & 0.0126 & 30.76 \\
\hline 21.88 & 0.0749 & 0.0080 & 31.45 \\
\hline 21.90 & 0.0743 & 0.0030 & 31.21 \\
\hline 16.69 & 0.0700 & 0.0028 & 31.86 \\
\hline 11.14 & 0.0653 & -0.0018 & 32.34 \\
\hline 11.17 & 0.0648 & 0.0021 & 31.82 \\
\hline 5.05 & 0.0591 & .0 .0044 & 32.94 \\
\hline 0.02 & 0.0496 & 0.0028 & 37.20 \\
\hline-0.04 & 0.0479 & 0.0031 & 34.08 \\
\hline 0.02 & 0.0488 & .0 .0025 & 35.21 \\
\hline 0.02 & 0.0487 & 0.0023 & 35.46 \\
\hline 0.02 & 0.0483 & 0.0023 & 35.42 \\
\hline
\end{tabular}

high Pressure test

\begin{tabular}{|c|c|c|c|}
\hline Pressure & Maxtmum & Minimum & Angle \\
\hline pstg & xStrain & xstrain & Degrees \\
\hline 9.89 & 0.0142 & -0.0022 & 18.16 \\
\hline 19.55 & 0.0239 & 0.0051 & 16.29 \\
\hline 29.57 & 0.0343 & 0.0136 & 14.23 \\
\hline 39.42 & 0.0444 & 0.0216 & 12.07 \\
\hline 49.16 & 0.0544 & 0.0289 & 9.15 \\
\hline 54.50 & 0.0603 & 0.0334 & 8.23 \\
\hline 59.57 & 0.0723 & 0.0354 & 10.58 \\
\hline 64.20 & 0.0866 & 0.0376 & 14.37 \\
\hline 69.32 & 0.1060 & 0.0395 & 16.23 \\
\hline 74.16 & 0.1243 & 0.0412 & 14.83 \\
\hline 80.16 & 0.1441 & 0.0468 & $11.9 ?$ \\
\hline 85.61 & 0.1687 & 0.0532 & 9.11 \\
\hline 90.58 & 0.1915 & 0.0613 & 5.88 \\
\hline 35.69 & 0.2190 & 0.0746 & 1.66 \\
\hline 100.92 & 0.2530 & 0.0828 & -1.25 \\
\hline 103.25 & 0.2661 & 0.0875 & 2.11 \\
\hline 106.11 & 0.2783 & 0.0909 & -2.79 \\
\hline 108.31 & 0.2886 & 0.0936 & .3 .11 \\
\hline 111.08 & 0.3024 & 0.0968 & -3.39 \\
\hline 113.24 & 0.3115 & 0.0993 & -3.38 \\
\hline 117.83 & 0.3289 & 0.1036 & -3.68 \\
\hline 120.92 & 0.3410 & 0.1070 & -3.11 \\
\hline 123.28 & 0.3504 & 0.1102 & -2.46 \\
\hline 122.97 & 0.3514 & 0.1106 & -2.32 \\
\hline 125.82 & 0.3608 & 0.1133 & -1.91 \\
\hline 125.60 & 0.3627 & 0.1139 & -1.77 \\
\hline 12.7 .84 & 0.3695 & 0.1157 & $=1.51$ \\
\hline 130.19 & 0.3806 & 0.1174 & -1.02 \\
\hline 132.53 & 0.3987 & 0.1199 & 0.49 \\
\hline 135.33 & 0.4431 & 0.1254 & 0.99 \\
\hline 134.42 & 0.4534 & 0.1258 & 1.45 \\
\hline 138.35 & 0.5256 & 0.1341 & 3.14 \\
\hline 140.16 & 0.6028 & 0.1396 & 4.17 \\
\hline 142.63 & 0.1175 & 0.1499 & 5.10 \\
\hline 145.78 & 0.8276 & 0.1655 & 4.92 \\
\hline 142.52 & 0.8585 & 0.1692 & 3.09 \\
\hline 0.22 & 0.5860 & 0.0298 & -0.08 \\
\hline
\end{tabular}




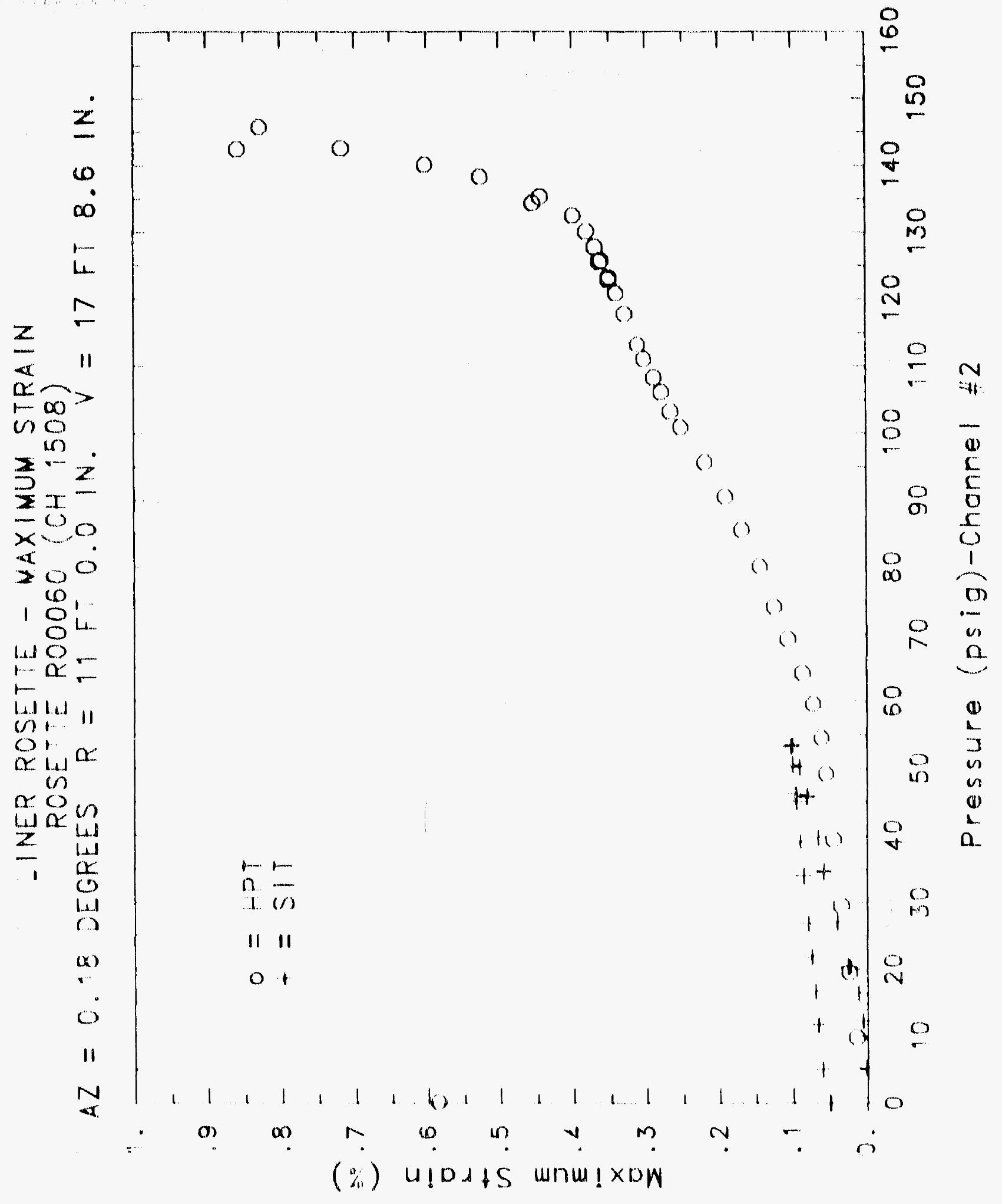




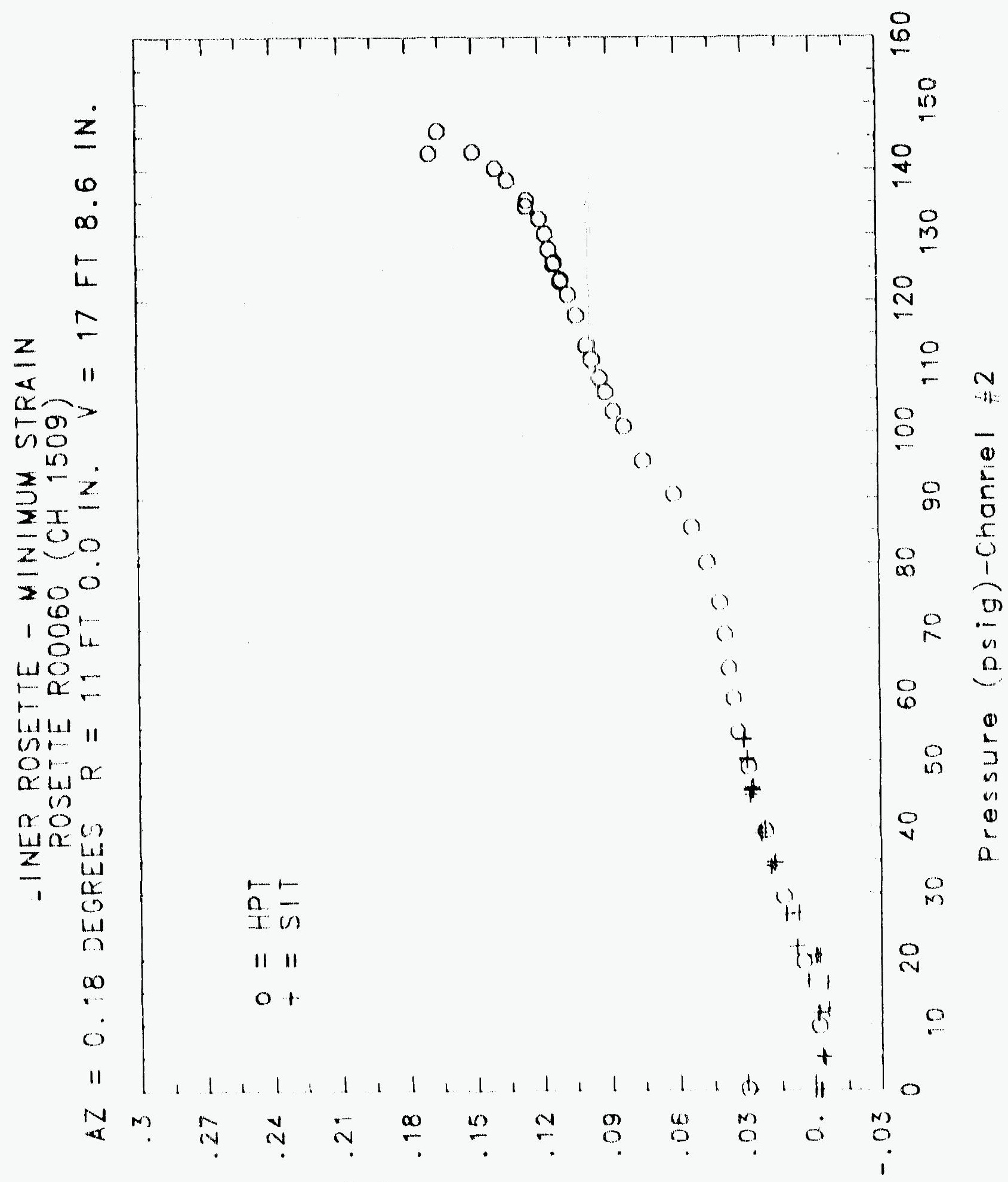

(\%) $4 ! 0 .+5$ wnแ! $4 ! w$ 


\section{Reinforced Concrete Test Data}

\section{Rosetre 61 Chamnels $1521 \quad 1522 \quad 1523$}

\section{STRUCTURAL INTEGRITY TEST}

\begin{tabular}{cccc}
\hline & & & \\
Pressure & Maximum & Minimum & Angle \\
psig & xStrain & \%Stratn & Degrees \\
-0.05 & 0.0001 & -0.0007 & 31.07 \\
5.33 & 0.0043 & -0.0002 & -10.68 \\
12.31 & 0.0112 & 0.0009 & -9.83 \\
16.44 & 0.0274 & 0.0006 & 0.57 \\
20.51 & 0.0452 & 0.0041 & 4.27 \\
20.46 & 0.0468 & 0.0063 & 5.10 \\
20.47 & 0.0470 & 0.0077 & 4.95 \\
27.05 & 0.0691 & 0.0159 & 6.42 \\
34.69 & 0.1001 & 0.0217 & 3.76 \\
34.53 & 0.1018 & 0.0227 & 3.74 \\
34.53 & 0.1022 & 0.0234 & 3.96 \\
39.70 & 0.1203 & 0.0283 & 3.06 \\
45.90 & 0.1453 & 0.0391 & 2.21 \\
45.65 & 0.1471 & 0.0396 & 2.27 \\
45.62 & 0.1477 & 0.0396 & 2.29 \\
50.10 & 0.1606 & 0.0408 & 0.51 \\
51.41 & 0.1740 & 0.0405 & -0.53 \\
53.29 & 0.1768 & 0.0403 & 0.58 \\
53.21 & 0.1180 & 0.0403 & -0.61 \\
50.49 & 0.1742 & 0.0398 & -0.82 \\
46.14 & 0.1648 & 0.0387 & 1.10 \\
46.13 & 0.1645 & 0.0383 & -1.13 \\
41.97 & 0.1617 & 0.0357 & -1.84 \\
38.98 & 0.1476 & 0.0337 & -2.55 \\
33.99 & 0.1344 & 0.0314 & -3.47 \\
33.96 & 0.1339 & 0.0313 & -3.50 \\
26.83 & 0.1143 & 0.0275 & -5.29 \\
21.88 & 0.1002 & 0.0243 & -6.82 \\
21.90 & 0.1002 & 0.0241 & -6.81 \\
16.69 & 0.0853 & 0.0201 & -9.34 \\
11.74 & 0.0705 & 0.0154 & -12.72 \\
11.77 & 0.0705 & 0.0153 & -12.55 \\
5.05 & 0.0570 & 0.0080 & -19.35 \\
0.02 & 0.0396 & 0.0010 & -26.97 \\
-0.04 & 0.0389 & -0.0014 & -25.48 \\
-0.02 & 0.0393 & -0.0031 & 25.91 \\
-0.02 & 0.0388 & 0.0034 & -26.22 \\
0.02 & 0.0384 & -0.0033 & -26.24
\end{tabular}

Hygh Pressure Test

\begin{tabular}{|c|c|c|c|}
\hline Pressure & Maximum & Minfrmum & Angle \\
\hline pstg & xstrain & xstrain & Degree \\
\hline 9.89 & 0.0314 & 0.0071 & 8.65 \\
\hline 19.55 & 0.0645 & 0.0141 & 8.83 \\
\hline 29.57 & 0.0963 & 0.0205 & 8.54 \\
\hline 39.42 & 0.1255 & 0.0260 & 8.13 \\
\hline 49.16 & 0.1538 & 0.0305 & 7.94 \\
\hline 54.50 & 0.1702 & 0.0345 & 7.20 \\
\hline 59.5 & 0.1869 & 0.0324 & -0.84 \\
\hline 64.2 & 0.2292 & 0.01 & -13.05 \\
\hline $6 y .3$ & & 96 & -19.15 \\
\hline 74.1 & 0.4 & -0.0 & -14.85 \\
\hline 80.16 & & -0.0 & 7.35 \\
\hline 85.61 & & -0.1 & 16.17 \\
\hline & & -0 & \\
\hline & & & \\
\hline 100. & 1.6 & $-0 . i^{A}$ & 15.44 \\
\hline 103.2 & 1.72. & -0.28 & 15.43 \\
\hline 106.11 & 1.793 & -0.3 & 15.47 \\
\hline & & -0 & 15.60 \\
\hline & & 0. & 15.72 \\
\hline & 2.0 & -0 . & 15.81 \\
\hline & 2.23 & -0.4503 & $15.9 \alpha$ \\
\hline 120.92 & 2.37 & -0.4938 & 16.02 \\
\hline 123.28 & 2.500 & -0.5322 & 16.15 \\
\hline & 2.5 & -0.5 & 16.18 \\
\hline & 2.6 & -0. & 16.2 \\
\hline .60 & 2.72 & -0.6 & 16.2 \\
\hline 127.84 & 2.85 & 0.6435 & 16.34 \\
\hline 130.19 & 3.0816 & -0.7159 & 16.41 \\
\hline 132.53 & 3.4001 & -0.8165 & 16.50 \\
\hline 135.33 & 3.8587 & -0.95 & 16.6 \\
\hline 134.42 & 3.9524 & -0.98 & 16.7 \\
\hline 138.35 & 4.60 & -1.11 & 16.9 \\
\hline 140.16 & 5.2080 & -1.3201 & 17.32 \\
\hline 142.63 & 6.2945 & -1.5867 & 18.2 \\
\hline 40 & 7.7082 & -1.9271 & 19.40 \\
\hline $142.5 ?$ & 8.2100 & -2.0605 & $19.7 ?$ \\
\hline 0.22 & 7.0378 & -1.8797 & 19.91 \\
\hline
\end{tabular}




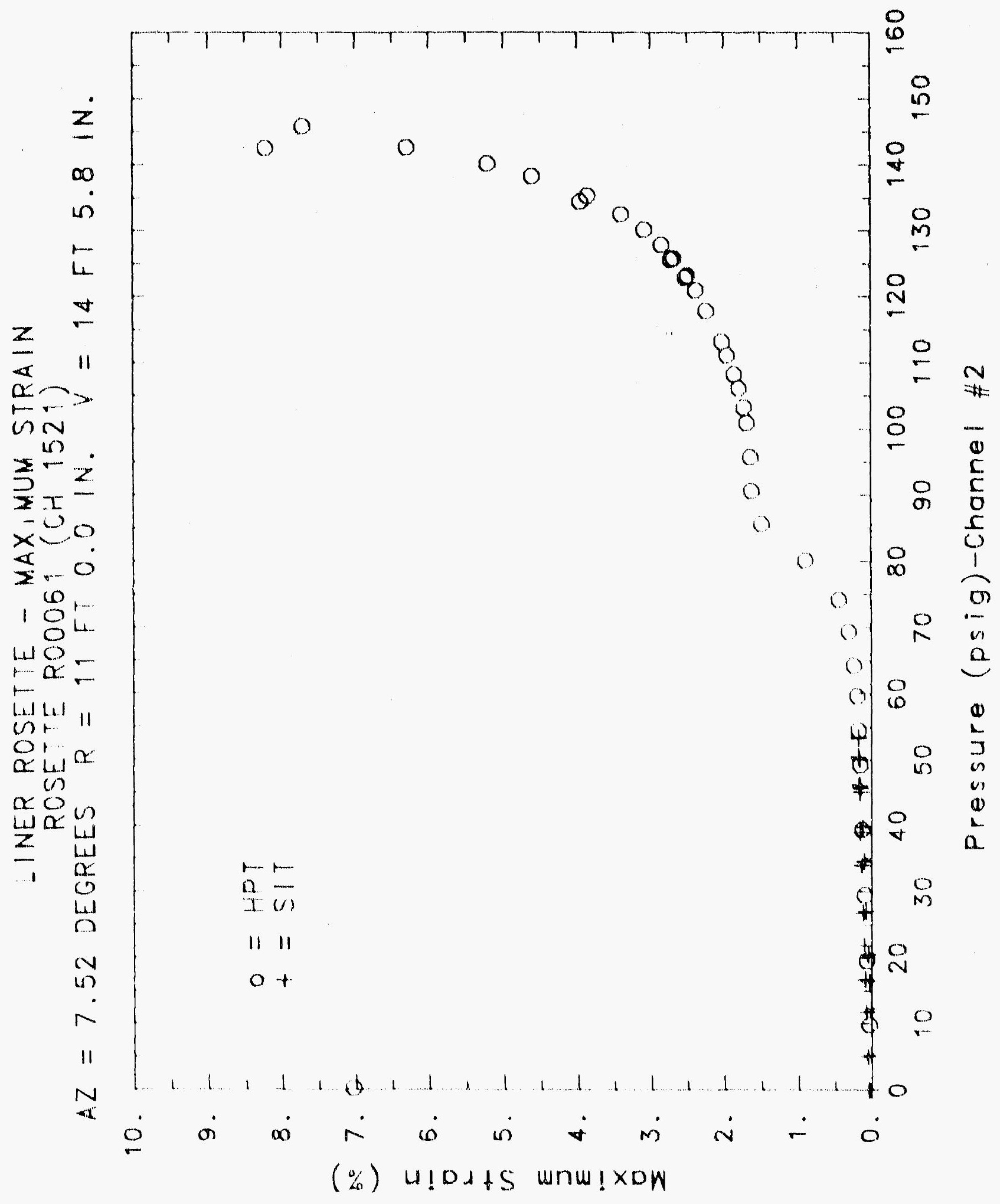




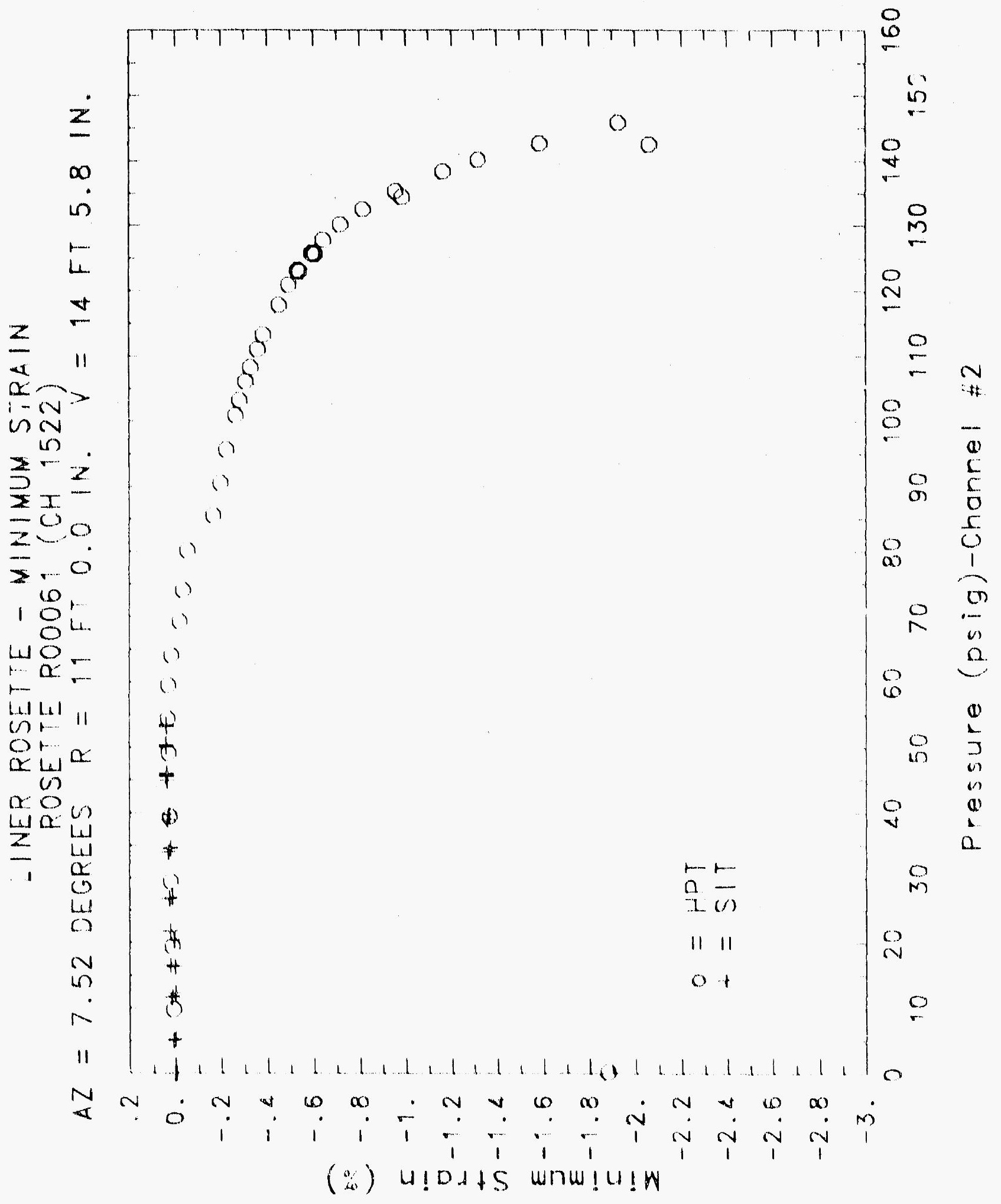




\title{
Reinforced Concrete Test Data
}

\author{
Rosette 62 Channels $1524 \quad 1525 \quad 1526$
}

StRUCTURAL INTEgRITY TEST
Ninimum

sistrain

0.0001

$-0.0041$

$0.05 \quad 0.0004$

$\begin{array}{lll}5.33 & -0.0029\end{array}$

12.310 .0018

$16.44 \quad 0.0054$

20.510 .0115

$20.46 \quad 0.0122$

$20.47 \quad 0.0128$

$2 \% .05 \quad 0.0248$

34.0190 .0410

$34.53 \quad 0.0422$

34.53

39.70

45.90

45.65

45.62

50.10

5.3 .47

53.29

53.21

50.49

46.14

46.13

$44.9 \%$

38.98

33.99

33.96

26.83

21.88

21.90

16.69

11.74

11.77

5.05

0.02

$-0.04$

$-0.02$

$-0.02$

0.02
$-0.0022$

$-0.0011$

0.0013

0.0026

0.0030

0.0079

0.0129

0.0135

0.0134

0.0154

0.0200

0.0195

0.0195

0.0222

0.0241

0.0241

0.0241

0.0234

0.0219

$0.021 \%$

0.0245

0.0223

0.0206

0.0205

0.0181

0.0163

0.0162

0.0143

0.0121

0.0113

0.0073

$-0.0015$

$-0.0001$

0.0000

$-0.0001$

0.0001

High Pressure Test

Angle Pressure Haximum

Degrees

8.16

$-27.70$

$-2.69$

$-1.01$

$-2.06$

$-0.69$

$-0.80$

$-5.91$

$-6.30$

$-6.38$

$-6.78$

$-5.72$

$-3.84$

$-4.39$

$-4.38$

.3 .68

$-3.36$

$-3.21$

$-3.25$

$-3.22$

$-3.61$

.3 .68

$-1.48$

$-1.18$

$-1.25$

$-1.68$

$-2.13$

$-3.29$

$-3.51$

$-5.43$

$-9.18$

$-10.50$

39.55

9.62

18.63

11. 32

10.13

8.10 psig

g. 89

19.55

29.57

39.42

49.16

54.50

$59.5 \%$

64.20

69.32

74.16

80.16

85.61

90.58

95.69

100.92

103.25

106.11

108.31

111.08

113.24

117.83

120.92

123.28

122.97

125.82

125.60

127.84

130.19

132.53

135.33

134.42

138.35

140.16

142.63

145.78

142.52

0.22

\section{strain}

0.0159

0.0305

0.0454

0.0593

0.0722

0.0798

0.0876

0.0963

0.1058

0.1154

0.1258

0.1353

0.1440

0.1533

0.1634

0.1685

0.1740

0.1784

0.1854

0.1917

0.2070

0.2192

0.2305

ก. 2326

0.2458

0.2498

0.2584

0.2768

0.3012

0.3364

0.3456

0.3960

0.4457

0.5348

$0.6 \% 55$

0.7414

0.5908
Minimum Angle

zstrain Degrees

$0.0042-0.44$

$0.0082 \quad 1.24$

$0.0122 \quad 1.42$

$0.0163 \quad 1.61$

$0.0201 \quad 1.10$

$0.0225 \quad 1.32$

$0.0246 \quad 1.20$

$0.0272 \quad 1.23$

$0.0296 \quad 1.25$

$0.0322 \quad 0.95$

$0.0331 \quad 0.03$

$0.0355 \quad-0.70$

$0.0374-1.65$

$0.0389 \quad-2.94$

$0.0401 \quad 4.46$

$0.0403 \quad-5.09$

$0.0407 \quad-5.79$

$0.0411-6.31$

$0.0412 \quad . .1 .04$

(1. $.0412-7.48$

$\begin{array}{ll}0.0412 & 8.40\end{array}$

$\begin{array}{ll}1.0414 & -8.85\end{array}$

$0.0414 \quad-9.02$

$0.0417-8.97$

$0.0422 \quad-9.09$

$0.0425 \quad-8.99$

$0.0435-8.99$

$0.0447 \quad-8.86$

$0.0469 \quad 8.70$

$0.0502 \quad-.8 .53$

$0.0500 \quad-8.43$

$0.0564 \quad-8.01$

$0.0591 \quad-7.71$

$0.0649-6.96$

$0.0709-6.33$

$0.0653 \quad-5.94$

$\begin{array}{ll}0.0399 & -8.13\end{array}$ 


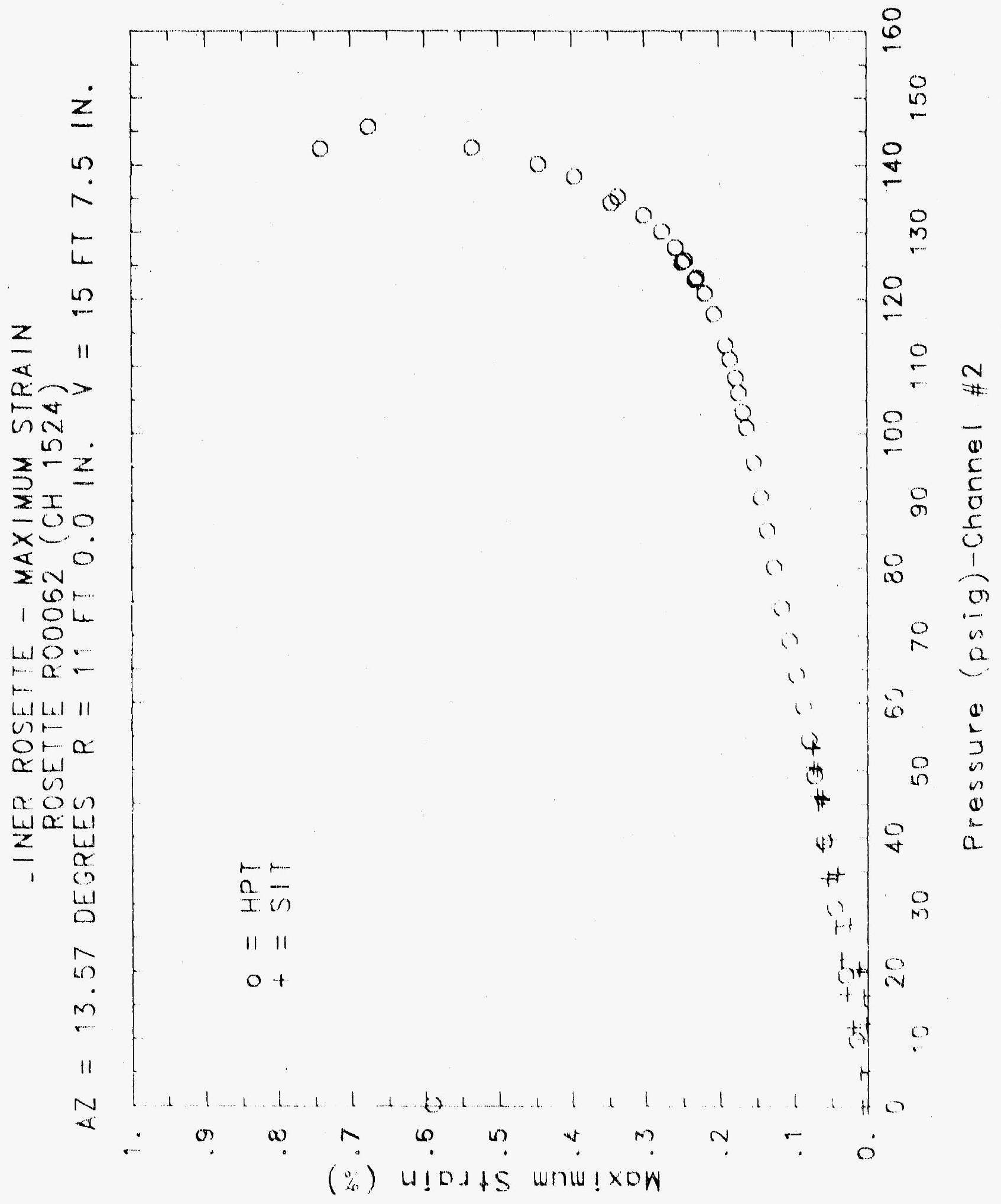




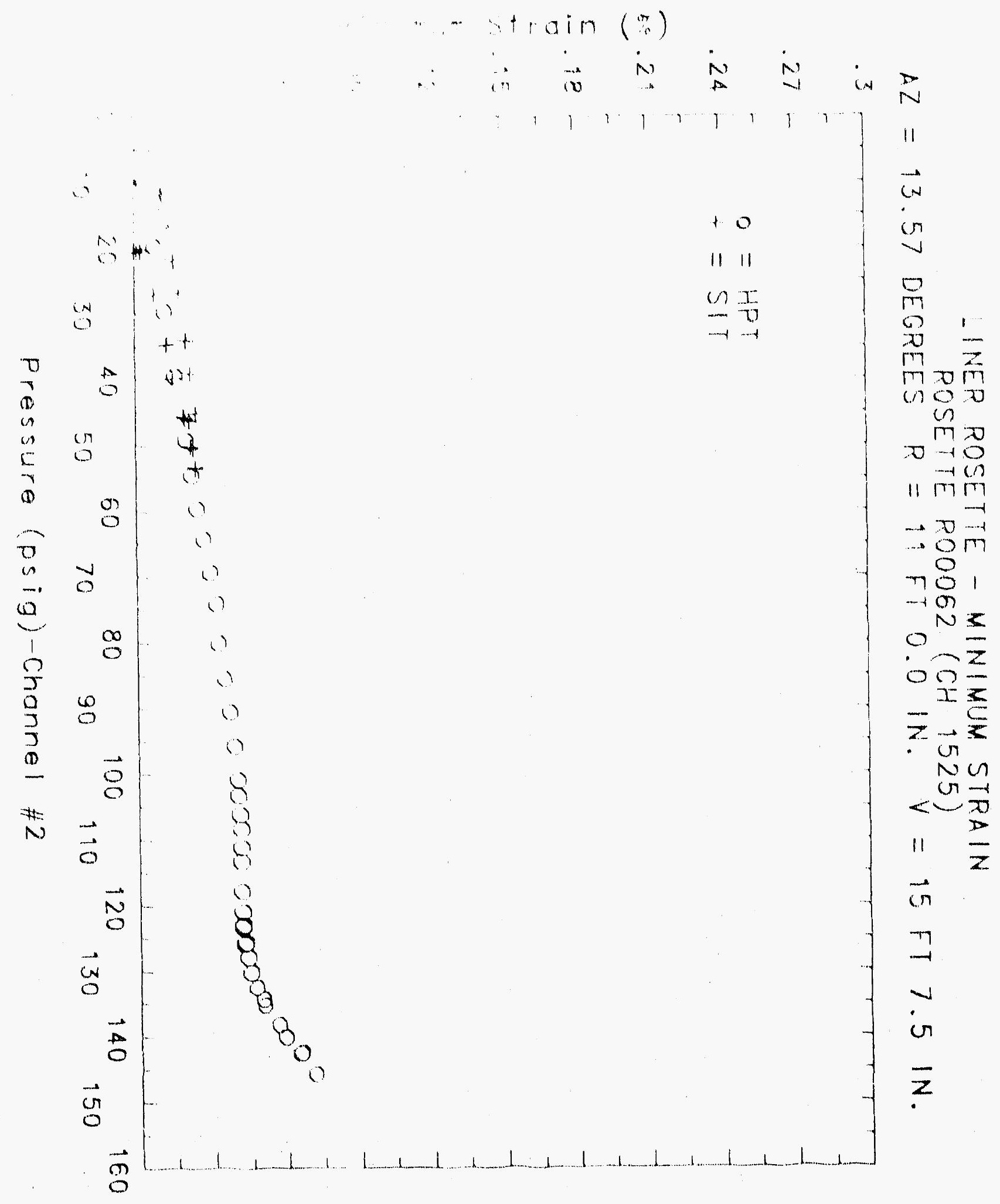




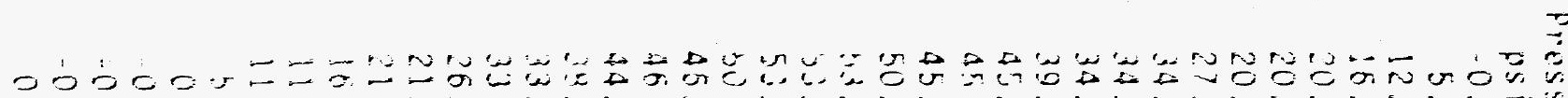

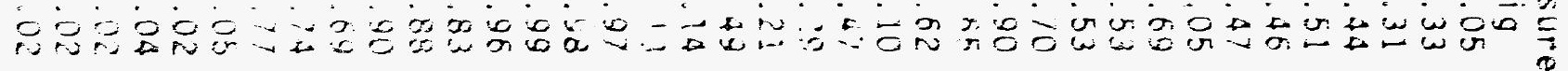
00000000000000000000000000000000000000 in

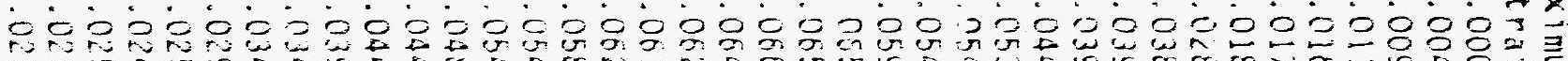

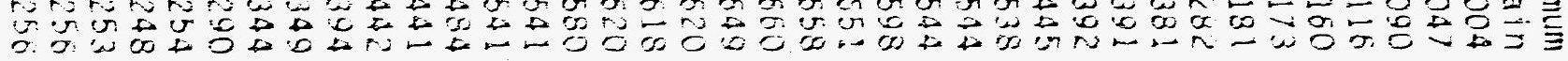

0000000000000000000000000000000000000003

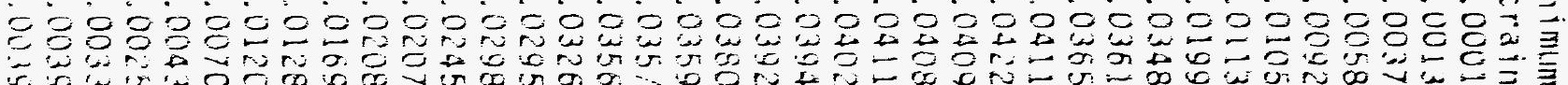

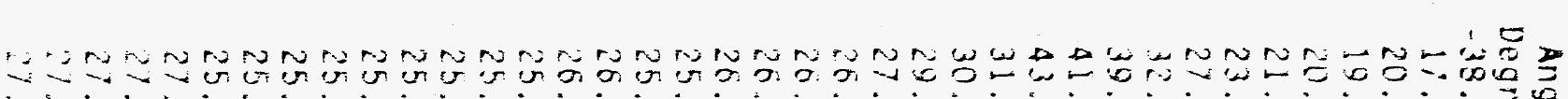

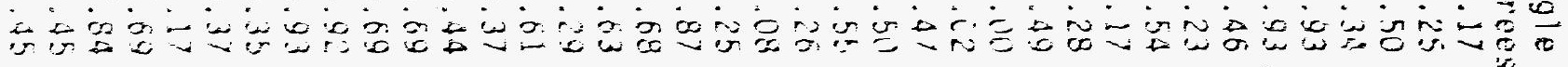

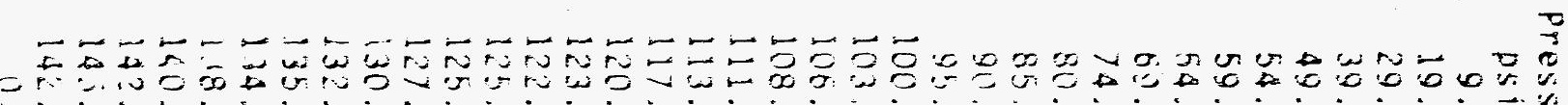

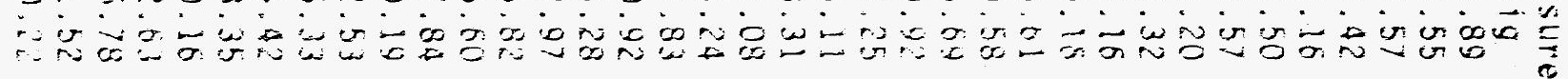

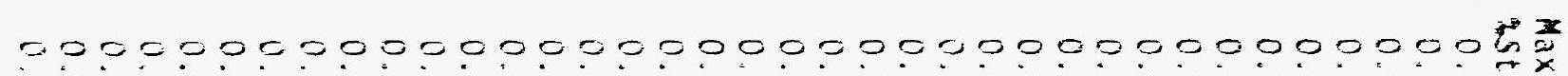

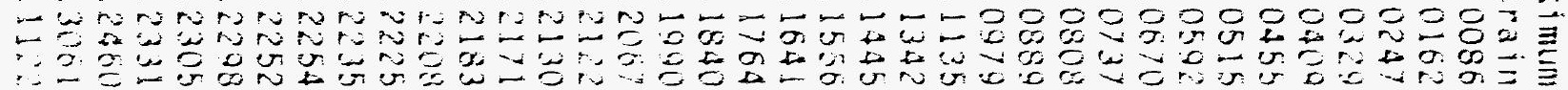

000000000000000000000000000000000000000

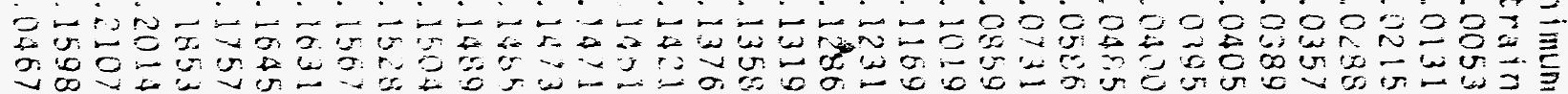

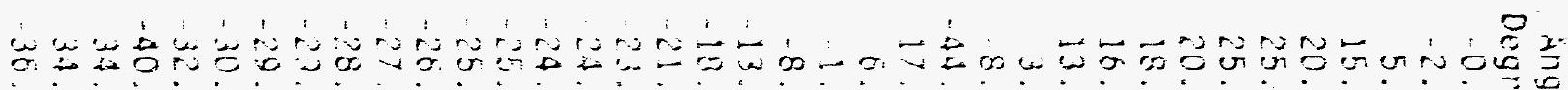

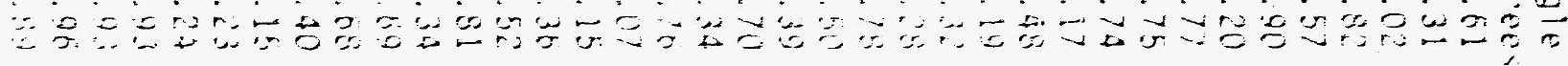




\section{IMAGE EVALUATION TEST TARGET (MT-3)}
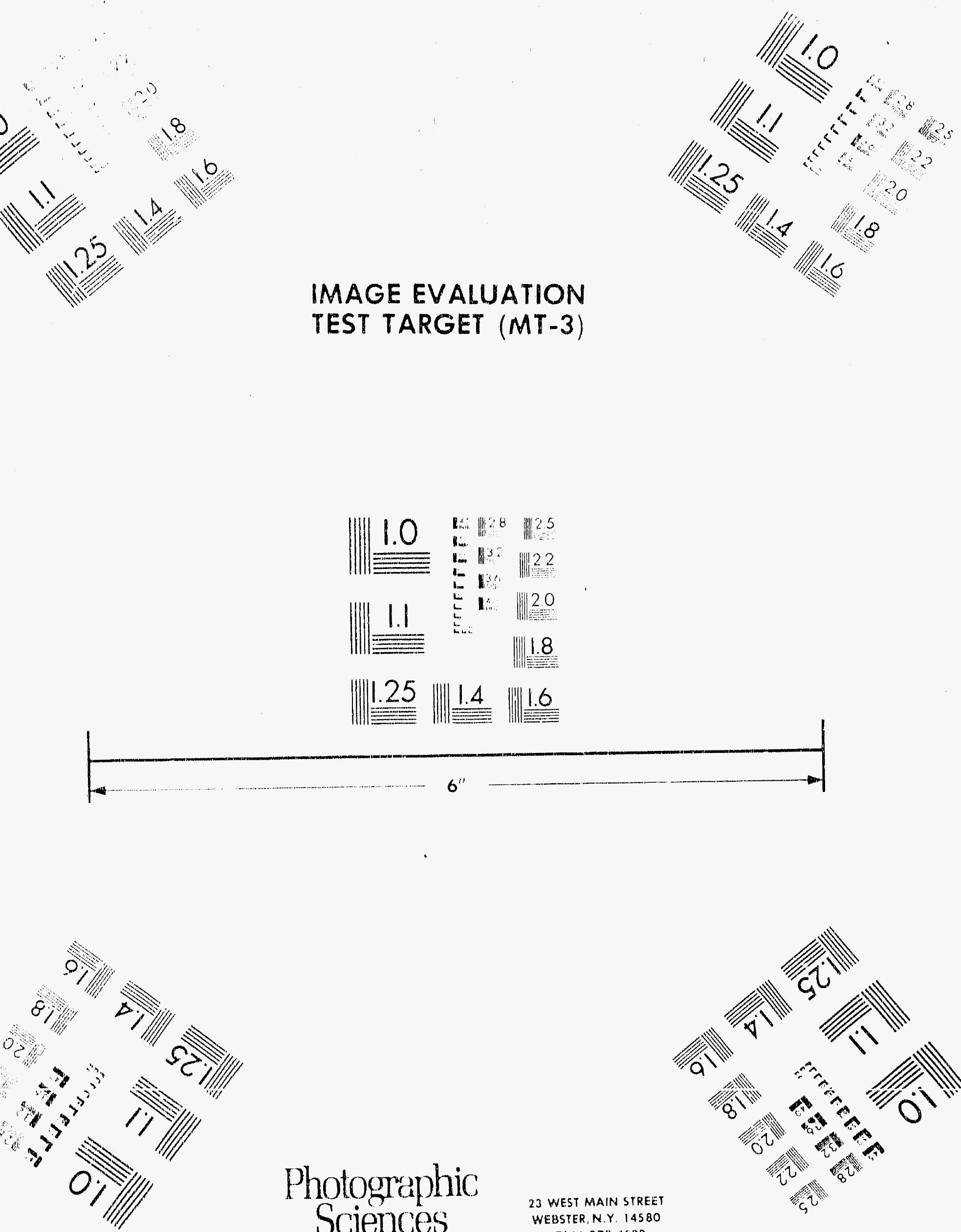

Photographic Sciences Corporation

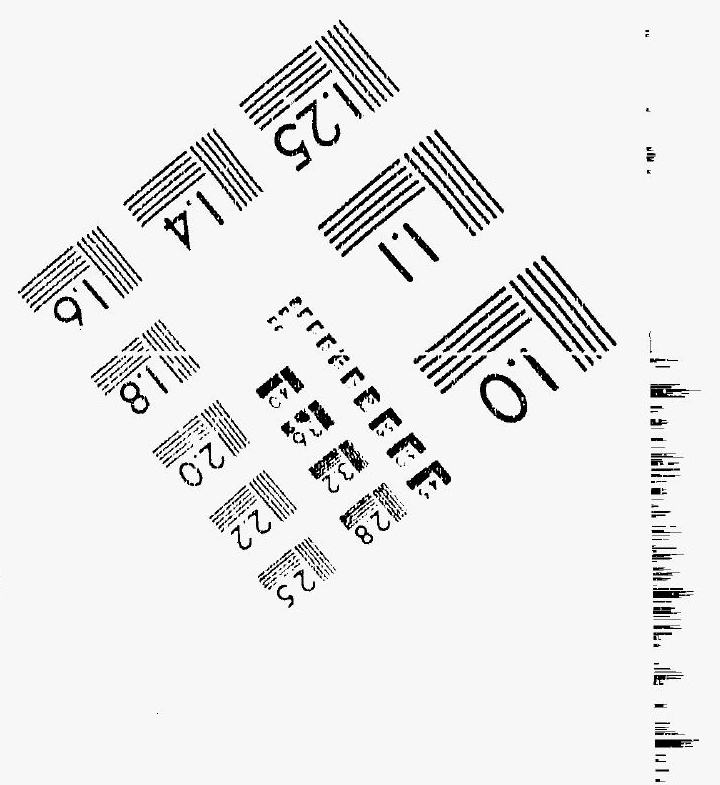




\section{SECURITY CLASSIFICATION}

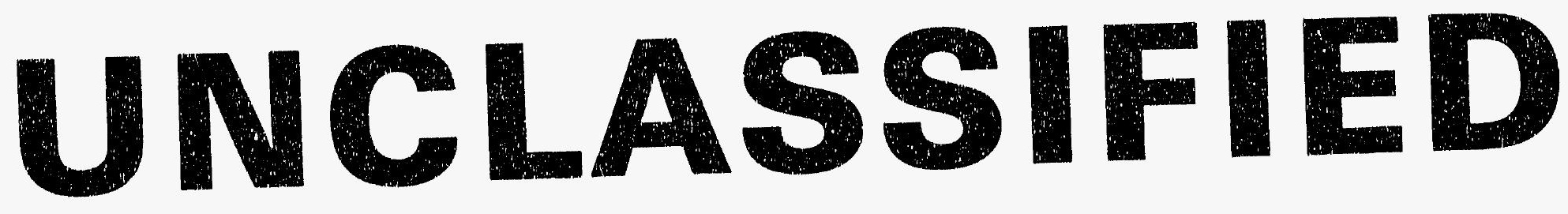

\section{DATE OF MICROFILMING $1-6-92$}

\section{MICROFILMED BY - errir hard}

SANDIA NATIONAL LABS 


\section{THIS DOCUMENT FILMED}

AT

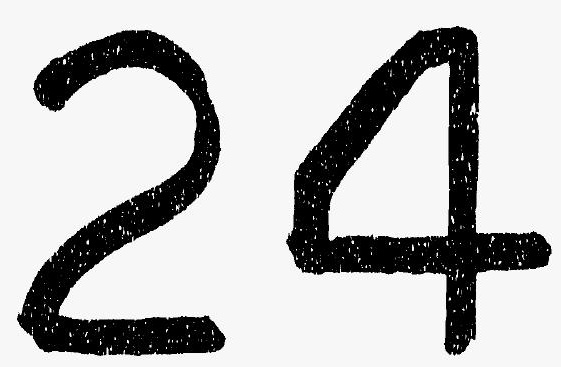

REDUCTION 


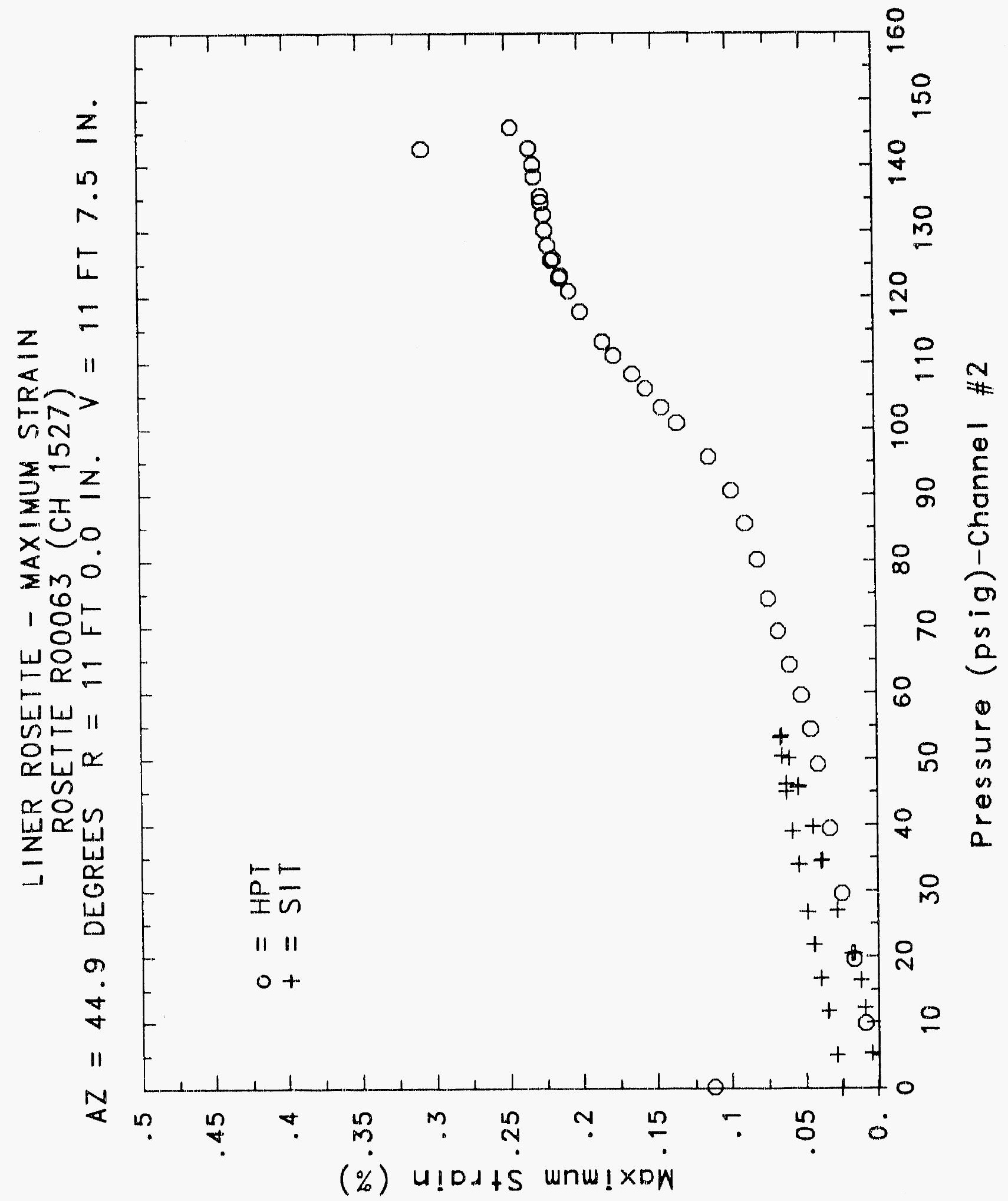




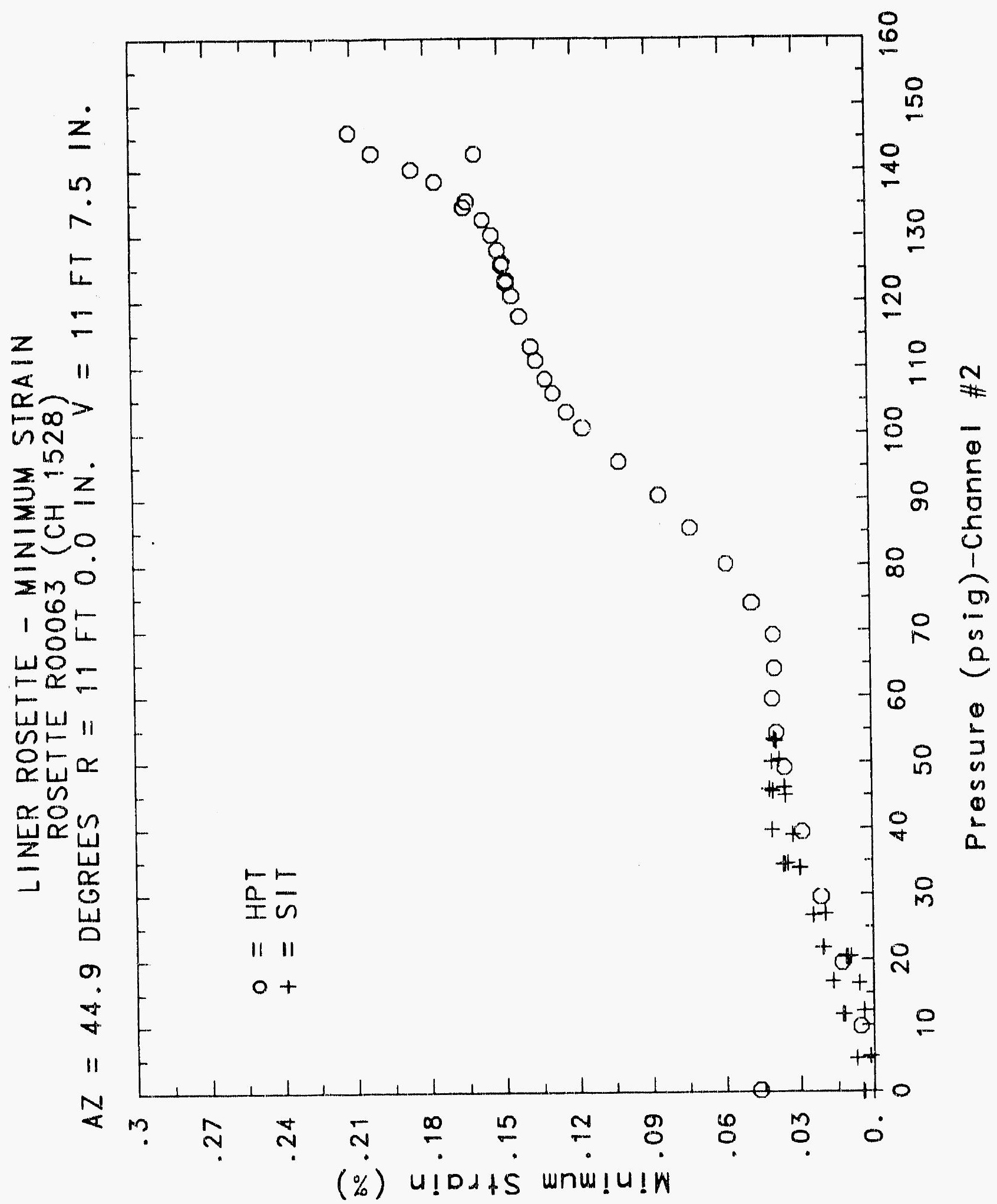




\title{
Reinforced Concrete Test Data
}

\author{
Rosette 64 Channels $1540 \quad 1541 \quad 1542$
}

\section{StRUCTURAL INTEgRITY TEST}

\begin{tabular}{|c|c|c|c|c|c|c|c|}
\hline $\begin{array}{c}\text { Pressure } \\
\text { psig } \\
-0.05 \\
5.33 \\
12.31 \\
16.44 \\
20.51 \\
20.46 \\
20.47 \\
27.05 \\
34.69 \\
34.53 \\
34.53 \\
39.70 \\
45.90 \\
45.65 \\
45.62 \\
50.10 \\
53.47 \\
53.29 \\
53.21 \\
50.49 \\
46.14 \\
46.13 \\
44.97 \\
38.98 \\
33.99 \\
33.96 \\
26.83 \\
21.88 \\
21.90 \\
16.69 \\
11.74 \\
11.77 \\
5.05 \\
0.02 \\
-0.04 \\
-0.02 \\
-0.02 \\
0.02\end{array}$ & $\begin{array}{c}\text { Maximum } \\
\text { \%Strain } \\
0.0003 \\
0.0028 \\
0.0072 \\
0.0111 \\
0.0178 \\
0.0192 \\
0.0201 \\
0.0302 \\
0.0419 \\
0.0425 \\
0.0426 \\
0.0485 \\
0.0567 \\
0.0570 \\
0.0570 \\
0.0618 \\
0.0659 \\
0.0661 \\
0.0662 \\
0.0642 \\
0.0607 \\
0.0605 \\
0.0595 \\
0.0542 \\
0.0493 \\
0.0494 \\
0.0424 \\
0.0374 \\
0.0374 \\
0.0320 \\
0.0267 \\
0.0264 \\
0.0195 \\
0.0145 \\
0.0137 \\
0.0140 \\
0.0142 \\
0.0143\end{array}$ & $\begin{array}{l}\text { Minimum } \\
\text { \%Strain } \\
0.0000 \\
0.0012 \\
0.0026 \\
0.0033 \\
0.0048 \\
0.0051 \\
0.0054 \\
0.0066 \\
0.0085 \\
0.0088 \\
0.0089 \\
0.0100 \\
0.0113 \\
0.0111 \\
0.0110 \\
0.0118 \\
0.0125 \\
0.0126 \\
0.0125 \\
0.0122 \\
0.0116 \\
0.0115 \\
0.0118 \\
0.0110 \\
0.0103 \\
0.0103 \\
0.0092 \\
0.0084 \\
0.0085 \\
0.0076 \\
0.0067 \\
0.0064 \\
0.0048 \\
0.0037 \\
0.0031 \\
0.0035 \\
0.0039 \\
0.0039\end{array}$ & $\begin{array}{c}\text { Angle } \\
\text { Degrees } \\
11.04 \\
8.72 \\
4.30 \\
1.10 \\
0.83 \\
0.97 \\
0.86 \\
-0.17 \\
-0.32 \\
-0.39 \\
-0.42 \\
-0.54 \\
-0.80 \\
-1.22 \\
-1.24 \\
-1.23 \\
-1.18 \\
-1.52 \\
-1.51 \\
-1.64 \\
-1.82 \\
-1.83 \\
-2.08 \\
-2.28 \\
-2.42 \\
-2.42 \\
-2.77 \\
-2.99 \\
-2.69 \\
-2.98 \\
-3.37 \\
-3.50 \\
-3.76 \\
-4.77 \\
-2.52 \\
-2.98 \\
-4.00 \\
-3.96\end{array}$ & $\begin{array}{c}\text { Pressure } \\
\text { psig } \\
9.89 \\
19.55 \\
29.57 \\
39.42 \\
49.16 \\
54.50 \\
59.57 \\
64.20 \\
69.32 \\
74.16 \\
80.16 \\
85.61 \\
90.58 \\
95.69 \\
100.92 \\
103.25 \\
106.11 \\
108.31 \\
111.08 \\
113.24 \\
117.83 \\
120.92 \\
123.28 \\
122.97 \\
125.82 \\
125.60 \\
127.84 \\
130.19 \\
132.53 \\
135.33 \\
134.42 \\
138.35 \\
140.16 \\
142.63 \\
145.78 \\
142.52 \\
0.22\end{array}$ & $\begin{array}{c}\text { Maximum } \\
\text { \%strain } \\
0.0102 \\
0.0206 \\
0.0312 \\
0.0412 \\
0.0510 \\
0.0564 \\
0.0617 \\
0.0670 \\
0.0726 \\
0.0773 \\
0.0819 \\
0.0869 \\
0.0914 \\
0.0954 \\
0.0990 \\
0.1007 \\
0.1024 \\
0.1037 \\
0.1053 \\
0.1064 \\
0.1086 \\
0.1096 \\
0.1104 \\
0.1101 \\
0.1115 \\
0.1113 \\
0.1125 \\
0.1137 \\
0.1160 \\
0.1206 \\
0.1204 \\
0.1322 \\
0.2471 \\
0.8956 \\
1.1933 \\
1.1428 \\
0.9607\end{array}$ & $\begin{array}{c}\text { Minimum } \\
\text { \%strain } \\
0.0024 \\
0.0043 \\
0.0060 \\
0.0076 \\
0.0093 \\
0.0102 \\
0.0113 \\
0.0122 \\
0.0135 \\
0.0151 \\
0.0159 \\
0.0174 \\
0.0189 \\
0.0207 \\
0.0228 \\
0.0237 \\
0.0246 \\
0.0254 \\
0.0265 \\
0.0271 \\
0.0284 \\
0.0290 \\
0.0294 \\
0.0294 \\
0.0299 \\
0.0299 \\
0.0303 \\
0.0307 \\
0.0312 \\
0.0320 \\
0.0321 \\
0.0328 \\
0.0205 \\
-0.0917 \\
-0.0413 \\
-0.0310 \\
-0.0572\end{array}$ & $\begin{array}{c}\text { Angle } \\
\text { Degrees } \\
-1.40 \\
-1.21 \\
-0.73 \\
-0.82 \\
-1.02 \\
-1.10 \\
-0.86 \\
-0.76 \\
-1.06 \\
-0.82 \\
-0.61 \\
-0.61 \\
-0.69 \\
-0.88 \\
-1.09 \\
-1.37 \\
-1.28 \\
-1.37 \\
-1.50 \\
-1.64 \\
-1.87 \\
-1.88 \\
-1.78 \\
-1.82 \\
-1.80 \\
-1.66 \\
-1.62 \\
-1.07 \\
-0.10 \\
1.92 \\
2.54 \\
5.58 \\
-10.10 \\
-18.42 \\
-8.69 \\
-7.20 \\
-7.80\end{array}$ \\
\hline
\end{tabular}

\author{
High Pressure Test
}


$015-3$

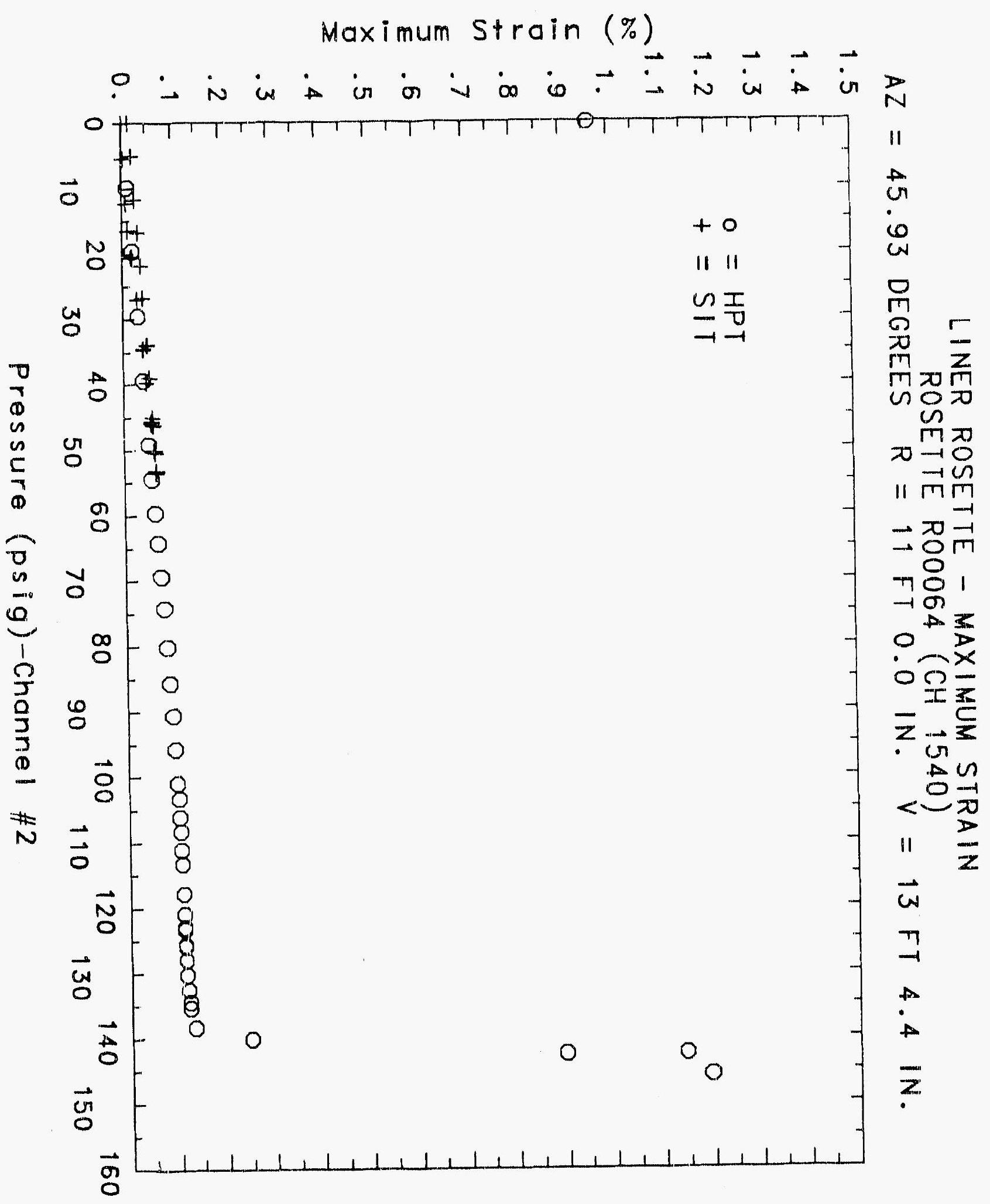




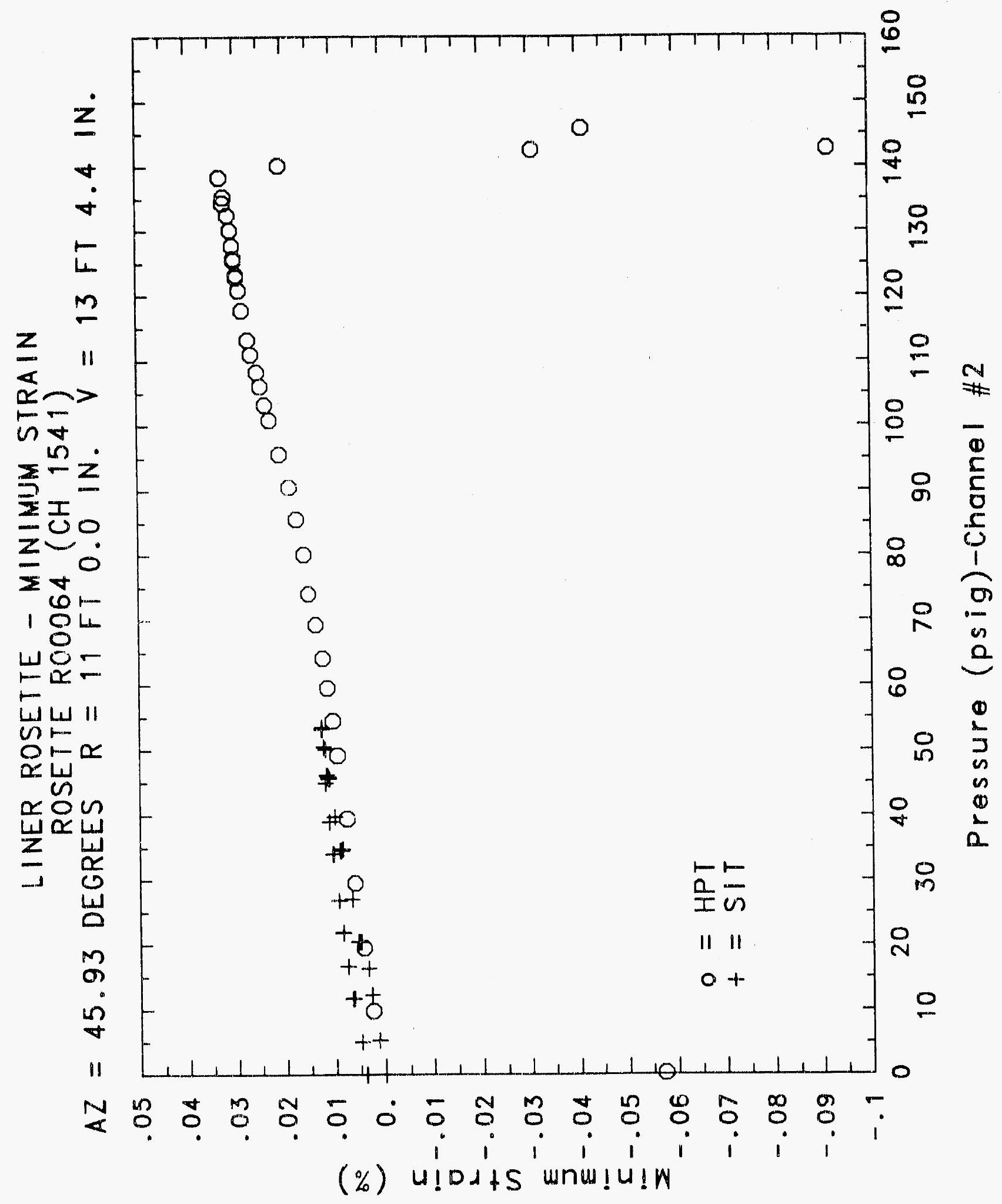




\title{
Reinforced Concrete Test Data
}

\author{
Rosette 65 Channels $1543 \quad 1544 \quad 1545$
}

Structural Integrity Test

\begin{tabular}{|c|c|c|c|c|c|c|c|}
\hline $\begin{array}{c}\text { Pressure } \\
\text { psig } \\
-0.05 \\
5.33 \\
12.31 \\
16.44 \\
20.51 \\
20.46 \\
20.47 \\
27.05 \\
34.69 \\
34.53 \\
34.53 \\
39.70 \\
45.90 \\
45.65 \\
45.62 \\
50.10 \\
53.47 \\
53.29 \\
53.21 \\
50.49 \\
46.14 \\
46.13 \\
44.97 \\
38.98 \\
33.99 \\
33.96 \\
26.83 \\
21.88 \\
21.90 \\
16.69 \\
11.74 \\
11.77 \\
5.05 \\
0.02 \\
-0.04 \\
-0.02 \\
-0.02 \\
0.02\end{array}$ & $\begin{array}{l}\text { Maximum } \\
\text { \%Strain } \\
0.0007 \\
0.0032 \\
0.0073 \\
0.0102 \\
0.0143 \\
0.0150 \\
0.0154 \\
0.0234 \\
0.0373 \\
0.0377 \\
0.0387 \\
0.0468 \\
0.0605 \\
0.0622 \\
0.0620 \\
0.0691 \\
0.0758 \\
0.0764 \\
0.0767 \\
0.0751 \\
0.0717 \\
0.0715 \\
0.0712 \\
0.0664 \\
0.0620 \\
0.0620 \\
0.0554 \\
0.0509 \\
0.0506 \\
0.0458 \\
0.0409 \\
0.0401 \\
0.0340 \\
0.0296 \\
0.0288 \\
0.0292 \\
0.0296 \\
0.0300\end{array}$ & $\begin{array}{l}\text { Minimum } \\
\text { \%Strain } \\
0.0002 \\
0.0019 \\
0.0047 \\
0.0068 \\
0.0106 \\
0.0119 \\
0.0128 \\
0.0199 \\
0.0323 \\
0.0331 \\
0.0334 \\
0.0356 \\
0.0385 \\
0.0386 \\
0.0387 \\
0.0407 \\
0.0424 \\
0.0424 \\
0.0425 \\
0.0415 \\
0.0395 \\
0.0394 \\
0.0393 \\
0.0369 \\
0.0346 \\
0.0345 \\
0.0316 \\
0.0291 \\
0.0292 \\
0.0267 \\
0.0243 \\
0.0238 \\
0.0204 \\
0.0175 \\
0.0162 \\
0.0166 \\
0.0166 \\
0.0168\end{array}$ & $\begin{array}{c}\text { Angle } \\
\text { Degrees } \\
-40.27 \\
-2.17 \\
3.31 \\
8.54 \\
10.00 \\
12.14 \\
12.31 \\
31.03 \\
-8.18 \\
-10.12 \\
-3.98 \\
0.66 \\
2.84 \\
4.83 \\
3.49 \\
3.65 \\
3.07 \\
4.05 \\
4.17 \\
3.99 \\
3.85 \\
3.74 \\
3.10 \\
2.98 \\
2.85 \\
3.03 \\
2.58 \\
2.07 \\
2.79 \\
3.33 \\
3.08 \\
2.70 \\
3.87 \\
6.67 \\
8.74 \\
8.49 \\
8.35 \\
9.06\end{array}$ & $\begin{array}{c}\text { Pressure } \\
\text { psig } \\
9.89 \\
19.55 \\
29.57 \\
39.42 \\
49.16 \\
54.50 \\
59.57 \\
64.20 \\
69.32 \\
74.16 \\
80.16 \\
85.61 \\
90.58 \\
95.69 \\
100.92 \\
103.25 \\
106.11 \\
108.31 \\
111.08 \\
113.24 \\
117.83 \\
120.92 \\
123.28 \\
122.97 \\
125.82 \\
125.60 \\
127.84 \\
130.19 \\
132.53 \\
135.33 \\
134.42 \\
138.35 \\
140.16 \\
142.63 \\
145.78 \\
142.52 \\
0.22\end{array}$ & $\begin{array}{l}\text { Maximum } \\
\text { XStrain } \\
0.0088 \\
0.0175 \\
0.0271 \\
0.0362 \\
0.0447 \\
0.0495 \\
0.0561 \\
0.0663 \\
0.0759 \\
0.0848 \\
0.0927 \\
0.1020 \\
0.1106 \\
0.1203 \\
0.1300 \\
0.1345 \\
0.1398 \\
0.1440 \\
0.1517 \\
0.1622 \\
0.2251 \\
0.2759 \\
0.3198 \\
0.3304 \\
0.3803 \\
0.3925 \\
0.4192 \\
0.4748 \\
0.5622 \\
0.6829 \\
0.7040 \\
0.8441 \\
0.9107 \\
0.9800 \\
1.0526 \\
1.0880 \\
0.8619\end{array}$ & $\begin{array}{l}\text { Minimum } \\
\text { \%Strain } \\
0.0061 \\
0.0108 \\
0.0157 \\
0.0207 \\
0.0256 \\
0.0285 \\
0.0306 \\
0.0312 \\
0.0329 \\
0.0348 \\
0.0362 \\
0.0379 \\
0.0398 \\
0.0427 \\
0.0470 \\
0.0497 \\
0.0538 \\
0.0585 \\
0.0661 \\
0.0715 \\
0.0693 \\
0.0643 \\
0.0654 \\
0.0665 \\
0.0709 \\
0.0712 \\
0.0746 \\
0.0834 \\
0.0958 \\
0.1250 \\
0.1291 \\
0.1696 \\
0.1969 \\
0.2789 \\
0.4049 \\
0.3570 \\
0.2836\end{array}$ & $\begin{array}{r}\text { Angle } \\
\text { Degrees } \\
-8.65 \\
-4.75 \\
-1.73 \\
-0.81 \\
-0.67 \\
-0.28 \\
0.80 \\
3.12 \\
4.17 \\
5.52 \\
5.14 \\
5.13 \\
4.82 \\
4.81 \\
4.13 \\
3.77 \\
3.04 \\
0.50 \\
-7.87 \\
-17.02 \\
-38.46 \\
44.54 \\
39.78 \\
38.61 \\
34.58 \\
33.73 \\
32.05 \\
28.63 \\
24.56 \\
19.48 \\
18.93 \\
15.38 \\
14.72 \\
15.71 \\
19.26 \\
22.45 \\
15.88\end{array}$ \\
\hline
\end{tabular}

High Pressure Test 


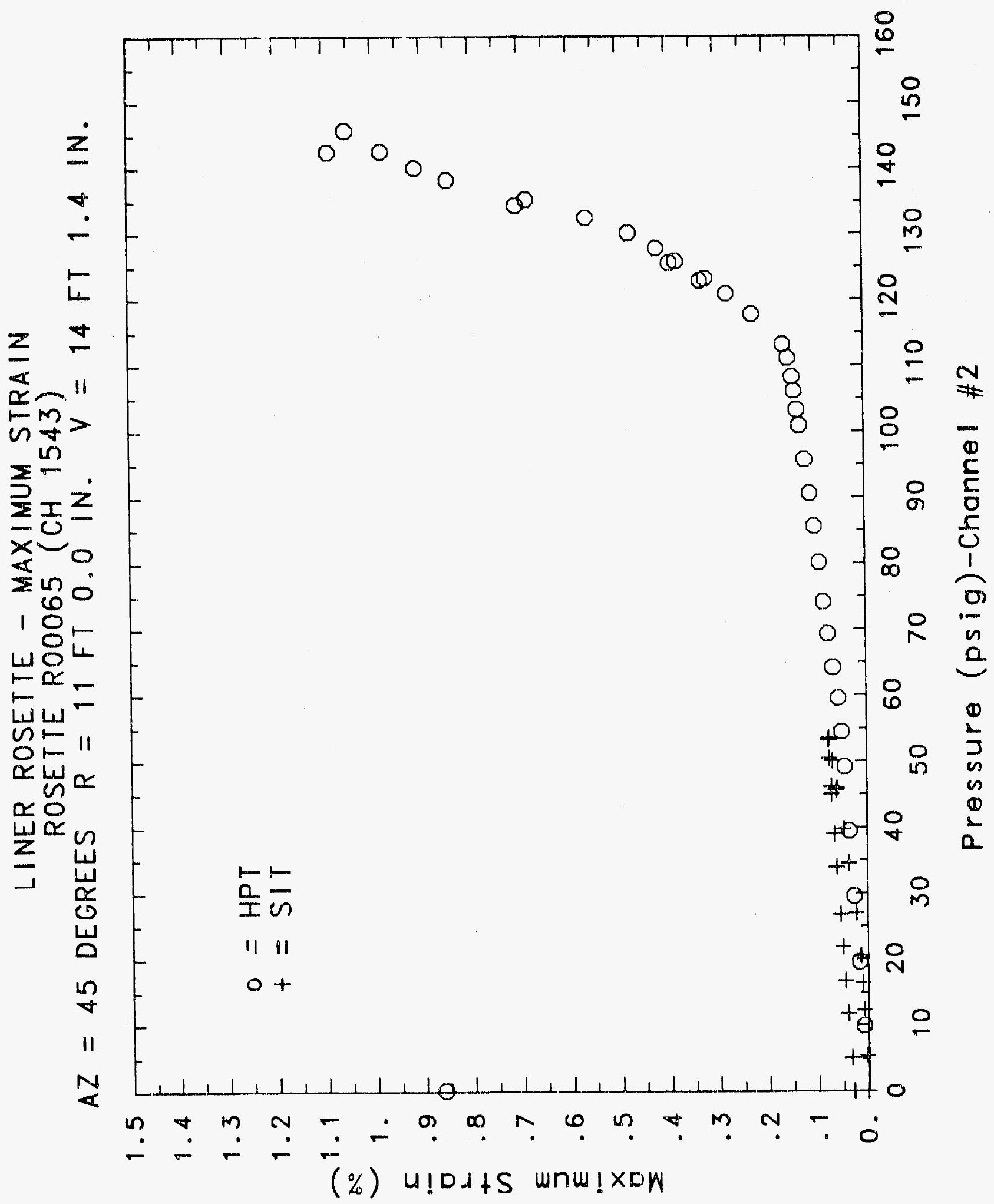




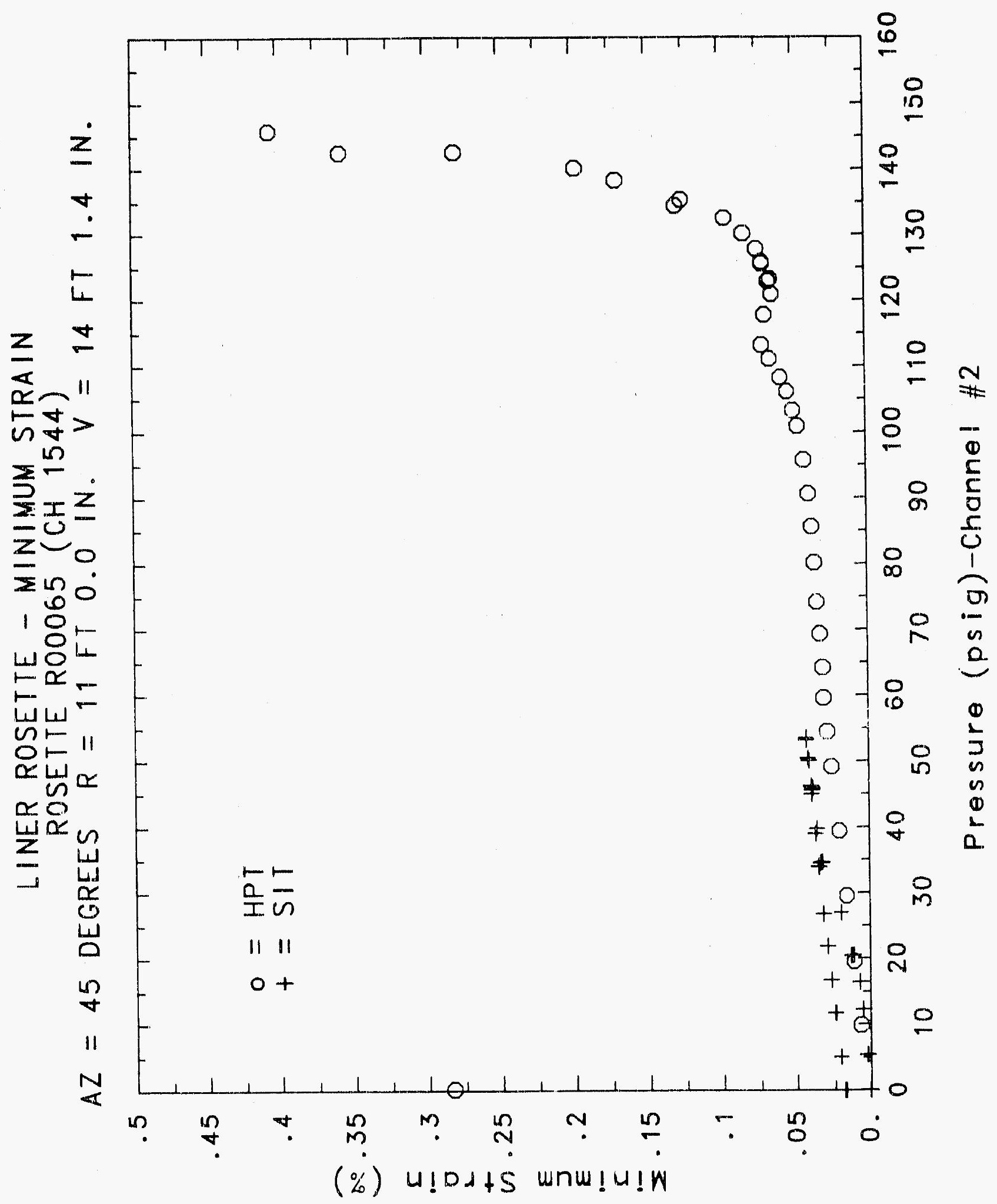




\title{
Reinforced Concrete Test Data
}

\author{
Rosette 66 Channels $1546 \quad 1547 \quad 1548$
}

STRUCTURAL INTEGRITY TEST

\begin{tabular}{|c|c|c|c|c|c|c|c|}
\hline $\begin{array}{c}\text { Pressure } \\
\text { psig } \\
-0.05 \\
5.33 \\
12.31 \\
16.44 \\
20.51 \\
20.46 \\
20.47 \\
27.05 \\
34.69 \\
34.53 \\
31.53 \\
39.70 \\
45.90 \\
45.65 \\
45.62 \\
50.10 \\
53.47 \\
53.29 \\
53.21 \\
50.49 \\
46.14 \\
46.13 \\
44.97 \\
38.98 \\
33.99 \\
33.96 \\
26.83 \\
21.88 \\
21.90 \\
16.69 \\
11.74 \\
11.77 \\
5.05 \\
0.02 \\
-0.04 \\
-0.02 \\
-0.02 \\
0.02\end{array}$ & $\begin{array}{c}\text { Maximum } \\
\% \text { Strain } \\
0.0003 \\
0.0036 \\
0.0634 \\
0.1118 \\
0.0167 \\
0.0174 \\
0.0176 \\
0.0261 \\
0.0383 \\
0.0394 \\
0.0395 \\
0.0460 \\
0.0557 \\
0.0562 \\
0.0564 \\
0.0617 \\
0.0666 \\
0.0670 \\
0.0670 \\
0.0652 \\
0.0614 \\
0.0613 \\
0.0615 \\
0.0555 \\
0.0505 \\
0.0503 \\
0.0428 \\
0.0378 \\
0.0378 \\
0.0325 \\
0.0280 \\
0.0280 \\
0.0232 \\
0.0198 \\
0.0182 \\
0.0191 \\
0.0189 \\
0.0184\end{array}$ & $\begin{array}{l}\text { Minimum } \\
\text { \% Strain } \\
-0.0002 \\
0.0009 \\
0.0027 \\
0.0038 \\
0.0062 \\
0.0074 \\
0.0081 \\
0.0150 \\
0.0257 \\
0.0258 \\
0.0258 \\
0.0309 \\
0.0368 \\
0.0375 \\
0.0377 \\
0.0413 \\
0.0448 \\
0.0454 \\
0.0458 \\
0.0448 \\
0.0430 \\
0.0428 \\
0.0431 \\
0.0410 \\
0.0385 \\
0.0384 \\
0.0357 \\
0.0319 \\
0.0318 \\
0.0283 \\
0.0233 \\
0.0225 \\
0.0158 \\
0.0107 \\
0.0086 \\
0.0085 \\
0.0094 \\
0.0100\end{array}$ & $\begin{array}{c}\text { Angle } \\
\text { Degrees } \\
\text {-29.37 } \\
16.80 \\
4.22 \\
5.10 \\
11.81 \\
9.58 \\
6.06 \\
13.25 \\
17.86 \\
20.25 \\
19.03 \\
19.20 \\
19.41 \\
20.75 \\
20.57 \\
19.28 \\
18.90 \\
18.54 \\
18.05 \\
18.97 \\
19.77 \\
19.96 \\
19.98 \\
20.18 \\
21.89 \\
21.45 \\
25.40 \\
31.73 \\
32.36 \\
-41.41 \\
-31.61 \\
-28.99 \\
-14.72 \\
-7.78 \\
-15.75 \\
-20.49 \\
-15.56 \\
-5.92\end{array}$ & $\begin{array}{c}\text { Pressure } \\
\text { psig } \\
9.89 \\
19.55 \\
29.57 \\
39.42 \\
49.16 \\
54.50 \\
59.57 \\
64.20 \\
69.32 \\
74.16 \\
80.16 \\
85.61 \\
90.58 \\
95.69 \\
100.92 \\
103.25 \\
106.11 \\
108.31 \\
111.08 \\
113.24 \\
117.83 \\
120.92 \\
123.28 \\
122.97 \\
125.82 \\
125.60 \\
127.84 \\
130.19 \\
132.53 \\
135.33 \\
134.42 \\
138.35 \\
140.16 \\
142.63 \\
145.78 \\
142.52 \\
0.22\end{array}$ & $\begin{array}{l}\text { Maximum } \\
\text { xStrain } \\
0.0126 \\
0.0239 \\
0.0356 \\
0.0470 \\
0.0578 \\
0.0641 \\
0.0709 \\
0.0772 \\
0.0843 \\
0.0920 \\
0.1018 \\
0.1113 \\
0.1204 \\
0.1309 \\
0.1423 \\
0.1484 \\
0.1549 \\
0.1602 \\
0.1682 \\
0.1713 \\
0.1838 \\
0.1995 \\
0.2094 \\
0.2110 \\
0.2230 \\
0.2246 \\
0.2276 \\
0.2473 \\
0.2767 \\
0.3437 \\
0.3631 \\
0.5065 \\
0.7131 \\
0.9988 \\
1.1943 \\
1.3345 \\
1.0746\end{array}$ & $\begin{array}{l}\text { Minimum } \\
\text { \%Strain } \\
0.0048 \\
0.0112 \\
0.0177 \\
0.0237 \\
0.0296 \\
0.0336 \\
0.0379 \\
0.0430 \\
0.0482 \\
0.0543 \\
0.0598 \\
0.0661 \\
0.0724 \\
0.0795 \\
0.0877 \\
0.0923 \\
0.0967 \\
0.1005 \\
0.1060 \\
0.1106 \\
0.1213 \\
0.1301 \\
0.1381 \\
0.1398 \\
0.1469 \\
0.1505 \\
0.1604 \\
0.1669 \\
0.1741 \\
0.1849 \\
0.1875 \\
0.2056 \\
0.2164 \\
0.2356 \\
0.2565 \\
0.2606 \\
0.1631\end{array}$ & $\begin{array}{c}\text { Angle } \\
\text { Degrees } \\
26.84 \\
22.78 \\
19.61 \\
17.67 \\
16.27 \\
15.89 \\
14.38 \\
12.98 \\
11.70 \\
11.15 \\
13.70 \\
13.91 \\
14.15 \\
15.11 \\
16.19 \\
16.84 \\
17.59 \\
17.98 \\
18.59 \\
18.85 \\
19.37 \\
19.24 \\
19.18 \\
19.13 \\
18.86 \\
17.70 \\
8.66 \\
10.17 \\
9.60 \\
7.77 \\
7.53 \\
5.00 \\
2.71 \\
1.77 \\
2.23 \\
-0.17 \\
0.69\end{array}$ \\
\hline
\end{tabular}

\section{High Pressure test}




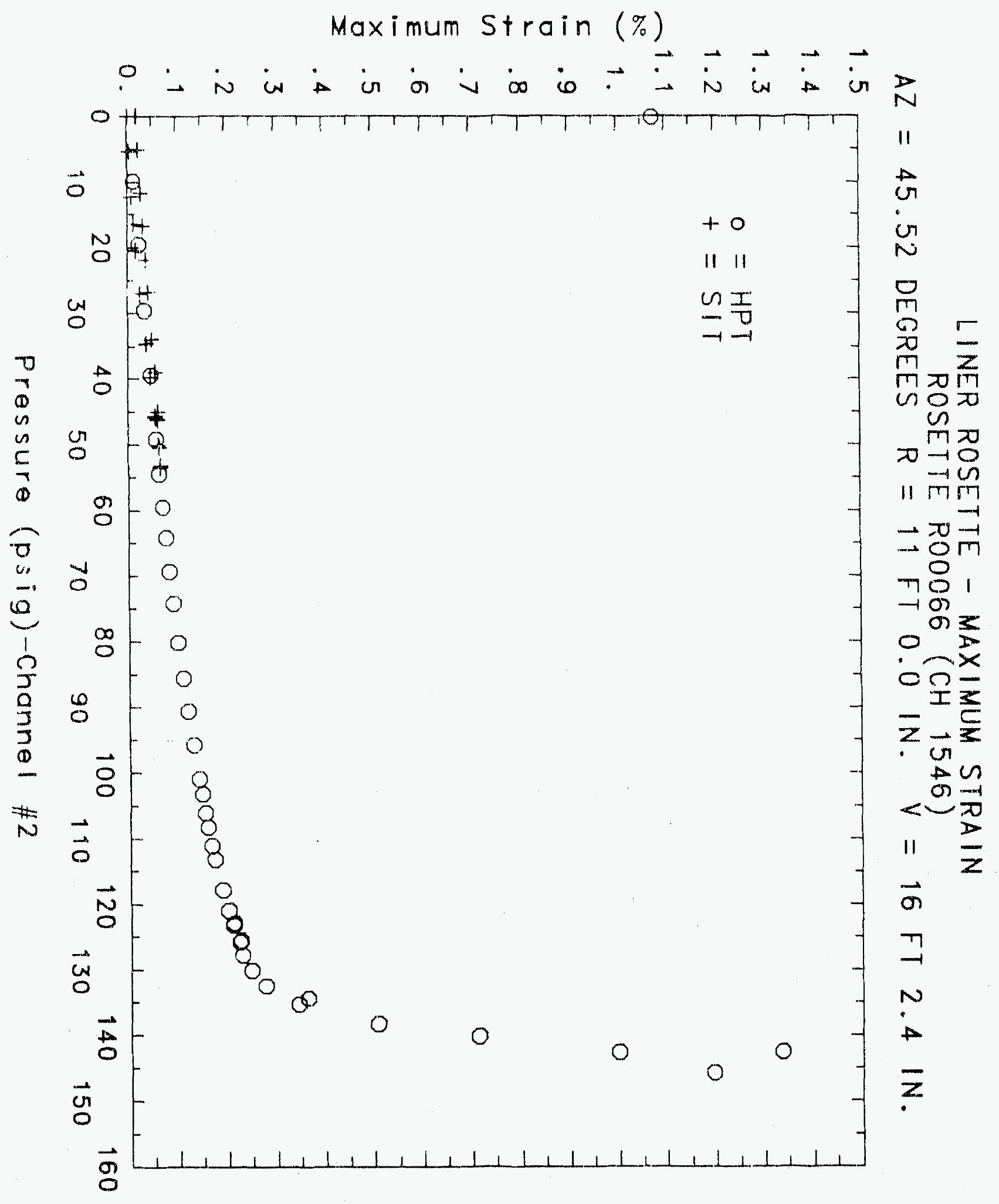




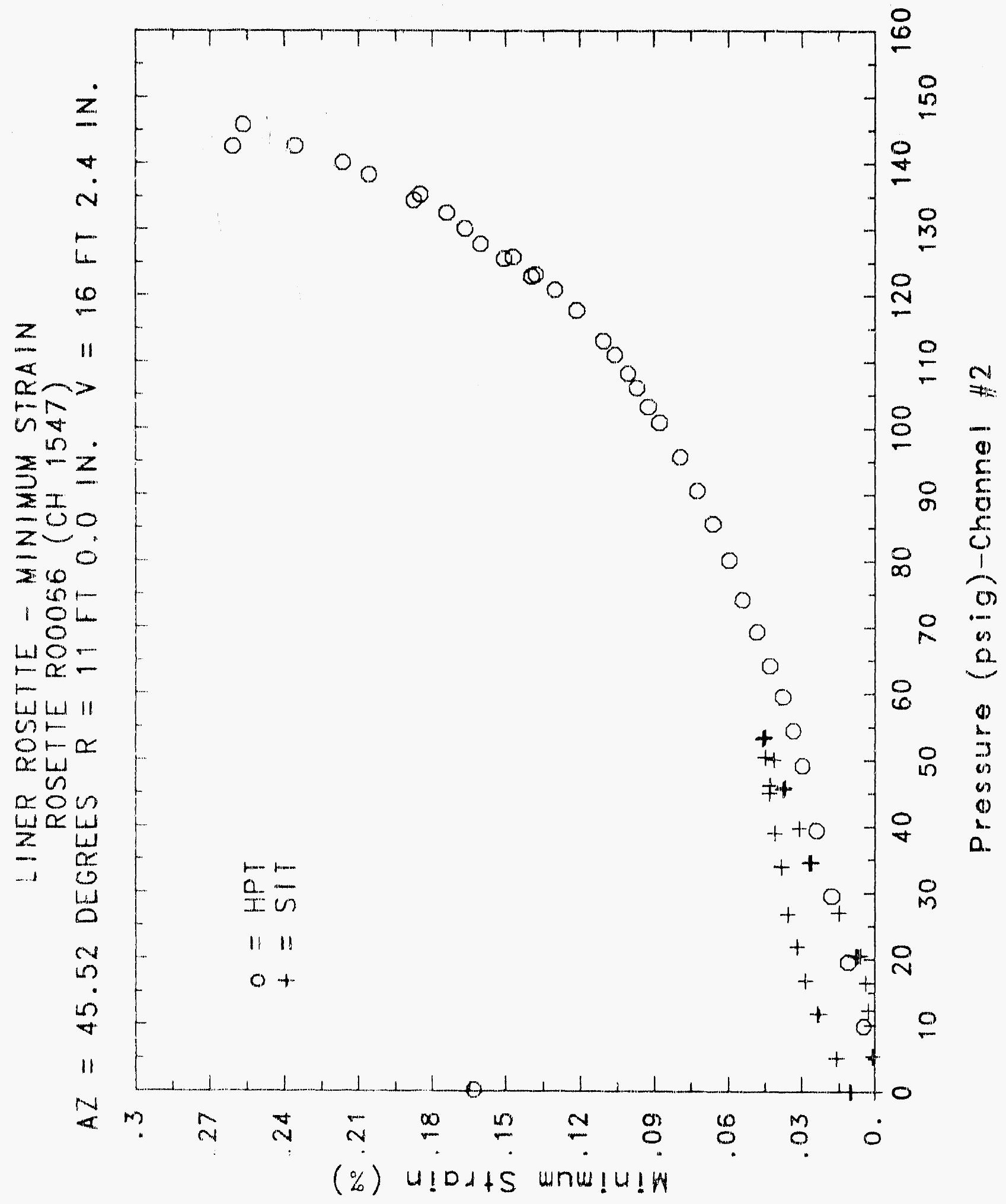




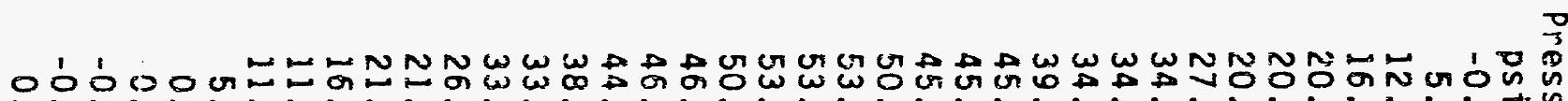

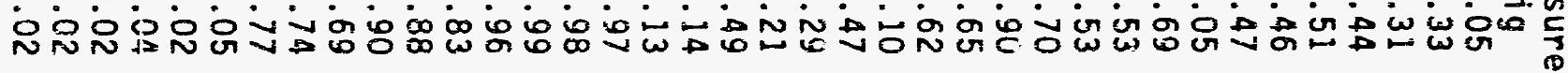

000000000000000000000000000000000000000

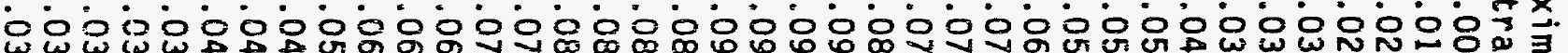

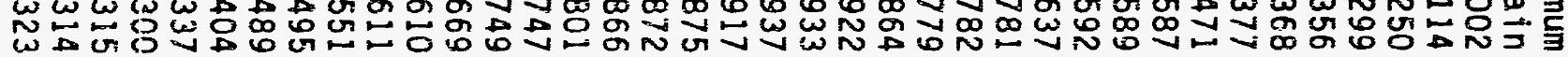

$00000000000000000000000000000000000000 \mathrm{f}$

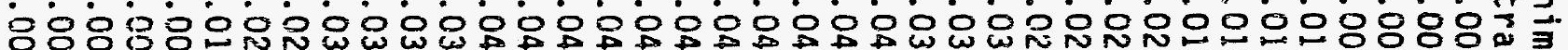

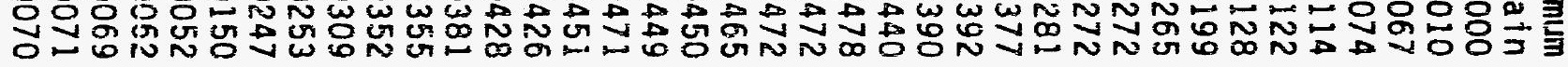

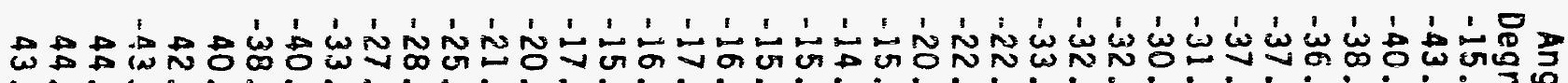

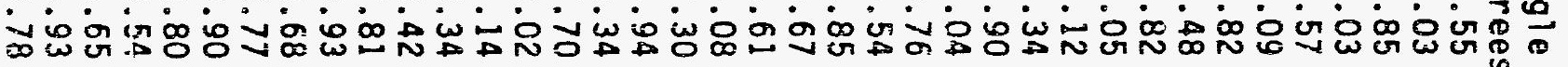

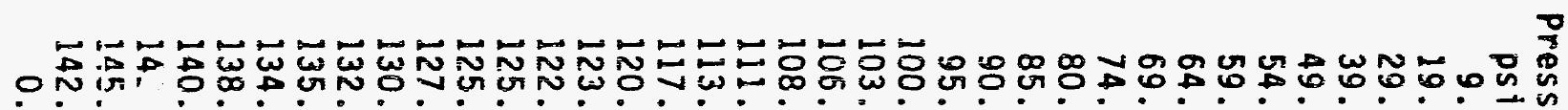

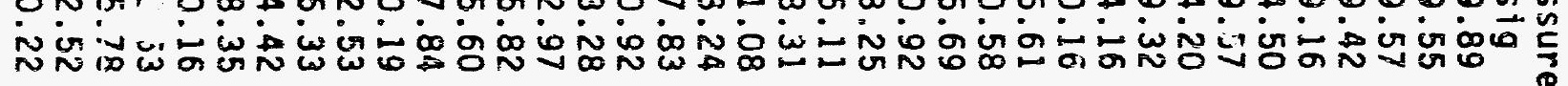
:

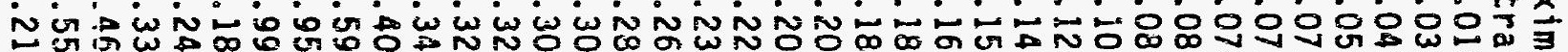

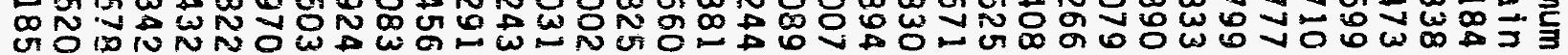

1000000000000000000000000000000000000节

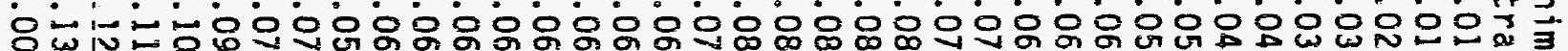

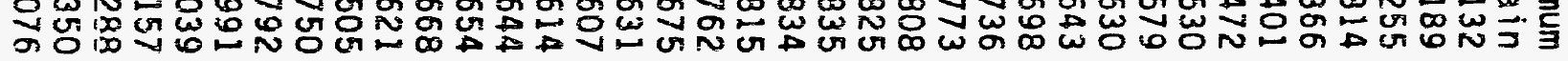

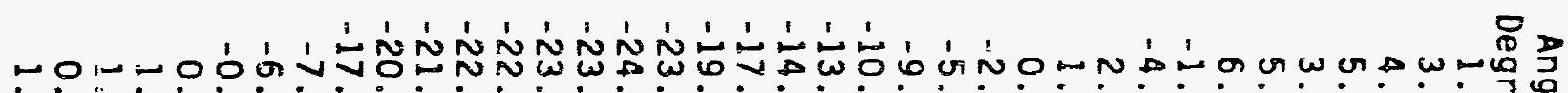

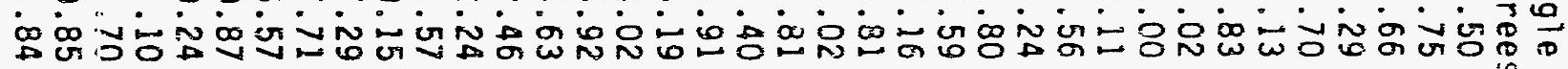




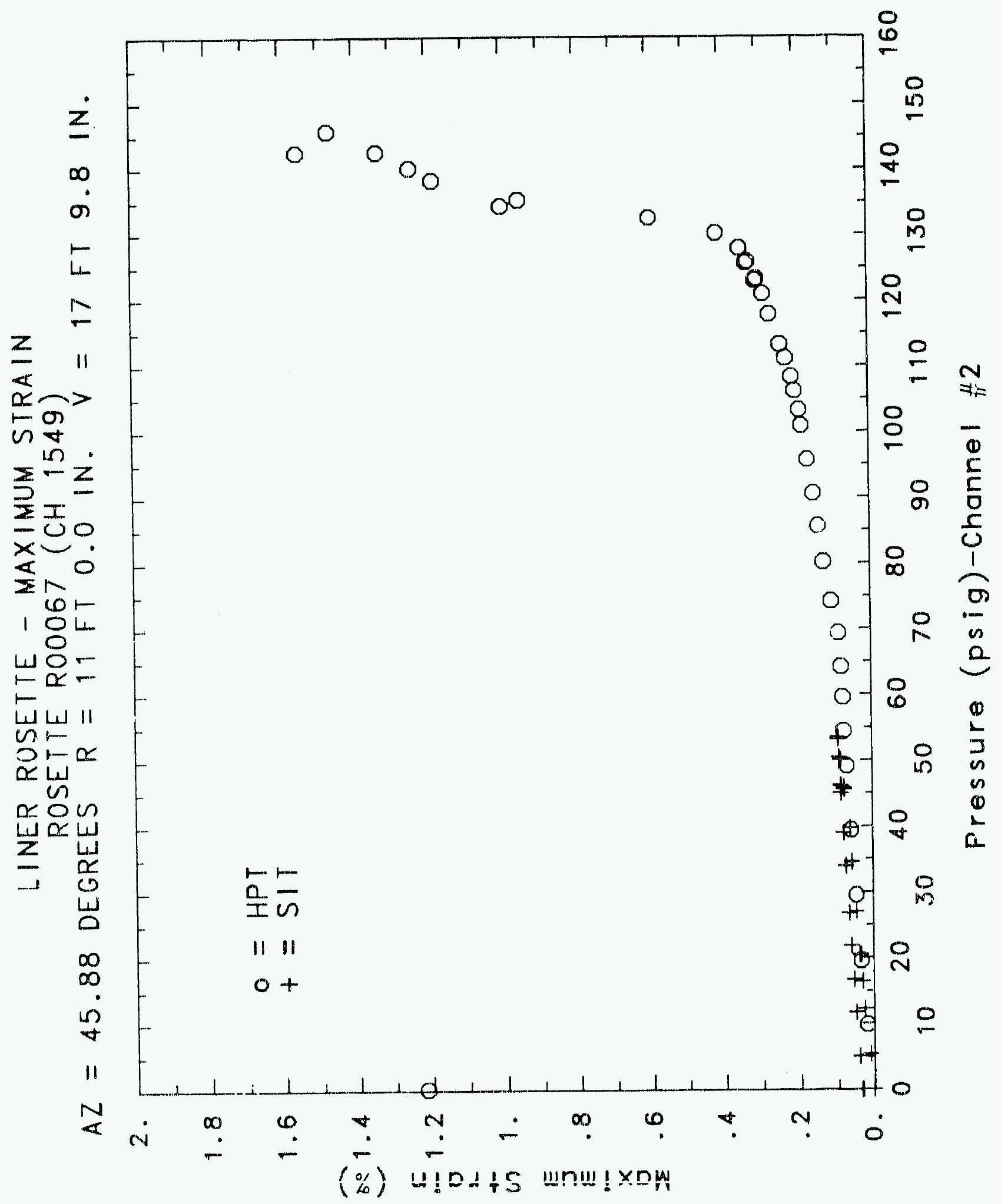




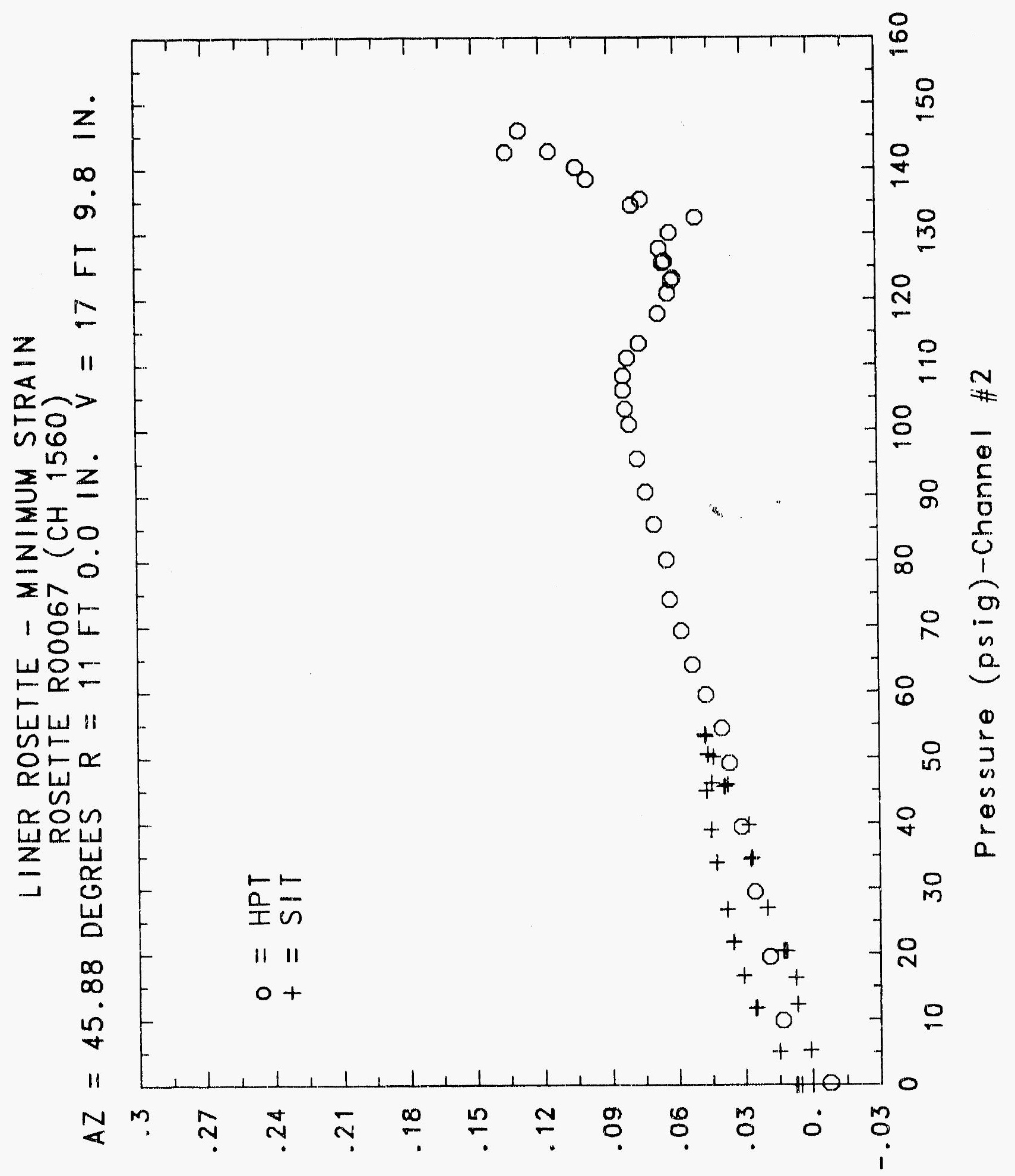

(\%) $u ! D d+5$ wnw! $u$ ! 


\section{Reinforced Concrete Test Data}

Rosette 68 Channels $1562 \quad 1563 \quad 1564$

StRUCTURAL INTEgRITY TEST

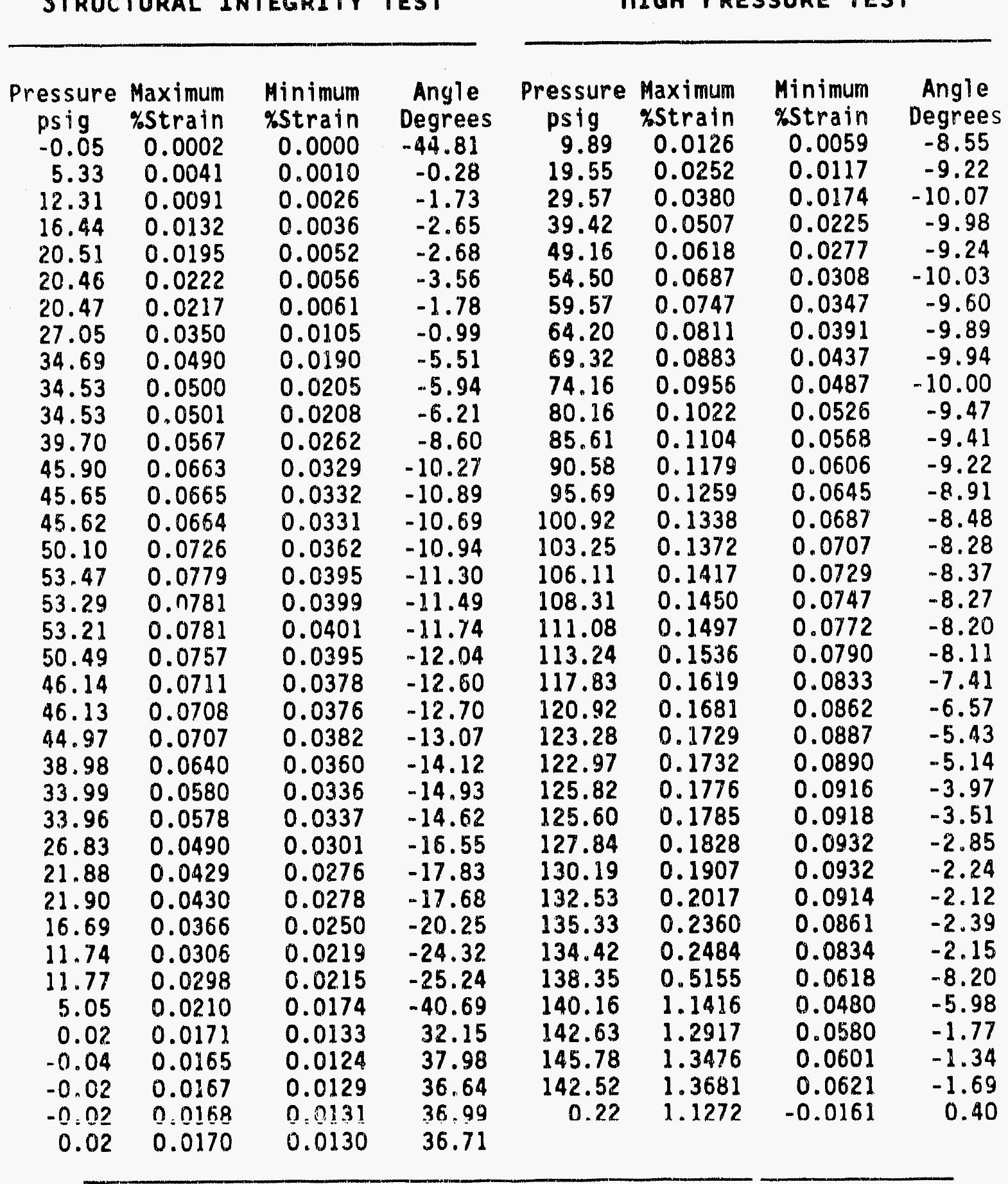

High Pressure test 


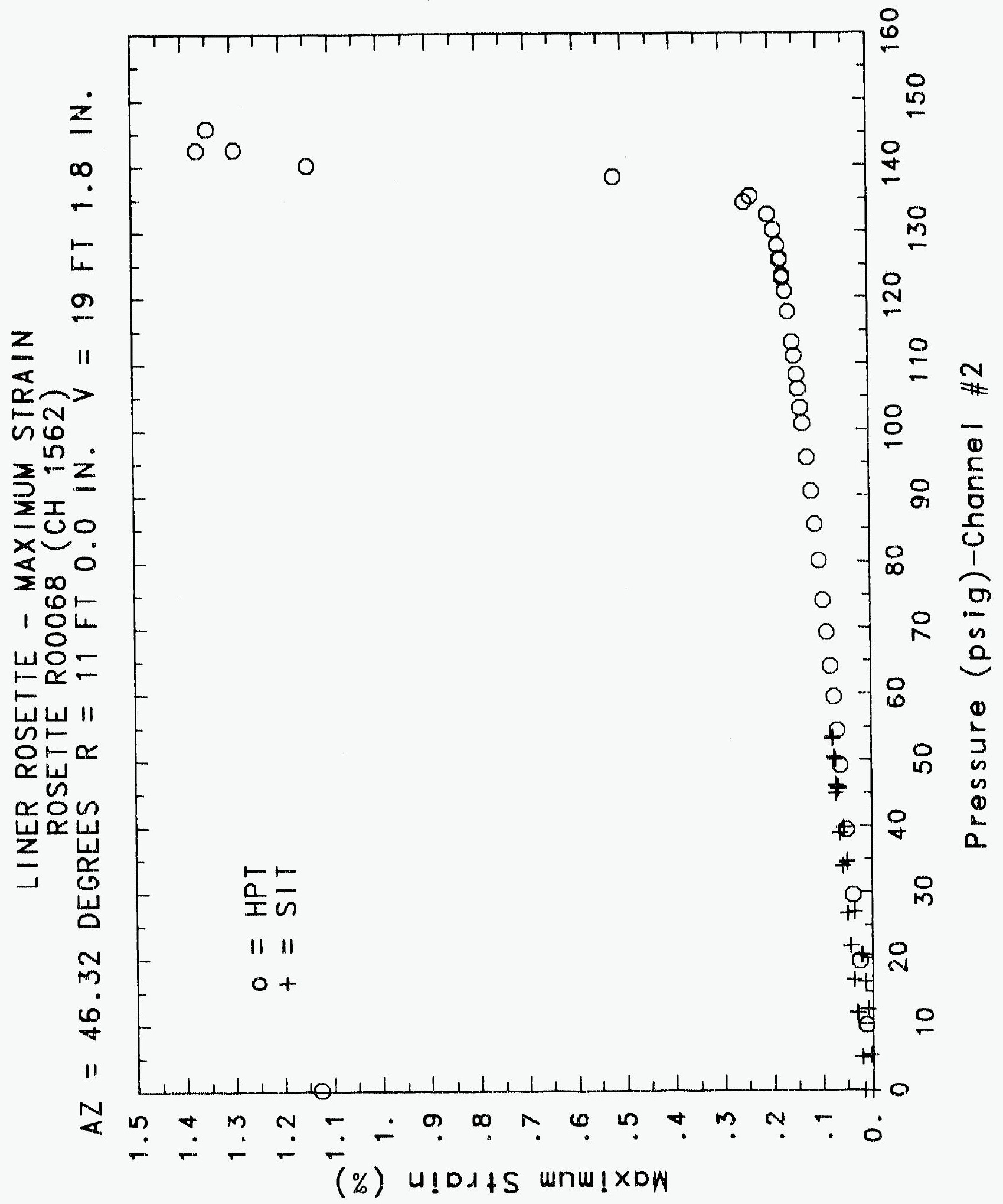




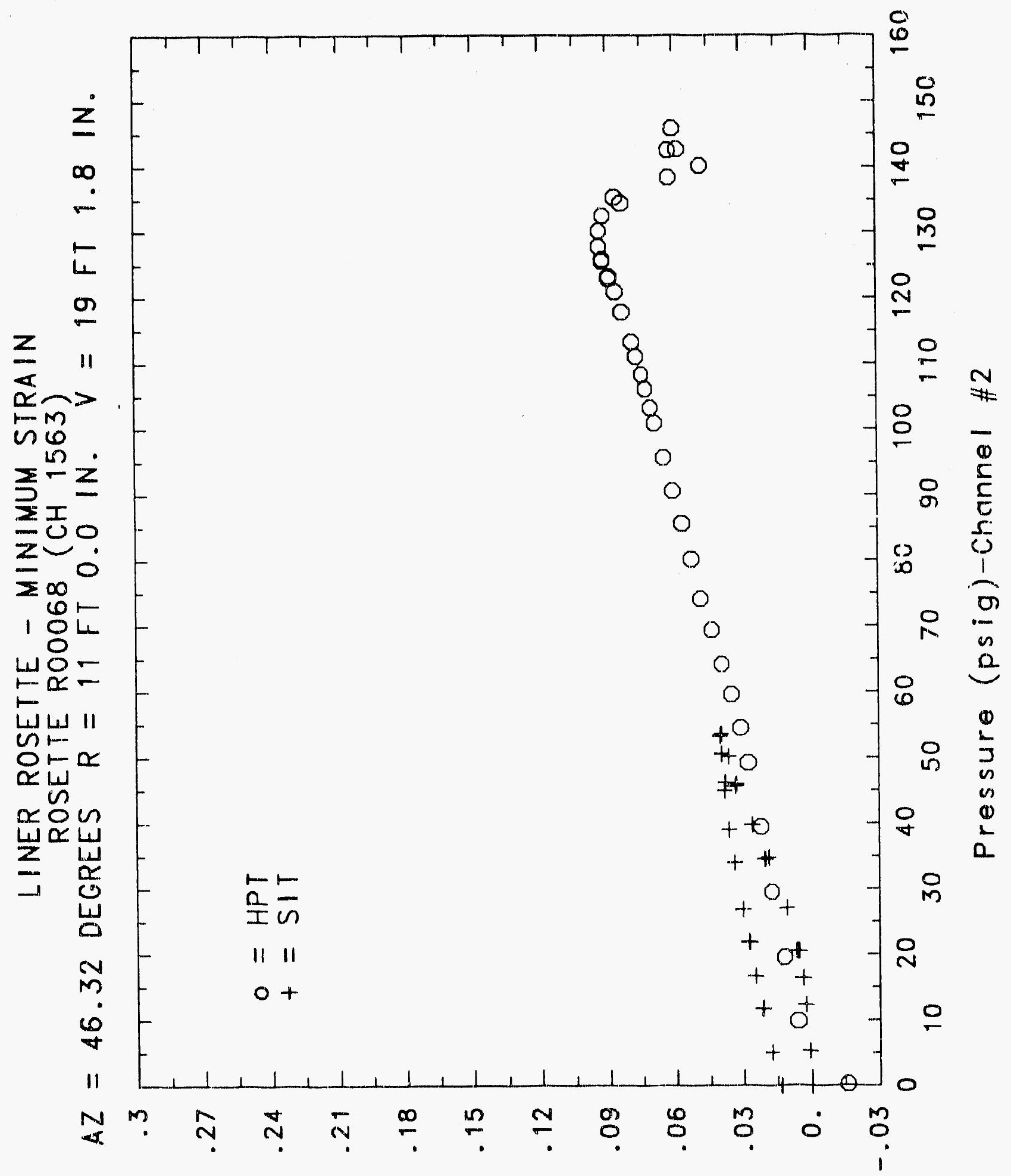

(\%) $u ! D d+5$ unw!u!w 


\title{
Reinforced Concrete Test Data
}

\author{
Rosette 69 Channels $1565 \quad 1566 \quad 1567$
}

Structural Integrity Test

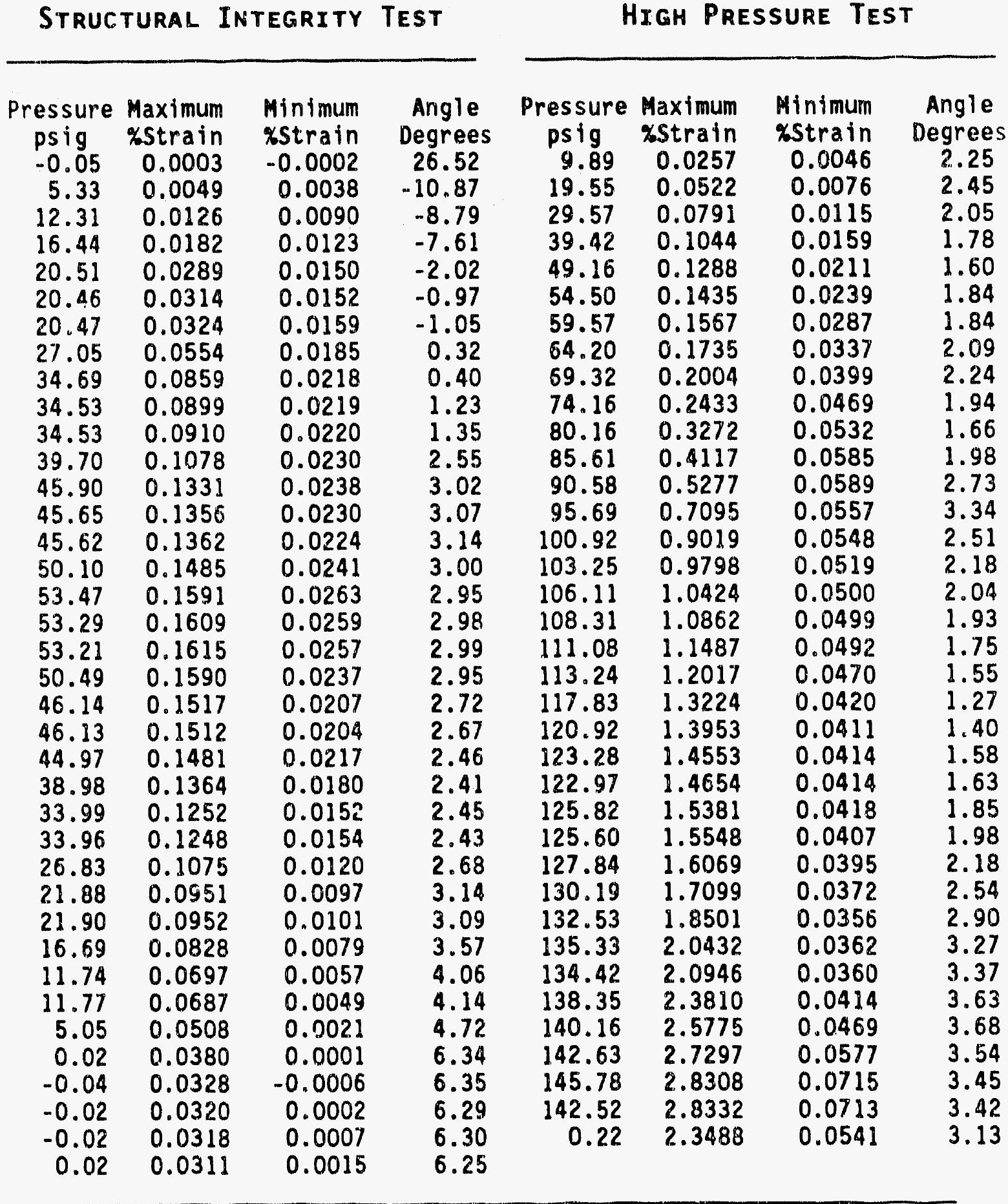

\section{High Pressure test}




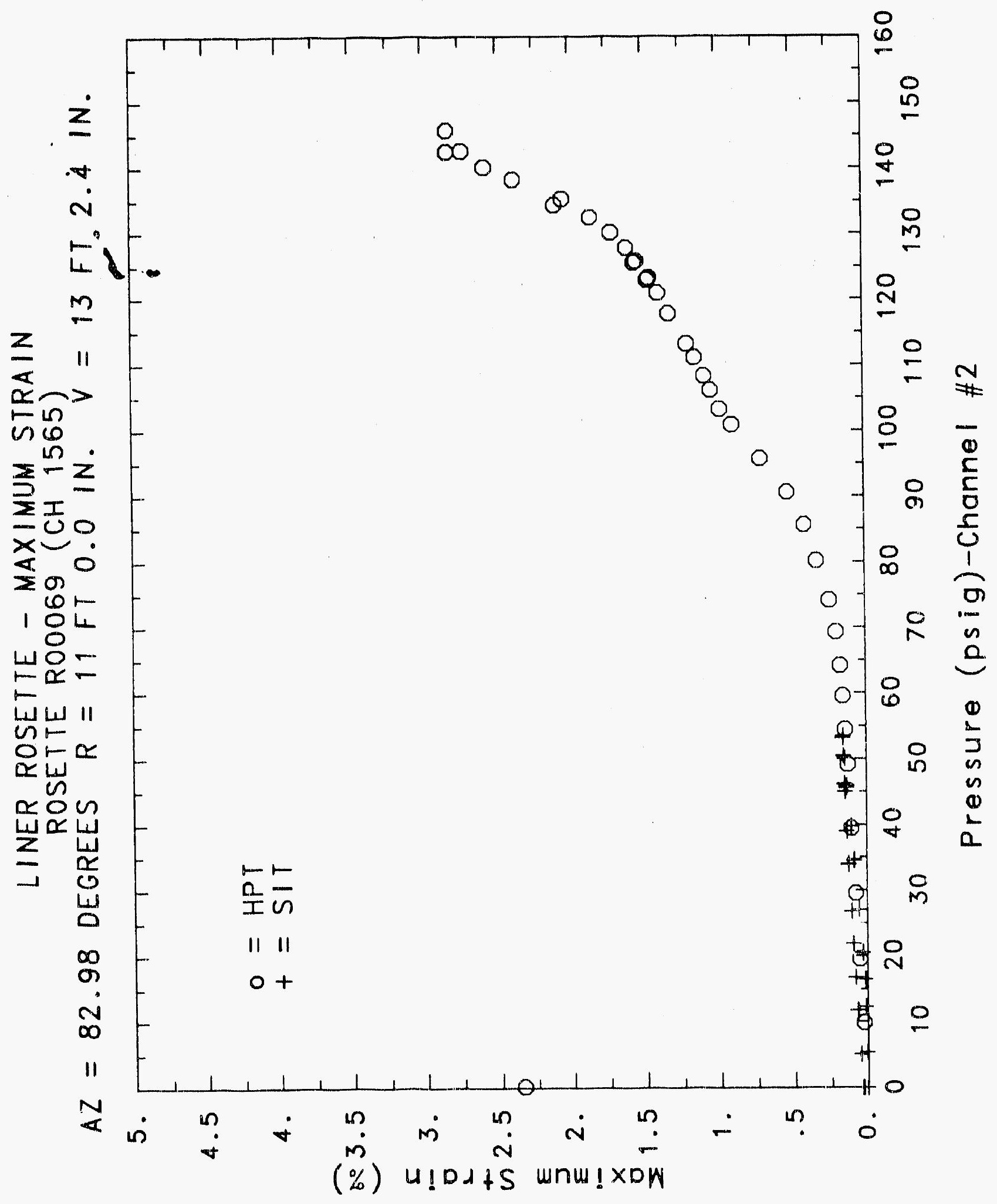




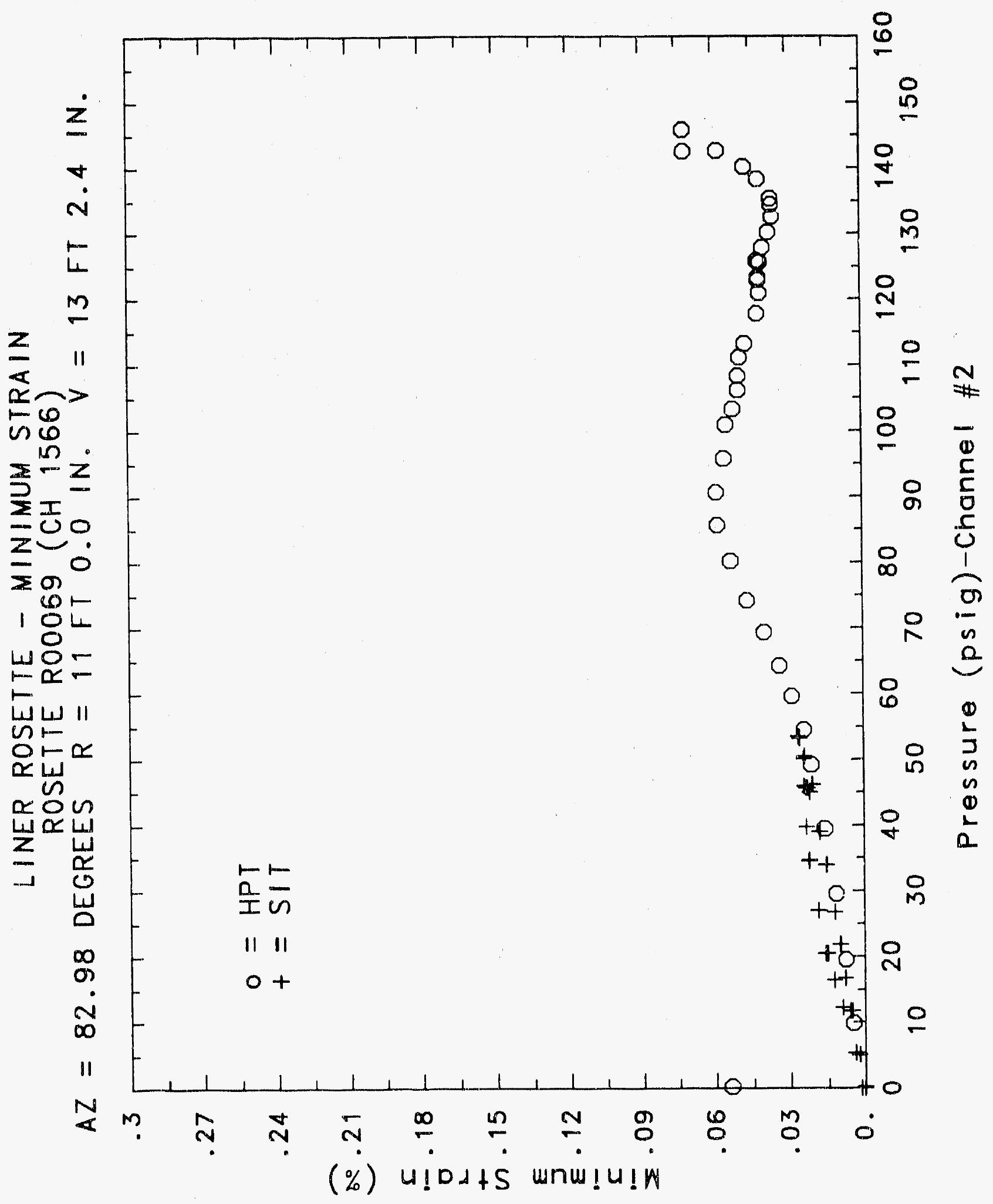




\section{Reinforced Concrete Test Data}

\section{Rosette 70 Channels $1568 \quad 15691580$}

\section{Structural INTEGRITY TEST}

\begin{tabular}{|c|c|c|c|c|c|c|c|}
\hline $\begin{array}{c}\text { Pressure } \\
\text { psig } \\
-0.05 \\
5.33 \\
12.31 \\
16.44 \\
20.51 \\
20.46 \\
20.47 \\
27.05 \\
34.09 \\
34.53 \\
34.53 \\
39.70 \\
45.90 \\
45.65 \\
45.62 \\
50.10 \\
53.47 \\
53.29 \\
53.21 \\
50.49 \\
46.14 \\
46.13 \\
44.97 \\
38.98 \\
33.99 \\
33.96 \\
26.83 \\
21.88 \\
21.90 \\
16.69 \\
11.74 \\
11.77 \\
5.05 \\
0.02 \\
-0.04 \\
-0.02 \\
-0.02 \\
0.02\end{array}$ & $\begin{array}{c}\text { Maximum } \\
\text { \%Strain } \\
0.0002 \\
0.0044 \\
0.0104 \\
0.0141 \\
0.0179 \\
0.0180 \\
0.0184 \\
0.0273 \\
0.0378 \\
0.0401 \\
0.0405 \\
0.0476 \\
0.0525 \\
0.0654 \\
0.0660 \\
0.0786 \\
0.0929 \\
0.0952 \\
0.0959 \\
0.0883 \\
0.0841 \\
0.0836 \\
0.0824 \\
0.0764 \\
0.0710 \\
0.0725 \\
0.0639 \\
0.0578 \\
0.0587 \\
0.0528 \\
0.0465 \\
0.0443 \\
0.0359 \\
0.0295 \\
0.0202 \\
0.0219 \\
0.0221 \\
0.0217\end{array}$ & $\begin{array}{l}\text { Minimum } \\
\text { \%Strain } \\
-0.0001 \\
0.0024 \\
0.0059 \\
0.0081 \\
0.0114 \\
0.0125 \\
0.0131 \\
0.0148 \\
0.0205 \\
0.0221 \\
0.0226 \\
0.0270 \\
0.0346 \\
0.0368 \\
0.0373 \\
0.0450 \\
0.0516 \\
0.0522 \\
0.0524 \\
0.0574 \\
0.0544 \\
0.0538 \\
0.0514 \\
0.0472 \\
0.0430 \\
0.0416 \\
0.0358 \\
0.0319 \\
0.0316 \\
0.0276 \\
0.0234 \\
0.0240 \\
0.0183 \\
0.0137 \\
0.0143 \\
0.0122 \\
0.0116 \\
0.0112\end{array}$ & $\begin{array}{c}\text { Angle } \\
\text { Degrees } \\
\text {-9.78 } \\
15.39 \\
14.19 \\
13.85 \\
13.46 \\
15.71 \\
12.67 \\
20.51 \\
22.55 \\
25.25 \\
26.33 \\
27.16 \\
30.77 \\
32.83 \\
32.98 \\
33.51 \\
31.22 \\
31.32 \\
31.40 \\
25.07 \\
25.14 \\
24.94 \\
23.00 \\
22.82 \\
24.03 \\
26.12 \\
26.91 \\
28.25 \\
29.45 \\
31.35 \\
33.96 \\
33.05 \\
37.46 \\
41.94 \\
32.47 \\
36.54 \\
36.06 \\
34.18\end{array}$ & $\begin{array}{c}\text { Pressure } \\
\text { psig } \\
9.89 \\
19.55 \\
29.57 \\
39.42 \\
49.16 \\
54.50 \\
59.57 \\
64.20 \\
69.32 \\
74.16 \\
80.16 \\
85.61 \\
90.58 \\
95.69 \\
100.92 \\
103.25 \\
106.11 \\
108.31 \\
111.08 \\
113.24 \\
117.83 \\
120.92 \\
123.28 \\
122.97 \\
125.82 \\
125.60 \\
127.84 \\
130.19 \\
132.53 \\
135.33 \\
134.42 \\
138.35 \\
140.16 \\
142.63 \\
145.78 \\
142.52 \\
0.22\end{array}$ & $\begin{array}{c}\text { Maximum } \\
\text { \%Strain } \\
0.0127 \\
0.0263 \\
0.0410 \\
0.0549 \\
0.0682 \\
0.0768 \\
0.0890 \\
0.1056 \\
0.1249 \\
0.1436 \\
0.1670 \\
0.1799 \\
0.1881 \\
0.1942 \\
0.1987 \\
0.2005 \\
0.2025 \\
0.2042 \\
0.2058 \\
0.2073 \\
0.2107 \\
0.2130 \\
0.2147 \\
0.2144 \\
0.2174 \\
0.2172 \\
0.2198 \\
0.2229 \\
0.2259 \\
0.2300 \\
0.2300 \\
0.2358 \\
0.2394 \\
0.2436 \\
0.2488 \\
0.2472 \\
0.0577\end{array}$ & $\begin{array}{c}\text { Minimum } \\
\text { XStrain } \\
0.0065 \\
0.0141 \\
0.0230 \\
0.0327 \\
0.0427 \\
0.0491 \\
0.0542 \\
0.0590 \\
0.0640 \\
0.0687 \\
0.0722 \\
0.0733 \\
0.0728 \\
0.0712 \\
0.0683 \\
0.0673 \\
0.0660 \\
0.0647 \\
0.0626 \\
0.0609 \\
0.0560 \\
0.0528 \\
0.0506 \\
0.0489 \\
0.0466 \\
0.0449 \\
0.0441 \\
0.0417 \\
0.0391 \\
0.0366 \\
0.0347 \\
0.0342 \\
0.0341 \\
0.0351 \\
0.0361 \\
0.0312 \\
-0.0872\end{array}$ & $\begin{array}{r}\text { Angle } \\
\text { Degrees } \\
21.31 \\
18.43 \\
18.16 \\
18.08 \\
18.48 \\
19.07 \\
20.87 \\
22.56 \\
23.04 \\
22.45 \\
24.48 \\
24.47 \\
24.13 \\
23.46 \\
22.65 \\
22.35 \\
22.00 \\
21.74 \\
21.31 \\
20.94 \\
20.18 \\
19.78 \\
19.25 \\
19.17 \\
18.88 \\
18.81 \\
18.72 \\
18.48 \\
18.32 \\
18.25 \\
18.28 \\
18.41 \\
18.48 \\
18.45 \\
18.33 \\
18.16 \\
14.59\end{array}$ \\
\hline
\end{tabular}

High Pressure Test 


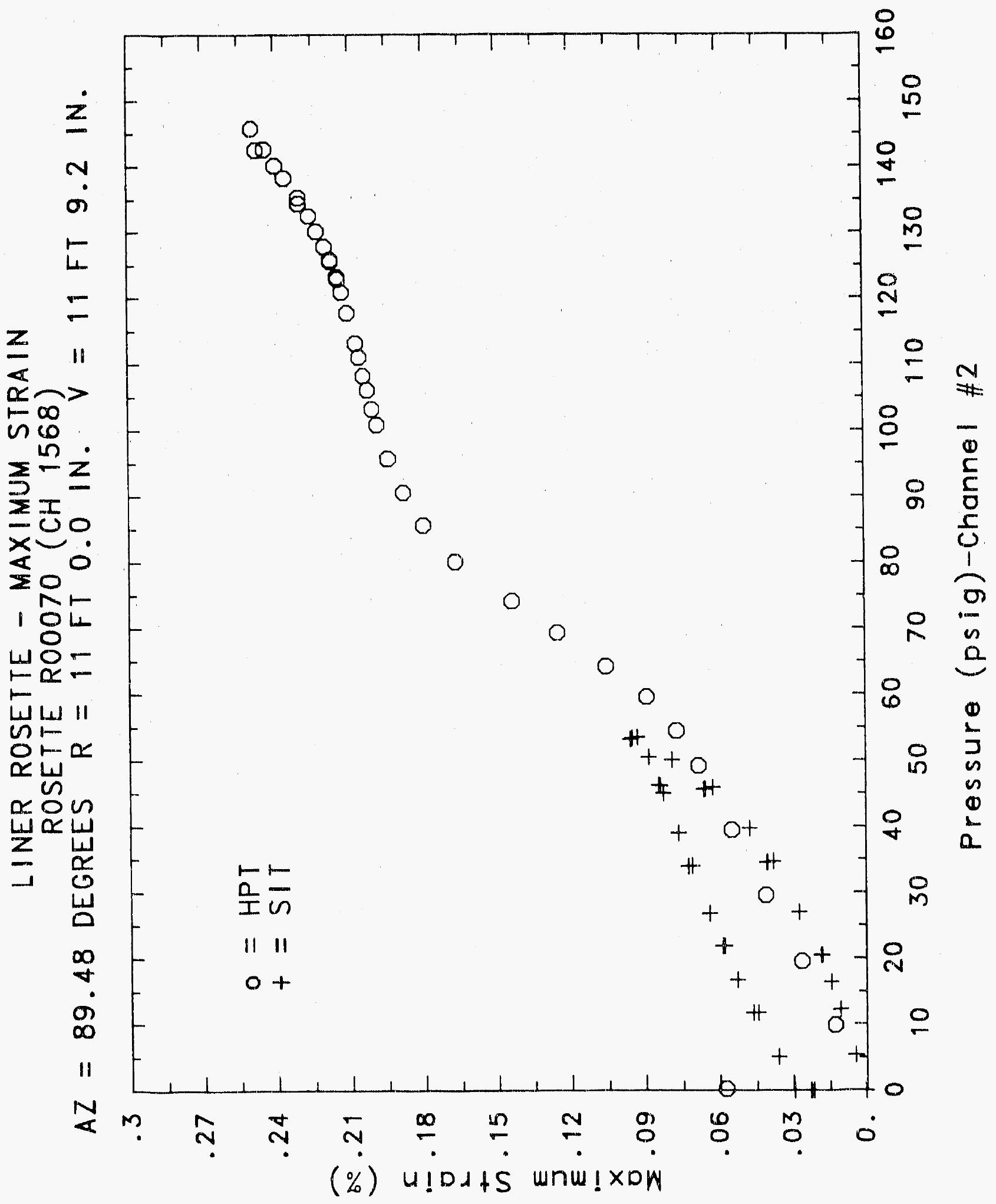




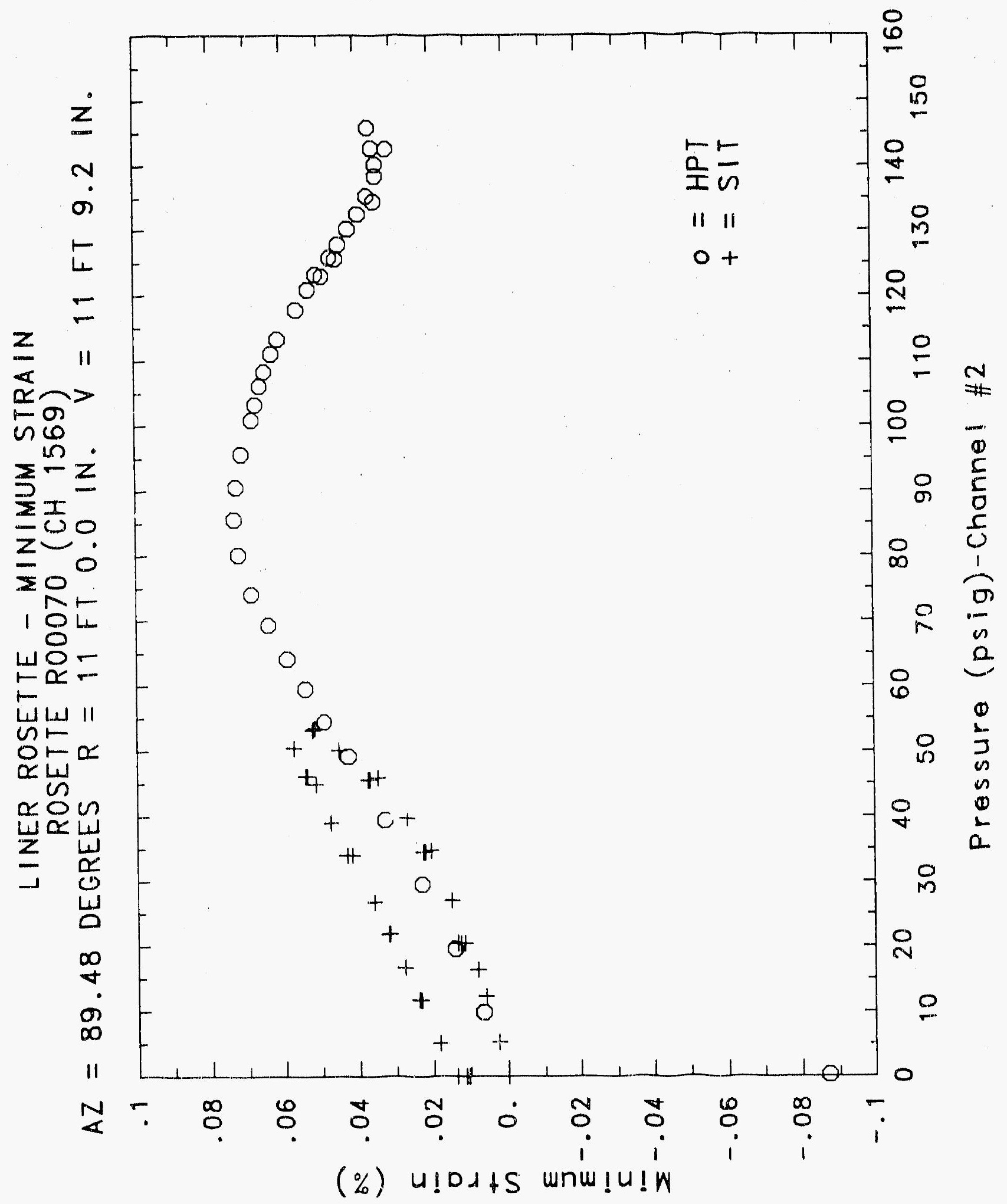

C-529 


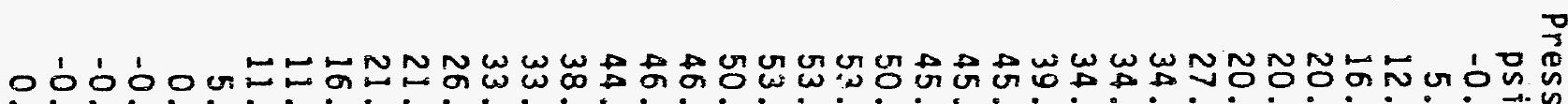

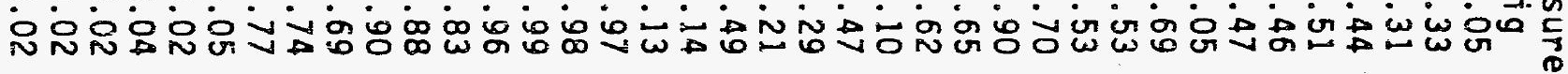

$00000000000000000000000000000000000000 \mathrm{~h}$

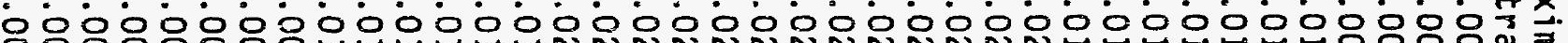

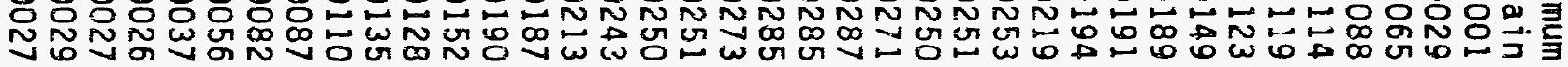

100010000000000000000000000000000000000

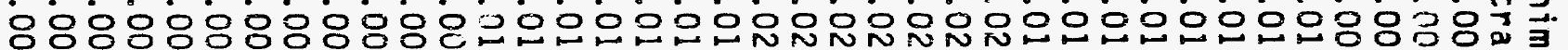

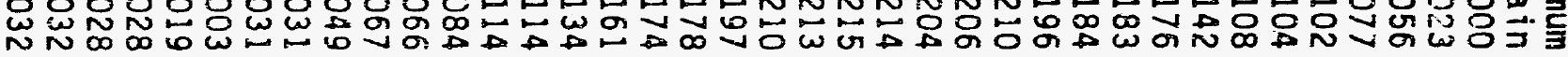

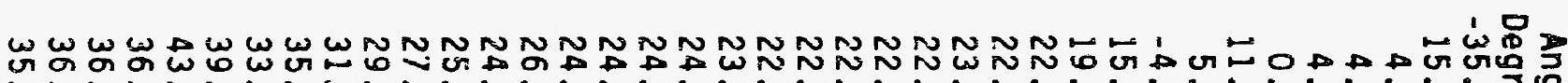
舟

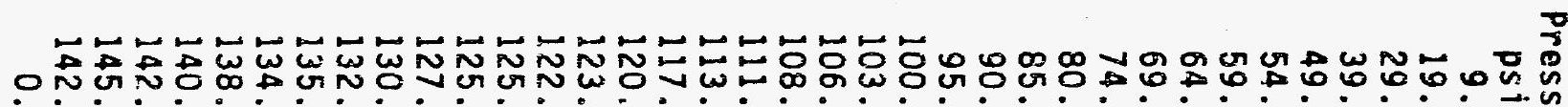

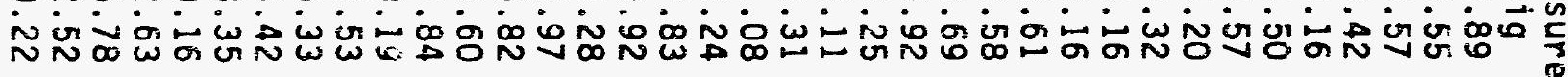
b000000000000000000000000000000000000発

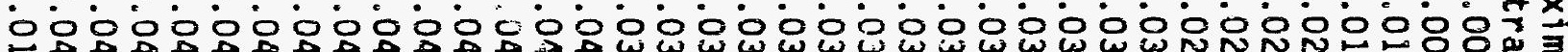

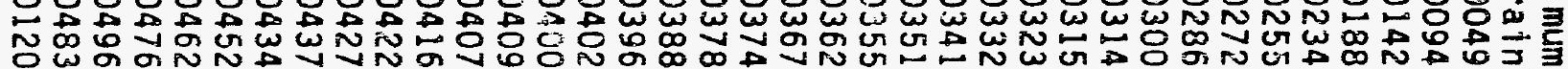

10000000000000000000000000000000000000永

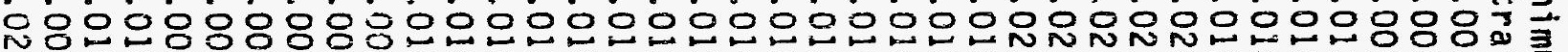

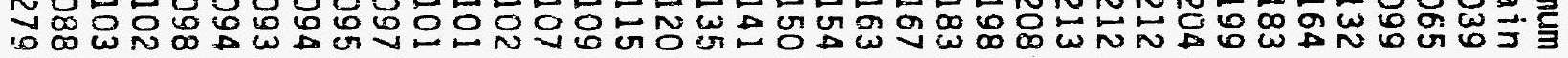

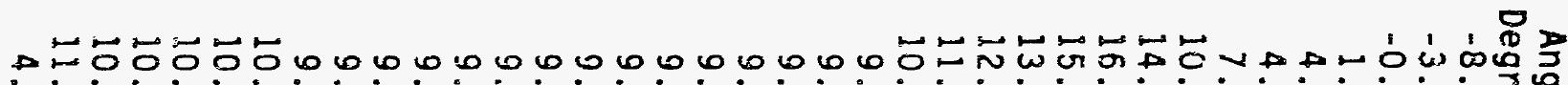

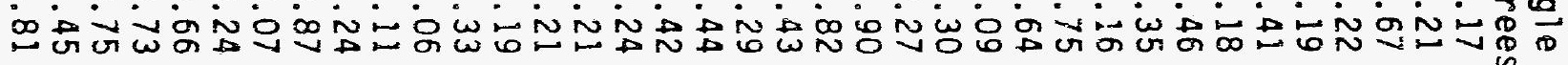




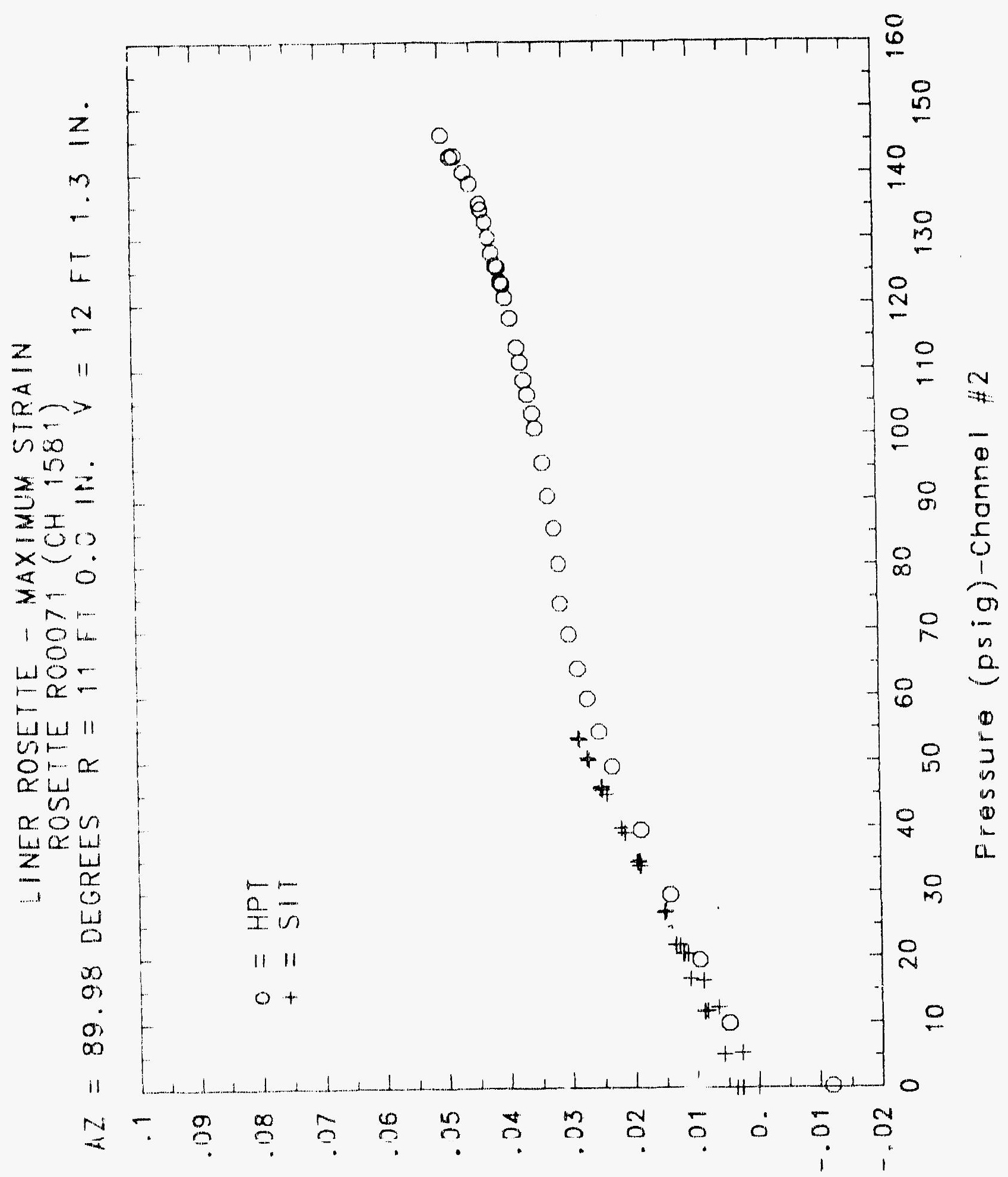

$(\%)$ u!Dits wnw! Xow 


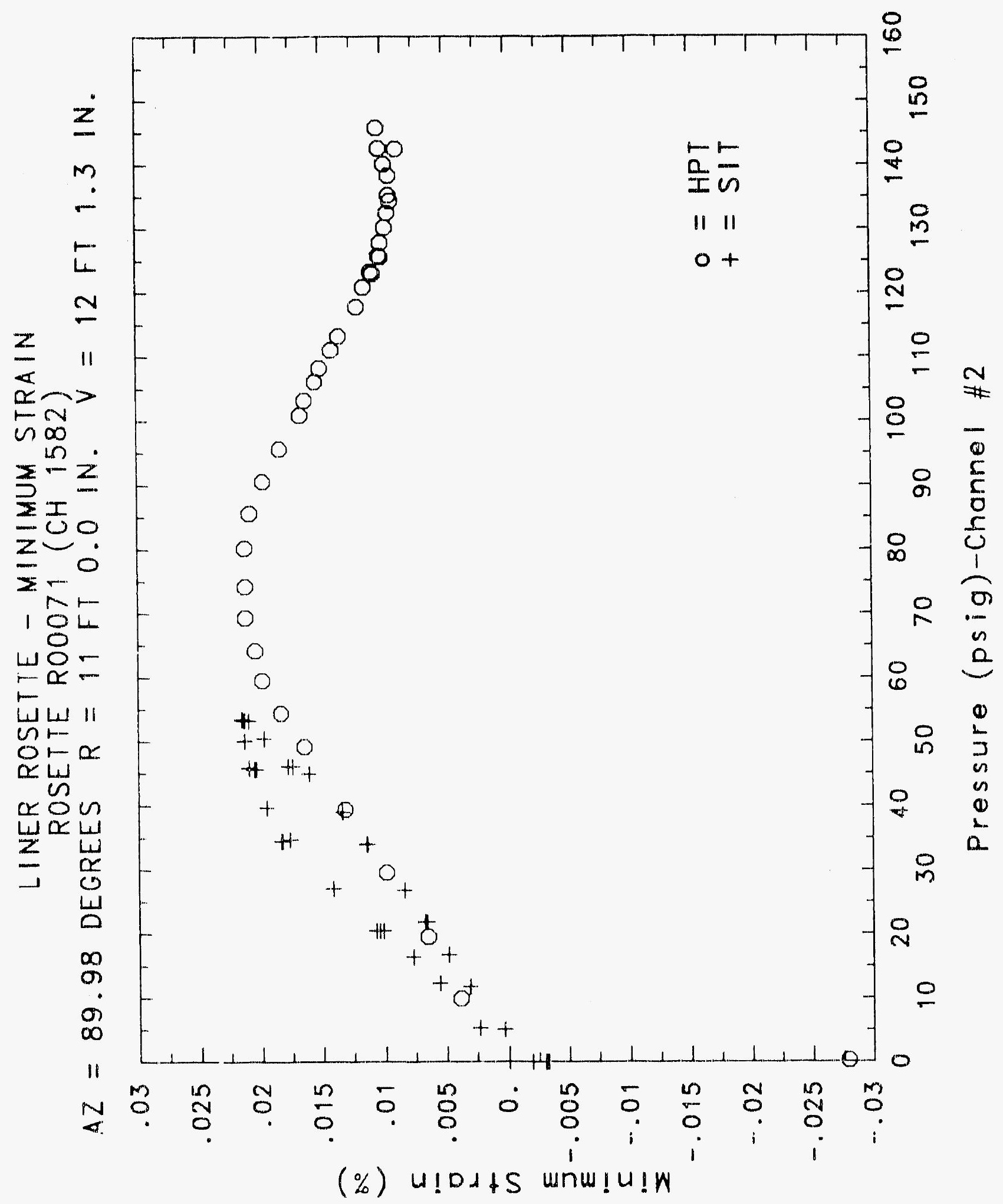




\title{
Reinforced Concrete Test Data
}

\author{
Rosette 72 Channels $1584 \quad 15851586$
}

\section{StruCtURAL INTEgRITY TEST}

\begin{tabular}{|c|c|c|c|c|c|c|c|}
\hline $\begin{array}{c}\text { Pressure } \\
\text { psig } \\
-0.05 \\
5.33 \\
12.31 \\
16.44 \\
20.51 \\
20.46 \\
20.47 \\
27.05 \\
34.69 \\
34.53 \\
34.53 \\
39.70 \\
45.90 \\
45.65 \\
45.62 \\
50.10 \\
53.47 \\
53.29 \\
53.21 \\
50.49 \\
46.14 \\
46.13 \\
44.97 \\
38.98 \\
33.99 \\
33.96 \\
26.83 \\
21.88 \\
21.90 \\
16.69 \\
11.74 \\
11.77 \\
5.05 \\
0.02 \\
-0.04 \\
-0.02 \\
-0.02 \\
0.02\end{array}$ & $\begin{array}{l}\text { Kaximum } \\
\text { \%Strain } \\
0.0002 \\
0.0033 \\
0.0071 \\
0.0097 \\
0.0143 \\
0.0151 \\
0.0167 \\
0.0208 \\
0.0242 \\
0.0243 \\
0.0289 \\
0.0322 \\
0.0363 \\
0.0399 \\
0.0410 \\
0.0438 \\
0.0470 \\
0.0482 \\
0.0490 \\
0.0493 \\
0.0484 \\
0.0504 \\
0.0725 \\
0.0831 \\
0.0827 \\
0.0831 \\
0.0812 \\
0.0800 \\
0.0847 \\
0.0878 \\
0.0908 \\
0.0939 \\
0.0915 \\
0.0890 \\
0.0945 \\
0.0946 \\
0.1050 \\
0.1282\end{array}$ & $\begin{array}{c}\text { Minimum } \\
\text { xStrain } \\
0.0001 \\
0.0024 \\
0.0049 \\
0.0076 \\
0.0094 \\
0.0095 \\
0.0096 \\
0.0138 \\
0.0185 \\
0.0184 \\
0.0182 \\
0.0205 \\
0.0236 \\
0.0226 \\
0.0222 \\
0.0243 \\
0.0258 \\
0.0253 \\
0.0251 \\
0.0235 \\
0.0211 \\
0.0206 \\
0.0157 \\
0.0100 \\
0.0070 \\
0.0072 \\
0.0028 \\
0.0002 \\
0.0001 \\
-0.0035 \\
-0.0070 \\
-0.0075 \\
-0.0114 \\
-0.0137 \\
-0.0147 \\
-0.0149 \\
-0.0167 \\
-0.0212\end{array}$ & $\begin{array}{c}\text { Angle } \\
\text { Degrees } \\
19.27 \\
-4.05 \\
34.85 \\
19.74 \\
9.06 \\
7.25 \\
1.55 \\
4.39 \\
4.23 \\
4.64 \\
-8.59 \\
-8.76 \\
-9.57 \\
-13.14 \\
-13.62 \\
-13.10 \\
-13.79 \\
-14.14 \\
-14.73 \\
-15.52 \\
-16.26 \\
-16.95 \\
-19.85 \\
-20.69 \\
-20.94 \\
-20.90 \\
-21.20 \\
-21.31 \\
-21.30 \\
-21.45 \\
-21.66 \\
-21.71 \\
-21.89 \\
-22.11 \\
-22.65 \\
-22.65 \\
-22.64 \\
-22.67\end{array}$ & $\begin{array}{c}\text { Pressure } \\
\text { psig } \\
9.89 \\
19.55 \\
29.57 \\
39.42 \\
49.16 \\
54.50 \\
59.57 \\
64.20 \\
69.32 \\
74.16 \\
80.16 \\
85.61 \\
90.58 \\
95.69 \\
100.92 \\
103.25 \\
106.11 \\
108.31 \\
111.08 \\
113.24 \\
117.83 \\
120.92 \\
123.28 \\
122.97 \\
125.82 \\
125.60 \\
127.84 \\
130.19 \\
132.53 \\
135.33 \\
134.42 \\
138.35 \\
140.16 \\
142.63 \\
145.78 \\
142.52 \\
0.22\end{array}$ & $\begin{array}{l}\text { Maximum } \\
\text { XStrain } \\
0.0118 \\
0.0185 \\
0.0564 \\
0.0625 \\
0.0665 \\
0.0683 \\
0.0685 \\
0.0740 \\
0.0738 \\
0.0801 \\
0.0927 \\
0.0969 \\
0.0994 \\
0.1003 \\
0.0995 \\
0.1050 \\
0.1032 \\
0.1043 \\
0.1074 \\
0.1087 \\
0.1072 \\
0.1094 \\
0.1104 \\
0.1101 \\
0.1100 \\
0.1092 \\
0.1096 \\
0.11119 \\
0.1122 \\
0.1131 \\
0.1137 \\
0.1158 \\
0.1161 \\
0.1200 \\
0.1226 \\
0.1216 \\
0.0219\end{array}$ & $\begin{array}{l}\text { Minimum } \\
\text { xStrain } \\
0.0045 \\
0.0096 \\
0.0093 \\
0.0143 \\
0.0195 \\
0.0222 \\
0.0250 \\
0.0262 \\
0.0285 \\
0.0293 \\
0.0272 \\
0.0272 \\
0.0274 \\
0.0274 \\
0.0279 \\
0.0268 \\
0.0275 \\
0.0276 \\
0.0272 \\
0.0271 \\
0.0275 \\
0.0273 \\
0.0274 \\
0.0271 \\
0.0277 \\
0.0275 \\
0.0277 \\
0.0272 \\
0.0275 \\
0.0280 \\
0.0273 \\
0.0278 \\
0.0285 \\
0.0292 \\
0.0303 \\
0.0290 \\
-0.2241\end{array}$ & $\begin{array}{l}\text { Angle } \\
\text { Degrees } \\
-20.91 \\
-16.48 \\
-20.62 \\
-19.92 \\
-19.06 \\
-18.63 \\
-17.83 \\
-18.19 \\
-17.80 \\
-18.79 \\
-19.85 \\
-20.53 \\
-20.99 \\
-21.45 \\
-22.07 \\
-22.48 \\
-22.68 \\
-22.89 \\
-23.31 \\
-23.58 \\
-24.31 \\
-24.68 \\
-25.04 \\
-25.31 \\
-25.72 \\
-25.96 \\
-25.56 \\
-25.45 \\
-25.71 \\
-26.01 \\
-25.81 \\
-25.44 \\
-25.21 \\
-25.33 \\
-25.23 \\
-25.17 \\
-22.01\end{array}$ \\
\hline
\end{tabular}

High Pressure test 


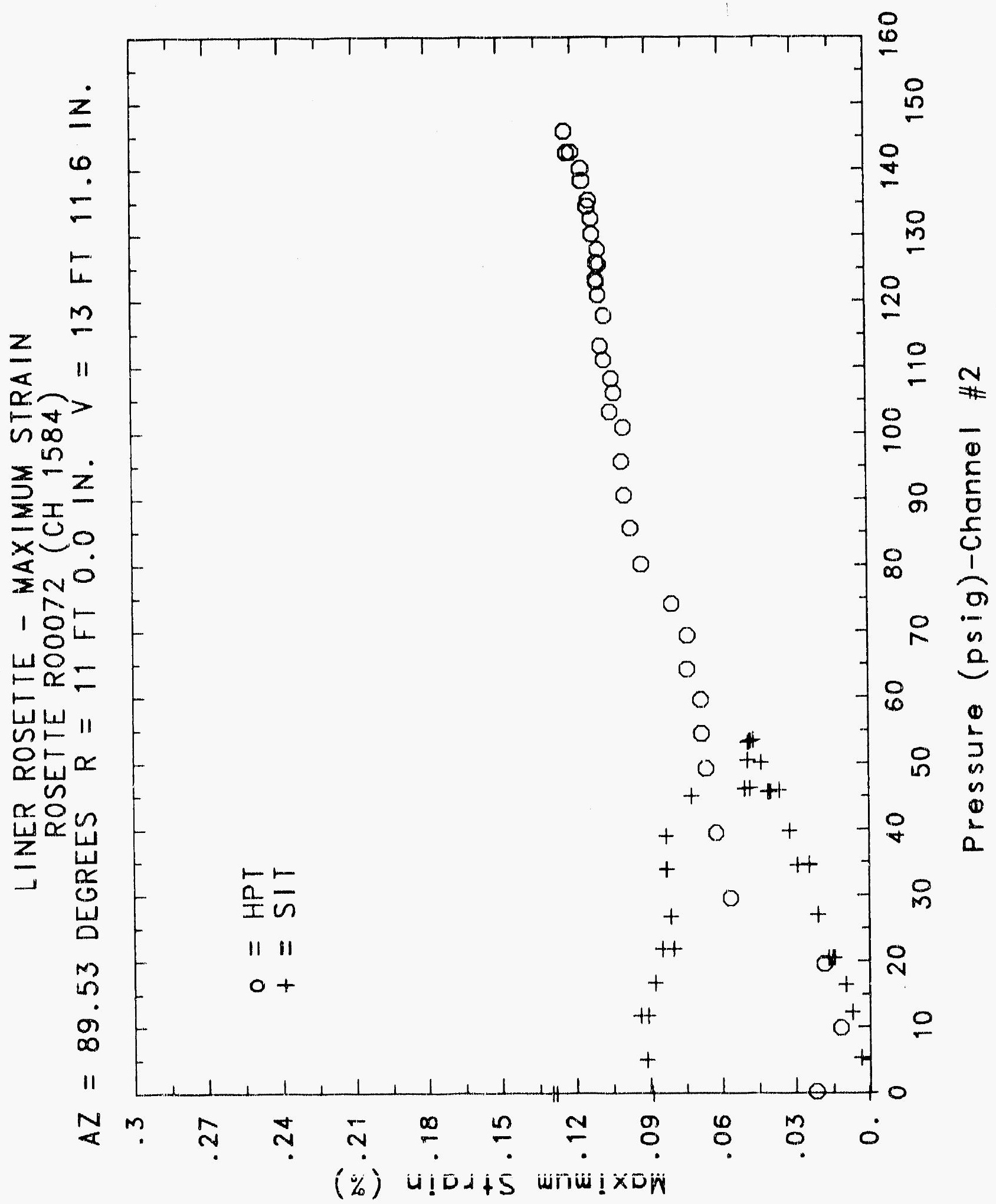




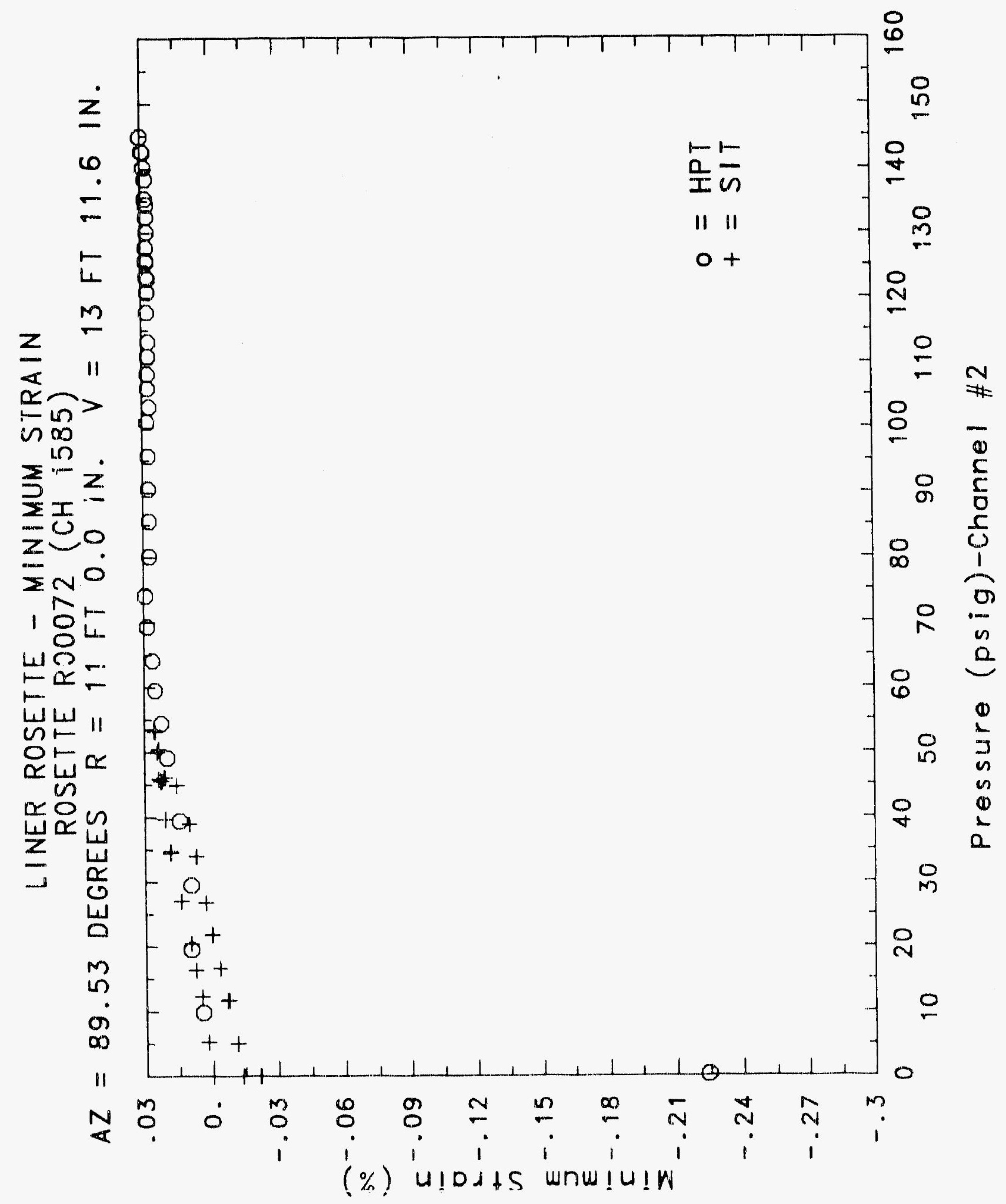




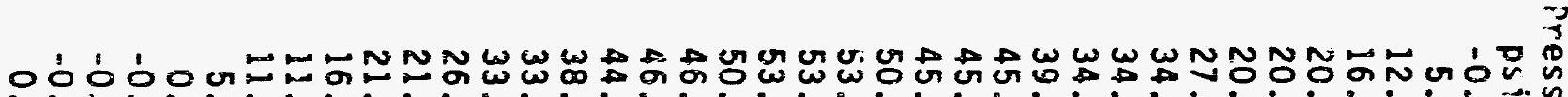

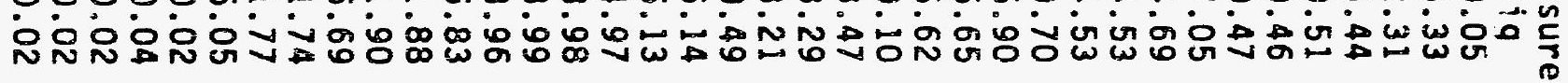
00000000000000000000000000000000000000界

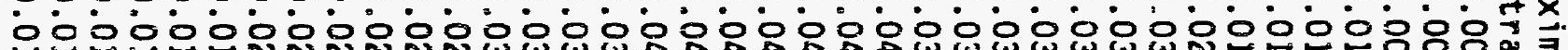

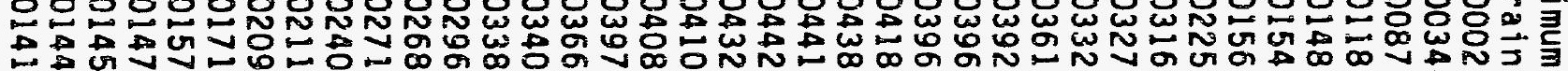

0000000000000000000000000000000000000043

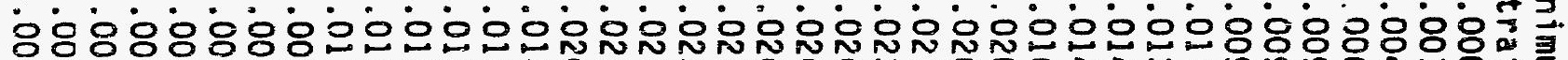

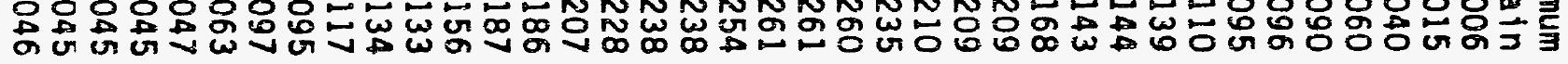

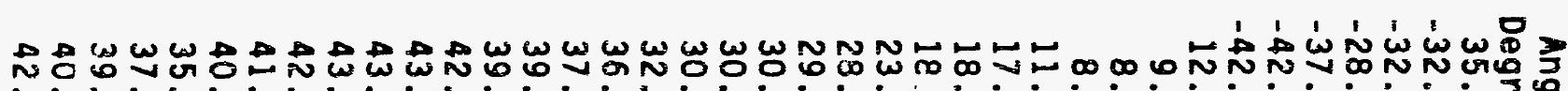
in

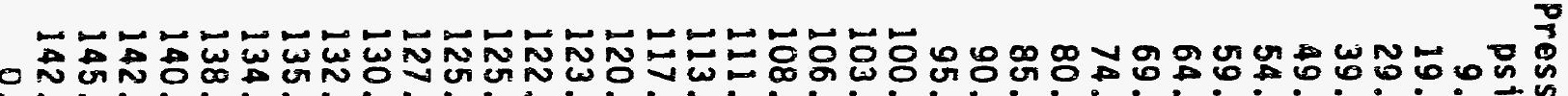

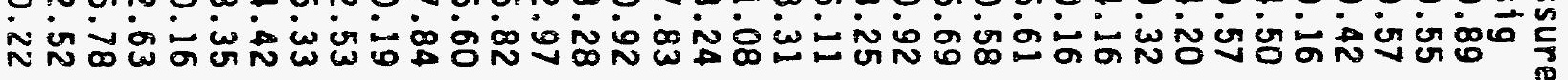
0.00000000000000000000000000000000000登

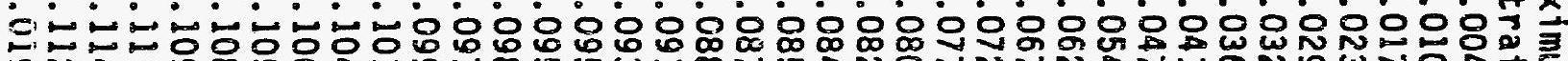

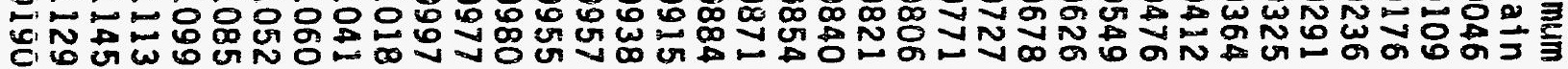

1000000000000000000000000000000000000癹

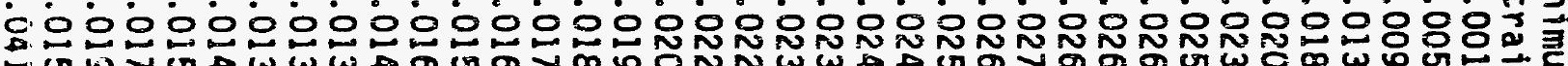

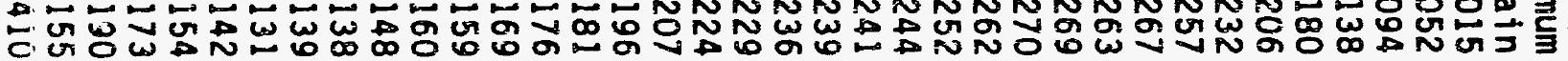

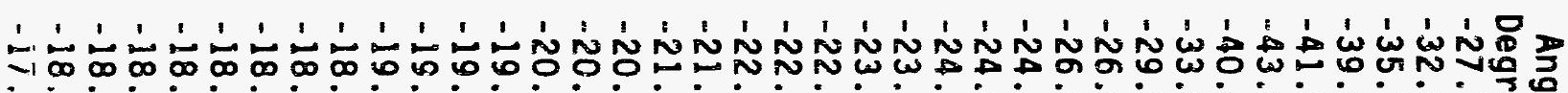

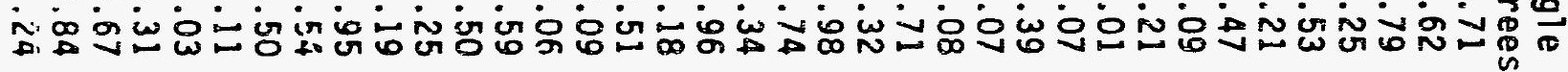




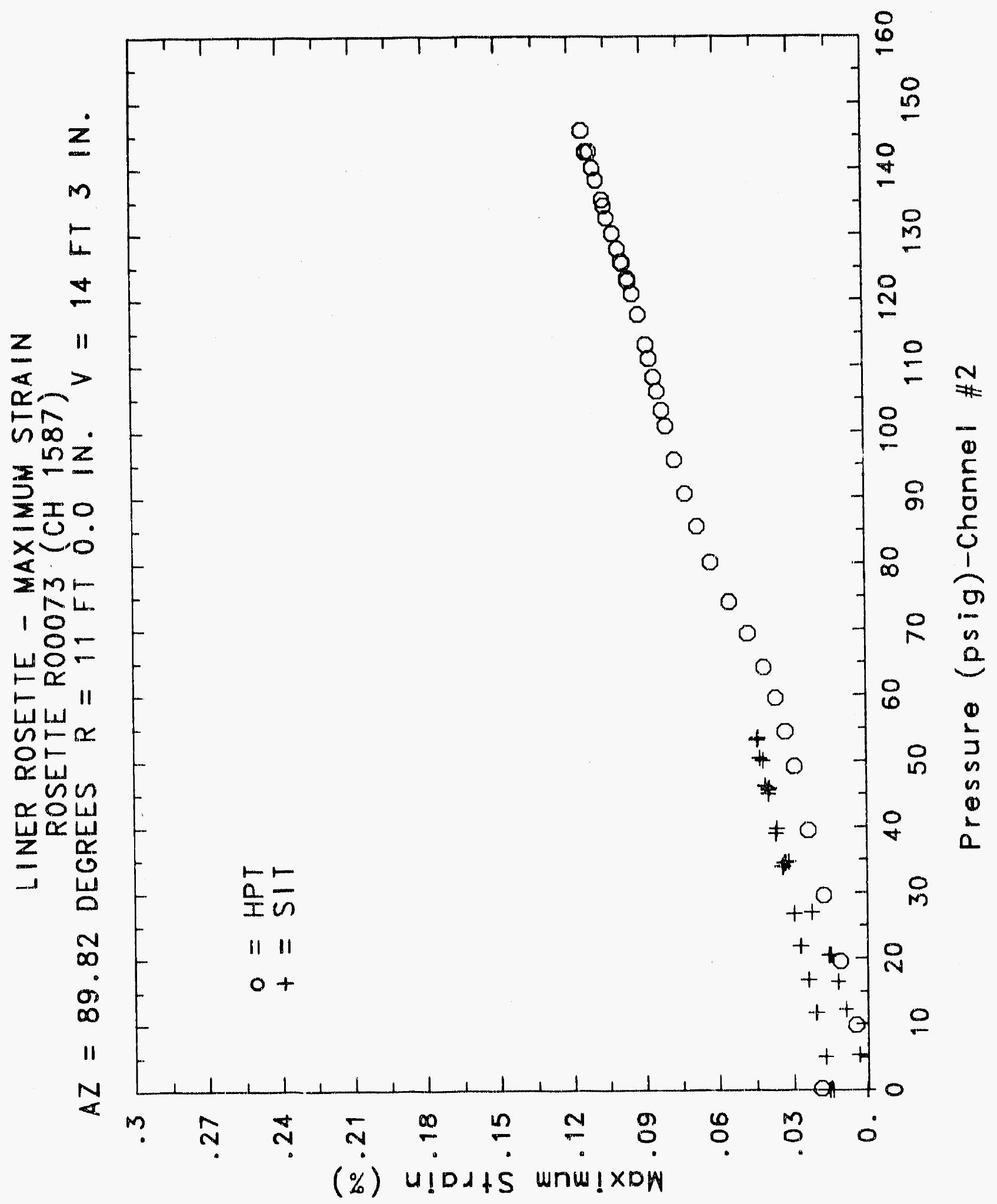




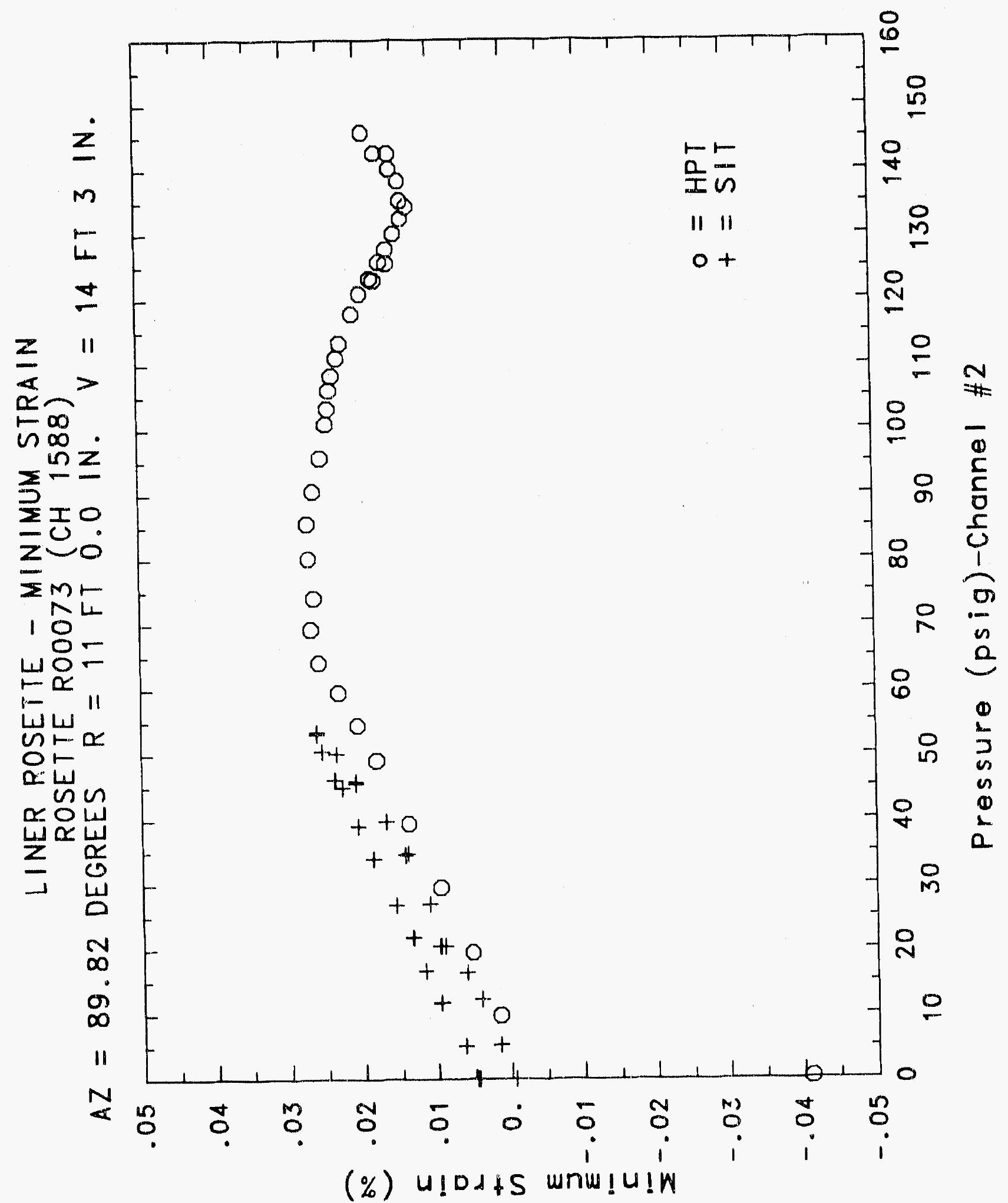




\title{
Reinforced Concrete Test Data
}

\author{
Rosette 74 Channels $1600 \quad 1601 \quad 1602$
}

Structural. Integrity Test

\begin{tabular}{|c|c|c|c|c|c|c|c|}
\hline $\begin{array}{c}\text { Pressure } \\
\text { psig } \\
\text {-0.05 } \\
5.33 \\
12.31 \\
16.44 \\
20.51 \\
20.46 \\
20.47 \\
27.05 \\
34.69 \\
34.53 \\
34.53 \\
39.70 \\
45.90 \\
45.65 \\
45.62 \\
50.10 \\
53.47 \\
53.29 \\
53.21 \\
50.49 \\
46.14 \\
46.13 \\
44.97 \\
38.98 \\
33.99 \\
33.96 \\
26.83 \\
21.88 \\
21.90 \\
16.69 \\
11.74 \\
11.77 \\
5.05 \\
0.02 \\
-0.04 \\
-0.02 \\
-0.02 \\
0.02\end{array}$ & $\begin{array}{l}\text { Maximum } \\
\text { \%Strain } \\
0.0003 \\
0.0046 \\
0.0110 \\
0.0153 \\
0.0201 \\
0.0203 \\
0.0204 \\
0.0299 \\
0.0429 \\
0.0447 \\
0.0450 \\
0.0529 \\
0.0646 \\
0.0646 \\
0.0644 \\
0.0707 \\
0.0772 \\
0.0776 \\
0.0776 \\
0.0754 \\
0.0710 \\
0.0708 \\
0.0695 \\
0.0630 \\
0.0572 \\
0.0572 \\
0.0482 \\
0.0419 \\
0.0428 \\
0.0366 \\
0.0301 \\
0.0300 \\
0.0217 \\
0.0158 \\
0.0152 \\
0.0151 \\
0.0152 \\
0.0146\end{array}$ & $\begin{array}{l}\text { Minimum } \\
\text { XStrain } \\
-0.0002 \\
0.0011 \\
0.0031 \\
0.0043 \\
0.0060 \\
0.0062 \\
0.0059 \\
0.0099 \\
0.0207 \\
0.0224 \\
0.0229 \\
0.0289 \\
0.0348 \\
0.0343 \\
0.0340 \\
0.0360 \\
0.0367 \\
0.0358 \\
0.0353 \\
0.0338 \\
0.0314 \\
0.0311 \\
0.0290 \\
0.0259 \\
0.0229 \\
0.0233 \\
0.0183 \\
0.0150 \\
0.0158 \\
0.0134 \\
0.0106 \\
0.0111 \\
0.0068 \\
0.0033 \\
0.0028 \\
0.0027 \\
0.0025 \\
0.0026\end{array}$ & $\begin{array}{c}\text { Angle } \\
\text { Degrees } \\
29.58 \\
5.09 \\
1.94 \\
0.88 \\
-1.78 \\
-2.91 \\
-2.89 \\
-5.58 \\
-9.01 \\
-9.44 \\
-9.72 \\
-10.40 \\
-10.38 \\
-12.47 \\
-13.07 \\
-13.35 \\
-14.45 \\
-15.34 \\
-15.94 \\
-16.49 \\
-16.95 \\
-16.88 \\
-16.45 \\
-16.88 \\
-17.30 \\
-16.83 \\
-17.46 \\
-18.07 \\
-17.12 \\
-18.13 \\
-20.17 \\
-20.55 \\
-24.98 \\
-29.67 \\
-24.32 \\
-23.09 \\
-22.15 \\
-19.54\end{array}$ & $\begin{array}{c}\text { Pressure } \\
\text { ps19 } \\
9.89 \\
19.55 \\
29.57 \\
39.42 \\
49.16 \\
54.50 \\
59.57 \\
64.20 \\
69.32 \\
74.16 \\
80.16 \\
85.61 \\
90.58 \\
95.69 \\
100.92 \\
103.25 \\
106.11 \\
108.31 \\
111.08 \\
113.24 \\
117.83 \\
120.92 \\
123.28 \\
122.97 \\
125.82 \\
125.60 \\
127.84 \\
130.19 \\
132.53 \\
135.33 \\
134.42 \\
138.35 \\
140.16 \\
142.63 \\
145.78 \\
142.52 \\
0.22\end{array}$ & $\begin{array}{l}\text { Maximum } \\
\text { xStrain } \\
0.0133 \\
0.0264 \\
0.0396 \\
0.0516 \\
0.0631 \\
0.0697 \\
0.0771 \\
0.0857 \\
0.0958 \\
0.1071 \\
0.1221 \\
0.1405 \\
0.1825 \\
0.2310 \\
0.2745 \\
0.2872 \\
0.3035 \\
0.3160 \\
0.3332 \\
0.3453 \\
0.3730 \\
0.3860 \\
0.3047 \\
0 \\
0 .\end{array}$ & $\begin{array}{c}\text { Minimum } \\
\text { XStrain } \\
0.0051 \\
0.0116 \\
0.0184 \\
0.0245 \\
0.0304 \\
0.0338 \\
0.0369 \\
0.0343 \\
0.0317 \\
0.0279 \\
0.0268 \\
0.0257 \\
0.0301 \\
0.0357 \\
0.0384 \\
0.0386 \\
0.0392 \\
0.0391 \\
0.0385 \\
0.0380 \\
0.0361 \\
0.0350 \\
0.0340 \\
0.0331 \\
0.0324 \\
0.0313 \\
0.0316 \\
0.0299 \\
0.0275 \\
0.0256 \\
0.0242 \\
0.0252 \\
0.0254 \\
0.0274 \\
0.0273 \\
0.0222 \\
-0.0988\end{array}$ & $\begin{array}{c}\text { Angle } \\
\text { Degrees } \\
-6.85 \\
-8.06 \\
-9.38 \\
-11.31 \\
-12.11 \\
-12.82 \\
-13.60 \\
-15.12 \\
-17.79 \\
-19.00 \\
-22.42 \\
-21.37 \\
-15.62 \\
-9.32 \\
-4.16 \\
-3.13 \\
-1.66 \\
-0.62 \\
0.69 \\
1.56 \\
3.43 \\
4.09 \\
4.47 \\
4.52 \\
5.12 \\
5.25 \\
5.48 \\
6.15 \\
7.06 \\
7.81 \\
7.90 \\
8.45 \\
8.79 \\
9.31 \\
10.02 \\
10.22 \\
9.65\end{array}$ \\
\hline
\end{tabular}

High Pressure Test 


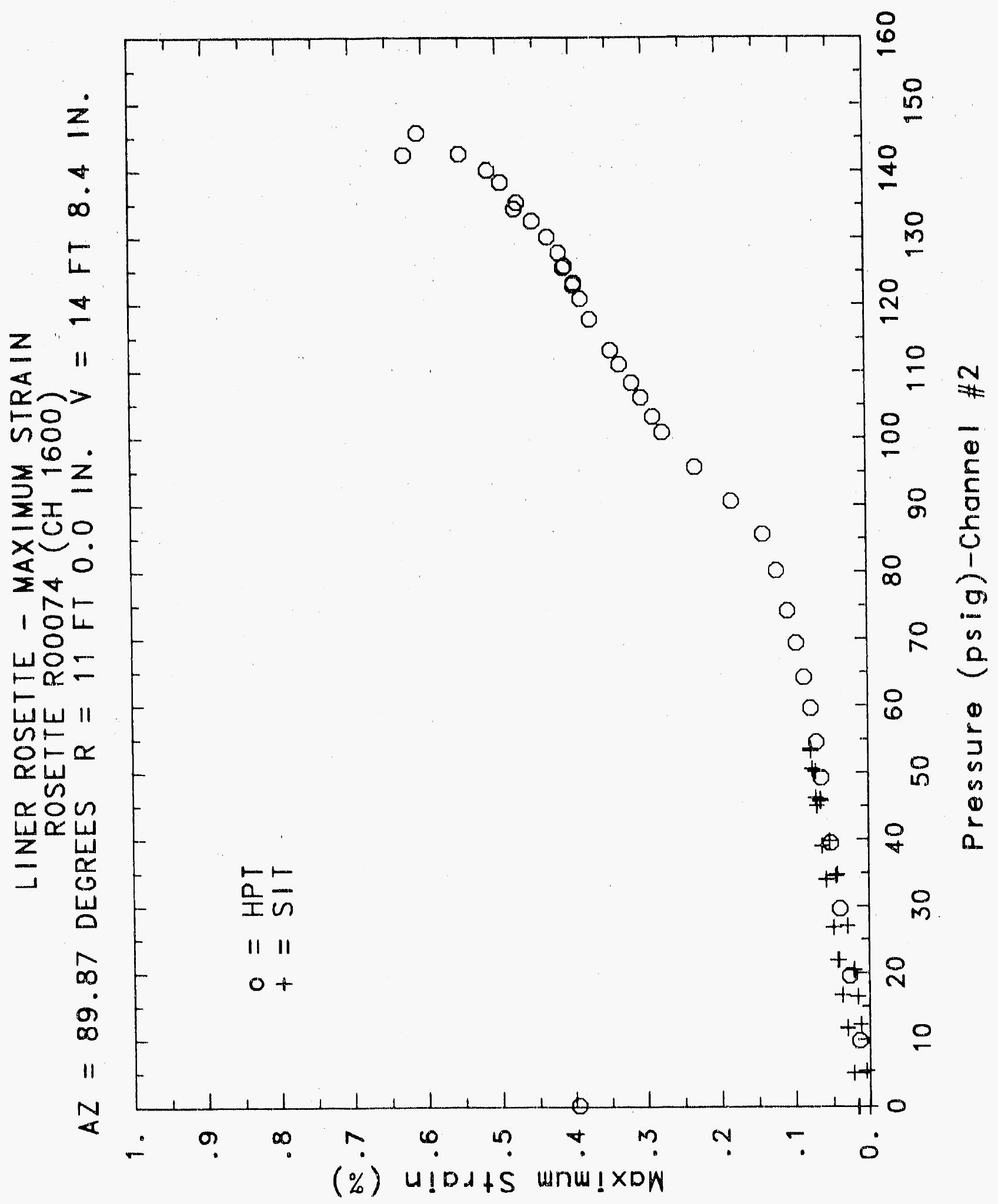




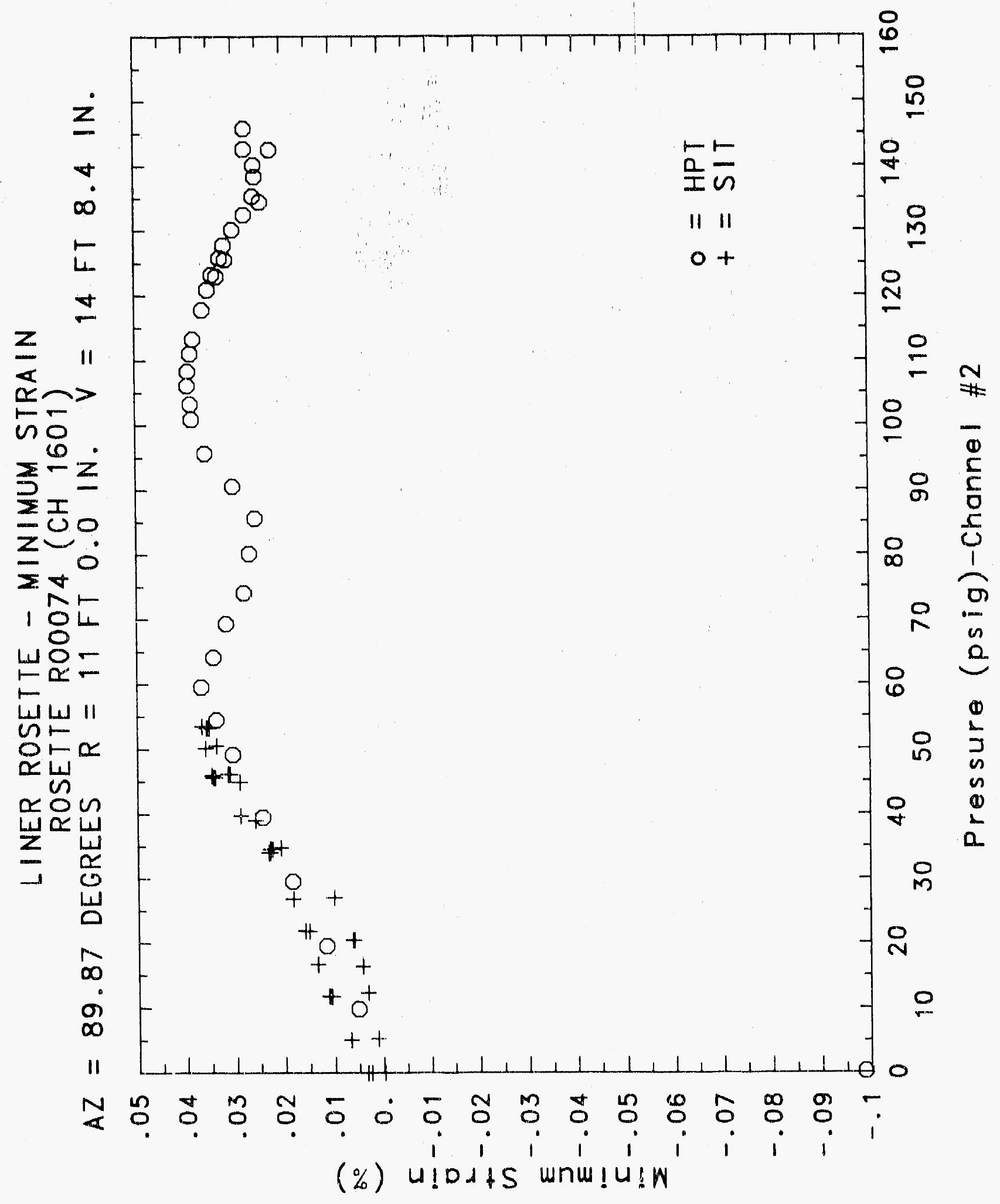




\section{Reinforced Concrete Test Data}

Rosette 75 Channels $1603 \quad 1604 \quad 1605$

\section{StRUCTURAL INTEGRITY TEST}

\begin{tabular}{|c|c|c|c|c|c|c|c|}
\hline $\begin{array}{c}\text { Pressure } \\
\text { psig } \\
-0.05 \\
5.33 \\
12.31 \\
16.44 \\
20.51 \\
20.46 \\
20.47 \\
27.05 \\
34.69 \\
34.53 \\
34.53 \\
39.70 \\
45.90 \\
45.65 \\
45.62 \\
50.10 \\
53.47 \\
53.29 \\
53.21 \\
50.49 \\
46.14 \\
46.13 \\
44.97 \\
38.98 \\
33.99 \\
33.96 \\
26.83 \\
21.88 \\
21.90 \\
16.69 \\
11.74 \\
11.77 \\
5.05 \\
0.02 \\
-0.04 \\
-0.02 \\
-0.02 \\
0.02\end{array}$ & $\begin{array}{l}\text { Maximum } \\
\text { \%Strain } \\
0.0012 \\
0.0298 \\
0.0556 \\
0.0661 \\
0.0768 \\
0.0806 \\
0.0833 \\
0.1023 \\
0.1269 \\
0.1350 \\
0.1361 \\
0.1491 \\
0.1702 \\
0.1730 \\
0.1739 \\
0.1854 \\
0.1969 \\
0.1988 \\
0.2003 \\
0.1997 \\
0.1963 \\
0.1985 \\
0.2029 \\
0.1993 \\
0.1945 \\
0.1960 \\
0.1901 \\
0.1854 \\
0.1885 \\
0.1825 \\
0.1769 \\
0.1758 \\
0.1575 \\
0.1282 \\
0.1174 \\
0.1181 \\
0.1181 \\
0.1217\end{array}$ & $\begin{array}{l}\text { Minimum } \\
\text { x.Strain } \\
-0.0001 \\
-0.0120 \\
-0.0120 \\
-0.0099 \\
-0.0071 \\
-0.0062 \\
-0.0056 \\
0.0006 \\
0.0106 \\
0.0123 \\
0.0128 \\
0.0172 \\
0.0235 \\
0.0236 \\
0.0236 \\
0.0268 \\
0.0299 \\
0.0301 \\
0.0301 \\
0.0294 \\
0.0274 \\
0.0271 \\
0.0271 \\
0.0243 \\
0.0212 \\
0.0212 \\
0.0160 \\
0.0119 \\
0.0121 \\
0.0082 \\
0.0035 \\
0.0021 \\
-0.0057 \\
-0.0049 \\
-0.0054 \\
-0.0049 \\
-0.0048 \\
-0.0052\end{array}$ & $\begin{array}{c}\text { Angle } \\
\text { Degrees } \\
-26.16 \\
10.30 \\
5.18 \\
1.88 \\
-0.13 \\
-1.12 \\
-2.10 \\
-2.74 \\
-0.48 \\
-0.97 \\
-0.89 \\
-0.32 \\
-0.10 \\
-0.55 \\
-0.68 \\
-0.50 \\
-0.08 \\
-0.02 \\
-0.14 \\
-0.42 \\
-0.77 \\
-1.17 \\
-1.65 \\
-2.34 \\
-2.65 \\
-2.90 \\
-3.53 \\
-3.87 \\
-4.27 \\
-4.62 \\
-5.21 \\
-5.40 \\
-5.09 \\
-8.44 \\
-8.05 \\
-7.52 \\
-7.42 \\
-8.06\end{array}$ & $\begin{array}{c}\text { Pressure } \\
\text { psig } \\
9.89 \\
19.55 \\
29.57 \\
39.42 \\
49.16 \\
54.50 \\
59.57 \\
64.20 \\
69.32 \\
74.16 \\
80.16 \\
85.61 \\
90.58 \\
95.69 \\
100.92 \\
103.25 \\
106.11 \\
108.31 \\
111.08 \\
113.24 \\
117.83 \\
120.92 \\
123.28 \\
122.97 \\
125.82 \\
125.60 \\
127.84 \\
130.19 \\
132.53 \\
135.33 \\
134.42 \\
138.35 \\
140.16 \\
142.63 \\
145.78 \\
142.52 \\
0.22\end{array}$ & $\begin{array}{c}\text { Maximum } \\
\text { xStrain } \\
0.0482 \\
0.0644 \\
0.0778 \\
0.0917 \\
0.1040 \\
0.1129 \\
0.1325 \\
0.1535 \\
0.1757 \\
0.2012 \\
0.2262 \\
0.2505 \\
0.2741 \\
0.3004 \\
0.3299 \\
0.3422 \\
0.3554 \\
0.3662 \\
0.3802 \\
0.3899 \\
0.4165 \\
0.4349 \\
0.4485 \\
0.4504 \\
0.4641 \\
0.4664 \\
0.4753 \\
0.4948 \\
0.5311 \\
0.5932 \\
0.6087 \\
0.6914 \\
0.7775 \\
0.9432 \\
1.0985 \\
1.1229 \\
0.7177\end{array}$ & $\begin{array}{l}\text { Minimum } \\
\text { XStrain } \\
0.0027 \\
0.0103 \\
0.0184 \\
0.0254 \\
0.0318 \\
0.0357 \\
0.0395 \\
0.0435 \\
0.0477 \\
0.0522 \\
0.0573 \\
0.0615 \\
0.0653 \\
0.0694 \\
0.0741 \\
0.0765 \\
0.0788 \\
0.0807 \\
0.0833 \\
0.0848 \\
0.0892 \\
0.0927 \\
0.0950 \\
0.0954 \\
0.0974 \\
0.0978 \\
0.0991 \\
0.1002 \\
0.1009 \\
0.1006 \\
0.1005 \\
0.1002 \\
0.0993 \\
0.0990 \\
0.1025 \\
0.1027 \\
-0.0022\end{array}$ & $\begin{array}{c}\text { Angle } \\
\text { Degrees } \\
4.26 \\
3.84 \\
4.55 \\
4.78 \\
6.16 \\
6.95 \\
5.99 \\
6.07 \\
6.76 \\
7.19 \\
8.53 \\
10.25 \\
11.55 \\
12.47 \\
12.81 \\
12.97 \\
13.02 \\
12.91 \\
12.78 \\
12.75 \\
12.04 \\
11.29 \\
10.75 \\
10.61 \\
9.95 \\
9.78 \\
9.34 \\
8.33 \\
6.77 \\
4.63 \\
4.03 \\
2.65 \\
1.50 \\
0.01 \\
-1.08 \\
-1.21 \\
-2.56\end{array}$ \\
\hline
\end{tabular}

High Pressure test 


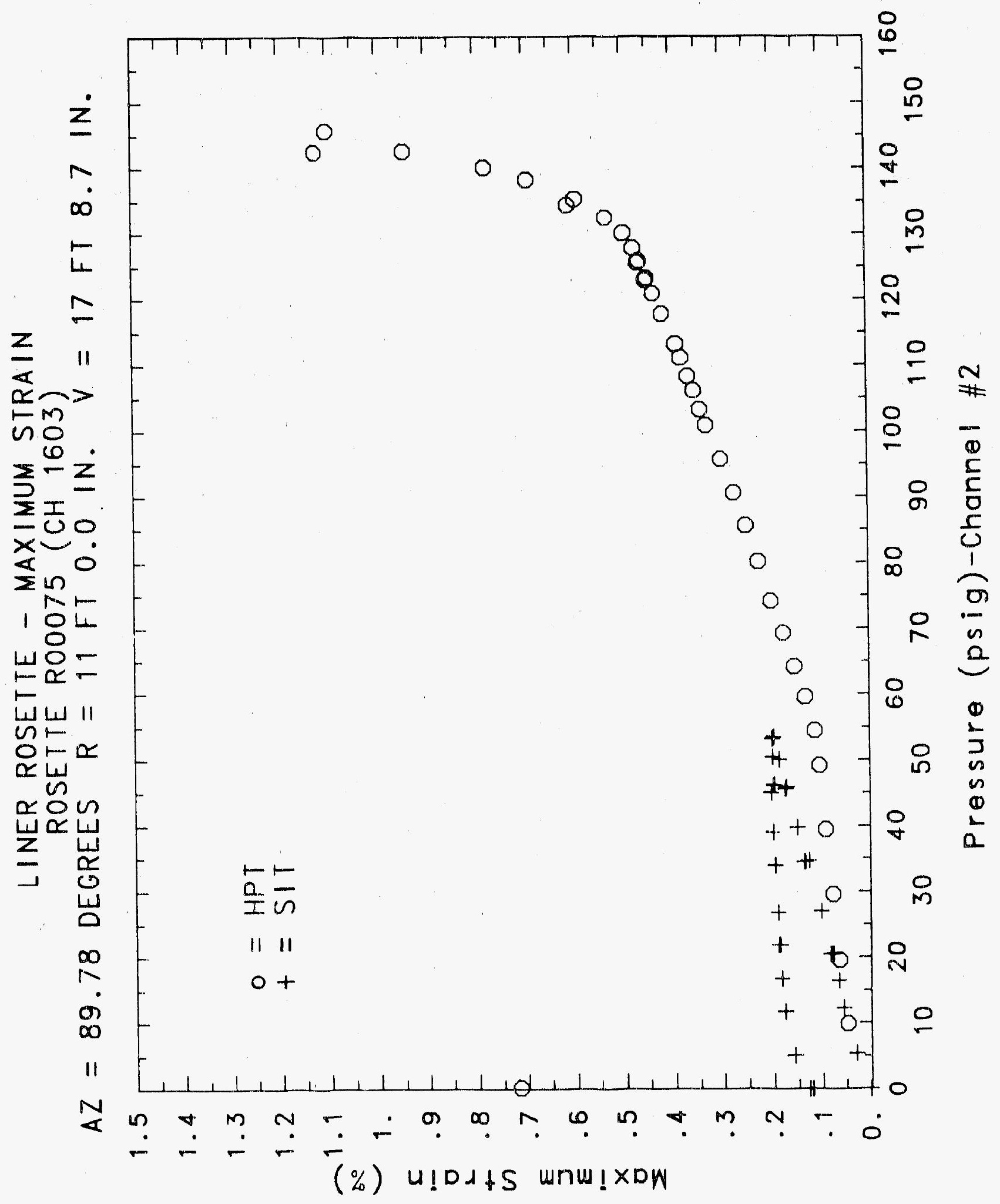


$+5-5$

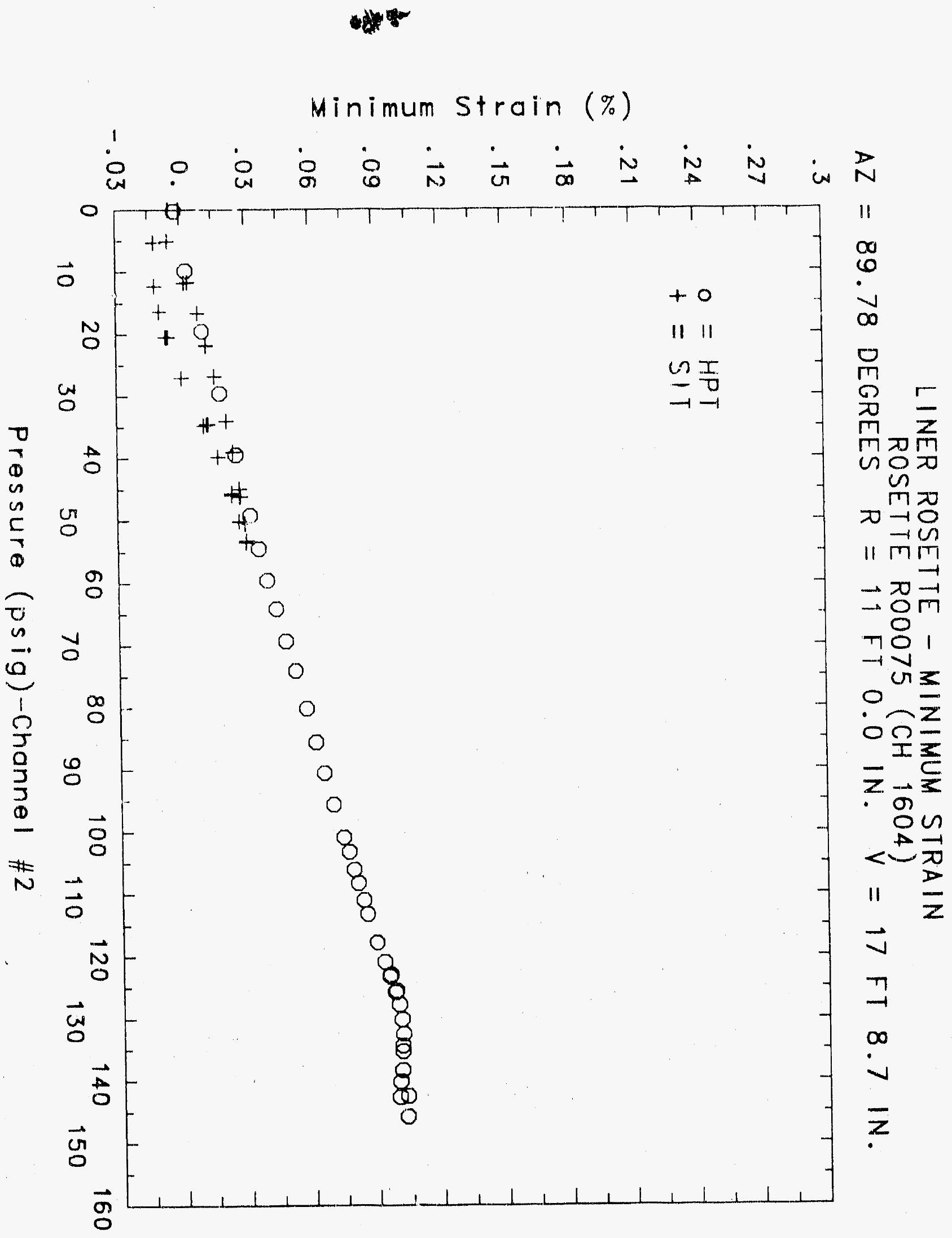




\title{
Reinforced Concrete Test Data
}

\author{
Rosette 76 Channels $1606 \quad 1607 \quad 1608$
}

\section{STRUCTURAL INTEGRITY TEST}

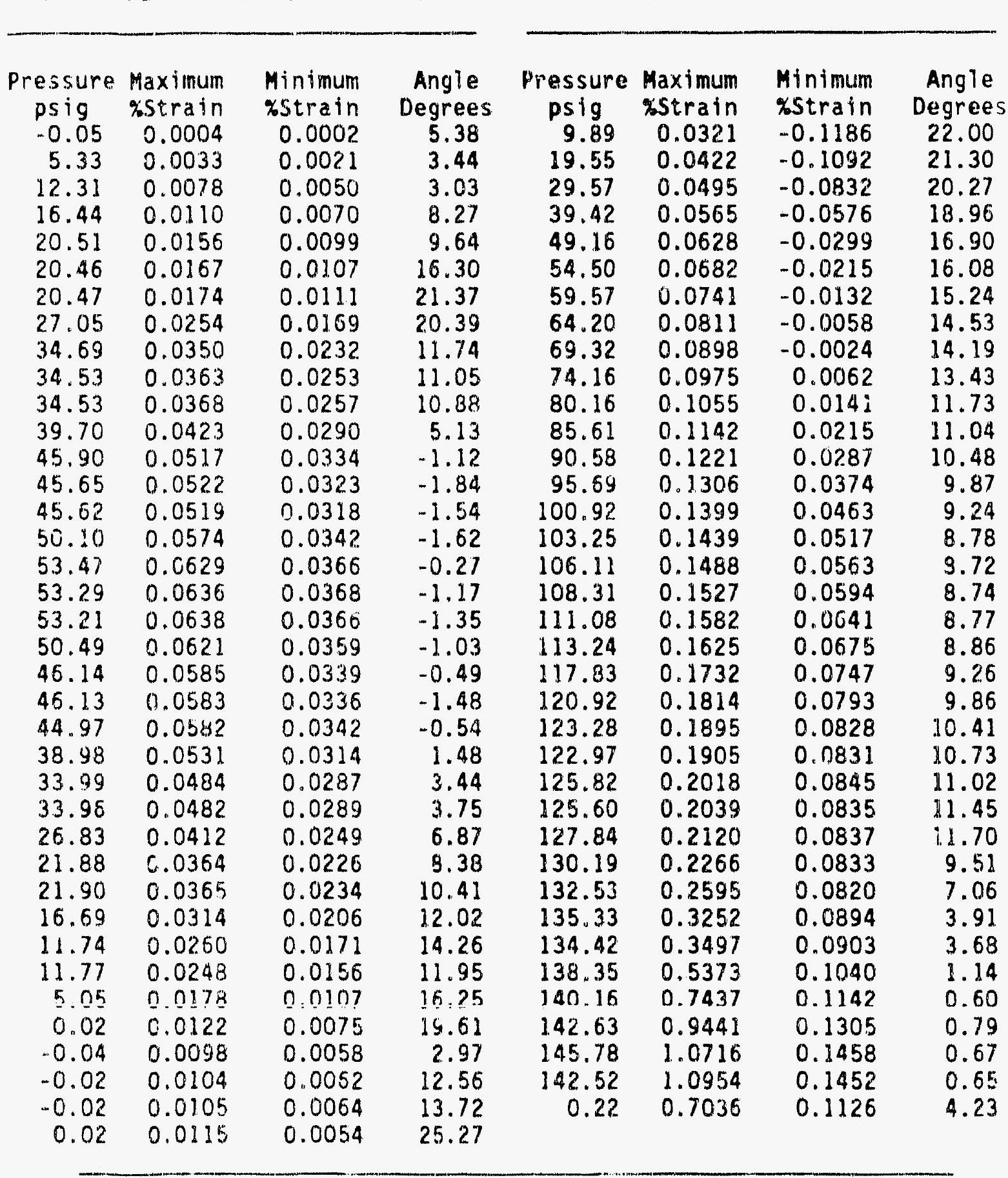

\section{High Pressure Test}




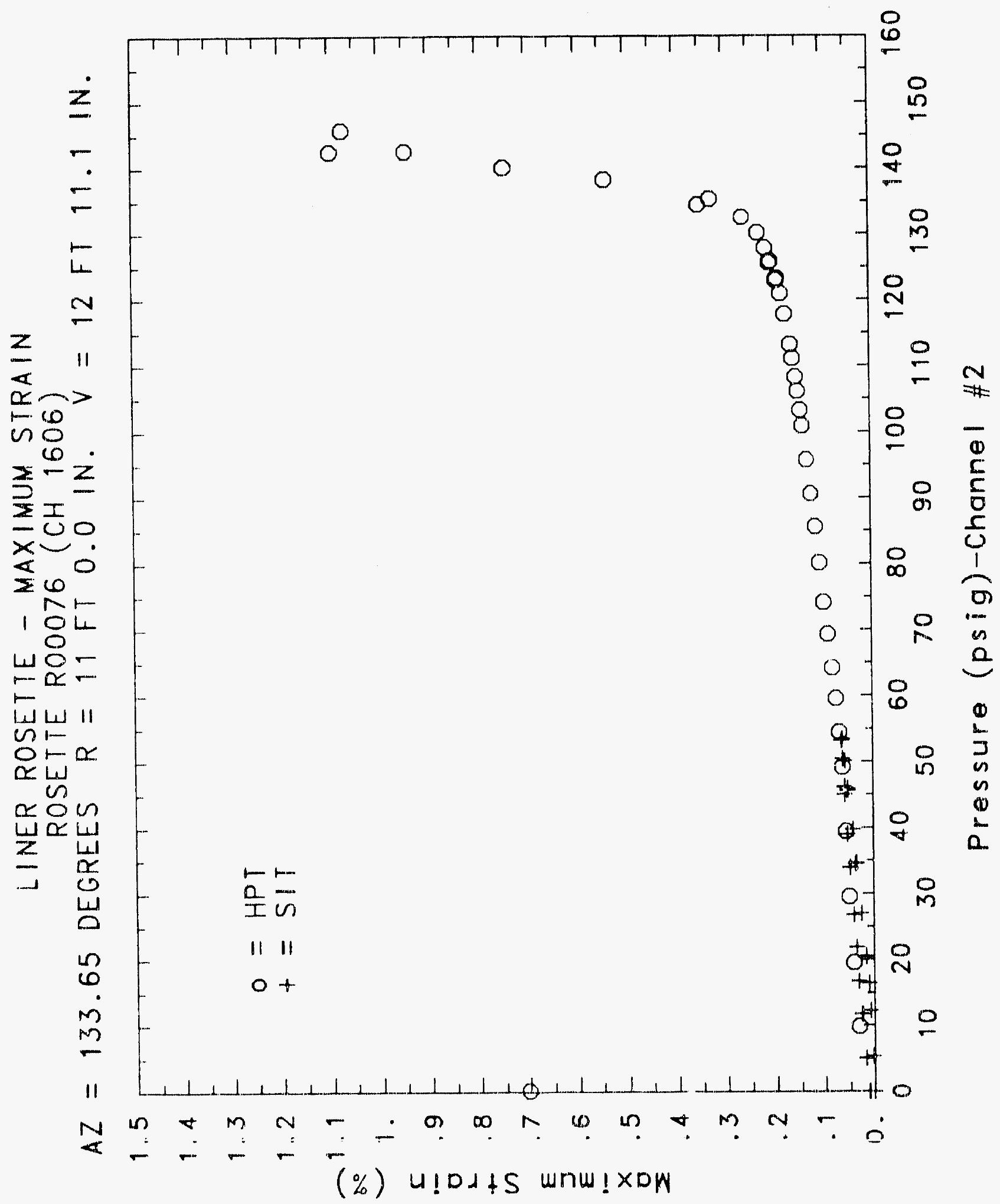




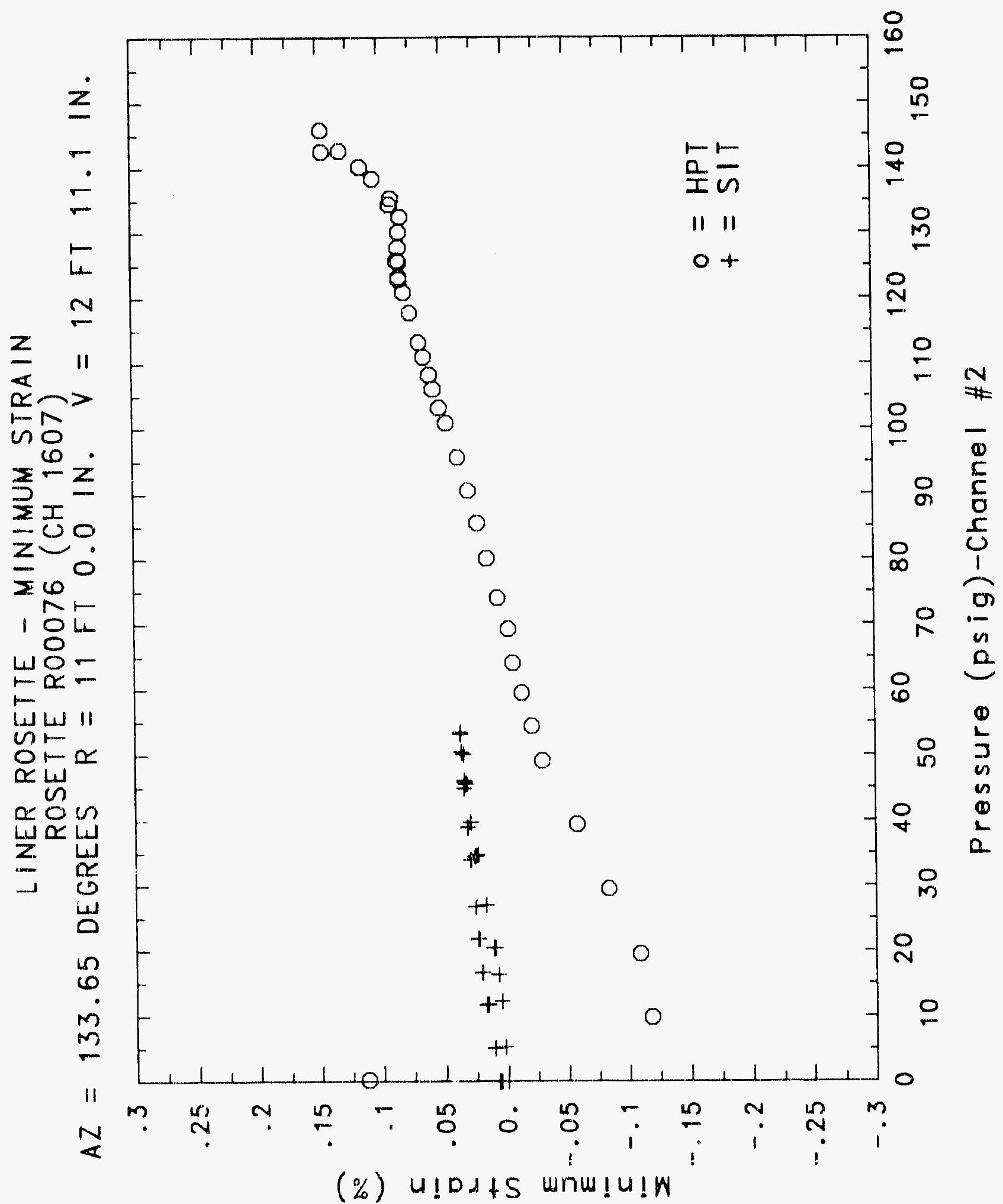




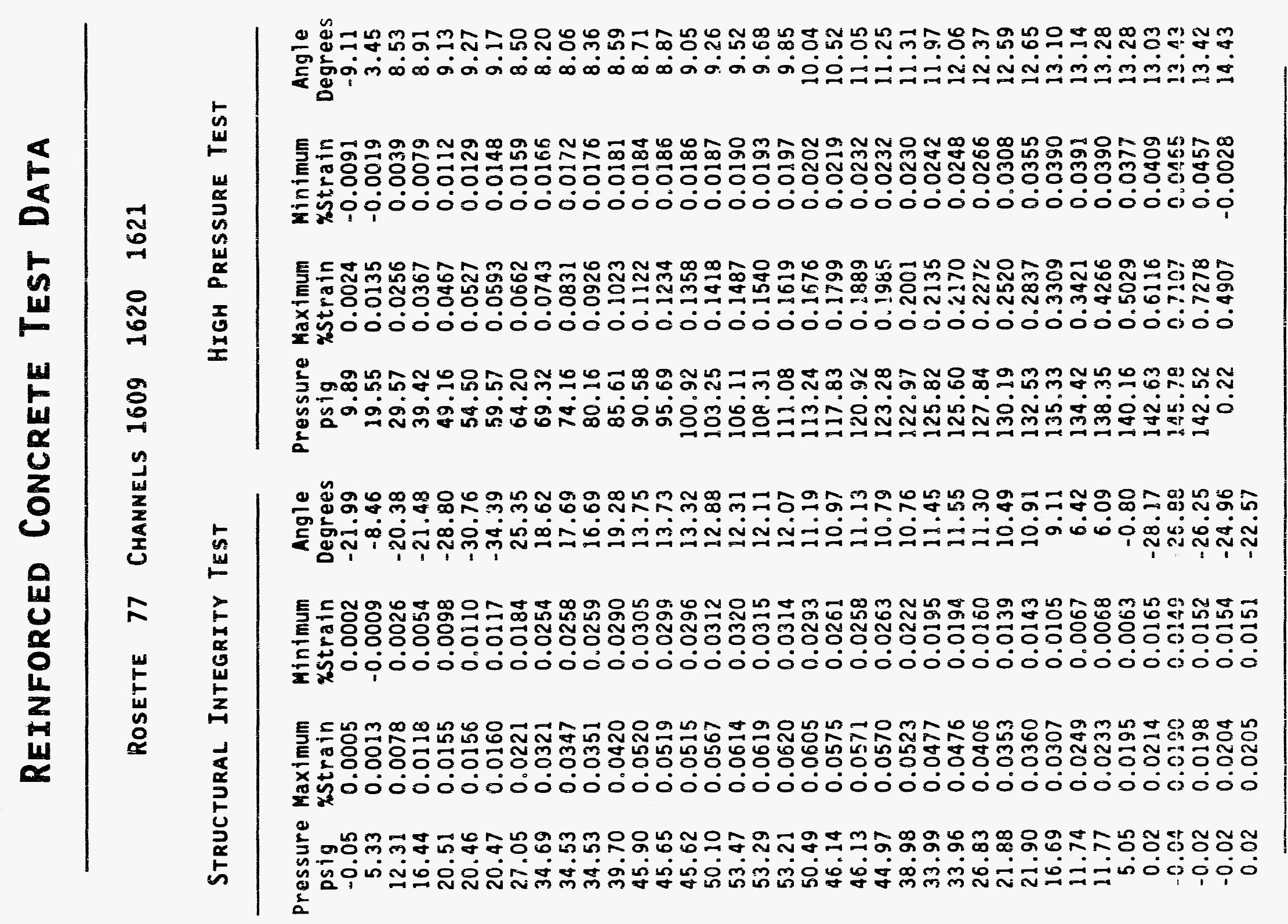




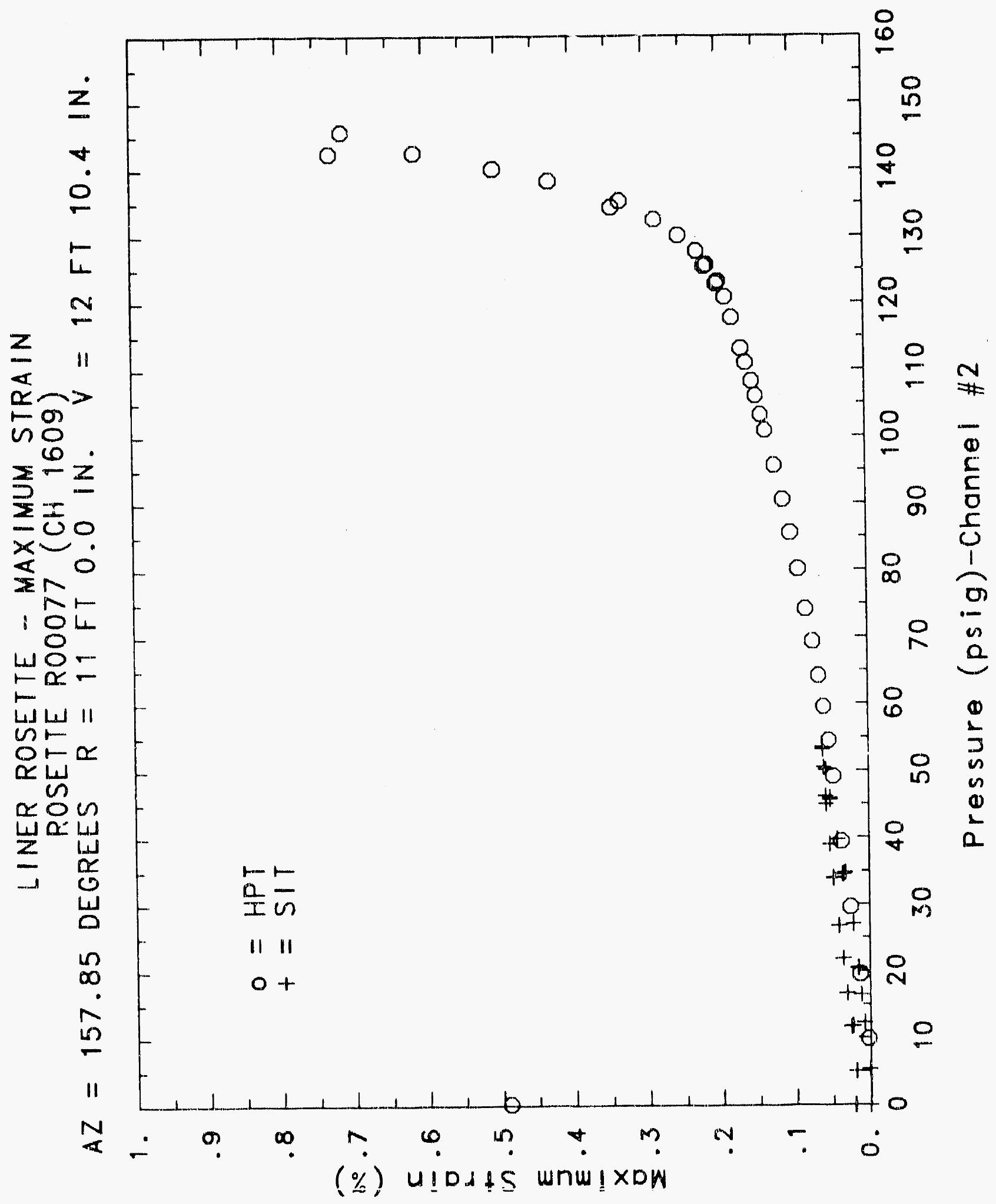




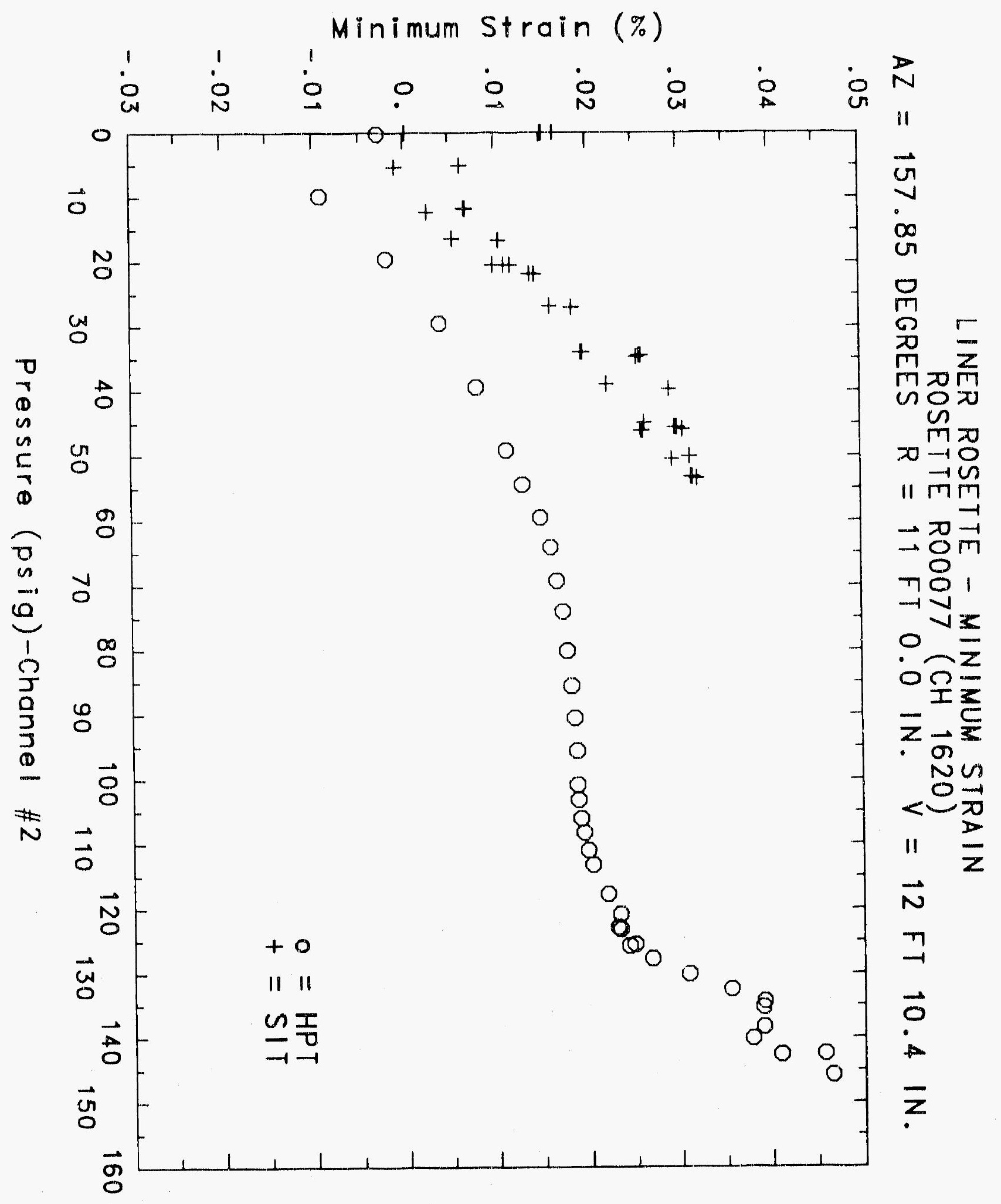




\title{
Reinforced Concrete Test Data
}

\author{
Rosette 78 Channels $301 \quad 302 \quad 303$
}

\section{Structural Integrity TEST}

\begin{tabular}{|c|c|c|c|c|c|c|c|}
\hline $\begin{array}{c}\text { Pressure } \\
\text { psig } \\
-0.05 \\
5.33 \\
12.31 \\
16.44 \\
20.51 \\
20.46 \\
20.47 \\
27.05 \\
34.69 \\
34.53 \\
34.53 \\
39.70 \\
45.90 \\
45.65 \\
45.62 \\
50.10 \\
53.47 \\
53.29 \\
53.21 \\
50.49 \\
46.14 \\
46.13 \\
44.97 \\
38.98 \\
33.99 \\
33.96 \\
26.83 \\
21.88 \\
21.90 \\
16.69 \\
11.74 \\
11.77 \\
5.05 \\
0.02 \\
-0.04 \\
-0.02 \\
-0.02 \\
0.02\end{array}$ & $\begin{array}{c}\text { Maximum } \\
\text { \%Strain } \\
0.0020 \\
0.0077 \\
0.0482 \\
0.0540 \\
0.0641 \\
0.0666 \\
0.0687 \\
0.0906 \\
0.1201 \\
0.1241 \\
0.1247 \\
0.1404 \\
0.1624 \\
0.1627 \\
0.1625 \\
0.1753 \\
0.1879 \\
0.1885 \\
0.1880 \\
0.1838 \\
0.1752 \\
0.1739 \\
0.1732 \\
0.1608 \\
0.1474 \\
0.1476 \\
0.1294 \\
0.1163 \\
0.1179 \\
0.1047 \\
0.0920 \\
0.0895 \\
0.0732 \\
0.0599 \\
0.0552 \\
0.0560 \\
0.0564 \\
0.0563\end{array}$ & $\begin{array}{l}\text { Minimum } \\
\text { \%Strain } \\
-0.0051 \\
-0.0025 \\
-0.2013 \\
-0.2005 \\
-0.2005 \\
-0.2020 \\
-0.2019 \\
-0.2072 \\
-0.2164 \\
-0.2169 \\
-0.2165 \\
-0.2233 \\
-0.2315 \\
-0.2300 \\
-0.2294 \\
-0.2324 \\
-0.2335 \\
-0.2324 \\
-0.2322 \\
-0.2296 \\
-0.2258 \\
-0.2251 \\
-0.2258 \\
-0.2196 \\
-0.2159 \\
-0.2157 \\
-0.2102 \\
-0.2062 \\
-0.2070 \\
-0.2031 \\
-0.2007 \\
-0.2006 \\
-0.1996 \\
-0.2009 \\
-0.2027 \\
-0.2028 \\
-0.2042 \\
-0.2021\end{array}$ & $\begin{array}{l}\text { Angle } \\
\text { Degrees } \\
-38.55 \\
-43.93 \\
-23.40 \\
-23.47 \\
-23.52 \\
-23.70 \\
-23.71 \\
-24.44 \\
-25.38 \\
-25.56 \\
-25.58 \\
-25.99 \\
-26.43 \\
-26.40 \\
-26.42 \\
-26.30 \\
-26.01 \\
-25.88 \\
-25.88 \\
-25.79 \\
-25.71 \\
-25.66 \\
-25.69 \\
-25.36 \\
-25.20 \\
-25.29 \\
-24.92 \\
-24.44 \\
-24.58 \\
-24.05 \\
-23.58 \\
-23.53 \\
-22.58 \\
-21.50 \\
-21.41 \\
-21.40 \\
-21.48 \\
-21.29\end{array}$ & $\begin{array}{c}\text { Pressure } \\
\text { psig } \\
9.89 \\
19.55 \\
29.57 \\
39.42 \\
49.16 \\
54.50 \\
59.57 \\
64.20 \\
69.32 \\
74.16 \\
80.16 \\
85.61 \\
90.58 \\
95.69 \\
100.92 \\
103.25 \\
106.11 \\
108.31 \\
111.08 \\
113.24 \\
117.83 \\
120.92 \\
123.28 \\
122.97 \\
125.82 \\
125.60 \\
127.84 \\
130.19 \\
132.53 \\
135.33 \\
134.42 \\
138.35 \\
140.16 \\
142.63 \\
145.78 \\
142.52 \\
0.22\end{array}$ & $\begin{array}{c}\text { Maximum } \\
\% \text { Strain } \\
0.0253 \\
0.0513 \\
0.0787 \\
0.1055 \\
0.1309 \\
0.1454 \\
0.1609 \\
0.1764 \\
0.1934 \\
0.2100 \\
0.2266 \\
0.2450 \\
0.2620 \\
0.2809 \\
0.3004 \\
0.3094 \\
0.3195 \\
0.3275 \\
0.3394 \\
0.3491 \\
0.3730 \\
0.3909 \\
0.4074 \\
0.4115 \\
0.4316 \\
0.4394 \\
0.4535 \\
0.4877 \\
0.5354 \\
0.6044 \\
0.6223 \\
0.7238 \\
0.8196 \\
0.9730 \\
1.1111 \\
1.1488 \\
0.8139\end{array}$ & $\begin{array}{l}\text { Minimum } \\
\% \text { Strain } \\
0.0015 \\
-0.0029 \\
-0.0121 \\
-0.0230 \\
-0.0342 \\
-0.0396 \\
-0.0369 \\
-0.0342 \\
-0.0346 \\
-0.0333 \\
-0.0356 \\
-0.0374 \\
-0.0383 \\
-0.0406 \\
-0.0431 \\
-0.0454 \\
-0.0470 \\
-0.0469 \\
-0.0496 \\
-0.0510 \\
-0.0527 \\
-0.0555 \\
-0.0571 \\
-0.0578 \\
-0.0608 \\
-0.0622 \\
-0.0674 \\
-0.0744 \\
-0.0845 \\
-0.0956 \\
-0.0965 \\
-0.1019 \\
-0.1031 \\
-0.1090 \\
-0.1145 \\
-0.1139 \\
-0.0734\end{array}$ & $\begin{array}{c}\text { Angle } \\
\text { Degrees } \\
-42.70 \\
-36.49 \\
-34.74 \\
-33.55 \\
-32.85 \\
-32.45 \\
-30.12 \\
-28.05 \\
-26.18 \\
-24.24 \\
-22.49 \\
-20.66 \\
-19.20 \\
-17.83 \\
-16.50 \\
-16.13 \\
-15.49 \\
-14.85 \\
-14.24 \\
-13.73 \\
-12.19 \\
-11.50 \\
-11.00 \\
-10.99 \\
-10.60 \\
-10.73 \\
-10.91 \\
-11.19 \\
-11.51 \\
-11.69 \\
-11.69 \\
-10.03 \\
-8.28 \\
-5.76 \\
-4.13 \\
-3.63 \\
0.41\end{array}$ \\
\hline
\end{tabular}




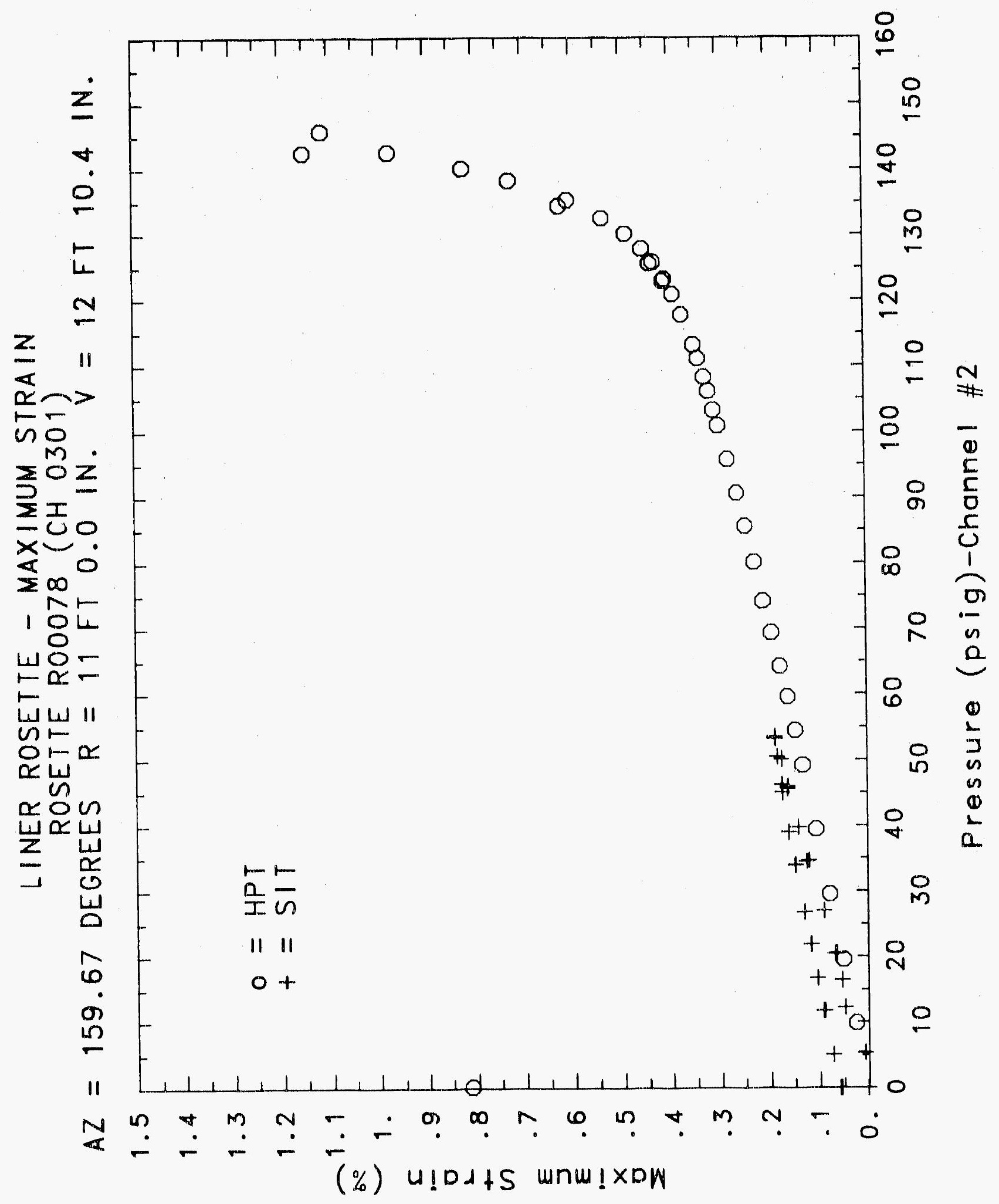




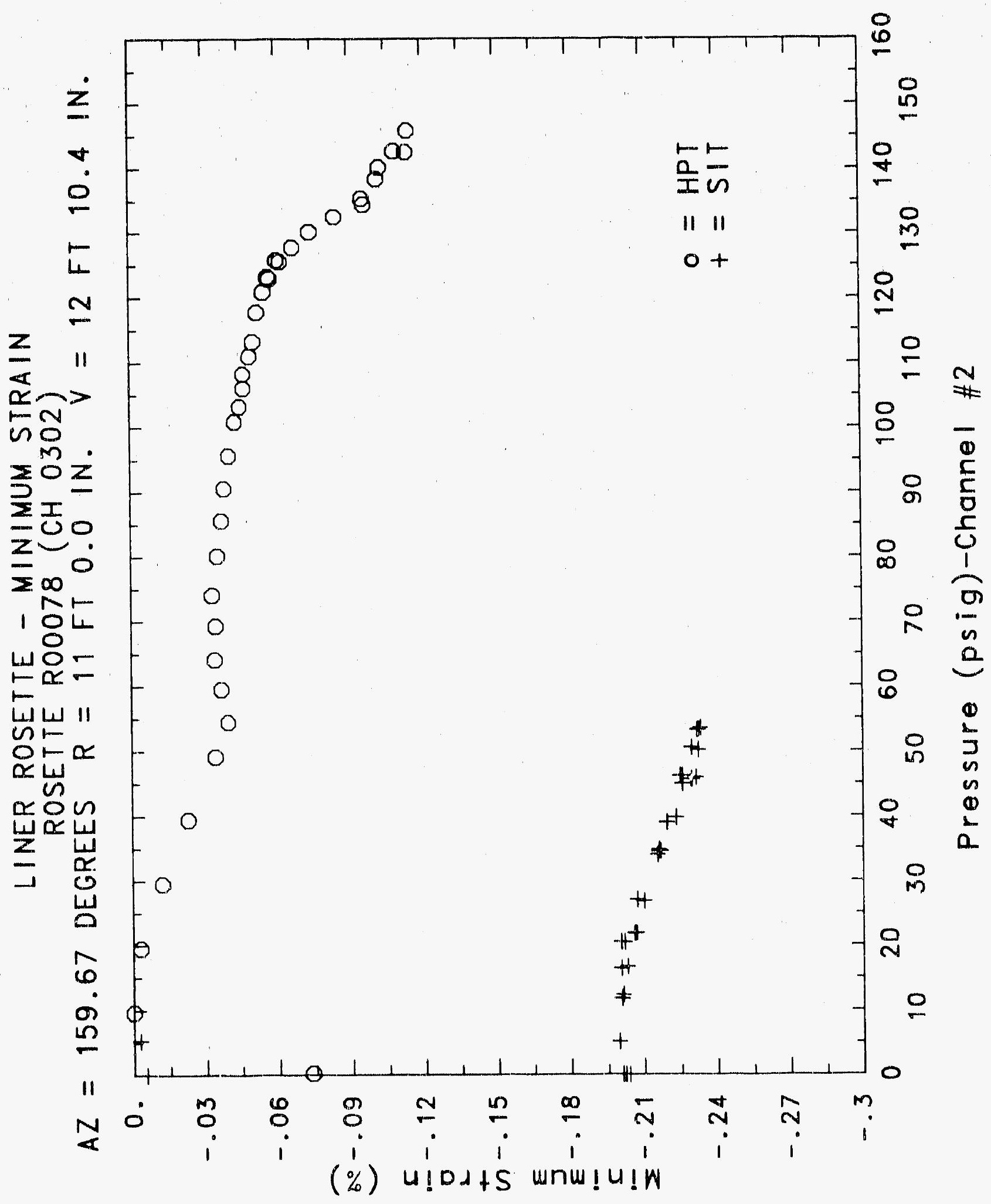


ㅇํㅇำ

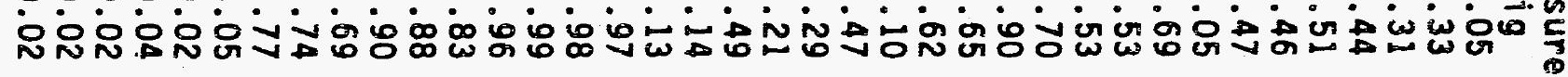
00000000000000000000000000000000000000 永

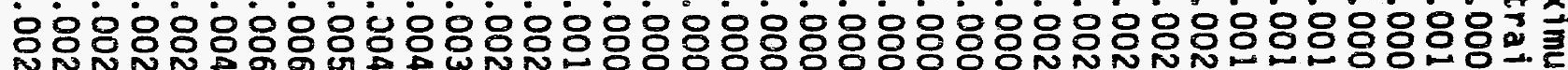

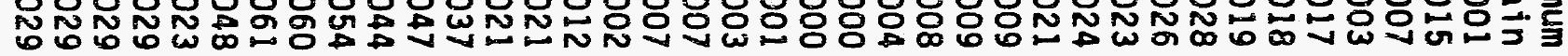

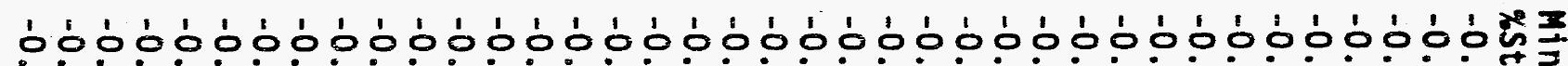

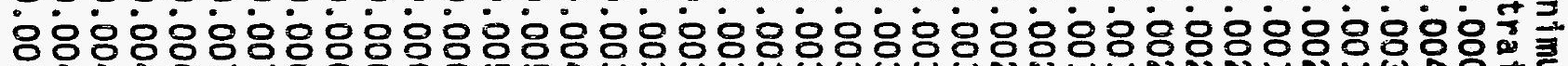

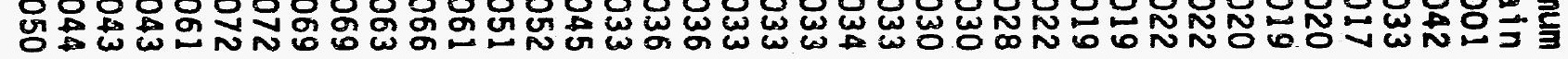

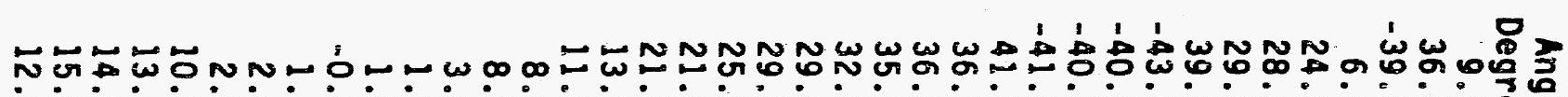

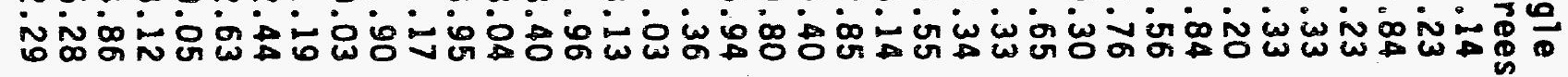

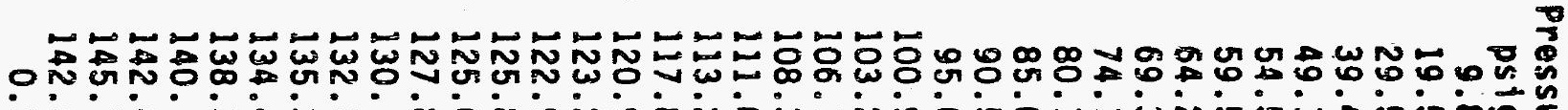

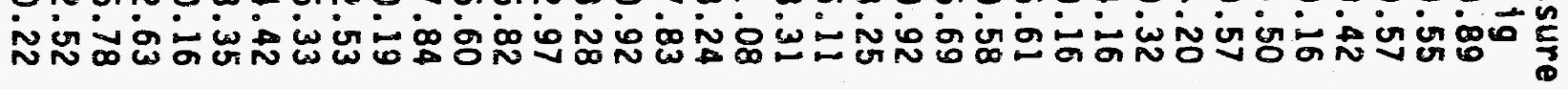

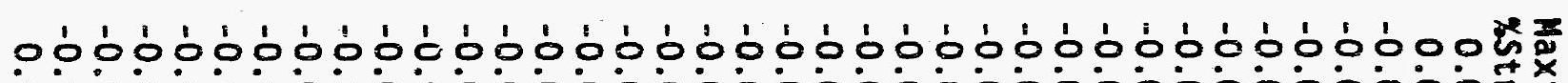

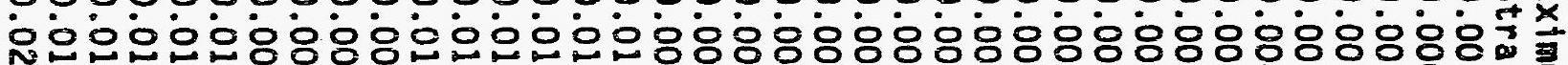

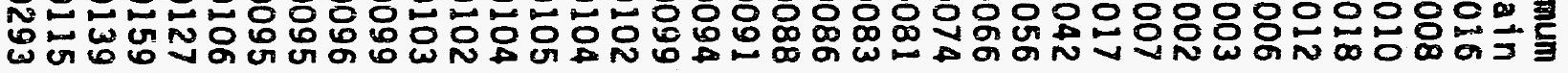

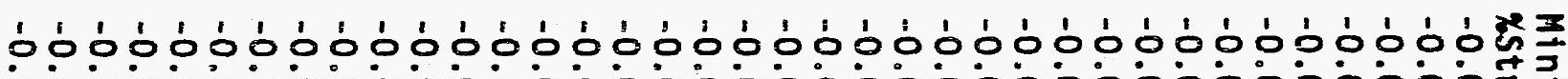

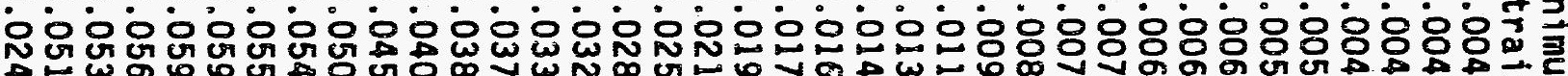

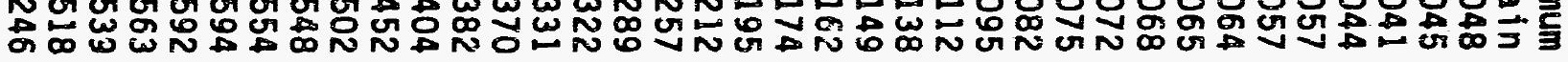

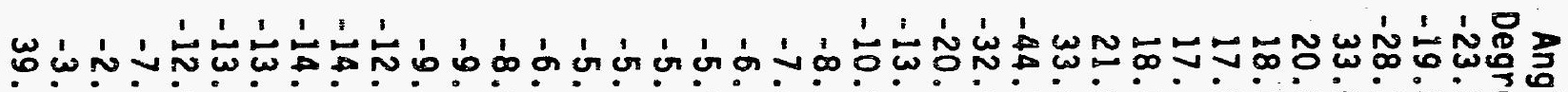

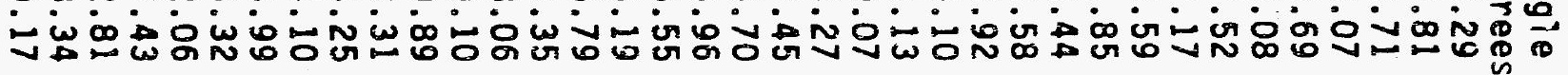




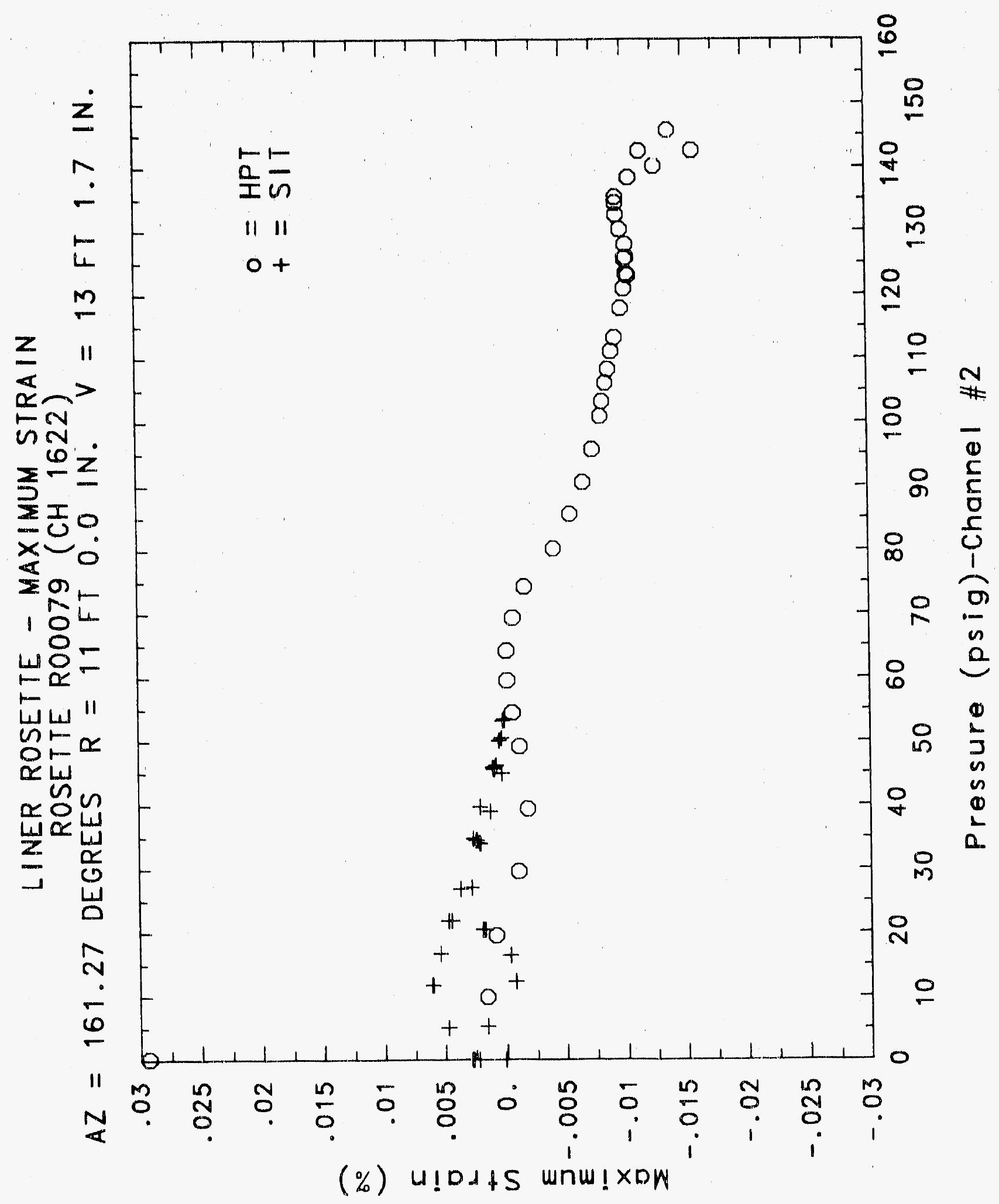




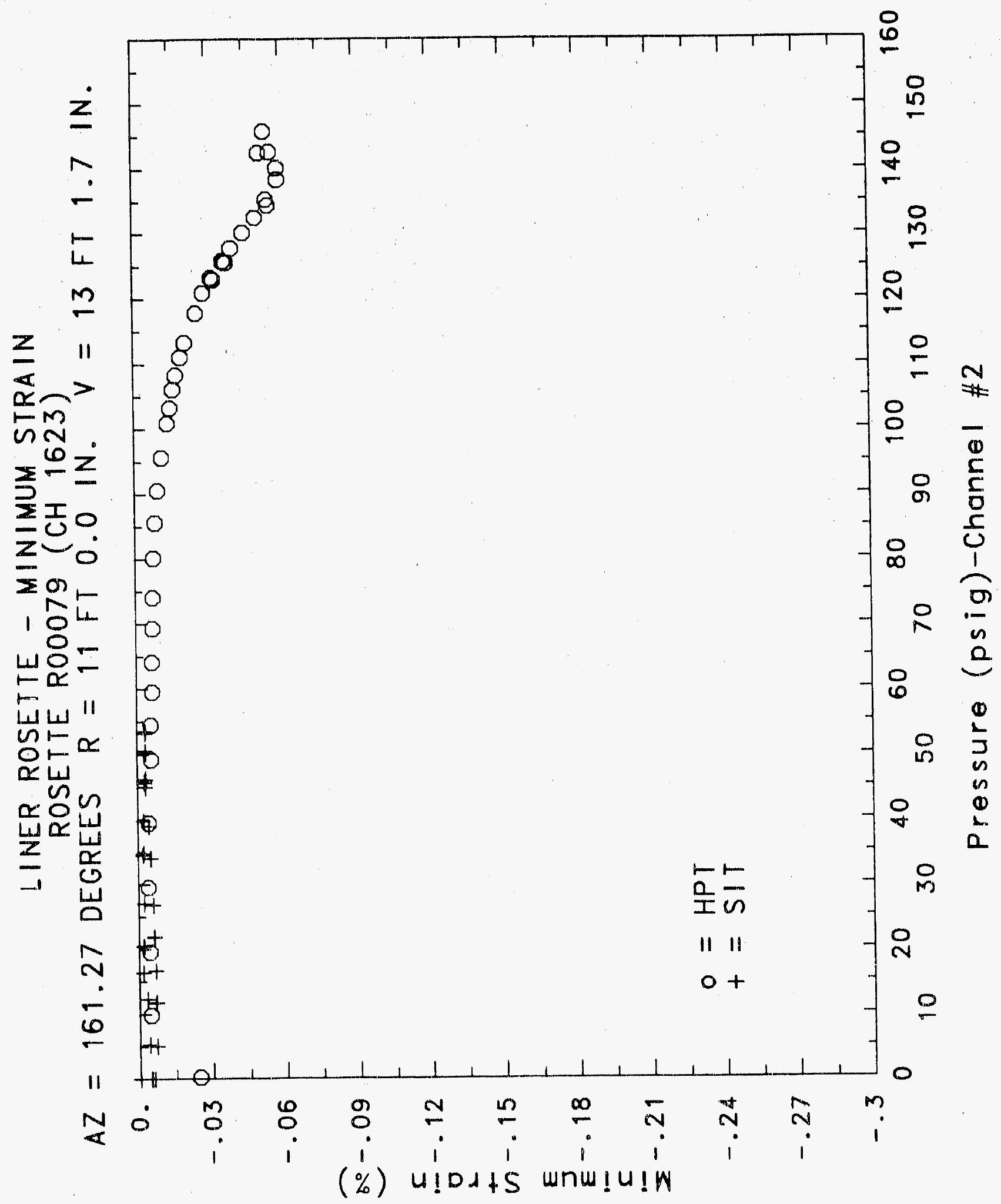




\title{
Reinforced Concrete Test Data
}

\author{
Rosette 80 Channels $1625 \quad 1625 \quad 1627$
}

\section{Structural Integrity TEST}

\begin{tabular}{|c|c|c|c|c|c|c|c|}
\hline $\begin{array}{c}\text { Pressure } \\
\text { psig } \\
-0.05 \\
5.33 \\
12.31 \\
16.44 \\
20.51 \\
20.46 \\
20.47 \\
27.05 \\
34.69 \\
34.53 \\
34.53 \\
39.70 \\
45.90 \\
45.65 \\
45.62 \\
50.10 \\
53.47 \\
53.29 \\
53.21 \\
50.49 \\
46.14 \\
46.13 \\
44.97 \\
38.98 \\
33.99 \\
33.96 \\
26.83 \\
21.88 \\
21.90 \\
16.69 \\
11.74 \\
11.77 \\
5.05 \\
0.02 \\
-0.04 \\
-0.02 \\
-0.02 \\
0.02\end{array}$ & $\begin{array}{c}\text { Maximum } \\
\% \text { Strain } \\
0.0006 \\
0.0016 \\
0.0027 \\
0.0026 \\
0.0024 \\
0.0033 \\
0.0032 \\
0.0015 \\
0.0018 \\
0.0013 \\
0.0013 \\
0.0024 \\
0.0034 \\
0.0026 \\
0.0030 \\
0.0040 \\
0.0049 \\
0.0044 \\
0.0045 \\
0.0036 \\
0.0026 \\
0.0030 \\
0.0013 \\
0.0014 \\
0.0010 \\
0.0010 \\
0.0000 \\
-0.0004 \\
-0.0004 \\
0.0001 \\
0.0018 \\
0.0015 \\
0.0039 \\
0.0034 \\
0.0050 \\
0.0055 \\
0.0050 \\
0.0023\end{array}$ & $\begin{array}{l}\text { Minimum } \\
\text { \%Strain } \\
-0.0009 \\
-0.0048 \\
-0.0050 \\
-0.0044 \\
-0.0038 \\
-0.0043 \\
-0.0045 \\
-0.0032 \\
-0.0046 \\
-0.0043 \\
-0.0043 \\
-0.0063 \\
-0.0092 \\
-0.0087 \\
-0.0090 \\
-0.0109 \\
-0.0121 \\
-0.0115 \\
-0.0115 \\
-0.0103 \\
-0.0094 \\
-0.0098 \\
-0.0072 \\
-0.0078 \\
-0.0078 \\
-0.0077 \\
-0.0069 \\
-0.0060 \\
-0.0061 \\
-0.0053 \\
-0.0049 \\
-0.0047 \\
-0.0044 \\
-0.0053 \\
-0.0080 \\
-0.0083 \\
-0.0078 \\
-0.0058\end{array}$ & $\begin{array}{c}\text { Angle } \\
\text { Degrees } \\
44.18 \\
-32.24 \\
-35.03 \\
-33.79 \\
-32.05 \\
-33.92 \\
-34.72 \\
-11.04 \\
21.87 \\
15.75 \\
15.86 \\
30.14 \\
38.77 \\
40.25 \\
41.21 \\
42.62 \\
44.27 \\
44.98 \\
-44.56 \\
-43.19 \\
-40.77 \\
-41.03 \\
-38.59 \\
-34.80 \\
-30.63 \\
-30.50 \\
-22.17 \\
-8.09 \\
-16.02 \\
-1.02 \\
14.82 \\
8.52 \\
19.87 \\
19.81 \\
28.31 \\
29.83 \\
28.47 \\
13.52\end{array}$ & $\begin{array}{c}\text { Pressure } \\
\text { Psig } \\
9.89 \\
19.55 \\
29.57 \\
39.42 \\
49.16 \\
54.50 \\
59.57 \\
64.20 \\
69.32 \\
74.16 \\
80.16 \\
85.61 \\
90.58 \\
95.69 \\
100.92 \\
103.25 \\
106.11 \\
108.31 \\
111.08 \\
113.24 \\
117.83 \\
120.92 \\
123.28 \\
122.97 \\
125.82 \\
125.60 \\
127.84 \\
134.19 \\
132.53 \\
135.33 \\
134.42 \\
138.35 \\
140.16 \\
142.63 \\
145.78 \\
142.52 \\
0.22\end{array}$ & $\begin{array}{c}\text { Maximum } \\
\text { zStrain } \\
0.0039 \\
0.0043 \\
0.0021 \\
0.0014 \\
0.0008 \\
0.0023 \\
0.0036 \\
0.0091 \\
0.0104 \\
0.0123 \\
0.0221 \\
0.0230 \\
0.0228 \\
0.0215 \\
0.0207 \\
0.0250 \\
0.0266 \\
0.0264 \\
0.0284 \\
0.0353 \\
0.0358 \\
0.0359 \\
0.0369 \\
0.0401 \\
0.0552 \\
0.0558 \\
0.0562 \\
0.0572 \\
0.0573 \\
0.0616 \\
0.0648 \\
0.0632 \\
0.0619 \\
0.0630 \\
0.0643 \\
0.0648 \\
0.0518\end{array}$ & $\begin{array}{l}\text { Minimum } \\
\text { XStrain } \\
-0.0031 \\
-0.0064 \\
-0.0058 \\
-0.0053 \\
-0.0045 \\
-0.0058 \\
-0.0073 \\
-0.0128 \\
-0.0151 \\
-0.0181 \\
-0.0306 \\
-0.0335 \\
-0.0350 \\
-0.0359 \\
-0.0376 \\
-0.0430 \\
-0.0460 \\
-0.0471 \\
-0.0509 \\
-0.0595 \\
-0.0633 \\
-0.0657 \\
-0.0689 \\
-0.0723 \\
-0.0896 \\
-0.0906 \\
-0.0923 \\
-0.0958 \\
-0.0985 \\
-0.1058 \\
-0.1095 \\
-0.1115 \\
-0.1115 \\
-0.1131 \\
-0.1139 \\
-0.1126 \\
-0.0248\end{array}$ & $\begin{array}{r}\text { Angle } \\
\text { Degrees } \\
-37.43 \\
-37.62 \\
-32.25 \\
-26.69 \\
5.68 \\
21.02 \\
27.26 \\
36.38 \\
38.53 \\
40.93 \\
44.78 \\
-44.33 \\
-43.31 \\
-42.03 \\
-40.70 \\
-40.63 \\
-40.32 \\
-40.01 \\
-39.66 \\
-40.10 \\
-39.24 \\
-38.55 \\
-37.94 \\
-38.13 \\
-38.98 \\
-38.86 \\
-38.56 \\
-38.04 \\
-37.43 \\
-37.27 \\
-37.41 \\
-36.85 \\
-36.63 \\
-36.89 \\
-36.98 \\
-36.89 \\
-37.66\end{array}$ \\
\hline
\end{tabular}

\section{High Pressure test}




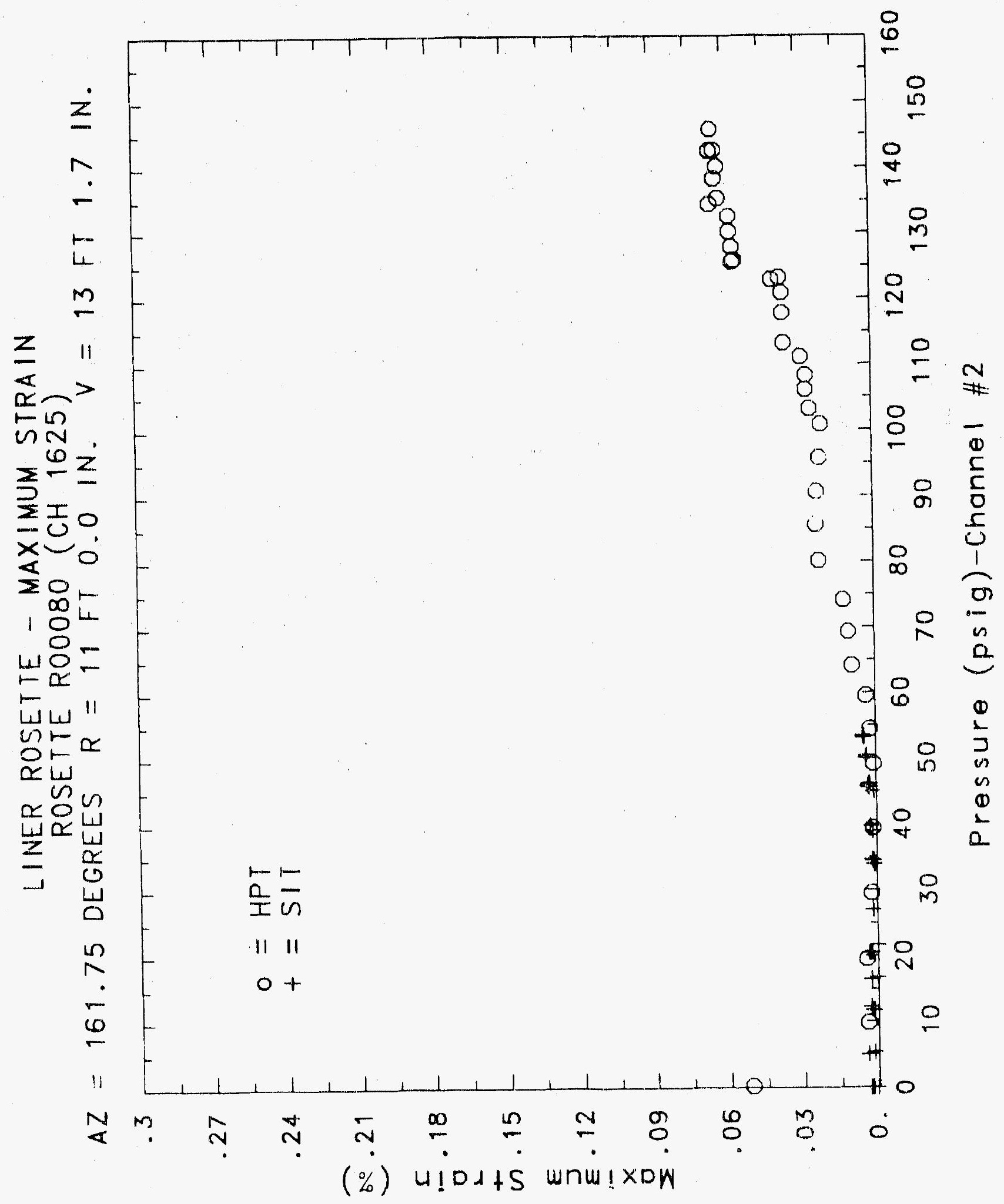


(1) 50$)$

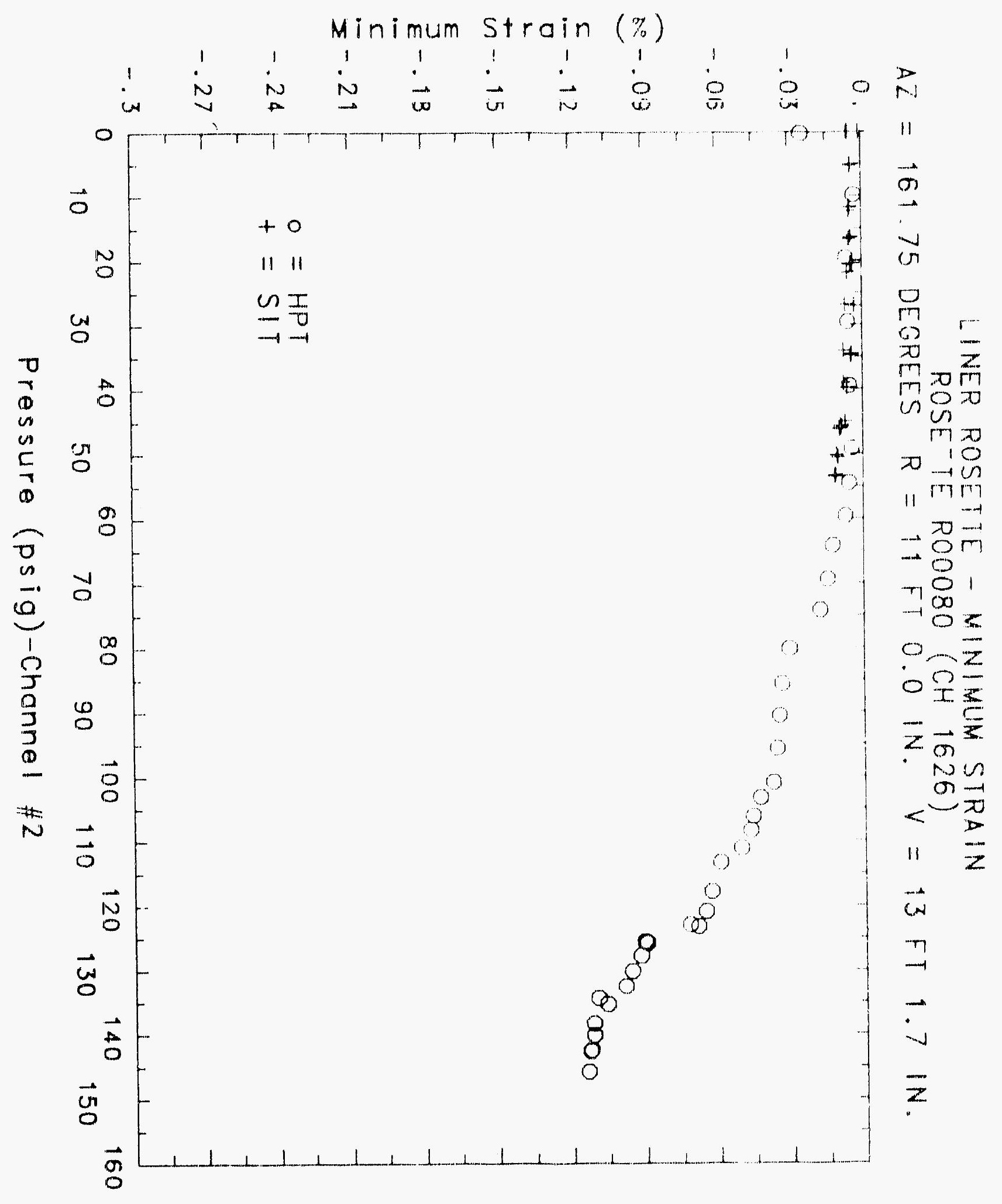




\title{
Reinforced Concrete Test Data
}

\author{
Rosette 81 Channels $1628 \quad 1629 \quad 1640$
}

\section{STRUCTURAL INTEGRITY TEST}

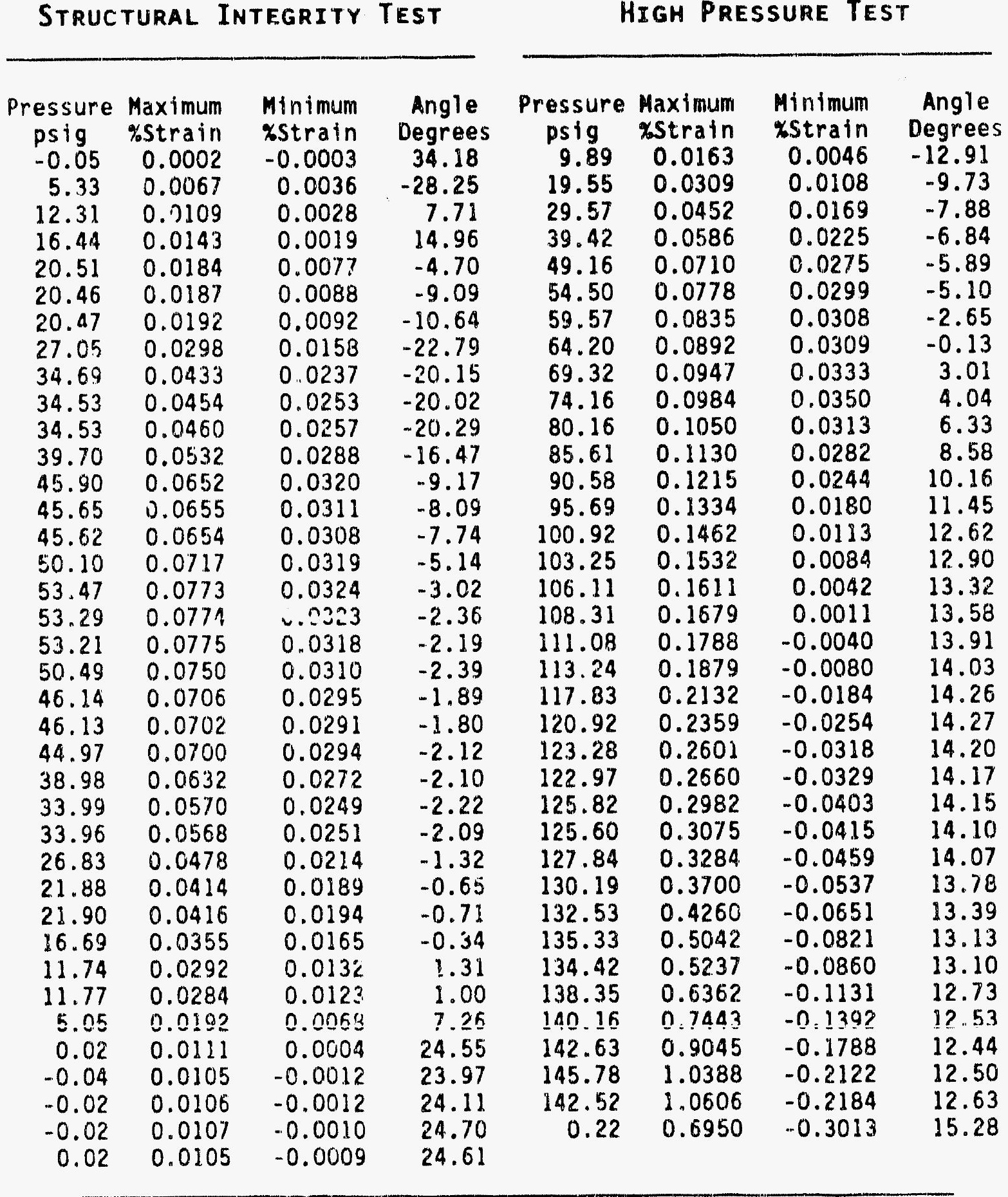

High Pressure test 


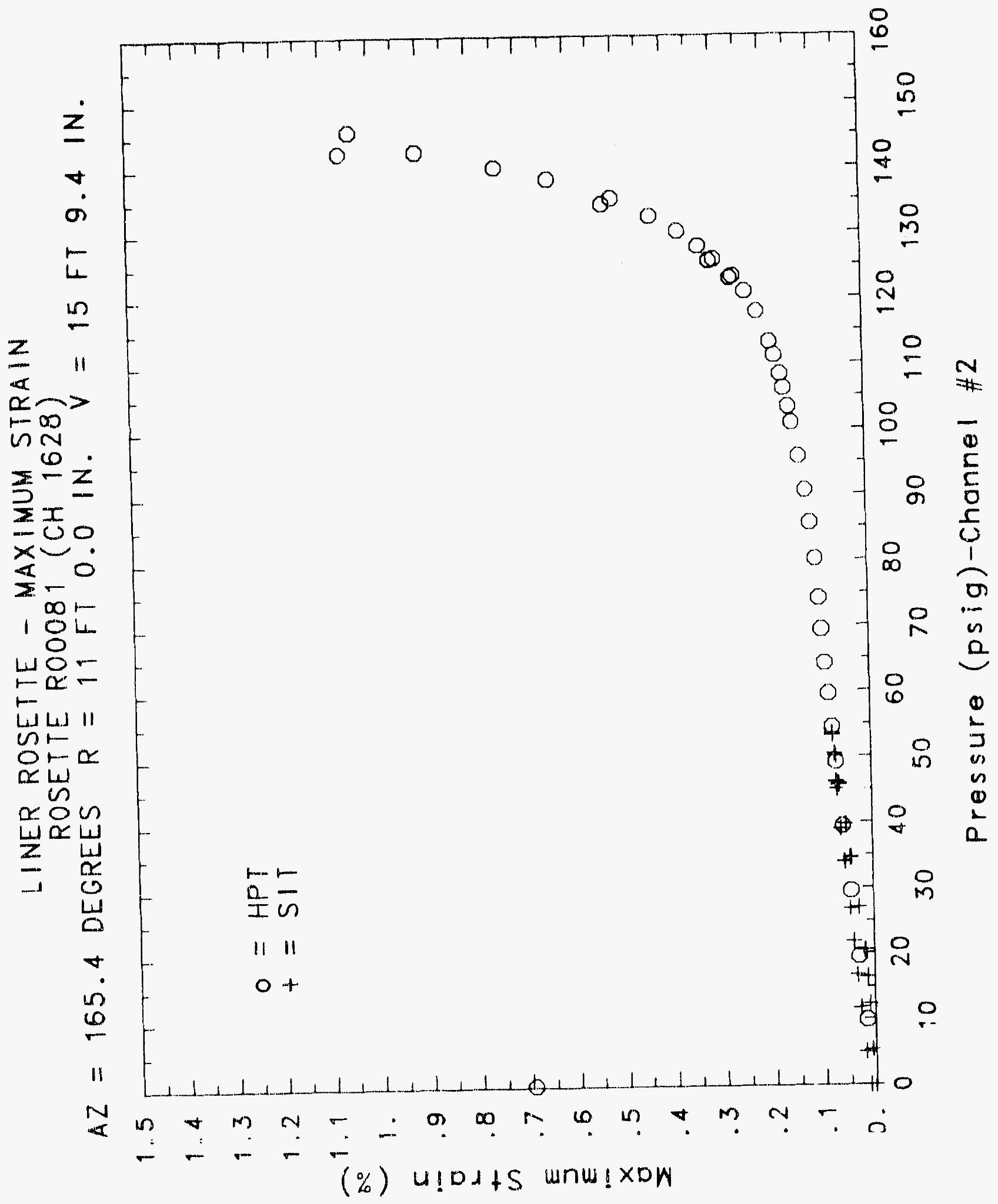




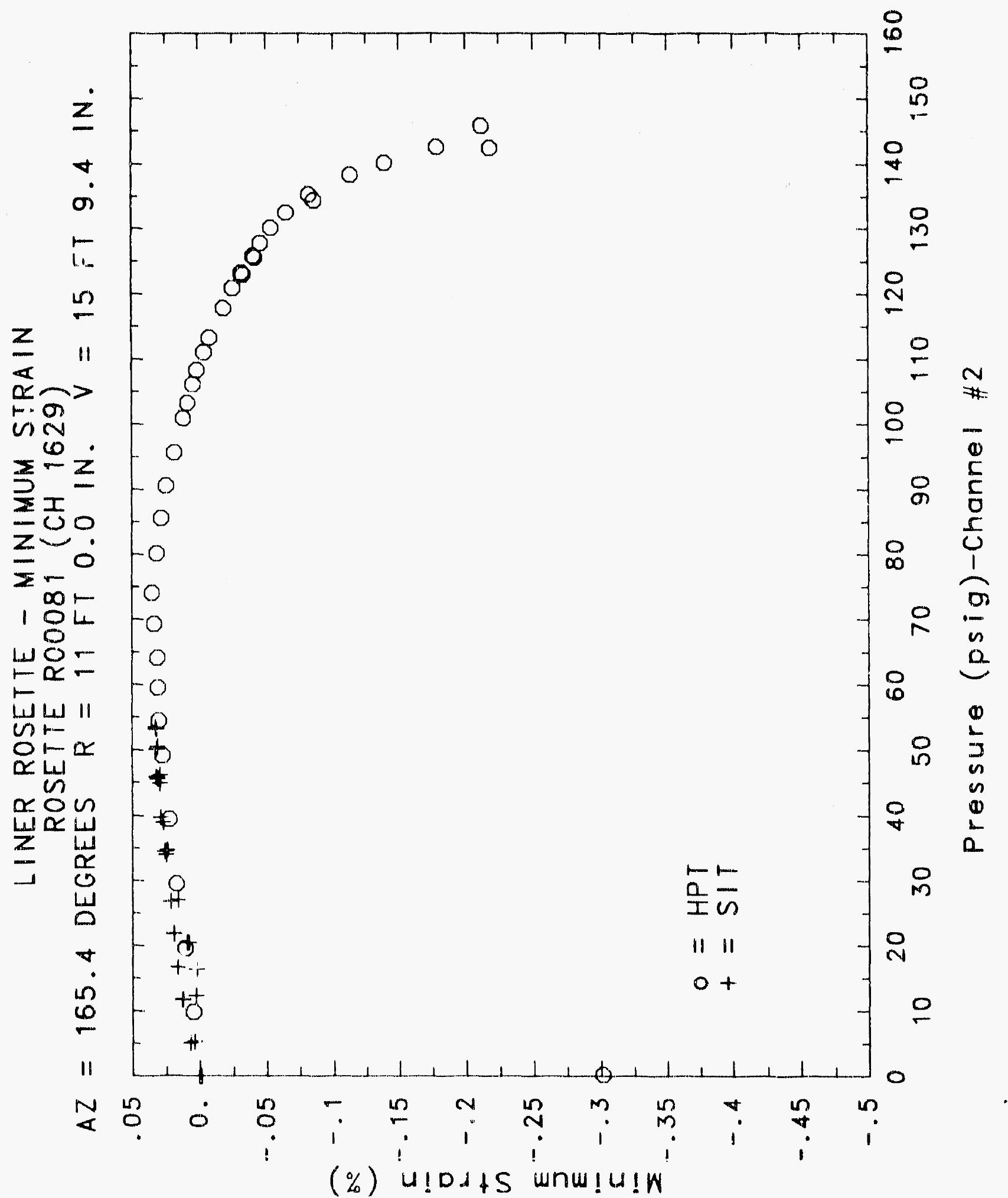




\title{
Reinforced Concrete Test Data
}

\author{
Rosette 82 Channels $1641 \quad 1642 \quad 1643$
}

STRUCTURAL INTEGRITY TEST

\begin{tabular}{|c|c|c|c|c|c|c|c|}
\hline $\begin{array}{c}\text { Pressure } \\
\text { psig } \\
-0.05 \\
5.33 \\
12.31 \\
16.44 \\
20.51 \\
20.46 \\
20.47 \\
27.05 \\
34.69 \\
34.53 \\
34.53 \\
39.70 \\
45.90 \\
45.65 \\
45.62 \\
50.10 \\
53.47 \\
53.29 \\
53.21 \\
50.49 \\
46.14 \\
46.13 \\
44.97 \\
38.98 \\
33.99 \\
33.96 \\
26.83 \\
21.88 \\
21.90 \\
16.69 \\
11.74 \\
11.77 \\
5.05 \\
0.02 \\
-0.04 \\
-0.02 \\
-0.02 \\
0.02\end{array}$ & $\begin{array}{c}\text { Maximum } \\
\text { xStrain } \\
0.0003 \\
-0.0007 \\
-0.0010 \\
0.0002 \\
0.0007 \\
0.0009 \\
0.0010 \\
0.0028 \\
0.0105 \\
0.0120 \\
0.0122 \\
0.0142 \\
0.0179 \\
0.0184 \\
0.0183 \\
0.0200 \\
0.0216 \\
0.0220 \\
0.0218 \\
0.0214 \\
0.0205 \\
0.0203 \\
0.0197 \\
0.0185 \\
0.0171 \\
0.0170 \\
0.0151 \\
0.0138 \\
0.0137 \\
0.0120 \\
0.0096 \\
0.0095 \\
0.0066 \\
0.0074 \\
0.0071 \\
0.0077 \\
0.0081 \\
0.0081\end{array}$ & $\begin{array}{l}\text { Minimum } \\
\text { XStrain } \\
-0.0001 \\
-0.0050 \\
-0.0109 \\
-0.0131 \\
-0.0153 \\
-0.0154 \\
-0.0153 \\
-0.0184 \\
-0.0218 \\
-0.0217 \\
-0.0217 \\
-0.0228 \\
-0.0250 \\
-0.0250 \\
-0.0247 \\
-0.0256 \\
-0.0262 \\
-0.0262 \\
-0.0260 \\
-0.0257 \\
-0.0251 \\
-0.0251 \\
-0.0251 \\
-0.0245 \\
-0.0236 \\
-0.0234 \\
-0.0219 \\
-0.0205 \\
-0.0205 \\
-0.0185 \\
-0.0148 \\
-0.0147 \\
-0.0089 \\
-0.0032 \\
-0.0022 \\
-0.0016 \\
-0.0016 \\
-0.0016\end{array}$ & $\begin{array}{c}\text { Angle } \\
\text { Degrees } \\
-30.59 \\
8.67 \\
7.57 \\
5.46 \\
3.75 \\
4.10 \\
3.11 \\
-0.80 \\
-10.94 \\
-12.10 \\
-12.11 \\
-13.27 \\
-14.08 \\
-14.51 \\
-14.55 \\
-15.45 \\
-16.42 \\
-16.65 \\
-16.72 \\
-16.75 \\
-16.86 \\
-16.98 \\
-17.46 \\
-17.22 \\
-17.08 \\
-17.26 \\
-16.85 \\
-16.56 \\
-16.38 \\
-16.38 \\
-14.65 \\
-14.13 \\
-9.45 \\
-14.20 \\
-22.16 \\
-26.07 \\
-26.65 \\
-26.65\end{array}$ & $\begin{array}{c}\text { Pressure } \\
\text { psig } \\
9.89 \\
19.55 \\
29.57 \\
39.42 \\
49.16 \\
54.50 \\
59.57 \\
64.20 \\
69.32 \\
74.16 \\
80.16 \\
85.61 \\
90.58 \\
95.69 \\
100.92 \\
103.25 \\
106.11 \\
108.31 \\
111.08 \\
113.24 \\
117.83 \\
120.92 \\
123.28 \\
122.97 \\
125.82 \\
125.60 \\
127.84 \\
130.19 \\
132.53 \\
135.33 \\
134.42 \\
138.35 \\
140.16 \\
142.63 \\
145.78 \\
142.52 \\
0.22\end{array}$ & $\begin{array}{c}\text { Maximum } \\
\text { xstrain } \\
0.0019 \\
0.0040 \\
0.0071 \\
0.0101 \\
0.0128 \\
0.0146 \\
0.0171 \\
0.0200 \\
0.0237 \\
0.0268 \\
0.0310 \\
0.0349 \\
0.0392 \\
0.0434 \\
0.0485 \\
0.0506 \\
0.0534 \\
0.0551 \\
0.0582 \\
0.0605 \\
0.0659 \\
0.0697 \\
0.0724 \\
0.0730 \\
0.0782 \\
0.0795 \\
0.0830 \\
0.0918 \\
0.1071 \\
0.1271 \\
0.1318 \\
0.1539 \\
0.1688 \\
0.1895 \\
0.2085 \\
0.2108 \\
0.0695\end{array}$ & $\begin{array}{l}\text { Minimum } \\
\text { xStrain } \\
-0.0160 \\
-0.0215 \\
-0.0257 \\
-0.0279 \\
-0.0289 \\
-0.0296 \\
-0.0298 \\
-0.0304 \\
-0.0309 \\
-0.0315 \\
-0.0327 \\
-0.0340 \\
-0.0357 \\
-0.0376 \\
-0.0403 \\
-0.0415 \\
-0.0432 \\
-0.0444 \\
-0.0464 \\
-0.0481 \\
-0.0524 \\
-0.0554 \\
-0.0581 \\
-0.0585 \\
-0.0622 \\
-0.0631 \\
-0.0657 \\
-0.0714 \\
-0.0789 \\
-0.0879 \\
-0.0899 \\
-0.0992 \\
-0.1046 \\
-0.1128 \\
-0.1210 \\
-0.1233 \\
-0.0448\end{array}$ & $\begin{array}{c}\text { Angle } \\
\text { Degrees } \\
5.95 \\
-3.50 \\
-8.00 \\
-10.02 \\
-11.72 \\
-12.39 \\
-14.08 \\
-15.66 \\
-18.10 \\
-19.41 \\
-21.70 \\
-23.11 \\
-24.47 \\
-25.65 \\
-26.86 \\
-27.34 \\
-27.91 \\
-28.26 \\
-28.77 \\
-29.23 \\
-30.06 \\
-30.51 \\
-30.86 \\
-31.00 \\
-31.22 \\
-31.25 \\
-31.17 \\
-30.77 \\
-29.86 \\
-28.63 \\
-28.35 \\
-27.56 \\
-27.29 \\
-27.71 \\
-28.63 \\
-29.16 \\
-21.16\end{array}$ \\
\hline
\end{tabular}

High Pressure test 


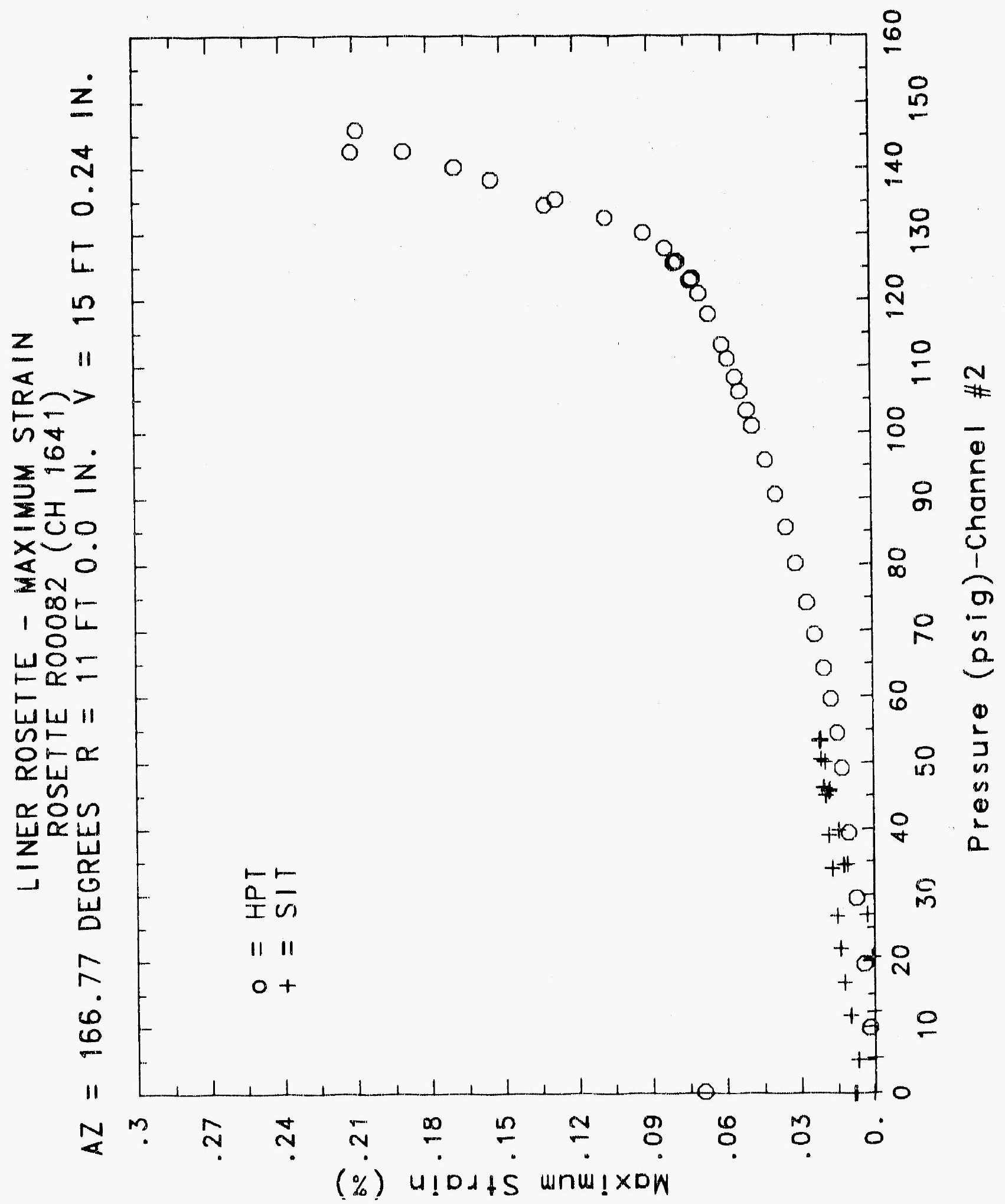




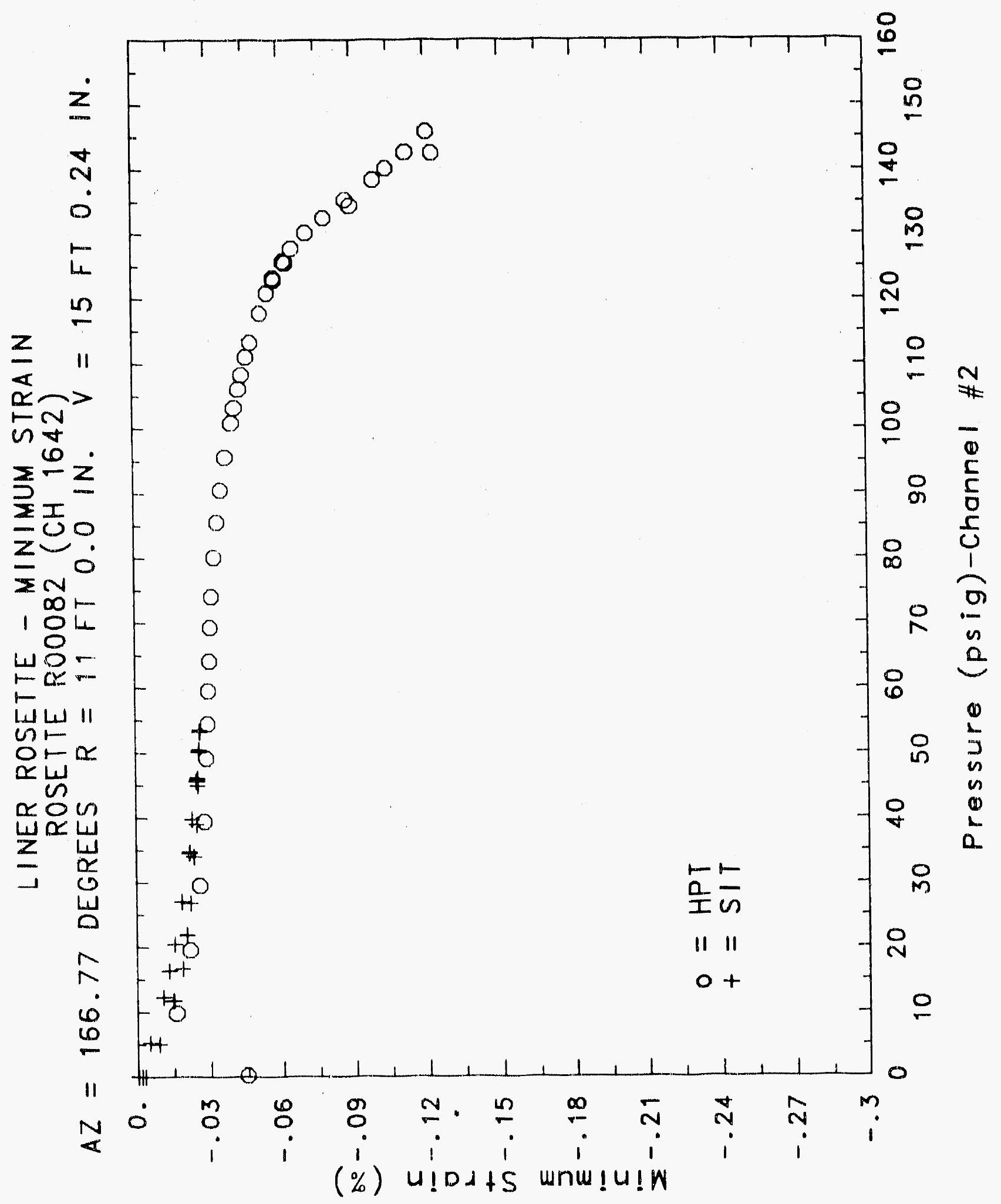

C. -56.5 


\section{Reinforced Concrete Test Data}

Rosette 83 Channels $1644 \quad 1645 \quad 1646$

Structural Integrity Test

\begin{tabular}{|c|c|c|c|c|c|c|c|}
\hline $\begin{array}{c}\text { Pressure } \\
\text { psig } \\
-0.05 \\
5.33 \\
12.31 \\
16.44 \\
20.51 \\
20.46 \\
20.47 \\
27.05 \\
34.69 \\
34.53 \\
34.53 \\
39.70 \\
45.90 \\
45.65 \\
45.62 \\
50.10 \\
53.47 \\
53.29 \\
53.21 \\
50.49 \\
46.14 \\
46.13 \\
44.97 \\
38.98 \\
33.99 \\
33.96 \\
26.83 \\
21.88 \\
21.90 \\
16.69 \\
11.74 \\
11.77 \\
5.05 \\
0.02 \\
-0.04 \\
-0.02 \\
-0.02 \\
0.02\end{array}$ & $\begin{array}{l}\text { Maximum } \\
\text { qStrain } \\
0.0000 \\
0.0028 \\
0.0034 \\
0.0037 \\
0.0040 \\
0.0041 \\
0.0041 \\
0.0057 \\
0.0140 \\
0.0155 \\
0.0156 \\
0.0175 \\
0.0219 \\
0.0222 \\
0.0225 \\
0.0237 \\
0.0251 \\
0.0254 \\
0.0255 \\
0.0252 \\
0.0246 \\
0.0247 \\
0.0240 \\
0.0232 \\
0.0223 \\
0.0221 \\
0.0204 \\
0.0187 \\
0.0188 \\
0.0170 \\
0.0151 \\
0.0148 \\
0.0124 \\
0.0097 \\
0.0093 \\
0.0093 \\
0.0094 \\
0.0089\end{array}$ & $\begin{array}{l}\text { Minimum } \\
\text { xStrain } \\
-0.0002 \\
-0.0014 \\
-0.0041 \\
-0.0052 \\
-0.0066 \\
-0.0063 \\
-0.0065 \\
-0.0087 \\
-0.0136 \\
-0.0140 \\
-0.0139 \\
-0.0157 \\
-0.0185 \\
-0.0183 \\
-0.0186 \\
-0.0194 \\
-0.0201 \\
-0.0197 \\
-0.0200 \\
-0.0196 \\
-0.0192 \\
-0.0193 \\
-0.0195 \\
-0.0189 \\
-0.0180 \\
-0.0177 \\
-0.0162 \\
-0.0142 \\
-0.0141 \\
-0.0117 \\
-0.0086 \\
-0.0082 \\
-0.0033 \\
-0.0016 \\
-0.0023 \\
-0.0023 \\
-0.0022 \\
-0.0022\end{array}$ & $\begin{array}{c}\text { Angle } \\
\text { Degrees } \\
-38.99 \\
-40.59 \\
-40.87 \\
-42.18 \\
-42.98 \\
-44.10 \\
-43.87 \\
42.62 \\
37.77 \\
36.88 \\
36.46 \\
35.30 \\
31.87 \\
31.33 \\
31.41 \\
30.95 \\
30.23 \\
29.84 \\
29.77 \\
29.53 \\
29.14 \\
29.24 \\
29.30 \\
28.74 \\
27.95 \\
28.07 \\
27.21 \\
26.94 \\
27.37 \\
27.72 \\
29.36 \\
29.93 \\
31.51 \\
21.11 \\
18.23 \\
18.18 \\
17.69 \\
17.03\end{array}$ & $\begin{array}{c}\text { Pressure } \\
\text { psig } \\
9.89 \\
19.55 \\
29.57 \\
39.42 \\
49.16 \\
54.50 \\
59.57 \\
64.20 \\
69.32 \\
74.16 \\
80.16 \\
85.61 \\
90.58 \\
95.69 \\
100.92 \\
103.25 \\
106.11 \\
108.31 \\
111.08 \\
113.24 \\
117.83 \\
120.92 \\
123.28 \\
122.97 \\
125.82 \\
125.60 \\
127.84 \\
130.19 \\
132.53 \\
135.33 \\
134.42 \\
138.35 \\
140.16 \\
142.63 \\
145.78 \\
142.52 \\
0.22\end{array}$ & $\begin{array}{l}\text { Maximum } \\
\text { XStrain } \\
0.0067 \\
0.0096 \\
0.0124 \\
0.0149 \\
0.0176 \\
0.0193 \\
0.0212 \\
0.0239 \\
0.0268 \\
0.0296 \\
0.0332 \\
0.0364 \\
0.0392 \\
0.0418 \\
0.0447 \\
0.0459 \\
0.0473 \\
0.0483 \\
0.0498 \\
0.0511 \\
0.0542 \\
0.0564 \\
0.0586 \\
0.0591 \\
0.0631 \\
0.0637 \\
0.0662 \\
0.0719 \\
0.0786 \\
0.0862 \\
0.0873 \\
0.0937 \\
0.0979 \\
0.1043 \\
0.1103 \\
0.1105 \\
0.0205\end{array}$ & $\begin{array}{l}\text { Minimum } \\
\text { XStrain } \\
-0.0051 \\
-0.0106 \\
-0.0143 \\
-0.0169 \\
-0.0188 \\
-0.0198 \\
-0.0208 \\
-0.0221 \\
-0.0234 \\
-0.0249 \\
-0.0259 \\
-0.0275 \\
-0.0284 \\
-0.0310 \\
-0.0332 \\
-0.0339 \\
-0.0347 \\
-0.0356 \\
-0.0366 \\
-0.0377 \\
-0.0408 \\
-0.0427 \\
-0.0449 \\
-0.0452 \\
-0.0480 \\
-0.0486 \\
-0.0499 \\
-0.0526 \\
-0.0593 \\
-0.0663 \\
-0.0666 \\
-0.0748 \\
-0.0804 \\
-0.0832 \\
-0.0833 \\
-0.0807 \\
-0.0709\end{array}$ & $\begin{array}{c}\text { Angle } \\
\text { Degrees } \\
-42.81 \\
39.63 \\
36.46 \\
34.78 \\
33.78 \\
33.32 \\
32.31 \\
30.74 \\
29.19 \\
27.95 \\
26.16 \\
25.01 \\
24.11 \\
24.50 \\
24.19 \\
23.90 \\
23.58 \\
23.34 \\
23.04 \\
22.84 \\
22.54 \\
22.49 \\
22.61 \\
22.59 \\
22.75 \\
22.79 \\
22.63 \\
21.99 \\
23.18 \\
24.02 \\
23.99 \\
25.46 \\
26.47 \\
26.98 \\
27.01 \\
26.82 \\
29.93\end{array}$ \\
\hline
\end{tabular}

High Pressure Test 


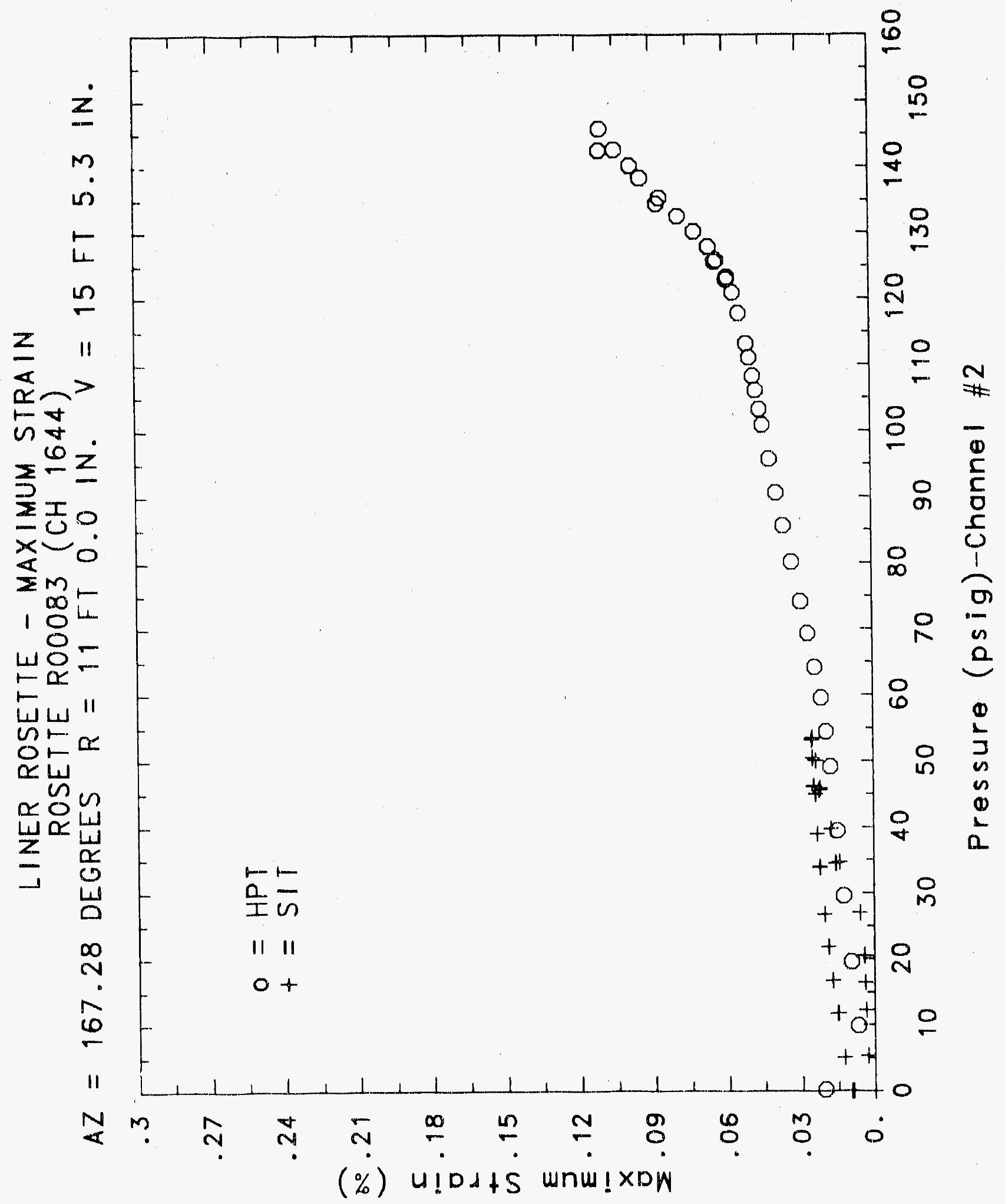




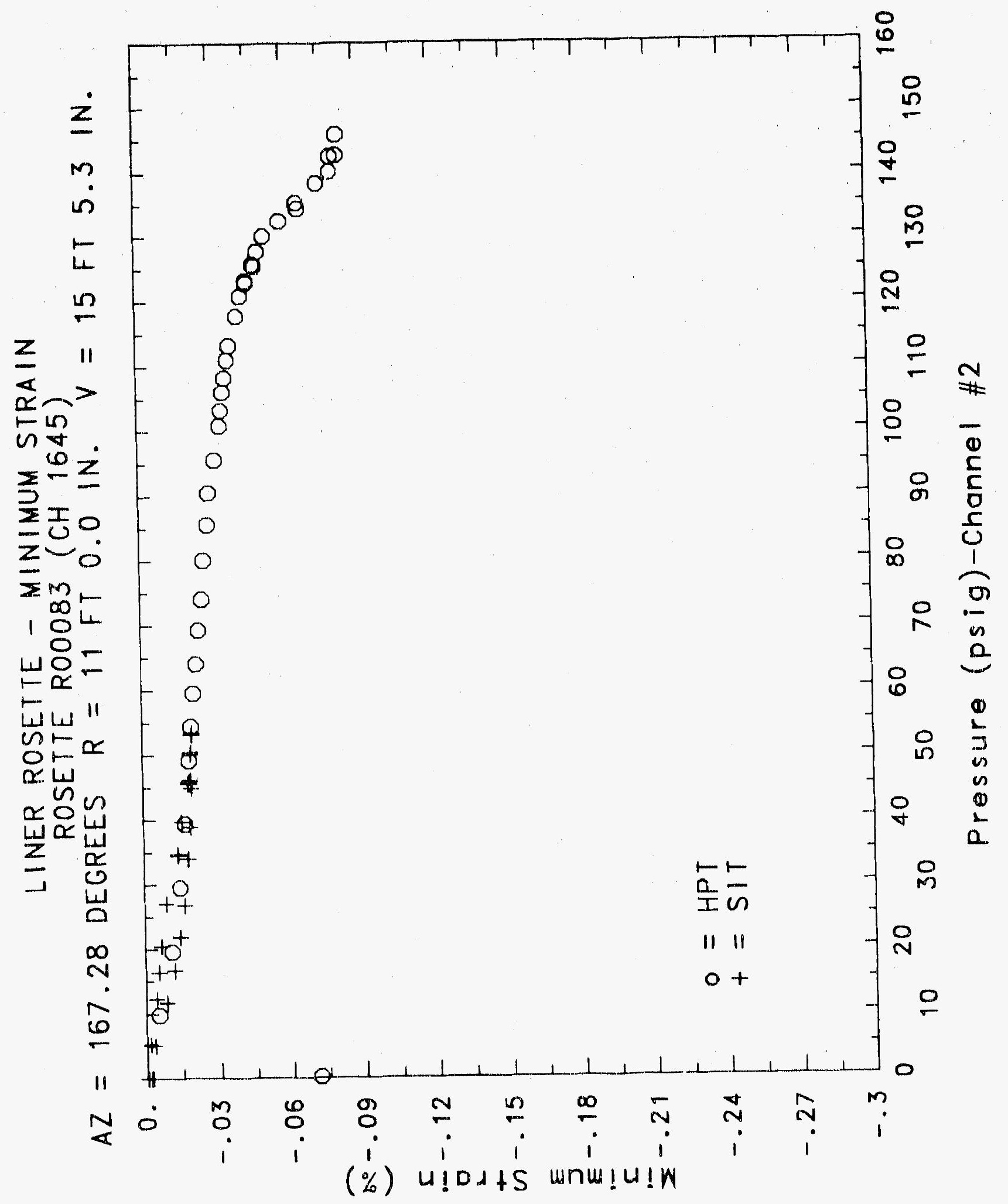




\section{Reinforced Concrete Test Data}

\section{Rosette 84 Channels $1647 \quad 1648 \quad 1649$}

\author{
Structural Integrity TEST
}

\begin{tabular}{|c|c|c|c|c|c|c|c|}
\hline $\begin{array}{c}\text { Pressure } \\
\text { psig } \\
-0.05 \\
5.33 \\
12.31 \\
16.44 \\
20.51 \\
20.46 \\
20.47 \\
27.05 \\
34.69 \\
34.53 \\
34.53 \\
39.70 \\
45.90 \\
45.65 \\
45.62 \\
50.10 \\
53.47 \\
53.29 \\
53.21 \\
50.49 \\
46.14 \\
46.13 \\
44.97 \\
38.98 \\
33.99 \\
33.96 \\
26.83 \\
21.88 \\
21.90 \\
16.69 \\
11.74 \\
11.77 \\
5.05 \\
0.02 \\
-0.04 \\
-0.02 \\
-0.02 \\
0.02\end{array}$ & $\begin{array}{l}\text { Maximum } \\
\text { \%Strain } \\
0.0038 \\
0.0073 \\
0.0208 \\
0.0223 \\
0.0230 \\
0.0233 \\
0.0236 \\
0.0257 \\
0.0469 \\
0.0476 \\
0.0481 \\
0.0581 \\
1.2425 \\
0.0218 \\
0.0140 \\
0.0233 \\
0.0560 \\
0.0541 \\
0.0610 \\
0.0626 \\
0.0696 \\
0.0734 \\
0.0669 \\
0.0497 \\
0.0341 \\
0.0340 \\
0.0390 \\
0.0408 \\
0.0462 \\
0.0451 \\
1.5684 \\
0.1203 \\
0.0154 \\
0.0051 \\
0.0506 \\
0.0700 \\
0.0397 \\
0.0376\end{array}$ & $\begin{array}{l}\text { Minimum } \\
\text { \%Strain } \\
-0.0219 \\
-0.0411 \\
-0.1159 \\
-0.1204 \\
-0.1222 \\
-0.1239 \\
-0.1250 \\
-0.1351 \\
-0.2431 \\
-0.2464 \\
-0.2490 \\
-0.3010 \\
-7.1982 \\
-0.0755 \\
-0.0135 \\
-0.0795 \\
-0.2717 \\
-0.2595 \\
-0.2996 \\
-0.3094 \\
-0.3523 \\
-0.3747 \\
-0.3415 \\
-0.2433 \\
-0.1538 \\
-0.1535 \\
-0.1875 \\
-0.2025 \\
-0.2346 \\
-0.2344 \\
-9.1185 \\
-0.0164 \\
-0.0779 \\
-0.0213 \\
-0.2818 \\
-0.0097 \\
-0.2169 \\
-0.2057\end{array}$ & $\begin{array}{c}\text { Angle } \\
\text { Degrees } \\
22.63 \\
23.03 \\
22.83 \\
22.86 \\
22.76 \\
22.71 \\
22.69 \\
22.34 \\
21.55 \\
21.47 \\
21.46 \\
21.29 \\
22.43 \\
16.54 \\
-0.69 \\
16.25 \\
20.37 \\
20.27 \\
20.57 \\
20.66 \\
20.92 \\
21.03 \\
20.89 \\
20.42 \\
19.50 \\
19.53 \\
20.52 \\
21.05 \\
21.26 \\
21.63 \\
22.49 \\
23.57 \\
21.86 \\
21.94 \\
22.54 \\
21.98 \\
22.72 \\
22.79\end{array}$ & $\begin{array}{c}\text { Pressure } \\
\text { psig } \\
9.89 \\
19.55 \\
29.57 \\
39.42 \\
49.16 \\
54.50 \\
59.57 \\
64.20 \\
69.32 \\
74.16 \\
80.16 \\
85.61 \\
90.58 \\
95.69 \\
100.92 \\
103.25 \\
106.11 \\
108.31 \\
111.08 \\
113.24 \\
117.83 \\
120.92 \\
123.28 \\
122.97 \\
125.82 \\
125.60 \\
127.84 \\
130.19 \\
132.53 \\
135.33 \\
134.42 \\
138.35 \\
140.16 \\
142.63 \\
145.78 \\
142.52 \\
0.22\end{array}$ & $\begin{array}{l}\text { Maximum } \\
\text { XStrain } \\
2.1506 \\
2.1249 \\
2.1392 \\
2.1284 \\
2.1224 \\
2.1244 \\
2.1259 \\
2.1247 \\
2.1228 \\
2.1217 \\
2.1101 \\
2.1081 \\
2.1071 \\
2.1001 \\
2.0934 \\
2.0783 \\
2.0685 \\
2.0682 \\
2.0561 \\
2.0533 \\
2.0357 \\
2.0228 \\
1.8902 \\
1.8683 \\
1.8543 \\
1.7967 \\
1.7851 \\
0.1867 \\
2.0674 \\
2.0766 \\
2.0657 \\
2.0439 \\
2.0126 \\
1.8906 \\
0.4577 \\
2.0264 \\
1.0412\end{array}$ & $\begin{array}{l}\text { Minimum } \\
\text { \%Strain } \\
-0.3802 \\
-0.3757 \\
-0.3776 \\
-0.3744 \\
-0.3728 \\
-0.3728 \\
-0.3730 \\
-0.3729 \\
-0.3728 \\
-0.3730 \\
-0.3711 \\
-0.3711 \\
-0.3711 \\
-0.3701 \\
-0.3691 \\
-0.3667 \\
-0.3650 \\
-0.3650 \\
-0.3631 \\
-0.3628 \\
-0.3600 \\
-0.3580 \\
-0.3347 \\
-0.3309 \\
-0.3286 \\
-0.3186 \\
-0.3165 \\
-0.0352 \\
-0.3669 \\
-0.3685 \\
-0.3668 \\
-0.3621 \\
-0.3567 \\
-0.3346 \\
-2.5542 \\
-0.3582 \\
-0.1755\end{array}$ & $\begin{array}{c}\text { Angle } \\
\text { Degrees } \\
22.54 \\
22.57 \\
22.65 \\
22.73 \\
22.78 \\
22.81 \\
22.83 \\
22.85 \\
22.87 \\
22.90 \\
22.92 \\
22.96 \\
22.98 \\
23.01 \\
23.04 \\
23.05 \\
23.07 \\
23.08 \\
23.11 \\
23.12 \\
23.16 \\
23.18 \\
23.24 \\
23.24 \\
23.27 \\
23.29 \\
23.32 \\
30.40 \\
23.26 \\
23.27 \\
23.27 \\
23.31 \\
23.31 \\
23.37 \\
21.90 \\
23.31 \\
21.12\end{array}$ \\
\hline
\end{tabular}

High Pressure test 


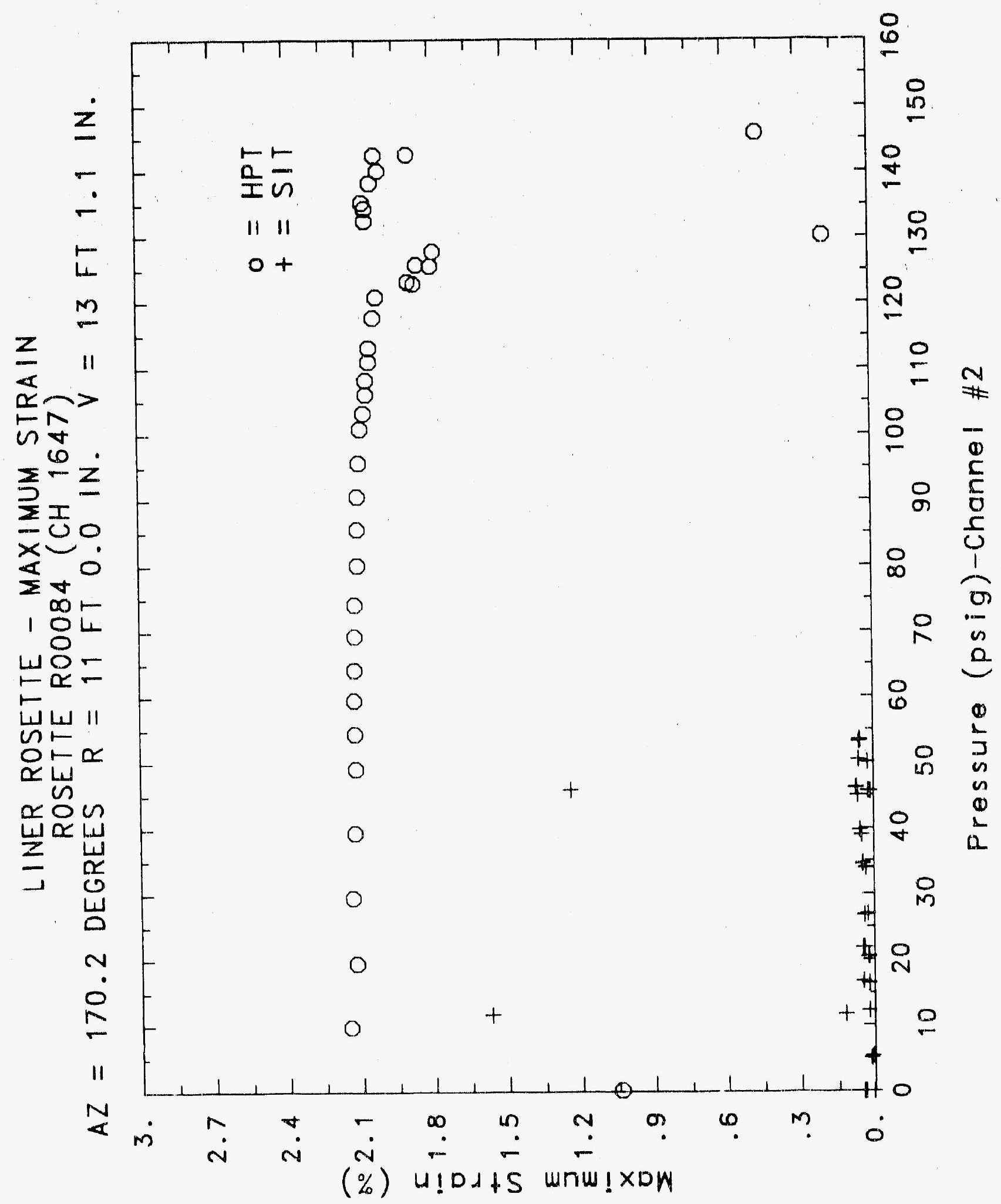




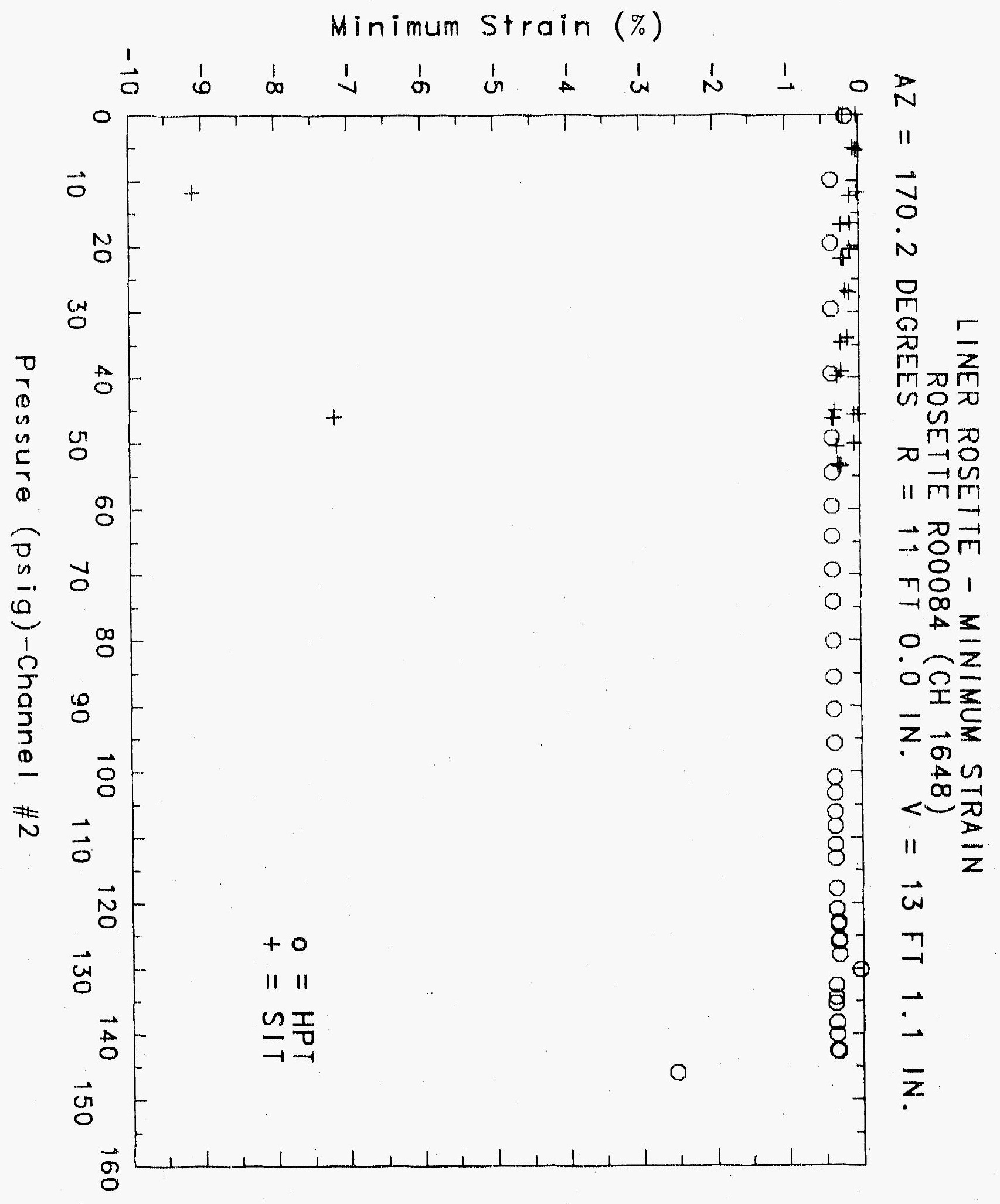




\title{
Reinforced Concrete Test Data
}

\author{
Rosette 85 Channels $16601661 \quad 1662$
}

\section{Structural Integrity Test}

\begin{tabular}{|c|c|c|c|c|c|c|c|}
\hline $\begin{array}{c}\text { Pressure } \\
\text { psig } \\
-0.05 \\
5.33 \\
12.31 \\
16.44 \\
20.51 \\
20.46 \\
20.47 \\
27.05 \\
34.69 \\
34.53 \\
34.53 \\
39.70 \\
45.90 \\
45.65 \\
45.62 \\
50.10 \\
53.47 \\
53.29 \\
53.21 \\
50.49 \\
46.14 \\
46.13 \\
44.97 \\
38.98 \\
33.99 \\
33.96 \\
26.83 \\
21.88 \\
21.90 \\
16.69 \\
11.74 \\
11.77 \\
5.05 \\
0.02 \\
-0.04 \\
-0.02 \\
-0.02 \\
0.02\end{array}$ & $\begin{array}{l}\text { Maximum } \\
\text { \%Strain } \\
0.0002 \\
0.0009 \\
0.0020 \\
0.0031 \\
0.0065 \\
0.0074 \\
0.0078 \\
0.0128 \\
0.0156 \\
0.0156 \\
0.0156 \\
0.0170 \\
0.0197 \\
0.0195 \\
0.0195 \\
0.0212 \\
0.0225 \\
0.0225 \\
0.0225 \\
0.0216 \\
0.0204 \\
0.0203 \\
0.0194 \\
0.0177 \\
0.0164 \\
0.0163 \\
0.0140 \\
0.0124 \\
0.0124 \\
0.0105 \\
0.0090 \\
0.0090 \\
0.0070 \\
0.0053 \\
0.0055 \\
0.0055 \\
0.0054 \\
0.0056\end{array}$ & $\begin{array}{r}\text { Minimum } \\
\text { \%Strain } \\
-0.0001 \\
-0.0001 \\
-0.0002 \\
0.0002 \\
0.0014 \\
0.0017 \\
0.0017 \\
0.0038 \\
0.0044 \\
0.0043 \\
0.0044 \\
0.0025 \\
-0.0012 \\
-0.0014 \\
-0.0017 \\
-0.0022 \\
-0.0022 \\
-0.0022 \\
-0.0023 \\
-0.0024 \\
-0.0025 \\
-0.0023 \\
-0.0023 \\
-0.0026 \\
-0.0026 \\
-0.0025 \\
-0.0025 \\
-0.0021 \\
-0.0022 \\
-0.0020 \\
-0.0016 \\
-0.0012 \\
-0.0010 \\
-0.0014 \\
-0.0004 \\
-0.0003 \\
-0.0002 \\
-0.0006\end{array}$ & $\begin{array}{c}\text { Angle } \\
\text { Degrees } \\
42.14 \\
-2.51 \\
-13.30 \\
-6.03 \\
-0.19 \\
-0.88 \\
3.77 \\
-3.52 \\
-11.92 \\
-12.29 \\
-11.78 \\
-13.79 \\
-16.20 \\
-15.36 \\
-15.63 \\
-14.32 \\
-14.15 \\
-14.50 \\
-14.56 \\
-15.14 \\
-15.99 \\
-15.82 \\
-16.10 \\
-17.85 \\
-19.17 \\
-18.66 \\
-21.53 \\
-21.31 \\
-21.08 \\
-21.79 \\
-23.24 \\
-22.27 \\
-24.73 \\
-27.20 \\
-24.55 \\
-21.88 \\
-20.92 \\
-23.86\end{array}$ & $\begin{array}{c}\text { Pressure } \\
\text { ps1g } \\
9.89 \\
19.55 \\
29.57 \\
39.42 \\
49.16 \\
54.50 \\
59.57 \\
64.20 \\
69.32 \\
74.16 \\
80.16 \\
85.61 \\
90.58 \\
95.69 \\
100.92 \\
103.25 \\
106.11 \\
108.31 \\
111.08 \\
113.24 \\
117.83 \\
120.92 \\
123.28 \\
122.97 \\
125.82 \\
125.60 \\
127.84 \\
130.19 \\
132.53 \\
135.33 \\
134.42 \\
138.35 \\
140.16 \\
142.63 \\
145.78 \\
142.52 \\
0.22\end{array}$ & $\begin{array}{l}\text { Maxiwum } \\
\text { \%Strain } \\
0.0026 \\
0.0050 \\
0.0077 \\
0.0107 \\
0.0140 \\
0.0165 \\
0.0189 \\
0.0218 \\
0.0245 \\
0.0276 \\
0.0329 \\
0.0366 \\
0.0401 \\
0.0440 \\
0.0478 \\
0.0501 \\
0.0521 \\
0.0534 \\
0.0551 \\
0.0564 \\
0.0588 \\
0.0608 \\
0.0626 \\
0.0628 \\
0.0547 \\
0.0651 \\
0.0668 \\
0.0688 \\
0.0708 \\
0.0733 \\
0.0729 \\
0.0761 \\
0.0780 \\
0.0802 \\
0.0833 \\
0.0811 \\
-0.0055\end{array}$ & $\begin{array}{r}\text { Minimum } \\
\text { xStrain } \\
0.0002 \\
-0.0014 \\
-0.0032 \\
-0.0037 \\
-0.0038 \\
-0.0038 \\
-0.0036 \\
-0.0037 \\
-0.0033 \\
-0.0030 \\
-0.0022 \\
-0.0019 \\
-0.0015 \\
-0.0009 \\
-0.0005 \\
-0.0001 \\
0.0001 \\
0.0002 \\
0.0004 \\
0.0006 \\
0.0011 \\
0.0013 \\
0.0016 \\
0.0018 \\
0.0019 \\
0.0020 \\
0.0021 \\
0.0023 \\
0.0020 \\
0.0017 \\
0.0013 \\
0.0007 \\
-0.6107 \\
-0.0053 \\
-0.0096 \\
-0.0115 \\
-0.0258\end{array}$ & $\begin{array}{c}\text { Angle } \\
\text { Degrees } \\
-32.60 \\
-27.44 \\
-27.06 \\
-26.19 \\
-23.16 \\
-21.60 \\
-19.73 \\
-17.48 \\
-15.65 \\
-13.47 \\
-10.04 \\
-8.52 \\
-7.41 \\
-5.83 \\
-5.03 \\
-4.11 \\
-3.73 \\
-3.41 \\
-2.97 \\
-2.69 \\
-2.15 \\
-1.81 \\
-1.49 \\
-1.36 \\
-1.41 \\
-1.35 \\
-1.35 \\
-1.22 \\
-1.64 \\
-2.01 \\
-2.16 \\
-2.38 \\
-2.74 \\
-2.89 \\
-3.10 \\
-3.25 \\
-8.76\end{array}$ \\
\hline
\end{tabular}

High Pressure test 


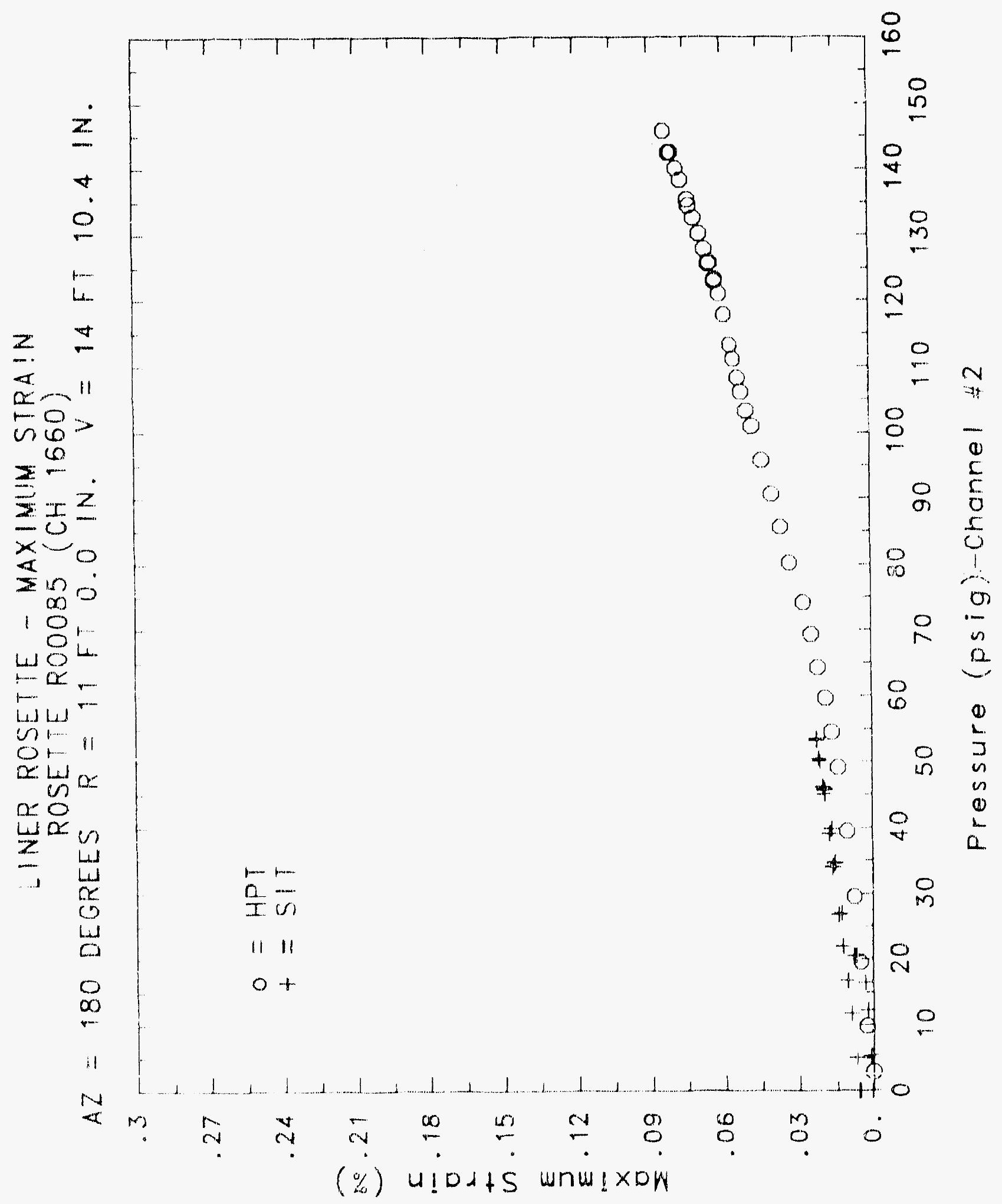




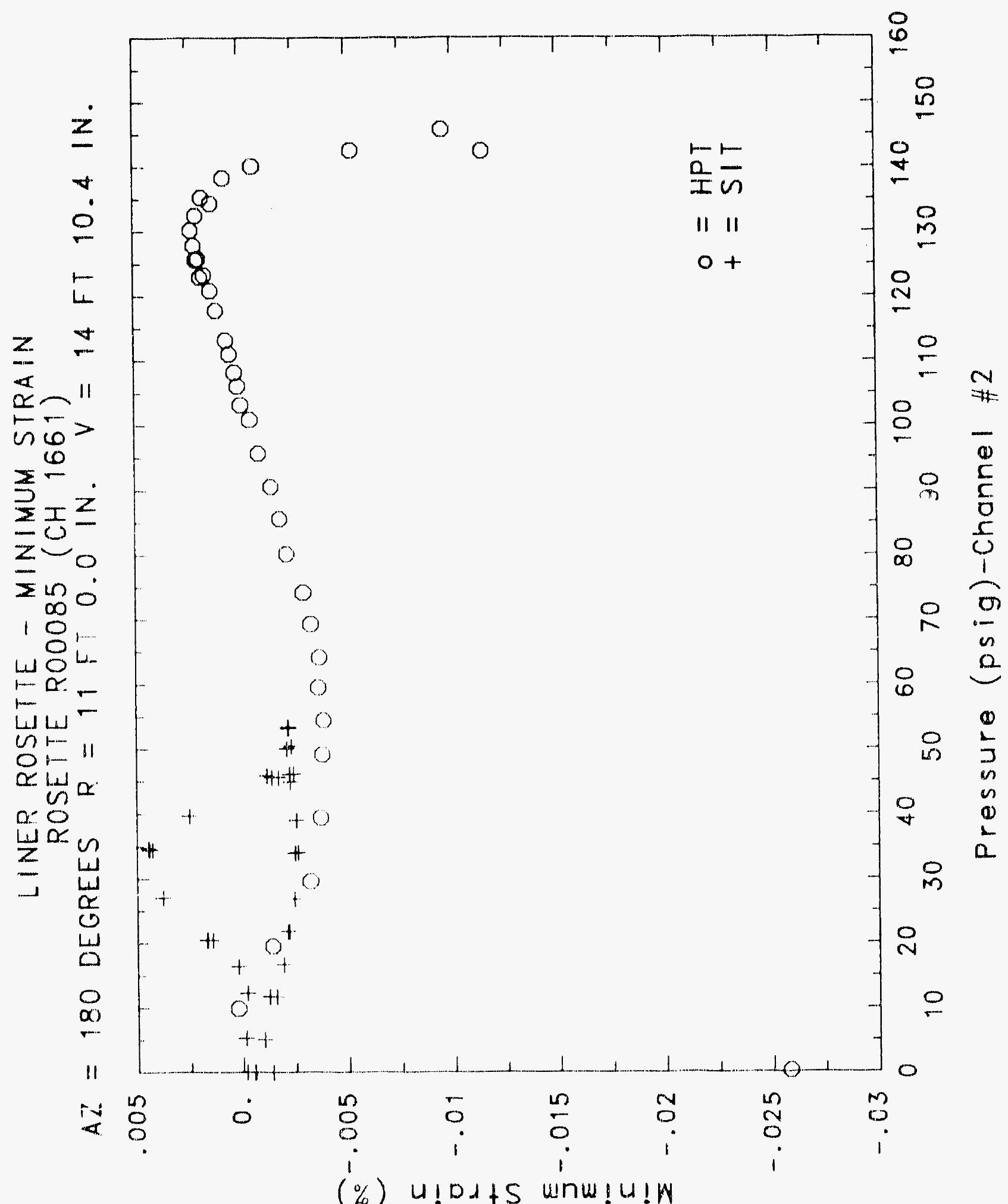




\title{
Reinforced Concrete Test Data
}

\author{
Rosette 86 Channels $1663 \quad 1664 \quad 1665$
}

STRUCTURAL INTEGRITY TEST

\begin{tabular}{|c|c|c|c|c|c|c|c|}
\hline $\begin{array}{c}\text { Pressure } \\
\text { psig } \\
-0.05 \\
5.33 \\
12.31 \\
16.44 \\
20.51 \\
20.46 \\
20.47 \\
27.05 \\
34.69 \\
34.53 \\
34.53 \\
39.70 \\
45.90 \\
45.65 \\
45.62 \\
50.10 \\
53.47 \\
53.29 \\
53.21 \\
50.49 \\
46.14 \\
46.13 \\
44.97 \\
38.98 \\
33.99 \\
33.96 \\
26.83 \\
21.88 \\
21.90 \\
16.69 \\
11.74 \\
11.77 \\
5.05 \\
0.02 \\
-0.04 \\
-0.02 \\
-0.02 \\
0.02\end{array}$ & $\begin{array}{r}\text { Maximum } \\
\text { \%5train } \\
0.0002 \\
-0.0008 \\
0.0003 \\
0.0007 \\
0.0013 \\
0.0019 \\
0.0021 \\
0.0044 \\
0.0083 \\
0.0087 \\
0.0087 \\
0.0099 \\
0.0119 \\
0.0123 \\
0.0123 \\
0.0132 \\
0.0139 \\
0.0143 \\
0.0142 \\
0.0140 \\
0.0134 \\
0.0132 \\
0.0125 \\
0.0119 \\
0.0114 \\
0.0114 \\
0.0103 \\
0.0097 \\
0.0097 \\
0.0089 \\
0.0092 \\
0.0092 \\
0.00911 \\
0.0089 \\
0.0083 \\
0.0082 \\
0.0084 \\
0.0080\end{array}$ & $\begin{array}{r}\text { Minimum } \\
\text { XStrain } \\
0.0001 \\
-0.0100 \\
-0.0135 \\
-0.0124 \\
-0.0091 \\
-0.0086 \\
-0.0081 \\
-0.0043 \\
-0.0021 \\
-0.0018 \\
-0.0018 \\
-0.0014 \\
-0.0003 \\
0.0002 \\
0.0003 \\
0.0007 \\
0.0016 \\
0.0017 \\
0.0018 \\
0.0021 \\
0.0020 \\
0.0020 \\
0.0014 \\
0.0013 \\
0.0013 \\
0.0013 \\
0.0012 \\
0.0011 \\
0.0011 \\
0.0010 \\
0.0010 \\
0.0011 \\
0.00034 \\
0.0069 \\
0.0057 \\
0.0057 \\
0.0056 \\
0.0056\end{array}$ & $\begin{array}{r}\text { Angle } \\
\text { Degrees } \\
36.25 \\
12.12 \\
9.30 \\
7.27 \\
6.97 \\
7.67 \\
6.85 \\
8.14 \\
9.36 \\
8.78 \\
10.02 \\
9.65 \\
8.52 \\
8.90 \\
9.41 \\
9.30 \\
9.25 \\
9.81 \\
9.56 \\
9.39 \\
9.92 \\
9.65 \\
8.42 \\
9.03 \\
9\end{array}$ & $\begin{array}{c}\text { Pressure } \\
\text { psig } \\
9.89 \\
19.55 \\
29.57 \\
39.42 \\
49.16 \\
54.50 \\
59.57 \\
64.20 \\
69.32 \\
74.16 \\
80.16 \\
85.61 \\
90.58 \\
95.69 \\
100.92 \\
103.25 \\
106.11 \\
108.31 \\
111.08 \\
113.24 \\
117.83 \\
120.92 \\
123.28 \\
122.97 \\
125.82 \\
125.60 \\
127.84 \\
130.19 \\
132.53 \\
135.33 \\
134.42 \\
138.35 \\
140.10 \\
142.63 \\
145.78 \\
142.52 \\
0.22\end{array}$ & $\begin{array}{c}\text { Maximum } \\
\text { x.Strain } \\
0.0017 \\
0.0037 \\
0.0059 \\
0.0075 \\
0.0089 \\
0.0100 \\
0.0111 \\
0.0119 \\
0.0128 \\
0.0137 \\
0.0148 \\
0.0156 \\
0.0165 \\
0.0175 \\
0.0182 \\
0.0184 \\
0.0187 \\
0.0188 \\
0.0188 \\
0.0189 \\
0.0185 \\
0.0185 \\
0.0186 \\
0.0187 \\
0.0191 \\
0.0193 \\
0.0197 \\
0.0211 \\
0.0232 \\
0.0262 \\
0.0271 \\
0.0308 \\
0.0336 \\
0.0381 \\
0.0419 \\
0.0427 \\
-0.0306\end{array}$ & $\begin{array}{l}\text { Minimum } \\
\text { XStrain } \\
-0.0056 \\
-0.0050 \\
-0.0051 \\
-0.0046 \\
-0.0040 \\
-0.0036 \\
-0.0026 \\
-0.0013 \\
0.0000 \\
0.0014 \\
0.0027 \\
0.0043 \\
0.0053 \\
0.0062 \\
0.0071 \\
0.0075 \\
0.0075 \\
0.0078 \\
0.0079 \\
0.0076 \\
0.0069 \\
0.0057 \\
0.0050 \\
0.0046 \\
0.0036 \\
0.0030 \\
0.0025 \\
0.0016 \\
0.0010 \\
0.0011 \\
0.0010 \\
0.0028 \\
0.0041 \\
0.0063 \\
0.0092 \\
0.0084 \\
-0.0330\end{array}$ & $\begin{array}{c}\text { Angle } \\
\text { Degrees } \\
-5.81 \\
15.55 \\
20.12 \\
19.57 \\
19.29 \\
18.53 \\
18.54 \\
21.03 \\
22.53 \\
23.69 \\
25.60 \\
26.79 \\
27.58 \\
30.14 \\
33.06 \\
34.88 \\
36.54 \\
38.19 \\
40.68 \\
43.01 \\
-39.75 \\
-34.6 \\
-30.27 \\
-29.63 \\
-25.54 \\
-24.22 \\
-23.02 \\
-19.72 \\
-17.52 \\
-15.15 \\
-14.39 \\
-12.30 \\
-10.55 \\
-7.48 \\
-6.63 \\
-5.93 \\
-5.42\end{array}$ \\
\hline
\end{tabular}

High Pressure Test

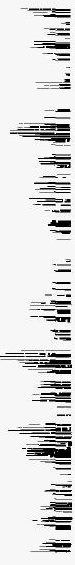




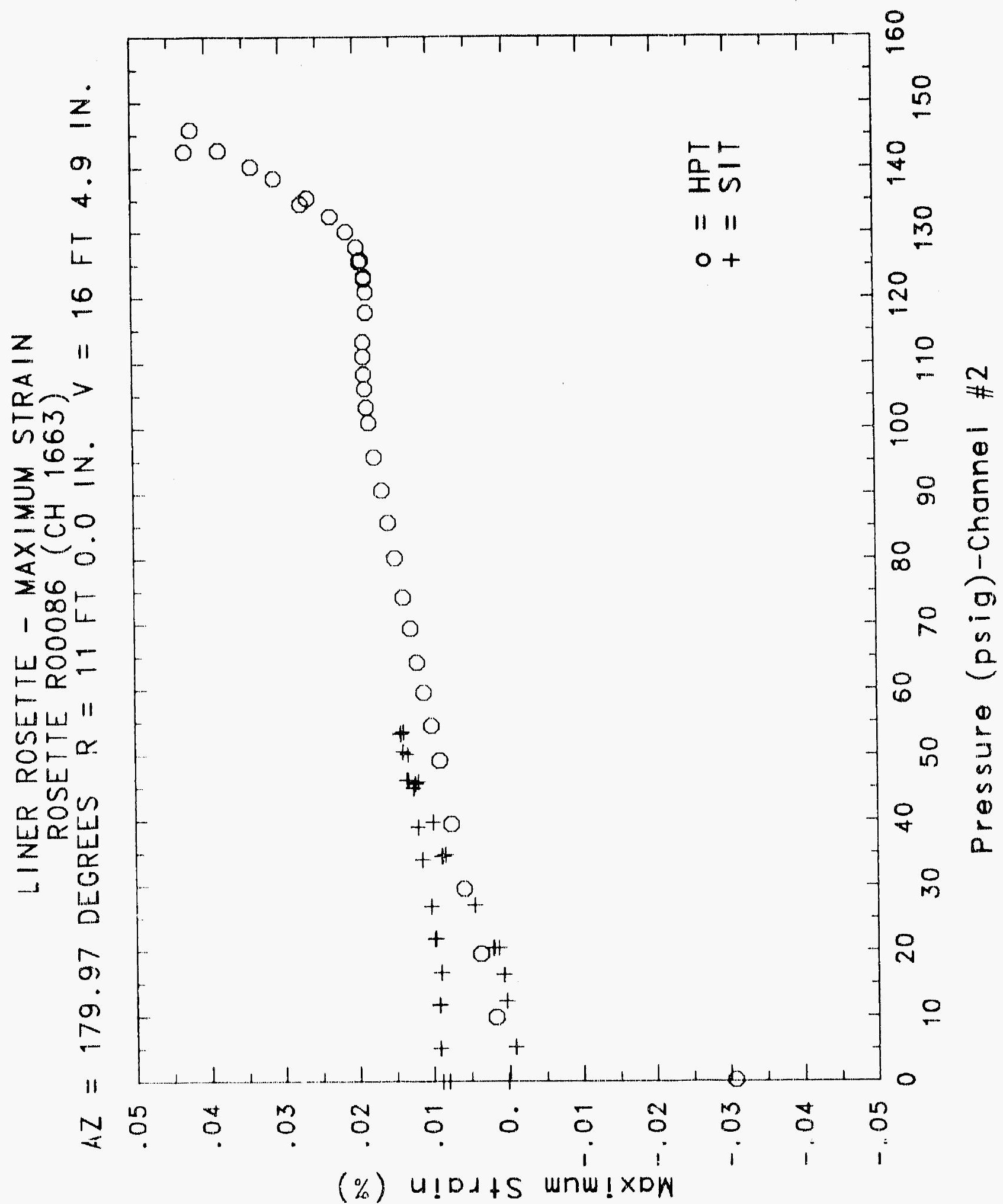




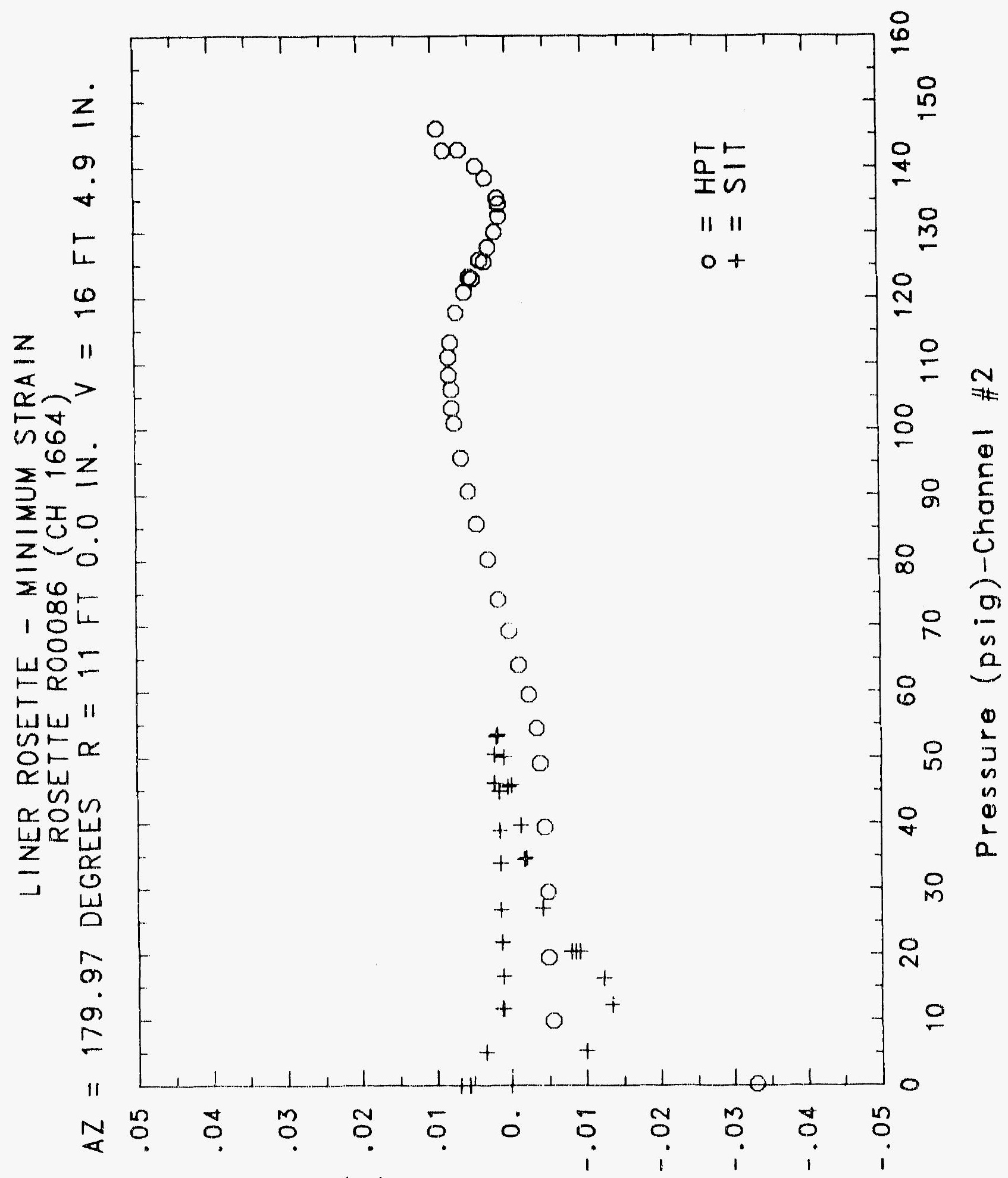

(\%) u!pdts unm!u! 


\section{Reinforced Concrete Test Data}

\section{$\begin{array}{llllll}\text { Rosette } & 87 & \text { Chanmels } & 1666 & 1667 & 1668\end{array}$}

\section{StRuctural InTEgrity Test}

\begin{tabular}{cccc}
\hline Pressure & Maximum & Minimum & Angle \\
psig & \%Strain & \%Strain & Degrees \\
-0.05 & 0.0005 & -0.0004 & 37.69 \\
5.33 & 0.0043 & -0.0153 & 37.79 \\
12.31 & 0.0016 & -0.0165 & 43.38 \\
16.44 & 0.0017 & -0.0151 & -38.89 \\
20.51 & 0.0028 & -0.0133 & -28.04 \\
20.46 & 0.0034 & -0.0128 & -26.80 \\
20.47 & 0.0031 & -0.0124 & -26.00 \\
27.05 & 0.0083 & -0.0109 & -15.90 \\
34.69 & 0.0175 & -0.0088 & -11.68 \\
34.53 & 0.0185 & -0.0083 & -11.98 \\
34.53 & 0.0186 & -0.0082 & -12.06 \\
39.70 & 0.0233 & -0.0070 & -13.19 \\
45.90 & 0.0302 & -0.0049 & -14.73 \\
45.65 & 0.0307 & -0.0044 & -14.68 \\
45.62 & 0.0309 & -0.0044 & -14.77 \\
50.10 & 0.0345 & -0.0037 & -15.63 \\
53.47 & 0.0375 & -0.0026 & -16.01 \\
53.29 & 0.0377 & -0.0022 & -15.76 \\
53.21 & 0.0377 & -0.0021 & -15.86 \\
50.49 & 0.0358 & -0.0018 & -15.53 \\
46.14 & 0.0331 & -0.0018 & -15.33 \\
46.13 & 0.0328 & -0.0018 & -15.26 \\
44.97 & 0.0295 & -0.0020 & -14.74 \\
38.98 & 0.0261 & -0.0024 & -13.74 \\
33.99 & 0.0237 & -0.0034 & -12.51 \\
33.96 & 0.0233 & -0.0032 & -12.50 \\
26.83 & 0.0202 & -0.0049 & -8.16 \\
21.88 & 0.0189 & -0.0059 & -4.41 \\
21.90 & 0.0185 & -0.0057 & -4.40 \\
16.69 & 0.0180 & -0.0060 & 0.73 \\
11.74 & 0.0172 & -0.0060 & 8.59 \\
11.77 & 0.0167 & -0.0059 & 9.22 \\
5.05 & 0.0192 & -0.0048 & 16.80 \\
0.02 & 0.0248 & -0.0082 & 29.22 \\
-0.04 & 0.0224 & -0.0063 & 27.55 \\
-0.02 & 0.0223 & -0.0061 & 27.29 \\
-0.02 & 0.0220 & -0.0059 & 27.24 \\
0.02 & 0.0211 & -0.0056 & 26.74
\end{tabular}

High Pressure test

\begin{tabular}{cccc}
\hline Pressure & Maximum & Minimum & \multicolumn{1}{c}{ Angle } \\
psig & xStrain & \%Strain & Degrees \\
9.89 & 0.0057 & -0.0128 & -43.7 .5 \\
19.55 & 0.0066 & -0.0149 & -40.43 \\
29.57 & 0.0110 & -0.0138 & -38.97 \\
39.42 & 0.0181 & -0.0125 & -36.54 \\
49.16 & 0.0263 & -0.0117 & -34.13 \\
54.50 & 0.0313 & -0.0109 & -32.73 \\
59.57 & 0.0359 & -0.0101 & -31.72 \\
64.20 & 0.0403 & -0.0087 & -31.13 \\
69.32 & 0.0450 & -0.0073 & -30.72 \\
74.16 & 0.0497 & -0.0057 & -30.13 \\
80.16 & 0.0561 & -0.0044 & -29.55 \\
85.61 & 0.0612 & -0.0032 & -29.11 \\
90.58 & 0.0650 & -0.0018 & -29.01 \\
95.69 & 0.0679 & -0.0003 & -28.76 \\
100.92 & 0.0702 & 0.0013 & -28.98 \\
103.25 & 0.0710 & 0.0019 & -29.17 \\
106.11 & 0.0720 & 0.0024 & -29.49 \\
108.31 & 0.0727 & 0.0029 & -29.66 \\
111.08 & 0.0735 & 0.0034 & -30.23 \\
113.24 & 0.0740 & 0.0039 & -30.65 \\
117.83 & 0.0747 & 0.0048 & -32.09 \\
120.92 & 0.0749 & 0.0054 & -33.41 \\
123.28 & 0.0747 & 0.0059 & -34.66 \\
122.97 & 0.0748 & 0.0059 & -35.08 \\
125.82 & 0.0748 & 0.0068 & -36.39 \\
125.60 & 0.0742 & 0.0070 & -36.92 \\
127.84 & 0.0750 & 0.0073 & -37.53 \\
130.19 & 0.0751 & 0.0089 & -39.21 \\
132.53 & 0.0765 & 0.0110 & -40.71 \\
135.33 & 0.0783 & 0.0138 & -42.14 \\
134.42 & 0.0783 & 0.0145 & -42.62 \\
138.35 & 0.0842 & 0.0175 & -43.97 \\
140.16 & 0.0881 & 0.0197 & -44.64 \\
142.63 & 0.0949 & 0.0227 & 44.69 \\
145.78 & 0.1028 & 0.0263 & -4.36 \\
142.52 & 0.1017 & 0.0263 & 43.45 \\
0.22 & -0.0138 & -0.0810 & 17.44 \\
& & &
\end{tabular}




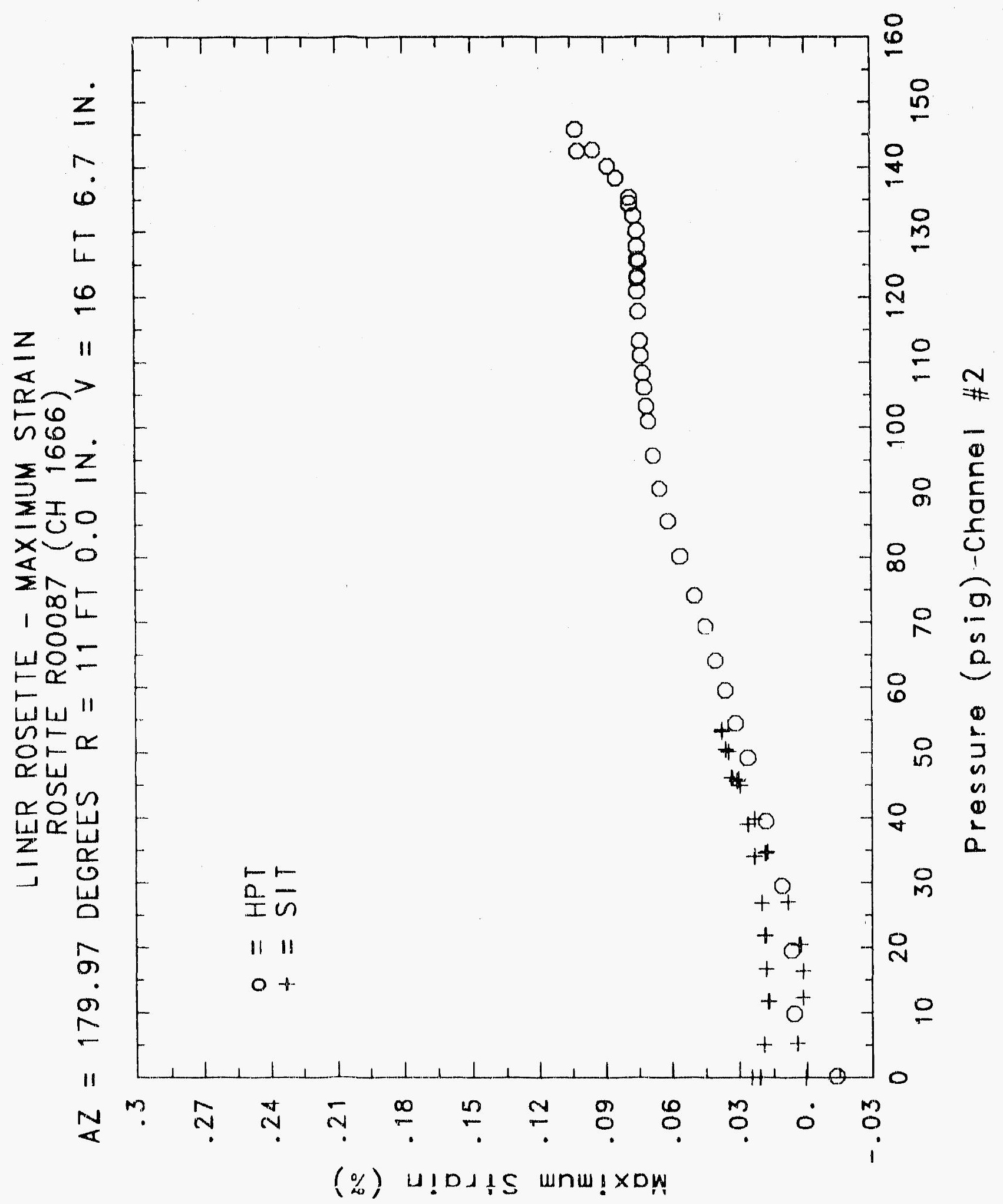




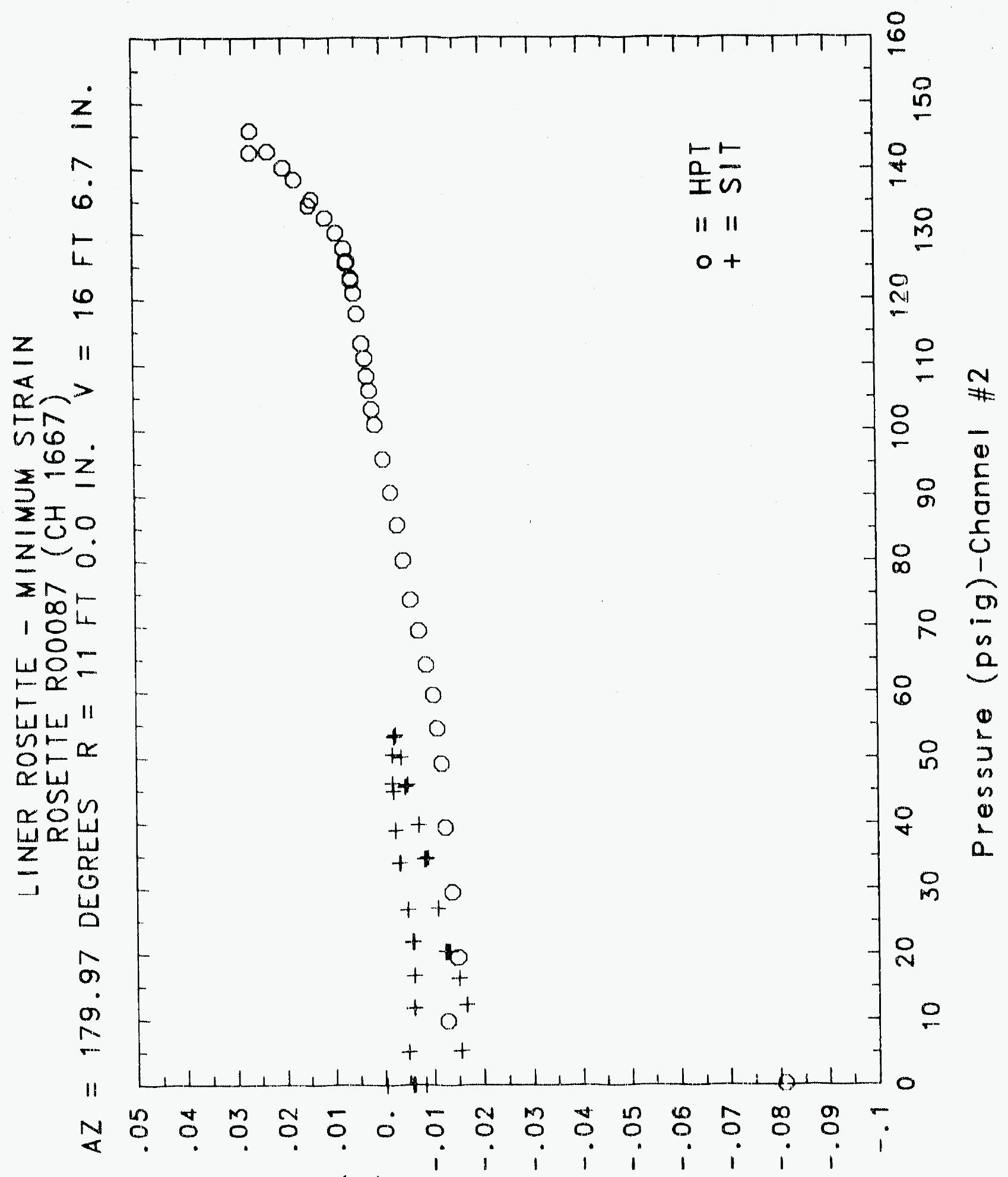

(\%) u!bdts unm!lu!w 


\title{
Reinforced Concrete Test Data
}

\author{
Rosette 88 Channels $1669 \quad 1680 \quad 1681$
}

\section{Structural InTEgRITY TEST}

\begin{tabular}{|c|c|c|c|c|c|c|c|}
\hline $\begin{array}{c}\text { Pressure } \\
\text { psig } \\
-0.05 \\
5.33 \\
12.31 \\
16.44 \\
20.51 \\
20.46 \\
20.47 \\
27.05 \\
34.69 \\
34.53 \\
34.53 \\
39.70 \\
45.90 \\
45.65 \\
45.62 \\
50.10 \\
53.47 \\
53.29 \\
53.21 \\
50.49 \\
46.14 \\
46.13 \\
44.97 \\
38.98 \\
33.99 \\
33.96 \\
26.83 \\
21.88 \\
21.90 \\
16.69 \\
11.74 \\
11.77 \\
5.05 \\
0.02 \\
-0.04 \\
-0.02 \\
-0.02 \\
0.02\end{array}$ & $\begin{array}{c}\text { Maximum } \\
\text { \%Strain } \\
0.0003 \\
0.0038 \\
0.0048 \\
0.0062 \\
0.0108 \\
0.0121 \\
0.0131 \\
0.0280 \\
0.0515 \\
0.0548 \\
0.0556 \\
0.0653 \\
0.0782 \\
0.0790 \\
0.0789 \\
0.0872 \\
0.0978 \\
0.1002 \\
0.1008 \\
0.0993 \\
0.0951 \\
0.0947 \\
0.0949 \\
0.0885 \\
0.0823 \\
0.0824 \\
0.0727 \\
0.0658 \\
0.0661 \\
0.0587 \\
0.0509 \\
0.0499 \\
0.0394 \\
0.0317 \\
0.0303 \\
0.0304 \\
0.0306 \\
0.0305\end{array}$ & $\begin{array}{l}\text { Minimum } \\
\text { xStrain } \\
-0.0003 \\
0.0010 \\
0.0011 \\
-0.0001 \\
-0.0017 \\
-0.0019 \\
-0.0026 \\
-0.0045 \\
-0.0087 \\
-0.0093 \\
-0.0095 \\
-0.0131 \\
-0.0183 \\
-0.0190 \\
-0.0191 \\
-0.0213 \\
-0.0231 \\
-0.0227 \\
-0.0222 \\
-0.0212 \\
-0.0199 \\
-0.0198 \\
-0.0175 \\
-0.0152 \\
-0.0136 \\
-0.0141 \\
-0.0117 \\
-0.0103 \\
-0.0106 \\
-0.0093 \\
-0.0085 \\
-0.0091 \\
-0.0088 \\
-0.0103 \\
-0.0104 \\
-0.0105 \\
-0.0108 \\
-0.0107\end{array}$ & $\begin{array}{c}\text { Angle } \\
\text { Degrees } \\
25.66 \\
21.07 \\
-30.92 \\
-9.42 \\
8.57 \\
9.65 \\
11.27 \\
19.91 \\
24.44 \\
24.90 \\
25.02 \\
25.06 \\
23.78 \\
23.33 \\
23.17 \\
22.21 \\
20.55 \\
20.00 \\
19.88 \\
19.85 \\
19.80 \\
19.77 \\
19.75 \\
19.67 \\
19.21 \\
19.29 \\
18.20 \\
16.99 \\
17.10 \\
15.92 \\
14.09 \\
13.66 \\
10.18 \\
4.63 \\
3.87 \\
4.70 \\
5.02 \\
4.94\end{array}$ & $\begin{array}{c}\text { Pressure } \\
\text { psig } \\
9.89 \\
19.55 \\
29.57 \\
39.42 \\
49.16 \\
54.50 \\
59.57 \\
64.20 \\
69.32 \\
74.16 \\
80.16 \\
85.61 \\
90.58 \\
95.69 \\
100.92 \\
103.25 \\
106.11 \\
108.31 \\
111.08 \\
113.24 \\
117.83 \\
120.92 \\
123.28 \\
122.97 \\
125.82 \\
125.60 \\
127.84 \\
130.19 \\
132.53 \\
135.33 \\
134.42 \\
138.35 \\
140.16 \\
142.63 \\
145.78 \\
142.52 \\
0.22\end{array}$ & $\begin{array}{c}\text { Maximum } \\
\text { \%Strain } \\
0.0161 \\
0.0340 \\
0.0504 \\
0.0656 \\
0.0802 \\
0.0889 \\
0.1021 \\
0.1201 \\
0.1416 \\
0.1660 \\
0.1976 \\
0.2241 \\
0.2507 \\
0.2795 \\
0.3085 \\
0.3200 \\
0.3328 \\
0.3433 \\
0.3578 \\
0.3695 \\
0.3958 \\
0.4123 \\
0.4244 \\
0.4268 \\
0.4431 \\
0.4476 \\
0.4583 \\
0.4765 \\
0.4994 \\
0.5291 \\
0.5326 \\
0.5661 \\
0.5926 \\
0.6346 \\
0.6659 \\
0.6667 \\
0.3090\end{array}$ & $\begin{array}{l}\text { Minimum } \\
\text { XStrain } \\
-0.0012 \\
-0.0049 \\
-0.0077 \\
-0.0105 \\
-0.0133 \\
-0.0148 \\
-0.0170 \\
-0.0203 \\
-0.0240 \\
-0.0287 \\
-0.0373 \\
-0.0435 \\
-0.0495 \\
-0.0570 \\
-0.0654 \\
-0.0684 \\
-0.0724 \\
-0.0757 \\
-0.0805 \\
-0.0841 \\
-0.0924 \\
-0.0979 \\
-0.1020 \\
-0.1026 \\
-0.1094 \\
-0.1118 \\
-0.1169 \\
-0.1232 \\
-0.1302 \\
-0.1399 \\
-0.1414 \\
-0.1571 \\
-0.1673 \\
-0.1834 \\
-0.1940 \\
-0.1968 \\
-0.2057\end{array}$ & $\begin{array}{c}\text { Angle } \\
\text { Degrees } \\
23.41 \\
25.90 \\
26.90 \\
27.38 \\
27.53 \\
27.19 \\
24.93 \\
22.69 \\
20.93 \\
19.74 \\
19.48 \\
18.76 \\
18.21 \\
17.83 \\
17.58 \\
17.54 \\
17.40 \\
17.32 \\
17.24 \\
17.16 \\
16.97 \\
17.00 \\
17.08 \\
17.11 \\
17.30 \\
17.61 \\
17.91 \\
17.90 \\
17.82 \\
17.85 \\
17.92 \\
17.93 \\
18.35 \\
18.85 \\
19.21 \\
19.35 \\
16.92\end{array}$ \\
\hline
\end{tabular}

\section{High Pressure test}




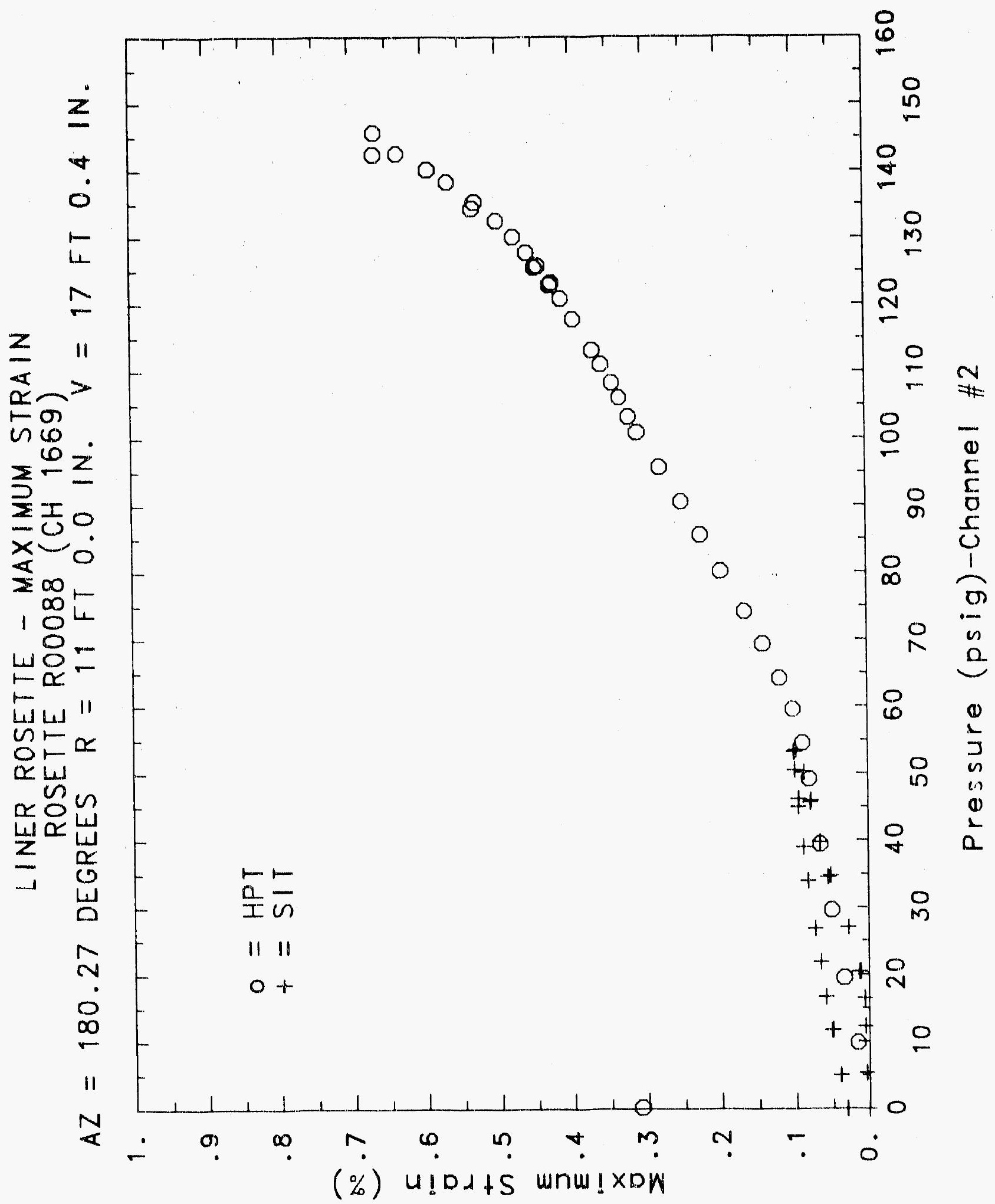

C. 582 


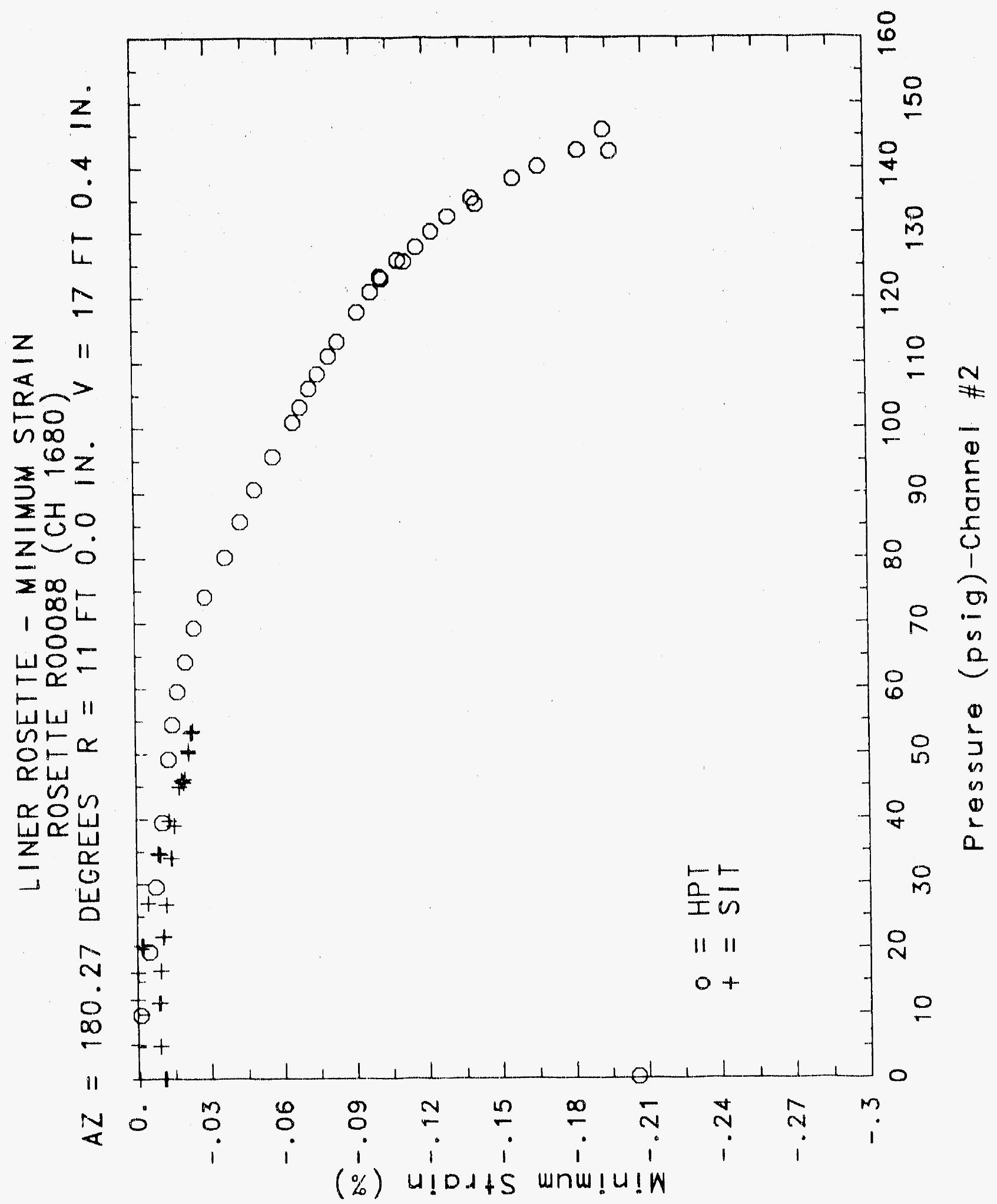




\title{
Reinforced Concrete Test Data
}

\author{
Rosette 89 Channels $1682 \quad 1683 \quad 1684$
}

\section{Structural IntEgrity TEST}

\begin{tabular}{|c|c|c|c|c|c|c|c|}
\hline $\begin{array}{c}\text { Pressure } \\
\text { psig } \\
-0.05 \\
5.33 \\
12.31 \\
16.44 \\
20.51 \\
20.46 \\
20.47 \\
27.05 \\
34.69 \\
34.53 \\
34.53 \\
39.70 \\
45.90 \\
45.65 \\
45.62 \\
50.10 \\
53.47 \\
53.29 \\
53.21 \\
50.49 \\
46.14 \\
46.13 \\
44.97 \\
38.98 \\
33.99 \\
33.96 \\
26.83 \\
21.88 \\
21.90 \\
16.69 \\
11.74 \\
11.77 \\
5.05 \\
0.02 \\
-0.04 \\
-0.02 \\
-0.02 \\
0.02\end{array}$ & $\begin{array}{c}\text { Maximum } \\
\text { xStrain } \\
0.0004 \\
0.0137 \\
0.0236 \\
0.0289 \\
0.0380 \\
0.0395 \\
0.0399 \\
0.0555 \\
0.1071 \\
0.1155 \\
0.1178 \\
0.1377 \\
0.1697 \\
0.1735 \\
0.1742 \\
0.1886 \\
0.2035 \\
0.2068 \\
0.2080 \\
0.2073 \\
0.2040 \\
0.2038 \\
0.2053 \\
0.2000 \\
0.1946 \\
0.1944 \\
0.1855 \\
0.1787 \\
0.1786 \\
0.1711 \\
0.1624 \\
0.1608 \\
0.1484 \\
0.1366 \\
0.1337 \\
0.1343 \\
0.1343 \\
0.1333\end{array}$ & $\begin{array}{l}\text { Minimum } \\
\text { \%Strain } \\
-0.0001 \\
0.0049 \\
0.0122 \\
0.0149 \\
0.0160 \\
0.0166 \\
0.0170 \\
0.0226 \\
0.0158 \\
0.0137 \\
0.0132 \\
0.0103 \\
0.0046 \\
0.0037 \\
0.0035 \\
0.0034 \\
0.0018 \\
0.0013 \\
0.0009 \\
-0.0004 \\
-0.0036 \\
-0.0039 \\
-0.0029 \\
-0.0076 \\
-0.0123 \\
-0.0125 \\
-0.0198 \\
-0.0252 \\
-0.0250 \\
-0.0311 \\
-0.0373 \\
-0.0375 \\
-0.0480 \\
-0.0602 \\
-0.0576 \\
-0.0568 \\
-0.0565 \\
-0.0570\end{array}$ & $\begin{array}{c}\text { Angle } \\
\text { Degrees } \\
\text {-18.97 } \\
-4.33 \\
-0.68 \\
-2.05 \\
-6.81 \\
-5.20 \\
-4.06 \\
17.82 \\
42.25 \\
43.74 \\
44.10 \\
-44.34 \\
-42.10 \\
-41.50 \\
-41.32 \\
-40.20 \\
-39.25 \\
-38.92 \\
-38.76 \\
-38.71 \\
-38.92 \\
-38.93 \\
-38.58 \\
-38.92 \\
-39.23 \\
-39.24 \\
-39.68 \\
-40.01 \\
-40.03 \\
-40.36 \\
-40.79 \\
-40.93 \\
-41.03 \\
-40.30 \\
-40.38 \\
-40.16 \\
-40.16 \\
-40.65\end{array}$ & $\begin{array}{c}\text { Pressure } \\
\text { psig } \\
9.89 \\
19.55 \\
29.57 \\
39.42 \\
49.16 \\
54.50 \\
59.57 \\
64.20 \\
69.32 \\
74.16 \\
80.16 \\
85.61 \\
90.58 \\
95.69 \\
100.92 \\
103.25 \\
106.11 \\
108.31 \\
111.08 \\
113.24 \\
117.83 \\
120.92 \\
123.28 \\
122.97 \\
125.82 \\
125.60 \\
127.84 \\
130.19 \\
132.53 \\
135.33 \\
134.42 \\
138.35 \\
140.16 \\
142.63 \\
145.78 \\
142.52 \\
0.22\end{array}$ & $\begin{array}{l}\text { Maximum } \\
\text { XStrain } \\
0.1164 \\
0.1329 \\
0.1498 \\
0.1620 \\
0.1750 \\
0.1828 \\
0.1920 \\
0.2020 \\
0.2174 \\
0.2392 \\
0.2717 \\
0.3031 \\
0.3395 \\
0.3862 \\
0.4387 \\
0.4625 \\
0.4907 \\
0.5134 \\
0.5455 \\
0.5689 \\
0.6208 \\
0.6522 \\
0.6765 \\
0.6817 \\
0.7056 \\
0.7116 \\
0.7260 \\
0.7617 \\
0.8125 \\
0.9068 \\
0.9241 \\
0.9807 \\
1.0226 \\
1.1398 \\
1.2028 \\
1.2179 \\
0.8828\end{array}$ & $\begin{array}{c}\text { Minimum } \\
\text { xStrain } \\
0.0025 \\
0.0153 \\
0.0262 \\
0.0353 \\
0.0425 \\
0.0468 \\
0.0523 \\
0.0576 \\
0.0617 \\
0.0628 \\
0.0619 \\
0.0608 \\
0.0601 \\
0.0596 \\
0.0591 \\
0.0588 \\
0.0582 \\
0.0574 \\
0.0559 \\
0.0544 \\
0.0505 \\
0.0480 \\
0.0458 \\
0.0453 \\
0.0430 \\
0.0420 \\
0.0407 \\
0.0351 \\
0.0262 \\
000110 \\
0.0076 \\
-0.0001 \\
-0.0059 \\
-0.0188 \\
-0.0248 \\
-0.0285 \\
-0.0350\end{array}$ & $\begin{array}{c}\text { Angle } \\
\text { Degrees } \\
23.54 \\
21.89 \\
20.07 \\
18.17 \\
16.54 \\
14.87 \\
12.13 \\
7.79 \\
3.25 \\
-0.50 \\
-2.77 \\
-4.07 \\
-3.96 \\
-2.77 \\
-0.97 \\
-0.12 \\
0.88 \\
1.67 \\
2.69 \\
3.33 \\
4.61 \\
5.24 \\
5.70 \\
5.85 \\
6.26 \\
6.40 \\
6.61 \\
7.18 \\
7.73 \\
8.30 \\
8.43 \\
7.91 \\
7.51 \\
7.74 \\
7.31 \\
7.32 \\
7.79\end{array}$ \\
\hline
\end{tabular}

\section{High Pressure Test}




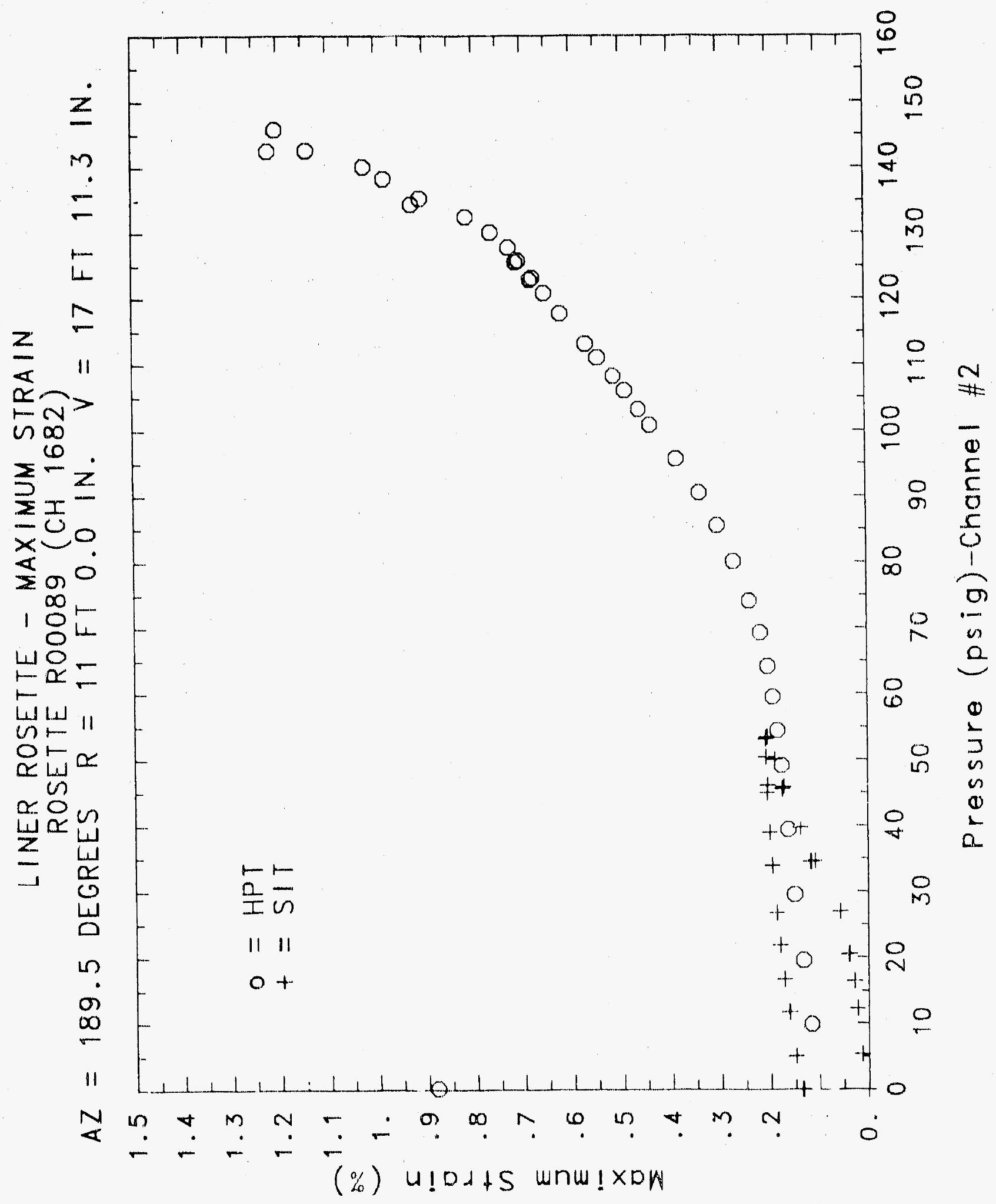




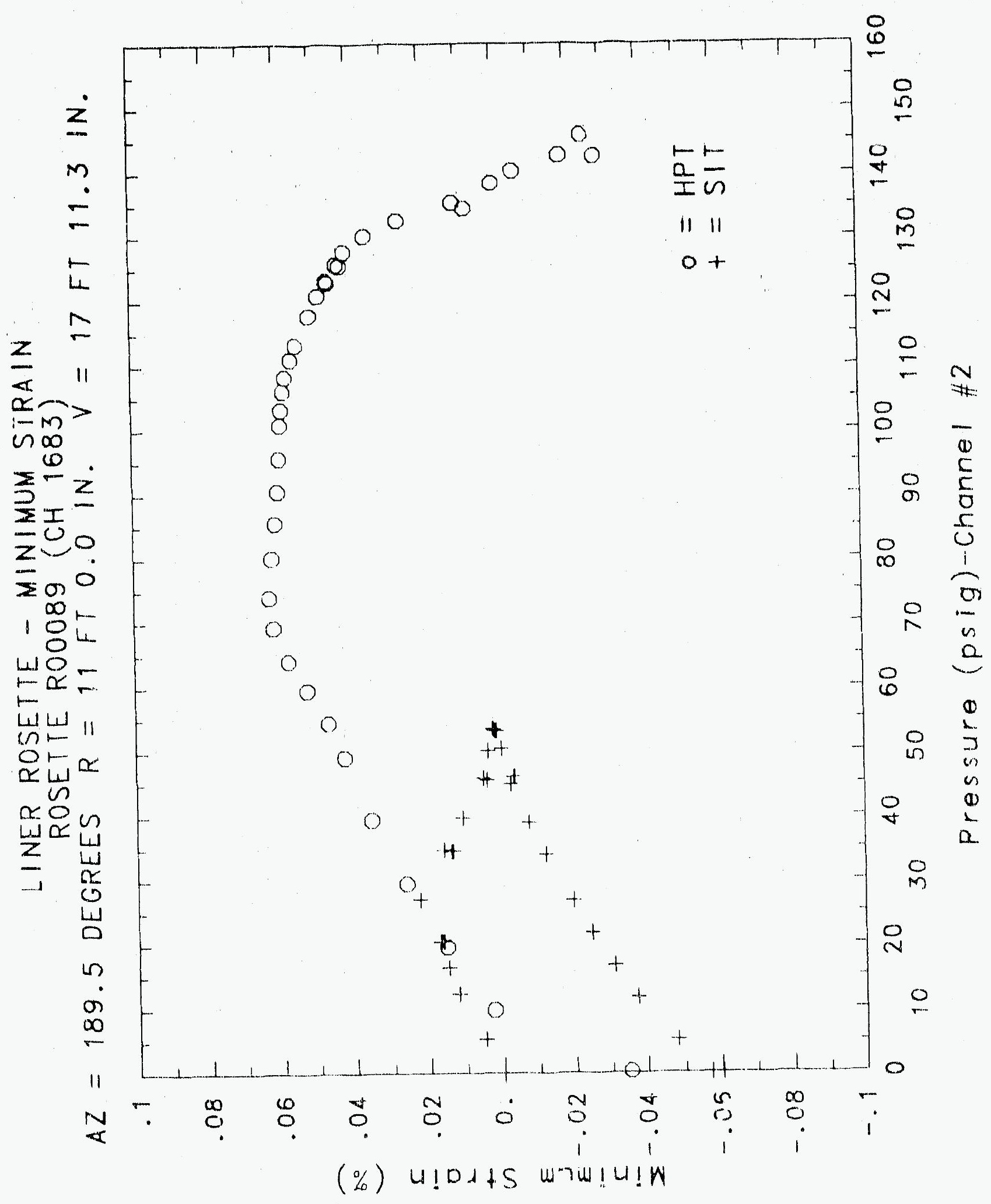

C.586 
O

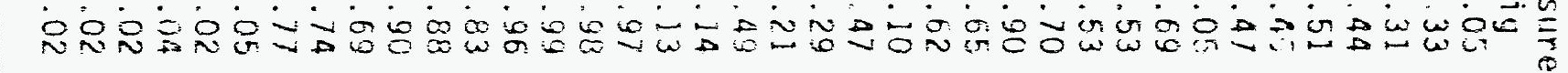
00000000000000000000000000000000000000 i้

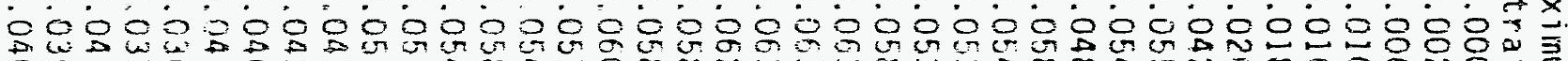

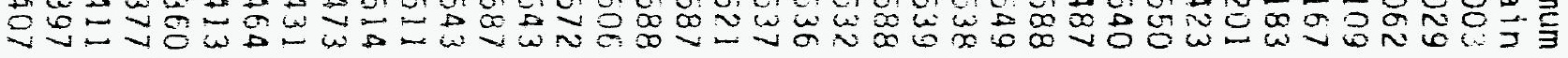

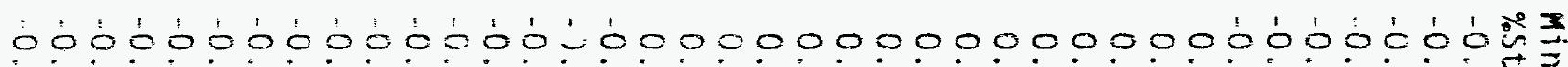

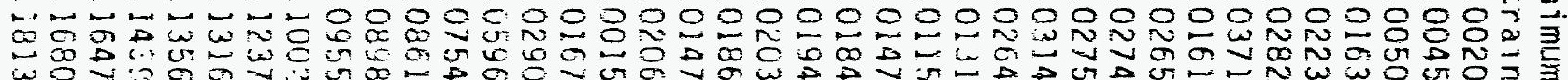

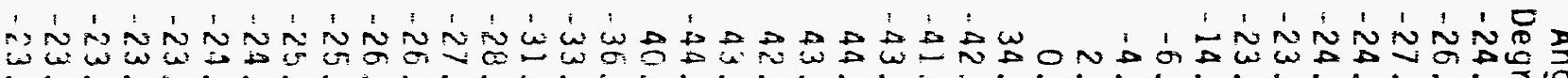

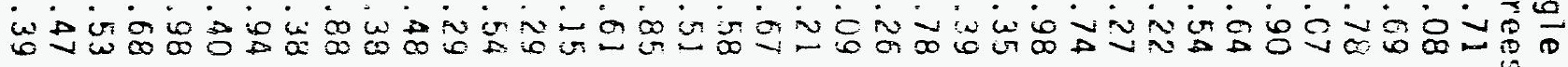

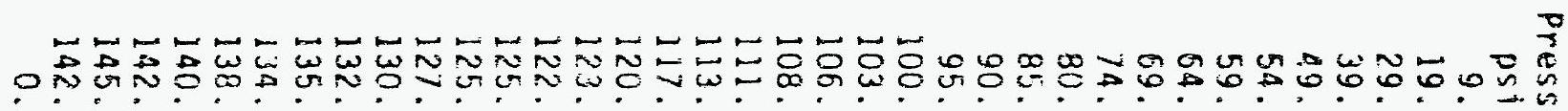

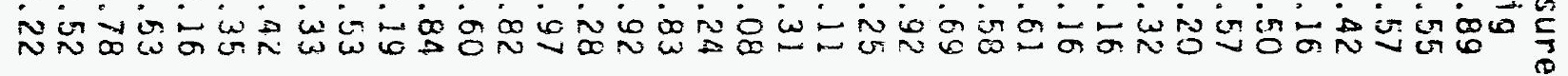
LHF-H-0000000000000000000000000000000

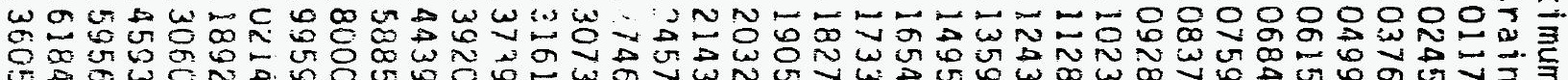

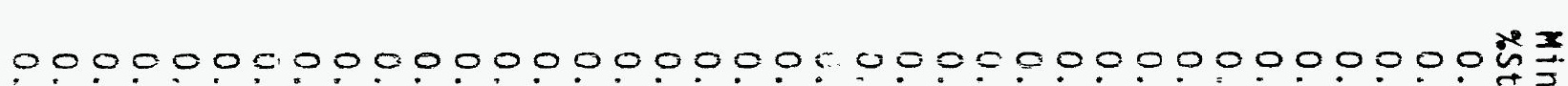

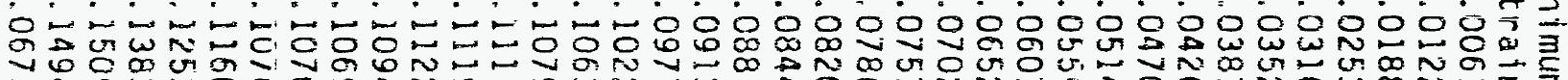

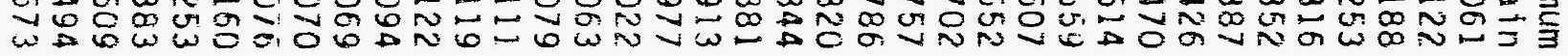

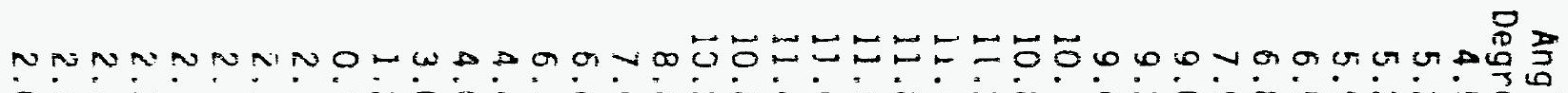

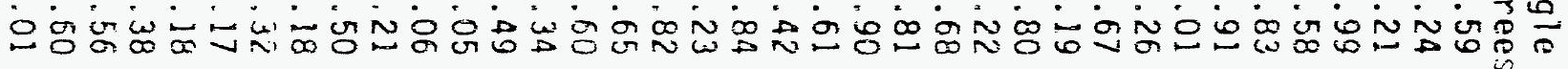


845.9

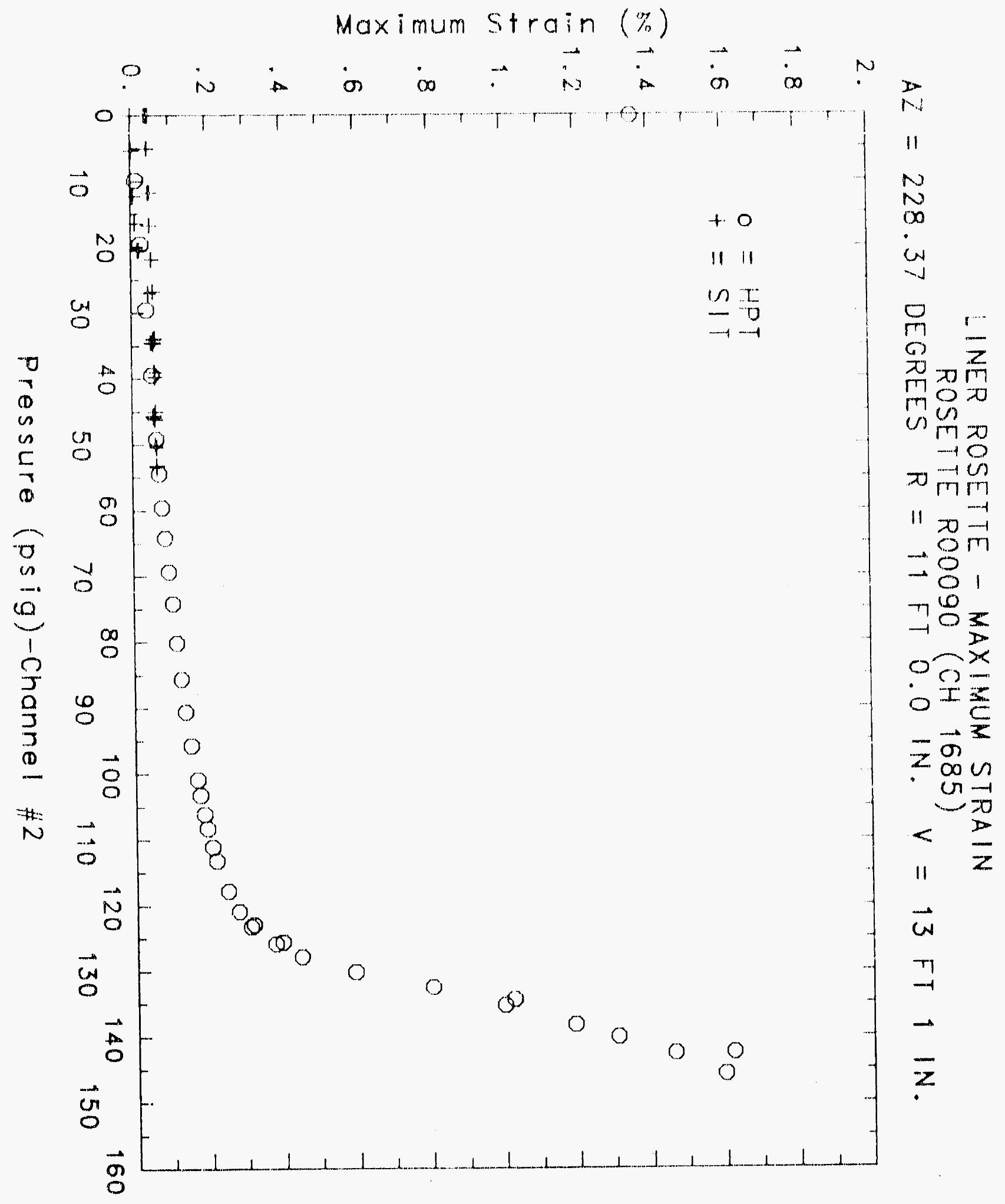




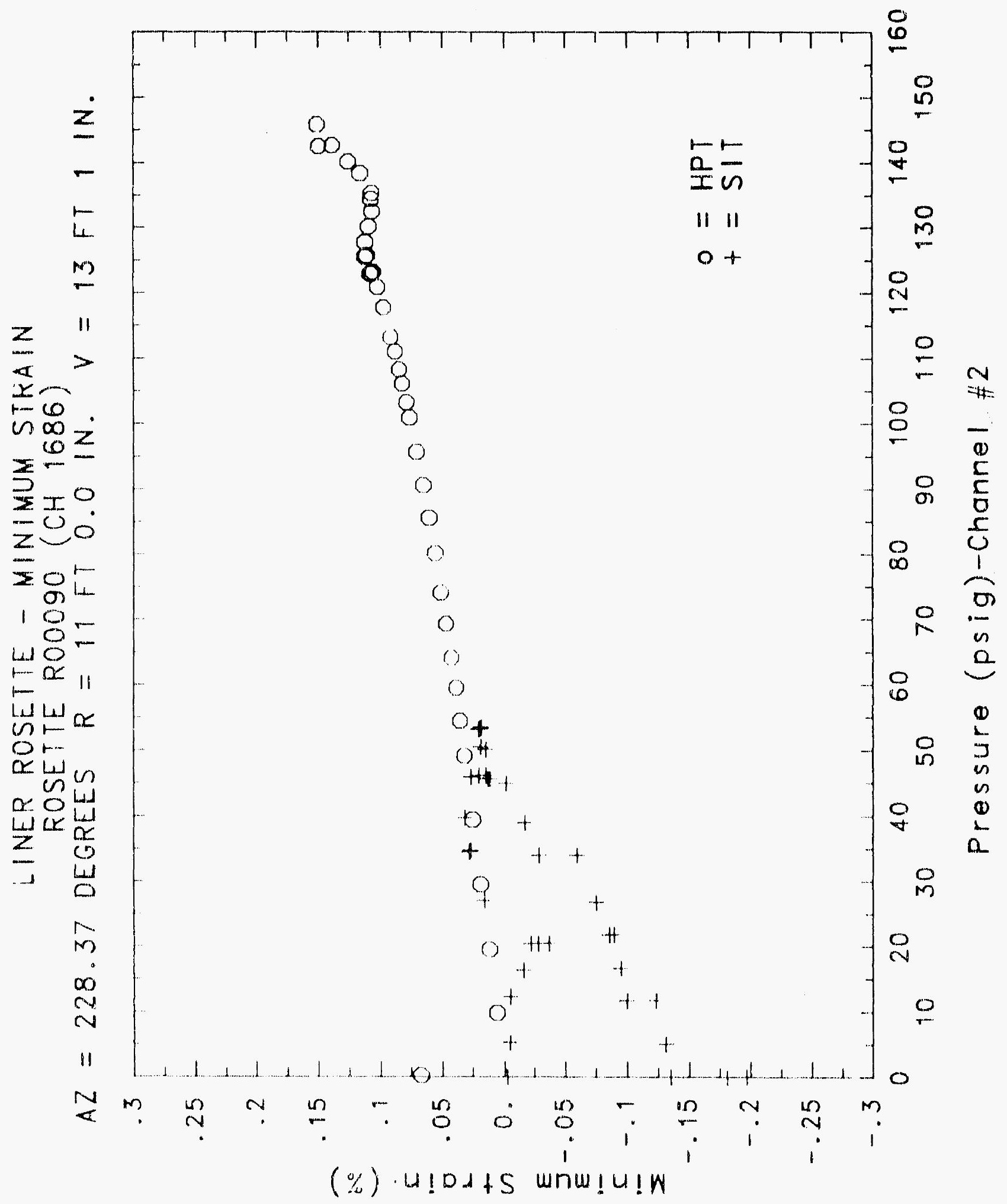




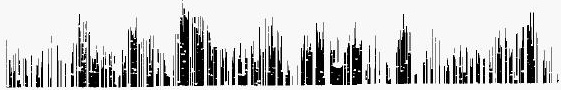

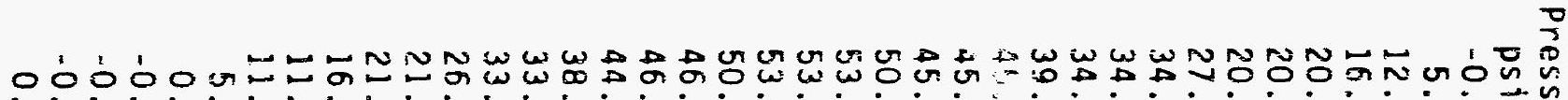

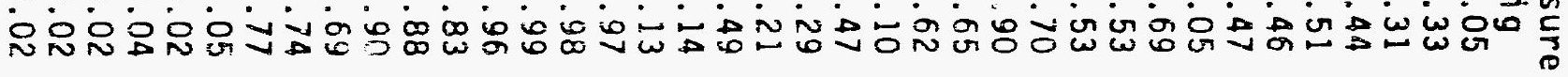

$000000000000000000000000000000000000003 \frac{1}{2}$

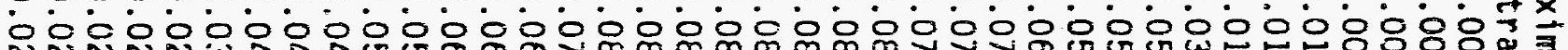

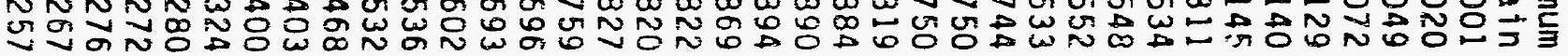

0000000000000000000000000000000000000043

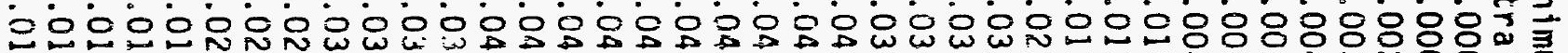

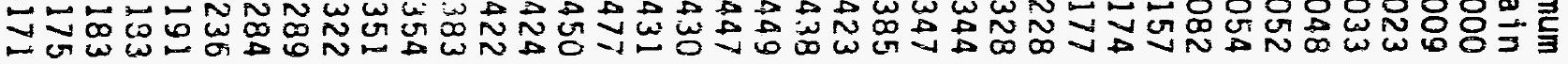

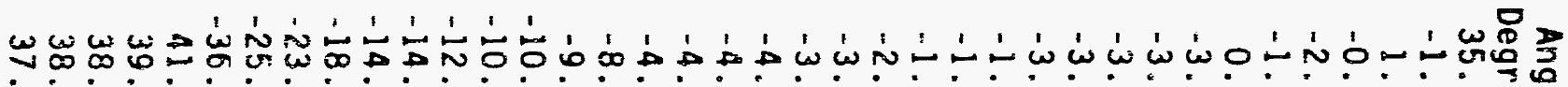

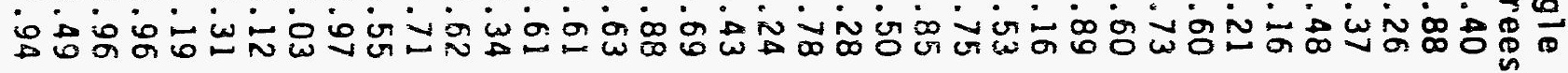

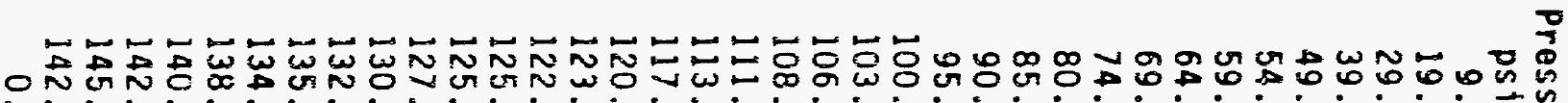

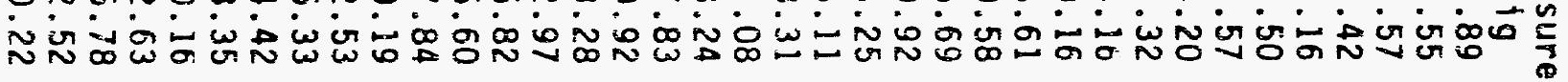

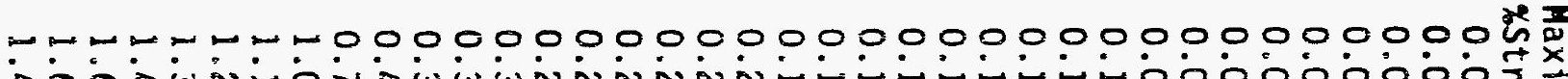

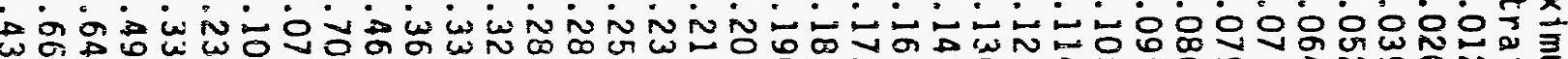

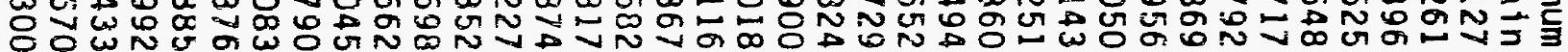

$00000000000000000000000000000000000006 \frac{13}{3}$

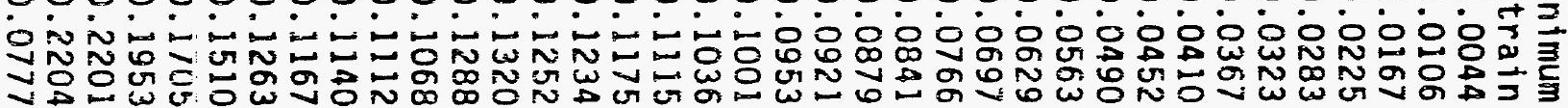

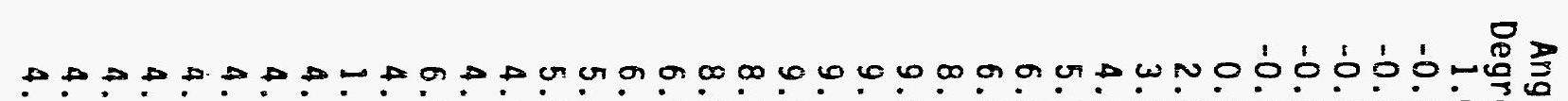

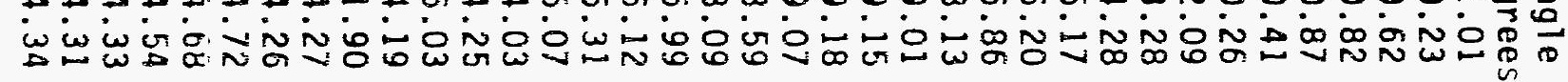


1650

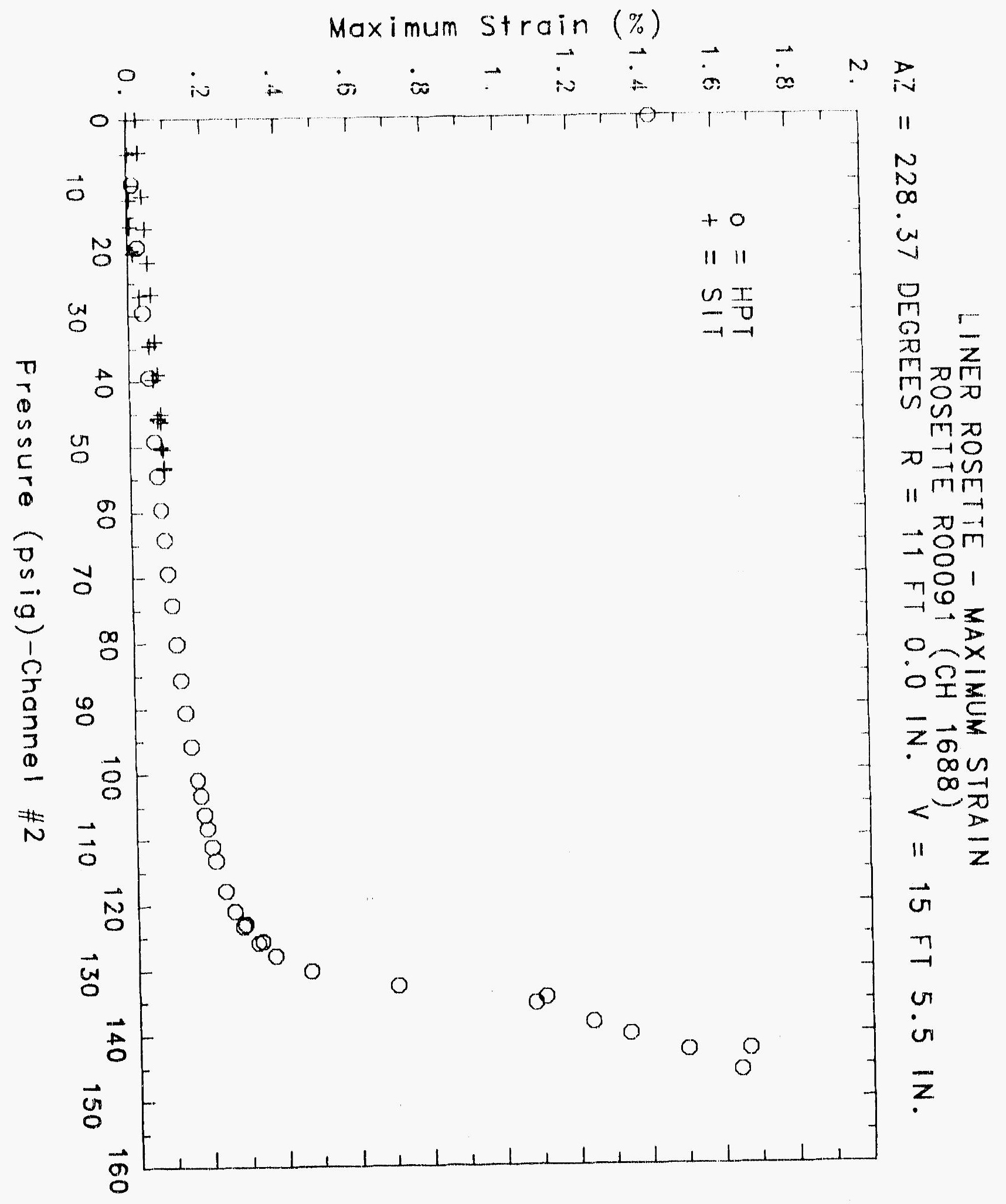




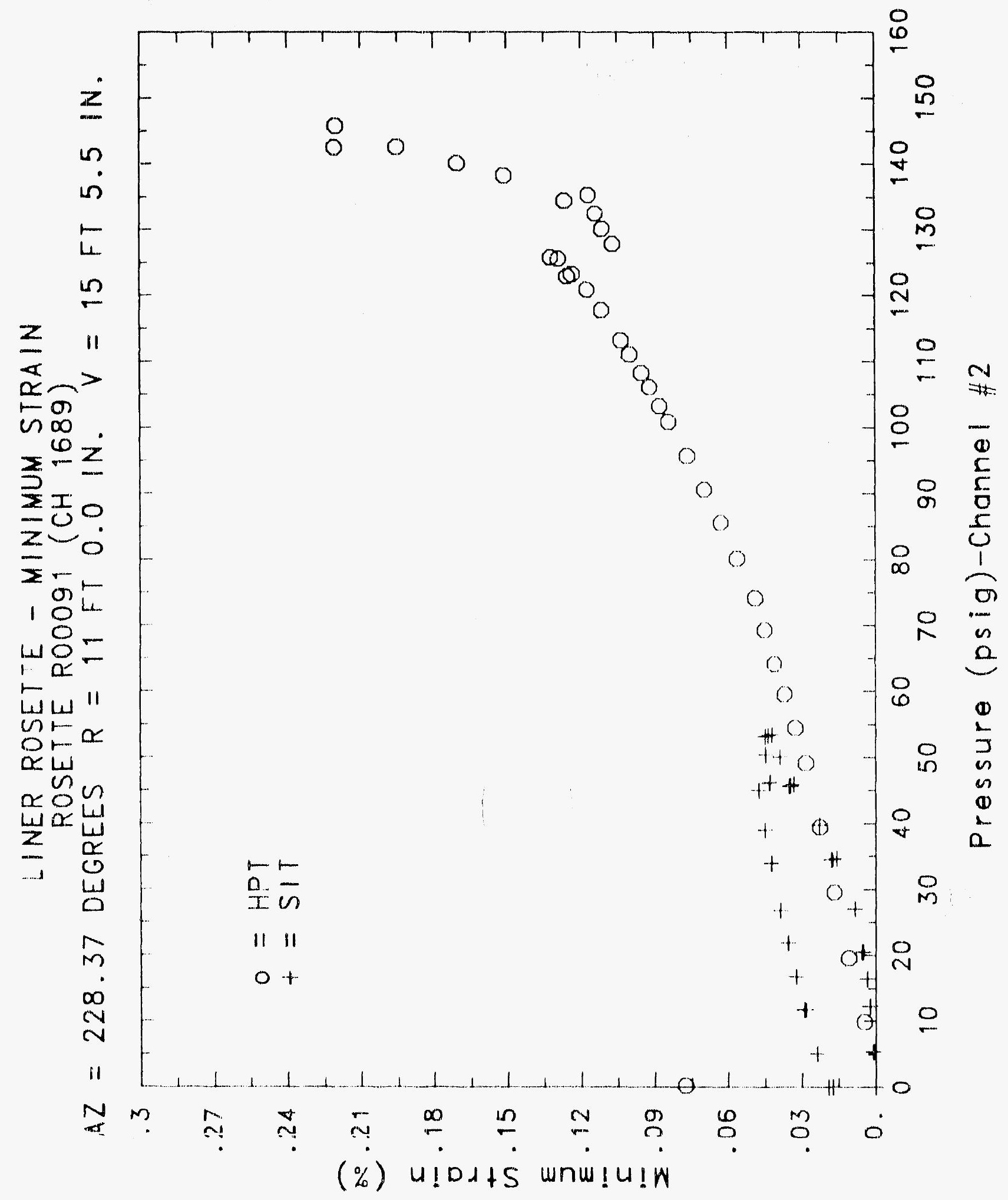


ㅇํㅇㅇำ

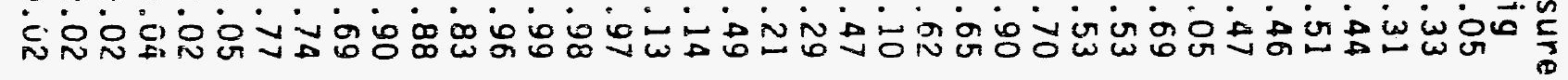
00000000000000000000000000000000000000热

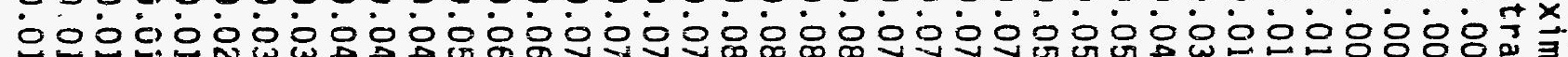

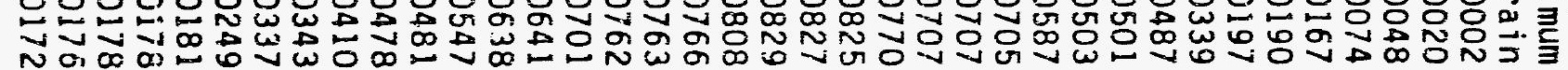

00000000000000000000000000000000000000

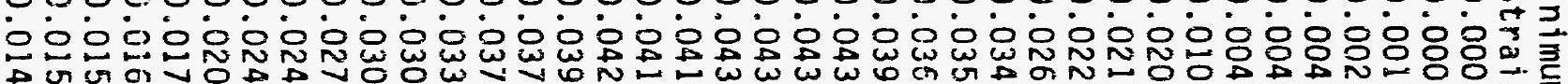

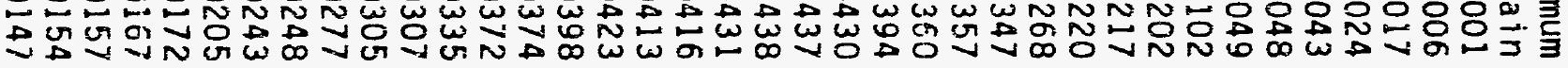

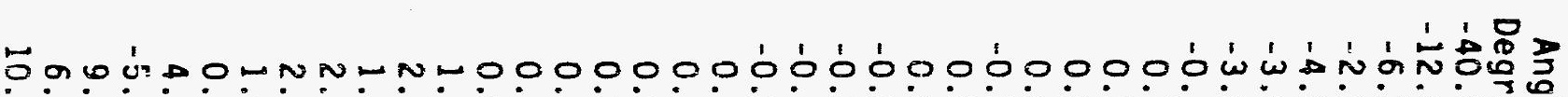

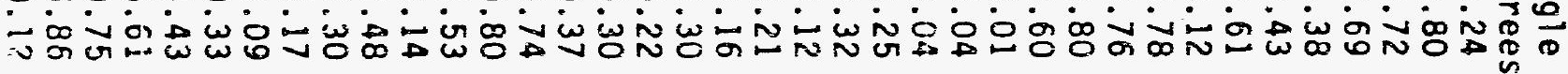

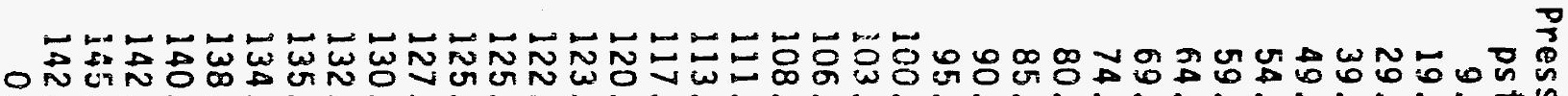
N

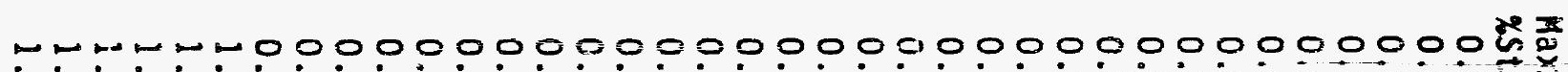

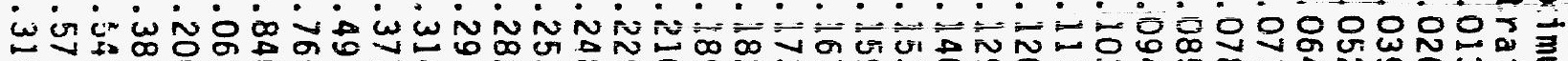
尚: 0000000000000000000000000000000000000苗

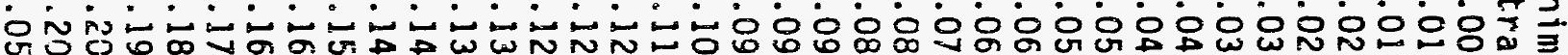

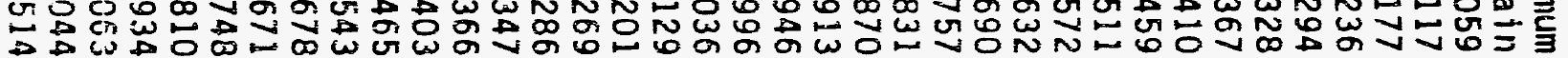

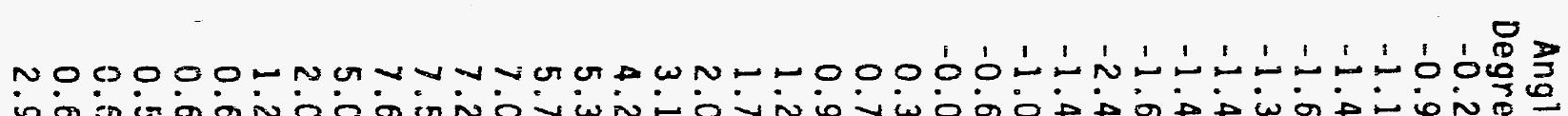
윗 


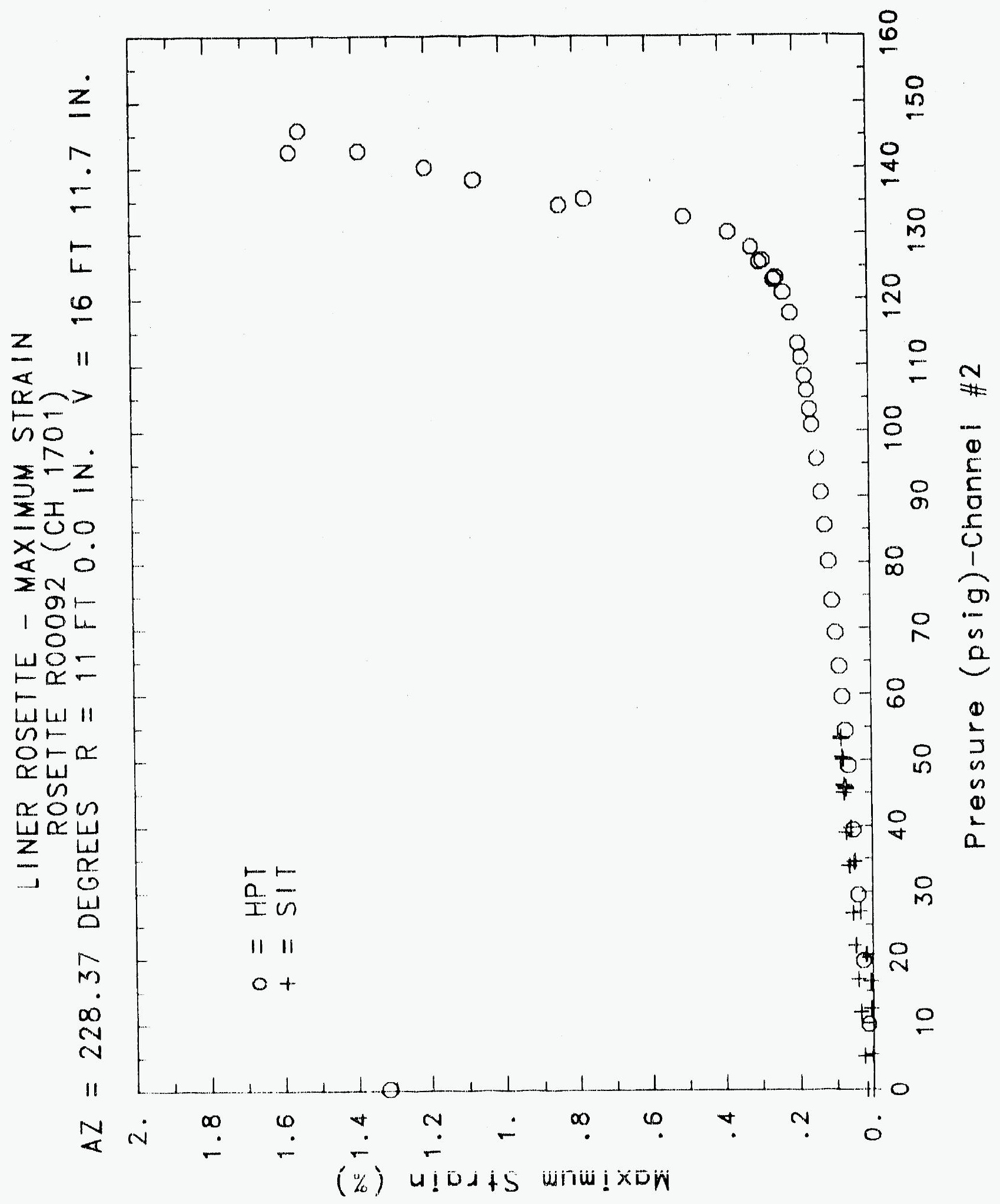




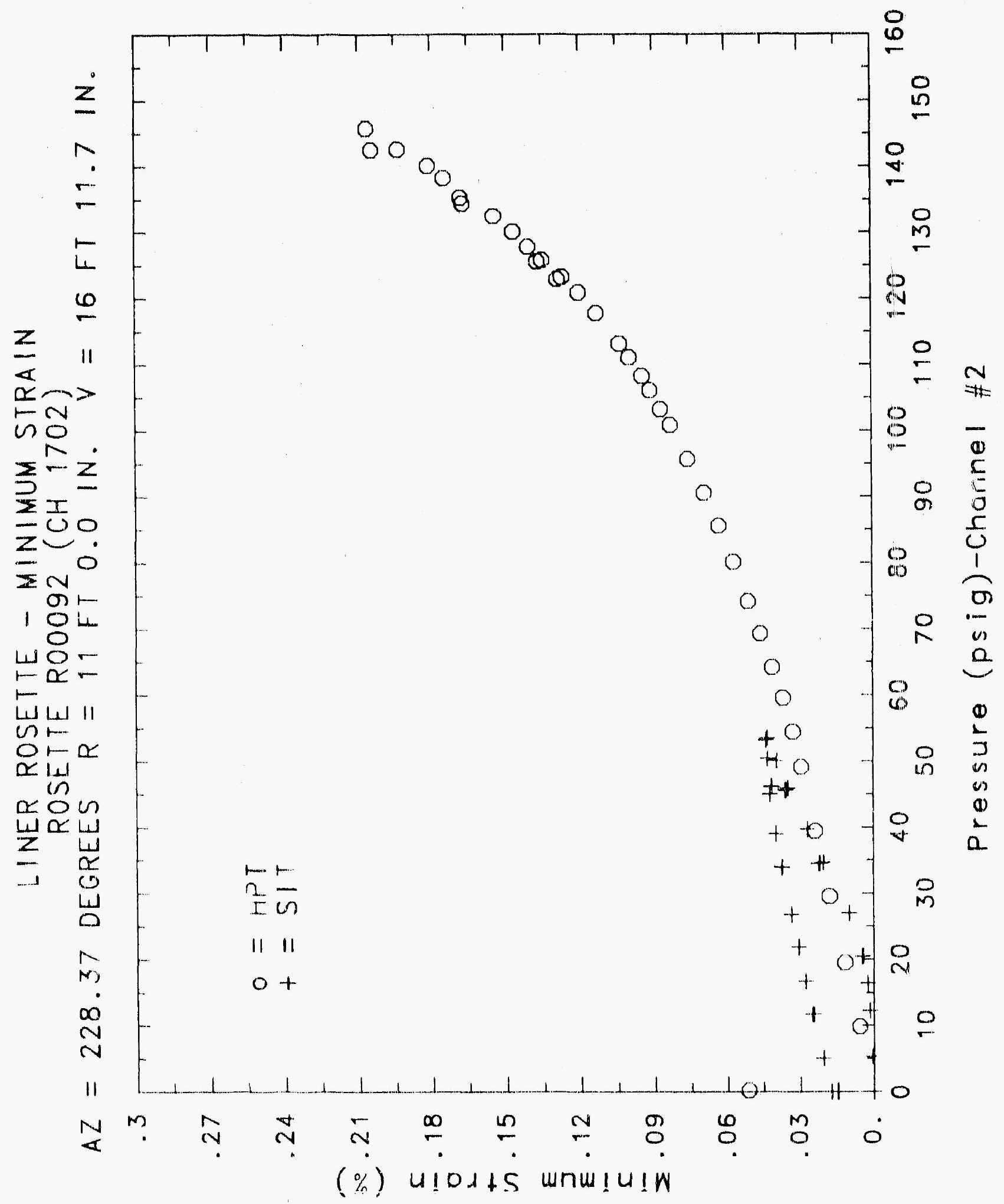




\title{
Reinforced Concrete Test Data
}

\author{
Rosette 93 Channels $1704 \quad 1705 \quad 1706$
}

StRUCTURAL INTEgRITY TEST

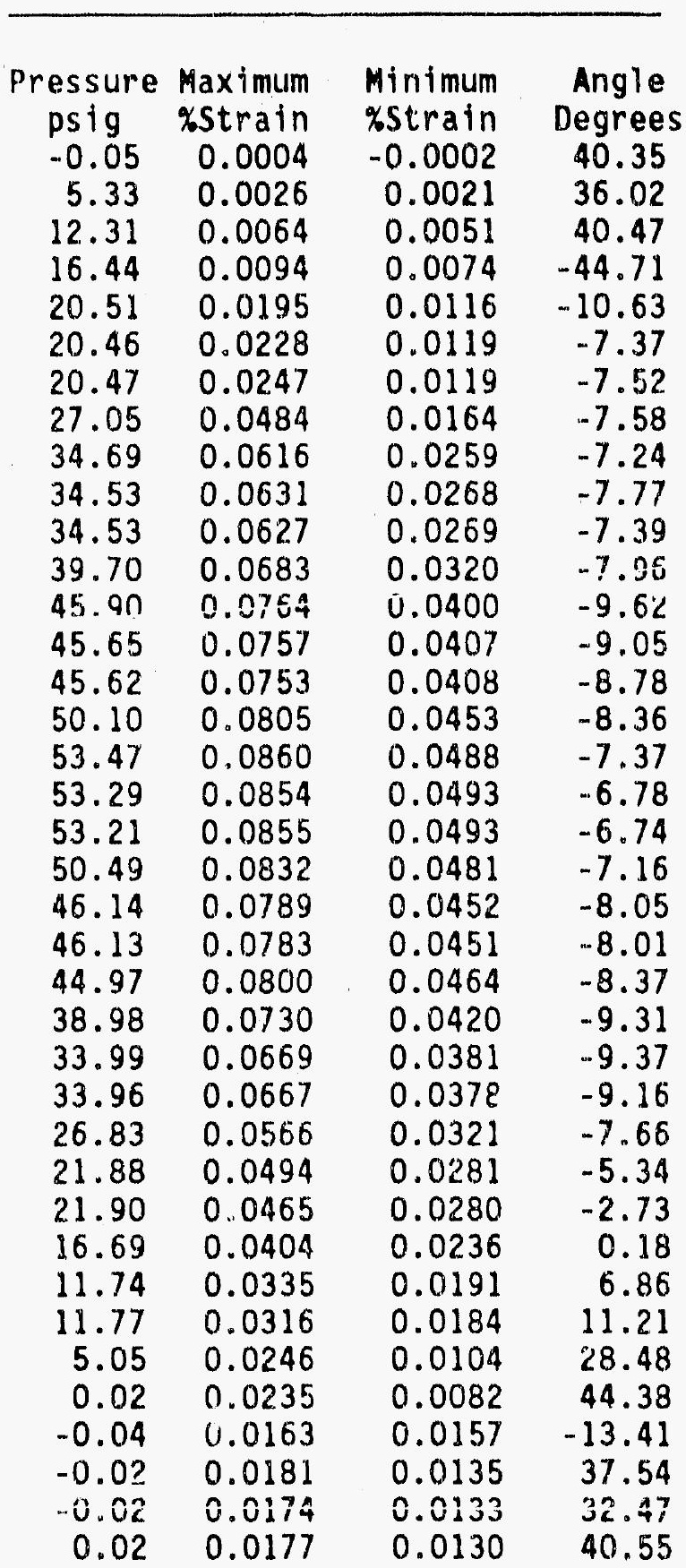

\section{High Pressure Test}

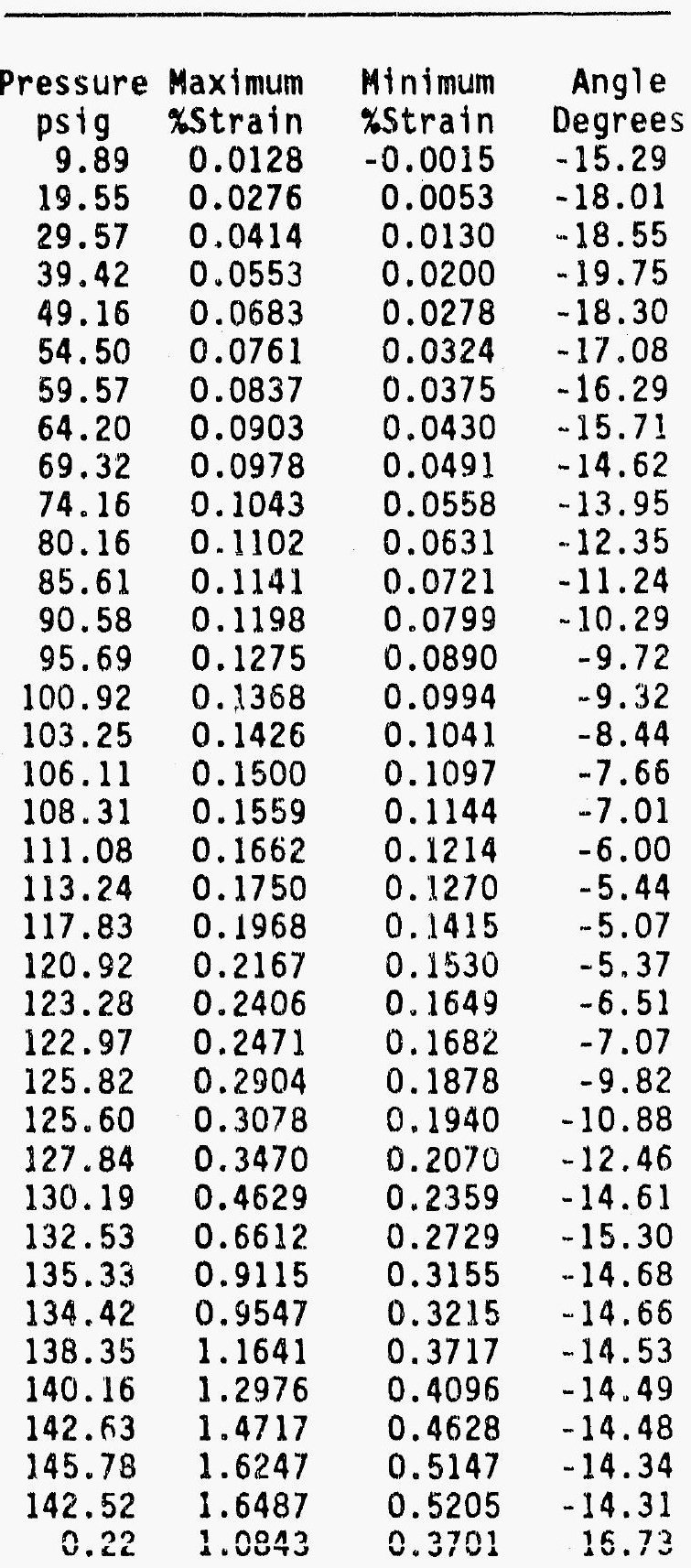




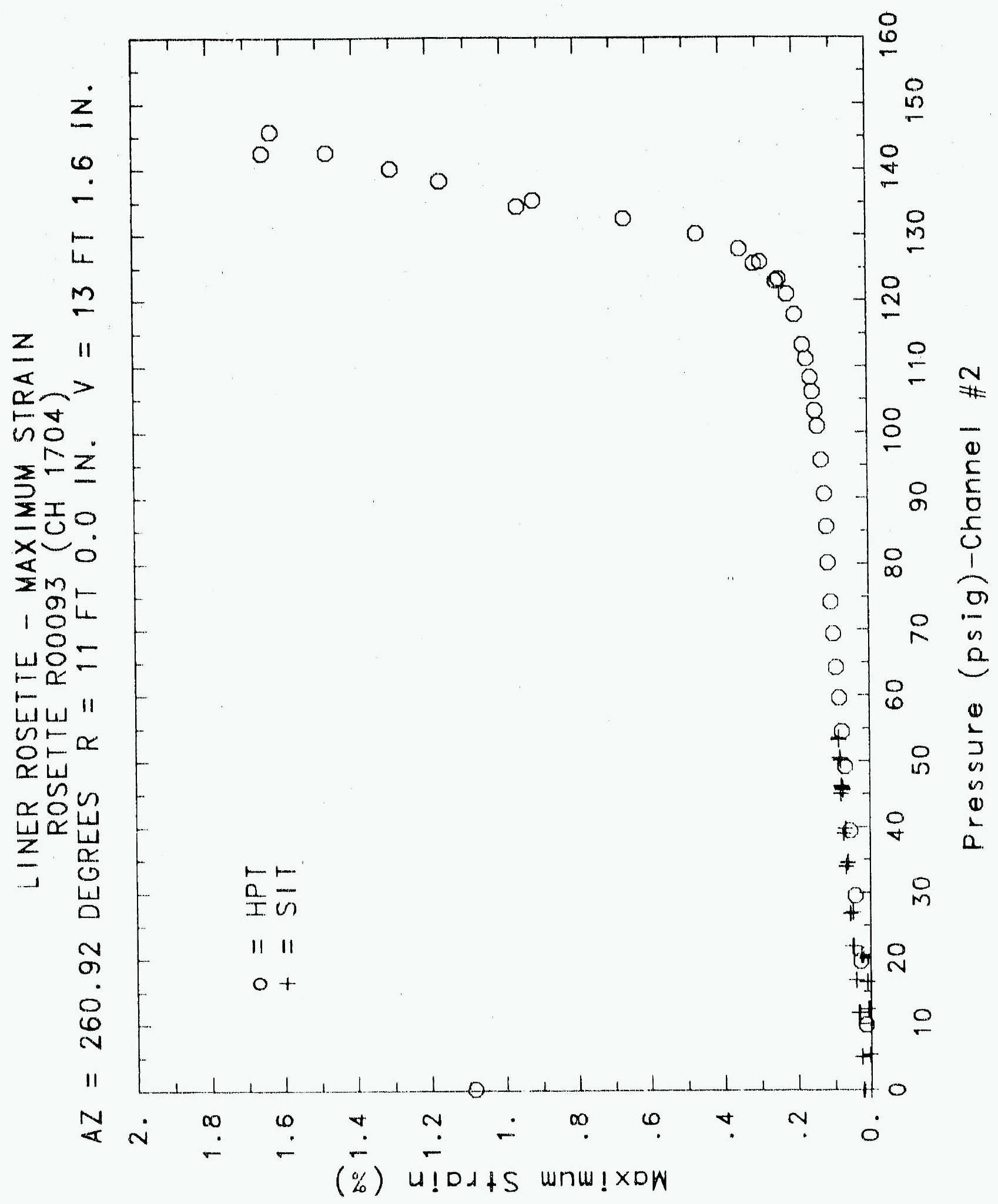




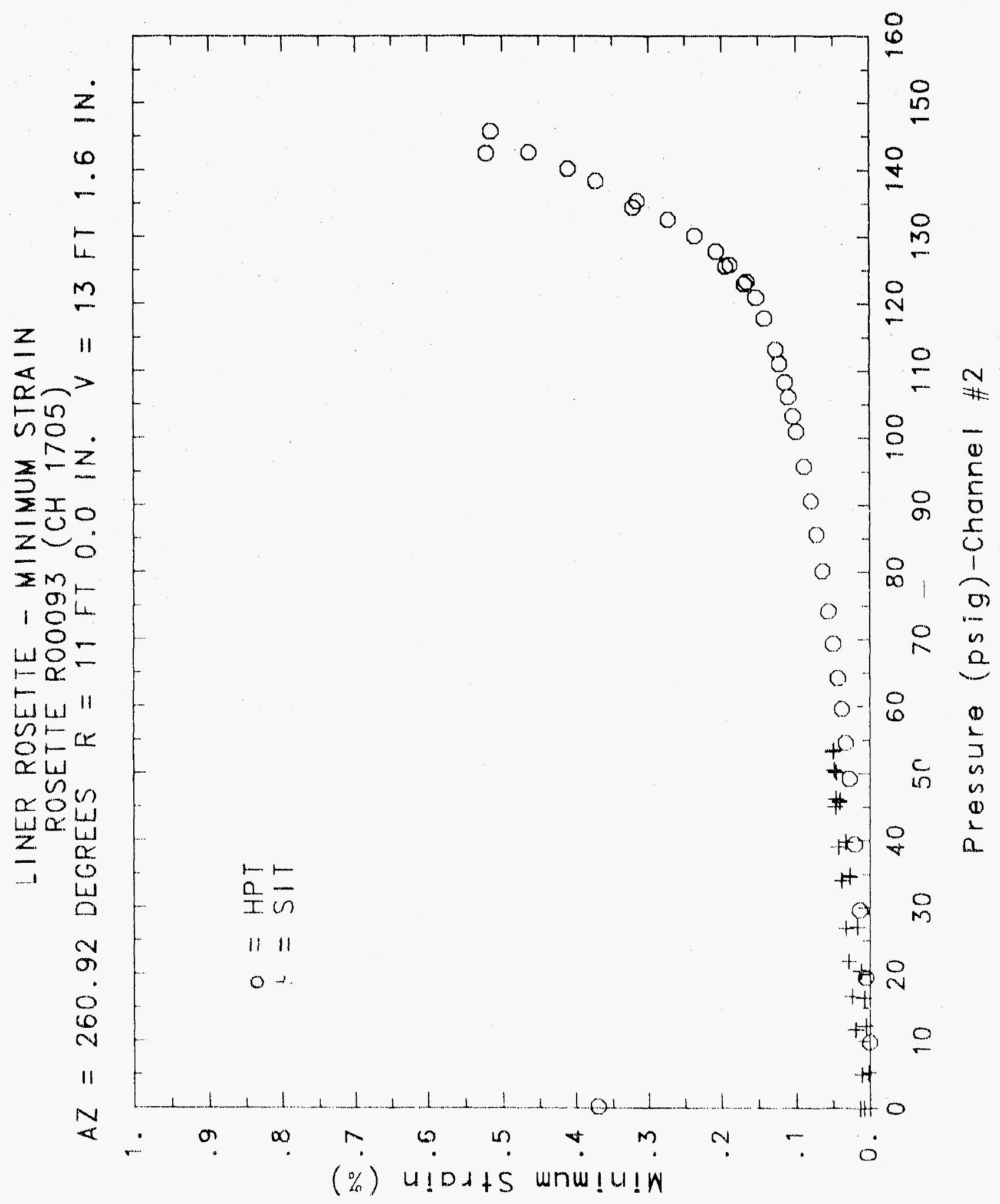




\title{
Reinforced Concrete Test Data
}

\author{
Rosette 94 Channels $1707 \quad 1708 \quad 1709$
}

StRUCTURAL InTEgRITY TEST

\begin{tabular}{|c|c|c|c|c|c|c|c|}
\hline Pressure & Maximum & Minimum & Angle & Pressure & Maximum & Minimum & Angle \\
\hline psig & \%strain & $\%$ strain & Degrees & psig & \%strain & \%strain & Degrees \\
\hline-0.05 & 0.0005 & 0.0000 & -0.02 & 9.89 & 0.0166 & 0.0082 & -14.48 \\
\hline 5.33 & 0.0058 & 0.0023 & -34.03 & 19.55 & 0.0330 & 0.0160 & -16.12 \\
\hline 12.31 & 0.0134 & 0.0055 & -36.64 & 29.57 & 0.0493 & 0.0244 & -17.21 \\
\hline 16.44 & 0.0175 & 0.0084 & -37.68 & 39.42 & 0.0651 & 0.0325 & -17.89 \\
\hline 20.51 & 0.0221 & 0.0146 & -34.26 & 49.16 & 0.0817 & 0.0401 & -18.40 \\
\hline 20.46 & 0.0227 & 0.0161 & -32.31 & 54.50 & 0.1006 & 0.0438 & -17.33 \\
\hline 20.47 & 0.0233 & 0.0165 & .30 .67 & 59. & 0.1738 & 0.0455 & -11.67 \\
\hline 27.05 & 0.0329 & 0.0261 & -14.10 & & 0.2742 & 0.0451 & -3.43 \\
\hline 34.69 & 0.0608 & 0.0341 & -17.14 & 69.32 & 0.3660 & 0.0474 & -8.56 \\
\hline 34.53 & 0.0660 & 0.0343 & -16.71 & 74. & 0.4715 & 0.0525 & -6.93 \\
\hline 34.53 & 0.0674 & 0.0343 & -16 & 80.16 & 0.5969 & 0.0600 & -4.95 \\
\hline 39.70 & 0.1042 & 0.0360 & -16.82 & 85.61 & 0.7220 & 0.0689 & -3.23 \\
\hline 45.90 & 0.1968 & 0.0346 & -14.46 & & 0.8190 & 0.11749 & -2.46 \\
\hline 45.65 & 0.2112 & 0.0332 & -14 & 95.69 & 0.9211 & 0.0771 & -2.21 \\
\hline 45.62 & 0.2143 & 0.0327 & -14. & 100.92 & 1.0514 & 0.06 & -2.50 \\
\hline 50.10 & 0.2691 & 0.0318 & -13.82 & 103. & 1.09 & 0.06 & -2.76 \\
\hline 53.47 & 0.3366 & 0.0290 & -13.18 & 106.11 & 1.1595 & 0.0641 & -3.04 \\
\hline 53.29 & 0.3504 & 0.0278 & -13.10 & 108.31 & 1.2129 & 0.0625 & -3.16 \\
\hline 53.21 & 0.3550 & 0.0272 & -13.08 & 111.08 & 1.2866 & 0.0605 & -3.15 \\
\hline 50.49 & 0.3563 & 0.0254 & -13.02 & 113.24 & 1.34 & 0.0 & -3.14 \\
\hline 46.14 & 0.3519 & 0.0223 & -12.96 & 117.83 & 1.4920 & 0.0604 & -2.75 \\
\hline 46.13 & 0.3518 & 0.0220 & -12.95 & 120.92 & 1.6017 & 0.0631 & $-2.6 ?$ \\
\hline 44.97 & 0.3507 & 0.0235 & -13.00 & 123.28 & 1.6980 & 0.0658 & -2.67 \\
\hline 38.98 & 0.3437 & 0.0188 & -12 & 122.97 & 1.7178 & 0.0666 & -2.67 \\
\hline 33.99 & 0.3370 & 0.0144 & -12.72 & 125.82 & 1.8508 & 0.07 & -2.44 \\
\hline 33.96 & 0.3369 & 0.0144 & -12.77 & 125.60 & 1.8766 & 0.07 & -2.46 \\
\hline 26.83 & 0.3263 & 0.0079 & -12.60 & 127.84 & 1.9677 & 0.0723 & -2.54 \\
\hline 21.88 & 0.3186 & 0.0033 & -12.50 & 130.19 & 2.1644 & 0.0761 & -2.25 \\
\hline 21.90 & 0.3180 & 0.0029 & -12.49 & 132.53 & 2.4301 & 0.0792 & -2.12 \\
\hline 16.69 & 0.3101 & -0.0019 & -12.40 & 135.33 & 2.8105 & 0.0766 & -2.35 \\
\hline 11.74 & 0.3018 & -0.0068 & -12.29 & 134.42 & 2.8807 & 0.0752 & -2.43 \\
\hline 11.77 & 0.3017 & -0.0075 & -12.32 & 138.35 & 3.3087 & 0.0711 & -2.58 \\
\hline 5.05 & 0.2901 & -0.0136 & -12.30 & 140.16 & 3.6509 & 0.0674 & -2.66 \\
\hline 0.02 & 0.2808 & -0.0181 & -12.30 & 142.63 & 4.1138 & 0.0624 & -2.54 \\
\hline-0.04 & 0.2797 & -0.0184 & .12 .26 & 145.78 & 4.6001 & 0.0581 & -2.32 \\
\hline-0.02 & 0.2788 & -0.0177 & -12.26 & 142.52 & 4.7251 & 0.0528 & -2.28 \\
\hline-0.02 & 0.2783 & -0.0183 & -12.18 & 0.22 & 4.2786 & -0.0274 & -2.13 \\
\hline 0.02 & $0.277 n$ & -0.0188 & -12.23 & & & & \\
\hline
\end{tabular}

\section{HIGH P .SSUKE TEST}




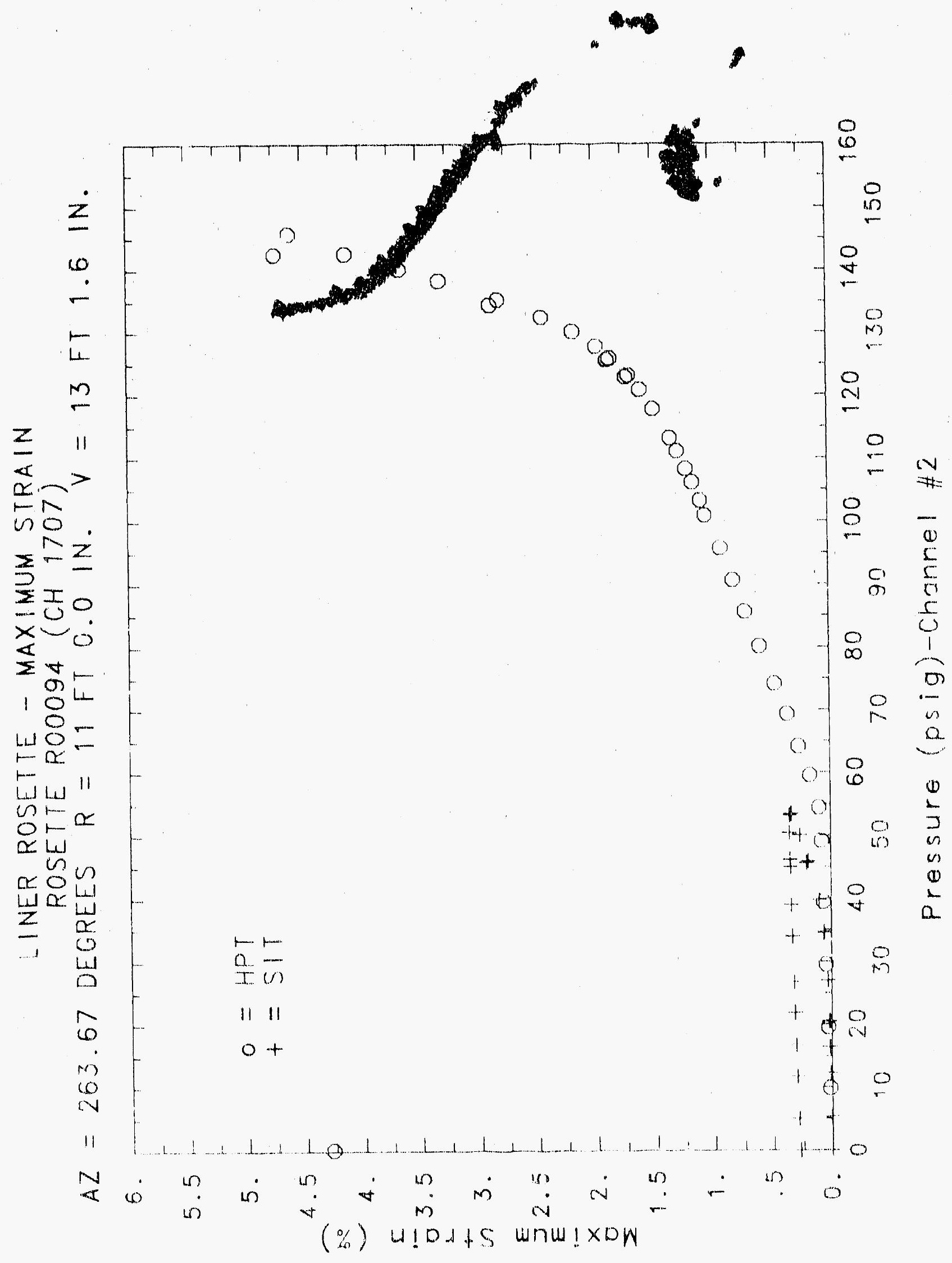




\section{SECURITY CLASSIFICATION}

\section{UNCLASSIFIED}

\section{DATE OF MICROFILMING $1-6-92$}

\section{MICROFILMED BY ERRY hARD}

SANDIA NATIONAL LABS 


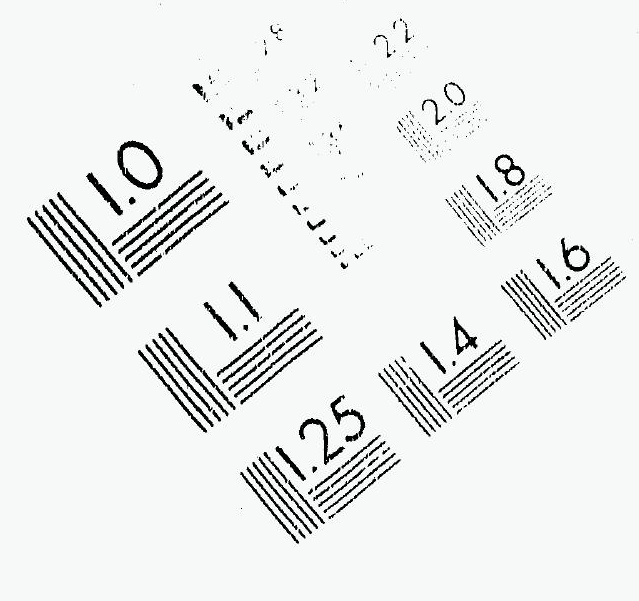

\section{IMAGE EVALUATION TEST TARGET (MT-3)}
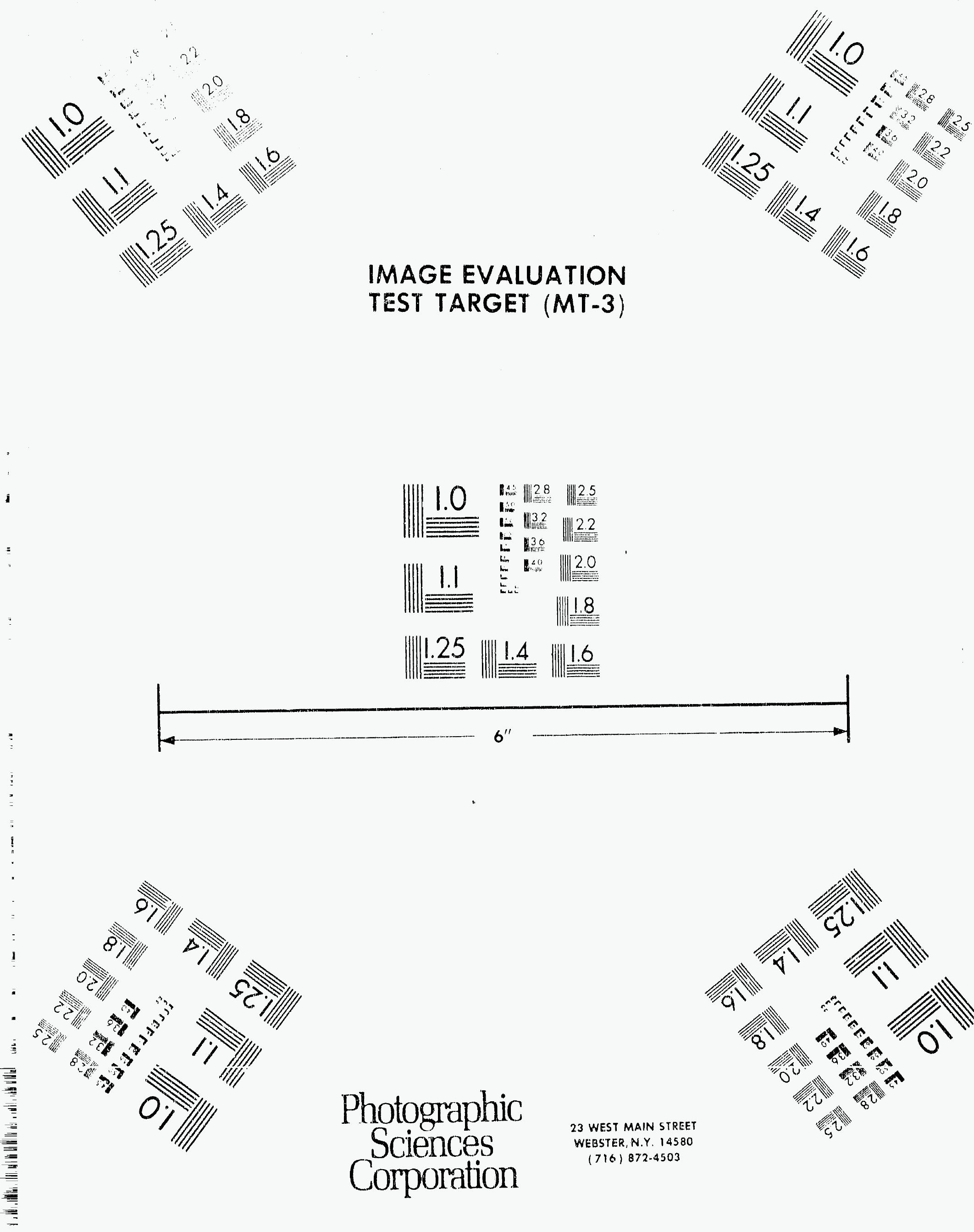

Photographic Sciences Corporation

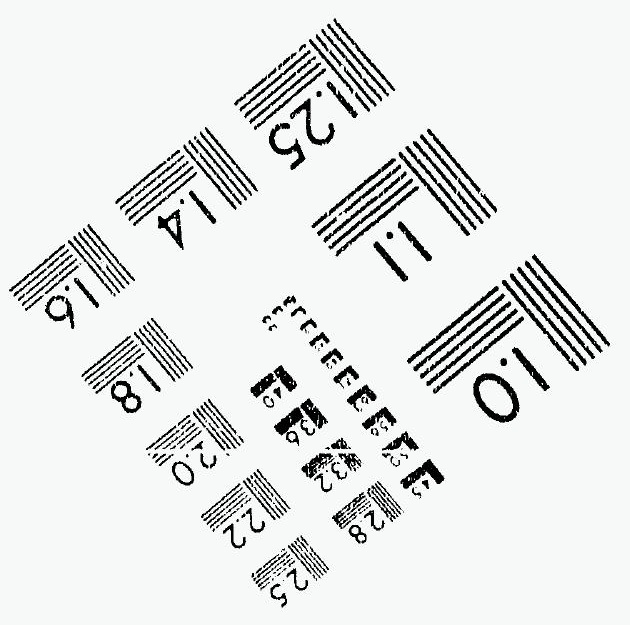




\section{THIS DOCUMENT FILMED}

AT

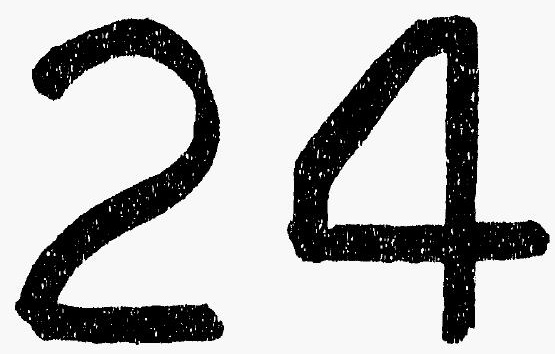

REDUCTION 


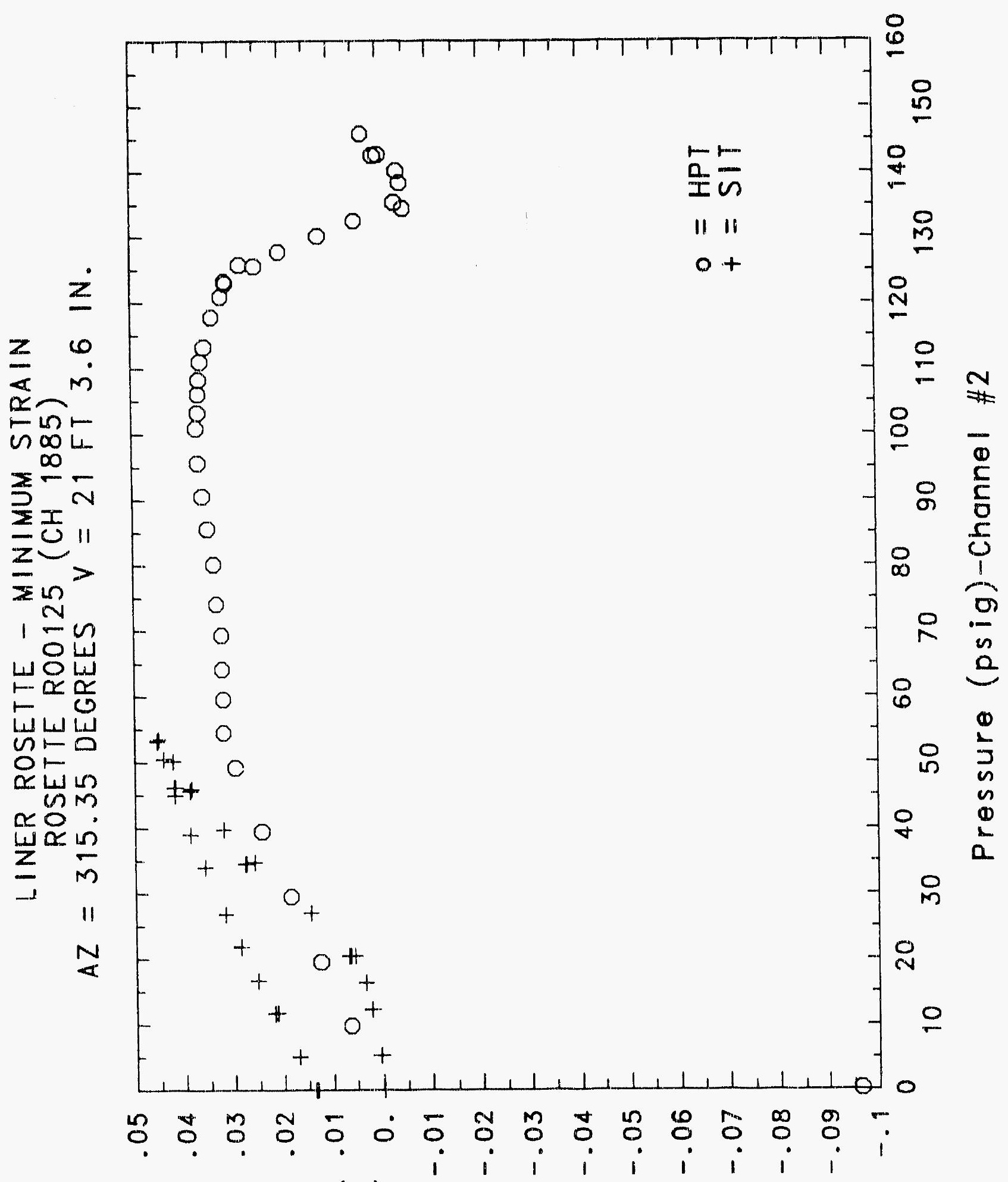

(\%) u!bdts wnw!u!w 


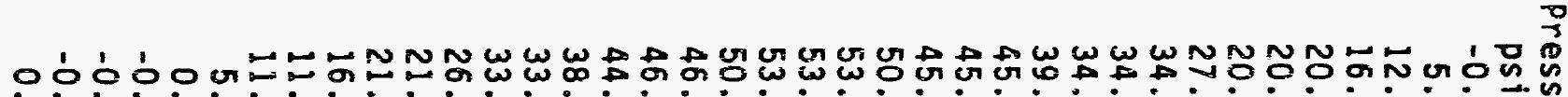

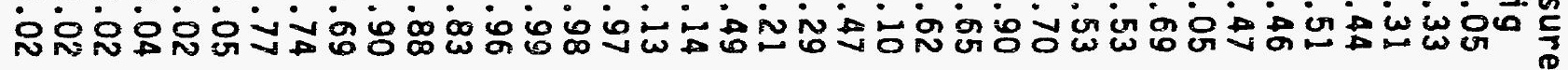

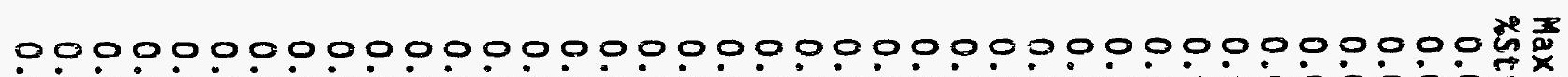

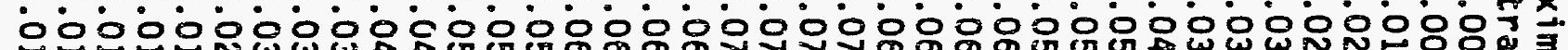

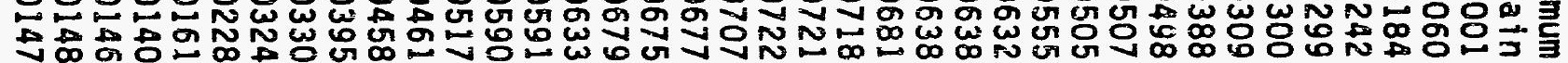

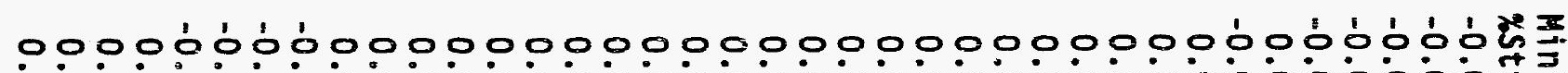

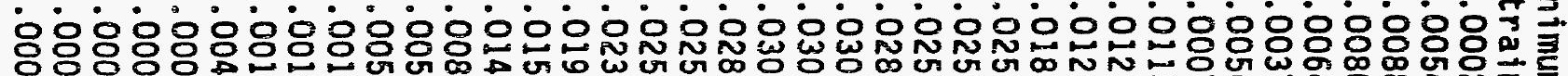

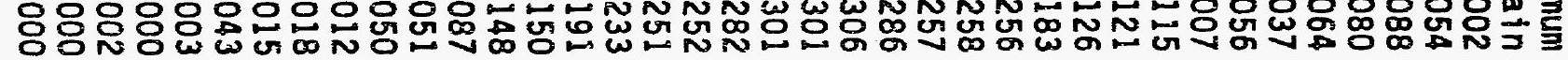

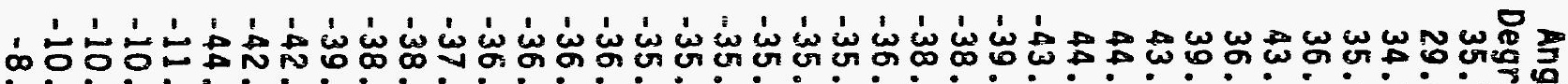
出

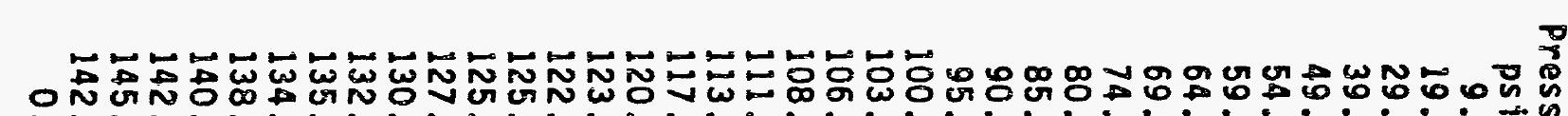
N - - - :- :000000000000000000000000000000\%

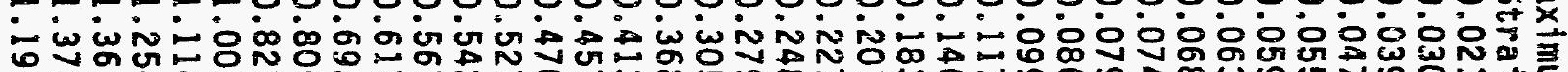

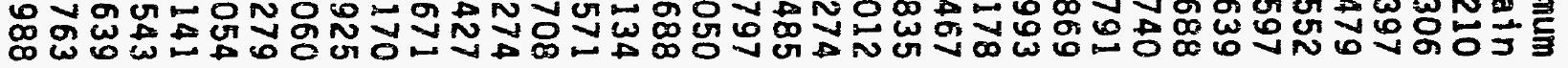
000000000000000000000000000000000000013

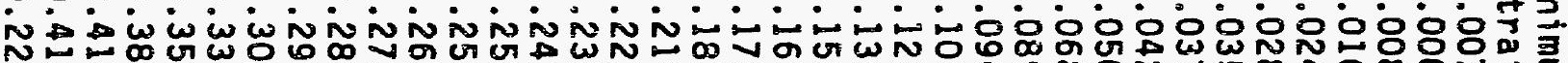

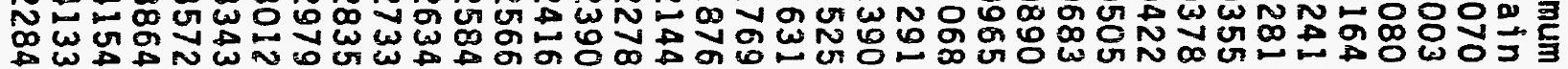

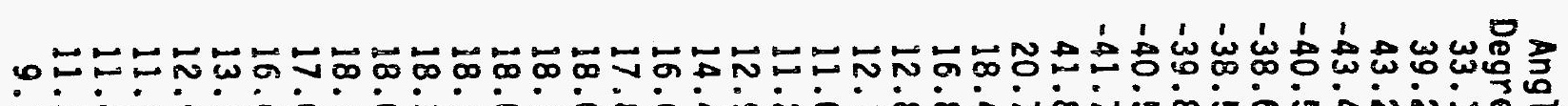

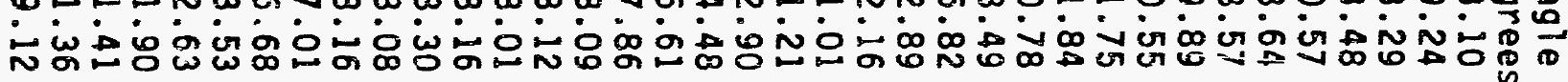




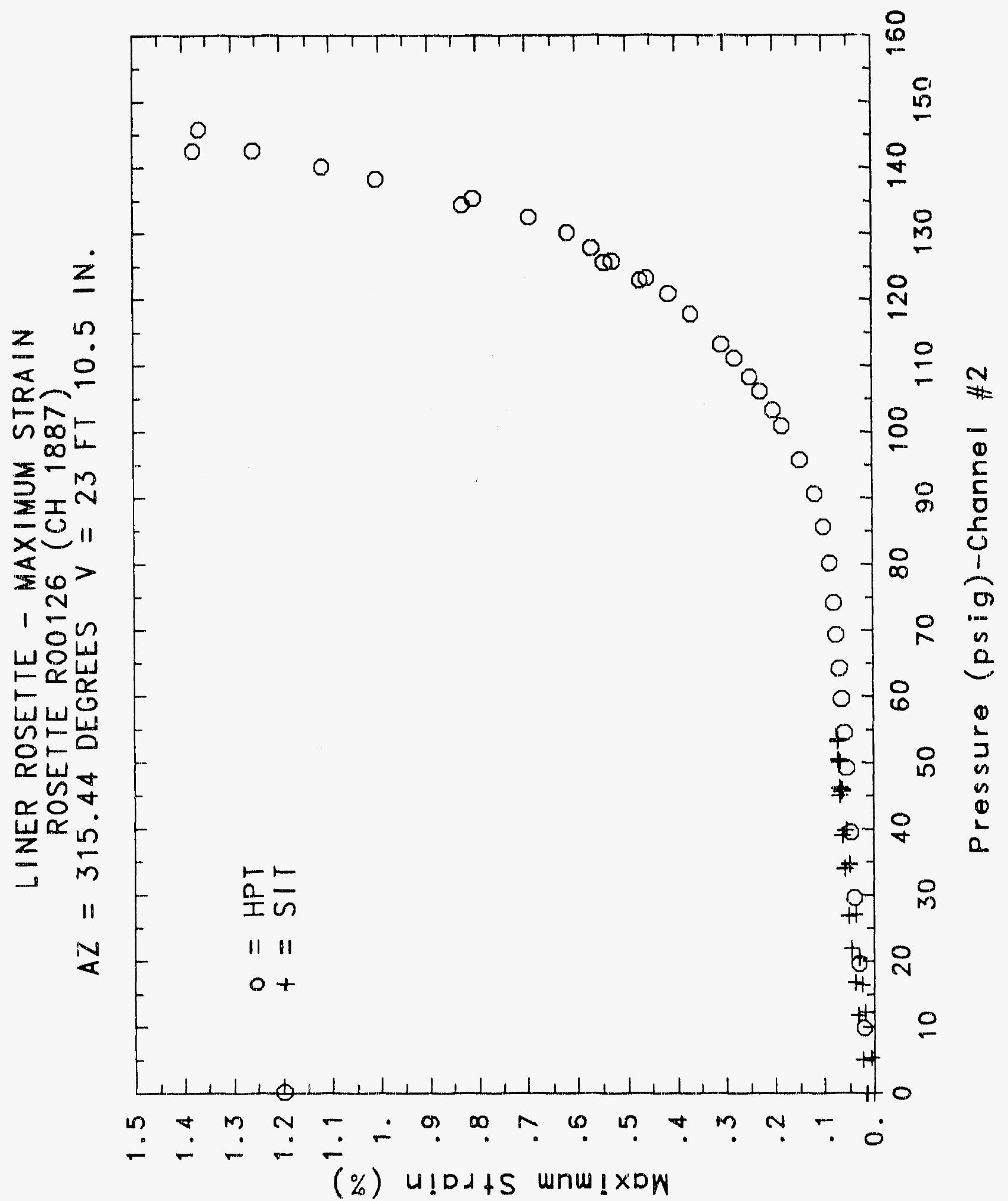




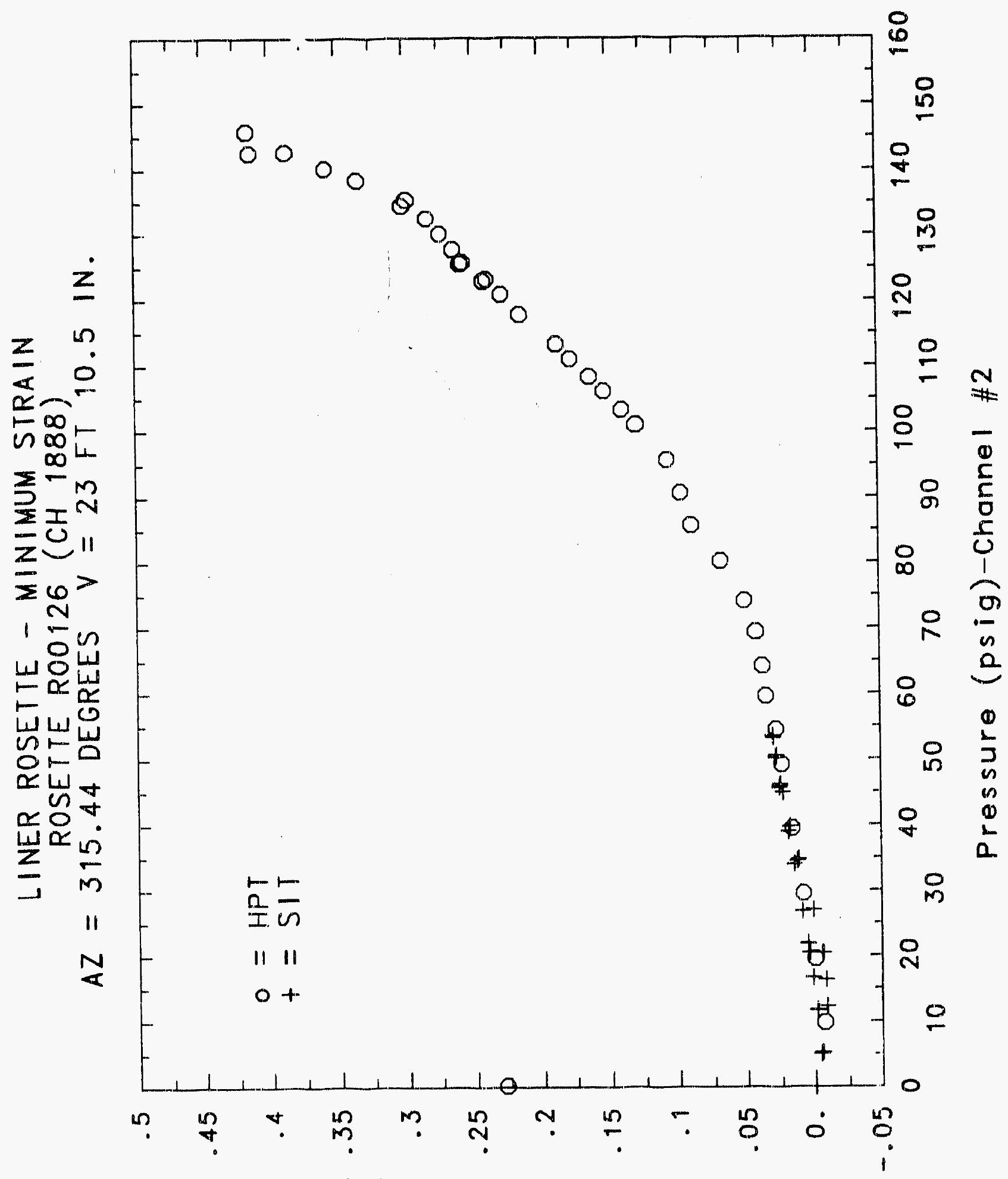

(\%) u!DdtS wnw!u!W 


\section{Reinforced Concrete Test Data}

Rosette 127 Channels 202020212022

STRUCTURAL INTEGRITY TEST

\begin{tabular}{|c|c|c|c|c|c|c|c|}
\hline $\begin{array}{c}\text { Pressure } \\
\text { psig } \\
-0.05 \\
5.33 \\
12.31 \\
16.44 \\
20.51 \\
20.46 \\
20.47 \\
27.05 \\
34.69 \\
34.53 \\
34.53 \\
39.70 \\
45.90 \\
45.65 \\
45.62 \\
50.10 \\
53.47 \\
53.29 \\
53.21 \\
50.49 \\
46.14 \\
46.13 \\
44.97 \\
38.98 \\
33.99 \\
33.96 \\
26.83 \\
21.88 \\
21.90 \\
16.69 \\
11.74 \\
11.77 \\
5.05 \\
0.02 \\
-0.04 \\
-0.02 \\
-0.02 \\
0.02\end{array}$ & $\begin{array}{c}\text { Maximum } \\
\% \text { Strain } \\
-0.0002 \\
0.0012 \\
0.0034 \\
0.0049 \\
0.0077 \\
0.0093 \\
0.0085 \\
0.0156 \\
0.0264 \\
0.0280 \\
0.0283 \\
0.0329 \\
0.0399 \\
0.0404 \\
0.0404 \\
0.0444 \\
0.0480 \\
0.0485 \\
0.0485 \\
0.0478 \\
0.0456 \\
0.0454 \\
0.0457 \\
0.0424 \\
0.0394 \\
0.0391 \\
0.0346 \\
0.0312 \\
0.0307 \\
0.0274 \\
0.0239 \\
0.0233 \\
0.0188 \\
0.0150 \\
0.0147 \\
0.0147 \\
0.0148 \\
0.0144\end{array}$ & $\begin{array}{l}\text { Minimum } \\
\text { \%Strain } \\
-0.0007 \\
0.0008 \\
0.0032 \\
0.0048 \\
0.0074 \\
0.0074 \\
0.0083 \\
0.0147 \\
0.0241 \\
0.0253 \\
0.0259 \\
0.0301 \\
0.0363 \\
0.0364 \\
0.0363 \\
0.0399 \\
0.0431 \\
0.0433 \\
0.0434 \\
0.0426 \\
0.0407 \\
0.0406 \\
0.0411 \\
0.0380 \\
0.0352 \\
0.0349 \\
0.0305 \\
0.0274 \\
0.0272 \\
0.0238 \\
0.0202 \\
0.0198 \\
0.0151 \\
0.0116 \\
0.0113 \\
0.0115 \\
0.0114 \\
0.0111\end{array}$ & $\begin{array}{c}\text { Angle } \\
\text { Degrees } \\
9.59 \\
-23.11 \\
41.25 \\
-0.52 \\
-44.27 \\
-14.08 \\
38.84 \\
5.17 \\
-2.94 \\
-2.54 \\
-3.51 \\
-4.91 \\
-2.17 \\
-2.29 \\
-4.00 \\
1.50 \\
-1.80 \\
-0.77 \\
-1.74 \\
0.18 \\
0.32 \\
-0.94 \\
3.39 \\
-0.96 \\
1.20 \\
-4.85 \\
-5.10 \\
-4.18 \\
-6.90 \\
-5.83 \\
-10.26 \\
-9.38 \\
-12.58 \\
-10.55 \\
-4.46 \\
-4.73 \\
-2.40 \\
-2.07\end{array}$ & $\begin{array}{c}\text { Pressure } \\
\text { psig } \\
9.89 \\
19.55 \\
29.57 \\
39.42 \\
49.16 \\
54.50 \\
59.57 \\
64.20 \\
69.32 \\
74.16 \\
80.16 \\
85.61 \\
90.58 \\
95.69 \\
100.92 \\
103.25 \\
106.11 \\
108.31 \\
111.08 \\
113.24 \\
117.83 \\
120.92 \\
123.28 \\
122.97 \\
125.82 \\
125.60 \\
127.84 \\
130.19 \\
132.53 \\
135.33 \\
134.42 \\
138.35 \\
140.16 \\
142.63 \\
145.78 \\
142.52 \\
0.22\end{array}$ & $\begin{array}{c}\text { Maximum } \\
\text { xStrain } \\
0.0068 \\
0.0137 \\
0.0204 \\
0.0272 \\
0.0338 \\
0.0376 \\
0.0419 \\
0.0461 \\
0.0515 \\
0.0564 \\
0.0618 \\
0.0674 \\
0.0722 \\
0.0775 \\
0.0824 \\
0.0843 \\
0.0869 \\
0.0881 \\
0.0890 \\
0.0895 \\
0.0891 \\
0.0898 \\
0.0905 \\
0.0900 \\
0.0917 \\
0.0911 \\
0.0932 \\
0.0960 \\
0.0991 \\
0.1028 \\
0.1028 \\
0.1069 \\
0.1099 \\
0.1135 \\
0.1181 \\
0.1134 \\
0.0032\end{array}$ & $\begin{array}{c}\text { Minimum } \\
\text { qStrain } \\
0.0055 \\
0.0122 \\
0.0191 \\
0.0252 \\
0.0313 \\
0.0352 \\
0.0388 \\
0.0426 \\
0.0461 \\
0.0498 \\
0.0533 \\
0.0564 \\
0.0598 \\
0.0629 \\
0.0668 \\
0.0695 \\
0.0710 \\
0.0728 \\
0.0741 \\
0.0756 \\
0.0787 \\
0.0830 \\
0.0865 \\
0.0871 \\
0.0899 \\
0.0906 \\
0.0921 \\
0.0932 \\
0.0937 \\
0.0940 \\
0.0932 \\
0.0940 \\
0.0938 \\
0.0934 \\
0.0940 \\
0.0875 \\
-0.0322\end{array}$ & $\begin{array}{r}\text { Angle } \\
\text { Degrees } \\
-29.57 \\
-20.71 \\
-11.11 \\
-7.93 \\
2.76 \\
3.46 \\
5.30 \\
1.21 \\
3.16 \\
-0.83 \\
0.32 \\
-1.38 \\
-1.92 \\
-1.47 \\
1.54 \\
2.19 \\
3.52 \\
4.38 \\
7.51 \\
12.78 \\
6.84 \\
11.15 \\
7.70 \\
2.57 \\
16.51 \\
21.05 \\
-20.37 \\
-14.10 \\
-10.12 \\
-5.95 \\
-6.44 \\
-4.39 \\
-4.30 \\
-3.09 \\
-4.05 \\
-2.32 \\
5.69\end{array}$ \\
\hline
\end{tabular}

high Pressure test 


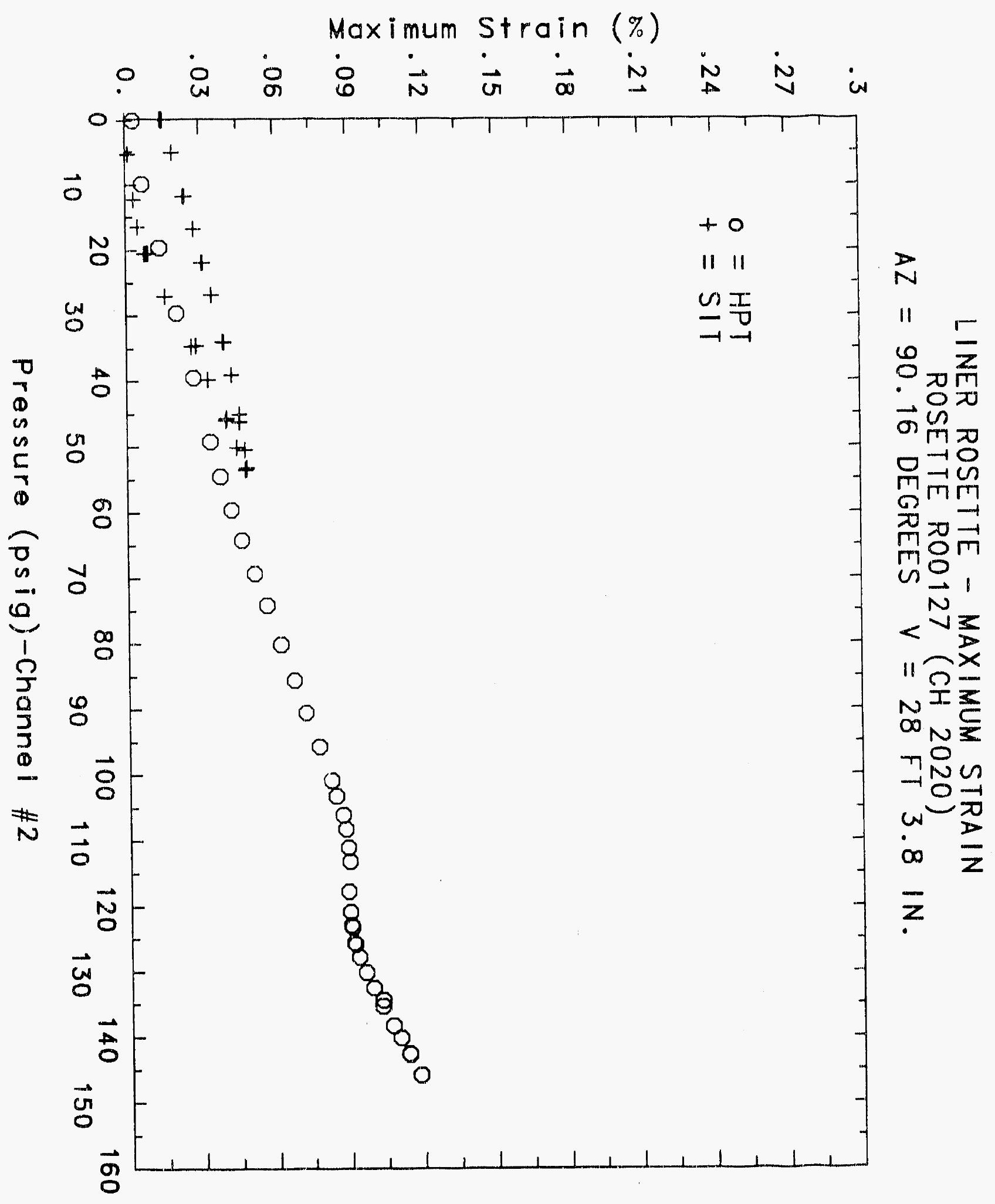


$00 L-J$

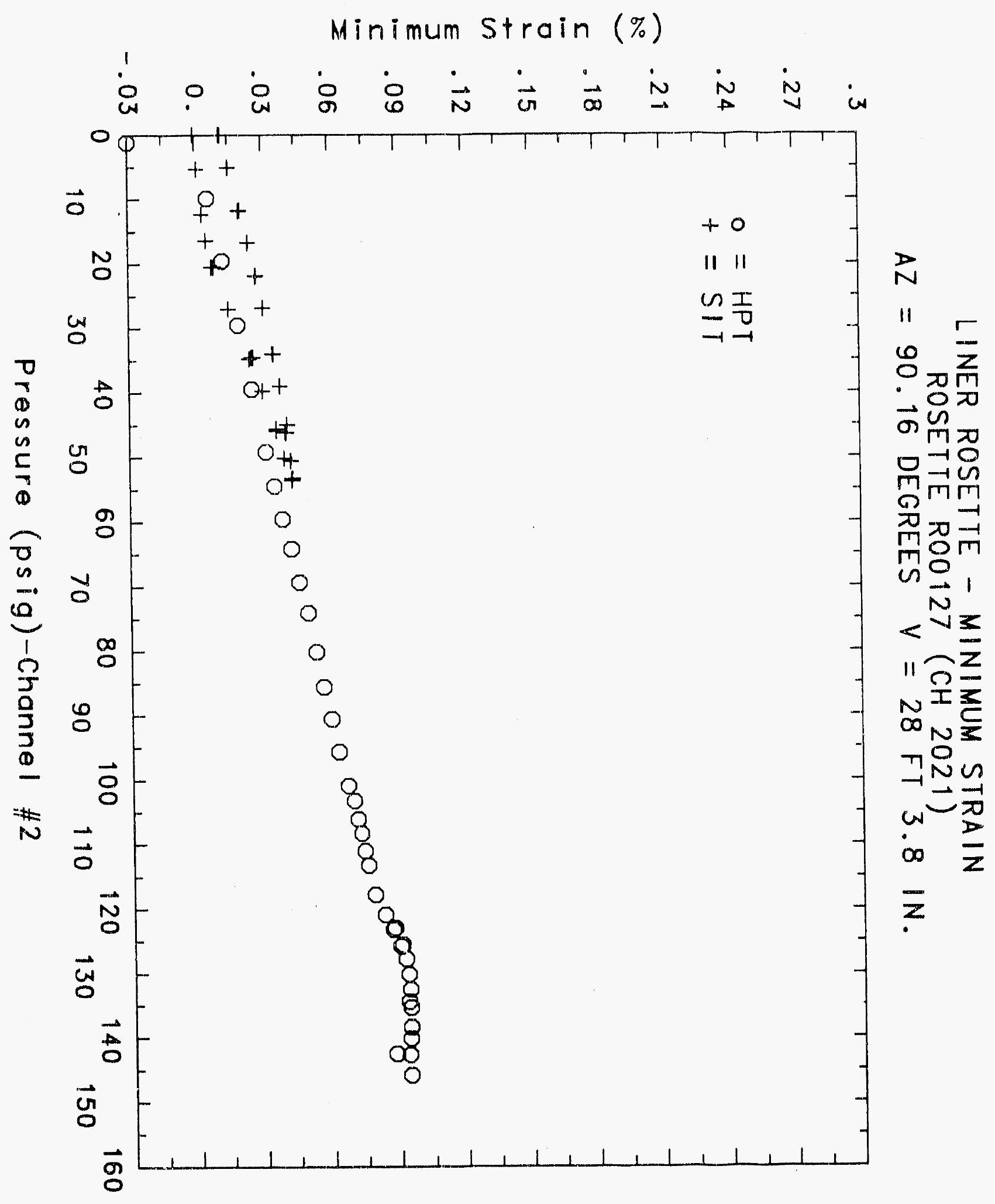




\section{Reinforced Concrete Test Data}

Rosette 128 Channels $2023 \quad 20242025$

\section{Structural Integrity Test}

\begin{tabular}{|c|c|c|c|c|c|c|c|}
\hline $\begin{array}{c}\text { Pressure } \\
\text { psig } \\
-0.05 \\
5.33 \\
12.31 \\
16.44 \\
20.51 \\
20.46 \\
20.47 \\
27.05 \\
34.69 \\
34.53 \\
34.53 \\
39.70 \\
45.90 \\
45.65 \\
45.62 \\
50.10 \\
53.47 \\
53.29 \\
53.21 \\
50.49 \\
46.14 \\
46.13 \\
44.97 \\
38.98 \\
33.99 \\
33.96 \\
26.83 \\
21.88 \\
21.90 \\
16.69 \\
11.74 \\
11.77 \\
5.05 \\
0.02 \\
-0.04 \\
-0.02 \\
-0.02 \\
0.02\end{array}$ & $\begin{array}{c}\text { Maximum } \\
\text { \%Strain } \\
0.0003 \\
0.0021 \\
0.0050 \\
0.0072 \\
0.0100 \\
0.0112 \\
0.0109 \\
0.0176 \\
0.0279 \\
0.0290 \\
0.0292 \\
0.0339 \\
0.0342 \\
0.0342 \\
0.0343 \\
0.0358 \\
0.0396 \\
0.0408 \\
0.0409 \\
0.0400 \\
0.0379 \\
0.0377 \\
0.0380 \\
0.0360 \\
0.0344 \\
0.0343 \\
0.0316 \\
0.0294 \\
0.0293 \\
0.0272 \\
0.0252 \\
0.0250 \\
0.0227 \\
0.0209 \\
0.0204 \\
0.0204 \\
0.0204 \\
0.0204\end{array}$ & $\begin{array}{c}\text { Minimum } \\
\text { \%Strain } \\
-0.0002 \\
0.0014 \\
0.0037 \\
0.0053 \\
0.0076 \\
0.0086 \\
0.0082 \\
0.0152 \\
0.0233 \\
0.0238 \\
0.0244 \\
0.0287 \\
0.0244 \\
0.0250 \\
0.0251 \\
0.0284 \\
0.0328 \\
0.0376 \\
0.0378 \\
0.0376 \\
0.0364 \\
0.0365 \\
0.0373 \\
0.0348 \\
0.0319 \\
0.0315 \\
0.0275 \\
0.0241 \\
0.0241 \\
0.0207 \\
0.0174 \\
0.0169 \\
0.0125 \\
0.0091 \\
0.0086 \\
0.0088 \\
0.0088 \\
0.0087\end{array}$ & $\begin{array}{c}\text { Angle } \\
\text { Degrees } \\
30.56 \\
-6.41 \\
10.10 \\
8.05 \\
16.89 \\
20.57 \\
22.60 \\
-35.82 \\
-13.20 \\
-16.92 \\
-12.01 \\
-15.46 \\
3.21 \\
2.99 \\
2.87 \\
6.53 \\
22.09 \\
10.28 \\
11.04 \\
12.79 \\
25.07 \\
19.89 \\
28.59 \\
-19.66 \\
-8.71 \\
-18.13 \\
-2.87 \\
-5.33 \\
-2.58 \\
-2.51 \\
-2.48 \\
0.80 \\
-0.36 \\
-0.97 \\
0.69 \\
-0.35 \\
-0.97 \\
0.49\end{array}$ & $\begin{array}{c}\text { Pressure } \\
\text { psig } \\
9.89 \\
19.55 \\
29.57 \\
39.42 \\
49.16 \\
54.50 \\
59.57 \\
64.20 \\
69.32 \\
74.16 \\
80.16 \\
85.61 \\
90.58 \\
95.69 \\
100.92 \\
103.25 \\
106.11 \\
168.31 \\
111.08 \\
113.24 \\
117.83 \\
120.92 \\
123.28 \\
122.97 \\
125.82 \\
125.60 \\
127.84 \\
130.19 \\
132.53 \\
135.33 \\
134.42 \\
138.35 \\
140.16 \\
142.63 \\
145.78 \\
142.52 \\
0.22\end{array}$ & $\begin{array}{c}\text { Maximum } \\
\text { xStrain } \\
0.0066 \\
0.0128 \\
0.0195 \\
0.0257 \\
0.0318 \\
0.0357 \\
0.0393 \\
0.0430 \\
0.0468 \\
0.0509 \\
0.0553 \\
0.0598 \\
0.0646 \\
0.0697 \\
0.0751 \\
0.0776 \\
0.0802 \\
0.0823 \\
0.0849 \\
0.0868 \\
0.0912 \\
0.0940 \\
0.0967 \\
0.0972 \\
0.0994 \\
0.0999 \\
0.1017 \\
0.1037 \\
0.1059 \\
0.1088 \\
0.1088 \\
0.1119 \\
0.1135 \\
0.1155 \\
0.1182 \\
0.1139 \\
0.0077\end{array}$ & $\begin{array}{c}\text { Minimum } \\
\text { \%Strain } \\
0.0041 \\
0.0073 \\
0.0121 \\
0.0166 \\
0.0210 \\
0.0237 \\
0.0273 \\
0.0310 \\
0.0352 \\
0.0392 \\
0.0428 \\
0.0466 \\
0.0501 \\
0.0538 \\
0.0574 \\
0.0592 \\
0.0609 \\
0.0624 \\
0.0644 \\
0.0662 \\
0.0691 \\
0.0715 \\
0.0731 \\
0.0731 \\
0.0747 \\
0.0754 \\
0.0763 \\
0.0776 \\
0.0782 \\
0.0794 \\
0.0792 \\
0.0797 \\
0.0799 \\
0.0792 \\
0.0803 \\
0.0751 \\
-0.0178\end{array}$ & $\begin{array}{c}\text { Angle } \\
\text { Degrees } \\
3.12 \\
5.65 \\
4.87 \\
6.06 \\
3.53 \\
5.86 \\
6.54 \\
10.52 \\
14.04 \\
20.42 \\
24.05 \\
29.67 \\
33.23 \\
36.71 \\
38.16 \\
37.82 \\
38.18 \\
37.53 \\
37.83 \\
36.59 \\
34.53 \\
32.88 \\
31.75 \\
31.71 \\
30.06 \\
29.61 \\
28.34 \\
26.88 \\
24.96 \\
22.29 \\
22.45 \\
20.35 \\
19.30 \\
18.26 \\
16.32 \\
16.90 \\
28.96\end{array}$ \\
\hline
\end{tabular}

High Pressure Test 


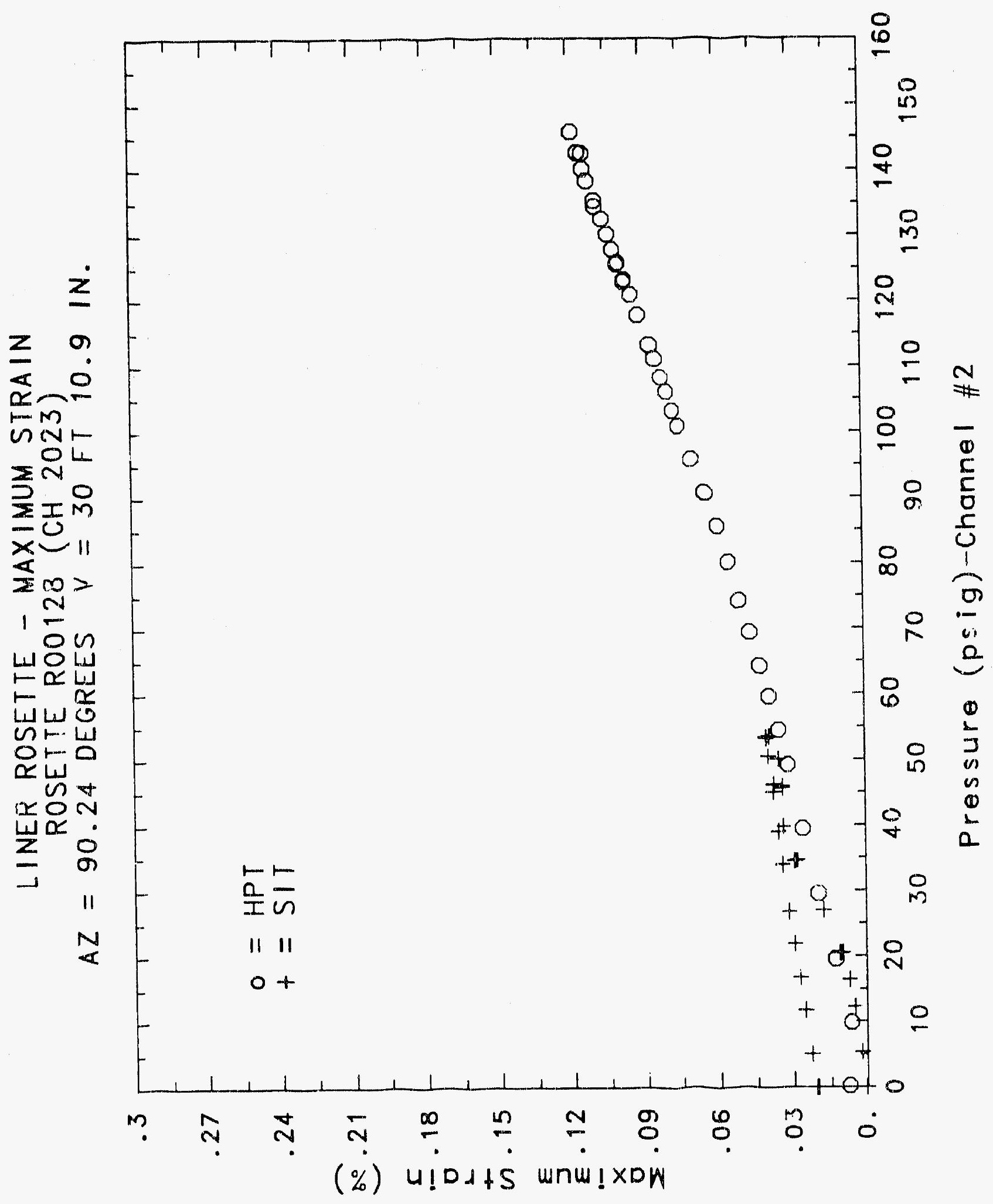




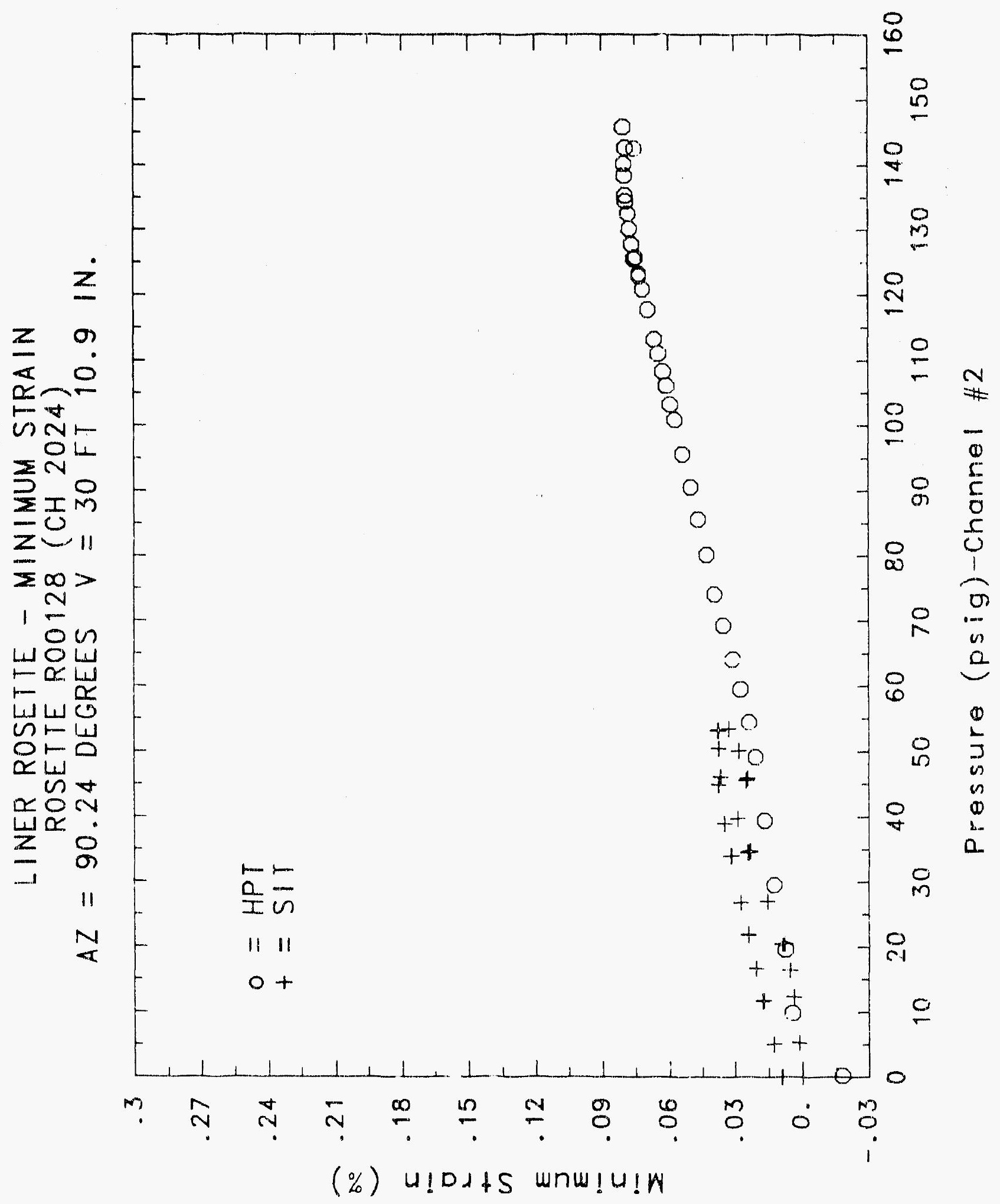




\section{Reinforced Concrete Test Data}

Rosette 129 Charinels $2026 \quad 2037 \quad 2028$

\section{STRUCTURAL INTEGRITY TEST}

\begin{tabular}{|c|c|c|c|c|c|c|c|}
\hline $\begin{array}{c}\text { Pressure } \\
\text { psig } \\
-0.05 \\
5.33 \\
12.31 \\
16.44 \\
20.51 \\
20.46 \\
20.47 \\
27.05 \\
34.69 \\
34.53 \\
34.53 \\
39.70 \\
45.90 \\
45.65 \\
45.62 \\
50.10 \\
53.47 \\
53.29 \\
53.21 \\
50.49 \\
46.14 \\
46.13 \\
44.97 \\
38.98 \\
33.99 \\
33.96 \\
26.83 \\
21.88 \\
21.90 \\
16.69 \\
11.74 \\
11.77 \\
5.05 \\
0.02 \\
-0.04 \\
-0.03 \\
.0 .02 \\
0.02\end{array}$ & $\begin{array}{l}\text { Maximum } \\
\text { \%Strain } \\
0.0000 \\
0.0025 \\
0.0062 \\
0.0085 \\
0.0115 \\
0.0122 \\
0.0124 \\
0.0186 \\
0.0274 \\
0.0280 \\
0.0280 \\
0.0325 \\
0.0399 \\
0.0401 \\
0.0404 \\
0.0444 \\
0.0477 \\
0.0482 \\
0.0483 \\
0.0471 \\
0.0444 \\
0.0441 \\
0.0444 \\
0.0403 \\
0.0368 \\
0.0367 \\
0.0309 \\
0.0273 \\
0.0274 \\
0.0232 \\
0.0192 \\
0.0185 \\
0.0133 \\
0.0098 \\
0.0090 \\
0.0034 \\
0.0097 \\
0.0094\end{array}$ & $\begin{array}{l}\text { Mininum } \\
\% \text { Strain } \\
-0.0006 \\
0.0015 \\
0.0040 \\
0.0057 \\
0.0078 \\
0.0079 \\
0.0082 \\
0.0117 \\
0.0172 \\
0.0179 \\
0.0183 \\
0.0210 \\
0.0249 \\
0.0243 \\
0.0243 \\
0.0267 \\
0.0292 \\
0.0296 \\
0.0297 \\
0.0290 \\
0.0275 \\
0.0273 \\
0.0290 \\
0.0271 \\
0.0249 \\
0.0244 \\
0.0214 \\
0.0195 \\
0.0195 \\
0.0170 \\
0.0144 \\
0.0134 \\
0.0102 \\
0.0076 \\
0.0078 \\
0.0080 \\
0.0081 \\
0.0074\end{array}$ & $\begin{array}{c}\text { Angle } \\
\text { Degrees } \\
34.35 \\
40.32 \\
30.97 \\
26.67 \\
23.74 \\
25.28 \\
26.43 \\
18.54 \\
15.49 \\
16.59 \\
16.34 \\
13.30 \\
10.42 \\
8.22 \\
8.58 \\
7.37 \\
6.70 \\
7.20 \\
7.35 \\
7.85 \\
8.05 \\
7.58 \\
10.72 \\
11.67 \\
12.32 \\
11.64 \\
12.57 \\
16.43 \\
18.98 \\
20.02 \\
23.41 \\
12.83 \\
17.02 \\
30.86 \\
16.09 \\
30.18 \\
36.87 \\
25.71\end{array}$ & $\begin{array}{c}\text { Pressure } \\
\text { psig } \\
9.89 \\
19.55 \\
29.57 \\
39.42 \\
49.16 \\
54.50 \\
59.57 \\
64.20 \\
69.32 \\
74.16 \\
80.16 \\
85.51 \\
90.58 \\
95.69 \\
100.92 \\
103.25 \\
106.11 \\
108.31 \\
111.08 \\
113.24 \\
117.83 \\
120.92 \\
123.28 \\
122.97 \\
125.82 \\
125.60 \\
127.84 \\
130.19 \\
132.53 \\
135.33 \\
134.42 \\
138.35 \\
140.16 \\
142.63 \\
145.78 \\
142.5 .2 \\
0.22\end{array}$ & $\begin{array}{l}\text { Haximum } \\
\text { \% Strain } \\
0.0078 \\
0.0155 \\
0.0236 \\
0.0310 \\
0.0383 \\
0.0427 \\
0.0476 \\
0.0527 \\
0.0564 \\
0.0520 \\
0.0514 \\
0.0534 \\
0.0566 \\
0.0614 \\
0.0665 \\
0.0695 \\
0.0721 \\
0.0742 \\
0.0770 \\
0.0791 \\
0.0835 \\
0.0868 \\
0.0898 \\
0.0903 \\
0.0932 \\
0.0937 \\
0.0958 \\
0.0986 \\
0.1014 \\
0.1051 \\
0.1060 \\
0.1114 \\
0.1120 \\
0.1151 \\
0.1186 \\
0.1145 \\
0.0223\end{array}$ & $\begin{array}{c}\text { Minimum } \\
\text { \%Strain } \\
0.0041 \\
0.0086 \\
0.0135 \\
0.0181 \\
0.0229 \\
0.0268 \\
0.0310 \\
0.0346 \\
0.0350 \\
0.0415 \\
0.0460 \\
0.0476 \\
0.0478 \\
0.0488 \\
0.0507 \\
0.0517 \\
0.0533 \\
0.0546 \\
0.0556 \\
0.0567 \\
0.0581 \\
0.0594 \\
0.0599 \\
0.0595 \\
0.0606 \\
0.0603 \\
0.0612 \\
0.0621 \\
0.0626 \\
0.0635 \\
0.0629 \\
0.0650 \\
0.0651 \\
0.0662 \\
0.0683 \\
0.0619 \\
0.0503\end{array}$ & $\begin{array}{c}\text { Angle } \\
\text { Degrees } \\
7.94 \\
6.98 \\
8.08 \\
6.09 \\
5.78 \\
8.25 \\
12.49 \\
12.87 \\
-4.79 \\
-8.16 \\
-25.02 \\
28.20 \\
16.39 \\
13.23 \\
11.54 \\
10.77 \\
10.59 \\
9.54 \\
9.17 \\
8.43 \\
7.48 \\
8.10 \\
8.21 \\
8.30 \\
7.91 \\
7.64 \\
8.03 \\
8.04 \\
7.91 \\
7.80 \\
7.07 \\
5.95 \\
8.23 \\
8.33 \\
8.79 \\
8.92 \\
3.07\end{array}$ \\
\hline
\end{tabular}

High Pressure test 


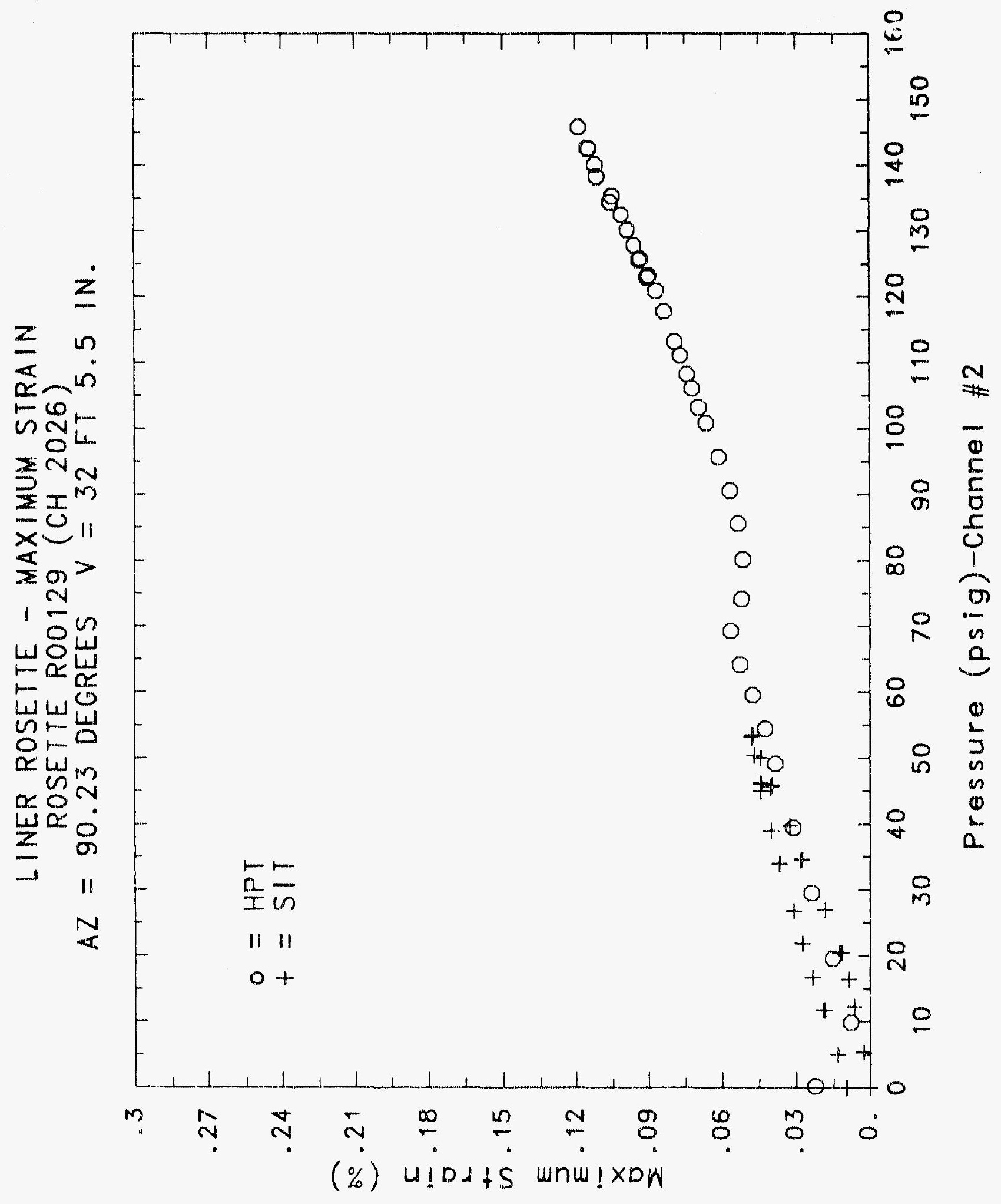




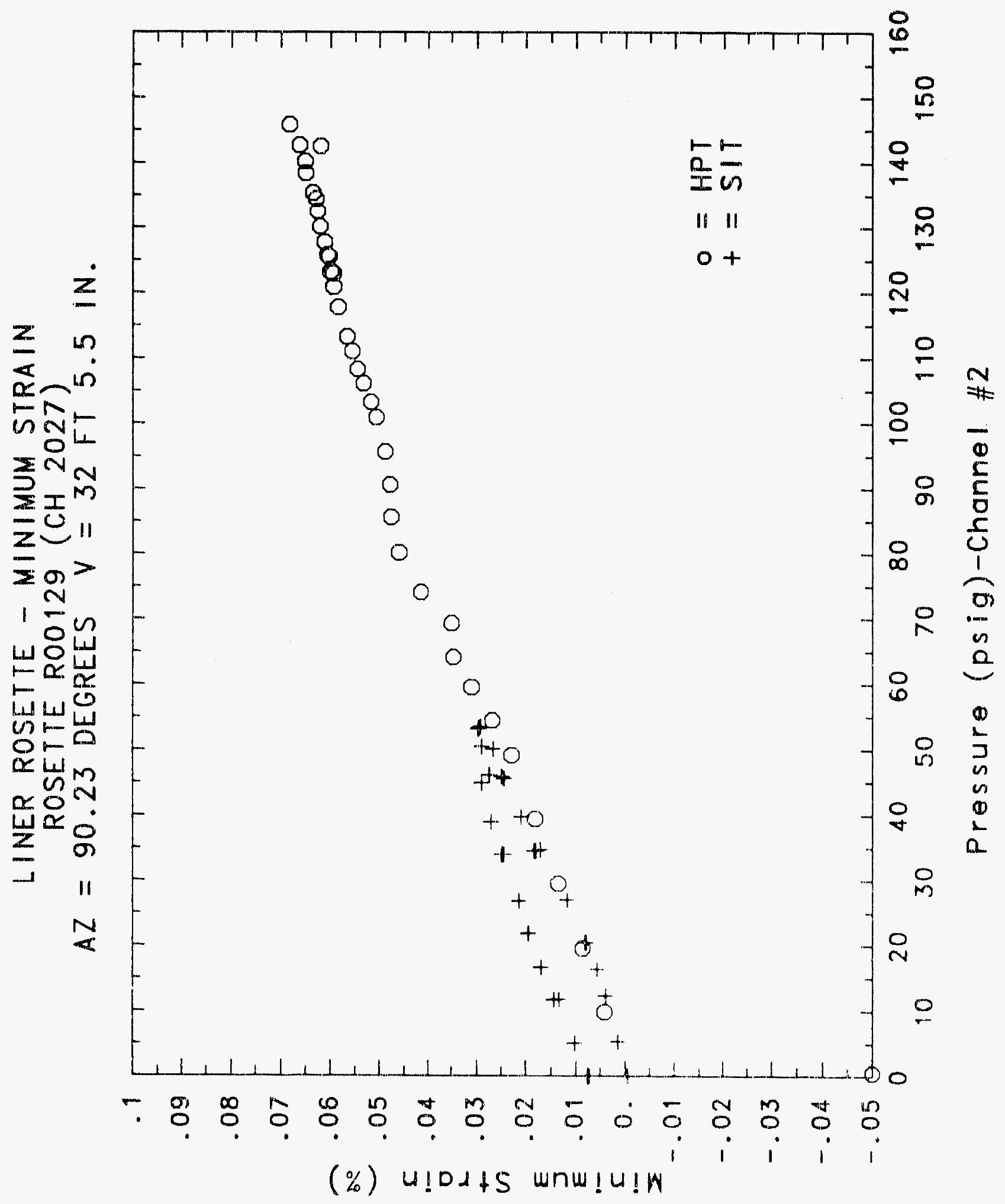




\title{
Reinforced Concrete Test Data
}

\author{
Rosette 130 Channels 202920402041
}

\section{STRUCTURAL INTEGRITY TEST}

\begin{tabular}{|c|c|c|c|c|c|c|c|}
\hline $\begin{array}{c}\text { Pressure } \\
\text { psig } \\
-0.05 \\
5.33 \\
12.31 \\
16.44 \\
20.51 \\
20.46 \\
20.47 \\
27.05 \\
34.69 \\
34.53 \\
34.53 \\
39.70 \\
45.90 \\
45.65 \\
45.62 \\
50.10 \\
53.47 \\
53.29 \\
53.21 \\
50.49 \\
46.14 \\
46.13 \\
44.97 \\
38.98 \\
33.99 \\
33.96 \\
26.83 \\
21.88 \\
21.90 \\
16.69 \\
11.74 \\
11.77 \\
5.05 \\
0.02 \\
-0.04 \\
-0.02 \\
-0.02 \\
0.02\end{array}$ & $\begin{array}{l}\text { Maximum } \\
\text { \%Strain } \\
0.0023 \\
0.0068 \\
0.0149 \\
0.0258 \\
0.0442 \\
0.0475 \\
0.0500 \\
0.0596 \\
0.0666 \\
0.0809 \\
0.0821 \\
0.0843 \\
0.0986 \\
0.1690 \\
0.1781 \\
0.1875 \\
0.1950 \\
0.2000 \\
0.2025 \\
0.2040 \\
0.2036 \\
0.2043 \\
0.2251 \\
0.2220 \\
0.2193 \\
0.2194 \\
0.2153 \\
0.2127 \\
0.2168 \\
0.2223 \\
0.2185 \\
0.2181 \\
0.2142 \\
0.2119 \\
0.1969 \\
0.1969 \\
0.1968 \\
0.1970\end{array}$ & $\begin{array}{r}\text { Minimum } \\
\text { \%Strain } \\
-0.0003 \\
0.0001 \\
0.0012 \\
0.0013 \\
0.0005 \\
0.0002 \\
-0.0001 \\
0.0022 \\
0.0065 \\
0.0047 \\
0.0045 \\
0.0071 \\
0.0093 \\
-0.0021 \\
-0.0037 \\
-0.0024 \\
-0.0010 \\
-0.0017 \\
-0.0021 \\
-0.0033 \\
-0.0050 \\
-0.0053 \\
-0.0091 \\
-0.0108 \\
-0.0124 \\
-0.0126 \\
-0.0150 \\
-0.0167 \\
-0.0175 \\
-0.0207 \\
-0.0224 \\
-0.0225 \\
-0.0246 \\
-0.0288 \\
-0.0266 \\
-0.0263 \\
-0.0283 \\
-0.00268\end{array}$ & $\begin{array}{c}\text { Angle } \\
\text { Degrees } \\
15.29 \\
19.12 \\
16.90 \\
17.67 \\
18.73 \\
18.86 \\
18.90 \\
17.87 \\
16.58 \\
17.96 \\
17.90 \\
17.29 \\
17.16 \\
19.72 \\
19.92 \\
19.74 \\
19.65 \\
19.73 \\
19.79 \\
19.92 \\
20.16 \\
20.19 \\
20.66 \\
20.98 \\
21.21 \\
21.21 \\
21.59 \\
21.88 \\
21.78 \\
22.11 \\
22.38 \\
22.36 \\
22.75 \\
22.97 \\
23.13 \\
23.14 \\
23.15 \\
23.11\end{array}$ & $\begin{array}{c}\text { Pressure } \\
\text { psig } \\
9.89 \\
19.55 \\
29.57 \\
39.42 \\
49.16 \\
54.50 \\
59.57 \\
64.20 \\
69.32 \\
74.16 \\
80.16 \\
85.61 \\
90.58 \\
95.69 \\
100.92 \\
103.25 \\
106.11 \\
108.31 \\
111.08 \\
113.24 \\
117.83 \\
120.92 \\
123.28 \\
122.97 \\
125.82 \\
125.60 \\
127.84 \\
130.19 \\
132.53 \\
135.33 \\
134.42 \\
138.35 \\
140.16 \\
142.63 \\
145.78 \\
142.52 \\
0.22\end{array}$ & $\begin{array}{l}\text { Maximum } \\
\text { \%Strain } \\
0.0075 \\
0.0155 \\
0.0238 \\
0.0272 \\
0.0334 \\
0.0360 \\
0.0386 \\
0.0415 \\
0.0455 \\
0.0496 \\
0.0623 \\
0.0695 \\
0.0761 \\
0.0837 \\
0.0921 \\
0.0962 \\
0.1015 \\
0.1057 \\
0.1093 \\
0.1122 \\
0.1188 \\
0.1235 \\
0.1278 \\
0.1284 \\
0.1326 \\
0.1335 \\
0.1366 \\
0.1415 \\
0.1462 \\
0.1555 \\
0.1173 \\
0.1244 \\
0.1292 \\
0.1354 \\
0.1527 \\
0.1741 \\
0.0535\end{array}$ & $\begin{array}{r}\text { Minimum } \\
\text { XStrain } \\
0.0034 \\
0.0054 \\
0.0078 \\
0.0079 \\
0.0099 \\
0.0093 \\
0.0062 \\
0.0009 \\
0.0031 \\
0.0030 \\
0.0246 \\
0.0281 \\
0.0311 \\
0.0341 \\
0.0372 \\
0.0389 \\
0.0402 \\
0.0416 \\
0.0426 \\
0.0438 \\
0.0460 \\
0.0476 \\
0.0491 \\
0.0492 \\
0.0506 \\
0.0505 \\
0.0519 \\
0.0529 \\
0.0541 \\
0.0557 \\
0.0473 \\
0.0497 \\
0.0513 \\
0.0530 \\
0.0587 \\
0.0593 \\
-0.0119\end{array}$ & $\begin{array}{c}\text { Angle } \\
\text { Degrees } \\
-15.12 \\
-10.41 \\
-8.70 \\
-22.90 \\
-24.79 \\
-30.84 \\
-37.38 \\
-43.92 \\
-42.97 \\
-43.58 \\
-16.26 \\
-12.52 \\
-10.58 \\
-8.22 \\
-5.82 \\
-4.50 \\
-3.24 \\
-2.05 \\
-2.18 \\
-1.83 \\
-1.65 \\
-1.59 \\
-1.13 \\
-0.95 \\
-0.74 \\
-0.71 \\
-0.48 \\
-0.23 \\
-0.28 \\
0.60 \\
-20.04 \\
-18.86 \\
-17.85 \\
-17.25 \\
-10.65 \\
-1.26 \\
8.74\end{array}$ \\
\hline
\end{tabular}

High Pressure Test 


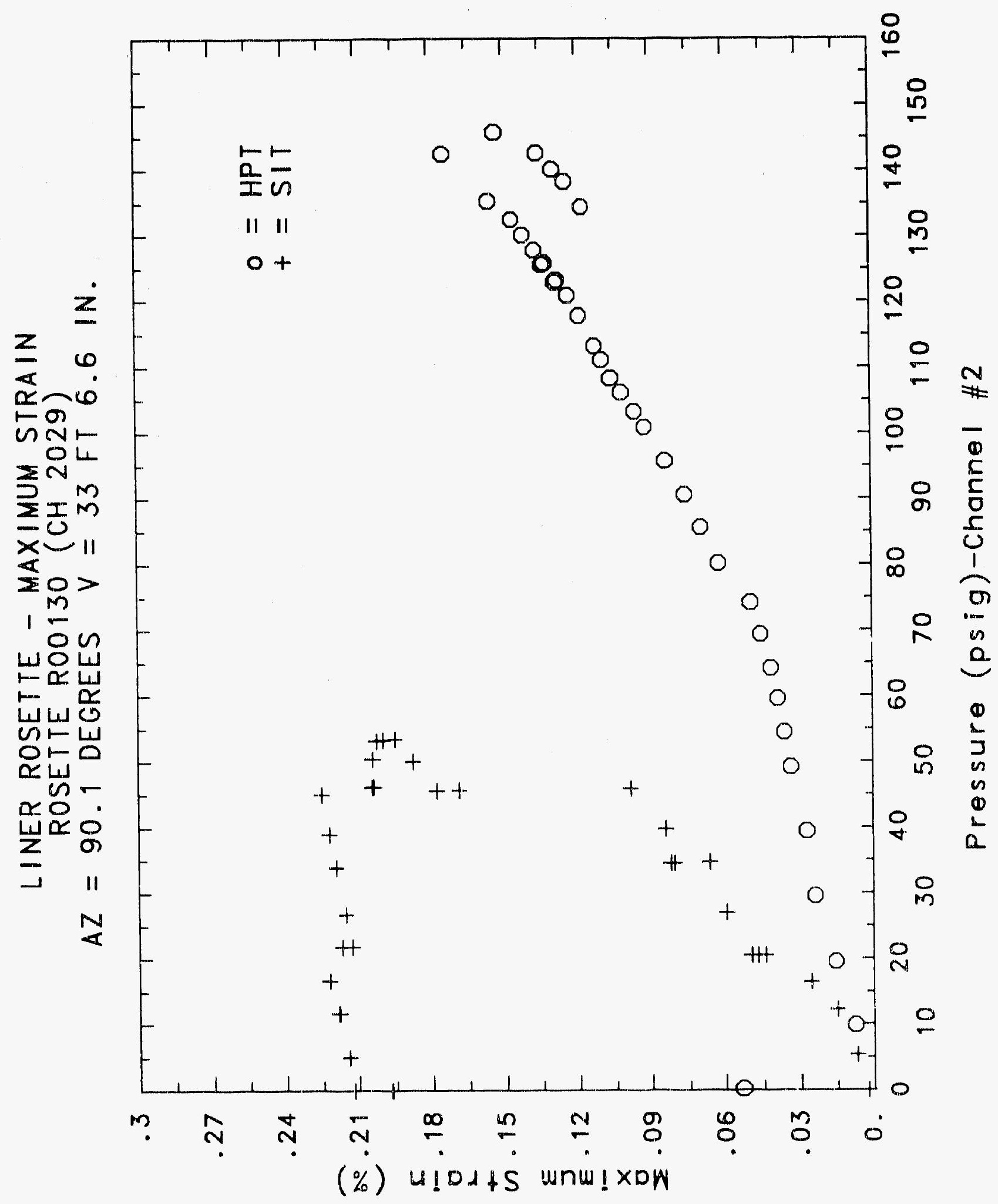

C-708 


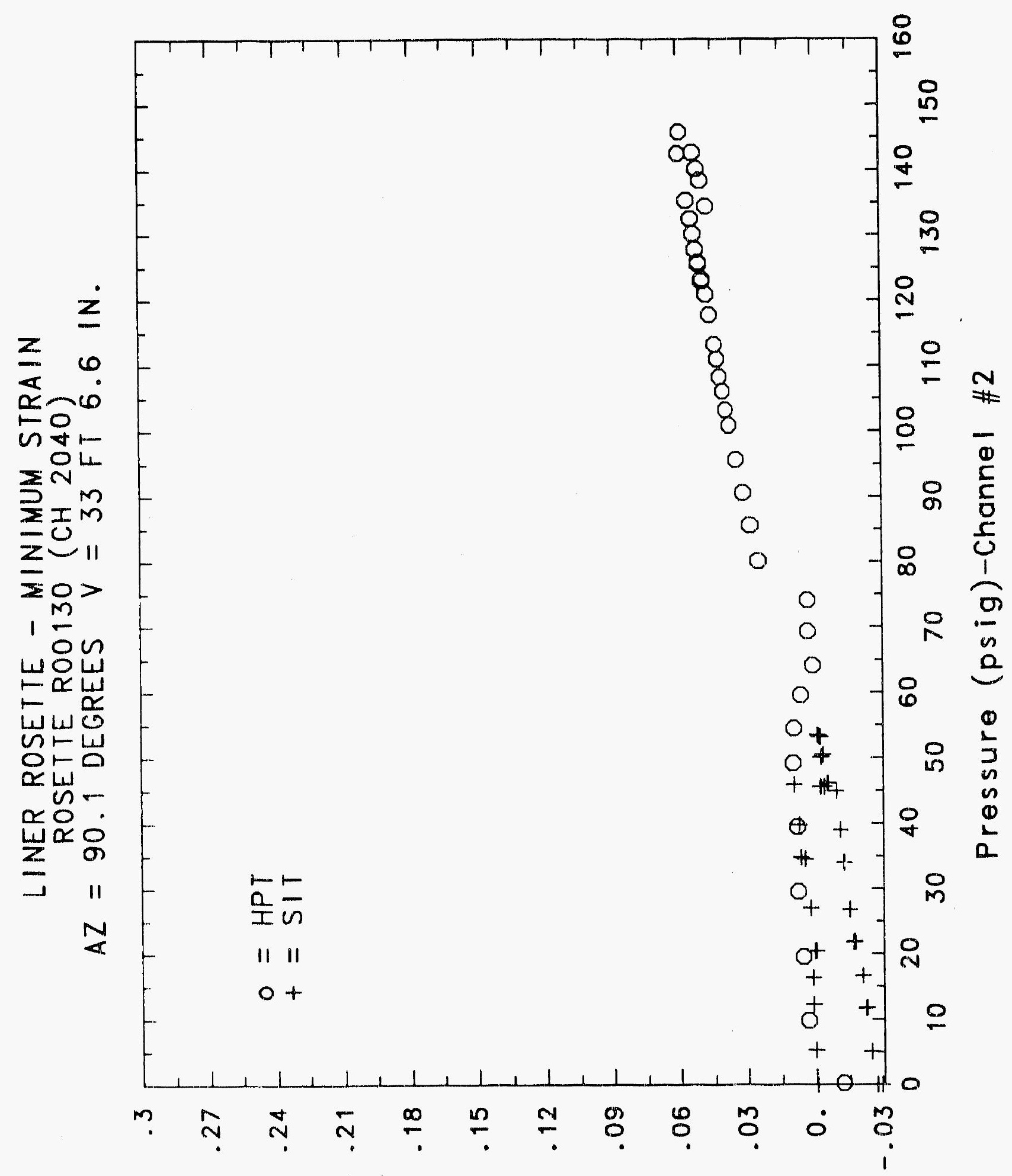

(\%) U!DdtS wnw!u!w 


\section{Reinforced Concrete Test Data}

Rosette 131 Channels $2042 \quad 20432044$

\section{Structural Integrity Test}

\begin{tabular}{|c|c|c|c|c|c|c|c|}
\hline $\begin{array}{c}\text { Pressure } \\
\text { psig } \\
-0.05 \\
5.33 \\
12.31 \\
16.44 \\
20.51 \\
20.46 \\
20.47 \\
27.05 \\
34.69 \\
34.53 \\
34.53 \\
39.70 \\
45.90 \\
45.65 \\
45.62 \\
50.10 \\
53.47 \\
53.29 \\
53.21 \\
50.49 \\
46.14 \\
46.13 \\
44.97 \\
38.98 \\
33.99 \\
33.96 \\
26.83 \\
21.88 \\
21.90 \\
16.69 \\
11.74 \\
11.77 \\
5.05 \\
0.02 \\
-0.04 \\
-0.02 \\
-0.02 \\
0.02\end{array}$ & $\begin{array}{c}\text { Maximum } \\
\text { \%Strain } \\
0.0003 \\
0.0033 \\
0.0073 \\
0.0102 \\
0.0134 \\
0.0137 \\
0.0136 \\
0.0195 \\
0.0286 \\
0.0289 \\
0.0291 \\
0.0343 \\
0.0424 \\
0.0428 \\
0.0429 \\
0.0470 \\
0.0509 \\
0.0513 \\
0.0513 \\
0.0500 \\
0.0473 \\
0.0470 \\
0.0465 \\
0.0423 \\
0.0390 \\
0.0390 \\
0.0335 \\
0.0294 \\
0.0290 \\
0.0248 \\
0.0209 \\
0.0213 \\
0.0154 \\
0.0110 \\
0.0098 \\
0.0102 \\
0.0103 \\
0.0100\end{array}$ & $\begin{array}{c}\text { Minimum } \\
\text { KStrain } \\
-0.0001 \\
0.0020 \\
0.0049 \\
0.0067 \\
0.0087 \\
0.0089 \\
0.0092 \\
0.0126 \\
0.0170 \\
0.0174 \\
0.0175 \\
0.0202 \\
0.0238 \\
0.0240 \\
0.0242 \\
0.0264 \\
0.0284 \\
0.0286 \\
0.0287 \\
0.0277 \\
0.0262 \\
0.0261 \\
0.0263 \\
0.0240 \\
0.0220 \\
0.0219 \\
0.0187 \\
0.0164 \\
0.0163 \\
0.0140 \\
0.0117 \\
0.0116 \\
0.0083 \\
0.0053 \\
0.0048 \\
0.0054 \\
0.0056 \\
0.0055\end{array}$ & $\begin{array}{c}\text { Angle } \\
\text { Degrees } \\
-22.54 \\
13.81 \\
9.32 \\
10.04 \\
7.11 \\
4.61 \\
3.90 \\
-0.49 \\
-2.66 \\
-5.00 \\
-4.78 \\
-4.89 \\
-5.42 \\
-6.29 \\
-6.24 \\
-6.60 \\
-6.75 \\
-6.37 \\
-6.33 \\
-6.76 \\
-6.55 \\
-6.81 \\
-5.67 \\
-6.17 \\
-5.92 \\
-6.61 \\
-7.12 \\
-7.68 \\
-9.27 \\
-8.83 \\
-8.50 \\
-6.01 \\
-7.57 \\
-8.61 \\
-12.28 \\
-11.05 \\
-10.00 \\
-9.91\end{array}$ & $\begin{array}{c}\text { Pressure } \\
\text { psig } \\
9.89 \\
19.55 \\
29.57 \\
39.42 \\
49.16 \\
54.50 \\
59.57 \\
64.20 \\
69.32 \\
74.16 \\
80.16 \\
85.61 \\
90.58 \\
95.69 \\
100.92 \\
103.25 \\
106.11 \\
108.31 \\
111.08 \\
113.24 \\
117.83 \\
120.99 \\
123.28 \\
122.97 \\
125.82 \\
125.60 \\
127.84 \\
130.19 \\
132.53 \\
135.33 \\
134.42 \\
138.35 \\
140.16 \\
142.63 \\
145.78 \\
142.52 \\
0.22\end{array}$ & $\begin{array}{c}\text { Maximum } \\
\text { xStrain } \\
0.0088 \\
0.0171 \\
0.0254 \\
0.0334 \\
0.0412 \\
0.0459 \\
0.0507 \\
0.0555 \\
0.0612 \\
0.0661 \\
0.0737 \\
0.0794 \\
0.0851 \\
0.0916 \\
0.0983 \\
0.1014 \\
0.1048 \\
0.1076 \\
0.1114 \\
0.1144 \\
0.1211 \\
0.1259 \\
0.1298 \\
0.1304 \\
0.1347 \\
0.1356 \\
0.1386 \\
0.1426 \\
0.1467 \\
0.1522 \\
0.1522 \\
0.1583 \\
0.1632 \\
0.1689 \\
0.1762 \\
0.1717 \\
0.0414\end{array}$ & $\begin{array}{c}\text { Minimum } \\
\text { \%Strain } \\
0.0048 \\
0.0092 \\
0.0135 \\
0.0179 \\
0.0223 \\
0.0250 \\
0.0273 \\
0.0299 \\
0.0328 \\
0.0361 \\
0.0390 \\
0.0426 \\
0.0462 \\
0.0493 \\
0.0528 \\
0.0546 \\
0.0562 \\
0.0576 \\
0.0594 \\
0.0610 \\
0.0638 \\
0.0659 \\
0.0682 \\
0.0684 \\
0.0704 \\
0.0707 \\
0.0723 \\
0.0740 \\
0.0757 \\
0.0782 \\
0.0774 \\
0.0799 \\
0.0816 \\
0.0830 \\
0.0856 \\
0.0817 \\
0.0026\end{array}$ & $\begin{array}{c}\text { Angle } \\
\text { Degrees } \\
-7.73 \\
-7.47 \\
-8.58 \\
-8.42 \\
-7.51 \\
-7.72 \\
-8.67 \\
-9.79 \\
-9.99 \\
-11.20 \\
-12.27 \\
-12.50 \\
-12.11 \\
-12.06 \\
-11.69 \\
-11.44 \\
-11.62 \\
-11.34 \\
-11.39 \\
-11.01 \\
-10.92 \\
-11.22 \\
-11.12 \\
-11.13 \\
-11.00 \\
-11.04 \\
-10.75 \\
-10.69 \\
-11.04 \\
-11.44 \\
-12.49 \\
-13.02 \\
-12.88 \\
-13.50 \\
-13.43 \\
-13.97 \\
-18.55\end{array}$ \\
\hline
\end{tabular}

High Pressure test 


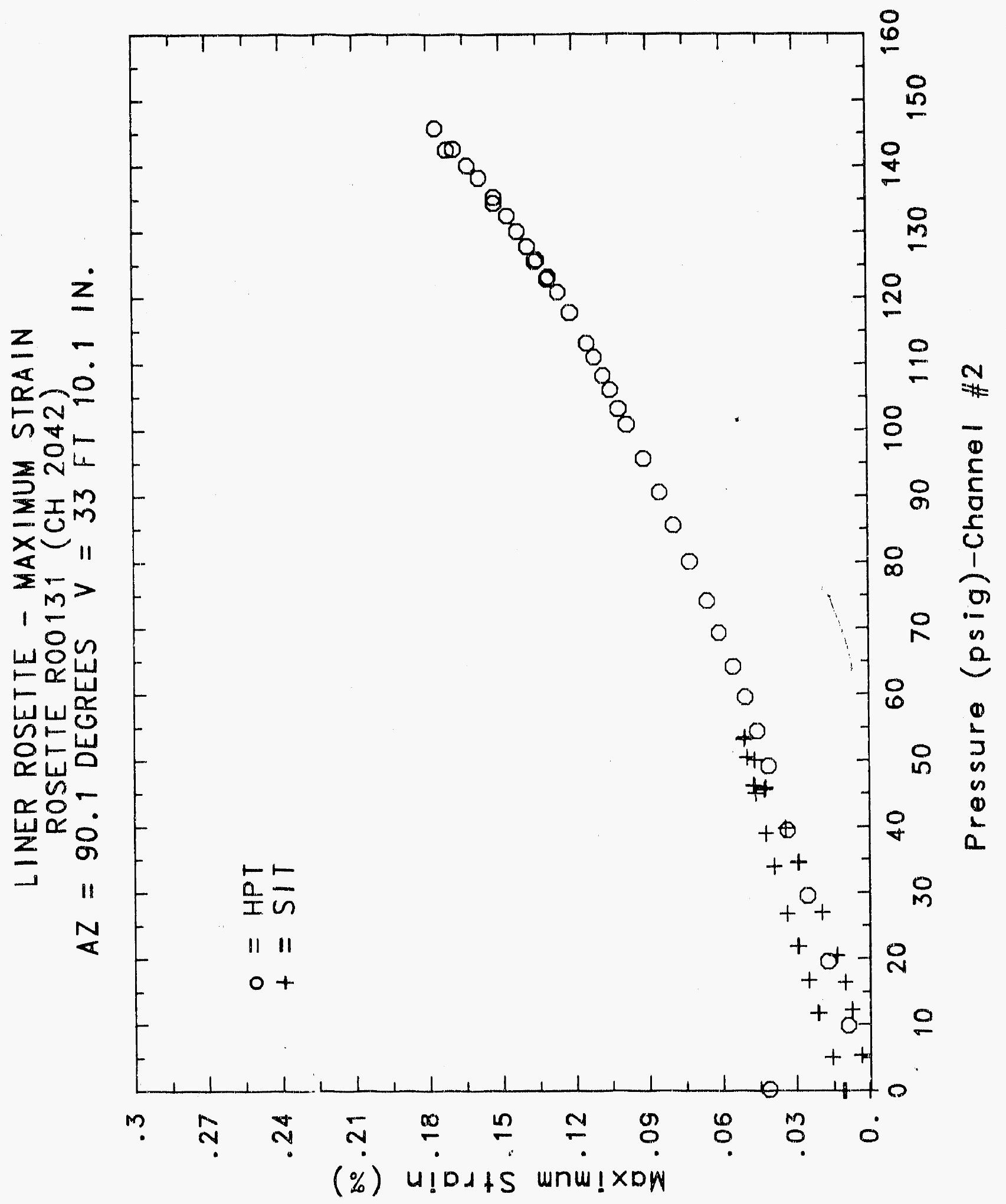




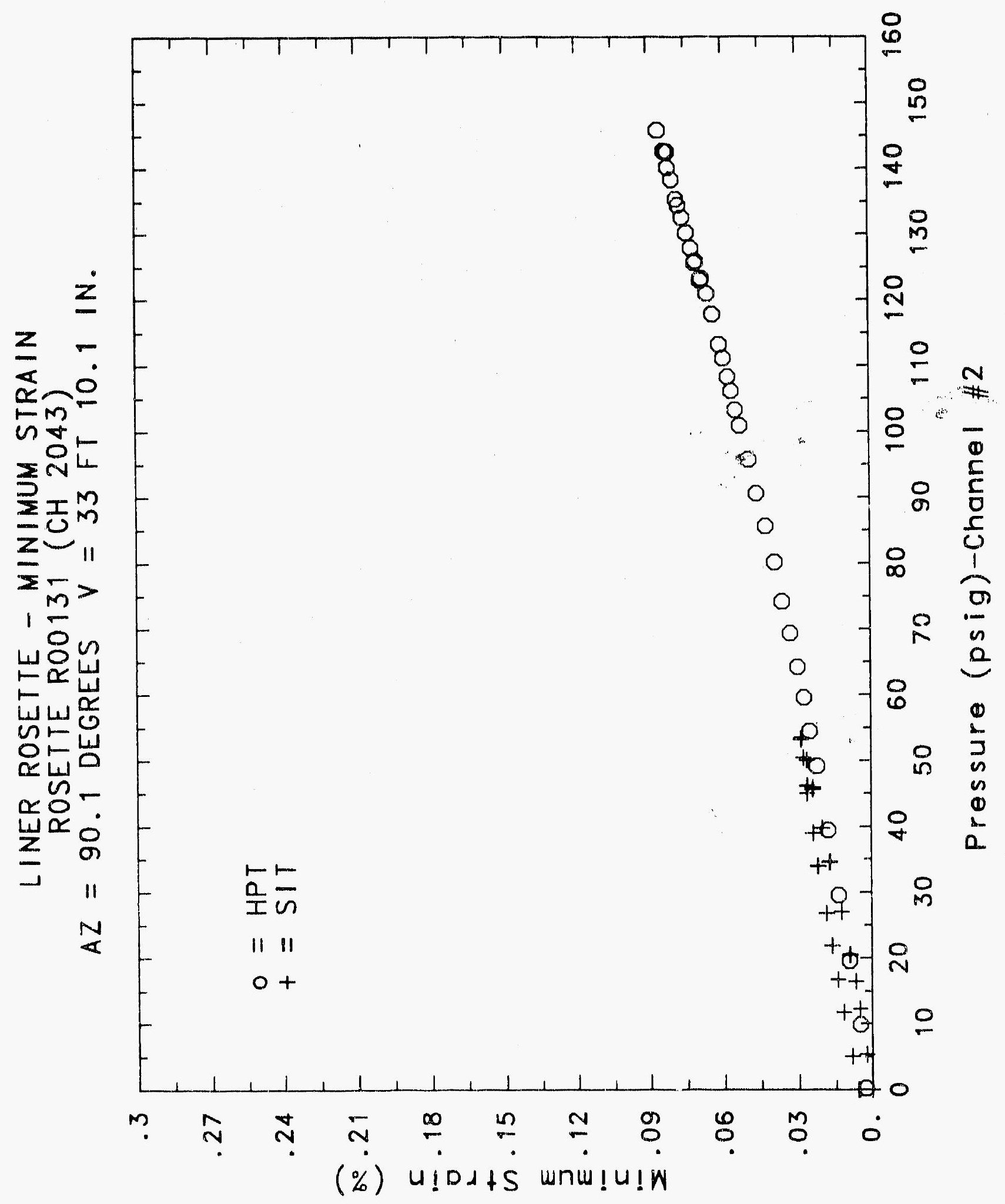




\section{Reinforced Concrete Test Data}

\section{Rosette 132 Channels $2045 \quad 20462047$}

$$
\text { Structural Integrity Test }
$$

\begin{tabular}{|c|c|c|c|c|c|c|c|}
\hline $\begin{array}{c}\text { Pressure } \\
\text { psig } \\
\text {-0.05 } \\
5.33 \\
12.31 \\
16.44 \\
20.51 \\
20.46 \\
20.47 \\
27.05 \\
34.69 \\
34.53 \\
34.53 \\
39.70 \\
45.90 \\
45.65 \\
45.62 \\
50.10 \\
53.47 \\
53.29 \\
53.21 \\
50.49 \\
46.14 \\
46.13 \\
44.97 \\
38.98 \\
33.99 \\
33.96 \\
26.83 \\
21.88 \\
21.90 \\
16.69 \\
11.74 \\
11.77 \\
5.05 \\
0.02 \\
-0.04 \\
-0.02 \\
-0.02 \\
0.02\end{array}$ & $\begin{array}{c}\text { Maximum } \\
\text { \%Strain } \\
0.0006 \\
0.0034 \\
0.0078 \\
0.0107 \\
0.0142 \\
0.0147 \\
0.0150 \\
0.0217 \\
0.0322 \\
0.0331 \\
0.0333 \\
0.0383 \\
0.0455 \\
0.0459 \\
0.0461 \\
0.0504 \\
0.0542 \\
0.0543 \\
0.0543 \\
0.0528 \\
0.0499 \\
0.0496 \\
0.0489 \\
0.0445 \\
0.0407 \\
0.0407 \\
0.0346 \\
0.0306 \\
0.0306 \\
0.0261 \\
0.0216 \\
0.0213 \\
0.0152 \\
0.0104 \\
0.0100 \\
0.0105 \\
0.0106 \\
0.0101\end{array}$ & $\begin{array}{c}\text { Minimum } \\
\text { XStrain } \\
-0.0002 \\
0.0021 \\
0.0050 \\
0.0066 \\
0.0087 \\
0.0088 \\
0.0087 \\
0.0116 \\
0.0152 \\
0.0153 \\
0.0155 \\
0.0182 \\
0.0231 \\
0.0236 \\
0.0238 \\
0.0265 \\
0.0289 \\
0.0294 \\
0.0295 \\
0.0285 \\
0.0266 \\
0.0265 \\
0.0268 \\
0.0242 \\
0.0218 \\
0.0215 \\
0.0178 \\
0.0150 \\
0.0149 \\
0.0119 \\
0.0092 \\
0.0091 \\
0.0054 \\
0.0023 \\
0.0023 \\
0.0027 \\
0.0025 \\
0.0024\end{array}$ & $\begin{array}{c}\text { Angle } \\
\text { Degrees } \\
-33.80 \\
-18.78 \\
-18.63 \\
-18.79 \\
-17.06 \\
-16.73 \\
-17.07 \\
-10.54 \\
-7.28 \\
-8.80 \\
-8.11 \\
-8.25 \\
-8.12 \\
-8.25 \\
-7.95 \\
-7.52 \\
-7.16 \\
-7.07 \\
-7.01 \\
-7.03 \\
-6.55 \\
-6.53 \\
-7.06 \\
-6.33 \\
-6.24 \\
-6.48 \\
-7.20 \\
-8.32 \\
-7.15 \\
-7.87 \\
-7.23 \\
-6.19 \\
-5.70 \\
-4.39 \\
-1.04 \\
-1.20 \\
-2.82 \\
-3.40\end{array}$ & $\begin{array}{c}\text { Pressure } \\
\text { psig } \\
9.89 \\
19.55 \\
29.57 \\
39.42 \\
49.16 \\
54.50 \\
59.57 \\
64.20 \\
69.32 \\
74.16 \\
80.16 \\
85.61 \\
90.58 \\
95.69 \\
100.92 \\
103.25 \\
106.11 \\
108.31 \\
111.08 \\
113.24 \\
117.83 \\
120.92 \\
123.28 \\
122.97 \\
125.82 \\
125.60 \\
127.84 \\
130.19 \\
132.53 \\
135.33 \\
134.42 \\
138.35 \\
140.16 \\
142.63 \\
145.78 \\
142.52 \\
0.22\end{array}$ & $\begin{array}{l}\text { Maximum } \\
\text { xStrain } \\
0.0091 \\
0.0176 \\
0.0261 \\
0.0344 \\
0.0424 \\
0.0471 \\
0.0517 \\
0.0566 \\
0.0617 \\
0.0669 \\
0.0727 \\
0.0784 \\
0.0837 \\
0.0894 \\
0.0955 \\
0.0980 \\
0.1012 \\
0.1037 \\
0.1070 \\
0.1098 \\
0.1156 \\
0.1198 \\
0.1233 \\
0.1235 \\
0.1272 \\
0.1276 \\
0.1302 \\
0.1336 \\
0.1373 \\
0.1423 \\
0.1427 \\
0.1487 \\
0.1522 \\
0.1570 \\
0.1631 \\
0.1575 \\
0.0230\end{array}$ & $\begin{array}{l}\text { Minimum } \\
\text { \%Strain } \\
0.0055 \\
0.0108 \\
0.0160 \\
0.0214 \\
0.0265 \\
0.0295 \\
0.0327 \\
0.0356 \\
0.0391 \\
0.0429 \\
0.0472 \\
0.0509 \\
0.0546 \\
0.0587 \\
0.0629 \\
0.0654 \\
0.0676 \\
0.0689 \\
0.0716 \\
0.0735 \\
0.0775 \\
0.0805 \\
0.0832 \\
0.0840 \\
0.0865 \\
0.0869 \\
0.0883 \\
0.0899 \\
0.0923 \\
0.0951 \\
0.0949 \\
0.0978 \\
0.1001 \\
0.1028 \\
0.1059 \\
0.1014 \\
0.0125\end{array}$ & $\begin{array}{c}\text { Angle } \\
\text { Degrees } \\
-9.88 \\
-12.11 \\
-13.37 \\
-12.11 \\
-12.73 \\
-12.31 \\
-12.48 \\
-11.86 \\
-11.53 \\
-10.99 \\
-10.37 \\
-11.25 \\
-11.18 \\
-11.44 \\
-11.40 \\
-11.26 \\
-10.84 \\
-11.41 \\
-10.90 \\
-10.64 \\
-10.59 \\
-10.95 \\
-10.98 \\
-10.88 \\
-10.65 \\
-10.76 \\
-10.78 \\
-11.14 \\
-10.90 \\
-11.21 \\
-11.53 \\
-11.63 \\
-11.70 \\
-11.51 \\
-11.66 \\
-11.18 \\
0.59\end{array}$ \\
\hline
\end{tabular}

High Pressure test 


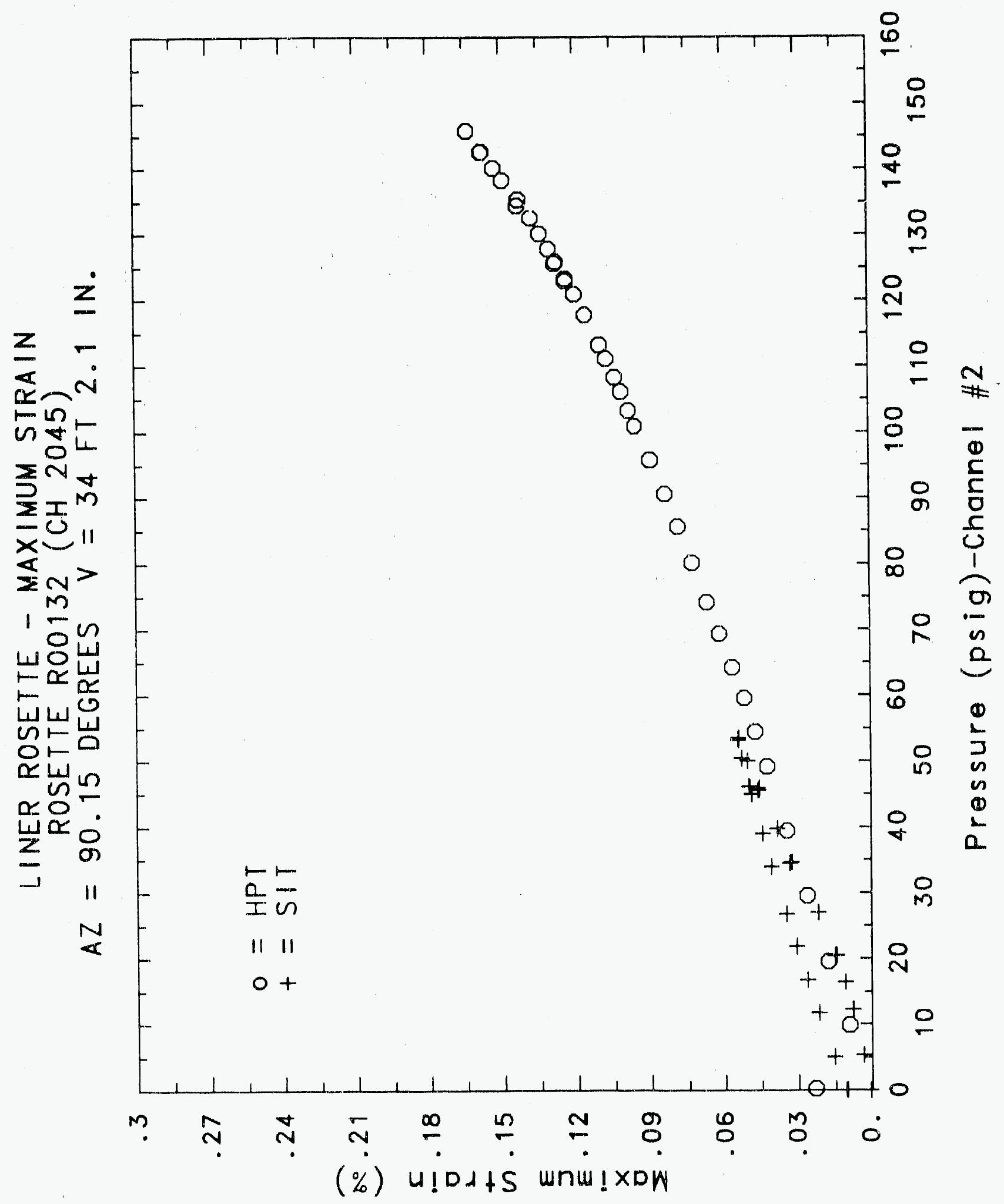




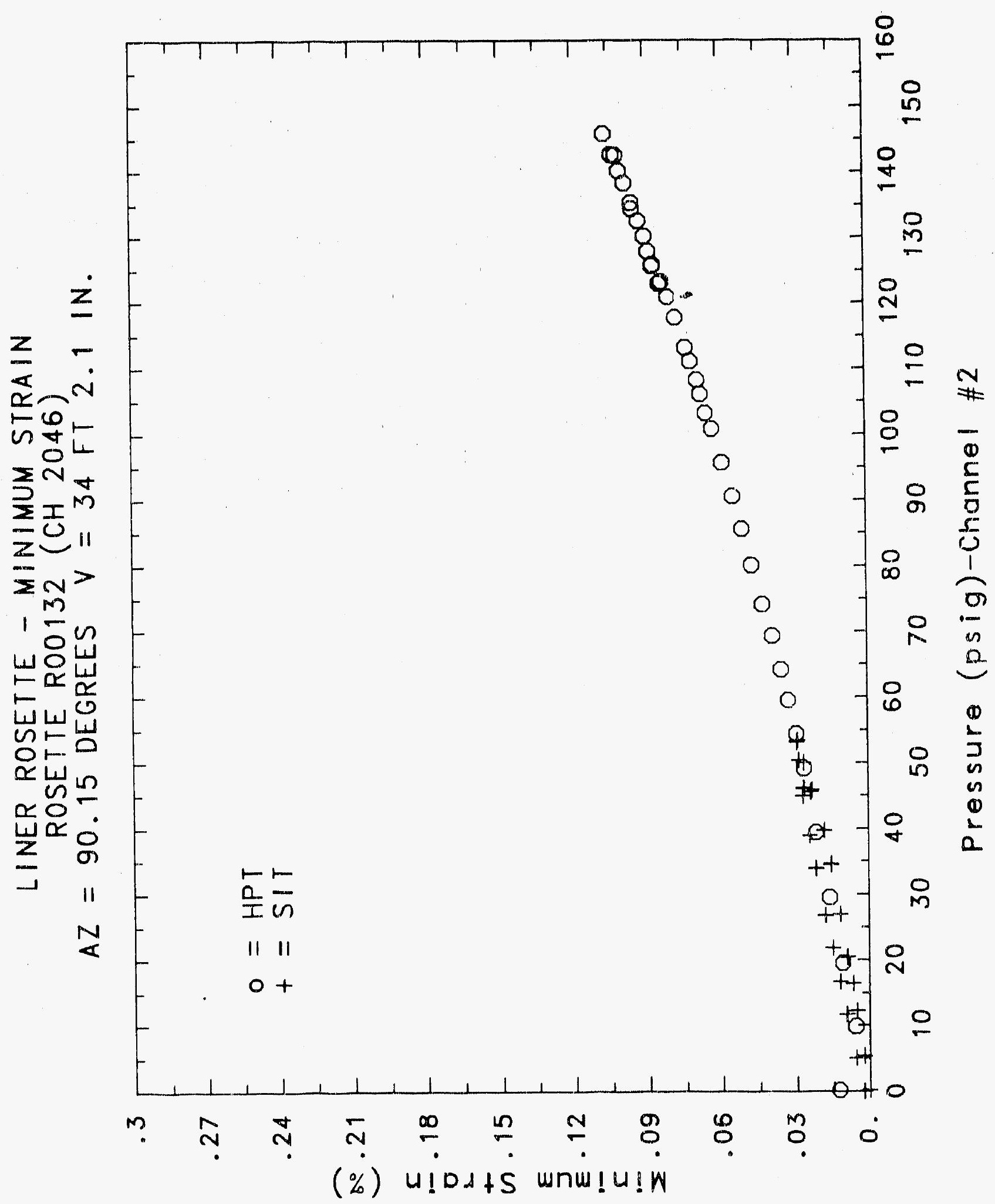

C.715 


\section{Reinforced Concrete Test Data}

\section{Rosette 133 Channels $2048 \quad 20492060$}

STRUCTURAL INTEGRITY TEST

\begin{tabular}{|c|c|c|c|c|c|c|c|}
\hline 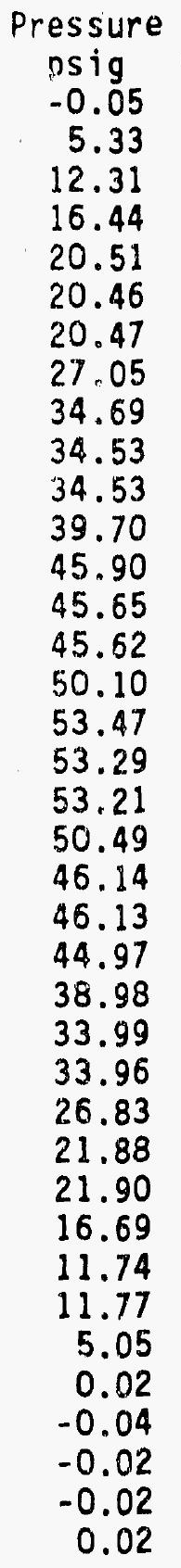 & $\begin{array}{c}\text { Maximum } \\
\text { \%Strain } \\
0.0000 \\
0.0039 \\
0.0078 \\
0.0103 \\
0.0135 \\
0.0145 \\
0.0145 \\
0.0202 \\
0.0283 \\
0.0319 \\
0.0319 \\
0.0355 \\
0.0404 \\
0.0402 \\
0.0404 \\
0.0444 \\
0.0482 \\
0.0486 \\
0.0487 \\
0.0473 \\
0.0443 \\
0.0442 \\
0.0446 \\
0.0402 \\
0.0365 \\
0.0365 \\
0.0314 \\
0.0287 \\
0.0299 \\
0.0262 \\
0.0220 \\
0.0207 \\
0.0149 \\
0.0122 \\
0.0115 \\
0.0122 \\
0.0124 \\
0.0123\end{array}$ & $\begin{array}{c}\text { Minimum } \\
\text { \% Strain } \\
-0.0002 \\
0.0013 \\
0.0039 \\
0.0058 \\
0.0078 \\
0.0045 \\
0.0077 \\
0.0113 \\
0.0165 \\
0.0140 \\
0.0140 \\
0.0176 \\
0.0227 \\
0.0235 \\
0.0233 \\
0.0259 \\
0.0278 \\
0.0278 \\
0.0278 \\
0.0270 \\
0.0251 \\
0.0251 \\
0.0258 \\
0.0233 \\
0.0206 \\
0.0203 \\
0.0159 \\
0.0121 \\
0.0107 \\
0.0074 \\
0.0049 \\
0.0055 \\
0.0027 \\
0.0014 \\
0.0012 \\
0.0015 \\
0.0011 \\
0.0007\end{array}$ & $\begin{array}{c}\text { Angle } \\
\text { Degrees } \\
-39.51 \\
-8.39 \\
-10.32 \\
-9.91 \\
-11.59 \\
-17.97 \\
-19.99 \\
-16.29 \\
-15.73 \\
-27.21 \\
27.52 \\
-24.97 \\
-17.19 \\
-13.05 \\
-12.81 \\
-11.93 \\
-11.68 \\
-11.78 \\
-11.61 \\
-11.58 \\
-12.58 \\
-11.57 \\
-12.16 \\
-12.24 \\
-13.00 \\
-14.26 \\
-17.29 \\
-21.63 \\
-25.96 \\
-27.61 \\
-27.23 \\
-24.18 \\
-23.91 \\
-27.32 \\
-26.74 \\
-27.72 \\
-29.60 \\
-30.84\end{array}$ & $\begin{array}{c}\text { Pressure } \\
\text { psig } \\
9.89 \\
19.55 \\
29.57 \\
39.42 \\
49.16 \\
54.50 \\
59.57 \\
64.20 \\
69.32 \\
74.16 \\
80.16 \\
85.61 \\
90.58 \\
95.69 \\
100.92 \\
103.25 \\
106.11 \\
108.31 \\
111.08 \\
113.24 \\
117.83 \\
120.92 \\
123.28 \\
122.97 \\
125.82 \\
125.60 \\
127.84 \\
130.19 \\
132.53 \\
135.33 \\
134.42 \\
138.35 \\
140.16 \\
142.63 \\
145.78 \\
142.52 \\
0.22\end{array}$ & $\begin{array}{c}\text { Maximum } \\
\text { XStrain } \\
0.0072 \\
0.0147 \\
0.0226 \\
0.0304 \\
0.0379 \\
0.0426 \\
0.0469 \\
0.0523 \\
0.0574 \\
0.0629 \\
0.0695 \\
0.0755 \\
0.0813 \\
0.0876 \\
0.0944 \\
0.0978 \\
0.1012 \\
0.1041 \\
0.1086 \\
0.1117 \\
0.1191 \\
0.1246 \\
0.1294 \\
0.1302 \\
0.1349 \\
0.1360 \\
0.1390 \\
0.1432 \\
0.1481 \\
0.1543 \\
0.1549 \\
0.1615 \\
0.1658 \\
0.1714 \\
0.1781 \\
0.1726 \\
0.0423\end{array}$ & $\begin{array}{c}\text { Minimum } \\
\text { zStrain } \\
0.0033 \\
0.0086 \\
0.0142 \\
0.0197 \\
0.0246 \\
0.0278 \\
0.0307 \\
0.0329 \\
0.0356 \\
0.0382 \\
0.0406 \\
0.0434 \\
0.0463 \\
0.0487 \\
0.0512 \\
0.0524 \\
0.0544 \\
0.0555 \\
0.0568 \\
0.0580 \\
0.0604 \\
0.0624 \\
0.0642 \\
0.0649 \\
0.0664 \\
0.0669 \\
0.0686 \\
0.0703 \\
0.0719 \\
0.0741 \\
0.0743 \\
0.0770 \\
0.0782 \\
0.0805 \\
0.0830 \\
0.0791 \\
-0.0118\end{array}$ & $\begin{array}{c}\text { Angle } \\
\text { Degrees } \\
-5.29 \\
-3.67 \\
-2.28 \\
-0.50 \\
-2.41 \\
-3.07 \\
-4.40 \\
-6.26 \\
-7.19 \\
-7.82 \\
-8.64 \\
-9.48 \\
-9.69 \\
-10.72 \\
-11.41 \\
-11.69 \\
-11.46 \\
-11.56 \\
-11.76 \\
-11.53 \\
-12.15 \\
-12.56 \\
-12.75 \\
-12.47 \\
-12.92 \\
-12.89 \\
-12.63 \\
-12.85 \\
-12.73 \\
-12.99 \\
-13.24 \\
-13.09 \\
-13.11 \\
-12.92 \\
-12.86 \\
-13.16 \\
-19.65\end{array}$ \\
\hline
\end{tabular}

High Pressure test 


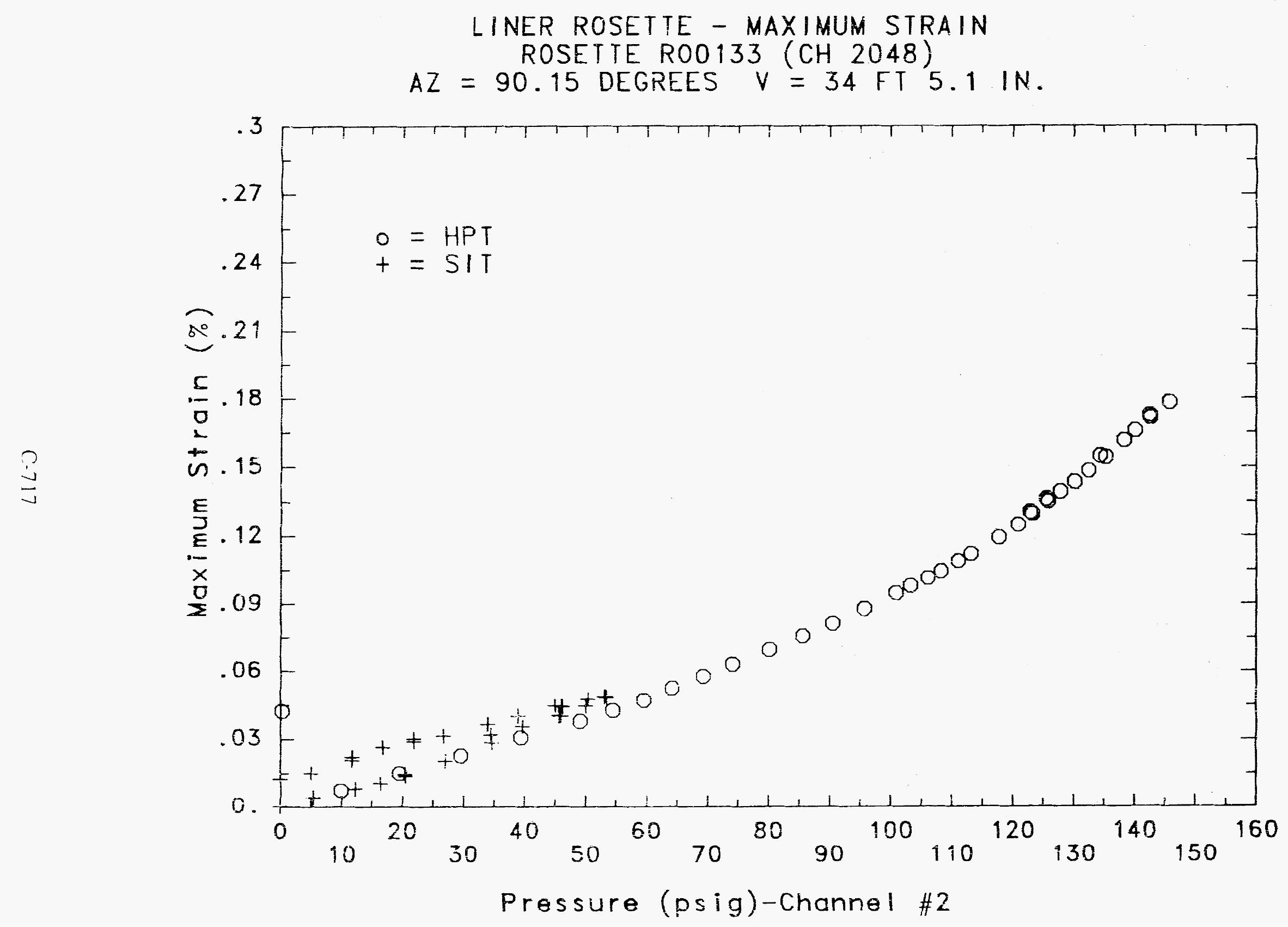


$8(i-5)$

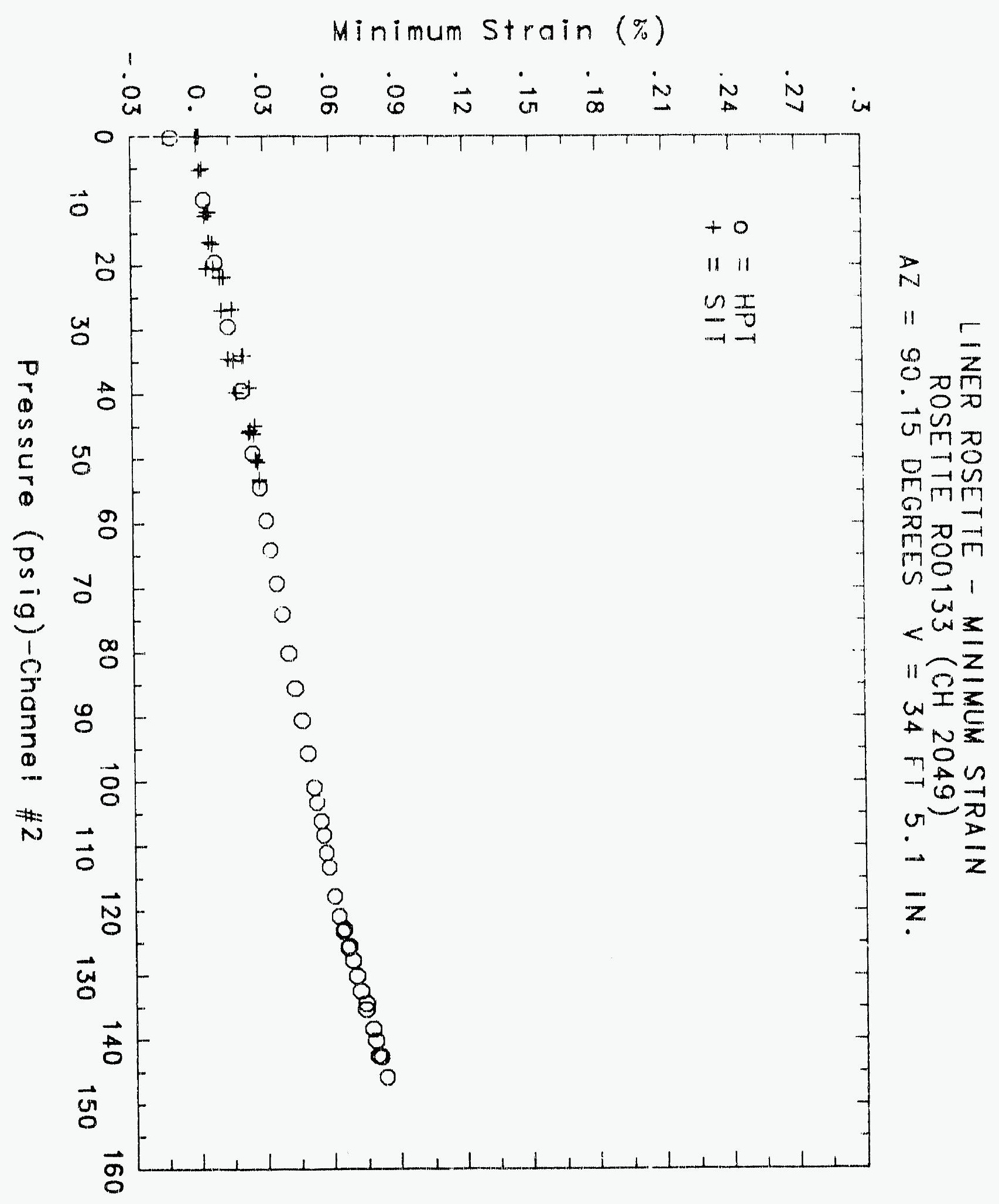




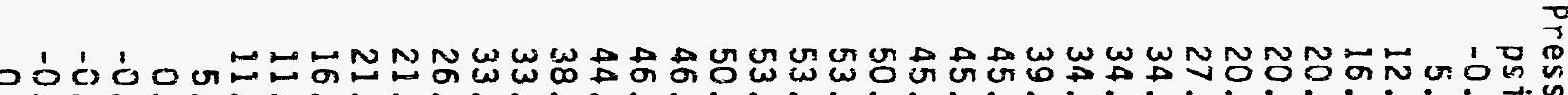

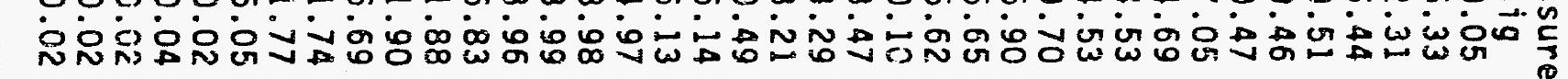
00000000000000000000000000000000000000 年

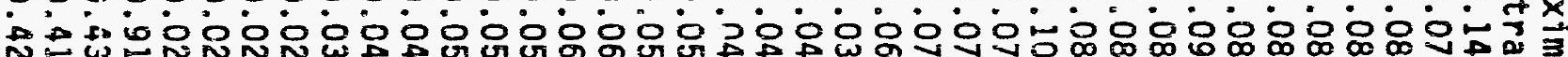

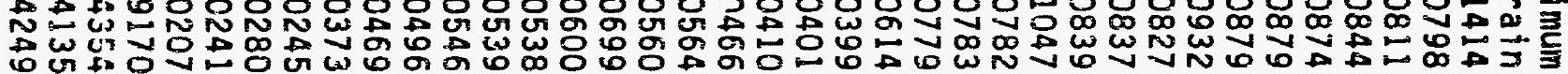

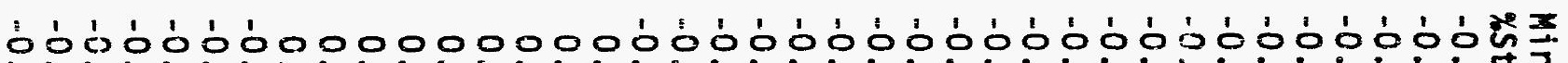

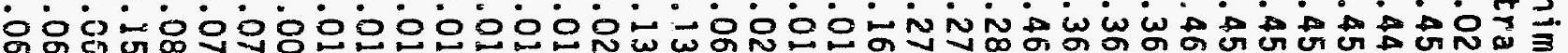

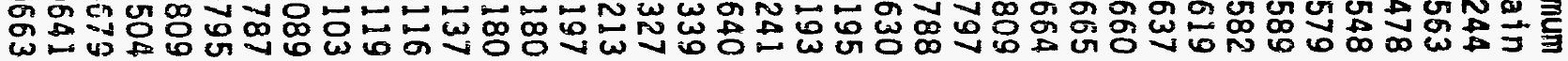

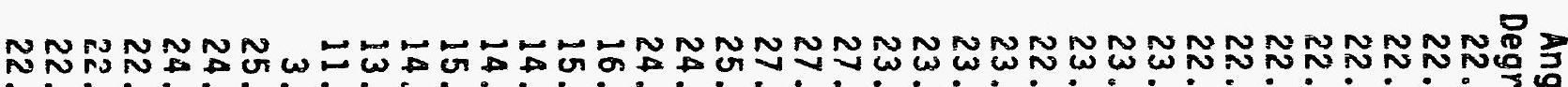
a

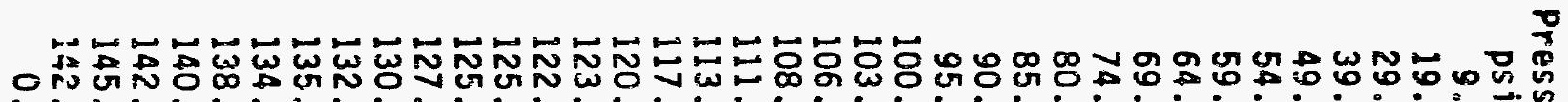

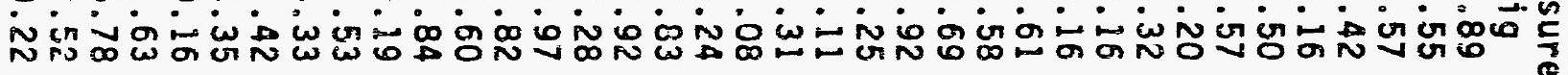
-100000000000000000000000000000000000018

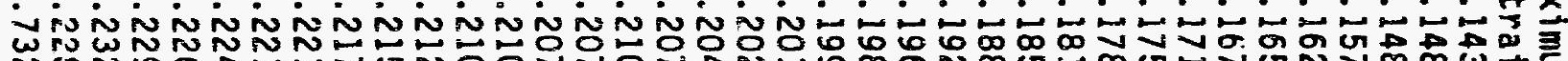

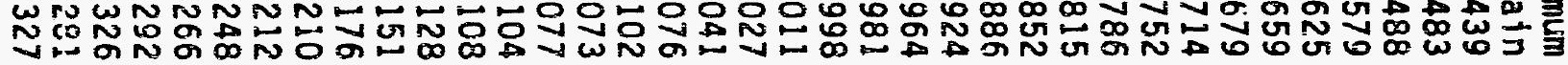

ட 윙

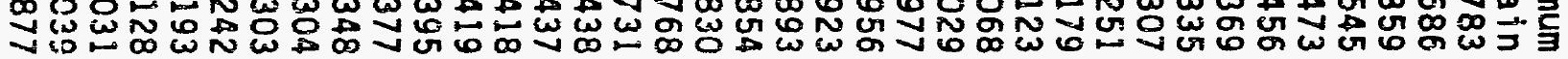

N

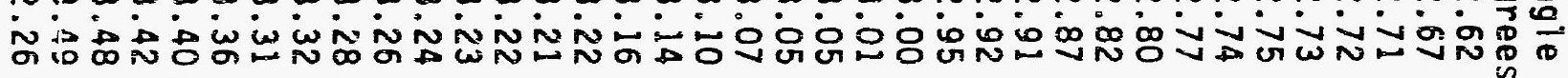




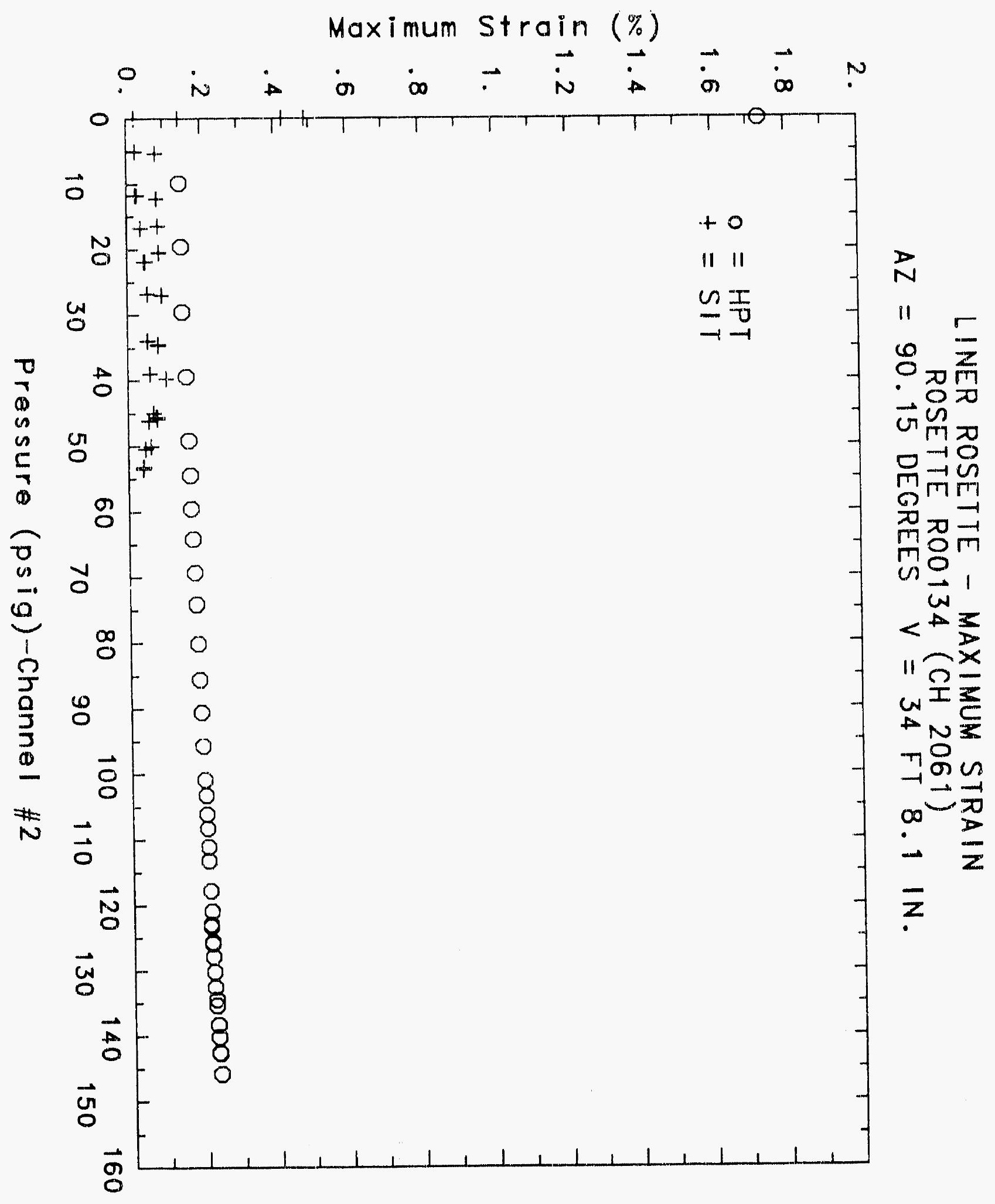




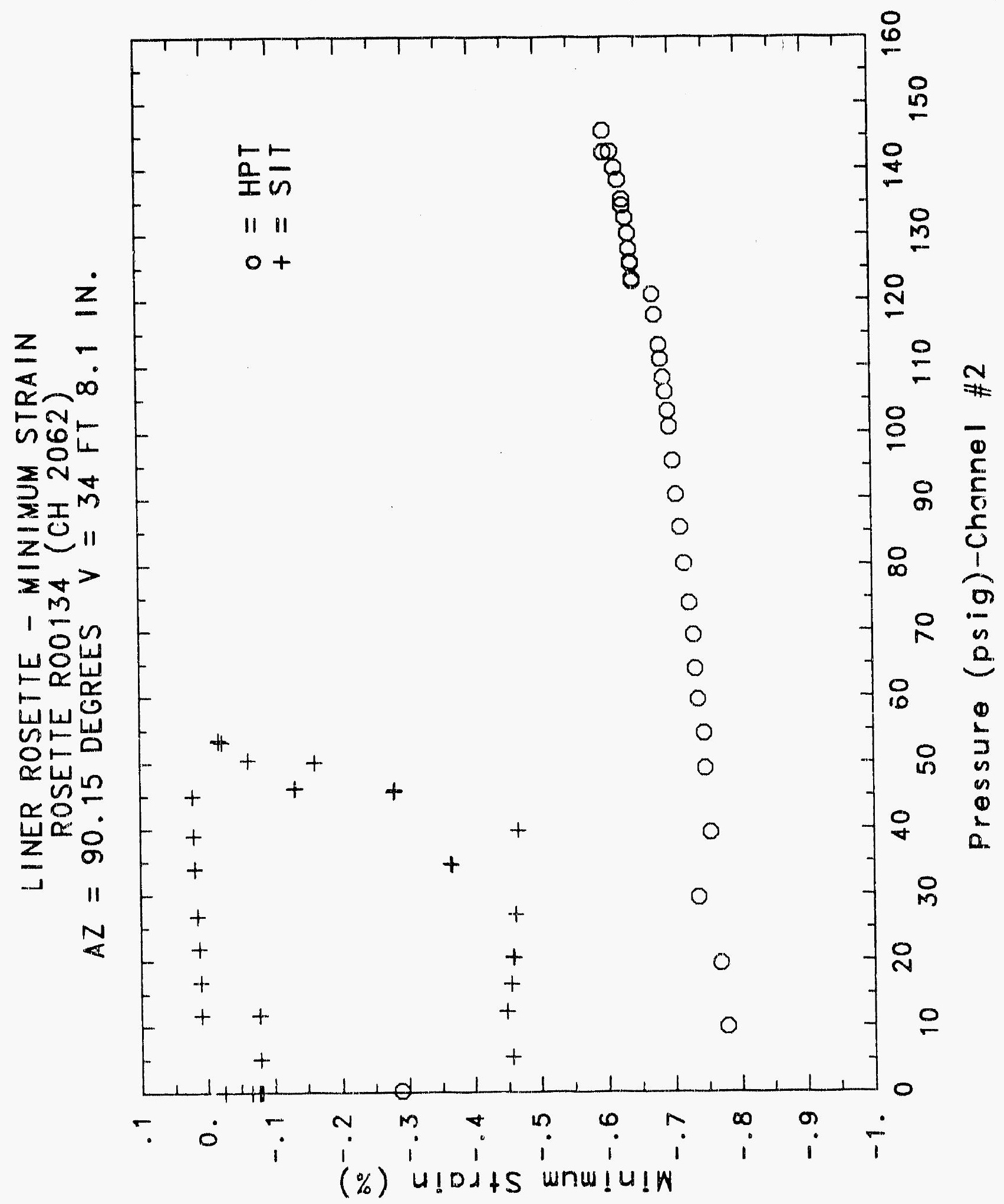




\section{Reinforced Concrete Test Data}

\section{nosette 135 Channels 206420652066}

STRUCTURAL INTEgRITY TEST

\begin{tabular}{|c|c|c|c|c|c|c|c|}
\hline $\begin{array}{c}\text { Pressure } \\
\text { psig } \\
-0.05 \\
5.33 \\
12.31 \\
16.44 \\
20.51 \\
20.46 \\
20.47 \\
27.05 \\
34.59 \\
34.53 \\
34.53 \\
39.70 \\
45.90 \\
45.65 \\
45.62 \\
50.10 \\
53.47 \\
53.29 \\
53.21 \\
50.49 \\
46.14 \\
46.13 \\
44.97 \\
38.98 \\
33.99 \\
33.96 \\
26.83 \\
21.88 \\
21.90 \\
16.69 \\
11.74 \\
11.77 \\
5.05 \\
0.02 \\
-0.04 \\
-0.02 \\
-0.02 \\
0.02\end{array}$ & $\begin{array}{c}\text { Maximum } \\
\text { xStrain } \\
0.0003 \\
0.0030 \\
0.0066 \\
0.0088 \\
0.0117 \\
0.0127 \\
0.0119 \\
0.0171 \\
0.0242 \\
0.0249 \\
0.0250 \\
0.0286 \\
0.0339 \\
0.0341 \\
0.0342 \\
0.0373 \\
0.0405 \\
0.0406 \\
0.0408 \\
0.0398 \\
0.0377 \\
0.0375 \\
0.0382 \\
0.0349 \\
0.0320 \\
0.0320 \\
0.0279 \\
0.0248 \\
0.0245 \\
0.0213 \\
0.0177 \\
0.0173 \\
0.0119 \\
0.0078 \\
0.0108 \\
0.0112 \\
0.01112 \\
0.0123\end{array}$ & $\begin{array}{l}\text { Minimum } \\
\text { \%Strain } \\
0.0000 \\
0.0012 \\
0.0030 \\
0.0048 \\
0.0072 \\
0.0076 \\
0.0058 \\
0.0094 \\
0.0153 \\
0.0138 \\
0.0138 \\
0.0170 \\
0.0237 \\
0.0250 \\
0.0250 \\
0.0277 \\
0.0308 \\
0.0312 \\
0.0314 \\
0.0306 \\
0.0287 \\
0.0288 \\
0.0303 \\
0.0286 \\
0.0263 \\
0.0263 \\
0.0220 \\
0.0188 \\
0.0182 \\
0.0150 \\
0.0113 \\
0.0114 \\
0.0077 \\
0.0067 \\
0.0053 \\
0.0056 \\
0.0057 \\
0.0052\end{array}$ & $\begin{array}{c}\text { Angle } \\
\text { Degrees } \\
-5.64 \\
11.78 \\
10.62 \\
11.07 \\
10.39 \\
10.46 \\
-3.38 \\
-6.76 \\
-6.42 \\
-9.89 \\
-9.27 \\
-9.05 \\
-7.98 \\
-4.60 \\
-6.16 \\
-5.51 \\
-2.65 \\
-2.79 \\
-2.63 \\
-3.08 \\
-5.60 \\
-3.97 \\
-0.46 \\
3.06 \\
1.25 \\
3.85 \\
-0.24 \\
-2.77 \\
-3.23 \\
-0.73 \\
-1.32 \\
-0.62 \\
3.34 \\
-33.76 \\
-20.23 \\
-22.73 \\
-22.98 \\
-23.64\end{array}$ & $\begin{array}{c}\text { Pressure } \\
\text { psig } \\
9.89 \\
19.55 \\
29.57 \\
39.42 \\
49.16 \\
54.50 \\
59.57 \\
64.20 \\
69.32 \\
74.16 \\
80.16 \\
85.61 \\
90.58 \\
95.69 \\
100.92 \\
103.25 \\
106.11 \\
108.31 \\
111.08 \\
113.24 \\
117.83 \\
120.92 \\
123.28 \\
122.97 \\
125.82 \\
125.60 \\
127.84 \\
130.19 \\
132.53 \\
135.33 \\
134.42 \\
138.35 \\
140.16 \\
142.63 \\
145.78 \\
142.52 \\
0.22\end{array}$ & $\begin{array}{l}\text { Maximum } \\
\text { xStrain } \\
0.0186 \\
0.0201 \\
0.0261 \\
0.0317 \\
0.0379 \\
0.0414 \\
0.0449 \\
0.0488 \\
0.0529 \\
0.0570 \\
0.0621 \\
0.0666 \\
0.0711 \\
0.0761 \\
0.0814 \\
0.0836 \\
0.0861 \\
0.0880 \\
0.0917 \\
0.0936 \\
0.0983 \\
0.1016 \\
0.1042 \\
0.1042 \\
0.1073 \\
0.1079 \\
0.1099 \\
0.1127 \\
0.1150 \\
0.1185 \\
0.1187 \\
0.1224 \\
0.1246 \\
0.1275 \\
0.1310 \\
0.1272 \\
0.3309\end{array}$ & $\begin{array}{c}\text { Minimum } \\
\text { \%Strain } \\
-0.0555 \\
-0.0183 \\
-0.0101 \\
-0.0014 \\
0.0042 \\
0.0071 \\
0.0111 \\
0.0143 \\
0.0181 \\
0.0220 \\
0.0240 \\
0.0278 \\
0.0308 \\
0.0333 \\
0.0369 \\
0.0378 \\
0.0390 \\
0.0402 \\
0.0407 \\
0.0418 \\
0.0451 \\
0.0467 \\
0.0480 \\
0.0485 \\
0.0505 \\
0.0489 \\
0.0503 \\
0.0514 \\
0.0530 \\
0.0555 \\
0.0558 \\
0.0588 \\
0.0610 \\
0.0635 \\
0.0671 \\
0.0628 \\
-0.0433\end{array}$ & $\begin{array}{l}\text { Angle } \\
\text { Degrees } \\
-21.23 \\
-20.20 \\
-20.13 \\
-18.53 \\
-17.81 \\
-17.96 \\
-16.80 \\
-16.57 \\
-16.76 \\
-15.83 \\
-16.48 \\
-16.14 \\
-15.88 \\
-15.34 \\
-15.22 \\
-15.30 \\
-15.36 \\
-14.44 \\
-13.94 \\
-13.83 \\
-13.58 \\
-13.07 \\
-12.92 \\
-12.99 \\
-12.68 \\
-13.13 \\
-12.88 \\
-12.86 \\
-12.92 \\
-12.60 \\
-12.48 \\
-12.74 \\
-12.24 \\
-12.32 \\
-12.09 \\
-12.65 \\
-23.21\end{array}$ \\
\hline
\end{tabular}

High Pressure test 


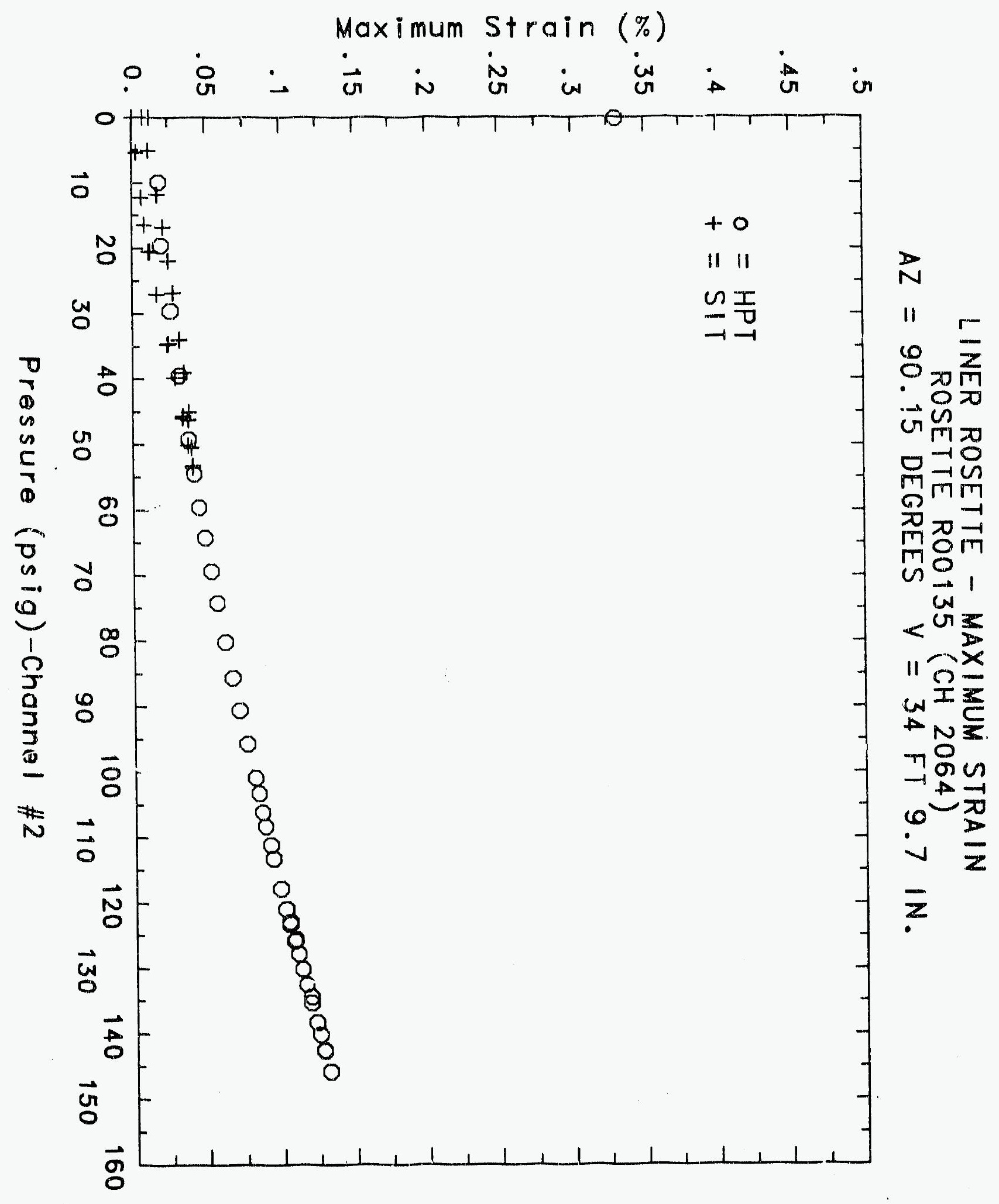




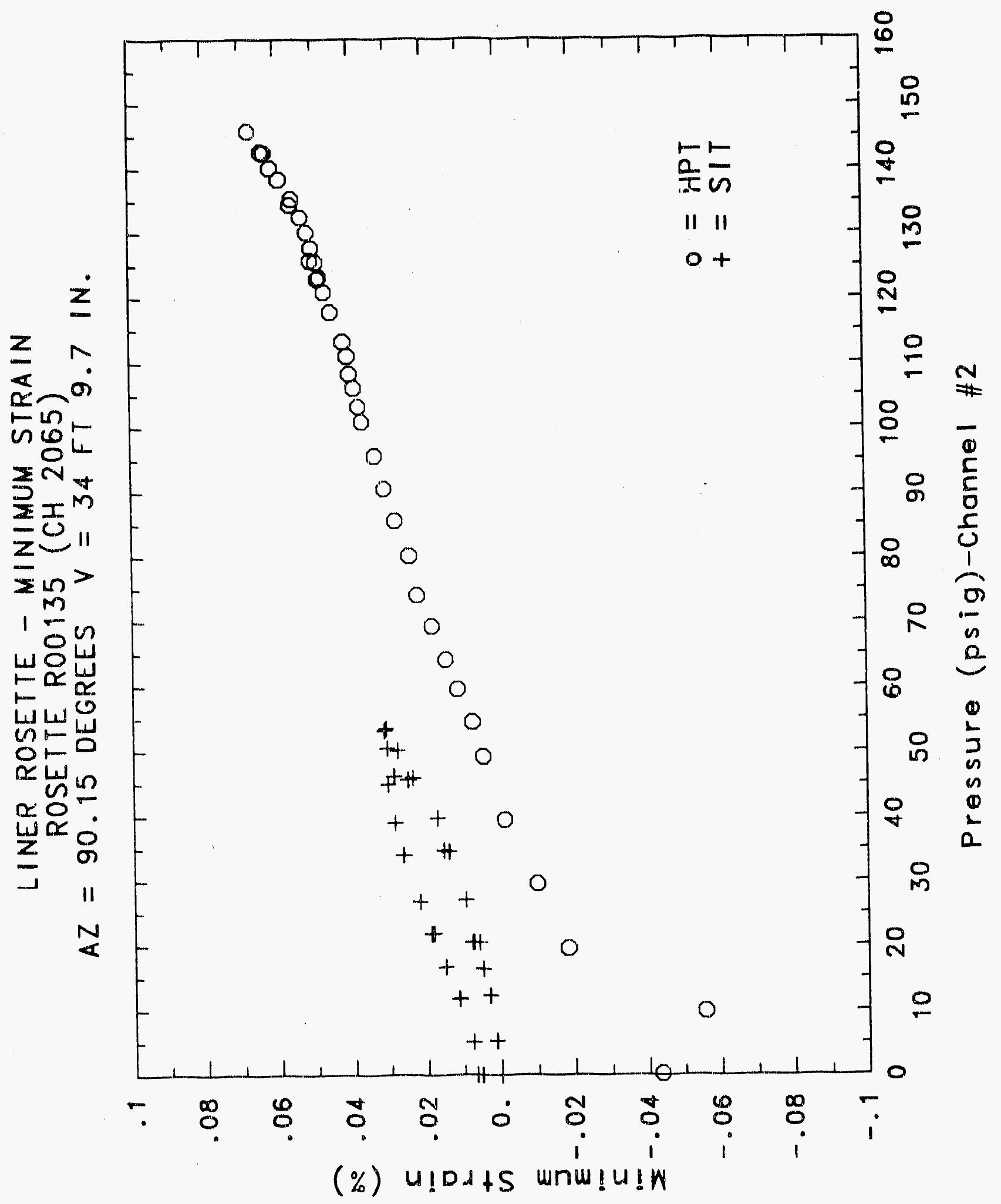




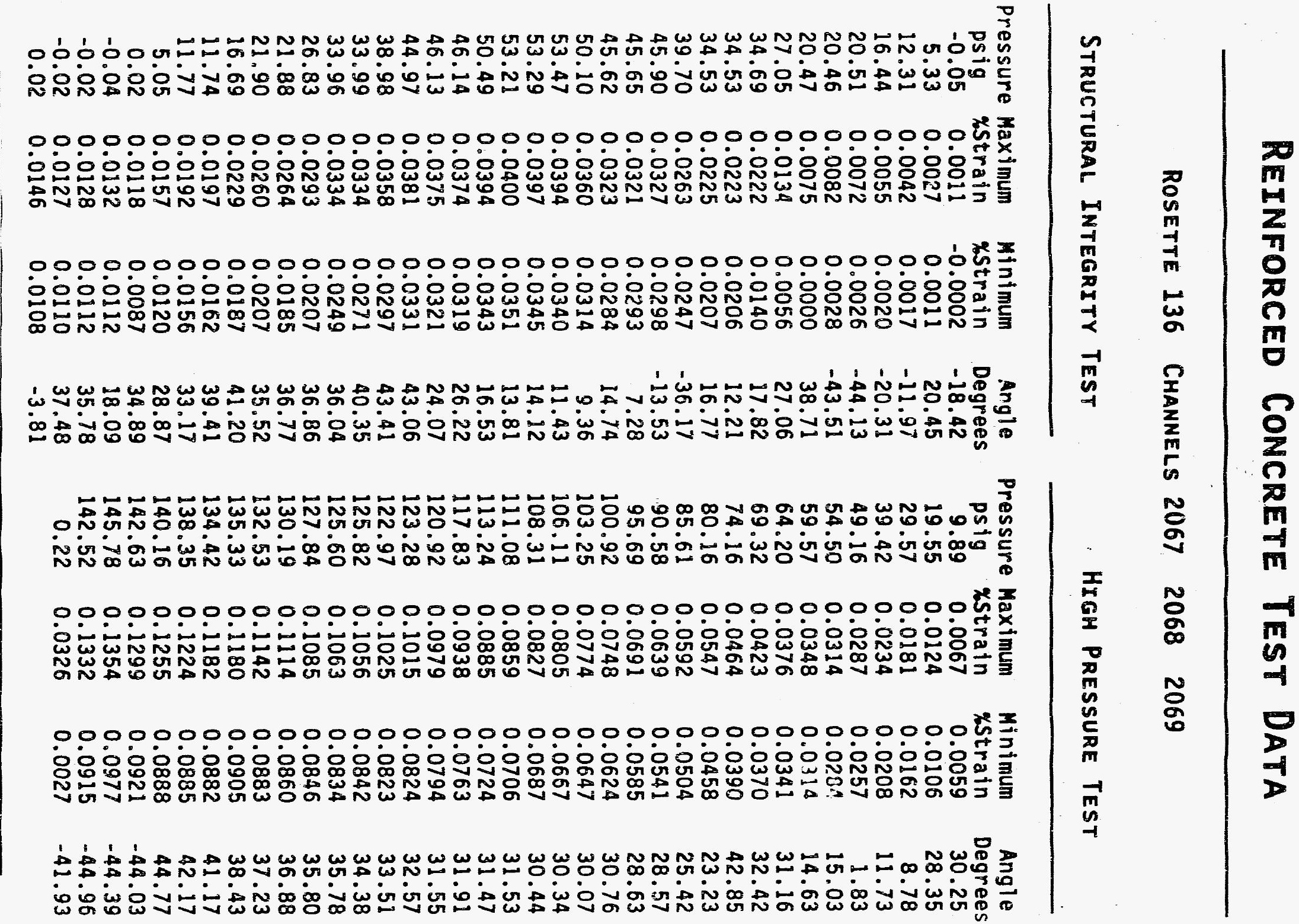




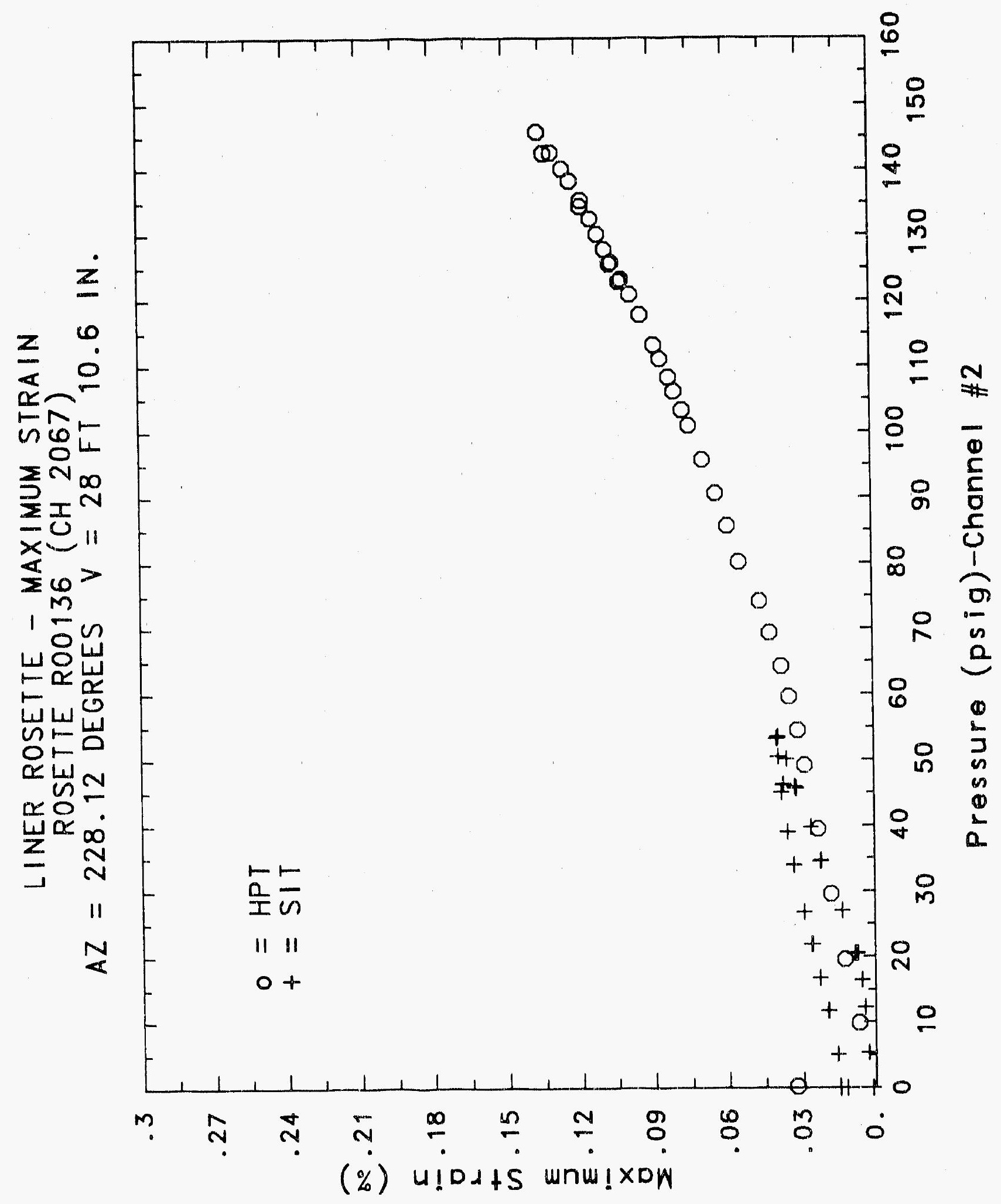




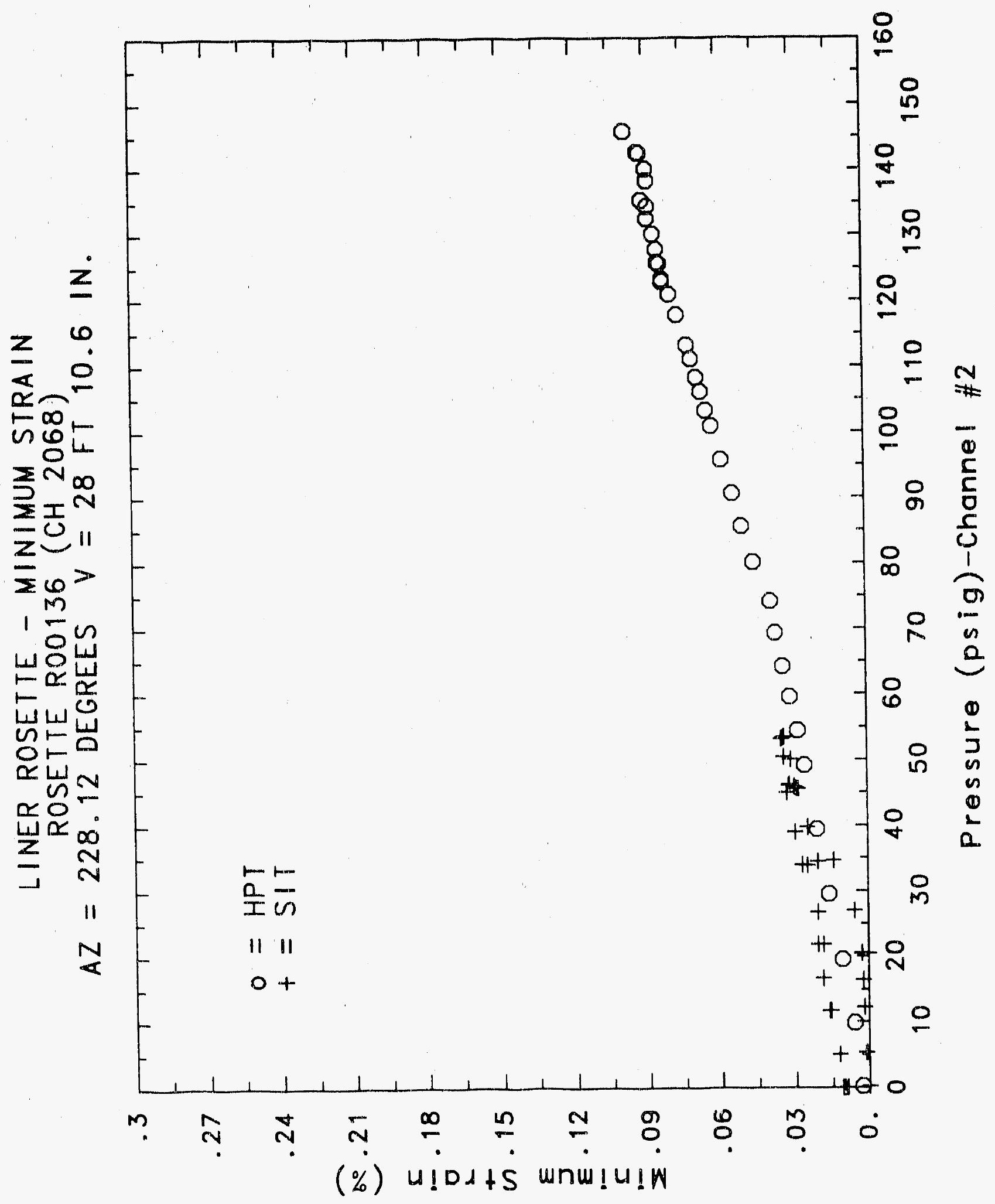




\section{Reinforced Concrete Test Data}

Rosette 137 Channels 208020812082

STRUCTURAL INTEGRITY TEST

\begin{tabular}{|c|c|c|c|c|c|c|c|}
\hline $\begin{array}{c}\text { Pressure } \\
\text { psig } \\
-0.05 \\
5.33 \\
12.31 \\
16.44 \\
20.51 \\
20.46 \\
20.47 \\
27.05 \\
34.69 \\
34.53 \\
34.53 \\
39.70 \\
45.90 \\
45.65 \\
45.62 \\
50.10 \\
53.47 \\
53.29 \\
53.21 \\
50.49 \\
46.14 \\
46.13 \\
44.97 \\
38.98 \\
33.99 \\
33.96 \\
26.83 \\
21.88 \\
21.90 \\
16.69 \\
11.74 \\
11.77 \\
5.05 \\
0.02 \\
-0.04 \\
-0.02 \\
-0.02 \\
0.02\end{array}$ & $\begin{array}{c}\text { Maximum } \\
\text { \%Strain } \\
0.0000 \\
0.0016 \\
0.0037 \\
0.0055 \\
0.0083 \\
0.0093 \\
0.0091 \\
0.0145 \\
0.0227 \\
0.0241 \\
0.0243 \\
0.0282 \\
0.0370 \\
0.0379 \\
0.0381 \\
0.0415 \\
0.0445 \\
0.0449 \\
0.0452 \\
0.0443 \\
0.0424 \\
0.0423 \\
0.0411 \\
0.0380 \\
0.0353 \\
0.0351 \\
0.0311 \\
0.0279 \\
0.0277 \\
0.0246 \\
0.0216 \\
0.0209 \\
0.0169 \\
0.0135 \\
0.0141 \\
0.0140 \\
0.0137 \\
0.0129\end{array}$ & $\begin{array}{c}\text { Minimum } \\
\text { \%Strain } \\
-0.0007 \\
0.0012 \\
0.0032 \\
0.0046 \\
0.0061 \\
0.0070 \\
0.0060 \\
0.0108 \\
0.0180 \\
0.0177 \\
0.0182 \\
0.0227 \\
0.0279 \\
0.0285 \\
0.0287 \\
0.0317 \\
0.0345 \\
0.0352 \\
0.0353 \\
0.0347 \\
0.0330 \\
0.0329 \\
0.0344 \\
0.0317 \\
0.0289 \\
0.0282 \\
0.0244 \\
0.0212 \\
0.0208 \\
0.0175 \\
0.0148 \\
0.0154 \\
0.0119 \\
0.0093 \\
0.0112 \\
0.0113 \\
0.0110 \\
0.0096\end{array}$ & $\begin{array}{c}\text { Argle } \\
\text { Degrees } \\
34.66 \\
1.60 \\
26.72 \\
9.38 \\
17.31 \\
15.93 \\
24.29 \\
18.42 \\
11.96 \\
20.53 \\
20.97 \\
18.69 \\
26.33 \\
27.32 \\
27.49 \\
27.48 \\
26.62 \\
25.87 \\
26.30 \\
26.64 \\
26.98 \\
27.32 \\
16.02 \\
16.08 \\
15.25 \\
16.47 \\
14.35 \\
11.69 \\
11.92 \\
14.16 \\
17.02 \\
14.65 \\
14.85 \\
14.40 \\
16.78 \\
13.83 \\
7.62 \\
-3.96\end{array}$ & $\begin{array}{c}\text { Pressure } \\
\text { psig } \\
9.89 \\
19.55 \\
29.57 \\
39.42 \\
49.16 \\
54.50 \\
59.57 \\
64.20 \\
69.32 \\
74.16 \\
80.16 \\
85.61 \\
90.58 \\
95.69 \\
100.92 \\
103.25 \\
106.11 \\
108.31 \\
111.08 \\
113.24 \\
117.83 \\
120.92 \\
123.28 \\
122.97 \\
125.82 \\
125.60 \\
127.84 \\
130.19 \\
132.53 \\
135.33 \\
134.42 \\
138.35 \\
140.16 \\
142.63 \\
145.78 \\
142.52 \\
0.22\end{array}$ & $\begin{array}{c}\text { Maximum } \\
\text { \%Strain } \\
0.0074 \\
0.0135 \\
0.0198 \\
0.0246 \\
0.0303 \\
0.0338 \\
0.0374 \\
0.0448 \\
0.0487 \\
0.0573 \\
0.0569 \\
0.0619 \\
0.0667 \\
0.0719 \\
0.0773 \\
0.0800 \\
0.0828 \\
0.0849 \\
0.0881 \\
0.0905 \\
0.0957 \\
0.0998 \\
0.1032 \\
0.1035 \\
0.1067 \\
0.1076 \\
0.1100 \\
0.1133 \\
0.1168 \\
0.1213 \\
0.1221 \\
0.1281 \\
0.1304 \\
0.1343 \\
0.1391 \\
0.1342 \\
0.0294\end{array}$ & $\begin{array}{c}\text { Minimum } \\
\text { xStrain } \\
0.0062 \\
0.0121 \\
0.0180 \\
0.0236 \\
0.0290 \\
0.0329 \\
0.0361 \\
0.0399 \\
0.0441 \\
0.0475 \\
0.0402 \\
0.0439 \\
0.0468 \\
0.0494 \\
0.0524 \\
0.0536 \\
0.0558 \\
0.0574 \\
0.0595 \\
0.0609 \\
0.0649 \\
0.0679 \\
0.0718 \\
0.0738 \\
0.0779 \\
0.0808 \\
0.0836 \\
0.0877 \\
0.0909 \\
0.0954 \\
0.1008 \\
0.1075 \\
0.1077 \\
0.1099 \\
0.1092 \\
0.1015 \\
0.0143\end{array}$ & $\begin{array}{r}\text { Angle } \\
\text { Degrees } \\
42.48 \\
-40.56 \\
-43.32 \\
-13.73 \\
-4.87 \\
-26.69 \\
-17.72 \\
30.78 \\
35.42 \\
30.99 \\
14.08 \\
13.20 \\
13.66 \\
12.72 \\
12.55 \\
12.61 \\
12.05 \\
11.50 \\
11.51 \\
11.39 \\
10.67 \\
10.44 \\
9.41 \\
8.39 \\
7.00 \\
5.06 \\
3.93 \\
2.17 \\
0.56 \\
-0.96 \\
-8.54 \\
-15.88 \\
-9.87 \\
-8.27 \\
-2.53 \\
0.79 \\
-5.82\end{array}$ \\
\hline
\end{tabular}

high Pressure test 


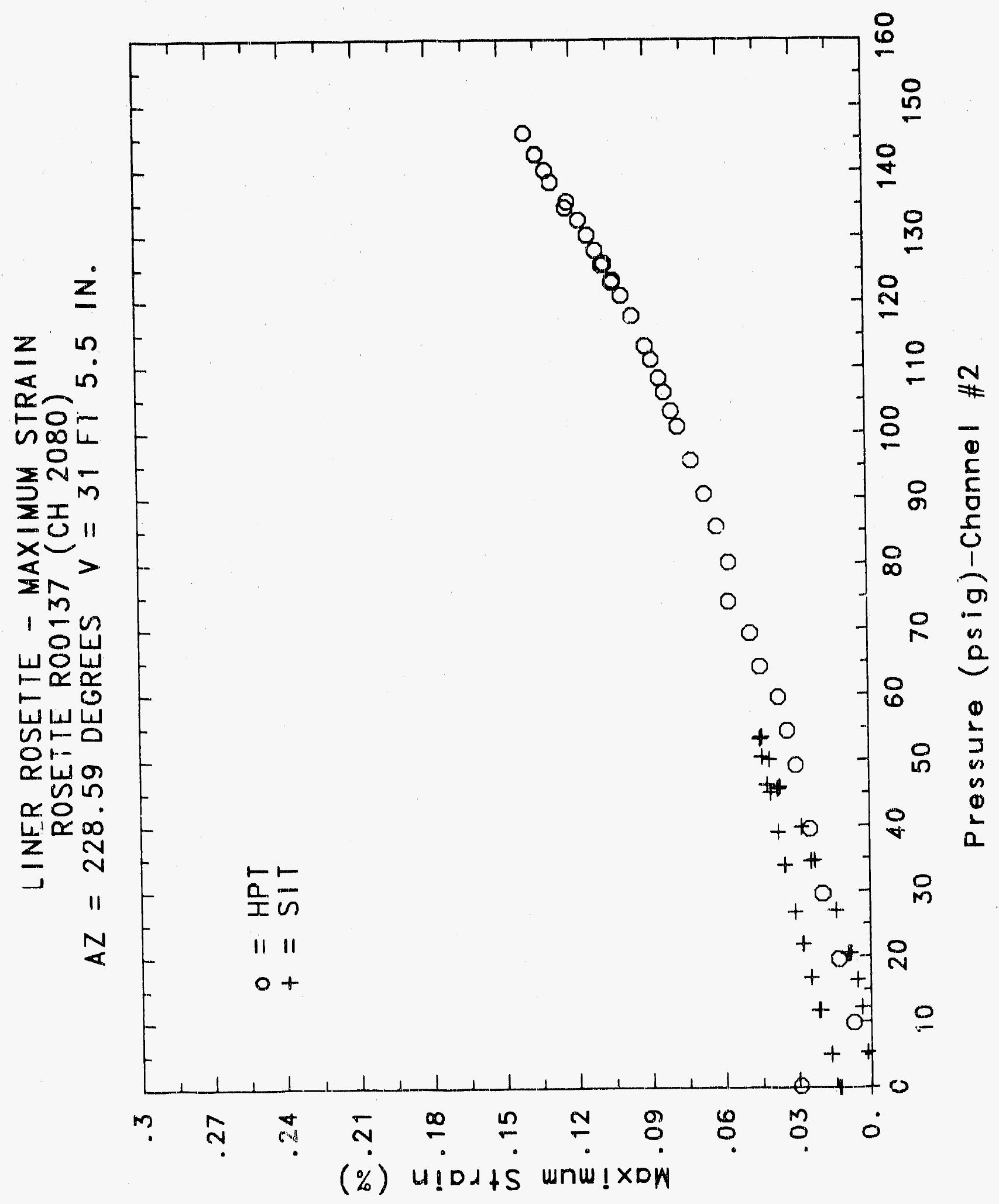




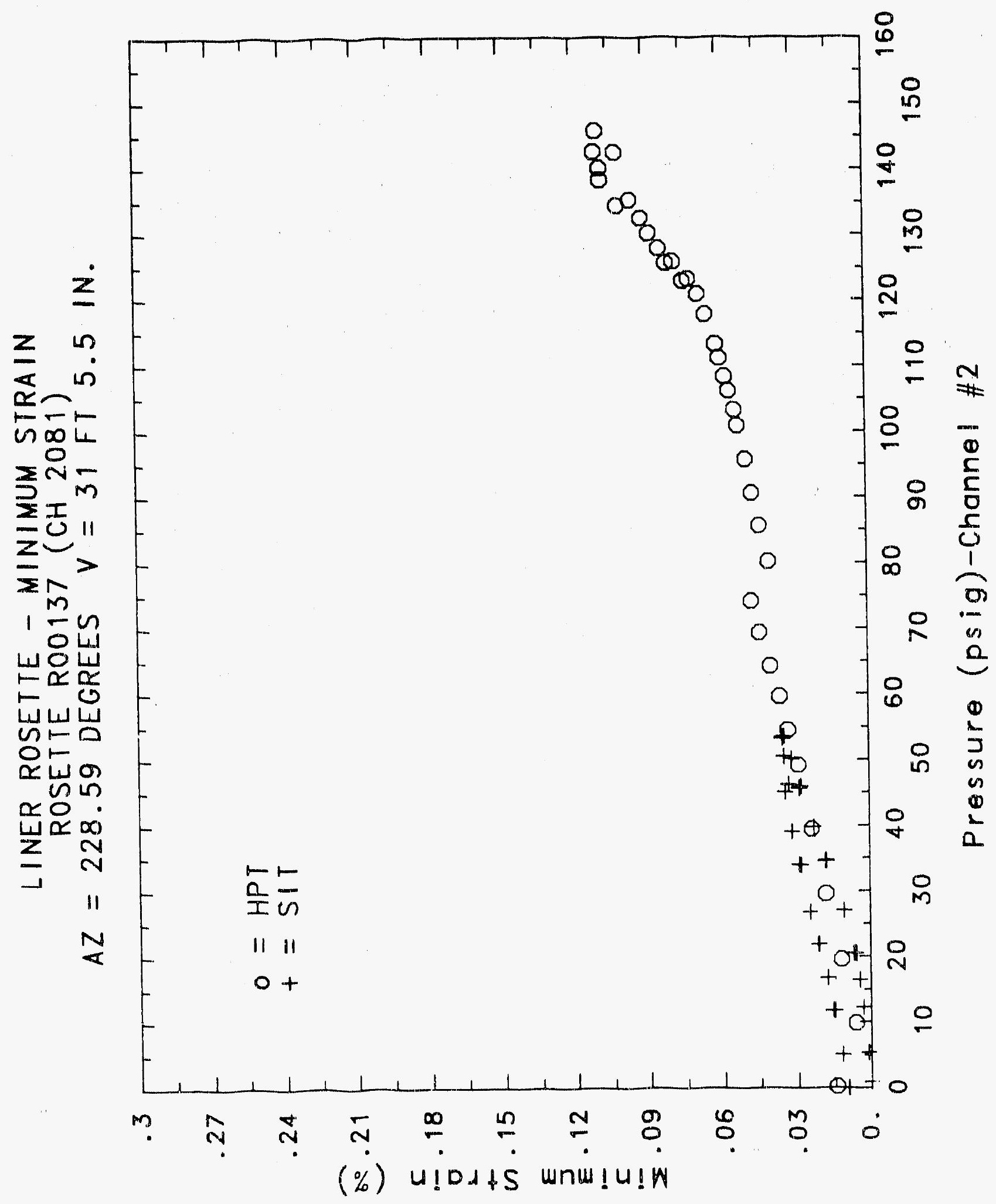




\section{Reinforced Concrete Test Data}

Rosette 138 Channels 208320842085

Structural Integrity Test

\begin{tabular}{|c|c|c|c|c|c|c|c|}
\hline $\begin{array}{c}\text { Pressure } \\
\text { psig } \\
-0.05 \\
5.33 \\
12.31 \\
16.44 \\
20.51 \\
20.46 \\
20.47 \\
27.05 \\
34.69 \\
34.53 \\
34.53 \\
39.70 \\
45.90 \\
45.65 \\
45.62 \\
50.10 \\
53.47 \\
53.29 \\
53.21 \\
50.49 \\
46.14 \\
46.13 \\
44.97 \\
38.98 \\
33.99 \\
33.96 \\
26.83 \\
21.88 \\
21.90 \\
16.69 \\
11.74 \\
11.77 \\
5.05 \\
0.02 \\
-0.04 \\
-0.02 \\
-0.02 \\
0.02\end{array}$ & $\begin{array}{c}\text { Maximum } \\
\text { \%Strain } \\
0.0001 \\
-0.0026 \\
-0.0015 \\
0.0004 \\
0.0037 \\
0.0042 \\
0.0045 \\
0.0108 \\
0.0188 \\
0.0199 \\
0.0198 \\
0.0231 \\
0.0277 \\
0.0278 \\
0.0279 \\
0.0308 \\
0.0334 \\
0.0335 \\
0.0336 \\
0.0324 \\
0.0301 \\
0.0301 \\
0.0308 \\
0.0272 \\
0.0245 \\
0.0246 \\
0.0196 \\
0.0166 \\
0.0162 \\
0.0126 \\
0.0086 \\
0.0080 \\
0.0036 \\
0.0039 \\
0.0036 \\
0.0038 \\
0.0038 \\
0.0036\end{array}$ & $\begin{array}{r}\text { Minimum } \\
\text { \%Strain } \\
0.0001 \\
-0.0077 \\
-0.0103 \\
-0.0099 \\
-0.0091 \\
-0.0088 \\
-0.0088 \\
-0.0063 \\
-0.0025 \\
-0.0021 \\
-0.0019 \\
0.0006 \\
0.0049 \\
0.0054 \\
0.0057 \\
0.0080 \\
0.0103 \\
0.0109 \\
0.0110 \\
0.0105 \\
0.0091 \\
0.0089 \\
0.0088 \\
0.0070 \\
0.0048 \\
0.0047 \\
0.0020 \\
0.0000 \\
-0.0001 \\
-0.0021 \\
-0.0037 \\
-0.0036 \\
-0.0047 \\
-0.0028 \\
-0.0020 \\
-0.0021 \\
-0.0022 \\
-0.0022\end{array}$ & $\begin{array}{c}\text { Angle } \\
\text { Degrees } \\
19.23 \\
-33.40 \\
-26.24 \\
-22.84 \\
-21.84 \\
-22.26 \\
-22.91 \\
-21.49 \\
-22.86 \\
-24.79 \\
-24.19 \\
-23.58 \\
-22.85 \\
-22.32 \\
-22.70 \\
-22.30 \\
-21.84 \\
-21.82 \\
-21.81 \\
-22.11 \\
-22.51 \\
-22.42 \\
-24.75 \\
-25.47 \\
-26.39 \\
-26.96 \\
-27.79 \\
-29.51 \\
-29.51 \\
-30.69 \\
-32.84 \\
-31.50 \\
-33.28 \\
-10.13 \\
-7.05 \\
-7.92 \\
-8.70 \\
-11.51\end{array}$ & $\begin{array}{c}\text { Pressure } \\
\text { psig } \\
9.89 \\
19.55 \\
29.57 \\
39.42 \\
49.16 \\
54.50 \\
59.57 \\
64.20 \\
69.32 \\
74.16 \\
80.16 \\
85.61 \\
90.58 \\
95.69 \\
100.92 \\
103.25 \\
106.11 \\
108.31 \\
111.08 \\
113.24 \\
117.83 \\
120.92 \\
123.28 \\
122.97 \\
125.82 \\
125.60 \\
127.84 \\
130.19 \\
132.53 \\
135.33 \\
134.42 \\
138.35 \\
140.16 \\
142.63 \\
145.78 \\
142.52 \\
0.22\end{array}$ & $\begin{array}{c}\text { Maximum } \\
\text { \%Strain } \\
0.0040 \\
0.0106 \\
0.0173 \\
0.0241 \\
0.0305 \\
0.0345 \\
0.0387 \\
0.0422 \\
0.0462 \\
0.0502 \\
0.0550 \\
0.0593 \\
0.0640 \\
0.0685 \\
0.0736 \\
0.0761 \\
0.0789 \\
0.0809 \\
0.0840 \\
0.0864 \\
0.0916 \\
0.0952 \\
0.0982 \\
0.0988 \\
0.1020 \\
0.1027 \\
0.1053 \\
0.1085 \\
0.1116 \\
0.1158 \\
0.1154 \\
0.1213 \\
0.1244 \\
0.1283 \\
0.1333 \\
0.1283 \\
0.0217\end{array}$ & $\begin{array}{c}\text { Ainimum } \\
\text { \%Strain } \\
-0.0028 \\
0.0007 \\
0.0047 \\
0.0087 \\
0.0126 \\
0.0151 \\
0.0175 \\
0.0207 \\
0.0238 \\
0.0272 \\
0.0308 \\
0.0342 \\
0.0373 \\
0.0407 \\
0.0442 \\
0.0460 \\
0.0477 \\
0.0492 \\
0.0512 \\
0.0526 \\
0.0558 \\
0.0584 \\
0.0606 \\
0.0611 \\
0.0632 \\
0.0637 \\
0.0652 \\
0.0671 \\
0.0690 \\
0.0714 \\
0.0717 \\
0.0747 \\
0.0763 \\
0.0785 \\
0.0814 \\
0.0774 \\
-0.0073\end{array}$ & $\begin{array}{c}\text { Angle } \\
\text { Degrees } \\
43.98 \\
-36.61 \\
-37.08 \\
-29.81 \\
-28.63 \\
-28.49 \\
-27.51 \\
-26.29 \\
-25.05 \\
-23.58 \\
-22.46 \\
-19.90 \\
-18.91 \\
-17.07 \\
-15.58 \\
-15.13 \\
-14.59 \\
-13.81 \\
-13.32 \\
-12.38 \\
-11.43 \\
-10.66 \\
-10.03 \\
-10.34 \\
-9.58 \\
-9.27 \\
-9.47 \\
-9.15 \\
-8.50 \\
-7.25 \\
-7.39 \\
-6.99 \\
-6.39 \\
-5.96 \\
-5.46 \\
-4.42 \\
27.93\end{array}$ \\
\hline
\end{tabular}

\section{High Pressure test}




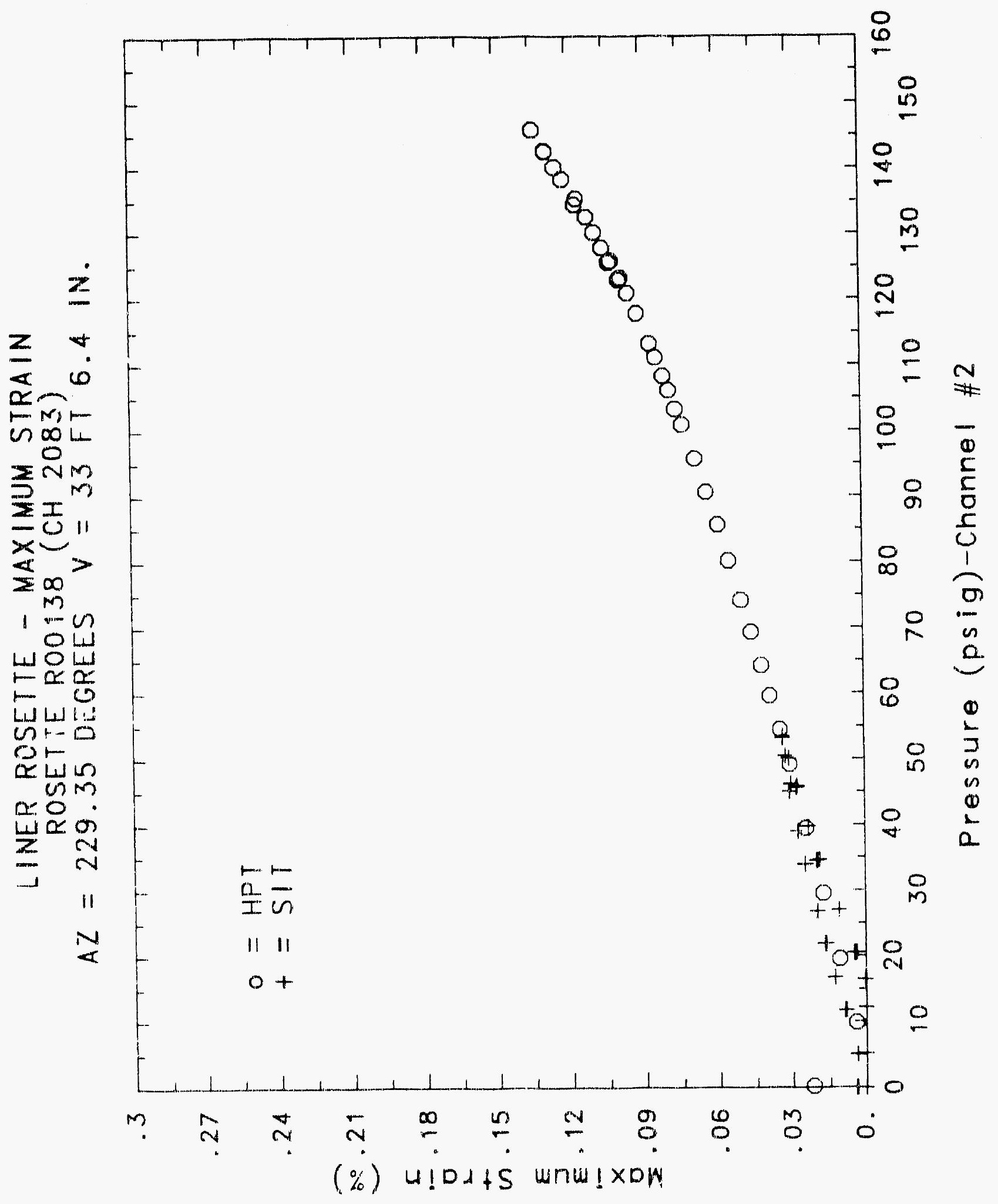

0.732 


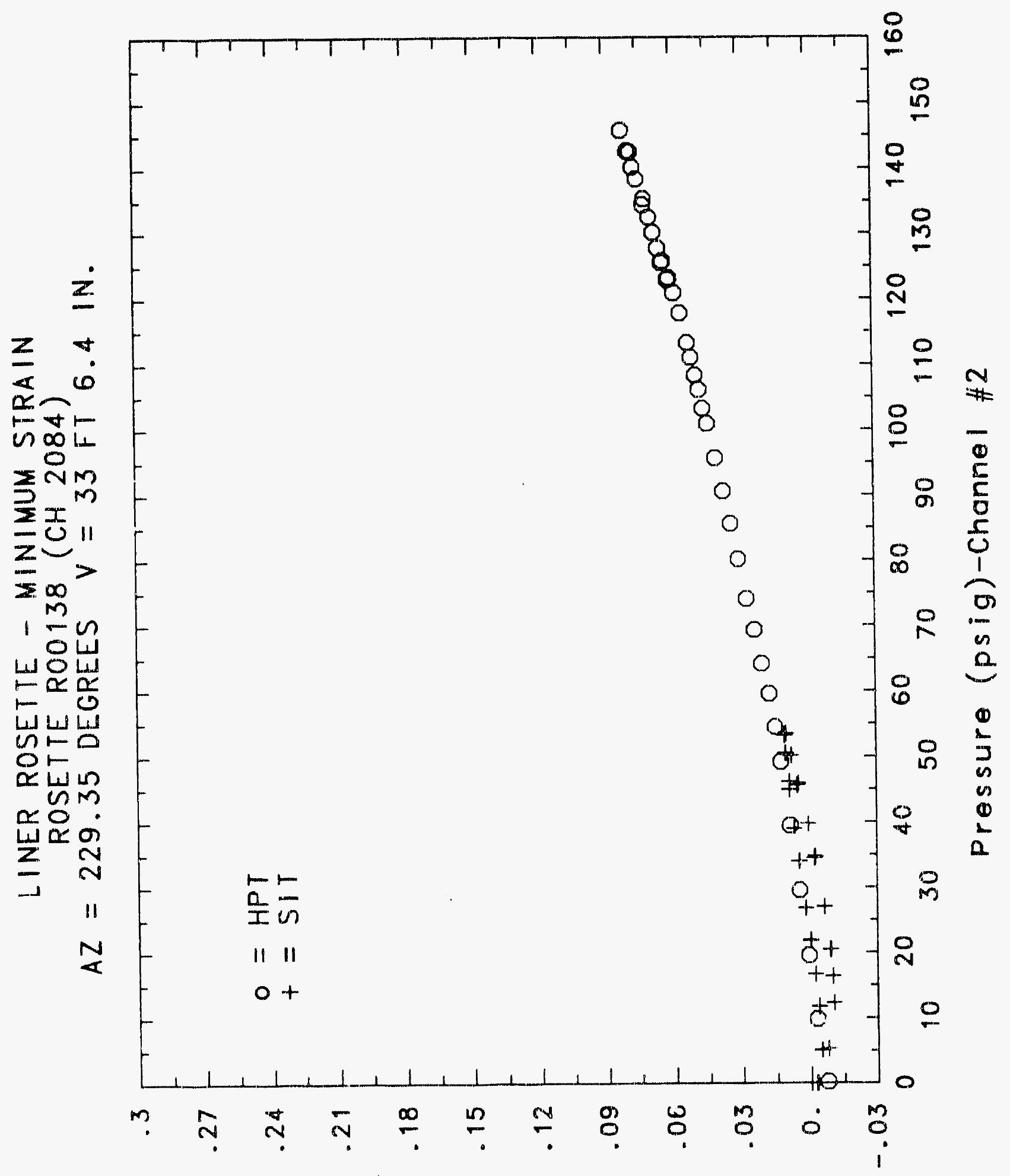

(\%) whodis whแ! แ! 


\section{Reinforced Concrete Test Data}

\section{Rosette 139 Channels 208620872088}

\section{StRUCTURAL INTEgRITY TEST}

\begin{tabular}{|c|c|c|c|c|c|c|c|}
\hline $\begin{array}{c}\text { Pressure } \\
\text { psig } \\
-0.05 \\
5.33 \\
12.31 \\
16.44 \\
20.51 \\
20.46 \\
20.47 \\
27.05 \\
34.69 \\
34.53 \\
34.53 \\
39.70 \\
45.90 \\
45.65 \\
45.62 \\
50.10 \\
53.47 \\
53.29 \\
53.21 \\
50.49 \\
46.14 \\
46.13 \\
44.97 \\
38.98 \\
33.99 \\
33.96 \\
26.83 \\
21.88 \\
21.90 \\
16.69 \\
11.74 \\
11.77 \\
5.05 \\
0.02 \\
-0.04 \\
0.02 \\
-0.02 \\
0.02\end{array}$ & $\begin{array}{c}\text { Maximum } \\
\text { \%.Strain } \\
0.0011 \\
0.0052 \\
0.0126 \\
0.0152 \\
0.0164 \\
0.0159 \\
0.0155 \\
0.0162 \\
0.0201 \\
0.0205 \\
0.0206 \\
0.0239 \\
0.0293 \\
0.0293 \\
0.0294 \\
0.0317 \\
0.0339 \\
0.0341 \\
0.0343 \\
0.0340 \\
0.0325 \\
0.0324 \\
0.0334 \\
0.0304 \\
0.0277 \\
0.0270 \\
0.0246 \\
0.0224 \\
0.0223 \\
0.0202 \\
0.0162 \\
0.0162 \\
0.0069 \\
0.0011 \\
0.0026 \\
0.0030 \\
0.0031 \\
0.0023\end{array}$ & $\begin{array}{l}\text { Minimum } \\
\text { \%Strain } \\
-0.0008 \\
-0.0078 \\
-0.0260 \\
-0.0362 \\
-0.0475 \\
-0.0480 \\
-0.0481 \\
-0.0564 \\
-0.0569 \\
-0.0563 \\
-0.0561 \\
-0.0547 \\
-0.0512 \\
-0.0509 \\
-0.0509 \\
-0.0470 \\
-0.0444 \\
-0.0443 \\
-0.0442 \\
-0.0448 \\
-0.0459 \\
-0.0459 \\
-0.0453 \\
-0.0470 \\
-0.0484 \\
-0.0482 \\
-0.0501 \\
-0.0516 \\
-0.0517 \\
-0.0534 \\
-0.0554 \\
-0.0557 \\
-0.0459 \\
-0.0252 \\
-0.0213 \\
-0.0222 \\
-0.0226 \\
-0.0230\end{array}$ & $\begin{array}{c}\text { Angle } \\
\text { Degrees } \\
-30.76 \\
4.79 \\
1.63 \\
0.48 \\
-0.28 \\
-0.39 \\
-0.35 \\
-2.06 \\
-3.65 \\
-3.81 \\
-3.83 \\
-4.03 \\
-4.61 \\
-4.68 \\
-4.72 \\
-4.68 \\
-4.61 \\
-4.65 \\
-4.68 \\
-4.73 \\
-4.72 \\
-4.71 \\
-4.99 \\
-4.62 \\
-4.51 \\
-4.24 \\
-4.82 \\
-4.91 \\
-4.97 \\
-5.49 \\
-5.64 \\
-5.75 \\
-4.71 \\
-10.65 \\
-10.83 \\
-10.35 \\
-10.67 \\
-10.52\end{array}$ & $\begin{array}{c}\text { Pressure } \\
\text { psig } \\
9.89 \\
19.55 \\
29.57 \\
39.42 \\
49.16 \\
54.50 \\
59.57 \\
64.20 \\
69.32 \\
74.16 \\
80.16 \\
85.61 \\
90.58 \\
95.69 \\
100.92 \\
103.25 \\
106.11 \\
108.31 \\
111.08 \\
113.24 \\
117.83 \\
120.92 \\
123.28 \\
122.97 \\
125.82 \\
125.60 \\
127.84 \\
130.19 \\
132.53 \\
135.33 \\
134.42 \\
138.35 \\
140.16 \\
142.63 \\
145.78 \\
142.52 \\
0.22\end{array}$ & $\begin{array}{c}\text { Maximum } \\
\text { xstrain } \\
0.0099 \\
0.0184 \\
0.0250 \\
0.0295 \\
0.0338 \\
0.0358 \\
0.0382 \\
0.0427 \\
0.0485 \\
0.0521 \\
0.0636 \\
0.0703 \\
0.0760 \\
0.0830 \\
0.0887 \\
0.0919 \\
0.0947 \\
0.0977 \\
0.1014 \\
0.1039 \\
0.1096 \\
0.1141 \\
0.1168 \\
0.1171 \\
0.1201 \\
0.1207 \\
0.1229 \\
0.1260 \\
0.1291 \\
0.1328 \\
0.1332 \\
0.1369 \\
0.1398 \\
0.1429 \\
0.1478 \\
0.1473 \\
0.0423\end{array}$ & $\begin{array}{l}\text { Minimum } \\
\text { XStrain } \\
-0.0315 \\
-0.0326 \\
-0.0258 \\
-0.0203 \\
-0.0147 \\
-0.0119 \\
-0.0087 \\
-0.0053 \\
-0.0024 \\
0.0006 \\
0.0047 \\
0.0084 \\
0.0115 \\
0.0150 \\
0.0186 \\
0.0198 \\
0.0216 \\
0.0224 \\
0.0288 \\
0.0296 \\
0.0323 \\
0.0349 \\
0.0365 \\
0.0370 \\
0.0390 \\
0.0395 \\
0.0407 \\
0.0427 \\
0.0444 \\
0.0470 \\
0.0474 \\
0.0500 \\
0.0519 \\
0.0539 \\
0.0564 \\
0.0538 \\
-0.0243\end{array}$ & $\begin{array}{c}\text { Angle } \\
\text { Degrees } \\
2.18 \\
-1.88 \\
-1.27 \\
-0.31 \\
0.26 \\
0.80 \\
1.03 \\
3.81 \\
5.00 \\
6.38 \\
1.65 \\
0.83 \\
0.35 \\
0.35 \\
0.04 \\
-0.08 \\
-0.31 \\
-0.19 \\
-2.05 \\
-1.99 \\
-2.51 \\
-2.60 \\
-2.39 \\
-2.63 \\
-2.45 \\
-2.49 \\
-2.37 \\
-2.51 \\
-2.40 \\
-2.32 \\
-2.33 \\
-1.90 \\
-1.94 \\
-1.72 \\
-1.30 \\
-1.83 \\
-6.12\end{array}$ \\
\hline
\end{tabular}

High Pressure Test 


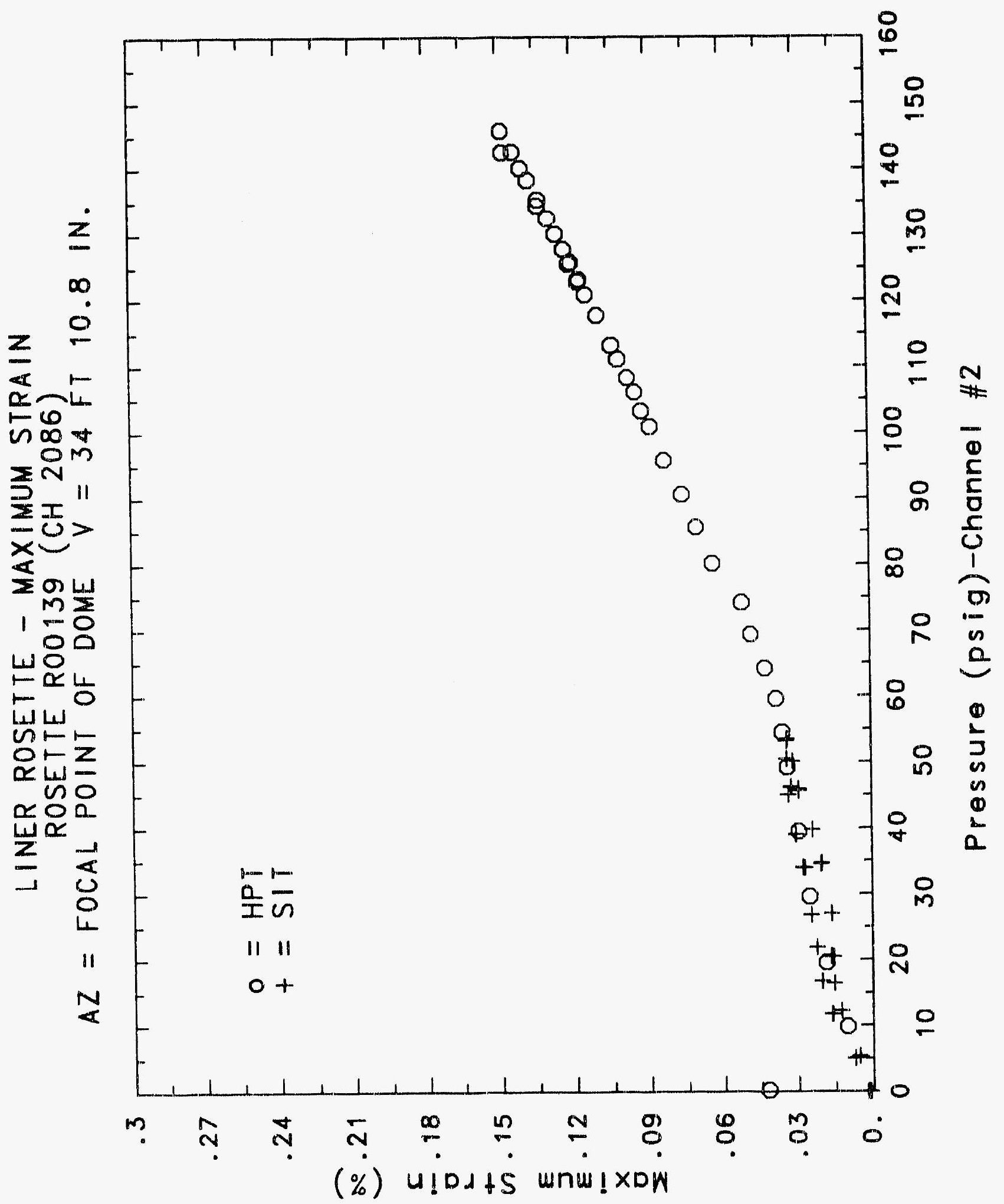




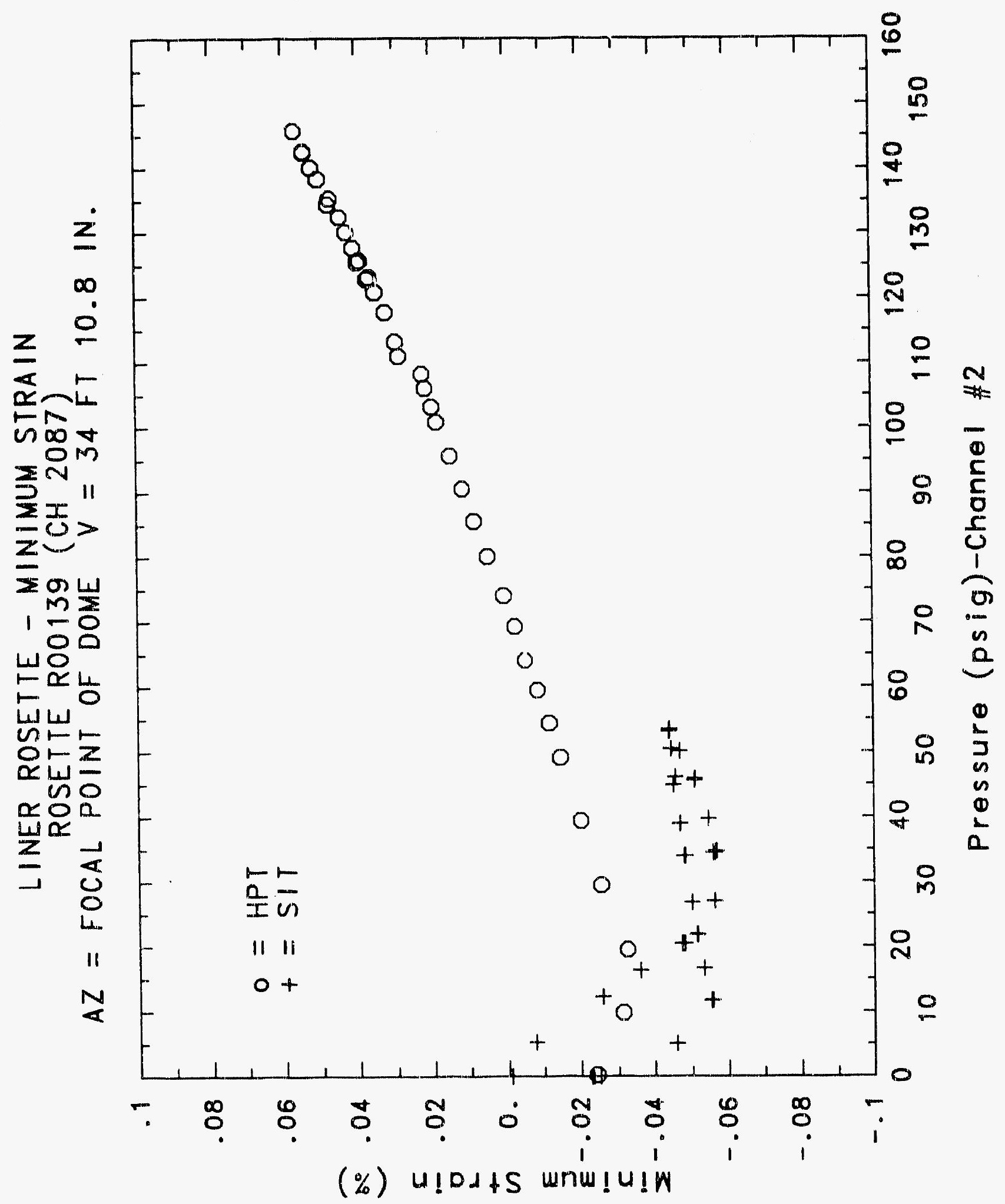

C-736 


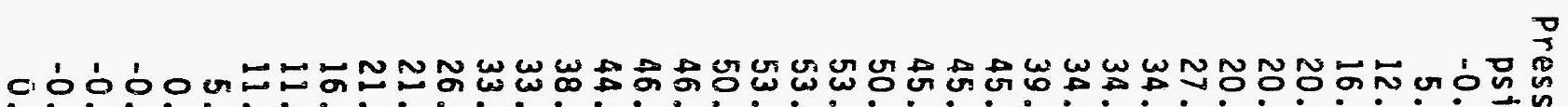

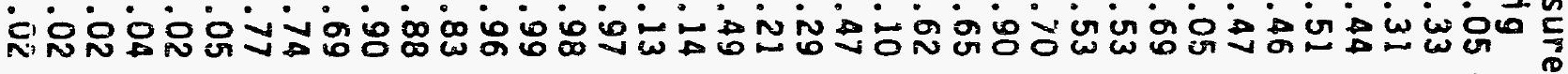

00000000000000000000000000000000000000 k

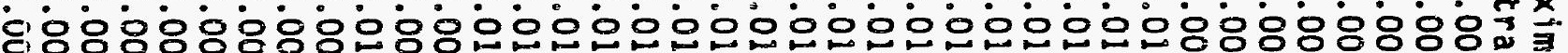

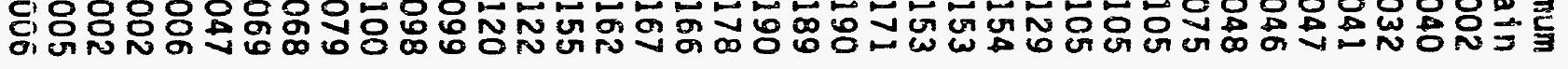

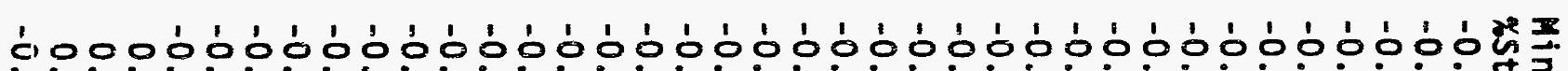

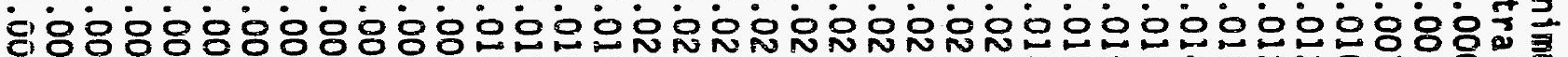

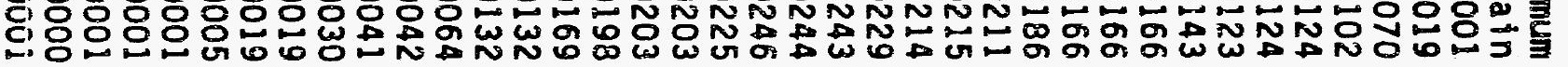

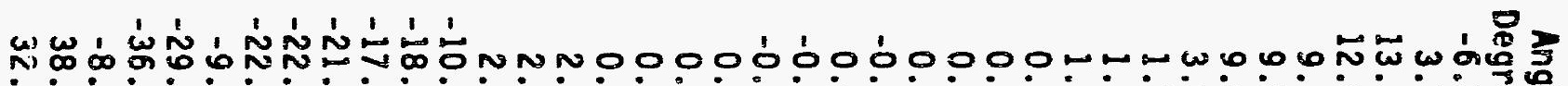

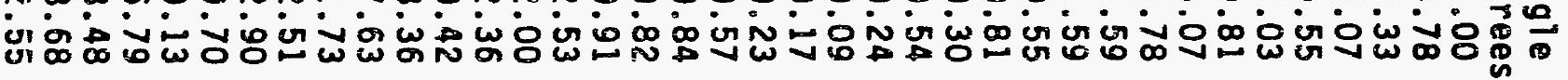

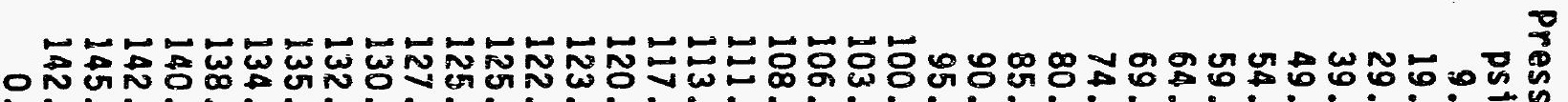
N 0000000000000000000000000000000000000 th o o o o o o

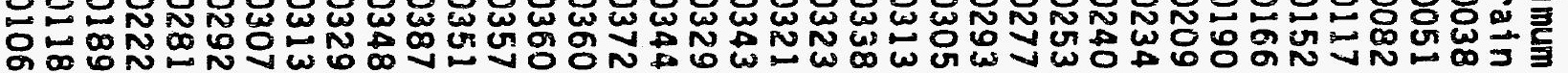

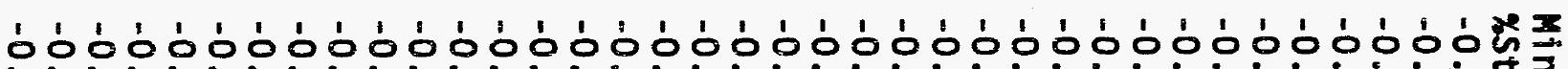
o o o o o o o o o o o

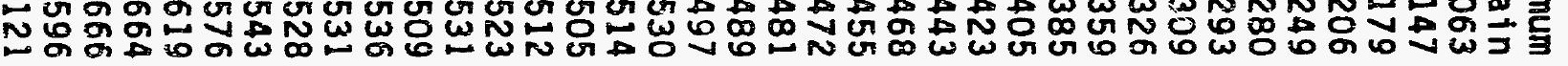

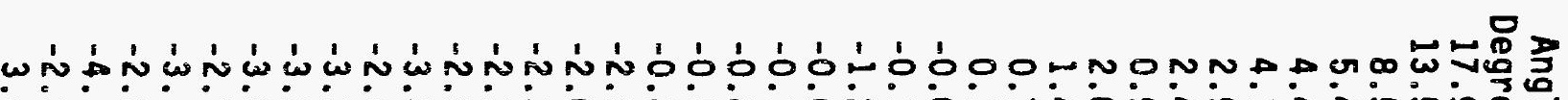

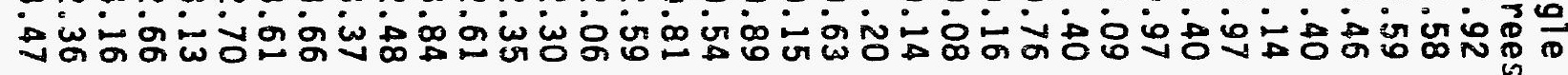




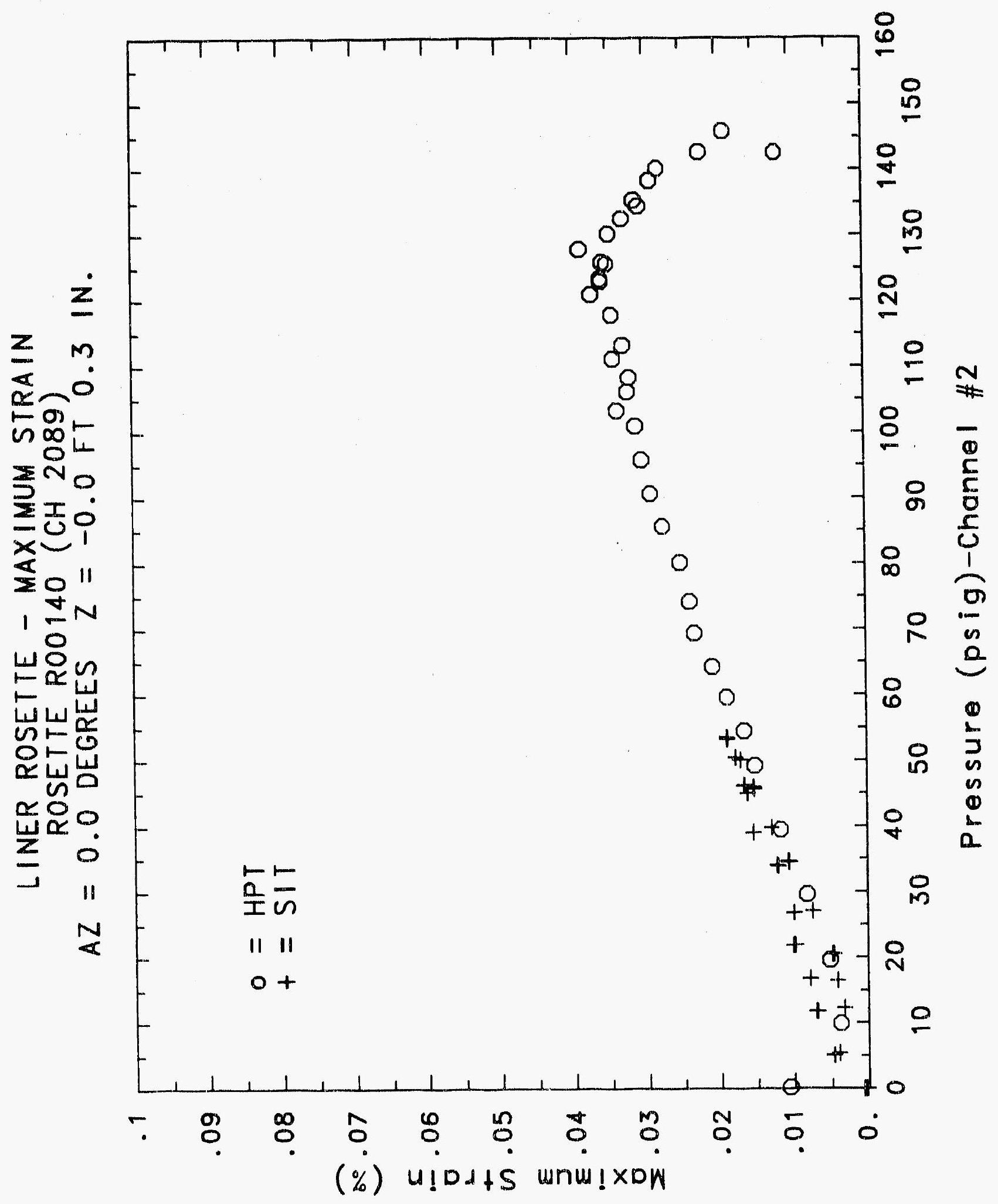




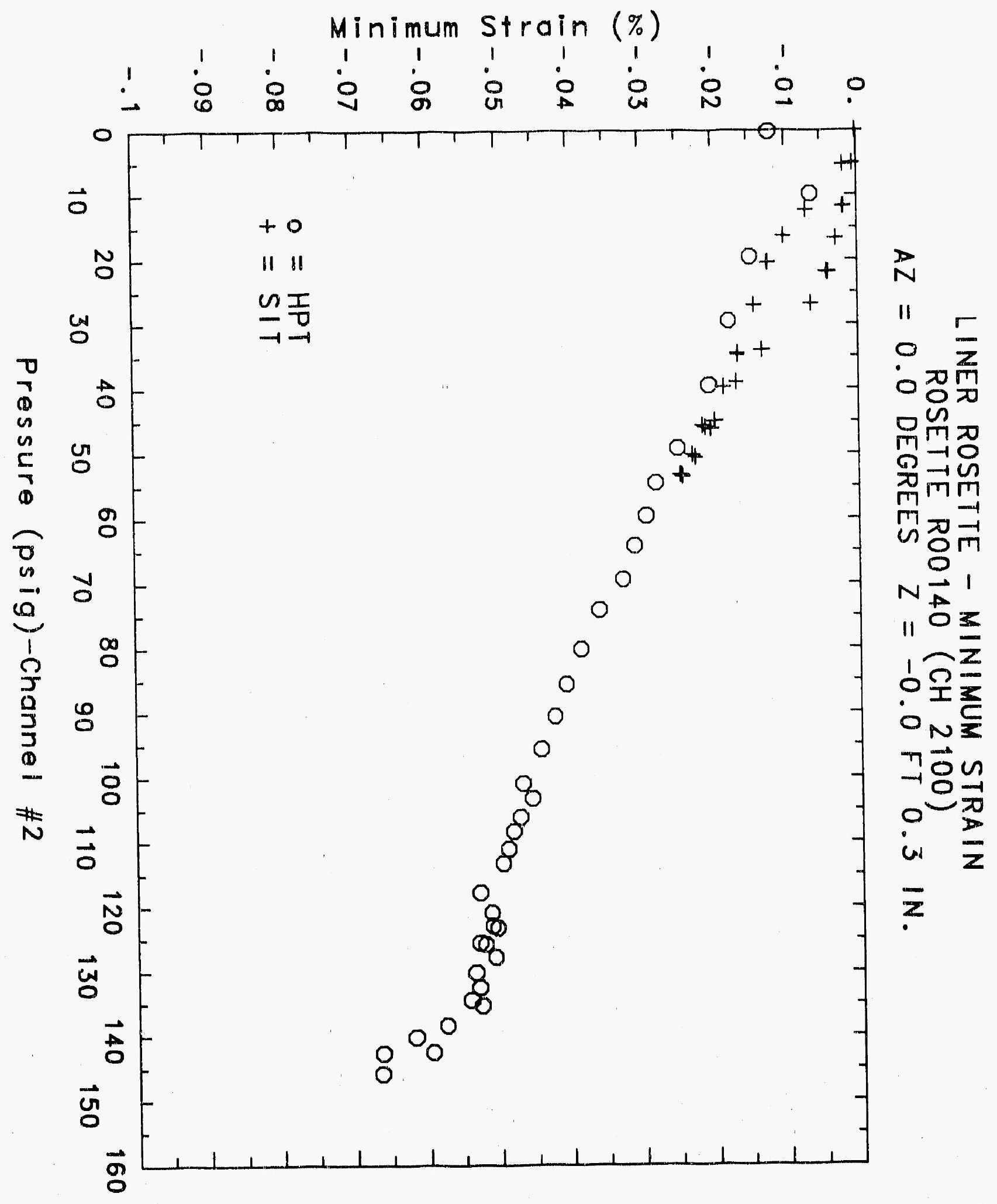




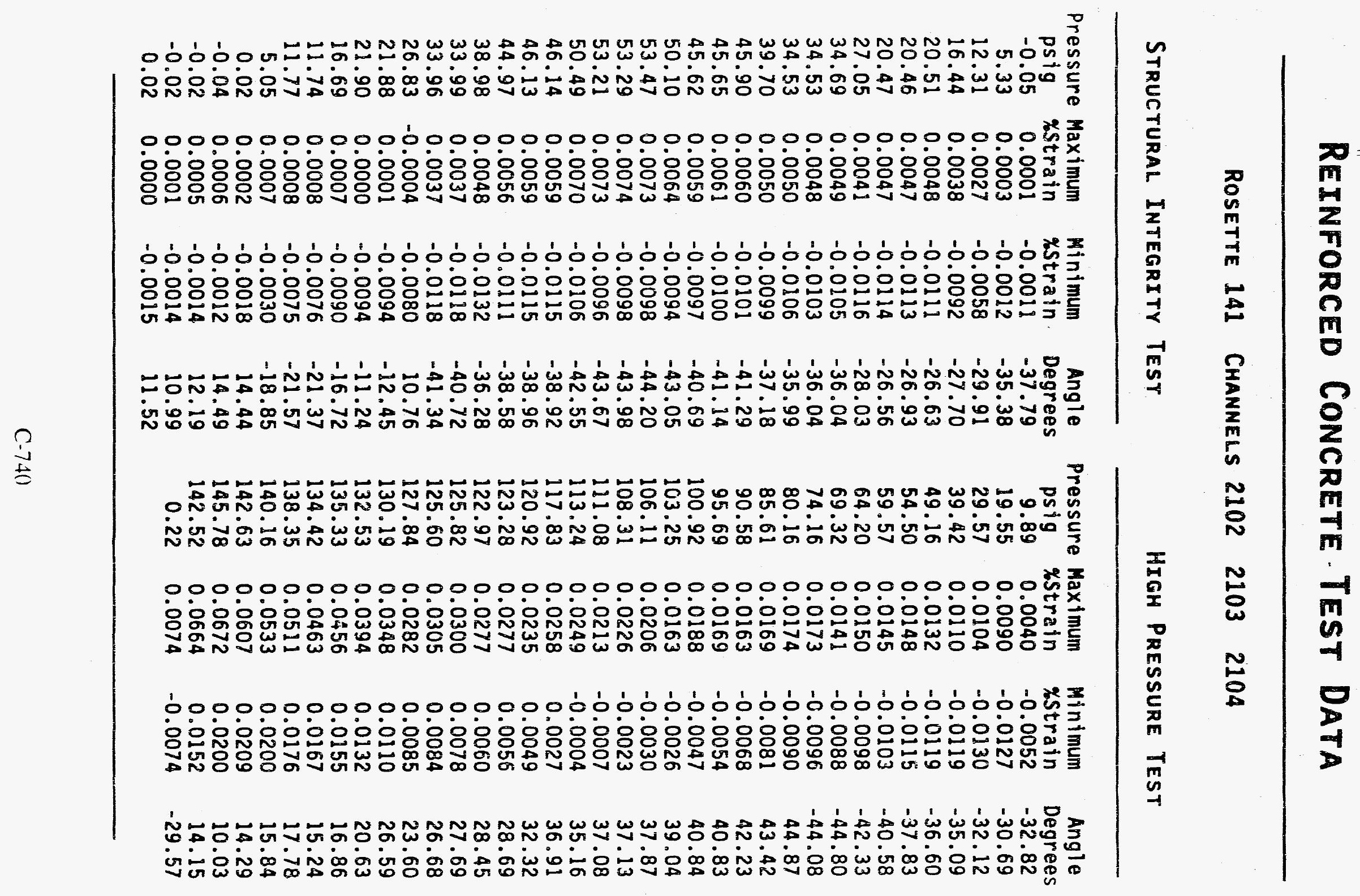




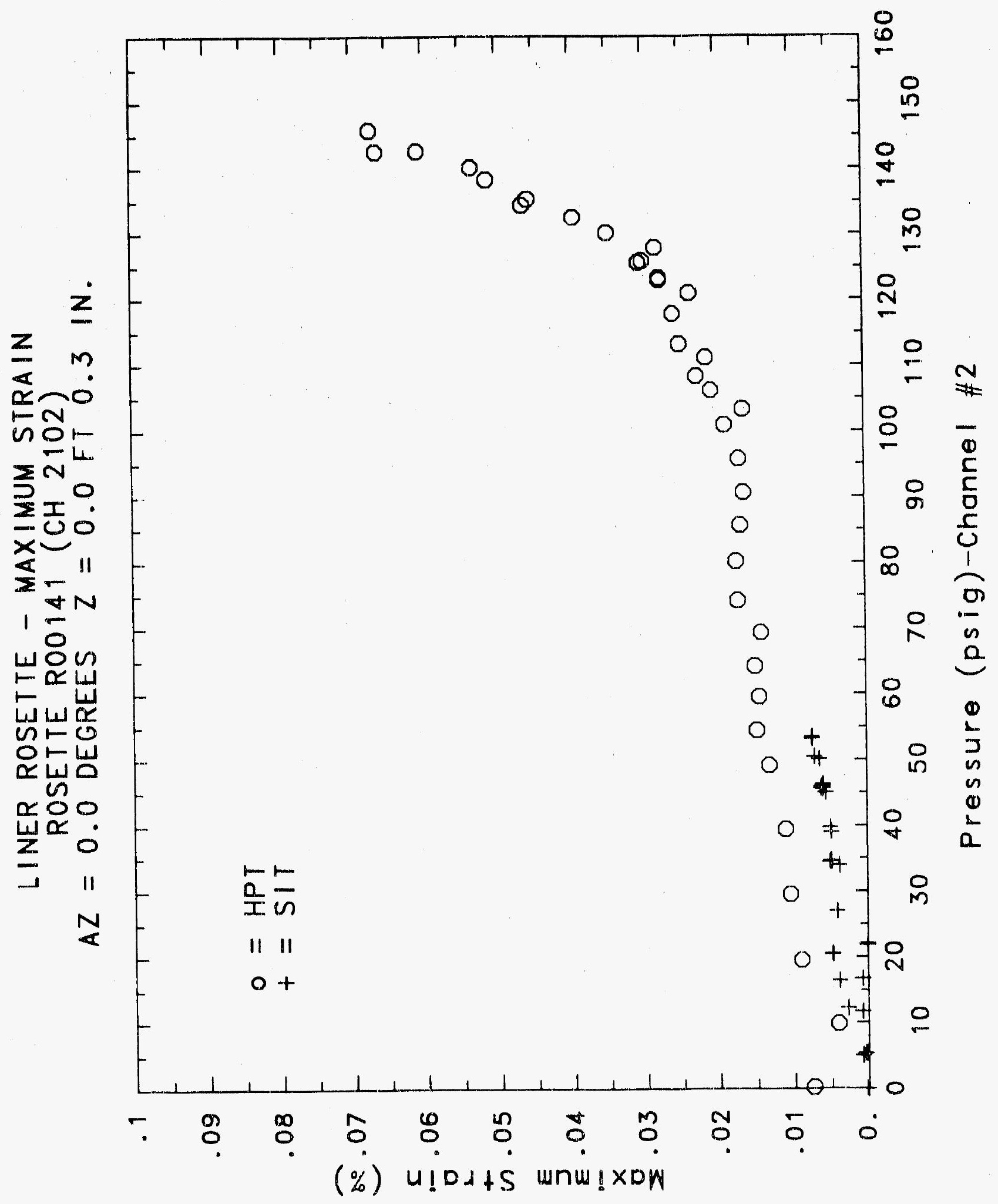




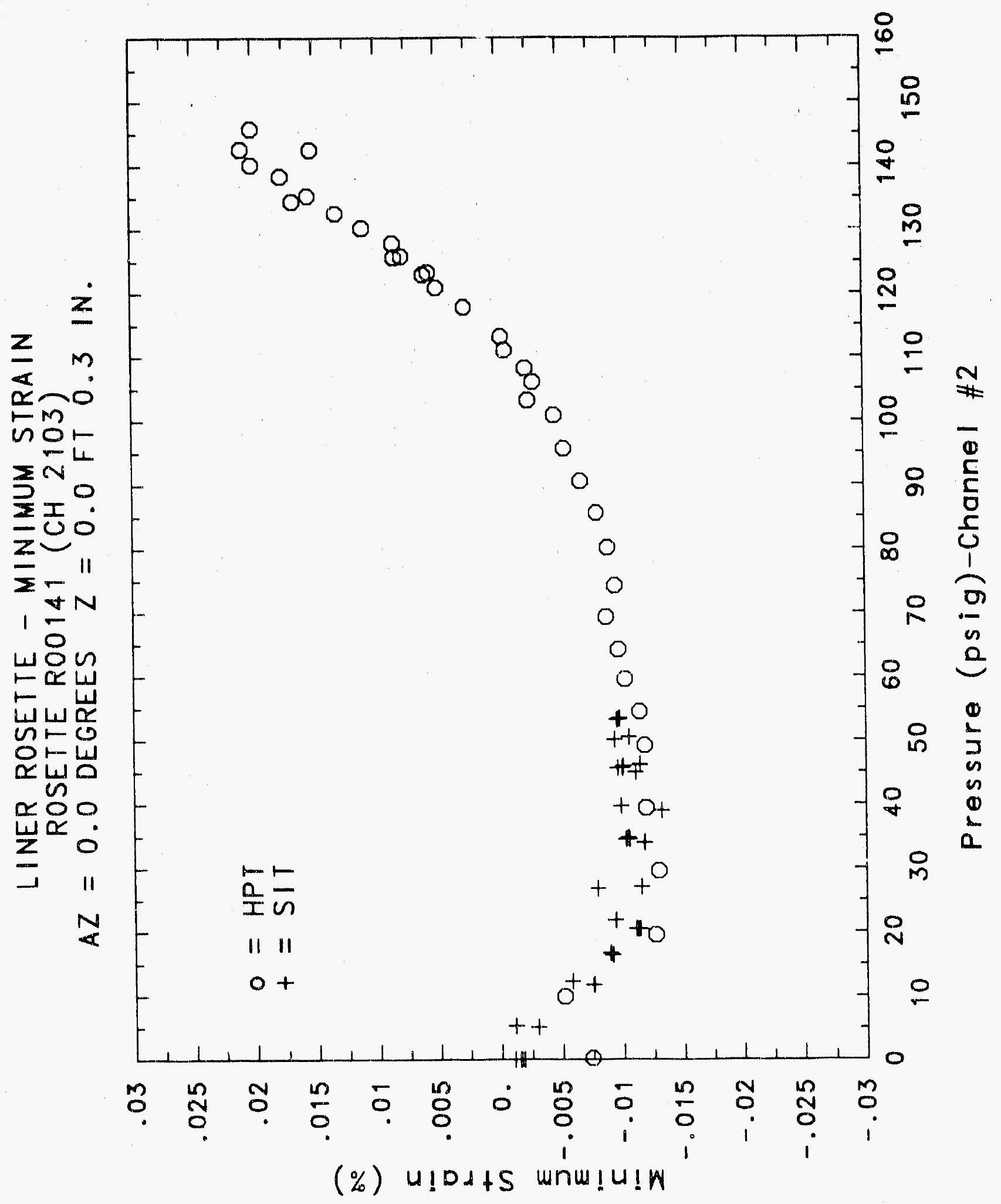




\section{Reinforced Concrete Test Data}

\section{Rosette 142 Channels $2105 \quad 2106 \quad 2107$}

Structural IntEgrity TEST

\begin{tabular}{|c|c|c|c|c|c|c|c|}
\hline $\begin{array}{c}\text { Pressure } \\
\text { psig } \\
-0.05 \\
5.33 \\
12.31 \\
16.44 \\
20.51 \\
20.46 \\
20.47 \\
27.05 \\
34.69 \\
34.53 \\
34.53 \\
39.70 \\
45.90 \\
45.65 \\
45.62 \\
50.10 \\
53.47 \\
53.29 \\
53.21 \\
50.49 \\
46.14 \\
46.13 \\
44.97 \\
38.98 \\
33.99 \\
33.96 \\
26.83 \\
21.88 \\
21.90 \\
16.69 \\
11.74 \\
11.77 \\
5.05 \\
0.02 \\
-0.04 \\
-0.02 \\
-0.02 \\
0.02\end{array}$ & $\begin{array}{c}\text { Maximum } \\
\text { \%Strain } \\
0.0004 \\
0.0011 \\
0.0028 \\
0.0036 \\
0.0045 \\
0.0046 \\
0.0046 \\
0.0080 \\
0.0132 \\
0.0143 \\
0.0143 \\
0.0174 \\
0.0236 \\
0.0245 \\
0.0248 \\
0.0281 \\
0.0311 \\
0.0318 \\
0.0320 \\
0.0324 \\
0.0327 \\
0.0325 \\
0.0325 \\
0.0324 \\
0.0321 \\
0.0323 \\
0.0303 \\
0.0267 \\
0.0266 \\
0.0231 \\
0.0199 \\
0.0198 \\
0.0152 \\
0.0110 \\
0.0110 \\
0.0110 \\
0.0107 \\
0.0106\end{array}$ & $\begin{array}{l}\text { Minimum } \\
\text { xStrain } \\
-0.0002 \\
-0.0008 \\
-0.0021 \\
-0.0027 \\
-0.0033 \\
-0.0032 \\
-0.0030 \\
-0.0038 \\
-0.0049 \\
-0.0048 \\
-0.0048 \\
-0.0057 \\
-0.0066 \\
-0.0068 \\
-0.0070 \\
-0.0076 \\
-0.0083 \\
-0.0082 \\
-0.0083 \\
-0.0081 \\
-0.0079 \\
-0.0080 \\
-0.0075 \\
-0.0073 \\
-0.0071 \\
-0.0071 \\
-0.0066 \\
-0.0057 \\
-0.0058 \\
-0.0049 \\
-0.0042 \\
-0.0042 \\
-0.0035 \\
-0.0025 \\
-0.0024 \\
-0.0024 \\
-0.0024 \\
-0.0025\end{array}$ & $\begin{array}{c}\text { Angle } \\
\text { Degrees } \\
-12.56 \\
-15.85 \\
-13.29 \\
-12.21 \\
-10.45 \\
-9.32 \\
-6.02 \\
-1.91 \\
0.93 \\
1.97 \\
1.08 \\
2.53 \\
4.36 \\
5.51 \\
5.27 \\
6.42 \\
6.16 \\
6.58 \\
6.37 \\
6.64 \\
7.08 \\
6.72 \\
7.03 \\
7.21 \\
7.09 \\
7.45 \\
7.38 \\
7.94 \\
7.84 \\
8.01 \\
10.22 \\
10.07 \\
12.23 \\
17.22 \\
15.90 \\
16.02 \\
15.31 \\
15.05\end{array}$ & $\begin{array}{c}\text { Pressure } \\
\text { psig } \\
9.89 \\
19.55 \\
29.57 \\
39.42 \\
49.16 \\
54.50 \\
59.57 \\
64.20 \\
69.32 \\
74.16 \\
80.16 \\
85.61 \\
90.58 \\
95.69 \\
100.92 \\
103.25 \\
106.11 \\
108.31 \\
111.08 \\
113.24 \\
117.83 \\
120.92 \\
123.28 \\
122.97 \\
125.82 \\
125.60 \\
127.84 \\
130.19 \\
132.53 \\
135.33 \\
134.42 \\
138.35 \\
140.16 \\
142.63 \\
145.78 \\
142.52 \\
0.22\end{array}$ & $\begin{array}{c}\text { Maximum } \\
\text { XStrain } \\
0.0014 \\
0.0095 \\
0.0189 \\
0.0288 \\
0.0389 \\
0.0454 \\
0.0492 \\
0.0535 \\
0.0572 \\
0.0618 \\
0.0653 \\
0.0684 \\
0.0708 \\
0.0736 \\
0.0767 \\
0.0781 \\
0.0790 \\
0.0800 \\
0.0808 \\
0.0820 \\
0.0837 \\
0.0845 \\
0.0858 \\
0.0860 \\
0.0874 \\
0.0882 \\
0.0894 \\
0.0906 \\
0.0916 \\
0.0933 \\
0.0933 \\
0.0944 \\
0.0946 \\
0.0955 \\
0.0961 \\
0.0923 \\
0.0203\end{array}$ & $\begin{array}{r}\text { Minimum } \\
\text { \%strain } \\
-0.0004 \\
0.0000 \\
0.0007 \\
0.0012 \\
0.0003 \\
-0.0006 \\
-0.0012 \\
-0.0018 \\
-0.0027 \\
-0.0040 \\
-0.0038 \\
-0.0042 \\
-0.0048 \\
-0.0048 \\
-0.0057 \\
-0.0059 \\
-0.0061 \\
-0.0062 \\
-0.0065 \\
-0.0065 \\
-0.0071 \\
-0.0072 \\
-0.0073 \\
-0.0073 \\
-0.0076 \\
-0.0077 \\
-0.0079 \\
-0.0083 \\
-0.0089 \\
-0.0089 \\
-0.0090 \\
-0.0094 \\
-0.0091 \\
-0.0091 \\
-0.0086 \\
-0.0089 \\
-0.0033\end{array}$ & $\begin{array}{c}\text { Angle } \\
\text { Degrees } \\
-44.90 \\
-41.77 \\
-39.71 \\
-36.08 \\
-32.47 \\
-31.22 \\
-30.47 \\
-30.23 \\
-29.48 \\
-29.32 \\
-28.00 \\
-27.93 \\
-27.57 \\
-27.49 \\
-27.27 \\
-27.29 \\
-27.05 \\
-27.09 \\
-26.81 \\
-26.84 \\
-26.59 \\
-26.41 \\
-26.30 \\
-26.30 \\
-26.17 \\
-26.27 \\
-26.51 \\
-26.37 \\
-26.13 \\
-26.39 \\
-26.48 \\
-26.37 \\
-26.13 \\
-26.02 \\
-25.98 \\
-25.58 \\
-17.53\end{array}$ \\
\hline
\end{tabular}

High Pressure Test 


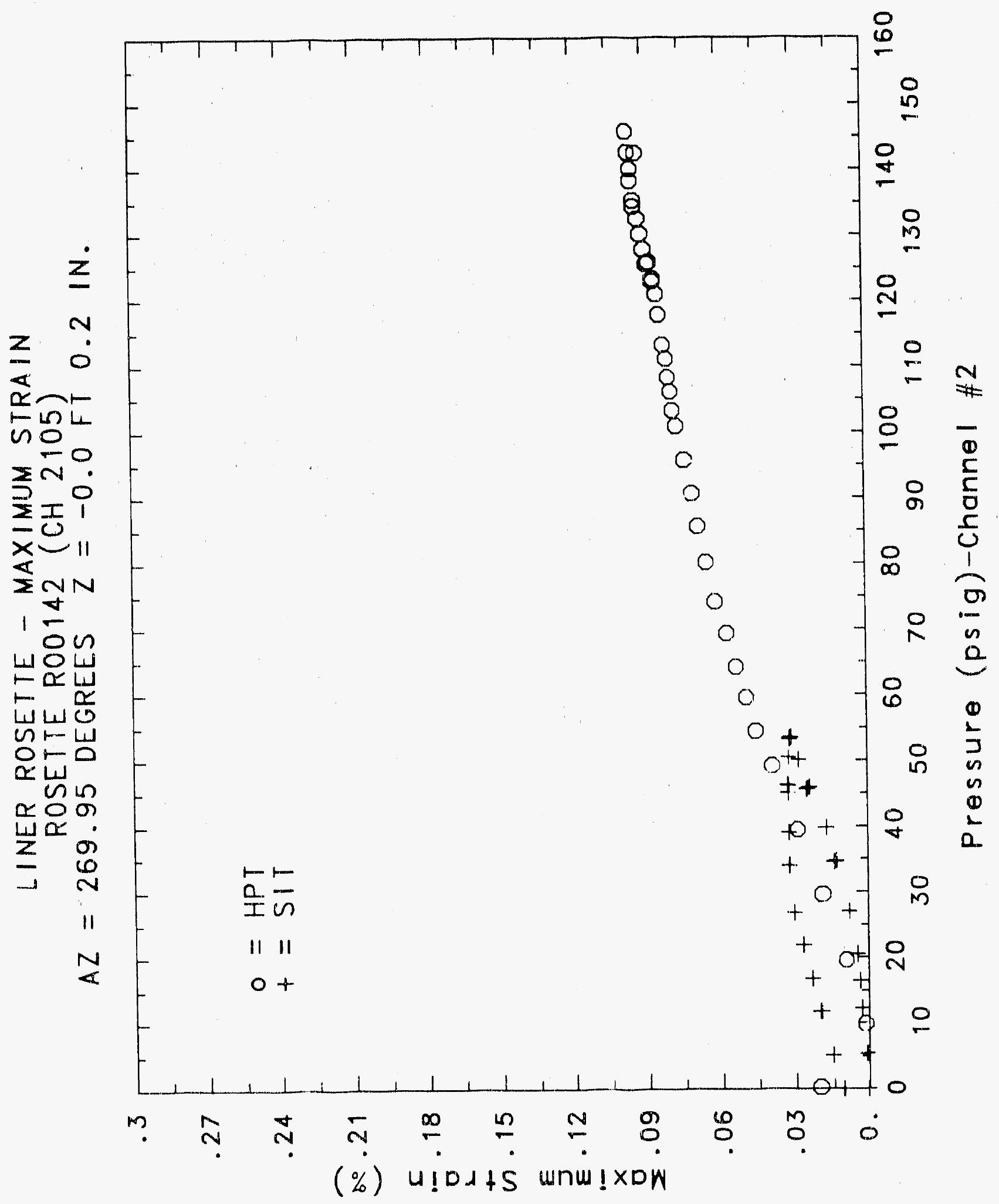




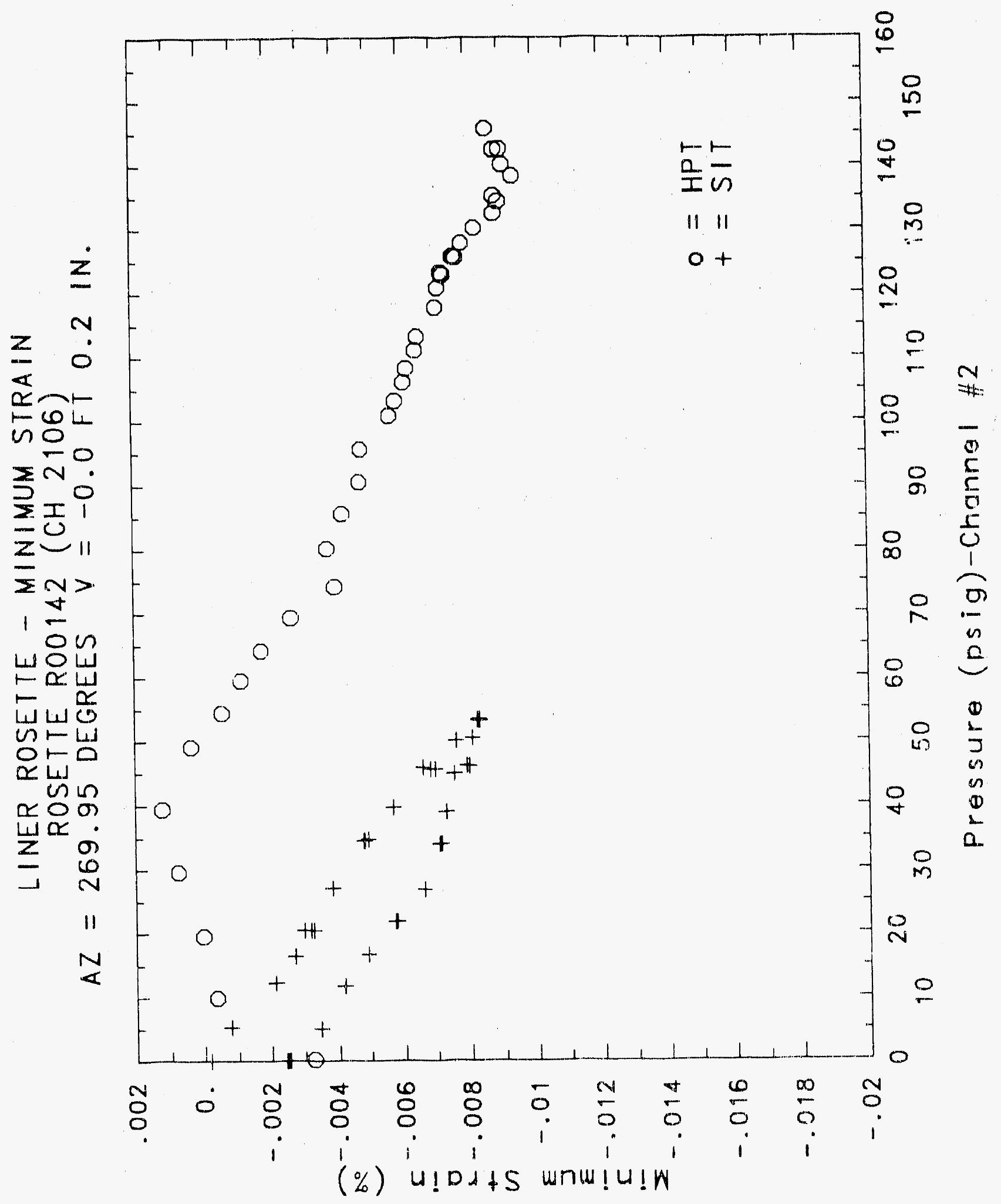




\title{
Reinforced Concrete Test Data
}

\author{
Rosette 143 Chanmels $2108 \quad 21092120$
}

StRUCTURAL INTEGRITY TEST

\begin{tabular}{|c|c|c|c|c|c|c|c|}
\hline $\begin{array}{l}\text { ressure } \\
\text { psig } \\
-0.05 \\
5.33 \\
12.31 \\
16.44 \\
20.51 \\
20.46 \\
20.47 \\
27.05 \\
34.69 \\
34.53 \\
34.53 \\
39.70 \\
45.90 \\
45.65 \\
45.62 \\
50.10 \\
53.47 \\
53.29 \\
53.21 \\
50.49 \\
46.14 \\
46.13 \\
44.97 \\
38.98 \\
33.99 \\
33.96 \\
26.83 \\
21.88 \\
21.90 \\
16.69 \\
11.74 \\
11.77 \\
37.05 \\
01.07 \\
-0.04 \\
-0.02 \\
-0.02 \\
0.02\end{array}$ & $\begin{array}{l}\text { Maximum } \\
\text { \%strain } \\
0.0012 \\
0.0015 \\
0.0031 \\
0.0037 \\
0.0042 \\
0.0039 \\
0.0044 \\
0.0027 \\
0.0016 \\
0.0019 \\
0.0017 \\
0.0019 \\
0.0020 \\
0.0021 \\
0.0020 \\
0.0022 \\
0.0024 \\
0.0022 \\
0.0022 \\
0.0022 \\
0.0022 \\
0.0020 \\
0.0020 \\
0.0018 \\
0.0018 \\
0.0019 \\
0.0020 \\
0.0021 \\
0.0025 \\
0.0026 \\
0.0027 \\
0.0026 \\
0.0028 \\
0.0024 \\
0.0021 \\
0.0023 \\
0.0023 \\
0.0027\end{array}$ & $\begin{array}{l}\text { Minimum } \\
\text { \%train } \\
-0.0006 \\
-0.0004 \\
-0.0006 \\
-0.0007 \\
-0.0011 \\
-0.0012 \\
-0.0016 \\
-0.0019 \\
-0.0049 \\
-0.0063 \\
-0.0061 \\
-0.0089 \\
-0.0150 \\
-0.0160 \\
0.0161 \\
-0.0186 \\
-0.0215 \\
-0.0221 \\
-0.0224 \\
-0.0227 \\
-0.0231 \\
-0.0231 \\
-0.0233 \\
-0.0238 \\
-0.0240 \\
-0.0239 \\
-0.0234 \\
-0.0207 \\
-0.0210 \\
-0.0191 \\
-0.0171 \\
-0.0168 \\
-0.0142 \\
-0.0112 \\
-0.0110 \\
-0.0109 \\
-0.0109 \\
-0.0111\end{array}$ & $\begin{array}{c}\text { Angle } \\
\text { Degrees } \\
44.38 \\
-35.64 \\
-32.73 \\
-32.20 \\
-37.48 \\
-39.14 \\
-41.19 \\
37.54 \\
11.88 \\
-18.31 \\
-14.48 \\
-11.92 \\
-7.47 \\
-8.51 \\
-7.19 \\
-5.52 \\
-5.51 \\
-4.52 \\
-5.02 \\
-4.70 \\
-5.40 \\
-5.70 \\
-6.55 \\
-7.14 \\
-7.31 \\
-0.76 \\
-7.02 \\
-9.02 \\
-10.95 \\
-12.49 \\
-11.84 \\
-10.96 \\
-11.80 \\
-12.03 \\
-15.29 \\
-14.73 \\
-14.50 \\
-13.99\end{array}$ & $\begin{array}{c}\text { Pressure } \\
\text { psig } \\
9.89 \\
19.55 \\
29.57 \\
39.42 \\
49.16 \\
54.50 \\
59.51 \\
64.20 \\
69.32 \\
74.16 \\
80.16 \\
85.61 \\
90.58 \\
95.69 \\
100.92 \\
103.25 \\
106.11 \\
108.31 \\
111.08 \\
113.24 \\
117.83 \\
120.92 \\
123.28 \\
122.97 \\
125.82 \\
125.60 \\
127.84 \\
130.19 \\
132.53 \\
135.33 \\
134.42 \\
138.35 \\
140.16 \\
142.63 \\
145.78 \\
142.52 \\
0.22\end{array}$ & $\begin{array}{c}\text { Maximum } \\
\text { \%Strain } \\
0.0010 \\
0.0026 \\
0.0040 \\
0.0049 \\
0.0058 \\
0.0063 \\
0.0067 \\
0.0073 \\
0.0081 \\
0.0102 \\
0.0114 \\
0.0124 \\
0.0135 \\
0.0145 \\
0.0157 \\
0.0162 \\
0.0168 \\
0.0174 \\
0.0179 \\
0.0185 \\
0.0198 \\
0.0205 \\
0.0212 \\
0.0212 \\
0.0222 \\
0.0222 \\
0.0228 \\
0.0236 \\
0.0242 \\
0.0252 \\
0.0256 \\
0.0265 \\
0.0267 \\
0.0281 \\
0.0296 \\
0.0291 \\
0.0048\end{array}$ & $\begin{array}{l}\text { Minimum } \\
\% \text { Strain } \\
-0.0040 \\
-0.0117 \\
-0.0181 \\
-0.0221 \\
-0.0254 \\
-0.0269 \\
-0.0289 \\
-0.0310 \\
-0.0333 \\
-0.0367 \\
-0.0400 \\
-0.0431 \\
-0.0458 \\
-0.0482 \\
-0.0510 \\
-0.0527 \\
-0.0540 \\
-0.0548 \\
-0.0566 \\
-0.0579 \\
-0.0609 \\
-0.0632 \\
-0.0652 \\
-0.0654 \\
-0.0675 \\
-0.0678 \\
-0.0695 \\
-0.0716 \\
-0.0734 \\
-0.0762 \\
-0.0764 \\
-0.0786 \\
-0.0796 \\
-0.0821 \\
-0.0850 \\
-0.0800 \\
-0.0062\end{array}$ & $\begin{array}{c}\text { Angle } \\
\text { Degrees } \\
-6.68 \\
-8.99 \\
-8.61 \\
-5.47 \\
-5.70 \\
-5.75 \\
-6.43 \\
-6.09 \\
-6.72 \\
-11.45 \\
-11.62 \\
-11.50 \\
-11.43 \\
-10.90 \\
-11.19 \\
-11.52 \\
-11.52 \\
-11.14 \\
-10.99 \\
-10.93 \\
-10.51 \\
-10.33 \\
-10.05 \\
-10.07 \\
-9.89 \\
-9.86 \\
-9.76 \\
-9.65 \\
-9.57 \\
-9.14 \\
-9.25 \\
-8.44 \\
-7.88 \\
-8.22 \\
-7.74 \\
-6.34 \\
20.47\end{array}$ \\
\hline
\end{tabular}

\section{High Pressure Test}




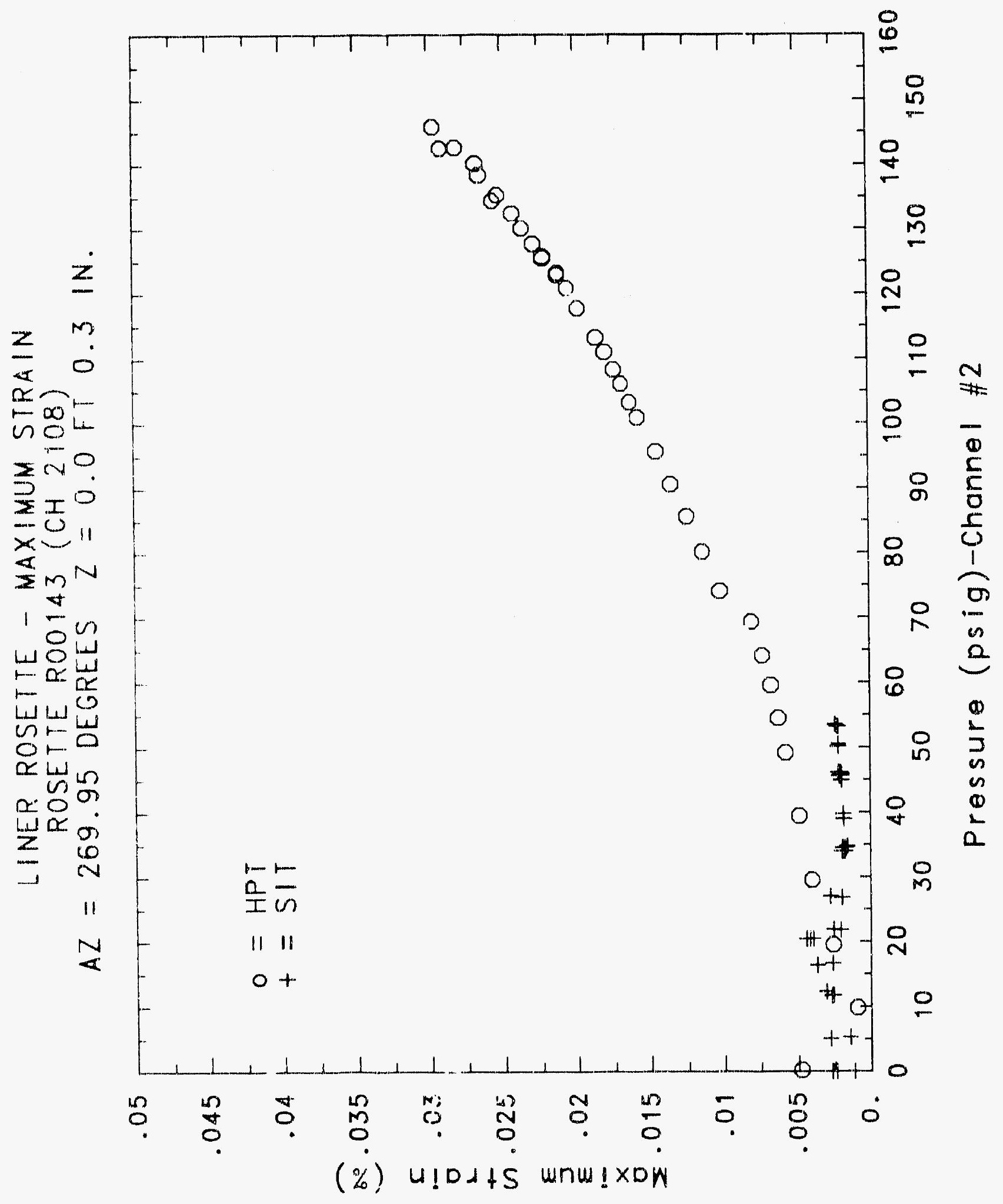




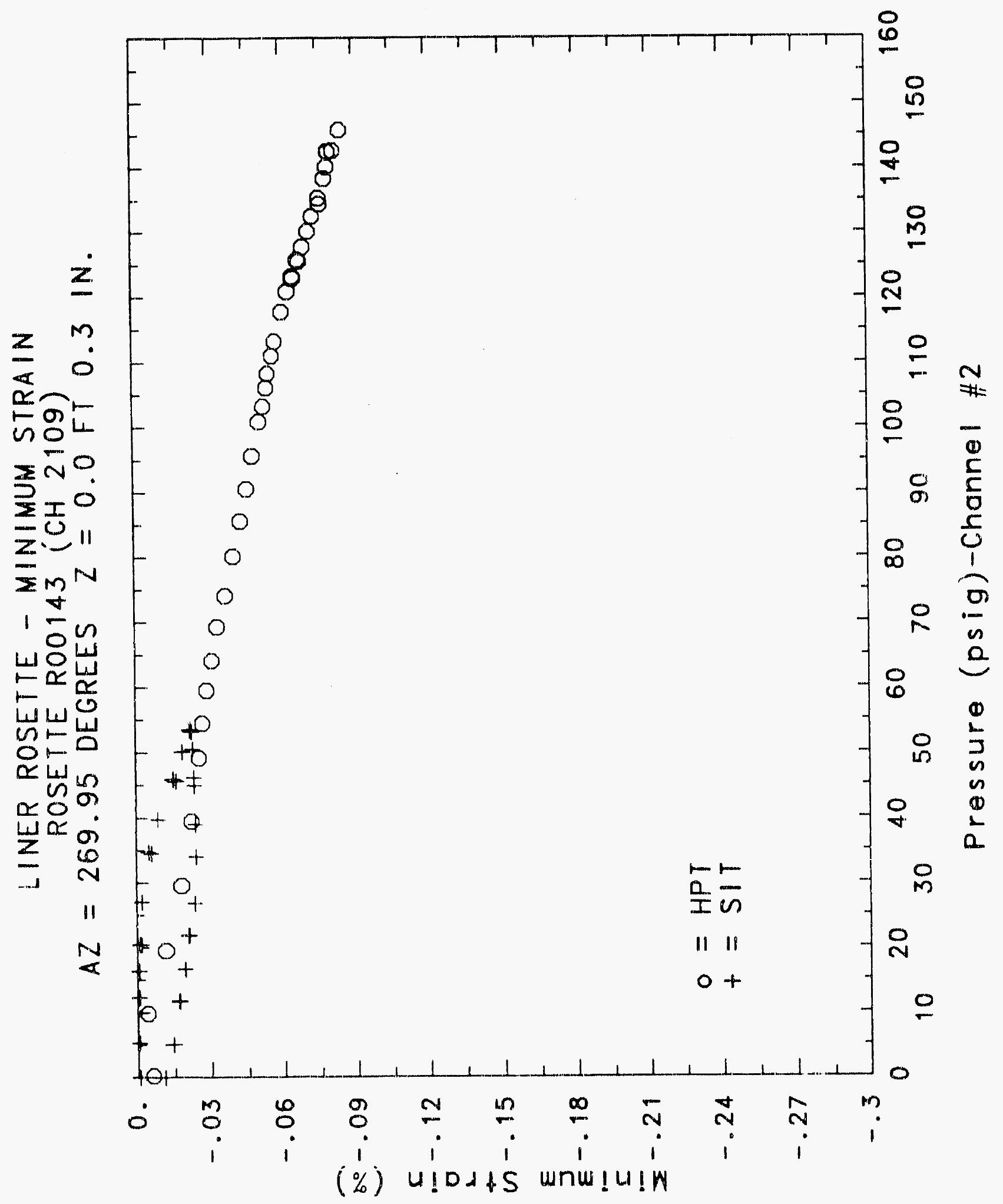




\begin{tabular}{|c|c|c|c|c|c|c|c|}
\hline 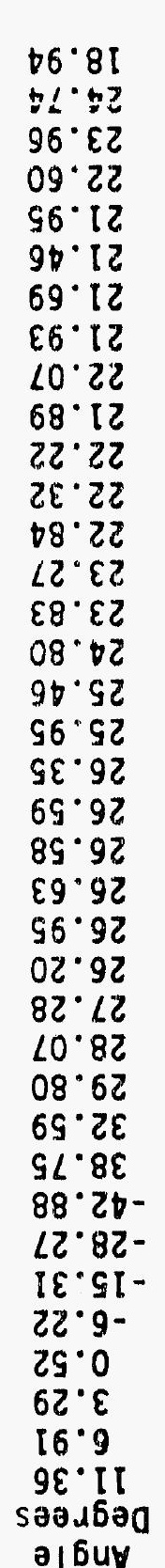 & 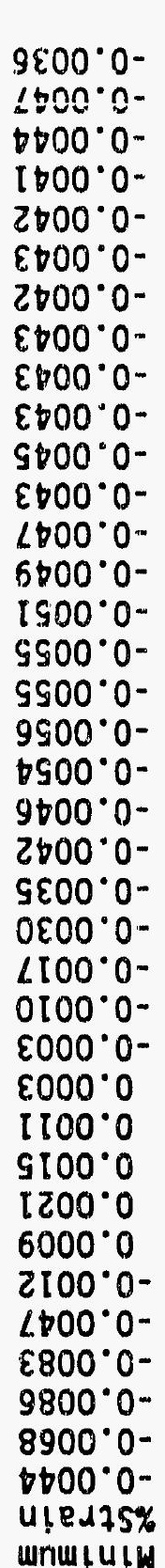 & 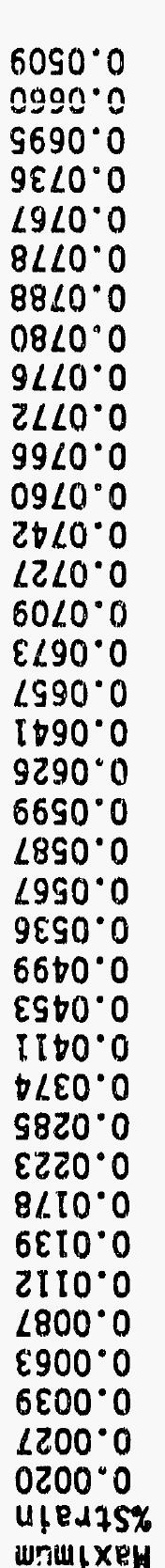 & 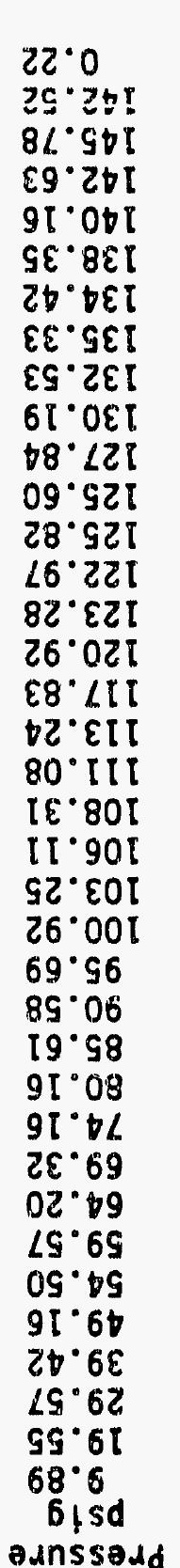 & 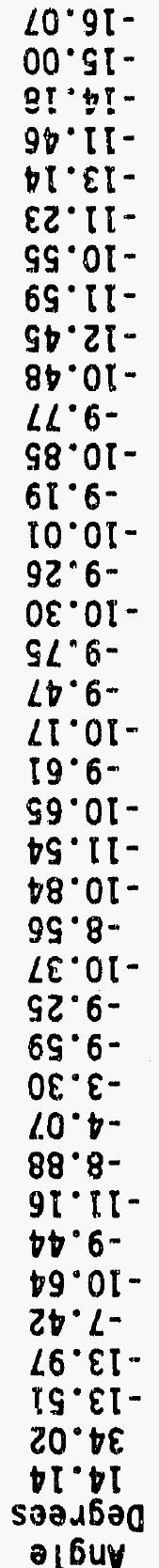 & 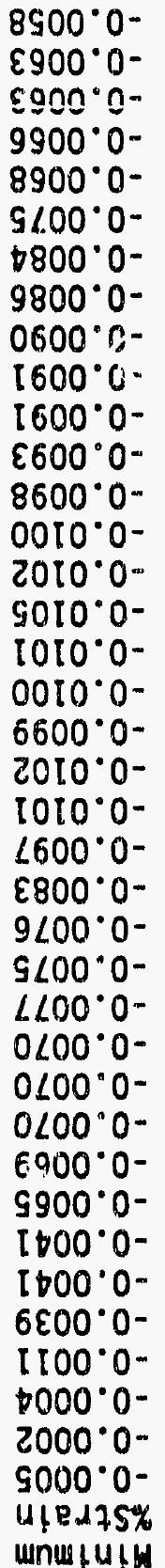 & 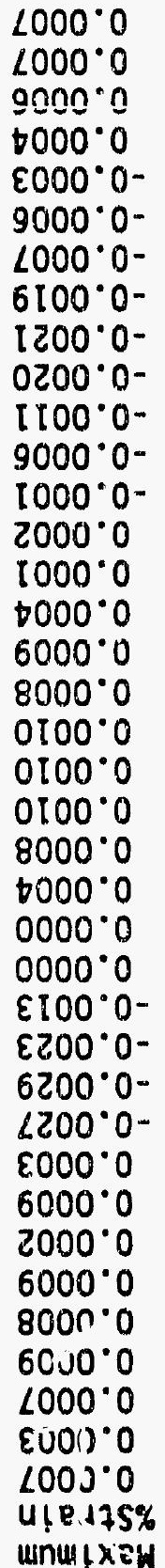 & 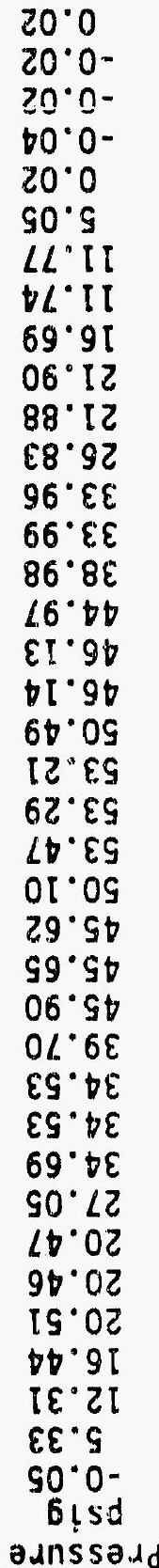 \\
\hline
\end{tabular}

$1 S \exists \perp$ JynSS $34 d$ HOIH

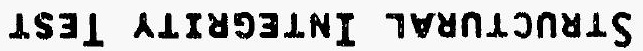

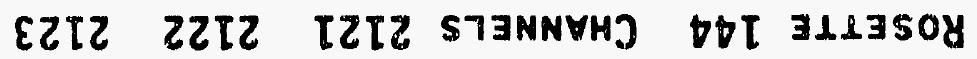

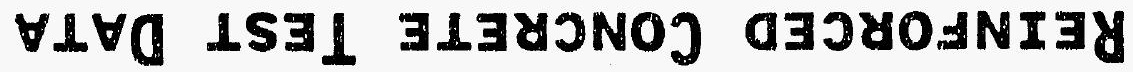




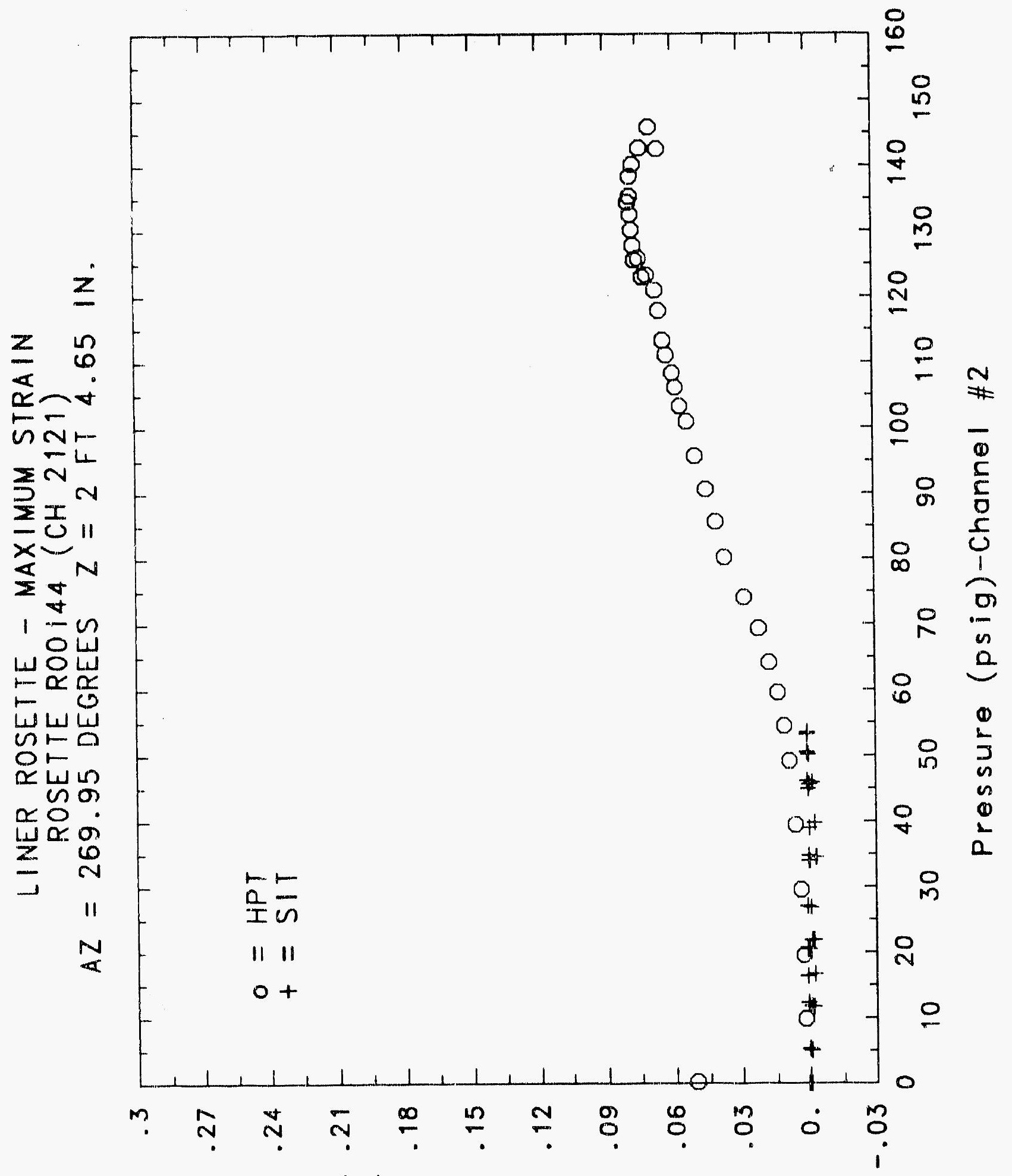

(\%) $u ! D d+S$ wnw!XDW 


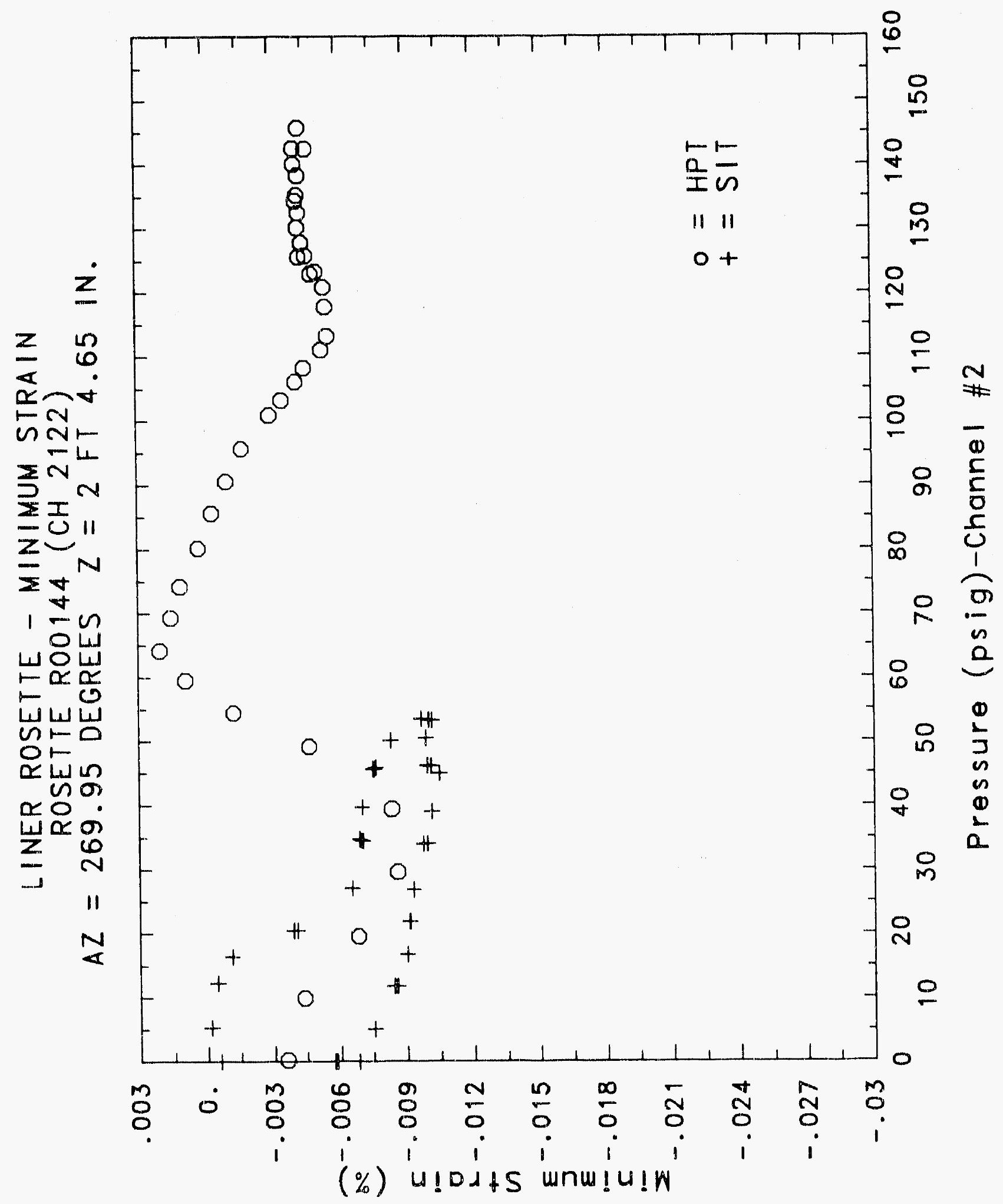




\section{Reinforced Concrete Test Data}

\section{Rosette 145 Channels $2124 \quad 2125 \quad 2126$}

STRUCTURAL INTEGRITY TEST

\begin{tabular}{|c|c|c|c|c|c|c|c|}
\hline $\begin{array}{c}\text { ressure } \\
\text { psig } \\
-0.05 \\
5.33 \\
12.31 \\
16.44 \\
20.51 \\
20.46 \\
20.47 \\
27.05 \\
34.69 \\
34.53 \\
34.53 \\
39.70 \\
45.90 \\
45.65 \\
45.62 \\
50.10 \\
53.47 \\
53.29 \\
53.21 \\
50.49 \\
46.14 \\
46.13 \\
44.97 \\
38.98 \\
33\end{array}$ & $\begin{array}{c}\text { Maximum } \\
\text { \%Strain } \\
0.0003 \\
0.0004 \\
0.0007 \\
0.0009 \\
0.0023 \\
0.0025 \\
0.0019 \\
0.0013 \\
0.0004 \\
-0.0005 \\
-0.0012 \\
-0.0002 \\
0.0012 \\
0.0021 \\
0.0024 \\
0.0029 \\
0.0033 \\
0.0035 \\
0.0036 \\
0.0035 \\
0.0033 \\
0.0033 \\
0.0022 \\
0.0022 \\
0.0018 \\
0.0019 \\
0.0011 \\
0.0011 \\
0.0002 \\
-0.0004 \\
0.0005 \\
0.0017 \\
0.0020 \\
0.0021 \\
0.0016 \\
0.0015 \\
0.0014 \\
0.0010\end{array}$ & $\begin{array}{r}\text { Minimum } \\
\text { \%Strain } \\
-0.0010 \\
-0.0006 \\
-0.0010 \\
-0.0005 \\
0.0007 \\
0.0010 \\
0.0005 \\
0.0007 \\
-0.0008 \\
-0.0021 \\
-0.0022 \\
-0.0017 \\
-0.0001 \\
0.0006 \\
0.0005 \\
0.0005 \\
0.0007 \\
0.0008 \\
0.0009 \\
0.0007 \\
0.0010 \\
0.0011 \\
0.0005 \\
0.0005 \\
0.0006 \\
0.0003 \\
-0.0003 \\
-0.0008 \\
-0.0010 \\
-0.0011 \\
-0.0009 \\
0.0002 \\
0.0002 \\
-0.0004 \\
-0.0003 \\
-0.0002 \\
-0.0001 \\
-0.00001\end{array}$ & $\begin{array}{c}\text { Angle } \\
\text { Degrees } \\
44.25 \\
-29.37 \\
-25.34 \\
-21.01 \\
26.52 \\
15.36 \\
18.73 \\
34.94 \\
-11.68 \\
13.56 \\
-7.11 \\
-6.81 \\
-14.84 \\
-33.57 \\
-37.32 \\
-33.37 \\
-38.04 \\
-40.61 \\
-44.25 \\
-43.02 \\
-44.65 \\
44.06 \\
-29.54 \\
-37.11 \\
43.91 \\
-40.34 \\
-39.73 \\
-40.82 \\
-37.09 \\
-33.38 \\
36.69 \\
33.31 \\
26.73 \\
31.55 \\
38.63 \\
38.90 \\
40.22 \\
38.62\end{array}$ & $\begin{array}{c}\text { Pressure } \\
\text { psig } \\
9.89 \\
19.55 \\
29.57 \\
39.42 \\
49.16 \\
54.50 \\
59.57 \\
64.20 \\
69.32 \\
74.16 \\
80.16 \\
85.61 \\
90.58 \\
95.69 \\
100.92 \\
103.25 \\
106.11 \\
108.31 \\
111.08 \\
113.24 \\
117.83 \\
120.92 \\
123.28 \\
122.97 \\
125.82 \\
125.60 \\
127.84 \\
130.19 \\
132.53 \\
135.33 \\
134.42 \\
138.35 \\
140.16 \\
142.63 \\
145.78 \\
142.52 \\
0.22\end{array}$ & $\begin{array}{l}\text { Maximum } \\
\text { XStrain } \\
0.0037 \\
0.0053 \\
0.0068 \\
0.0084 \\
0.0102 \\
0.0114 \\
0.0126 \\
0.0136 \\
0.0149 \\
0.0159 \\
0.0186 \\
0.0207 \\
0.0224 \\
0.0247 \\
0.0264 \\
0.0279 \\
0.0289 \\
0.0298 \\
0.0311 \\
0.0318 \\
0.0335 \\
0.0345 \\
0.0357 \\
0.0359 \\
0.0368 \\
0.0368 \\
0.0374 \\
0.0379 \\
0.0386 \\
0.0395 \\
0.0391 \\
0.0396 \\
0.0404 \\
0.0412 \\
0.0424 \\
0.0414 \\
0.0161\end{array}$ & $\begin{array}{r}\text { Minimum } \\
\text { XStrain } \\
-0.0009 \\
-0.0011 \\
-0.0025 \\
-0.0043 \\
-0.0064 \\
-0.0076 \\
-0.0091 \\
-0.0117 \\
-0.0144 \\
-0.0173 \\
-0.0198 \\
-0.0231 \\
-0.0260 \\
-0.0292 \\
-0.0316 \\
-0.0325 \\
-0.0338 \\
-0.0349 \\
-0.0368 \\
-0.0383 \\
-0.0402 \\
-0.0410 \\
-0.0421 \\
-0.0420 \\
-0.0427 \\
-0.0423 \\
-0.0430 \\
-0.0431 \\
-0.0435 \\
-0.0433 \\
-0.0428 \\
-0.0423 \\
-0.0407 \\
-0.0390 \\
-0.0381 \\
-0.0356 \\
0.0061\end{array}$ & $\begin{array}{c}\text { Angle } \\
\text { Degrees } \\
17.88 \\
6.35 \\
3.18 \\
4.23 \\
2.21 \\
2.22 \\
0.65 \\
-0.05 \\
-0.84 \\
-1.41 \\
-1.47 \\
-1.26 \\
-0.57 \\
-0.07 \\
-0.18 \\
-0.19 \\
-0.24 \\
-0.20 \\
-0.30 \\
-0.32 \\
0.54 \\
1.04 \\
1.35 \\
1.52 \\
1.75 \\
2.04 \\
2.03 \\
2.18 \\
2.30 \\
2.23 \\
2.14 \\
2.25 \\
2.27 \\
2.01 \\
1.88 \\
1.68 \\
-35.38\end{array}$ \\
\hline
\end{tabular}

High Pressure test 


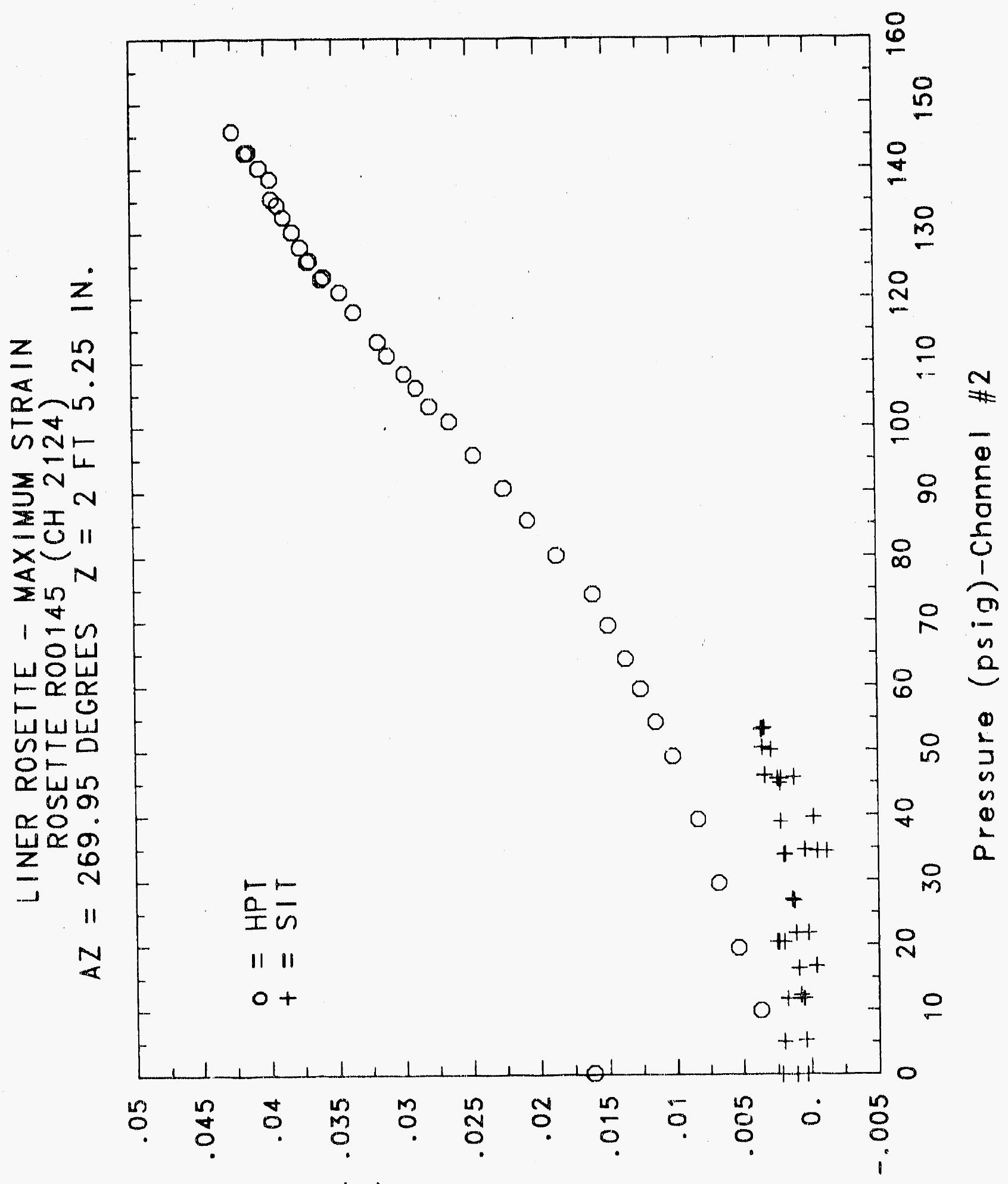

(\%) U!Ddis wnu! $\times D W$ 


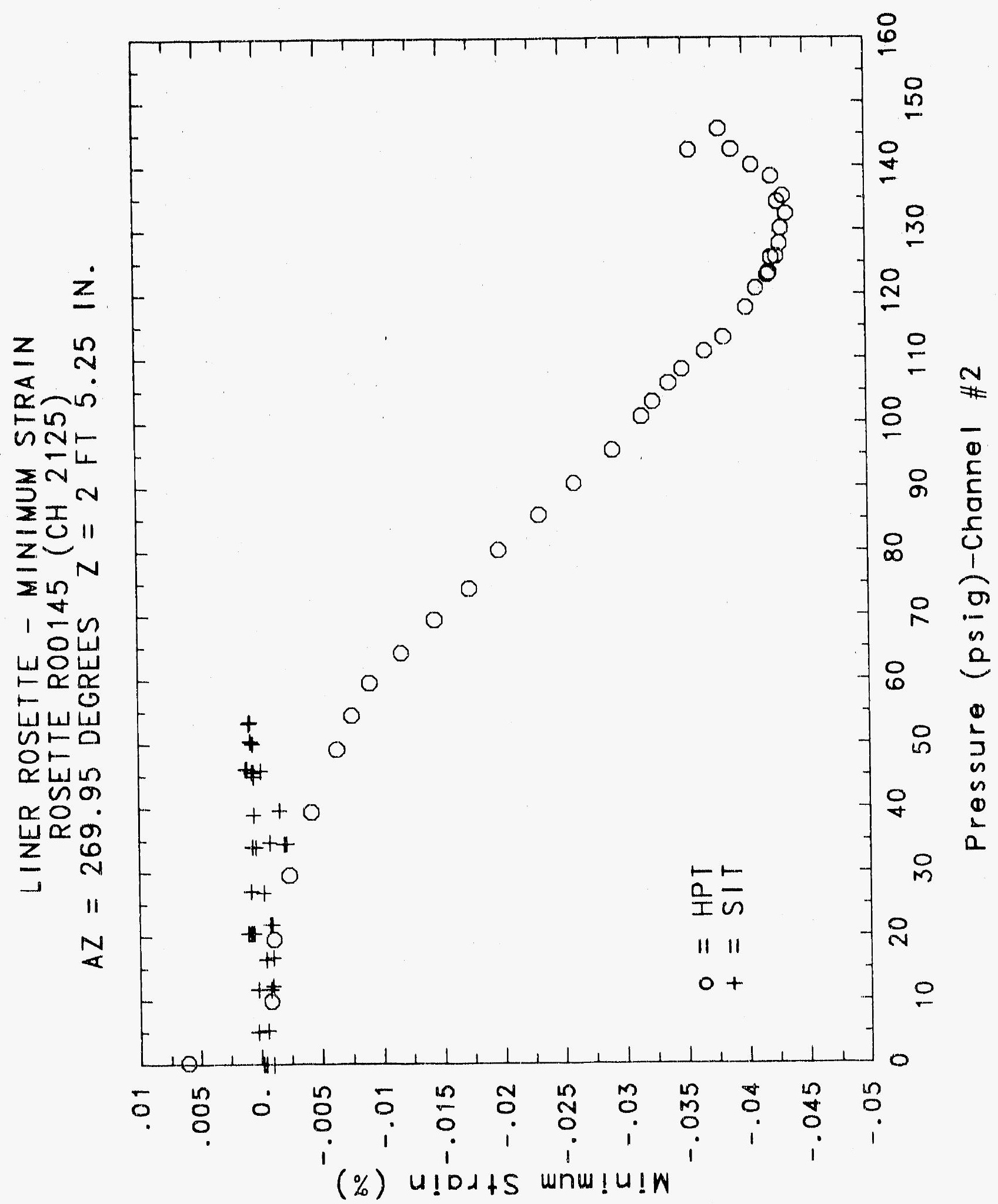




\section{Reinforced Concrete Test Data}

Rosette 146 Channels $2127 \quad 2128 \quad 2129$

STRUCTURAL INTEgRITY TEST

\begin{tabular}{|c|c|c|c|c|c|c|c|}
\hline $\begin{array}{c}\text { Pressure } \\
\text { psig } \\
-0.05 \\
5.33 \\
12.31 \\
16.44 \\
20.51 \\
20.46 \\
20.47 \\
27.05 \\
34.69 \\
34.53 \\
34.53 \\
39.70 \\
45.90 \\
45.65 \\
45.62 \\
50.10 \\
53.47 \\
53.29 \\
53.21 \\
50.49 \\
46.14 \\
46.13 \\
44.97 \\
38.98 \\
33.99 \\
33.96 \\
26.83 \\
21.88 \\
21.90 \\
16.69 \\
11.74 \\
11.77 \\
5.05 \\
0.02 \\
-0.04 \\
-0.02 \\
-0.02 \\
0.02\end{array}$ & $\begin{array}{c}\text { Maximum } \\
\text { xStrain } \\
0.0002 \\
0.0014 \\
0.0032 \\
0.0042 \\
0.0052 \\
0.0052 \\
0.0052 \\
0.0039 \\
0.0064 \\
0.0065 \\
0.0064 \\
0.0080 \\
0.0099 \\
0.0102 \\
0.0101 \\
0.0111 \\
0.0119 \\
0.0119 \\
0.0118 \\
0.0110 \\
0.0097 \\
0.0095 \\
0.0085 \\
0.0065 \\
0.0053 \\
0.0053 \\
0.0055 \\
0.0060 \\
0.0060 \\
0.0067 \\
0.0076 \\
0.0079 \\
0.0097 \\
0.0075 \\
0.0070 \\
0.0071 \\
0.0071 \\
0.0073\end{array}$ & $\begin{array}{l}\text { Minimum } \\
\text { \&Strain } \\
-0.0004 \\
-0.0012 \\
-0.0029 \\
-0.0043 \\
-0.0051 \\
-0.0053 \\
-0.0051 \\
-0.0070 \\
-0.0092 \\
-0.0093 \\
-0.0090 \\
-0.0093 \\
-0.0091 \\
-0.0089 \\
-0.0088 \\
-0.0094 \\
-0.0100 \\
-0.0101 \\
-0.0099 \\
-0.0092 \\
-0.0080 \\
-0.0080 \\
-0.0086 \\
-0.0068 \\
-0.0054 \\
-0.0051 \\
-0.0034 \\
-0.0024 \\
-0.0024 \\
-0.0014 \\
0.0002 \\
0.0001 \\
0.0013 \\
0.0017 \\
0.0012 \\
0.0014 \\
0.0015 \\
0.0013\end{array}$ & $\begin{array}{c}\text { Angle } \\
\text { Degrees } \\
19.60 \\
0.01 \\
-1.58 \\
-3.82 \\
-3.30 \\
-3.92 \\
-2.68 \\
-5.96 \\
-1.09 \\
-1.40 \\
0.23 \\
-0.65 \\
-5.09 \\
-3.98 \\
-3.84 \\
-5.62 \\
-5.71 \\
-5.48 \\
-4.97 \\
-5.35 \\
-5.58 \\
-5.86 \\
-5.84 \\
-7.69 \\
-7.79 \\
-7.33 \\
-7.50 \\
-8.30 \\
-7.84 \\
-6.95 \\
-7.89 \\
-8.27 \\
-14.69 \\
-24.47 \\
-22.13 \\
-22.39 \\
-23.05 \\
-24.67\end{array}$ & $\begin{array}{c}\text { Pressure } \\
\text { psig } \\
9.89 \\
19.55 \\
29.57 \\
39.42 \\
49.16 \\
54.50 \\
59.57 \\
64.20 \\
69.32 \\
74.16 \\
80.16 \\
85.61 \\
90.58 \\
95.69 \\
100.92 \\
103.25 \\
106.11 \\
108.31 \\
111.08 \\
113.24 \\
117.83 \\
120.92 \\
123.28 \\
122.97 \\
125.82 \\
125.60 \\
127.84 \\
130.19 \\
132.53 \\
135.33 \\
134.42 \\
138.35 \\
140.16 \\
142.63 \\
145.78 \\
142.52 \\
0.22\end{array}$ & $\begin{array}{r}\text { Maximum } \\
\text { xStrain } \\
-0.0001 \\
-0.0001 \\
-0.0001 \\
0.0003 \\
0.0006 \\
0.0007 \\
0.0004 \\
0.0003 \\
0.0003 \\
0.0001 \\
0.0004 \\
0.0002 \\
0.0004 \\
-0.0001 \\
0.0004 \\
0.0004 \\
0.0001 \\
0.0000 \\
0.0000 \\
0.0001 \\
0.0001 \\
0.0000 \\
-0.0001 \\
-0.0002 \\
-0.0005 \\
-0.0001 \\
-0.0005 \\
-0.0008 \\
-0.0006 \\
-0.0009 \\
-0.0007 \\
-0.0005 \\
-0.0004 \\
0.0000 \\
0.0000 \\
0.0013 \\
0.0079\end{array}$ & $\begin{array}{l}\text { Minimum } \\
\text { Xtrain } \\
-0.0006 \\
-0.0036 \\
-0.0065 \\
-0.0073 \\
-0.0072 \\
-0.0073 \\
-0.0075 \\
-0.0069 \\
-0.0066 \\
-0.0064 \\
-0.0056 \\
-0.0053 \\
-0.0053 \\
-0.0052 \\
-0.0053 \\
-0.0052 \\
-0.0052 \\
-0.0050 \\
-0.0049 \\
-0.0050 \\
-0.0049 \\
-0.0048 \\
-0.0051 \\
-0.0052 \\
-0.0054 \\
-0.0061 \\
-0.0056 \\
-0.0057 \\
-0.0058 \\
-0.0058 \\
-0.0055 \\
-0.0052 \\
-0.0050 \\
-0.0049 \\
-0.0050 \\
-0.0039 \\
0.0005\end{array}$ & $\begin{array}{c}\text { Angle } \\
\text { Degrees } \\
-14.51 \\
4.33 \\
-1.56 \\
-2.92 \\
-4.54 \\
-6.23 \\
-6.13 \\
-9.46 \\
-12.26 \\
-14.75 \\
-22.39 \\
-23.83 \\
-25.11 \\
-24.54 \\
-29.63 \\
-28.65 \\
-28.69 \\
-29.50 \\
-31.92 \\
-31.84 \\
-34.86 \\
-33.20 \\
-35.29 \\
-34.04 \\
-33.47 \\
-35.87 \\
-33.81 \\
-32.30 \\
-30.48 \\
-29.59 \\
-31.62 \\
-28.40 \\
-25.40 \\
-22.02 \\
-18.22 \\
-20.84 \\
10.34\end{array}$ \\
\hline
\end{tabular}

High Pressure test 


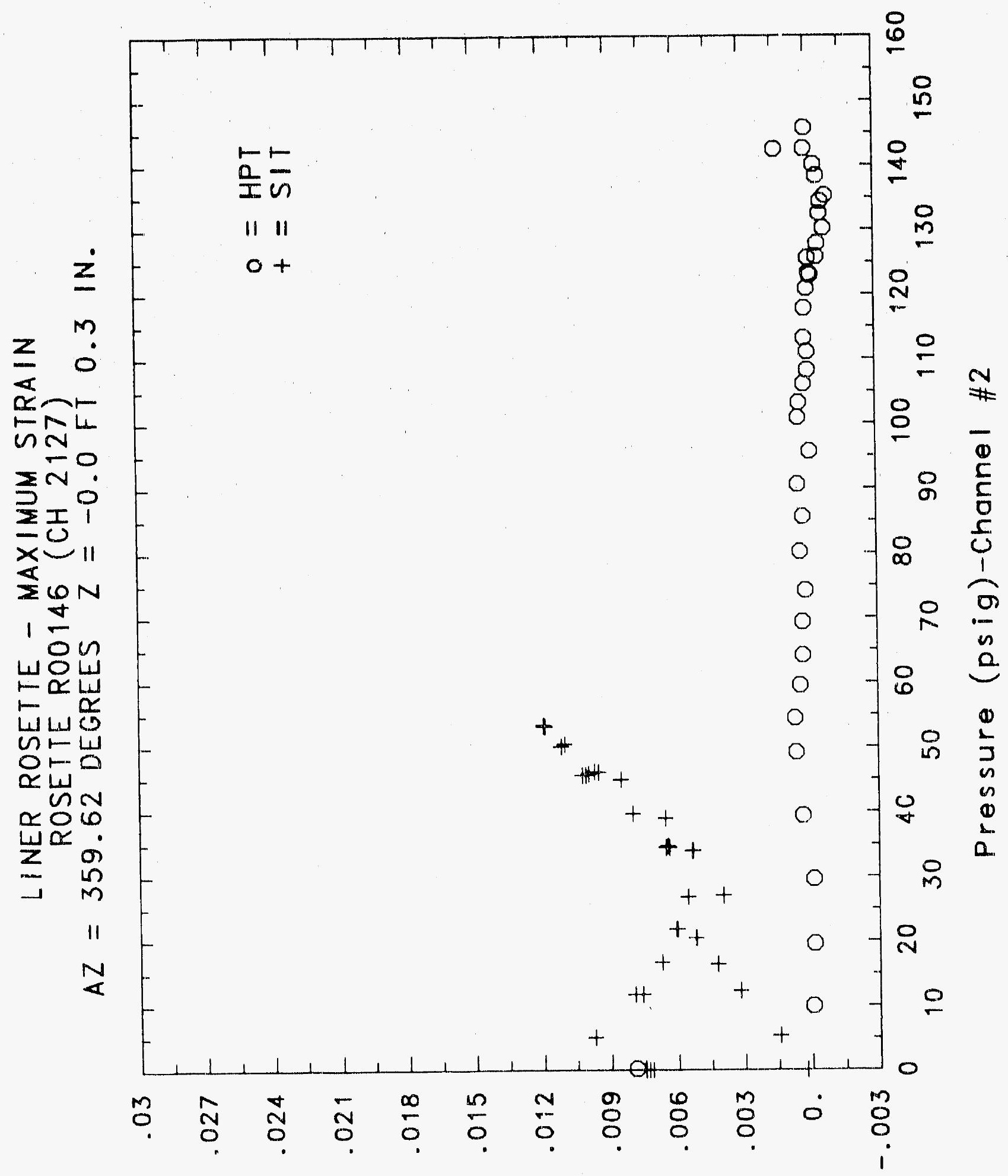

(\%) U!DdtS WnW!XDW 


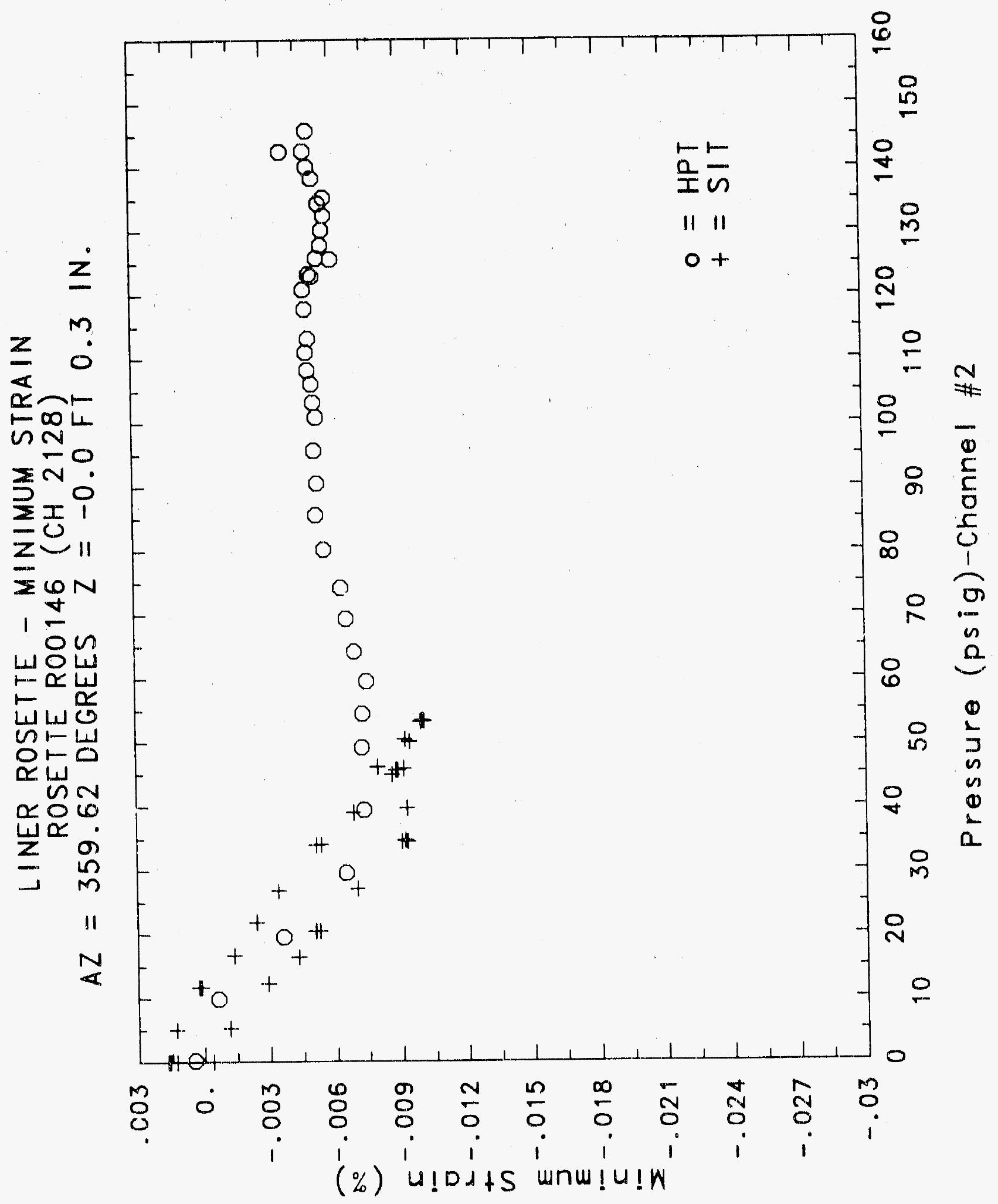




\section{Reinforced Concrete Test Data}

Rosette 147 Channel.s $2140 \quad 2141 \quad 2142$

STRUCTURAL INTEgRITY TEST

\begin{tabular}{|c|c|c|c|c|c|c|c|}
\hline $\begin{array}{c}\text { Pressure } \\
\text { psig } \\
-0.05 \\
5.33 \\
12.31 \\
16.44 \\
20.51 \\
20.46 \\
20.47 \\
27.05 \\
34.69 \\
34.53 \\
34.53 \\
39.70 \\
45.90 \\
45.65 \\
45.62 \\
50.10 \\
53.47 \\
53.29 \\
53.21 \\
50.49 \\
46.14 \\
46.13 \\
44.97 \\
38.98 \\
33.99 \\
33.96 \\
26.83 \\
21.88 \\
21.90 \\
16.69 \\
11.74 \\
11.77 \\
5.05 \\
0.02 \\
-0.04 \\
-0.02 \\
-0.02 \\
0.02\end{array}$ & $\begin{array}{c}\text { Maximum } \\
\text { \%Strain } \\
0.0000 \\
0.0014 \\
0.0034 \\
0.0046 \\
0.0066 \\
0.0072 \\
0.0074 \\
0.0123 \\
0.0113 \\
0.0113 \\
0.0113 \\
0.0124 \\
0.0152 \\
0.0149 \\
0.0150 \\
0.0169 \\
0.0186 \\
0.0185 \\
0.0185 \\
0.0178 \\
0.0164 \\
0.0164 \\
0.0161 \\
0.0145 \\
0.0129 \\
0.0130 \\
0.0092 \\
0.0066 \\
0.0065 \\
0.0034 \\
0.0002 \\
-0.0001 \\
-0.0005 \\
-0.0002 \\
-0.0006 \\
-0.0005 \\
-0.0005 \\
-0.0008\end{array}$ & $\begin{array}{c}\text { Minimum } \\
\text { xStrain } \\
-0.0001 \\
0.0001 \\
0.0004 \\
0.0005 \\
0.0009 \\
0.0011 \\
0.0011 \\
0.0025 \\
0.0053 \\
0.0048 \\
0.0049 \\
0.0043 \\
0.0051 \\
0.0051 \\
0.0053 \\
0.0053 \\
0.0057 \\
0.0059 \\
0.0061 \\
0.0059 \\
0.0054 \\
0.0054 \\
0.0046 \\
0.0041 \\
0.0035 \\
0.0034 \\
0.0023 \\
0.0014 \\
0.0011 \\
0.0000 \\
-0.0007 \\
-0.0007 \\
-0.0057 \\
-0.0059 \\
-0.0057 \\
-0.0065 \\
-0.0063 \\
-0.0061\end{array}$ & $\begin{array}{c}\text { Angle } \\
\text { Degrees } \\
-23.24 \\
-21.20 \\
-17.45 \\
-14.92 \\
-16.44 \\
-16.31 \\
-14.49 \\
-11.10 \\
-2.76 \\
3.59 \\
3.07 \\
2.75 \\
-4.87 \\
-1.43 \\
-2.83 \\
-0.44 \\
-0.47 \\
-0.29 \\
-0.78 \\
-0.33 \\
0.73 \\
0.28 \\
-2.43 \\
-1.30 \\
-0.06 \\
0.33 \\
1.41 \\
0.84 \\
1.72 \\
7.20 \\
-20.37 \\
-15.48 \\
21.16 \\
26.38 \\
24.95 \\
24.71 \\
25.99 \\
27.04\end{array}$ & $\begin{array}{c}\text { Pressure } \\
\text { ps } 19 \\
9.89 \\
19.55 \\
29.57 \\
39.42 \\
49.16 \\
54.50 \\
59.57 \\
64.20 \\
69.32 \\
74.16 \\
80.16 \\
85.61 \\
90.58 \\
95.69 \\
100.92 \\
103.25 \\
106.11 \\
108.31 \\
111.08 \\
113.24 \\
117.83 \\
120.92 \\
123.28 \\
122.97 \\
125.82 \\
125.60 \\
127.84 \\
130.19 \\
132.53 \\
135.33 \\
134.42 \\
138.35 \\
140.16 \\
142.63 \\
145.78 \\
142.52 \\
0.22\end{array}$ & $\begin{array}{c}\text { Maximum } \\
\text { XStrain } \\
0.0030 \\
0.0070 \\
0.0113 \\
0.0135 \\
0.0137 \\
0.0143 \\
0.0149 \\
0.0146 \\
0.0149 \\
0.0152 \\
0.0153 \\
0.0160 \\
0.0167 \\
0.0175 \\
0.0185 \\
0.0187 \\
0.0188 \\
0.0190 \\
0.0191 \\
0.0195 \\
0.0197 \\
0.0201 \\
0.0205 \\
0.0204 \\
0.0209 \\
0.0215 \\
0.0211 \\
0.0214 \\
0.0217 \\
0.0221 \\
0.0224 \\
0.0230 \\
0.0239 \\
0.0243 \\
0.0251 \\
0.0207 \\
0.0029\end{array}$ & $\begin{array}{l}\text { Minimum } \\
\text { \%strain } \\
0.0010 \\
0.0022 \\
0.0031 \\
0.0033 \\
0.0035 \\
0.0034 \\
0.0034 \\
0.0035 \\
0.0036 \\
0.0036 \\
0.0038 \\
0.0037 \\
0.0037 \\
0.0037 \\
0.0038 \\
0.0036 \\
0.0035 \\
0.0038 \\
0.0036 \\
0.0037 \\
0.0038 \\
0.0038 \\
0.0036 \\
0.0038 \\
0.0037 \\
0.0037 \\
0.0036 \\
0.0034 \\
0.0035 \\
0.0034 \\
0.0036 \\
0.0036 \\
0.0036 \\
0.0035 \\
0.0040 \\
0.0 \Gamma 06 \\
-0.0 .16\end{array}$ & $\begin{array}{c}\text { Angle } \\
\text { Degrees } \\
-5.87 \\
5.67 \\
13.44 \\
14.06 \\
12.57 \\
13.22 \\
12.58 \\
11.54 \\
9.42 \\
8.45 \\
4.40 \\
3.57 \\
2.60 \\
0.64 \\
-2.16 \\
-2.17 \\
-2.88 \\
-4.09 \\
-3.94 \\
-4.87 \\
-4.68 \\
-4.49 \\
-4.08 \\
-3.95 \\
-4.07 \\
-5.47 \\
-5.37 \\
-5.79 \\
-6.78 \\
-5.03 \\
-5.59 \\
-4.60 \\
-4.84 \\
-4.94 \\
-5.41 \\
-2.49 \\
15.80\end{array}$ \\
\hline
\end{tabular}

High Pressure Test 


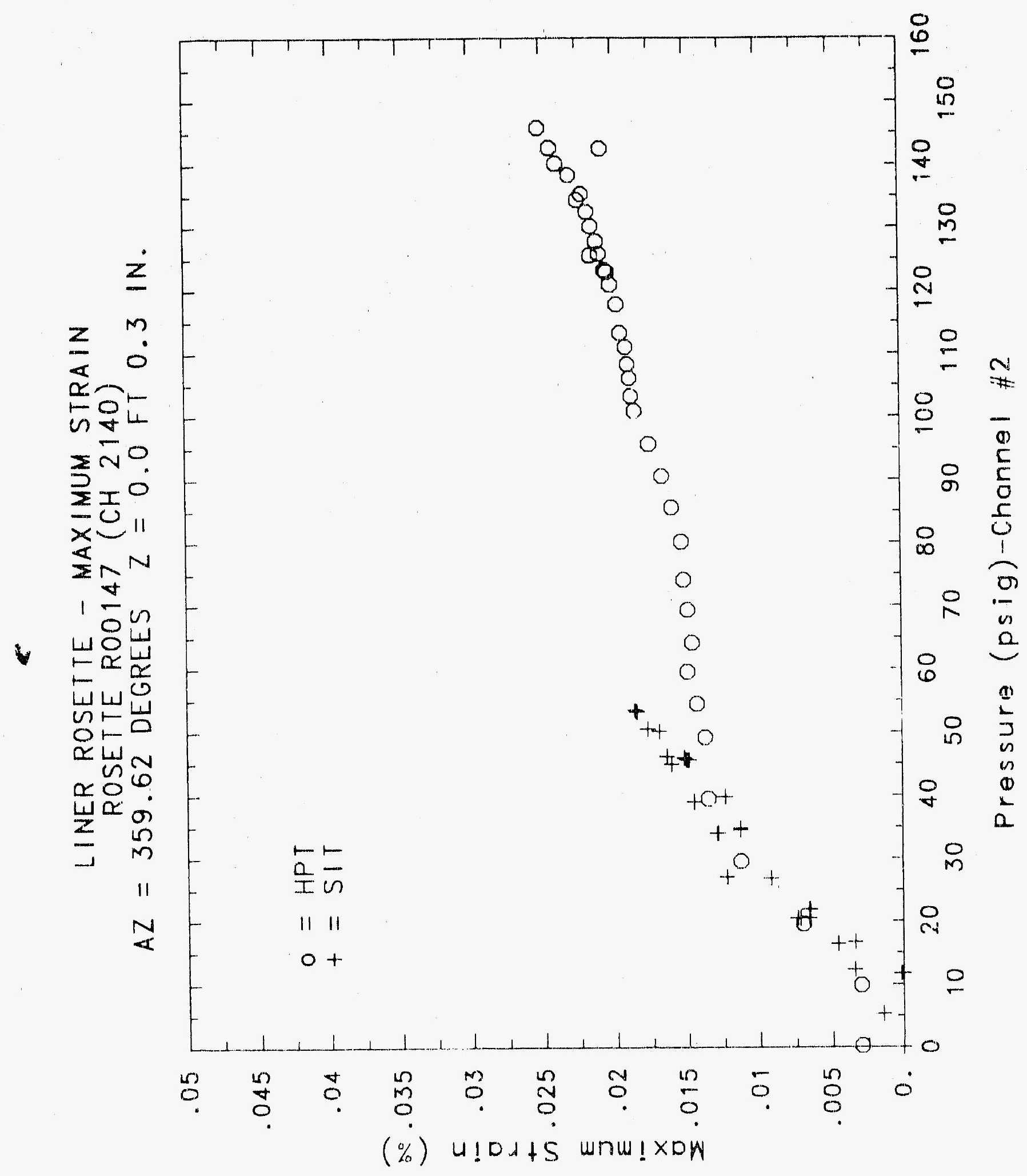




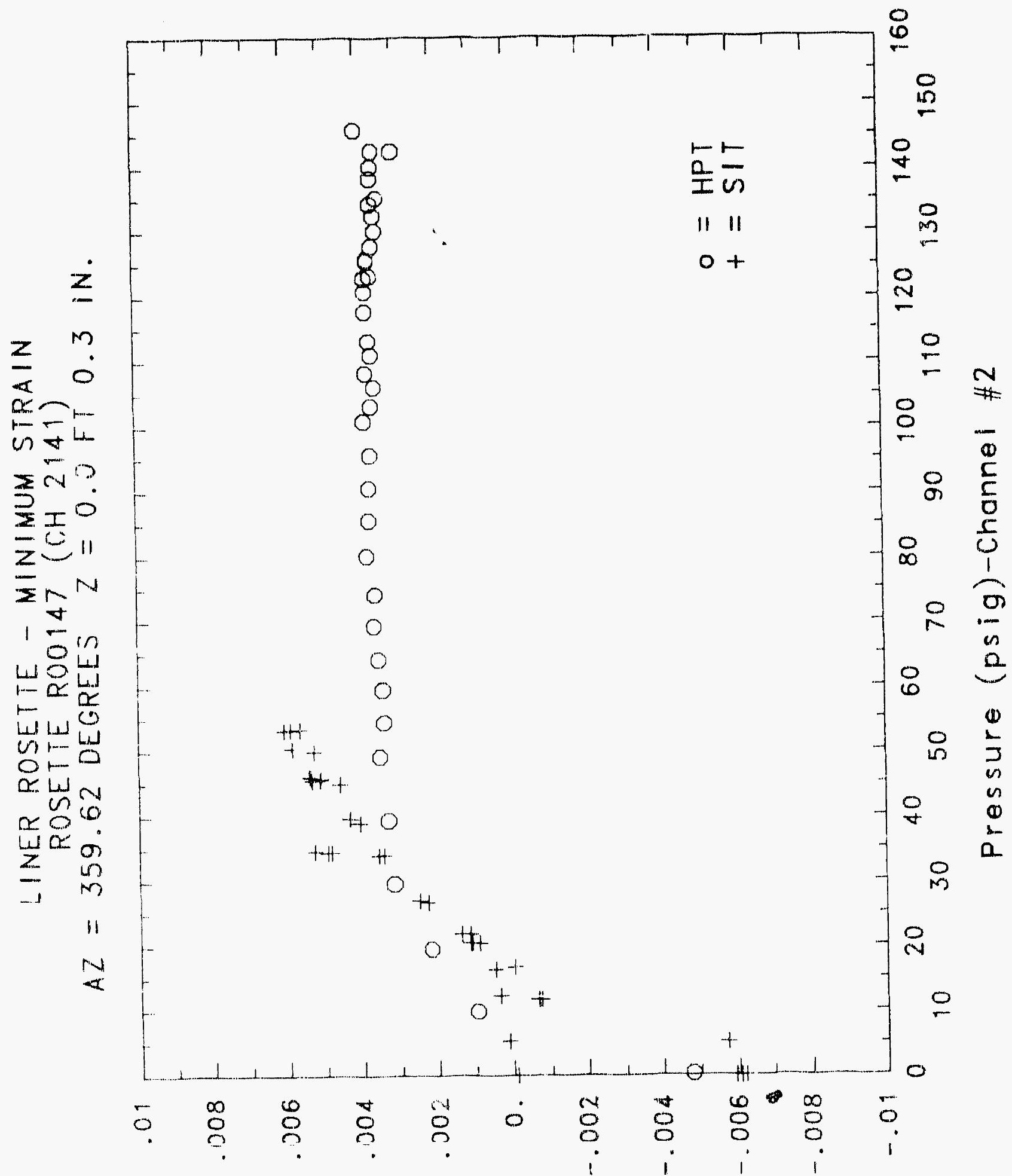

$(\%)$ U!Dd+S unw!U!W 


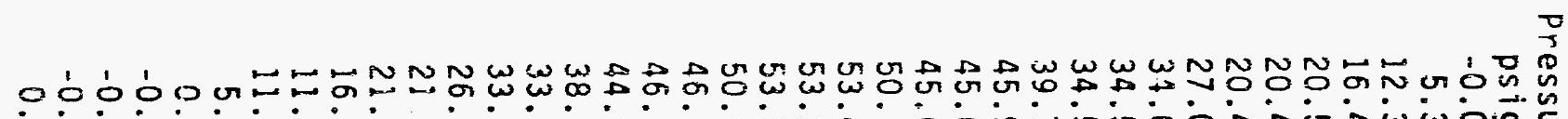

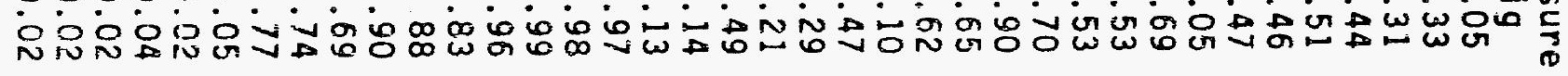
$00000000000000000000000000000.000 .000 . \mathrm{H}^{\mathrm{x}}$

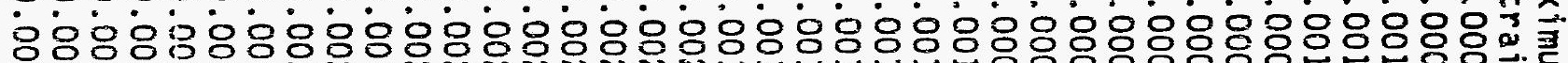

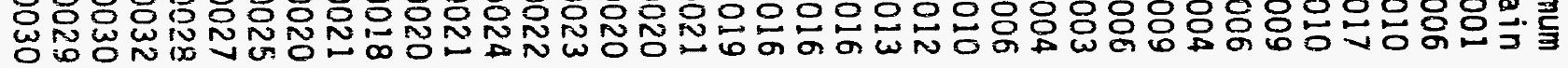

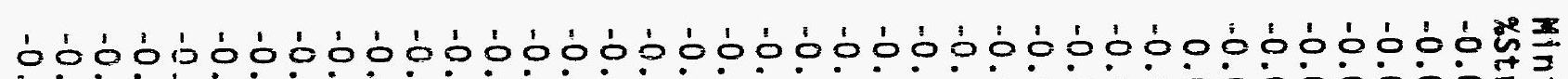
-

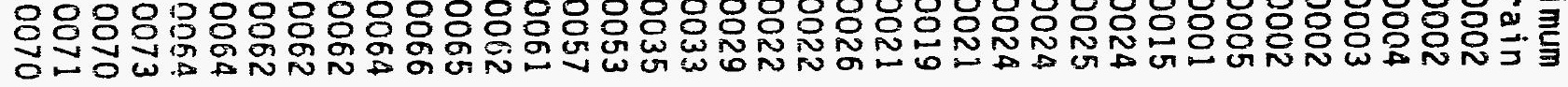

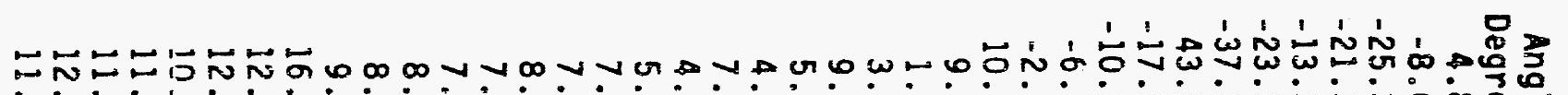

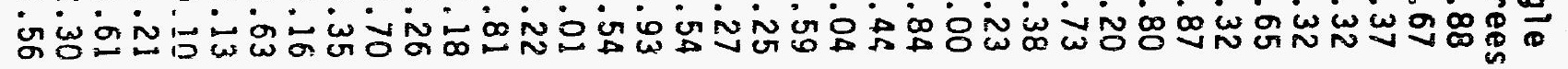

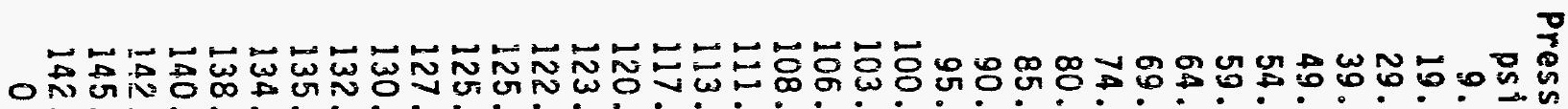

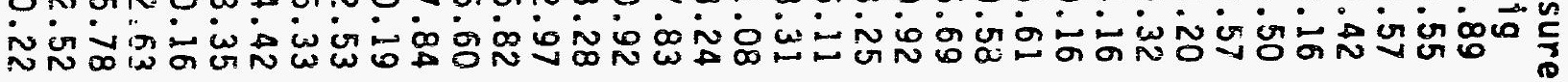
0000000000000000000000000000000000000聯 -

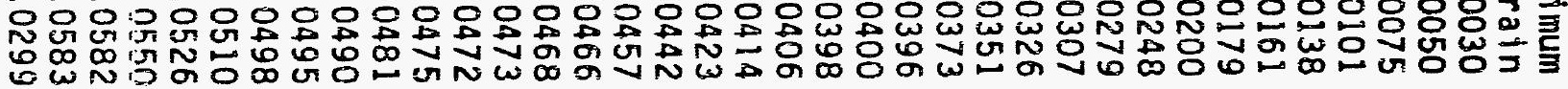
000000000000000000000000000000000000043 ㅇㅇㅇㅇㅇㅇㅇㅇㅇㅇㅇㅇㅇㅇㅇㅇㅇㅇㅇㅇㅇㅇㅇㅇㅇㅇㅇㅇㅇㅇㅇㅇㅇㅇㅇㅇㅇㅇㅇ영

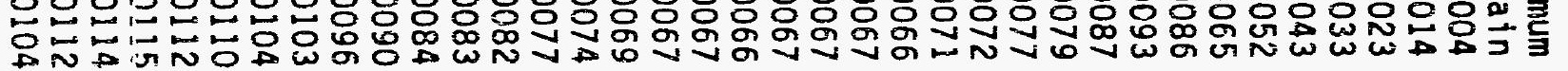

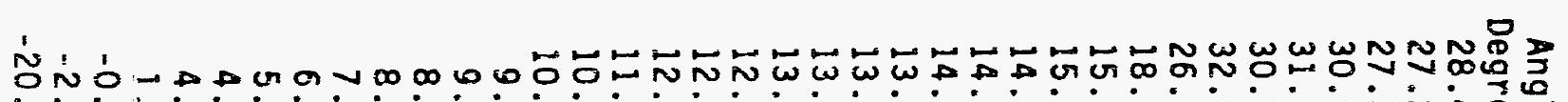
в 


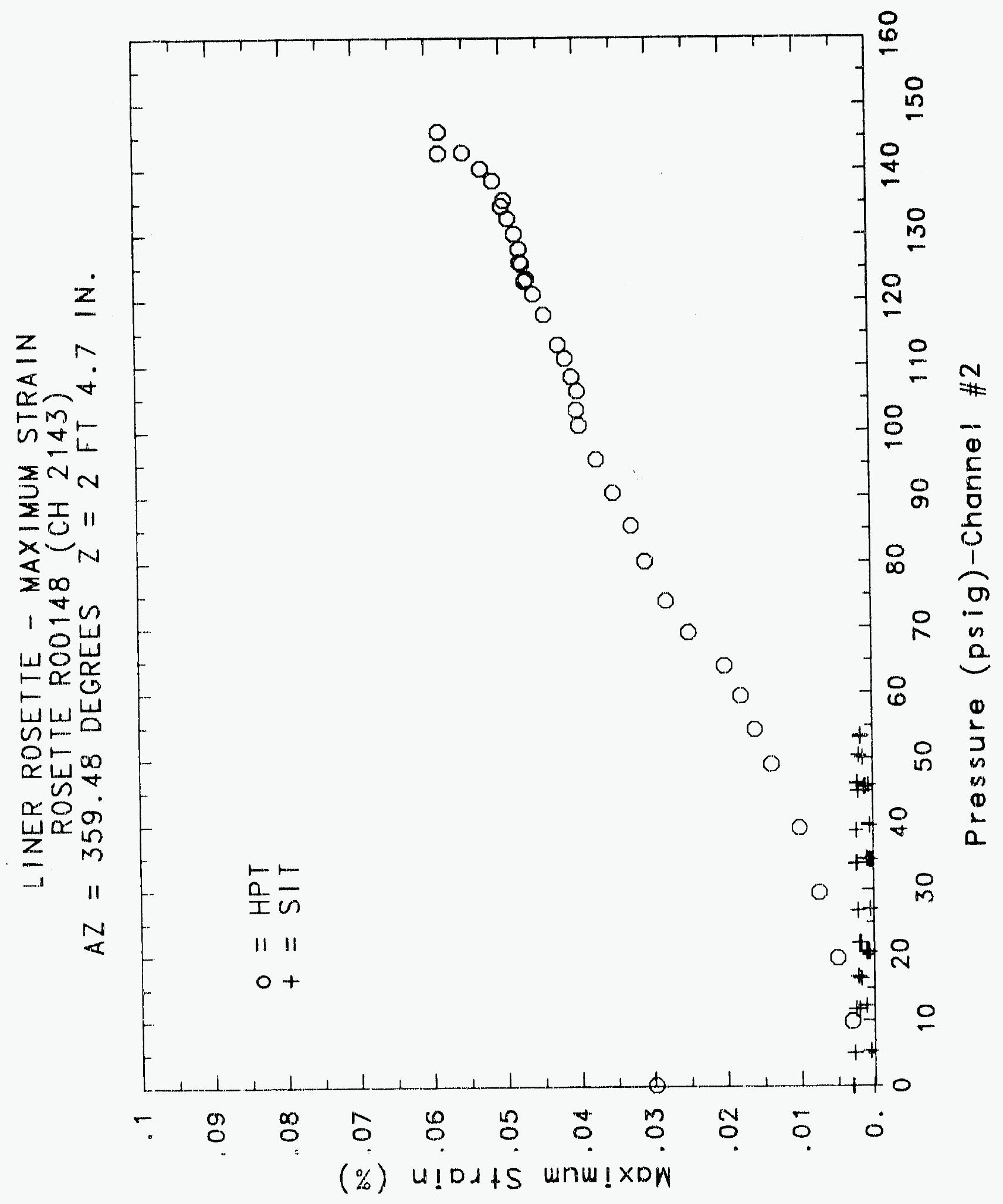

C. 762 


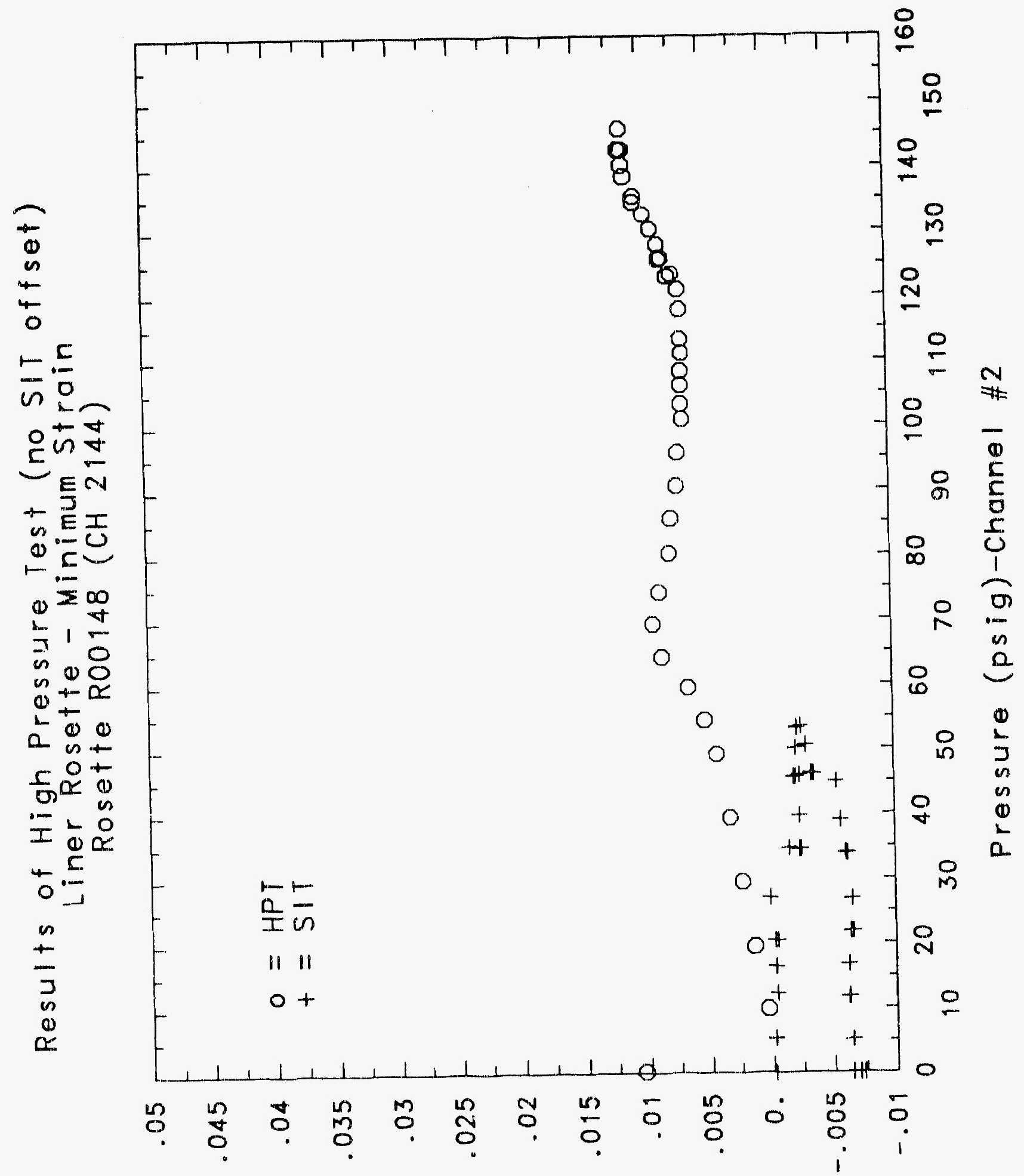

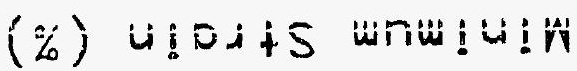




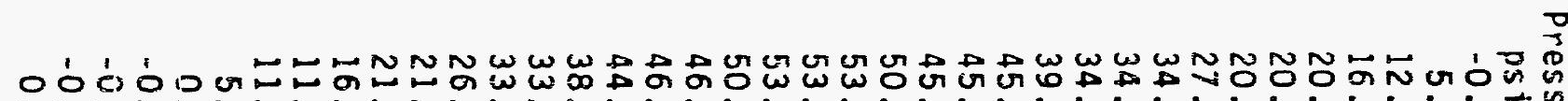

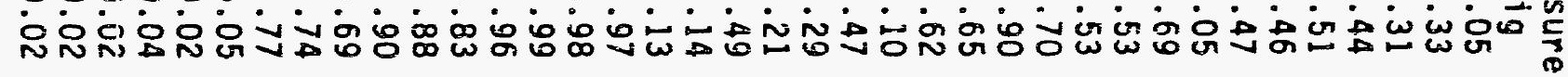

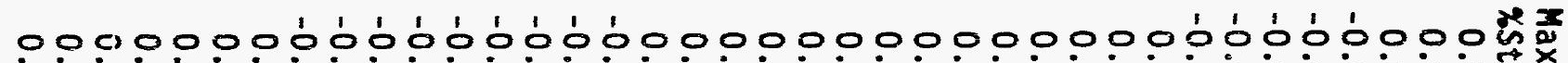
80808080808080808080880808080808003 等品

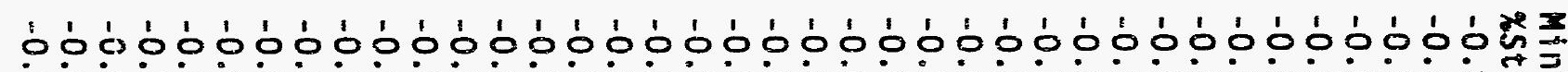

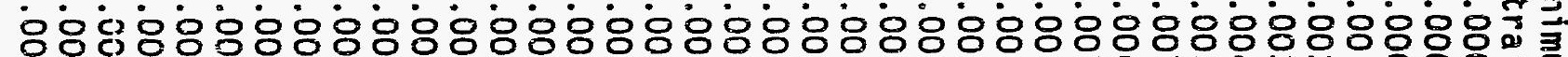

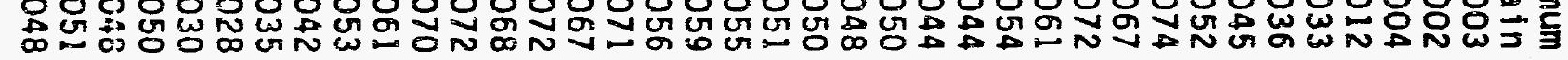

ڤب

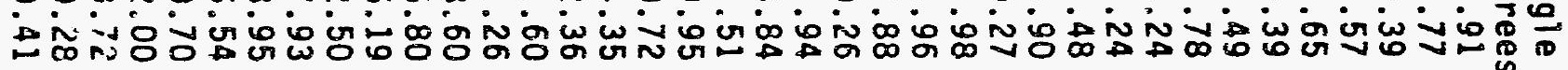

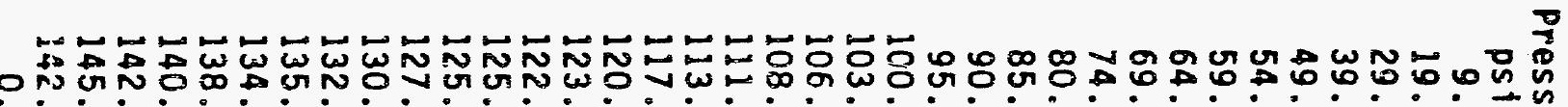

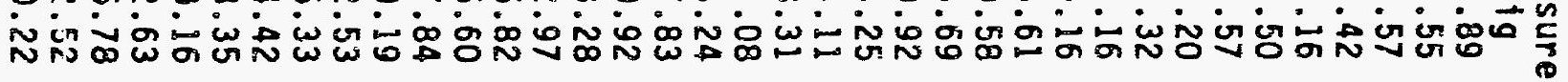
$0000000000000000000000000000000000000 \%$ o

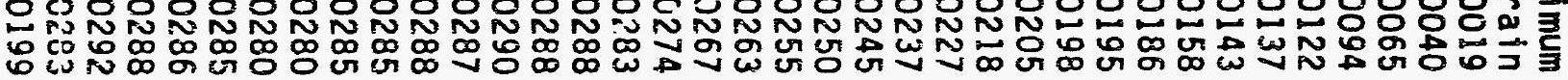

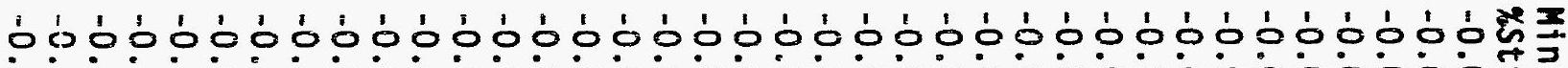

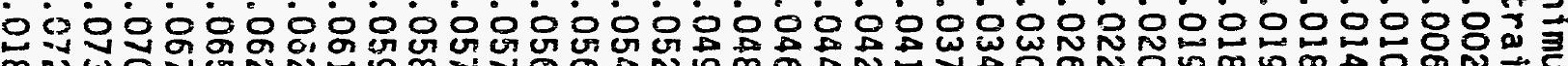

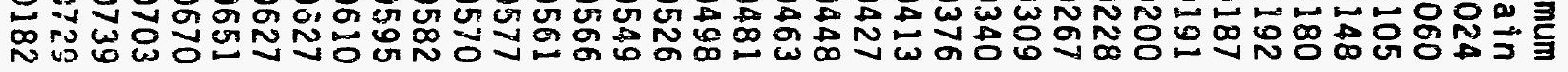

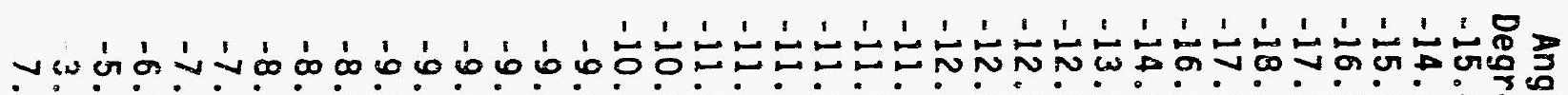

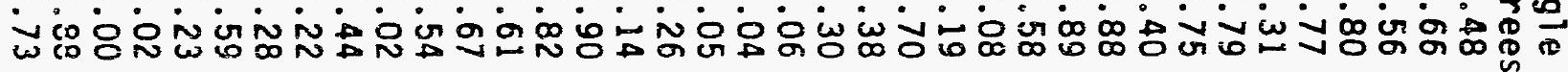




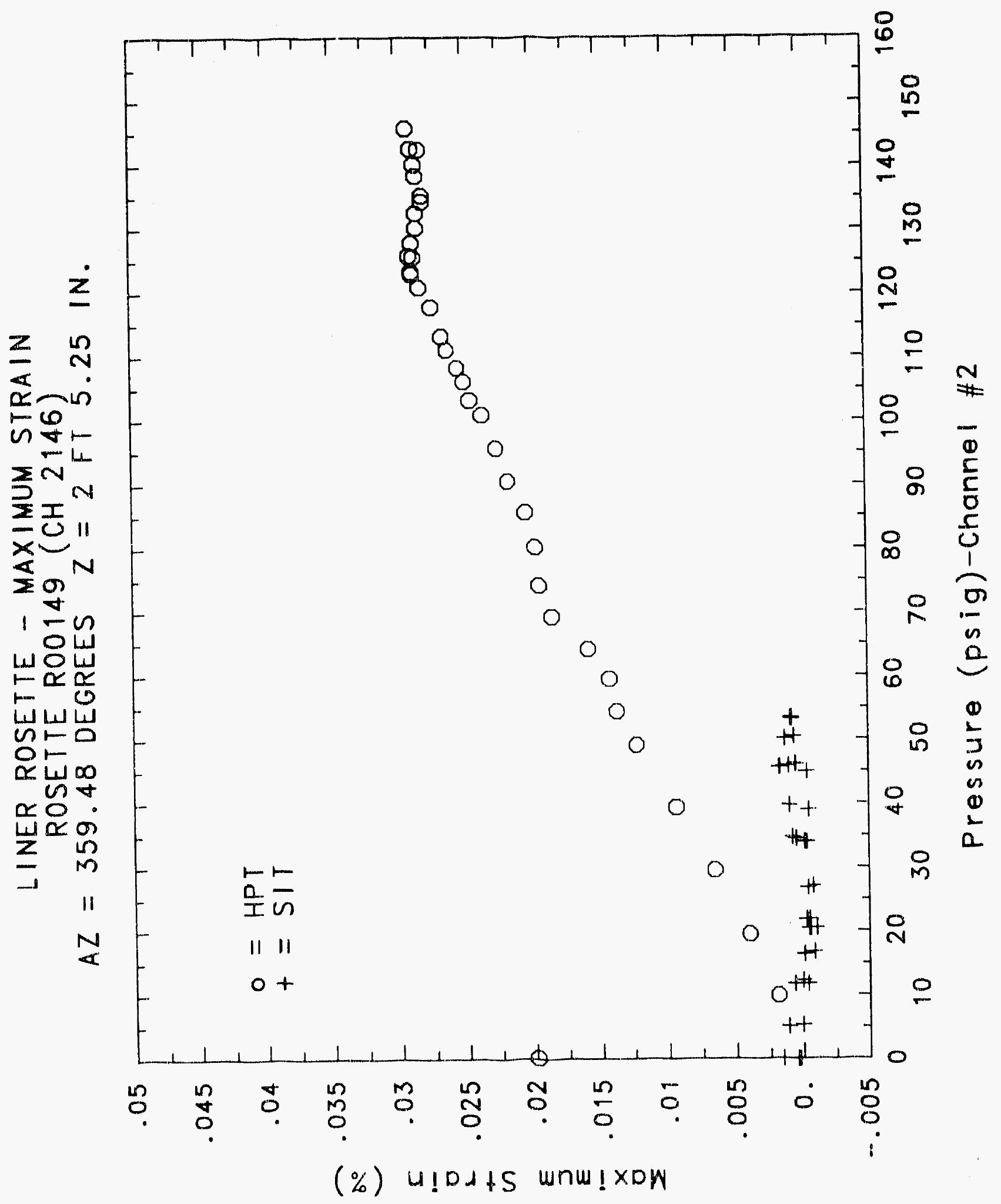




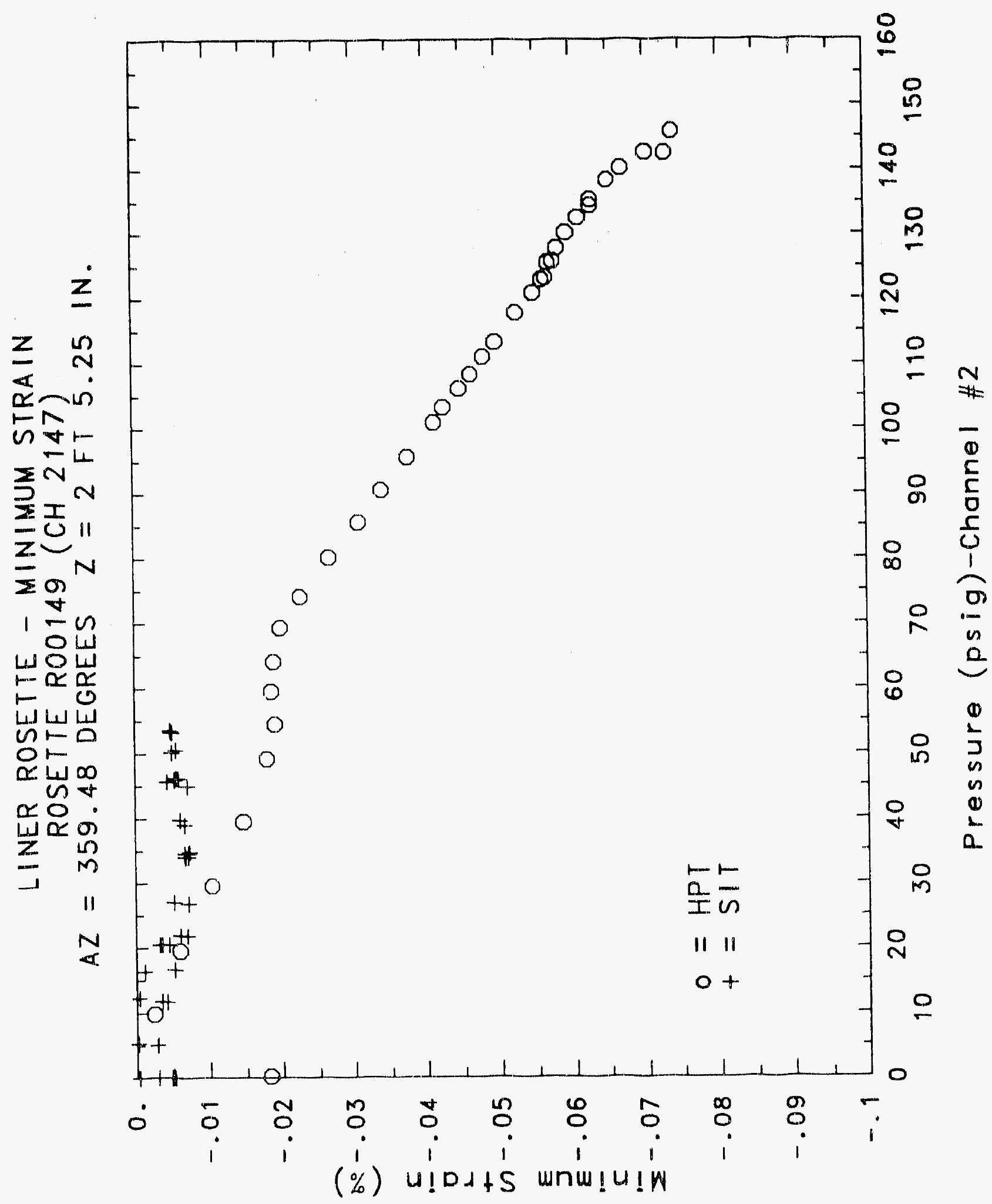




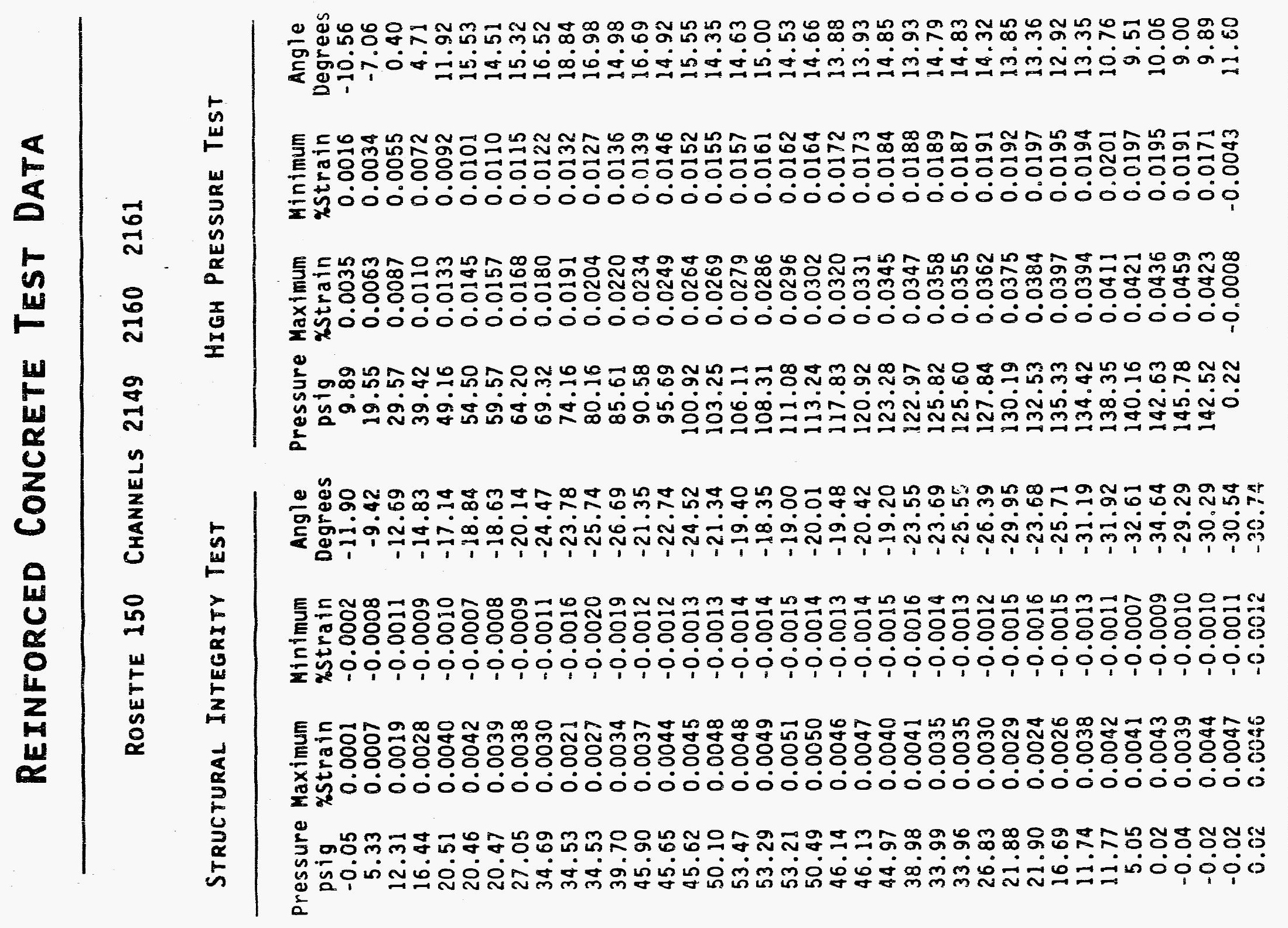




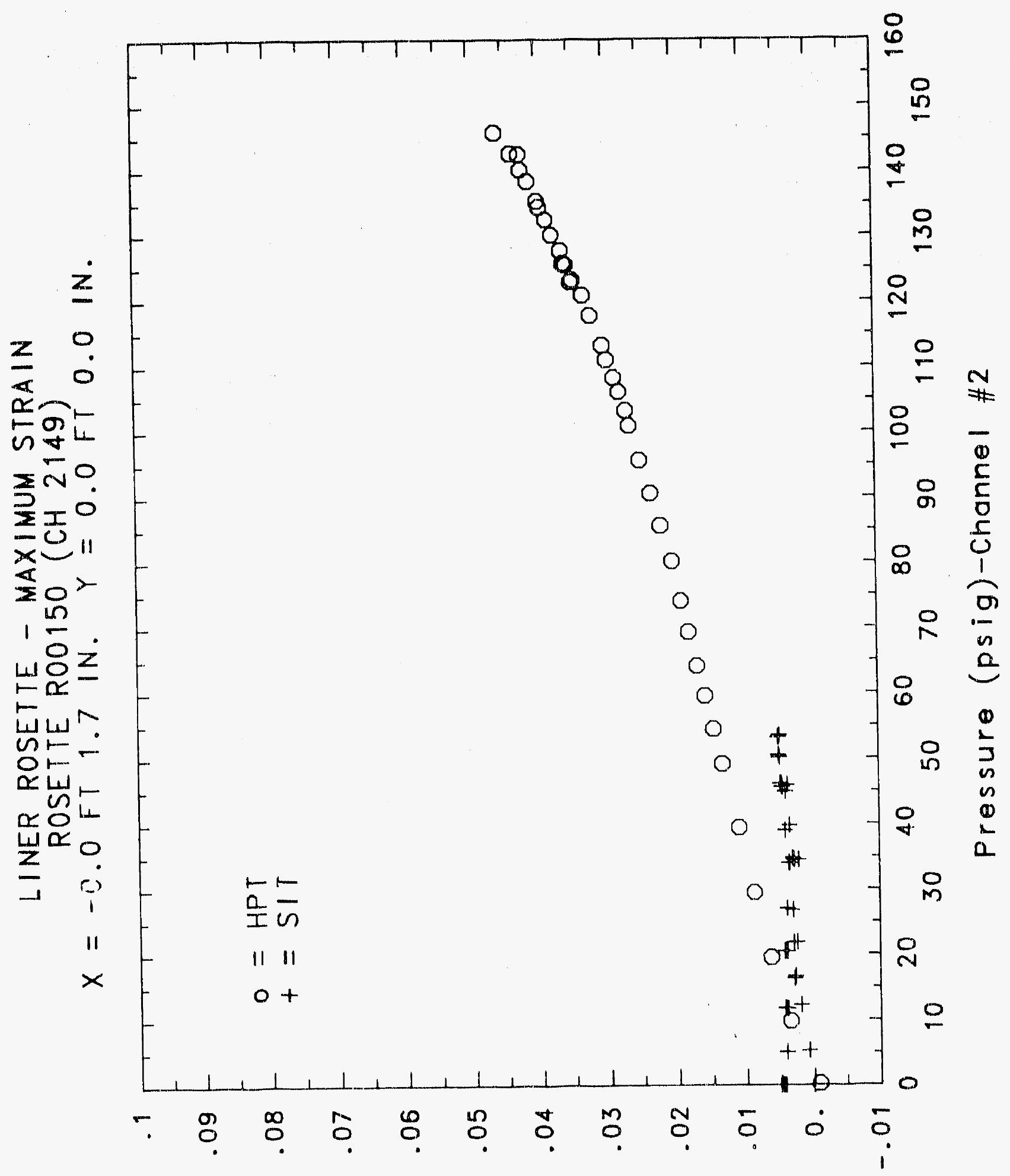

(\%) $4 ! 0 d+5$ unw! XDW 


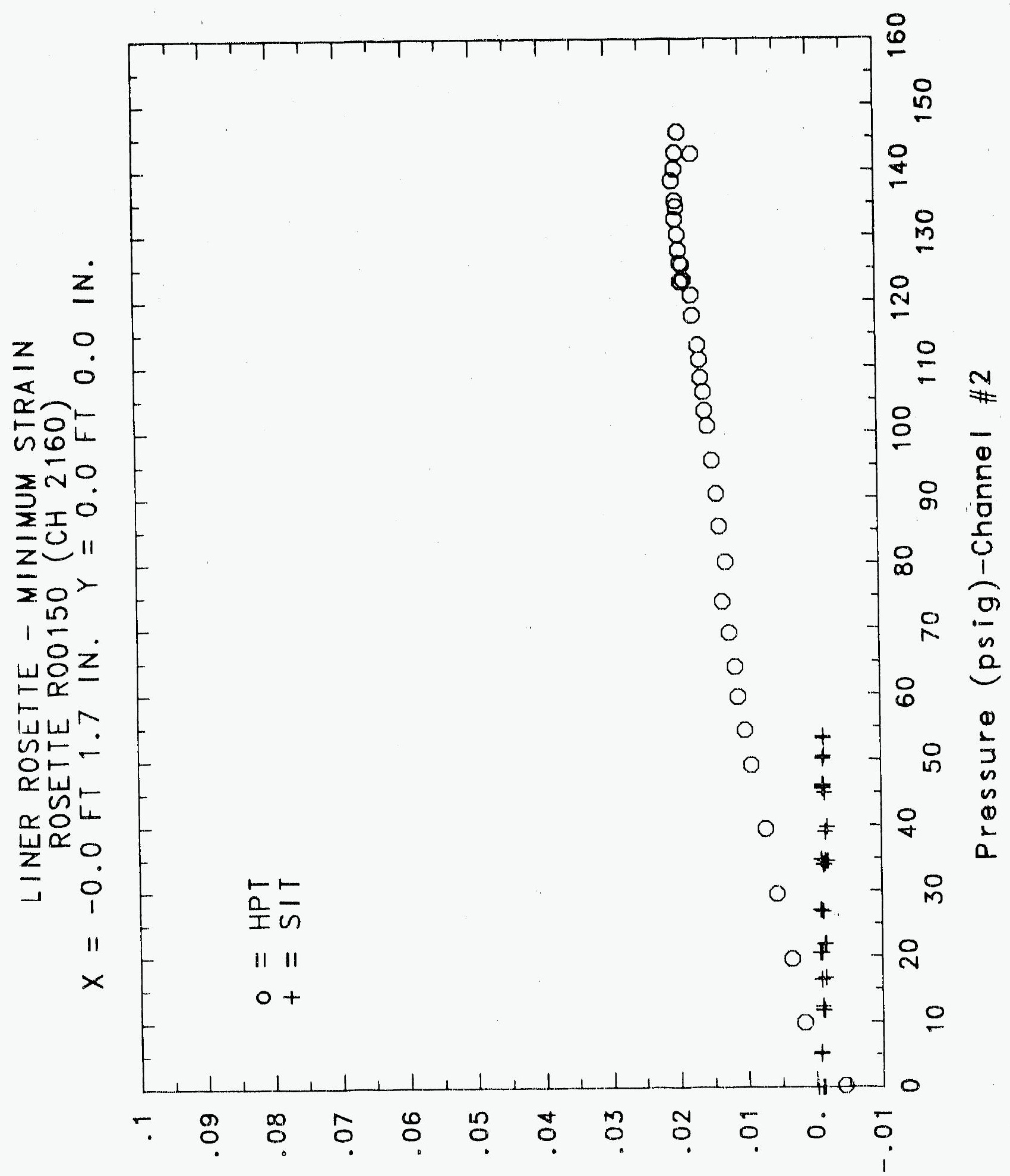

(\%) u!Ddts wnw!u!w 


\section{Reinforced Concrete Test Data}

Rosette 151 Channels $2162 \quad 21632164$

Structural Integrity TEST

\begin{tabular}{|c|c|c|c|c|c|c|c|}
\hline $\begin{array}{c}\text { Pressure } \\
\text { psig } \\
\text {-0.05 } \\
5.33 \\
12.31 \\
16.44 \\
20.51 \\
20.46 \\
20.47 \\
27.05 \\
34.69 \\
34.53 \\
34.53 \\
39.70 \\
45.90 \\
45.65 \\
45.62 \\
50.10 \\
53.47 \\
53.29 \\
53.21 \\
50.49 \\
46.14 \\
46.13 \\
44.97 \\
38.98 \\
33.99 \\
33.96 \\
26.83 \\
21.88 \\
21.90 \\
16.69 \\
11.74 \\
11.77 \\
5.05 \\
0.02 \\
-0.04 \\
-0.02 \\
-0.02 \\
0.02\end{array}$ & $\begin{array}{l}\text { Maximum } \\
\text { xStrain } \\
0.0002 \\
0.0066 \\
0.0123 \\
0.0150 \\
0.0183 \\
0.0184 \\
0.0183 \\
0.0245 \\
0.0313 \\
0.0312 \\
0.0313 \\
0.0343 \\
0.0389 \\
0.0387 \\
0.0388 \\
0.0416 \\
0.0439 \\
0.0439 \\
0.0441 \\
0.0435 \\
0.0417 \\
0.0415 \\
0.0411 \\
0.0370 \\
0.0333 \\
0.0333 \\
0.0275 \\
0.0223 \\
0.0225 \\
0.0176 \\
0.0141 \\
0.0141 \\
0.0091 \\
0.0022 \\
0.0023 \\
0.0025 \\
0.0025 \\
0.0024\end{array}$ & $\begin{array}{l}\text { Minimum } \\
\text { WStrain } \\
-0.0003 \\
-0.0020 \\
-0.0039 \\
-0.0049 \\
-0.0057 \\
-0.0059 \\
-0.0058 \\
-0.0077 \\
-0.0097 \\
-0.0097 \\
-0.0096 \\
-0.0109 \\
-0.0124 \\
-0.0121 \\
-0.0122 \\
-0.0134 \\
-0.0141 \\
-0.0139 \\
-0.0138 \\
-0.0141 \\
-0.0137 \\
-0.0138 \\
-0.0130 \\
-0.0123 \\
-0.0115 \\
-0.0114 \\
-0.0106 \\
-0.0084 \\
-0.0086 \\
-0.0054 \\
-0.0033 \\
-0.0036 \\
-0.0019 \\
0.0001 \\
-0.0004 \\
-0.0003 \\
-0.0002 \\
-0.0002\end{array}$ & $\begin{array}{c}\text { Angle } \\
\text { Degrees } \\
42.67 \\
-6.15 \\
-5.35 \\
-4.49 \\
-3.33 \\
-4.92 \\
-4.74 \\
-5.30 \\
-4.55 \\
-5.17 \\
-5.20 \\
-4.76 \\
-4.17 \\
-3.97 \\
-4.00 \\
-3.73 \\
-3.73 \\
-3.68 \\
-3.68 \\
-3.31 \\
-3.22 \\
-3.38 \\
-3.11 \\
-3.86 \\
-3.83 \\
-3.86 \\
-1.92 \\
-0.62 \\
-0.97 \\
-3.33 \\
-5.11 \\
-5.79 \\
-8.78 \\
15.14 \\
21.96 \\
20.72 \\
18.16 \\
10.98\end{array}$ & $\begin{array}{c}\text { Pressure } \\
\text { psig } \\
9.89 \\
19.55 \\
29.57 \\
39.42 \\
49.16 \\
54.50 \\
59.57 \\
64.20 \\
69.32 \\
74.16 \\
80.16 \\
85.61 \\
90.58 \\
95.69 \\
100.92 \\
103.25 \\
106.11 \\
108.31 \\
111.08 \\
113.24 \\
117.83 \\
120.92 \\
123.28 \\
122.97 \\
125.82 \\
125.60 \\
127.84 \\
130.19 \\
132.53 \\
135.33 \\
134.42 \\
138.35 \\
140.16 \\
142.63 \\
145.78 \\
142.52 \\
0.22\end{array}$ & $\begin{array}{c}\text { Maximum } \\
\text { xStrain } \\
0.0121 \\
0.0207 \\
0.0273 \\
0.0332 \\
0.0391 \\
0.0423 \\
0.0458 \\
0.0492 \\
0.0531 \\
0.0570 \\
0.0617 \\
0.0661 \\
0.0708 \\
0.0752 \\
0.0801 \\
0.0824 \\
0.0850 \\
0.0869 \\
0.0897 \\
0.0913 \\
0.0957 \\
0.0994 \\
0.1021 \\
0.1024 \\
0.1054 \\
0.1057 \\
0.1080 \\
0.1107 \\
0.1142 \\
0.1190 \\
0.1192 \\
0.1262 \\
0.1307 \\
0.1357 \\
0.1427 \\
0.1430 \\
0.0150\end{array}$ & $\begin{array}{l}\text { Minimum } \\
\text { XStrain } \\
-0.0040 \\
-0.0062 \\
-0.0087 \\
-0.0106 \\
-0.0125 \\
-0.0132 \\
-0.0145 \\
-0.0152 \\
-0.0165 \\
-0.0177 \\
-0.0204 \\
-0.0222 \\
-0.0239 \\
-0.0252 \\
-0.0271 \\
-0.0278 \\
-0.0287 \\
-0.0294 \\
-0.0303 \\
-0.0307 \\
-0.0314 \\
-0.0319 \\
-0.0322 \\
-0.0317 \\
-0.0321 \\
-0.0316 \\
-0.0321 \\
-0.0317 \\
-0.0309 \\
-0.0301 \\
-0.0291 \\
-0.0289 \\
-0.0287 \\
-0.0279 \\
-0.0278 \\
-0.0276 \\
0.0018\end{array}$ & $\begin{array}{c}\text { Angle } \\
\text { Degrees } \\
-11.00 \\
-6.50 \\
-5.80 \\
-5.27 \\
-5.04 \\
-4.97 \\
-4.29 \\
-4.08 \\
-3.74 \\
-3.65 \\
-2.19 \\
-1.87 \\
-1.50 \\
-1.10 \\
-0.82 \\
-0.45 \\
-0.13 \\
-0.08 \\
0.25 \\
0.46 \\
0.72 \\
0.81 \\
1.25 \\
1.48 \\
1.33 \\
1.29 \\
1.23 \\
1.39 \\
1.53 \\
1.18 \\
1.05 \\
0.92 \\
0.73 \\
0.30 \\
0.60 \\
0.76 \\
10.60\end{array}$ \\
\hline
\end{tabular}

High Pressure Test 


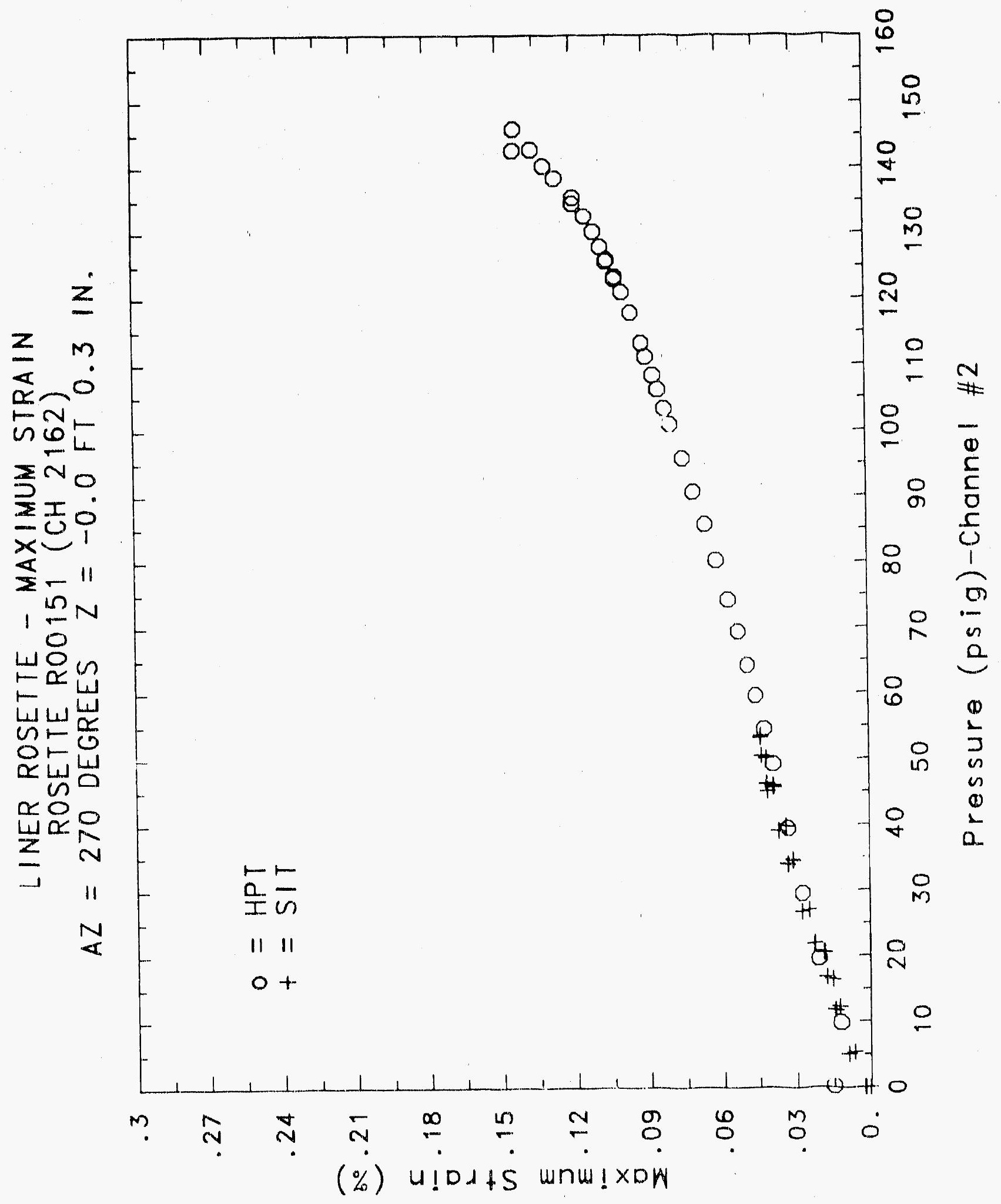




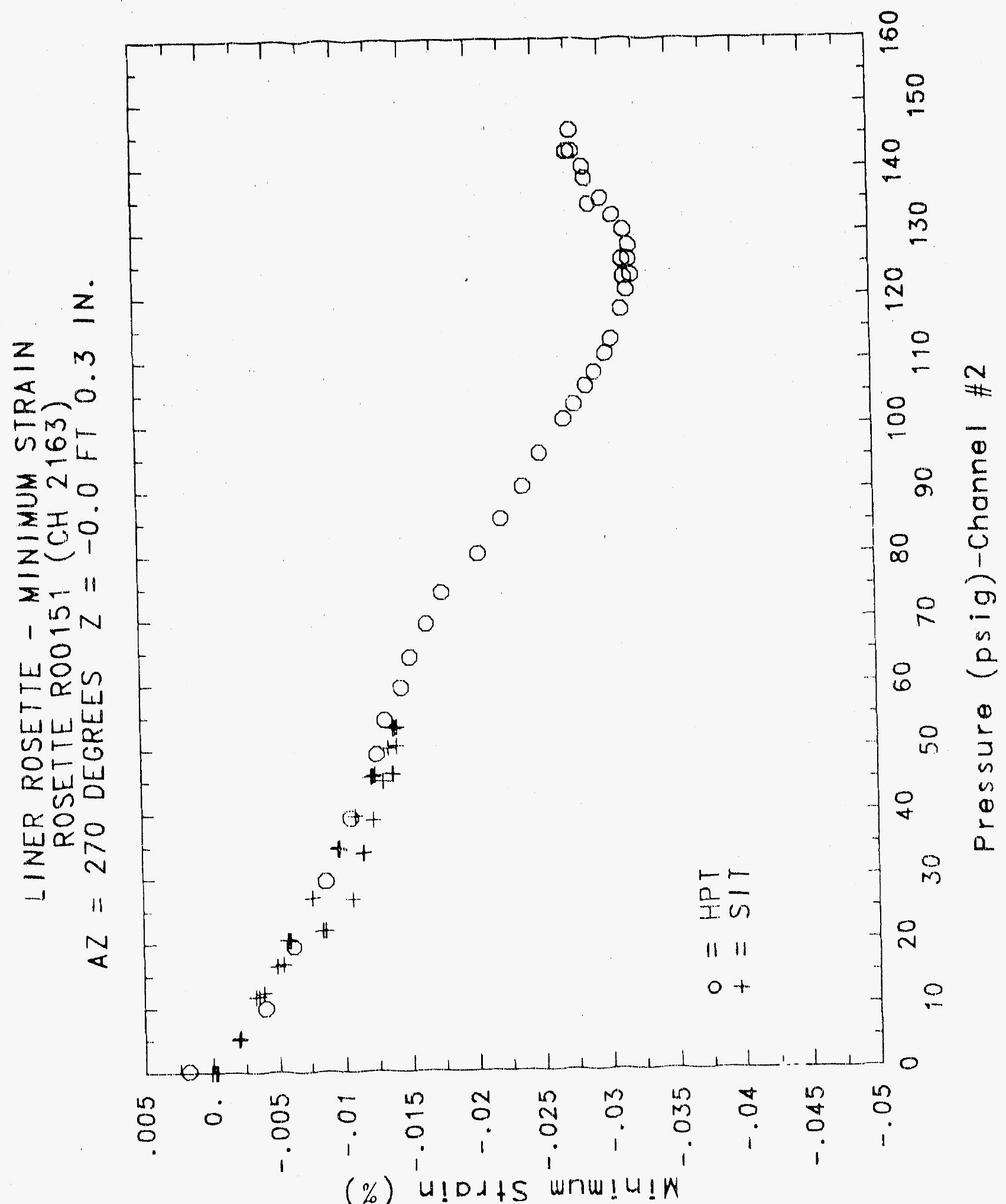




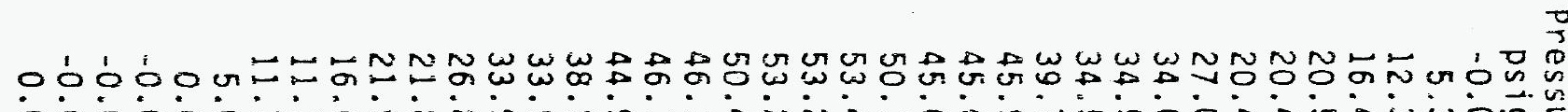

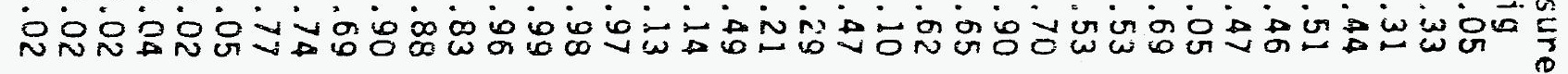

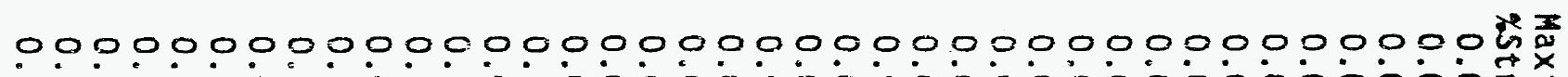
-

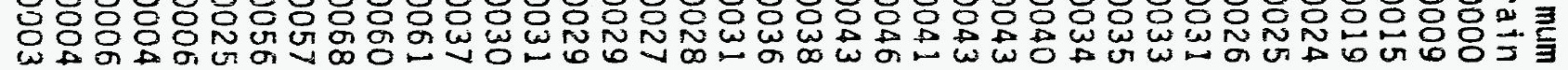

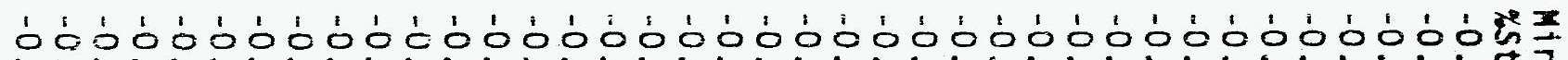

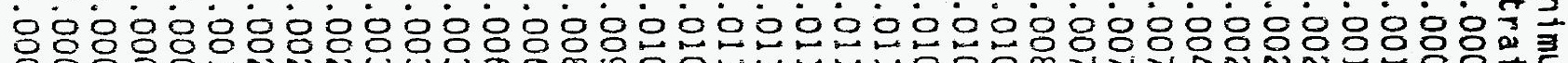

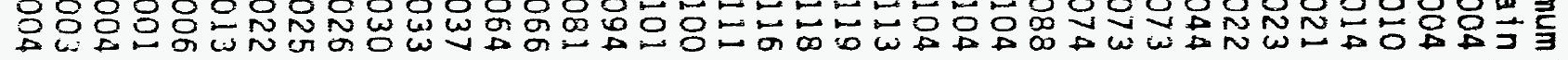

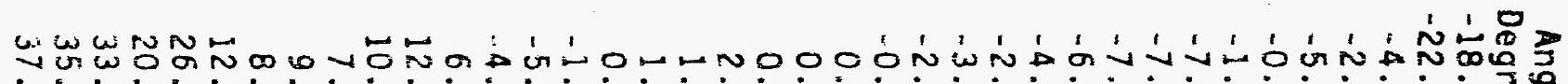

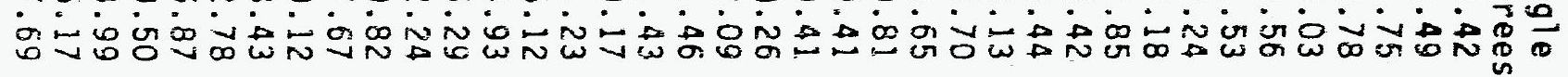

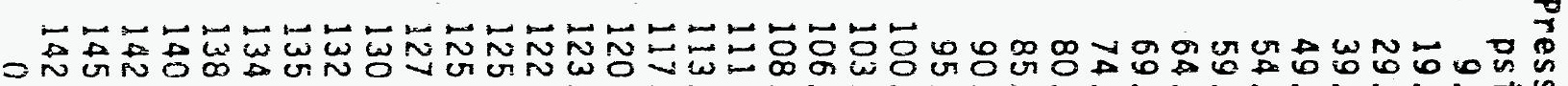
N

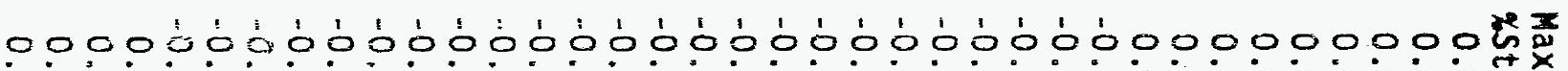

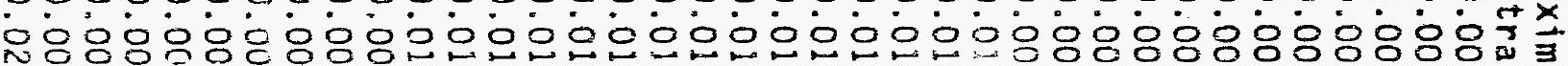

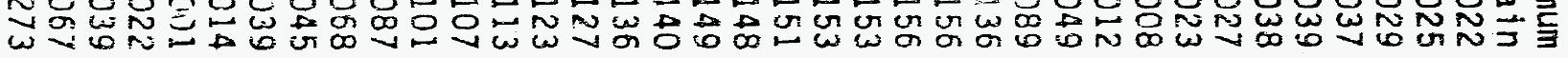

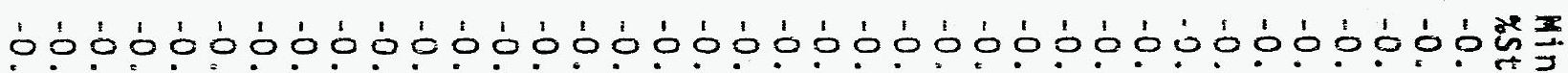

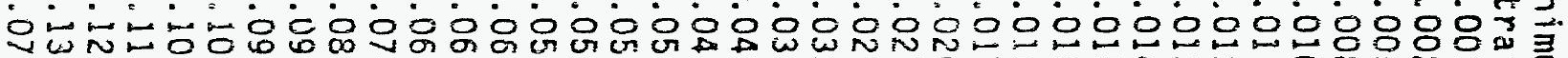

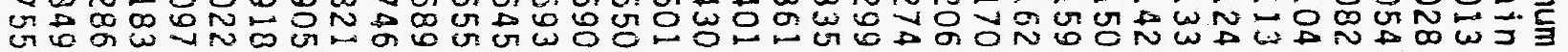

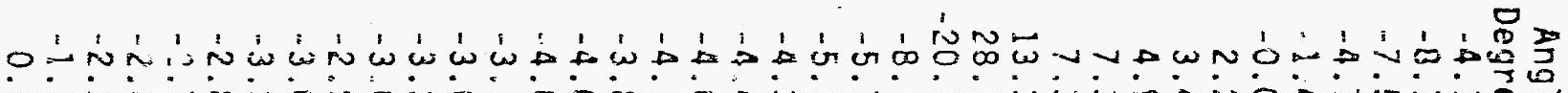

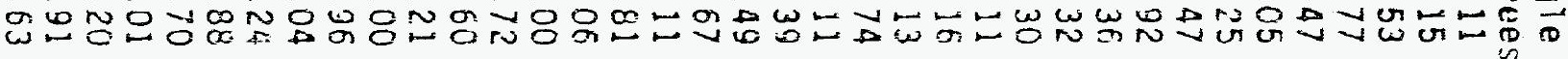




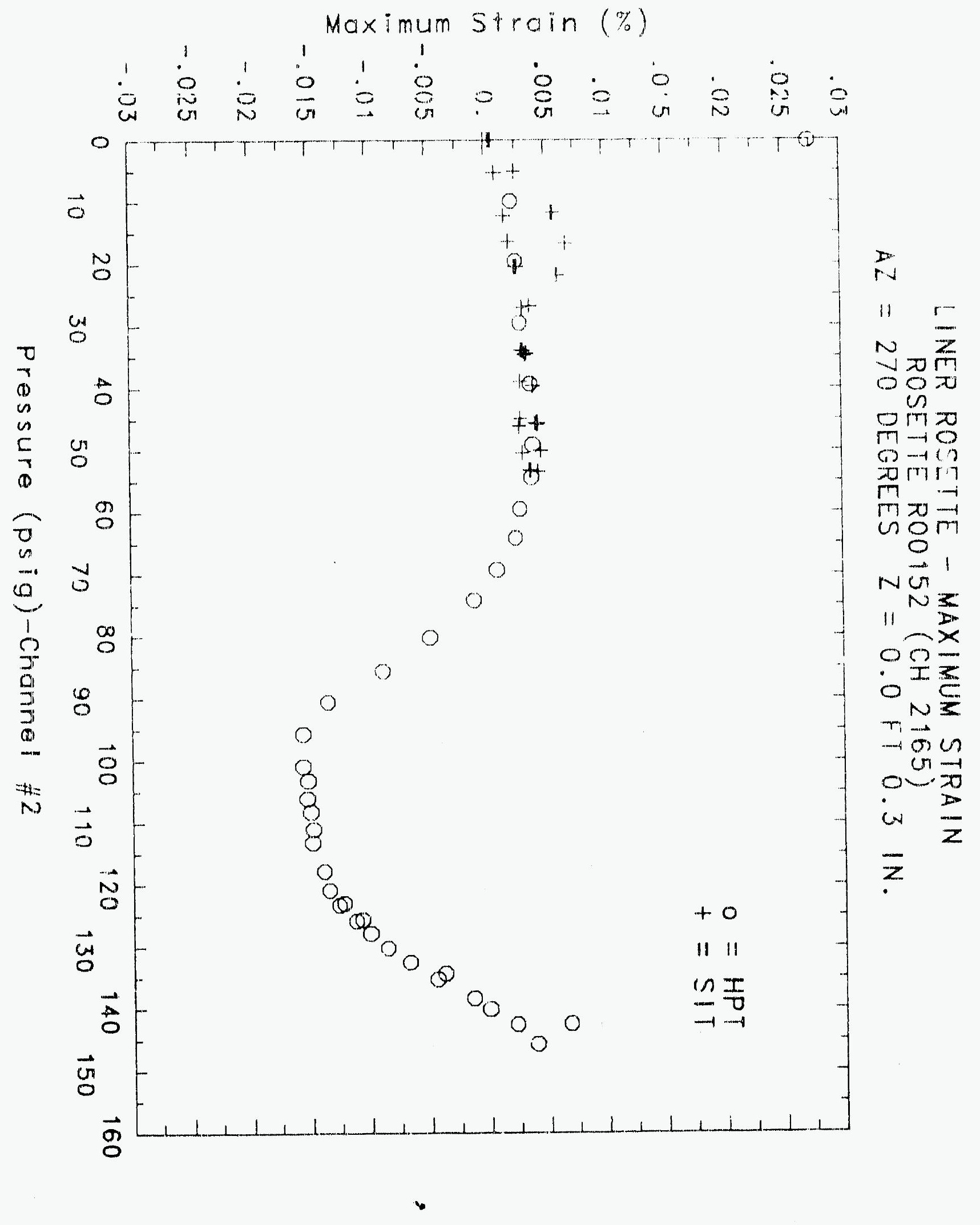




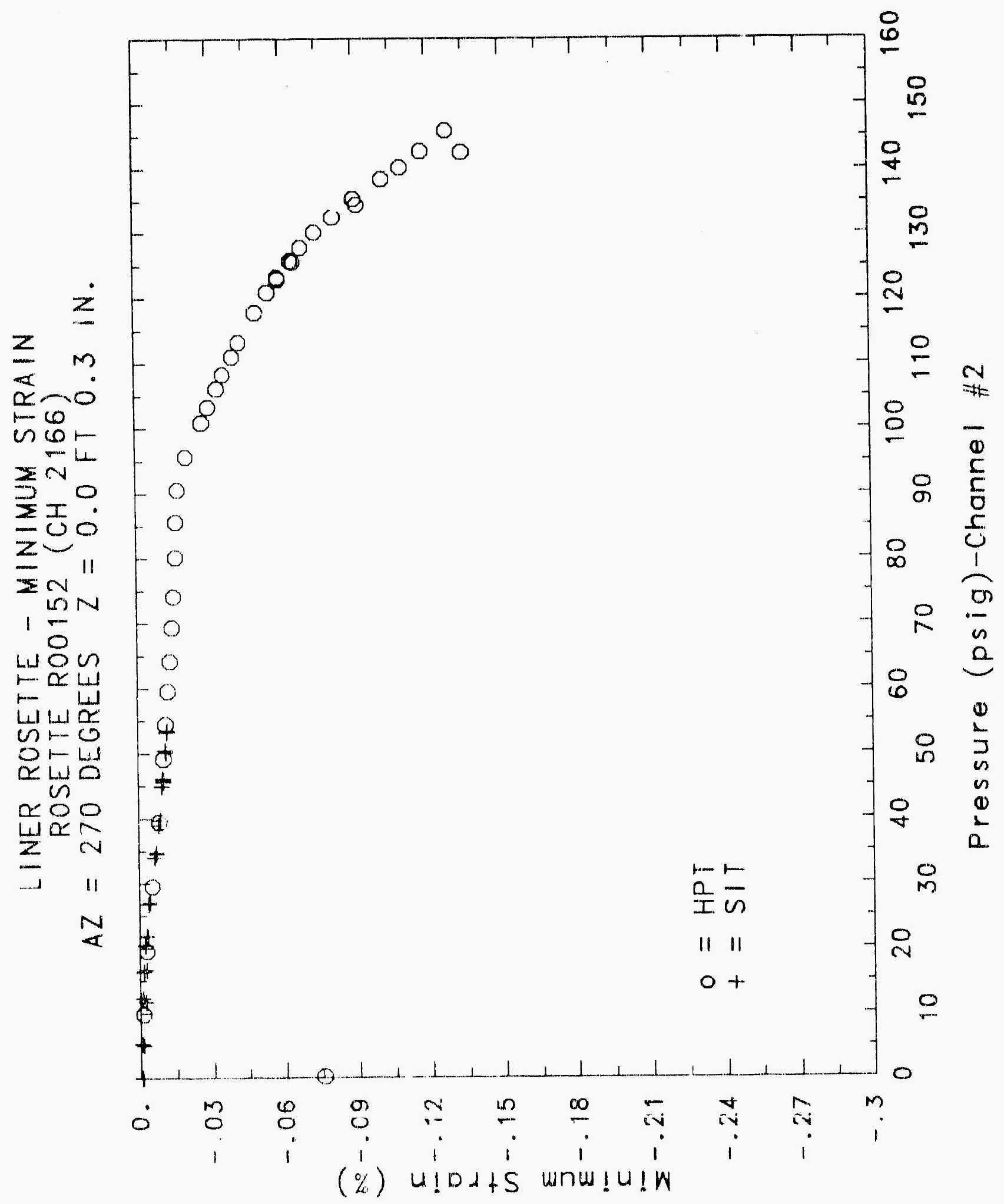




\title{
Reinforced Concrete Test Data
}

\author{
Rosette 153 Channels $2168 \quad 21692180$
}

\section{STRUCTURAL INTEGRITY TEST}

\begin{tabular}{|c|c|c|c|c|c|c|c|}
\hline $\begin{array}{c}\text { Pressure } \\
\text { psig } \\
-0.05 \\
5.33 \\
12.31 \\
16.44 \\
20.51 \\
2046 \\
20.47 \\
27.05 \\
34.69 \\
34.53 \\
34.53 \\
39.70 \\
45.90 \\
45.65 \\
45.62 \\
50.10 \\
53.47 \\
53.29 \\
53.21 \\
50.49 \\
46.14 \\
46.13 \\
44.97 \\
38.98 \\
33.99 \\
33.96 \\
26.83 \\
21.88 \\
21.90 \\
16.69 \\
11.74 \\
11.77 \\
5.05 \\
0.02 \\
-0.04 \\
-0.02 \\
-0.02 \\
0.02\end{array}$ & $\begin{array}{l}\text { Maximum } \\
\text { \%Strain } \\
0.0003 \\
0.0034 \\
0.0048 \\
0.0060 \\
0.0111 \\
0.0129 \\
0.0138 \\
0.0303 \\
0.0532 \\
0.0568 \\
0.0578 \\
0.0700 \\
0.0871 \\
0.0873 \\
0.0874 \\
0.0957 \\
0.1047 \\
0.1058 \\
0.1060 \\
0.1048 \\
0.1009 \\
0.1006 \\
0.1008 \\
0.0948 \\
0.0880 \\
0.0877 \\
0.0766 \\
0.0681 \\
0.0681 \\
0.0597 \\
0.0517 \\
0.0503 \\
0.0412 \\
0.052 \\
0.0302 \\
0.0313 \\
0.0302 \\
0.0302\end{array}$ & $\begin{array}{l}\text { Minimum } \\
4 \text { Strain } \\
0.0001 \\
-0.0167 \\
-0.0303 \\
0.0341 \\
-0.0389 \\
-0.0384 \\
-0.0381 \\
-0.0396 \\
-0.0367 \\
-0.0352 \\
-0.0350 \\
-0.0328 \\
-0.0323 \\
-0.0324 \\
-0.0326 \\
-0.0324 \\
-0.0325 \\
-0.0326 \\
-0.0328 \\
-0.0339 \\
-0.0356 \\
-0.0357 \\
-0.0345 \\
-0.0363 \\
-0.0372 \\
-0.0371 \\
-0.0384 \\
-0.0389 \\
-0.0385 \\
-0.0381 \\
-0.0339 \\
-0.0340 \\
-0.0203 \\
-0.0042 \\
-0.0042 \\
-0.0044 \\
-0.0043 \\
-0.0042\end{array}$ & $\begin{array}{c}\text { Angle } \\
\text { Degrees } \\
-4.92 \\
20.02 \\
15.22 \\
14.33 \\
14.54 \\
14.19 \\
14.42 \\
12.07 \\
9.66 \\
9.01 \\
8.85 \\
7.51 \\
6.01 \\
5.85 \\
5.90 \\
5.53 \\
4.99 \\
4.87 \\
4.92 \\
4.75 \\
4.74 \\
4.72 \\
4.68 \\
4.76 \\
4.80 \\
4.80 \\
5.20 \\
5.50 \\
5.43 \\
5.90 \\
6.80 \\
7.04 \\
9.23 \\
1.79 \\
1.91 \\
2.59 \\
2.15 \\
1.01\end{array}$ & $\begin{array}{c}\text { Pressure } \\
\text { psig } \\
9.89 \\
19.55 \\
29.57 \\
39.42 \\
49.16 \\
54.50 \\
59.57 \\
64.20 \\
69.32 \\
74.16 \\
80.16 \\
85.61 \\
90.58 \\
95.69 \\
100.92 \\
103.25 \\
106.11 \\
108.31 \\
111.08 \\
113.24 \\
117.83 \\
120.92 \\
123.28 \\
122.97 \\
125.82 \\
125.60 \\
127.84 \\
130.19 \\
132.53 \\
135.33 \\
134.42 \\
138.35 \\
140.16 \\
142.63 \\
145.78 \\
142.52 \\
0.22\end{array}$ & $\begin{array}{c}\text { Maximum } \\
\text { zStrain } \\
0.0151 \\
0.0304 \\
0.0482 \\
0.0644 \\
0.0783 \\
0.0869 \\
0.0967 \\
0.1095 \\
0.1228 \\
0.1365 \\
0.1496 \\
0.1623 \\
0.1734 \\
0.1837 \\
0.1924 \\
0.1961 \\
0.1992 \\
0.2020 \\
0.2048 \\
0.2070 \\
0.2106 \\
0.2126 \\
0.2141 \\
0.2138 \\
0.2153 \\
0.2147 \\
0.2163 \\
0.2174 \\
0.2190 \\
0.2232 \\
0.2233 \\
0.2310 \\
0.2375 \\
0.2477 \\
0.2622 \\
0.2505 \\
0.0260\end{array}$ & $\begin{array}{l}\text { Minimum } \\
\text { \%strain } \\
-0.0263 \\
-0.0323 \\
-0.0319 \\
-0.0310 \\
-0.0295 \\
-0.0287 \\
-0.0283 \\
-0.0282 \\
-0.0286 \\
-0.0292 \\
-0.0307 \\
-0.0310 \\
-0.0323 \\
-0.0333 \\
-0.0342 \\
-0.0350 \\
-0.0359 \\
-0.0360 \\
-0.0367 \\
-0.0372 \\
0.0381 \\
-0.0385 \\
-0.0387 \\
-0.0389 \\
-0.0391 \\
-0.0391 \\
-0.0393 \\
-0.0400 \\
-0.0406 \\
-0.0424 \\
-0.0436 \\
-0.0501 \\
-0.0550 \\
-0.0643 \\
-0.0730 \\
-0.0798 \\
-0.0571\end{array}$ & $\begin{array}{r}\text { Angle } \\
\text { Degrees } \\
13.57 \\
8.36 \\
6.74 \\
5.65 \\
5.30 \\
4.79 \\
3.98 \\
3.08 \\
2.69 \\
2.24 \\
1.21 \\
0.56 \\
0.22 \\
-0.30 \\
-0.66 \\
-0.80 \\
-0.86 \\
-1.04 \\
-0.99 \\
-0.96 \\
-0.87 \\
-0.76 \\
-0.67 \\
-0.58 \\
-0.50 \\
-0.35 \\
-0.24 \\
0.06 \\
0.40 \\
0.65 \\
0.71 \\
0.99 \\
1.53 \\
2.21 \\
2.72 \\
2.61 \\
-9.53\end{array}$ \\
\hline
\end{tabular}

\section{High Pressure Test}




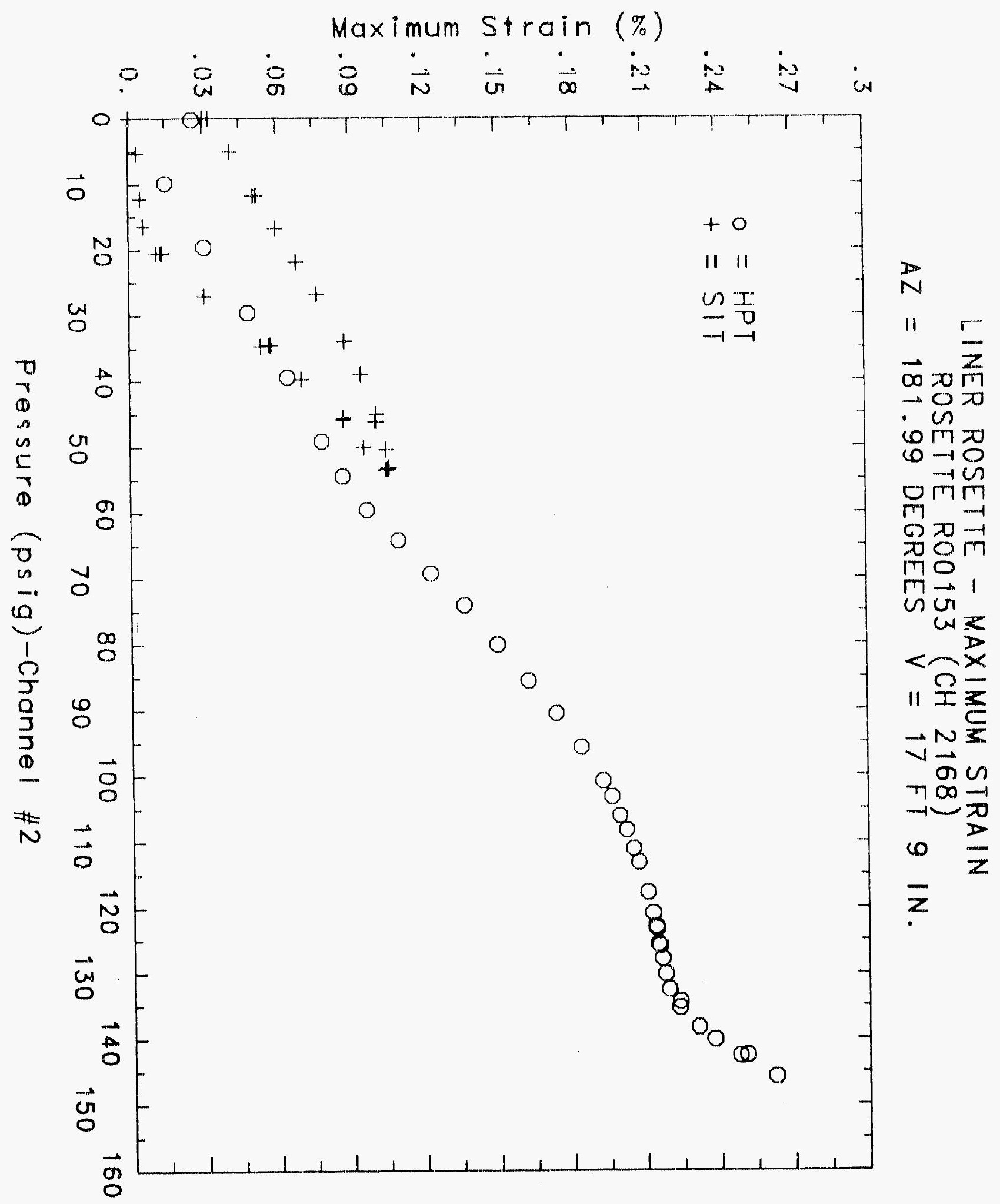




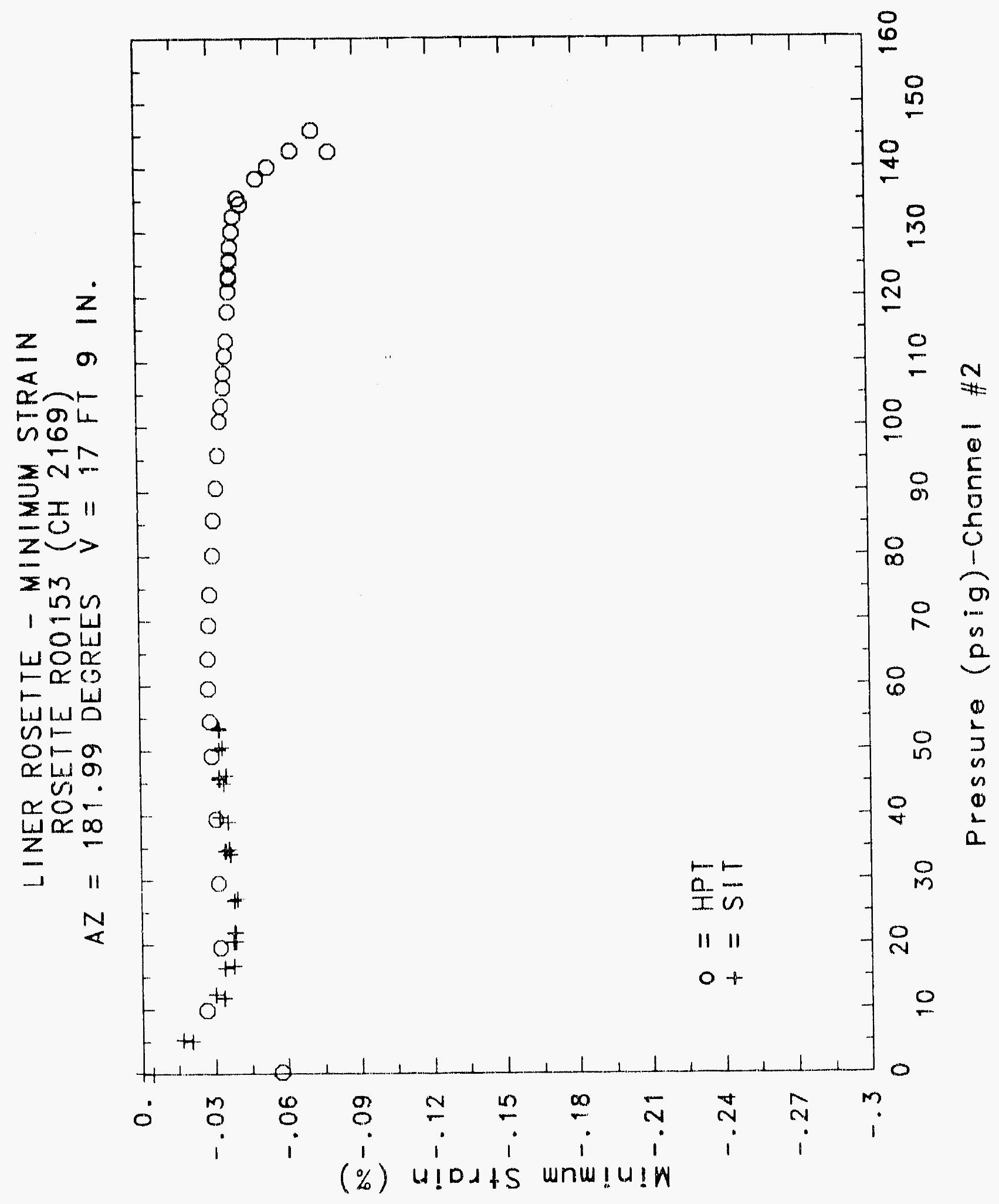

0.778 


\title{
Reinforced Concrete Test Data
}

\author{
Rosette 154 Channels $2181 \quad 218 \% 2183$
}

Structural Integrity Test

\begin{tabular}{|c|c|c|c|c|c|c|c|}
\hline $\begin{array}{c}\text { Pressure } \\
\text { psig } \\
-0.05 \\
5.33 \\
12.31 \\
16.44 \\
20.51 \\
20.46 \\
20.47 \\
27.05 \\
34.69 \\
34.53 \\
34.53 \\
39.70 \\
45.90 \\
45.65 \\
45.62 \\
50.10 \\
53.47 \\
53.29 \\
53.21 \\
50.49 \\
46.14 \\
46.13 \\
44.97 \\
38.98 \\
33.99 \\
33.96 \\
26.83 \\
21.88 \\
21.90 \\
16.69 \\
11.74 \\
11.77 \\
5.05 \\
0.02 \\
-0.04 \\
-0.02 \\
-0.02 \\
0.02\end{array}$ & $\begin{array}{l}\text { Maximum } \\
\text { \%Strain } \\
0.0004 \\
0.0003 \\
0.0006 \\
0.0010 \\
0.0012 \\
0.0016 \\
0.0019 \\
0.0026 \\
0.0044 \\
0.0055 \\
0.0056 \\
0.0060 \\
0.0068 \\
0.0072 \\
0.0072 \\
0.0073 \\
0.0074 \\
0.0077 \\
0.0077 \\
0.0073 \\
0.0064 \\
0.0063 \\
0.0057 \\
0.0044 \\
0.0035 \\
0.0033 \\
0.0023 \\
0.0024 \\
0.0029 \\
0.0031 \\
0.0028 \\
0.0019 \\
0.0022 \\
0.0028 \\
0.0021 \\
0.0016 \\
0.0015 \\
0.0016\end{array}$ & $\begin{array}{r}\text { Minimum } \\
\text { \%Strain } \\
0.0000 \\
-0.0015 \\
-0.0021 \\
-0.0023 \\
-0.0019 \\
-0.0014 \\
-0.0019 \\
-0.0011 \\
-0.0011 \\
-0.0009 \\
-0.0003 \\
-0.0008 \\
-0.0011 \\
-0.0009 \\
-0.0010 \\
-0.0014 \\
-0.0016 \\
-0.0015 \\
-0.0016 \\
-0.0014 \\
-0.0012 \\
-0.0014 \\
-0.0018 \\
-0.0018 \\
-0.0012 \\
-0.0016 \\
-0.0013 \\
-0.0014 \\
-0.0018 \\
-0.0016 \\
-0.0007 \\
0.0001 \\
0.0007 \\
0.0010 \\
0.0007 \\
0.0007 \\
0.0004 \\
-0.0004\end{array}$ & $\begin{array}{c}\text { Angle } \\
\text { Degrees } \\
-44.71 \\
-23.48 \\
-18.13 \\
-20.55 \\
-28.35 \\
-29.10 \\
-36.43 \\
33.15 \\
15.10 \\
19.56 \\
18.14 \\
13.90 \\
5.96 \\
-0.07 \\
-0.37 \\
-2.15 \\
-4.97 \\
-6.58 \\
-6.52 \\
-7.30 \\
-8.06 \\
-8.77 \\
-4.31 \\
0.91 \\
0.39 \\
8.93 \\
14.59 \\
26.76 \\
29.83 \\
36.65 \\
37.36 \\
34.53 \\
41.39 \\
32.84 \\
-25.26 \\
-3.10 \\
16.80 \\
34.13\end{array}$ & $\begin{array}{c}\text { Pressure } \\
\text { psig } \\
9.89 \\
19.55 \\
29.57 \\
39.42 \\
49.16 \\
54.50 \\
59.57 \\
64.20 \\
69.32 \\
74.16 \\
80.16 \\
85.61 \\
90.58 \\
95.69 \\
100.92 \\
103.25 \\
106.11 \\
108.31 \\
111.08 \\
113.24 \\
117.83 \\
120.92 \\
123.28 \\
122.97 \\
125.82 \\
125.60 \\
127.84 \\
130.19 \\
132.53 \\
135.33 \\
134.42 \\
138.35 \\
140.16 \\
142.63 \\
145.78 \\
142.52 \\
0.22\end{array}$ & $\begin{array}{c}\text { Maximum } \\
\text { xStrain } \\
0.0163 \\
0.0306 \\
0.0438 \\
0.0574 \\
0.0701 \\
0.0774 \\
0.0839 \\
0.0915 \\
0.0979 \\
0.1039 \\
0.1105 \\
0.1177 \\
0.1253 \\
0.1338 \\
0.1419 \\
0.1459 \\
0.1502 \\
0.1535 \\
0.1580 \\
0.1618 \\
0.1686 \\
0.1730 \\
0.1767 \\
0.1773 \\
0.1803 \\
0.1811 \\
0.1837 \\
0.1879 \\
0.1926 \\
0.2007 \\
0.2019 \\
0.2139 \\
0.2277 \\
0.2663 \\
0.3266 \\
0.3393 \\
0.1406\end{array}$ & $\begin{array}{l}\text { Minimum } \\
\text { xStrain } \\
-0.0035 \\
-0.0060 \\
-0.0076 \\
-0.0087 \\
-0.0093 \\
-0.0097 \\
-0.0114 \\
-0.0140 \\
-0.0168 \\
-0.0198 \\
-0.0233 \\
-0.0270 \\
-0.0302 \\
-0.0336 \\
-0.0368 \\
-0.0383 \\
-0.0397 \\
-0.0410 \\
-0.0425 \\
-0.0438 \\
-0.0464 \\
-0.0478 \\
-0.0488 \\
-0.0489 \\
-0.0498 \\
-0.0501 \\
-0.0507 \\
-0.0514 \\
-0.0522 \\
-0.0535 \\
-0.0536 \\
-0.0554 \\
-0.0573 \\
-0.0638 \\
-0.0747 \\
-0.0801 \\
-0.0336\end{array}$ & $\begin{array}{c}\text { Angle } \\
\text { Degrees } \\
15.91 \\
-22.72 \\
-5.86 \\
-8.51 \\
-6.27 \\
-5.24 \\
-5.89 \\
-6.20 \\
-3.06 \\
-2.69 \\
-13.09 \\
-14.52 \\
-9.52 \\
-11.38 \\
-10.62 \\
-10.69 \\
-9.97 \\
-8.48 \\
-4.73 \\
-2.76 \\
-2.54 \\
-2.09 \\
-1.40 \\
-1.81 \\
-0.94 \\
-0.12 \\
0.35 \\
0.47 \\
1.24 \\
1.19 \\
1.56 \\
2.20 \\
2.33 \\
1.60 \\
1.80 \\
2.32 \\
-6.38\end{array}$ \\
\hline
\end{tabular}

High Pressure Test 
(1) 8 L- J

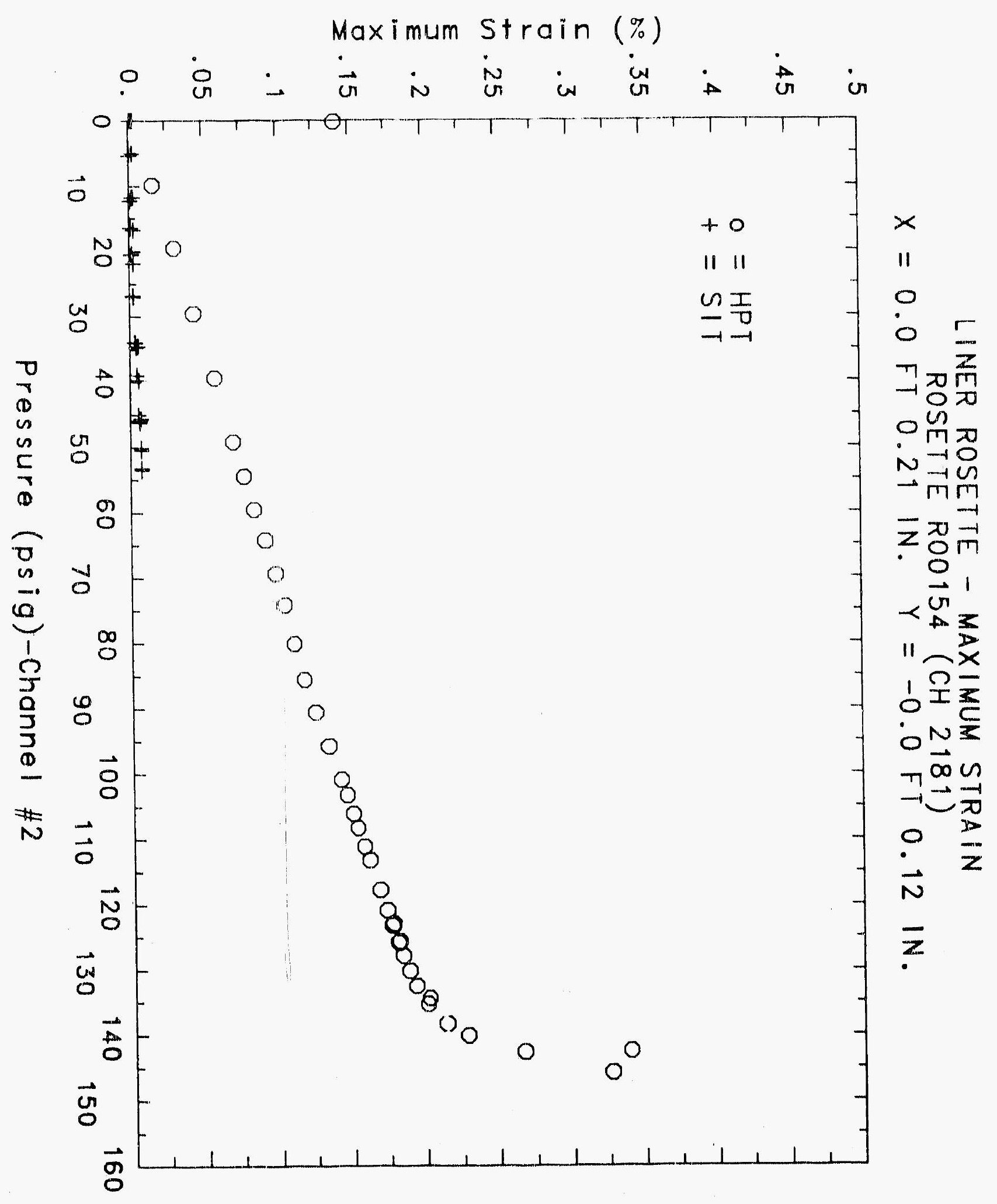




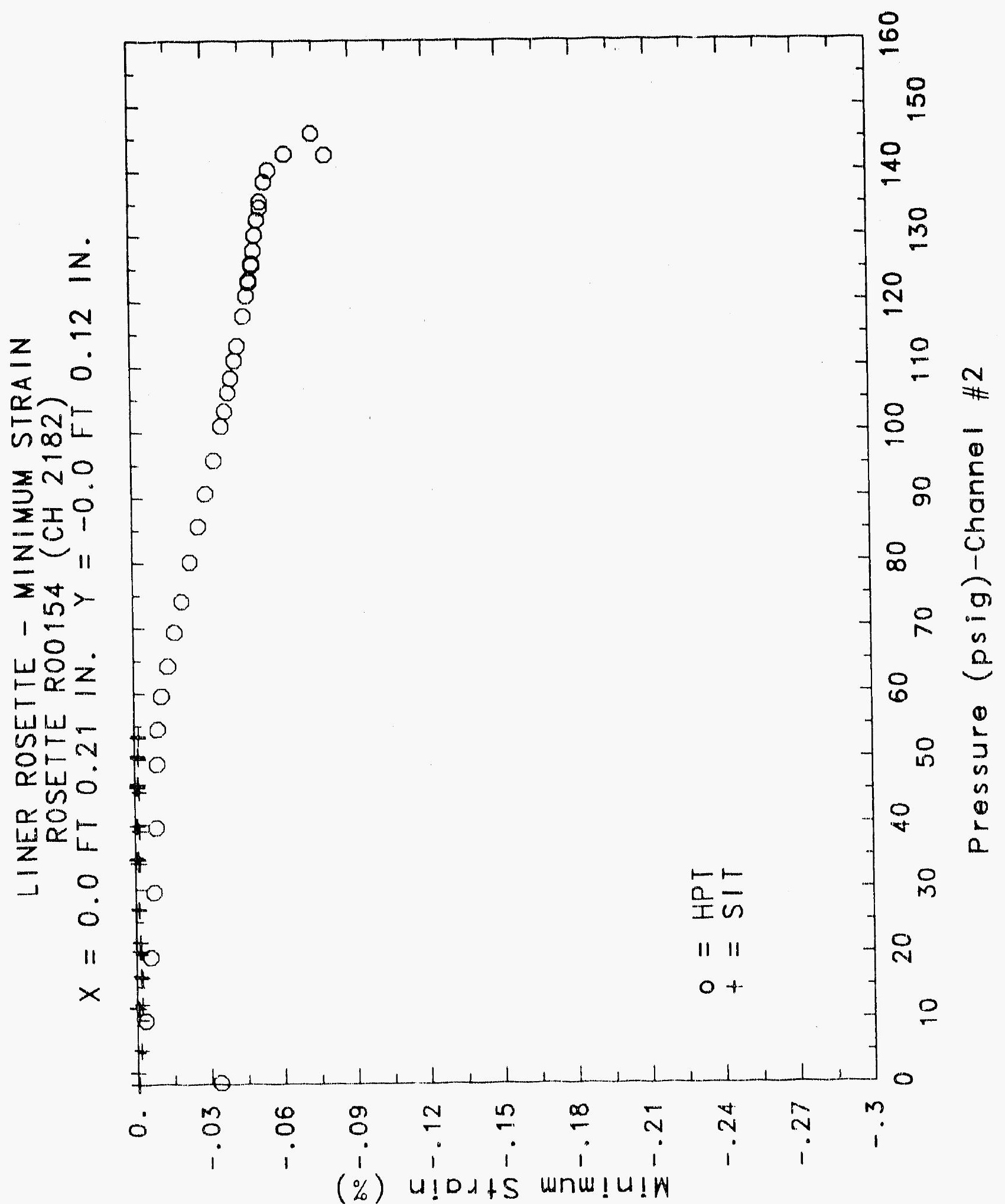




\section{Reinforced Concrete Test Data}

\section{Rosette 155 Channels $345 \quad 346 \quad 347$}

\section{Structural Integrity Test}

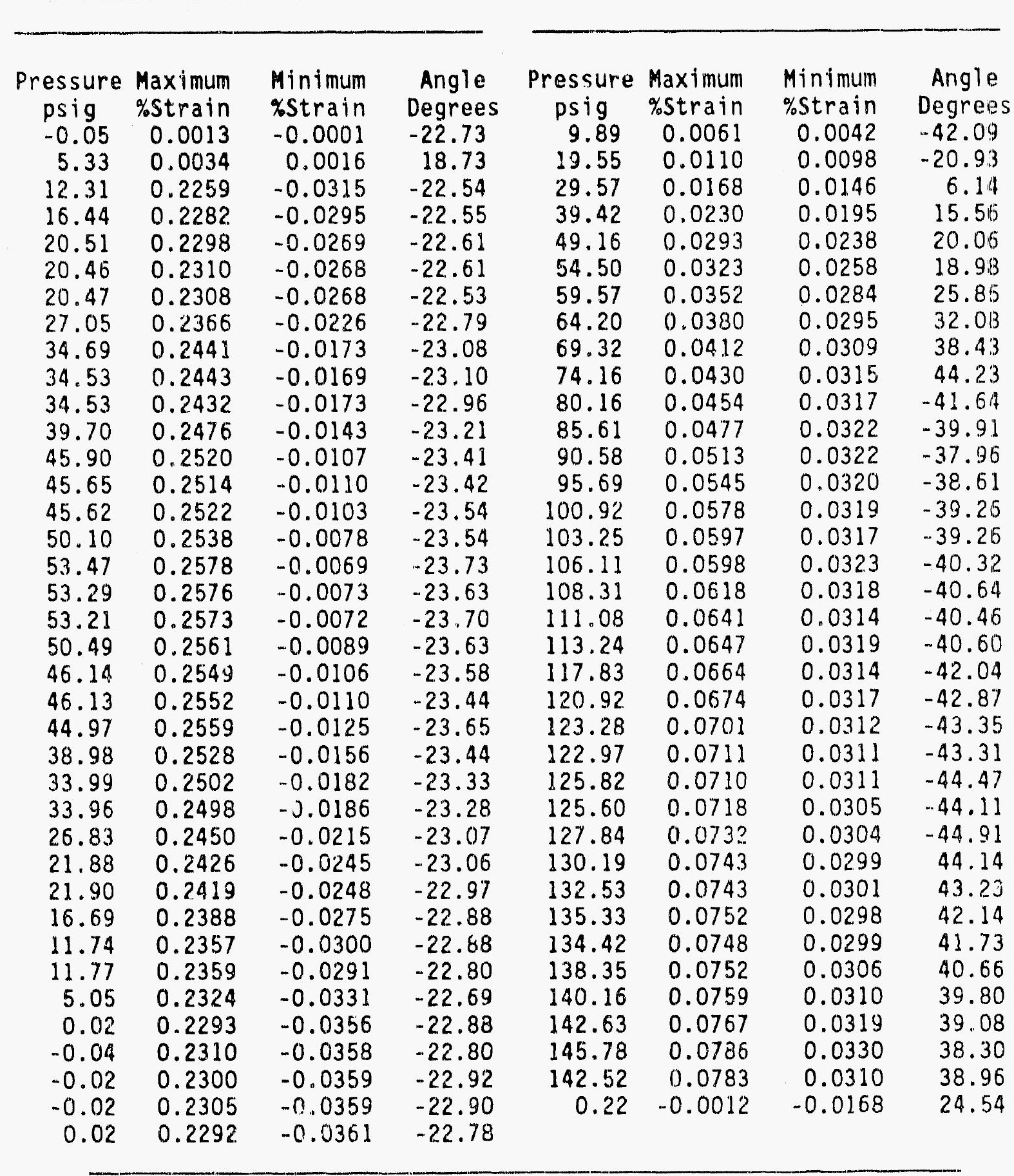

\section{High Pressure Test}




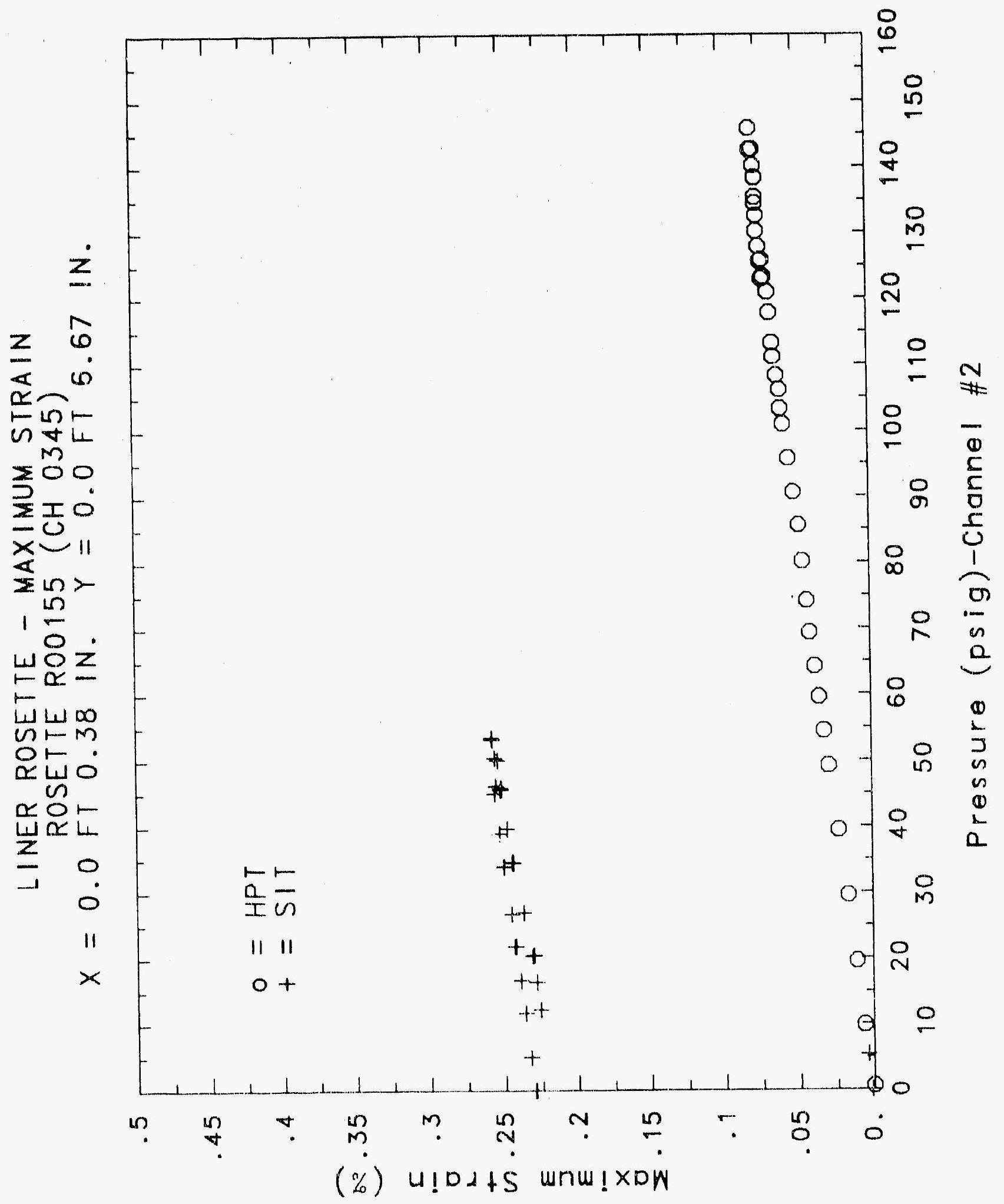




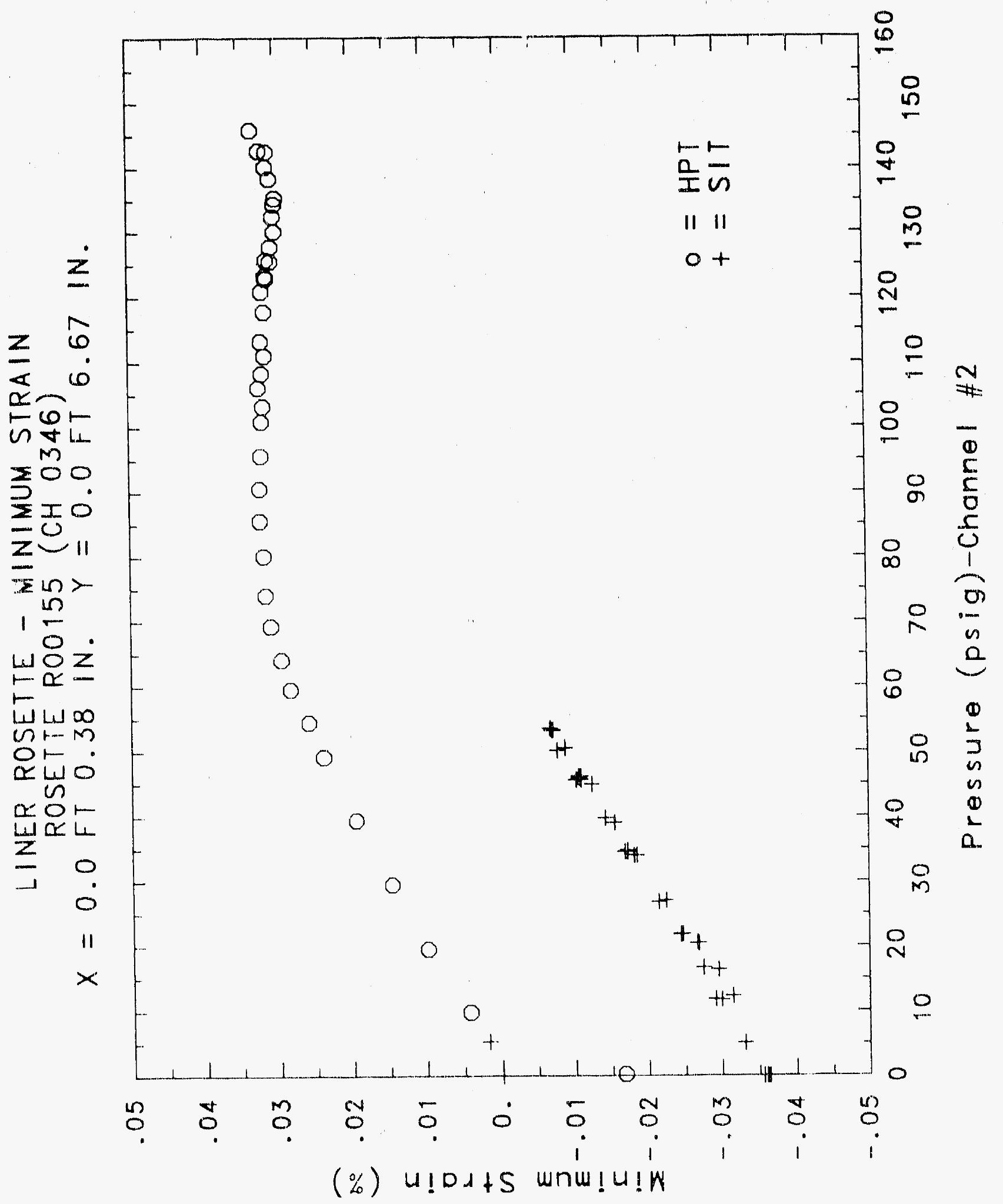




\section{Reinforced Concrete Test Data}

Rosette 156 Channels $348 \quad 349 \quad 360$

Structural Integrity Test

\begin{tabular}{|c|c|c|c|c|c|c|c|}
\hline Pressure & Maximum & Minimum & Angle & Pressure & Maximum & Minimum & Angle \\
\hline psig & $\%$ Strain & \%strain & Degrees & psig & \%strain & \%strain & Degrees \\
\hline-0.05 & 0.0023 & -0.0009 & -6.46 & 9.89 & 0.0050 & 0.0010 & 20.87 \\
\hline 5.33 & 0.0052 & -0.0003 & -43.04 & 19.55 & 0.0095 & 0.0006 & 20.43 \\
\hline 12.31 & 0.0076 & 0.0022 & 42.97 & 29.57 & 0.0143 & 0.0010 & 19.79 \\
\hline 16.44 & 0.0129 & 0.0030 & -37.22 & 39.42 & 0.0192 & 0.0012 & 21.39 \\
\hline 20.51 & 0.2099 & -0.1882 & -44.58 & 49.16 & 0.0246 & 0.0014 & 22.03 \\
\hline 20.46 & 0.2096 & -0.1820 & -44.13 & 54.50 & 0.0272 & 0.0016 & 22.60 \\
\hline 20.47 & 0.2105 & -0.1537 & -41.63 & 59.57 & 0.0297 & 0.0022 & 22.97 \\
\hline 27.05 & 0.2150 & -0.1484 & -41.49 & 64.20 & 0.0301 & 0.0014 & 21.81 \\
\hline 34.69 & 0.2196 & -0.1427 & -41.47 & 69.32 & 0.0332 & 0.0006 & 22.13 \\
\hline 34.53 & 0.2299 & -0.0736 & -32.70 & 74.16 & 0.0340 & -0.0001 & 22.06 \\
\hline 34.53 & 0.2312 & -0.0703 & -32.13 & 80.16 & 0.0351 & 0.0010 & 22.69 \\
\hline 39.70 & 0.2344 & -0.0701 & -32.42 & 85.61 & 0.0346 & 0.0015 & 21.04 \\
\hline 45.90 & 0.2342 & -0.0793 & -34.47 & 90.58 & 0.0361 & 0.0009 & 21.90 \\
\hline 45.65 & 0.2318 & -0.0884 & -35.87 & 95.69 & 0.0377 & 0.0002 & 22.52 \\
\hline 45.62 & 0.2307 & -0.0891 & -35.86 & 100.92 & 0.0390 & -0.0005 & 21.75 \\
\hline 50.10 & 0.2327 & -0.0389 & -36.29 & 103.25 & 0.0391 & -0.0010 & 21.63 \\
\hline 53.47 & 0.2344 & -0.0891 & -36.52 & 105.11 & 0.0407 & -0.0016 & 22.12 \\
\hline 53.29 & 0.2346 & -0.0899 & -36.63 & $1 \cup \varepsilon .31$ & 0.0412 & -0.0018 & 21.94 \\
\hline 53.21 & 0.2340 & -0.0905 & -36.67 & 111.08 & 0.0418 & -0.0026 & 21.96 \\
\hline 50.49 & 0.2333 & -0.0917 & -36.62 & 113.24 & 0.0433 & -0.0030 & 22.27 \\
\hline 46.14 & 0.2310 & -0.0919 & -36.36 & 117.83 & 0.0441 & -0.0044 & 22.16 \\
\hline 46.13 & 0.2307 & -0.0927 & -36.44 & 120.92 & 0.0466 & -0.0059 & 22.08 \\
\hline 44.97 & 0.1913 & -0.0514 & -33.13 & 123.28 & 0.0482 & -0.0068 & 22.79 \\
\hline 38.98 & 0.2004 & -0.0565 & -32.50 & 122.97 & 0.0480 & -0.0066 & 22.70 \\
\hline 33.99 & 0.2281 & -0.0845 & -34.46 & 125.82 & 0.0482 & -0.0076 & 21.71 \\
\hline 33.96 & 0.2291 & -0.0831 & -34.21 & 125.60 & 0.0489 & -0.0083 & 22.31 \\
\hline 26.83 & 0.2272 & -0.0821 & -33.44 & 127.84 & 0.0495 & -0.0089 & 21.79 \\
\hline 21.88 & 0.2271 & -0.0770 & -32.14 & 130.19 & 0.0509 & -0.0101 & 22.50 \\
\hline 21.90 & 0.2300 & -0.0697 & -30.90 & 132.53 & 0.0522 & -0.0111 & 22.10 \\
\hline 16.69 & 0.2279 & -0.0692 & -30.34 & 135.33 & 0.0529 & -0.0117 & 22.24 \\
\hline 11.74 & 0.2264 & -0.0711 & -30.39 & 134.42 & 0.0527 & -0.0118 & 22.07 \\
\hline 11.77 & 0.2206 & -0.0863 & -33.11 & 138.35 & 0.0556 & -0.0145 & 22.27 \\
\hline 5.05 & 0.2185 & -0.0892 & -33.28 & 140.16 & 0.0564 & -0.0149 & 22.11 \\
\hline 0.02 & 0.2159 & -0.0900 & -33.23 & 142.63 & 0.0578 & -0.0152 & 22.30 \\
\hline-0.04 & 0.2176 & $\ldots 0.0928$ & -33.53 & 145.78 & 0.0594 & -0.0153 & 22.16 \\
\hline-0.02 & 0.2173 & -0.0906 & -33.47 & 142.52 & 0.0587 & -0.0170 & 22.13 \\
\hline-0.02 & 0.2174 & -0.0916 & -33.51 & 0.22 & -0.0040 & -0.0187 & 23.70 \\
\hline 0.02 & 0.2190 & -0.0848 & -32.22 & & & & \\
\hline
\end{tabular}

High Pressure test 


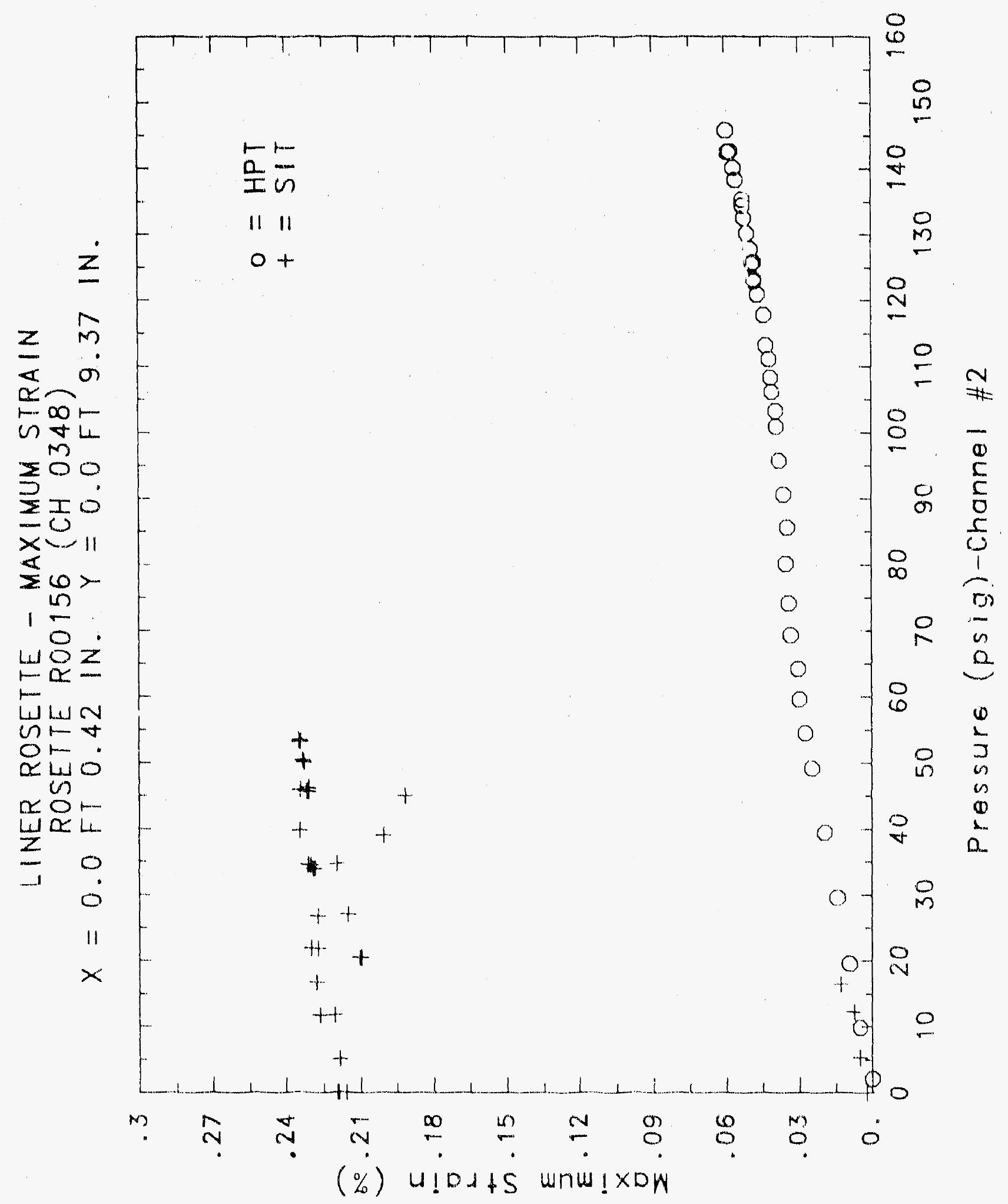


$1.9(.$.

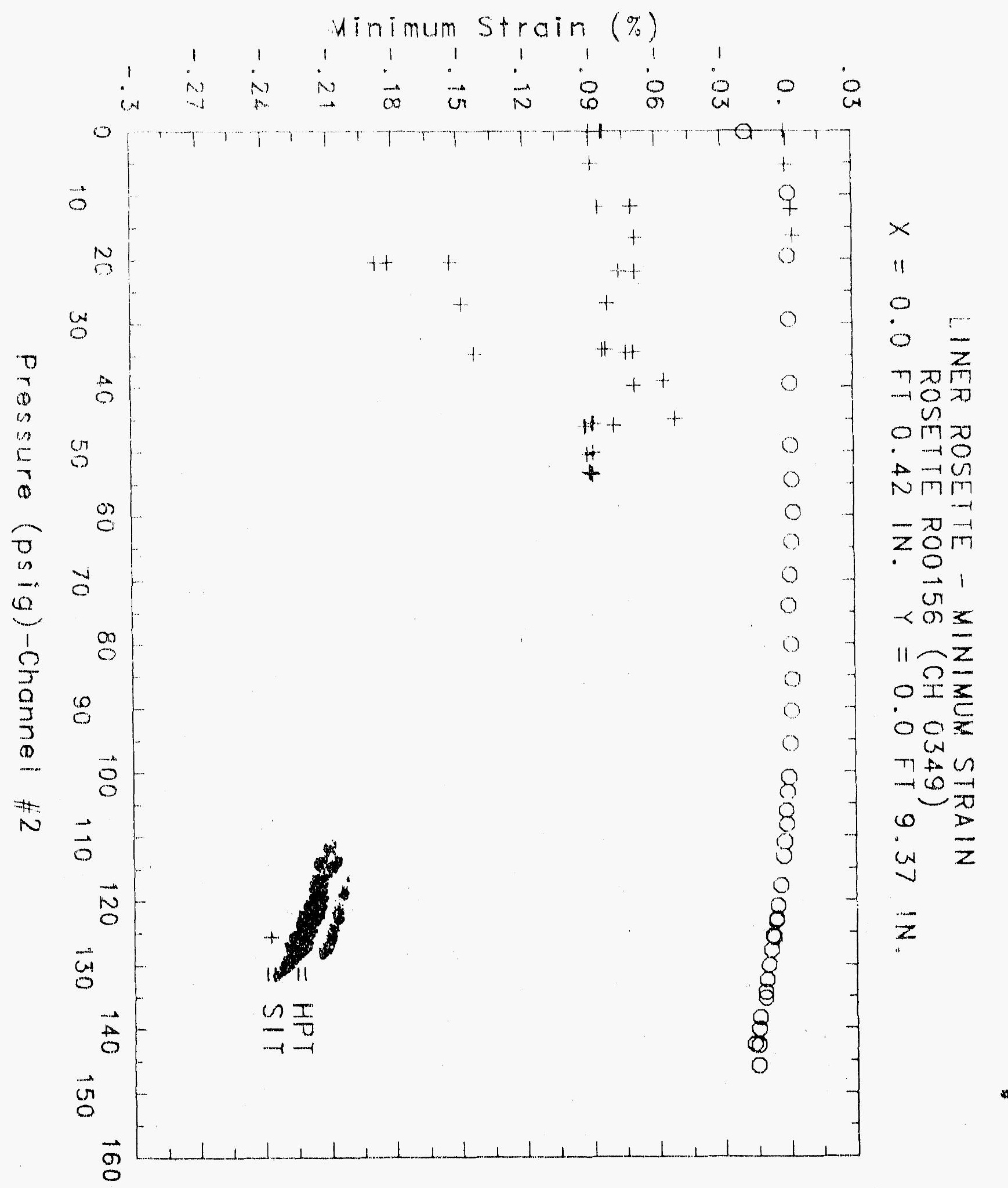




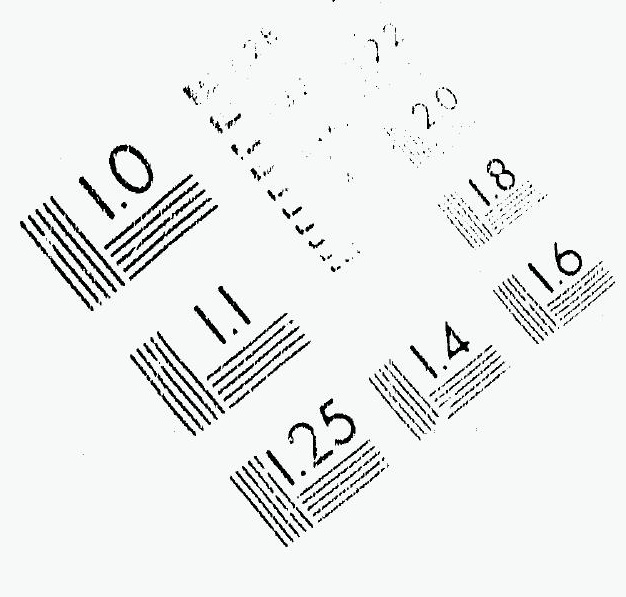

\section{IMAGE EVALUATION TEST TARGET (MT-3)}
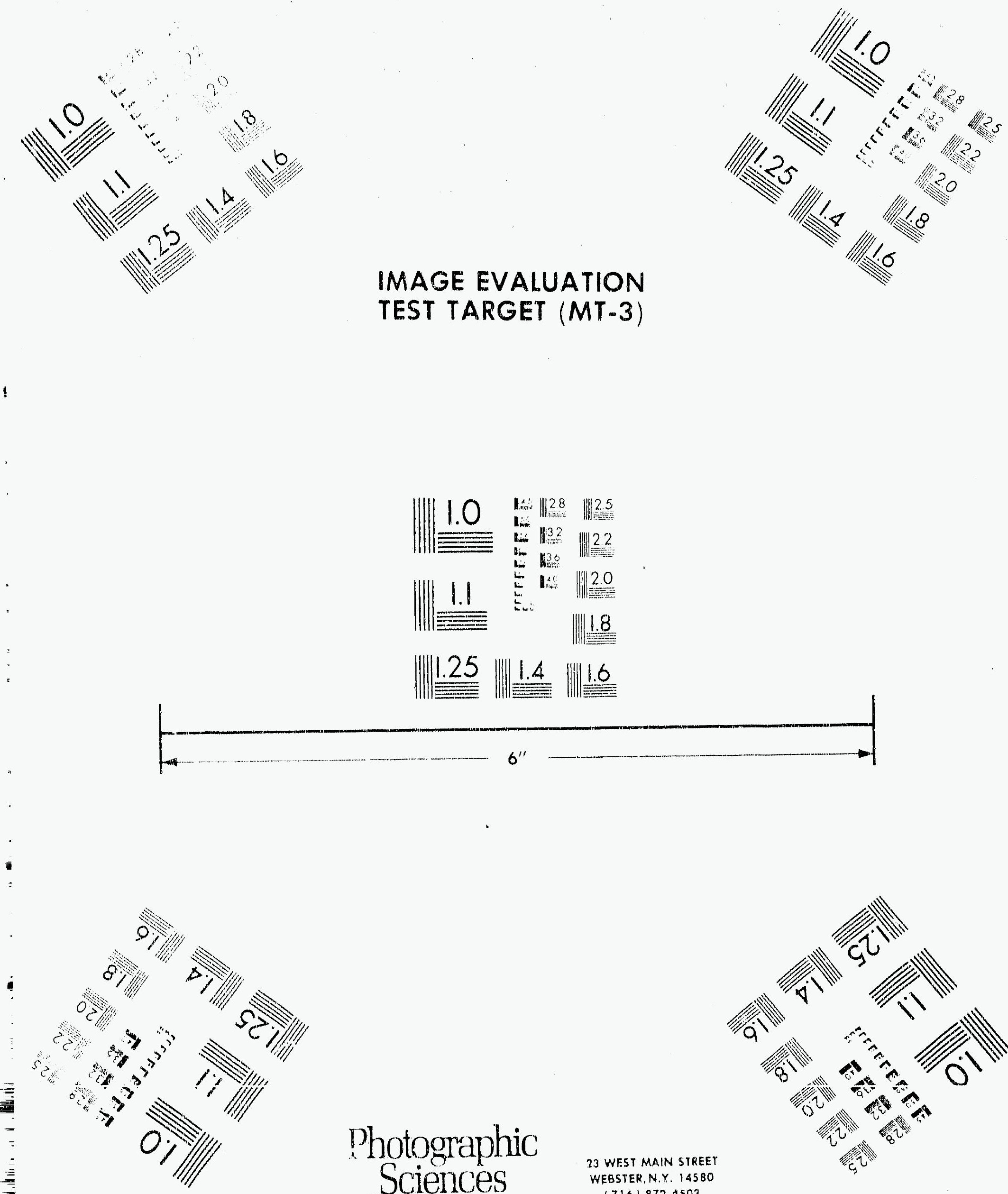

Photographic Sciences Corporation

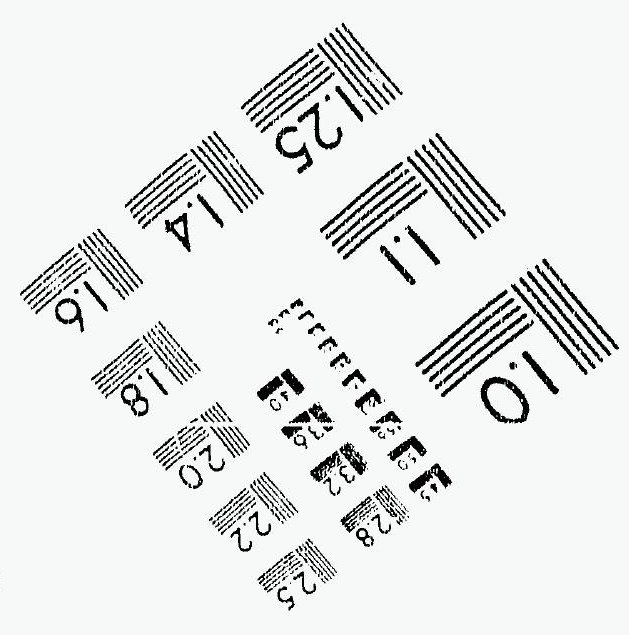

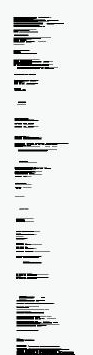




\section{SECURITY CLASSIFICATION}
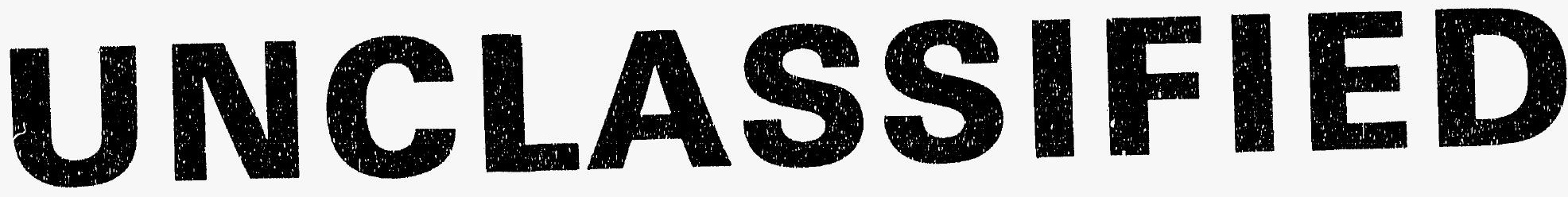

\section{DATE OF MICROFILMING $1-6-92$}

\section{MICROFILMED BY - errar hasd}




\section{THIS DOCUMENT FILMED}

AT

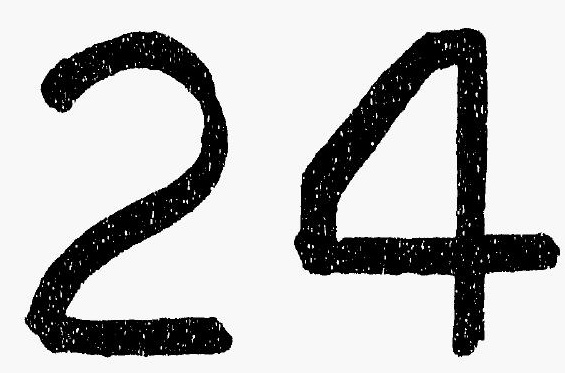

REDUCTION 


\section{Reinforced Concrete Test Data}

\section{Rosette 157 Channels $361 \quad 362 \quad 363$}

\section{STRUCTURAL INTEGRITY TEST}

\begin{tabular}{|c|c|c|c|c|c|c|c|}
\hline $\begin{array}{c}\text { Pressure } \\
\text { psig } \\
-0.05 \\
5.33 \\
12.31 \\
16.44 \\
20.51 \\
20.46 \\
20.47 \\
27.05 \\
34.69 \\
34.53 \\
34.53 \\
39.70 \\
45.90 \\
45.65 \\
45.62 \\
50.10 \\
53.47 \\
53.29 \\
53.21 \\
50.49 \\
46.14 \\
46.13 \\
44.97 \\
38.98 \\
33.99 \\
33.96 \\
26.83 \\
21.88 \\
21.90 \\
16.69 \\
11.74 \\
11.77 \\
5.05 \\
0.02 \\
-0.04 \\
-0.02 \\
-0.02 \\
0.02\end{array}$ & $\begin{array}{r}\text { Maximum } \\
\text { \%.Strain } \\
0.0016 \\
0.0009 \\
-0.0001 \\
0.4182 \\
0.4186 \\
0.4191 \\
0.4176 \\
0.4223 \\
0.4261 \\
0.4252 \\
0.4252 \\
0.4277 \\
0.4307 \\
0.4302 \\
0.4311 \\
0.4333 \\
0.4343 \\
0.4345 \\
0.4345 \\
0.4330 \\
0.4301 \\
0.4312 \\
0.4302 \\
0.4287 \\
0.4253 \\
0.4253 \\
0.4217 \\
0.4194 \\
0.4197 \\
0.4166 \\
0.4155 \\
0.4157 \\
0.4141 \\
0.4115 \\
0.4115 \\
0.4131 \\
0.4133 \\
0.4130\end{array}$ & $\begin{array}{l}\text { Minimum } \\
\text { \%Strain } \\
-0.0048 \\
-0.0085 \\
-0.0069 \\
-0.0735 \\
-0.0747 \\
-0.0749 \\
-0.0747 \\
-0.0755 \\
-0.0771 \\
-0.0772 \\
-0.0775 \\
-0.0783 \\
-0.0790 \\
-0.0787 \\
-0.0788 \\
-0.0797 \\
-0.0798 \\
-0.0793 \\
-0.0801 \\
-0.0789 \\
-0.0786 \\
-0.0782 \\
-0.0788 \\
-0.0770 \\
-0.0766 \\
-0.0759 \\
-0.0751 \\
-0.0743 \\
-0.0749 \\
-0.0741 \\
-0.0730 \\
-0.0731 \\
-0.0715 \\
-0.0708 \\
-0.0695 \\
-0.0702 \\
-0.0695 \\
-0.0700\end{array}$ & $\begin{array}{l}\text { Angle } \\
\text { Degrees } \\
-14.11 \\
-20.43 \\
-31.90 \\
-22.45 \\
-22.35 \\
-22.35 \\
-22.33 \\
-22.25 \\
-21.99 \\
-22.07 \\
-22.09 \\
-22.02 \\
-21.79 \\
-21.72 \\
-21.81 \\
-21.70 \\
-21.65 \\
-21.62 \\
-21.61 \\
-21.63 \\
-21.72 \\
-21.76 \\
-21.77 \\
-21.96 \\
-22.02 \\
-21.96 \\
-22.11 \\
-22.17 \\
-22.25 \\
-22.35 \\
-22.39 \\
-22.39 \\
-22.46 \\
-22.50 \\
-22.54 \\
-22.49 \\
-22.55 \\
-22.57\end{array}$ & $\begin{array}{c}\text { Pressure } \\
\text { psig } \\
9.89 \\
19.55 \\
29.57 \\
39.42 \\
49.16 \\
54.50 \\
59.57 \\
64.20 \\
69.32 \\
74.16 \\
80.16 \\
85.61 \\
90.58 \\
95.69 \\
100.92 \\
103.25 \\
106.11 \\
108.31 \\
111.08 \\
113.24 \\
117.83 \\
120.92 \\
123.28 \\
122.97 \\
125.82 \\
125.60 \\
127.84 \\
130.19 \\
122.53 \\
135.33 \\
134.42 \\
138.35 \\
140.16 \\
142.63 \\
145.78 \\
142.52 \\
0.22\end{array}$ & $\begin{array}{c}\text { Maximum } \\
\text { \%Strain } \\
0.0046 \\
0.0093 \\
0.0134 \\
0.0180 \\
0.0220 \\
0.0248 \\
0.0261 \\
0.0272 \\
0.0284 \\
0.0303 \\
0.0305 \\
0.0321 \\
0.0321 \\
0.0332 \\
0.0341 \\
0.0345 \\
0.0354 \\
0.0359 \\
0.0365 \\
0.0370 \\
0.0386 \\
0.0390 \\
0.0398 \\
0.0400 \\
0.0412 \\
0.0410 \\
0.0421 \\
0.0428 \\
0.0431 \\
0.0442 \\
0.0439 \\
0.0452 \\
0.0464 \\
0.0477 \\
0.0494 \\
0.0483 \\
0.0111\end{array}$ & $\begin{array}{l}\text { Minimum } \\
\% \text { Strain } \\
-0.0016 \\
-0.0032 \\
-0.0049 \\
-0.0066 \\
-0.0082 \\
-0.0096 \\
-0.0103 \\
-0.0106 \\
-0.0129 \\
-0.0120 \\
-0.0125 \\
-0.0127 \\
-0.0125 \\
-0.0119 \\
-0.0111 \\
-0.0112 \\
-0.0109 \\
-0.0112 \\
-0.0108 \\
-0.0106 \\
-0.0099 \\
-0.0097 \\
-0.0092 \\
-0.0093 \\
-0.0085 \\
-0.0089 \\
-0.0094 \\
-0.0082 \\
-0.0083 \\
-0.0087 \\
-0.0090 \\
-0.0087 \\
-0.0091 \\
-0.0102 \\
-0.0114 \\
-0.0098 \\
-0.0117\end{array}$ & $\begin{array}{c}\text { Angle } \\
\text { Degrees } \\
-6.66 \\
-10.70 \\
-7.66 \\
-7.99 \\
-7.72 \\
-8.59 \\
-6.52 \\
-6.78 \\
-5.26 \\
-7.11 \\
-6.20 \\
-7.00 \\
-5.13 \\
-4.85 \\
-4.60 \\
-4.59 \\
-4.60 \\
-4.15 \\
-3.86 \\
-3.73 \\
-3.06 \\
-2.60 \\
-1.95 \\
-2.31 \\
-2.35 \\
-1.42 \\
-1.27 \\
-0.80 \\
-0.58 \\
0.25 \\
0.94 \\
0.32 \\
1.00 \\
1.22 \\
0.59 \\
2.66 \\
-27.84\end{array}$ \\
\hline
\end{tabular}

High Pressure test 


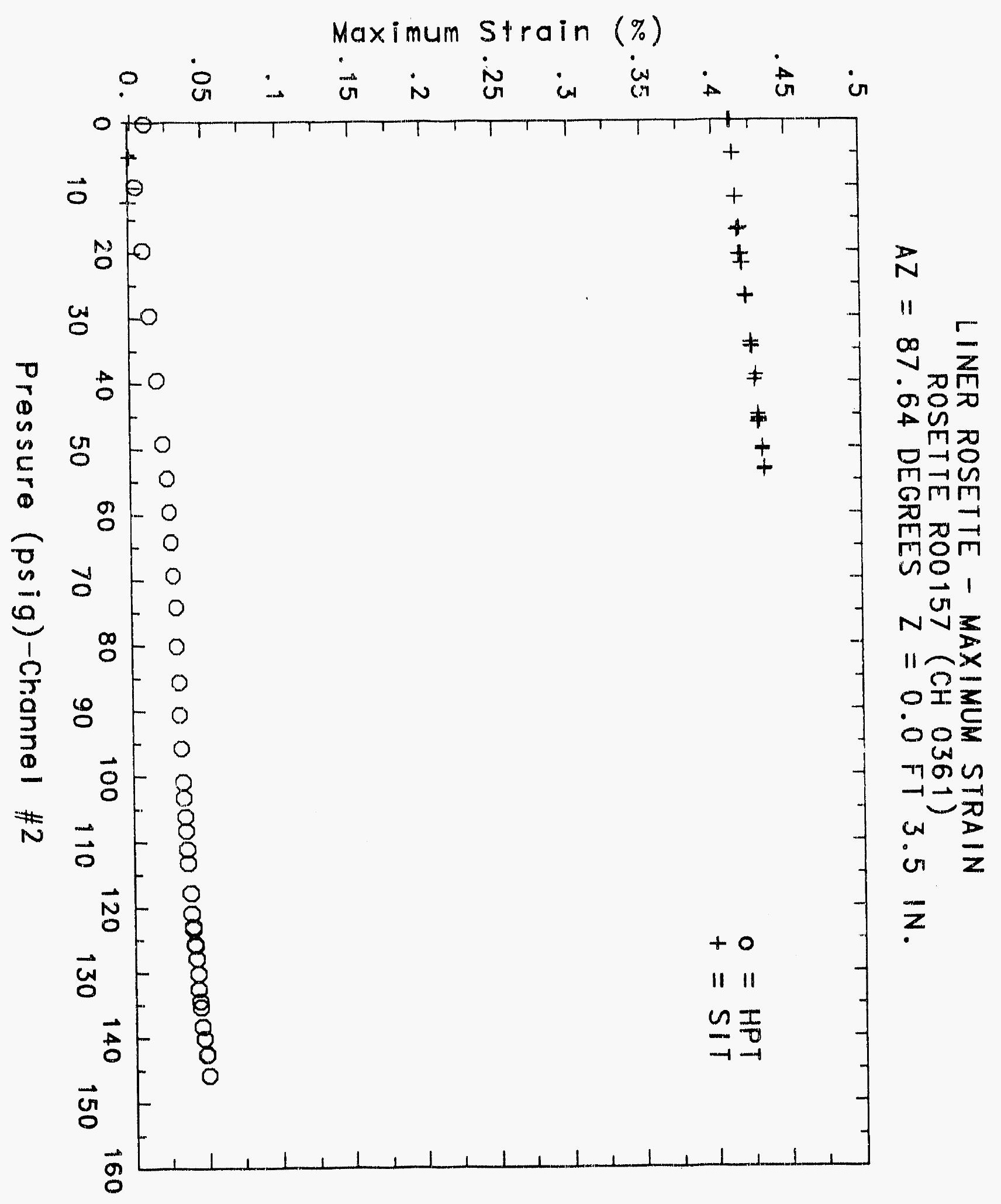




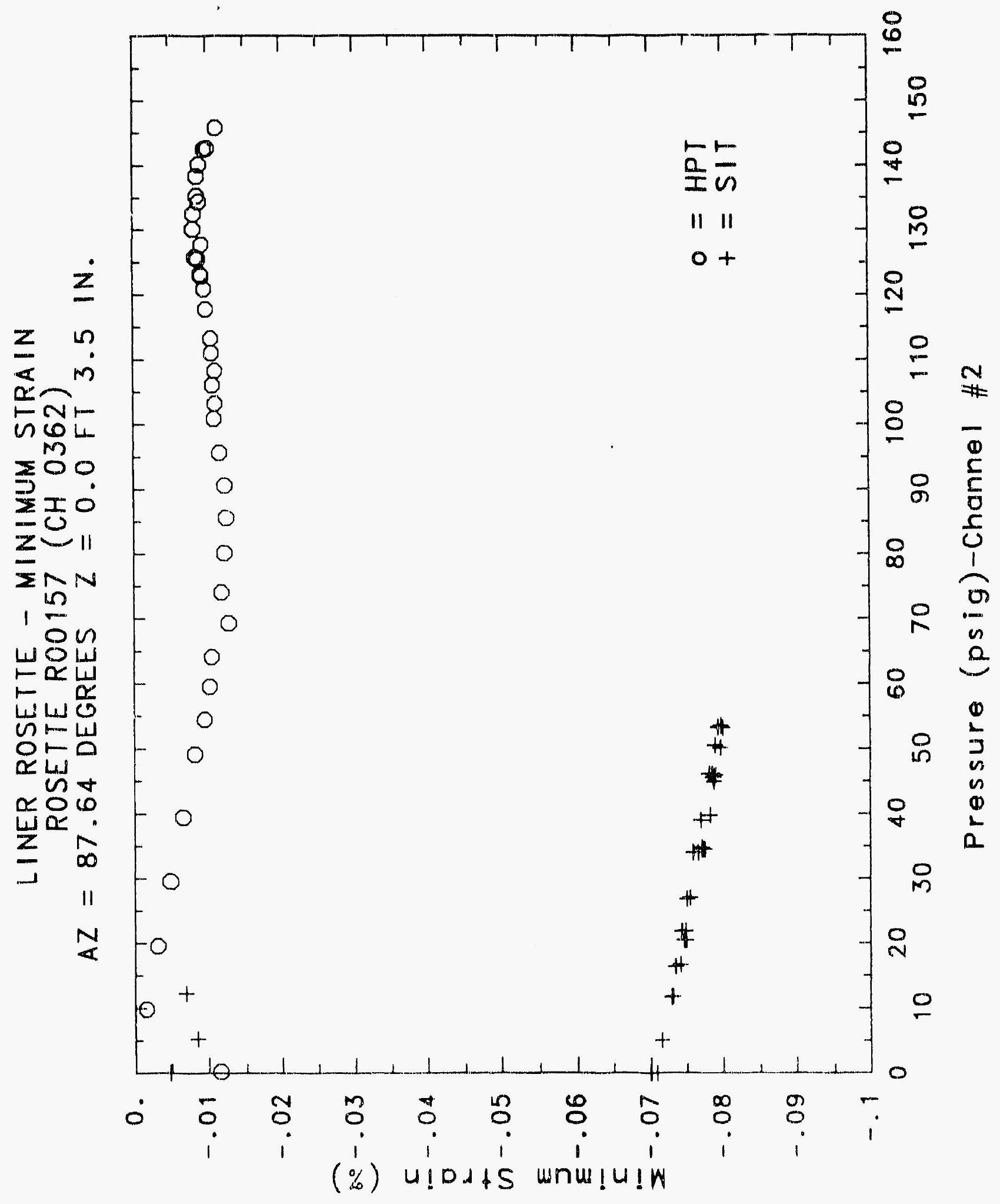




\section{Reinforced Concrete Test Data.}

\section{Rosette 158 Channels $364 \quad 365 \quad 366$}

\section{STRUCTURAL INTEGRITY TEST}

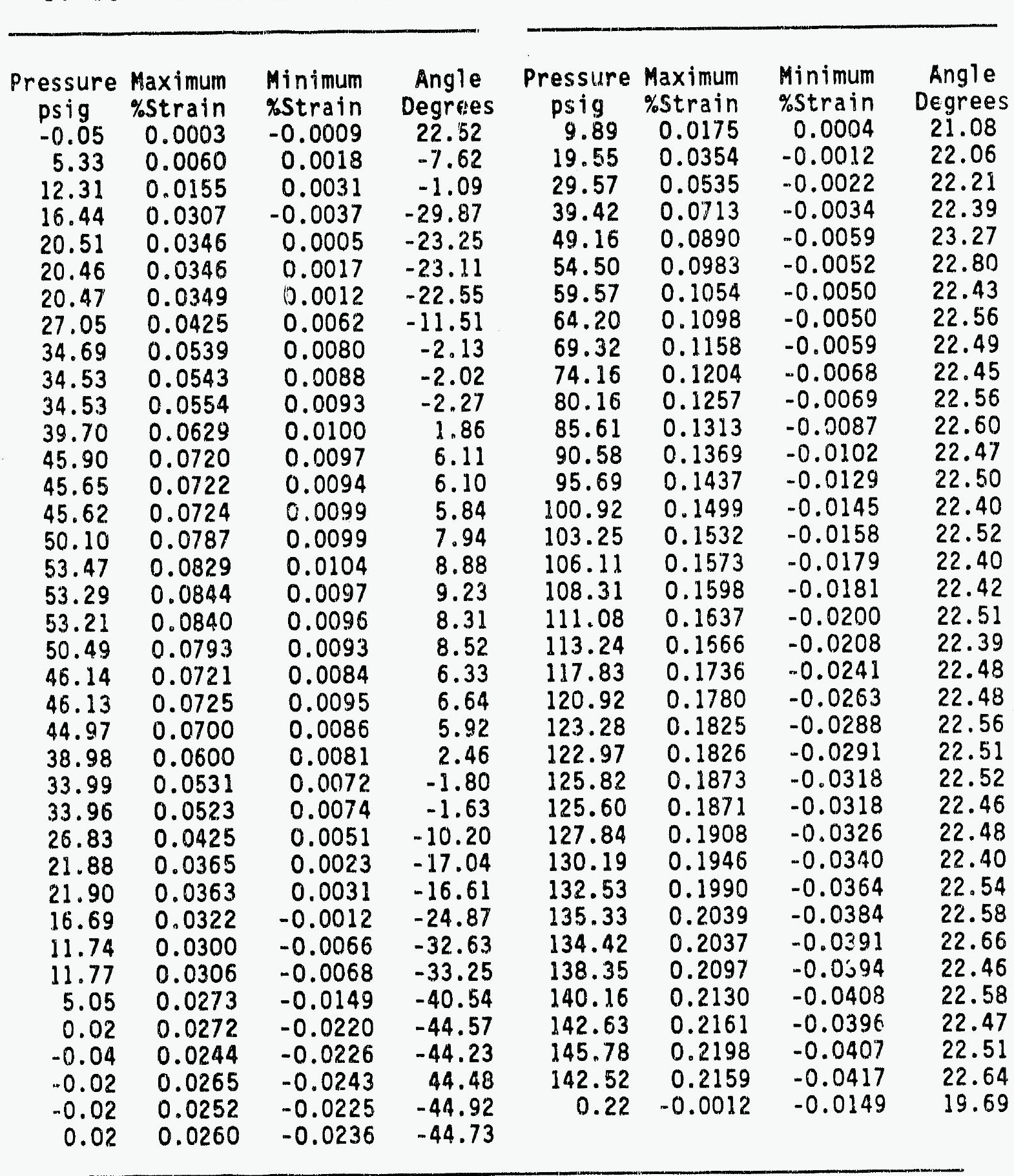

High Pressure Test 


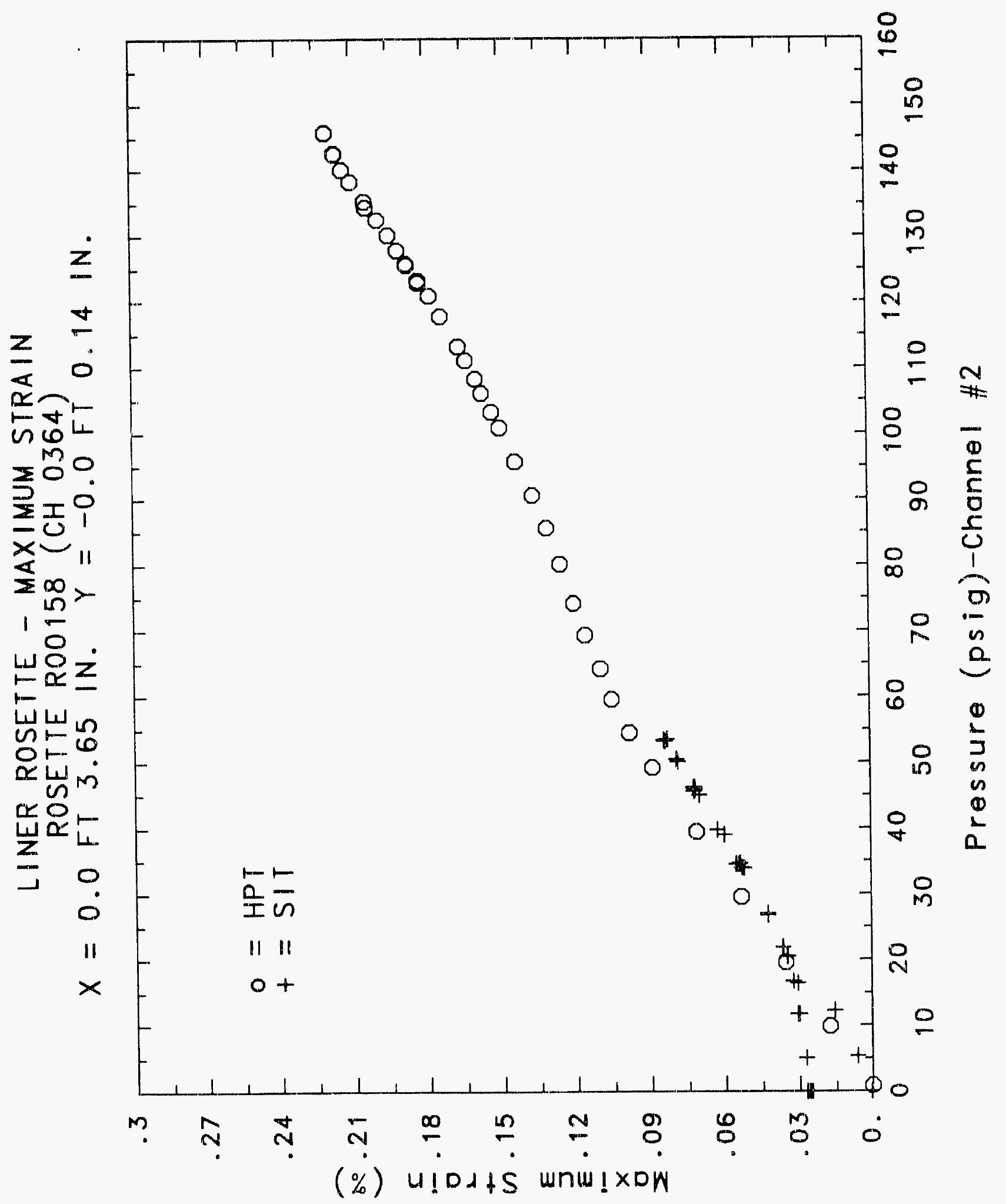




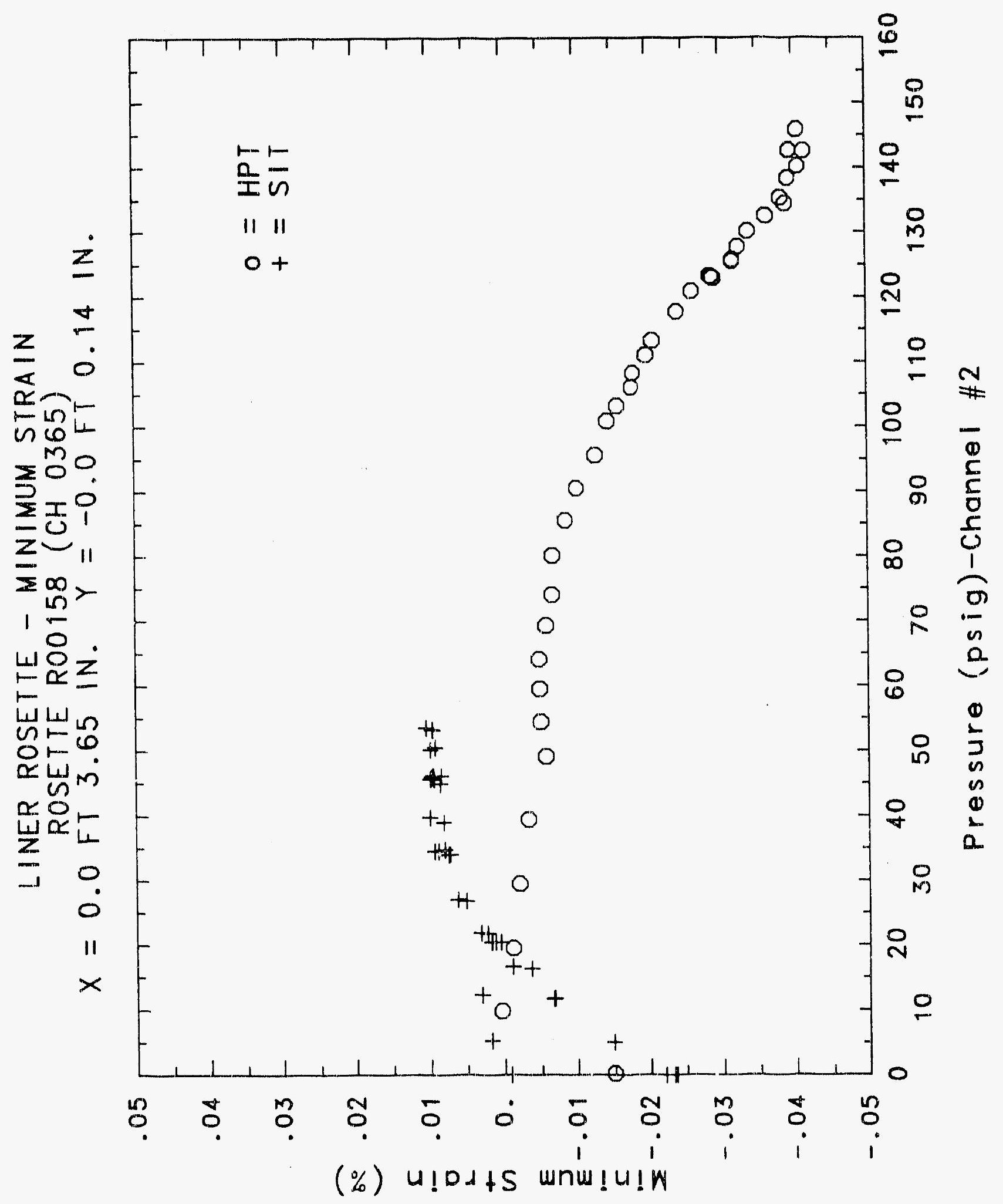




\section{Reinforced Concrete Test Data}

Rosette 159 Channels $367 \quad 368 \quad 369$

ITRUCTURAL INTEGRITY TEST

\begin{tabular}{|c|c|c|c|c|c|c|c|}
\hline $\begin{array}{c}\text { Pressure } \\
\text { psig } \\
-0.05 \\
5.33 \\
12.31 \\
16.44 \\
20.51 \\
20.46 \\
20.47 \\
27.05 \\
34.69 \\
34.53 \\
34.53 \\
39.70 \\
45.90 \\
45.65 \\
45.62 \\
50.10 \\
53.47 \\
53.29 \\
53.21 \\
50.49 \\
46.14 \\
46.13 \\
44.97 \\
38.98 \\
33.99 \\
33.96 \\
26.83 \\
21.88 \\
21.90 \\
16.69 \\
11.74 \\
11.77 \\
5.05 \\
0.02 \\
-0.04 \\
-0.02 \\
-0.02 \\
0.02\end{array}$ & $\begin{array}{c}\text { Maximum } \\
\% \text { Strain } \\
0.0016 \\
0.0053 \\
0.0086 \\
0.0111 \\
0.0204 \\
0.0212 \\
0.0211 \\
0.0226 \\
0.0245 \\
0.0239 \\
0.0236 \\
0.0250 \\
0.0259 \\
0.0303 \\
0.0300 \\
0.0311 \\
0.0319 \\
0.0315 \\
0.0314 \\
0.0318 \\
0.0298 \\
0.0296 \\
0.0445 \\
0.0421 \\
0.0263 \\
0.0263 \\
0.0248 \\
0.0242 \\
0.0251 \\
0.0248 \\
0.0237 \\
0.0260 \\
0.0246 \\
0.0243 \\
0.0226 \\
0.0242 \\
0.0236 \\
0.0246\end{array}$ & $\begin{array}{r}\text { Minimum } \\
\% \text { Strain } \\
-0.0024 \\
-0.0001 \\
0.0014 \\
0.0029 \\
-0.0768 \\
-0.0768 \\
-0.0767 \\
-0.0744 \\
-0.0725 \\
-0.0735 \\
-0.0730 \\
-0.0730 \\
-0.0709 \\
-0.0677 \\
-0.0675 \\
-0.0660 \\
-0.0655 \\
-0.0653 \\
-0.0660 \\
-0.0669 \\
-0.0671 \\
-0.0674 \\
0.0068 \\
0.0040 \\
-0.0725 \\
-0.0723 \\
-0.0745 \\
-0.0758 \\
-0.0770 \\
-0.0775 \\
-0.0784 \\
-0.0775 \\
-0.0791 \\
-0.0797 \\
-0.0801 \\
-0.0810 \\
-0.0815 \\
-0.0819\end{array}$ & $\begin{array}{c}\text { Angle } \\
\text { Degrees } \\
-30.07 \\
43.98 \\
38.71 \\
35.45 \\
20.73 \\
20.63 \\
19.91 \\
20.45 \\
21.74 \\
19.62 \\
19.23 \\
19.83 \\
20.82 \\
19.09 \\
18.80 \\
19.00 \\
19.43 \\
19.74 \\
20.09 \\
19.56 \\
19.25 \\
19.12 \\
35.48 \\
38.41 \\
16.00 \\
15.51 \\
14.58 \\
13.63 \\
13.40 \\
12.59 \\
12.45 \\
12.18 \\
11.78 \\
11.56 \\
11.37 \\
11.59 \\
11.17 \\
10.19\end{array}$ & $\begin{array}{c}\text { Pressure } \\
\text { psig } \\
9.89 \\
19.55 \\
29.57 \\
39.42 \\
49.16 \\
54.50 \\
59.57 \\
64.20 \\
69.32 \\
74.16 \\
80.16 \\
85.61 \\
90.58 \\
95.69 \\
100.92 \\
103.25 \\
106.11 \\
108.31 \\
111.08 \\
113.24 \\
117.83 \\
120.92 \\
123.28 \\
122.97 \\
125.82 \\
125.60 \\
127.84 \\
130.19 \\
132.53 \\
135.33 \\
134.42 \\
138.35 \\
140.16 \\
142.63 \\
145.78 \\
142.52 \\
0.22\end{array}$ & $\begin{array}{c}\text { Maximum } \\
\% \text { Strain } \\
0.0096 \\
0.0151 \\
0.0153 \\
0.0201 \\
0.0214 \\
0.0223 \\
0.0232 \\
0.0238 \\
0.0256 \\
0.0256 \\
0.0220 \\
0.0219 \\
0.0204 \\
0.0189 \\
0.0164 \\
0.0158 \\
0.0152 \\
0.0146 \\
0.0139 \\
0.0140 \\
0.0117 \\
0.0110 \\
0.0102 \\
0.0104 \\
0.0099 \\
0.0093 \\
0.0088 \\
0.0083 \\
0.0077 \\
0.0070 \\
0.0071 \\
0.0072 \\
0.0078 \\
0.0089 \\
0.0095 \\
0.0081 \\
-0.0099\end{array}$ & $\begin{array}{c}\text { Minimum } \\
\% \text { Strain } \\
0.0020 \\
0.0048 \\
0.0086 \\
0.0115 \\
0.0147 \\
0.0172 \\
0.0181 \\
0.0186 \\
0.0187 \\
0.0192 \\
0.0192 \\
0.0182 \\
0.0175 \\
0.0166 \\
0.0162 \\
0.0153 \\
0.0148 \\
0.0141 \\
0.0126 \\
0.0108 \\
0.0092 \\
0.0077 \\
0.0055 \\
0.0050 \\
0.0045 \\
0.0050 \\
0.0035 \\
0.0034 \\
0.0027 \\
0.0023 \\
0.0029 \\
0.0022 \\
0.0017 \\
0.0023 \\
0.0002 \\
-0.0015 \\
-0.0208\end{array}$ & $\begin{array}{c}\text { Angle } \\
\text { Degrees } \\
-21.50 \\
-22.13 \\
-18.18 \\
-20.23 \\
-17.45 \\
-13.91 \\
-24.73 \\
-20.46 \\
-25.64 \\
-17.35 \\
-10.68 \\
-19.04 \\
-20.06 \\
-14.79 \\
27.04 \\
43.20 \\
-16.05 \\
19.84 \\
-26.03 \\
-34.29 \\
-25.13 \\
-22.68 \\
-30.70 \\
-27.57 \\
-24.44 \\
-21.81 \\
-25.42 \\
-22.36 \\
-22.32 \\
-15.93 \\
-24.84 \\
-31.62 \\
-24.55 \\
-18.68 \\
-23.25 \\
-25.44 \\
-22.46\end{array}$ \\
\hline
\end{tabular}

\section{High Pressure test}




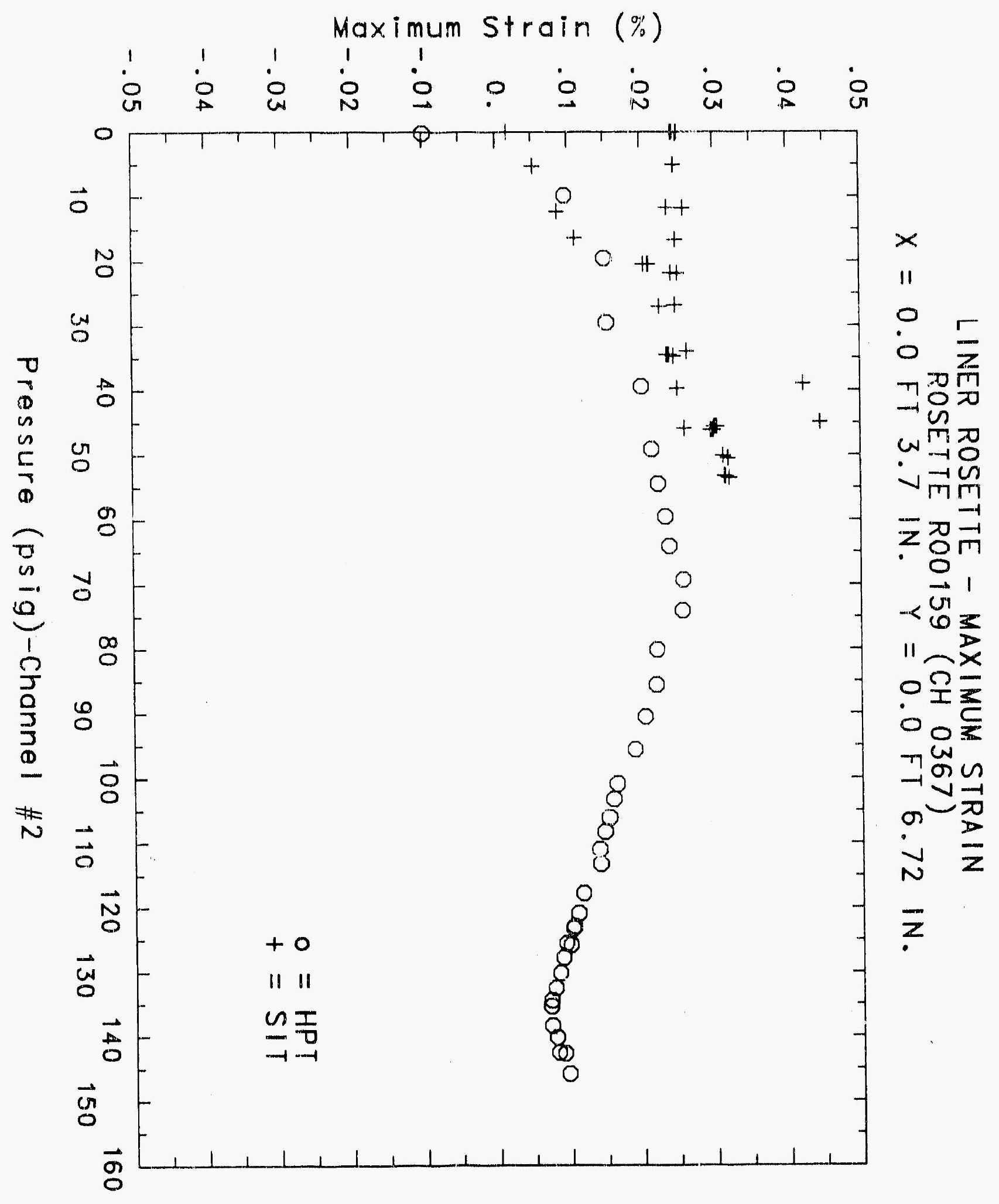




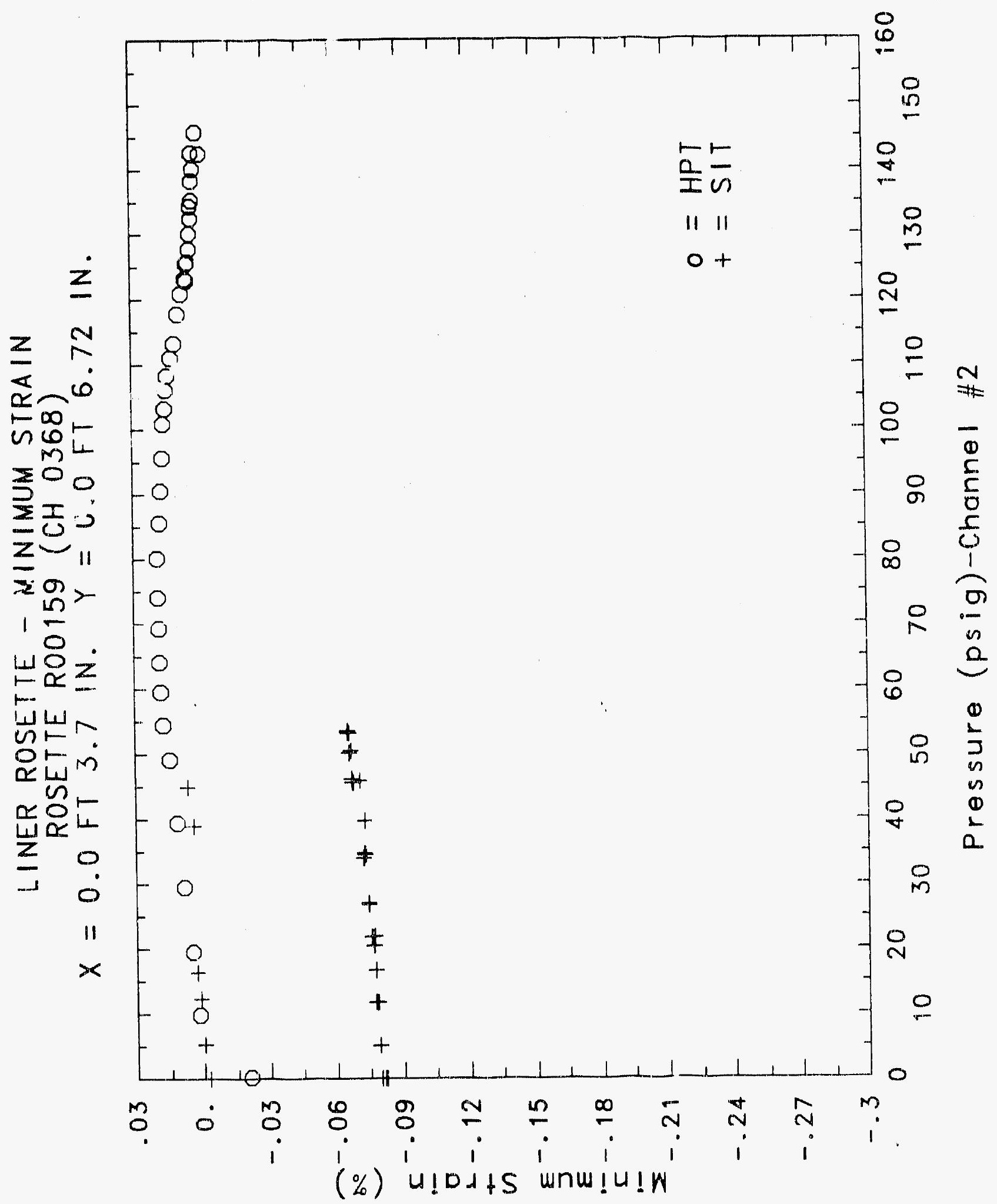




\section{Reinforced Concrete Test Data}

Rosette 160 Channels $380 \quad 381382$

STRUCTURAL INTEGRITY TEST

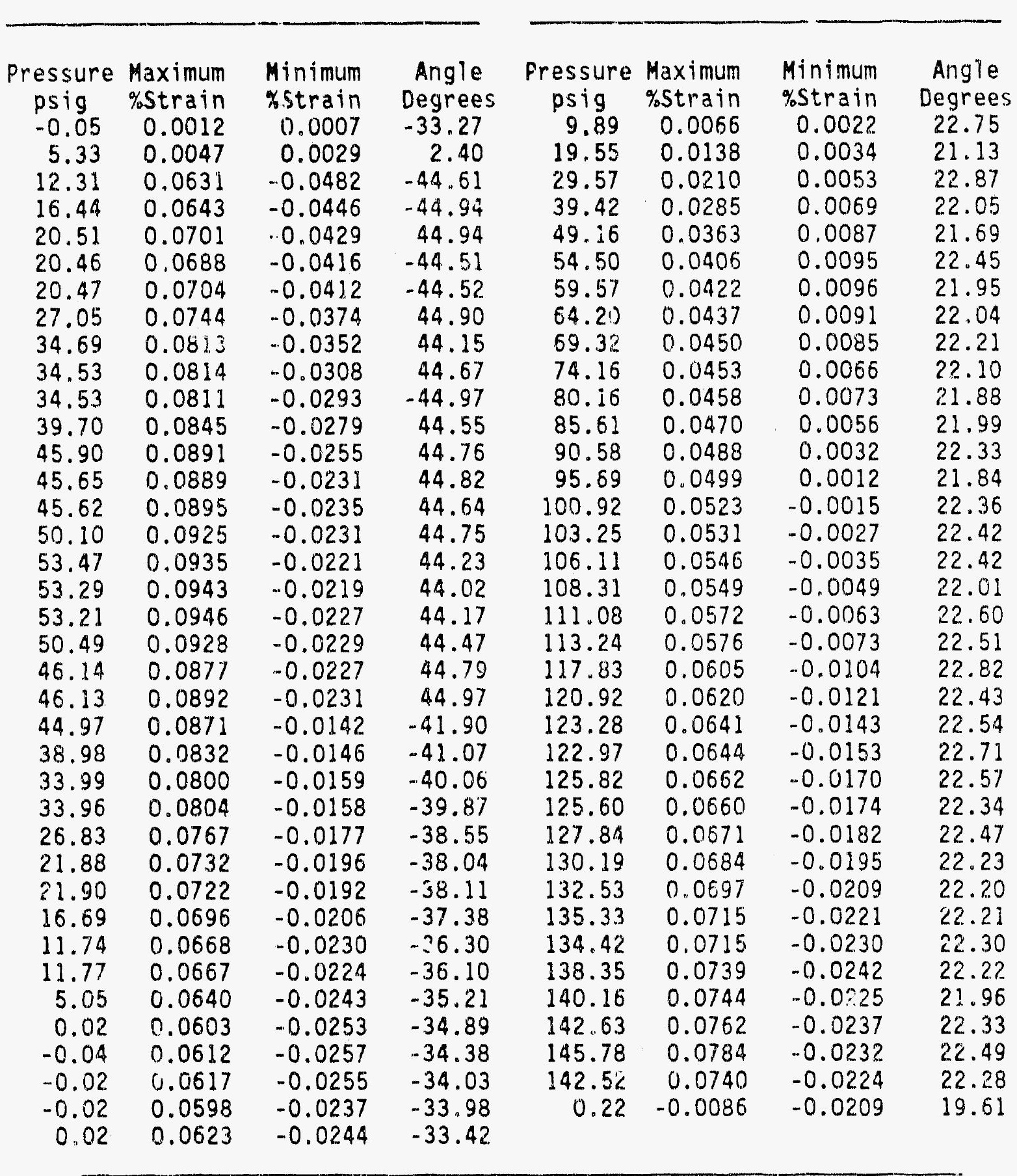

\section{High Pressure Test}




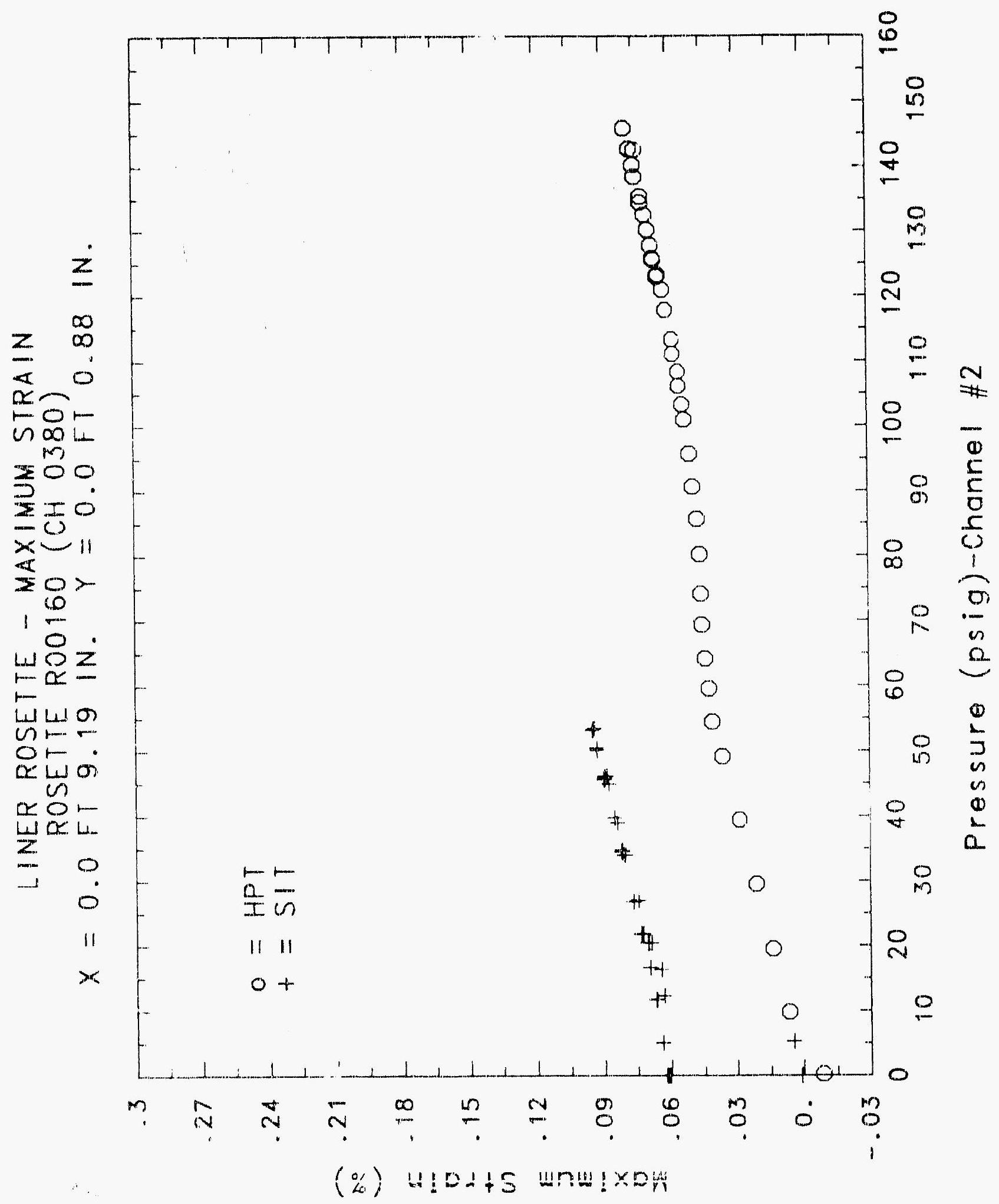




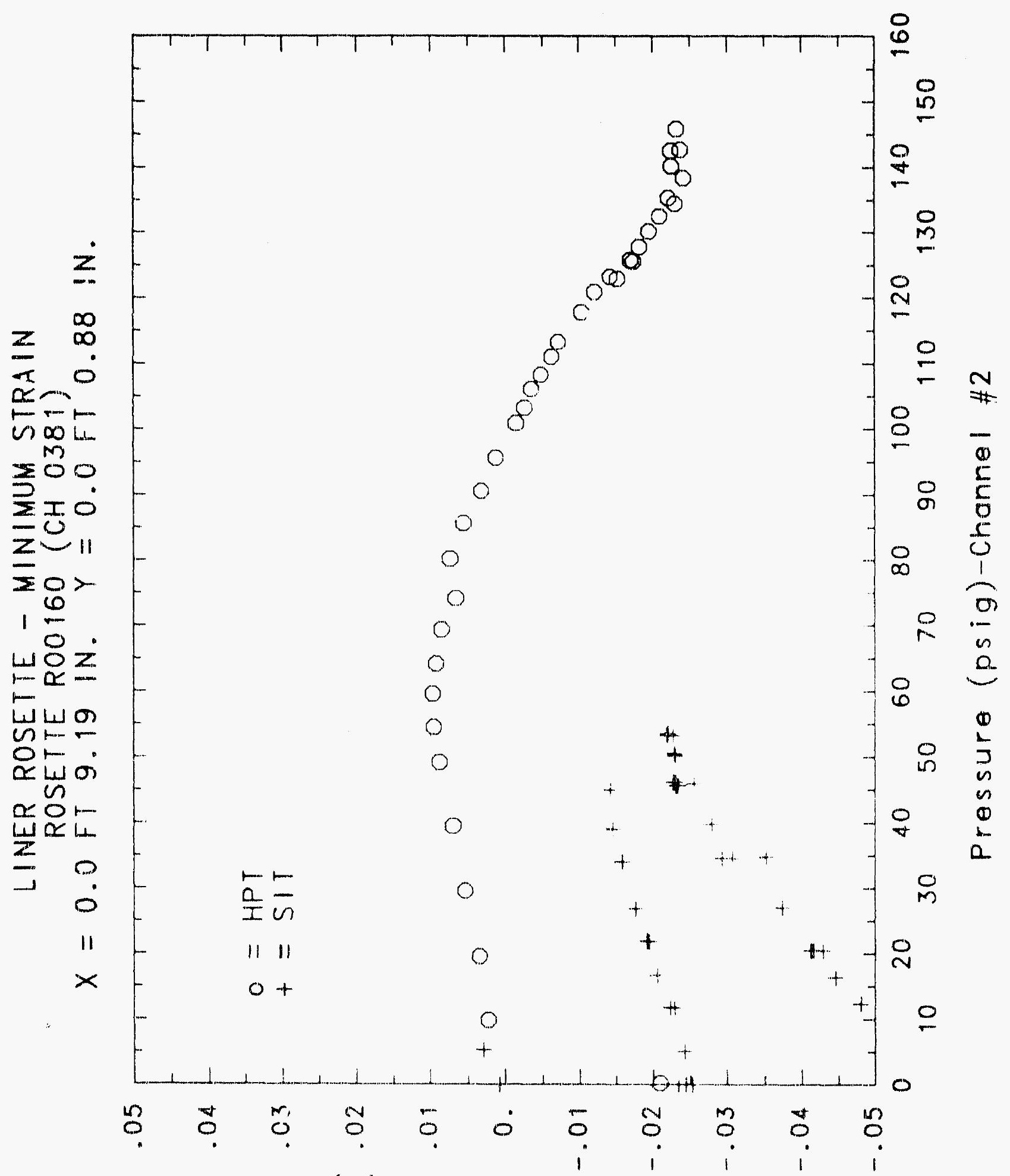

(\%) U! D.tS wnw! u! w 
○ำ

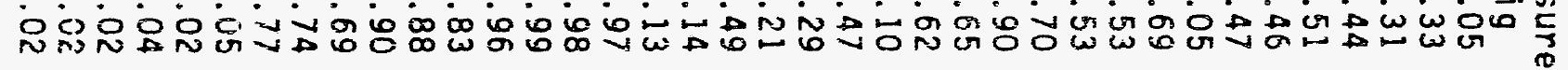
00000000000000000000000000000000000000 in 영

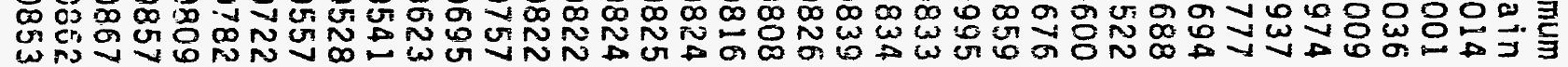

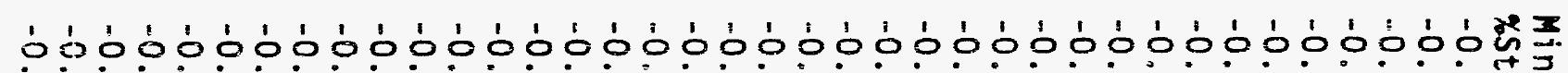

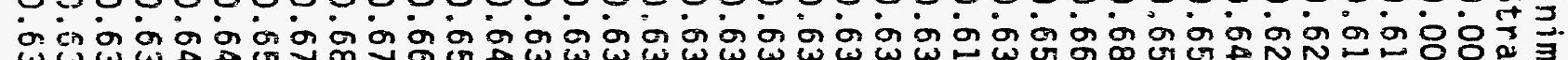

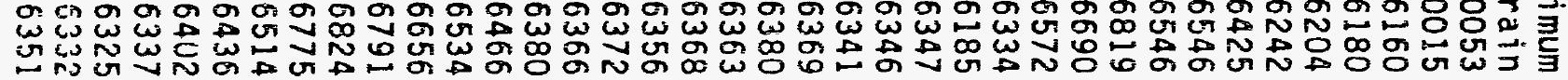

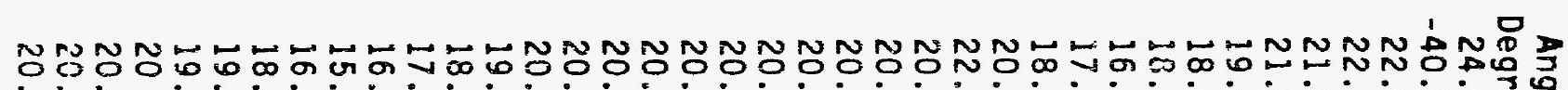
б

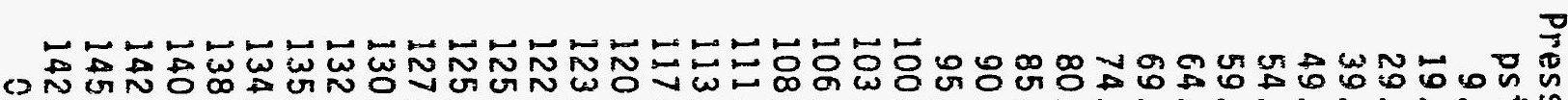
ruㅆ

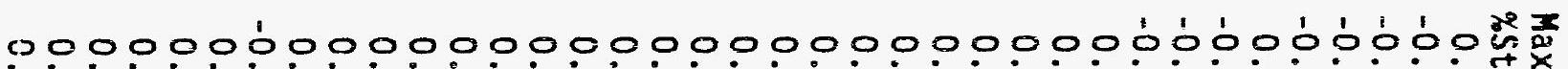

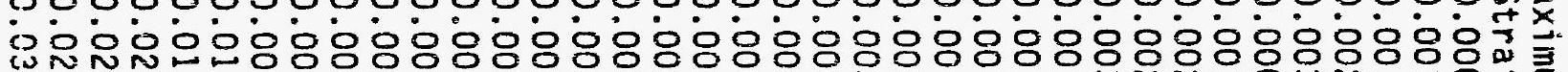

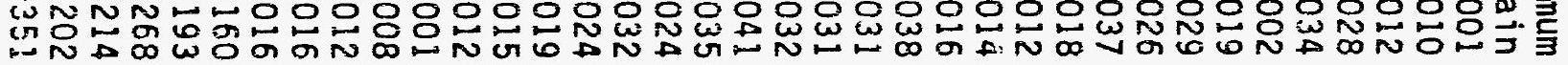

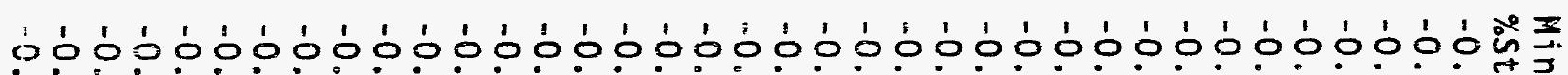

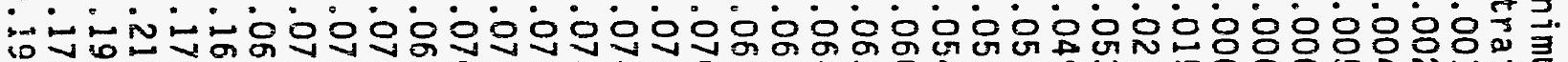
出

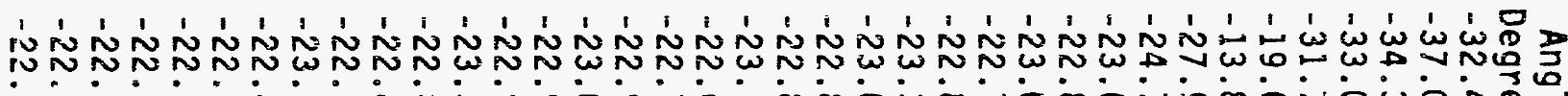
行 


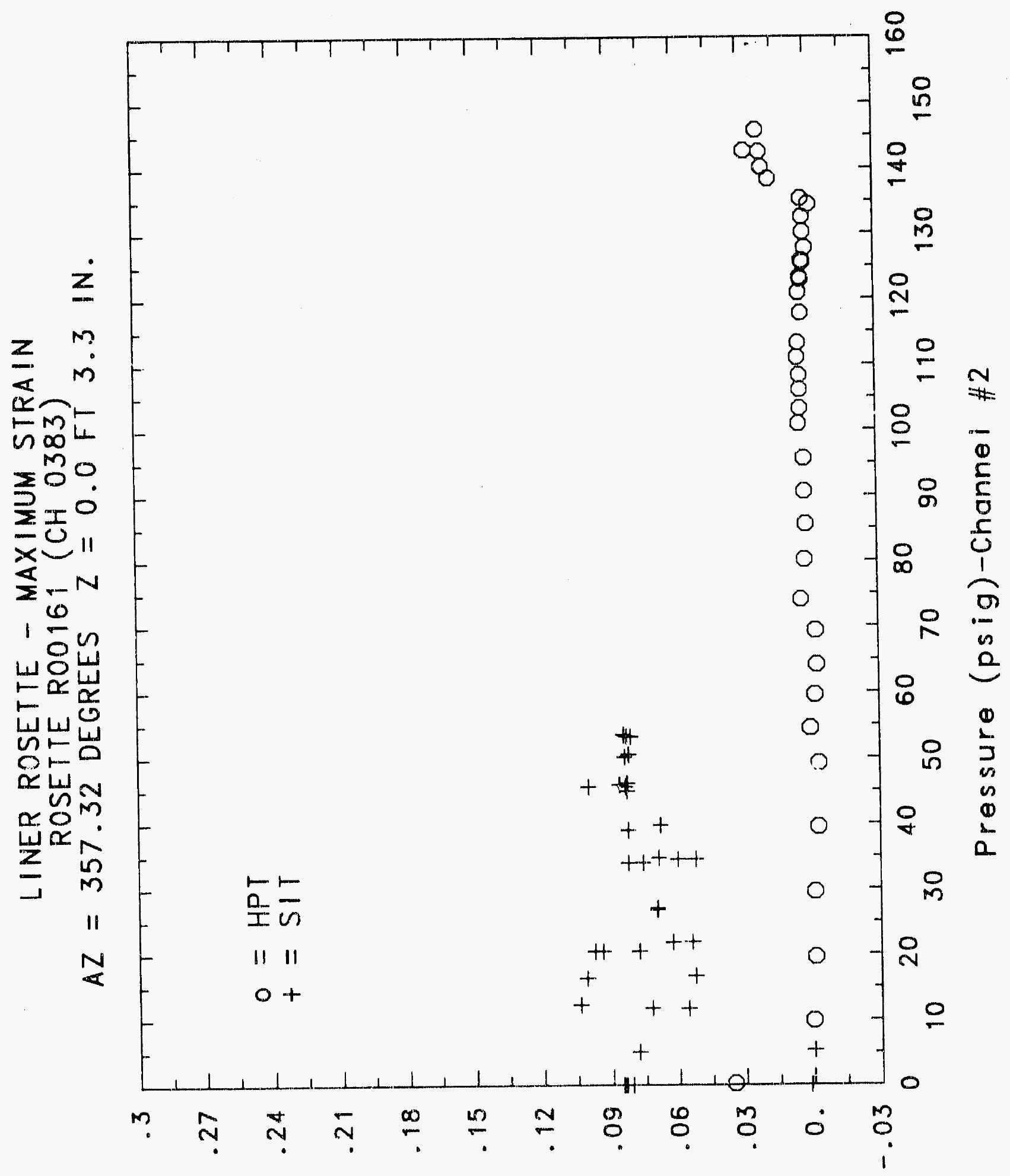

(\%) $u ! D d+S$ wnw! $\times D W$ 


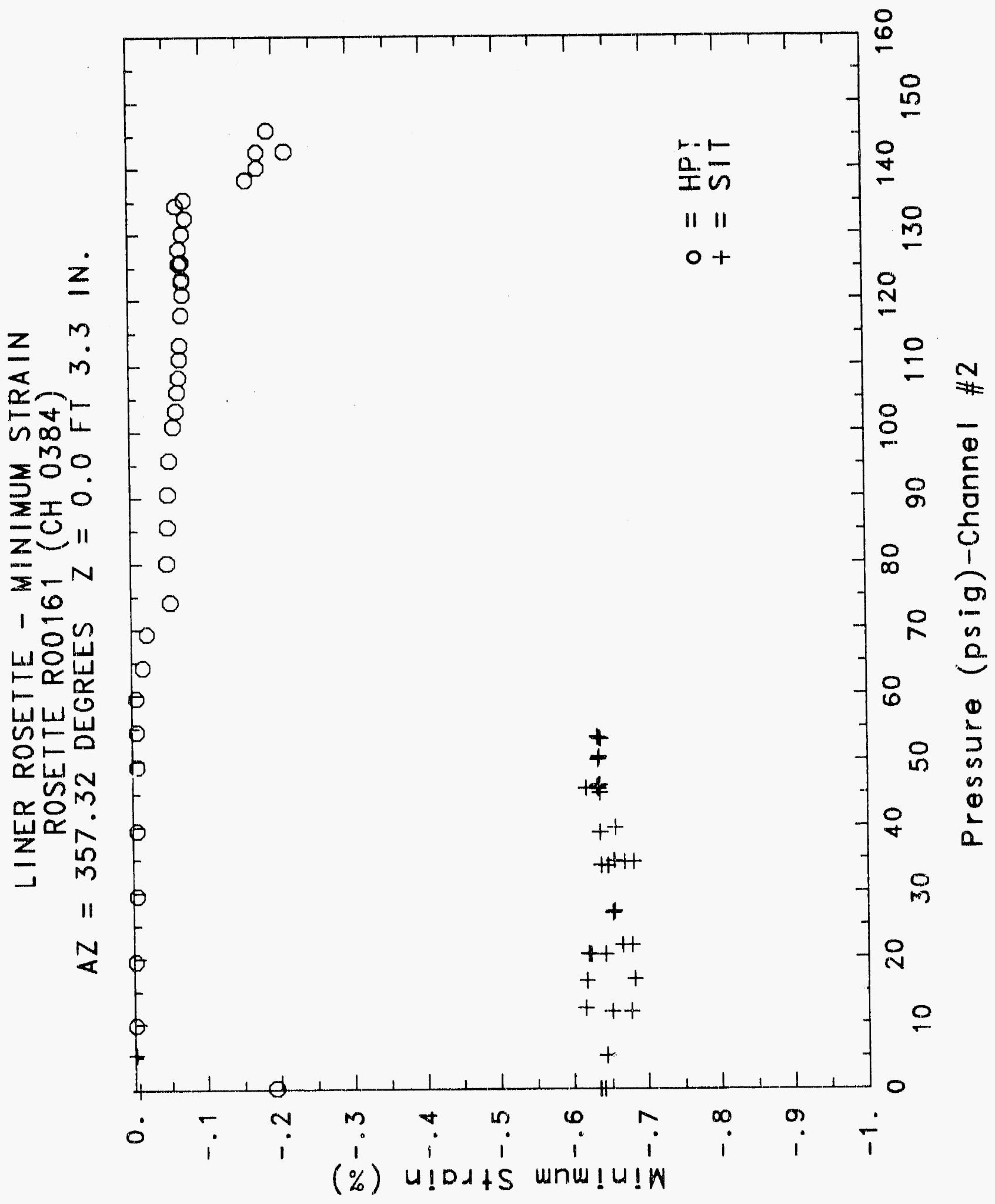




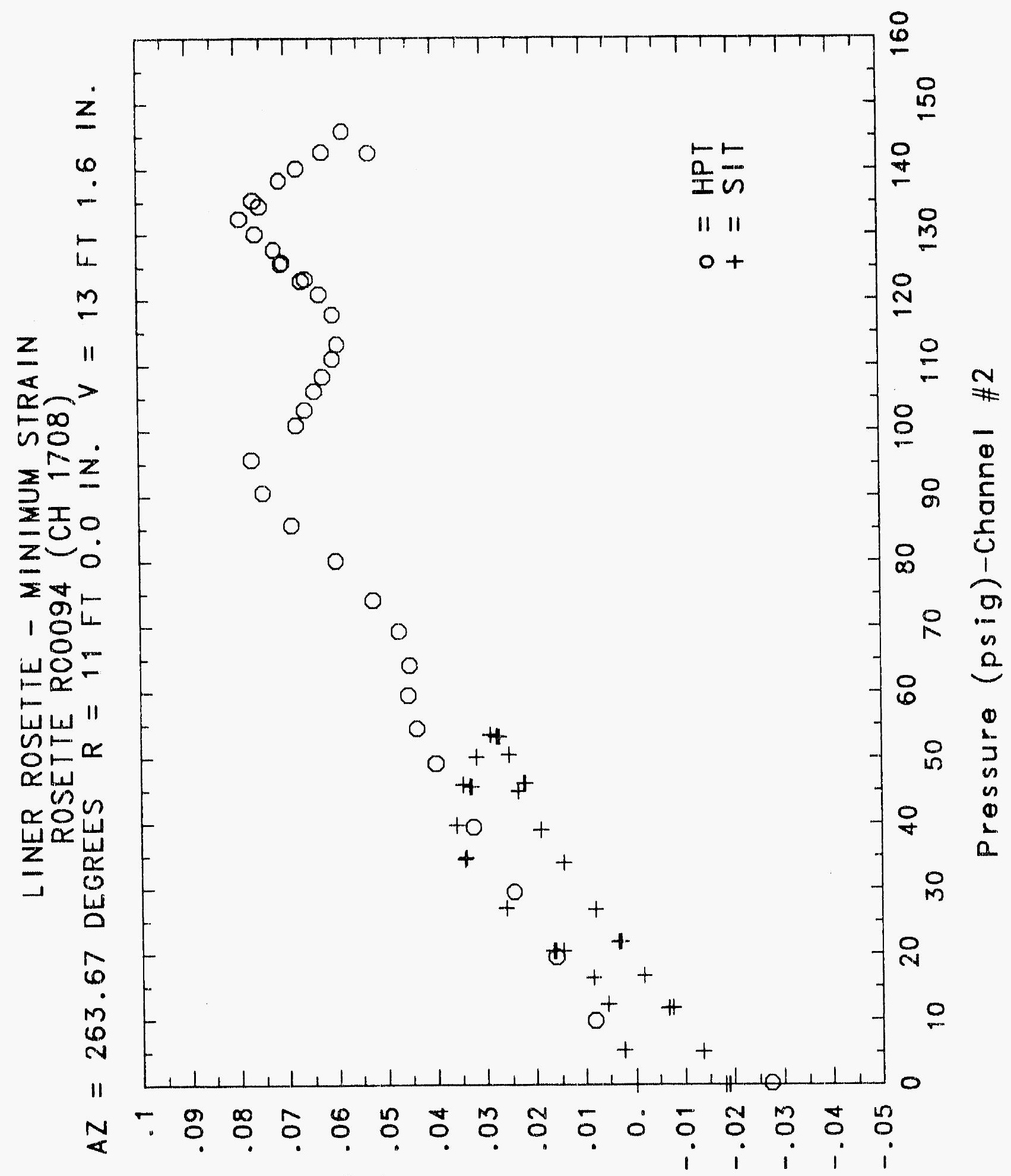

(\%) $u ! D d+5$ wnw!u!W 


\title{
Reinforced Concrete Test Data
}

\author{
Rosette 95 Channels $1720 \quad 1721 \quad 1722$
}

\section{Structural InTEgrity TEST}

\begin{tabular}{|c|c|c|c|c|c|c|c|}
\hline $\begin{array}{c}\text { Pressure } \\
\text { psig } \\
-0.05 \\
5.33 \\
12.31 \\
16.44 \\
20.51 \\
20.46 \\
20.47 \\
27.05 \\
34.69 \\
34.53 \\
34.53 \\
39.70 \\
45.90 \\
45.65 \\
45.62 \\
50.10 \\
53.47 \\
53.29 \\
53.21 \\
50.49 \\
46.14 \\
46.13 \\
44.97 \\
38.98 \\
33.99 \\
33.96 \\
26.83 \\
21.88 \\
21.90 \\
16.69 \\
11.74 \\
11.77 \\
5.05 \\
0.02 \\
-0.04 \\
-0.02 \\
-0.02 \\
0.02\end{array}$ & $\begin{array}{l}\text { Maximum } \\
\text { xStrain } \\
0.0004 \\
0.0053 \\
0.0119 \\
0.0160 \\
0.0240 \\
0.0253 \\
0.0257 \\
0.0395 \\
0.0534 \\
0.0544 \\
0.0546 \\
0.0623 \\
0.0750 \\
0.0772 \\
0.0775 \\
0.0866 \\
0.0971 \\
0.0996 \\
0.1005 \\
0.0989 \\
0.0944 \\
0.0941 \\
0.0943 \\
0.0875 \\
0.0811 \\
0.0805 \\
0.0709 \\
0.0637 \\
0.0628 \\
0.0556 \\
0.0485 \\
0.0482 \\
0.0393 \\
0.0326 \\
0.0302 \\
0.0303 \\
0.0298 \\
0.0287\end{array}$ & $\begin{array}{l}\text { Minimum } \\
\text { \%Strain } \\
-0.0002 \\
0.0028 \\
0.0066 \\
0.0090 \\
0.0034 \\
-0.0006 \\
-0.0014 \\
-0.0076 \\
-0.0148 \\
-0.0158 \\
-0.0161 \\
-0.0189 \\
-0.0243 \\
-0.0251 \\
-0.0252 \\
-0.0279 \\
-0.0314 \\
-0.0322 \\
-0.0323 \\
-0.0323 \\
-0.0313 \\
-0.0309 \\
-0.0302 \\
-0.0290 \\
-0.0269 \\
-0.0266 \\
-0.0234 \\
-0.0216 \\
-0.0212 \\
-0.0201 \\
-0.0195 \\
-0.0191 \\
-0.0199 \\
-0.0212 \\
-0.0182 \\
-0.0177 \\
-0.0177 \\
-0.0177\end{array}$ & $\begin{array}{c}\text { Angle } \\
\text { Degrees } \\
42.90 \\
-33.92 \\
-35.73 \\
-34.28 \\
-22.48 \\
-18.54 \\
-18.13 \\
-6.01 \\
-3.36 \\
-3.03 \\
-2.93 \\
-1.83 \\
0.08 \\
0.88 \\
0.97 \\
2.09 \\
3.11 \\
3.54 \\
3.73 \\
4.00 \\
4.32 \\
4.42 \\
4.71 \\
5.17 \\
5.77 \\
5.78 \\
7.00 \\
7.79 \\
7.66 \\
8.78 \\
9.96 \\
9.99 \\
11.70 \\
12.70 \\
12.00 \\
12.04 \\
12.01 \\
12.09\end{array}$ & $\begin{array}{c}\text { Pressure } \\
\text { psig } \\
9.89 \\
19.55 \\
29.57 \\
39.42 \\
49.16 \\
54.50 \\
59.57 \\
64.20 \\
69.32 \\
74.16 \\
80.16 \\
85.61 \\
90.58 \\
95.69 \\
100.92 \\
103.25 \\
106.11 \\
108.31 \\
111.08 \\
113.24 \\
117.83 \\
120.92 \\
123.28 \\
122.97 \\
125.82 \\
125.60 \\
127.84 \\
130.19 \\
132.53 \\
135.33 \\
134.42 \\
138.35 \\
140.16 \\
142.63 \\
145.78 \\
142.52 \\
0.22\end{array}$ & $\begin{array}{l}\text { Maximum } \\
\text { xStrain } \\
0.0127 \\
0.0264 \\
0.0416 \\
0.0570 \\
0.0720 \\
0.0826 \\
0.0961 \\
0.1074 \\
0.1261 \\
0.1488 \\
0.1744 \\
0.1894 \\
0.2006 \\
0.2137 \\
0.2261 \\
0.2311 \\
0.2373 \\
0.2423 \\
0.2491 \\
0.2538 \\
0.2650 \\
0.2720 \\
0.2776 \\
0.2784 \\
0.2858 \\
0.2874 \\
0.2926 \\
0.3011 \\
0.3112 \\
0.3252 \\
0.3272 \\
0.3443 \\
0.3577 \\
0.3749 \\
0.3948 \\
0.3930 \\
0.2068\end{array}$ & $\begin{array}{l}\text { Minimum } \\
\text { xStrain } \\
0.0031 \\
0.0011 \\
-0.0037 \\
-0.0091 \\
-0.0144 \\
-0.0182 \\
-0.0244 \\
-0.0337 \\
-0.0465 \\
-0.0566 \\
-0.0654 \\
-0.0683 \\
-0.0693 \\
-0.0704 \\
-0.0703 \\
-0.0702 \\
-0.0701 \\
-0.0700 \\
-0.0700 \\
-0.0700 \\
-0.0709 \\
-0.0716 \\
-0.0722 \\
-0.0725 \\
-0.0734 \\
-0.0738 \\
-0.0744 \\
-0.0761 \\
-0.0789 \\
-0.0829 \\
-0.0844 \\
-0.0883 \\
-0.0919 \\
-0.0956 \\
-0.0990 \\
-0.1004 \\
-0.1267\end{array}$ & $\begin{array}{r}\text { Angle } \\
\text { Degree } \\
2.49 \\
3.25 \\
3.91 \\
4.27 \\
4.31 \\
4.46 \\
1.70 \\
-2.93 \\
-5.96 \\
-5.96 \\
-5.18 \\
-4.87 \\
-4.64 \\
-4.33 \\
-3.96 \\
-3.84 \\
-3.68 \\
-3.58 \\
-3.50 \\
-3.52 \\
-3.72 \\
-3.86 \\
-3.98 \\
-4.00 \\
-4.08 \\
-4.06 \\
-4.09 \\
-4.15 \\
-4.40 \\
-4.61 \\
-4.68 \\
-4.71 \\
-4.67 \\
-4.49 \\
-4.24 \\
-4.08 \\
-7.22\end{array}$ \\
\hline
\end{tabular}

High Pressure Test

Aree

3.25

3.91

4.31

4.46

$-5.96$

$-5.96$

4.87

$-4.64$

$-4.33$

$-3.96$

$-3.84$

$-3.68$

3.50

$-3.52$

$-3.72$

$-3.86$

3.98

4.00

4.06

$-4.09$

$-4.15$

$-4.40$

$-4.61$

4.68

$-4.67$

$-4.49$

$-4.24$

$-4.08$

$-7.22$ 


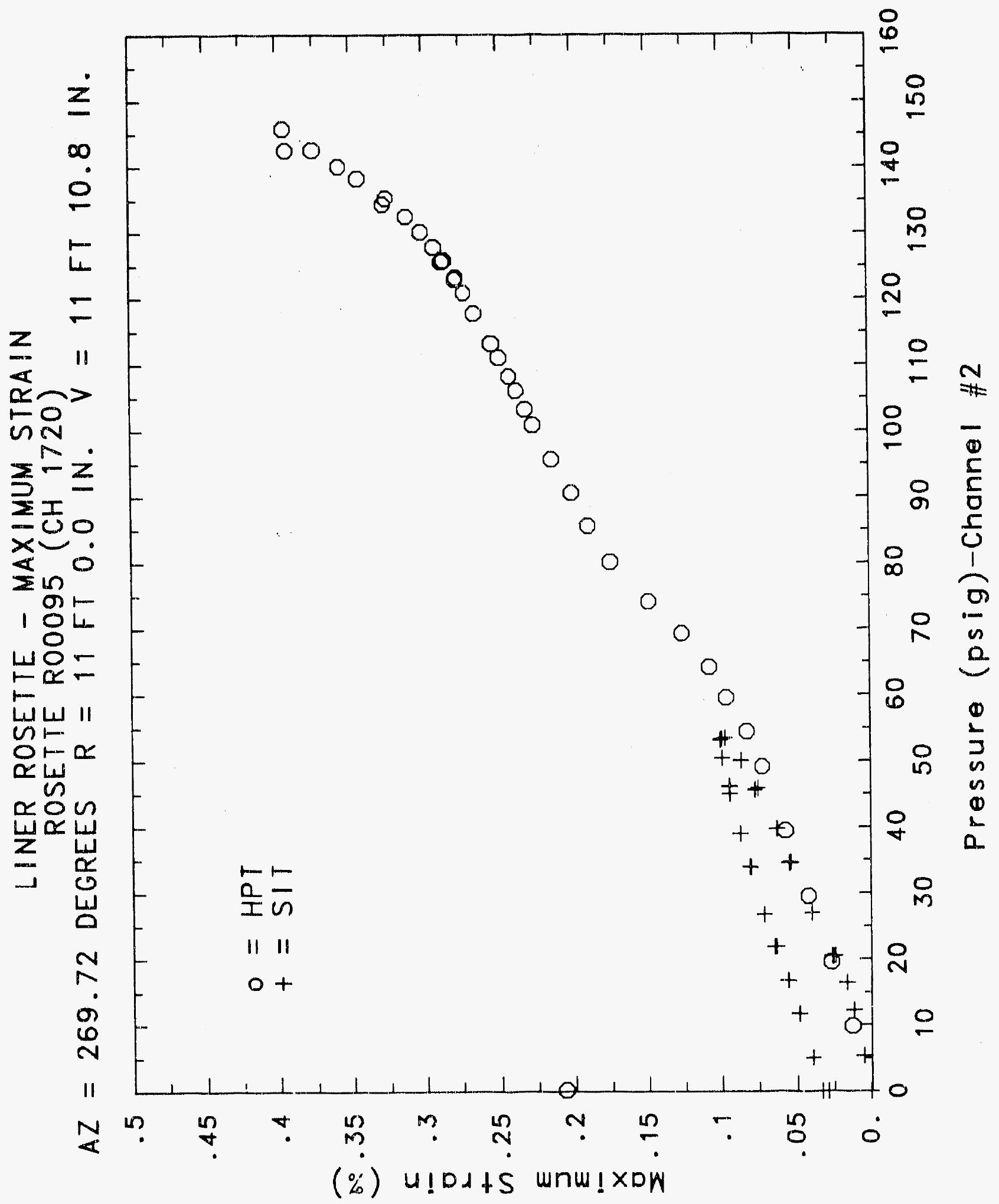

C-603 


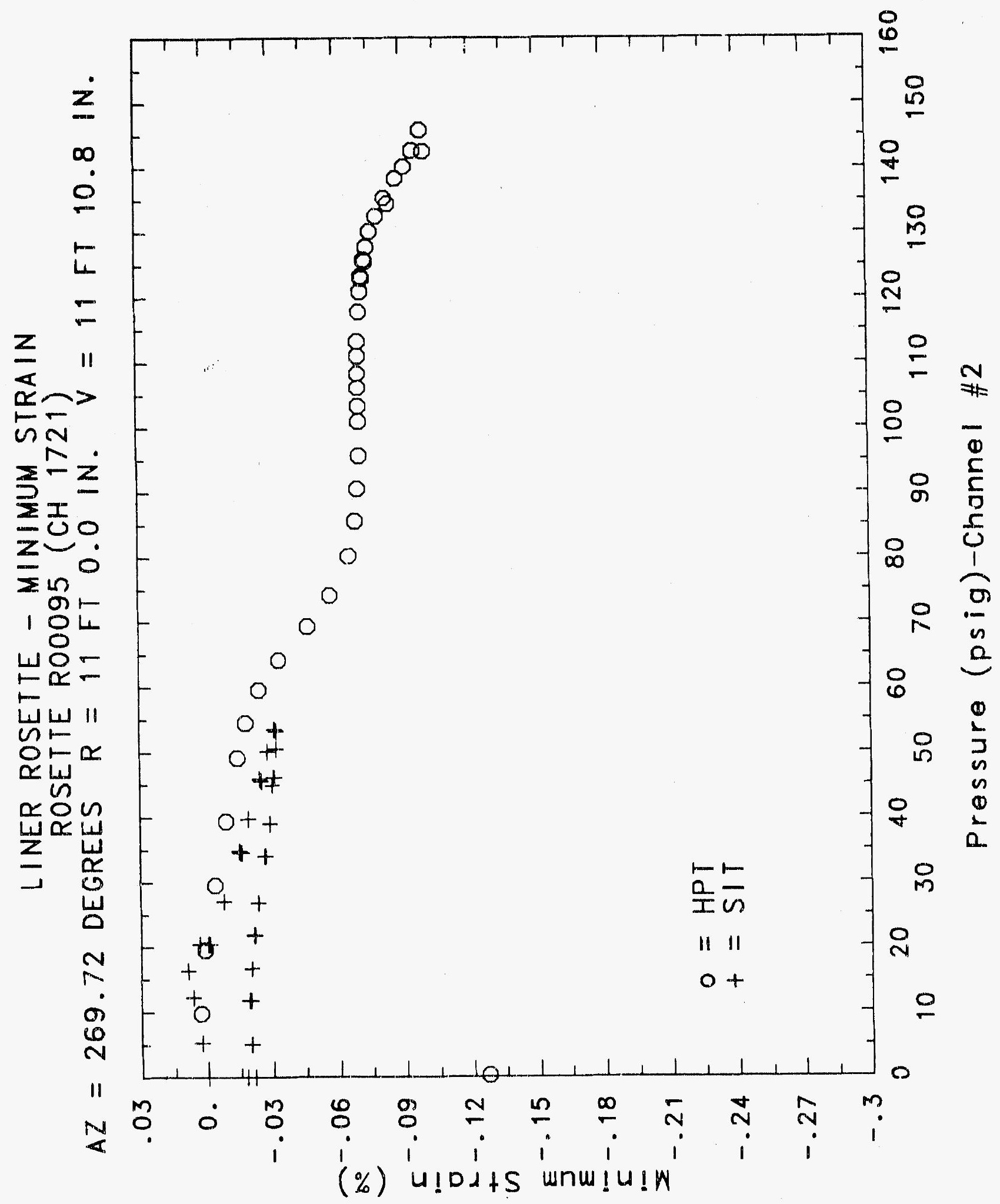




\section{Reinforced Concrete Test Data}

Rosette 96 Chanmels $1723 \quad 1724 \quad 1725$

Structural Integrity Test

\begin{tabular}{|c|c|c|c|c|c|c|c|}
\hline $\begin{array}{c}\text { Pressure } \\
\text { psig } \\
-0.05 \\
5.33 \\
12.31 \\
16.44 \\
20.51 \\
20.46 \\
20.47 \\
27.05 \\
34.69 \\
34.53 \\
34.53 \\
39.70 \\
45.90 \\
45.65 \\
45.62 \\
50.10 \\
53.47 \\
53.29 \\
53.21 \\
50.49 \\
46.14 \\
46.13 \\
44.97 \\
38.98 \\
33.99 \\
33.96 \\
26.83 \\
21.88 \\
21.90 \\
16.69 \\
11.74 \\
11.77 \\
5.05 \\
0.02 \\
-0.04 \\
-0.02 \\
-0.02 \\
0.02\end{array}$ & $\begin{array}{c}\text { Maximum } \\
\text { \% Strain } \\
0.0003 \\
0.0016 \\
0.0071 \\
0.0105 \\
0.0167 \\
0.0176 \\
0.0179 \\
0.0294 \\
0.0444 \\
0.0453 \\
0.0455 \\
0.0539 \\
0.0662 \\
0.0675 \\
0.0678 \\
0.0743 \\
0.0804 \\
0.0813 \\
0.0816 \\
0.0802 \\
0.0769 \\
0.0766 \\
0.0759 \\
0.0708 \\
0.0655 \\
0.0652 \\
0.0555 \\
0.0473 \\
0.0467 \\
0.0379 \\
0.0296 \\
0.0291 \\
0.0195 \\
0.0151 \\
0.0136 \\
0.0139 \\
0.0140 \\
0.0136\end{array}$ & $\begin{array}{r}\text { Minimum } \\
\% \text { Strain } \\
0.0000 \\
-0.0001 \\
0.0016 \\
0.0029 \\
0.0043 \\
0.0046 \\
0.0048 \\
0.0067 \\
0.0087 \\
0.0087 \\
0.0084 \\
0.0087 \\
0.0091 \\
0.0089 \\
0.0087 \\
0.0108 \\
0.0130 \\
0.0130 \\
0.0129 \\
0.0121 \\
0.0100 \\
0.0099 \\
0.0103 \\
0.0073 \\
0.0048 \\
0.0049 \\
0.0019 \\
0.0006 \\
0.0006 \\
-0.0011 \\
-0.0035 \\
-0.0039 \\
-0.0081 \\
-0.0090 \\
-0.0089 \\
-0.0085 \\
-0.0086 \\
-0.0087\end{array}$ & $\begin{array}{c}\text { Angle } \\
\text { Degrees } \\
-28.34 \\
19.51 \\
17.98 \\
17.74 \\
18.54 \\
18.49 \\
18.40 \\
18.09 \\
17.11 \\
17.15 \\
17.23 \\
18.36 \\
20.44 \\
20.64 \\
20.77 \\
21.91 \\
23.33 \\
23.70 \\
23.80 \\
23.80 \\
23.89 \\
23.98 \\
24.13 \\
24.22 \\
24.60 \\
24.53 \\
25.20 \\
25.30 \\
25.26 \\
25.38 \\
25.53 \\
25.49 \\
25.02 \\
23.14 \\
24.63 \\
25.03 \\
24.10 \\
24.65\end{array}$ & $\begin{array}{c}\text { Pressure } \\
\text { psig } \\
9.89 \\
19.55 \\
29.57 \\
39.42 \\
49.16 \\
54.50 \\
59.57 \\
64.20 \\
69.32 \\
74.16 \\
80.16 \\
85.61 \\
90.58 \\
95.69 \\
100.92 \\
103.25 \\
106.11 \\
108.31 \\
111.08 \\
113.24 \\
117.83 \\
120.92 \\
123.28 \\
122.97 \\
125.82 \\
125.60 \\
127.84 \\
130.19 \\
132.53 \\
135.33 \\
134.42 \\
138.35 \\
140.16 \\
142.63 \\
145.78 \\
142.52 \\
0.22\end{array}$ & $\begin{array}{l}\text { Maximum } \\
\text { XStrain } \\
0.0098 \\
0.0220 \\
0.0351 \\
0.0483 \\
0.0603 \\
0.0670 \\
0.0764 \\
0.0910 \\
0.1139 \\
0.1380 \\
0.1624 \\
0.1792 \\
0.1954 \\
0.2077 \\
0.2161 \\
0.2200 \\
0.2238 \\
0.2263 \\
0.2300 \\
0.2328 \\
0.2375 \\
0.2420 \\
0.2465 \\
0.2472 \\
0.2527 \\
0.2553 \\
0.2600 \\
0.2689 \\
0.2765 \\
0.2900 \\
0.2940 \\
0.3108 \\
0.3257 \\
0.3448 \\
0.3643 \\
0.3652 \\
0.1948\end{array}$ & $\begin{array}{r}\text { Minimum } \\
\text { zStrain } \\
0.0016 \\
0.0071 \\
0.0122 \\
0.0169 \\
0.0219 \\
0.0248 \\
0.0277 \\
0.0289 \\
0.0204 \\
0.0092 \\
0.0071 \\
0.0154 \\
0.0228 \\
0.0260 \\
0.0259 \\
0.0252 \\
0.0246 \\
0.0240 \\
0.0226 \\
0.0214 \\
0.0185 \\
0.0158 \\
0.0128 \\
0.0119 \\
0.0087 \\
0.0073 \\
0.0059 \\
0.0020 \\
-0.0022 \\
-0.0084 \\
-0.0115 \\
-0.0170 \\
-0.0223 \\
-0.0298 \\
-0.0353 \\
-0.0420 \\
-0.0656\end{array}$ & $\begin{array}{c}\text { Angle } \\
\text { Degrees } \\
32.34 \\
30.33 \\
27.94 \\
25.77 \\
24.14 \\
23.94 \\
27.03 \\
30.97 \\
33.19 \\
33.81 \\
30.28 \\
25.28 \\
20.26 \\
16.93 \\
15.33 \\
14.89 \\
14.48 \\
14.29 \\
13.95 \\
13.74 \\
13.39 \\
13.24 \\
13.15 \\
13.12 \\
13.16 \\
13.06 \\
13.00 \\
12.89 \\
13.03 \\
13.02 \\
13.05 \\
12.72 \\
12.39 \\
12.10 \\
11.75 \\
11.57 \\
7.66\end{array}$ \\
\hline
\end{tabular}

high Pressure Test 


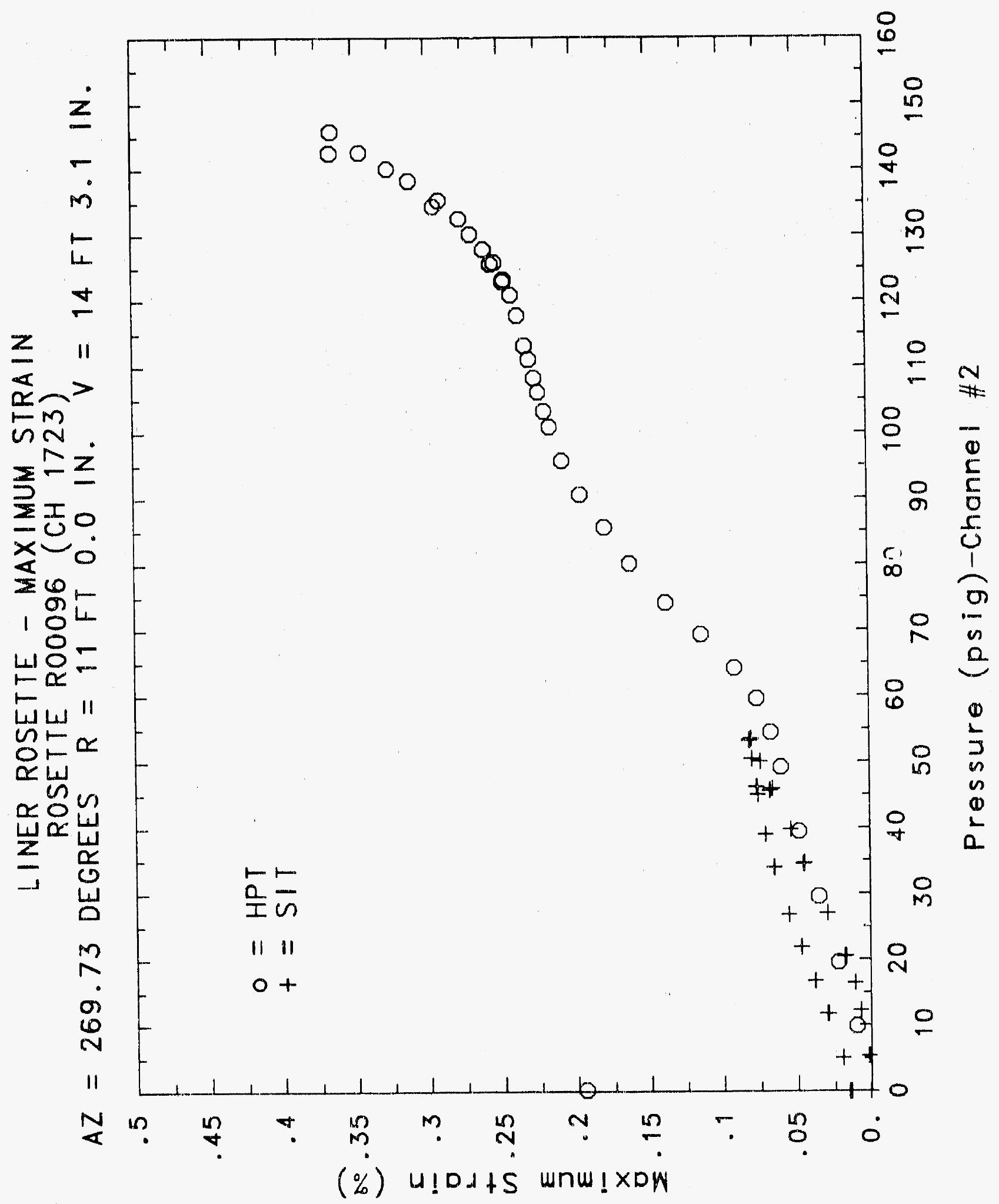




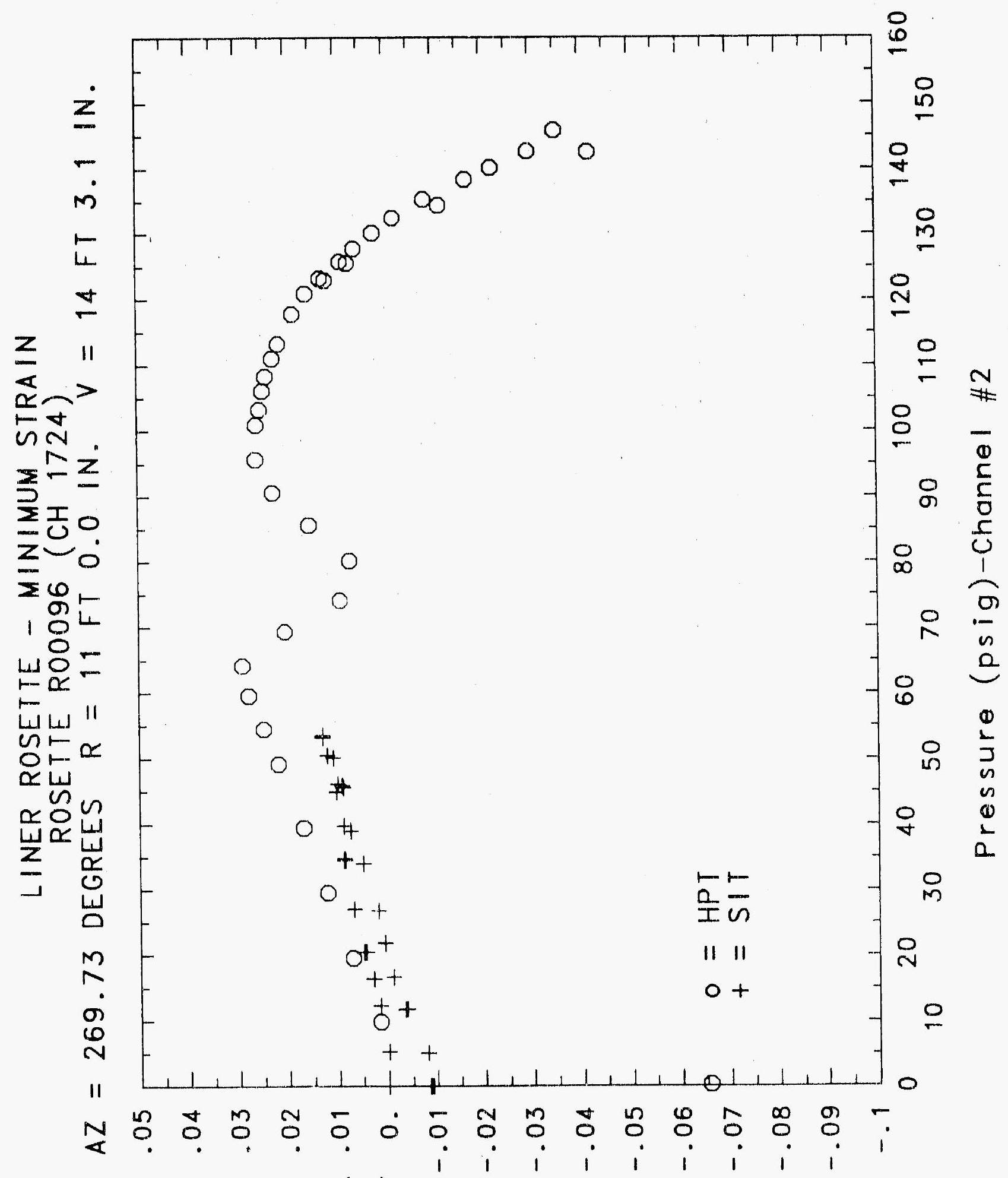

(\%) U!Dd+S wnw!u!W 


\section{Reinforced Concrete Test Data}

\section{$\begin{array}{llllll}\text { Rosette } & 97 & \text { Channels } & 1726 & 1727 & 1728\end{array}$}

StRUCTURAL InTEgRity Test

\begin{tabular}{|c|c|c|c|c|c|c|c|}
\hline $\begin{array}{c}\text { Pressure } \\
\text { psig } \\
-0.05 \\
5.33 \\
12.31 \\
16.44 \\
20.51 \\
20.46 \\
20.47 \\
27.05 \\
34.69 \\
34.53 \\
34.53 \\
39.70 \\
45.90 \\
45.65 \\
45.62 \\
50.10 \\
53.47 \\
53.29 \\
53.21 \\
50.49 \\
46.14 \\
46.13 \\
44.97 \\
38.98 \\
33.99 \\
33.96 \\
26.83 \\
21.88 \\
21.90 \\
16.69 \\
11.74 \\
11.77 \\
5.05 \\
0.02 \\
-0.04 \\
-0.02 \\
-0.02 \\
0.02\end{array}$ & $\begin{array}{l}\text { Maximum } \\
\text { \%Strain } \\
0.0003 \\
0.0026 \\
0.0063 \\
0.0091 \\
0.0147 \\
0.0158 \\
0.0163 \\
0.0261 \\
0.0396 \\
0.0407 \\
0.0410 \\
0.0486 \\
0.0593 \\
0.0602 \\
0.0603 \\
0.0663 \\
0.0720 \\
0.0728 \\
0.0729 \\
0.0711 \\
0.0672 \\
0.0670 \\
0.0669 \\
0.0615 \\
0.0563 \\
0.0561 \\
0.0487 \\
0.0435 \\
0.0430 \\
0.0379 \\
0.0332 \\
0.0329 \\
0.0276 \\
0.0243 \\
0.0222 \\
0.0232 \\
0.0231 \\
0.0228\end{array}$ & $\begin{array}{c}\text { Minimum } \\
\text { \%Strain } \\
-0.0002 \\
0.0017 \\
0.0035 \\
0.0046 \\
0.0066 \\
0.0071 \\
0.0073 \\
0.0122 \\
0.0221 \\
0.0234 \\
0.0237 \\
0.0286 \\
0.0328 \\
0.0326 \\
0.0325 \\
0.0343 \\
0.0359 \\
0.0356 \\
0.0356 \\
0.0346 \\
0.0322 \\
0.0322 \\
0.0326 \\
0.0287 \\
0.0254 \\
0.0252 \\
0.0203 \\
0.0162 \\
0.0163 \\
0.0123 \\
0.0079 \\
0.0073 \\
0.0016 \\
-0.0029 \\
-0.0035 \\
-0.0036 \\
-0.0035 \\
-0.0039\end{array}$ & $\begin{array}{c}\text { Angle } \\
\text { Degrees } \\
30.55 \\
-24.78 \\
-16.67 \\
-12.60 \\
-13.32 \\
-14.26 \\
-14.80 \\
-17.61 \\
-26.01 \\
-26.07 \\
-25.76 \\
-22.40 \\
-24.83 \\
-25.74 \\
-25.90 \\
-25.37 \\
-25.12 \\
-25.42 \\
-25.51 \\
-25.94 \\
-26.75 \\
-26.68 \\
-26.24 \\
-27.87 \\
-29.29 \\
-29.46 \\
-31.67 \\
-33.48 \\
-33.48 \\
-35.93 \\
-38.49 \\
-38.37 \\
-42.30 \\
-44.86 \\
-43.42 \\
-43.00 \\
-43.36 \\
-43.53\end{array}$ & $\begin{array}{c}\text { Pressure } \\
\text { psig } \\
9.89 \\
19.55 \\
29.57 \\
39.42 \\
49.16 \\
54.50 \\
59.57 \\
64.20 \\
69.32 \\
74.16 \\
80.16 \\
85.61 \\
90.58 \\
95.69 \\
100.92 \\
103.25 \\
106.11 \\
108.31 \\
111.08 \\
113.24 \\
117.83 \\
120.92 \\
123.28 \\
122.97 \\
125.82 \\
125.60 \\
127.84 \\
130.19 \\
132.53 \\
135.33 \\
134.42 \\
138.35 \\
140.16 \\
142.63 \\
145.78 \\
142.52 \\
0.22\end{array}$ & $\begin{array}{c}\text { Maximum } \\
\text { x.Strain } \\
0.0096 \\
0.0195 \\
0.0301 \\
0.0406 \\
0.0507 \\
0.0570 \\
0.0633 \\
0.0702 \\
0.0778 \\
0.0856 \\
0.0966 \\
0.1071 \\
0.1163 \\
0.1251 \\
0.1336 \\
0.1369 \\
0.1404 \\
0.1426 \\
0.1459 \\
0.1487 \\
0.1523 \\
0.1546 \\
0.1563 \\
0.1559 \\
0.1567 \\
0.1568 \\
0.1590 \\
0.1616 \\
0.1623 \\
0.1637 \\
0.1632 \\
0.1665 \\
0.1670 \\
0.1697 \\
0.1775 \\
0.1737 \\
0.0318\end{array}$ & $\begin{array}{l}\text { Minimum } \\
\text { xStrain } \\
0.0048 \\
0.0112 \\
0.0182 \\
0.0252 \\
0.0323 \\
0.0368 \\
0.0402 \\
0.0433 \\
0.0499 \\
0.0574 \\
0.0655 \\
0.0731 \\
0.0798 \\
0.0851 \\
0.0886 \\
0.0904 \\
0.0924 \\
0.0941 \\
0.0956 \\
0.0975 \\
0.1026 \\
0.1053 \\
0.1081 \\
0.1083 \\
0.1113 \\
0.1122 \\
0.1153 \\
0.1178 \\
0.1200 \\
0.1220 \\
0.1210 \\
0.1218 \\
0.1228 \\
0.1253 \\
0.1307 \\
0.1255 \\
0.0046\end{array}$ & $\begin{array}{c}\text { Angle } \\
\text { Degrees } \\
11.27 \\
7.60 \\
3.72 \\
0.87 \\
-2.18 \\
-3.60 \\
-6.06 \\
-11.90 \\
-14.76 \\
-16.83 \\
-21.63 \\
-25.30 \\
-28.31 \\
-32.02 \\
-34.45 \\
-34.68 \\
-35.16 \\
-35.75 \\
-35.59 \\
-34.57 \\
-32.14 \\
-31.68 \\
-31.31 \\
-31.21 \\
-30.18 \\
-29.46 \\
-27.16 \\
-25.45 \\
-24.94 \\
-26.17 \\
-26.84 \\
-28.68 \\
-30.03 \\
-33.33 \\
-37.18 \\
-41.79 \\
-34.94\end{array}$ \\
\hline
\end{tabular}

High Pressure test 


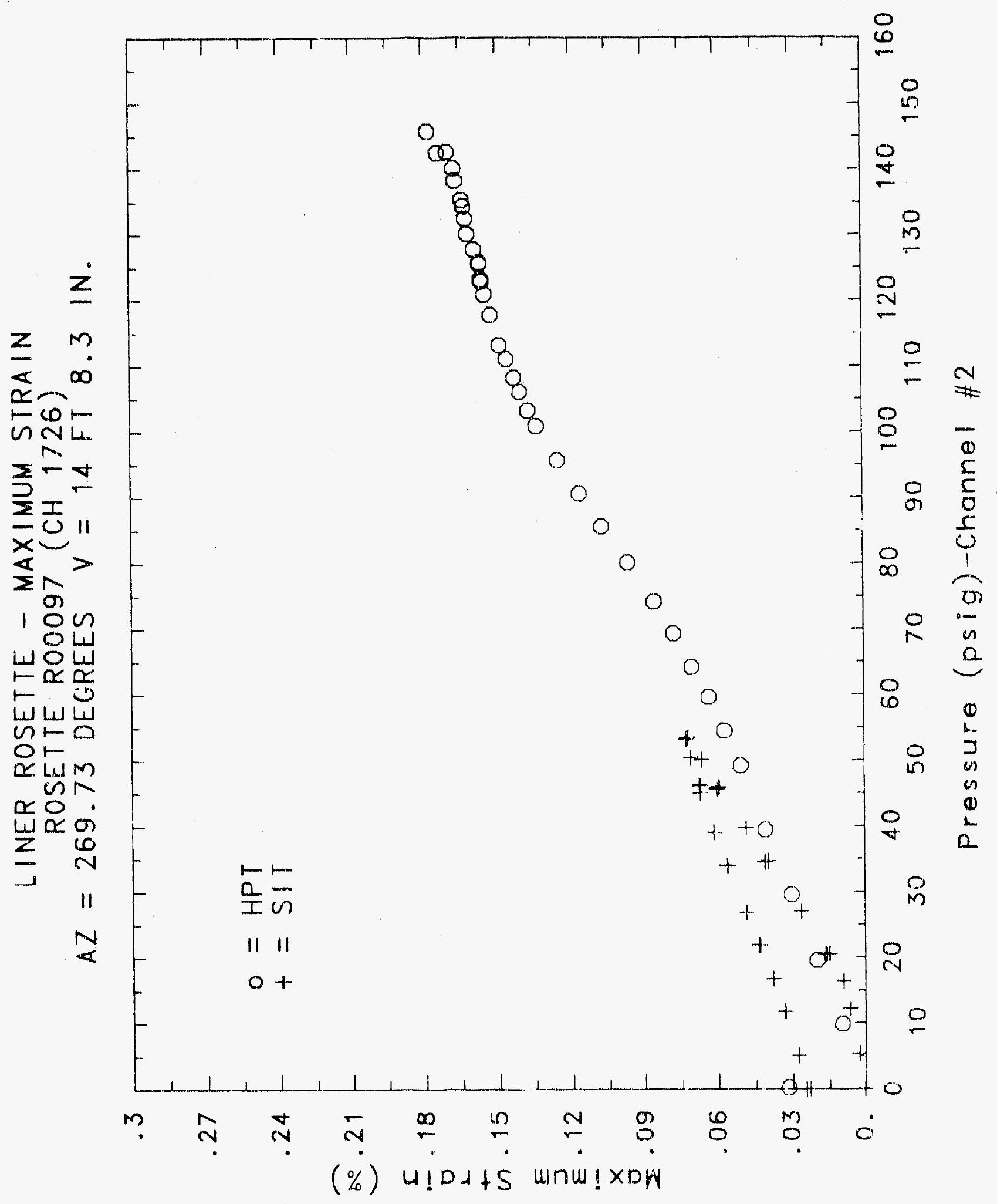




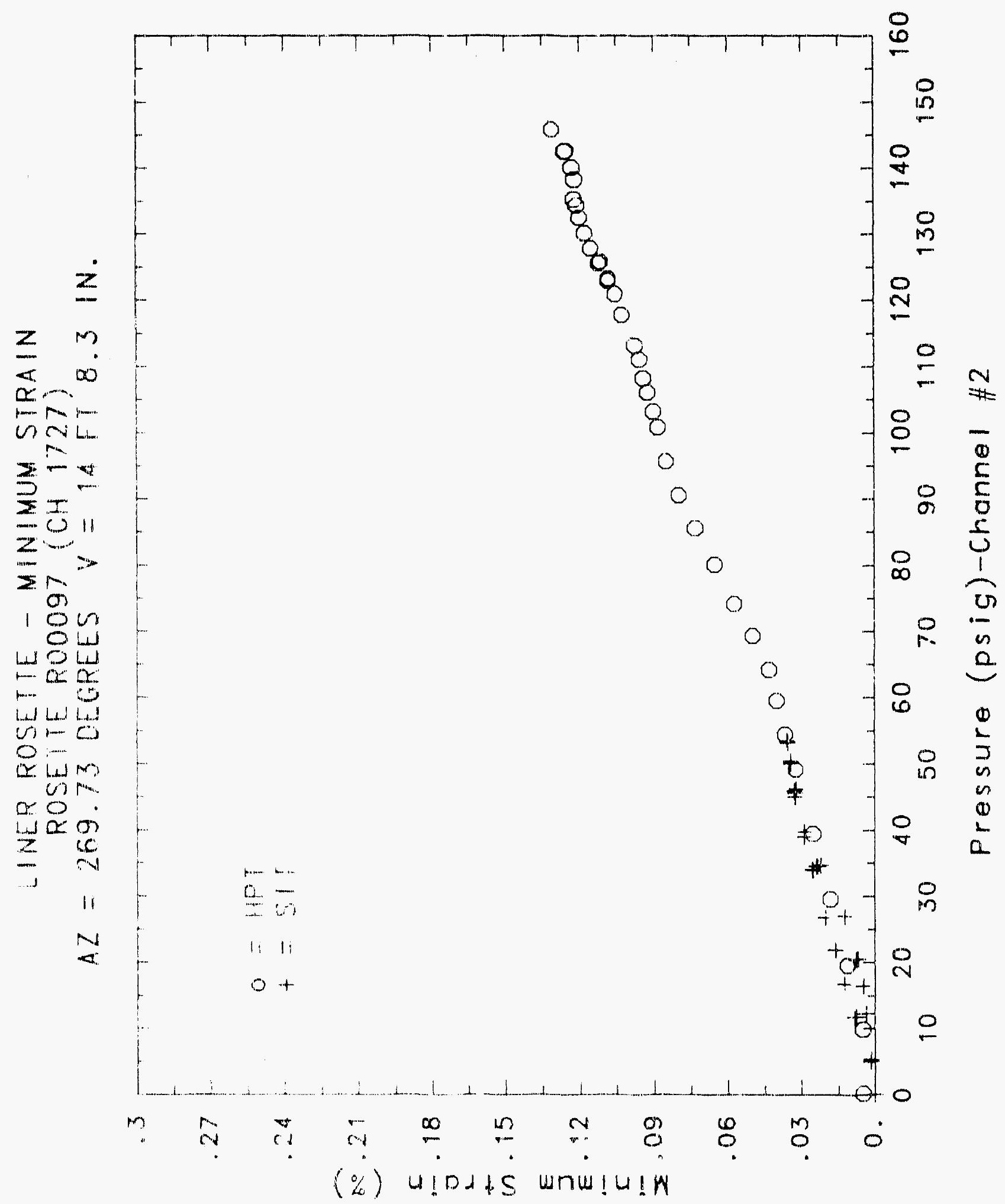

$(-6)(1)$ 


\title{
Reinforced Concrete Test Data
}

\author{
Rosette 98 Channels $1729 \quad 1740 \quad 1741$
}

\section{STRUCTURAL INTEGRITY TEST}

\begin{tabular}{|c|c|c|c|c|c|c|c|}
\hline \multicolumn{2}{|c|}{ STRUCTURAL } & \multicolumn{2}{|c|}{ NTEGRITY } & \multicolumn{4}{|c|}{ High Pressure Test } \\
\hline $\begin{array}{c}\text { Pressure } \\
\text { psig } \\
-0.05 \\
5.33 \\
12.31 \\
16.44 \\
20.51 \\
20.46 \\
20.47 \\
27.05 \\
34.69 \\
34.53 \\
34.53 \\
39.70 \\
45.90 \\
45.65 \\
45.62 \\
50.10 \\
53.47 \\
53.29 \\
53.21 \\
50.41 \\
46.14 \\
46.13 \\
44.97 \\
38.98 \\
33.99 \\
33.96 \\
26.83 \\
21.88 \\
21.90 \\
16.69 \\
11.74 \\
11.77 \\
5.05 \\
0.02 \\
-0.04 \\
-0.02 \\
-0.02 \\
0.02\end{array}$ & $\begin{array}{l}\text { Maximum } \\
\% \text { Strain } \\
0.0000 \\
0.0026 \\
0.0063 \\
0.0101 \\
0.0204 \\
0.0218 \\
0.0230 \\
0.0364 \\
0.0531 \\
0.0525 \\
0.0526 \\
0.0609 \\
0.0737 \\
0.0752 \\
0.0757 \\
0.0831 \\
0.0902 \\
0.0907 \\
0.0909 \\
0.0891 \\
0.0837 \\
0.0833 \\
0.0850 \\
0.0777 \\
0.0707 \\
0.0703 \\
0.0603 \\
0.0522 \\
0.0519 \\
0.0444 \\
0.0371 \\
0.0362 \\
0.0274 \\
0.0208 \\
0.0214 \\
0.0216 \\
0.0209 \\
0.0198\end{array}$ & $\begin{array}{r}\text { Minimum } \\
\text { KStrain } \\
-0.0006 \\
0.0014 \\
0.0058 \\
0.0090 \\
0.0010 \\
0.0009 \\
0.0009 \\
0.0034 \\
0.0086 \\
0.0087 \\
0.0087 \\
0.0119 \\
0.0145 \\
0.0141 \\
0.0141 \\
0.0170 \\
0.0193 \\
0.0193 \\
0.0193 \\
0.0178 \\
0.0151 \\
0.0150 \\
0.0181 \\
0.0137 \\
0.0104 \\
0.0102 \\
0.0059 \\
0.0028 \\
0.0027 \\
-0.0004 \\
-0.0035 \\
-0.0041 \\
-0.0079 \\
-0.0102 \\
-0.0080 \\
-0.0069 \\
-0.0084 \\
-0.0099\end{array}$ & $\begin{array}{c}\text { Angle } \\
\text { Degrees } \\
-2.30 \\
-18.15 \\
41.41 \\
34.12 \\
-2.50 \\
-1.61 \\
-2.57 \\
-0.12 \\
0.24 \\
1.15 \\
1.17 \\
1.13 \\
-0.10 \\
-0.42 \\
-0.71 \\
-0.68 \\
-0.75 \\
-0.60 \\
-0.60 \\
-0.82 \\
-0.59 \\
-0.51 \\
-0.92 \\
-0.67 \\
-0.57 \\
-0.53 \\
-0.20 \\
0.64 \\
0.73 \\
1.10 \\
1.77 \\
2.08 \\
2.43 \\
1.95 \\
0.37 \\
1.43 \\
0.95 \\
1.14\end{array}$ & $\begin{array}{c}\text { Pressure } \\
\text { psig } \\
9.89 \\
19.55 \\
29.57 \\
39.42 \\
49.16 \\
54.50 \\
59.57 \\
64.20 \\
69.32 \\
74.16 \\
80.16 \\
85.61 \\
90.58 \\
95.69 \\
100.92 \\
103.25 \\
106.11 \\
108.31 \\
111.08 \\
113.24 \\
117.83 \\
120.92 \\
123.28 \\
122.97 \\
125.82 \\
125.60 \\
127.84 \\
130.19 \\
132.53 \\
135.33 \\
134.42 \\
138.35 \\
140.16 \\
142.63 \\
145.78 \\
142.52 \\
0.22\end{array}$ & $\begin{array}{l}\text { Maximum } \\
\text { xStrain } \\
0.0117 \\
0.0256 \\
0.0397 \\
0.0533 \\
0.0668 \\
0.0751 \\
0.0844 \\
0.0951 \\
0.1073 \\
0.1195 \\
0.1396 \\
0.1574 \\
0.1786 \\
0.2111 \\
0.2600 \\
0.2839 \\
0.3151 \\
0.3428 \\
0.3904 \\
0.4316 \\
0.5492 \\
0.6576 \\
0.7703 \\
0.7953 \\
0.9299 \\
0.9509 \\
1.0058 \\
1.0755 \\
1.1563 \\
1.2863 \\
1.3021 \\
1.55879 \\
1.7853 \\
2.0770 \\
2.3567 \\
2.4117 \\
2.1350\end{array}$ & $\begin{array}{c}\text { Minimum } \\
\text { \%Strain } \\
0.0051 \\
0.0107 \\
0.0167 \\
0.0226 \\
0.0285 \\
0.0317 \\
0.0344 \\
0.0364 \\
0.0385 \\
0.0403 \\
0.0418 \\
0.0445 \\
0.0475 \\
0.0504 \\
0.0541 \\
0.0564 \\
0.0590 \\
0.0613 \\
0.0641 \\
0.0664 \\
0.0711 \\
0.0737 \\
0.0767 \\
0.0779 \\
0.0809 \\
0.0820 \\
0.0828 \\
0.0813 \\
0.0788 \\
0.0770 \\
0.0766 \\
0.0738 \\
0.0726 \\
0.0690 \\
0.0663 \\
0.0625 \\
-0.0107\end{array}$ & $\begin{array}{c}\text { Angle } \\
\text { Jegrees } \\
11.12 \\
4.83 \\
3.08 \\
2.62 \\
1.81 \\
1.55 \\
1.51 \\
1.40 \\
1.75 \\
2.55 \\
1.19 \\
1.38 \\
1.30 \\
0.94 \\
0.42 \\
0.05 \\
-0.20 \\
-0.38 \\
-0.66 \\
-0.93 \\
-1.36 \\
-1.93 \\
-2.21 \\
-2.15 \\
-1.75 \\
-1.53 \\
-1.13 \\
-0.90 \\
-0.92 \\
-1.07 \\
-1.06 \\
-0.74 \\
-0.34 \\
-0.10 \\
-0.23 \\
-0.28 \\
-0.33\end{array}$ \\
\hline
\end{tabular}

High Pressure test 


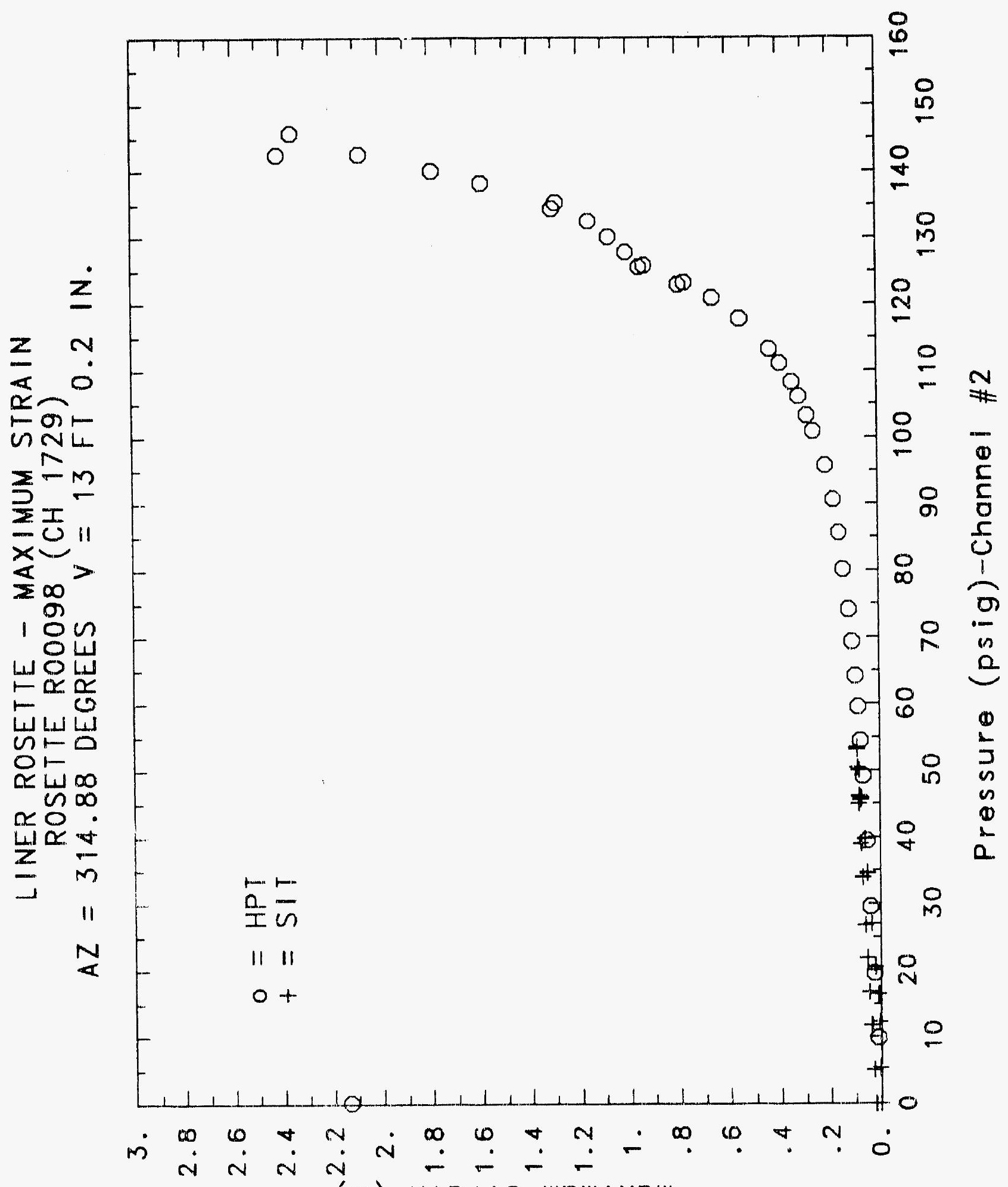

(\%) U!DdtS WnW!XDW 


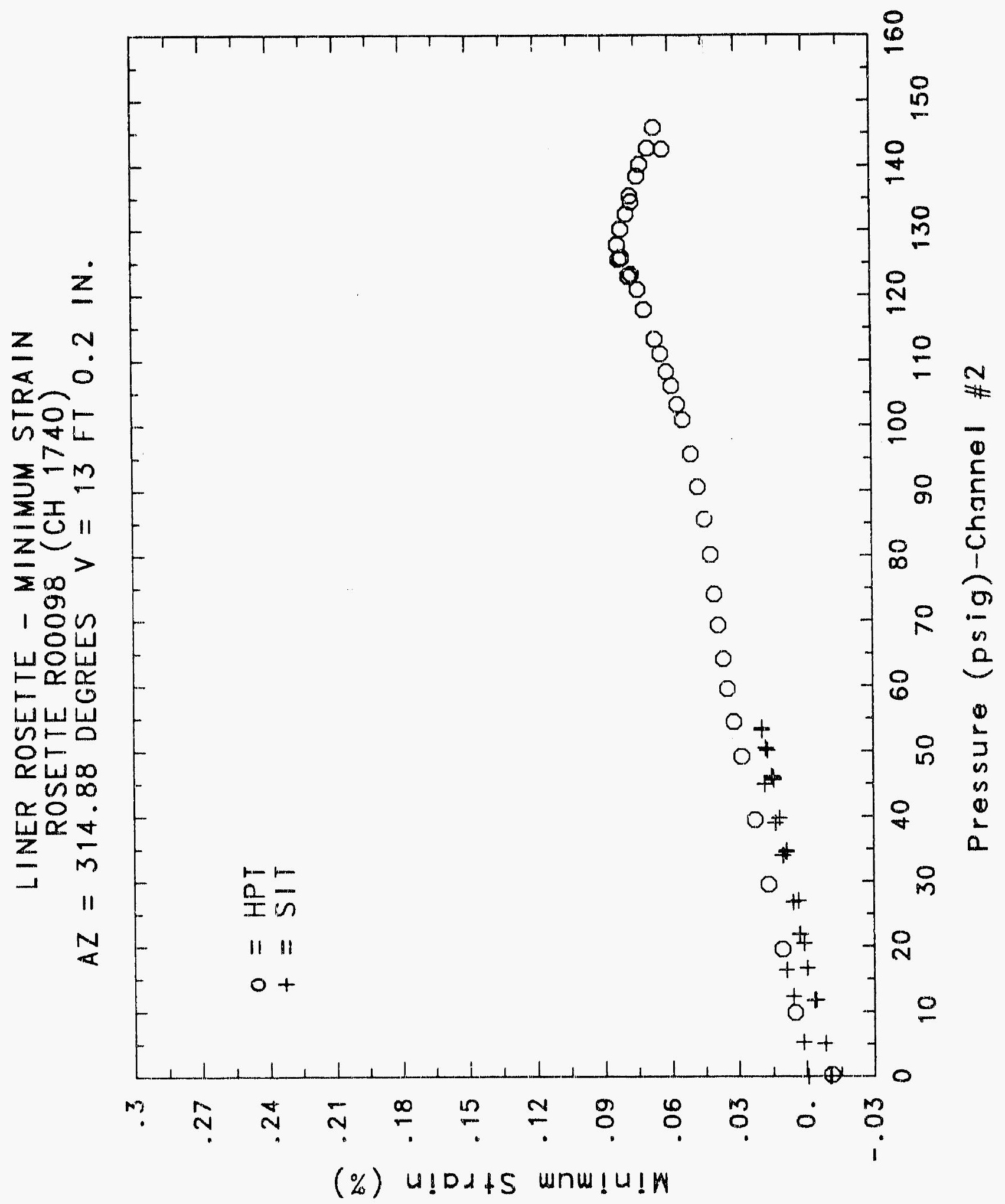




\section{Reinforced Concrete Test Data}

\section{Rosette 99 Channels $1742 \quad 1743 \quad 1744$}

\section{StRuCTURAL INTEgRITY TEST}

\begin{tabular}{|c|c|c|c|c|c|c|c|}
\hline $\begin{array}{c}\text { Pressure } \\
\text { psig } \\
-0.05 \\
5.33 \\
12.31 \\
16.44 \\
20.51 \\
20.46 \\
20.47 \\
27.05 \\
34.69 \\
34.53 \\
34.53 \\
39.70 \\
45.90 \\
45.65 \\
45.62 \\
50.10 \\
53.47 \\
53.29 \\
53.21 \\
50.49 \\
46.14 \\
46.13 \\
44.97 \\
38.98 \\
33.99 \\
33.96 \\
26.83 \\
21.88 \\
21.90 \\
16.69 \\
11.74 \\
11.77 \\
5.05 \\
0.02 \\
-0.04 \\
-0.02 \\
-0.02 \\
0.02\end{array}$ & $\begin{array}{c}\text { Maximum } \\
\text { \%Strain } \\
0.0005 \\
0.0023 \\
0.0055 \\
0.0082 \\
0.0126 \\
0.0139 \\
0.0145 \\
0.0280 \\
0.0404 \\
0.010 \\
0.0410 \\
0.0469 \\
0.0558 \\
0.0560 \\
0.0560 \\
0.0609 \\
0.0652 \\
0.0656 \\
0.0656 \\
0.0639 \\
0.0605 \\
0.0604 \\
0.0614 \\
0.0564 \\
0.0519 \\
0.0517 \\
0.0447 \\
0.0399 \\
0.0395 \\
0.0346 \\
0.0295 \\
0.0289 \\
0.0230 \\
0.0193 \\
0.0186 \\
0.0194 \\
0.0192 \\
0.0188\end{array}$ & $\begin{array}{l}\text { Minimum } \\
\text { \%Strain } \\
-0.0003 \\
0.0004 \\
0.0011 \\
0.0019 \\
0.0030 \\
0.0036 \\
0.0037 \\
0.0076 \\
0.0171 \\
0.0182 \\
0.0183 \\
0.0220 \\
0.0291 \\
0.0298 \\
0.0299 \\
0.0331 \\
0.0369 \\
0.0377 \\
0.0379 \\
0.0376 \\
0.0364 \\
0.0361 \\
0.0376 \\
0.0357 \\
0.0339 \\
0.0339 \\
0.0310 \\
0.0287 \\
0.0285 \\
0.0263 \\
0.0237 \\
0.0233 \\
0.0196 \\
0.0157 \\
0.0146 \\
0.0150 \\
0.0152 \\
0.0153\end{array}$ & $\begin{array}{c}\text { Angle } \\
\text { Degrees } \\
44.99 \\
-1.71 \\
-1.19 \\
-3.26 \\
-1.67 \\
-0.47 \\
0.57 \\
13.79 \\
14.30 \\
13.82 \\
13.56 \\
11.81 \\
10.68 \\
10.65 \\
10.51 \\
10.47 \\
10.33 \\
10.46 \\
11.00 \\
10.96 \\
11.38 \\
11.52 \\
13.90 \\
14.54 \\
15.14 \\
14.88 \\
16.71 \\
19.03 \\
19.10 \\
21.01 \\
27.01 \\
26.24 \\
43.54 \\
-24.97 \\
-28.56 \\
-31.31 \\
-30.86 \\
-30.58\end{array}$ & $\begin{array}{c}\text { Pressure } \\
\text { psig } \\
9.89 \\
19.55 \\
29.57 \\
39.42 \\
49.16 \\
54.50 \\
59.57 \\
64.20 \\
69.32 \\
74.16 \\
80.16 \\
85.61 \\
90.58 \\
95.69 \\
100.92 \\
103.25 \\
106.11 \\
108.31 \\
111.08 \\
113.24 \\
117.83 \\
120.92 \\
123.28 \\
122.97 \\
125.82 \\
125.60 \\
127.84 \\
130.19 \\
132.53 \\
135.33 \\
134.42 \\
138.35 \\
140.16 \\
142.63 \\
145.78 \\
142.52 \\
0.22\end{array}$ & $\begin{array}{l}\text { Maximuin } \\
\text { \%Strain } \\
0.0096 \\
0.0198 \\
0.0303 \\
0.0400 \\
0.0494 \\
0.0547 \\
0.0602 \\
0.0665 \\
0.0781 \\
0.0911 \\
0.0986 \\
0.1101 \\
0.1221 \\
0.1343 \\
0.1479 \\
0.1539 \\
0.1587 \\
0.1639 \\
0.1696 \\
0.1747 \\
0.1811 \\
0.1844 \\
0.1860 \\
0.1864 \\
0.1882 \\
0.1894 \\
0.1917 \\
0.1941 \\
0.1958 \\
0.1962 \\
0.1958 \\
0.2014 \\
0.2127 \\
0.2528 \\
0.3313 \\
0.3507 \\
0.1893\end{array}$ & $\begin{array}{c}\text { Minimum } \\
\text { xStrain } \\
0.0046 \\
0.0096 \\
0.0145 \\
0.0190 \\
0.0235 \\
0.0263 \\
0.0298 \\
0.0342 \\
0.0388 \\
0.0418 \\
0.0432 \\
0.0441 \\
0.0453 \\
0.0462 \\
0.0474 \\
0.0480 \\
0.0491 \\
0.0497 \\
0.0508 \\
0.0516 \\
0.0536 \\
0.0547 \\
0.0560 \\
0.0556 \\
0.0572 \\
0.0572 \\
0.0587 \\
0.0599 \\
0.0609 \\
0.0614 \\
0.0600 \\
0.0599 \\
0.0578 \\
0.0552 \\
0.0553 \\
0.0515 \\
-0.0306\end{array}$ & $\begin{array}{c}\text { Angie } \\
\text { Degrees } \\
6.24 \\
5.50 \\
5.75 \\
6.18 \\
6.32 \\
6.28 \\
5.37 \\
2.94 \\
-3.43 \\
-8.88 \\
-10.56 \\
-12.32 \\
-12.76 \\
-12.92 \\
-12.79 \\
-12.43 \\
-11.82 \\
-11.73 \\
-11.23 \\
-10.77 \\
-9.27 \\
-8.50 \\
-6.74 \\
-6.63 \\
-4.96 \\
-4.89 \\
-3.83 \\
-2.87 \\
-3.06 \\
-4.30 \\
-5.03 \\
-5.95 \\
-6.59 \\
-7.36 \\
-6.47 \\
-7.49 \\
-7.51\end{array}$ \\
\hline
\end{tabular}

Hxgh Pressure test 


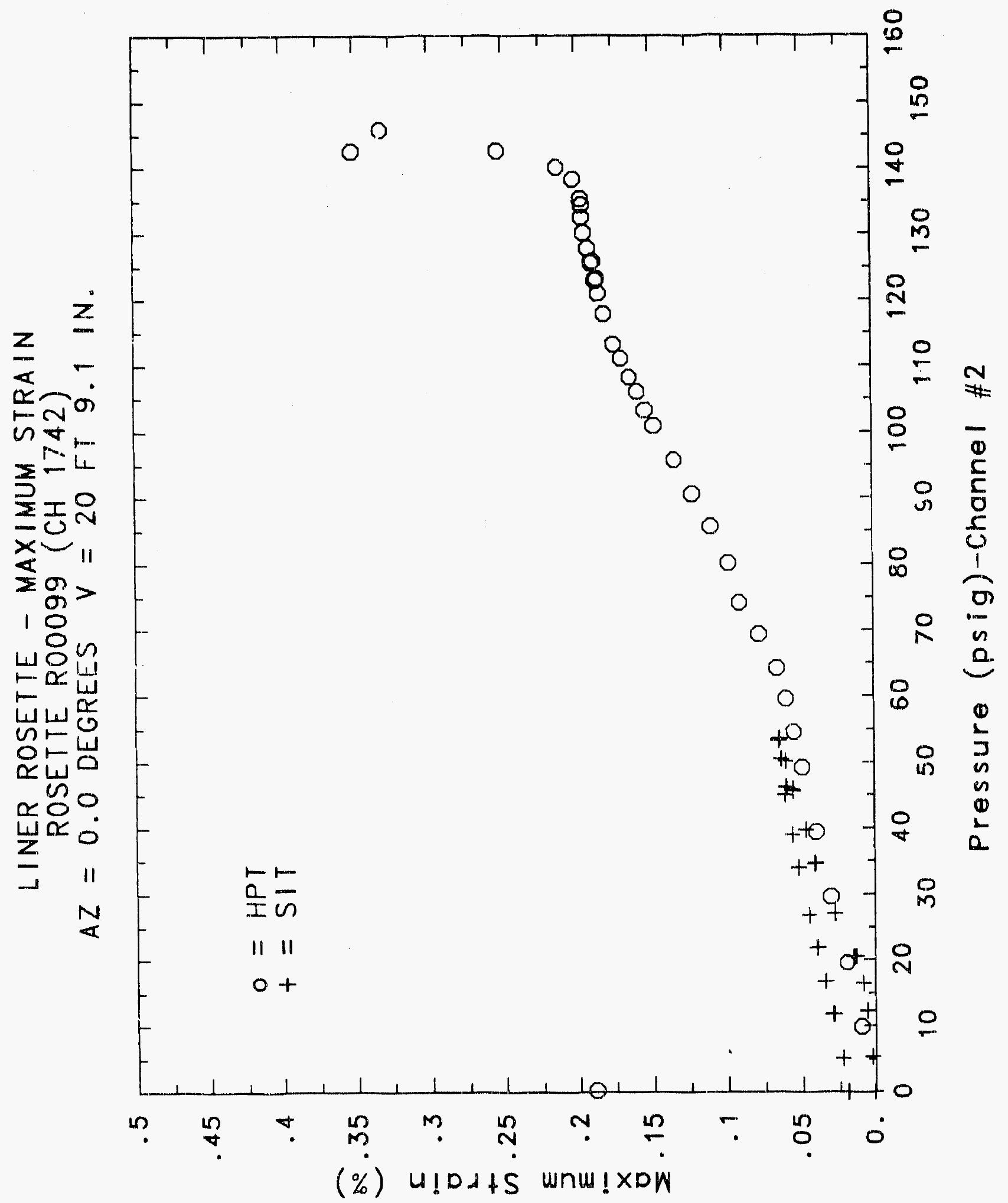




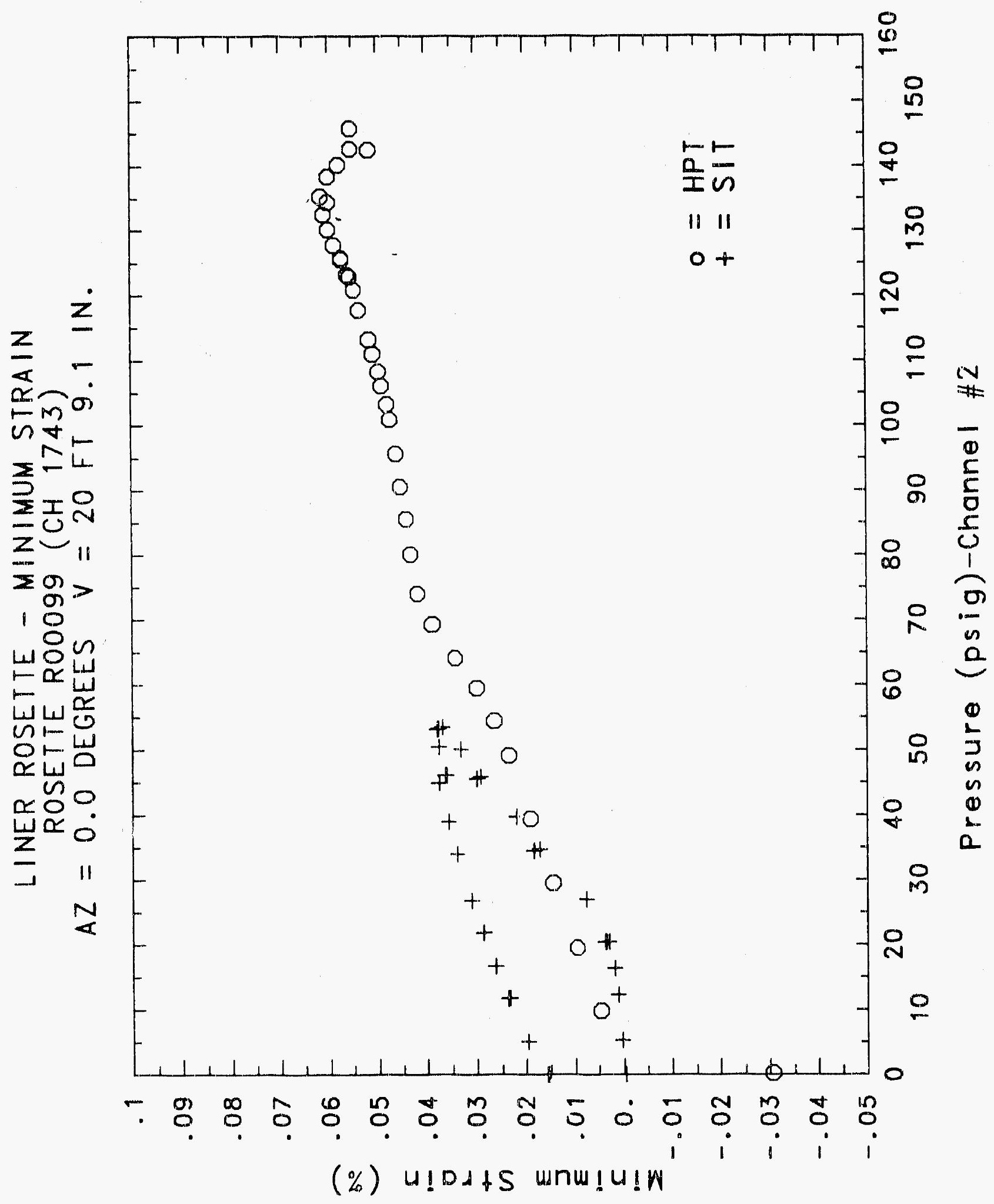

C-616 


\title{
Reinforced Concrete Test Data
}

\author{
Rosette 100 Channels $1745 \quad 1746 \quad 1747$
}

\section{STRUCTURAL INTEGRITY TEST}

\begin{tabular}{|c|c|c|c|c|c|c|c|}
\hline $\begin{array}{c}\text { Pressure } \\
\text { psig } \\
-0.05 \\
5.33 \\
12.31 \\
16.44 \\
20.51 \\
20.46 \\
20.47 \\
27.05 \\
34.69 \\
34.53 \\
34.53 \\
39.70 \\
45.90 \\
45.65 \\
45.62 \\
50.10 \\
53.47 \\
53.29 \\
53.21 \\
50.49 \\
46.14 \\
46.13 \\
44.97 \\
38.98 \\
33.99 \\
33.96 \\
26.83 \\
21.88 \\
21.90 \\
16.59 \\
11.74 \\
11.77 \\
5.05 \\
0.02 \\
-0.04 \\
-0.02 \\
-0.01 \\
0.02\end{array}$ & $\begin{array}{c}\text { Maximum } \\
\text { \%Strain } \\
0.0003 \\
0.0112 \\
0.0185 \\
0.0229 \\
0.0287 \\
0.0295 \\
0.0297 \\
0.0399 \\
0.0517 \\
0.0524 \\
0.0527 \\
0.0571 \\
0.0634 \\
0.0636 \\
0.0637 \\
0.0671 \\
0.0708 \\
0.0711 \\
0.0712 \\
0.0701 \\
0.0677 \\
0.0675 \\
0.0686 \\
0.0648 \\
0.0615 \\
0.0616 \\
0.0561 \\
0.0521 \\
0.0520 \\
0.0472 \\
0.0411 \\
0.0405 \\
0.0288 \\
0.0138 \\
0.0143 \\
0.0145 \\
0.0145 \\
0.0143\end{array}$ & $\begin{array}{r}\text { Minimum } \\
\text { xStrain } \\
-0.0002 \\
-0.0046 \\
-0.0107 \\
-0.0119 \\
-0.0143 \\
-0.0142 \\
-0.0140 \\
-0.0161 \\
-0.0145 \\
-0.0143 \\
-0.0142 \\
-0.0103 \\
-0.0038 \\
-0.0035 \\
-0.0037 \\
0.0011 \\
0.0051 \\
0.0054 \\
0.0054 \\
0.0035 \\
-0.0001 \\
-0.0002 \\
0.0012 \\
-0.0044 \\
-0.0090 \\
-0.0087 \\
-0.0146 \\
-0.0181 \\
-0.0176 \\
-0.0196 \\
-0.0191 \\
-0.0188 \\
-0.0150 \\
-0.0214 \\
-0.0181 \\
-0.0192 \\
-0.0195 \\
-0.0192\end{array}$ & $\begin{array}{c}\text { Angle } \\
\text { Degrees } \\
15.71 \\
-35.13 \\
.42 .11 \\
-44.39 \\
43.14 \\
42.75 \\
42.64 \\
41.21 \\
40.38 \\
40.02 \\
40.08 \\
39.64 \\
38.66 \\
38.48 \\
38.48 \\
38.27 \\
33.09 \\
37.95 \\
37.92 \\
37.80 \\
37.49 \\
37.48 \\
37.67 \\
37.13 \\
36.55 \\
36.50 \\
35.95 \\
35.99 \\
35.87 \\
36.02 \\
36.73 \\
36.66 \\
37.88 \\
22.19 \\
24.84 \\
24.85 \\
24.69 \\
24.72\end{array}$ & $\begin{array}{c}\text { Pressure } \\
\text { psig } \\
9.89 \\
19.55 \\
29.57 \\
39.42 \\
49.16 \\
54.50 \\
59.57 \\
64.20 \\
69.32 \\
74.16 \\
80.16 \\
85.61 \\
90.58 \\
95.69 \\
100.92 \\
103.25 \\
106.11 \\
108.31 \\
111.08 \\
113.24 \\
117.83 \\
120.92 \\
123.28 \\
122.97 \\
125.82 \\
125.60 \\
127.84 \\
130.19 \\
132.53 \\
135.33 \\
134.42 \\
138.35 \\
140.16 \\
142.63 \\
145.78 \\
142.52 \\
0.22\end{array}$ & $\begin{array}{c}\text { Maximum } \\
\text { xStrain } \\
0.0265 \\
0.0355 \\
0.0429 \\
0.0503 \\
0.0567 \\
0.0608 \\
0.0650 \\
0.0691 \\
0.0731 \\
0.0765 \\
0.0796 \\
0.0837 \\
0.0878 \\
0.0926 \\
0.0987 \\
0.1021 \\
0.1072 \\
0.1120 \\
0.1210 \\
0.1295 \\
0.1559 \\
0.1794 \\
0.2020 \\
0.2082 \\
0.2406 \\
0.2484 \\
0.2667 \\
0.3092 \\
0.3808 \\
0.5839 \\
0.6242 \\
0.9389 \\
1.0700 \\
1.2374 \\
1.3852 \\
1.4143 \\
1.2009\end{array}$ & $\begin{array}{l}\text { Minimum } \\
\text { \%Strain } \\
-0.0032 \\
0.0019 \\
0.0060 \\
0.0153 \\
0.0242 \\
0.0286 \\
0.0340 \\
0.0389 \\
0.0450 \\
0.0513 \\
0.0567 \\
0.0629 \\
0.0686 \\
0.0750 \\
0.0825 \\
0.0858 \\
0.0886 \\
0.0913 \\
0.0941 \\
0.0958 \\
0.0972 \\
0.0975 \\
0.0983 \\
0.0983 \\
0.1006 \\
0.1018 \\
0.1059 \\
0.1203 \\
0.1361 \\
0.1488 \\
0.1503 \\
0.1660 \\
0.1797 \\
0.1966 \\
0.2142 \\
0.2103 \\
0.0582\end{array}$ & $\begin{array}{r}\text { Angle } \\
\text { Degrees } \\
-37.41 \\
-44.71 \\
44.60 \\
-43.28 \\
-40.41 \\
-38.91 \\
-36.48 \\
-34.82 \\
-33.29 \\
-32.87 \\
-33.11 \\
-33.67 \\
-32.59 \\
-30.56 \\
-23.49 \\
-18.52 \\
-10.49 \\
-4.47 \\
3.95 \\
8.73 \\
14.76 \\
15.54 \\
15.41 \\
15.58 \\
14.54 \\
14.25 \\
13.43 \\
9.43 \\
2.55 \\
0.74 \\
1.47 \\
4.57 \\
4.60 \\
4.74 \\
5.30 \\
5.31 \\
6.28\end{array}$ \\
\hline
\end{tabular}

\section{High Pressure test}




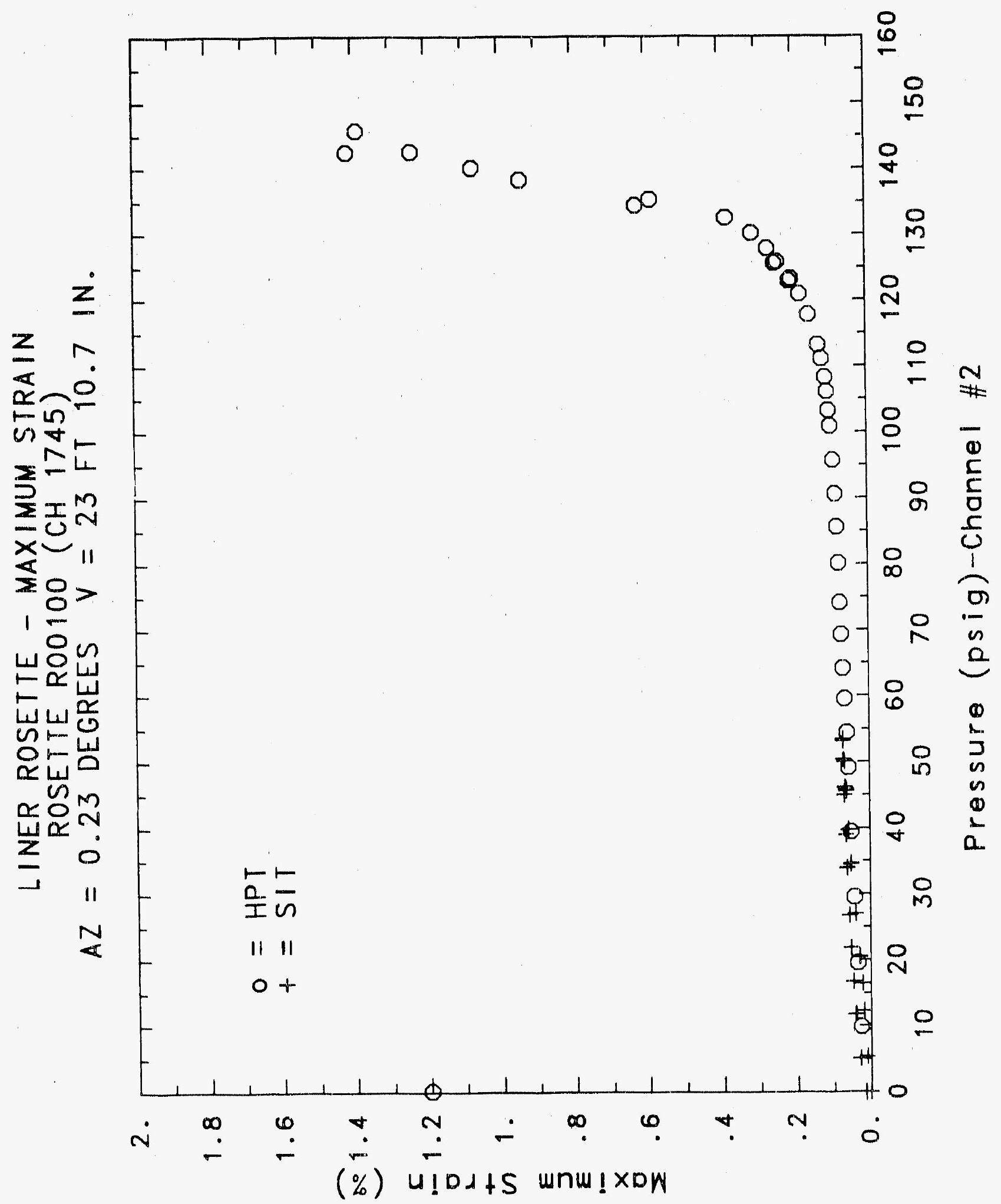




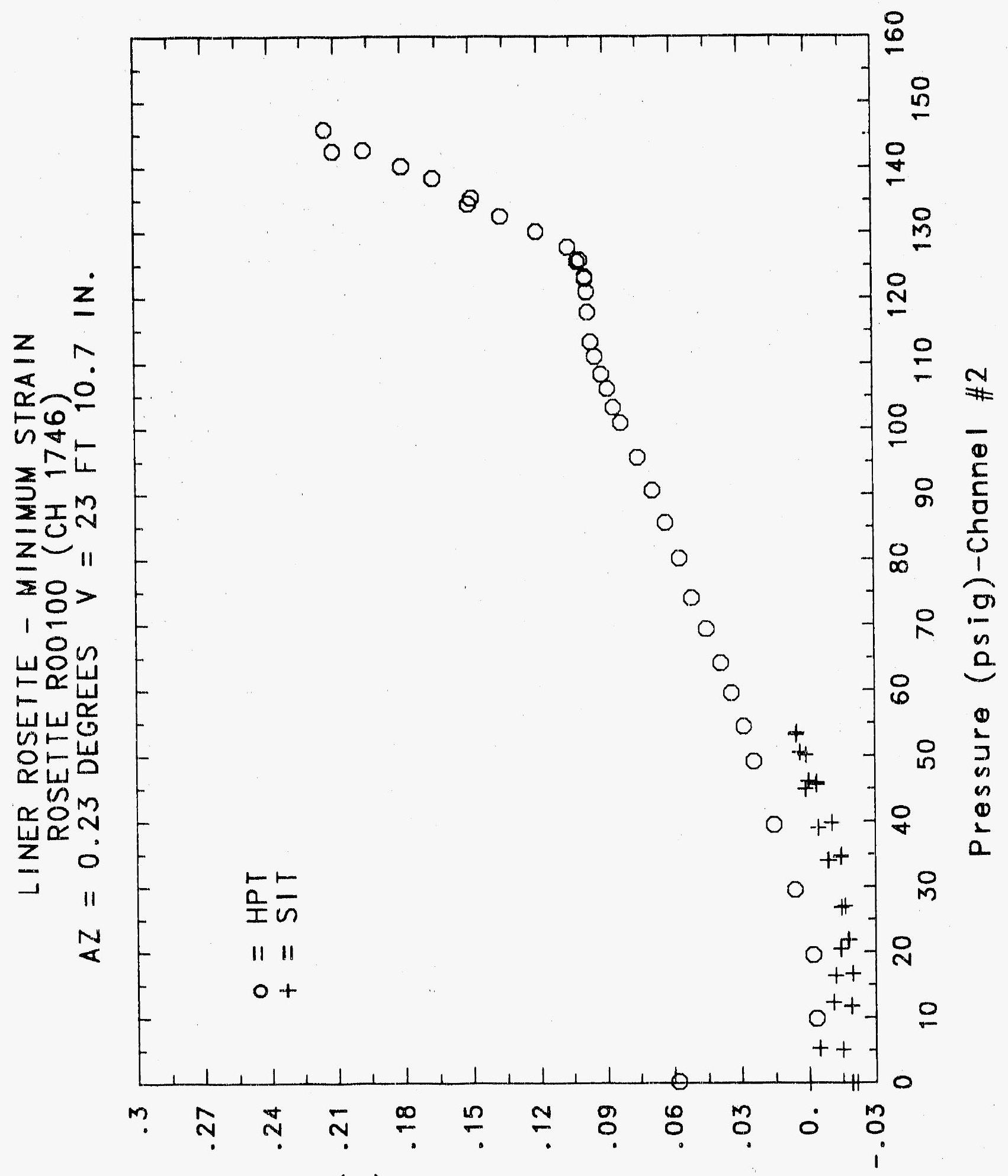

(\%) $u ! D d+s$ wnw!u!w 


\section{Reinforced Concrete Test Data}

Rosette 101 Channels $1748 \quad 1749 \quad 1760$

STRUCTURAL INTEGRITY TEST

\begin{tabular}{|c|c|c|c|c|c|c|c|}
\hline $\begin{array}{c}\text { Pressure } \\
\text { psig } \\
-0.05 \\
5.33 \\
12.31 \\
16.44 \\
20.51 \\
20.46 \\
20.47 \\
27.05 \\
34.69 \\
34.53 \\
34.53 \\
39.70 \\
45.90 \\
45.65 \\
45.62 \\
50.10 \\
53.47 \\
53.29 \\
53.21 \\
50.49 \\
46.14 \\
46.13 \\
44.97 \\
38.98 \\
33.99 \\
33.96 \\
26.83 \\
21.88 \\
21.90 \\
16.69 \\
11.74 \\
11.77 \\
5.05 \\
0.02 \\
-0.04 \\
-0.02 \\
-0.02 \\
0.02\end{array}$ & $\begin{array}{l}\text { Maximum } \\
\text { \%Strain } \\
0.0002 \\
0.0022 \\
0.0059 \\
0.0091 \\
0.0158 \\
0.0170 \\
0.0175 \\
0.0233 \\
0.0397 \\
0.0460 \\
0.0473 \\
0.0642 \\
0.0826 \\
0.0834 \\
0.0834 \\
0.0925 \\
0.1010 \\
0.1018 \\
0.1021 \\
0.1001 \\
0.0952 \\
0.0949 \\
0.0971 \\
0.0899 \\
0.0828 \\
0.0826 \\
0.0717 \\
0.0639 \\
0.0648 \\
0.0567 \\
0.0478 \\
0.0456 \\
0.0346 \\
0.0267 \\
0.0232 \\
0.0239 \\
0.0244 \\
0.0247\end{array}$ & $\begin{array}{c}\text { Minimum } \\
\text { xStrain } \\
0.0000 \\
0.0011 \\
0.0028 \\
0.0044 \\
0.0071 \\
0.0079 \\
0.0085 \\
0.0088 \\
0.0258 \\
0.0272 \\
0.0276 \\
0.0339 \\
0.0422 \\
0.0426 \\
0.0426 \\
0.0468 \\
0.0506 \\
0.0511 \\
0.0512 \\
0.0504 \\
0.0483 \\
0.0480 \\
0.0499 \\
0.0465 \\
0.0432 \\
0.0428 \\
0.0371 \\
0.0326 \\
0.0316 \\
0.0266 \\
0.0210 \\
0.0199 \\
0.0124 \\
0.0053 \\
0.0027 \\
0.0033 \\
0.0034 \\
0.0033\end{array}$ & $\begin{array}{c}\text { Angle } \\
\text { Degrees } \\
-0.68 \\
41.85 \\
-31.15 \\
-28.48 \\
-32.74 \\
-34.14 \\
-35.29 \\
41.87 \\
-9.51 \\
1.17 \\
2.12 \\
2.68 \\
3.55 \\
3.45 \\
3.37 \\
3.73 \\
4.21 \\
4.37 \\
4.37 \\
4.75 \\
5.17 \\
4.98 \\
5.61 \\
6.61 \\
7.63 \\
8.85 \\
11.68 \\
14.68 \\
17.29 \\
20.90 \\
25.11 \\
24.88 \\
31.14 \\
39.61 \\
39.27 \\
39.78 \\
40.34 \\
40.60\end{array}$ & $\begin{array}{c}\text { Pressure } \\
\text { psig } \\
9.89 \\
19.55 \\
29.57 \\
39.42 \\
49.16 \\
54.50 \\
59.57 \\
64.20 \\
69.32 \\
74.16 \\
80.16 \\
85.61 \\
90.58 \\
95.69 \\
100.92 \\
103.25 \\
106.11 \\
108.31 \\
111.08 \\
113.24 \\
117.83 \\
120.92 \\
123.28 \\
122.97 \\
125.82 \\
125.60 \\
127.84 \\
130.19 \\
132.53 \\
135.33 \\
134.42 \\
138.35 \\
140.16 \\
142.63 \\
145.78 \\
142.52 \\
0.22\end{array}$ & $\begin{array}{l}\text { Maximum } \\
\text { XStrain } \\
0.0172 \\
0.0369 \\
0.0557 \\
0.0726 \\
0.0880 \\
0.0972 \\
0.1068 \\
0.1168 \\
0.1282 \\
0.1392 \\
0.1512 \\
0.1639 \\
0.1764 \\
0.1899 \\
0.2033 \\
0.2090 \\
0.2157 \\
0.2209 \\
0.2283 \\
0.2341 \\
0.2462 \\
0.2552 \\
0.2640 \\
0.2657 \\
0.2740 \\
0.2773 \\
0.2832 \\
0.2949 \\
0.3224 \\
0.4246 \\
0.4506 \\
0.6289 \\
0.7244 \\
0.8538 \\
0.9773 \\
1.0678 \\
0.7869\end{array}$ & $\begin{array}{l}\text { Minimum } \\
\text { XStrain } \\
0.0097 \\
0.0187 \\
0.0274 \\
0.0354 \\
0.0433 \\
0.0475 \\
0.0516 \\
0.0555 \\
0.0594 \\
0.0652 \\
0.0682 \\
0.0722 \\
0.0757 \\
0.0800 \\
0.0849 \\
0.0871 \\
0.0889 \\
0.0904 \\
0.0932 \\
0.0950 \\
0.0986 \\
0.1017 \\
0.1068 \\
0.1082 \\
0.1179 \\
0.1201 \\
0.1284 \\
0.1518 \\
0.1831 \\
0.2099 \\
0.2102 \\
0.1988 \\
0.1903 \\
0.1790 \\
0.1674 \\
0.1542 \\
0.0312\end{array}$ & $\begin{array}{c}\text { Angle } \\
\text { Degrees } \\
-6.23 \\
-1.90 \\
-0.88 \\
-0.88 \\
-0.91 \\
-0.61 \\
0.80 \\
2.91 \\
4.65 \\
6.98 \\
8.37 \\
9.83 \\
10.95 \\
11.91 \\
12.79 \\
13.11 \\
13.81 \\
14.35 \\
14.98 \\
15.32 \\
15.70 \\
15.50 \\
15.10 \\
14.89 \\
14.16 \\
13.76 \\
13.25 \\
10.65 \\
5.32 \\
-7.46 \\
-8.77 \\
-12.85 \\
-13.29 \\
-13.80 \\
-14.40 \\
-15.07 \\
-16.33\end{array}$ \\
\hline
\end{tabular}

High Pressure Test 


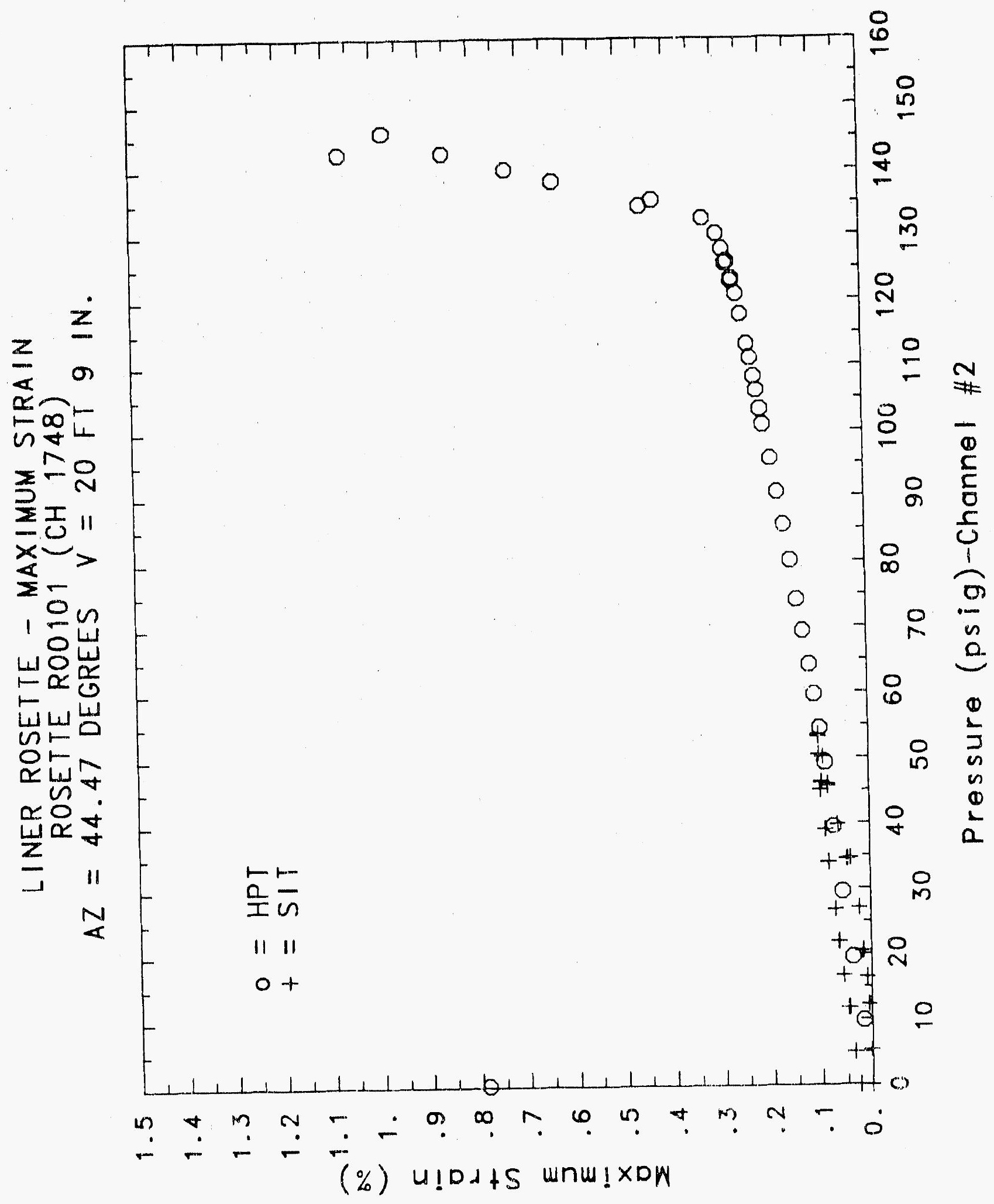




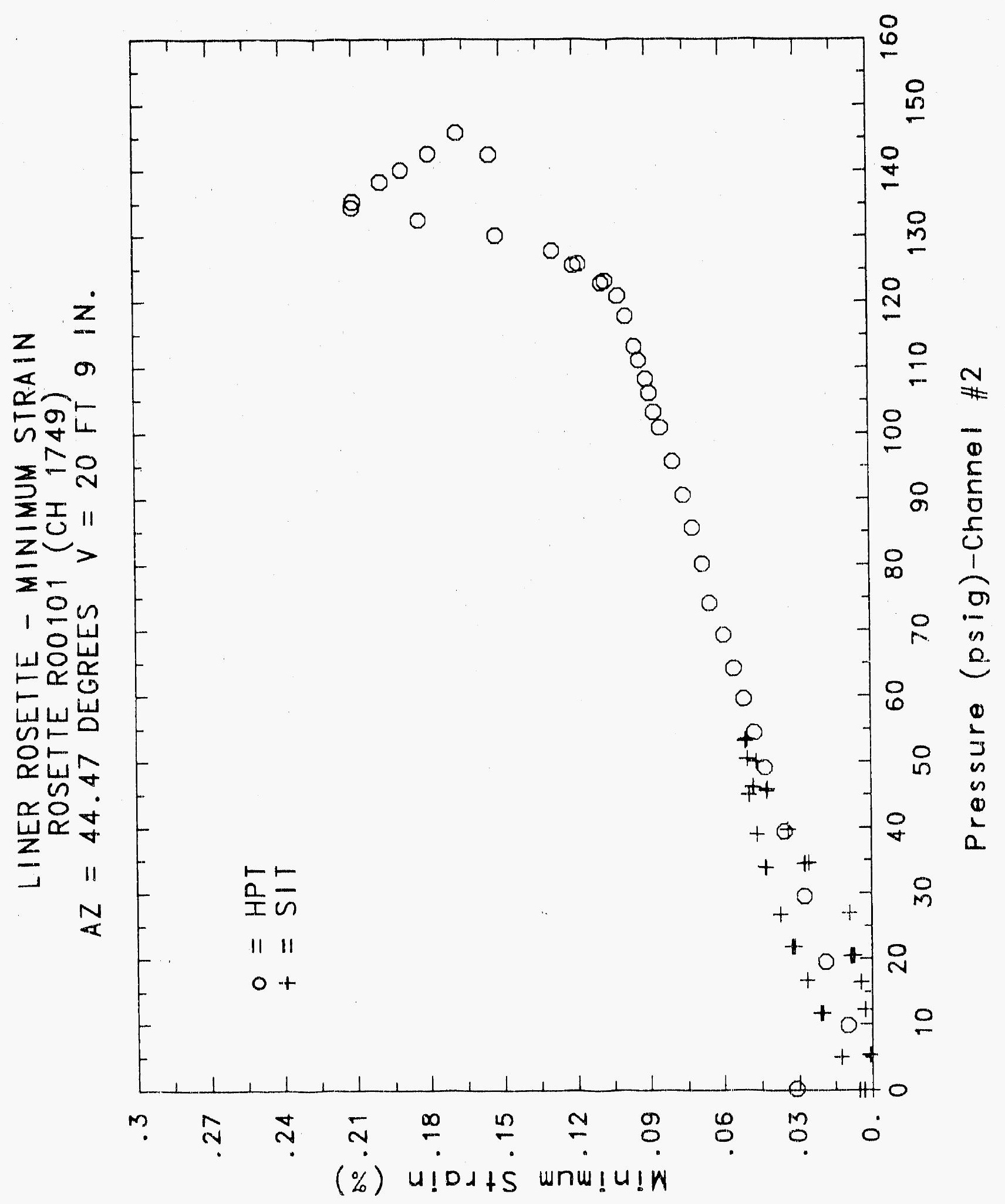


Oப்

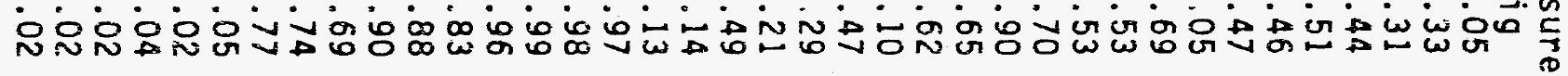
00000000000000000000000000000000000000 hén

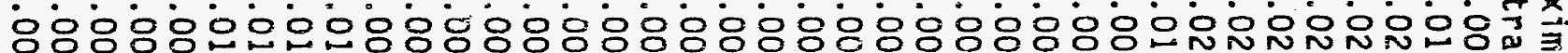

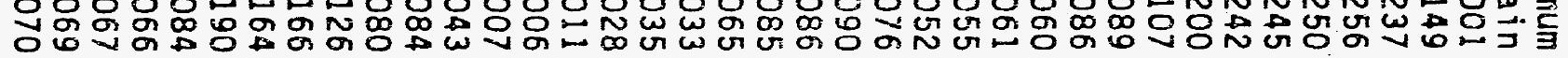

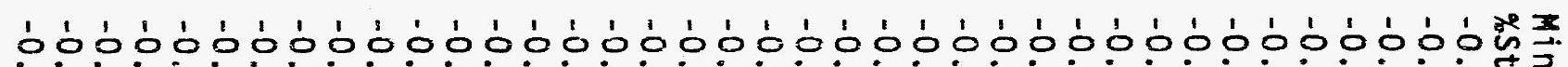

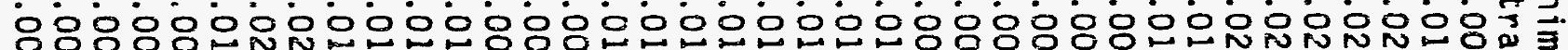

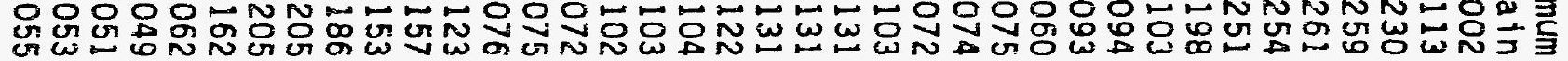

P뮤.

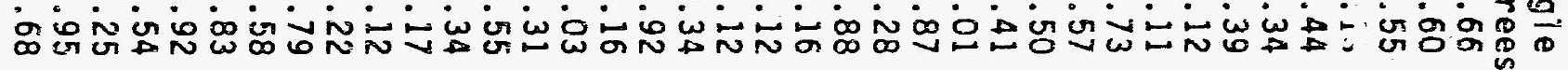

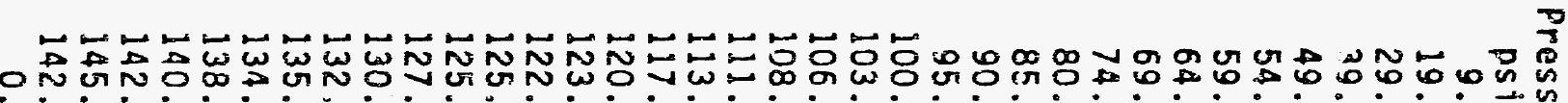
Nin 00000000000000000000000000000000000003 क o o in w

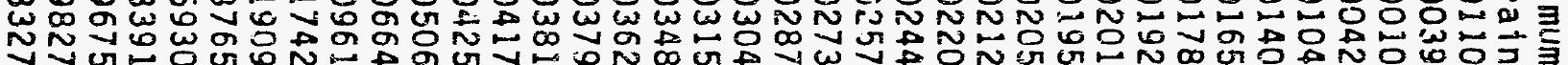

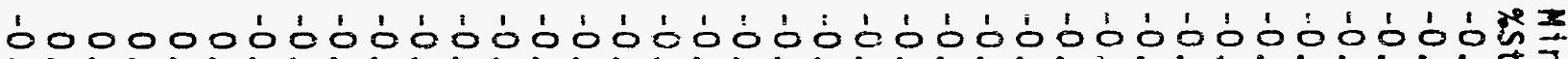

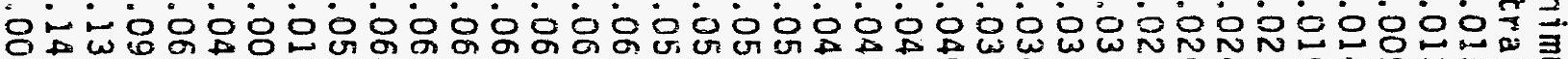

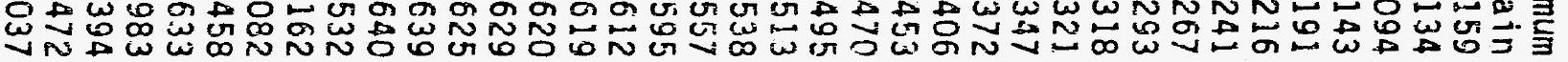

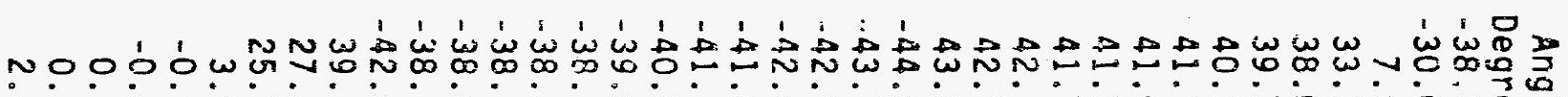

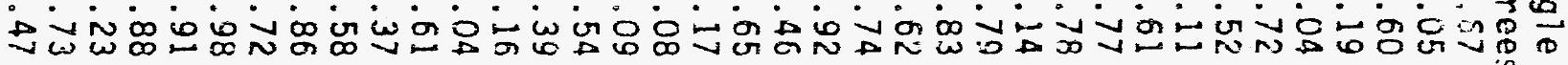




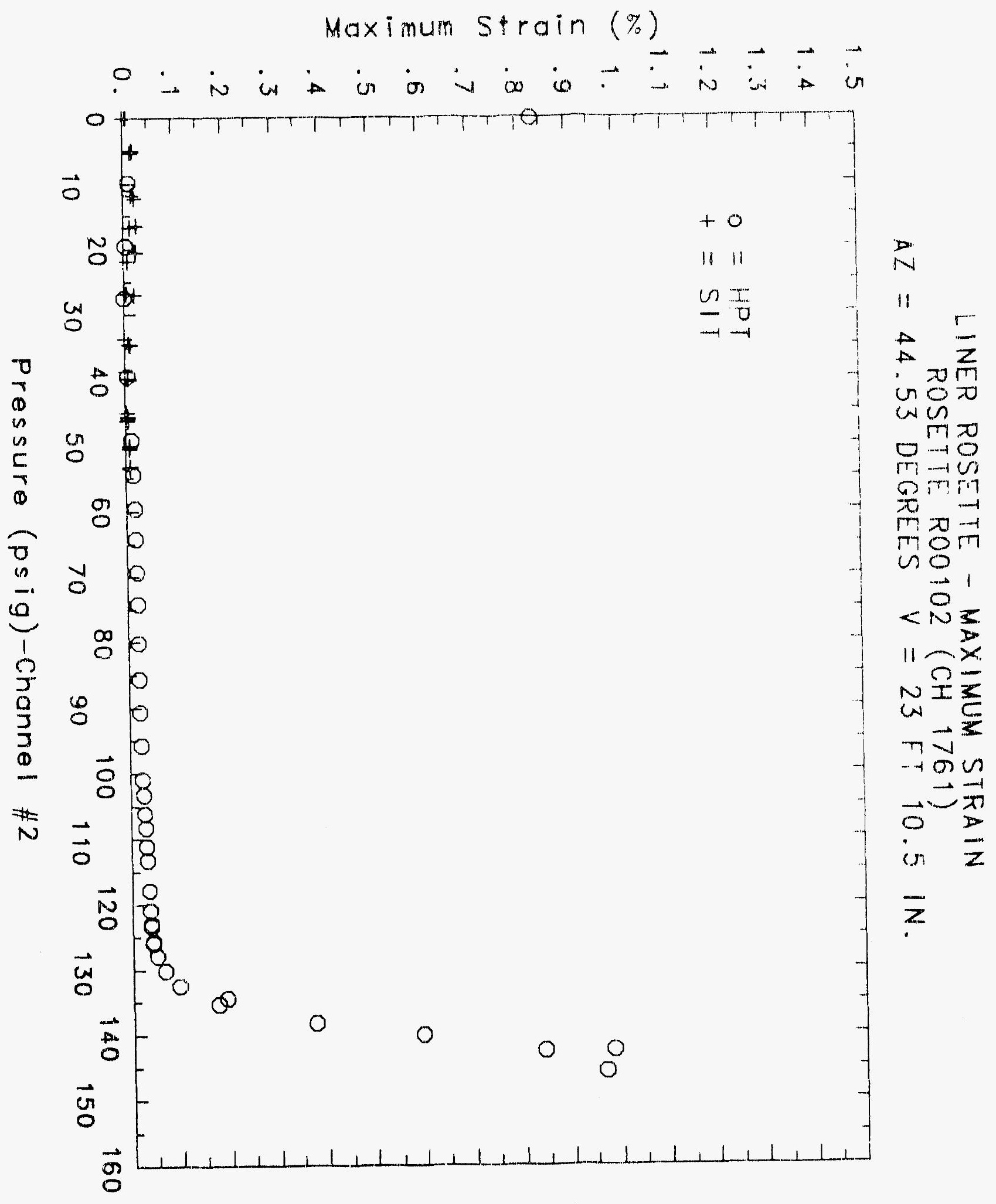




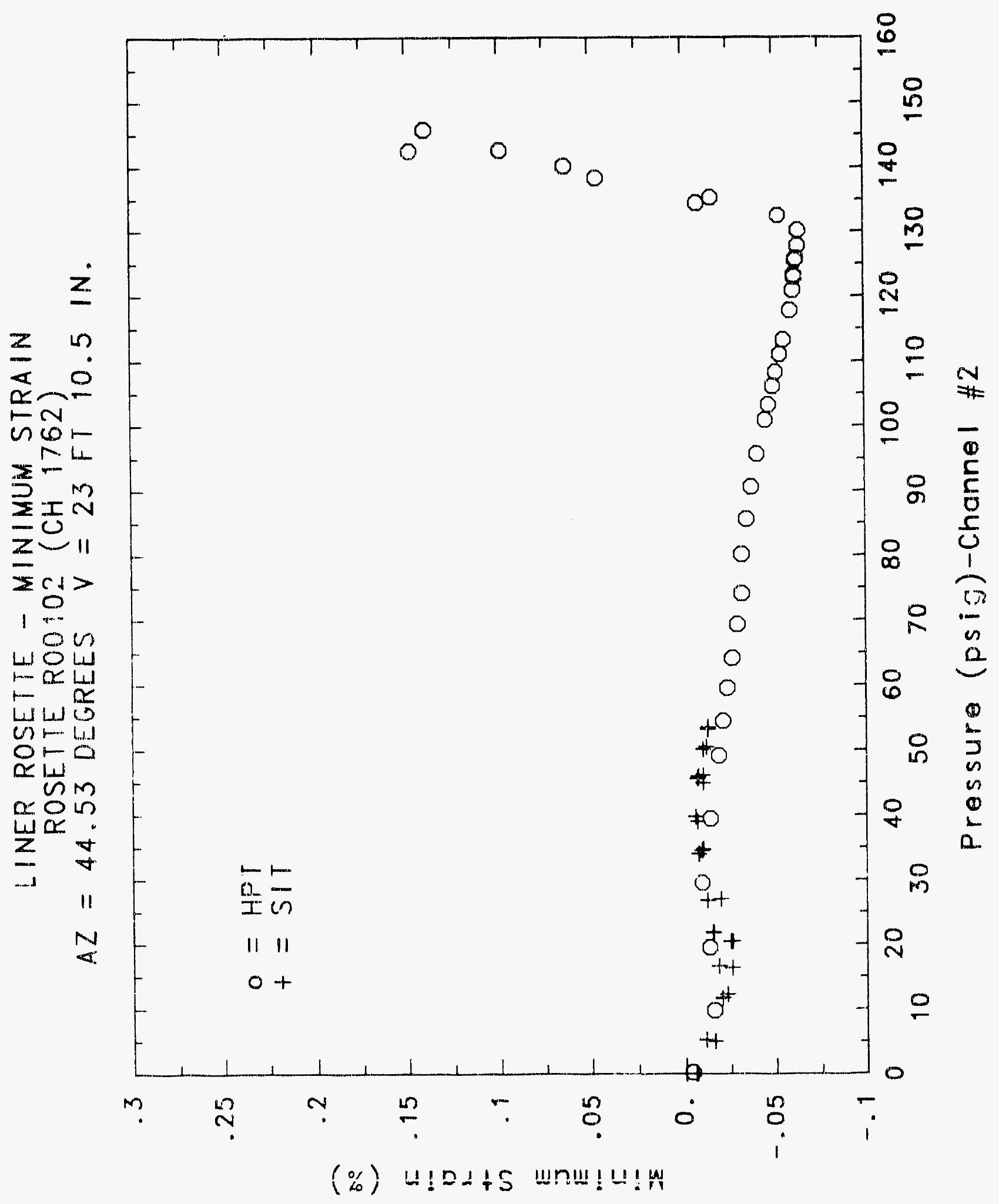


ㅇํㅇำ

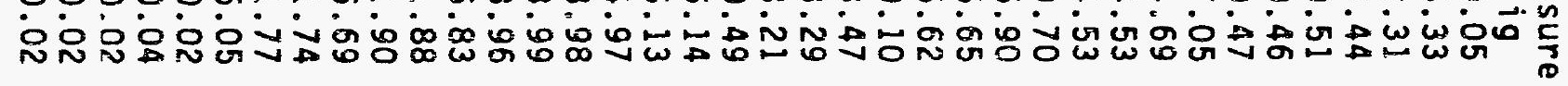
00000000000000000000000000000000000000 槅

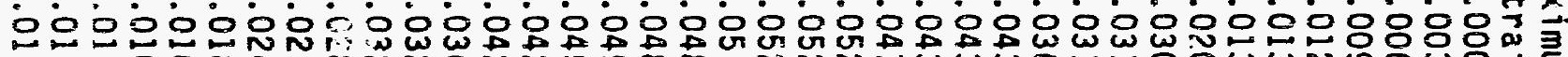

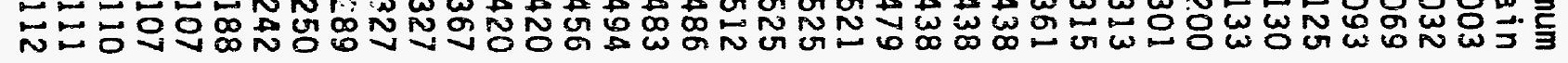

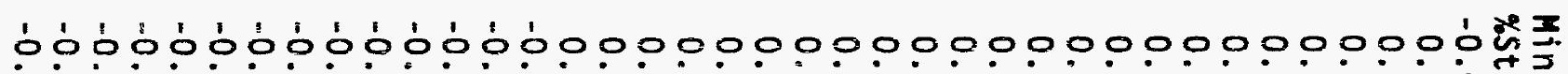

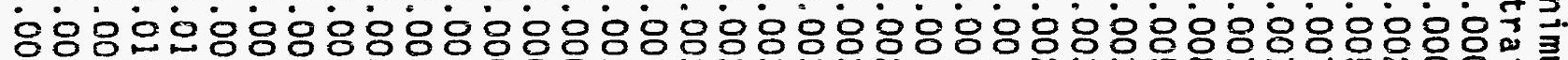

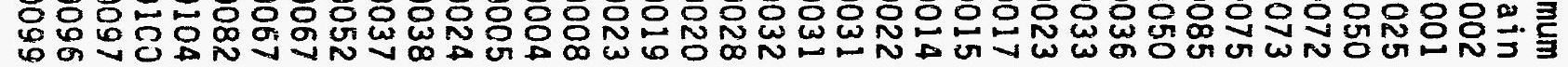

ON Oก๊

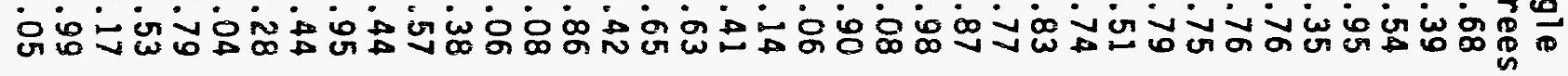

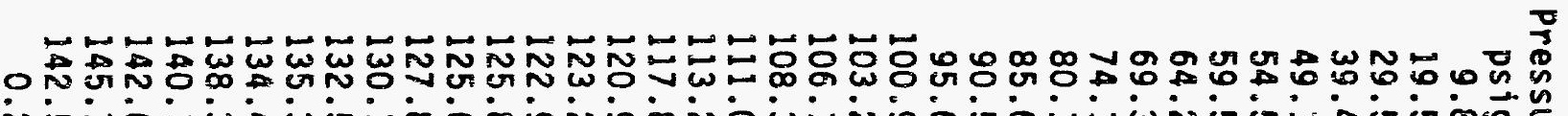

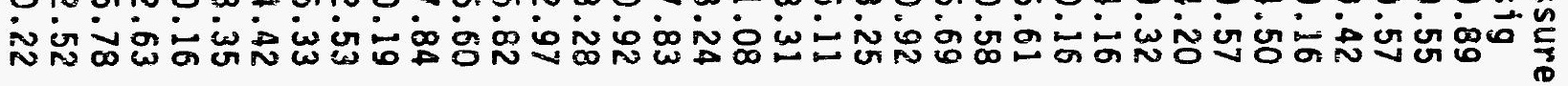
$0000000000000000000000000000000000000 \frac{12}{2}$

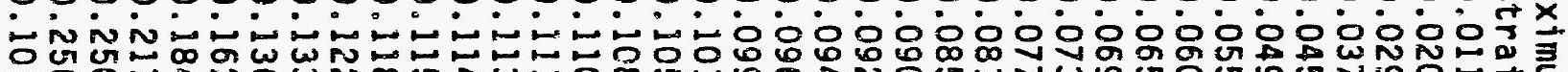

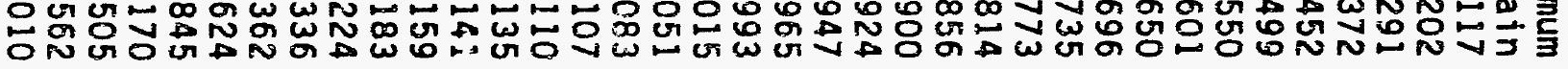

10000000000000000000000000000000000000势

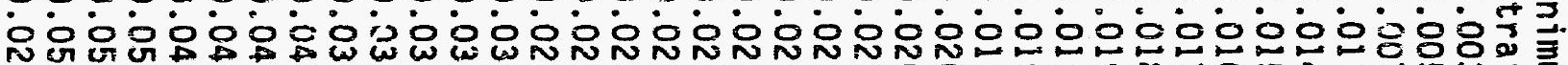

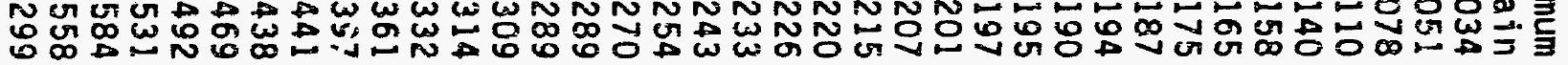

Oด

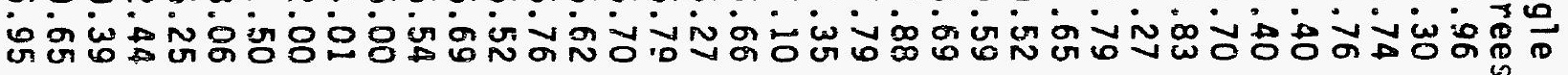




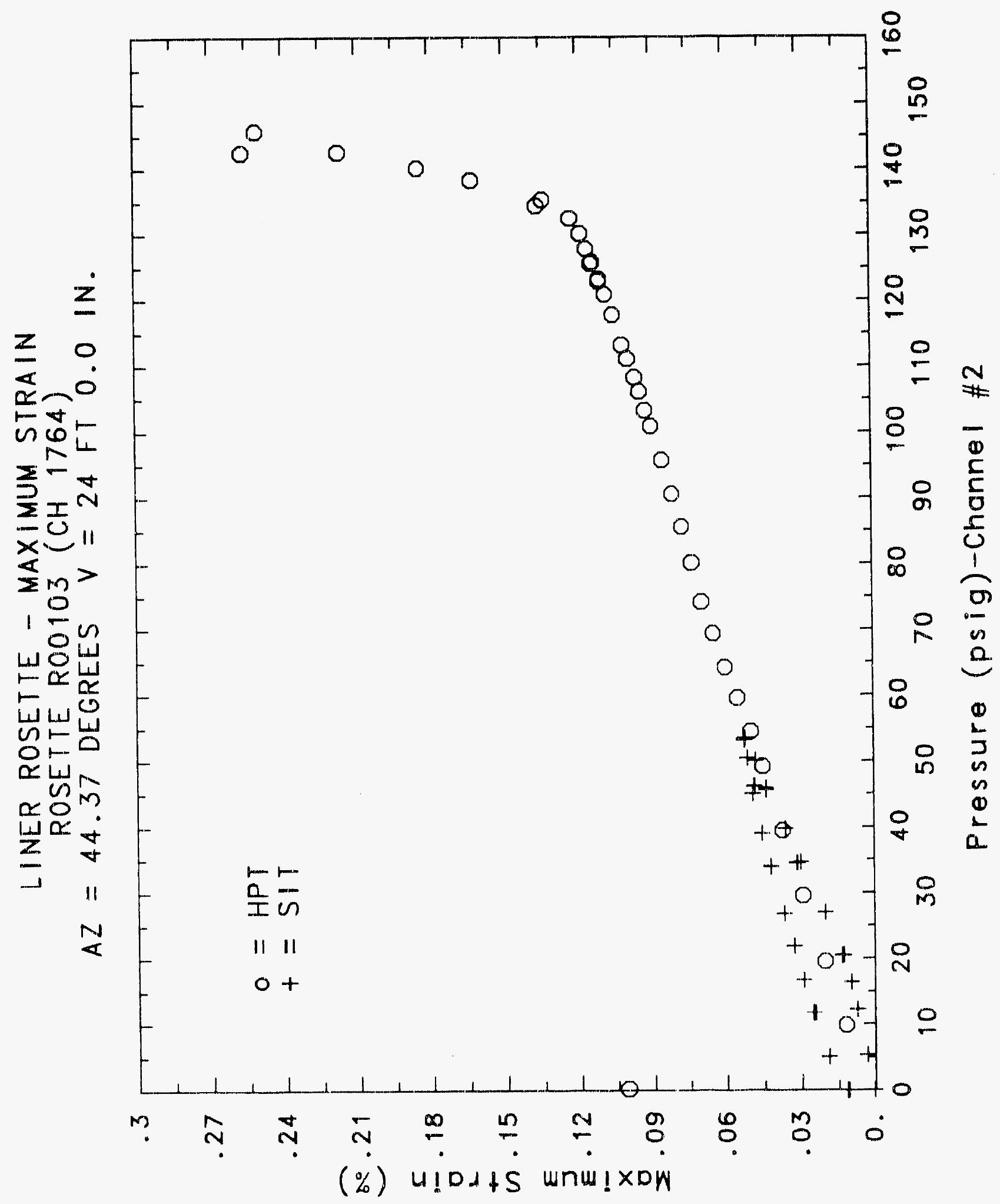




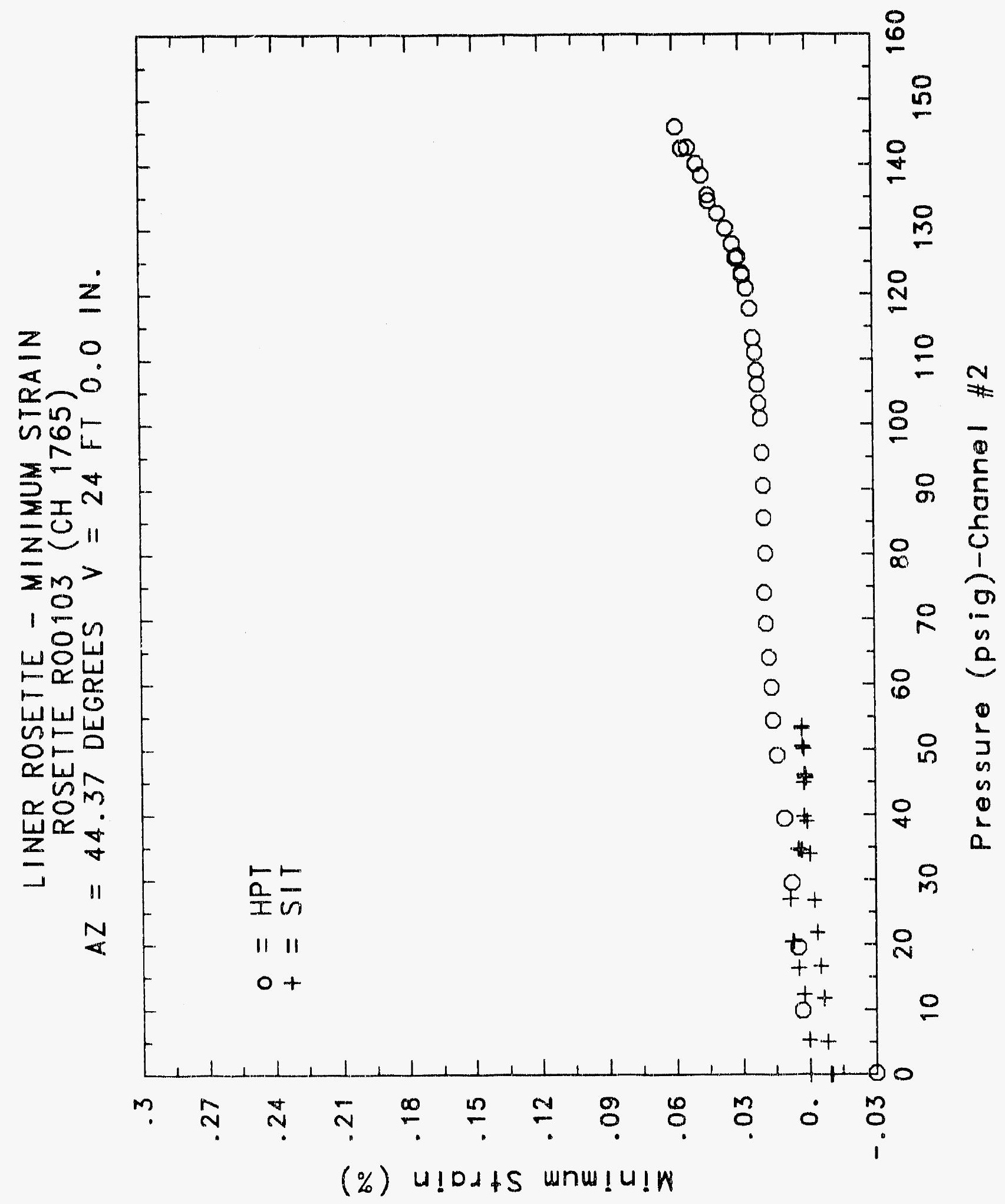




\section{Reinforced Concrete Test Data}

\section{Rosette 104 Channels $1767 \quad 1768 \quad 1769$}

StRUCTURAL INTEgRITY TEST

\begin{tabular}{|c|c|c|c|c|c|c|c|}
\hline $\begin{array}{c}\text { Pressure } \\
\text { psig } \\
-0.05 \\
5.33 \\
12.31 \\
16.44 \\
20.51 \\
20.46 \\
20.47 \\
27.05 \\
34.69 \\
34.53 \\
34.53 \\
39.70 \\
45.90 \\
45.65 \\
45.62 \\
50.10 \\
53.47 \\
53.29 \\
53.21 \\
50.49 \\
46.14 \\
46.13 \\
44.97 \\
38.98 \\
33.99 \\
33.96 \\
26.83 \\
21.88 \\
21.90 \\
16.69 \\
11.74 \\
11.77 \\
5.05 \\
0.02 \\
-0.04 \\
-0.02 \\
-0.02 \\
0.02\end{array}$ & $\begin{array}{l}\text { Maximum } \\
\text { \%strain } \\
0.0002 \\
0.0019 \\
0.0044 \\
0.0060 \\
0.0092 \\
0.0094 \\
0.0100 \\
0.0175 \\
0.0289 \\
0.0304 \\
0.0305 \\
0.0351 \\
0.0425 \\
0.0423 \\
0.0424 \\
0.0464 \\
0.0503 \\
0.0507 \\
0.0512 \\
0.0496 \\
0.0473 \\
0.0471 \\
0.0474 \\
0.0437 \\
0.0404 \\
0.0404 \\
0.0351 \\
0.0315 \\
0.0313 \\
0.0278 \\
0.0243 \\
0.0235 \\
0.0190 \\
0.0153 \\
0.0152 \\
0.0156 \\
0.0156 \\
0.0154\end{array}$ & $\begin{array}{l}\text { Minimum } \\
\text { xStrain } \\
-0.0001 \\
0.0014 \\
0.0039 \\
0.0053 \\
0.0085 \\
0.0092 \\
0.0095 \\
0.0157 \\
0.0247 \\
0.0254 \\
0.0259 \\
0.0301 \\
0.0365 \\
0.0373 \\
0.0374 \\
0.0411 \\
0.0446 \\
0.0451 \\
0.0452 \\
0.0445 \\
0.0424 \\
0.0424 \\
0.0431 \\
0.0402 \\
0.0372 \\
0.0371 \\
0.0327 \\
0.0292 \\
0.0290 \\
0.0255 \\
0.0219 \\
0.0215 \\
0.0166 \\
0.0127 \\
0.0120 \\
0.0123 \\
0.0126 \\
0.012\end{array}$ & $\begin{array}{c}\text { Angle } \\
\text { Degrees } \\
\text {-41.27 } \\
-42.65 \\
-14.06 \\
-32.43 \\
7.31 \\
7.70 \\
24.21 \\
28.93 \\
36.47 \\
39.43 \\
38.60 \\
37.32 \\
37.88 \\
38.15 \\
38.55 \\
37.65 \\
37.32 \\
37.35 \\
37.74 \\
38.68 \\
40.88 \\
40.36 \\
41.34 \\
-44.96 \\
-43.85 \\
-41.98 \\
-34.72 \\
-27.88 \\
-28.60 \\
-22.08 \\
-18.80 \\
-17.38 \\
-8.03 \\
-6.44 \\
-6.98 \\
-9.66 \\
-12.48 \\
-11.74\end{array}$ & $\begin{array}{c}\text { Pressure } \\
\text { ps1g } \\
9.89 \\
19.55 \\
29.57 \\
39.42 \\
49.16 \\
54.50 \\
59.57 \\
64.20 \\
69.32 \\
74.16 \\
80.16 \\
85.61 \\
90.58 \\
95.69 \\
100.92 \\
103.25 \\
106.11 \\
108.31 \\
111.08 \\
113.24 \\
117.83 \\
120.92 \\
123.28 \\
122.97 \\
125.82 \\
125.60 \\
127.84 \\
130.19 \\
132.53 \\
135.33 \\
134.42 \\
138.35 \\
140.16 \\
142.63 \\
145.78 \\
142.52 \\
0.22\end{array}$ & $\begin{array}{c}\text { Maximum } \\
\text { xStrain } \\
0.0080 \\
0.0159 \\
0.0238 \\
0.0310 \\
0.0381 \\
0.0423 \\
0.0466 \\
0.0510 \\
0.0564 \\
0.0615 \\
0.0668 \\
0.0720 \\
0.0779 \\
0.0843 \\
0.0912 \\
0.0944 \\
0.0979 \\
0.1004 \\
0.1043 \\
0.1073 \\
0.1141 \\
0.1194 \\
0.1243 \\
0.1251 \\
0.1203 \\
0.1316 \\
0.1352 \\
0.1409 \\
0.1477 \\
0.1587 \\
0.1607 \\
0.1764 \\
0.1894 \\
0.2085 \\
0.2289 \\
0.2281 \\
0.0922\end{array}$ & $\begin{array}{l}\text { Minimum } \\
\text { XStrain } \\
0.0065 \\
0.0131 \\
0.0199 \\
0.0264 \\
0.0325 \\
0.0362 \\
0.0402 \\
0.0444 \\
0.0490 \\
0.0539 \\
0.0585 \\
0.0639 \\
0.0689 \\
0.0743 \\
0.0795 \\
0.0821 \\
0.0849 \\
0.0872 \\
0.0901 \\
0.0924 \\
0.0980 \\
0.1022 \\
0.1058 \\
0.1064 \\
0.1100 \\
0.1109 \\
0.1133 \\
0.1166 \\
0.1200 \\
0.1248 \\
0.1252 \\
0.1318 \\
0.1368 \\
0.1447 \\
0.1544 \\
0.1522 \\
0.0262\end{array}$ & $\begin{array}{c}\text { Angle } \\
\text { Degrees } \\
27.15 \\
25.20 \\
26.18 \\
24.71 \\
24.18 \\
23.96 \\
22.58 \\
18.66 \\
18.76 \\
13.12 \\
9.18 \\
-8.58 \\
-14.51 \\
-16.60 \\
-17.40 \\
-17.03 \\
-17.14 \\
-15.89 \\
-14.41 \\
-13.85 \\
-12.70 \\
-11.41 \\
-10.41 \\
-9.59 \\
-8.55 \\
-8.10 \\
-7.64 \\
-6.58 \\
-5.68 \\
-4.32 \\
-4.18 \\
-4.06 \\
-4.24 \\
-4.48 \\
-4.44 \\
-4.01 \\
-1.52\end{array}$ \\
\hline
\end{tabular}

High Pressure test 


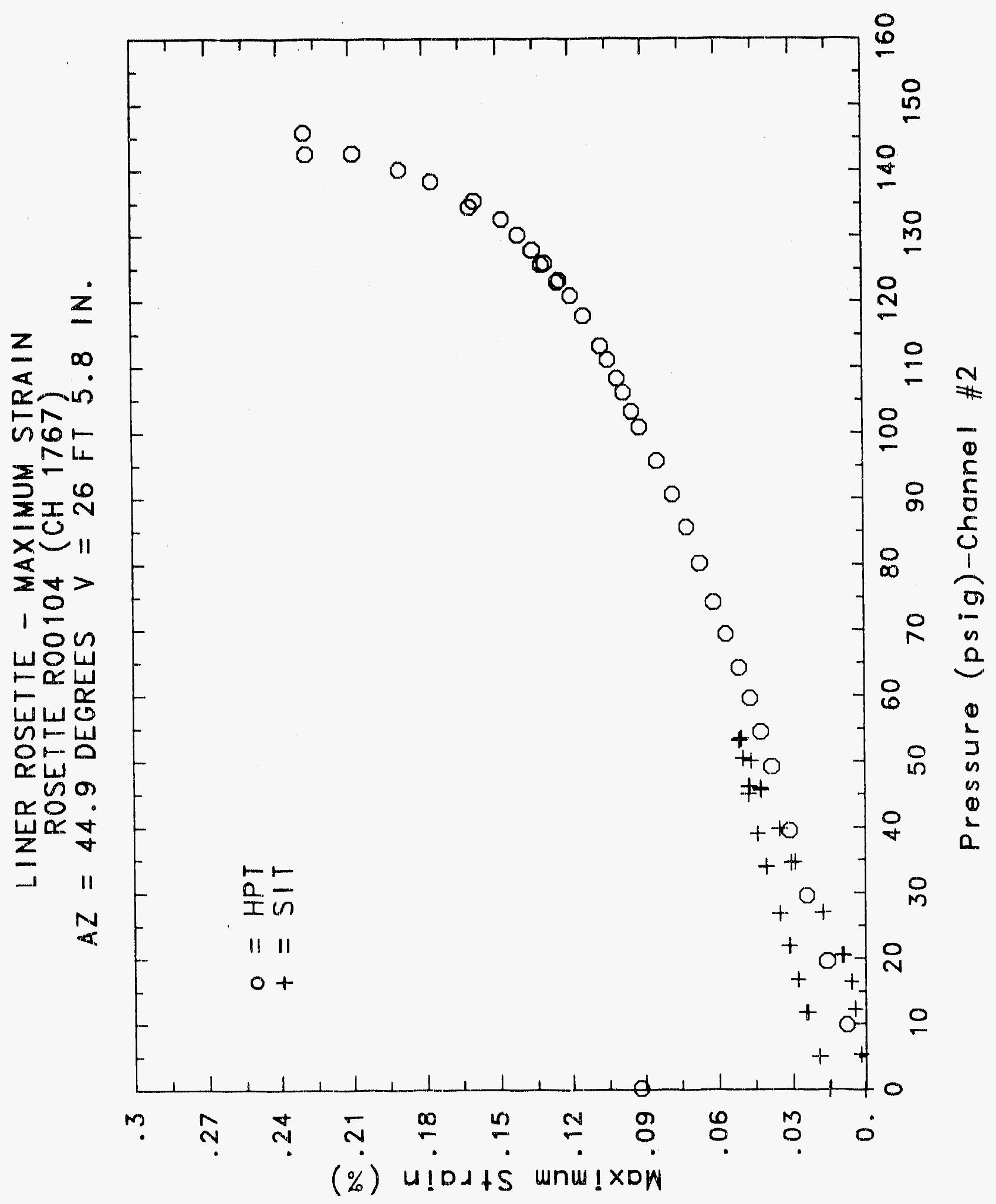




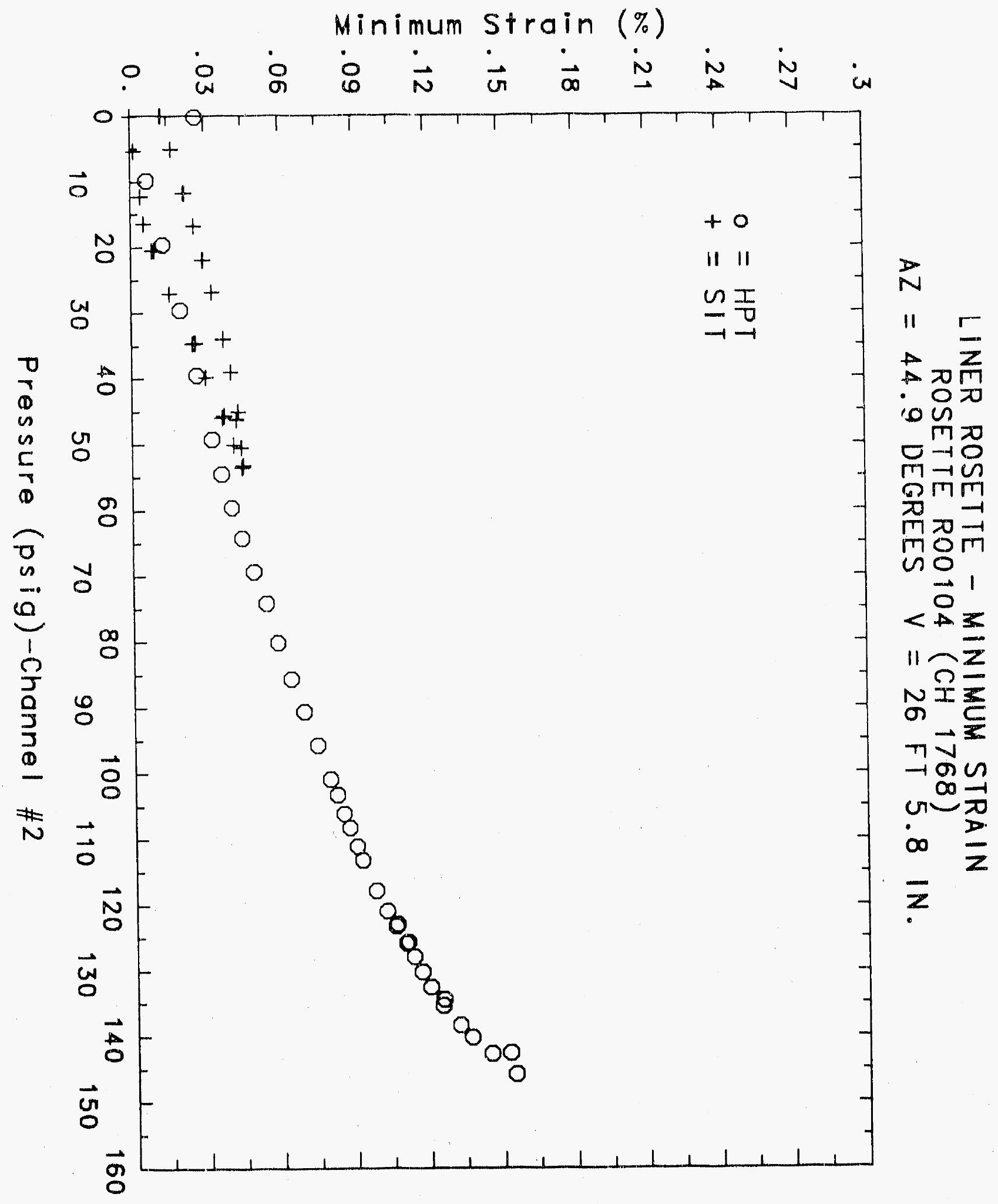




\section{Reinforced Concrete Test Data}

Rosette 105 Channels $1780 \quad 1781 \quad 1782$

Structural InTEgrity TEST

\begin{tabular}{|c|c|c|c|c|c|c|c|}
\hline $\begin{array}{c}\text { Pressure } \\
\text { psig } \\
-0.05 \\
5.33 \\
12.31 \\
16.44 \\
20.51 \\
20.46 \\
20.47 \\
27.05 \\
34.69 \\
34.53 \\
34.53 \\
39.70 \\
45.90 \\
45.65 \\
45.62 \\
50.10 \\
53.47 \\
53.29 \\
53.21 \\
50.49 \\
46.14 \\
46.13 \\
44.97 \\
38.98 \\
33.99 \\
33.96 \\
26.83 \\
21.88 \\
21.90 \\
16.69 \\
11.74 \\
11.77 \\
5.05 \\
0.02 \\
-0.04 \\
-0.02 \\
-0.02 \\
0.02\end{array}$ & $\begin{array}{l}\text { Maximum } \\
\text { \%Strain } \\
0.0004 \\
0.0037 \\
0.0084 \\
0.0119 \\
0.0176 \\
0.0185 \\
0.0189 \\
0.0302 \\
0.0449 \\
0.0469 \\
0.0472 \\
0.0541 \\
0.0607 \\
0.0595 \\
0.0590 \\
0.0716 \\
0.0882 \\
0.0883 \\
0.0882 \\
0.0858 \\
0.0815 \\
0.0812 \\
0.0808 \\
0.0748 \\
0.0693 \\
0.0694 \\
0.0611 \\
0.0554 \\
0.0563 \\
0.0506 \\
0.0446 \\
0.0440 \\
0.0363 \\
0.0308 \\
0.0312 \\
0.0319 \\
0.0318 \\
0.0318\end{array}$ & $\begin{array}{l}\text { Minimum } \\
\text { \%Strain } \\
0.0000 \\
0.0008 \\
0.0022 \\
0.0033 \\
0.0058 \\
0.0070 \\
0.0075 \\
0.0150 \\
0.0279 \\
0.0300 \\
0.0308 \\
0.0361 \\
0.0435 \\
0.0433 \\
0.0432 \\
0.0472 \\
0.0494 \\
0.0495 \\
0.0495 \\
0.0486 \\
0.0466 \\
0.0463 \\
0.0471 \\
0.0442 \\
0.0413 \\
0.0413 \\
0.0369 \\
0.0339 \\
0.0342 \\
0.0312 \\
0.0276 \\
0.0267 \\
0.0218 \\
0.0181 \\
0.0164 \\
0.0167 \\
0.0169 \\
0.0170\end{array}$ & $\begin{array}{c}\text { Angle } \\
\text { Degrees } \\
\text {-31.72 } \\
1.97 \\
3.43 \\
4.82 \\
6.97 \\
7.28 \\
9.16 \\
8.14 \\
4.96 \\
7.81 \\
7.88 \\
6.16 \\
11.25 \\
12.65 \\
12.53 \\
-0.65 \\
-7.34 \\
-6.57 \\
-6.40 \\
-6.41 \\
-6.82 \\
-6.51 \\
-5.41 \\
-6.58 \\
-7.43 \\
-7.13 \\
-8.03 \\
-8.64 \\
-7.40 \\
-8.39 \\
-10.23 \\
-10.49 \\
-14.07 \\
-17.33 \\
-16.72 \\
-17.21 \\
-17.23 \\
-16.84\end{array}$ & $\begin{array}{c}\text { Pressure } \\
\text { psig } \\
9.89 \\
19.55 \\
29.57 \\
39.42 \\
49.16 \\
54.50 \\
59.57 \\
64.20 \\
69.32 \\
74.16 \\
80.16 \\
85.61 \\
90.58 \\
95.69 \\
100.92 \\
103.25 \\
106.11 \\
108.31 \\
111.08 \\
113.24 \\
117.83 \\
120.92 \\
123.28 \\
122.97 \\
125.82 \\
125.60 \\
127.84 \\
130.19 \\
132.53 \\
135.33 \\
134.42 \\
138.35 \\
140.16 \\
142.63 \\
145.78 \\
142.52 \\
0.22\end{array}$ & $\begin{array}{l}\text { Maximum } \\
\text { \%Strain } \\
0.0124 \\
0.0249 \\
0.0373 \\
0.0488 \\
0.0595 \\
0.0656 \\
0.0661 \\
0.0720 \\
0.0755 \\
0.0813 \\
0.0910 \\
0.1102 \\
0.1210 \\
0.1299 \\
0.1414 \\
0.1445 \\
0.1482 \\
0.1511 \\
0.1550 \\
0.1611 \\
0.1752 \\
0.1887 \\
0.2009 \\
0.2023 \\
0.2121 \\
0.2138 \\
0.2182 \\
0.2244 \\
0.2320 \\
0.2476 \\
0.2500 \\
0.2804 \\
0.3152 \\
0.4083 \\
0.5061 \\
0.5234 \\
0.3155\end{array}$ & $\begin{array}{l}\text { Minimum } \\
\text { XStrain } \\
0.0072 \\
0.0142 \\
0.0211 \\
0.0275 \\
0.0337 \\
0.0376 \\
0.0397 \\
0.0392 \\
0.0385 \\
0.0384 \\
0.0481 \\
0.0581 \\
0.0647 \\
0.0711 \\
0.0799 \\
0.0837 \\
0.0878 \\
0.0910 \\
0.0951 \\
0.0990 \\
0.1072 \\
0.1123 \\
0.1161 \\
0.1164 \\
0.1205 \\
0.1213 \\
0.1239 \\
0.1283 \\
0.1323 \\
0.1326 \\
0.1316 \\
0.1355 \\
0.1404 \\
0.1471 \\
0.1532 \\
0.1523 \\
0.0660\end{array}$ & $\begin{array}{r}\text { Angle } \\
\text { Degrees } \\
1.31 \\
1.67 \\
1.43 \\
1.65 \\
2.03 \\
2.10 \\
8.57 \\
21.47 \\
30.27 \\
34.13 \\
24.71 \\
13.71 \\
11.21 \\
11.68 \\
9.36 \\
10.38 \\
10.59 \\
10.09 \\
10.65 \\
8.80 \\
2.95 \\
-0.74 \\
-2.79 \\
-2.91 \\
-4.85 \\
-5.04 \\
-5.65 \\
-8.18 \\
-11.01 \\
-13.59 \\
-13.90 \\
-14.00 \\
-13.74 \\
-11.94 \\
-11.42 \\
-10.73 \\
-15.48\end{array}$ \\
\hline
\end{tabular}

High Pressure test 


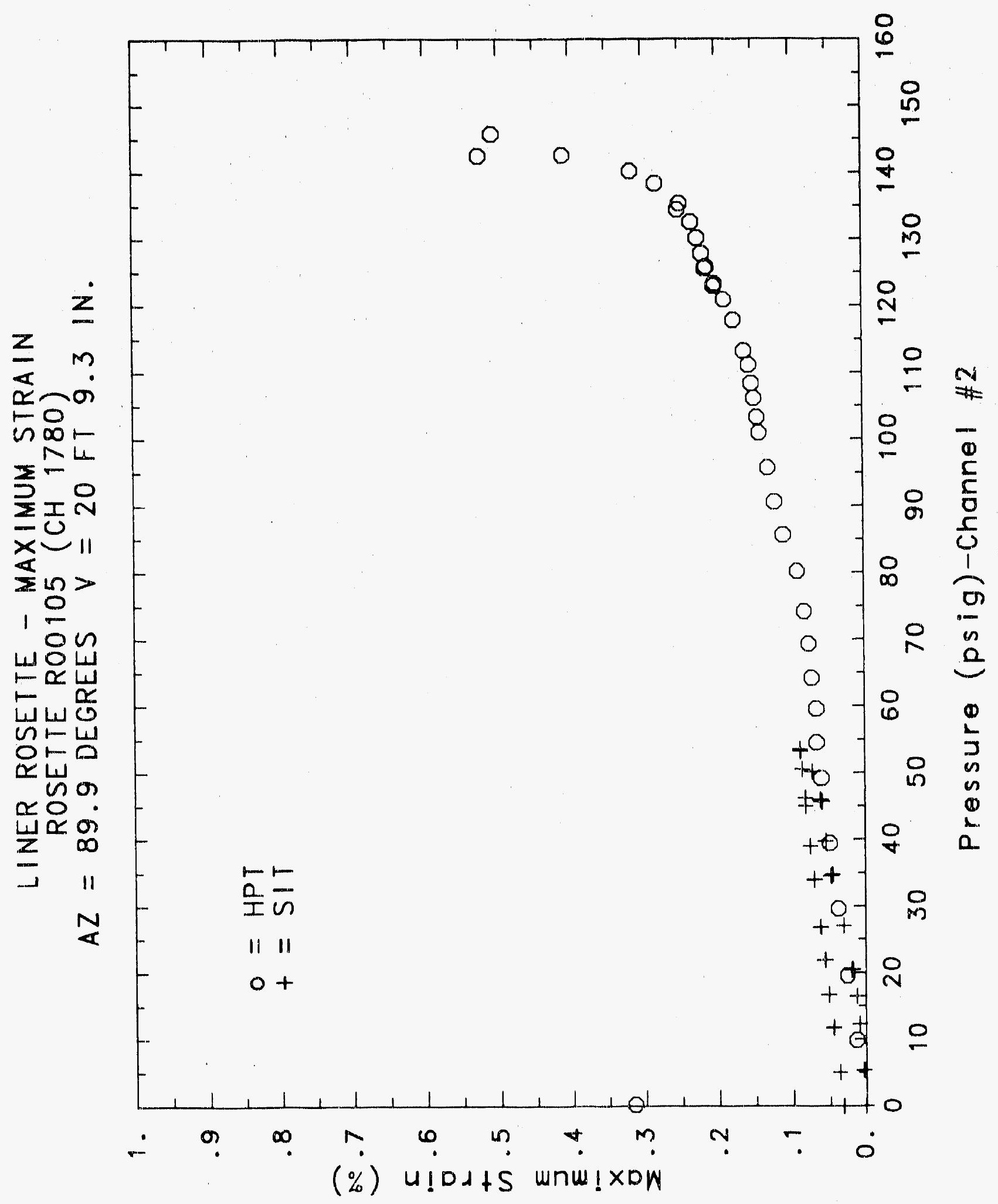




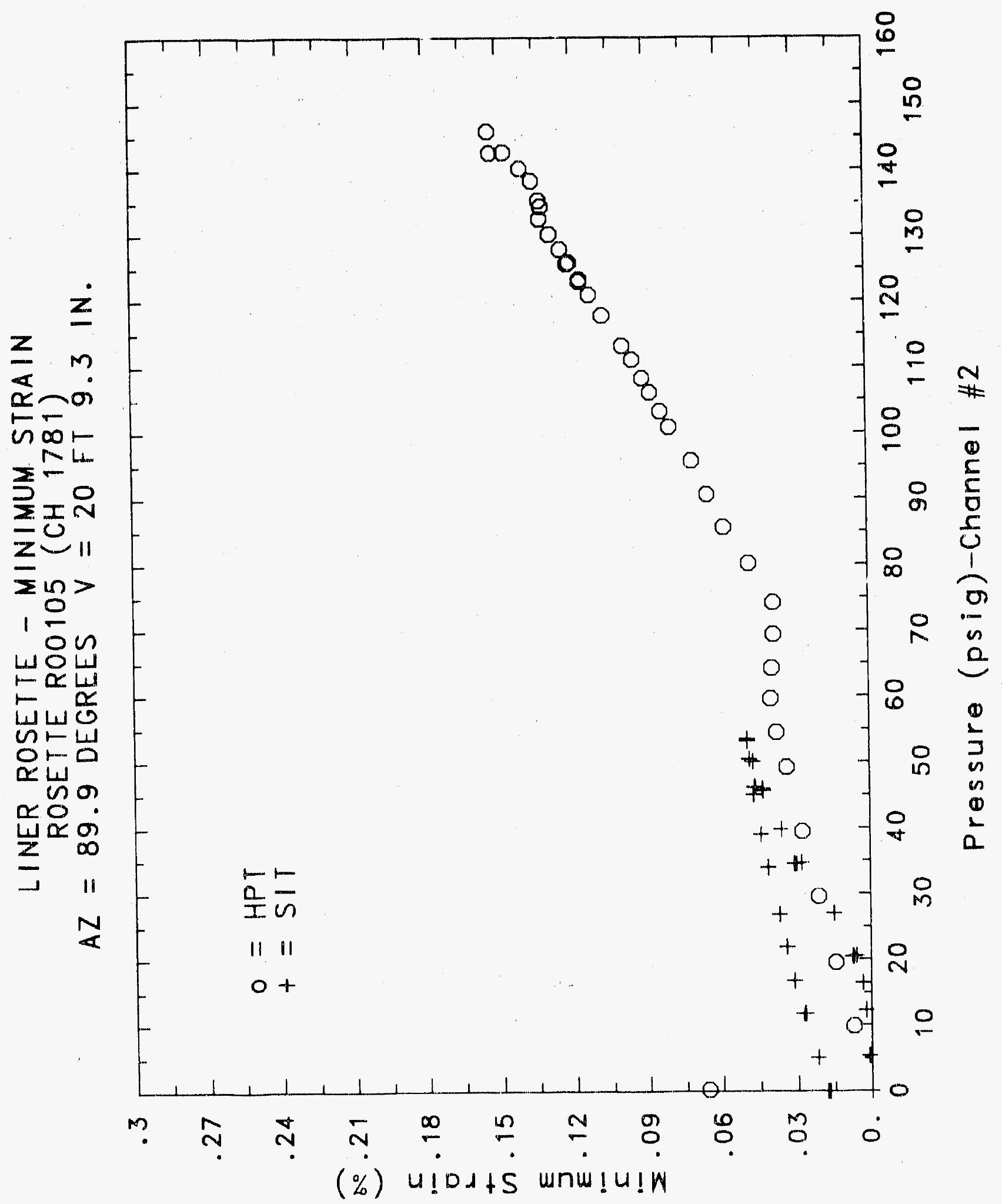




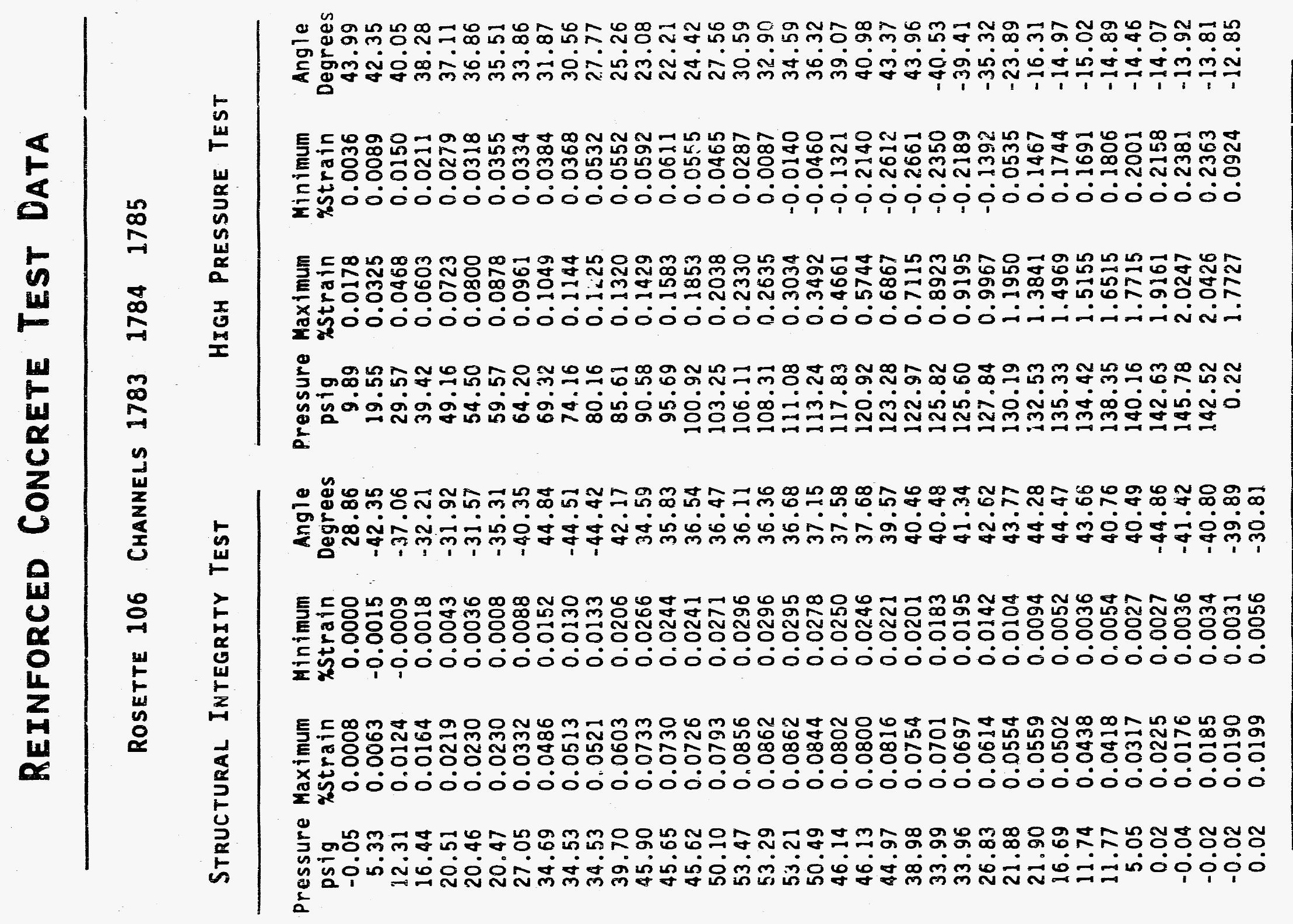




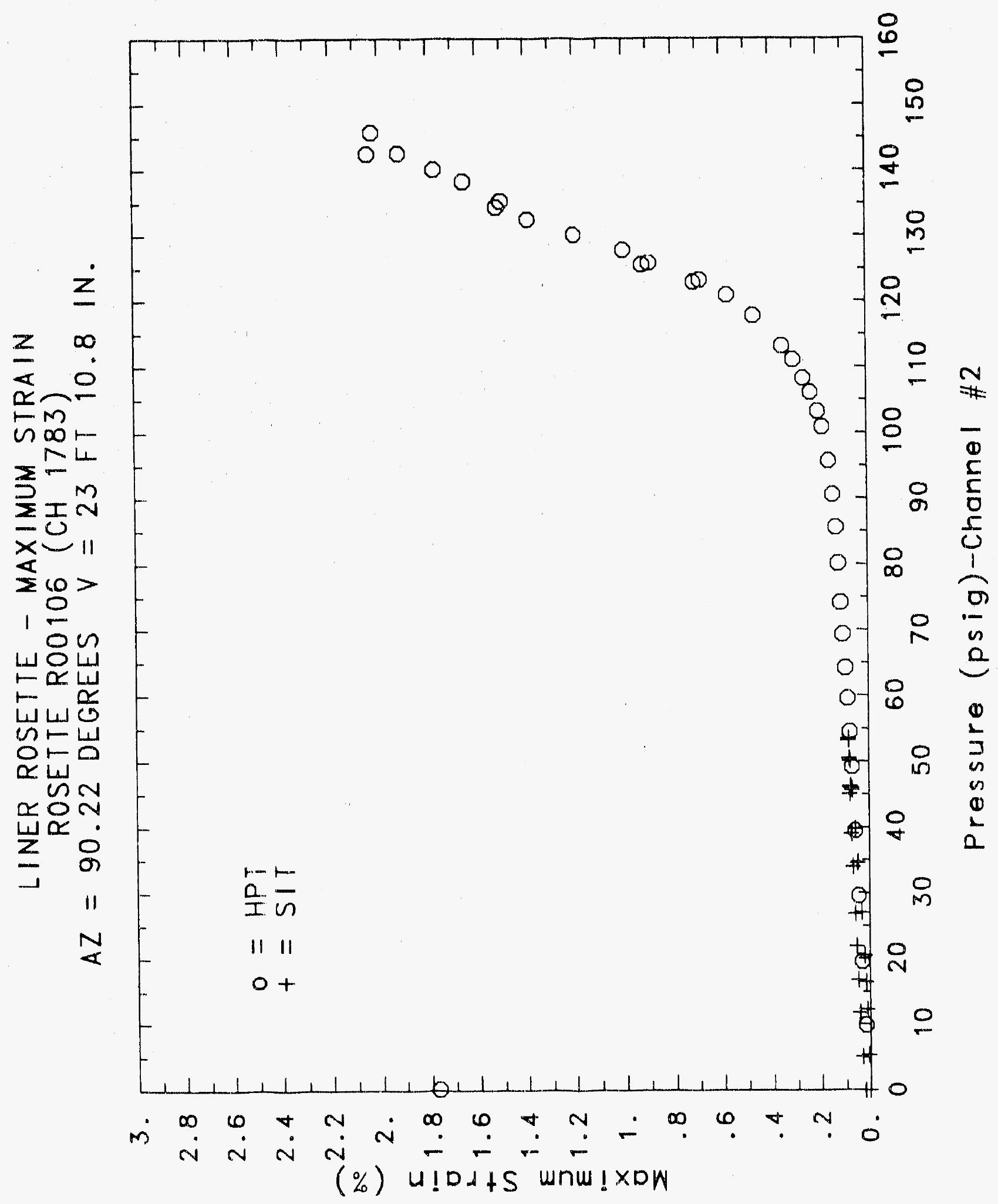




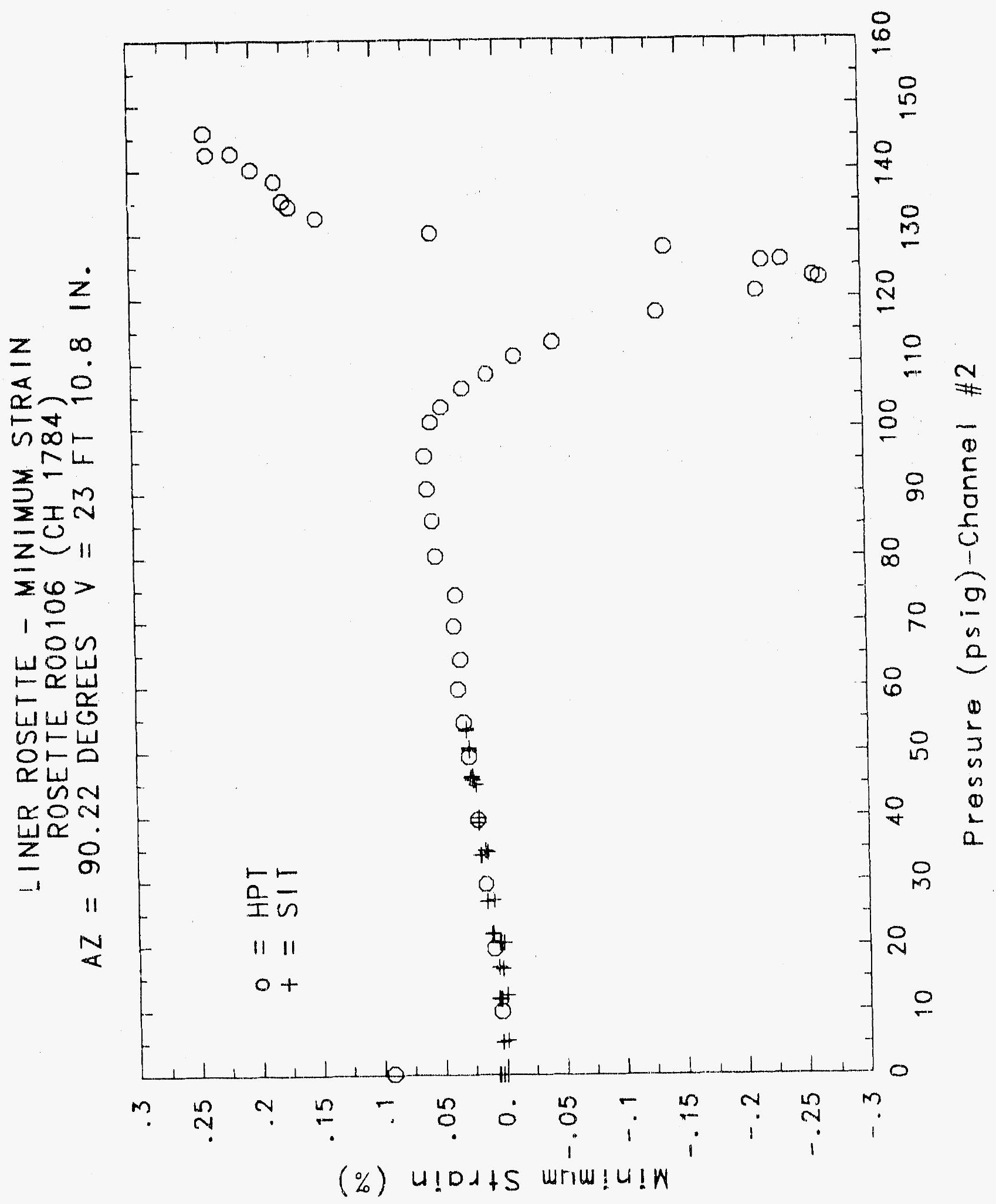




\title{
Reinforced Concrete Test Data
}

\author{
Rosette 107 Channels $1786 \quad 1787 \quad 1788$
}

StRUCTURAL INTEgRITY TEST

\begin{tabular}{|c|c|c|c|c|c|c|c|}
\hline $\begin{array}{c}\text { Pressure } \\
\text { Psig } \\
.0 .05 \\
5.33 \\
12.31 \\
16.44 \\
20.51 \\
20.46 \\
20.47 \\
27.05 \\
34.69 \\
34.53 \\
34.53 \\
39.75 \\
45.90 \\
45.65 \\
45.62 \\
50.10 \\
53.47 \\
53.29 \\
53.21 \\
50.49 \\
46.14 \\
46.13 \\
44.97 \\
38.98 \\
33.99 \\
33.96 \\
26.83 \\
21.83 \\
21.90 \\
16.69 \\
11.74 \\
11.77 \\
5.05 \\
0.06 \\
-0.04 \\
-0.02 \\
-0.02 \\
0.02\end{array}$ & $\begin{array}{l}\text { Maximum } \\
\% \text { Strain } \\
0.0102 \\
0.0057 \\
0.0109 \\
0.0137 \\
0.0168 \\
0.0169 \\
0.0169 \\
0.0225 \\
0.0295 \\
0.0304 \\
0.0310 \\
0.0349 \\
0.0399 \\
0.0395 \\
0.0393 \\
0.0426 \\
0.0451 \\
0.0452 \\
0.0452 \\
0.0439 \\
0.0413 \\
0.0413 \\
0.0427 \\
0.0390 \\
0.0356 \\
0.0358 \\
0.0304 \\
0.0267 \\
0.0273 \\
0.0237 \\
0.0193 \\
0.0184 \\
0.0139 \\
0.01005 \\
0.0111 \\
0.0113 \\
0.0114 \\
0.0112\end{array}$ & $\begin{array}{l}\text { Minimum } \\
\text { \% Strain } \\
\text {-0.0003 } \\
0.0009 \\
0.0033 \\
0.0051 \\
0.0079 \\
0.0084 \\
0.0083 \\
0.0140 \\
0.0218 \\
0.0230 \\
0.0231 \\
0.0271 \\
0.0330 \\
0.0321 \\
0.0320 \\
0.0346 \\
0.0388 \\
0.0388 \\
0.0387 \\
0.0374 \\
0.0353 \\
0.0354 \\
0.0349 \\
0.0316 \\
0.0287 \\
0.0288 \\
0.0251 \\
0.0222 \\
0.0218 \\
0.0188 \\
0.0163 \\
0.0175 \\
0.0115 \\
0.0052 \\
0.0051 \\
0.0056 \\
0.0057 \\
0.0057\end{array}$ & $\begin{array}{c}\text { Angle } \\
\text { Degrees } \\
-26.26 \\
-3.25 \\
0.80 \\
1.19 \\
0.77 \\
-0.07 \\
-0.91 \\
0.60 \\
0.24 \\
0.71 \\
1.27 \\
2.11 \\
-5.20 \\
-7.58 \\
-6.63 \\
-4.93 \\
4.02 \\
3.66 \\
2.06 \\
0.07 \\
-2.81 \\
-0.60 \\
-11.48 \\
-14.00 \\
-18.11 \\
-16.14 \\
-20.52 \\
-22.68 \\
-23.93 \\
-23.25 \\
-21.78 \\
3.60 \\
-32.04 \\
-16.12 \\
-16.76 \\
-17.80 \\
-18.42 \\
-19.78\end{array}$ & $\begin{array}{c}\text { Pressure } \\
\text { psig } \\
9.89 \\
19.55 \\
29.57 \\
39.42 \\
49.16 \\
54.50 \\
59.57 \\
64.20 \\
69.32 \\
74.16 \\
80.16 \\
85.61 \\
90.58 \\
95.69 \\
100.92 \\
103.25 \\
106.11 \\
108.31 \\
111.08 \\
113.24 \\
117.83 \\
120.92 \\
123.28 \\
122.97 \\
125.82 \\
125.60 \\
1127.84 \\
130.19 \\
132.53 \\
135.33 \\
134.42 \\
138.35 \\
140.16 \\
142.63 \\
145.78 \\
142.52 \\
0.22\end{array}$ & $\begin{array}{l}\text { Maximum } \\
\text { xStrain } \\
0.0112 \\
0.0192 \\
0.0276 \\
0.0353 \\
0.0425 \\
0.0467 \\
0.0491 \\
0.0506 \\
0.0527 \\
0.0549 \\
0.0580 \\
0.0624 \\
0.0662 \\
0.0693 \\
0.0682 \\
0.0690 \\
0.0708 \\
0.0713 \\
0.0728 \\
0.0741 \\
0.0764 \\
0.0769 \\
0.0775 \\
0.0771 \\
0.0780 \\
0.0779 \\
0.0791 \\
0.0794 \\
0.0805 \\
0.0823 \\
0.0816 \\
0.0863 \\
0.0888 \\
0.03920 \\
0.0368 \\
0.0913 \\
-0.0222\end{array}$ & $\begin{array}{l}\text { Minimum } \\
\text { xStrain } \\
0.0067 \\
0.0122 \\
0.0166 \\
0.0209 \\
0.0258 \\
0.0284 \\
0.0304 \\
0.0338 \\
0.0330 \\
0.0409 \\
0.0457 \\
0.0483 \\
0.0435 \\
0.0511 \\
0.0523 \\
0.0531 \\
0.0529 \\
0.0528 \\
0.0525 \\
0.0519 \\
0.0511 \\
0.0511 \\
0.0511 \\
0.0509 \\
0.0514 \\
0.0511 \\
0.0519 \\
0.0525 \\
0.0527 \\
0.0528 \\
0.0521 \\
0.0521 \\
0.0517 \\
0.0513 \\
0.0532 \\
0.0490 \\
-0.0868\end{array}$ & $\begin{array}{c}\text { Angle } \\
\text { Degrees } \\
-3.16 \\
-6.92 \\
-8.91 \\
-9.76 \\
-9.62 \\
-10.17 \\
-8.26 \\
-2.92 \\
3.18 \\
7.94 \\
27.57 \\
43.73 \\
-36.16 \\
-27.99 \\
-17.72 \\
-13.54 \\
-9.28 \\
-5.12 \\
-1.93 \\
-0.85 \\
1.84 \\
5.33 \\
6.70 \\
7.12 \\
5.53 \\
5.70 \\
4.63 \\
2.42 \\
0.32 \\
1.74 \\
3.37 \\
6.82 \\
8.37 \\
3.86 \\
8.83 \\
9.13 \\
5.99\end{array}$ \\
\hline
\end{tabular}

High Pressure test 


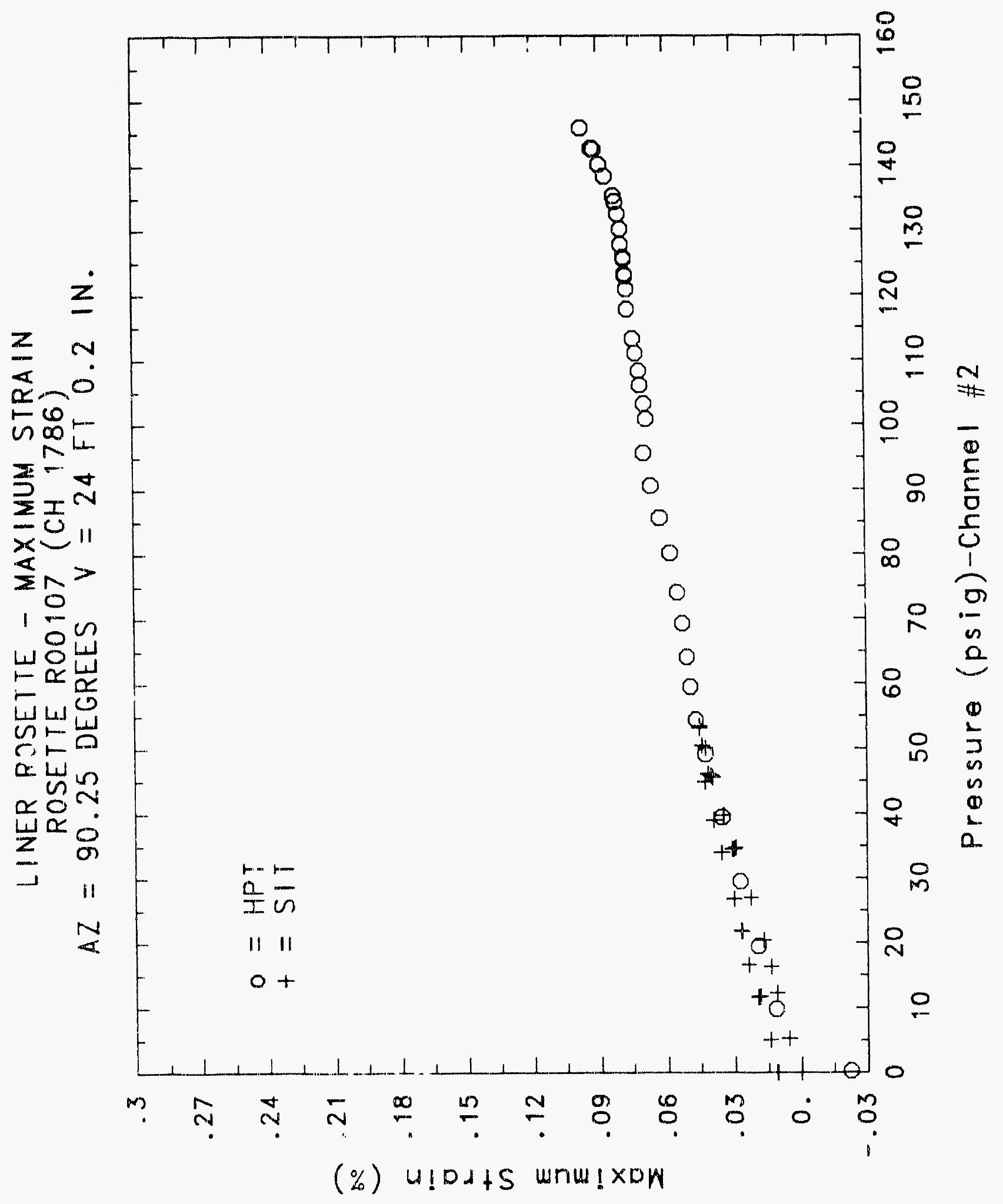

C. 639 


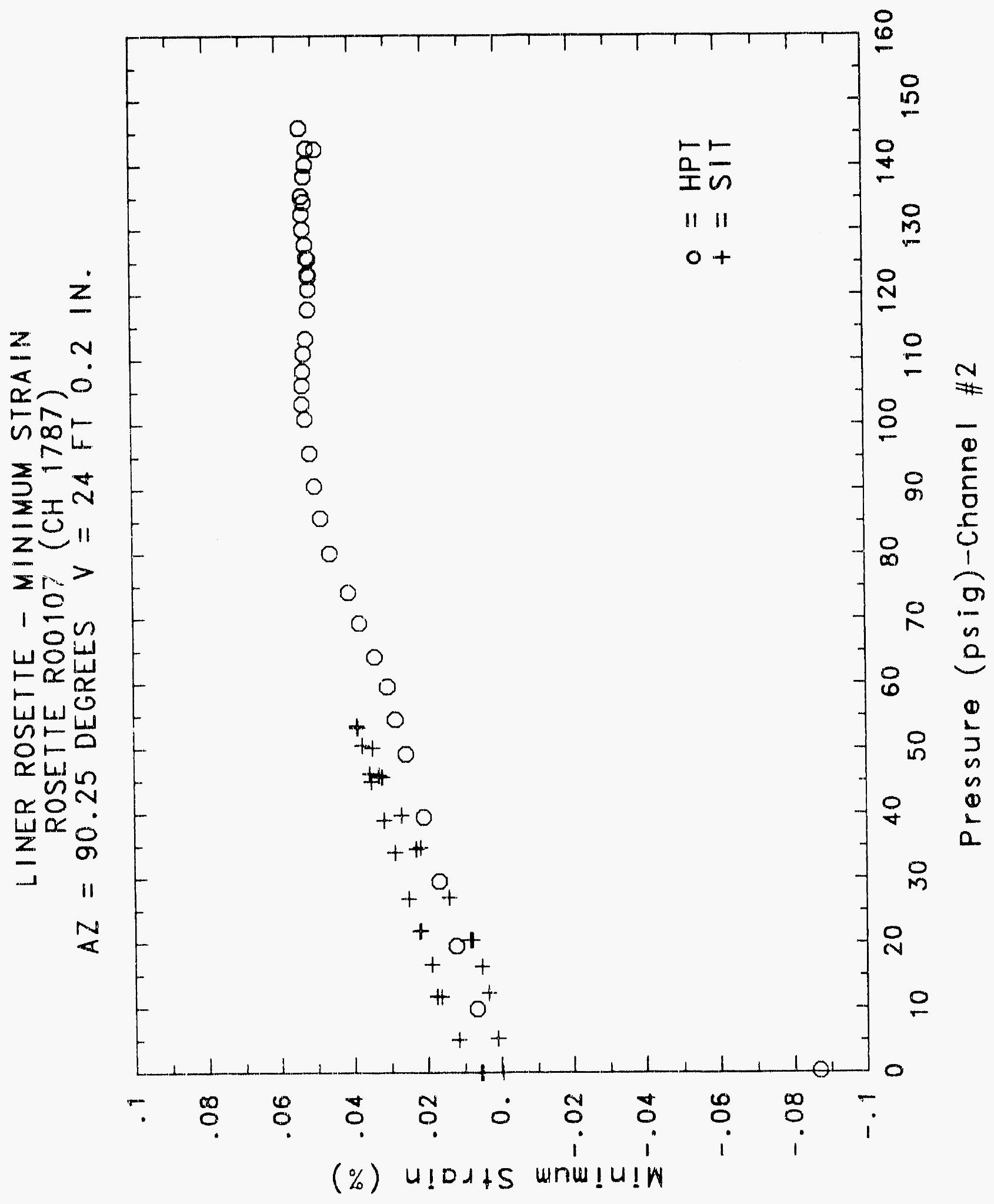




\section{Reinforced Concrete Test Data}

Rosette 108 Channels $17891800 \quad 1801$

\section{Structural IntEgrity TEST}

\begin{tabular}{|c|c|c|c|c|c|c|c|}
\hline $\begin{array}{c}\text { Pressure } \\
\text { psig } \\
\text {-0.05 } \\
5.33 \\
12.31 \\
16.44 \\
20.51 \\
20.46 \\
20.47 \\
27.05 \\
34.69 \\
34.53 \\
34.53 \\
39.70 \\
45.90 \\
45.65 \\
45.62 \\
50.10 \\
53.47 \\
53.29 \\
53.21 \\
50.49 \\
46.14 \\
46.13 \\
44.97 \\
38.98 \\
33.99 \\
33.96 \\
10.83 \\
21.88 \\
21.90 \\
16.69 \\
11.74 \\
11.77 \\
5.05 \\
0.02 \\
-0.04 \\
-0.02 \\
-0.02 \\
0.02\end{array}$ & $\begin{array}{c}\text { Maximum } \\
\text { XStrain } \\
0.0004 \\
0.0013 \\
-0.0004 \\
-0.0006 \\
0.0004 \\
0.0011 \\
0.0013 \\
0.0055 \\
0.0132 \\
0.0145 \\
0.0152 \\
0.0187 \\
0.0240 \\
0.0239 \\
0.0239 \\
0.0270 \\
0.0297 \\
0.0300 \\
0.0300 \\
0.0288 \\
0.0273 \\
0.0271 \\
0.0284 \\
0.0257 \\
0.0234 \\
0.0233 \\
0.0202 \\
0.0184 \\
0.0183 \\
0.0173 \\
0.0163 \\
0.0153 \\
0.0134 \\
0.0118 \\
0.0106 \\
0.0112 \\
0.0113 \\
0.0109\end{array}$ & $\begin{array}{r}\text { Kinimum } \\
\text { XStrain } \\
0.0001 \\
-0.0147 \\
-0.0234 \\
-0.0268 \\
-0.0284 \\
-0.0276 \\
-0.0271 \\
-0.0272 \\
-0.0227 \\
-0.0201 \\
-0.0193 \\
-0.0185 \\
-0.0148 \\
-0.0149 \\
-0.0149 \\
-0.0125 \\
-0.0094 \\
-0.0090 \\
-0.0088 \\
-0.0096 \\
-0.0104 \\
-0.0106 \\
-0.0088 \\
-0.0083 \\
-0.0083 \\
-0.0083 \\
-0.0086 \\
-0.0091 \\
-0.0092 \\
-0.0096 \\
-0.0091 \\
-0.0101 \\
-0.0054 \\
0.0021 \\
0.0021 \\
0.0023 \\
0.0022 \\
0.0021\end{array}$ & $\begin{array}{c}\text { Angle } \\
\text { Degrees } \\
-28.30 \\
6.46 \\
2.98 \\
0.73 \\
-0.64 \\
-0.64 \\
-0.82 \\
-2.48 \\
-4.66 \\
-4.76 \\
-4.52 \\
-5.87 \\
-7.10 \\
-7.06 \\
-7.04 \\
-8.15 \\
-9.10 \\
-9.25 \\
-9.09 \\
-8.64 \\
-7.45 \\
-7.39 \\
-7.01 \\
-5.57 \\
-4.09 \\
-4.29 \\
-2.48 \\
-1.65 \\
-1.43 \\
-0.66 \\
-0.55 \\
0.10 \\
-4.06 \\
-28.24 \\
-27.94 \\
-29.06 \\
-28.90 \\
-29.91\end{array}$ & $\begin{array}{c}\text { Pressure } \\
\text { psig } \\
9.89 \\
19.55 \\
29.57 \\
39.42 \\
49.16 \\
54.50 \\
59.57 \\
64.20 \\
69.32 \\
74.16 \\
80.16 \\
85.61 \\
90.58 \\
95.69 \\
100.92 \\
103.25 \\
106.11 \\
108.31 \\
111.08 \\
113.24 \\
117.83 \\
120.92 \\
123.28 \\
122.97 \\
125.82 \\
125.60 \\
127.84 \\
130.19 \\
132.53 \\
135.33 \\
134.42 \\
138.35 \\
140.16 \\
142.63 \\
145.78 \\
142.52 \\
0.22\end{array}$ & $\begin{array}{l}\text { Maximum } \\
\text { xstrain } \\
0.0051 \\
0.0078 \\
0.0114 \\
0.0160 \\
0.0208 \\
0.0247 \\
0.0285 \\
0.0318 \\
0.0360 \\
0.0418 \\
0.0481 \\
0.0551 \\
0.0628 \\
0.0702 \\
0.0767 \\
0.0800 \\
0.0824 \\
0.0849 \\
0.0890 \\
0.0920 \\
0.0975 \\
0.1013 \\
0.1052 \\
0.1058 \\
0.1094 \\
0.1110 \\
0.1132 \\
0.1164 \\
0.1209 \\
0.1282 \\
0.1303 \\
0.1439 \\
0.1553 \\
0.1751 \\
0.1968 \\
0.1988 \\
0.0668\end{array}$ & $\begin{array}{l}\text { Minimum } \\
\text { \%Strain } \\
-0.0149 \\
-0.0165 \\
-0.0152 \\
-0.0138 \\
-0.0106 \\
-0.0070 \\
-0.0030 \\
0.0018 \\
0.0068 \\
0.0118 \\
0.0161 \\
0.0208 \\
0.0244 \\
0.0299 \\
0.0359 \\
0.0391 \\
0.0434 \\
0.0461 \\
0.0497 \\
0.0519 \\
0.0572 \\
0.0615 \\
0.0648 \\
0.0655 \\
0.0685 \\
0.0689 \\
0.0713 \\
0.0743 \\
0.0779 \\
0.0836 \\
0.0842 \\
0.0905 \\
0.0949 \\
0.1000 \\
0.1059 \\
0.0999 \\
-0.0082\end{array}$ & $\begin{array}{c}\text { Angle } \\
\text { Degrees } \\
11.52 \\
8.02 \\
3.58 \\
0.15 \\
-3.64 \\
-4.75 \\
-7.51 \\
-11.78 \\
-16.06 \\
-22.29 \\
-28.03 \\
-32.67 \\
-34.96 \\
-35.75 \\
-37.75 \\
-38.03 \\
-39.07 \\
-39.73 \\
-40.73 \\
-41.69 \\
-43.37 \\
-43.80 \\
-43.93 \\
-43.87 \\
-43.93 \\
-43.83 \\
-43.59 \\
-42.24 \\
-39.73 \\
-34.52 \\
-33.17 \\
-25.94 \\
-21.76 \\
-17.58 \\
-14.58 \\
-13.81 \\
-9.80\end{array}$ \\
\hline
\end{tabular}

High Pressure test 


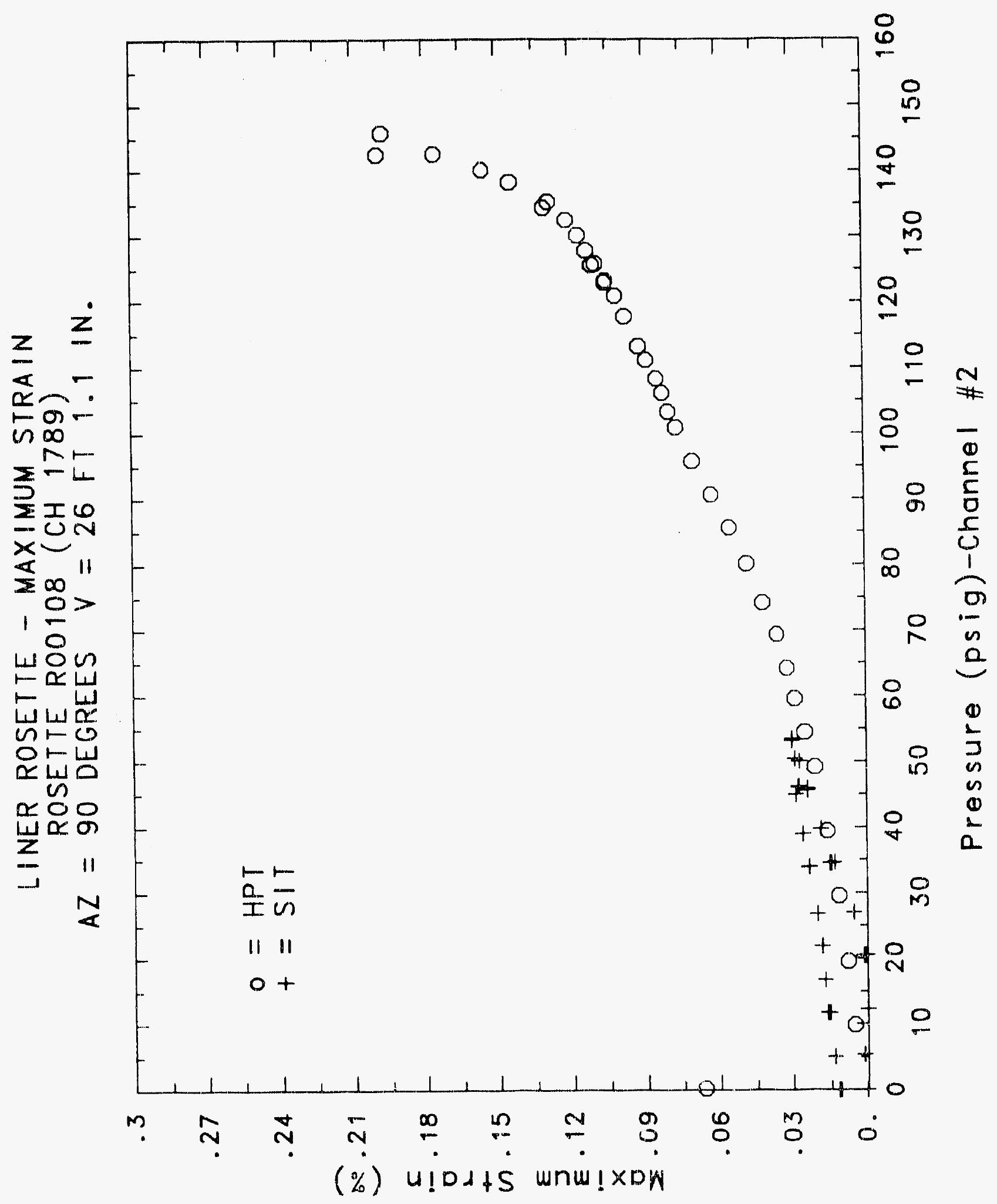




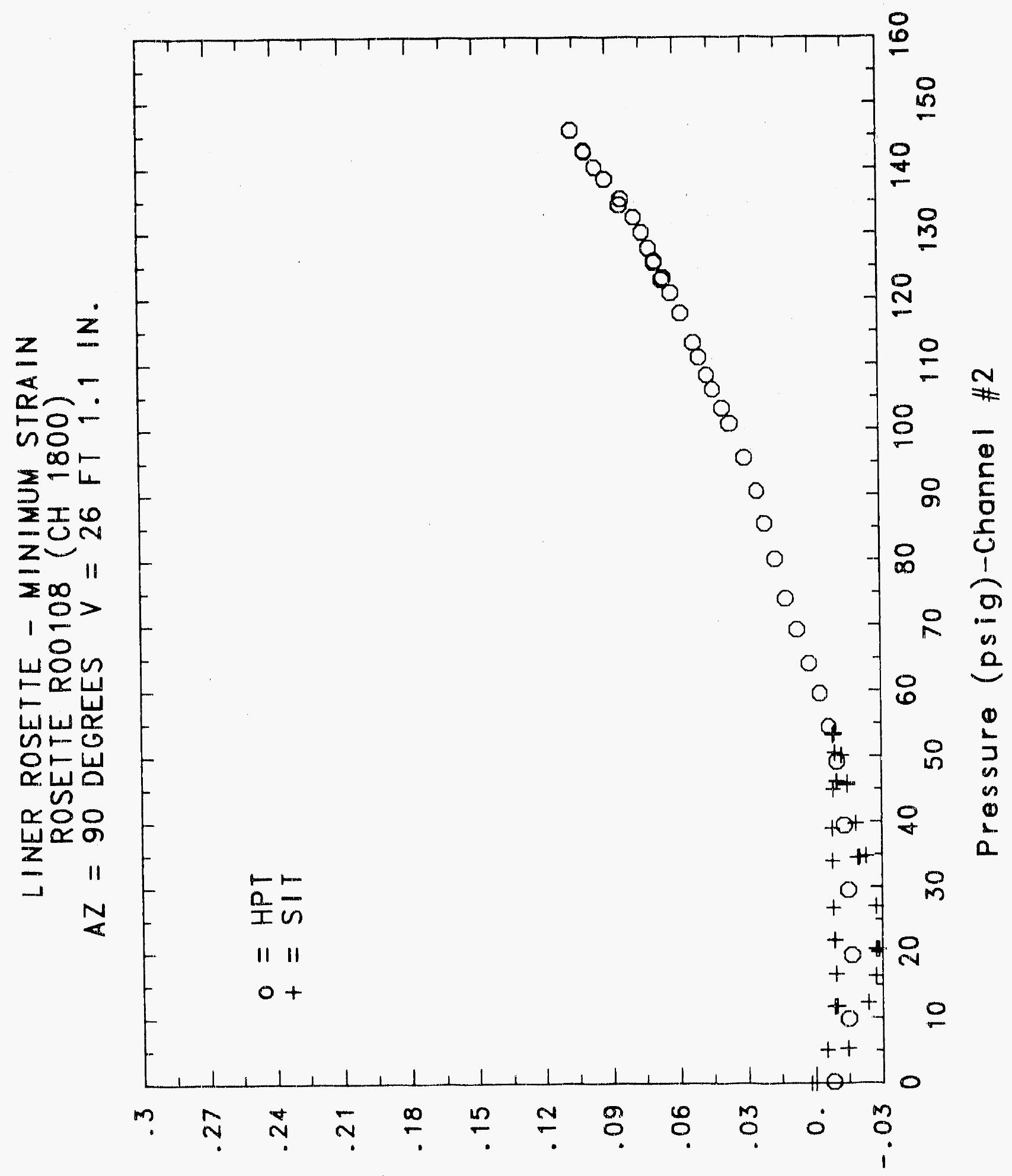

(\%) $u ! D d+S$ unw!u!W 


\section{Reinforced Concrete Test Data}

Rosette 109 Channels $1802 \quad 1803 \quad 1804$

StRUCTURAL INTEGRITY TEST

\begin{tabular}{|c|c|c|c|c|c|c|c|}
\hline $\begin{array}{c}\text { Pressure } \\
\text { psig } \\
\text {-0.05 } \\
5.33 \\
12.31 \\
16.44 \\
20.51 \\
20.46 \\
20.47 \\
27.05 \\
34.69 \\
34.53 \\
34.53 \\
39.70 \\
45.90 \\
45.65 \\
45.62 \\
50.10 \\
53.47 \\
53.29 \\
53.21 \\
50.49 \\
46.14 \\
46.13 \\
44.97 \\
38.98 \\
33.99 \\
33.96 \\
26.83 \\
21.88 \\
21.90 \\
16.69 \\
11.74 \\
11.77 \\
5.05 \\
0.02 \\
-0.04 \\
-0.02 \\
-0.02 \\
0.02\end{array}$ & $\begin{array}{l}\text { Maximum } \\
\text { xStrain } \\
0.0008 \\
0.0046 \\
0.0098 \\
0.0135 \\
0.0197 \\
0.0210 \\
0.0217 \\
0.0340 \\
0.0499 \\
0.0524 \\
0.0529 \\
0.0607 \\
0.0712 \\
0.0709 \\
0.0706 \\
0.0762 \\
0.0815 \\
0.0816 \\
0.0816 \\
0.0793 \\
0.0748 \\
0.0743 \\
0.0743 \\
0.0677 \\
0.0615 \\
0.0614 \\
0.0521 \\
0.0456 \\
0.0460 \\
0.0395 \\
0.0325 \\
0.0309 \\
0.0222 \\
0.0161 \\
0.0146 \\
0.0148 \\
0.0149 \\
0.0150\end{array}$ & $\begin{array}{l}\text { Minimum } \\
\text { \%Strain } \\
-0.0001 \\
0.0012 \\
0.0030 \\
0.0044 \\
0.0065 \\
0.0072 \\
0.0076 \\
0.0124 \\
0.0205 \\
0.0229 \\
0.0234 \\
0.0270 \\
0.0323 \\
0.0319 \\
0.0314 \\
0.0340 \\
0.0367 \\
0.0369 \\
0.0370 \\
0.0363 \\
0.0348 \\
0.0346 \\
0.0359 \\
0.0336 \\
0.0313 \\
0.0314 \\
0.0279 \\
0.0255 \\
0.0259 \\
0.0239 \\
0.0908 \\
0.0144 \\
0.0152 \\
0.0115 \\
0.0099 \\
0.0105 \\
0.0109 \\
0.0101\end{array}$ & $\begin{array}{c}\text { Angle } \\
\text { Degrees } \\
-40.95 \\
-16.31 \\
-14.53 \\
-12.90 \\
-10.09 \\
-9.76 \\
-9.46 \\
-4.85 \\
-1.89 \\
-2.15 \\
-2.10 \\
-1.32 \\
-0.50 \\
-0.38 \\
0.12 \\
0.22 \\
0.34 \\
0.48 \\
0.45 \\
0.52 \\
0.78 \\
0.99 \\
1.37 \\
1.74 \\
2.28 \\
1.81 \\
3.19 \\
3.66\end{array}$ & $\begin{array}{c}\text { Pressure } \\
\text { psig } \\
9.89 \\
19.55 \\
29.57 \\
39.42 \\
49.16 \\
54.50 \\
59.57 \\
64.20 \\
59.32 \\
74.16 \\
80.16 \\
85.61 \\
90.58 \\
95.69 \\
100.92 \\
103.25 \\
106.11 \\
108.31 \\
111.08 \\
113.24 \\
117.83 \\
120.92 \\
123.28 \\
122.97 \\
125.82 \\
125.60 \\
127.84 \\
130.19 \\
132.53 \\
135.33 \\
134.42 \\
138.35 \\
140.16 \\
142.63 \\
145.78 \\
142.52 \\
0.22\end{array}$ & $\begin{array}{l}\text { Maximum } \\
\text { xStrain } \\
0.0135 \\
0.0274 \\
0.0419 \\
0.0555 \\
0.0683 \\
0.0759 \\
0.0832 \\
0.0910 \\
0.0993 \\
0.1079 \\
0.1173 \\
0.1285 \\
0.1396 \\
0.1506 \\
0.1561 \\
0.1568 \\
0.1568 \\
0.1567 \\
0.1563 \\
0.1558 \\
0.1546 \\
0.1548 \\
0.1547 \\
0.1548 \\
0.1555 \\
0.1553 \\
0.1558 \\
0.1557 \\
0.1558 \\
0.1564 \\
0.1559 \\
0.1594 \\
0.1623 \\
0.2085 \\
0.4956 \\
0.5784 \\
0.3814\end{array}$ & $\begin{array}{l}\text { Minimum } \\
\text { xStrain } \\
0.0053 \\
0.0106 \\
0.0161 \\
0.0216 \\
0.0267 \\
0.0302 \\
0.0335 \\
0.0374 \\
0.0402 \\
0.0438 \\
0.0476 \\
0.0524 \\
0.0572 \\
0.0616 \\
0.0670 \\
0.0698 \\
0.0727 \\
0.0749 \\
0.0782 \\
0.0806 \\
0.0859 \\
0.0897 \\
0.0928 \\
0.0934 \\
0.0969 \\
0.0975 \\
0.0999 \\
0.1041 \\
0.1097 \\
0.1147 \\
0.1143 \\
0.1170 \\
0.1181 \\
0.1216 \\
0.1235 \\
0.1213 \\
0.0224\end{array}$ & $\begin{array}{c}\text { Angle } \\
\text { Degrees } \\
-2.38 \\
-1.84 \\
-1.95 \\
-2.63 \\
-2.53 \\
-2.75 \\
-2.83 \\
-3.02 \\
-1.33 \\
0.22 \\
0.53 \\
1.13 \\
0.60 \\
0.48 \\
-1.15 \\
-2.07 \\
-2.80 \\
-3.02 \\
-3.80 \\
-3.86 \\
-4.72 \\
-5.60 \\
-6.18 \\
-6.58 \\
-7.53 \\
-7.46 \\
-7.88 \\
-8.71 \\
-10.57 \\
-11.35 \\
-11.13 \\
-10.16 \\
-10.42 \\
-1.96 \\
0.96 \\
1.00 \\
0.45\end{array}$ \\
\hline
\end{tabular}

high Pressure test 


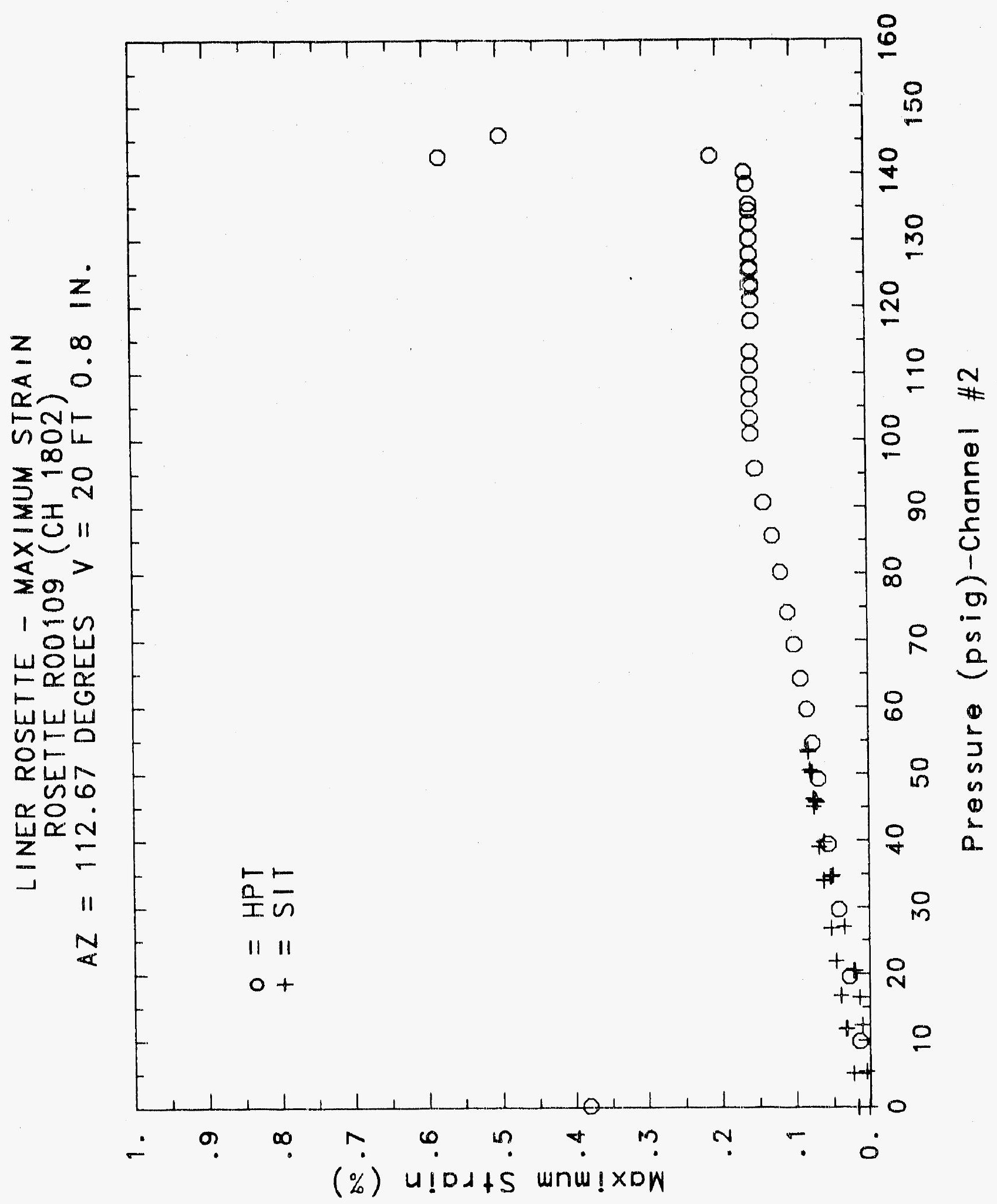

C- 64.5 


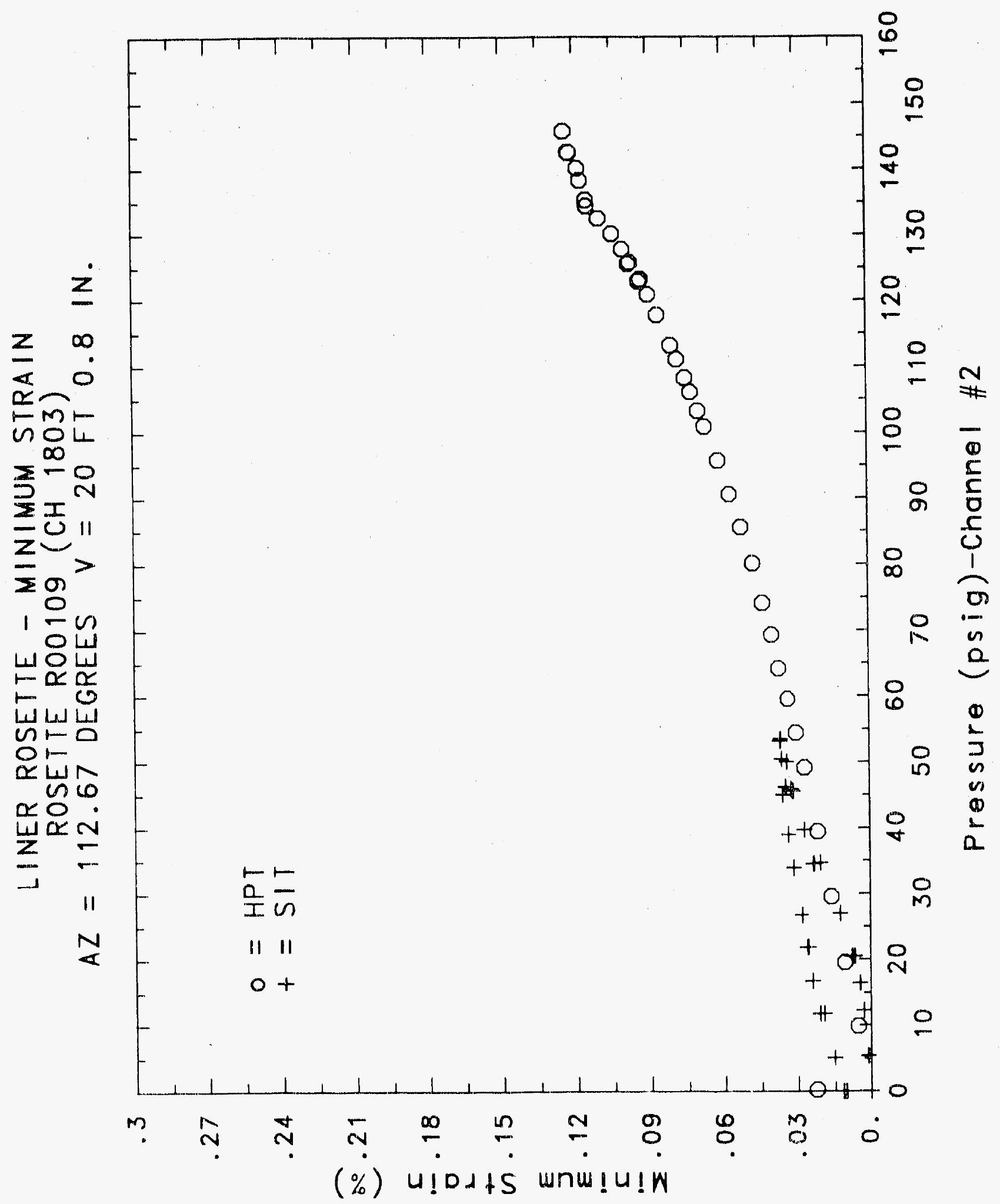




\section{Reinforced Concrete Test Data}

\begin{tabular}{|c|c|c|c|c|c|c|c|}
\hline $\begin{array}{c}\text { Pressure } \\
\text { psig } \\
-0.05 \\
5.33 \\
12.31 \\
16.44 \\
20.51 \\
20.46 \\
20.47 \\
27.05 \\
34.69 \\
34.53 \\
34.53 \\
39.70 \\
45.90 \\
45.65 \\
45.62 \\
50.10 \\
53.47 \\
53.29 \\
53.21 \\
50.49 \\
46.14 \\
46.13 \\
44.97 \\
38.98 \\
33.99 \\
33.96 \\
26.83 \\
21.88 \\
21.90 \\
16.69 \\
11.74 \\
11.77 \\
5.05 \\
0.02 \\
-0.04 \\
-0.02 \\
-0.02 \\
0.02\end{array}$ & $\begin{array}{c}\text { Maximum } \\
\text { \%Strain } \\
0.0003 \\
0.0082 \\
0.0191 \\
0.0266 \\
0.0367 \\
0.0379 \\
0.0385 \\
0.0432 \\
0.0581 \\
0.0772 \\
0.0785 \\
0.0937 \\
0.1168 \\
0.1158 \\
0.1152 \\
0.1278 \\
0.1397 \\
0.1403 \\
0.1404 \\
0.1365 \\
0.1288 \\
0.1283 \\
0.1292 \\
0.1182 \\
0.1080 \\
0.1077 \\
0.0925 \\
0.0822 \\
0.0832 \\
0.0734 \\
0.0627 \\
0.0600 \\
0.0465 \\
0.0375 \\
0.0345 \\
0.0352 \\
0.0355 \\
0.0358\end{array}$ & $\begin{array}{r}\text { Minimum } \\
\text { xStrain } \\
0.0000 \\
0.0004 \\
0.0009 \\
0.0014 \\
0.0020 \\
0.0022 \\
0.0024 \\
-0.0049 \\
-0.0175 \\
-0.0267 \\
-0.0273 \\
-0.0312 \\
-0.0359 \\
-0.0353 \\
-0.0349 \\
-0.0358 \\
-0.0360 \\
-0.0356 \\
-0.0355 \\
-0.0361 \\
-0.0367 \\
-0.0363 \\
-0.0378 \\
-0.0387 \\
-0.0389 \\
-0.0386 \\
-0.0380 \\
-0.0377 \\
-0.0385 \\
-0.0393 \\
-0.0391 \\
-0.0364 \\
-0.0377 \\
-0.0476 \\
-0.0420 \\
-0.0430 \\
-0.0434 \\
-0.0439\end{array}$ & $\begin{array}{c}\text { Angle } \\
\text { Degrees } \\
35.82 \\
4.64 \\
5.08 \\
5.10 \\
5.36 \\
5.55 \\
5.21 \\
25.01 \\
34.96 \\
35.57 \\
35.83 \\
35.35 \\
35.07 \\
34.80 \\
34.71 \\
34.13 \\
33.63 \\
33.64 \\
33.58 \\
34.12 \\
35.04 \\
34.97 \\
35.53 \\
37.02 \\
38.36 \\
38.36 \\
40.50 \\
42.30 \\
42.52 \\
44.81 \\
-42.53 \\
-42.88 \\
-37.92 \\
-31.02 \\
-31.21 \\
-31.12 \\
-31.07 \\
-31.03\end{array}$ & $\begin{array}{c}\text { Pressure } \\
\text { psig } \\
9.89 \\
19.55 \\
29.57 \\
39.42 \\
49.16 \\
54.50 \\
59.57 \\
64.20 \\
69.32 \\
74.16 \\
80.16 \\
85.61 \\
90.58 \\
95.69 \\
100.92 \\
103.25 \\
106.11 \\
108.31 \\
111.08 \\
113.24 \\
117.83 \\
120.92 \\
123.28 \\
122.97 \\
125.82 \\
125.60 \\
127.84 \\
130.19 \\
132.53 \\
135.33 \\
134.42 \\
138.35 \\
140.16 \\
142.63 \\
145.78 \\
142.52 \\
0.22\end{array}$ & $\begin{array}{c}\text { Maximum } \\
\text { xstrain } \\
0.0327 \\
0.0585 \\
0.0831 \\
0.1060 \\
0.1279 \\
0.1405 \\
0.1579 \\
0.1793 \\
0.2071 \\
0.2527 \\
0.3948 \\
0.6249 \\
0.8314 \\
0.9947 \\
1.1071 \\
1.1464 \\
1.1907 \\
1.2229 \\
1.2644 \\
1.2893 \\
1.3497 \\
1.4044 \\
1.4564 \\
1.4718 \\
1.5870 \\
1.6078 \\
1.6636 \\
1.8143 \\
2.0245 \\
2.3189 \\
2.3748 \\
2.7224 \\
3.0199 \\
3.5113 \\
4.0328 \\
4.1531 \\
3.7266\end{array}$ & $\begin{array}{r}\text { Minimum } \\
\text { XStrain } \\
-0.0030 \\
-0.0071 \\
-0.0104 \\
-0.0129 \\
-0.0146 \\
-0.0151 \\
-0.0132 \\
-0.0087 \\
-0.0036 \\
0.0060 \\
0.0133 \\
0.0132 \\
0.0127 \\
0.0091 \\
0.0025 \\
-0.0009 \\
-0.0034 \\
-0.0038 \\
-0.0026 \\
0.0009 \\
0.0102 \\
0.0144 \\
0.0169 \\
0.0174 \\
0.0228 \\
0.0234 \\
0.0248 \\
0.0249 \\
0.0204 \\
0.0102 \\
0.0075 \\
-0.0065 \\
-0.0180 \\
-0.0326 \\
-0.0436 \\
-0.0489 \\
-0.0841\end{array}$ & $\begin{array}{c}\text { Angle } \\
\text { Degrees } \\
15.70 \\
19.66 \\
20.87 \\
21.14 \\
21.01 \\
20.91 \\
21.52 \\
20.99 \\
19.09 \\
14.23 \\
5.96 \\
0.37 \\
-1.14 \\
1.60 \\
3.49 \\
4.58 \\
5.17 \\
5.16 \\
4.90 \\
4.62 \\
3.51 \\
3.06 \\
2.90 \\
2.78 \\
1.85 \\
1.65 \\
1.11 \\
-0.06 \\
-0.85 \\
-1.46 \\
-1.52 \\
-1.70 \\
-1.55 \\
-1.12 \\
-0.91 \\
-0.94 \\
-1.96\end{array}$ \\
\hline
\end{tabular}

High Pressure test

\section{Structural Integrity Test}

\section{Rosette 110 Channels $1805 \quad 1806 \quad 1807$}




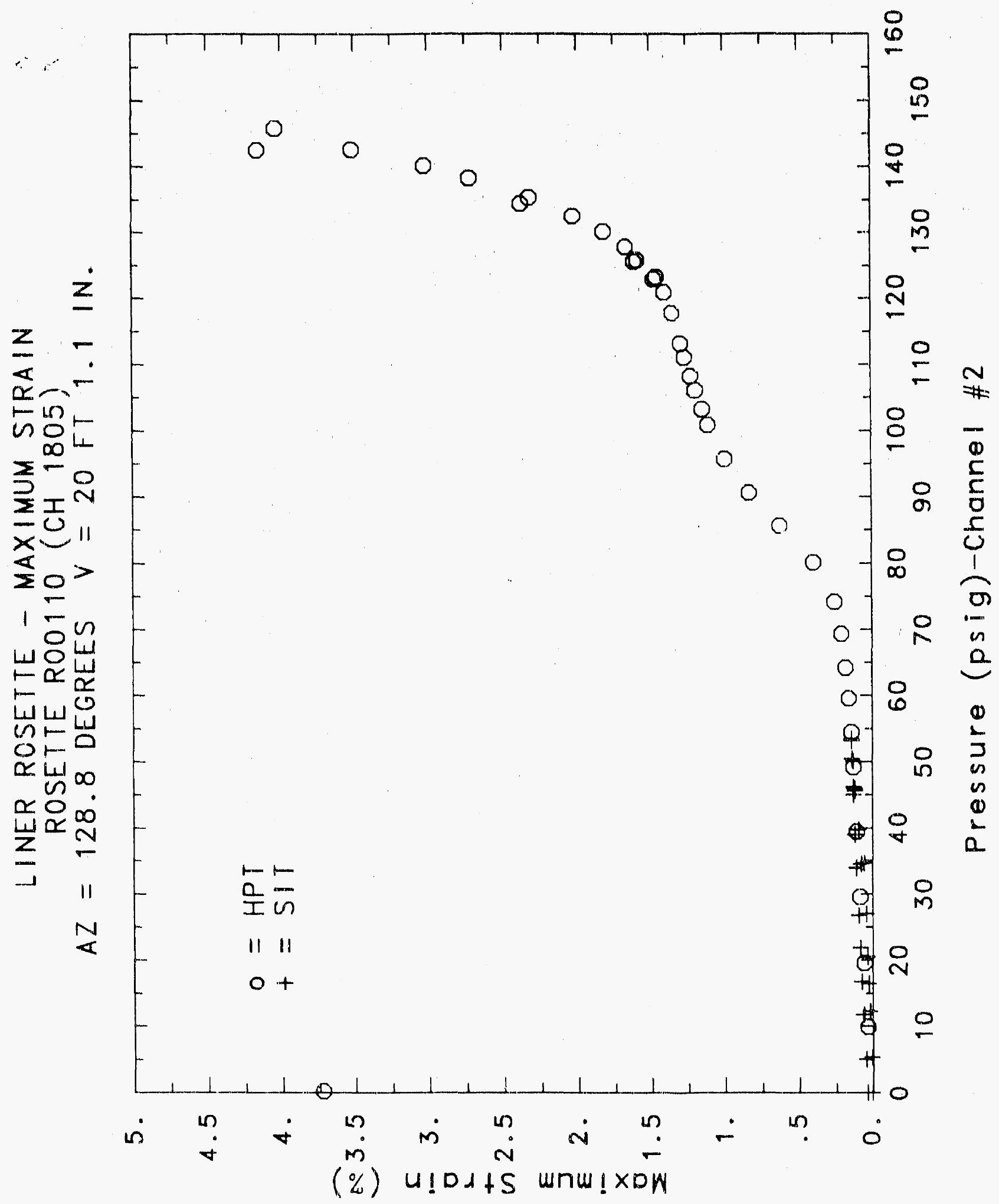




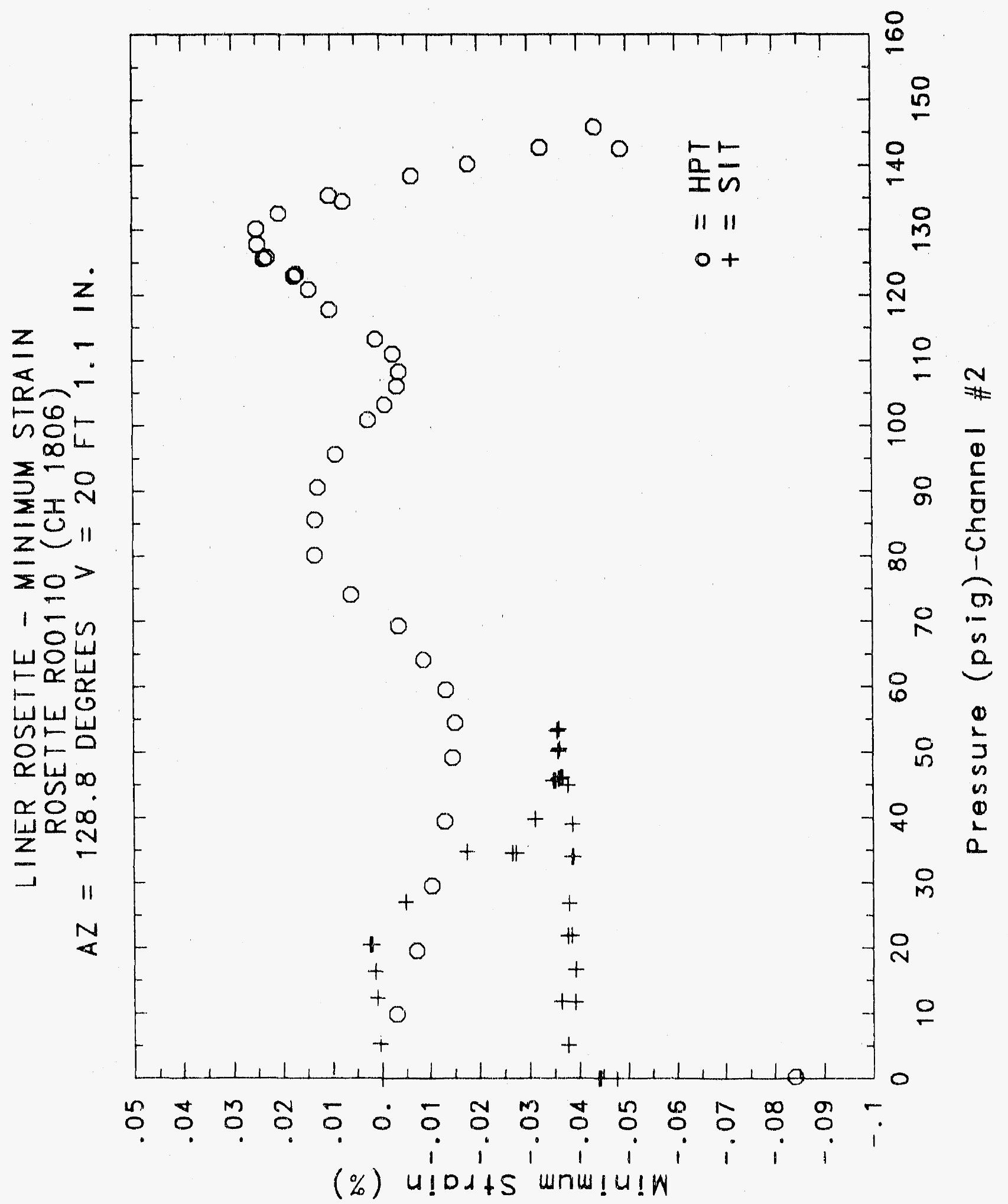




\section{Reinforced Concrete Test Data}

Rosette 111 Channels $1808 \quad 18091820$

StruCtURAL INTEGRITY TEST

\begin{tabular}{|c|c|c|c|c|c|c|c|}
\hline $\begin{array}{c}\text { Pressure } \\
\text { psig } \\
-0.05 \\
5.33 \\
12.31 \\
16.44 \\
20.51 \\
20.46 \\
20.47 \\
27.05 \\
34.69 \\
34.53 \\
34.53 \\
39.70 \\
45.90 \\
45.65 \\
45.62 \\
50.10 \\
53.47 \\
53.29 \\
53.21 \\
50.49 \\
46.14 \\
46.13 \\
44.97 \\
38.98 \\
33.99 \\
33.96 \\
26.83 \\
21.88 \\
21.90 \\
16.69 \\
11.74 \\
11.77 \\
5.05 \\
0.02 \\
-0.04 \\
-0.02 \\
-0.02 \\
0.02\end{array}$ & $\begin{array}{l}\text { Maximun } \\
\text { \%Strain } \\
0.0003 \\
0.0041 \\
0.0090 \\
0.0122 \\
0.0164 \\
0.0171 \\
0.0170 \\
0.0262 \\
0.0366 \\
0.0378 \\
0.0380 \\
0.0433 \\
0.0476 \\
0.0463 \\
0.0461 \\
0.0494 \\
0.0519 \\
0.0519 \\
0.0520 \\
0.0500 \\
0.0467 \\
0.0467 \\
0.0476 \\
0.0437 \\
0.0405 \\
0.0405 \\
0.0353 \\
0.0314 \\
0.0327 \\
0.0296 \\
0.0259 \\
0.0236 \\
0.0171 \\
0.0112 \\
0.0086 \\
0.0094 \\
0.0100 \\
0.0102\end{array}$ & $\begin{array}{l}\text { Minimum } \\
\text { XStrain } \\
-0.0001 \\
0.0025 \\
0.0062 \\
0.0086 \\
0.0117 \\
0.0120 \\
0.0121 \\
0.0180 \\
0.0281 \\
0.0308 \\
0.0314 \\
0.0362 \\
0.0425 \\
0.0414 \\
0.0408 \\
0.0440 \\
0.0469 \\
0.0469 \\
0.0468 \\
0.0457 \\
0.0428 \\
0.0425 \\
0.0436 \\
0.0389 \\
0.0348 \\
0.0347 \\
0.0283 \\
0.0242 \\
0.0247 \\
0.0205 \\
0.0157 \\
0.0144 \\
0.0093 \\
0.0056 \\
0.0036 \\
0.0036 \\
0.0039 \\
0.0044\end{array}$ & $\begin{array}{c}\text { Angle } \\
\text { Degrees } \\
11.01 \\
-39.05 \\
-36.81 \\
-36.12 \\
-40.13 \\
-40.71 \\
-41.16 \\
44.29 \\
-43.16 \\
-41.83 \\
-40.49 \\
-37.38 \\
-27.69 \\
-23.04 \\
-25.24 \\
-24.18 \\
-19.69 \\
-17.36 \\
-16.55 \\
-11.14 \\
0.49 \\
-2.07 \\
12.34 \\
25.76 \\
29.17 \\
29.30 \\
33.55 \\
36.21 \\
38.01 \\
41.44 \\
41.50 \\
35.74 \\
39.29 \\
-40.64 \\
-39.62 \\
-38.56 \\
-37.83 \\
-40.36\end{array}$ & $\begin{array}{c}\text { Pressure } \\
\text { psig } \\
9.89 \\
19.55 \\
29.57 \\
39.42 \\
49.16 \\
54.50 \\
59.57 \\
64.20 \\
69.32 \\
74.16 \\
80.16 \\
85.61 \\
90.58 \\
95.69 \\
100.92 \\
103.25 \\
106.11 \\
108.31 \\
111.08 \\
113.24 \\
117.83 \\
120.92 \\
123.28 \\
122.97 \\
125.82 \\
125.60 \\
127.84 \\
130.19 \\
132.53 \\
135.33 \\
134.42 \\
138.35 \\
140.16 \\
142.63 \\
145.78 \\
142.52 \\
0.22\end{array}$ & $\begin{array}{c}\text { Maximum } \\
\text { wStrain } \\
0.0096 \\
0.0193 \\
0.0284 \\
0.0369 \\
0.0453 \\
0.0503 \\
0.0549 \\
0.0661 \\
0.0758 \\
0.0925 \\
0.1087 \\
0.1208 \\
0.1285 \\
0.1421 \\
0.1583 \\
0.1688 \\
0.1786 \\
0.1843 \\
0.1906 \\
0.1961 \\
0.2068 \\
0.2147 \\
0.2195 \\
0.2208 \\
0.2273 \\
0.2294 \\
0.2341 \\
0.2451 \\
0.2741 \\
0.3682 \\
0.3880 \\
0.5878 \\
0.7310 \\
0.8987 \\
1.0015 \\
1.0091 \\
0.8097\end{array}$ & $\begin{array}{l}\text { Minimum } \\
\text { zStrain } \\
0.0069 \\
0.0146 \\
0.0228 \\
0.0307 \\
0.0387 \\
0.0434 \\
0.0472 \\
0.0495 \\
0.0529 \\
0.0553 \\
0.0571 \\
0.0605 \\
0.0639 \\
0.0684 \\
0.0750 \\
0.0791 \\
0.0850 \\
0.0899 \\
0.0962 \\
0.0999 \\
0.1016 \\
0.1026 \\
0.1046 \\
0.1044 \\
0.1057 \\
0.1064 \\
0.1088 \\
0.1112 \\
0.1198 \\
0.1706 \\
0.1805 \\
0.2379 \\
0.2833 \\
0.3509 \\
0.4118 \\
0.4184 \\
0.2156\end{array}$ & $\begin{array}{c}\text { Angle } \\
\text { Degrees } \\
22.85 \\
21.27 \\
17.35 \\
9.08 \\
0.84 \\
-2.52 \\
-6.68 \\
-7.65 \\
-4.72 \\
-5.79 \\
-4.89 \\
-0.89 \\
2.84 \\
4.74 \\
4.09 \\
3.30 \\
0.76 \\
-0.07 \\
-3.01 \\
-5.01 \\
-7.80 \\
-8.71 \\
-9.53 \\
-9.82 \\
-11.48 \\
-12.15 \\
-13.06 \\
-15.08 \\
-19.13 \\
-38.29 \\
-40.78 \\
34.03 \\
28.76 \\
23.87 \\
21.25 \\
20.69 \\
19.25\end{array}$ \\
\hline
\end{tabular}

high Pressure test 


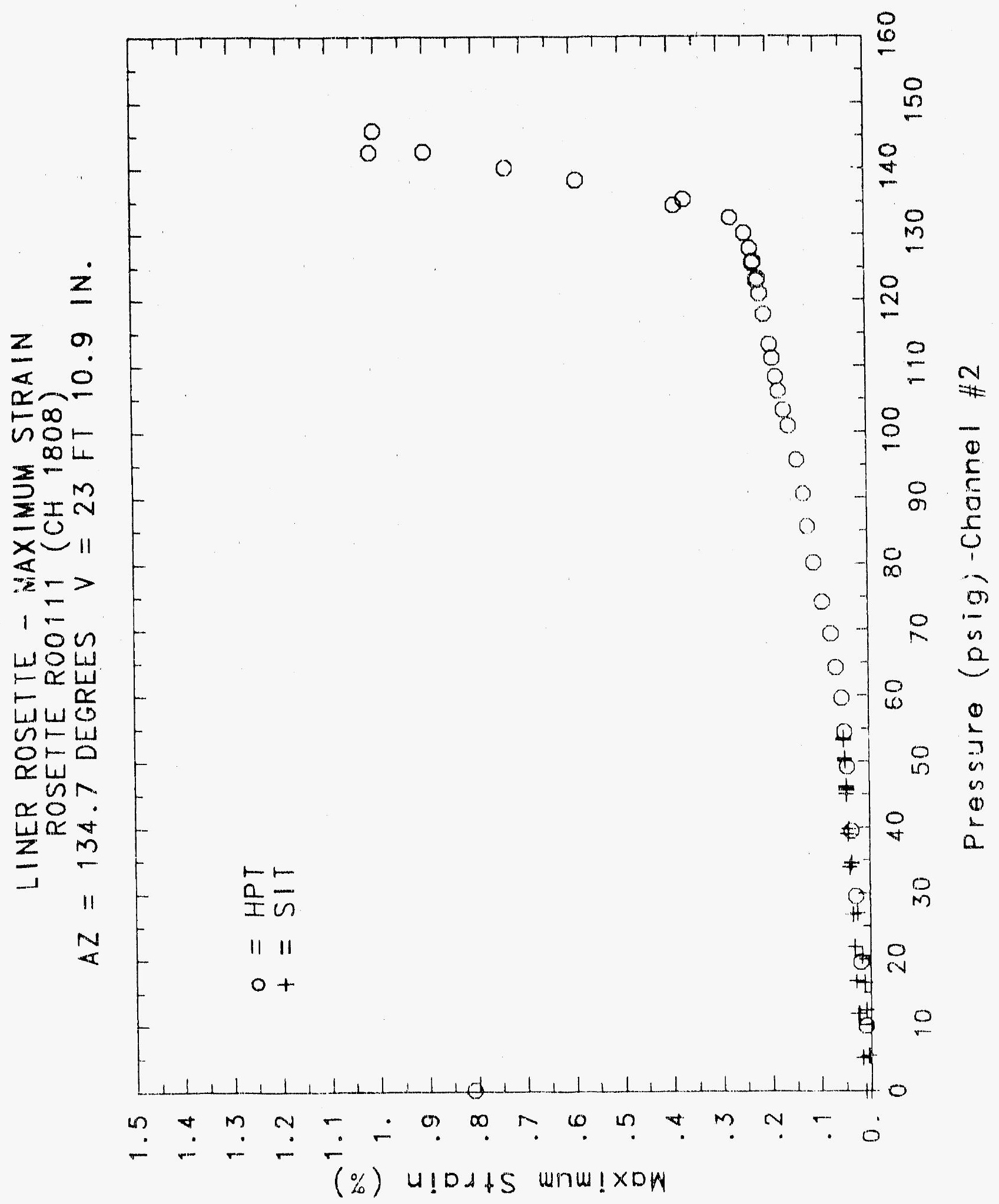




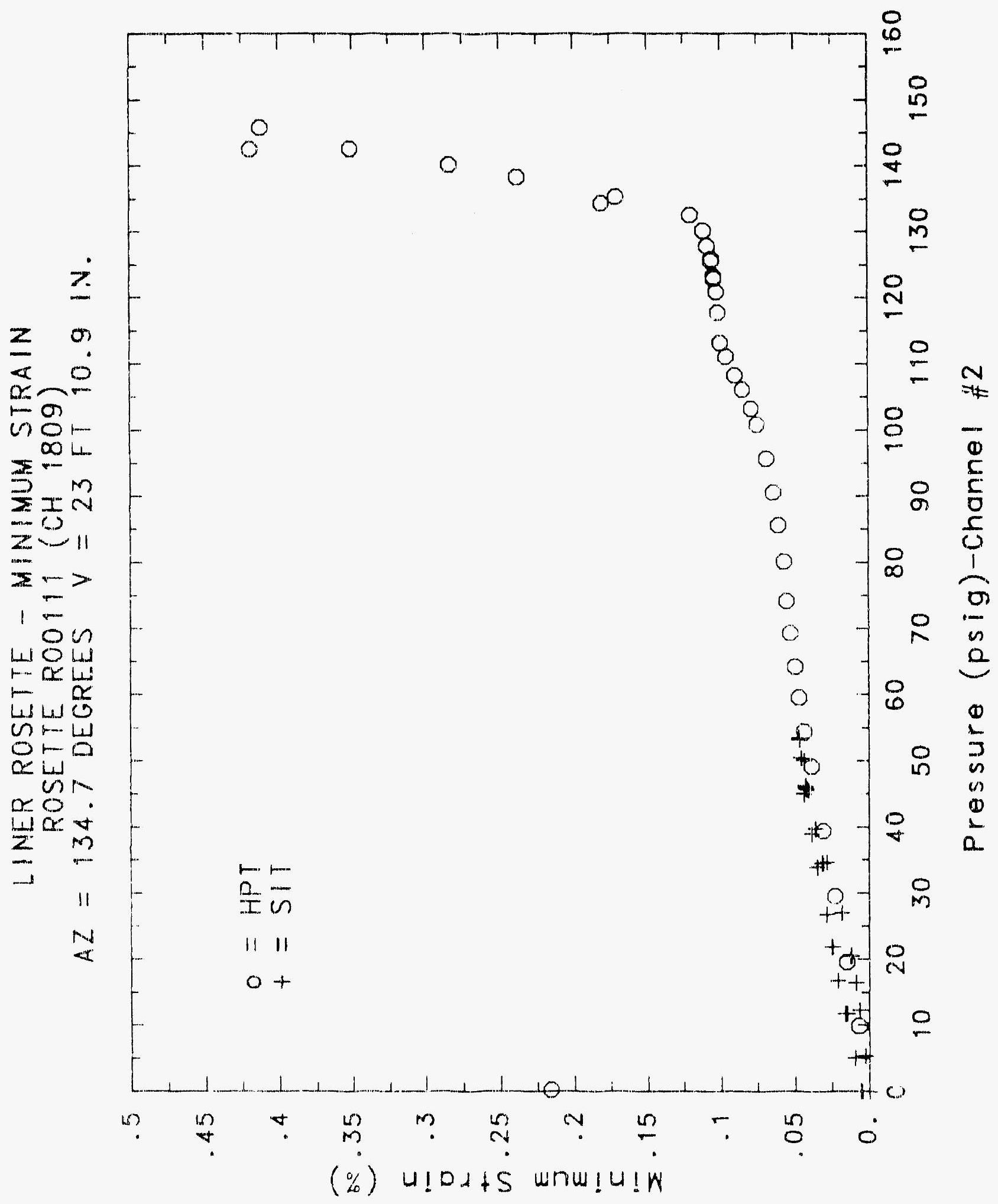




\section{Reinforced Concrete Test Data}

\section{Rosette 112 Channels $1821 \quad 1822 \quad 1823$}

\section{Structural InTEgrity TEST}

\begin{tabular}{|c|c|c|c|c|c|c|c|}
\hline $\begin{array}{c}\text { Pressure } \\
\text { psig } \\
\text {-0.05 } \\
5.33 \\
12.31 \\
16.44 \\
20.51 \\
20.46 \\
20.47 \\
27.05 \\
34.69 \\
34.53 \\
34.53 \\
39.70 \\
45.90 \\
45.65 \\
45.62 \\
50.10 \\
53.47 \\
53.29 \\
53.21 \\
50.49 \\
46.14 \\
46.13 \\
44.97 \\
38.98 \\
33.99 \\
33.96 \\
26.83 \\
21.88 \\
21.90 \\
16.69 \\
11.74 \\
11.77 \\
5.05 \\
0.02 \\
-0.04 \\
-0.02 \\
-0.02 \\
0.02\end{array}$ & $\begin{array}{l}\text { Maximum } \\
\text { \%Strain } \\
0.0006 \\
0.0043 \\
0.0101 \\
0.0128 \\
0.0184 \\
0.0197 \\
0.0203 \\
0.0330 \\
0.0501 \\
0.0517 \\
0.0519 \\
0.0586 \\
0.0703 \\
0.0689 \\
0.0679 \\
0.0732 \\
0.0779 \\
0.0786 \\
0.0784 \\
0.0763 \\
0.0721 \\
0.0722 \\
0.0692 \\
0.0630 \\
0.0577 \\
0.0574 \\
0.0505 \\
0.0459 \\
0.0461 \\
0.0419 \\
0.0374 \\
0.0362 \\
0.0308 \\
0.0276 \\
0.0421 \\
0.0428 \\
0.0411 \\
0.0358\end{array}$ & $\begin{array}{r}\text { Minimum } \\
\text { xStrain } \\
-0.0024 \\
0.0104 \\
-0.0209 \\
-0.0201 \\
-0.0182 \\
-0.0170 \\
-0.0171 \\
-0.0088 \\
-0.0019 \\
-0.0012 \\
0.0017 \\
0.0085 \\
0.0137 \\
0.0166 \\
0.0182 \\
0.0220 \\
0.0271 \\
0.0207 \\
0.0194 \\
0.0176 \\
0.0162 \\
0.0118 \\
0.0044 \\
0.0007 \\
-0.0025 \\
0.0003 \\
-0.0055 \\
-0.0088 \\
-0.0078 \\
-0.0119 \\
-0.0171 \\
-0.0185 \\
-0.0255 \\
-0.0297 \\
-0.0238 \\
-0.0253 \\
-0.0268 \\
-0.0354\end{array}$ & $\begin{array}{c}\text { Angle } \\
\text { Degrees } \\
20.25 \\
22.39 \\
21.24 \\
20.83 \\
19.66 \\
19.57 \\
19.91 \\
18.31 \\
18.26 \\
18.80 \\
18.99 \\
18.58 \\
18.05 \\
19.01 \\
19.56 \\
20.06 \\
20.46 \\
21.94 \\
22.69 \\
23.79 \\
24.81 \\
25.17 \\
33.50 \\
37.42 \\
40.47 \\
41.69 \\
-44.69 \\
-41.58 \\
-40.18 \\
-36.71 \\
-33.90 \\
-33.44 \\
-30.69 \\
-27.00 \\
-9.55 \\
-9.98 \\
-11.49 \\
-17.05\end{array}$ & $\begin{array}{c}\text { Pressure } \\
\text { psig } \\
9.89 \\
19.55 \\
29.57 \\
39.42 \\
49.16 \\
54.50 \\
59.57 \\
64.20 \\
69.32 \\
74.16 \\
80.16 \\
85.61 \\
90.58 \\
95.69 \\
100.92 \\
103.25 \\
106.11 \\
108.31 \\
111.08 \\
113.24 \\
117.83 \\
120.92 \\
123.28 \\
122.97 \\
125.82 \\
125.60 \\
127.84 \\
130.19 \\
132.53 \\
135.33 \\
134.42 \\
138.35 \\
140.16 \\
142.63 \\
145.78 \\
142.52 \\
0.22\end{array}$ & $\begin{array}{l}\text { Maximum } \\
\text { Xstrain } \\
0.0157 \\
0.0314 \\
0.0503 \\
0.0732 \\
0.0877 \\
0.0965 \\
0.1058 \\
0.1153 \\
0.1256 \\
0.1391 \\
0.1566 \\
0.1699 \\
0.1826 \\
0.2031 \\
0.2210 \\
0.2287 \\
0.2336 \\
0.2365 \\
0.2438 \\
0.2466 \\
0.2505 \\
0.2547 \\
0.2605 \\
0.2636 \\
0.2678 \\
0.2731 \\
0.2769 \\
0.2925 \\
0.2974 \\
0.3067 \\
0.3102 \\
0.3426 \\
0.3950 \\
0.5640 \\
0.8315 \\
0.8846 \\
0.6101\end{array}$ & $\begin{array}{l}\text { Minimum } \\
\text { xStrain } \\
-0.0082 \\
-0.0104 \\
-0.0251 \\
-0.0399 \\
-0.0419 \\
-0.0453 \\
-0.0512 \\
-0.0548 \\
-0.0577 \\
-0.0780 \\
-0.1126 \\
-0.1245 \\
-0.1344 \\
-0.1819 \\
-0.2000 \\
-0.2152 \\
-0.2191 \\
-0.2200 \\
-0.2434 \\
-0.2478 \\
-0.2513 \\
-0.2577 \\
-0.2749 \\
-0.2911 \\
-0.2967 \\
-0.3235 \\
-0.3278 \\
-0.3947 \\
-0.3971 \\
-0.3994 \\
-0.4053 \\
-0.4444 \\
-0.4368 \\
-0.42444 \\
-0.4270 \\
-0.4431 \\
-0.0378\end{array}$ & $\begin{array}{c}\text { Angle } \\
\text { Degrees } \\
15.20 \\
14.03 \\
14.69 \\
13.01 \\
13.05 \\
13.32 \\
13.75 \\
14.00 \\
14.14 \\
1311 \\
16.01 \\
16.13 \\
16.16 \\
16.88 \\
16.69 \\
16.70 \\
16.60 \\
16.57 \\
16.83 \\
16.92 \\
17.05 \\
17.16 \\
17.47 \\
17.65 \\
17.79 \\
18.05 \\
18.10 \\
18.64 \\
18.64 \\
18.54 \\
18.51 \\
18.13 \\
16.55 \\
13.09 \\
10.13 \\
10.00 \\
2.05\end{array}$ \\
\hline
\end{tabular}

High Pressure Test 


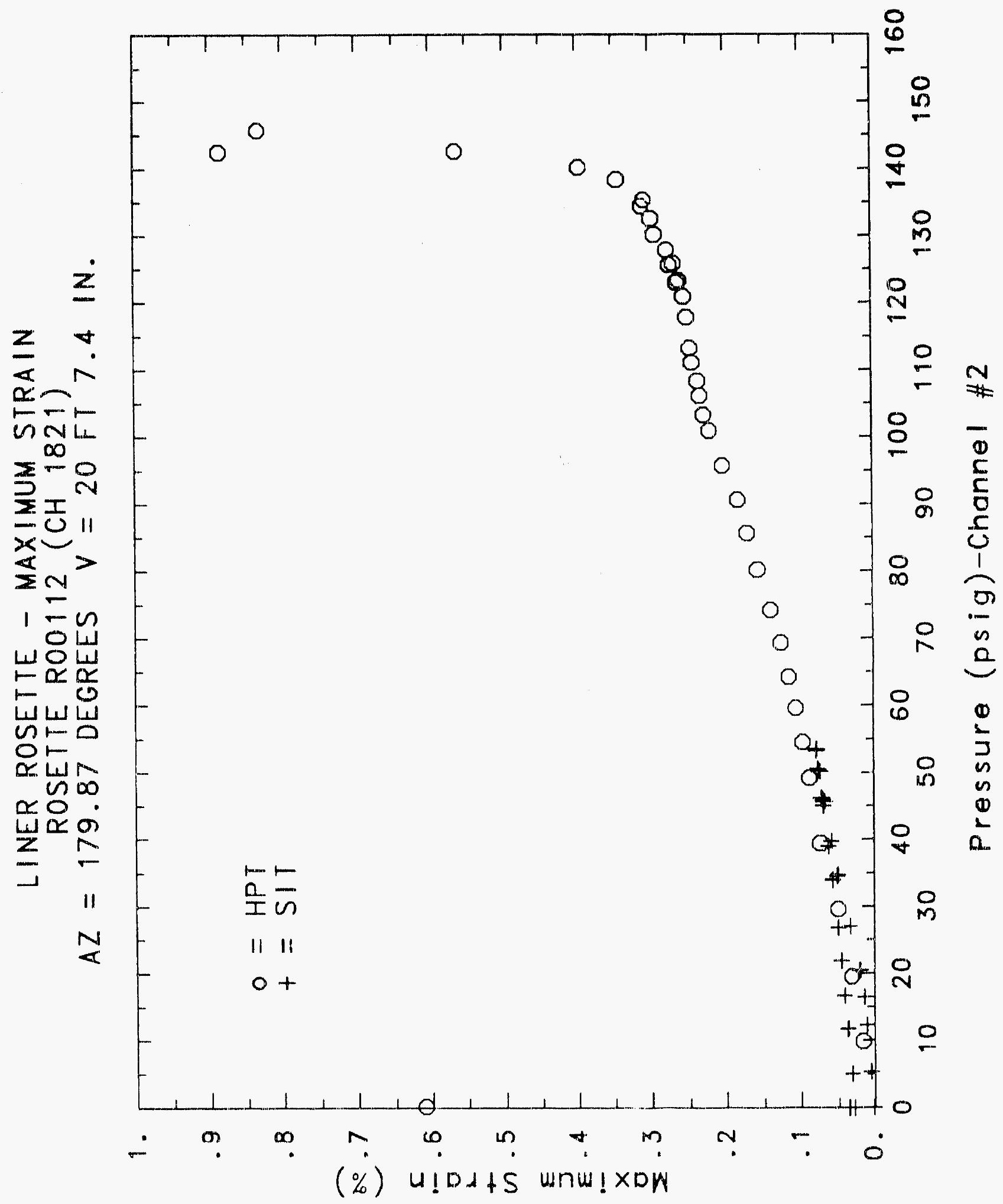




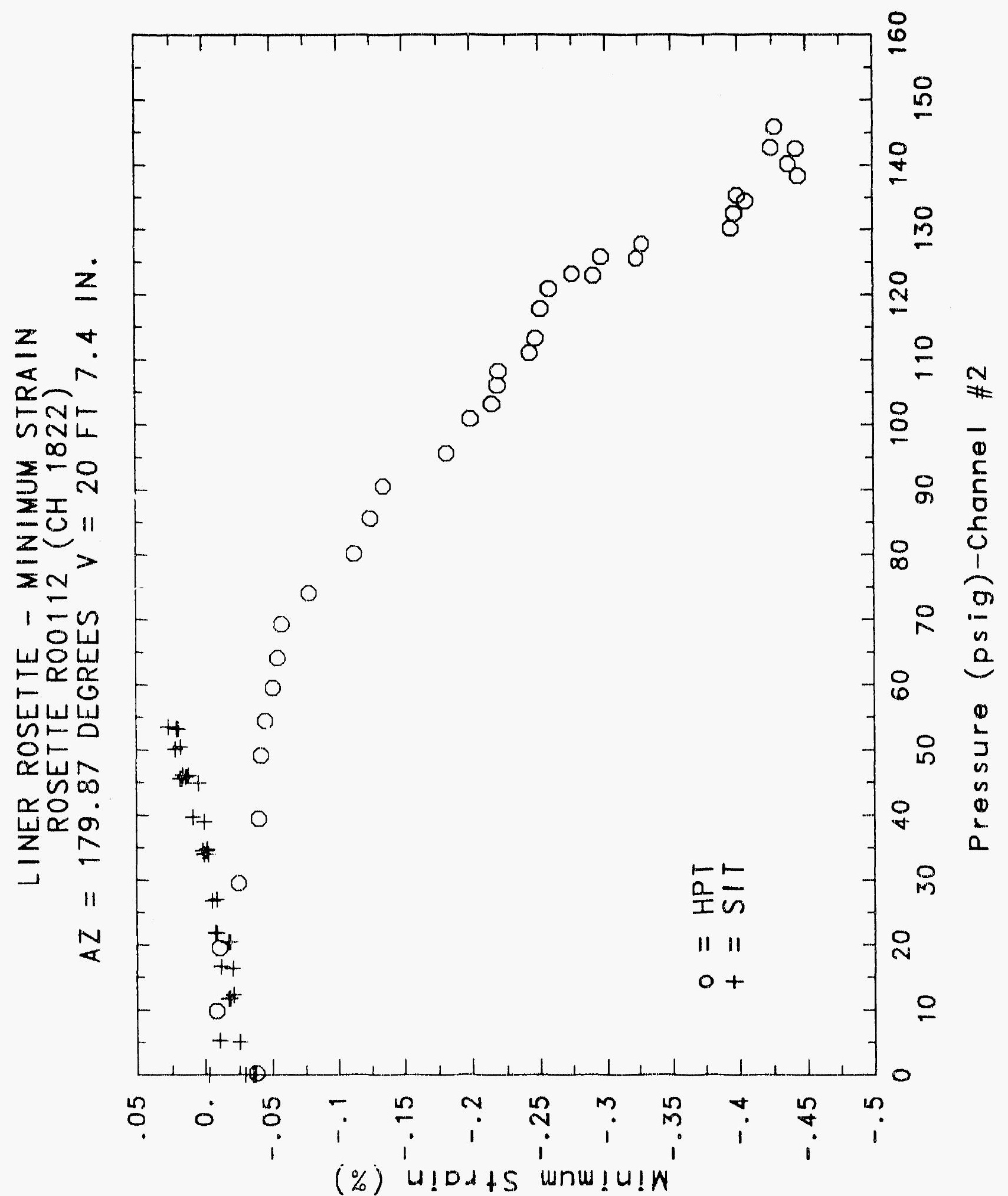


ㅇํㅇำ

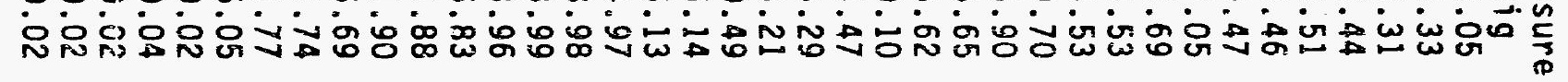
$00000000000000000000000000000000000000 \mathrm{~h}$

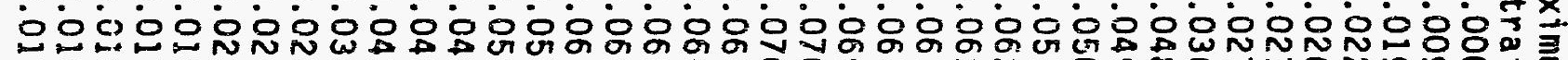

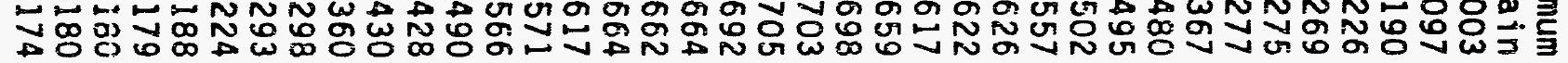

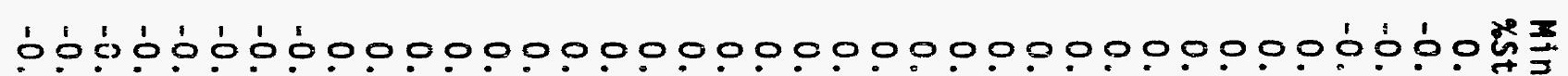

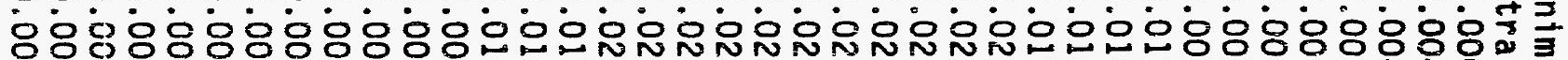

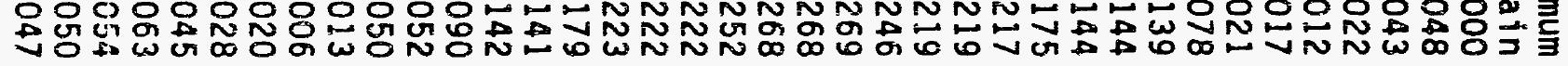

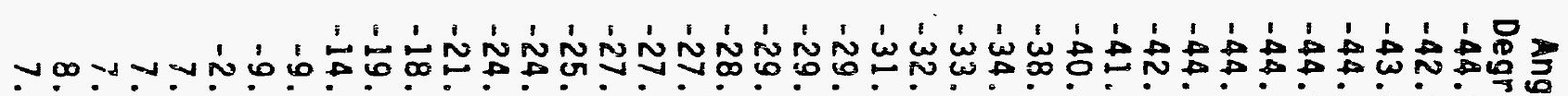

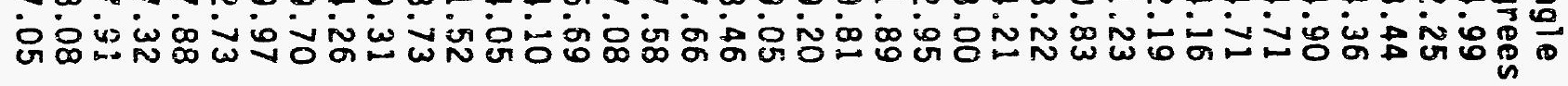

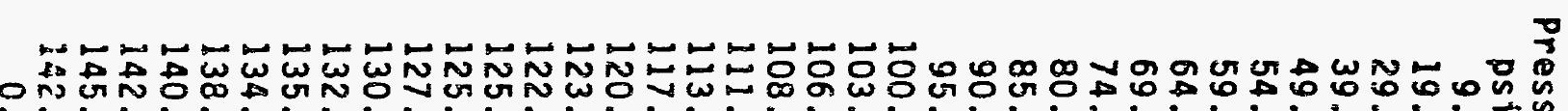
Nin

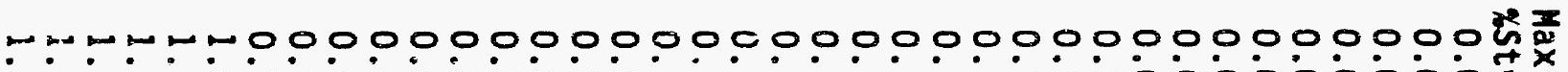

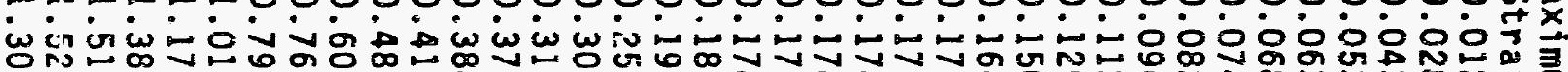

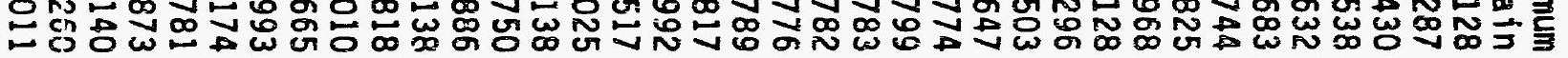

0000000000000000000000000000000000000月永

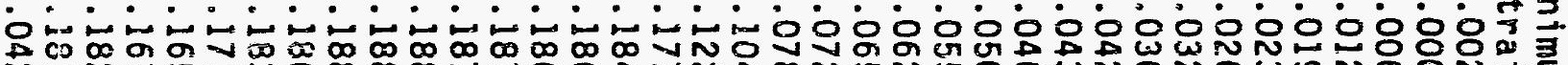

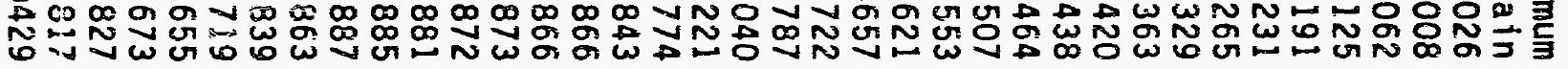

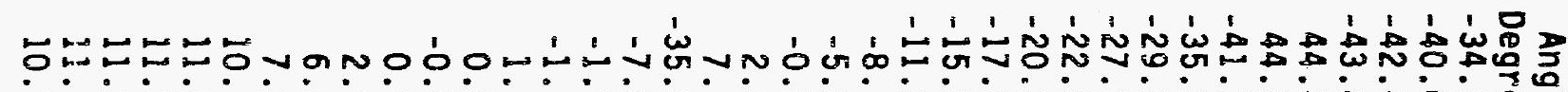

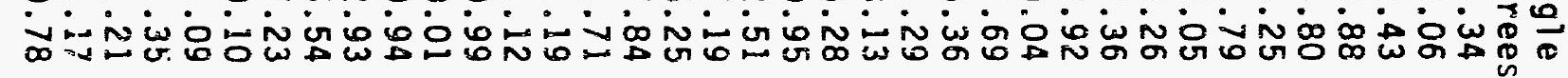




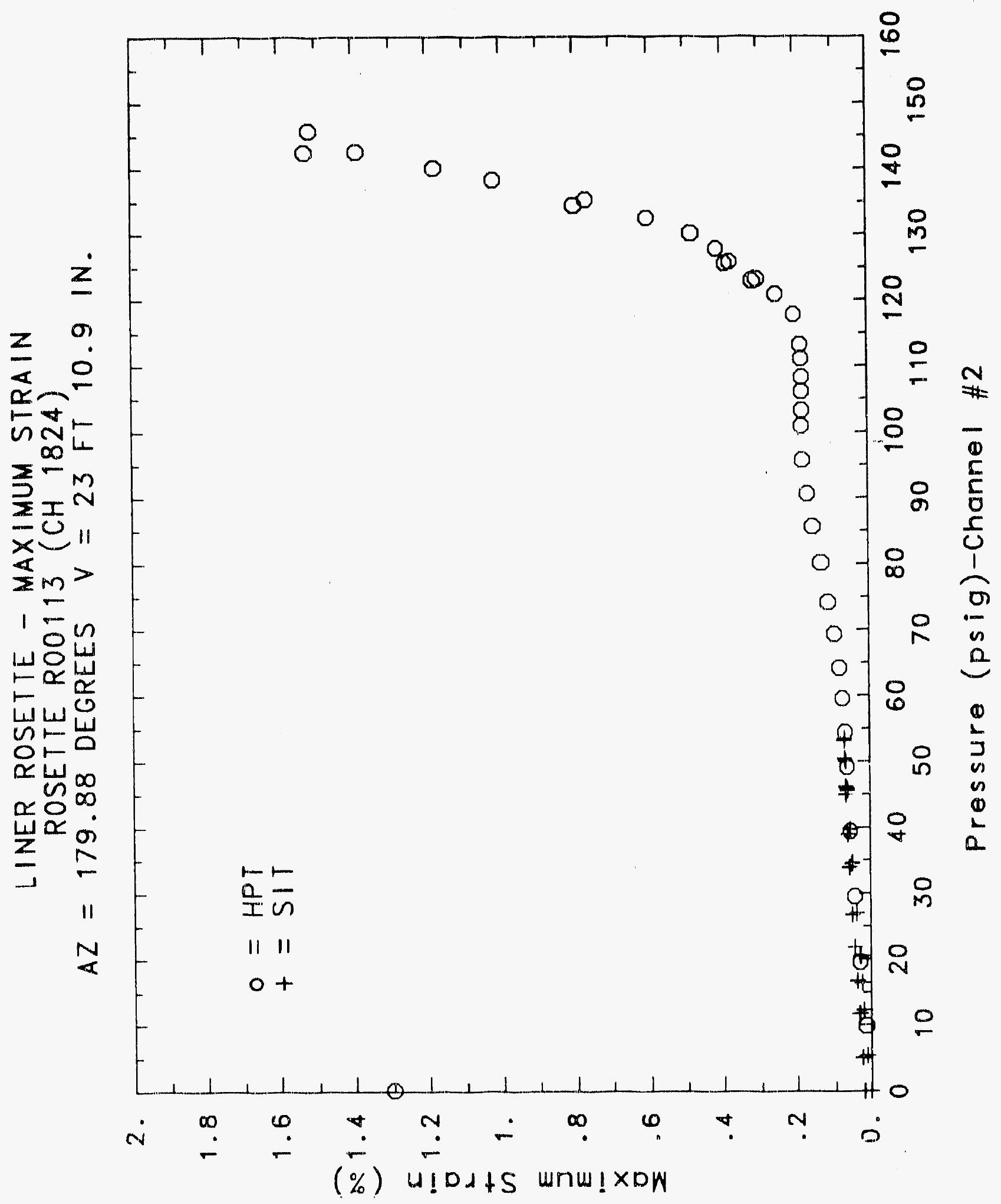




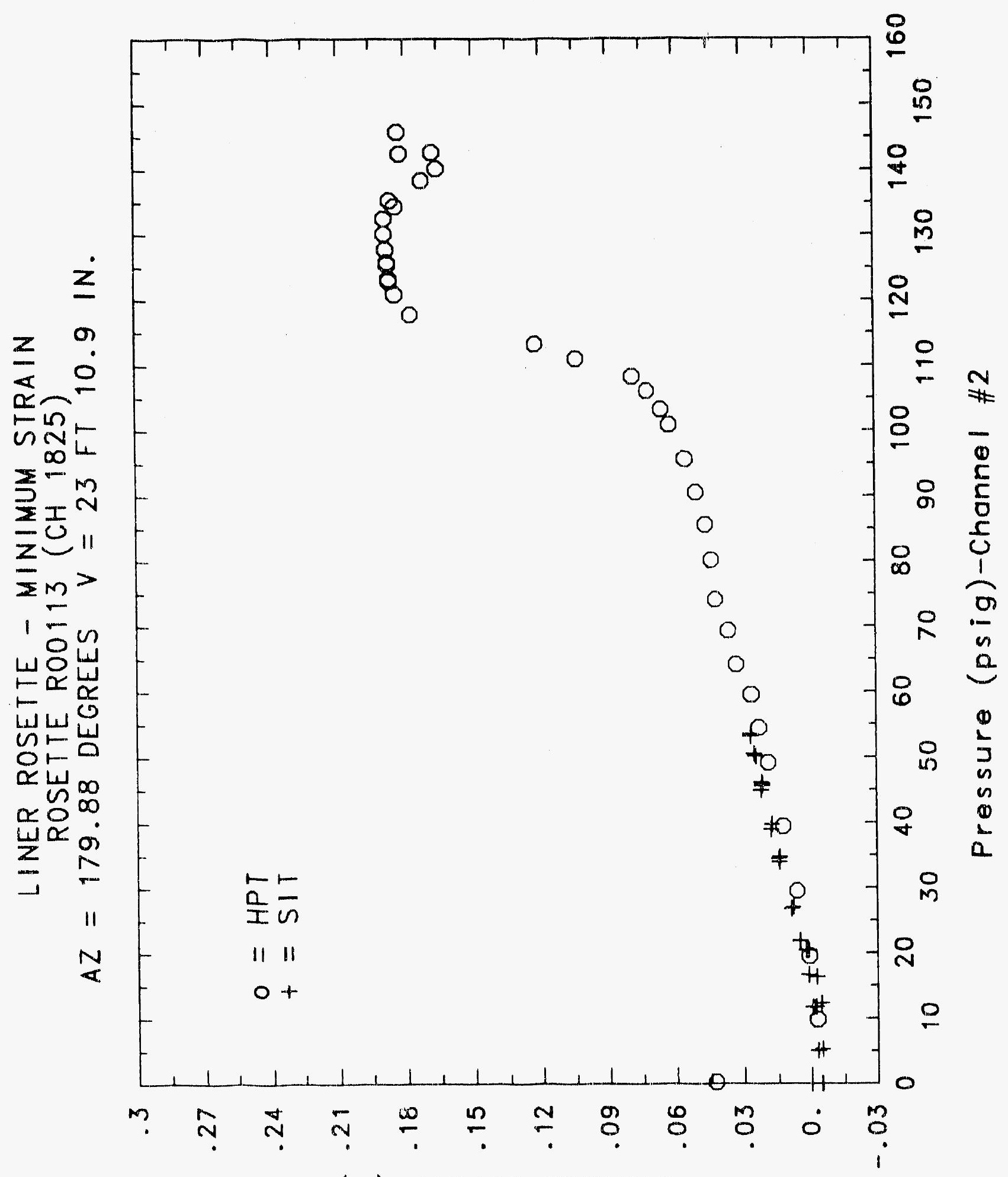

(\%) $u ! D d+S$ wnw!u!W 
ㅇํㅇำ

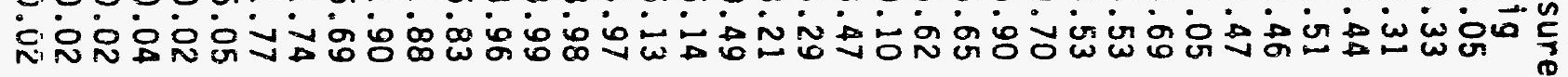

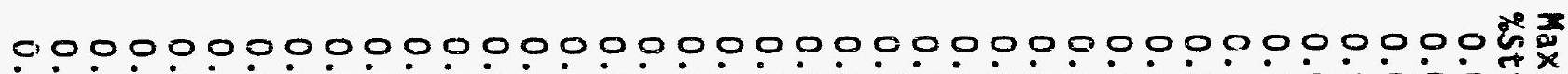

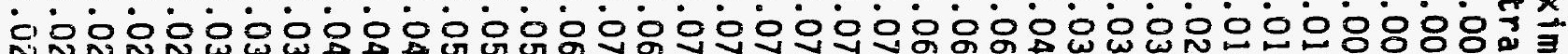
岱出出

10000000000000000000000000000000000000

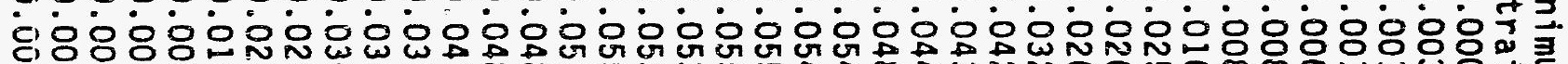

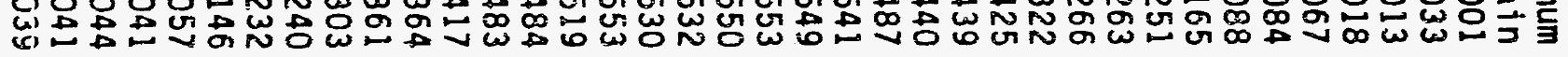

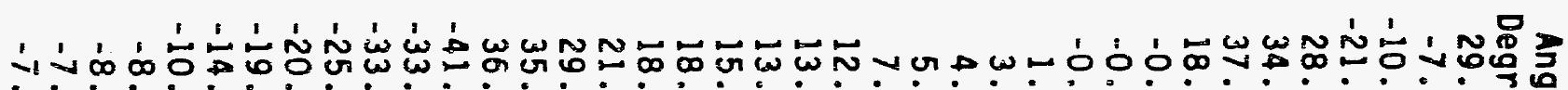

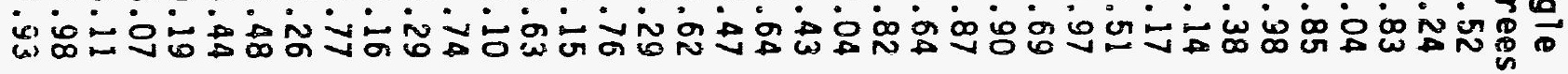

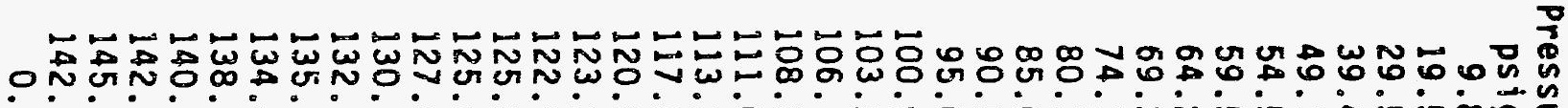

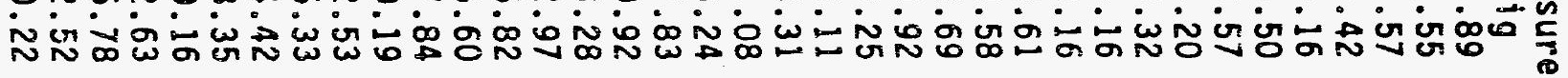

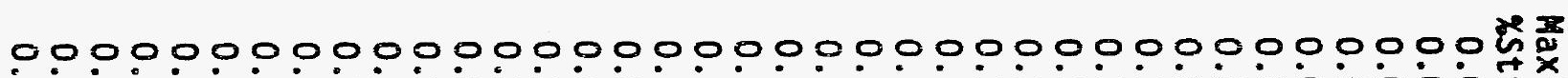

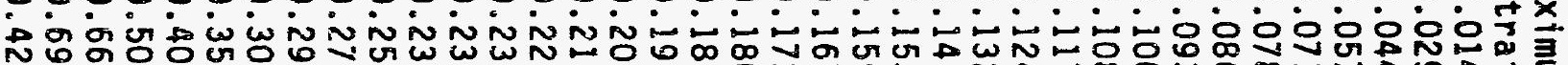

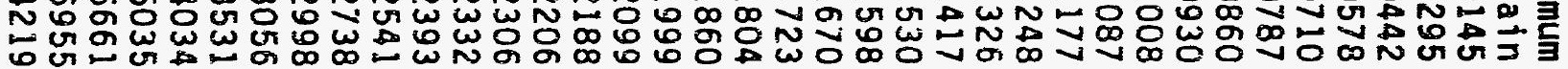

$00000000000000000000000000000000000004 \frac{1}{5}$

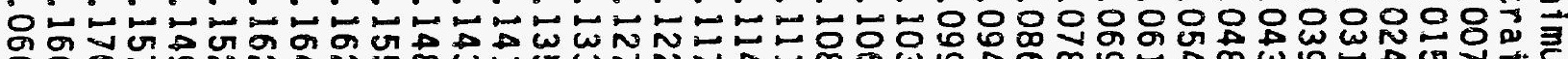

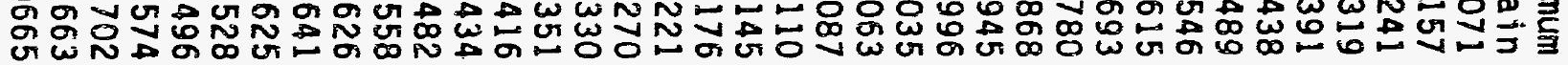

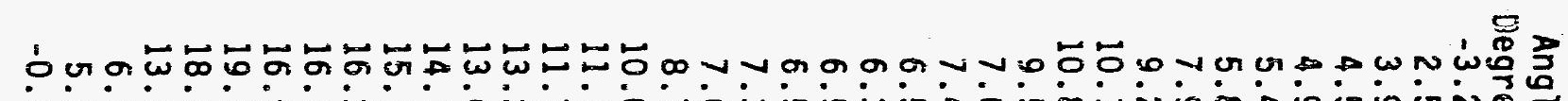

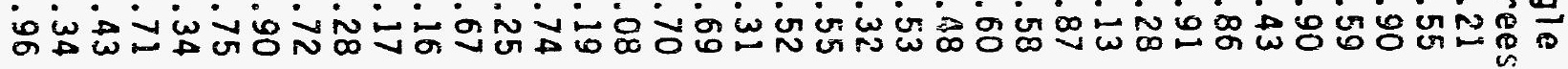




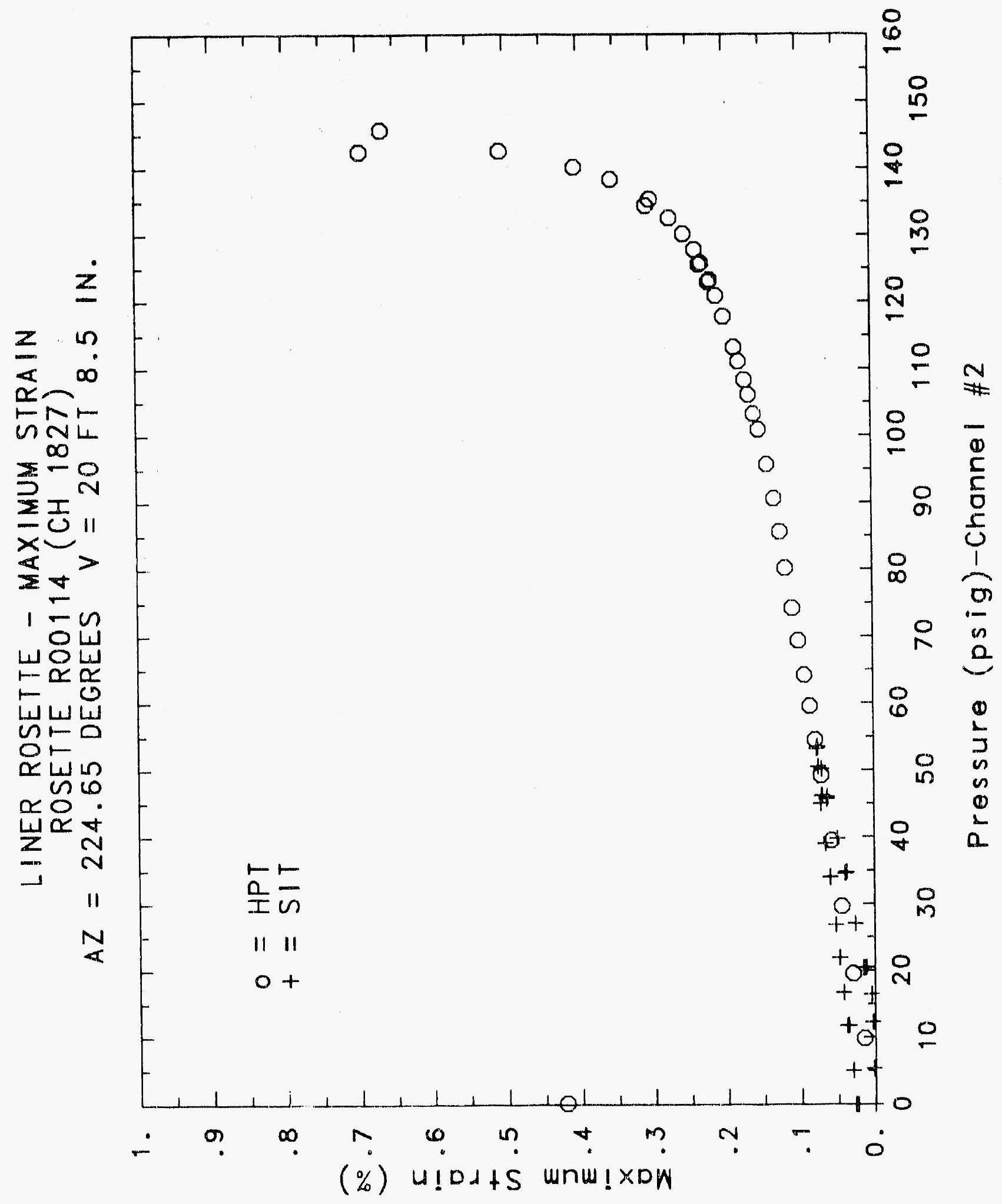




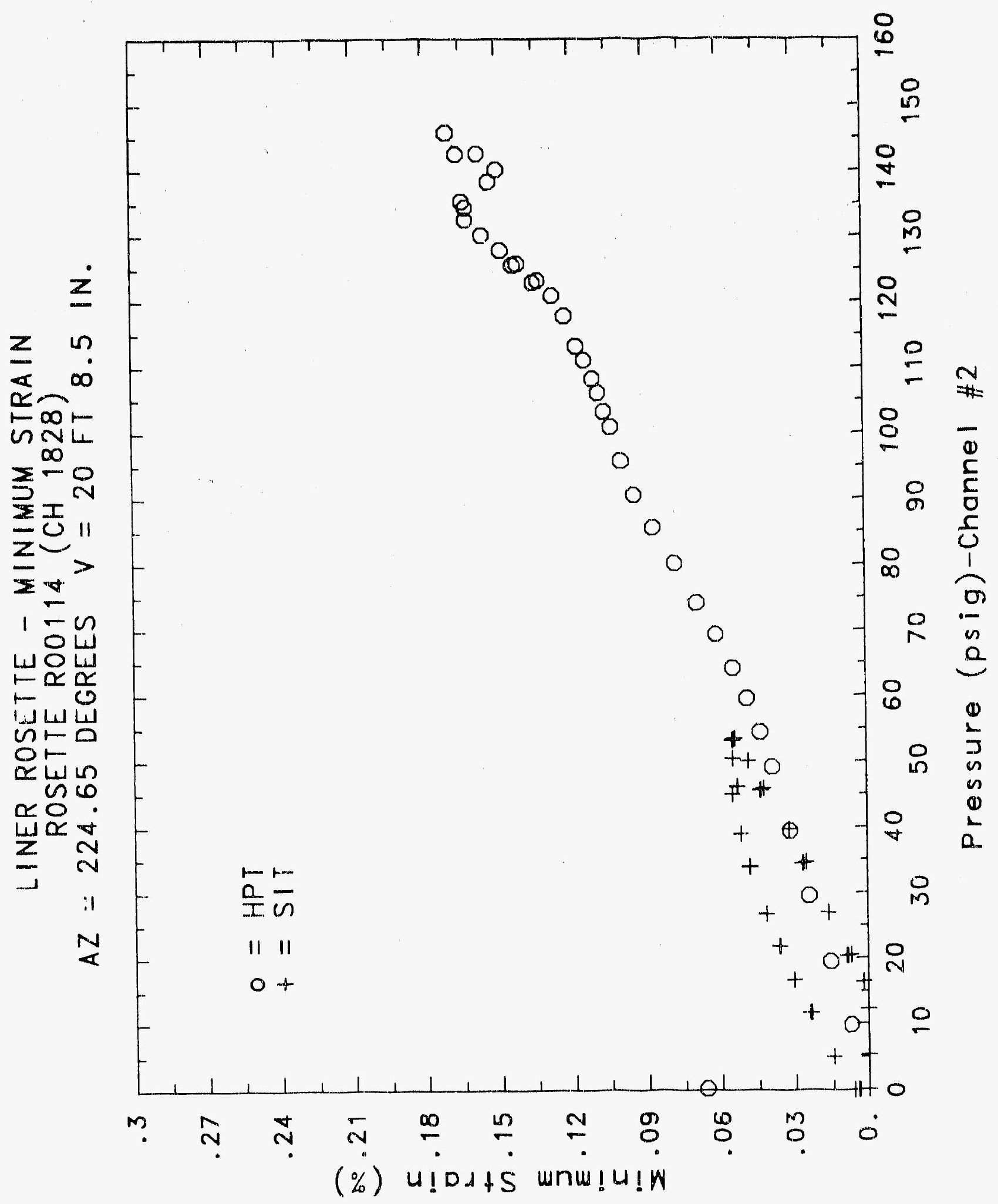




\section{Reinforced Concrete Test Data}

Rosette 115 Channels $1840 \quad 1841 \quad 1842$

STRUCTURAL INTEGRITY TEST

\begin{tabular}{|c|c|c|c|c|c|c|c|}
\hline $\begin{array}{c}\text { Pressure } \\
\text { psig } \\
\text {-0.05 } \\
5.33 \\
12.31 \\
16.44 \\
20.51 \\
20.46 \\
20.47 \\
27.05 \\
34.69 \\
34.53 \\
34.53 \\
39.70 \\
45.90 \\
45.65 \\
45.62 \\
50.10 \\
53.47 \\
53.29 \\
53.21 \\
50.49 \\
46.14 \\
46.13 \\
44.97 \\
38.98 \\
33.99 \\
33.96 \\
26.83 \\
21.88 \\
21.90 \\
16.69 \\
11.74 \\
11.77 \\
5.05 \\
0.02 \\
-0.04 \\
-0.02 \\
-0.02 \\
0.02\end{array}$ & $\begin{array}{l}\text { Maximum } \\
\text { \%Strain } \\
0.0005 \\
0.0161 \\
0.0183 \\
0.0207 \\
0.0249 \\
0.0259 \\
0.0263 \\
0.0342 \\
0.0450 \\
0.0456 \\
0.0457 \\
0.0510 \\
0.0587 \\
0.0590 \\
0.0589 \\
0.0640 \\
0.0690 \\
0.0693 \\
0.0691 \\
0.0673 \\
0.0638 \\
0.0635 \\
0.0644 \\
0.0590 \\
0.0546 \\
0.0545 \\
0.0484 \\
0.0436 \\
0.0433 \\
0.0364 \\
0.0285 \\
0.0283 \\
0.0186 \\
0.0029 \\
0.0030 \\
0.0027 \\
0.0026 \\
0.0024\end{array}$ & $\begin{array}{r}\text { Minimum } \\
\text { KStrain } \\
-0.0006 \\
-0.0146 \\
-0.0149 \\
-0.0131 \\
-0.0097 \\
-0.0090 \\
-0.0087 \\
-0.0021 \\
0.0064 \\
0.0070 \\
0.0072 \\
0.0107 \\
0.0149 \\
0.0149 \\
0.0149 \\
0.0174 \\
0.0199 \\
0.0200 \\
0.0200 \\
0.0190 \\
0.0167 \\
0.0166 \\
0.0171 \\
0.0138 \\
0.0105 \\
0.0104 \\
0.0053 \\
0.0014 \\
0.0012 \\
-0.0028 \\
-0.0054 \\
-0.0061 \\
-0.0052 \\
0.0012 \\
0.0009 \\
0.0009 \\
0.0009 \\
0.0006\end{array}$ & $\begin{array}{c}\text { Angle } \\
\text { Degrees } \\
40.66 \\
39.71 \\
40.64 \\
42.16 \\
44.92 \\
-44.53 \\
-44.29 \\
-40.85 \\
-38.48 \\
-38.45 \\
-38.58 \\
-38.71 \\
-40.03 \\
-40.63 \\
-40.70 \\
-40.97 \\
-41.02 \\
-41.34 \\
-41.54 \\
-41.89 \\
-42.31 \\
-42.31 \\
-42.10 \\
-42.70 \\
-43.25 \\
-43.28 \\
-44.53 \\
44.13 \\
44.12 \\
42.75 \\
43.76 \\
43.29 \\
-44.82 \\
-10.84 \\
-17.06 \\
-17.93 \\
-23.60 \\
-25.99\end{array}$ & $\begin{array}{c}\text { Pressure } \\
\text { psig } \\
9.89 \\
19.55 \\
29.57 \\
39.42 \\
49.16 \\
54.50 \\
59.57 \\
64.20 \\
69.32 \\
74.16 \\
80.16 \\
85.61 \\
90.58 \\
95.69 \\
100.92 \\
103.25 \\
106.11 \\
108.31 \\
111.08 \\
113.24 \\
117.83 \\
120.92 \\
123.28 \\
122.97 \\
125.82 \\
125.60 \\
127.84 \\
130.19 \\
132.53 \\
135.33 \\
134.42 \\
138.35 \\
140.16 \\
142.63 \\
145.78 \\
142.52 \\
0.22\end{array}$ & $\begin{array}{l}\text { Maximum } \\
\text { \%Strain } \\
0.0225 \\
0.0358 \\
0.0476 \\
0.0578 \\
0.0667 \\
0.0724 \\
0.0796 \\
0.0860 \\
0.0929 \\
0.0998 \\
0.1074 \\
0.1165 \\
0.1246 \\
0.1339 \\
0.1412 \\
0.1438 \\
0.1482 \\
0.1521 \\
0.1554 \\
0.1580 \\
0.1638 \\
0.1702 \\
0.1804 \\
0.1836 \\
0.1976 \\
0.2018 \\
0.2105 \\
0.2337 \\
0.2808 \\
0.3763 \\
0.3958 \\
0.5515 \\
0.7063 \\
0.9205 \\
1.0450 \\
1.0578 \\
0.8690\end{array}$ & $\begin{array}{l}\text { Minimum } \\
\text { xStrain } \\
-0.0084 \\
-0.0029 \\
0.0049 \\
0.0124 \\
0.0198 \\
0.0239 \\
0.0283 \\
0.0317 \\
0.0345 \\
0.0374 \\
0.0407 \\
0.0438 \\
0.0471 \\
0.0502 \\
0.0545 \\
0.0568 \\
0.0592 \\
0.0608 \\
0.0644 \\
0.0667 \\
0.0730 \\
0.0773 \\
0.0798 \\
0.0804 \\
0.0837 \\
0.0848 \\
0.0888 \\
0.0987 \\
0.1167 \\
0.1543 \\
0.1629 \\
0.2289 \\
0.2805 \\
0.3630 \\
0.4308 \\
0.4381 \\
0.2633\end{array}$ & $\begin{array}{c}\text { Angle } \\
\text { Degrees } \\
42.01 \\
41.47 \\
43.73 \\
-44.31 \\
-42.91 \\
-41.96 \\
-40.62 \\
-40.91 \\
-41.47 \\
-41.54 \\
-41.71 \\
-41.19 \\
-40.46 \\
-39.24 \\
-38.40 \\
-37.79 \\
-37.07 \\
-36.67 \\
-35.73 \\
-34.87 \\
-32.25 \\
-30.12 \\
-28.71 \\
-28.51 \\
-28.06 \\
-28.83 \\
-29.80 \\
-33.68 \\
-40.11 \\
39.08 \\
37.27 \\
26.44 \\
20.03 \\
12.63 \\
9.12 \\
8.53 \\
7.91\end{array}$ \\
\hline
\end{tabular}

High Pressure test 


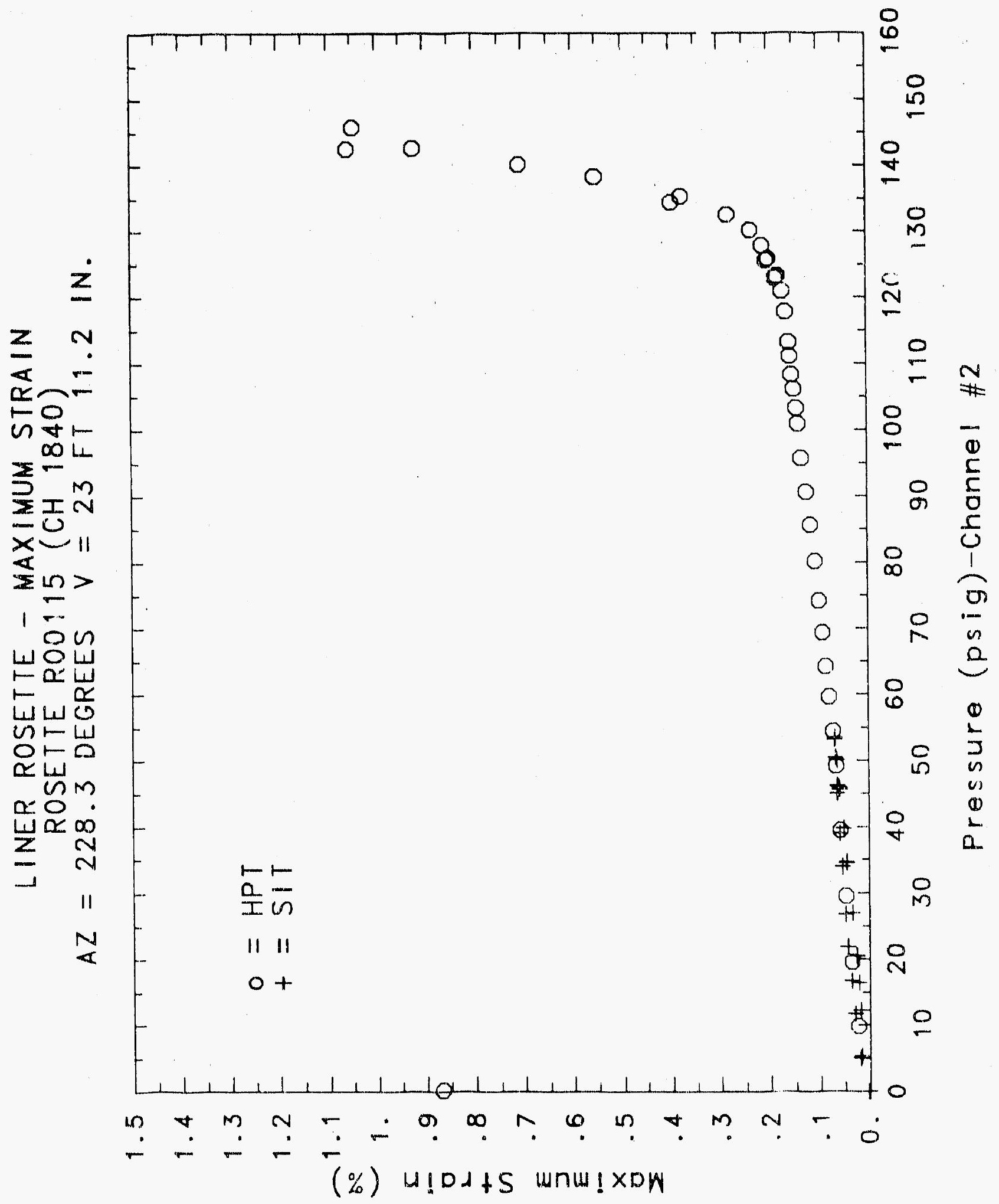

C. 66.3 


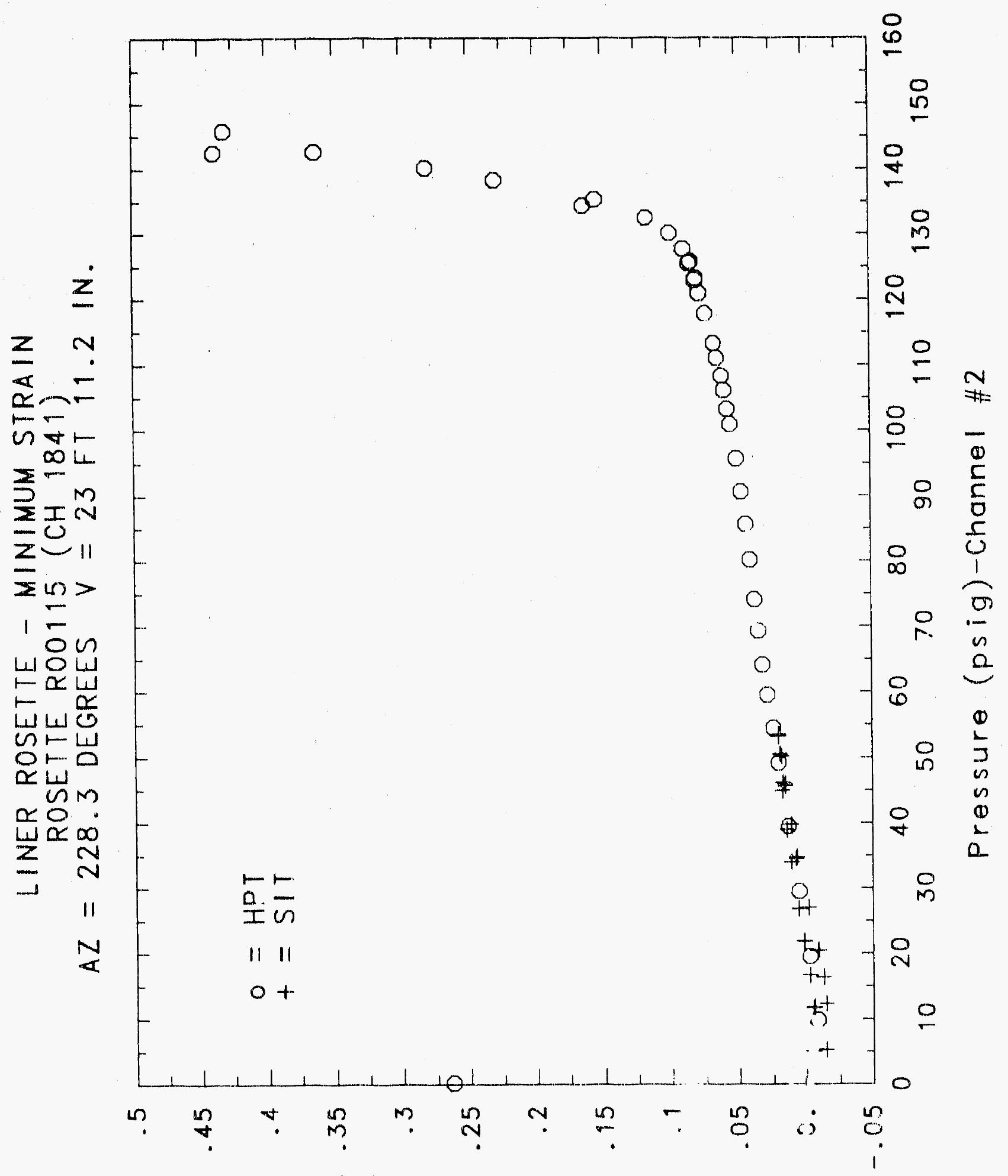

(\%) $u ! D d+5$ unw! $w ! w$ 


\section{Reinforced Concrete Test Data}

\section{Rosette 116 Channels $1843 \quad 1844 \quad 1845$}

\section{Structural Integrity Test}

\begin{tabular}{|c|c|c|c|c|c|c|c|}
\hline Pressure & Maximum & Mirimum & Angle & Presslire & Maximum & Minimum & Angle \\
\hline psig & \%strain & *strain & Degrees & psig & xstrain & xstrain & Degree: \\
\hline-0.05 & 0.0005 & -0.0001 & 22.52 & 9.89 & 0.0064 & -0.0036 & $-21 \cdot 15$ \\
\hline 5.33 & 0.0048 & -0.0038 & -10.47 & 19.55 & 0.0160 & -0.0018 & -16.95 \\
\hline 12.31 & 0.0089 & -0.0004 & -11.30 & 29.57 & 0.0281 & 0.0050 & -18.14 \\
\hline 16.44 & 0.0119 & 0.0019 & -10.36 & 39.42 & 0.0367 & 0.0139 & -19.92 \\
\hline 20.51 & 0.0146 & 0.0072 & -16.65 & 49.16 & 0.0449 & 0.0215 & -18.71 \\
\hline 20.46 & 0.0146 & 0.0082 & -20.53 & 54.50 & 0.0499 & 0.0252 & -17.36 \\
\hline 20.47 & 0.0146 & 0.0087 & -21.82 & 59.57 & 0.0557 & 0.0294 & -16.61 \\
\hline 27.05 & 0.0211 & 0.0156 & 39.06 & 64.20 & 0.0624 & 0.0339 & -14.62 \\
\hline 34.59 & 0.0311 & $0.02: 9$ & 20.0 & 69. & 0.0711 & 0.0377 & -13.56 \\
\hline 34.53 & 0.0329 & 0.0222 & 15.04 & 74.16 & 0.0793 & 0.0407 & -12.96 \\
\hline 34.53 & 0.0332 & 0.0220 & 15.29 & 80.16 & 0.0893 & 0.0439 & -12.81 \\
\hline 39.70 & 0.0411 & 0.0259 & 8.89 & 85.61 & 0.0980 & 0.0471 & -12.80 \\
\hline 45.90 & 0.0535 & 0.0304 & 5.39 & 90. & 0.1057 & 0.0499 & -12.30 \\
\hline 45.65 & 0.0546 & 0.0305 & 5.1 & 95. & 0.1146 & 0.0525 & $=12.47$ \\
\hline 45.62 & 0.0547 & 0.0302 & 5.58 & 100.92 & 0.1228 & 0.055 & -12.38 \\
\hline 50.10 & 0.0606 & 0.0333 & 4,59 & 103. & 0.1274 & 0.1 & -12.71 \\
\hline 53.47 & 0.0673 & 0.0358 & 3.79 & 106.11 & 0.1325 & 0.0580 & -12.93 \\
\hline 53.29 & 0.0681 & 0.0358 & 3.56 & 108.31 & 0.1367 & 0.0593 & -13.08 \\
\hline 53.21 & 0.0681 & 0.0359 & 3. & 111.08 & 0.1436 & 0.0611 & -13.81 \\
\hline 50.89 & 0.1 & & 3. & & 0.14 & & $-14,28$ \\
\hline 46.14 & 0.0639 & 0. & 3. & & 0.100 & & -14.62 \\
\hline 46.13 & 0.0637 & 0.0318 & 3.54 & 120.92 & 0.1694 & 0.0690 & -14.82 \\
\hline 44.97 & 0.0645 & 0.0316 & 3.05 & 123.28 & 0.1776 & 0.0716 & .14 .94 \\
\hline 38.98 & 0.0605 & 0.0255 & 3.34 & 122.97 & 0.1797 & 0.07 & -14.73 \\
\hline 33.99 & 0.0570 & 0.0202 & 4.14 & 125.82 & 0.1901 & 0.0743 & -14.66 \\
\hline 33.96 & 0.0570 & 0.0190 & 4.27 & 1.25 .60 & 0.1922 & 0.0743 & -14.74 \\
\hline 26.83 & 0.0516 & 0.0124 & 5.59 & .84 & 0.1978 & 0.0766 & -14.66 \\
\hline 21.88 & 0.0465 & $0.00 \% 6$ & 7.22 & 130.19 & 0.2100 & 0.0796 & -14.40 \\
\hline 21.90 & 0.0463 & 0.0075 & 6.99 & 132.53 & 0.2288 & 0.0834 & -13.98 \\
\hline 16.69 & 0.0384 & 0.0045 & 9.87 & 135.33 & 0.2527 & 0.0910 & -14.03 \\
\hline 11.74 & 0.0327 & 0.0036 & 12.27 & 134.42 & 0.2573 & 0.0912 & -14.14 \\
\hline 11.77 & 0.0323 & 0.0034 & 12.07 & 138.35 & 0.2863 & 0.1037 & $\ldots 18$ \\
\hline 5.05 & 0.0300 & 0.0044 & 18.14 & 140.16 & 0.3116 & 0.1148 & -14.03 \\
\hline 0.02 & 0.0301 & 0.0045 & 25.02 & 142.63 & 0.3507 & 0.1326 & -14.20 \\
\hline-0.04 & 0.0294 & 0.0050 & 23.39 & 145.78 & 0.1920 & 0.1475 & -13.68 \\
\hline-0.02 & 0.0292 & 0.0047 & 23.11 & 142.52 & 0.3947 & 0.1436 & .13 .61 \\
\hline-0.02 & 0.0292 & 0.0044 & 23.81 & 0.22 & 0.2359 & 0.0217 & -16.88 \\
\hline 0.02 & 0.0291 & 0.0041 & 23.64 & & & & \\
\hline
\end{tabular}

High Pressure test esslire Raximum

$29.57 \quad 0.0281$

54.50

59.57

74.16

85.61

90.58

106.11

122.97

127.84

132.53

135.33

1420.63

45.78

5.52

0.22
Minimum

0.0036

0.0139

0.0252

0.0294

0.0499

0.056

$-12.71$

$-12.93$

$-13.08$

$-13.81$

14.62

14.82

4.66

14.66

13.98

14.03

4.03

13.68

13.61

16.88 


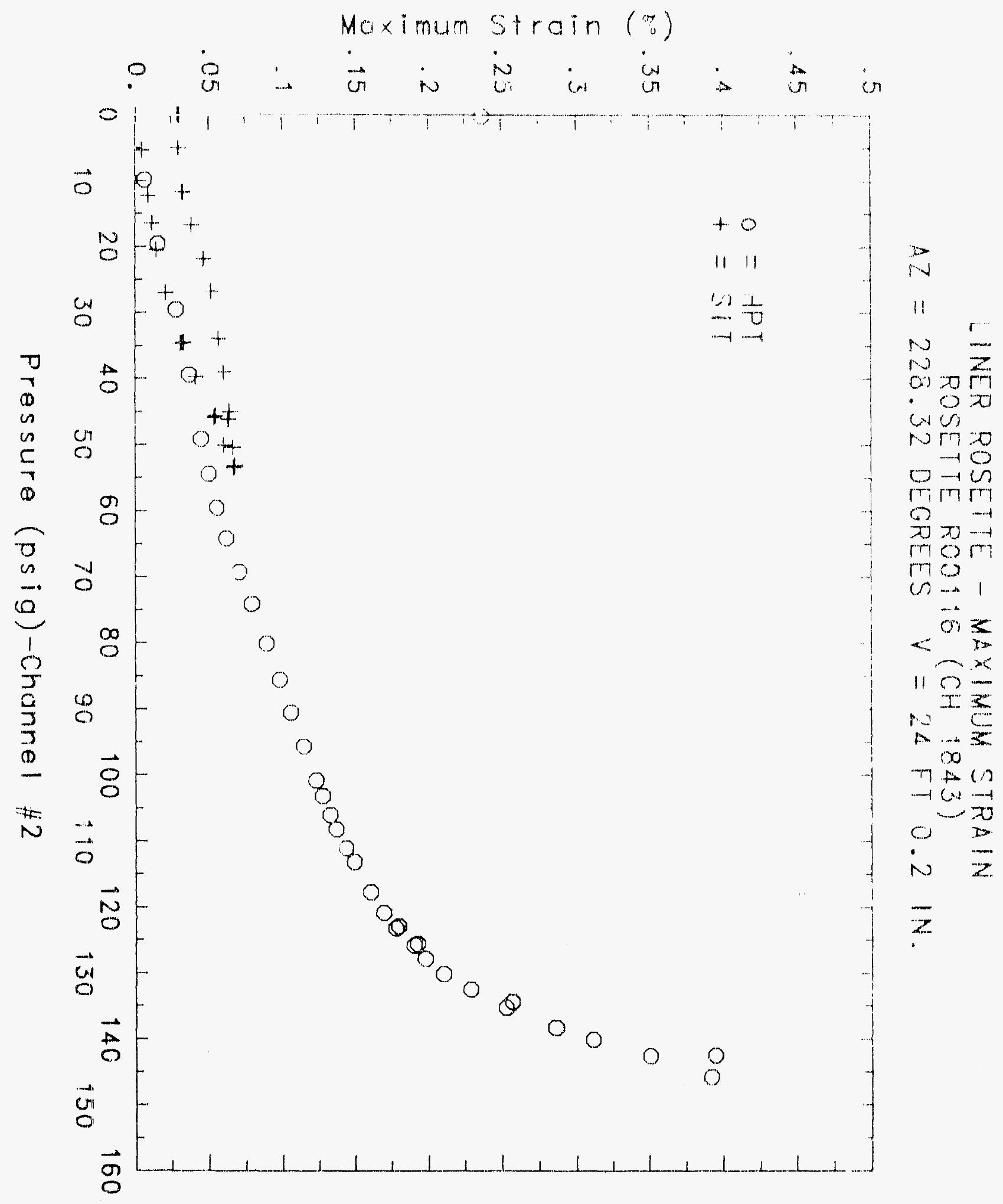




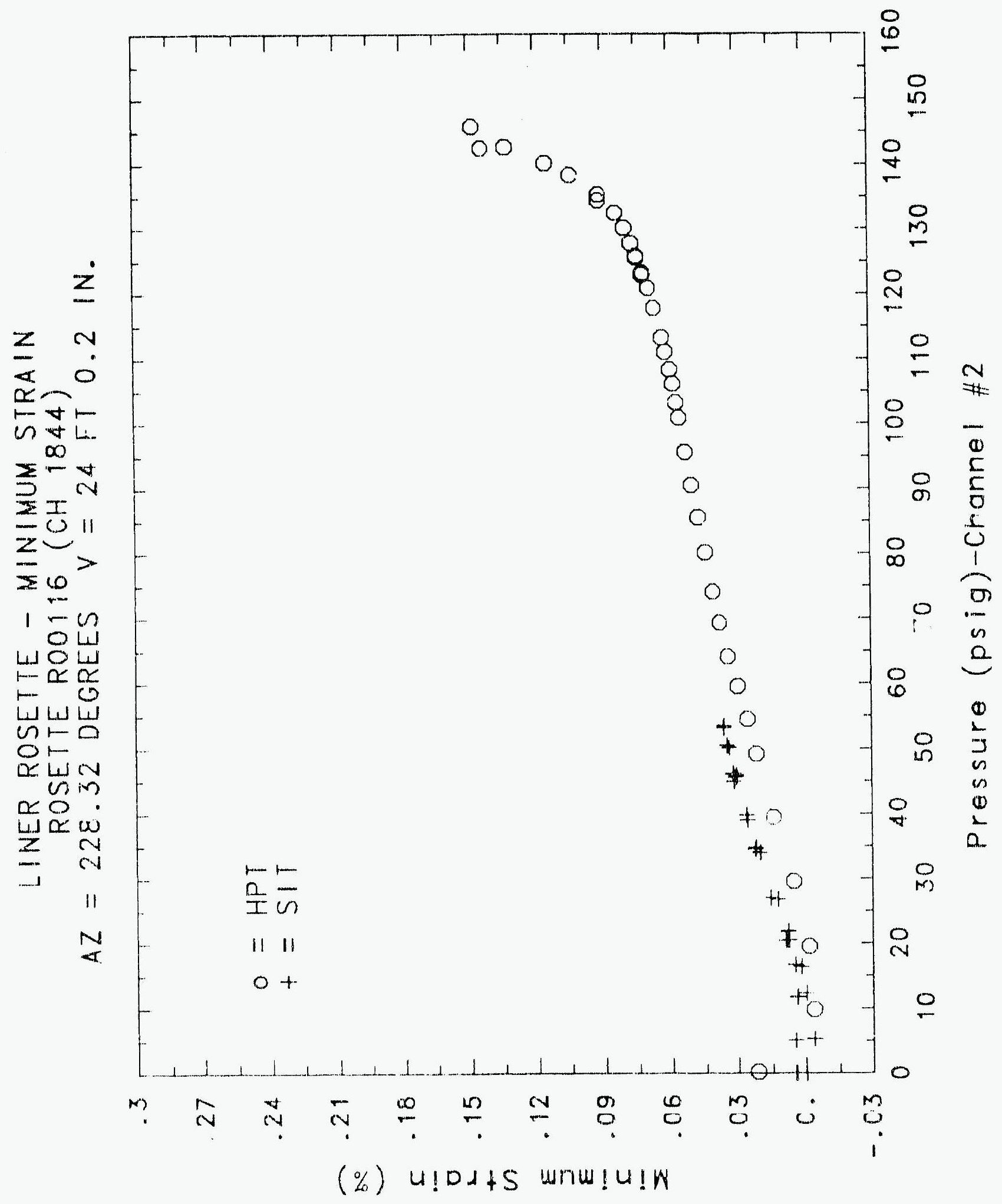

C- $-6,67$ 


\title{
Reinforced Concrete Test Data
}

\author{
Rosette 117 Channels $1846 \quad 1847 \quad 1848$
}

\section{STRUCTURAL INTEGRITY TEST}

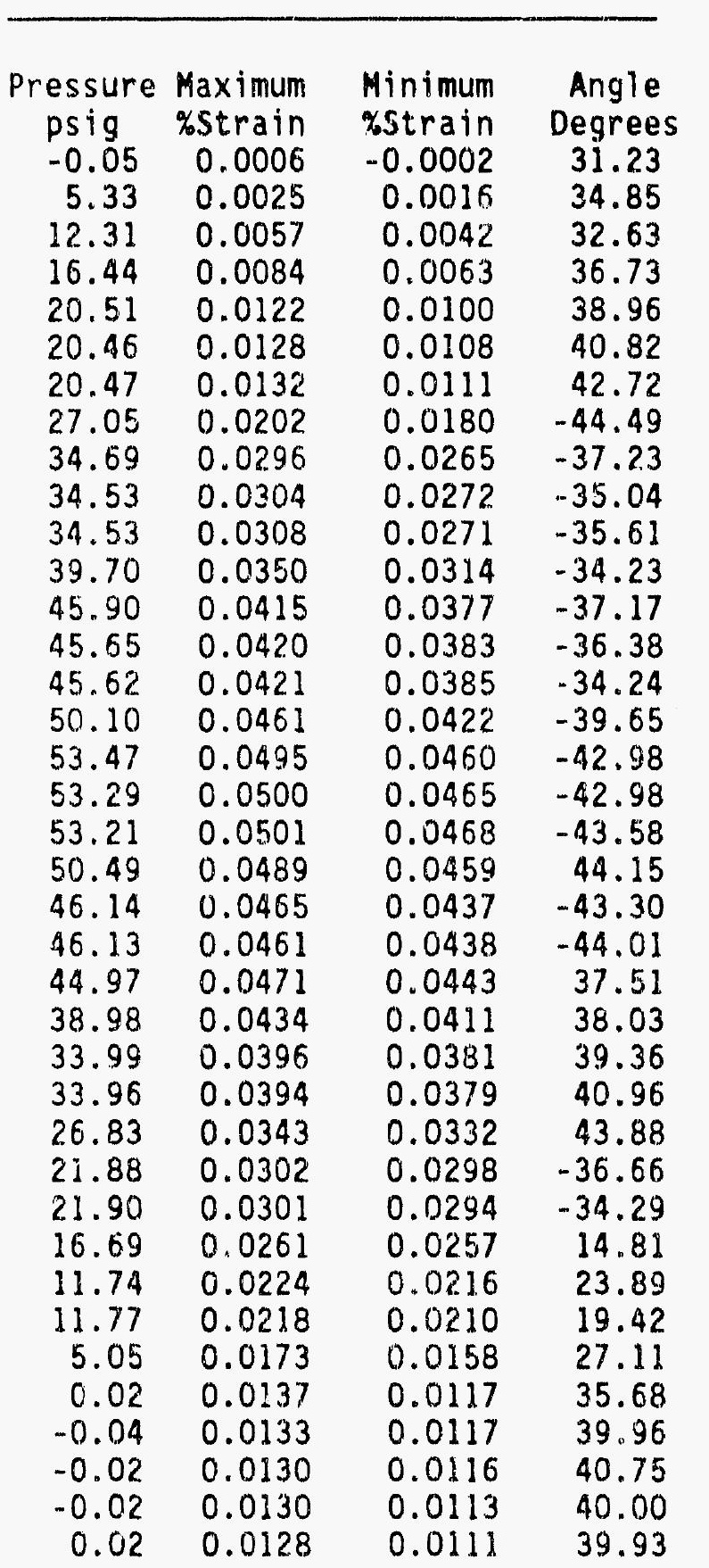

\section{High Pressure Test}

\begin{tabular}{|c|c|c|c|}
\hline Pressure & Maximum & Minimum & Angle \\
\hline psig & xstrain & xstrain & Degrees \\
\hline 9.89 & 0.0079 & 0.0066 & -41.58 \\
\hline 19.55 & 0.0158 & 0.0134 & 43.58 \\
\hline 29.57 & 0.0239 & 0.0205 & 41.94 \\
\hline 39.42 & 0.0315 & 0.0276 & 39.77 \\
\hline 49.16 & 0.0392 & 0.0345 & 38.04 \\
\hline 54.50 & 0.0440 & 0.0387 & 36.13 \\
\hline 59.57 & 0.0486 & 0.0430 & 34.54 \\
\hline 64.20 & 0.0535 & 0.0478 & 31.84 \\
\hline 69.32 & 0.0590 & 0.0529 & 30.78 \\
\hline 74.16 & 0.0645 & 0.0582 & 30.56 \\
\hline 80.16 & 0.0712 & 0.0647 & 31.49 \\
\hline 85.61 & 0.0774 & 0.0706 & 31.48 \\
\hline 90.58 & 0.0832 & 0.0763 & 31.13 \\
\hline 95.69 & 0.0887 & 0.0830 & 28.30 \\
\hline 100.92 & 0.0955 & 0.0893 & 31.57 \\
\hline 103.25 & 0.0986 & 0.0925 & 29.66 \\
\hline 106.11 & 0.1018 & 0.0960 & 29.21 \\
\hline 108.31 & 0.1045 & 0.0985 & 28.43 \\
\hline 111.08 & 0.1083 & 0.1022 & 27.72 \\
\hline 113.24 & 0.1113 & 0.1050 & 26.46 \\
\hline 117.83 & 0.1178 & 0.1111 & 22.97 \\
\hline 120.92 & 0.1231 & 0.1155 & 20.51 \\
\hline 123.28 & 0.1284 & 0.1196 & 16.15 \\
\hline $12 \% .97$ & 0.1300 & 0.1206 & 15.10 \\
\hline 125.82 & 0.1356 & 0.1243 & 13.13 \\
\hline 125.60 & 0.1360 & 0.1246 & 11.43 \\
\hline 127.84 & 0.1396 & 0.1269 & 11.01 \\
\hline 130.19 & 0.1463 & 0.1303 & 9.63 \\
\hline 132.53 & 0.1559 & 0.1342 & 7.73 \\
\hline 135.33 & 0.1716 & 0.1398 & 6.79 \\
\hline 134.42 & 0.1745 & 0.1403 & 6.53 \\
\hline 138.35 & 0.1984 & 0.1489 & 5.79 \\
\hline 140.16 & 0.2180 & 0.1557 & 4.78 \\
\hline 142.63 & 0.2466 & 0.1665 & 3.71 \\
\hline 145.78 & 0.2751 & 0.1811 & 3.12 \\
\hline 142.52 & 0.2755 & 0.1778 & 2.73 \\
\hline 0.22 & 0.1308 & 0.0439 & 7.08 \\
\hline
\end{tabular}




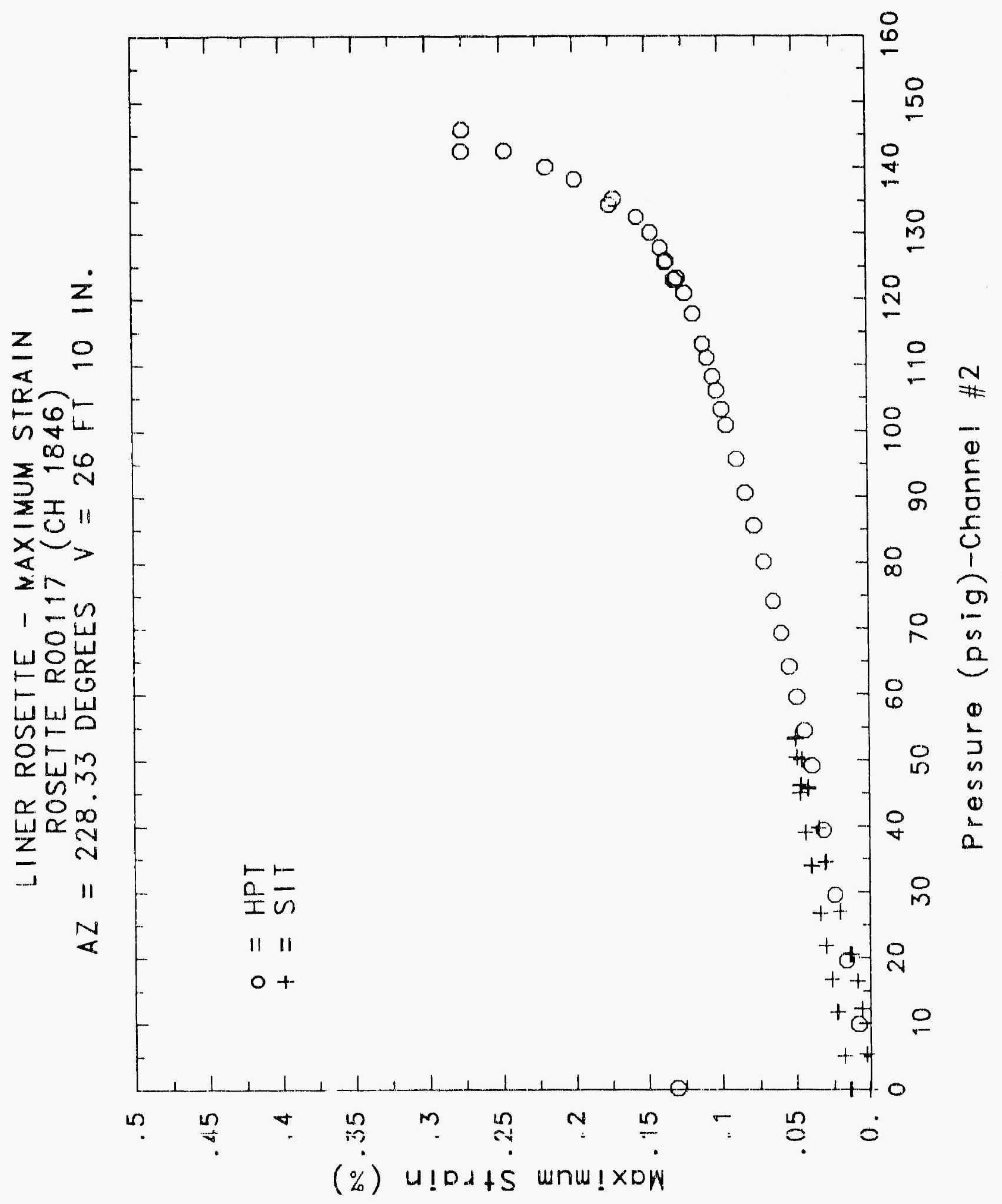

C. -669 


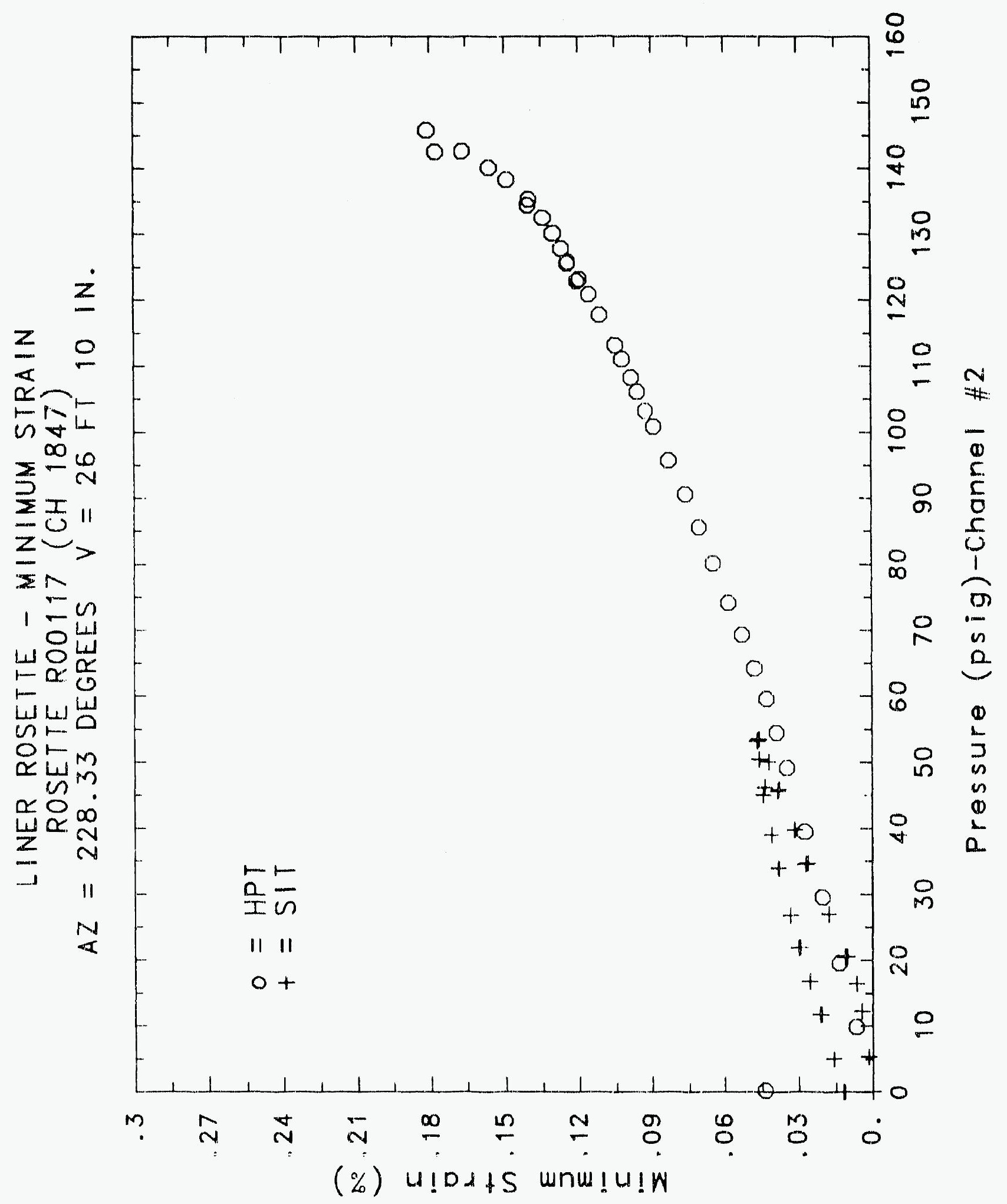




\section{Reinforced Concrete Test Data}

\section{Rosette 118 Channels $1849 \quad 1860 \quad 1861$}

\section{Structural INTEgRity TEST}

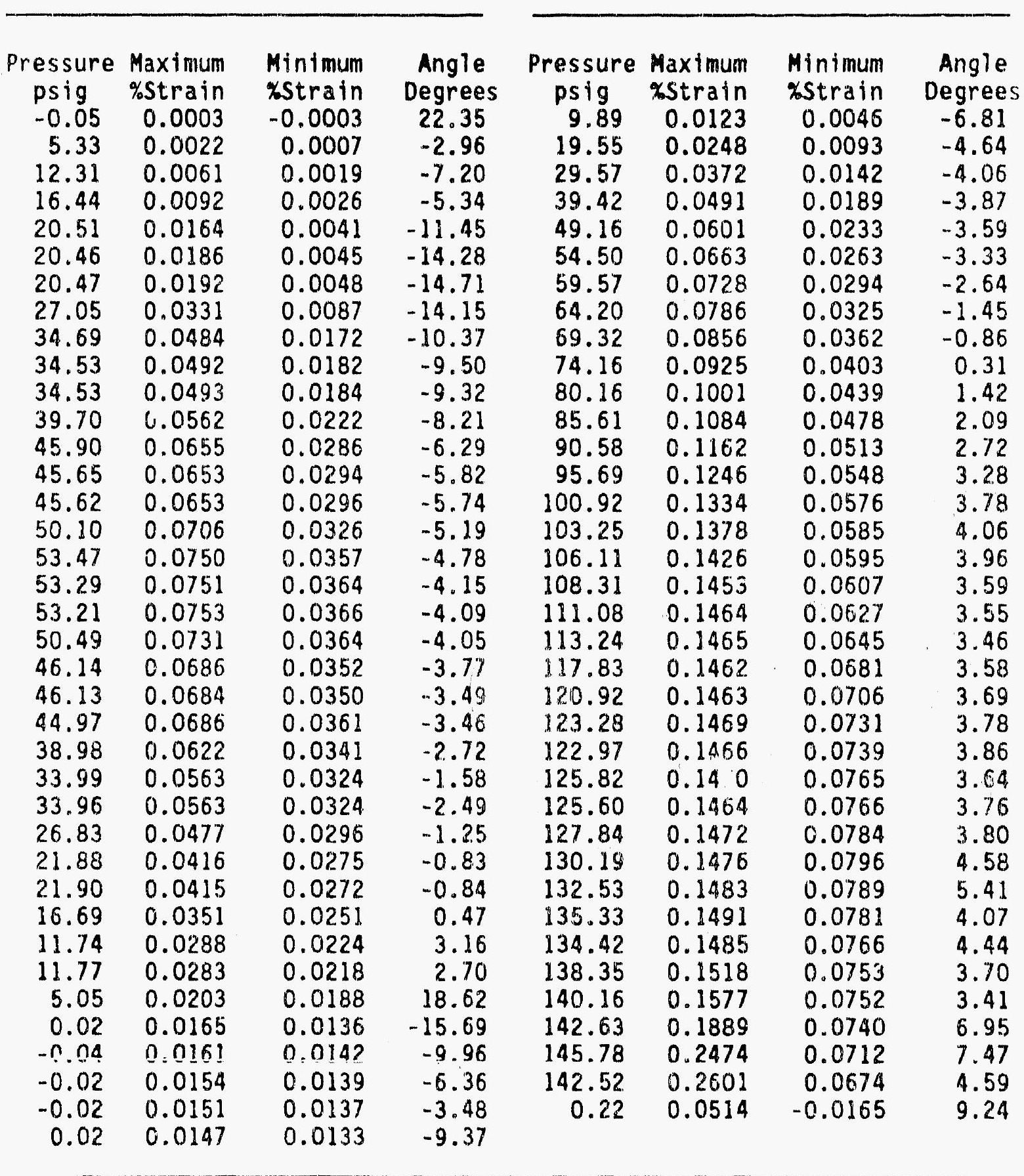

High Pressure test 


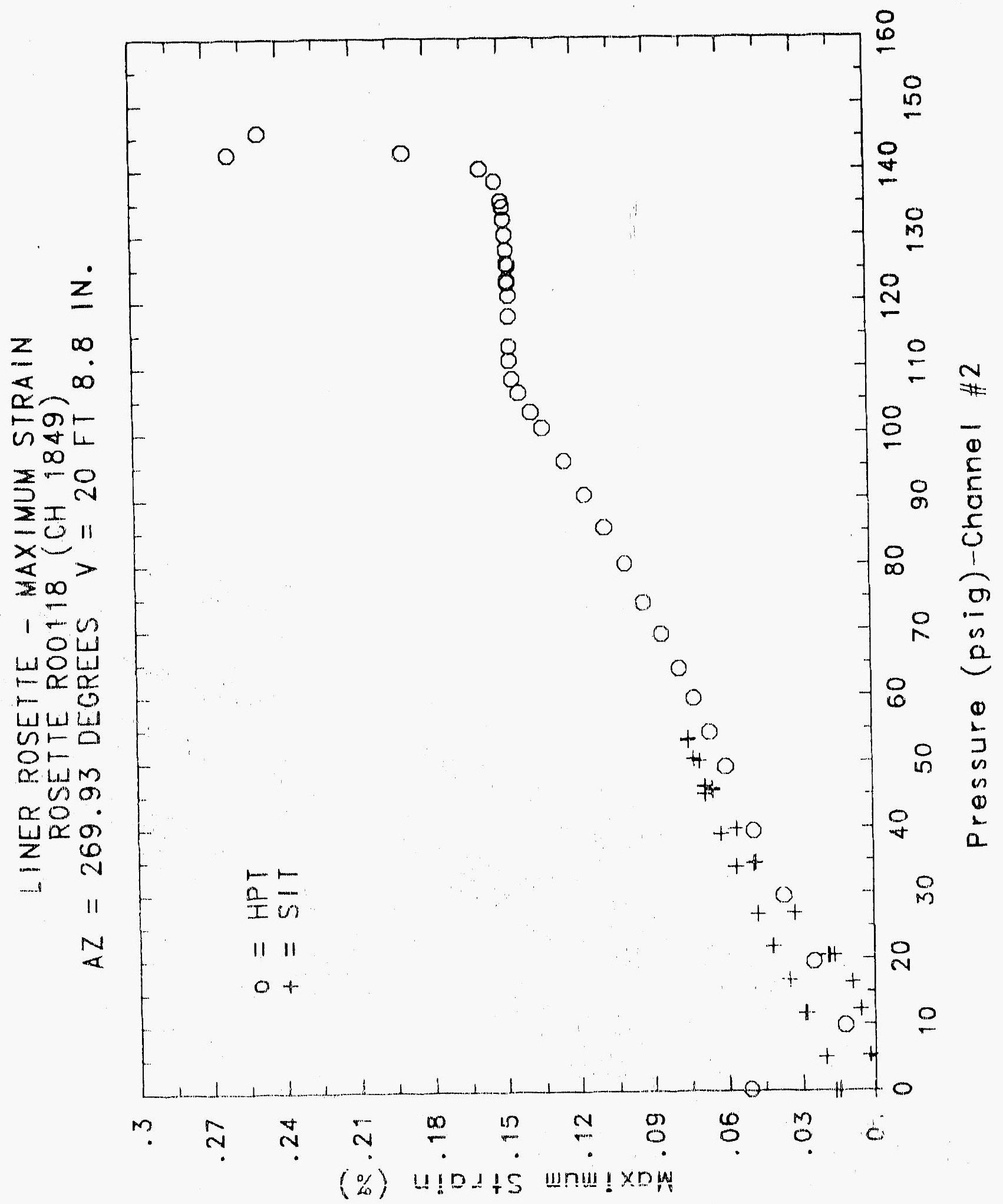




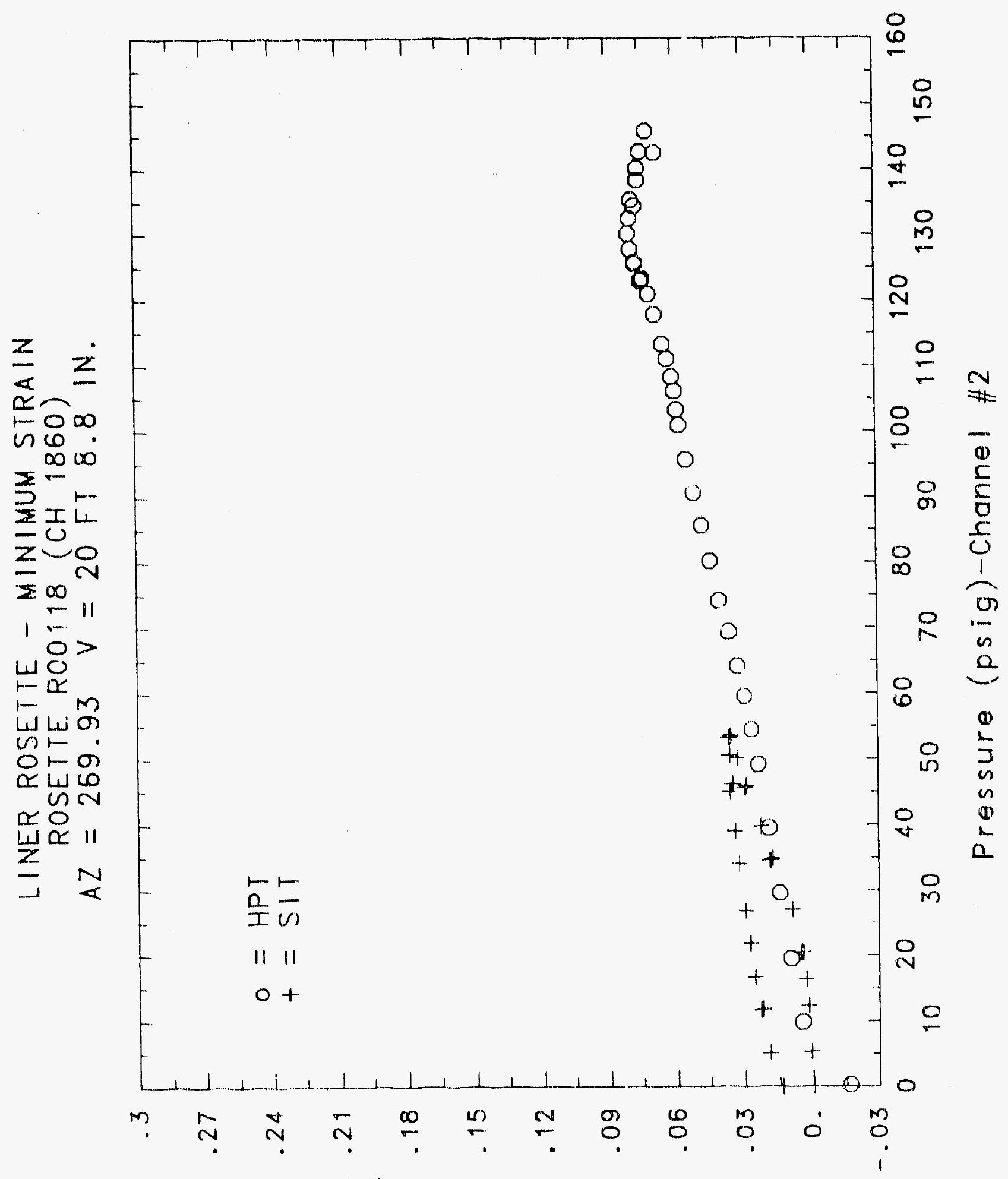

(\%) $u ! D d+S$ wnw! $u ! W$ 
ㅇํㅇำ

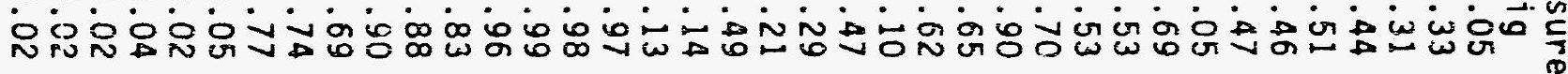
$00000000000000000000000000000000000000 \mathrm{w}$

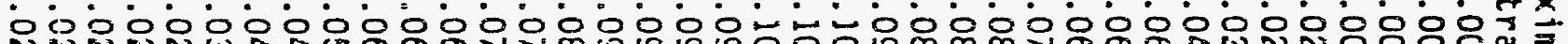

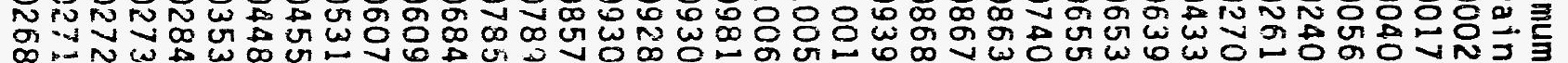

00000000000000000000000000000000000000 留

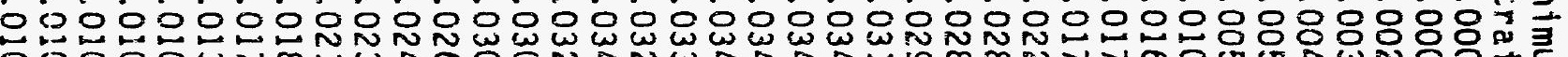

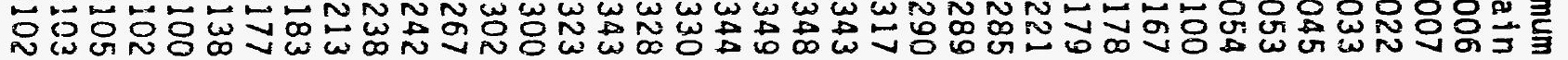

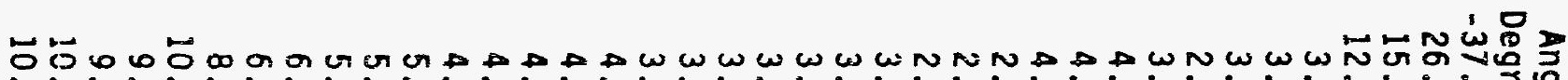

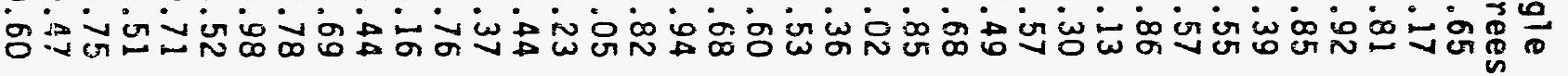

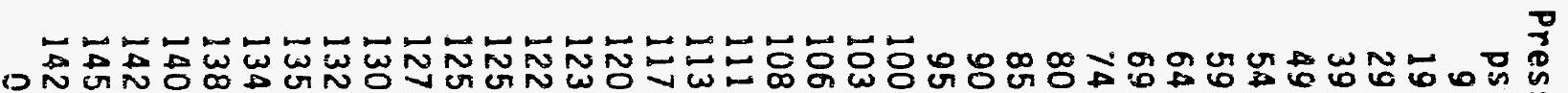

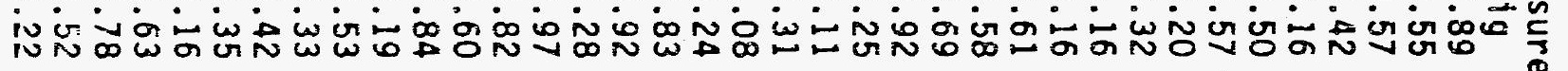

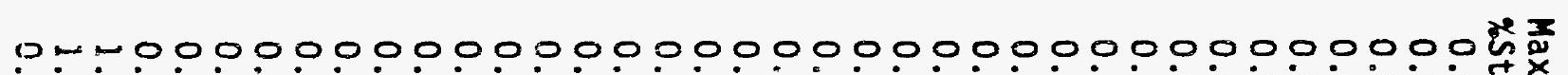

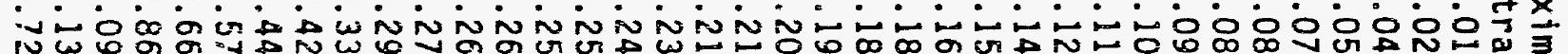

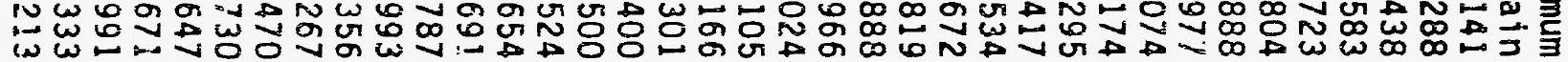

10000000000000000000000000000000000000年

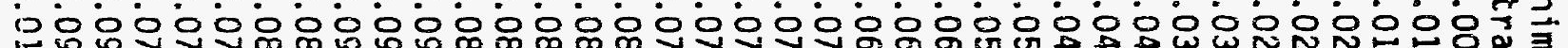

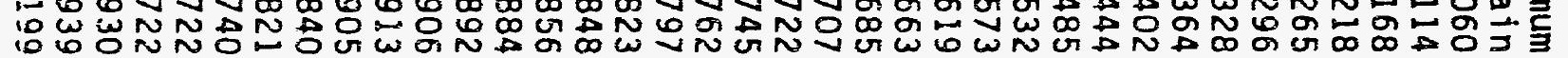

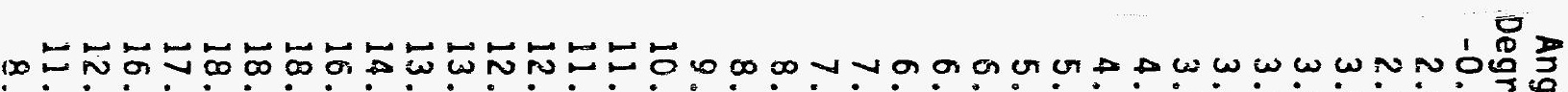

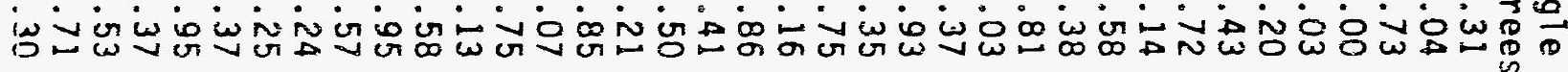




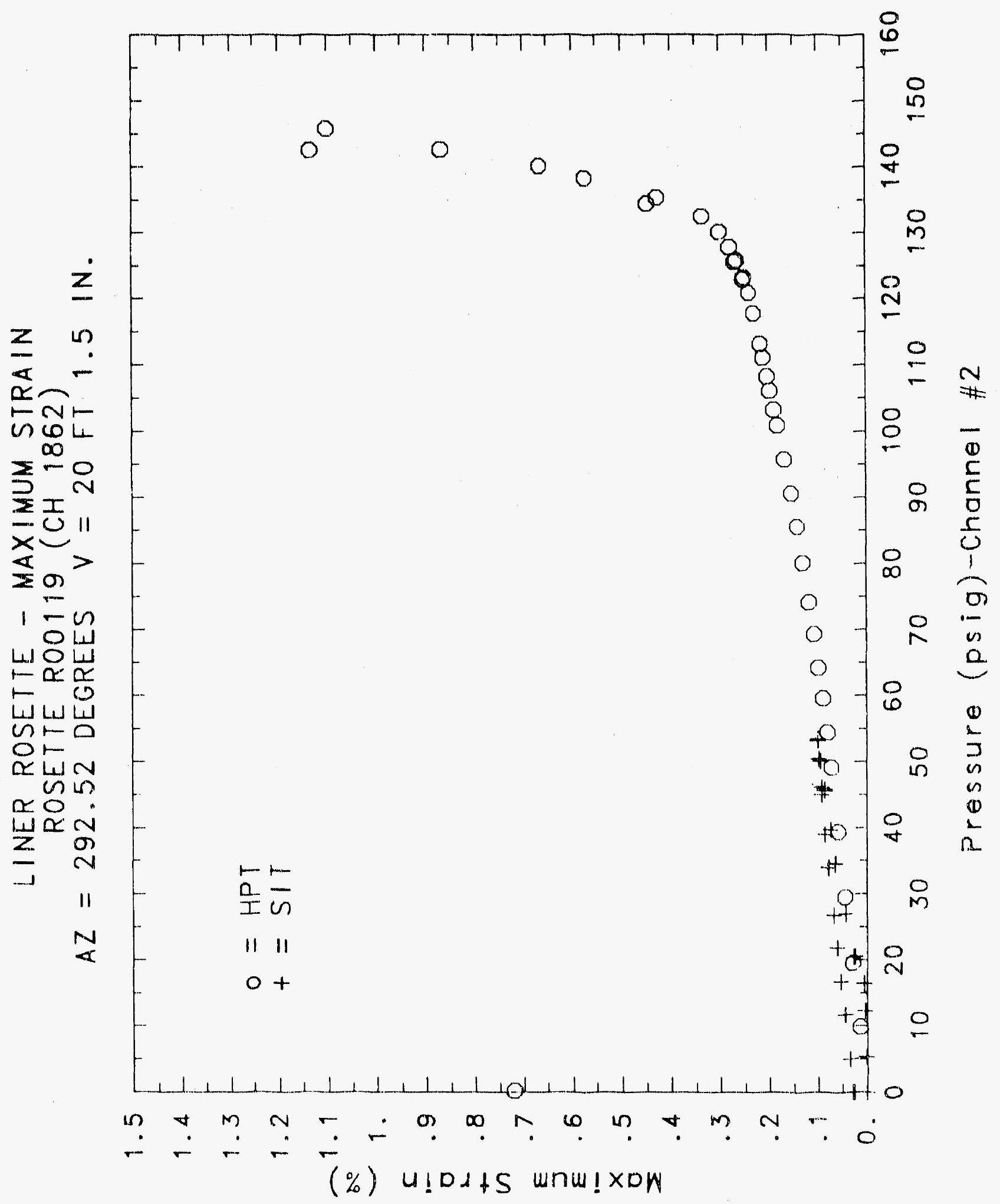




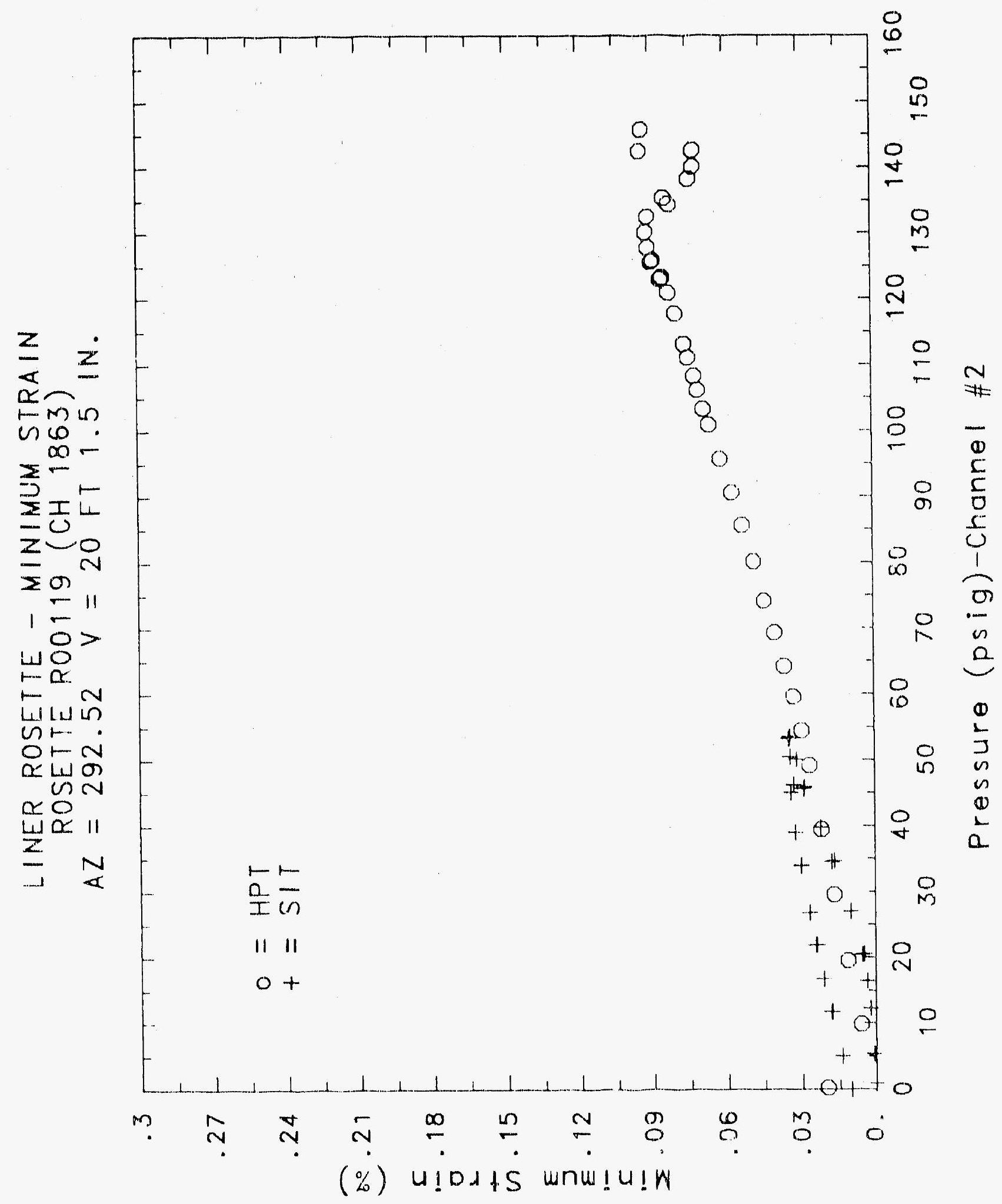




\title{
Reinforced Concrete Test Data
}

\author{
Rosette 120 Channel.s $1865 \quad 1866 \quad 1867$
}

Structural Integrity TEST

\begin{tabular}{|c|c|c|c|c|c|c|c|}
\hline Pressure & Maximum & Minimum & Angle & Pressure & Maximum & Minimum & Angle \\
\hline ncio & \%strain & \%strain & Degrees & psig & xstrain & xstrain & Degrees \\
\hline-0.05 & 0.0003 & -0.0006 & -20.19 & 9.89 & 0,1523 & -0.1388 & -43.98 \\
\hline 5.33 & 0.0121 & -0.0088 & -16.93 & 19.55 & 0.1593 & -0.1362 & 44.87 \\
\hline 12.31 & 0.0150 & -0.0121 & -10.54 & 29.57 & 0.1660 & -0.1303 & 43.88 \\
\hline .44 & 0.0133 & -0.0122 & -7.47 & 39.42 & 0.1712 & -0.1227 & 42.67 \\
\hline .51 & 0.0102 & -0.0113 & -7.54 & 49.16 & 0.1786 & -0.1171 & 41.33 \\
\hline .46 & 0.0163 & -0.0167 & -26.85 & 54.50 & 0.1815 & -0.1128 & 40.54 \\
\hline .47 & 0.0099 & -0.0100 & -8.07 & 59.57 & 0.1837 & -0.1096 & 39.85 \\
\hline .05 & 0.0067 & -0.0044 & -26.87 & 64.20 & 0.1843 & -0.1062 & 39.59 \\
\hline .69 & 158 & -0.0 & 30.98 & 69.32 & 0.1865 & -0.1 & 39. \\
\hline 34.53 & 165 & 012 & 2.9 .80 & 74.16 & 0.1888 & -0.1005 & 39.28 \\
\hline .53 & 0.0167 & -0.0012 & 29.13 & 80.16 & 0.1916 & -0.0963 & 38.68 \\
\hline .70 & 229 & -0.0 & 22.51 & 85.61 & 0.1992 & -0.0926 & 38.22 \\
\hline & 0.0367 & -0.0 & 20.25 & 90.58 & 0.2080 & -0.0882 & 37.57 \\
\hline 45.65 & 0.0373 & -0.0011 & 19.65 & 95.69 & 0.2211 & -0.0822 & 36.37 \\
\hline 45.62 & 0.0373 & -0.0012 & 19.71 & 100.92 & 0.2430 & -0.0763 & 34.70 \\
\hline .10 & 0.0443 & -0.0014 & 16. & 10.3 .25 & 0.2574 & -0.0717 & 33.40 \\
\hline 53.47 & 0.0502 & -0.1 & & & 0.2 & -0 & 31.84 \\
\hline 53.29 & 0.0507 & -0 & & 10 & 0.2948 & -0. & 30.69 \\
\hline 53.21 & 0.0508 & -0.0006 & 12.77 & 111.08 & 0.3280 & -0 & 28.85 \\
\hline 50.49 & 0.0483 & -0.0003 & 12.7 & 113.24 & 0.3599 & -0.0574 & 27.43 \\
\hline 46.14 & 0.0434 & -0.0004 & 13.75 & 117.83 & 0.4683 & -0.0558 & 24.09 \\
\hline 46.13 & 0.0434 & -0.0006 & 14.06 & 120.92 & 0.5832 & -0.0543 & 21.75 \\
\hline 44.97 & 0.0436 & 0.0018 & 10,21 & 123.28 & 0.72 .52 & -0.0402 & 18.71 \\
\hline 38.98 & 0.0372 & 0.0010 & 13.47 & 122.97 & 0.7679 & -0. & 17.79 \\
\hline 33.99 & 0.0321 & -0.0001 & 16.67 & 125.82 & 0.9839 & -0.1 & 14.13 \\
\hline 33.96 & 0.0318 & -0.0002 & 16.81 & 125.60 & 1.0293 & -0.0135 & 13.31 \\
\hline 26.83 & 0.0252 & -0.0024 & 22. & 127.84 & 1.1046 & -0.8 & 12.10 \\
\hline 21.88 & 0.0215 & -0.0047 & 26.94 & 130.19 & 1.2051 & 0.0007 & 10.55 \\
\hline 21.90 & 0.0217 & -0.0048 & 27.40 & 132.53 & 1.3218 & 0.0059 & 9.39 \\
\hline 16.69 & 0.0191 & -0.0072 & 32.7 & 135.33 & 1.4507 & 0.0085 & 8.67 \\
\hline 11.74 & 0.0166 & .0 .0090 & 39.23 & 134.42 & 1.4726 & 0.0089 & 8.46 \\
\hline 11.77 & 0.0168 & -0.0099 & 39.97 & 138.35 & 1.6734 & 0.0100 & 7.72 \\
\hline 5.05 & 0.0154 & -0.0155 & 40.78 & 140.16 & 1.8361 & 0.0094 & 7.14 \\
\hline 0.02 & 0.0157 & -0.0225 & 30.58 & 142.63 & 2.0838 & 0.0066 & 6.51 \\
\hline-0.04 & 0.0141 & -0.0192 & 29.79 & 145.78 & 2.3455 & 0.0063 & 5.97 \\
\hline-0.02 & 0.0134 & -0.0179 & 29.17 & 142.52 & 2.3922 & 0.0032 & 5.82 \\
\hline-0.02 & & -0.0186 & 29.22 & 0.22 & 2.0358 & -0.0526 & -8.22 \\
\hline & 0.0153 & -0.0207 & & & & & \\
\hline
\end{tabular}

\section{High Pressure test}




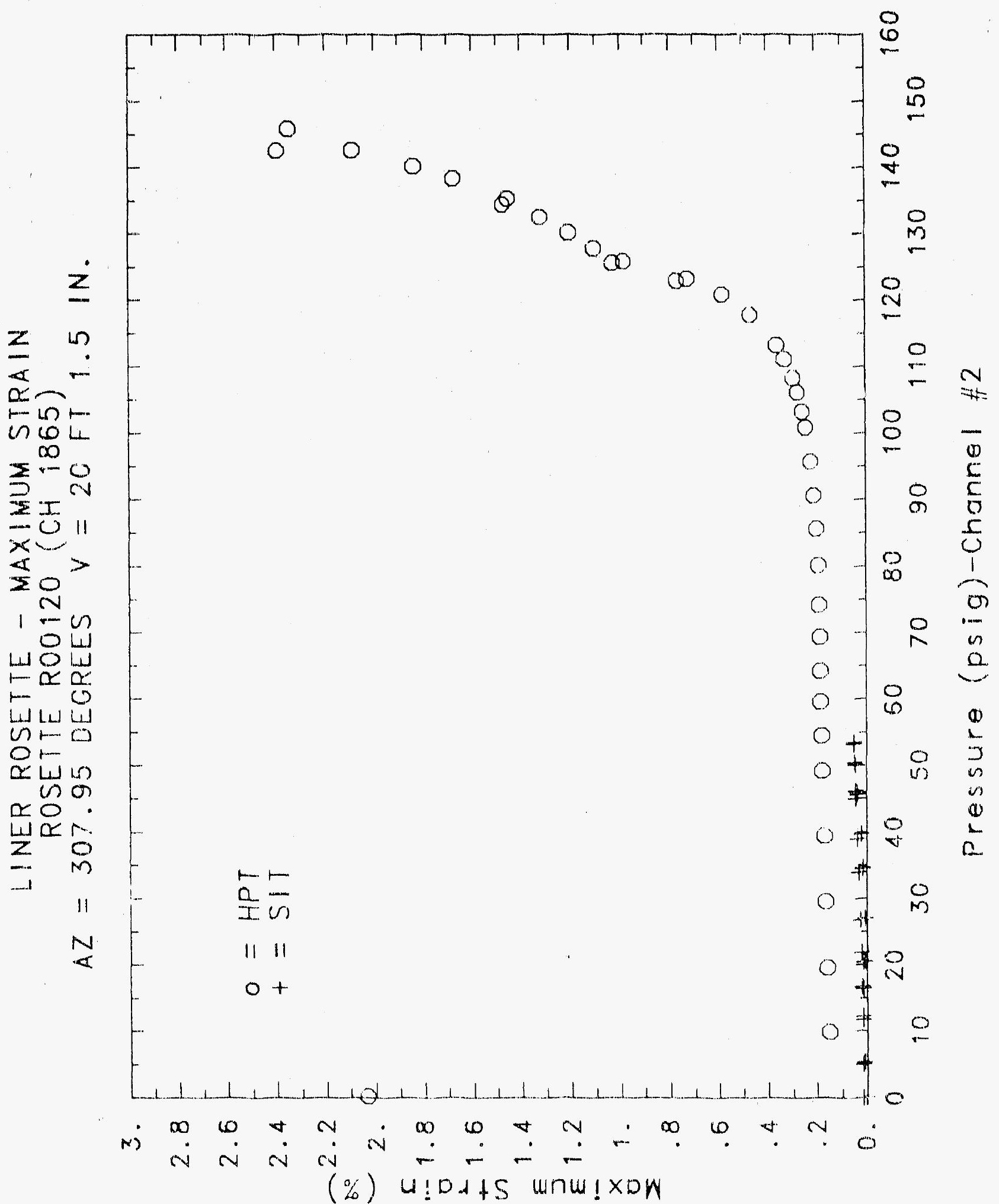




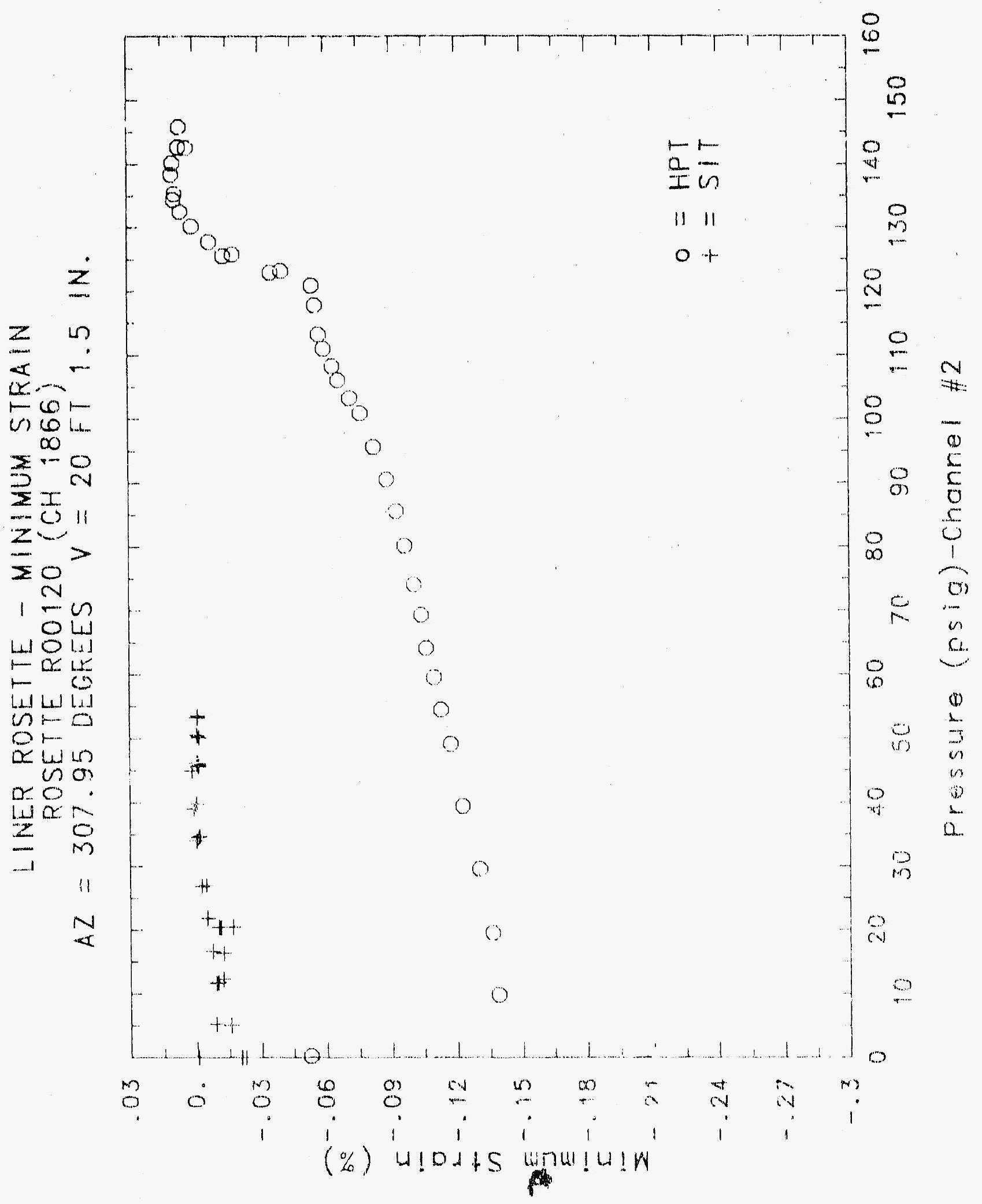

$(679)$ 
IMAGE EVALUATION

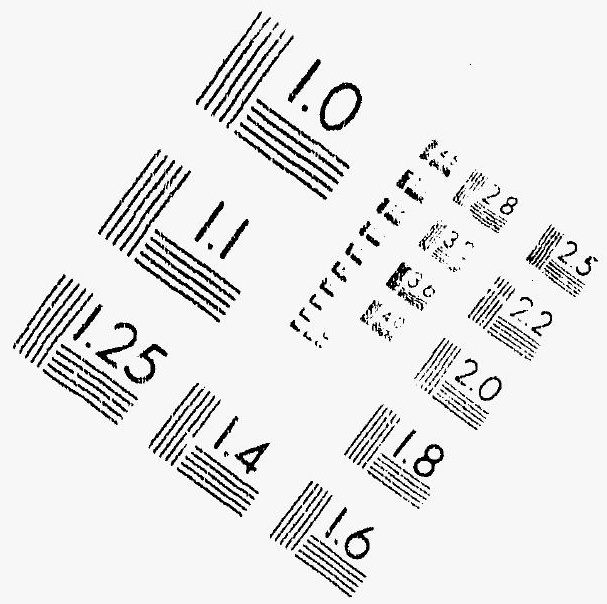

TEST TARGET (MT-3)
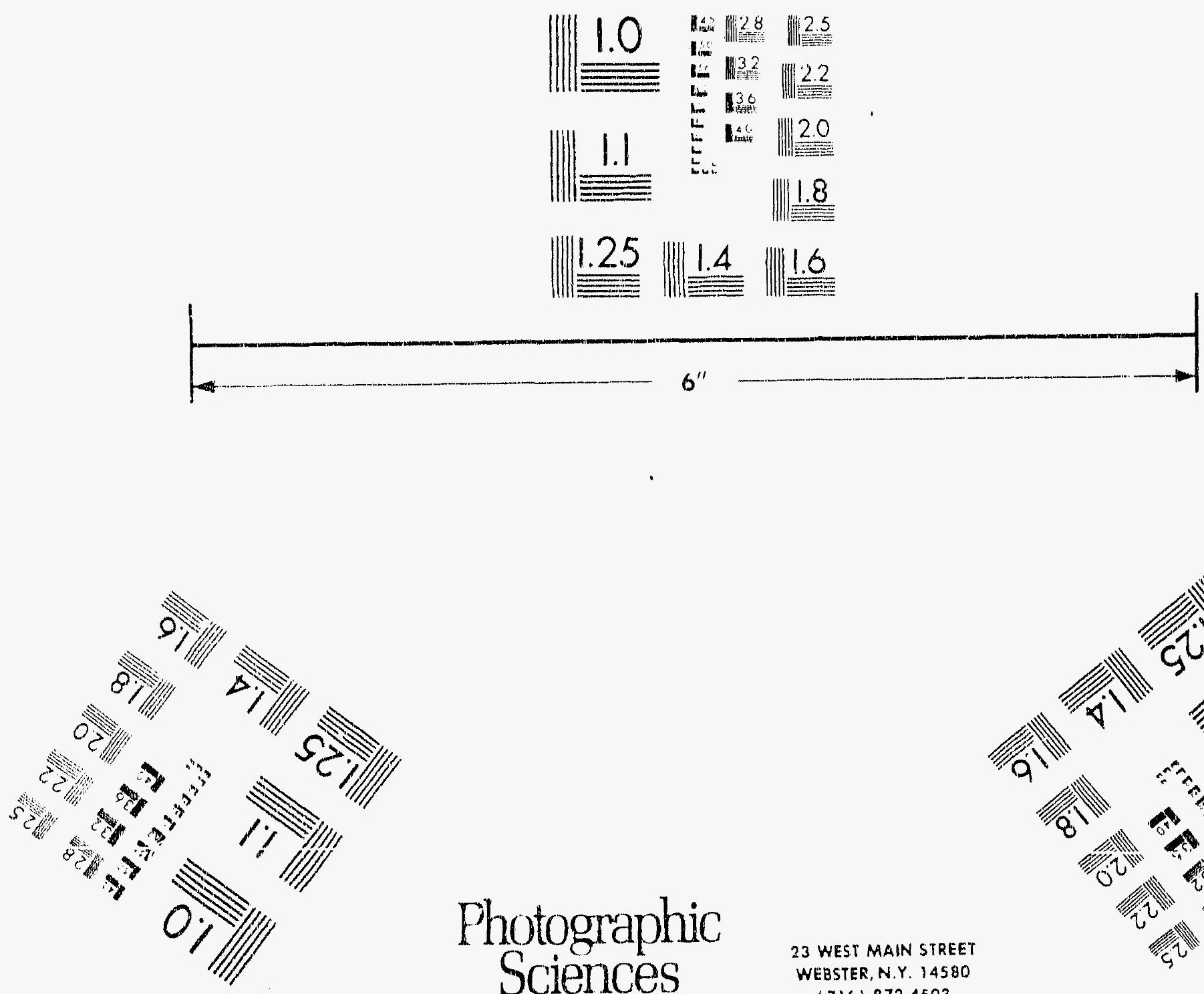

Photographic Sciences Corporation
23 WEST MAIN STREET

WEBSTER, N.Y. 14580 (716) 872.4503

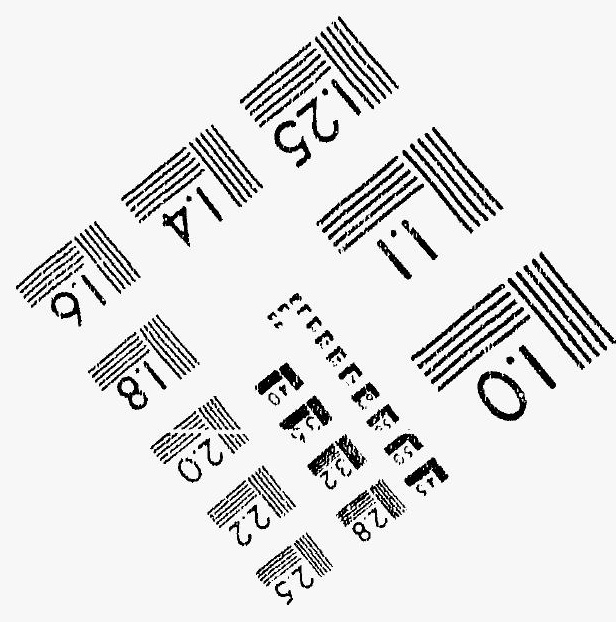


SECURITY CLASSIFICATION

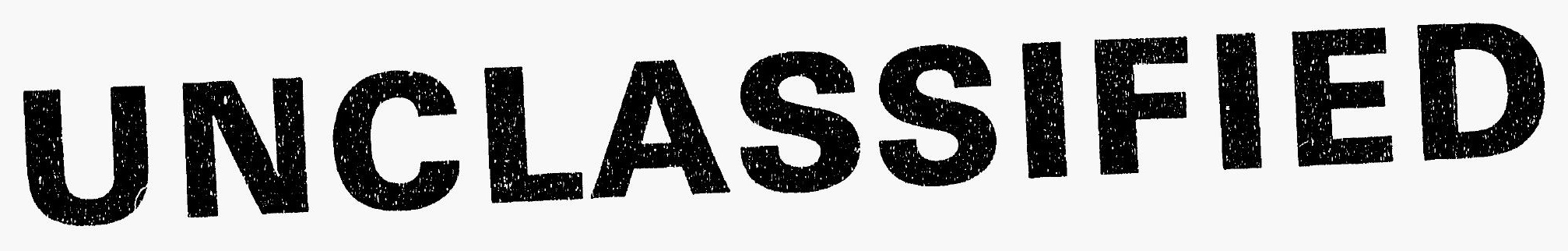

\section{DATE OF MICROFILMING $1-6-92$}

\section{MICROFILMED BY ERrY Ward}

SANDIA NATIONAL LABS 


\section{THIS DOCUMENT FILMED}

AT

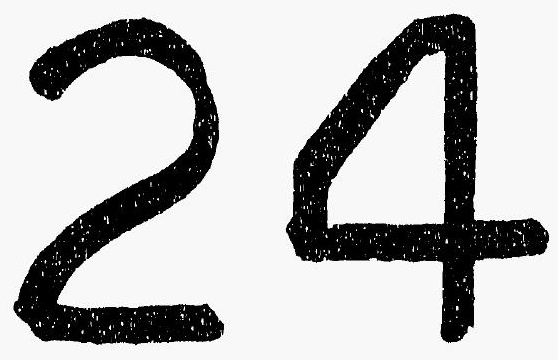

REDUCTION 


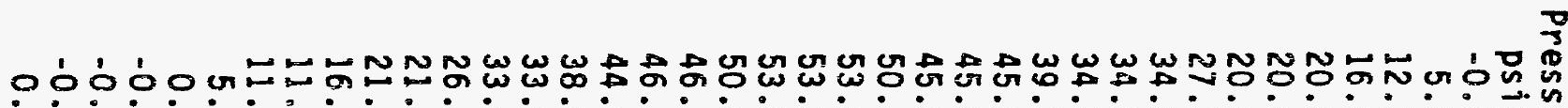

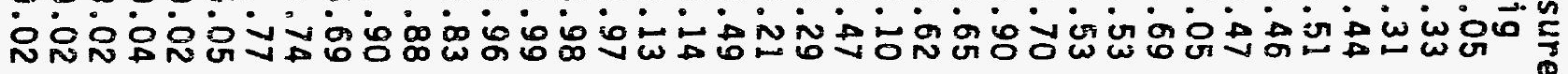
00000000000000000000000000000000000000苦

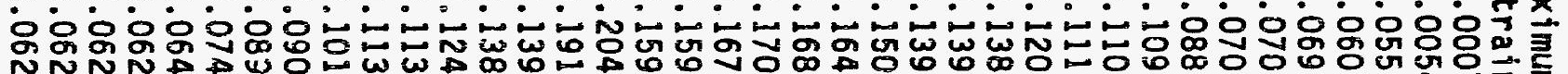

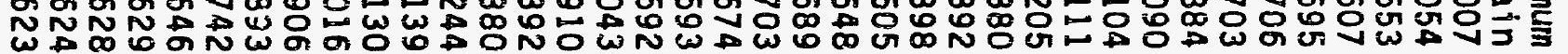

$000000000000000000000000000000000000005 \overline{1}$

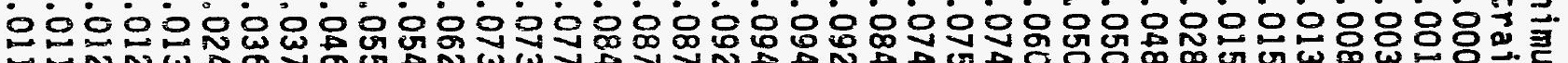
N

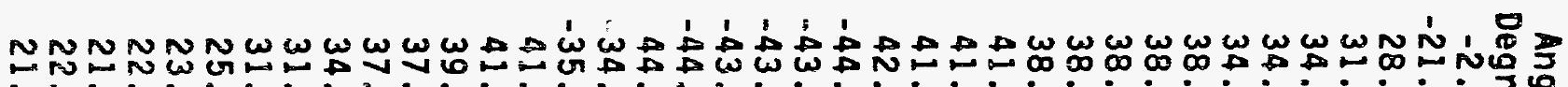

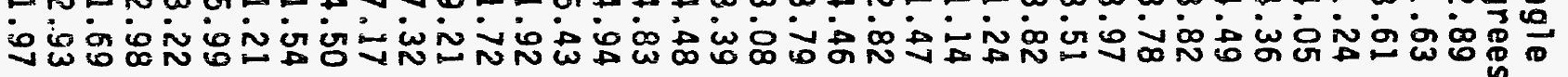

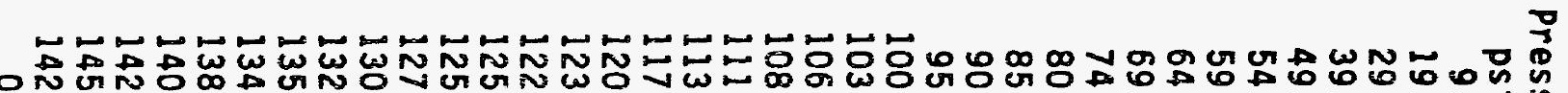

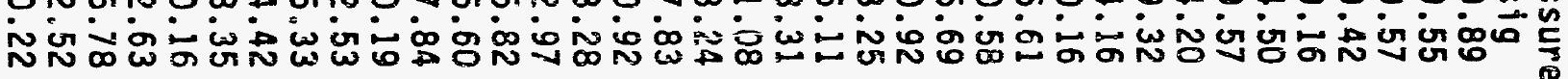
W $\Rightarrow$ F

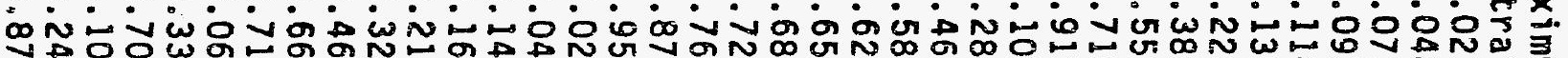

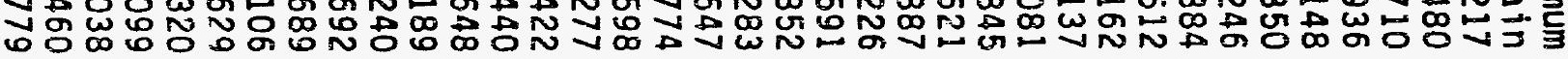

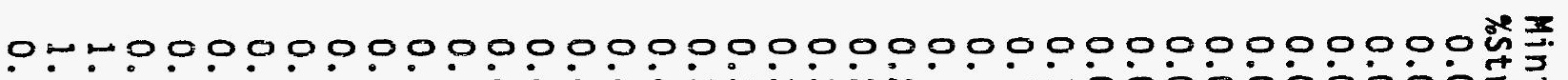

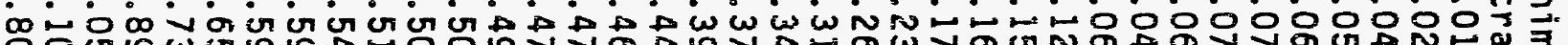

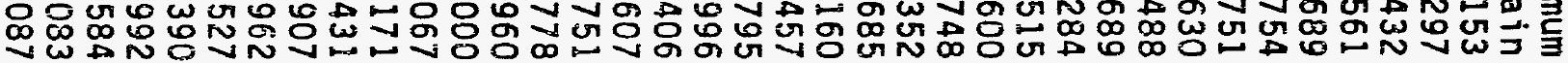

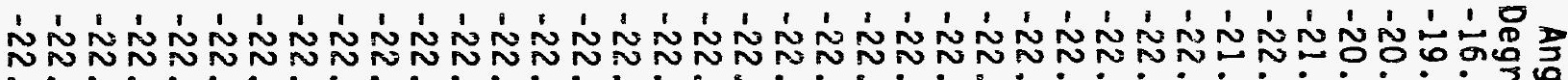

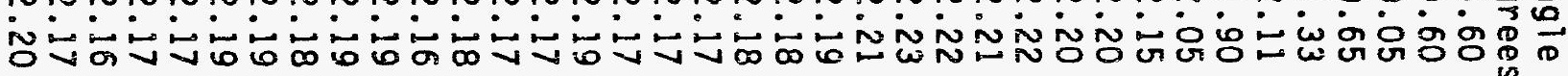




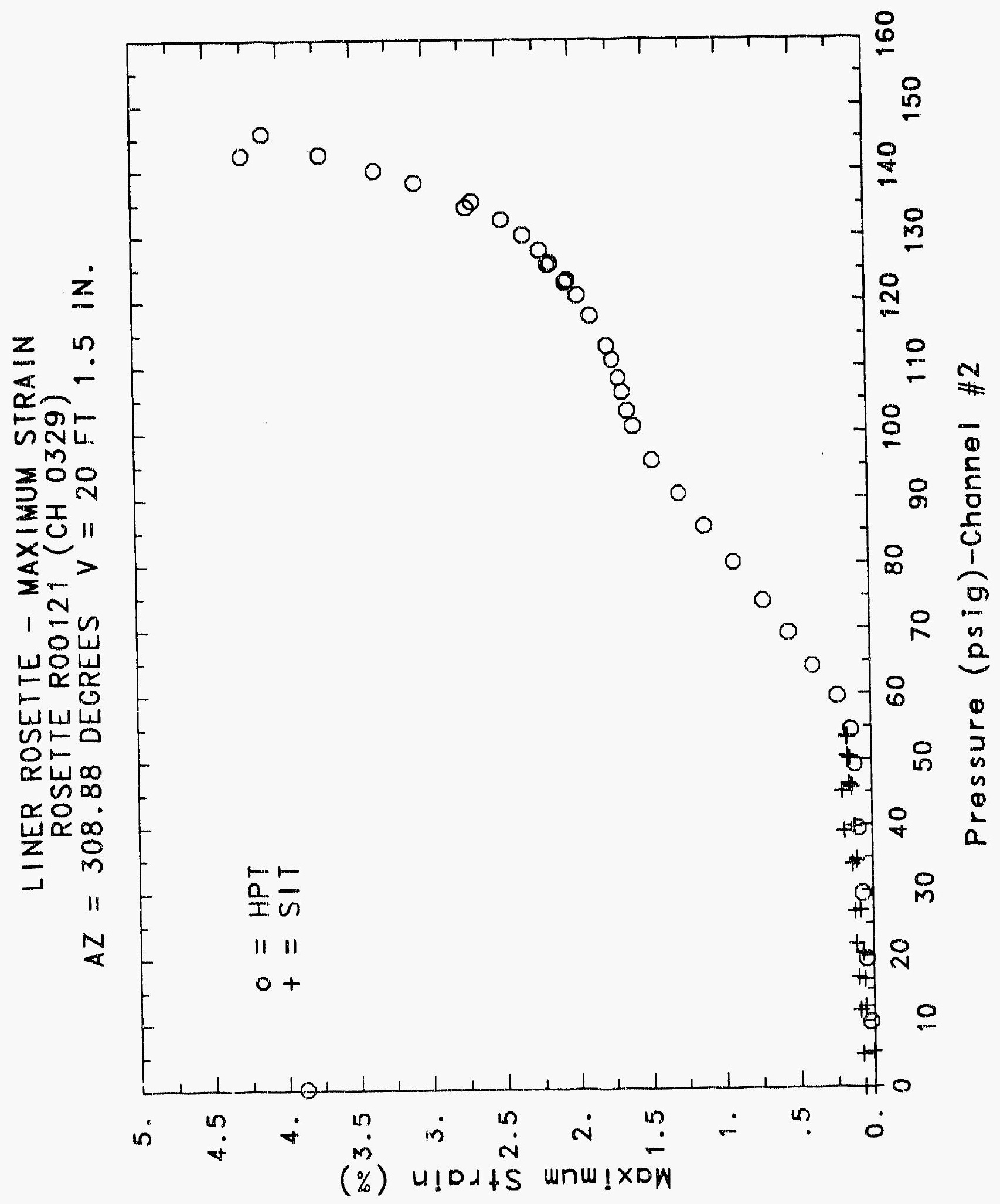

C. $i 81$ 


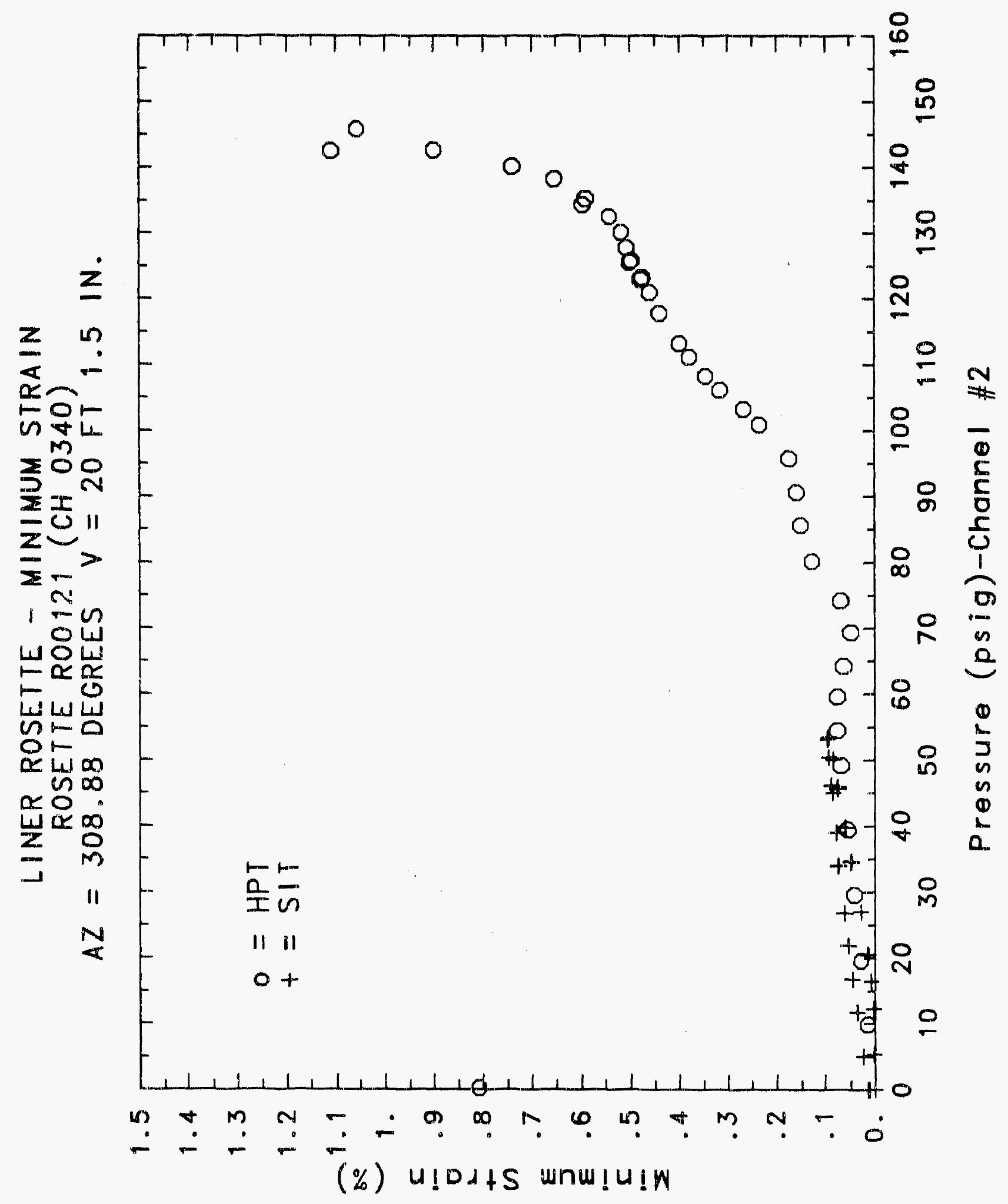




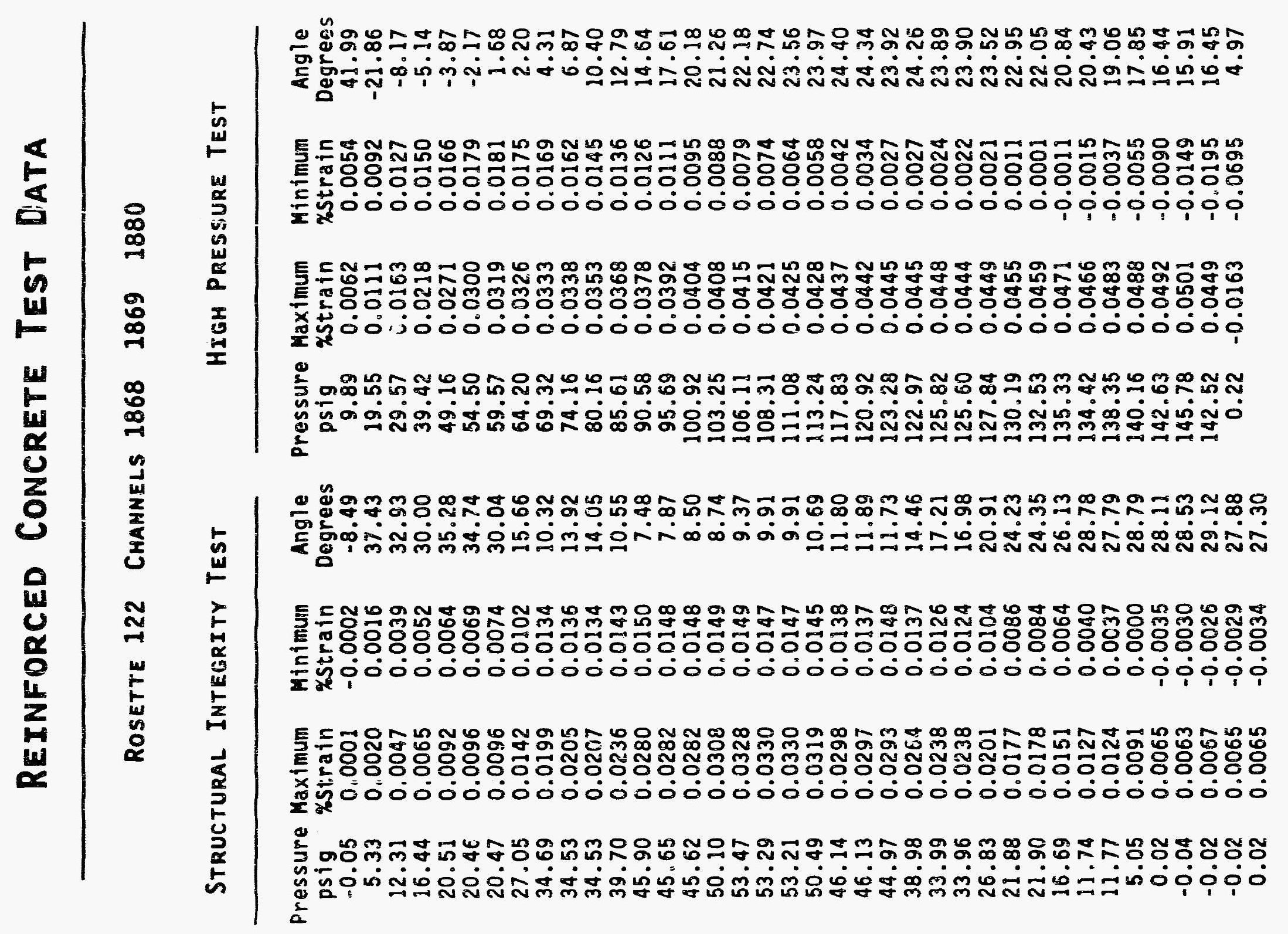




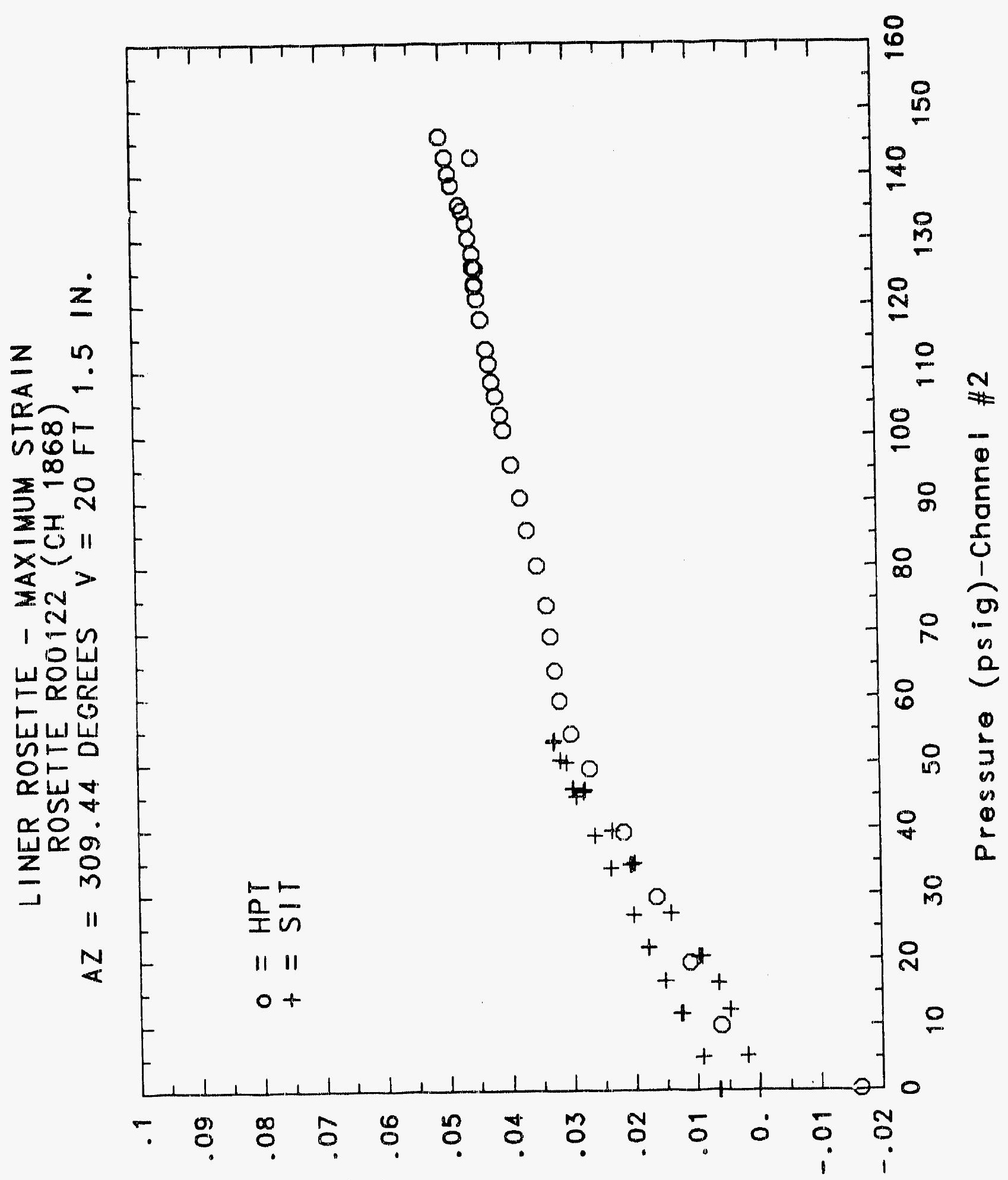

(\%) $u ! D d+S$ wnw! XDW 


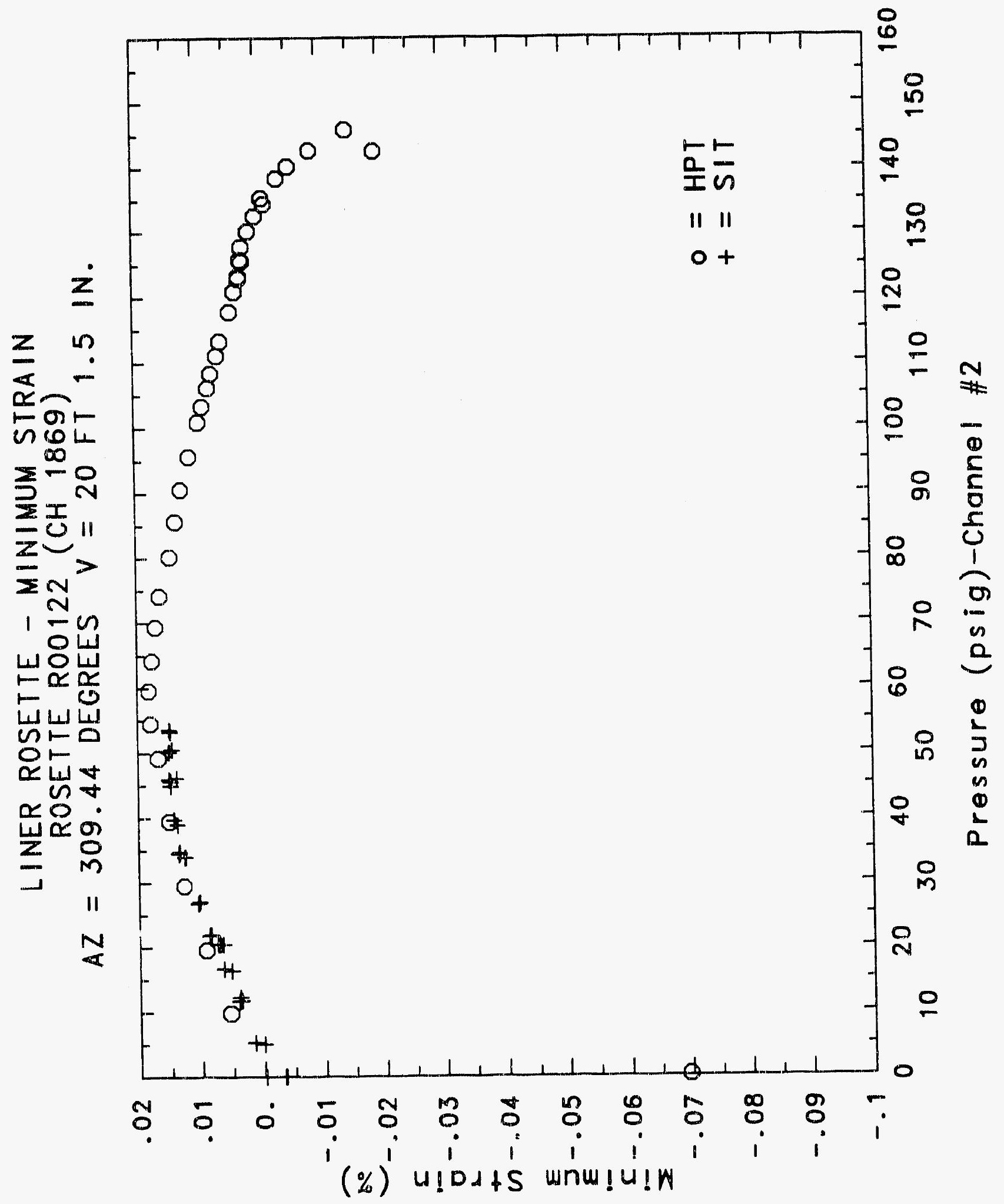

C. 685 


\section{Reinforced Concrete Test Data}

Rosette 123 Channels $342 \quad 343 \quad 344$

\author{
StRUCTURAL InTEgrity TEST
}

\begin{tabular}{|c|c|c|c|c|c|c|c|}
\hline $\begin{array}{c}\text { Pressure } \\
\text { psig } \\
-0.05 \\
5.33 \\
12.31 \\
16.44 \\
20.51 \\
20.46 \\
20.47 \\
27.05 \\
34.69 \\
34.53 \\
34.53 \\
39.70 \\
45.90 \\
45.65 \\
45.62 \\
50.10 \\
53.47 \\
53.29 \\
53.21 \\
50.49 \\
46.14 \\
46.13 \\
44.97 \\
38.98 \\
33.99 \\
33.96 \\
26.83 \\
21.88 \\
21.90 \\
16.69 \\
11.74 \\
11.77 \\
5.05 \\
0.02 \\
-0.04 \\
-0.02 \\
-0.02 \\
0.02\end{array}$ & $\begin{array}{c}\text { Maximum } \\
\text { \%Strain } \\
-0.0008 \\
0.0026 \\
0.0042 \\
0.0055 \\
0.0077 \\
0.0084 \\
0.0089 \\
0.0135 \\
0.0192 \\
0.0209 \\
0.0210 \\
0.0249 \\
0.0299 \\
0.0307 \\
0.0308 \\
0.0335 \\
0.0358 \\
0.0366 \\
0.0370 \\
0.0359 \\
0.0350 \\
0.0348 \\
0.0340 \\
0.0310 \\
0.0282 \\
0.0281 \\
0.0251 \\
0.0224 \\
0.0217 \\
0.0191 \\
0.0160 \\
0.0160 \\
0.0128 \\
0.0095 \\
0.0122 \\
0.0138 \\
0.0154 \\
0.0157\end{array}$ & $\begin{array}{l}\text { Minimum } \\
\text { KStrain } \\
-0.0015 \\
0.0002 \\
0.0030 \\
0.0038 \\
0.0065 \\
0.0065 \\
0.0071 \\
0.0109 \\
0.0173 \\
0.0164 \\
0.0172 \\
0.0189 \\
0.0224 \\
0.0220 \\
0.0217 \\
0.0245 \\
0.0265 \\
0.0268 \\
0.0275 \\
0.0254 \\
0.0241 \\
0.0244 \\
0.0220 \\
0.0200 \\
0.0169 \\
0.0165 \\
0.0134 \\
0.0104 \\
0.0111 \\
0.0091 \\
0.0059 \\
0.0060 \\
0.0023 \\
0.0003 \\
-0.0213 \\
-0.0308 \\
-0.0373 \\
-0.0413\end{array}$ & $\begin{array}{c}\text { Angle } \\
\text { Degrees } \\
-26.85 \\
10.74 \\
2.28 \\
27.73 \\
28.88 \\
32.94 \\
42.44 \\
-43.91 \\
36.24 \\
42.10 \\
41.42 \\
37.58 \\
36.44 \\
35.79 \\
38.44 \\
33.11 \\
33.39 \\
33.17 \\
29.13 \\
31.53 \\
28.03 \\
30.31 \\
43.95 \\
43.99 \\
39.34 \\
42.66 \\
41.17 \\
41.42 \\
39.46 \\
42.39 \\
42.23 \\
40.39 \\
42.85 \\
43.60 \\
-28.69 \\
-26.88 \\
-25.42 \\
-26.21\end{array}$ & $\begin{array}{c}\text { Pressure } \\
\text { Psig } \\
9.89 \\
19.55 \\
29.57 \\
39.42 \\
49.16 \\
54.50 \\
59.57 \\
64.20 \\
69.32 \\
74.16 \\
80.16 \\
85.61 \\
90.58 \\
95.69 \\
100.92 \\
103.25 \\
106.11 \\
108.31 \\
111.08 \\
113.24 \\
117.83 \\
120.92 \\
123.28 \\
122.97 \\
125.82 \\
125.60 \\
127.84 \\
130.19 \\
132.53 \\
135.33 \\
134.42 \\
138.35 \\
140.16 \\
142.63 \\
145.78 \\
142.52 \\
0.22\end{array}$ & $\begin{array}{r}\text { Maximum } \\
\text { \%Strain } \\
0.0056 \\
0.0109 \\
0.0167 \\
0.0219 \\
0.0272 \\
0.0307 \\
0.0339 \\
0.0369 \\
0.0404 \\
0.0437 \\
0.0478 \\
0.0510 \\
0.0543 \\
0.0568 \\
0.0591 \\
0.0603 \\
0.0616 \\
0.0625 \\
0.0630 \\
0.0639 \\
0.0647 \\
0.0658 \\
0.0665 \\
0.0663 \\
0.0665 \\
0.0663 \\
0.0658 \\
0.0658 \\
0.0647 \\
0.0653 \\
0.0639 \\
0.0644 \\
0.0632 \\
0.0617 \\
0.0609 \\
0.0605 \\
-0.0129\end{array}$ & $\begin{array}{c}\text { Minimum } \\
\% \text { Strain } \\
0.0038 \\
0.0082 \\
0.0123 \\
0.0162 \\
0.0211 \\
0.0229 \\
0.0251 \\
0.0277 \\
0.0299 \\
0.0318 \\
0.0341 \\
0.0375 \\
0.0390 \\
0.0422 \\
0.0445 \\
0.0442 \\
0.0452 \\
0.0454 \\
0.0472 \\
0.0476 \\
0.0477 \\
0.0481 \\
0.0483 \\
0.0488 \\
0.0491 \\
0.0487 \\
0.0503 \\
0.0486 \\
0.0491 \\
0.0479 \\
0.0471 \\
0.0477 \\
0.0468 \\
0.0476 \\
0.0478 \\
0.0440 \\
-0.0162\end{array}$ & $\begin{array}{c}\text { Angle } \\
\text { Degrees } \\
41.78 \\
41.37 \\
41.09 \\
42.42 \\
43.63 \\
-43.83 \\
44.51 \\
42.21 \\
41.26 \\
40.09 \\
38.80 \\
36.34 \\
34.41 \\
31.25 \\
28.44 \\
28.04 \\
26.79 \\
26.41 \\
25.04 \\
23.46 \\
24.40 \\
25.10 \\
22.94 \\
23.19 \\
21.94 \\
21.62 \\
20.41 \\
21.48 \\
20.26 \\
21.92 \\
22.92 \\
23.08 \\
21.83 \\
25.45 \\
28.95 \\
33.71 \\
-32.35\end{array}$ \\
\hline
\end{tabular}

\section{High Pressure test}




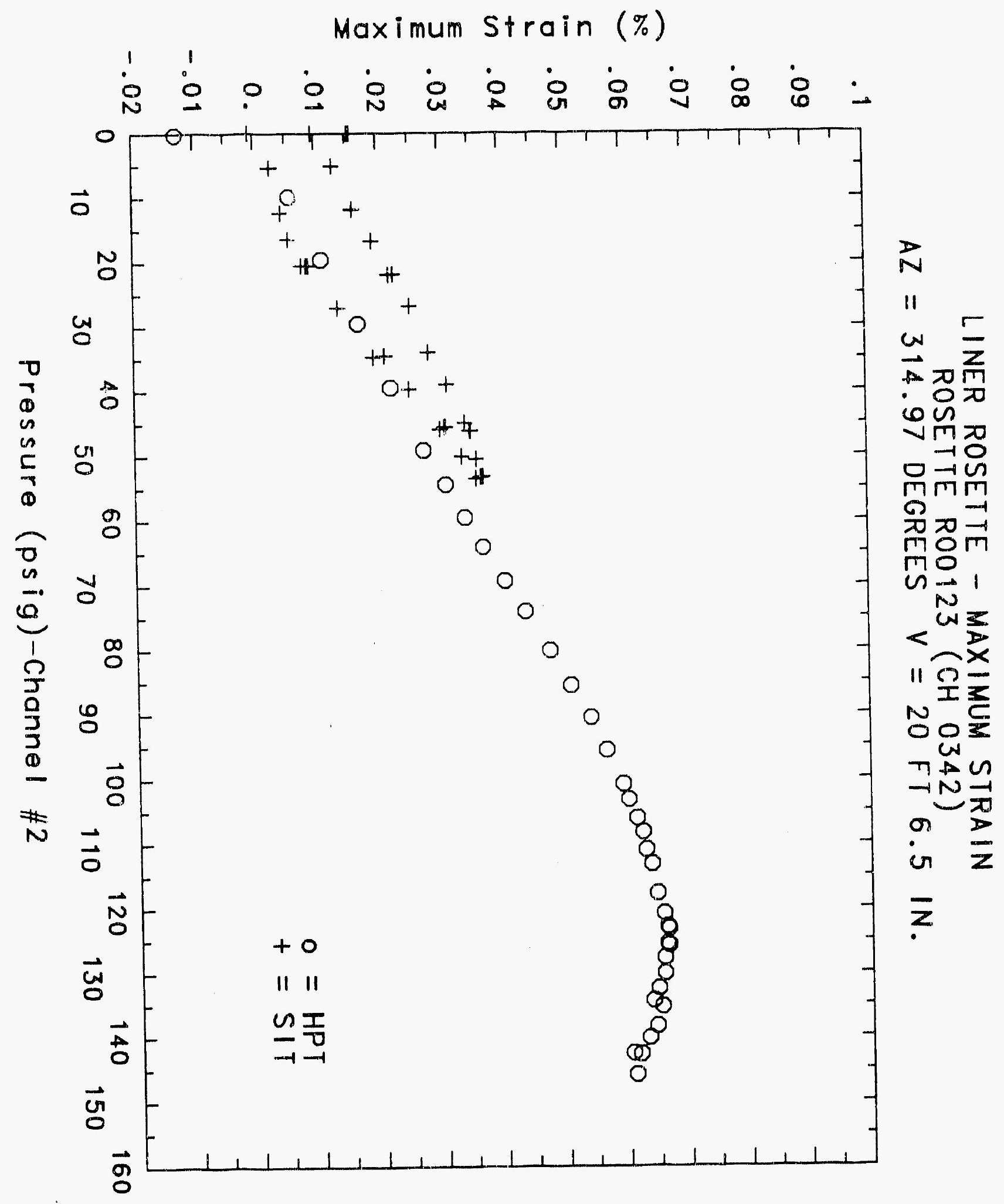




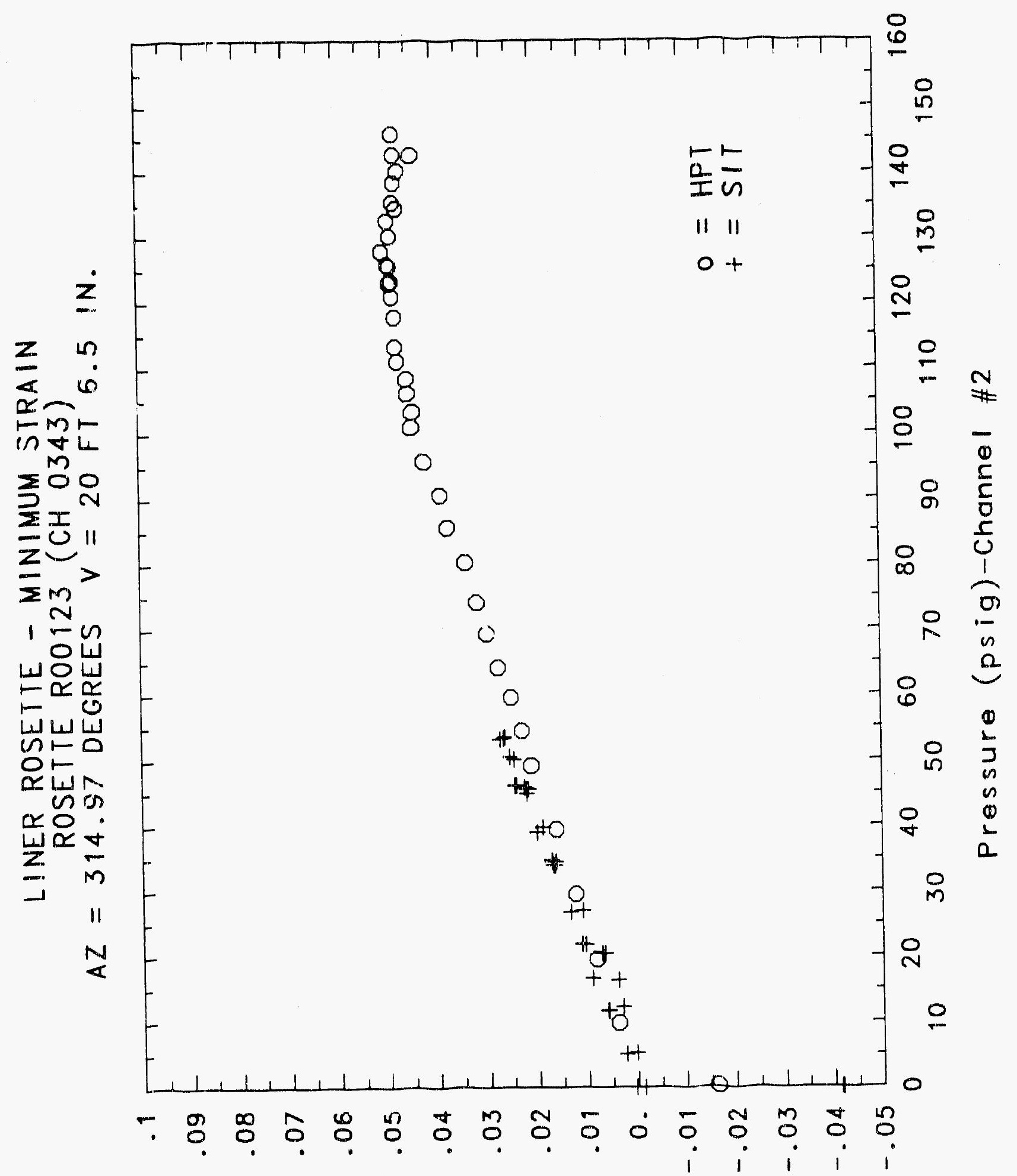

(\%) U!Dd+S wnw!U!W 


\title{
REINFORCEO CONCRETE TEST DATA
}

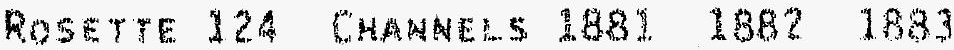

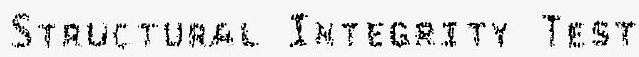

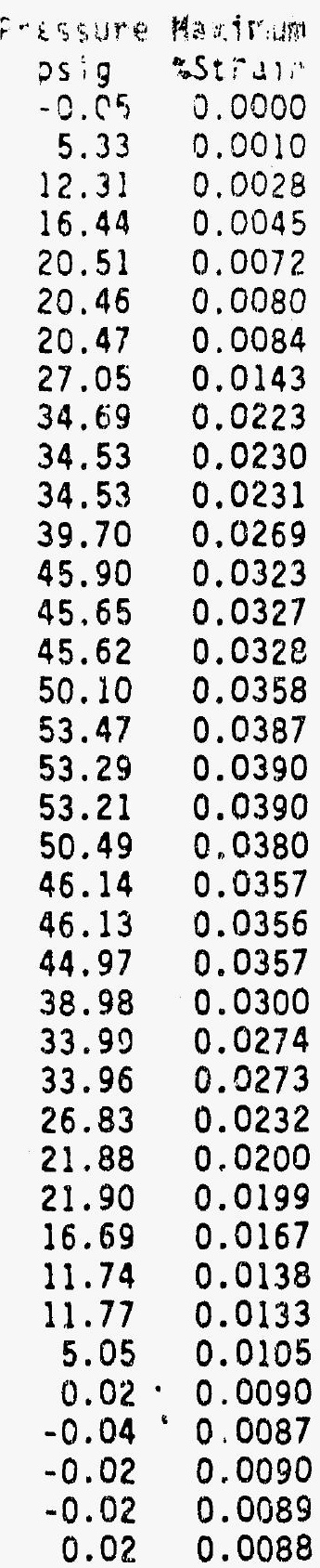

\begin{abstract}
Minl
as irait:

$-0.000 \%$

0.000

$0.001 \%$

0.0021

0.0037

0.0033

0.0036

$0.00 \% 1$

0.0107

0.0128

0.0128

0.0147

0.0175

0.0184

0.0185

0.0199

0.0212

0.0210

0.0209

0.0205

0.0198

0.0197

0.0195

0.0187

0.0176

0.0176

0.0157

0.0145

0.0149

0.0135

0.0118

0.0116

0.0089

0.0060

0.0045

0.0050

0.0051

0.0051
\end{abstract}

Argla

nom-ees

9.23

$-20.48$

$-10.03$

$-7.79$

$-1.27$

$-2.77$

$-5.32$

0.65

$-0.96$

3.81

3.21

3.75

2.42

4.12

4.17

3.91

4.03

3.01

2.77

3.03

3.11

2.91

3.00

$-2.05$

$-1.53$

$-1.76$

$-2.47$

$-4.77$

$-3.22$

$-6.96$

$-16.26$

$-21.97$

31.21

19.65

22.70

21.92

22.92

22.72

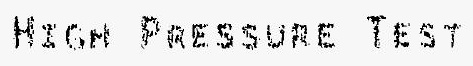

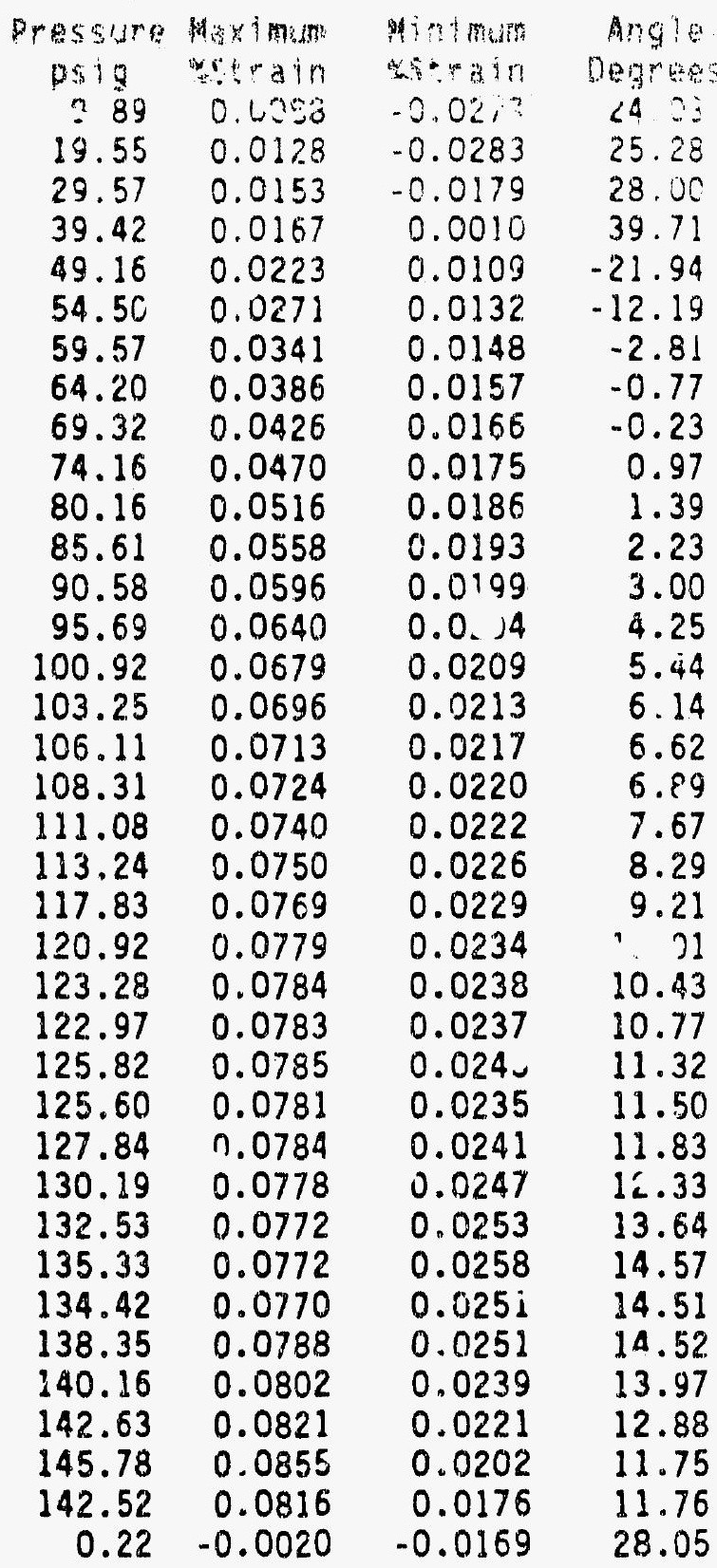




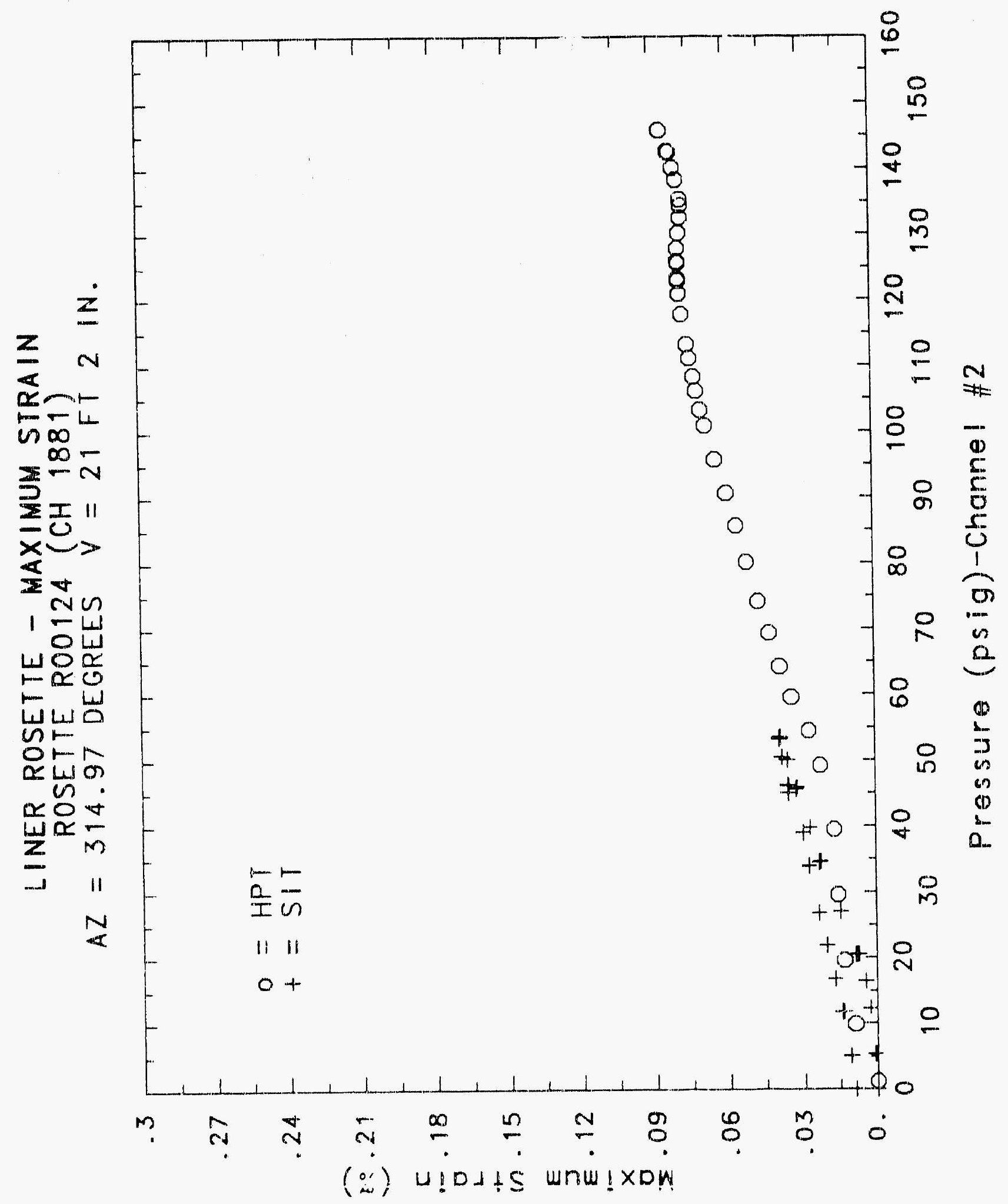


[69-J

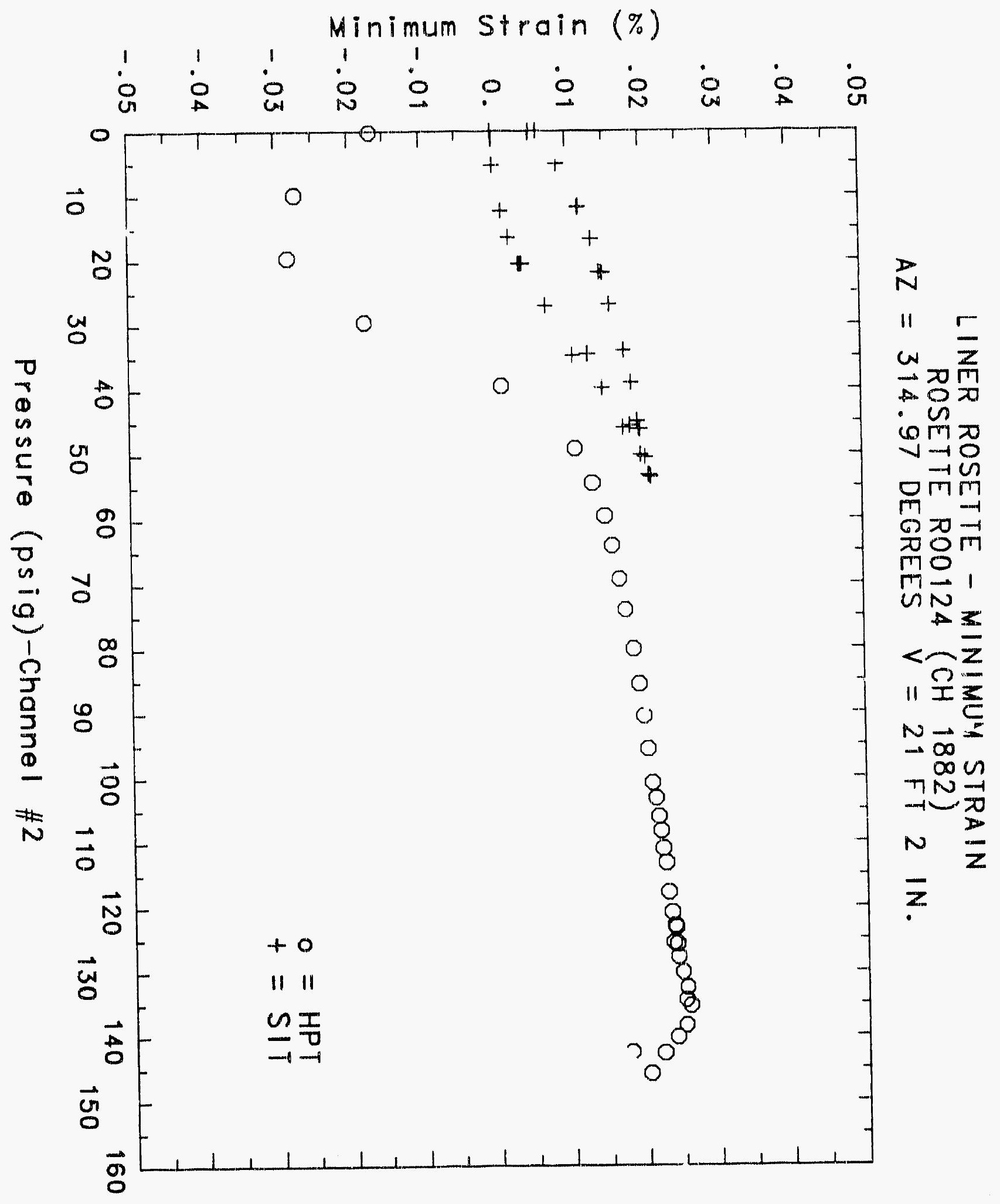




\section{Reinforced Concrete Test Data}

Rosette 125 Channels $1884 \quad 18851886$

StRUCTURAL INTEGRITY TEST

\begin{tabular}{|c|c|c|c|c|c|c|c|}
\hline $\begin{array}{c}\text { Pressure } \\
\text { psig } \\
-0.05 \\
5.33 \\
12.31 \\
16.44 \\
20.51 \\
20.46 \\
20.47 \\
27.05 \\
34.69 \\
34.53 \\
34.53 \\
39.70 \\
45.90 \\
45.65 \\
45.62 \\
50.10 \\
53.47 \\
53.29 \\
53.21 \\
50.49 \\
46.14 \\
46.13 \\
44.97 \\
38.98 \\
33.99 \\
33.96 \\
26.83 \\
21.88 \\
21.90 \\
16.69 \\
11.74 \\
11.77 \\
5.05 \\
0.02 \\
-0.04 \\
-0.02 \\
-0.02 \\
0.02\end{array}$ & $\begin{array}{l}\text { Maximum } \\
\text { \%Strain } \\
0.0001 \\
0.0016 \\
0.0039 \\
0.0059 \\
0.0087 \\
0.0095 \\
0.0100 \\
0.0167 \\
0.0288 \\
0.0308 \\
0.0313 \\
0.0372 \\
0.0485 \\
0.0501 \\
0.0504 \\
0.0555 \\
0.0603 \\
0.0612 \\
0.0614 \\
0.0607 \\
0.0588 \\
0.0585 \\
0.0583 \\
0.0554 \\
0.0524 \\
0.0523 \\
0.0476 \\
0.0442 \\
0.0438 \\
0.0400 \\
0.0360 \\
0.0353 \\
0.0300 \\
0.0255 \\
0.0238 \\
0.0243 \\
0.0241 \\
0.0237\end{array}$ & $\begin{array}{l}\text { Minimuni } \\
\text { XStrain } \\
-0.0001 \\
0.0004 \\
0.0020 \\
0.0032 \\
0.0055 \\
0.0063 \\
0.0067 \\
0.0144 \\
0.0258 \\
0.0274 \\
0.0276 \\
0.0320 \\
0.0385 \\
0.0388 \\
0.0389 \\
0.0423 \\
0.0453 \\
0.0455 \\
0.0454 \\
0.0442 \\
0.0421 \\
0.0418 \\
0.0419 \\
0.0389 \\
0.0360 \\
0.0360 \\
0.0318 \\
0.0286 \\
0.0286 \\
0.0253 \\
0.0219 \\
0.0213 \\
0.0170 \\
0.0137 \\
0.0126 \\
0.0132 \\
0.0132 \\
0.0133\end{array}$ & $\begin{array}{c}\text { Angle } \\
\text { Degrees } \\
22.51 \\
25.57 \\
23.59 \\
24.63 \\
24.89 \\
25.95 \\
24.31 \\
26.36 \\
-19.39 \\
-10.15 \\
-9.27 \\
-8.93 \\
-6.32 \\
-6.94 \\
-7.21 \\
-6.56 \\
-5.50 \\
-3.70 \\
-3.41 \\
-2.24 \\
-2.03 \\
-2.04 \\
-4.85 \\
-4.32 \\
-4.00 \\
-3.65 \\
-2.74 \\
-1.55 \\
-1.59 \\
-0.49 \\
0.93 \\
0.34 \\
2.44 \\
2.88 \\
-0.64 \\
1.51 \\
1.20 \\
1.86\end{array}$ & $\begin{array}{c}\text { Pressure } \\
\text { psig } \\
9.89 \\
19.55 \\
29.57 \\
39.42 \\
49.16 \\
54.50 \\
59.57 \\
64.20 \\
69.32 \\
74.16 \\
80.16 \\
85.61 \\
90.58 \\
95.69 \\
100.92 \\
103.25 \\
106.11 \\
108.31 \\
111.08 \\
113.24 \\
117.83 \\
120.92 \\
123.28 \\
122.97 \\
125.82 \\
125.60 \\
127.84 \\
130.19 \\
132.53 \\
135.33 \\
134.42 \\
138.35 \\
140.16 \\
142.63 \\
145.78 \\
142.52 \\
0.22\end{array}$ & $\begin{array}{l}\text { Maximum } \\
\text { \%Strain } \\
0.0083 \\
0.0164 \\
0.0241 \\
0.0317 \\
0.0389 \\
0.0445 \\
0.0516 \\
0.0572 \\
0.0647 \\
0.0712 \\
0.0792 \\
0.0864 \\
0.0932 \\
0.1004 \\
0.1080 \\
0.1118 \\
0.1155 \\
0.1184 \\
0.1225 \\
0.1257 \\
0.1321 \\
0.1367 \\
0.1408 \\
0.1419 \\
0.1520 \\
0.1546 \\
0.1621 \\
0.1713 \\
0.1819 \\
0.1972 \\
0.1984 \\
0.2071 \\
0.2093 \\
0.2079 \\
0.2062 \\
0.1976 \\
0.0729\end{array}$ & $\begin{array}{r}\text { Minimum } \\
\text { xStrain } \\
0.0063 \\
0.0125 \\
0.0184 \\
0.0243 \\
0.0296 \\
0.0319 \\
0.0319 \\
0.03 \\
0.0322 \\
0.0333 \\
0.0338 \\
0.0350 \\
0.0350 \\
0.0367 \\
0.0371 \\
0.0367 \\
0.0366 \\
0.0365 \\
0.0362 \\
0.0354 \\
0.0338 \\
0.0318 \\
0.0311 \\
0.0308 \\
0.0279 \\
0.0250 \\
0.0200 \\
0.0119 \\
0.0046 \\
-0.0034 \\
-0.0052 \\
-0.0045 \\
-0.0039 \\
-0.0003 \\
0.0030 \\
0.0007 \\
-0.0964\end{array}$ & $\begin{array}{c}\text { Angle } \\
\text { Degrees } \\
-7.50 \\
-6.29 \\
-2.34 \\
3.34 \\
6.04 \\
9.19 \\
11.46 \\
7.76 \\
2.47 \\
0.47 \\
-1.11 \\
-2.47 \\
-4.00 \\
-5.91 \\
-7.27 \\
-8.18 \\
-8.69 \\
-9.24 \\
-9.86 \\
-10.69 \\
-11.66 \\
-12.52 \\
-12.46 \\
-11.98 \\
-15.19 \\
-16.57 \\
-18.83 \\
-21.37 \\
-22.86 \\
-23.85 \\
-24.12 \\
-24.28 \\
-24.85 \\
-25.31 \\
-25.83 \\
-25.88 \\
-38.96\end{array}$ \\
\hline
\end{tabular}

High Pressure Test 


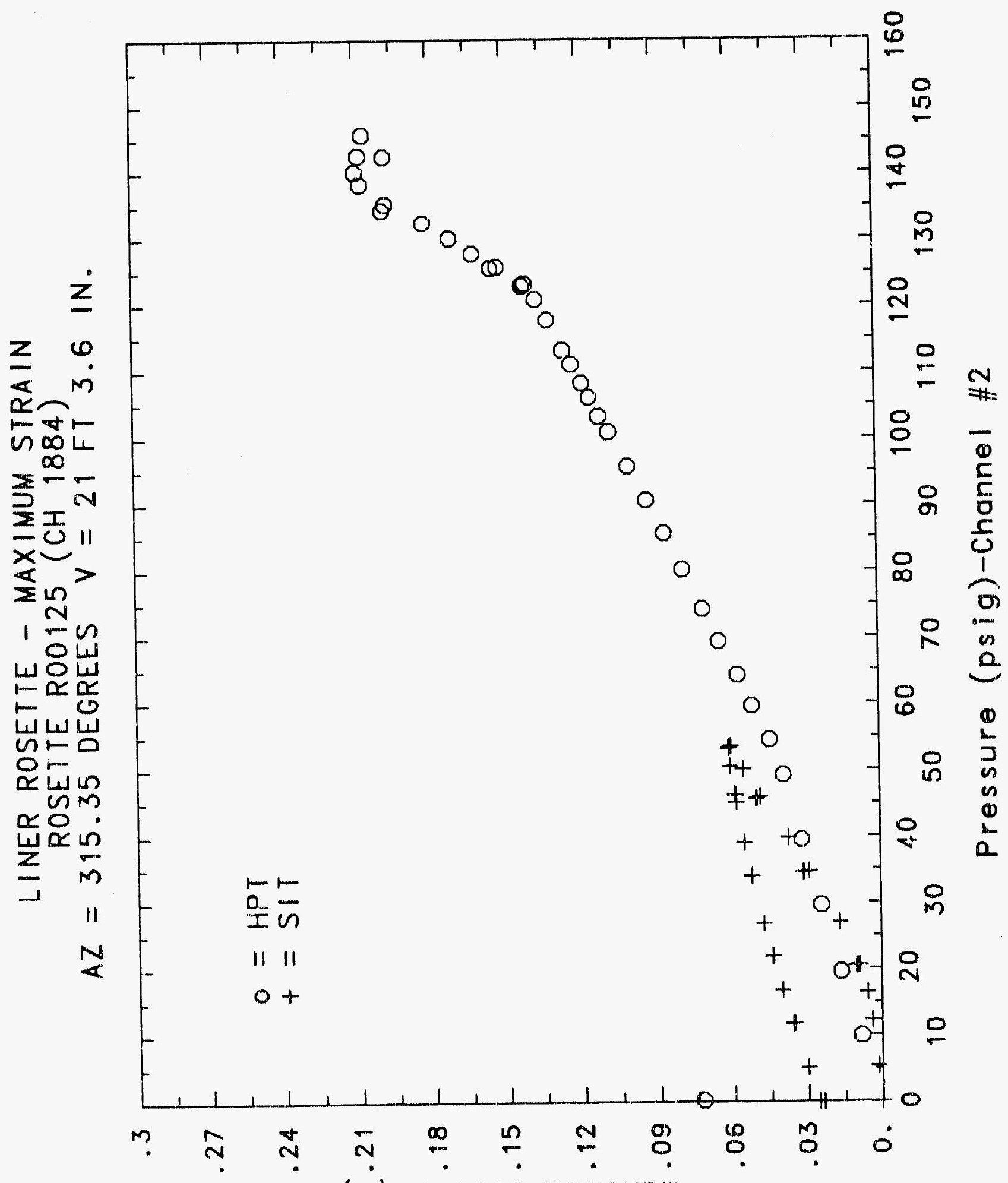

(\%) $4 ! D d+S$ WnW! XDW 

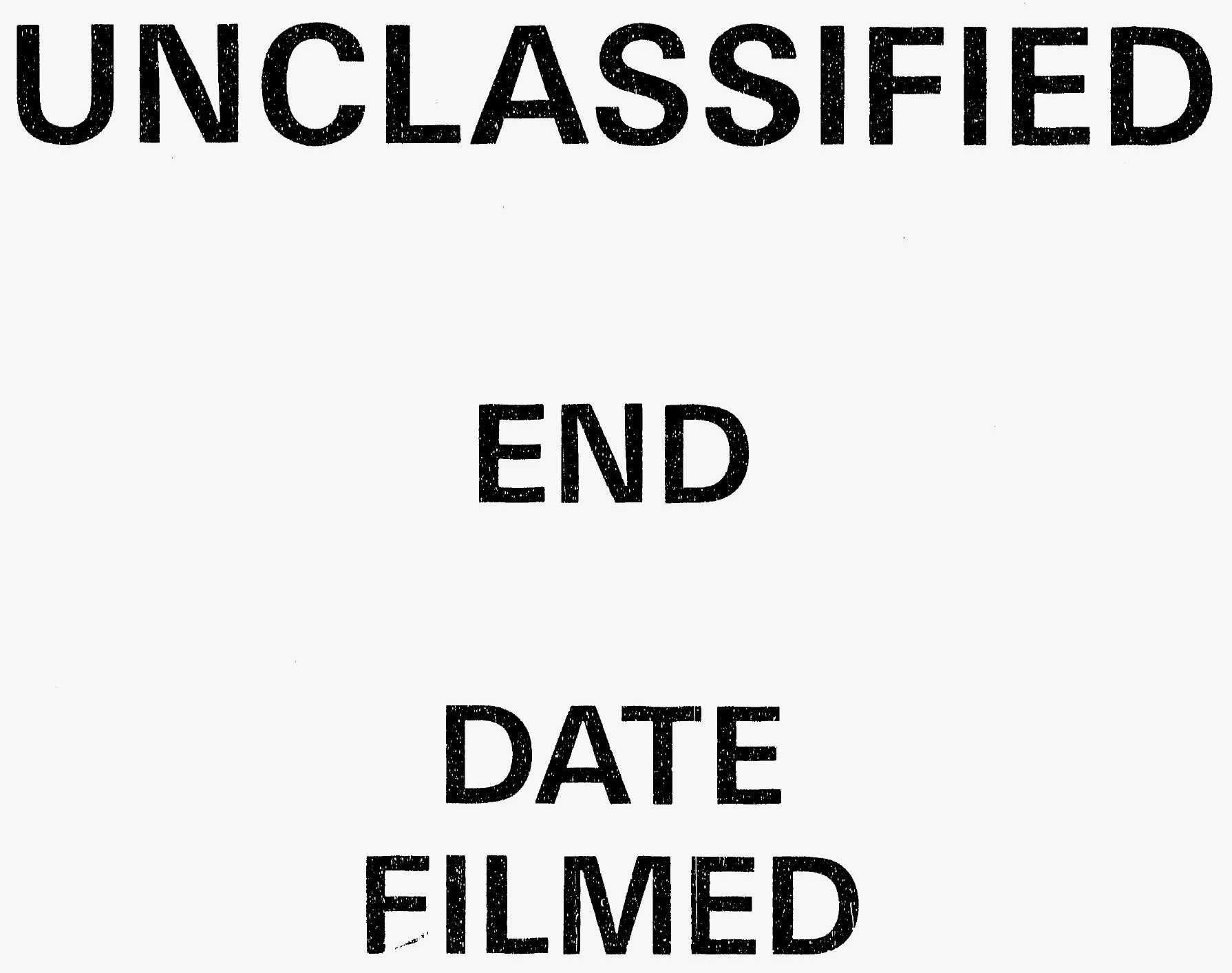

$$
1-6-92
$$

SANDIA NATIONAL LABS 


\section{IMAGE EVALUATION}

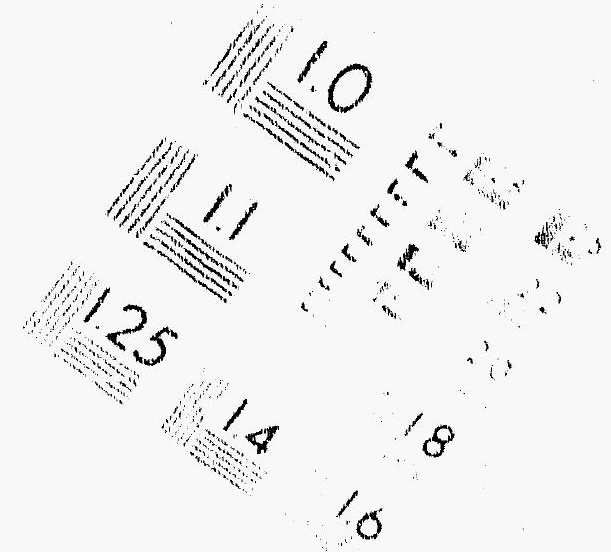

TEST TARGET (MT-3)
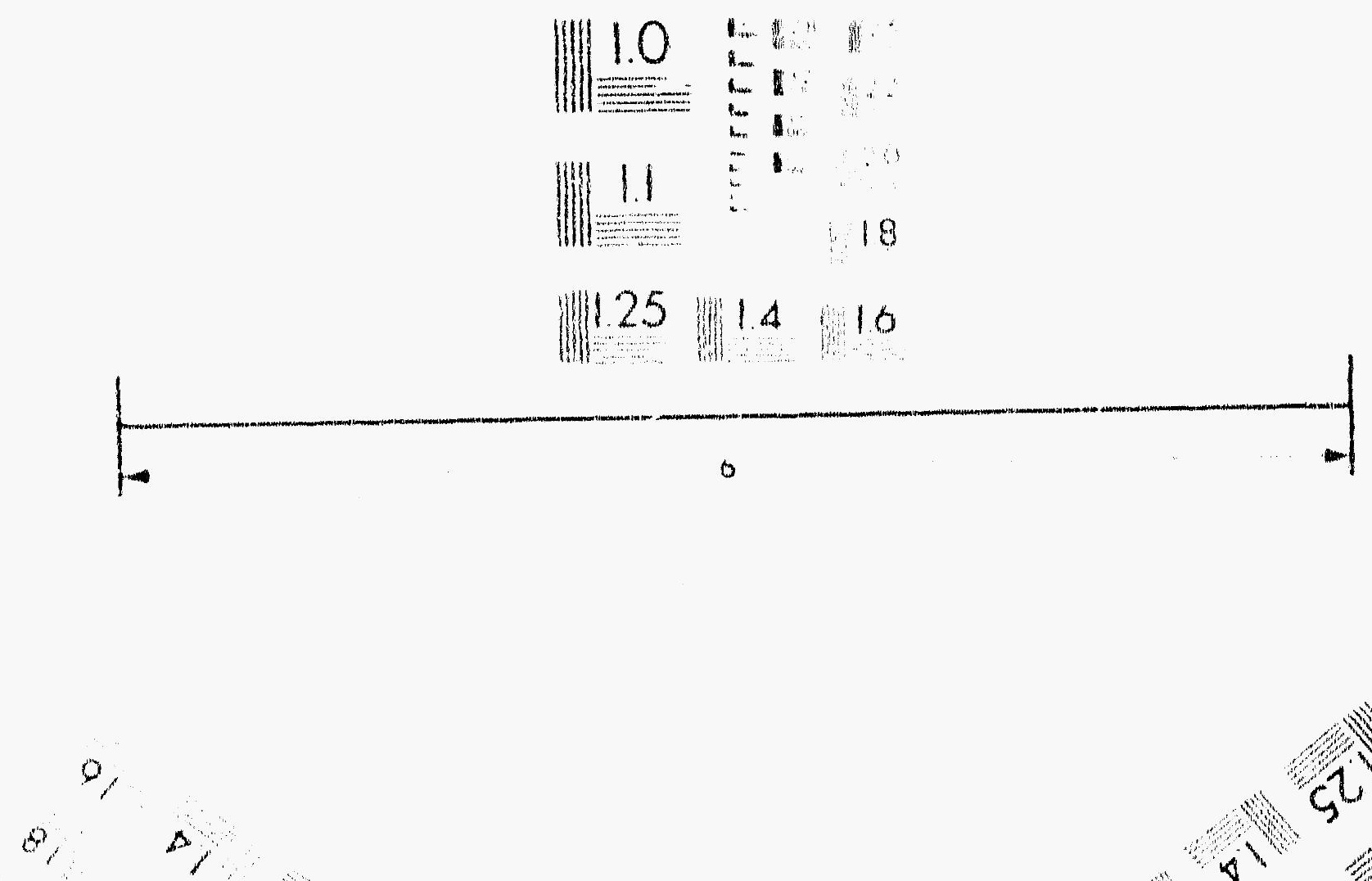

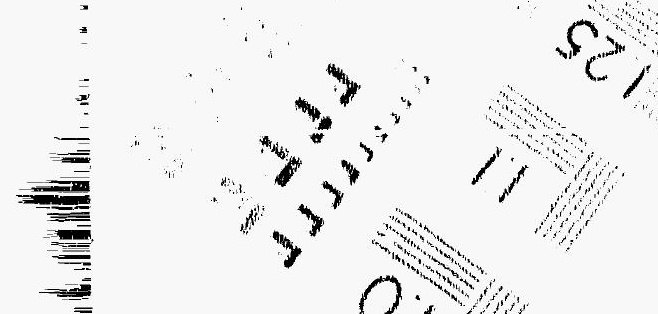

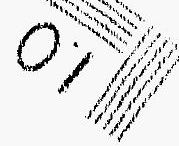

Photogrymis:

Sciences

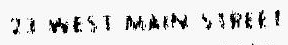

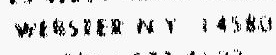

Corporation

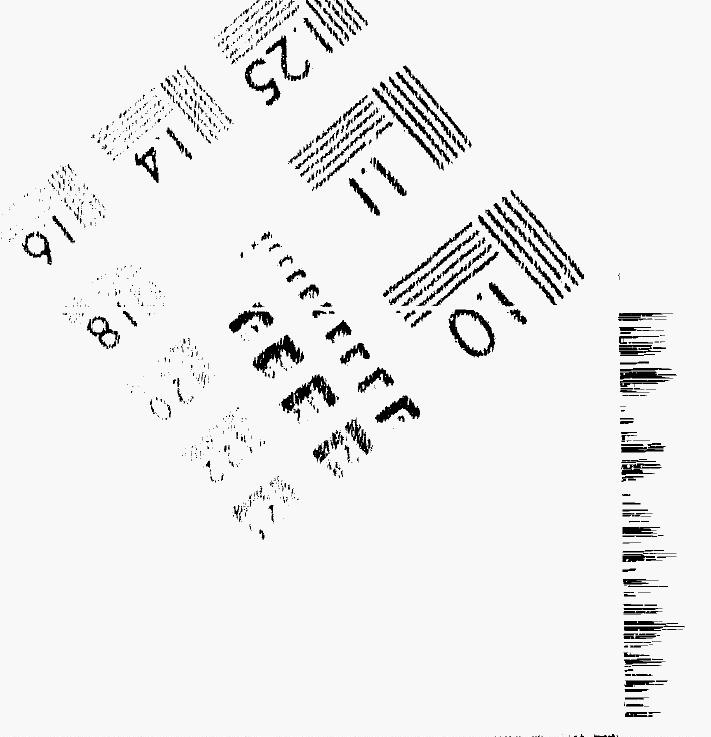




\section{SECURITY CLASSIFICATION}
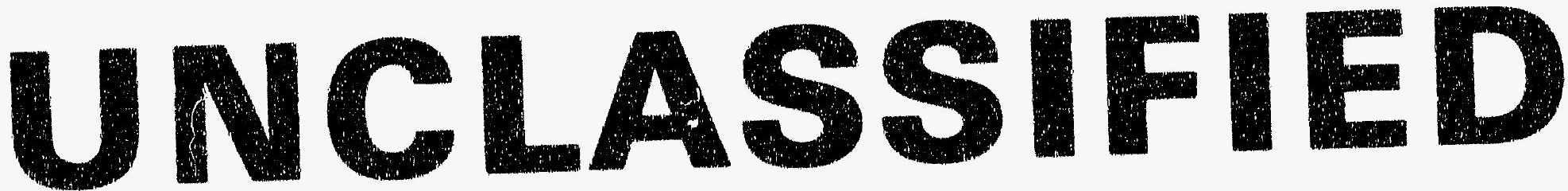

\section{DATE OF MICROFILMING $1-6-92$}

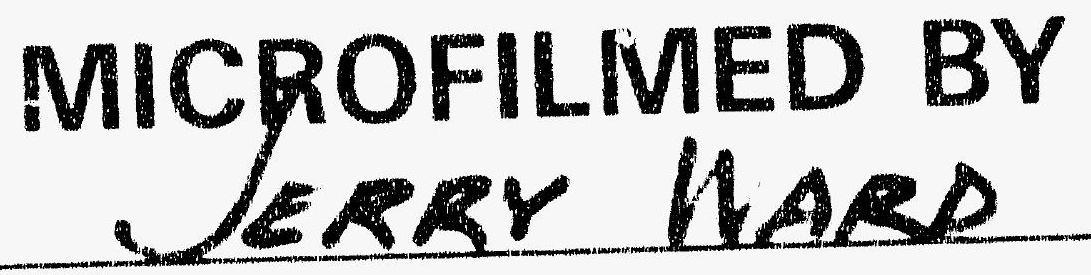

SANDIA NATIONAL LABS 
THIS DOCUMENT FILMED

AT

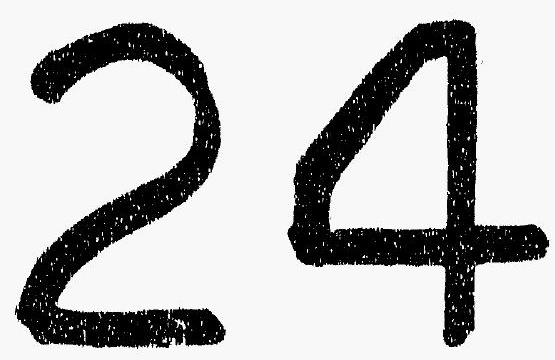

REDUCTION 


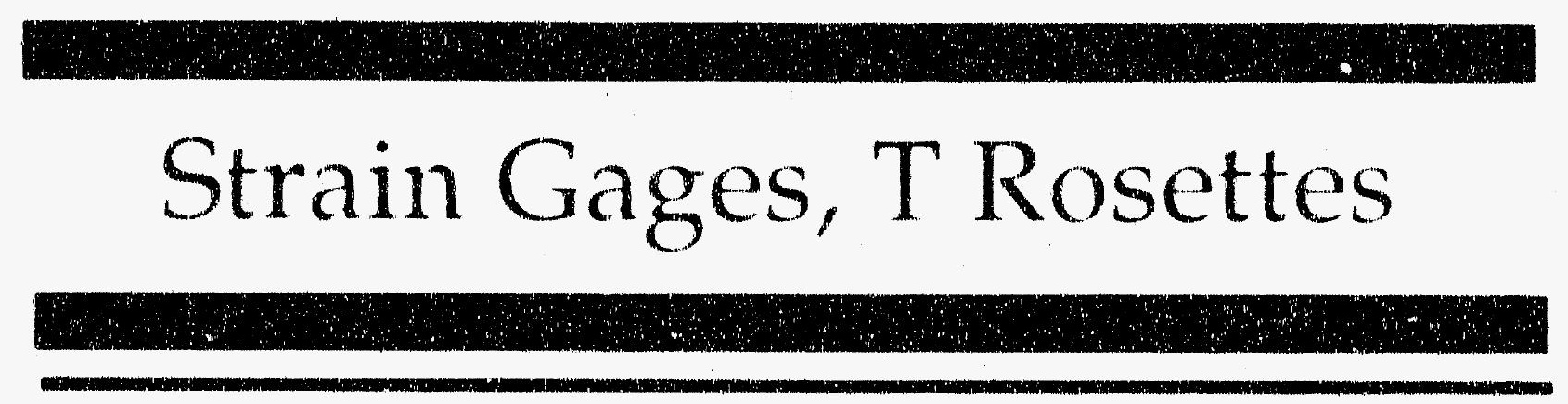




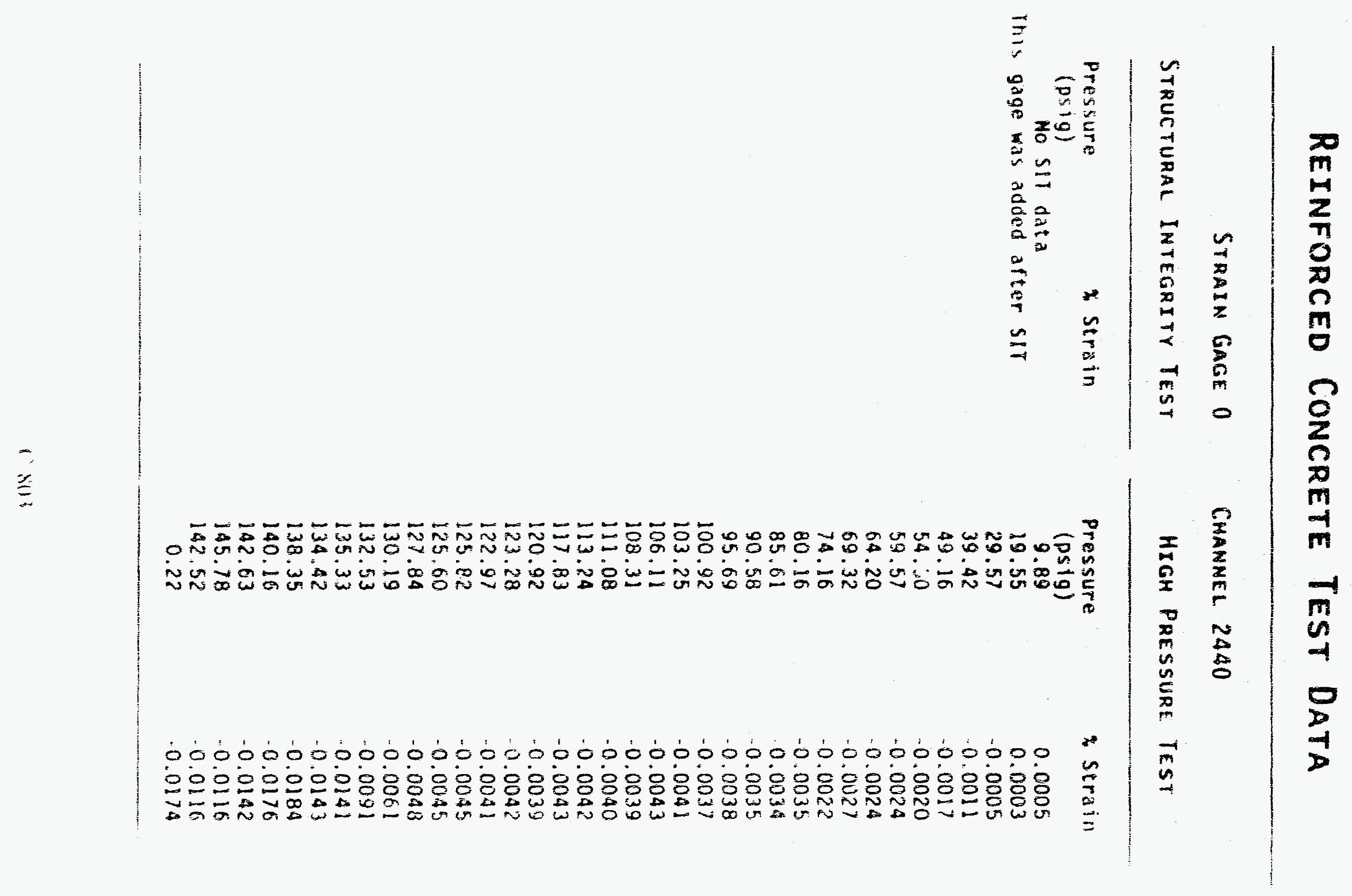




\section{Reinforced Concrete Test Data}

Strayn Gage 0

Structural Integrity test

Pressure

(psig)

Ho sit data

Inis gage was added after SIT
* Strain 


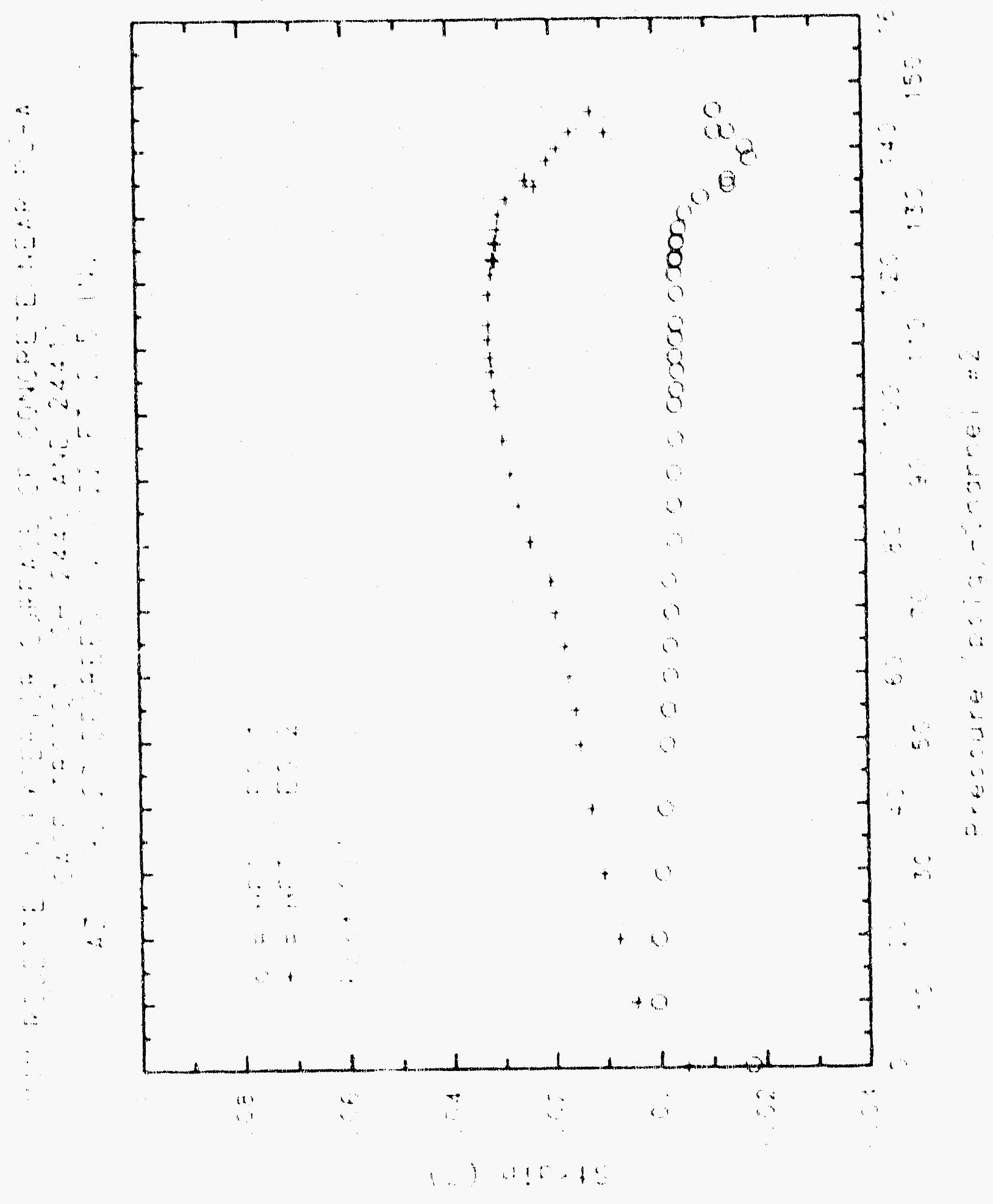

$(2.815$ 


\section{Reinforced Concrete Test Data}

Strain Gage 0
StructuaAl Integrity Test
$\begin{gathered}\text { Pressure } \\ \text { (psig) } \\ \text { No SIT data }\end{gathered}$

Channel 2442

High pressure test

inis gaye was added after SIT

\begin{tabular}{cc} 
Pressure & 2 Strain \\
(psig) & \\
9.89 & -0.0013 \\
19.55 & -0.0024 \\
29.57 & -0.0041 \\
39.42 & -0.0054 \\
49.16 & -0.0066 \\
54.50 & -0.0074 \\
59.57 & -0.0082 \\
64.20 & -0.0094 \\
69.32 & -0.0097 \\
74.16 & -0.0105 \\
80.16 & -0.0094 \\
85.61 & -0.0096 \\
90.58 & -0.0095 \\
95.69 & -0.0093 \\
100.92 & -0.0098 \\
103.25 & -0.0098 \\
106.11 & -0.0100 \\
108.31 & -0.0099 \\
111.08 & -0.0098 \\
113.24 & -0.0097 \\
117.83 & -0.0097 \\
120.92 & -0.0095 \\
123.28 & -0.0093 \\
122.97 & -0.0096 \\
125.82 & -0.0094 \\
125.60 & -0.0096 \\
127.84 & -0.0099 \\
130.19 & -0.0099 \\
132.53 & -0.0098 \\
135.33 & -0.0101 \\
134.42 & -0.0105 \\
138.35 & -0.0108 \\
140.16 & -0.0997 \\
142.63 & -0.0074 \\
145.78 & -0.0045 \\
142.52 & -0.0033 \\
0.22 & -0.0048 \\
& \\
\hline & \\
\hline & \\
132 &
\end{tabular}




\section{Reinforced Concrete Test Data}

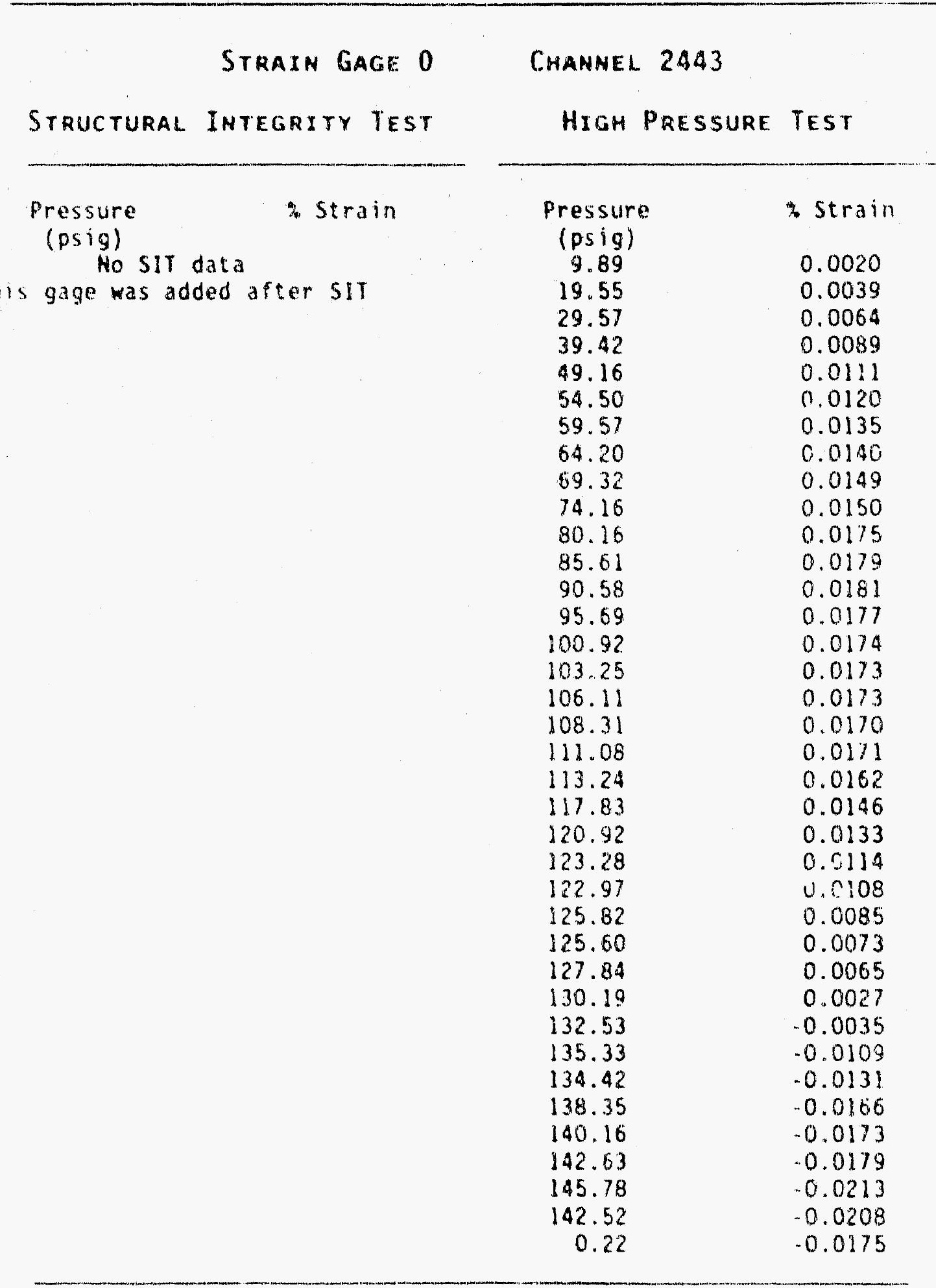




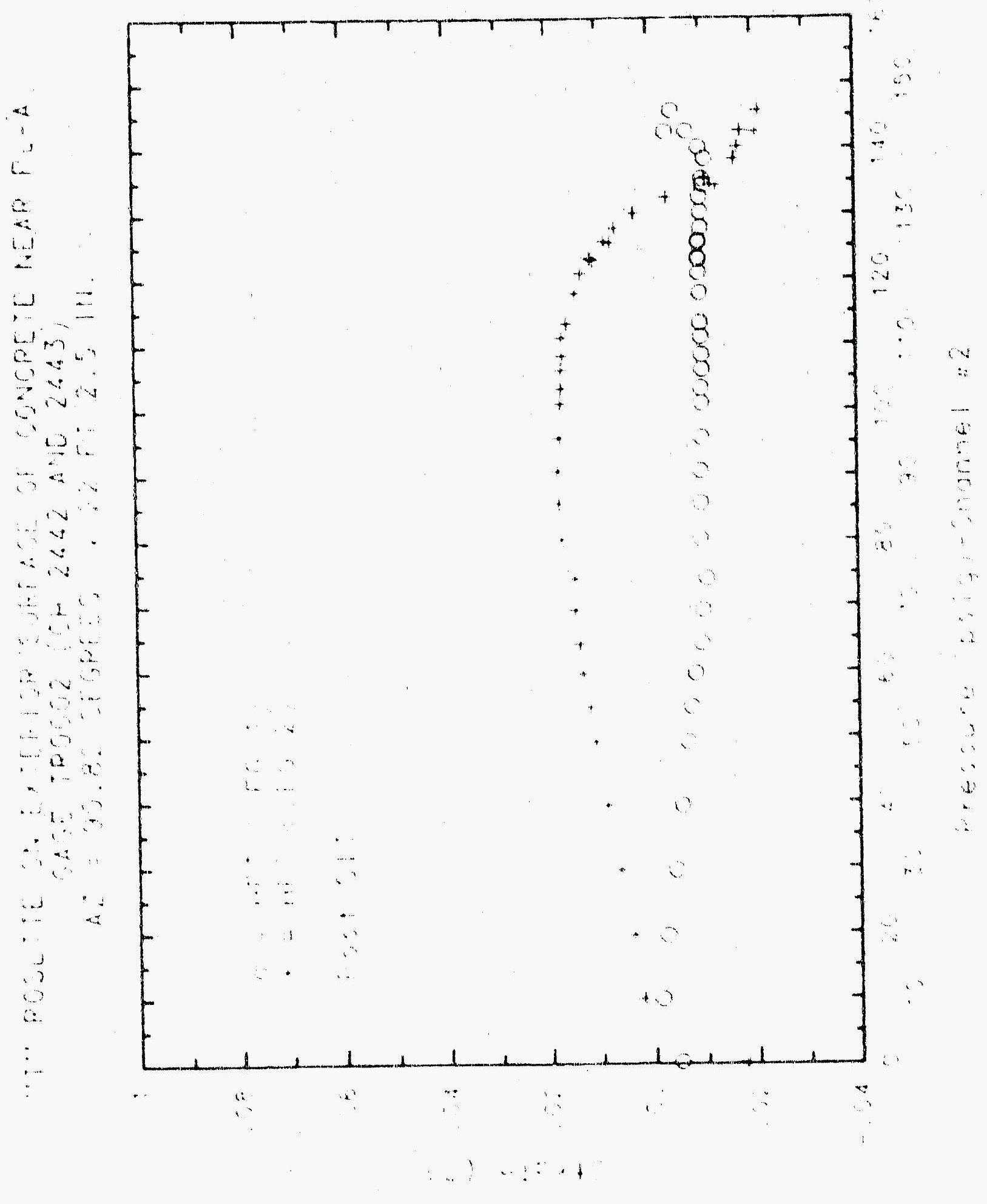

C.sus 


\section{Reinforced Concrete Test Data}

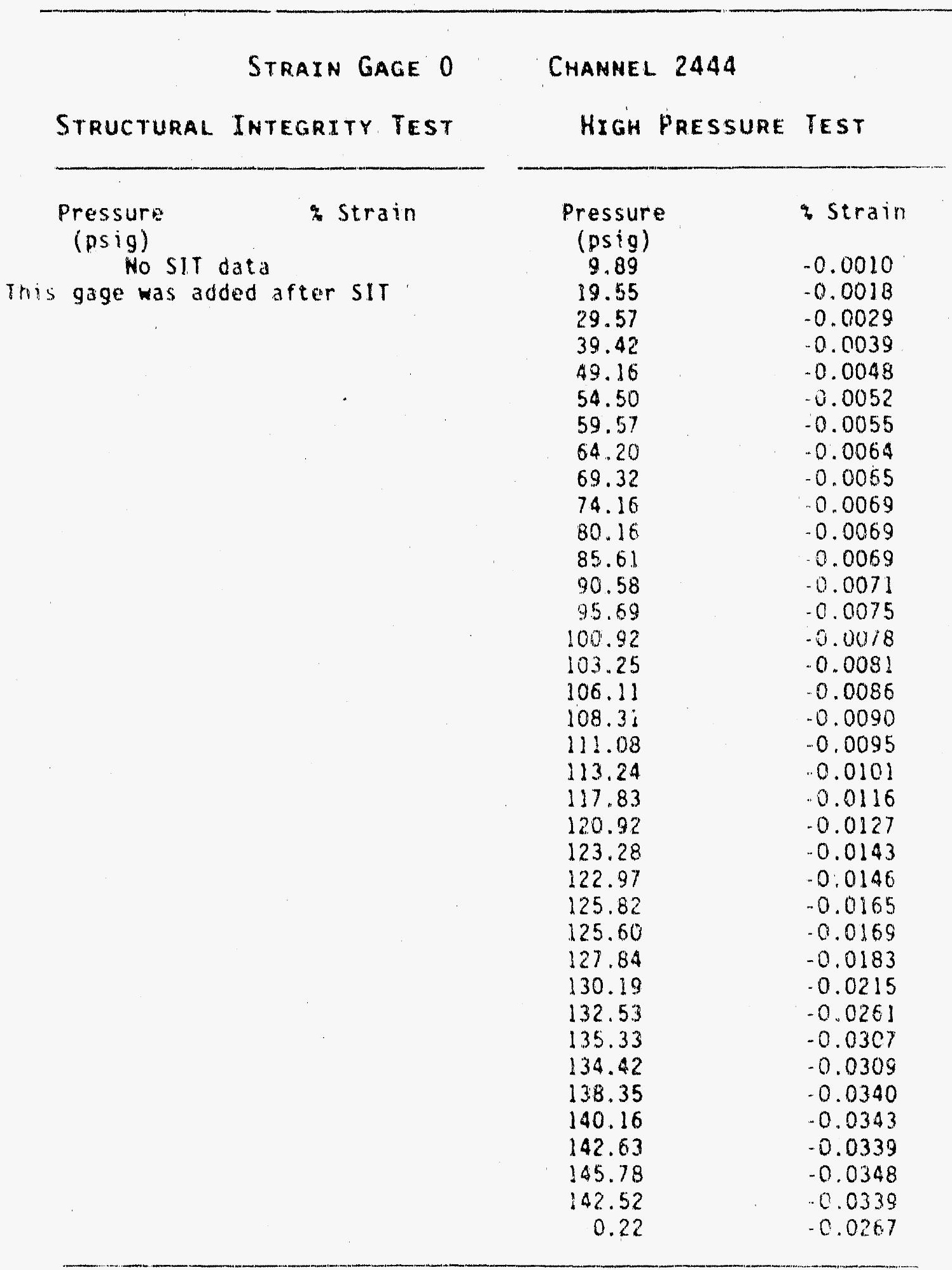




\section{Reinforced Concrete test Data}

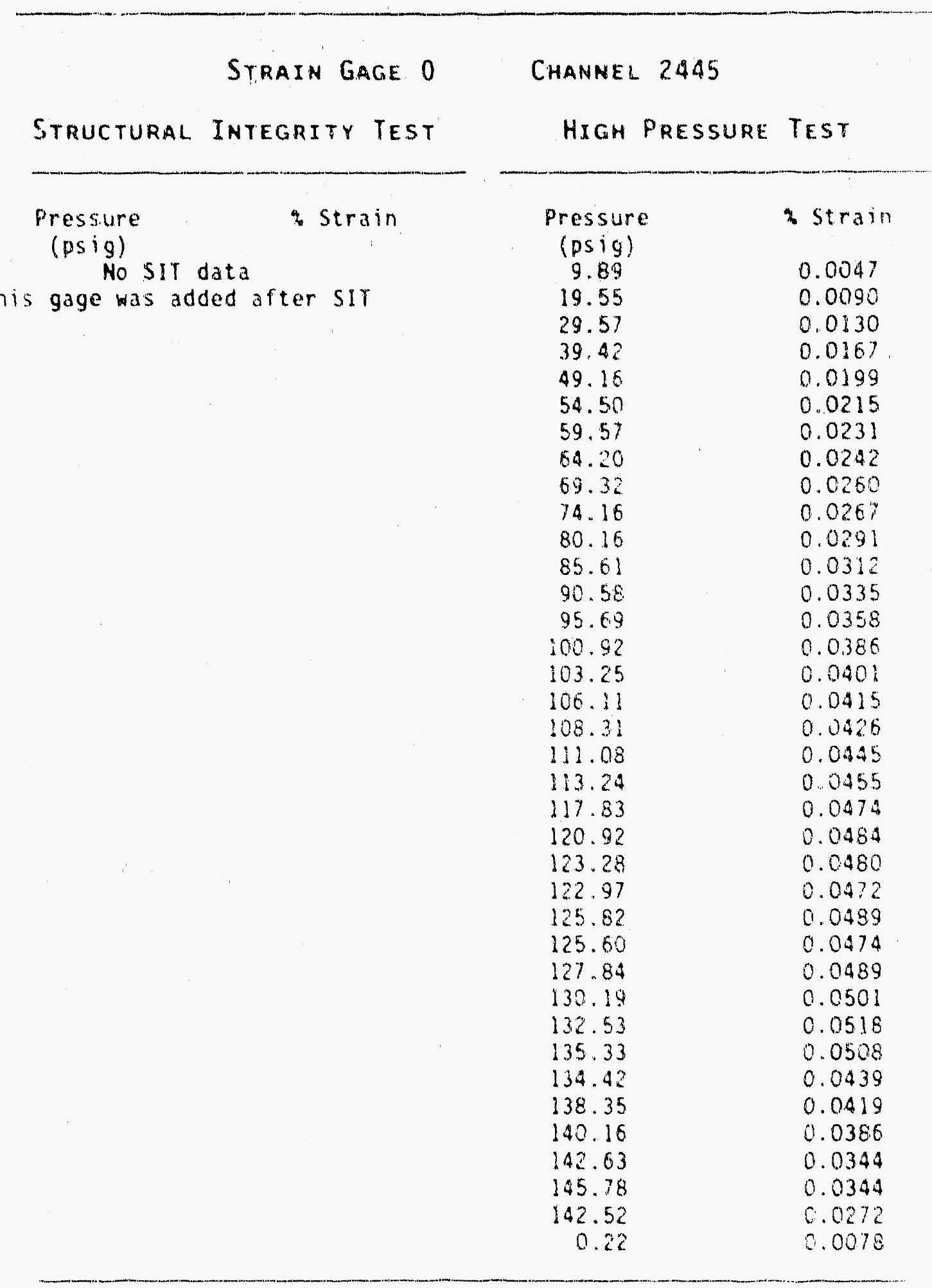




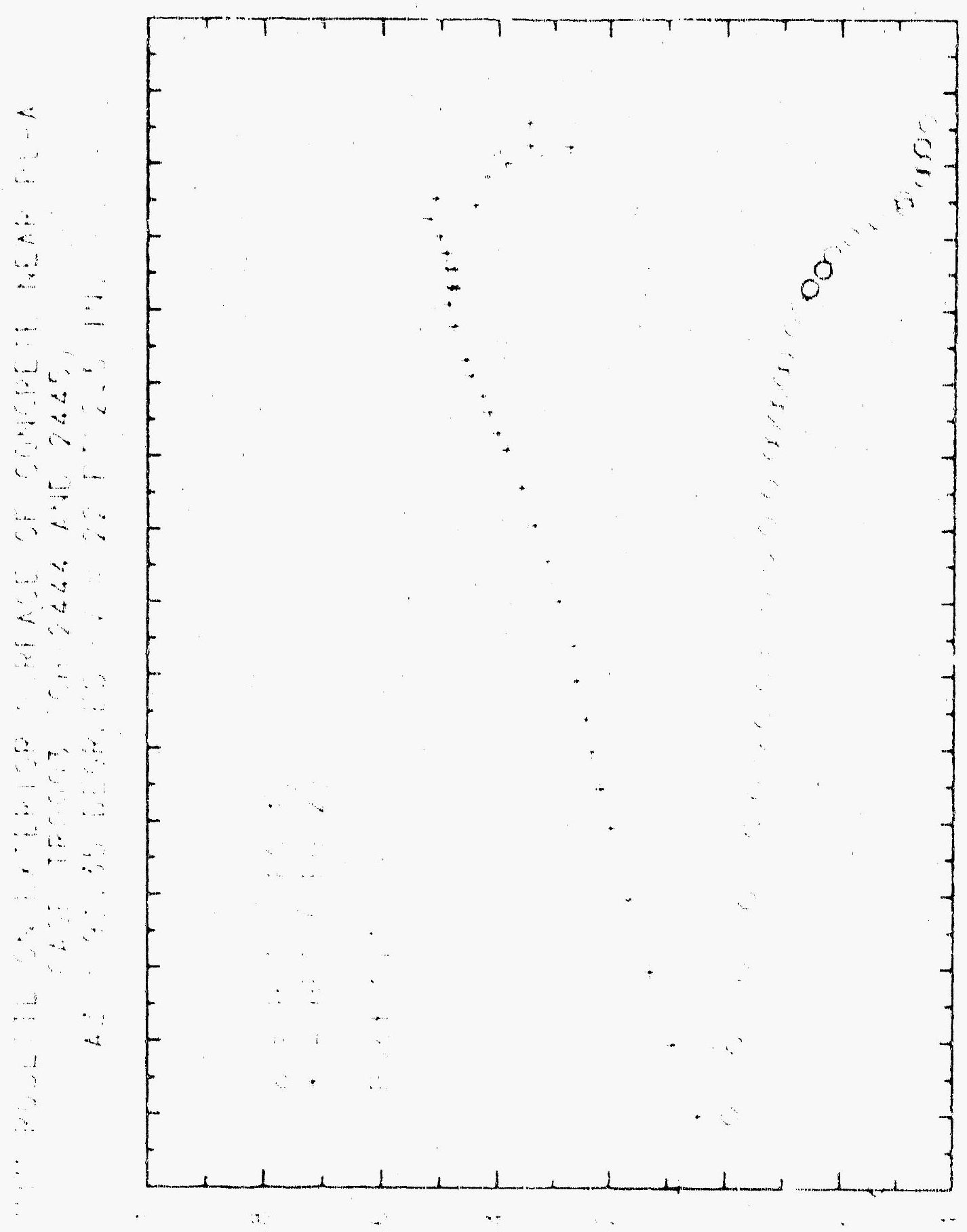


Reinforced Concrete Teri Data

Strain race 0

Structural Integrator test

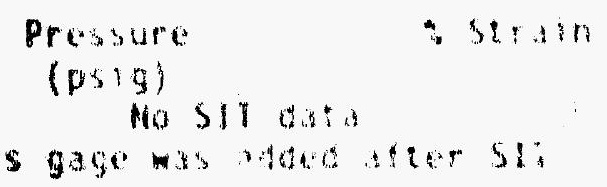

LANNER 2446

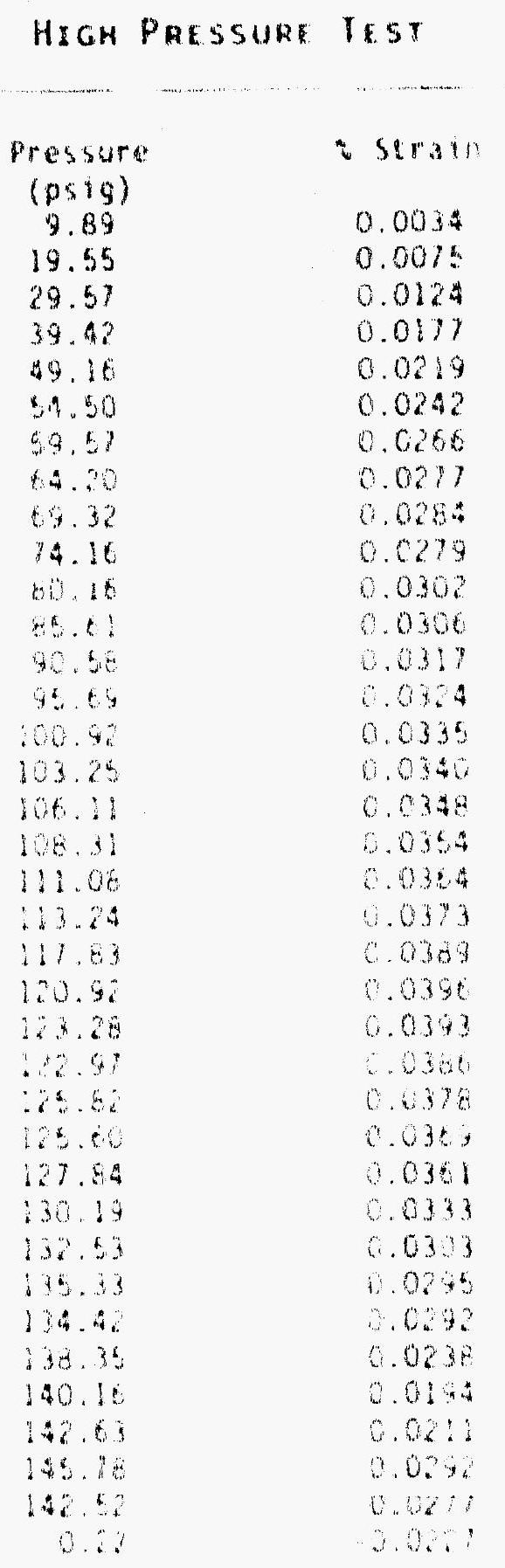




\section{Reinforced Concrete test Data}

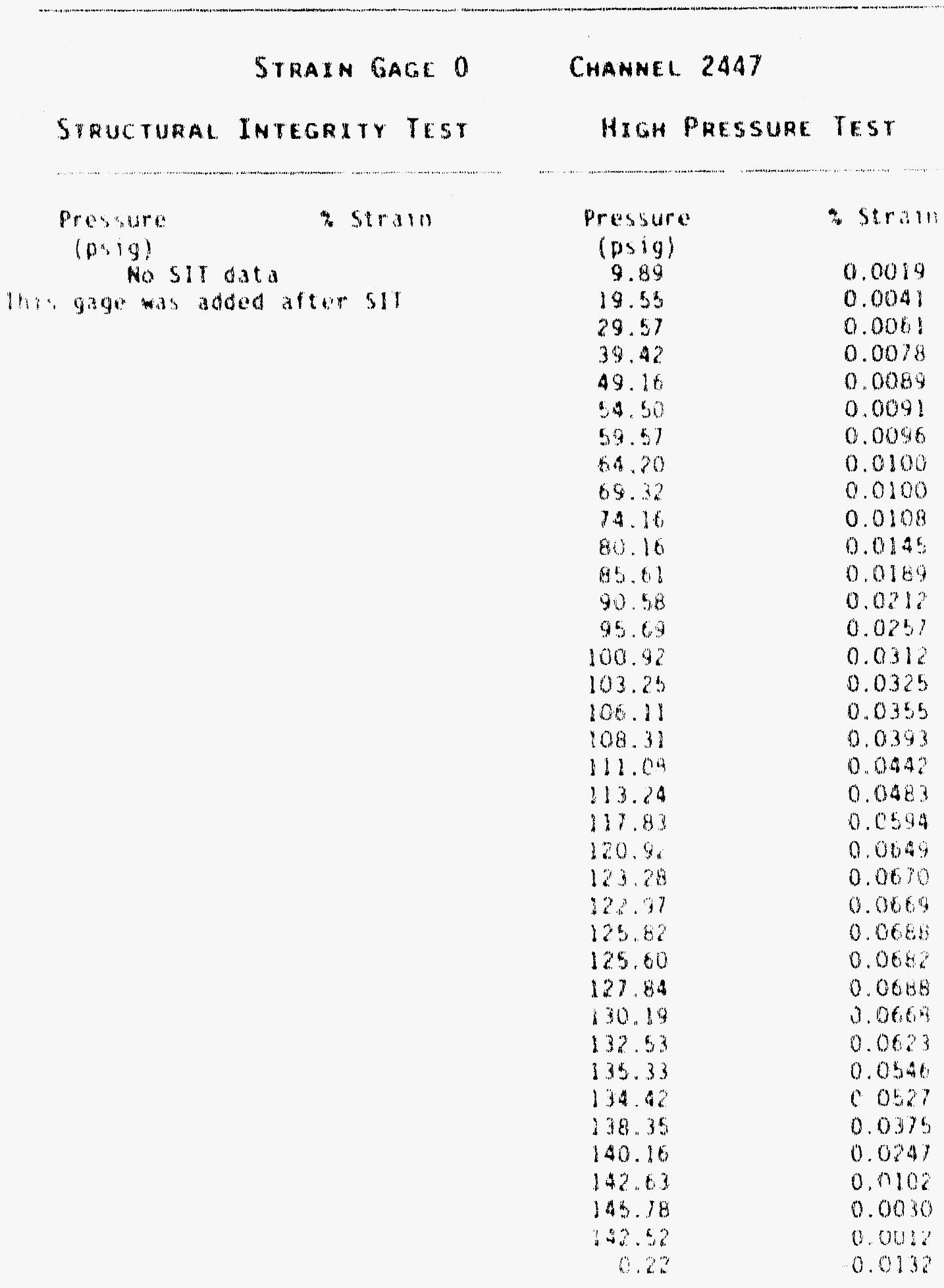




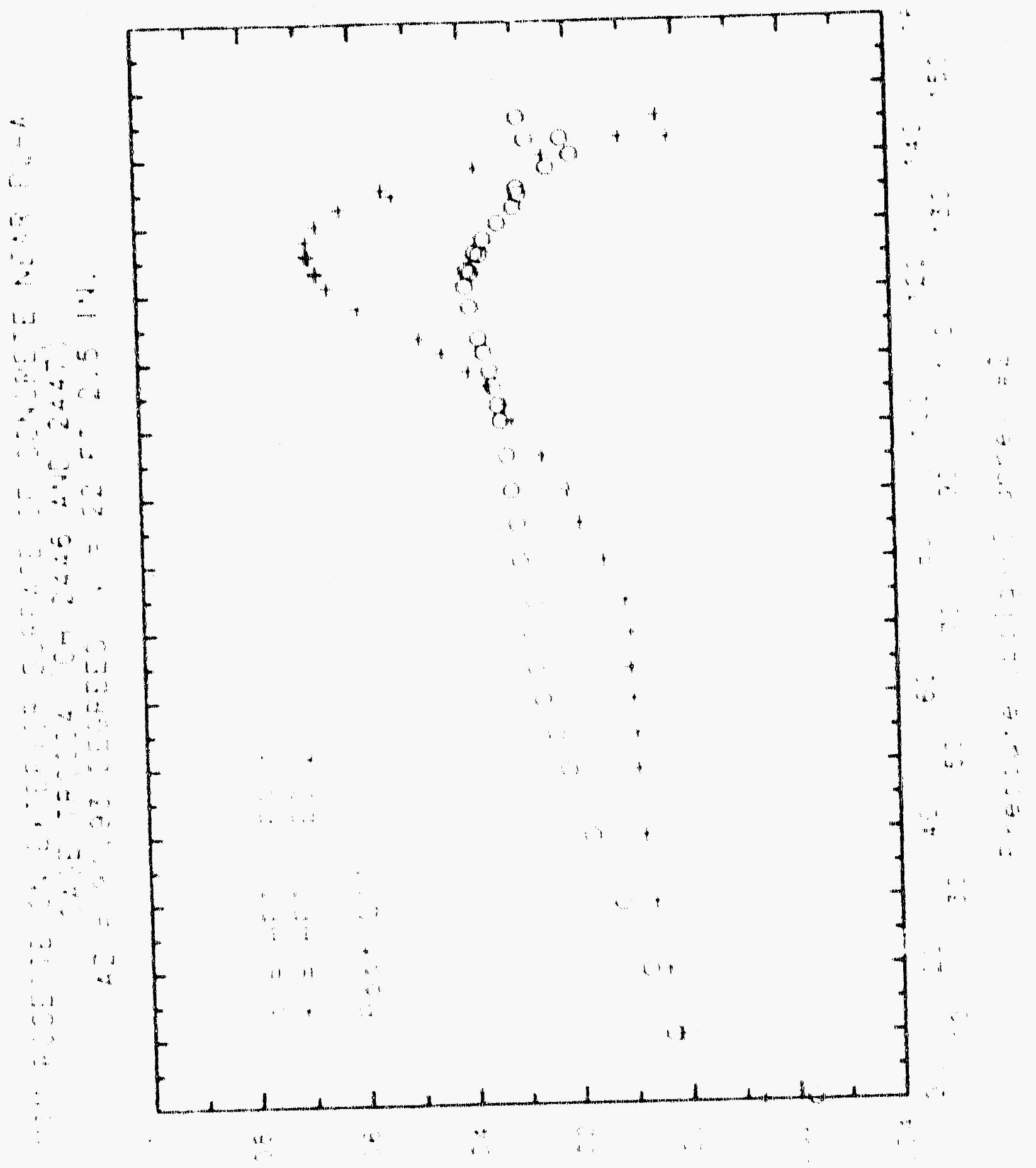

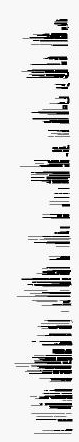



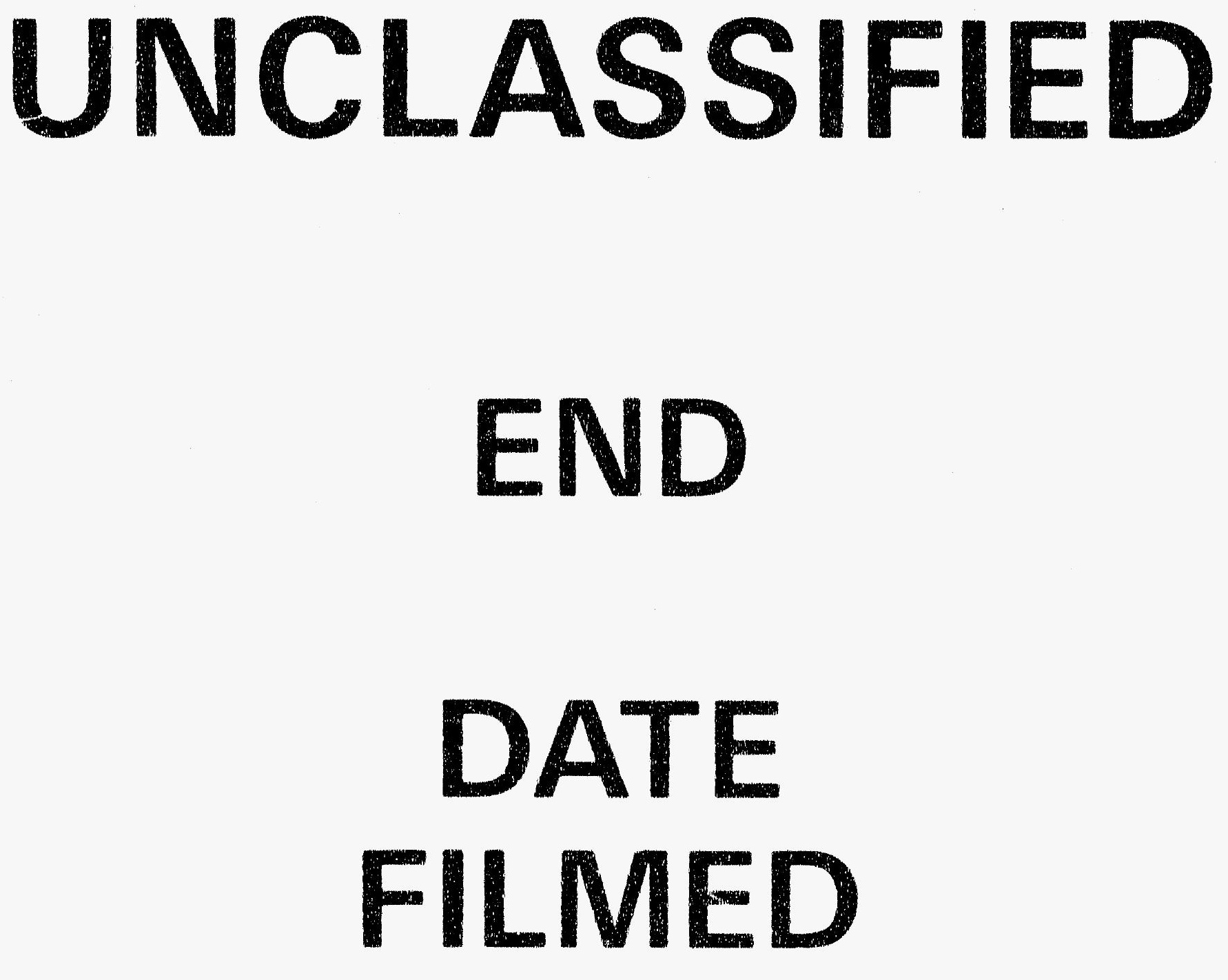

$1-6-92$

SANDIA NATIONAL LABS 

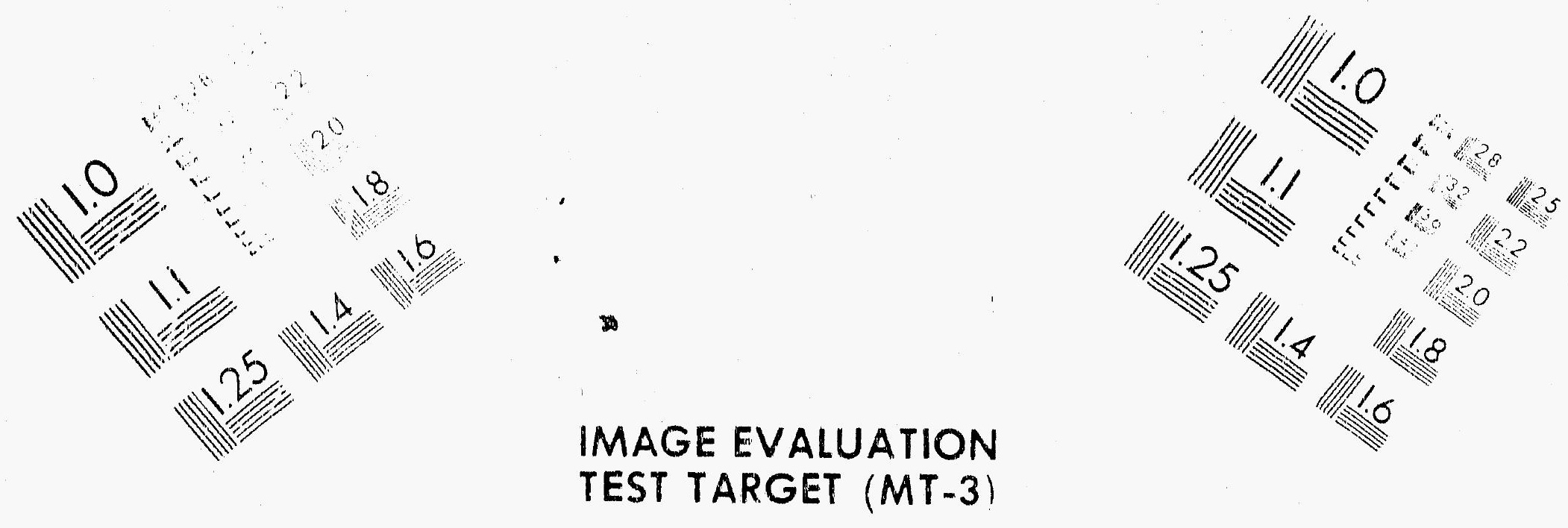

TEST TARGET (MT-3)
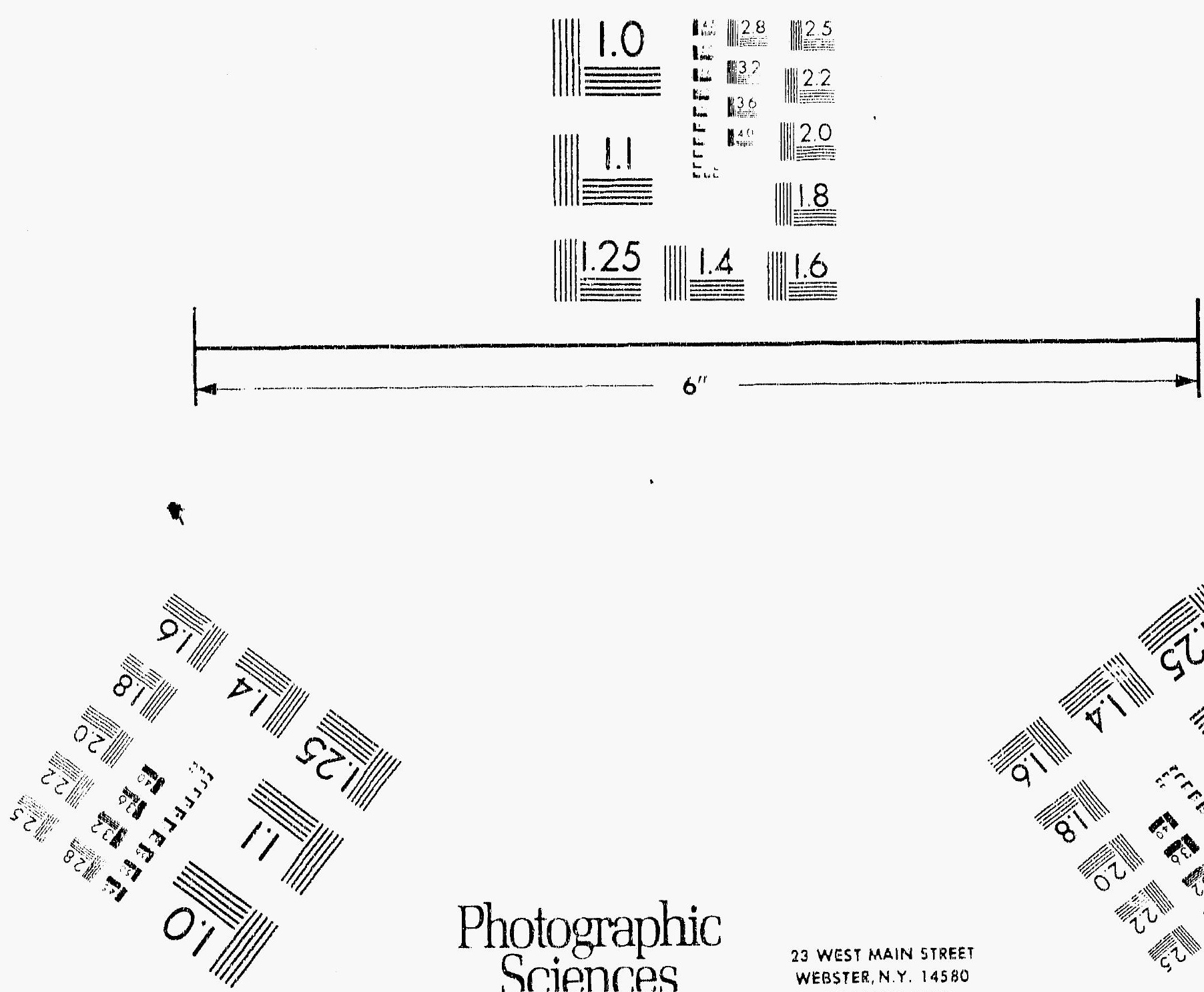

Photographic Sciences Corporation

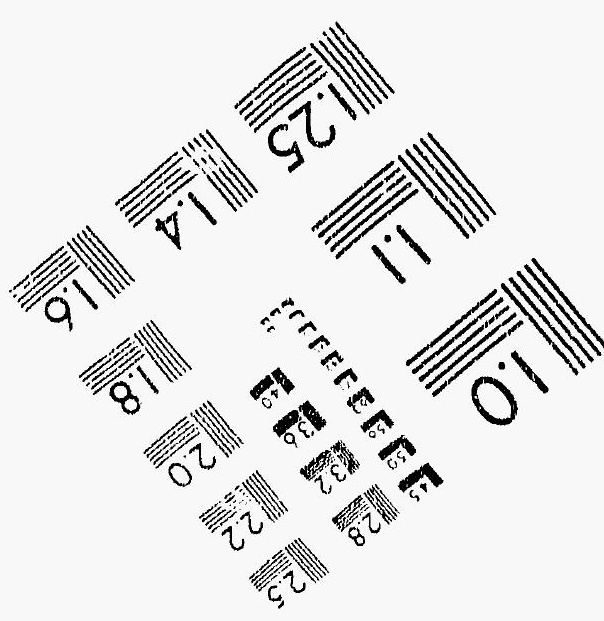




\section{SECURITY CLASSIFICATION}

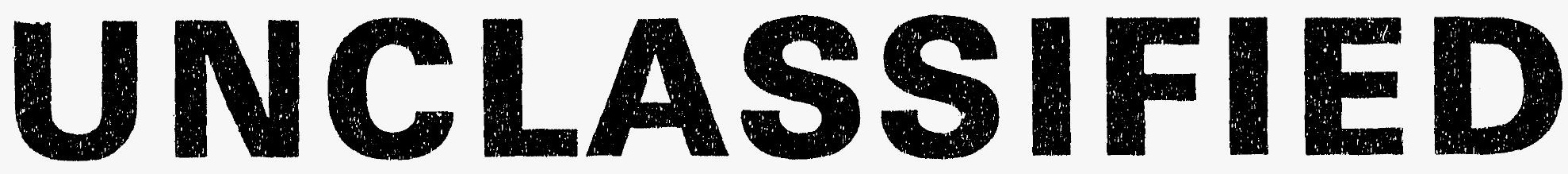

\section{DATE OF MICROFILMING $1-6-92$}

\section{MICROFILMED BY ERRY HARS}

SANDIA NATIONAL LABS 


\section{THIS DOCUMENT FILMED}

AT

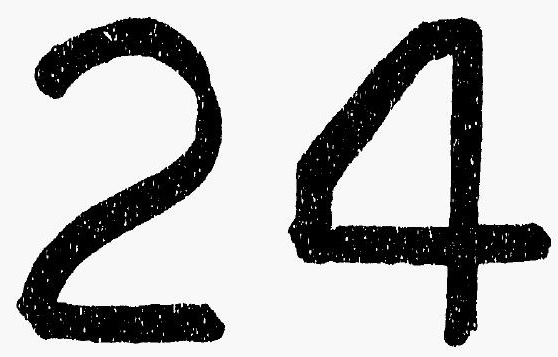

REDUCTION 


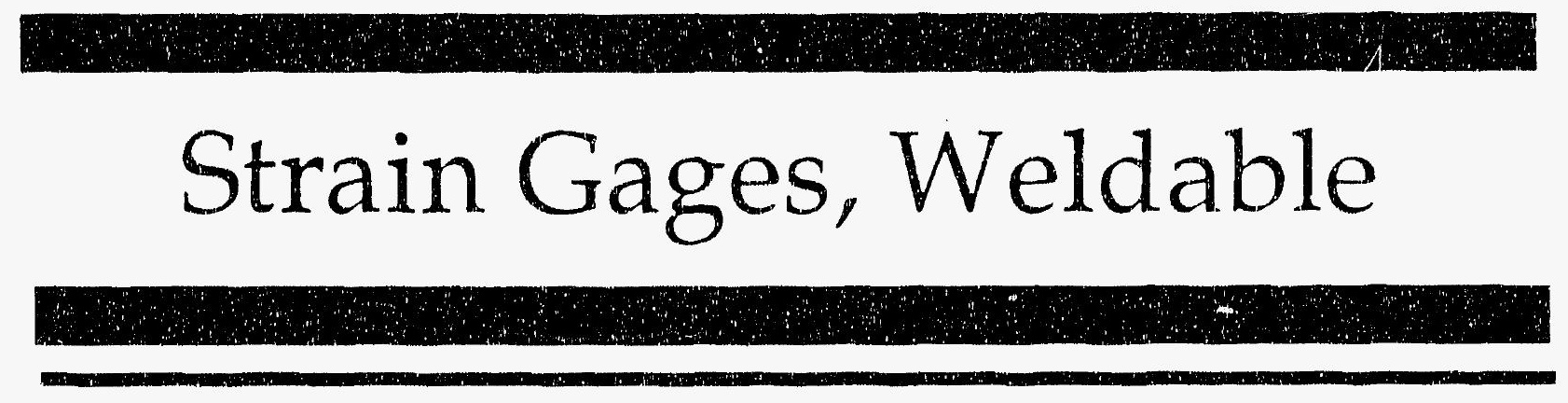




\section{Reinforced Concrete Test Data}

Weldable Gage 1 Channel 400

STRUCTURAL InTEGRITY TEST

Hrgh Pressure test

\begin{tabular}{|c|c|c|c|}
\hline $\begin{array}{c}\text { Pressure } \\
(p s i g) \\
-0.05 \\
5.33 \\
12.31 \\
16.44 \\
20.51 \\
20.46 \\
20.47 \\
27.05 \\
34.69 \\
34.53 \\
34.53 \\
39.70 \\
45.90 \\
45.65 \\
45.62 \\
50.10 \\
53.47 \\
53.29 \\
53.21 \\
50.49 \\
46.14 \\
46.13 \\
44.97 \\
38.98 \\
33.99 \\
33.96 \\
26.83 \\
21.88 \\
21.90 \\
16.69 \\
11.74 \\
11.77 \\
5.05 \\
0.02 \\
-0.04 \\
-0.02 \\
-0.02 \\
0.02 \\
\end{array}$ & $\begin{array}{l}\text { \% Strain } \\
0.0010 \\
0.0009 \\
0.0010 \\
0.0009 \\
0.0014 \\
0.0013 \\
0.0011 \\
0.0020 \\
0.0024 \\
0.0019 \\
0.0020 \\
0.0024 \\
0.0028 \\
0.0027 \\
0.0025 \\
0.0034 \\
0.0033 \\
0.0032 \\
0.0037 \\
0.0034 \\
0.0030 \\
0.0031 \\
0.0035 \\
0.0036 \\
0.0033 \\
0.0031 \\
0.0026 \\
0.0024 \\
0.0022 \\
0.0017 \\
0.0018 \\
0.0017 \\
0.0007 \\
0.0004 \\
0.0006 \\
0.0005 \\
0.0008 \\
0.0004\end{array}$ & $\begin{array}{c}\text { Pressure } \\
\text { (psig) } \\
9.89 \\
19.55 \\
29.57 \\
39.42 \\
49.16 \\
54.50 \\
59.57 \\
64.20 \\
69.32 \\
74.16 \\
80.16 \\
85.61 \\
90.58 \\
95.69 \\
100.92 \\
103.25 \\
106.11 \\
108.31 \\
111.08 \\
113.24 \\
117.83 \\
120.92 \\
123.28 \\
122.97 \\
125.82 \\
125.60 \\
127.84 \\
130.19 \\
132.53 \\
135.33 \\
134.42 \\
138.35 \\
140.16 \\
142.63 \\
145.78 \\
142.52 \\
0.22 \\
\end{array}$ & $\begin{array}{l}\text { \% Strain } \\
-0.0041 \\
-0.0034 \\
-0.0027 \\
-0.0024 \\
-0.0021 \\
-0.0020 \\
-0.0022 \\
0.0002 \\
0.0034 \\
0.0106 \\
0.0228 \\
0.0315 \\
0.0411 \\
0.0517 \\
0.0644 \\
0.0733 \\
0.0787 \\
0.0821 \\
0.0846 \\
0.0843 \\
0.0830 \\
0.0826 \\
0.0832 \\
0.0835 \\
0.0834 \\
0.0839 \\
0.0843 \\
0.0848 \\
0.0848 \\
0.0852 \\
0.0854 \\
0.0856 \\
0.0857 \\
0.0858 \\
0.0858 \\
0.0852 \\
0.0015\end{array}$ \\
\hline
\end{tabular}




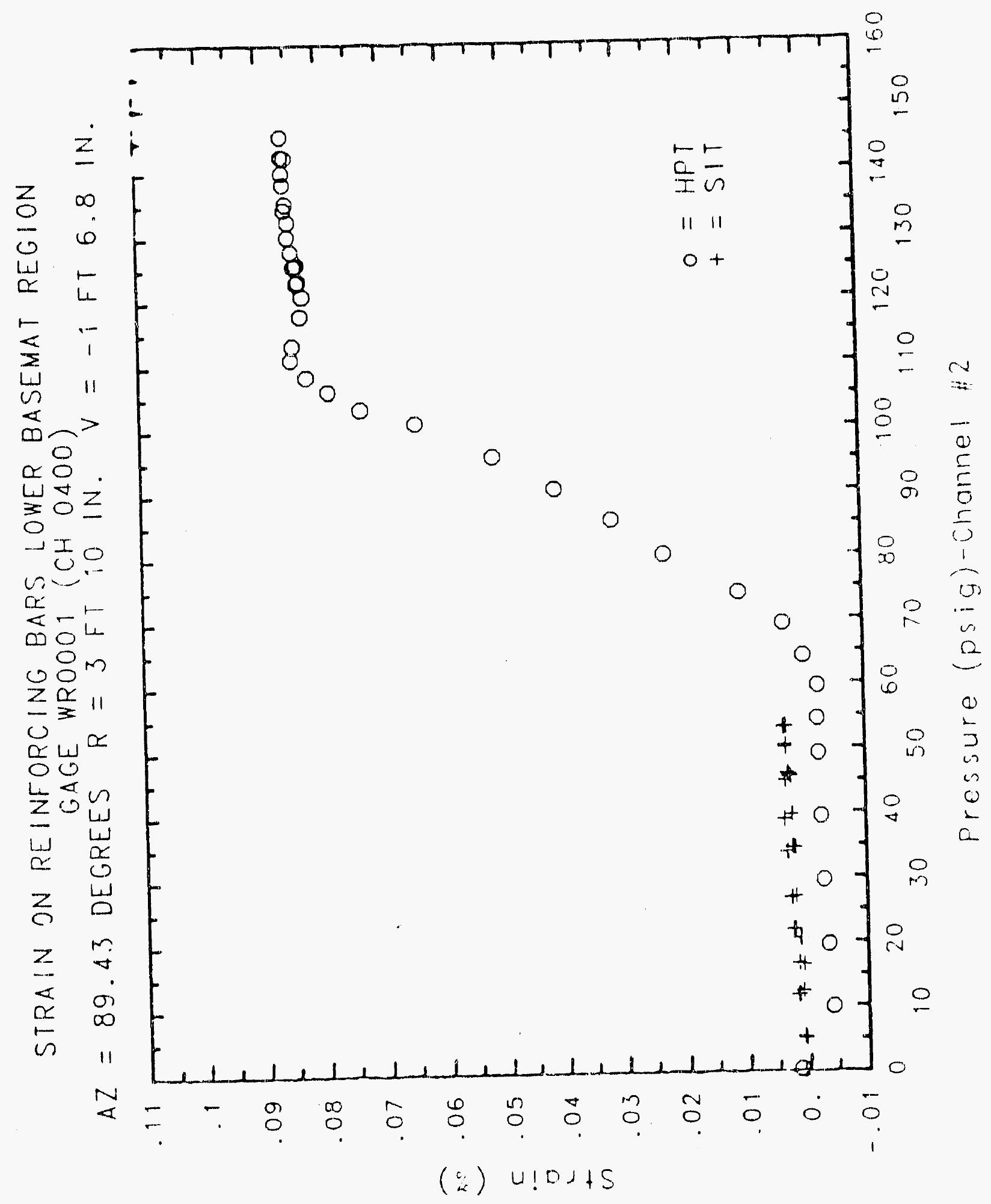

C. 816 


\section{Reinforced Concrete Test Data}

Weldable Gage 2 Channel 401

Structural Integrity Test

\begin{tabular}{|c|c|c|c|}
\hline $\begin{array}{c}\text { Pressure } \\
\text { (psig) } \\
-0.05 \\
5.33 \\
12.31 \\
16.44 \\
20.51 \\
20.46 \\
20.47 \\
27.05 \\
34.69 \\
34.53 \\
34.53 \\
39.70 \\
45.90 \\
45.65 \\
45.62 \\
50.10 \\
53.47 \\
53.29 \\
53.21 \\
50.49 \\
46.14 \\
46.13 \\
44.97 \\
38.98 \\
33.99 \\
33.96 \\
26.83 \\
21.88 \\
21.90 \\
16.69 \\
11.74 \\
11.77 \\
5.05 \\
0.02 \\
-0.04 \\
-0.02 \\
-0.02 \\
0.02\end{array}$ & $\begin{array}{c}\text { \% Strain } \\
-0.0004 \\
-0.0002 \\
0.1044 \\
0.1044 \\
0.1046 \\
0.1047 \\
0.1048 \\
0.1054 \\
0.1053 \\
0.1056 \\
0.1060 \\
0.1059 \\
0.1062 \\
0.1064 \\
0.1060 \\
0.1063 \\
0.1064 \\
0.1061 \\
0.1068 \\
0.1066 \\
0.1065 \\
0.1066 \\
0.1070 \\
0.1069 \\
0.1066 \\
0.1065 \\
0.1060 \\
0.1056 \\
0.1056 \\
0.1055 \\
0.1052 \\
0.1048 \\
0.1042 \\
0.1039 \\
0.1037 \\
0.1043 \\
0.1043 \\
0.1039\end{array}$ & $\begin{array}{c}\text { Pressure } \\
(\text { psig) } \\
9.89 \\
19.55 \\
29.57 \\
39.42 \\
49.16 \\
54.50 \\
59.57 \\
64.20 \\
69.32 \\
74.16 \\
80.16 \\
85.61 \\
90.58 \\
95.69 \\
100.92 \\
103.25 \\
106.11 \\
.08 .31 \\
11.08 \\
113.24 \\
117.83 \\
120.92 \\
123.28 \\
122.97 \\
125.82 \\
125.60 \\
127.84 \\
130.19 \\
132.53 \\
135.33 \\
134.42 \\
138.35 \\
140.16 \\
142.63 \\
145.78 \\
142.52 \\
0.22\end{array}$ & $\begin{array}{c}\% \text { Strain } \\
\text {-0.0040 } \\
-0.0037 \\
-0.0034 \\
-0.0023 \\
-0.0018 \\
-0.0019 \\
-0.0021 \\
-0.0002 \\
0.0036 \\
0.0104 \\
0.0228 \\
0.0317 \\
0.0406 \\
0.0519 \\
0.0646 \\
0.0729 \\
0.0783 \\
0.0818 \\
0.0848 \\
0.0841 \\
0.0826 \\
0.0828 \\
0.0833 \\
0.0837 \\
0.0835 \\
0.0840 \\
0.0840 \\
0.0847 \\
0.0848 \\
0.0850 \\
0.0854 \\
0.0858 \\
0.0857 \\
0.0853 \\
0.0859 \\
0.0853 \\
0.0008\end{array}$ \\
\hline
\end{tabular}

High Pressure test

Pressure

(psig)

19.55

54.50

64.20

69.32

74.16

80.16

95.69

100.92

103.25

11.08

120.92

23.28

22.97

125.82

125.60

127.84

135.33

140.16

142.63

145.78

42.52

0.22
$\%$ Strain

0.0040

$-0.0037$

$-0.0034$

0.0023

$-0.0018$

0.0002

0.0036

0.0104

0.0228

0.0317

0.0646

0.0729

0.0783

0.0818

0.0837

0.0835

0.0840

0.0840

0.0850

0.0858

0.0857

0.0853

0.0859

0.0853

0.1043 


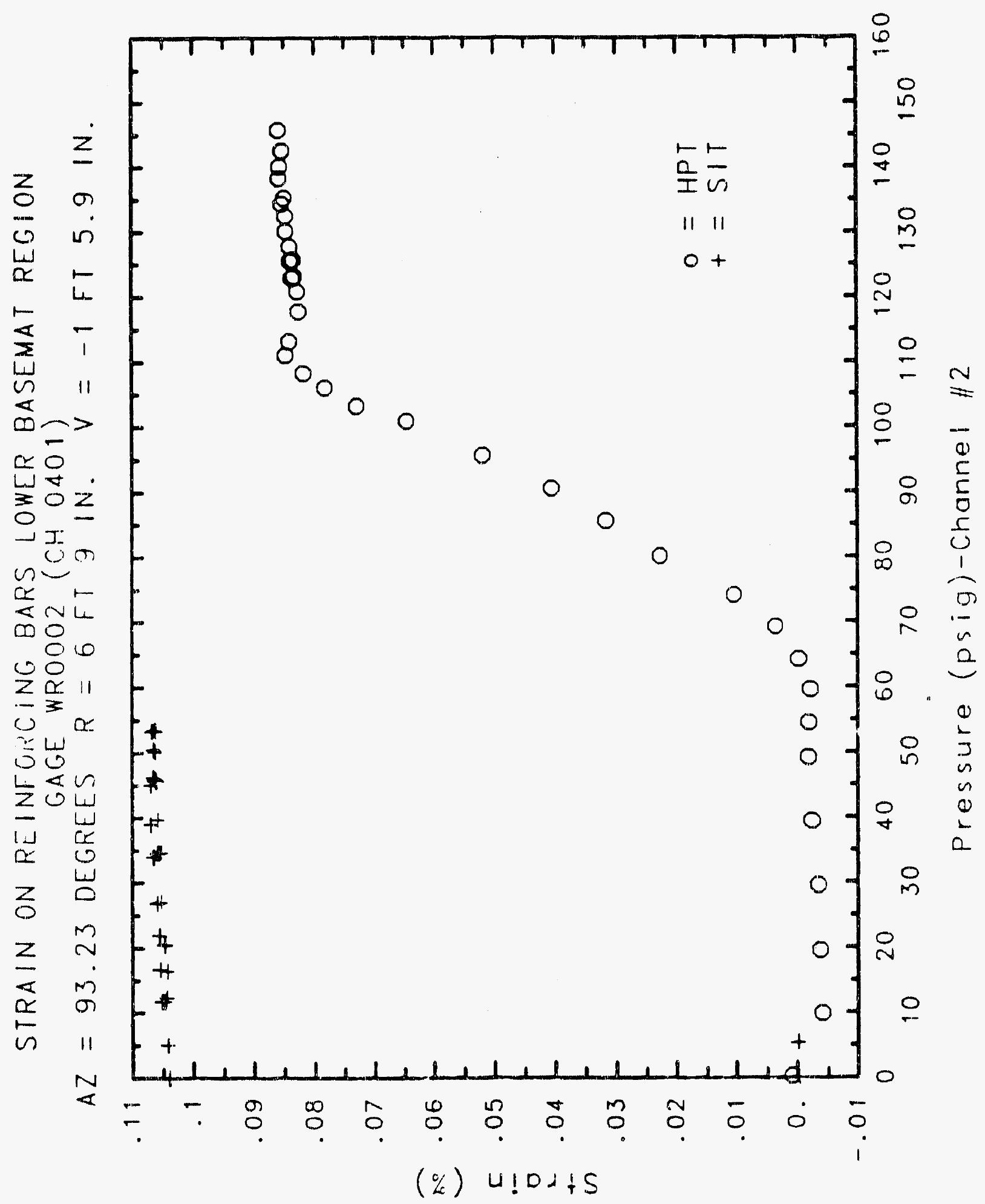




\section{Reinforced Concrete Test Data}

Weldable Gage 3 Channel 402

StRUCTURAL INTEgRITY TEST

High Pressure. Test

\begin{tabular}{|c|c|c|c|}
\hline $\begin{array}{c}\text { Pressure } \\
\text { (psig) } \\
-0.05 \\
5.33 \\
12.31 \\
16.44 \\
20.51 \\
20.46 \\
20.47 \\
27.05 \\
34.69 \\
34.53 \\
34.53 \\
39.70 \\
45.90 \\
45.65 \\
45.62 \\
50.10 \\
53.47 \\
53.29 \\
53.21 \\
50.49 \\
46.14 \\
46.13 \\
44.97 \\
38.98 \\
33.99 \\
33.96 \\
26.83 \\
21.88 \\
21.90 \\
16.69 \\
11.74 \\
11.77 \\
5.05 \\
0.02 \\
-0.04 \\
-0.02 \\
-0.02 \\
0.02 \\
\end{array}$ & $\begin{array}{l}\text { Strain } \\
-0.0001 \\
-0.0013 \\
-0.0002 \\
-0.0004 \\
-0.0004 \\
-0.0005 \\
0.0000 \\
0.0002 \\
0.0002 \\
0.0001 \\
0.0001 \\
0.0002 \\
0.0002 \\
0.0002 \\
0.0006 \\
0.0002 \\
0.0000 \\
0.0006 \\
0.0004 \\
0.0004 \\
0.0001 \\
0.0002 \\
0.0004 \\
0.0001 \\
-0.0001 \\
-0.0003 \\
-0.0007 \\
-0.0011 \\
-0.0010 \\
-0.0005 \\
-0.0015 \\
-0.0016 \\
-0.0013 \\
-0.0021 \\
-0.0023 \\
-0.0017 \\
-0.0014 \\
-0.0018\end{array}$ & $\begin{array}{c}\text { Pressure } \\
\text { (psig) } \\
9.89 \\
19.55 \\
29.57 \\
39.42 \\
49.16 \\
54.50 \\
59.57 \\
64.20 \\
69.32 \\
74.16 \\
80.16 \\
85.61 \\
90.58 \\
95.59 \\
100.92 \\
103.25 \\
106.11 \\
108.31 \\
111.08 \\
113.24 \\
117.83 \\
120.92 \\
123.28 \\
122.97 \\
125.82 \\
125.60 \\
127.84 \\
130.19 \\
132.53 \\
135.33 \\
134.42 \\
138.35 \\
140.16 \\
142.63 \\
145.78 \\
142.52 \\
0.22 \\
\end{array}$ & $\begin{array}{l}\text { \% Strain } \\
-0.0035 \\
-0.0027 \\
-0.0026 \\
-0.0028 \\
-0.0024 \\
-0.0020 \\
-0.0020 \\
-0.0029 \\
-0.0039 \\
-0.0053 \\
-0.0118 \\
-0.0029 \\
0.0103 \\
0.0279 \\
0.0482 \\
0.0608 \\
0.0715 \\
0.0785 \\
0.0873 \\
0.0937 \\
0.1038 \\
0.1104 \\
0.1160 \\
0.1170 \\
0.1211 \\
0.1231 \\
0.1266 \\
0.1339 \\
0.1406 \\
0.1508 \\
0.1517 \\
0.1637 \\
0.1717 \\
0.1804 \\
0.1903 \\
0.1915 \\
0.0134\end{array}$ \\
\hline
\end{tabular}




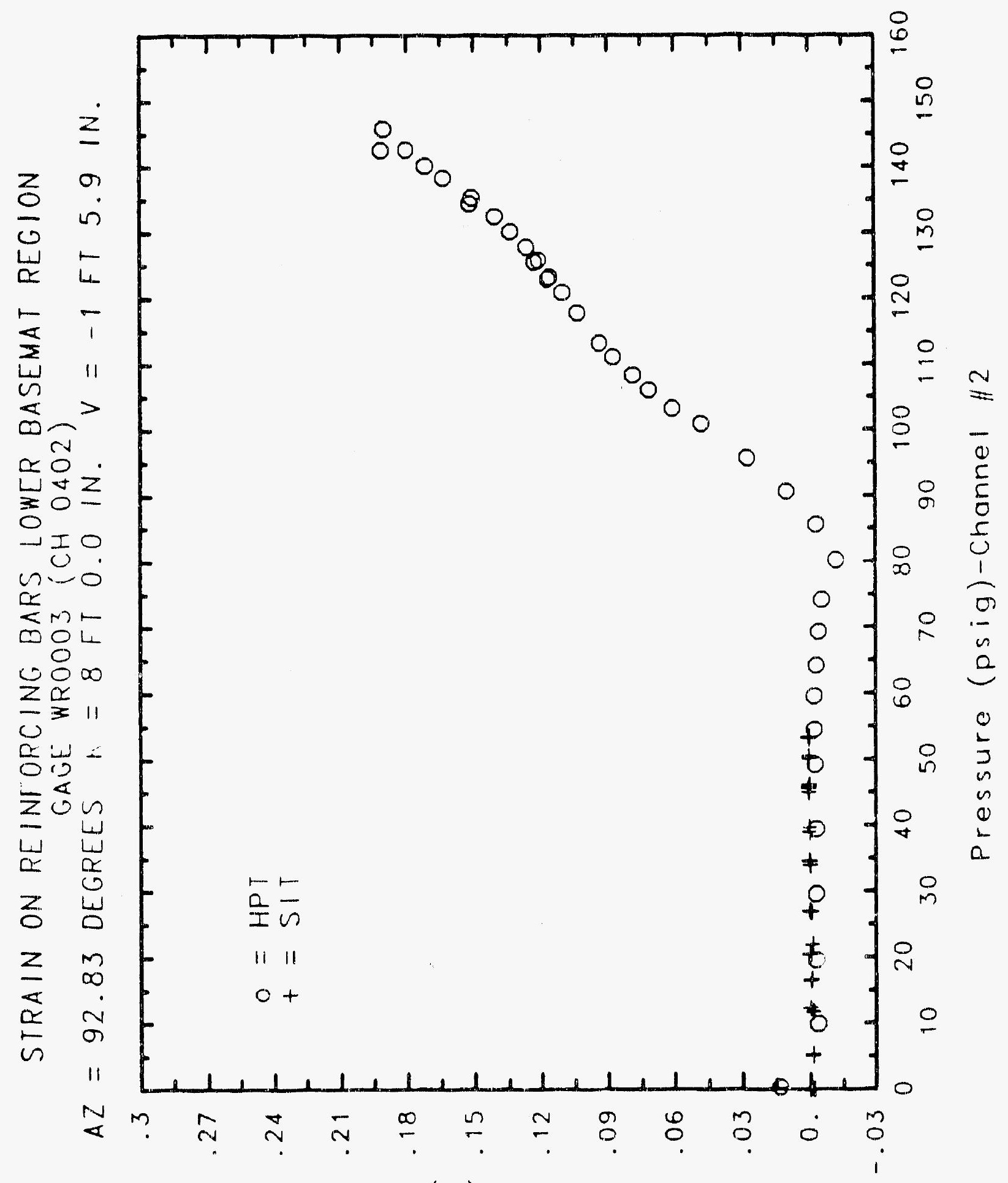

(\%) $u ! D+5$ 


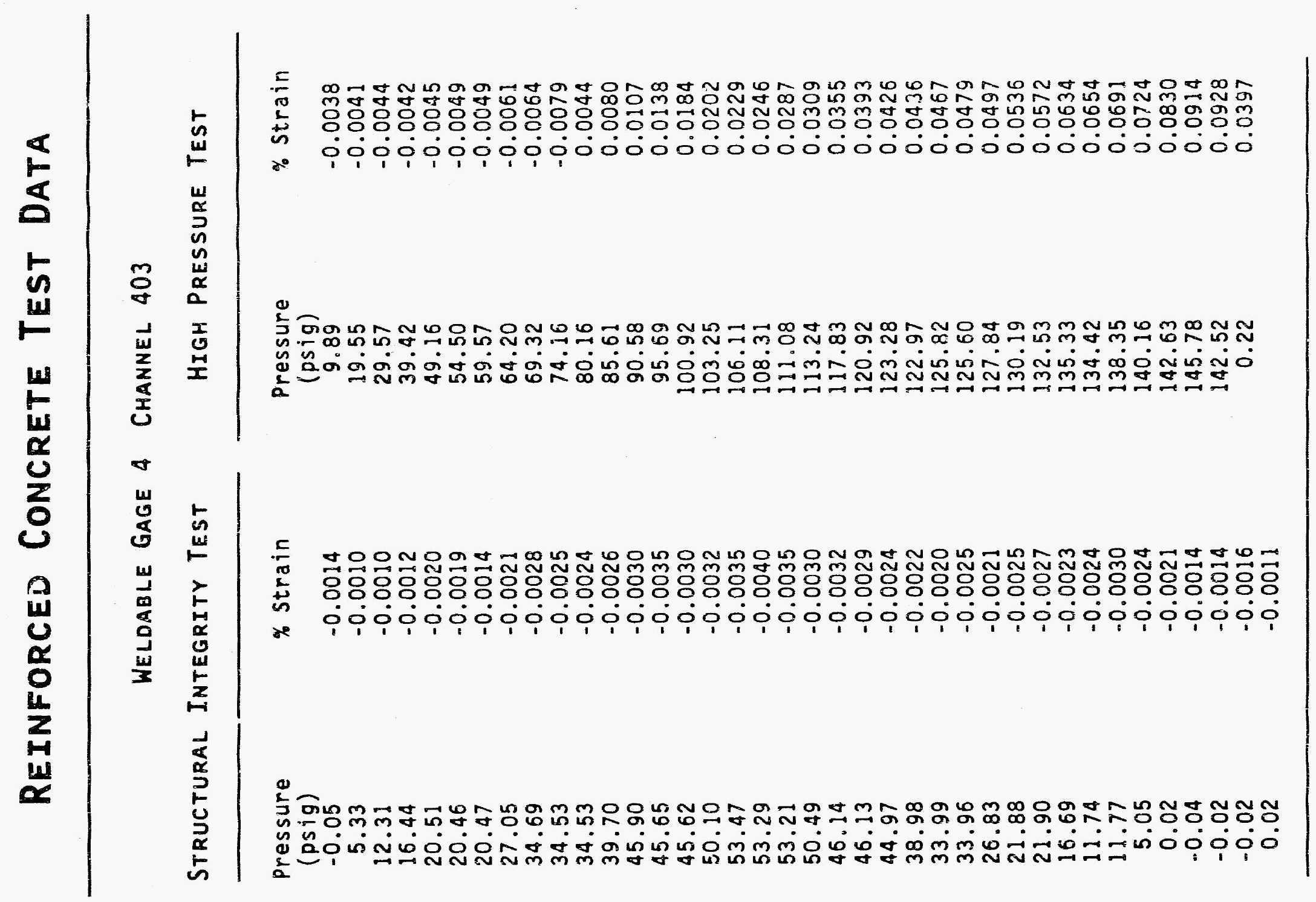




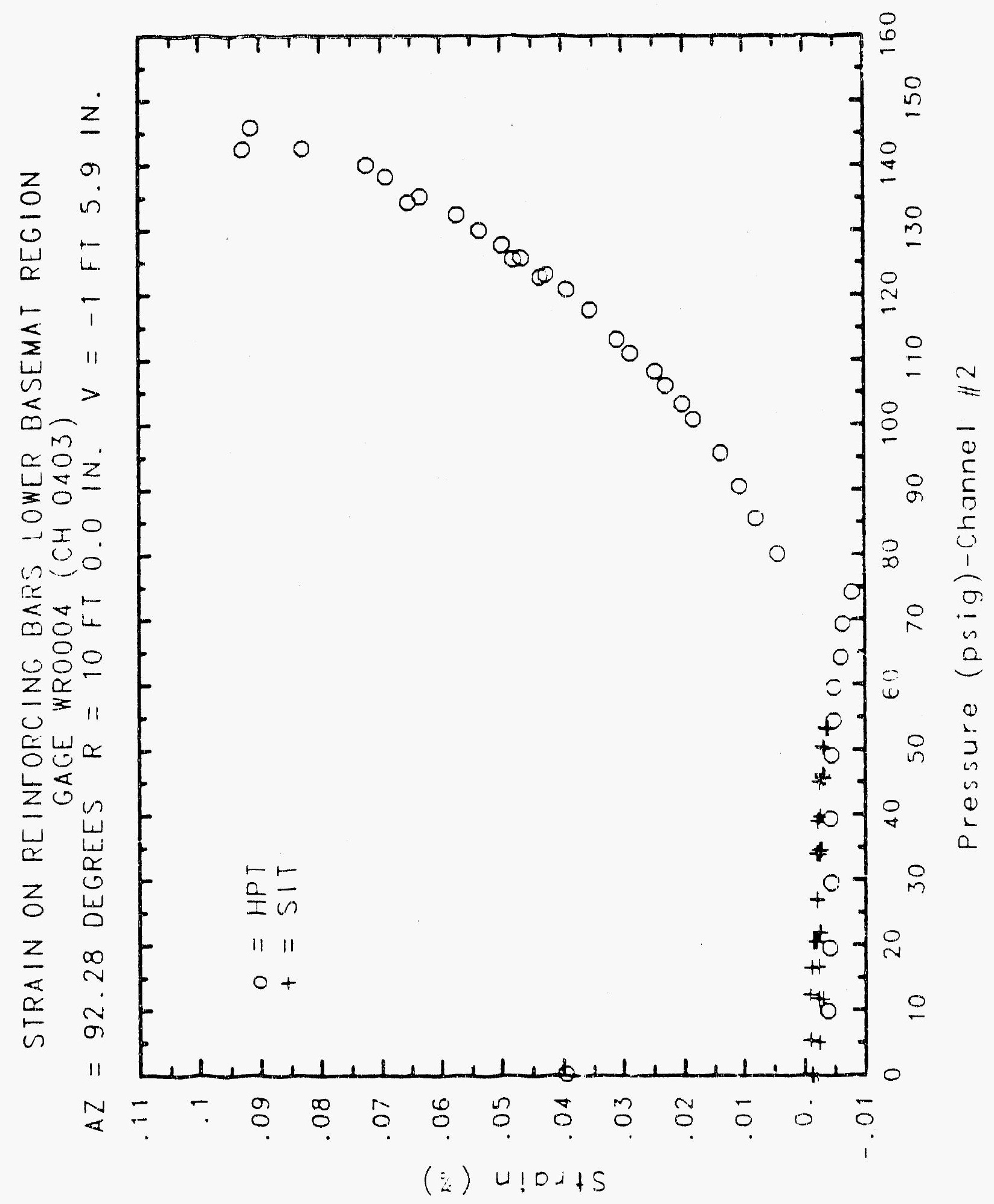

C-1.22 


\section{Reinforced Concrete test data}

Weldable gage 5 Channel 404

STRUCTLRAL INTEGRITY TEST

\begin{tabular}{|c|c|c|c|}
\hline $\begin{array}{c}\text { Pressure } \\
\text { (psig) } \\
-0.05 \\
5.33 \\
12.31 \\
16.44 \\
20.51 \\
20.40 \\
20.47 \\
27.05 \\
34.69 \\
34.53 \\
34.53 \\
39.70 \\
45.90 \\
45.55 \\
45.62 \\
50.10 \\
53.47 \\
53.29 \\
53.21 \\
50.49 \\
46.14 \\
46.13 \\
44.97 \\
38.98 \\
33.99 \\
33.96 \\
26.83 \\
21.88 \\
21.90 \\
10.59 \\
11.74 \\
11.77 \\
5.05 \\
0.02 \\
-0.04 \\
-0.02 \\
-0.02 \\
11.02 \\
\end{array}$ & $\begin{array}{l}\text { Strain } \\
-0.0007 \\
-0.0004 \\
-0.0007 \\
-0.0014 \\
-0.0012 \\
-0.0019 \\
-0.0021 \\
-0.0030 \\
-0.0035 \\
-0.0046 \\
-0.0038 \\
-0.0045 \\
-0.0048 \\
-0.0048 \\
-0.0045 \\
-0.0043 \\
-0.0044 \\
-0.0039 \\
-0.0041 \\
-0.0038 \\
-0.0038 \\
-0.0032 \\
-0.0022 \\
-0.0020 \\
-0.0026 \\
-0.0031 \\
-0.0028 \\
-0.0034 \\
-0.0035 \\
-0.0035 \\
-0.0034 \\
-0.0033 \\
-0.0030 \\
-0.0026 \\
-0.0607 \\
-0.0009 \\
-0.0012 \\
-0.0011\end{array}$ & $\begin{array}{c}\text { Pressure } \\
(\text { psig) } \\
9.89 \\
19.55 \\
29.57 \\
39.42 \\
49.16 \\
54.50 \\
59.57 \\
64.20 \\
69.32 \\
74.16 \\
80.16 \\
85.61 \\
90.58 \\
95.69 \\
100.92 \\
103.25 \\
106.11 \\
108.31 \\
111.08 \\
113.24 \\
117.83 \\
120.92 \\
123.28 \\
122.97 \\
125.82 \\
125.60 \\
127.84 \\
130.19 \\
132.53 \\
135.33 \\
134.42 \\
138.35 \\
140.16 \\
142.63 \\
145.78 \\
142.52 \\
0.22\end{array}$ & $\begin{array}{l}\% \text { Strain } \\
-0.0046 \\
-0.0049 \\
-0.0054 \\
-0.0058 \\
-0.0063 \\
-0.0066 \\
-0.0069 \\
-0.0078 \\
-0.0090 \\
0.0103 \\
-0.0130 \\
-0.0130 \\
-0.0123 \\
-0.0124 \\
-0.0124 \\
-0.0122 \\
-0.0120 \\
-0.0123 \\
-0.0126 \\
-0.0123 \\
-0.0123 \\
-0.0122 \\
-0.0117 \\
-0.0116 \\
-0.0112 \\
-0.0103 \\
-0.0104 \\
-0.0094 \\
-0.0083 \\
-0.0056 \\
-0.0044 \\
-0.0025 \\
0.0003 \\
0.0071 \\
0.0118 \\
0.0147 \\
-0.0014\end{array}$ \\
\hline
\end{tabular}

\section{High Pressure test}




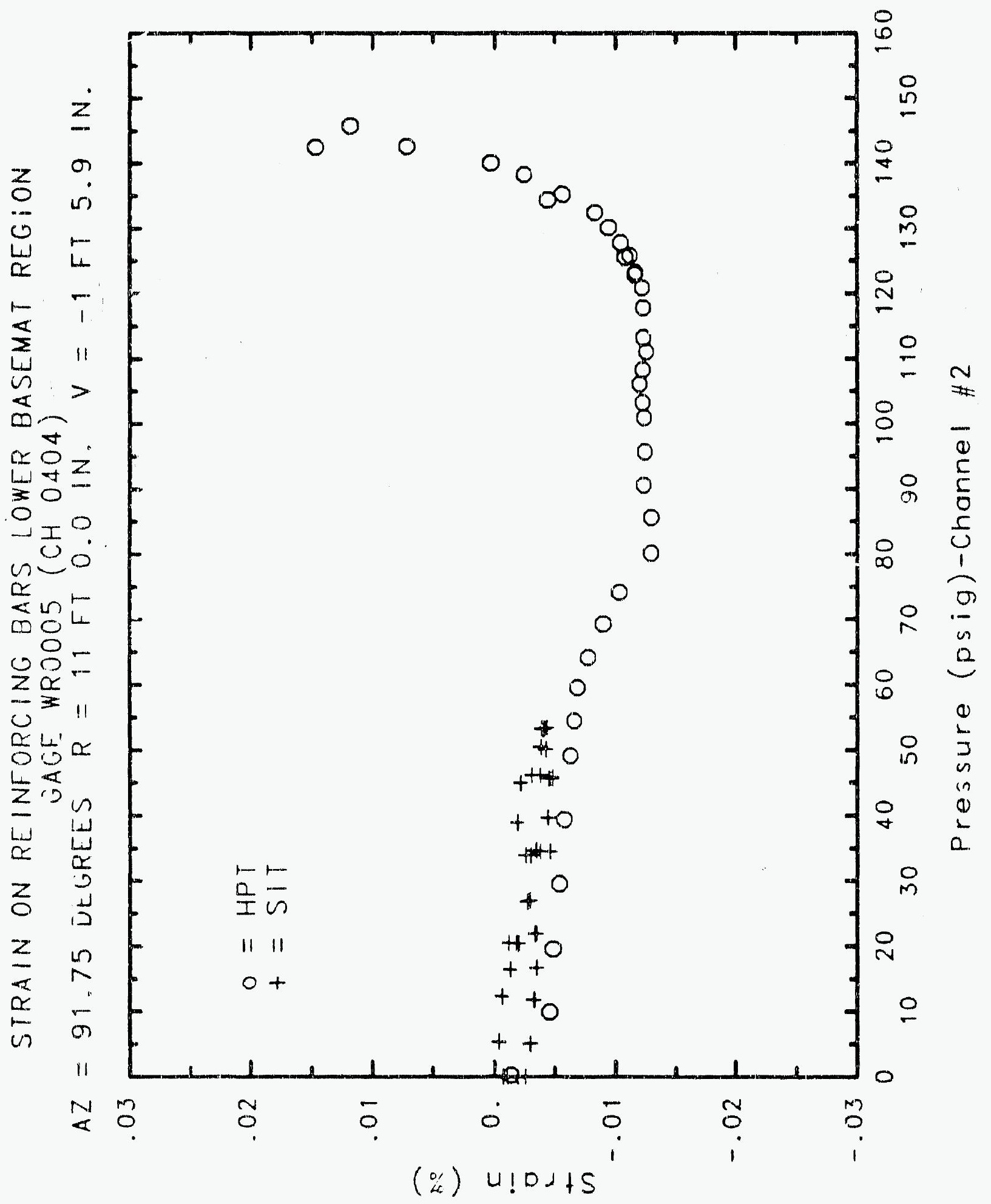




\section{Reinforced Concrete Test Data}

Weldable Gage 6 Chamnel 405

STRUCTURAL INTEGRITY TEST

\begin{tabular}{|c|c|c|c|}
\hline $\begin{array}{c}\text { Pressure } \\
\text { (psig) } \\
-0.05 \\
5.33 \\
12.31 \\
16.44 \\
20.51 \\
20.46 \\
20.47 \\
27.05 \\
34.69 \\
34.53 \\
34.53 \\
39.70 \\
45.90 \\
45.65 \\
45.62 \\
50.10 \\
53.47 \\
53.29 \\
53.21 \\
50.49 \\
46.14 \\
46.13 \\
44.97 \\
38.98 \\
33.99 \\
33.96 \\
26.83 \\
21.88 \\
21.90 \\
16.69 \\
11.74 \\
11.77 \\
5.05 \\
0.02 \\
-0.04 \\
-0.02 \\
-0.02 \\
0.02 \\
\end{array}$ & $\begin{array}{l}\% \text { Strain } \\
0.0009 \\
0.0005 \\
-0.1481 \\
-0.1481 \\
-0.1484 \\
-0.1488 \\
-0.1494 \\
-0.1503 \\
-0.1506 \\
-0.1507 \\
-0.1509 \\
-0.1519 \\
-0.1518 \\
-0.1516 \\
-0.1518 \\
-0.1517 \\
-0.1514 \\
-0.1515 \\
-0.1513 \\
-0.1507 \\
-0.1506 \\
-0.1506 \\
-0.1495 \\
-0.1495 \\
-0.1497 \\
-0.1501 \\
-0.1501 \\
-0.1506 \\
-0.1504 \\
-0.1506 \\
-0.1505 \\
-0.1509 \\
-0.1503 \\
-0.1499 \\
-0.1484 \\
-0.1479 \\
-0.1483 \\
-0.1484\end{array}$ & $\begin{array}{c}\text { Pressure } \\
(\text { psig) } \\
9.89 \\
19.55 \\
29.57 \\
39.42 \\
49.16 \\
54.50 \\
59.57 \\
64.20 \\
69.32 \\
74.16 \\
80.16 \\
85.61 \\
90.58 \\
95.69 \\
100.92 \\
103.25 \\
106.11 \\
108.31 \\
111.08 \\
113.24 \\
117.83 \\
120.92 \\
123.28 \\
122.97 \\
125.82 \\
125.60 \\
127.84 \\
130.19 \\
132.53 \\
135.33 \\
134.42 \\
138.35 \\
140.16 \\
142.63 \\
145.78 \\
142.52 \\
0.22 \\
\end{array}$ & $\begin{array}{l}\% \text { Strain } \\
-0.0045 \\
-0.0053 \\
-0.0057 \\
-0.0058 \\
-0.0063 \\
-0.0071 \\
-0.0068 \\
-0.0081 \\
-0.0092 \\
-0.0107 \\
-0.0133 \\
-0.0130 \\
-0.0129 \\
-0.0127 \\
-0.0124 \\
-0.0125 \\
-0.0127 \\
-0.0125 \\
-0.0125 \\
-0.0126 \\
-0.0127 \\
-0.0125 \\
-0.0118 \\
-0.0116 \\
-0.0115 \\
-0.0109 \\
-0.0108 \\
-0.0098 \\
-0.0085 \\
-0.0059 \\
-0.0046 \\
-0.0027 \\
-0.0002 \\
0.0066 \\
0.0120 \\
0.0143 \\
-0.0021\end{array}$ \\
\hline
\end{tabular}

High Pressure test

(psig)

$9.89 \quad-0.0045$

$19.55 \quad-0.0053$

$29.57-0.0057$

$-0.0058$

$-0.0063$

64.20

$69.32 \quad-0.0092$

$74.16 \quad-0.0107$

$80.16 \quad-0.0133$

$-0.0130$

$-0.0129$

$-0.0124$

$-0.0127$

$-0.0125$

$-0.0125$

$-0.0126$

17.83

27.84

30.19

134.42

138.35

140.16

142.63

45.78

0.22

$-0.0116$

$-0.0115$

$-0.0109$

0085

$-0.0027$

0.0002

0.0066

0.0120

0.0143
-0.0021
$-0.0046$ 


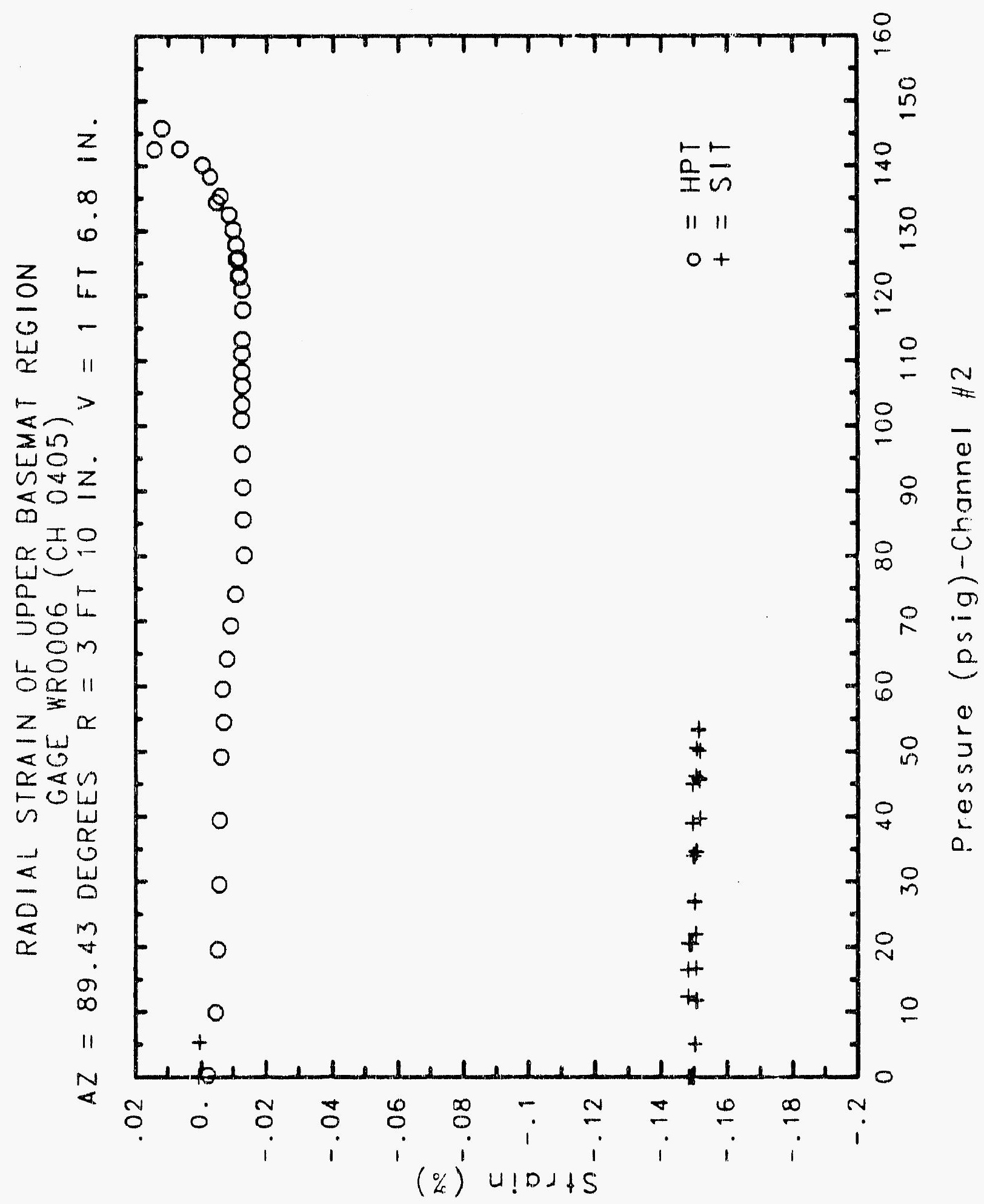




\section{Reinforced Concrete Test Data}

Weldable Gage 7 Channel 405

STRUCTURAL INTEgRITY TEST

High Pressure Test

\begin{tabular}{|c|c|c|c|}
\hline $\begin{array}{c}\text { Pressure } \\
\text { (psig) } \\
-0.05 \\
5.33 \\
12.31 \\
16.44 \\
20.51 \\
20.46 \\
20.47 \\
27.05 \\
34.69 \\
34.53 \\
34.53 \\
39.70 \\
45.90 \\
45.65 \\
45.62 \\
50.10 \\
53.47 \\
53.29 \\
53.21 \\
50.49 \\
46.14 \\
46.13 \\
44.97 \\
38.98 \\
33.99 \\
33.96 \\
26.83 \\
21.98 \\
21.90 \\
16.69 \\
11.74 \\
11.77 \\
5.05 \\
0.02 \\
-0.04 \\
-0.02 \\
-0.02 \\
0.02 \\
\end{array}$ & $\begin{array}{l}\text { \% Strain } \\
-0.0006 \\
-0.0002 \\
-0.0007 \\
-0.0005 \\
-0.0007 \\
-0.0011 \\
-0.0011 \\
-0.0017 \\
-0.0018 \\
-0.0018 \\
-0.0022 \\
-0.0022 \\
-0.0024 \\
-0.0024 \\
-0.0023 \\
-0.0025 \\
-0.0030 \\
-0.0027 \\
-0.0032 \\
-0.0028 \\
-0.0028 \\
-0.0031 \\
-0.0028 \\
-0.0027 \\
-0.0028 \\
-0.0025 \\
-0.0021 \\
-0.0022 \\
-0.0018 \\
-0.0015 \\
-0.0015 \\
-0.0014 \\
-0.0014 \\
-0.0009 \\
-0.0009 \\
-0.0010 \\
-0.0012 \\
-0.0007\end{array}$ & $\begin{array}{c}\text { Pressure } \\
(\text { psig) } \\
9.89 \\
19.55 \\
29.57 \\
39.42 \\
49.16 \\
54.50 \\
59.57 \\
64.20 \\
69.32 \\
74.16 \\
80.16 \\
85.61 \\
90.58 \\
95.65 \\
100.92 \\
103.25 \\
106.11 \\
108.31 \\
111.08 \\
113.24 \\
117.83 \\
120.92 \\
123.28 \\
122.97 \\
125.82 \\
125.60 \\
127.84 \\
130.19 \\
132.53 \\
135.33 \\
134.42 \\
138.35 \\
140.16 \\
142.63 \\
145.78 \\
142.52 \\
0.22 \\
\end{array}$ & $\begin{array}{l}\% \text { Strain } \\
-0.0043 \\
-0.0049 \\
-0.0059 \\
-0.0058 \\
-0.0061 \\
-0.0063 \\
-0.0061 \\
-0.0067 \\
-0.0062 \\
-0.0058 \\
-0.0072 \\
-0.0074 \\
-0.0075 \\
-0.0084 \\
-0.0092 \\
-0.0090 \\
-0.0090 \\
-0.0087 \\
-0.0087 \\
-0.0084 \\
-0.0077 \\
-0.0077 \\
-0.0073 \\
-0.0070 \\
-0.0072 \\
-0.0070 \\
-0.0068 \\
-0.0065 \\
-0.0065 \\
-0.0067 \\
-0.0070 \\
-0.0068 \\
-0.0067 \\
-0.0065 \\
-0.0064 \\
-0.0059 \\
-0.0031\end{array}$ \\
\hline
\end{tabular}




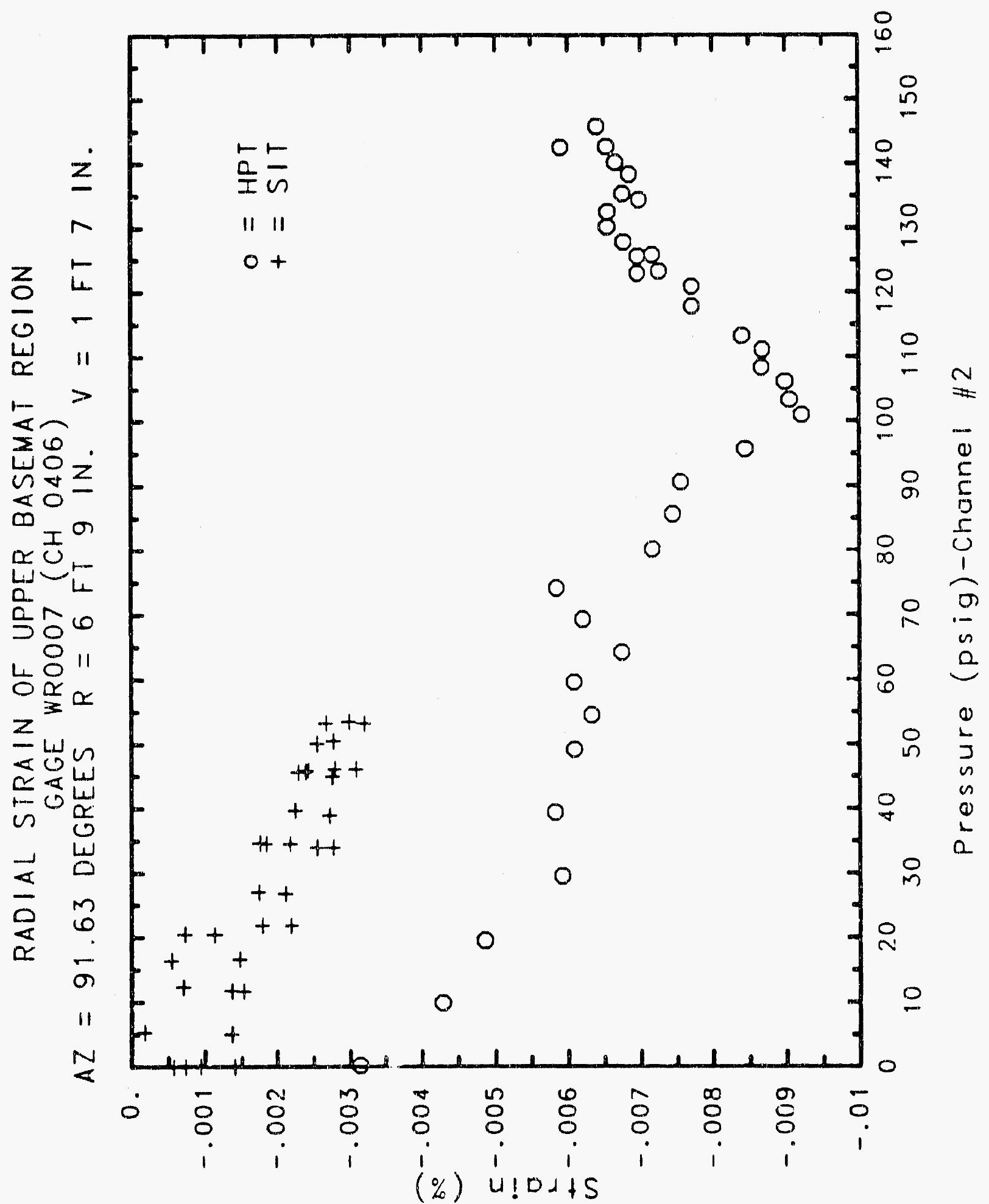




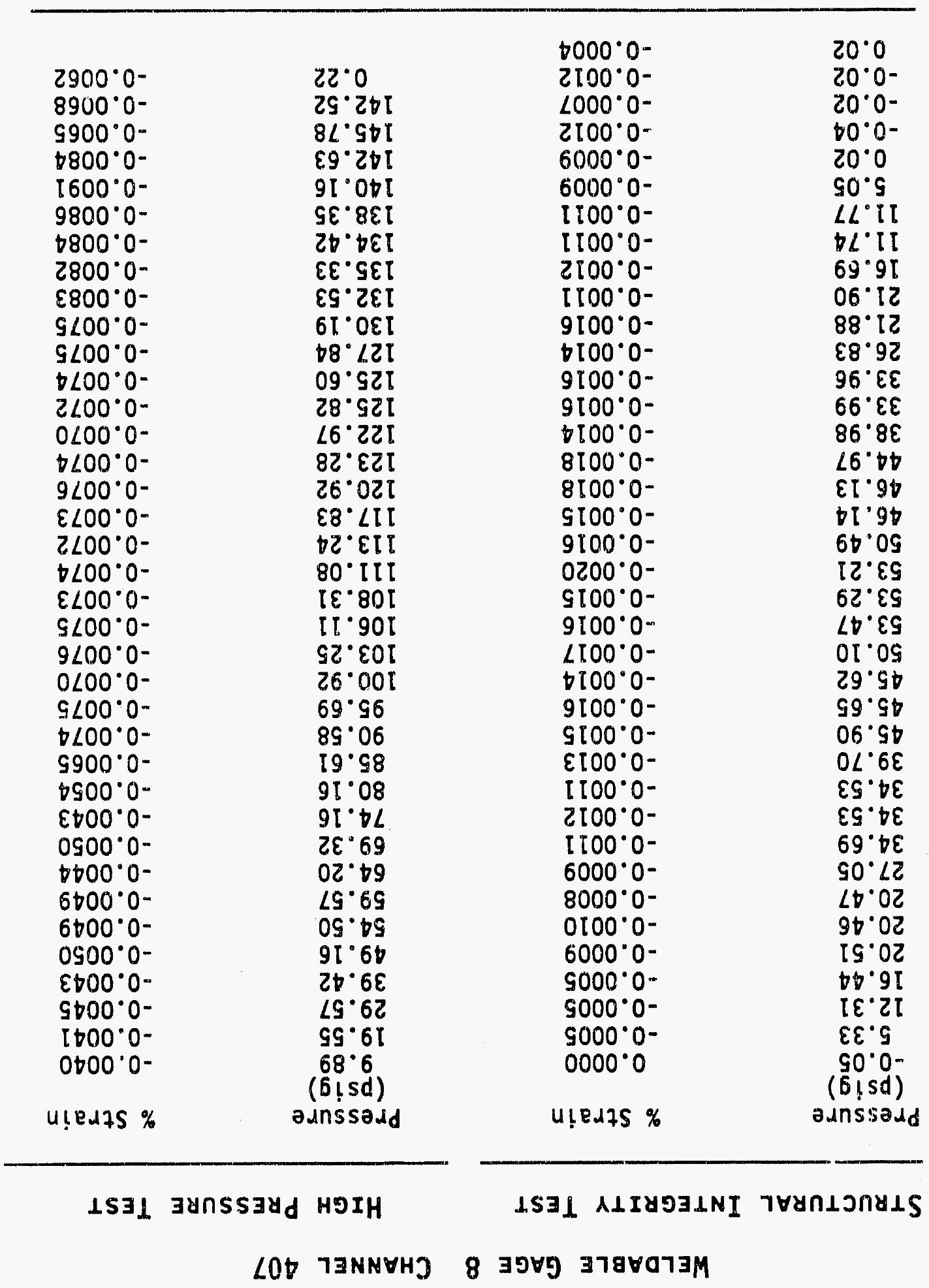

\section{$\forall \perp \forall 0 \perp S \exists \perp$ ILIVJNOJ GJJYOJNIJY}




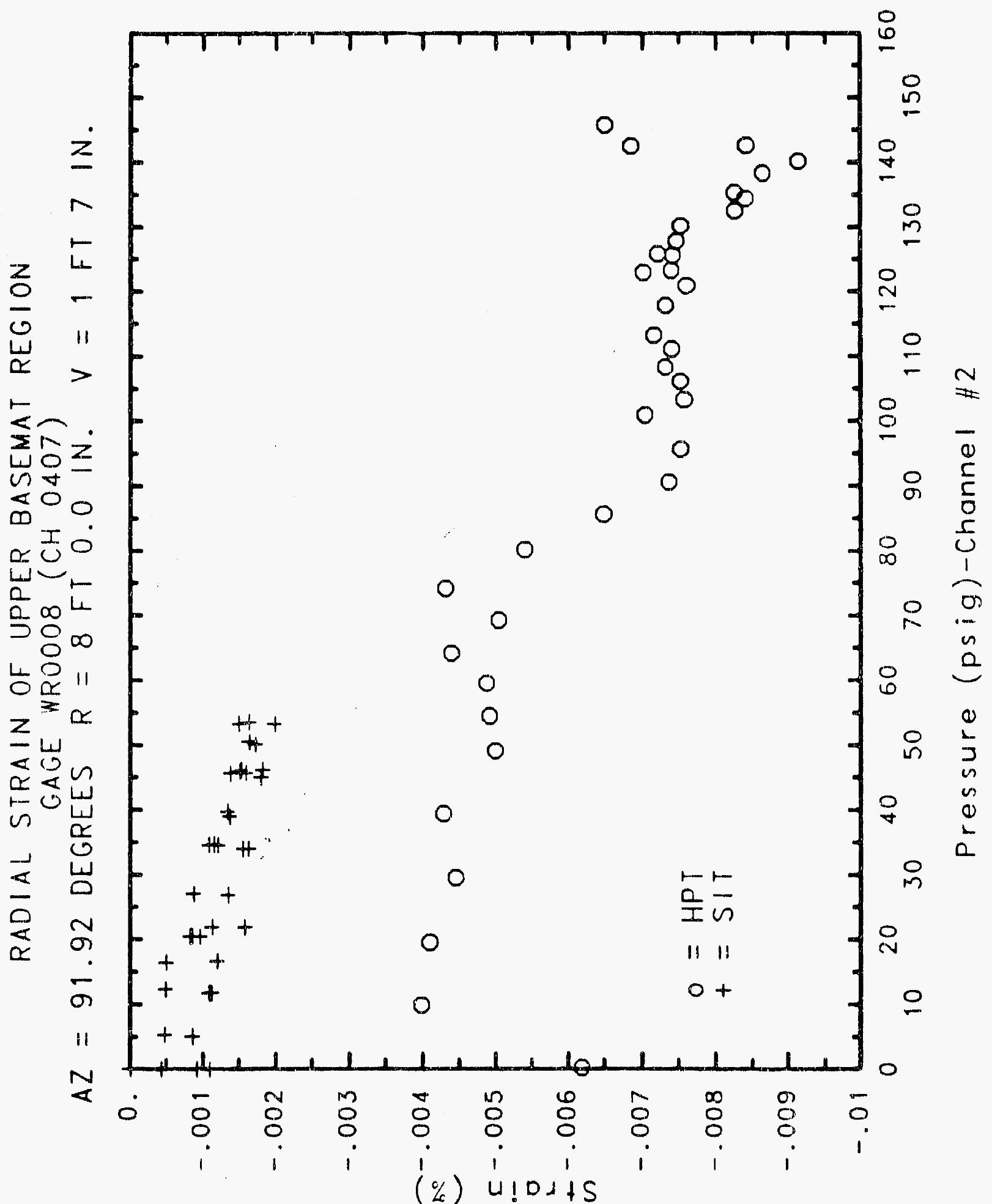




\section{Reinforced Concrete Test Data}

Weldable" Gage 9 Channel 408

Structural Integr?ty tést

\begin{tabular}{|c|c|c|c|}
\hline $\begin{array}{c}\text { Pressure } \\
(p s i g) \\
-0.05 \\
5.33 \\
12.31 \\
16.44 \\
20.51 \\
20.46 \\
20.47 \\
27.05 \\
34.69 \\
34.53 \\
34.53 \\
39.70 \\
45.90 \\
45.65 \\
45.62 \\
50.10 \\
53.47 \\
53.29 \\
53.21 \\
50.49 \\
46.14 \\
46.13 \\
44.97 \\
38.98 \\
33.99 \\
33.96 \\
26.83 \\
21.88 \\
21.90 \\
16.69 \\
11.74 \\
11.77 \\
5.05 \\
0.02 \\
-0.04 \\
-0.02 \\
-0.02 \\
0.02 \\
\end{array}$ & $\begin{array}{r}\text { \% Strain } \\
\\
-0.0005 \\
0.0001 \\
-0.0001 \\
-0.0002 \\
-0.0003 \\
-0.0003 \\
-0.0003 \\
-0.0009 \\
-0.0002 \\
0.0000 \\
-0.0007 \\
-0.0003 \\
-0.0001 \\
-0.0002 \\
-0.0004 \\
-0.0001 \\
-0.0007 \\
-0.0003 \\
-0.0003 \\
-0.0004 \\
-0.0007 \\
-0.0011 \\
-0.0006 \\
-0.0004 \\
-0.0006 \\
-0.0009 \\
-0.0010 \\
-0.0013 \\
-0.0010 \\
-0.0014 \\
-0.0018 \\
-0.0013 \\
-0.0019 \\
-0.0017 \\
-0.0019 \\
-0.0015 \\
-0.0014 \\
-0.0010\end{array}$ & $\begin{array}{c}\text { Pressure } \\
(\text { psig) } \\
9.89 \\
19.55 \\
29.57 \\
39.42 \\
49.16 \\
54.50 \\
59.57 \\
64.20 \\
69.32 \\
74.16 \\
80.16 \\
85.61 \\
90.58 \\
95.69 \\
100.92 \\
103.25 \\
106.11 \\
108.31 \\
111.08 \\
113.24 \\
117.83 \\
120.92 \\
123.28 \\
122.97 \\
125.82 \\
125.60 \\
127.84 \\
130.19 \\
132.53 \\
135.33 \\
134.42 \\
138.35 \\
140.16 \\
142.63 \\
145.78 \\
142.52 \\
0.22 \\
\end{array}$ & $\begin{array}{l}\% \text { Strain } \\
-0.0039 \\
-0.0037 \\
-0.0034 \\
-0.0035 \\
-0.0031 \\
-0.0029 \\
-0.0031 \\
-0.0028 \\
-0.0024 \\
-0.0016 \\
-0.0016 \\
-0.0015 \\
0.0010 \\
0.0058 \\
0.0090 \\
0.0108 \\
0.0122 \\
0.0130 \\
0.0149 \\
0.0158 \\
0.0189 \\
0.0210 \\
0.0231 \\
0.0236 \\
0.0249 \\
0.0258 \\
0.0265 \\
0.0281 \\
0.0292 \\
0.0314 \\
0.0322 \\
0.0346 \\
0.0377 \\
0.0414 \\
0.0453 \\
0.0470 \\
0.0117\end{array}$ \\
\hline
\end{tabular}

High Pressure Test

19.55

49.16

54.50

59.57

64.20

69.32

85.61

90.58

100.92

103.25

106.11

108.3

11.08

13.24

20.92

123.28

122.97

125.82

25.60

127.84

32.53

135.33

134.42

138.35

140.16

142.63

45.78

142.52

0.0470

0.0117
Pressure \% Strain 


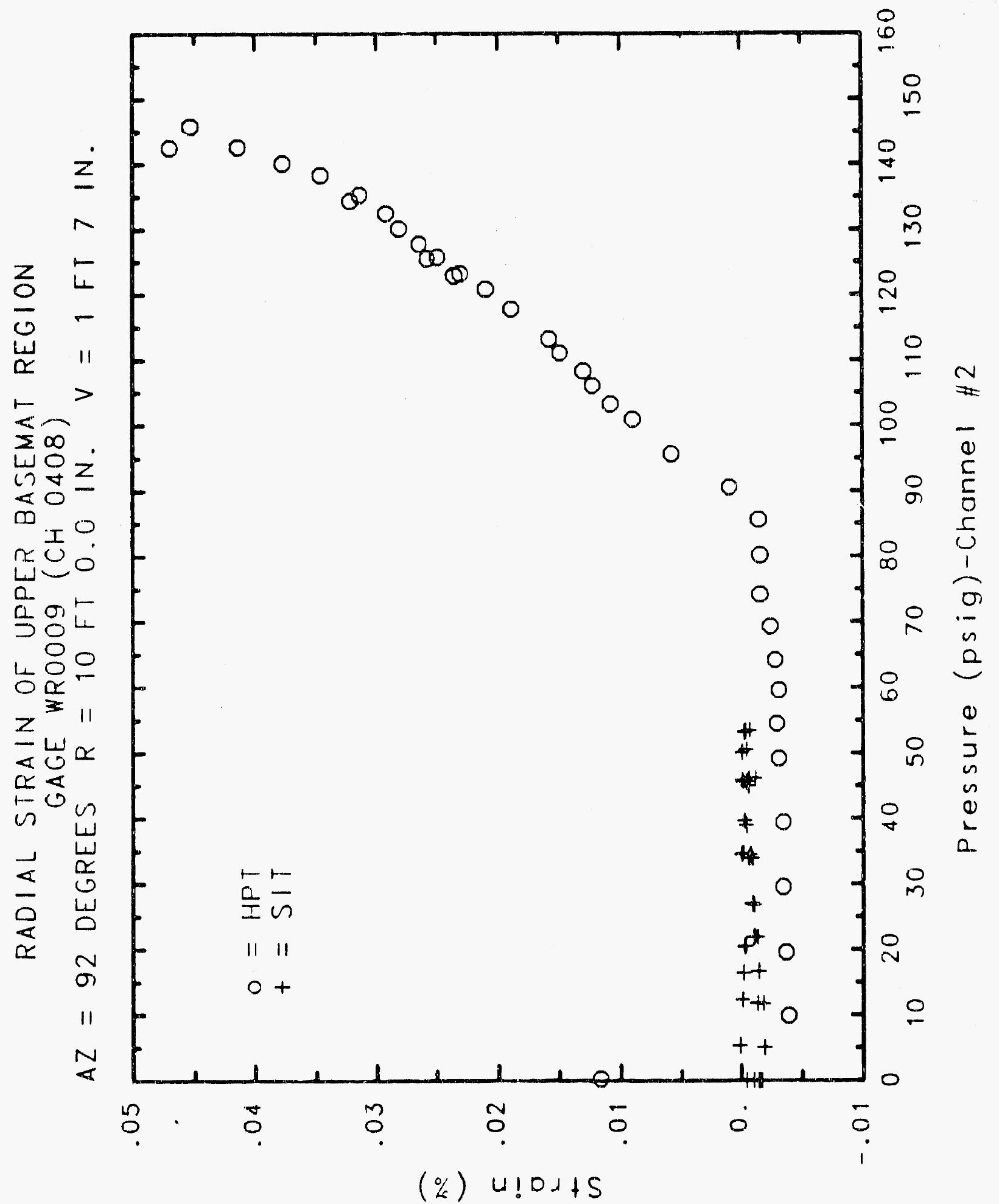




\section{Reinforced Concrete Test Data}

Weldable gage 10 Channel 409

Structural Interarity Test

High Pressure test

\begin{tabular}{|c|c|c|c|c|}
\hline $\begin{array}{c}\text { Pressure } \\
\text { (psig) } \\
-0.05 \\
5.33 \\
12.31 \\
16.44 \\
20.51 \\
20.46 \\
20.47 \\
27.05 \\
34.69 \\
34.53 \\
34.53 \\
39.70 \\
45.90 \\
45.65 \\
45.62 \\
50.10 \\
53.47 \\
53.29 \\
53.21 \\
50.49 \\
46.14 \\
46.13 \\
44.97 \\
38.98 \\
33.99 \\
33.96 \\
26.83 \\
21.88 \\
21.90 \\
16.69 \\
11.74 \\
11.77 \\
5.05 \\
0.02 \\
-0.04 \\
-0.02 \\
-0.02 \\
0.02\end{array}$ & $\begin{array}{c}\text { \% Strain } \\
0.0017 \\
0.0013 \\
0.0008 \\
0.0003 \\
0.2444 \\
0.2442 \\
0.2442 \\
0.2414 \\
0.2443 \\
0.2441 \\
0.2437 \\
0.2442 \\
0.2441 \\
0.2444 \\
0.2440 \\
0.2441 \\
0.2439 \\
0.2440 \\
0.2439 \\
0.2437 \\
0.2438 \\
0.2433 \\
-0.0023 \\
-0.0022 \\
0.2437 \\
0.2435 \\
0.2435 \\
0.2428 \\
0.2432 \\
0.2430 \\
0.2427 \\
0.2431 \\
0.2424 \\
0.2428 \\
0.2426 \\
0.2430 \\
0.2434 \\
0.2434\end{array}$ & & $\begin{array}{c}\text { Pressure } \\
\text { (psig) } \\
9.89 \\
19.55 \\
29.57 \\
39.42 \\
49.16 \\
54.50 \\
59.57 \\
64.20 \\
69.32 \\
74.16 \\
80.16 \\
85.61 \\
90.58 \\
95.69 \\
100.92 \\
103.25 \\
106.11 \\
108.31 \\
111.08 \\
113.24 \\
117.83 \\
120.92 \\
123.28 \\
122.97 \\
125.82 \\
125.60 \\
127.84 \\
130.19 \\
132.53 \\
135.33 \\
134.42 \\
138.35 \\
140.16 \\
142.63 \\
145.78 \\
142.52 \\
0.22\end{array}$ & $\begin{array}{l}\text { \% Strain } \\
-0.0038 \\
-0.0036 \\
-0.0035 \\
-0.0034 \\
-0.0031 \\
-0.0037 \\
-0.0031 \\
-0.0028 \\
-0.0025 \\
-0.0017 \\
-0.0020 \\
-0.0013 \\
0.0006 \\
0.0053 \\
0.0094 \\
0.0107 \\
0.0118 \\
0.0131 \\
0.0148 \\
0.0162 \\
0.0186 \\
0.0209 \\
0.0229 \\
0.0236 \\
0.0248 \\
0.0258 \\
0.0265 \\
0.0279 \\
0.0291 \\
0.0312 \\
0.0320 \\
0.0347 \\
0.0372 \\
0.0412 \\
0.0454 \\
0.0470 \\
0.0110\end{array}$ \\
\hline
\end{tabular}




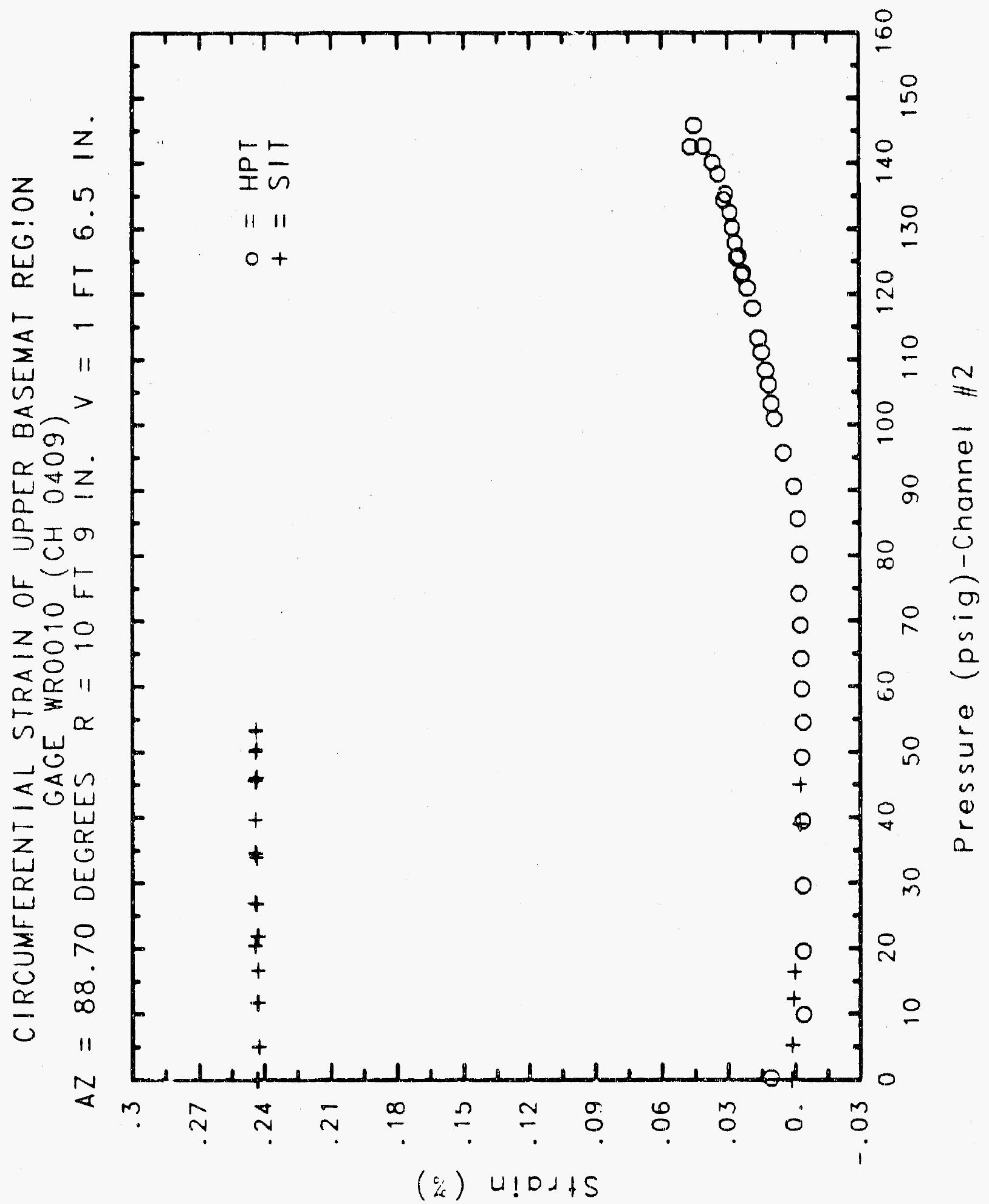




\title{
Reinforced Concrete Test Data
}

Weldable gage 11 Channel 420

StRUCTURAL IntEgrity TEST-

\author{
High Pressure Test
}

\begin{tabular}{|c|c|c|c|}
\hline $\begin{array}{c}\text { Pressure } \\
\text { (psig) } \\
-0.05 \\
5.33 \\
12.31 \\
16.44 \\
20.51 \\
20.46 \\
20.47 \\
27.05 \\
34.69 \\
34.53 \\
34.53 \\
39.70 \\
45.90 \\
45.65 \\
45.62 \\
50.10 \\
53.47 \\
53.29 \\
53.21 \\
50.49 \\
46.14 \\
46.13 \\
44.97 \\
38.98 \\
33.99 \\
33.96 \\
26.83 \\
21.88 \\
21.90 \\
16.69 \\
11.74 \\
11.77 \\
5.05 \\
0.02 \\
-0.04 \\
-0.02 \\
-0.02 \\
0.02 \\
\end{array}$ & $\begin{array}{l}\text { \% Strain } \\
0.0004 \\
0.0002 \\
0.0003 \\
0.0005 \\
0.0006 \\
0.0005 \\
0.0002 \\
0.0011 \\
0.0020 \\
0.0024 \\
0.0023 \\
0.0028 \\
0.0048 \\
0.0050 \\
0.0051 \\
0.0060 \\
0.0073 \\
0.0078 \\
0.0077 \\
0.0077 \\
0.0071 \\
0.0072 \\
0.0064 \\
0.0052 \\
0.0045 \\
0.0044 \\
0.0030 \\
0.0020 \\
0.0020 \\
0.0015 \\
0.0011 \\
0.0014 \\
0.0006 \\
0.0007 \\
0.0014 \\
0.0004 \\
0.0009 \\
0.0000\end{array}$ & $\begin{array}{c}\text { Pressure } \\
(\text { psig) } \\
9.89 \\
19.55 \\
29.57 \\
39.42 \\
49.16 \\
54.50 \\
59.57 \\
64.20 \\
69.32 \\
74.16 \\
80.16 \\
85.61 \\
90.58 \\
95.69 \\
100.92 \\
103.25 \\
106.11 \\
108.31 \\
111.08 \\
113.24 \\
117.83 \\
120.92 \\
123.28 \\
122.97 \\
125.82 \\
125.60 \\
127.84 \\
130.19 \\
132.53 \\
135.33 \\
134.42 \\
138.35 \\
140.16 \\
142.63 \\
145.78 \\
142.52 \\
0.22 \\
\end{array}$ & $\begin{array}{l}\text { \% Strain } \\
\text {-0.0032 } \\
-0.0022 \\
-0.0008 \\
0.0004 \\
0.0023 \\
0.0030 \\
0.0037 \\
0.0061 \\
0.0079 \\
0.0102 \\
0.0139 \\
0.0187 \\
0.0243 \\
0.0303 \\
0.0351 \\
0.0380 \\
0.0400 \\
0.0419 \\
0.0454 \\
0.0473 \\
0.0526 \\
0.0566 \\
0.0597 \\
0.0602 \\
0.0635 \\
0.0647 \\
0.0661 \\
0.0698 \\
0.0733 \\
0.0772 \\
0.0779 \\
0.0823 \\
0.0866 \\
0.0918 \\
0.0959 \\
0.0954 \\
0.0232\end{array}$ \\
\hline
\end{tabular}




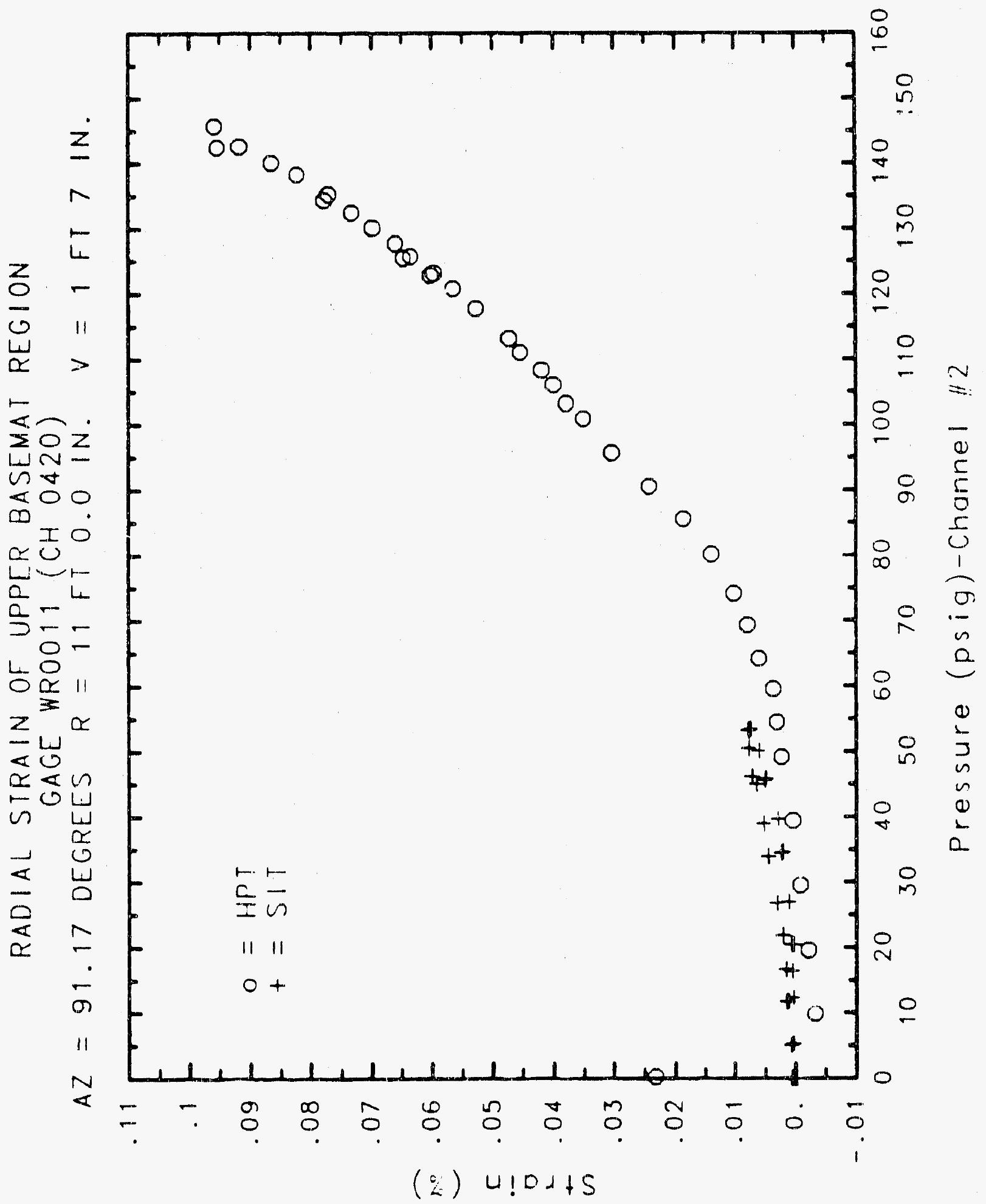




\section{Reinforced Concrete Test Data}

Weldable gage 12 Channel 421

STRUIT TURAL INTEGRITY TEST

High Pressure test

\begin{tabular}{|c|c|c|c|}
\hline $\begin{array}{c}\text { Fressure } \\
\text { (psig) } \\
-0.05 \\
5.33 \\
12.31 \\
16.44 \\
20.51 \\
20.46 \\
20.47 \\
27.05 \\
34.69 \\
34.53 \\
34.53 \\
39.70 \\
45.90 \\
45.65 \\
45.62 \\
50.10 \\
53.47 \\
53.29 \\
53.21 \\
50.49 \\
46.14 \\
46.13 \\
44.97 \\
38.98 \\
33.99 \\
33.96 \\
26.83 \\
21.88 \\
21.90 \\
16.69 \\
11.74 \\
11.77 \\
5.05 \\
0.02 \\
-0.04 \\
-0.02 \\
-0.02 \\
0.02 \\
\end{array}$ & $\begin{array}{l}\text { Strain } \\
0.0005 \\
-0.0006 \\
-0.1128 \\
-0.1126 \\
-0.1123 \\
-0.1125 \\
-0.1126 \\
-0.1120 \\
-0.1107 \\
-0.1107 \\
-0.1106 \\
-0.1100 \\
-0.1083 \\
-0.1083 \\
-0.1077 \\
-0.1071 \\
-0.1055 \\
-0.1057 \\
-0.1054 \\
-0.1052 \\
-0.1056 \\
-0.1056 \\
-0.1068 \\
-0.1076 \\
-0.1089 \\
-0.1085 \\
-0.1098 \\
-0.1105 \\
-0.1111 \\
-0.1117 \\
-0.1119 \\
-0.1117 \\
-0.1119 \\
-0.1126 \\
-0.1115 \\
-0.1120 \\
-0.1118 \\
-0.1127\end{array}$ & $\begin{array}{c}\text { Pressure } \\
(\text { psig) } \\
9.89 \\
19.55 \\
29.57 \\
39.42 \\
49.16 \\
54.50 \\
59.57 \\
64.20 \\
69.32 \\
74.16 \\
80.16 \\
85.61 \\
90.58 \\
95.69 \\
100.92 \\
103.25 \\
106.11 \\
108.31 \\
111.08 \\
113.24 \\
117.83 \\
120.92 \\
123.28 \\
122.97 \\
125.82 \\
125.60 \\
127.84 \\
130.19 \\
132.53 \\
135.33 \\
134.42 \\
138.35 \\
140.16 \\
142.63 \\
145.78 \\
142.52 \\
0.22\end{array}$ & $\begin{array}{c}\text { \% Strain } \\
-0.0030 \\
-0.0017 \\
-0.0004 \\
0.0010 \\
0.0027 \\
0.0033 \\
0.0043 \\
0.0064 \\
0.0681 \\
0.0106 \\
0.0145 \\
0.0189 \\
0.0243 \\
0.0303 \\
0.0354 \\
0.0378 \\
0.0404 \\
0.0423 \\
0.0454 \\
0.0477 \\
0.0531 \\
0.0568 \\
0.0601 \\
0.0609 \\
0.0634 \\
0.0645 \\
0.0666 \\
0.0701 \\
0.0734 \\
0.0778 \\
0.0784 \\
0.0828 \\
0.0865 \\
0.0919 \\
0.0963 \\
0.0956 \\
0.0233\end{array}$ \\
\hline
\end{tabular}




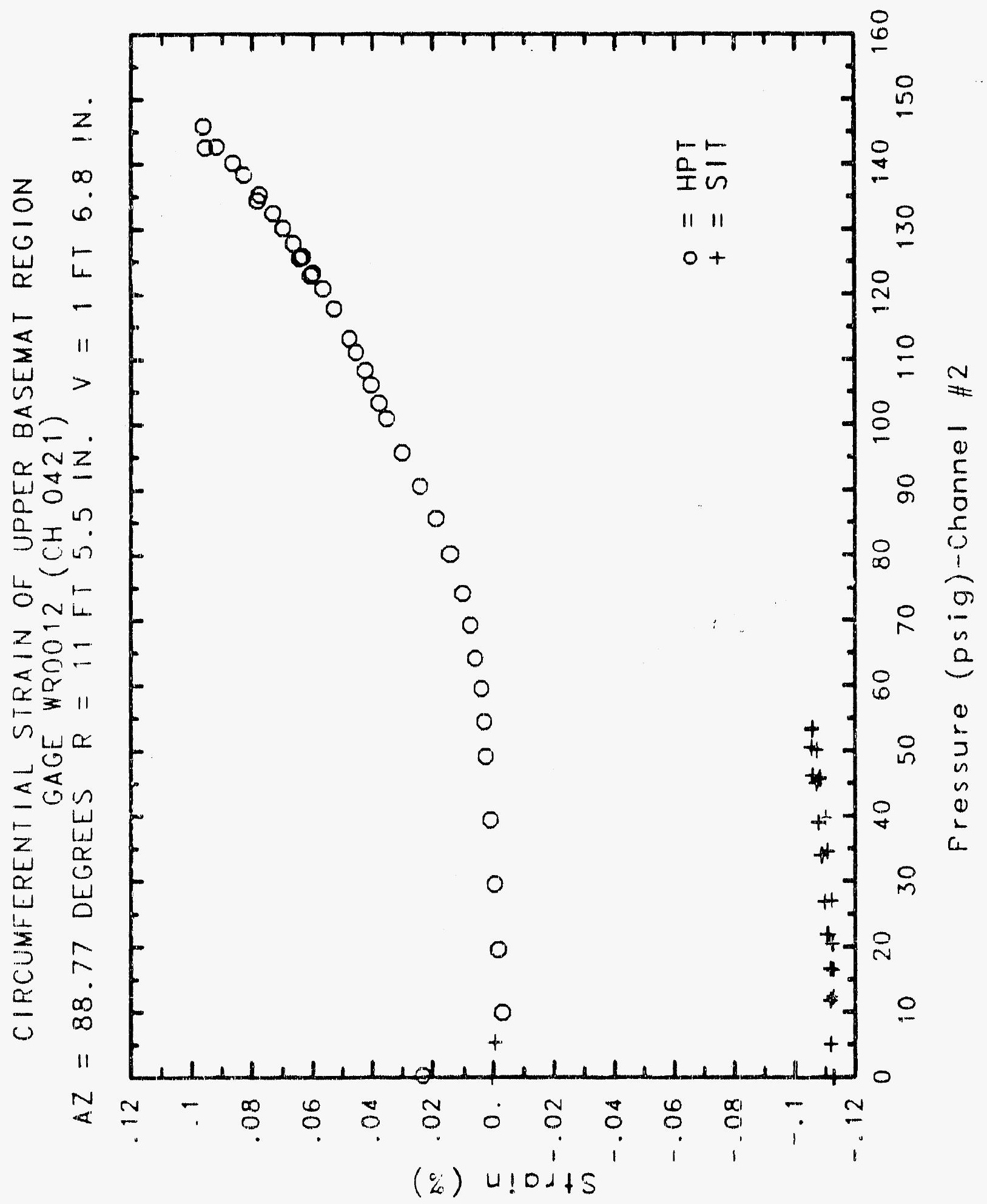




\section{Reinforced Concrete Test Data}

Weldable gage 13 Channel 422

StruCtURAL INTEgRITY TEST

\begin{tabular}{|c|c|c|c|}
\hline $\begin{array}{c}\text { Pressure } \\
\text { (psig) } \\
-0.05 \\
5.33 \\
12.31 \\
16.44 \\
20.51 \\
20.46 \\
20.47 \\
27.05 \\
34.69 \\
34.53 \\
34.53 \\
39.70 \\
45.90 \\
45.65 \\
45.62 \\
50.10 \\
53.47 \\
53.29 \\
53.21 \\
50.49 \\
46.14 \\
46.13 \\
44.97 \\
38.98 \\
33.99 \\
33.96 \\
26.83 \\
21.88 \\
21.90 \\
16.69 \\
11.74 \\
11.77 \\
5.05 \\
0.02 \\
-0.04 \\
-0.02 \\
-0.02 \\
0.02 \\
\end{array}$ & $\begin{array}{c}\text { \% Strain } \\
-0.0002 \\
0.0030 \\
0.0061 \\
0.0085 \\
0.0122 \\
0.0135 \\
0.0144 \\
0.0199 \\
0.0275 \\
0.0288 \\
0.0291 \\
0.0337 \\
0.0419 \\
0.0431 \\
0.0438 \\
0.0486 \\
0.0529 \\
0.0534 \\
0.0538 \\
0.0529 \\
0.0502 \\
0.0502 \\
0.0493 \\
0.0457 \\
0.0426 \\
0.0433 \\
0.0384 \\
0.0347 \\
0.0350 \\
0.0312 \\
0.0279 \\
0.0276 \\
0.0231 \\
0.0186 \\
0.0167 \\
0.0163 \\
0.0165 \\
0.0170\end{array}$ & $\begin{array}{c}\text { Pressure } \\
(\text { psig) } \\
9.89 \\
19.55 \\
29.57 \\
39.42 \\
49.16 \\
54.50 \\
59.57 \\
64.20 \\
69.32 \\
74.16 \\
80.16 \\
85.61 \\
90.58 \\
95.69 \\
100.92 \\
103.25 \\
106.11 \\
108.31 \\
111.08 \\
113.24 \\
117.83 \\
120.92 \\
123.28 \\
122.97 \\
125.82 \\
125.60 \\
127.84 \\
130.19 \\
132.53 \\
135.33 \\
134.42 \\
138.35 \\
140.16 \\
142.63 \\
145.78 \\
142.52 \\
0.22 \\
\end{array}$ & $\begin{array}{l}\text { Strain } \\
0.0038 \\
0.0120 \\
0.0198 \\
0.0278 \\
0.0354 \\
0.0399 \\
0.0448 \\
0.0534 \\
0.0616 \\
0.0690 \\
0.0846 \\
0.0957 \\
0.1063 \\
0.1182 \\
0.1285 \\
0.1356 \\
0.1387 \\
0.1422 \\
0.1485 \\
0.1518 \\
0.1582 \\
0.1627 \\
0.1670 \\
0.1667 \\
0.1702 \\
0.1706 \\
0.1737 \\
0.1747 \\
0.1723 \\
0.1697 \\
0.1672 \\
0.1678 \\
0.1681 \\
0.1719 \\
0.1824 \\
0.1824 \\
0.0380\end{array}$ \\
\hline
\end{tabular}




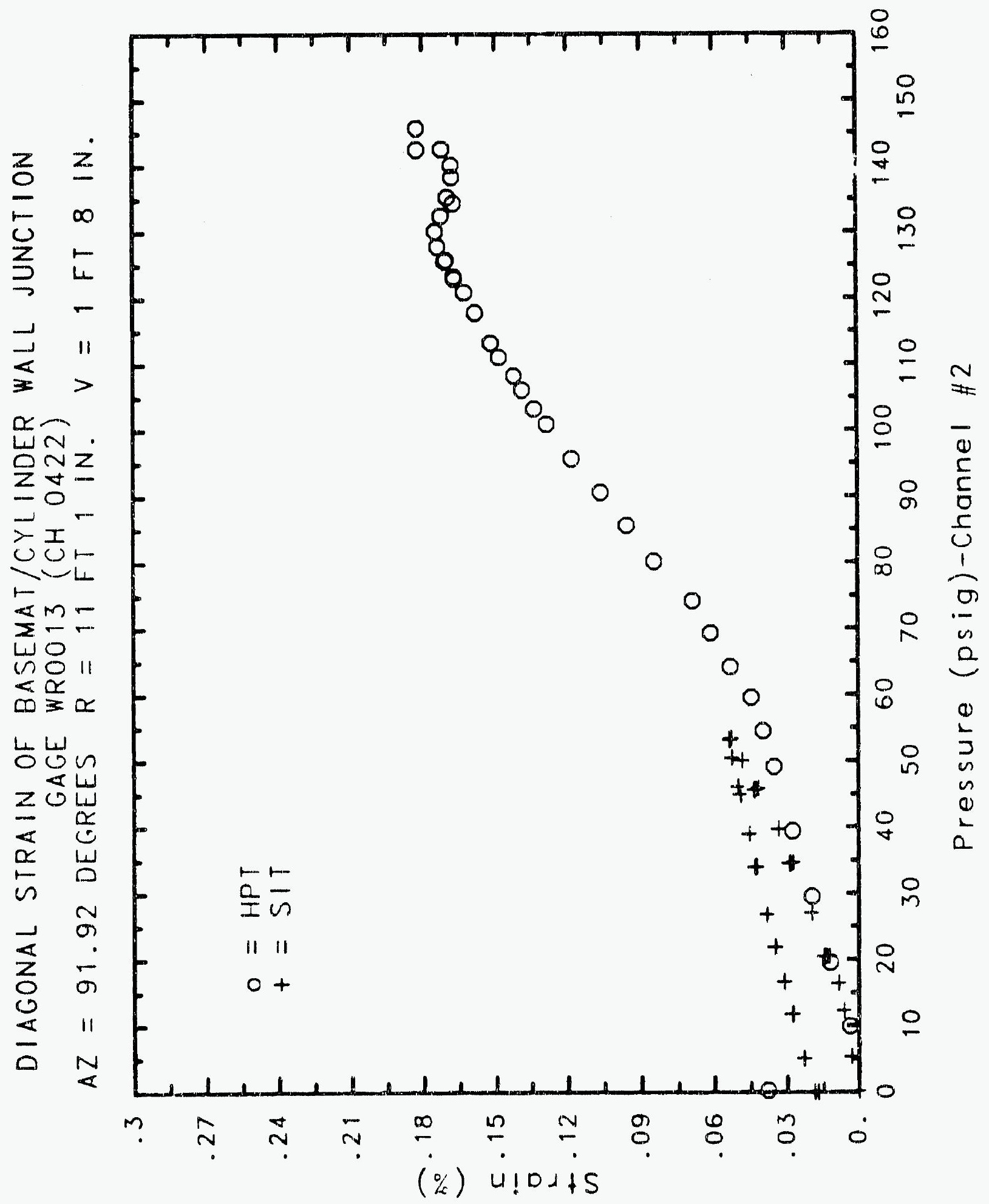

C. 840 


\section{Reinforced Concrete Test Data}

Weldable Gage 14 Channel 423

StRuCtURAL Integrity TEST

\begin{tabular}{|c|c|c|c|}
\hline $\begin{array}{c}\text { Pressure } \\
\text { (psig) } \\
-0.05 \\
5.33 \\
12.31 \\
16.44 \\
20.51 \\
20.46 \\
20.47 \\
27.05 \\
34.69 \\
34.53 \\
34.53 \\
39.70 \\
45.90 \\
45.65 \\
45.62 \\
50.10 \\
53.47 \\
53.29 \\
53.21 \\
50.49 \\
46.14 \\
46.13 \\
44.97 \\
38.98 \\
33.99 \\
33.96 \\
26.83 \\
21.88 \\
21.90 \\
16.69 \\
11.74 \\
11.77 \\
5.05 \\
0.02 \\
10.04 \\
-0.02 \\
-0.02 \\
0.02 \\
\end{array}$ & $\begin{array}{l}\text { Strain } \\
-0.0010 \\
0.0035 \\
0.0097 \\
0.0135 \\
0.0187 \\
0.0200 \\
0.0206 \\
0.0296 \\
0.0397 \\
0.0408 \\
0.0412 \\
0.0469 \\
0.0552 \\
0.0551 \\
0.0553 \\
0.0607 \\
0.0651 \\
0.0650 \\
0.0647 \\
0.0626 \\
0.0582 \\
0.0576 \\
0.0551 \\
0.0492 \\
0.0446 \\
0.0455 \\
0.0384 \\
0.0337 \\
0.0336 \\
0.0281 \\
0.0233 \\
0.0225 \\
0.0159 \\
0.0103 \\
0.0042 \\
0.0037 \\
0.0046 \\
0.0052\end{array}$ & $\begin{array}{c}\text { Pressure } \\
\text { (psig) } \\
9.89 \\
19.55 \\
29.57 \\
39.42 \\
49.16 \\
54.50 \\
59.57 \\
64.20 \\
69.32 \\
74.16 \\
80.16 \\
85.61 \\
90.58 \\
95.69 \\
100.92 \\
103.25 \\
106.11 \\
108.31 \\
111.08 \\
113.24 \\
117.83 \\
120.92 \\
123.28 \\
122.97 \\
125.82 \\
125.60 \\
127.84 \\
130.19 \\
132.53 \\
135.33 \\
134.42 \\
138.35 \\
140.16 \\
142.63 \\
145.78 \\
142.52 \\
0.22 \\
\end{array}$ & $\begin{array}{l}\text { \% Strain } \\
0.0080 \\
0.0201 \\
0.0323 \\
0.0436 \\
0.0548 \\
0.0605 \\
0.0670 \\
0.0755 \\
0.0828 \\
0.0897 \\
0.1006 \\
0.1062 \\
0.1100 \\
0.1154 \\
0.1204 \\
0.1219 \\
0.1247 \\
0.1272 \\
0.1302 \\
0.1331 \\
0.1388 \\
0.1447 \\
0.1506 \\
0.1510 \\
0.1556 \\
0.1558 \\
0.1607 \\
0.1713 \\
0.1792 \\
0.1901 \\
0.1914 \\
0.2044 \\
0.2049 \\
0.2084 \\
0.2162 \\
0.2102 \\
-0.0053\end{array}$ \\
\hline
\end{tabular}

Hign Pressure Test

Pressur

19.55

29.57

54.50

69.32

90.58

100.92

103.25

113.24

120.92

123.28

125.60

135.33

134.42

138.35

140.16

142.63

145.78

42.52

0.22 ain

0.0080

$0.03 ? 3$

.

0.0605

0.0670

0.0755

0.0828

.1062

0.1154

0.1204

0.1219

0.1447

.1558

0.1713

0.1792

0.1901

0.1914

0.2044

0.2049

0.2162

0.2102

0.0053 


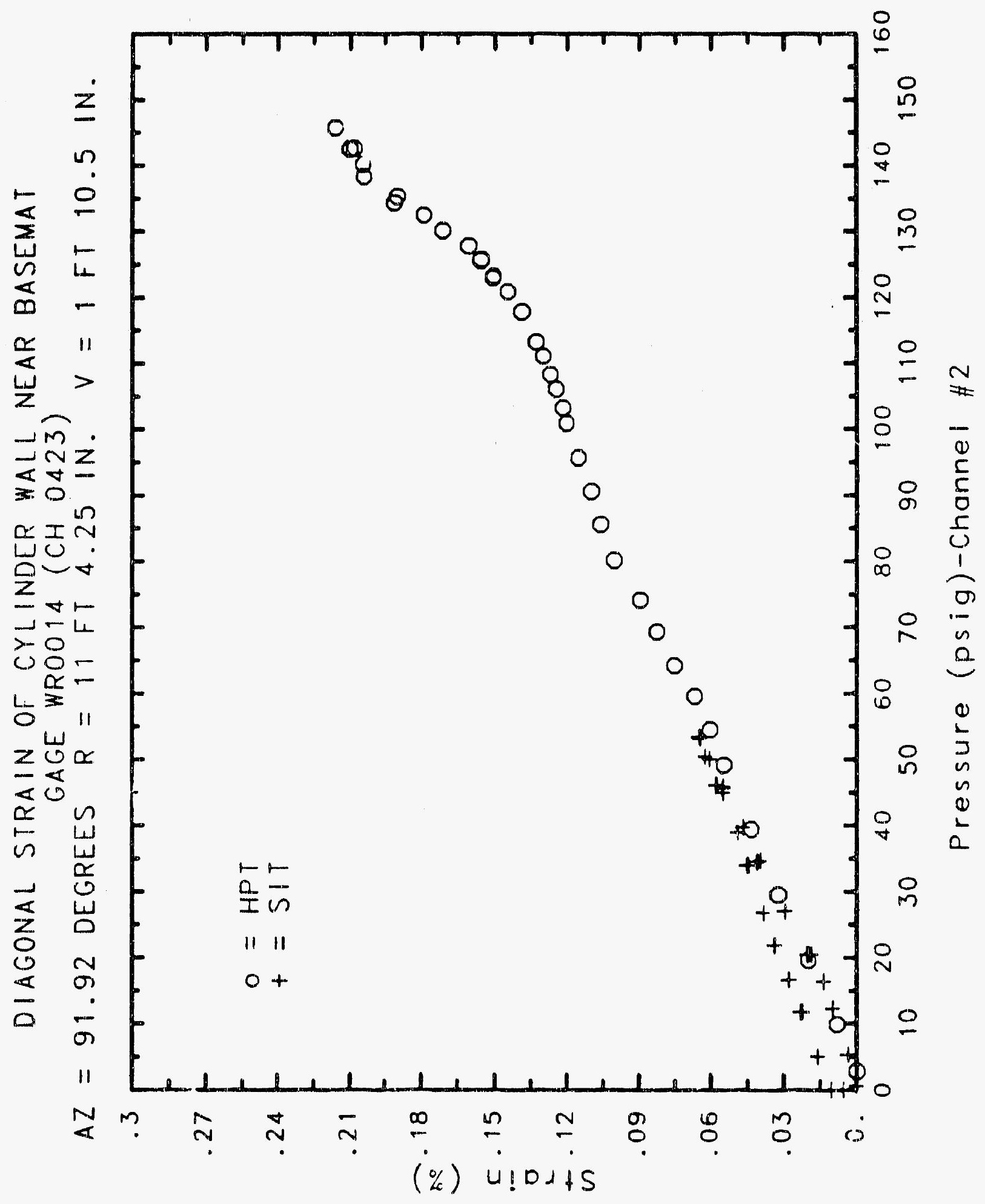




\section{Reinforced Concrete Test Data}

Weldable gage 15 Channel 424

StRUCTURAL INTEGRITY TEST

High Pressure test

\begin{tabular}{|c|c|c|c|}
\hline $\begin{array}{c}\text { Pressure } \\
\text { (psig) } \\
-0.05 \\
5.33 \\
12.31 \\
16.44 \\
20.51 \\
20.46 \\
20.47 \\
27.05 \\
34.69 \\
34.53 \\
34.53 \\
39.70 \\
45.90 \\
45.65 \\
45.62 \\
50.10 \\
53.47 \\
53.29 \\
53.21 \\
50.49 \\
46.14 \\
46.13 \\
44.97 \\
38.98 \\
33.99 \\
33.96 \\
26.83 \\
21.88 \\
21.90 \\
16.69 \\
11.74 \\
11.77 \\
5.05 \\
0.02 \\
-0.04 \\
-0.02 \\
-0.02 \\
0.02 \\
\end{array}$ & $\begin{array}{l}\text { \% Strain } \\
-0.0006 \\
0.0013 \\
0.0035 \\
0.0056 \\
0.0254 \\
-0.6762 \\
-0.5946 \\
-0.5545 \\
-0.5246 \\
-0.4904 \\
-0.5085 \\
-0.5787 \\
-0.8406 \\
-1.4109 \\
-1.4548 \\
-1.5099 \\
-1.4503 \\
-1.4695 \\
-1.4411 \\
-1.4452 \\
-1.4498 \\
-1.4278 \\
-1.6008 \\
-1.6645 \\
-1.6822 \\
-1.6211 \\
-1.6309 \\
-1.6053 \\
-1.5952 \\
-1.5343 \\
-1.5061 \\
-1.4605 \\
-1.3920 \\
-1.3790 \\
-1.4173 \\
-1.0859 \\
-1.2828 \\
-1.1955\end{array}$ & $\begin{array}{c}\text { Pressure } \\
\text { (psig) } \\
9.89 \\
19.55 \\
29.57 \\
39.42 \\
49.16 \\
54.50 \\
59.57 \\
64.20 \\
69.32 \\
74.16 \\
80.16 \\
85.61 \\
90.58 \\
95.69 \\
100.92 \\
103.25 \\
106.11 \\
108.31 \\
111.08 \\
113.24 \\
117.83 \\
120.92 \\
123.28 \\
122.97 \\
125.82 \\
125.60 \\
127.84 \\
130.19 \\
132.53 \\
135.33 \\
134.42 \\
138.35 \\
140.16 \\
142.63 \\
145.78 \\
142.52 \\
0.22\end{array}$ & $\begin{array}{r}\% \text { Strain } \\
-0.0012 \\
0.0010 \\
-0.0032 \\
-0.0010 \\
0.0063 \\
0.0102 \\
0.0160 \\
0.0229 \\
0.0289 \\
0.0354 \\
0.0518 \\
0.0636 \\
0.0744 \\
0.0865 \\
0.0999 \\
0.1064 \\
0.1130 \\
0.1150 \\
0.1247 \\
0.1289 \\
0.1377 \\
0.1432 \\
0.1477 \\
0.1482 \\
0.1506 \\
0.1504 \\
0.1518 \\
0.1514 \\
0.1505 \\
0.1535 \\
0.1523 \\
0.1557 \\
0.1494 \\
0.1327 \\
0.1112 \\
0.0922 \\
-0.0697\end{array}$ \\
\hline
\end{tabular}




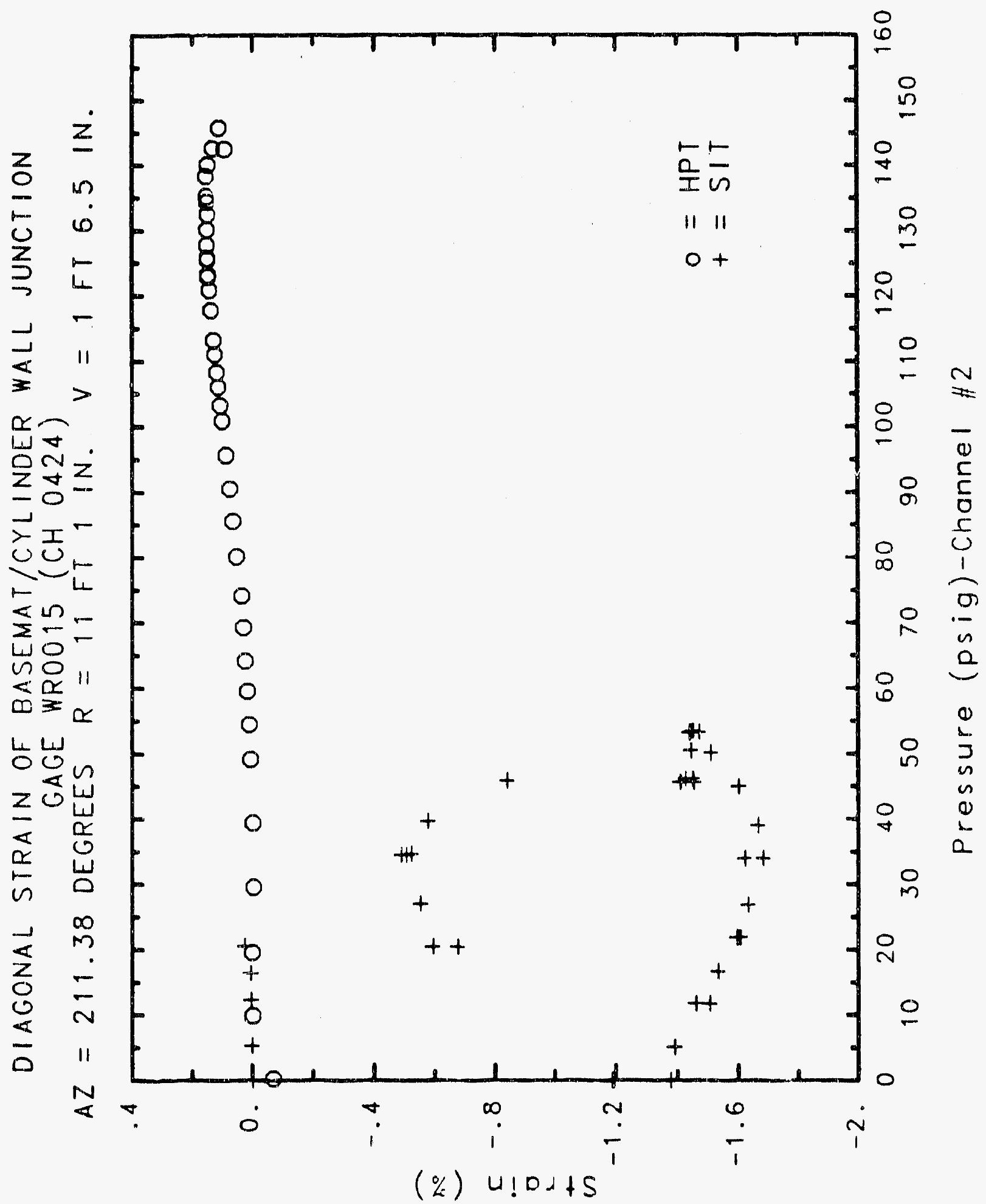




\section{Reinforced Concrete Test Data}

Weldable gage 16 Channel 425

Structural IntEgrity TEST

\begin{tabular}{|c|c|c|c|}
\hline $\begin{array}{c}\text { Pressure } \\
\text { (psig) } \\
-0.05 \\
5.33 \\
12.31 \\
16.44 \\
20.51 \\
20.46 \\
20.47 \\
27.05 \\
34.69 \\
34.53 \\
34.53 \\
39.70 \\
45.90 \\
45.65 \\
45.62 \\
50.10 \\
53.47 \\
53.29 \\
53.21 \\
50.49 \\
46.14 \\
46.13 \\
44.97 \\
38.98 \\
33.99 \\
33.96 \\
26.83 \\
21.88 \\
21.90 \\
16.69 \\
11.74 \\
11.77 \\
5.05 \\
0.02 \\
-0.04 \\
-0.02 \\
-0.02 \\
0.02 \\
\end{array}$ & $\begin{array}{l}\text { Strain } \\
0.0002 \\
0.0021 \\
0.0052 \\
-1.3022 \\
-1.2826 \\
-1.9656 \\
-1.8873 \\
-1.8478 \\
-1.8190 \\
-1.7848 \\
-1.8034 \\
-1.8723 \\
-2.1280 \\
-2.6815 \\
-2.7256 \\
-2.7763 \\
-2.7217 \\
-2.7401 \\
-2.7143 \\
-2.7165 \\
-2.7224 \\
-2.6972 \\
-2.8673 \\
-2.9294 \\
-2.9468 \\
-2.8864 \\
-2.8939 \\
-2.8725 \\
-2.8626 \\
-2.8010 \\
-2.7745 \\
-2.7309 \\
-2.6636 \\
-2.6513 \\
-2.6883 \\
-2.3649 \\
-2.5582 \\
-2.4731\end{array}$ & $\begin{array}{c}\text { Pressure } \\
\text { (psig) } \\
9.89 \\
19.55 \\
29.57 \\
39.42 \\
49.16 \\
54.50 \\
59.57 \\
64.20 \\
69.32 \\
74.16 \\
80.16 \\
85.61 \\
90.58 \\
95.69 \\
100.92 \\
103.25 \\
106.11 \\
108.31 \\
111.08 \\
113.24 \\
117.83 \\
120.92 \\
123.28 \\
122.97 \\
125.82 \\
125.60 \\
127.84 \\
130.19 \\
132.53 \\
135.33 \\
134.42 \\
138.35 \\
140.16 \\
142.63 \\
145.78 \\
142.52 \\
0.22 \\
\end{array}$ & $\begin{array}{r}\text { Strain } \\
-0.0011 \\
0.0008 \\
-0.0029 \\
-0.0008 \\
0.0064 \\
0.0106 \\
0.0160 \\
0.0234 \\
0.0289 \\
0.0353 \\
0.0520 \\
0.0639 \\
0.0744 \\
0.0864 \\
0.0999 \\
0.1060 \\
0.1129 \\
0.1181 \\
0.1247 \\
0.1293 \\
0.1378 \\
0.1432 \\
0.1479 \\
0.1481 \\
0.1507 \\
0.1501 \\
0.1522 \\
0.1512 \\
0.1508 \\
0.1539 \\
0.1524 \\
0.1559 \\
0.1495 \\
0.1326 \\
0.1112 \\
0.0922 \\
-0.0694\end{array}$ \\
\hline
\end{tabular}

High Pressure test

Pressur

9.89

19.55

49.16

54.50

59.57

74.16

90.58

100.92

103.25

111.08

120.92

123.28

122.97

125.82

132.53

135.33

134.42

142.63

5.5

0.22
0.0011

.0008

0.0106

0.0289

.0639

0.0864

0.0999

0.1060

0.1247

0.1378

0.1432

0.1479

0.1481

0.1522

0.1508

0.1539

0.1524

0.1559

0.1495

.1326

$-0.0694$
$-0.0029$ 


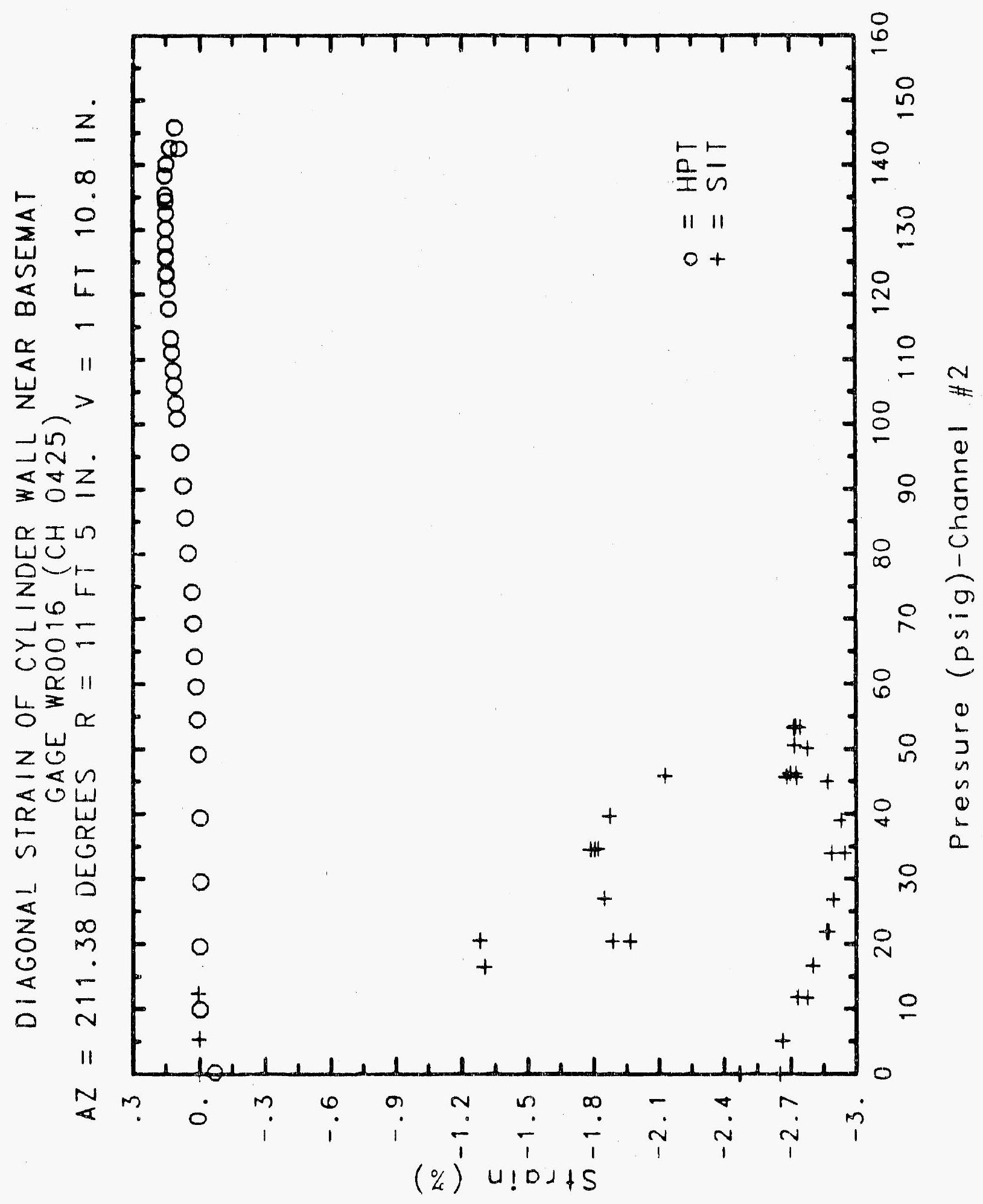




\section{Reinforced Concrete Test Data}

Weldable gage 17 Channel 426

Structural Integrity Test

High Pressure test

\begin{tabular}{|c|c|c|c|}
\hline $\begin{array}{c}\text { Pressure } \\
\text { (psig) } \\
-0.05 \\
5.33 \\
12.31 \\
16.44 \\
20.51 \\
20.46 \\
20.47 \\
27.05 \\
34.69 \\
34.53 \\
34.53 \\
39.70 \\
45.90 \\
45.65 \\
45.62 \\
50.10 \\
53.47 \\
53.29 \\
53.21 \\
50.49 \\
46.14 \\
46.13 \\
44.97 \\
38.98 \\
33.99 \\
33.96 \\
26.83 \\
21.88 \\
21.90 \\
16.69 \\
11.74 \\
11.77 \\
5.05 \\
0.02 \\
-0.04 \\
.0 .02 \\
-0.02 \\
0.02 \\
\end{array}$ & $\begin{array}{l}\text { Strain } \\
0.0007 \\
0.0027 \\
0.0056 \\
0.0074 \\
0.0100 \\
0.0106 \\
0.0107 \\
0.0162 \\
0.0243 \\
0.0254 \\
0.0256 \\
0.0313 \\
0.0422 \\
0.0428 \\
0.0433 \\
0.0481 \\
0.0491 \\
0.0494 \\
0.0495 \\
0.0477 \\
0.0444 \\
0.0440 \\
0.0434 \\
0.0396 \\
0.0359 \\
0.0359 \\
0.0301 \\
0.0266 \\
0.0262 \\
0.0227 \\
0.0185 \\
0.0183 \\
0.0134 \\
0.0100 \\
0.0068 \\
0.0075 \\
0.0075 \\
0.0087\end{array}$ & $\begin{array}{c}\text { Pressure } \\
\text { (psig) } \\
9.89 \\
19.55 \\
29.57 \\
39.42 \\
49.16 \\
54.50 \\
59.57 \\
64.20 \\
69.32 \\
74.16 \\
80.16 \\
85.61 \\
90.58 \\
95.69 \\
100.92 \\
103.25 \\
106.11 \\
108.31 \\
111.08 \\
113.24 \\
117.83 \\
120.92 \\
123.28 \\
122.97 \\
125.82 \\
125.60 \\
127.84 \\
130.19 \\
132.53 \\
135.33 \\
134.42 \\
138.35 \\
140.16 \\
142.63 \\
145.78 \\
142.52 \\
0.22\end{array}$ & $\begin{array}{c}\text { \% Strain } \\
0.0035 \\
0.0111 \\
0.0188 \\
0.0273 \\
0.0353 \\
0.0403 \\
0.0452 \\
0.0512 \\
0.0586 \\
0.0687 \\
0.0835 \\
0.0965 \\
0.1055 \\
0.1168 \\
0.1291 \\
0.1352 \\
0.1419 \\
0.1470 \\
0.1541 \\
0.1580 \\
0.1643 \\
0.1656 \\
0.1669 \\
0.1663 \\
0.1661 \\
0.1641 \\
0.1602 \\
0.1515 \\
0.1416 \\
0.1390 \\
0.1369 \\
0.1350 \\
0.1349 \\
0.1355 \\
0.1399 \\
0.1380 \\
0.0123\end{array}$ \\
\hline
\end{tabular}




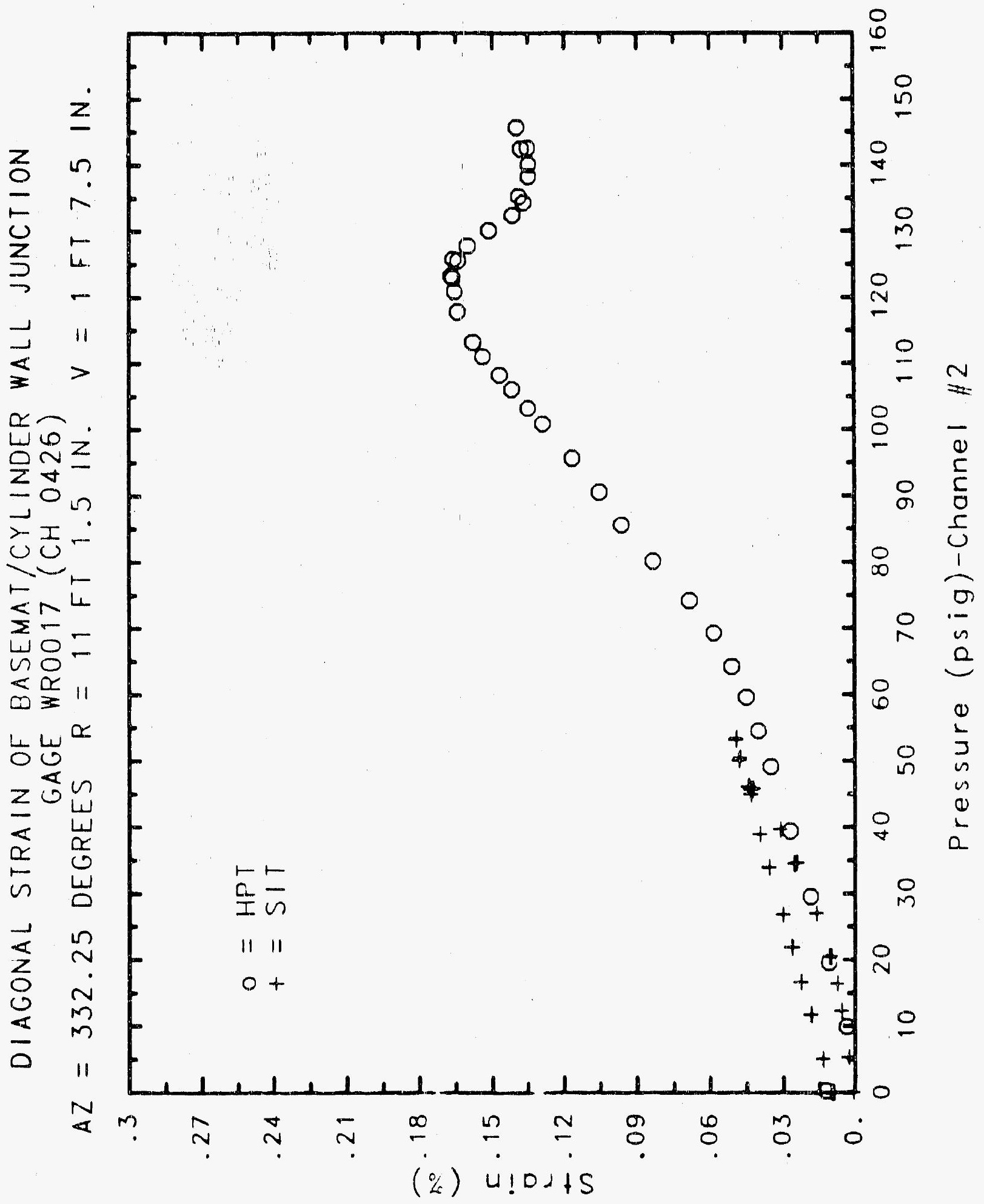




\section{Reinforced Concrete Test Data}

Weldable Gage 18 Channel 427

Structural Integrity Test

High Pressure Test

\begin{tabular}{|c|c|c|c|}
\hline $\begin{array}{c}\text { Pressure } \\
\text { (psig) } \\
-0.05 \\
5.33 \\
12.31 \\
16.44 \\
20.51 \\
20.46 \\
20.47 \\
27.05 \\
34.69 \\
34.53 \\
34.53 \\
39.70 \\
45.90 \\
45.65 \\
45.62 \\
50.10 \\
53.47 \\
53.29 \\
53.21 \\
50.49 \\
46.14 \\
46.13 \\
44.97 \\
38.98 \\
33.99 \\
33.96 \\
26.83 \\
21.88 \\
21.90 \\
16.69 \\
11.74 \\
11.77 \\
5.05 \\
0.02 \\
-0.04 \\
-0.02 \\
-0.02 \\
0.02 \\
\end{array}$ & $\begin{array}{l}\text { \% Strain } \\
0.0003 \\
0.0027 \\
0.0060 \\
0.0077 \\
0.0108 \\
0.0114 \\
0.0118 \\
0.0171 \\
0.0237 \\
0.0243 \\
0.0243 \\
0.0279 \\
0.0344 \\
0.0350 \\
0.0351 \\
0.0387 \\
0.0404 \\
0.0410 \\
0.0411 \\
0.0399 \\
0.0375 \\
0.0373 \\
0.0382 \\
0.0346 \\
0.0316 \\
0.0314 \\
0.0260 \\
0.0227 \\
0.0222 \\
0.0188 \\
0.0153 \\
0.0150 \\
0.0104 \\
0.0075 \\
0.0065 \\
0.0069 \\
0.0066 \\
0.0068\end{array}$ & $\begin{array}{c}\text { Pressure } \\
(\text { psig) } \\
9.89 \\
19.55 \\
29.57 \\
39.42 \\
49.16 \\
54.50 \\
59.57 \\
64.20 \\
69.32 \\
74.16 \\
80.16 \\
85.61 \\
90.58 \\
95.69 \\
100.92 \\
102.25 \\
16.11 \\
108.31 \\
111.08 \\
113.24 \\
117.83 \\
120.92 \\
123.28 \\
122.97 \\
125.82 \\
125.60 \\
127.84 \\
130.19 \\
132.53 \\
135.33 \\
134.42 \\
138.35 \\
140.16 \\
142.63 \\
145.78 \\
142.52 \\
0.22\end{array}$ & $\begin{array}{l}\% \text { Strain } \\
0.0024 \\
0.0082 \\
0.0155 \\
0.0227 \\
0.0302 \\
0.0338 \\
0.0382 \\
0.0436 \\
0.0490 \\
0.0562 \\
0.0666 \\
0.0754 \\
0.0814 \\
0.0892 \\
0.0989 \\
0.1038 \\
0.1094 \\
0.1136 \\
0.1201 \\
0.1258 \\
0.1390 \\
0.1504 \\
0.1601 \\
0.1626 \\
0.1734 \\
0.1779 \\
0.1898 \\
0.2065 \\
0.2213 \\
0.2305 \\
0.2302 \\
0.2414 \\
0.2497 \\
0.2621 \\
0.2770 \\
0.2775 \\
0.1284\end{array}$ \\
\hline
\end{tabular}




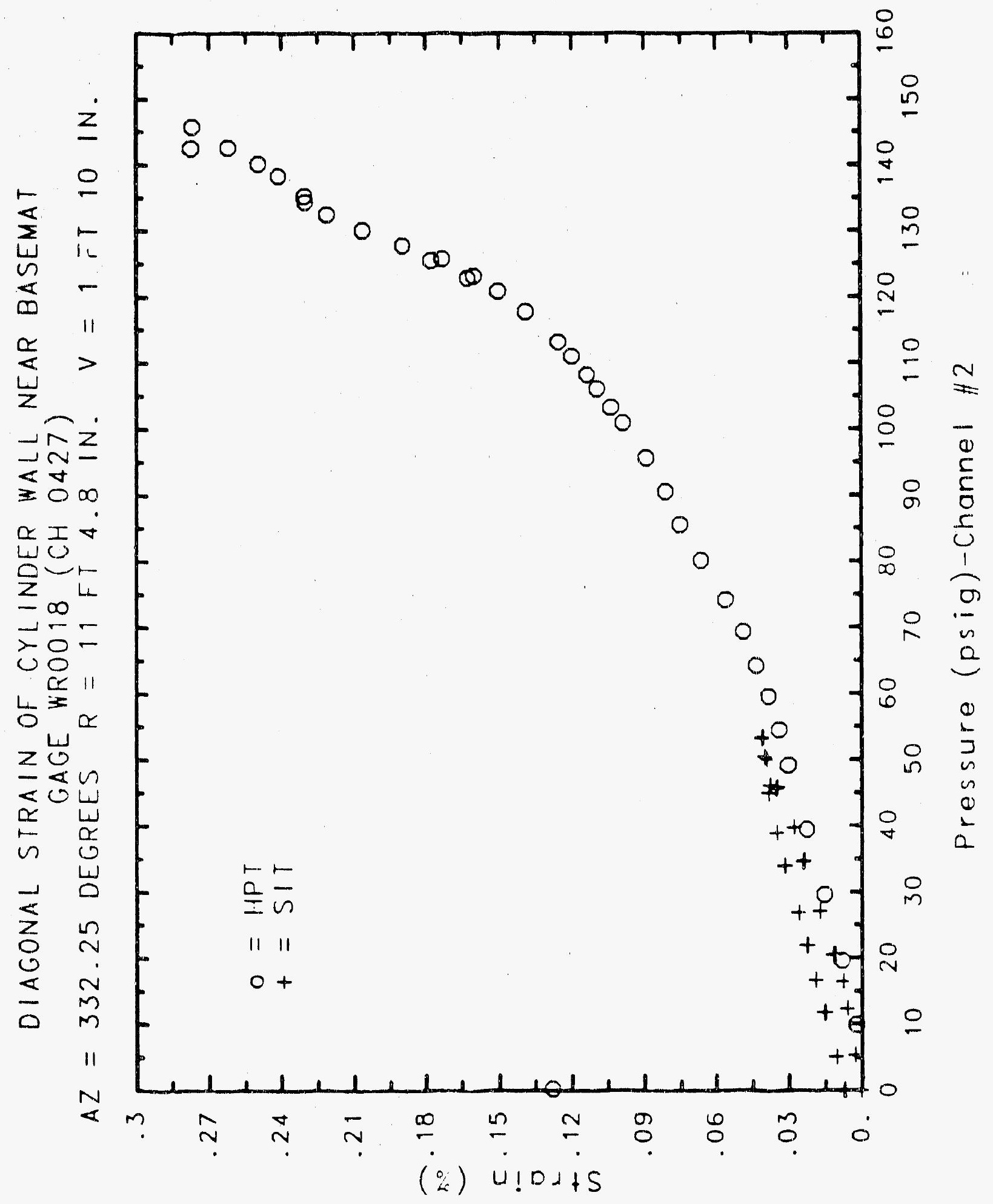




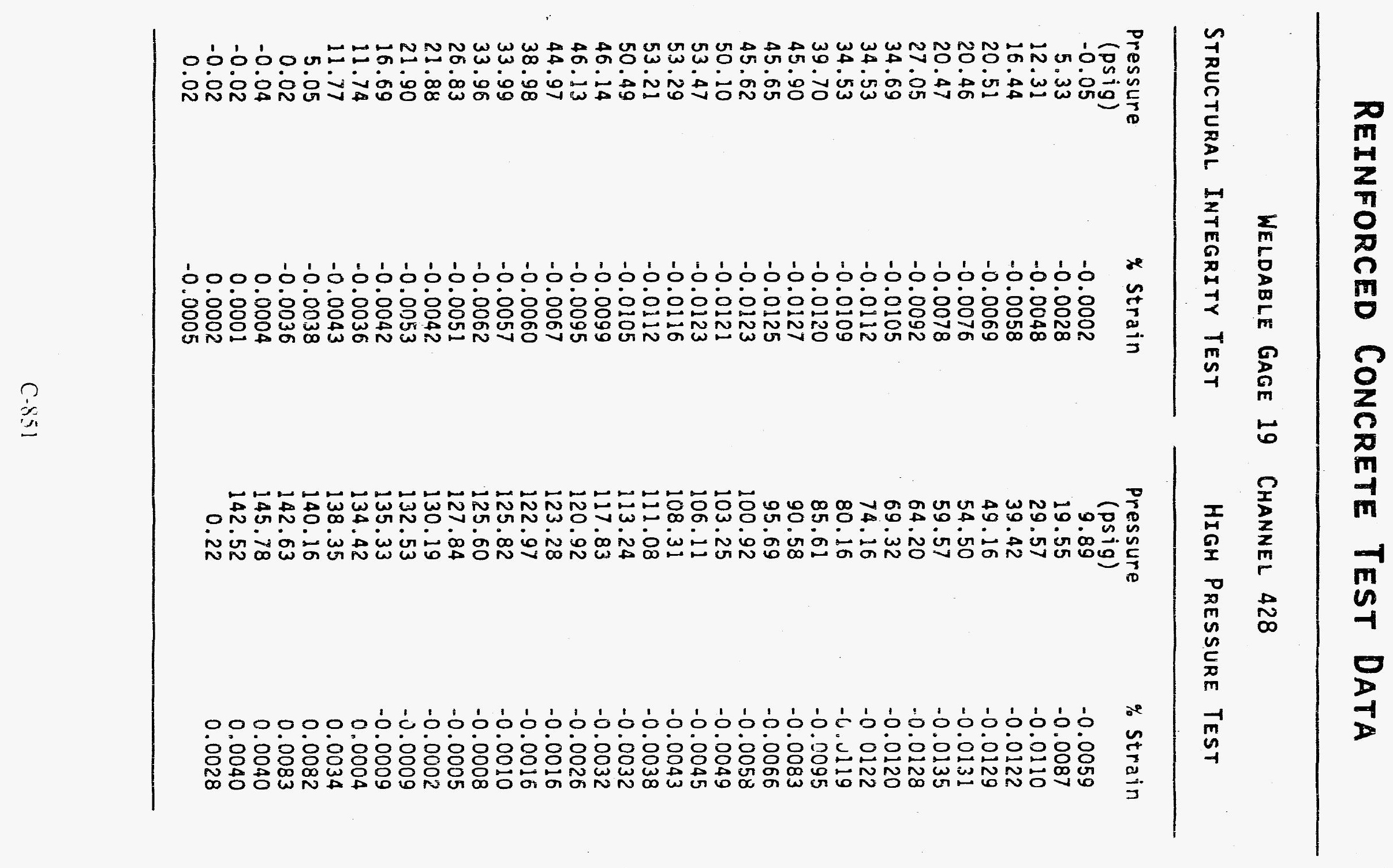




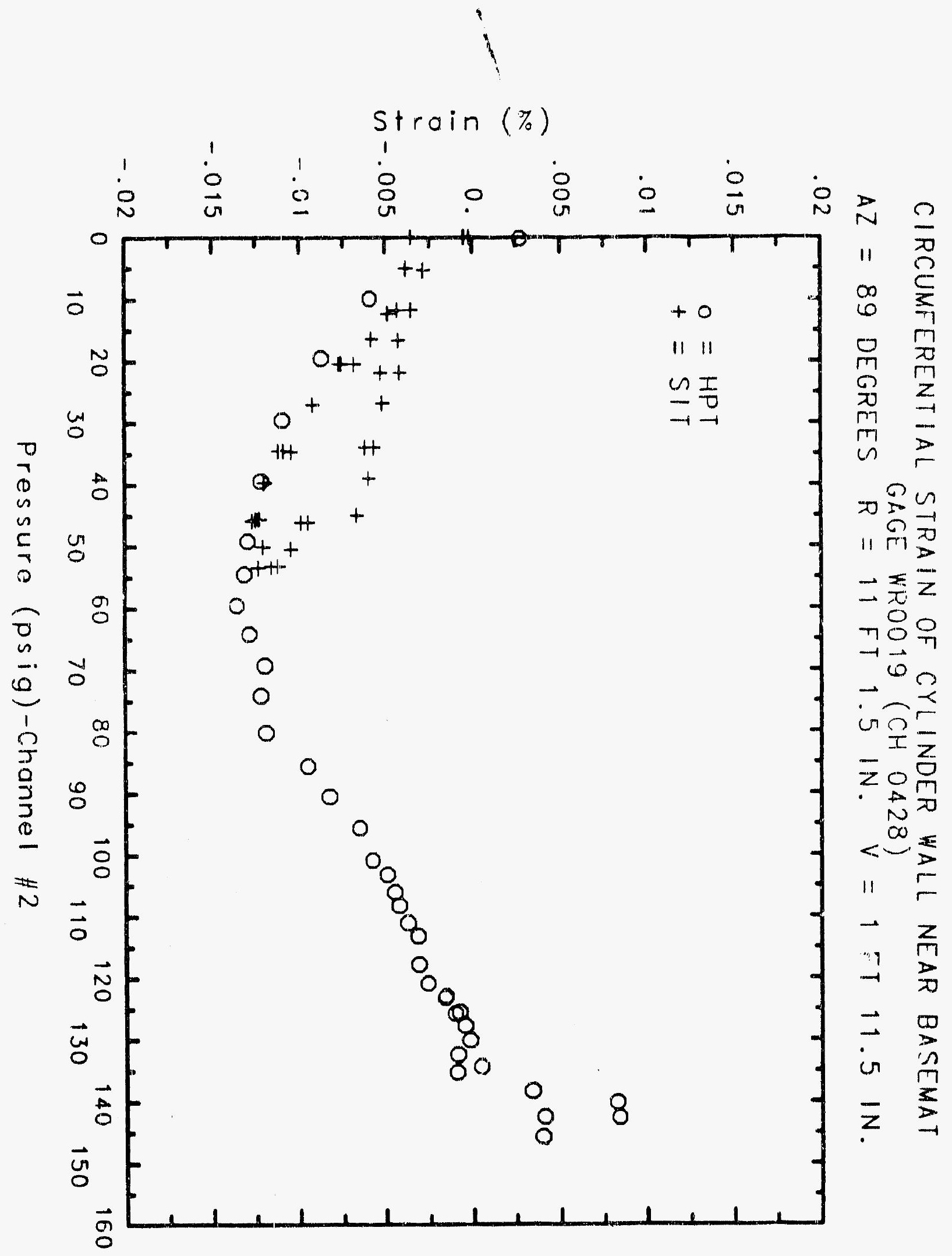




\section{Reinforced Concrete Test Data}

Weldable gage 20 Channel 429

StRUCTURAL InTEgrity TEST

\begin{tabular}{|c|c|}
\hline $\begin{array}{c}\text { Pressure } \\
\text { (psig) } \\
-0.05 \\
5.33 \\
12.31 \\
16.44 \\
20.51 \\
20.46 \\
20.47 \\
27.05 \\
34.69 \\
34.53 \\
34.53 \\
39.70 \\
45.90 \\
45.65 \\
45.62 \\
50.10 \\
53.47 \\
53.29 \\
53.21 \\
50.49 \\
46.14 \\
46.13 \\
44.97 \\
38.98 \\
33.99 \\
33.96 \\
26.83 \\
21.88 \\
21.90 \\
16.69 \\
11.74 \\
11.77 \\
5.05 \\
0.02 \\
-0.04 \\
-0.02 \\
-0.02 \\
0.02\end{array}$ & $\begin{array}{l}\text { \% strain } \\
-0.0010 \\
-0.0002 \\
0.0008 \\
0.0007 \\
-0.2784 \\
-0.2795 \\
-0.2797 \\
-0.2809 \\
-0.2821 \\
-0.2825 \\
-0.2830 \\
-0.2835 \\
-0.2846 \\
-0.2841 \\
-0.2842 \\
-0.2844 \\
-0.2837 \\
-0.2835 \\
-0.2830 \\
-0.2825 \\
-0.2818 \\
-0.2813 \\
0.0128 \\
0.0117 \\
-0.2774 \\
-0.2780 \\
-0.2767 \\
-0.2761 \\
-0.2773 \\
-0.2761 \\
-0.2756 \\
-0.2761 \\
-0.2756 \\
-0.2751 \\
-0.2717 \\
-0.2718 \\
-0.2716 \\
-0.2728\end{array}$ \\
\hline
\end{tabular}

High Pressure Test

\begin{tabular}{|c|c|}
\hline $\begin{array}{c}\text { Pressure } \\
\text { (psig) } \\
9.89 \\
19.55 \\
29.57 \\
39.42 \\
49.16 \\
54.50 \\
59.57 \\
64.20 \\
69.32 \\
74.16 \\
80.16 \\
85.61 \\
90.58 \\
95.69 \\
100.92 \\
103.25 \\
106.11 \\
108.31 \\
111.08 \\
113.24 \\
117.83 \\
120.92 \\
123.28 \\
122.97 \\
125.82 \\
125.60 \\
127.84 \\
130.19 \\
132.53 \\
135.33 \\
134.42 \\
138.35 \\
140.16 \\
142.63 \\
145.78 \\
142.52 \\
0.22 \\
\end{array}$ & $\begin{array}{l}\% \text { Strain } \\
-0.0059 \\
-0.0085 \\
-0.0107 \\
-0.0122 \\
-0.0128 \\
-0.0133 \\
-0.0133 \\
-0.0129 \\
-0.0117 \\
-0.0119 \\
-0.0116 \\
-0.0093 \\
-0.0083 \\
-0.0066 \\
-0.0055 \\
-0.0051 \\
-0.0045 \\
-0.0040 \\
-0.0033 \\
-0.0028 \\
-0.0026 \\
-0.0020 \\
-0.0017 \\
-0.0015 \\
-0.0009 \\
-0.0008 \\
-0.0003 \\
-0.0002 \\
-0.0008 \\
-0.0010 \\
0.0003 \\
0.0036 \\
0.0080 \\
0.0079 \\
0.0041 \\
0.0042 \\
0.0033\end{array}$ \\
\hline
\end{tabular}




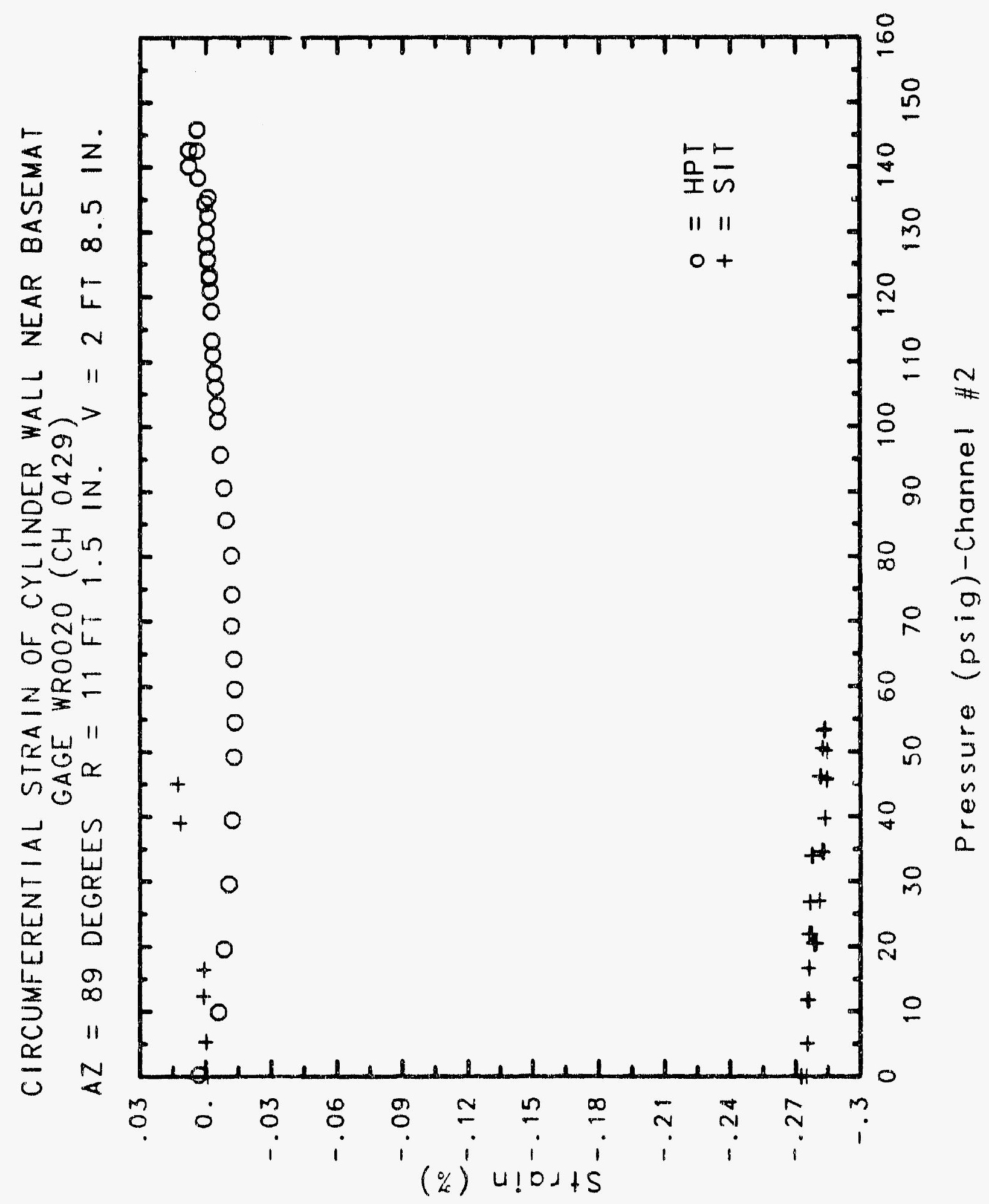




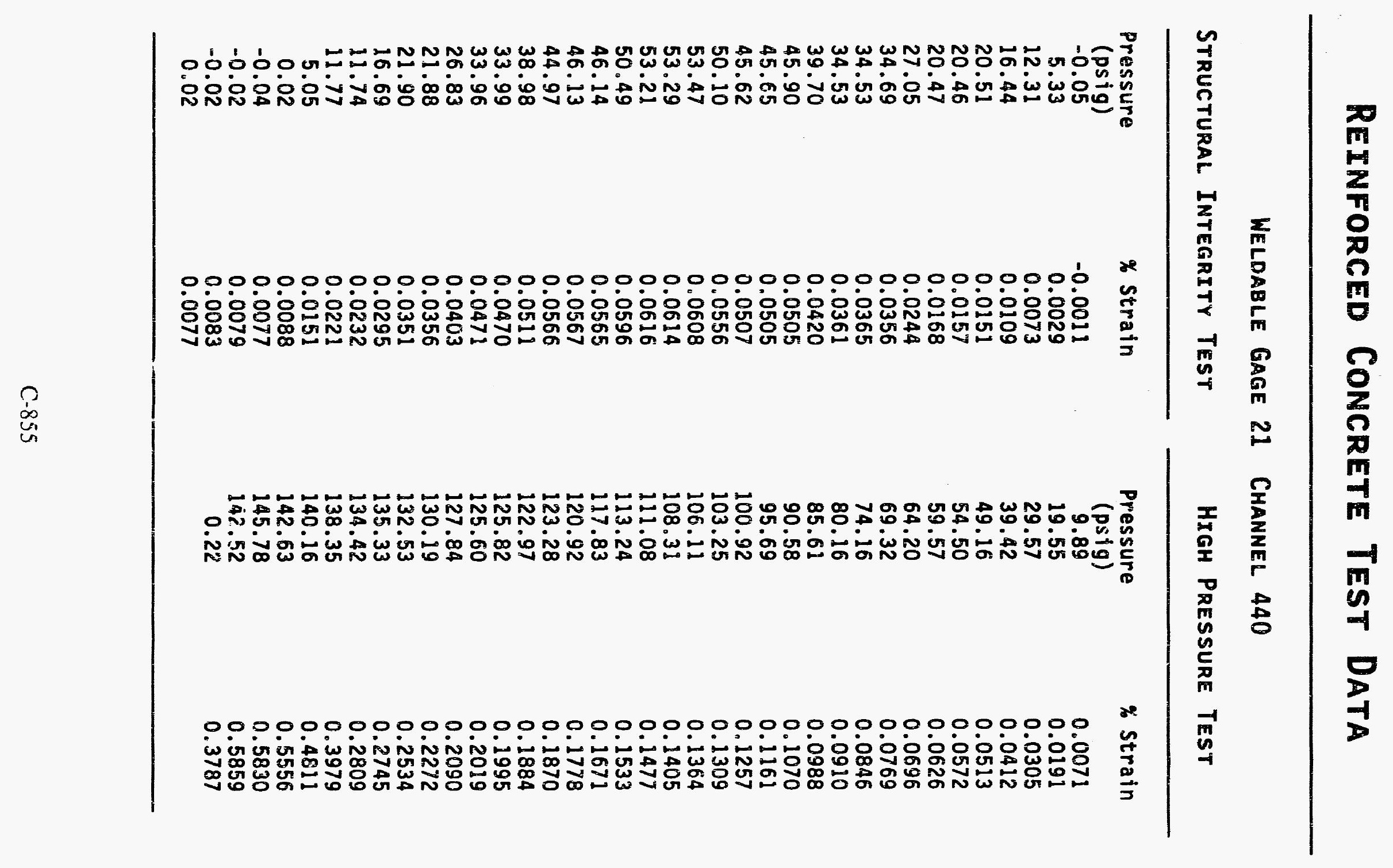




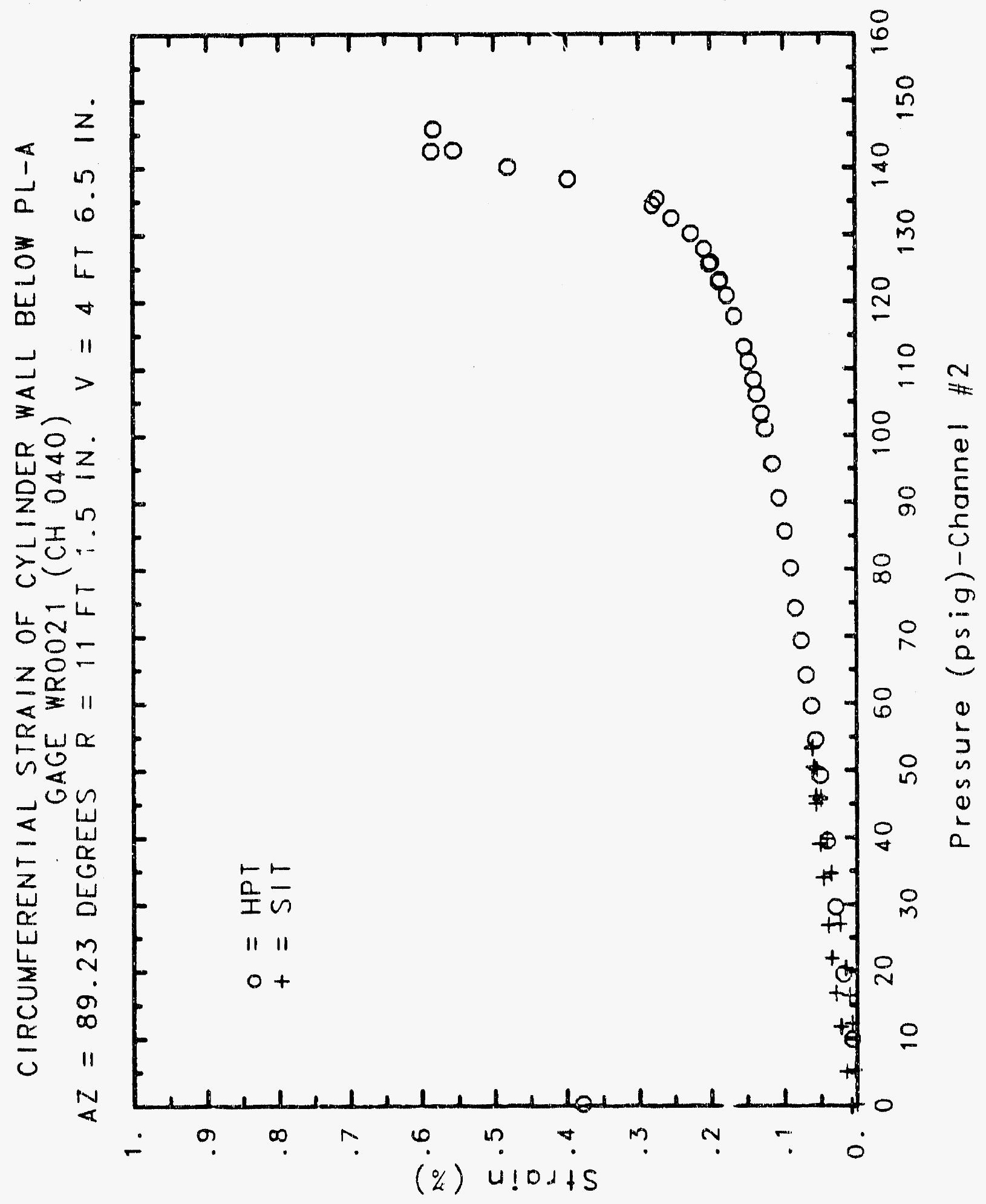

C- 856 


\section{Reinforced Concrete Test Data}

Weldable gage 22 Channel 441

Structural Integrity Test

High Pressure test

\begin{tabular}{|c|c|c|c|}
\hline $\begin{array}{c}\text { Pressure } \\
\text { (psig) } \\
-0.05 \\
5.33 \\
12.31 \\
16.44 \\
20.51 \\
20.46 \\
20.47 \\
27.05 \\
34.69 \\
34.53 \\
34.53 \\
39.70 \\
45.90 \\
45.65 \\
45.62 \\
50.10 \\
53.47 \\
53.29 \\
53.21 \\
50.49 \\
46.14 \\
46.13 \\
44.97 \\
38.98 \\
33.99 \\
33.96 \\
26.83 \\
21.88 \\
21.90 \\
16.69 \\
11.74 \\
11.77 \\
5.05 \\
0.02 \\
-0.04 \\
-0.02 \\
-0.02 \\
0.02\end{array}$ & $\begin{array}{c}\text { \% Strain } \\
-0.0007 \\
0.0054 \\
0.0130 \\
0.0663 \\
0.0707 \\
0.0716 \\
0.0723 \\
0.0795 \\
0.0912 \\
0.0920 \\
0.0919 \\
0.0976 \\
0.1060 \\
0.1061 \\
0.1060 \\
0.1117 \\
0.1165 \\
0.1168 \\
0.1169 \\
0.1156 \\
0.1122 \\
0.1123 \\
0.1123 \\
0.1070 \\
0.1026 \\
0.1030 \\
0.0959 \\
0.0912 \\
0.0911 \\
0.0852 \\
0.0790 \\
0.0778 \\
0.0707 \\
0.0647 \\
0.0633 \\
0.0633 \\
0.0640 \\
0.0634\end{array}$ & $\begin{array}{c}\text { Pressure } \\
\text { (psig) } \\
9.89 \\
19.55 \\
29.57 \\
39.42 \\
49.16 \\
54.50 \\
59.57 \\
64.20 \\
69.32 \\
74.16 \\
80.16 \\
85.61 \\
90.58 \\
95.69 \\
100.92 \\
103.25 \\
106.11 \\
108.31 \\
111.08 \\
113.24 \\
117.83 \\
120.92 \\
123.28 \\
122.97 \\
125.82 \\
125.60 \\
127.84 \\
130.19 \\
132.53 \\
135.33 \\
134.42 \\
138.35 \\
140.16 \\
142.63 \\
145.78 \\
142.52 \\
0.22\end{array}$ & $\begin{array}{l}\text { Strain } \\
0.0077 \\
0.0193 \\
0.0309 \\
0.0418 \\
0.0517 \\
0.0577 \\
0.0639 \\
0.0706 \\
0.0779 \\
0.0861 \\
0.0918 \\
0.0999 \\
0.1076 \\
0.1173 \\
0.1276 \\
0.1321 \\
0.1380 \\
0.1427 \\
0.1499 \\
0.1553 \\
0.1695 \\
0.1800 \\
0.1888 \\
0.1904 \\
0.2014 \\
0.2040 \\
0.2115 \\
0.2300 \\
0.2561 \\
0.2778 \\
0.2844 \\
0.4023 \\
0.4860 \\
0.5614 \\
0.5888 \\
0.5923 \\
0.3826\end{array}$ \\
\hline
\end{tabular}




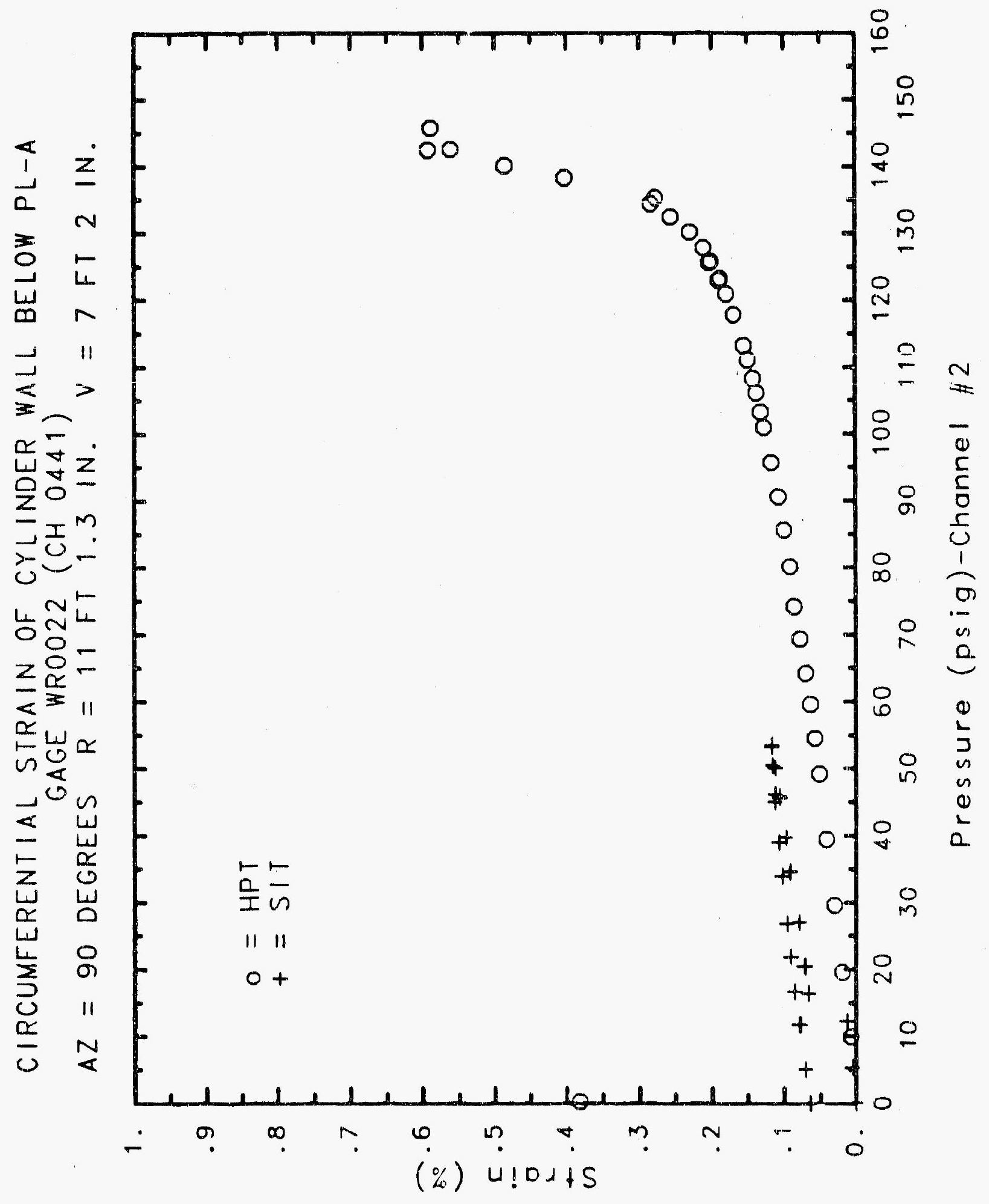

C- 858 


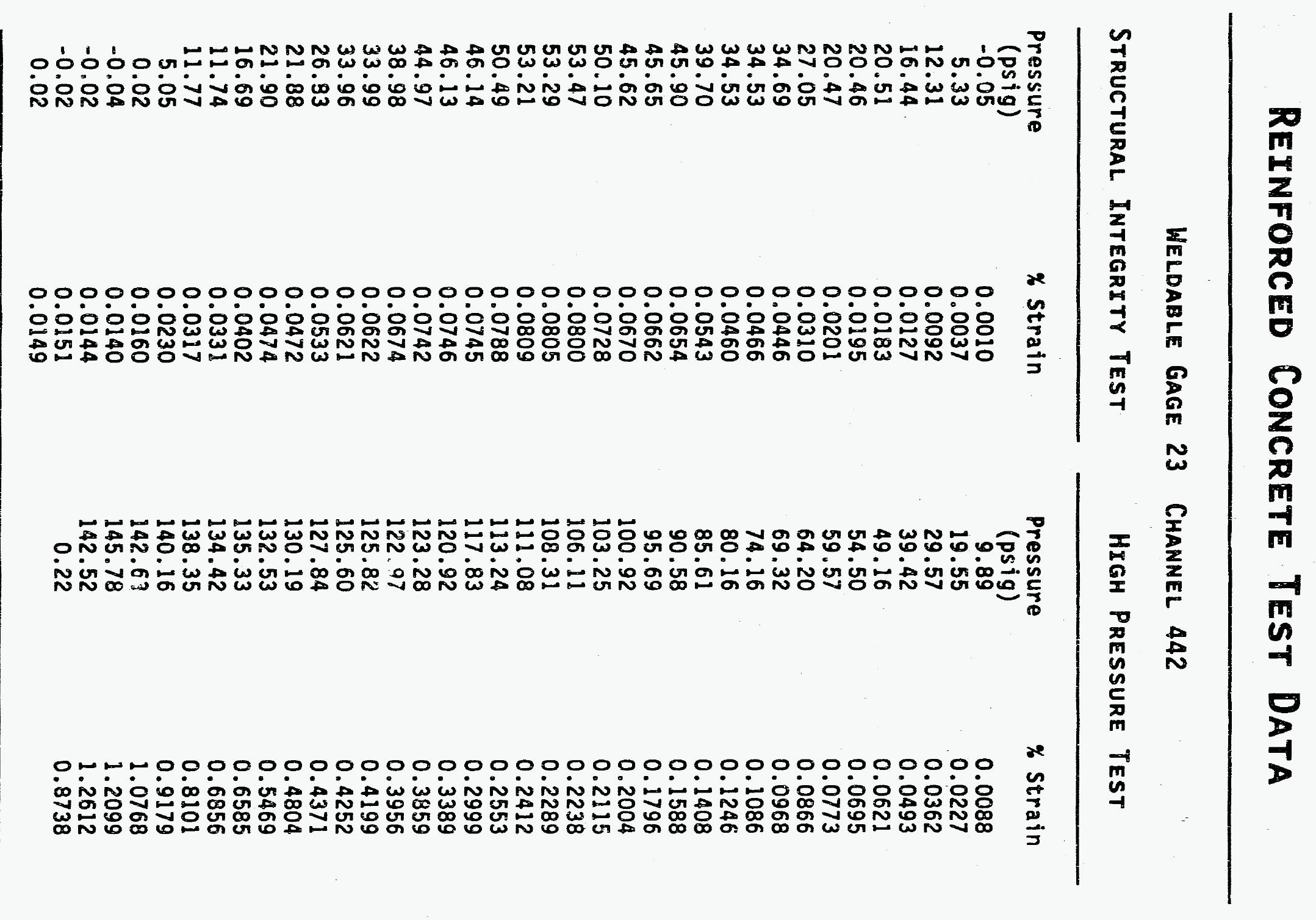




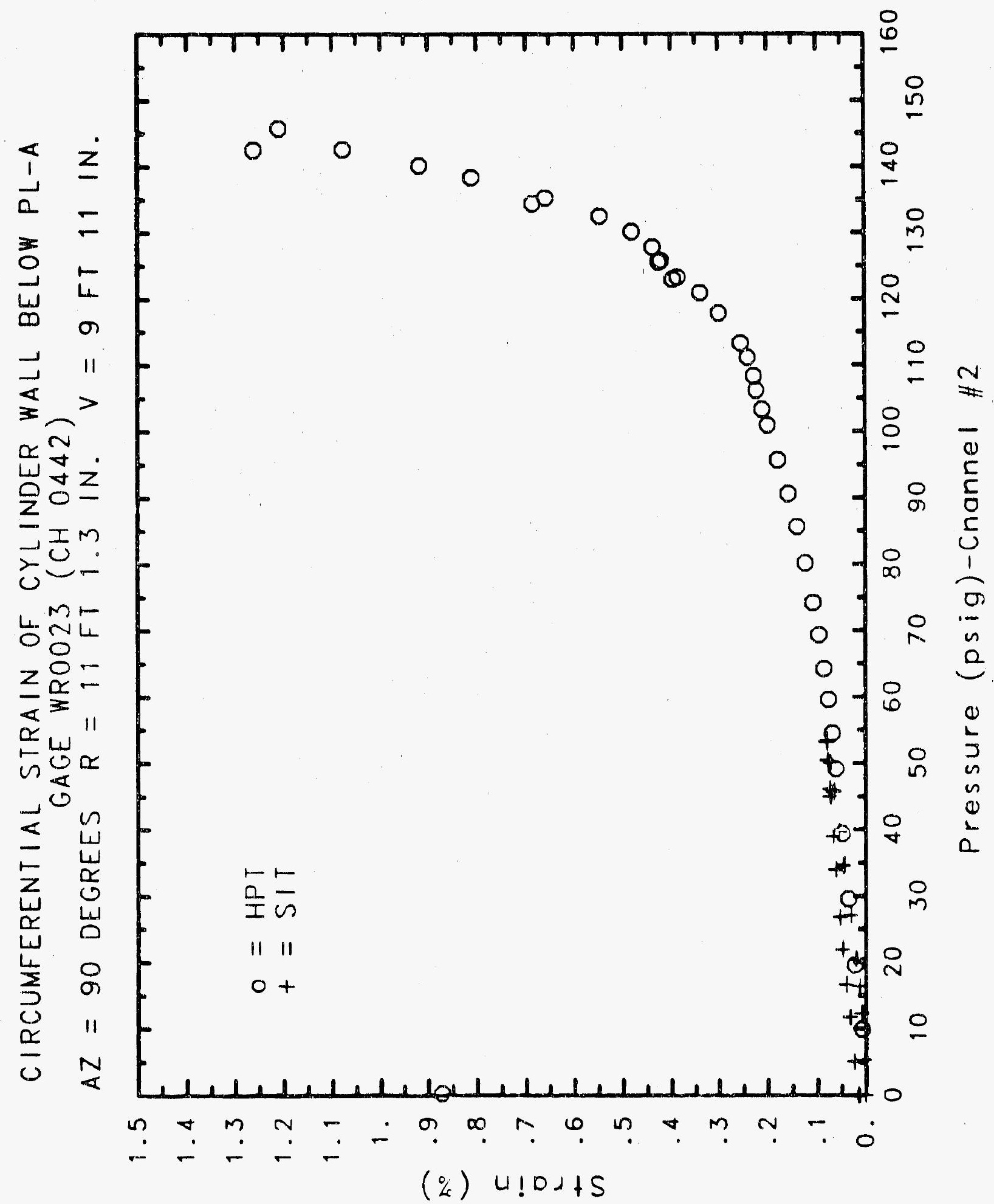




\section{Reinforced Concrete Test Data}

Weldable gage 24 Channel 443

Structural Integrity Test

High Pressure test

\begin{tabular}{|c|c|c|c|}
\hline $\begin{array}{c}\text { Pressure } \\
\text { (psig) } \\
-0.05 \\
5.33 \\
12.31 \\
16.44 \\
20.51 \\
20.46 \\
20.47 \\
27.05 \\
34.69 \\
34.53 \\
34.53 \\
39.70 \\
45.90 \\
45.65 \\
45.62 \\
50.10 \\
53.47 \\
53.29 \\
53.21 \\
50.49 \\
46.14 \\
46.13 \\
44.97 \\
38.98 \\
33.99 \\
33.96 \\
26.83 \\
21.88 \\
21.90 \\
16.69 \\
11.74 \\
11.77 \\
5.05 \\
0.02 \\
-0.04 \\
-0.02 \\
-0.02 \\
0.02 \\
\end{array}$ & $\begin{array}{l}\text { \% Strain } \\
0.0008 \\
0.0037 \\
0.0081 \\
0.0106 \\
0.0164 \\
0.0175 \\
0.0181 \\
0.0301 \\
0.0458 \\
0.0483 \\
0.0490 \\
0.0565 \\
0.0689 \\
0.0704 \\
0.0703 \\
0.0778 \\
0.0841 \\
0.0850 \\
0.0854 \\
0.0838 \\
0.0794 \\
0.0798 \\
0.0803 \\
0.0744 \\
0.0691 \\
0.0688 \\
0.0602 \\
0.0541 \\
0.0541 \\
0.0479 \\
0.0408 \\
0.0396 \\
0.0307 \\
0.0242 \\
0.0198 \\
0.0203 \\
0.0206 \\
0.0213\end{array}$ & $\begin{array}{c}\text { Pressure } \\
(\text { psig) } \\
9.89 \\
19.55 \\
29.57 \\
39.42 \\
49.16 \\
54.50 \\
59.57 \\
64.20 \\
69.32 \\
74.16 \\
80.16 \\
85.61 \\
90.58 \\
95.69 \\
100.92 \\
103.25 \\
106.11 \\
108.31 \\
111.08 \\
113.24 \\
117.83 \\
120.92 \\
123.28 \\
122.97 \\
125.82 \\
125.60 \\
127.84 \\
130.19 \\
132.53 \\
135.33 \\
134.42 \\
138.35 \\
140.16 \\
142.63 \\
145.78 \\
142.52 \\
0.22 \\
\end{array}$ & $\begin{array}{l}\text { \% Strain } \\
0.0087 \\
0.0221 \\
0.0361 \\
0.0493 \\
0.0624 \\
0.0700 \\
0.0793 \\
0.0895 \\
0.1015 \\
0.1136 \\
0.1293 \\
0.1450 \\
0.1618 \\
0.1815 \\
0.2026 \\
0.2136 \\
0.2255 \\
0.2350 \\
0.2497 \\
0.2619 \\
0.2897 \\
0.3126 \\
0.3347 \\
0.3422 \\
0.3710 \\
0.3804 \\
0.3976 \\
0.4401 \\
0.4934 \\
0.5717 \\
0.5954 \\
0.7009 \\
0.7945 \\
0.9316 \\
1.0629 \\
1.1198 \\
0.7476\end{array}$ \\
\hline
\end{tabular}




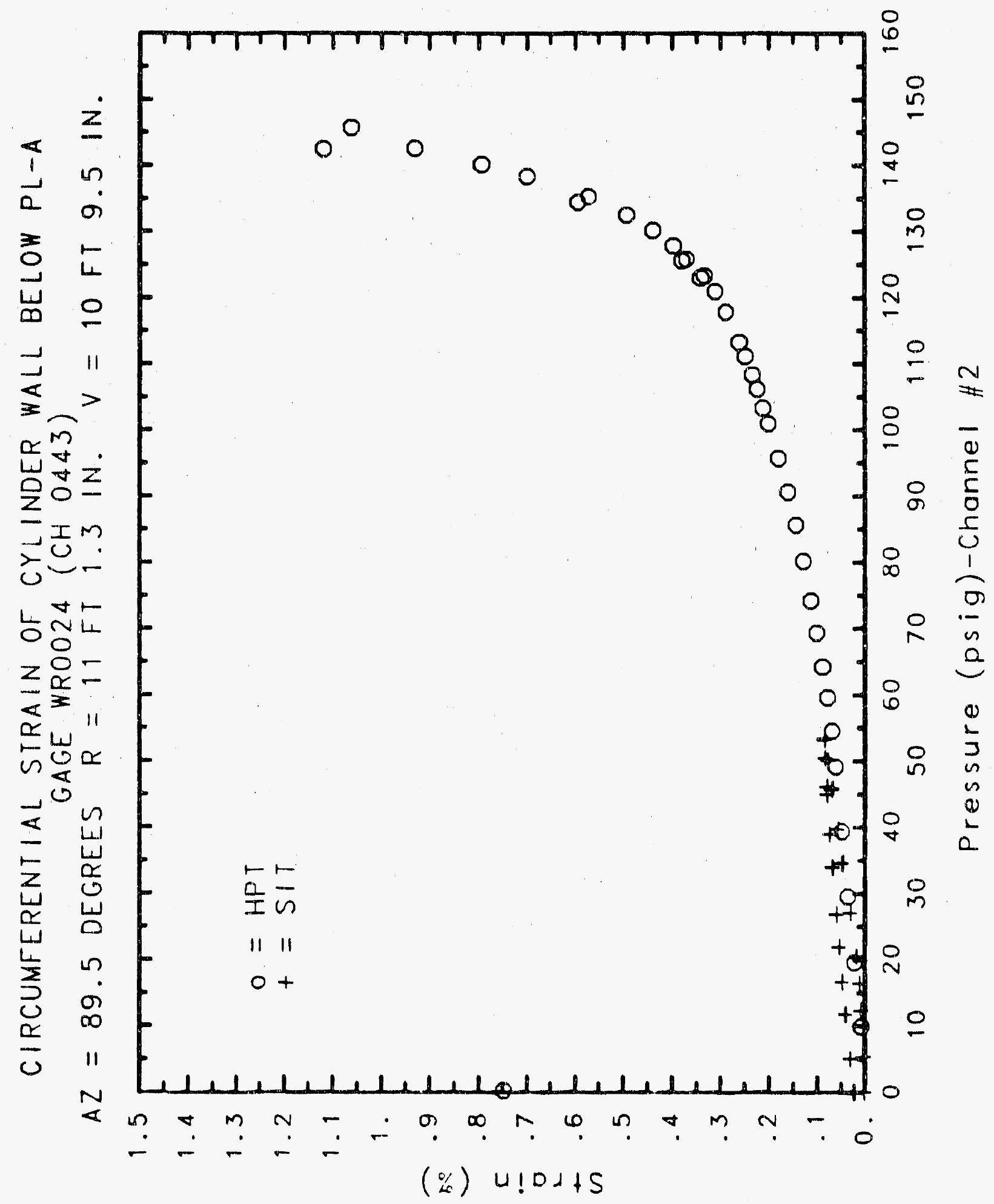




\section{Reinforced Concrete Test Data}

Weldable gage 25 Channel. 444

Structural InTEgrity TEST

\begin{tabular}{|c|c|c|c|}
\hline $\begin{array}{c}\text { Pressure } \\
\text { (psig) } \\
-0.05 \\
5.33 \\
12.31 \\
10.44 \\
20.51 \\
20.46 \\
20.47 \\
27.05 \\
34.69 \\
34.53 \\
34.53 \\
39.70 \\
45.90 \\
45.65 \\
45.62 \\
50.10 \\
53.47 \\
53.29 \\
53.21 \\
50.49 \\
46.14 \\
46.13 \\
44.97 \\
38.98 \\
33.99 \\
33.96 \\
26.83 \\
21.88 \\
21.90 \\
16.69 \\
11.74 \\
11.77 \\
5.05 \\
0.02 \\
-0.04 \\
-0.02 \\
-0.02 \\
0.02\end{array}$ & $\begin{array}{l}\text { Strain } \\
0.0003 \\
0.0033 \\
0.0076 \\
0.0105 \\
0.0153 \\
0.0166 \\
0.0169 \\
0.0241 \\
0.0321 \\
0.0332 \\
0.0333 \\
0.0388 \\
0.0458 \\
0.0459 \\
0.0460 \\
0.0503 \\
0.0546 \\
0.0543 \\
0.0552 \\
0.0528 \\
0.0497 \\
0.0498 \\
0.0503 \\
0.0457 \\
0.0416 \\
0.0414 \\
0.0355 \\
0.0310 \\
0.0311 \\
0.0266 \\
0.0215 \\
0.0206 \\
0.0144 \\
0.0095 \\
0.0068 \\
0.0069 \\
0.0068 \\
0.0079\end{array}$ & $\begin{array}{c}\text { Pressure } \\
\text { (psig) } \\
9.89 \\
19.55 \\
29.57 \\
39.42 \\
49.16 \\
54.50 \\
59.57 \\
64.20 \\
69.32 \\
74.16 \\
80.16 \\
85.61 \\
90.58 \\
95.69 \\
100.92 \\
103.25 \\
106.11 \\
108.31 \\
111.08 \\
113.24 \\
117.83 \\
120.92 \\
123.28 \\
122.97 \\
125.82 \\
125.60 \\
127.84 \\
130.19 \\
132.53 \\
135.33 \\
134.42 \\
138.35 \\
140.16 \\
142.63 \\
145.78 \\
142.52 \\
0.22\end{array}$ & $\begin{array}{c}\% \text { Strain } \\
0.0042 \\
0.0135 \\
0.0233 \\
0.0324 \\
0.0415 \\
0.0470 \\
0.0530 \\
0.0604 \\
0.0682 \\
0.0775 \\
0.0887 \\
0.0989 \\
0.1081 \\
0.1190 \\
0.1304 \\
0.1360 \\
0.1419 \\
0.1468 \\
0.1540 \\
0.1594 \\
0.1728 \\
0.1825 \\
0.1916 \\
0.1933 \\
0.2026 \\
0.2051 \\
0.2106 \\
0.2212 \\
0.2331 \\
0.2484 \\
0.2521 \\
0.2728 \\
0.2895 \\
0.3187 \\
0.3495 \\
0.3664 \\
0.1642\end{array}$ \\
\hline
\end{tabular}

High Pressure tejt

Pressure

0.0135

0.0233

0.0324

0.0415

0.0604

0.0887

0.0989

0.1081

0.1360

0.1419

0.1468

0.1540

0.1594

0.1825

0.1916

0.1933

0.2026

0.2051

0.2106

0.2484

0.2521

0.2728

0.2895

0.3187

0.1642 


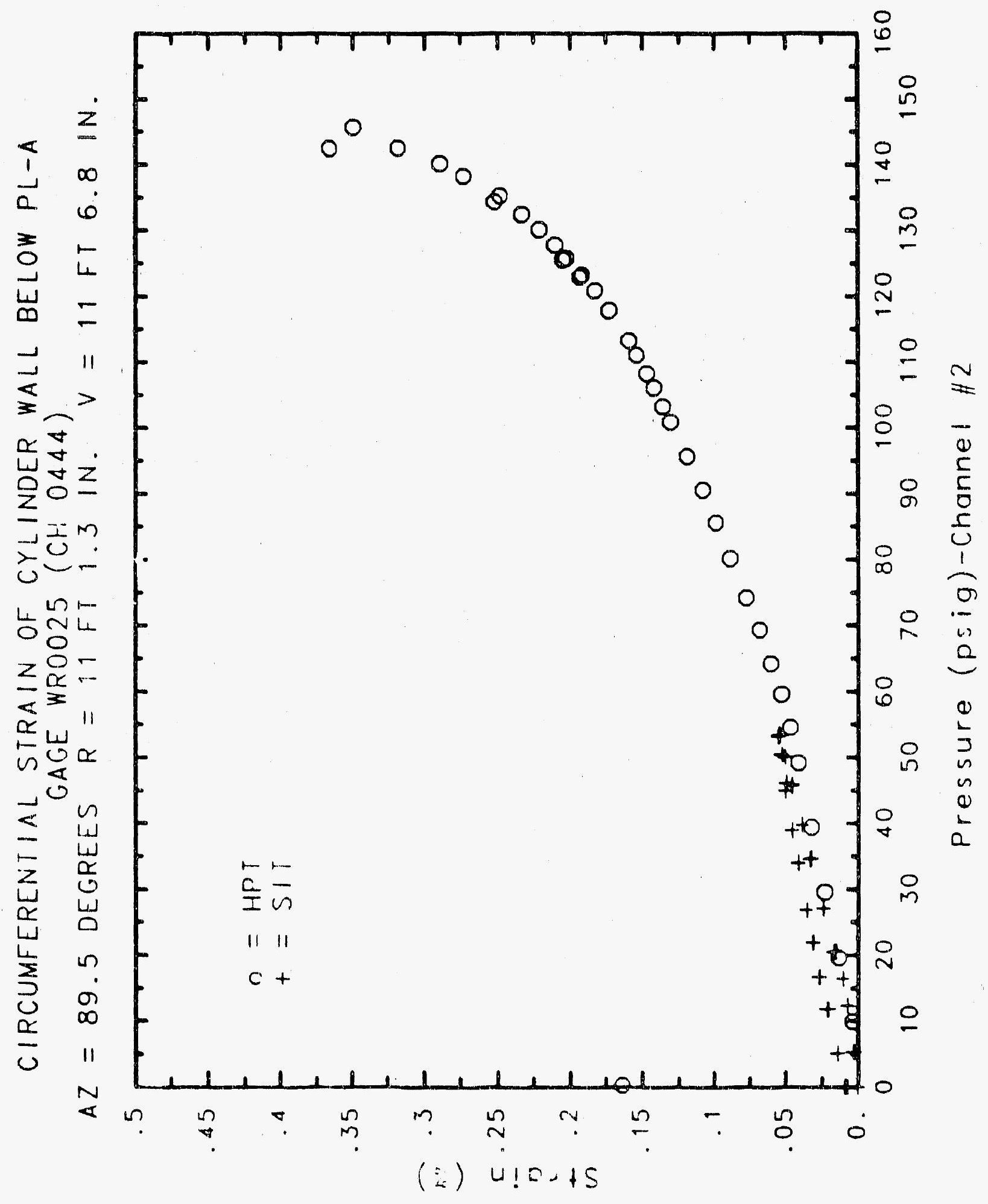

C.stot 


\section{Reinforced Concrete Test Data}

\section{Weldable gage 26 Channel 445}

Structural Integrity Test

High Pressure Test

\begin{tabular}{|c|c|c|c|}
\hline $\begin{array}{c}\text { Pressure } \\
\text { (psig) } \\
-0.05 \\
5.33 \\
12.31 \\
16.44 \\
20.51 \\
20.46 \\
20.47 \\
27.05 \\
34.69 \\
34.53 \\
34.53 \\
39.70 \\
45.90 \\
45.65 \\
45.62 \\
50.10 \\
53.47 \\
53.29 \\
53.21 \\
50.49 \\
46.14 \\
46.13 \\
44.97 \\
38.98 \\
33.99 \\
33.96 \\
26.83 \\
21.88 \\
21.90 \\
16.69 \\
11.74 \\
11.77 \\
5.05 \\
0.02 \\
-0.04 \\
-0.02 \\
-0.02 \\
0.02 \\
\end{array}$ & $\begin{array}{l}\text { \% Strain } \\
\text {-0.0007 } \\
0.0026 \\
0.0078 \\
0.2914 \\
0.2960 \\
0.2973 \\
0.2977 \\
0.3047 \\
0.3128 \\
0.3140 \\
0.3142 \\
0.3198 \\
0.3266 \\
0.3270 \\
0.3269 \\
0.3314 \\
0.3353 \\
0.3360 \\
0.3359 \\
0.3340 \\
0.3306 \\
0.3303 \\
0.3312 \\
0.3268 \\
0.3220 \\
0.3224 \\
0.3164 \\
0.3118 \\
0.3120 \\
0.3072 \\
0.3025 \\
0.3012 \\
0.2950 \\
0.2904 \\
0.2867 \\
0.2877 \\
0.2873 \\
0.2883\end{array}$ & $\begin{array}{c}\text { Pressure } \\
(\text { psig) } \\
9.89 \\
19.55 \\
29.57 \\
39.42 \\
49.16 \\
54.50 \\
59.57 \\
64.20 \\
69.32 \\
74.16 \\
80.16 \\
85.61 \\
90.58 \\
95.69 \\
100.92 \\
103.25 \\
106.11 \\
108.31 \\
111.08 \\
113.24 \\
117.83 \\
120.92 \\
123.28 \\
122.97 \\
125.82 \\
125.60 \\
127.84 \\
130.19 \\
132.53 \\
135.33 \\
134.42 \\
138.35 \\
140.16 \\
142.63 \\
145.78 \\
142.52 \\
0.22\end{array}$ & $\begin{array}{l}\text { Strain } \\
0.0042 \\
0.0132 \\
0.0232 \\
0.0322 \\
0.0414 \\
0.0464 \\
0.0533 \\
0.0602 \\
0.0683 \\
0.0775 \\
0.0886 \\
0.0983 \\
0.1080 \\
0.1191 \\
0.1302 \\
0.1359 \\
0.1418 \\
0.1468 \\
0.1538 \\
0.1596 \\
0.1726 \\
0.182 .7 \\
0.1912 \\
0.1931 \\
0.2021 \\
0.2049 \\
0.2107 \\
0.2212 \\
0.2331 \\
0.2482 \\
0.2522 \\
0.2726 \\
0.2894 \\
0.3185 \\
0.3491 \\
0.3665 \\
0.1641\end{array}$ \\
\hline
\end{tabular}




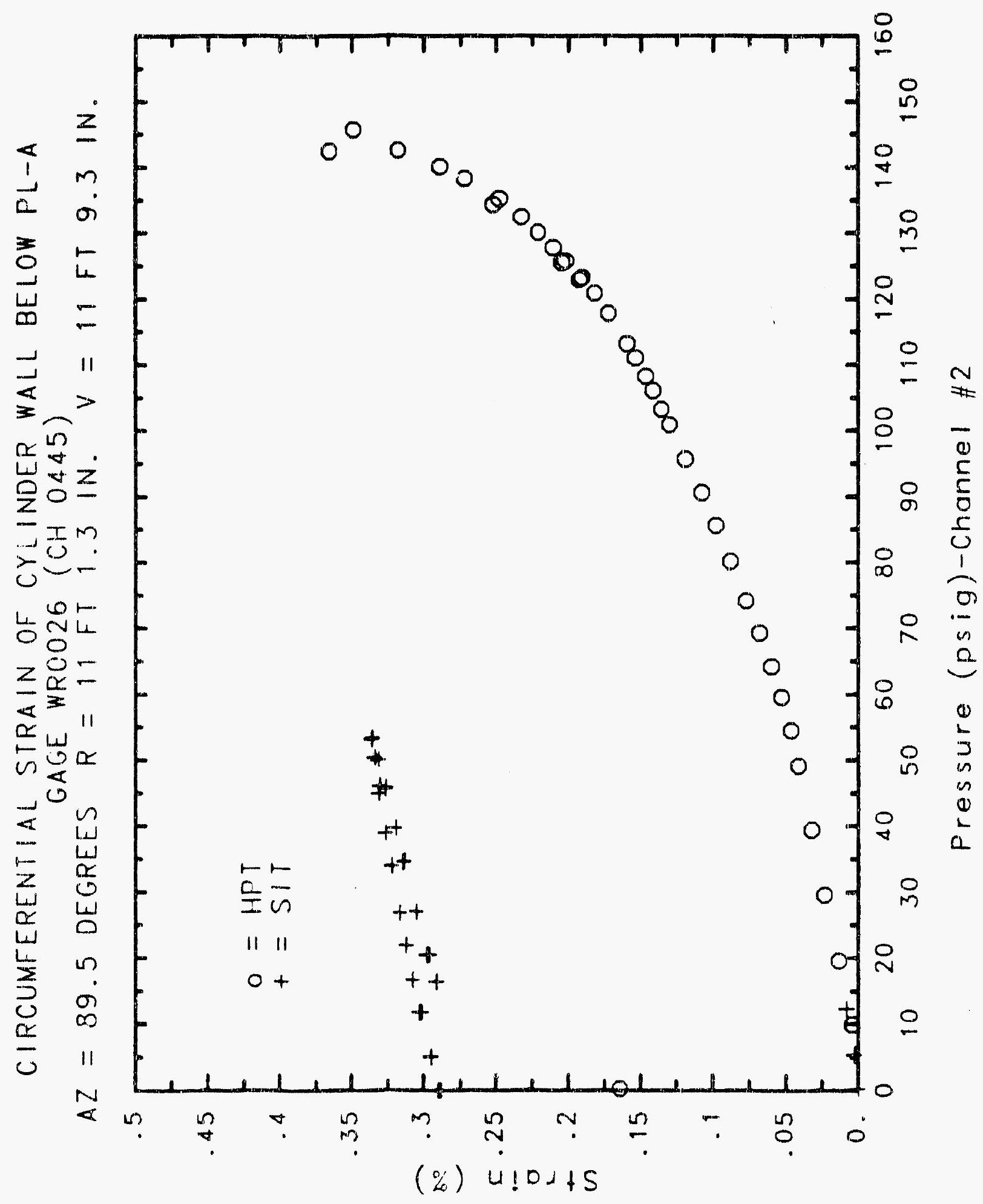




\section{Reinforced Concrete Test Data}

\begin{tabular}{|c|c|c|c|}
\hline STRUCTURAL & $\begin{array}{l}\text { WELDABLE GAGE } 27 \\
\text { INTEGRITY TEST }\end{array}$ & $\begin{array}{l}\text { Channel } 446 \\
\text { High Pressure }\end{array}$ & TEST \\
\hline $\begin{array}{c}\text { Pressure } \\
\text { (psig) } \\
-0.05 \\
5.33 \\
12.31 \\
16.44 \\
20.51 \\
20.46 \\
20.47 \\
27.05 \\
34.69 \\
34.53 \\
34.53 \\
39.70 \\
45.90 \\
45.65 \\
45.62 \\
50.10 \\
53.47 \\
53.29 \\
53.21 \\
50.49 \\
46.14 \\
46.13 \\
44.97 \\
38.98 \\
33.99 \\
33.96 \\
26.83 \\
21.88 \\
21.90 \\
16.69 \\
11.74 \\
11.77 \\
5.05 \\
0.02 \\
-0.04 \\
-0.02 \\
-0.02 \\
0.02 \\
\end{array}$ & $\begin{array}{l}\text { \% Strain } \\
0.0002 \\
0.0049 \\
0.0124 \\
0.0176 \\
0.0255 \\
0.0269 \\
0.0266 \\
0.0406 \\
0.0552 \\
0.0567 \\
0.0570 \\
0.0651 \\
0.0765 \\
0.0759 \\
0.0759 \\
0.0827 \\
0.0879 \\
0.0884 \\
0.0882 \\
0.0855 \\
0.0196 \\
0.0791 \\
0.0788 \\
0.0707 \\
0.0629 \\
0.0629 \\
0.0515 \\
0.0445 \\
0.0447 \\
0.0368 \\
0.0286 \\
0.0272 \\
0.0172 \\
0.0093 \\
0.0062 \\
0.0067 \\
0.0066 \\
0.0074\end{array}$ & $\begin{array}{c}\text { Pressure } \\
\text { (psig) } \\
9.89 \\
19.55 \\
29.57 \\
39.42 \\
49.16 \\
54.50 \\
59.57 \\
64.20 \\
69.32 \\
74.16 \\
80.16 \\
85.61 \\
90.58 \\
95.69 \\
100.92 \\
103.25 \\
106.11 \\
108.31 \\
111.08 \\
113.24 \\
117.83 \\
120.92 \\
123.28 \\
122.97 \\
125.82 \\
125.60 \\
127.84 \\
130.19 \\
132.53 \\
135.33 \\
134.42 \\
138.35 \\
140.16 \\
142.63 \\
145.78 \\
142.52 \\
0.22 \\
\end{array}$ & $\begin{array}{l}\text { \% Strain } \\
0.0119 \\
0.0287 \\
0.0457 \\
0.0613 \\
0.0762 \\
0.0848 \\
0.0928 \\
0.1023 \\
0.1119 \\
0.1228 \\
0.1348 \\
0.1481 \\
0.1606 \\
0.1764 \\
0.1943 \\
0.2035 \\
0.2140 \\
0.2224 \\
0.2377 \\
0.2492 \\
0.2779 \\
0.2959 \\
0.3317 \\
0.3343 \\
0.3627 \\
0.3756 \\
0.4045 \\
0.4559 \\
0.6256 \\
0.7815 \\
0.8041 \\
0.8701 \\
0.9476 \\
1.0604 \\
1.1592 \\
1.1934 \\
0.8622\end{array}$ \\
\hline
\end{tabular}




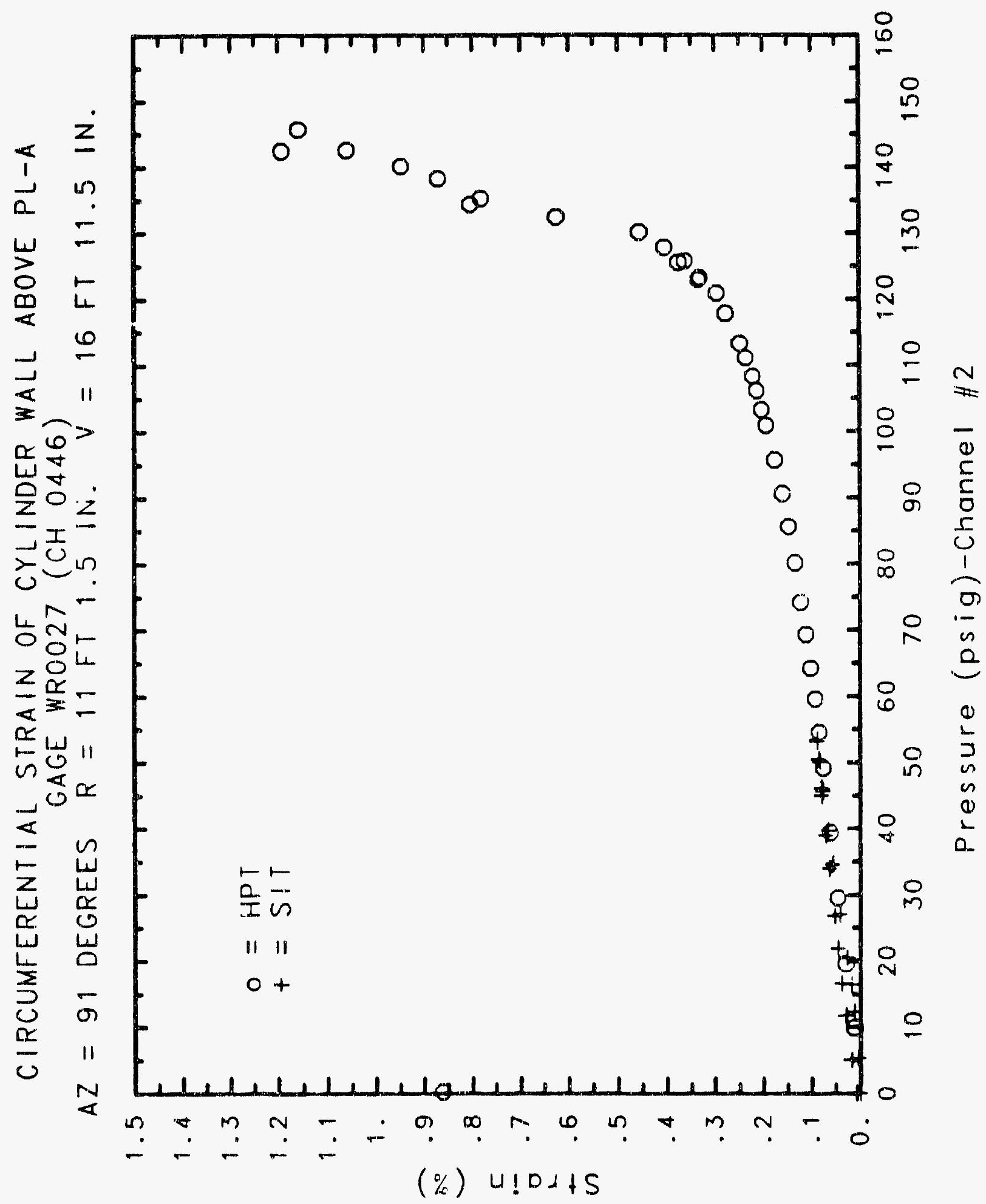




\section{Reinforced Concrete Test Data}

Weldable gage 28 Channel 447

Structural Integrity Test

\begin{tabular}{|c|c|}
\hline $\begin{array}{c}\text { Pressure } \\
\text { (psig) } \\
-0.05 \\
5.33 \\
12.31 \\
16.44 \\
20.51 \\
20.46 \\
20.47 \\
27.05 \\
34.69 \\
34.53 \\
34.53 \\
39.70 \\
45.90 \\
45.65 \\
45.62 \\
50.10 \\
53.47 \\
53.29 \\
53.21 \\
50.49 \\
46.14 \\
46.13 \\
44.97 \\
38.98 \\
33.99 \\
33.96 \\
26.83 \\
21.88 \\
21.90 \\
16.69 \\
11.74 \\
11.77 \\
5.05 \\
0.02 \\
-0.04 \\
-0.02 \\
-0.02 \\
0.02 \\
\end{array}$ & $\begin{array}{l}\text { \% Strain } \\
0.0003 \\
0.0040 \\
0.0080 \\
0.0108 \\
0.0143 \\
0.0149 \\
0.0146 \\
0.0224 \\
0.0347 \\
0.0365 \\
0.0373 \\
0.0430 \\
0.0518 \\
0.0516 \\
0.0513 \\
0.0564 \\
0.0612 \\
0.0619 \\
0.0621 \\
0.0604 \\
0.0570 \\
0.0568 \\
0.0599 \\
0.0547 \\
0.0499 \\
0.0491 \\
0.0418 \\
0.0355 \\
0.0360 \\
0.0307 \\
0.0249 \\
0.0231 \\
0.0155 \\
0.0098 \\
0.0079 \\
0.0084 \\
0.0084 \\
0.0089\end{array}$ \\
\hline
\end{tabular}

High Pressure Test

Pressure

\% Strain

9.89

19.55

29.57

39.42

49.16

54.50

59.57

64.20

69.32

74.16

80.16

85.61

90.58

95.69

$100.9 ?$

103.25

106.11

108.31

111.08

113.24

117.83

120.92

123.28

122.97

125.82

125.60

127.84

130.19

132.53

135.33

134.42

138.35

140.16

142.63

145.78

142.52

0.22
0.0079

0.0192

0.0314

0.0424

0.0535

0.0600

0.0666

0.0742

0.0821

0.0904

0.0984

0.1080

0.1169

0.1270

0.1376

0.1435

0.1500

0.1550

0.1626

0.1687

0.1820

0.1910

0.1996

0.2011

0.2101

0.2125

0.2181

0.2277

0.2413

0.2651

0.2702

0.2932

0.3339

0.4634

0.5876

0.6225

0.3700 


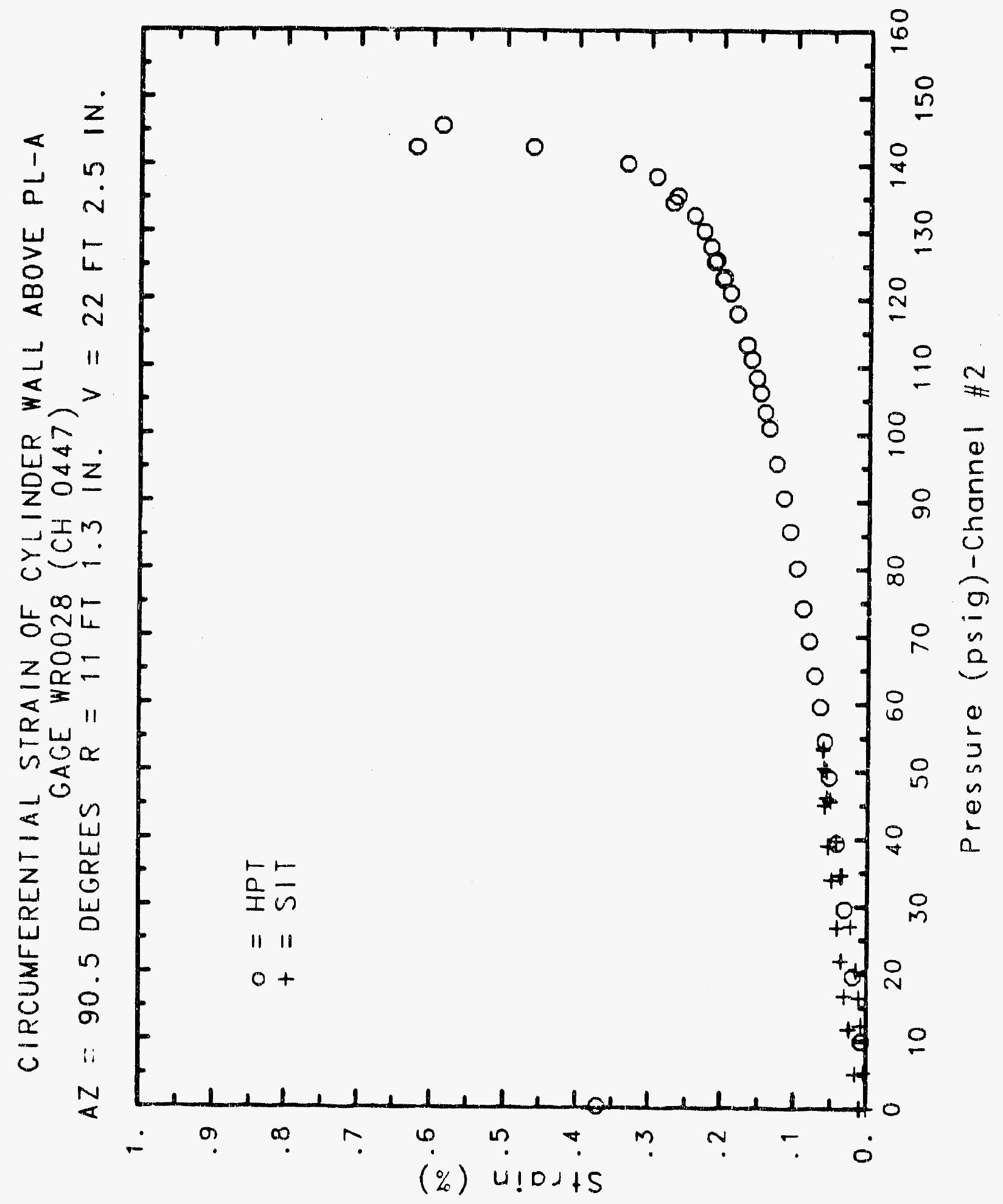

C. 870 


\section{Reinforced Concrete Test Data}

Weldable gage 29 Channel 448

Structural Integraty Test

High Pressure test

\begin{tabular}{|c|c|c|c|}
\hline $\begin{array}{c}\text { Pressure } \\
\text { (psig) } \\
-0.05 \\
5.33 \\
12.31 \\
16.44 \\
20.51 \\
20.46 \\
20.47 \\
27.05 \\
34.69 \\
34.53 \\
34.53 \\
39.70 \\
45.90 \\
45.65 \\
45.62 \\
50.10 \\
53.47 \\
53.29 \\
53.21 \\
50.49 \\
46.14 \\
46.13 \\
44.97 \\
38.98 \\
33.99 \\
33.96 \\
26.83 \\
21.88 \\
21.90 \\
16.69 \\
11.74 \\
11.77 \\
5.05 \\
0.02 \\
-0.04 \\
-0.02 \\
-0.02 \\
0.02 \\
\end{array}$ & $\begin{array}{c}\text { \% Strain } \\
\text {-0.0002 } \\
0.0038 \\
0.0079 \\
0.0108 \\
0.0148 \\
0.0156 \\
0.0161 \\
0.0235 \\
0.0346 \\
0.0363 \\
0.0366 \\
0.0419 \\
0.0489 \\
0.0485 \\
0.0479 \\
0.0522 \\
0.0557 \\
0.0559 \\
0.0560 \\
0.0540 \\
0.0508 \\
0.0506 \\
0.0514 \\
0.0474 \\
0.0430 \\
0.0428 \\
0.0362 \\
0.0312 \\
0.0312 \\
0.0267 \\
0.0222 \\
0.0207 \\
0.0142 \\
0.0097 \\
0.0081 \\
0.0083 \\
0.0085 \\
0.0089\end{array}$ & $\begin{array}{c}\text { Pressure } \\
(\text { psig) } \\
9.89 \\
19.55 \\
29.57 \\
39.42 \\
49.16 \\
54.50 \\
59.57 \\
64.20 \\
69.32 \\
74.16 \\
80.16 \\
85.61 \\
90.58 \\
95.69 \\
100.92 \\
103.25 \\
106.11 \\
108.31 \\
111.08 \\
113.24 \\
117.83 \\
120.92 \\
123.28 \\
122.97 \\
125.82 \\
125.60 \\
127.84 \\
130.19 \\
132.53 \\
135.33 \\
134.42 \\
138.35 \\
140.16 \\
142.63 \\
145.78 \\
142.52 \\
0.22 \\
\end{array}$ & $\begin{array}{l}\text { Strain } \\
0.0054 \\
0.0151 \\
0.0249 \\
0.0346 \\
0.0435 \\
0.0493 \\
0.0541 \\
0.0597 \\
0.0654 \\
0.0717 \\
0.0771 \\
0.0842 \\
0.0901 \\
0.0967 \\
0.1048 \\
0.1078 \\
0.1122 \\
0.1157 \\
0.1209 \\
0.1246 \\
0.1335 \\
0.1395 \\
0.1449 \\
0.1458 \\
0.1515 \\
0.1530 \\
0.1566 \\
0.1630 \\
0.1710 \\
0.1853 \\
0.1886 \\
0.2141 \\
0.2359 \\
0.2769 \\
0.3208 \\
0.3431 \\
0.1709\end{array}$ \\
\hline
\end{tabular}


CIRCUMFERENTIAL STRAIN OF DOME NEAR SPRING LINE GAGE WR0029 ( $\mathrm{CH} \mathrm{0448)}$
$A Z=93$ DEGREES $R=11 \mathrm{FT} 1.8 \mathrm{IN} . \mathrm{V}=24 \mathrm{FT} 2.8 \mathrm{IN}$.

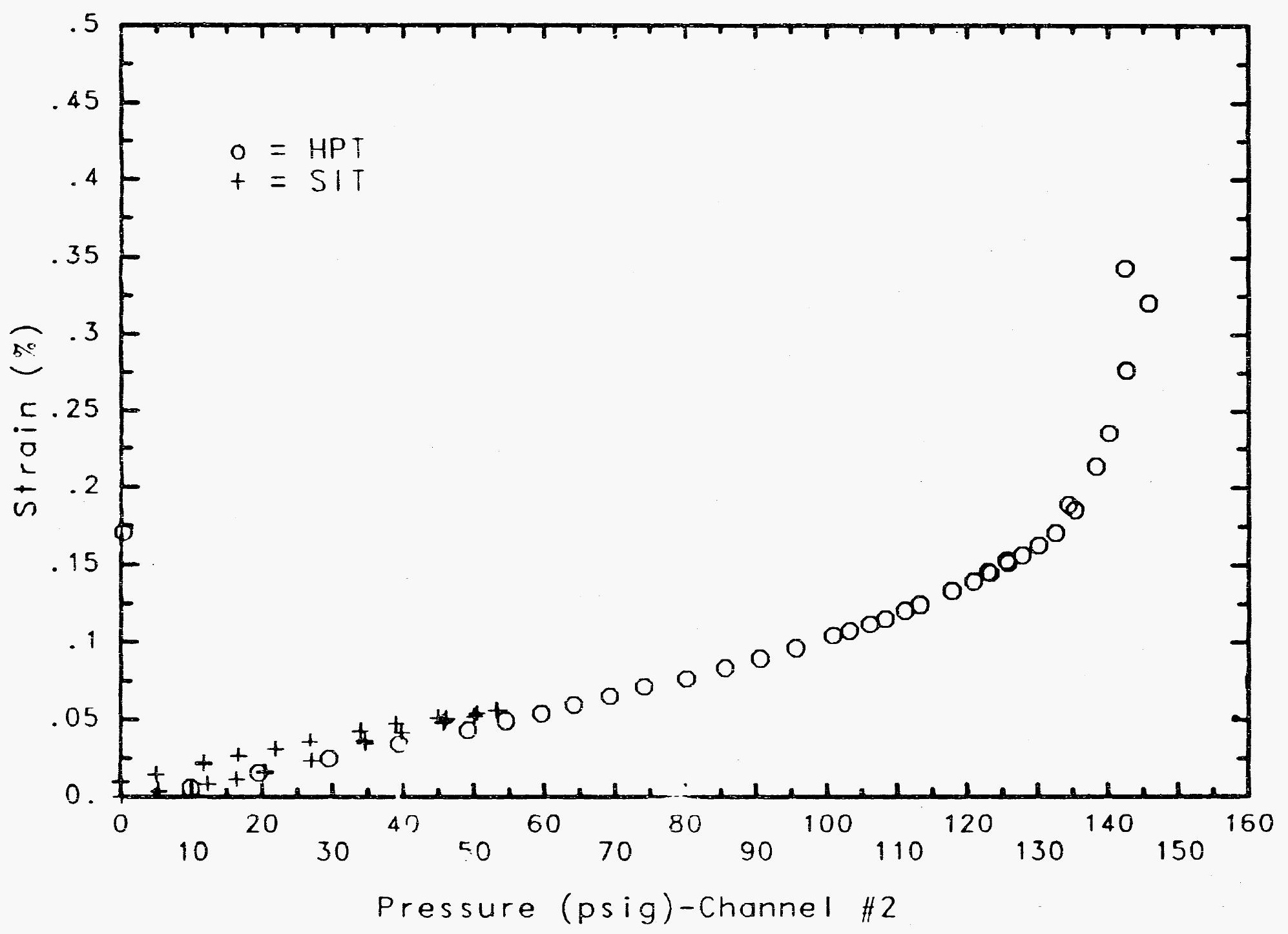




\section{Reinforced Concrete Test Data}

Weldable gage 30 Channel 449

StRUCTURAL INTEGRTTY TEST

High Pressure test

\begin{tabular}{|c|c|c|c|}
\hline $\begin{array}{c}\text { Pressure } \\
\text { (psig) } \\
-0.05 \\
5.33 \\
12.31 \\
16.44 \\
20.51 \\
20.46 \\
20.47 \\
27.05 \\
34.69 \\
34.53 \\
34.53 \\
39.70 \\
45.90 \\
45.65 \\
45.62 \\
50.10 \\
53.47 \\
53.29 \\
53.21 \\
50.49 \\
46.14 \\
46.13 \\
44.97 \\
38.98 \\
33.99 \\
33.96 \\
26.83 \\
21.88 \\
21.90 \\
16.69 \\
11.74 \\
11.77 \\
5.05 \\
0.02 \\
-0.04 \\
-0.02 \\
-0.02 \\
0.02 \\
\end{array}$ & $\begin{array}{c}\text { \% Strain } \\
\text {-0.0007 } \\
0.0030 \\
0.0069 \\
0.0099 \\
0.2760 \\
0.2767 \\
0.2774 \\
0.2851 \\
0.2962 \\
0.2982 \\
0.2983 \\
0.3037 \\
0.3107 \\
0.3099 \\
0.3090 \\
0.3135 \\
0.3173 \\
0.3177 \\
0.3174 \\
0.3158 \\
0.3128 \\
0.3124 \\
0.0451 \\
0.0400 \\
0.3044 \\
0.3043 \\
0.2978 \\
0.2926 \\
0.2928 \\
0.2883 \\
0.2836 \\
0.2827 \\
0.2760 \\
0.2709 \\
0.2695 \\
0.2697 \\
0.2701 \\
0.2704\end{array}$ & $\begin{array}{c}\text { Pressure } \\
(\text { psig) } \\
9.89 \\
19.55 \\
29.57 \\
39.42 \\
49.16 \\
54.50 \\
59.57 \\
64.20 \\
69.32 \\
74.16 \\
80.16 \\
85.61 \\
90.58 \\
95.69 \\
100.92 \\
103.25 \\
106.11 \\
108.31 \\
111.08 \\
113.24 \\
117.83 \\
120.92 \\
123.28 \\
122.97 \\
125.82 \\
125.60 \\
127.84 \\
130.19 \\
132.53 \\
135.33 \\
134.42 \\
138.35 \\
140.16 \\
142.63 \\
145.78 \\
142.52 \\
0.22\end{array}$ & $\begin{array}{l}\text { \% Strain } \\
0.0052 \\
0.0146 \\
0.0247 \\
0.0341 \\
0.0436 \\
0.0485 \\
0.0540 \\
0.0593 \\
0.0656 \\
0.0714 \\
0.0769 \\
0.0836 \\
0.0898 \\
0.0967 \\
0.1044 \\
0.1079 \\
0.1124 \\
0.1158 \\
0.1206 \\
0.1247 \\
0.1330 \\
0.1398 \\
0.1446 \\
0.1454 \\
0.1511 \\
0.1524 \\
0.1566 \\
0.1628 \\
0.1706 \\
0.1853 \\
0.1886 \\
0.2137 \\
0.2351 \\
0.2768 \\
0.3209 \\
0.3427 \\
0.1709\end{array}$ \\
\hline
\end{tabular}




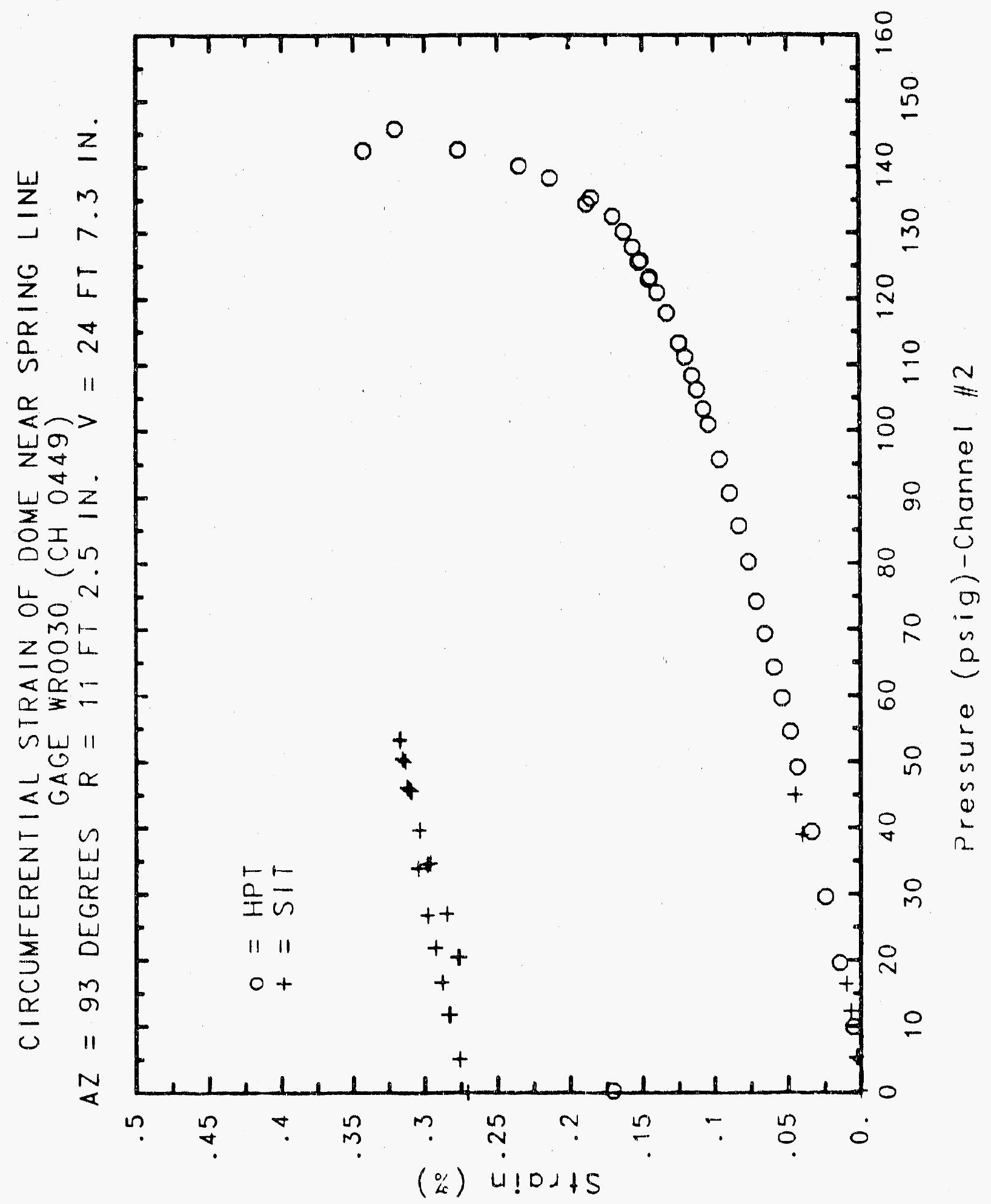




\section{Reinforced Concrete Test Data}

Weldable gage 31 Channel 460

Structural Integrity Test

High Pressure test

\begin{tabular}{|c|c|c|c|}
\hline $\begin{array}{c}\text { Pressure } \\
\text { (psig) } \\
-0.05 \\
5.33 \\
12.31 \\
16.44 \\
20.51 \\
20.46 \\
20.47 \\
27.05 \\
34.69 \\
34.53 \\
34.53 \\
39.70 \\
45.90 \\
45.65 \\
45.62 \\
50.10 \\
53.47 \\
53.29 \\
53.21 \\
50.49 \\
46.14 \\
46.13 \\
44.97 \\
38.98 \\
33.99 \\
33.96 \\
26.83 \\
21.88 \\
21.90 \\
16.69 \\
11.74 \\
11.77 \\
5.05 \\
0.02 \\
-0.04 \\
-0.02 \\
-0.02 \\
0.02\end{array}$ & $\begin{array}{l}\text { X Strain } \\
0.0003 \\
0.0028 \\
0.0064 \\
0.0087 \\
0.0119 \\
0.0125 \\
0.0129 \\
0.0197 \\
0.0292 \\
0.0308 \\
0.0306 \\
0.0355 \\
0.0421 \\
0.0414 \\
0.0414 \\
0.0451 \\
0.0485 \\
0.0488 \\
0.0488 \\
0.0475 \\
0.0449 \\
0.0445 \\
0.0453 \\
0.0407 \\
0.0374 \\
0.0369 \\
0.0315 \\
0.0272 \\
0.0267 \\
0.0228 \\
0.0189 \\
0.0182 \\
0.0122 \\
0.0083 \\
0.0081 \\
0.0082 \\
0.0084 \\
0.0081\end{array}$ & $\begin{array}{c}\text { Pressure } \\
\text { (psig) } \\
9.89 \\
19.55 \\
29.57 \\
39.42 \\
49.16 \\
54.50 \\
59.57 \\
64.20 \\
69.32 \\
74.16 \\
80.16 \\
85.61 \\
90.58 \\
95.69 \\
100.92 \\
103.25 \\
106.11 \\
108.31 \\
111.08 \\
113.24 \\
117.83 \\
120.92 \\
123.28 \\
122.97 \\
125.82 \\
125.60 \\
127.84 \\
130.19 \\
132.53 \\
135.33 \\
134.42 \\
138.35 \\
140.16 \\
142.63 \\
145.78 \\
142.52 \\
0.22 \\
\end{array}$ & $\begin{array}{l}\text { \% Strain } \\
0.0040 \\
0.0122 \\
0.0206 \\
0.0286 \\
0.0364 \\
0.0411 \\
0.0454 \\
0.0502 \\
0.0550 \\
0.0600 \\
0.0651 \\
0.0703 \\
0.0749 \\
0.0798 \\
0.0852 \\
0.0880 \\
0.0908 \\
0.0929 \\
0.0964 \\
0.0993 \\
0.1047 \\
0.1084 \\
0.1122 \\
0.1124 \\
0.1155 \\
0.1165 \\
0.1188 \\
0.1220 \\
0.1255 \\
0.1314 \\
0.1320 \\
0.1414 \\
0.1486 \\
0.1599 \\
0.1710 \\
0.1720 \\
0.0345\end{array}$ \\
\hline
\end{tabular}




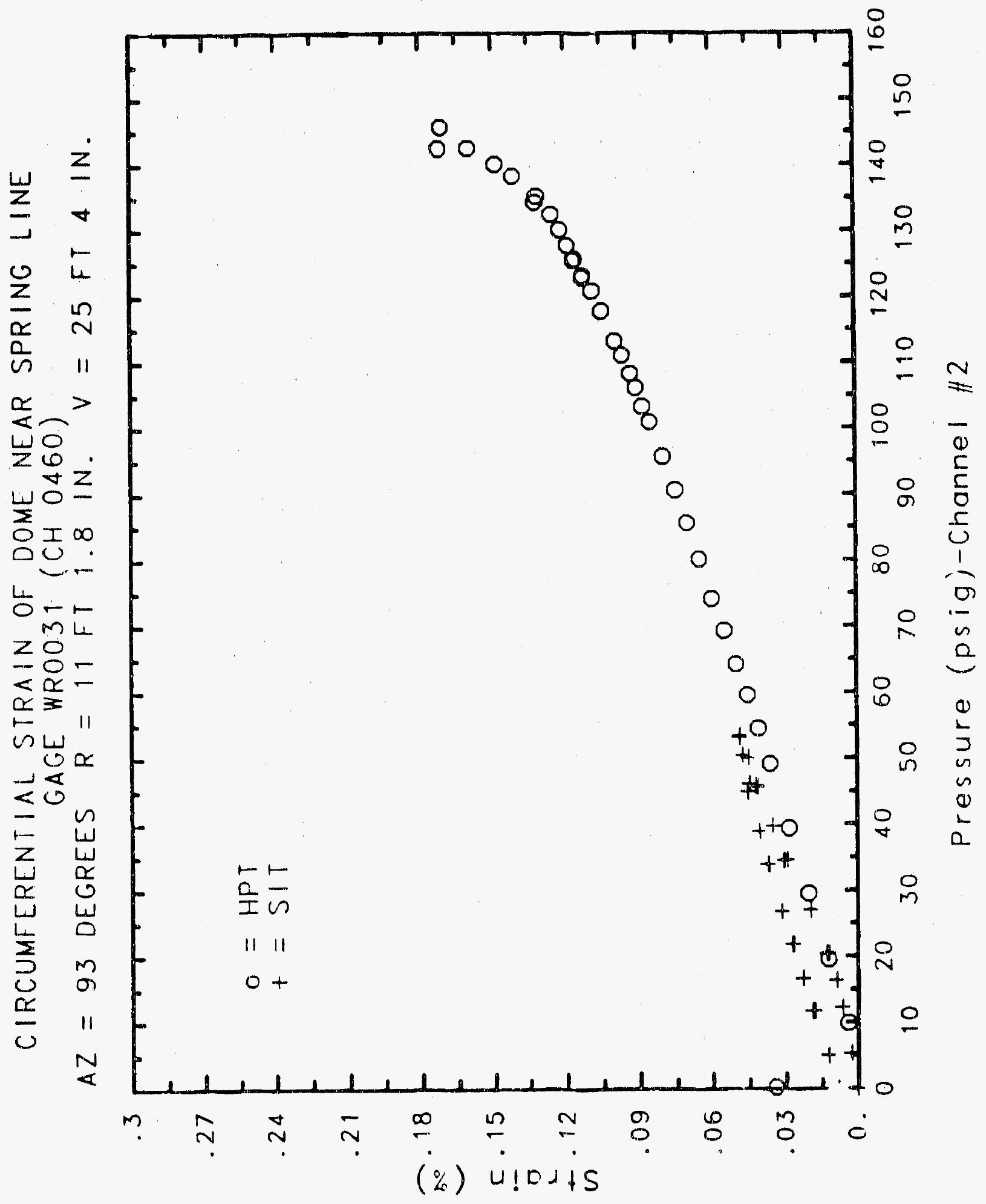




\section{Reinforced Concrete Test Data}

Weldable gage 32 Chanmel 461

Structural IntEgrity TEST

\begin{tabular}{|c|c|c|c|}
\hline $\begin{array}{c}\text { Pressure } \\
\text { (psig) } \\
-0.05 \\
5.33 \\
12.31 \\
16.44 \\
20.51 \\
20.46 \\
20.47 \\
27.05 \\
34.69 \\
34.53 \\
34.53 \\
39.70 \\
45.90 \\
45.65 \\
45.62 \\
50.10 \\
53.47 \\
53.29 \\
53.21 \\
50.49 \\
46.14 \\
46.13 \\
44.97 \\
38.98 \\
33.99 \\
33.96 \\
26.83 \\
21.88 \\
21.90 \\
16.69 \\
11.74 \\
11.77 \\
5.05 \\
0.02 \\
-0.04 \\
-0.02 \\
-0.02 \\
0.02 \\
\end{array}$ & $\begin{array}{l}\text { X Strain } \\
-0.0003 \\
0.0014 \\
0.0037 \\
-0.0802 \\
-0.0772 \\
-0.0766 \\
-0.0761 \\
-0.0694 \\
-0.0599 \\
-0.0588 \\
-0.0585 \\
-0.0534 \\
-0.0473 \\
-0.0479 \\
-0.0475 \\
-0.0440 \\
-0.0404 \\
-0.0404 \\
-0.0404 \\
-0.0416 \\
-0.0446 \\
-0.0445 \\
0.0477 \\
-0.0478 \\
-0.0520 \\
-0.0519 \\
-0.0579 \\
-0.0618 \\
-0.0625 \\
.0 .0666 \\
-0.0703 \\
-0.0713 \\
-0.0769 \\
-0.0806 \\
-0.0812 \\
-0.0807 \\
-0.0806 \\
-0.0809\end{array}$ & $\begin{array}{c}\text { Pressure } \\
(\text { psig) } \\
9.89 \\
19.55 \\
29.57 \\
39.42 \\
49.16 \\
54.50 \\
59.57 \\
64.20 \\
69.32 \\
71.16 \\
80.16 \\
85.61 \\
90.58 \\
95.69 \\
100.92 \\
103.25 \\
106.11 \\
108.31 \\
111.08 \\
113.24 \\
117.83 \\
120.92 \\
123.28 \\
122.97 \\
125.82 \\
125.60 \\
127.84 \\
130.19 \\
132.53 \\
135.33 \\
134.42 \\
138.35 \\
140.16 \\
142.63 \\
145.78 \\
142.52 \\
0.22\end{array}$ & $\begin{array}{l}\text { \% Strain } \\
0.0039 \\
0.0118 \\
0.0207 \\
0.0282 \\
0.0363 \\
0.0406 \\
0.0454 \\
0.0497 \\
0.0546 \\
0.0597 \\
0.0646 \\
0.0698 \\
0.0747 \\
0.0793 \\
0.0849 \\
0.0874 \\
0.0903 \\
0.0928 \\
0.0961 \\
0.0989 \\
0.1044 \\
0.1084 \\
0.1116 \\
0.1119 \\
0.1153 \\
0.1160 \\
0.1183 \\
0.1215 \\
0.1250 \\
0.1309 \\
0.1316 \\
0.1409 \\
0.1484 \\
0.1594 \\
0.1713 \\
0.1716 \\
0.0345\end{array}$ \\
\hline
\end{tabular}

High Pressure test

Pressure

0.89

39.42

49.16

59.57

64.20

69.32

79.16

90.58

100.92

103.25

106.11

113.24

123.28

122.97

125.82

125.60

127.84

132.53

135.33

134.42

138.35

140.16

145.78

42.52

0.22
0.0039

0.0118

0.0207

0.0282

0.0454

0.0497

0.0546

0.0597

0.0747

0.0793

0.0849

0.0874

0.0961

0.1044

0.1084

0.1116

0.1160

0.1215

0.1250

0.1309

0.1484

0.1716

0.0345 


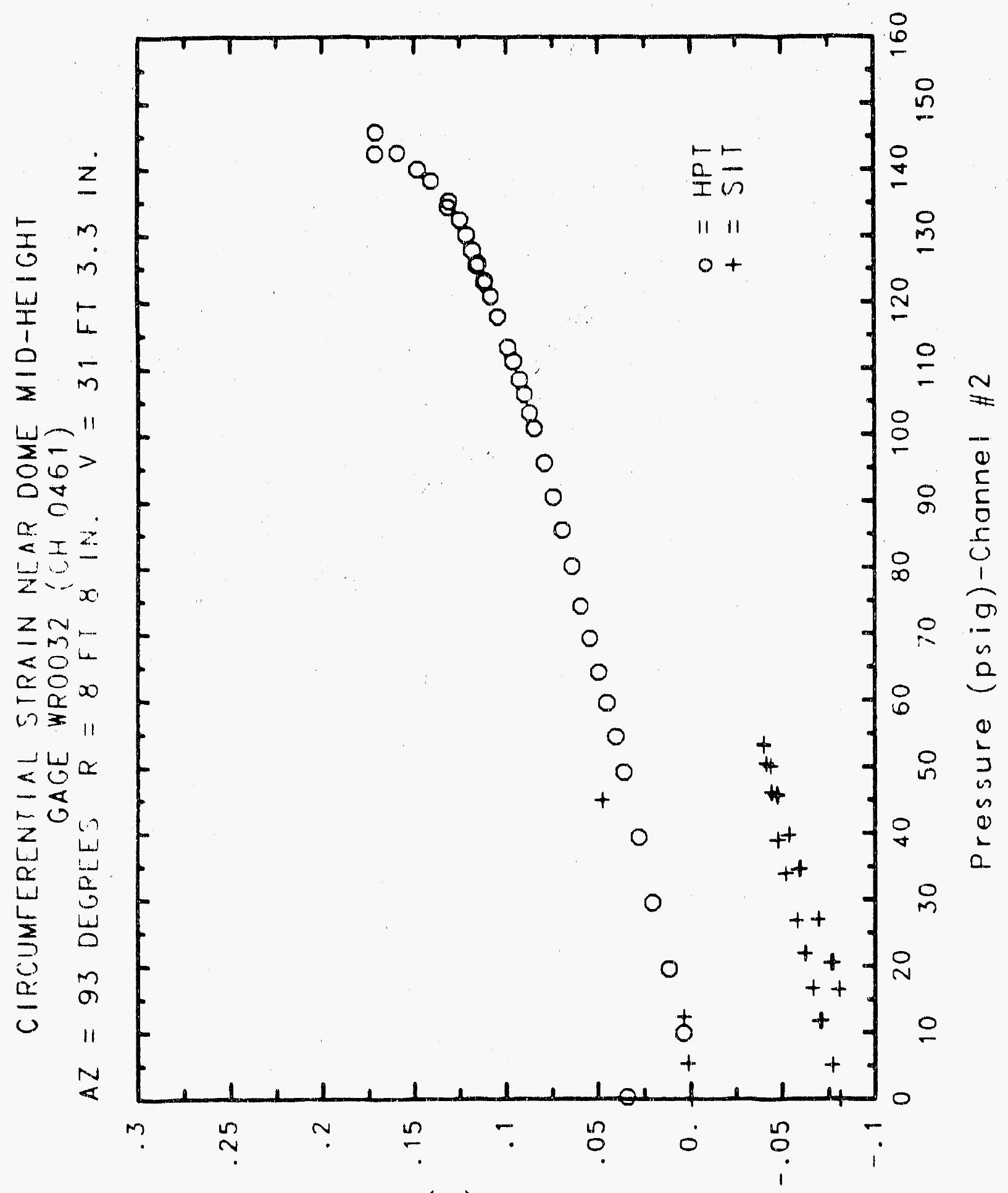

(a) $4 ! 0 \lambda+S$ 


\section{Reinforced Concrete Test Data}

Weldable gage 33 Channel 462

Structural INTEgRITY TEST

High Pressure test

\begin{tabular}{|c|c|c|c|}
\hline $\begin{array}{c}\text { Pressure } \\
\text { (psig) } \\
-0.05 \\
5.33 \\
12.31 \\
16.44 \\
20.51 \\
20.46 \\
20.47 \\
27.05 \\
34.69 \\
34.53 \\
34.53 \\
39.70 \\
45.90 \\
45.65 \\
45.62 \\
50.10 \\
53.47 \\
53.29 \\
53.21 \\
50.49 \\
46.14 \\
46.13 \\
44.97 \\
38.98 \\
33.99 \\
33.96 \\
26.83 \\
21.88 \\
21.90 \\
16.69 \\
11.74 \\
11.77 \\
5.05 \\
0.02 \\
-0.04 \\
-0.02 \\
-0.02 \\
0.02 \\
\end{array}$ & $\begin{array}{c}\text { \% Strain } \\
\text {-0.0006 } \\
0.0028 \\
0.0071 \\
0.0099 \\
0.0135 \\
0.0136 \\
0.0142 \\
0.0209 \\
0.0287 \\
0.0301 \\
0.0300 \\
0.0350 \\
0.0410 \\
0.0408 \\
0.0409 \\
0.0450 \\
0.0485 \\
0.0484 \\
0.0486 \\
0.0470 \\
0.0439 \\
0.0435 \\
0.0434 \\
0.0392 \\
0.0349 \\
0.0350 \\
0.0285 \\
0.0241 \\
0.0243 \\
0.0198 \\
0.0152 \\
0.0144 \\
0.0084 \\
0.0033 \\
0.0018 \\
0.0024 \\
0.0027 \\
0.0027\end{array}$ & $\begin{array}{c}\text { Pressure } \\
(\text { psig) } \\
9.89 \\
19.55 \\
29.57 \\
39.42 \\
49.16 \\
54.50 \\
59.57 \\
64.20 \\
69.32 \\
74.16 \\
80.16 \\
85.61 \\
90.58 \\
95.69 \\
100.92 \\
103.25 \\
106.11 \\
108.31 \\
111.08 \\
113.24 \\
117.83 \\
120.92 \\
123.28 \\
122.97 \\
125.82 \\
125.60 \\
127.84 \\
130.19 \\
132.53 \\
135.33 \\
134.42 \\
138.35 \\
140.16 \\
142.63 \\
145.78 \\
142.52 \\
0.22\end{array}$ & $\begin{array}{l}\text { * Strain } \\
0.0051 \\
0.0151 \\
0.0244 \\
0.0335 \\
0.0420 \\
0.0468 \\
0.0515 \\
0.0564 \\
0.0617 \\
0.0672 \\
0.0737 \\
0.0799 \\
0.0858 \\
0.0922 \\
0.0986 \\
0.1019 \\
0.1052 \\
0.1075 \\
0.1105 \\
0.1133 \\
0.1180 \\
0.1217 \\
0.1247 \\
0.1251 \\
0.1284 \\
0.1289 \\
0.1316 \\
0.1363 \\
0.1426 \\
0.1500 \\
0.1509 \\
0.1602 \\
0.1695 \\
0.1844 \\
0.1965 \\
0.1975 \\
0.0416\end{array}$ \\
\hline
\end{tabular}




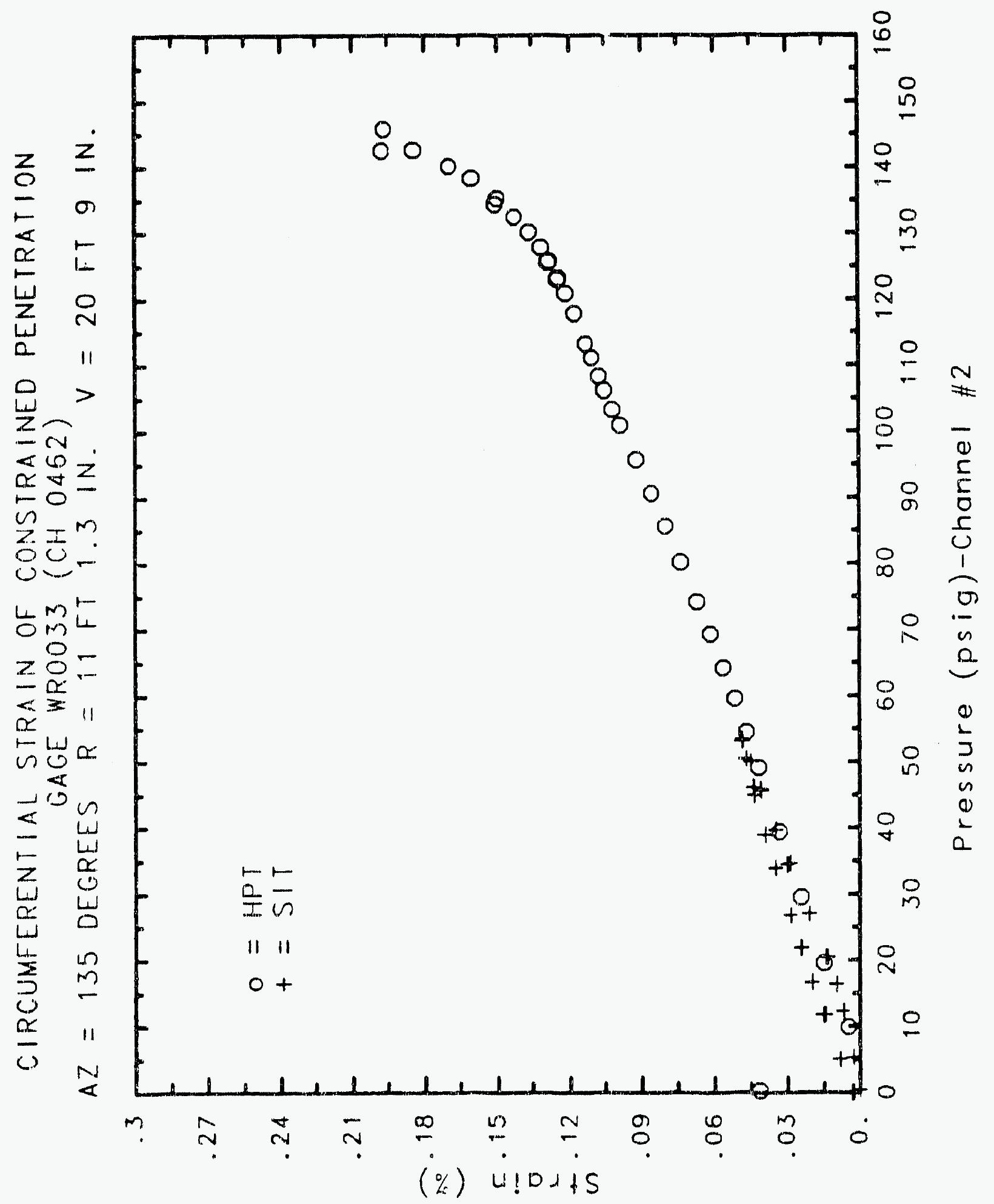




\section{Reinforced Concrete Test Data}

Weldable gage 34 Channel 463

StRUCTURAL InTEgrity TEST

\begin{tabular}{|c|c|c|c|}
\hline $\begin{array}{c}\text { Pressure } \\
\text { (psig) } \\
-0.05 \\
5.33 \\
12.31 \\
16.44 \\
20.51 \\
20.46 \\
20.47 \\
27.05 \\
34.69 \\
34.53 \\
34.53 \\
39.70 \\
45.90 \\
45.65 \\
45.62 \\
50.10 \\
53.47 \\
53.29 \\
53.21 \\
50.49 \\
46.14 \\
46.13 \\
44.97 \\
38.98 \\
33.99 \\
33.96 \\
26.83 \\
21.88 \\
21.90 \\
16.69 \\
11.74 \\
11.77 \\
5.05 \\
0.02 \\
-0.04 \\
-0.02 \\
-0.02 \\
0.02\end{array}$ & $\begin{array}{l}\text { Strain } \\
0.0005 \\
0.0030 \\
0.0054 \\
0.0074 \\
0.0100 \\
0.0106 \\
0.0108 \\
0.0160 \\
0.0231 \\
0.0244 \\
0.0248 \\
0.0294 \\
0.0359 \\
0.0360 \\
0.0363 \\
0.0397 \\
0.0425 \\
0.0427 \\
0.0431 \\
0.0417 \\
0.0392 \\
0.0389 \\
0.0390 \\
0.0356 \\
0.0323 \\
0.0324 \\
0.0279 \\
0.0245 \\
0.0247 \\
0.0215 \\
0.0178 \\
0.0172 \\
0.0125 \\
0.0088 \\
0.0062 \\
0.0068 \\
0.0067 \\
0.0074\end{array}$ & $\begin{array}{c}\text { Pressure } \\
(\text { psig) } \\
9.89 \\
19.55 \\
29.57 \\
39.42 \\
49.16 \\
54.50 \\
59.57 \\
64.20 \\
69.32 \\
74.16 \\
80.16 \\
85.61 \\
90.58 \\
95.69 \\
100.92 \\
103.25 \\
106.11 \\
108.31 \\
111.08 \\
113.24 \\
117.83 \\
120.92 \\
123.28 \\
122.97 \\
125.82 \\
125.60 \\
127.84 \\
130.19 \\
132.53 \\
135.33 \\
134.42 \\
138.35 \\
140.16 \\
142.63 \\
145.78 \\
142.52 \\
0.22 \\
\end{array}$ & $\begin{array}{l}\text { \% Strain } \\
0.0032 \\
0.0110 \\
0.0193 \\
0.0270 \\
0.0350 \\
0.0390 \\
0.0438 \\
0.0485 \\
0.0542 \\
0.0597 \\
0.0659 \\
0.0721 \\
0.0780 \\
0.0846 \\
0.0911 \\
0.0948 \\
0.1982 \\
0.1008 \\
0.1045 \\
0.1070 \\
0.1125 \\
0.1174 \\
0.1209 \\
0.1216 \\
0.1263 \\
0.1270 \\
0.1301 \\
0.1354 \\
0.1426 \\
0.1512 \\
0.1525 \\
0.1641 \\
0.1747 \\
0.1888 \\
0.1996 \\
0.1992 \\
0.0380\end{array}$ \\
\hline
\end{tabular}

High Pressure test

9.89

19.55

39.42

49.16

(54.

69.32

85.61

90.58

100.92

103.25

106.11

11.08

113.24

117.83

120.92

123.28

125.82

125.60

127.84

135.33

134.42

45.78

2.52

0.1992

0.0380 


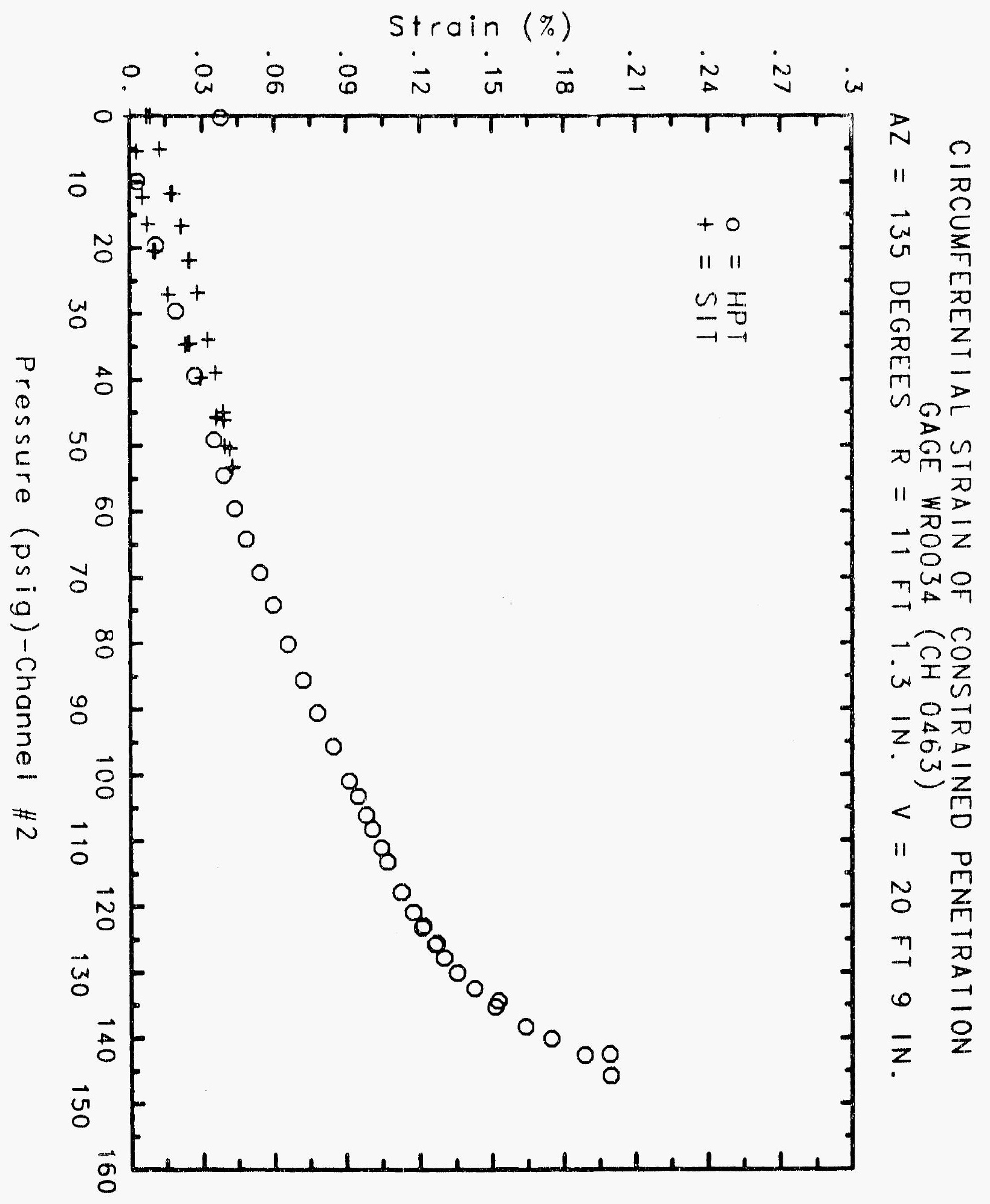




\section{Reinforced Concrete Test Data}

Weldable gage 35 Channel 464

Structural Integrity Test

\begin{tabular}{c} 
Pressure \\
\hline psig) \\
-0.05 \\
5.33 \\
12.31 \\
16.44 \\
20.51 \\
20.46 \\
20.47 \\
27.05 \\
34.69 \\
34.53 \\
34.53 \\
39.70 \\
45.90 \\
45.65 \\
45.62 \\
50.10 \\
53.47 \\
53.29 \\
53.21 \\
50.49 \\
46.14 \\
46.13 \\
44.97 \\
38.98 \\
33.99 \\
33.96 \\
26.83 \\
21.88 \\
21.90 \\
16.69 \\
11.74 \\
11.77 \\
5.05 \\
0.02 \\
-0.04 \\
-0.02 \\
-0.02 \\
0.02 \\
\end{tabular}

* Strain

0.0001

0.0013

0.0036

0.0062

0.0113

0.0129

0.0134

0.0234

0.0351

0.0364

0.0368

0.0423

0.0504

0.0506

0.0507

0.0556

0.0594

0.0597

0.0600

0.0587

0.0557

0.0556

0.0568

0.0528

0.0486

0.0480

0.0419

0.0376

0.0371

0.0326

0.0282

0.0276

0.0217

0.0173

0.0160

0.0161

0.0165

0.0165
High Pressure Test

Pressure

(psig)
9.89

19.55

29.57

39.42

49.16

54.50

59.57

64.20

69.32

74.16

80.16

85.61

90.58

95.69

100.92

103.25

106.11

108.31

111.08

113.24

117.83

120.92

123.28

122.97

125.82

125.60

127.84

130.19

132.53

135.33

134.42

138.35

140.16

142.63

145.78

142.52

0.22
\% Strain

0.0043

0.0139

0.0229

0.0326

0.0410

0.0461

0.0514

0.0568

0.0629

0.0691

0.0763

0.0836

0.0903

0.0979

0.1080

0.1098

0.1140

0.1173

0.1226

0.1260

0.1342 .

0.1401

0.1461

0.1472

0.1539

0.1550

0.1588

0.1667

0.1780

0.1974

0.2009

0.2264

0.2428

0.2602

0.3807

0.4105

0.2304 


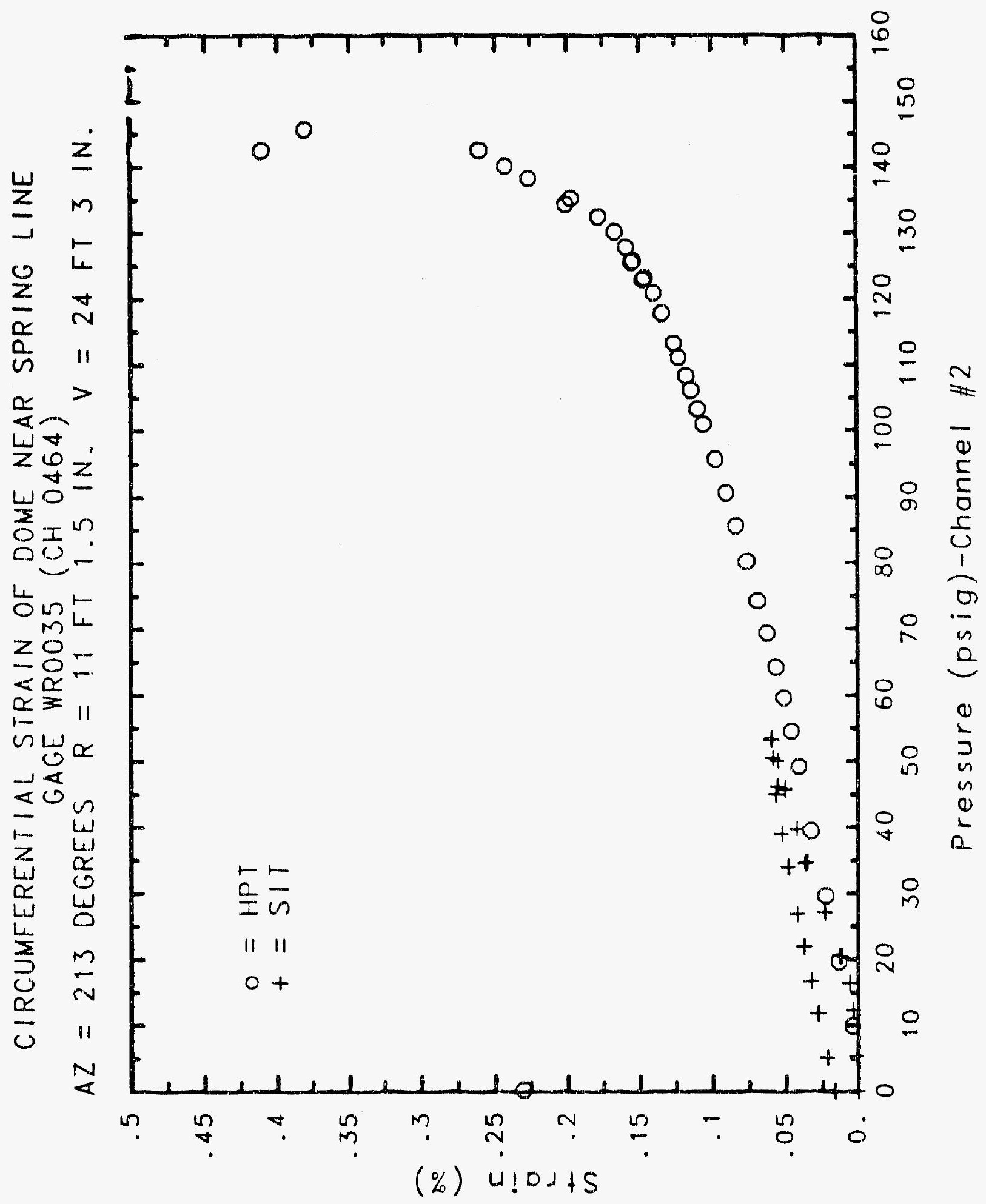

C- 884 


\section{Reinforced Concrete Test Data}

Weldable gage 36 Channel 465

Structural Integrity Test

High Pressure test

\begin{tabular}{|c|c|c|c|}
\hline $\begin{array}{c}\text { Pressure } \\
\text { (psig) } \\
-0.05 \\
5.33 \\
12.31 \\
16.44 \\
20.51 \\
20.46 \\
20.47 \\
27.05 \\
34.69 \\
34.53 \\
34.53 \\
39.70 \\
45.90 \\
45.65 \\
45.62 \\
50.10 \\
53.47 \\
53.29 \\
53.21 \\
50.49 \\
46.14 \\
46.13 \\
44.97 \\
38.98 \\
33.99 \\
33.96 \\
26.83 \\
21.88 \\
21.90 \\
16.69 \\
11.74 \\
11.77 \\
5.05 \\
0.02 \\
-0.04 \\
-0.02 \\
-0.02 \\
0.02 \\
\end{array}$ & $\begin{array}{l}\text { \% Strain } \\
0.0001 \\
0.0032 \\
0.0090 \\
0.2812 \\
0.2868 \\
0.2879 \\
0.2886 \\
0.2990 \\
0.3102 \\
0.3116 \\
0.3120 \\
0.3174 \\
0.3256 \\
0.3259 \\
0.3258 \\
0.3305 \\
0.3348 \\
0.3352 \\
0.3355 \\
0.3342 \\
0.3311 \\
0.3309 \\
0.0847 \\
0.3278 \\
0.3237 \\
0.3233 \\
0.3173 \\
0.3126 \\
0.3126 \\
0.3079 \\
0.3033 \\
0.3027 \\
0.2966 \\
0.2922 \\
0.2918 \\
0.2915 \\
0.2914 \\
0.2916\end{array}$ & $\begin{array}{c}\text { Pressure } \\
\text { (psig) } \\
9.89 \\
19.55 \\
29.57 \\
39.42 \\
49.16 \\
54.50 \\
59.57 \\
64.20 \\
69.32 \\
74.16 \\
80.16 \\
85.61 \\
90.58 \\
95.69 \\
100.92 \\
103.25 \\
106.11 \\
108.31 \\
111.08 \\
113.24 \\
117.83 \\
120.92 \\
123.28 \\
122.97 \\
125.82 \\
125.60 \\
127.84 \\
130.19 \\
132.53 \\
135.33 \\
134.42 \\
138.35 \\
140.16 \\
142.63 \\
145.78 \\
142.52 \\
0.22 \\
\end{array}$ & $\begin{array}{l}\text { Strain } \\
0.0048 \\
0.0140 \\
0.0237 \\
0.0326 \\
0.0413 \\
0.0463 \\
0.0518 \\
0.0572 \\
0.0635 \\
0.0694 \\
0.0766 \\
0.0838 \\
0.0906 \\
0.0980 \\
0.1061 \\
0.1100 \\
0.1144 \\
0.1178 \\
0.1226 \\
0.1264 \\
0.1347 \\
0.1408 \\
0.1462 \\
0.1476 \\
0.1540 \\
0.1553 \\
0.1592 \\
0.1665 \\
0.1785 \\
0.1975 \\
0.2015 \\
0.2267 \\
0.2427 \\
0.2603 \\
0.3816 \\
0.4106 \\
0.2310\end{array}$ \\
\hline
\end{tabular}




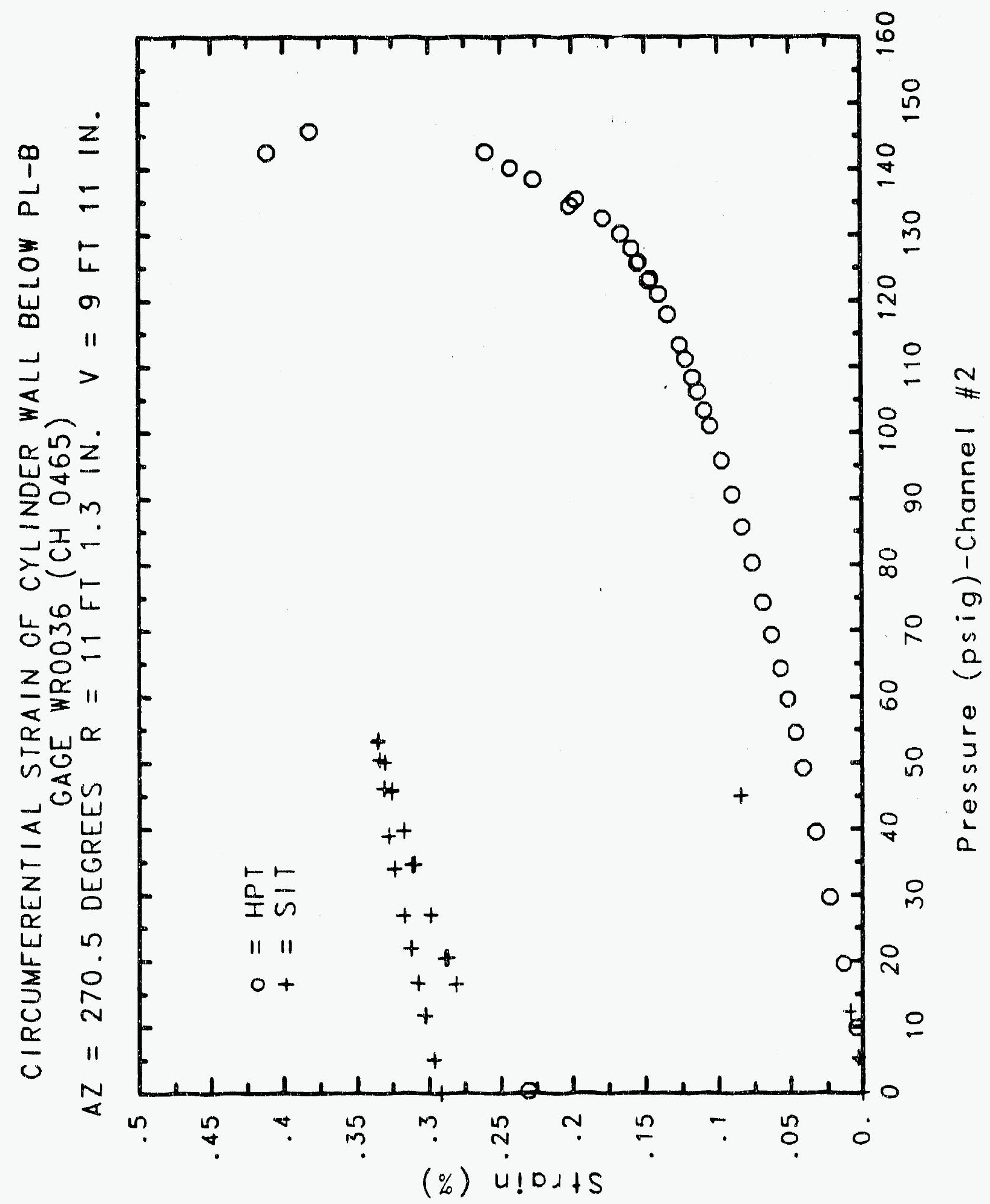




\section{Reinforced Concrete Test Data}

Weldable gage 37 Chaminel 466

Structural Integrity Test

High Pressure test

\begin{tabular}{|c|c|c|c|}
\hline $\begin{array}{c}\text { Pressure } \\
\text { (psig) } \\
-0.05 \\
5.33 \\
12.31 \\
16.44 \\
20.51 \\
20.46 \\
20.47 \\
27.05 \\
34.69 \\
34.53 \\
34.53 \\
39.70 \\
45.90 \\
45.65 \\
45.62 \\
50.10 \\
53.47 \\
53.29 \\
53.21 \\
50.49 \\
46.14 \\
46.13 \\
44.97 \\
38.98 \\
33.99 \\
33.96 \\
26.83 \\
21.88 \\
21.90 \\
16.69 \\
11.74 \\
11.77 \\
5.05 \\
0.02 \\
-0.04 \\
-0.02 \\
-0.02 \\
0.02 \\
\end{array}$ & $\begin{array}{c}\text { \% Strain } \\
-0.0009 \\
0.0032 \\
0.0081 \\
0.0118 \\
0.0187 \\
0.0202 \\
0.0207 \\
0.0347 \\
0.0522 \\
0.0536 \\
0.0540 \\
0.0627 \\
0.0761 \\
0.0774 \\
0.0776 \\
0.0858 \\
0.0934 \\
0.0943 \\
0.0947 \\
0.0919 \\
0.0867 \\
0.0864 \\
0.0881 \\
0.0803 \\
0.0734 \\
0.0726 \\
0.0622 \\
0.0548 \\
0.0542 \\
0.0465 \\
0.0388 \\
0.0382 \\
0.0285 \\
0.0213 \\
0.0199 \\
0.0195 \\
0.0191 \\
0.0189\end{array}$ & $\begin{array}{c}\text { Pressure } \\
(\text { psig) } \\
9.89 \\
19.55 \\
29.57 \\
39.42 \\
49.16 \\
54.50 \\
59.57 \\
64.20 \\
69.32 \\
74.16 \\
80.16 \\
85.61 \\
90.58 \\
95.69 \\
100.92 \\
103.25 \\
106.11 \\
108.31 \\
111.08 \\
113.24 \\
117.83 \\
120.92 \\
123.28 \\
122.97 \\
125.82 \\
125.60 \\
127.84 \\
130.19 \\
132.53 \\
135.33 \\
134.42 \\
138.35 \\
140.16 \\
142.63 \\
145.78 \\
142.52 \\
0.22\end{array}$ & $\begin{array}{l}\text { \% Strain } \\
0.0106 \\
0.0255 \\
0.0409 \\
0.0559 \\
0.0709 \\
0.0801 \\
0.0898 \\
0.1007 \\
0.1133 \\
0.1269 \\
0.1456 \\
0.1648 \\
0.1855 \\
0.2097 \\
0.2359 \\
0.2493 \\
0.2632 \\
0.2746 \\
0.2918 \\
0.3058 \\
0.3405 \\
0.3675 \\
0.3936 \\
0.4009 \\
0.4294 \\
0.4402 \\
0.4596 \\
0.5009 \\
0.5656 \\
0.6625 \\
0.6889 \\
0.7970 \\
0.8875 \\
1.0151 \\
1.1381 \\
1.1793 \\
0.8196\end{array}$ \\
\hline
\end{tabular}




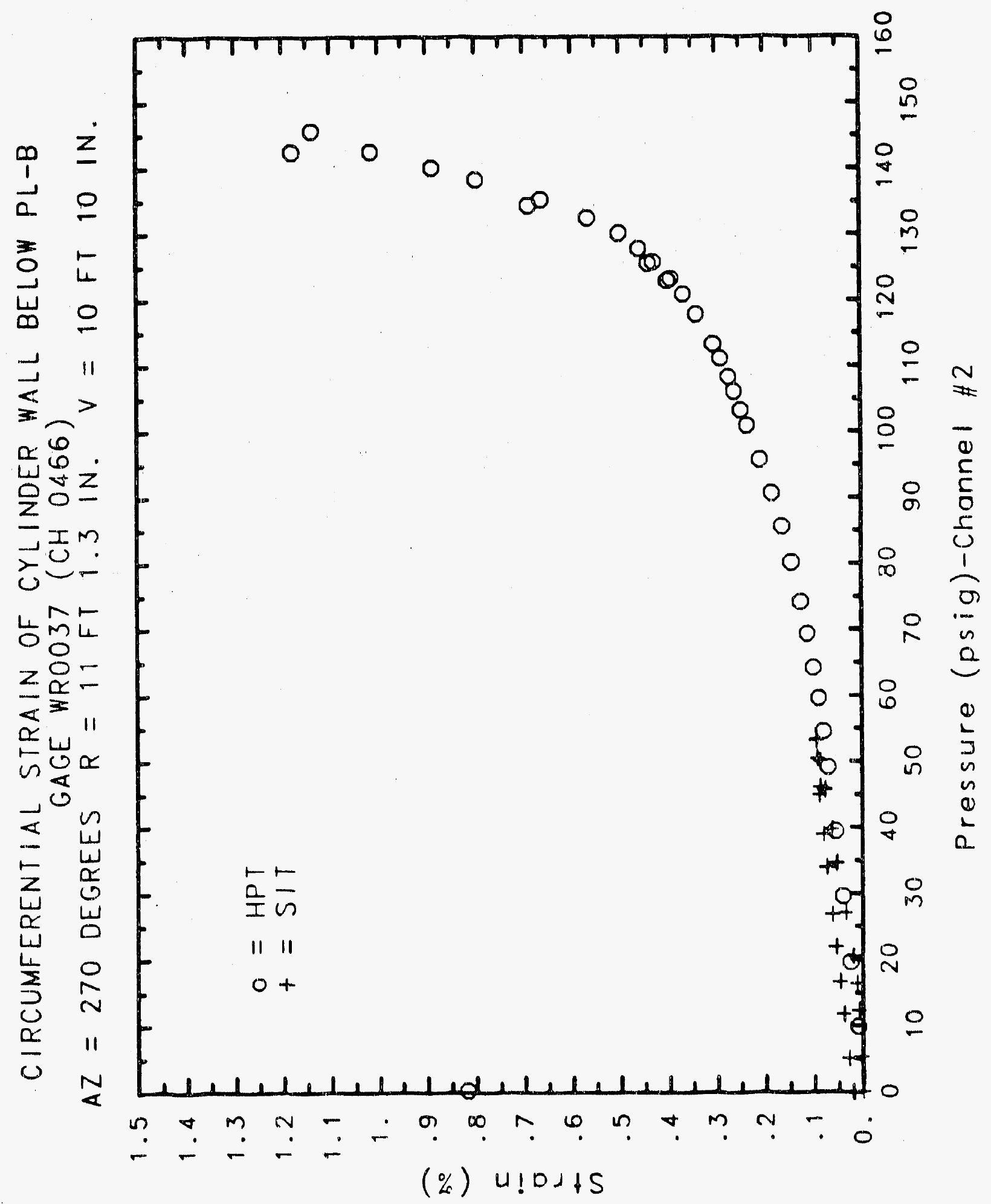




\section{Reinforced Concrete Test Data}

Weldable gage 38 Chammel 467

StRUCTURAL INTEgRITY TEST

High Pressure Test

\begin{tabular}{|c|c|c|c|}
\hline $\begin{array}{c}\text { Pressure } \\
\text { (psig) } \\
-0.05 \\
5.33 \\
12.31 \\
16.44 \\
20.51 \\
20.46 \\
20.47 \\
27.05 \\
34.69 \\
34.53 \\
34.53 \\
39.70 \\
45.90 \\
45.65 \\
45.62 \\
50.10 \\
53.47 \\
53.29 \\
53.21 \\
50.49 \\
46.14 \\
46.13 \\
44.97 \\
38.98 \\
33.99 \\
33.96 \\
26.83 \\
21.88 \\
21.90 \\
16.69 \\
11.74 \\
11.77 \\
5.05 \\
0.02 \\
-0.04 \\
-0.02 \\
-0.02 \\
0.02 \\
\end{array}$ & $\begin{array}{c}\text { X Strain } \\
\text {-0.0011 } \\
0.0027 \\
0.0073 \\
0.0107 \\
0.0153 \\
0.0157 \\
0.0157 \\
0.0232 \\
0.0328 \\
0.0331 \\
0.0333 \\
0.0389 \\
0.0480 \\
0.0486 \\
0.0486 \\
0.0540 \\
0.0591 \\
0.0596 \\
0.0594 \\
0.0577 \\
0.0539 \\
0.0541 \\
0.0554 \\
0.0498 \\
0.0450 \\
0.0447 \\
0.0375 \\
0.0319 \\
0.0316 \\
0.0259 \\
0.0203 \\
0.0192 \\
0.0122 \\
0.0064 \\
0.0054 \\
0.0054 \\
0.0053 \\
0.0050\end{array}$ & $\begin{array}{c}\text { Pressure } \\
(\text { psig) } \\
9.89 \\
19.55 \\
29.57 \\
39.42 \\
49.16 \\
54.50 \\
59.57 \\
64.20 \\
69.32 \\
74.16 \\
80.16 \\
85.61 \\
90.58 \\
95.69 \\
100.92 \\
103.25 \\
106.11 \\
108.31 \\
111.08 \\
113.24 \\
117.83 \\
120.92 \\
123.28 \\
122.97 \\
125.82 \\
125.60 \\
127.84 \\
130.19 \\
132.53 \\
135.33 \\
134.42 \\
138.35 \\
140.16 \\
142.63 \\
145.78 \\
142.52 \\
0.22\end{array}$ & $\begin{array}{l}\text { \% Strain } \\
0.0059 \\
0.0171 \\
0.0284 \\
0.0391 \\
0.0493 \\
0.0551 \\
0.0620 \\
0.0696 \\
0.0784 \\
0.0877 \\
0.0980 \\
0.1074 \\
0.1160 \\
0.1253 \\
0.1357 \\
0.1413 \\
0.1468 \\
0.1514 \\
0.1583 \\
0.1639 \\
0.1769 \\
0.1877 \\
0.1974 \\
0.1997 \\
0.2103 \\
0.2141 \\
0.2205 \\
0.2334 \\
0.2476 \\
0.2648 \\
0.2684 \\
0.2868 \\
0.3016 \\
0.3216 \\
0.3465 \\
0.3564 \\
0.1604\end{array}$ \\
\hline
\end{tabular}




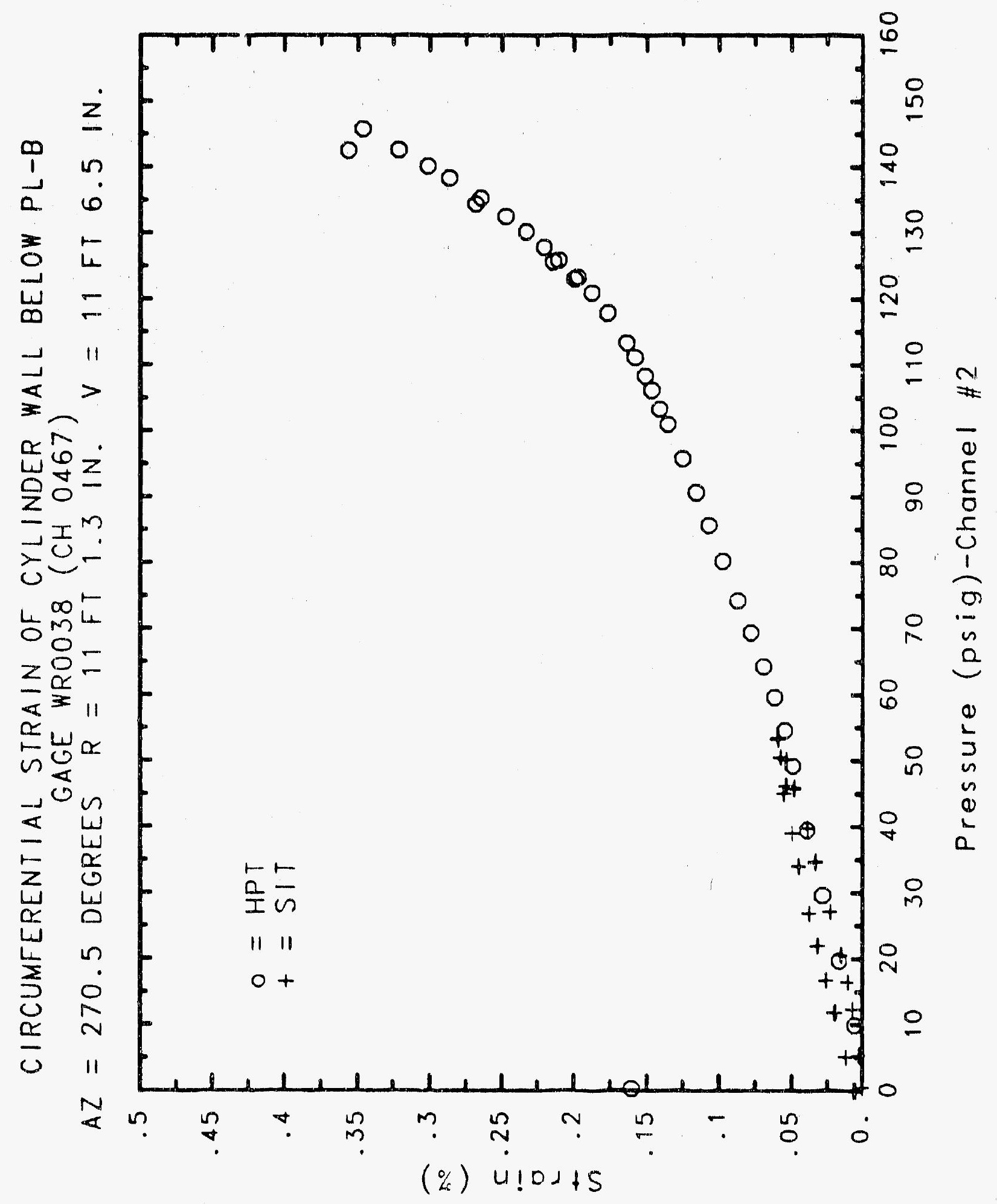




\section{Reinforced Concrete Test Data}

Weldable Gage 39 Channel 468

Structural Integrity Test

\begin{tabular}{|c|c|c|c|}
\hline $\begin{array}{c}\text { Pressure } \\
\text { (psig) } \\
-0.05 \\
5.33 \\
12.31 \\
16.44 \\
20.51 \\
20.46 \\
20.47 \\
27.05 \\
34.69 \\
34.53 \\
34.53 \\
39.70 \\
45.90 \\
45.65 \\
45.62 \\
50.10 \\
53.47 \\
53.29 \\
53.21 \\
50.49 \\
46.14 \\
46.13 \\
44.97 \\
38.98 \\
33.99 \\
33.96 \\
26.83 \\
21.88 \\
21.90 \\
16.69 \\
11.74 \\
11.77 \\
5.05 \\
0.02 \\
-0.04 \\
-0.02 \\
-0.02 \\
0.02 \\
\end{array}$ & $\begin{array}{c}\text { \% strain } \\
-0.0002 \\
0.0040 \\
0.0091 \\
0.0129 \\
0.0182 \\
0.0189 \\
0.0190 \\
0.0268 \\
0.0348 \\
0.0349 \\
0.0350 \\
0.0397 \\
0.0462 \\
0.0463 \\
0.0466 \\
0.0518 \\
0.0561 \\
0.0565 \\
0.0565 \\
0.0548 \\
0.0515 \\
0.0513 \\
0.0532 \\
0.0481 \\
0.0439 \\
0.0429 \\
0.0364 \\
0.0312 \\
0.0307 \\
0.0256 \\
0.0207 \\
0.0197 \\
0.0135 \\
0.0081 \\
0.0073 \\
0.0076 \\
0.0078 \\
0.0079\end{array}$ & $\begin{array}{c}\text { Pressure } \\
(\text { psig) } \\
9.89 \\
19.55 \\
29.57 \\
39.42 \\
49.16 \\
54.50 \\
59.57 \\
64.20 \\
69.32 \\
74.16 \\
80.16 \\
85.61 \\
90.58 \\
95.69 \\
100.92 \\
103.25 \\
106.11 \\
108.31 \\
111.08 \\
113.24 \\
117.83 \\
120.92 \\
123.28 \\
122.97 \\
125.82 \\
125.60 \\
127.84 \\
130.19 \\
132.53 \\
135.33 \\
134.42 \\
138.35 \\
140.16 \\
142.63 \\
145.78 \\
142.52 \\
0.22\end{array}$ & $\begin{array}{l}\text { \% Strain } \\
0.0056 \\
0.0146 \\
0.0244 \\
0.0338 \\
0.0432 \\
0.0486 \\
0.0542 \\
0.0606 \\
0.0676 \\
0.0748 \\
0.0841 \\
0.0924 \\
0.1006 \\
0.1106 \\
0.1210 \\
0.1269 \\
0.1323 \\
0.1363 \\
0.1429 \\
0.1485 \\
0.1610 \\
0.1705 \\
0.1800 \\
0.1822 \\
0.1919 \\
0.1951 \\
0.2015 \\
0.2126 \\
0.2248 \\
0.2405 \\
0.2448 \\
0.2607 \\
0.2722 \\
0.2871 \\
0.3015 \\
0.3056 \\
0.1221\end{array}$ \\
\hline
\end{tabular}

\section{high Pressure test}

Pressure

9.89

19.55

29.57

49.16

54.50

59.57

64.20

80.16

90.58

95.69

106.11

108.31

111.08

113.24

120.92

123.28

122.97

127.84

130.19

132.53

135.33

134.42

0.2607

0.2722

0.2871

0.3056

0.1221 


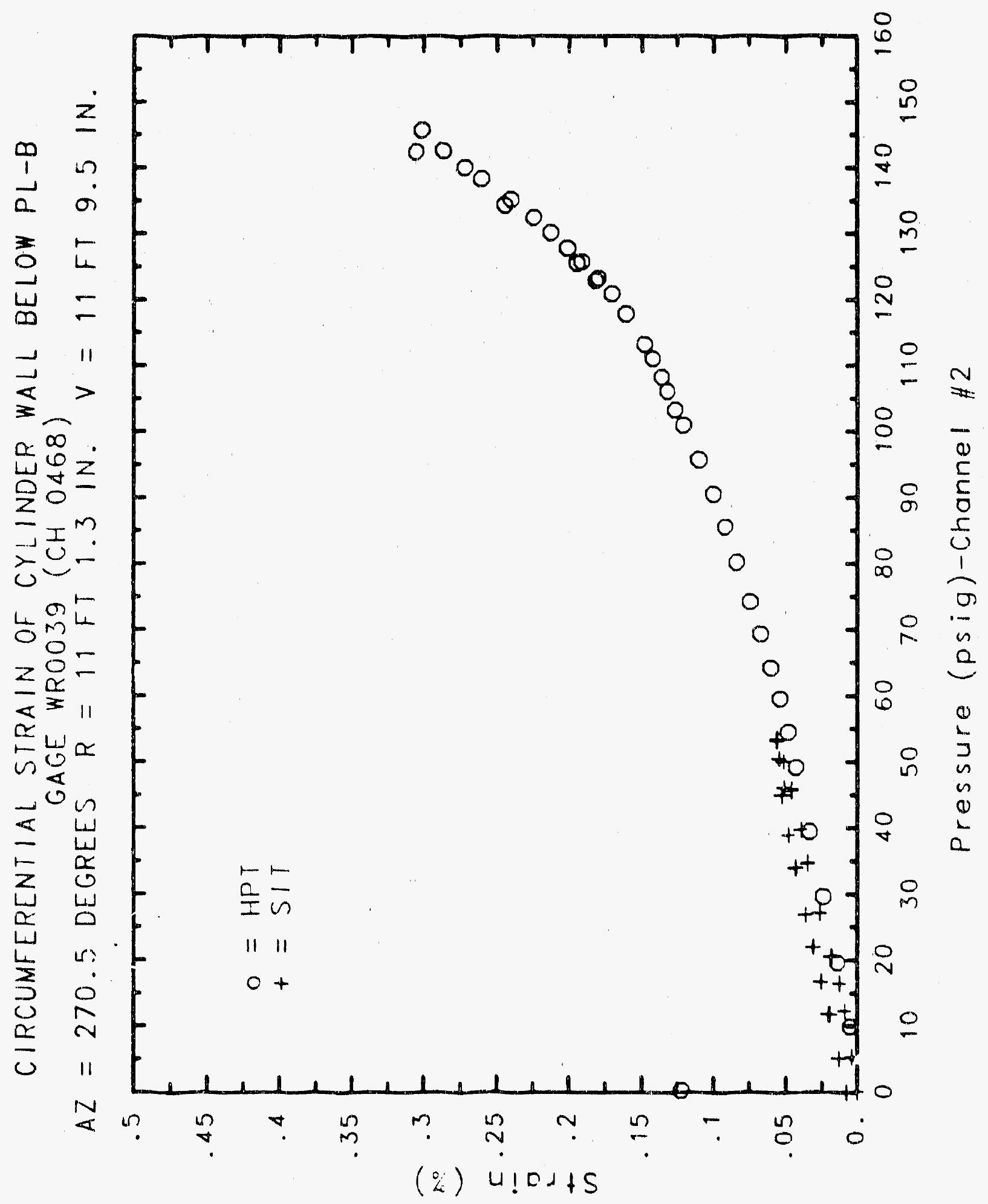




\section{Reinforced Concrete Test Data}

Weldable gage 40 Channel 469

Structural InTEgRITY TEST

\begin{tabular}{|c|c|c|c|}
\hline $\begin{array}{c}\text { Pressure } \\
(\text { psig) } \\
-0.05 \\
5.33 \\
12.31 \\
16.44 \\
20.51 \\
20.46 \\
20.47 \\
27.05 \\
34.69 \\
34.53 \\
34.53 \\
39.70 \\
45.90 \\
45.65 \\
45.62 \\
50.10 \\
53.47 \\
53.29 \\
53.21 \\
50.49 \\
46.14 \\
46.13 \\
44.97 \\
38.98 \\
33.99 \\
33.96 \\
26.83 \\
21.88 \\
21.90 \\
16.69 \\
11.74 \\
11.77 \\
5.05 \\
0.02 \\
-0.04 \\
-0.02 \\
-0.02 \\
0.02 \\
\end{array}$ & $\begin{array}{l}x \text { Strain } \\
0.0008 \\
0.0025 \\
0.0061 \\
0.0082 \\
-0.1828 \\
-0.1824 \\
-0.1821 \\
-0.1742 \\
-0.1667 \\
-0.1663 \\
-0.1661 \\
-0.1615 \\
-0.1550 \\
-0.1550 \\
-0.1546 \\
-0.1500 \\
-0.1451 \\
-0.1448 \\
-0.1449 \\
-0.1462 \\
-0.1498 \\
-0.1497 \\
0.0467 \\
0.0432 \\
-0.1577 \\
-0.1582 \\
-0.1647 \\
-0.1698 \\
-0.1704 \\
-0.1754 \\
-0.1805 \\
-0.1813 \\
-0.1879 \\
-0.1926 \\
-0.1939 \\
-0.1924 \\
-0.1927 \\
-0.1934\end{array}$ & $\begin{array}{c}\text { Pressure } \\
\text { (psig) } \\
9.89 \\
19.55 \\
29.57 \\
39.42 \\
49.16 \\
54.50 \\
59.57 \\
64.20 \\
69.32 \\
74.16 \\
80.16 \\
85.61 \\
90.58 \\
95.69 \\
100.92 \\
103.25 \\
106.11 \\
108.31 \\
111.08 \\
113.24 \\
117.83 \\
120.92 \\
123.28 \\
122.97 \\
125.82 \\
125.50 \\
127.84 \\
130.19 \\
132.53 \\
135.33 \\
134.42 \\
136.35 \\
140.16 \\
142.63 \\
145.78 \\
142.52 \\
0.22\end{array}$ & $\begin{array}{l}\text { \% Strain } \\
0.0052 \\
0.0149 \\
0.0246 \\
0.0340 \\
0.0434 \\
0.0483 \\
0.0542 \\
0.0603 \\
0.0672 \\
0.0744 \\
0.0839 \\
0.0922 \\
0.1004 \\
0.1103 \\
0.1210 \\
0.1266 \\
0.1322 \\
0.1365 \\
0.1430 \\
0.1483 \\
0.1611 \\
0.1705 \\
0.1796 \\
0.1821 \\
0.1915 \\
0.1952 \\
0.2010 \\
0.2124 \\
0.2246 \\
0.2402 \\
0.2444 \\
0.2604 \\
0.2723 \\
0.2867 \\
0.3016 \\
0.3053 \\
0.1214\end{array}$ \\
\hline
\end{tabular}

\section{High Pressure test}

9.89

39.42

49.16

74.16

80.16

85.61

103.25

111.08

13.24

20.92

123.28

122.97

130.19

40.16

45.78

0.52

0.1927

0.22
0.3053

0.0052

0.0246

0.0434

.

0.0744

0.0839

0.1266

0.1322

0.1796

0.1952

0.2246

0.2402

0.2444

0.2604

0.2723

0.2867

0.1214 
568-3

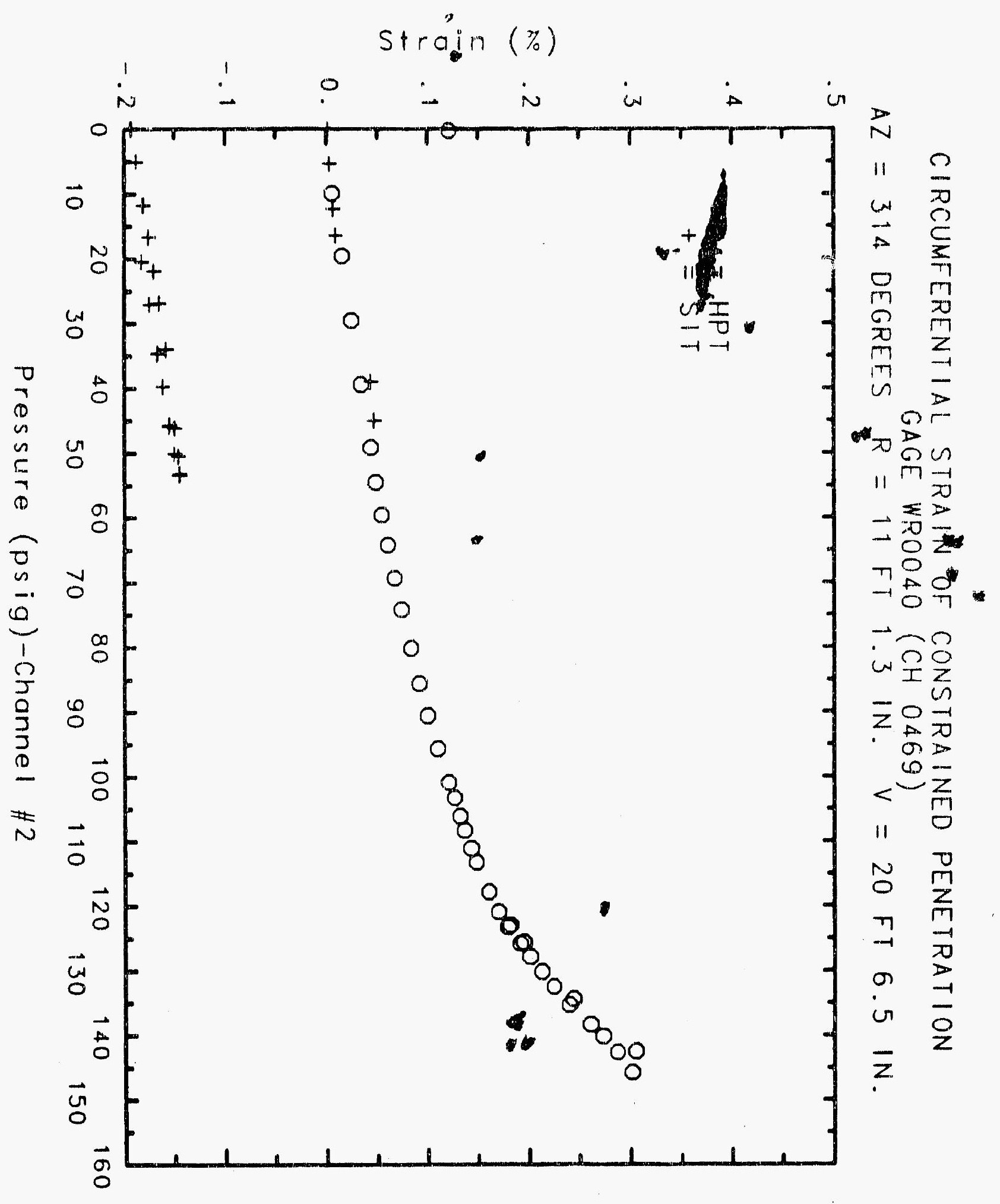




\section{Reinforced Conirete Test Data}

Weldable gage 42 Channel 481

STRUCTURAL INTEGRITY TEST

\begin{tabular}{|c|c|c|c|}
\hline $\begin{array}{c}\text { Pressure } \\
\text { (psig) } \\
-0.05 \\
5.33 \\
12.31 \\
16.44 \\
20.51 \\
20.46 \\
20.47 \\
27.05 \\
34.69 \\
34.53 \\
34.53 \\
39.70 \\
45.90 \\
45.65 \\
45.62 \\
50.10 \\
53.47 \\
53.29 \\
53.21 \\
50.49 \\
46.14 \\
46.13 \\
44.97 \\
38.98 \\
33.99 \\
33.96 \\
26.83 \\
21.88 \\
21.90 \\
16.69 \\
11.74 \\
11.77 \\
5.05 \\
0.02 \\
-0.04 \\
.0 .02 \\
-0.02 \\
0.02 \\
\end{array}$ & $\begin{array}{l}\text { \% Strain } \\
0.0004 \\
0.0025 \\
0.0065 \\
-0.3542 \\
-0.3515 \\
-0.3510 \\
-0.3510 \\
-0.3449 \\
-0.3355 \\
-0.3341 \\
-0.3338 \\
-0.3295 \\
-0.3218 \\
-0.3211 \\
-0.3208 \\
-0.3170 \\
-0.3131 \\
-0.3124 \\
-0.3121 \\
-0.3132 \\
-0.3153 \\
-0.3153 \\
-0.3148 \\
-0.3180 \\
-0.3213 \\
-0.3221 \\
-0.3272 \\
-0.3311 \\
-0.3310 \\
-0.3356 \\
-0.3398 \\
-0.3402 \\
-0.3451 \\
-0.3489 \\
-0.3500 \\
-0.3500 \\
-0.3500 \\
-0.3504\end{array}$ & $\begin{array}{c}\text { Pressure } \\
\text { (psig) } \\
9.89 \\
19.55 \\
29.57 \\
39.42 \\
49.16 \\
54.50 \\
59.57 \\
64.20 \\
63.32 \\
74.16 \\
80.16 \\
85.61 \\
90.58 \\
95.69 \\
100.92 \\
103.25 \\
106.11 \\
108.31 \\
111.08 \\
113.24 \\
117.83 \\
120.92 \\
123.28 \\
122.97 \\
125.82 \\
125.60 \\
127.84 \\
130.19 \\
132.53 \\
135.33 \\
134.42 \\
138.35 \\
140.16 \\
142.63 \\
145.78 \\
142.52 \\
0.22 \\
\end{array}$ & $\begin{array}{c}\text { \% Strain } \\
-0.0028 \\
-0.0022 \\
-0.0041 \\
-0.0028 \\
-0.0040 \\
-0.0050 \\
-0.0050 \\
0.0011 \\
-0.0050 \\
-0.0050 \\
0.0018 \\
-0.0017 \\
-0.0003 \\
-0.0049 \\
-0.0020 \\
-0.0006 \\
-0.0028 \\
-0.0004 \\
-0.0050 \\
-0.0050 \\
-0.0001 \\
-0.0001 \\
-0.0018 \\
-0.0028 \\
-0.0002 \\
-0.0028 \\
-0.0016 \\
0.0011 \\
-0.0019 \\
-0.0025 \\
-0.0041 \\
-0.0018 \\
-0.0019 \\
-0.0006 \\
-0.0028 \\
-0.0017 \\
-0.0003\end{array}$ \\
\hline
\end{tabular}

High Pressure test

(psig)

(psig)

39.42

49.16

64.20

83.32

74.16

80.16

95.69

100.92

108.31

111.08

113.24

120.92

125.60

127.84

134.42

138.35

140.16

142.63

145.78

2.52

0.22

\section{.}




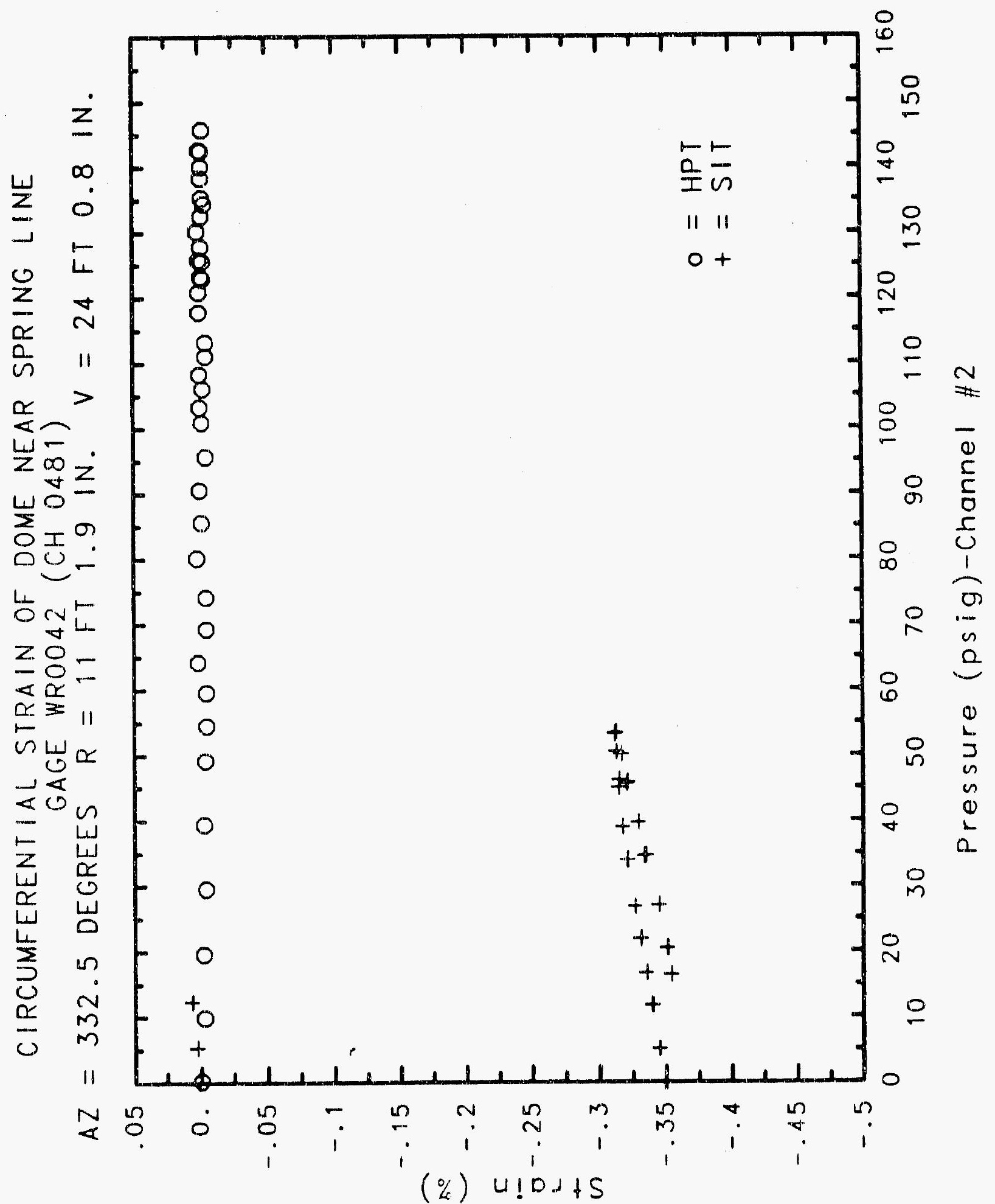

C. 896 


\section{Reinforced Concrete Test Data}

Weldable gage 43 Channel 482

Structural Integrity Test

\begin{tabular}{|c|c|c|c|}
\hline $\begin{array}{c}\text { Pressure } \\
\text { (psig) } \\
-0.05 \\
5.33 \\
12.31 \\
16.44 \\
20.51 \\
20.46 \\
20.47 \\
27.05 \\
34.69 \\
34.53 \\
34.53 \\
39.70 \\
45.90 \\
45.65 \\
45.62 \\
50.10 \\
53.47 \\
53.29 \\
53.21 \\
50.49 \\
46.14 \\
46.13 \\
44.97 \\
38.98 \\
33.99 \\
33.96 \\
26.83 \\
21.88 \\
21.90 \\
16.69 \\
11.74 \\
11.77 \\
5.05 \\
0.02 \\
-0.04 \\
-0.02 \\
-0.02 \\
0.02 \\
\end{array}$ & $\begin{array}{r}\text { Strain } \\
-0.0003 \\
-0.0008 \\
-0.0006 \\
-0.0006 \\
-0.0005 \\
-0.0005 \\
-0.0004 \\
-0.0005 \\
-0.0001 \\
-0.0002 \\
-0.0001 \\
-0.0005 \\
0.0000 \\
0.0000 \\
-0.0002 \\
0.0001 \\
-0.0004 \\
0.0000 \\
-0.0001 \\
-0.0002 \\
-0.0003 \\
-0.0002 \\
-0.0004 \\
-0.0006 \\
-0.0004 \\
-0.0007 \\
-0.0005 \\
-0.0004 \\
-0.0005 \\
-0.0001 \\
-0.0004 \\
0.0000 \\
-0.0001 \\
0.0000 \\
-0.0006 \\
-0.0010 \\
-0.0007 \\
-0.0017\end{array}$ & $\begin{array}{c}\text { Pressure } \\
(\text { psig) } \\
9.89 \\
19.55 \\
29.57 \\
39.42 \\
49.16 \\
54.50 \\
59.57 \\
64.20 \\
69.32 \\
74.16 \\
80.16 \\
85.61 \\
90.58 \\
95.69 \\
100.92 \\
103.25 \\
106.11 \\
108.31 \\
111.08 \\
113.24 \\
117.83 \\
120.92 \\
123.28 \\
122.97 \\
125.82 \\
125.60 \\
127.84 \\
130.19 \\
132.53 \\
135.33 \\
134.42 \\
138.35 \\
140.16 \\
142.63 \\
145.78 \\
142.52 \\
0.22 \\
\end{array}$ & $\begin{array}{l}\text { \% Strain } \\
-0.0041 \\
-0.0040 \\
-0.0041 \\
-0.0040 \\
-0.0040 \\
-0.0038 \\
-0.0036 \\
-0.0040 \\
-0.0040 \\
-0.0039 \\
-0.0031 \\
-0.0036 \\
-0.0030 \\
-0.0021 \\
-0.0012 \\
-0.0009 \\
-0.0003 \\
-0.0002 \\
0.0005 \\
0.0007 \\
0.0020 \\
0.0034 \\
0.0047 \\
0.0047 \\
0.0064 \\
0.0072 \\
0.0076 \\
0.0097 \\
0.0106 \\
0.0131 \\
0.0144 \\
0.0173 \\
0.0197 \\
0.0238 \\
0.0289 \\
0.0321 \\
0.0146\end{array}$ \\
\hline
\end{tabular}

High Pressure Test

Pre 


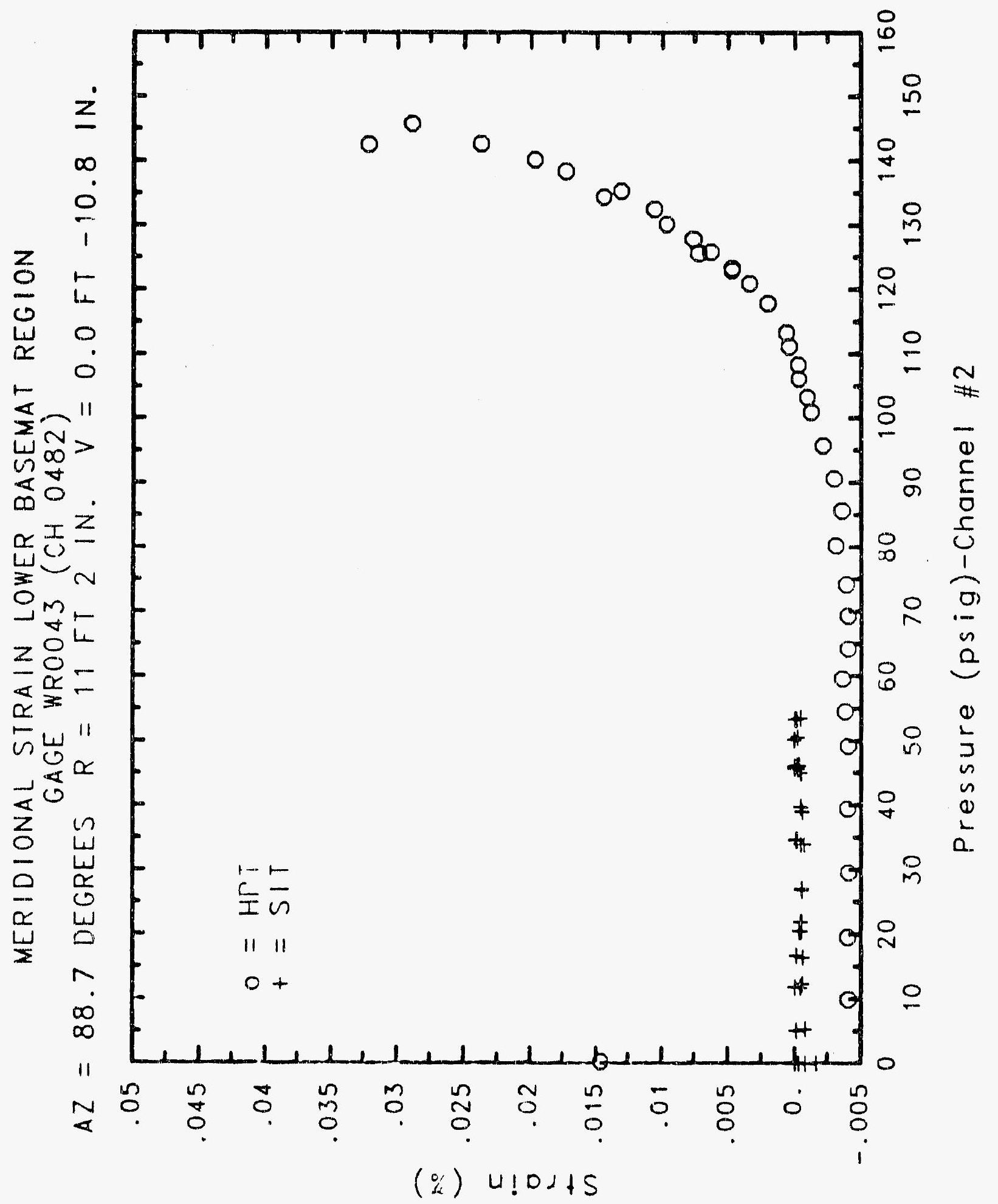




\section{Reinforced Concrete Test Data}

Weldable gage 44 Channel 483

Structural Integrity test

High Pressure test

\begin{tabular}{|c|c|c|c|}
\hline $\begin{array}{c}\text { Pressure } \\
\text { (psig) } \\
-0.05 \\
5.33 \\
12.31 \\
16.44 \\
20.51 \\
20.46 \\
20.47 \\
27.05 \\
34.69 \\
34.53 \\
34.53 \\
39.70 \\
45.90 \\
45.65 \\
45.62 \\
50.10 \\
53.47 \\
53.29 \\
53.21 \\
50.49 \\
46.14 \\
46.13 \\
44.97 \\
38.98 \\
33.99 \\
33.96 \\
26.83 \\
21.88 \\
21.90 \\
16.69 \\
11.74 \\
11.77 \\
5.05 \\
0.02 \\
-0.04 \\
-0.02 \\
-0.02 \\
0.02 \\
\end{array}$ & $\begin{array}{l}\text { \% Strain } \\
0.0001 \\
0.0002 \\
0.0025 \\
0.0040 \\
0.0051 \\
0.0055 \\
0.0062 \\
0.0089 \\
0.0133 \\
0.0146 \\
0.0147 \\
0.0179 \\
0.0219 \\
0.0232 \\
0.0236 \\
0.0263 \\
0.0293 \\
0.0301 \\
0.0310 \\
0.0303 \\
0.0291 \\
0.0293 \\
0.0301 \\
0.0278 \\
0.0261 \\
0.0266 \\
0.0240 \\
0.0219 \\
0.0217 \\
0.0201 \\
0.0177 \\
0.0171 \\
0.0136 \\
0.0110 \\
0.0078 \\
0.0071 \\
0.0076 \\
0.0066\end{array}$ & $\begin{array}{c}\text { Pressure } \\
(\text { psig) } \\
9.89 \\
19.55 \\
29.57 \\
39.42 \\
49.16 \\
54.50 \\
59.57 \\
64.20 \\
69.32 \\
74.16 \\
80.16 \\
85.61 \\
90.58 \\
95.69 \\
100.92 \\
103.25 \\
106.11 \\
108.31 \\
111.08 \\
113.24 \\
117.83 \\
120.92 \\
123.28 \\
122.97 \\
125.82 \\
125.60 \\
127.84 \\
130.19 \\
132.53 \\
135.3 \\
134.42 \\
138.35 \\
140.16 \\
142.63 \\
145.78 \\
142.52 \\
0.22\end{array}$ & $\begin{array}{c}\text { \% Strain } \\
-0.0003 \\
0.0036 \\
0.0087 \\
0.0132 \\
0.0185 \\
0.0218 \\
0.0261 \\
0.0413 \\
0.0519 \\
0.0646 \\
0.0826 \\
0.0968 \\
0.1101 \\
0.1247 \\
0.1379 \\
0.1449 \\
0.1516 \\
0.1569 \\
0.1650 \\
0.1710 \\
0.1810 \\
0.1877 \\
0.1930 \\
0.1932 \\
0.1986 \\
0.1999 \\
0.2038 \\
0.2090 \\
0.2131 \\
0.2179 \\
0.2175 \\
0.2249 \\
0.2294 \\
0.2355 \\
0.2444 \\
0.2412 \\
0.0203\end{array}$ \\
\hline
\end{tabular}




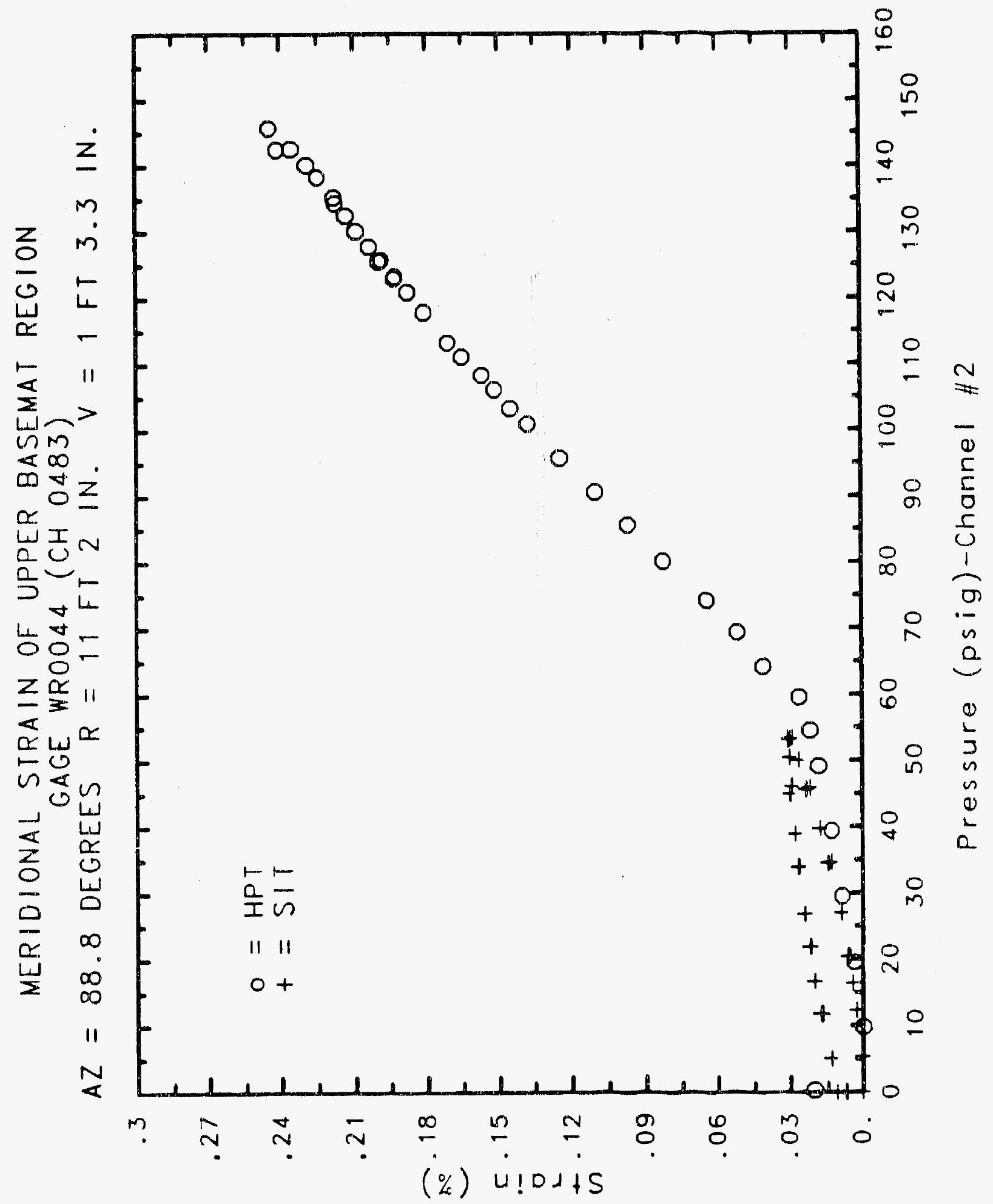




\section{Reinforced Concrete Test Dáta}

Weldable gage 45 Channel 484

Structural Integrity Test

High Pressure test

\begin{tabular}{|c|c|c|c|}
\hline $\begin{array}{c}\text { Pressure } \\
\text { (psig) } \\
-0.05 \\
5.33 \\
12.31 \\
16.44 \\
20.51 \\
20.46 \\
20.47 \\
27.05 \\
34.69 \\
34.53 \\
34.53 \\
39.70 \\
45.90 \\
45.65 \\
45.62 \\
50.10 \\
53.47 \\
53.29 \\
53.21 \\
50.49 \\
46.14 \\
46.13 \\
44.97 \\
38.98 \\
33.99 \\
33.96 \\
26.83 \\
21.88 \\
21.90 \\
16.69 \\
11.74 \\
11.77 \\
5.05 \\
0.02 \\
-0.04 \\
-0.02 \\
-0.02 \\
0.02 \\
\end{array}$ & $\begin{array}{l}\text { X Strain } \\
0.0010 \\
0.0037 \\
0.0088 \\
0.0120 \\
0.0162 \\
0.0177 \\
0.0192 \\
0.0281 \\
0.0412 \\
0.0434 \\
0.0438 \\
0.0514 \\
0.0616 \\
0.0623 \\
0.0624 \\
0.0688 \\
0.0751 \\
0.0755 \\
0.0758 \\
0.0727 \\
0.0689 \\
0.0679 \\
0.0652 \\
0.0600 \\
0.0550 \\
0.0560 \\
0.0492 \\
0.0441 \\
0.0443 \\
0.0384 \\
0.0323 \\
0.0308 \\
0.0224 \\
0.0156 \\
0.0095 \\
0.0087 \\
0.0091 \\
0.0097\end{array}$ & $\begin{array}{c}\text { Pressure } \\
(\text { psig) } \\
9.89 \\
19.55 \\
29.57 \\
39.42 \\
49.16 \\
54.50 \\
59.57 \\
64.20 \\
69.32 \\
74.16 \\
80.16 \\
85.61 \\
90.58 \\
95.69 \\
100.92 \\
103.25 \\
106.11 \\
108.31 \\
111.08 \\
113.24 \\
117.83 \\
120.92 \\
123.28 \\
122.97 \\
125.82 \\
125.60 \\
127.84 \\
130.19 \\
132.53 \\
135.33 \\
134.42 \\
138.35 \\
140.16 \\
142.63 \\
145.78 \\
142.52 \\
0.22 \\
\end{array}$ & $\begin{array}{l}\text { \% Strain } \\
0.0076 \\
0.0196 \\
0.0332 \\
0.0464 \\
0.0594 \\
0.0678 \\
0.0764 \\
0.0871 \\
0.0965 \\
0.1075 \\
0.1238 \\
0.1356 \\
0.1455 \\
0.1548 \\
0.1647 \\
0.1697 \\
0.1759 \\
0.1813 \\
0.1890 \\
0.1963 \\
0.2077 \\
0.2140 \\
0.2188 \\
0.2185 \\
0.2239 \\
0.2249 \\
0.2287 \\
0.2346 \\
0.2397 \\
0.2454 \\
0.2456 \\
0.2479 \\
0.3336 \\
0.6366 \\
0.8789 \\
0.9242 \\
0.5542\end{array}$ \\
\hline
\end{tabular}




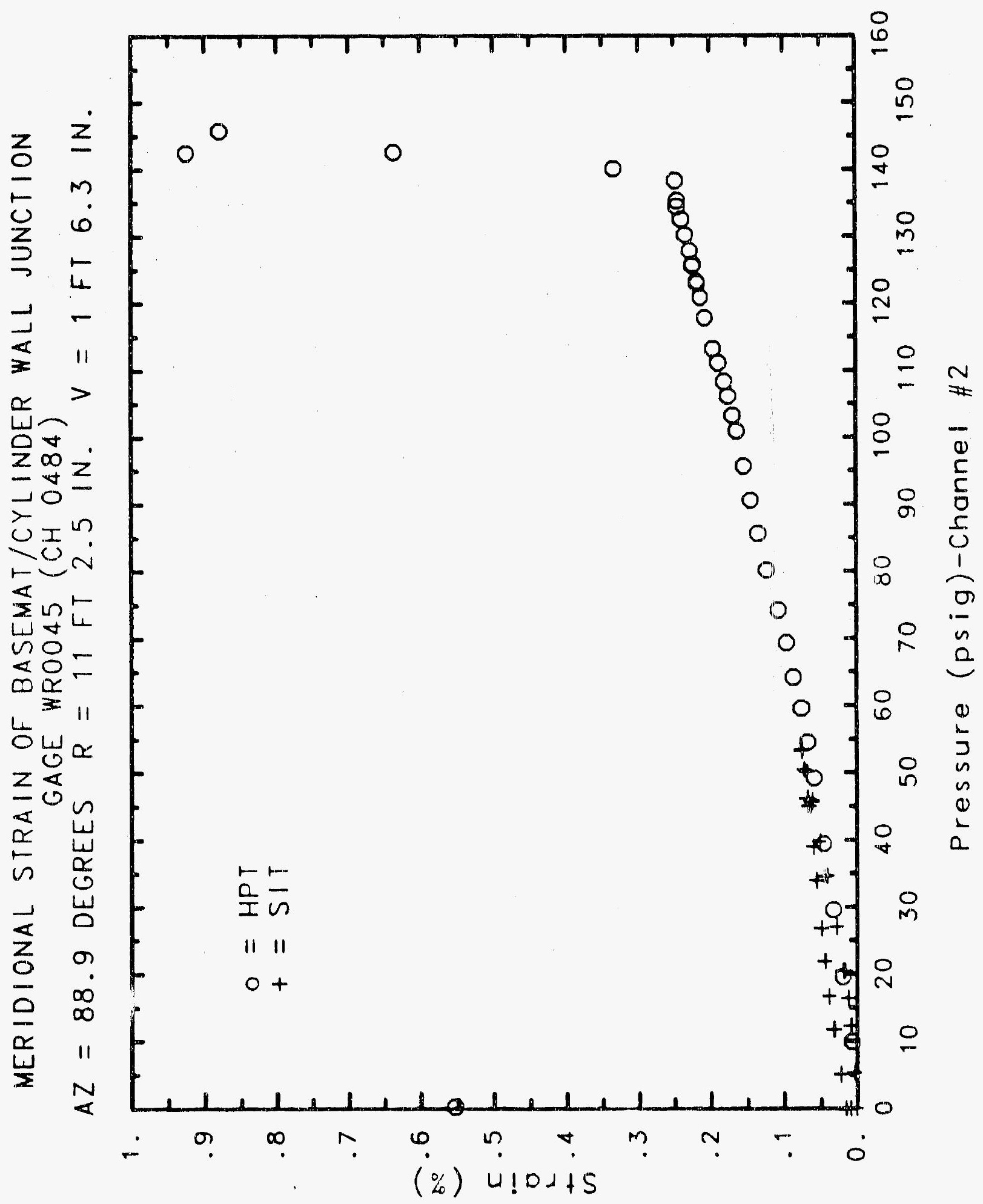

C. $-9(12$ 


\section{Reinforced Concrete Test Data}

Weldable Gage 46 Channel 485

Structural. Integrity Test

\begin{tabular}{|c|c|c|c|}
\hline $\begin{array}{c}\text { Pressure } \\
(\text { psig) } \\
-0.05 \\
5.33 \\
12.31 \\
16.44 \\
20.51 \\
20.46 \\
20.47 \\
27.05 \\
34.69 \\
34.53 \\
34.53 \\
39.70 \\
45.90 \\
45.65 \\
45.62 \\
50.10 \\
53.47 \\
53.29 \\
53.21 \\
50.49 \\
46.14 \\
46.13 \\
44.97 \\
38.98 \\
33.99 \\
33.96 \\
26.83 \\
21.88 \\
21.90 \\
16.69 \\
11.74 \\
11.77 \\
5.05 \\
0.02 \\
-0.04 \\
-0.02 \\
-0.02 \\
0.02 \\
\end{array}$ & $\begin{array}{l}x \text { Strain } \\
0.0000 \\
0.0051 \\
0.0107 \\
-0.4637 \\
-0.4595 \\
-0.4578 \\
-0.4562 \\
-0.4475 \\
-0.4345 \\
-0.4322 \\
-0.4318 \\
-0.4244 \\
-0.4143 \\
-0.4134 \\
-0.4131 \\
-0.4070 \\
-0.4005 \\
-0.4004 \\
-0.4008 \\
-0.4028 \\
-0.4070 \\
-0.4077 \\
-0.4105 \\
-0.4161 \\
-0.4209 \\
-0.4197 \\
-0.4263 \\
-0.4317 \\
-0.4315 \\
-0.4374 \\
-0.4433 \\
-0.4448 \\
-0.4528 \\
-0.4594 \\
-0.4663 \\
-0.4671 \\
-0.4663 \\
-0.4655\end{array}$ & $\begin{array}{c}\text { Pressure } \\
\text { (psig) } \\
9.89 \\
19.55 \\
29.57 \\
39.42 \\
49.16 \\
54.50 \\
59.57 \\
64.20 \\
69.32 \\
74.16 \\
80.16 \\
85.61 \\
90.58 \\
95.69 \\
100.92 \\
103.25 \\
106.11 \\
108.31 \\
111.08 \\
113.24 \\
117.83 \\
120.92 \\
123.26 \\
122.97 \\
125.82 \\
125.60 \\
127.84 \\
130.19 \\
132.53 \\
135.33 \\
131.42 \\
138.35 \\
140.16 \\
142.63 \\
145.78 \\
142.52 \\
0.22 \\
\end{array}$ & $\begin{array}{l}\text { Strain } \\
0.0071 \\
0.0197 \\
0.0330 \\
0.0459 \\
0.0595 \\
0.0674 \\
0.0761 \\
0.0866 \\
0.0965 \\
0.1078 \\
0.1235 \\
0.1357 \\
0.1455 \\
0.1547 \\
0.1648 \\
0.1698 \\
0.1758 \\
0.1810 \\
0.1886 \\
0.1961 \\
0.2078 \\
0.2139 \\
0.2188 \\
0.2188 \\
0.2236 \\
0.2247 \\
0.2289 \\
0.2341 \\
0.2399 \\
0.2456 \\
0.2455 \\
0.2478 \\
0.3329 \\
0.6366 \\
0.8787 \\
0.9241 \\
0.5541\end{array}$ \\
\hline
\end{tabular}

High Pressure test

Pressure

9.89

0.0197

0.0459

0.0595

0.0674

0.0866

0.0965

0.1235

0.1357

0.1648

0.1758

0.1810

0.2078

0.2188

0.2188

0.2236

0.2247

0.2399

0.2456

0.2455

0.2478

0.8787

0.9241

0.5541 


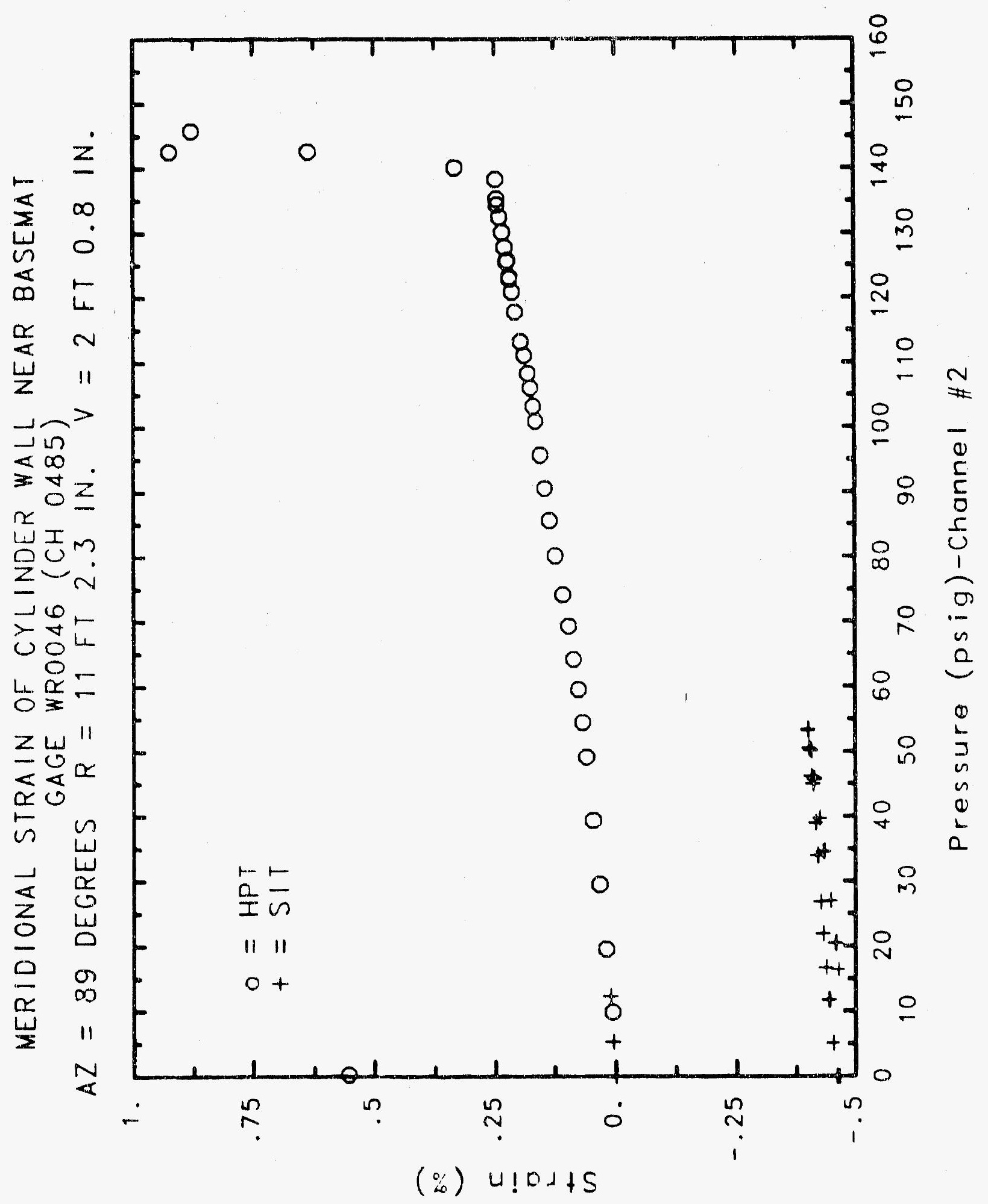




\section{Reinforced Concrete Test Data}

Weldable gage 47 Channel 486

StRUCTURAL INTEGRITY TEST

\begin{tabular}{|c|c|c|c|}
\hline $\begin{array}{c}\text { Pressure } \\
\text { (psig) } \\
-0.05 \\
5.33 \\
12.31 \\
16.44 \\
20.51 \\
20.46 \\
20.47 \\
27.05 \\
34.69 \\
34.53 \\
34.53 \\
39.70 \\
45.90 \\
45.65 \\
45.62 \\
50.10 \\
53.47 \\
53.29 \\
53.21 \\
50.49 \\
46.14 \\
46.13 \\
44.97 \\
38.98 \\
33.99 \\
33.96 \\
26.83 \\
21.88 \\
21.90 \\
16.69 \\
11.74 \\
11.77 \\
5.05 \\
0.02 \\
-0.04 \\
-0.02 \\
-0.02 \\
0.02\end{array}$ & $\begin{array}{l}\text { X Strain } \\
0.0002 \\
0.0021 \\
0.0052 \\
0.0067 \\
0.0093 \\
0.0108 \\
0.0120 \\
0.0211 \\
0.0340 \\
0.0361 \\
0.0366 \\
0.0434 \\
0.0532 \\
0.0539 \\
0.0538 \\
0.0591 \\
0.0641 \\
0.0645 \\
0.0641 \\
0.0624 \\
0.0593 \\
0.0591 \\
0.0573 \\
0.0533 \\
0.0492 \\
0.0505 \\
0.0442 \\
0.0400 \\
0.0402 \\
0.0349 \\
0.0294 \\
0.0276 \\
0.0211 \\
0.0160 \\
0.0122 \\
0.0121 \\
0.0123 \\
0.0132\end{array}$ & $\begin{array}{c}\text { Pressure } \\
\text { (psig) } \\
9.89 \\
19.55 \\
29.57 \\
39.42 \\
49.16 \\
54.50 \\
59.57 \\
64.20 \\
69.32 \\
74.16 \\
80.16 \\
85.61 \\
90.58 \\
95.69 \\
100.92 \\
103.25 \\
106.11 \\
108.31 \\
111.08 \\
113.24 \\
117.83 \\
120.92 \\
123.28 \\
122.97 \\
125.82 \\
125.60 \\
127.84 \\
130.19 \\
132.53 \\
135.33 \\
134.42 \\
138.35 \\
140.16 \\
142.63 \\
145.78 \\
142.52 \\
0.22\end{array}$ & $\begin{array}{l}\text { \% Strain } \\
0.0041 \\
0.0140 \\
0.0254 \\
0.0363 \\
0.0469 \\
0.0537 \\
0.0598 \\
0.0692 \\
0.0781 \\
0.0893 \\
0.1032 \\
0.1167 \\
0.1290 \\
0.1405 \\
0.1508 \\
0.1567 \\
0.1638 \\
0.1689 \\
0.1765 \\
0.1816 \\
0.1914 \\
0.1972 \\
0.2017 \\
0.2013 \\
0.2061 \\
0.2068 \\
0.2107 \\
0.2162 \\
0.2203 \\
0.2234 \\
0.2228 \\
0.2325 \\
0.2394 \\
0.2398 \\
0.3052 \\
0.3129 \\
0.0743\end{array}$ \\
\hline
\end{tabular}

High Pressure test

9.89

39.42

54.50

59.57

8.16

85.61

90.58

103.25

106.11

113.24

122.97

127.84

132.53

135.33

140.16

42.52

0.22
0.0041

0.0254

0.0469

0.0537

0.0598

(1)

0.1167

0.1290

0.1405

1638

0.1765

0.1816

0.1914

0.1972

0.2017

0.2068

0.2162

0.2203

0.2234

0.2394

0.3052

0.3129

0.0743 
9()$(-)$

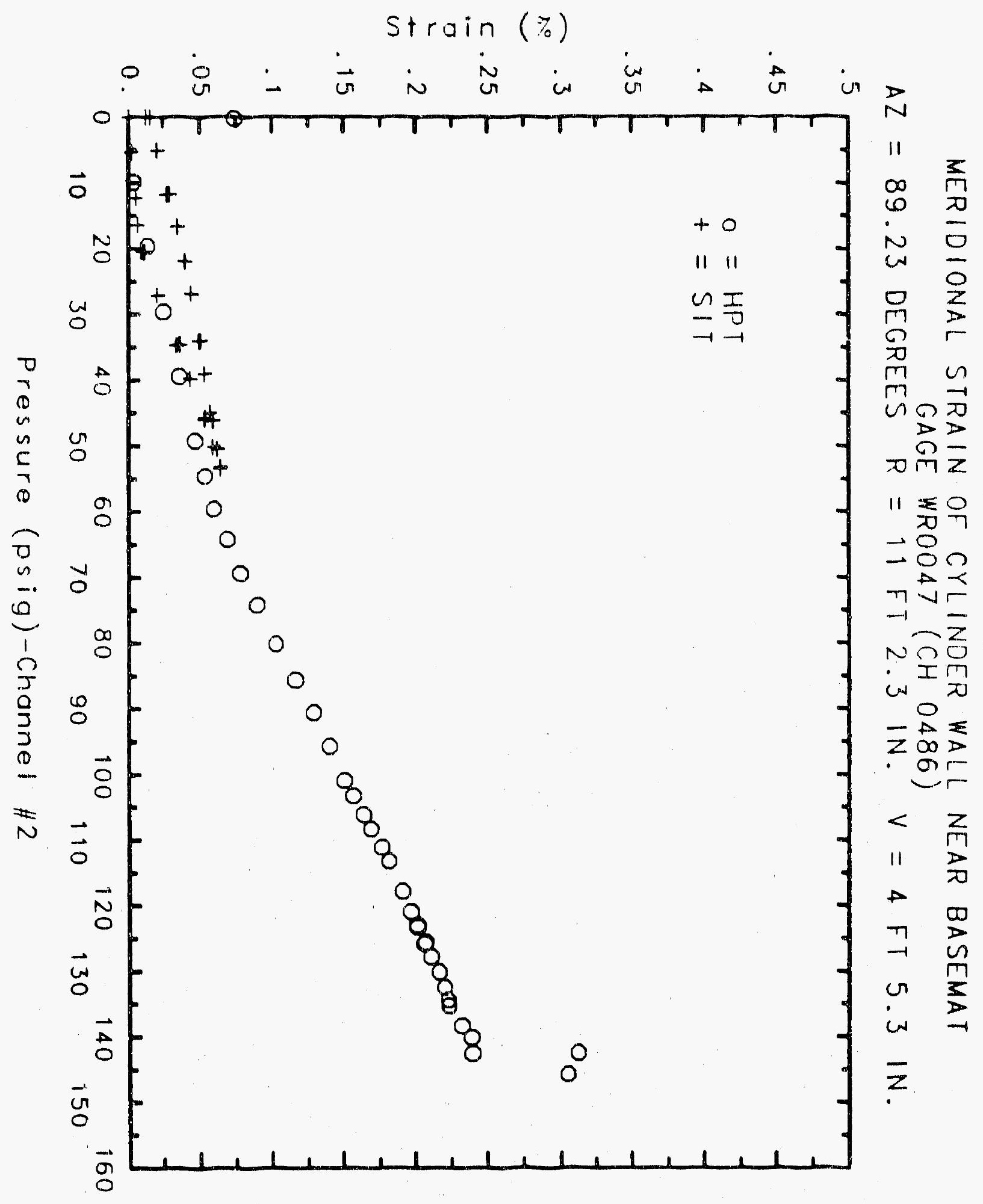


Reinforced Concrete Test Data

Weldable gage 48 Channel 487

StRuCtURAL INTEgRITY TEST

\author{
High Pressure test
}

\begin{tabular}{|c|c|c|c|}
\hline $\begin{array}{c}\text { Pressure } \\
\text { (psig) } \\
-0.05 \\
5.33 \\
12.31 \\
16.44 \\
20.51 \\
20.46 \\
20.47 \\
27.05 \\
34.69 \\
34.53 \\
34.53 \\
39.70 \\
45.90 \\
45.65 \\
45.62 \\
50.10 \\
53.47 \\
53.29 \\
53.21 \\
50.49 \\
46.14 \\
46.13 \\
44.97 \\
38.98 \\
33.99 \\
33.96 \\
26.83 \\
21.88 \\
21.90 \\
16.69 \\
11.74 \\
11.77 \\
5.05 \\
0.02 \\
-0.04 \\
-0.02 \\
-0.02 \\
0.02 \\
\end{array}$ & $\begin{array}{l}\text { \% Sirain } \\
0.0005 \\
0.0052 \\
0.0118 \\
0.0160 \\
0.0198 \\
0.0209 \\
0.0223 \\
0.0278 \\
0.0355 \\
0.0362 \\
0.0367 \\
0.0411 \\
0.0457 \\
0.0442 \\
0.0436 \\
0.0464 \\
0.0480 \\
0.0468 \\
0.0458 \\
0.0441 \\
0.0411 \\
0.0410 \\
0.0419 \\
0.0382 \\
0.0341 \\
0.0347 \\
0.0293 \\
0.0252 \\
0.0260 \\
0.0214 \\
0.0164 \\
0.0154 \\
0.0085 \\
0.0032 \\
-0.0016 \\
-0.0008 \\
-0.0006 \\
0.0020\end{array}$ & $\begin{array}{c}\text { Pressure } \\
(\text { psig) } \\
9.89 \\
19.55 \\
29.57 \\
39.42 \\
49.16 \\
54.50 \\
59.57 \\
64.20 \\
69.32 \\
74.16 \\
80.16 \\
85.61 \\
90.58 \\
95.69 \\
100.92 \\
103.25 \\
106.11 \\
108.31 \\
111.08 \\
113.24 \\
117.83 \\
120.92 \\
123.28 \\
122.97 \\
125.82 \\
125.60 \\
127.84 \\
130.19 \\
132.53 \\
135.33 \\
134.42 \\
138.35 \\
140.16 \\
142.63 \\
145.78 \\
142.52 \\
0.22\end{array}$ & $\begin{array}{l}\text { \% Strain } \\
0.0058 \\
0.0149 \\
0.0247 \\
0.0330 \\
0.0416 \\
0.0458 \\
0.0502 \\
0.0546 \\
0.0602 \\
0.0650 \\
0.0701 \\
0.0773 \\
0.0836 \\
0.0901 \\
0.0972 \\
0.1003 \\
0.1044 \\
0.1079 \\
0.1132 \\
0.1177 \\
0.1295 \\
0.1393 \\
0.1496 \\
0.1517 \\
0.1627 \\
0.1740 \\
0.1823 \\
0.2026 \\
0.2288 \\
0.2715 \\
0.2873 \\
0.3433 \\
0.3953 \\
0.4623 \\
0.5174 \\
0.5473 \\
0.3786\end{array}$ \\
\hline
\end{tabular}


IMAGE EVALUATION

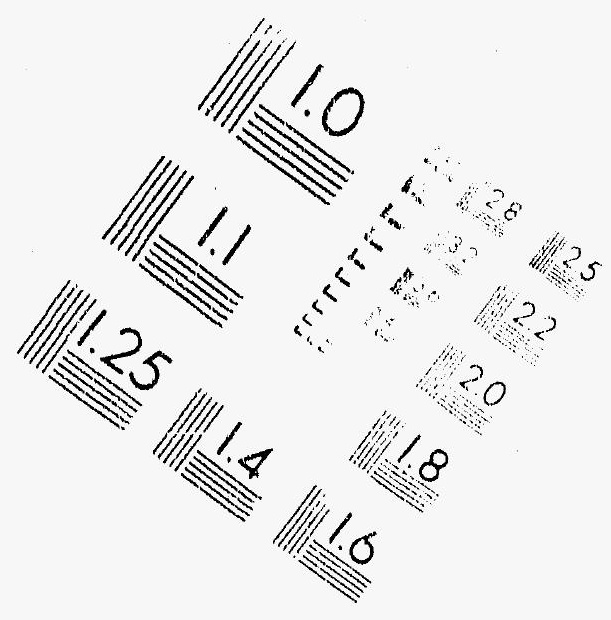

TEST TARGET (MT-3)
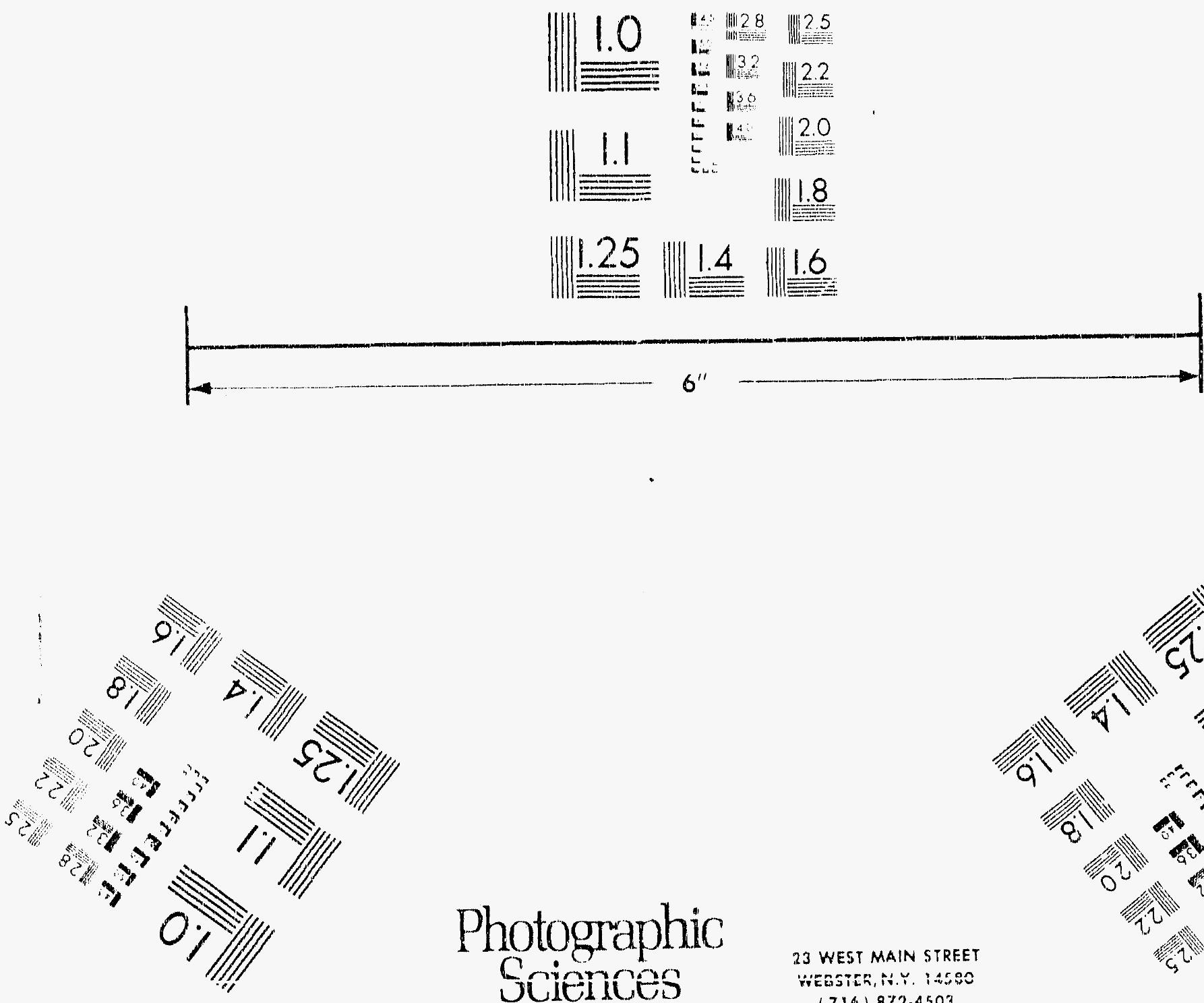

Photographic Sciences 


\section{SECURITY CLASSIFICATION}

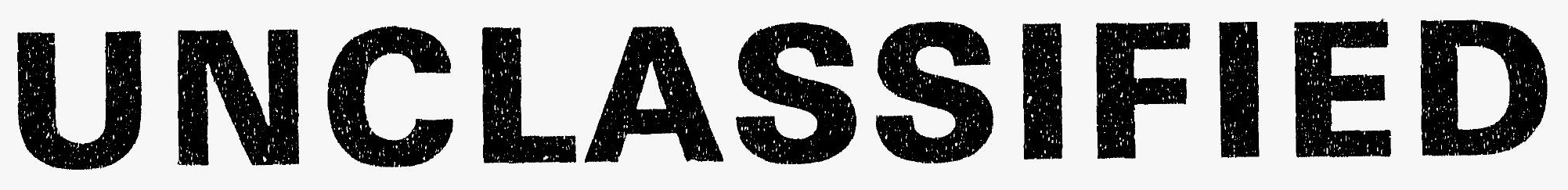

\section{DATE OF MICROFILMIING $1-6-92$}

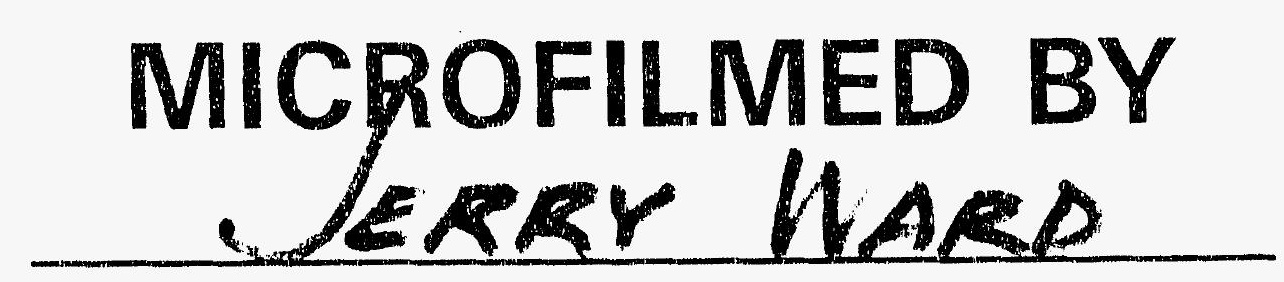

SANDIA NATIONAL LABS 


\section{THIS DOCUMENT FILMED}

AT
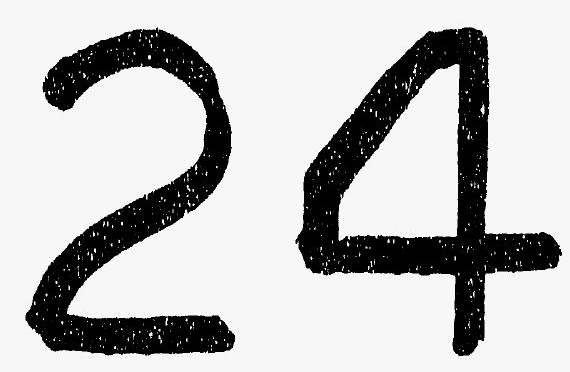

REDUCTION 


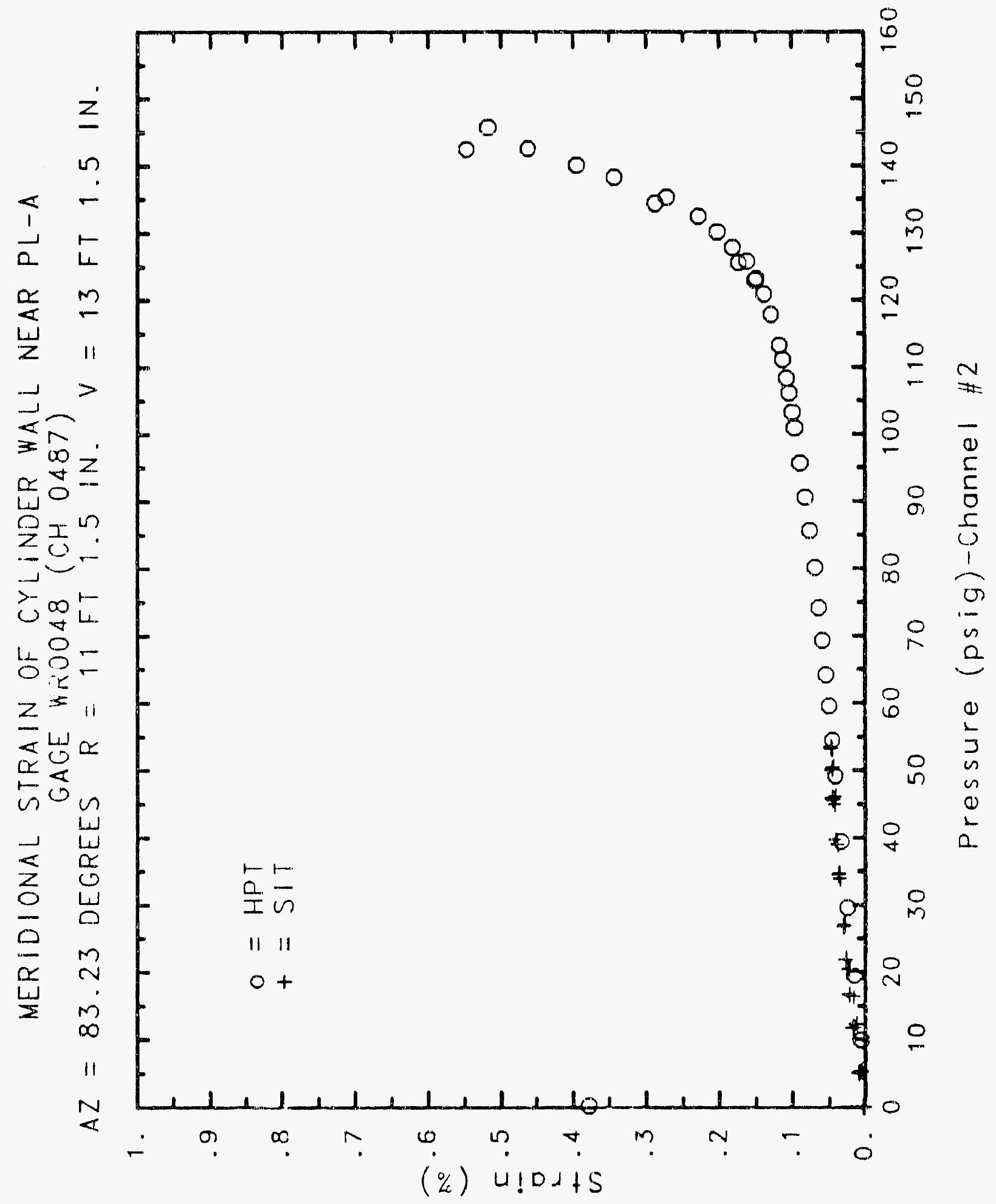




\section{Reinforced Concrete Test Data}

Weldable gage 49 Channel 488

Structural Integrity Test

\begin{tabular}{|c|c|c|c|}
\hline $\begin{array}{c}\text { Pressure } \\
\text { (psig) } \\
-0.05 \\
5.33 \\
12.31 \\
16.44 \\
20.51 \\
20.46 \\
20.47 \\
27.05 \\
34.69 \\
34.53 \\
34.53 \\
39.70 \\
45.90 \\
45.65 \\
45.62 \\
50.10 \\
53.47 \\
53.29 \\
53.21 \\
50.49 \\
46.14 \\
46.13 \\
44.97 \\
38.98 \\
33.99 \\
33.96 \\
26.83 \\
21.88 \\
21.90 \\
16.69 \\
11.74 \\
11.77 \\
5.05 \\
0.02 \\
-0.04 \\
-0.02 \\
-0.02 \\
0.02 \\
\end{array}$ & $\begin{array}{l}\text { Strain } \\
0.0003 \\
0.0044 \\
0.0094 \\
0.0128 \\
0.0156 \\
0.0153 \\
0.0166 \\
0.0174 \\
0.0168 \\
0.0165 \\
0.0165 \\
0.0181 \\
0.0209 \\
0.0199 \\
0.0194 \\
0.0220 \\
0.0254 \\
0.0254 \\
0.0256 \\
0.0240 \\
0.0222 \\
0.0221 \\
0.0234 \\
0.0217 \\
0.0190 \\
0.0192 \\
0.0157 \\
0.0134 \\
0.0138 \\
0.0105 \\
0.0072 \\
0.0063 \\
0.0015 \\
-0.0023 \\
-0.0055 \\
-0.0047 \\
-0.0046 \\
-0.0025\end{array}$ & $\begin{array}{c}\text { Pressure } \\
(\text { psig) } \\
9.89 \\
19.55 \\
29.57 \\
39.42 \\
49.16 \\
54.50 \\
59.57 \\
64.20 \\
69.32 \\
74.16 \\
80.16 \\
85.61 \\
90.58 \\
95.69 \\
100.92 \\
103.25 \\
106.11 \\
108.31 \\
111.08 \\
113.24 \\
117.83 \\
120.92 \\
123.28 \\
122.97 \\
125.82 \\
125.60 \\
127.84 \\
130.19 \\
132.53 \\
135.33 \\
134.42 \\
138.35 \\
140.16 \\
142.63 \\
145.78 \\
142.52 \\
0.22 \\
\end{array}$ & $\begin{array}{l}\text { Strain } \\
0.0015 \\
0.0080 \\
0.0153 \\
0.0213 \\
0.0278 \\
0.0318 \\
0.0370 \\
0.0437 \\
0.0520 \\
0.0610 \\
0.0698 \\
0.0798 \\
0.0901 \\
0.1020 \\
0.1137 \\
0.1194 \\
0.1259 \\
0.1308 \\
0.1378 \\
0.1439 \\
0.1640 \\
0.1830 \\
0.2112 \\
0.2173 \\
0.2393 \\
0.2493 \\
0.2624 \\
0.2927 \\
0.3242 \\
0.3601 \\
0.3713 \\
0.4122 \\
0.4488 \\
0.5042 \\
0.5627 \\
0.5966 \\
0.3924\end{array}$ \\
\hline
\end{tabular}

Hxgh Pressure test

0.0015

0.0153

0.0213

0.0370

0.0520

0.0610

0.0698

0.0798

0.1137

0.1194

0.1259

0.1439

0.2112

0.2173

0.2624

0.2927

0.3242

0.3601

0.3713

0.5042

0.5627

0.5966

0.3924 


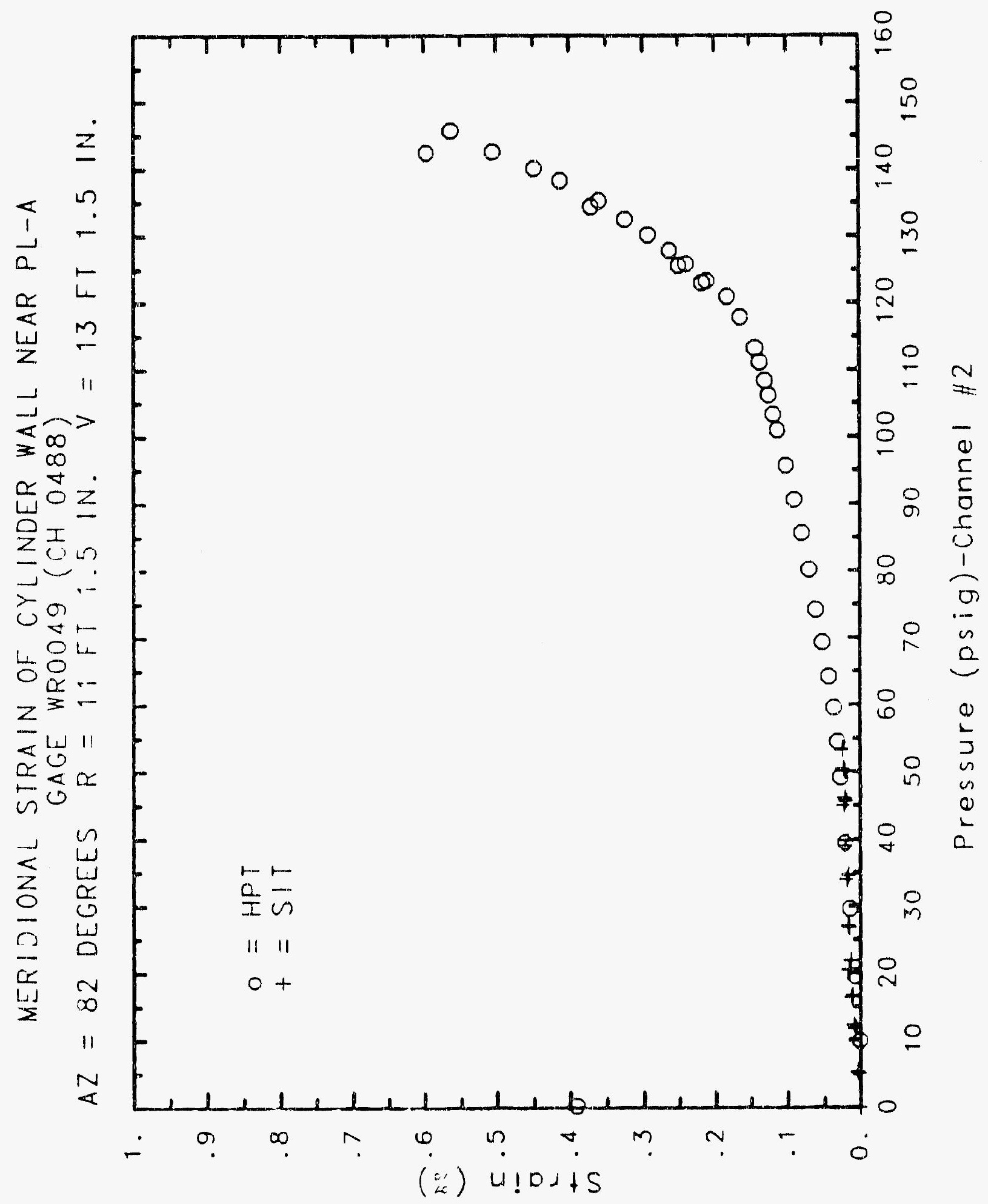

C.-910 


\section{Reinforced Concrete Test Data}

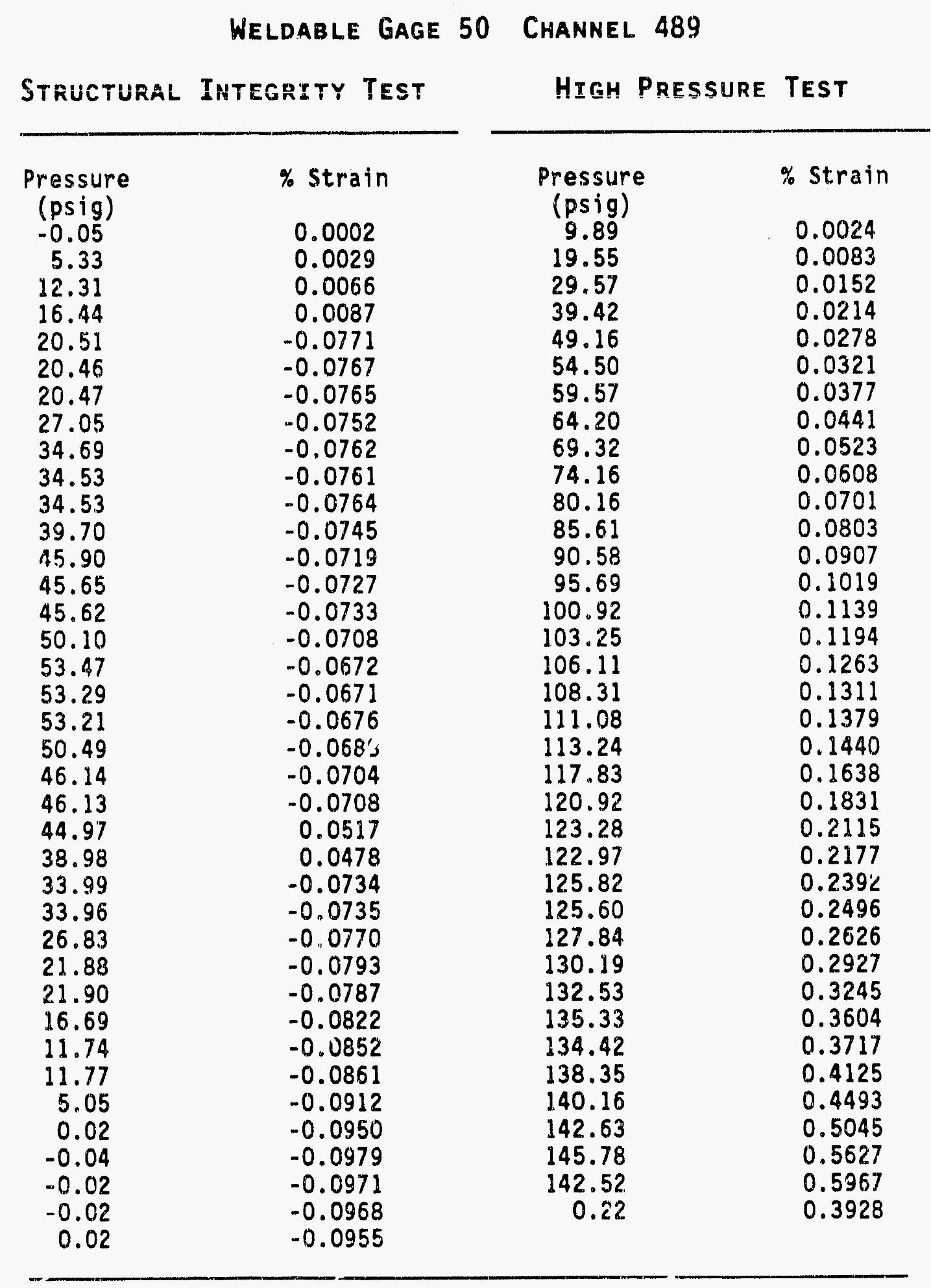




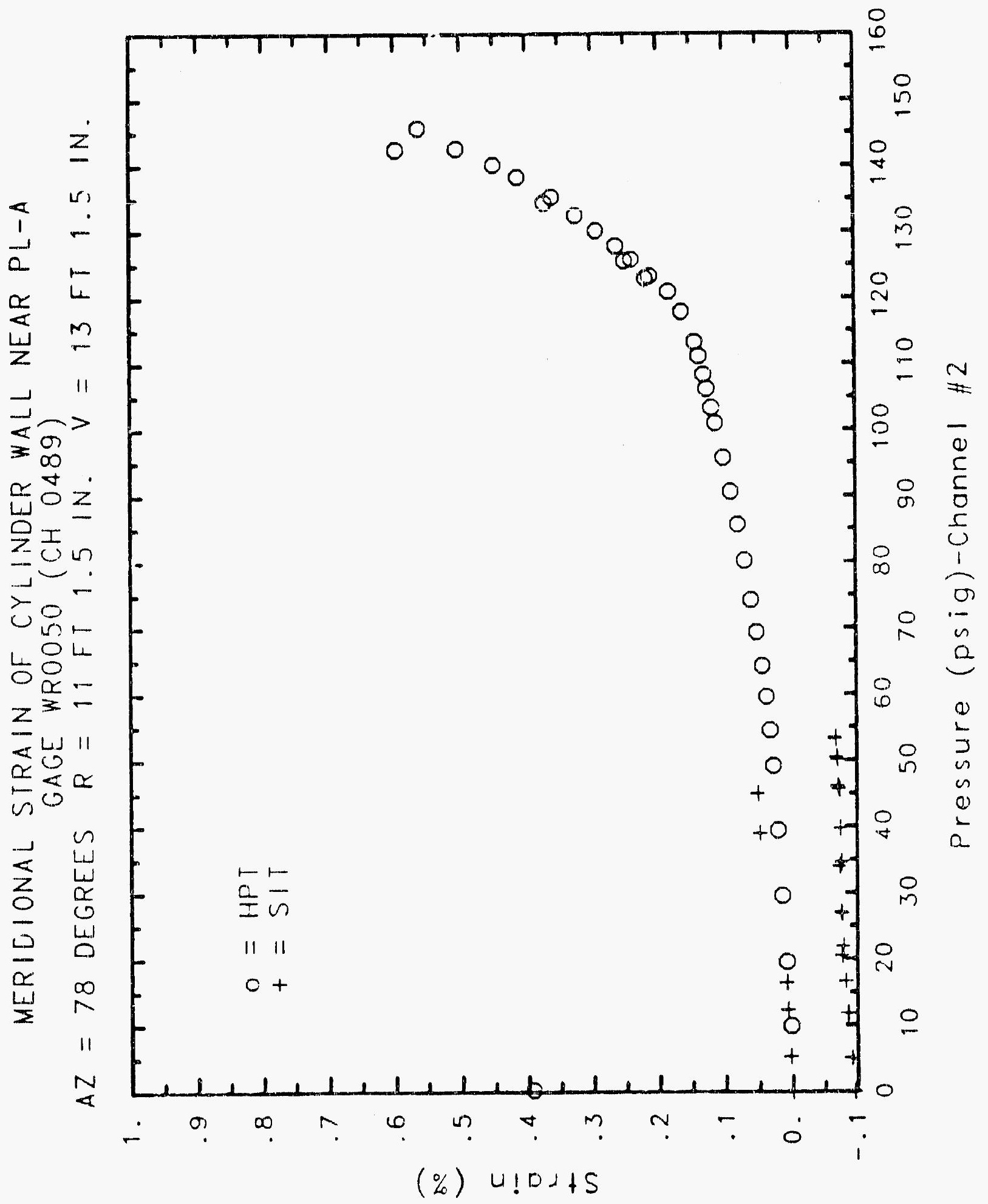




\section{Reinforced Concrete Test Data}

Weldable gage 51 Channel 500

Structural INTEgRITY TEST

\begin{tabular}{|c|c|c|c|}
\hline $\begin{array}{c}\text { Pressure } \\
\text { (psig) } \\
-0.05 \\
5.33 \\
12.31 \\
16.44 \\
20.51 \\
20.46 \\
20.47 \\
27.05 \\
34.69 \\
34.53 \\
34.53 \\
39.70 \\
45.90 \\
45.65 \\
45.62 \\
50.10 \\
53.47 \\
53.29 \\
53.21 \\
50.49 \\
46.14 \\
46.13 \\
44.97 \\
38.98 \\
33.99 \\
33.96 \\
26.83 \\
21.88 \\
21.90 \\
16.69 \\
11.74 \\
11.77 \\
5.05 \\
0.02 \\
-0.04 \\
-0.02 \\
-0.02 \\
0.02 \\
\end{array}$ & $\begin{array}{c}\text { \% Strain } \\
-0.0002 \\
0.0072 \\
0.0149 \\
0.0185 \\
0.0230 \\
0.0230 \\
0.0235 \\
0.0306 \\
0.0393 \\
0.0403 \\
0.0407 \\
0.0453 \\
0.0513 \\
0.0504 \\
0.0500 \\
0.0542 \\
0.0577 \\
0.0574 \\
0.0574 \\
0.0551 \\
0.0514 \\
0.0514 \\
0.0519 \\
0.0466 \\
0.0415 \\
0.0410 \\
0.0336 \\
0.0282 \\
0.0285 \\
0.0230 \\
0.0181 \\
0.0168 \\
0.0096 \\
0.0036 \\
0.0021 \\
0.0024 \\
0.0026 \\
0.0041\end{array}$ & $\begin{array}{c}\text { Pressure } \\
(\text { psig) } \\
9.89 \\
19.55 \\
29.57 \\
39.42 \\
49.16 \\
54.50 \\
59.57 \\
64.20 \\
69.32 \\
74.16 \\
80.16 \\
85.61 \\
90.58 \\
95.69 \\
100.92 \\
103.25 \\
106.11 \\
108.31 \\
111.08 \\
113.24 \\
117.83 \\
120.92 \\
123.28 \\
122.97 \\
125.82 \\
125.60 \\
127.84 \\
130.19 \\
132.53 \\
135.33 \\
134.42 \\
138.35 \\
140.16 \\
142.63 \\
145.78 \\
142.52 \\
0.26\end{array}$ & $\begin{array}{l}\text { \% sirain } \\
0.0082 \\
0.0187 \\
0.0288 \\
0.0383 \\
0.0476 \\
0.0532 \\
0.0576 \\
0.0628 \\
0.0683 \\
0.0735 \\
0.0792 \\
0.0856 \\
0.0917 \\
0.0988 \\
0.1063 \\
0.1097 \\
0.1138 \\
0.1172 \\
0.1221 \\
0.1257 \\
0.1349 \\
0.1415 \\
0.1482 \\
0.1497 \\
0.1580 \\
0.1595 \\
0.1658 \\
0.1780 \\
0.1965 \\
0.2274 \\
0.2345 \\
0.3116 \\
0.4027 \\
0.5822 \\
0.6551 \\
0.6784 \\
0.4971\end{array}$ \\
\hline
\end{tabular}




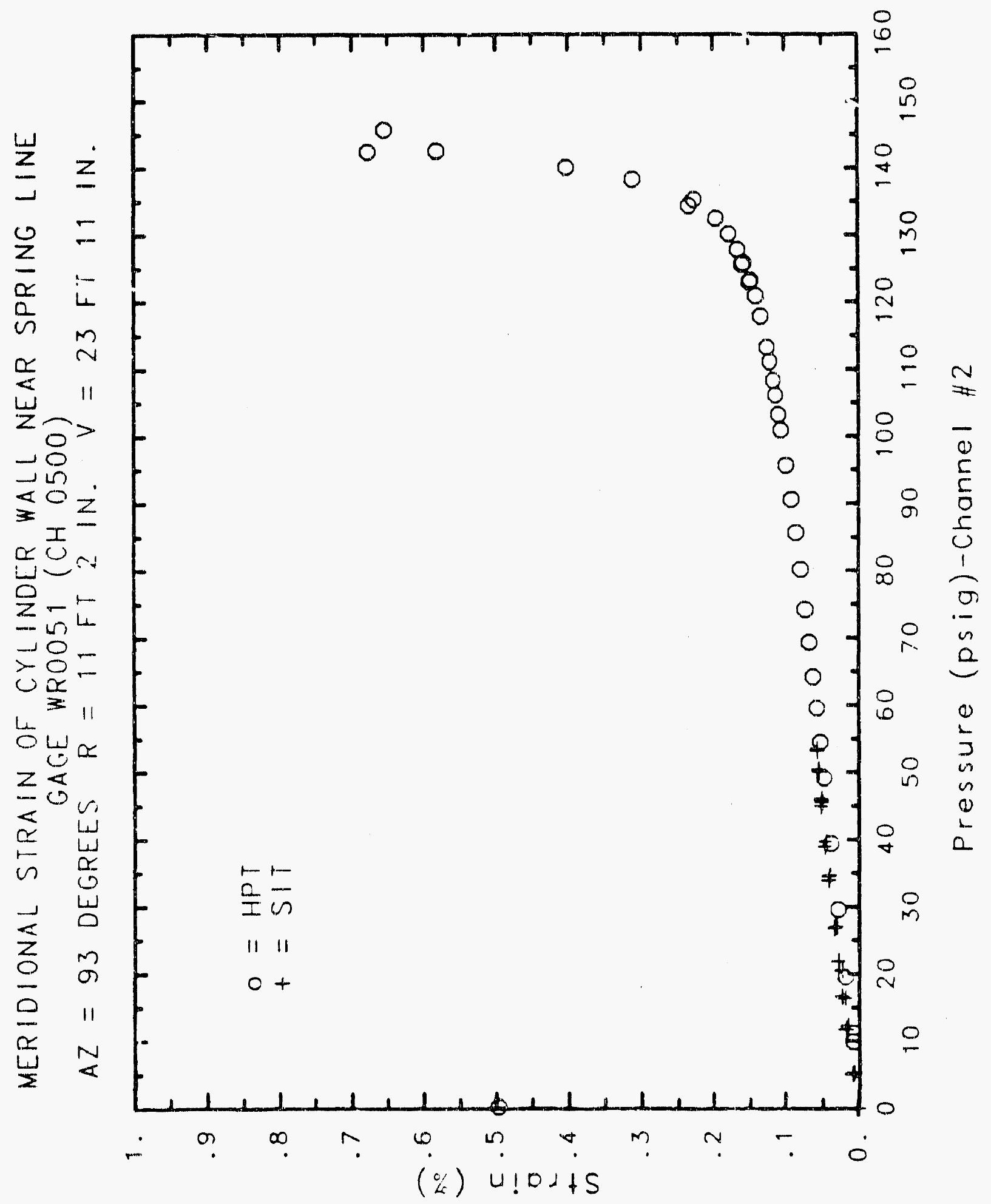




\section{Reinforced Concrete Test Data}

Weldable gage 52 Channel 501

Structural InTEgrity TEST

\begin{tabular}{|c|c|c|c|}
\hline $\begin{array}{c}\text { Pressure } \\
\text { (psig) } \\
-0.05 \\
5.33 \\
12.31 \\
16.44 \\
20.51 \\
20.46 \\
20.47 \\
27.05 \\
34.69 \\
34.53 \\
34.53 \\
39.70 \\
45.90 \\
45.65 \\
45.62 \\
50.10 \\
53.47 \\
53.29 \\
53.21 \\
50.49 \\
46.14 \\
46.13 \\
44.97 \\
38.98 \\
33.99 \\
33.96 \\
26.83 \\
21.88 \\
21.90 \\
16.69 \\
11.74 \\
11.77 \\
5.05 \\
0.02 \\
-0.04 \\
-0.02 \\
-0.02 \\
0.02 \\
\end{array}$ & $\begin{array}{l}\text { \% Strain } \\
0.0007 \\
0.0033 \\
0.0066 \\
0.0086 \\
0.0128 \\
0.0138 \\
0.0137 \\
0.0212 \\
0.0314 \\
0.0332 \\
0.0338 \\
0.0387 \\
0.0454 \\
0.0452 \\
0.0453 \\
0.0489 \\
0.0516 \\
0.0521 \\
0.0521 \\
0.0511 \\
0.0491 \\
0.0490 \\
0.0488 \\
0.0458 \\
0.0431 \\
0.0428 \\
0.0378 \\
0.0340 \\
0.0339 \\
0.0302 \\
0.0257 \\
0.0250 \\
0.0188 \\
0.0138 \\
0.0114 \\
0.0115 \\
0.0121 \\
0.0122\end{array}$ & $\begin{array}{c}\text { Pressure } \\
(\text { psig) } \\
9.89 \\
19.55 \\
29.57 \\
39.42 \\
49.16 \\
54.50 \\
59.57 \\
64.20 \\
69.32 \\
74.16 \\
80.16 \\
85.61 \\
90.58 \\
95.69 \\
100.92 \\
103.25 \\
106.11 \\
108.31 \\
111.08 \\
113.24 \\
117.83 \\
120.92 \\
123.28 \\
122.97 \\
125.82 \\
125.60 \\
127.84 \\
130.19 \\
132.53 \\
135.33 \\
134.42 \\
138.35 \\
140.16 \\
142.63 \\
145.78 \\
142.52 \\
0.22\end{array}$ & $\begin{array}{l}\text { \% Strain } \\
0.0051 \\
0.0139 \\
0.0224 \\
0.0302 \\
0.0374 \\
0.0419 \\
0.0463 \\
0.0512 \\
0.0565 \\
0.0619 \\
0.0674 \\
0.0737 \\
0.0795 \\
0.0861 \\
0.0930 \\
0.0959 \\
0.0999 \\
0.1029 \\
0.1070 \\
0.1100 \\
0.1180 \\
0.1240 \\
0.1296 \\
0.1304 \\
0.1381 \\
0.1400 \\
0.1448 \\
0.1555 \\
0.1704 \\
0.1925 \\
0.1962 \\
0.2226 \\
0.2336 \\
0.2451 \\
0.2480 \\
0.2469 \\
0.1031\end{array}$ \\
\hline
\end{tabular}

High Pressure Test

Pressure

9.89

0.0224

0.0302

0.0374

0.0419

0.0463

0.0512

0.0619

0.0674

0.0737

0.0795

0.086

0.0999

0.1029

0.1070

0.1240

0.1304

0.1381

0.1555

.

0.1962

0.2226

0.2336

0.2451

.2480

0.0121

. 52

0.1031 


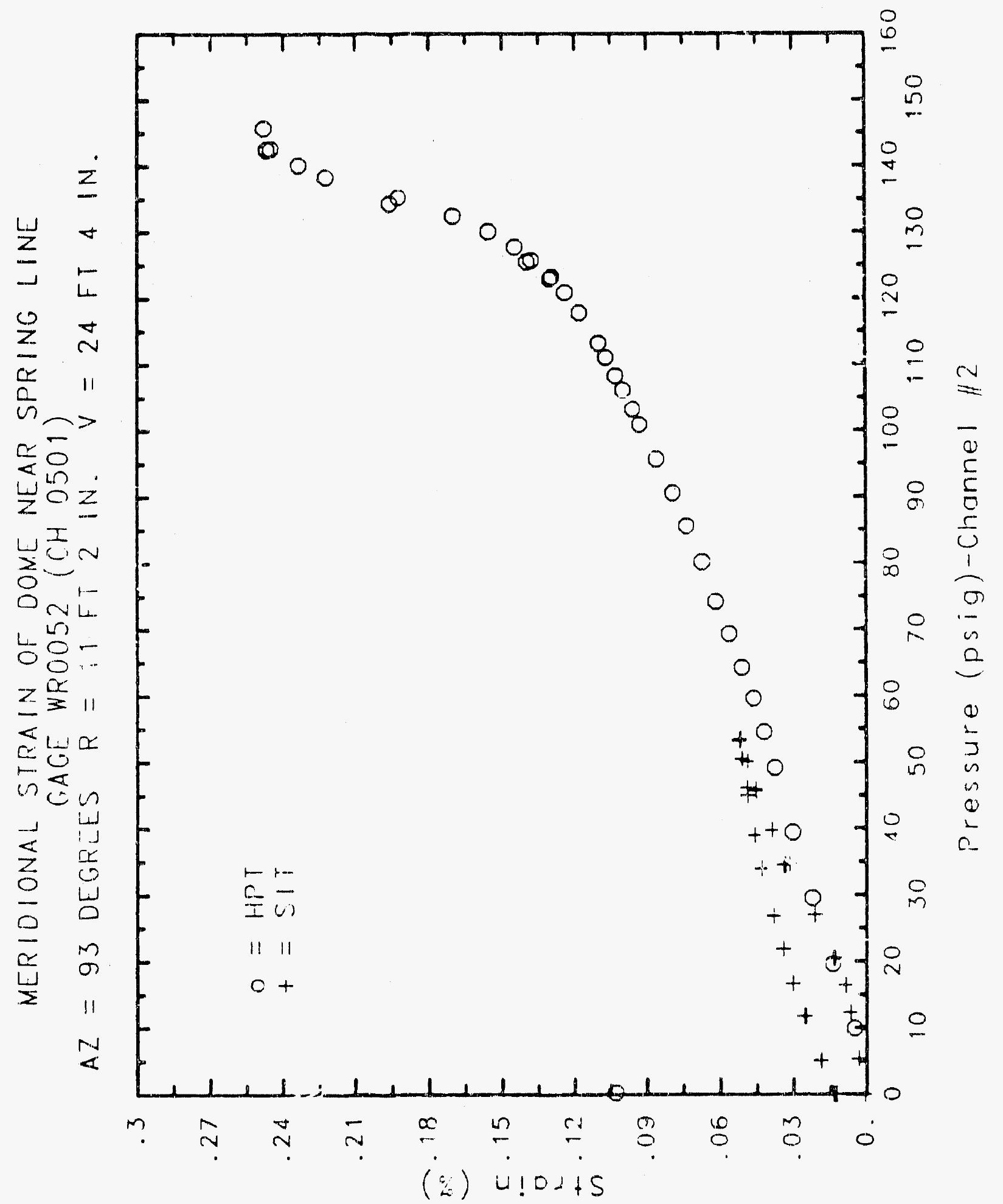

C.916 


\section{Reinforced Concrete Test Data}

Weluable gage 53 Channel 502

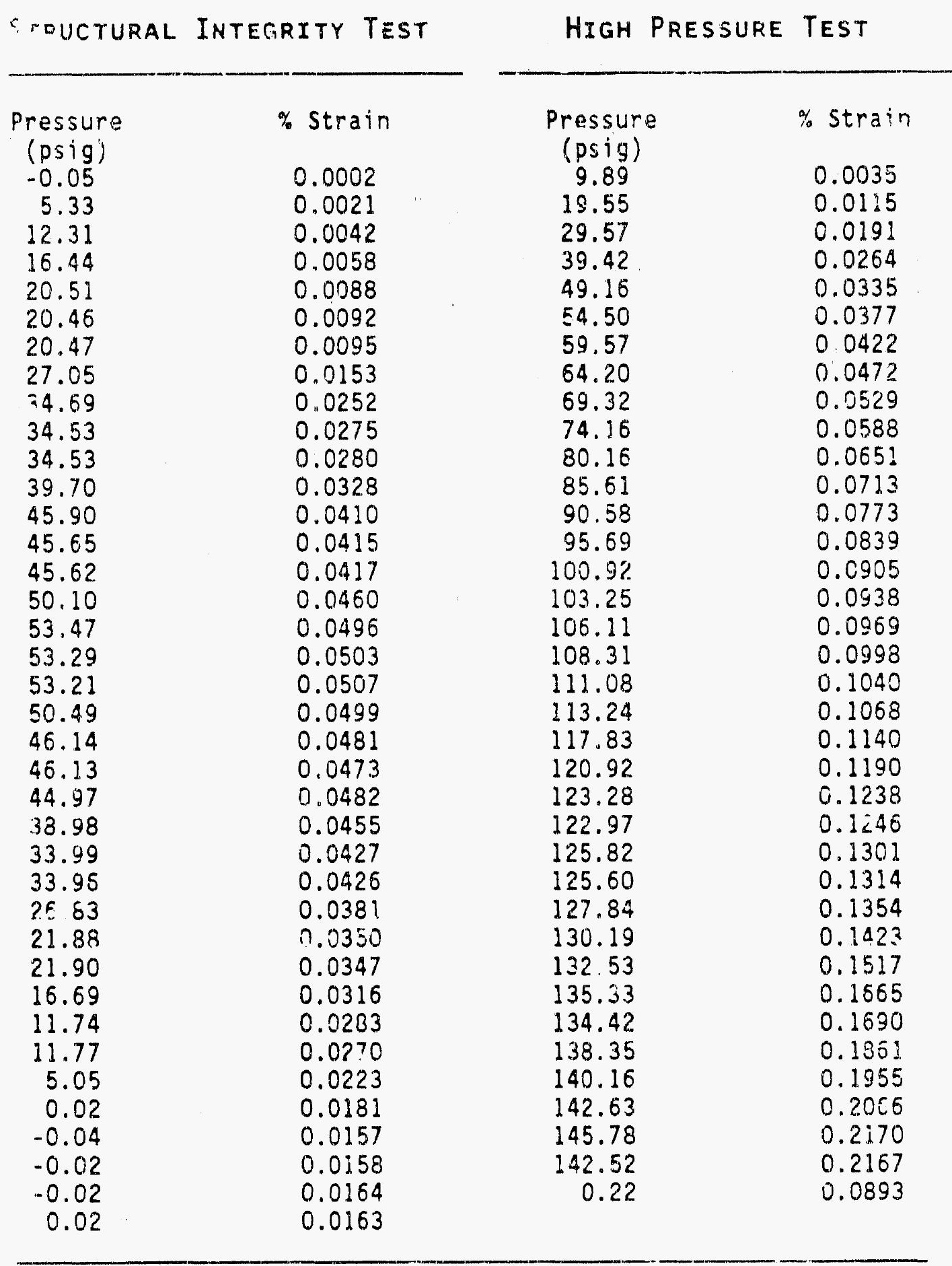




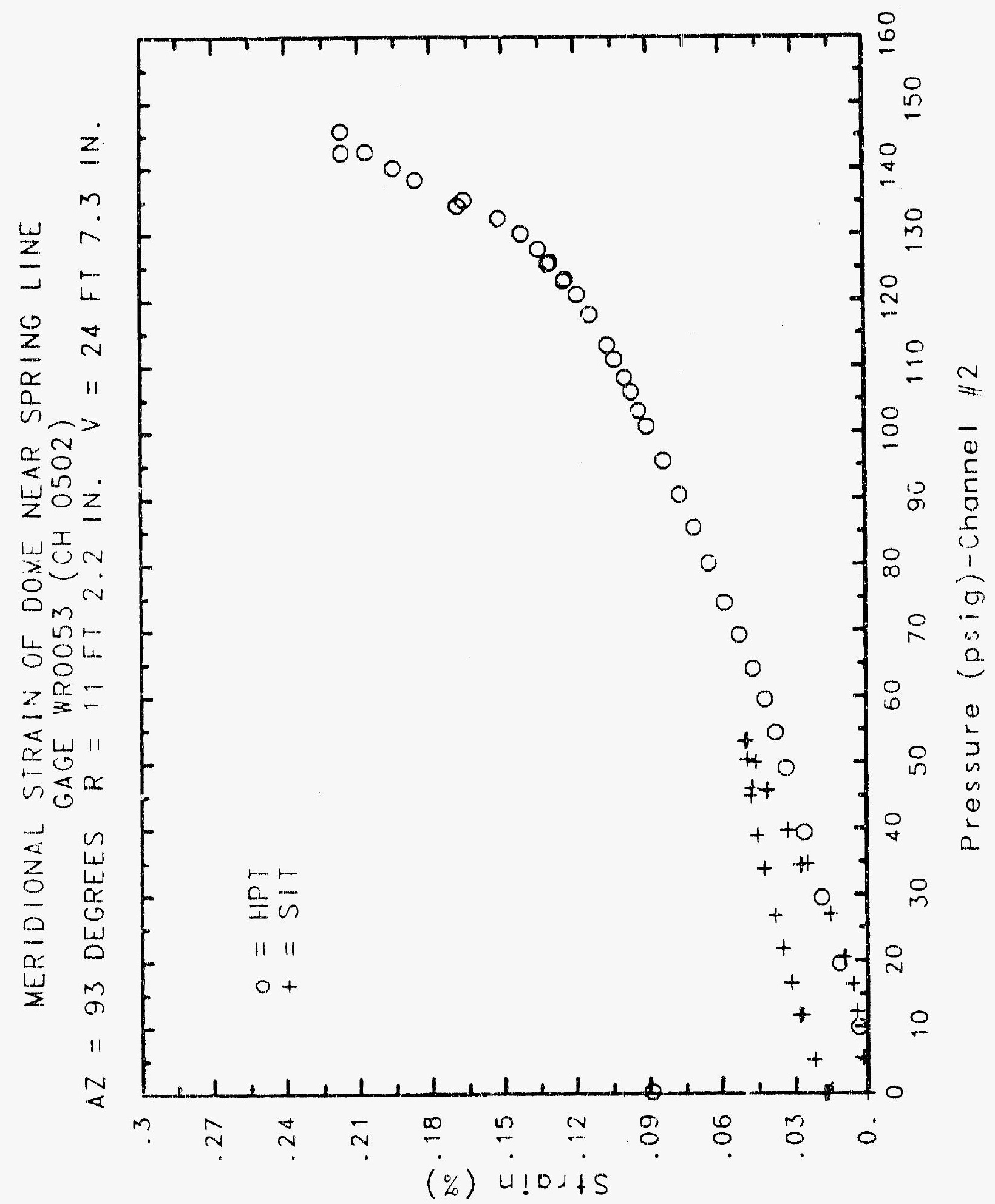




\section{Reinforced Concrete Test Data}

Weldable gage 54 Channel 503

Structural Integrity Test

\begin{tabular}{|c|c|c|c|}
\hline $\begin{array}{c}\text { Pressure } \\
\text { (psig) } \\
-0.05 \\
5.33 \\
12.31 \\
16.44 \\
20.51 \\
20.46 \\
20.47 \\
27.05 \\
34.69 \\
34.53 \\
34.53 \\
39.70 \\
45.90 \\
45.65 \\
45.62 \\
50.10 \\
53.47 \\
53.29 \\
53.21 \\
50.49 \\
46.14 \\
46.13 \\
44.97 \\
38.98 \\
33.99 \\
33.96 \\
26.83 \\
21.88 \\
21.90 \\
16.69 \\
11.74 \\
11.77 \\
5.05 \\
0.02 \\
-0.04 \\
.0 .02 \\
-0.02 \\
0.02 \\
\end{array}$ & $\begin{array}{l}\text { \% Strain } \\
-0.0006 \\
0.0019 \\
0.0043 \\
0.0065 \\
0.0093 \\
0.0100 \\
0.0059 \\
0.0155 \\
0.0231 \\
0.0244 \\
0.0251 \\
0.0292 \\
0.0347 \\
0.0352 \\
0.0351 \\
0.0391 \\
0.0426 \\
0.0425 \\
0.0429 \\
0.0417 \\
0.0395 \\
0.0392 \\
0.0401 \\
0.0368 \\
0.0334 \\
0.0337 \\
0.0284 \\
0.0252 \\
0.0251 \\
0.0219 \\
0.0185 \\
0.0177 \\
0.0132 \\
0.0097 \\
0.0082 \\
0.0090 \\
0.0092 \\
0.0088\end{array}$ & $\begin{array}{c}\text { Pressure } \\
(\text { psig) } \\
9.89 \\
19.55 \\
29.57 \\
39.42 \\
49.16 \\
54.50 \\
59.57 \\
64.20 \\
69.32 \\
74.16 \\
80.16 \\
85.61 \\
90.58 \\
95.69 \\
100.92 \\
103.25 \\
106.11 \\
108.31 \\
111.08 \\
113.24 \\
117.83 \\
120.92 \\
123.28 \\
122.97 \\
125.82 \\
125.60 \\
127.84 \\
130.19 \\
132.53 \\
135.33 \\
134.42 \\
138.35 \\
140.16 \\
142.63 \\
145.78 \\
142.52 \\
0.22\end{array}$ & $\begin{array}{l}\% \text { Strain } \\
0.0023 \\
0.0095 \\
0.0168 \\
0.0243 \\
0.0308 \\
0.0350 \\
0.0392 \\
0.0435 \\
0.0486 \\
0.0534 \\
0.0594 \\
0.0652 \\
0.0706 \\
0.0762 \\
0.0820 \\
0.0851 \\
0.0884 \\
0.0911 \\
0.0945 \\
0.0974 \\
0.1040 \\
0.1086 \\
0.1133 \\
0.1137 \\
0.1185 \\
0.1197 \\
0.1225 \\
0.1280 \\
0.1353 \\
0.1467 \\
0.1490 \\
0.1639 \\
0.1739 \\
0.1882 \\
0.2021 \\
0.2043 \\
0.0812\end{array}$ \\
\hline
\end{tabular}

High Pressure test

Pres?

(psig)

19.55

29.57

49.16

69.32

74.16

90.58

95.69

106.11

113.24

117.83

125.82

25.60

130.19

132.53

135.33

34.42

138.35

40.16

45.78

42.52

0.22
0.0023

0.0095

0.0308

0.0392

0.0435

0.0486

0.0652

.0706

0.0820

0.0851

0.0884

0.0911

0.0974

0.1040

0.1086

0.1133

0.1137

0.1225

0.1280

0.1353

0.1467

0.1490

0.1639

0.1739

0.2021

0.2043

0.0812 


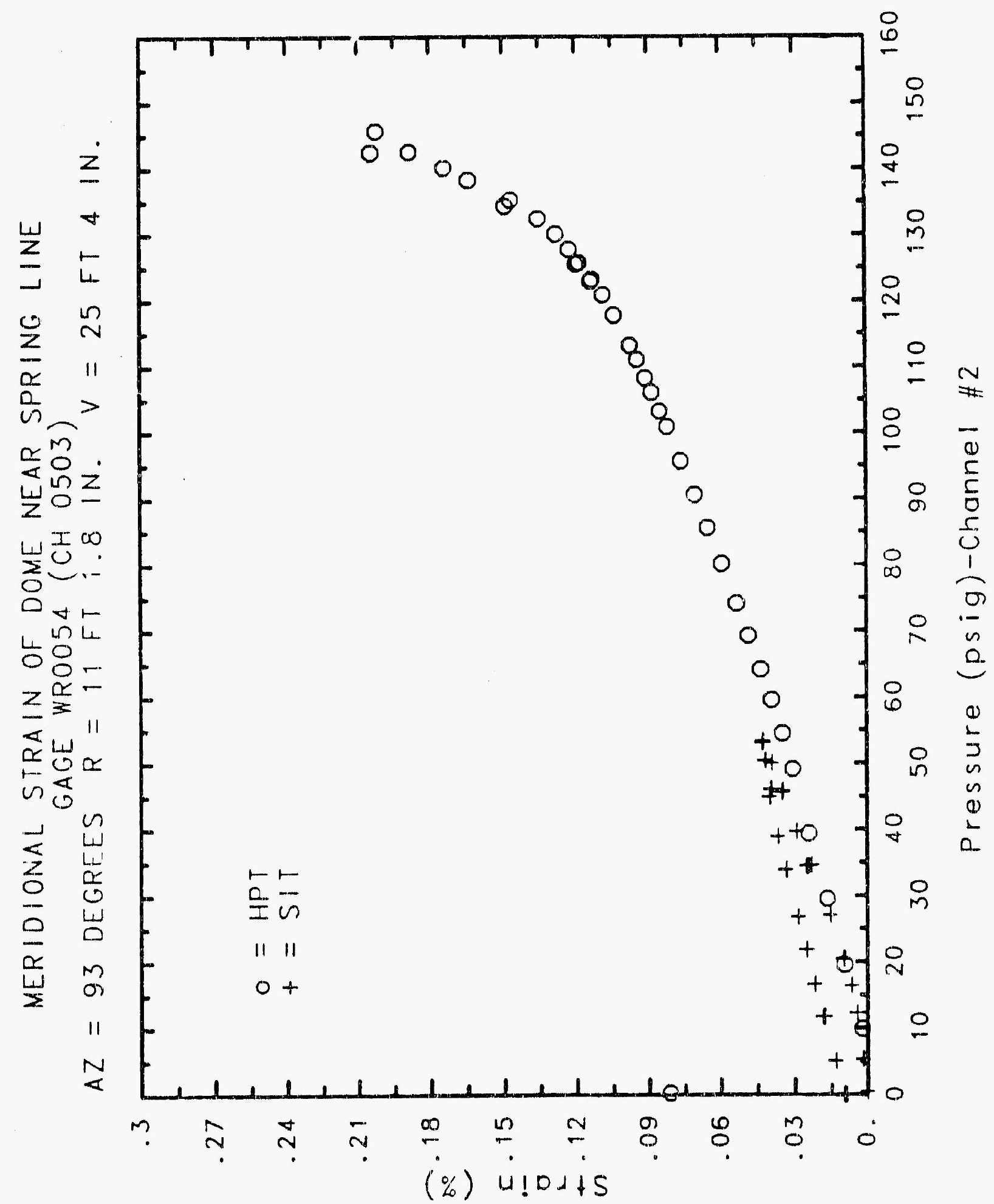




\section{Reinforced Concrete Test Data}

Weldable Gage 55 Channel 504

Structural INTEGRITY TEST

\begin{tabular}{|c|c|c|c|}
\hline $\begin{array}{c}\text { Pressure } \\
\text { (psig) } \\
-0.05 \\
5.33 \\
12.31 \\
16.44 \\
20.51 \\
20.46 \\
20.47 \\
27.05 \\
34.69 \\
34.53 \\
34.53 \\
39.70 \\
45.90 \\
45.65 \\
45.62 \\
50.10 \\
53.47 \\
53.29 \\
53.21 \\
50.49 \\
46.14 \\
46.13 \\
44.97 \\
38.98 \\
33.99 \\
33.96 \\
26.83 \\
21.88 \\
21.90 \\
16.69 \\
11.74 \\
11.77 \\
5.05 \\
0.02 \\
-0.04 \\
-0.02 \\
-0.02 \\
0.02 \\
\end{array}$ & $\begin{array}{c}\text { \% Strain } \\
-0.0011 \\
0.0013 \\
0.0029 \\
0.0044 \\
0.0067 \\
0.0073 \\
0.0071 \\
0.0138 \\
0.0232 \\
0.0239 \\
0.0243 \\
0.0297 \\
0.0344 \\
0.0351 \\
0.0352 \\
0.0389 \\
0.0425 \\
0.0428 \\
0.0424 \\
0.0418 \\
0.0399 \\
0.0393 \\
0.0353 \\
0.0365 \\
0.0337 \\
0.0338 \\
0.0294 \\
0.0263 \\
0.0265 \\
0.0232 \\
0.0201 \\
0.0200 \\
0.0157 \\
0.0129 \\
0.0118 \\
0.0123 \\
0.0124 \\
0.0122\end{array}$ & $\begin{array}{c}\text { Pressure } \\
\text { (psig) } \\
9.89 \\
19.55 \\
29.57 \\
39.42 \\
49.16 \\
54.50 \\
59.57 \\
64.20 \\
69.32 \\
74.16 \\
80.16 \\
85.61 \\
90.58 \\
95.69 \\
100.92 \\
103.25 \\
106.11 \\
108.31 \\
111.08 \\
113.24 \\
117.83 \\
120.92 \\
123.28 \\
122.97 \\
125.82 \\
125.60 \\
127.84 \\
130.19 \\
132.53 \\
135.33 \\
134.42 \\
138.35 \\
140.16 \\
142.63 \\
145.78 \\
142.52 \\
0.22\end{array}$ & $\begin{array}{l}\% \text { Strain } \\
0.0017 \\
0.0081 \\
0.0149 \\
0.0209 \\
0.0272 \\
0.0307 \\
0.0344 \\
0.0385 \\
0.0430 \\
0.0476 \\
0.0528 \\
0.0578 \\
0.0628 \\
0.0683 \\
0.0736 \\
0.0764 \\
0.0790 \\
0.0814 \\
0.0845 \\
0.0869 \\
0.0921 \\
0.0958 \\
0.0990 \\
0.0993 \\
0.1026 \\
0.1028 \\
0.1055 \\
0.1086 \\
0.1115 \\
0.1153 \\
0.1154 \\
0.1203 \\
0.1227 \\
0.1263 \\
0.1304 \\
0.1293 \\
0.0160\end{array}$ \\
\hline
\end{tabular}

High Pressure test

Pressure

0.0017

0.0081

0.0149

0.0307

0.0344

0.0385

0.0528

0.0578

0.0628

0.0683

0.0790

0.0814

0.0845

0.0869

0.0921

0.0958

0.0993

0.1026

0.1028

0.1115

0.1153

0.1154

0.1203

0.1227

0.1263

0.1304

0.1293

0.0160 


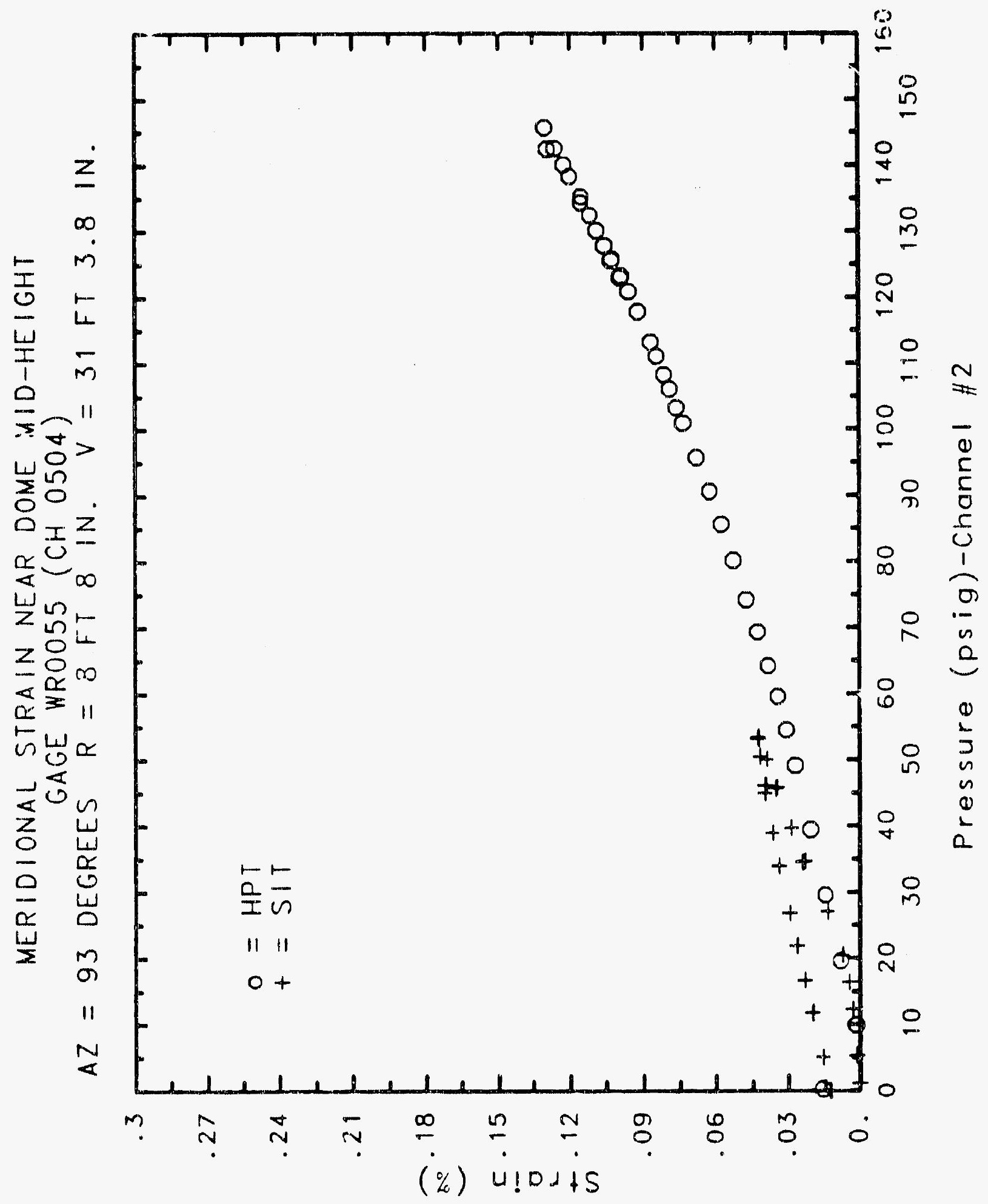

C. 922 


\section{Reinforced Concrete Test Data}

\begin{tabular}{|c|c|c|c|}
\hline STRUCTURAL & $\begin{array}{l}\text { WELDABLE GAGE } 56 \\
\text { INTEGRITY TEST }\end{array}$ & $\begin{array}{l}\text { Channel } 505 \\
\text { High Pressure }\end{array}$ & TEST \\
\hline $\begin{array}{c}\text { Pressure } \\
\text { (psig) } \\
-0.05 \\
5.33 \\
12.31 \\
16.44 \\
20.51 \\
20.46 \\
20.47 \\
27.05 \\
34.69 \\
34.53 \\
34.53 \\
39.70 \\
45.90 \\
45.65 \\
45.62 \\
50.10 \\
53.47 \\
53.29 \\
53.21 \\
50.49 \\
46.14 \\
46.13 \\
44.97 \\
38.98 \\
33.99 \\
33.96 \\
26.83 \\
21.88 \\
21.90 \\
16.69 \\
11.74 \\
11.77 \\
5.05 \\
0.02 \\
-0.04 \\
-0.02 \\
-0.02 \\
0.02 \\
\end{array}$ & $\begin{array}{l}\text { Strain } \\
0.0010 \\
0.0042 \\
0.0082 \\
0.0111 \\
0.0161 \\
0.0168 \\
0.0172 \\
0.0242 \\
0.0345 \\
0.0350 \\
0.0348 \\
0.0408 \\
0.0487 \\
0.0507 \\
0.0519 \\
0.0565 \\
0.0608 \\
0.0617 \\
0.0638 \\
0.0639 \\
0.0610 \\
0.0609 \\
0.0682 \\
0.0645 \\
0.0611 \\
0.0617 \\
0.0558 \\
0.0513 \\
0.0513 \\
0.0466 \\
0.0421 \\
0.0423 \\
0.0363 \\
0.0325 \\
0.0390 \\
0.0402 \\
0.0406 \\
0.0406\end{array}$ & $\begin{array}{c}\text { Pressure } \\
\text { (psig) } \\
9.89 \\
19.55 \\
29.57 \\
39.42 \\
49.16 \\
54.50 \\
59.57 \\
64.20 \\
69.32 \\
74.16 \\
80.16 \\
85.61 \\
90.58 \\
95.69 \\
100.92 \\
103.25 \\
106.11 \\
108.31 \\
111.08 \\
113.24 \\
117.83 \\
120.92 \\
123.28 \\
122.97 \\
125.82 \\
125.60 \\
127.84 \\
130.19 \\
132.53 \\
135.33 \\
134.42 \\
138.35 \\
140.16 \\
142.63 \\
145.78 \\
142.52 \\
0.22 \\
\end{array}$ & $\begin{array}{l}\text { \% Strain } \\
0.0069 \\
0.0157 \\
0.0247 \\
0.0331 \\
0.0412 \\
0.0463 \\
0.0512 \\
0.0564 \\
0.0620 \\
0.0680 \\
0.0772 \\
0.0846 \\
0.0914 \\
0.0986 \\
0.1062 \\
0.1097 \\
0.1137 \\
0.1171 \\
0.1214 \\
0.1244 \\
0.1327 \\
0.1382 \\
0.1430 \\
0.1433 \\
0.1486 \\
0.1490 \\
0.1528 \\
0.1574 \\
0.1625 \\
0.1691 \\
0.1696 \\
0.1771 \\
0.1820 \\
0.1891 \\
0.1960 \\
0.1951 \\
0.0272\end{array}$ \\
\hline
\end{tabular}




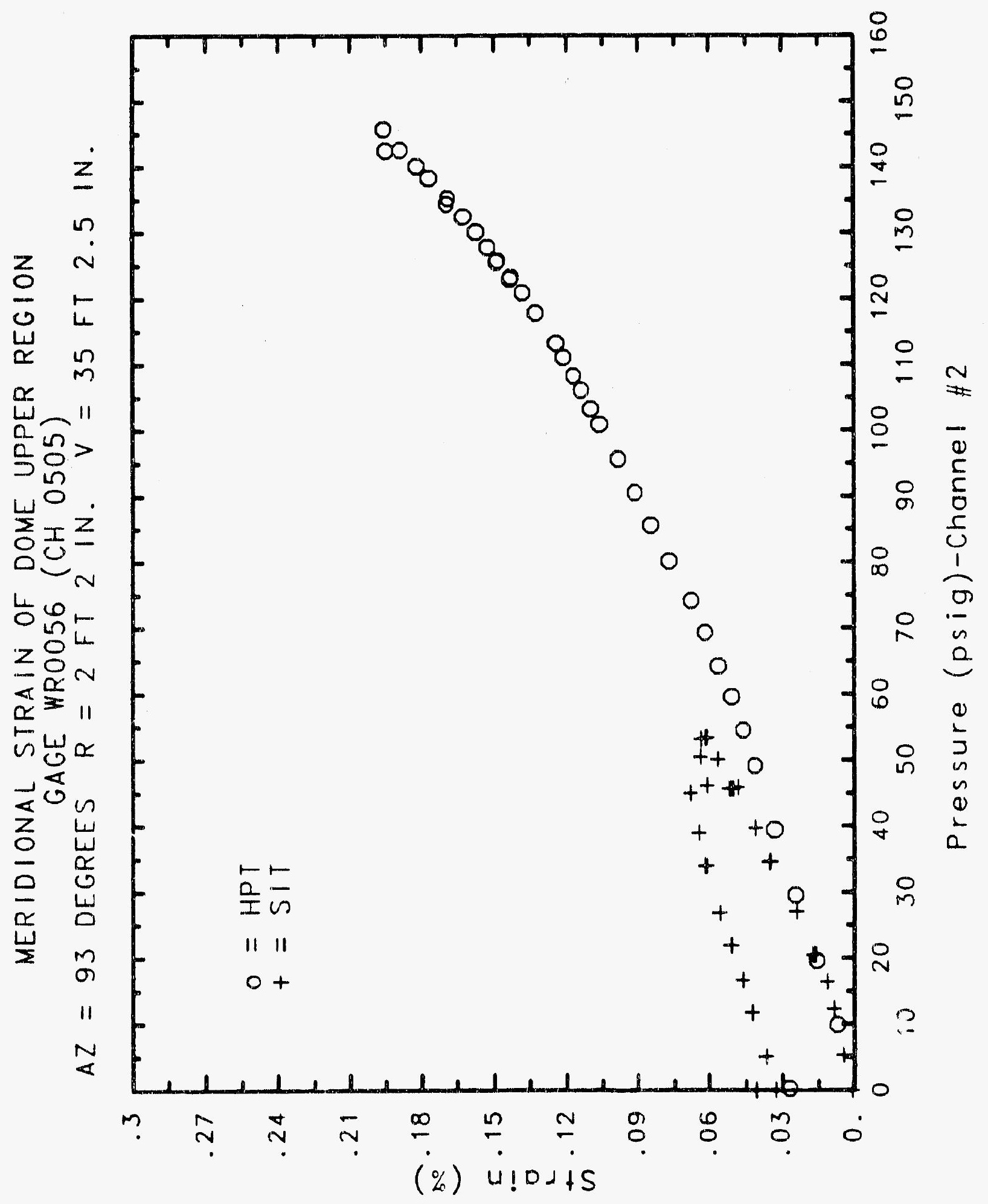




\section{Reinforced Concrete Test Data}

Weldable Gage 57 Channel 506

Strultural InTEgrity TEST

\begin{tabular}{|c|c|c|c|}
\hline $\begin{array}{c}\text { Pressure } \\
\text { (psig) } \\
-0.05 \\
5.33 \\
12.31 \\
16.44 \\
20.51 \\
20.46 \\
20.47 \\
27.05 \\
34.69 \\
34.53 \\
34.53 \\
39.70 \\
45.90 \\
45.65 \\
45.62 \\
50.10 \\
53.47 \\
53.29 \\
53.21 \\
50.49 \\
46.14 \\
46.13 \\
44.97 \\
38.98 \\
33.99 \\
33.96 \\
26.83 \\
21.88 \\
21.90 \\
16.69 \\
11.74 \\
11.77 \\
5.05 \\
0.02 \\
-0.04 \\
-0.02 \\
-0.02 \\
0.02\end{array}$ & $\begin{array}{c}\text { \% Strain } \\
\text {-0.0003 } \\
0.0014 \\
0.0032 \\
0.0045 \\
0.0067 \\
0.0072 \\
0.0081 \\
0.0137 \\
0.0216 \\
0.0232 \\
0.0237 \\
0.0274 \\
0.0325 \\
0.0319 \\
0.0316 \\
0.0340 \\
0.0365 \\
0.0358 \\
0.0359 \\
0.0344 \\
0.0323 \\
0.0322 \\
0.0324 \\
0.0297 \\
0.0279 \\
0.0278 \\
0.0247 \\
0.0222 \\
0.0227 \\
0.0203 \\
0.0177 \\
0.0168 \\
0.0127 \\
0.0097 \\
0.0062 \\
0.0069 \\
0.0071 \\
0.0079\end{array}$ & $\begin{array}{c}\text { Pressure } \\
\text { (psig) } \\
9.89 \\
19.55 \\
29.57 \\
39.42 \\
49.16 \\
54.50 \\
59.57 \\
64.20 \\
69.32 \\
74.16 \\
80.16 \\
85.61 \\
90.58 \\
95.69 \\
100.92 \\
103.25 \\
106.11 \\
108.31 \\
111.08 \\
113.24 \\
117.83 \\
120.92 \\
123.28 \\
122.97 \\
125.92 \\
125.60 \\
127.84 \\
130.19 \\
132.53 \\
135.33 \\
134.42 \\
138.35 \\
140.16 \\
142.63 \\
145.78 \\
142.52 \\
0.22\end{array}$ & $\begin{array}{l}\text { \% Strain } \\
0.0026 \\
0.0091 \\
0.0152 \\
0.0211 \\
0.0266 \\
0.0298 \\
0.0328 \\
0.0366 \\
0.0405 \\
0.0446 \\
0.0475 \\
0.0518 \\
0.0554 \\
0.0593 \\
0.0641 \\
0.0657 \\
0.0681 \\
0.0698 \\
0.0724 \\
0.0750 \\
0.0797 \\
0.0840 \\
0.0861 \\
0.0875 \\
0.0919 \\
0.0933 \\
0.0962 \\
0.1011 \\
0.1062 \\
0.1125 \\
0.1139 \\
0.1210 \\
0.1260 \\
0.1331 \\
0.1407 \\
0.1413 \\
0.0333\end{array}$ \\
\hline
\end{tabular}

\section{High Pressure test}

Pressure

(psig)

19.55

0.0091

0.0152

0.0211

0.0298

0.0328

0.0366

0.0405

0.0446

0.0554

0.0593

0.0641

0.0657

0681

0.0724

0.0750

0.0797

0.0840

0.0861

0.0919

0.0933

0.0962

0.1011

0.1062

0.1210

0.1260

0.1331

0.1407

0.1413

0.0333 


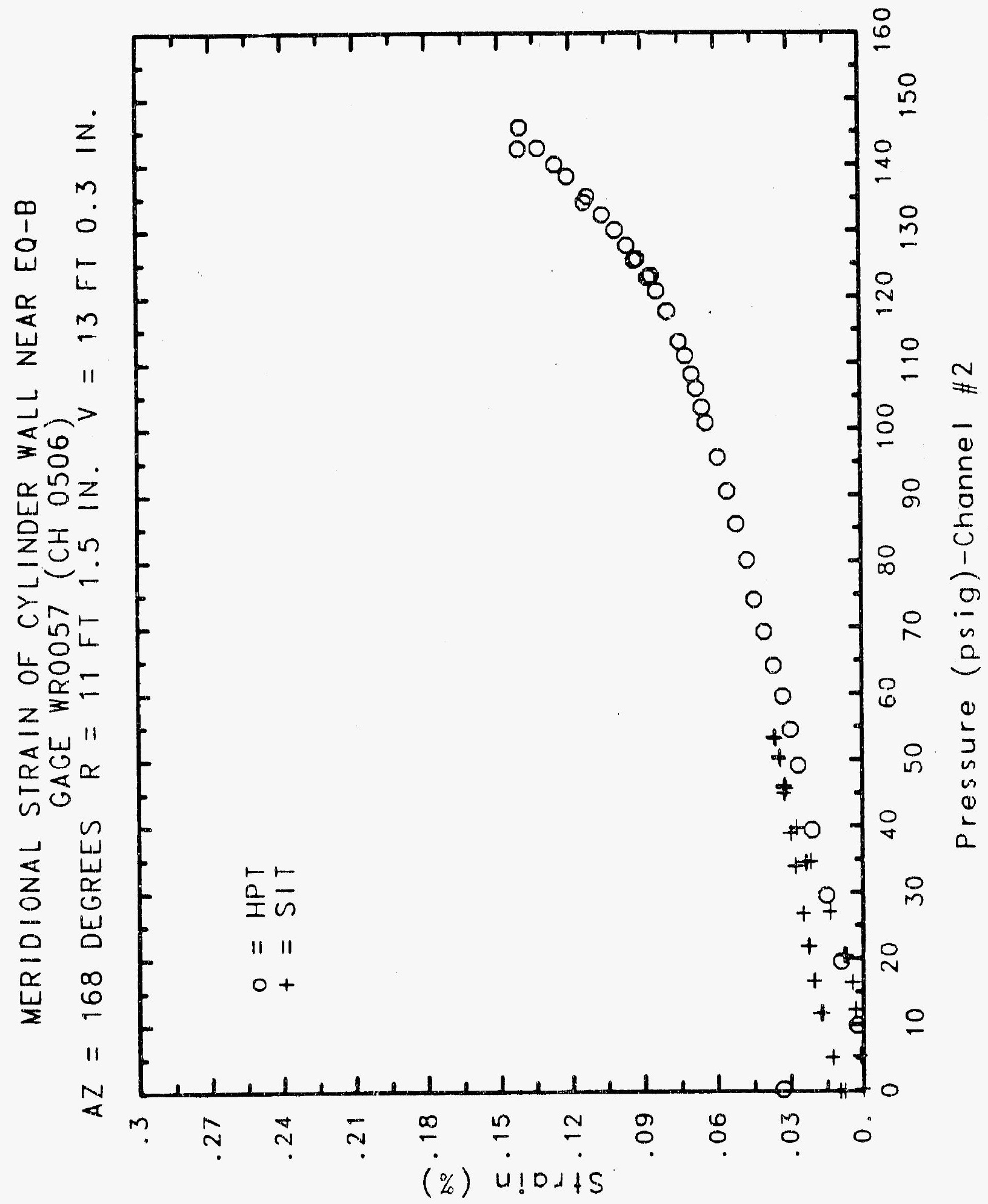

"C.926 


\section{Reinforced Concrete Test Data}

Weldable gage 58 Channel 507

STEIJCTURAL INTEGRITY TEST

High Pressure test

\begin{tabular}{|c|c|c|c|}
\hline $\begin{array}{c}\text { Pressure } \\
\text { (psig) } \\
-0.05 \\
5.33 \\
12.31 \\
16.44 \\
20.51 \\
20.46 \\
20.47 \\
27.05 \\
34.69 \\
34.53 \\
34.53 \\
39.70 \\
45.90 \\
45.65 \\
45.62 \\
50.10 \\
53.47 \\
53.29 \\
53.21 \\
50.49 \\
46.14 \\
46.13 \\
44.97 \\
38.98 \\
33.99 \\
33.96 \\
26.83 \\
21.88 \\
21.90 \\
16.69 \\
11.74 \\
11.77 \\
5.05 \\
0.02 \\
-0.04 \\
-0.02 \\
-0.02 \\
0.02 \\
\end{array}$ & $\begin{array}{l}\text { \% Strain } \\
0.0007 \\
0.0018 \\
0.0040 \\
0.0052 \\
0.0070 \\
0.0083 \\
0.0085 \\
0.0139 \\
0.0215 \\
0.0235 \\
0.0240 \\
0.0270 \\
0.0332 \\
0.0327 \\
0.0324 \\
0.0353 \\
0.0384 \\
0.0382 \\
0.0380 \\
0.0373 \\
0.0347 \\
0.0344 \\
0.0360 \\
0.0340 \\
0.0314 \\
0.0315 \\
0.0280 \\
0.0253 \\
0.0256 \\
0.0231 \\
0.0208 \\
0.0193 \\
0.0153 \\
0.0115 \\
0.0072 \\
0.0075 \\
0.0080 \\
0.0089\end{array}$ & $\begin{array}{c}\text { Pressure } \\
\text { (psig) } \\
9.89 \\
19.55 \\
29.57 \\
39.42 \\
49.16 \\
54.50 \\
59.57 \\
64.20 \\
69.32 \\
74.16 \\
80.16 \\
85.61 \\
90.58 \\
95.69 \\
100.92 \\
103.25 \\
106.11 \\
108.31 \\
111.08 \\
113.24 \\
117.83 \\
120.92 \\
123.28 \\
122.97 \\
125.82 \\
125.60 \\
127.84 \\
130.19 \\
132.53 \\
135.33 \\
134.42 \\
138.35 \\
140.16 \\
142.63 \\
145.78 \\
142.52 \\
0.22\end{array}$ & $\begin{array}{l}\text { \% Strain } \\
0.0024 \\
0.0090 \\
0.0157 \\
0.0221 \\
0.0284 \\
0.0322 \\
0.0363 \\
0.0406 \\
0.0448 \\
0.0496 \\
0.0550 \\
0.0605 \\
0.0655 \\
0.0709 \\
0.0772 \\
0.0804 \\
0.0835 \\
0.0861 \\
0.0899 \\
0.0927 \\
0.0990 \\
0.1046 \\
0.1101 \\
0.1109 \\
0.1169 \\
0.1190 \\
0.1231 \\
0.1284 \\
0.1348 \\
0.1422 \\
0.1441 \\
0.1521 \\
0.1580 \\
0.1659 \\
0.1741 \\
0.1753 \\
0.0500\end{array}$ \\
\hline
\end{tabular}




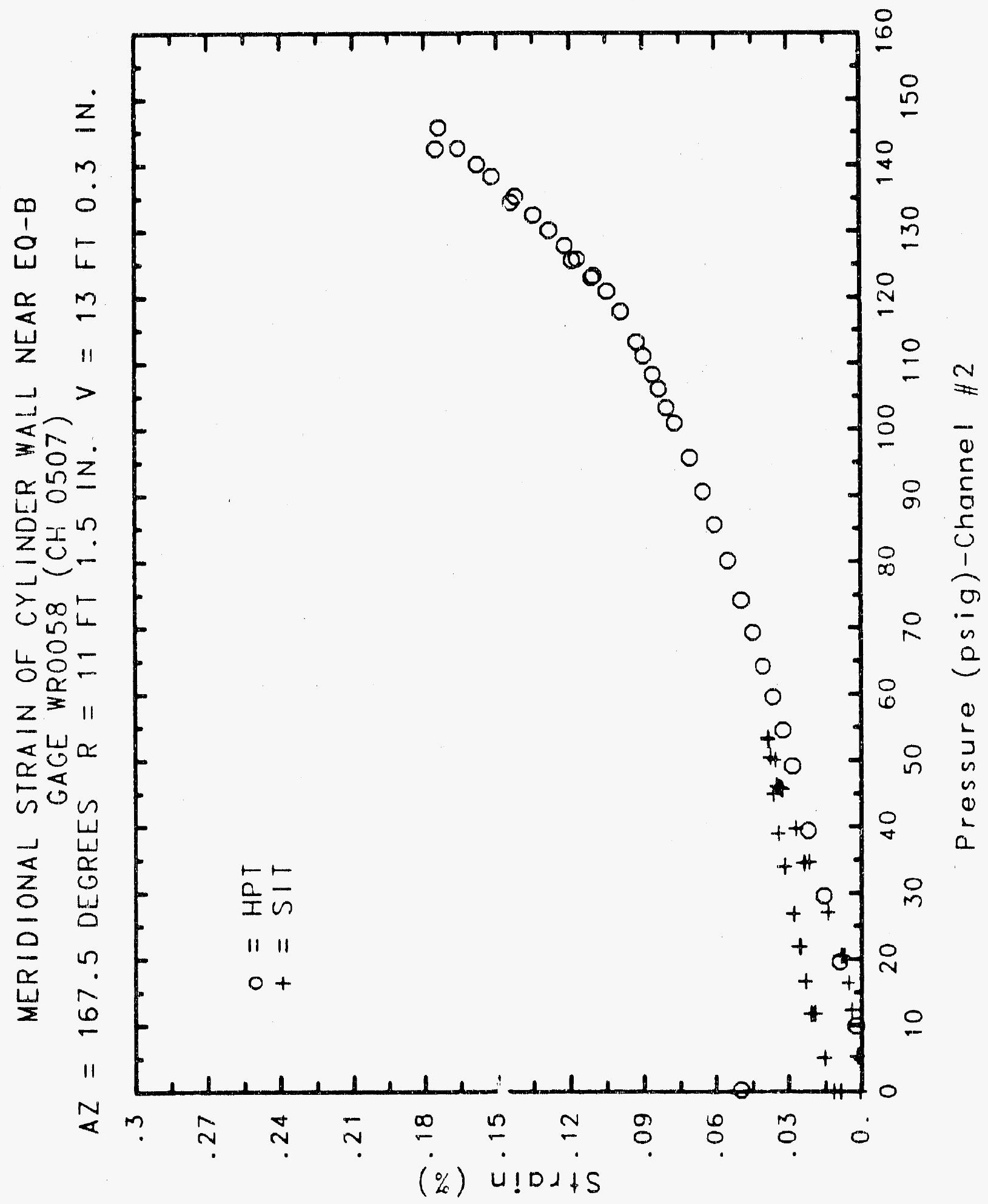

C-928 


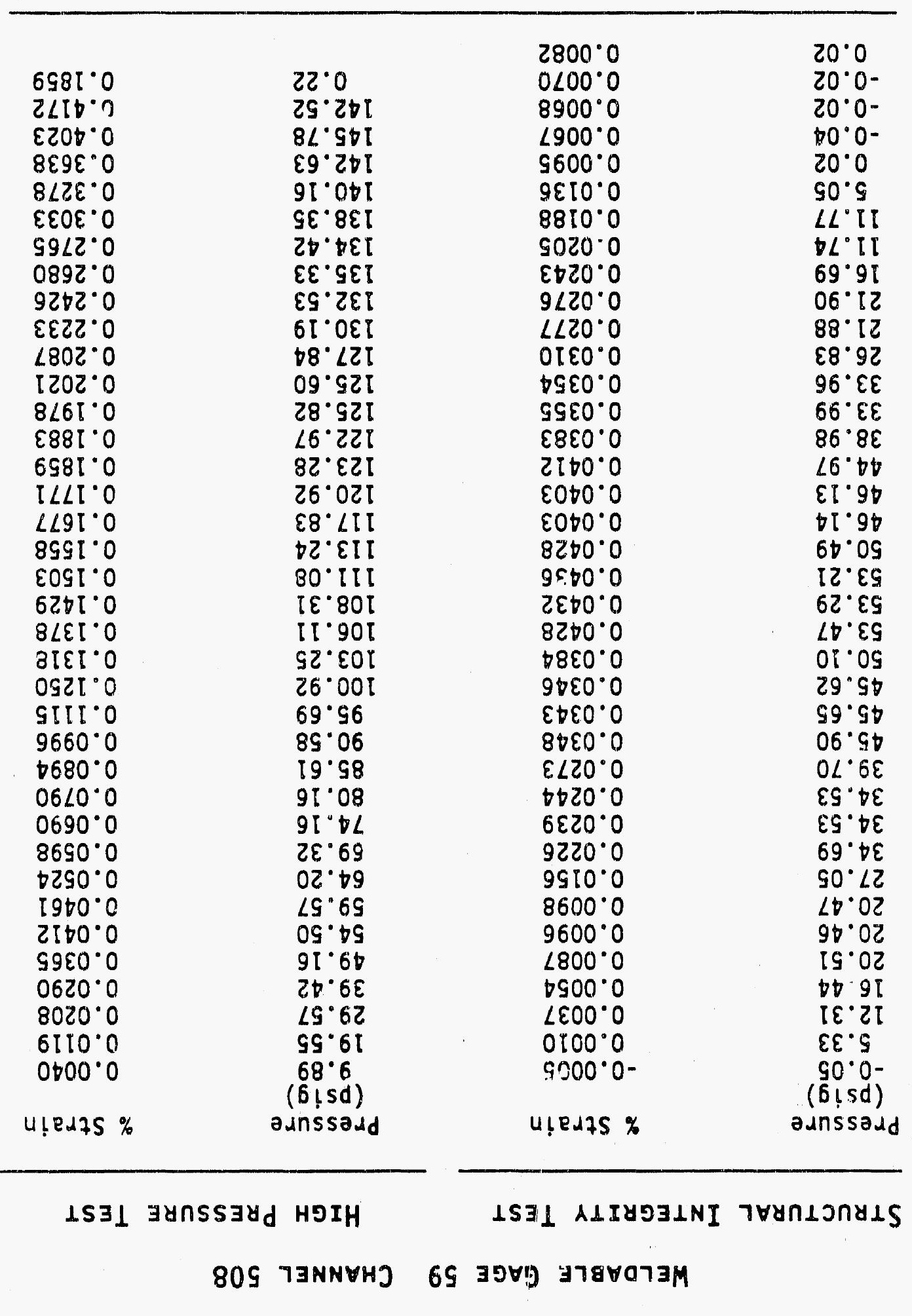

\section{$\forall \perp \forall O \perp S \exists \perp$ JIJYJNOJ GJJUOANIJY}




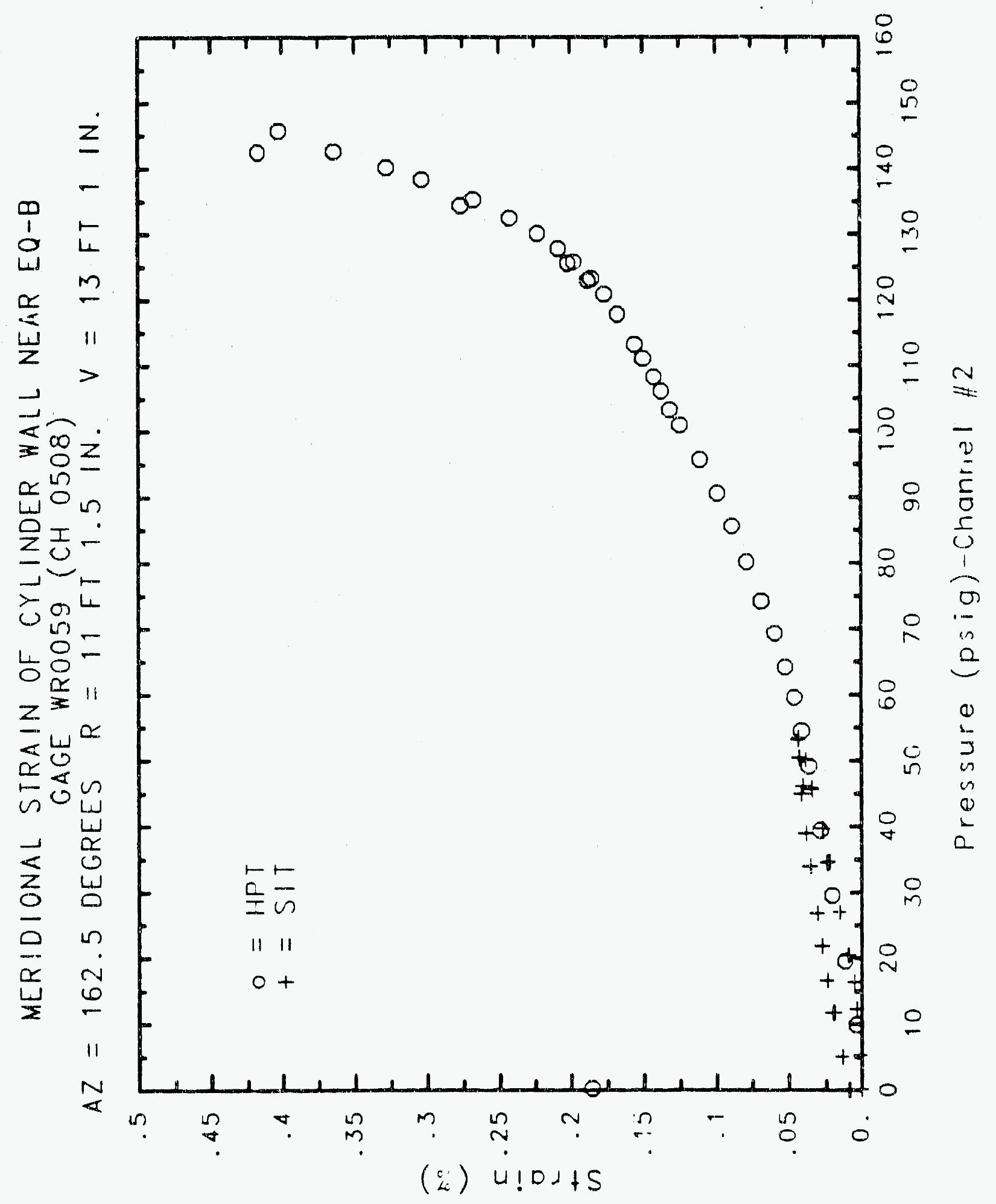




\section{Reinforced Concrete Test Data}

Weldable Gage 60 Channel 509

STRUCTURAL INTEGRITY TEST

High Pressure test

\begin{tabular}{|c|c|c|c|}
\hline $\begin{array}{c}\text { Pressure } \\
\text { (psig) } \\
-0.05 \\
5.37 \\
12.31 \\
16.44 \\
20.51 \\
20.46 \\
20.47 \\
27.05 \\
34.69 \\
34.53 \\
34.53 \\
39.70 \\
45.90 \\
45.65 \\
45.62 \\
50.10 \\
53.17 \\
53.29 \\
53.21 \\
50.49 \\
46.14 \\
46.13 \\
44.97 \\
38.9 \\
33\end{array}$ & $\begin{array}{l}\text { \% Strain } \\
0.0100 \\
0.0019 \\
0.0067 \\
0.0096 \\
0.0147 \\
0.0161 \\
0.0167 \\
0.0298 \\
0.0446 \\
0.0472 \\
0.0475 \\
0.0536 \\
0.0644 \\
0.0642 \\
0.0642 \\
0.0704 \\
0.0764 \\
0.0775 \\
0.0782 \\
0.0768 \\
0.0733 \\
0.0734 \\
0.0743 \\
0.0690 \\
0.0634 \\
0.0629 \\
0.0546 \\
0.0485 \\
0.0482 \\
0.0423 \\
0.0352 \\
0.0323 \\
0.0225 \\
0.0148 \\
0.0119 \\
0.0120 \\
0.0122 \\
0.0129\end{array}$ & $\begin{array}{c}\text { Pressure } \\
(p 5 i g) \\
9.89 \\
19.55 \\
29.57 \\
39.42 \\
49.16 \\
54.50 \\
59.57 \\
64.20 \\
69.32 \\
74.16 \\
80.16 \\
85.61 \\
90.58 \\
95.69 \\
100.92 \\
103.25 \\
100.11 \\
108.31 \\
111.08 \\
113.24 \\
117.83 \\
120.92 \\
123.28 \\
122.97 \\
125.82 \\
125.60 \\
127.84 \\
130.19 \\
132.53 \\
135.33 \\
134.42 \\
138.35 \\
140.16 \\
142.63 \\
145.78 \\
142.52 \\
0.22 \\
\end{array}$ & $\begin{array}{l}\% \text { Strain } \\
0.0096 \\
0.0243 \\
0.0386 \\
0.0524 \\
0.0644 \\
0.0719 \\
0.0793 \\
0.0875 \\
0.0968 \\
0.1069 \\
0.1183 \\
0.1291 \\
0.1398 \\
0.1515 \\
0.1630 \\
0.1688 \\
0.1747 \\
0.1789 \\
0.1849 \\
0.1895 \\
0.1992 \\
0.2061 \\
0.2144 \\
0.2167 \\
0.2271 \\
0.2301 \\
0.2371 \\
0.2493 \\
0.2627 \\
0.2818 \\
0.2887 \\
0.3183 \\
0.3455 \\
0.3854 \\
0.4242 \\
0.4352 \\
0.2127\end{array}$ \\
\hline
\end{tabular}




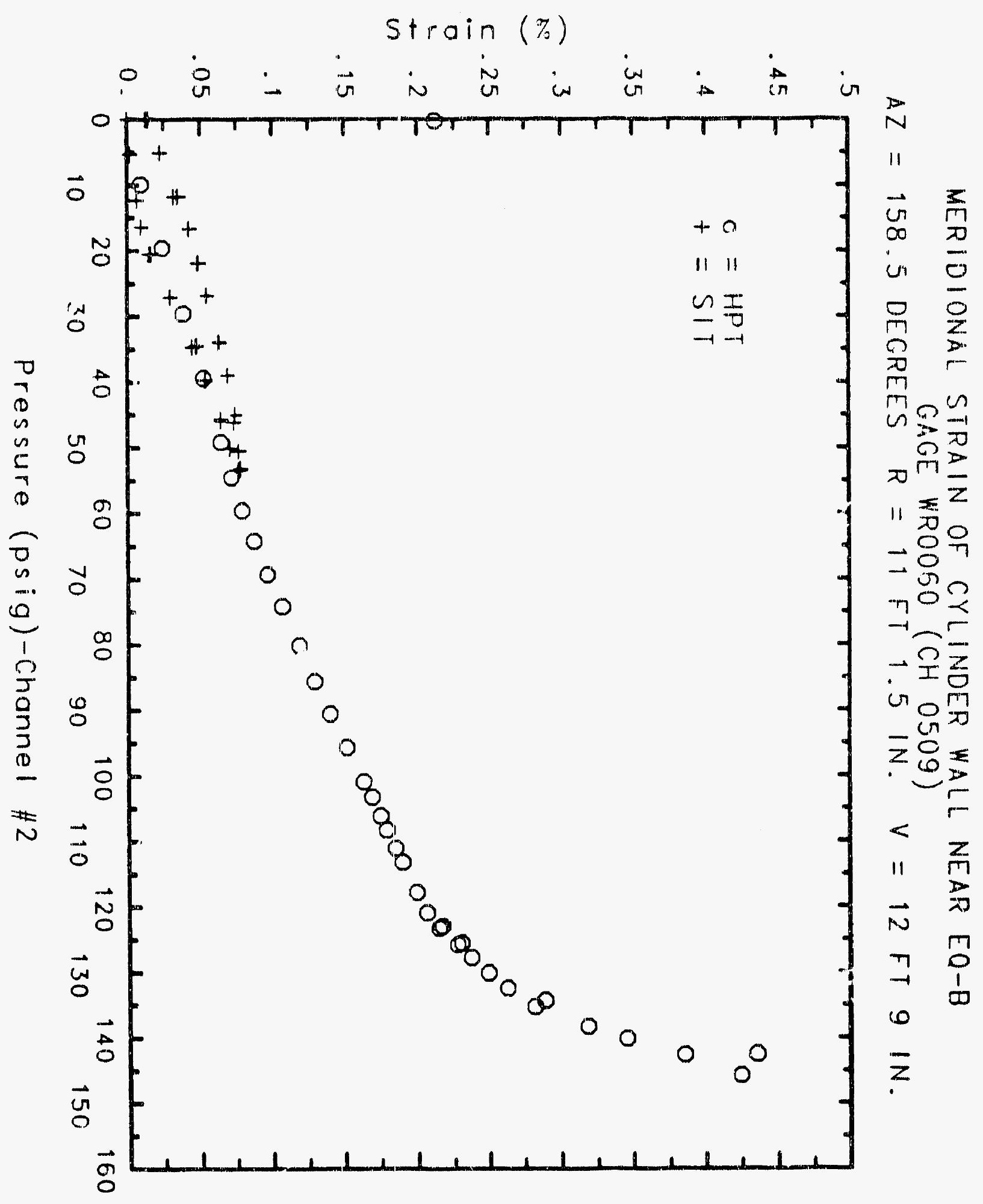




\section{Reinforced Concrete Test Data}

Weldable gage 61 Channel 520

Structural Integrity Test

High Pressure test

\begin{tabular}{|c|c|c|c|}
\hline $\begin{array}{c}\text { Pressure } \\
\text { (psig) } \\
-0.05 \\
5.33 \\
12.31 \\
16.44 \\
20.51 \\
20.46 \\
20.47 \\
27.05 \\
34.09 \\
34.53 \\
34.53 \\
39.70 \\
45.90 \\
45.65 \\
45.62 \\
50.10 \\
53.47 \\
53.29 \\
53.21 \\
50.49 \\
46.14 \\
46.13 \\
44.97 \\
38.98 \\
33.94 \\
33.96 \\
26.83 \\
21.88 \\
21.90 \\
16.69 \\
11.74 \\
11.77 \\
5.05 \\
0.02 \\
-0.04 \\
-0.02 \\
-0.02 \\
0.02 \\
\end{array}$ & $\begin{array}{c}\text { \% Strain } \\
-0.0005 \\
0.0027 \\
0.0075 \\
0.0106 \\
0.0141 \\
0.0154 \\
0.0160 \\
0.0237 \\
0.0356 \\
0.0373 \\
0.0377 \\
0.0449 \\
0.0575 \\
0.0589 \\
0.0591 \\
0.0672 \\
0.0744 \\
0.0760 \\
0.0761 \\
0.0740 \\
0.0694 \\
0.0688 \\
0.0687 \\
0.0628 \\
0.0568 \\
0.0565 \\
0.0482 \\
0.0416 \\
0.0416 \\
0.0355 \\
0.0292 \\
0.0284 \\
0.0205 \\
0.0147 \\
0.0097 \\
0.0098 \\
0.0104 \\
0.0109\end{array}$ & $\begin{array}{c}\text { Pressure } \\
\text { (psig) } \\
9.89 \\
19.55 \\
29.57 \\
39.42 \\
49.16 \\
54.50 \\
59.57 \\
64.20 \\
69.32 \\
74.16 \\
80.16 \\
85.61 \\
90.58 \\
95.69 \\
100.92 \\
103.25 \\
106.11 \\
108.31 \\
111.08 \\
113.24 \\
117.83 \\
120.92 \\
123.28 \\
122.97 \\
125.82 \\
125.60 \\
127.84 \\
130.19 \\
132.53 \\
135.33 \\
134.42 \\
138.35 \\
140.16 \\
142.63 \\
145.78 \\
142.52 \\
0.22\end{array}$ & $\begin{array}{l}\text { \% Strain } \\
0.0057 \\
0.0185 \\
0.0329 \\
0.0465 \\
0.0603 \\
0.0693 \\
0.0791 \\
0.0904 \\
0.0982 \\
0.1087 \\
0.1300 \\
0.1436 \\
0.1556 \\
0.1675 \\
0.1810 \\
0.1882 \\
0.1975 \\
0.2047 \\
0.2182 \\
0.2307 \\
0.2565 \\
0.2686 \\
0.2741 \\
0.2748 \\
0.2780 \\
0.2793 \\
0.2824 \\
0.2855 \\
0.2907 \\
0.3613 \\
0.4397 \\
0.8934 \\
0.9457 \\
1.0194 \\
1.1022 \\
1.1171 \\
0.7289\end{array}$ \\
\hline
\end{tabular}




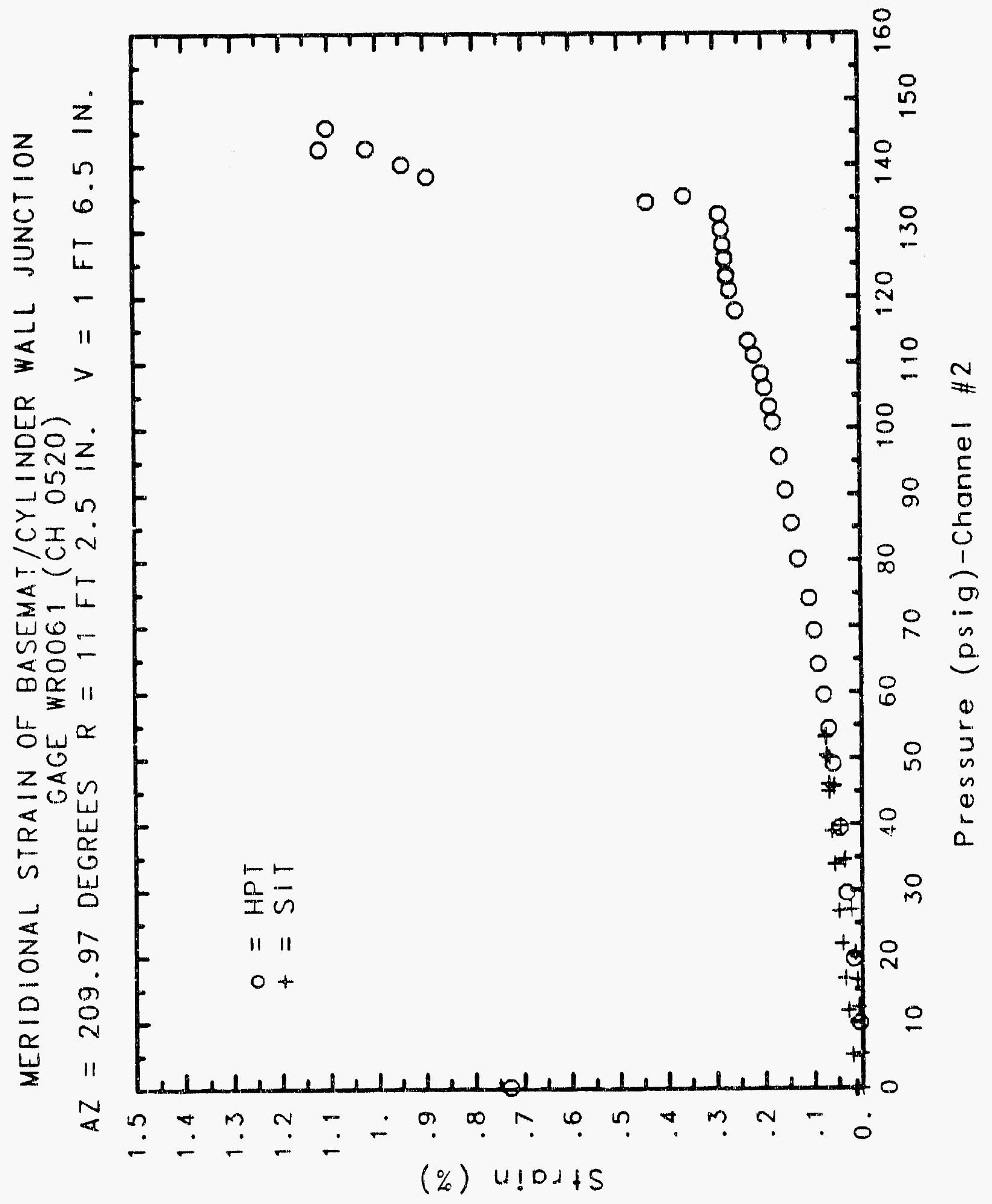




\section{Reinforced Concrete Test Data}

Weldable gage 62 Channel 521

StRUCTURAL INTEgRITY TEST

\begin{tabular}{|c|c|c|c|}
\hline $\begin{array}{c}\text { Pressure } \\
\text { (psig) } \\
-0.05 \\
5.33 \\
12.31 \\
16.44 \\
20.51 \\
20.46 \\
20.47 \\
27.05 \\
34.69 \\
34.53 \\
34.53 \\
39.70 \\
45.90 \\
45.65 \\
45.62 \\
50.10 \\
53.47 \\
53.29 \\
53.21 \\
50.49 \\
46.14 \\
46.13 \\
44.97 \\
38.98 \\
33.99 \\
33.96 \\
26.83 \\
21.88 \\
21.90 \\
16.69 \\
11.74 \\
11.77 \\
5.05 \\
0.02 \\
-0.04 \\
-0.02 \\
-0.02 \\
0.02 \\
\end{array}$ & $\begin{array}{c}\text { \% Strain } \\
-0.0006 \\
0.0015 \\
0.0051 \\
0.0072 \\
0.0115 \\
0.0120 \\
0.0123 \\
0.0232 \\
0.0393 \\
0.0410 \\
0.0413 \\
0.0493 \\
0.0609 \\
0.0611 \\
0.0612 \\
0.0675 \\
0.0731 \\
0.0733 \\
0.0730 \\
0.0708 \\
0.0667 \\
0.0658 \\
0.0654 \\
0.0602 \\
0.0542 \\
0.0546 \\
0.0463 \\
0.0406 \\
0.0408 \\
0.0350 \\
0.0288 \\
0.0281 \\
0.0209 \\
0.0153 \\
0.0128 \\
0.0128 \\
0.0123 \\
0.0126\end{array}$ & $\begin{array}{c}\text { Pressure } \\
(\text { psi }) \\
9.85 \\
19.55 \\
29.57 \\
39.42 \\
49.16 \\
54.50 \\
59.57 \\
64.20 \\
69.32 \\
74.16 \\
80.16 \\
85.61 \\
90.58 \\
95.69 \\
100.92 \\
103.25 \\
106.11 \\
108.31 \\
111.08 \\
113.24 \\
117.83 \\
120.92 \\
123.28 \\
122.97 \\
125.82 \\
125.60 \\
127.84 \\
130.19 \\
132.53 \\
135.33 \\
134.42 \\
138.35 \\
140.16 \\
142.63 \\
145.78 \\
142.52 \\
0.22\end{array}$ & $\begin{array}{l}\% \text { Strain } \\
0.0063 \\
0.0191 \\
0.0330 \\
0.0463 \\
0.0589 \\
0.0661 \\
0.0742 \\
0.0831 \\
0.0907 \\
0.1014 \\
0.1218 \\
0.1349 \\
0.1463 \\
0.1582 \\
0.1701 \\
0.1763 \\
0.1826 \\
0.1878 \\
0.1942 \\
0.1987 \\
0.2087 \\
0.2124 \\
0.2143 \\
0.2139 \\
0.2147 \\
0.2136 \\
0.2143 \\
0.2144 \\
0.2097 \\
0.2038 \\
0.2309 \\
0.5329 \\
0.7686 \\
0.8922 \\
0.9830 \\
1.0115 \\
0.6516\end{array}$ \\
\hline
\end{tabular}

High Pressure Test

Pres

9.83

19.55

49.16

54.50

59.57

64.20

69.32

85.61

90.58

95.69

100.92

103.25

111.08

113.24

120.92

123.28

125.60

127.84

132.53

135.33

134.42

138.35

140.16

142.63

145.78

2.52

0.22
0.0063

0.0191

0.0330

0.0589

0.0661

0.0742

0.0831

0.1218

0.1463

0.1582

0.1701

0.1763

0.1942

0.1987

0.2087

0.2124

0.2143

0.2136

0.2144

0.2097

0.2038

0.2309

0.8922

.9830

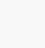




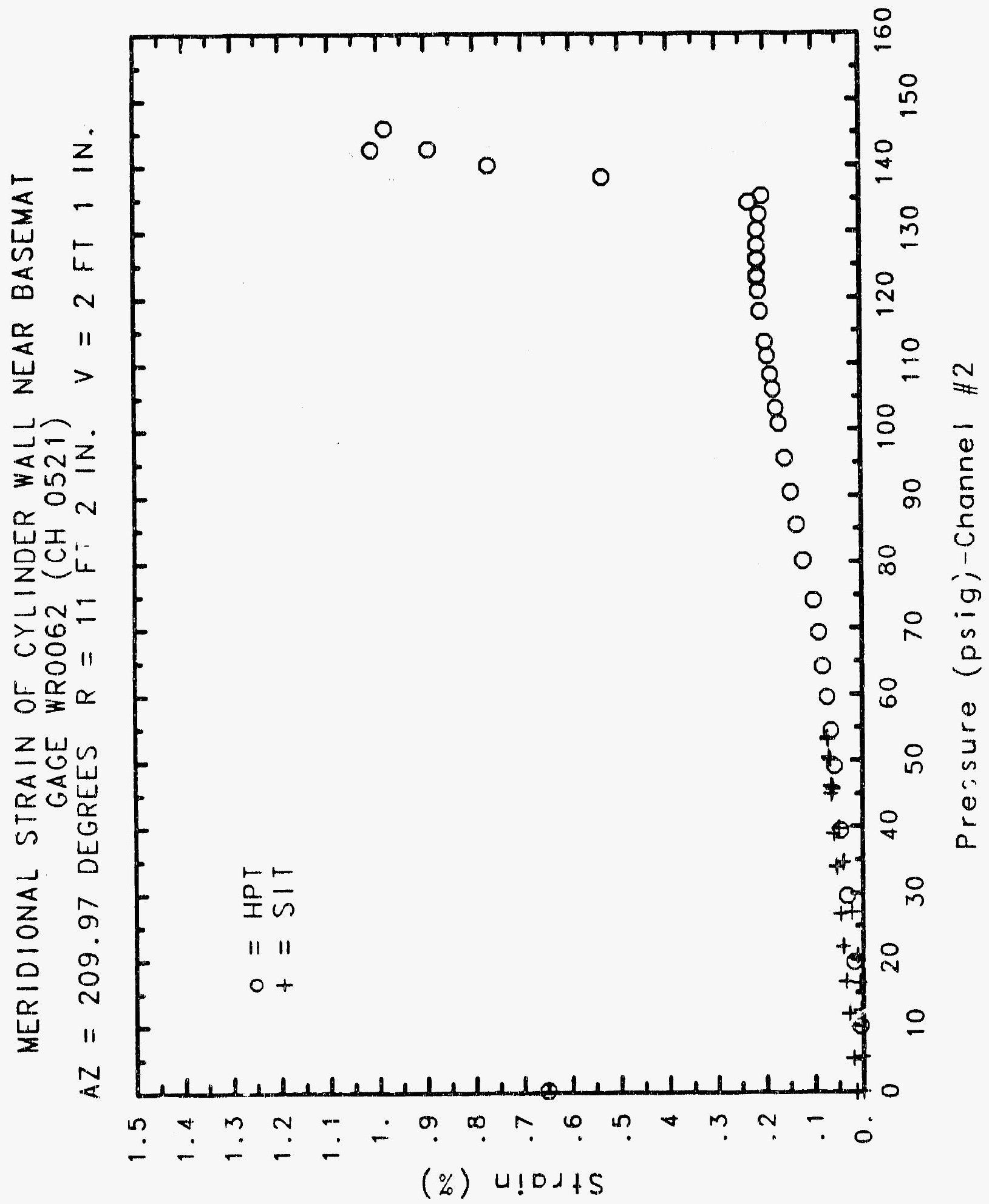

C- 936 


\section{Reinforced Concrete Test Data}

Weldable gage 63 Channel 522

Structural Integrity Test

\begin{tabular}{c} 
Pressure \\
(psig) \\
-0.05 \\
5.33 \\
12.31 \\
16.44 \\
20.51 \\
20.46 \\
20.47 \\
27.05 \\
34.69 \\
34.53 \\
34.53 \\
39.71 \\
45.90 \\
45.65 \\
45.62 \\
50.10 \\
53.47 \\
53.29 \\
53.21 \\
50.49 \\
46.14 \\
46.13 \\
44.97 \\
38.98 \\
33.99 \\
33.96 \\
26.83 \\
21.88 \\
21.90 \\
16.69 \\
11.74 \\
11.77 \\
5.05 \\
0.02 \\
-0.04 \\
-0.02 \\
-0.02 \\
0.02 \\
7 \\
\hline
\end{tabular}

High Pressure test

Pressure \% Strain

(psig)

9.89

19.55

29.57

39.42

49.16

54.50

59.57

64.20

69.32

74.16

80.16

85.61

90.58

95.69

100.92

103.25

106.11

108.31

111.08

113.24

117.83

120.92

123.28

122.97

125.82

125.60

127.84

130.19

132.53

135.33

134.42

138.35

140.16

142.63

145.78

142.52

0.22
0.0022

0.0106

0.0197

0.0276

0.0357

$0.04 C 4$

0.0459

0.0516

0.0575

0.0647

0.0815

0.0932

0.1049

0.1187

0.1337

0.1413

0.1493

0.1552

0.1635

0.1688

0.1794

0.1869

0.1929

0.1938

0.1978

0.1975

0.1989

0.1997

0.2008

0.2036

0.2023

0.2085

0.2127

0.2197

0.2240

0.2217

0.0134 


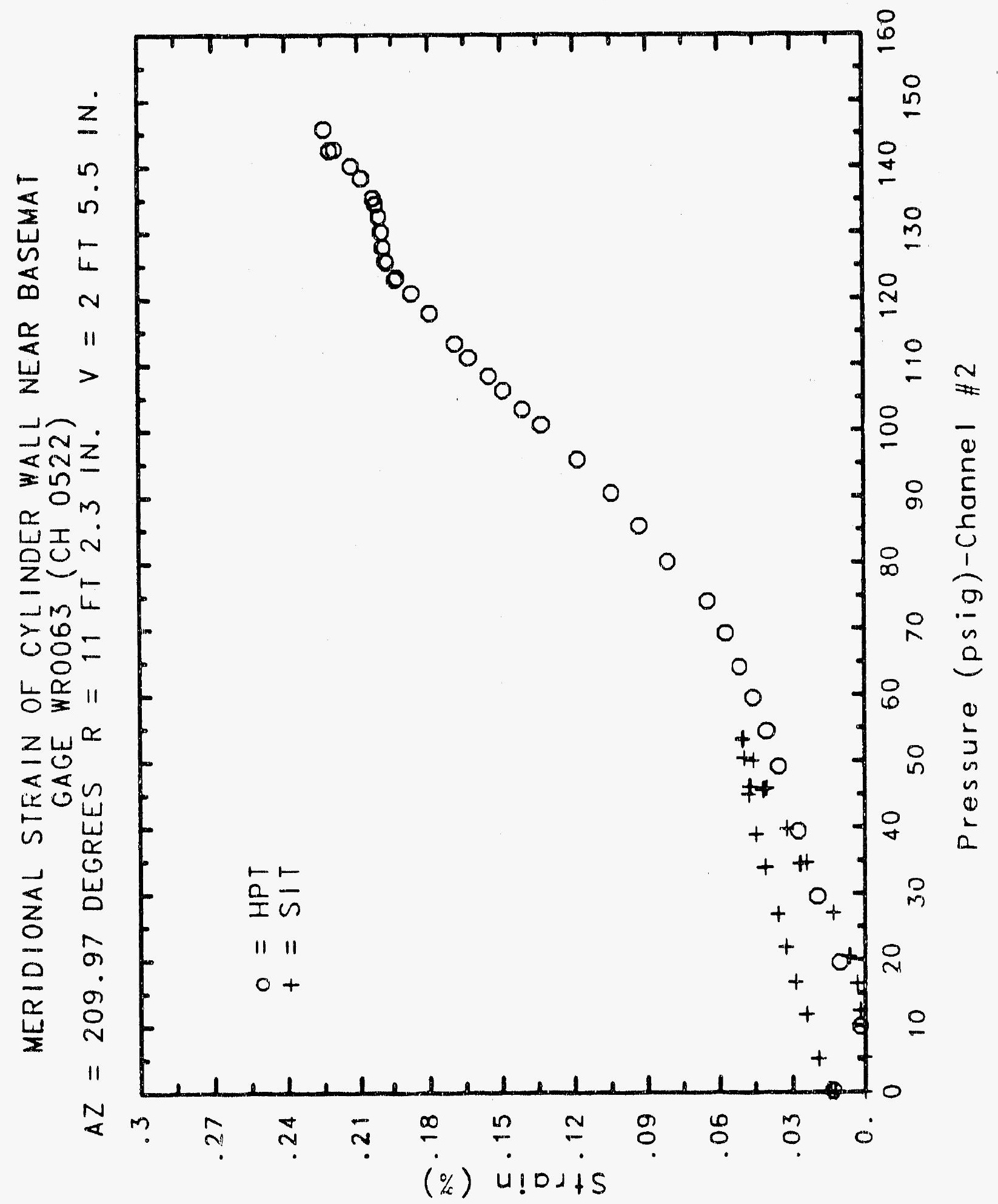

C- 938 


\section{Reinforced Concrete Test Data}

Weldable gage 64 Channel 523

Structural Integrity Test

High Pressure Test

\begin{tabular}{|c|c|c|c|}
\hline $\begin{array}{c}\text { Pressure } \\
\text { (psig) } \\
-0.05 \\
5.33 \\
12.31 \\
16.44 \\
20.51 \\
20.46 \\
20.47 \\
27.05 \\
34.69 \\
34.53 \\
34.53 \\
39.70 \\
45.90 \\
45.65 \\
45.62 \\
50.10 \\
53.47 \\
53.29 \\
53.21 \\
50.49 \\
46.14 \\
46.13 \\
44.97 \\
38.98 \\
33.99 \\
33.96 \\
26.83 \\
21.88 \\
21.90 \\
16.69 \\
11.74 \\
11.77 \\
5.05 \\
0.02 \\
-0.04 \\
-0.02 \\
-0.02 \\
0.02\end{array}$ & $\begin{array}{c}\text { \% Strain } \\
-0.0004 \\
0.0029 \\
0.0066 \\
0.0091 \\
0.0127 \\
0.0129 \\
0.0129 \\
0.0195 \\
0.0286 \\
0.0287 \\
0.0291 \\
0.0335 \\
0.0402 \\
0.0402 \\
0.0403 \\
0.0446 \\
0.0479 \\
0.0478 \\
0.0480 \\
0.0469 \\
0.0443 \\
0.0441 \\
0.0451 \\
0.0411 \\
0.0379 \\
0.0376 \\
0.0322 \\
0.0280 \\
0.0280 \\
0.0240 \\
0.0194 \\
0.0189 \\
0.0141 \\
0.0094 \\
0.0090 \\
0.0083 \\
0.0084 \\
0.0079\end{array}$ & $\begin{array}{c}\text { Pressure } \\
\text { (psig) } \\
9.89 \\
19.55 \\
29.57 \\
39.42 \\
49.16 \\
54.50 \\
59.57 \\
64.20 \\
69.32 \\
74.16 \\
80.16 \\
85.61 \\
90.58 \\
95.69 \\
100.92 \\
103.25 \\
106.11 \\
108.31 \\
111.08 \\
113.24 . . \\
117.83 \\
120.92 \\
123.28 \\
122.97 \\
125.82 \\
125.60 \\
127.84 \\
130.19 \\
132.53 \\
135.33 \\
134.42 \\
138.35 \\
140.16 \\
142.63 \\
145.78 \\
142.52 \\
0.22 \\
0\end{array}$ & $\begin{array}{l}\% \text { Strain } \\
0.0043 \\
0.0122 \\
0.0204 \\
0.0278 \\
0.0350 \\
0.0391 \\
0.0442 \\
0.0489 \\
0.0538 \\
0.0591 \\
0.0644 \\
0.0695 \\
0.0745 \\
0.0809 \\
0.0888 \\
0.0920 \\
0.0958 \\
0.0991 \\
0.1036 \\
0.1074 \\
0.1159 \\
0.1214 \\
0.1274 \\
0.1289 \\
0.1366 \\
0.1375 \\
0.1421 \\
0.1522 \\
0.1670 \\
0.1855 \\
0.1882 \\
0.2062 \\
0.2044 \\
0.1993 \\
0.2236 \\
0.2324 \\
0.0893\end{array}$ \\
\hline
\end{tabular}




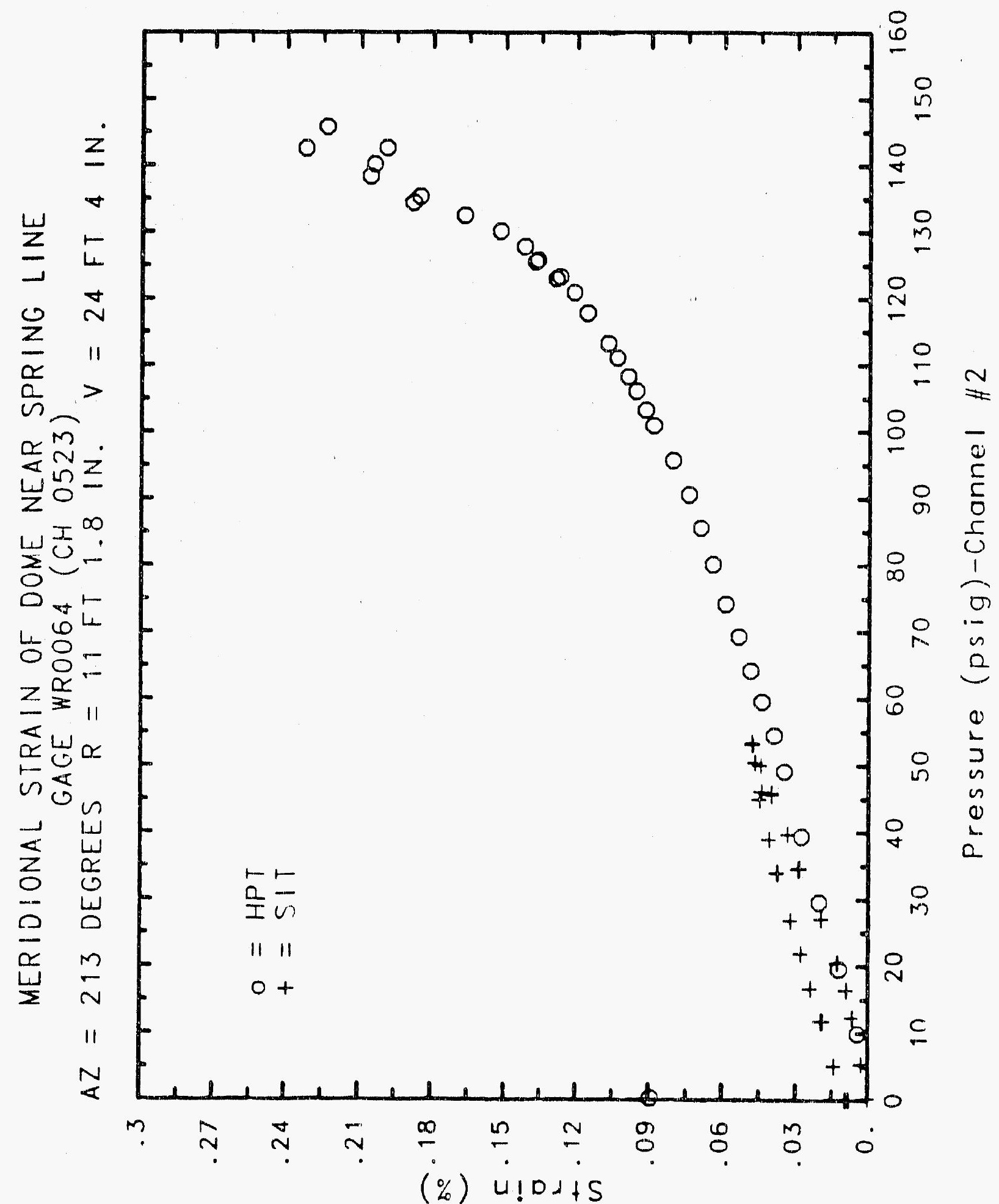




\section{Reinforced Concrete Test Data}

Weldable Gage 65 Channel 524

Structural INTEgRITy TEST

\begin{tabular}{|c|c|c|c|}
\hline $\begin{array}{c}\text { Pressure } \\
\text { (psig) } \\
-0.05 \\
5.33 \\
12.31 \\
16.44 \\
20.51 \\
20.46 \\
20.47 \\
27.05 \\
34.69 \\
34.53 \\
34.53 \\
39.70 \\
45.90 \\
45.65 \\
45.62 \\
50.10 \\
53.47 \\
53.29 \\
53.21 \\
50.49 \\
46.14 \\
46.13 \\
44.97 \\
38.98 \\
33.99 \\
33.96 \\
26.83 \\
21.88 \\
21.90 \\
16.69 \\
11.74 \\
11.77 \\
5.05 \\
0.02 \\
-0.04 \\
-0.02 \\
-0.02 \\
0.02\end{array}$ & $\begin{array}{l}\text { \% Strain } \\
0.0010 \\
0.0018 \\
0.0044 \\
0.0066 \\
0.0101 \\
0.0105 \\
0.0109 \\
0.0186 \\
0.0284 \\
0.0295 \\
0.0298 \\
0.0341 \\
0.0408 \\
0.0414 \\
0.0416 \\
0.0452 \\
0.0492 \\
0.0494 \\
0.0500 \\
0.0490 \\
0.0466 \\
0.0469 \\
0.0475 \\
0.0435 \\
0.0406 \\
0.0404 \\
0.0355 \\
0.0322 \\
0.0319 \\
0.0283 \\
0.0239 \\
0.0235 \\
0.0184 \\
0.0139 \\
0.0144 \\
0.0135 \\
0.0139 \\
0.0136\end{array}$ & $\begin{array}{c}\text { Pressure } \\
(\text { psig) } \\
9.89 \\
19.55 \\
29.57 \\
39.42 \\
49.16 \\
54.50 \\
59.57 \\
64.20 \\
69.32 \\
74.16 \\
80.16 \\
85.61 \\
90.58 \\
95.69 \\
100.92 \\
103.25 \\
106.11 \\
108.31 \\
111.08 \\
113.24 \\
117.83 \\
120.92 \\
123.28 \\
122.97 \\
125.82 \\
125.60 \\
127.84 \\
130.19 \\
132.53 \\
135.33 \\
134.42 \\
138.35 \\
140.16 \\
142.63 \\
145.78 \\
142.52 \\
0.22\end{array}$ & $\begin{array}{l}\text { \% Strain } \\
0.0033 \\
0.0116 \\
0.0191 \\
0.0262 \\
0.0340 \\
0.0377 \\
0.0422 \\
0.0465 \\
0.0519 \\
0.0571 \\
0.0632 \\
0.0689 \\
0.0747 \\
0.0812 \\
0.0879 \\
0.0905 \\
0.0949 \\
0.0977 \\
0.1022 \\
0.1051 \\
0.1125 \\
0.1176 \\
0.1233 \\
0.1252 \\
0.1306 \\
0.1314 \\
0.1350 \\
0.1416 \\
0.1518 \\
0.1677 \\
0.1708 \\
0.1971 \\
0.2212 \\
0.2710 \\
0.3233 \\
0.3356 \\
0.2000\end{array}$ \\
\hline
\end{tabular}

High Pressure test

0.0033

0.0116

0.0191

0.0262

0.0377

0.0465

0.0519

0.0571

0.0632

0.0689

0.0747

0.0879

0.0905

0.0949

0.0977

0.1022

0.1051

0.1233

0.1252

0.1306

0.1314

0.1350

0.1416

0.1708

0.1971

0.2212

0.2710

0.3233

0.3356 ressure 


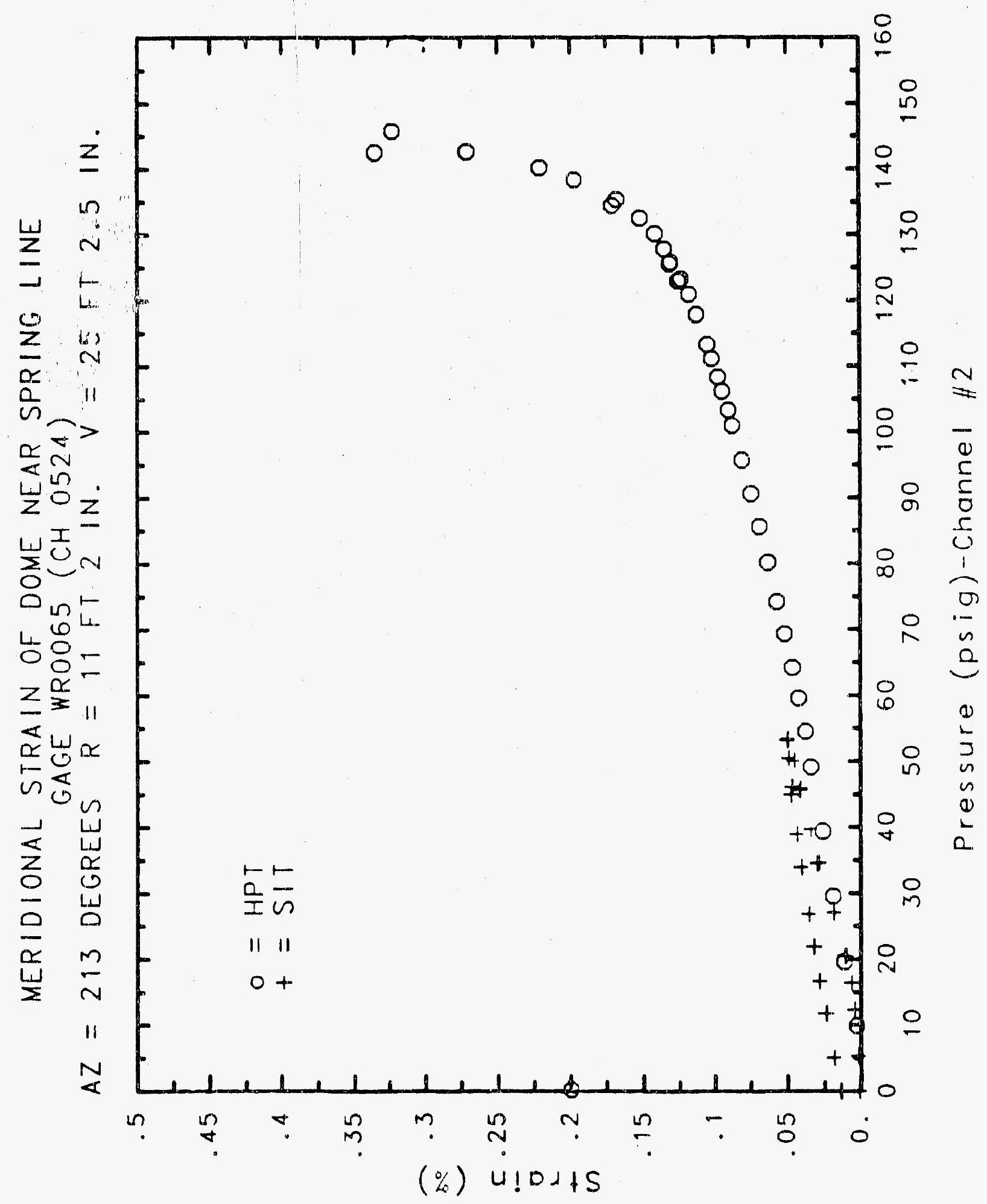




\section{Reinforced Concrete Test Data}

Weldable gage 66 Channel 525

STRUCTURAL INTEGRITY TEST

High Pressure Test

\begin{tabular}{|c|c|c|c|}
\hline $\begin{array}{c}\text { Pressure } \\
\text { (psig) } \\
-0.05 \\
5.33 \\
12.31 \\
16.44 \\
20.51 \\
20.46 \\
20.47 \\
27.05 \\
34.69 \\
34.53 \\
34.53 \\
39.70 \\
45.90 \\
45.65 \\
45.62 \\
50.10 \\
53.47 \\
53.29 \\
53.21 \\
50.49 \\
46.14 \\
46.13 \\
44.97 \\
38.98 \\
33.99 \\
33.96 \\
26.83 \\
21.88 \\
21.90 \\
16.69 \\
11.74 \\
11.77 \\
5.05 \\
0.02 \\
-0.04 \\
-0.02 \\
-0.02 \\
0.02\end{array}$ & $\begin{array}{l}\text { \% Strain } \\
0.0007 \\
0.0028 \\
0.0059 \\
0.0085 \\
0.0128 \\
0.0134 \\
0.0140 \\
0.0224 \\
0.0284 \\
0.0284 \\
0.0284 \\
0.0318 \\
0.0348 \\
0.0345 \\
0.0341 \\
0.0252 \\
0.0517 \\
0.0375 \\
0.0374 \\
0.0361 \\
0.0337 \\
0.0340 \\
0.0363 \\
0.0327 \\
0.0305 \\
0.0298 \\
0.0256 \\
0.0222 \\
0.0220 \\
0.0181 \\
0.0148 \\
0.0137 \\
0.0086 \\
0.0046 \\
0.0047 \\
0.0059 \\
0.0053 \\
0.0044\end{array}$ & $\begin{array}{c}\text { Pressure } \\
\text { (psig) } \\
9.89 \\
19.55 \\
29.57 \\
39.42 \\
49.16 \\
54.50 \\
59.57 \\
64.20 \\
69.32 \\
74.16 \\
80.16 \\
85.61 \\
90.58 \\
95.69 \\
100.92 \\
103.25 \\
106.11 \\
108.31 \\
111.08 \\
113.24 \\
117.83 \\
120.92 \\
123.28 \\
122.97 \\
125.82 \\
125.60 \\
127.84 \\
130.19 \\
132.53 \\
135.33 \\
134.42 \\
138.35 \\
140.16 \\
142.63 \\
145.78 \\
142.52 \\
0.22\end{array}$ & $\begin{array}{l}\text { Strain } \\
0.0031 \\
0.0087 \\
0.0150 \\
0.0209 \\
0.0258 \\
0.0291 \\
0.0315 \\
0.0349 \\
0.0383 \\
0.0425 \\
0.0473 \\
0.0545 \\
0.0617 \\
0.0698 \\
0.0798 \\
0.0854 \\
0.0910 \\
0.0958 \\
0.1030 \\
0.1098 \\
0.1275 \\
0.1447 \\
0.1638 \\
0.1711 \\
0.1926 \\
0.2028 \\
0.2147 \\
0.2405 \\
0.2710 \\
0.3073 \\
0.3194 \\
0.3548 \\
0.3841 \\
0.4207 \\
0.4541 \\
0.4654 \\
0.3146\end{array}$ \\
\hline
\end{tabular}




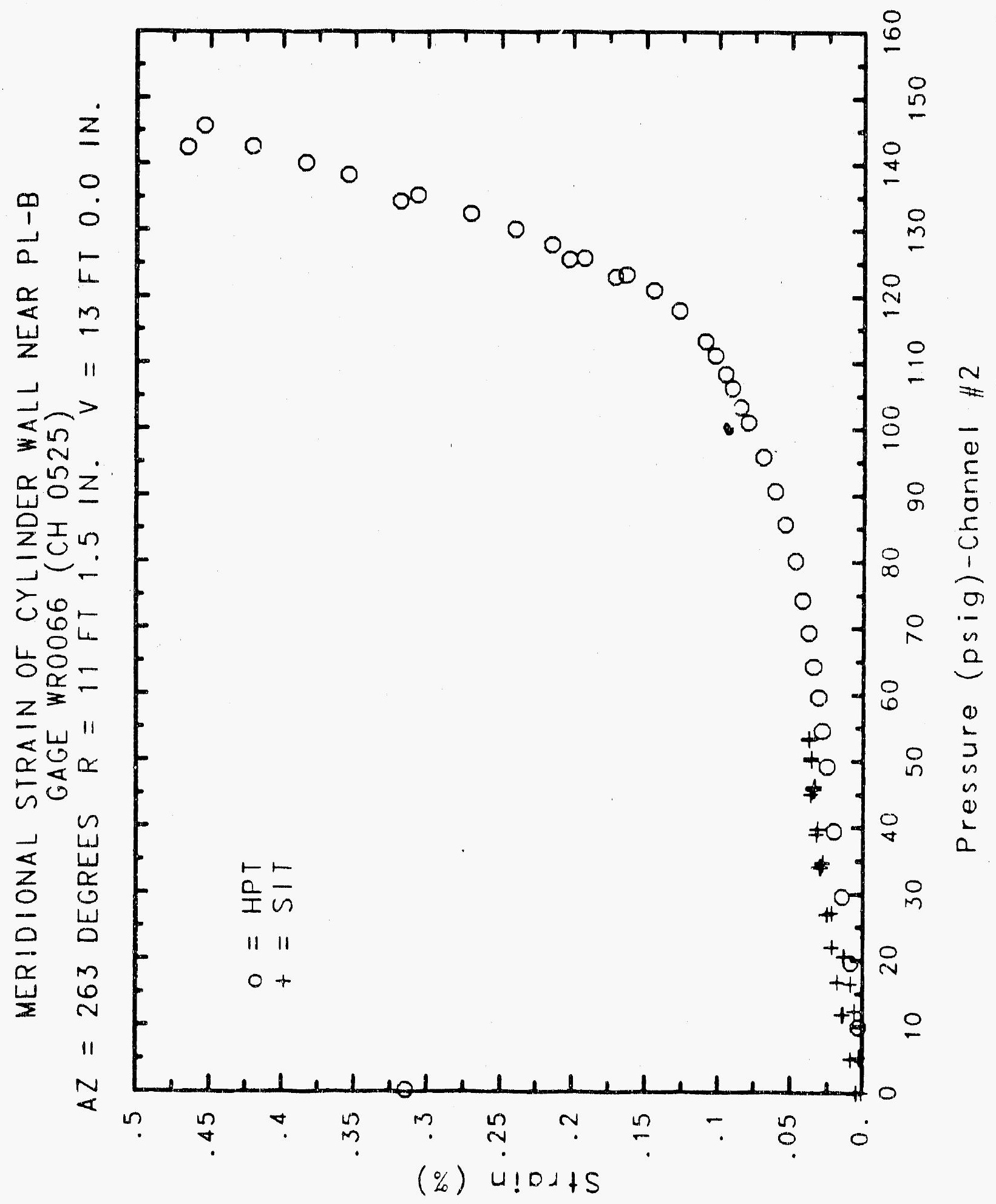




\section{Reinforced Concrete Test Data}

Weldable gage 67 Channel 526

STRUCTURAL INTEGRITY TEST

\begin{tabular}{|c|c|c|c|}
\hline $\begin{array}{c}\text { Pressure } \\
\text { (psig) } \\
-0.05 \\
5.33 \\
12.31 \\
16.44 \\
20.51 \\
20.46 \\
20.47 \\
27.05 \\
34.69 \\
34.53 \\
34.53 \\
39.70 \\
45.90 \\
45.65 \\
45.62 \\
50.10 \\
53.47 \\
53.29 \\
53.21 \\
50.49 \\
46.14 \\
46.13 \\
44.97 \\
38.98 \\
33.99 \\
33.96 \\
26.83 \\
21.88 \\
21.90 \\
16.69 \\
11.74 \\
11.77 \\
5.05 \\
0.02 \\
-0.04 \\
-0.02 \\
-0.02 \\
0.02\end{array}$ & $\begin{array}{l}\text { \% Strain } \\
0.0002 \\
0.0058 \\
0.0121 \\
0.0160 \\
0.0220 \\
0.0232 \\
0.0232 \\
0.0404 \\
0.0572 \\
0.0582 \\
0.0585 \\
0.0677 \\
0.0811 \\
0.0808 \\
0.0804 \\
0.0872 \\
0.0925 \\
0.0928 \\
0.0927 \\
0.0888 \\
0.0822 \\
0.0818 \\
0.0859 \\
0.0754 \\
0.0678 \\
0.0675 \\
0.0561 \\
0.0479 \\
0.0480 \\
0.0413 \\
0.0299 \\
0.0296 \\
0.0186 \\
0.0116 \\
0.0115 \\
0.0120 \\
0.0121 \\
0.0103\end{array}$ & $\begin{array}{c}\text { Pressure } \\
(p s i g) \\
9.89 \\
19.55 \\
29.57 \\
39.42 \\
49.16 \\
54.50 \\
59.57 \\
64.20 \\
69.32 \\
74.15 \\
80.10 \\
85.61 \\
90.58 \\
95.69 \\
100.92 \\
103.25 \\
106.11 \\
108.31 \\
111.08 \\
113.24 \\
117.83 \\
120.92 \\
123.28 \\
122.97 \\
125.82 \\
125.60 \\
127.84 \\
130.19 \\
132.53 \\
135.33 \\
134.42 \\
138.35 \\
140.16 \\
142.63 \\
145.78 \\
142.52 \\
0.22\end{array}$ & $\begin{array}{l}\text { \% Strain } \\
0.0136 \\
0.0309 \\
0.0487 \\
0.0653 \\
0.0817 \\
0.0907 \\
0.1567 \\
0.1892 \\
0.3021 \\
0.347 n \\
0.35 \\
0.2383 \\
0.2472 \\
0.1860 \\
0.2067 \\
0.2098 \\
0.2225 \\
0.6307 \\
0.2411 \\
0.2459 \\
0.2616 \\
0.2628 \\
0.4098 \\
0.4691 \\
0.7917 \\
0.8985 \\
0.9772 \\
1.0079 \\
1.0314 \\
1.3607 \\
1.3792 \\
1.4067 \\
1.4205 \\
1.4918 \\
1.5892 \\
1.6065 \\
1.1719\end{array}$ \\
\hline
\end{tabular}

High Pressure fest

Pressure

(p.89

39.42

59.57

74.15

90.58

103.25

111.08

120.92

125.82

25.60

130.19

132.53

.

0.22
0.0309

0.0487

0.0817

0.0907

0.1567

$0.347 n$

0.35

0.2472

0.1860

0.2098

0.2225

0.241

0.2459

0.2628

0.4098

0.8985

. 3607

.3792

.4918

1.1719 


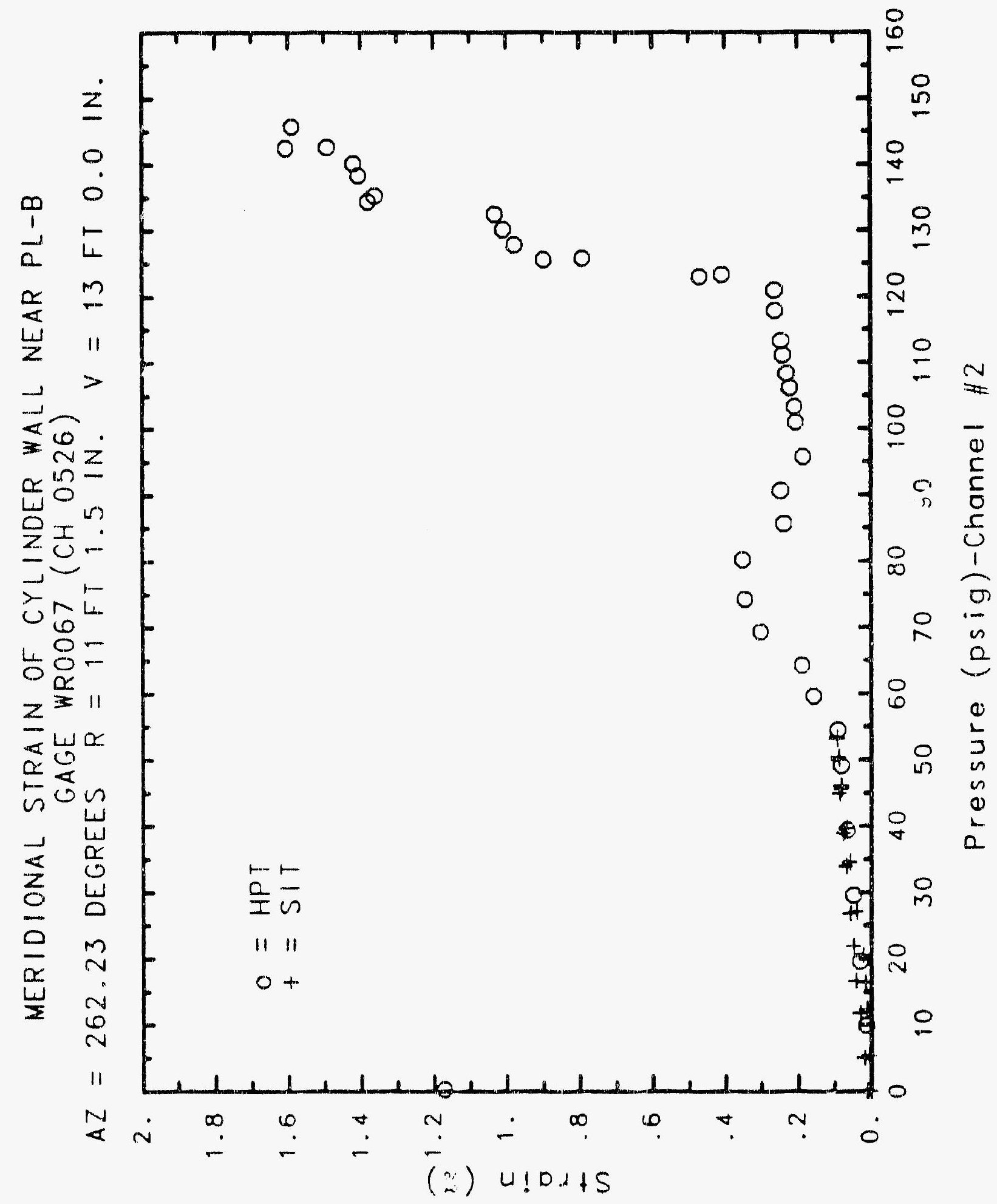

C- 946 


\section{Reinforced Concrete Test Data}

Weldable gage 68 Channel 527

Structural Integrity Test

\begin{tabular}{|c|c|}
\hline $\begin{array}{c}\text { Pressure } \\
\text { (psig) } \\
-0.05 \\
5.33 \\
12.31 \\
16.44 \\
20.51 \\
20.46 \\
20.47 \\
27.05 \\
34.69 \\
34.53 \\
34.53 \\
39.70 \\
45.90 \\
45.65 \\
45.62 \\
50.10 \\
53.47 \\
53.29 \\
53.21 \\
50.49 \\
46.14 \\
46.13 \\
44.97 \\
38.98 \\
33.99 \\
33.96 \\
26.83 \\
21.88 \\
21.90 \\
16.69 \\
11.74 \\
11.77 \\
5.05 \\
0.02 \\
-0.04 \\
-0.02 \\
-0.02 \\
0.02 \\
\end{array}$ & $\begin{array}{c}\text { \% Strain } \\
\\
-0.0003 \\
0.0017 \\
0.0051 \\
0.0072 \\
0.0103 \\
0.0109 \\
0.0110 \\
0.0176 \\
0.0293 \\
0.0306 \\
0.0308 \\
0.0371 \\
0.0473 \\
0.0477 \\
0.0482 \\
0.0539 \\
0.0594 \\
0.0598 \\
0.0599 \\
0.0588 \\
0.0556 \\
0.0555 \\
0.0566 \\
0.0515 \\
0.0470 \\
0.0471 \\
0.0398 \\
0.0354 \\
0.0352 \\
0.0301 \\
0.0256 \\
0.0252 \\
0.0183 \\
0.0135 \\
0.0123 \\
0.0115 \\
0.0116 \\
0.0117\end{array}$ \\
\hline
\end{tabular}

High Pressure test

Pressure

(psig)

9.89

19.55

29.57

39.42

49.16

54.50

59.57

64.20

59.32

74.16

80.16

85.61

90.58

95.69

100.92

103.25

106.11

108.31

111.08

113.24

117.83

120.92

123.28

122.97

125.82

125.60

127.84

130.19

132.53

135.33

134.42

138.35

140.16

142.63

145.78

142.52

0.22
\% Strain

0.0059

0.0159

0.0270

0.0375

0.0472

0.0531

0.0592

0.0659

0.0727

0.0807

0.0305

0.1007

0.1095

0.1198

0.1303

0.1351

0.1406

0.1448

0.1518

0.1578

0.1699

0.1792

0.1888

0.1912

0.2044

0.2075

0.2170

0.2364

0.2669

0.3120

0.3180

0.3581

0.3937

0.4585

0.5233

0.5452

0.3153 


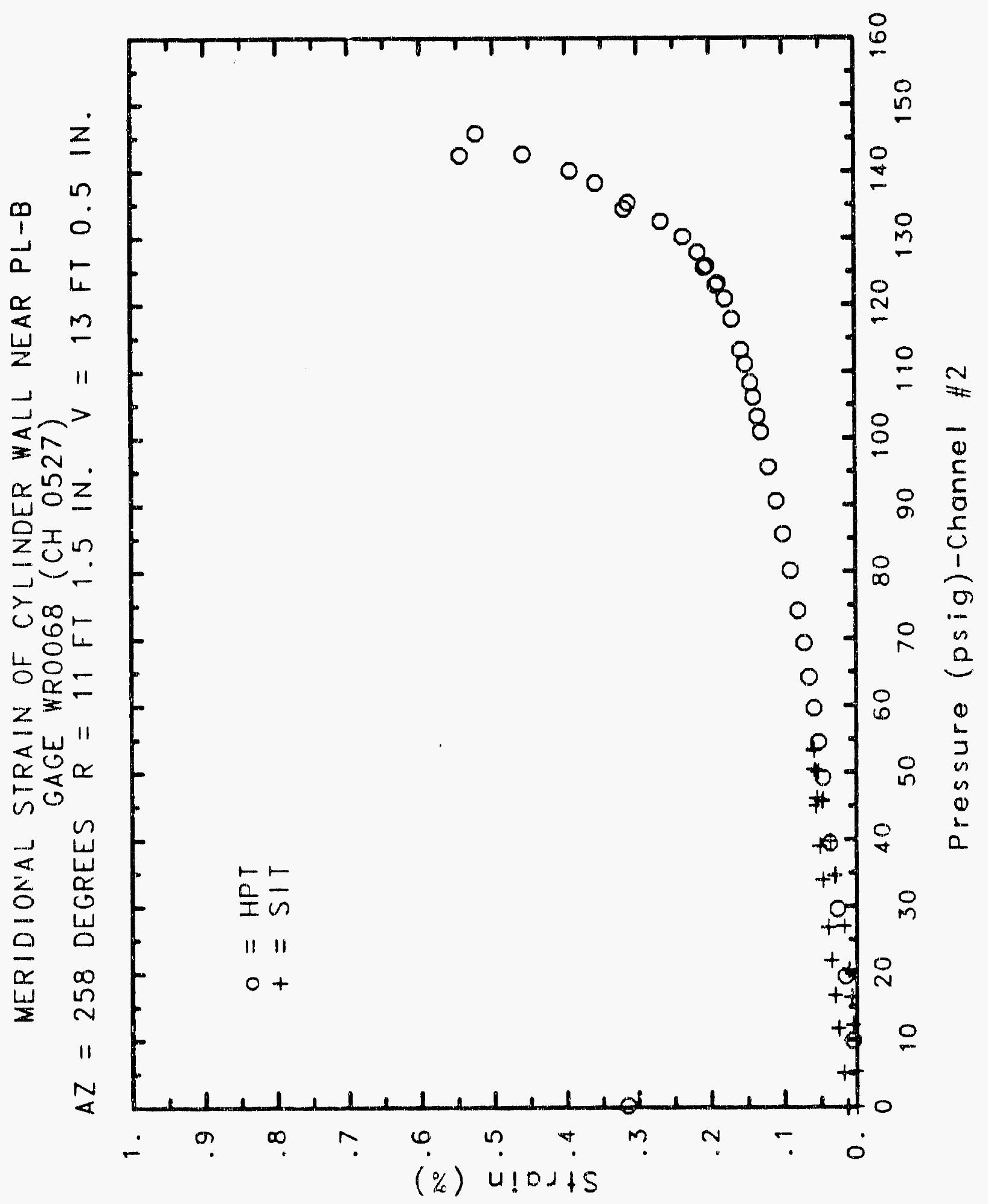




\section{Reinforced Concrete Test Data}

Weldable Gage 69 Channel 528

İTRUCTURAL INTEGRITY TEST

High Pressure test

\begin{tabular}{|c|c|c|c|}
\hline $\begin{array}{c}\text { Pressure } \\
\text { (psig) } \\
-0.05 \\
5.33 \\
12.31 \\
16.44 \\
20.51 \\
20.46 \\
20.47 \\
27.05 \\
34.69 \\
34.53 \\
34.53 \\
39.70 \\
45.90 \\
45.65 \\
45.62 \\
50.10 \\
53.47 \\
53.29 \\
53.21 \\
50.49 \\
46.14 \\
46.13 \\
44.97 \\
38.98 \\
33.99 \\
33.96 \\
26.83 \\
21.88 \\
21.90 \\
16.69 \\
11.74 \\
11.77 \\
5.05 \\
0.02 \\
-0.04 \\
-0.02 \\
-0.02 \\
0.02\end{array}$ & $\begin{array}{c}\text { \% Strain } \\
-0.0002 \\
0.0028 \\
0.0061 \\
0.0087 \\
0.0136 \\
0.0149 \\
0.0154 \\
0.0254 \\
0.0354 \\
0.0359 \\
0.0358 \\
0.0415 \\
0.0495 \\
0.0495 \\
0.0493 \\
0.0546 \\
0.0594 \\
0.0601 \\
0.0598 \\
0.0584 \\
0.0556 \\
0.0561 \\
0.0585 \\
0.0540 \\
0.0496 \\
0.0491 \\
0.0426 \\
0.0378 \\
0.0377 \\
0.0322 \\
0.0271 \\
0.0269 \\
0.0199 \\
0.0143 \\
0.0138 \\
0.0145 \\
0.0136 \\
0.0135\end{array}$ & $\begin{array}{c}\text { Pressure } \\
(\text { psig) } \\
9.89 \\
19.55 \\
29.57 \\
39.42 \\
49.16 \\
54.50 \\
59.57 \\
64.20 \\
69.32 \\
74.16 \\
80.16 \\
85.61 \\
90.58 \\
95.69 \\
100.92 \\
103.25 \\
106.11 \\
108.31 \\
111.08 \\
113.24 \\
117.83 \\
120.92 \\
123.28 \\
122.97 \\
125.82 \\
125.60 \\
127.84 \\
130.19 \\
132.53 \\
135.33 \\
134.42 \\
138.35 \\
140.16 \\
142.63 \\
145.78 \\
142.52 \\
0.82 \\
\end{array}$ & $\begin{array}{l}\text { \% Strain } \\
0.0068 \\
0.0170 \\
0.0269 \\
0.0363 \\
0.0454 \\
0.0505 \\
0.0560 \\
0.0619 \\
0.0681 \\
0.0744 \\
0.0801 \\
0.0878 \\
0.0949 \\
0.1033 \\
0.1131 \\
0.1185 \\
0.1243 \\
0.1284 \\
0.1347 \\
0.1395 \\
0.1501 \\
0.1596 \\
0.1711 \\
0.1758 \\
0.1931 \\
0.2014 \\
0.2124 \\
0.2339 \\
0.2570 \\
0.2868 \\
0.2972 \\
0.3267 \\
0.3515 \\
0.3836 \\
0.4196 \\
0.4410 \\
0.2451\end{array}$ \\
\hline
\end{tabular}




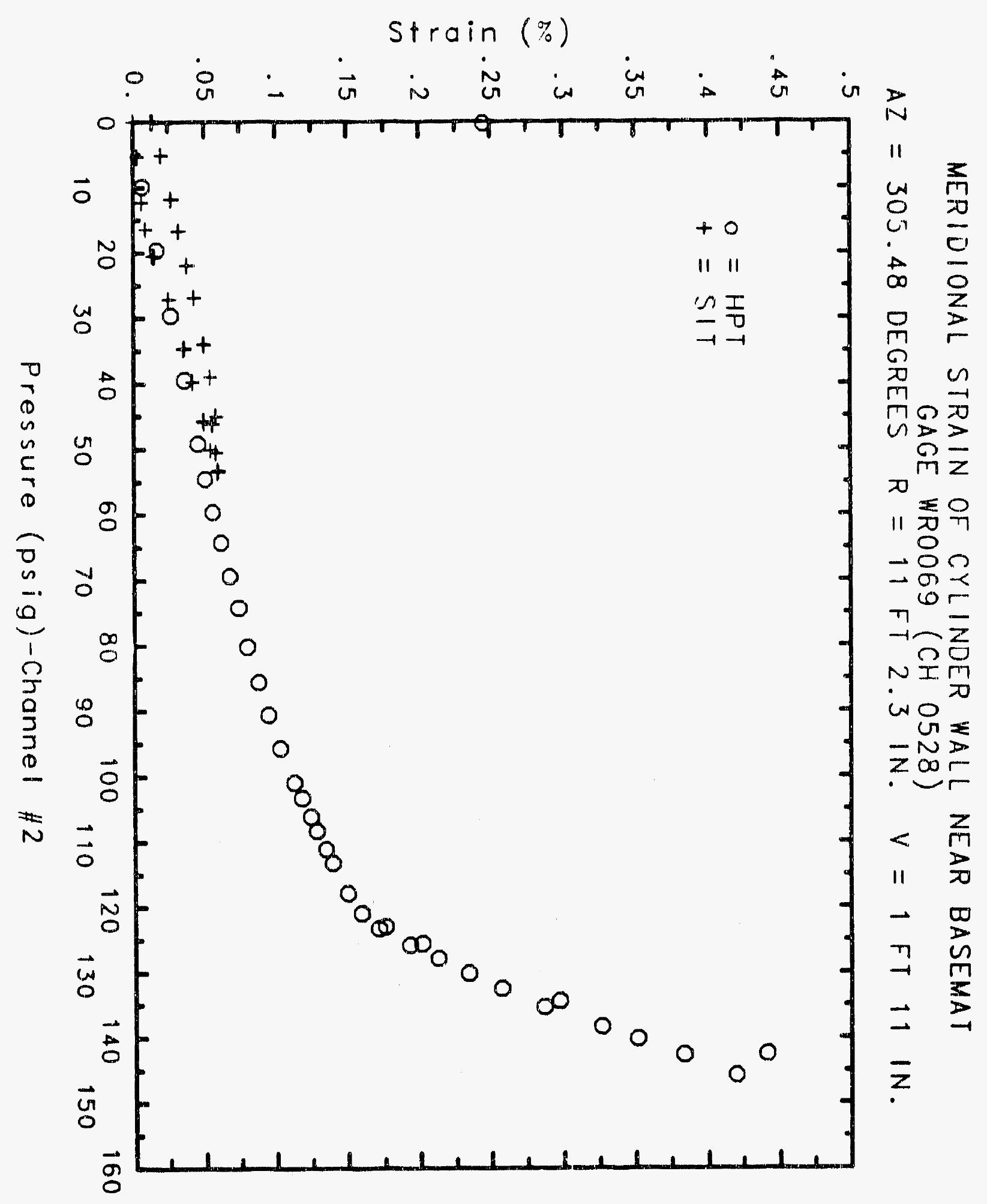




\section{Reinforced Concrete Test Data}

Weldable gage 70 Channel 529

StRUCTURAL INTEgRITY TEST

High Pressure Test

\begin{tabular}{|c|c|c|c|}
\hline $\begin{array}{c}\text { Pressure } \\
\text { (psig) } \\
-0.05 \\
5.33 \\
12.31 \\
16.44 \\
20.51 \\
20.46 \\
20.47 \\
27.05 \\
34.69 \\
34.53 \\
34.53 \\
39.70 \\
45.90 \\
45.65 \\
45.62 \\
50.10 \\
53.47 \\
53.29 \\
53.21 \\
50.49 \\
46.14 \\
46.13 \\
44.97 \\
38.98 \\
33.99 \\
33.96 \\
26.83 \\
21.88 \\
21.90 \\
16.69 \\
11.74 \\
11.77 \\
5.05 \\
0.02 \\
-0.04 \\
-0.02 \\
-0.02 \\
0.02 \\
\end{array}$ & $\begin{array}{c}\text { \% Strain } \\
-0.0003 \\
0.0016 \\
0.0057 \\
0.0088 \\
0.0124 \\
0.0128 \\
0.0130 \\
0.0191 \\
0.0268 \\
0.0268 \\
0.0263 \\
0.0310 \\
0.0369 \\
0.0361 \\
0.0365 \\
0.0402 \\
0.0429 \\
0.0432 \\
0.0428 \\
0.0413 \\
0.0386 \\
0.0384 \\
0.0391 \\
0.0348 \\
0.0297 \\
0.0295 \\
0.0226 \\
0.0178 \\
0.0172 \\
0.0121 \\
0.0071 \\
0.0064 \\
-0.0005 \\
-0.0058 \\
-0.0023 \\
-0.0008 \\
-0.0037 \\
-0.0053\end{array}$ & $\begin{array}{c}\text { Pressure } \\
\text { (psig) } \\
9.89 \\
19.55 \\
29.57 \\
39.42 \\
49.16 \\
54.50 \\
59.57 \\
64.20 \\
69.32 \\
74.16 \\
80.16 \\
85.61 \\
90.58 \\
95.69 \\
100.92 \\
103.25 \\
106.11 \\
108.31 \\
111.08 \\
113.24 \\
117.83 \\
120.92 \\
123.28 \\
122.97 \\
125.82 \\
125.60 \\
127.84 \\
130.19 \\
132.53 \\
135.33 \\
134.42 \\
138.35 \\
140.16 \\
142.63 \\
145.78 \\
142.52 \\
0.22 \\
\end{array}$ & $\begin{array}{l}\text { \% Strain } \\
0.0045 \\
0.0126 \\
0.0212 \\
0.0298 \\
0.0383 \\
0.0430 \\
0.0466 \\
0.0511 \\
0.0561 \\
0.0605 \\
0.0658 \\
0.0713 \\
0.0764 \\
0.0828 \\
0.0889 \\
0.0914 \\
0.0941 \\
0.0964 \\
0.0999 \\
0.1028 \\
0.1076 \\
0.1111 \\
0.1137 \\
0.1146 \\
0.1184 \\
0.1198 \\
0.1221 \\
0.1285 \\
0.1369 \\
0.1476 \\
0.1477 \\
0.1673 \\
0.1858 \\
0.2080 \\
0.2256 \\
0.2258 \\
0.0869\end{array}$ \\
\hline
\end{tabular}




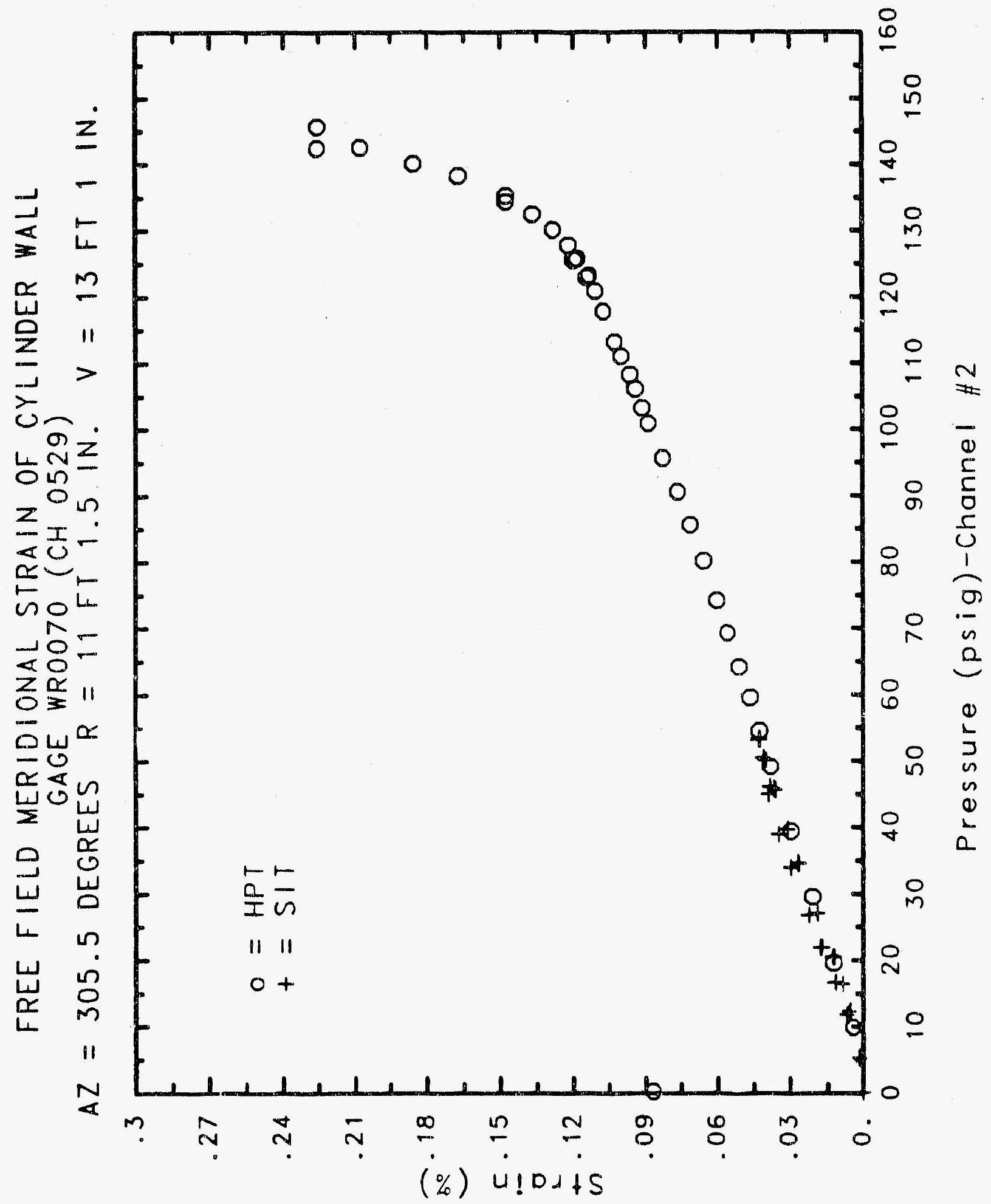


Reinforced Concrete Test Data

Weldable Gage 71 Channel 540

StRUCTURAL INTEgRITY TEST

\begin{tabular}{|c|c|c|c|}
\hline $\begin{array}{c}\text { Pressure } \\
\text { (psig) } \\
-0.05 \\
5.33 \\
12.31 \\
16.44 \\
20.51 \\
20.46 \\
20.47 \\
27.05 \\
34.69 \\
34.53 \\
34.53 \\
39.70 \\
45.90 \\
45.65 \\
45.62 \\
50.10 \\
53.47 \\
53.29 \\
53.21 \\
50.49 \\
46.14 \\
46.13 \\
44.97 \\
38.98 \\
33.99 \\
33.96 \\
26.83 \\
21.88 \\
21.90 \\
16.69 \\
11.74 \\
11.77 \\
5.05 \\
0.02 \\
-0.04 \\
-0.02 \\
-0.02 \\
0.02 \\
\end{array}$ & $\begin{array}{l}\text { \% Strain } \\
-0.0003 \\
0.0002 \\
0.0004 \\
-0.0011 \\
-0.0068 \\
-0.0265 \\
-0.0306 \\
-0.0319 \\
-0.0238 \\
-0.0228 \\
-0.0230 \\
-0.0224 \\
-0.0188 \\
-0.0200 \\
-0.0241 \\
-0.0271 \\
-0.0267 \\
-0.0445 \\
-0.0649 \\
-0.0868 \\
-0.0879 \\
-0.0857 \\
-0.0499 \\
-0.0512 \\
-0.0606 \\
-0.1322 \\
-0.1187 \\
-0.1186 \\
-0.1183 \\
-0.1209 \\
-0.1228 \\
-0.1460 \\
-0.1557 \\
-0.1484 \\
-0.0943 \\
-0.2635 \\
-0.1607 \\
-0.122 .6\end{array}$ & $\begin{array}{c}\text { Pressure } \\
(\text { psig) } \\
9.89 \\
19.55 \\
29.57 \\
39.42 \\
49.16 \\
54.50 \\
59.57 \\
64.20 \\
69.32 \\
74.16 \\
80.16 \\
85.61 \\
90.58 \\
95.69 \\
100.92 \\
103.25 \\
106.11 \\
108.31 \\
111.08 \\
113.24 \\
117.83 \\
120.92 \\
123.28 \\
122.97 \\
125.82 \\
125.60 \\
127.84 \\
130.19 \\
132.53 \\
135.33 \\
134.42 \\
138.35 \\
140.16 \\
142.63 \\
145.78 \\
142.52 \\
0.22 \\
\end{array}$ & $\begin{array}{l}\text { Strain } \\
0.0017 \\
0.0075 \\
0.0129 \\
0.0177 \\
0.0226 \\
0.0251 \\
0.0281 \\
0.0320 \\
0.0362 \\
0.0404 \\
0.0453 \\
0.0504 \\
0.0549 \\
0.0601 \\
0.0662 \\
0.0692 \\
0.0724 \\
0.0746 \\
0.0782 \\
0.0809 \\
0.0870 \\
0.0910 \\
0.0952 \\
0.0964 \\
0.1000 \\
0.1009 \\
0.1030 \\
0.1061 \\
0.1090 \\
0.1140 \\
0.1138 \\
0.1207 \\
0.1284 \\
0.1422 \\
0.1614 \\
0.1654 \\
0.0408\end{array}$ \\
\hline
\end{tabular}

High Pressure test

Pressure

(psig)

39.42

49.16

64.20

74.16

80.16

95.69

103.25

106.11

113.24

120.92

123.28

125.60

127.84

130.19

134.42

138.35

142.63

45.78

42.52

0.22
0.0017

0.0120

0.0177

0.0226

0.0320

0.0362

0.0404

0.0453

0.0601

0.0692

0.0724

0.0809

0.0952

(1009

0.1061

0.1090

0.1207

0.1422

0.1614

0.1654

0.0408 


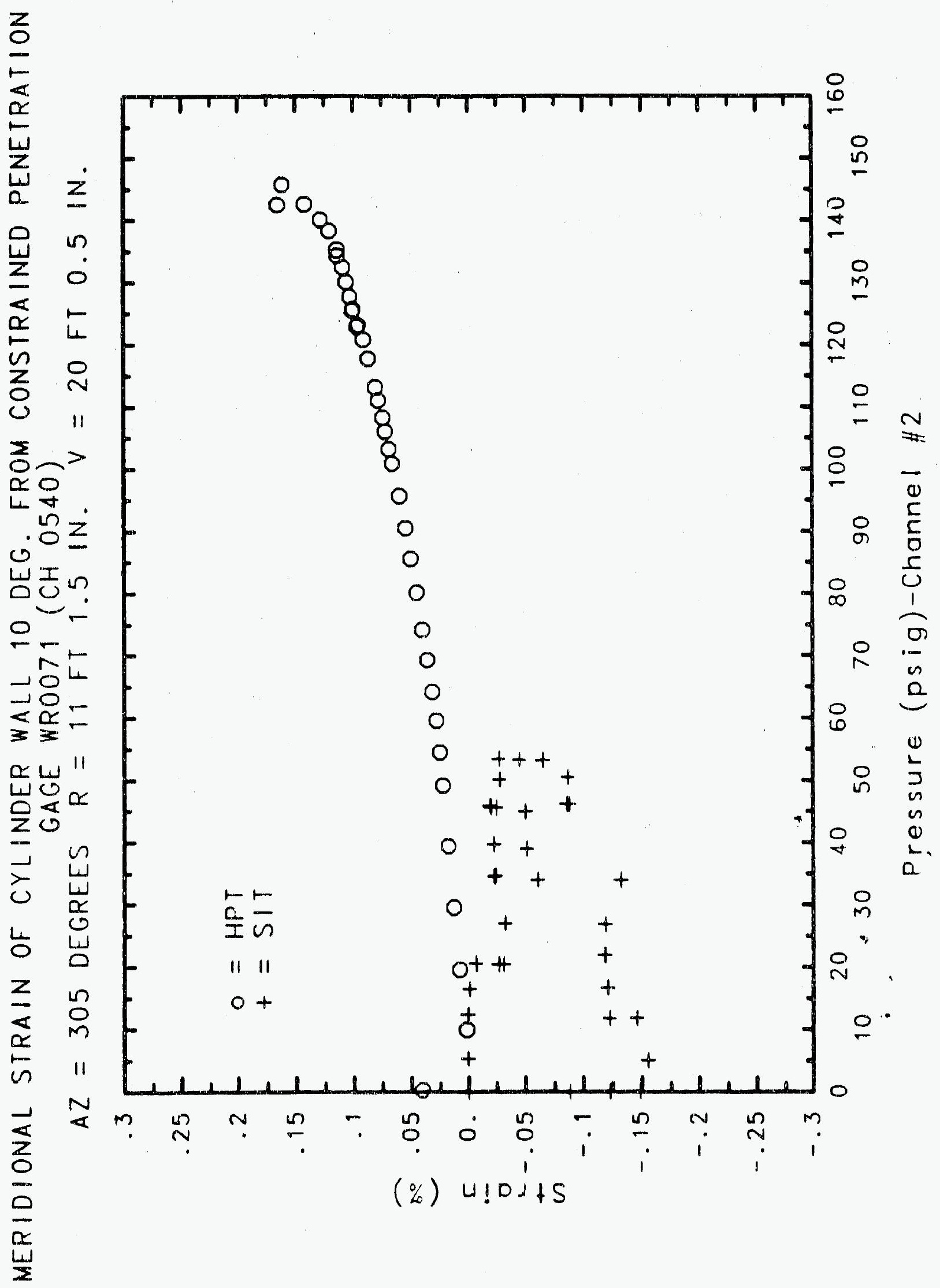




\section{Reinforced Concrete Test Data}

Weldable Gage 72 Channel. 541

Structural Integrity Test

\begin{tabular}{|c|c|c|c|}
\hline $\begin{array}{c}\text { Pressure } \\
\text { (psig) } \\
-0.05 \\
5.33 \\
12.31 \\
16.44 \\
20.51 \\
20.46 \\
20.47 \\
27.05 \\
34.69 \\
34.53 \\
34.53 \\
39.70 \\
45.90 \\
45.65 \\
45.62 \\
50.10 \\
53.47 \\
53.29 \\
53.21 \\
50.49 \\
46.14 \\
46.13 \\
44.97 \\
38.98 \\
33.99 \\
33.96 \\
26.83 \\
21.88 \\
21.90 \\
16.69 \\
11.74 \\
11.77 \\
5.05 \\
0.02 \\
-0.04 \\
-0.02 \\
-0.02 \\
0.02 \\
\end{array}$ & $\begin{array}{c}\text { \% Strain } \\
-0.0006 \\
0.0000 \\
0.0012 \\
0.0014 \\
0.0027 \\
0.0026 \\
0.0025 \\
0.0048 \\
0.0070 \\
0.0079 \\
0.0082 \\
0.0094 \\
0.0118 \\
0.0121 \\
0.0122 \\
0.0133 \\
0.0148 \\
0.0147 \\
0.0146 \\
0.0144 \\
0.0136 \\
0.0142 \\
0.0142 \\
0.0136 \\
0.0123 \\
0.0128 \\
0.0108 \\
0.0097 \\
0.0097 \\
0.0087 \\
0.0075 \\
0.0072 \\
0.0057 \\
0.0042 \\
0.0034 \\
0.0046 \\
0.0036 \\
0.0041\end{array}$ & $\begin{array}{c}\text { Pressure } \\
\text { (psig) } \\
9.89 \\
19.55 \\
29.57 \\
39.42 \\
49.16 \\
54.50 \\
59.57 \\
64.20 \\
69.32 \\
74.16 \\
80.16 \\
85.61 \\
90.58 \\
95.69 \\
100.92 \\
103.25 \\
106.11 \\
108.31 \\
111.08 \\
113.24 \\
117.83 \\
120.92 \\
123.28 \\
122.97 \\
125.82 \\
125.60 \\
127.84 \\
130.19 \\
132.53 \\
135.33 \\
134.42 \\
138.35 \\
140.16 \\
142.63 \\
145.78 \\
142.52 \\
0.22\end{array}$ & $\begin{array}{l}\text { \% Strain } \\
\text {-0.0016 } \\
0.0011 \\
0.0034 \\
0.0057 \\
0.0081 \\
0.0099 \\
0.0113 \\
0.0129 \\
0.0153 \\
0.0170 \\
0.0190 \\
0.0215 \\
0.0237 \\
0.0257 \\
0.0288 \\
0.0297 \\
0.0309 \\
0.0323 \\
0.0333 \\
0.0347 \\
0.0369 \\
0.0392 \\
0.0408 \\
0.0413 \\
0.0431 \\
0.0435 \\
0.0445 \\
0.0460 \\
0.0440 \\
0.0513 \\
0.0517 \\
0.0576 \\
0.0622 \\
0.0697 \\
0.0790 \\
0.0820 \\
0.0244\end{array}$ \\
\hline
\end{tabular}

High Pressure test

Pressure

19.55

39.42

49.16

54.50

69.32

74.16

80.16

103.25

111.08

3.24

20.92

123.28

125.60

27.84

132.53

35.33

134.42

140.16

142.63

0.0820

0.0036

0.0041 


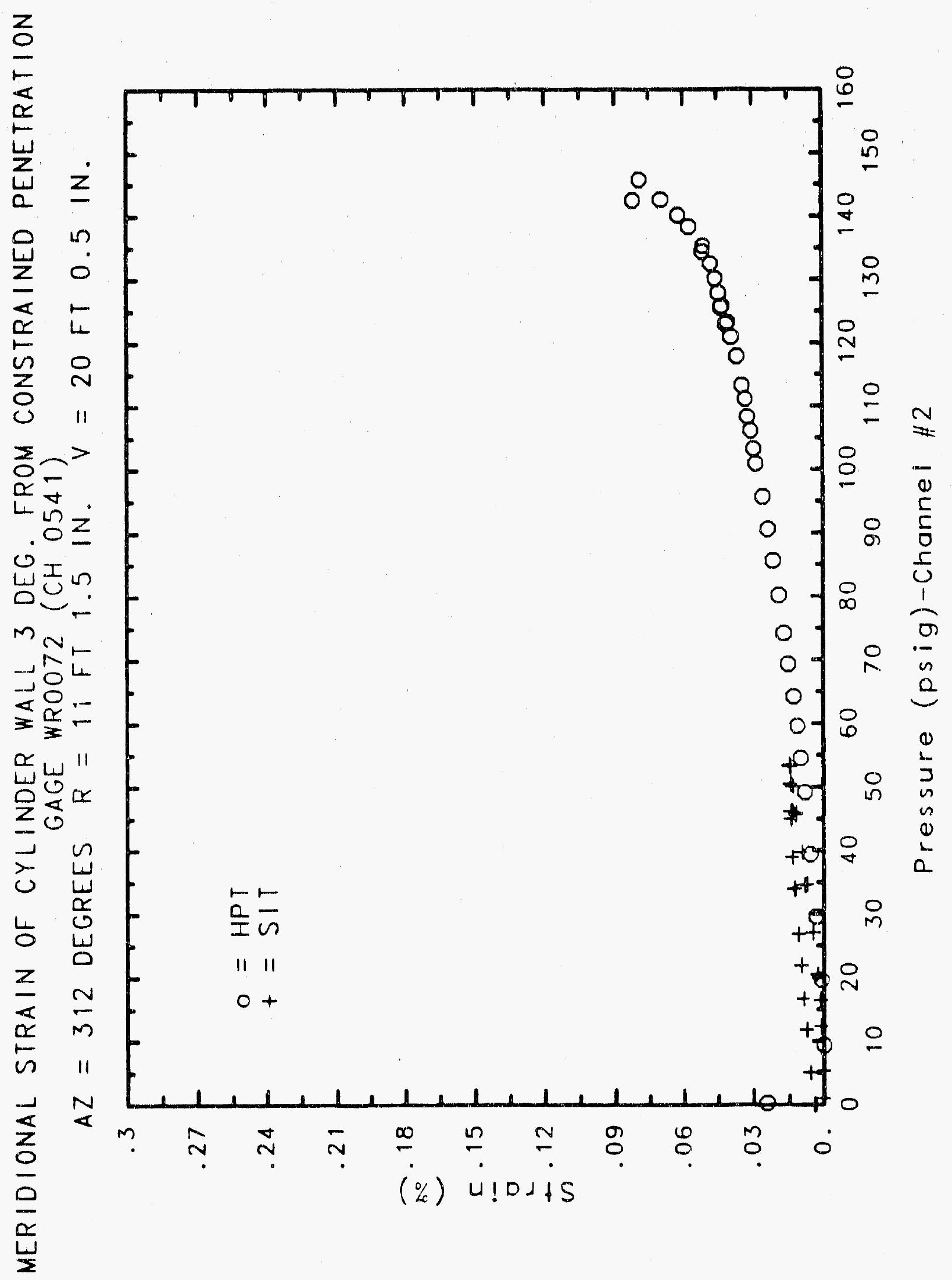




\section{Reinforced Concrete Test Data}

Weldable gage 73 Channel 542

StRUCTURAL INTEgRITY TEST

\begin{tabular}{|c|c|c|c|}
\hline $\begin{array}{c}\text { Pressure } \\
\text { (psig) } \\
-0.05 \\
5.33 \\
12.31 \\
16.44 \\
20.51 \\
20.46 \\
20.47 \\
27.05 \\
34.69 \\
34.53 \\
34.53 \\
39.70 \\
45.90 \\
45.65 \\
45.62 \\
50.10 \\
53.47 \\
53.29 \\
53.21 \\
50.49 \\
46.14 \\
46.13 \\
44.97 \\
38.98 \\
33.99 \\
33.96 \\
26.83 \\
21.88 \\
21.90 \\
16.69 \\
11.74 \\
11.77 \\
5.05 \\
0.02 \\
-0.04 \\
-0.02 \\
-0.02 \\
0.02 \\
\end{array}$ & $\begin{array}{c}\text { \% Strain } \\
-0.0003 \\
0.0003 \\
0.0014 \\
0.0018 \\
0.0031 \\
0.0030 \\
0.0036 \\
0.0064 \\
0.0096 \\
0.0096 \\
0.0100 \\
0.0115 \\
0.0143 \\
0.0145 \\
0.0146 \\
0.0162 \\
0.0178 \\
0.0178 \\
0.0181 \\
0.0174 \\
0.0165 \\
0.0163 \\
0.0164 \\
0.0154 \\
0.0142 \\
0.0141 \\
0.0115 \\
0.0103 \\
0.0101 \\
0.0088 \\
0.0075 \\
0.0074 \\
0.0053 \\
0.0036 \\
0.0028 \\
0.0033 \\
0.0032 \\
0.0031\end{array}$ & $\begin{array}{c}\text { Pressure } \\
(\text { psig) } \\
9.89 \\
19.55 \\
29.57 \\
39.42 \\
49.16 \\
54.50 \\
59.57 \\
64.20 \\
69.32 \\
74.16 \\
80.16 \\
85.61 \\
90.58 \\
95.69 \\
100.92 \\
103.25 \\
106.11 \\
108.31 \\
111.08 \\
113.24 \\
117.83 \\
120.92 \\
123.28 \\
122.97 \\
125.82 \\
125.60 \\
127.84 \\
130.19 \\
132.53 \\
135.33 \\
134.42 \\
138.35 \\
140.16 \\
142.63 \\
145.78 \\
142.52 \\
0.22 \\
\end{array}$ & $\begin{array}{c}\text { \% Strain } \\
\text { - } .0016 \\
0.0016 \\
0.0049 \\
0.0080 \\
0.0112 \\
0.0128 \\
0.0148 \\
0.0167 \\
0.0188 \\
0.0218 \\
0.0240 \\
0.0268 \\
0.0292 \\
0.0313 \\
0.0346 \\
0.0359 \\
0.0372 \\
0.0384 \\
0.0401 \\
0.0413 \\
0.0445 \\
0.0464 \\
0.0484 \\
0.0487 \\
0.0501 \\
0.0507 \\
0.0512 \\
0.0543 \\
0.0570 \\
0.0606 \\
0.0612 \\
0.0654 \\
0.0698 \\
0.0766 \\
0.0845 \\
0.0869 \\
0.0314\end{array}$ \\
\hline
\end{tabular}

\section{High Pressure Test}

Pressure

9.89

19.55

29.57

54.50

69.32

74.16

90.58

95.69

106.11

108.31

11.08

117.83

120.92

123.28

122.97

125.82

25.60

132.53

135.33

134.42

138.35

140.16

2.52

2.52
4.0016

0.0080

0.0128

0.0148

0.0167

0.0188

0.0218

0.0292

0.0313

0.0346

0.0359

0.0372

0.0384

0.0413

0.0445

0.0464

0.0484

0.0487

0.0512

0.0570

0.0606

0.0612

0.0766

0.0869

0.0314 


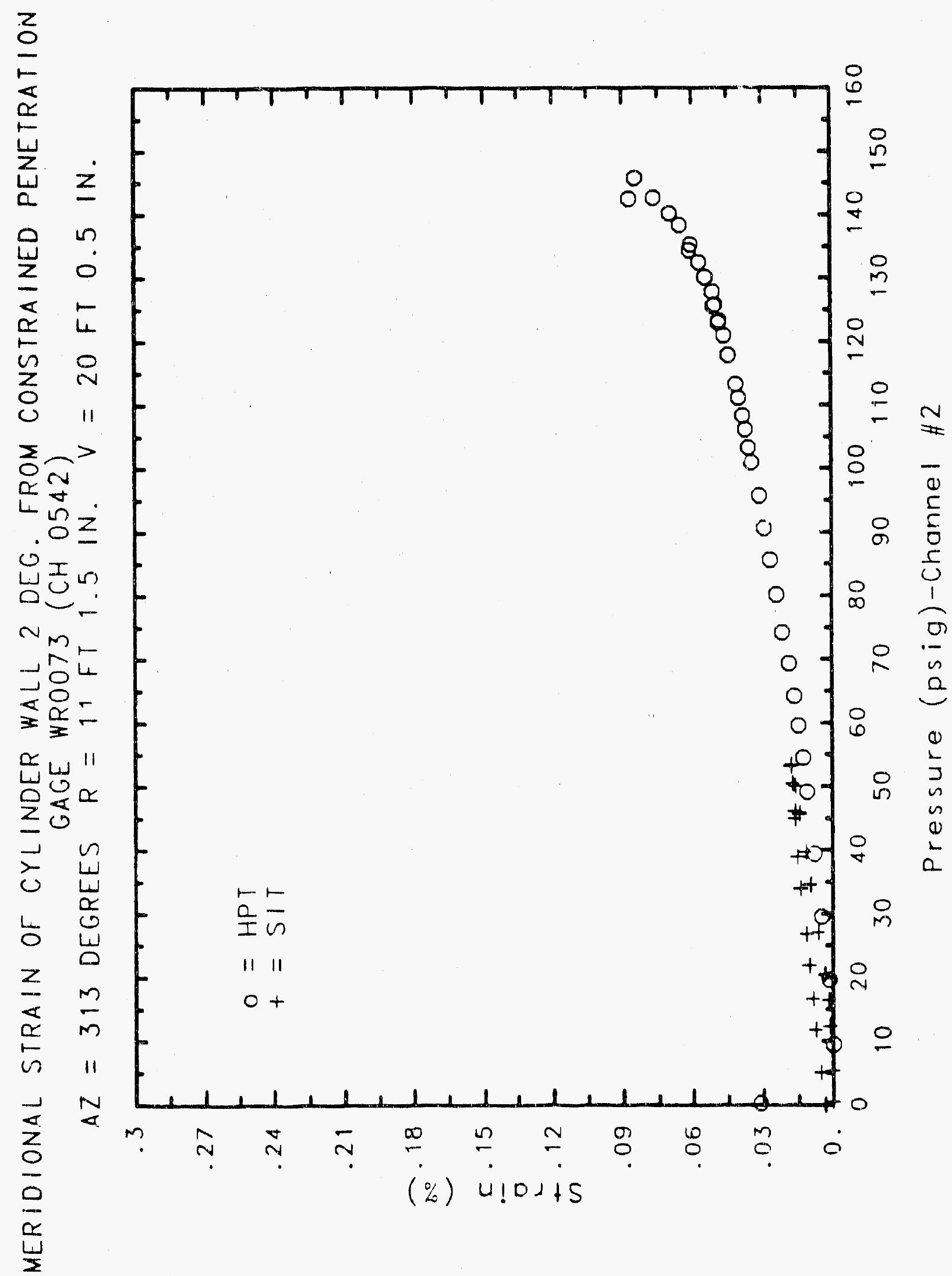




\section{Reinforced Concrete Test Data}

Weldable Gage 74 Channel 543

StRUCTURAL InTEgrity TEst

\begin{tabular}{|c|c|c|c|}
\hline $\begin{array}{c}\text { Pressure } \\
(p 5 i g) \\
-0.05 \\
5.33 \\
12.31 \\
16.44 \\
20.51 \\
20.46 \\
20.47 \\
27.05 \\
34.69 \\
34.53 \\
34.53 \\
39.70 \\
45.90 \\
45.65 \\
45.62 \\
50.10 \\
53.47 \\
53.29 \\
53.21 \\
50.49 \\
46.14 \\
46.13 \\
44.97 \\
38.98 \\
33.99 \\
33.96 \\
26.83 \\
21.88 \\
21.90 \\
16.69 \\
11.74 \\
11.77 \\
5.07 \\
0\end{array}$ & $\begin{array}{l}\text { \% Strain } \\
\\
0.0003 \\
0.0031 \\
0.0069 \\
0.0090 \\
0.0127 \\
0.0132 \\
0.0137 \\
0.0209 \\
0.0309 \\
0.0318 \\
0.0321 \\
0.0382 \\
0.0489 \\
0.0499 \\
0.0502 \\
0.0564 \\
0.0618 \\
0.0623 \\
0.0625 \\
0.0605 \\
0.0569 \\
0.0564 \\
0.0578 \\
0.0531 \\
0.0483 \\
0.0478 \\
0.0407 \\
0.0357 \\
0.0352 \\
0.0299 \\
0.0249 \\
0.0245 \\
0.0176 \\
0.0124 \\
0.0087 \\
0.0105 \\
0.0109 \\
0.0109\end{array}$ & $\begin{array}{c}\text { Pressure } \\
\text { (psig) } \\
9.89 \\
19.55 \\
29.57 \\
39.42 \\
49.16 \\
54.50 \\
59.57 \\
64.20 \\
69.32 \\
74.16 \\
80.16 \\
85.61 \\
90.58 \\
95.69 \\
100.92 \\
103.25 \\
106.11 \\
108.31 \\
111.08 \\
113.24 \\
117.83 \\
120.92 \\
123.28 \\
122.97 \\
125.82 \\
125.60 \\
127.84 \\
130.19 \\
132.53 \\
135.33 \\
134.42 \\
138.35 \\
140.16 \\
142.63 \\
145.78 \\
142.52 \\
0.22\end{array}$ & $\begin{array}{l}\text { \% Strain } \\
\\
0.0061 \\
0.0167 \\
0.0276 \\
0.0385 \\
0.0492 \\
0.0555 \\
0.0617 \\
0.0704 \\
0.0793 \\
0.0914 \\
0.1066 \\
0.1193 \\
0.1295 \\
0.1397 \\
0.1488 \\
0.1516 \\
0.1533 \\
0.1539 \\
0.1526 \\
0.1512 \\
0.1481 \\
0.1481 \\
0.1492 \\
0.1492 \\
0.1506 \\
0.1515 \\
0.1540 \\
0.1537 \\
0.1522 \\
0.1568 \\
0.1564 \\
0.1617 \\
0.1740 \\
0.2346 \\
0.3599 \\
0.4686 \\
0.1847\end{array}$ \\
\hline
\end{tabular}

High Pressure test

Pressure

0.0061

0.0276

0.0385

0.0555

0.0617

0704

0.1066

0.1193

0.1295

0.1488

0.1516

0.1512

0.1481

0.1492

0.1540

0.1568

0.1564

0.1617

0.4686

0.1847 


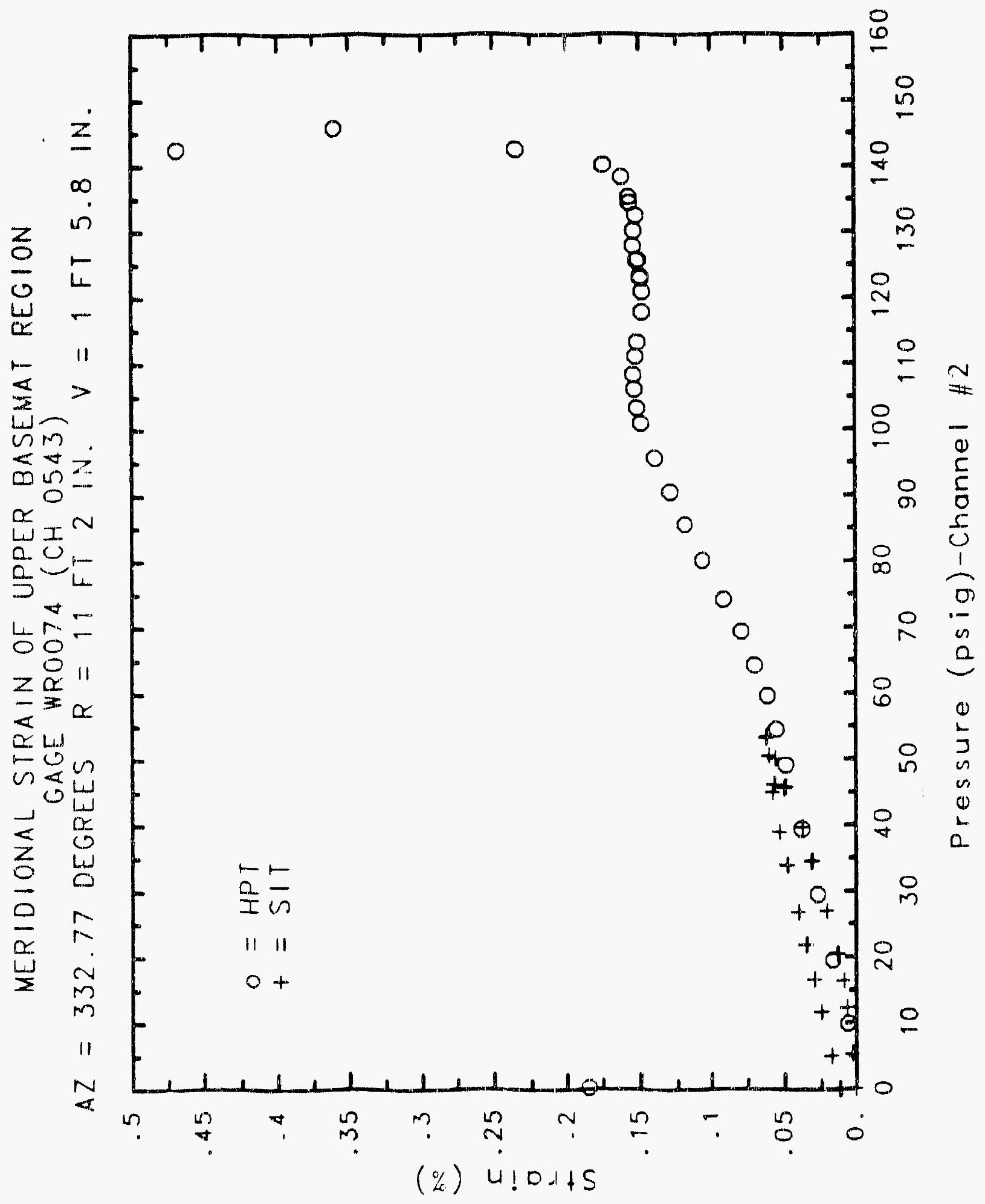




\section{Reinforced Concrete Test Dáta}

\section{Weldable gage 75 Channel 544}

Structural INTEgRITY TEST

\begin{tabular}{|c|c|c|c|}
\hline $\begin{array}{c}\text { Pressure } \\
\text { (psig) } \\
-0.05 \\
5.33 \\
12.31 \\
16.44 \\
20.51 \\
20.46 \\
20.47 \\
27.05 \\
34.69 \\
34.53 \\
34.53 \\
39.70 \\
45.90 \\
45.65 \\
45.62 \\
50.10 \\
53.47 \\
53.29 \\
53.21 \\
50.19 \\
46.14 \\
46.13 \\
44.97 \\
38.98 \\
33.99 \\
33.96 \\
26.83 \\
21.88 \\
21.90 \\
16.69 \\
11.74 \\
11.77 \\
5.05 \\
0.02 \\
-0.04 \\
-0.02 \\
-0.02 \\
0.02 \\
\end{array}$ & $\begin{array}{c}\text { \% Strain } \\
-0.0002 \\
0.0035 \\
0.0081 \\
0.0111 \\
0.0175 \\
0.0193 \\
0.0212 \\
0.0323 \\
0.0487 \\
0.0483 \\
0.0451 \\
0.0494 \\
0.0605 \\
0.0599 \\
0.0595 \\
0.0663 \\
0.0719 \\
0.0725 \\
0.0730 \\
0.0705 \\
0.0656 \\
0.0654 \\
0.0774 \\
0.0704 \\
0.0645 \\
0.0630 \\
0.0538 \\
0.0467 \\
0.0464 \\
0.0394 \\
0.0330 \\
0.0322 \\
0.0229 \\
0.0162 \\
0.0026 \\
0.0085 \\
0.0092 \\
0.0091\end{array}$ & $\begin{array}{c}\text { Pressure } \\
(\text { psig) } \\
9.89 \\
19.55 \\
29.57 \\
39.42 \\
49.16 \\
54.50 \\
59.57 \\
64.20 \\
69.32 \\
74.16 \\
80.16 \\
85.61 \\
90.58 \\
95.69 \\
100.92 \\
103.25 \\
106.11 \\
108.31 \\
111.08 \\
113.24 \\
117.83 \\
120.92 \\
123.28 \\
122.97 \\
125.82 \\
125.60 \\
127.84 \\
130.19 \\
132.53 \\
135.33 \\
134.42 \\
138.35 \\
140.16 \\
142.63 \\
145.78 \\
142.52 \\
0.22\end{array}$ & $\begin{array}{l}\text { \% Strain } \\
0.0103 \\
0.0255 \\
0.0414 \\
0.0565 \\
0.0711 \\
0.0785 \\
0.0870 \\
0.0972 \\
0.1078 \\
0.1222 \\
0.1373 \\
0.1515 \\
0.1622 \\
0.1748 \\
0.1887 \\
0.1945 \\
0.2012 \\
0.2060 \\
0.2134 \\
0.2186 \\
0.2282 \\
0.2299 \\
0.2326 \\
0.2343 \\
0.2364 \\
0.2395 \\
0.2427 \\
0.2440 \\
0.2460 \\
0.2430 \\
0.2508 \\
0.16593 \\
0.9386 \\
1.0789 \\
1.2057 \\
1.2220 \\
0.8252\end{array}$ \\
\hline
\end{tabular}

High Pressure Test

Pressure

(psig)

19.55

29.57

54.50

64.20

69.32

74.16

80.16

95.69

103.25

106.11

108,31

117.83

125.82

130.19

132.53

135.33

134.42

145.78

2.52

.2220

0.8252 


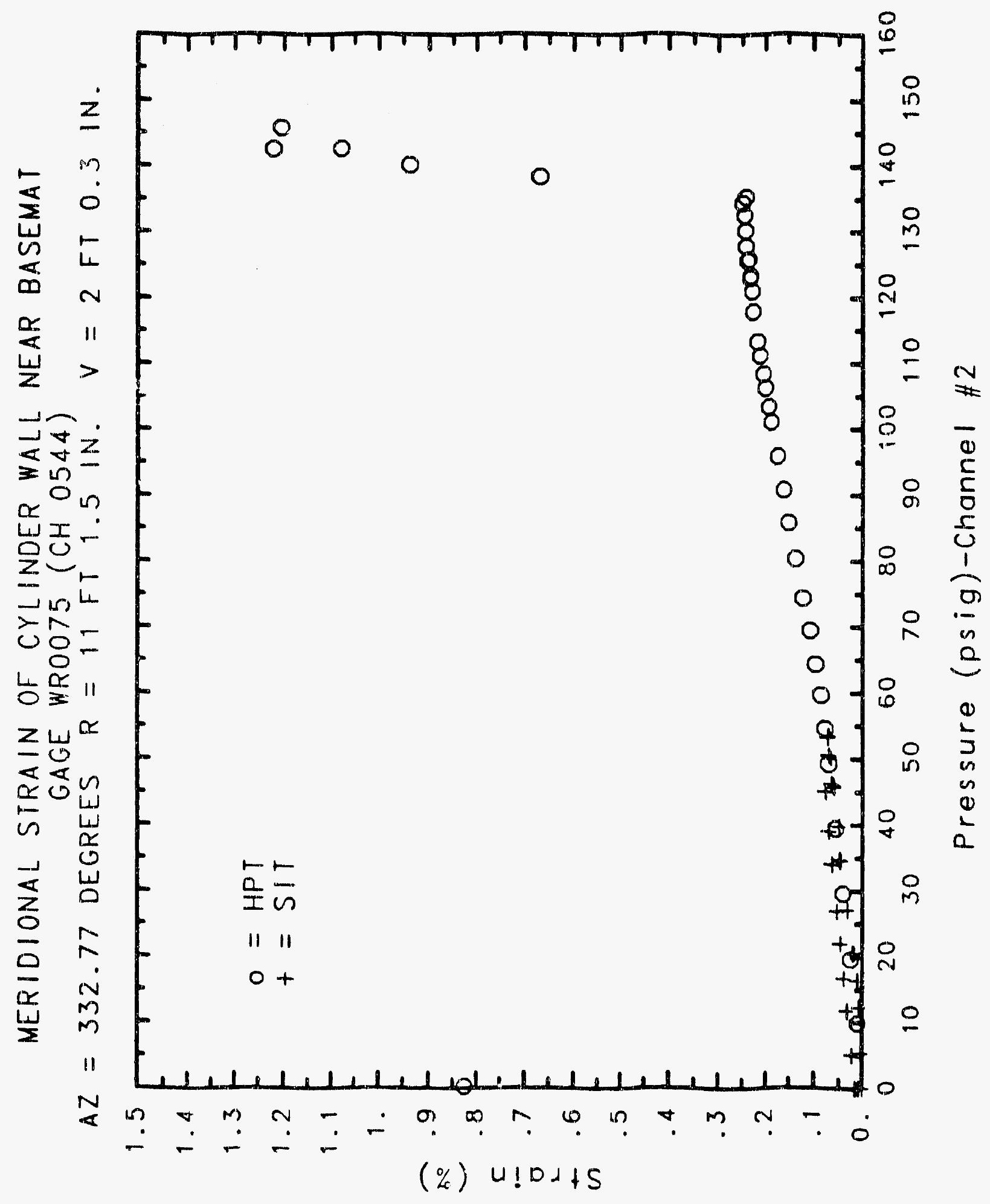




\section{Reinforced Concrete Test Data}

Weldable gage 76 Channel 545

Structural INTEgRITY TEST

High Pressure test

\begin{tabular}{|c|c|c|c|}
\hline $\begin{array}{c}\text { Pressure } \\
\text { (psig) } \\
-0.05 \\
5.33 \\
12.31 \\
16.44 \\
20.51 \\
20.46 \\
20.47 \\
27.05 \\
34.69 \\
34.53 \\
34.53 \\
39.70 \\
45.90 \\
45.65 \\
45.62 \\
50.10 \\
53.47 \\
53.29 \\
53.21 \\
50.49 \\
46.14 \\
46.13 \\
44.97 \\
38.98 \\
33.99 \\
33.96 \\
26.83 \\
21.88 \\
21.90 \\
16.69 \\
11.74 \\
11.77 \\
5.05 \\
0.02 \\
-0.04 \\
-0.02 \\
-0.02 \\
0.02\end{array}$ & $\begin{array}{l}\text { \% Strain } \\
0.0009 \\
0.0019 \\
0.0047 \\
0.0061 \\
0.0094 \\
0.0106 \\
0.0105 \\
0.0194 \\
0.0335 \\
0.0349 \\
0.0358 \\
0.0429 \\
0.0552 \\
0.0564 \\
0.0566 \\
0.0632 \\
0.0686 \\
0.0695 \\
0.0696 \\
0.0680 \\
0.0640 \\
0.0639 \\
0.0670 \\
0.0614 \\
0.0565 \\
0.0559 \\
0.0485 \\
0.0427 \\
0.0424 \\
0.0365 \\
0.0308 \\
0.0301 \\
0.0229 \\
0.0179 \\
0.0150 \\
0.0165 \\
0.0164 \\
0.0160\end{array}$ & $\begin{array}{c}\text { Pressure } \\
\text { (psig) } \\
9.89 \\
19.55 \\
29.57 \\
39.42 \\
49.16 \\
54.50 \\
59.57 \\
64.20 \\
69.32 \\
74.16 \\
80.16 \\
85.61 \\
90.58 \\
95.69 \\
100.92 \\
103.25 \\
106.11 \\
108.31 \\
111.08 \\
113.24 \\
117.83 \\
120.92 \\
123.28 \\
122.97 \\
125.82 \\
125.60 \\
127.84 \\
130.19 \\
132.53 \\
135.33 \\
134.42 \\
138.35 \\
140.16 \\
142.63 \\
145.78 \\
142.52 \\
0.22\end{array}$ & $\begin{array}{l}\text { \% Strain } \\
0.0053 \\
0.0175 \\
0.0297 \\
0.0418 \\
0.0533 \\
0.0603 \\
0.0673 \\
0.0768 \\
0.0862 \\
0.0997 \\
0.1157 \\
0.1301 \\
0.1413 \\
0.1554 \\
0.1697 \\
0.1774 \\
0.1844 \\
0.1896 \\
0.1969 \\
0.2022 \\
0.2133 \\
0.2192 \\
0.2246 \\
0.2259 \\
0.2311 \\
0.2329 \\
0.2347 \\
0.2344 \\
0.2354 \\
0.2350 \\
0.2338 \\
0.2384 \\
0.2428 \\
0.3441 \\
0.6394 \\
0.7663 \\
0.4621\end{array}$ \\
\hline
\end{tabular}




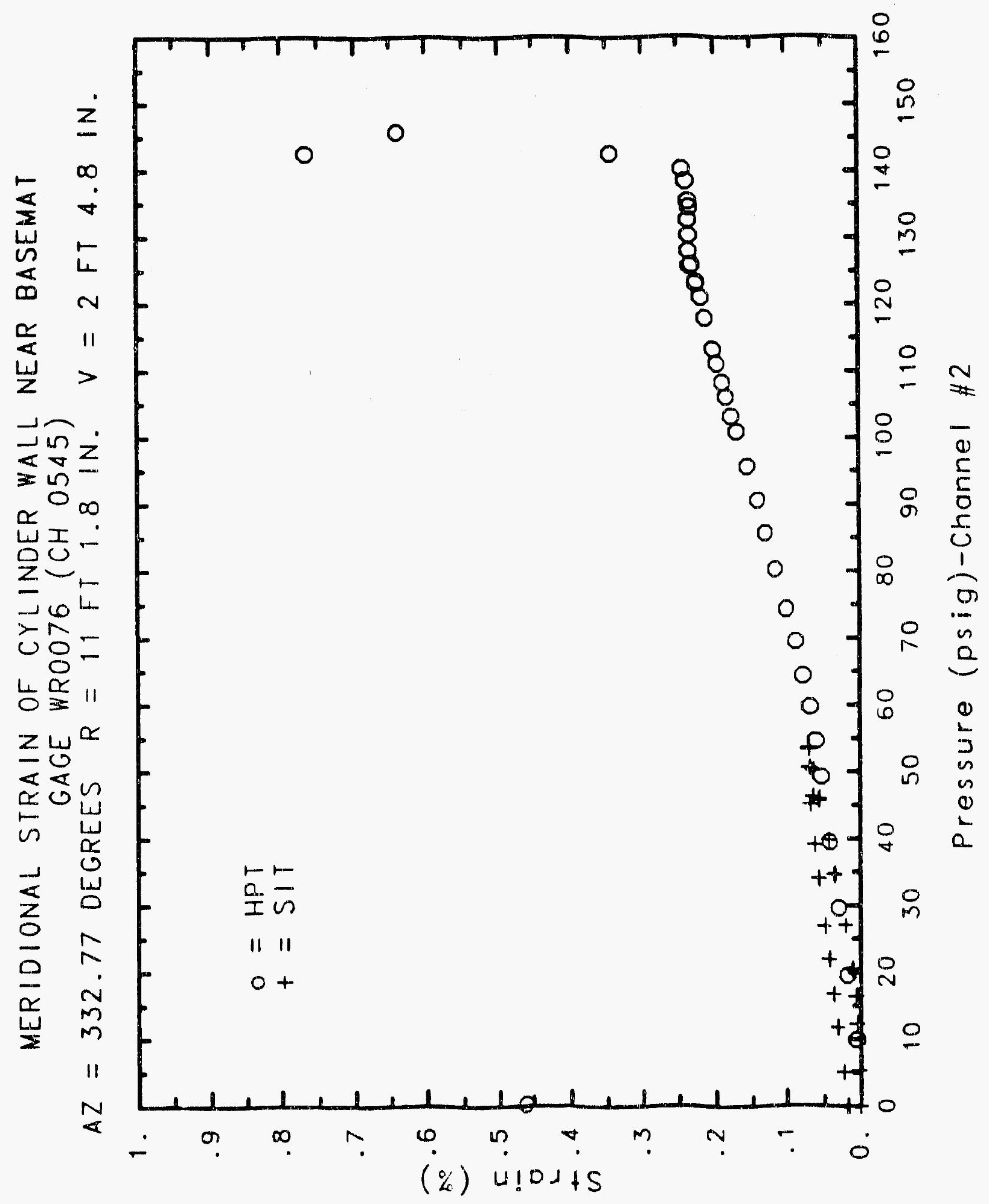




\section{Reinforced Concrete Test Data}

Weldable gage 77 Channel 546

Structural Integrity TEST

High Pressure test

\begin{tabular}{|c|c|c|c|}
\hline $\begin{array}{c}\text { Pressure } \\
\text { (psig) } \\
-0.05 \\
5.33 \\
12.31 \\
16.44 \\
20.51 \\
20.46 \\
20.47 \\
27.05 \\
34.69 \\
34.53 \\
34.53 \\
39.70 \\
45.90 \\
45.65 \\
45.62 \\
50.10 \\
53.47 \\
53.29 \\
53.21 \\
50.49 \\
46.14 \\
46.13 \\
44.97 \\
38.98 \\
33.99 \\
33.96 \\
26.83 \\
21.88 \\
21.90 \\
16.69 \\
11.74 \\
11.77 \\
5.05 \\
0.02 \\
-0.04 \\
-0.02 \\
-0.02 \\
0.02\end{array}$ & $\begin{array}{c}\text { \% Strain } \\
-0.0006 \\
0.0035 \\
0.0080 \\
0.0106 \\
0.0146 \\
0.0146 \\
0.0144 \\
0.0207 \\
0.0291 \\
0.0291 \\
0.0295 \\
0.0335 \\
0.0404 \\
0.0403 \\
0.0401 \\
0.0438 \\
0.0471 \\
0.0469 \\
0.0473 \\
0.0459 \\
0.0431 \\
0.0431 \\
0.0434 \\
0.0396 \\
0.0360 \\
0.0358 \\
0.0301 \\
0.0256 \\
0.0258 \\
0.0212 \\
0.0166 \\
0.0167 \\
0.0109 \\
0.0065 \\
0.0067 \\
0.0066 \\
0.0063 \\
0.0067\end{array}$ & $\begin{array}{c}\text { Pressure } \\
\text { (psig) } \\
9.89 \\
19.55 \\
29.57 \\
39.42 \\
49.16 \\
54.50 \\
59.57 \\
64.20 \\
69.32 \\
74.16 \\
80.16 \\
85.61 \\
90.58 \\
95.69 \\
100.92 \\
103.25 \\
106.11 \\
108.31 \\
111.08 \\
113.24 \\
117.83 \\
120.92 \\
123.28 \\
122.97 \\
125.82 \\
125.60 \\
127.84 \\
130.19 \\
132.53 \\
135.33 \\
134.42 \\
138.35 \\
140.16 \\
142.63 \\
145.78 \\
142.52 \\
0.22\end{array}$ & $\begin{array}{l}\text { \% Strain } \\
0.0038 \\
0.0121 \\
0.0206 \\
0.0285 \\
0.0361 \\
0.0401 \\
0.0449 \\
0.0496 \\
0.0552 \\
0.0608 \\
0.0654 \\
0.0723 \\
0.0784 \\
0.0845 \\
0.0921 \\
0.0948 \\
0.0988 \\
0.1020 \\
0.1061 \\
0.1087 \\
0.1166 \\
0.1214 \\
0.1264 \\
0.1277 \\
0.1339 \\
0.1355 \\
0.1388 \\
0.1450 \\
0.1537 \\
0.1688 \\
0.1709 \\
0.1954 \\
0.2140 \\
0.2451 \\
0.2571 \\
0.2533 \\
0.1109\end{array}$ \\
\hline
\end{tabular}




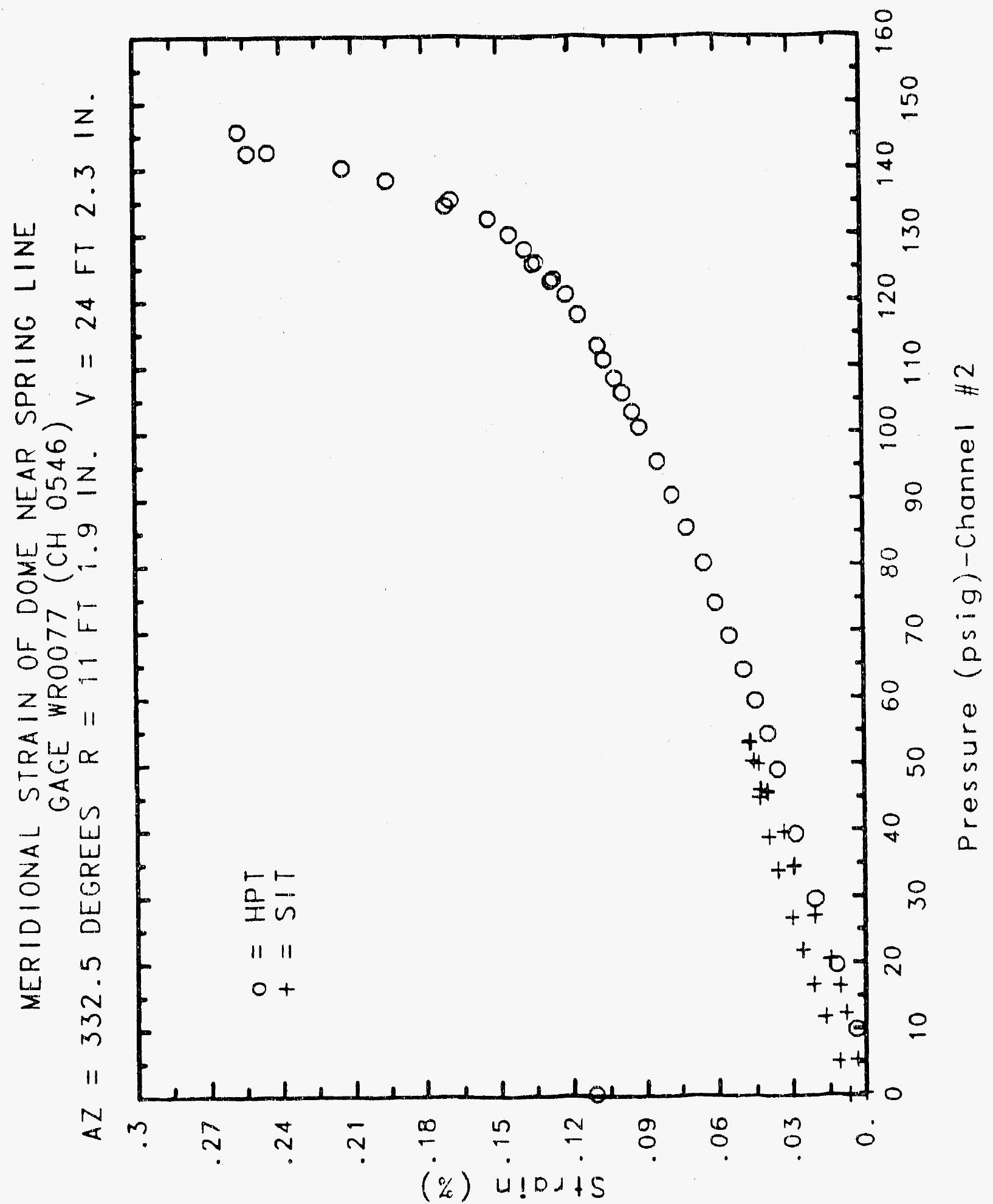




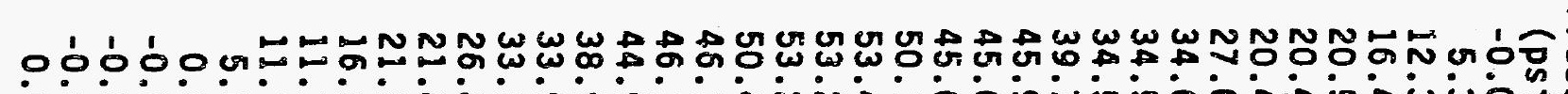

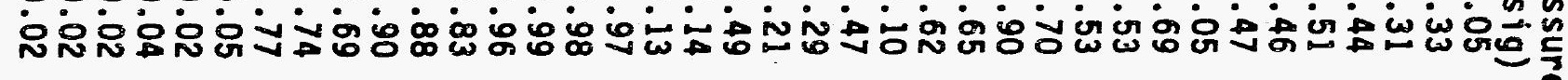

00000000000000000000000000000000000000

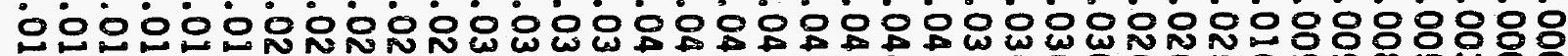

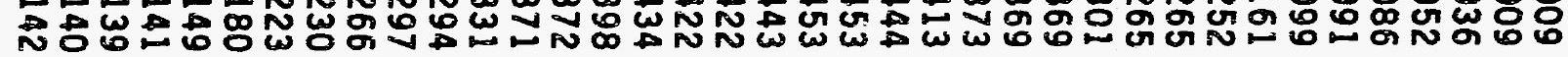

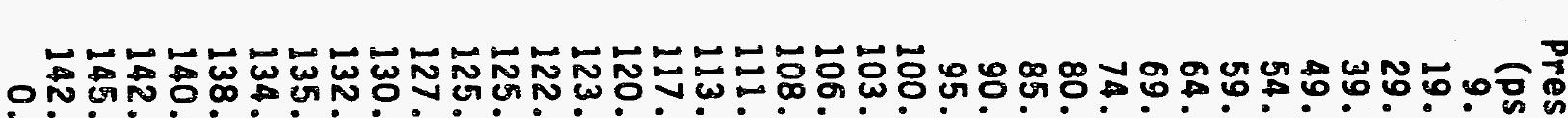

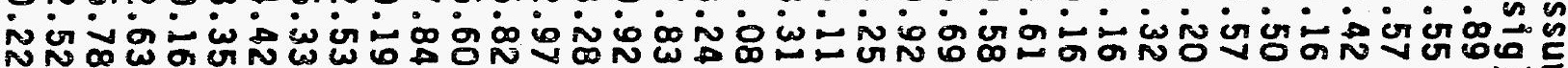

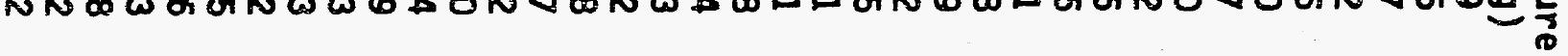

0000000000000000000000000000000000000 onitini

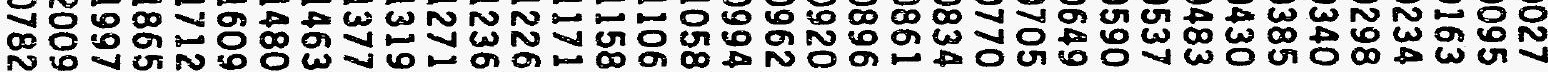




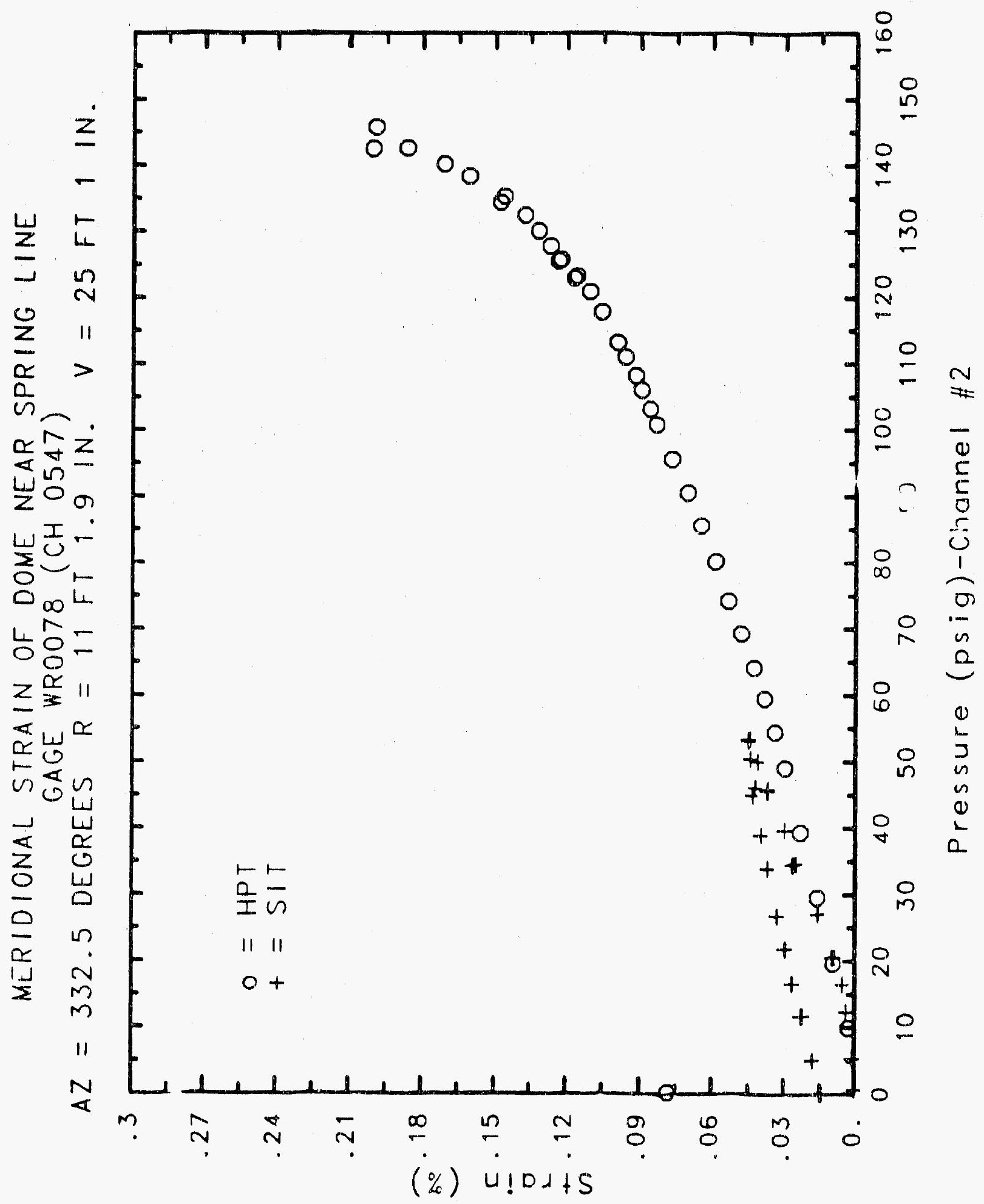




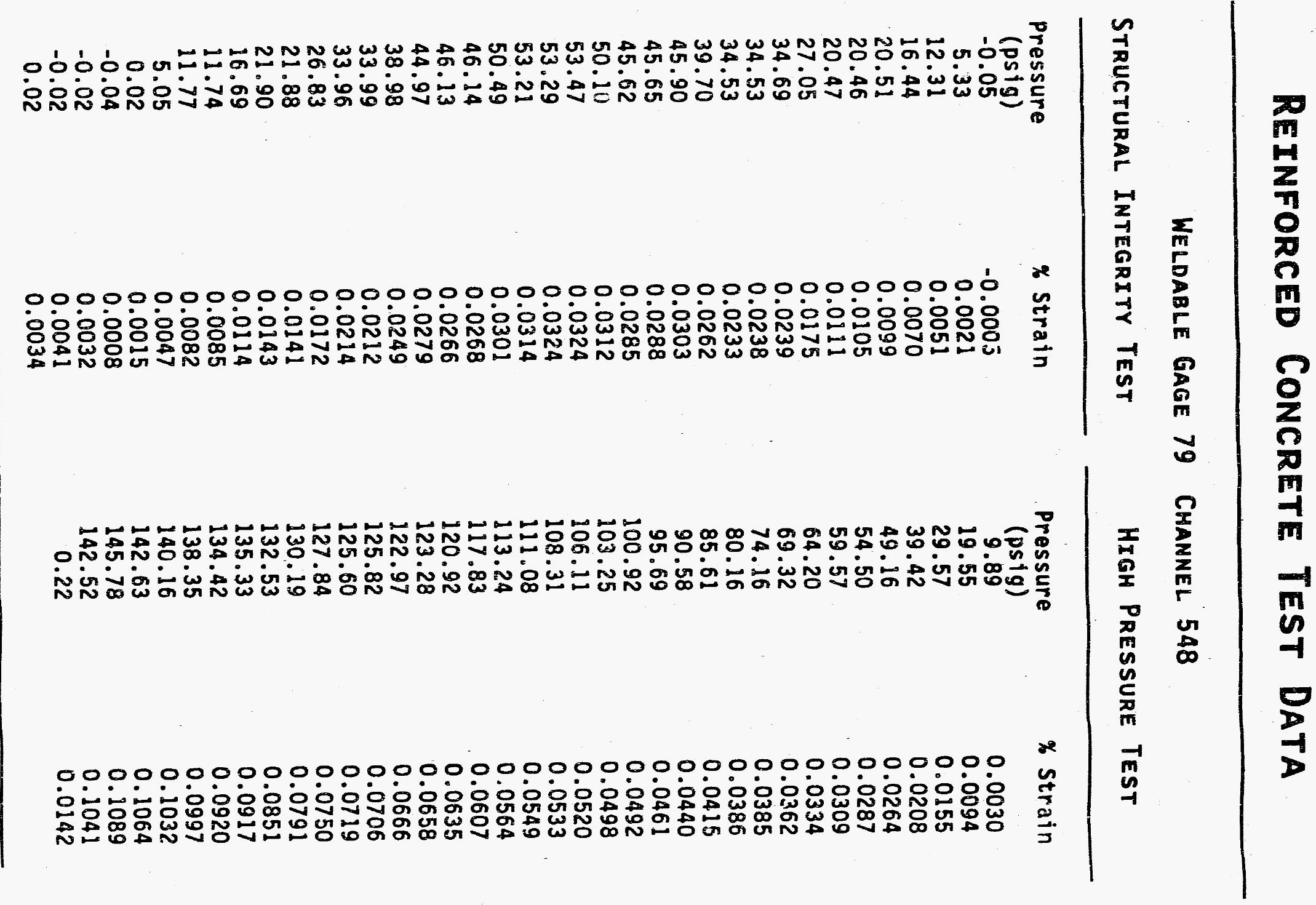




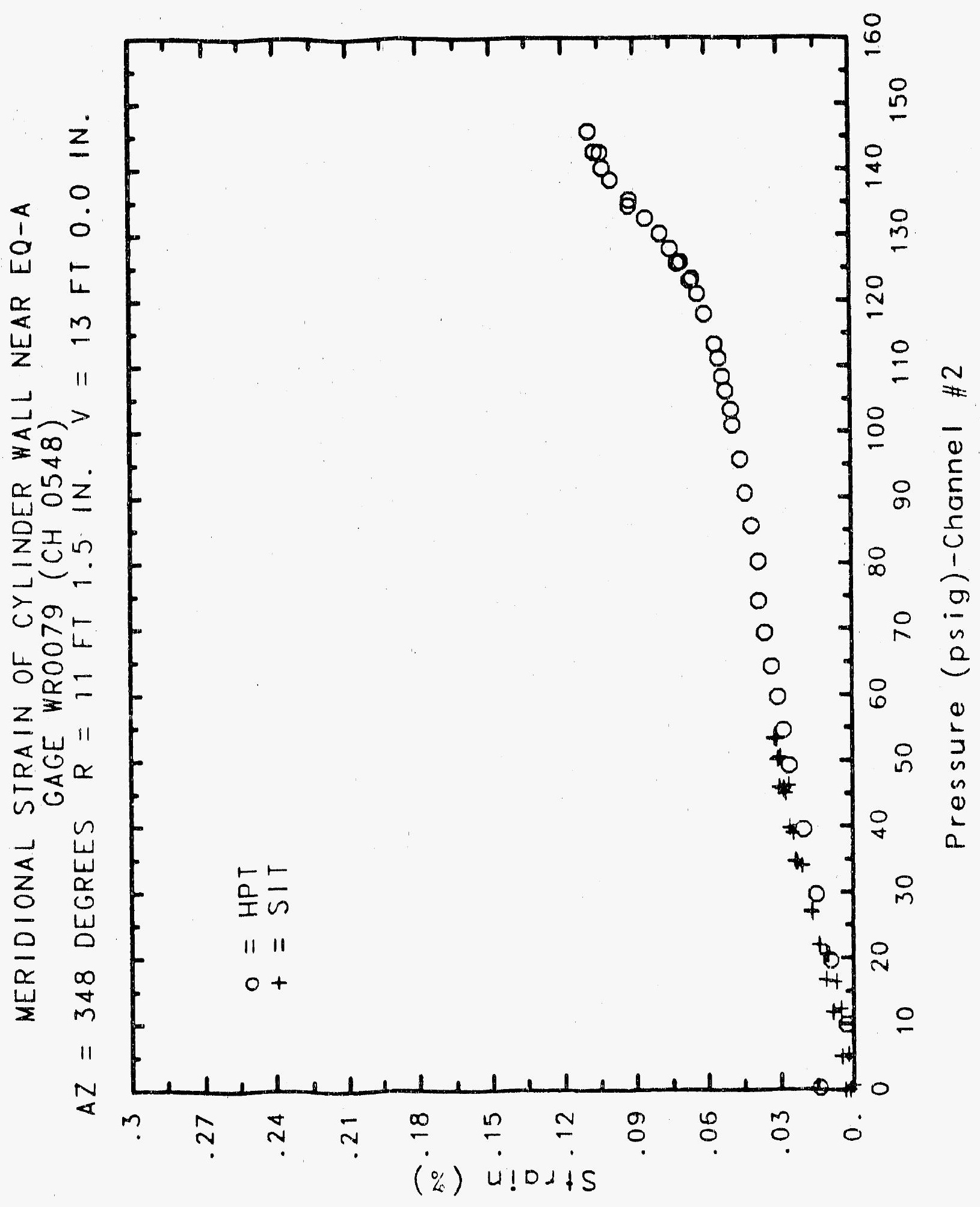




\section{Reinforced Concrete Test Data}

Weldable gage 80 Channel 549

STRUCTURAL. INTEGRITY TEST

\begin{tabular}{|c|c|c|c|}
\hline $\begin{array}{c}\text { Pressure } \\
\text { (psig) } \\
-0.05 \\
5.33 \\
12.31 \\
16.44 \\
20.51 \\
20.46 \\
20.47 \\
27.05 \\
34.69 \\
34.53 \\
34.53 \\
39.70 \\
45.90 \\
45.65 \\
45.62 \\
50.10 \\
53.47 \\
53.29 \\
53.21 \\
50.49 \\
46.14 \\
46.13 \\
44.97 \\
38.98 \\
33.99 \\
33.96 \\
26.83 \\
21.88 \\
21.90 \\
16.59 \\
11.74 \\
11.77 \\
5.05 \\
0.02 \\
-0.04 \\
-0.02 \\
-0.02 \\
0.02\end{array}$ & $\begin{array}{c}\text { \% Strain } \\
-0.0002 \\
0.0030 \\
0.0071 \\
0.0098 \\
0.0141 \\
0.0153 \\
0.0160 \\
0.0234 \\
0.0318 \\
0.031 \\
0.0315 \\
0.0351 \\
0.0393 \\
0.0389 \\
0.0384 \\
0.0414 \\
0.0435 \\
0.0436 \\
0.0432 \\
0.0414 \\
0.0381 \\
0.0381 \\
0.0429 \\
0.0385 \\
0.0344 \\
0.0338 \\
0.0285 \\
0.0243 \\
0.0239 \\
0.0198 \\
0.0157 \\
0.0148 \\
0.0096 \\
0.0061 \\
0.0033 \\
0.0071 \\
0.0077 \\
0.0071\end{array}$ & $\begin{array}{c}\text { Pressure } \\
\text { (psig) } \\
9.89 \\
19.55 \\
29.57 \\
39.42 \\
49.16 \\
54.50 \\
59.57 \\
64.20 \\
69.32 \\
74.16 \\
80.16 \\
85.61 \\
90.58 \\
95.69 \\
100.92 \\
103.25 \\
106.11 \\
108.31 \\
111.08 \\
113.24 \\
117.83 \\
120.92 \\
123.28 \\
122.97 \\
125.82 \\
125.60 \\
127.84 \\
130.19 \\
132.53 \\
135.33 \\
134.42 \\
138.35 \\
140.16 \\
142.63 \\
145.78 \\
142.52 \\
0.22\end{array}$ & $\begin{array}{c}\text { \% Strain } \\
0.0049 \\
0.0124 \\
0.0205 \\
0.0289 \\
0.0367 \\
0.0413 \\
0.0455 \\
0.0498 \\
0.0538 \\
0.0574 \\
0.0599 \\
0.0647 \\
0.0694 \\
0.0755 \\
0.0817 \\
0.0855 \\
0.0890 \\
0.0916 \\
0.0963 \\
0.0996 \\
0.1069 \\
0.1136 \\
0.1213 \\
0.1242 \\
0.1320 \\
0.1364 \\
0.1414 \\
0.1508 \\
0.1624 \\
0.1820 \\
0.1894 \\
0.2151 \\
0.2401 \\
0.2744 \\
0.3084 \\
0.3260 \\
0.1492\end{array}$ \\
\hline
\end{tabular}

High Pressure test

(psig)

9.89

0.0049

0.0124

0.0205

0.0289

0.0367

0.0413

0.0455

0.0498

0.0538

0.0574

0.0599

0.0647

0.075

0.0817

0.0855

0.0916

0.1069

0.1213

0.1242

0.1414

0.1508

0.1624

0.1820

0.1894

0.2151

0.2744

0.3084

0.3260

0.1492
0.0890 


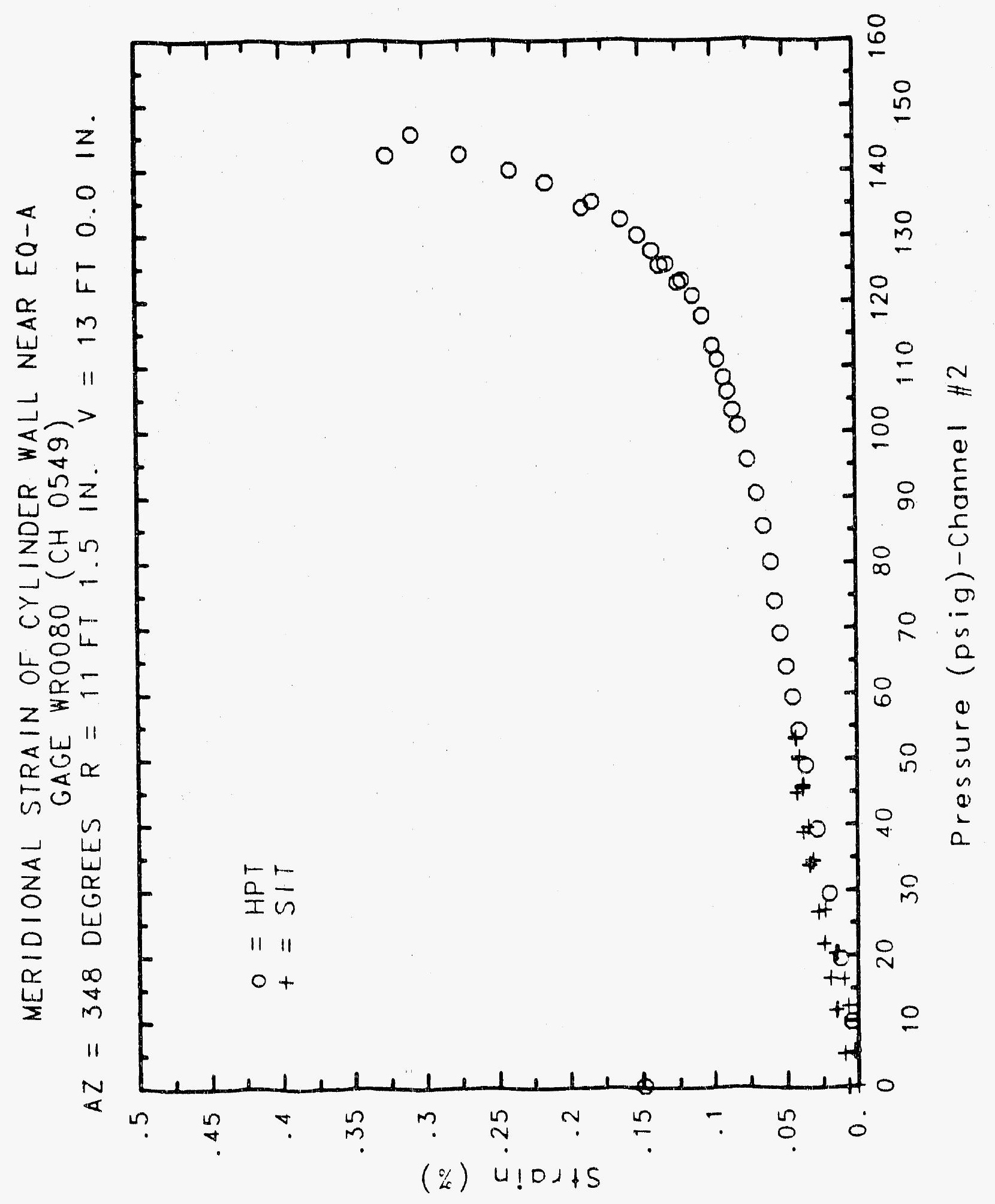

C. -972 


\section{Reinforced Concrete Test Data}

Weldable gage 81 Channel 560

StRUCTURAL INTEgRITY TEST

high Pressure test

\begin{tabular}{|c|c|c|c|}
\hline 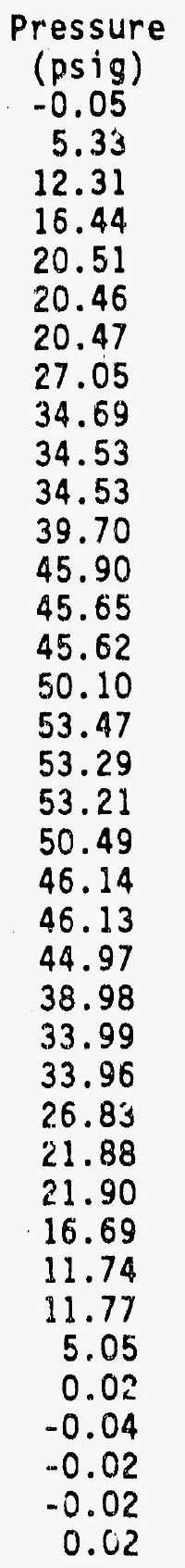 & $\begin{array}{l}\text { \% Strain } \\
0.0014 \\
0.0029 \\
0.0060 \\
0.0075 \\
0.0102 \\
0.0103 \\
0.0103 \\
0.0147 \\
0.0201 \\
0.0208 \\
0.0205 \\
0.0245 \\
0.0313 \\
0.0316 \\
0.0320 \\
0.0355 \\
0.0399 \\
0.0403 \\
0.0405 \\
0.0397 \\
0.0374 \\
0.0374 \\
0.0385 \\
0.0357 \\
0.0324 \\
0.0323 \\
0.0279 \\
0.0240 \\
0.0243 \\
0.0204 \\
0.0172 \\
0.0161 \\
0.0123 \\
0.0089 \\
0.0074 \\
0.0086 \\
0.0086 \\
0.0078\end{array}$ & $\begin{array}{c}\text { Pressure } \\
(\text { psig) } \\
9.89 \\
19.55 \\
29.57 \\
39.42 \\
49.16 \\
54.50 \\
59.57 \\
64.20 \\
69.32 \\
74.16 \\
80.16 \\
85.61 \\
90.58 \\
95.69 \\
100.92 \\
103.25 \\
106.11 \\
108.31 \\
111.08 \\
113.24 \\
117.83 \\
120.92 \\
123.28 \\
122.97 \\
125.82 \\
125.60 \\
127.84 \\
130.19 \\
132.53 \\
135.33 \\
134.42 \\
138.35 \\
140.16 \\
142.63 \\
145.78 \\
142.52 \\
0.22\end{array}$ & $\begin{array}{l}\text { \% Strain } \\
\\
0.0029 \\
0.0102 \\
0.0177 \\
0.0257 \\
0.0334 \\
0.0381 \\
0.0425 \\
0.0488 \\
0.0557 \\
0.0623 \\
0.0705 \\
0.0782 \\
0.0860 \\
0.0950 \\
0.1052 \\
0.1115 \\
0.1180 \\
0.1229 \\
0.1314 \\
0.1375 \\
0.1531 \\
0.1659 \\
0.1791 \\
0.1836 \\
0.1961 \\
0.2025 \\
0.2095 \\
0.2218 \\
0.2375 \\
0.2595 \\
0.2661 \\
0.2939 \\
0.3207 \\
0.3647 \\
0.4169 \\
0.4412 \\
0.2372\end{array}$ \\
\hline
\end{tabular}




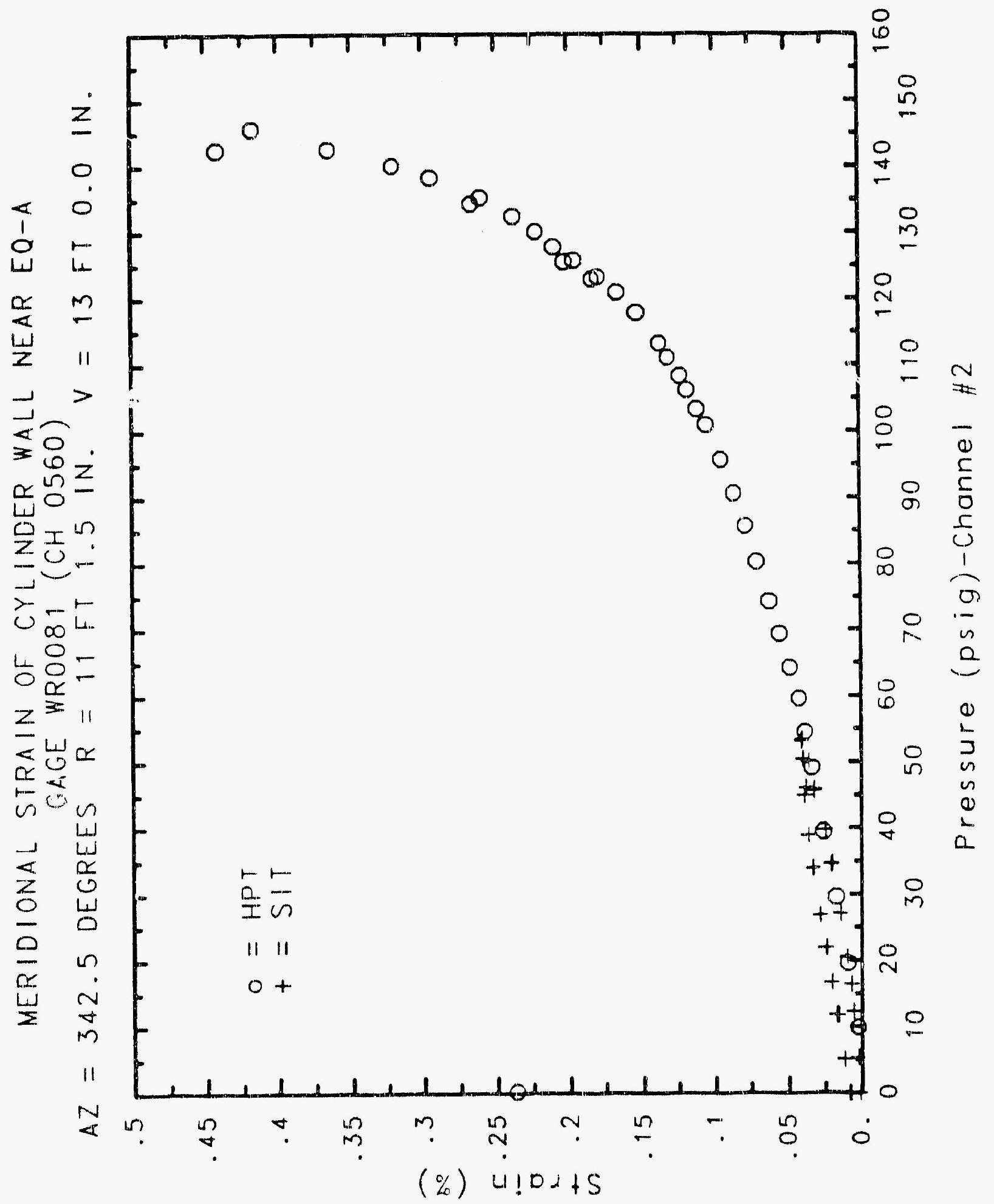




\section{Reinforced Concrete Test Data}

Weldable gage 82 Channel 561

Structural Integrity Test

\begin{tabular}{|c|c|c|c|}
\hline $\begin{array}{c}\text { Pressure } \\
\text { (psig) } \\
-0.05 \\
5.33 \\
12.31 \\
16.44 \\
20.51 \\
20.46 \\
20.47 \\
27.05 \\
34.69 \\
34.53 \\
34.53 \\
39.70 \\
45.90 \\
45.65 \\
45.62 \\
50.10 \\
53.47 \\
53.29 \\
53.21 \\
50.49 \\
46.14 \\
46.13 \\
44.37 \\
38.98 \\
33.99 \\
33.96 \\
26.83 \\
21.88 \\
21.90 \\
16.69 \\
11.74 \\
11.77 \\
5.05 \\
0.02 \\
-0.04 \\
-0.02 \\
-0.02 \\
0.02 \\
\end{array}$ & $\begin{array}{c}\text { \% Strain } \\
\text {-0.0002 } \\
0.0010 \\
0.0031 \\
0.0047 \\
0.0069 \\
0.0076 \\
0.0073 \\
0.0123 \\
0.0204 \\
0.0211 \\
0.0207 \\
0.0248 \\
0.0312 \\
0.0313 \\
0.0315 \\
0.0351 \\
0.0386 \\
0.0387 \\
0.0391 \\
0.0382 \\
0.0353 \\
0.0351 \\
0.0356 \\
0.0323 \\
0.0283 \\
0.0276 \\
0.0228 \\
0.0194 \\
0.0193 \\
0.0149 \\
0.0112 \\
0.0104 \\
0.0067 \\
0.0038 \\
0.0035 \\
0.0046 \\
0.0038 \\
0.0020\end{array}$ & $\begin{array}{c}\text { Pressure } \\
\text { (psig) } \\
9.89 \\
19.55 \\
29.57 \\
39.42 \\
49.16 \\
54.50 \\
59.57 \\
64.20 \\
69.32 \\
74.16 \\
80.16 \\
85.61 \\
90.58 \\
95.69 \\
100.92 \\
103.25 \\
106.11 \\
108.31 \\
111.08 \\
113.24 \\
117.83 \\
120.92 \\
123.28 \\
122.97 \\
125.82 \\
125.60 \\
127.84 \\
130.19 \\
132.53 \\
135.33 \\
134.42 \\
138.35 \\
140.16 \\
142.63 \\
145.78 \\
142.52 \\
0.22\end{array}$ & $\begin{array}{l}\text { \% Strain } \\
0.0022 \\
0.0096 \\
0.0176 \\
0.0250 \\
0.0323 \\
0.0364 \\
0.0405 \\
0.0460 \\
0.0507 \\
0.0552 \\
0.0612 \\
0.0676 \\
0.0728 \\
0.0790 \\
0.0859 \\
0.0889 \\
0.0923 \\
0.0950 \\
0.0992 \\
0.1022 \\
0.1090 \\
0.1142 \\
0.1195 \\
0.1206 \\
0.1273 \\
0.1288 \\
0.1330 \\
0.1411 \\
0.1505 \\
0.1636 \\
0.1653 \\
0.1782 \\
0.1906 \\
0.2069 \\
0.2262 \\
0.2351 \\
0.0961\end{array}$ \\
\hline
\end{tabular}

High Pressure Test
Pressure

8.89

19.55

29.57

54.50

64.20

69.32

85.61

95.69

10092

103.25

120.92

123.28

25.60

132.53

138.35

140.16

2.52

0.22
.

0.0323

0.0364

0.0405

0.0460

0.0507

0.0676

0.0728

0.0790

0.0859

0.0889

0.0992

0.1142

1288

0.141

0.1505

0.1636

0.1653

0.2069

0.2351

0.0961 


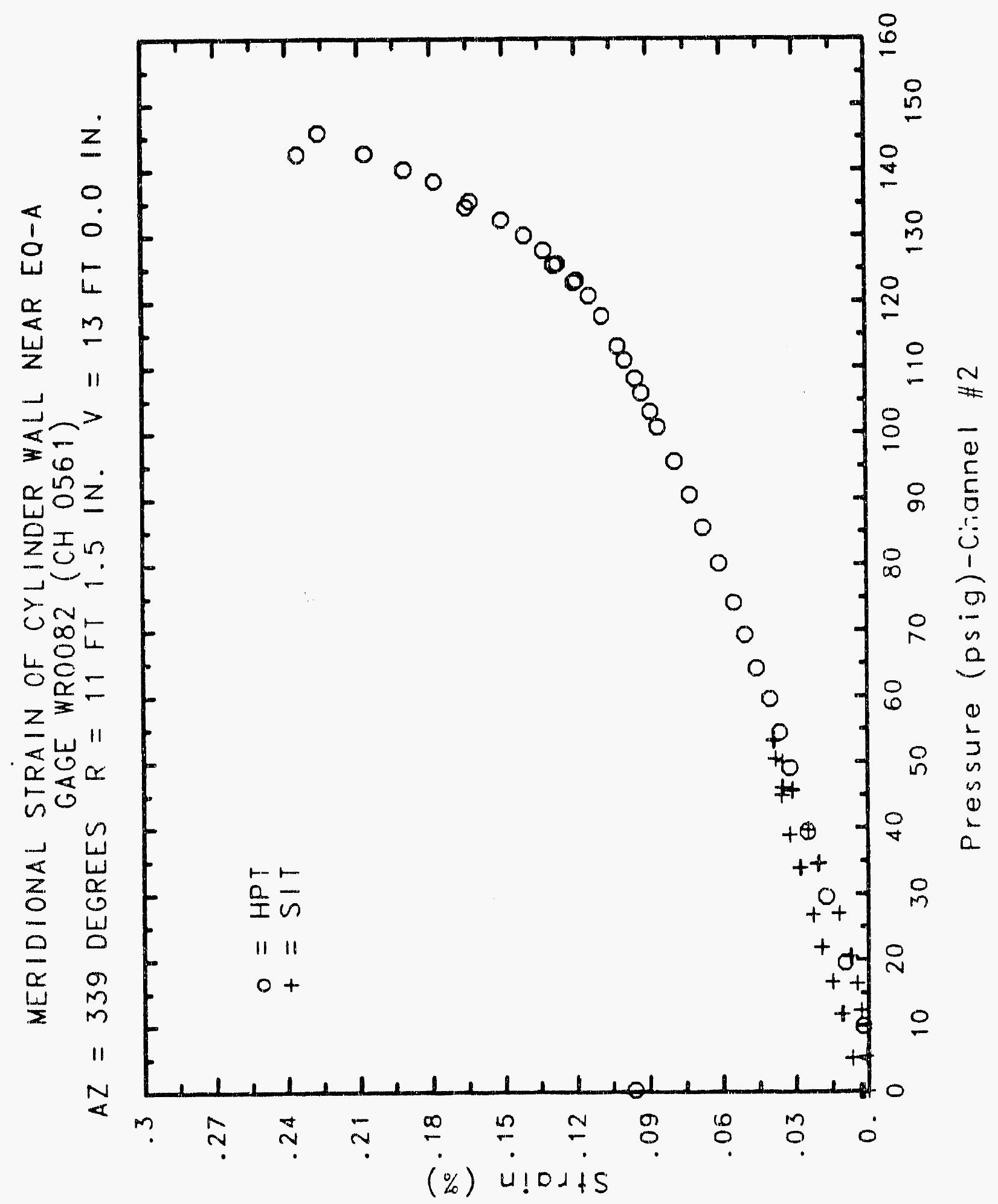

C-976 


\section{Reinforced Concrete Test Data}

Weldable gage 83 Channel 562

Structural Integrity test

High Pressure test

\begin{tabular}{|c|c|c|c|}
\hline $\begin{array}{c}\text { Pressure } \\
\text { (psig) } \\
-0.05 \\
5.33 \\
12.31 \\
16.44 \\
20.51 \\
20.46 \\
20.47 \\
27.05 \\
34.69 \\
34.53 \\
34.53 \\
39.70 \\
45.90 \\
45.65 \\
45.62 \\
50.10 \\
53.47 \\
53.29 \\
53.21 \\
50.49 \\
46.14 \\
46.13 \\
44.97 \\
38.98 \\
33.99 \\
33.96 \\
26.83 \\
21.88 \\
21.90 \\
16.69 \\
11.74 \\
11.77 \\
5.05 \\
0.02 \\
-0.04 \\
-0.02 \\
-0.02 \\
0.02 \\
\end{array}$ & $\begin{array}{l}\text { \% Strain } \\
0.0003 \\
0.0008 \\
0.0013 \\
0.0021 \\
0.0022 \\
0.0032 \\
0.0034 \\
0.0047 \\
0.0077 \\
0.0089 \\
0.0084 \\
0.0101 \\
0.0135 \\
0.0142 \\
0.0146 \\
0.0157 \\
0.0174 \\
0.0175 \\
0.0181 \\
0.0177 \\
0.0167 \\
0.0168 \\
0.0174 \\
0.0163 \\
0.0154 \\
0.0146 \\
0.0135 \\
0.0126 \\
0.0123 \\
0.0113 \\
0.0099 \\
0.0100 \\
0.0082 \\
0.0071 \\
0.0064 \\
0.0063 \\
0.0063 \\
0.0066\end{array}$ & $\begin{array}{c}\text { Pressure } \\
\text { (psig) } \\
9.89 \\
19.55 \\
29.57 \\
39.42 \\
49.16 \\
54.50 \\
59.57 \\
64.20 \\
69.32 \\
74.16 \\
80.16 \\
85.61 \\
90.58 \\
95.69 \\
100.92 \\
103.25 \\
106.11 \\
108.31 \\
111.08 \\
113.24 \\
117.83 \\
120.92 \\
123.28 \\
122.97 \\
125.82 \\
125.60 \\
127.84 \\
130.19 \\
132.53 \\
135.33 \\
134.42 \\
138.35 \\
140.16 \\
142.63 \\
145.78 \\
142.52 \\
0.22\end{array}$ & $\begin{array}{c}\text { \% Strain } \\
-0.0016 \\
0.0005 \\
0.0026 \\
0.0049 \\
0.0075 \\
0.0093 \\
0.0109 \\
0.0127 \\
0.0150 \\
0.0192 \\
0.0241 \\
0.0273 \\
0.0302 \\
0.0336 \\
0.0386 \\
0.0414 \\
0.0438 \\
0.0455 \\
0.0418 \\
0.0493 \\
0.0533 \\
0.0565 \\
0.0596 \\
0.0604 \\
0.0631 \\
0.0646 \\
0.0664 \\
0.0691 \\
0.0730 \\
0.0774 \\
0.0797 \\
0.0868 \\
0.0921 \\
0.1014 \\
0.1093 \\
0.1132 \\
0.0445\end{array}$ \\
\hline
\end{tabular}




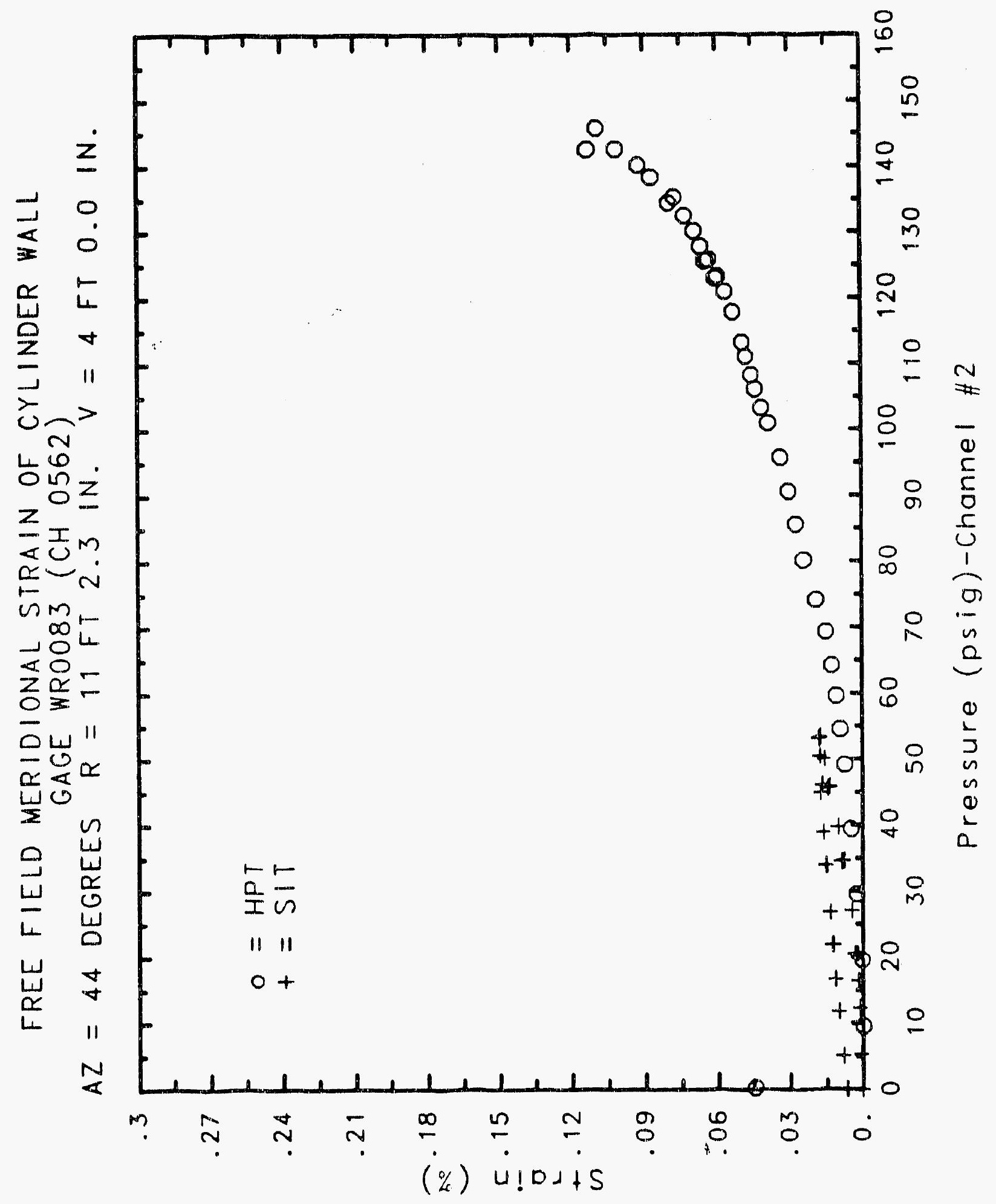




\section{Reinforced Concrete Test Data}

Weldable Gage 84 Channel 563

Structural Integrity Test

\begin{tabular}{|c|c|c|c|}
\hline $\begin{array}{c}\text { Pressure } \\
\text { (psig) } \\
-0.05 \\
5.33 \\
12.31 \\
16.44 \\
20.51 \\
20.46 \\
20.47 \\
27.05 \\
34.69 \\
34.53 \\
34.53 \\
39.70 \\
45.90 \\
45.65 \\
45.62 \\
50.10 \\
53.47 \\
53.29 \\
53.21 \\
50.49 \\
46.14 \\
46.13 \\
44.97 \\
38.98 \\
33.99 \\
33.96 \\
26.83 \\
21.88 \\
21.90 \\
16.69 \\
11.74 \\
11.77 \\
5.05 \\
0.02 \\
-0.04 \\
-0.02 \\
-0.02 \\
0.02 \\
\end{array}$ & $\begin{array}{c}\text { Strain } \\
-0.0001 \\
0.0017 \\
0.0053 \\
0.0082 \\
0.0127 \\
0.0134 \\
0.0140 \\
0.0216 \\
0.0319 \\
0.0336 \\
0.0335 \\
0.0394 \\
0.0472 \\
0.0479 \\
0.0482 \\
0.0529 \\
0.0568 \\
0.0569 \\
0.0574 \\
0.0558 \\
0.0523 \\
0.0525 \\
0.0527 \\
0.0480 \\
0.0444 \\
0.0441 \\
0.0381 \\
0.0338 \\
0.0335 \\
0.0290 \\
0.0243 \\
0.0238 \\
0.0177 \\
0.0127 \\
0.0099 \\
0.0103 \\
0.0111 \\
0.0110\end{array}$ & $\begin{array}{c}\text { Pressure } \\
\text { (psig) } \\
9.89 \\
19.55 \\
29.57 \\
39.42 \\
49.16 \\
54.50 \\
59.57 \\
64.20 \\
69.32 \\
74.16 \\
80.16 \\
85.61 \\
90.58 \\
95.69 \\
100.92 \\
103.25 \\
106.11 \\
108.31 \\
111.08 \\
113.24 \\
117.83 \\
120.92 \\
123.28 \\
122.97 \\
125.82 \\
125.60 \\
127.84 \\
130.19 \\
132.53 \\
135.33 \\
134.42 \\
138.35 \\
140.16 \\
142.63 \\
145.78 \\
142.52 \\
0.22\end{array}$ & $\begin{array}{l}\text { \% Strain } \\
0.0054 \\
0.0149 \\
0.0250 \\
0.0340 \\
0.0427 \\
0.0482 \\
0.0533 \\
0.0590 \\
0.0655 \\
0.0726 \\
0.0803 \\
0.0878 \\
0.0946 \\
0.1023 \\
0.1107 \\
0.1146 \\
0.1187 \\
0.1217 \\
0.1266 \\
0.1305 \\
0.1374 \\
0.1434 \\
0.1479 \\
0.1482 \\
0.1533 \\
0.1535 \\
0.1573 \\
0.1636 \\
0.1715 \\
0.1821 \\
0.1833 \\
0.2022 \\
0.2235 \\
0.2535 \\
0.2616 \\
0.2420 \\
0.0797\end{array}$ \\
\hline
\end{tabular}

High Pressure test

Pressure

9.89

39.42

49.16

54.50

59.57

64.20

80.16

85.61

90.58

103.25

106.11

111.08

113.24

117.83

122.97

25.82

127.84

130.19

132.53

135.33

134.42

140.16

142.63

145.78

0.22 strain

0.0054

0.0340

0.0482

0.0533

0.0590

0.0655

0.0878

0.0946

0.1023

0.1107

0.1217

0.1266

0.1305

0.1374

0.1434

0.1479

0.1482

0.1535

0.1573

0.1636

0.1715

0.2022

0.2235

0.2535

0.2616

0.2420

0.0797 


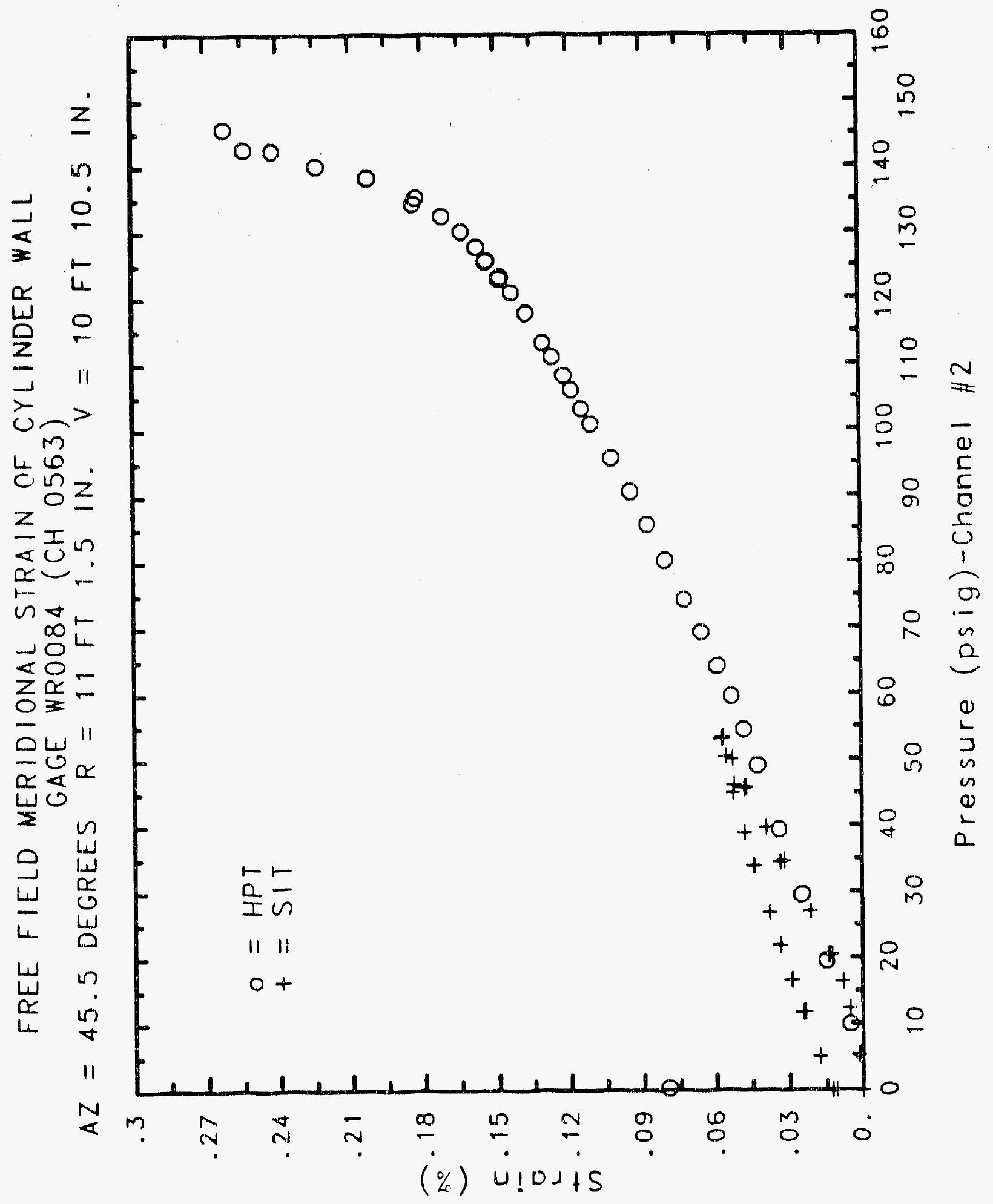

C-980 


\section{Reinforced Concrete Test Data}

Weldable gage 85 Channel 564

Structural Integrity Test

\begin{tabular}{|c|c|c|c|}
\hline $\begin{array}{c}\text { Pressure } \\
\text { (psig) } \\
-0.05 \\
5.33 \\
12.31 \\
16.44 \\
20.51 \\
20.46 \\
20.47 \\
27.05 \\
34.69 \\
34.53 \\
34.53 \\
39.70 \\
45.90 \\
45.65 \\
45.62 \\
50.10 \\
53.47 \\
53.29 \\
53.21 \\
50.49 \\
46.14 \\
46.13 \\
44.97 \\
38.98 \\
33.99 \\
33.96 \\
26.83 \\
21.88 \\
21.90 \\
16.69 \\
11.74 \\
11.77 \\
5.05 \\
0.02 \\
-0.04 \\
-0.02 \\
-0.02 \\
0.02 \\
\end{array}$ & $\begin{array}{c}\text { \% Strain } \\
-0.0014 \\
0.0002 \\
0.0002 \\
0.0007 \\
0.0039 \\
0.0052 \\
0.0053 \\
0.0157 \\
0.0299 \\
0.0315 \\
0.0318 \\
0.0372 \\
0.0453 \\
0.0459 \\
0.0458 \\
0.0503 \\
0.0542 \\
0.0547 \\
0.0552 \\
0.0541 \\
0.0514 \\
0.0518 \\
0.0533 \\
0.0497 \\
0.0466 \\
0.0464 \\
0.0421 \\
0.0386 \\
0.0383 \\
0.0346 \\
0.0319 \\
0.0315 \\
0.0274 \\
0.0247 \\
0.0222 \\
0.0223 \\
0.0227 \\
0.0233\end{array}$ & $\begin{array}{c}\text { Pressure } \\
(\text { psig) } \\
9.89 \\
19.55 \\
29.57 \\
39.42 \\
49.16 \\
54.50 \\
59.57 \\
64.20 \\
69.32 \\
74.16 \\
80.16 \\
85.61 \\
90.58 \\
95.69 \\
100.92 \\
103.25 \\
106.11 \\
108.31 \\
111.08 \\
113.24 \\
117.83 \\
120.92 \\
123.28 \\
122.97 \\
125.82 \\
125.60 \\
127.84 \\
130.19 \\
132.53 \\
135.33 \\
134.42 \\
138.35 \\
140.16 \\
142.63 \\
145.78 \\
142.52 \\
0.22\end{array}$ & $\begin{array}{l}\text { \% Strain } \\
0.0027 \\
0.0105 \\
0.0182 \\
0.0257 \\
0.0326 \\
0.0367 \\
0.0413 \\
0.0464 \\
0.0517 \\
0.0574 \\
0.0637 \\
0.0695 \\
0.0758 \\
0.0829 \\
0.0907 \\
0.0946 \\
0.0992 \\
0.1024 \\
0.1077 \\
0.1115 \\
0.1203 \\
0.1268 \\
0.1316 \\
0.1319 \\
0.1359 \\
0.1372 \\
0.1403 \\
0.1450 \\
0.1513 \\
0.1620 \\
0.1642 \\
0.1840 \\
0.2003 \\
0.2170 \\
0.2282 \\
0.2254 \\
0.0779\end{array}$ \\
\hline
\end{tabular}

High Pressure test

Pressure

9.89

19.55

39.42

49.16

69.32

80.16

95.69

106.11

108.31

117.83

122.97

125.82

130.19

134.42

138.35

142.63

45.78

42.52

0.2282

0.2254

0.0227

0.0233 


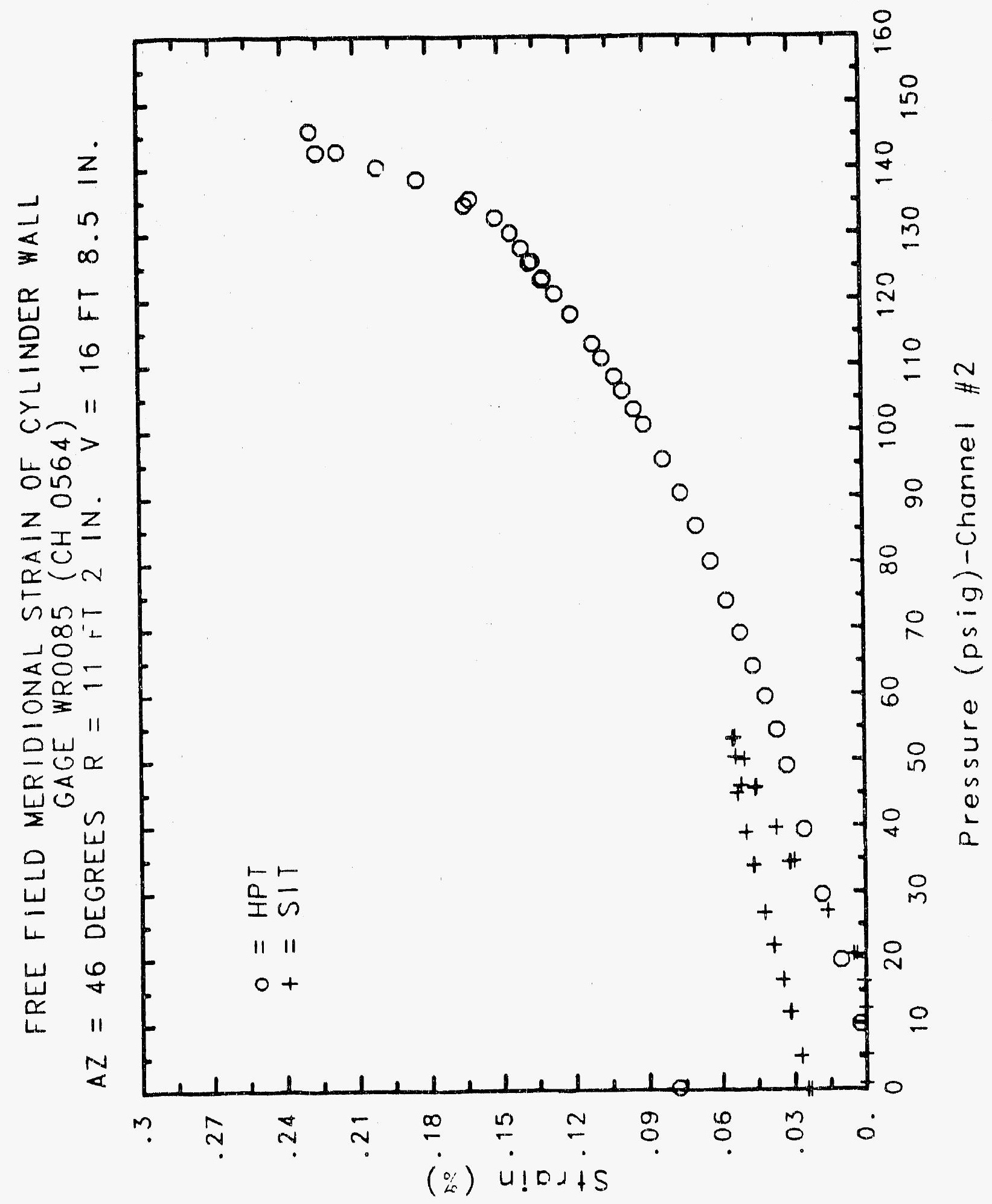




\section{Reinforced Concrete Test Data}

Weldable gage 86 Channel 565

StRUCTURAL InTEGRITY TEST

\begin{tabular}{|c|c|c|c|}
\hline $\begin{array}{c}\text { Pressure } \\
\text { (psig) } \\
-0.05 \\
5.33 \\
12.31 \\
16.44 \\
20.51 \\
20.46 \\
20.47 \\
27.05 \\
34.69 \\
34.53 \\
34.53 \\
39.70 \\
45.90 \\
45.65 \\
45.62 \\
50.10 \\
53.47 \\
53.29 \\
53.21 \\
50.49 \\
46.14 \\
46.13 \\
44.97 \\
38.98 \\
33.99 \\
33.96 \\
26.83 \\
21.88 \\
21.90 \\
16.69 \\
11.74 \\
11.77 \\
5.05 \\
0.02 \\
-0.04 \\
-0.02 \\
-0.02 \\
0.02 \\
\end{array}$ & $\begin{array}{l}\text { \% Strain } \\
-0.0001 \\
0.0041 \\
0.0081 \\
0.0118 \\
0.0164 \\
0.0170 \\
0.0171 \\
0.0259 \\
0.0355 \\
0.0363 \\
0.0362 \\
0.0402 \\
0.0472 \\
0.0471 \\
0.0467 \\
0.0510 \\
0.0545 \\
0.0545 \\
0.0544 \\
0.0531 \\
0.0500 \\
0.0499 \\
0.0505 \\
0.0457 \\
0.0418 \\
0.0412 \\
0.0347 \\
0.0302 \\
0.0300 \\
0.0258 \\
0.0200 \\
0.0193 \\
0.0127 \\
0.0076 \\
0.0066 \\
0.0067 \\
0.0074 \\
0.0080\end{array}$ & $\begin{array}{c}\text { Pressure } \\
(\text { psig) } \\
9.89 \\
19.55 \\
29.57 \\
39.42 \\
49.16 \\
54.50 \\
59.57 \\
64.20 \\
69.32 \\
74.16 \\
80.16 \\
85.61 \\
90.58 \\
95.69 \\
100.92 \\
103.25 \\
106.11 \\
108.31 \\
111.08 \\
113.24 \\
117.83 \\
120.92 \\
123.28 \\
122.97 \\
125.82 \\
125.60 \\
127.84 \\
130.19 \\
132.53 \\
135.33 \\
134.42 \\
138.35 \\
140.16 \\
142.63 \\
145.78 \\
142.52 \\
0.22 \\
\end{array}$ & $\begin{array}{l}\text { \% Strain } \\
0.0056 \\
0.0155 \\
0.0257 \\
0.0348 \\
0.0431 \\
0.0477 \\
0.0526 \\
0.0573 \\
0.0631 \\
0.0688 \\
0.0746 \\
0.0806 \\
0.0863 \\
0.0928 \\
0.0996 \\
0.1031 \\
0.1066 \\
0.1092 \\
0.1140 \\
0.1165 \\
0.1236 \\
0.1287 \\
0.1335 \\
0.1342 \\
0.1401 \\
0.1409 \\
0.1448 \\
0.1516 \\
0.1599 \\
0.1737 \\
0.1763 \\
0.1916 \\
0.2003 \\
0.2119 \\
0.2219 \\
0.2247 \\
0.0885\end{array}$ \\
\hline
\end{tabular}

High Pressure test

Pres

(psig)

19.55

59.57

64.20

80.16

95.69

100.92

103.25

13.24

117.83

120.92

123.28

22.97

25.82

25.60

127.84

32.53

135.33

134.42

138.35

40.16

42.63

145.78

2.52

0.22
0.0056

0.0155

0.0257

0.0477

0.0526

0.0573

0.0746

0.0863

0.0928

0.0996

0.1092

0.1236

0.1287

0.1335

0.1342

0.1401

0.1409

0.1516

0.1599

0.1737

0.1763

0.1916

0.2003

0.2219

0.2247

0.0885 


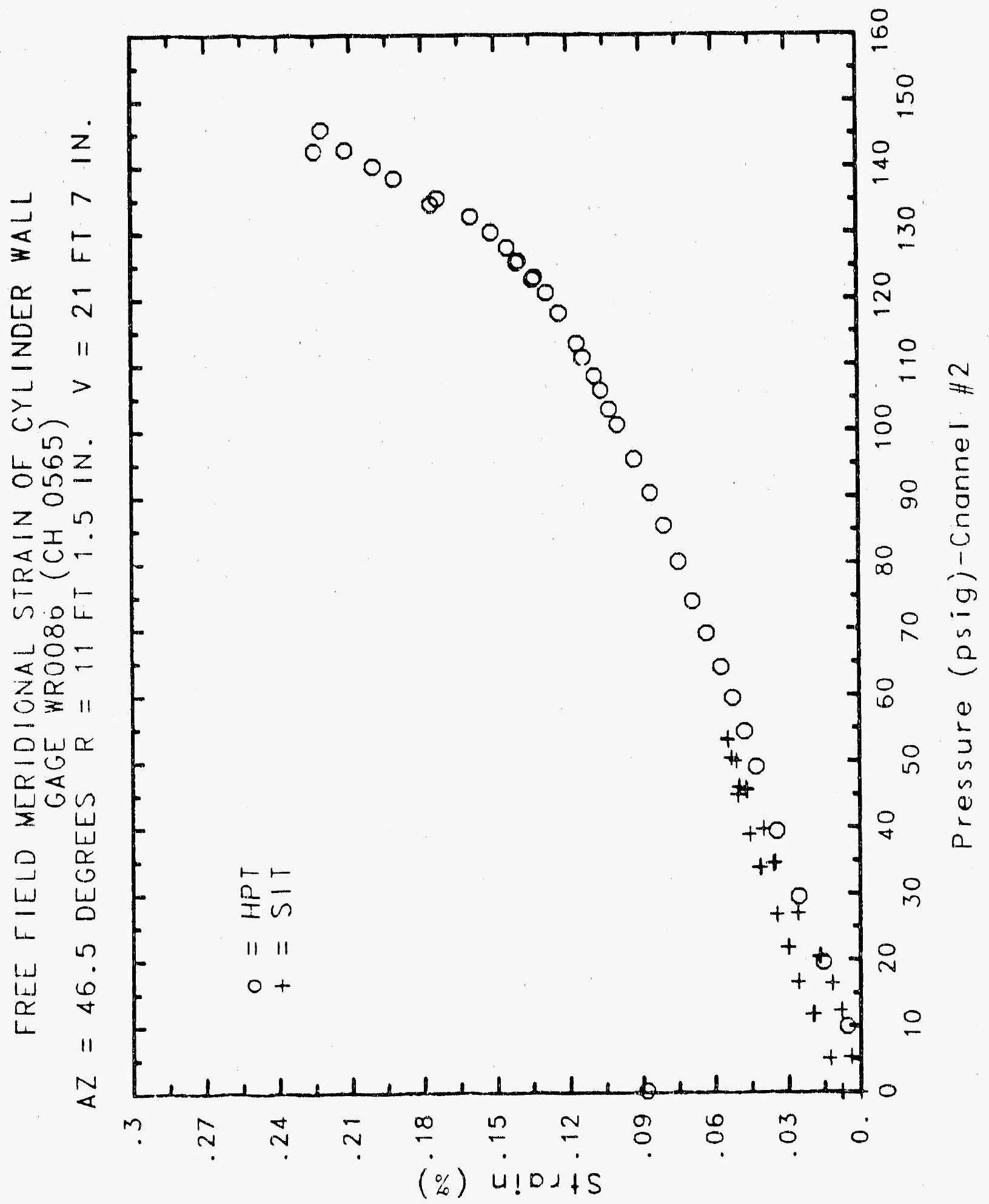




\section{Reinforced Concrete Test Data}

Weldable gage 87 Channel 566

STRIJCTURAL IN'EGRITY TEST

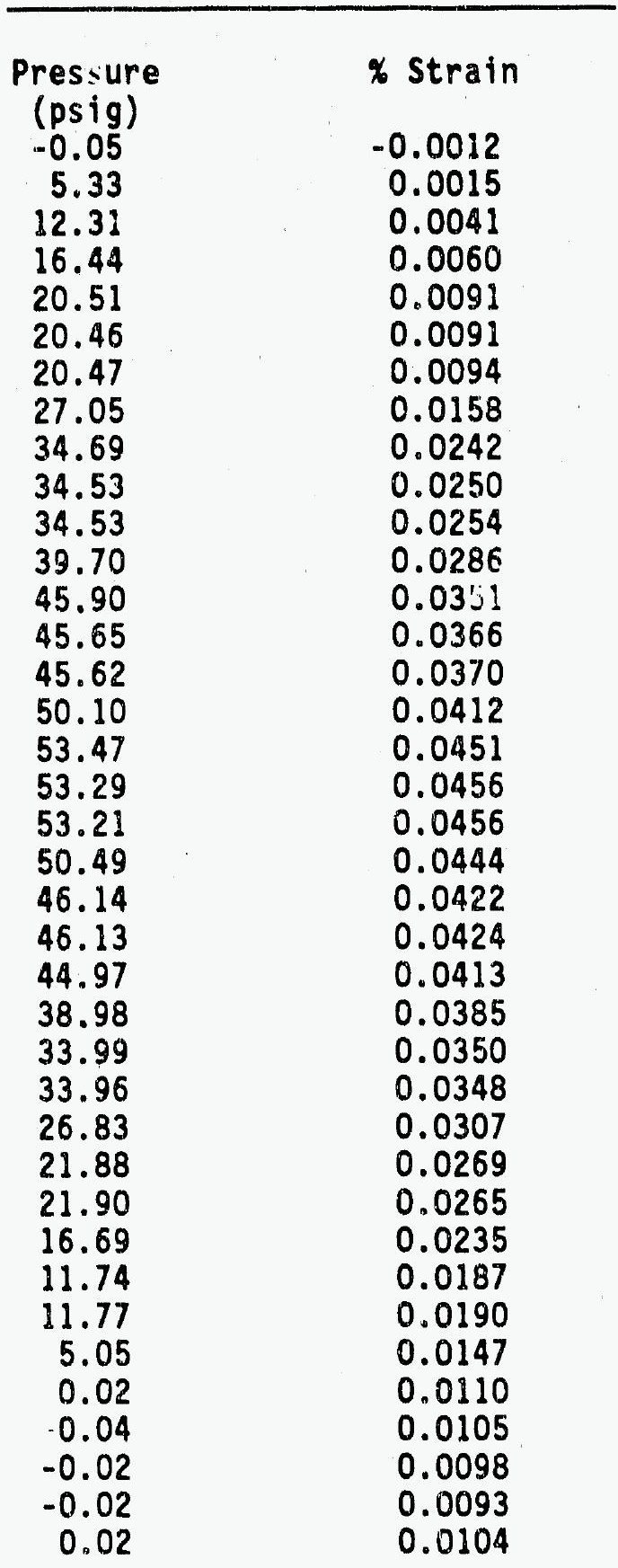

High Pressure test

$\begin{array}{cc}\text { Pressure } & \text { \% Strain } \\ \text { (psig) } & \\ 9.89 & 0.0035 \\ 19.55 & 0.0107 \\ 29.57 & 0.0178 \\ 39.42 & 0.0256 \\ 49.16 & 0.0330 \\ 54.50 & 0.0371 \\ 59.57 & 0.0411 \\ 64.20 & 0.0457 \\ 69.32 & 0.0504 \\ 74.16 & 0.0555 \\ 80.16 & 0.0623 \\ 85.61 & 0.0679 \\ 90.58 & 0.0732 \\ 95.69 & 0.0789 \\ 100.92 & 0.0846 \\ 103.25 & 0.0878 \\ 106.11 & 0.0913 \\ 108.31 & 0.0939 \\ 111.08 & 0.0973 \\ 113.24 & 0.1001 \\ 117.83 & 0.1069 \\ 120.92 & 0.1117 \\ 123.28 & 0.1159 \\ 122.97 & 0.1164 \\ 125.82 & 0.1206 \\ 125.60 & 0.1218 \\ 127.84 & 0.1245 \\ 130.19 & 0.1292 \\ 132.53 & 0.1357 \\ 135.33 & 0.1470 \\ 134.42 & 0.1498 \\ 138.35 & 0.1663 \\ 140.16 & 0.1792 \\ 142.63 & 0.1967 \\ 145.78 & 0.2124 \\ 142.52 & 0.2133 \\ 0.22 & 0.0668 \\ & \\ & \end{array}$




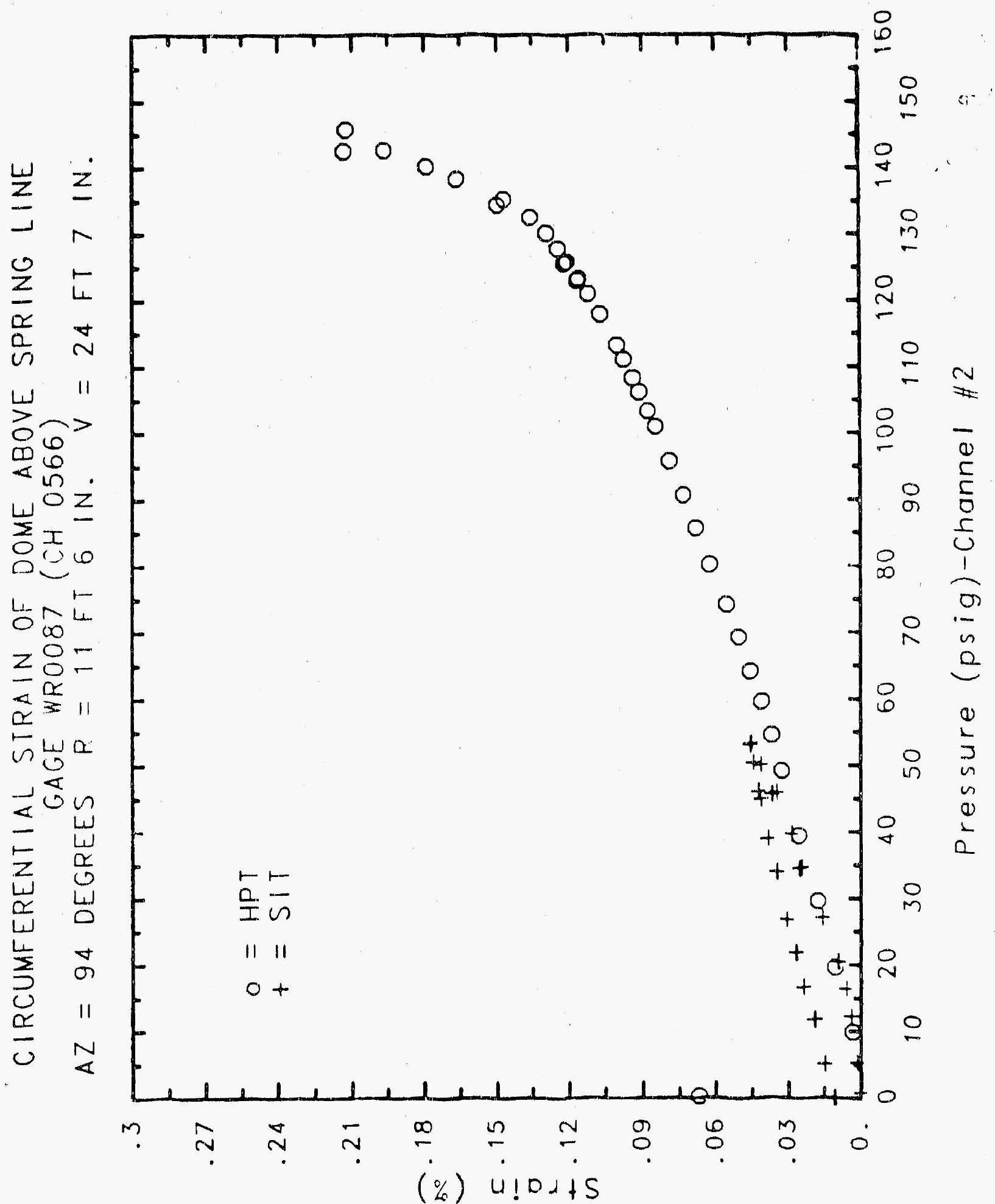


Reinforced Concrete test Data

Weldable gage 89 Channel 568

Structural Integrity test

High Pressure test

\begin{tabular}{|c|c|c|c|}
\hline $\begin{array}{c}\text { Pressure } \\
\text { (psig) } \\
-0.05 \\
5.33 \\
: 2.31 \\
16.44 \\
20.51 \\
20.46 \\
20.47 \\
27.05 \\
34.69 \\
34.53 \\
34.53 \\
39.70 \\
45.90 \\
45.65 \\
45.62 \\
50.10 \\
53.47 \\
53.29 \\
53.21 \\
50.49 \\
46.14 \\
46.13 \\
44.97 \\
38.98 \\
33.99 \\
33.96 \\
26.83 \\
21.88 \\
21.90 \\
16.69 \\
11.74 \\
11.77 \\
5.05 \\
0.02 \\
-0.04 \\
-0.02 \\
-0.02 \\
0.02\end{array}$ & $\begin{array}{c}\text { \% Strain } \\
-0.0004 \\
0.0011 \\
0.0043 \\
0.0066 \\
0.0098 \\
0.0103 \\
0.0103 \\
0.0168 \\
0.0251 \\
0.0249 \\
0.0259 \\
0.0298 \\
0.0353 \\
0.0359 \\
0.0356 \\
0.0401 \\
0.0434 \\
0.0434 \\
0.0432 \\
0.0424 \\
0.0396 \\
0.0396 \\
0.0380 \\
0.0345 \\
0.0306 \\
0.0303 \\
0.0257 \\
0.0217 \\
0.0218 \\
0.0178 \\
0.0143 \\
0.0142 \\
0.0092 \\
0.0051 \\
0.0045 \\
0.0047 \\
0.0047 \\
0.0043\end{array}$ & $\begin{array}{c}\text { Pressure } \\
\text { (psig) } \\
9.89 \\
19.55 \\
29.57 \\
39.42 \\
49.16 \\
54.50 \\
59.57 \\
64.20 \\
69.32 \\
74.16 \\
80.16 \\
85.61 \\
90.58 \\
95.69 \\
100.92 \\
103.25 \\
106.11 \\
108.31 \\
111.08 \\
113.24 \\
117.83 \\
120.92 \\
123.28 \\
122.97 \\
125.82 \\
125.60 \\
127.84 \\
130.19 \\
132.53 \\
135.33 \\
134.42 \\
138.35 \\
140.16 \\
142.63 \\
145.78 \\
142.52 \\
0.22\end{array}$ & $\begin{array}{l}\% \text { Strain } \\
0.0031 \\
0.0112 \\
0.0191 \\
0.0273 \\
0.0349 \\
0.0394 \\
0.0438 \\
0.0485 \\
0.0534 \\
0.0584 \\
0.0654 \\
0.0714 \\
0.0767 \\
0.0829 \\
0.0891 \\
0.0920 \\
0.0952 \\
0.0982 \\
0.1014 \\
0.1045 \\
0.1104 \\
0.1154 \\
0.1194 \\
0.1190 \\
0.1229 \\
0.1236 \\
0.1262 \\
0.1298 \\
0.1336 \\
0.1388 \\
0.1390 \\
0.1451 \\
0.1494 \\
0.1545 \\
0.1601 \\
0.1596 \\
0.0240\end{array}$ \\
\hline
\end{tabular}




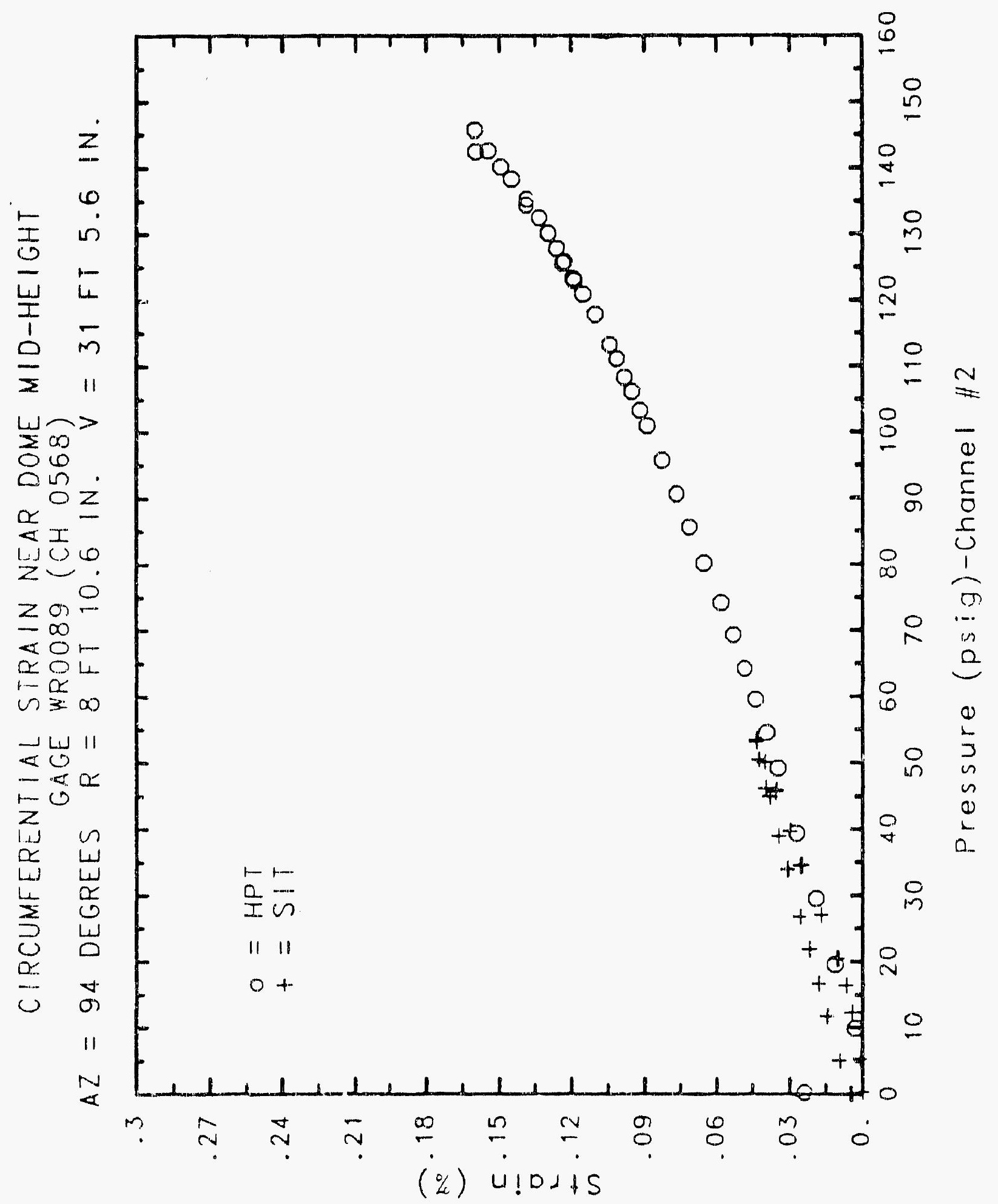




\section{Reinforced Concrete Test Data}

Weldable gage 90 Channel 569

Structural INTEgRITY TEST

High Pressure test

\begin{tabular}{|c|c|c|c|}
\hline $\begin{array}{c}\text { Pressure } \\
\text { (psig) } \\
-0.05 \\
5.33 \\
12.31 \\
16.44 \\
20.51 \\
20.46 \\
20.47 \\
27.05 \\
34.69 \\
34.53 \\
34.53 \\
39.70 \\
45.90 \\
45.65 \\
45.62 \\
50.10 \\
53.47 \\
53.29 \\
53.21 \\
50.49 \\
46.14 \\
46.13 \\
44.97 \\
38.98 \\
33.99 \\
33.96 \\
26.83 \\
21.88 \\
21.90 \\
16.69 \\
11.74 \\
11.77 \\
5.05 \\
0.02 \\
-0.04 \\
-0.02 \\
-0.02 \\
0.02 \\
\end{array}$ & $\begin{array}{l}\text { Strain } \\
0.0003 \\
-0.0011 \\
-0.0021 \\
-0.0027 \\
-0.0034 \\
-0.0040 \\
-0.0044 \\
-0.0031 \\
-0.0011 \\
-0.0007 \\
-0.0002 \\
0.0014 \\
0.0033 \\
0.0029 \\
0.0038 \\
0.0044 \\
0.0058 \\
0.0063 \\
0.0061 \\
0.0054 \\
0.0050 \\
0.0049 \\
0.0043 \\
0.0032 \\
0.0021 \\
0.0023 \\
0.0010 \\
0.0002 \\
0.0004 \\
-0.0001 \\
-0.0004 \\
-0.0005 \\
-0.0009 \\
-0.0005 \\
0.0024 \\
0.0024 \\
0.0018 \\
0.0001\end{array}$ & $\begin{array}{c}\text { Pressure } \\
\text { (psig) } \\
9.89 \\
19.55 \\
29.57 \\
39.42 \\
49.16 \\
54.50 \\
59.57 \\
64.20 \\
69.32 \\
74.16 \\
80.16 \\
85.61 \\
90.58 \\
95.69 \\
100.92 \\
103.25 \\
106.11 \\
108.31 \\
111.08 \\
113.24 \\
117.83 \\
120.92 \\
123.28 \\
122.97 \\
125.82 \\
125.60 \\
127.84 \\
130.19 \\
132.53 \\
135.33 \\
134.42 \\
138.35 \\
140.16 \\
142.63 \\
145.78 \\
142.52 \\
0.22\end{array}$ & $\begin{array}{l}\text { \% Strain } \\
-0.0049 \\
-0.0048 \\
-0.0034 \\
-0.0016 \\
0.0005 \\
0.0024 \\
0.0040 \\
0.0076 \\
0.0108 \\
0.0132 \\
0.0177 \\
0.0212 \\
0.0244 \\
0.0277 \\
0.0310 \\
0.0322 \\
0.0335 \\
0.0348 \\
0.0360 \\
0.0367 \\
0.0390 \\
0.0400 \\
0.0404 \\
0.0398 \\
0.0410 \\
0.0405 \\
0.0421 \\
0.0448 \\
0.0489 \\
0.0540 \\
0.0556 \\
0.0649 \\
0.0724 \\
0.0843 \\
0.0975 \\
0.1015 \\
0.0295\end{array}$ \\
\hline
\end{tabular}




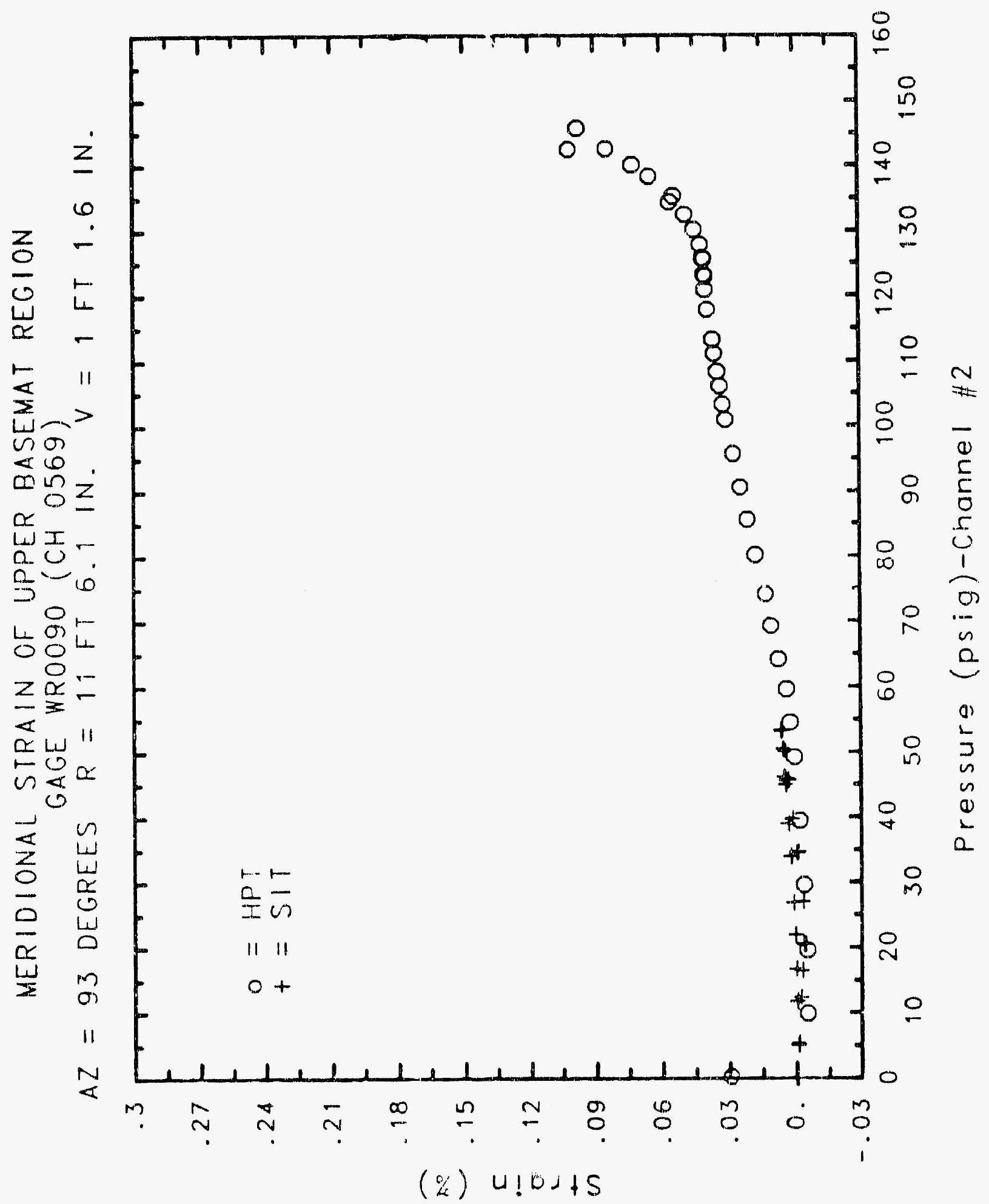




\section{Reinforced Concrete Test Data}

\begin{tabular}{|c|c|c|c|}
\hline STRUCTURAL & $\begin{array}{l}\text { WeLdABLE GAge } 92 \\
\text { INTEGRITY TEST }\end{array}$ & $\begin{array}{l}\text { Channel } 5,80 \\
\text { High Pressure }\end{array}$ & TEST \\
\hline $\begin{array}{c}\text { Pressure } \\
\text { (psig) } \\
-0.05 \\
5.33 \\
12.31 \\
16.44 \\
20.51 \\
20.46 \\
20.47 \\
27.05 \\
34.69 \\
34.53 \\
34.53 \\
39.70 \\
45.90 \\
45.65 \\
45.62 \\
50.10 \\
53.47 \\
53.29 \\
53.21 \\
50.49 \\
46.14 \\
46.13 \\
44.97 \\
38.98 \\
33.99 \\
33.96 \\
26.83 \\
21.88 \\
21.90 \\
16.69 \\
11.74 \\
11.77 \\
5.05 \\
0.02 \\
-0.04 \\
-0.02 \\
-0.02 \\
0.02 \\
\end{array}$ & $\begin{array}{c}\text { \% Strain } \\
0.0003 \\
-0.0008 \\
-0.0015 \\
-0.0021 \\
-0.0027 \\
-0.0034 \\
-0.0043 \\
-0.0035 \\
-0.0015 \\
-0.0007 \\
-0.0001 \\
0.0013 \\
0.0032 \\
0.0033 \\
0.0034 \\
0.0044 \\
0.0059 \\
0.0060 \\
0.0061 \\
0.0054 \\
0.0045 \\
0.0046 \\
0.0042 \\
0.0033 \\
0.0029 \\
0.0026 \\
0.0013 \\
0.0000 \\
-0.0001 \\
-0.0005 \\
-0.0006 \\
0.0001 \\
-0.0002 \\
0.0002 \\
0.0023 \\
0.0022 \\
0.0021 \\
0.0007\end{array}$ & $\begin{array}{c}\text { Pressure } \\
(\text { psig) } \\
9.89 \\
19.55 \\
29.57 \\
39.42 \\
49.16 \\
54.50 \\
59.57 \\
64.20 \\
69.32 \\
74.16 \\
80.16 \\
85.61 \\
90.58 \\
95.69 \\
100.92 \\
103.25 \\
106.11 \\
108.31 \\
111.08 \\
113.24 \\
117.83 \\
120.92 \\
123.28 \\
122.97 \\
125.82 \\
125.60 \\
127.84 \\
130.19 \\
132.53 \\
135.33 \\
134.42 \\
138.35 \\
140.16 \\
142.63 \\
145.78 \\
142.52 \\
0.22 \\
\end{array}$ & $\begin{array}{c}\text { \% Strain } \\
-0.0048 \\
-0.0042 \\
-0.0035 \\
-0.0019 \\
0.0001 \\
0.0011 \\
0.0026 \\
0.0053 \\
0.0063 \\
0.0057 \\
0.0089 \\
0.0107 \\
0.0123 \\
0.0140 \\
0.0149 \\
0.0151 \\
0.0159 \\
0.0168 \\
0.0182 \\
0.0198 \\
0.0249 \\
0.0278 \\
0.0301 \\
0.0300 \\
0.0335 \\
0.0332 \\
0.0359 \\
0.0410 \\
0.0481 \\
0.0597 \\
0.0608 \\
0.0772 \\
0.0854 \\
0.0984 \\
0.1087 \\
0.1070 \\
0.0406\end{array}$ \\
\hline
\end{tabular}




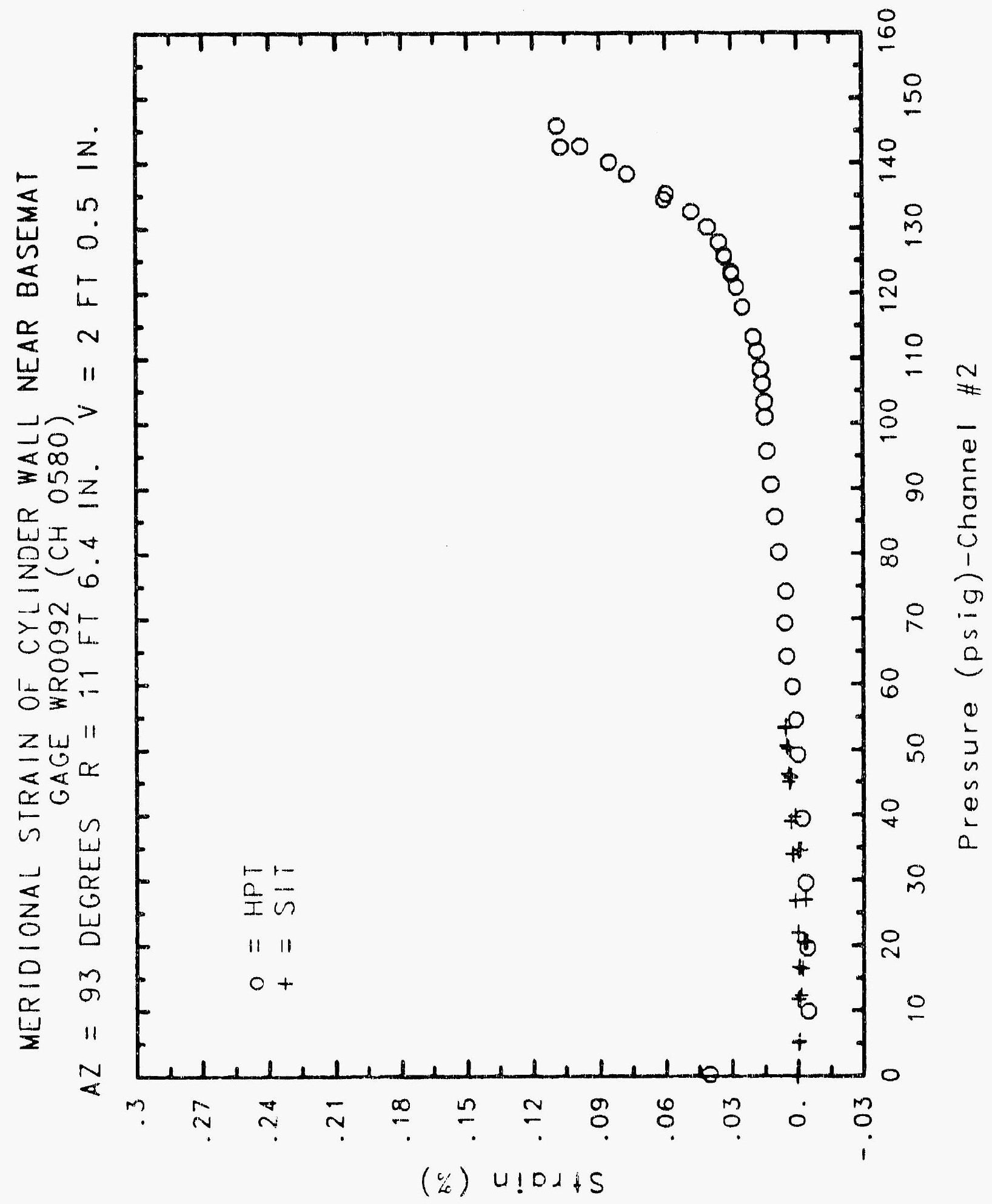

C.-992 


\section{Reinforced Concrete Test Data}

Weldable Gage 93 Channel 581

Structural Integrity test

High Pressure test

\begin{tabular}{|c|c|c|c|}
\hline $\begin{array}{c}\text { Pressure } \\
\text { (psig) } \\
-0.05 \\
5.33 \\
12.31 \\
16.44 \\
20.51 \\
20.46 \\
20.47 \\
27.05 \\
34.69 \\
34.53 \\
34.53 \\
39.70 \\
45.90 \\
45.65 \\
45.62 \\
50.10 \\
53.47 \\
53.29 \\
53.21 \\
50.49 \\
46.14 \\
46.13 \\
44.97 \\
38.98 \\
33.99 \\
33.96 \\
26.83 \\
21.88 \\
21.90 \\
16.69 \\
11.74 \\
11.77 \\
5.05 \\
0.02 \\
-0.04 \\
-0.02 \\
-0.02 \\
0.02 \\
\end{array}$ & $\begin{array}{l}\text { Strain } \\
-0.0003 \\
-0.0006 \\
0.0004 \\
0.0004 \\
0.0007 \\
0.0004 \\
0.0002 \\
0.0014 \\
0.0042 \\
0.0054 \\
0.0057 \\
0.0072 \\
0.0094 \\
0.0100 \\
0.0098 \\
0.0105 \\
0.0117 \\
0.0118 \\
0.0119 \\
0.0116 \\
0.0111 \\
0.0106 \\
0.0104 \\
0.0096 \\
0.0092 \\
0.0086 \\
0.0075 \\
0.0064 \\
0.0065 \\
0.0063 \\
0.0058 \\
0.0062 \\
0.0017 \\
0.0040 \\
0.0034 \\
0.0031 \\
0.0033 \\
0.0033\end{array}$ & $\begin{array}{c}\text { Pressure } \\
\text { (psig) } \\
9.89 \\
19.55 \\
29.57 \\
39.42 \\
49.16 \\
54.50 \\
59.57 \\
64.20 \\
69.32 \\
74.16 \\
80.16 \\
85.61 \\
90.58 \\
95.69 \\
100.92 \\
103.25 \\
106.11 \\
108.31 \\
111.08 \\
113.24 \\
117.83 \\
120.92 \\
123.28 \\
122.97 \\
125.82 \\
125.60 \\
127.84 \\
130.19 \\
132.53 \\
135.33 \\
134.42 \\
138.35 \\
140.16 \\
142.63 \\
145.78 \\
142.52 \\
0.22\end{array}$ & $\begin{array}{l}\text { Strain } \\
-0.0021 \\
-0.0004 \\
0.0014 \\
0.0034 \\
0.0052 \\
0.0065 \\
0.0079 \\
0.0097 \\
0.0105 \\
0.0099 \\
0.0133 \\
0.0156 \\
0.0182 \\
0.0214 \\
0.0244 \\
0.0262 \\
0.0282 \\
0.0294 \\
0.0316 \\
0.0339 \\
0.0391 \\
0.0428 \\
0.0465 \\
0.0471 \\
0.0510 \\
0.0516 \\
0.0544 \\
0.0610 \\
0.0698 \\
0.0816 \\
0.0831 \\
0.0984 \\
0.1073 \\
0.1205 \\
0.1314 \\
0.1307 \\
0.0438\end{array}$ \\
\hline
\end{tabular}


$566-3$

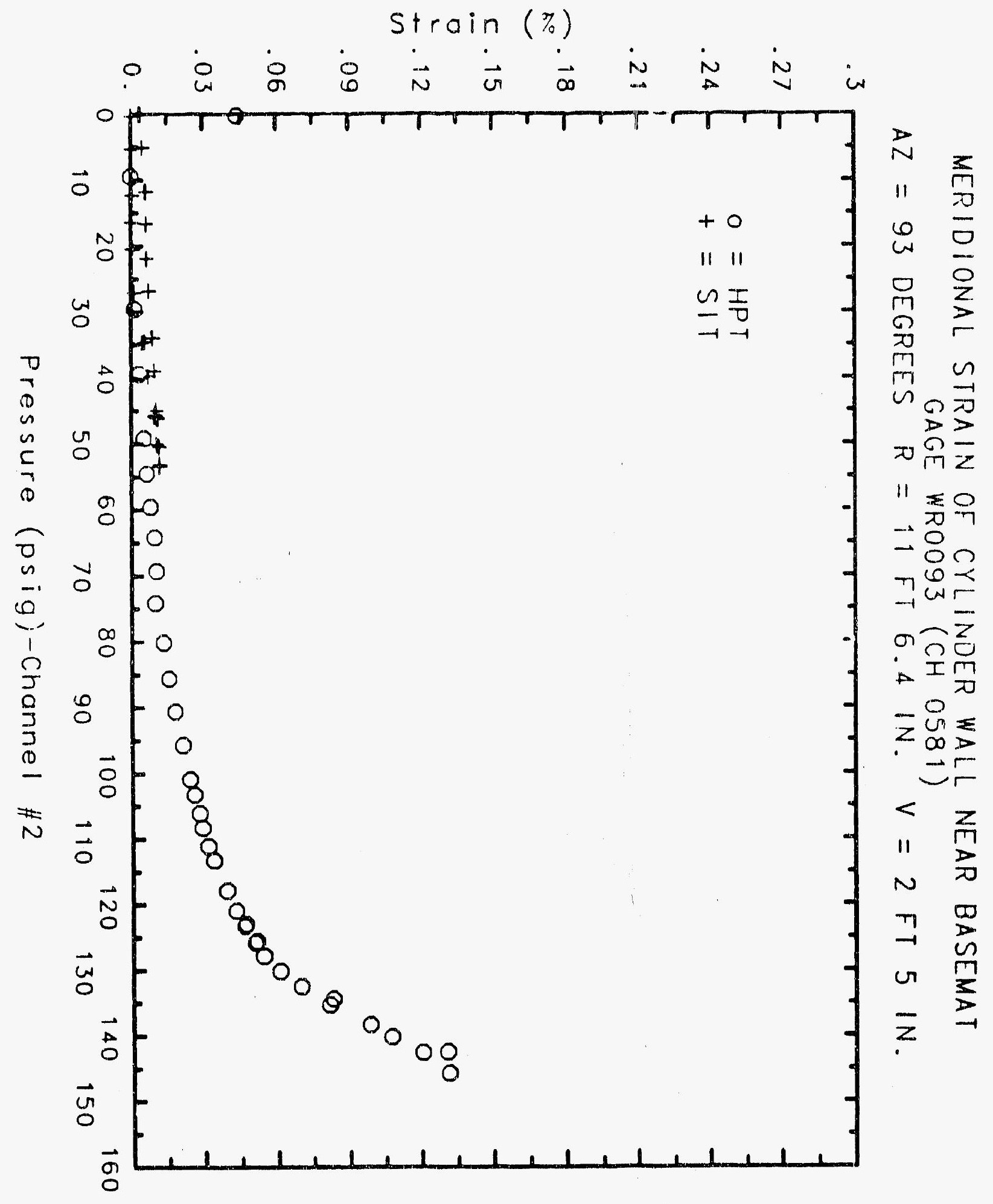




\section{Reinforced Concrete Test Data}

Weldable gage 94 Channel 582

Structural INTEgRITY TEST

\begin{tabular}{|c|c|c|c|}
\hline $\begin{array}{c}\text { Pressure } \\
\text { (psig) } \\
-0.05 \\
5.33 \\
12.31 \\
16.44 \\
20.51 \\
20.46 \\
20.47 \\
27.05 \\
34.69 \\
34.53 \\
34.53 \\
39.70 \\
45.90 \\
45.65 \\
45.62 \\
50.10 \\
53.47 \\
53.29 \\
53.21 \\
50.49 \\
46.14 \\
46.13 \\
44.97 \\
38.98 \\
33.99 \\
33.96 \\
26.83 \\
21.88 \\
21.90 \\
16.69 \\
11.74 \\
11.77 \\
5.05 \\
0.02 \\
-0.04 \\
-0.02 \\
-0.02 \\
0.02 \\
\end{array}$ & $\begin{array}{c}\text { * Strain } \\
-0.0001 \\
0.0031 \\
0.0059 \\
0.0086 \\
0.0113 \\
0.0115 \\
0.0122 \\
0.0152 \\
0.0168 \\
0.0168 \\
0.0163 \\
0.0178 \\
0.0171 \\
0.0163 \\
0.0161 \\
0.0171 \\
0.0181 \\
0.0177 \\
0.0174 \\
0.0164 \\
0.0156 \\
0.0152 \\
0.0154 \\
0.0135 \\
0.0125 \\
0.0129 \\
0.0104 \\
0.0093 \\
0.0095 \\
0.0075 \\
0.0055 \\
0.0048 \\
0.0015 \\
-0.0005 \\
-0.0026 \\
-0.0026 \\
-0.0020 \\
-0.0010\end{array}$ & $\begin{array}{c}\text { Pressure } \\
(\text { psig) } \\
9.89 \\
19.55 \\
29.57 \\
39.42 \\
49.16 \\
54.50 \\
59.57 \\
64.20 \\
69.32 \\
74.16 \\
80.16 \\
85.61 \\
90.58 \\
95.69 \\
100.92 \\
103.25 \\
106.11 \\
108.31 \\
111.08 \\
113.24 \\
117.83 \\
120.92 \\
123.28 \\
122.97 \\
125.82 \\
125.60 \\
127.84 \\
130.19 \\
132.53 \\
135.33 \\
134.42 \\
138.35 \\
140.16 \\
142.63 \\
145.78 \\
142.52 \\
0.22 \\
\end{array}$ & $\begin{array}{l}\text { \% Strain } \\
0.0013 \\
0.0056 \\
0.0098 \\
0.0135 \\
0.0175 \\
0.0196 \\
0.0222 \\
0.0260 \\
0.0315 \\
0.0367 \\
0.0404 \\
0.0465 \\
0.0518 \\
0.0587 \\
0.0668 \\
0.0709 \\
0.0753 \\
0.0790 \\
0.0835 \\
0.0874 \\
0.1038 \\
0.1174 \\
0.1288 \\
0.1309 \\
0.1407 \\
0.1430 \\
0.1481 \\
0.1596 \\
0.1742 \\
0.1926 \\
0.1963 \\
0.2274 \\
0.2484 \\
0.2779 \\
0.3057 \\
0.3219 \\
0.1731\end{array}$ \\
\hline
\end{tabular}

High Pressure test

Press

9.89

19.55

39.42

49.16

54.50

59.57

74.16

90.58

103.25

106.11

108.31

117.83

120.92

123.28

122.97

125.82

125.60

132.53

135.33

134.42

138.35

140.16

45.78

42.52

0.22
0.0013

0.0135

0.0175

0.0196

0.0222

0.0367

0.0404

0.0465

0.0668

0.0790

0.1038

0.1288

0.1309

0.1481

0.1742

0.1926

0.1963

0.2779

0.3219

0.1731 


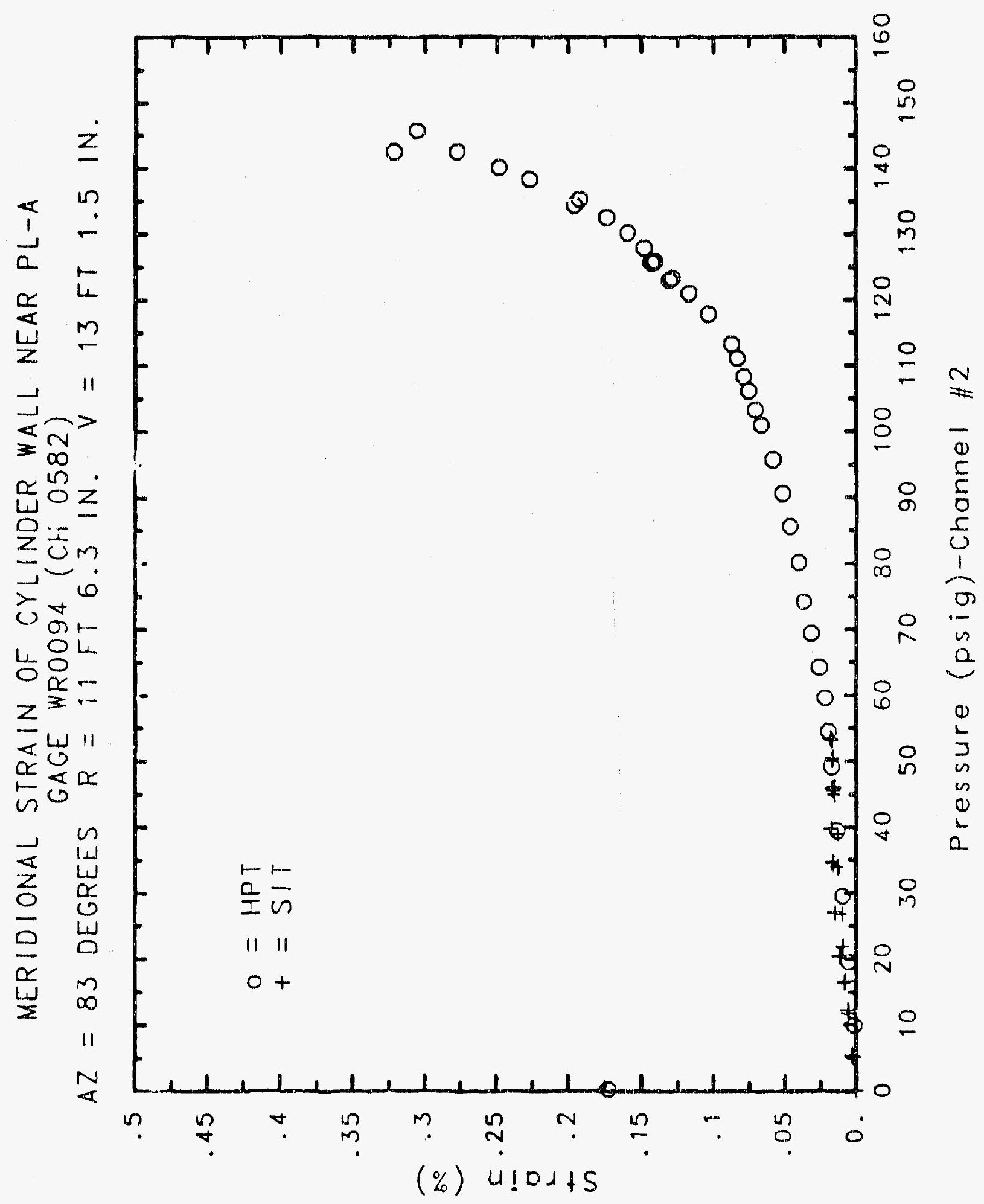




\section{Reinforced Concrete Test Data}

Weldable gage 95 Channel 583

Structural Integrity TEST

\begin{tabular}{|c|c|c|c|}
\hline $\begin{array}{c}\text { Pressure } \\
\text { (psig) } \\
.0 .05 \\
5.33 \\
12.31 \\
16.44 \\
20.51 \\
20.46 \\
20.47 \\
27.05 \\
34.69 \\
34.53 \\
34.53 \\
39.70 \\
45.90 \\
45.65 \\
45.62 \\
50.10 \\
53.47 \\
53.29 \\
53.21 \\
50.49 \\
46.14 \\
46.13 \\
44.97 \\
38.98 \\
33.99 \\
33.96 \\
26.83 \\
21.88 \\
21.90 \\
16.69 \\
11.74 \\
11.77 \\
5.05 \\
0.02 \\
-0.04 \\
-0.02 \\
-0.02 \\
0.02\end{array}$ & $\begin{array}{c}\text { \% Strain } \\
\text {-0.0004 } \\
0.0027 \\
0.0059 \\
0.0078 \\
0.0104 \\
0.0106 \\
0.0115 \\
0.0147 \\
0.0168 \\
0.0170 \\
0.0166 \\
0.0181 \\
0.0214 \\
0.0214 \\
0.0209 \\
0.0238 \\
0.0270 \\
0.0271 \\
0.0273 \\
0.0265 \\
0.0249 \\
0.0249 \\
0.0252 \\
0.0232 \\
0.0214 \\
0.0218 \\
0.0187 \\
0.0171 \\
0.0175 \\
0.0150 \\
0.0118 \\
0.0109 \\
0.0070 \\
0.0038 \\
0.0020 \\
0.0022 \\
0.0028 \\
0.0040\end{array}$ & $\begin{array}{c}\text { Pressure } \\
(\text { psig) } \\
9.89 \\
19.55 \\
29.57 \\
39.42 \\
49.16 \\
54.50 \\
59.57 \\
64.20 \\
69.32 \\
74.16 \\
80.16 \\
85.61 \\
90.58 \\
95.69 \\
100.92 \\
103.25 \\
106.11 \\
108.31 \\
111.08 \\
113.24 \\
117.83 \\
120.92 \\
123.28 \\
122.97 \\
125.82 \\
125.60 \\
127.84 \\
130.19 \\
132.53 \\
135.33 \\
134.42 \\
138.35 \\
140.16 \\
142.63 \\
145.78 \\
142.52 \\
0.22\end{array}$ & $\begin{array}{l}\text { \% Strain } \\
0.0026 \\
0.0082 \\
0.0134 \\
0.0184 \\
0.0228 \\
0.0255 \\
0.0283 \\
0.0324 \\
0.0379 \\
0.0440 \\
0.0500 \\
0.0573 \\
0.0656 \\
0.0745 \\
0.0845 \\
0.0892 \\
0.0945 \\
0.0990 \\
0.1050 \\
0.1107 \\
0.1227 \\
0.1303 \\
0.1377 \\
0.1391 \\
0.1484 \\
0.1511 \\
0.1580 \\
0.1711 \\
0.1877 \\
0.2081 \\
0.2141 \\
0.2448 \\
0.2726 \\
0.3097 \\
0.3477 \\
0.3771 \\
0.2137\end{array}$ \\
\hline
\end{tabular}

High Pressure Test

ressure

0.0026

0.0082

0.0134

0.0184

0.0228

0.0255

0.0379

0.0440

0.0500

0.0573

0.0656

0.0745

0.0845

0.0945

0.0990

0.1050

0.1107

0.1227

0.1303

.

0.1484

0.1511

0.1580

0.1711

0.1877

0.2081

0.2448

0.2726

0.3097

0.3477

0.3771

0.0028

42.52

0.2137 


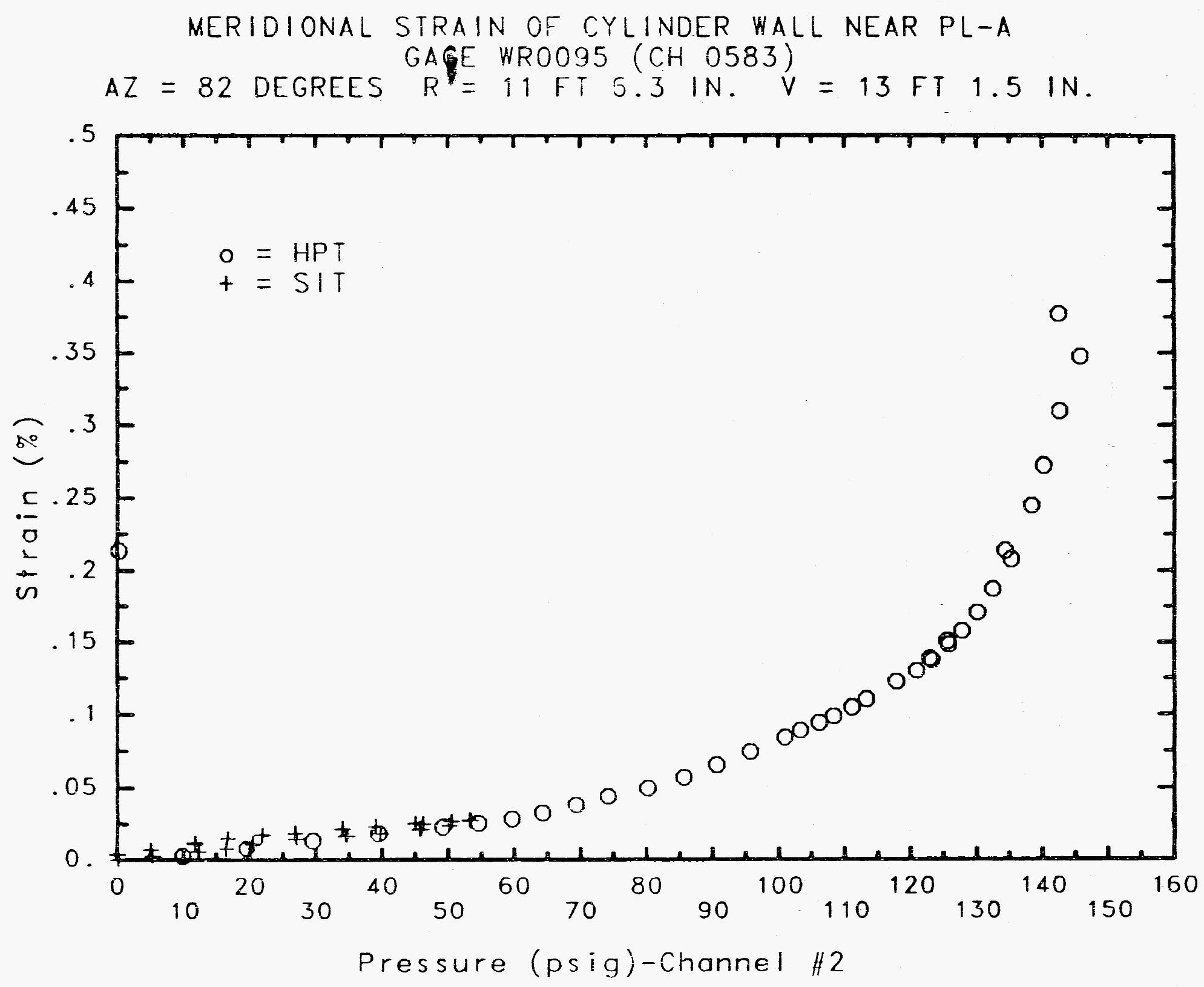




\section{Reinforced Concrete Test Data}

Weldable gage 96 Channel 584

Structural Integrity Test

High Pressure test

\begin{tabular}{|c|c|c|c|}
\hline $\begin{array}{c}\text { Pressure } \\
\text { (psig) } \\
-0.05 \\
5.33 \\
12.31 \\
16.44 \\
20.51 \\
20.46 \\
20.47 \\
27.05 \\
34.69 \\
34.53 \\
34.53 \\
39.70 \\
45.90 \\
45.65 \\
45.62 \\
50.10 \\
53.47 \\
53.29 \\
53.21 \\
50.49 \\
46.14 \\
46.13 \\
44.97 \\
38.98 \\
33.99 \\
33.96 \\
26.83 \\
21.88 \\
21.90 \\
16.69 \\
11.74 \\
11.77 \\
5.05 \\
0.02 \\
-0.04 \\
-0.02 \\
-0.02 \\
0.02\end{array}$ & $\begin{array}{c}\text { \% Strain } \\
-0.0001 \\
0.0008 \\
0.0017 \\
0.0021 \\
0.0033 \\
0.0036 \\
0.0033 \\
0.0059 \\
0.0112 \\
0.0120 \\
0.0120 \\
0.0153 \\
0.0206 \\
0.0233 \\
0.0239 \\
0.0274 \\
0.0312 \\
0.0321 \\
0.0321 \\
0.0328 \\
0.0314 \\
0.0318 \\
0.0300 \\
0.0284 \\
0.0272 \\
0.0264 \\
0.0229 \\
0.0204 \\
0.0184 \\
0.0160 \\
0.0131 \\
0.0133 \\
0.0115 \\
0.0088 \\
0.0094 \\
0.0083 \\
0.0083 \\
0.0081\end{array}$ & $\begin{array}{c}\text { Pressure } \\
\text { (psig) } \\
9.89 \\
19.55 \\
29.57 \\
39.42 \\
49.16 \\
54.50 \\
59.57 \\
64.20 \\
69.32 \\
74.16 \\
80.16 \\
85.61 \\
90.58 \\
95.69 \\
100.92 \\
103.25 \\
106.11 \\
108.31 \\
111.08 \\
113.24 \\
117.83 \\
120.92 \\
123.28 \\
122.97 \\
125.82 \\
125.60 \\
127.84 \\
130.19 \\
132.53 \\
135.33 \\
134.42 \\
138.35 \\
140.16 \\
142.63 \\
145.78 \\
142.52 \\
0.22 \\
\end{array}$ & $\begin{array}{l}\text { \% Strain } \\
0.0014 \\
0.1063 \\
0.0114 \\
0.0157 \\
0.0204 \\
0.0231 \\
0.0266 \\
0.0305 \\
0.0358 \\
0.0408 \\
0.0514 \\
0.0587 \\
0.0653 \\
0.0733 \\
0.0818 \\
0.0861 \\
0.0904 \\
0.0936 \\
0.0989 \\
0.1030 \\
0.1135 \\
0.1214 \\
0.1296 \\
0.1314 \\
0.1420 \\
0.1450 \\
0.1516 \\
0.1685 \\
0.1903 \\
0.2081 \\
0.2102 \\
0.2217 \\
0.2298 \\
0.2410 \\
0.2529 \\
0.2555 \\
0.1184\end{array}$ \\
\hline
\end{tabular}




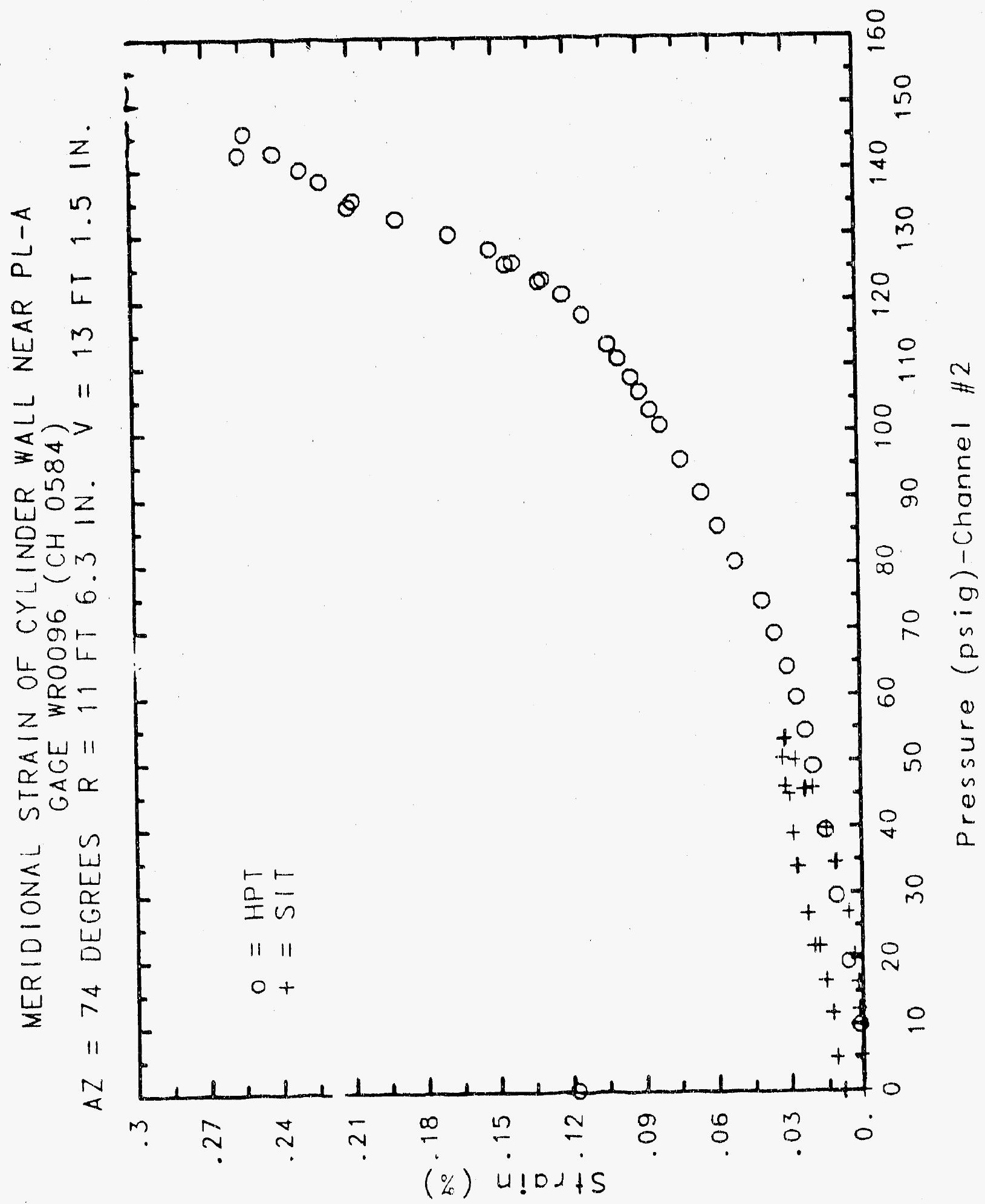

C-10no 
Reinforced Concrete Test Data

Weldable Gage 97 Channel 585

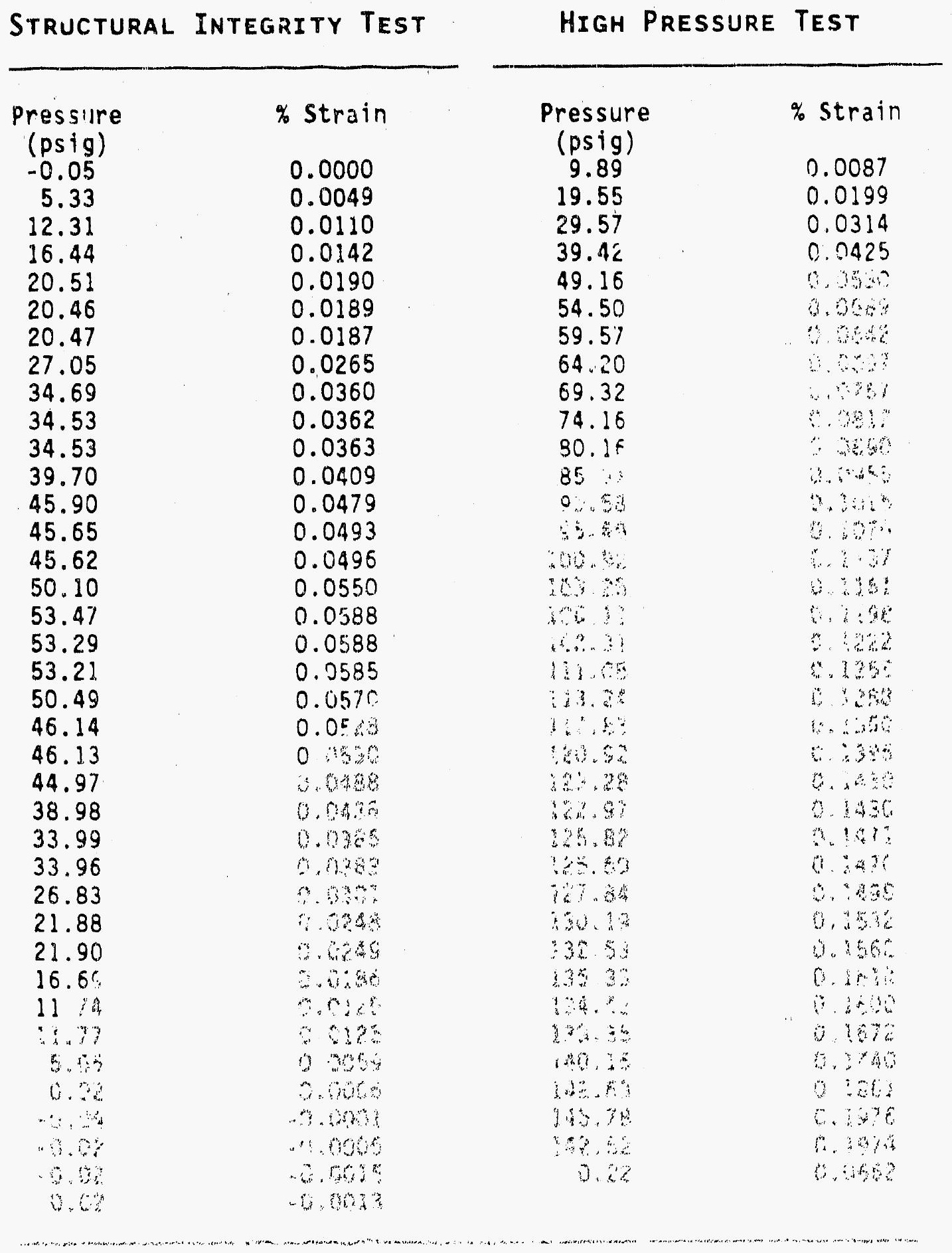




\section{IMAGE EVALUATION TEST TARGET (MT-3)}
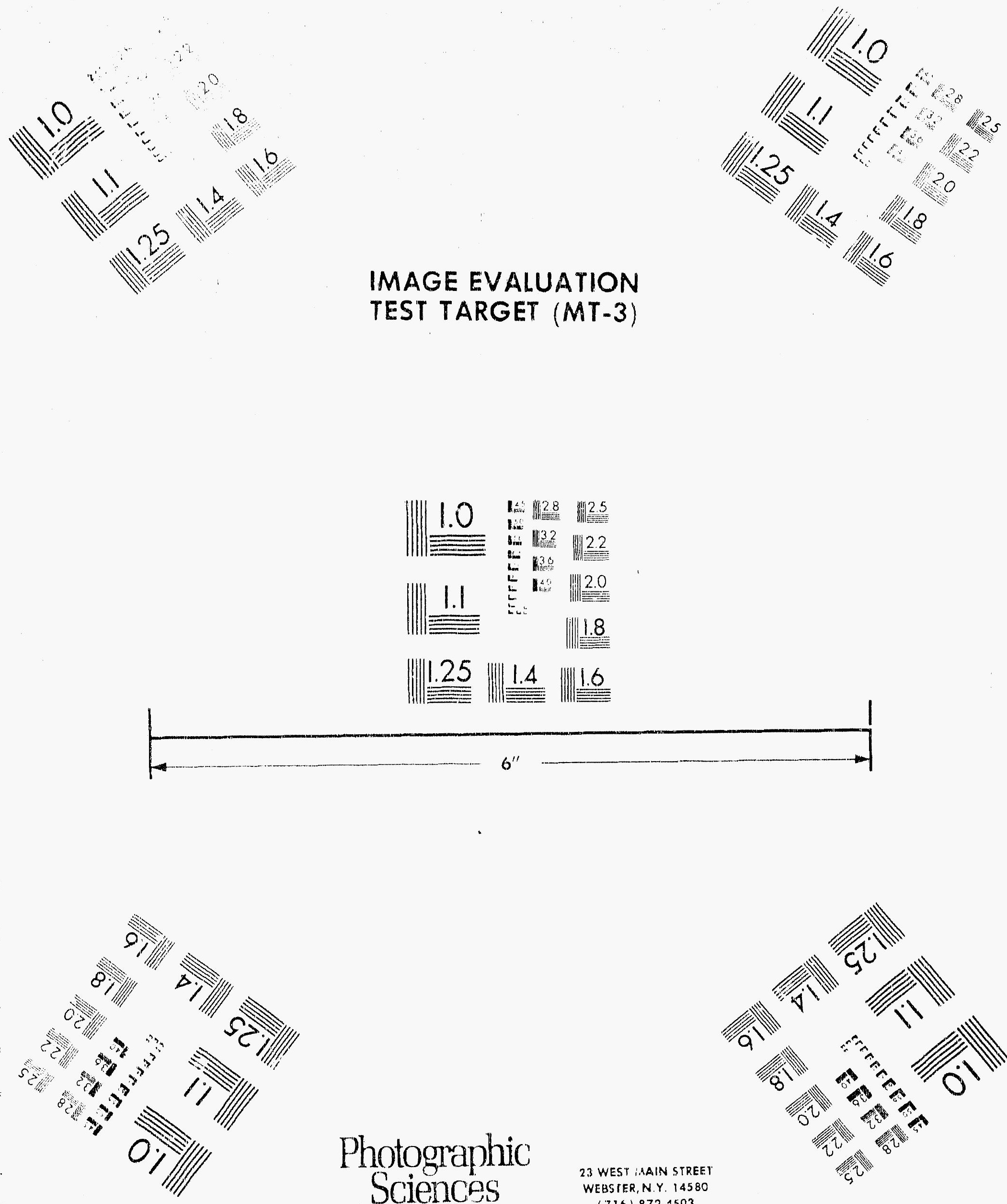

Photographic Sciences Corporation

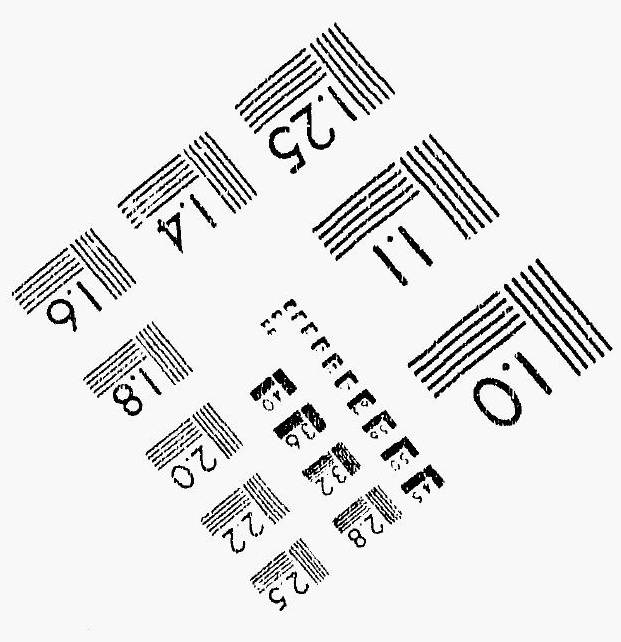




\section{SECURITY CLASSIFICATION}

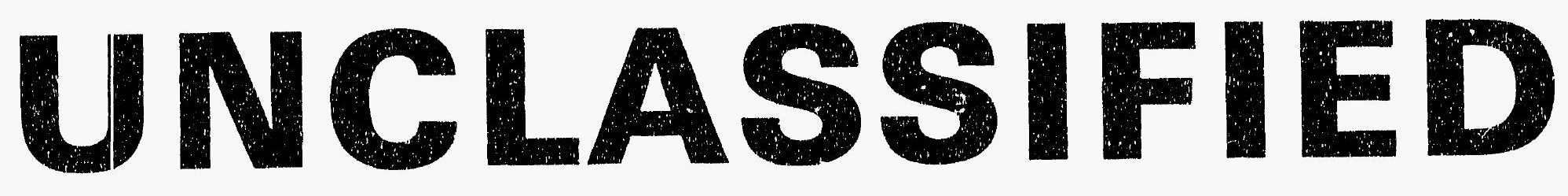

\section{DATE OF MICROFILMING $1-6-92$}

MICROFILMED BY uerri hard

SANDIA NATIONAL LABS 
THIS DOCUMENT FILMED AT

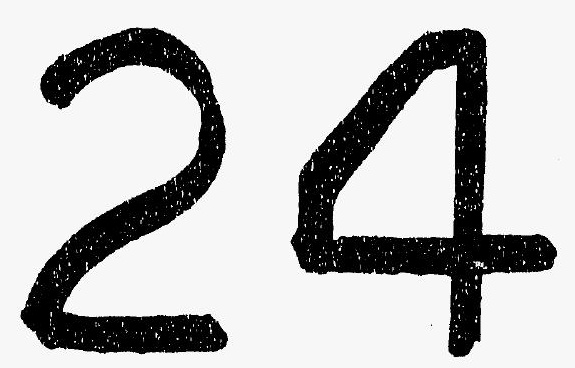

REDUCTION 


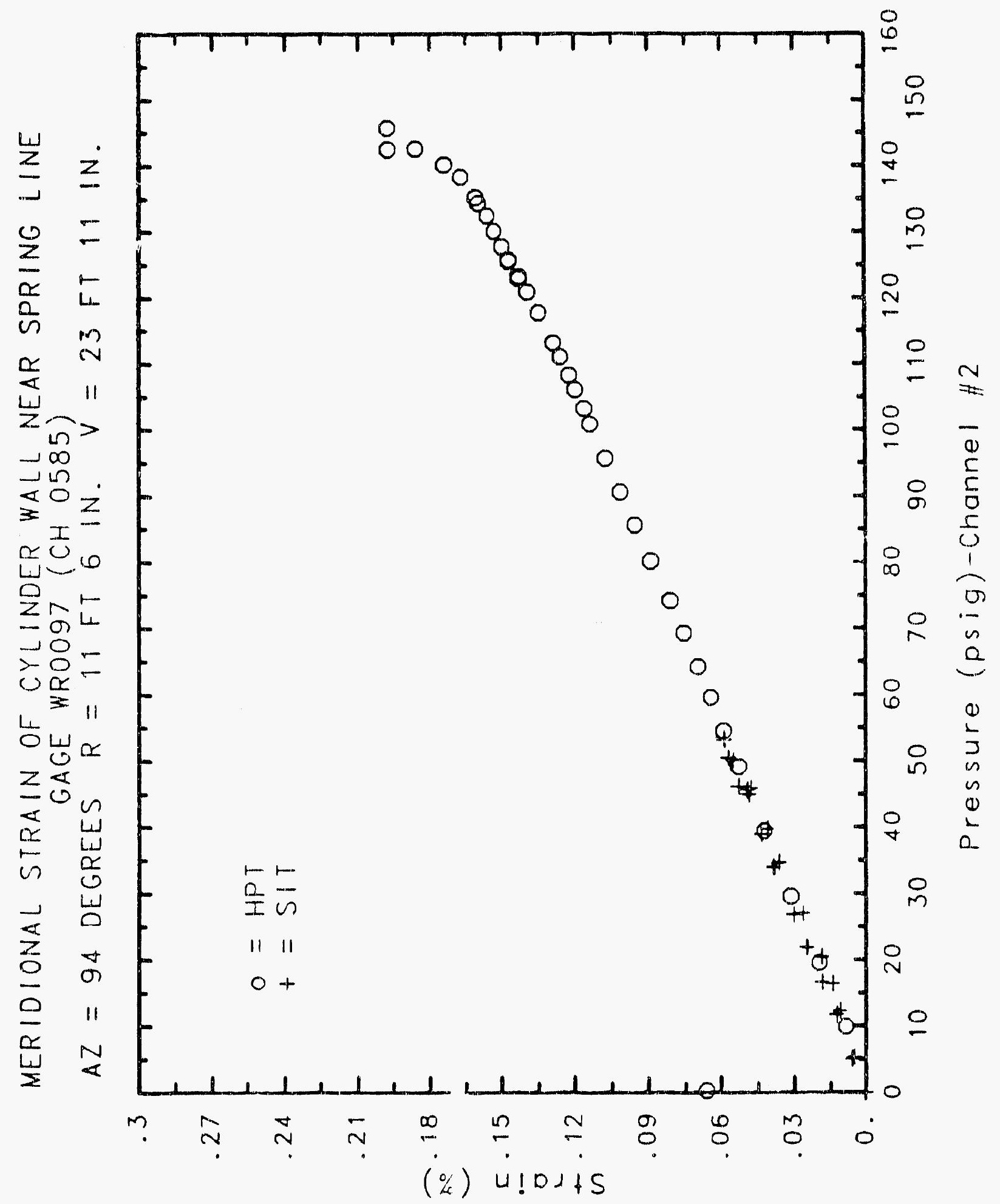




\section{Reinforced Concrete Te:t Data}

Weldable gage 98 Channel 586

Structural Integrity Test

\begin{tabular}{|c|c|c|c|}
\hline $\begin{array}{c}\text { Pressure } \\
\text { (psig) } \\
-0.05 \\
5.33 \\
12.31 \\
16.44 \\
20.51 \\
20.46 \\
20.47 \\
27.05 \\
34.69 \\
34.53 \\
34.53 \\
39.70 \\
45.90 \\
45.65 \\
45.62 \\
50.10 \\
53.47 \\
53.29 \\
53.21 \\
50.49 \\
46.14 \\
46.13 \\
44.97 \\
38.98 \\
33.99 \\
33.96 \\
26.83 \\
21.88 \\
21.90 \\
16.69 \\
11.74 \\
11.77 \\
5.05 \\
0.02 \\
-0.04 \\
-0.02 \\
-0.02 \\
0.02 \\
\end{array}$ & $\begin{array}{c}\text { \% Strain } \\
-0.0008 \\
0.0007 \\
0.0021 \\
0.0026 \\
0.0043 \\
0.0 n 45 \\
0.0044 \\
0.0081 \\
0.0150 \\
0.0161 \\
0.0165 \\
0.0195 \\
0.0247 \\
0.0266 \\
0.0272 \\
0.0304 \\
0.0339 \\
0.0349 \\
0.0350 \\
0.0352 \\
0.0340 \\
0.0339 \\
0.0325 \\
0.0311 \\
0.0289 \\
0.0291 \\
0.0262 \\
0.0242 \\
0.0243 \\
0.0218 \\
0.0192 \\
0.0191 \\
0.0167 \\
0.0147 \\
0.0144 \\
0.0143 \\
0.0136 \\
0.0138\end{array}$ & $\begin{array}{c}\text { Pressure } \\
(\text { psig) } \\
9.89 \\
19.55 \\
29.57 \\
39.42 \\
49.16 \\
54.50 \\
59.57 \\
64.20 \\
69.32 \\
74.16 \\
80.16 \\
85.61 \\
90.58 \\
95.69 \\
100.92 \\
103.25 \\
106.11 \\
108.31 \\
111.08 \\
113.24 \\
117.83 \\
120.92 \\
123.28 \\
122.97 \\
125.82 \\
125.60 \\
127.84 \\
130.19 \\
132.53 \\
135.33 \\
134.42 \\
138.35 \\
140.16 \\
142.63 \\
145.78 \\
142.52 \\
0.22 \\
\end{array}$ & $\begin{array}{l}0.0003 \\
0.0050 \\
0.0103 \\
0.0151 \\
0.0201 \\
0.0231 \\
0.0259 \\
0.0296 \\
0.0328 \\
0.0371 \\
0.0441 \\
0.0490 \\
0.0537 \\
0.0585 \\
0.0641 \\
0.0668 \\
0.0691 \\
0.0714 \\
0.0744 \\
0.0764 \\
0.0818 \\
0.0852 \\
0.0885 \\
0.0890 \\
0.0915 \\
0.0924 \\
0.0936 \\
0.0965 \\
0.0989 \\
0.1025 \\
0.1032 \\
0.1081 \\
0.1122 \\
0.1213 \\
0.1299 \\
0.1312 \\
0.0386\end{array}$ \\
\hline
\end{tabular}

High Pressu're test

Pressure

9.89

19.55

29.57

54.50

59.57

64.20

69.32

85.61

90.58

100.92

103.25

111.08

113.24

120.92

123.28

122.97

125.82

130.19

134.42

138.35

45.78

2.52

.1312

0.0386

0.0050

0.0151

0.0231

0.0296

0.0371

0.0490

0.0585

0.0641

0.0691

0.0744

0764

0.0852

0.0890

0.0924

0.0936

0.0989

0.1081

.1122

.1213 


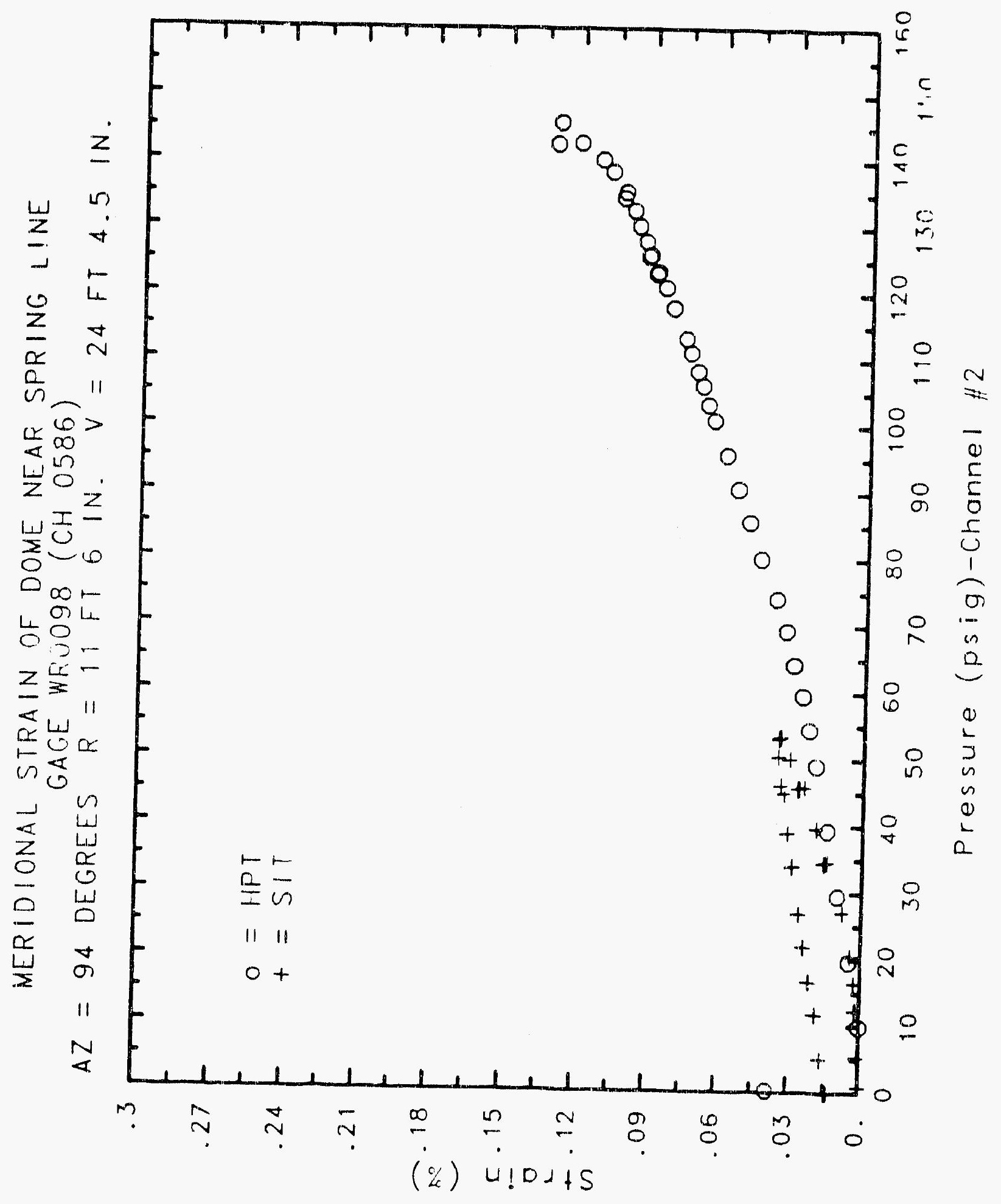




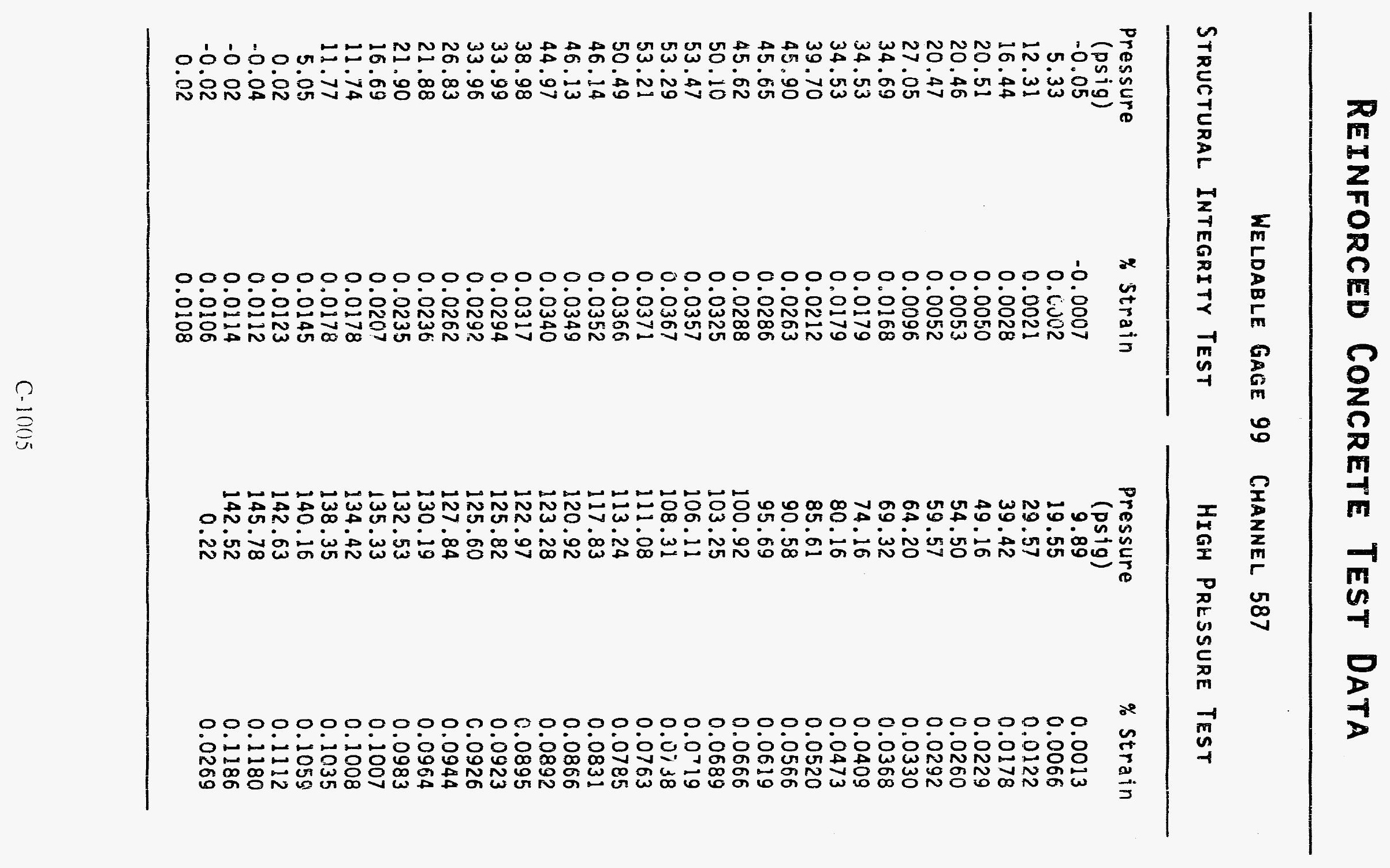




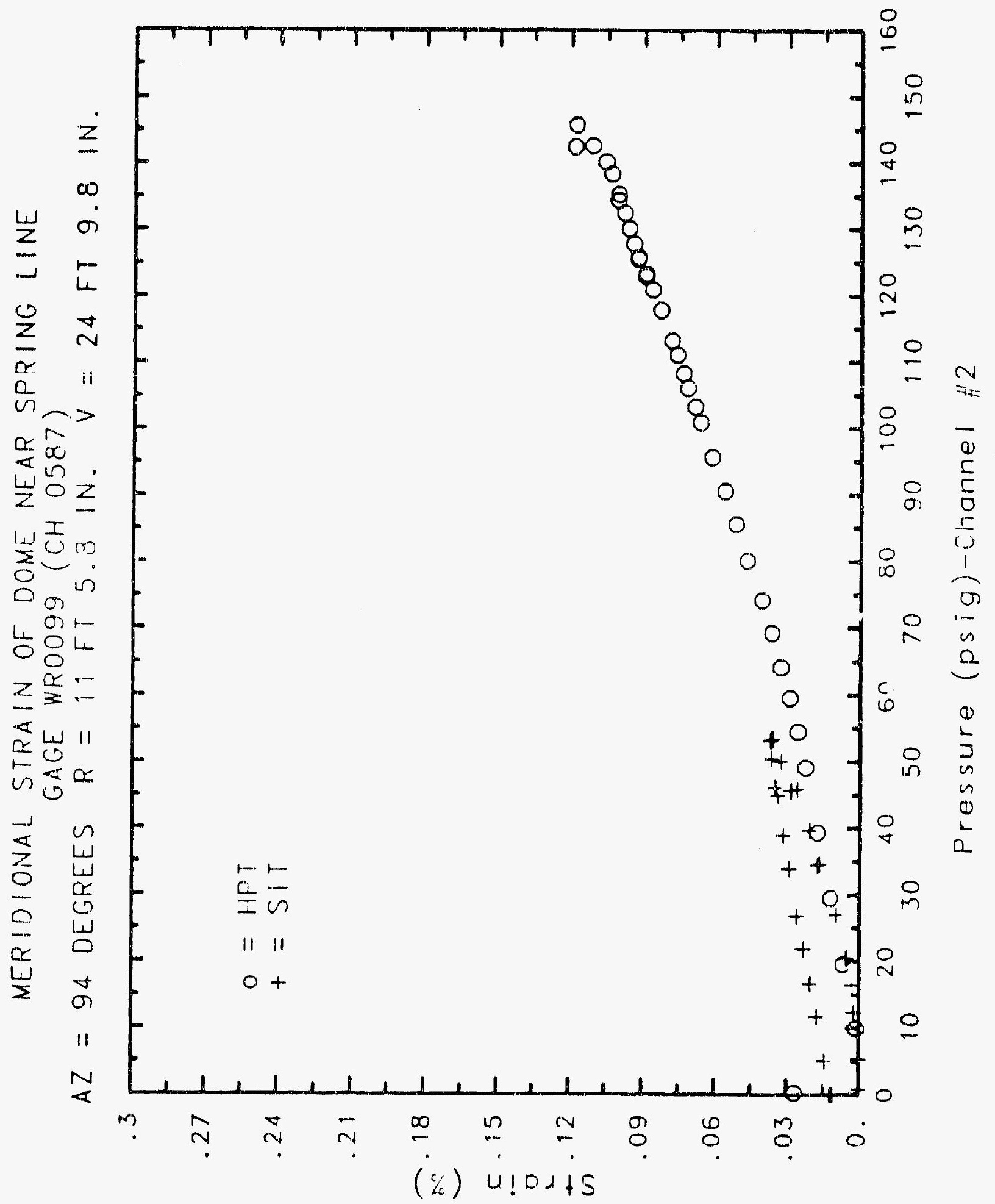




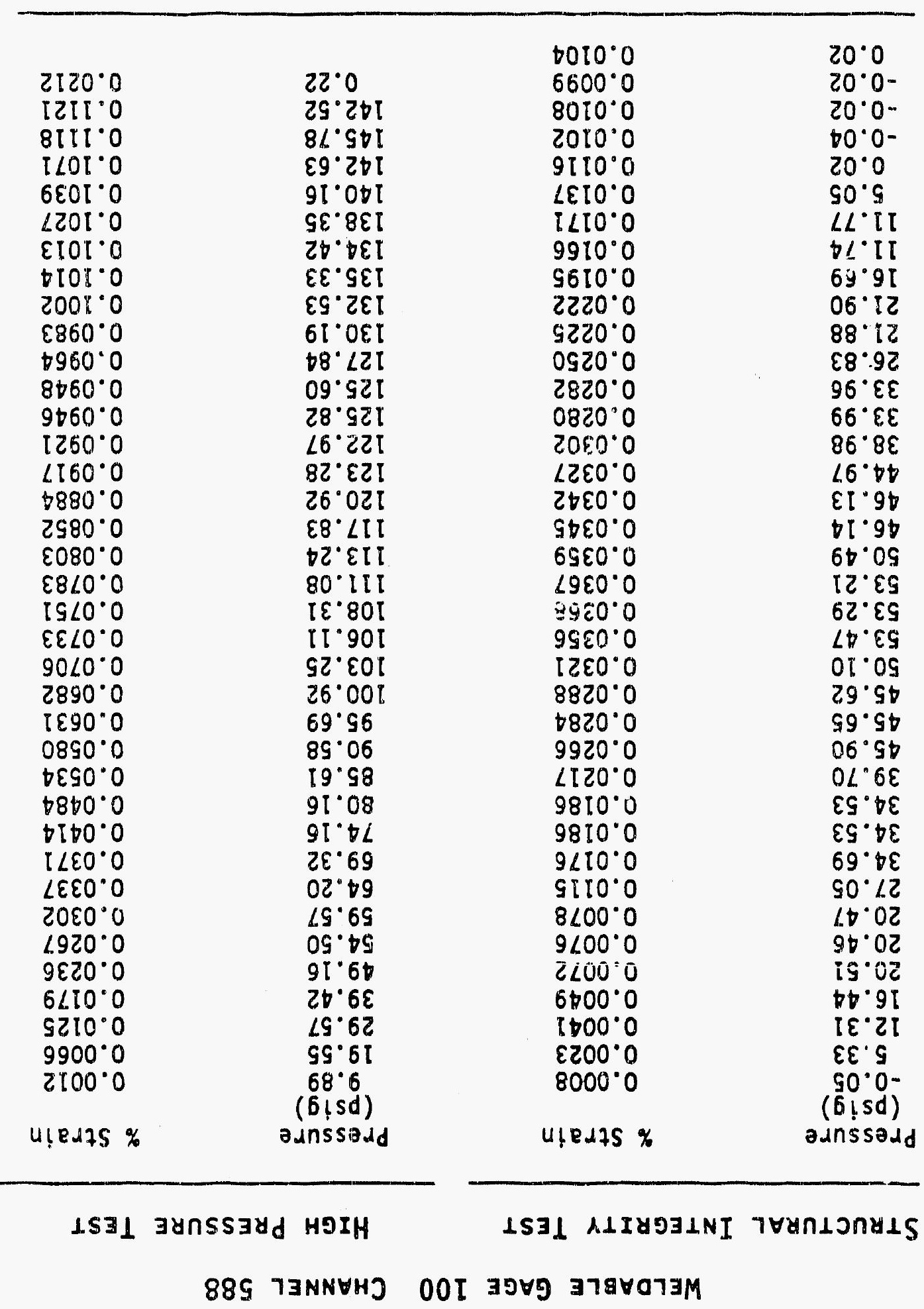

\section{$\forall \perp \forall 0 \perp S \exists \perp$ ILIUSNOJ OJJYOJNIJY}




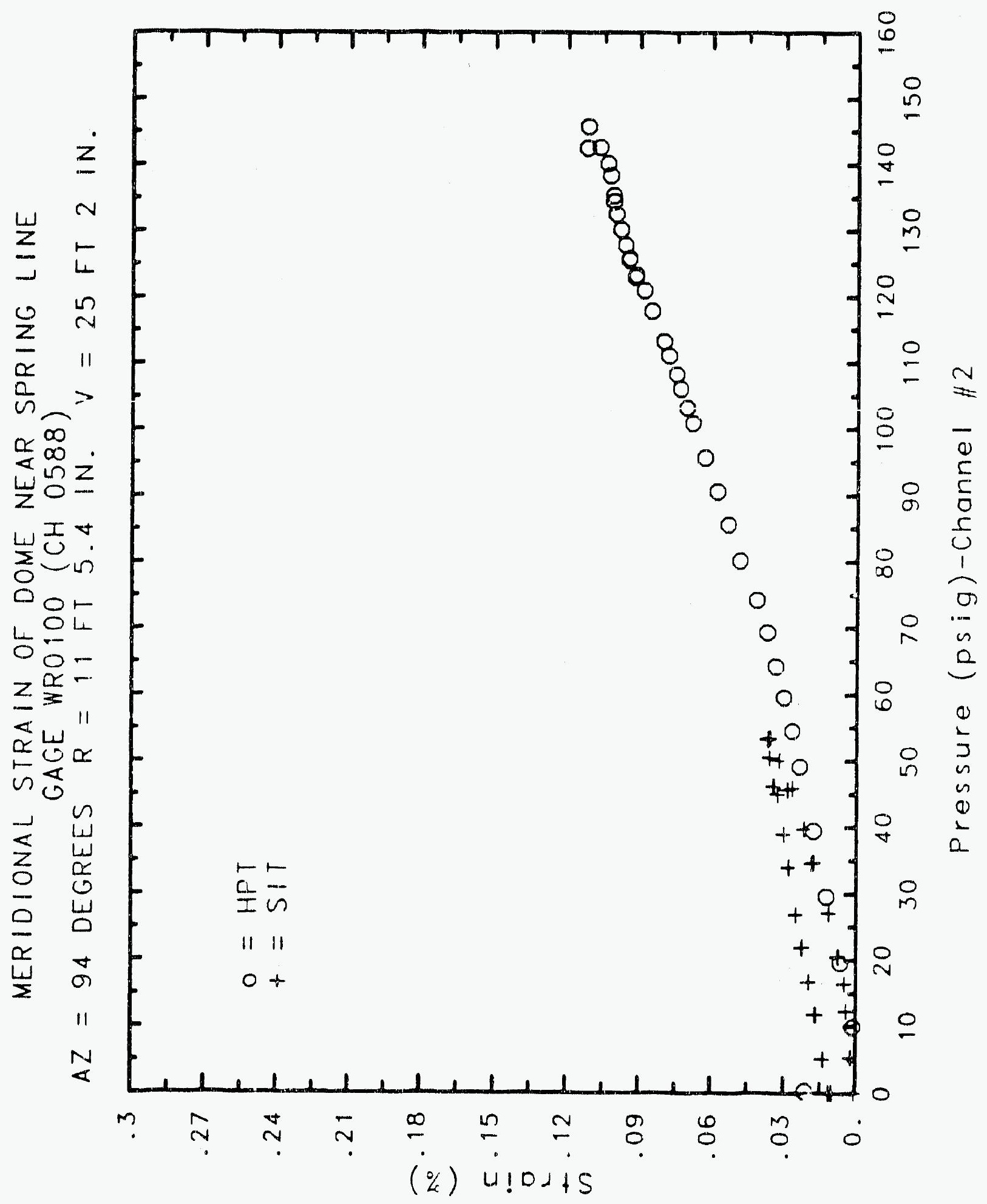




\section{Reinforced Concrete Test Data}

Weldable Gage 101 Channel 589

StRUCTURAL INTEGRITY TEST

High Pressure Test

\begin{tabular}{|c|c|c|c|}
\hline $\begin{array}{c}\text { Pressure } \\
(\text { psig) } \\
-0.05 \\
5.33 \\
12.31 \\
16.44 \\
20.51 \\
20.46 \\
20.47 \\
27.05 \\
34.69 \\
34.53 \\
34.53 \\
39.70 \\
45.90 \\
45.65 \\
45.62 \\
50.10 \\
53.47 \\
53.29 \\
53.21 \\
50.49 \\
46.14 \\
46.13 \\
44.97 \\
38.98 \\
33.99 \\
33.96 \\
26.83 \\
21.88 \\
21.90 \\
16.69 \\
11.74 \\
11.77 \\
5.05 \\
0.02 \\
-0.04 \\
-0.02 \\
-0.02 \\
0.02\end{array}$ & $\begin{array}{l}\text { \% Strain } \\
0.0007 \\
0.0014 \\
0.0032 \\
0.0046 \\
0.0070 \\
0.0077 \\
0.0078 \\
0.0146 \\
0.0239 \\
0.0247 \\
0.0248 \\
0.0283 \\
0.0359 \\
0.0377 \\
0.0384 \\
0.0422 \\
0.0456 \\
0.0460 \\
0.0465 \\
0.0459 \\
0.0440 \\
0.0436 \\
0.0409 \\
0.0381 \\
0.0357 \\
0.0354 \\
0.0317 \\
0.0285 \\
0.0284 \\
0.0253 \\
0.0219 \\
0.0225 \\
0.0183 \\
0.0155 \\
0.0146 \\
0.0136 \\
0.0134 \\
0.0133\end{array}$ & $\begin{array}{c}\text { Pressure } \\
(\text { psig) } \\
9.89 \\
19.55 \\
29.57 \\
39.42 \\
49.16 \\
54.50 \\
53.57 \\
64.20 \\
69.32 \\
74.16 \\
80.16 \\
85.61 \\
90.58 \\
95.69 \\
100.92 \\
103.25 \\
106.11 \\
108.31 \\
111.08 \\
113.24 \\
117.83 \\
120.92 \\
123.28 \\
12.92 .97 \\
125.82 \\
125.60 \\
127.84 \\
130.19 \\
132.53 \\
135.33 \\
134.42 \\
138.35 \\
140.16 \\
142.63 \\
145.78 \\
142.52 \\
0.22 \\
\end{array}$ & $\begin{array}{l}\text { \% Strain } \\
0.0026 \\
0.0094 \\
0.0161 \\
0.0228 \\
0.0289 \\
0.0324 \\
0.0365 \\
0.0407 \\
0.0455 \\
0.0502 \\
0.0579 \\
0.0631 \\
0.0681 \\
0.0732 \\
0.0788 \\
0.0817 \\
0.0840 \\
0.0870 \\
0.0898 \\
0.0922 \\
0.0976 \\
0.1018 \\
0.1047 \\
0.1046 \\
0.1080 \\
0.1079 \\
0.1102 \\
0.1134 \\
0.1167 \\
0.1210 \\
0.1207 \\
0.1264 \\
0.1305 \\
0.1351 \\
0.1402 \\
0.1403 \\
0.0213\end{array}$ \\
\hline
\end{tabular}




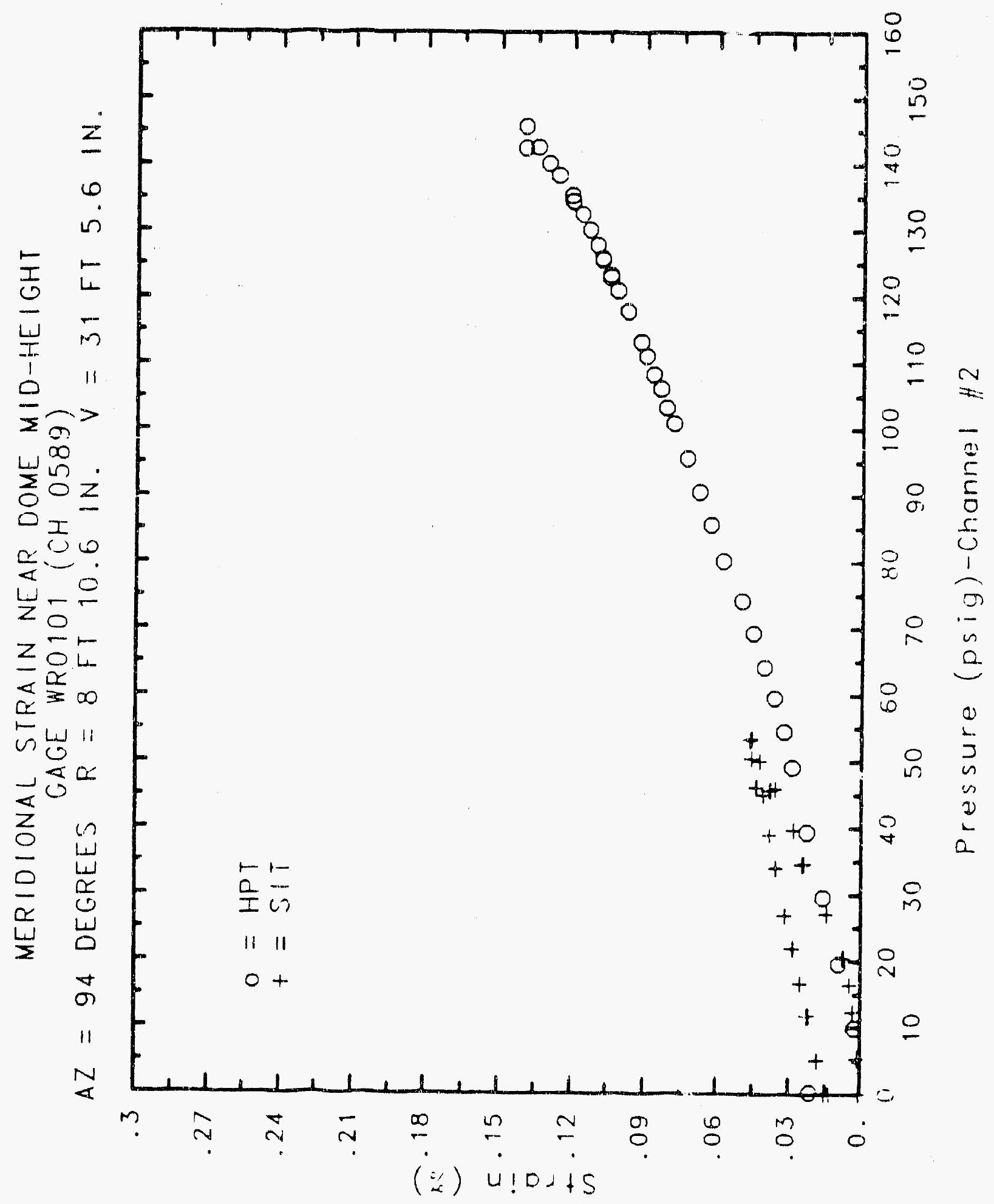




\title{
Reinforced Concrete Test Data
}

\author{
Weldable gage 102 Channel 600
}

STRUCTURAL INTEGRITY TEST

\begin{tabular}{|c|c|c|c|}
\hline $\begin{array}{c}\text { Pressure } \\
(p 5 i g) \\
-0.05 \\
5.33 \\
12.31 \\
16.44 \\
20.51 \\
20.46 \\
20.47 \\
27.05 \\
34.69 \\
34.53 \\
34.53 \\
39.70 \\
45.90 \\
45.65 \\
45.62 \\
30.10 \\
53.47 \\
53.29 \\
53.21 \\
50.49 \\
46.14 \\
46.13 \\
41.97 \\
38.98 \\
33.99 \\
33.95 \\
26.83 \\
21.88 \\
21.90 \\
16.69 \\
11.74 \\
11.77 \\
5.05 \\
0.02 \\
.0 .04 \\
-0.02 \\
-0.02 \\
0.02 \\
\end{array}$ & $\begin{array}{c}\text { z Strain } \\
-0.0011 \\
0.0018 \\
0.0042 \\
0.0061 \\
0.0082 \\
0.0084 \\
0.0085 \\
0.0133 \\
0.0202 \\
0.0208 \\
0.0211 \\
0.0250 \\
0.0314 \\
0.0329 \\
0.0335 \\
0.0372 \\
0.0403 \\
0.0406 \\
0.0416 \\
0.0406 \\
0.0389 \\
0.0383 \\
0.0362 \\
0.0326 \\
0.0307 \\
0.0306 \\
0.0272 \\
0.0239 \\
0.0240 \\
0.0211 \\
0.0181 \\
0.0186 \\
0.0145 \\
0.0112 \\
0.0105 \\
0.0088 \\
0.0089 \\
0.0086\end{array}$ & $\begin{array}{c}\text { Pressure } \\
(\text { psig) } \\
9.89 \\
19.55 \\
29.57 \\
39.42 \\
49.15 \\
54.50 \\
59.57 \\
64.20 \\
69.32 \\
74.16 \\
80.16 \\
85.61 \\
90.58 \\
95.69 \\
100.92 \\
103.2 \\
106.11 \\
108.31 \\
111.08 \\
113.24 \\
117.83 \\
120.92 \\
123.28 \\
122.97 \\
125.82 \\
125.60 \\
127.84 \\
130.19 \\
132.53 \\
135.33 \\
134.42 \\
138.35 \\
140.16 \\
142.63 \\
145.78 \\
142.52 \\
0.22\end{array}$ & $\begin{array}{c}\% \text { strain } \\
0.0024 \\
0.0087 \\
0.0156 \\
0.0219 \\
0.0282 \\
0.0319 \\
0.0352 \\
0.0388 \\
0.0440 \\
0.0483 \\
0.0611 \\
0.0694 \\
0.0761 \\
0.0836 \\
0.0913 \\
0.0948 \\
0.0984 \\
0.1007 \\
0.1056 \\
0.1087 \\
0.1152 \\
0.1198 \\
0.1229 \\
0.1229 \\
0.1259 \\
0.1260 \\
0.1282 \\
0.1312 \\
0.1350 \\
0.1377 \\
0.1375 \\
221.8900 \\
0.1464 \\
0.1505 \\
0.1564 \\
0.1559 \\
0.0161\end{array}$ \\
\hline
\end{tabular}




\section{2ioi-J}

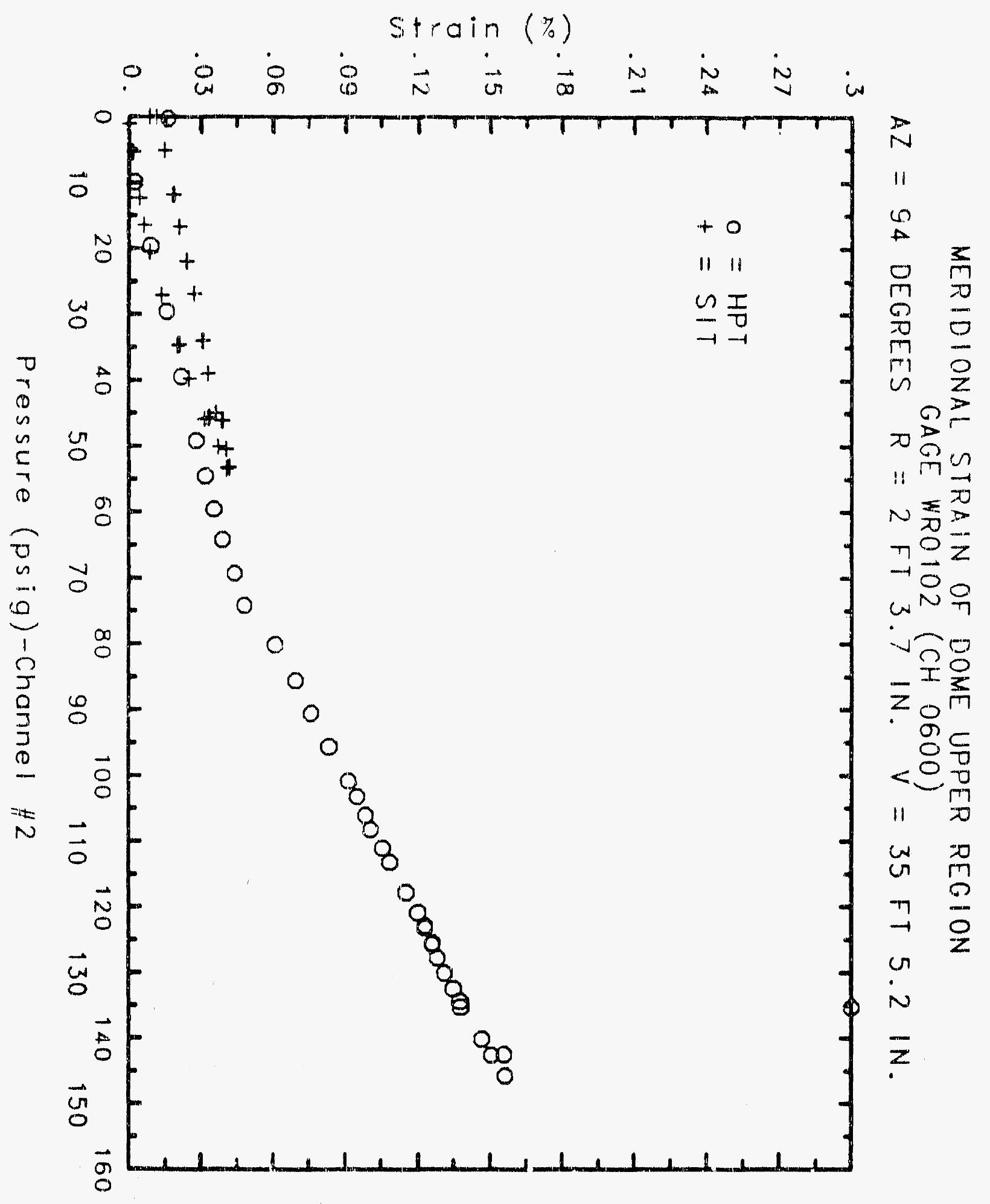




\section{Reinforced Concrete Test Data}

Weldable Gage 103 Channel 601

Structural Integrity Test

High Pressure Test

\begin{tabular}{|c|c|c|c|}
\hline $\begin{array}{c}\text { Pressure } \\
\text { (psig) } \\
-0.05 \\
5.33 \\
12.31 \\
16.44 \\
20.51 \\
20.46 \\
20.47 \\
27.05 \\
34.69 \\
34.53 \\
34.53 \\
39.70 \\
45.90 \\
45.65 \\
45.62 \\
50.10 \\
53.47 \\
53.29 \\
53.21 \\
50.49 \\
46.14 \\
46.13 \\
44.97 \\
38.98 \\
33.99 \\
33.96 \\
26.83 \\
21.88 \\
21.90 \\
16.69 \\
11.74 \\
11.77 \\
5.05 \\
0.02 \\
-0.04 \\
-0.02 \\
-0.02 \\
0.02 \\
\end{array}$ & $\begin{array}{l}\text { \% Strain } \\
0.0001 \\
0.0018 \\
0.0036 \\
0.0054 \\
0.0074 \\
0.0081 \\
0.0086 \\
0.0136 \\
0.0213 \\
0.0230 \\
0.0235 \\
0.0274 \\
0.0343 \\
0.0343 \\
0.0345 \\
0.0380 \\
0.0414 \\
0.0415 \\
0.0414 \\
0.0408 \\
0.0387 \\
0.0379 \\
0.0375 \\
0.0351 \\
0.0328 \\
0.0328 \\
0.0290 \\
0.0262 \\
0.0264 \\
0.0236 \\
0.0203 \\
0.0195 \\
0.0147 \\
0.0110 \\
0.0103 \\
0.0100 \\
0.0106 \\
0.0102\end{array}$ & $\begin{array}{c}\text { Pressure } \\
\text { (psig) } \\
9.89 \\
19.55 \\
29.57 \\
39.42 \\
49.16 \\
54.50 \\
59.57 \\
64.20 \\
69.32 \\
74.16 \\
80.16 \\
85.61 \\
90.58 \\
95.69 \\
100.92 \\
103.25 \\
106.11 \\
108.31 \\
111.08 \\
113.24 \\
117.83 \\
120.92 \\
123.28 \\
122.97 \\
125.82 \\
125.60 \\
127.84 \\
130.19 \\
132.53 \\
135.33 \\
134.42 \\
138.35 \\
140.16 \\
142.63 \\
145.78 \\
142.52 \\
0.22 \\
\end{array}$ & $\begin{array}{l}\text { Strain } \\
0.0025 \\
0.0094 \\
0.0164 \\
0.0225 \\
0.0280 \\
0.0316 \\
0.0352 \\
0.0397 \\
0.0437 \\
0.0496 \\
0.0544 \\
0.0584 \\
0.0607 \\
0.0634 \\
0.0659 \\
0.0665 \\
0.0680 \\
0.0694 \\
0.0699 \\
0.0706 \\
0.0739 \\
0.0759 \\
0.0770 \\
0.0171 \\
0.0805 \\
0.0801 \\
0.0820 \\
0.0850 \\
0.0880 \\
0.0912 \\
0.0904 \\
0.0945 \\
0.0962 \\
0.0991 \\
0.1024 \\
0.1010 \\
0.0097\end{array}$ \\
\hline
\end{tabular}




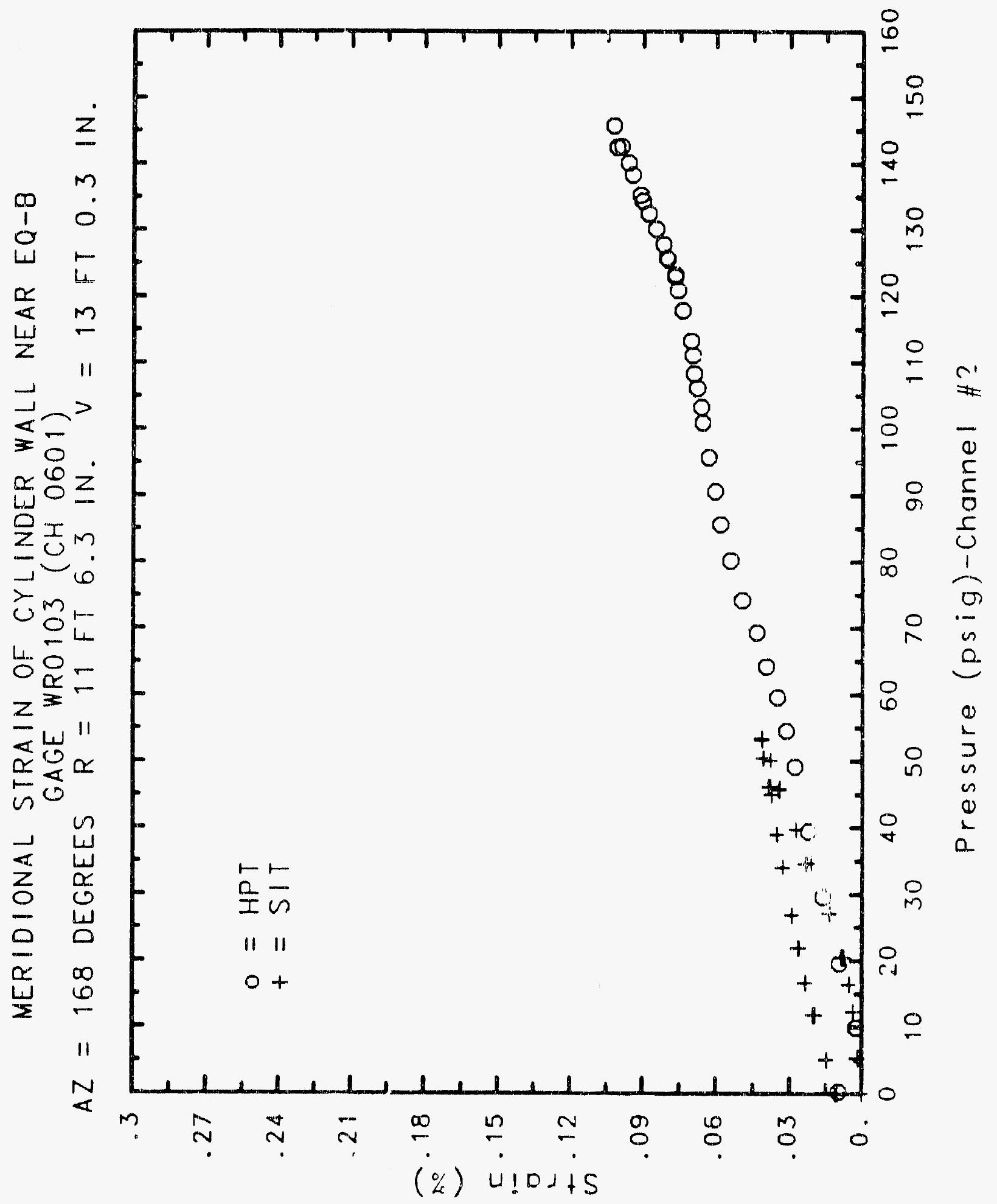




\section{Reinforced Concrete Test Data}

Weldable Gage 104 Channel 602

Structural Integrity Test

High Pressure Test

\begin{tabular}{|c|c|c|c|}
\hline $\begin{array}{c}\text { Pressure } \\
\text { (psig) } \\
-0.05 \\
5.33 \\
12.31 \\
16.44 \\
20.51 \\
20.46 \\
20.47 \\
27.05 \\
34.69 \\
34.53 \\
34.53 \\
39.70 \\
45.90 \\
45.65 \\
45.62 \\
50.10 \\
53.47 \\
53.29 \\
53.21 \\
50.49 \\
46.14 \\
46.13 \\
44.97 \\
38.98 \\
33.99 \\
33.96 \\
26.83 \\
21.88 \\
21.90 \\
16.69 \\
11.74 \\
11.77 \\
5.05 \\
0.02 \\
-0.04 \\
-0.02 \\
-0.02 \\
0.02 \\
\end{array}$ & $\begin{array}{l}\text { \% Strain } \\
0.0000 \\
0.0021 \\
0.0042 \\
0.0059 \\
0.0079 \\
0.0090 \\
0.0098 \\
0.0151 \\
0.0227 \\
0.0244 \\
0.0251 \\
0.0295 \\
0.0378 \\
0.0385 \\
0.0381 \\
0.0425 \\
0.0465 \\
0.0474 \\
0.0469 \\
0.0465 \\
0.0441 \\
0.0438 \\
0.0450 \\
0.0414 \\
0.0384 \\
0.0383 \\
0.0337 \\
0.0306 \\
0.0306 \\
0.0273 \\
0.0233 \\
0.0223 \\
0.0174 \\
0.0141 \\
0.0112 \\
0.0118 \\
0.0122 \\
0.0115\end{array}$ & $\begin{array}{c}\text { Pressure } \\
\text { (psig) } \\
9.89 \\
19.55 \\
29.57 \\
39.42 \\
49.16 \\
54.50 \\
59.57 \\
64.20 \\
69.32 \\
14.16 \\
80.16 \\
85.61 \\
90.58 \\
95.69 \\
100.92 \\
103.25 \\
106.11 \\
108.31 \\
111.08 \\
113.24 \\
117.83 \\
120.92 \\
123.28 \\
122.97 \\
125.82 \\
125.60 \\
127.84 \\
130.19 \\
132.53 \\
135.33 \\
134.42 \\
138.35 \\
140.16 \\
142.63 \\
145.78 \\
142.52 \\
0.22\end{array}$ & $\begin{array}{l}\text { \% Strain } \\
0.0029 \\
0.0109 \\
0.0195 \\
0.0278 \\
0.0361 \\
0.0409 \\
0.0459 \\
0.0512 \\
0.0575 \\
0.0639 \\
0.0722 \\
0.0793 \\
0.0862 \\
0.0937 \\
0.1012 \\
0.1049 \\
0.1097 \\
0.1126 \\
0.1178 \\
0.1212 \\
0.1301 \\
0.1359 \\
0.1422 \\
0.1431 \\
0.1497 \\
0.1513 \\
0.1553 \\
0.1623 \\
0.1698 \\
0.1778 \\
0.1793 \\
0.1873 \\
0.1933 \\
0.2011 \\
0.2090 \\
0.2089 \\
0.0457\end{array}$ \\
\hline
\end{tabular}




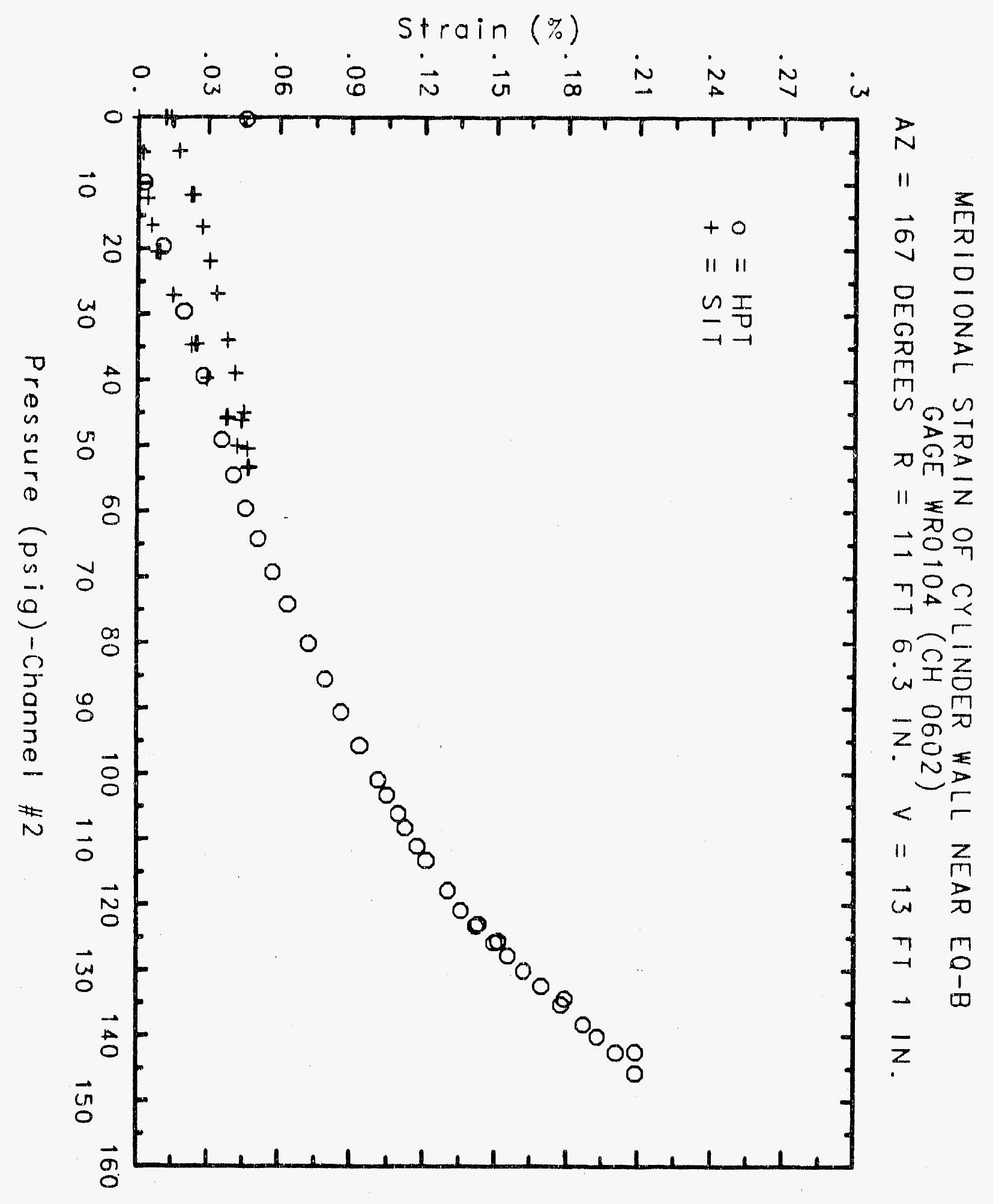




\section{Reinforced Concrete Test Dhta}

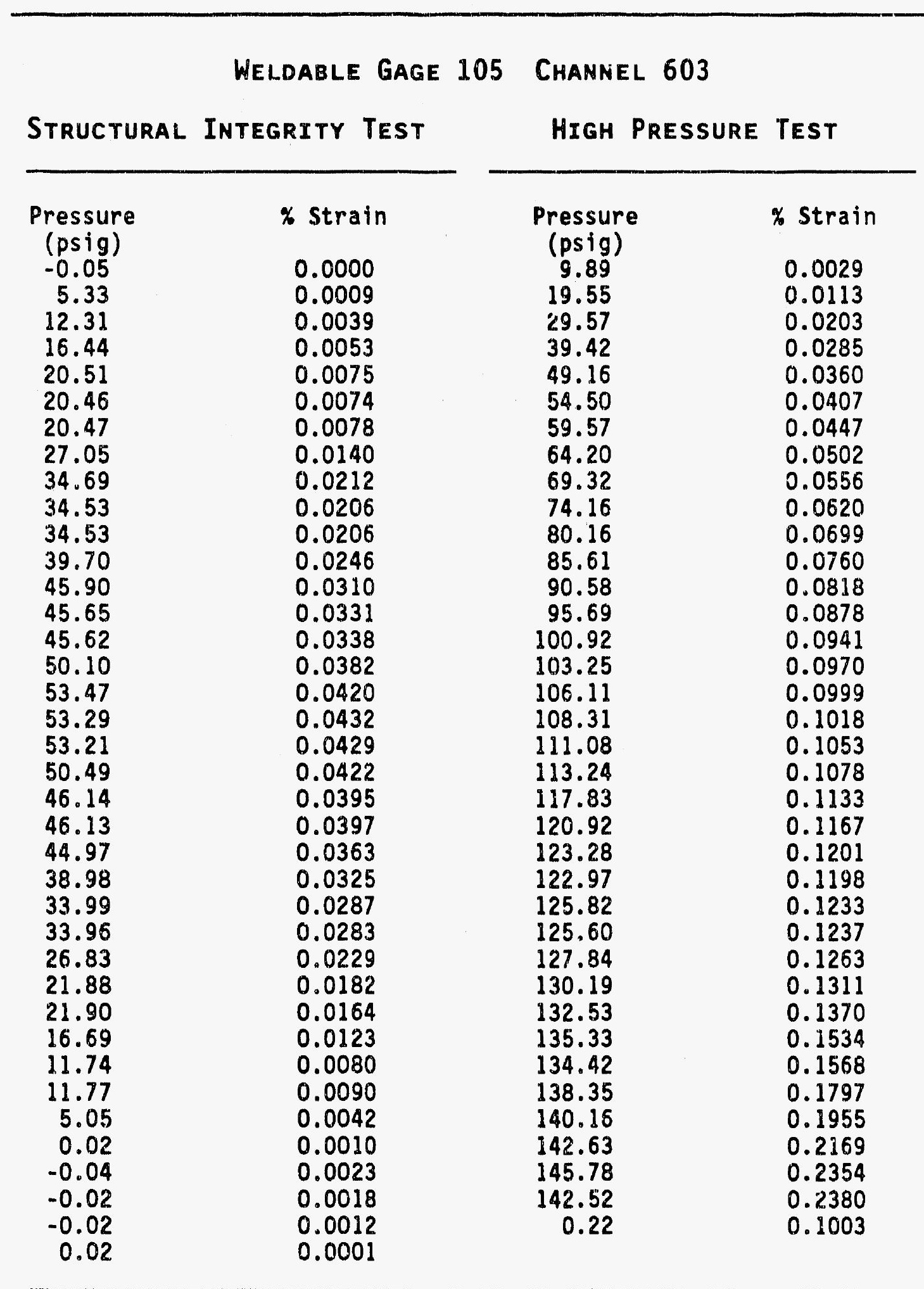




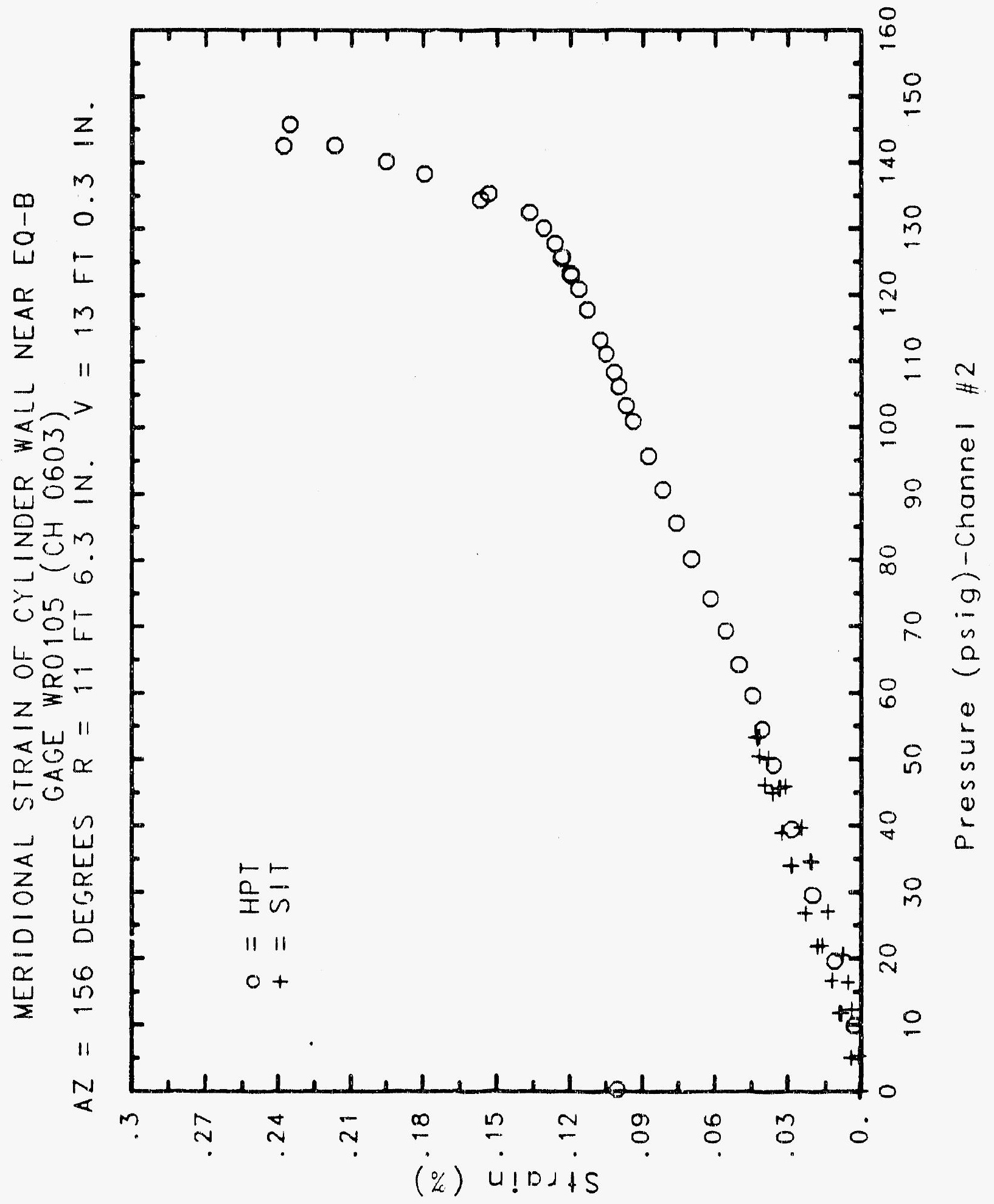

C- 1018 


\section{Reinforced Concrete Test Data}

Weldable gage 106 Channel 604

Structural Integrity Test

High Pressure Test

\begin{tabular}{|c|c|c|c|}
\hline $\begin{array}{c}\text { Pressure } \\
\text { (psig) } \\
-0.05 \\
5.33 \\
12.31 \\
16.44 \\
20.51 \\
20.46 \\
20.47 \\
27.05 \\
34.69 \\
34.53 \\
34.53 \\
39.70 \\
45.90 \\
45.65 \\
45.62 \\
50.10 \\
53.47 \\
53.29 \\
53.21 \\
50.49 \\
46.14 \\
46.13 \\
44.97 \\
38.98 \\
33.99 \\
33.96 \\
26.83 \\
21.88 \\
21.90 \\
16.69 \\
12.74 \\
11.77 \\
5.05 \\
0.02 \\
-0.04 \\
-0.02 \\
-0.02 \\
0.02 \\
\end{array}$ & $\begin{array}{c}\text { \% Strain } \\
-0.0011 \\
0.0014 \\
0.0066 \\
0.0085 \\
0.0128 \\
0.0149 \\
0.0163 \\
0.0209 \\
0.0274 \\
0.0284 \\
0.0286 \\
0.0321 \\
0.0357 \\
0.0310 \\
0.0286 \\
0.0306 \\
0.0334 \\
0.0308 \\
0.0343 \\
0.0359 \\
0.0337 \\
0.0336 \\
0.0387 \\
0.0375 \\
0.0352 \\
0.0364 \\
0.0336 \\
0.0318 \\
0.0318 \\
0.0298 \\
0.0269 \\
0.0263 \\
0.0230 \\
0.0193 \\
0.0135 \\
0.0161 \\
0.0146 \\
0.0169\end{array}$ & $\begin{array}{c}\text { Pressure } \\
\text { (psig) } \\
9.89 \\
19.55 \\
29.57 \\
39.42 \\
49.16 \\
54.50 \\
59.57 \\
64.20 \\
69.32 \\
74.16 \\
80.16 \\
85.61 \\
90.58 \\
95.69 \\
100.92 \\
103.25 \\
106.11 \\
108.31 \\
111.08 \\
113.24 \\
117.83 \\
120.92 \\
123.28 \\
122.97 \\
125.82 \\
125.60 \\
127.84 \\
130.19 \\
132.53 \\
135.33 \\
134.42 \\
138.35 \\
140.16 \\
142.63 \\
145.78 \\
142.52 \\
0.22 \\
\end{array}$ & $\begin{array}{l}\text { \% Strain } \\
0.0004 \\
0.0051 \\
0.0104 \\
0.0155 \\
0.0206 \\
0.0234 \\
0.0267 \\
0.0295 \\
0.0333 \\
0.0363 \\
0.0431 \\
0.0474 \\
0.0513 \\
0.0545 \\
0.0580 \\
0.0602 \\
0.0629 \\
0.0643 \\
0.0667 \\
0.0692 \\
0.0737 \\
0.0774 \\
0.0804 \\
0.0856 \\
0.0830 \\
0.0816 \\
0.0849 \\
0.0880 \\
0.0911 \\
0.0937 \\
0.0944 \\
0.0991 \\
0.1051 \\
0.1163 \\
0.1285 \\
0.1309 \\
0.0473\end{array}$ \\
\hline
\end{tabular}




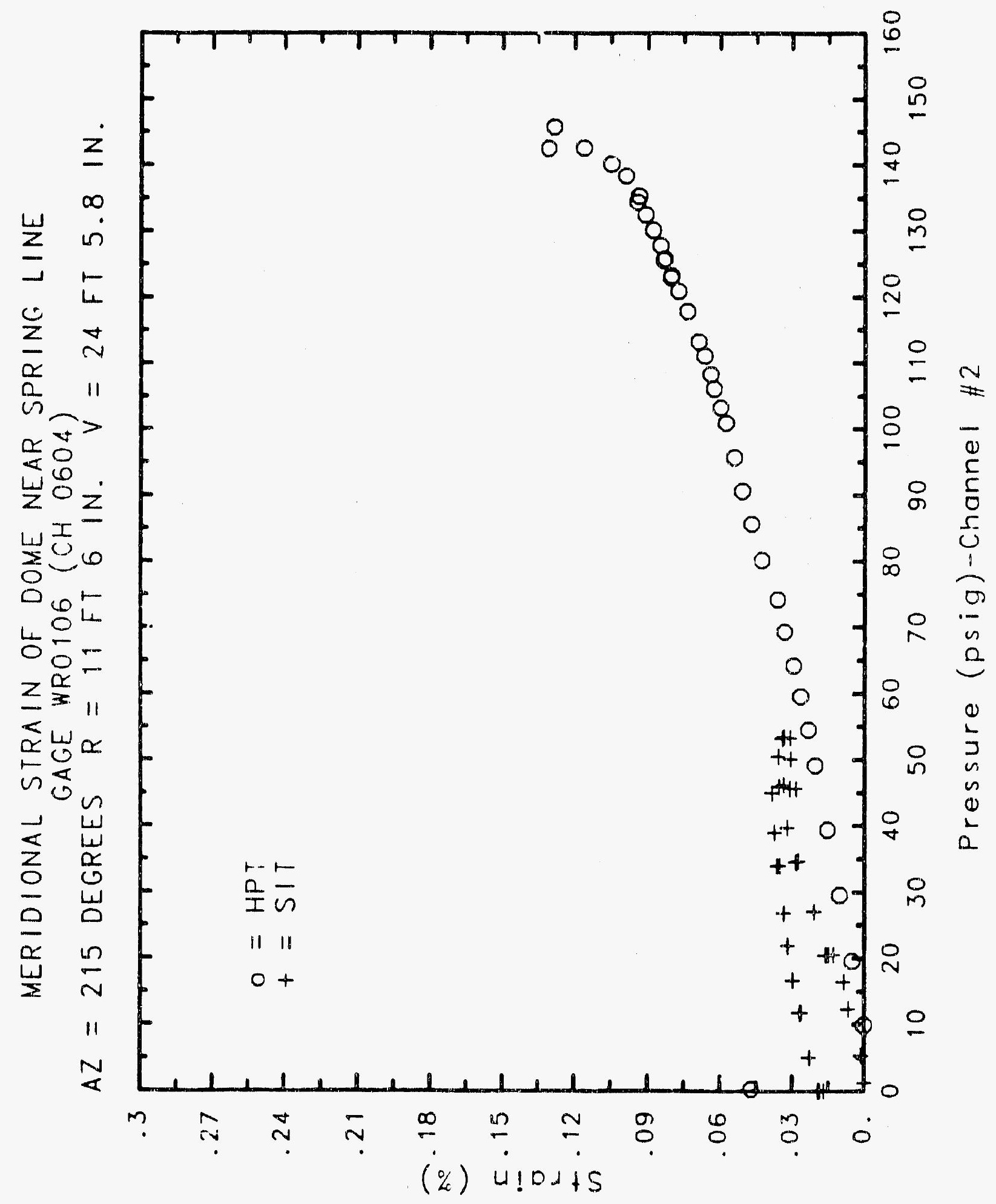




\section{Reinforced Concrete Test Cata}

Weldable Gage 107 Channel 605

Structural InTEgrity TEST

High Pressure Test

\begin{tabular}{|c|c|c|c|}
\hline $\begin{array}{c}\text { Pressure } \\
\text { (psig) } \\
-0.05 \\
5.33 \\
12.31 \\
16.44 \\
20.51 \\
20.46 \\
20.47 \\
27.05 \\
34.69 \\
34.53 \\
34.53 \\
39.70 \\
45.90 \\
45.65 \\
45.62 \\
50.10 \\
53.47 \\
53.29 \\
53.21 \\
50.49 \\
46.14 \\
46.13 \\
44.97 \\
38.98 \\
33.99 \\
33.96 \\
26.83 \\
21.88 \\
21.90 \\
16.69 \\
11.74 \\
11.77 \\
5.05 \\
0.02 \\
-0.04 \\
-0.02 \\
-0.02 \\
0.02 \\
\end{array}$ & $\begin{array}{l}\text { \% Strain } \\
-0.0005 \\
0.0013 \\
0.0037 \\
0.0052 \\
0.0072 \\
0.0077 \\
0.0081 \\
0.0135 \\
0.0207 \\
0.0207 \\
0.0206 \\
0.0244 \\
0.0294 \\
0.0302 \\
0.0303 \\
0.0336 \\
0.0359 \\
0.0368 \\
0.0369 \\
0.0361 \\
0.0342 \\
0.0341 \\
0.0328 \\
0.0298 \\
0.0269 \\
0.0267 \\
0.0231 \\
0.0203 \\
0.0200 \\
0.0174 \\
0.0143 \\
0.0143 \\
0.0103 \\
0.0081 \\
0.0058 \\
0.0066 \\
0.0060 \\
0.0066\end{array}$ & $\begin{array}{c}\text { Pressure } \\
(\text { psig) } \\
9.89 \\
19.55 \\
29.57 \\
39.42 \\
49.16 \\
54.50 \\
59.57 \\
64.20 \\
69.32 \\
74.16 \\
80.16 \\
85.61 \\
90.58 \\
95.69 \\
100.92 \\
103.25 \\
106.11 \\
108.31 \\
111.08 \\
113.24 \\
117.83 \\
120.92 \\
123.28 \\
122.97 \\
125.82 \\
125.60 \\
127.84 \\
130.19 \\
132.53 \\
135.33 \\
134.42 \\
138.35 \\
140.16 \\
142.63 \\
145.78 \\
142.52 \\
0.22 \\
\end{array}$ & $\begin{array}{l}\text { \% Strain } \\
0.0012 \\
0.0075 \\
0.0132 \\
0.0198 \\
0.0253 \\
0.0286 \\
0.0320 \\
0.0352 \\
0.0389 \\
0.0428 \\
0.0489 \\
0.0532 \\
0.0573 \\
0.0611 \\
0.0659 \\
0.0681 \\
0.0702 \\
0.0723 \\
0.0748 \\
0.0769 \\
0.0814 \\
0.0848 \\
0.0872 \\
0.0875 \\
0.0896 \\
0.0897 \\
0.0911 \\
0.0942 \\
0.0967 \\
0.0996 \\
0.0997 \\
0.1047 \\
0.1087 \\
0.1165 \\
0.1255 \\
0.1273 \\
0.0367\end{array}$ \\
\hline
\end{tabular}




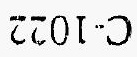

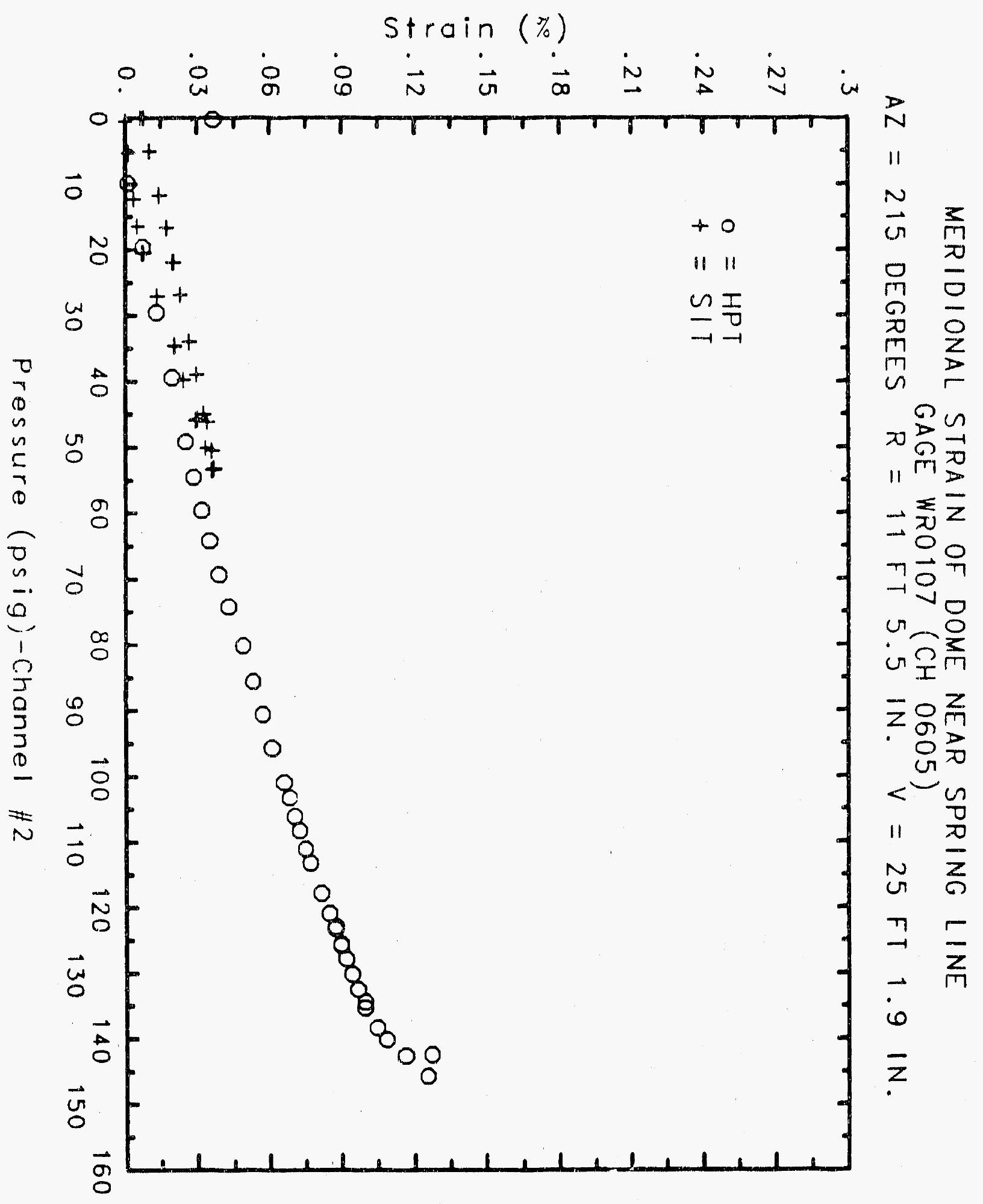




\section{Reinforced Concrete Test Data}

Weldable Gage 108 Channel 606

StRUCTURAL INTEgRITY TEST

High Pressure test

\begin{tabular}{|c|c|c|c|}
\hline $\begin{array}{c}\text { Pressure } \\
\text { (psig) } \\
-0.05 \\
5.33 \\
12.31 \\
16.44 \\
20.51 \\
20.46 \\
20.47 \\
27.05 \\
34.69 \\
34.53 \\
34.53 \\
39.70 \\
45.90 \\
45.65 \\
45.62 \\
50.10 \\
53.47 \\
53.29 \\
53.21 \\
50.49 \\
46.14 \\
46.13 \\
44.97 \\
38.98 \\
33.99 \\
33.96 \\
26.83 \\
21.88 \\
21.90 \\
16.69 \\
11.74 \\
11.77 \\
5.05 \\
0.02 \\
-0.04 \\
-0.02 \\
-0.02 \\
0.02 \\
\end{array}$ & $\begin{array}{l}\text { \% Strain } \\
0.0014 \\
0.0020 \\
0.0044 \\
0.0059 \\
0.0082 \\
0.0088 \\
0.0090 \\
0.0146 \\
0.0201 \\
0.0206 \\
0.0202 \\
0.0225 \\
0.0245 \\
0.0243 \\
0.0240 \\
0.0259 \\
0.0265 \\
0.0268 \\
0.0269 \\
0.0258 \\
0.0246 \\
0.0243 \\
0.0254 \\
0.0236 \\
0.0215 \\
0.0218 \\
0.0190 \\
0.0173 \\
0.0172 \\
0.0151 \\
0.0131 \\
0.0128 \\
0.0102 \\
0.0076 \\
0.0677 \\
0.0073 \\
0.0069 \\
0.0077\end{array}$ & $\begin{array}{c}\text { Pressure } \\
(\text { psig) } \\
9.89 \\
19.55 \\
29.57 \\
39.42 \\
49.16 \\
54.50 \\
59.57 \\
64.20 \\
69.32 \\
74.16 \\
80.16 \\
85.61 \\
90.58 \\
95.69 \\
100.92 \\
103.25 \\
106.11 \\
108.31 \\
111.08 \\
113.24 \\
117.83 \\
120.92 \\
123.28 \\
122.97 \\
125.82 \\
125.60 \\
127.84 \\
130.19 \\
132.53 \\
135.33 \\
134.42 \\
138.35 \\
140.16 \\
142.63 \\
145.78 \\
142.52 \\
0.22 \\
\end{array}$ & $\begin{array}{l}\text { \% strain } \\
0.0001 \\
0.0037 \\
0.0079 \\
0.0112 \\
0.0149 \\
0.0170 \\
0.0187 \\
0.0200 \\
0.0227 \\
0.0250 \\
0.0272 \\
0.0319 \\
0.0362 \\
0.0407 \\
0.0454 \\
0.0479 \\
0.0503 \\
0.0524 \\
0.0551 \\
0.0580 \\
0.0655 \\
0.0712 \\
0.0781 \\
0.0824 \\
0.0829 \\
0.0826 \\
0.0833 \\
0.0837 \\
0.0823 \\
0.0847 \\
0.0869 \\
0.0939 \\
0.1036 \\
0.1147 \\
0.1275 \\
0.1341 \\
0.0593\end{array}$ \\
\hline
\end{tabular}




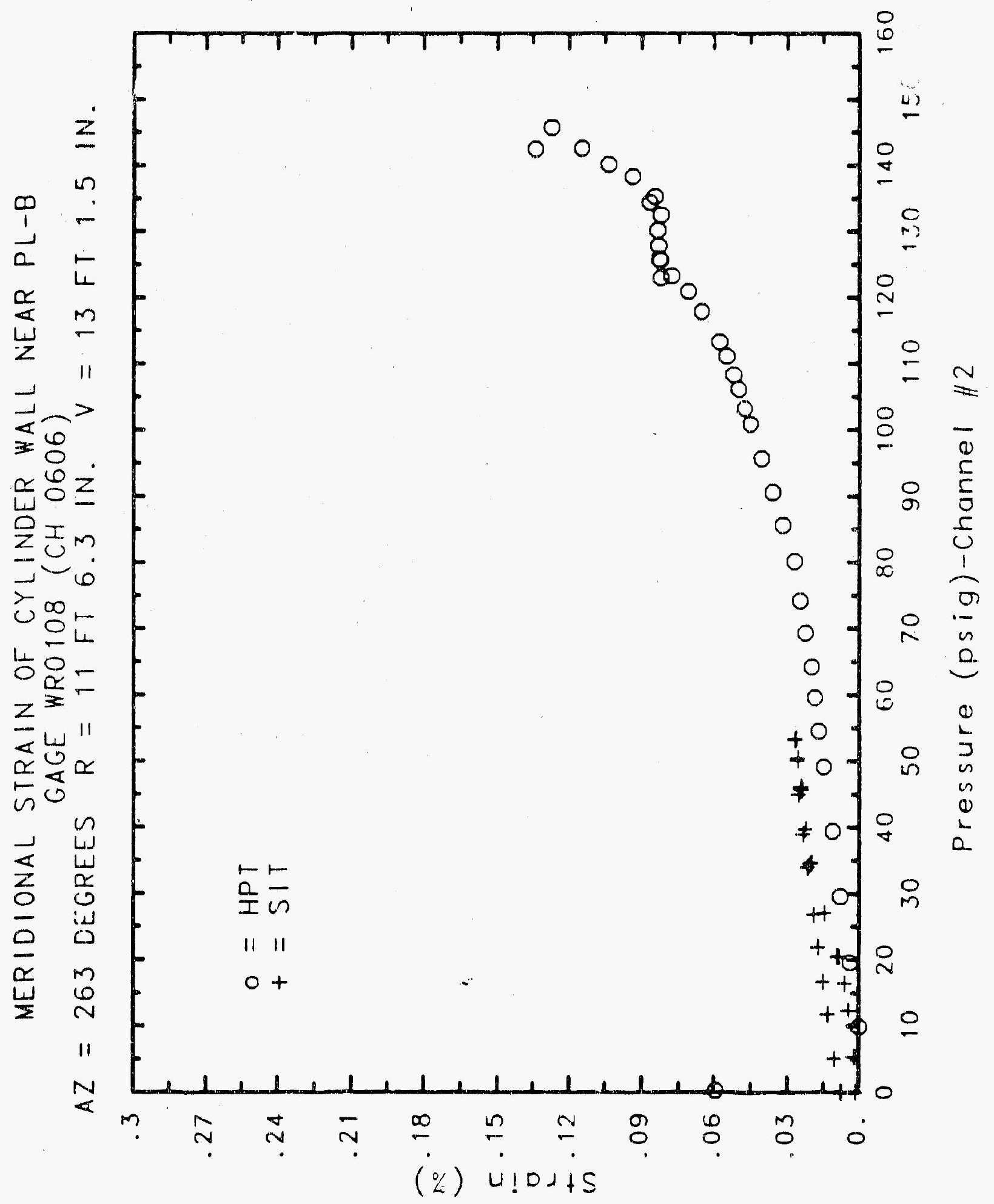

C. 1024 


\section{Reinforced Concrete test Data}

Weldable Gage 109 Channel 607

StRUCTURAL INTEgRITY TEST

High Pressure test

\begin{tabular}{|c|c|c|c|}
\hline $\begin{array}{c}\text { Pressure } \\
(p \text { pig) } \\
-0.05 \\
5.33 \\
12.31 \\
16.44 \\
20.51 \\
20.46 \\
20.47 \\
27.05 \\
34.69 \\
34.53 \\
34.53 \\
39.70 \\
45.90 \\
45.65 \\
45.62 \\
50.10 \\
53.47 \\
53.29 \\
53.21 \\
50.49 \\
46.14 \\
46.13 \\
44.97 \\
38.98 \\
33.99 \\
33.96 \\
26.83 \\
21.88 \\
21.90 \\
16.69 \\
11.74 \\
11.77 \\
5.05 \\
0.02 \\
-0.04 \\
-0.02 \\
-0.02 \\
0.02\end{array}$ & $\begin{array}{l}\text { \% Strain } \\
0.0009 \\
0.0018 \\
0.0036 \\
0.0053 \\
0.0082 \\
0.0087 \\
0.0083 \\
0.0147 \\
0.0179 \\
0.0172 \\
0.0168 \\
0.0183 \\
0.0229 \\
0.0230 \\
0.0227 \\
0.0252 \\
0.0270 \\
0.0274 \\
0.0271 \\
0.0264 \\
0.0246 \\
0.0247 \\
0.0252 \\
0.0226 \\
0.0207 \\
0.0203 \\
0.0170 \\
0.0147 \\
0.0148 \\
0.0125 \\
0.0100 \\
0.0095 \\
0.0068 \\
0.0043 \\
0.0043 \\
0.0038 \\
0.0035 \\
0.0041\end{array}$ & $\begin{array}{c}\text { Pressure } \\
(\text { psig) } \\
9.89 \\
19.55 \\
29.57 \\
39.42 \\
49.16 \\
54.50 \\
59.57 \\
64.20 \\
69.32 \\
74.16 \\
80.16 \\
85.61 \\
90.58 \\
95.69 \\
100.92 \\
103.25 \\
106.11 \\
108.31 \\
111.08 \\
113.24 \\
117.83 \\
120.92 \\
123.28 \\
122.97 \\
125.82 \\
125.60 \\
127.84 \\
130.19 \\
132.53 \\
135.33 \\
134.42 \\
138.35 \\
140.16 \\
142.63 \\
145.78 \\
142.52 \\
0.22\end{array}$ & $\begin{array}{l}\text { \% Strain } \\
\\
0.0007 \\
0.0060 \\
0.0110 \\
0.0158 \\
0.0201 \\
0.0228 \\
0.0251 \\
0.0279 \\
0.0314 \\
0.0358 \\
0.0394 \\
0.0467 \\
0.0526 \\
0.0591 \\
0.0661 \\
0.0693 \\
0.0727 \\
0.0754 \\
0.0796 \\
0.0826 \\
0.0910 \\
0.0982 \\
0.1074 \\
0.1126 \\
0.1342 \\
0.1421 \\
0.1530 \\
0.1791 \\
0.2204 \\
0.2747 \\
0.2928 \\
0.3377 \\
0.3742 \\
0.4192 \\
0.4610 \\
0.4797 \\
0.3022\end{array}$ \\
\hline
\end{tabular}




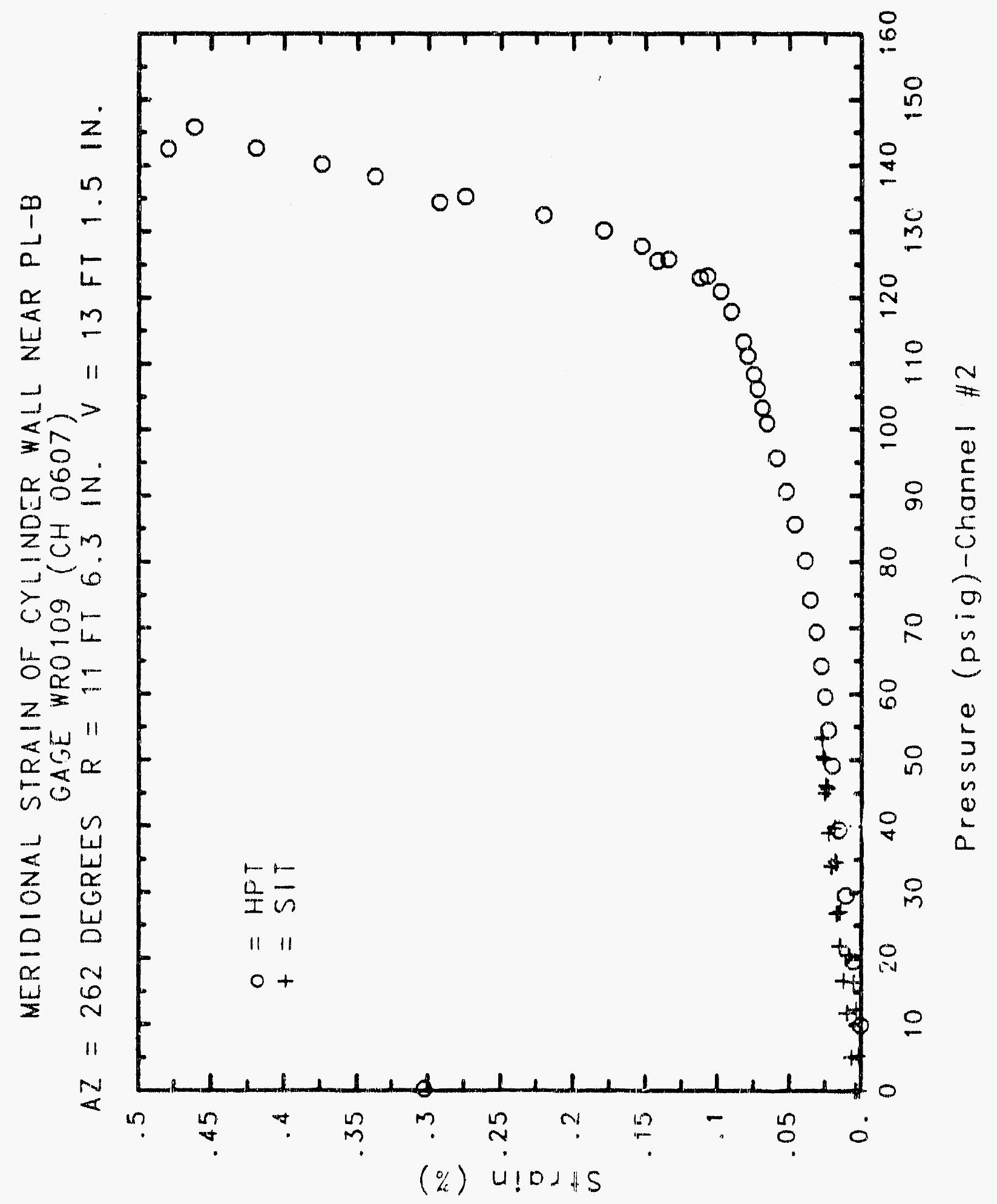




\section{Reinforced Concrete Test Data}

Weldable Gage 110 Channel 608

Structural Integrity Test

High Pressure test

\begin{tabular}{|c|c|c|c|}
\hline $\begin{array}{c}\text { Pressure } \\
\text { (psig) } \\
-0.05 \\
5.33 \\
12.31 \\
16.44 \\
20.51 \\
20.46 \\
20.47 \\
27.05 \\
34.69 \\
34.53 \\
34.53 \\
39.70 \\
45.90 \\
45.65 \\
45.62 \\
50.10 \\
53.47 \\
53.29 \\
53.21 \\
50.49 \\
46.14 \\
46.13 \\
44.97 \\
38.98 \\
33.99 \\
33.96 \\
26.83 \\
21.88 \\
21.90 \\
16.69 \\
11.74 \\
11.77 \\
5.05 \\
0.02 \\
-0.04 \\
-0.02 \\
-0.02 \\
0.02 \\
\end{array}$ & $\begin{array}{l}\text { S Strain } \\
0.0017 \\
0.0048 \\
0.0081 \\
0.0098 \\
0.0135 \\
0.0143 \\
0.0144 \\
0.0213 \\
0.0305 \\
0.0308 \\
0.0313 \\
0.0358 \\
0.0424 \\
0.0432 \\
0.0434 \\
0.0476 \\
0.0504 \\
0.0510 \\
0.0517 \\
0.0502 \\
0.0479 \\
0.0478 \\
0.0440 \\
0.0401 \\
0.0370 \\
0.0371 \\
0.0317 \\
0.0281 \\
0.0281 \\
0.0247 \\
0.0208 \\
0.0206 \\
0.0161 \\
0.0121 \\
0.0052 \\
0.0041 \\
0.0066 \\
0.0086\end{array}$ & $\begin{array}{c}\text { Pressure } \\
\text { (psig) } \\
9.89 \\
19.55 \\
29.57 \\
39.42 \\
49.16 \\
54.50 \\
59.57 \\
64.20 \\
69.32 \\
74.16 \\
80.16 \\
85.61 \\
90.58 \\
95.69 \\
100.92 \\
103.25 \\
106.11 \\
108.31 \\
111.08 \\
113.24 \\
117.83 \\
120.92 \\
123.28 \\
122.97 \\
125.82 \\
125.60 \\
127.84 \\
130.19 \\
132.53 \\
135.33 \\
134.42 \\
138.35 \\
140.16 \\
142.63 \\
145.78 \\
142.52 \\
0.22 \\
\end{array}$ & $\begin{array}{l}\text { S Strain } \\
0.0049 \\
0.0129 \\
0.0215 \\
0.0299 \\
0.0379 \\
0.0422 \\
0.0474 \\
0.0525 \\
0.0591 \\
0.0654 \\
0.0754 \\
0.0850 \\
0.0927 \\
0.1015 \\
0.1107 \\
0.1150 \\
0.1200 \\
0.1238 \\
0.1290 \\
0.1333 \\
0.1433 \\
0.1516 \\
0.1574 \\
0.1577 \\
0.1658 \\
0.1678 \\
0.1760 \\
0.1913 \\
0.2072 \\
0.2229 \\
0.2279 \\
0.2317 \\
0.2435 \\
0.2465 \\
0.2513 \\
0.2501 \\
0.1074\end{array}$ \\
\hline
\end{tabular}




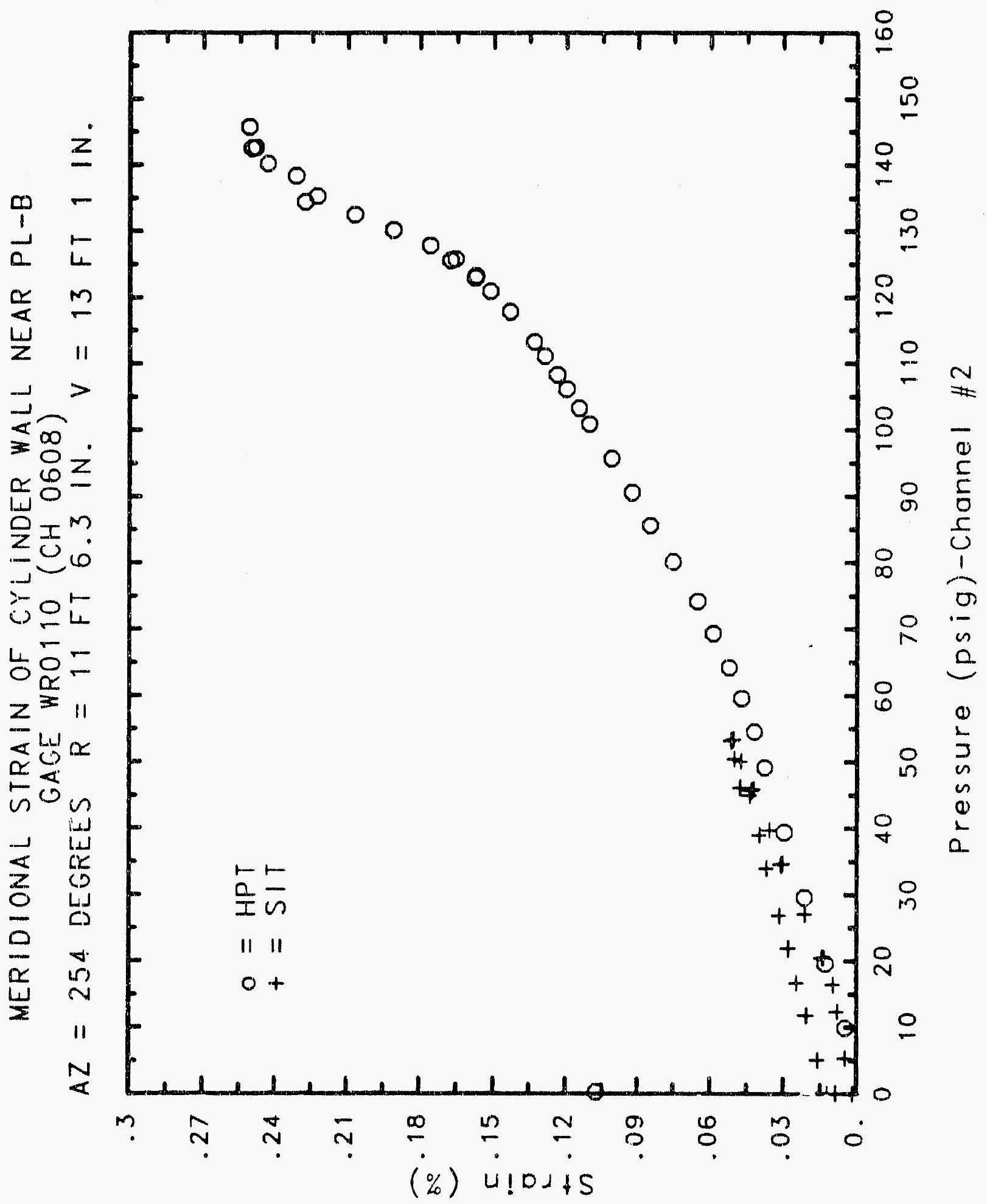




\section{Reinforced Concrete Test Data}

Weldoable Gage 111 Channel 609

Structural INTEgRITY TEST

\begin{tabular}{|c|c|c|c|}
\hline $\begin{array}{c}\text { Pressure } \\
(\text { psig) } \\
-0.05 \\
5.33 \\
12.31 \\
16.44 \\
20.51 \\
20.46 \\
20.47 \\
27.05 \\
34.69 \\
34.53 \\
34.53 \\
39.70 \\
45.90 \\
45.65 \\
45.62 \\
50.10 \\
53.47 \\
53.29 \\
53.21 \\
50.49 \\
46.14 \\
46.13 \\
44.97 \\
38.98 \\
33.99 \\
33.96 \\
26.83 \\
21.88 \\
21.90 \\
16.69 \\
11.74 \\
11.77 \\
5.05 \\
0.02 \\
-0.04 \\
-0.02 \\
-0.02 \\
0.02 \\
\end{array}$ & $\begin{array}{l}\text { \% Strain } \\
-0.0028 \\
-0.0021 \\
-0.0019 \\
-0.0016 \\
-0.0002 \\
-0.0001 \\
-0.0001 \\
0.0022 \\
0.0068 \\
0.0070 \\
0.0068 \\
0.0094 \\
0.0136 \\
0.0137 \\
0.0145 \\
0.0166 \\
0.0191 \\
0.0189 \\
0.0198 \\
0.0189 \\
0.0182 \\
0.0181 \\
0.0162 \\
0.0144 \\
0.0131 \\
0.0125 \\
0.0108 \\
0.0091 \\
0.0087 \\
0.0072 \\
0.0057 \\
0.0055 \\
0.0050 \\
0.0052 \\
0.0066 \\
0.0019 \\
0.0019 \\
0.0024\end{array}$ & $\begin{array}{c}\text { Pressure } \\
(\text { psig) } \\
9.89 \\
19.55 \\
29.57 \\
39.42 \\
49.16 \\
54.50 \\
59.57 \\
64.20 \\
69.32 \\
74.16 \\
80.16 \\
85.61 \\
90.58 \\
95.69 \\
100.92 \\
103.25 \\
106.11 \\
108.31 \\
111.08 \\
113.24 \\
117.83 \\
120.92 \\
123.28 \\
122.97 \\
125.82 \\
125.60 \\
127.84 \\
130.19 \\
132.53 \\
135.33 \\
134.42 \\
138.35 \\
140.16 \\
142.63 \\
145.78 \\
142.52 \\
0.22 \\
\end{array}$ & $\begin{array}{l}\text { \% Strain } \\
-0.0035 \\
-0.0001 \\
0.0032 \\
0.0069 \\
0.0103 \\
0.0128 \\
0.0148 \\
0.0179 \\
0.0215 \\
0.0260 \\
0.0312 \\
0.0363 \\
0.0408 \\
0.0460 \\
0.0519 \\
0.0546 \\
0.0578 \\
0.0603 \\
0.0642 \\
0.0671 \\
0.0732 \\
0.0775 \\
0.0816 \\
0.0823 \\
0.0882 \\
0.0896 \\
0.0953 \\
0.1026 \\
0.1147 \\
0.1297 \\
0.1304 \\
0.1494 \\
0.1623 \\
0.1814 \\
0.1984 \\
0.1979 \\
0.1096\end{array}$ \\
\hline
\end{tabular}




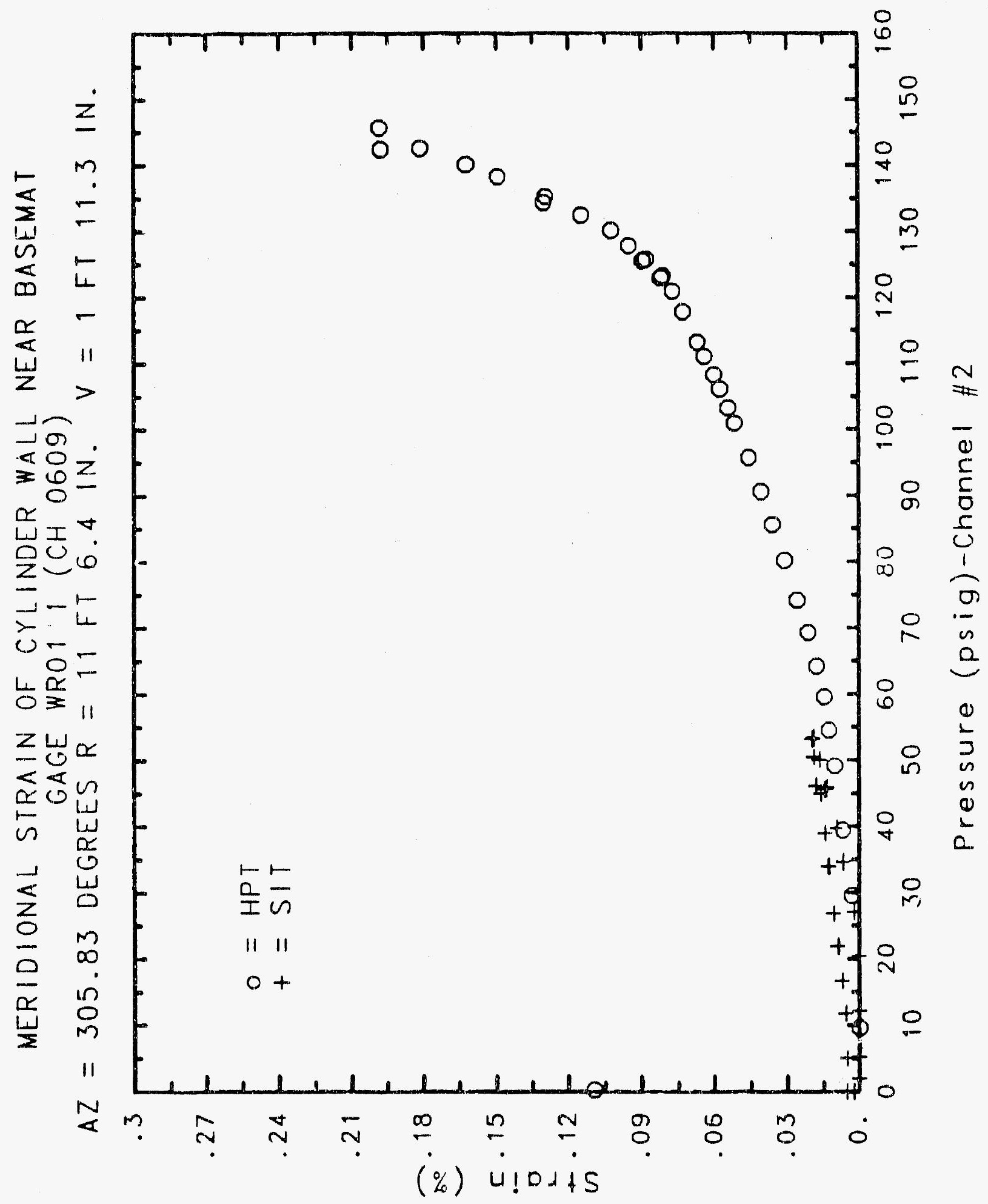

C. 1030 


\section{Reinforced Concrete Test Data}

Weldable gage 112 Channel 620

Structural IntEgrity TEST

\begin{tabular}{|c|c|c|c|}
\hline $\begin{array}{c}\text { Pressure } \\
\text { (psig) } \\
-0.05 \\
5.33 \\
12.31 \\
16.44 \\
20.51 \\
20.46 \\
20.47 \\
27.05 \\
34.69 \\
34.53 \\
34.53 \\
39.70 \\
45.90 \\
45.65 \\
45.62 \\
50.10 \\
53.47 \\
53.29 \\
53.21 \\
50.49 \\
46.14 \\
46.13 \\
44.97 \\
38.98 \\
33.99 \\
33.96 \\
26.83 \\
21.88 \\
21.90 \\
16.69 \\
11.74 \\
11.77 \\
5.05 \\
0.02 \\
-0.04 \\
-0.02 \\
-0.02 \\
0.02 \\
\end{array}$ & $\begin{array}{c}\text { \% Strain } \\
\text {-0.0009 } \\
0.0060 \\
0.0119 \\
0.0148 \\
0.0180 \\
0.0182 \\
0.0183 \\
0.0276 \\
0.0367 \\
0.0364 \\
0.0368 \\
0.0420 \\
0.0499 \\
0.0504 \\
0.0510 \\
0.0564 \\
0.0597 \\
0.0602 \\
0.0601 \\
0.0586 \\
0.0551 \\
0.0553 \\
0.0470 \\
0.0421 \\
0.0376 \\
0.0382 \\
0.0311 \\
0.0256 \\
0.0257 \\
0.0202 \\
0.0148 \\
0.0156 \\
0.0092 \\
0.0038 \\
0.0009 \\
-0.0037 \\
-0.0025 \\
0.0000\end{array}$ & $\begin{array}{c}\text { Pressure } \\
(\text { psig) } \\
9.89 \\
19.55 \\
29.57 \\
39.42 \\
49.16 \\
54.50 \\
59.57 \\
64.20 \\
69.32 \\
74.16 \\
80.16 \\
85.61 \\
90.58 \\
95.69 \\
100.92 \\
103.25 \\
106.11 \\
108.31 \\
111.08 \\
113.24 \\
117.83 \\
120.92 \\
123.28 \\
122.97 \\
125.82 \\
125.60 \\
127.84 \\
130.19 \\
132.53 \\
135.33 \\
134.42 \\
138.35 \\
140.16 \\
142.63 \\
145.78 \\
142.52 \\
0.22\end{array}$ & $\begin{array}{l}\text { \% Strain } \\
0.0060 \\
0.0171 \\
0.0286 \\
0.0397 \\
0.0496 \\
0.0551 \\
0.0608 \\
0.0659 \\
0.0718 \\
0.0772 \\
0.0885 \\
0.0955 \\
0.1024 \\
0.1100 \\
0.1177 \\
0.1213 \\
0.1251 \\
0.1282 \\
0.1328 \\
0.1365 \\
0.1447 \\
0.1504 \\
0.1551 \\
0.1539 \\
0.1579 \\
0.1558 \\
0.1592 \\
0.1693 \\
0.1881 \\
0.2151 \\
0.2194 \\
0.2572 \\
0.2854 \\
0.3003 \\
0.3045 \\
0.3020 \\
0.1541\end{array}$ \\
\hline
\end{tabular}

High Pressure test 


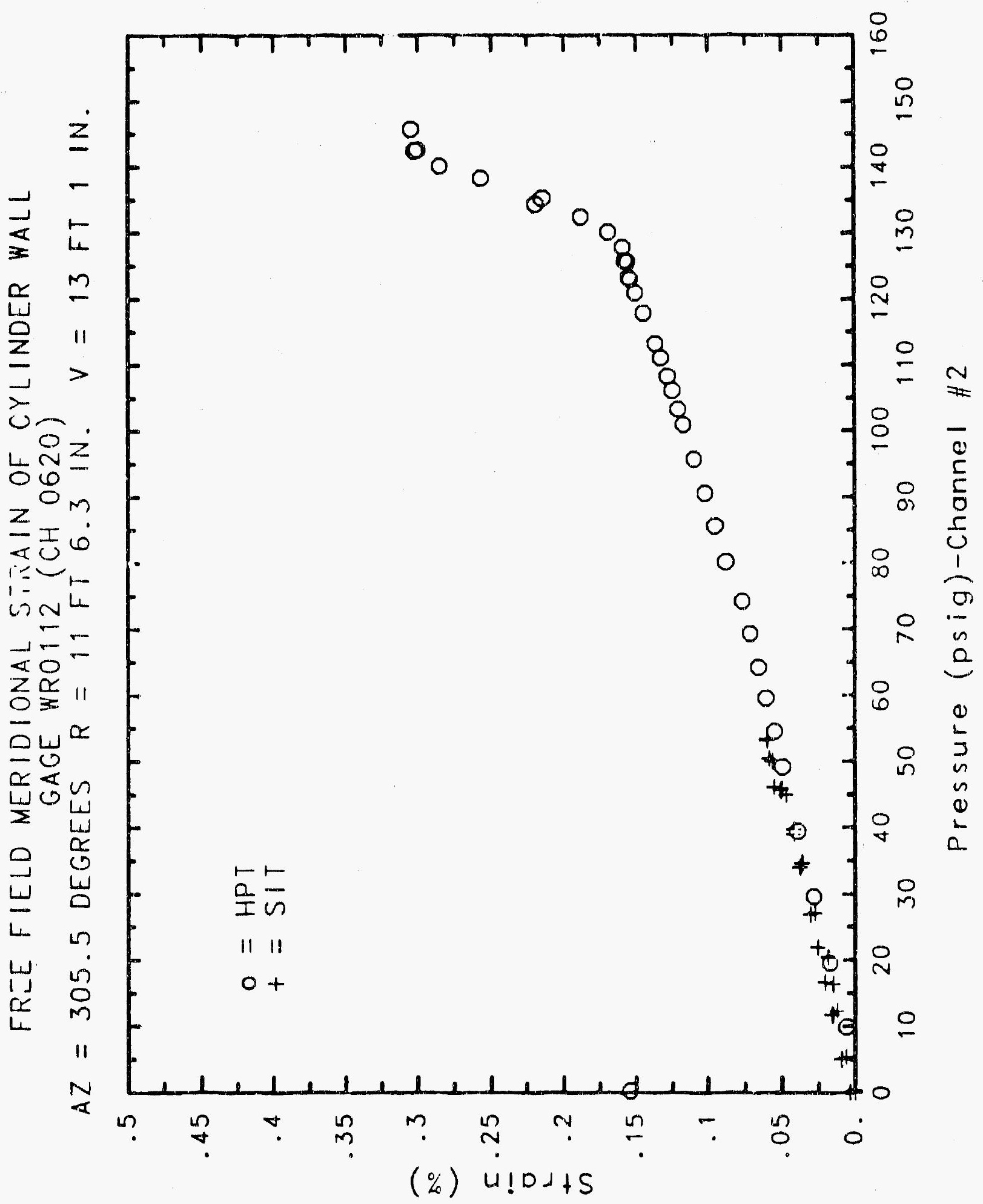




\section{Reinforced Concrete Test Data}

Weldable Gage 113 Channel 621

StRuctural IntEgrity TEST

High Pressure test

\begin{tabular}{|c|c|c|c|}
\hline $\begin{array}{c}\text { Pressure } \\
(\text { psig) } \\
-0.05 \\
5.33 \\
12.31 \\
16.44 \\
20.51 \\
20.46 \\
20.47 \\
27.05 \\
34.69 \\
34.53 \\
34.53 \\
39.70 \\
45.90 \\
45.65 \\
45.62 \\
50.10 \\
53.47 \\
53.29 \\
53.21 \\
50.49 \\
46.14 \\
46.13 \\
44.97 \\
38.98 \\
33.99 \\
33.96 \\
26.83 \\
21.88 \\
21.90 \\
16.69 \\
11.74 \\
11.77 \\
5.05 \\
0.02 \\
-0.04 \\
-0.02 \\
-0.02 \\
0.02 \\
\end{array}$ & $\begin{array}{c}\text { \% Strain } \\
-0.0003 \\
0.0029 \\
0.0057 \\
0.0077 \\
0.0125 \\
0.0130 \\
0.0132 \\
0.0216 \\
0.0302 \\
0.0304 \\
0.0306 \\
0.0354 \\
0.0412 \\
0.0419 \\
0.0424 \\
0.0464 \\
0.0495 \\
0.0497 \\
0.0495 \\
0.0484 \\
0.0453 \\
0.0448 \\
0.0409 \\
0.0368 \\
0.0328 \\
0.0328 \\
0.0267 \\
0.0225 \\
0.0224 \\
0.0175 \\
0.0131 \\
0.0135 \\
0.0079 \\
0.0042 \\
0.0015 \\
0.0008 \\
0.0008 \\
0.0016\end{array}$ & $\begin{array}{c}\text { Pressure } \\
(\text { psig) } \\
9.89 \\
19.55 \\
29.57 \\
39.42 \\
49.16 \\
54.50 \\
59.57 \\
64.20 \\
69.32 \\
74.16 \\
80.16 \\
85.61 \\
90.58 \\
95.69 \\
100.92 \\
103.25 \\
106.11 \\
108.31 \\
111.08 \\
113.24 \\
117.83 \\
120.92 \\
123.28 \\
122.97 \\
125.82 \\
125.60 \\
127.84 \\
130.19 \\
132.53 \\
135.33 \\
134.42 \\
138.35 \\
140.16 \\
142.63 \\
145.78 \\
142.52 \\
0.22 \\
\end{array}$ & $\begin{array}{l}\text { \% Strain } \\
0.0038 \\
0.0126 \\
0.0218 \\
0.0307 \\
0.0394 \\
0.0443 \\
0.0491 \\
0.0538 \\
0.0582 \\
0.0630 \\
0.0709 \\
0.0758 \\
0.0807 \\
0.0865 \\
0.0922 \\
0.0950 \\
0.0982 \\
0.1011 \\
0.1052 \\
0.1084 \\
0.1158 \\
0.1208 \\
0.1246 \\
0.1242 \\
0.1292 \\
0.1292 \\
0.1330 \\
0.1407 \\
0.1501 \\
0.1637 \\
0.1662 \\
0.1880 \\
0.2065 \\
0.2264 \\
0.2318 \\
0.2298 \\
0.0816\end{array}$ \\
\hline
\end{tabular}




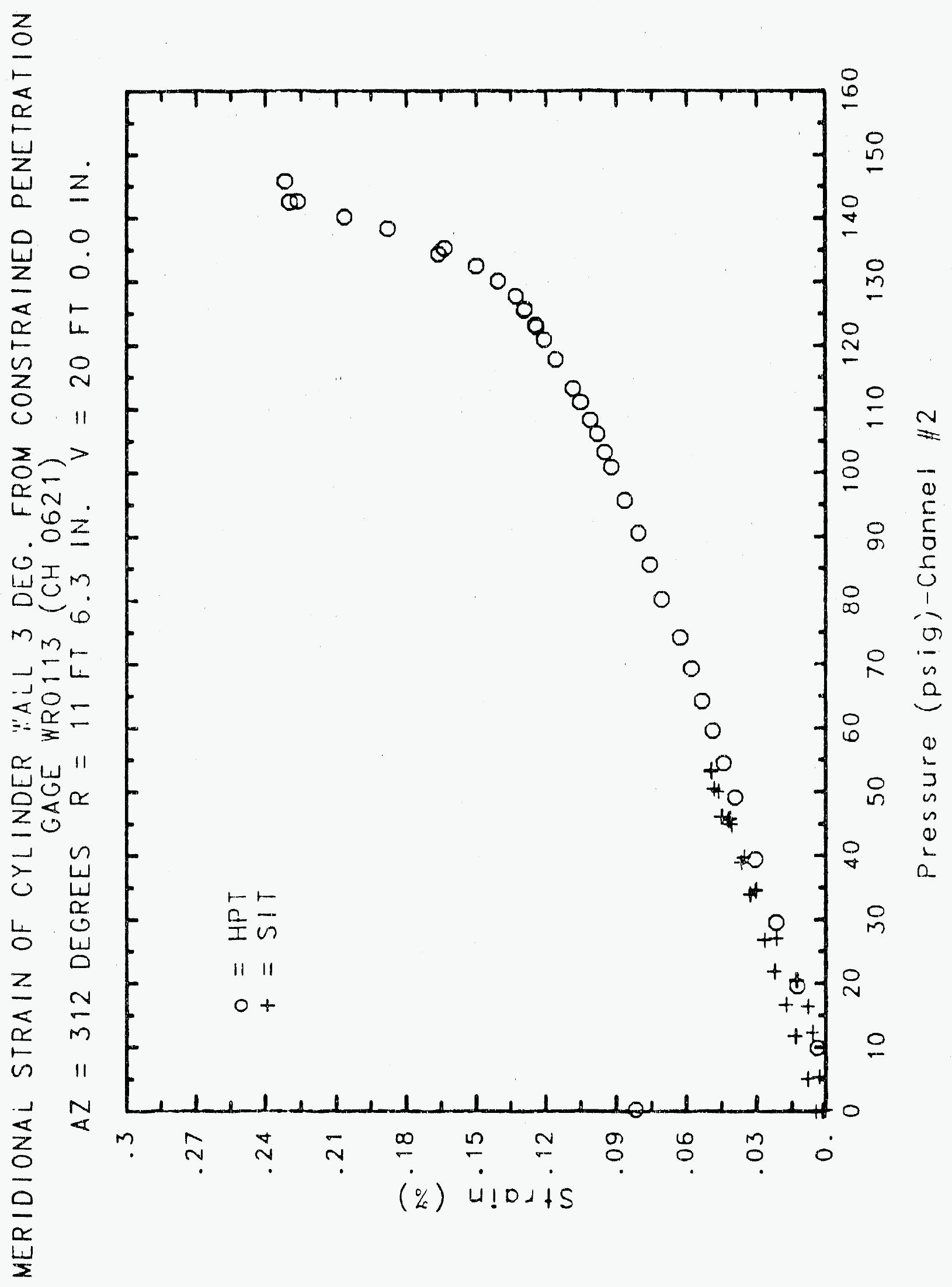




\section{Reinforced Concrete Test Data}

Weldable Gage 114 Channel 622

Structural Integrity Test

\begin{tabular}{|c|c|c|c|}
\hline $\begin{array}{c}\text { Pressure } \\
\text { (psig) } \\
-0.05 \\
5.33 \\
12.31 \\
16.44 \\
20.51 \\
20.46 \\
20.47 \\
27.05 \\
34.69 \\
34.53 \\
34.53 \\
39.70 \\
45.90 \\
45.65 \\
45.62 \\
50.10 \\
53.47 \\
53.29 \\
53.21 \\
50.49 \\
46.14 \\
46.13 \\
44.97 \\
38.98 \\
33.99 \\
33.96 \\
26.83 \\
21.88 \\
21.90 \\
16.69 \\
11.74 \\
11.77 \\
5.05 \\
0.02 \\
-0.04 \\
-0.02 \\
-0.02 \\
0.02 \\
\end{array}$ & $\begin{array}{l}\text { \% Strain } \\
0.0004 \\
0.0008 \\
0.0016 \\
0.0023 \\
0.0039 \\
0.0041 \\
0.0039 \\
0.0079 \\
0.0145 \\
0.0157 \\
0.0159 \\
0.0197 \\
0.0264 \\
0.0283 \\
0.0288 \\
0.0323 \\
0.0359 \\
0.0372 \\
0.0373 \\
0.0372 \\
0.0362 \\
0.0365 \\
0.0354 \\
0.0334 \\
0.0315 \\
0.0315 \\
0.0284 \\
0.0259 \\
0.0256 \\
0.0236 \\
0.0209 \\
0.0213 \\
0.0181 \\
0.0164 \\
0.0155 \\
0.0146 \\
0.0141 \\
0.0141\end{array}$ & $\begin{array}{c}\text { Pressure } \\
\text { (psig) } \\
9.89 \\
19.55 \\
29.57 \\
39.42 \\
49.16 \\
54.50 \\
59.57 \\
64.20 \\
69.32 \\
74.16 \\
80.16 \\
85.61 \\
90.58 \\
95.69 \\
100.92 \\
103.25 \\
106.11 \\
108.31 \\
111.08 \\
113.24 \\
117.83 \\
120.92 \\
123.28 \\
122.97 \\
125.82 \\
125.60 \\
127.84 \\
130.19 \\
132.53 \\
135.33 \\
134.42 \\
138.35 \\
140.16 \\
142.63 \\
145.78 \\
142.52 \\
0.22 \\
\end{array}$ & $\begin{array}{c}\% \text { Strain } \\
-0.0004 \\
0.0041 \\
0.0094 \\
0.0147 \\
0.0199 \\
0.0233 \\
0.0268 \\
0.0308 \\
0.0350 \\
0.0394 \\
0.0469 \\
0.0524 \\
0.0582 \\
0.0643 \\
0.0705 \\
0.0735 \\
0.0773 \\
0.0798 \\
0.0834 \\
0.0864 \\
0.0930 \\
0.0975 \\
0.1014 \\
0.1018 \\
0.1054 \\
0.1061 \\
0.1086 \\
0.1126 \\
0.1183 \\
0.1284 \\
0.1301 \\
0.1483 \\
0.1670 \\
0.1969 \\
0.2144 \\
0.2160 \\
0.0809\end{array}$ \\
\hline
\end{tabular}

high Pressure test 


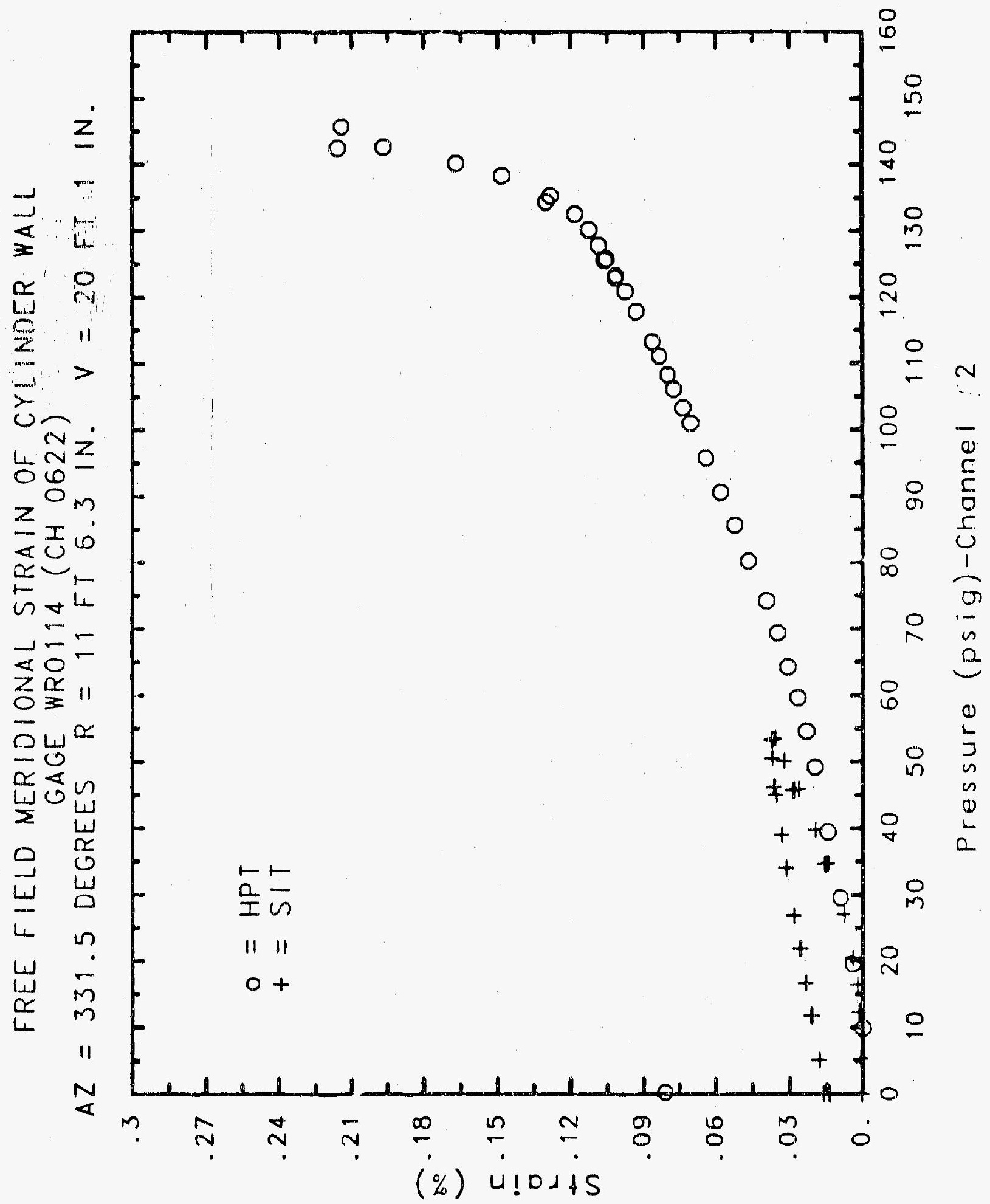

C-1036 


\section{Reinforced Concrete Test Data}

Weldable Gage 115 Channel 623

Structural Integrity TEst

\begin{tabular}{|c|c|c|c|}
\hline $\begin{array}{c}\text { Pressure } \\
\text { (psig) } \\
-0.05 \\
5.33 \\
12.31 \\
16.44 \\
20.51 \\
20.46 \\
20.47 \\
27.05 \\
34.69 \\
34.53 \\
34.53 \\
39.70 \\
45.90 \\
45.65 \\
45.62 \\
50.10 \\
53.47 \\
53.29 \\
53.21 \\
50.49 \\
46.14 \\
46.13 \\
44.97 \\
38.98 \\
33.99 \\
33.96 \\
26.83 \\
21.88 \\
21.90 \\
16.69 \\
11.74 \\
11.77 \\
5.05 \\
0.02 \\
-0.04 \\
-0.02 \\
-0.02 \\
0.02 \\
\end{array}$ & $\begin{array}{l}\text { \% Strain } \\
0.0009 \\
0.0025 \\
0.0043 \\
0.0061 \\
0.0103 \\
0.0113 \\
0.0115 \\
0.0196 \\
0.0284 \\
0.0285 \\
0.0291 \\
0.0338 \\
0.0402 \\
0.0410 \\
0.0411 \\
0.7450 \\
0.0484 \\
0.0489 \\
0.0487 \\
0.0480 \\
0.0453 \\
0.0452 \\
0.0422 \\
0.0386 \\
0.0348 \\
0.0349 \\
0.0294 \\
0.0252 \\
0.0252 \\
0.0211 \\
0.0171 \\
0.0173 \\
0.0122 \\
0.0088 \\
0.0073 \\
0.0056 \\
0.0061 \\
0.0064\end{array}$ & $\begin{array}{c}\text { Pressure } \\
(p s i g) \\
9.89 \\
19.55 \\
29.57 \\
39.42 \\
49.16 \\
54.50 \\
59.57 \\
64.20 \\
69.32 \\
74.16 \\
80.16 \\
85.61 \\
90.58 \\
95.69 \\
100.92 \\
103.25 \\
106.11 \\
108.31 \\
111.08 \\
113.24 \\
117.83 \\
120.92 \\
123.28 \\
122.97 \\
125.82 \\
125.60 \\
127.84 \\
130.19 \\
132.53 \\
135.33 \\
134.42 \\
138.35 \\
140.16 \\
142.63 \\
145.78 \\
142.52 \\
0.22 \\
\end{array}$ & $\begin{array}{l}\text { \% Strain } \\
0.0026 \\
0.0107 \\
0.0193 \\
0.0272 \\
0.0354 \\
0.0398 \\
0.0443 \\
0.0490 \\
0.0538 \\
0.0582 \\
0.0655 \\
0.0711 \\
0.0759 \\
0.0820 \\
0.0878 \\
0.0905 \\
0.0937 \\
0.0966 \\
0.1005 \\
0.1036 \\
0.1106 \\
0.1152 \\
0.1190 \\
0.1183 \\
0.1227 \\
0.1224 \\
0.1258 \\
0.1318 \\
0.1389 \\
0.1494 \\
0.1503 \\
0.1647 \\
0.1731 \\
0.1847 \\
0.1932 \\
0.1917 \\
0.0705\end{array}$ \\
\hline
\end{tabular}

High Pressure test

Pressure

9.89

19.55

39.42

49.16

64.20

74.16

80.16

85.61

90.58

103.25

106.11

111.08

113.24

117.83

120.92

123.28

22.97

25.82

130.19

132.53

35.33

134.42

138.35

40.16

2.52

0.1917

0.0064 


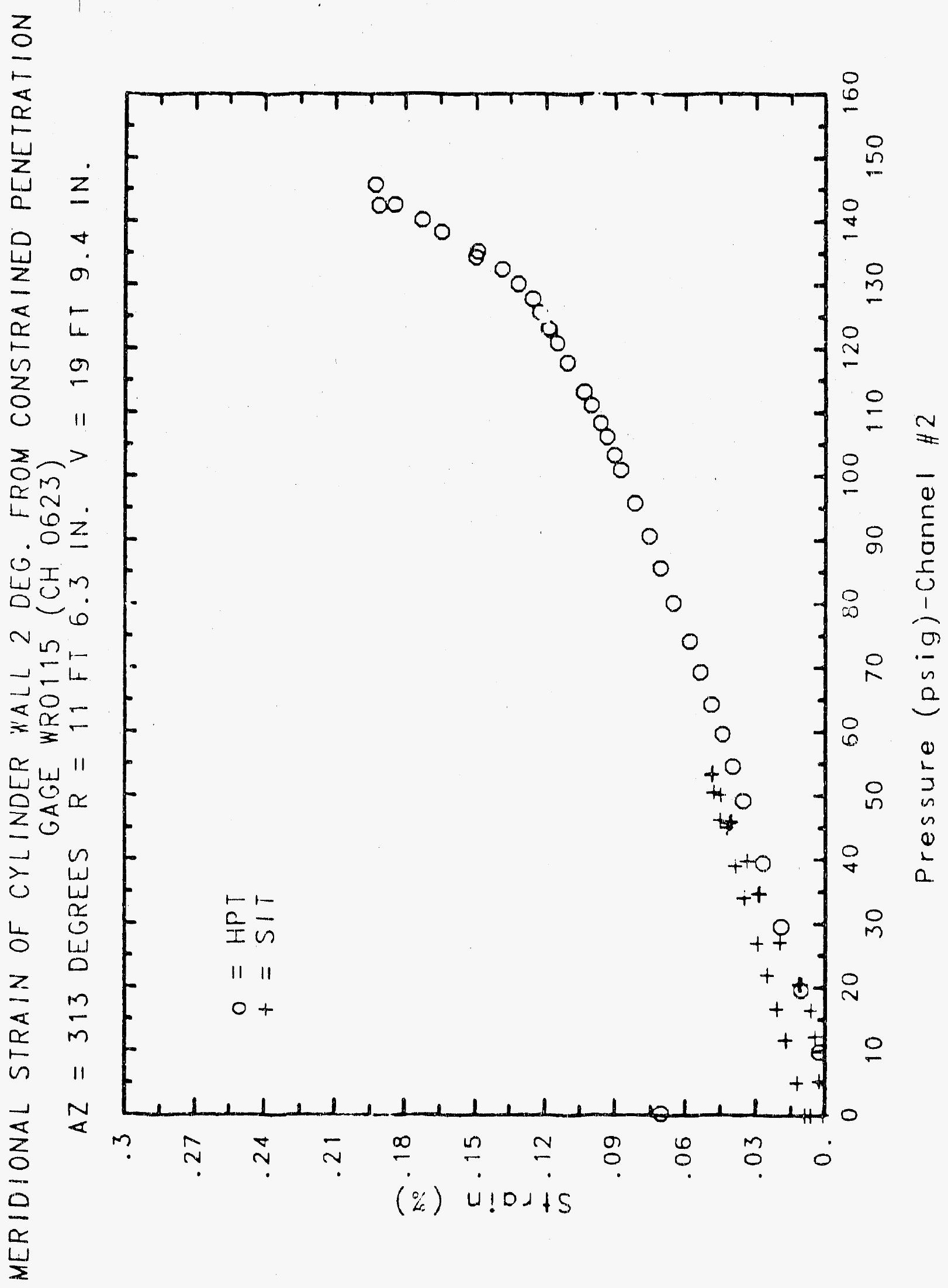




\section{Reinforced Concrete Test Data}

Weldable Gage 116 Channel 624

StRUCTURAL INTEGRITY TEST

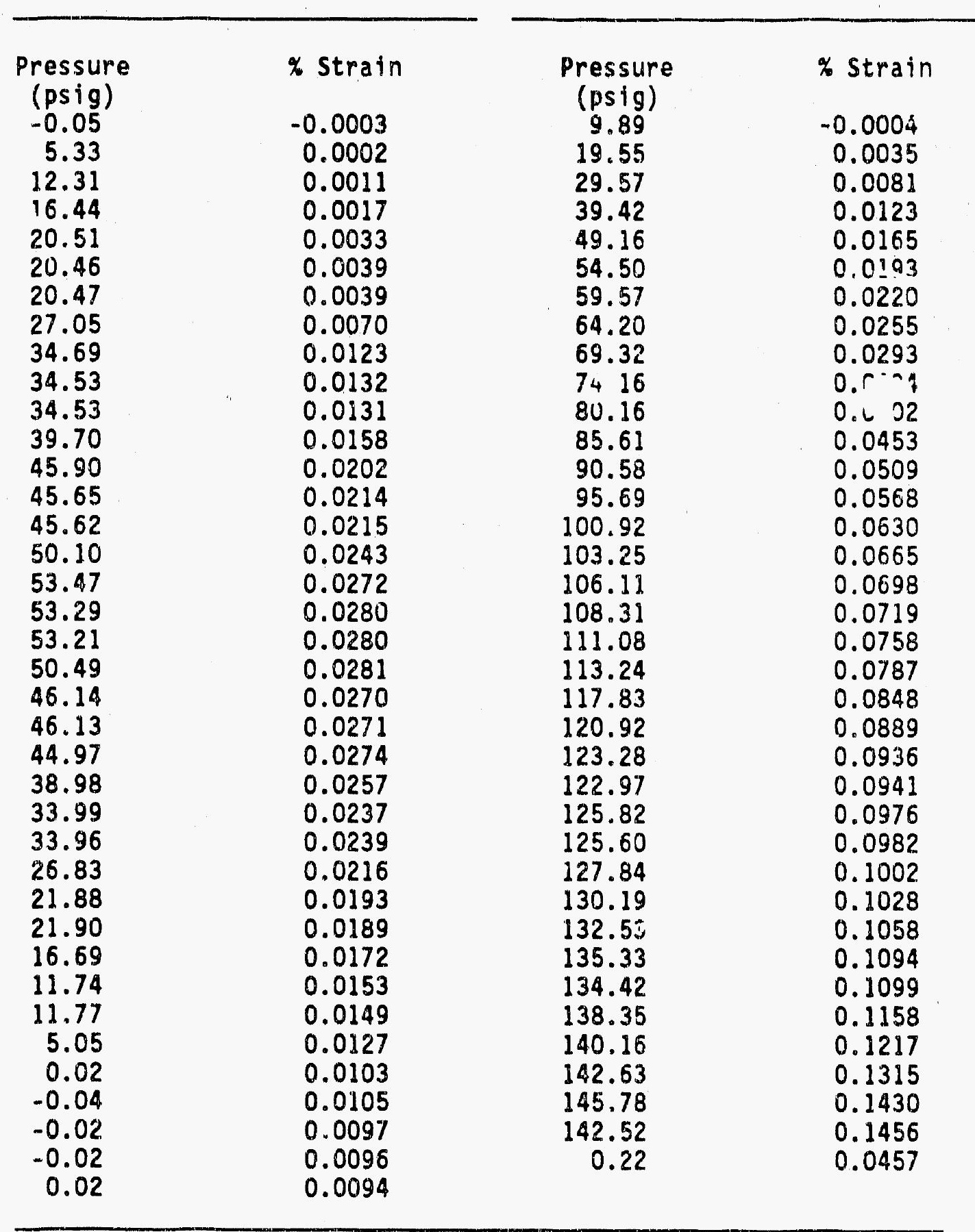

\section{High Pressure Test}

Pressure

9.89

9.55

39.42

49.16

59.57

64.20

74.16

80.16

95.69

100.92

103.25

113.24

117.83

120.92

123.28

127.84

130.19

$132.5 \mathrm{i}$

142.63

145.78

42.52

0.1456

0.0094 


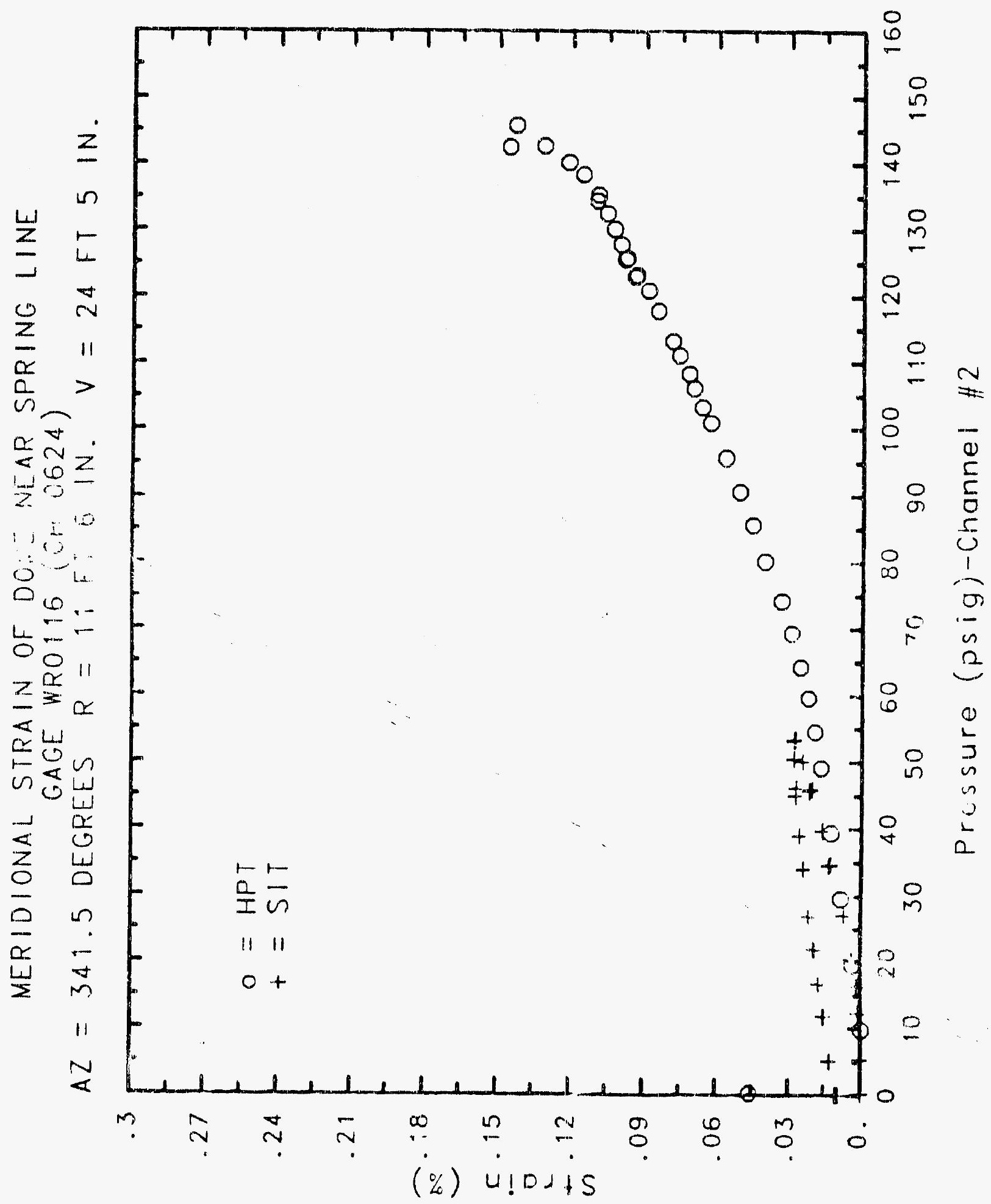




\section{Reinforced Concrete Test Data}

Weloable Gage 117 Channel 625

STRUCTURAL INTEGRITY TEST

\begin{tabular}{|c|c|c|c|}
\hline $\begin{array}{c}\text { Pressure } \\
(\text { psig) } \\
-0.05 \\
5.33 \\
12.31 \\
16.44 \\
20.51 \\
20.46 \\
20.47 \\
27.05 \\
34.69 \\
34.53 \\
34.53 \\
39.70 \\
45.90 \\
45.65 \\
45.62 \\
50.10 \\
53.47 \\
53.29 \\
53.21 \\
50.49 \\
46.14 \\
46.13 \\
44.97 \\
38.98 \\
33.99 \\
33.96 \\
26.83 \\
21.88 \\
21.90 \\
16.69 \\
11.74 \\
11.77 \\
5.05 \\
0.02 \\
-0.04 \\
-0.02 \\
-0.02 \\
0.02 \\
\end{array}$ & $\begin{array}{l}\text { \% Strain } \\
0.0001 \\
0.0008 \\
0.0019 \\
0.0029 \\
0.0040 \\
0.0044 \\
0.0046 \\
0.0080 \\
0.0142 \\
0.0152 \\
0.0153 \\
0.0185 \\
0.0241 \\
0.0256 \\
0.0259 \\
0.0294 \\
0.0323 \\
0.0333 \\
0.0339 \\
0.0334 \\
0.0323 \\
0.0323 \\
0.0324 \\
0.0307 \\
0.0290 \\
0.0289 \\
0.0263 \\
0.0239 \\
0.0240 \\
0.0220 \\
0.0194 \\
0.0197 \\
0.0166 \\
0.0147 \\
0.0139 \\
0.0134 \\
0.0135 \\
0.0134\end{array}$ & $\begin{array}{c}\text { Pressure } \\
(\text { psig) } \\
9.89 \\
19.55 \\
29.57 \\
39.42 \\
49.16 \\
54.50 \\
59.57 \\
64.20 \\
69.32 \\
74.16 \\
80.16 \\
85.61 \\
90.58 \\
95.69 \\
100.92 \\
103.25 \\
106.11 \\
108.31 \\
111.08 \\
113.24 \\
117.83 \\
120.92 \\
123.28 \\
122.97 \\
125.82 \\
125.60 \\
127.84 \\
130.19 \\
132.53 \\
135.33 \\
134.42 \\
138.35 \\
140.16 \\
142.63 \\
145.78 \\
142.52 \\
0.22 \\
\end{array}$ & $\begin{array}{c}\text { Strain } \\
-0.0005 \\
0.0042 \\
0.0091 \\
0.0134 \\
0.0182 \\
0.0213 \\
0.0245 \\
0.0282 \\
0.0320 \\
0.0366 \\
0.0436 \\
0.0490 \\
0.0540 \\
0.0597 \\
0.0655 \\
0.0687 \\
0.0713 \\
0.0739 \\
0.0767 \\
0.0794 \\
0.0844 \\
0.0880 \\
0.0918 \\
0.0927 \\
0.0952 \\
0.0949 \\
0.0966 \\
0.0993 \\
0.1016 \\
0.1040 \\
0.1040 \\
0.1070 \\
0.1089 \\
0.1125 \\
0.1171 \\
0.1172 \\
0.0221\end{array}$ \\
\hline
\end{tabular}




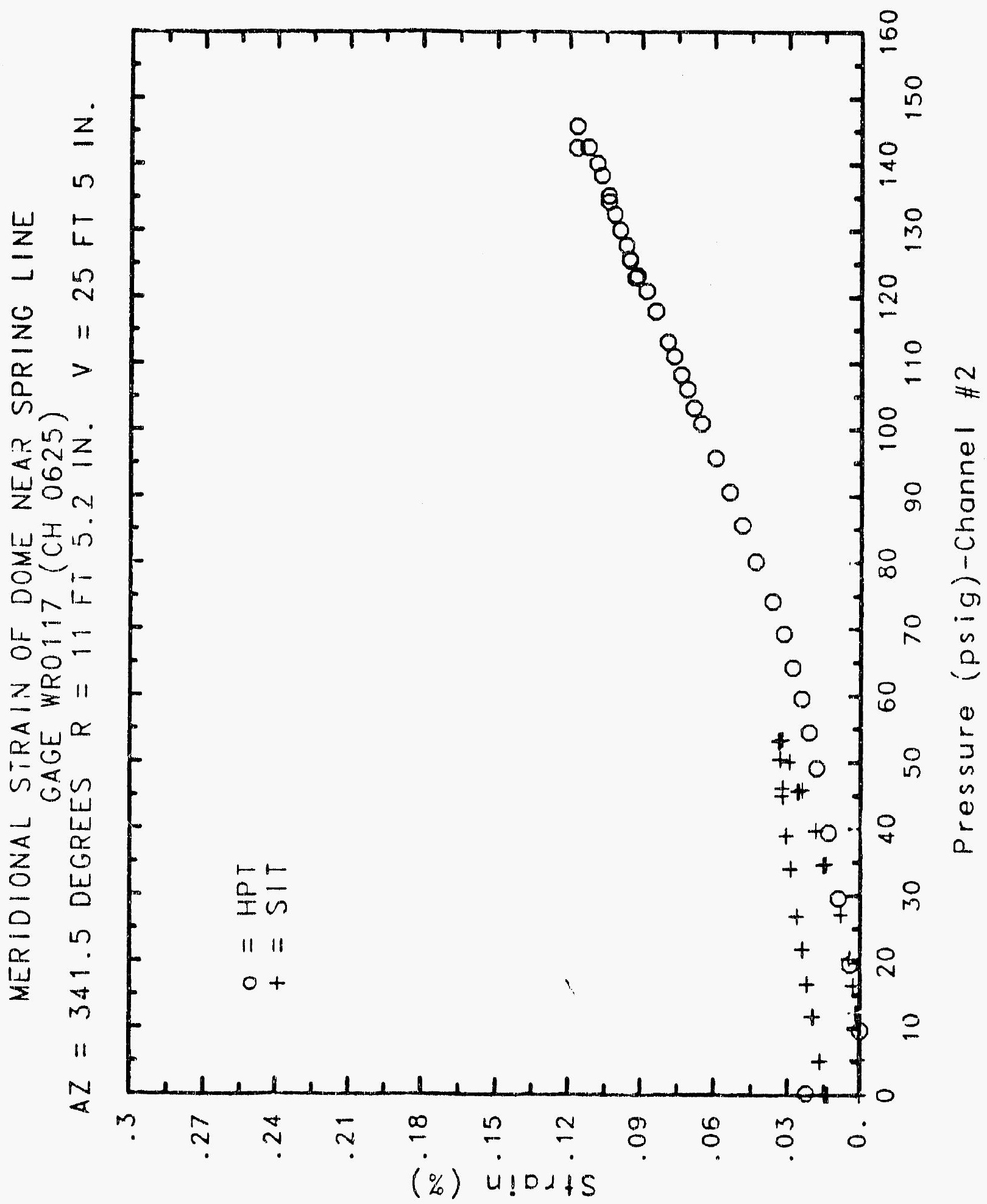




\section{Reinforced Concrete Test Data}

Weldable gage 118 Channel 626

Structural Integrity Test

High Pressure test

\begin{tabular}{|c|c|c|c|}
\hline $\begin{array}{c}\text { Pressure } \\
(\text { psig) } \\
-0.05 \\
5.33 \\
12.31 \\
16.44 \\
20.51 \\
20.46 \\
20.47 \\
27.05 \\
34.69 \\
34.53 \\
34.53 \\
39.70 \\
45.90 \\
45.65 \\
45.62 \\
50.10 \\
53.47 \\
53.29 \\
53.21 \\
50.49 \\
46.14 \\
46.13 \\
44.97 \\
38.98 \\
33.99 \\
33.96 \\
26.83 \\
21.88 \\
21.90 \\
16.69 \\
11.74 \\
11.77 \\
5.05 \\
0.02 \\
-0.04 \\
-0.02 \\
-0.02 \\
0.02\end{array}$ & $\begin{array}{l}\text { \% Strain } \\
\\
0.0002 \\
0.0000 \\
0.0001 \\
0.0000 \\
-0.0006 \\
-0.0005 \\
-0.0004 \\
-0.0003 \\
-0.0005 \\
-0.0002 \\
-0.0003 \\
-0.0006 \\
-0.0006 \\
-0.0010 \\
-0.0005 \\
-0.0009 \\
-0.0011 \\
-0.0009 \\
-0.0009 \\
-0.0007 \\
-0.0009 \\
-0.0005 \\
0.0001 \\
-0.0001 \\
-0.0001 \\
0.0000 \\
0.0002 \\
-0.0002 \\
-0.0005 \\
-0.0004 \\
-0.0002 \\
-0.0001 \\
-0.0003 \\
-0.0001 \\
-0.0017 \\
-0.0008 \\
-0.0005 \\
-0.0005\end{array}$ & $\begin{array}{c}\text { Pressure } \\
\text { (psig) } \\
9.89 \\
19.55 \\
29.57 \\
39.42 \\
49.16 \\
54.50 \\
59.57 \\
64.20 \\
69.32 \\
74.16 \\
80.16 \\
85.61 \\
90.58 \\
95.69 \\
100.92 \\
103.25 \\
106.11 \\
108.31 \\
111.08 \\
113.24 \\
117.83 \\
120.92 \\
123.28 \\
122.97 \\
125.82 \\
125.60 \\
127.84 \\
130.19 \\
132.53 \\
135.33 \\
134.42 \\
138.35 \\
140.16 \\
142.63 \\
145.78 \\
142.52 \\
0.22\end{array}$ & $\begin{array}{l}\% \text { strain } \\
-0.0044 \\
-0.0044 \\
-0.0049 \\
-0.0048 \\
-0.0047 \\
-0.0048 \\
-0.0049 \\
-0.0052 \\
-0.0054 \\
-0.0055 \\
-0.0055 \\
-0.0058 \\
-0.0061 \\
-0.0060 \\
-0.0081 \\
-0.0087 \\
-0.0086 \\
-0.0088 \\
-0.0088 \\
-0.0093 \\
-0.0093 \\
-0.0101 \\
-0.0101 \\
-0.0105 \\
-0.0104 \\
-0.0098 \\
-0.0098 \\
-0.0098 \\
-0.0098 \\
-0.0094 \\
-0.0090 \\
-0.0091 \\
-0.0084 \\
-0.0079 \\
-0.0066 \\
-0.0061 \\
-0.0093\end{array}$ \\
\hline
\end{tabular}




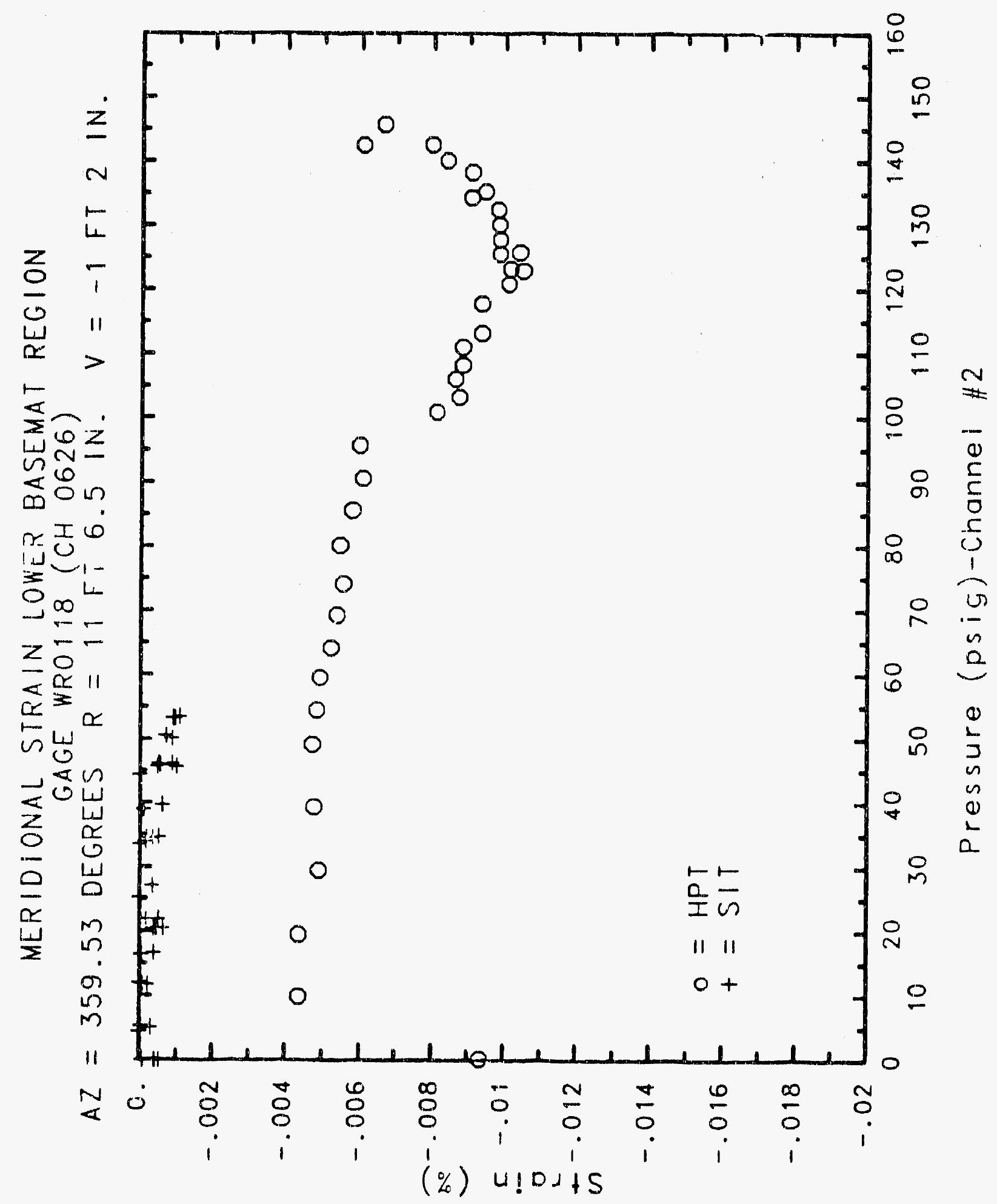




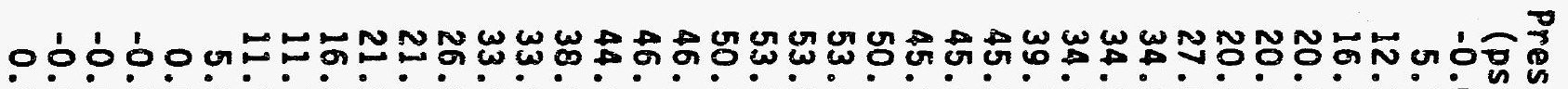

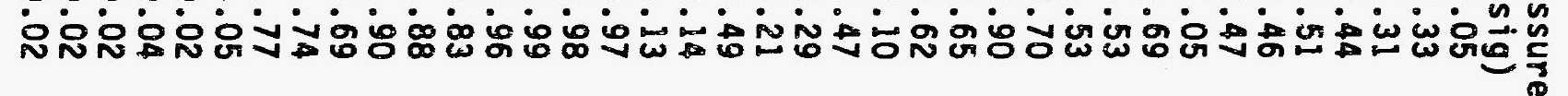

00000000000000000000000000000000000000

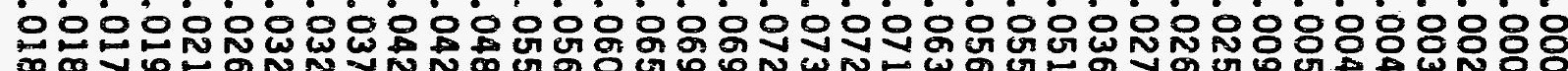

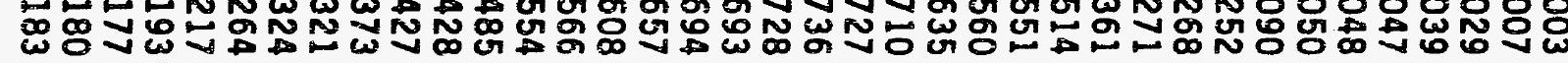

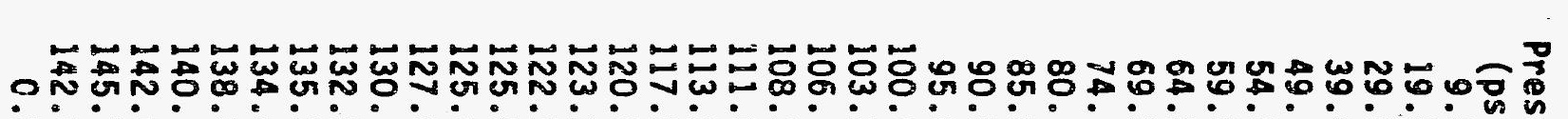
N

0000000000000000000000000000000000000 웅

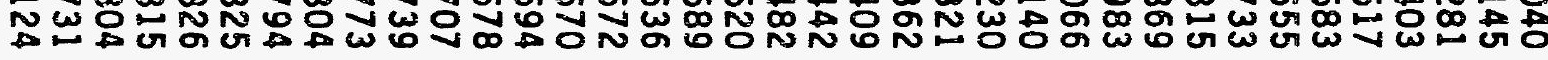

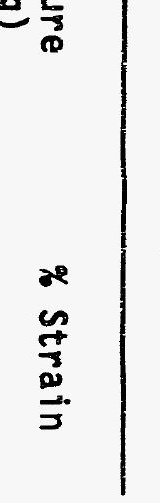




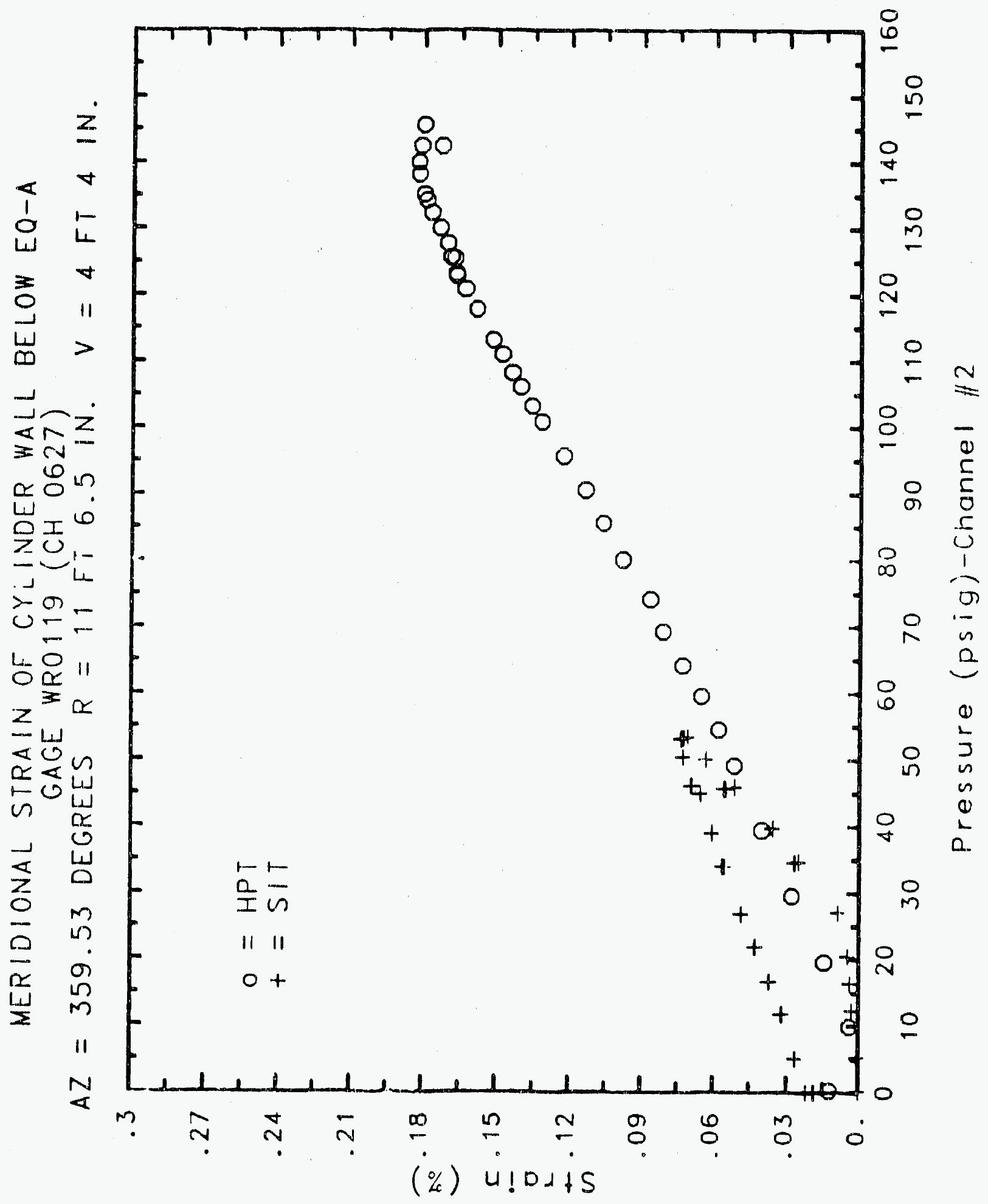




\section{Reinforced Concrete Test Data}

Weldable gage 120 Chanmel 628

Structural Integrity Test

High Pressure Test

\begin{tabular}{|c|c|c|c|}
\hline $\begin{array}{c}\text { Pressure } \\
\text { (psig) } \\
-0.05 \\
5.33 \\
12.31 \\
16.44 \\
20.51 \\
20.46 \\
20.47 \\
27.05 \\
34.69 \\
34.53 \\
34.53 \\
39.70 \\
45.90 \\
45.65 \\
45.62 \\
50.10 \\
53.47 \\
53.29 \\
53.21 \\
50.49 \\
46.14 \\
46.13 \\
44.97 \\
38.98 \\
33.99 \\
33.96 \\
26.83 \\
21.88 \\
21.90 \\
16.69 \\
11.74 \\
11.77 \\
5.05 \\
0.02 \\
-0.04 \\
-0.02 \\
-0.02 \\
0.02 \\
\end{array}$ & $\begin{array}{l}\text { \% Strain } \\
\text {-0.0001 } \\
0.0024 \\
0.0067 \\
0.0095 \\
0.0134 \\
0.0130 \\
0.0135 \\
0.0245 \\
0.0405 \\
0.0416 \\
0.0416 \\
0.0488 \\
0.0600 \\
0.0614 \\
0.0618 \\
0.0683 \\
0.0738 \\
0.0743 \\
0.0749 \\
0.0733 \\
0.0697 \\
0.0695 \\
0.0667 \\
0.0617 \\
0.0571 \\
0.0565 \\
0.0490 \\
0.0436 \\
0.0435 \\
0.0373 \\
0.0314 \\
0.0318 \\
0.0242 \\
0.0177 \\
0.0158 \\
0.0147 \\
0.0141 \\
0.0151\end{array}$ & $\begin{array}{c}\text { Pressure } \\
\text { (psig) } \\
9.89 \\
19.55 \\
29.57 \\
39.42 \\
49.16 \\
54.50 \\
59.57 \\
64.20 \\
69.32 \\
74.16 \\
80.16 \\
85.61 \\
90.58 \\
95.69 \\
100.92 \\
103.25 \\
106.11 \\
108.31 \\
111.08 \\
113.24 \\
117.83 \\
120.92 \\
123.28 \\
122.97 \\
125.82 \\
125.60 \\
127.84 \\
130.19 \\
132.53 \\
135.33 \\
134.42 \\
138.35 \\
140.16 \\
142.63 \\
145.78 \\
142.52 \\
0.22 \\
\end{array}$ & $\begin{array}{l}\text { \% Strain } \\
0.0086 \\
0.0216 \\
0.0345 \\
0.0464 \\
0.0577 \\
0.0639 \\
0.0708 \\
0.0777 \\
0.0852 \\
0.0905 \\
0.0993 \\
0.1067 \\
0.1129 \\
0.1207 \\
0.1285 \\
0.1325 \\
0.1361 \\
0.1394 \\
0.1433 \\
0.1468 \\
0.1535 \\
0.1581 \\
0.1615 \\
0.1617 \\
0.1653 \\
0.1649 \\
0.1681 \\
0.1720 \\
0.1762 \\
0.1813 \\
0.1811 \\
0.1866 \\
0.1876 \\
0.1880 \\
0.1871 \\
0.1821 \\
0.0129\end{array}$ \\
\hline
\end{tabular}




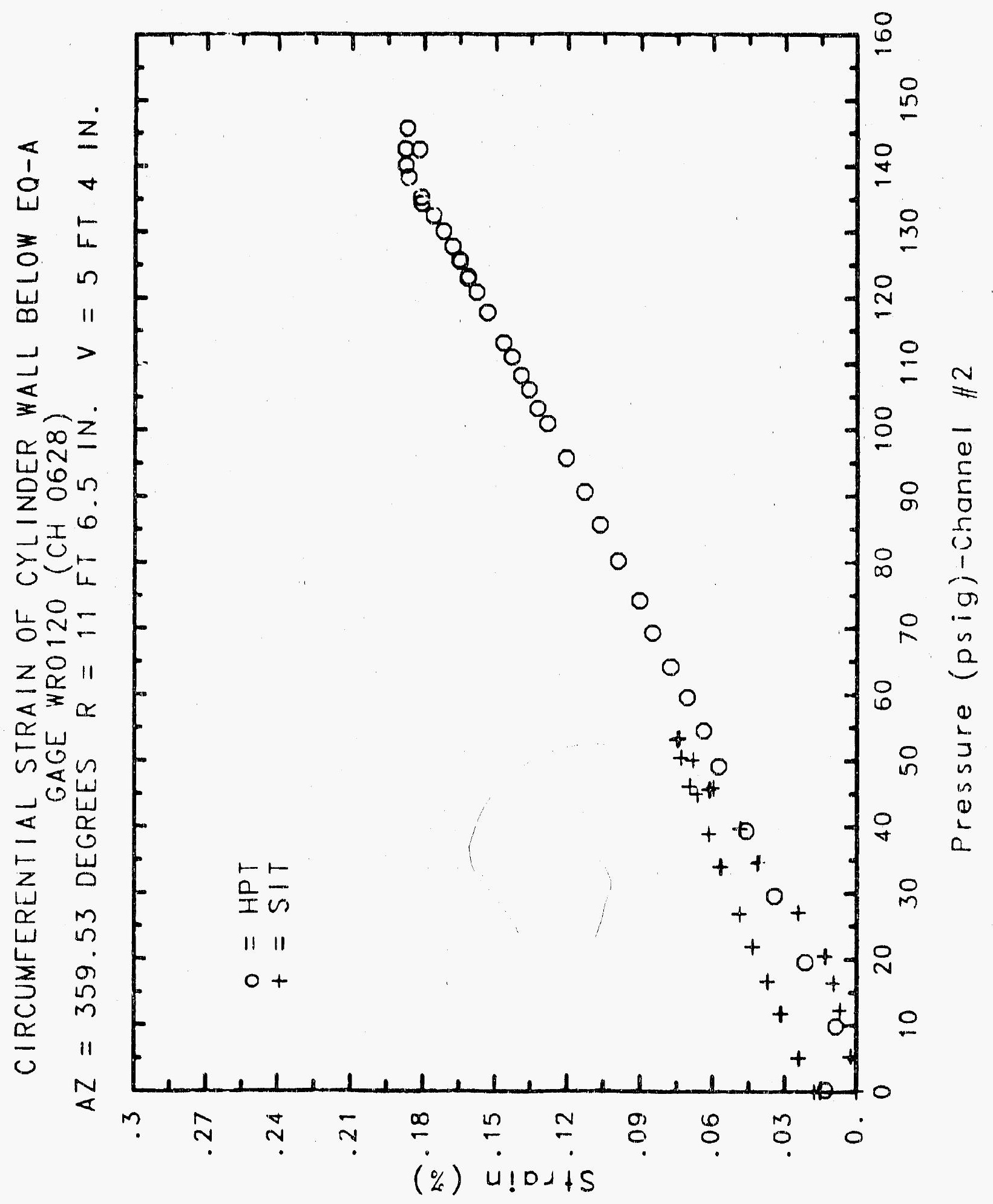




\section{Reinforced Concrete Test Data}

\begin{tabular}{|c|c|c|c|}
\hline STRUCTURAL & $\begin{array}{l}\text { WeLdABLE GAGE } 121 \\
\text { INTEGRITY TEST }\end{array}$ & $\begin{array}{l}\text { Channel } 629 \\
\text { High Pressure }\end{array}$ & TEST \\
\hline $\begin{array}{c}\text { Pressure } \\
\text { (psig) } \\
-0.05 \\
5.33 \\
12.31 \\
16.44 \\
20.51 \\
20.46 \\
20.47 \\
27.05 \\
34.69 \\
34.53 \\
34.53 \\
39.70 \\
45.90 \\
45.65 \\
45.62 \\
50.10 \\
53.47 \\
53.29 \\
53.21 \\
50.49 \\
46.14 \\
46.13 \\
44.97 \\
38.98 \\
33.99 \\
33.96 \\
26.83 \\
21.88 \\
21.90 \\
16.69 \\
11.74 \\
11.77 \\
5.05 \\
0.02 \\
-0.04 \\
-0.02 \\
-0.02 \\
0.02 \\
\end{array}$ & $\begin{array}{l}\text { X Strain } \\
\\
0.0000 \\
0.0023 \\
0.0044 \\
0.0060 \\
0.0085 \\
0.0089 \\
0.0091 \\
0.0142 \\
0.0207 \\
0.0205 \\
0.0201 \\
0.0230 \\
0.0266 \\
0.0258 \\
0.0258 \\
0.0281 \\
0.0298 \\
0.0300 \\
0.0300 \\
0.0288 \\
0.0267 \\
0.0266 \\
0.0302 \\
0.0276 \\
0.0250 \\
0.0244 \\
0.0204 \\
0.0175 \\
0.0177 \\
0.0145 \\
0.0116 \\
0.0109 \\
0.0074 \\
0.0044 \\
0.0045 \\
0.0069 \\
0.0068 \\
0.0059\end{array}$ & $\begin{array}{c}\text { Pressure } \\
(\text { psig) } \\
9.89 \\
19.55 \\
29.57 \\
39.42 \\
49.16 \\
54.50 \\
59.57 \\
64.20 \\
69.32 \\
74.16 \\
80.16 \\
85.61 \\
90.58 \\
95.69 \\
100.92 \\
103.25 \\
106.11 \\
108.31 \\
111.08 \\
113.24 \\
117.83 \\
120.92 \\
123.28 \\
122.97 \\
125.82 \\
125.60 \\
127.84 \\
130.19 \\
132.53 \\
135.33 \\
134.42 \\
138.35 \\
140.16 \\
142.53 \\
145.78 \\
142.52 \\
0.22 \\
\end{array}$ & $\begin{array}{l}\text { \% Strain } \\
0.0023 \\
0.0079 \\
0.0130 \\
0.0183 \\
0.0227 \\
0.0252 \\
0.0273 \\
0.0303 \\
0.0333 \\
0.0360 \\
0.0377 \\
0.0415 \\
0.0455 \\
0.0497 \\
0.0546 \\
0.0567 \\
0.0596 \\
0.0616 \\
0.0642 \\
0.0663 \\
0.0701 \\
0.0731 \\
0.0753 \\
0.0763 \\
0.0797 \\
0.0823 \\
0.0858 \\
0.0918 \\
0.0979 \\
0.1064 \\
0.1076 \\
0.1167 \\
0.1230 \\
0.1315 \\
0.1406 \\
0.1439 \\
0.0428\end{array}$ \\
\hline
\end{tabular}




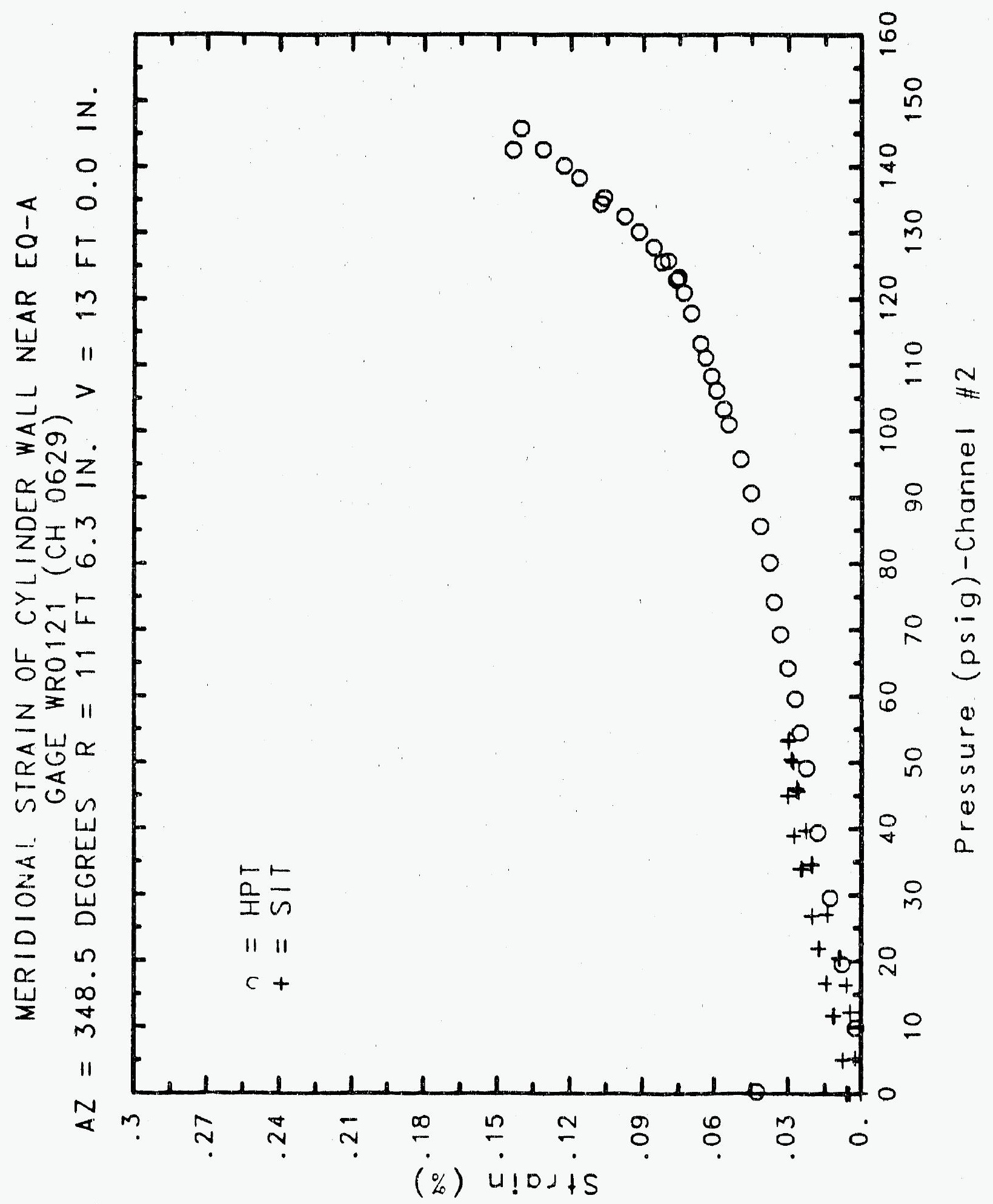




\section{Reinforced Concrete Test Data}

Weldable gage 122 Channel 640

StRUCTURAL INTEgRITY TEST

\begin{tabular}{|c|c|c|c|}
\hline $\begin{array}{c}\text { Pressure } \\
\text { (psig) } \\
-0.05 \\
5.33 \\
12.31 \\
16.44 \\
20.51 \\
20.46 \\
20.47 \\
27.05 \\
34.69 \\
34.53 \\
34.53 \\
39.70 \\
45.90 \\
45.65 \\
45.62 \\
50.10 \\
53.47 \\
53.29 \\
53.21 \\
50.49 \\
46.14 \\
46.13 \\
44.97 \\
38.98 \\
33.99 \\
33.96 \\
26.83 \\
21.88 \\
21.90 \\
16.69 \\
11.74 \\
11.77 \\
5.05 \\
0.02 \\
-0.04 \\
-0.02 \\
-0.02 \\
0.02 \\
\end{array}$ & $\begin{array}{l}\text { \% Strain } \\
0.0002 \\
0.0015 \\
0.0047 \\
0.0061 \\
0.0095 \\
0.0103 \\
0.0101 \\
0.0150 \\
0.0220 \\
0.0224 \\
0.0221 \\
0.0249 \\
0.0291 \\
0.0286 \\
0.0287 \\
0.0309 \\
0.0336 \\
0.0329 \\
0.0333 \\
0.0322 \\
0.0301 \\
0.0296 \\
0.0321 \\
0.0300 \\
0.0274 \\
0.0267 \\
0.0226 \\
0.0199 \\
0.0199 \\
0.0167 \\
0.0139 \\
0.0127 \\
0.0091 \\
0.0058 \\
0.0045 \\
0.0068 \\
0.0070 \\
0.0066\end{array}$ & $\begin{array}{c}\text { Pressure } \\
(\text { psig) } \\
9.89 \\
19.55 \\
29.57 \\
39.42 \\
49.16 \\
54.50 \\
59.57 \\
64.20 \\
69.32 \\
74.16 \\
80.16 \\
85.61 \\
90.58 \\
95.69 \\
100.92 \\
103.25 \\
106.11 \\
108.31 \\
111.08 \\
113.24 \\
117.83 \\
120.92 \\
123.28 \\
122.97 \\
125.82 \\
125.60 \\
127.84 \\
130.19 \\
132.53 \\
135.33 \\
134.42 \\
138.35 \\
140.16 \\
142.63 \\
145.78 \\
142.52 \\
0.22\end{array}$ & $\begin{array}{l}\text { \% Strain } \\
0.0029 \\
0.0093 \\
0.0157 \\
0.0216 \\
0.0269 \\
0.0297 \\
0.0326 \\
0.0359 \\
0.0397 \\
0.0431 \\
0.0451 \\
0.0494 \\
0.0534 \\
0.0586 \\
0.0638 \\
0.0653 \\
0.0689 \\
0.0716 \\
0.0754 \\
0.0779 \\
0.0851 \\
0.0902 \\
0.0958 \\
0.0971 \\
0.1030 \\
0.1057 \\
0.1092 \\
0.1156 \\
0.1236 \\
0.1341 \\
0.1359 \\
0.1481 \\
0.1580 \\
0.1712 \\
0.1843 \\
0.1889 \\
0.0670\end{array}$ \\
\hline
\end{tabular}

High Pressure test 


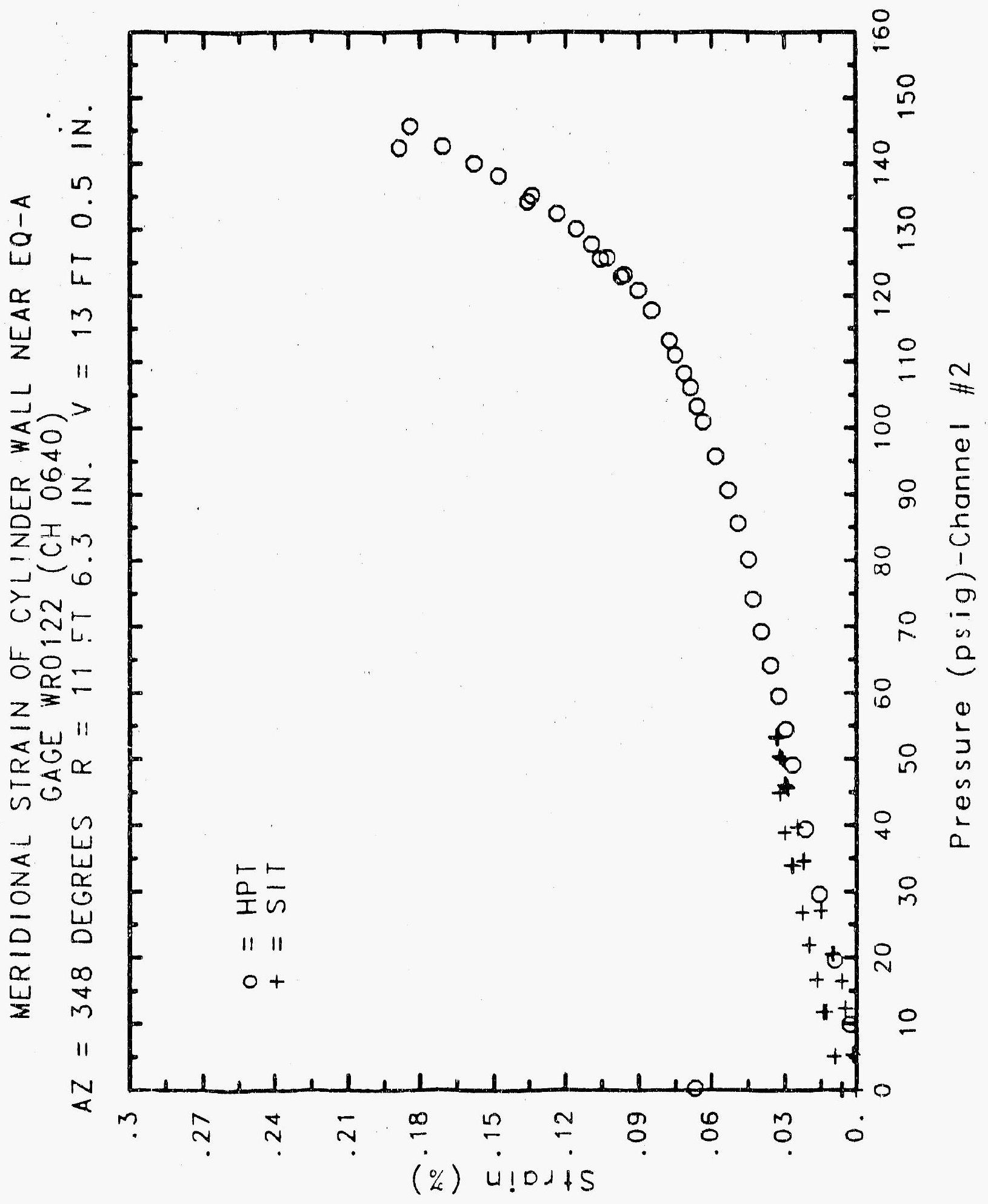




\section{Reinforced Concrete Test Data}

\begin{tabular}{|c|c|c|c|}
\hline STRUCTURAL & $\begin{array}{l}\text { Weldable Gage } 123 \\
\text { INTEgRIty TESt }\end{array}$ & $\begin{array}{l}\text { Channel } 641 \\
\text { High Pressure }\end{array}$ & TEST \\
\hline $\begin{array}{l}\text { Pressure } \\
\text { (psig) } \\
-0.05 \\
5.33 \\
12.31 \\
16.44 \\
20.51 \\
20.46 \\
20.47 \\
27.05 \\
34.69 \\
34.53 \\
34.53 \\
39.70 \\
45.90 \\
45.65 \\
45.62 \\
50.10 \\
53.47 \\
53.29 \\
53.21 \\
50.49 \\
46.14 \\
46.13 \\
44.97 \\
38.98 \\
33.99 \\
33.96 \\
26.83 \\
21.88 \\
21.90 \\
16.69 \\
11.74 \\
11.77 \\
5.05 \\
0.02 \\
-0.04 \\
-0.02 \\
-0.02 \\
0.02 \\
\end{array}$ & $\begin{array}{c}\text { \% Strain } \\
-0.0005 \\
0.0021 \\
0.0059 \\
0.0081 \\
0.0111 \\
0.0110 \\
0.0109 \\
0.0180 \\
0.0261 \\
0.0262 \\
0.0266 \\
0.0305 \\
0.0366 \\
0.0376 \\
0.0376 \\
0.0418 \\
0.0455 \\
0.0458 \\
0.0458 \\
0.0448 \\
0.0422 \\
0.0422 \\
0.0374 \\
0.0332 \\
0.0298 \\
0.0295 \\
0.0239 \\
0.0202 \\
0.0199 \\
0.0152 \\
0.0116 \\
0.0116 \\
0.0067 \\
0.0030 \\
0.0034 \\
0.0010 \\
0.0003 \\
0.0000\end{array}$ & $\begin{array}{c}\text { Pressure } \\
\text { (psig) } \\
9.89 \\
19.55 \\
29.57 \\
39.42 \\
49.16 \\
54.50 \\
59.57 \\
64.20 \\
69.32 \\
74.16 \\
80.16 \\
85.61 \\
90.58 \\
95.69 \\
100.92 \\
103.25 \\
106.11 \\
108.31 \\
111.08 \\
113.24 \\
117.83 \\
120.92 \\
123.28 \\
122.97 \\
125.82 \\
125.60 \\
127.84 \\
130.19 \\
132.53 \\
135.33 \\
134.42 \\
138.35 \\
140.16 \\
142.63 \\
145.78 \\
142.52 \\
0.22\end{array}$ & $\begin{array}{l}\text { * Strain } \\
0.0030 \\
0.0106 \\
0.0190 \\
0.0269 \\
0.0354 \\
0.0397 \\
0.0444 \\
0.0499 \\
0.0550 \\
0.0603 \\
0.0696 \\
0.0765 \\
0.0831 \\
0.0897 \\
0.0969 \\
0.0998 \\
0.1039 \\
0.1068 \\
0.1108 \\
0.1141 \\
0.1216 \\
0.1277 \\
0.1336 \\
0.1344 \\
0.1420 \\
0.1420 \\
0.1482 \\
0.1634 \\
0.1806 \\
0.1993 \\
0.2025 \\
0.2126 \\
0.2101 \\
0.2084 \\
0.2118 \\
0.2104 \\
0.0875\end{array}$ \\
\hline
\end{tabular}




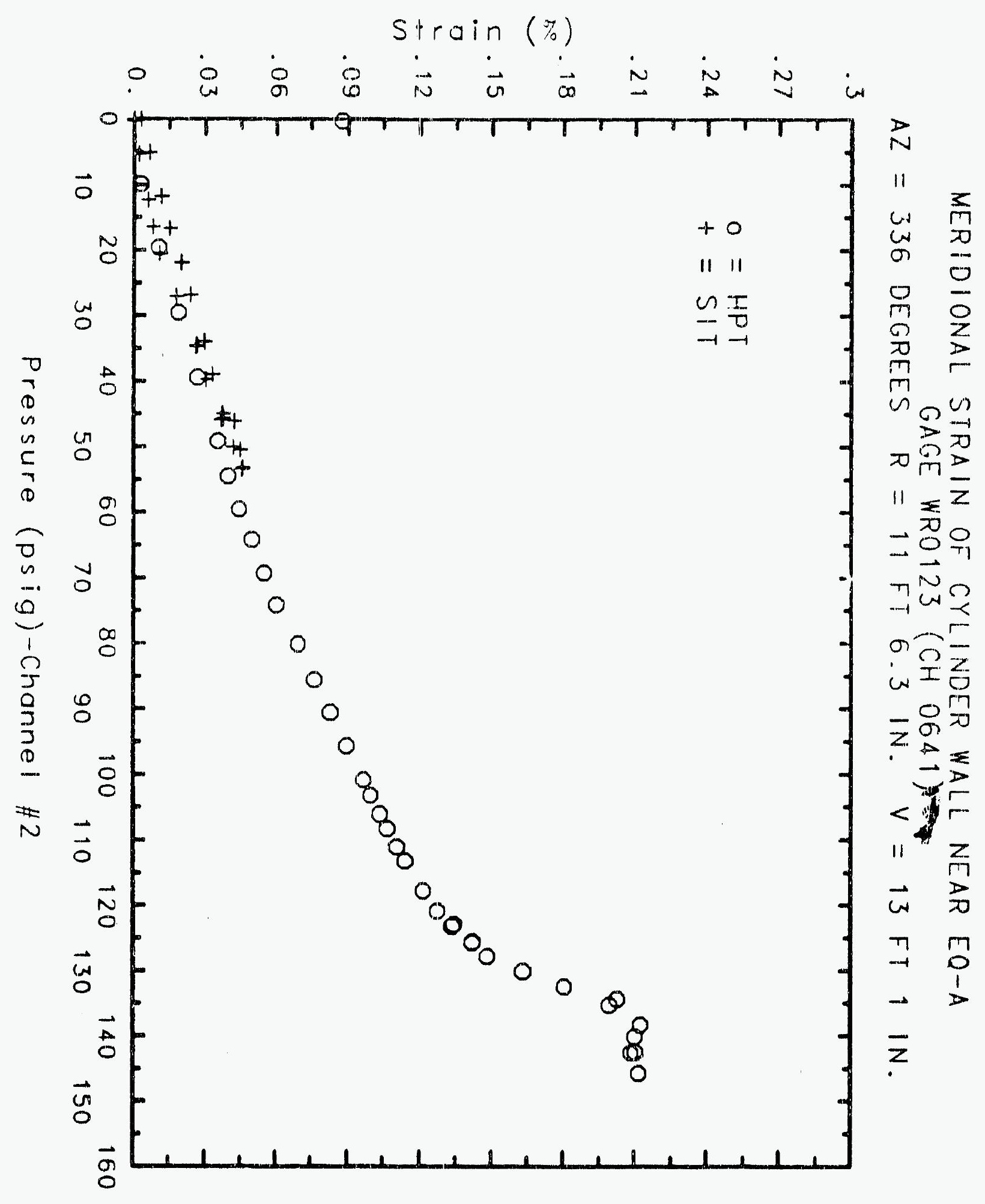




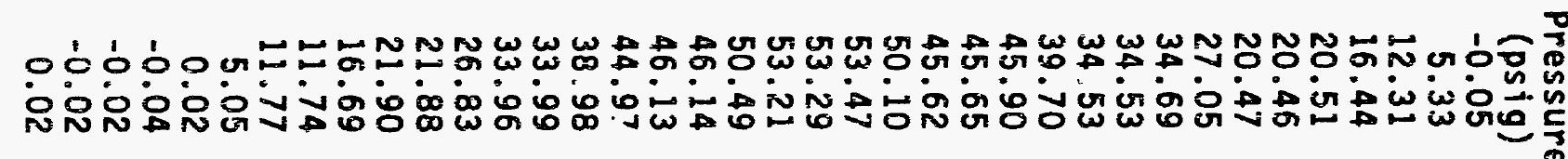

00000000000000000000000000000000000000

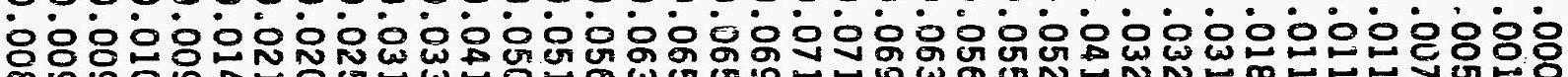
吅路。

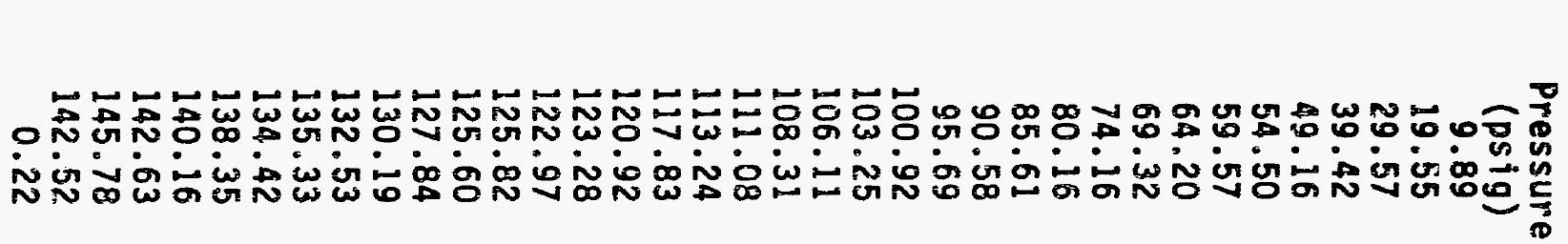

0000000000000000000000000000000000000

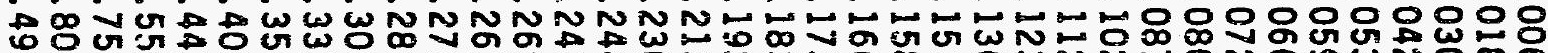

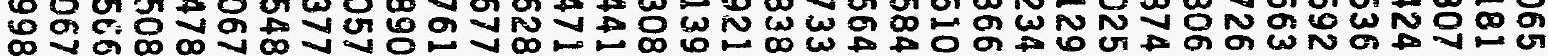

$\mid$




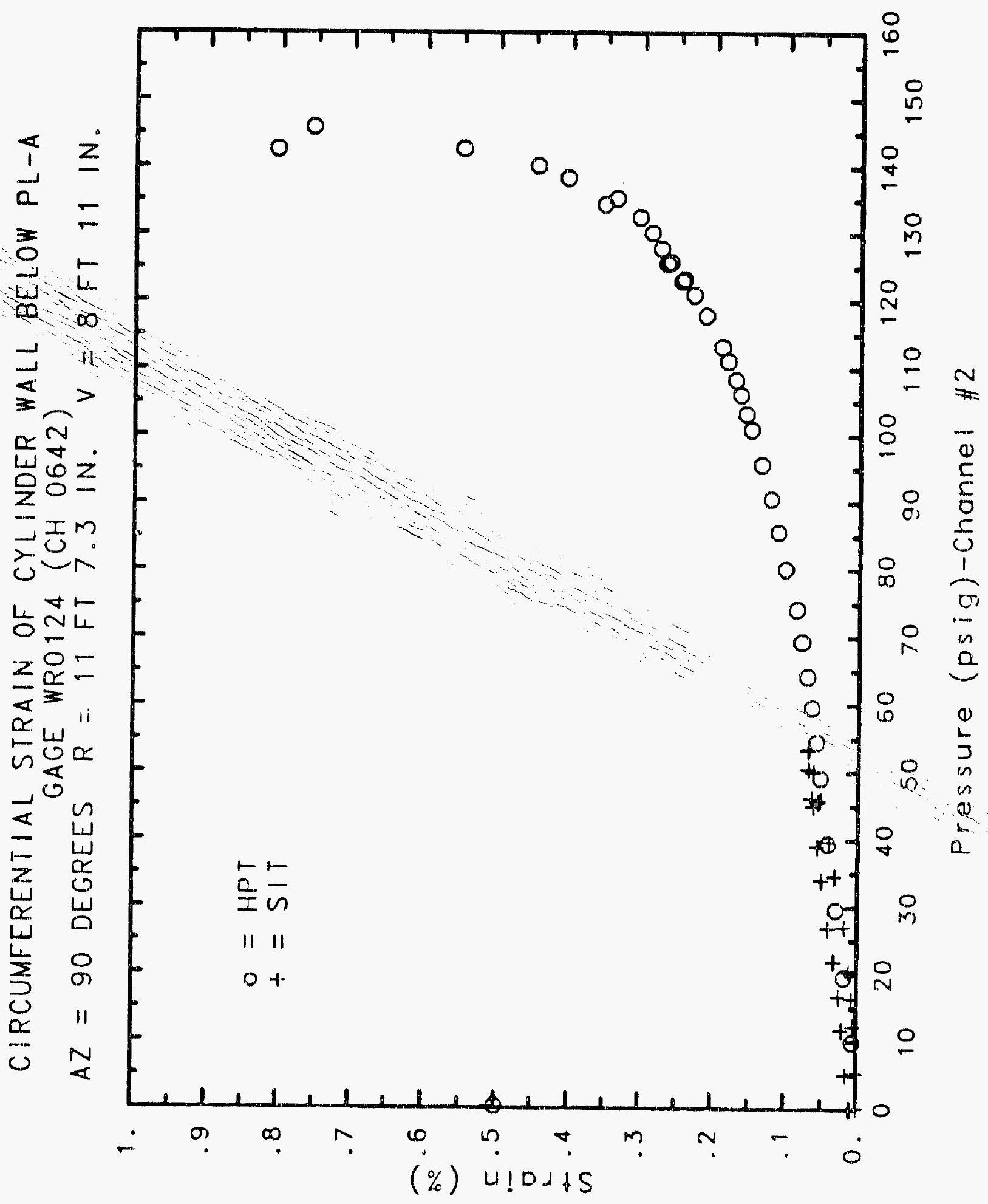




\section{Reinforced Concrete Test Data}

Weldable Gage 125 Channel 643

StRuCtURAL INTEgRITY TEST

High Pressure test

\begin{tabular}{|c|c|c|c|}
\hline $\begin{array}{c}\text { Pressure } \\
\text { (psig) } \\
-0.05 \\
5.33 \\
12.31 \\
16.44 \\
20.51 \\
20.46 \\
20.47 \\
27.05 \\
34.69 \\
34.53 \\
34.53 \\
39.70 \\
45.90 \\
45.65 \\
45.62 \\
50.10 \\
53.47 \\
53.29 \\
53.21 \\
50.49 \\
46.14 \\
46.13 \\
44.97 \\
38.98 \\
33.99 \\
33.96 \\
26.83 \\
21.88 \\
21.90 \\
16.69 \\
11.74 \\
11.77 \\
5.05 \\
0.02 \\
-0.04 \\
-0.02 \\
-0.02 \\
0.02 \\
\end{array}$ & $\begin{array}{l}\text { \% Strain } \\
0.0009 \\
0.0028 \\
0.0060 \\
0.0079 \\
0.0107 \\
0.0113 \\
0.0115 \\
0.0161 \\
0.0215 \\
0.0222 \\
0.0220 \\
0.0253 \\
0.0304 \\
0.0310 \\
0.0306 \\
0.0340 \\
0.0377 \\
0.0381 \\
0.0388 \\
0.0371 \\
0.0353 \\
0.0350 \\
0.0370 \\
0.0336 \\
0.0310 \\
0.0308 \\
0.0269 \\
0.0245 \\
0.0242 \\
0.0207 \\
0.0167 \\
0.0151 \\
0.0108 \\
0.0075 \\
0.0055 \\
0.0059 \\
0.0065 \\
0.0073\end{array}$ & $\begin{array}{c}\text { Pressure } \\
(\text { psig) } \\
9.89 \\
19.55 \\
29.57 \\
39.42 \\
49.16 \\
54.50 \\
59.57 \\
64.20 \\
69.32 \\
74.16 \\
80.16 \\
85.61 \\
90.58 \\
95.69 \\
100.92 \\
103.25 \\
106.11 \\
108.31 \\
111.08 \\
113.24 \\
117.83 \\
120.92 \\
123.28 \\
122.97 \\
125.82 \\
125.60 \\
127.84 \\
130.19 \\
132.53 \\
135.33 \\
134.42 \\
138.35 \\
140.16 \\
142.63 \\
145.78 \\
142.52 \\
0.22 \\
\end{array}$ & $\begin{array}{l}\text { \% Strain } \\
0.0031 \\
0.0101 \\
0.0169 \\
0.0235 \\
0.0303 \\
0.0337 \\
0.0379 \\
0.0430 \\
0.0479 \\
0.0540 \\
0.0597 \\
0.0665 \\
0.0725 \\
0.0800 \\
0.0873 \\
0.0912 \\
0.0960 \\
0.0997 \\
0.1048 \\
0.1088 \\
0.1187 \\
0.1266 \\
0.1333 \\
0.1348 \\
0.1428 \\
0.1449 \\
0.1498 \\
0.1591 \\
0.1713 \\
0.1863 \\
0.1899 \\
0.2090 \\
0.2237 \\
0.2444 \\
0.2646 \\
0.2723 \\
0.1311\end{array}$ \\
\hline
\end{tabular}




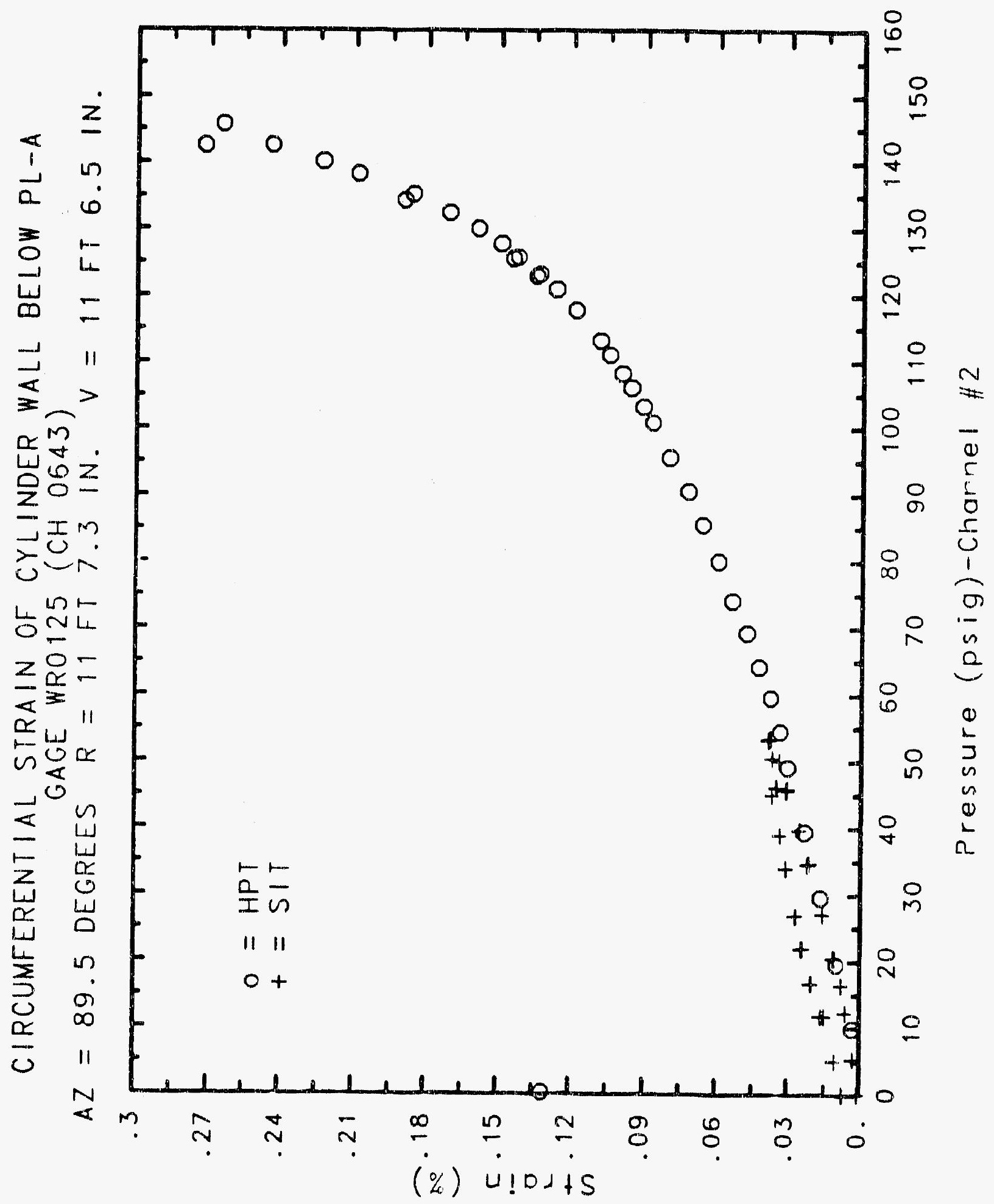

C. 1058 


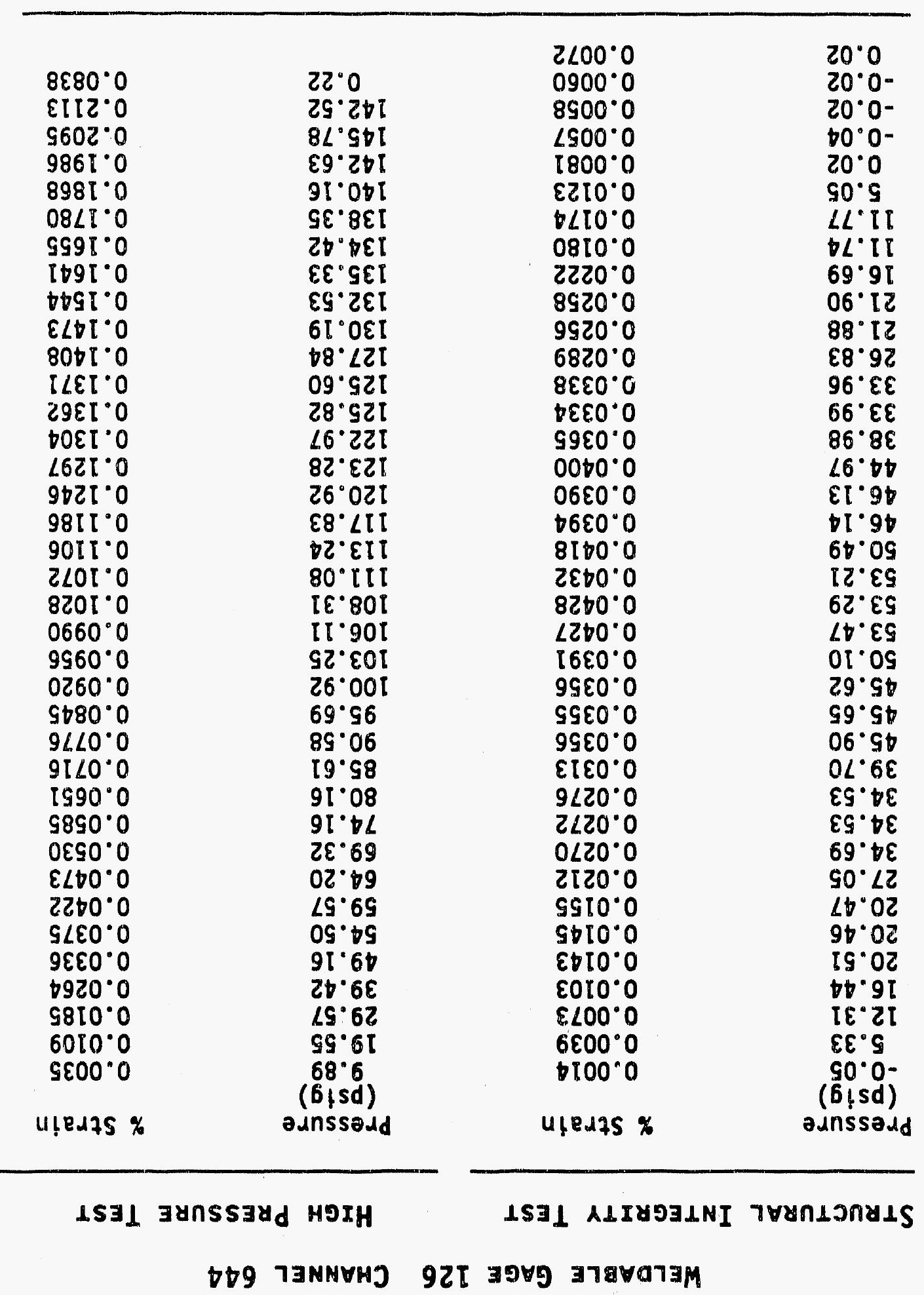

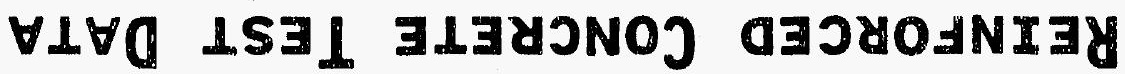




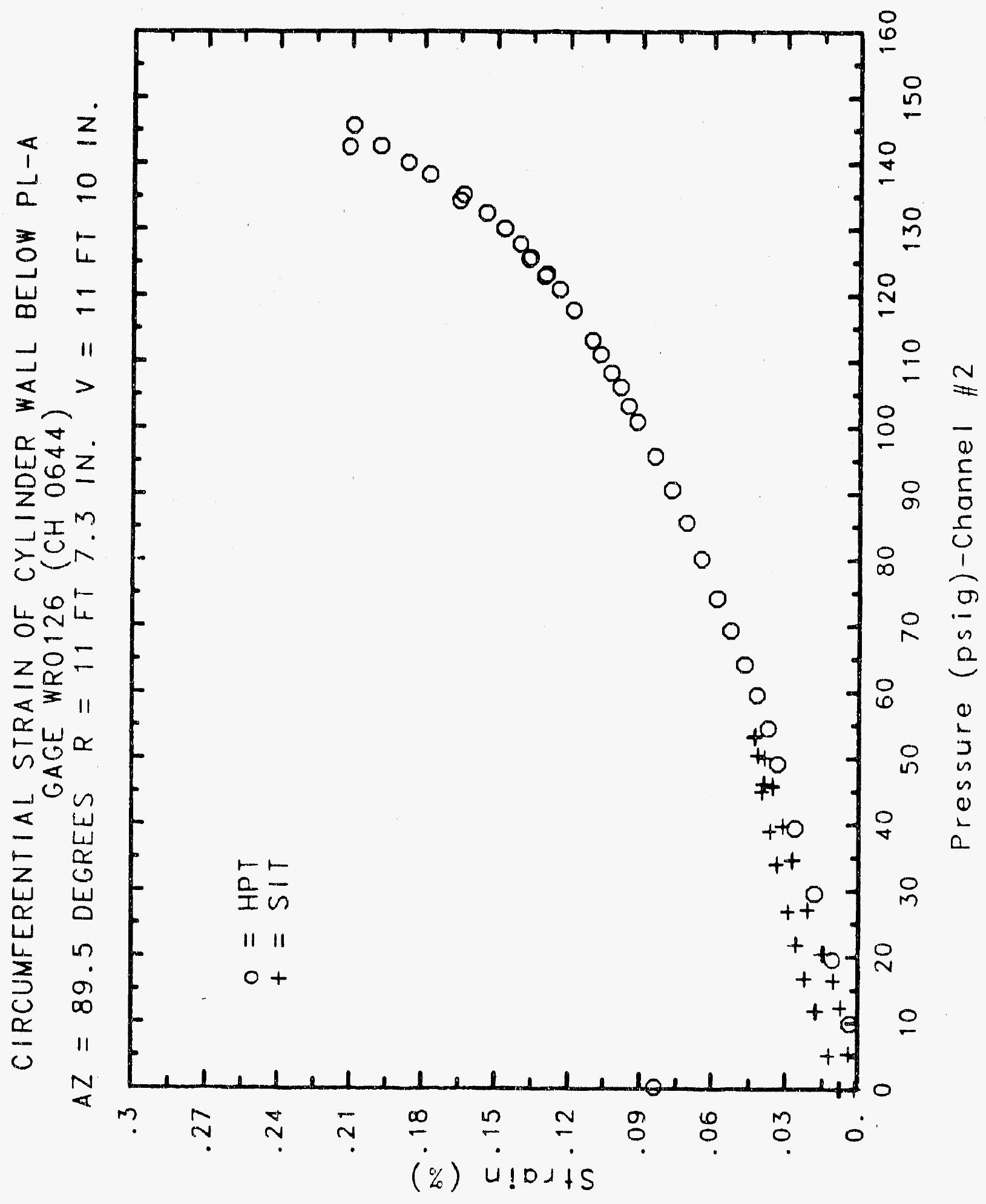




\section{Reinforced Concrete Test Data}

Weldable Gage 127 Channel 645

Structural InTEgrity Test

\begin{tabular}{|c|c|c|c|}
\hline $\begin{array}{c}\text { Pressure } \\
\text { (psig) } \\
-0.05 \\
5.33 \\
12.31 \\
16.44 \\
20.51 \\
20.46 \\
20.47 \\
27.05 \\
34.69 \\
34.53 \\
34.53 \\
39.70 \\
45.90 \\
45.65 \\
45.62 \\
50.10 \\
53.47 \\
53.29 \\
53.21 \\
50.49 \\
46.14 \\
46.13 \\
44.97 \\
38.98 \\
33.99 \\
33.96 \\
26.83 \\
21.88 \\
21.90 \\
16.69 \\
11.74 \\
11.77 \\
5.05 \\
0.02 \\
-0.04 \\
-0.02 \\
-0.02 \\
0.02 \\
\end{array}$ & $\begin{array}{l}\text { \% Strain } \\
0.0009 \\
0.0019 \\
0.0047 \\
0.0068 \\
0.0105 \\
0.0105 \\
0.0109 \\
0.0204 \\
0.0328 \\
0.0322 \\
0.0326 \\
0.0385 \\
0.0487 \\
0.0525 \\
0.0533 \\
0.0595 \\
0.0641 \\
0.0655 \\
0.0659 \\
0.0644 \\
0.0611 \\
0.0612 \\
0.0578 \\
0.0526 \\
0.0487 \\
0.0479 \\
0.0414 \\
0.0368 \\
0.0356 \\
0.0302 \\
0.0245 \\
0.0256 \\
0.0200 \\
0.0158 \\
0.0153 \\
0.0140 \\
0.0131 \\
0.0131\end{array}$ & $\begin{array}{c}\text { Pressure } \\
(\text { psig) } \\
9.89 \\
19.55 \\
29.57 \\
39.42 \\
49.16 \\
54.50 \\
59.57 \\
64.20 \\
69.32 \\
74.16 \\
80.16 \\
85.61 \\
90.58 \\
95.69 \\
100.92 \\
103.25 \\
106.11 \\
108.31 \\
111.08 \\
113.24 \\
117.83 \\
120.92 \\
123.28 \\
122.97 \\
125.82 \\
125.60 \\
127.84 \\
130.19 \\
132.53 \\
135.33 \\
134.42 \\
138.35 \\
140.16 \\
142.63 \\
145.78 \\
142.52 \\
0.22 \\
\end{array}$ & $\begin{array}{l}\text { Strain } \\
0.0055 \\
0.0158 \\
0.0267 \\
0.0373 \\
0.0473 \\
0.0531 \\
0.0588 \\
0.0645 \\
0.0707 \\
0.0767 \\
0.0879 \\
0.0951 \\
0.1018 \\
0.1087 \\
0.1154 \\
0.1190 \\
0.1231 \\
0.1262 \\
0.1303 \\
0.1344 \\
0.1427 \\
0.1480 \\
0.1532 \\
0.1533 \\
0.1594 \\
0.1600 \\
0.1645 \\
0.1725 \\
0.1851 \\
0.2039 \\
0.2077 \\
0.2361 \\
0.2596 \\
0.3143 \\
0.44069 \\
0.4467 \\
0.2585\end{array}$ \\
\hline
\end{tabular}

high Pressure test

Pressure

9.89

39.42

59.57

69.32

74.16

80.16

95.69

106.11

108.31

117.83

125.82

125.60

130.19

132.53

135.33

134.42

142.63

145.78

2.52

0.22
0.0055

0.0158

0.0267

0.0373

0.0473

0.0588

0.0645

0.0707

0.0767

0.0879

0.0951

0.1018

0.1154

0.1190

0.1231

0.1262

0.1427

0.1480

0.1532

0.1533

0.1594

0.1725

0.1851

0.2039

0.2077

0.2361

0.2596

0.4059

0.4467

0.2585 


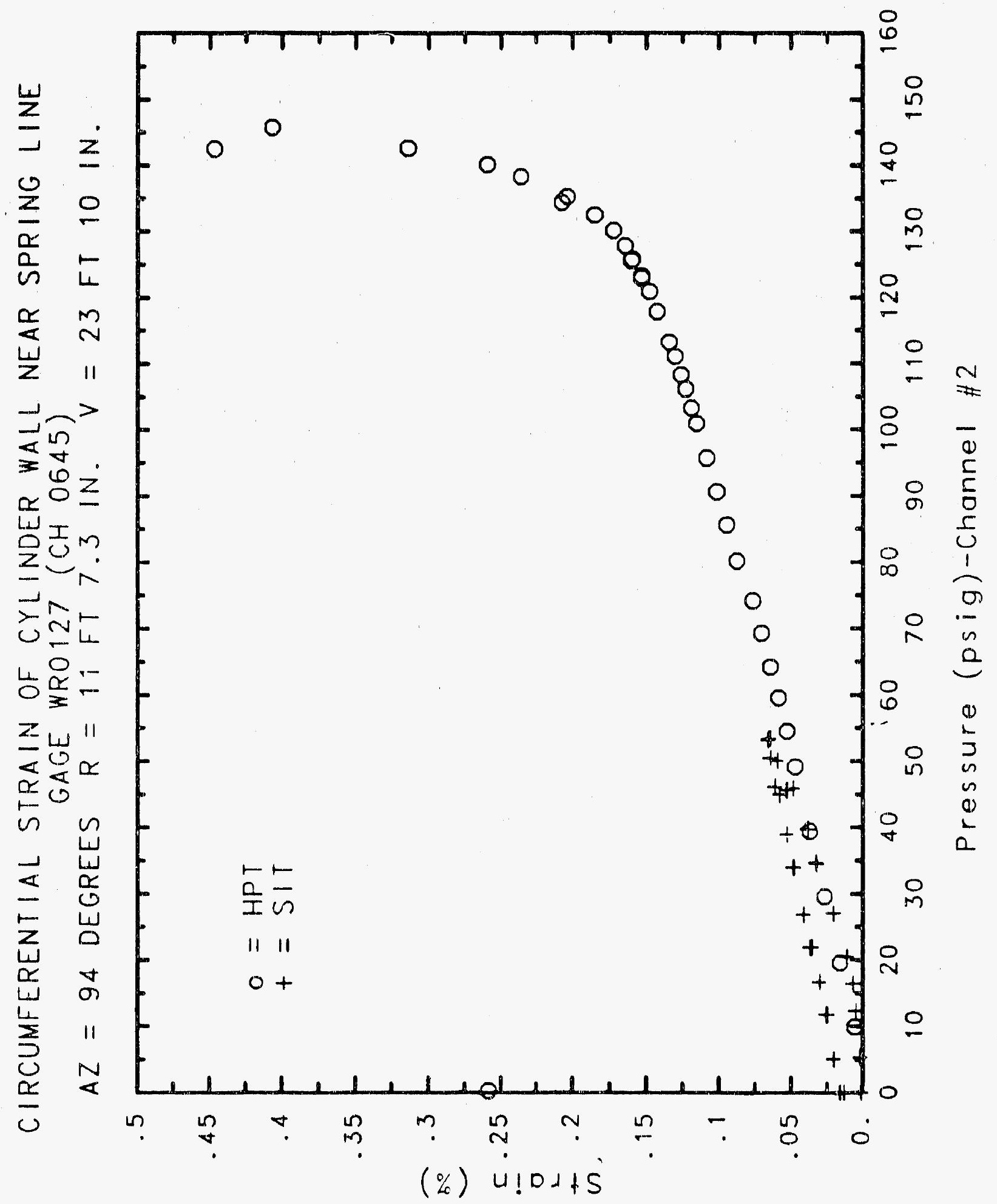




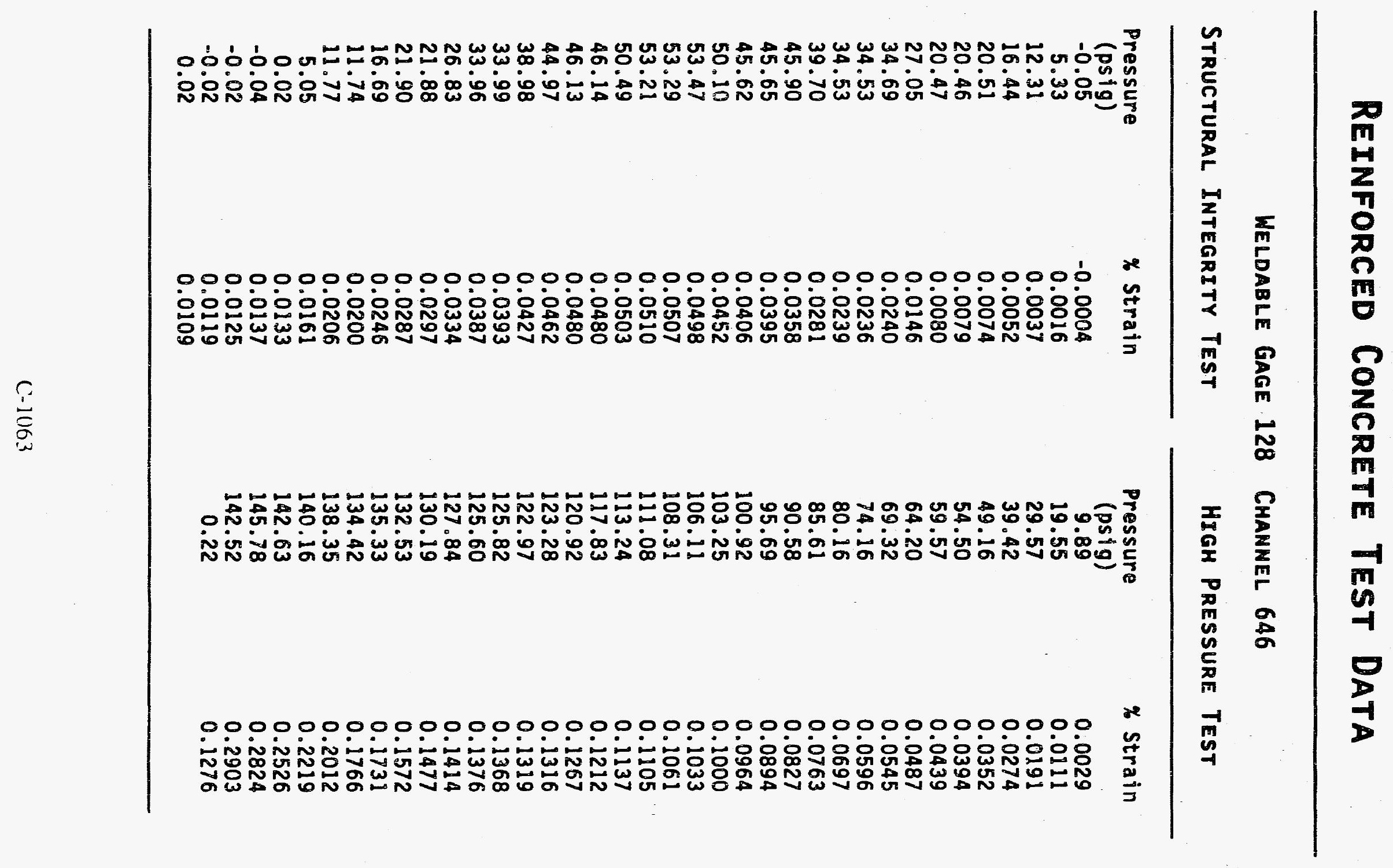




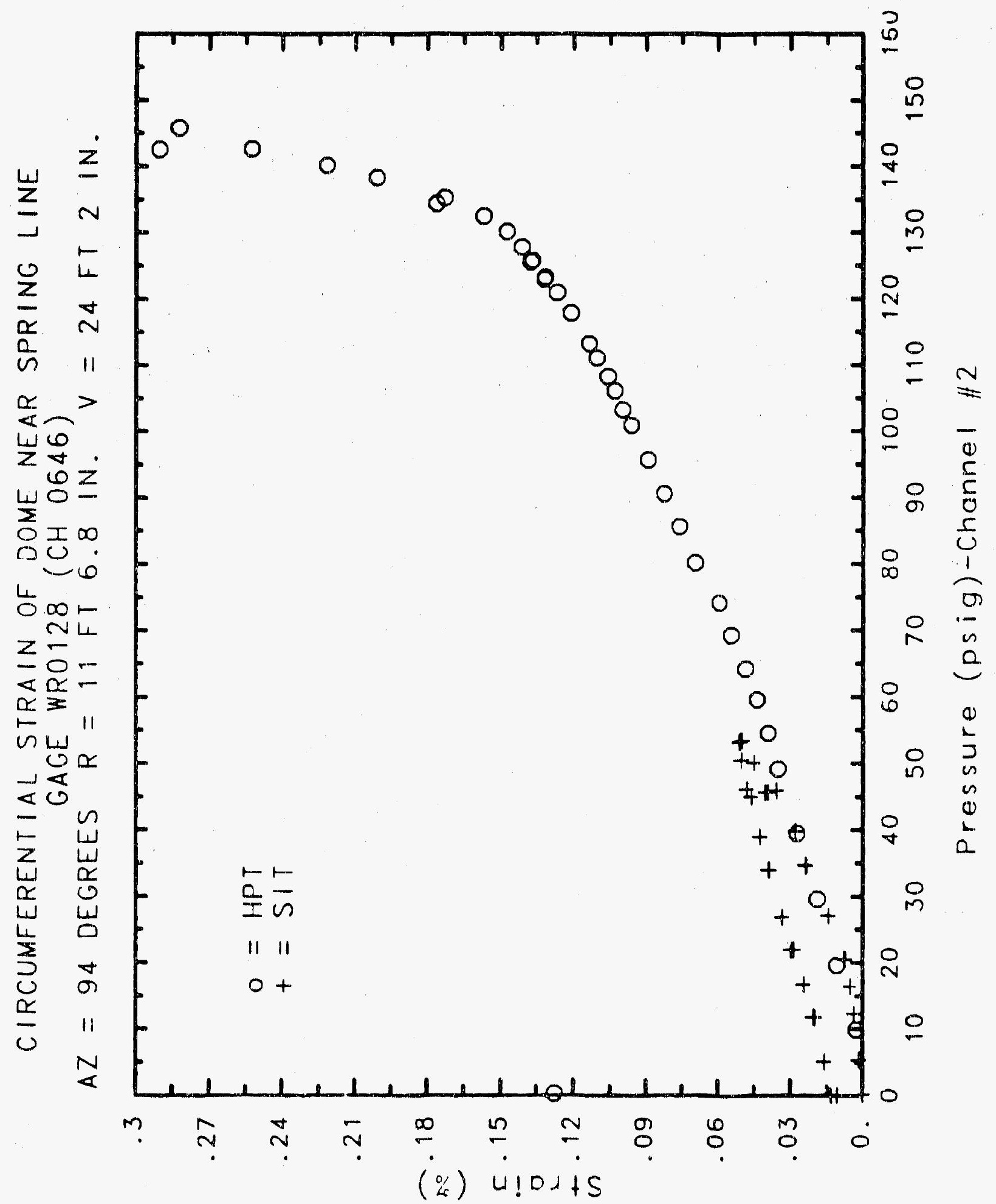




\section{Reinforced Concrete Test Data}

Weldable Gage 129 Channel 647

STRUCTURAL INTEGRITY TEST

high Pressure test

\begin{tabular}{|c|c|c|c|}
\hline $\begin{array}{c}\text { Pressure } \\
\text { (psig) } \\
-0.05 \\
5.33 \\
12.31 \\
16.44 \\
20.51 \\
20.46 \\
20.47 \\
27.05 \\
34.69 \\
34.53 \\
34.53 \\
39.70 \\
45.90 \\
45.65 \\
45.62 \\
50.10 \\
53.47 \\
53.29 \\
53.21 \\
50.49 \\
46.14 \\
46.13 \\
44.97 \\
38.98 \\
33.99 \\
33.96 \\
26.83 \\
21.88 \\
21.90 \\
16.69 \\
11.74 \\
11.77 \\
5.05 \\
0.02 \\
-0.04 \\
-0.02 \\
-0.02 \\
0.02 \\
\end{array}$ & $\begin{array}{l}\text { \% Strain } \\
0.0000 \\
0.0019 \\
0.0036 \\
0.0056 \\
0.0107 \\
0.0116 \\
0.0124 \\
0.0229 \\
0.0343 \\
0.0551 \\
0.0354 \\
0.0407 \\
0.0524 \\
0.0532 \\
0.0528 \\
0.0585 \\
0.0635 \\
0.0640 \\
0.0641 \\
0.0630 \\
0.0594 \\
0.0589 \\
0.0586 \\
0.0542 \\
0.0495 \\
0.0493 \\
0.0421 \\
0.0377 \\
0.0374 \\
0.0326 \\
0.0278 \\
0.0267 \\
0.0201 \\
0.0158 \\
0.0150 \\
0.0151 \\
0.0149 \\
0.0151\end{array}$ & $\begin{array}{c}\text { Pressure } \\
(\text { psig) } \\
9.89 \\
19.55 \\
29.57 \\
39.42 \\
49.16 \\
54.50 \\
59.57 \\
64.20 \\
69.32 \\
74.16 \\
80.16 \\
85.61 \\
90.58 \\
95.69 \\
100.92 \\
103.25 \\
106.11 \\
108.31 \\
111.08 \\
113.24 \\
117.83 \\
120.92 \\
123.28 \\
122.97 \\
125.82 \\
125.60 \\
127.84 \\
130.19 \\
132.53 \\
135.33 \\
134.42 \\
138.35 \\
140.16 \\
142.63 \\
145.78 \\
142.52 \\
0.22 \\
\end{array}$ & $\begin{array}{l}\text { Strain } \\
0.0048 \\
0.0157 \\
0.0272 \\
0.0378 \\
0.0487 \\
0.0543 \\
0.0609 \\
0.0680 \\
0.0757 \\
0.0843 \\
0.0935 \\
0.1029 \\
0.1122 \\
0.1218 \\
0.1329 \\
0.1385 \\
0.1438 \\
0.1482 \\
0.1545 \\
0.1596 \\
0.1702 \\
0.1781 \\
0.1859 \\
0.1871 \\
0.1943 \\
0.1962 \\
0.2003 \\
0.2070 \\
0.2134 \\
0.2216 \\
0.2233 \\
0.2327 \\
0.2394 \\
0.2480 \\
0.2572 \\
0.2571 \\
0.0606\end{array}$ \\
\hline
\end{tabular}




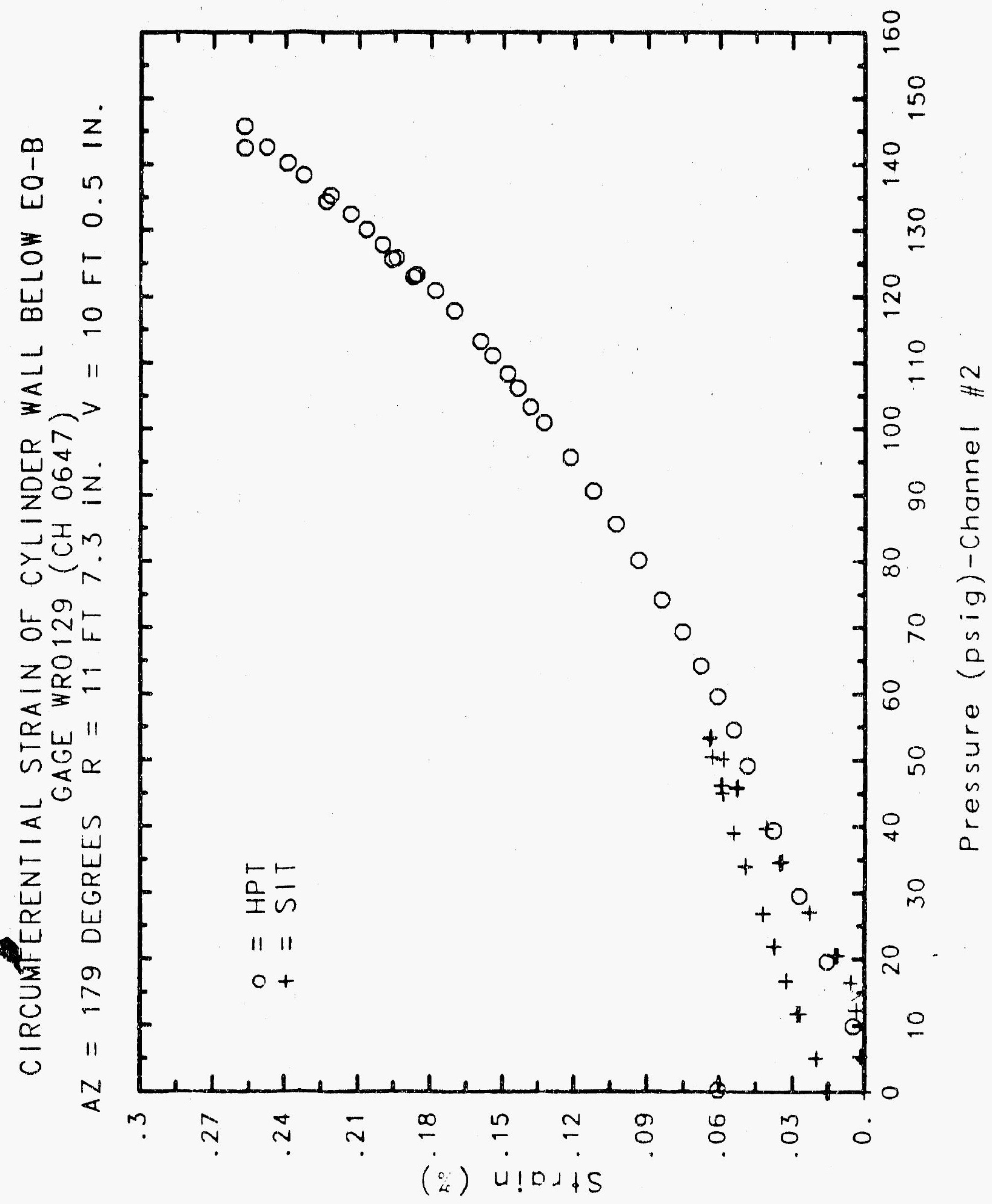

C 1066 


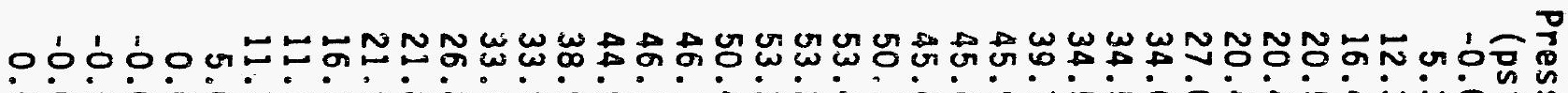

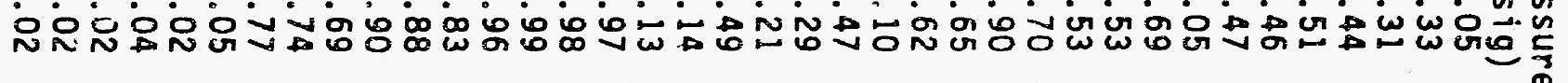

00000000000000000000000000000000000000

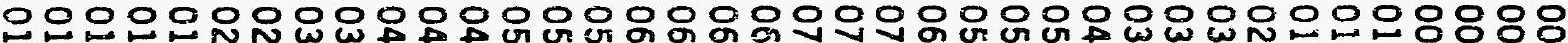

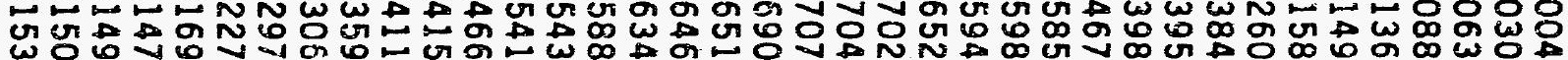

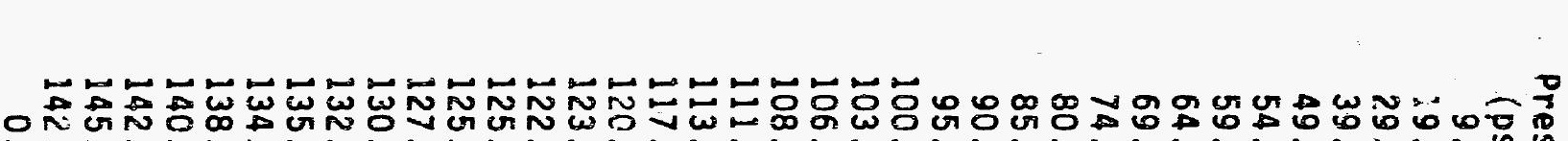

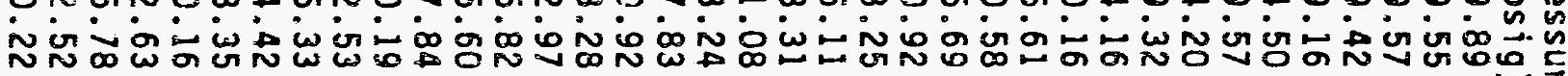
N

0000000000000000000000000000000000000

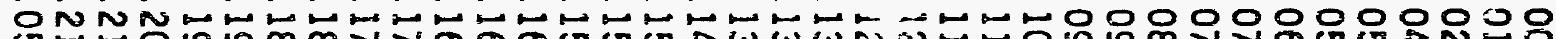

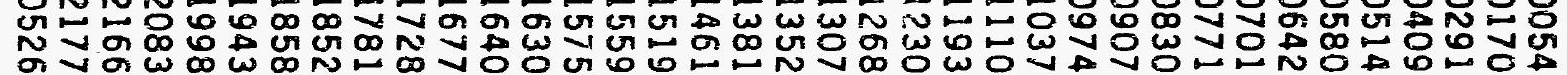




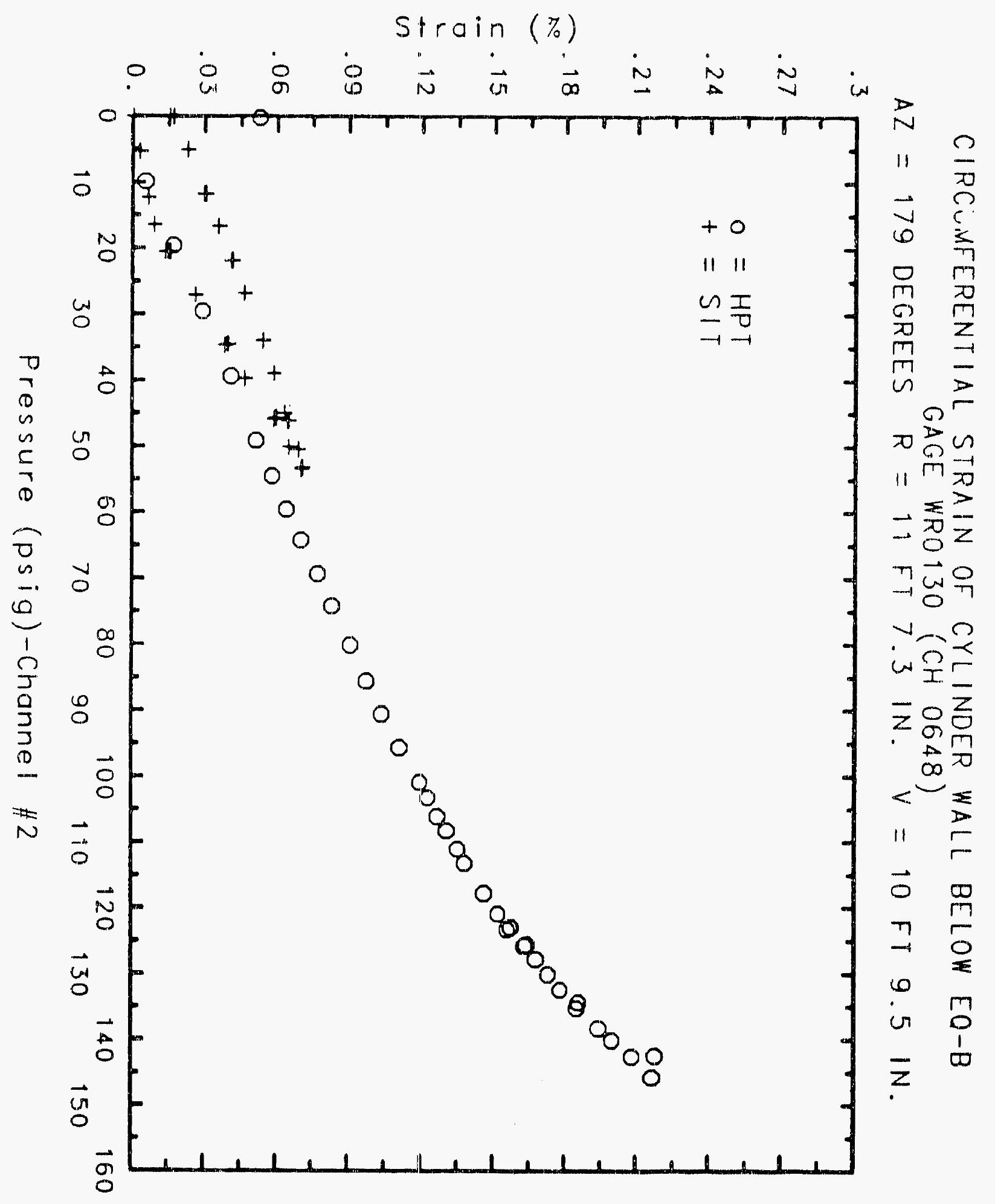




\section{Reinforced Concrete Test Data}

Weldable Gage 131 Channel 649

StRUCTURAL INTEgRITY TEST

High Pressure test

\begin{tabular}{|c|c|c|c|}
\hline $\begin{array}{c}\text { Pressure } \\
\text { (psig) } \\
-0.05 \\
5.33 \\
12.31 \\
16.44 \\
20.51 \\
20.46 \\
20.47 \\
27.05 \\
34.69 \\
34.53 \\
34.53 \\
39.70 \\
45.90 \\
45.65 \\
45.62 \\
50.10 \\
53.47 \\
53.29 \\
53.21 \\
50.49 \\
46.14 \\
46.13 \\
44.97 \\
38.98 \\
33.99 \\
33.96 \\
26.83 \\
21.88 \\
21.90 \\
16.69 \\
11.74 \\
11.77 \\
5.05 \\
0.02 \\
-0.04 \\
-0.02 \\
-0.02 \\
0.02 \\
\end{array}$ & $\begin{array}{c}\text { \% Strain } \\
-0.0013 \\
0.0011 \\
0.0041 \\
0.0066 \\
0.0114 \\
0.0120 \\
0.0130 \\
0.0230 \\
0.0348 \\
0.0360 \\
0.0364 \\
0.0419 \\
0.0537 \\
0.0542 \\
0.0545 \\
0.0590 \\
0.0640 \\
0.0638 \\
0.0640 \\
0.0619 \\
0.0583 \\
0.0582 \\
0.0566 \\
0.0517 \\
0.0470 \\
0.0465 \\
0.0393 \\
0.0344 \\
0.0342 \\
0.0293 \\
0.0234 \\
0.0228 \\
0.0163 \\
0.0108 \\
0.0094 \\
0.0099 \\
0.0101 \\
0.0100\end{array}$ & $\begin{array}{c}\text { Pressure } \\
(\text { psig) } \\
9.89 \\
19.55 \\
29.57 \\
39.42 \\
49.16 \\
54.50 \\
59.57 \\
64.20 \\
69.32 \\
74.16 \\
80.16 \\
85.61 \\
90.58 \\
95.69 \\
100.92 \\
103.25 \\
106.11 \\
108.31 \\
111.08 \\
113.24 \\
117.83 \\
120.92 \\
123.28 \\
122.97 \\
125.82 \\
125.60 \\
127.84 \\
130.19 \\
132.53 \\
135.33 \\
134.42 \\
138.35 \\
140.16 \\
142.63 \\
145.78 \\
142.52 \\
0.22 \\
\end{array}$ & $\begin{array}{l}\% \text { Strain } \\
0.0059 \\
0.0169 \\
0.0291 \\
0.0401 \\
0.0504 \\
0.0558 \\
0.0616 \\
0.0676 \\
0.0732 \\
0.0788 \\
0.0840 \\
0.0899 \\
0.0943 \\
0.0991 \\
0.1042 \\
0.1062 \\
0.1094 \\
0.1113 \\
0.1143 \\
0.1167 \\
0.1219 \\
0.1253 \\
0.1276 \\
0.1272 \\
0.1315 \\
0.1308 \\
0.1334 \\
0.1370 \\
0.1410 \\
0.1451 \\
0.1444 \\
0.1502 \\
0.1541 \\
0.1592 \\
0.1644 \\
0.1626 \\
0.0293\end{array}$ \\
\hline
\end{tabular}




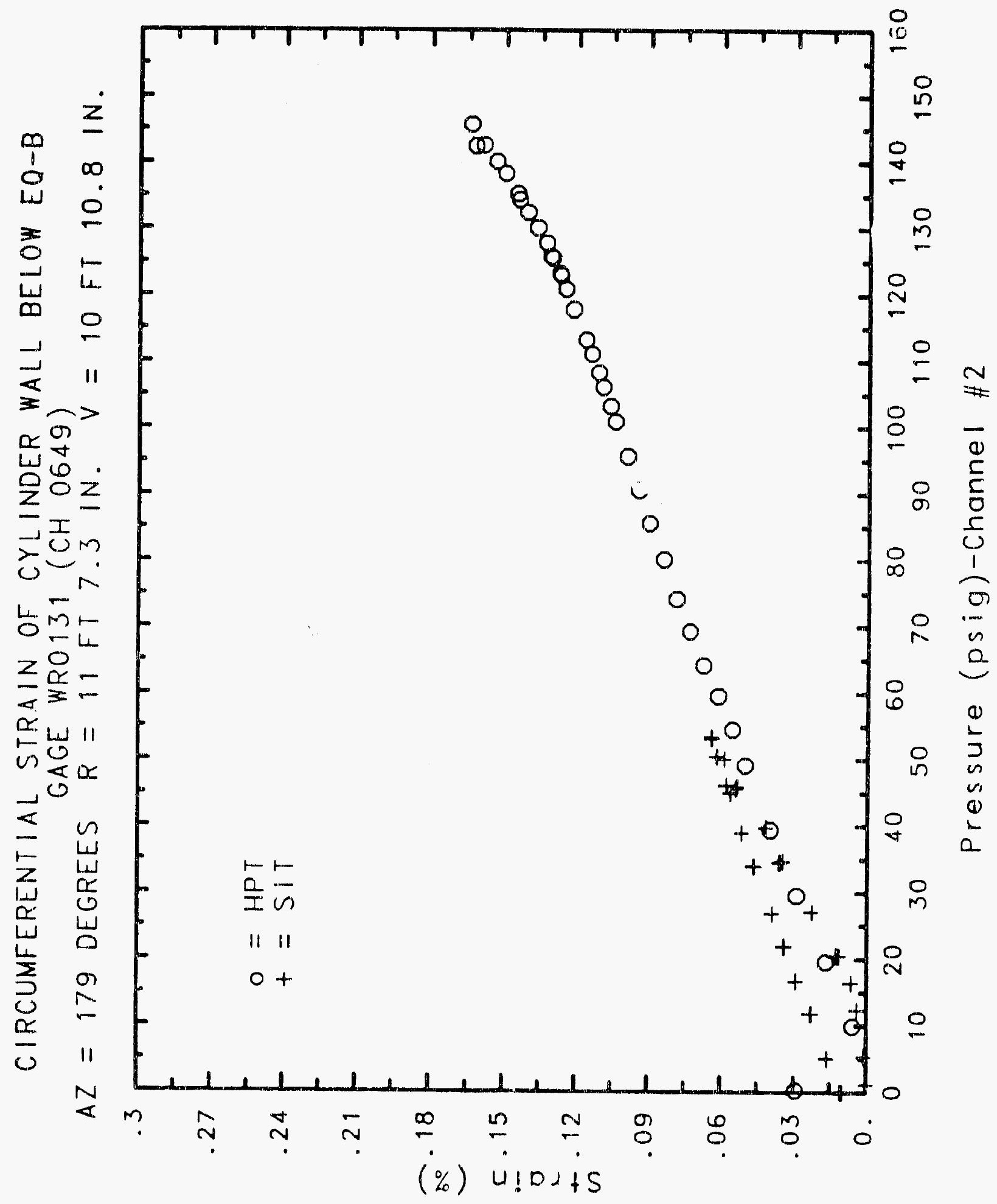




\section{Reinforced Concrete Test Data}

Weldable Gage 132 Channel 660

Structural Integrity Test

\begin{tabular}{|c|c|c|c|}
\hline $\begin{array}{c}\text { Pressure } \\
\text { (psig) } \\
-0.05 \\
5.33 \\
11.31 \\
16.44 \\
20.51 \\
20.46 \\
20.47 \\
27.05 \\
34.69 \\
34.53 \\
34.53 \\
39.70 \\
45.90 \\
45.65 \\
45.62 \\
50.10 \\
53.47 \\
53.29 \\
53.21 \\
50.49 \\
46.14 \\
46.13 \\
44.97 \\
38.98 \\
33.99 \\
33.96 \\
26.83 \\
21.88 \\
21.90 \\
16.69 \\
11.74 \\
11.77 \\
5.05 \\
0.02 \\
-0.04 \\
-0.02 \\
-0.02 \\
0.02 \\
\end{array}$ & $\begin{array}{l}\text { \% Strain } \\
\text { O. } 0005 \\
0.0014 \\
0.0034 \\
0.0053 \\
0.0100 \\
0.0107 \\
0.0110 \\
0.0186 \\
0.0277 \\
0.0280 \\
0.0278 \\
0.0324 \\
0.0395 \\
0.0408 \\
0.0412 \\
0.0466 \\
0.0507 \\
0.0511 \\
0.0513 \\
0.0501 \\
0.0471 \\
0.0470 \\
0.0454 \\
0.0409 \\
0.0374 \\
0.0372 \\
0.0316 \\
0.0277 \\
0.0273 \\
0.0236 \\
0.0195 \\
0.0196 \\
0.0149 \\
0.0114 \\
0.0102 \\
0.0100 \\
0.0103 \\
0.0102\end{array}$ & $\begin{array}{c}\text { Pressure } \\
\text { (psig) } \\
9.89 \\
19.55 \\
29.57 \\
39.42 \\
49.16 \\
54.50 \\
59.57 \\
64.20 \\
69.32 \\
74.16 \\
80.16 \\
85.61 \\
90.58 \\
95.69 \\
100.92 \\
103.25 \\
106.11 \\
108.31 \\
111.08 \\
113.24 \\
117.83 \\
120.92 \\
123.28 \\
122.97 \\
125.82 \\
125.60 \\
127.84 \\
130.19 \\
132.53 \\
135.33 \\
134.42 \\
138.35 \\
140.16 \\
142.63 \\
145.78 \\
142.52 \\
0.22 \\
\end{array}$ & $\begin{array}{l}\text { \% Strain } \\
0.0030 \\
0.0113 \\
0.0202 \\
0.0288 \\
0.0377 \\
0.0426 \\
0.0475 \\
0.0526 \\
0.0584 \\
0.0640 \\
0.0729 \\
0.0801 \\
0.0863 \\
0.0935 \\
0.1009 \\
0.1038 \\
0.1080 \\
0.1114 \\
0.1156 \\
0.1185 \\
0.1264 \\
0.1316 \\
0.1357 \\
0.1359 \\
0.1402 \\
0.1409 \\
0.1449 \\
0.1516 \\
0.1618 \\
0.1783 \\
0.1822 \\
0.2110 \\
0.2384 \\
0.2797 \\
0.3179 \\
0.3371 \\
0.1685\end{array}$ \\
\hline
\end{tabular}

High Pressure Test 


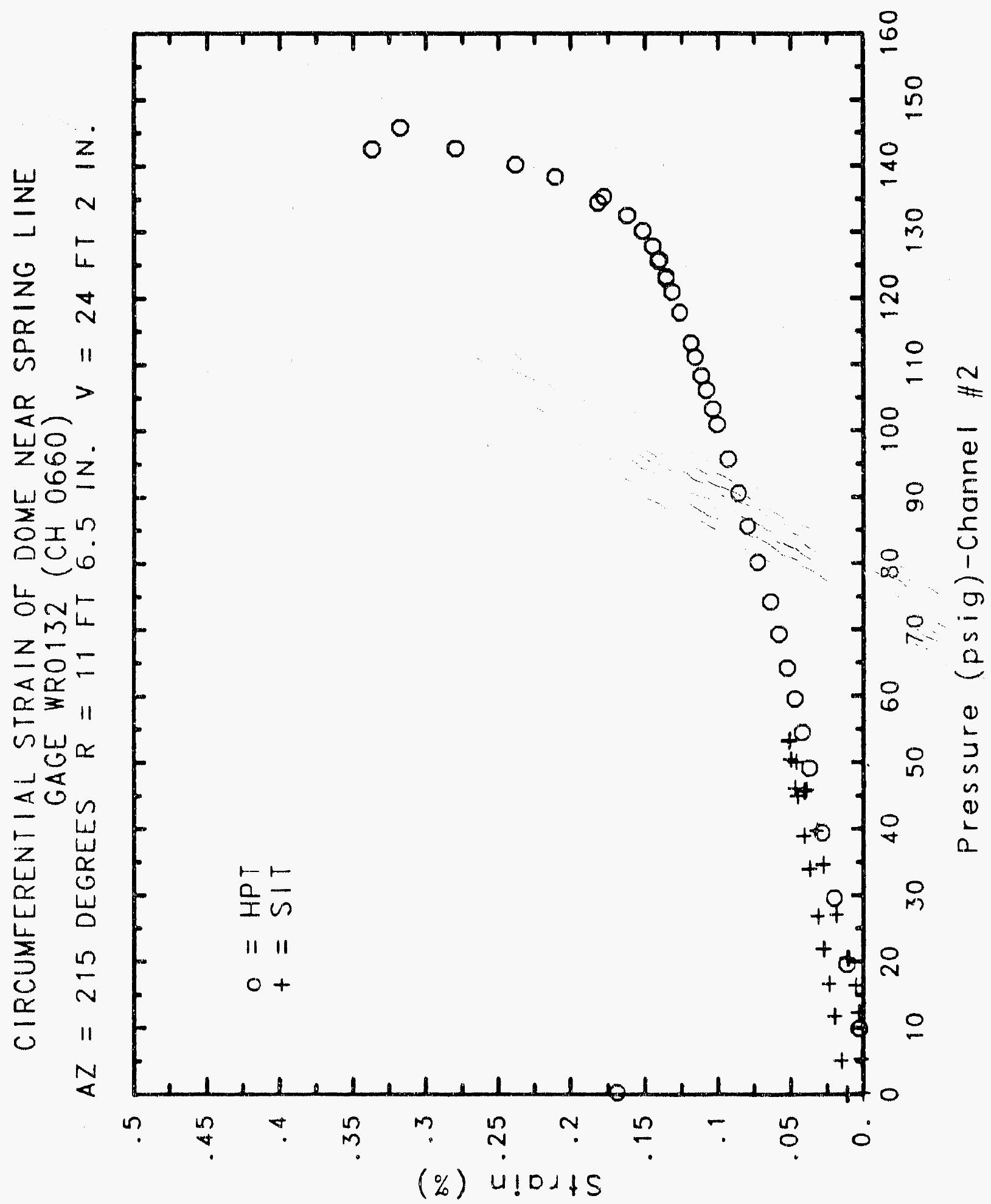

C-1072 


\section{Reinforced Concrete Test Data}

Weldable Gage 133 Channel 661

STRUCTURAL INTEgRITY TEST

High Pressure Test

\begin{tabular}{|c|c|c|c|}
\hline $\begin{array}{c}\text { Pressure } \\
\text { (psig) } \\
-0.05 \\
5.33 \\
12.31 \\
16.44 \\
20.51 \\
20.46 \\
20.47 \\
27.05 \\
34.69 \\
34.53 \\
34.53 \\
39.70 \\
45.90 \\
45.65 \\
45.62 \\
50.10 \\
53.47 \\
53.29 \\
53.21 \\
50.49 \\
46.14 \\
46.13 \\
44.97 \\
38.98 \\
33.99 \\
33.96 \\
26.83 \\
21.88 \\
21.90 \\
16.69 \\
11.74 \\
11.77 \\
5.05 \\
0.02 \\
-0.04 \\
-0.02 \\
-0.02 \\
0.02 \\
\end{array}$ & $\begin{array}{l}\text { \% Strain } \\
0.0002 \\
0.0038 \\
0.0087 \\
0.0119 \\
0.0170 \\
0.0175 \\
0.0180 \\
0.0288 \\
0.0426 \\
0.0434 \\
0.0439 \\
0.0515 \\
0.0634 \\
0.0653 \\
0.0661 \\
0.0730 \\
0.0794 \\
0.0809 \\
0.0810 \\
0.0791 \\
0.0749 \\
0.0747 \\
0.0704 \\
0.0643 \\
0.0584 \\
0.0588 \\
0.0496 \\
0.0436 \\
0.0430 \\
0.0365 \\
0.0298 \\
0.0303 \\
0.0222 \\
0.0159 \\
0.0074 \\
0.0087 \\
0.0109 \\
0.0124\end{array}$ & $\begin{array}{c}\text { Pressure } \\
(\text { psig) } \\
9.89 \\
19.55 \\
29.57 \\
39.42 \\
49.16 \\
54.50 \\
59.57 \\
64.20 \\
69.32 \\
74.16 \\
80.16 \\
85.61 \\
90.58 \\
95.69 \\
100.92 \\
103.25 \\
106.11 \\
108.31 \\
111.08 \\
113.24 \\
117.83 \\
120.92 \\
123.28 \\
122.97 \\
125.82 \\
125.60 \\
127.84 \\
130.19 \\
132.53 \\
135.33 \\
134.42 \\
138.35 \\
140.16 \\
142.63 \\
145.78 \\
142.52 \\
0.22 \\
\\
130\end{array}$ & $\begin{array}{l}\text { \% Strain } \\
0.0094 \\
0.0235 \\
0.0376 \\
0.0509 \\
0.0639 \\
0.0712 \\
0.0788 \\
0.0868 \\
0.0962 \\
0.1060 \\
0.1213 \\
0.1330 \\
0.1442 \\
0.1572 \\
0.1717 \\
0.1793 \\
0.1877 \\
0.1944 \\
0.2080 \\
0.2176 \\
0.2409 \\
0.2583 \\
0.2726 \\
0.2734 \\
0.2904 \\
0.2955 \\
0.3083 \\
0.3322 \\
0.3631 \\
0.3988 \\
0.4052 \\
0.4484 \\
0.4928 \\
0.5542 \\
0.6461 \\
0.6805 \\
0.3699\end{array}$ \\
\hline
\end{tabular}




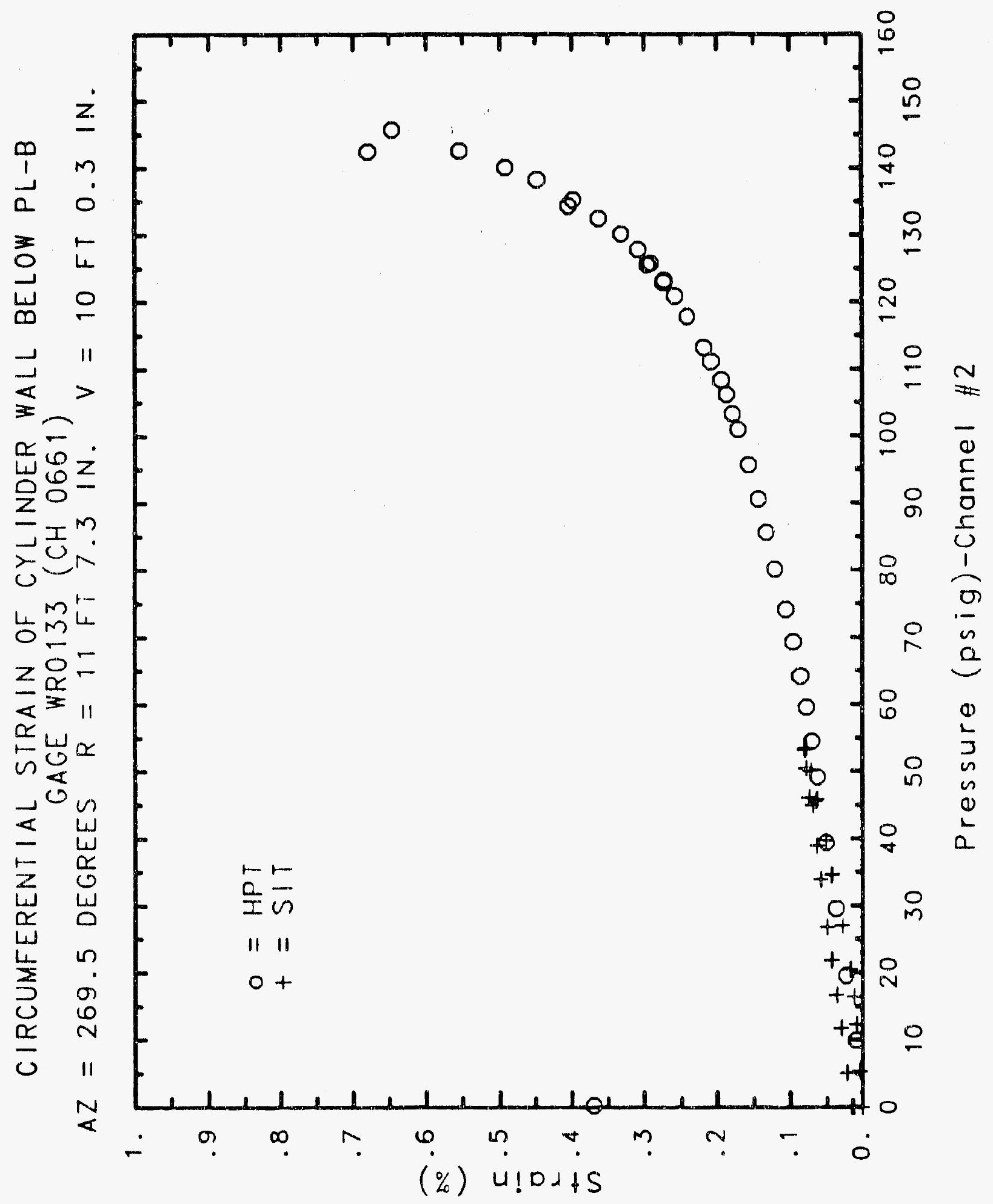




\section{Reinforced Concrete Test Data}

\section{Weldable Gage 134 Channel. 662}

Structural Integrity TEST

\begin{tabular}{|c|c|c|c|}
\hline $\begin{array}{c}\text { Pressure } \\
\text { (psig) } \\
-0.05 \\
5.33 \\
12.31 \\
16.44 \\
20.51 \\
20.46 \\
20.47 \\
27.05 \\
34.69 \\
34.53 \\
34.53 \\
39.70 \\
45.90 \\
45.65 \\
45.62 \\
50.10 \\
53.47 \\
53.29 \\
53.21 \\
50.49 \\
46.14 \\
46.13 \\
44.97 \\
38.98 \\
33.99 \\
33.96 \\
26.83 \\
21.88 \\
21.90 \\
16.69 \\
11.74 \\
11.77 \\
5.05 \\
0.02 \\
-0.04 \\
-0.02 \\
-0.02 \\
0.02 \\
\end{array}$ & $\begin{array}{c}\text { X Strain } \\
-0.0001 \\
0.0025 \\
0.0052 \\
0.0075 \\
0.0104 \\
0.0108 \\
0.0110 \\
0.0151 \\
0.0208 \\
0.0217 \\
0.0217 \\
0.0250 \\
0.0306 \\
0.0310 \\
0.0315 \\
0.0351 \\
0.0382 \\
0.0390 \\
0.0393 \\
0.0378 \\
0.0355 \\
0.0353 \\
0.0368 \\
0.0332 \\
0.0299 \\
0.0301 \\
0.0253 \\
0.0224 \\
0.0217 \\
0.0185 \\
0.0155 \\
0.0150 \\
0.0107 \\
0.0072 \\
0.0071 \\
0.0069 \\
0.0069 \\
0.0065\end{array}$ & $\begin{array}{c}\text { Pressure } \\
\text { (psig) } \\
9.89 \\
19.55 \\
29.57 \\
39.42 \\
49.16 \\
54.50 \\
59.57 \\
64.20 \\
69.32 \\
74.16 \\
80.16 \\
85.61 \\
90.58 \\
95.69 \\
100.92 \\
103.25 \\
106.11 \\
108.31 \\
111.08 \\
113.24 \\
117.83 \\
120.92 \\
123.28 \\
122.97 \\
125.82 \\
125.60 \\
127.84 \\
130.19 \\
132.53 \\
135.33 \\
134.42 \\
138.35 \\
140.16 \\
142.63 \\
145.78 \\
142.52 \\
0.22\end{array}$ & $\begin{array}{l}\text { \% Strain } \\
0.0019 \\
0.0083 \\
0.0150 \\
0.0219 \\
0.0284 \\
0.0328 \\
0.0371 \\
0.0416 \\
0.0465 \\
0.0515 \\
0.0577 \\
0.0647 \\
0.0706 \\
0.0779 \\
0.0854 \\
0.0892 \\
0.0931 \\
0.0965 \\
0.1016 \\
0.1048 \\
0.1138 \\
0.1206 \\
0.1272 \\
0.1293 \\
0.1367 \\
0.1381 \\
0.1426 \\
0.1500 \\
0.1584 \\
0.1695 \\
0.1709 \\
0.1819 \\
0.1898 \\
0.1996 \\
0.2102 \\
0.2121 \\
0.0808\end{array}$ \\
\hline
\end{tabular}

High Pressure test

Pressure

9.89

19.55

29.57

39.42

59.57

69.32

74.16

80.16

103.25

108.31

111.08

113.24

117.83

120.92

123.28

127.84

130.19

132.53

135.33

134.42

140.16

142.63

145.78

42.52

0.22
\% Strain

0.0019

0.0083

0.0150

0.0219

0.0371

0.0465

0.0515

0.0577

0.0647

0.0706

0.0779

0.0892

0.093 .

0.0965

0.1016

0.1048

0.1138

0.1293

0.1367

0.1381

0.1426

0.1500

0.1584

0.1819

0.1898

0.1996

0.2102

0.2121

0.0808 


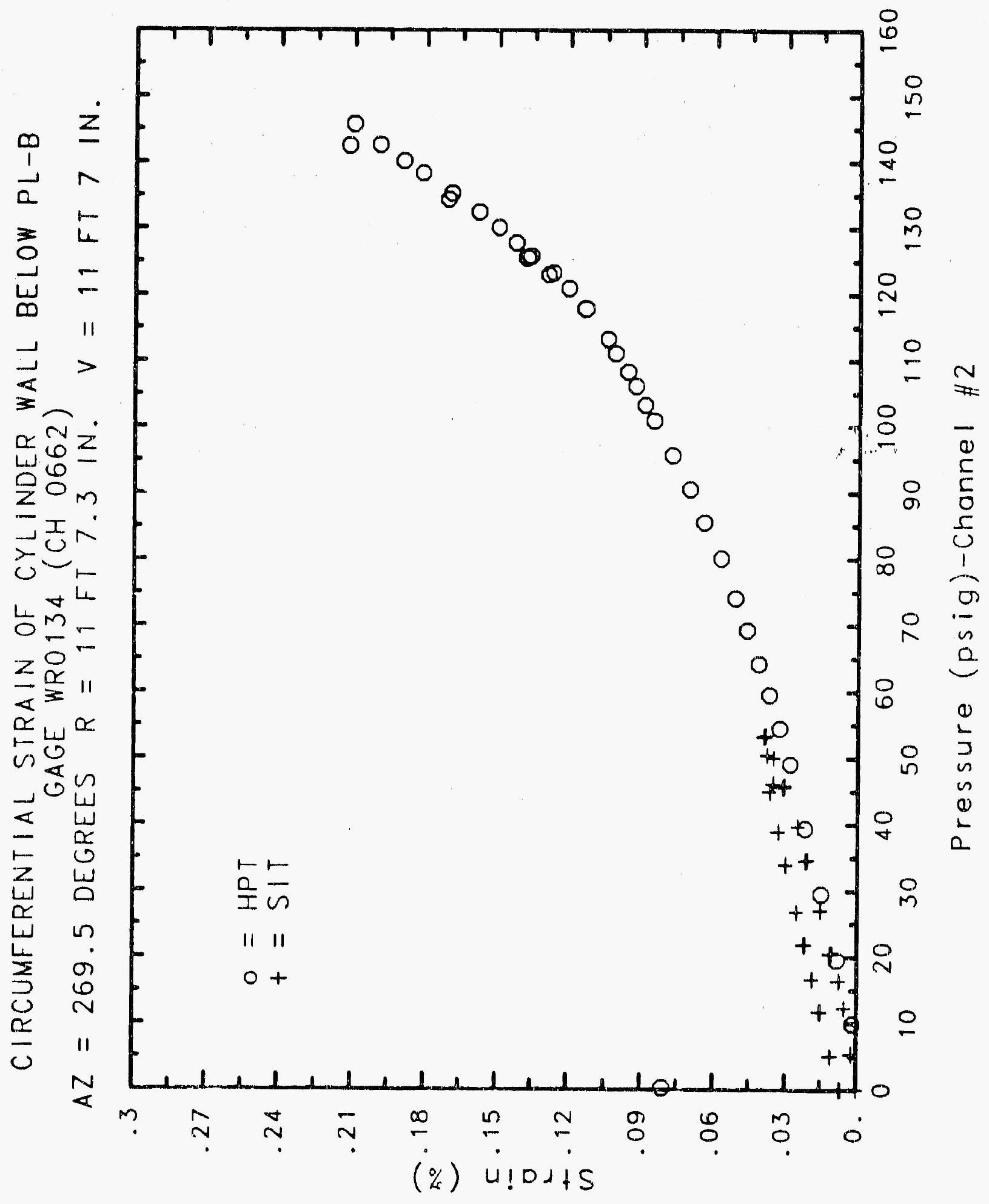




\section{Reinforced Concrete Test Data}

Weldable gage 135 Channel 663

Structural Integrity test

High Pressure test

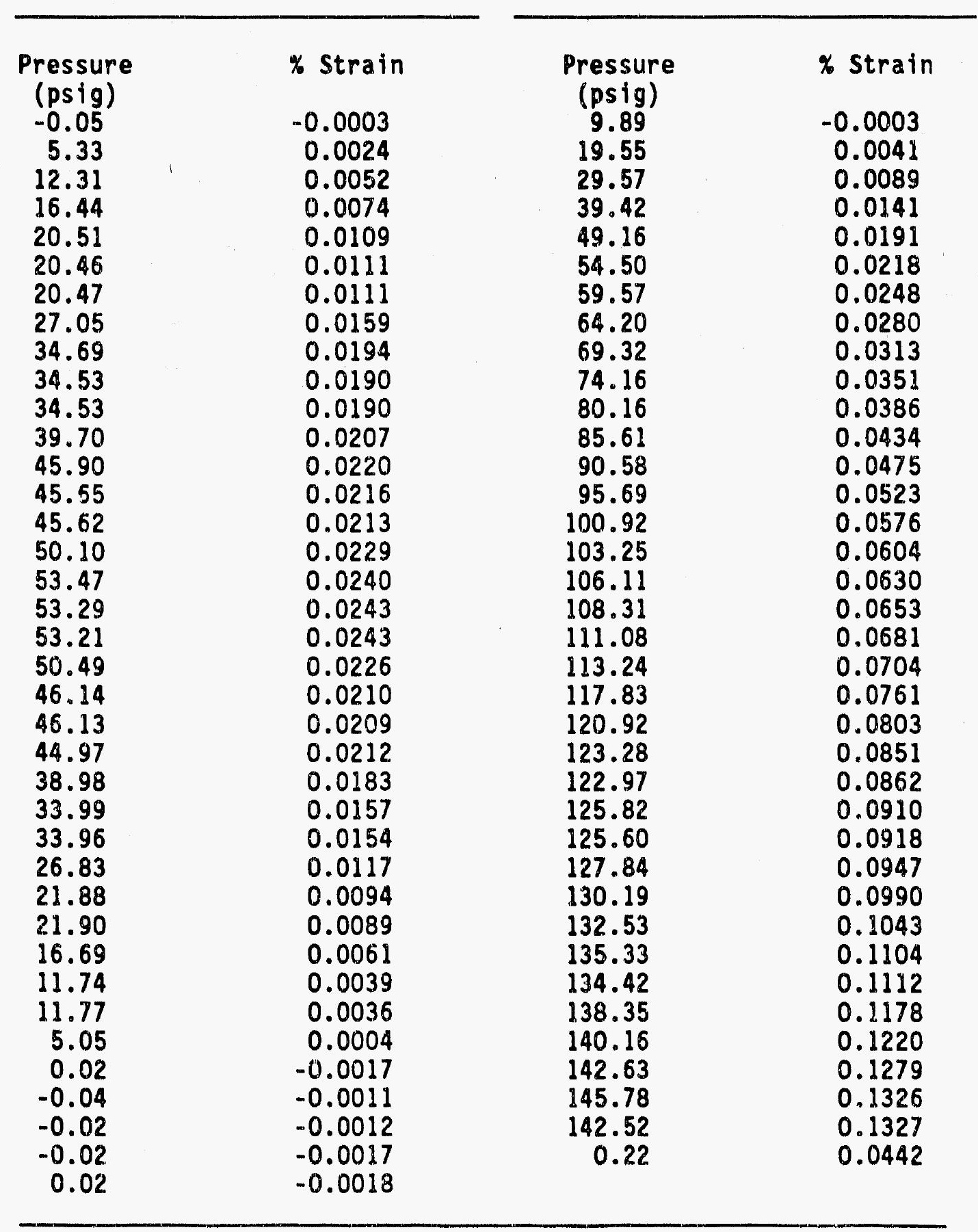




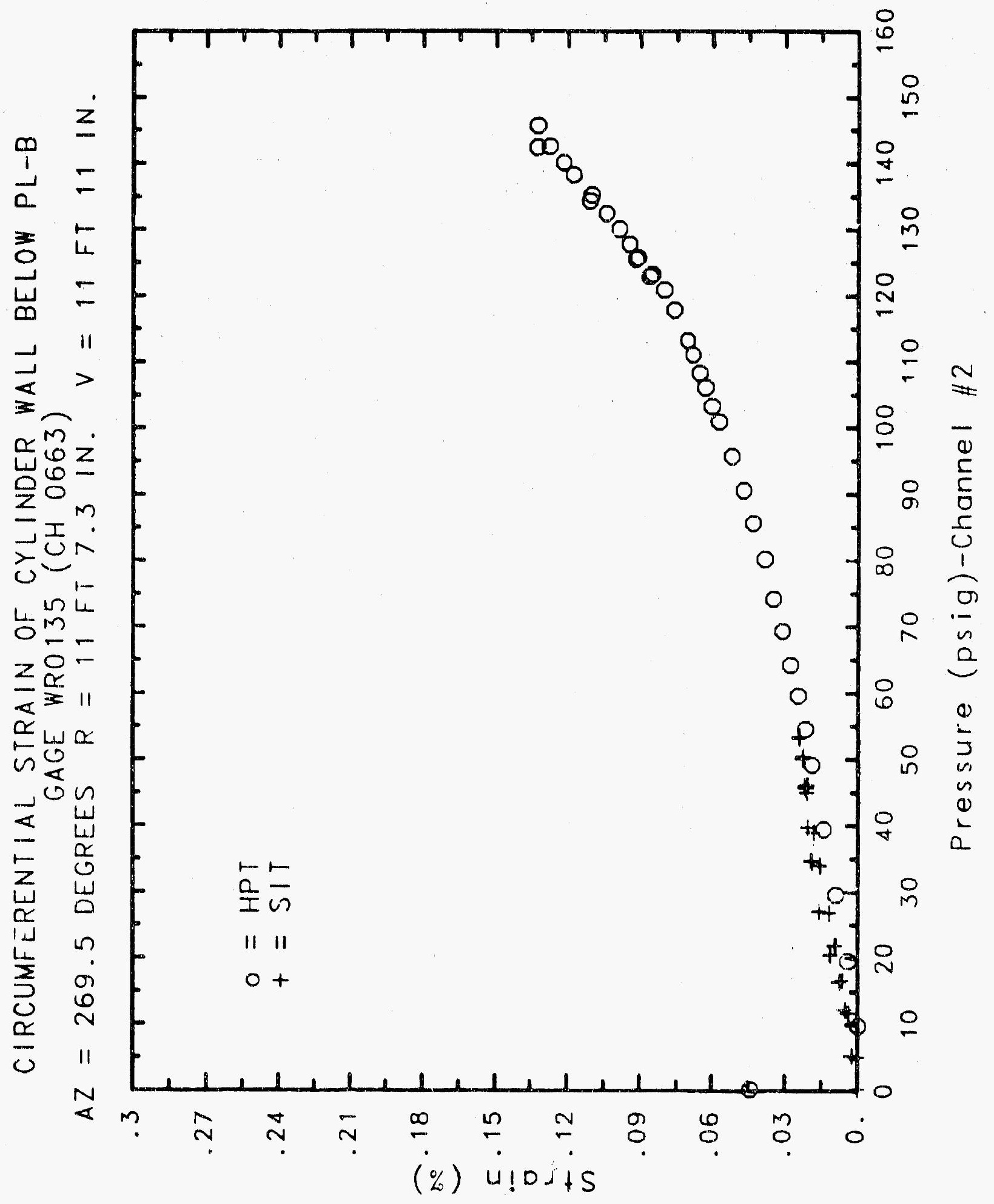




\section{Reinforced Concrete Test Data}

Weldable Gage 136 Channel 664

Structural In REgRITY TEST

High Pressure test

\begin{tabular}{cccc}
\hline Pressure & \% strain & $\begin{array}{c}\text { Pressure } \\
\text { (psig) }\end{array}$ & $\begin{array}{c}\text { (psig) } \\
9.89\end{array}$ \\
-0.05 & 0.0005 & 19.55 & 0.0073 \\
5.33 & 0.0032 & 29.57 & 0.0195 \\
12.31 & 0.0072 & 39.42 & 0.0328 \\
16.44 & 0.0108 & 49.16 & 0.0459 \\
20.51 & 0.0164 & 54.50 & 0.0588 \\
20.46 & 0.0174 & 59.57 & 0.0657 \\
20.47 & 0.0174 & 64.20 & 0.0728 \\
27.05 & 0.0297 & 69.32 & 0.0794 \\
34.69 & 0.0431 & 74.16 & 0.0870 \\
34.53 & 0.0434 & 80.16 & 0.0936 \\
34.53 & 0.0437 & 85.61 & 0.1046 \\
39.70 & 0.0506 & 90.58 & 0.1126 \\
45.90 & 0.0601 & 95.69 & 0.1205 \\
45.65 & 0.0611 & 100.92 & 0.1294 \\
45.62 & 0.0614 & 103.25 & 0.1380 \\
50.10 & 0.0671 & 106.11 & 0.1423 \\
53.47 & 0.0715 & 108.31 & 0.1473 \\
53.29 & 0.0719 & 111.08 & 0.1509 \\
53.21 & 0.0719 & 113.24 & 0.1568 \\
50.49 & 0.0699 & 117.83 & 0.1609 \\
46.14 & 0.0656 & 120.92 & 0.1714 \\
46.13 & 0.0653 & 123.28 & 0.1787 \\
44.97 & 0.0617 & 122.97 & 0.1842 \\
38.98 & 0.0560 & 125.82 & 0.1846 \\
33.99 & 0.0504 & 125.60 & 0.1930 \\
33.96 & 0.0500 & 127.84 & 0.1945 \\
26.83 & 0.0414 & 130.19 & 0.2011 \\
21.88 & 0.0354 & 132.53 & 0.2129 \\
21.90 & 0.0347 & 135.33 & 0.2276 \\
16.69 & 0.0284 & 134.42 & 0.2504 \\
11.74 & 0.0220 & 138.35 & 0.2524 \\
11.77 & 0.0220 & 142.63 & 0.2696 \\
5.05 & 0.0140 & 145.78 & 0.2797 \\
0.02 & 0.0085 & 142.52 & 0.2905 \\
-0.04 & 0.0054 & 0.22 & 0.2950 \\
-0.02 & 0.0051 & & 0.2936 \\
-0.02 & 0.0050 & & 0.0824 \\
0.02 & 0.0050 & & \\
\hline & & & \\
\hline
\end{tabular}




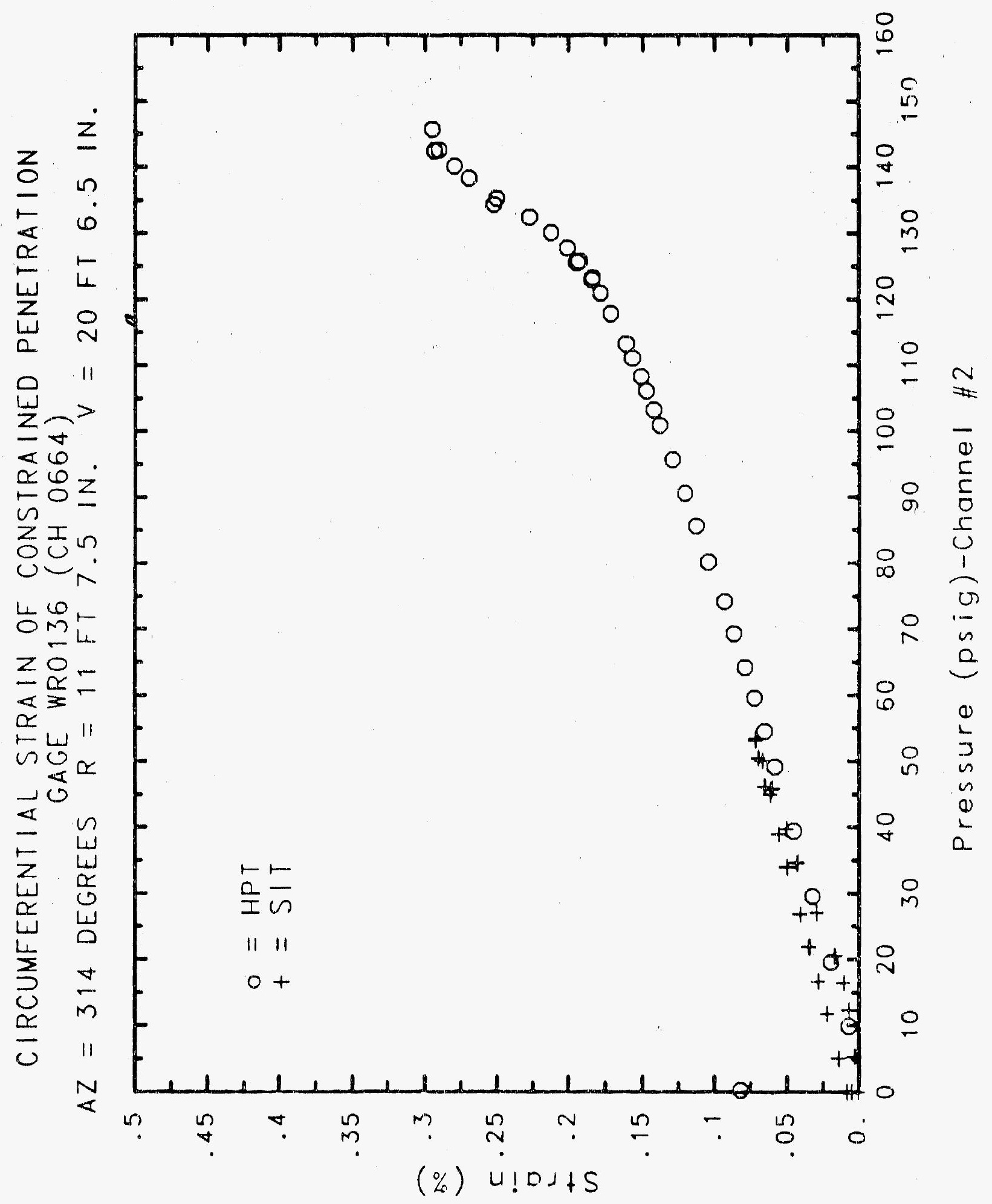

C- 1080 


\section{Reinforced Concrete test Data}

Weldable gage 137 Channel 665

StRUCTURAL INTEgRITY TEST

\begin{tabular}{|c|c|c|c|}
\hline $\begin{array}{c}\text { Pressure } \\
\text { (psig) } \\
-0.05 \\
5.33 \\
12.31 \\
16.44 \\
20.51 \\
20.46 \\
20.47 \\
27.05 \\
34.69 \\
34.53 \\
34.53 \\
39.70 \\
45.90 \\
45.65 \\
45.62 \\
50.10 \\
53.47 \\
53.29 \\
53.21 \\
50.49 \\
46.14 \\
46.13 \\
44.97 \\
38.98 \\
33.99 \\
33.96 \\
26.83 \\
21.88 \\
21.90 \\
16.69 \\
11.74 \\
11.77 \\
5.05 \\
0.02 \\
-0.04 \\
-0.02 \\
-0.02 \\
0.02 \\
\end{array}$ & $\begin{array}{l}\text { \% Strain } \\
0.0005 \\
0.0028 \\
0.0068 \\
0.0101 \\
0.0149 \\
0.0158 \\
0.0164 \\
0.0260 \\
0.0389 \\
0.0398 \\
0.0399 \\
0.0470 \\
0.0570 \\
0.0586 \\
0.0591 \\
0.0652 \\
0.0705 \\
0.0715 \\
0.0718 \\
0.0700 \\
0.0651 \\
0.0658 \\
0.0631 \\
0.0575 \\
0.0526 \\
0.0525 \\
0.0447 \\
0.0390 \\
0.0386 \\
0.0328 \\
0.0269 \\
0.0263 \\
0.0191 \\
0.0138 \\
0.0105 \\
0.0100 \\
0.0099 \\
0.0098\end{array}$ & $\begin{array}{c}\text { Pressure } \\
\text { (psig) } \\
9.89 \\
19.55 \\
29.57 \\
39.42 \\
49.16 \\
54.50 \\
59.57 \\
64.20 \\
69.32 \\
74.16 \\
80.16 \\
85.61 \\
90.58 \\
95.69 \\
100.92 \\
103.25 \\
106.11 \\
108.31 \\
111.08 \\
113.24 \\
117.83 \\
120.92 \\
123.28 \\
122.97 \\
125.82 \\
125.60 \\
127.84 \\
130.19 \\
132.53 \\
135.33 \\
134.42 \\
138.35 \\
140.16 \\
142.63 \\
145.78 \\
142.52 \\
0.22\end{array}$ & $\begin{array}{l}\text { \% Strain } \\
0.0075 \\
0.0196 \\
0.0331 \\
0.0455 \\
0.0573 \\
0.0641 \\
0.0709 \\
0.0782 \\
0.0864 \\
0.0950 \\
0.1088 \\
0.1191 \\
0.1284 \\
0.1387 \\
0.1496 \\
0.1556 \\
0.1611 \\
0.1658 \\
0.1729 \\
0.1781 \\
0.1905 \\
0.1996 \\
0.2073 \\
0.2077 \\
0.2171 \\
0.2188 \\
0.2260 \\
0.2388 \\
0.2500 \\
0.2617 \\
0.2631 \\
0.2704 \\
0.2741 \\
0.2794 \\
0.2845 \\
0.2832 \\
0.0499\end{array}$ \\
\hline
\end{tabular}




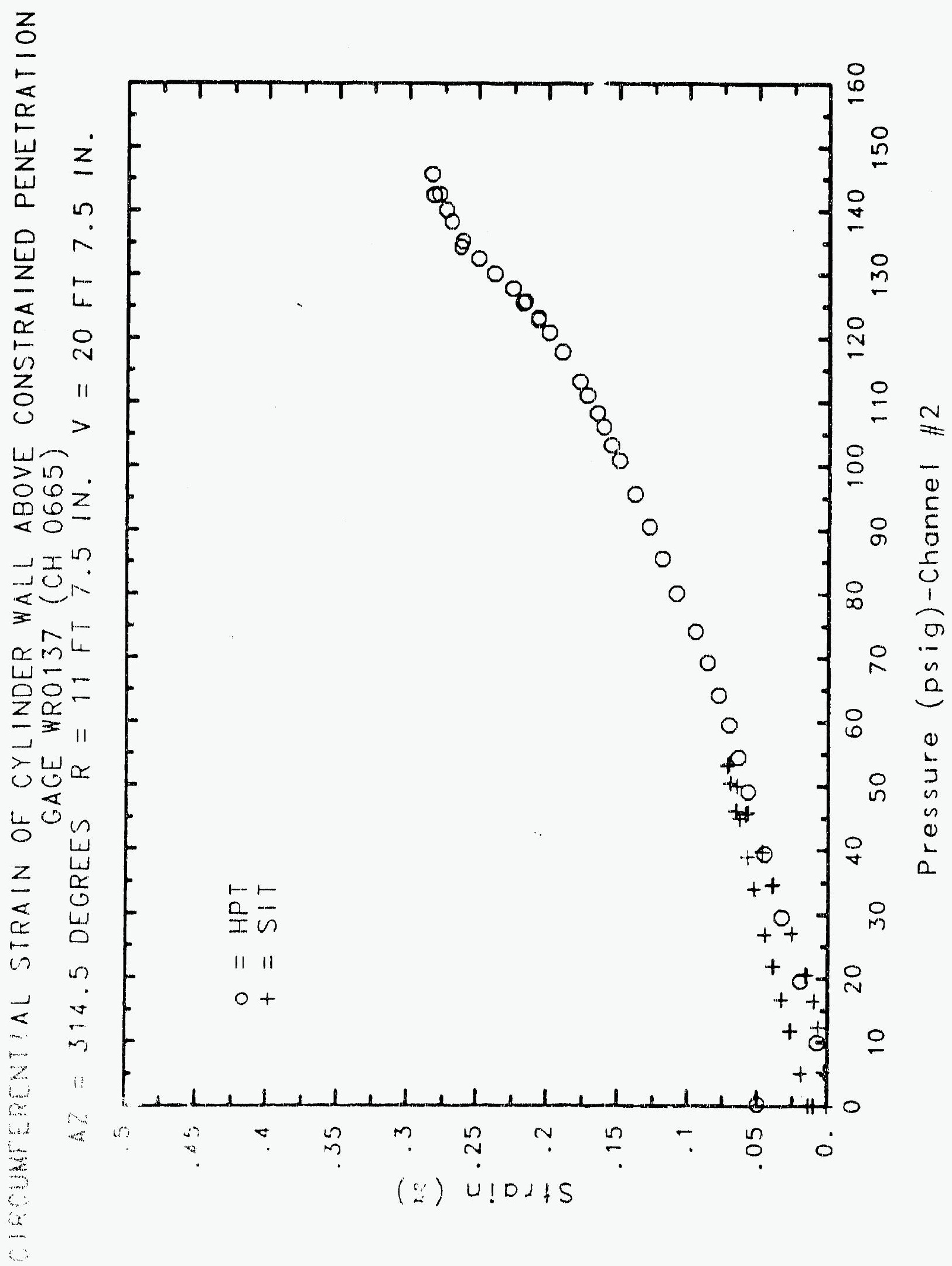




\section{Reinforced Concrete Test Data}

Weldable Gage 138 Channel 666

StruCtural IntEgrItY TEST

High Pressure test

\begin{tabular}{|c|c|c|c|}
\hline $\begin{array}{c}\text { Pressure } \\
\text { (psig) } \\
-0.05 \\
5.33 \\
12.31 \\
16.44 \\
20.51 \\
20.46 \\
20.47 \\
27.05 \\
34.69 \\
34.53 \\
34.53 \\
39.70 \\
45.90 \\
45.65 \\
45.62 \\
50.10 \\
53.47 \\
53.29 \\
53.21 \\
50.49 \\
46.14 \\
46.13 \\
44.97 \\
38.98 \\
33.99 \\
33.96 \\
26.83 \\
21.88 \\
21.90 \\
16.69 \\
11.74 \\
11.77 \\
5.05 \\
0.02 \\
-0.04 \\
-0.02 \\
-0.02 \\
0.02 \\
\end{array}$ & $\begin{array}{c}\text { \% Strain } \\
\text {-0.0013 } \\
0.0009 \\
0.0030 \\
0.0050 \\
0.008 \text { C } \\
0.0088 \\
0.0090 \\
0.0171 \\
0.0282 \\
0.0293 \\
0.0294 \\
0.0342 \\
0.0419 \\
0.0435 \\
0.0440 \\
0.0482 \\
0.0524 \\
0.0532 \\
0.0534 \\
0.0524 \\
0.0501 \\
0.0497 \\
0.0487 \\
0.0449 \\
0.0419 \\
0.0415 \\
0.0364 \\
0.0328 \\
0.0325 \\
0.0286 \\
0.0247 \\
0.0247 \\
0.0199 \\
0.0161 \\
0.0141 \\
0.0137 \\
0.0134 \\
0.0140\end{array}$ & $\begin{array}{c}\text { Pressure } \\
\text { (psig) } \\
9.89 \\
19.55 \\
29.57 \\
39.42 \\
49.16 \\
54.50 \\
59.57 \\
64.20 \\
69.32 \\
74.16 \\
180.16 \\
85.61 \\
90.58 \\
95.59 \\
100.92 \\
103.25 \\
106.11 \\
108.31 \\
111.08 \\
113.24 \\
117.83 \\
120.92 \\
123.28 \\
122.97 \\
125.82 \\
125.60 \\
127.84 \\
130.19 \\
132.53 \\
135.33 \\
134.42 \\
138.35 \\
140.16 \\
142.63 \\
145.78 \\
142.52 \\
0.22\end{array}$ & $\begin{array}{l}\text { \% Strain } \\
0.0036 \\
0.0118 \\
0.0204 \\
0.0284 \\
0.0363 \\
0.0407 \\
0.0458 \\
0.0508 \\
0.0560 \\
0.0615 \\
0.0697 \\
0.0757 \\
0.0819 \\
0.0885 \\
0.0948 \\
0.0982 \\
0.1019 \\
0.1048 \\
0.1089 \\
0.1120 \\
0.1201 \\
0.1258 \\
0.1303 \\
0.1313 \\
0.1356 \\
0.1361 \\
0.1397 \\
0.1450 \\
0.1511 \\
0.1598 \\
0.1610 \\
0.1750 \\
0.1884 \\
0.2082 \\
0.2287 \\
0.2325 \\
0.0811\end{array}$ \\
\hline
\end{tabular}




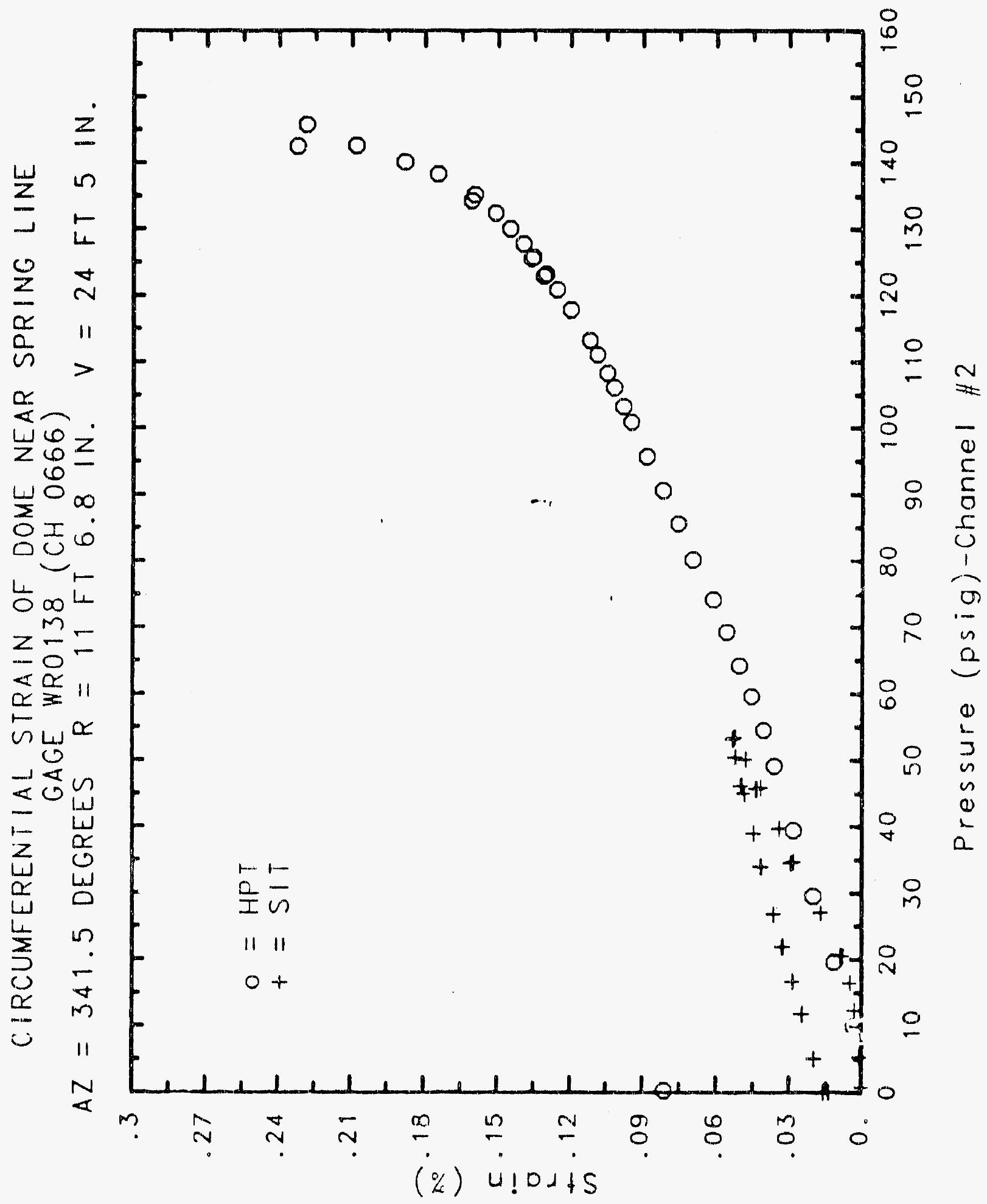




\section{Reinforced Concrete Test Data}

Weldable Gage 139 Channel 667

Structural Integrity Test

high Pressure test

\begin{tabular}{|c|c|c|c|}
\hline $\begin{array}{c}\text { Pressure } \\
\text { (psig) } \\
-0.05 \\
5.33 \\
12.31 \\
16.44 \\
20.51 \\
20.46 \\
20.47 \\
27.05 \\
34.69 \\
34.53 \\
34.53 \\
39.70 \\
45.90 \\
45.65 \\
45.62 \\
50.10 \\
53.47 \\
53.29 \\
53.21 \\
50.49 \\
46.14 \\
46.13 \\
44.97 \\
38.98 \\
33.99 \\
33.96 \\
26.83 \\
21.88 \\
21.90 \\
16.69 \\
11.74 \\
11.77 \\
5.05 \\
0.02 \\
-0.04 \\
.0 .02 \\
-0.02 \\
0.02 \\
\end{array}$ & $\begin{array}{l}\text { \% Strain } \\
0.0001 \\
0.0025 \\
0.0050 \\
0.0065 \\
0.0085 \\
0.0087 \\
0.0088 \\
0.0136 \\
0.0215 \\
0.0223 \\
0.0221 \\
0.0264 \\
0.0324 \\
0.0335 \\
0.0339 \\
0.0376 \\
0.0423 \\
0.0429 \\
0.0432 \\
0.0422 \\
0.0397 \\
0.0396 \\
0.0410 \\
0.0376 \\
0.0344 \\
0.0344 \\
0.0298 \\
0.0264 \\
0.0263 \\
0.0227 \\
0.0192 \\
0.0188 \\
0.0140 \\
0.0104 \\
0.0088 \\
0.0095 \\
0.0101 \\
0.0100\end{array}$ & $\begin{array}{c}\text { Pressure } \\
\text { (psig) } \\
9.89 \\
19.55 \\
29.57 \\
39.42 \\
49.16 \\
54.50 \\
59.57 \\
64.20 \\
69.32 \\
74.16 \\
80.16 \\
85.61 \\
90.58 \\
95.69 \\
100.92 \\
103.25 \\
106.11 \\
108.31 \\
111.08 \\
113.24 \\
117.83 \\
120.92 \\
123.28 \\
122.97 \\
125.82 \\
125.60 \\
127.84 \\
130.19 \\
132.53 \\
135.33 \\
134.42 \\
138.35 \\
140.16 \\
142.63 \\
145.78 \\
142.52 \\
0.22\end{array}$ & $\begin{array}{l}\% \text { Strain } \\
0.0030 \\
0.0099 \\
0.0168 \\
0.0238 \\
0.0311 \\
0.0362 \\
0.0413 \\
0.0484 \\
0.0562 \\
0.0647 \\
0.0736 \\
0.0836 \\
0.0933 \\
0.1042 \\
0.1156 \\
0.1217 \\
0.1274 \\
0.1322 \\
0.1394 \\
0.1445 \\
0.1568 \\
0.1660 \\
0.1740 \\
0.1758 \\
0.1841 \\
0.1865 \\
0.1922 \\
0.2009 \\
0.2113 \\
0.2256 \\
0.2287 \\
0.2494 \\
0.2654 \\
0.2921 \\
0.3201 \\
0.3307 \\
0.1474\end{array}$ \\
\hline
\end{tabular}




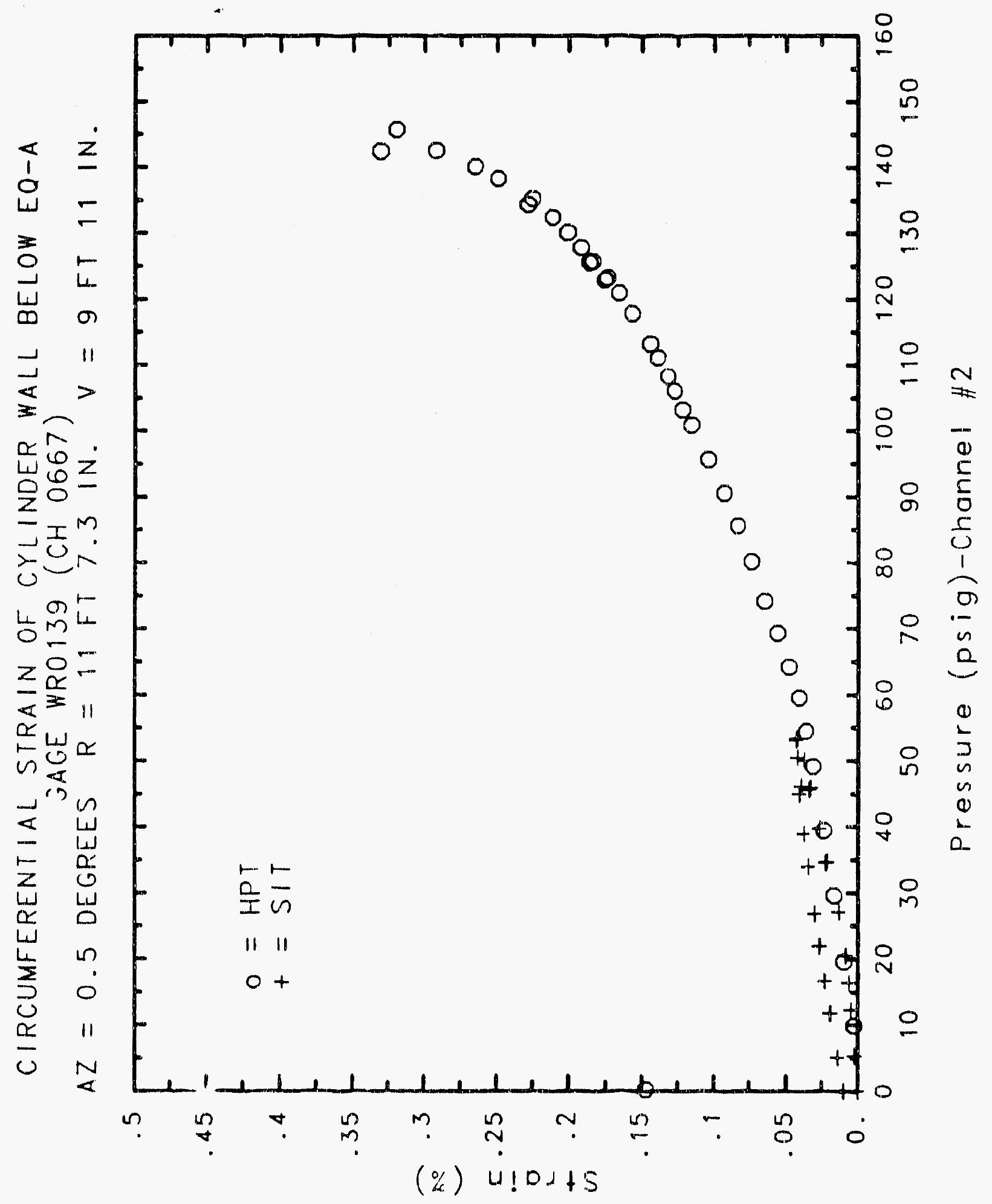




\section{Reinforced Concrete Test Data}

Weldable Gage 140 Chamnel 668

StRUCTURAL INTEgRITY TEST

High Pressure Test

\begin{tabular}{|c|c|c|c|}
\hline $\begin{array}{c}\text { Pressure } \\
(\text { psig) } \\
-0.05 \\
5.33 \\
12.31 \\
16.44 \\
20.51 \\
20.46 \\
20.47 \\
27.05 \\
34.69 \\
34.53 \\
34.53 \\
39.70 \\
45.90 \\
45.65 \\
45.62 \\
50.10 \\
53.47 \\
53.29 \\
53.21 \\
50.49 \\
46.14 \\
46.13 \\
44.97 \\
38.98 \\
33.99 \\
33.96 \\
26.83 \\
21.88 \\
21.90 \\
16.69 \\
11.74 \\
11.77 \\
5.05 \\
0.02 \\
-0.04 \\
-0.02 \\
-0.02 \\
0.02 \\
\end{array}$ & $\begin{array}{l}\text { \% Strain } \\
0.0009 \\
0.0040 \\
0.0084 \\
0.0110 \\
0.0150 \\
0.0153 \\
0.0151 \\
0.0218 \\
0.0291 \\
0.0291 \\
0.0290 \\
0.0336 \\
0.0397 \\
0.0392 \\
0.0387 \\
0.0439 \\
0.0514 \\
0.0518 \\
0.0518 \\
0.0504 \\
0.0467 \\
0.0467 \\
0.0476 \\
0.0424 \\
0.0382 \\
0.0380 \\
0.0312 \\
0.0266 \\
0.0263 \\
0.0210 \\
0.0162 \\
0.0156 \\
0.0091 \\
0.0042 \\
0.0026 \\
0.0041 \\
0.0043 \\
0.0041\end{array}$ & $\begin{array}{c}\text { Pressure } \\
(\text { ps } 1 \text { g) } \\
9.89 \\
19.55 \\
29.57 \\
39.42 \\
49.16 \\
54.50 \\
59.57 \\
64.20 \\
69.32 \\
74.16 \\
80.16 \\
85.61 \\
90.58 \\
95.69 \\
100.92 \\
103.25 \\
106.11 \\
108.31 \\
111.08 \\
113.24 \\
117.83 \\
120.92 \\
123.28 \\
122.97 \\
125.82 \\
125.60 \\
127.84 \\
130.19 \\
132.53 \\
135.33 \\
134.42 \\
138.35 \\
140.16 \\
142.63 \\
145.78 \\
142.52 \\
0.22 \\
\end{array}$ & $\begin{array}{l}\text { \% Strain } \\
0.0050 \\
0.0149 \\
0.0250 \\
0.0349 \\
0.0442 \\
0.0498 \\
0.0560 \\
0.0631 \\
0.0713 \\
0.0785 \\
0.0867 \\
0.0950 \\
0.1035 \\
0.1125 \\
0.1233 \\
0.1287 \\
0.1349 \\
0.1396 \\
0.1471 \\
0.1531 \\
0.1676 \\
0.1785 \\
0.1880 \\
0.1901 \\
0.2013 \\
0.2042 \\
0.2122 \\
0.2251 \\
0.2433 \\
0.2685 \\
0.2736 \\
0.3063 \\
0.3339 \\
0.3703 \\
0.4214 \\
0.4562 \\
0.2446\end{array}$ \\
\hline
\end{tabular}




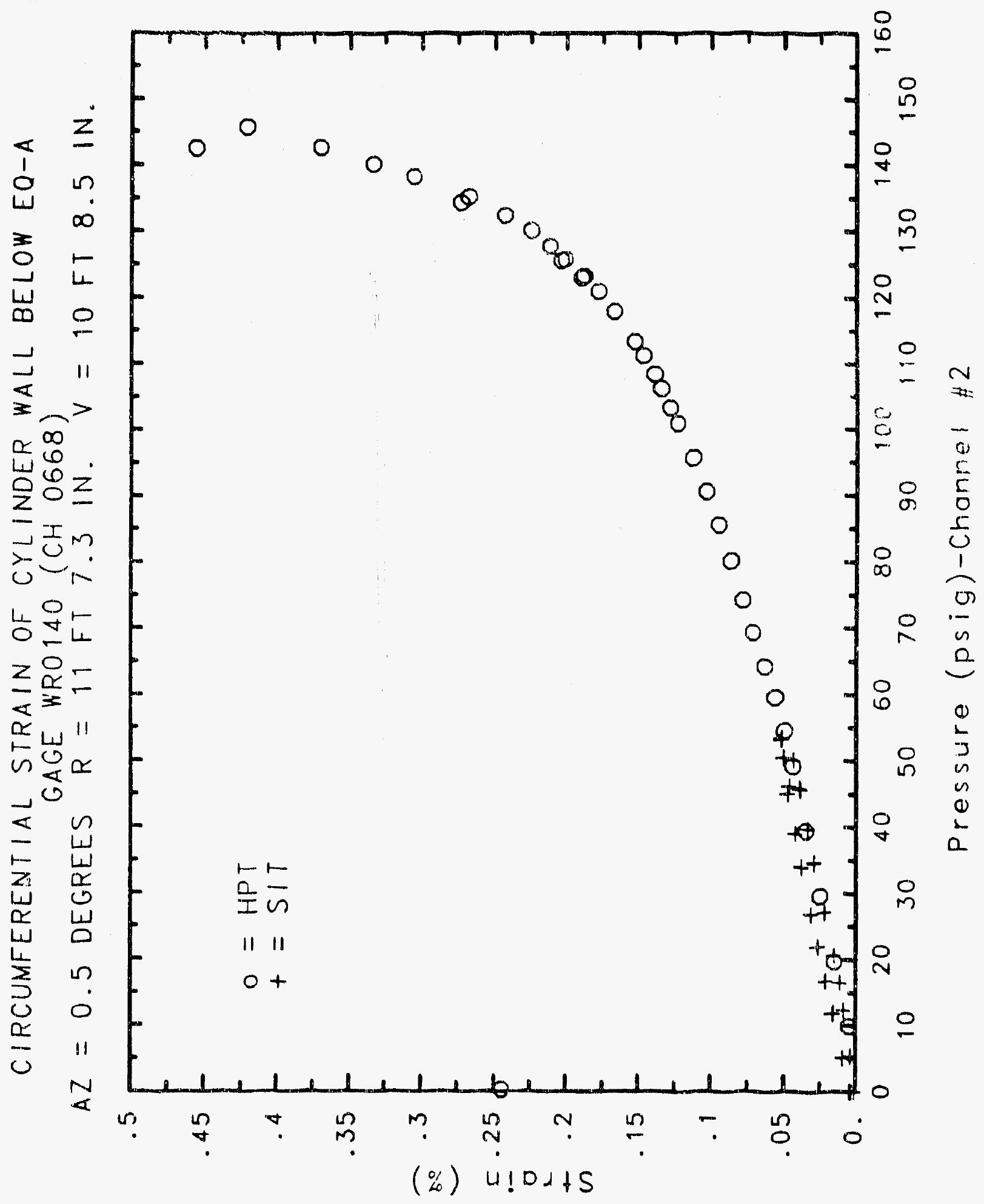

C. -1088 


\section{Reinforced Concrete Test Data}

Weldable Gage 141 Channel 669

StRUCTURAL IntEgrity TEST

\begin{tabular}{cc} 
Pressure & \\
(psig) & Strain \\
-0.05 & \\
5.33 & 0.0002 \\
12.31 & 0.0029 \\
16.44 & 0.0077 \\
20.51 & 0.0103 \\
20.46 & 0.0144 \\
20.47 & 0.0146 \\
27.05 & 0.0151 \\
34.69 & 0.0207 \\
34.53 & 0.0283 \\
34.53 & 0.0281 \\
39.70 & 0.0277 \\
45.90 & 0.0315 \\
45.65 & 0.0362 \\
45.62 & 0.0355 \\
50.10 & 0.0350 \\
53.47 & 0.0390 \\
53.29 & 0.0443 \\
53.21 & 0.0445 \\
50.49 & 0.0444 \\
46.14 & 0.0429 \\
46.13 & 0.0397 \\
44.97 & 0.0398 \\
38.98 & 0.0404 \\
33.99 & 0.0359 \\
33.96 & 0.0322 \\
26.83 & 0.0323 \\
21.88 & 0.0268 \\
21.90 & 0.0228 \\
16.69 & 0.0226 \\
11.74 & 0.0181 \\
11.77 & 0.0133 \\
5.05 & 0.0133 \\
0.02 & 0.0076 \\
-0.04 & 0.0033 \\
-0.02 & 0.0028 \\
-0.02 & 0.0038 \\
0.02 & 0.0036 \\
& 0.0035 \\
\hline
\end{tabular}

High Pressure test

\begin{tabular}{|c|c|}
\hline $\begin{array}{c}\text { Pressure } \\
\text { (psig) } \\
9.89 \\
19.55 \\
29.57 \\
39.42 \\
49.16 \\
54.50 \\
59.57 \\
64.20 \\
69.32 \\
74.16 \\
80.16 \\
85.61 \\
90.58 \\
95.69 \\
100.92 \\
103.25 \\
106.11 \\
108.31 \\
111.08 \\
113.24 \\
117.83 \\
120.92 \\
123.28 \\
122.97 \\
125.82 \\
125.60 \\
127.84 \\
130.19 \\
132.53 \\
135.33 \\
134.42 \\
138.35 \\
140.16 \\
142.63 \\
145.78 \\
142.52 \\
0.22\end{array}$ & $\begin{array}{l}\text { \% Strain } \\
0.0039 \\
0.0122 \\
0.0203 \\
0.0283 \\
0.0364 \\
0.0410 \\
0.0461 \\
0.0527 \\
0.0587 \\
0.0650 \\
0.0720 \\
0.0794 \\
0.0864 \\
0.0940 \\
0.1025 \\
0.1068 \\
0.1118 \\
0.1161 \\
0.1227 \\
1.1278 \\
0.1417 \\
0.1530 \\
0.1639 \\
0.1664 \\
0.1791 \\
0.1829 \\
0.1917 \\
0.2082 \\
0.2310 \\
0.2655 \\
0.2750 \\
0.3226 \\
0.3676 \\
0.4228 \\
0.4791 \\
0.5090 \\
0.3234\end{array}$ \\
\hline
\end{tabular}




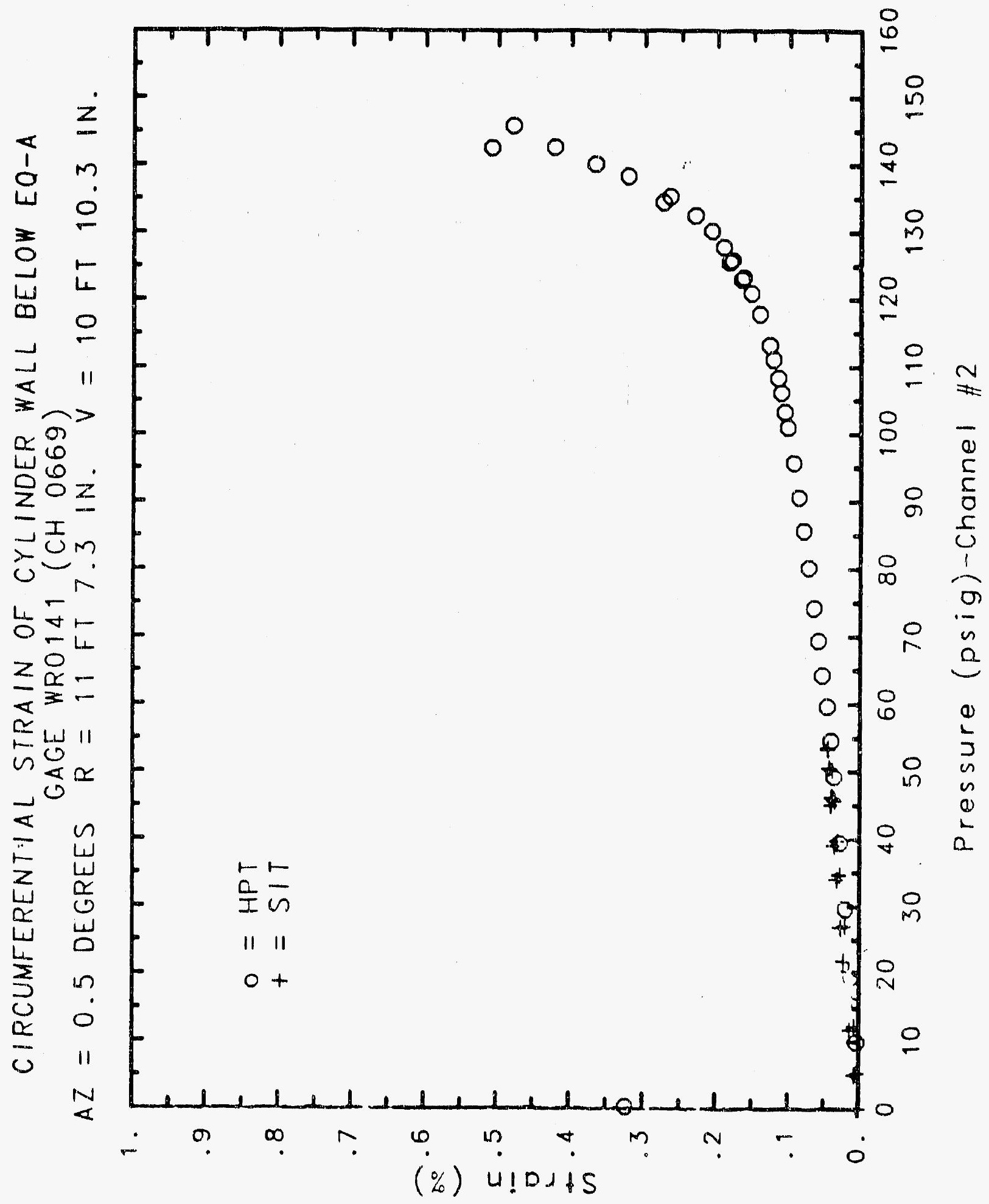

C-1090) 


\section{Reinforced Concrete Test Data}

Weldable gage 142 Channel 680

Structural INTEgRITY TEST

\begin{tabular}{|c|c|c|c|}
\hline $\begin{array}{c}\text { Pressure } \\
\text { (psig) } \\
-0.05 \\
5.33 \\
12.31 \\
16.44 \\
20.51 \\
20.46 \\
20.47 \\
27.05 \\
34.69 \\
34.53 \\
34.53 \\
39.70 \\
45.90 \\
45.65 \\
45.62 \\
50.10 \\
53.47 \\
53.29 \\
53.21 \\
50.49 \\
46.14 \\
46.13 \\
44.97 \\
38.98 \\
33.99 \\
33.96 \\
26.83 \\
21.88 \\
21.90 \\
16.69 \\
11.74 \\
11.77 \\
5.05 \\
0.02 \\
-0.04 \\
-0.02 \\
-0.02 \\
0.02 \\
\end{array}$ & $\begin{array}{c}\text { X Strain } \\
\\
-0.0010 \\
0.0017 \\
0.0062 \\
0.0083 \\
0.0117 \\
0.0103 \\
0.0098 \\
0.0183 \\
0.0308 \\
0.0310 \\
0.0318 \\
0.0394 \\
0.0496 \\
0.0515 \\
0.0522 \\
0.0580 \\
0.0612 \\
0.0625 \\
0.0620 \\
0.0606 \\
0.0572 \\
0.0576 \\
0.0538 \\
0.0483 \\
0.0428 \\
0.0407 \\
0.0335 \\
0.0285 \\
0.0284 \\
0.0227 \\
0.0167 \\
0.0182 \\
0.0109 \\
0.0050 \\
0.0066 \\
0.0055 \\
0.0046 \\
0.0030\end{array}$ & $\begin{array}{c}\text { Pressure } \\
(\text { psig) } \\
9.89 \\
19.55 \\
29.57 \\
39.42 \\
49.16 \\
54.50 \\
59.57 \\
64.20 \\
69.32 \\
74.16 \\
80.16 \\
85.61 \\
90.58 \\
95.69 \\
100.92 \\
103.25 \\
106.11 \\
108.31 \\
111.08 \\
113.24 \\
117.83 \\
120.92 \\
123.28 \\
122.97 \\
125.82 \\
125.60 \\
127.84 \\
130.19 \\
132.53 \\
135.33 \\
134.42 \\
138.35 \\
140.16 \\
142.63 \\
145.78 \\
142.52 \\
0.22 \\
\end{array}$ & $\begin{array}{l}\text { \% Strain } \\
0.0084 \\
0.0205 \\
0.0322 \\
0.0419 \\
0.0515 \\
0.0560 \\
0.0608 \\
0.0653 \\
0.0708 \\
0.0757 \\
0.0853 \\
0.0908 \\
0.0974 \\
0.1036 \\
0.1104 \\
0.1133 \\
0.1171 \\
0.1199 \\
0.1236 \\
0.1271 \\
0.1341 \\
0.1394 \\
0.1436 \\
0.1439 \\
0.1500 \\
0.1511 \\
0.1545 \\
0.1619 \\
0.1724 \\
0.1872 \\
0.1893 \\
0.2098 \\
0.2305 \\
0.2718 \\
0.3094 \\
0.3384 \\
0.1856\end{array}$ \\
\hline
\end{tabular}

High Pressure test

ressur

9.89

39.42

54.50

59.57

74.16

85.61

90.58

95.69

106.11

108.31

111.08

113.24

123.28

122.97

127.84

130.19

132.53

134.42

138.35

140.16

42.52

0.3384

0.1856 


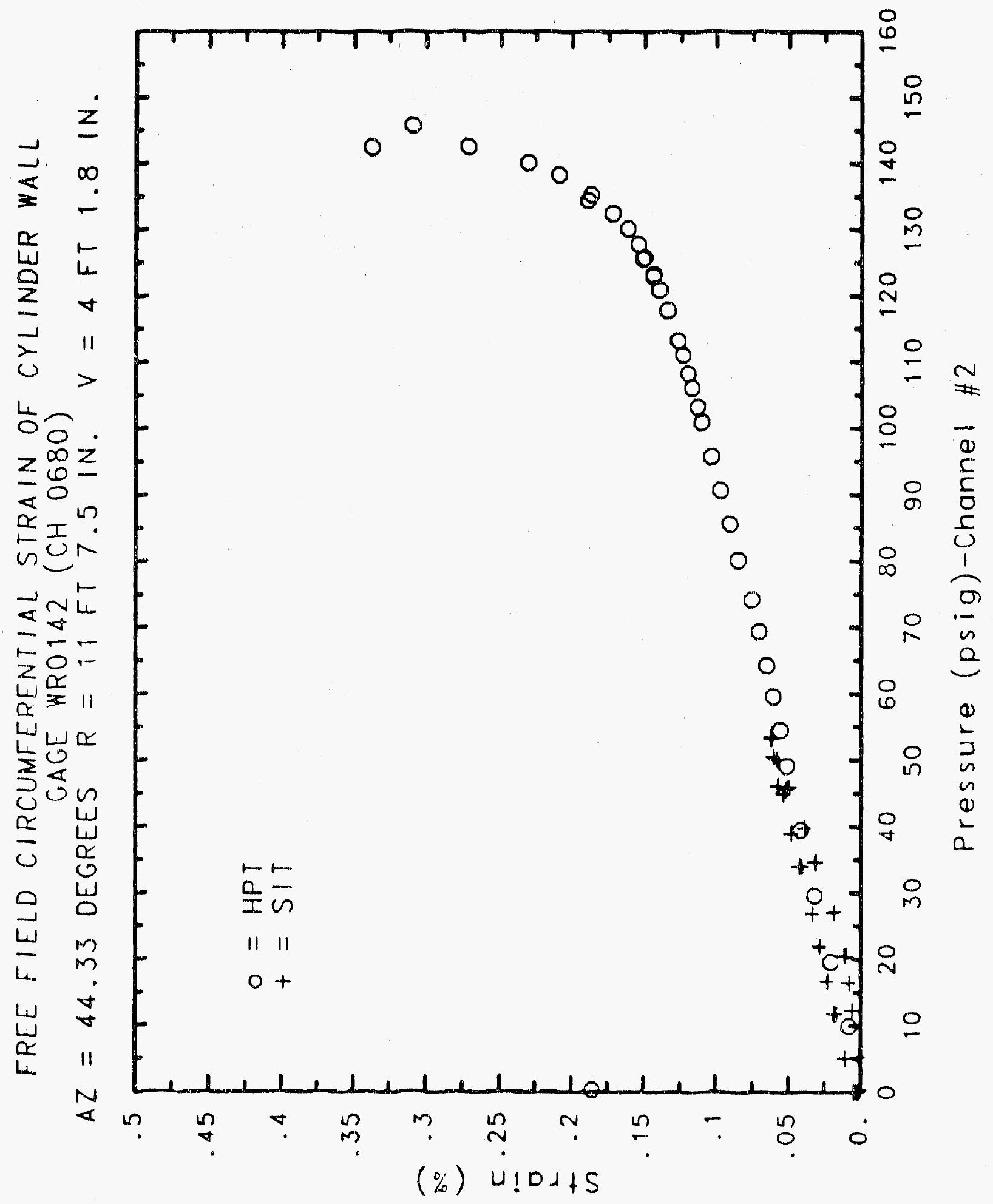




\section{Reinforced Concrete Test Data}

Weldable Gage 143 Chanmel 681

Structural Integrity Test

\begin{tabular}{|c|c|c|c|}
\hline $\begin{array}{c}\text { Pressure } \\
\text { (psig) } \\
-0.05 \\
5.33 \\
12.31 \\
16.44 \\
20.51 \\
20.46 \\
20.47 \\
27.05 \\
34.69 \\
34.53 \\
34.53 \\
39.70 \\
45.90 \\
45.65 \\
45.62 \\
50.10 \\
53.47 \\
53.29 \\
53.21 \\
50.49 \\
46.14 \\
46.13 \\
44.97 \\
38.98 \\
33.99 \\
33.96 \\
26.83 \\
21.88 \\
21.90 \\
16.69 \\
11.74 \\
11.77 \\
5.05 \\
0.02 \\
-0.04 \\
-0.02 \\
-0.02 \\
0.02 \\
\end{array}$ & $\begin{array}{c}\text { S Strain } \\
\text {-0.0005 } \\
0.0026 \\
0.0080 \\
0.0112 \\
0.0165 \\
0.0169 \\
0.0169 \\
0.0270 \\
0.0420 \\
0.0424 \\
0.0429 \\
0.0512 \\
0.0628 \\
0.0644 \\
0.0654 \\
0.0718 \\
0.0782 \\
0.0790 \\
0.0791 \\
0.0769 \\
0.0723 \\
0.0721 \\
0.0698 \\
0.0631 \\
0.0571 \\
0.0560 \\
0.0468 \\
0.0100 \\
0.0399 \\
0.0330 \\
0.0262 \\
0.0267 \\
0.0186 \\
0.0120 \\
0.0114 \\
0.0105 \\
0.0100 \\
0.0100\end{array}$ & $\begin{array}{c}\text { Pressure } \\
(\text { psig) } \\
9.89 \\
19.55 \\
29.57 \\
39.42 \\
49.16 \\
54.50 \\
59.57 \\
64.20 \\
69.32 \\
74.16 \\
80.16 \\
85.61 \\
90.58 \\
95.69 \\
100.92 \\
103.25 \\
106.11 \\
108.31 \\
111.08 \\
113.24 \\
117.83 \\
120.92 \\
123.28 \\
122.97 \\
125.82 \\
125.60 \\
127.84 \\
130.19 \\
132.53 \\
135.33 \\
134.42 \\
138.35 \\
140.16 \\
142.63 \\
145.78 \\
142.52 \\
0.22 \\
\end{array}$ & $\begin{array}{l}\text { \% Strain } \\
0.0088 \\
0.0227 \\
0.0367 \\
0.0508 \\
0.0629 \\
0.0705 \\
0.0779 \\
0.0855 \\
0.0948 \\
0.1036 \\
0.1180 \\
0.1291 \\
0.1400 \\
0.1524 \\
0.1664 \\
0.1734 \\
0.1809 \\
0.1866 \\
0.1963 \\
0.2032 \\
0.2224 \\
0.2391 \\
0.2533 \\
0.2563 \\
0.2788 \\
0.2826 \\
0.2937 \\
0.3175 \\
0.3481 \\
0.3807 \\
0.3856 \\
0.4507 \\
0.5042 \\
0.5314 \\
0.6096 \\
0.6598 \\
0.3772\end{array}$ \\
\hline
\end{tabular}




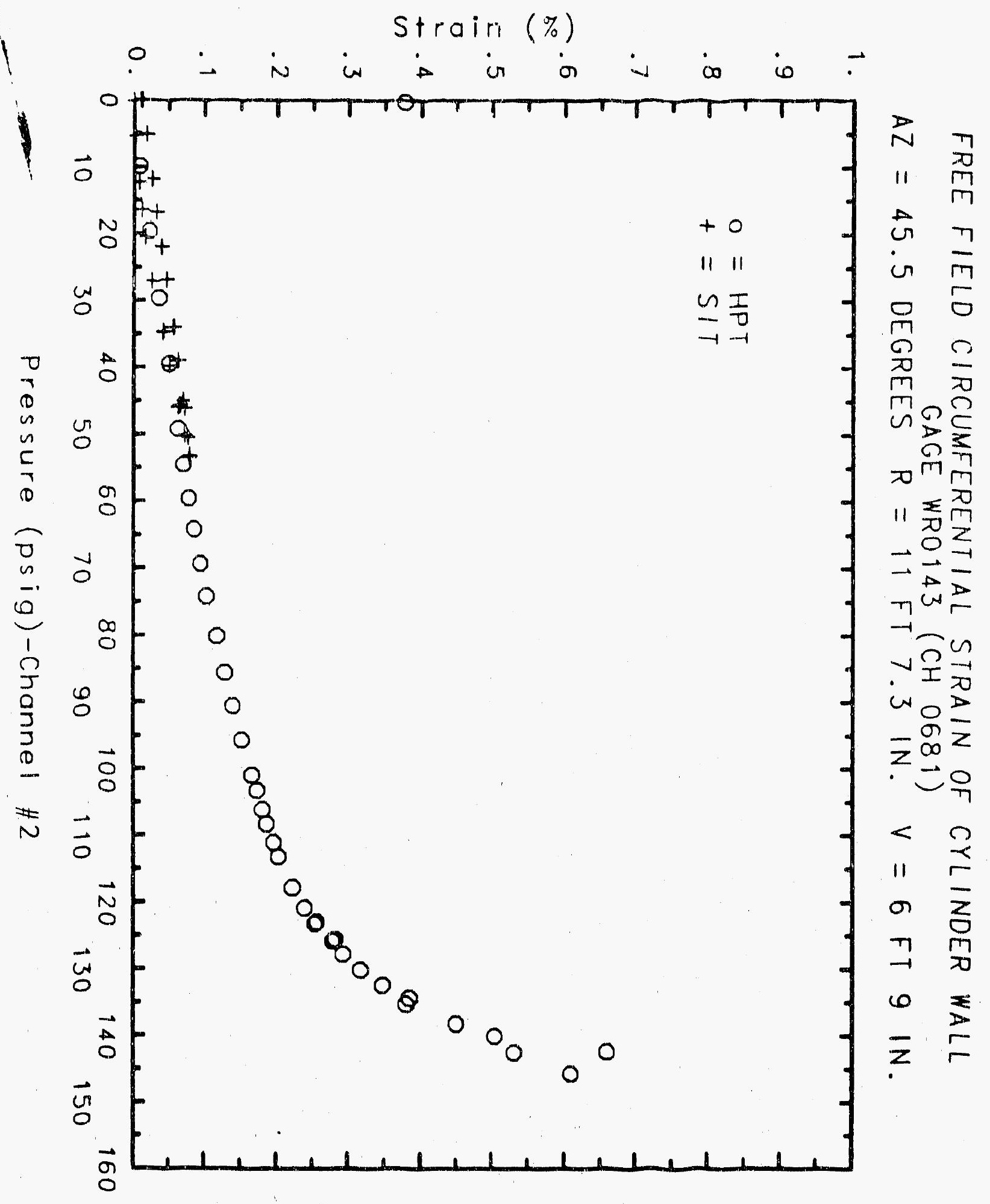


34:

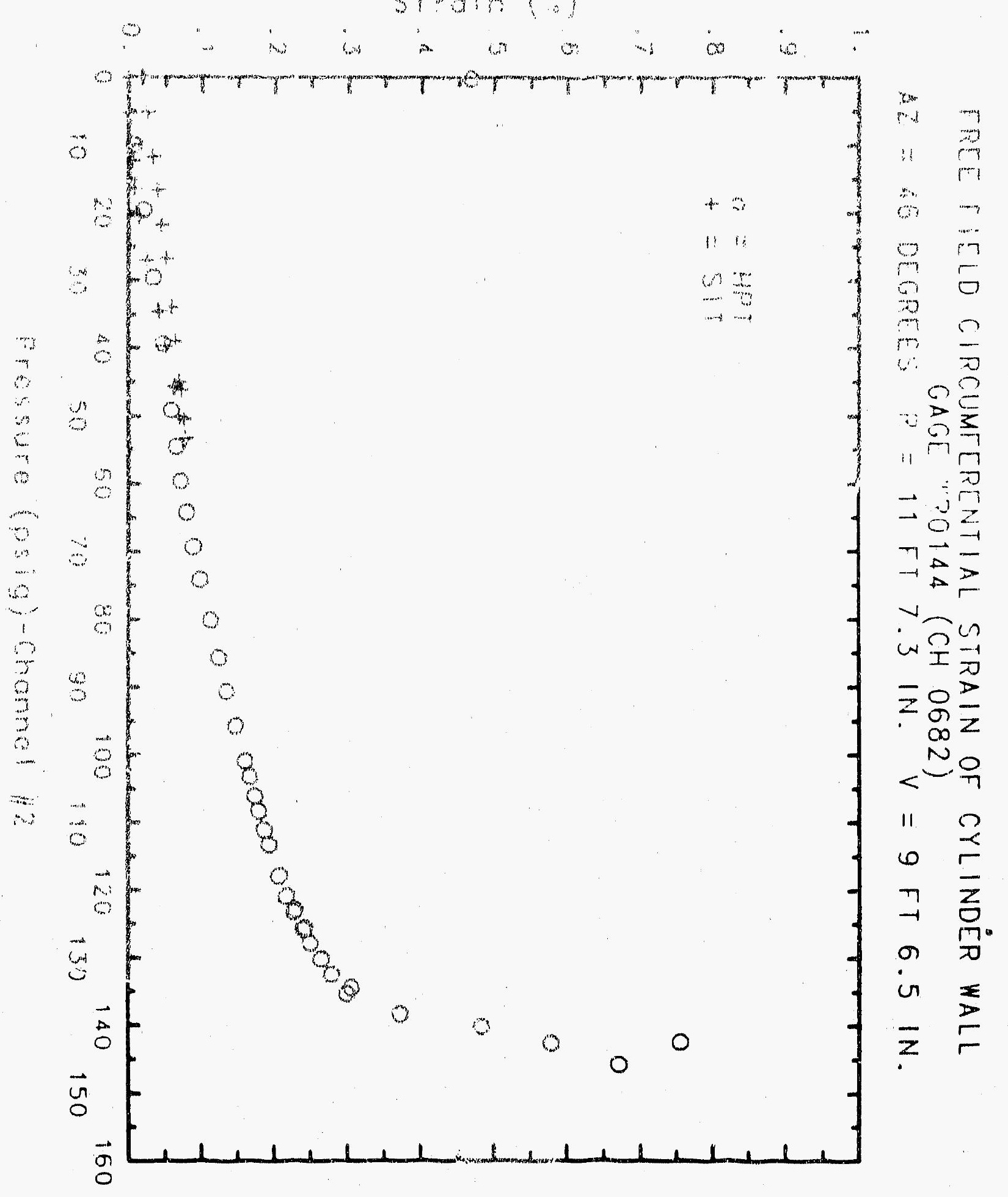




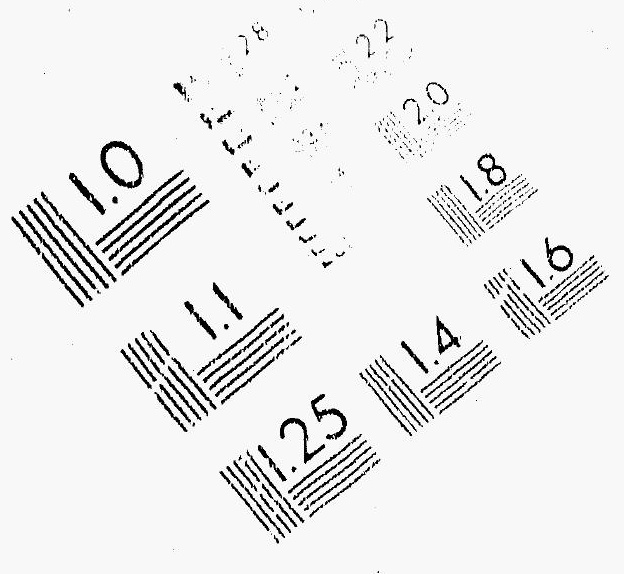

\section{IMAGE EVALUATION TEST TARGET (MT-3)}
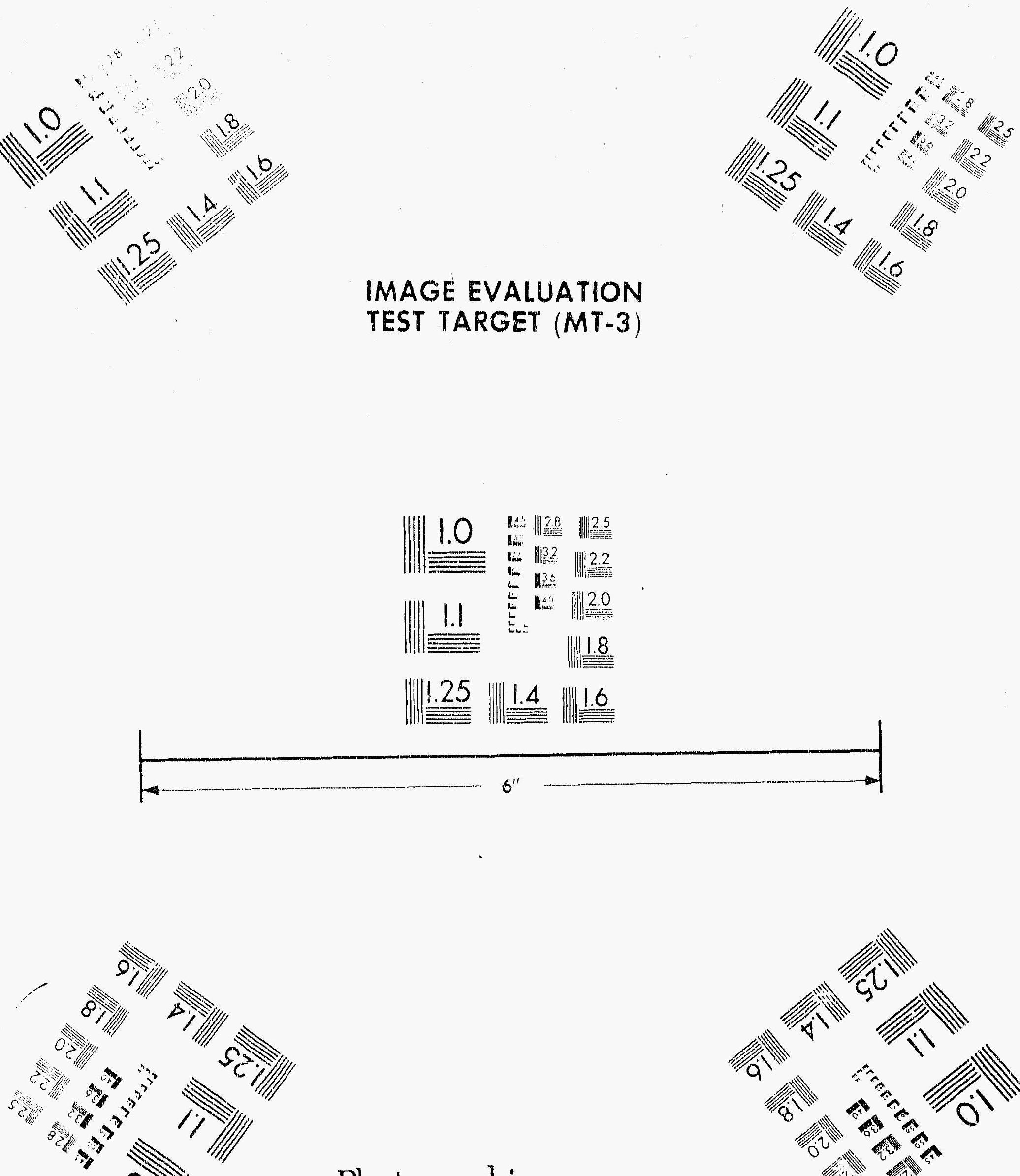

$0, \mid$

Photographic Sciences Corporation
23 WEST MAIN STREET WEBSTER, N.Y. 14580 ( 716 ) B72.4503

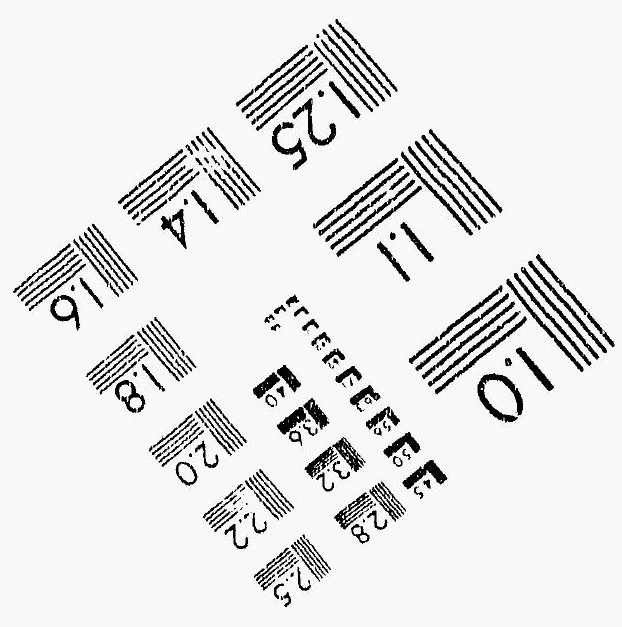




\section{SECURITY CLASSIFICATION}

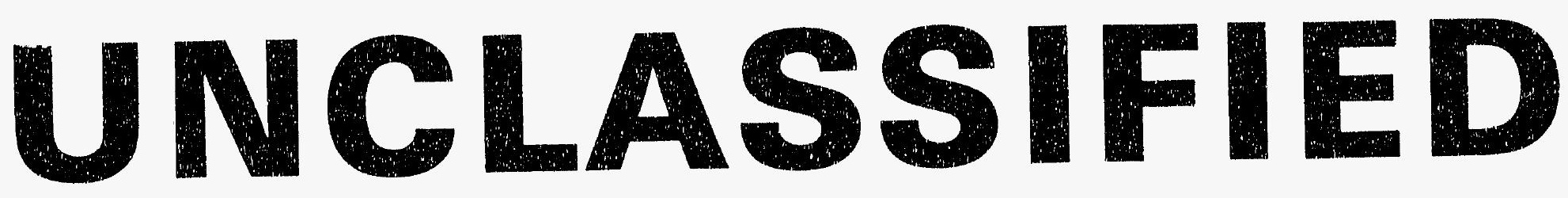

\section{DATE OF MICROFILMING $1-6-92$}

MICROFILMED BY - Lerrar hard

SANDIA NATIONAL LABS 


\section{THIS DOCUMENT FILMED}

AT

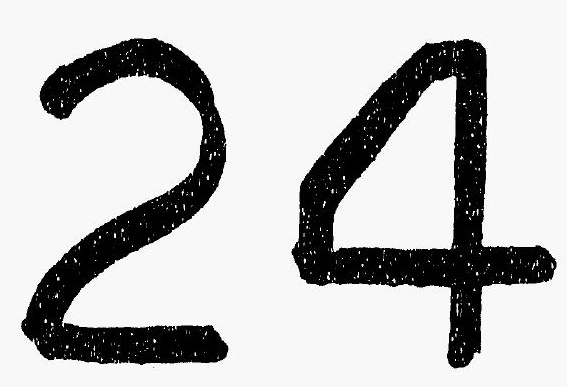

REDUCTION 


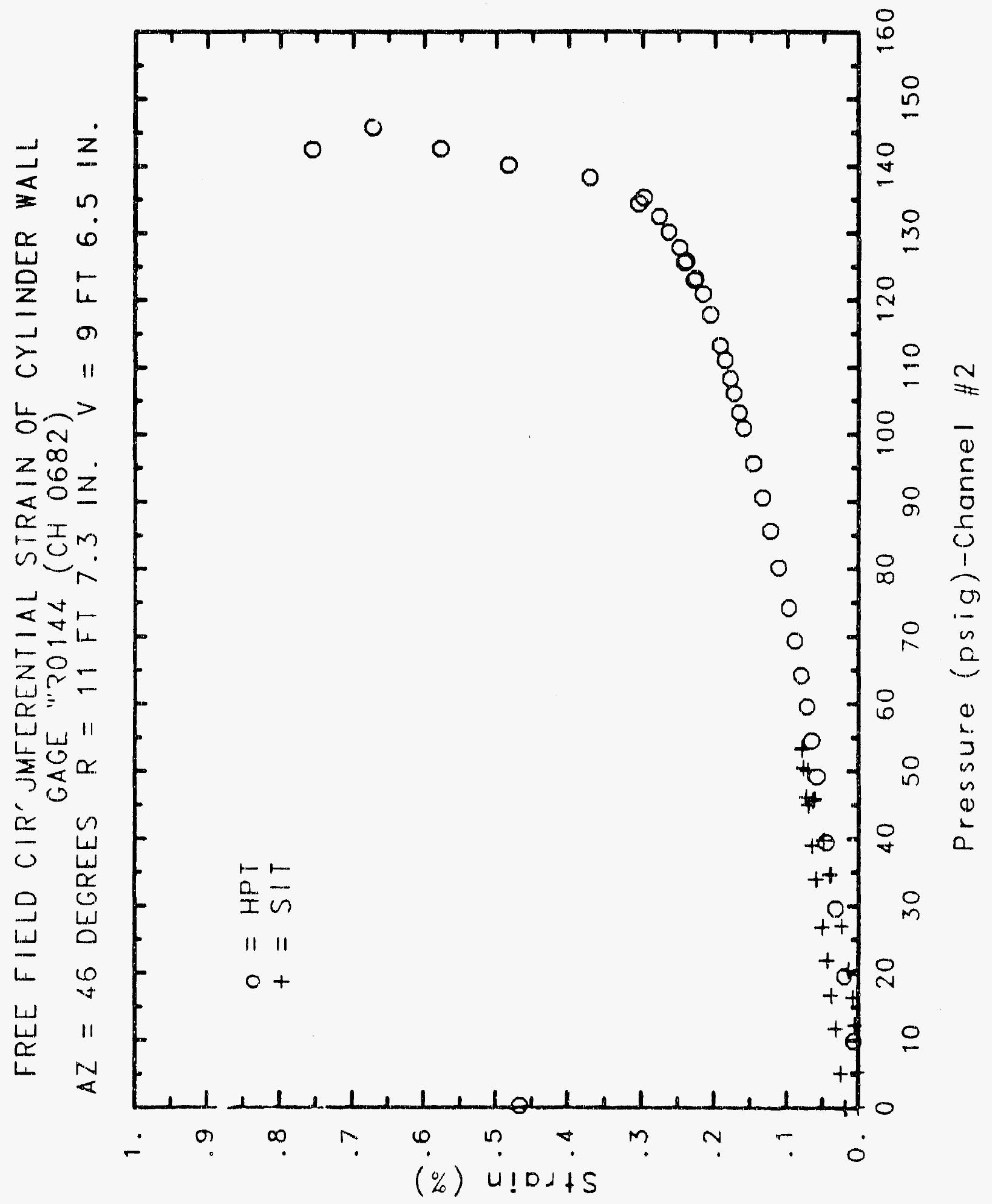




\section{Reinforced Concrete Test Data}

\begin{tabular}{|c|c|c|c|}
\hline STRUCTURAL & $\begin{array}{l}\text { WELDABLE GAGE } 145 \\
\text { INTEGRITY TEST }\end{array}$ & $\begin{array}{l}\text { Channel } 683 \\
\text { High Pressure }\end{array}$ & TEST \\
\hline $\begin{array}{c}\text { Pressure } \\
\text { (psig) } \\
-0.05 \\
5.33 \\
12.31 \\
16.44 \\
20.51 \\
20.46 \\
20.47 \\
27.05 \\
34.69 \\
34.53 \\
34.53 \\
39.70 \\
45.90 \\
45.65 \\
45.62 \\
50.10 \\
53.47 \\
53.29 \\
53.21 \\
50.49 \\
46.14 \\
46.13 \\
44.97 \\
38.98 \\
33.99 \\
33.96 \\
26.83 \\
21.88 \\
21.90 \\
16.69 \\
11.74 \\
11.77 \\
5.05 \\
0.02 \\
-0.04 \\
-0.02 \\
-0.02 \\
0.02 \\
\end{array}$ & $\begin{array}{l}\text { Strain } \\
\text { S.0014 } \\
0.0028 \\
0.0072 \\
0.0102 \\
0.0167 \\
0.0174 \\
0.0182 \\
0.0317 \\
0.0466 \\
0.0471 \\
0.0475 \\
0.0553 \\
0.0662 \\
0.0677 \\
0.0686 \\
0.0746 \\
0.0800 \\
0.0807 \\
0.0812 \\
0.0788 \\
0.0741 \\
0.0741 \\
0.0700 \\
0.0637 \\
0.0579 \\
0.0574 \\
0.0489 \\
0.0422 \\
0.0423 \\
0.0357 \\
0.0293 \\
0.0296 \\
0.0220 \\
0.0162 \\
0.0141 \\
0.0135 \\
0.0126 \\
0.0127\end{array}$ & $\begin{array}{c}\text { Pressure } \\
\text { (psig) } \\
9.89 \\
19.55 \\
29.57 \\
39.42 \\
49.16 \\
54.50 \\
59.57 \\
64.20 \\
69.32 \\
74.16 \\
80.16 \\
85.61 \\
90.58 \\
95.69 \\
100.92 \\
103.25 \\
106.11 \\
108.31 \\
111.08 \\
113.24 \\
117.83 \\
120.92 \\
123.28 \\
122.97 \\
125.82 \\
125.60 \\
127.84 \\
130.19 \\
132.53 \\
135.33 \\
134.42 \\
138.35 \\
140.16 \\
142.63 \\
145.78 \\
142.52 \\
0.22 \\
\end{array}$ & $\begin{array}{l}\text { \% Strain } \\
0.0084 \\
0.0215 \\
0.0352 \\
0.0486 \\
0.0611 \\
0.0679 \\
0.0752 \\
0.0822 \\
0.0894 \\
0.0966 \\
0.1089 \\
0.1171 \\
0.1250 \\
0.1333 \\
0.1421 \\
0.1466 \\
0.1517 \\
0.1555 \\
0.1614 \\
0.1658 \\
0.1764 \\
0.1843 \\
0.1912 \\
0.1921 \\
0.2002 \\
0.2012 \\
0.2072 \\
0.2186 \\
0.2322 \\
0.2474 \\
0.2518 \\
0.2788 \\
0.3110 \\
0.3600 \\
0.4517 \\
0.4688 \\
0.2169\end{array}$ \\
\hline
\end{tabular}




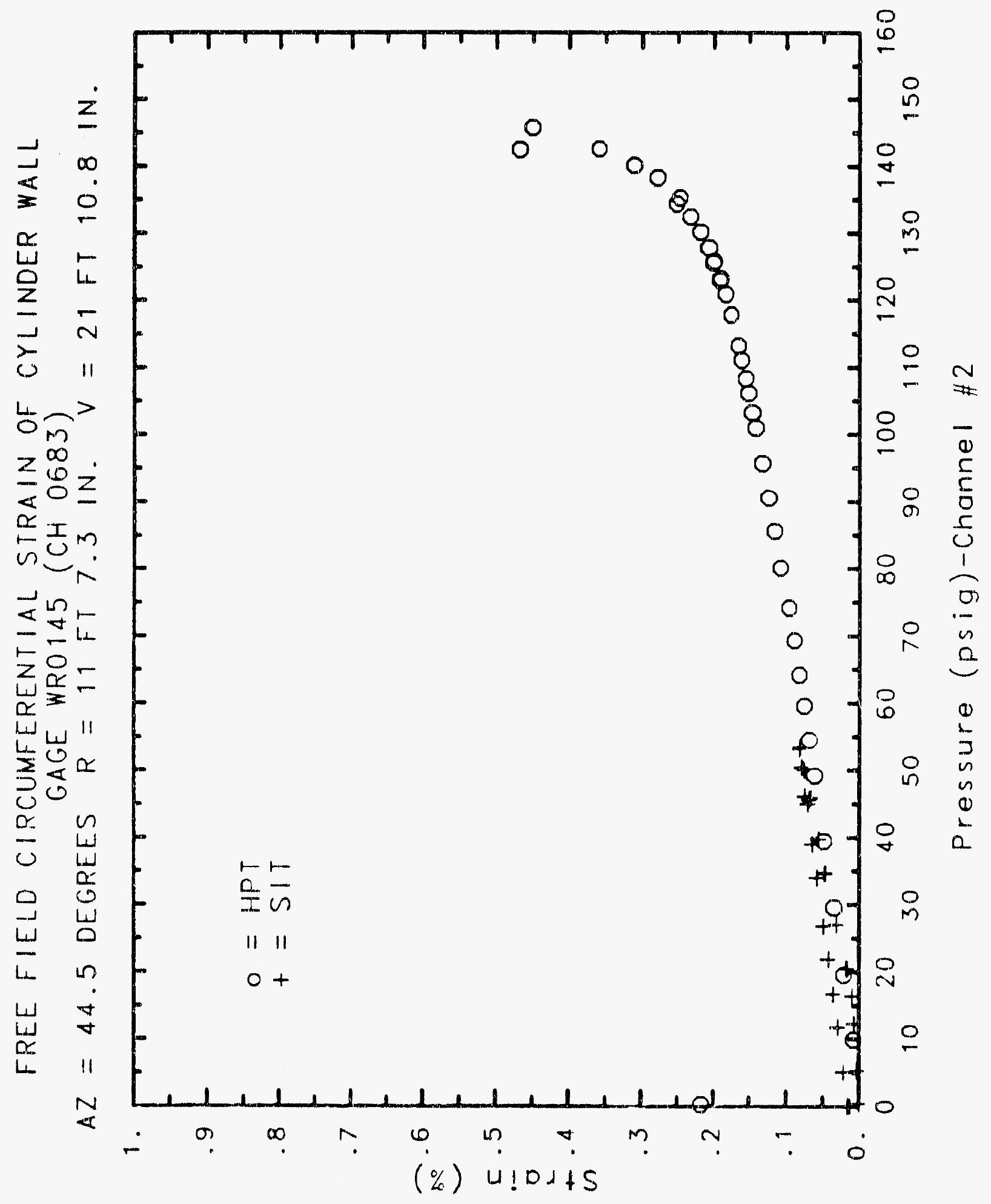




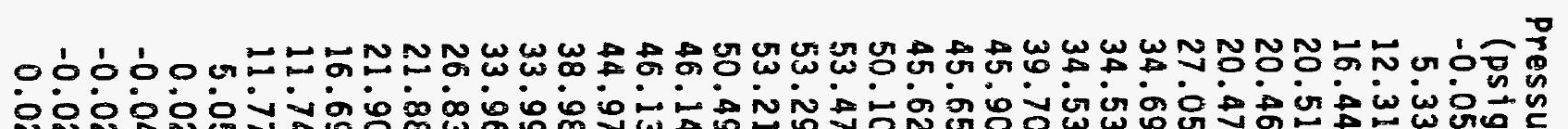

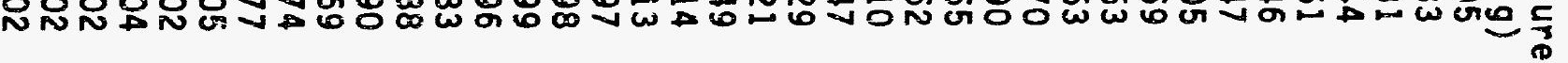

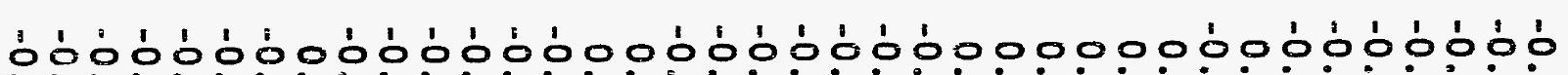

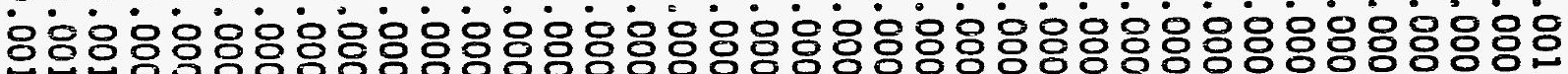

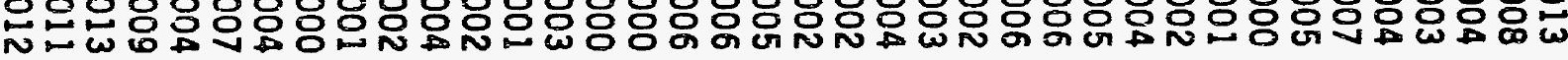

$000000000000000000000000000 \dot{0} 0 \dot{0} 0 \dot{0} 0 \dot{0}$

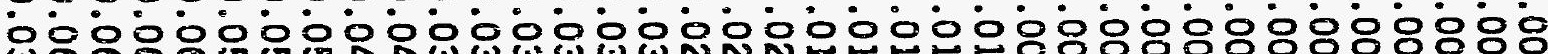

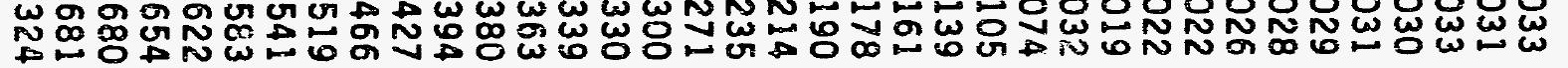

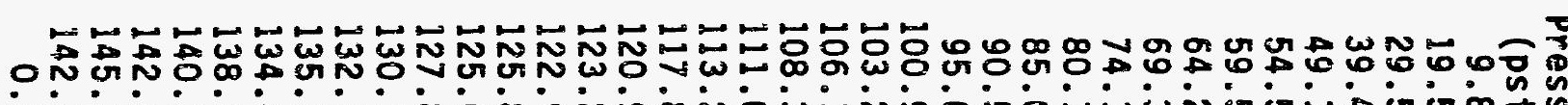

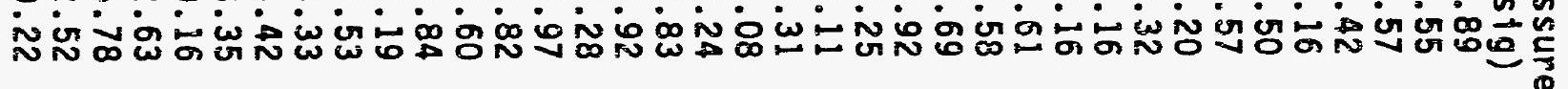






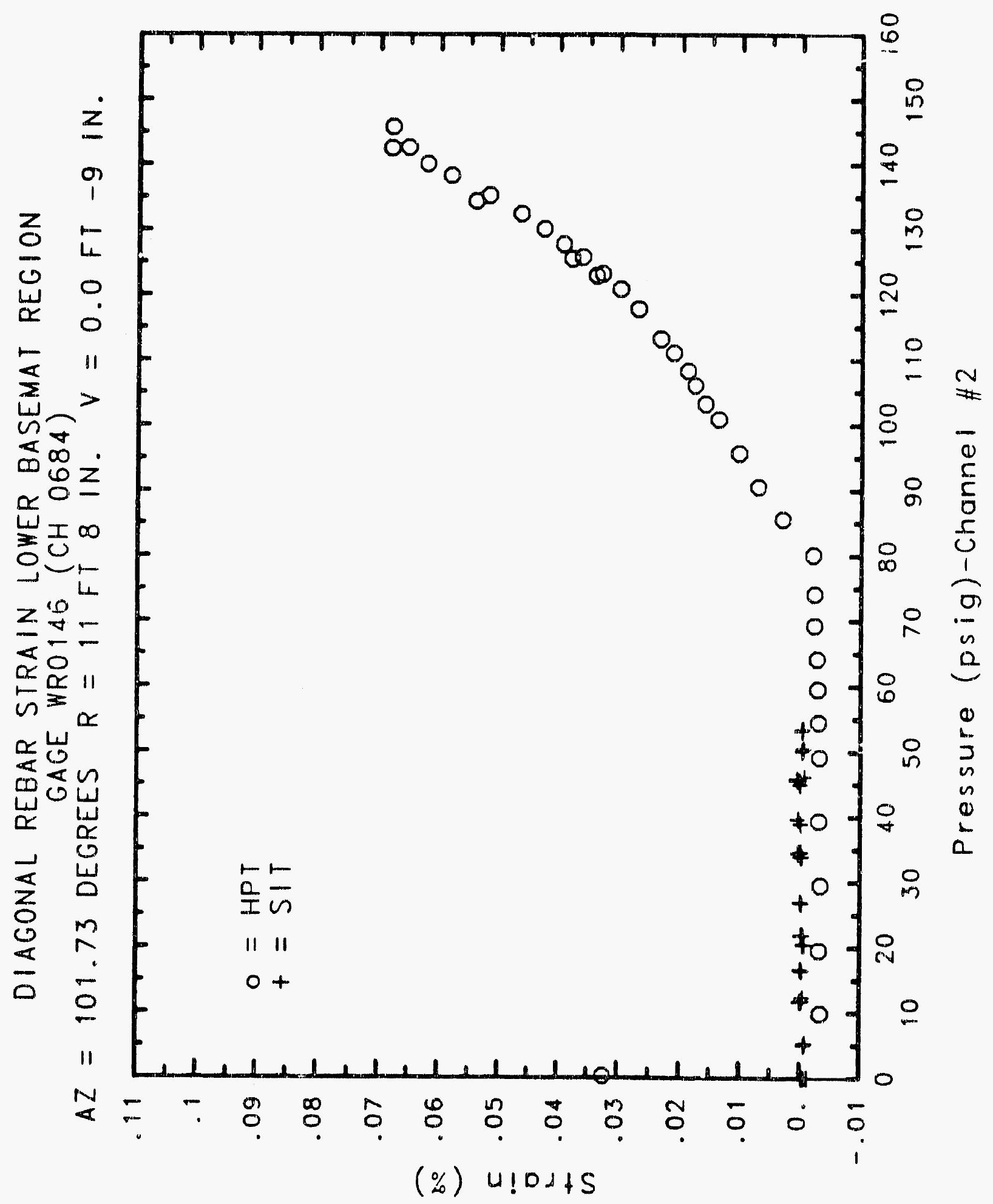




\section{Reinforced Concrete Test Data}

Weldable gage 147 Channel 685

STRUCTURAL INTEGRITY TEST

\begin{tabular}{|c|c|c|c|}
\hline $\begin{array}{c}\text { Pressure } \\
\text { (psig) } \\
-0.05 \\
5.33 \\
12.31 \\
16.44 \\
20.51 \\
20.46 \\
20.47 \\
27.05 \\
34.69 \\
34.53 \\
34.53 \\
39.70 \\
45.90 \\
45.65 \\
45.62 \\
50.10 \\
53.47 \\
53.29 \\
53.21 \\
50.49 \\
46.14 \\
46.13 \\
44.97 \\
38.98 \\
33.99 \\
33.96 \\
26.83 \\
21.88 \\
21.50 \\
16.69 \\
11.74 \\
11.77 \\
5.05 \\
0.02 \\
-0.04 \\
-0.02 \\
-0.02 \\
0.02 \\
\end{array}$ & $\begin{array}{l}\text { \% Strain } \\
-0.0002 \\
-0.0002 \\
-0.0018 \\
-0.0032 \\
-0.0053 \\
-0.0062 \\
-0.0071 \\
-0.0081 \\
-0.0090 \\
-0.0097 \\
-0.0087 \\
-0.0087 \\
-0.0088 \\
-0.0088 \\
-0.0086 \\
-0.0090 \\
-0.0088 \\
-0.0085 \\
-0.0085 \\
-0.0081 \\
-0.0085 \\
-0.0085 \\
-0.0081 \\
-0.0081 \\
-0.0081 \\
-0.0087 \\
-0.0088 \\
-0.0093 \\
-0.0099 \\
-0.0104 \\
-0.0095 \\
-0.0091 \\
-0.0073 \\
-0.0051 \\
0.0004 \\
-0.0002 \\
-0.0004 \\
-0.0014\end{array}$ & $\begin{array}{c}\text { Pressure } \\
(\text { psig) } \\
9.89 \\
19.55 \\
29.57 \\
39.42 \\
49.16 \\
54.50 \\
59.57 \\
64.20 \\
69.32 \\
74.16 \\
80.16 \\
85.61 \\
90.58 \\
95.69 \\
100.92 \\
103.25 \\
106.11 \\
108.31 \\
111.08 \\
113.24 \\
117.83 \\
120.92 \\
123.28 \\
122.97 \\
125.82 \\
125.60 \\
127.84 \\
130.19 \\
132.53 \\
135.33 \\
134.42 \\
138.35 \\
140.16 \\
142.63 \\
145.78 \\
142.52 \\
0.22 \\
\end{array}$ & $\begin{array}{l}\text { \% Strain } \\
-0.0068 \\
-0.0087 \\
-0.0099 \\
-0.0104 \\
-0.0111 \\
-0.0109 \\
-0.0109 \\
-0.0096 \\
-0.0094 \\
-0.0094 \\
-0.0055 \\
-0.0040 \\
-0.0021 \\
0.0007 \\
0.0027 \\
0.0046 \\
0.0051 \\
0.0063 \\
0.0081 \\
0.0090 \\
0.0115 \\
0.0138 \\
0.0153 \\
0.0155 \\
0.0169 \\
0.0168 \\
0.0179 \\
0.0206 \\
0.0237 \\
0.0273 \\
0.0284 \\
0.0333 \\
0.0366 \\
0.0417 \\
0.0456 \\
0.0448 \\
0.0067\end{array}$ \\
\hline
\end{tabular}

High Pressure test

Pressure \% Strain

psig)

$-0.0087$

$-0.0099$

$-0.0104$

$-0.0094$

$-0.0094$

$-0.0055$

$-0.0040$

$-0.0021$

0.0007

0.0046

0.0081

0.0090

0.0138

0.0153

0.0168

0.0237

0.0273

0.0284

0.0333

0366

0.0456

0.0448

0.0067 


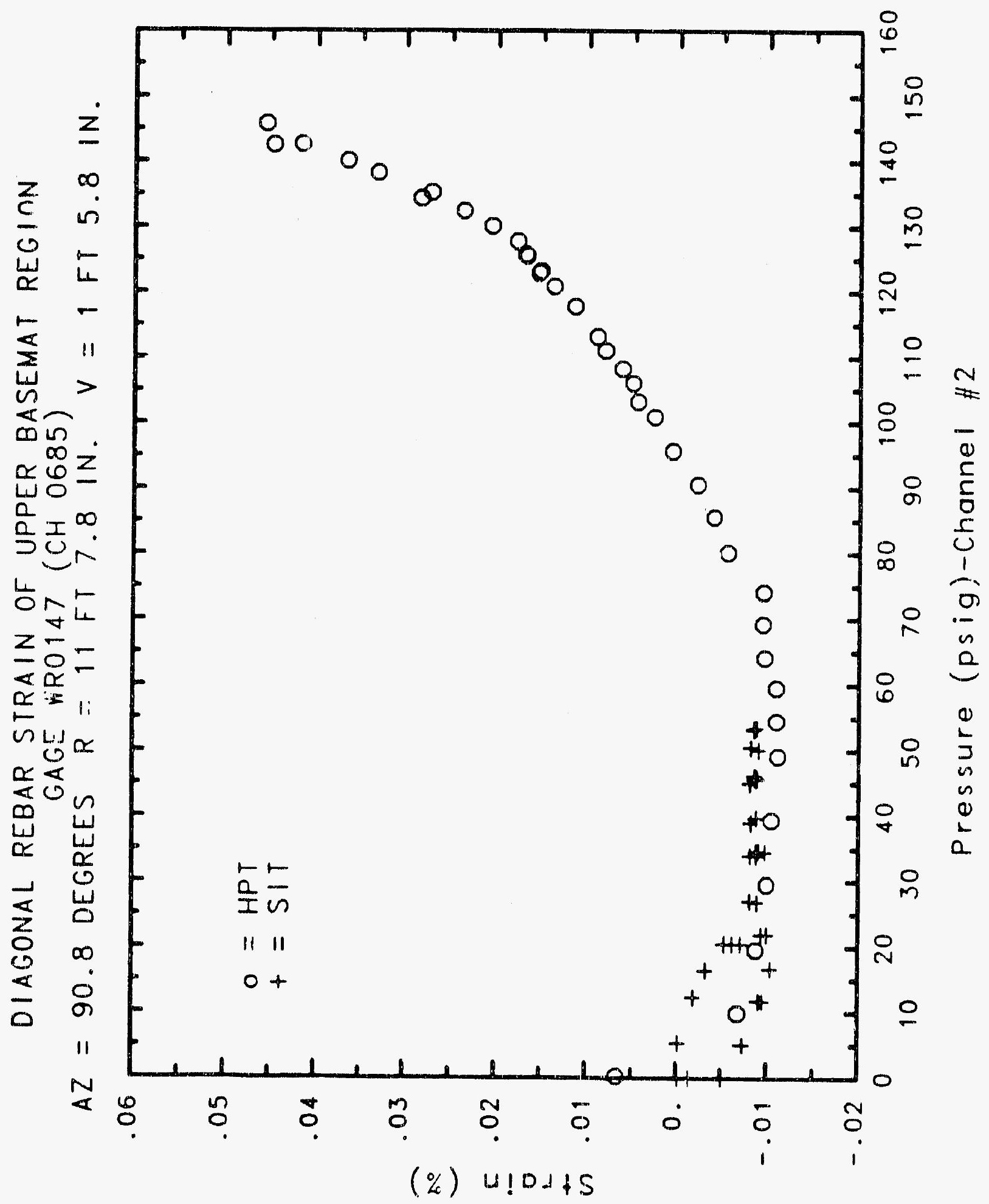




\section{Reinforced Concrete Test Data}

\begin{tabular}{|c|c|c|c|}
\hline STRUCTURAL & $\begin{array}{l}\text { WELDABLE GAGE } 148 \\
\text { INTEGRITY TEST }\end{array}$ & $\begin{aligned} \text { Channel } 686 \\
\text { High Pressure }\end{aligned}$ & TEST \\
\hline $\begin{array}{l}\text { Pressure } \\
\text { (psig) } \\
-0.05 \\
5.33 \\
12.31 \\
16.44 \\
20.51 \\
20.46 \\
20.47 \\
27.05 \\
34.69 \\
34.53 \\
34.53 \\
39.70 \\
45.90 \\
45.65 \\
45.62 \\
50.10 \\
53.47 \\
53.29 \\
53.21 \\
50.49 \\
46.14 \\
46.13 \\
44.97 \\
38.98 \\
33.99 \\
33.96 \\
26.83 \\
21.88 \\
21.90 \\
16.69 \\
11.74 \\
11.77 \\
5.05 \\
0.02 \\
-0.04 \\
-0.02 \\
-0.02 \\
0.02 \\
\end{array}$ & $\begin{array}{l}\text { Strain } \\
0.0002 \\
-0.0005 \\
-0.0023 \\
-0.0035 \\
-0.0045 \\
-0.0051 \\
-0.0053 \\
-0.0059 \\
-0.0058 \\
-0.0051 \\
-0.0051 \\
-0.0049 \\
-0.0044 \\
-0.0040 \\
-0.0040 \\
-0.0037 \\
-0.0032 \\
-0.0034 \\
-0.0030 \\
-0.0029 \\
-0.0035 \\
-0.0036 \\
-0.0044 \\
-0.0045 \\
-0.0049 \\
-0.0046 \\
-0.0047 \\
-0.0046 \\
-0.0049 \\
-0.0051 \\
-0.0052 \\
-0.0055 \\
-0.0049 \\
-0.0032 \\
-0.0001 \\
-0.0004 \\
-0.0015 \\
-0.0016\end{array}$ & $\begin{array}{c}\text { Pressure } \\
(\text { psig) } \\
9.89 \\
19.55 \\
29.57 \\
39.42 \\
49.16 \\
54.50 \\
59.57 \\
64.20 \\
69.32 \\
74.16 \\
80.16 \\
85.61 \\
90.58 \\
95.69 \\
100.92 \\
103.25 \\
106.11 \\
108.31 \\
111.08 \\
113.24 \\
117.83 \\
120.92 \\
123.28 \\
122.97 \\
125.82 \\
125.60 \\
127.84 \\
130.19 \\
132.53 \\
135.33 \\
134.42 \\
138.35 \\
140.16 \\
142.63 \\
145.78 \\
142.52 \\
0.22 \\
\end{array}$ & $\begin{array}{l}\text { \% Strain } \\
-0.0064 \\
-0.0072 \\
-0.0069 \\
-0.0065 \\
-0.0058 \\
-0.0060 \\
-0.0055 \\
-0.0050 \\
-0.0047 \\
-0.0049 \\
-0.0024 \\
-0.0017 \\
-0.0012 \\
-0.0002 \\
0.0006 \\
0.0009 \\
0.0011 \\
0.0018 \\
0.0019 \\
0.0029 \\
0.0049 \\
0.0058 \\
0.0067 \\
0.0070 \\
0.0079 \\
0.0085 \\
0.0097 \\
0.0123 \\
0.0156 \\
0.0207 \\
0.0208 \\
0.0273 \\
0.0309 \\
0.0366 \\
0.0431 \\
0.0436 \\
-0.0002\end{array}$ \\
\hline
\end{tabular}




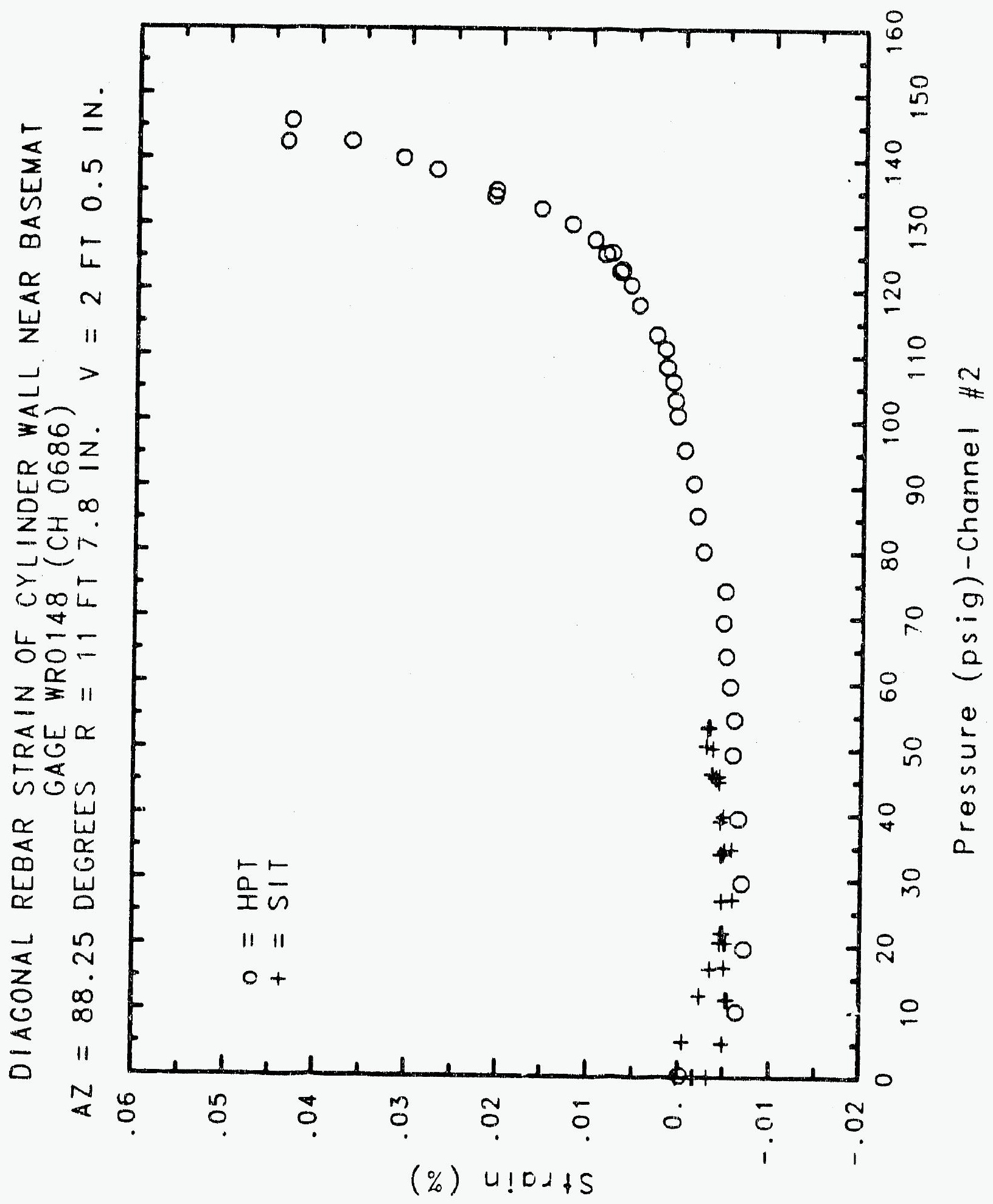




\section{Reinforced Concrete Test Data}

\begin{tabular}{|c|c|c|c|}
\hline STRUCTURAL & $\begin{array}{l}\text { WELDABLE GAGE } 149 \\
\text { INTEGRITY TEST }\end{array}$ & $\begin{array}{l}\text { Channel } 687 \\
\text { Higm Pressure }\end{array}$ & TEST \\
\hline $\begin{array}{l}\text { Pressure } \\
\text { (psig) } \\
-0.05 \\
5.33 \\
12.31 \\
16.44 \\
20.51 \\
20.46 \\
20.47 \\
27.05 \\
34.69 \\
34.53 \\
34.53 \\
39.70 \\
45.90 \\
45.65 \\
45.62 \\
50.10 \\
53.47 \\
53.29 \\
53.21 \\
50.49 \\
46.14 \\
46.13 \\
44.97 \\
38.98 \\
33.99 \\
33.96 \\
26.83 \\
21.88 \\
21.90 \\
16.69 \\
11.74 \\
11.77 \\
5.05 \\
0.02 \\
-0.04 \\
-0.02 \\
-0.02 \\
0.02 \\
\end{array}$ & $\begin{array}{c}\% \text { Strain } \\
0.0003 \\
-0.0002 \\
0.0003 \\
-0.0005 \\
-0.0015 \\
-0.0030 \\
-0.0058 \\
-0.0060 \\
-0.0046 \\
-0.0046 \\
-0.0050 \\
-0.0046 \\
-0.0019 \\
0.0010 \\
0.0016 \\
0.0035 \\
0.0054 \\
0.0064 \\
0.0073 \\
0.0076 \\
0.0073 \\
0.0078 \\
0.0076 \\
0.0061 \\
0.0042 \\
0.0004 \\
-0.0031 \\
-0.0060 \\
-0.0080 \\
-0.0071 \\
-0.0074 \\
-0.0045 \\
-0.0048 \\
-0.0050 \\
0.0018 \\
0.0015 \\
0.0008 \\
-0.0015\end{array}$ & $\begin{array}{l}\text { Pressure } \\
\text { (psig) } \\
9.89 \\
19.55 \\
29.57 \\
39.42 \\
49.16 \\
54.50 \\
59.57 \\
64.20 \\
69.32 \\
74.16 \\
80.16 \\
35.61 \\
90.58 \\
95.69 \\
100.92 \\
103.25 \\
106.11 \\
108.31 \\
111.08 \\
113.24 \\
117.83 \\
120.92 \\
123.28 \\
122.97 \\
125.82 \\
125.60 \\
127.84 \\
130.19 \\
132.53 \\
135.33 \\
134.42 \\
138.35 \\
140.16 \\
142.63 \\
145.78 \\
142.52 \\
0.22 \\
\end{array}$ & 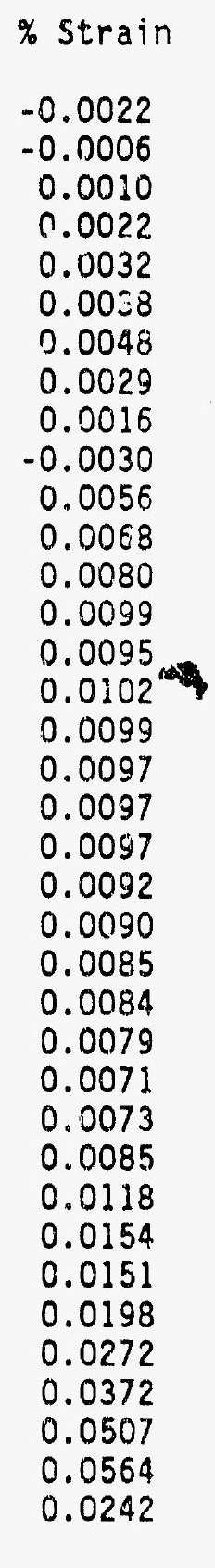 \\
\hline
\end{tabular}




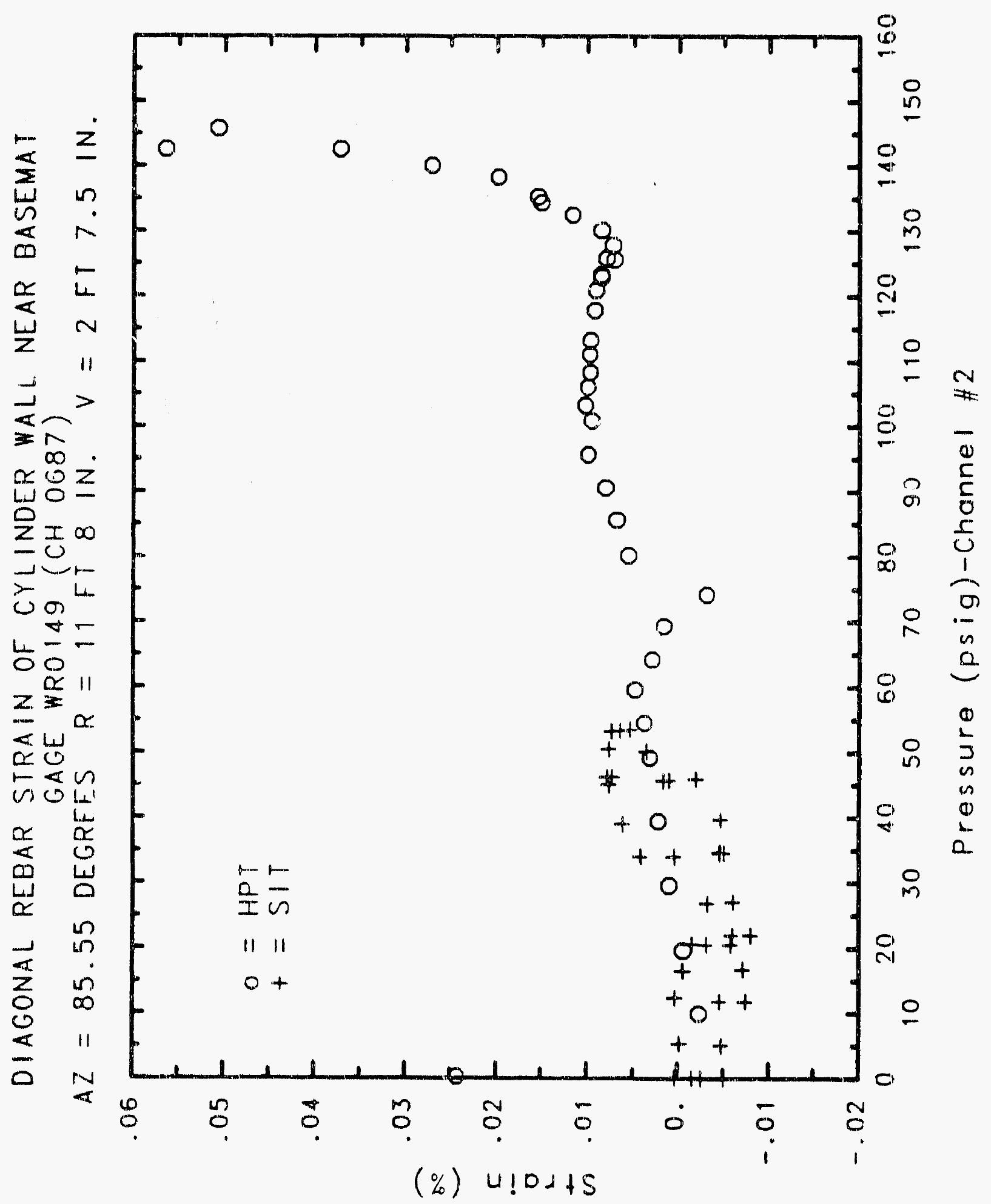




\section{Reinforced Concrete Test Data}

Weldable Gage 150 Channel. 688

Structural InTEgrity TEST

\begin{tabular}{|c|c|c|c|}
\hline $\begin{array}{c}\text { Pressure } \\
\text { (psig) } \\
-0.05 \\
5.33 \\
12.31 \\
16.44 \\
20.51 \\
20.46 \\
20.47 \\
27.05 \\
34.69 \\
34.53 \\
34.53 \\
39.70 \\
45.90 \\
45.65 \\
45.62 \\
50.10 \\
53.47 \\
53.29 \\
53.21 \\
50.49 \\
46.14 \\
46.13 \\
44.97 \\
38.98 \\
33.99 \\
33.96 \\
26.83 \\
21.88 \\
21.90 \\
16.69 \\
11.74 \\
11.77 \\
5.05 \\
0.02 \\
-0.04 \\
-0.02 \\
-0.02 \\
0.02 \\
\end{array}$ & $\begin{array}{l}\text { \% Strain } \\
-0.0003 \\
0.0014 \\
0.0039 \\
0.0053 \\
0.0069 \\
0.0067 \\
0.0055 \\
0.0112 \\
0.0218 \\
0.0230 \\
0.0242 \\
0.0309 \\
0.0419 \\
0.0453 \\
0.0452 \\
0.0515 \\
0.0565 \\
0.0578 \\
0.0580 \\
0.0573 \\
0.0545 \\
0.0549 \\
0.0523 \\
0.0477 \\
0.0444 \\
0.0412 \\
0.0347 \\
0.0292 \\
0.0274 \\
0.0245 \\
0.0214 \\
0.0233 \\
0.0187 \\
0.0143 \\
0.0131 \\
0.0125 \\
0.0118 \\
0.0108\end{array}$ & $\begin{array}{c}\text { Pressure } \\
\text { (psig) } \\
9.89 \\
19.55 \\
29.57 \\
39.42 \\
49.16 \\
54.50 \\
59.57 \\
64.20 \\
69.32 \\
74.16 \\
80.16 \\
85.61 \\
90.58 \\
95.69 \\
100.92 \\
103.25 \\
106.11 \\
108.31 \\
111.08 \\
113.24 \\
117.83 \\
120.92 \\
123.28 \\
122.97 \\
125.82 \\
125.60 \\
127.84 \\
130.19 \\
132.53 \\
135.33 \\
134.42 \\
138.35 \\
140.16 \\
142.63 \\
145.78 \\
142.52 \\
0.22 \\
\end{array}$ & $\begin{array}{l}\text { \% Strain } \\
0.0057 \\
0.0158 \\
0.0256 \\
0.0347 \\
0.0431 \\
0.0476 \\
0.0521 \\
0.0559 \\
0.0604 \\
0.0626 \\
0.0757 \\
0.0825 \\
0.0883 \\
0.0952 \\
0.1014 \\
0.1041 \\
0.1067 \\
0.1091 \\
0.1118 \\
0.1144 \\
0.1191 \\
0.1224 \\
0.1254 \\
0.1254 \\
0.1288 \\
0.1292 \\
0.1319 \\
0.1384 \\
0.1493 \\
0.1628 \\
0.1643 \\
0.1807 \\
0.2028 \\
0.2305 \\
0.2484 \\
0.2477 \\
0.1138\end{array}$ \\
\hline
\end{tabular}

High Pressure Test

Pressure

9.89

19.55

29.57

39.42

59.57

64.20

69.32

74.16

80.16

95.69

100.92

106.11

108.31

111.08

113.24

117.83

120.92

123.28

122.97

125.60

127.84

130.19

132.53

135.33

134.42

138.35

140.16

142.63

145.78

42.52

0.22
0.0057

0.0158

0.0256

0.0347

0.0431

0.0476

.

0.0559

0.0604

0.0626

0.0757

0.0825

0.0883

0.0952

0.1014

0.1118

0.1144

0.1191

0.1224

0.1254

0.1254

0.1292

0.1493

0.1628

0.1643

0.1807

0.2028

0.2484

0.2477

0.1138 


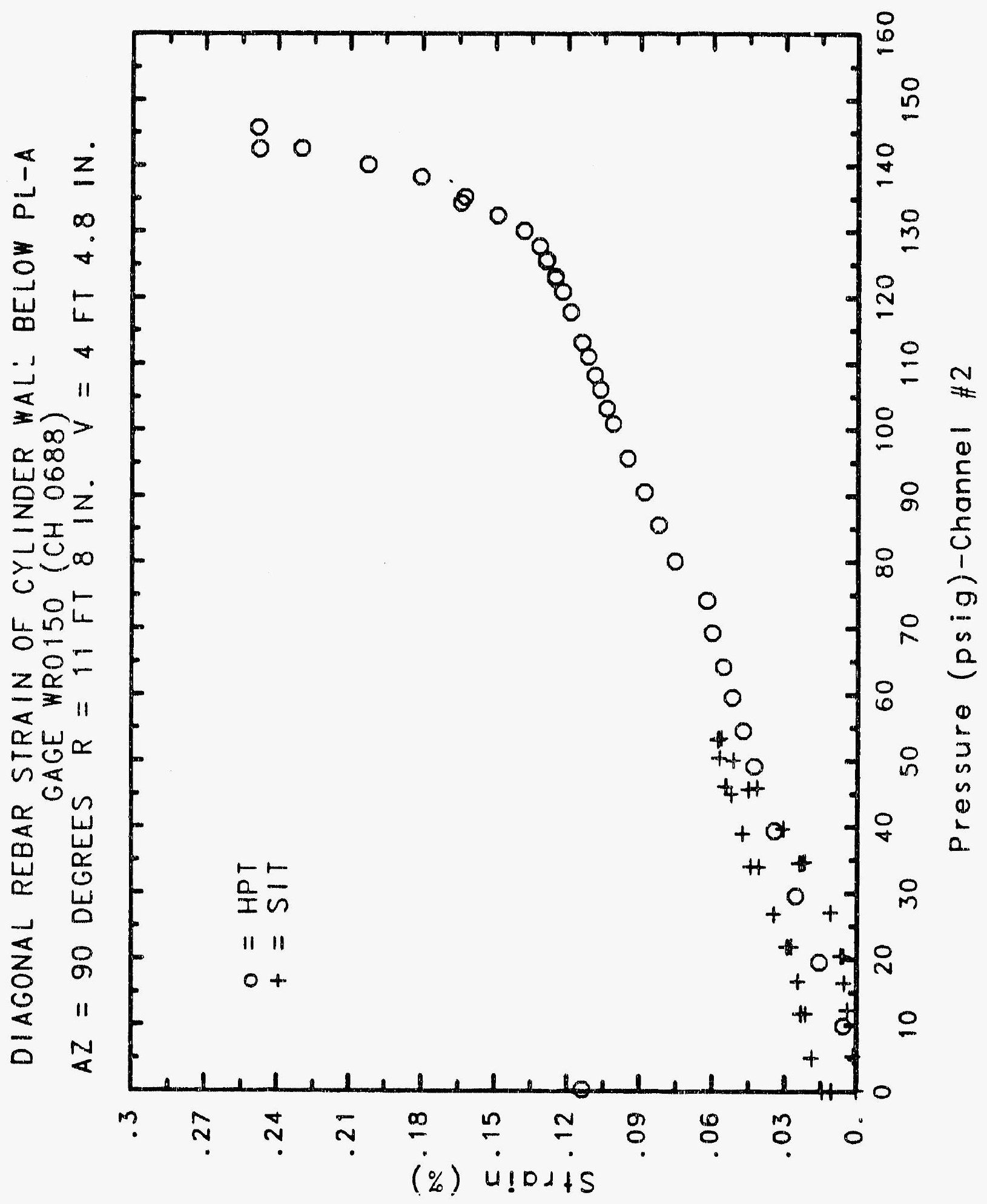




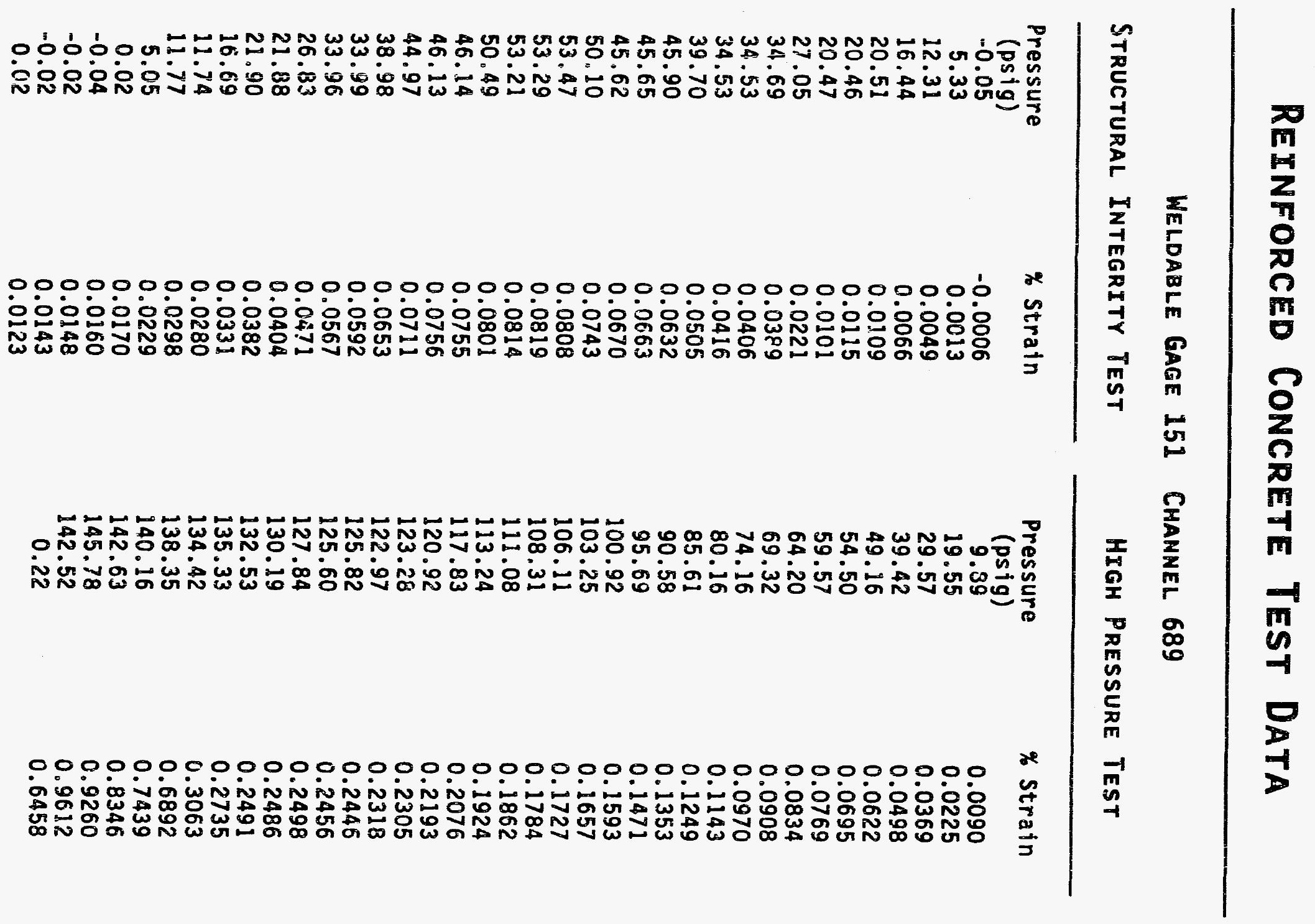




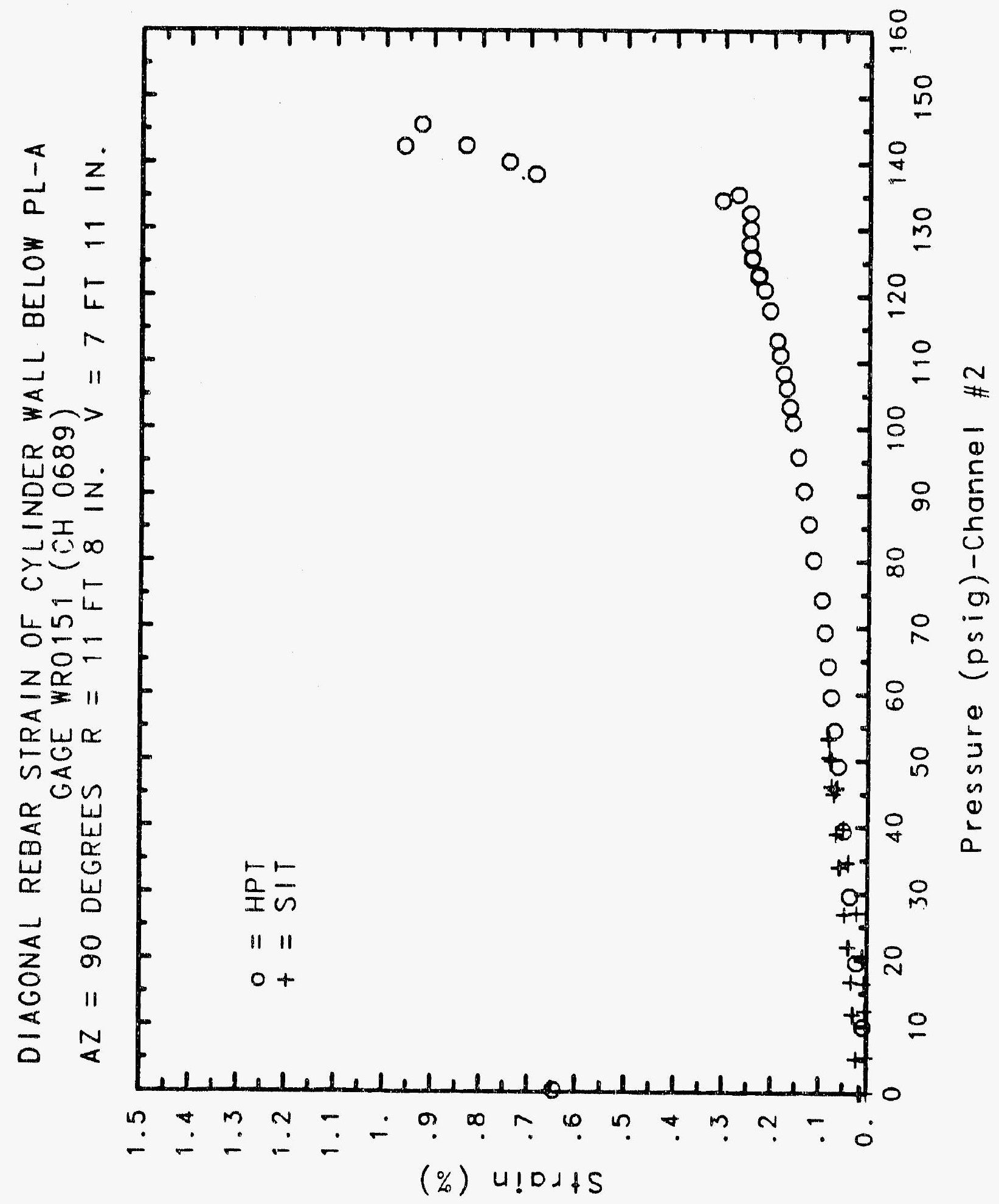




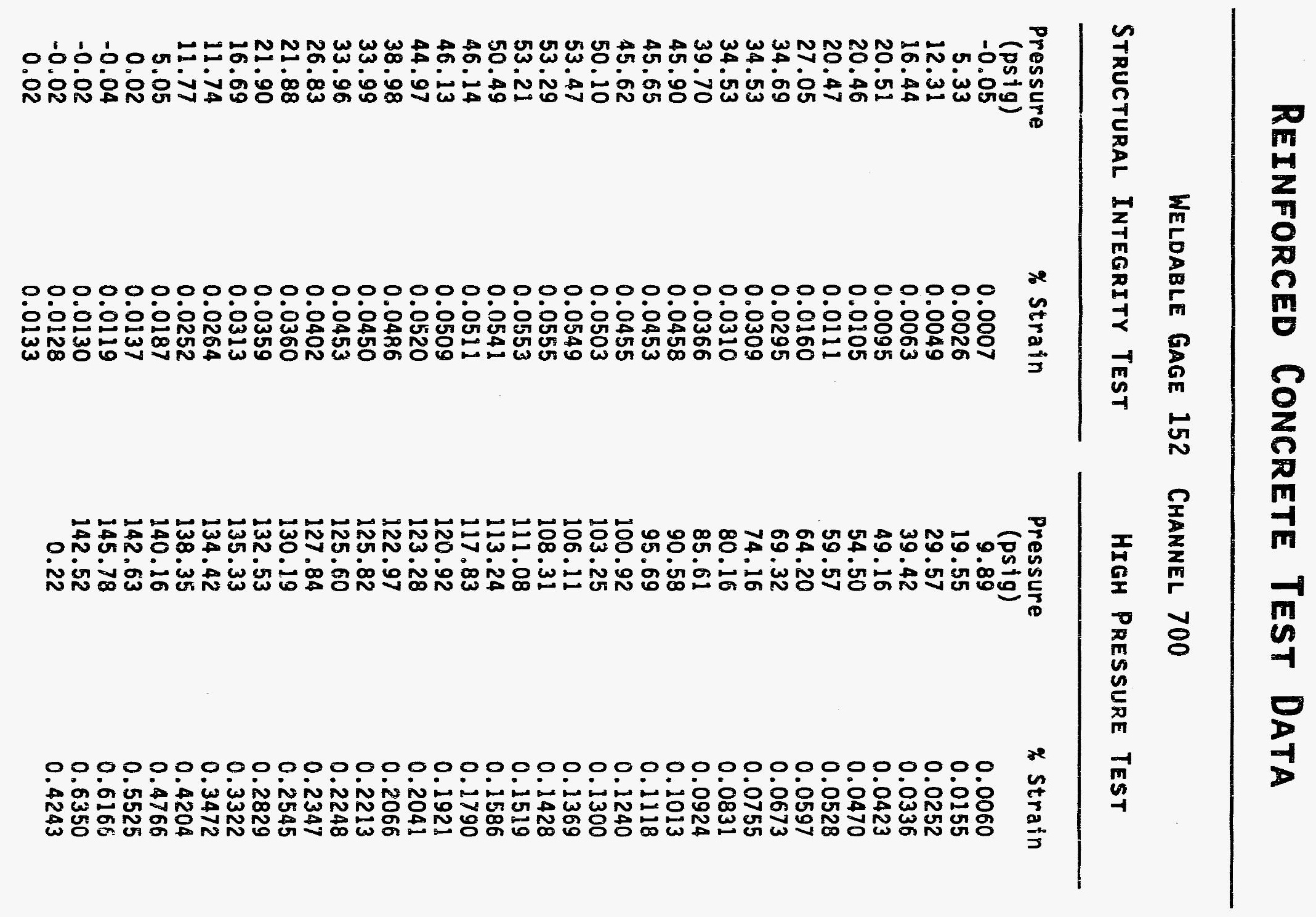




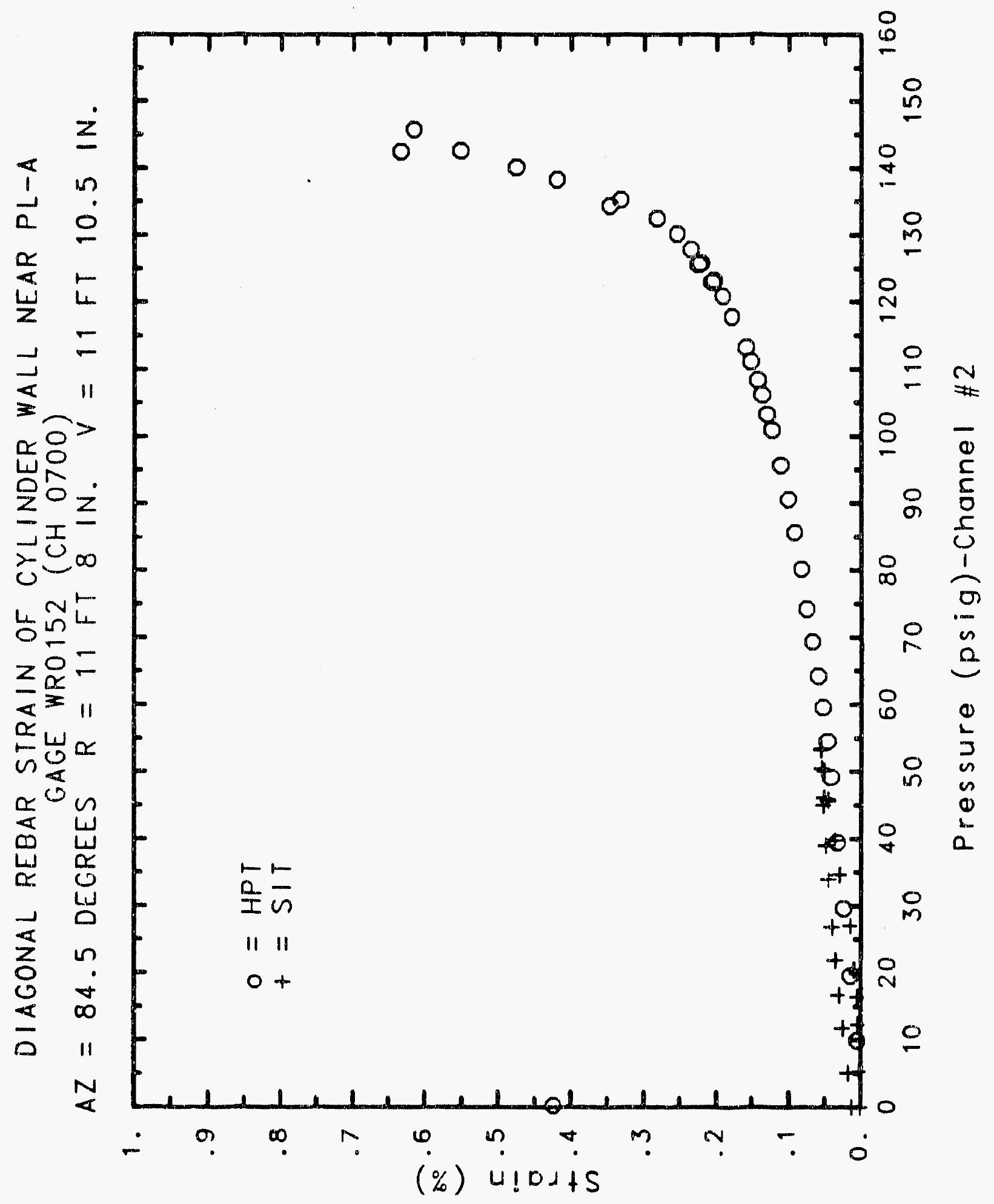




\section{Reinforced Concrete Test Data}

Weldable Gage 153 Channel 701

Structural Integrity Test

\begin{tabular}{|c|c|c|c|}
\hline $\begin{array}{c}\text { Pressure } \\
\text { (psig) } \\
-0.05 \\
5.33 \\
12.31 \\
16.44 \\
20.51 \\
20.46 \\
20.47 \\
27.05 \\
34.69 \\
34.53 \\
34.53 \\
39.70 \\
45.90 \\
45.65 \\
45.62 \\
50.10 \\
53.47 \\
53.29 \\
53.21 \\
50.49 \\
46.14 \\
46.13 \\
44.97 \\
38.98 \\
33.99 \\
33.96 \\
26.83 \\
21.88 \\
21.90 \\
16.69 \\
11.74 \\
11.77 \\
5.05 \\
0.02 \\
-0.04 \\
-0.02 \\
-0.02 \\
0.02 \\
\end{array}$ & $\begin{array}{l}\text { \% Strain } \\
0.0014 \\
0.0029 \\
0.0060 \\
0.0079 \\
0.0115 \\
0.0127 \\
0.0134 \\
0.0205 \\
0.0352 \\
0.0371 \\
0.0374 \\
0.0438 \\
0.0545 \\
0.0546 \\
0.0551 \\
0.0612 \\
0.0671 \\
0.0679 \\
0.0682 \\
0.0666 \\
0.0638 \\
0.0633 \\
0.0648 \\
0.0609 \\
0.0572 \\
0.0575 \\
0.0521 \\
0.0476 \\
0.0475 \\
0.0426 \\
0.0368 \\
0.0359 \\
0.0289 \\
0.0238 \\
0.0210 \\
0.0217 \\
0.0220 \\
0.0228\end{array}$ & $\begin{array}{c}\text { Pressure } \\
(\text { psig) } \\
9.89 \\
19.55 \\
29.57 \\
39.42 \\
49.16 \\
54.50 \\
59.57 \\
64.20 \\
69.32 \\
74.16 \\
80.16 \\
85.61 \\
90.58 \\
95.69 \\
100.92 \\
103.25 \\
106.11 \\
108.31 \\
111.08 \\
113.24 \\
117.83 \\
120.92 \\
123.28 \\
122.97 \\
125.82 \\
125.60 \\
127.84 \\
130.19 \\
132.53 \\
135.33 \\
134.42 \\
138.35 \\
140.16 \\
142.63 \\
145.78 \\
142.52 \\
0.22 \\
\end{array}$ & $\begin{array}{l}\text { \% Strain } \\
0.0068 \\
0.0170 \\
0.0274 \\
0.0369 \\
0.0463 \\
0.0524 \\
0.0594 \\
0.0692 \\
0.0804 \\
0.0929 \\
0.1059 \\
0.1212 \\
0.1363 \\
0.1551 \\
0.1760 \\
0.1870 \\
0.1998 \\
0.2105 \\
0.2274 \\
0.2407 \\
0.2739 \\
0.2988 \\
0.3196 \\
0.3243 \\
0.3470 \\
0.3518 \\
0.3640 \\
0.3889 \\
0.4210 \\
0.4688 \\
0.4797 \\
0.5461 \\
0.5994 \\
0.6785 \\
0.7508 \\
0.7733 \\
0.5299\end{array}$ \\
\hline
\end{tabular}

High Pressure Test

Pressure

(psig)

19.55

29.57

54.50

59.57

64.20

69.32

74.16

80.16

85.61

90.58

100.92

103.25

106.11

108.31

111.08

113.24

117.83

120.92

122.97

125.82

125.60

127.84

130.19

132.53

135.33

134.42

140.16

142.63

145.78

42.52

0.22
0.0170

0.0274

0.0369

0.0594

0.0692

0.0804

0.0929

0.1059

0.1212

0.1551

0.1760

0.1870

0.1998

0.2105

0.2274

0.2407

0.3196

0.3243

0.3470

0.3518

0.3640

0.3889

0.4210

0.4688

0.5994

0.6785

0.7508

0.7733
0.5299 


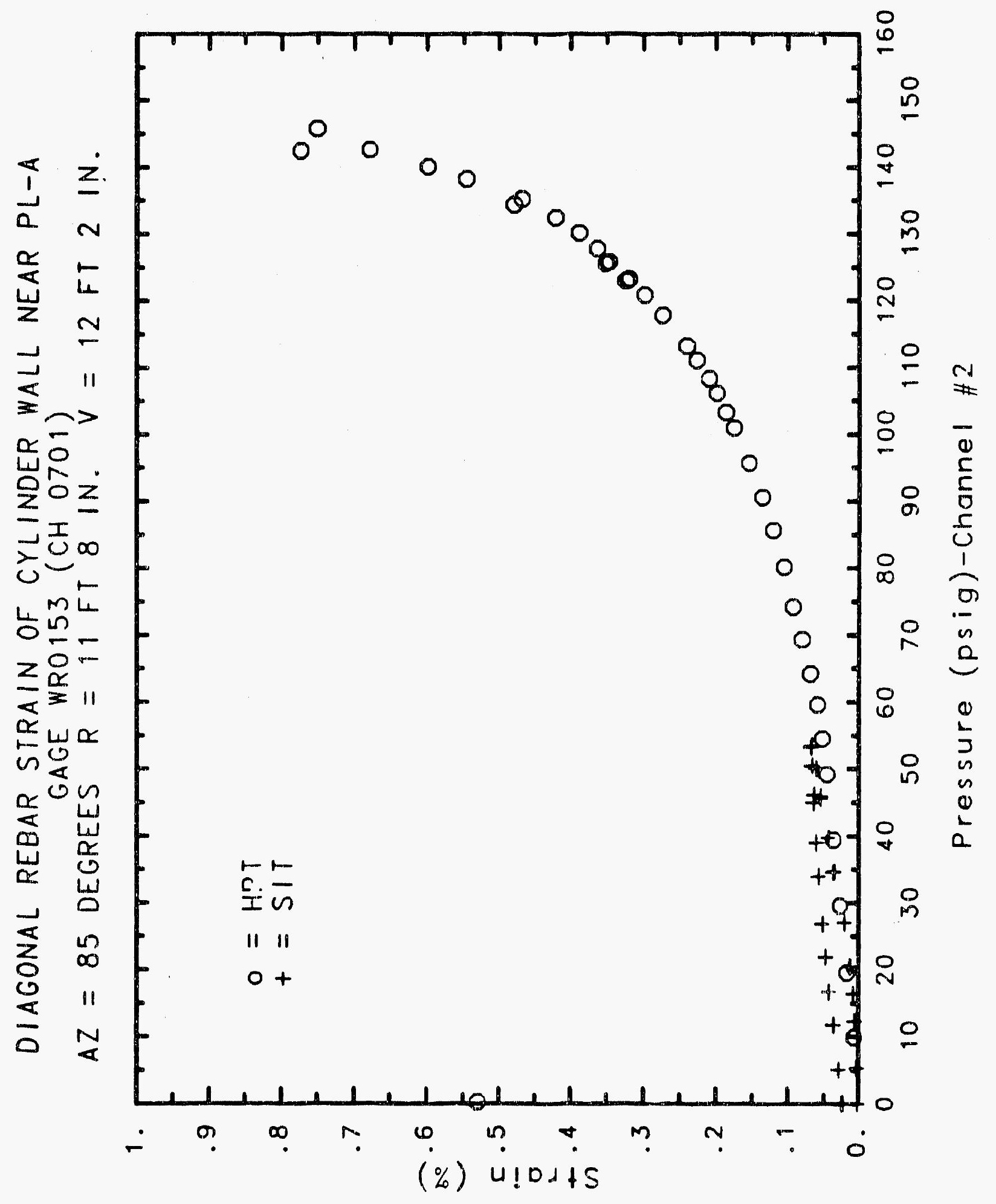

C- 1114 


\section{Reinforced Concrete Test Data}

Weldable gage 154 Channel 702

STRUCTURAL INTEgRITY TEST

\begin{tabular}{|c|c|c|c|}
\hline $\begin{array}{c}\text { Pressure } \\
\text { (psig) } \\
-0.05 \\
5.33 \\
12.31 \\
16.44 \\
20.51 \\
20.46 \\
20.47 \\
27.05 \\
36.69 \\
34.53 \\
34.53 \\
39.70 \\
45.90 \\
45.65 \\
45.62 \\
50.10 \\
53.47 \\
53.29 \\
53.21 \\
50.49 \\
46.14 \\
46.13 \\
44.97 \\
38.98 \\
33.99 \\
33.96 \\
26.83 \\
21.88 \\
21.90 \\
16.69 \\
11.74 \\
11.77 \\
5.05 \\
0.02 \\
-0.04 \\
-0.02 \\
-0.02 \\
0.02 \\
\end{array}$ & $\begin{array}{c}\text { \% Strain } \\
\\
-0.0006 \\
0.0012 \\
0.0030 \\
0.0042 \\
0.0060 \\
0.0065 \\
0.0064 \\
0.0104 \\
0.0155 \\
0.0158 \\
0.0151 \\
0.0183 \\
0.0222 \\
0.0248 \\
0.0257 \\
0.0286 \\
0.0318 \\
0.0325 \\
0.0326 \\
0.0318 \\
0.0303 \\
0.0303 \\
0.0287 \\
0.0267 \\
0.0247 \\
0.0246 \\
0.0218 \\
0.0197 \\
0.0198 \\
0.0167 \\
0.0138 \\
0.0148 \\
0.0122 \\
0.0104 \\
0.0092 \\
0.0085 \\
0.0086 \\
0.0087\end{array}$ & $\begin{array}{c}\text { Pressure } \\
\text { (psig) } \\
9.89 \\
19.55 \\
29.57 \\
39.42 \\
49.1 j \\
54.50 \\
59.57 \\
64.20 \\
69.32 \\
74.16 \\
80.16 \\
85.61 \\
90.58 \\
95.69 \\
100.92 \\
103.25 \\
106.11 \\
108.31 \\
111.08 \\
113.24 \\
117.83 \\
120.92 \\
123.28 \\
122.97 \\
125.82 \\
125.60 \\
127.84 \\
130.19 \\
132.53 \\
135.33 \\
134.42 \\
138.35 \\
140.16 \\
142.63 \\
145.78 \\
142.52 \\
0.22 \\
\end{array}$ & $\begin{array}{l}\text { \% Strain } \\
0.0007 \\
0.0053 \\
0.0102 \\
0.0154 \\
0.0202 \\
0.0230 \\
0.0257 \\
0.0287 \\
0.0321 \\
0.0353 \\
0.0421 \\
0.0463 \\
0.0501 \\
0.0546 \\
0.0587 \\
0.0608 \\
0.0630 \\
0.0644 \\
0.0669 \\
0.0688 \\
0.0728 \\
0.0754 \\
0.0776 \\
0.0777 \\
0.0799 \\
0.0797 \\
0.0816 \\
0.0833 \\
0.0853 \\
0.0880 \\
0.0882 \\
0.0927 \\
0.0970 \\
0.1030 \\
0.1102 \\
0.1114 \\
0.0204\end{array}$ \\
\hline
\end{tabular}

High Pressure Test

Pressure Strain

$\begin{array}{ll}9.89 & 0.0007\end{array}$

$19.55 \quad 0.0053$

$29.57 \quad 0.0102$

$39.42 \quad 0.0154$

$59.57 \quad 0.0257$

$64.20 \quad 0.0287$

$69.32 \quad 0.0321$

$74.16 \quad 0.0353$

$80.16 \quad 0.0421$

$85.61 \quad 0.0463$

0.0501

$106.11 \quad 0.0630$

$108.31 \quad 0.0644$

$111.08 \quad 0.0669$

$\begin{array}{ll}113.24 & 0.0688\end{array}$

$117.83 \quad 0.0728$

0.0754

0.0776

0.0777

25.60

$\begin{array}{ll}127.84 & 0.0816\end{array}$

$130.19 \quad 0.0833$

$\begin{array}{ll}32.53 & 0.0853\end{array}$

$\begin{array}{ll}35.33 & 0.0880\end{array}$

0.0882

0.0927

0.0970

2.52

0.0204 


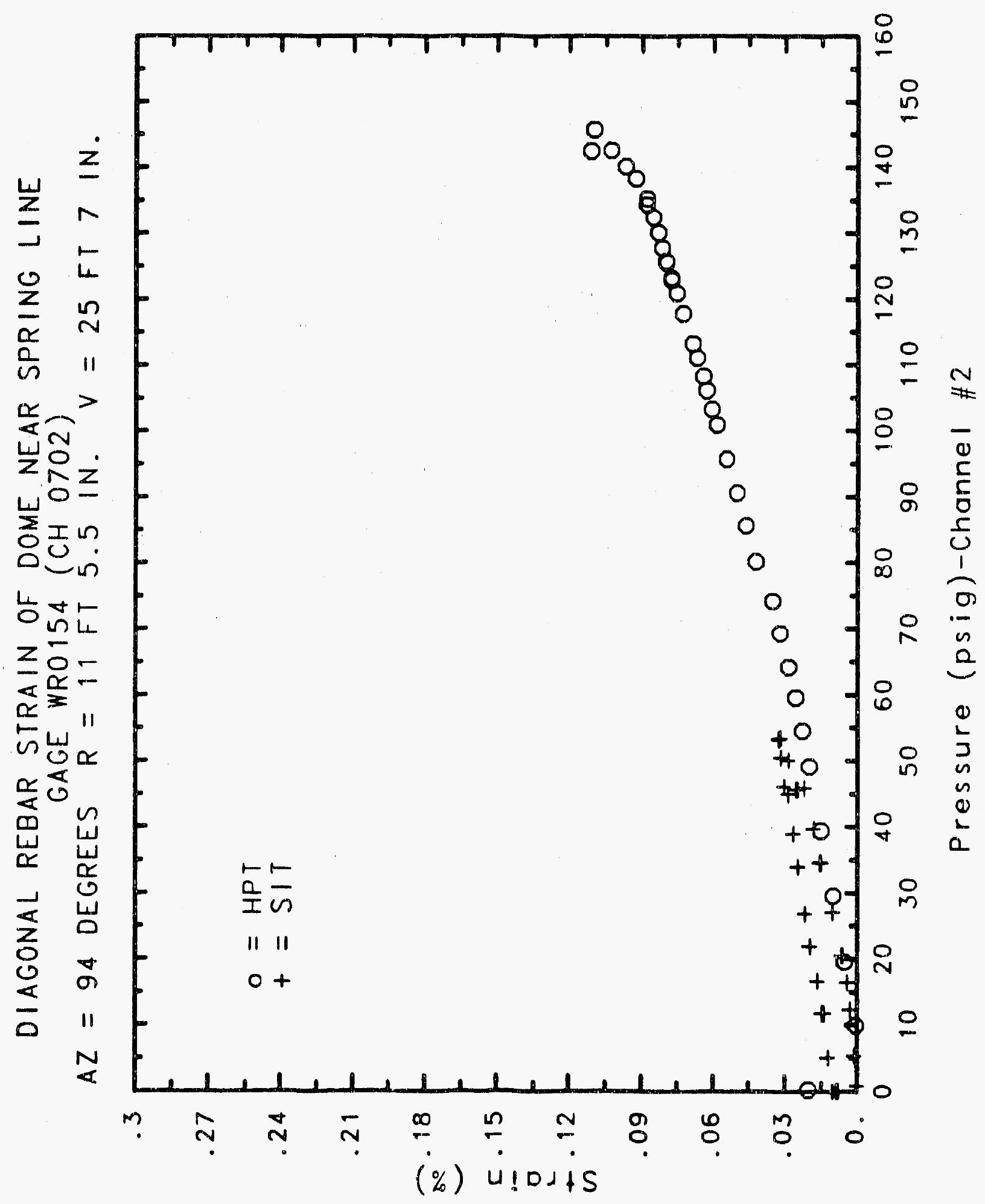




\section{Reinforced Concrete Test Data}

Weldable Gage 155 Channel 703

Structural IntEgrity Test

High Pressure test

\begin{tabular}{|c|c|c|c|}
\hline $\begin{array}{c}\text { Pressure } \\
\text { (psig) } \\
-0.05 \\
5.33 \\
12.31 \\
16.44 \\
20.51 \\
20.46 \\
20.47 \\
27.05 \\
34.69 \\
34.53 \\
34.53 \\
39.70 \\
45.90 \\
45.65 \\
45.62 \\
50.10 \\
53.47 \\
53.29 \\
53.21 \\
50.49 \\
46.14 \\
46.13 \\
44.97 \\
38.98 \\
33.99 \\
33.96 \\
26.83 \\
21.88 \\
21.90 \\
16.69 \\
11.74 \\
11.77 \\
5.05 \\
0.02 \\
-0.04 \\
-0.02 \\
-0.02 \\
0.02 \\
\end{array}$ & $\begin{array}{c}\text { \% Strain } \\
\\
-0.0004 \\
0.0024 \\
0.0054 \\
0.0079 \\
0.0127 \\
0.0131 \\
0.0142 \\
0.0238 \\
0.0357 \\
0.0372 \\
0.0372 \\
0.0434 \\
0.0541 \\
0.0548 \\
0.0548 \\
0.0611 \\
0.0673 \\
0.0683 \\
0.0686 \\
0.0669 \\
0.0633 \\
0.0629 \\
0.0620 \\
0.0573 \\
0.0521 \\
0.0519 \\
0.0450 \\
0.0397 \\
0.0398 \\
0.0345 \\
0.0286 \\
0.0279 \\
0.0207 \\
0.0152 \\
0.0120 \\
0.0121 \\
0.0125 \\
0.0126\end{array}$ & $\begin{array}{c}\text { Pressure } \\
\text { (psig) } \\
9.89 \\
19.55 \\
29.57 \\
39.42 \\
49.16 \\
54.50 \\
59.57 \\
64.20 \\
69.32 \\
74.16 \\
80.16 \\
85.61 \\
90.58 \\
95.69 \\
100.92 \\
103.25 \\
106.11 \\
108.31 \\
111.08 \\
113.24 \\
117.83 \\
120.92 \\
123.28 \\
122.97 \\
125.82 \\
125.60 \\
127.84 \\
130.19 \\
132.53 \\
135.33 \\
134.42 \\
138.35 \\
140.16 \\
142.63 \\
145.78 \\
142.52 \\
0.22 \\
\end{array}$ & $\begin{array}{l}\text { Strain } \\
0.0060 \\
0.0175 \\
0.0295 \\
0.0407 \\
0.0522 \\
0.0590 \\
0.0658 \\
0.0728 \\
0.0804 \\
0.0877 \\
0.0956 \\
0.1030 \\
0.1107 \\
0.1192 \\
0.1289 \\
0.1340 \\
0.1401 \\
0.1444 \\
0.1513 \\
0.1571 \\
0.1713 \\
0.1826 \\
0.1924 \\
0.1945 \\
0.2038 \\
0.2064 \\
0.2114 \\
0.2182 \\
0.2265 \\
0.2377 \\
0.2414 \\
0.2559 \\
0.2679 \\
0.2840 \\
0.2994 \\
0.3049 \\
0.1303\end{array}$ \\
\hline
\end{tabular}




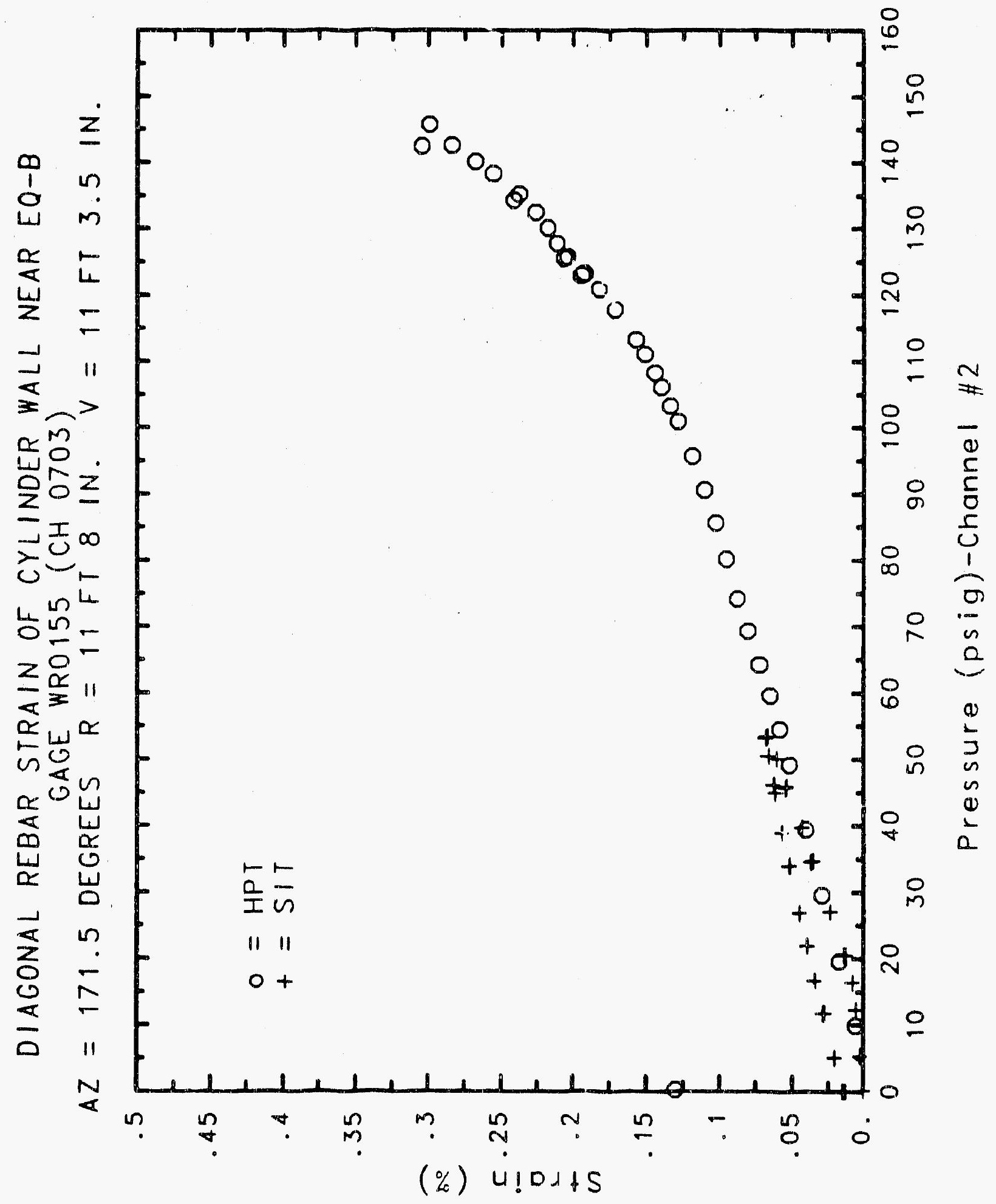




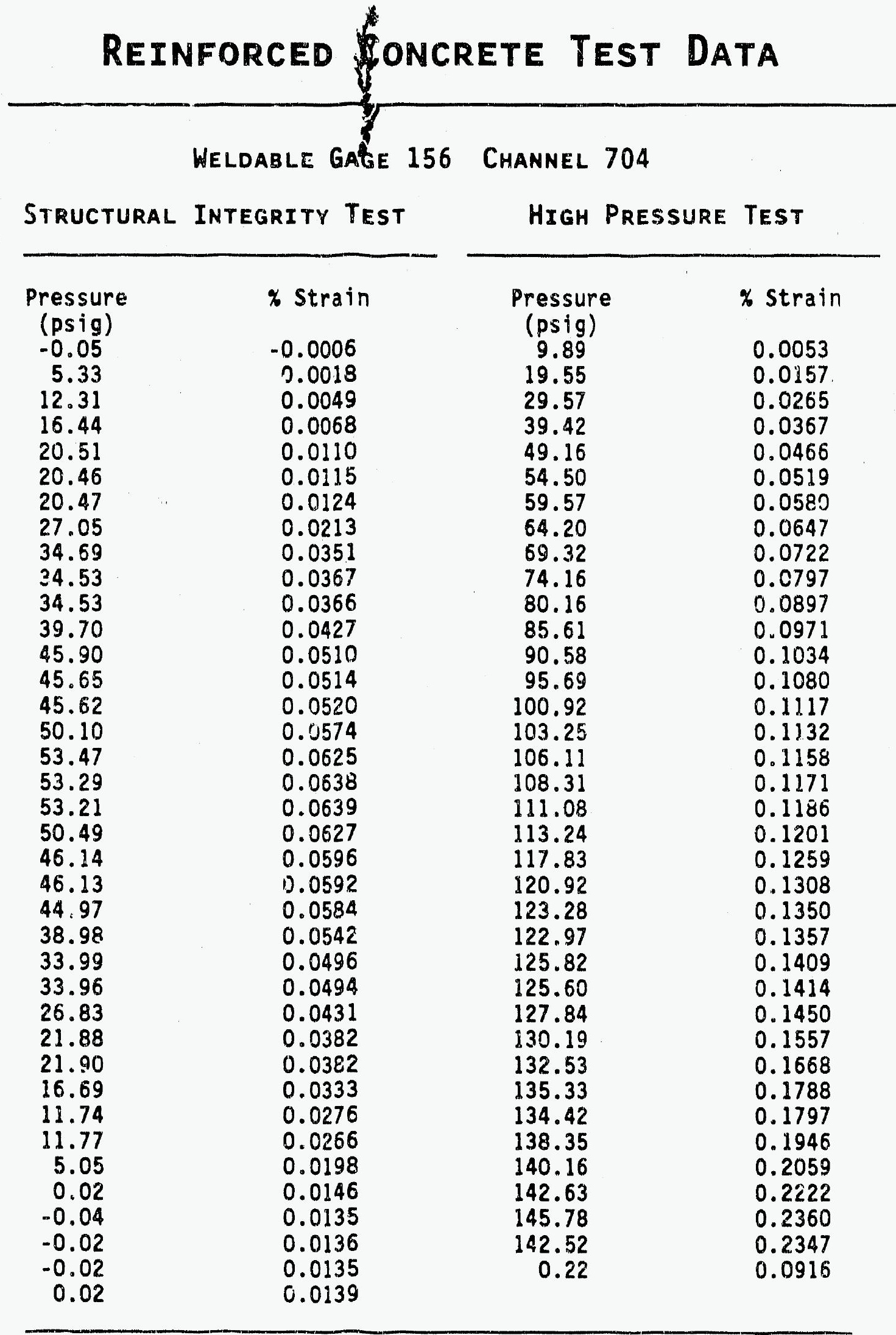




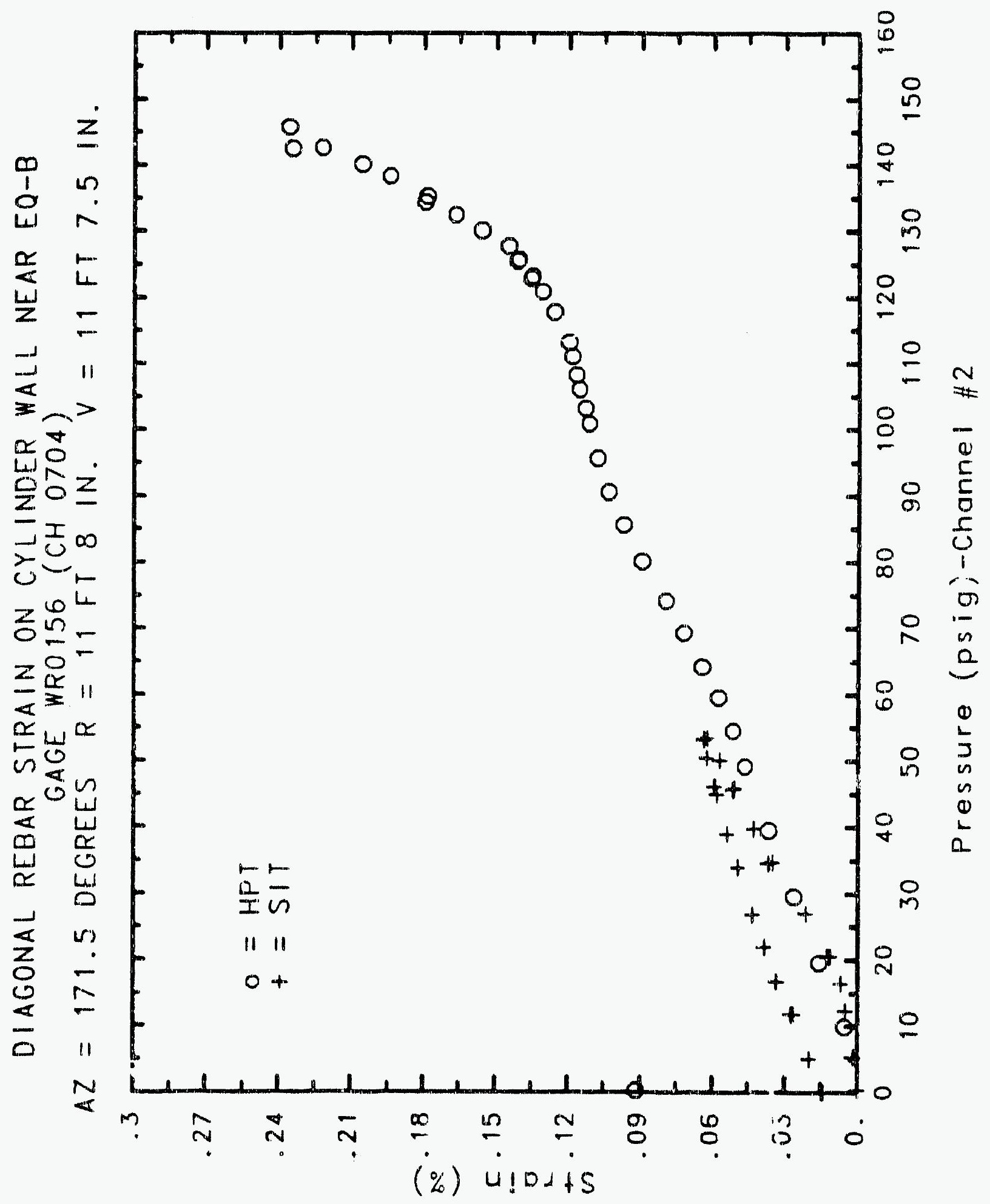




\section{Reinforced Concrete Test Data}

Weldable gage 157 Channel 705

STRUCTURAL INTEGRITY TEST

\begin{tabular}{|c|c|c|c|}
\hline $\begin{array}{c}\text { Pressure } \\
\text { (psig) } \\
-0.05 \\
5.33 \\
12.31 \\
16.44 \\
20.51 \\
20.46 \\
20.47 \\
27.05 \\
34.69 \\
34.53 \\
34.53 \\
39.70 \\
45.90 \\
45.65 \\
45.62 \\
50.10 \\
53.47 \\
53.29 \\
53.21 \\
50.49 \\
46.14 \\
46.13 \\
44.97 \\
38.98 \\
33.99 \\
33.96 \\
26.83 \\
21.88 \\
21.90 \\
16.69 \\
11.74 \\
11.77 \\
5.05 \\
0.02 \\
-0.04 \\
-0.02 \\
-0.02 \\
0.02 \\
\end{array}$ & $\begin{array}{l}\text { \% Strain } \\
\text {-0.0003 } \\
0.0003 \\
0.0020 \\
0.0035 \\
0.0060 \\
0.0074 \\
0.0082 \\
0.0217 \\
0.0412 \\
0.0422 \\
0.0420 \\
0.0485 \\
0.0591 \\
0.0588 \\
0.0588 \\
0.0647 \\
0.0689 \\
0.0687 \\
0.0685 \\
0.0663 \\
0.0624 \\
0.0619 \\
0.0602 \\
0.0549 \\
0.0503 \\
0.0498 \\
0.0430 \\
0.0382 \\
0.0384 \\
0.0333 \\
0.0284 \\
0.0281 \\
0.0217 \\
0.0179 \\
0.0183 \\
0.0179 \\
0.0172 \\
0.0179\end{array}$ & $\begin{array}{c}\text { Pressure } \\
(\text { psig) } \\
9.89 \\
19.55 \\
29.57 \\
39.42 \\
49.16 \\
54.50 \\
59.57 \\
64.20 \\
69.32 \\
74.16 \\
80.16 \\
85.61 \\
90.58 \\
95.69 \\
100.92 \\
103.25 \\
106.11 \\
108.31 \\
111.08 \\
113.24 \\
117.83 \\
120.92 \\
123.28 \\
122.97 \\
125.82 \\
125.60 \\
127.84 \\
130.19 \\
132.53 \\
135.33 \\
134.42 \\
138.35 \\
140.16 \\
142.63 \\
145.78 \\
142.52 \\
0.22 \\
\end{array}$ & $\begin{array}{l}\text { \% Strain } \\
0.0054 \\
0.0157 \\
0.0262 \\
0.0362 \\
0.0454 \\
0.0508 \\
0.0560 \\
0.0605 \\
0.0660 \\
0.0710 \\
0.0757 \\
0.0819 \\
0.0873 \\
0.0925 \\
0.0986 \\
0.1007 \\
0.1044 \\
0.1075 \\
0.1110 \\
0.1146 \\
0.1223 \\
0.1278 \\
0.1322 \\
0.1327 \\
0.1378 \\
0.1380 \\
0.1413 \\
0.1465 \\
0.1523 \\
0.1587 \\
0.1589 \\
0.1672 \\
0.1722 \\
0.1804 \\
0.1885 \\
0.1866 \\
0.0446\end{array}$ \\
\hline
\end{tabular}

High Pressure test

(psig)

9.89

0.0054

0.0262

0.0362

0.0454

0.0508

0.0660

0.0710

0.0757

0.0819

0.0873

0.1007

0.1044

0.1075

0.1110

0.1146

0.1223

0.1327

0.1378

0.1380

0.1413

0.1465

0.1523

0.1672

0.1722

0.1804

0.1885

0.1866

0.0446 


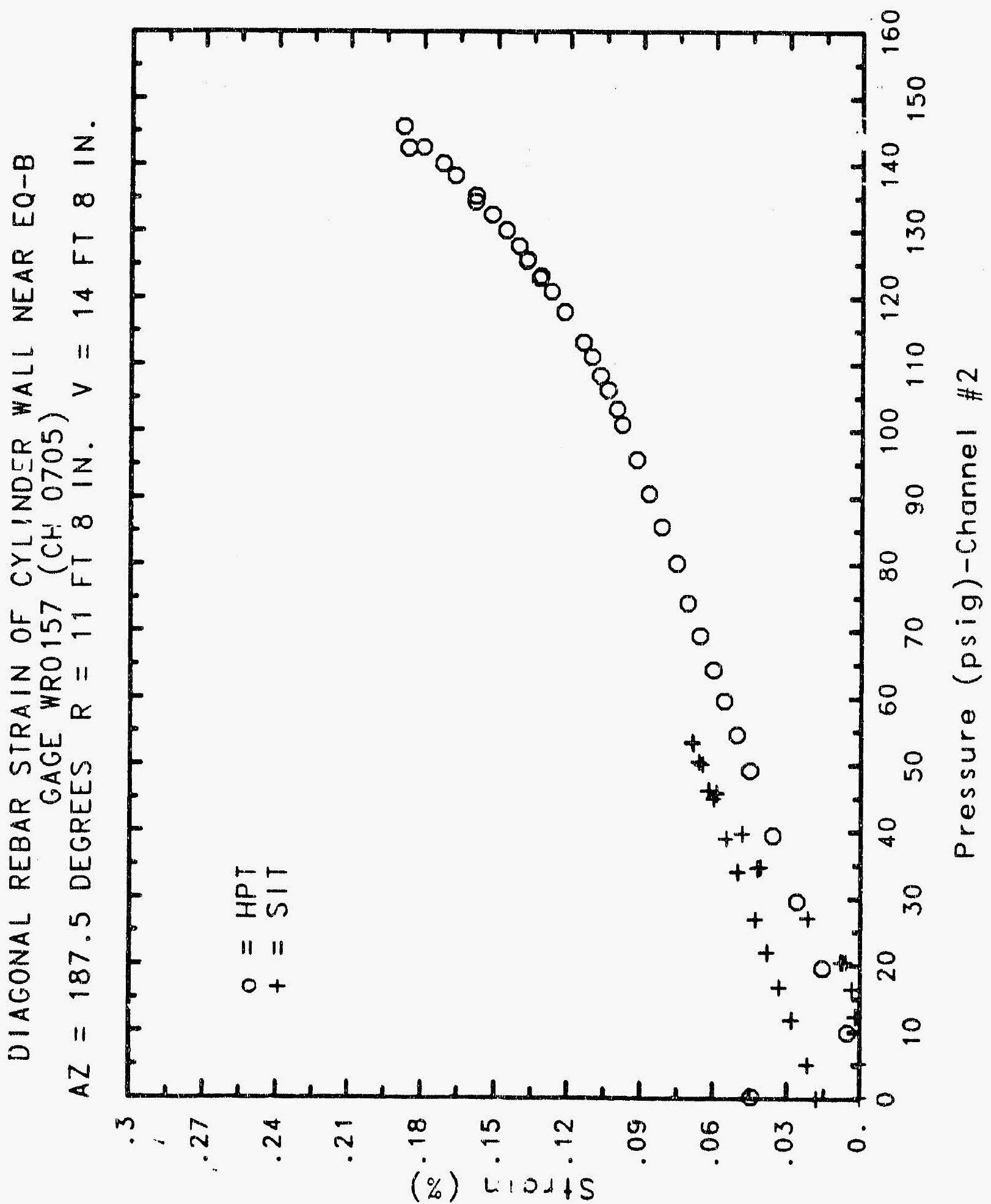




\section{Reinforced Concrete Test Data}

Weldable Gage 158 Chammel 706

STRUCTURAL INTEGRITY TEST

\begin{tabular}{|c|c|c|c|}
\hline $\begin{array}{c}\text { Pressure } \\
\text { (psig) } \\
-0.05 \\
5.33 \\
12.31 \\
16.44 \\
20.51 \\
20.46 \\
20.47 \\
27.05 \\
34.69 \\
34.53 \\
34.53 \\
39.70 \\
45.90 \\
45.65 \\
45.62 \\
50.10 \\
53.47 \\
53.29 \\
53.21 \\
50.49 \\
46.14 \\
46.13 \\
44.97 \\
38.98 \\
33.99 \\
33.96 \\
26.83 \\
21.88 \\
21.90 \\
16.69 \\
11.74 \\
11.77 \\
5.05 \\
0.02 \\
-0.04 \\
-0.02 \\
-0.02 \\
0.02 \\
\end{array}$ & $\begin{array}{c}\text { \% Strain } \\
\text {-0.0004 } \\
0.0008 \\
0.0024 \\
0.0039 \\
0.0070 \\
0.0087 \\
0.0095 \\
0.0215 \\
0.0386 \\
0.0400 \\
0.0404 \\
0.0465 \\
0.0571 \\
0.0583 \\
0.0583 \\
0.0647 \\
0.0705 \\
0.0707 \\
0.0709 \\
0.0690 \\
0.0658 \\
0.0654 \\
0.0644 \\
0.0596 \\
0.0555 \\
0.0549 \\
0.0482 \\
0.0429 \\
0.0429 \\
0.0380 \\
0.0326 \\
0.0316 \\
0.0249 \\
0.0200 \\
0.0183 \\
0.0182 \\
0.0177 \\
0.0183\end{array}$ & $\begin{array}{c}\text { Pressure } \\
(\text { psig) } \\
9.89 \\
19.55 \\
29.57 \\
39.42 \\
49.16 \\
54.50 \\
59.57 \\
64.20 \\
69.32 \\
74.16 \\
80.16 \\
85.61 \\
90.58 \\
95.69 \\
100.92 \\
103.25 \\
106.11 \\
108.31 \\
111.08 \\
113.24 \\
117.83 \\
120.92 \\
123.28 \\
122.97 \\
125.82 \\
125.60 \\
127.84 \\
130.19 \\
132.53 \\
135.33 \\
134.42 \\
138.35 \\
140.16 \\
142.63 \\
145.78 \\
142.52 \\
0.22 \\
\end{array}$ & $\begin{array}{l}\text { Strain } \\
0.0060 \\
0.0169 \\
0.0278 \\
0.0383 \\
0.0488 \\
0.0544 \\
0.0604 \\
0.0667 \\
0.0736 \\
0.0806 \\
0.0885 \\
0.0957 \\
0.1026 \\
0.1107 \\
0.1198 \\
0.1242 \\
0.1293 \\
0.1335 \\
0.1395 \\
0.1440 \\
0.1551 \\
0.1643 \\
0.1728 \\
0.1750 \\
0.1839 \\
0.1859 \\
0.1919 \\
0.2012 \\
0.2107 \\
0.2216 \\
0.2231 \\
0.2354 \\
0.2436 \\
0.2553 \\
0.2693 \\
0.2711 \\
0.1052\end{array}$ \\
\hline
\end{tabular}

High Pressure test

ressur

9.89

19.55

54.50

59.57

64.20

69.32

74.16

80.16

95.69

100.92

103.25

111.08

113.24

125.82

125.60

127.84

130.19

135.33

134.42

138.35

140.16

142.63

145.78

42.52

0.22
Strain

0.0060

0.0169

0.0488

0.0604

0.0667

0.0736

0.0806

0.1026

0.1198

0.1242

0.1293

0.1551

0.1728

0.1750

0.1839

0.1859

0.2107

0.2216

0.2231

0.2354

0.2436

0.2553

0.2693

0.2711

0.1052 


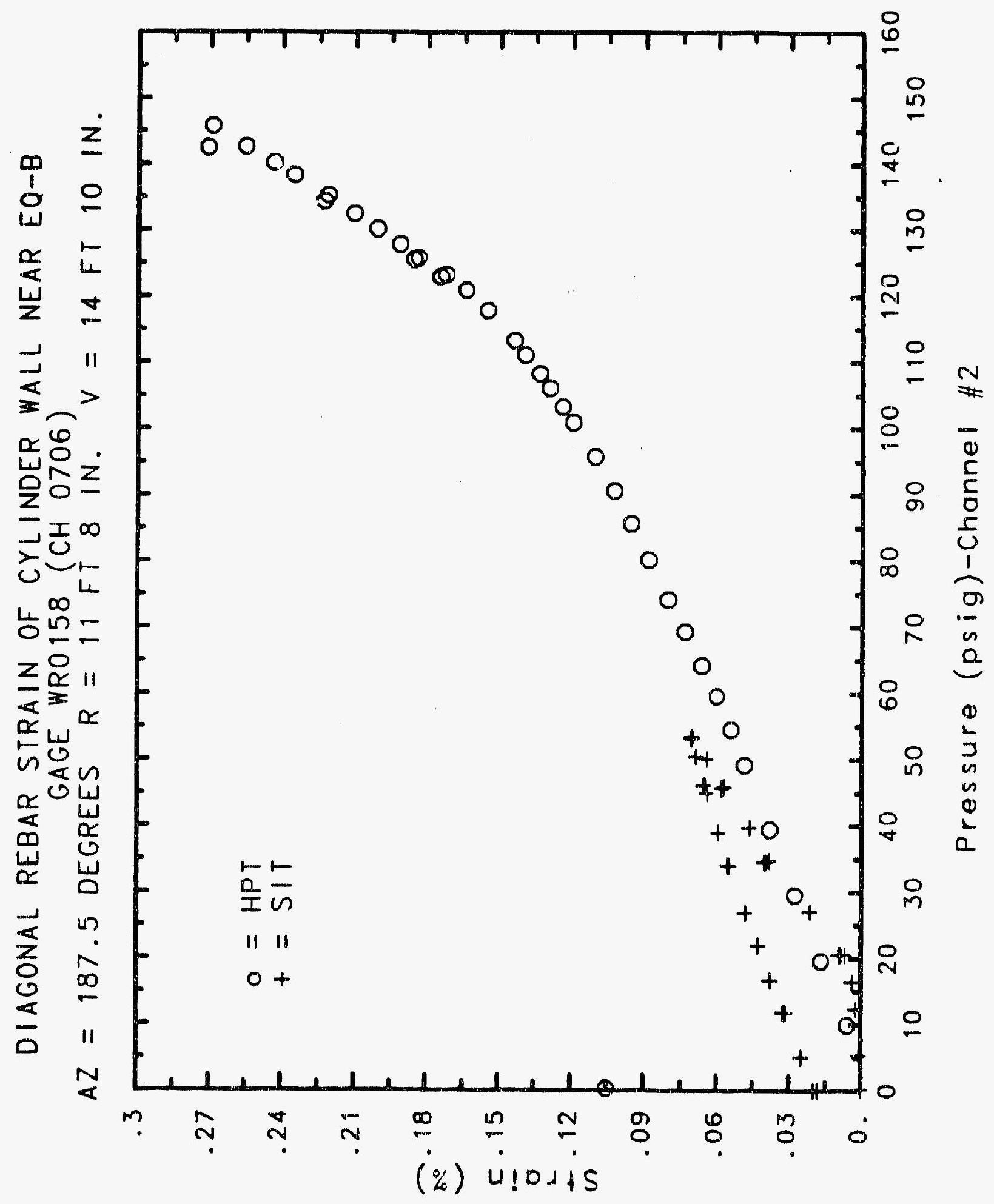




\section{Reinforced Concrete Test Data}

Weldable gage 159 Channel 707

Structural Integrity Test

high Pressure Test

\begin{tabular}{rrrr}
\hline Pressure & \% Strain & $\begin{array}{c}\text { Pressure } \\
\text { (psig) }\end{array}$ & \% strain \\
-0.05 & -0.0002 & 9.89 & 0.0026 \\
5.33 & 0.0008 & 19.55 & 0.0106 \\
12.31 & 0.0019 & 29.57 & 0.0186 \\
16.44 & 0.0028 & 39.42 & 0.0261 \\
20.51 & 0.0042 & 49.16 & 0.0336 \\
20.46 & 0.0046 & 54.50 & 0.0382 \\
20.47 & 0.0047 & 59.57 & 0.0425 \\
27.05 & 0.0093 & 64.20 & 0.0470 \\
34.69 & 0.0325 & 69.32 & 0.0516 \\
34.53 & 0.0336 & 74.16 & 0.0565 \\
34.53 & 0.0343 & 80.16 & 0.0625 \\
39.70 & 0.0390 & 85.61 & 0.0689 \\
45.90 & 0.0458 & 90.58 & 0.0752 \\
45.65 & 0.0460 & 95.69 & 0.0842 \\
45.62 & 0.0459 & 100.92 & 0.0917 \\
50.10 & 0.0496 & 103.25 & 0.0957 \\
53.47 & 0.0525 & 106.11 & 0.1000 \\
53.29 & 0.0528 & 108.31 & 0.1030 \\
53.21 & 0.0526 & 111.08 & 0.1079 \\
50.49 & 0.0514 & 113.24 & 0.1118 \\
46.14 & 0.0489 & 117.83 & 0.1201 \\
46.13 & 0.0485 & 120.92 & 0.1264 \\
44.97 & 0.0473 & 123.28 & 0.1324 \\
38.98 & 0.0440 & 122.97 & 0.1338 \\
33.99 & 0.0407 & 125.82 & 0.1395 \\
33.96 & 0.0408 & 125.60 & 0.1407 \\
26.83 & 0.0357 & 127.84 & 0.1443 \\
21.88 & 0.0323 & 130.19 & 0.1509 \\
21.90 & 0.0322 & 132.53 & 0.1594 \\
16.69 & 0.0285 & 135.33 & 0.1713 \\
11.74 & 0.0251 & 134.42 & 0.1751 \\
11.77 & 0.0245 & 138.35 & 0.1916 \\
5.05 & 0.0201 & 140.16 & 0.2247 \\
0.02 & 0.0172 & 145.78 & 0.2431 \\
-0.04 & 0.0159 & 142.52 & 0.089 \\
-0.02 & 0.0156 & 0.22 & \\
-0.02 & 0.0150 & & \\
0.02 & 0.0154 & & \\
\hline & & & \\
\hline
\end{tabular}




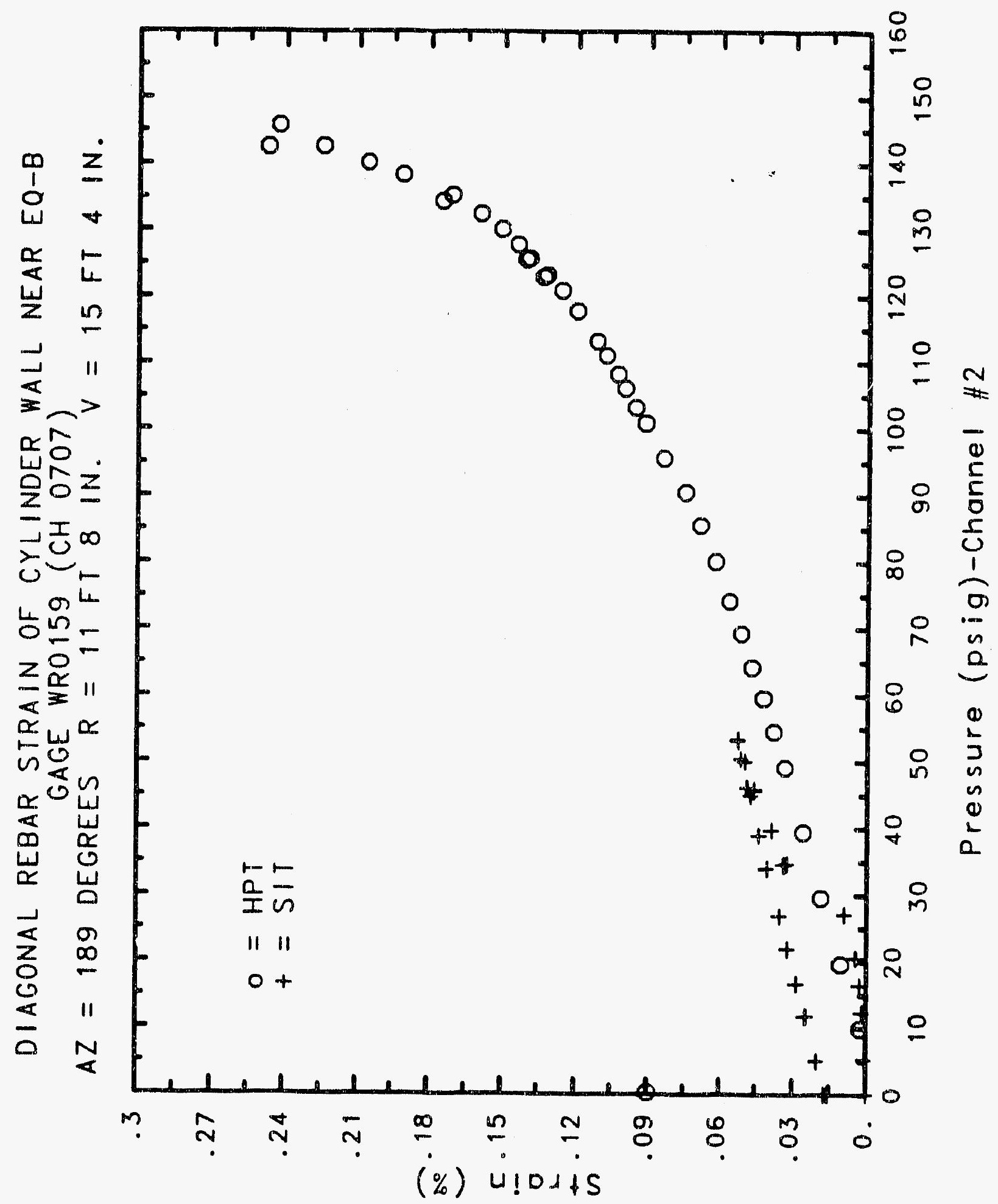




\section{Reinforced Concrete Test Data}

\begin{tabular}{|c|c|c|c|}
\hline STRUCTURAL & $\begin{array}{l}\text { WeLdABLE GAGE } 160 \\
\text { IntEGRITY TEST }\end{array}$ & $\begin{aligned} & \text { Channel } 708 \\
& \text { High Pressure }\end{aligned}$ & TEST \\
\hline $\begin{array}{c}\text { Pressure } \\
\text { (psig) } \\
-0.05 \\
5.33 \\
12.31 \\
16.44 \\
20.51 \\
20.46 \\
20.47 \\
27.05 \\
34.69 \\
34.53 \\
34.53 \\
39.70 \\
45.90 \\
45.65 \\
45.62 \\
50.10 \\
53.47 \\
53.29 \\
53.21 \\
50.49 \\
46.14 \\
46.13 \\
44.97 \\
38.98 \\
33.99 \\
33.96 \\
26.83 \\
21.88 \\
21.90 \\
16.69 \\
11.74 \\
11.77 \\
5.05 \\
0.02 \\
-0.04 \\
-0.02 \\
-0.02 \\
0.02 \\
\end{array}$ & $\begin{array}{c}\text { Strain } \\
\text {-0.0006 } \\
0.0010 \\
0.0041 \\
0.0061 \\
0.0086 \\
0.0091 \\
0.0094 \\
0.0118 \\
0.0182 \\
0.0183 \\
0.0185 \\
0.0226 \\
0.0283 \\
0.0284 \\
0.0280 \\
0.0298 \\
0.0311 \\
0.0310 \\
0.0306 \\
0.0299 \\
0.0280 \\
0.0273 \\
0.0276 \\
0.0242 \\
0.0217 \\
0.0215 \\
0.0174 \\
0.0146 \\
0.0141 \\
0.0112 \\
0.0078 \\
0.0071 \\
0.0031 \\
0.0002 \\
-0.0003 \\
-0.0002 \\
-0.0007 \\
-0.0010\end{array}$ & $\begin{array}{c}\text { Pressure } \\
\text { (psig) } \\
9.89 \\
19.55 \\
29.57 \\
39.42 \\
49.16 \\
54.50 \\
59.57 \\
64.20 \\
69.32 \\
74.16 \\
80.16 \\
85.61 \\
90.58 \\
95.69 \\
100.92 \\
103.25 \\
106.11 \\
108.31 \\
111.08 \\
113.24 \\
117.83 \\
120.92 \\
123.28 \\
122.97 \\
125.82 \\
125.60 \\
127.84 \\
130.19 \\
132.53 \\
135.33 \\
134.42 \\
138.35 \\
140.16 \\
142.63 \\
145.78 \\
142.52 \\
0.22 \\
\text { (125 }\end{array}$ & $\begin{array}{l}\text { \% Strain } \\
0.0020 \\
0.0082 \\
0.0145 \\
0.0206 \\
0.0267 \\
0.0298 \\
0.0333 \\
0.0378 \\
0.0432 \\
0.0489 \\
0.0561 \\
0.0680 \\
0.0774 \\
0.0867 \\
0.0967 \\
0.1020 \\
0.1070 \\
0.1109 \\
0.1174 \\
0.1222 \\
0.1360 \\
0.1483 \\
0.1605 \\
0.1640 \\
0.1768 \\
0.1808 \\
0.1881 \\
0.2033 \\
0.2223 \\
0.2516 \\
0.2701 \\
0.3173 \\
0.3609 \\
0.4196 \\
0.4722 \\
0.4917 \\
0.3125\end{array}$ \\
\hline
\end{tabular}




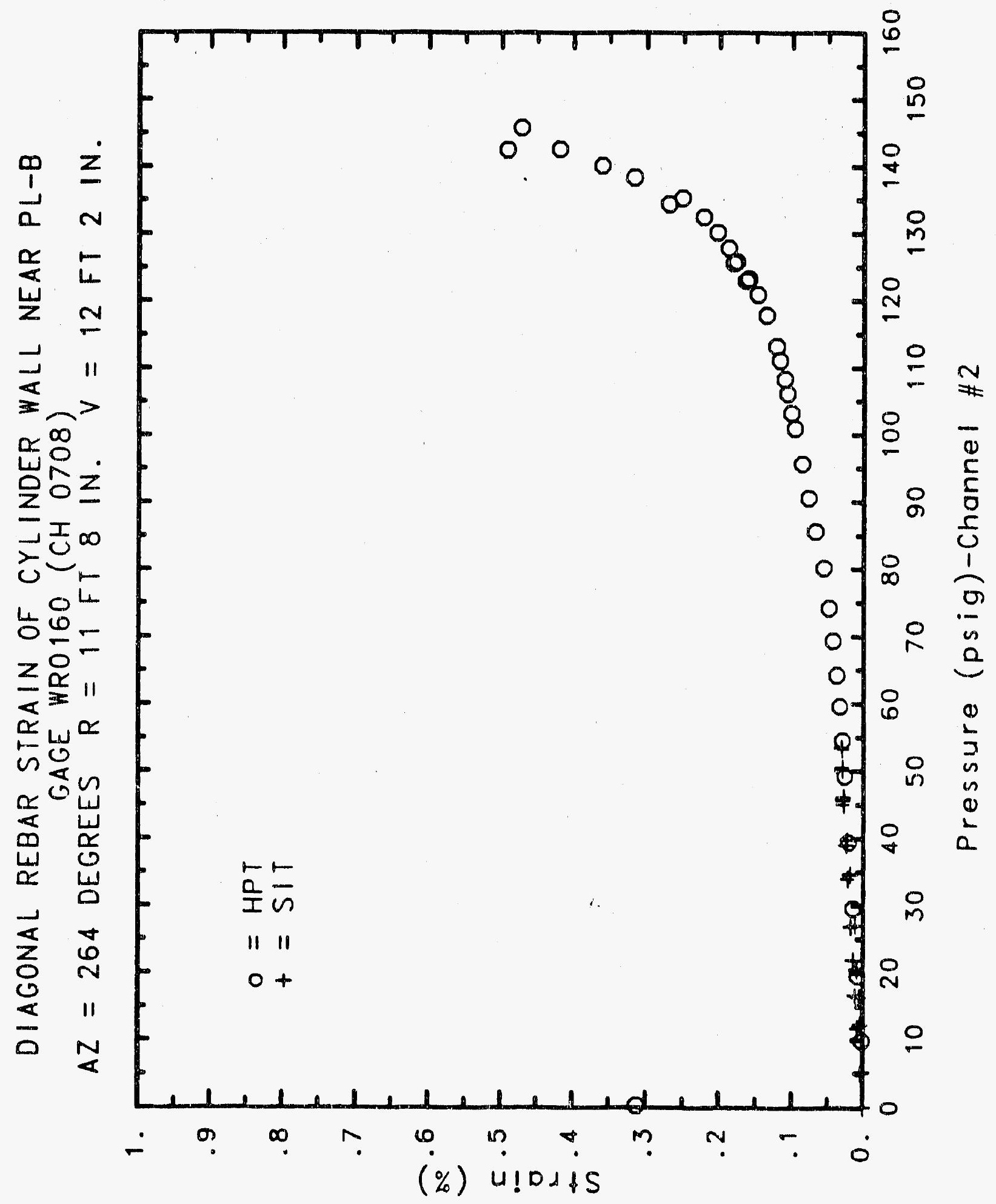




\section{Reinforced Concrete Test Data}

Weldable Gage 161 Channel 709

Structural Integrity Test

High Pressure test

\begin{tabular}{|c|c|c|c|}
\hline $\begin{array}{c}\text { Pressure } \\
\text { (psig) } \\
-0.05 \\
5.33 \\
12.31 \\
16.44 \\
20.51 \\
20.46 \\
20.47 \\
27.05 \\
34.69 \\
34.53 \\
34.53 \\
39.70 \\
45.90 \\
45.65 \\
45.62 \\
50.10 \\
53.47 \\
53.29 \\
53.21 \\
50.49 \\
46.14 \\
46.13 \\
44.97 \\
38.98 \\
33.99 \\
33.96 \\
26.83 \\
21.88 \\
21.90 \\
16.69 \\
11.74 \\
11.77 \\
5.05 \\
0.02 \\
-0.04 \\
-0.02 \\
-0.02 \\
0.02 \\
\end{array}$ & $\begin{array}{l}\text { Strain } \\
0.0012 \\
0.0026 \\
0.0065 \\
0.0092 \\
0.0128 \\
0.0136 \\
0.0137 \\
0.0196 \\
0.0292 \\
0.0299 \\
0.0302 \\
0.0346 \\
0.0422 \\
0.0428 \\
0.0429 \\
0.0468 \\
0.0509 \\
0.0513 \\
0.0508 \\
0.0499 \\
0.0475 \\
0.0471 \\
0.0484 \\
0.0443 \\
0.0405 \\
0.0401 \\
0.0346 \\
0.0303 \\
0.0304 \\
0.0261 \\
0.0218 \\
0.0213 \\
0.0157 \\
0.0113 \\
0.0105 \\
0.0108 \\
0.0106 \\
0.0100\end{array}$ & $\begin{array}{c}\text { Pressure } \\
(\text { psig) } \\
9.89 \\
19.55 \\
29.57 \\
39.42 \\
49.16 \\
54.50 \\
59.57 \\
64.20 \\
69.32 \\
74.16 \\
80.16 \\
85.61 \\
90.58 \\
95.69 \\
100.92 \\
103.25 \\
106.11 \\
108.31 \\
111.08 \\
113.24 \\
117.83 \\
120.92 \\
123.28 \\
122.97 \\
125.82 \\
125.60 \\
127.84 \\
130.19 \\
132.53 \\
135.33 \\
134.42 \\
138.35 \\
140.16 \\
142.63 \\
145.78 \\
142.52 \\
0.22 \\
\end{array}$ & $\begin{array}{l}\text { \% Strain } \\
0.0040 \\
0.0125 \\
0.0214 \\
0.0297 \\
0.0381 \\
0.0430 \\
0.0477 \\
0.0540 \\
0.0608 \\
0.0686 \\
0.0798 \\
0.0995 \\
0.1145 \\
0.1296 \\
0.1460 \\
0.1537 \\
0.1632 \\
0.1706 \\
0.1817 \\
0.1905 \\
0.2125 \\
0.2306 \\
0.2506 \\
0.2573 \\
0.2780 \\
0.2838 \\
0.2948 \\
0.3182 \\
0.3462 \\
0.3868 \\
0.3981 \\
0.4437 \\
0.4836 \\
0.5406 \\
0.5987 \\
0.6185 \\
0.4129\end{array}$ \\
\hline
\end{tabular}




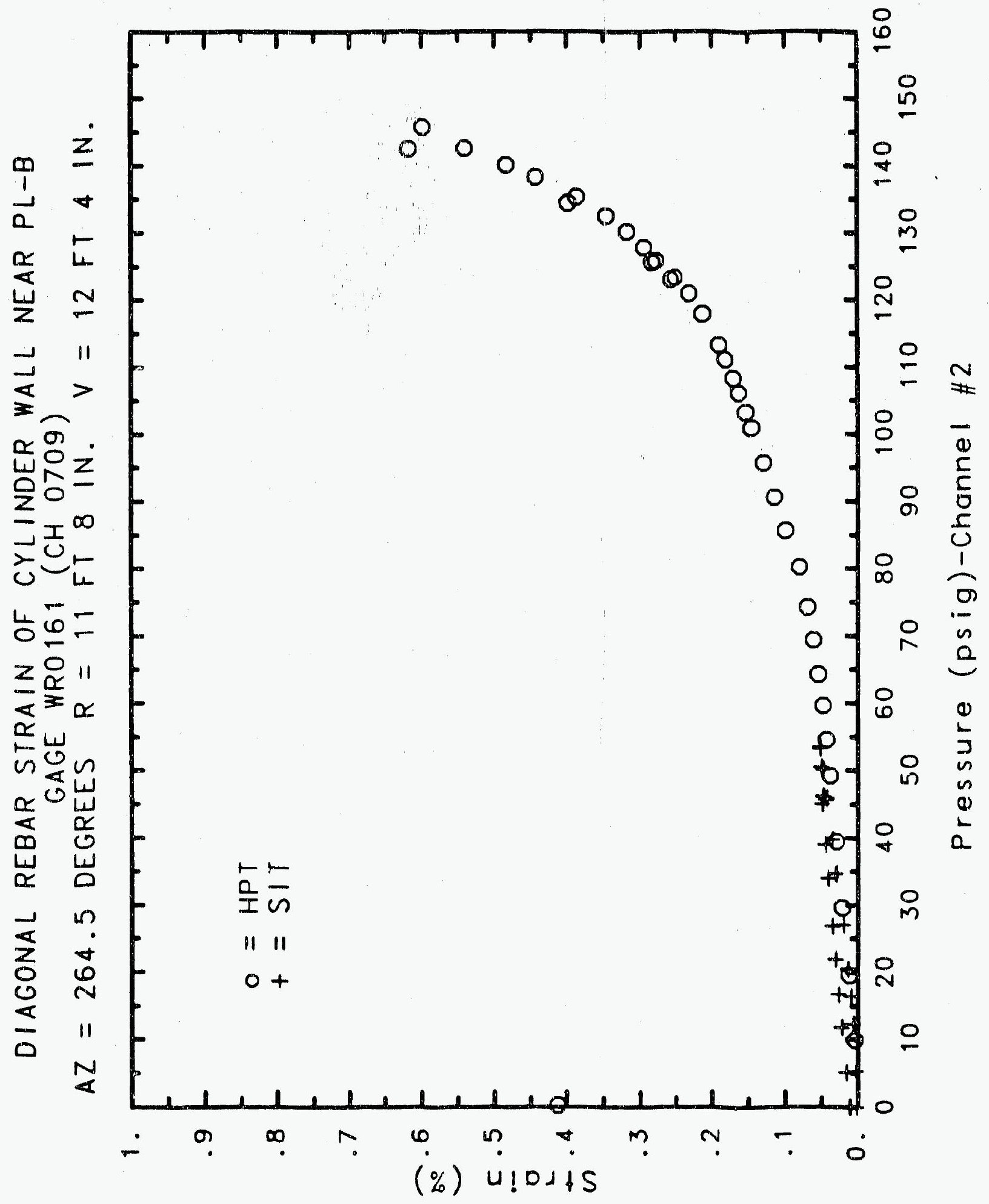




\section{Reinforced Concrete Test Data}

Weldable gage 162 Channel 720

Structural INTEgRITY TEST

High Pressure test

\begin{tabular}{|c|c|c|c|}
\hline $\begin{array}{c}\text { Pressure } \\
\text { (psig) } \\
-0.05 \\
5.33 \\
12.31 \\
16.44 \\
20.51 \\
20.46 \\
20.47 \\
27.05 \\
34.69 \\
34.53 \\
34.53 \\
39.70 \\
45.90 \\
45.65 \\
45.62 \\
50.10 \\
53.47 \\
53.29 \\
53.21 \\
50.49 \\
46.14 \\
46.13 \\
44.97 \\
38.98 \\
33.99 \\
33.96 \\
26.83 \\
21.88 \\
21.90 \\
16.69 \\
11.74 \\
11.77 \\
5.05 \\
0.02 \\
-0.04 \\
-0.02 \\
-0.02 \\
0.02 \\
\end{array}$ & $\begin{array}{c}\text { Strain } \\
-0.0004 \\
0.0018 \\
0.0051 \\
0.0074 \\
0.0104 \\
0.0111 \\
0.0109 \\
0.0165 \\
0.0258 \\
0.0263 \\
0.0269 \\
0.0309 \\
0.0366 \\
0.0366 \\
0.0371 \\
0.0400 \\
0.0432 \\
0.0432 \\
0.0432 \\
0.0420 \\
0.0398 \\
0.0395 \\
0.0410 \\
0.0380 \\
0.0348 \\
0.0350 \\
0.0303 \\
0.0264 \\
0.0266 \\
0.0228 \\
0.0195 \\
0.0190 \\
0.0138 \\
0.0100 \\
0.0074 \\
0.0086 \\
0.0098 \\
0.0092\end{array}$ & $\begin{array}{c}\text { Pressure } \\
(\text { psig) } \\
9.89 \\
19.55 \\
29.57 \\
39.42 \\
49.16 \\
54.50 \\
59.57 \\
64.20 \\
69.32 \\
74.16 \\
80.16 \\
85.61 \\
90.58 \\
95.69 \\
100.92 \\
103.25 \\
106.11 \\
108.31 \\
111.08 \\
113.24 \\
117.83 \\
120.92 \\
123.28 \\
122.97 \\
125.82 \\
125.60 \\
127.84 \\
130.19 \\
132.53 \\
135.33 \\
134.42 \\
138.35 \\
140.16 \\
142.63 \\
145.78 \\
142.52 \\
0.22\end{array}$ & $\begin{array}{l}\text { \% Strain } \\
0.0035 \\
0.0101 \\
0.0181 \\
0.0252 \\
0.0319 \\
0.0361 \\
0.0404 \\
0.0457 \\
0.0524 \\
0.0600 \\
0.0701 \\
0.0812 \\
0.0925 \\
0.1054 \\
0.1203 \\
0.1274 \\
0.1355 \\
0.1412 \\
0.1509 \\
0.1579 \\
0.1739 \\
0.1854 \\
0.1975 \\
0.2009 \\
0.2149 \\
0.2196 \\
0.2287 \\
0.2460 \\
0.2695 \\
0.3057 \\
0.3156 \\
0.3585 \\
0.3942 \\
0.4492 \\
0.5044 \\
0.5299 \\
0.3426\end{array}$ \\
\hline
\end{tabular}




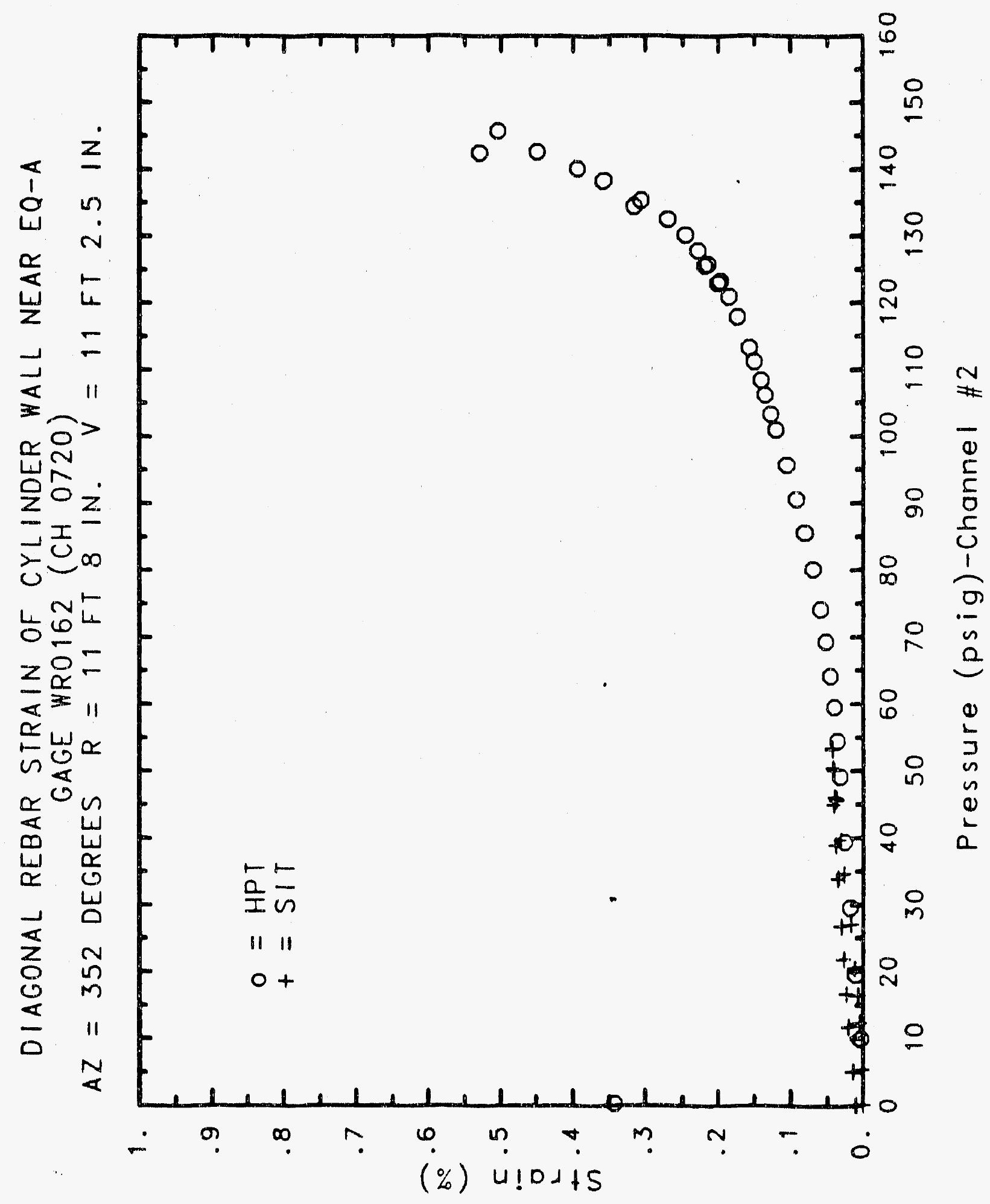




\section{Reinforced Concrete Test Data}

Weldable gage 163 Channel 7201

Structural IntegrtTy Test

\begin{tabular}{|c|c|c|c|}
\hline $\begin{array}{c}\text { Pressure } \\
\text { (psig) } \\
-0.05 \\
5.33 \\
12.31 \\
16.44 \\
20.51 \\
20.46 \\
20.47 \\
27.05 \\
34.69 \\
34.53 \\
34.53 \\
39.70 \\
45.90 \\
45.65 \\
45.62 \\
50.10 \\
53.47 \\
53.29 \\
53.21 \\
50.49 \\
46.14 \\
46.13 \\
44.97 \\
38.98 \\
33.99 \\
33.96 \\
26.83 \\
21.88 \\
21.90 \\
16.69 \\
11.74 \\
11.77 \\
5.05 \\
0.02 \\
-0.04 \\
-0.02 \\
-0.02 \\
0.02 \\
\end{array}$ & $\begin{array}{l}\text { \% Strain } \\
\text {-0.0010 } \\
0.0017 \\
0.0043 \\
0.0061 \\
0.0090 \\
0.0099 \\
0.0094 \\
0.0156 \\
0.0217 \\
0.0216 \\
0.0225 \\
0.0249 \\
0.0289 \\
0.0285 \\
0.0289 \\
0.0312 \\
0.0340 \\
0.0339 \\
0.0339 \\
0.0326 \\
0.0305 \\
0.0305 \\
0.0319 \\
0.0291 \\
0.0260 \\
0.0263 \\
0.0221 \\
0.0188 \\
0.0188 \\
0.0160 \\
0.0126 \\
0.0120 \\
0.0079 \\
0.0042 \\
0.0027 \\
0.0045 \\
0.0055 \\
0.0044\end{array}$ & $\begin{array}{c}\text { Pressure } \\
(\text { ps } 1 \text { g) } \\
9.89 \\
19.55 \\
29.57 \\
39.42 \\
49.16 \\
54.50 \\
59.57 \\
64.20 \\
69.32 \\
74.16 \\
80.16 \\
85.61 \\
90.58 \\
95.69 \\
100.92 \\
103.25 \\
106.11 \\
108.31 \\
111.08 \\
113.24 \\
117.83 \\
120.92 \\
123.28 \\
122.97 \\
125.82 \\
125.60 \\
127.84 \\
130.19 \\
132.53 \\
135.33 \\
134.42 \\
138.35 \\
140.16 \\
142.63 \\
145.78 \\
142.52 \\
0.22\end{array}$ & $\begin{array}{c}\text { \% Strain } \\
0.0023 \\
0.0085 \\
0.0142 \\
0.0202 \\
0.0260 \\
0.0285 \\
0.0324 \\
0.357 \\
0.0399 \\
0.0450 \\
0.0498 \\
0.0561 \\
0.0618 \\
0.0679 \\
0.0755 \\
0.0799 \\
0.0845 \\
0.0890 \\
0.0962 \\
0.1015 \\
0.1148 \\
0.1252 \\
0.1354 \\
0.1379 \\
0.1488 \\
0.1534 \\
0.1605 \\
0.1747 \\
0.1906 \\
0.2110 \\
0.2166 \\
0.2412 \\
0.2631 \\
0.2977 \\
0.3393 \\
0.3667 \\
0.1699\end{array}$ \\
\hline
\end{tabular}

\section{High Pressure test}

Pressure

19.55

49.16

54.50

74.16

80.16

90.58

95.69

106.11

08.31

1.08

117.83

120.92

123.28

122.97

125.82

25.60

132.53

135.33

134.42

138.35

140.16

2.52

0.3667

0.1699 


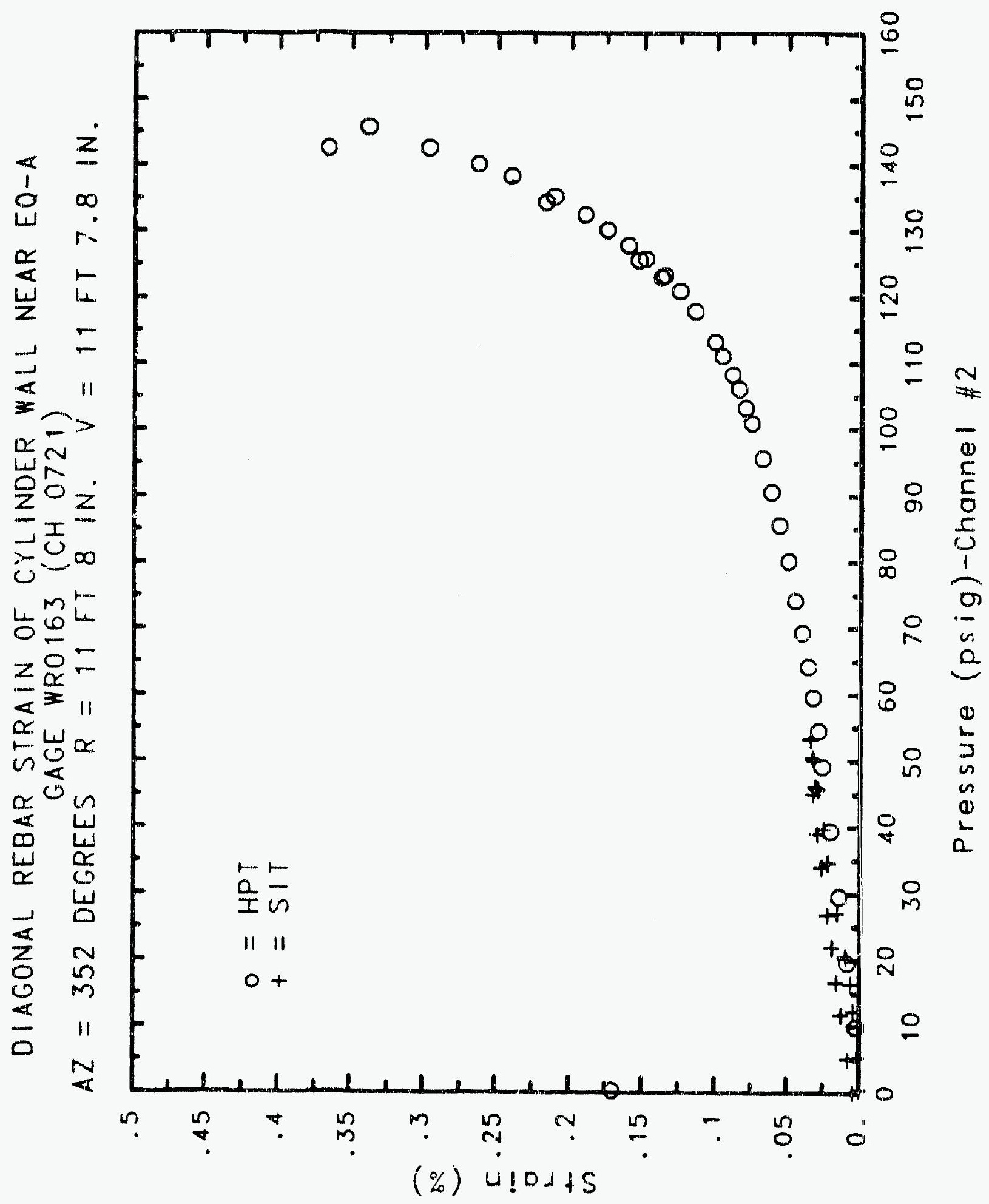




\section{Reinforced Concrete Test Data}

Weldable gage 164 Channel 722

Structural Integrity test

High Pressure test

\begin{tabular}{|c|c|c|c|}
\hline $\begin{array}{c}\text { Pressure } \\
\text { (psig) } \\
-0.05 \\
5.33 \\
12.31 \\
16.44 \\
20.51 \\
20.46 \\
20.47 \\
27.05 \\
34.69 \\
34.53 \\
34.53 \\
39.70 \\
45.90 \\
45.65 \\
45.62 \\
50.10 \\
53.47 \\
53.29 \\
53.21 \\
50.49 \\
46.14 \\
46.13 \\
44.97 \\
38.98 \\
33.99 \\
33.96 \\
26.83 \\
21.88 \\
21.90 \\
16.69 \\
11.74 \\
11.77 \\
5.05 \\
0.02 \\
-0.04 \\
-0.02 \\
-0.02 \\
0.02 \\
\end{array}$ & $\begin{array}{c}\text { \% Strain } \\
-0.0010 \\
0.0022 \\
0.0046 \\
0.0068 \\
0.0098 \\
0.0107 \\
0.0110 \\
0.0171 \\
0.0252 \\
0.0260 \\
0.0263 \\
0.0301 \\
0.0363 \\
0.0363 \\
0.0363 \\
0.0395 \\
0.0432 \\
0.0430 \\
0.0428 \\
0.0418 \\
0.0389 \\
0.0386 \\
0.0391 \\
0.0360 \\
0.0322 \\
0.0327 \\
0.0277 \\
0.0242 \\
0.0238 \\
0.0204 \\
0.0167 \\
0.0163 \\
0.0114 \\
0.0081 \\
0.0065 \\
0.0076 \\
0.0081 \\
0.0075\end{array}$ & $\begin{array}{c}\text { Pressure } \\
(\text { psig) } \\
9.89 \\
19.55 \\
29.57 \\
39.42 \\
49.16 \\
54.50 \\
59.57 \\
64.20 \\
69.32 \\
74.16 \\
80.16 \\
85.61 \\
90.58 \\
95.69 \\
100.92 \\
103.25 \\
106.11 \\
108.31 \\
111.08 \\
113.24 \\
117.83 \\
120.92 \\
123.28 \\
122.97 \\
125.82 \\
125.60 \\
127.84 \\
130.19 \\
132.53 \\
135.33 \\
134.42 \\
138.35 \\
140.16 \\
142.63 \\
145.78 \\
142.52 \\
0.22 \\
\end{array}$ & $\begin{array}{l}\text { \% Strain } \\
0.0029 \\
0.0104 \\
0.0182 \\
0.0258 \\
0.0328 \\
0.0369 \\
0.0404 \\
0.0451 \\
0.0493 \\
0.0531 \\
0.0567 \\
0.0619 \\
0.0678 \\
0.0734 \\
0.0786 \\
0.0811 \\
0.0854 \\
0.0885 \\
0.0940 \\
0.0983 \\
0.1109 \\
0.1201 \\
0.1283 \\
0.1298 \\
0.1393 \\
0.1418 \\
0.1484 \\
0.1631 \\
0.1838 \\
0.2145 \\
0.2205 \\
0.2629 \\
0.2976 \\
0.3485 \\
0.3896 \\
0.4025 \\
0.2392\end{array}$ \\
\hline
\end{tabular}




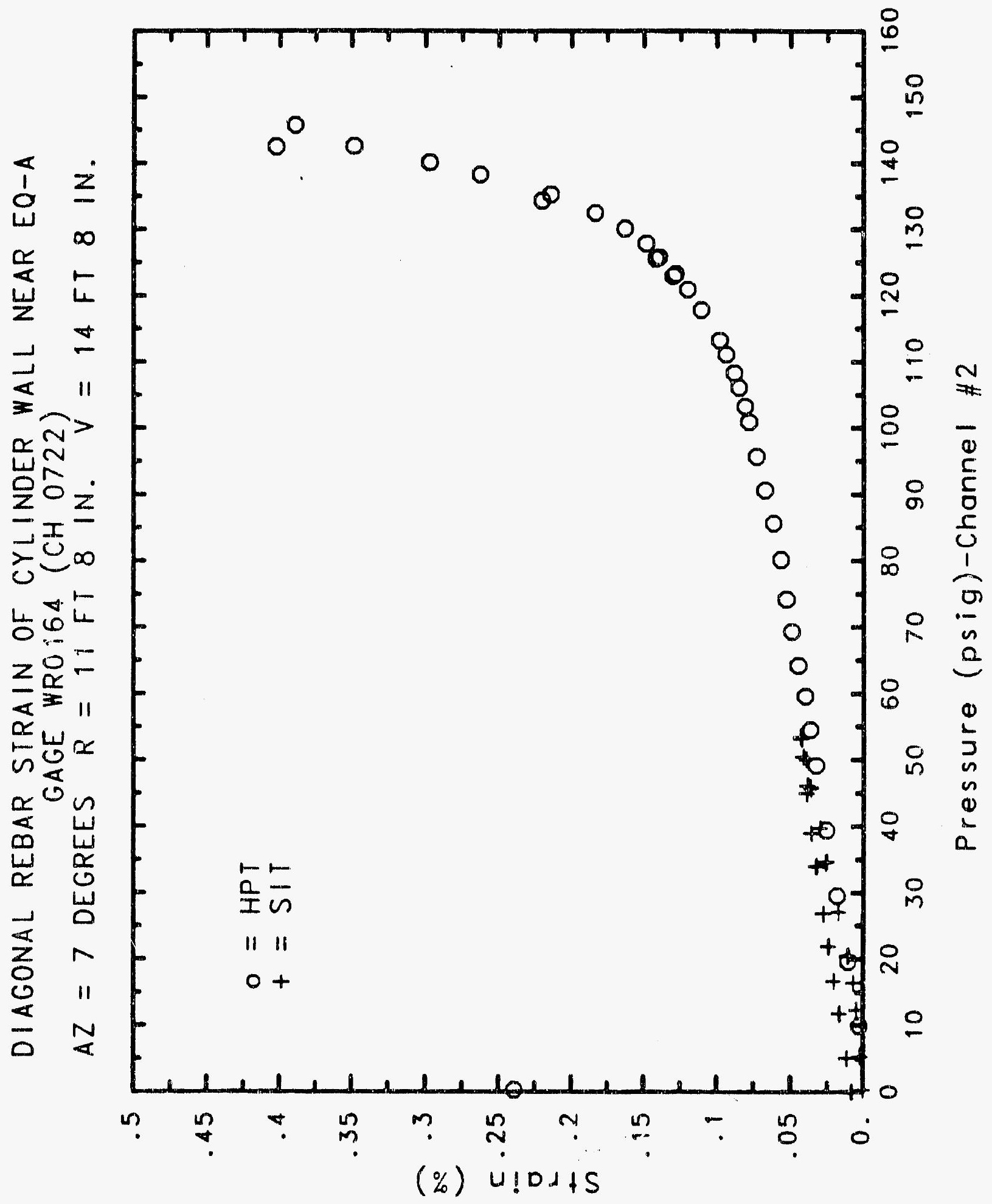




\section{Reinforced Concrete Test Data}

Weldable gage 165 Channel 723

STRUCTURAL INTEGRITY TEST

High Pressure test

\begin{tabular}{|c|c|c|c|}
\hline $\begin{array}{c}\text { Pressure } \\
\text { (psig) } \\
-0.05 \\
5.33 \\
12.31 \\
16.44 \\
20.51 \\
20.46 \\
20.47 \\
27.05 \\
34.69 \\
34.53 \\
34.53 \\
39.70 \\
45.90 \\
45.65 \\
45.62 \\
50.10 \\
53.47 \\
53.29 \\
53.21 \\
50.49 \\
46.14 \\
46.13 \\
44.97 \\
38.98 \\
33.99 \\
33.96 \\
26.83 \\
21.88 \\
21.90 \\
16.69 \\
11.74 \\
11.77 \\
5.05 \\
0.02 \\
-0.04 \\
-0.02 \\
-0.02 \\
0.02 \\
\end{array}$ & $\begin{array}{l}\text { Strain } \\
0.0000 \\
0.0018 \\
0.0041 \\
0.0059 \\
0.0074 \\
0.0083 \\
0.0085 \\
0.0136 \\
0.0201 \\
0.0212 \\
0.0212 \\
0.0254 \\
0.0321 \\
0.0320 \\
0.0315 \\
0.0352 \\
0.0389 \\
0.0390 \\
0.0396 \\
0.0385 \\
0.0358 \\
0.0357 \\
0.0373 \\
0.0340 \\
0.0309 \\
0.0311 \\
0.0259 \\
0.0226 \\
0.0222 \\
0.0186 \\
0.0151 \\
0.0146 \\
0.0101 \\
0.0067 \\
0.0050 \\
0.0062 \\
0.0069 \\
0.0061\end{array}$ & $\begin{array}{c}\text { Pressure } \\
\text { (psig) } \\
9.89 \\
19.55 \\
29.57 \\
39.42 \\
49.16 \\
54.50 \\
59.57 \\
64.20 \\
69.32 \\
74.16 \\
80.16 \\
85.61 \\
90.58 \\
95.69 \\
100.92 \\
103.25 \\
106.11 \\
108.31 \\
111.08 \\
113.24 \\
117.83 \\
120.92 \\
123.28 \\
122.97 \\
125.82 \\
125.60 \\
127.84 \\
130.19 \\
132.53 \\
135.33 \\
134.42 \\
138.35 \\
140.16 \\
142.63 \\
145.78 \\
142.52 \\
0.22 \\
\end{array}$ & $\begin{array}{l}\text { x Strain } \\
0.0028 \\
0.0107 \\
0.0181 \\
0.0256 \\
0.0321 \\
0.0357 \\
0.0400 \\
0.0449 \\
0.0510 \\
0.0576 \\
0.0635 \\
0.0717 \\
0.0796 \\
0.0898 \\
0.0996 \\
0.1051 \\
0.1107 \\
0.1158 \\
0.1232 \\
0.1299 \\
0.1472 \\
0.1614 \\
0.1746 \\
0.1782 \\
0.1941 \\
0.2009 \\
0.2106 \\
0.2323 \\
0.2976 \\
0.3495 \\
0.3620 \\
0.4160 \\
0.4623 \\
0.5223 \\
0.5762 \\
0.5935 \\
0.4051\end{array}$ \\
\hline
\end{tabular}




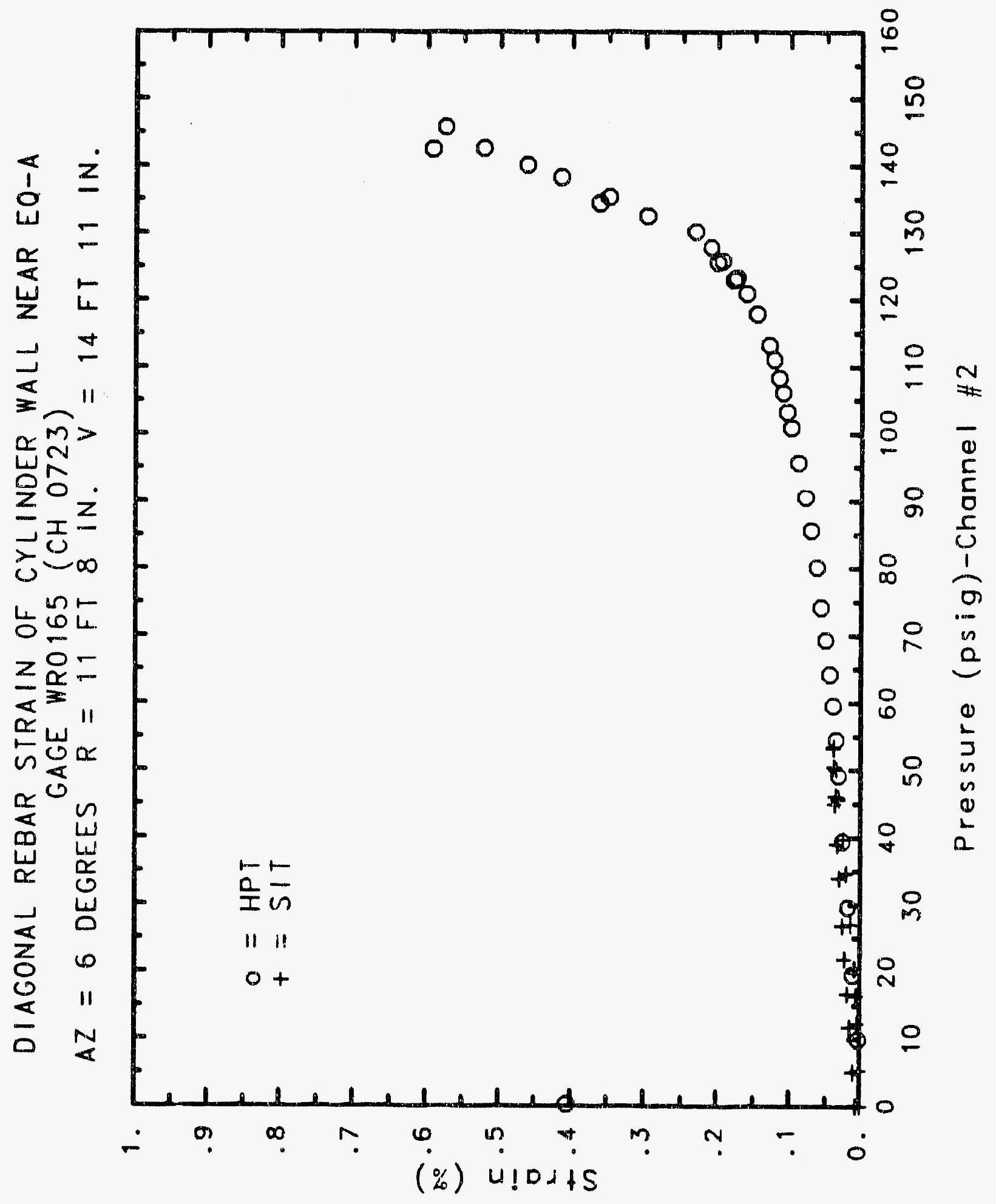




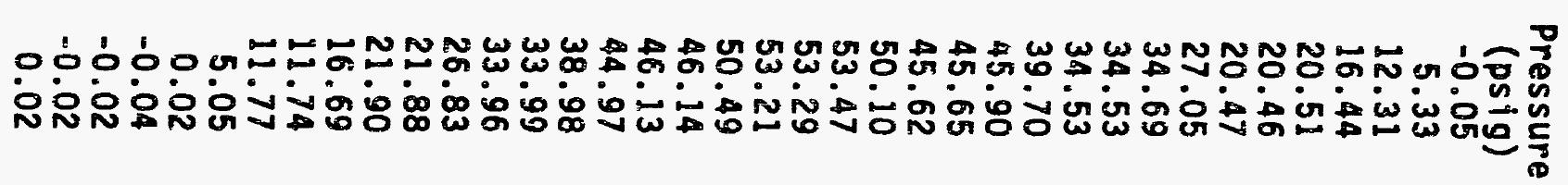

00000000000000000000000000000000000000

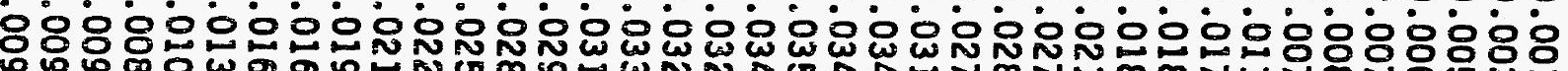

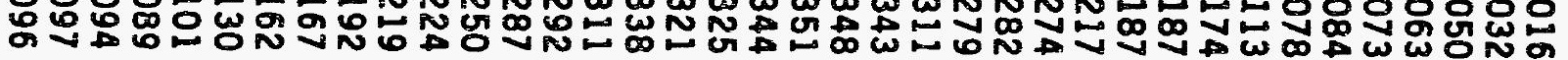

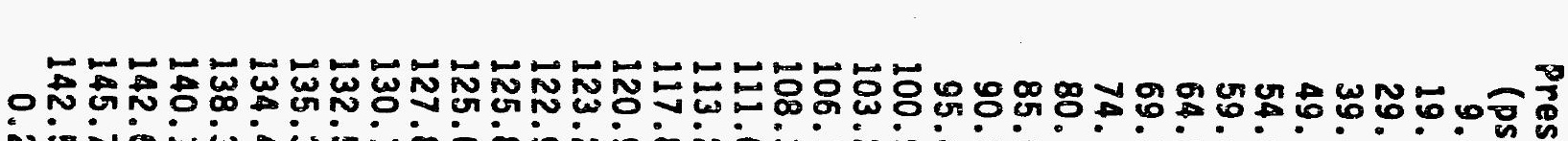
N

0000000000000000000000000000000000000

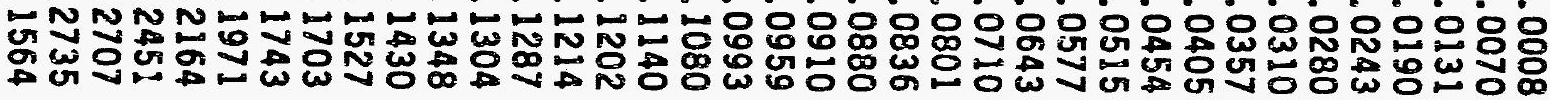




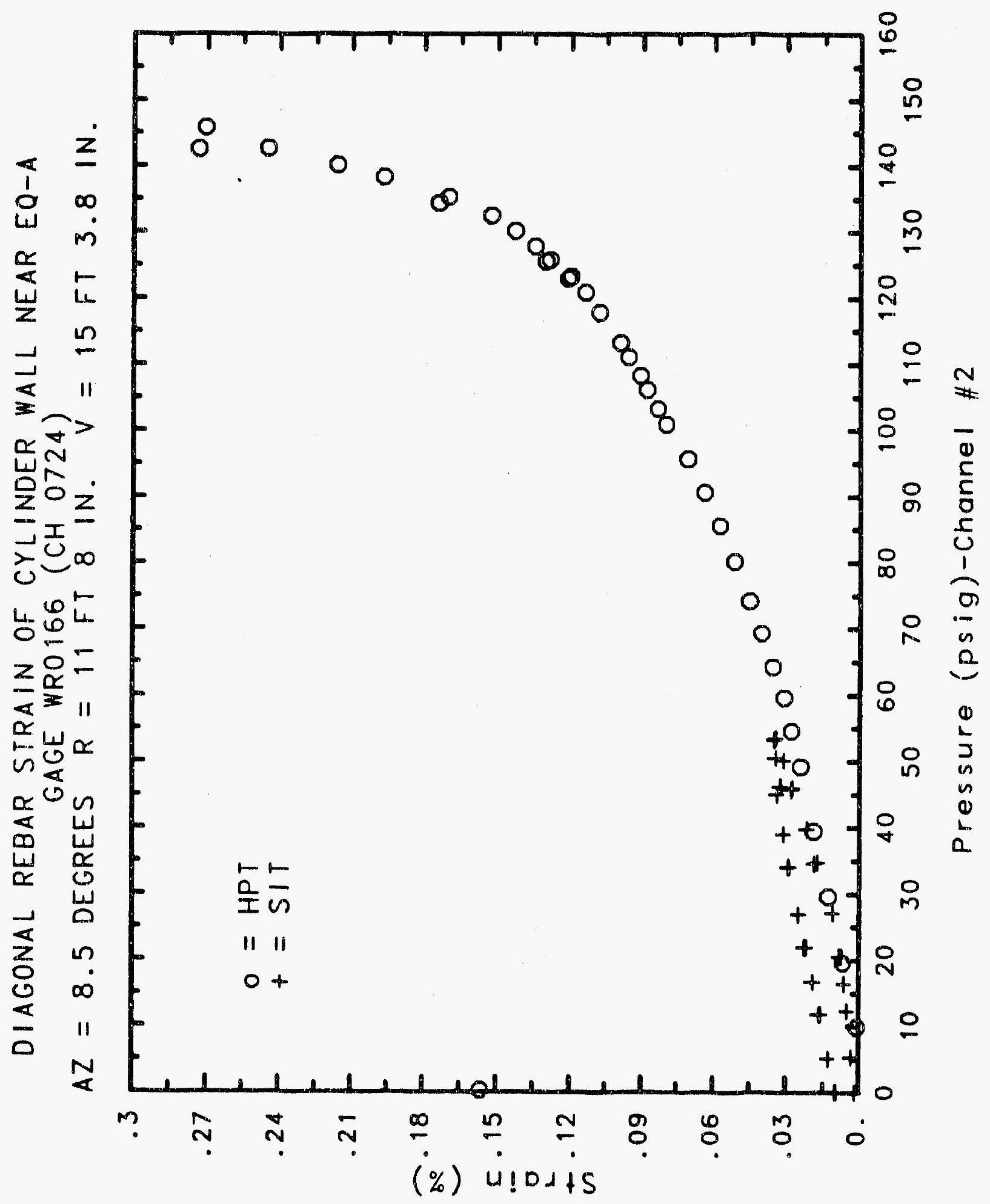




\section{Reinforced Concrete Test Data}

Weldable Gage 167 Chammel 725

StRUCTURAL INTEGRITY TEST

High Pressure test

\begin{tabular}{|c|c|c|c|}
\hline $\begin{array}{c}\text { Pressure } \\
(\text { psig) } \\
-0.05 \\
5.33 \\
12.31 \\
16.44 \\
20.51 \\
20.46 \\
20.47 \\
27.05 \\
34.69 \\
34.53 \\
34.53 \\
39.70 \\
45.90 \\
45.65 \\
45.62 \\
50.10 \\
53.47 \\
53.29 \\
53.21 \\
50.49 \\
46.14 \\
46.13 \\
44.97 \\
38.98 \\
33.99 \\
33.96 \\
26.83 \\
21.88 \\
21.90 \\
16.69 \\
11.74 \\
11.77 \\
5.05 \\
0.02 \\
-0.04 \\
-0.02 \\
-0.02 \\
0.02 \\
0\end{array}$ & $\begin{array}{l}\text { \% Strain } \\
0.0010 \\
0.0009 \\
0.0010 \\
0.0013 \\
0.0014 \\
0.0018 \\
0.0020 \\
0.0023 \\
0.0023 \\
0.0024 \\
0.0025 \\
0.0022 \\
0.0022 \\
0.0026 \\
0.0024 \\
0.0024 \\
0.0022 \\
0.0020 \\
0.0027 \\
0.0023 \\
0.0020 \\
0.0022 \\
0.0020 \\
0.0018 \\
0.0019 \\
0.0020 \\
0.0017 \\
0.0019 \\
0.0021 \\
0.0018 \\
0.0016 \\
0.0017 \\
0.0011 \\
0.0012 \\
0.0013 \\
0.0006 \\
0.0007 \\
0.0011\end{array}$ & $\begin{array}{c}\text { Pressure } \\
(\text { psig) } \\
9.89 \\
19.55 \\
29.57 \\
39.42 \\
49.16 \\
54.50 \\
59.57 \\
64.20 \\
69.32 \\
74.16 \\
80.16 \\
85.61 \\
90.58 \\
95.69 \\
100.92 \\
103.25 \\
106.11 \\
108.31 \\
111.08 \\
113.24 \\
117.83 \\
120.92 \\
123.28 \\
122.91 \\
125.82 \\
125.60 \\
127.84 \\
130.19 \\
132.53 \\
135.33 \\
134.42 \\
138.35 \\
140.16 \\
142.63 \\
145.78 \\
142.52 \\
0.22 \\
\end{array}$ & $\begin{array}{l}\text { \% Strain } \\
-0.0032 \\
-0.0029 \\
-0.0029 \\
-0.0020 \\
-0.0019 \\
-0.0021 \\
-0.0016 \\
-0.0008 \\
-0.0011 \\
-0.0006 \\
-0.0008 \\
-0.0006 \\
-0.0009 \\
-0.0007 \\
-0.0018 \\
-0.0015 \\
-0.0014 \\
-0.0009 \\
-0.0006 \\
0.0004 \\
0.0018 \\
0.0028 \\
0.0035 \\
0.0043 \\
0.0046 \\
0.0049 \\
0.0054 \\
0.0061 \\
0.0072 \\
0.0084 \\
0.0090 \\
0.0110 \\
0.0130 \\
0.0143 \\
0.0164 \\
0.0177 \\
0.0084\end{array}$ \\
\hline
\end{tabular}




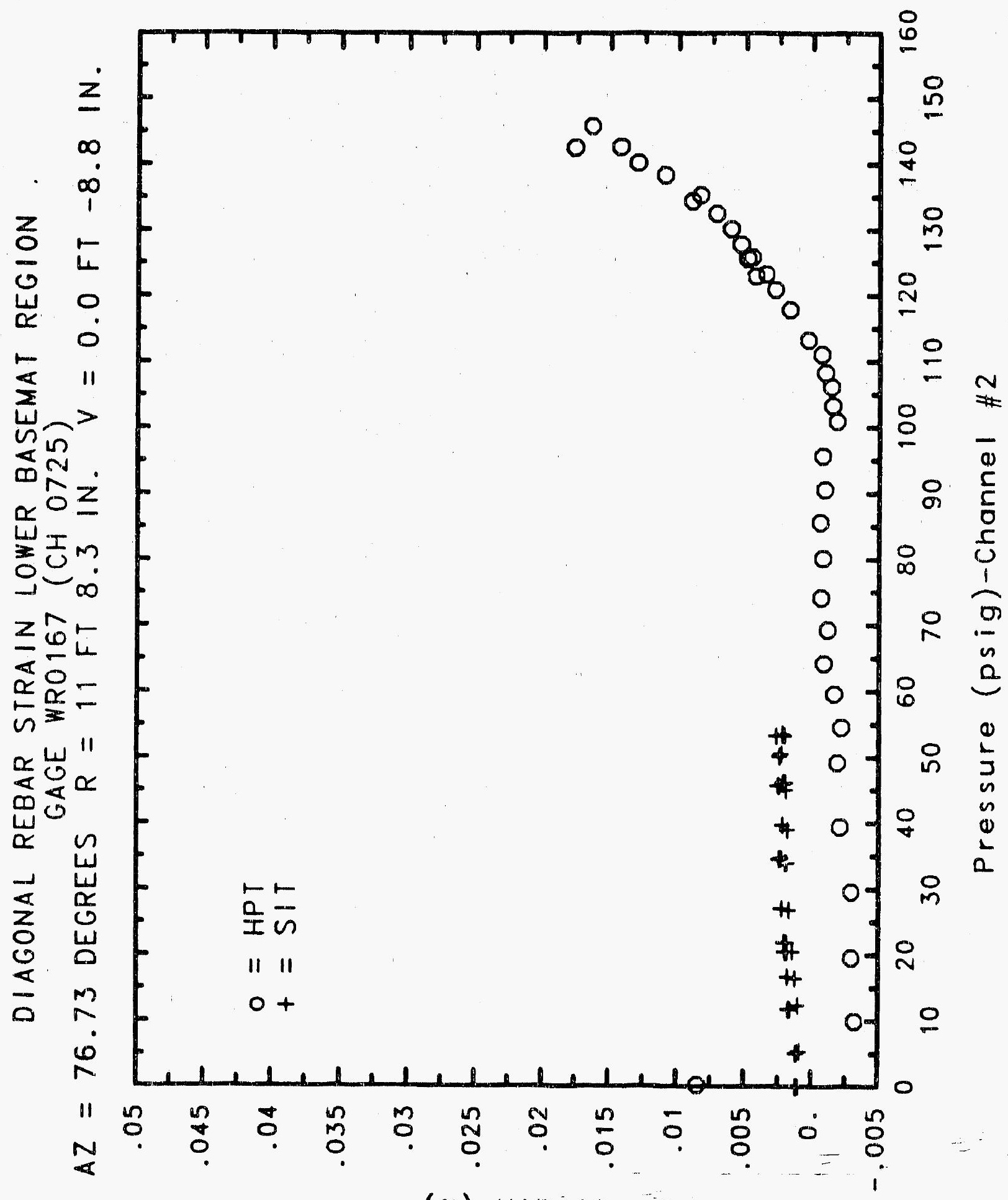

(\%) $4 ! 0+5$ 


\section{Reinforced Concrete Test Data}

Weldable gage 168 Channel 726

Structural Integrity Test

High Pressure test

\begin{tabular}{|c|c|c|c|}
\hline 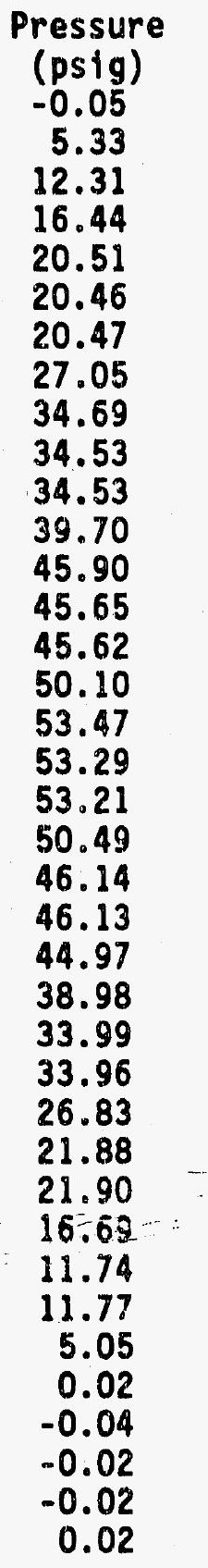 & $\begin{array}{c}\% \text { Strain } \\
0.0010 \\
0.0003 \\
-0.0009 \\
-0.0021 \\
-0.0028 \\
-0.0038 \\
-0.0046 \\
-0.0050 \\
-0.0058 \\
-0.0058 \\
-0.0063 \\
-0.0066 \\
-0.0070 \\
-0.0067 \\
-0.0065 \\
-0.0071 \\
-0.0067 \\
-0.0074 \\
-0.0067 \\
-0.0067 \\
-0.0065 \\
-0.0067 \\
-0.0060 \\
-0.0063 \\
-0.0060 \\
-0.0067 \\
-0.0069 \\
-0.0065 \\
-0.0072 \\
-0.0072 \\
-0.0064 \\
-0.0059 \\
-0.0052 \\
-0.0041 \\
-0.0005 \\
-0.0002 \\
-0.0012 \\
-0.0016\end{array}$ & $\begin{array}{c}\text { Pressure } \\
\text { (psig) } \\
9.89 \\
19.55 \\
29.57 \\
39.42 \\
49.16 \\
54.50 \\
59.57 \\
64.20 \\
69.32 \\
74.16 \\
80.16 \\
85.61 \\
90.58 \\
95.69 \\
100.92 \\
103.25 \\
106.11 \\
108.31 \\
111.08 \\
113.24 \\
117.83 \\
120.92 \\
123.28 \\
122.97 \\
125.82 \\
125.60 \\
127.84 \\
130.19 \\
132.53 \\
135.33 \\
134.42 \\
138.35 \\
140.16 \\
142.63 \\
145.78 \\
142.52 \\
0.22 \\
=\end{array}$ & $\begin{array}{l}\text { \% Strain } \\
-0.0058 \\
-0.0069 \\
-0.0077 \\
-0.0086 \\
-0.0090 \\
-0.0090 \\
-0.0095 \\
-0.0089 \\
-0.0092 \\
-0.0104 \\
-0.0094 \\
-0.0083 \\
-0.0073 \\
-0.0062 \\
-0.0049 \\
-0.0047 \\
-0.0040 \\
-0.0035 \\
-0.0022 \\
-0.0013 \\
-0.0030 \\
-0.0038 \\
-0.0040 \\
-0.0047 \\
-0.0044 \\
-0.0049 \\
-0.0051 \\
-0.0039 \\
-0.0035 \\
-0.0030 \\
-0.0037 \\
-0.0022 \\
-0.0005 \\
0.0027 \\
0.0067 \\
0.0078 \\
-0.0105\end{array}$ \\
\hline
\end{tabular}




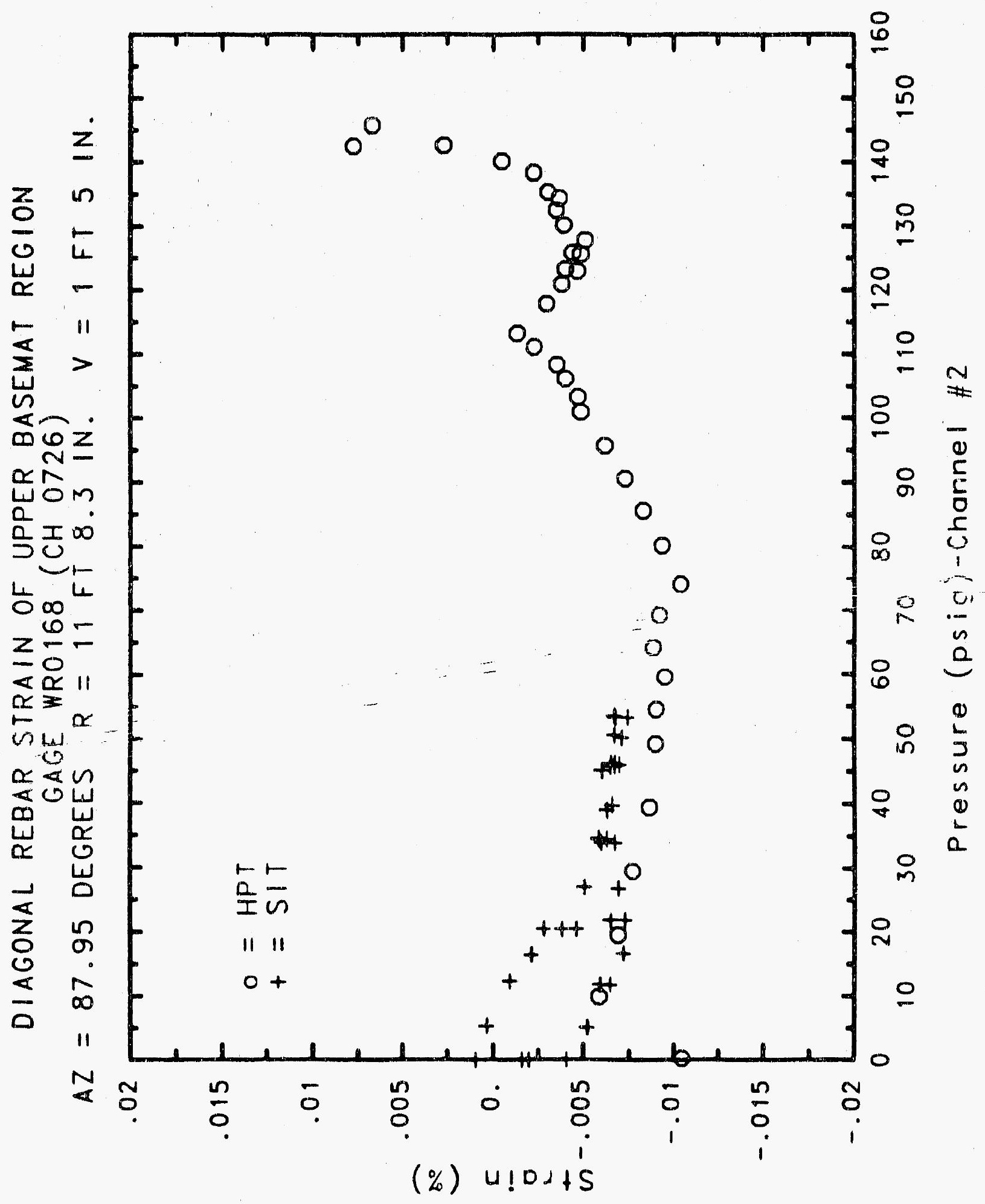




\section{Reinforced Concrete Test Data}

Weldable Gage 169 Channel 727

Structural Integrity Test

High Pressure test

\begin{tabular}{|c|c|c|c|}
\hline $\begin{array}{c}\text { Pressure } \\
\text { (psig) } \\
-0.05 \\
5.33 \\
12.31 \\
16.44 \\
20.51 \\
20.46 \\
20.47 \\
21.05 \\
34.69 \\
34.53 \\
34.53 \\
39.70 \\
45.90 \\
45.65 \\
45.62 \\
50.10 \\
53.47 \\
53.29 \\
53.21 \\
50.49 \\
46.14 \\
46.13 \\
44.97 \\
38.98 \\
33.99 \\
33.96 \\
26.83 \\
21.88 \\
21.90 \\
16.69 \\
11.74 \\
11.77 \\
5.05 \\
0.02 \\
-0.04 \\
-0.02 \\
-0.02 \\
0.02 \\
\end{array}$ & $\begin{array}{r}\text { X strain } \\
-0.0004 \\
-0.0018 \\
-0.0038 \\
-0.0036 \\
-0.0019 \\
0.0375 \\
0.0101 \\
0.0080 \\
0.0071 \\
-0.0139 \\
-0.0136 \\
-0.0146 \\
-0.0149 \\
0.0443 \\
-0.0130 \\
-0.0133 \\
-0.0124 \\
-0.0125 \\
0.0190 \\
0.0466 \\
0.0471 \\
-0.0061 \\
0.0487 \\
-0.0060 \\
-0.0058 \\
-0.0128 \\
-0.0148 \\
-0.0156 \\
-0.0156 \\
-0.0144 \\
-0.0132 \\
-0.0116 \\
-0.0099 \\
0.0525 \\
0.0099 \\
0.0603 \\
0.0592 \\
0.0328\end{array}$ & $\begin{array}{c}\text { Pressure } \\
(\text { psig) } \\
9.89 \\
19.55 \\
29.57 \\
39.42 \\
49.16 \\
54.50 \\
59.57 \\
64.20 \\
69.32 \\
74.16 \\
80.16 \\
85.61 \\
90.58 \\
95.69 \\
100.92 \\
103.25 \\
106.11 \\
108.31 \\
111.08 \\
113.24 \\
117.83 \\
120.92 \\
123.28 \\
122.97 \\
125.82 \\
125.60 \\
127.84 \\
130.19 \\
132.53 \\
135.33 \\
134.42 \\
138.35 \\
140.16 \\
142.63 \\
145.78 \\
142.52 \\
0.22 \\
\end{array}$ & $\begin{array}{l}\text { \% Strain } \\
-0.0062 \\
-0.0082 \\
-0.0099 \\
-0.0113 \\
-0.0123 \\
-0.0131 \\
-0.0138 \\
-0.0150 \\
-0.0164 \\
-0.0197 \\
-0.0175 \\
-0.0172 \\
-0.0176 \\
-0.0167 \\
-0.0171 \\
-0.0168 \\
-0.0173 \\
-0.0174 \\
-0.0178 \\
-0.0178 \\
-0.0184 \\
-0.0185 \\
-0.0186 \\
-0.0185 \\
-0.0184 \\
-0.0183 \\
-0.0183 \\
-0.0183 \\
-0.0183 \\
-0.0178 \\
0.0354 \\
-0.0212 \\
-0.0214 \\
-0.0171 \\
-0.0188 \\
-0.0190 \\
-0.0226\end{array}$ \\
\hline
\end{tabular}




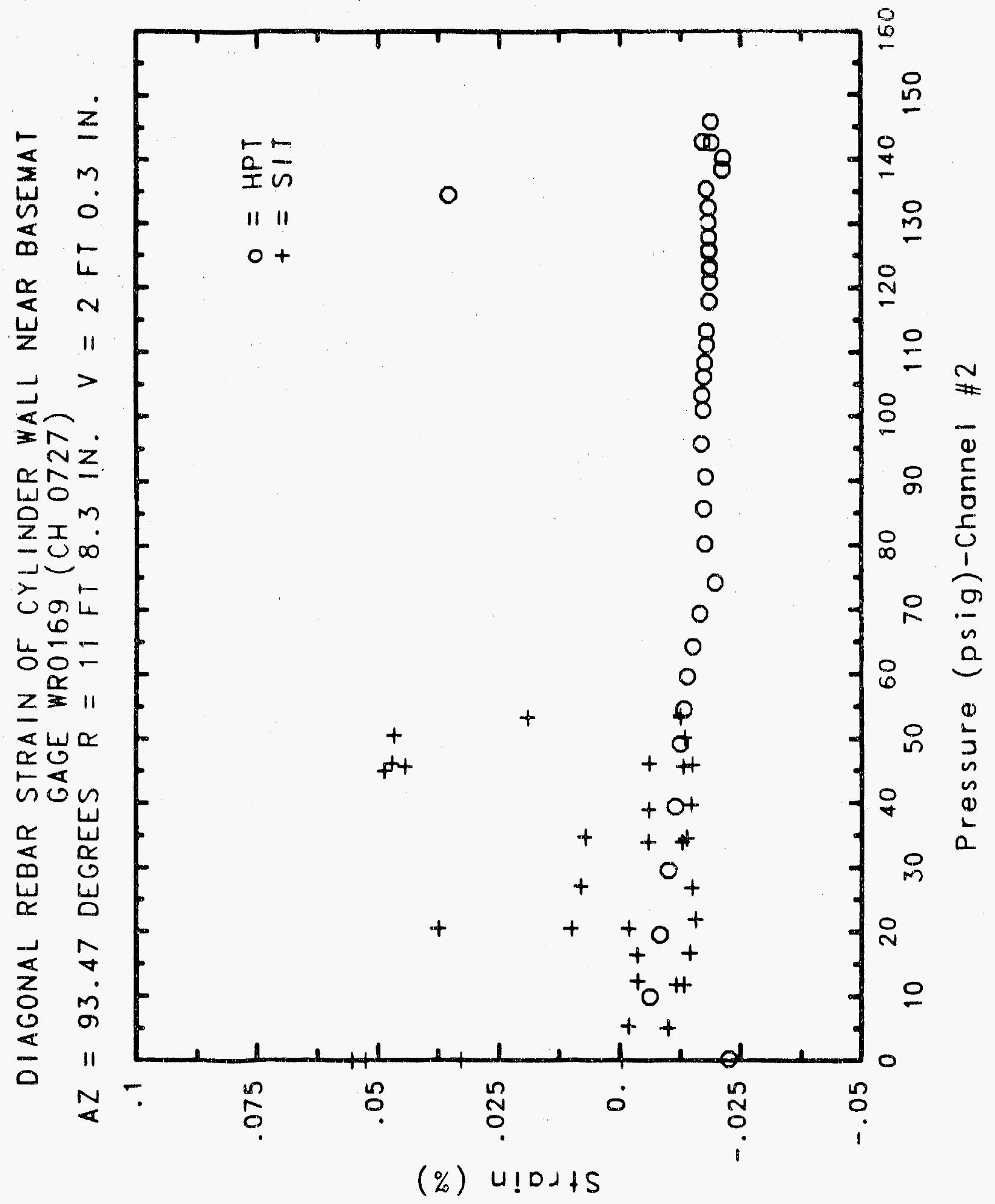




\section{Reinforced Concrete Test Data}

Weldable Gage 170 Channel 728

Structural Integrity Tesi"

High Pressure test

\begin{tabular}{|c|c|c|c|}
\hline $\begin{array}{c}\text { Pressure } \\
\text { (psig) } \\
-0.05 \\
5.33 \\
12.31 \\
16.44 \\
20.51 \\
20.46 \\
20.47 \\
27.05 \\
34.69 \\
34.53 \\
34.53 \\
39.70 \\
45.90 \\
45.65 \\
45.62 \\
50.10 \\
53.47 \\
53.29 \\
53.21 \\
50.49 \\
46.14 \\
46.13 \\
44.97 \\
38.98 \\
33.99 \\
33.96 \\
26.83 \\
21.88 \\
21.90 \\
16.69 \\
11.74 \\
11.77 \\
5.05 \\
0.02 \\
-0.04 \\
-0.02 \\
-0.02 \\
0.02 \\
\end{array}$ & $\begin{array}{l}\text { \% strain } \\
-0.0012 \\
-0.0011 \\
-0.0013 \\
-0.0019 \\
-0.0032 \\
-0.0062 \\
-0.0092 \\
-0.0089 \\
-0.0073 \\
-0.0079 \\
-0.0084 \\
-0.0069 \\
-0.0058 \\
-0.0025 \\
-0.0015 \\
-0.0003 \\
0.0005 \\
0.0021 \\
0.0024 \\
0.0029 \\
0.0029 \\
0.0032 \\
0.0027 \\
0.0014 \\
0.0002 \\
-0.0055 \\
-0.0081 \\
-0.0100 \\
-0.0078 \\
-0.0082 \\
-0.0084 \\
-0.0062 \\
-0.0055 \\
-0.0052 \\
0.0001 \\
-0.0007 \\
-0.0018 \\
.0 .0031\end{array}$ & $\begin{array}{c}\text { Pressure } \\
(\text { psig) } \\
9.89 \\
19.55 \\
29.57 \\
39.42 \\
49.16 \\
54.50 \\
59.57 \\
64.20 \\
69.32 \\
74.16 \\
80.16 \\
85.61 \\
90.58 \\
95.69 \\
100.92 \\
103.25 \\
106.11 \\
108.31 \\
111.08 \\
113.24 \\
117.83 \\
120.92 \\
123.28 \\
122.97 \\
125.82 \\
125.60 \\
127.84 \\
130.19 \\
132.53 \\
135.33 \\
134.42 \\
138.35 \\
140.16 \\
142.63 \\
145.78 \\
142.52 \\
0.22 \\
\end{array}$ & $\begin{array}{l}\% \text { Strain } \\
-0.0030 \\
-0.0019 \\
-0.0013 \\
-0.0009 \\
-0.0005 \\
-0.0007 \\
-0.0003 \\
-0.002 .4 \\
-0.0036 \\
-0.0103 \\
-0.0007 \\
-0.0008 \\
-0.0007 \\
-0.0003 \\
-0.0011 \\
-0.0020 \\
-0.0024 \\
-0.0031 \\
-0.0043 \\
-0.0049 \\
-0.0067 \\
-0.0090 \\
-0.0107 \\
-0.0117 \\
-0.0146 \\
-0.0157 \\
-0.0175 \\
-0.0221 \\
-0.0263 \\
-0.0301 \\
-0.0342 \\
-0.0401 \\
-0.0403 \\
-0.0415 \\
-0.0410 \\
-0.0391 \\
-0\end{array}$ \\
\hline
\end{tabular}




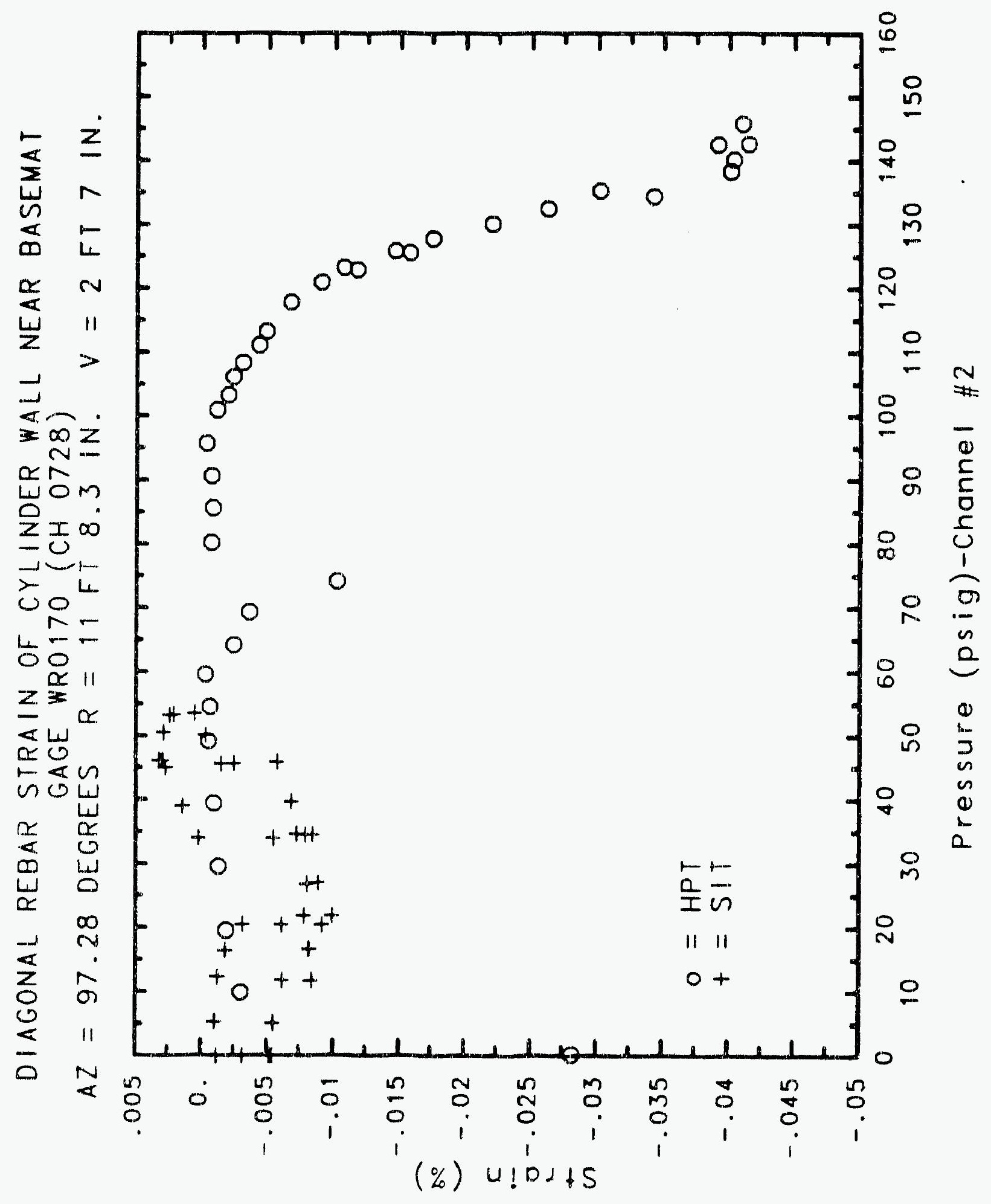




\section{Reinforced Concrete Test Data}

\section{Weldarle gage 171 Channel 729}

STRUCTURAL INTEGRITY TEST

High Pressure Test

\begin{tabular}{|c|c|c|c|}
\hline $\begin{array}{c}\text { Pressure } \\
\text { (psig) } \\
-0.05 \\
5.33 \\
12.31 \\
16.44 \\
20.51 \\
20.46 \\
20.47 \\
27.05 \\
34.69 \\
34.53 \\
34.53 \\
39.70 \\
45.90 \\
45.65 \\
45.62 \\
50.10 \\
53.47 \\
53.29 \\
53.21 \\
50.49 \\
46.14 \\
46.13 \\
44.97 \\
38.98 \\
33.99 \\
33.96 \\
26.83 \\
21.88 \\
21.90 \\
16.69 \\
11.74 \\
11.77 \\
5.05 \\
0.02 \\
-0.04 \\
-0.02 \\
-0.02 \\
0.02 \\
\end{array}$ & $\begin{array}{l}\text { X Strain } \\
\text {-0.0011 } \\
0.0009 \\
0.0030 \\
0.0045 \\
0.0087 \\
0.0103 \\
0.0124 \\
0.0217 \\
0.0306 \\
0.0315 \\
0.0311 \\
0.0358 \\
0.0433 \\
0.0432 \\
0.0436 \\
0.0490 \\
0.0545 \\
0.0553 \\
0.0554 \\
0.0548 \\
0.0529 \\
0.0519 \\
0.0547 \\
0.0517 \\
0.1184 \\
0.0488 \\
0.0450 \\
0.0422 \\
0.0415 \\
0.0370 \\
0.0323 \\
0.0306 \\
0.0248 \\
0.0205 \\
0.0199 \\
0.0200 \\
0.0201 \\
0.0212\end{array}$ & $\begin{array}{c}\text { Pressure } \\
\text { (psig) } \\
9.89 \\
19.55 \\
29.57 \\
39.42 \\
49.16 \\
54.50 \\
59.57 \\
64.20 \\
69.32 \\
74.16 \\
80.16 \\
85.61 \\
90.58 \\
95.69 \\
100.92 \\
103.25 \\
106.11 \\
108.31 \\
111.08 \\
113.24 \\
117.83 \\
120.92 \\
123.28 \\
122.97 \\
125.82 \\
125.60 \\
127.84 \\
130.19 \\
132.53 \\
135.33 \\
134.42 \\
138.35 \\
140.16 \\
142.63 \\
145.78 \\
142.52 \\
0.22 \\
\end{array}$ & $\begin{array}{l}\text { \% Strain } \\
0.0040 \\
0.0118 \\
0.0200 \\
0.0278 \\
0.0351 \\
0.0392 \\
0.0445 \\
0.0506 \\
0.0584 \\
0.0661 \\
0.0712 \\
0.0780 \\
0.0854 \\
0.0930 \\
0.1012 \\
0.1052 \\
0.1093 \\
0.1130 \\
0.1181 \\
0.1216 \\
0.1303 \\
0.1359 \\
0.1415 \\
0.1425 \\
0.1487 \\
0.1508 \\
0.1543 \\
0.1615 \\
0.1685 \\
0.1802 \\
0.1827 \\
0.1995 \\
0.2145 \\
0.2349 \\
0.2557 \\
0.2608 \\
0.1450\end{array}$ \\
\hline
\end{tabular}




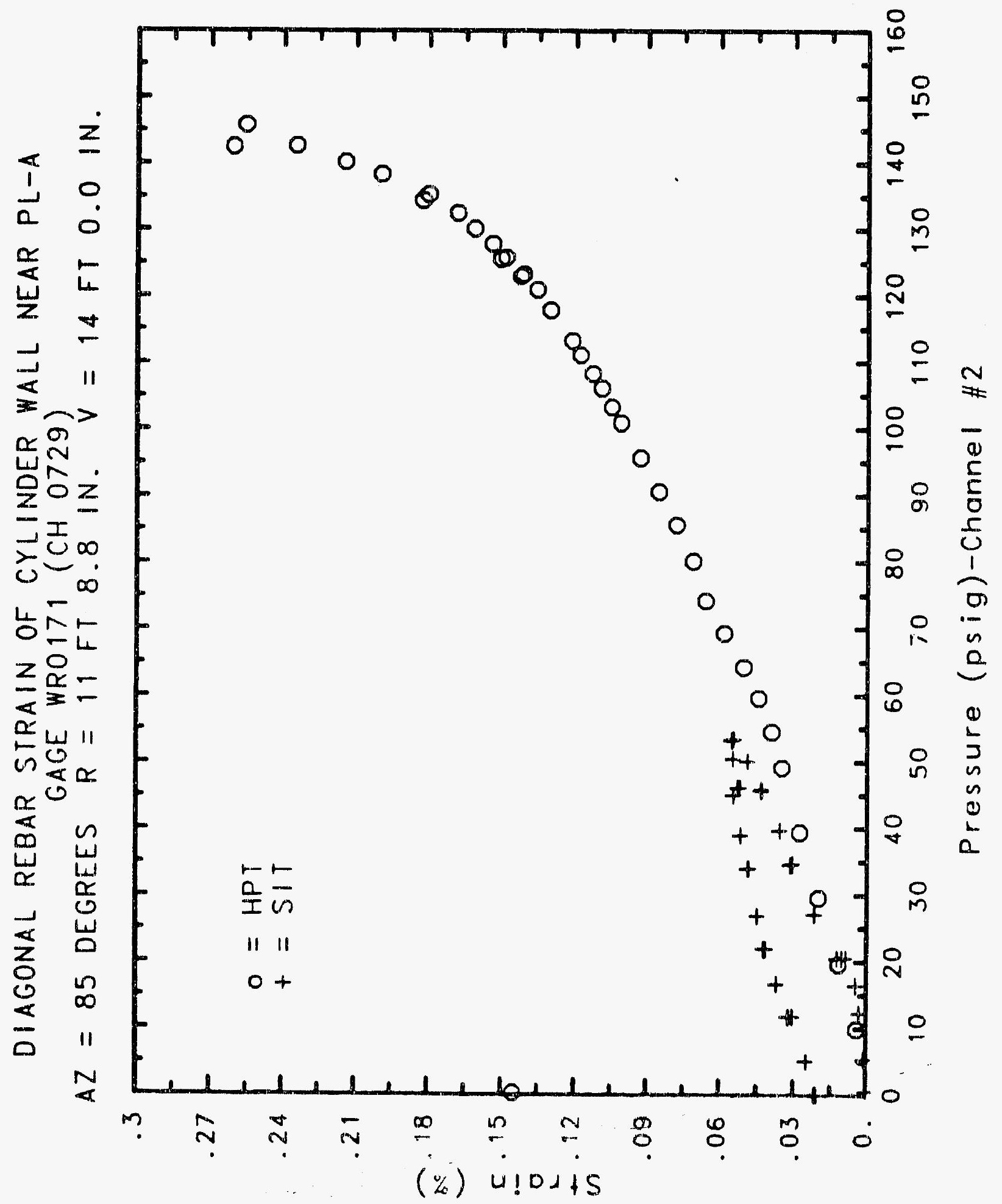




\section{Reinforced Concrete Test Data}

Weldable Gage 172 Channel 740

STRUCTURAL INTEGRITY TEST

High Pressure test

\begin{tabular}{|c|c|c|c|}
\hline $\begin{array}{c}\text { Pressure } \\
(\text { psig) } \\
-0.05 \\
5.33 \\
12.31 \\
16.44 \\
20.51 \\
20.46 \\
20.47 \\
27.05 \\
34.69 \\
34.53 \\
34.53 \\
39.70 \\
45.90 \\
45.65 \\
45.62 \\
50.10 \\
53.47 \\
53.29 \\
53.21 \\
50.49 \\
46.14 \\
46.13 \\
44.97 \\
38.98 \\
33.99 \\
33.96 \\
26.83 \\
21.88 \\
21.90 \\
16.69 \\
11.74 \\
11.77 \\
5.05 \\
0.02 \\
-0.04 \\
-0.02 \\
-0.02 \\
0.02 \\
\end{array}$ & $\begin{array}{l}\text { \% Strain } \\
\text {-0.0016 } \\
0.0001 \\
0.0019 \\
0.0025 \\
0.0050 \\
0.0058 \\
0.0072 \\
0.0122 \\
0.0164 \\
0.0174 \\
0.0174 \\
0.0198 \\
0.0222 \\
0.0219 \\
0.0223 \\
0.0235 \\
0.0260 \\
0.0261 \\
0.0259 \\
0.0257 \\
0.0249 \\
0.0246 \\
0.0251 \\
0.0240 \\
0.0222 \\
0.0230 \\
0.0216 \\
0.0202 \\
0.0200 \\
0.0182 \\
0.0156 \\
0.0149 \\
0.0122 \\
0.0098 \\
0.0095 \\
0.0092 \\
0.0099 \\
0.0105\end{array}$ & $\begin{array}{c}\text { Pressure } \\
\text { (psig) } \\
9.89 \\
19.55 \\
29.57 \\
39.42 \\
49.16 \\
54.50 \\
59.57 \\
64.20 \\
69.32 \\
74.16 \\
80.16 \\
85.61 \\
90.58 \\
95.69 \\
100.92 \\
103.25 \\
106.11 \\
108.31 \\
111.08 \\
113.24 \\
117.83 \\
120.92 \\
123.28 \\
122.97 \\
125.82 \\
125.60 \\
127.84 \\
130.19 \\
132.53 \\
135.33 \\
134.42 \\
138.35 \\
140.16 \\
142.63 \\
145.78 \\
142.52 \\
0.22 \\
\end{array}$ & $\begin{array}{l}\text { \% Strain } \\
-0.0002 \\
0.0035 \\
0.0071 \\
0.0106 \\
0.0140 \\
0.0163 \\
0.0183 \\
0.0213 \\
0.0245 \\
0.0290 \\
0.0325 \\
0.0383 \\
0.0444 \\
0.0518 \\
0.0593 \\
0.0639 \\
0.0681 \\
0.0720 \\
0.0773 \\
0.0813 \\
0.0922 \\
0.0994 \\
0.1069 \\
0.1088 \\
0.1161 \\
0.1194 \\
0.1247 \\
0.1363 \\
0.1528 \\
0.1740 \\
0.1808 \\
0.2089 \\
0.2326 \\
0.2710 \\
0.3042 \\
0.3174 \\
0.2265\end{array}$ \\
\hline
\end{tabular}




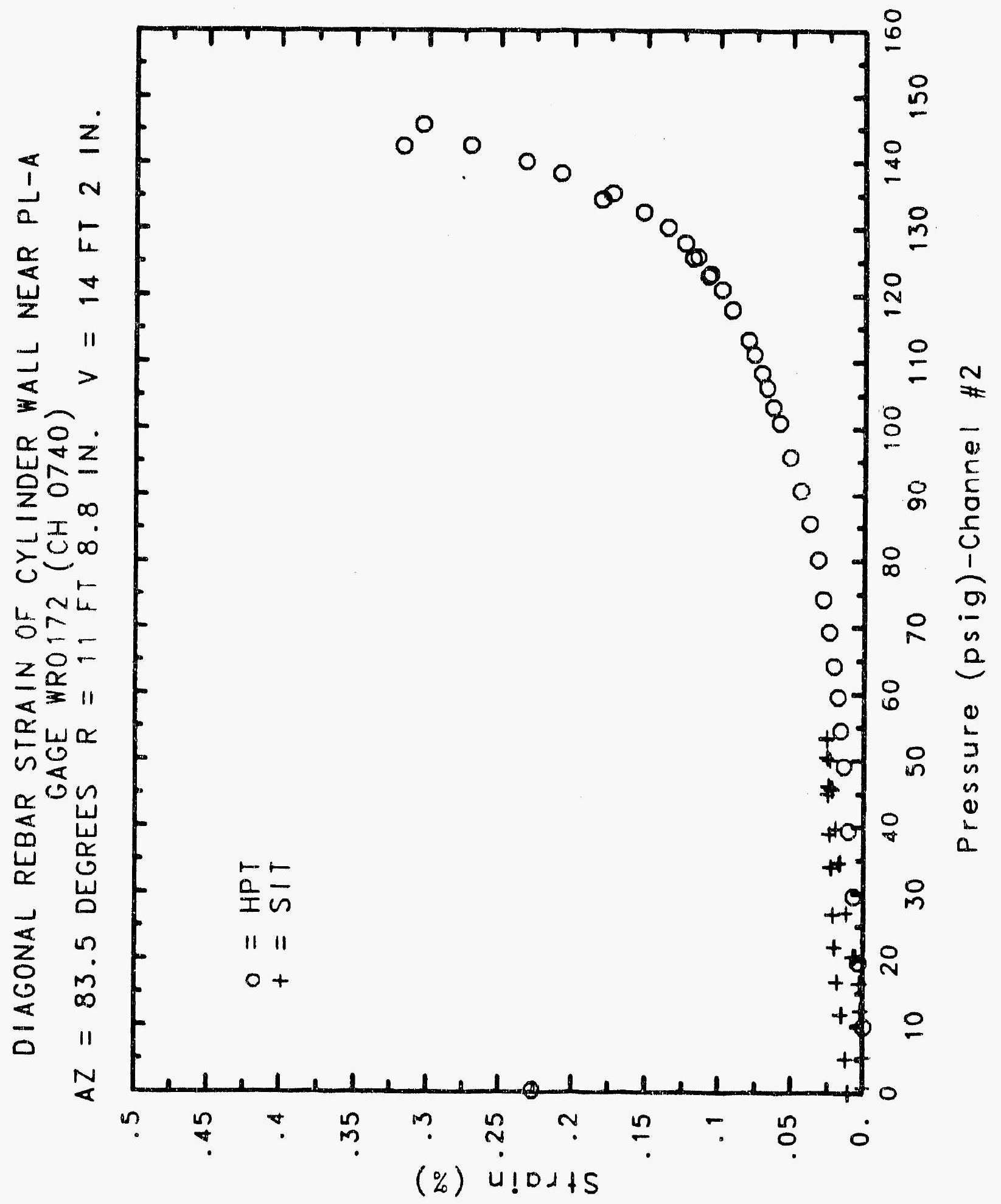




\section{Reinforced Concrete Test Data}

Weldable gage 173 Channel 741

Structural Integrity Test

\begin{tabular}{|c|c|c|c|}
\hline $\begin{array}{c}\text { Pressure } \\
\text { (psig) } \\
-0.05 \\
5.33 \\
12.31 \\
16.44 \\
20.51 \\
20.46 \\
20.47 \\
27.05 \\
34.69 \\
34.53 \\
34.53 \\
39.70 \\
45.90 \\
45.65 \\
45.62 \\
50.10 \\
53.47 \\
53.29 \\
53.21 \\
50.49 \\
46.14 \\
46.13 \\
44.97 \\
38.98 \\
33.99 \\
33.96 \\
26.83 \\
21.88 \\
21.90 \\
16.69 \\
11.74 \\
11.77 \\
5.05 \\
0.02 \\
-0.04 \\
-0.02 \\
-0.02 \\
0.02 \\
\end{array}$ & $\begin{array}{c}\text { \% Strain } \\
\text {-0.0008 } \\
0.0012 \\
0.0035 \\
0.0047 \\
0.0068 \\
0.0067 \\
0.0066 \\
0.0115 \\
0.0182 \\
0.0178 \\
0.0182 \\
0.0209 \\
0.0265 \\
0.0296 \\
0.0301 \\
0.0339 \\
0.0376 \\
0.0380 \\
0.0385 \\
0.0375 \\
0.0358 \\
0.0355 \\
0.0328 \\
0.0299 \\
0.0273 \\
0.0272 \\
0.0234 \\
0.0202 \\
0.0198 \\
0.0161 \\
0.0129 \\
0.0140 \\
0.0105 \\
0.0078 \\
0.0072 \\
0.0065 \\
0.0062 \\
0.0058\end{array}$ & $\begin{array}{c}\text { Pressure } \\
\text { (psig) } \\
9.89 \\
19.55 \\
29.57 \\
39.42 \\
49.16 \\
54.50 \\
59.57 \\
64.20 \\
69.32 \\
74.16 \\
80.16 \\
85.61 \\
90.58 \\
95.69 \\
100.92 \\
103.25 \\
106.11 \\
108.31 \\
111.08 \\
113.24 \\
117.83 \\
120.92 \\
123.28 \\
122.97 \\
125.82 \\
125.60 \\
127.84 \\
130.19 \\
132.53 \\
135.33 \\
134.42 \\
138.35 \\
140.16 \\
142.63 \\
145.78 \\
142.52 \\
0.22 \\
\end{array}$ & $\begin{array}{l}\% \text { Strain } \\
0.0021 \\
0.0079 \\
0.0145 \\
0.0210 \\
0.0272 \\
0.0305 \\
0.0341 \\
0.0375 \\
0.0413 \\
0.0453 \\
0.0534 \\
0.0579 \\
0.0624 \\
0.0673 \\
0.0720 \\
0.0747 \\
0.0772 \\
0.0794 \\
0.0817 \\
0.0843 \\
0.0891 \\
0.0928 \\
0.0953 \\
0.0951 \\
0.0980 \\
0.0982 \\
0.1001 \\
0.1032 \\
0.1058 \\
0.1082 \\
0.1078 \\
0.1095 \\
0.1107 \\
0.1136 \\
0.1189 \\
0.1188 \\
0.0081\end{array}$ \\
\hline
\end{tabular}

Hygh Pressure test

ressure

19.55

29.57

54.50

59.57

64.20

69.32

74.16

90.58

103.25

106.11

108.31

111.08

120.92

123.28

122.97

125.82

125.60

132.53

135.33

134.42

138.35

140.16

142.63

145.78

42.52

0.0081 


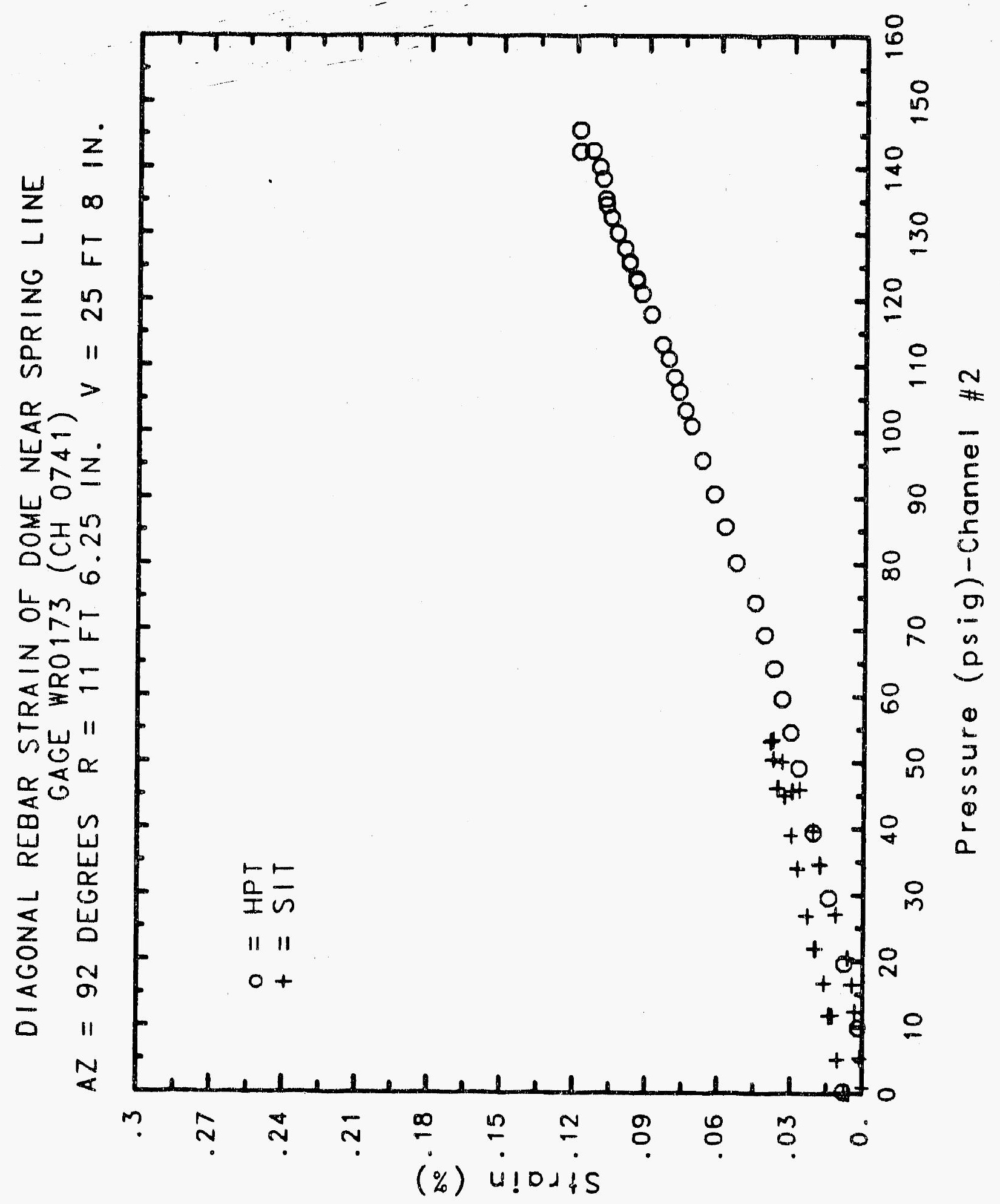




\section{Reinforced Concrete Test Data}

Weldable Gage 174 Channel 742

Structural Integrity TEst

High Pressure test

\begin{tabular}{|c|c|c|c|}
\hline $\begin{array}{c}\text { Pressure } \\
\text { (psig) } \\
-0.05 \\
5.33 \\
12.31 \\
16.44 \\
20.51 \\
20.46 \\
20.47 \\
27.05 \\
34.69 \\
34.53 \\
34.53 \\
39.70 \\
45.90 \\
45.65 \\
45.62 \\
50.10 \\
53.47 \\
53.29 \\
53.21 \\
50.49 \\
46.14 \\
46.13 \\
44.97 \\
38.98 \\
33.99 \\
33.96 \\
26.83 \\
21.88 \\
21.90 \\
16.69 \\
11.74 \\
11.77 \\
5.05 \\
0.02 \\
-0.04 \\
-0.02 \\
-0.02 \\
0.02 \\
\end{array}$ & $\begin{array}{l}\text { \% Strain } \\
\text {-0.0002 } \\
0.0015 \\
0.0044 \\
0.0059 \\
0.0095 \\
0.0099 \\
0.0106 \\
0.0193 \\
0.0358 \\
0.0396 \\
0.0405 \\
0.0485 \\
0.0648 \\
0.0654 \\
0.0652 \\
0.0714 \\
0.0763 \\
0.0768 \\
0.0770 \\
0.0755 \\
0.0722 \\
0.0722 \\
0.0714 \\
0.0666 \\
0.0623 \\
0.0622 \\
0.0550 \\
0.0498 \\
0.0496 \\
0.0445 \\
0.0384 \\
0.0366 \\
0.0294 \\
0.0238 \\
0.0218 \\
0.0220 \\
0.0219 \\
0.0218\end{array}$ & $\begin{array}{c}\text { Pressure } \\
(\text { psig) } \\
9.89 \\
19.55 \\
29.57 \\
39.42 \\
49.16 \\
54.50 \\
59.57 \\
64.20 \\
69.32 \\
74.16 \\
80.16 \\
85.61 \\
90.58 \\
95.69 \\
100.92 \\
103.25 \\
106.11 \\
108.31 \\
111.08 \\
113.24 \\
117.83 \\
120.92 \\
123.28 \\
122.97 \\
125.82 \\
125.60 \\
127.84 \\
130.19 \\
132.53 \\
135.33 \\
134.42 \\
138.35 \\
140.16 \\
142.63 \\
145.78 \\
142.52 \\
0.22 \\
\end{array}$ & $\begin{array}{l}\text { X Strain } \\
0.0047 \\
0.0158 \\
0.0281 \\
0.0395 \\
0.0510 \\
0.0575 \\
0.0638 \\
0.0701 \\
0.0767 \\
0.0830 \\
0.0904 \\
0.0978 \\
0.1044 \\
0.1122 \\
0.1202 \\
0.1235 \\
0.1274 \\
0.1305 \\
0.1350 \\
0.1385 \\
0.1467 \\
0.1522 \\
0.1573 \\
0.1584 \\
0.1630 \\
0.1643 \\
0.1677 \\
0.1723 \\
0.1778 \\
0.1853 \\
0.1863 \\
0.1941 \\
0.1996 \\
0.2072 \\
0.2159 \\
0.2173 \\
0.0743\end{array}$ \\
\hline
\end{tabular}




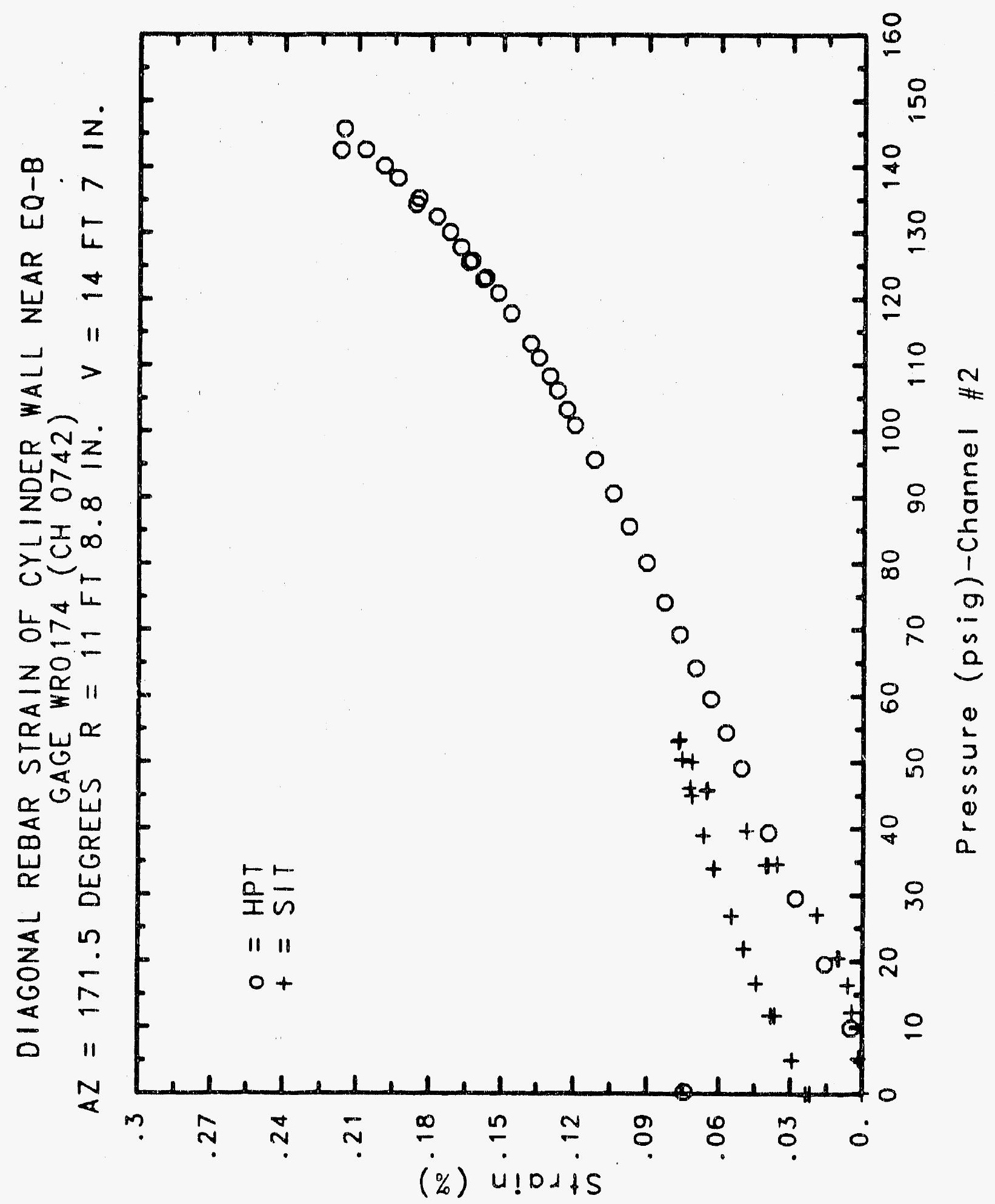




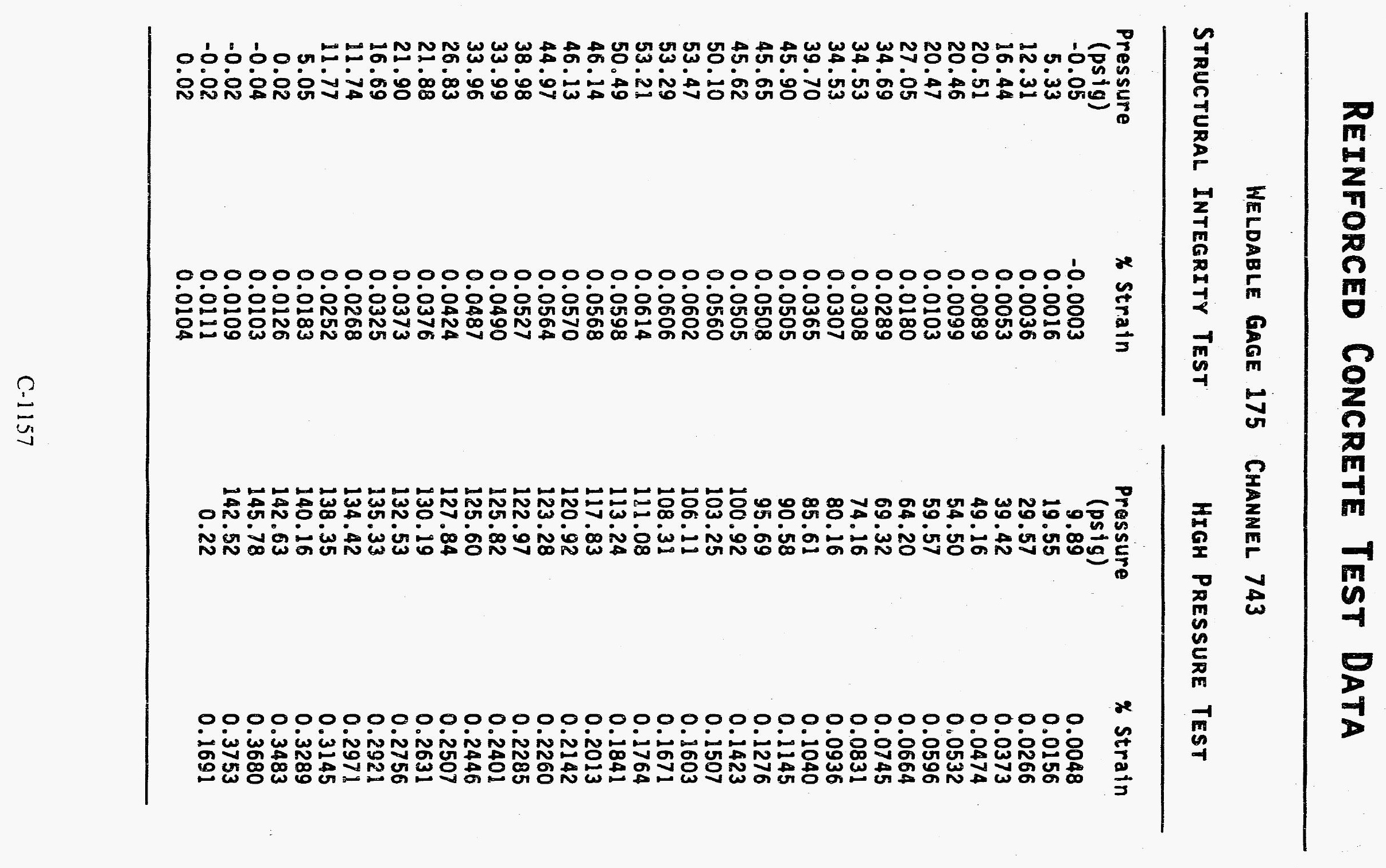




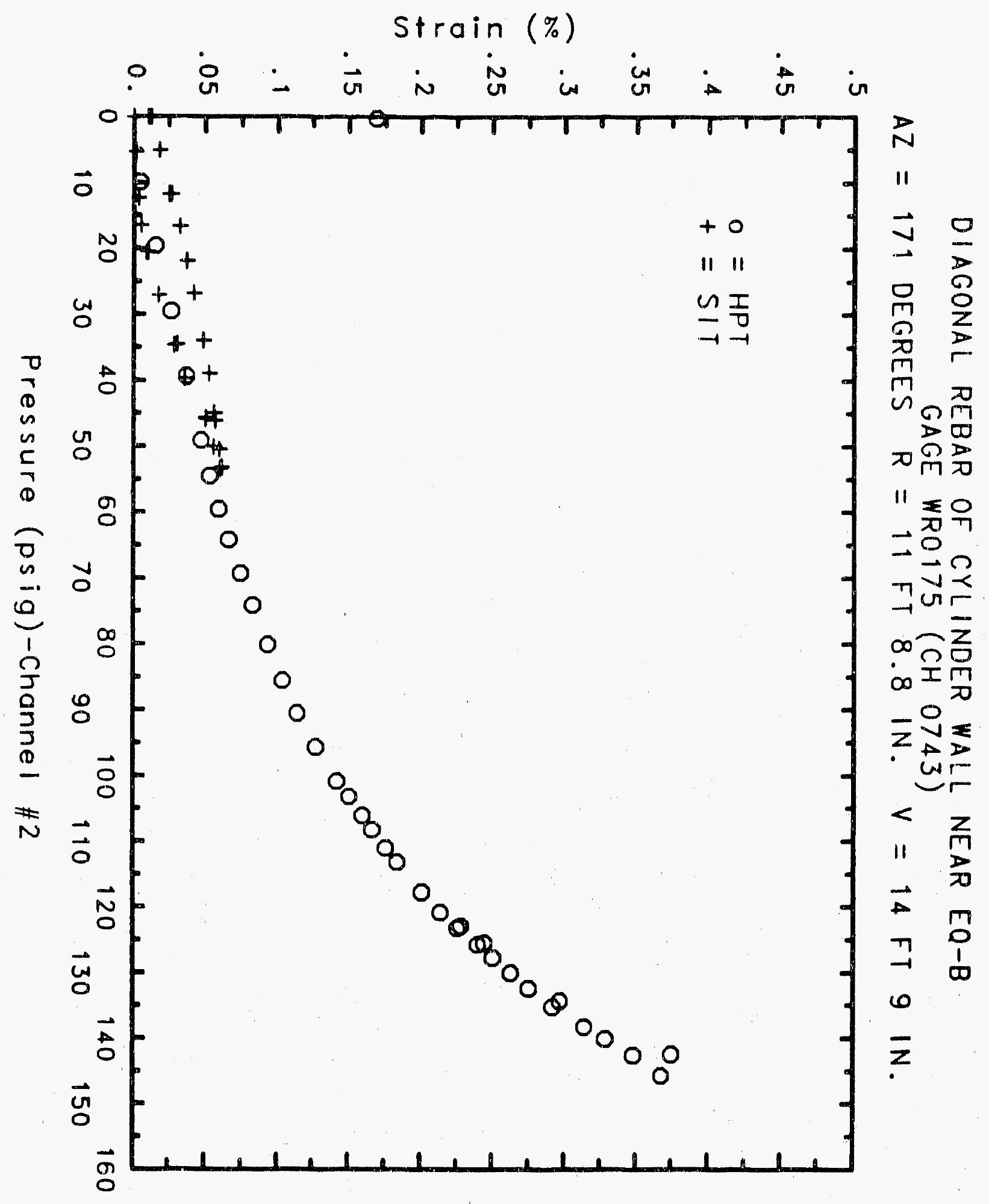




\section{Reinforced Concrete Test Data}

\begin{tabular}{|c|c|c|c|}
\hline STRUCTURAL & $\begin{array}{l}\text { WELDABLE GAgE } 176 \\
\text { INTEGRITY TEST }\end{array}$ & $\begin{array}{l}\text { Channel } 744 \\
\text { High Pressure }\end{array}$ & TEST \\
\hline $\begin{array}{c}\text { Pressure } \\
\text { (psig) } \\
-0.05 \\
5.33 \\
12.31 \\
16.44 \\
20.51 \\
20.46 \\
20.47 \\
27.05 \\
34.69 \\
34.53 \\
34.53 \\
39.70 \\
45.90 \\
45.65 \\
45.62 \\
50.10 \\
53.47 \\
53.29 \\
53.21 \\
50.49 \\
46.14 \\
46.13 \\
44.97 \\
38.98 \\
33.99 \\
33.96 \\
26.83 \\
21.88 \\
21.90 \\
16.69 \\
11.74 \\
11.77 \\
5.05 \\
0.02 \\
-0.04 \\
-0.02 \\
-0.02 \\
0.02 \\
\end{array}$ & $\begin{array}{c}\text { \% Strain } \\
\text {-0.0003 } \\
0.0012 \\
0.0035 \\
0.0049 \\
0.0077 \\
0.0084 \\
0.0092 \\
0.0166 \\
0.0279 \\
0.0292 \\
0.0296 \\
0.0334 \\
0.0372 \\
0.0366 \\
0.0366 \\
0.0395 \\
0.0411 \\
0.0411 \\
0.0414 \\
0.0398 \\
0.0373 \\
0.0376 \\
0.0369 \\
0.0338 \\
0.0307 \\
0.0308 \\
0.0261 \\
0.0231 \\
0.0230 \\
0.0193 \\
0.0160 \\
0.0150 \\
0.0108 \\
0.0076 \\
0.0066 \\
0.0071 \\
0.0067 \\
0.0065\end{array}$ & $\begin{array}{c}\text { Pressure } \\
(\text { psig) } \\
9.89 \\
19.55 \\
29.57 \\
39.42 \\
49.16 \\
54.50 \\
59.57 \\
64.20 \\
69.32 \\
74.16 \\
80.16 \\
85.61 \\
90.58 \\
95.69 \\
100.92 \\
103.25 \\
106.11 \\
108.31 \\
111.08 \\
113.24 \\
117.83 \\
120.92 \\
123.28 \\
122.97 \\
125.82 \\
125.60 \\
127.84 \\
130.19 \\
132.53 \\
135.33 \\
134.42 \\
138.35 \\
140.16 \\
142.63 \\
195.78 \\
142.52 \\
0.22 \\
\end{array}$ & $\begin{array}{l}\text { X Strain } \\
0.0021 \\
0.0093 \\
0.0161 \\
0.0225 \\
0.0287 \\
0.0325 \\
0.0362 \\
0.0403 \\
0.0445 \\
0.0487 \\
0.0537 \\
0.0590 \\
0.0649 \\
0.0717 \\
0.0788 \\
0.0825 \\
0.0864 \\
0.0895 \\
0.0946 \\
0.0991 \\
0.1086 \\
0.1156 \\
0.1218 \\
0.1234 \\
0.1304 \\
0.1326 \\
0.1371 \\
0.1459 \\
0.1565 \\
0.1709 \\
0.1743 \\
0.1895 \\
0.2003 \\
0.2136 \\
0.2281 \\
0.2305 \\
0.0627\end{array}$ \\
\hline
\end{tabular}




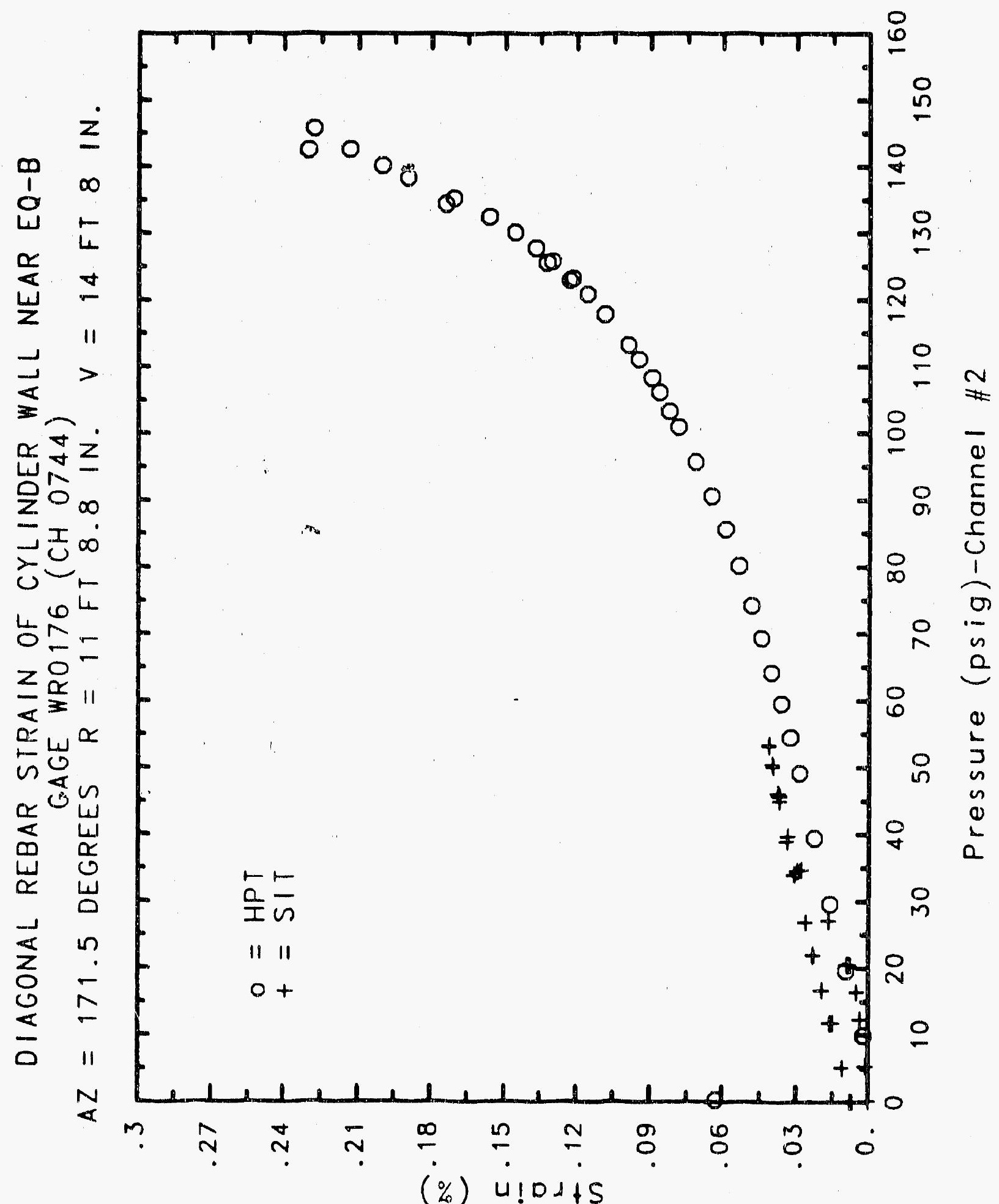




\section{Reinforced Concrete Test Data}

Weldable gage 177 Channel 745

Structural Integrity Test

High Pressure test

\begin{tabular}{cccc}
\hline $\begin{array}{c}\text { Pressure } \\
\text { (psig) }\end{array}$ & \% Strain & $\begin{array}{c}\text { Pressure } \\
(\text { psig) }\end{array}$ & \% Strain \\
0.05 & 0.0008 & 9.89 & 0.0054 \\
5.33 & 0.0018 & 19.55 & 0.0169 \\
12.31 & 0.0038 & 29.57 & 0.0292 \\
16.44 & 0.0055 & 39.42 & 0.0406 \\
20.51 & 0.0102 & 49.16 & 0.0516 \\
20.46 & 0.0114 & 54.50 & 0.0576 \\
20.47 & 0.0117 & 59.57 & 0.0640 \\
27.05 & 0.0210 & 64.20 & 0.0699 \\
34.69 & 0.0399 & 69.32 & 0.0765 \\
34.53 & 0.0430 & 74.16 & 0.0839 \\
34.53 & 0.0432 & 80.16 & 0.0935 \\
39.70 & 0.0531 & 85.61 & 0.1005 \\
45.90 & 0.0631 & 90.58 & 0.1067 \\
45.65 & 0.0639 & 95.69 & 0.1144 \\
45.62 & 0.0646 & 100.92 & 0.1218 \\
50.10 & 0.0701 & 103.25 & 0.1261 \\
53.47 & 0.0742 & 106.11 & 0.1308 \\
53.29 & 0.0741 & 108.31 & 0.1347 \\
53.21 & 0.0747 & 111.08 & 0.1398 \\
50.49 & 0.0725 & 113.24 & 0.1442 \\
46.14 & 0.0690 & 117.83 & 0.1533 \\
46.13 & 0.0689 & 120.92 & 0.1606 \\
44.97 & 0.0676 & 123.28 & 0.1689 \\
38.98 & 0.0618 & 122.97 & 0.1709 \\
33.99 & 0.0574 & 125.82 & 0.1794 \\
33.96 & 0.0571 & 125.60 & 0.1848 \\
26.83 & 0.0499 & 127.84 & 0.1906 \\
21.88 & 0.0443 & 130.19 & 0.2039 \\
21.90 & 0.0438 & 132.53 & 0.2175 \\
16.69 & 0.0381 & 135.33 & 0.2388 \\
11.74 & 0.0323 & 134.42 & 0.2489 \\
11.77 & 0.0314 & 138.35 & 0.3043 \\
5.05 & 0.0241 & 140.16 & 0.3384 \\
0.02 & 0.0193 & 142.63 & 0.3639 \\
-0.04 & 0.0179 & 145.78 & 0.3722 \\
-0.02 & 0.0172 & 0.22 & \\
-0.02 & 0.0173 & & \\
0.02 & 0.0168 & & \\
\hline & & & \\
\hline
\end{tabular}




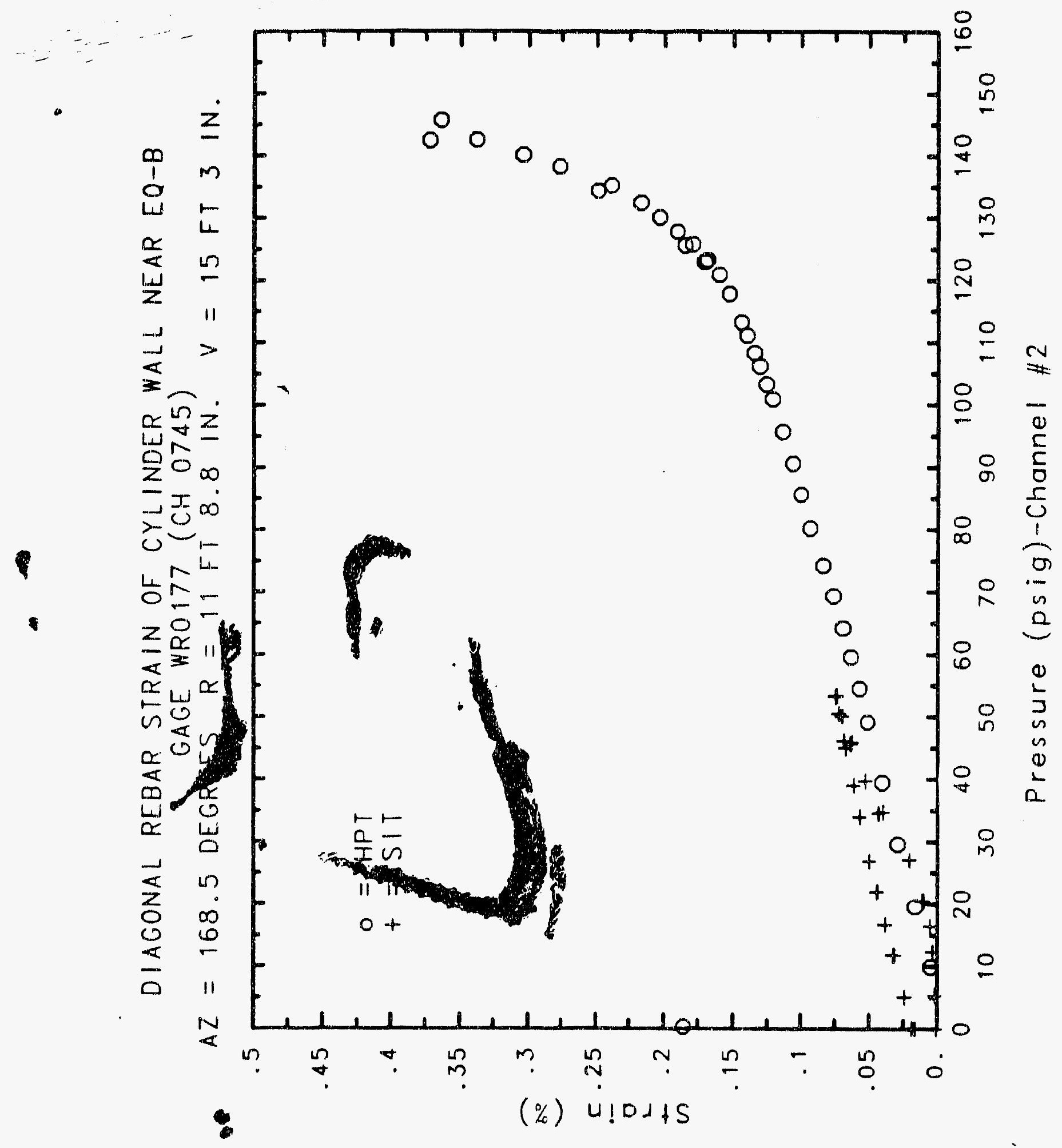




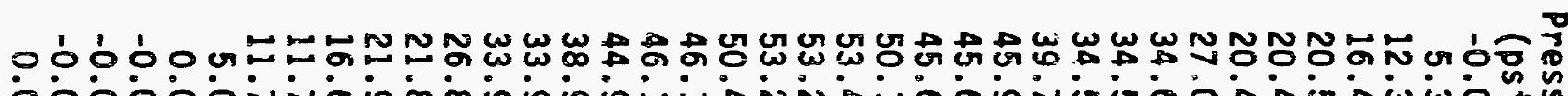

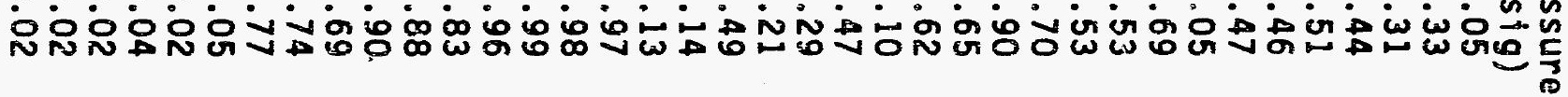

00000000000000000000000000000000000000

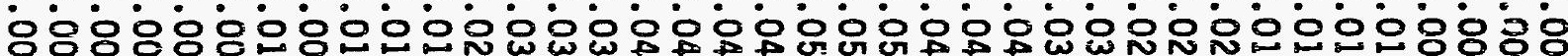

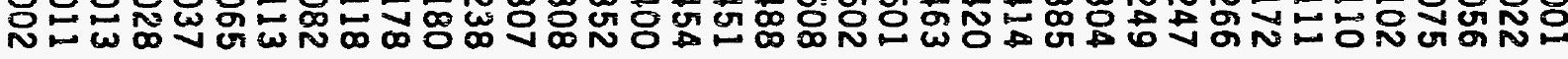

0000000000000000000000000000000000000

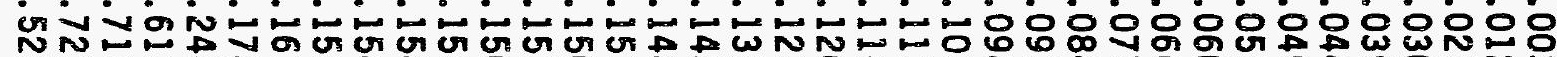
N

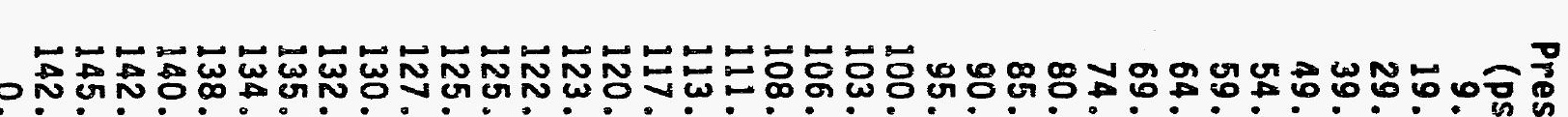

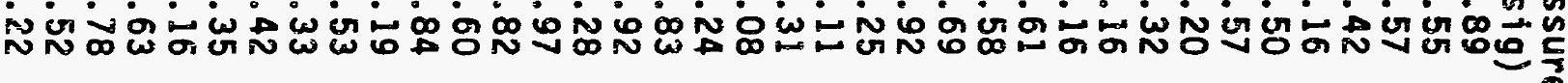




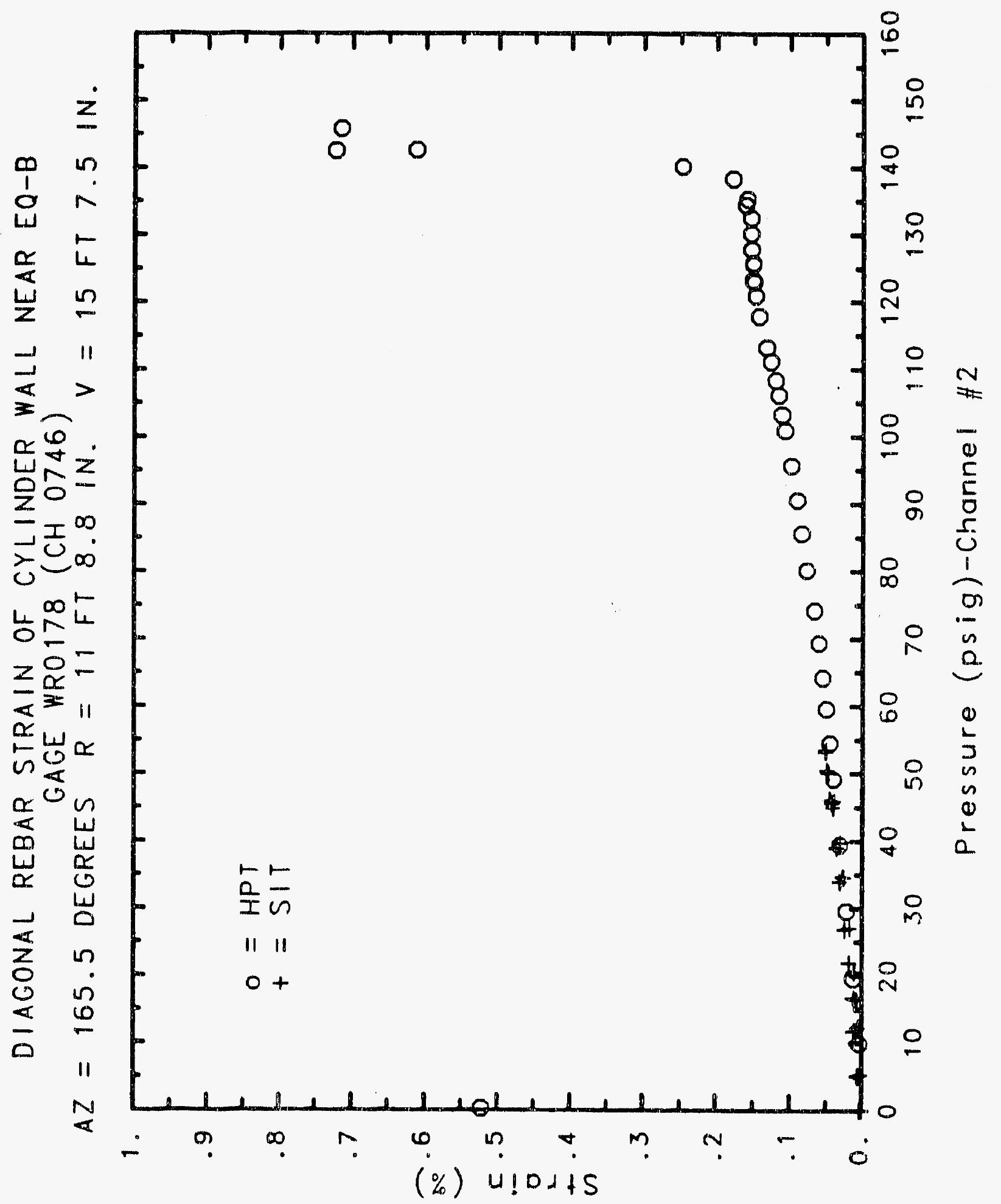




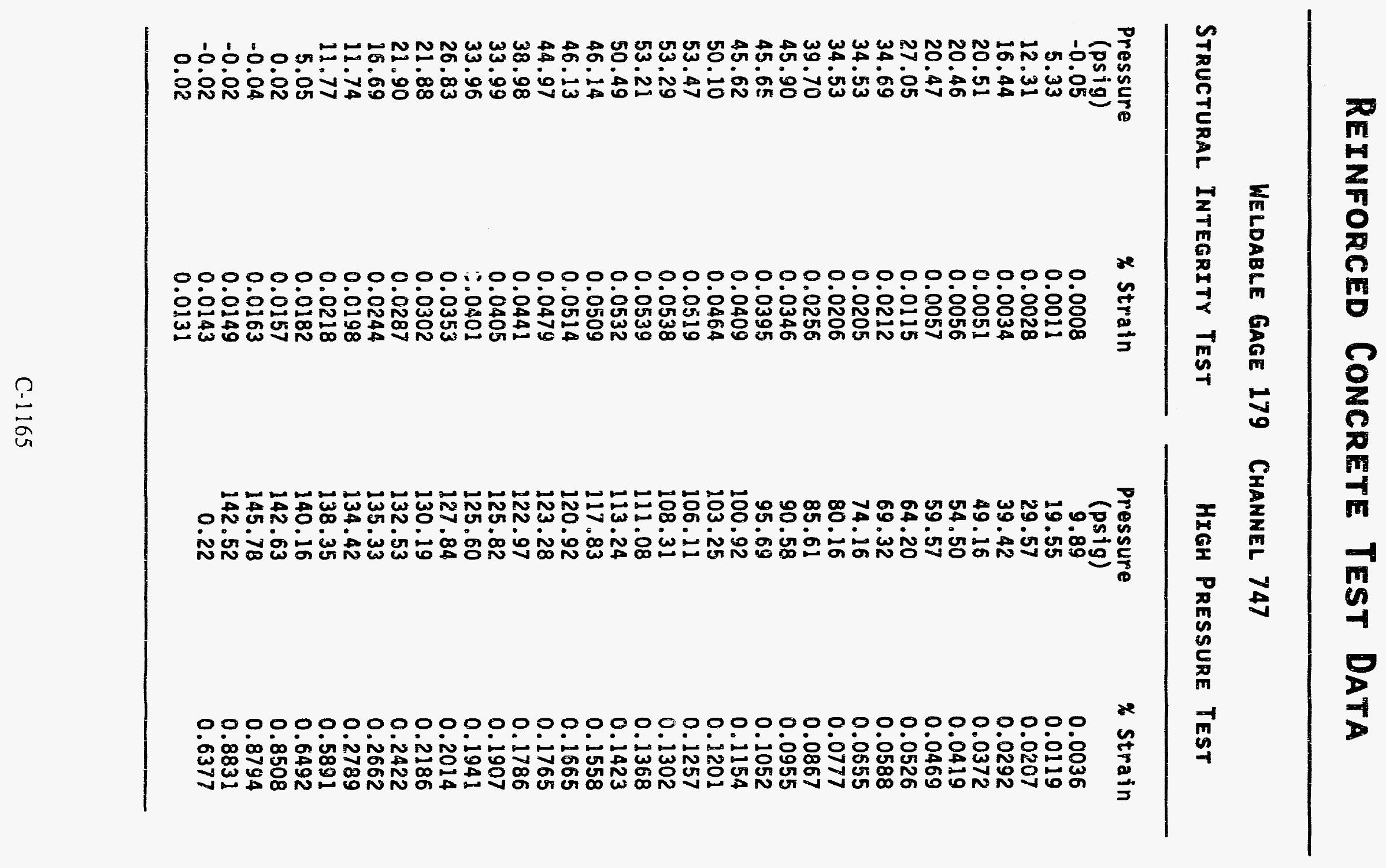




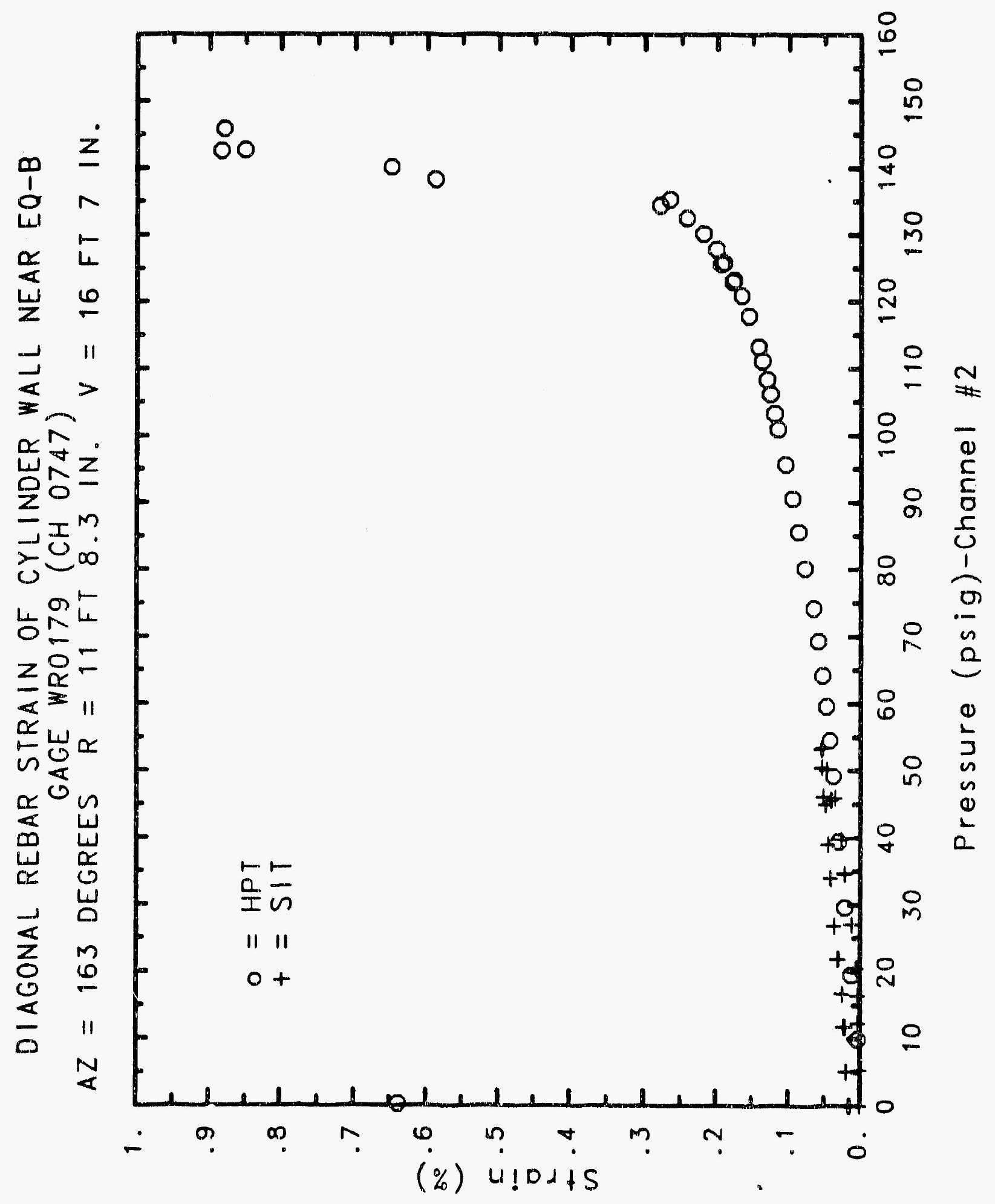

C-1166 
Oல்

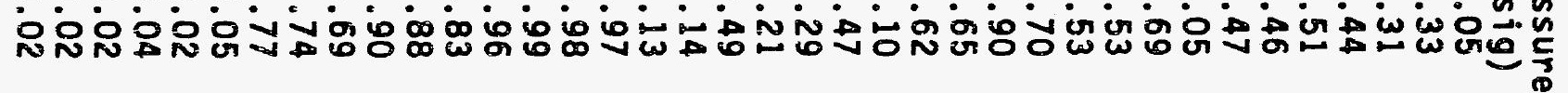

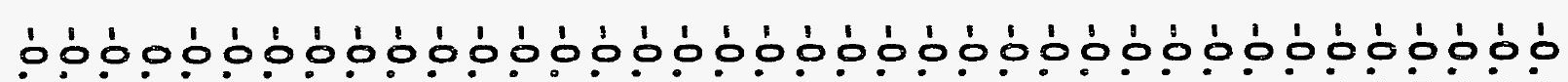

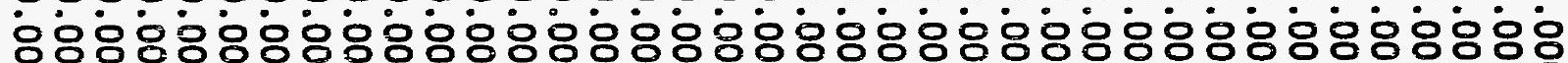

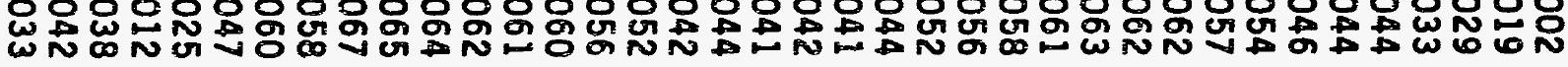

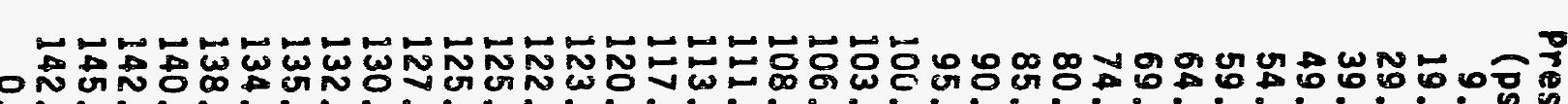

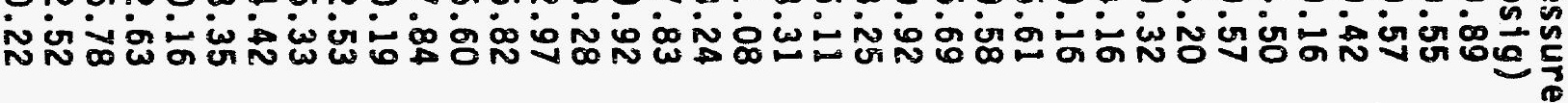

000000000000000000000000000000000000்

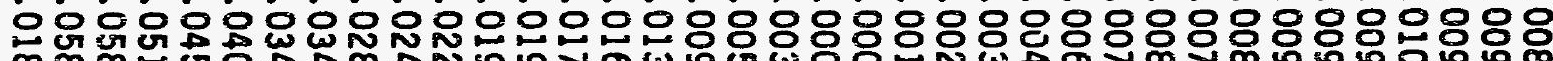

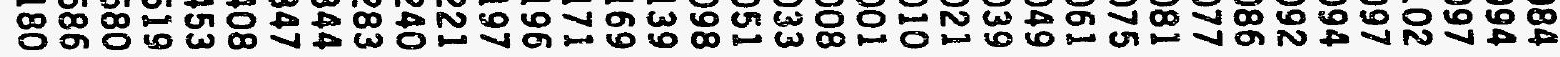

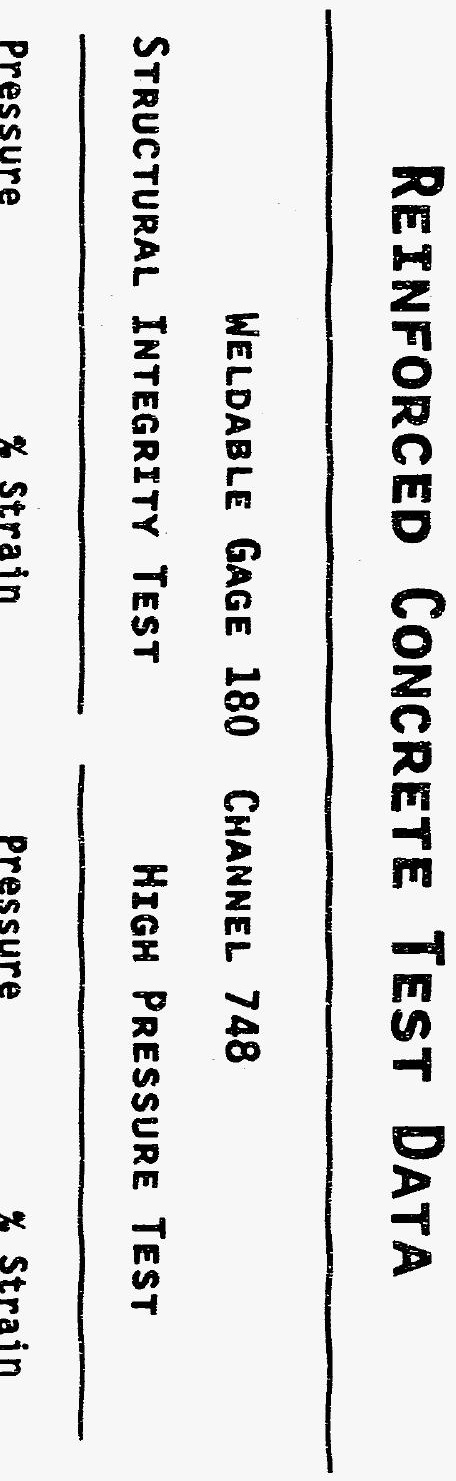




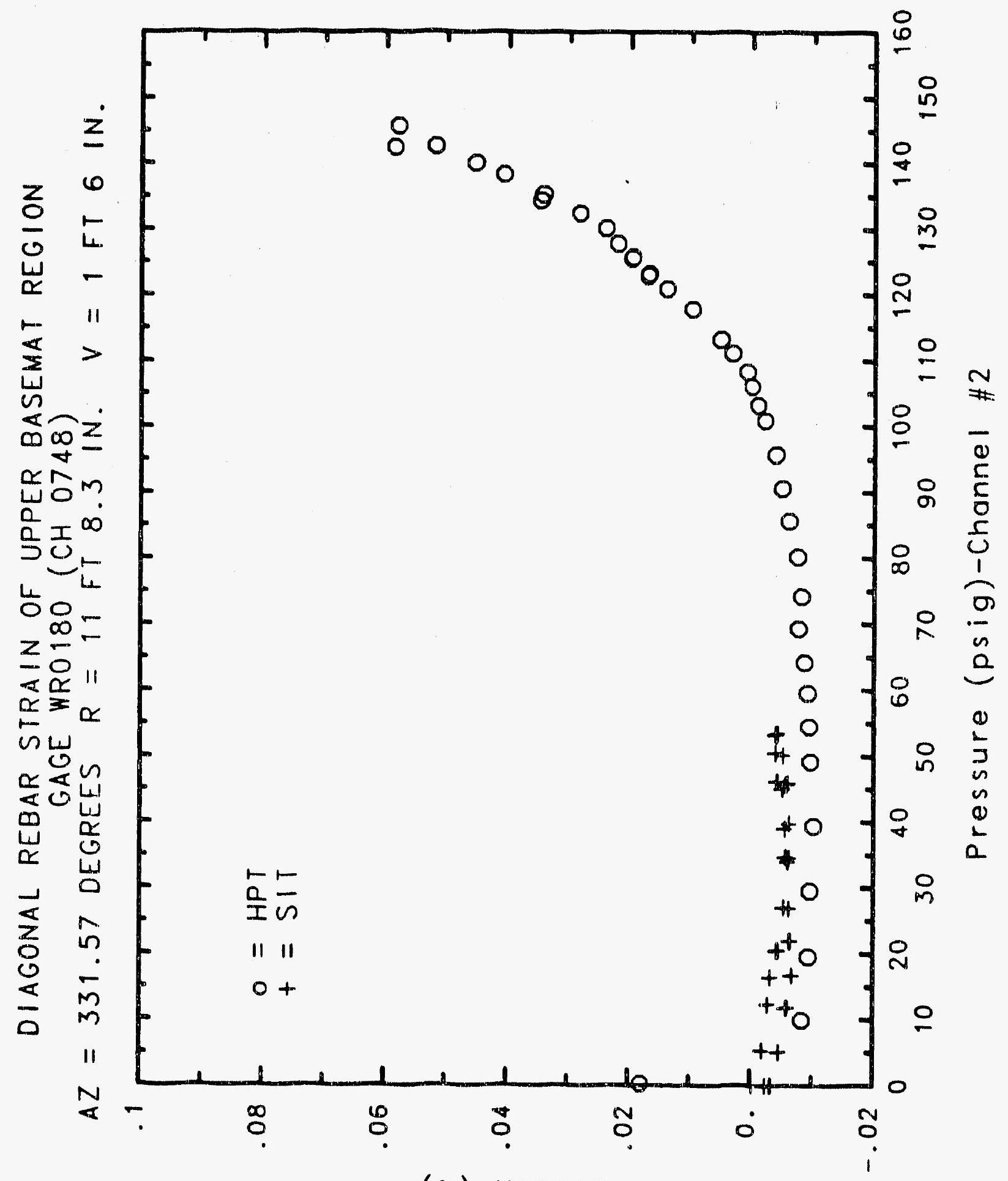

(\%) $4 ! 0215$ 


\section{Reinforced Concrete Test Data}

Weldable gage 181 Channel 749

Structural INTEGRITY TEST

High Pressure test

\begin{tabular}{|c|c|c|c|}
\hline $\begin{array}{c}\text { Pressure } \\
\text { (psig) } \\
-0.05 \\
5.33 \\
12.31 \\
16.44 \\
20.51 \\
20.46 \\
20.47 \\
27.05 \\
34.69 \\
34.53 \\
34.53 \\
39.70 \\
45.90 \\
45.65 \\
45.62 \\
50.10 \\
53.47 \\
53.29 \\
53.21 \\
50.49 \\
46.14 \\
46.13 \\
44.97 \\
38.98 \\
33.99 \\
33.96 \\
26.83 \\
21.88 \\
21.90 \\
16.69 \\
11.74 \\
11.77 \\
5.05 \\
0.02 \\
-0.04 \\
-0.02 \\
-0.02 \\
0.02 \\
\end{array}$ & $\begin{array}{l}\text { Strain } \\
-0.0002 \\
-0.0003 \\
0.0002 \\
-0.0001 \\
-0.0004 \\
-0.0007 \\
-0.0007 \\
-0.0014 \\
-0.0025 \\
-0.0027 \\
-0.0030 \\
-0.0029 \\
-0.0035 \\
-0.0032 \\
-0.0032 \\
-0.0032 \\
-0.0035 \\
-0.0032 \\
-0.0032 \\
-0.0029 \\
-0.0030 \\
-0.0029 \\
-0.0047 \\
-0.0044 \\
-0.0045 \\
-0.0042 \\
-0.0032 \\
-0.0031 \\
-0.0033 \\
-0.0026 \\
-0.0016 \\
-0.0018 \\
-0.0006 \\
0.0001 \\
0.0006 \\
-0.0011 \\
-0.0019 \\
-0.0006\end{array}$ & $\begin{array}{c}\text { Pressure } \\
\text { (psig) } \\
9.89 \\
19.55 \\
29.57 \\
39.42 \\
49.16 \\
54.50 \\
59.57 \\
64.20 \\
69.32 \\
74.16 \\
80.16 \\
85.61 \\
90.58 \\
95.69 \\
100.92 \\
103.25 \\
106.11 \\
108.31 \\
111.08 \\
113.24 \\
117.83 \\
120.92 \\
123.28 \\
122.97 \\
125.82 \\
125.60 \\
127.84 \\
130.19 \\
132.53 \\
135.33 \\
134.42 \\
138.35 \\
140.16 \\
142.63 \\
145.78 \\
142.52 \\
0.22 \\
\end{array}$ & $\begin{array}{l}\text { \% Strain } \\
-0.0059 \\
-0.0075 \\
-0.0084 \\
-0.0089 \\
-0.0093 \\
-0.0094 \\
-0.0091 \\
-0.0093 \\
-0.0091 \\
-0.0095 \\
-0.0092 \\
-0.0089 \\
-0.0091 \\
-0.0089 \\
-0.0090 \\
-0.0087 \\
-0.0086 \\
-0.0082 \\
-0.0073 \\
-0.0065 \\
-0.0032 \\
-0.0009 \\
0.0010 \\
0.0008 \\
0.0027 \\
0.0034 \\
0.0059 \\
0.0121 \\
0.0210 \\
0.0307 \\
0.0329 \\
0.0403 \\
0.0454 \\
0.0538 \\
0.0630 \\
0.0641 \\
0.0210\end{array}$ \\
\hline
\end{tabular}




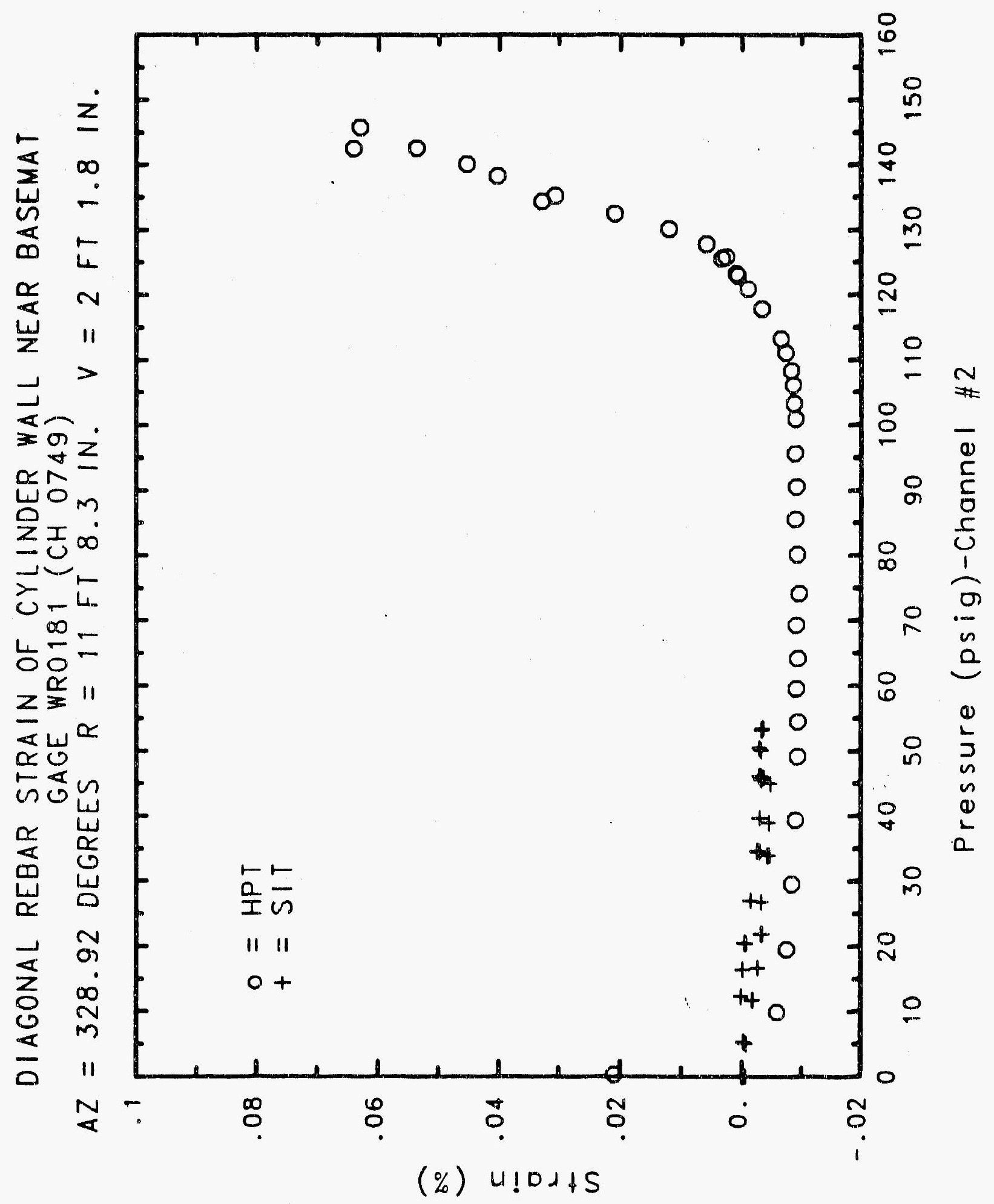




\section{Reinforced Concrete Test Data}

Weldable gage 182 Channel 760

Structural IntEgrity Test

High Pressure test

\begin{tabular}{|c|c|c|c|}
\hline $\begin{array}{c}\text { Pressure } \\
\text { (psig) } \\
-0.05 \\
5.33 \\
12.31 \\
16.44 \\
20.51 \\
20.46 \\
20.47 \\
27.05 \\
34.69 \\
34.53 \\
34.53 \\
39.70 \\
45.90 \\
45.65 \\
45.62 \\
50.10 \\
53.47 \\
53.29 \\
53.21 \\
50.49 \\
46.14 \\
46.13 \\
44.97 \\
38.98 \\
33.99 \\
33.96 \\
26.83 \\
21.88 \\
21.90 \\
16.69 \\
11.74 \\
11.77 \\
5.05 \\
0.02 \\
-0.04 \\
-0.02 \\
-0.02 \\
0.02 \\
\end{array}$ & $\begin{array}{c}\text { \% Strain } \\
\\
-0.0004 \\
0.0016 \\
0.0044 \\
0.0056 \\
0.0052 \\
0.0054 \\
0.0053 \\
0.0061 \\
0.0061 \\
0.0061 \\
0.0063 \\
0.0074 \\
0.0087 \\
0.0099 \\
0.0101 \\
0.0111 \\
0.0126 \\
0.0135 \\
0.0135 \\
0.0141 \\
0.0134 \\
0.0136 \\
0.0090 \\
0.0089 \\
0.0090 \\
0.0090 \\
0.0084 \\
0.0074 \\
0.0074 \\
0.0067 \\
0.0062 \\
0.0063 \\
0.0056 \\
0.0042 \\
0.0021 \\
-0.0011 \\
-0.0008 \\
0.0024\end{array}$ & $\begin{array}{c}\text { Pressure } \\
(\text { ps } 1 \mathrm{c}) \\
9.89 \\
19.55 \\
29.57 \\
39.42 \\
49.16 \\
54.50 \\
59.57 \\
64.20 \\
69.32 \\
74.16 \\
80.16 \\
85.61 \\
90.58 \\
95.69 \\
100.92 \\
103.25 \\
106.11 \\
108.31 \\
111.08 \\
113.24 \\
117.83 \\
120.92 \\
123.28 \\
122.97 \\
125.82 \\
125.60 \\
127.84 \\
130.19 \\
132.53 \\
135.33 \\
134.42 \\
138.35 \\
140.16 \\
142.63 \\
145.78 \\
142.52 \\
0.22 \\
\end{array}$ & $\begin{array}{c}\text { \% Strain } \\
-0.0035 \\
-0.0017 \\
0.0004 \\
0.0024 \\
0.0044 \\
0.0056 \\
0.0067 \\
0.0080 \\
0.0089 \\
0.0093 \\
0.0132 \\
0.0143 \\
0.0138 \\
0.0137 \\
0.0142 \\
0.0147 \\
0.0153 \\
0.0147 \\
0.0155 \\
0.0155 \\
0.0163 \\
0.0163 \\
0.0160 \\
0.0147 \\
0.0139 \\
0.0119 \\
0.0122 \\
0.0124 \\
0.0148 \\
0.0183 \\
0.0204 \\
0.0302 \\
0.0394 \\
0.0549 \\
0.0754 \\
0.0827 \\
0.0279\end{array}$ \\
\hline
\end{tabular}




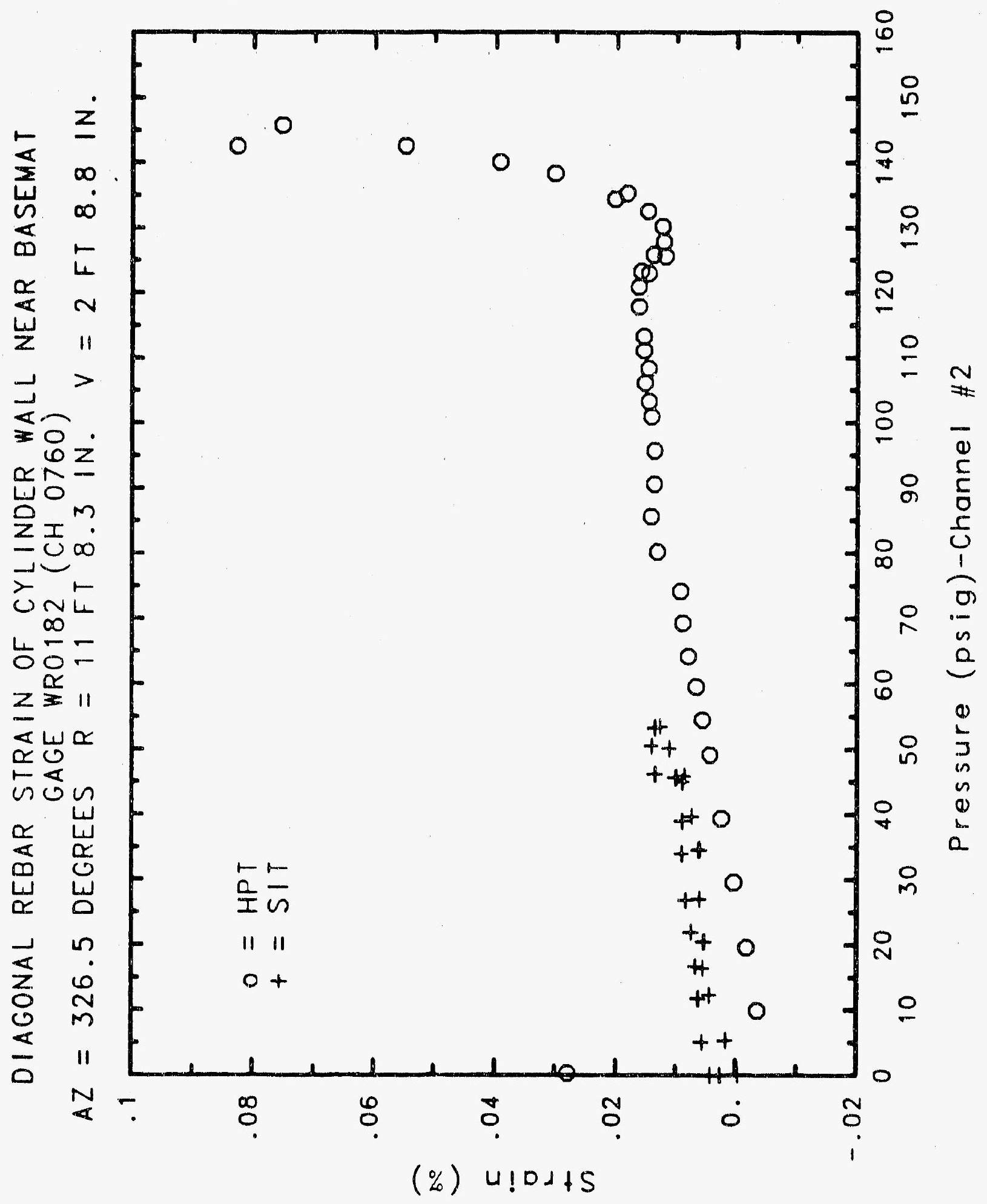




\section{Reinforced Concrete Test Data}

Weldable gage 183 Channel 761

Structural Integrity Test

high Pressure test

\begin{tabular}{|c|c|c|c|}
\hline $\begin{array}{c}\text { Pressure } \\
\text { (psig) } \\
-0.05 \\
5.33 \\
12.31 \\
16.44 \\
20.51 \\
20.46 \\
20.47 \\
27.05 \\
34.69 \\
34.53 \\
34.53 \\
39.70 \\
45.90 \\
45.65 \\
45.62 \\
50.10 \\
53.47 \\
53.29 \\
53.21 \\
50.49 \\
46.14 \\
46.13 \\
44.97 \\
38.98 \\
33.99 \\
33.96 \\
26.83 \\
21.88 \\
21.90 \\
16.69 \\
11.74 \\
11.77 \\
5.05 \\
0.02 \\
-0.04 \\
-0.02 \\
-0.02 \\
0.02 \\
\end{array}$ & $\begin{array}{l}\text { \% Strain } \\
0.0005 \\
0.0016 \\
0.0044 \\
0.0062 \\
0.0098 \\
0.0106 \\
0.0109 \\
0.0165 \\
0.0229 \\
0.0238 \\
0.0239 \\
0.0273 \\
0.0316 \\
0.0320 \\
0.0318 \\
0.0345 \\
0.0375 \\
0.0377 \\
0.0381 \\
0.0373 \\
0.0353 \\
0.0353 \\
0.0362 \\
0.0338 \\
0.0319 \\
0.0317 \\
0.0288 \\
0.0256 \\
0.0256 \\
0.0231 \\
0.0203 \\
0.0200 \\
0.0164 \\
0.0132 \\
0.0139 \\
0.0131 \\
0.0119 \\
0.0125\end{array}$ & $\begin{array}{c}\text { Pressure } \\
(\text { psig) } \\
9.89 \\
19.55 \\
29.57 \\
39.42 \\
49.16 \\
54.50 \\
59.57 \\
64.20 \\
69.32 \\
74.16 \\
80.16 \\
85.61 \\
90.58 \\
95.69 \\
100.92 \\
103.25 \\
106.11 \\
108.31 \\
111.08 \\
113.24 \\
117.83 \\
120.92 \\
123.28 \\
122.97 \\
125.82 \\
125.60 \\
127.84 \\
130.19 \\
132.53 \\
135.33 \\
134.42 \\
138.35 \\
140.16 \\
142.63 \\
145.78 \\
142.52 \\
0.22 \\
\end{array}$ & $\begin{array}{l}\text { Strain } \\
0.0002 \\
0.0053 \\
0.0105 \\
0.0165 \\
0.0222 \\
0.0256 \\
0.0291 \\
0.0346 \\
0.0404 \\
0.0471 \\
0.0556 \\
0.0709 \\
0.0839 \\
0.0967 \\
0.1100 \\
0.1162 \\
0.1234 \\
0.1298 \\
0.1398 \\
0.1477 \\
0.1676 \\
0.1839 \\
0.1991 \\
0.2038 \\
0.2193 \\
0.2224 \\
0.2297 \\
0.2448 \\
0.2627 \\
0.2875 \\
0.2922 \\
0.3202 \\
0.3421 \\
0.3701 \\
0.3895 \\
0.3946 \\
0.2468\end{array}$ \\
\hline
\end{tabular}




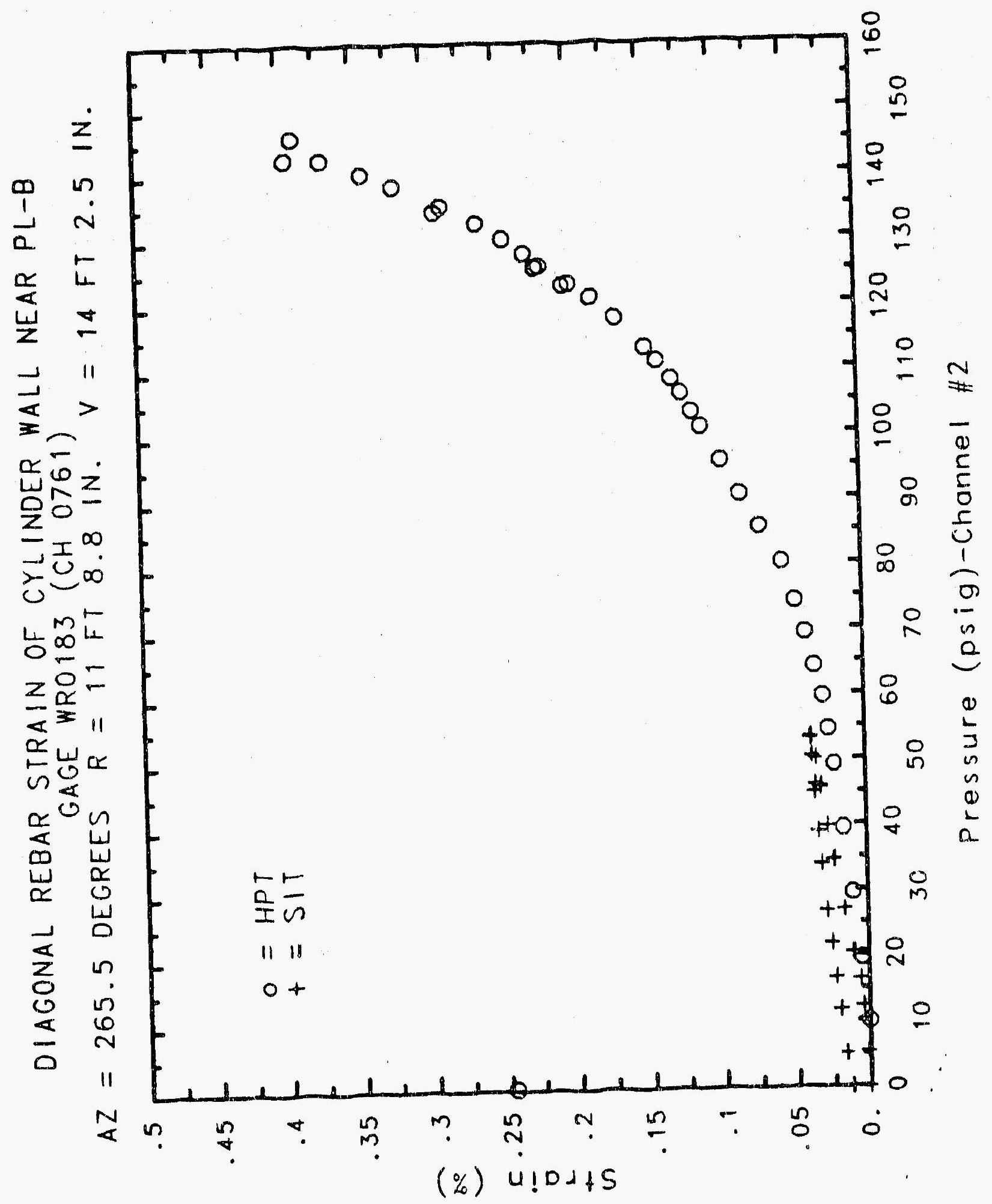




\section{Reinforced Concrete Test Data}

Weldable Gage 184 Channel 762

StRuCtURAL InTEGRITY TEST

High Pressure test

\begin{tabular}{|c|c|c|c|}
\hline $\begin{array}{c}\text { Pressure } \\
\text { (psig) } \\
-0.05 \\
5.33 \\
12.31 \\
16.44 \\
20.51 \\
20.46 \\
20.47 \\
27.05 \\
34.69 \\
34.53 \\
34.53 \\
39.70 \\
45.90 \\
45.65 \\
45.62 \\
50.10 \\
53.47 \\
53.29 \\
53.21 \\
50.49 \\
46.14 \\
46.13 \\
44.97 \\
38.98 \\
33.99 \\
33.96 \\
26.83 \\
21.88 \\
21.90 \\
16.69 \\
11.74 \\
11.77 \\
5.05 \\
0.02 \\
-0.04 \\
-0.02 \\
-0.02 \\
0.02 \\
\end{array}$ & $\begin{array}{l}\text { Strain } \\
0.0016 \\
0.0018 \\
0.0042 \\
0.0054 \\
0.0096 \\
0.0105 \\
0.0109 \\
0.0196 \\
0.0303 \\
0.0310 \\
0.0313 \\
0.0362 \\
0.0418 \\
0.0421 \\
0.0421 \\
0.0458 \\
0.0486 \\
0.0494 \\
0.0495 \\
0.0486 \\
0.0459 \\
0.0461 \\
0.0465 \\
0.0425 \\
0.0394 \\
0.0390 \\
0.0346 \\
0.0309 \\
0.0304 \\
0.0272 \\
0.0230 \\
0.0229 \\
0.0181 \\
0.0144 \\
0.0153 \\
0.0135 \\
0.0131 \\
0.0136\end{array}$ & $\begin{array}{c}\text { Pressure } \\
\text { (psig) } \\
9.89 \\
19.55 \\
29.57 \\
39.42 \\
49.16 \\
54.50 \\
59.57 \\
64.20 \\
69.32 \\
74.16 \\
80.16 \\
85.61 \\
90.58 \\
95.69 \\
100.92 \\
103.25 \\
106.11 \\
108.31 \\
111.08 \\
113.24 \\
117.83 \\
120.92 \\
123.28 \\
122.97 \\
125.82 \\
125.60 \\
127.84 \\
130.19 \\
132.53 \\
135.33 \\
134.42 \\
138.35 \\
140.16 \\
142.63 \\
145.78 \\
142.52 \\
0.22\end{array}$ & $\begin{array}{l}\text { \% Strain } \\
0.0024 \\
0.0099 \\
0.0178 \\
0.0258 \\
0.0336 \\
0.0384 \\
0.0437 \\
0.0505 \\
0.0548 \\
0.0587 \\
0.0670 \\
0.0772 \\
0.0873 \\
0.0978 \\
0.1092 \\
0.1150 \\
0.1205 \\
0.1253 \\
0.1331 \\
0.1387 \\
0.1536 \\
0.1677 \\
0.1822 \\
0.1869 \\
0.2011 \\
0.2047 \\
0.2123 \\
0.2267 \\
0.2484 \\
0.2825 \\
0.2917 \\
0.3334 \\
0.3643 \\
0.4060 \\
0.4414 \\
0.4527 \\
0.3030\end{array}$ \\
\hline
\end{tabular}




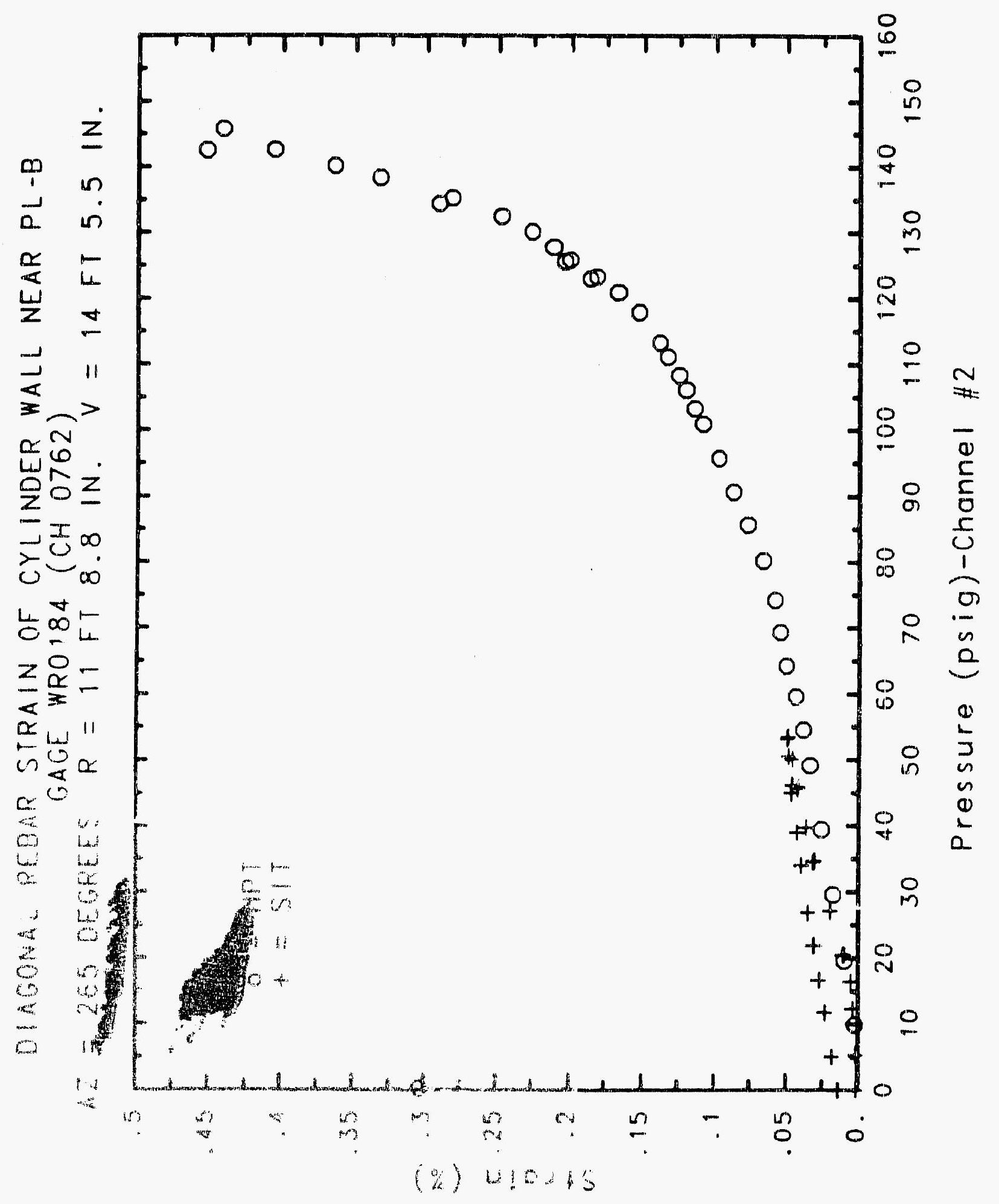




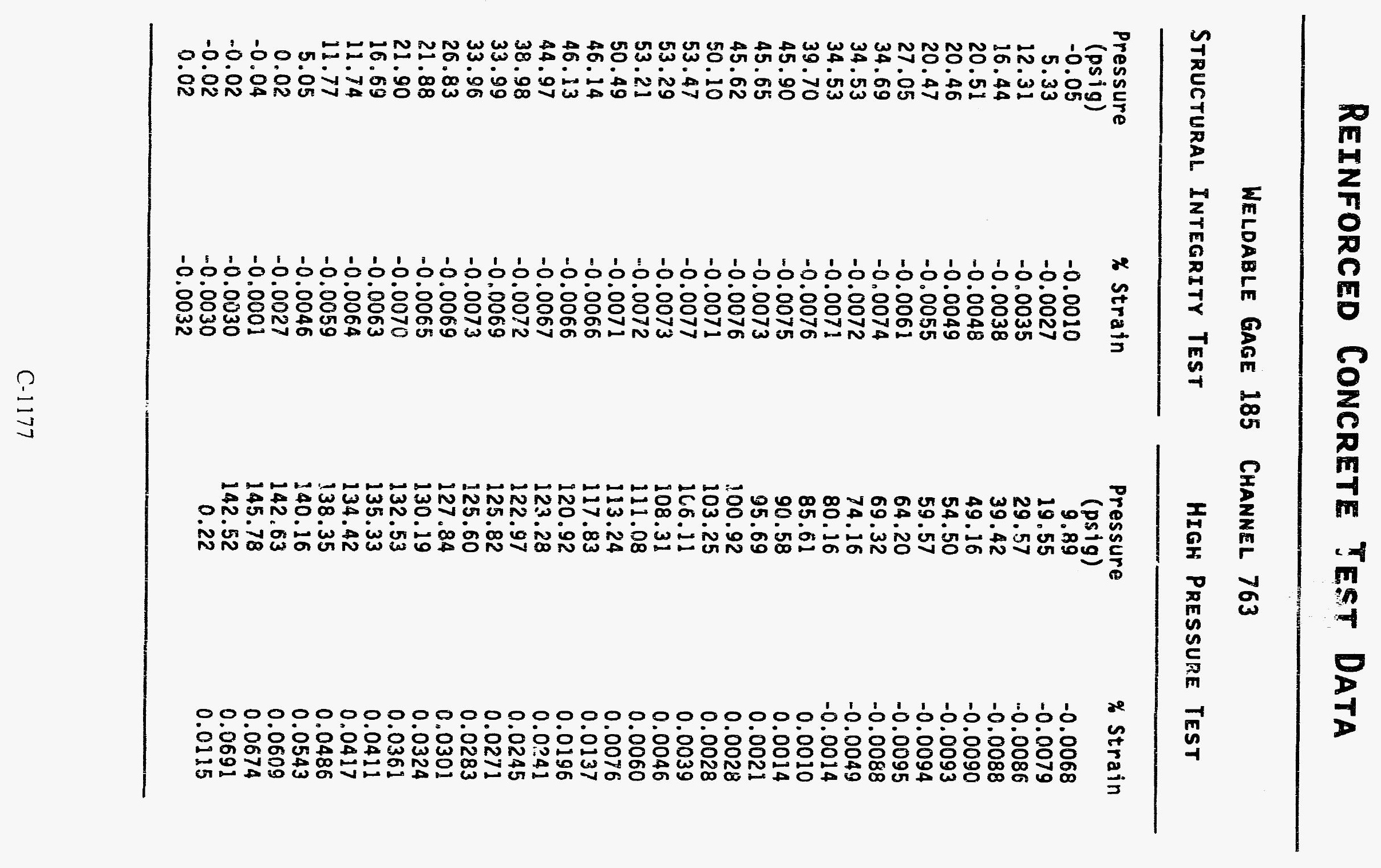




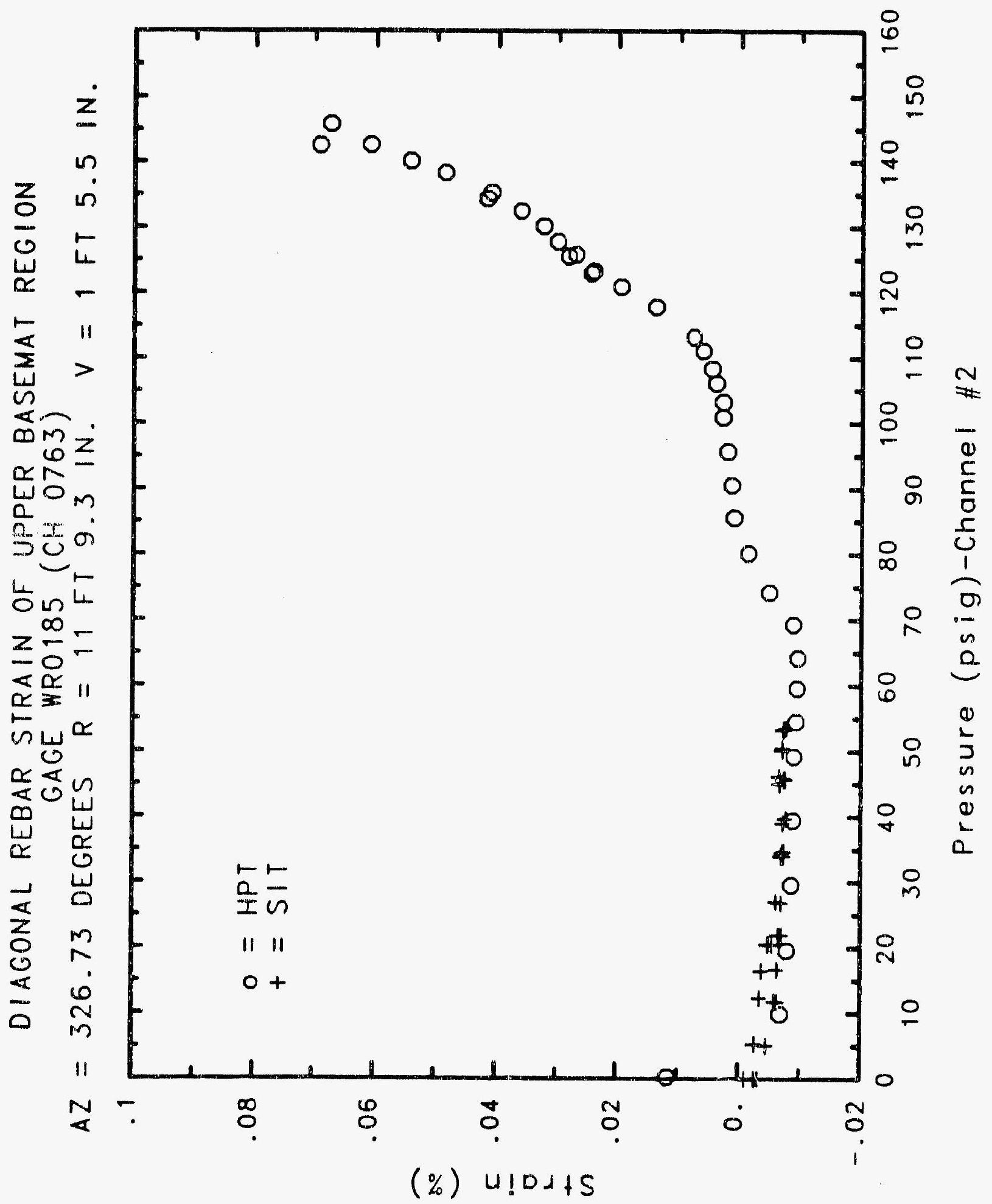




\section{Reinforced Concrete Test Data}

Weldable Gage 186 Channel 764

Structural InTEgrity TEST

high Pressure Test

\begin{tabular}{|c|c|c|c|}
\hline $\begin{array}{c}\text { Pressure } \\
\text { (psig) } \\
-0.05 \\
5.33 \\
12.31 \\
16.44 \\
20.51 \\
20.46 \\
20.47 \\
27.05 \\
34.69 \\
34.53 \\
34.53 \\
39.70 \\
45.90 \\
45.65 \\
45.62 \\
50.10 \\
53.47 \\
53.29 \\
53.21 \\
50.49 \\
46.14 \\
46.13 \\
44.97 \\
38.98 \\
33.99 \\
33.96 \\
26.83 \\
21.88 \\
21.90 \\
16.69 \\
11.74 \\
11.77 \\
5.05 \\
0.02 \\
-0.04 \\
-0.02 \\
-0.02 \\
0.02 \\
\end{array}$ & $\begin{array}{l}\text { X Strain } \\
-0.0004 \\
-0.0011 \\
-0.0005 \\
-0.0007 \\
-0.0023 \\
-0.0030 \\
-0.0032 \\
-0.0042 \\
-0.0066 \\
-0.0063 \\
-0.0060 \\
-0.0071 \\
-0.0070 \\
-0.0060 \\
-0.0054 \\
-0.0051 \\
-0.0053 \\
-0.0048 \\
-0.0040 \\
-0.0035 \\
-0.0034 \\
-0.0025 \\
-0.0053 \\
-0.0045 \\
-0.0039 \\
-0.0041 \\
-0.0031 \\
-0.0025 \\
-0.0029 \\
-0.0023 \\
-0.0015 \\
-0.0007 \\
0.0011 \\
0.0027 \\
0.0030 \\
-0.0015 \\
-0.0008 \\
0.0019\end{array}$ & $\begin{array}{c}\text { Pressure } \\
(\text { psig) } \\
9.89 \\
19.55 \\
29.57 \\
39.42 \\
49.16 \\
54.50 \\
59.57 \\
64.20 \\
69.32 \\
74.16 \\
80.16 \\
85.61 \\
90.58 \\
95.69 \\
100.92 \\
103.25 \\
106.11 \\
108.31 \\
111.08 \\
113.24 \\
117.83 \\
120.92 \\
123.28 \\
122.97 \\
125.82 \\
125.60 \\
127.84 \\
130.19 \\
132.53 \\
135.33 \\
134.42 \\
138.35 \\
140.16 \\
142.63 \\
145.78 \\
142.52 \\
0.22 \\
\end{array}$ & $\begin{array}{l}\text { \% Strain } \\
-0.0074 \\
-0.0085 \\
-0.0095 \\
-0.0101 \\
-0.0108 \\
-0.0110 \\
-0.0114 \\
-0.0120 \\
-0.0126 \\
-0.0123 \\
-0.0079 \\
-0.0073 \\
-0.0057 \\
-0.0049 \\
-0.0036 \\
-0.0024 \\
-0.0013 \\
-0.0005 \\
0.0010 \\
0.0029 \\
0.0057 \\
0.0079 \\
0.0092 \\
0.0085 \\
0.0089 \\
0.0088 \\
0.0096 \\
0.0128 \\
0.0156 \\
0.0195 \\
0.0209 \\
0.0280 \\
0.0321 \\
0.0403 \\
0.0493 \\
0.0521 \\
0.0072\end{array}$ \\
\hline
\end{tabular}




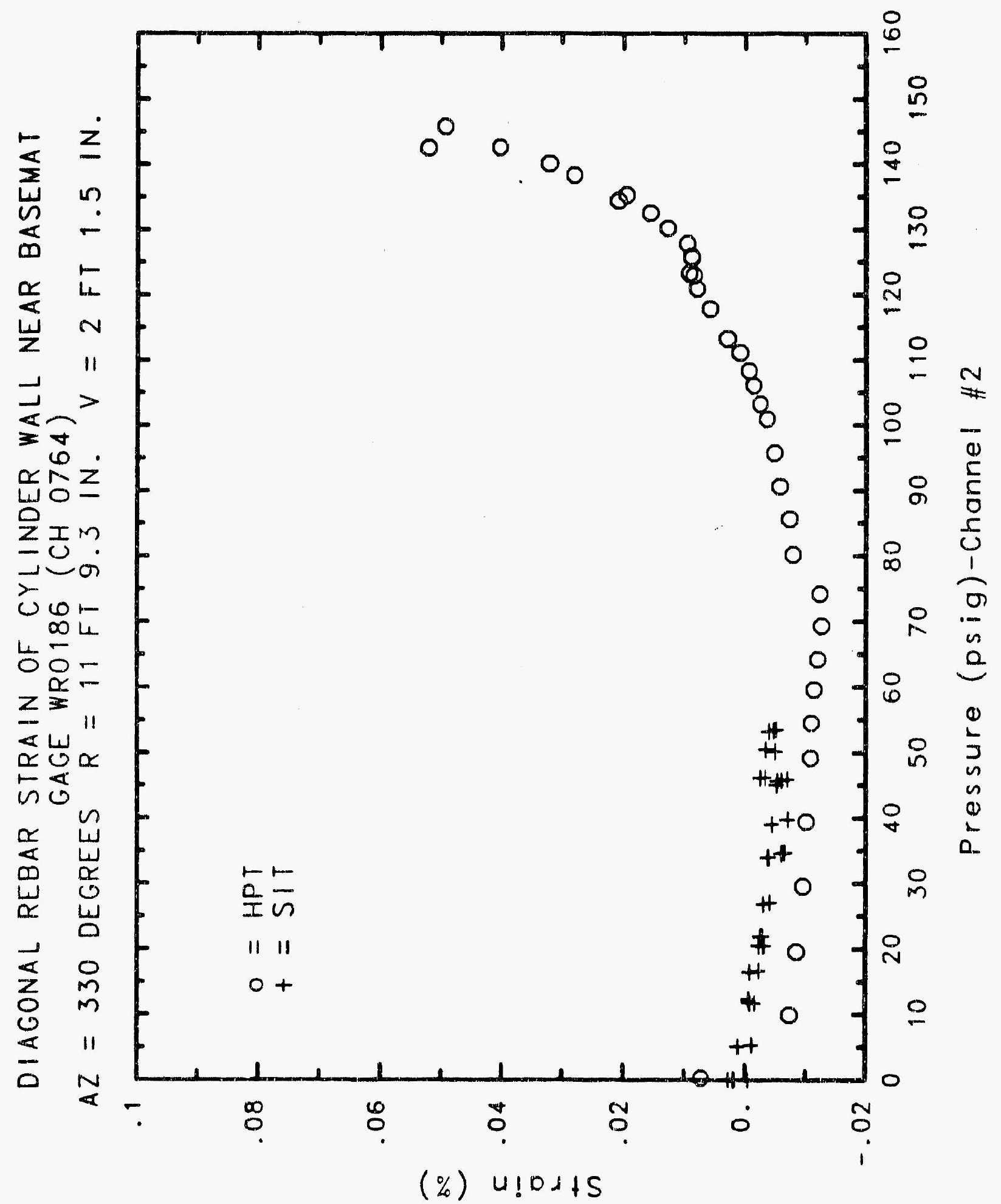

C- 1180 


\section{Reinforced Concrete Test Data}

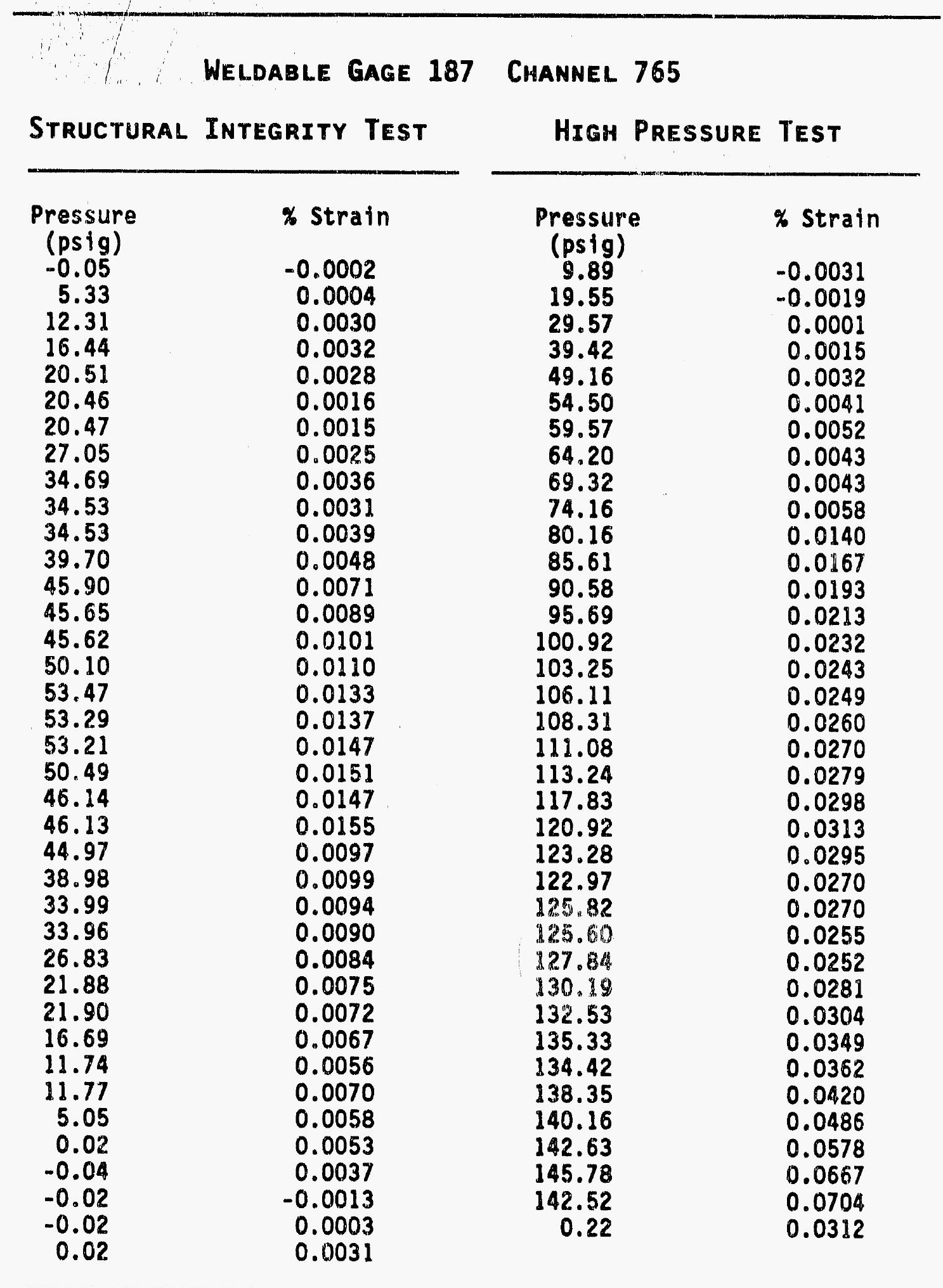




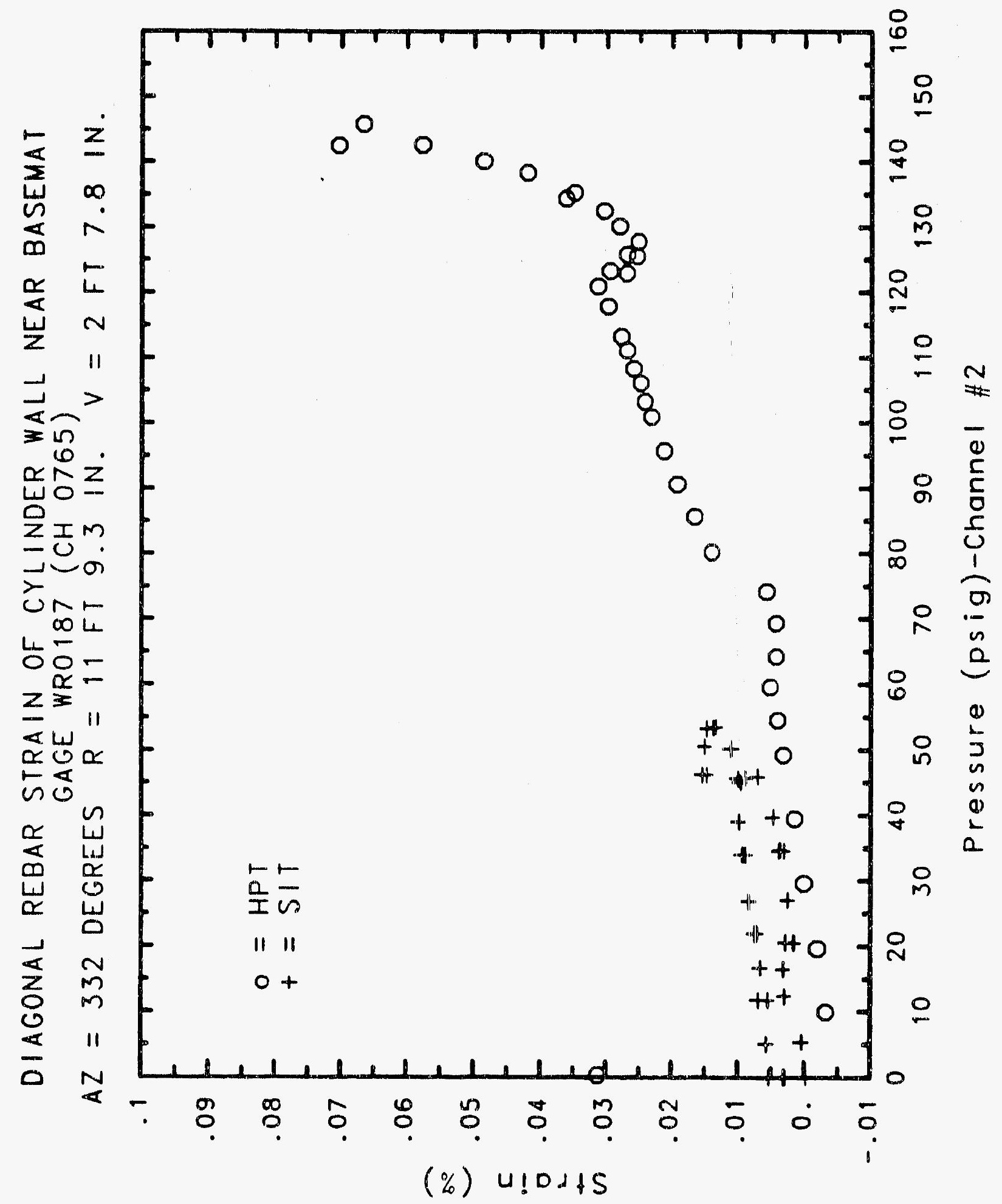

C-1182 


\section{Reinforced Concrete Test Data}

Weldable Gage 188 Channel 766

Structural. Integrity Test

High Pressure test

\begin{tabular}{|c|c|c|c|}
\hline $\begin{array}{c}\text { Pressure } \\
(\text { psig) } \\
-0.05 \\
5.33 \\
12.31 \\
16.44 \\
20.51 \\
20.46 \\
20.47 \\
27.05 \\
34.69 \\
34.53 \\
34.53 \\
39.70 \\
45.90 \\
45.65 \\
45.62 \\
50.10 \\
53.47 \\
53.29 \\
53.21 \\
50.49 \\
46.14 \\
46.13 \\
44.97 \\
38.98 \\
33.99 \\
33.96 \\
26.83 \\
21.88 \\
21.90 \\
16.69 \\
11.74 \\
11.77 \\
5.05 \\
0.02 \\
10.04 \\
-0.02 \\
1.0 .02 \\
0.02 \\
\end{array}$ & $\begin{array}{l}\text { \% Strain } \\
\text {-0.0002 } \\
0.0009 \\
0.0036 \\
0.0055 \\
0.0097 \\
0.0104 \\
0.0104 \\
0.0166 \\
0.0231 \\
0.0231 \\
0.0233 \\
0.0260 \\
0.0302 \\
0.0303 \\
0.0302 \\
0.0331 \\
0.0358 \\
0.0357 \\
0.0360 \\
0.0344 \\
0.0323 \\
0.0326 \\
0.0347 \\
0.0320 \\
0.0288 \\
0.0287 \\
0.0247 \\
0.0218 \\
0.0212 \\
0.0182 \\
0.0145 \\
0.0144 \\
0.0099 \\
0.0066 \\
0.0058 \\
0.0078 \\
0.0082 \\
0.0071\end{array}$ & $\begin{array}{c}\text { Pressure } \\
(\text { psig) } \\
9.89 \\
19.55 \\
29.57 \\
39.42 \\
49.16 \\
54.50 \\
59.57 \\
64.20 \\
69.32 \\
74.16 \\
80.16 \\
85.61 \\
90.58 \\
95.69 \\
100.92 \\
103.25 \\
106.11 \\
108.31 \\
111.08 \\
113.24 \\
117.83 \\
120.92 \\
123.28 \\
122.97 \\
125.82 \\
125.60 \\
127.84 \\
130.19 \\
132.53 \\
135.33 \\
134.42 \\
138.35 \\
140.16 \\
142.63 \\
145.78 \\
142.52 \\
0.22 \\
\end{array}$ & $\begin{array}{l}\text { \% Strain } \\
0.0016 \\
0.0071 \\
0.0136 \\
0.0196 \\
0.0253 \\
0.0283 \\
0.0315 \\
0.0353 \\
0.0399 \\
0.0449 \\
0.0507 \\
0.0574 \\
0.0637 \\
0.0711 \\
0.0797 \\
0.0840 \\
0.0884 \\
0.0923 \\
0.0975 \\
0.1021 \\
0.1129 \\
0.1206 \\
0.1283 \\
0.1308 \\
0.1397 \\
0.1427 \\
0.1477 \\
0.1570 \\
0.1698 \\
0.1874 \\
0.1931 \\
0.2138 \\
0.2334 \\
0.2622 \\
0.2927 \\
0.3074 \\
0.1411\end{array}$ \\
\hline
\end{tabular}




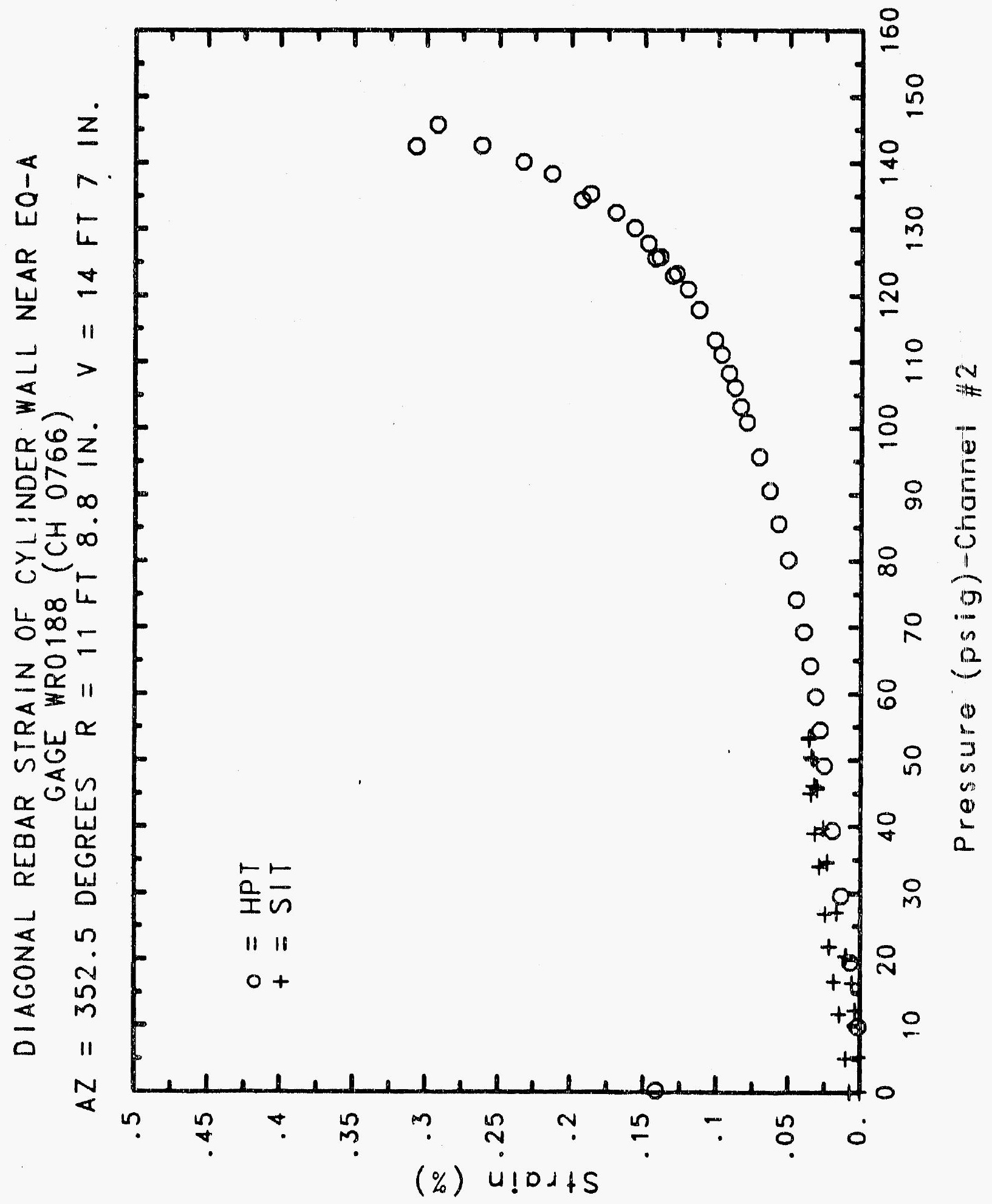




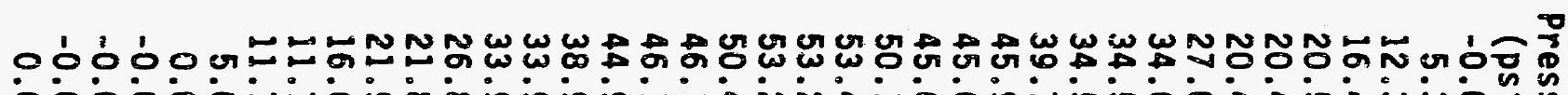

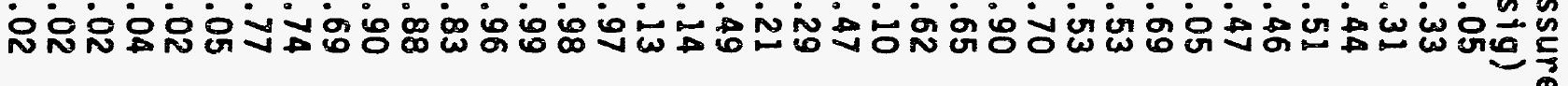

00000000000000000000000000000000000000

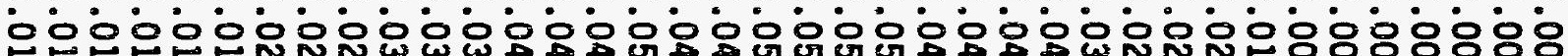

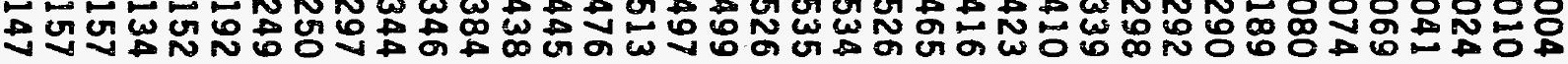

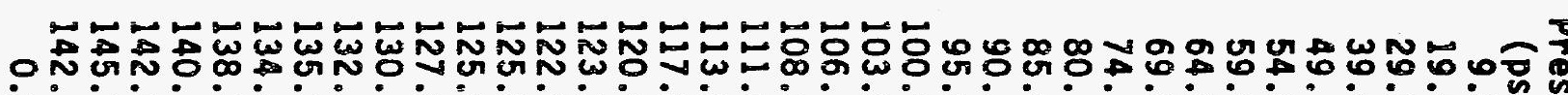

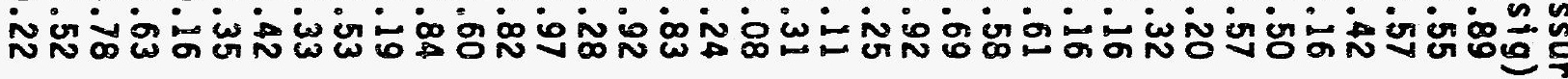




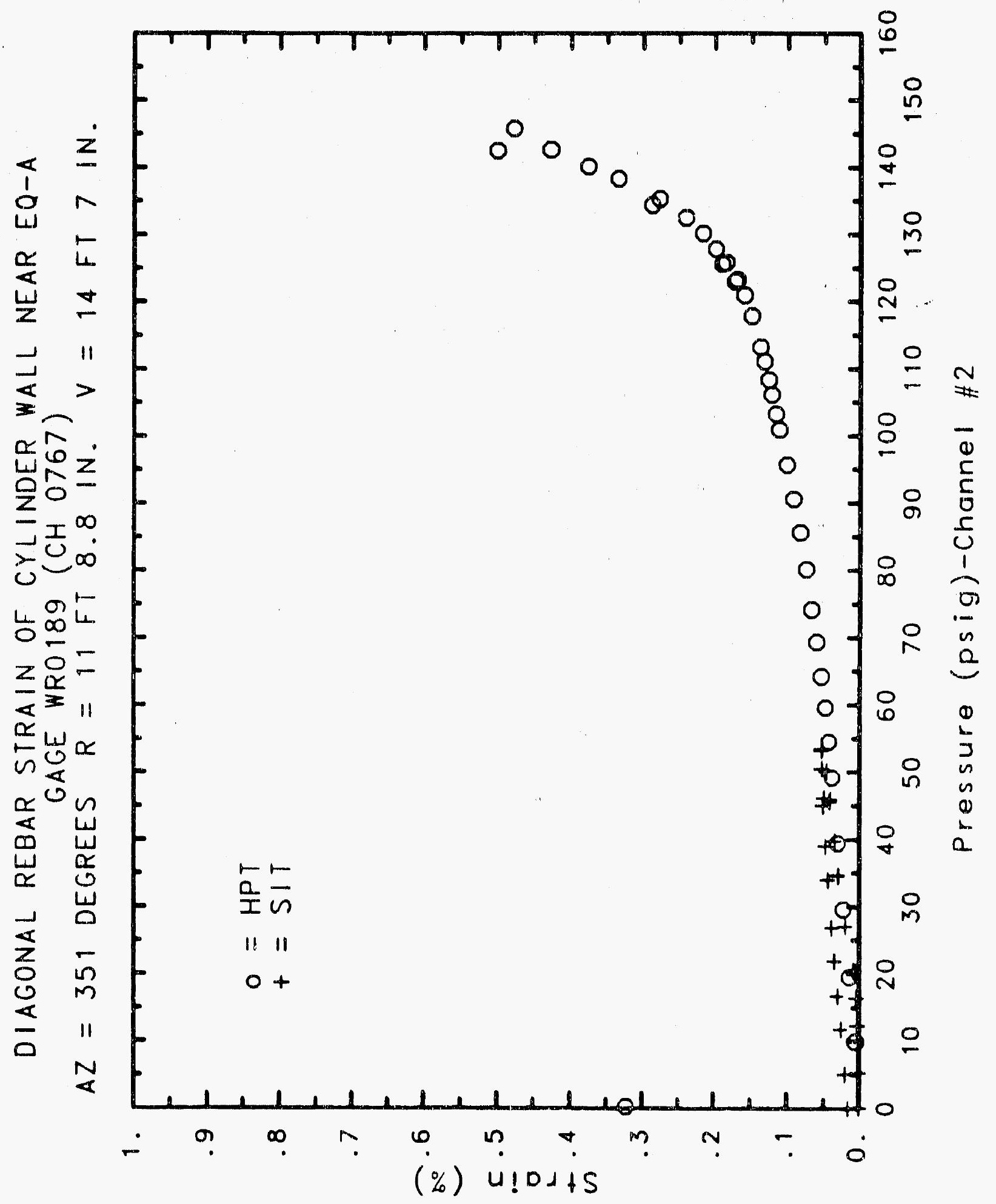




\section{Reinforced Concrete Test Data}

Weldable Gage 190 Channel 768

STRUCTURAL INTEGRITY TEST

high Pressure test

\begin{tabular}{|c|c|c|c|}
\hline $\begin{array}{c}\text { Pressure } \\
\text { (psig) } \\
-0.05 \\
5.33 \\
12.31 \\
16.44 \\
20.51 \\
20.46 \\
20.47 \\
27.05 \\
34.69 \\
34.53 \\
34.53 \\
39.70 \\
45.90 \\
45.65 \\
45.62 \\
50.10 \\
53.47 \\
53.29 \\
53.21 \\
50.49 \\
46.14 \\
46.13 \\
44.97 \\
38.98 \\
33.99 \\
33.96 \\
26.83 \\
21.88 \\
21.90 \\
16.69 \\
11.74 \\
11.77 \\
5.05 \\
0.02 \\
-0.04 \\
-0.02 \\
-0.02 \\
0.02 \\
\end{array}$ & $\begin{array}{l}\text { \% Strain } \\
-0.0007 \\
0.0015 \\
0.0036 \\
0.0060 \\
0.0101 \\
0.0103 \\
0.0110 \\
0.0172 \\
0.0228 \\
0.0229 \\
0.0229 \\
0.0260 \\
0.0314 \\
0.0320 \\
0.0314 \\
0.0354 \\
0.0396 \\
0.0403 \\
0.0400 \\
0.0391 \\
0.0365 \\
0.0370 \\
0.0383 \\
0.0354 \\
0.0323 \\
0.0319 \\
0.0277 \\
0.0247 \\
0.0243 \\
0.0211 \\
0.0173 \\
0.0171 \\
0.0122 \\
0.0090 \\
0.0071 \\
0.0097 \\
0.0093 \\
0.0085\end{array}$ & $\begin{array}{c}\text { Pressure } \\
(\text { psig) } \\
9.89 \\
19.55 \\
29.57 \\
39.42 \\
49.16 \\
54.50 \\
59.57 \\
64.20 \\
69.32 \\
74.16 \\
80.16 \\
85.61 \\
90.58 \\
95.69 \\
100.92 \\
103.25 \\
106.11 \\
108.31 \\
111.08 \\
113.24 \\
117.83 \\
120.92 \\
123.28 \\
122.97 \\
125.82 \\
125.60 \\
127.84 \\
130.19 \\
132.53 \\
135.33 \\
134.42 \\
138.35 \\
140.16 \\
142.63 \\
145.78 \\
142.52 \\
0.22 \\
\end{array}$ & $\begin{array}{l}\text { \% Strain } \\
0.0027 \\
0.0091 \\
0.0167 \\
0.0236 \\
0.0302 \\
0.0342 \\
0.0376 \\
0.0421 \\
0.0477 \\
0.0542 \\
0.0626 \\
0.0719 \\
0.0800 \\
0.0894 \\
0.0997 \\
0.1045 \\
0.1101 \\
0.1143 \\
0.1206 \\
0.1256 \\
0.1371 \\
0.1455 \\
0.1537 \\
0.1561 \\
0.1655 \\
0.1683 \\
0.1739 \\
0.1838 \\
0.1973 \\
0.2160 \\
0.2214 \\
0.2435 \\
0.2637 \\
0.2940 \\
0.3302 \\
0.3478 \\
0.1706\end{array}$ \\
\hline
\end{tabular}




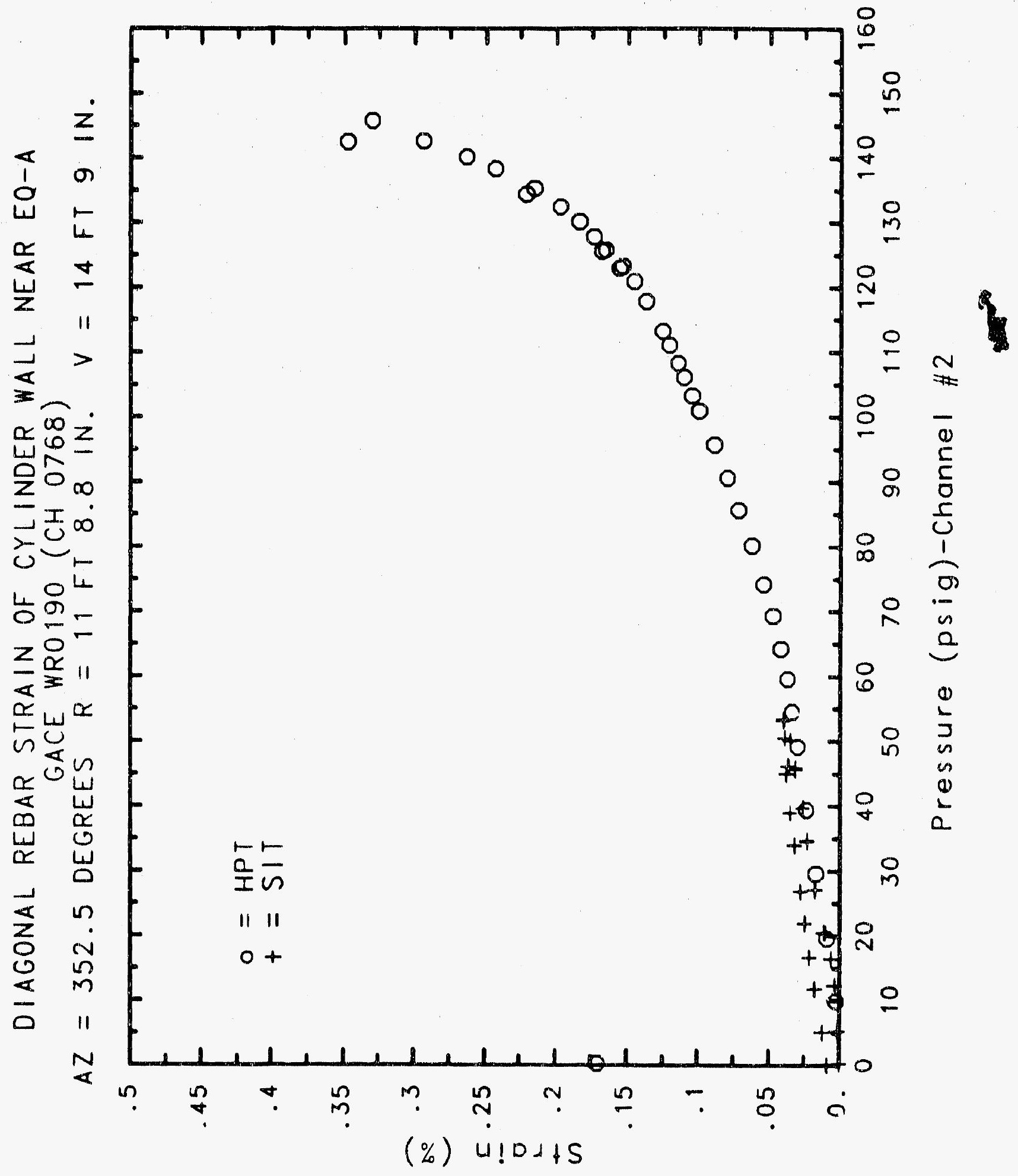




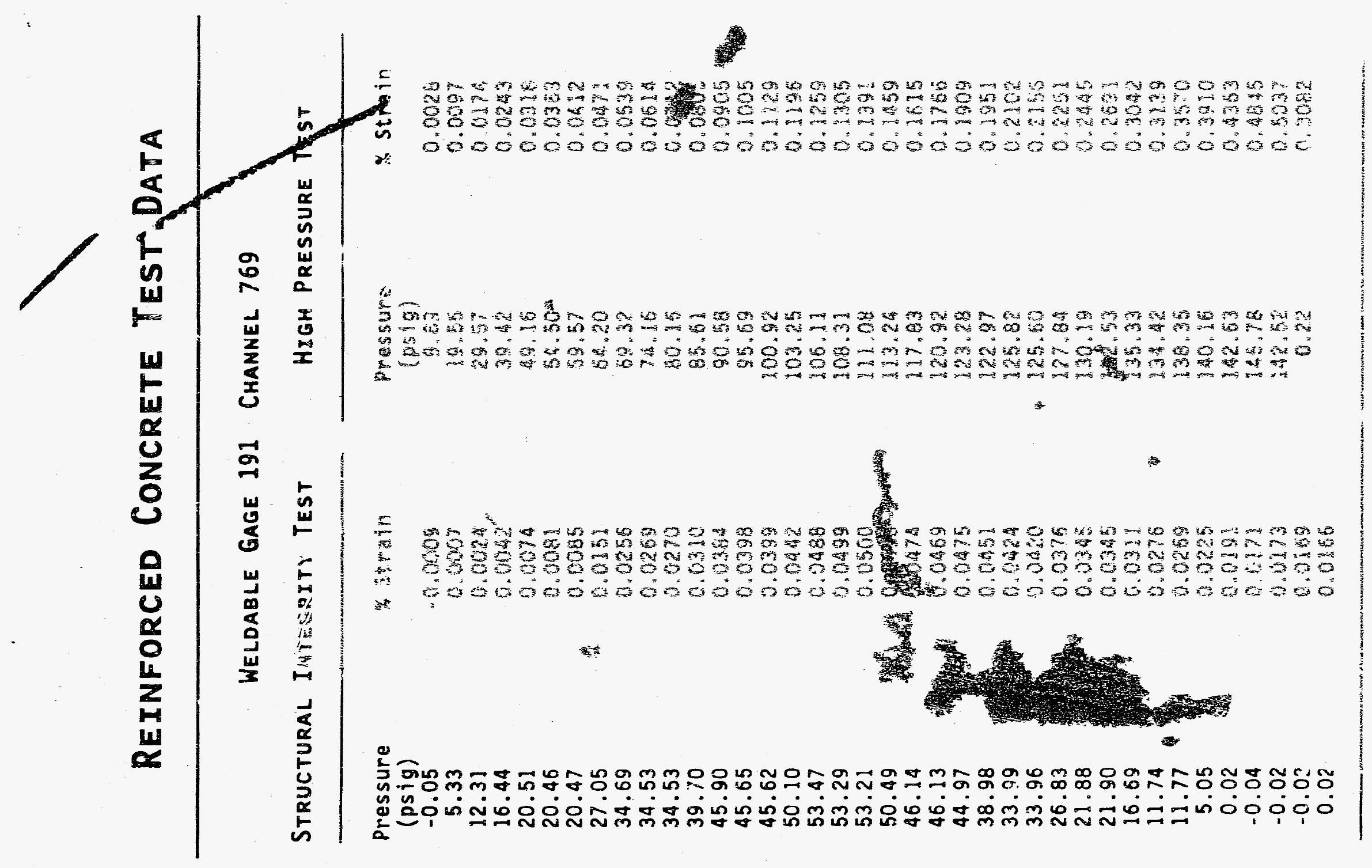

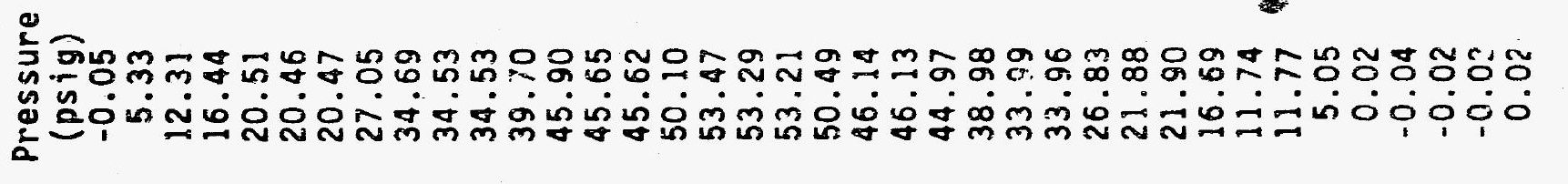

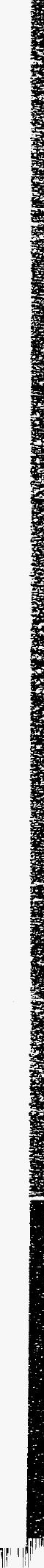




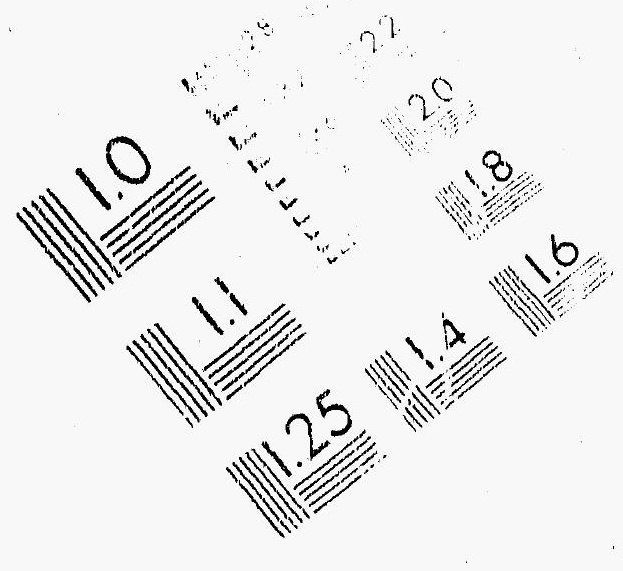

\section{IMAGE EVALUATION TEST TARGET (MT-3)}
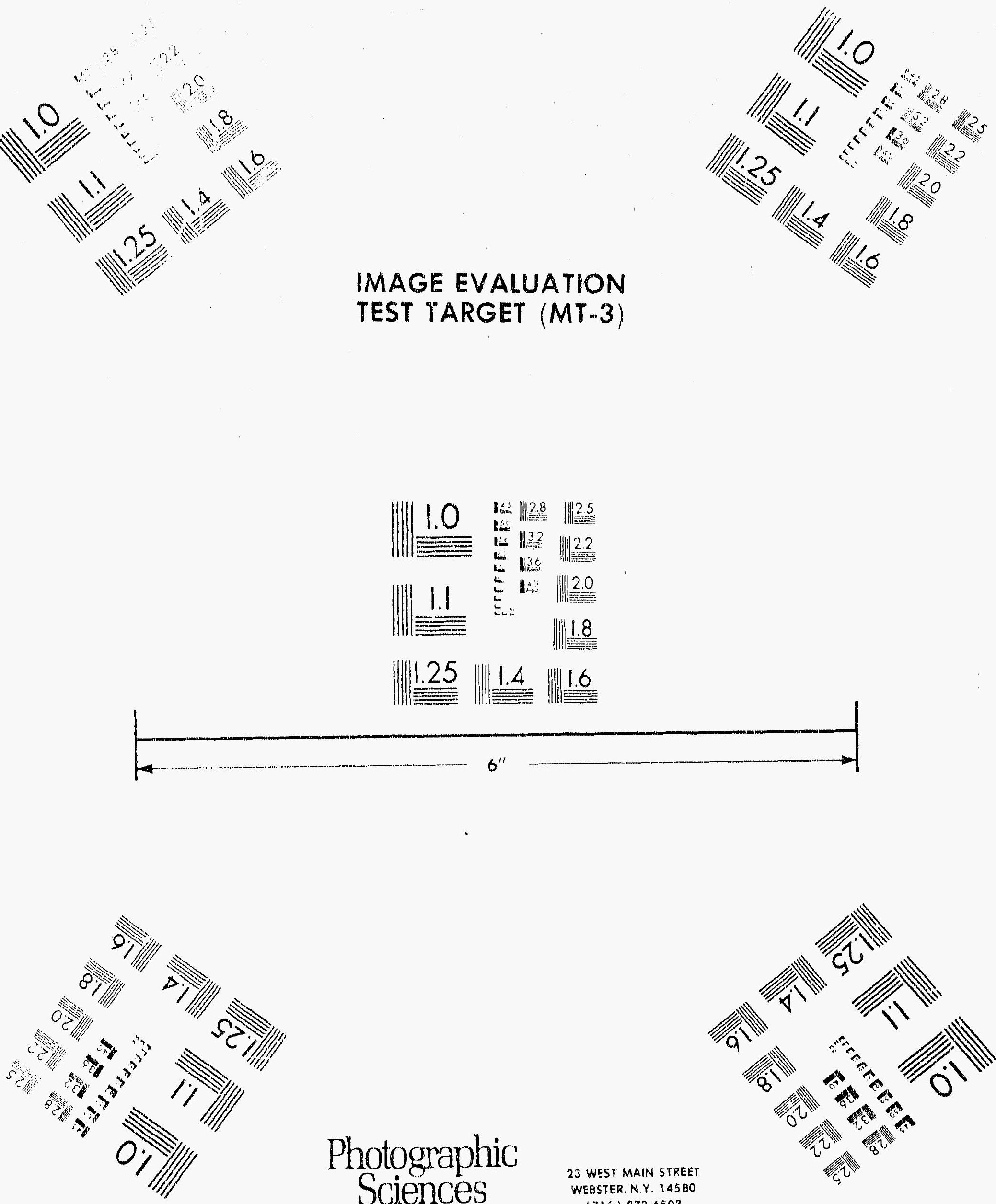

Photographic Sciences Corporation

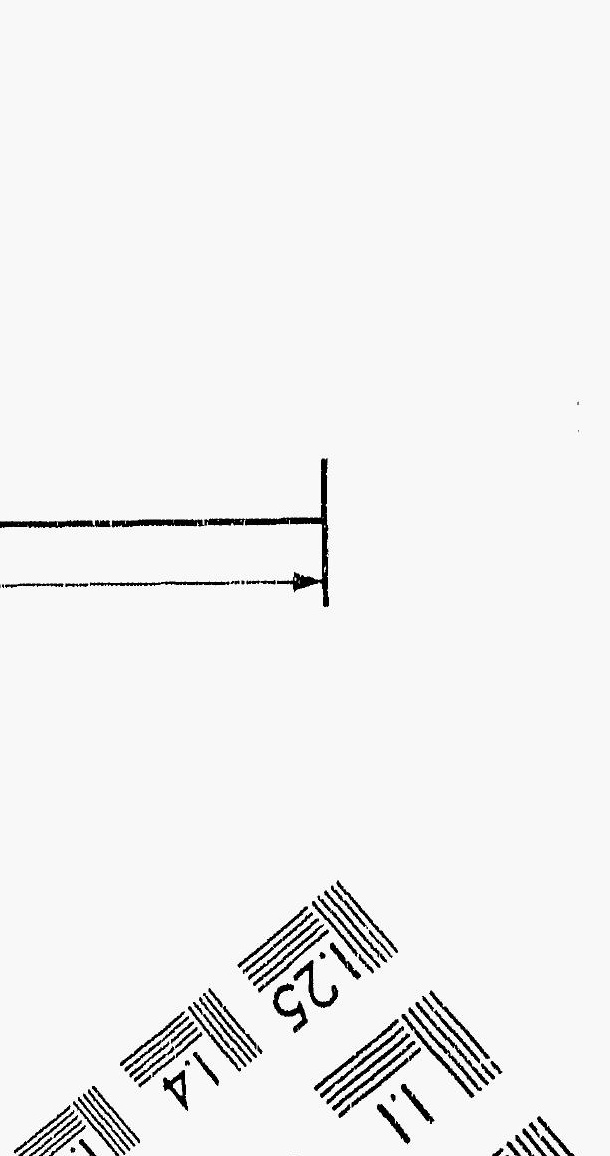

a

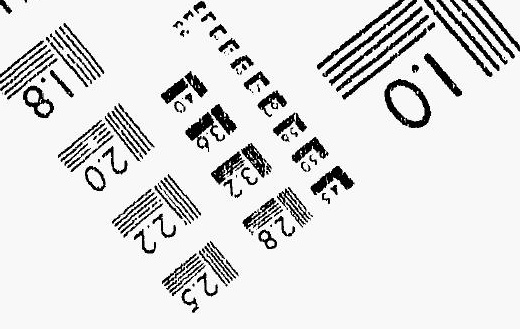




\section{SECURITY CLASSIFICATION}

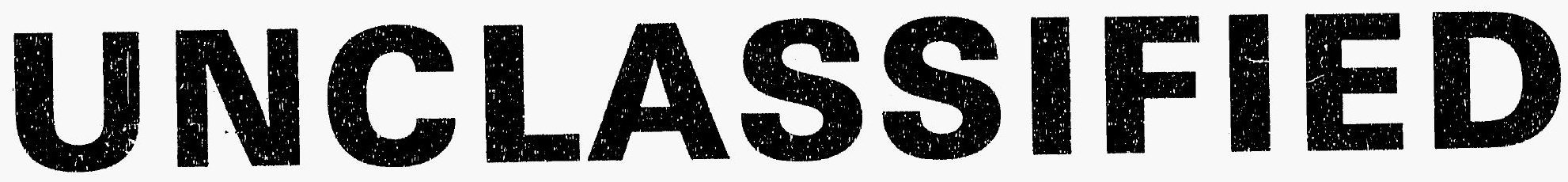

\section{DATE OF MICROFILMING $1-6-92$}

\section{MICROFILMED BY ERrY hard}

SANDIA NATIONAL LABS 


\section{THIS DOCUMENT FILMED}

AT

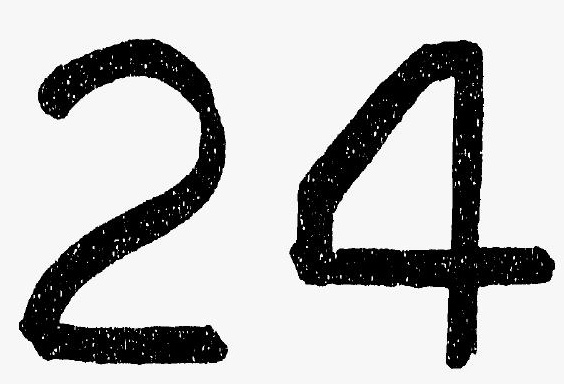

REDUCTION 


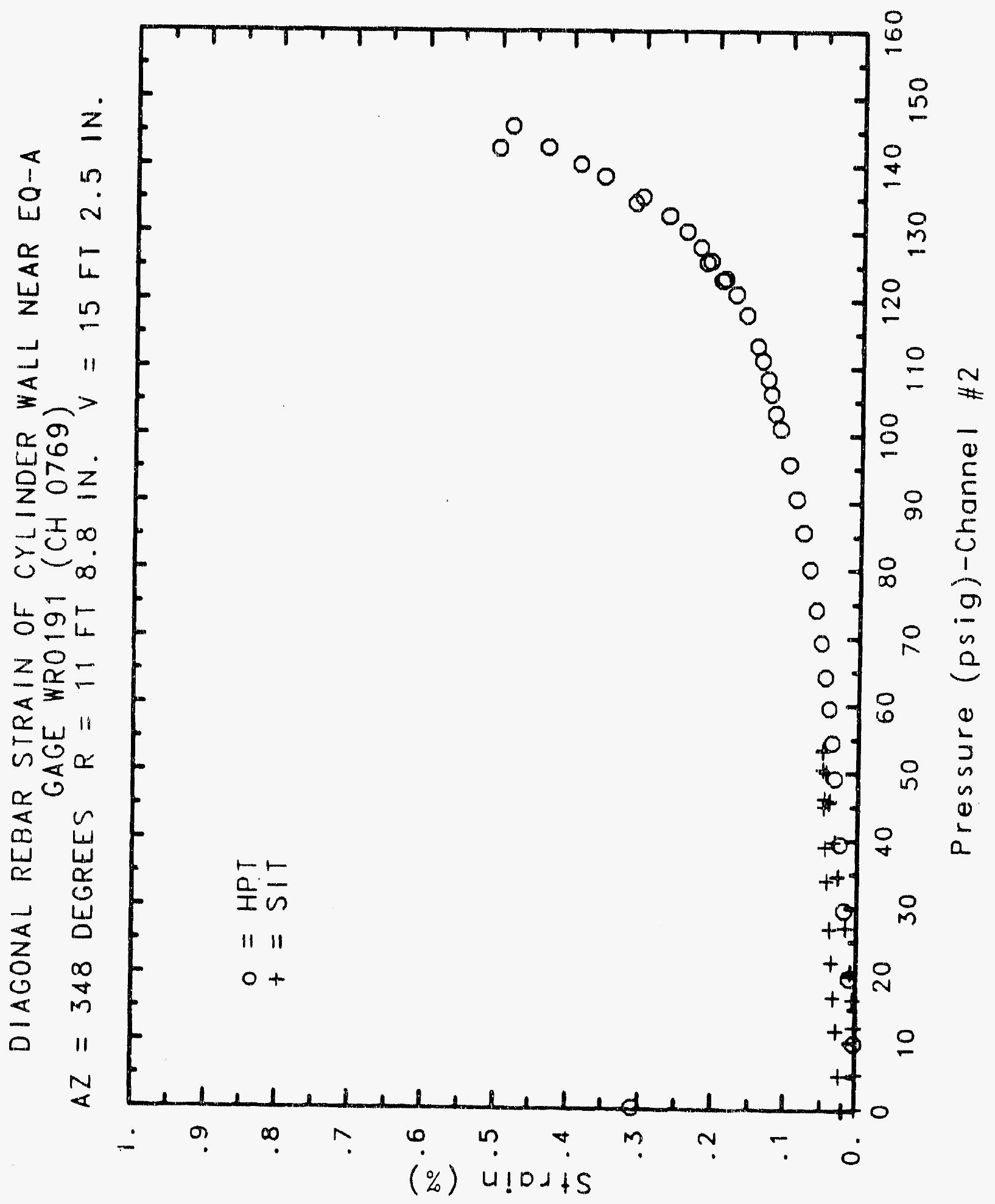




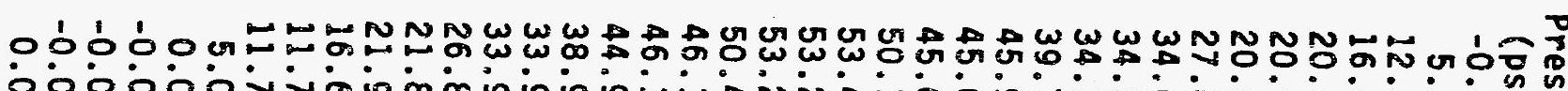

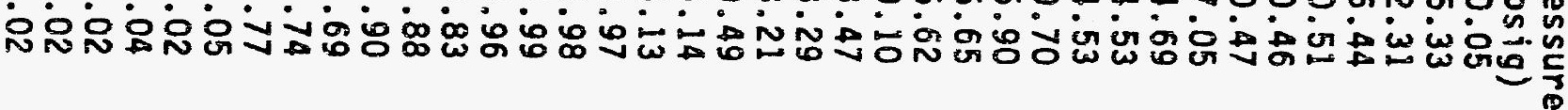

00000000000000000000000000000000000000

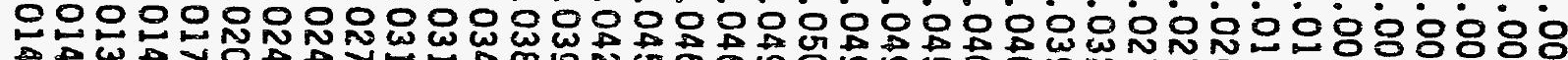

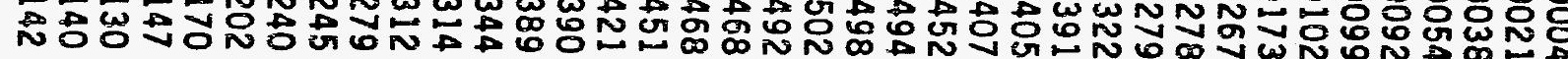

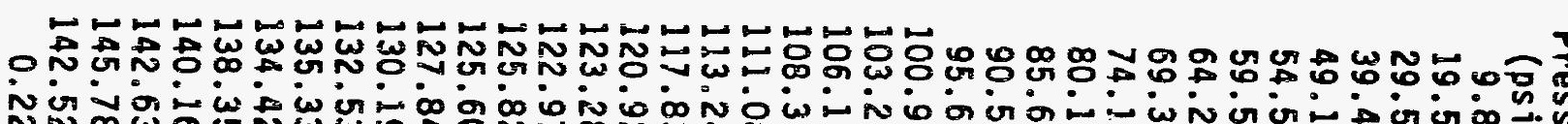

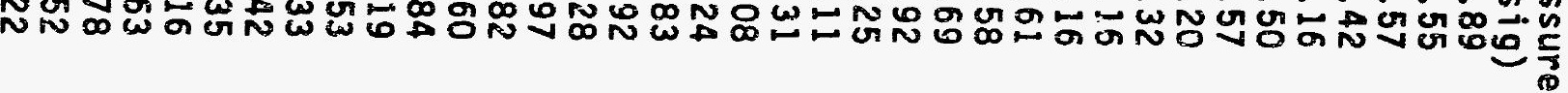




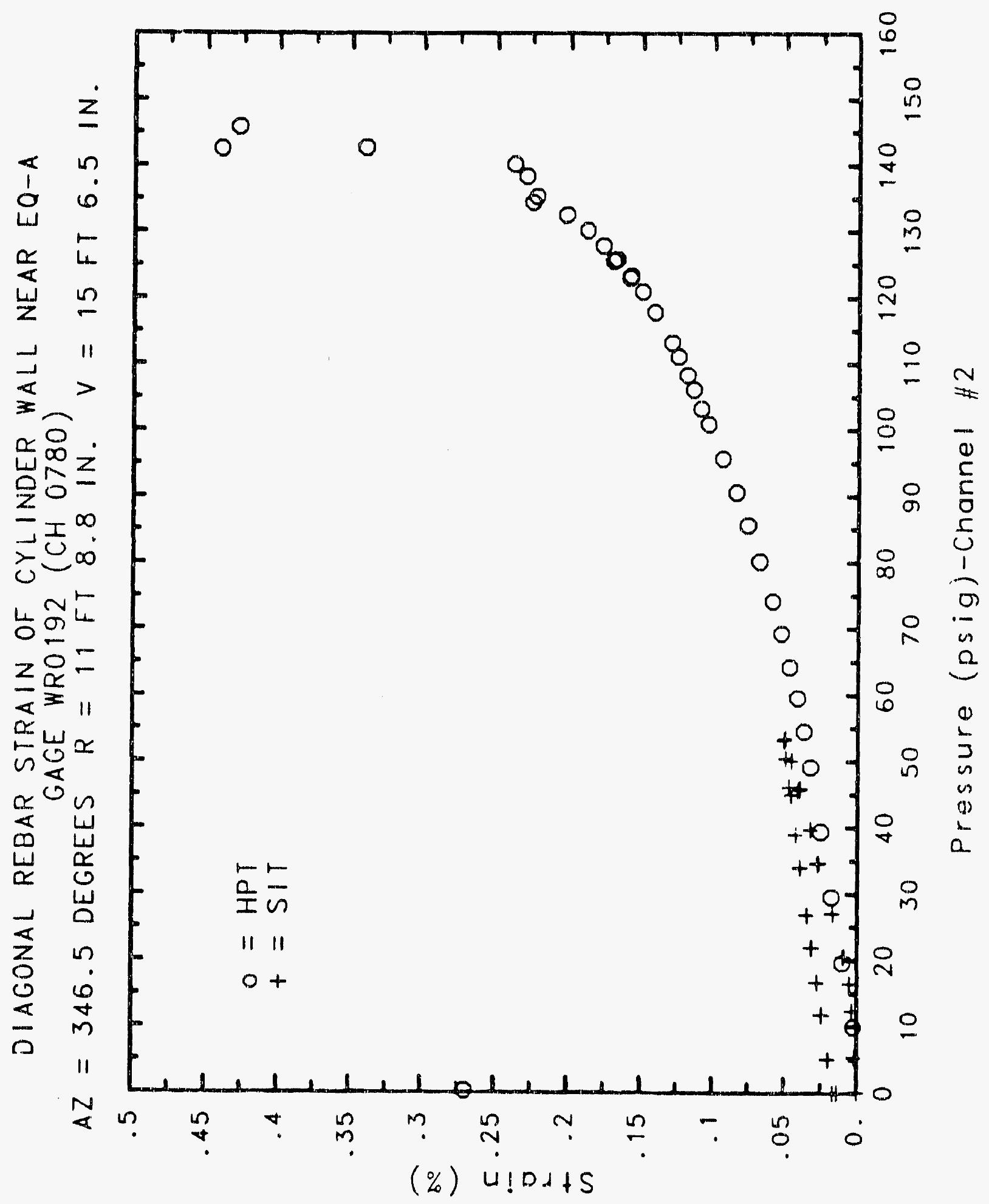




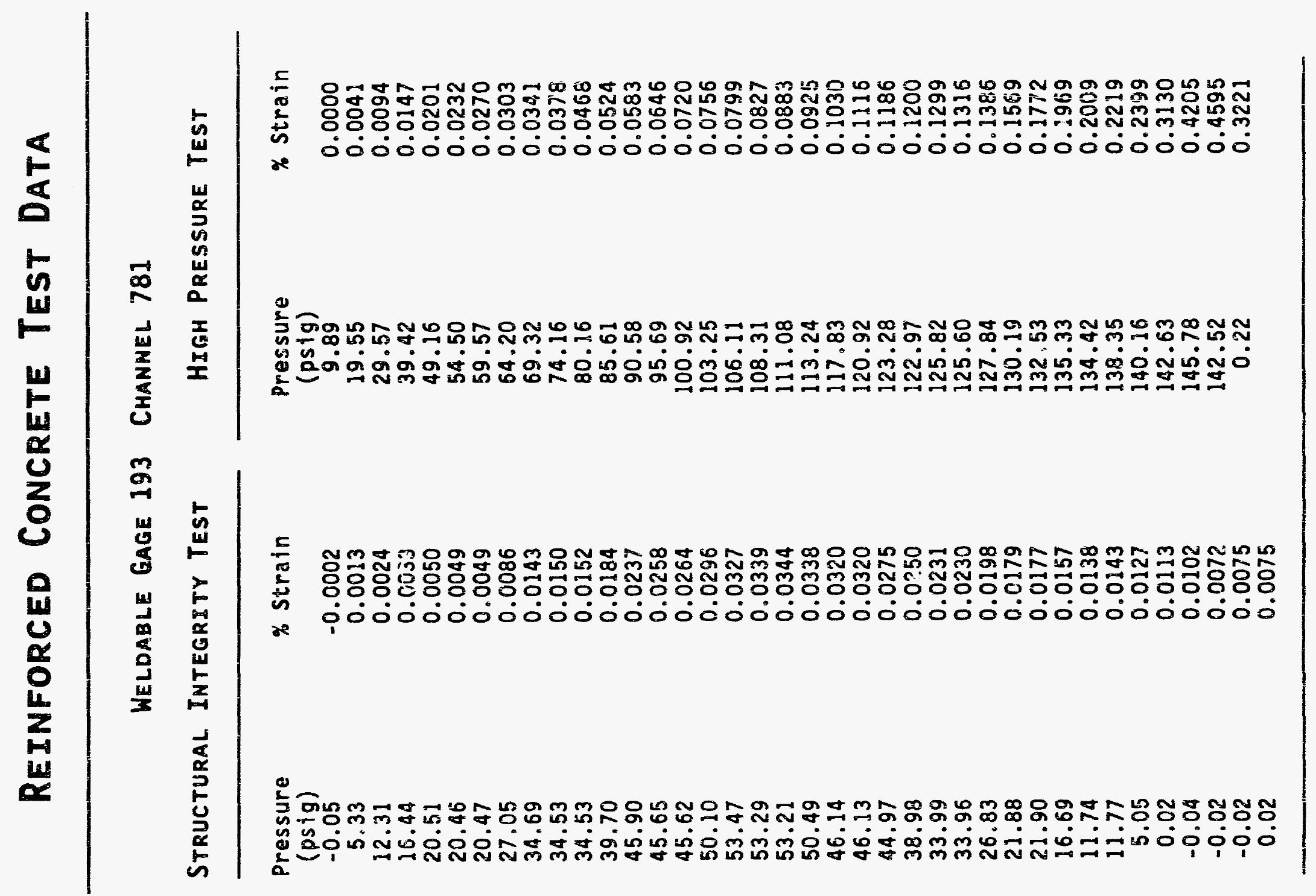




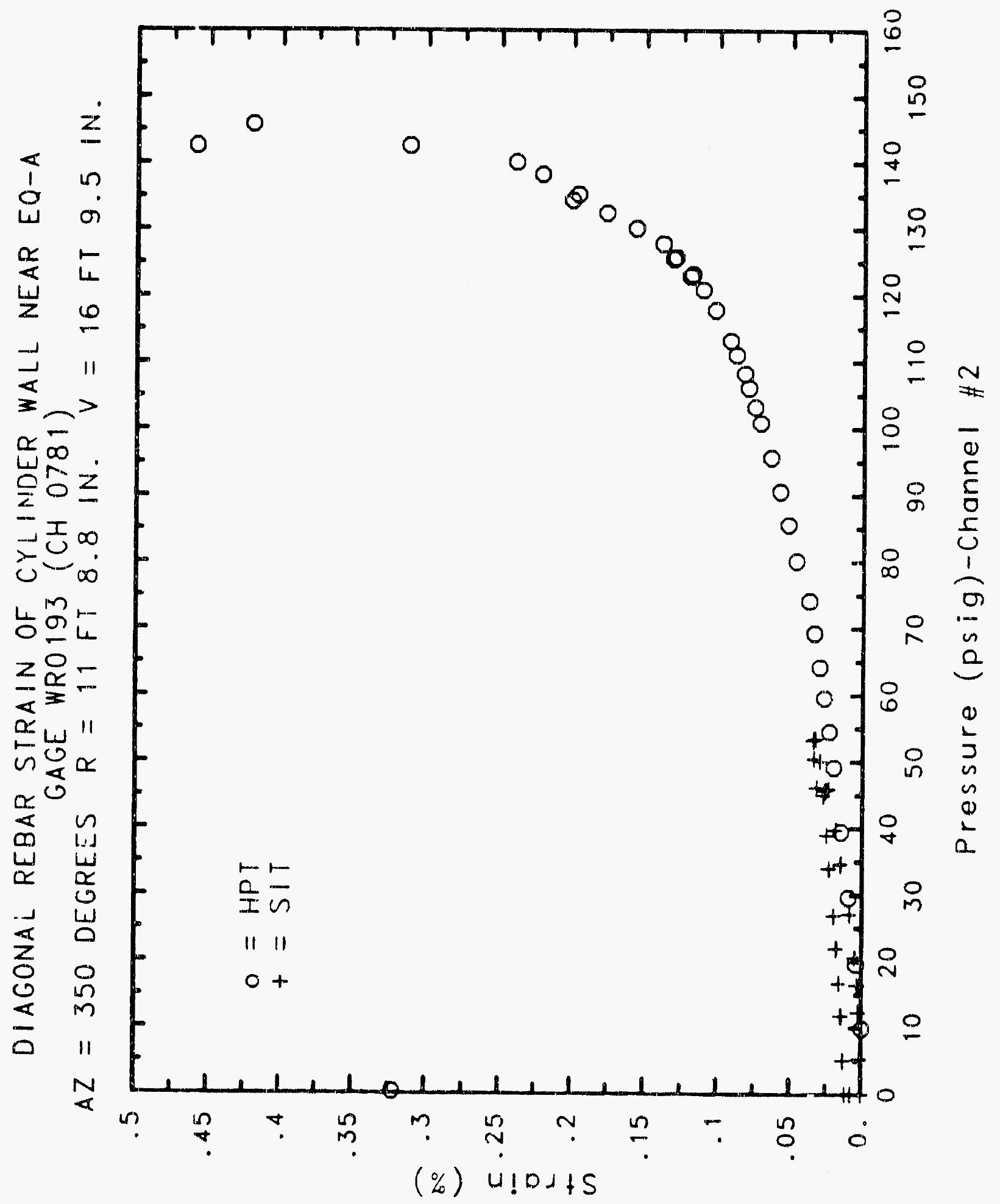




\section{Reinforced Concrete Test Data}

Weldable gage 194 Channel 782

STRUCTURAL INTEGRITY TEST

High Pressure test

\begin{tabular}{|c|c|c|c|}
\hline $\begin{array}{c}\text { Pressure } \\
\text { (psig) } \\
-0.05 \\
5.33 \\
12.31 \\
16.44 \\
20.51 \\
20.46 \\
20.47 \\
27.05 \\
34.69 \\
34.53 \\
34.53 \\
39.70 \\
45.90 \\
45.65 \\
45.62 \\
50.10 \\
53.47 \\
53.29 \\
53.21 \\
50.49 \\
46.14 \\
46.13 \\
44.97 \\
38.98 \\
33.99 \\
33.96 \\
26.83 \\
21.88 \\
21.90 \\
16.69 \\
11.74 \\
11.77 \\
5.05 \\
0.02 \\
-0.04 \\
-0.02 \\
-0.02 \\
0.02 \\
\end{array}$ & $\begin{array}{c}\text { Strain } \\
-0.0004 \\
0.0018 \\
0.0035 \\
0.0044 \\
0.0050 \\
0.0044 \\
0.0042 \\
0.0093 \\
0.0178 \\
0.0191 \\
0.0198 \\
0.0260 \\
0.0353 \\
0.0363 \\
0.0392 \\
0.0433 \\
0.0481 \\
0.0497 \\
0.0501 \\
0.0497 \\
0.0477 \\
0.0475 \\
0.0450 \\
0.0418 \\
0.0373 \\
0.0348 \\
0.0316 \\
0.0287 \\
0.0278 \\
0.0247 \\
0.0218 \\
0.0226 \\
0.0183 \\
0.0139 \\
0.0140 \\
0.0119 \\
0.0119 \\
0.0101\end{array}$ & $\begin{array}{c}\text { Pressure } \\
(\text { psig) } \\
9.89 \\
19.55 \\
29.57 \\
39.42 \\
49.16 \\
54.50 \\
59.57 \\
64.20 \\
69.32 \\
74.16 \\
80.16 \\
85.61 \\
90.58 \\
95.69 \\
100.92 \\
103.25 \\
106.11 \\
108.31 \\
111.08 \\
113.24 \\
117.83 \\
120.92 \\
123.28 \\
122.97 \\
125.82 \\
125.60 \\
127.84 \\
130.19 \\
132.53 \\
135.33 \\
134.42 \\
138.35 \\
140.16 \\
142.63 \\
145.78 \\
142.52 \\
0.22 \\
\end{array}$ & $\begin{array}{l}\text { * Strain } \\
0.0041 \\
0.0126 \\
0.0212 \\
0.0283 \\
0.0348 \\
0.0382 \\
0.0424 \\
0.0435 \\
0.0476 \\
0.0541 \\
0.0670 \\
0.0728 \\
0.0781 \\
0.0838 \\
0.0900 \\
0.0931 \\
0.0956 \\
0.0978 \\
0.1003 \\
0.1022 \\
0.1056 \\
0.1085 \\
0.1104 \\
0.1099 \\
0.1112 \\
0.1111 \\
0.1122 \\
0.1124 \\
0.1124 \\
0.1119 \\
0.1098 \\
0.1087 \\
0.1123 \\
0.1181 \\
0.1272 \\
0.1281 \\
0.0175\end{array}$ \\
\hline
\end{tabular}




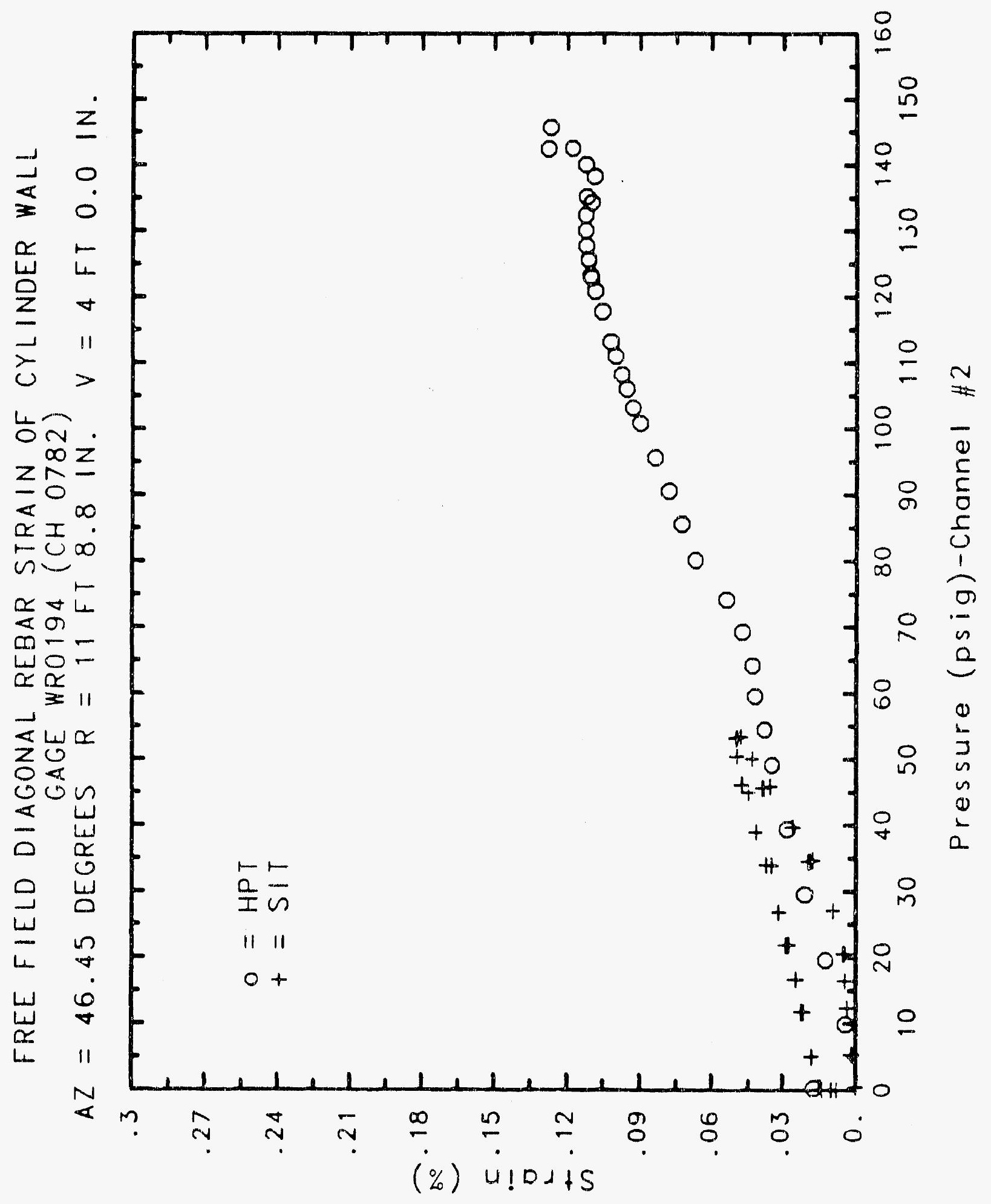




\section{Reinforced Concrete Test Data}

\begin{tabular}{|c|c|c|c|}
\hline STRUCTURAL & $\begin{array}{l}\text { WeLdable Gage } 195 \\
\text { INTEGRITY TEST }\end{array}$ & $\begin{array}{l}\text { Chanhel } 783 \\
\text { High Pressure }\end{array}$ & TEST \\
\hline $\begin{array}{l}\text { Pressure } \\
\text { (psig) } \\
-0.05 \\
5.33 \\
12.31 \\
16.44 \\
20.51 \\
20.46 \\
20.47 \\
27.05 \\
34.69 \\
34.53 \\
34.53 \\
39.70 \\
45.90 \\
45.65 \\
45.62 \\
50.10 \\
53.47 \\
53.29 \\
53.21 \\
50.49 \\
46.14 \\
46.13 \\
44.97 \\
38.98 \\
33.99 \\
33.96 \\
26.83 \\
21.88 \\
21.90 \\
16.69 \\
11.74 \\
11.77 \\
5.05 \\
0.02 \\
-0.04 \\
-0.02 \\
-0.02 \\
0.02 \\
\end{array}$ & $\begin{array}{l}\text { Strain } \\
\text {-0.0004 } \\
0.0050 \\
0.0114 \\
0.0155 \\
0.0221 \\
0.0220 \\
0.0213 \\
0.0347 \\
0.0483 \\
0.0479 \\
0.0479 \\
0.0569 \\
0.0671 \\
0.0675 \\
0.0684 \\
0.0748 \\
0.0800 \\
0.0802 \\
0.0802 \\
0.0769 \\
0.0709 \\
0.0708 \\
0.0548 \\
0.0561 \\
0.0489 \\
0.0472 \\
0.0363 \\
0.0296 \\
0.0298 \\
0.0221 \\
0.0152 \\
0.0166 \\
0.0078 \\
0.0014 \\
0.0013 \\
-0.0004 \\
-0.0008 \\
-0.0012\end{array}$ & $\begin{array}{c}\text { Pressure } \\
(\text { psig) } \\
9.89 \\
19.55 \\
29.57 \\
39.42 \\
49.16 \\
54.50 \\
59.57 \\
64.20 \\
69.32 \\
74.16 \\
80.16 \\
85.61 \\
90.58 \\
95.69 \\
100.92 \\
103.25 \\
106.11 \\
108.31 \\
111.08 \\
113.24 \\
117.83 \\
120.92 \\
123.28 \\
122.97 \\
125.82 \\
125.60 \\
127.84 \\
130.19 \\
132.53 \\
135.33 \\
134.42 \\
138.35 \\
140.16 \\
142.63 \\
145.78 \\
142.52 \\
0.22 \\
20\end{array}$ & $\begin{array}{l}\text { \% Strain } \\
0.0106 \\
0.0250 \\
0.0399 \\
0.0545 \\
0.0695 \\
0.0769 \\
0.0851 \\
0.0916 \\
0.1001 \\
0.1086 \\
0.1242 \\
0.1349 \\
0.1455 \\
0.1571 \\
0.1696 \\
0.1757 \\
0.1831 \\
0.1887 \\
0.1972 \\
0.2042 \\
0.2215 \\
0.2357 \\
0.2501 \\
0.2520 \\
0.2705 \\
0.2752 \\
0.2891 \\
0.3239 \\
0.4631 \\
0.6748 \\
0.6957 \\
0.7460 \\
0.7698 \\
0.7786 \\
0.7866 \\
0.8520 \\
0.5964\end{array}$ \\
\hline
\end{tabular}




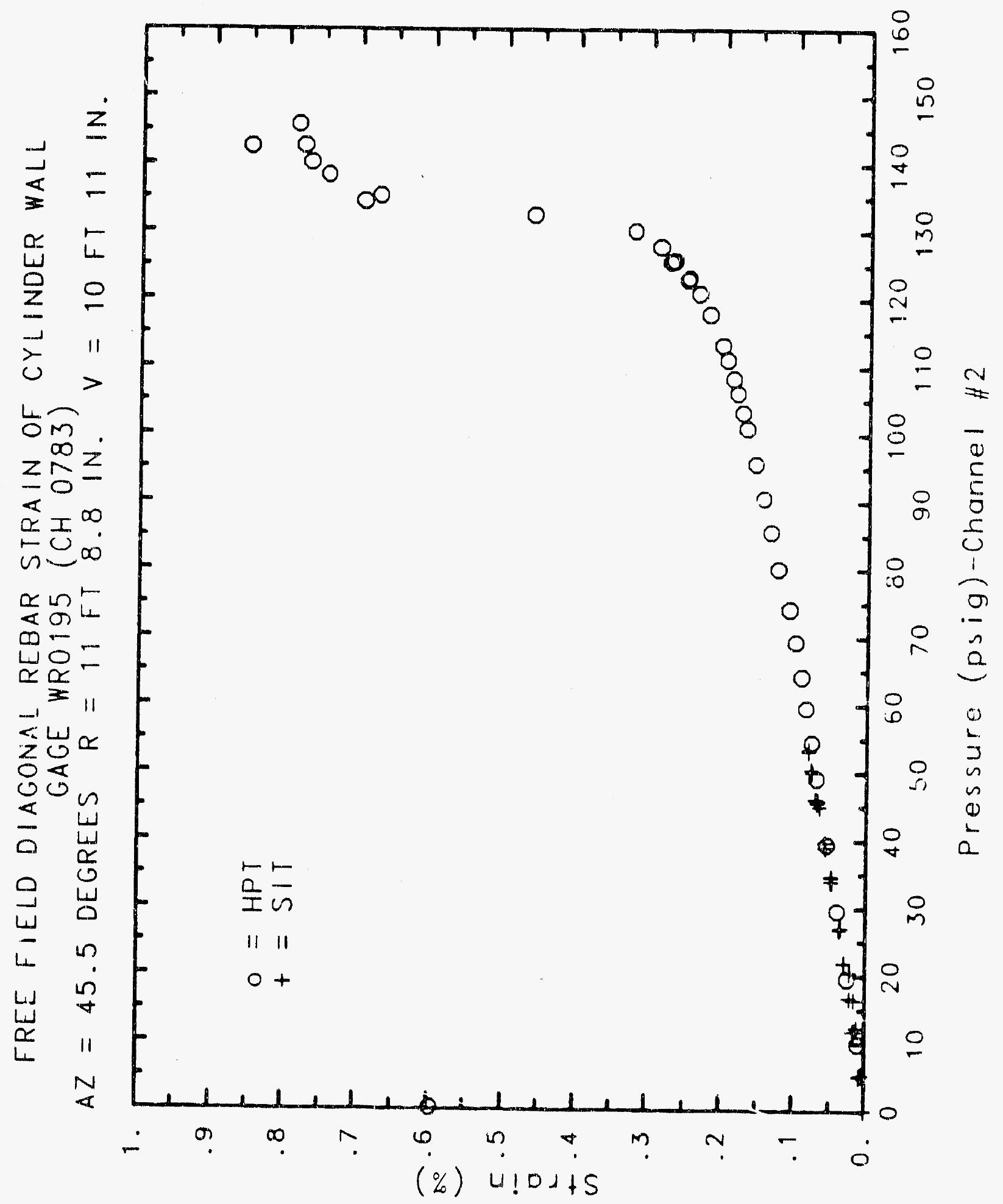

C. 1198 


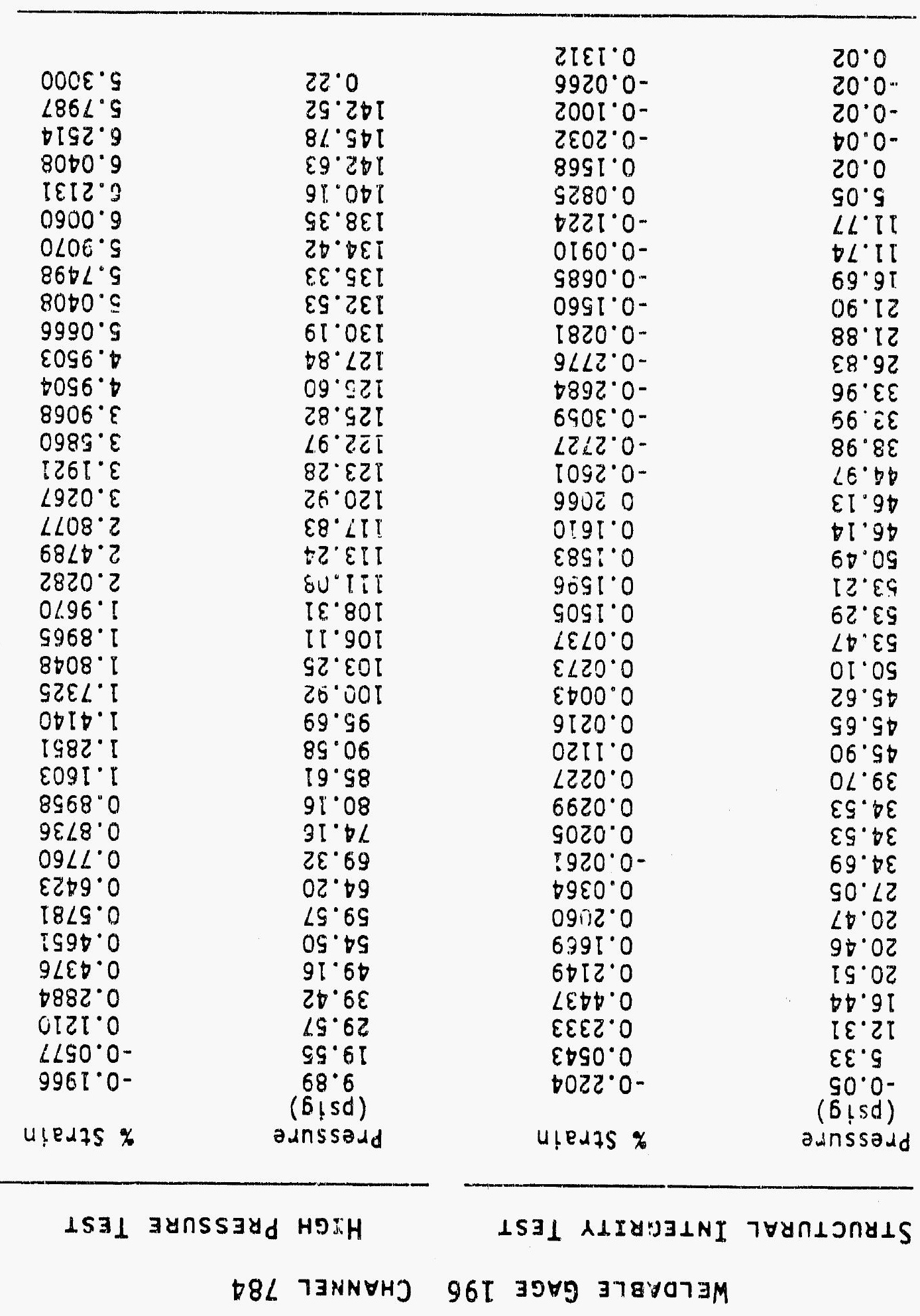

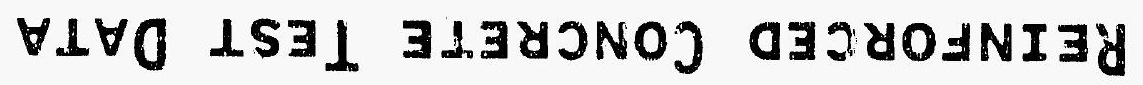




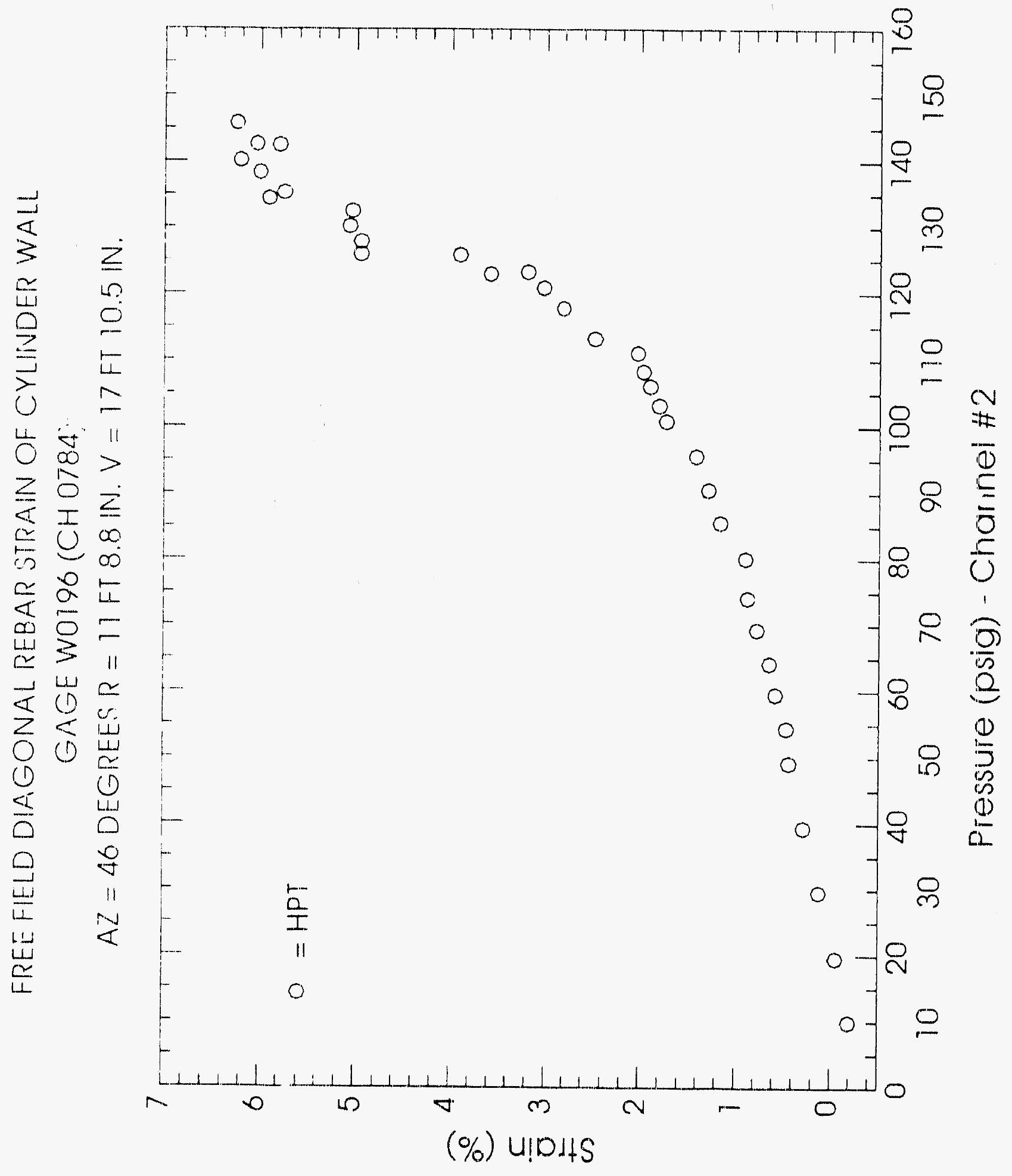




\section{Reinforced Concrete Test Data}

Weldable gage 197 Channel 785

Structural IntEgrity TEST

High Pressure test

\begin{tabular}{|c|c|c|c|}
\hline $\begin{array}{c}\text { Pressure } \\
\text { (psig) } \\
-0.05 \\
5.33 \\
12.31 \\
16.44 \\
20.51 \\
20.46 \\
20.47 \\
27.05 \\
34.69 \\
34.53 \\
34.53 \\
39.70 \\
45.90 \\
45.65 \\
45.62 \\
50.10 \\
53.47 \\
53.29 \\
53.21 \\
50.49 \\
46.14 \\
46.13 \\
44.97 \\
38.98 \\
33.99 \\
33.96 \\
26.83 \\
21.88 \\
21.90 \\
16.69 \\
11.74 \\
11.77 \\
5.05 \\
0.02 \\
-0.04 \\
-0.02 \\
-0.02 \\
0.02 \\
\end{array}$ & $\begin{array}{l}\text { Strain } \\
-0.0010 \\
0.0009 \\
0.0044 \\
0.0066 \\
0.0115 \\
0.0117 \\
0.0120 \\
0.0229 \\
0.0367 \\
0.0374 \\
0.0380 \\
0.0453 \\
0.0564 \\
0.0586 \\
0.0584 \\
0.0651 \\
0.0704 \\
0.0709 \\
0.0716 \\
0.0700 \\
0.0661 \\
0.0658 \\
0.0622 \\
0.0562 \\
0.0512 \\
0.0508 \\
0.0435 \\
0.0381 \\
0.0370 \\
0.0316 \\
0.0263 \\
0.0265 \\
0.0203 \\
0.0150 \\
0.0136 \\
0.0123 \\
0.0112 \\
0.0106\end{array}$ & $\begin{array}{c}\text { Pressure } \\
\text { (psig) } \\
9.89 \\
19.55 \\
29.57 \\
39.42 \\
49.16 \\
54.50 \\
59.57 \\
64.20 \\
69.32 \\
74.16 \\
80.16 \\
85.61 \\
90.58 \\
95.69 \\
100.92 \\
103.25 \\
106.11 \\
108.31 \\
111.08 \\
113.24 \\
117.83 \\
120.92 \\
123.28 \\
122.97 \\
125.82 \\
125.60 \\
127.84 \\
130.19 \\
132.53 \\
135.33 \\
134.42 \\
138.35 \\
140.16 \\
142.63 \\
145.78 \\
142.52 \\
0.22 \\
\end{array}$ & $\begin{array}{l}\text { Strain } \\
0.0060 \\
0.0187 \\
0.0309 \\
0.0432 \\
0.0549 \\
0.0613 \\
0.0681 \\
0.0750 \\
0.0828 \\
0.0904 \\
0.1038 \\
0.1131 \\
0.1214 \\
0.1301 \\
0.1399 \\
0.1441 \\
0.1494 \\
0.1532 \\
0.1591 \\
0.1634 \\
0.1743 \\
0.1822 \\
0.1885 \\
0.1887 \\
0.1967 \\
0.1975 \\
0.2030 \\
0.2135 \\
0.2298 \\
0.2635 \\
0.2707 \\
0.2950 \\
0.3776 \\
0.5209 \\
0.6091 \\
0.6261 \\
0.4097\end{array}$ \\
\hline
\end{tabular}




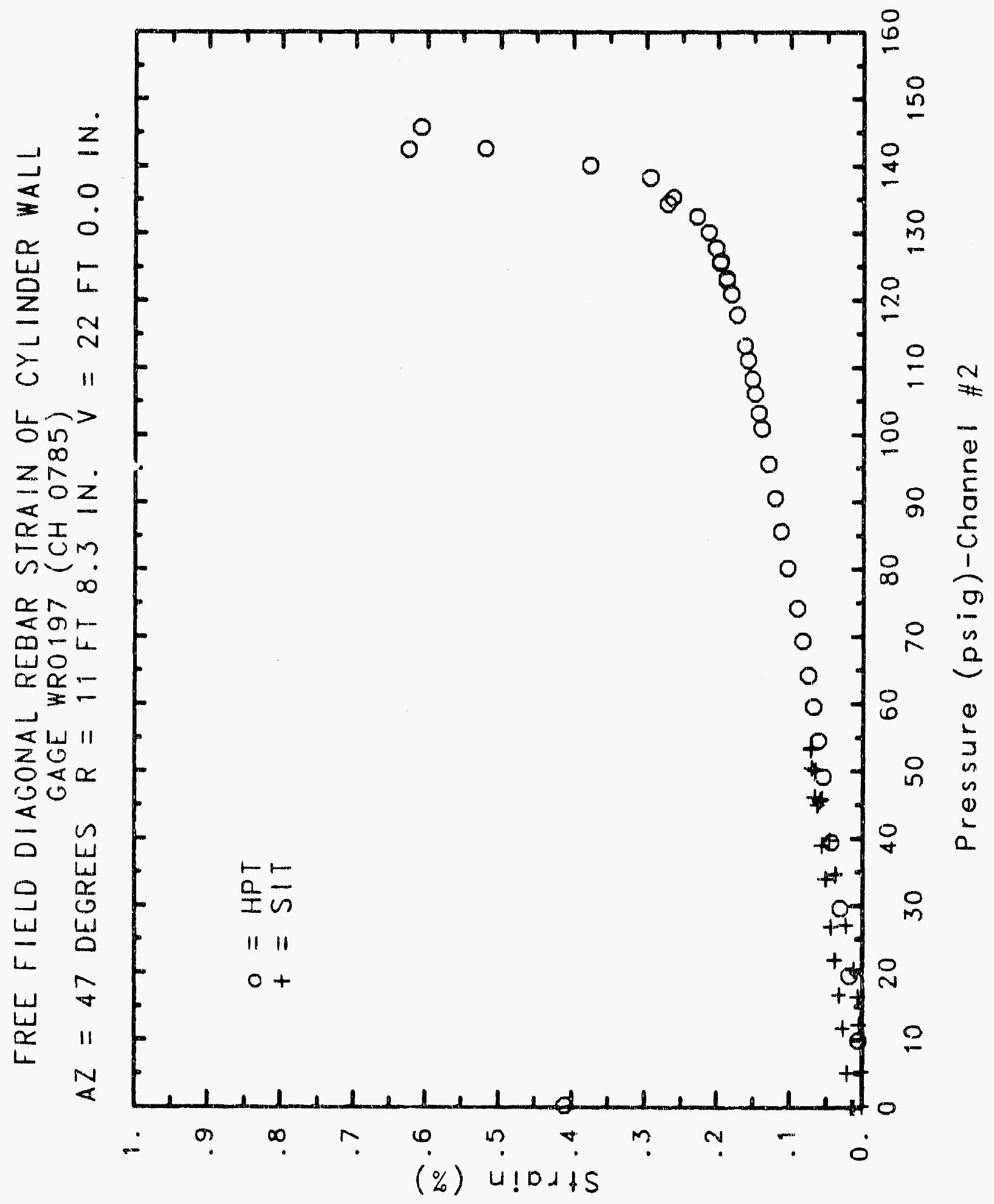




\section{Reinforced Concrete Test Data}

\begin{tabular}{|c|c|c|c|}
\hline JTRUCTURAL & $\begin{array}{l}\text { WELDABLE GAGE } 198 \\
\text { INTEGRITY TEST }\end{array}$ & $\begin{array}{r}\text { Channel } 786 \\
\text { High Pressure }\end{array}$ & TEST \\
\hline $\begin{array}{c}\text { Pressure } \\
\text { (psig) } \\
-0.05 \\
5.33 \\
12.31 \\
16.44 \\
20.51 \\
20.46 \\
20.47 \\
27.05 \\
34.69 \\
34.53 \\
34.53 \\
39.70 \\
45.90 \\
45.65 \\
45.62 \\
50.10 \\
53.47 \\
53.29 \\
53.21 \\
50.49 \\
46.14 \\
46.13 \\
44.97 \\
38.98 \\
33.99 \\
33.96 \\
26.83 \\
21.88 \\
21.90 \\
16.69 \\
11.74 \\
11.77 \\
5.05 \\
0.02 \\
-0.04 \\
-0.02 \\
-0.02 \\
0.02 \\
\end{array}$ & $\begin{array}{l}\text { S Strain } \\
\text {-0.0002 } \\
0.0004 \\
0.0018 \\
0.0030 \\
0.0047 \\
0.00052 \\
0.0054 \\
0.0089 \\
0.0174 \\
0.0186 \\
0.0194 \\
0.0234 \\
0.0300 \\
0.0307 \\
0.0303 \\
0.0336 \\
0.0368 \\
0.0368 \\
0.0374 \\
0.0365 \\
0.0347 \\
0.0347 \\
0.0353 \\
0.0328 \\
0.0306 \\
0.0305 \\
0.0275 \\
0.0251 \\
0.0254 \\
0.0232 \\
0.0207 \\
0.0197 \\
0.0163 \\
0.0134 \\
0.0111 \\
0.0113 \\
0.0109 \\
0.0113\end{array}$ & $\begin{array}{c}\text { Pressure } \\
(\text { psig) } \\
3.89 \\
19.55 \\
29.57 \\
39.42 \\
49.16 \\
54.50 \\
59.57 \\
64.20 \\
69.32 \\
74.16 \\
80.16 \\
85.61 \\
90.58 \\
95.69 \\
100.92 \\
103.25 \\
106.11 \\
108.31 \\
111.08 \\
113.24 \\
117.83 \\
120.92 \\
123.28 \\
122.97 \\
125.82 \\
125.60 \\
127.84 \\
130.19 \\
132.53 \\
135.33 \\
134.42 \\
138.35 \\
140.16 \\
142.63 \\
145.78 \\
142.52 \\
0.22 \\
\end{array}$ & $\begin{array}{l}\text { Strain } \\
0.0011 \\
0.0069 \\
0.0130 \\
0.0187 \\
0.0247 \\
0.0283 \\
0.0317 \\
0.0364 \\
0.0413 \\
0.0466 \\
0.0519 \\
0.0576 \\
0.0628 \\
0.0688 \\
0.0750 \\
0.0779 \\
0.0814 \\
0.0840 \\
0.0882 \\
0.0910 \\
0.0983 \\
0.1037 \\
0.1082 \\
0.1090 \\
0.1145 \\
0.1155 \\
0.1194 \\
0.1264 \\
0.1353 \\
0.1478 \\
0.1500 \\
0.1682 \\
0.1815 \\
0.1926 \\
0.1992 \\
0.1987 \\
0.0832\end{array}$ \\
\hline
\end{tabular}




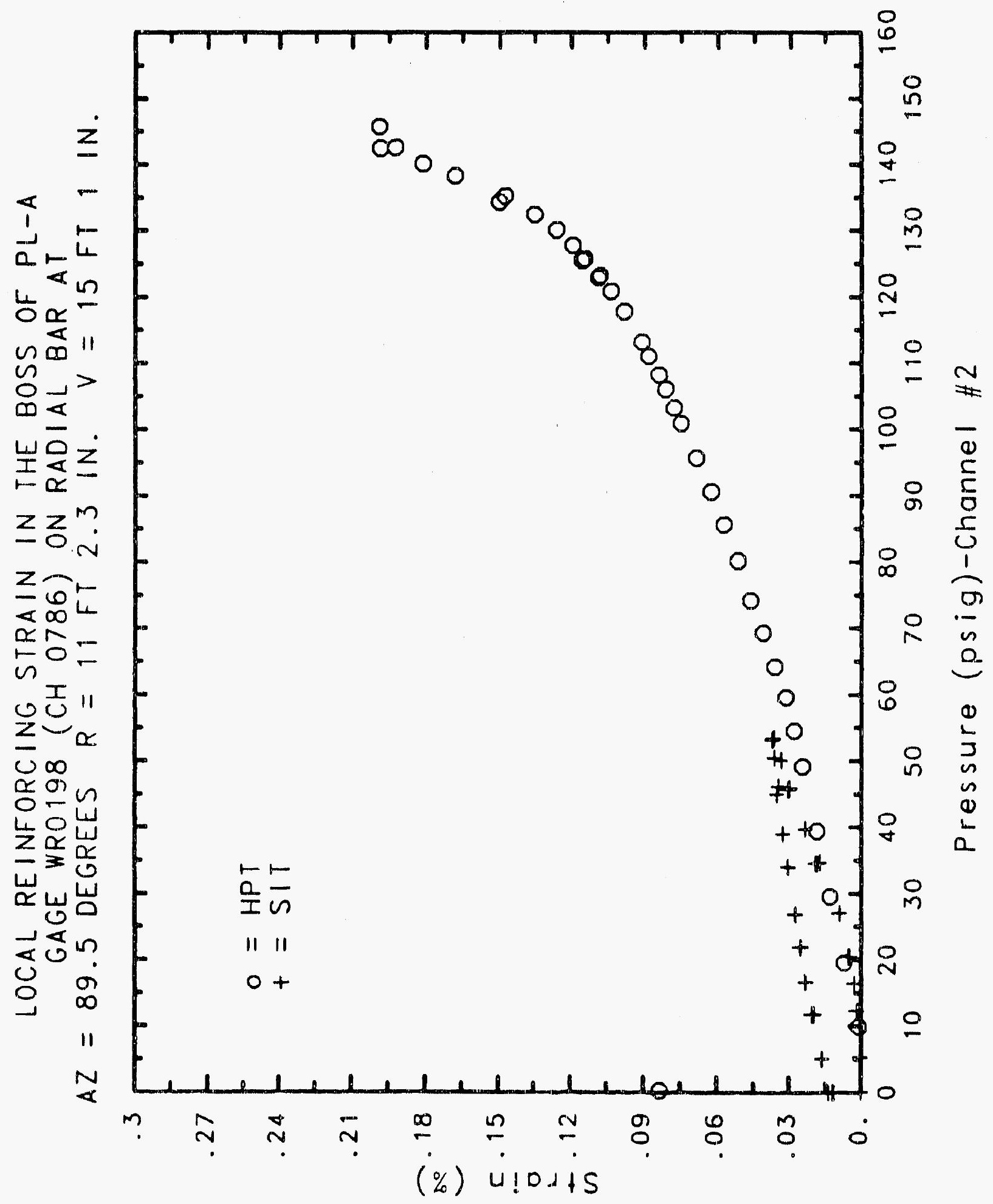




\section{Reinforced Concrete Test Data}

Weldable Gage 199 Chanmel 787

Structural Integrity Test

High Pressure test

\begin{tabular}{|c|c|c|c|}
\hline $\begin{array}{c}\text { Pressure } \\
(\text { psig) } \\
-0.05 \\
5.33 \\
12.31 \\
16.44 \\
20.51 \\
20.46 \\
20.47 \\
27.05 \\
34.69 \\
34.53 \\
34.53 \\
39.70 \\
45.90 \\
45.65 \\
45.62 \\
50.10 \\
53.47 \\
53.29 \\
53.21 \\
50.49 \\
46.14 \\
46.13 \\
44.97 \\
38.98 \\
33.99 \\
33.96 \\
26.83 \\
21.88 \\
21.90 \\
16.69 \\
11.74 \\
11.77 \\
5.05 \\
0.02 \\
-0.04 \\
-0.02 \\
-0.02 \\
0.02 \\
\end{array}$ & $\begin{array}{l}\text { \% Strain } \\
0.0006 \\
0.0008 \\
0.0023 \\
0.0032 \\
0.0052 \\
0.0058 \\
0.0060 \\
0.0098 \\
0.0156 \\
0.0160 \\
0.0164 \\
0.0189 \\
0.0233 \\
0.0255 \\
0.0258 \\
0.0289 \\
0.0314 \\
0.0326 \\
0.0329 \\
0.0327 \\
0.0313 \\
0.0311 \\
0.0297 \\
0.0282 \\
0.0264 \\
0.0265 \\
0.0239 \\
0.0214 \\
0.0206 \\
0.0183 \\
0.0164 \\
0.0172 \\
0.0150 \\
0.0131 \\
0.0128 \\
0.0124 \\
0.0119 \\
0.0115\end{array}$ & $\begin{array}{c}\text { Pressure } \\
(\text { psig) } \\
9.89 \\
19.55 \\
29.57 \\
39.42 \\
49.16 \\
54.50 \\
59.57 \\
64.20 \\
69.32 \\
74.16 \\
80.16 \\
85.61 \\
90.58 \\
95.69 \\
100.92 \\
103.25 \\
106.11 \\
108.21 \\
111.08 \\
113.24 \\
117.83 \\
120.92 \\
123.28 \\
122.97 \\
125.82 \\
125.60 \\
127.84 \\
130.19 \\
132.53 \\
135.33 \\
134.42 \\
138.35 \\
140.16 \\
142.63 \\
145.78 \\
142.52 \\
0.22 \\
130\end{array}$ & $\begin{array}{c}\text { \% Strain } \\
\text {-0.0001 } \\
0.0046 \\
0.0091 \\
0.0139 \\
0.0185 \\
0.0213 \\
0.0242 \\
0.0278 \\
0.0309 \\
0.0336 \\
0.0387 \\
0.0405 \\
0.0426 \\
0.0446 \\
0.0475 \\
0.0487 \\
0.0505 \\
0.0516 \\
0.0534 \\
0.0547 \\
0.0571 \\
0.0591 \\
0.0615 \\
0.0617 \\
0.0631 \\
0.0633 \\
0.0644 \\
0.0642 \\
0.0634 \\
0.0636 \\
0.0636 \\
0.0655 \\
0.0672 \\
0.0715 \\
0.0769 \\
0.0760 \\
-0.0023\end{array}$ \\
\hline
\end{tabular}




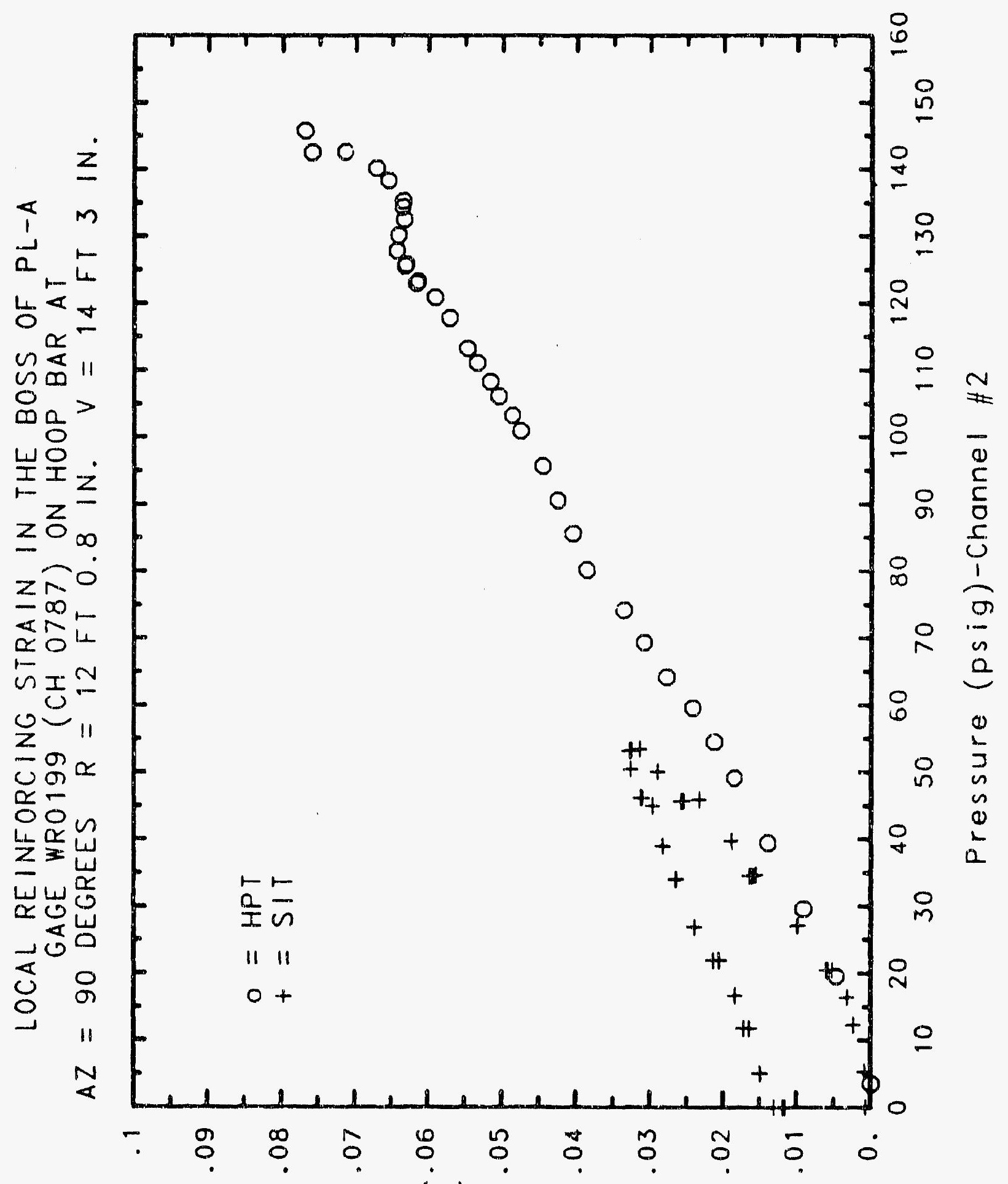

(\%) $4 ! 0+15$ 


\section{Reinforced Concrete Test Data}

Weldable gacie 200 Channel 788

Structural Integrity TEST

\begin{tabular}{c} 
Pressure \\
(psig) \\
-0.05 \\
5.33 \\
12.31 \\
16.44 \\
20.51 \\
20.46 \\
20.47 \\
27.05 \\
34.69 \\
34.53 \\
34.53 \\
39.70 \\
45.90 \\
45.65 \\
45.62 \\
50.10 \\
53.47 \\
53.29 \\
53.21 \\
50.49 \\
46.14 \\
46.13 \\
44.97 \\
38.98 \\
33.99 \\
33.96 \\
26.83 \\
21.88 \\
21.90 \\
16.69 \\
11.74 \\
11.77 \\
5.05 \\
0.02 \\
-0.04 \\
-0.02 \\
-0.02 \\
0.02 \\
\\
\hline
\end{tabular}

\% Strain

0.0000

0.0043

0.0095

0.0130

0.0181

0.0189

0.0199

0.0304

0.0457

0.0477

0.0478

0.0551

0.0665

0.0670

0.0665

0.0737

0.0798

0.0801

0.0800

0.0782

0.0728

0.0724

0.0725

0.0660

0.0600

0.0596

0.0510

0.0441

0.0438

0.0371

0.0300

0.0289

0.0197

0.0137

0.0113

0.0113

0.0116

0.0121
High Pressure test

Pressure

* Strain

9.89

19.55

29.57

39.42

49.16

54.50

59.57

64.20

69.32

74.16

80.16

85.61

90.58

95.69

100.92

103.25

106.11

108.31

111.08

113.24

117.83

120.92

123.28

122.97

125.82

125.60

127.84

130.19

132.53

135.33

134.42

138.35

140.16

142.63

145.78

142.52

0.22
0.0091

0.0237

0.0386

0.0516

0.0654

0.0733

0.0809

0.0892

0.0978

0.1080

0.1193

0.1317

0.1438

0.1576

0.1740

0.1819

0.1912

0.1986

0.2100

0.2189

0.2437

0.2646

0.2850

0.2895

0.3156

0.3226

0.3407

0.4614

0.6263

0.8592

0.9072

1.1735

1.3537

1.5858

1.7681

1.8159

1.4445 


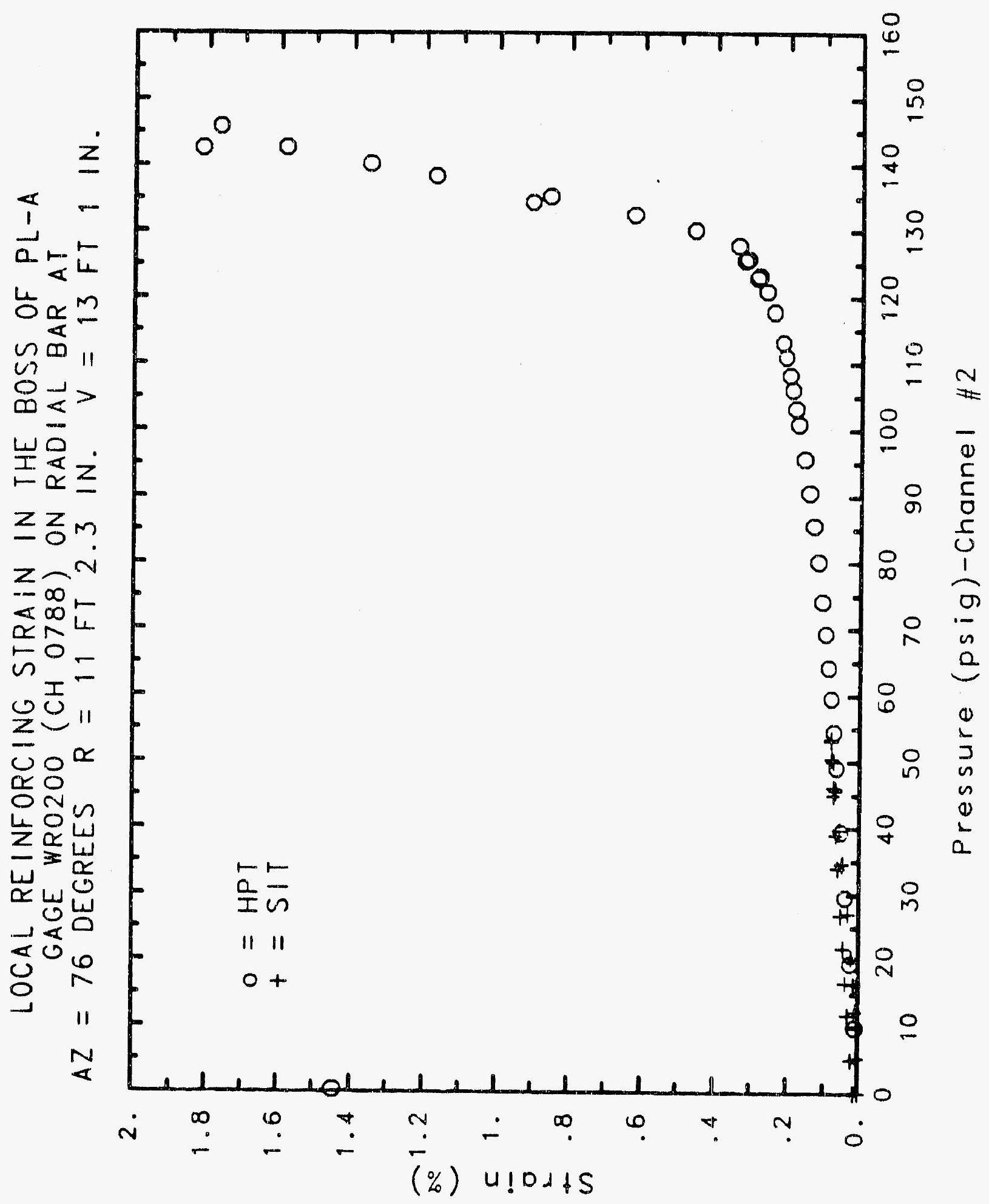




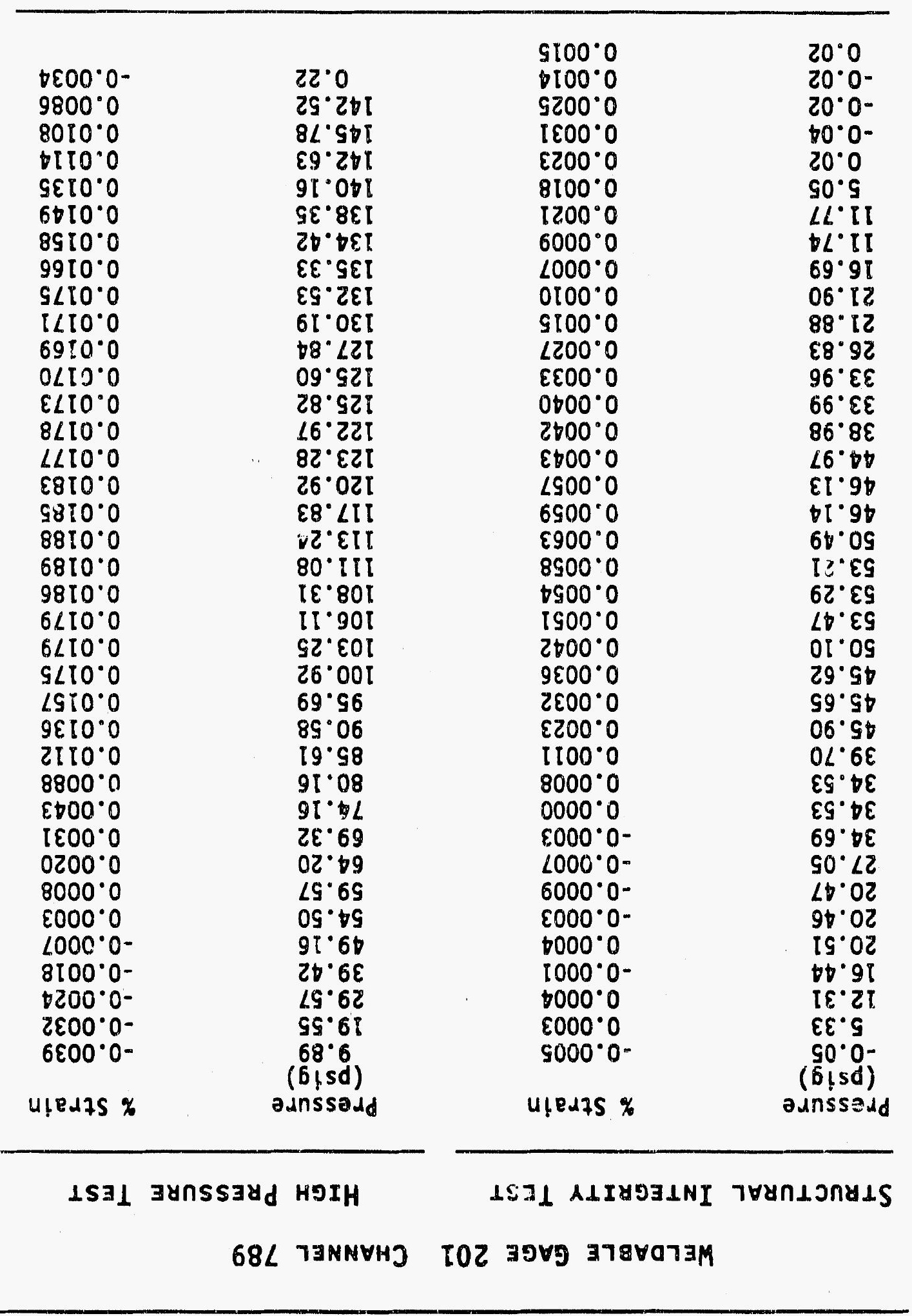

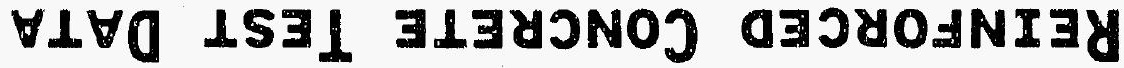




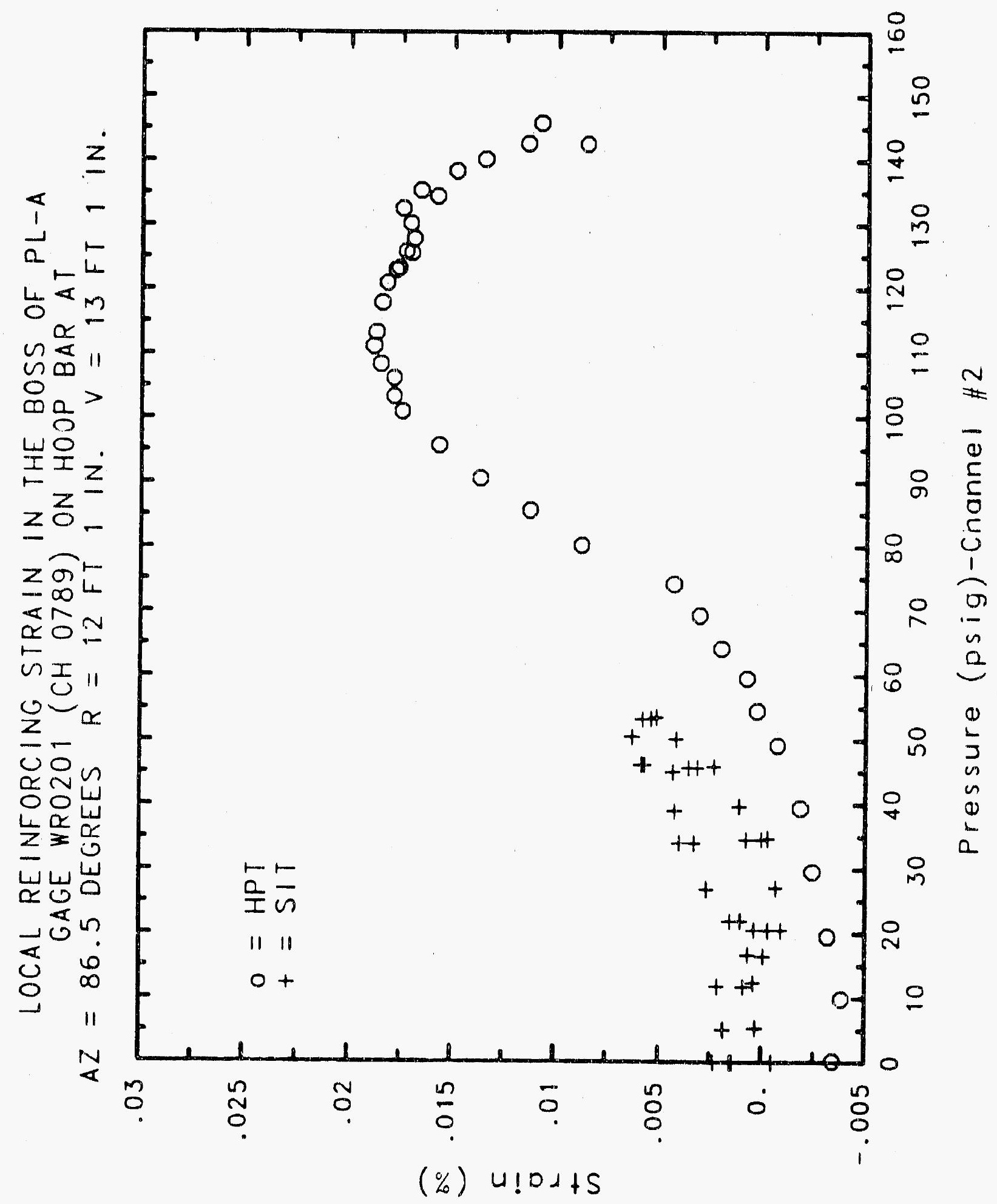




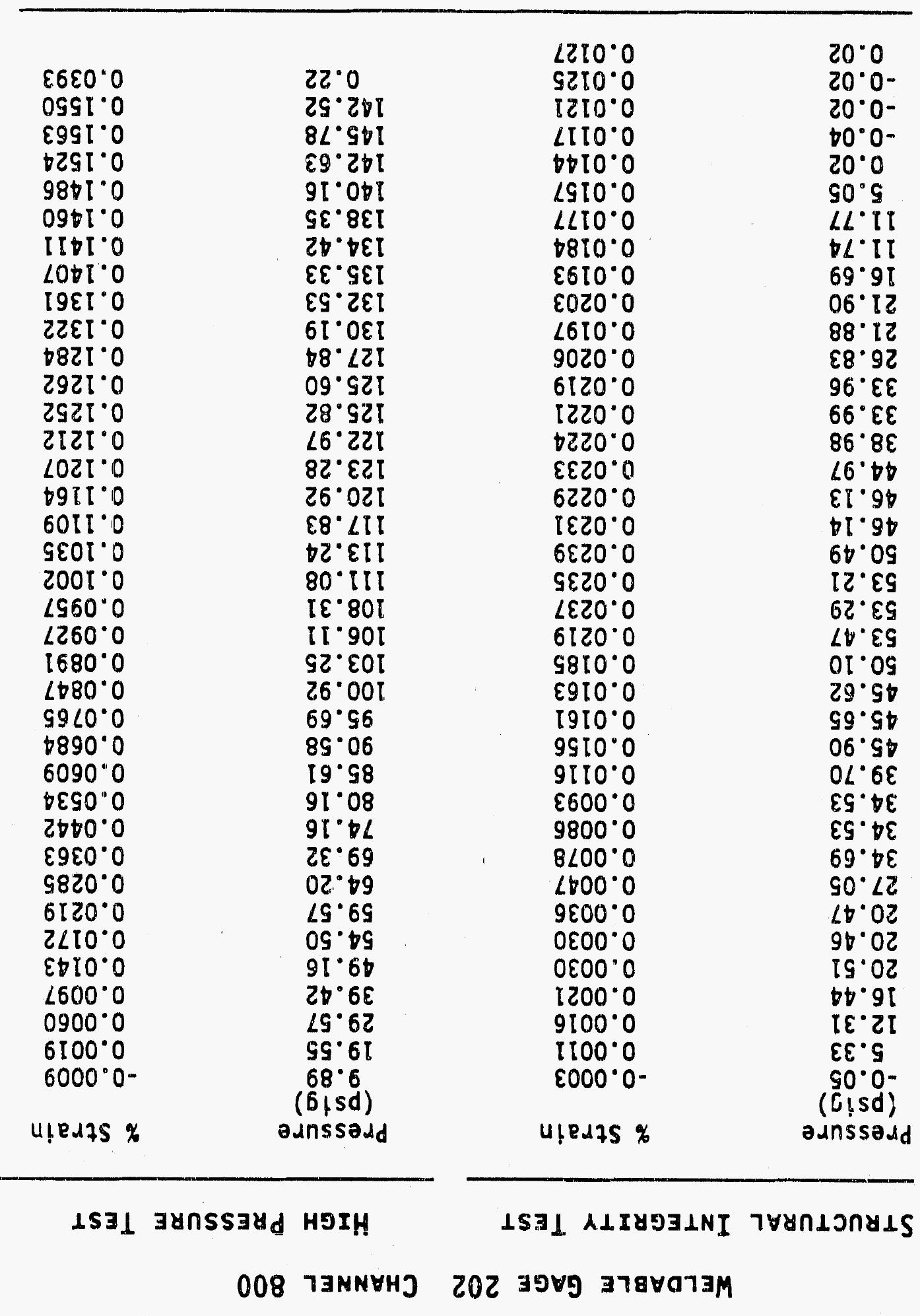

\section{$\forall \perp \forall 0$ LISI ILJYJNOJ dJJYOANIJY}




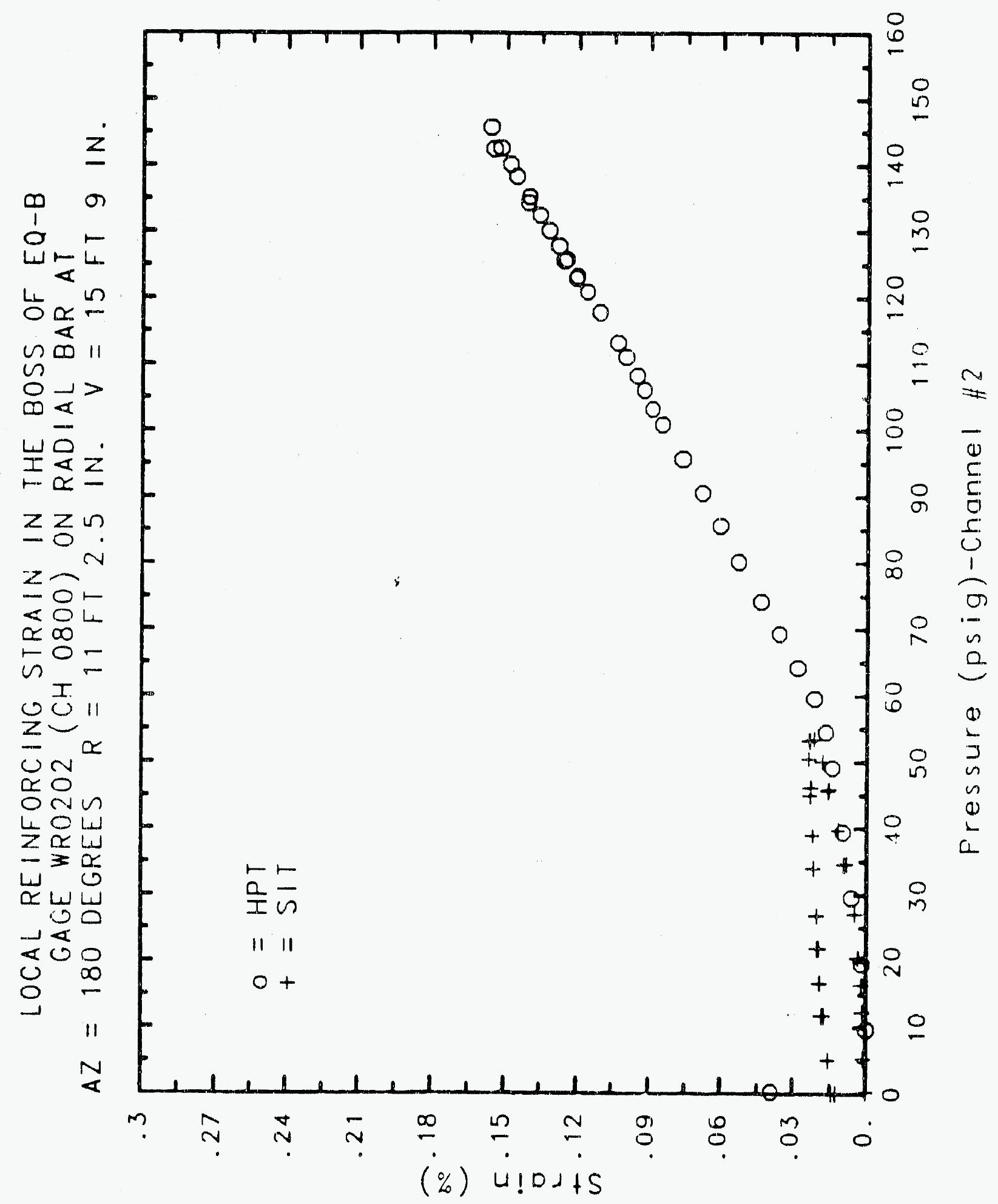


Reinforced Concrete Test Data

'weldable Gage 203 Channel 801

STRUCTURAL INTEGRITY TEST

\begin{tabular}{|c|c|c|c|}
\hline $\begin{array}{c}\text { Pressure } \\
\text { (psig) } \\
-0.05 \\
5.33 \\
12.31 \\
16.44 \\
20.51 \\
20.46 \\
20.47 \\
27.05 \\
34.69 \\
34.53 \\
34.53 \\
39.70 \\
45.90 \\
45.65 \\
45.62 \\
50.10 \\
53.47 \\
53.29 \\
53.21 \\
50.49 \\
46.14 \\
46.13 \\
44.97 \\
38.98 \\
33.99 \\
33.96 \\
26.83 \\
21.88 \\
21.90 \\
16.69 \\
11.74 \\
11.77 \\
5.05 \\
0.02 \\
-0.04 \\
-0.02 \\
-0.02 \\
0.02 \\
\end{array}$ & $\begin{array}{l}\text { \% Strain } \\
\text {-0.0003 } \\
0.0015 \\
0.0023 \\
0.0039 \\
0.0055 \\
0.0055 \\
0.0061 \\
0.0096 \\
0.0154 \\
0.0174 \\
0.0182 \\
0.0215 \\
0.0263 \\
0.0261 \\
0.0264 \\
0.0290 \\
0.0311 \\
0.0319 \\
0.0310 \\
0.0305 \\
0.0296 \\
0.0290 \\
0.0287 \\
0.0270 \\
0.0252 \\
0.0251 \\
0.0225 \\
0.0203 \\
0.0210 \\
0.0187 \\
0.0161 \\
0.0156 \\
0.0126 \\
0.0100 \\
0.0080 \\
0.0085 \\
0.0090 \\
0.0098\end{array}$ & $\begin{array}{c}\text { Pressure } \\
(\text { psig) } \\
9.89 \\
19.55 \\
29.57 \\
39.42 \\
49.16 \\
54.50 \\
59.57 \\
64.20 \\
69.32 \\
74.16 \\
80.16 \\
85.61 \\
90.58 \\
95.69 \\
100.92 \\
103.25 \\
106.11 \\
108.31 \\
111.08 \\
113.24 \\
117.83 \\
120.92 \\
123.28 \\
122.97 \\
125.82 \\
125.60 \\
127.84 \\
130.19 \\
132.53 \\
135.33 \\
134.42 \\
138.35 \\
140.16 \\
142.63 \\
145.78 \\
142.52 \\
0.22 \\
\end{array}$ & $\begin{array}{l}0.0008 \\
0.0058 \\
0.0105 \\
0.0156 \\
0.0198 \\
0.0225 \\
0.0249 \\
0.0279 \\
0.0309 \\
0.0351 \\
0.0405 \\
0.0425 \\
0.0434 \\
0.0441 \\
0.0446 \\
0.0443 \\
0.0448 \\
0.0453 \\
0.0444 \\
0.0424 \\
0.0428 \\
0.0441 \\
0.0443 \\
0.0451 \\
0.0477 \\
0.0482 \\
0.0496 \\
0.0531 \\
0.0558 \\
0.0587 \\
0.0588 \\
0.0620 \\
0.0630 \\
0.0648 \\
0.0670 \\
0.0644 \\
-0.0075\end{array}$ \\
\hline
\end{tabular}




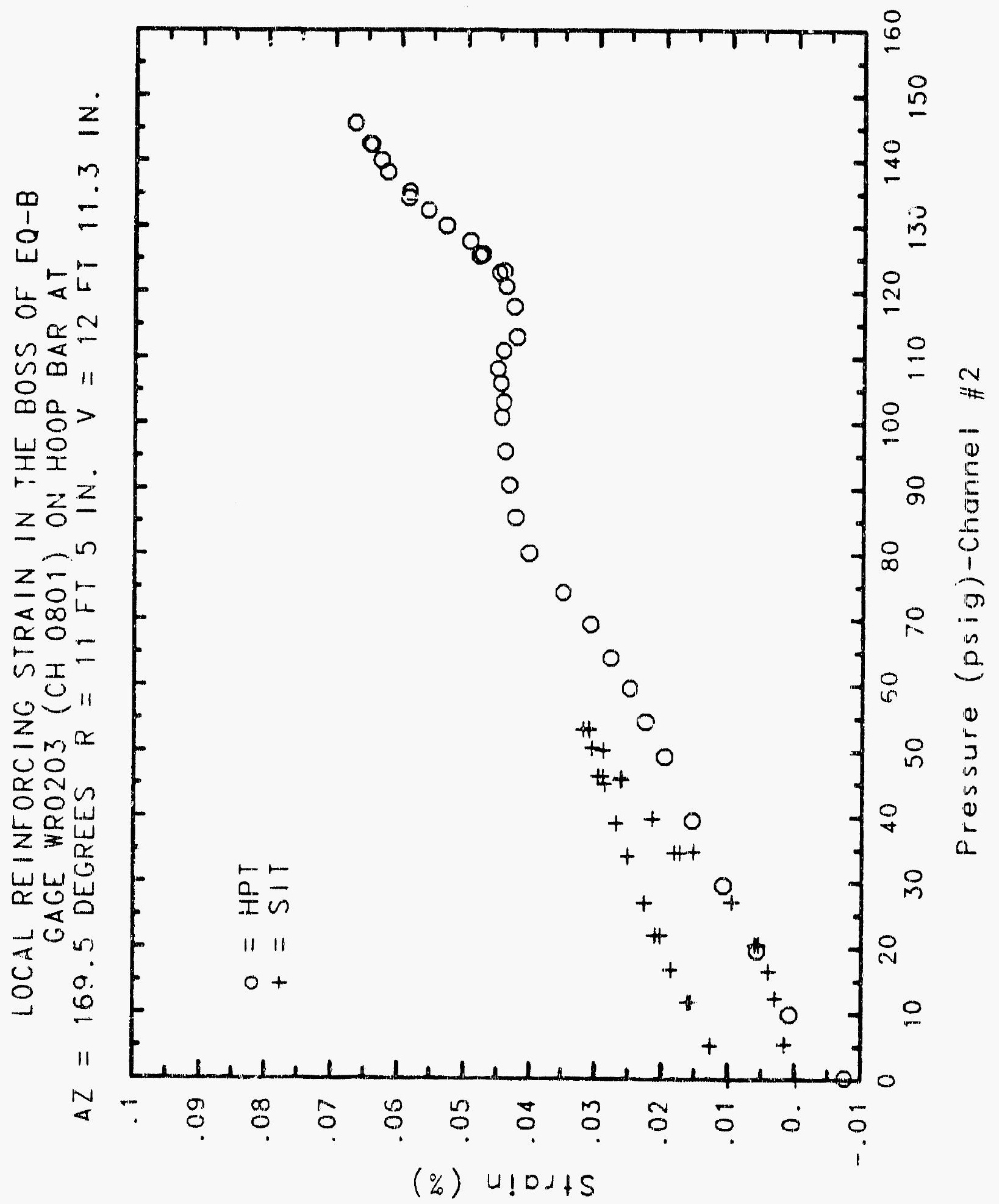




\section{Reinforced Concrete Test Data}

Weldable Gage 204 Channel 802

STRUCTURAL INTEGRITY TEST

High Pressure test

\begin{tabular}{|c|c|c|c|}
\hline $\begin{array}{c}\text { Pressure } \\
\text { (psig) } \\
-0.05 \\
5.33 \\
12.31 \\
16.44 \\
20.51 \\
20.46 \\
20.47 \\
27.05 \\
34.69 \\
34.53 \\
34.53 \\
39.70 \\
45.90 \\
45.65 \\
45.62 \\
50.10 \\
53.47 \\
53.29 \\
53.21 \\
50.49 \\
46.14 \\
46.13 \\
44.97 \\
38.98 \\
33.99 \\
33.96 \\
26.83 \\
21.88 \\
21.90 \\
16.69 \\
11.74 \\
11.77 \\
5.05 \\
0.02 \\
-0.04 \\
-0.02 \\
-0.02 \\
0.02 \\
\end{array}$ & $\begin{array}{l}\text { \% Strain } \\
\text {-0.0003 } \\
0.0002 \\
0.0003 \\
0.0005 \\
0.0016 \\
0.0021 \\
0.0024 \\
0.0040 \\
0.0071 \\
0.0062 \\
0.0063 \\
0.0085 \\
0.0125 \\
0.0159 \\
0.0171 \\
0.0198 \\
0.0234 \\
0.0255 \\
0.0261 \\
0.0259 \\
0.0258 \\
0.0258 \\
0.0246 \\
0.0238 \\
0.0234 \\
0.0231 \\
0.0226 \\
0.0208 \\
0.0204 \\
0.0192 \\
0.0182 \\
0.0196 \\
0.0189 \\
0.0189 \\
0.0174 \\
0.0170 \\
0.0171 \\
0.0171\end{array}$ & $\begin{array}{c}\text { Pressure } \\
\text { (psig) } \\
9.89 \\
19.55 \\
29.57 \\
39.42 \\
49.16 \\
54.50 \\
59.57 \\
64.20 \\
69.32 \\
74.16 \\
80.16 \\
85.61 \\
90.58 \\
95.69 \\
100.92 \\
103.25 \\
106.11 \\
108.31 \\
111.08 \\
113.24 \\
117.83 \\
120.92 \\
123.28 \\
122.97 \\
125.82 \\
125.60 \\
127.84 \\
130.19 \\
132.53 \\
135.33 \\
134.42 \\
138.35 \\
140.16 \\
142.63 \\
145.78 \\
142.52 \\
0.22 \\
\end{array}$ & $\begin{array}{c}\% \text { Strain } \\
-0.0016 \\
0.0006 \\
0.0036 \\
0.0067 \\
0.0102 \\
0.0123 \\
0.0166 \\
0.0232 \\
0.0297 \\
0.0363 \\
0.0462 \\
0.0515 \\
0.0563 \\
0.0606 \\
0.0651 \\
0.0671 \\
0.0 r 77 \\
0.0712 \\
0.0735 \\
0.0751 \\
0.0779 \\
0.0791 \\
0.0800 \\
0.0794 \\
0.0807 \\
0.0804 \\
0.0820 \\
0.0831 \\
0.0845 \\
0.0857 \\
0.0850 \\
0.0863 \\
0.0861 \\
0.0860 \\
0.0858 \\
0.0841 \\
0.0152\end{array}$ \\
\hline
\end{tabular}




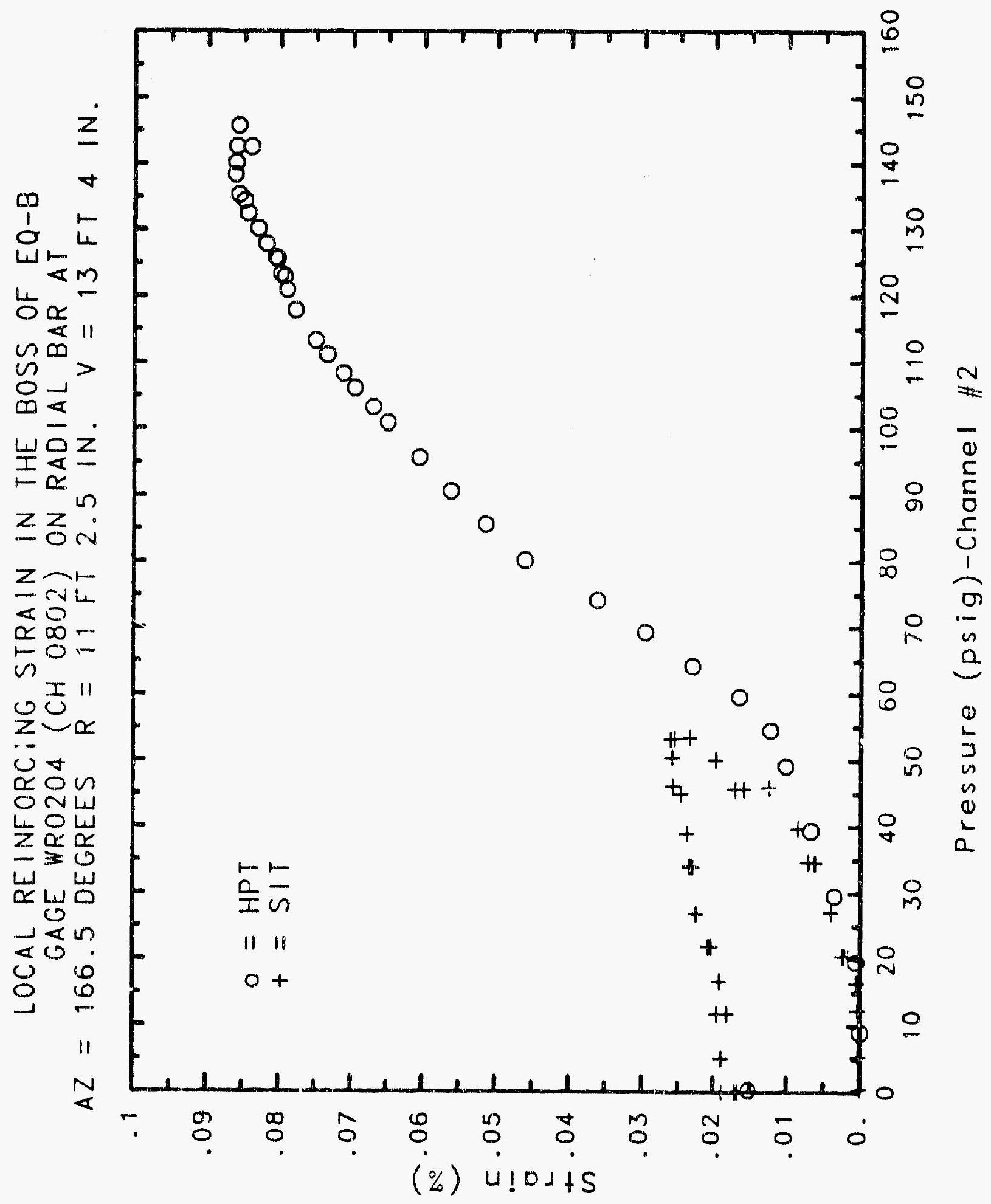




\section{Reinforced Concrete Test Data}

Weldable Gage 205 Channel 803

Structural Integryty Test

\begin{tabular}{|c|c|c|c|}
\hline STRUCTURAL & $\begin{array}{l}\text { WELDABLE GAGE } 205 \\
\text { INTEGRITY TEST }\end{array}$ & $\begin{array}{r}\text { Channel } 803 \\
\text { High Pressure }\end{array}$ & TEST \\
\hline $\begin{array}{c}\text { Pressure } \\
\text { (psig) } \\
-0.05 \\
5.33 \\
12.31 \\
16.44 \\
20.51 \\
20.46 \\
20.47 \\
27.05 \\
34.69 \\
34.53 \\
34.53 \\
39.70 \\
45.90 \\
45.65 \\
45.62 \\
50.10 \\
53.47 \\
53.29 \\
53.21 \\
50.49 \\
46.14 \\
46.13 \\
44.97 \\
38.98 \\
33.99 \\
33.96 \\
26.83 \\
21.88 \\
21.90 \\
16.69 \\
11.74 \\
11.77 \\
5.05 \\
0.02 \\
-0.04 \\
-0.02 \\
-0.02 \\
0.02 \\
\end{array}$ & $\begin{array}{l}\text { \% Strain } \\
\text {-0.0003 } \\
0.0003 \\
0.0010 \\
0.0015 \\
0.0022 \\
0.0024 \\
0.0025 \\
0.0034 \\
0.0049 \\
0.0056 \\
0.0063 \\
0.0083 \\
0.0127 \\
0.0136 \\
0.0141 \\
0.0160 \\
0.0197 \\
0.0209 \\
0.0213 \\
0.0211 \\
0.0203 \\
0.0207 \\
0.0208 \\
0.0198 \\
0.0191 \\
0.0189 \\
0.0180 \\
0.0175 \\
0.0176 \\
0.0166 \\
0.0154 \\
0.0149 \\
0.0132 \\
0.0116 \\
0.0092 \\
0.0094 \\
0.0097 \\
0.0093\end{array}$ & $\begin{array}{c}\text { Pressure } \\
(\text { psig) } \\
9.89 \\
19.55 \\
29.57 \\
39.42 \\
49.16 \\
54.50 \\
59.57 \\
64.20 \\
69.32 \\
74.16 \\
80.16 \\
85.61 \\
90.58 \\
95.69 \\
100.92 \\
103.25 \\
106.11 \\
108.31 \\
111.08 \\
113.24 \\
117.83 \\
120.92 \\
123.28 \\
122.97 \\
125.82 \\
125.60 \\
127.84 \\
130.19 \\
132.53 \\
135.33 \\
134.42 \\
138.35 \\
140.16 \\
142.63 \\
145.78 \\
142.52 \\
0.22 \\
\end{array}$ & $\begin{array}{c}\text { \% strain } \\
-0.0009 \\
0.0025 \\
0.0058 \\
0.0087 \\
0.0119 \\
0.0142 \\
0.0164 \\
0.0203 \\
0.0251 \\
0.0315 \\
0.0406 \\
0.0486 \\
0.0566 \\
0.0657 \\
0.0760 \\
0.0815 \\
0.0864 \\
0.0901 \\
0.0957 \\
0.1004 \\
0.1097 \\
0.1167 \\
0.1229 \\
0.1245 \\
0.1296 \\
0.1317 \\
0.1346 \\
0.1409 \\
0.1472 \\
0.1556 \\
0.1578 \\
0.1675 \\
0.1747 \\
0.1817 \\
0.1883 \\
0.1877 \\
0.0412\end{array}$ \\
\hline
\end{tabular}

High Pressure Test

Pres 


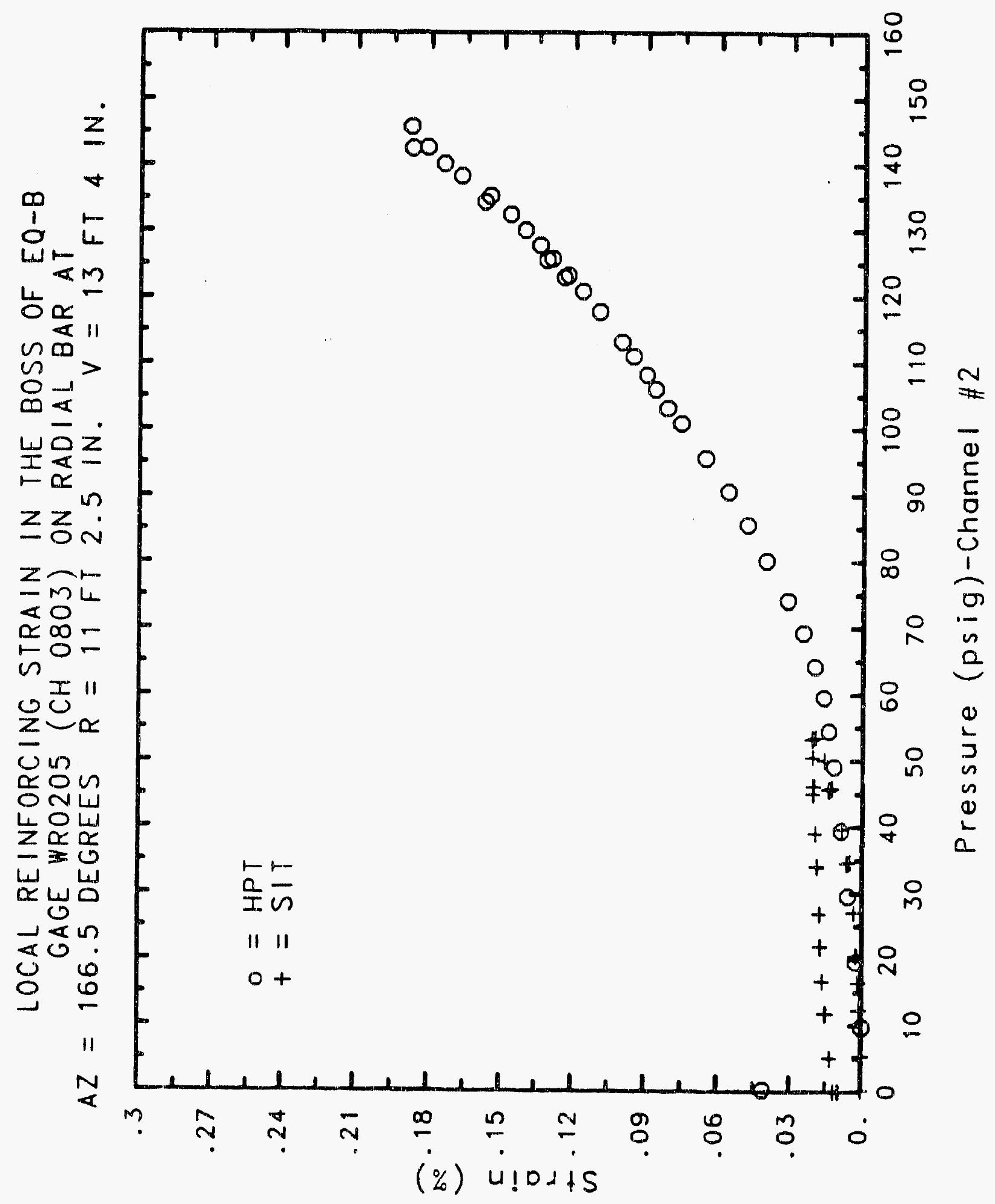




\section{Reinforced Concrete Test Data}

Weldable Gage 206 Channel 804

Structural Integrity Test

\begin{tabular}{|c|c|}
\hline $\begin{array}{c}\text { Pressure } \\
\text { (psig) } \\
-0.05 \\
5.33 \\
12.31 \\
16.44 \\
20.51 \\
20.46 \\
20.47 \\
27.05 \\
34.69 \\
34.53 \\
34.53 \\
39.70 \\
45.90 \\
45.65 \\
45.62 \\
50.10 \\
53.47 \\
53.29 \\
53.21 \\
50.49 \\
46.14 \\
46.13 \\
44.97 \\
38.98 \\
33.99 \\
33.96 \\
26.83 \\
21.88 \\
21.90 \\
16.69 \\
11.74 \\
11.77 \\
5.05 \\
0.02 \\
-0.04 \\
-0.02 \\
-0.02 \\
0.02 \\
\end{array}$ & $\begin{array}{l}\text { X Strain } \\
\text {-0.0004 } \\
0.0019 \\
0.0055 \\
0.0091 \\
0.0161 \\
0.0174 \\
0.0181 \\
0.0291 \\
0.0419 \\
0.0422 \\
0.0420 \\
0.0474 \\
0.0548 \\
0.0539 \\
0.0542 \\
0.0596 \\
0.0640 \\
0.0638 \\
0.0637 \\
0.0611 \\
0.0572 \\
0.0570 \\
0.0563 \\
0.0502 \\
0.0446 \\
0.0447 \\
0.0365 \\
0.0311 \\
0.0307 \\
0.0247 \\
0.0181 \\
0.0169 \\
0.0092 \\
0.0040 \\
0.0033 \\
0.0043 \\
0.0044 \\
0.0045\end{array}$ \\
\hline
\end{tabular}

high Pressure test

Pressure

(psig)

9.89

19.55

29.57

39.42

49.16

54.50

59.57

64.20

69.32

74.16

80.16

85.61

90.58

95.69

100.92

103.25

106.11

108.31

111.08

113.24

117.83

120.92

123.28

122.97

125.82

125.60

127.84

130.19

132.53

135.33

134.42

138.35

140.16

142.63

145.78

142.52

0.22
* Strain

0.0066

0.0181

0.0307

0.0434

0.0541

0.0534

$0.05 \% 6$

0.0472

0.0477

0.0504

0.0546

0.0594

0.0631

0.0678

0.0729

0.0751

0.0776

0.0797

0.0824

0.0843

0.0891

0.0925

0.0955

0.0954

0.0984

0.0988

0.1014

0.1048

0.1089

0.1143

0.1144

0.1198

0.1237

0.1282

0.1323

0.1307

0.0219 


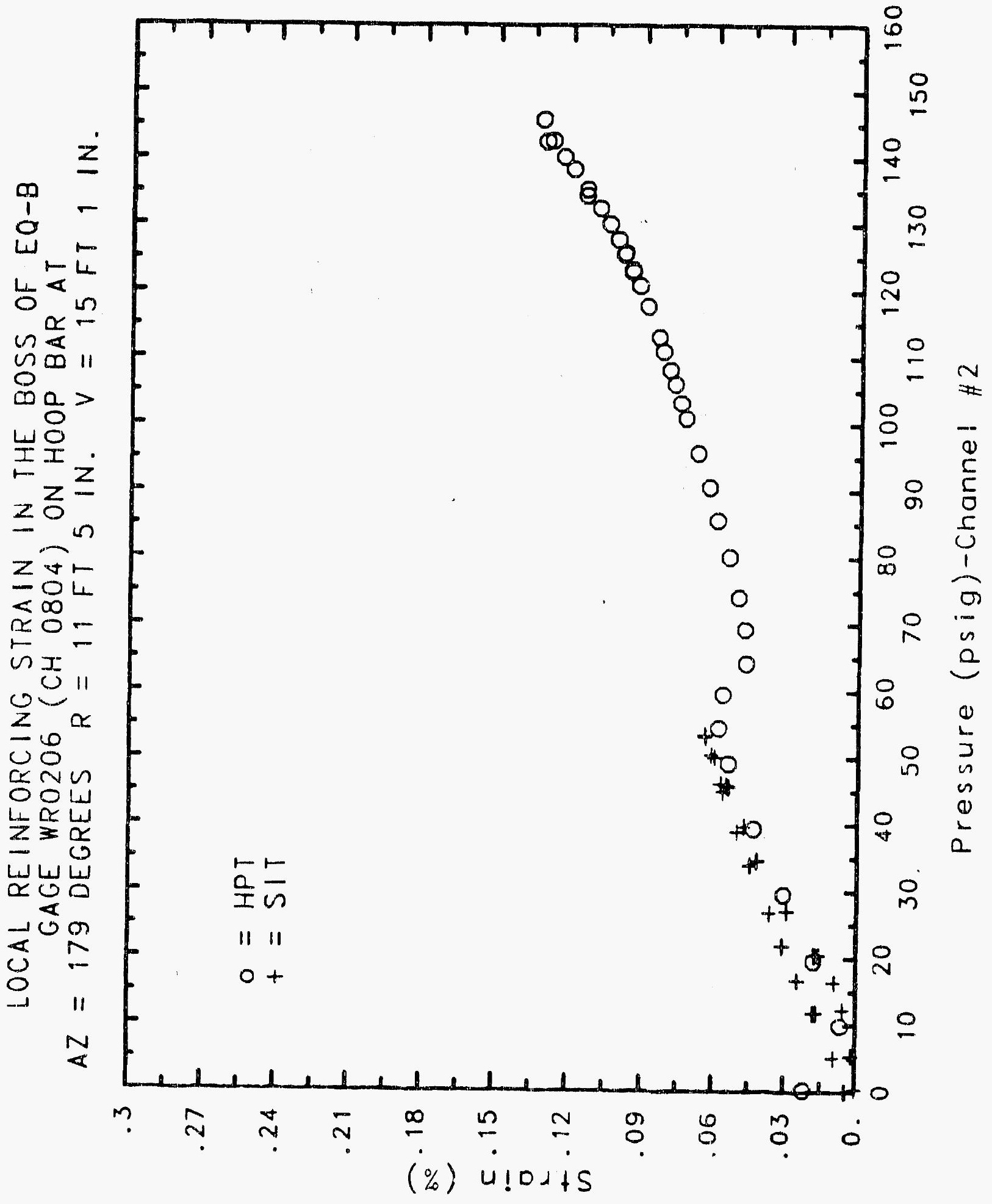




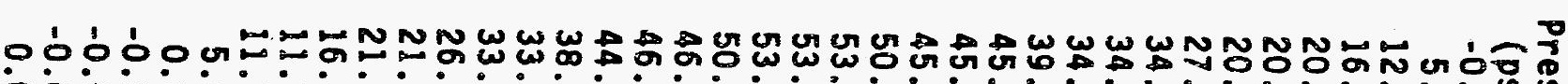

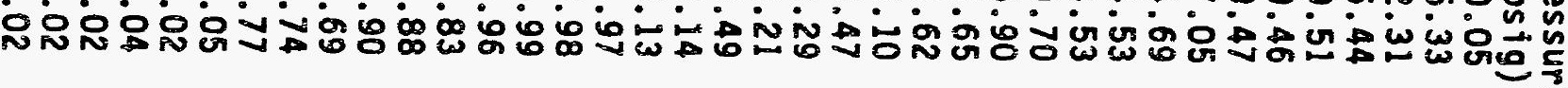

.00000000000000000000000000000000000000

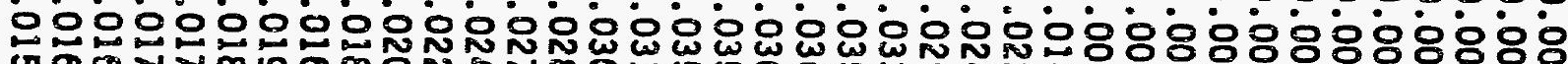

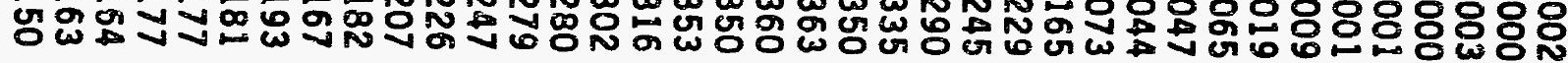




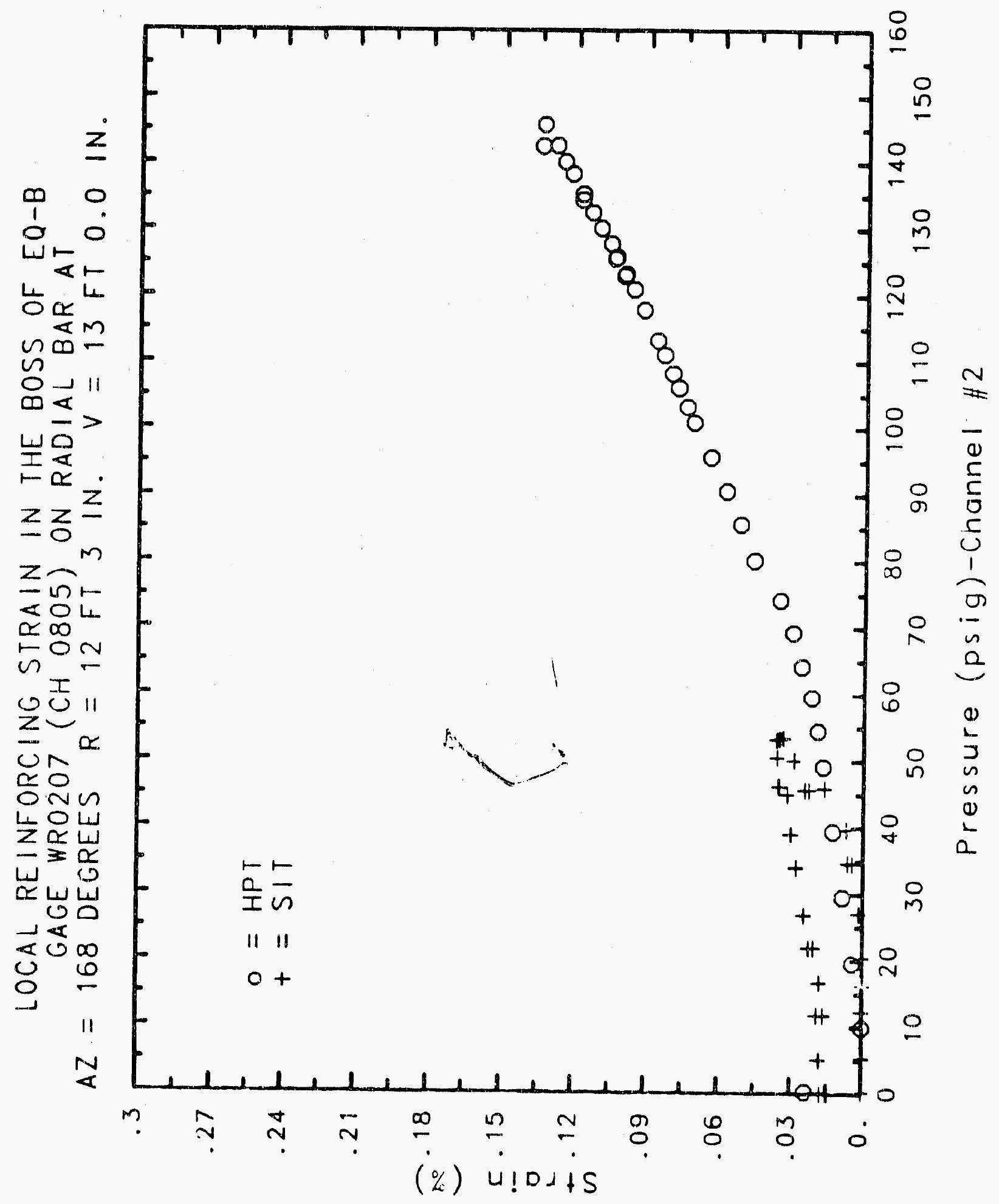




\section{Reinforced Concrete Test Data}

Weldable gage 208 Channel 806

Structural Integrity test

High Pressure test

\begin{tabular}{|c|c|c|c|}
\hline $\begin{array}{c}\text { Pressure } \\
\text { (psig) } \\
-0.05 \\
5.33 \\
12.31 \\
16.44 \\
20.51 \\
20.46 \\
20.47 \\
27.05 \\
34.69 \\
34.53 \\
34.53 \\
39.70 \\
45.90 \\
45.65 \\
45.62 \\
50.10 \\
53.47 \\
53.29 \\
53.21 \\
50.49 \\
46.14 \\
46.13 \\
44.97 \\
38.98 \\
33.99 \\
33.96 \\
26.83 \\
21.88 \\
21.90 \\
16.69 \\
11.74 \\
11.77 \\
5.05 \\
0.02 \\
-0.04 \\
-0.02 \\
-0.02 \\
0.02 \\
\end{array}$ & $\begin{array}{l}\text { \% Strain } \\
0.0006 \\
0.0008 \\
0.0020 \\
0.0030 \\
0.0046 \\
0.0052 \\
0.0052 \\
0.0107 \\
0.0204 \\
0.0218 \\
0.0218 \\
0.0274 \\
0.0365 \\
0.0386 \\
0.0383 \\
0.0435 \\
0.0480 \\
0.0491 \\
0.0496 \\
0.0480 \\
0.0458 \\
0.0461 \\
0.0460 \\
0.0435 \\
0.0399 \\
0.0399 \\
0.0358 \\
0.0326 \\
0.0323 \\
0.0291 \\
0.0258 \\
0.0251 \\
0.0213 \\
0.0175 \\
0.0157 \\
0.0160 \\
0.0161 \\
0.0160\end{array}$ & $\begin{array}{c}\text { Pressure } \\
(\text { psig) } \\
9.89 \\
19.55 \\
29.57 \\
39.42 \\
49.16 \\
54.50 \\
59.57 \\
64.20 \\
69.32 \\
74.16 \\
80.16 \\
85.61 \\
90.58 \\
95.69 \\
100.92 \\
103.25 \\
106.11 \\
108.31 \\
111.08 \\
113.24 \\
117.83 \\
120.92 \\
123.28 \\
122.97 \\
125.82 \\
125.60 \\
127.84 \\
130.19 \\
132.53 \\
135.33 \\
134.42 \\
138.35 \\
140.16 \\
142.63 \\
145.78 \\
142.52 \\
0.22 \\
\end{array}$ & $\begin{array}{l}\text { \% Strain } \\
0.0036 \\
0.0109 \\
0.0188 \\
0.0268 \\
0.0346 \\
0.0390 \\
0.0443 \\
0.0501 \\
0.0567 \\
0.0642 \\
0.0722 \\
0.0802 \\
0.0881 \\
0.0971 \\
0.1066 \\
0.1109 \\
0.1161 \\
0.1204 \\
0.1266 \\
0.1315 \\
0.1428 \\
0.1514 \\
0.1599 \\
0.1621 \\
0.1722 \\
0.1751 \\
0.1810 \\
0.1928 \\
0.1993 \\
0.2062 \\
0.2057 \\
0.2123 \\
0.2142 \\
0.2182 \\
0.2223 \\
0.2188 \\
0.0616\end{array}$ \\
\hline
\end{tabular}




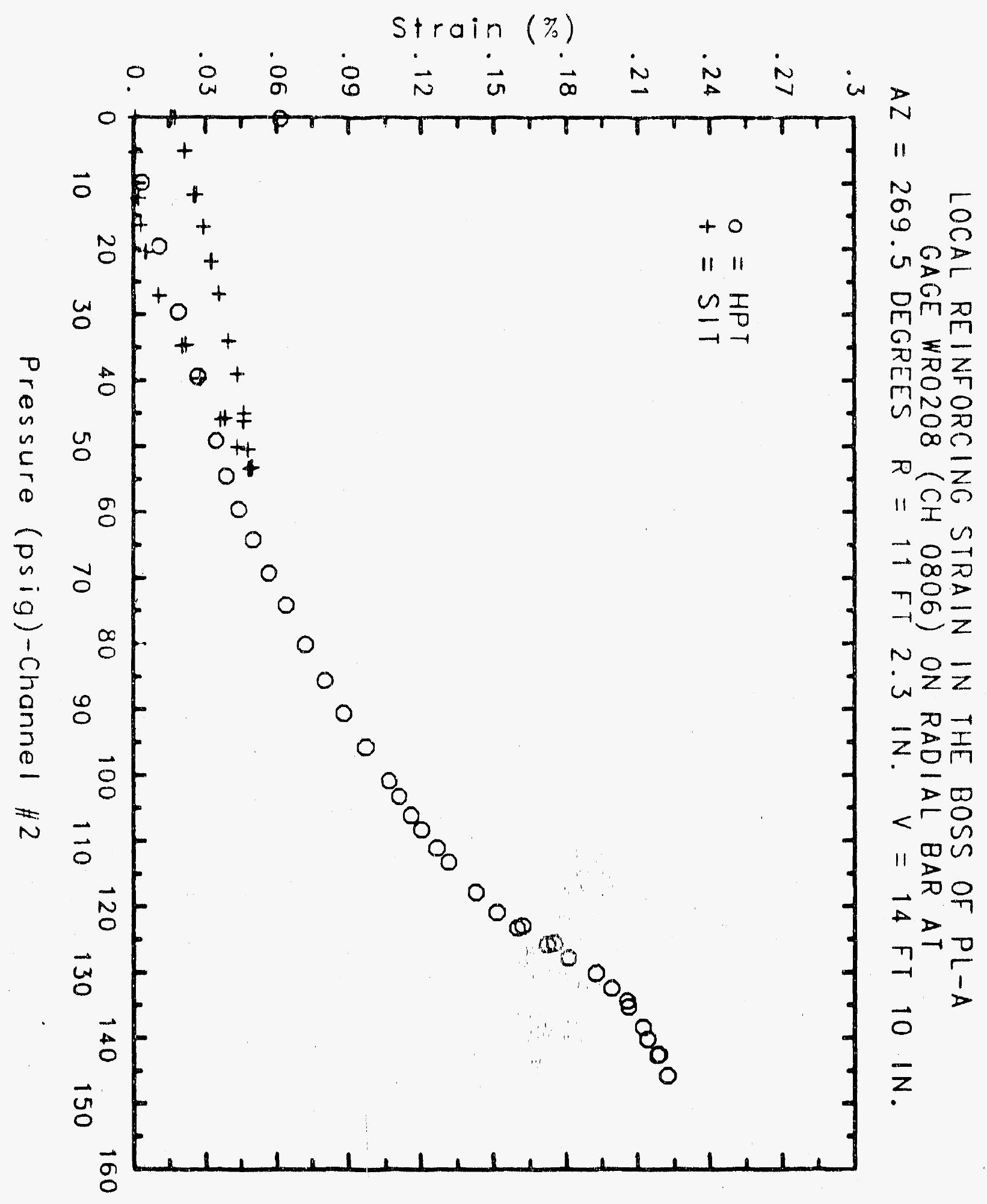




\section{Reinforced Concrete Test Data}

Weldable Gage 209 Chanmel 807

Structural. Integrity Test

High Pressure Test

\begin{tabular}{rc}
\hline Pressure & \\
(psig) & \% strain \\
-0.05 & \\
5.33 & -0.0016 \\
12.31 & 0.0010 \\
16.44 & 0.0035 \\
20.51 & 0.0048 \\
20.46 & 0.0074 \\
20.47 & 0.0086 \\
27.05 & 0.0086 \\
34.69 & 0.0151 \\
34.53 & 0.0230 \\
34.53 & 0.0240 \\
39.70 & 0.0242 \\
45.90 & 0.0282 \\
45.65 & 0.0333 \\
45.62 & 0.0345 \\
50.10 & 0.0348 \\
53.47 & 0.0375 \\
53.29 & 0.0398 \\
53.21 & 0.0402 \\
50.49 & 0.0400 \\
46.14 & 0.0394 \\
46.13 & 0.0372 \\
44.97 & 0.0371 \\
38.98 & 0.0348 \\
33.99 & 0.0320 \\
33.96 & 0.0303 \\
26.83 & 0.0301 \\
21.88 & 0.0264 \\
21.90 & 0.0243 \\
16.69 & 0.0239 \\
11.74 & 0.0215 \\
11.77 & 0.0193 \\
5.05 & 0.0189 \\
0.02 & 0.0155 \\
-0.04 & 0.0133 \\
-0.02 & 0.0099 \\
-0.02 & 0.0093 \\
0.02 & 0.0107 \\
& 0.0106 \\
& \\
\hline 5 &
\end{tabular}

Pressure

(psig)

9.89

19.55

29.57

39.42

49.16

54.50

59.57

64.20

69.32

74.16

80.16

85.61

90.58

95.69

100.92

103.25

106.11

108.31

111.08

113.24

117.83

120.92

123.28

122.97

125.82

125.60

127.84

130.19

132.53

135.33

134.42

138.35

140.16

142.63

145.78

142.52

0.22
* Strain

0.0010

0.0068

0.0127

0.0191

0.0248

0.0286

0.0321

0.0421

0.0519

0.0584

0.0655

0.0698

0.0732

0.0774

0.0820

0.0837

0.0865

0.0882

0.0904

0.0919

0.0952

0.0976

$0.099 \varepsilon$

0.0993

0.1017

0.1013

0.1031

0.1049

0.1063

0.1089

0.1103

0.1148

0.1200

0.1259

0.1339

0.1357

0.0202 


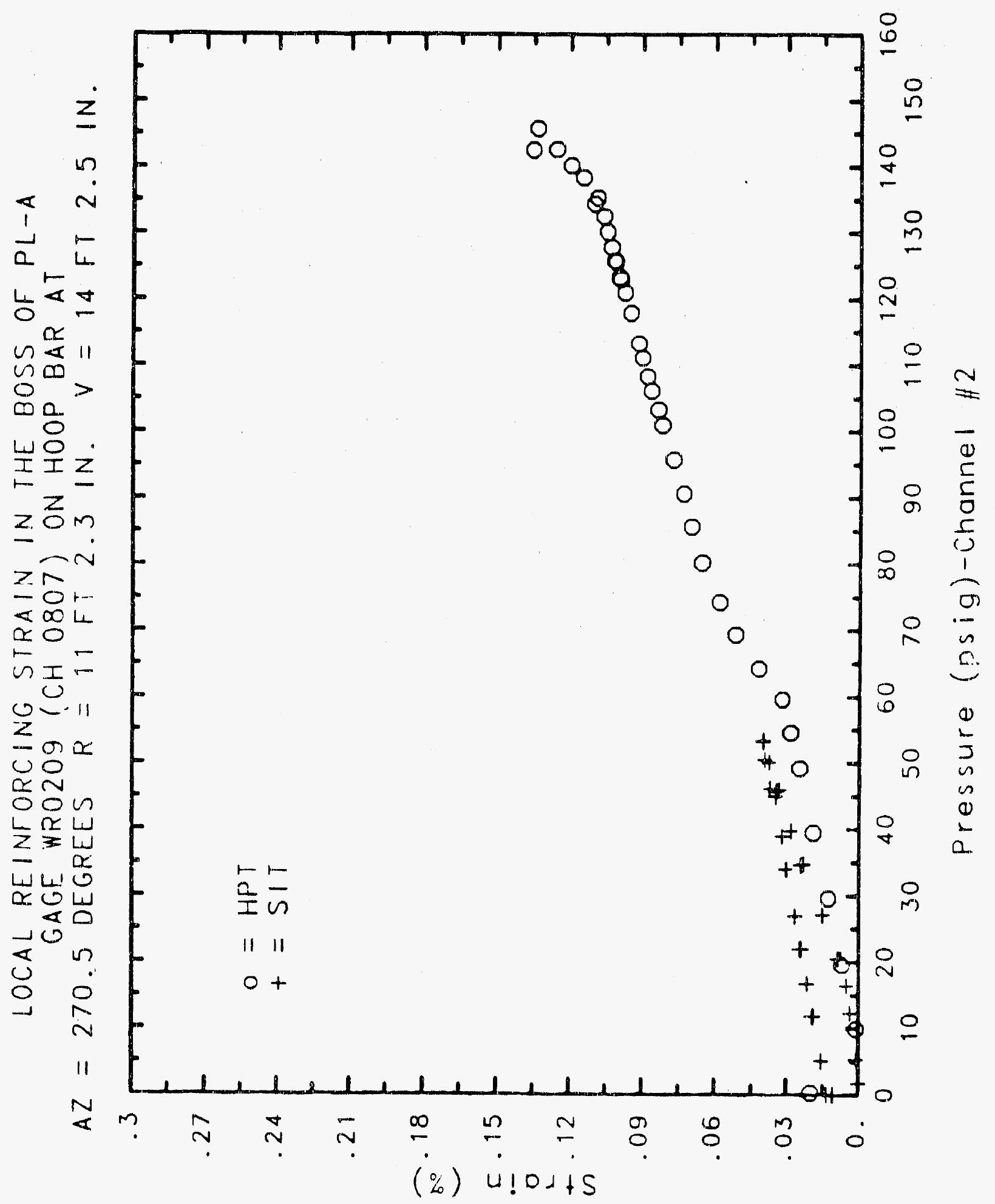




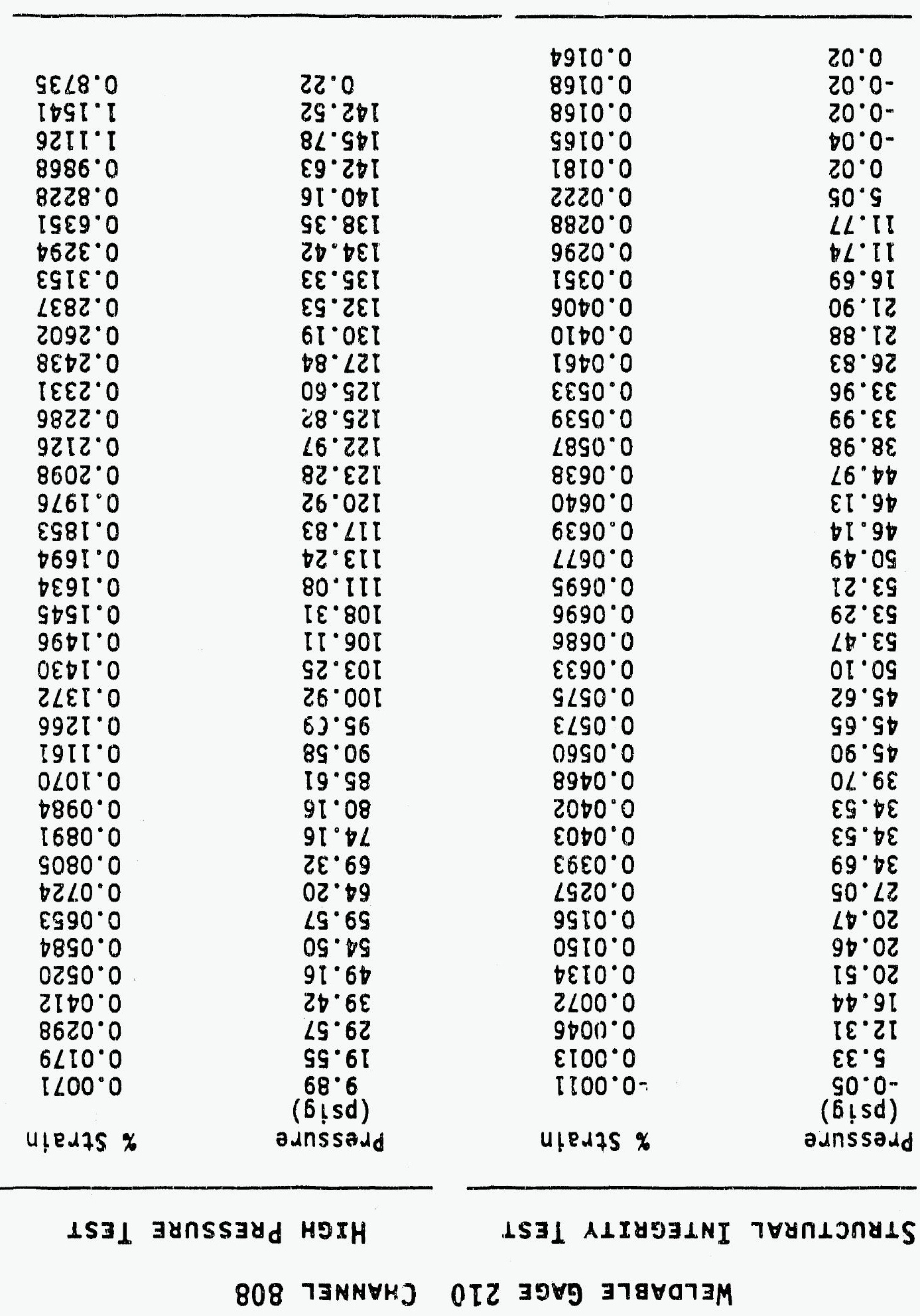

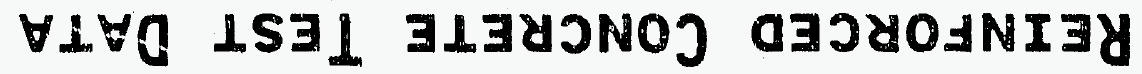




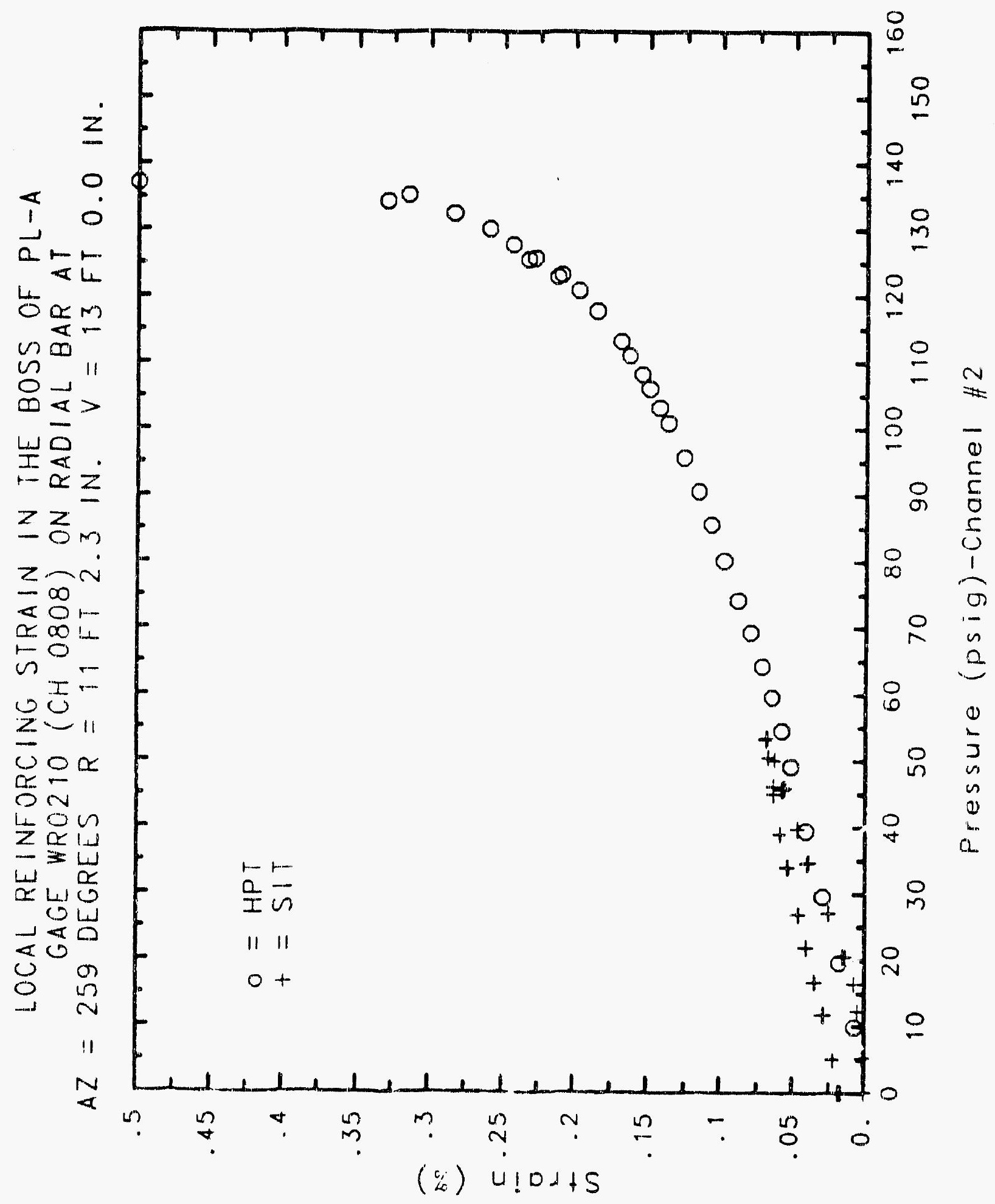




\section{Reinforced Concrete Test Data}

Weldable Gage 211 Chanmel 809

Structural Integrity Test

High Pressure Test

\begin{tabular}{|c|c|c|c|}
\hline $\begin{array}{c}\text { Pressure } \\
\text { (psig) } \\
-0.05 \\
5.33 \\
12.31 \\
16.44 \\
20.51 \\
20.46 \\
20.47 \\
27.05 \\
34.69 \\
34.53 \\
34.53 \\
39.70 \\
45.90 \\
45.65 \\
45.62 \\
50.10 \\
53.47 \\
53.29 \\
53.21 \\
50.49 \\
46.14 \\
46.13 \\
44.97 \\
38.98 \\
33.99 \\
33.96 \\
26.83 \\
21.88 \\
21.90 \\
16.69 \\
11.74 \\
11.77 \\
5.05 \\
0.02 \\
-0.04 \\
-0.02 \\
-0.02 \\
0.02 \\
\end{array}$ & $\begin{array}{c}\text { Strain } \\
-0.0005 \\
0.0005 \\
0.0014 \\
0.0016 \\
0.0018 \\
0.0020 \\
0.0024 \\
0.0024 \\
0.0041 \\
0.0044 \\
0.0046 \\
0.0050 \\
0.0068 \\
0.0076 \\
0.0082 \\
0.0090 \\
0.0098 \\
0.0104 \\
0.0102 \\
0.0107 \\
0.0101 \\
0.0099 \\
0.0088 \\
0.0082 \\
0.0083 \\
0.0086 \\
0.0079 \\
0.0078 \\
0.0079 \\
0.0073 \\
0.0070 \\
0.0069 \\
0.0065 \\
0.0065 \\
0.0029 \\
0.0037 \\
0.0047 \\
0.0052\end{array}$ & $\begin{array}{c}\text { Pressure } \\
(\text { psig) } \\
9.89 \\
19.55 \\
29.57 \\
39.42 \\
49.16 \\
54.50 \\
59.57 \\
64.20 \\
69.32 \\
74.16 \\
80.16 \\
85.61 \\
90.58 \\
95.69 \\
100.92 \\
103.25 \\
106.11 \\
108.31 \\
111.08 \\
113.24 \\
117.83 \\
120.92 \\
123.28 \\
122.97 \\
125.82 \\
125.60 \\
127.84 \\
130.19 \\
132.53 \\
135.33 \\
134.42 \\
138.35 \\
140.16 \\
142.63 \\
145.78 \\
142.52 \\
0.22\end{array}$ & $\begin{array}{l}-0.0026 \\
-0.0012 \\
-0.0002 \\
0.0013 \\
0.0025 \\
0.0037 \\
0.0043 \\
0.0056 \\
0.0074 \\
0.0090 \\
0.0124 \\
0.0148 \\
0.0178 \\
0.0211 \\
0.0246 \\
0.0263 \\
0.0281 \\
0.0292 \\
0.0310 \\
0.0321 \\
0.0350 \\
0.0372 \\
0.0385 \\
0.0380 \\
0.0380 \\
0.0378 \\
0.0389 \\
0.0398 \\
0.0393 \\
0.0395 \\
0.0395 \\
0.0408 \\
0.0418 \\
0.0431 \\
0.0448 \\
0.0463 \\
0.0211\end{array}$ \\
\hline
\end{tabular}


g6Z2I-ว

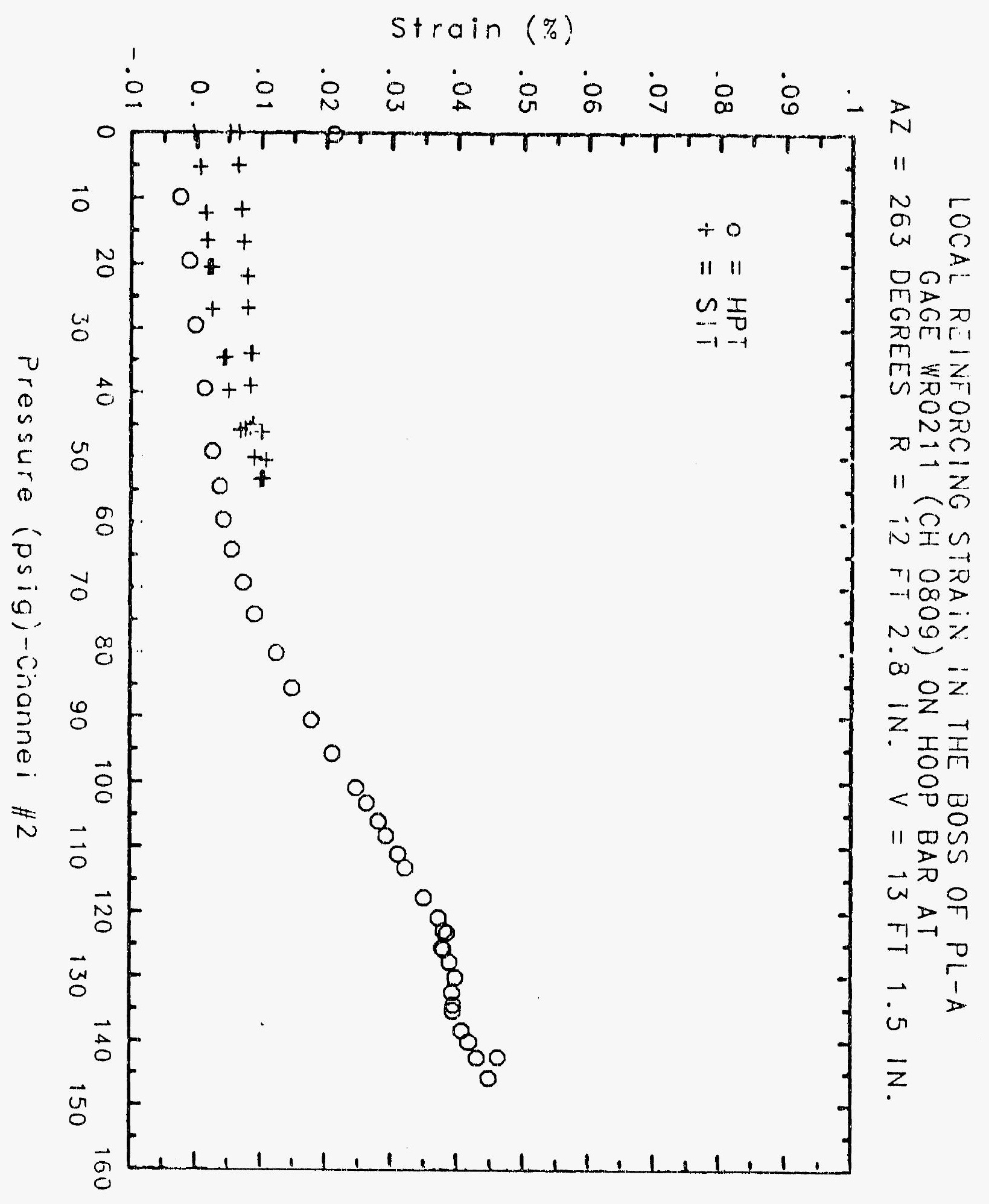




\section{Reinforced Concrete Test Data}

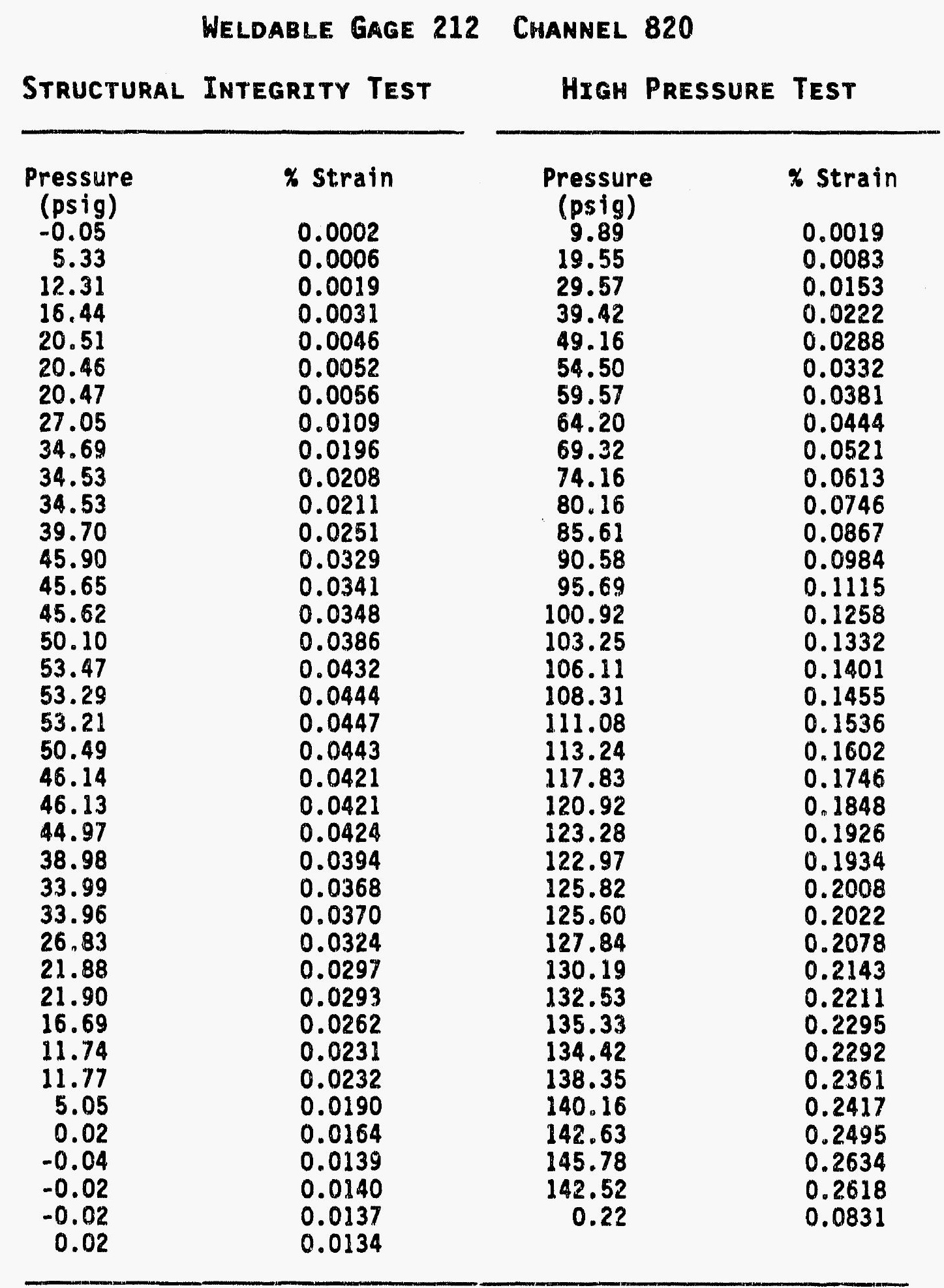




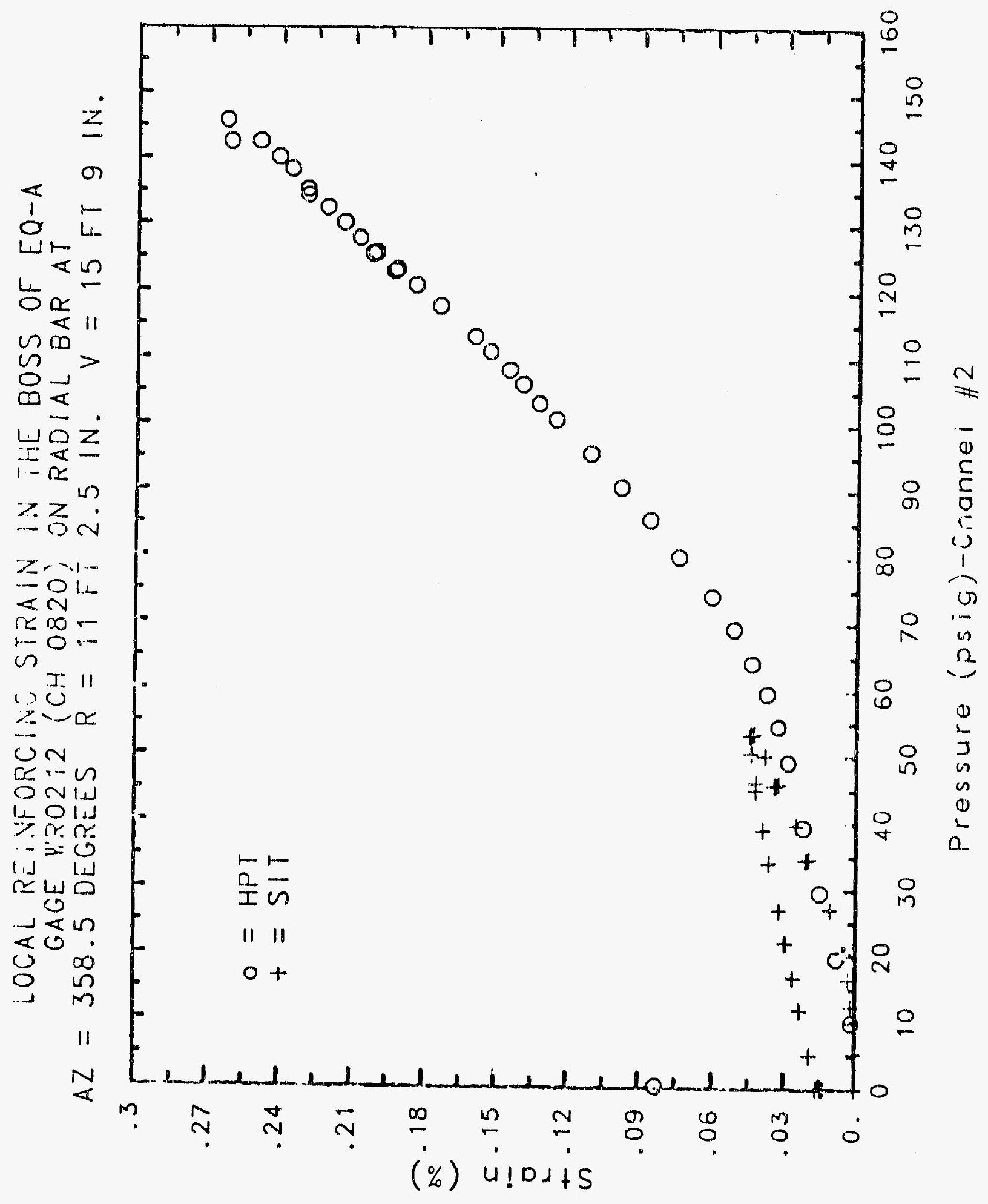




\section{Reinforced Concrete Test Data}

Weldable Gage 213 Channel 821

Structural Integrity Test

\begin{tabular}{|c|c|c|c|}
\hline $\begin{array}{c}\text { Pressure } \\
\text { (psig) } \\
-0.05 \\
5.33 \\
12.31 \\
16.44 \\
20.51 \\
20.46 \\
20.47 \\
27.05 \\
34.69 \\
34.53 \\
34.53 \\
39.70 \\
45.90 \\
45.65 \\
45.62 \\
50.10 \\
53.47 \\
53.29 \\
53.21 \\
50.49 \\
46.14 \\
46.13 \\
44.97 \\
38.98 \\
33.99 \\
33.96 \\
26.83 \\
21.88 \\
21.90 \\
16.69 \\
11.74 \\
11.77 \\
5.05 \\
0.02 \\
-0.04 \\
-0.02 \\
-0.02 \\
0.02 \\
\end{array}$ & $\begin{array}{l}\text { \% Strain } \\
\text {-0.0002 } \\
0.0024 \\
0.0070 \\
0.0096 \\
0.0139 \\
0.0142 \\
0.0148 \\
0.0269 \\
0.0423 \\
0.0429 \\
0.0432 \\
0.0495 \\
0.0575 \\
0.0573 \\
0.0575 \\
0.0624 \\
0.0659 \\
0.0656 \\
0.0656 \\
0.0633 \\
0.0592 \\
0.0587 \\
0.0583 \\
0.0522 \\
0.0470 \\
0.0468 \\
0.0386 \\
0.0329 \\
0.0326 \\
0.0265 \\
0.0210 \\
0.0208 \\
0.0136 \\
0.0083 \\
0.0069 \\
0.0079 \\
0.0071 \\
0.0074\end{array}$ & $\begin{array}{c}\text { Pressure } \\
(\text { psig) } \\
9.89 \\
19.55 \\
29.57 \\
39.42 \\
49.16 \\
54.50 \\
54.57 \\
64.20 \\
69.32 \\
74.16 \\
80.16 \\
85.61 \\
90.58 \\
95.69 \\
100.92 \\
103.25 \\
106.11 \\
108.31 \\
111.08 \\
113.24 \\
117.83 \\
120.92 \\
123.28 \\
122.97 \\
125.82 \\
125.60 \\
127.84 \\
130.19 \\
132.53 \\
135.33 \\
134.42 \\
138.35 \\
140.16 \\
142.63 \\
145.78 \\
142.52 \\
0.22 \\
\end{array}$ & $\begin{array}{l}\text { \% Strain } \\
0.0059 \\
0.0167 \\
0.0277 \\
0.0384 \\
0.0480 \\
0.0529 \\
0.0581 \\
0.0670 \\
0.0759 \\
0.0847 \\
0.0847 \\
0.0942 \\
0.1032 \\
0.1144 \\
0.1252 \\
0.1288 \\
0.1326 \\
0.1344 \\
0.1314 \\
0.1324 \\
0.1367 \\
0.1391 \\
0.1406 \\
0.1401 \\
0.1442 \\
0.1438 \\
0.1485 \\
0.1535 \\
0.1608 \\
0.1690 \\
0.1680 \\
0.1788 \\
0.1812 \\
0.1858 \\
0.1978 \\
0.1949 \\
0.0905\end{array}$ \\
\hline
\end{tabular}

High Pressure Test

Pressure

9.89

0.0167

0.0277

0.0384

0.0480

0.0529

0.0581

0.0670

0.0847

0.0942

0.1032

0.1144

0.1252

0.1288

0.1344

0.1314

0.1324

0.1367

0.1391

0.1442

0.1485

0.1535

0.1608

0.1690

0.1680

0.1858

0.1978

0.1949

0.0905 


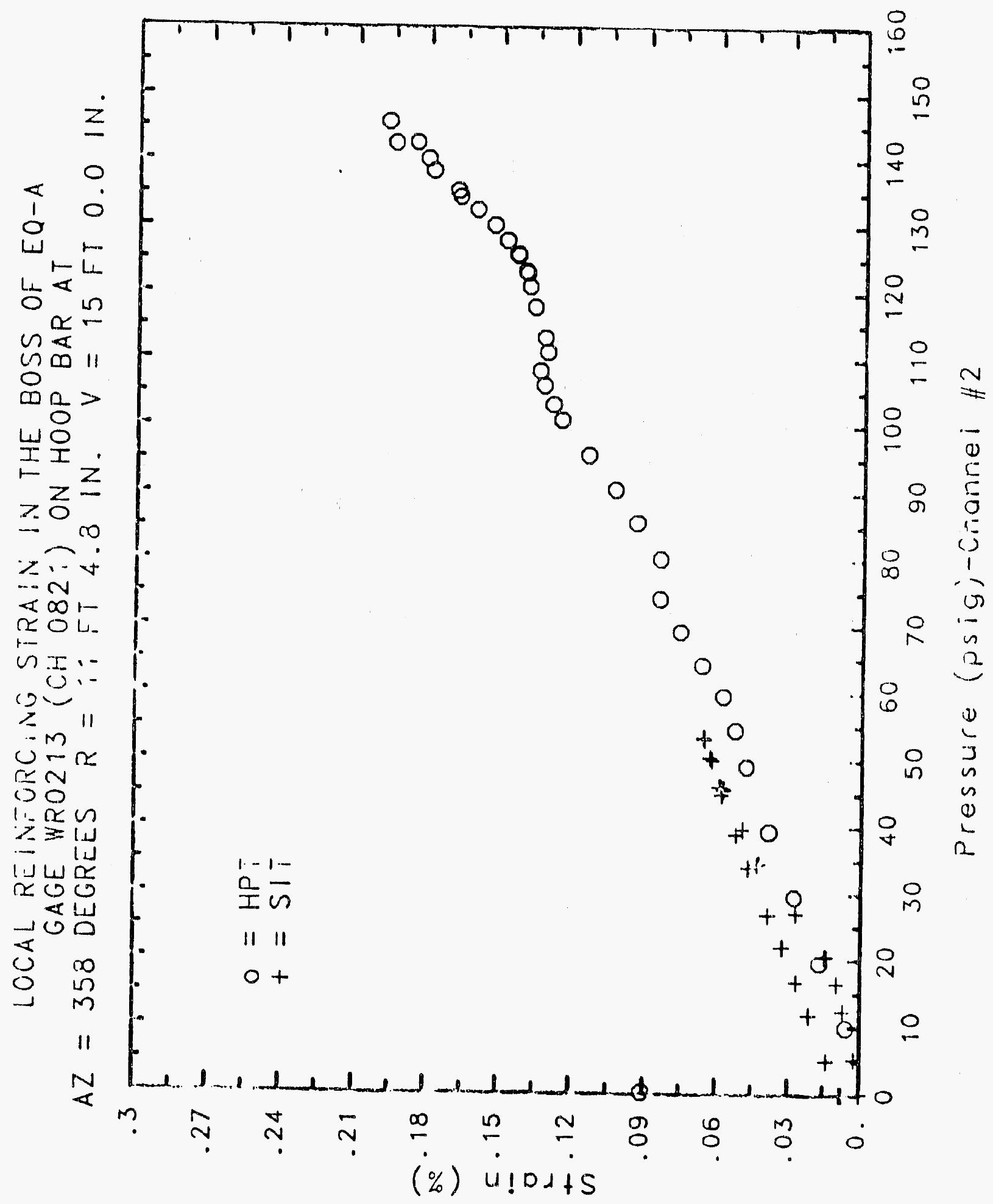




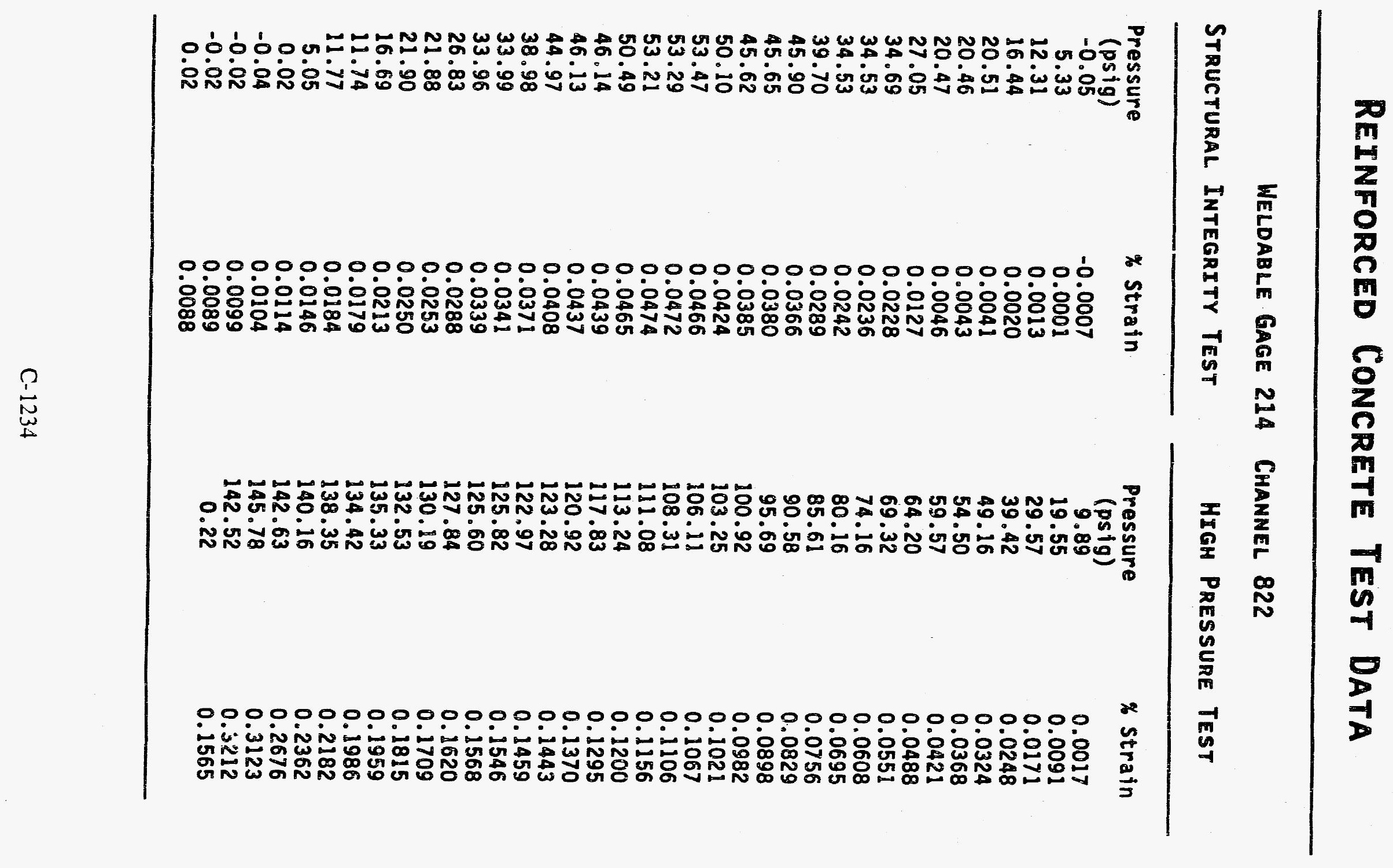


SEZI-D

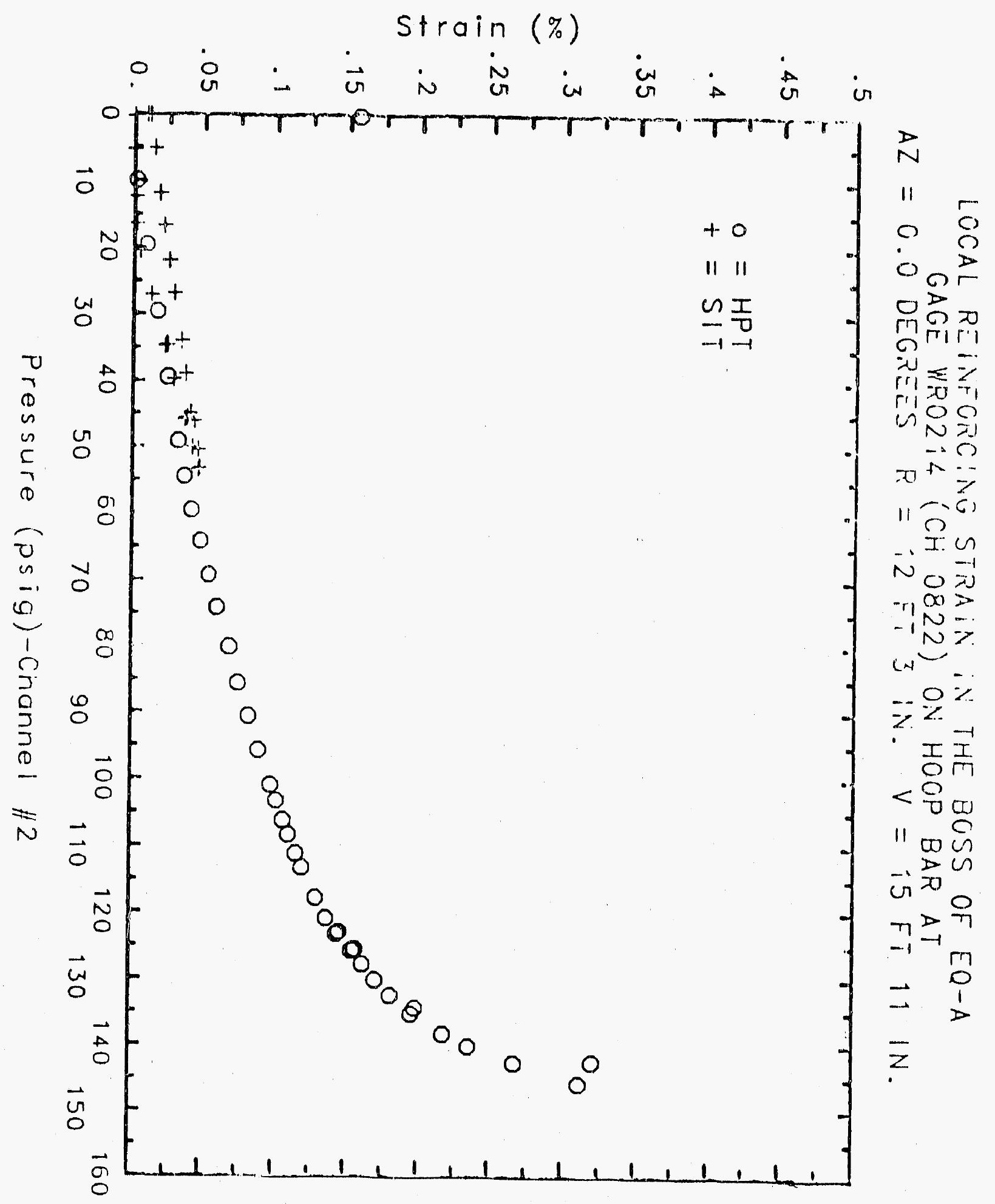




\section{Reinforced Concrete Test Data}

Weldable gage 215 Channel 823

Structural IntEgrity TEST

High Pressure. Test

\begin{tabular}{rrrr}
\hline $\begin{array}{c}\text { Pressure } \\
\text { (psig) }\end{array}$ & \% Strain & $\begin{array}{c}\text { Pressure } \\
\text { (psig) }\end{array}$ & \% strain \\
-0.05 & -0.0003 & 9.89 & -0.0039 \\
5.33 & -0.0004 & 19.55 & -0.0036 \\
12.31 & -0.0002 & 29.57 & -0.0034 \\
16.44 & -0.0005 & 39.42 & -0.0030 \\
20.51 & 0.0002 & 49.16 & -0.0012 \\
20.46 & -0.0001 & 54.50 & 0.0041 \\
20.47 & 0.0000 & 59.57 & 0.0140 \\
27.05 & 0.0008 & 64.20 & 0.0240 \\
34.69 & 0.0008 & 69.32 & 0.0307 \\
34.53 & 0.0012 & 74.16 & 0.0371 \\
34.53 & 0.0011 & 80.16 & 0.0464 \\
39.70 & 0.0008 & 85.61 & 0.0533 \\
45.90 & 0.0010 & 90.58 & 0.0599 \\
45.65 & 0.0015 & 95.69 & 0.0670 \\
45.62 & 0.0010 & 100.92 & 0.0734 \\
50.10 & 0.0015 & 103.25 & 0.0765 \\
53.47 & 0.0018 & 106.11 & 0.0792 \\
53.29 & 0.0020 & 108.31 & 0.0818 \\
53.21 & 0.0021 & 111.08 & 0.0847 \\
50.49 & 0.0025 & 113.24 & 0.0873 \\
46.14 & 0.0028 & 117.83 & 0.0933 \\
46.13 & 0.0026 & 120.92 & 0.0973 \\
44.97 & 0.0031 & 123.28 & 0.1009 \\
38.98 & 0.0036 & 122.97 & 0.1014 \\
33.99 & 0.0036 & 125.82 & 0.1039 \\
33.96 & 0.0036 & 125.60 & 0.1040 \\
26.83 & 0.0035 & 127.84 & 0.1059 \\
21.88 & 0.0039 & 130.19 & 0.1083 \\
21.90 & 0.0036 & 132.53 & 0.1115 \\
16.69 & 0.0041 & 135.33 & 0.1152 \\
11.74 & 0.0039 & 134.42 & 0.1153 \\
11.77 & 0.0040 & 138.35 & 0.1207 \\
5.05 & 0.0038 & 140.16 & 0.1243 \\
0.02 & 0.0039 & 142.63 & 0.1296 \\
-0.04 & 0.0036 & 145.78 & 0.1347 \\
-0.02 & 0.0032 & 0.22 & 0.1345 \\
-0.02 & 0.0033 & & 0.0667 \\
0.02 & 0.0038 & & \\
\hline & & 142.52 & \\
\hline & & & \\
\hline
\end{tabular}




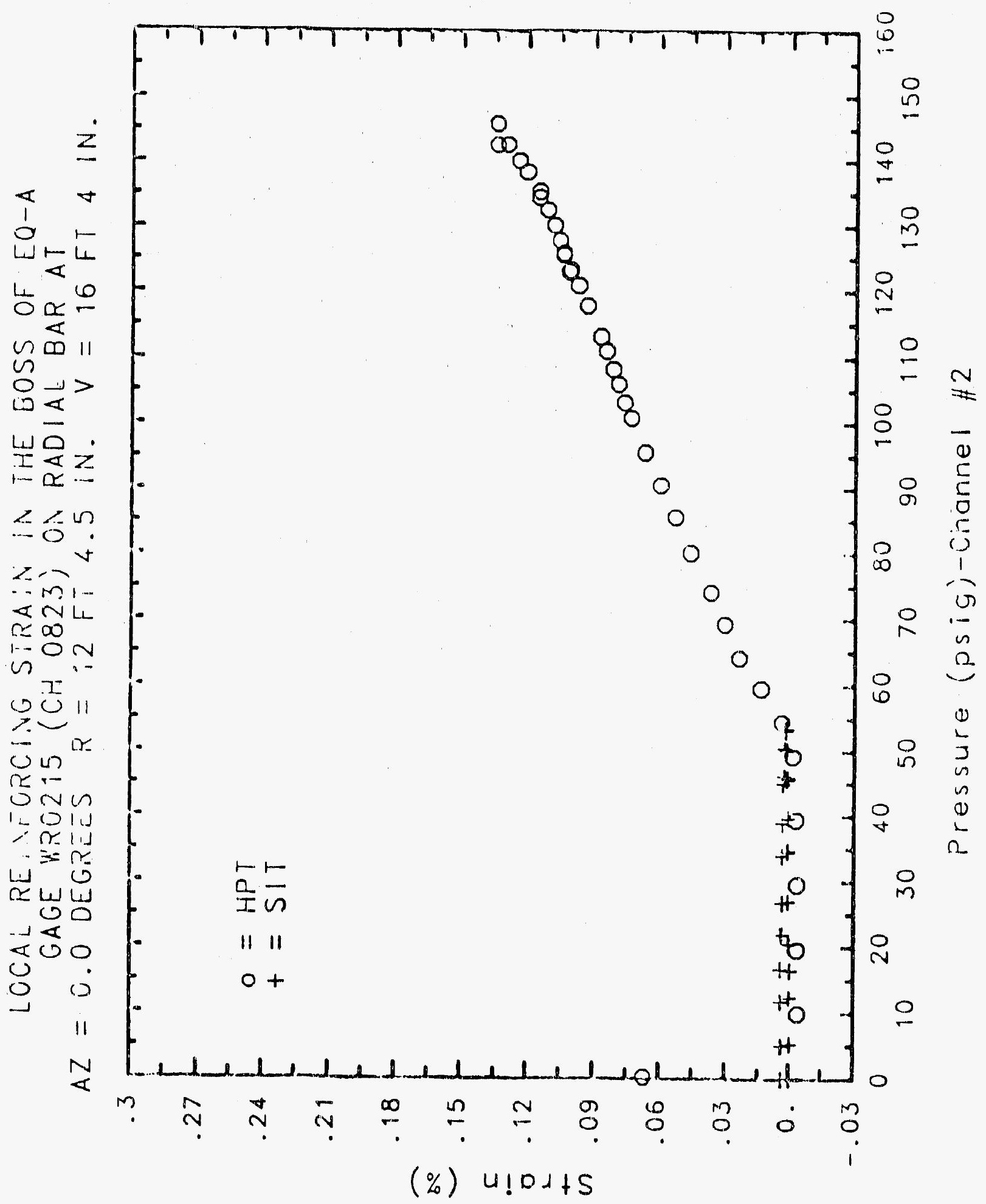




\section{Reinforced Concrete Test Data}

Weldable gage 216 Channel 824

Structural Integrity test

\begin{tabular}{|c|c|c|c|}
\hline $\begin{array}{c}\text { Pressure } \\
\text { (psig) } \\
-0.05 \\
5.33 \\
12.31 \\
16.44 \\
20.51 \\
20.46 \\
20.47 \\
27.05 \\
34.69 \\
34.53 \\
34.53 \\
39.70 \\
45.90 \\
45.65 \\
45.62 \\
50.10 \\
53.47 \\
53.29 \\
53.21 \\
50.49 \\
46.14 \\
46.13 \\
44.97 \\
38.98 \\
33.99 \\
33.96 \\
26.83 \\
21.88 \\
21.90 \\
16.69 \\
11.74 \\
11.77 \\
5.05 \\
0.02 \\
-0.04 \\
-0.02 \\
-0.02 \\
0.02 \\
\end{array}$ & $\begin{array}{l}\text { \% Strain } \\
0.0001 \\
0.0031 \\
0.0054 \\
0.0062 \\
0.0075 \\
0.0076 \\
0.0079 \\
0.0123 \\
0.0184 \\
0.0191 \\
0.0192 \\
0.0229 \\
0.0283 \\
0.0297 \\
0.0300 \\
0.0329 \\
0.0356 \\
0.0359 \\
0.0363 \\
0.0361 \\
0.0350 \\
0.0350 \\
0.0267 \\
0.0252 \\
0.0237 \\
0.0245 \\
0.0211 \\
0.0188 \\
0.0186 \\
0.0163 \\
0.0133 \\
0.0145 \\
0.0107 \\
0.0083 \\
0.0020 \\
-0.0018 \\
0.0001 \\
0.0034\end{array}$ & $\begin{array}{c}\text { Pressure } \\
(\text { ps } 1 \text { g) } \\
9.89 \\
19.55 \\
29.57 \\
39.42 \\
49.16 \\
54.50 \\
59.57 \\
64.20 \\
69.32 \\
74.16 \\
80.16 \\
85.61 \\
90.58 \\
95.69 \\
100.92 \\
103.25 \\
106.11 \\
108.31 \\
111.08 \\
113.24 \\
117.83 \\
120.92 \\
123.28 \\
122.97 \\
125.82 \\
125.60 \\
127.84 \\
130.19 \\
132.53 \\
135.33 \\
134.42 \\
138.35 \\
140.16 \\
142.63 \\
145.78 \\
142.52 \\
0.22 \\
\end{array}$ & $\begin{array}{l}\text { \% Strain } \\
0.0024 \\
0.0081 \\
0.0140 \\
0.0197 \\
0.0247 \\
0.0280 \\
0.0318 \\
0.0362 \\
0.0411 \\
0.0460 \\
0.0558 \\
0.0625 \\
0.0695 \\
0.0769 \\
0.0854 \\
0.0896 \\
0.0940 \\
0.0972 \\
0.1026 \\
0.1063 \\
0.1158 \\
0.1232 \\
0.1266 \\
0.1250 \\
0.1306 \\
0.1305 \\
0.1368 \\
0.1493 \\
0.1637 \\
0.1827 \\
0.1861 \\
0.2110 \\
0.2299 \\
0.2557 \\
0.2842 \\
0.2938 \\
0.1652\end{array}$ \\
\hline
\end{tabular}

high Pressure test

Pressure

0.89

19.55

0.0197

0.0247

0.0318

0.0362

0.0411

0.0460

0.0558

0.0625

0.0769

0.0854

0.0896

0.0940

0.0972

0.1026

0.1063

0.1232

0.1266

0.1250

0.1306

0.1305

0.1368

0.1827

0.1861

0.2110

0.2299

0.2557

0.2842

0.2938

0.1652 


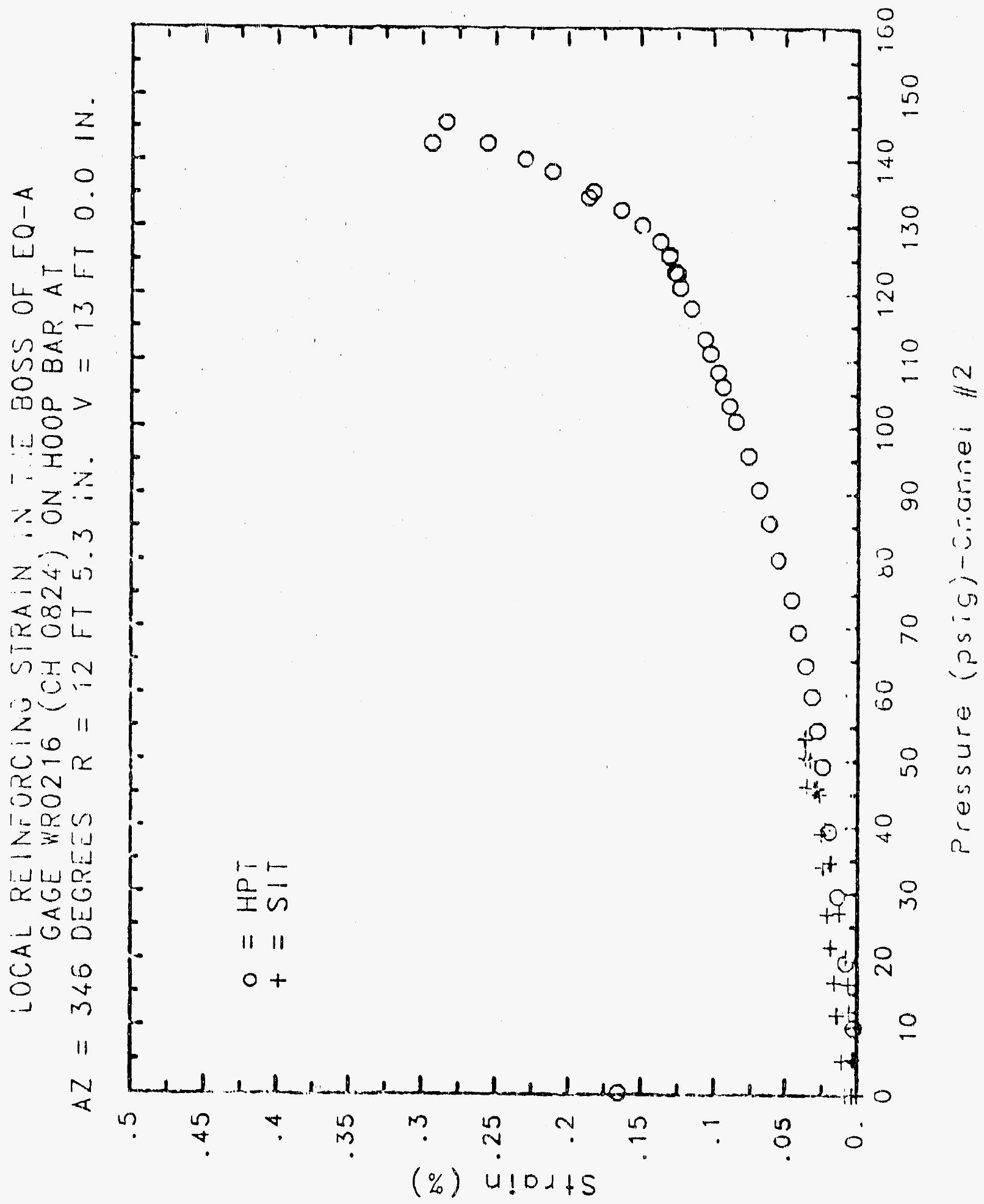




\section{Reinforced Concrete Test Data}

Weldable gajge 217 Channel 825

StRUCTURAL INTEGRITY TEST

High Pressure test

\begin{tabular}{|c|c|c|c|}
\hline $\begin{array}{c}\text { Pressure } \\
(\text { psig) } \\
-0.05 \\
5.33 \\
12.31 \\
16.44 \\
20.51 \\
20.46 \\
20.47 \\
27.05 \\
34.69 \\
34.53 \\
34.53 \\
39.70 \\
45.90 \\
45.65 \\
45.62 \\
50.10 \\
53.47 \\
53.29 \\
53.21 \\
50.49 \\
46.14 \\
46.13 \\
44.97 \\
38.98 \\
33.99 \\
33.96 \\
26.83 \\
21.88 \\
21.90 \\
16.69 \\
11.74 \\
11.77 \\
5.05 \\
0.02 \\
-0.04 \\
-0.02 \\
-0.02 \\
0.02 \\
\end{array}$ & $\begin{array}{l}\text { \% Strain } \\
0.0005 \\
0.0015 \\
0.0025 \\
0.0034 \\
0.0054 \\
0.0062 \\
0.0063 \\
0.0142 \\
0.0232 \\
0.0242 \\
0.0247 \\
0.0287 \\
0.0362 \\
0.0374 \\
0.0373 \\
0.0412 \\
0.0450 \\
0.0451 \\
0.0454 \\
0.0445 \\
0.0422 \\
0.0419 \\
0.0433 \\
0.0402 \\
0.0374 \\
0.0373 \\
0.0333 \\
0.0301 \\
0.0299 \\
0.0268 \\
0.0237 \\
0.0232 \\
0.0197 \\
0.0164 \\
0.0146 \\
0.0150 \\
0.0155 \\
0.0155\end{array}$ & $\begin{array}{c}\text { Pressure } \\
(\text { psig) } \\
9.89 \\
19.55 \\
29.57 \\
39.42 \\
49.16 \\
54.50 \\
59.57 \\
64.20 \\
69.32 \\
74.16 \\
80.16 \\
85.61 \\
90.58 \\
95.69 \\
100.92 \\
103.25 \\
106.11 \\
108.31 \\
111.08 \\
113.24 \\
117.83 \\
120.92 \\
123.28 \\
122.97 \\
125.82 \\
125.60 \\
127.84 \\
130.19 \\
132.53 \\
135.33 \\
134.42 \\
138.35 \\
140.16 \\
142.63 \\
145.78 \\
142.52 \\
0.22 \\
\end{array}$ & $\begin{array}{l}\text { X Strain } \\
0.0020 \\
0.0086 \\
0.0152 \\
0.0217 \\
0.0285 \\
0.0324 \\
0.0366 \\
0.0404 \\
0.0449 \\
0.0496 \\
0.0545 \\
0.0593 \\
0.0642 \\
0.0702 \\
0.0754 \\
0.0782 \\
0.0812 \\
0.0837 \\
0.0872 \\
0.0899 \\
0.0963 \\
0.1011 \\
0.1056 \\
0.1068 \\
0.1113 \\
0.1128 \\
0.1162 \\
0.1218 \\
0.1276 \\
0.1365 \\
0.1379 \\
0.1505 \\
0.1607 \\
0.1743 \\
0.1879 \\
0.1891 \\
0.0478\end{array}$ \\
\hline
\end{tabular}




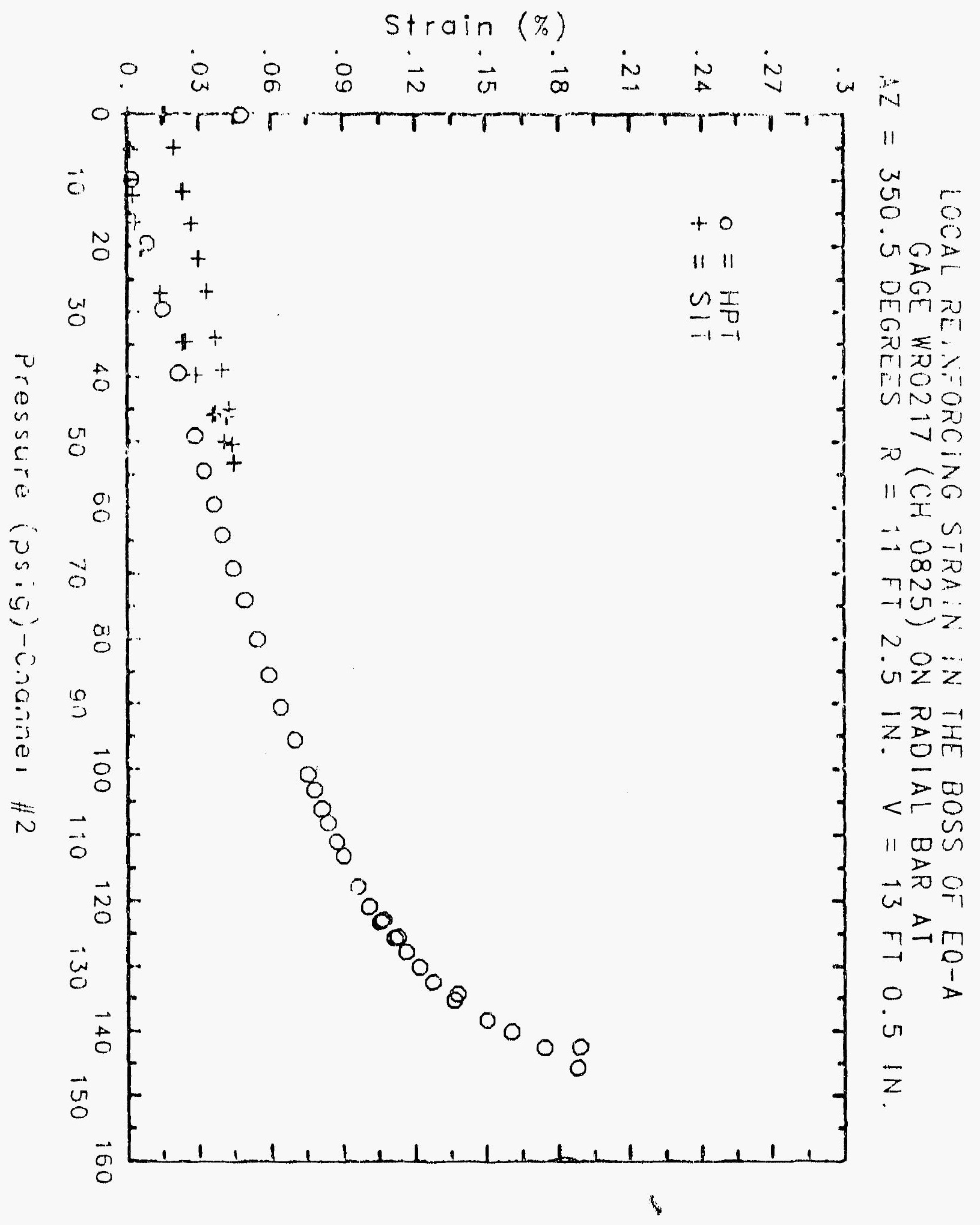




\section{Reinforced Concrete Test Data}

\section{Weldable Gage 218 Channel 826}

Structural IntEgRITY TEST

high Pressure Test

\begin{tabular}{|c|c|c|c|}
\hline $\begin{array}{c}\text { Pressure } \\
\text { (psig) } \\
-0.05 \\
5.33 \\
12.31 \\
16.44 \\
20.51 \\
20.46 \\
20.47 \\
27.05 \\
34.69 \\
34.53 \\
34.53 \\
39.70 \\
45.90 \\
45.65 \\
45.62 \\
50.10 \\
53.47 \\
53.29 \\
53.21 \\
50.49 \\
46.14 \\
46.13 \\
44.97 \\
38.98 \\
33.99 \\
33.96 \\
26.83 \\
21.88 \\
21.90 \\
16.69 \\
11.74 \\
11.77 \\
5.05 \\
0.02 \\
-0.04 \\
-0.02 \\
-0.02 \\
0.02 \\
\end{array}$ & $\begin{array}{l}\text { Strain } \\
0.0005 \\
0.0022 \\
0.0040 \\
0.0048 \\
0.0076 \\
0.0078 \\
0.0080 \\
0.0118 \\
0.0153 \\
0.0155 \\
0.0155 \\
0.0171 \\
0.0197 \\
0.0191 \\
0.0192 \\
0.0211 \\
0.0225 \\
0.0223 \\
0.0224 \\
0.0215 \\
0.0197 \\
0.0197 \\
0.0229 \\
0.0211 \\
0.0187 \\
0.0185 \\
0.0159 \\
0.0136 \\
0.0136 \\
0.0115 \\
0.0095 \\
0.0091 \\
0.0066 \\
0.0042 \\
0.0052 \\
0.0066 \\
0.0067 \\
0.0057\end{array}$ & $\begin{array}{c}\text { Pressure } \\
(\text { psig) } \\
9.89 \\
19.55 \\
29.57 \\
39.42 \\
49.16 \\
54.50 \\
59.57 \\
64.20 \\
69.32 \\
74.16 \\
80.16 \\
85.61 \\
90.58 \\
95.69 \\
100.92 \\
103.25 \\
106.11 \\
108.31 \\
111.08 \\
113.24 \\
117.83 \\
120.92 \\
123.28 \\
122.97 \\
125.82 \\
125.60 \\
127.84 \\
130.19 \\
132.53 \\
135.33 \\
134.42 \\
138.35 \\
140.16 \\
142.63 \\
145.78 \\
142.52 \\
0.22 \\
\end{array}$ & $\begin{array}{l}\text { \% Strain } \\
0.0006 \\
0.0046 \\
0.0093 \\
0.0129 \\
0.0164 \\
0.0184 \\
0.0205 \\
0.0223 \\
0.0238 \\
0.0252 \\
0.0255 \\
0.0274 \\
0.0294 \\
0.0322 \\
0.0351 \\
0.0373 \\
0.0390 \\
0.0404 \\
0.0428 \\
0.0441 \\
0.0472 \\
0.0500 \\
0.0531 \\
0.0549 \\
0.0579 \\
0.0583 \\
0.0583 \\
0.0606 \\
0.0652 \\
0.0703 \\
0.0722 \\
0.0779 \\
0.0837 \\
0.0959 \\
0.1044 \\
0.1071 \\
0.0260\end{array}$ \\
\hline
\end{tabular}




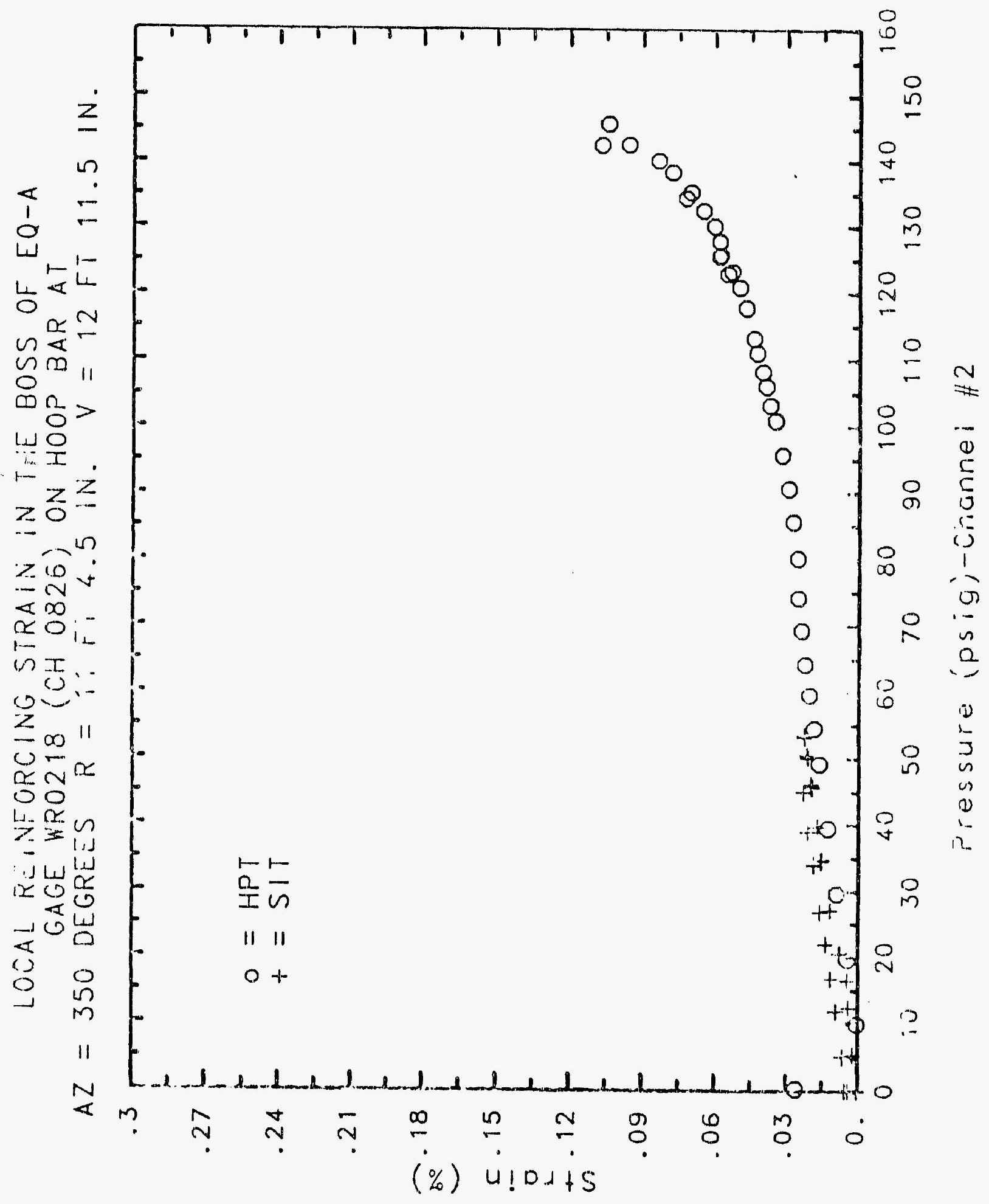




\section{Reinforced Concrete Test Data}

Weldable gage 219 Channel 827

Structural Integrity Test

High Pressure test

\begin{tabular}{|c|c|c|c|}
\hline $\begin{array}{c}\text { Pressure } \\
(\text { psig) } \\
-0.05 \\
5.33 \\
12.31 \\
16.44 \\
20.51 \\
20.46 \\
20.47 \\
27.05 \\
34.69 \\
34.53 \\
34.53 \\
39.70 \\
45.90 \\
45.65 \\
45.62 \\
50.10 \\
53.47 \\
53.29 \\
53.21 \\
50.49 \\
46.14 \\
46.13 \\
44.97 \\
38.98 \\
33.99 \\
33.96 \\
26.83 \\
21.88 \\
21.90 \\
16.69 \\
11.74 \\
11.77 \\
5.05 \\
0.02 \\
-0.04 \\
-0.02 \\
-0.02 \\
0.02 \\
50\end{array}$ & $\begin{array}{c}\text { \% Strain } \\
-0.0005 \\
0.0006 \\
0.0004 \\
0.0003 \\
-0.0003 \\
-0.0004 \\
-0.0004 \\
-0.0011 \\
-0.0002 \\
-0.0001 \\
0.0003 \\
0.0007 \\
0.0027 \\
0.0039 \\
0.0045 \\
0.0050 \\
0.0067 \\
0.0076 \\
0.0083 \\
0.0089 \\
0.0095 \\
0.0099 \\
0.0098 \\
0.0098 \\
0.0103 \\
0.0103 \\
0.0104 \\
0.0100 \\
0.0102 \\
0.0100 \\
0.0095 \\
0.0100 \\
0.0092 \\
0.0087 \\
0.0083 \\
0.0074 \\
0.0071 \\
0.0071\end{array}$ & $\begin{array}{c}\text { Pressure } \\
\text { (psig) } \\
9.89 \\
19.55 \\
29.57 \\
39.42 \\
49.16 \\
54.50 \\
59.57 \\
64.20 \\
69.32 \\
74.16 \\
80.16 \\
85.61 \\
90.58 \\
95.69 \\
100.92 \\
103.25 \\
106.11 \\
108.31 \\
111.08 \\
113.24 \\
117.83 \\
120.92 \\
123.28 \\
122.97 \\
125.82 \\
125.60 \\
127.84 \\
130.19 \\
132.53 \\
135.33 \\
134.42 \\
138.35 \\
140.16 \\
142.63 \\
145.78 \\
142.52 \\
0.22 \\
\end{array}$ & $\begin{array}{l}\text { X Strain } \\
-0.0033 \\
-0.0029 \\
-0.002 .6 \\
-0.0017 \\
-0.0006 \\
0.0002 \\
0.0012 \\
0.0028 \\
0.0046 \\
0.0071 \\
0.0134 \\
0.0179 \\
0.0226 \\
0.0282 \\
0.0344 \\
0.0378 \\
0.0403 \\
0.0426 \\
0.0465 \\
0.0493 \\
0.0559 \\
0.0603 \\
0.0642 \\
0.0650 \\
0.0680 \\
0.0684 \\
0.0705 \\
0.0735 \\
0.0758 \\
0.0782 \\
0.0780 \\
0.0819 \\
0.0847 \\
0.0899 \\
0.0943 \\
0.0984 \\
0.0311\end{array}$ \\
\hline
\end{tabular}




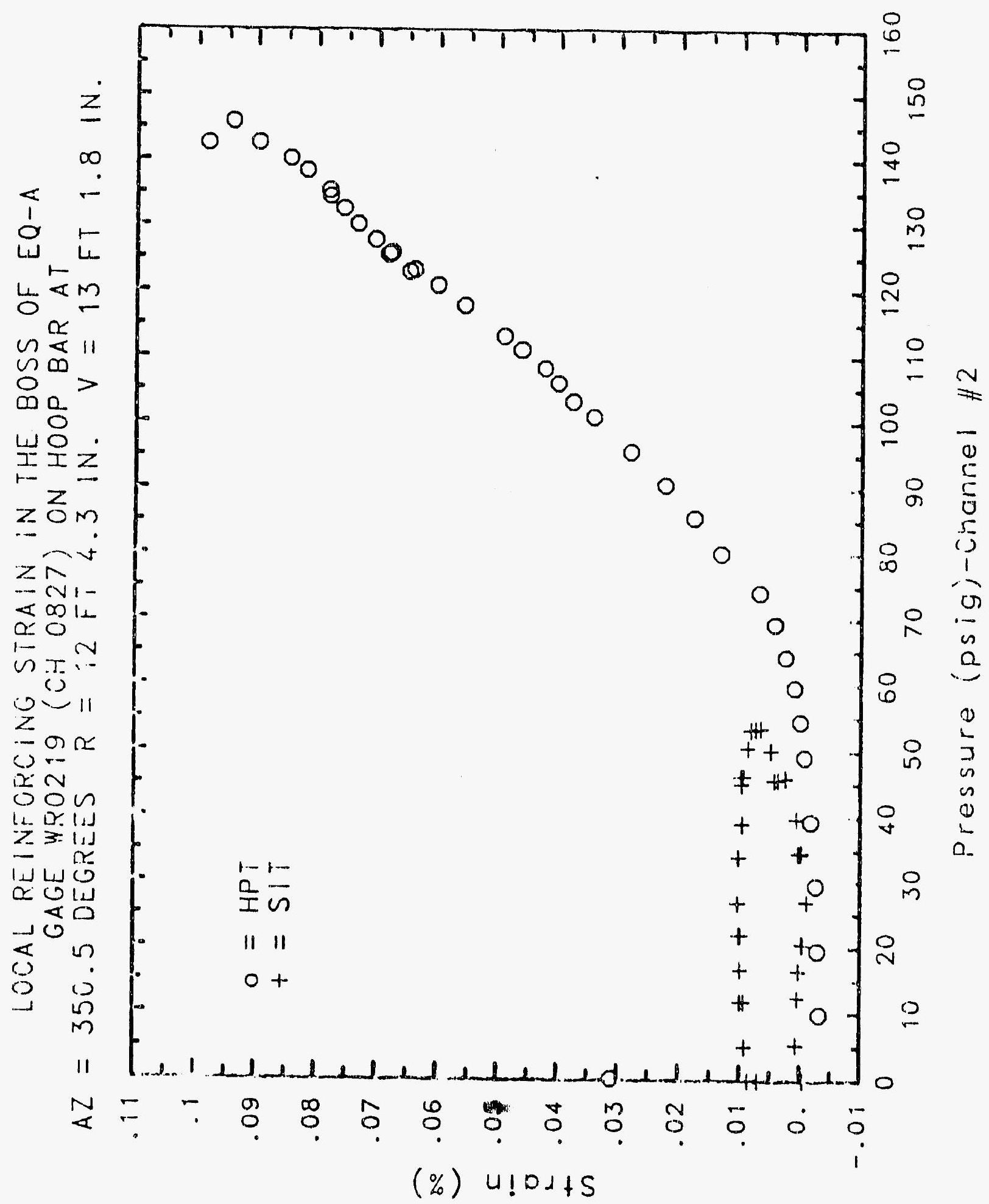




\section{Reinforced Concrete Test Data}

Weldable gage 220 Channel 828

Structural Integrity Test

high Pressure test

\begin{tabular}{lc}
\hline Pressure & \\
(psig) & \% strain \\
-0.05 & \\
5.33 & -0.0005 \\
12.31 & 0.0004 \\
16.44 & 0.0004 \\
20.51 & 0.0005 \\
20.46 & -0.0001 \\
20.47 & 0.0000 \\
27.05 & 0.0005 \\
34.69 & -0.0002 \\
34.53 & -0.0004 \\
34.53 & -0.0004 \\
39.70 & 0.0000 \\
45.90 & -0.0004 \\
45.65 & 0.0001 \\
45.62 & 0.0006 \\
50.10 & 0.0005 \\
53.47 & 0.0003 \\
53.29 & 0.0001 \\
53.21 & 0.0004 \\
50.49 & 0.0003 \\
46.14 & 0.0006 \\
46.13 & 0.0009 \\
44.97 & 0.0007 \\
38.98 & 0.0009 \\
33.99 & 0.0013 \\
33.96 & 0.0021 \\
26.83 & 0.0023 \\
21.88 & 0.0030 \\
21.90 & 0.0033 \\
16.69 & 0.0038 \\
11.74 & 0.0039 \\
11.77 & 0.0044 \\
5.05 & 0.0046 \\
0.02 & 0.0049 \\
-0.04 & 0.0053 \\
-0.02 & 0.0026 \\
-0.02 & 0.0030 \\
0.02 & 0.0039 \\
& 0.0047 \\
\hline
\end{tabular}

Pressure

(psig)

9.89

19.55

29.57

39.42

49.16

54.50

59.57

64.20

69.32

74.16

80.16

85.61

90.58

95.69

100.92

103.25

106.11

108.31

111.08

113.24

117.83

120.92

123.28

122.97

125.82

125.60

127.84

130.19

132.53

135.33

134.42

138.35

140.16

142.63

145.78

142.52

0.22
* Strain

$-0.0046$

$-0.0052$

$-0.0056$

$-0.0063$

$-0.0067$

$-0.0069$

$-0.0073$

$-0.0072$

$-0.0073$

$-0.0071$

$-0.0065$

$-0.0071$

$-0.0071$

$-0.0069$

$-0.0069$

$-0.0065$

$-0.0065$

$-0.0063$

$-0.0061$

$-0.0056$

$-0.0052$

$-0.0048$

$-0.0039$

$-0.0035$

$-0.0029$

$-0.0026$

$-0.0021$

$-0.0024$

$-0.0008$

$-0.0003$

0.0007

0.0008

0.0020

0.0071

0.0122

0.0167

0.0054 


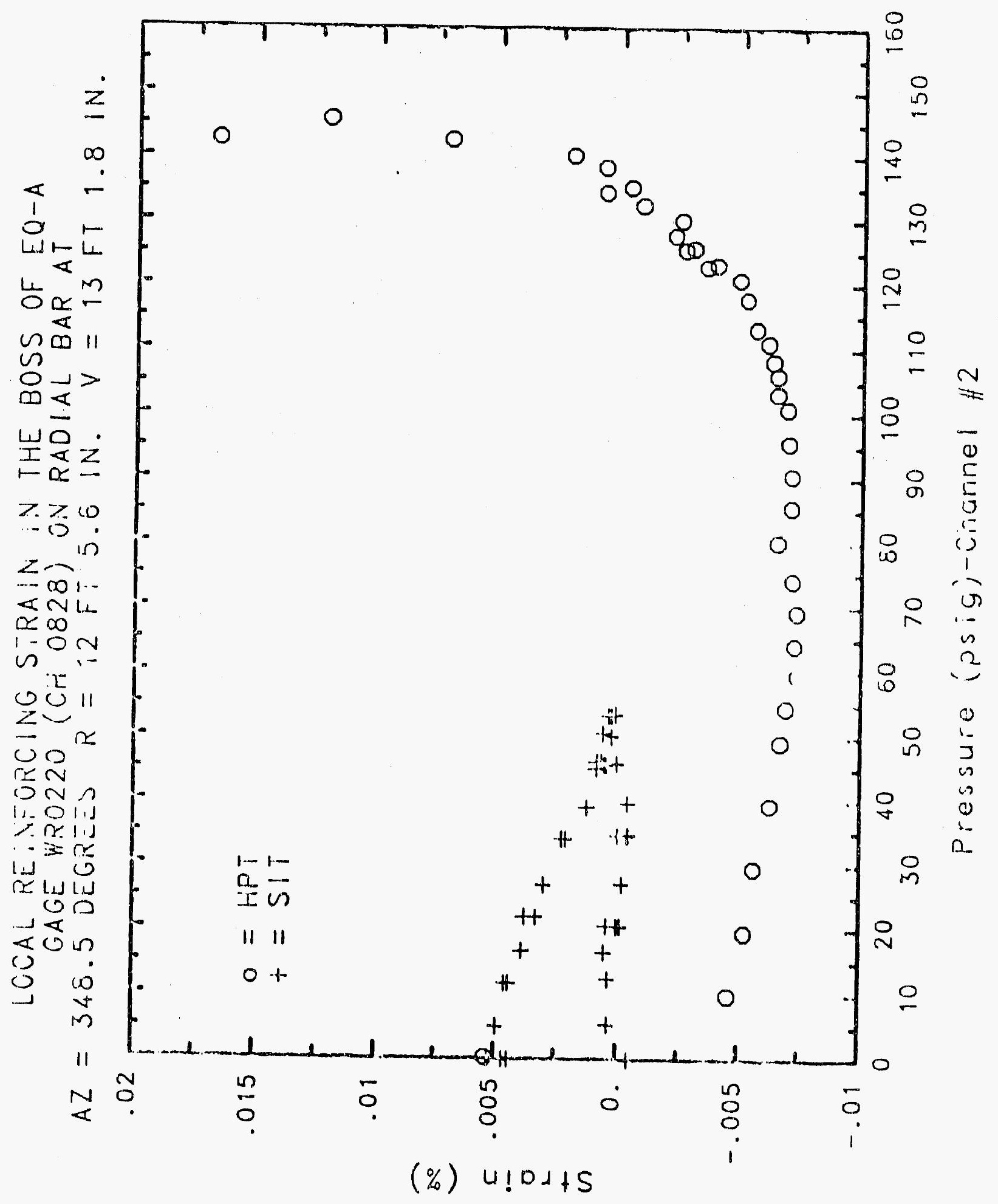




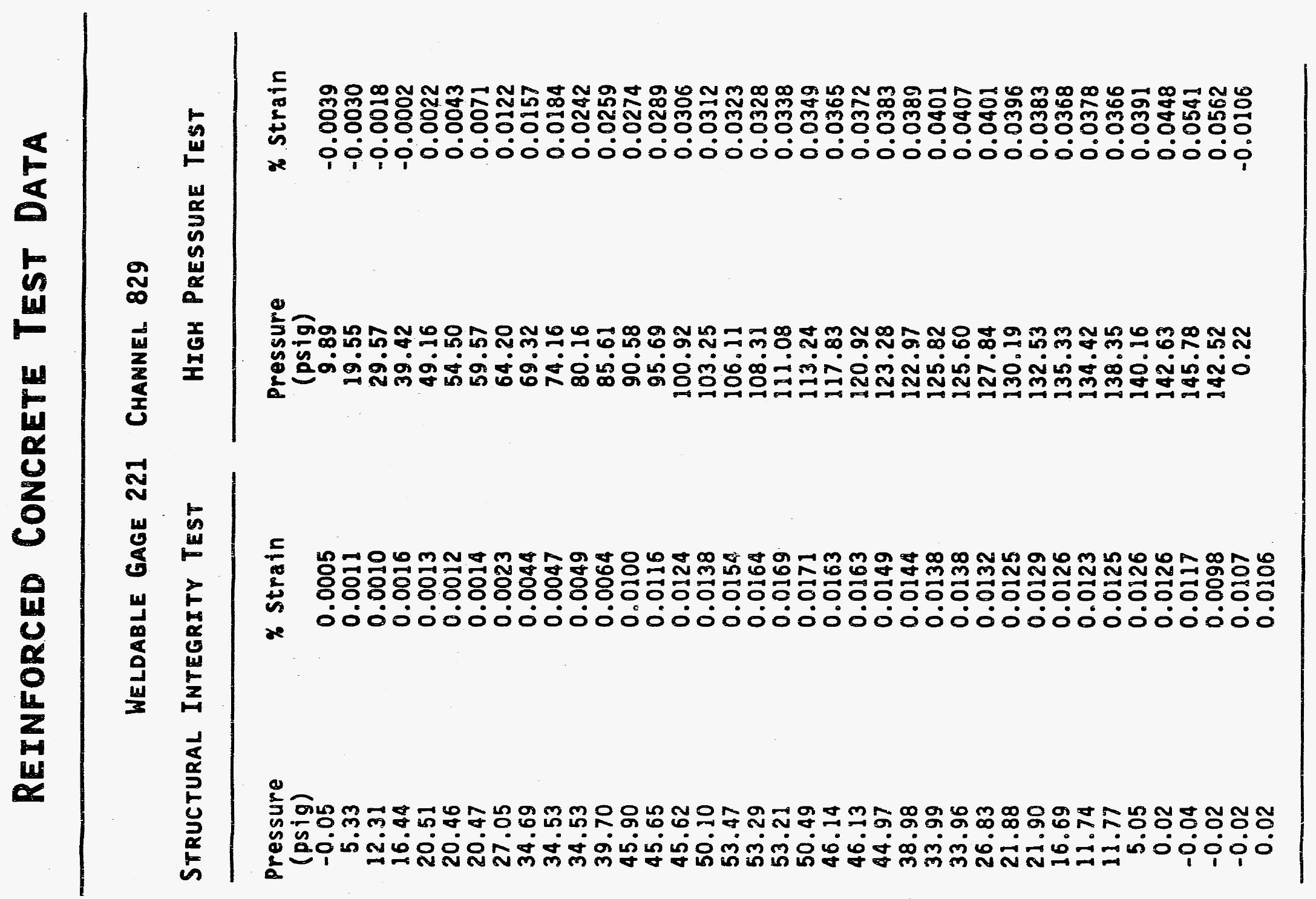




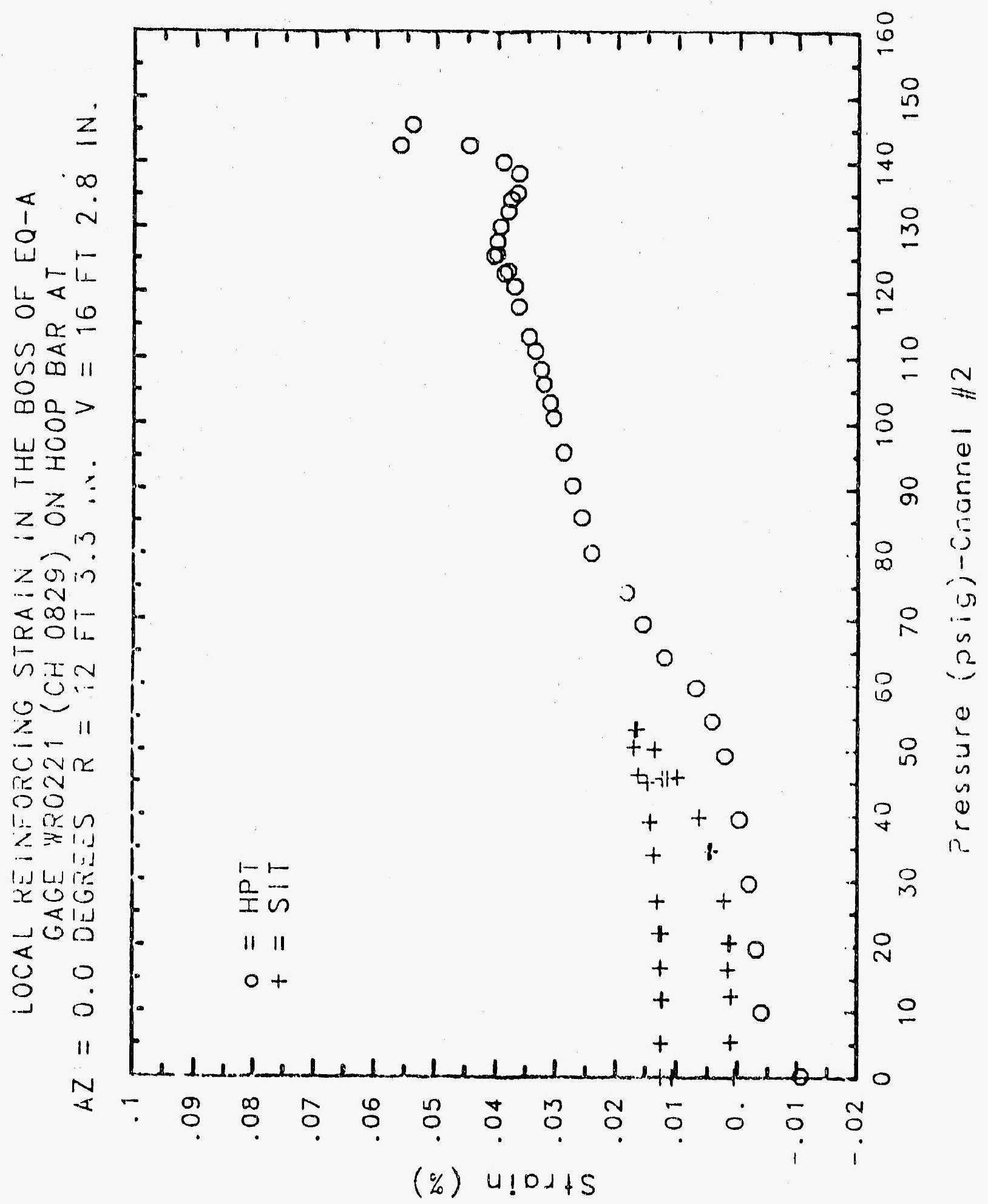




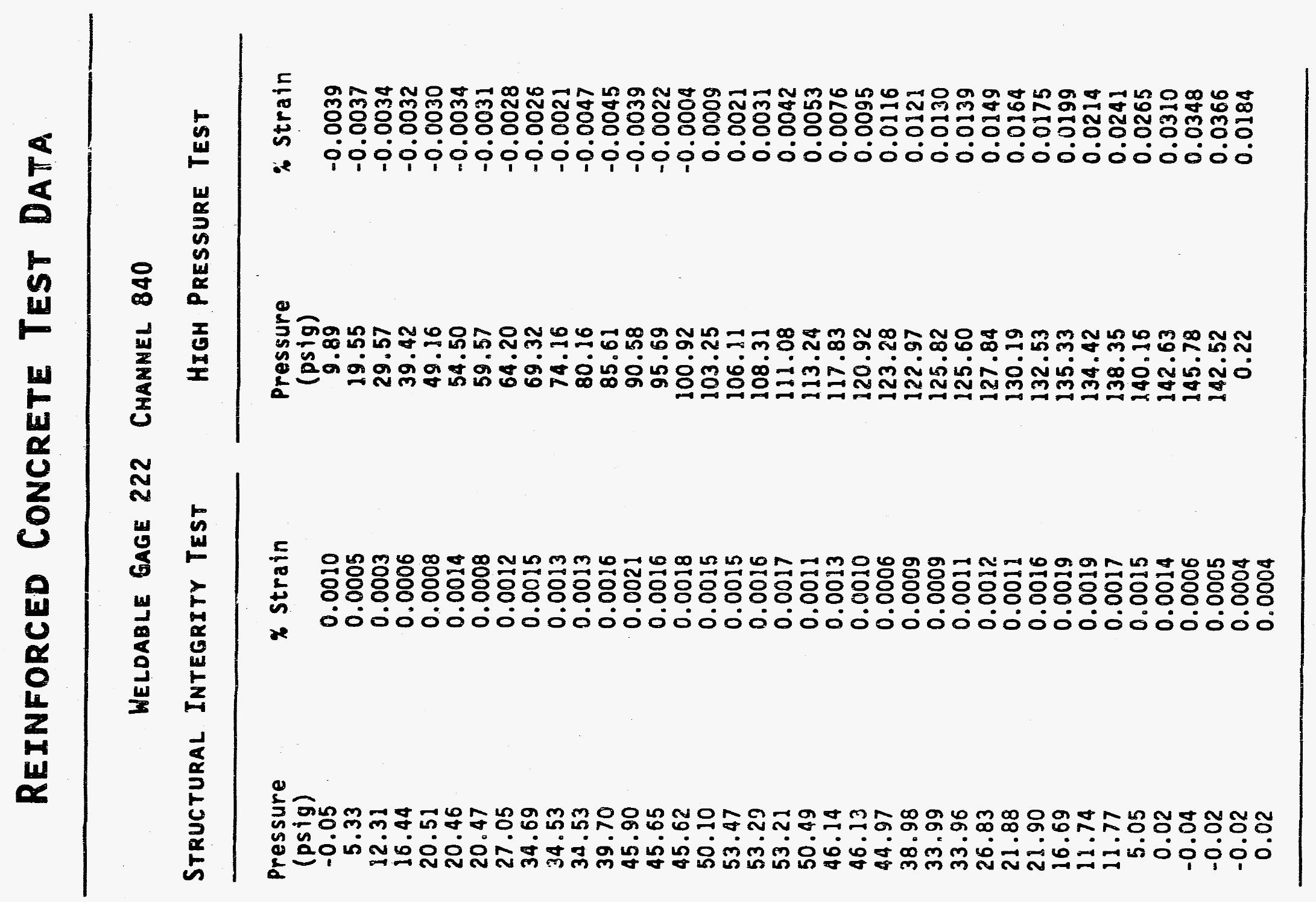




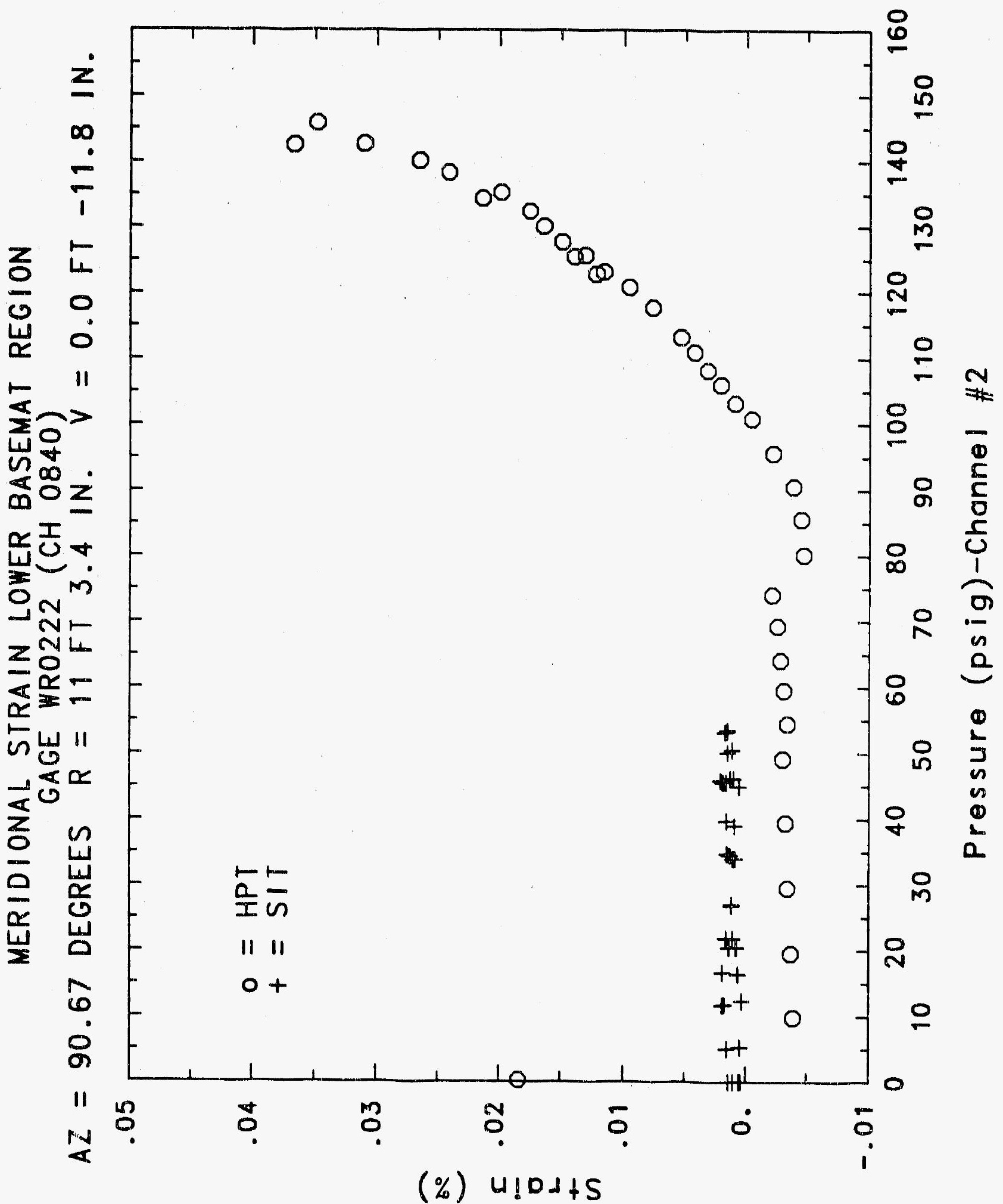




\section{Reinforced Concrete Test Data}

Weldable gage 223 Channel 841

Structural INTEgRITY TEST

High Pressure test

\begin{tabular}{|c|c|c|c|}
\hline $\begin{array}{c}\text { Pressure } \\
\text { (psig) } \\
-0.05 \\
5.33 \\
12.31 \\
16.44 \\
20.51 \\
20.46 \\
20.47 \\
27.05 \\
34.69 \\
34.53 \\
34.53 \\
39.70 \\
45.90 \\
45.65 \\
45.62 \\
50.10 \\
53.47 \\
53.29 \\
53.21 \\
50.49 \\
46.14 \\
46.13 \\
44.97 \\
38.98 \\
33.99 \\
33.96 \\
26.83 \\
21.88 \\
21.90 \\
16.69 \\
11.74 \\
11.77 \\
5.05 \\
0.02 \\
-0.04 \\
-0.02 \\
-0.02 \\
0.02 \\
\end{array}$ & $\begin{array}{l}\text { \% Strain } \\
0.0001 \\
0.0002 \\
0.0008 \\
0.0012 \\
0.0015 \\
0.0016 \\
0.0015 \\
0.0027 \\
0.0052 \\
0.0050 \\
0.0058 \\
0.0072 \\
0.0090 \\
0.0096 \\
0.0099 \\
0.0107 \\
0.0124 \\
0.0126 \\
0.0128 \\
0.0123 \\
0.0118 \\
0.0118 \\
0.0109 \\
0.0107 \\
0.0100 \\
0.0100 \\
0.0087 \\
0.0080 \\
0.0083 \\
0.0076 \\
0.0070 \\
0.0067 \\
0.0062 \\
0.0054 \\
0.0043 \\
0.0033 \\
0.0040 \\
0.0031\end{array}$ & $\begin{array}{c}\text { Pressure } \\
(\text { psig) } \\
9.89 \\
19.55 \\
29.57 \\
39.42 \\
49.16 \\
54.50 \\
59.57 \\
64.20 \\
69.32 \\
74.16 \\
80.16 \\
85.61 \\
90.58 \\
95.69 \\
100.92 \\
103.25 \\
106.11 \\
108.31 \\
111.08 \\
113.24 \\
117.83 \\
120.92 \\
123.28 \\
122.97 \\
125.82 \\
125.60 \\
127.84 \\
130.19 \\
132.53 \\
135.33 \\
134.42 \\
138.35 \\
140.16 \\
142.63 \\
145.78 \\
142.52 \\
0.22 \\
\end{array}$ & $\begin{array}{l}\text { \% Strain } \\
-0.0026 \\
-0.0008 \\
0.0011 \\
0.0033 \\
0.0052 \\
0.0067 \\
0.0076 \\
0.0120 \\
0.0155 \\
0.0230 \\
0.0389 \\
0.0470 \\
0.0545 \\
0.0621 \\
0.0670 \\
0.0698 \\
0.0725 \\
0.0742 \\
0.0777 \\
0.0807 \\
0.0871 \\
0.0926 \\
0.0973 \\
0.0985 \\
0.1032 \\
0.1043 \\
0.1076 \\
0.1143 \\
0.1214 \\
0.1290 \\
0.1300 \\
0.1369 \\
0.1418 \\
0.1479 \\
0.1551 \\
0.1554 \\
0.0333\end{array}$ \\
\hline
\end{tabular}




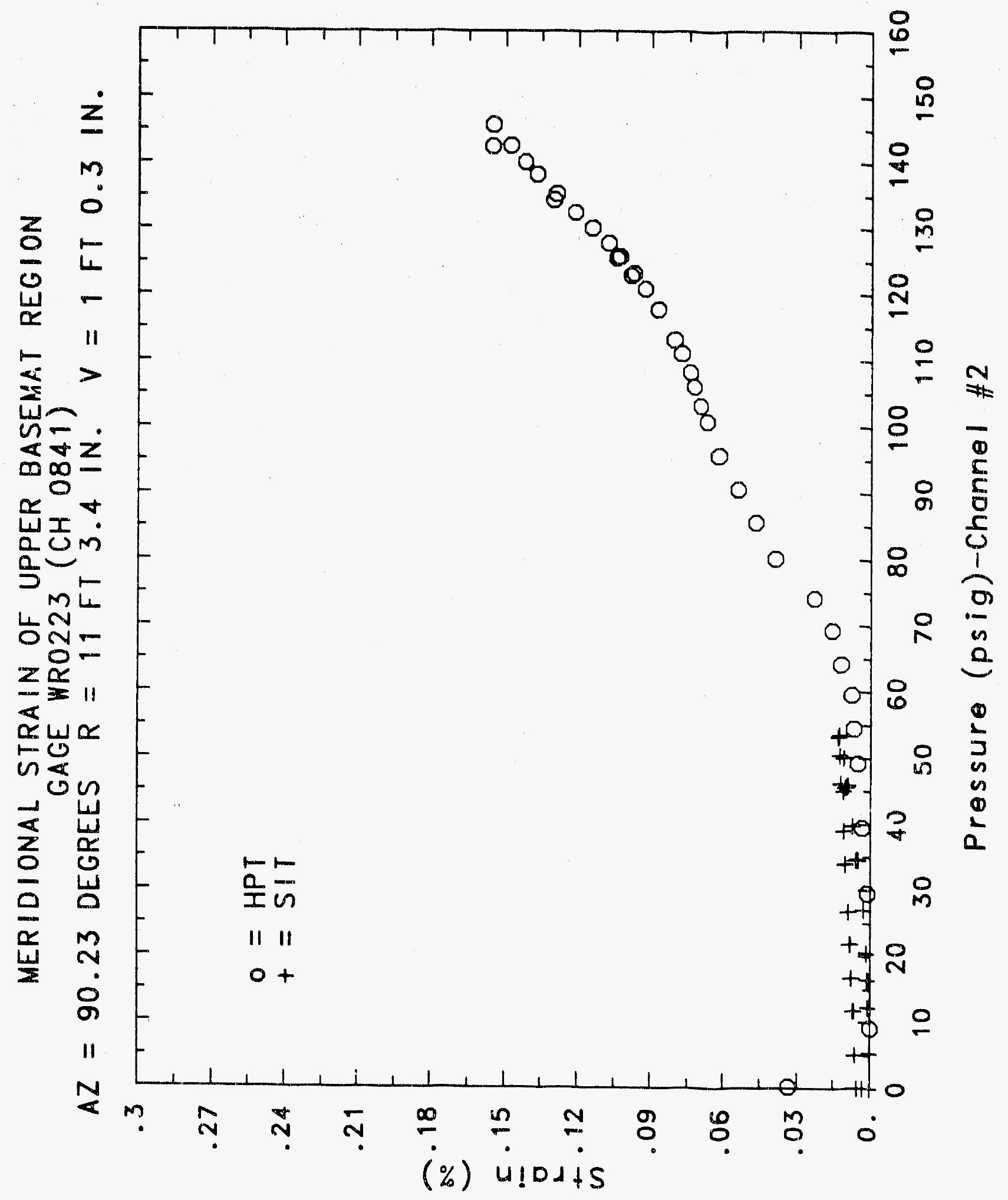




\section{Reinforced Concrete Test Data}

Weldable Gage 224 Channel 842

STRUCTURAL INTEGRITY TEST

High Pressure Test

\begin{tabular}{|c|c|c|c|}
\hline $\begin{array}{c}\text { Pressure } \\
\text { (psig) } \\
-0.05 \\
5.33 \\
12.31 \\
16.44 \\
20.51 \\
20.46 \\
20.47 \\
27.05 \\
34.69 \\
34.53 \\
34.53 \\
39.70 \\
45.90 \\
45.65 \\
45.62 \\
50.10 \\
53.47 \\
53.29 \\
53.21 \\
50.49 \\
46.14 \\
46.13 \\
44.97 \\
38.98 \\
33.99 \\
33.96 \\
26.83 \\
21.88 \\
21.90 \\
16.69 \\
11.74 \\
11.77 \\
5.05 \\
0.02 \\
-0.04 \\
-0.02 \\
-0.02 \\
0.02 \\
\end{array}$ & $\begin{array}{l}\text { \% strain } \\
-0.0001 \\
0.0011 \\
0.0038 \\
0.0056 \\
0.0080 \\
0.0092 \\
0.0097 \\
0.0160 \\
0.0253 \\
0.0271 \\
0.0270 \\
0.0329 \\
0.0413 \\
0.0417 \\
0.0410 \\
0.0457 \\
0.0511 \\
0.0515 \\
0.0514 \\
0.0493 \\
0.0464 \\
0.0463 \\
0.0441 \\
0.0404 \\
0.0372 \\
0.0380 \\
0.0332 \\
0.0295 \\
0.0294 \\
0.0251 \\
0.0214 \\
0.0206 \\
0.0153 \\
0.0113 \\
0.0072 \\
0.0063 \\
0.0067 \\
0.0064\end{array}$ & $\begin{array}{c}\text { Pressure } \\
(\text { psig) } \\
9.89 \\
19.55 \\
29.57 \\
39.42 \\
49.16 \\
54.50 \\
59.57 \\
64.20 \\
69.32 \\
74.16 \\
80.16 \\
85.61 \\
90.58 \\
95.69 \\
100.92 \\
103.25 \\
106.11 \\
108.31 \\
111.08 \\
113.24 \\
117.83 \\
120.92 \\
123.28 \\
122.97 \\
125.82 \\
125.60 \\
127.84 \\
130.19 \\
132.53 \\
135.33 \\
124.42 \\
138.35 \\
140.16 \\
142.63 \\
145.78 \\
142.52 \\
0.22 \\
\end{array}$ & $\begin{array}{l}\% \text { Strain } \\
0.0025 \\
0.0111 \\
0.0208 \\
0.0297 \\
0.0390 \\
0.0447 \\
0.0506 \\
0.0600 \\
0.0668 \\
0.0743 \\
0.0864 \\
0.0974 \\
0.1078 \\
0.1196 \\
0.1294 \\
0.1343 \\
0.1391 \\
0.1432 \\
0.1489 \\
0.1533 \\
0.1619 \\
0.1673 \\
0.1713 \\
0.1712 \\
0.1768 \\
0.1769 \\
0.1822 \\
0.1917 \\
0.2035 \\
0.2136 \\
0.2110 \\
0.2174 \\
0.2223 \\
0.2208 \\
0.2342 \\
0.3301 \\
0.1537\end{array}$ \\
\hline
\end{tabular}




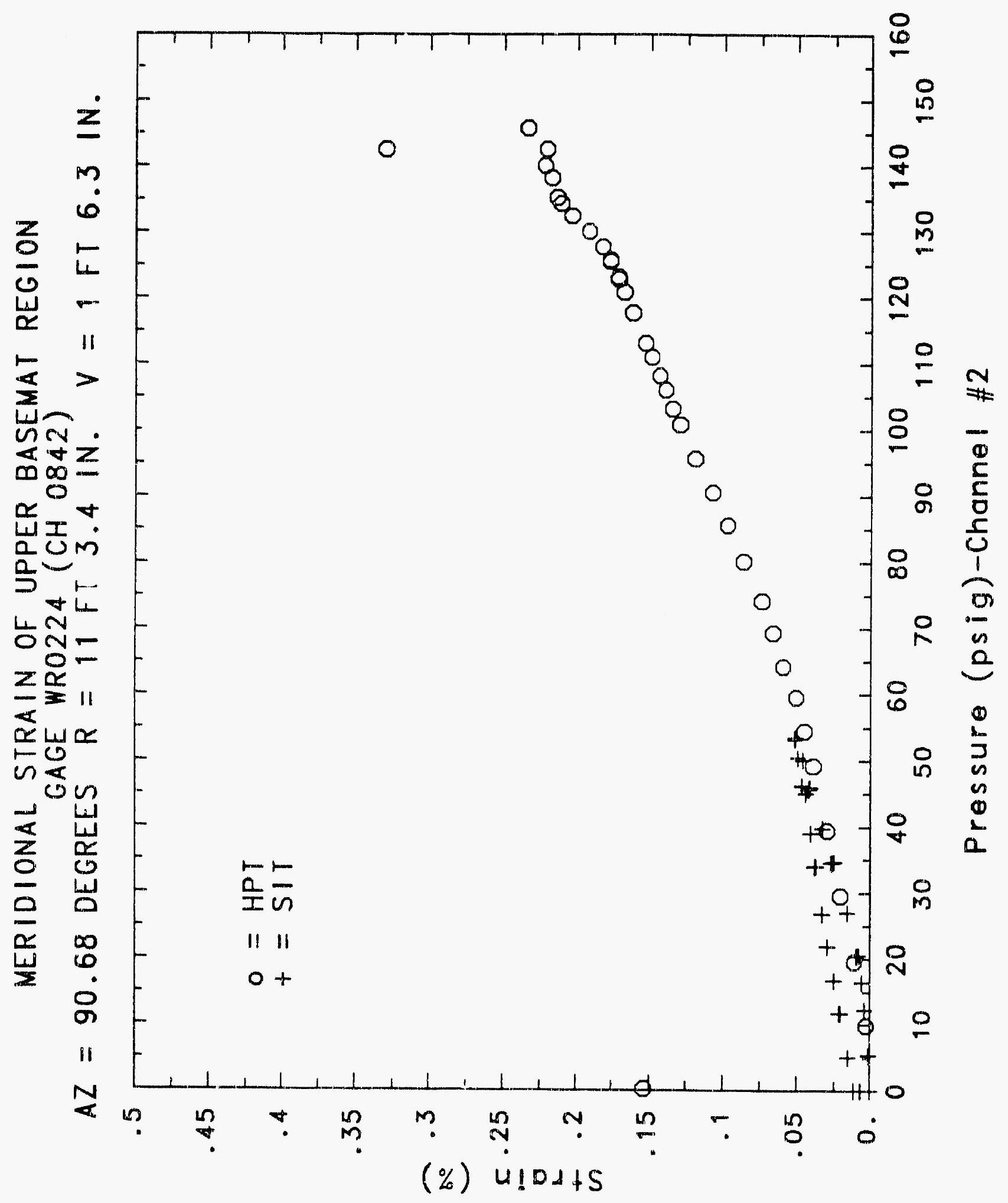




\section{Reinforced Concrete Test Data}

Weldable gage 225 Channel 843

Structural Integrity Test

\begin{tabular}{|c|c|c|c|}
\hline $\begin{array}{c}\text { Pressure } \\
\text { (psig) } \\
-0.05 \\
5.33 \\
12.31 \\
16.44 \\
20.51 \\
20.46 \\
20.47 \\
27.05 \\
34.69 \\
34.53 \\
34.53 \\
39.70 \\
45.90 \\
45.65 \\
45.62 \\
50.10 \\
53.47 \\
53.29 \\
53.21 \\
50.49 \\
46.14 \\
46.13 \\
44.97 \\
38.98 \\
33.99 \\
33.96 \\
26.83 \\
21.88 \\
21.90 \\
16.69 \\
11.74 \\
11.77 \\
5.05 \\
0.02 \\
-0.04 \\
-0.02 \\
-0.02 \\
0.02 \\
\end{array}$ & $\begin{array}{c}\text { \% Strain } \\
\text {-0.0004 } \\
0.0025 \\
0.0075 \\
0.0108 \\
0.0150 \\
0.0160 \\
0.0170 \\
0.0279 \\
0.0419 \\
0.0436 \\
0.0440 \\
0.0515 \\
0.0619 \\
0.0614 \\
0.0607 \\
0.0671 \\
0.0724 \\
0.0723 \\
0.0717 \\
0.0693 \\
0.0643 \\
0.0636 \\
0.0616 \\
0.0557 \\
0.0502 \\
0.0523 \\
0.0442 \\
0.0389 \\
0.0392 \\
0.0324 \\
0.0266 \\
0.0253 \\
0.0174 \\
0.0111 \\
0.0079 \\
0.0074 \\
0.0080 \\
0.0081\end{array}$ & $\begin{array}{c}\text { Pressure } \\
(\text { ps } 1 \text { g) } \\
9.89 \\
19.55 \\
29.57 \\
39.42 \\
49.16 \\
54.50 \\
59.57 \\
64.20 \\
69.32 \\
74.16 \\
80.16 \\
85.61 \\
90.58 \\
95.69 \\
100.92 \\
103.25 \\
106.11 \\
108.31 \\
111.08 \\
113.24 \\
117.83 \\
120.92 \\
123.28 \\
122.97 \\
125.82 \\
125.60 \\
127.84 \\
130.19 \\
132.53 \\
135.33 \\
134.42 \\
138.35 \\
140.16 \\
142.63 \\
145.78 \\
142.52 \\
0.22 \\
\end{array}$ & $\begin{array}{l}\text { \% Strain } \\
0.0072 \\
0.0200 \\
0.0335 \\
0.0472 \\
0.0596 \\
0.0671 \\
0.0743 \\
0.0827 \\
0.0901 \\
0.0984 \\
0.1100 \\
0.1201 \\
0.1281 \\
0.1365 \\
0.1457 \\
0.1509 \\
0.1568 \\
0.1623 \\
0.1709 \\
0.1800 \\
0.2019 \\
0.2173 \\
0.2313 \\
0.2336 \\
0.2553 \\
0.2605 \\
0.2799 \\
0.3498 \\
0.5162 \\
0.6828 \\
0.7098 \\
0.8120 \\
0.8866 \\
0.9836 \\
1.0554 \\
1.0758 \\
0.7259\end{array}$ \\
\hline
\end{tabular}

High Pressure test

ressure

9.89

19.55

29.57

49.16

54.50

59.57

64.20

69.32

85.61

90.58

103.25

106.11

11.08

113.24

117.83

120.92

123.28

125.60

127.84

130.19

32.53

35.33

40.16

142.63

45.78

2.52

0.0080

0.0081
1.0758

strain

0.0072

0.0200

0.0472

0.0671

0.0743

0.0827

0.0901

0.1100

0.1365

0.1457

0.1623

0.1800

0.2019

0.2173

0.2313

0.2605

0.2799

0.3498

0.5162

0.6828

0.7098

0.9836

0.7259 


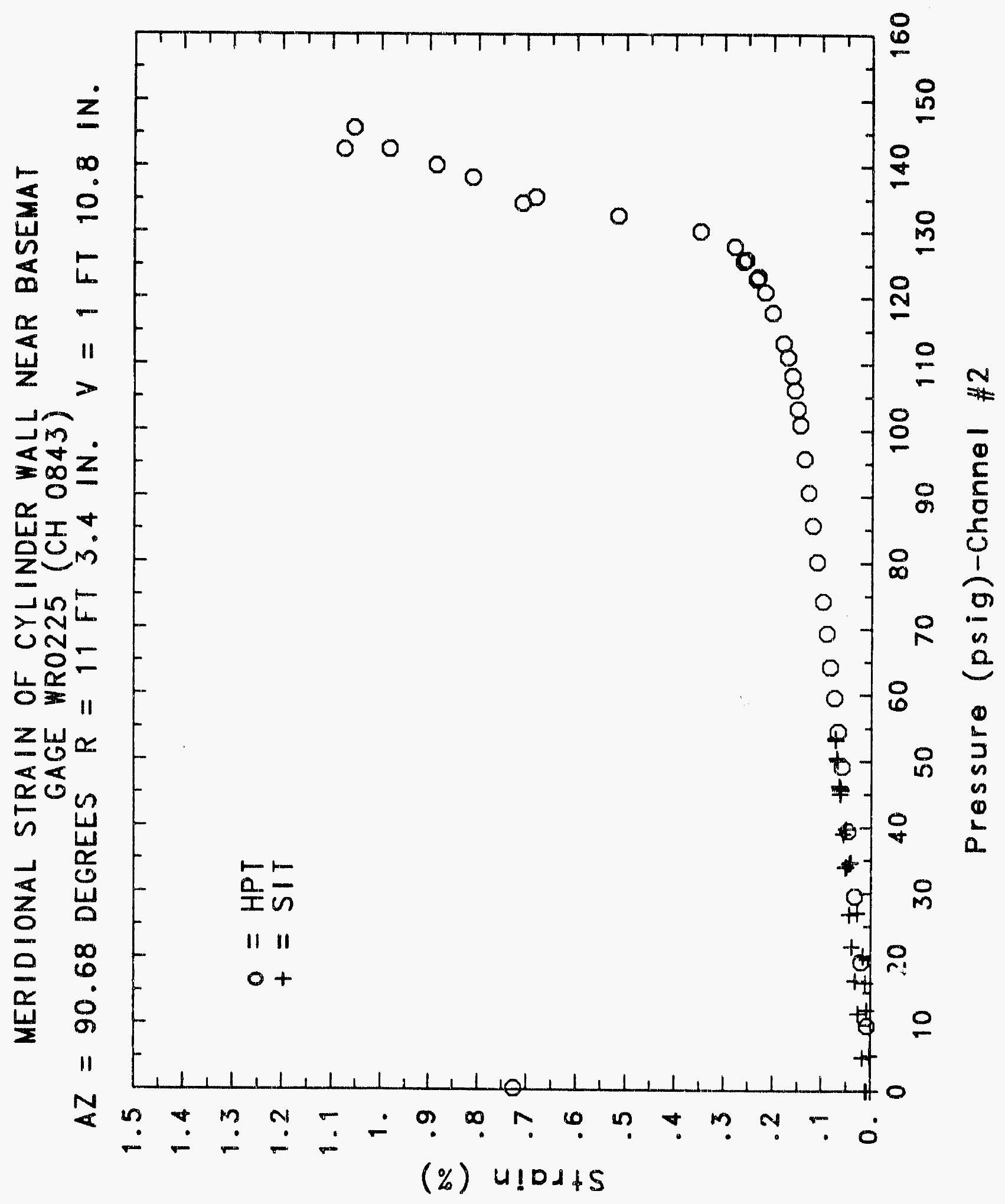




\section{Reinforced Concrete Test Data}

Weldable Gage 226 Chanmel 844

Structural Integrity Test

\begin{tabular}{|c|c|}
\hline $\begin{array}{c}\text { Pressure } \\
\text { (psig) } \\
-0.05 \\
5.33 \\
12.31 \\
16.44 \\
20.51 \\
20.46 \\
20.47 \\
27.05 \\
34.69 \\
34.53 \\
34.53 \\
39.70 \\
45.90 \\
45.65 \\
45.62 \\
50.10 \\
53.47 \\
53.29 \\
53.21 \\
50.49 \\
46.14 \\
46.13 \\
44.97 \\
38.98 \\
33.99 \\
33.96 \\
26.83 \\
21.88 \\
21.90 \\
16.69 \\
11.74 \\
11.77 \\
5.05 \\
0.02 \\
-0.04 \\
-0.02 \\
-0.02 \\
0.02 \\
\end{array}$ & $\begin{array}{l}\text { \% Strain } \\
0.0005 \\
0.0006 \\
0.0028 \\
0.0038 \\
0.0054 \\
0.0059 \\
0.0068 \\
0.0117 \\
0.0207 \\
0.0224 \\
0.0229 \\
0.0267 \\
0.0341 \\
0.0344 \\
0.0342 \\
0.0378 \\
0.0418 \\
0.0421 \\
0.0421 \\
0.0407 \\
0.0389 \\
0.0385 \\
0.0385 \\
0.0353 \\
0.0326 \\
0.0333 \\
0.0293 \\
0.0262 \\
0.0251 \\
0.0216 \\
0.0181 \\
0.0179 \\
0.0137 \\
0.0101 \\
0.0093 \\
0.0093 \\
0.0085 \\
0.0095\end{array}$ \\
\hline
\end{tabular}

High Pressure Test

\begin{tabular}{|c|c|}
\hline $\begin{array}{c}\text { Pressure } \\
\text { (psig) } \\
9.89 \\
19.55 \\
29.57 \\
39.42 \\
49.16 \\
54.50 \\
59.57 \\
64.20 \\
69.32 \\
74.16 \\
80.16 \\
85.61 \\
90.58 \\
95.69 \\
100.92 \\
103.25 \\
106.11 \\
108.31 \\
111.08 \\
113.24 \\
117.83 \\
120.92 \\
123.28 \\
122.97 \\
125.82 \\
125.60 \\
127.84 \\
130.19 \\
132.53 \\
135.33 \\
134.42 \\
138.35 \\
140.16 \\
142.63 \\
145.78 \\
142.52 \\
0.22 \\
\end{array}$ & $\begin{array}{l}\text { \% Strain } \\
0.0011 \\
0.0075 \\
0.0144 \\
0.0211 \\
0.0280 \\
0.0319 \\
0.0369 \\
0.0421 \\
0.0472 \\
0.0521 \\
0.0616 \\
0.0699 \\
0.0769 \\
0.0846 \\
0.0919 \\
0.0958 \\
0.0997 \\
0.1030 \\
0.1076 \\
0.1109 \\
0.1170 \\
0.1217 \\
0.1253 \\
0.1259 \\
0.1313 \\
0.1321 \\
0.1361 \\
0.1440 \\
0.1529 \\
0.1609 \\
0.1610 \\
0.1697 \\
0.1746 \\
0.1827 \\
0.1912 \\
0.1905 \\
0.0433\end{array}$ \\
\hline
\end{tabular}




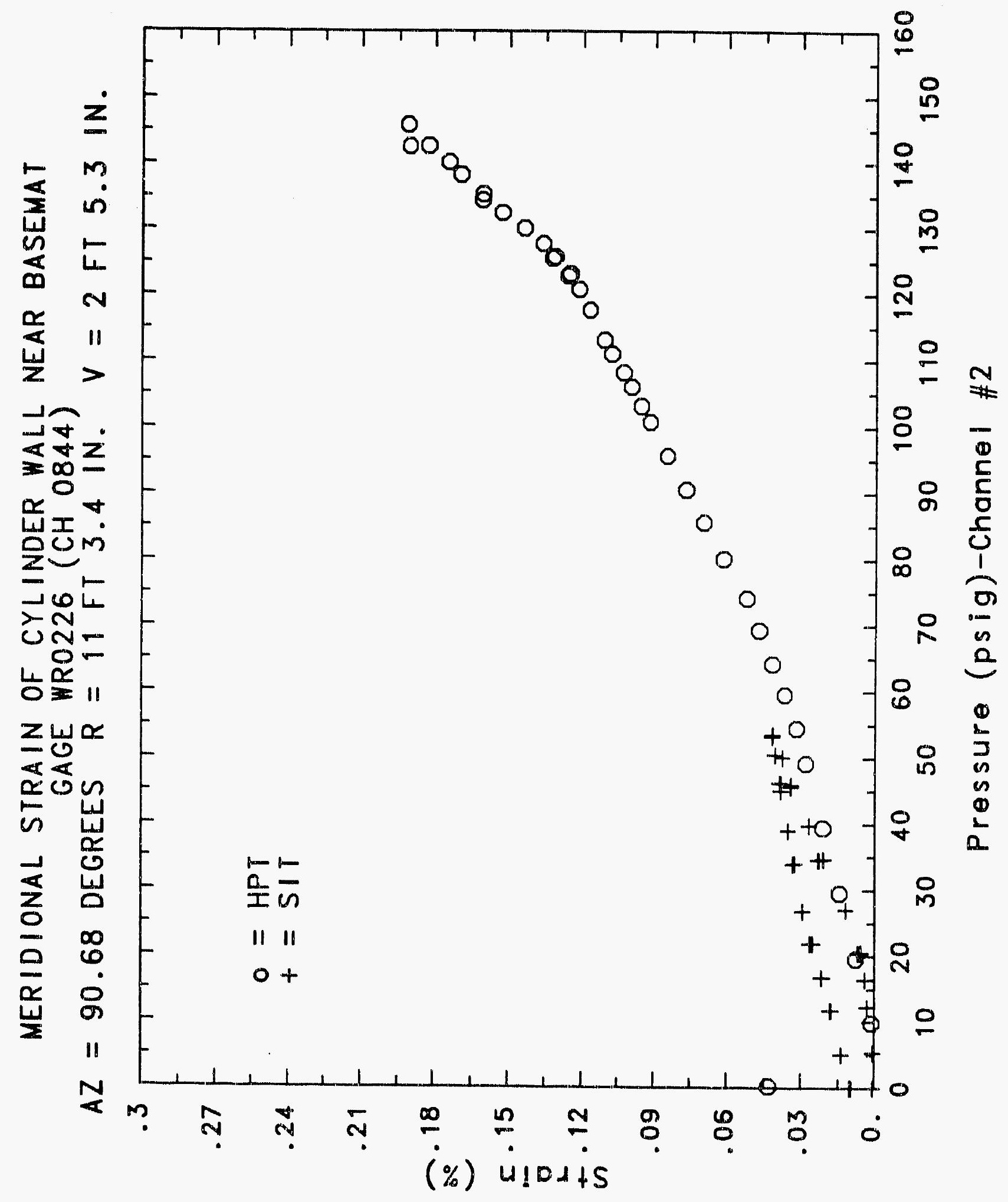




\section{Reinforced Concrete Test Data}

Weldable gage 227 Chanmel 845

StRUCTURAL INTEgRITY TEST

high Pressure test

\begin{tabular}{|c|c|c|c|}
\hline $\begin{array}{c}\text { Pressure } \\
(\text { psig) } \\
-0.05 \\
5.33 \\
12.31 \\
16.44 \\
20.51 \\
20.46 \\
20.47 \\
27.05 \\
34.69 \\
34.53 \\
34.53 \\
39.70 \\
45.90 \\
45.65 \\
45.62 \\
50.10 \\
53.47 \\
53.29 \\
53.21 \\
50.49 \\
46.14 \\
46.13 \\
44.97 \\
38.98 \\
33.99 \\
33.96 \\
26.83 \\
21.88 \\
21.90 \\
16.69 \\
11.74 \\
11.77 \\
5.05 \\
0.02 \\
-0.04 \\
-0.02 \\
-0.02 \\
0.02 \\
\end{array}$ & $\begin{array}{l}\text { \% Strain } \\
0.0002 \\
0.0001 \\
0.0022 \\
0.0025 \\
0.0038 \\
0.0035 \\
0.0036 \\
0.0059 \\
0.0096 \\
0.0094 \\
0.0096 \\
0.0105 \\
0.0145 \\
0.0148 \\
0.0149 \\
0.0172 \\
0.0190 \\
0.0190 \\
0.0193 \\
0.0190 \\
0.0175 \\
0.0176 \\
0.0171 \\
0.0152 \\
0.0136 \\
0.0133 \\
0.0105 \\
0.0080 \\
0.0068 \\
0.0050 \\
0.0035 \\
0.0041 \\
0.0023 \\
0.0008 \\
0.0025 \\
0.0031 \\
0.0023 \\
0.0024\end{array}$ & $\begin{array}{c}\text { Pressure } \\
\text { (psig) } \\
9.89 \\
19.55 \\
29.57 \\
39.42 \\
49.16 \\
54.50 \\
59.57 \\
64.20 \\
69.32 \\
74.16 \\
80.16 \\
85.61 \\
90.58 \\
95.69 \\
100.92 \\
103.25 \\
106.11 \\
108.31 \\
111.08 \\
113.24 \\
117.83 \\
120.92 \\
123.28 \\
122.97 \\
125.82 \\
125.60 \\
127.84 \\
130.19 \\
132.53 \\
135.33 \\
134.42 \\
138.35 \\
140.16 \\
142.63 \\
145.78 \\
142.52 \\
0.22 \\
\end{array}$ & $\begin{array}{c}\text { \% Strain } \\
-0.0006 \\
0.0027 \\
0.0053 \\
0.0081 \\
0.0107 \\
0.0128 \\
0.0139 \\
0.0160 \\
0.0177 \\
0.0188 \\
0.0220 \\
0.0241 \\
0.0254 \\
0.0267 \\
0.0275 \\
0.0283 \\
0.0290 \\
0.0295 \\
0.0302 \\
0.0312 \\
0.0328 \\
0.0345 \\
0.0352 \\
0.0359 \\
0.0369 \\
0.0376 \\
0.0389 \\
0.0403 \\
0.0432 \\
0.0459 \\
0.0471 \\
0.0513 \\
0.0540 \\
0.0580 \\
0.0605 \\
0.0602 \\
0.0051\end{array}$ \\
\hline
\end{tabular}




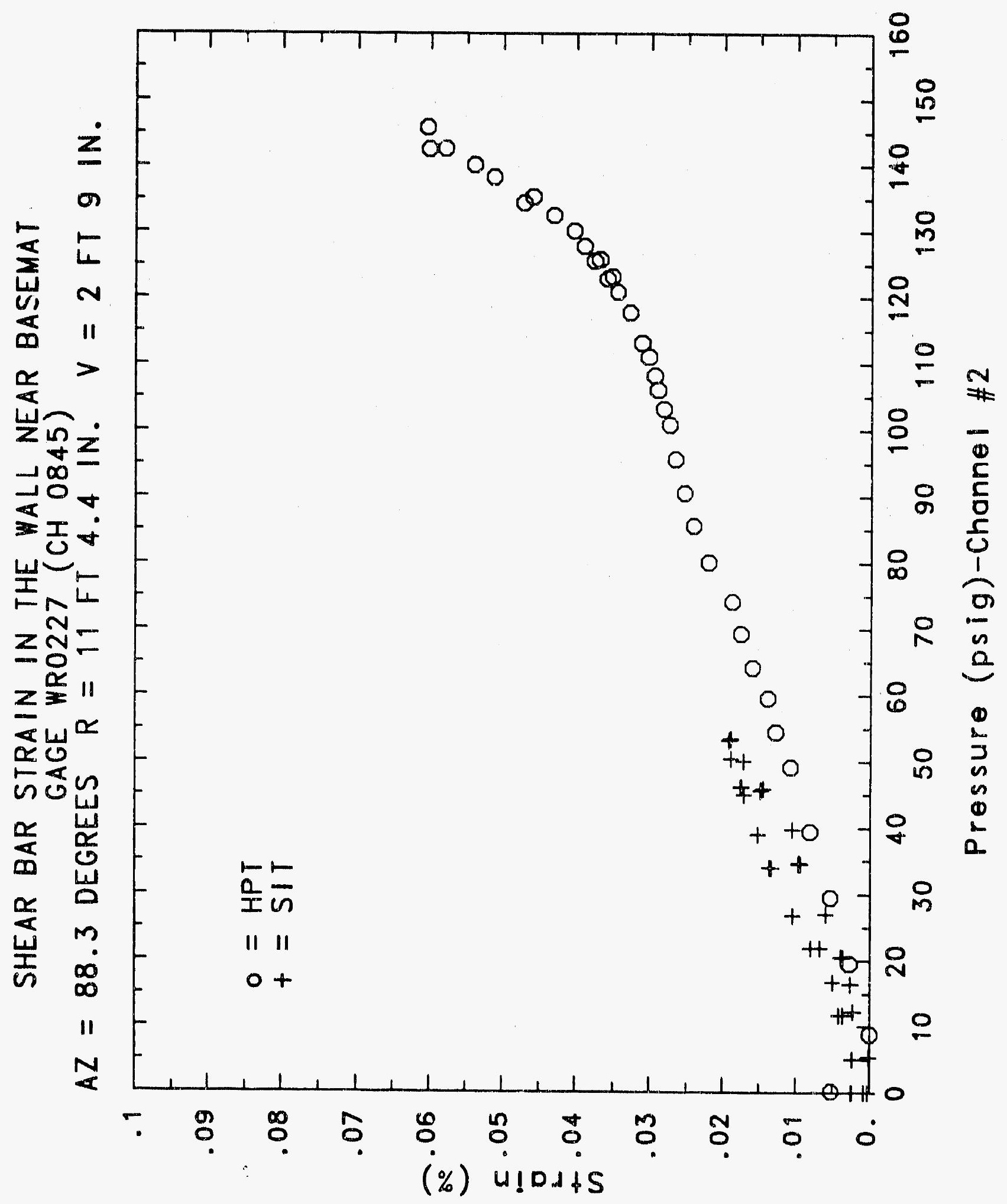




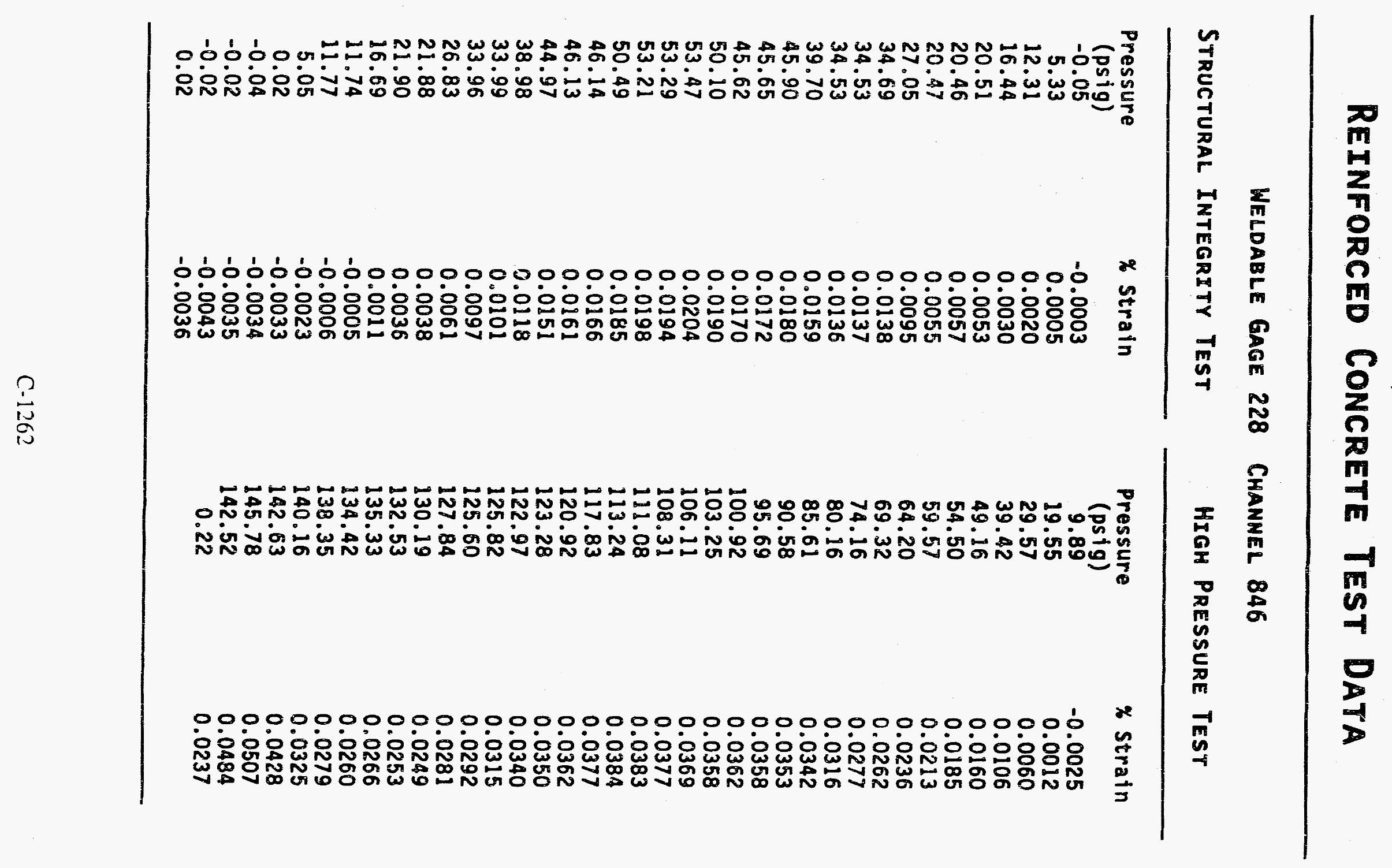




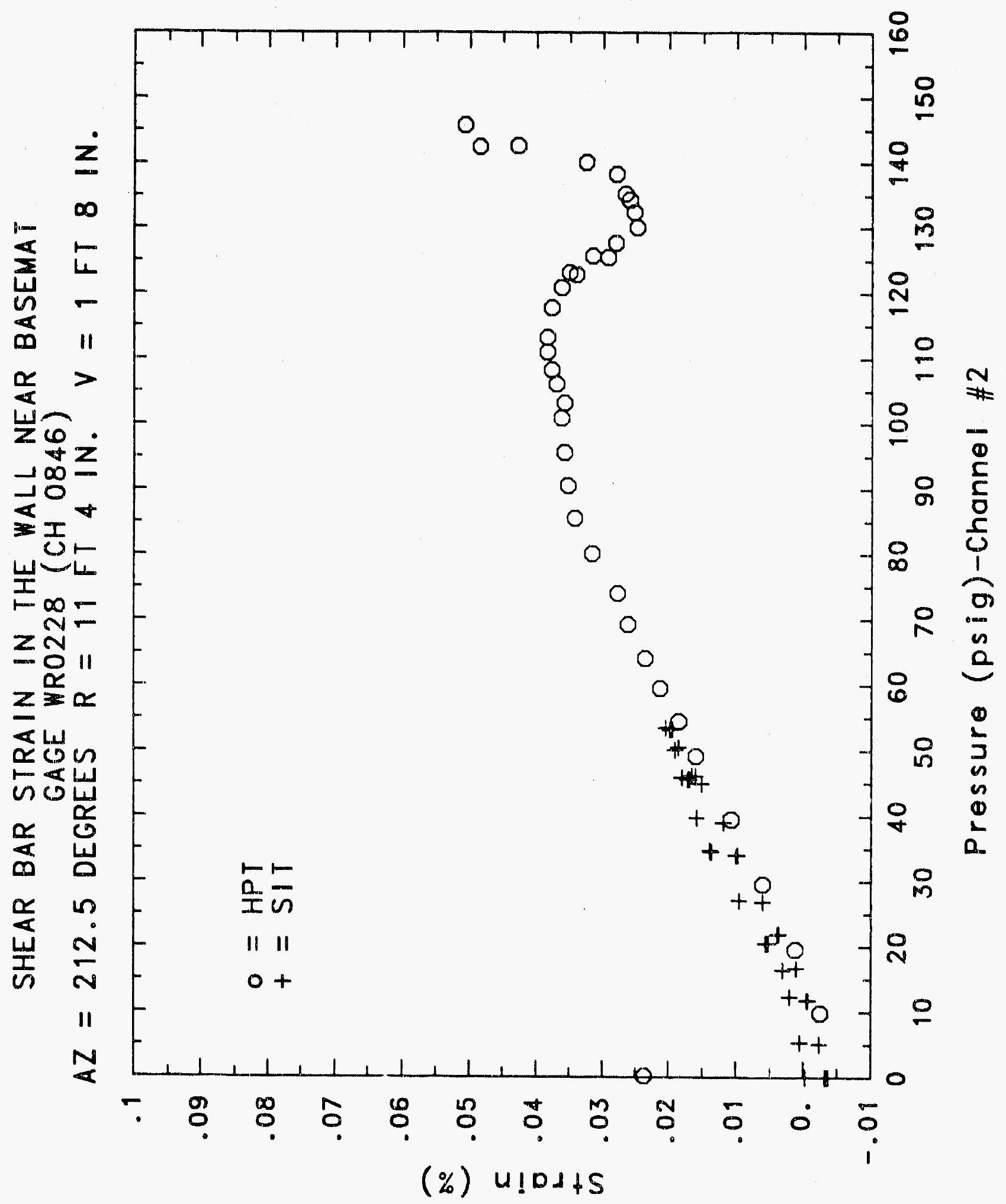




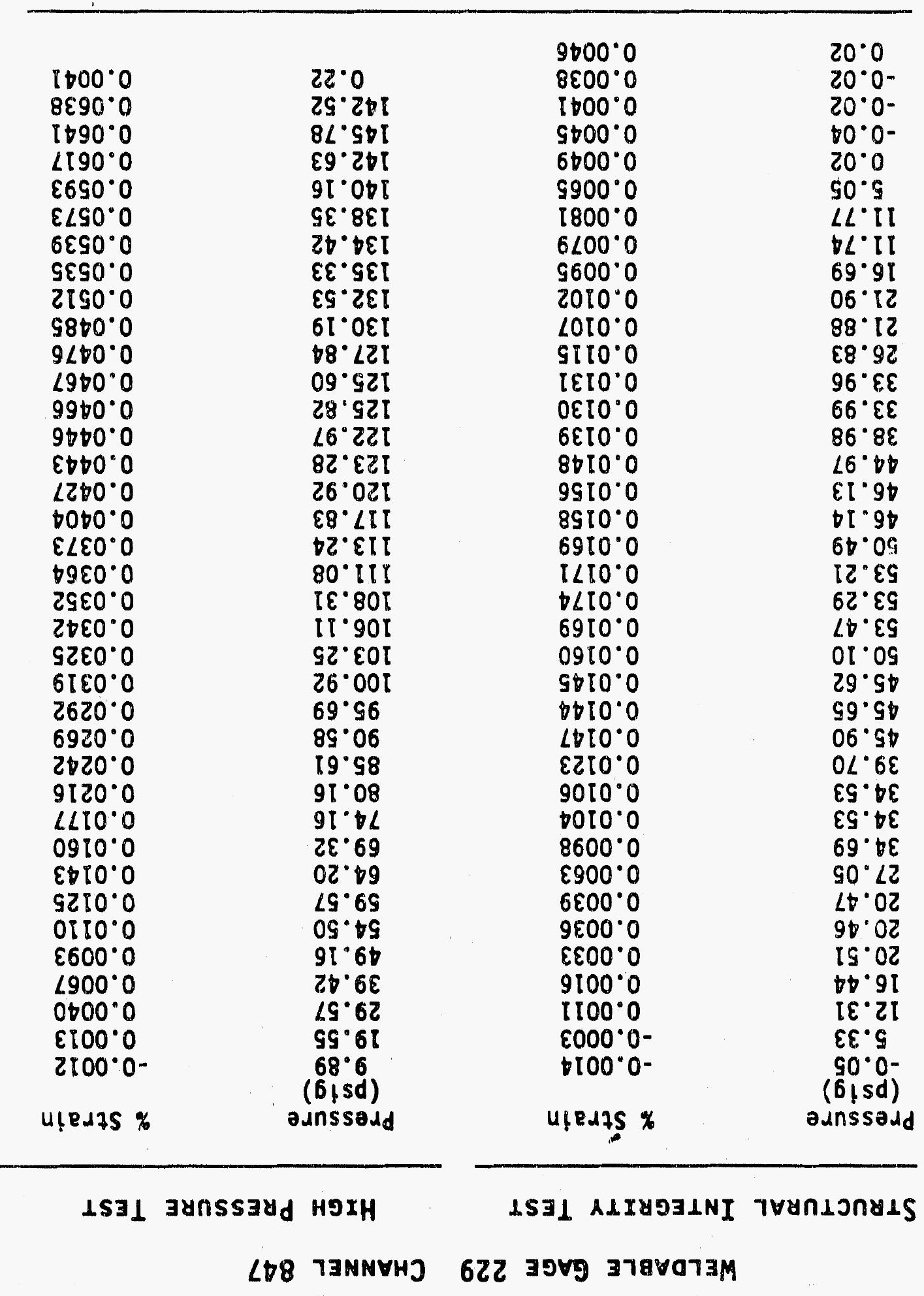

\section{$\forall \perp \forall Q \perp S \exists \perp \exists \perp \exists \& J N O J$ ajJUOJNIJY}




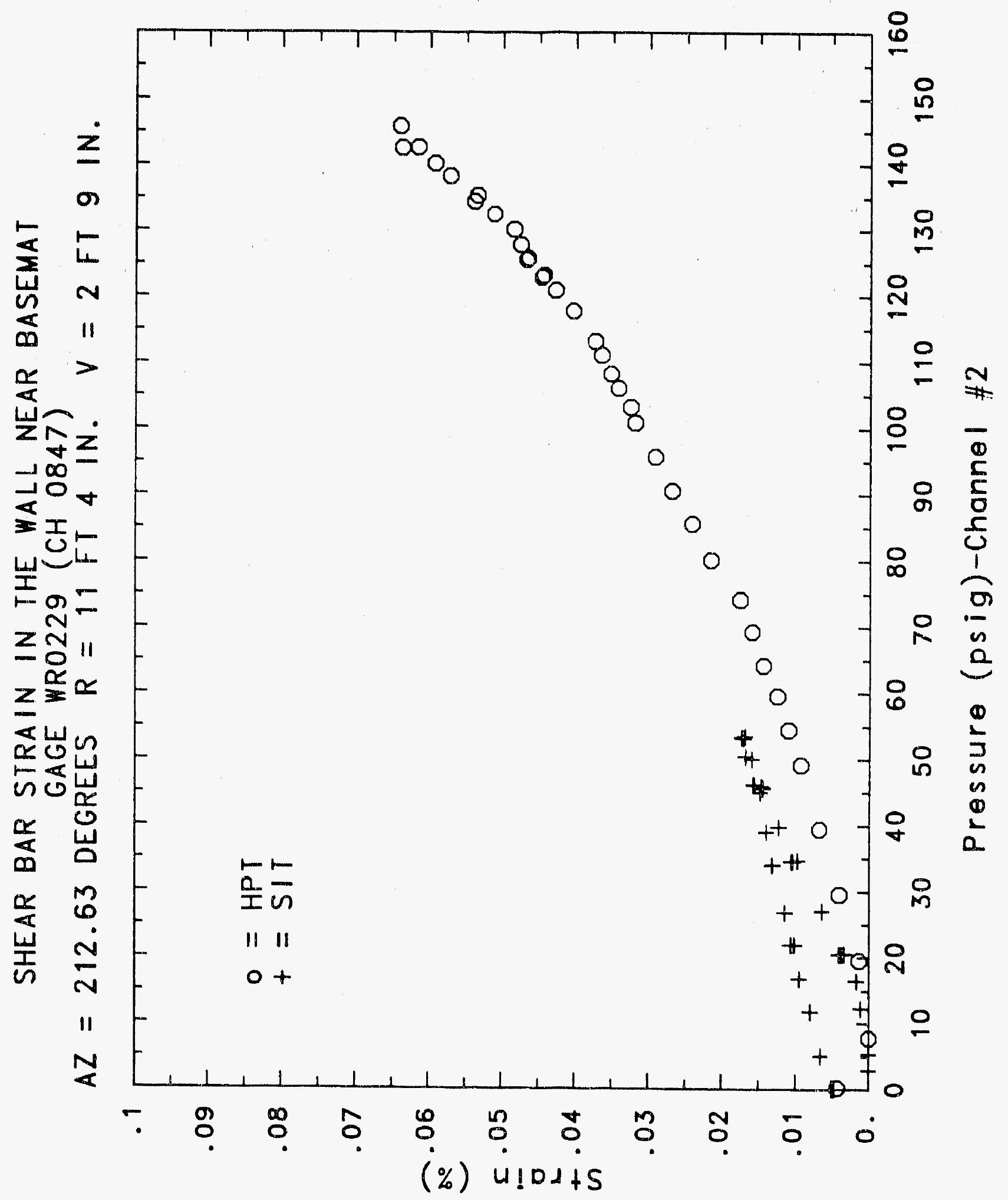




\section{Reinforced Conclete Test Data}

Weldable Gage 230 Channel 848

Structural Integrity Test

High Pressure test

\begin{tabular}{|c|c|c|c|}
\hline $\begin{array}{c}\text { Pressure } \\
\text { (psig) } \\
-0.05 \\
5.33 \\
12.31 \\
16.44 \\
20.51 \\
20.46 \\
20.47 \\
27.05 \\
34.69 \\
34.53 \\
34.53 \\
39.70 \\
45.90 \\
45.65 \\
45.62 \\
50.10 \\
53.47 \\
53.29 \\
53.21 \\
50.49 \\
46.14 \\
46.13 \\
44.97 \\
38.98 \\
33.99 \\
33.96 \\
26.83 \\
21.88 \\
21.90 \\
16.69 \\
11.74 \\
11.77 \\
5.05 \\
0.02 \\
-0.04 \\
-0.02 \\
-0.02 \\
0.02 \\
\end{array}$ & $\begin{array}{c}\text { \% Strain } \\
-0.0009 \\
0.0003 \\
0.0026 \\
0.0044 \\
0.0076 \\
0.0078 \\
0.0081 \\
0.0139 \\
0.0194 \\
0.0196 \\
0.0193 \\
0.0226 \\
0.0256 \\
0.0253 \\
0.0254 \\
0.0279 \\
0.0294 \\
0.0292 \\
0.0290 \\
0.0279 \\
0.0255 \\
0.0258 \\
0.0234 \\
0.0205 \\
0.0177 \\
0.0174 \\
0.0135 \\
0.0102 \\
0.0100 \\
0.0071 \\
0.0050 \\
0.0050 \\
0.0020 \\
0.0011 \\
0.0008 \\
-0.0012 \\
-0.0012 \\
0.0001\end{array}$ & $\begin{array}{c}\text { Pressure } \\
\text { (psig) } \\
9.89 \\
19.55 \\
29.57 \\
39.42 \\
49.16 \\
54.50 \\
59.57 \\
64.20 \\
69.32 \\
74.16 \\
80.16 \\
85.61 \\
90.58 \\
95.69 \\
100.92 \\
103.25 \\
106.11 \\
108.31 \\
111.08 \\
113.24 \\
117.83 \\
120.92 \\
123.28 \\
122.97 \\
125.82 \\
125.60 \\
127.84 \\
130.19 \\
132.53 \\
135.33 \\
134.42 \\
138.35 \\
140.16 \\
142.63 \\
145.78 \\
142.52 \\
0.22 \\
\end{array}$ & $\begin{array}{l}\text { * Strain } \\
0.0007 \\
0.0061 \\
0.0120 \\
0.0169 \\
0.0222 \\
0.0247 \\
0.0274 \\
0.0300 \\
0.0318 \\
0.0348 \\
0.0378 \\
0.0411 \\
0.0421 \\
0.0445 \\
0.0468 \\
0.0475 \\
0.0482 \\
0.0490 \\
0.0494 \\
0.0497 \\
0.0498 \\
0.0495 \\
0.0496 \\
0.0487 \\
0.0506 \\
0.0501 \\
0.0525 \\
0.0547 \\
0.0587 \\
0.0645 \\
0.0642 \\
0.0688 \\
0.0714 \\
0.0723 \\
0.0726 \\
0.0686 \\
0.0434\end{array}$ \\
\hline
\end{tabular}




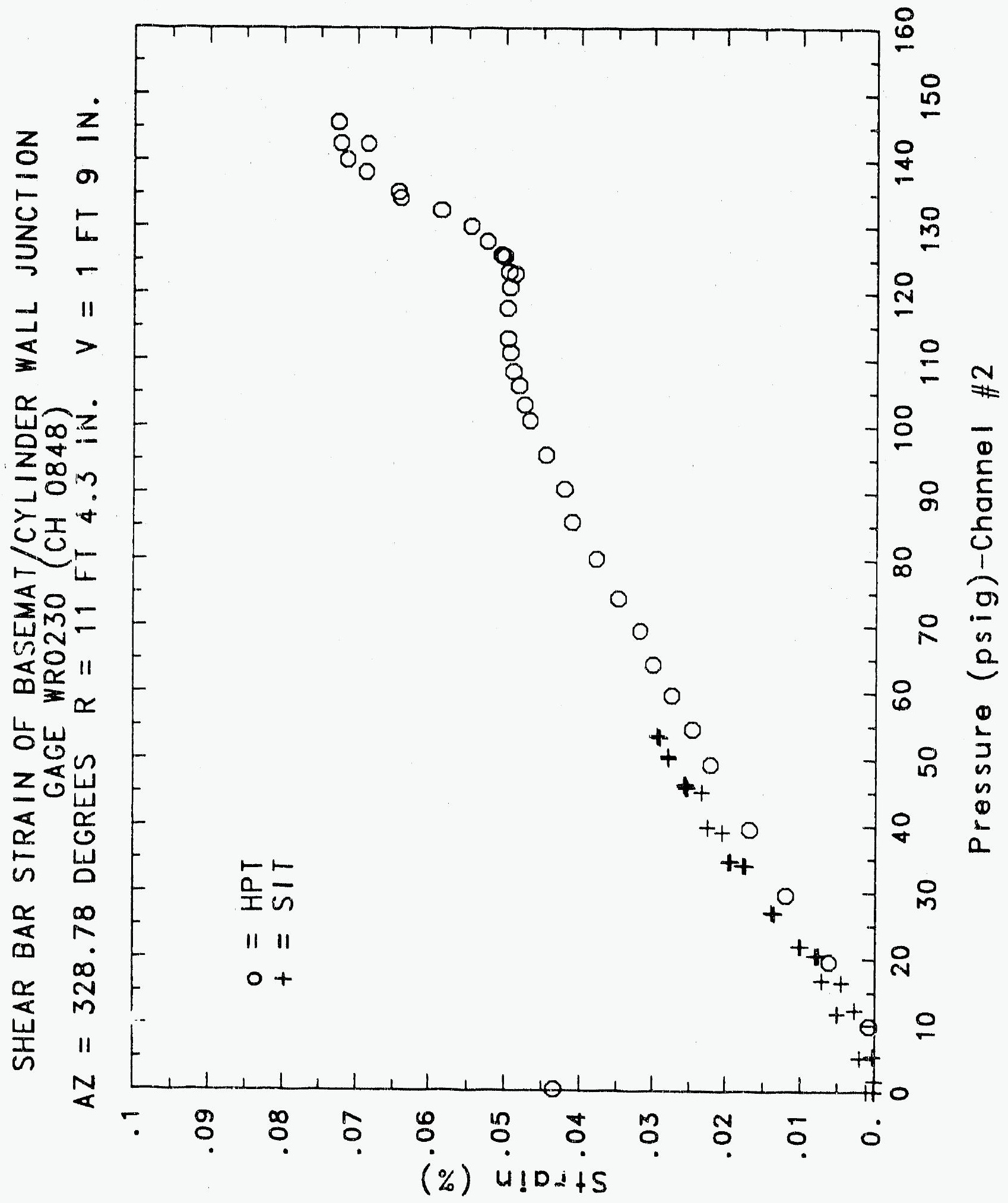




\section{Reinforced Concrete Test Data}

\begin{tabular}{|c|c|c|c|}
\hline STRUCTURAL & $\begin{array}{l}\text { WELDABLE GAGE } 231 \\
\text { INTEGRITY TEST }\end{array}$ & $\begin{array}{r}\text { Channel } 849 \\
\text { High Pressure }\end{array}$ & TEST \\
\hline $\begin{array}{c}\text { Pressure } \\
\text { (psig) } \\
-0.05 \\
5.33 \\
12.31 \\
16.44 \\
20.51 \\
20.46 \\
20.47 \\
27.05 \\
34.69 \\
34.53 \\
34.53 \\
39.70 \\
45.90 \\
45.65 \\
45.62 \\
50.10 \\
53.47 \\
53.29 \\
53.21 \\
50.49 \\
46.14 \\
46.13 \\
44.97 \\
38.98 \\
33.99 \\
33.96 \\
26.83 \\
21.88 \\
21.90 \\
16.69 \\
11.74 \\
11.77 \\
5.05 \\
0.02 \\
-0.04 \\
-0.02 \\
-0.02 \\
0.02 \\
\end{array}$ & $\begin{array}{l}\text { Strain } \\
0.0011 \\
0.0031 \\
0.0049 \\
0.0054 \\
0.0064 \\
0.0068 \\
0.0068 \\
0.0080 \\
0.0101 \\
0.0110 \\
0.0110 \\
0.0123 \\
0.0141 \\
0.0138 \\
0.0142 \\
0.0147 \\
0.0157 \\
0.0158 \\
0.0154 \\
0.0153 \\
0.0140 \\
0.0136 \\
0.0148 \\
0.0141 \\
0.0135 \\
0.0135 \\
0.0121 \\
0.0112 \\
0.0111 \\
0.0103 \\
0.0096 \\
0.0095 \\
0.0079 \\
0.0072 \\
0.0037 \\
0.0050 \\
0.0069 \\
0.0073\end{array}$ & $\begin{array}{c}\text { Pressure } \\
\text { (psig) } \\
9.89 \\
19.55 \\
29.57 \\
39.42 \\
49.16 \\
54.50 \\
5.57 \\
54.20 \\
69.32 \\
74.16 \\
80.16 \\
85.61 \\
90.58 \\
95.69 \\
100.92 \\
103.25 \\
106.11 \\
108.31 \\
111.08 \\
113.24 \\
117.83 \\
120.92 \\
123.28 \\
122.97 \\
125.82 \\
125.60 \\
127.84 \\
130.19 \\
132.53 \\
135.33 \\
134.42 \\
138.35 \\
140.16 \\
142.63 \\
145.78 \\
142.52 \\
0.21 \\
63\end{array}$ & $\begin{array}{l}\text { \% Strain } \\
\text {-0.0007 } \\
0.0014 \\
0.0033 \\
0.0059 \\
0.0078 \\
0.0096 \\
0.0108 \\
0.0122 \\
0.0134 \\
0.0155 \\
0.0173 \\
0.0185 \\
0.0205 \\
0.0232 \\
0.0265 \\
0.0279 \\
0.0297 \\
0.0314 \\
0.0333 \\
0.0350 \\
0.0387 \\
0.0423 \\
0.0459 \\
0.0470 \\
0.0501 \\
0.0524 \\
0.0540 \\
0.0572 \\
0.0597 \\
0.0630 \\
0.0640 \\
0.0692 \\
0.0724 \\
0.0785 \\
0.0840 \\
0.0841 \\
0.0086\end{array}$ \\
\hline
\end{tabular}




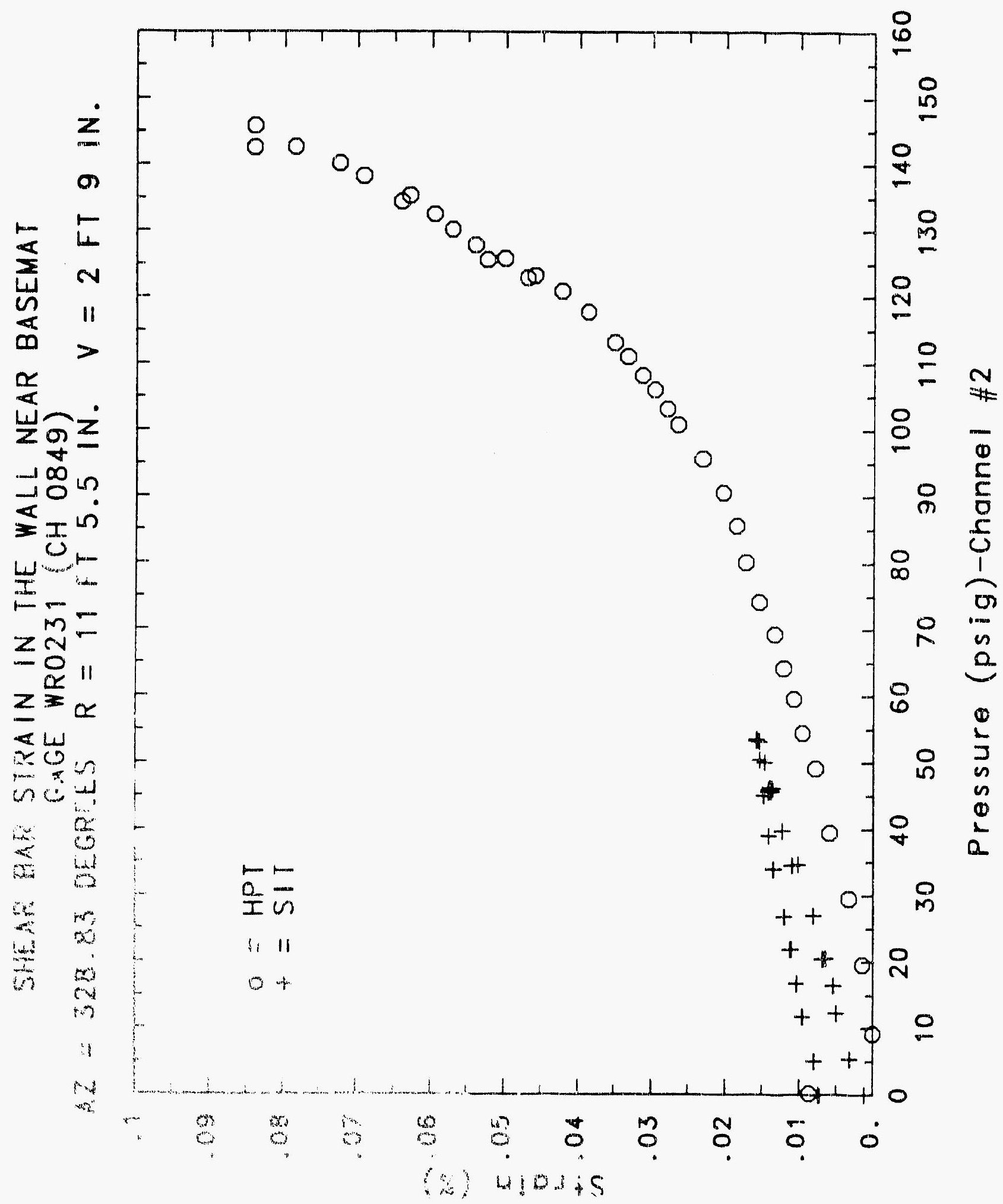




\section{Reinforced Concrete Test Data}

Weldable Gage 232 Channel 860

Structural Integrity Test

High Pressure test

\begin{tabular}{|c|c|c|c|}
\hline $\begin{array}{c}\text { Pressure } \\
\text { (psig) } \\
-0.05 \\
5.33 \\
12.31 \\
16.44 \\
20.51 \\
20.46 \\
20.47 \\
27.05 \\
34.69 \\
34.53 \\
34.53 \\
39.70 \\
45.90 \\
45.65 \\
45.62 \\
50.10 \\
53.47 \\
53.29 \\
53.21 \\
50.49 \\
46.14 \\
46.13 \\
44.97 \\
38.98 \\
33.99 \\
33.96 \\
26.83 \\
21.88 \\
21.90 \\
16.69 \\
11.74 \\
11.77 \\
5.05 \\
0.02 \\
-0.04 \\
-0.02 \\
-0.02 \\
0.02 \\
\end{array}$ & $\begin{array}{l}\text { \% Strain } \\
-0.0004 \\
-0.0027 \\
-0.0041 \\
-0.0051 \\
-0.0049 \\
-0.0048 \\
-0.0052 \\
-0.0035 \\
0.0001 \\
0.0006 \\
0.0007 \\
0.0033 \\
0.0086 \\
0.0090 \\
0.0092 \\
0.0119 \\
0.0149 \\
0.0153 \\
0.0151 \\
0.0139 \\
0.0126 \\
0.0124 \\
0.0133 \\
0.0106 \\
0.0089 \\
0.0092 \\
0.0063 \\
0.0046 \\
0.0047 \\
0.0027 \\
0.0019 \\
0.0018 \\
0.0017 \\
0.0023 \\
0.0058 \\
0.0039 \\
0.0027 \\
0.0024\end{array}$ & $\begin{array}{c}\text { Pressurs } \\
(\text { psig) } \\
9.89 \\
19.55 \\
29.57 \\
39.42 \\
49.16 \\
54.50 \\
59.57 \\
64.20 \\
69.32 \\
74.16 \\
80.16 \\
85.61 \\
90.58 \\
95.69 \\
100.92 \\
103.25 \\
106.11 \\
108.31 \\
111.08 \\
113.24 \\
117.83 \\
120.92 \\
123.28 \\
122.97 \\
125.82 \\
125.60 \\
127.644 \\
130.19 \\
132.53 \\
135.33 \\
134.42 \\
138.35 \\
140.16 \\
142.63 \\
145.78 \\
142.52 \\
0.22 \\
\end{array}$ & $\begin{array}{l}\text { \% Strain } \\
-0.0057 \\
-0.0038 \\
0.0002 \\
0.0041 \\
0.0084 \\
0.0112 \\
0.0140 \\
0.0177 \\
0.0217 \\
0.0238 \\
0.0252 \\
0.0288 \\
0.0311 \\
0.0349 \\
0.0384 \\
0.0399 \\
0.0417 \\
0.0436 \\
0.0457 \\
0.0471 \\
0.0509 \\
0.0523 \\
0.0537 \\
0.0539 \\
0.0578 \\
0.0582 \\
0.0609 \\
0.0669 \\
0.0760 \\
0.0866 \\
0.0874 \\
0.1017 \\
0.1119 \\
0.1275 \\
0.1432 \\
0.1441 \\
0.0421\end{array}$ \\
\hline
\end{tabular}




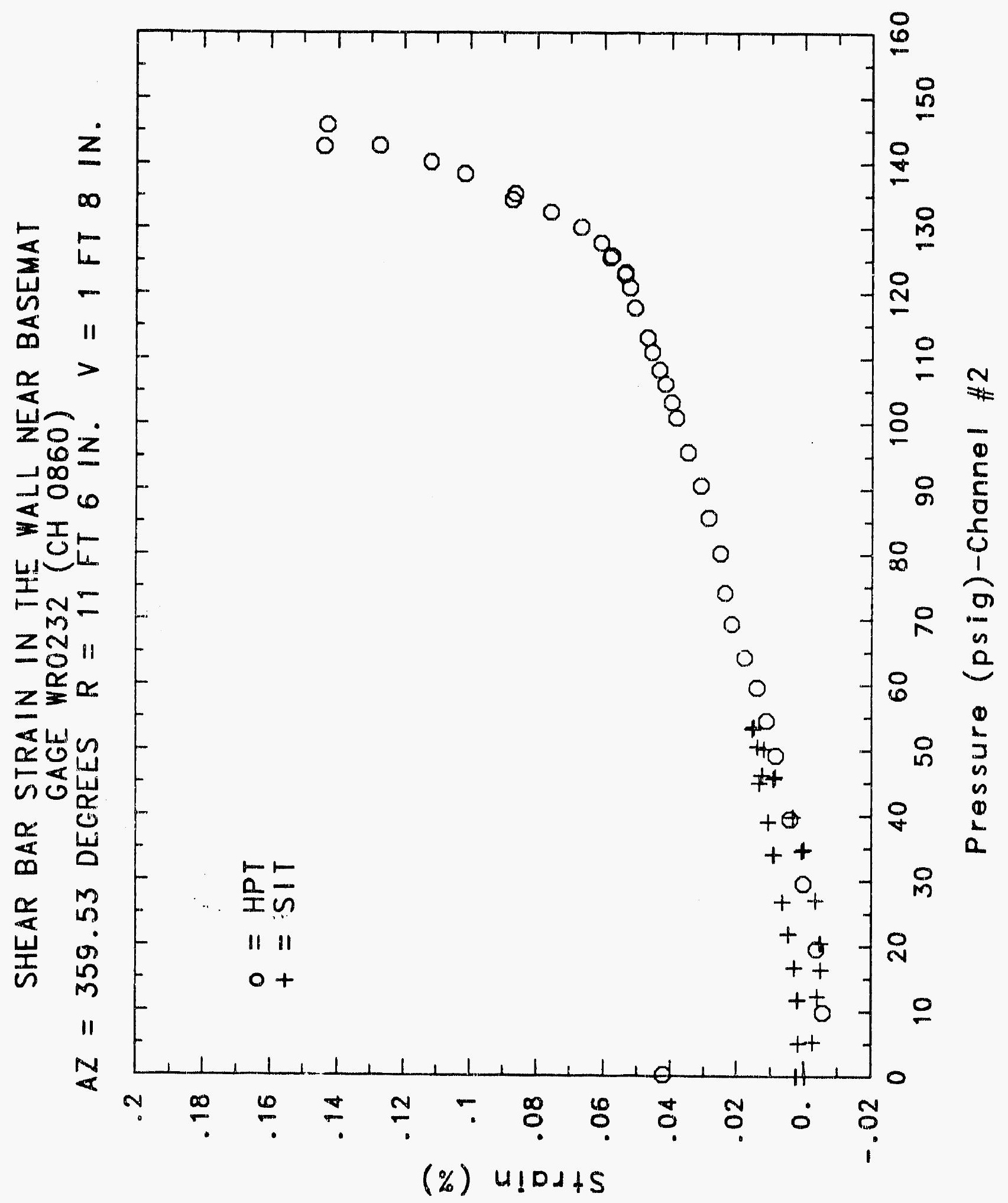




\section{Reinforced Concrete Test Data}

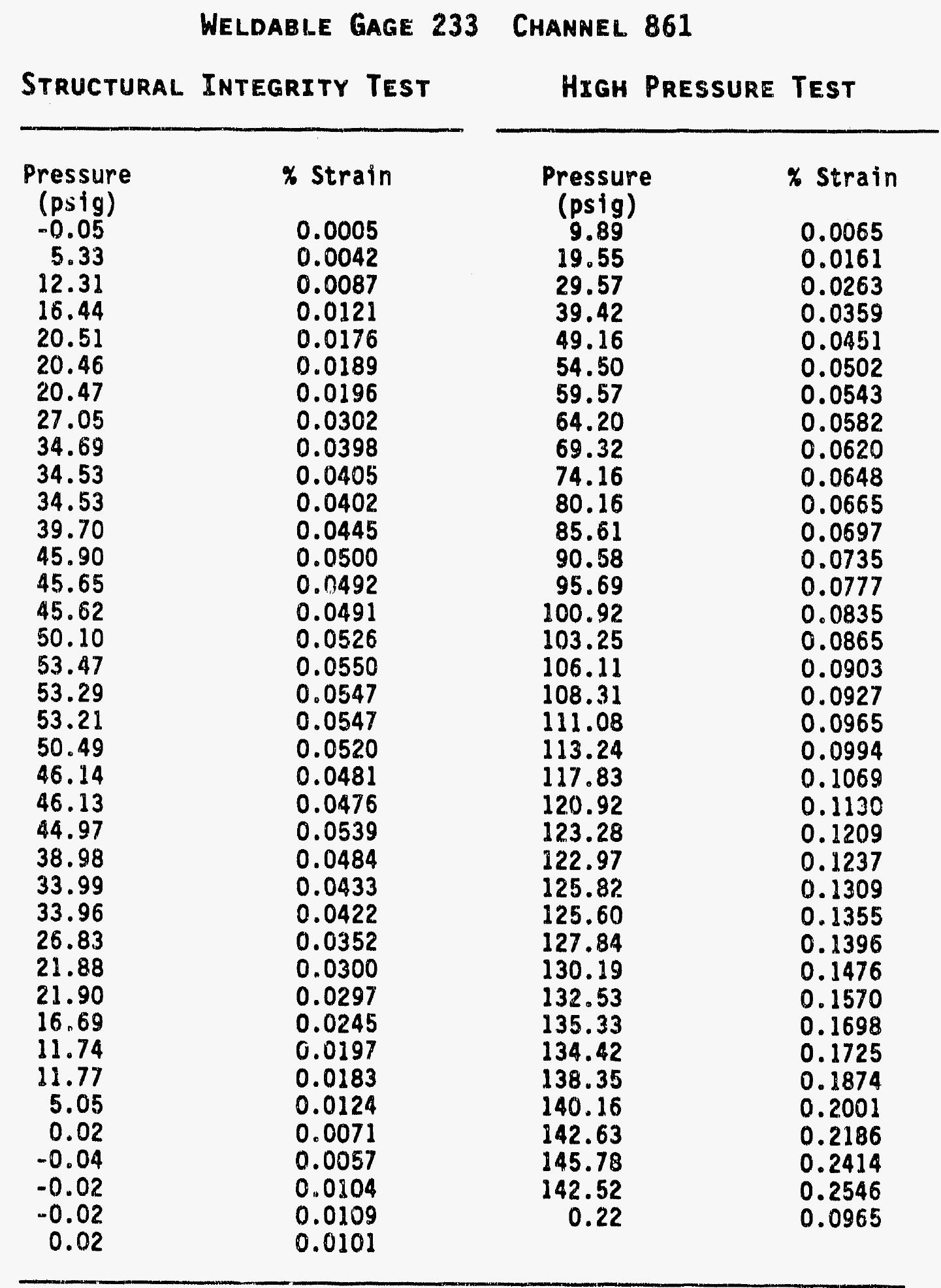




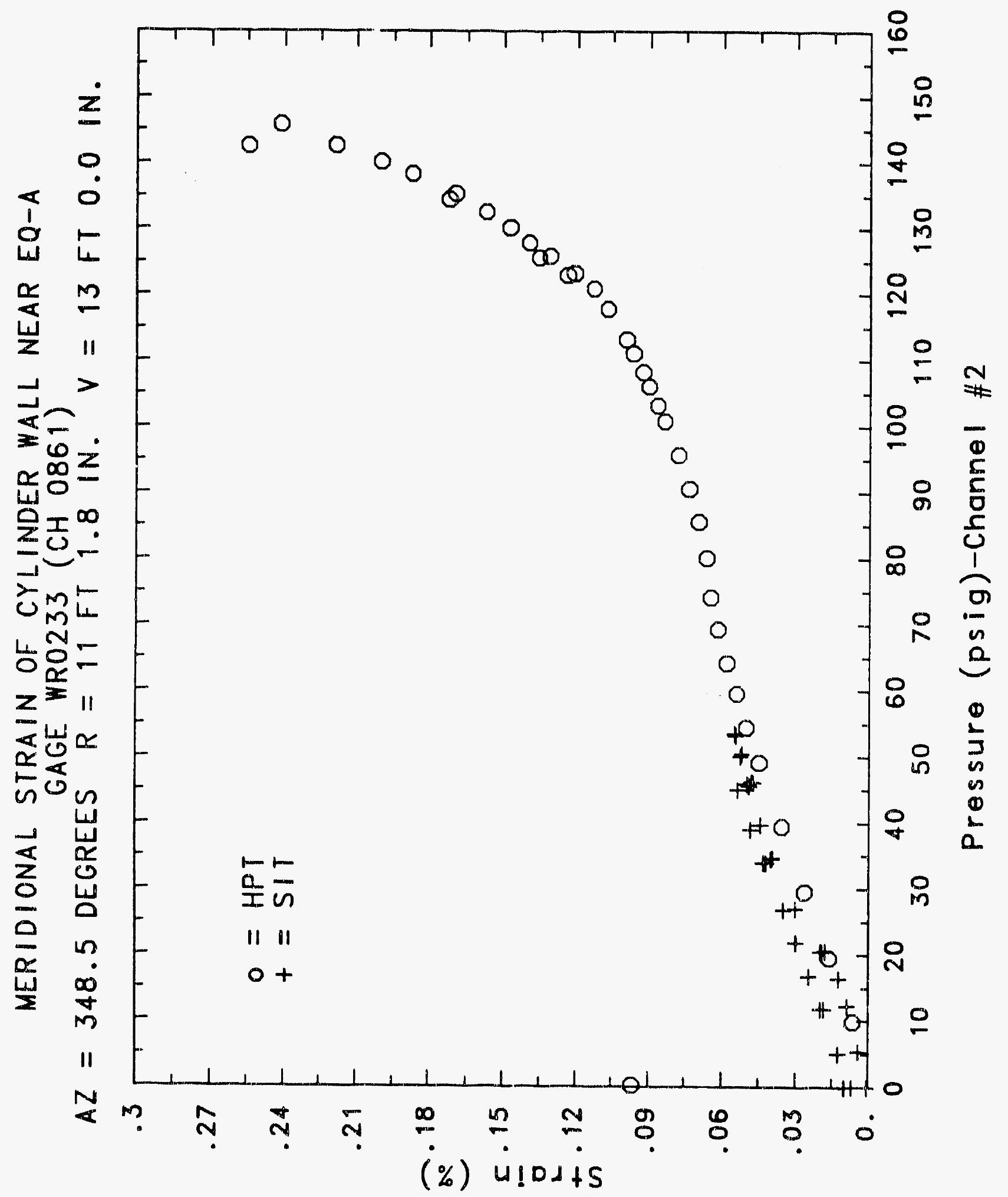


Reinforced Concrete Test Data

\begin{tabular}{|c|c|c|c|}
\hline Structurdi & $\begin{array}{l}\text { WHDABLE GAGE } 234 \\
\text { WNTEGRYTY TEST }\end{array}$ & $\begin{aligned} \text { Channel } 862 \\
\text { Hrgh Pressure }\end{aligned}$ & TEST \\
\hline $\begin{array}{c}\text { Pressure } \\
\text { (psig) } \\
-0.05 \\
5.33 \\
12.31 \\
16.44 \\
20.51 \\
20.46 \\
20.47 \\
27.05 \\
34.69 \\
34.53 \\
34.53 \\
39.70 \\
45.90 \\
45.65 \\
45.62 \\
50.10 \\
53.47 \\
53.29 \\
53.21 \\
50.49 \\
46.14 \\
46.13 \\
44.97 \\
38.98 \\
33.99 \\
33.96 \\
26.83 \\
21.88 \\
21.90 \\
16.69 \\
11.74 \\
11.77 \\
5.05 \\
0.02 \\
-0.04 \\
-0.02 \\
-0.02 \\
0.02 \\
\end{array}$ & $\begin{array}{l}\text { Strain } \\
0.0008 \\
0.0024 \\
0.0048 \\
0.0059 \\
0.0080 \\
0.0089 \\
0.0097 \\
0.0151 \\
0.0231 \\
0.0253 \\
0.0260 \\
0.0296 \\
0.0357 \\
0.0352 \\
0.0343 \\
0.0374 \\
0.0401 \\
0.0397 \\
0.0397 \\
0.0389 \\
0.0363 \\
0.0356 \\
0.0372 \\
0.0346 \\
0.0324 \\
0.0326 \\
0.0293 \\
0.0266 \\
0.0273 \\
0.0250 \\
0.0222 \\
0.0210 \\
0.0169 \\
0.0131 \\
0.0096 \\
0.0107 \\
0.0111 \\
0.0119 \\
\end{array}$ & $\begin{array}{c}\text { Pressure } \\
(\text { psig) } \\
9.89 \\
19.55 \\
29.57 \\
39.42 \\
49.16 \\
54.50 \\
59.57 \\
64.20 \\
69.32 \\
74.16 \\
80.16 \\
85.61 \\
90.58 \\
95.69 \\
100.92 \\
103.25 \\
106.11 \\
108.31 \\
111.08 \\
113.24 \\
117.83 \\
120.92 \\
123.28 \\
122.97 \\
125.82 \\
125.60 \\
127.84 \\
130.19 \\
132.53 \\
135.33 \\
134.42 \\
138.35 \\
140.16 \\
142.63 \\
145.78 \\
142.52 \\
0.22 \\
\end{array}$ & $\begin{array}{l}\text { \% Strain } \\
0.0027 \\
0.0094 \\
0.0160 \\
0.0226 \\
0.0288 \\
0.0319 \\
0.0358 \\
0.0398 \\
0.0440 \\
0.0493 \\
0.0544 \\
0.0596 \\
0.0645 \\
0.0690 \\
0.0744 \\
0.0766 \\
0.0793 \\
0.0820 \\
0.0855 \\
0.0881 \\
0.0933 \\
0.0971 \\
0.1005 \\
0.1012 \\
0.1052 \\
0.1054 \\
0.1081 \\
0.1118 \\
0.1156 \\
0.1204 \\
0.1198 \\
0.1252 \\
0.128 \\
0.1336 \\
0.1390 \\
0.1383 \\
0.0283\end{array}$ \\
\hline
\end{tabular}




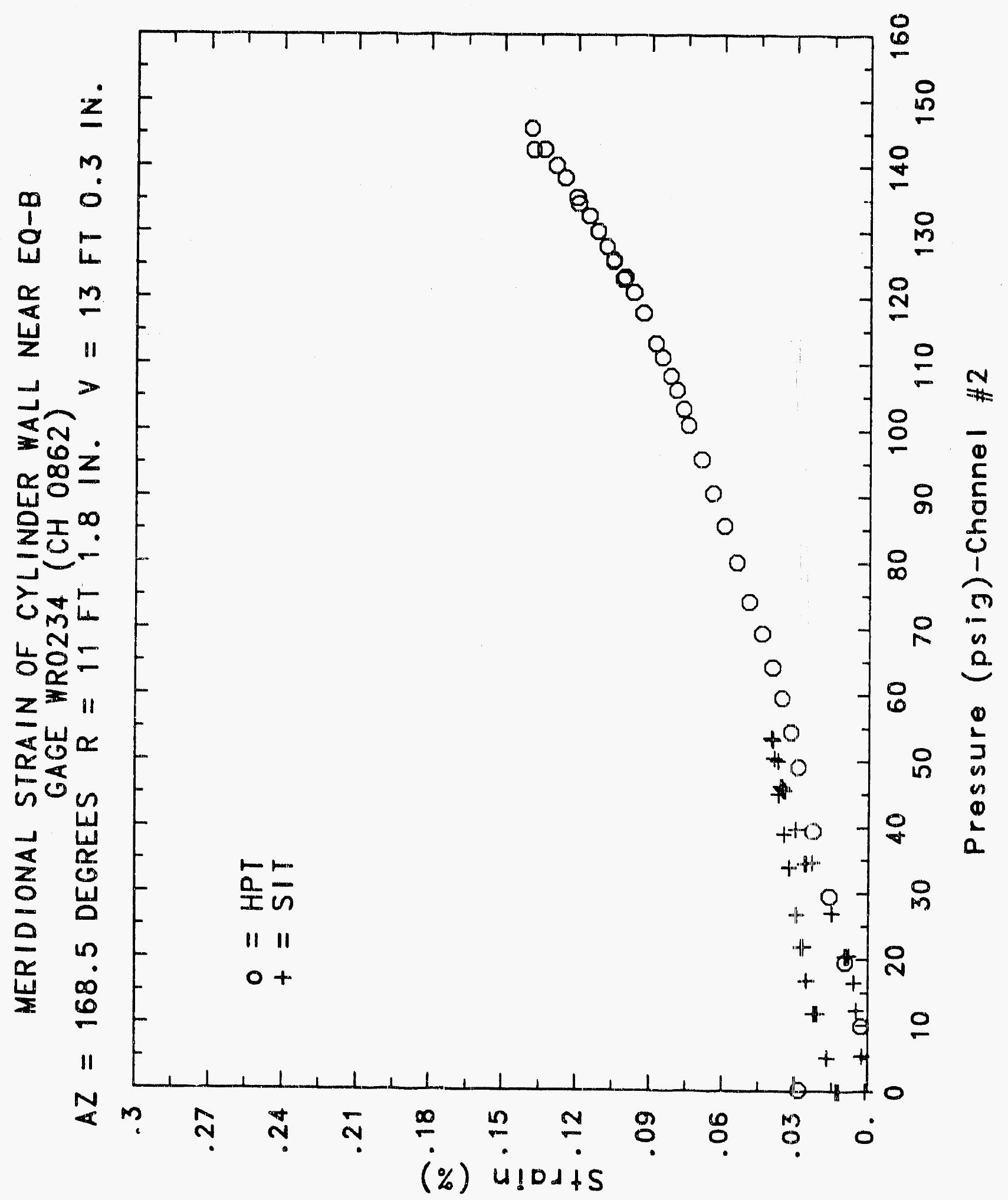


Reinforced Concrete Test Data

\begin{tabular}{|c|c|c|c|}
\hline STRUCTURAL & $\begin{array}{l}\text { WELDABLE GAGE } 235 \\
\text { INTEGRITY TEST }\end{array}$ & $\begin{array}{r}\text { Channel } 863 \\
\text { High Pressure }\end{array}$ & TEST \\
\hline $\begin{array}{c}\text { Pressure } \\
\text { (psig) } \\
-0.05 \\
5.33 \\
12.31 \\
16.44 \\
20.51 \\
20.46 \\
20.47 \\
27.05 \\
34.69 \\
34.53 \\
34.53 \\
39.70 \\
45.90 \\
45.65 \\
45.62 \\
50.10 \\
53.47 \\
53.29 \\
53.21 \\
50.49 \\
46.14 \\
46.13 \\
44.97 \\
38.98 \\
33.99 \\
33.96 \\
26.83 \\
21.88 \\
21.90 \\
16.69 \\
11.74 \\
11.77 \\
5.05 \\
0.02 \\
-0.04 \\
-0.02 \\
-0.02 \\
0.02 \\
\end{array}$ & $\begin{array}{l}\text { Strain } \\
0.0013 \\
0.0026 \\
0.0050 \\
0.0062 \\
0.0092 \\
0.0097 \\
0.0098 \\
0.0155 \\
0.0214 \\
0.0217 \\
0.0215 \\
0.0241 \\
0.0279 \\
0.0271 \\
0.0271 \\
0.0291 \\
0.0312 \\
0.0311 \\
0.0310 \\
0.0300 \\
0.0276 \\
0.0275 \\
0.0311 \\
0.0282 \\
0.0258 \\
0.0255 \\
0.0222 \\
0.0195 \\
0.0194 \\
0.0166 \\
0.0140 \\
0.0130 \\
0.0097 \\
0.0068 \\
0.0059 \\
0.0090 \\
0.0090 \\
0.0078\end{array}$ & $\begin{array}{c}\text { Pressure } \\
\text { (psig) } \\
9.89 \\
19.55 \\
29.57 \\
39.42 \\
49.16 \\
54.50 \\
59.57 \\
64.20 \\
69.32 \\
74.16 \\
80.16 \\
85.61 \\
90.58 \\
95.69 \\
100.92 \\
103.25 \\
106.11 \\
108.31 \\
111.08 \\
113.24 \\
117.83 \\
120.92 \\
123.28 \\
122.97 \\
125.82 \\
125.60 \\
127.84 \\
130.19 \\
132.53 \\
135.33 \\
134.42 \\
138.35 \\
140.16 \\
142.63 \\
145.78 \\
142.52 \\
0.22 \\
0.92\end{array}$ & $\begin{array}{l}\text { X Strain } \\
0.0024 \\
0.0080 \\
0.0135 \\
0.0189 \\
0.0239 \\
0.0267 \\
0.0301 \\
0.0319 \\
0.0351 \\
0.0382 \\
0.0388 \\
0.0423 \\
0.0461 \\
0.0501 \\
0.0553 \\
0.0578 \\
0.0606 \\
0.0625 \\
0.0655 \\
0.0678 \\
0.0726 \\
0.0770 \\
0.0819 \\
0.0835 \\
0.0893 \\
0.0925 \\
0.0954 \\
0.1011 \\
0.1085 \\
0.1172 \\
0.1187 \\
0.1279 \\
0.1343 \\
0.1428 \\
0.1519 \\
0.1563 \\
0.0408\end{array}$ \\
\hline
\end{tabular}




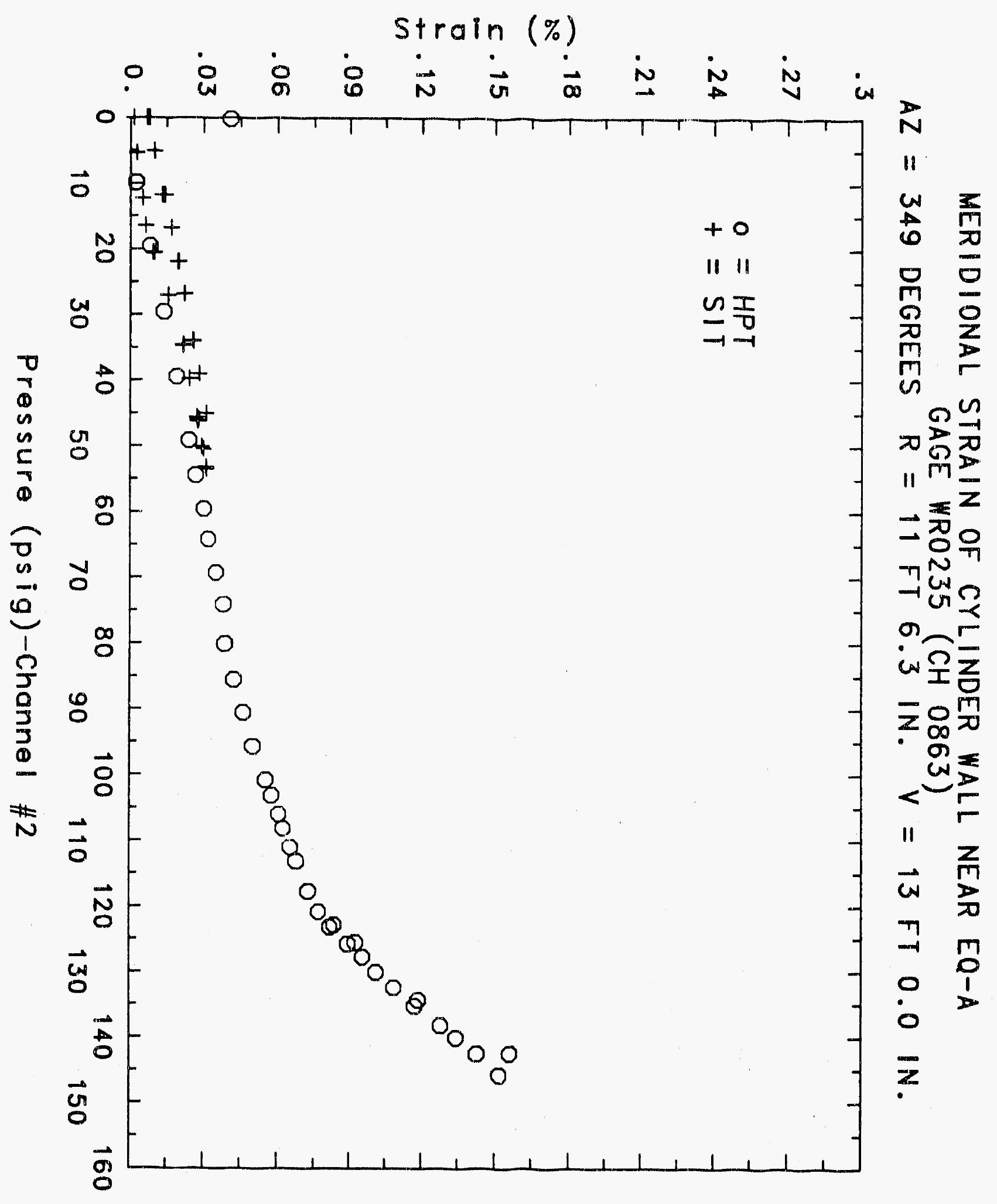




\section{Reinforced Concrete Test Data}

Weldable Gage 236 Channel 864

Structural Integrity Test

High Pressure test

\begin{tabular}{|c|c|c|c|}
\hline $\begin{array}{c}\text { Pressure } \\
\text { (psig) } \\
-0.05 \\
5.33 \\
12.31 \\
16.44 \\
20.51 \\
20.46 \\
20.47 \\
27.05 \\
34.69 \\
34.53 \\
34.53 \\
39.70 \\
45.90 \\
45.65 \\
45.62 \\
50.10 \\
53.47 \\
53.29 \\
53.2 .1 \\
50.49 \\
46.14 \\
46.13 \\
44.97 \\
38.98 \\
33.99 \\
33.96 \\
26.83 \\
21.88 \\
21.90 \\
16.69 \\
11.74 \\
11.77 \\
5.05 \\
0.02 \\
-0.04 \\
-0.02 \\
-0.02 \\
0.02 \\
\end{array}$ & $\begin{array}{l}\text { \% strain } \\
0.0002 \\
0.0018 \\
0.0036 \\
0.0050 \\
0.0072 \\
0.0073 \\
0.0082 \\
0.0127 \\
0.0217 \\
0.0234 \\
0.0238 \\
0.0283 \\
0.0365 \\
0.0367 \\
0.0370 \\
0.0412 \\
0.0454 \\
0.0461 \\
0.0467 \\
0.0457 \\
0.0438 \\
0.0438 \\
0.0457 \\
0.0423 \\
0.0391 \\
0.0393 \\
0.0352 \\
0.0316 \\
0.0319 \\
0.0283 \\
0.0244 \\
0.0228 \\
0.0181 \\
0.0140 \\
0.0131 \\
0.0138 \\
0.0140 \\
0.0137\end{array}$ & $\begin{array}{c}\text { Pressure } \\
(\text { psig) } \\
9.89 \\
19.55 \\
29.57 \\
39.42 \\
49.16 \\
54.50 \\
59.57 \\
64.20 \\
69.32 \\
74.16 \\
80.16 \\
85.61 \\
90.58 \\
95.69 \\
100.92 \\
103.25 \\
106.11 \\
108.31 \\
111.08 \\
113.24 \\
117.83 \\
120.92 \\
123.28 \\
122.97 \\
125.82 \\
125.60 \\
127.84 \\
130.19 \\
132.53 \\
135.33 \\
134.42 \\
138.35 \\
140.16 \\
142.63 \\
145.78 \\
142.52 \\
0.22 \\
\end{array}$ & $\begin{array}{l}\text { \% Strain } \\
0.0027 \\
0.0101 \\
0.0181 \\
0.0257 \\
0.0328 \\
0.0359 \\
0.0413 \\
0.0460 \\
0.0511 \\
0.0575 \\
0.0665 \\
0.0743 \\
0.0823 \\
0.0905 \\
0.0989 \\
0.1034 \\
0.1071 \\
0.1103 \\
0.1154 \\
0.1191 \\
0.1258 \\
0.1312 \\
0.1368 \\
0.1378 \\
0.1434 \\
0.1446 \\
0.1482 \\
0.1537 \\
0.1593 \\
0.1655 \\
0.1660 \\
0.1718 \\
0.1757 \\
0.1801 \\
0.1850 \\
0.1836 \\
0.0385\end{array}$ \\
\hline
\end{tabular}




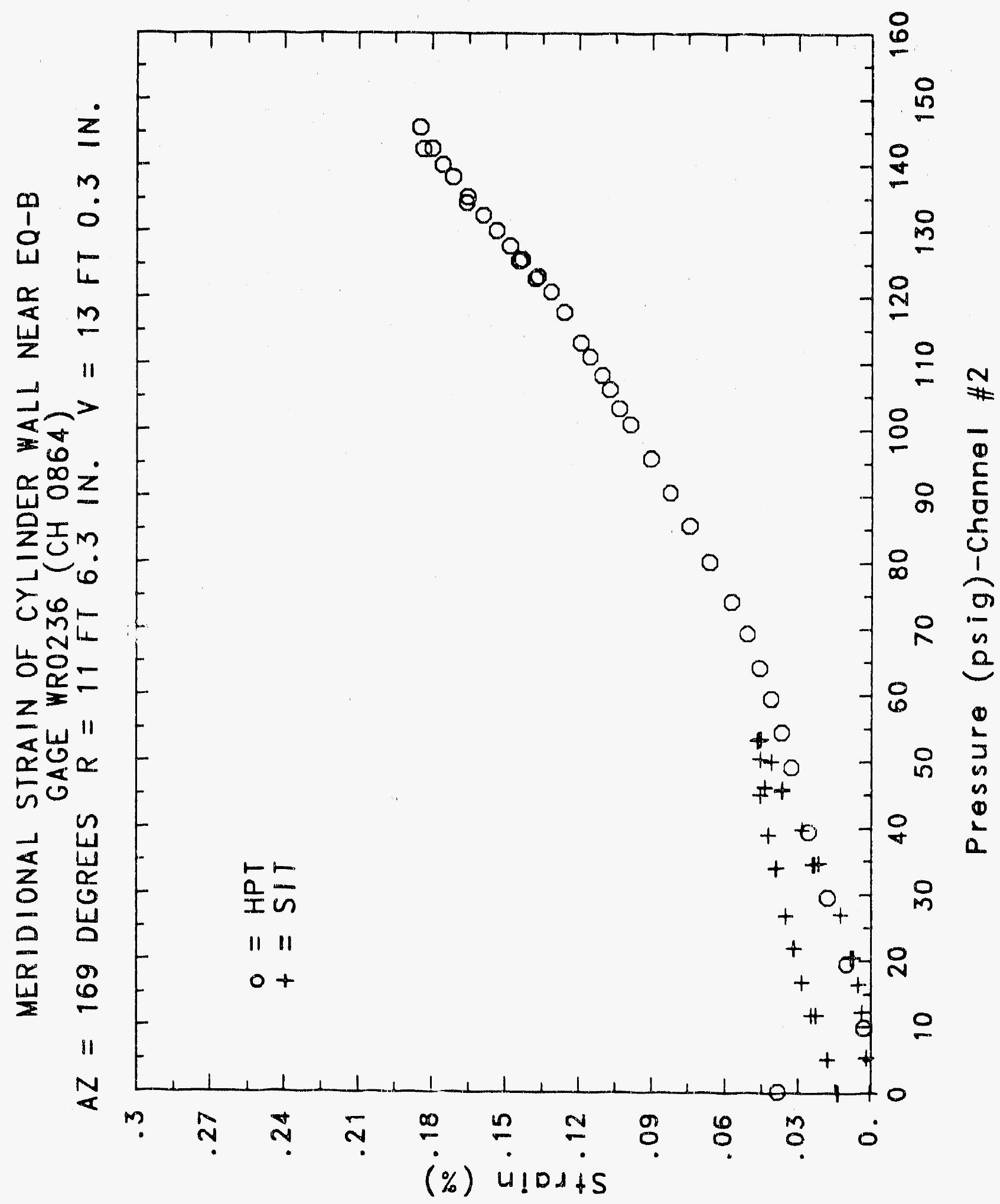




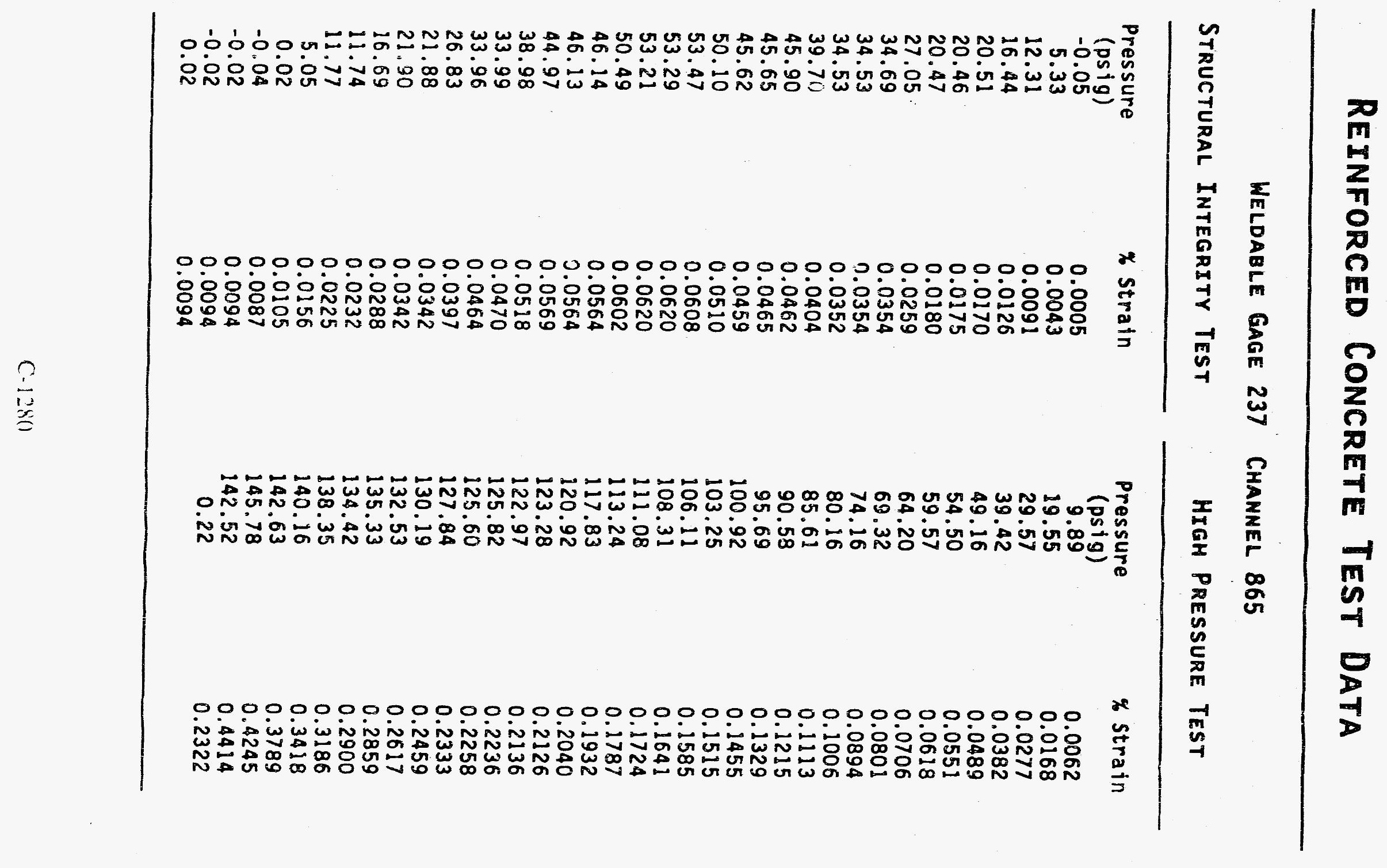




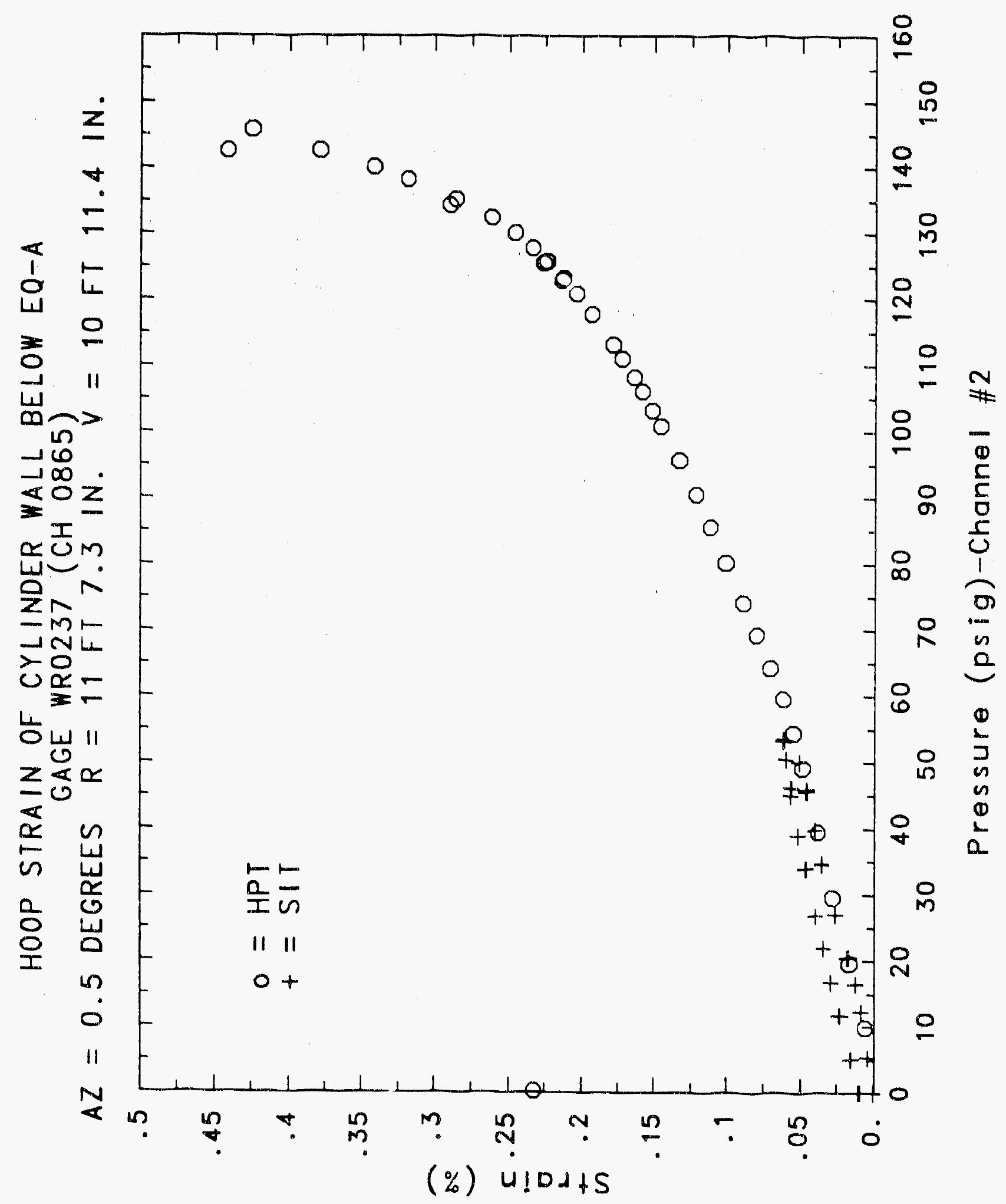




\section{Reinforced Concrete Test Data}

\section{Weldable Gage 238 Channel 866}

Structural Integrity Test

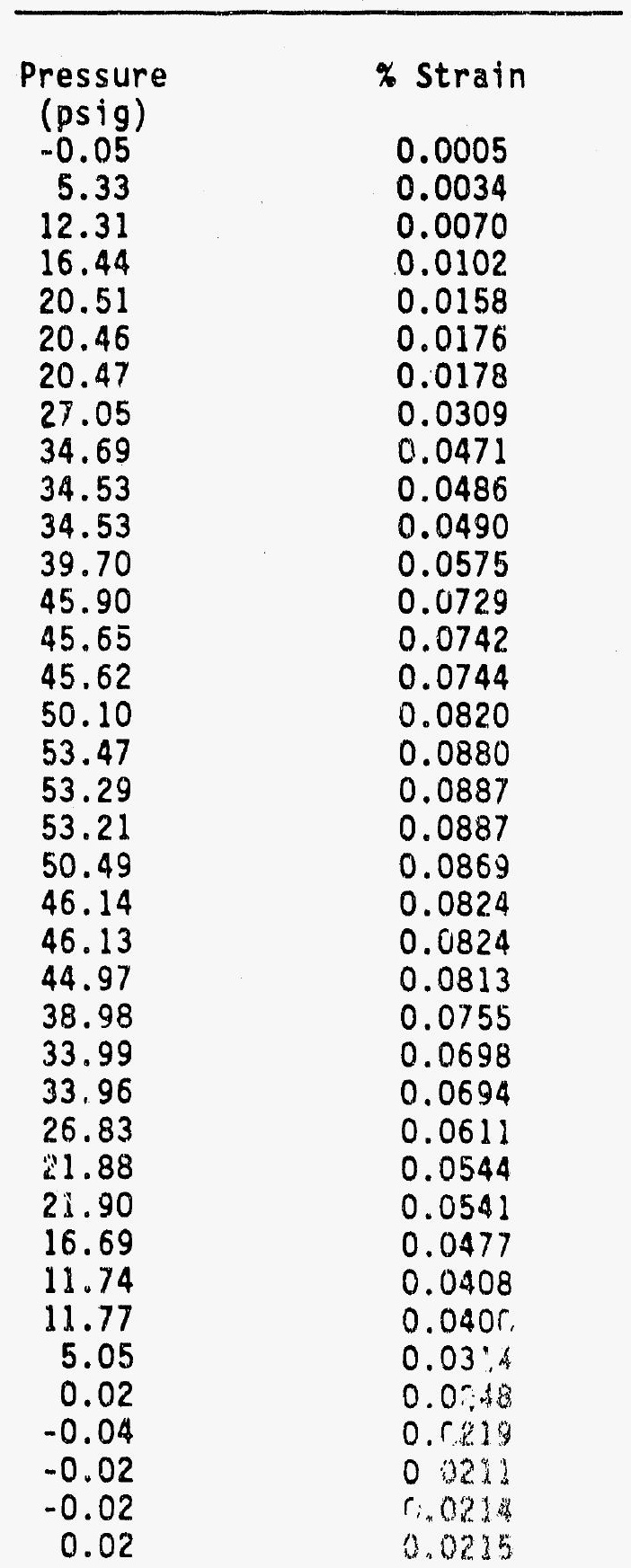

\section{high Pressure test}

Pressure

(psig)

9.89

19.55

29.57

39.42

49.16

54.50

59.57

64.20

69.32

74.16

80.16

85.61

90.58

$95.6 ?$

$100 . ?$ ?

103.15

106.11

$10,3.31$

?.1.

33.24

11). 53

120.32

123. 24

122.67

15.5.

3.t. 6

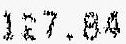

230.0

532.5

135.33

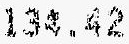

130.35

140.35

10.2 .5

4.45 .98

14.4.5.

4. 2
* Stran

$\because .0074$

0.0218

0.035

0.050 .3

0.0635

0.0709

0.079

0.0868

0.034

0.9030

0.1176

a. $3 a^{2}$

0.1313

0.3409

0.1507

0.1555

0.1606

$0.165 \%$

a. 2713

0.1755

0.3010

0. 2945

$0.80: 3$

0.2028

0.2100

0.21 .4

4. $2 y^{3}$

0.293

0.2325

A. $23 \%$

b.

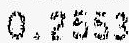

0.2534

0.274

0. 2898

0.2836

0.0936 


\section{IMAGE EVALUATION TEST TARGET (MT-3)}
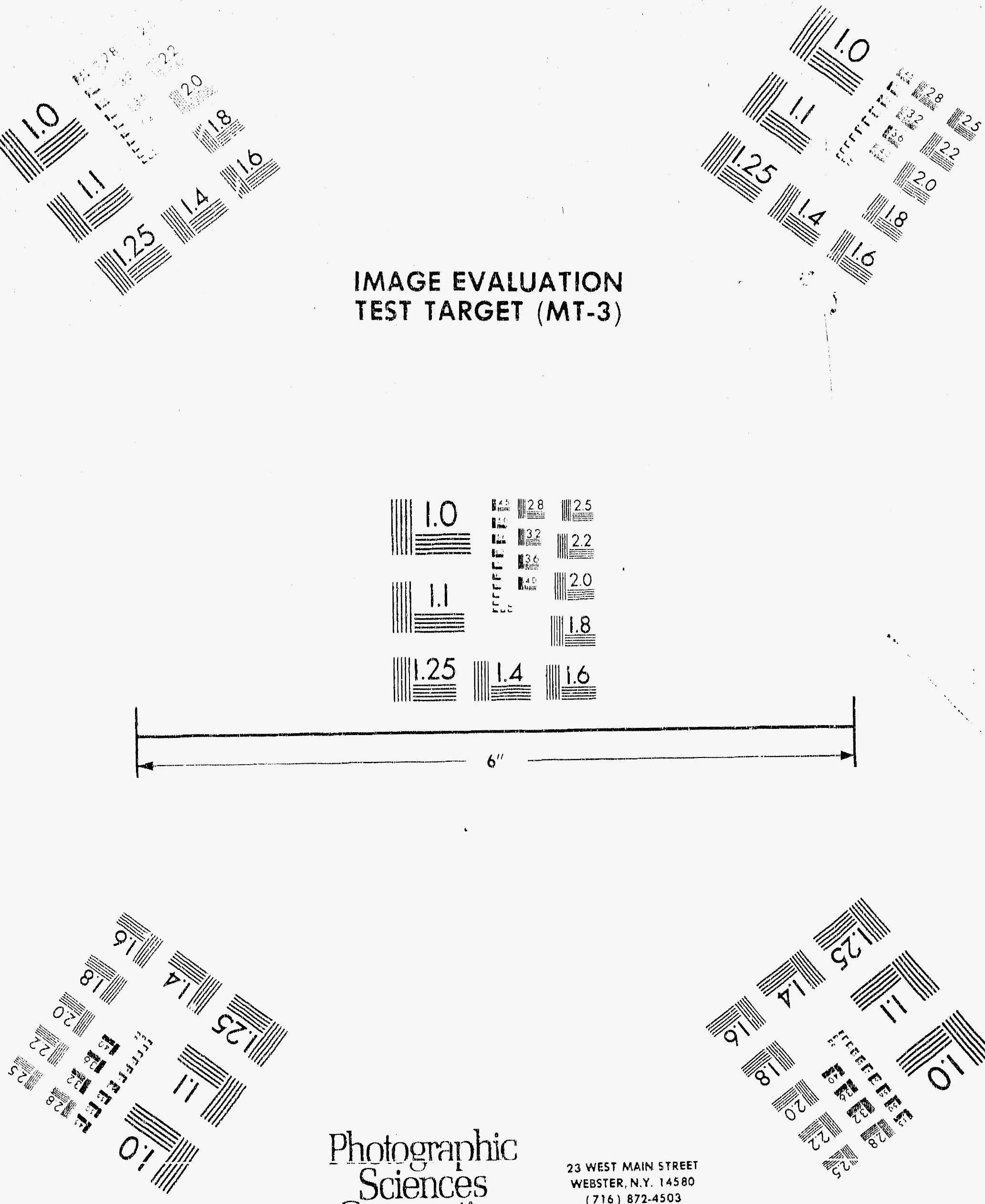

Photogranhic Sciences Corporation

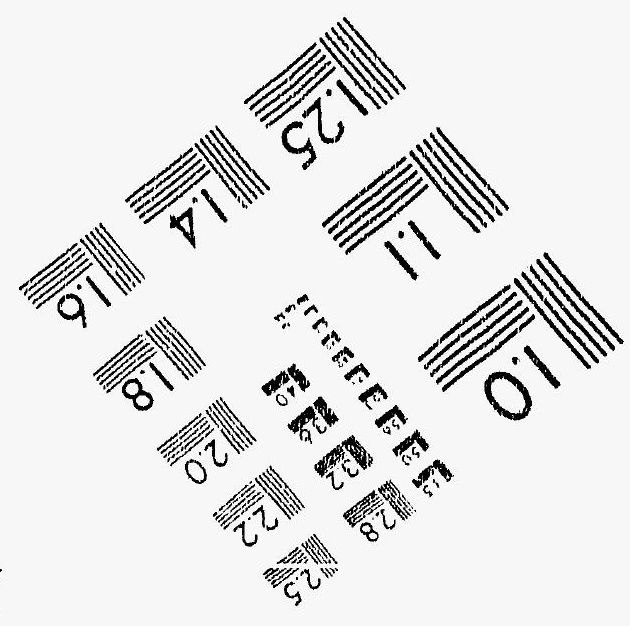




\section{SECURITY CLASSIFICATION}
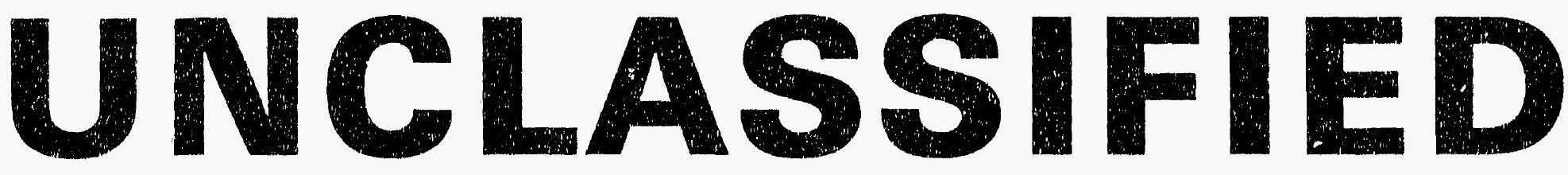

\section{DATE OF MICROFILMING $1-6-92$}

\section{MICROFILMED BY ERrY hard}

SANDIA NATIONAL LABS 


\section{THIS DOCUMENT FILMED}

AT

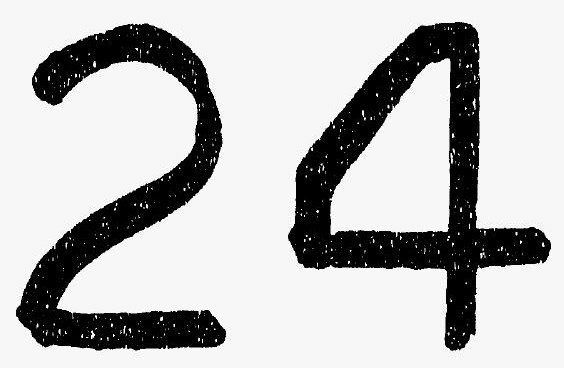

REDUCTION 


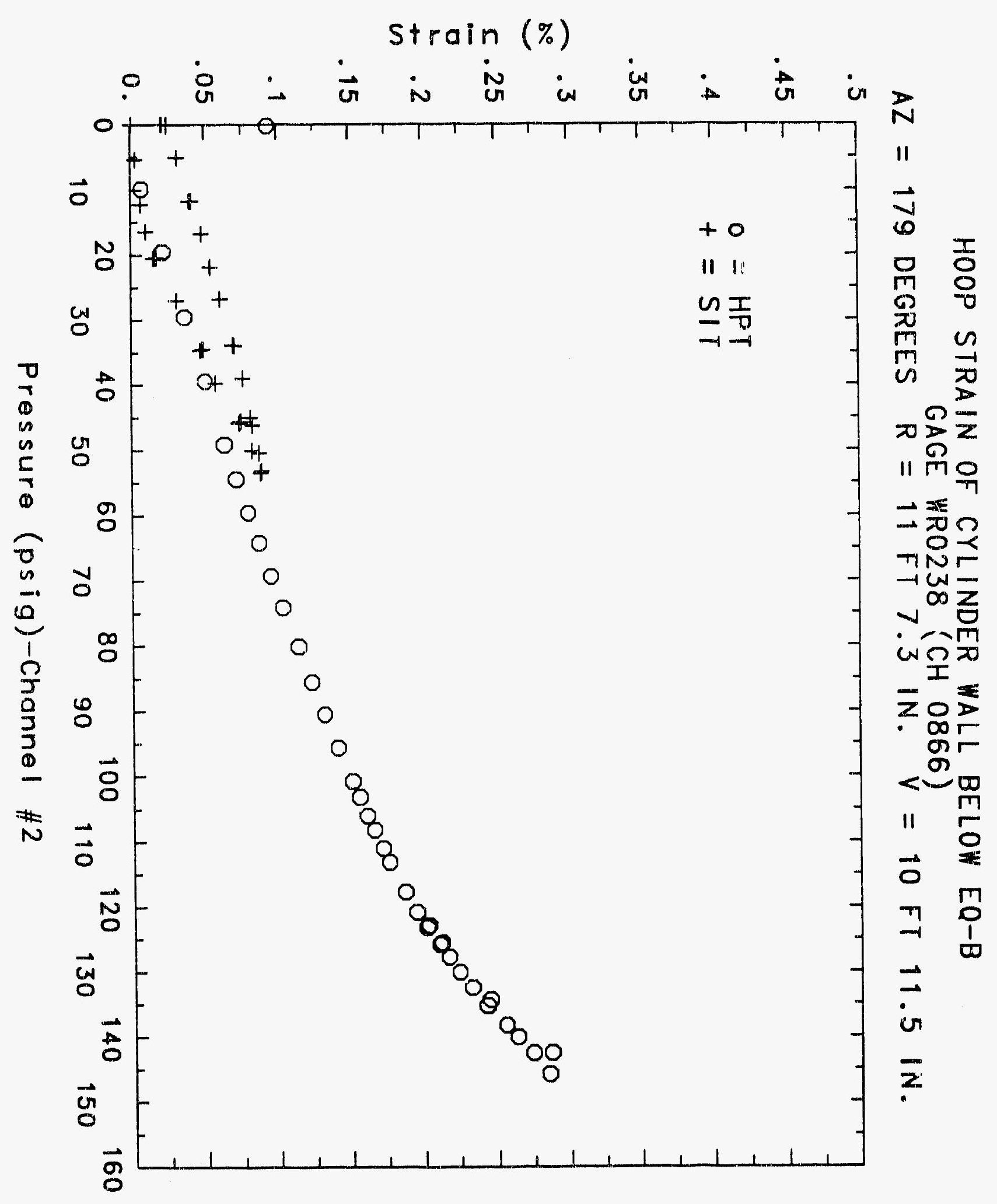




\section{Reinforced Concrete Test Data}

Weldable gage 239 Chanhel 867

Structural InTEgrity TEST

high Pressure test

\begin{tabular}{|c|c|c|c|}
\hline $\begin{array}{c}\text { Pressure } \\
(\text { psig) } \\
-0.05 \\
5.33 \\
12.31 \\
16.44 \\
20.51 \\
20.46 \\
20.47 \\
27.05 \\
34.69 \\
34.53 \\
34.53 \\
39.70 \\
45.90 \\
45.65 \\
45.62 \\
50.10 \\
53.47 \\
53.29 \\
53.21 \\
50.49 \\
46.14 \\
46.13 \\
44.97 \\
38.98 \\
33.99 \\
33.96 \\
26.83 \\
21.88 \\
21.90 \\
16.69 \\
11.74 \\
11.77 \\
5.05 \\
0.02 \\
-0.04 \\
-0.02 \\
-0.02 \\
0.02\end{array}$ & $\begin{array}{c}\text { \% Strain } \\
0.0009 \\
0.0066 \\
0.0131 \\
0.0170 \\
0.0210 \\
0.0216 \\
0.0213 \\
0.0285 \\
0.0376 \\
0.0384 \\
0.0387 \\
0.0439 \\
0.0501 \\
0.0489 \\
0.0493 \\
0.0530 \\
0.0564 \\
0.0566 \\
0.0567 \\
0.0546 \\
0.0516 \\
0.0514 \\
0.0515 \\
0.0472 \\
0.0423 \\
0.0416 \\
0.0348 \\
0.0294 \\
0.0297 \\
0.0246 \\
0.0193 \\
0.0186 \\
0.0110 \\
0.0046 \\
0.0034 \\
0.0035 \\
0.0043 \\
0.0047\end{array}$ & $\begin{array}{c}\text { Pressure } \\
\text { (psig) } \\
9.89 \\
19.55 \\
29.57 \\
39.42 \\
49.16 \\
54.50 \\
59.57 \\
64.20 \\
69.32 \\
74.16 \\
80.16 \\
85.61 \\
90.58 \\
95.69 \\
100.92 \\
103.25 \\
106.11 \\
108.31 \\
111.08 \\
113.24 \\
117.83 \\
120.92 \\
123.28 \\
122.97 \\
125.82 \\
125.60 \\
127.84 \\
130.19 \\
132.53 \\
135.33 \\
134.42 \\
138.35 \\
140.16 \\
142.63 \\
145.78 \\
142.52 \\
0.22\end{array}$ & $\begin{array}{l}\text { Strain } \\
0.0077 \\
0.0173 \\
0.0271 \\
0.0361 \\
0.0452 \\
0.0503 \\
0.0559 \\
0.0611 \\
0.0665 \\
0.0723 \\
0.0783 \\
0.0850 \\
0.0916 \\
0.0979 \\
0.1050 \\
0.1079 \\
0.1118 \\
0.1149 \\
0.1189 \\
0.1222 \\
0.1303 \\
0.1360 \\
0.1417 \\
0.1426 \\
0.1506 \\
0.1520 \\
0.1575 \\
0.1689 \\
0.1864 \\
0.2175 \\
0.2232 \\
0.2772 \\
0.3827 \\
0.5689 \\
0.6315 \\
0.6540 \\
0.4947\end{array}$ \\
\hline
\end{tabular}




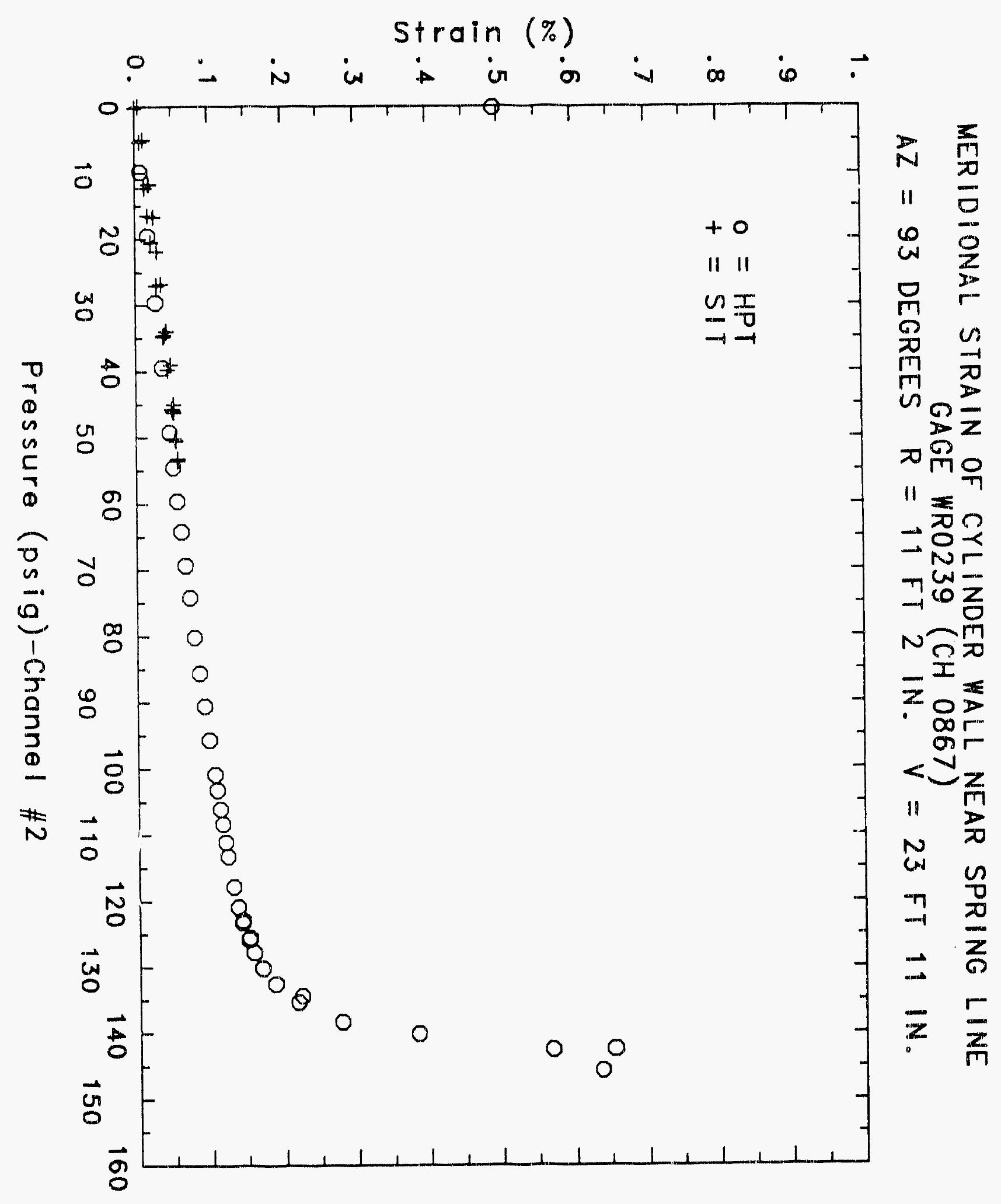




\section{Reinforced Concrete Test Data}

Weldable gage 240 Channel 868

Structural. Integrity Test

\begin{tabular}{|c|c|c|c|}
\hline $\begin{array}{c}\text { Pressure } \\
\text { (psig) } \\
-0.05 \\
5.33 \\
12.31 \\
16.44 \\
20.51 \\
20.46 \\
20.47 \\
27.05 \\
34.69 \\
34.53 \\
34.53 \\
39.70 \\
45.90 \\
45.65 \\
45.62 \\
50.10 \\
53.47 \\
53.29 \\
53.21 \\
50.49 \\
46.14 \\
46.13 \\
44.97 \\
38.98 \\
33.99 \\
33.96 \\
26.83 \\
21.88 \\
21.90 \\
16.69 \\
11.74 \\
11.77 \\
5.05 \\
0.02 \\
-0.04 \\
-0.02 \\
-0.02 \\
0.02 \\
\end{array}$ & $\begin{array}{c}\text { x Strain } \\
0.0000 \\
0.0013 \\
0.0035 \\
0.0052 \\
0.0074 \\
0.0084 \\
0.0085 \\
0.0145 \\
0.0235 \\
0.0251 \\
0.0260 \\
0.0306 \\
0.0376 \\
0.0376 \\
0.0380 \\
0.0418 \\
0.0460 \\
0.0465 \\
0.0464 \\
0.0459 \\
0.0439 \\
0.0438 \\
0.0416 \\
0.0417 \\
0.0390 \\
0.0390 \\
0.0348 \\
0.0317 \\
0.0316 \\
0.0282 \\
0.0245 \\
0.0242 \\
0.0193 \\
0.0151 \\
0.0133 \\
0.0136 \\
0.0141 \\
0.0145\end{array}$ & $\begin{array}{c}\text { Pressure } \\
\text { (psig) } \\
9.89 \\
19.55 \\
29.57 \\
39.42 \\
49.16 \\
54.50 \\
59.57 \\
64.20 \\
69.32 \\
74.16 \\
80.16 \\
85.61 \\
90.58 \\
95.69 \\
100.92 \\
103.25 \\
106.11 \\
108.31 \\
111.08 \\
113.24 \\
117.83 \\
120.92 \\
123.28 \\
122.97 \\
125.82 \\
125.60 \\
127.84 \\
130.19 \\
132.53 \\
135.33 \\
134.42 \\
138.35 \\
140.16 \\
142.63 \\
145.78 \\
142.52 \\
0.22\end{array}$ & $\begin{array}{l}\text { \% Strain } \\
0.0034 \\
0.0108 \\
0.0181 \\
0.0254 \\
0.0319 \\
0.0359 \\
0.0401 \\
0.0449 \\
0.0503 \\
0.0557 \\
0.0623 \\
0.0689 \\
0.0753 \\
0.0820 \\
0.0891 \\
0.0935 \\
0.0970 \\
0.1003 \\
0.1050 \\
0.1085 \\
0.1165 \\
0.1226 \\
0.1282 \\
0.1292 \\
0.1353 \\
0.1367 \\
0.1410 \\
0.1490 \\
0.1602 \\
0.1773 \\
0.1808 \\
0.2009 \\
0.2135 \\
0.2294 \\
0.2436 \\
0.2454 \\
0.1032\end{array}$ \\
\hline
\end{tabular}

High Pressure test

Pressure

8.89

19.55

29.57

39.42

59.57

64.20

74.16

80.16

85.61

90.58

00.92

103.25

106.11

108.31

111.08

120.83

123.28

127.84

130.19

132.53

135.33

134.42

138.35

140.16

142.63

145.78

42.52

0.22
0.0034

0.0108

0.0181

0.0254

0.0319

0.0401

0.0449

0.0503

0.0557

0.0623

0.0820

0.0935

0.0970

0.1085

0.1226

0.1282

0.1292

0.1353

0.1367

0.1602

0.1808

0.2009

0.2135

0.2294

0.2436

0.2454

0.1032 


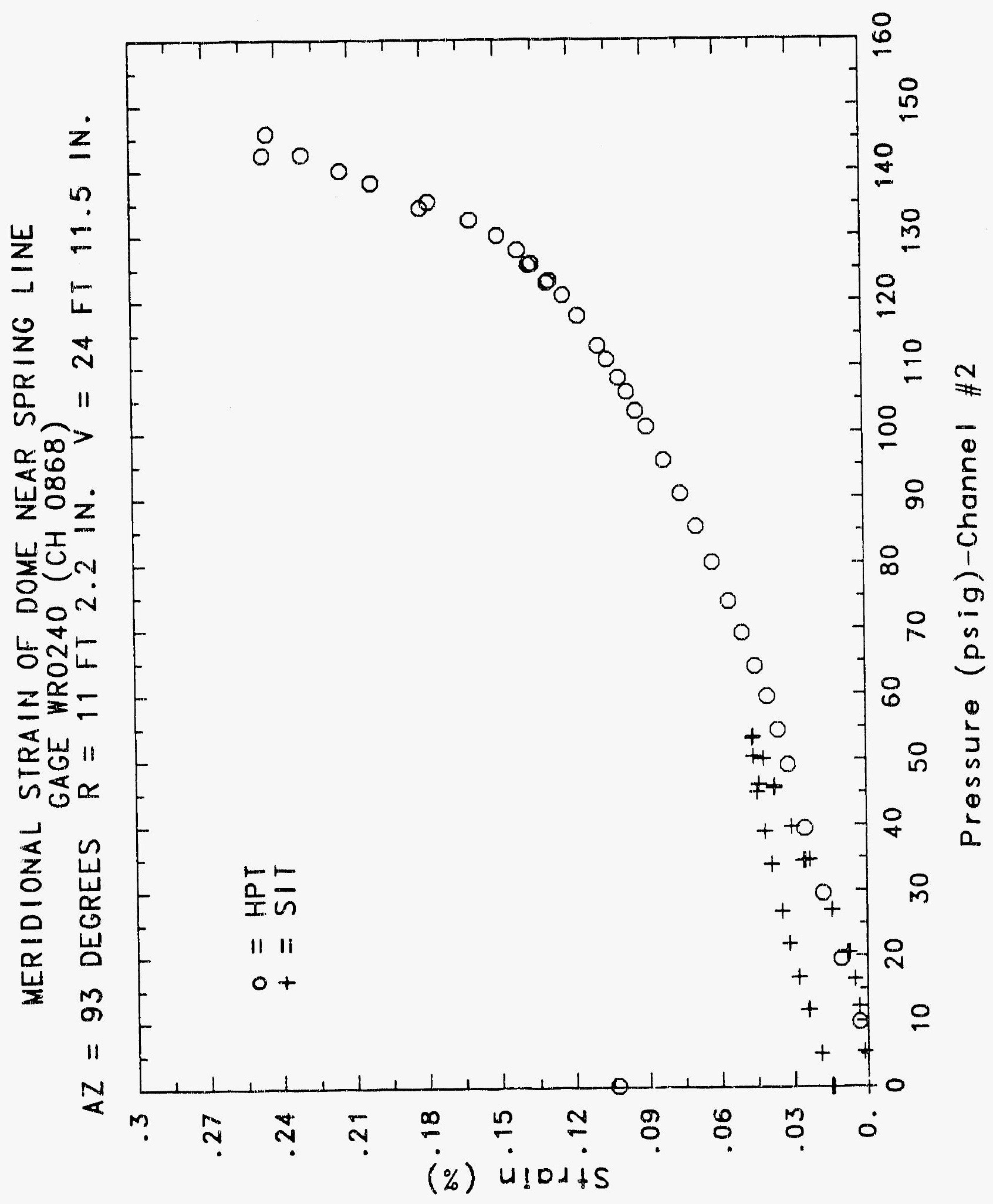




\section{Reinforced Concrete Test Data}

Weldable gage 241 Channel 869

Structural Integrity test

High Pressure test

\begin{tabular}{|c|c|c|c|}
\hline $\begin{array}{c}\text { Pressure } \\
\text { (psig) } \\
-0.05 \\
5.33 \\
12.31 \\
16.44 \\
20.51 \\
20.46 \\
20.47 \\
27.05 \\
34.69 \\
34.53 \\
34.53 \\
39.70 \\
45.90 \\
45.65 \\
45.62 \\
50.10 \\
53.47 \\
53.29 \\
53.21 \\
50.49 \\
46.14 \\
46.13 \\
44.97 \\
38.98 \\
33.99 \\
33.96 \\
26.83 \\
21.88 \\
21.90 \\
16.69 \\
11.74 \\
11.77 \\
5.05 \\
0.02 \\
-0.04 \\
-0.02 \\
-0.02 \\
0.02\end{array}$ & $\begin{array}{c}\text { \% Strain } \\
-0.0003 \\
0.0025 \\
0.0073 \\
0.0112 \\
0.0217 \\
0.0232 \\
0.0243 \\
0.0368 \\
0.0494 \\
0.0499 \\
0.0496 \\
0.0564 \\
0.0665 \\
0.0691 \\
0.0699 \\
0.0777 \\
0.0838 \\
0.0840 \\
0.0848 \\
0.0821 \\
0.0773 \\
0.0761 \\
0.0725 \\
0.0647 \\
0.0572 \\
0.0568 \\
0.0458 \\
0.0382 \\
0.0357 \\
0.0287 \\
0.0206 \\
0.0209 \\
0.0118 \\
0.0062 \\
0.0038 \\
0.0036 \\
0.0031 \\
0.0031\end{array}$ & $\begin{array}{c}\text { Pressure } \\
(\text { ps } 1 g) \\
9.89 \\
19.55 \\
29.57 \\
39.42 \\
49.16 \\
54.50 \\
59.57 \\
64.20 \\
69.32 \\
74.16 \\
80.16 \\
85.61 \\
90.58 \\
95.69 \\
100.92 \\
103.25 \\
106.11 \\
108.31 \\
111.08 \\
113.24 \\
117.83 \\
120.92 \\
123.28 \\
122.97 \\
125.82 \\
125.60 \\
127.84 \\
130.19 \\
132.53 \\
135.33 \\
134.42 \\
138.35 \\
140.16 \\
142.63 \\
145.78 \\
142.52 \\
0.22\end{array}$ & $\begin{array}{c}\text { * Strain } \\
0.0079 \\
0.0232 \\
0.0404 \\
0.0576 \\
0.0750 \\
0.0850 \\
0.0963 \\
0.1075 \\
0.1186 \\
0.1291 \\
0.1441 \\
0.1566 \\
0.1683 \\
0.1817 \\
0.1960 \\
0.2027 \\
0.2109 \\
0.2174 \\
0.2267 \\
0.2341 \\
0.2513 \\
0.2643 \\
0.2766 \\
0.2783 \\
0.2906 \\
0.2934 \\
0.3007 \\
0.3151 \\
0.3308 \\
0.3504 \\
0.3543 \\
0.3756 \\
0.3929 \\
0.4171 \\
0.4413 \\
0.4480 \\
0.1872\end{array}$ \\
\hline
\end{tabular}




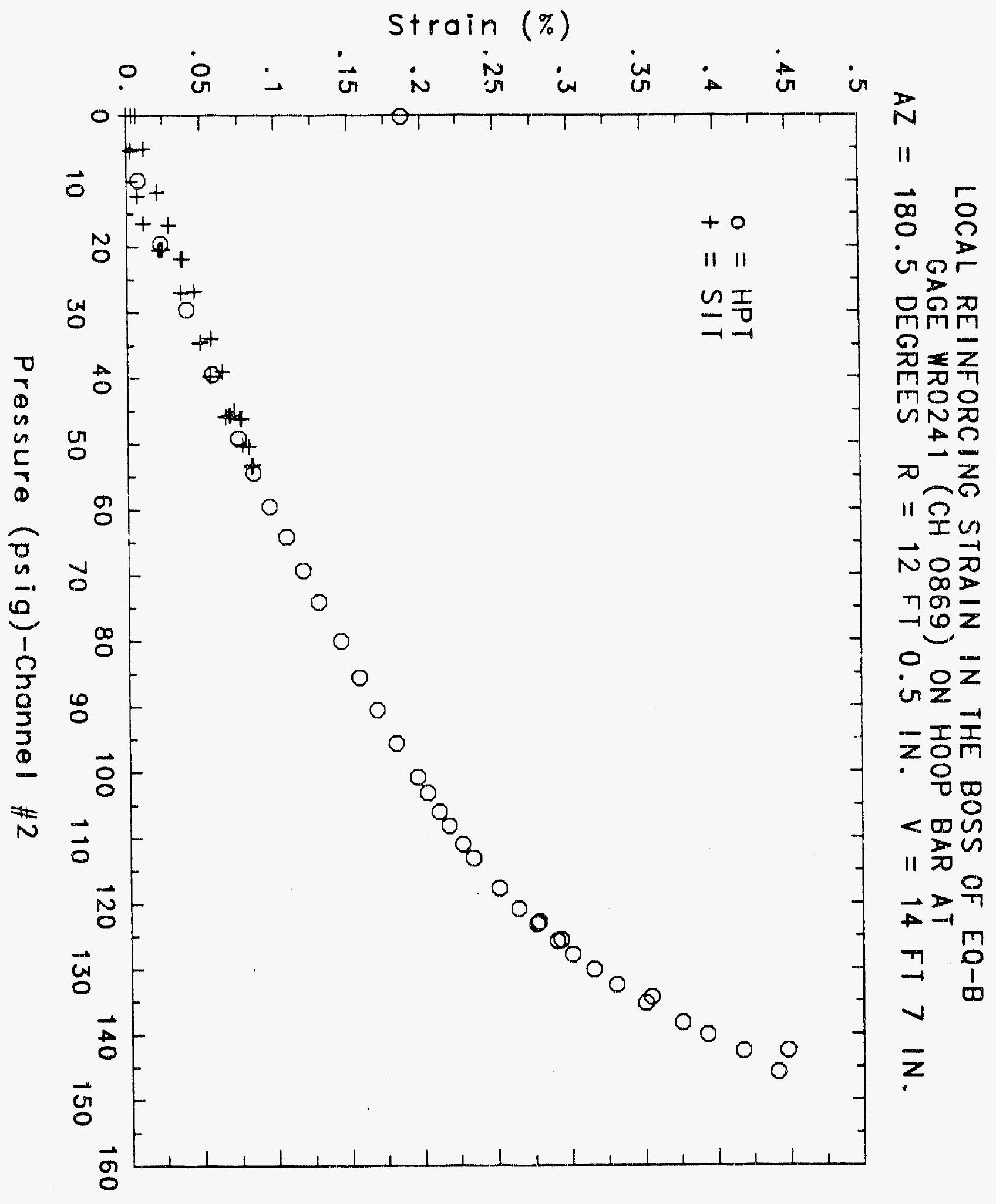




\section{Reinforced Concrete Test Data}

\begin{tabular}{|c|c|c|c|}
\hline STRUCTURAL & $\begin{array}{l}\text { WELDABLE GIAGE } 242 \\
\text { INTEGRITY TEST }\end{array}$ & $\begin{aligned} \text { Chanhel } 880 \\
\text { High Pressure }\end{aligned}$ & TEST \\
\hline $\begin{array}{l}\text { Pressure } \\
\text { (psig) } \\
-0.05 \\
5.33 \\
12.31 \\
16.44 \\
20.51 \\
20.46 \\
20.47 \\
27.05 \\
34.69 \\
34.53 \\
34.53 \\
39.70 \\
45.90 \\
45.65 \\
45.62 \\
50.10 \\
53.47 \\
53.29 \\
53.21 \\
50.49 \\
46.14 \\
46.13 \\
44.97 \\
38.98 \\
33.99 \\
33.96 \\
26.83 \\
21.88 \\
21.90 \\
16.69 \\
11.74 \\
11.77 \\
5.05 \\
0.02 \\
-0.04 \\
-0.02 \\
-0.02 \\
0.02\end{array}$ & 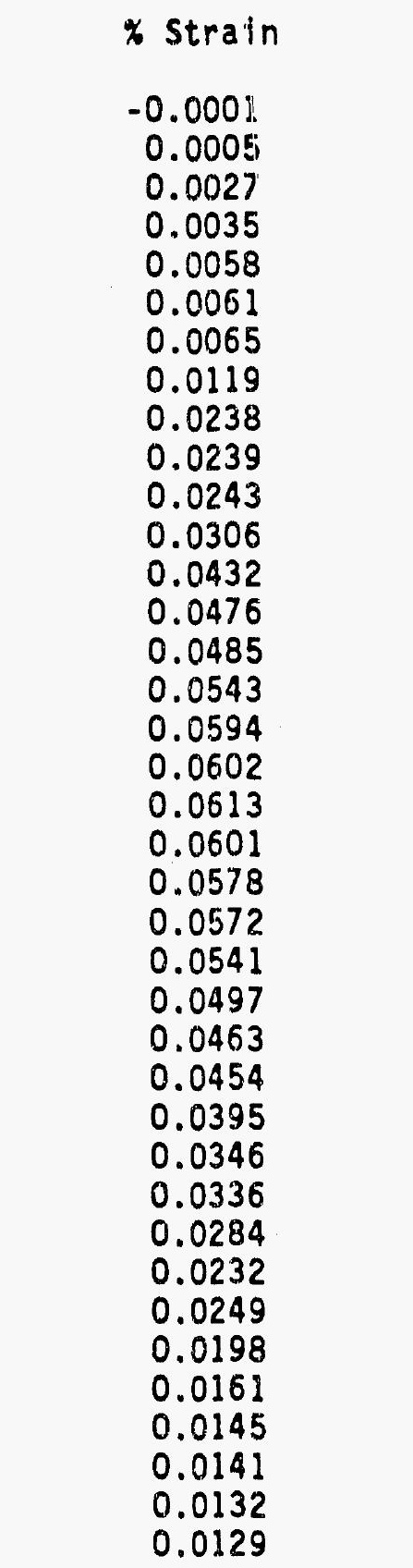 & $\begin{array}{c}\text { Pressure } \\
(\text { psig) } \\
9.89 \\
19.55 \\
29.57 \\
39.42 \\
49.16 \\
54.50 \\
59.57 \\
64.20 \\
69.32 \\
74.16 \\
80.16 \\
85.61 \\
90.58 \\
95.69 \\
100.92 \\
103.25 \\
106.11 \\
108.31 \\
111.08 \\
113.24 \\
117.83 \\
120.92 \\
123.28 \\
122.97 \\
125.82 \\
125.60 \\
127.84 \\
130.19 \\
132.53 \\
135.33 \\
134.42 \\
138.35 \\
140.16 \\
142.63 \\
145.78 \\
142.52 \\
0.22 \\
\end{array}$ & $\begin{array}{l}\text { \% Strain } \\
0.0038 \\
0.0133 \\
0.0234 \\
0.0328 \\
0.0416 \\
0.0467 \\
0.0525 \\
0.0584 \\
0.0651 \\
0.0739 \\
0.0857 \\
0.0928 \\
0.0986 \\
0.1046 \\
0.1106 \\
0.1136 \\
0.1168 \\
0.1193 \\
0.1228 \\
0.1252 \\
0.1305 \\
0.1343 \\
0.1373 \\
0.1377 \\
0.1416 \\
0.1416 \\
0.1444 \\
0.1480 \\
0.1521 \\
0.1557 \\
0.1555 \\
0.1593 \\
0.1612 \\
0.1635 \\
0.1666 \\
0.1648 \\
0.0192\end{array}$ \\
\hline
\end{tabular}




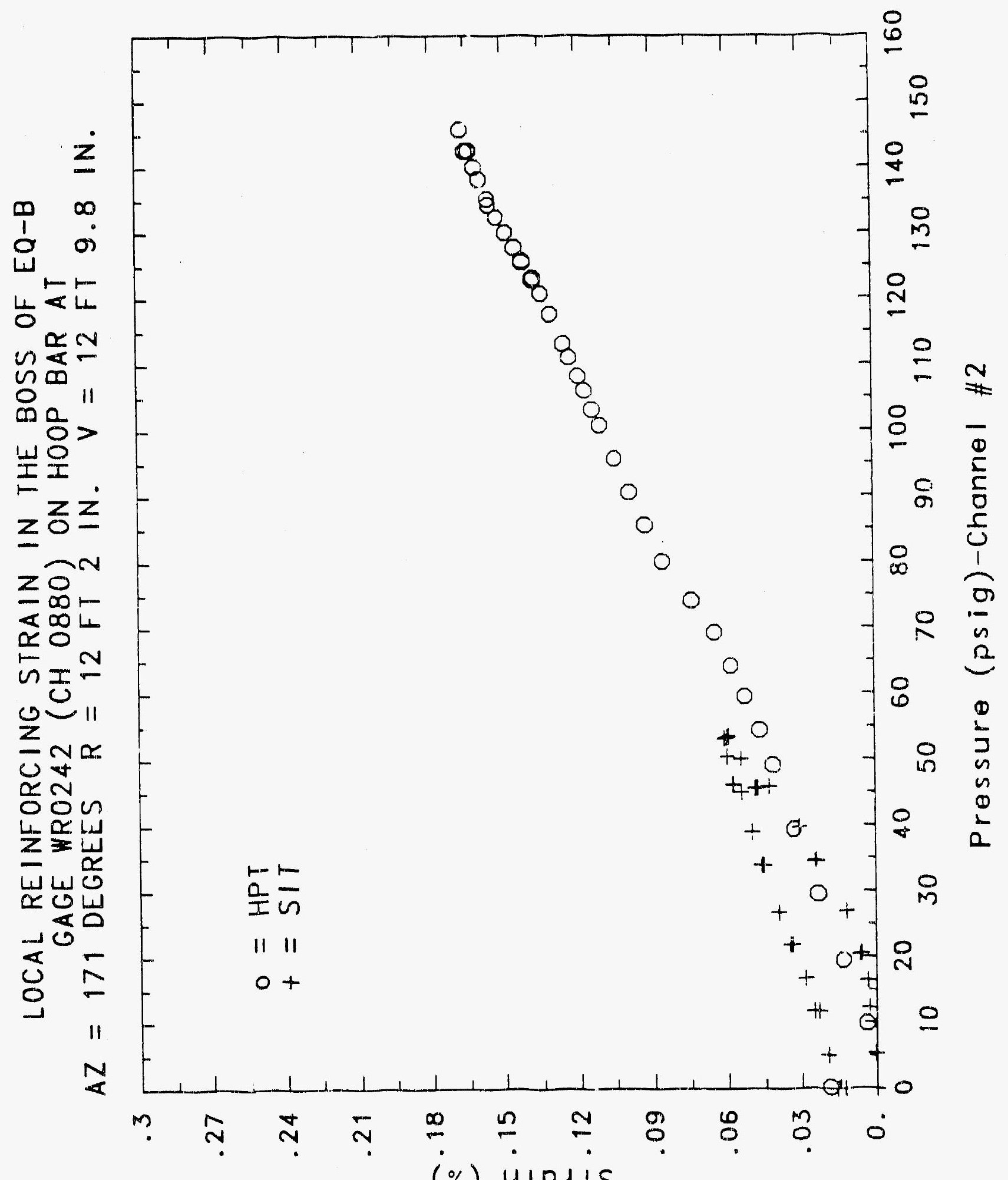

(\%) $4 ! 0 d+5$ 


\section{Reinforced Concrete test Data}

Weldable gage 243 Channel 881

STRUCTURAL INTEGRITY TEST

\begin{tabular}{|c|c|c|c|}
\hline $\begin{array}{c}\text { Pressure } \\
(p s i g) \\
-0.05 \\
5.33 \\
12.31 \\
16.44 \\
20.51 \\
20.46 \\
20.47 \\
27.05 \\
34.69 \\
34.53 \\
34.53 \\
39.70 \\
45.90 \\
45.65 \\
45.62 \\
50.10 \\
53.47 \\
53.29 \\
53.21 \\
50.49 \\
46.14 \\
46.19 \\
44.37 \\
38.98 \\
33.95 \\
33.96 \\
26.83 \\
21.83 \\
21.90 \\
10.69 \\
11.74 \\
11.77 \\
5.05 \\
0.02 \\
-0.04 \\
-0.02 \\
-0.02 \\
0.02\end{array}$ & $\begin{array}{l}\text { Strain } \\
0.0000 \\
-0.0024 \\
-0.0069 \\
-0.0115 \\
-0.0182 \\
-0.0199 \\
-0.0207 \\
-0.0291 \\
-0.0368 \\
-0.0369 \\
-0.0371 \\
-0.0413 \\
-0.0464 \\
-0.0464 \\
-0.0461 \\
-0.0504 \\
-0.0538 \\
-0.0539 \\
-0.0537 \\
-0.0519 \\
-0.0475 \\
-0.0476 \\
-0.0466 \\
-0.0415 \\
-0.0372 \\
-0.0372 \\
-0.0308 \\
-0.0263 \\
-0.0268 \\
-0.0209 \\
-0.0149 \\
-0.0141 \\
-0.0049 \\
0.0024 \\
0.0038 \\
0.0040 \\
0.0031 \\
0.0032\end{array}$ & $\begin{array}{c}\text { Pressure } \\
(p s i g) \\
9.89 \\
19.55 \\
29.57 \\
39.42 \\
49.16 \\
54.50 \\
59.57 \\
64.20 \\
69.32 \\
74.16 \\
80.16 \\
85.61 \\
90.58 \\
95.59 \\
100.92 \\
103.25 \\
106.11 \\
108.31 \\
111.08 \\
113.24 \\
117.83 \\
120.92 \\
123.28 \\
122.97 \\
125.82 \\
125.60 \\
127.84 \\
130.19 \\
132.53 \\
135.33 \\
134.42 \\
138.35 \\
140.16 \\
142.63 \\
145.78 \\
142.52 \\
0.22 \\
\end{array}$ & $\begin{array}{l}\% \text { Strain } \\
-0.0129 \\
-0.0260 \\
-0.0367 \\
-0.0461 \\
-0.0556 \\
-0.0603 \\
-0.0652 \\
-0.0716 \\
-0.0771 \\
-0.0834 \\
-0.0916 \\
-0.0988 \\
-0.1051 \\
-0.1116 \\
-0.1158 \\
-0.1183 \\
-0.1216 \\
-0.1239 \\
-0.1277 \\
-0.1315 \\
-0.1376 \\
-0.1423 \\
-0.1460 \\
-0.1465 \\
-0.1513 \\
-0.1515 \\
-0.1546 \\
-0.1598 \\
-0.1666 \\
-0.1748 \\
-0.1754 \\
-0.1870 \\
-0.1946 \\
-0.2069 \\
-0.2228 \\
-0.2238 \\
-0.0492\end{array}$ \\
\hline
\end{tabular}

High Pressure test

Pressure

9.89

49.16

54.50

64.20

69.32

74.16

80.16

0.58

35.59

100.92

103.25

106.11

108.31

111.08

120.92

123.28

122.97

127.84

130.19

132.53

135.33

134.42

138.35

142.63

145.78

42.52

0.22
$-0.0129$

$-0.0260$

$-0.0367$

$-0.0461$

$-0.0652$

$-0.0716$

$-0.0771$

$-0.0834$

0.0916

0.1051

$-0.1116$

$-0.1158$

0.1183

$-0.1277$

$-0.1315$

$-0.1376$

$-0.1423$

$-0.1460$

$-0.1515$

$-0.1546$

$-0.1598$

$-0.1666$

$-0.1748$

$-0.1946$

$-0.2069$

$-0.2228$

$-0.2238$

$-0.0492$ 


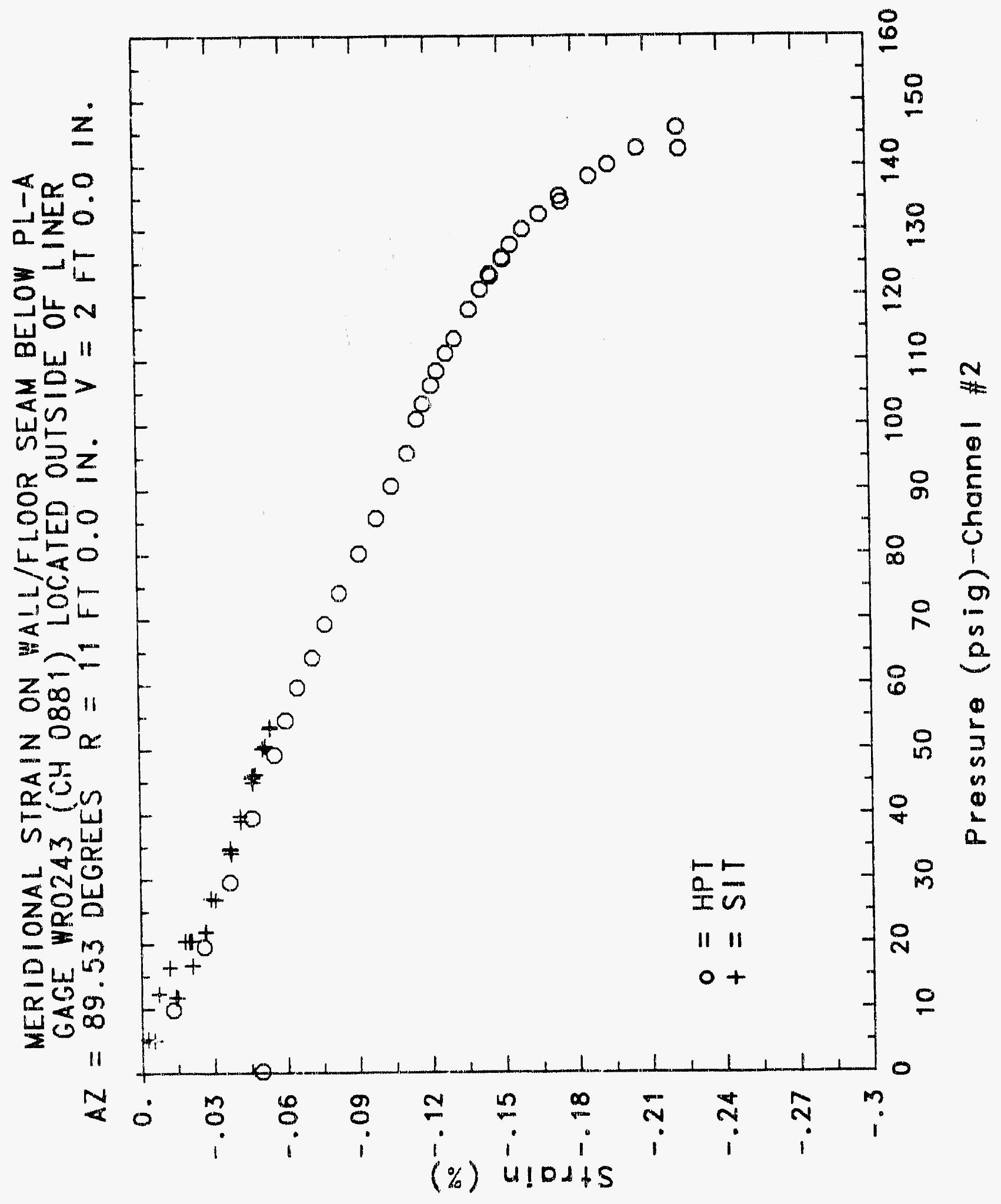




\section{Reinforced Concrete Test Data}

Weldable Gage 244 Channel 882

STRUCTURAL INTEGRITY TEST

\begin{tabular}{|c|c|c|c|}
\hline $\begin{array}{c}\text { Pressure } \\
\text { (psig) } \\
-0.05 \\
5.33 \\
12.31 \\
16.44 \\
20.51 \\
20.46 \\
20.47 \\
27.05 \\
34.69 \\
34.53 \\
34.53 \\
39.70 \\
45.90 \\
45.65 \\
45.62 \\
50.10 \\
53.47 \\
53.29 \\
53.21 \\
50.49 \\
46.14 \\
46.13 \\
44.97 \\
38.93 \\
33.99 \\
33.96 \\
26.83 \\
21.88 \\
21.90 \\
16.69 \\
11.74 \\
11.77 \\
5.05 \\
0.02 \\
-0.04 \\
-0.02 \\
-0.02 \\
0.02 \\
\end{array}$ & $\begin{array}{l}\text { \% Strain } \\
-0.0003 \\
-0.0016 \\
-0.0031 \\
-0.0029 \\
-0.0026 \\
-0.0025 \\
-0.0022 \\
-0.0028 \\
-0.0043 \\
-0.0044 \\
-0.0044 \\
-0.0057 \\
-0.0071 \\
-0.0071 \\
-0.0073 \\
-0.0080 \\
-0.0083 \\
-0.0084 \\
-0.0081 \\
-0.0079 \\
-0.0075 \\
-0.0075 \\
-0.0057 \\
-0.0054 \\
-0.0053 \\
-0.0049 \\
-0.0046 \\
-0.0040 \\
-0.0048 \\
-0.0051 \\
-0.0050 \\
-0.0054 \\
-0.0052 \\
-0.0044 \\
-0.0012 \\
-0.0014 \\
-0.0016 \\
-0.0014\end{array}$ & $\begin{array}{c}\text { Pressure } \\
(\text { psig) } \\
9.89 \\
19.55 \\
29.57 \\
39.42 \\
49.16 \\
54.50 \\
59.57 \\
64.20 \\
59.32 \\
74.16 \\
80.16 \\
85.61 \\
90.58 \\
95.69 \\
100.92 \\
103.25 \\
106.11 \\
108.31 \\
111.08 \\
113.24 \\
117.83 \\
120.92 \\
123.28 \\
122.97 \\
125.82 \\
125.60 \\
127.84 \\
130.19 \\
132.53 \\
135.33 \\
134.42 \\
138.35 \\
140.16 \\
142.63 \\
142.78 \\
142.52 \\
0.22 \\
\end{array}$ & $\begin{array}{l}\text { \% Strain } \\
-0.0052 \\
-0.0060 \\
-0.0066 \\
-0.0079 \\
-0.0088 \\
-0.0097 \\
-0.0102 \\
-0.0106 \\
-0.0109 \\
-0.0117 \\
-0.0143 \\
-0.0155 \\
-0.0167 \\
-0.0183 \\
-0.0195 \\
-0.0199 \\
-0.0205 \\
-0.0211 \\
-0.0219 \\
-0.0215 \\
-0.0228 \\
-0.0230 \\
-0.0241 \\
-0.0240 \\
-0.0249 \\
-0.0249 \\
-0.0252 \\
-0.0265 \\
-0.0272 \\
-0.0292 \\
-0.0293 \\
-0.0324 \\
-0.0355 \\
-0.0406 \\
-0.0462 \\
-0.0479 \\
-0.0193\end{array}$ \\
\hline
\end{tabular}

High Pressure test

$9.89 \quad-0.0052$

$-0.0060$

$49.16 \quad-0.0088$

$54.50 \quad-0.0097$

$59.57 \quad-0.0102$

64.20

$-0.0109$

80.16

85.61

$90.58 \quad-0.0167$

$95.69 \quad-0.0183$

$-0.0195$

$-0.0199$

$-0.0205$

111.08

$113.24-0.0215$

$117.83 \quad-0.0228$

$120.92-0.0230$

$-0.0241$

$-0.0240$

0249

$-0.0265$

$132.53 \quad-0.0272$

$-0.0292$

$-0.0293$

40.16

$142.63 \quad-0.0406$

$142.78 \quad-0.0462$

$\begin{array}{ll}0.22 & -0.0193\end{array}$ 


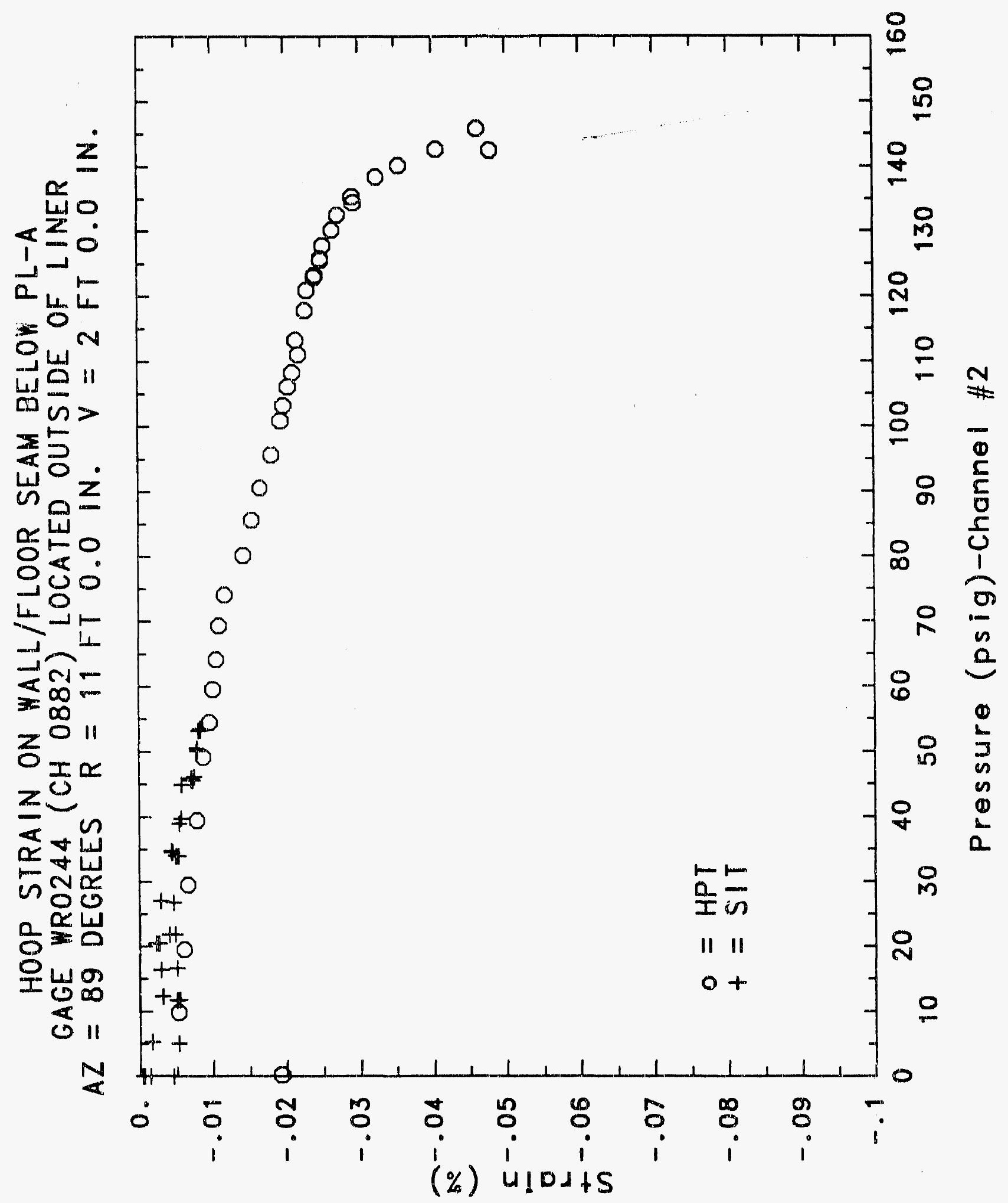




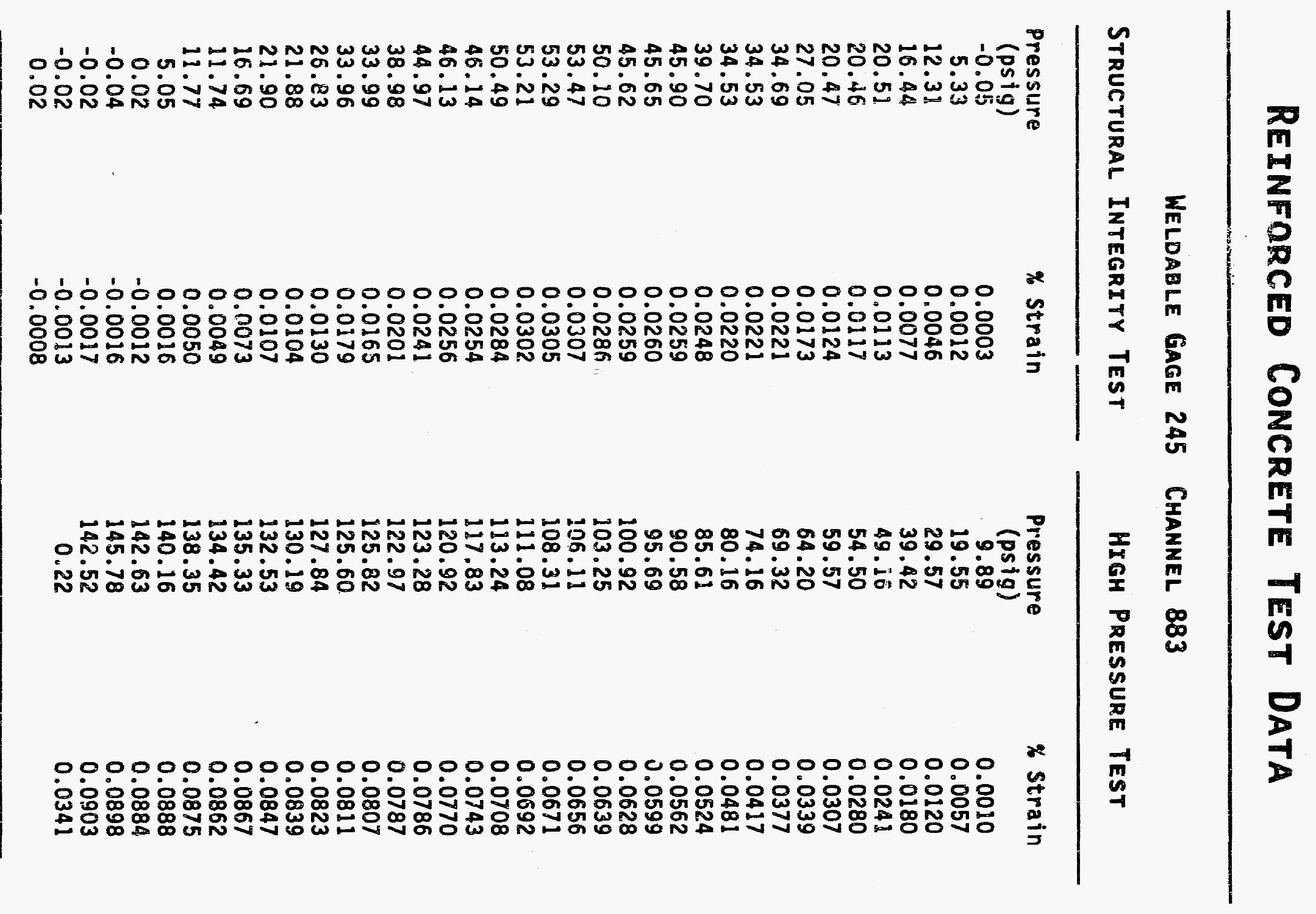




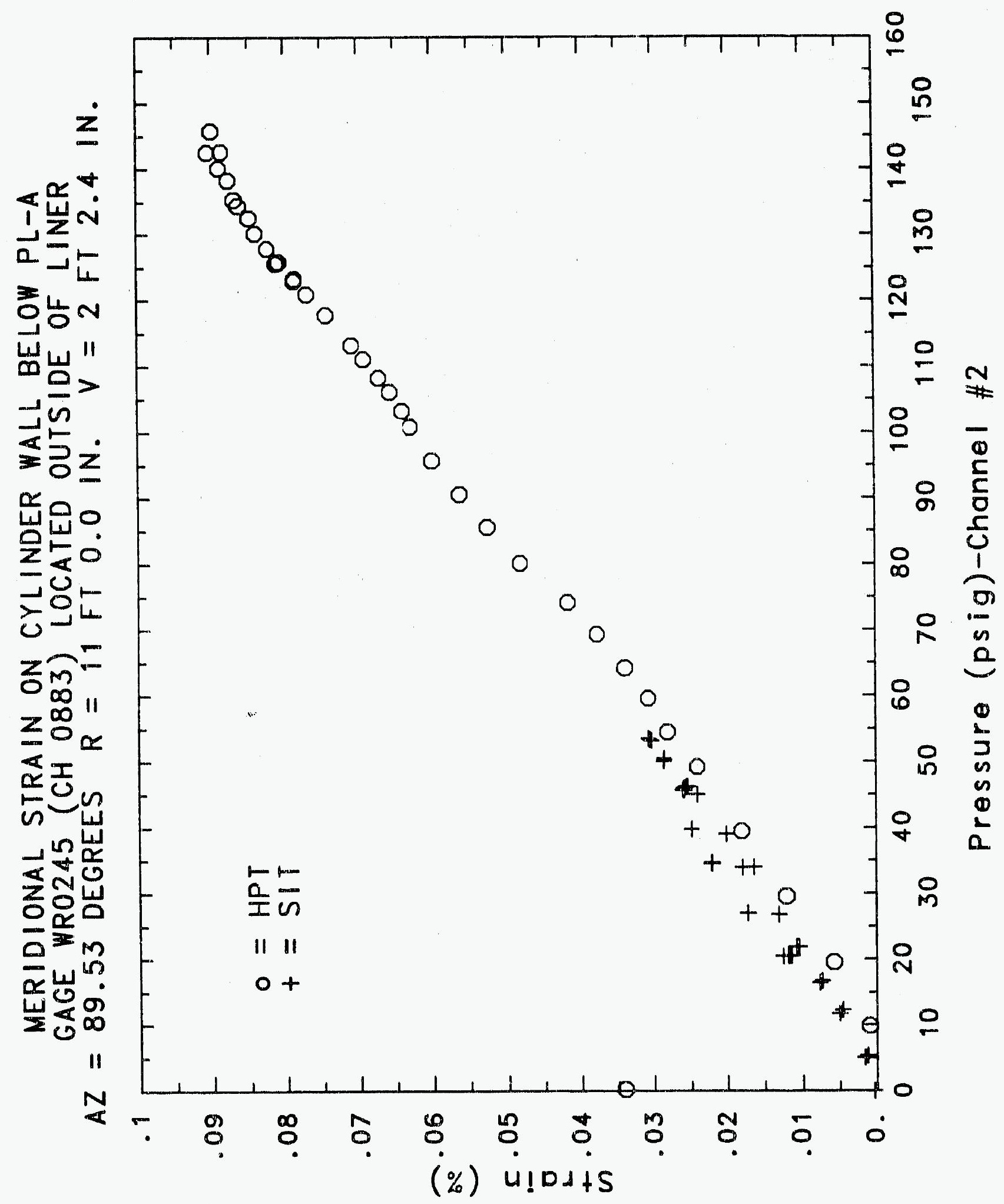




\section{Reinforced Concrete Test Data}

Weldable gage 246 Channel 884

Structural INTEgRITY TEST

\begin{tabular}{|c|c|c|c|}
\hline $\begin{array}{c}\text { Pressure } \\
\text { (psig) } \\
-0.05 \\
5.33 \\
12.31 \\
16.44 \\
20.51 \\
20.46 \\
20.47 \\
27.05 \\
34.69 \\
34.53 \\
34.53 \\
39.70 \\
45.90 \\
45.65 \\
45.62 \\
50.10 \\
53.47 \\
53.29 \\
53.21 \\
50.49 \\
46.14 \\
46.13 \\
44.97 \\
38.98 \\
33.99 \\
33.96 \\
26.83 \\
21.88 \\
21.90 \\
16.69 \\
11.74 \\
11.77 \\
5.05 \\
0.02 \\
-0.04 \\
-0.02 \\
-0.02 \\
0.02 \\
\end{array}$ & $\begin{array}{c}\text { \% Strain } \\
-0.0009 \\
0.0000 \\
0.0018 \\
0.0019 \\
0.0022 \\
0.0019 \\
0.0016 \\
0.0013 \\
-0.0012 \\
-0.0016 \\
-0.0017 \\
-0.0023 \\
-0.0029 \\
-0.0028 \\
-0.0033 \\
-0.0030 \\
-0.0030 \\
-0.0027 \\
-0.0029 \\
-0.0024 \\
-0.0020 \\
-0.0019 \\
-0.0008 \\
-0.0008 \\
-0.0009 \\
-0.0010 \\
-0.0016 \\
-0.0017 \\
-0.0024 \\
-0.0027 \\
-0.0030 \\
-0.0032 \\
-0.0031 \\
-0.0024 \\
0.0002 \\
0.0001 \\
0.0004 \\
0.0001\end{array}$ & $\begin{array}{c}\text { Pressure } \\
(\text { psig) } \\
9.89 \\
19.55 \\
29.57 \\
39.42 \\
49.16 \\
54.50 \\
59.57 \\
64.20 \\
69.32 \\
74.16 \\
80.16 \\
85.61 \\
90.58 \\
95.69 \\
100.92 \\
103.25 \\
106.11 \\
108.31 \\
111.08 \\
113.24 \\
117.83 \\
120.92 \\
123.28 \\
122.97 \\
125.82 \\
125.60 \\
127.84 \\
130.19 \\
132.53 \\
135.33 \\
134.42 \\
138.35 \\
140.16 \\
142.63 \\
145.78 \\
142.52 \\
0.22\end{array}$ & $\begin{array}{l}\text { \% Strain } \\
-0.0045 \\
-0.0043 \\
-0.0038 \\
-0.0036 \\
-0.0036 \\
-0.0042 \\
-0.0040 \\
-0.0047 \\
-0.0051 \\
-0.0059 \\
-0.0078 \\
-0.0087 \\
-0.0089 \\
-0.0092 \\
-0.0110 \\
-0.0112 \\
-0.0123 \\
-0.0129 \\
-0.0130 \\
-0.0133 \\
-0.0136 \\
-0.0149 \\
-0.0153 \\
-0.0154 \\
-0.0160 \\
-0.0164 \\
-0.0166 \\
-0.0176 \\
-0.0179 \\
-0.0199 \\
-0.0203 \\
-0.0226 \\
-0.0251 \\
-0.0273 \\
-0.0307 \\
-0.0309 \\
-0.0178\end{array}$ \\
\hline
\end{tabular}

High Pressure Test

(psig)

9.89

39.42

54.50

59.57

80.16

85.6 .1

$90 ., 58$

95.69

108.31

111.08

125.82

125.60

127.84

130.19

132.53

138.35

140.16

142.63

145.78

42.52

$-0.0309$ 


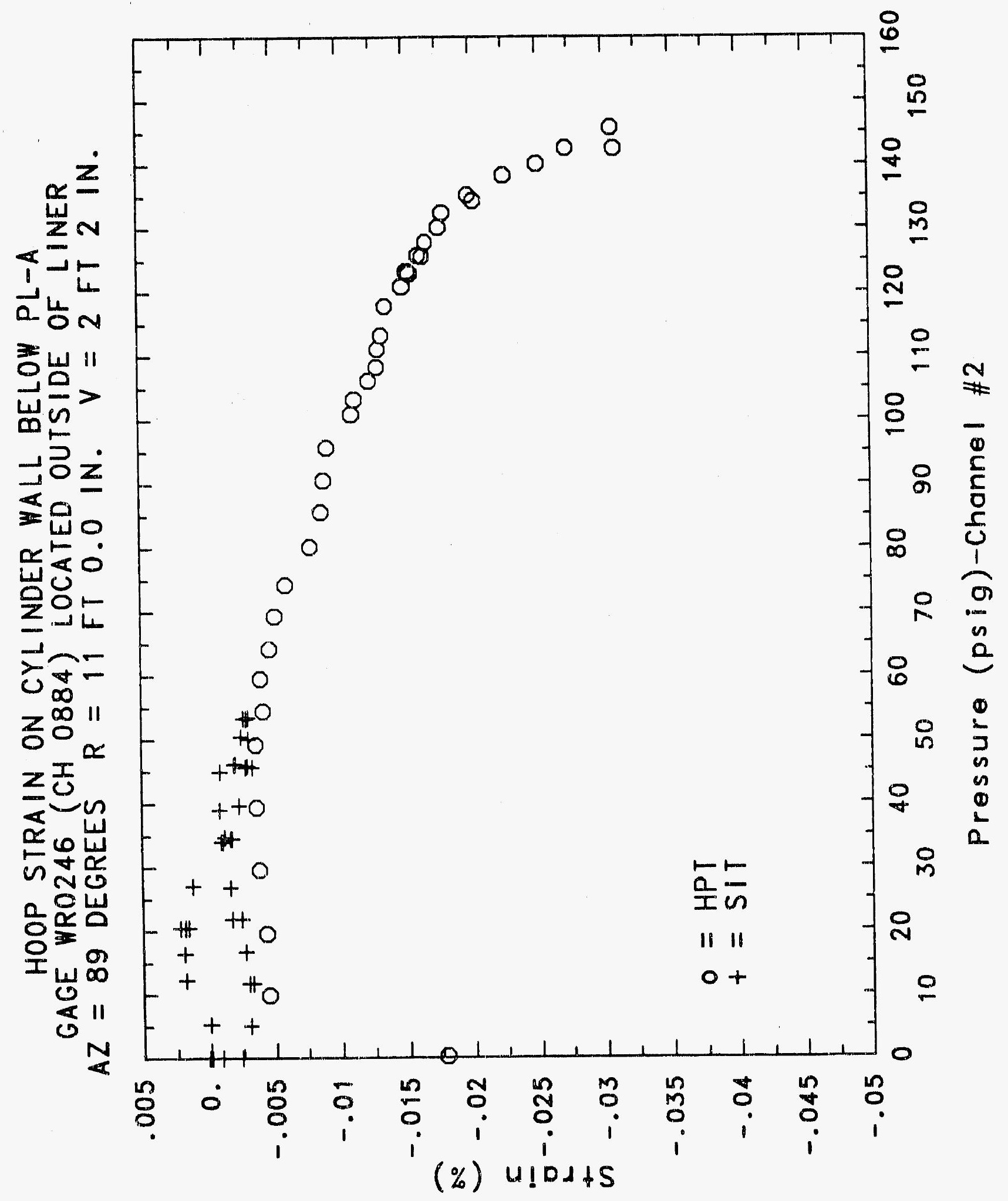




\section{Reinforced Concrete Test Data}

Weldable Gage 247 Channel 885

StRUCTURAL INTEgRITy TEST

\begin{tabular}{|c|c|c|c|}
\hline STRUCTURAL & $\begin{array}{l}\text { WeLdabLe GAGE } 247 \\
\text { INTEGRITY TEST }\end{array}$ & $\begin{array}{r}\text { Channel } 885 \\
\text { High Pressure }\end{array}$ & TEST \\
\hline $\begin{array}{c}\text { Pressure } \\
\text { (psig) } \\
-0.05 \\
5.33 \\
12.31 \\
16.44 \\
20.51 \\
20.46 \\
20.47 \\
27.05 \\
34.69 \\
34.53 \\
34.53 \\
39.70 \\
45.90 \\
45.65 \\
45.62 \\
50.10 \\
53.47 \\
53.29 \\
53.21 \\
50.49 \\
46.14 \\
46.13 \\
44.97 \\
38.98 \\
33.99 \\
33.96 \\
26.83 \\
21.88 \\
21.90 \\
16.69 \\
11.74 \\
11.77 \\
5.05 \\
0.02 \\
-0.04 \\
-0.02 \\
-0.02 \\
0.02 \\
\end{array}$ & $\begin{array}{c}\text { \% Strain } \\
-0.0013 \\
0.0018 \\
0.0045 \\
0.0070 \\
0.0100 \\
0.0109 \\
0.0120 \\
0.0179 \\
0.0226 \\
0.0229 \\
0.0226 \\
0.0252 \\
0.0268 \\
0.0269 \\
0.0266 \\
0.0279 \\
0.0295 \\
0.0291 \\
0.0292 \\
0.0280 \\
0.0258 \\
0.0255 \\
0.0247 \\
0.0226 \\
0.0208 \\
0.0214 \\
0.0181 \\
0.0167 \\
0.0167 \\
0.0149 \\
0.0126 \\
0.0115 \\
0.0089 \\
0.0060 \\
0.0044 \\
0.0041 \\
0.0048 \\
0.0046\end{array}$ & $\begin{array}{c}\text { Pressure } \\
(\text { psig) } \\
9.89 \\
19.55 \\
29.57 \\
39.42 \\
49.16 \\
54.50 \\
59.57 \\
64.20 \\
69.32 \\
74.16 \\
80.16 \\
85.61 \\
90.58 \\
95.69 \\
100.92 \\
103.25 \\
106.11 \\
108.31 \\
111.08 \\
113.24 \\
117.83 \\
120.92 \\
123.28 \\
122.97 \\
125.82 \\
125.60 \\
127.84 \\
130.19 \\
132.53 \\
135.33 \\
134.42 \\
138.35 \\
140.16 \\
142.63 \\
145.78 \\
142.52 \\
0.22\end{array}$ & $\begin{array}{l}\text { \% Strain } \\
0.0027 \\
0.0075 \\
0.0111 \\
0.0147 \\
0.0183 \\
0.0211 \\
0.0231 \\
0.0251 \\
0.0272 \\
0.0291 \\
0.0321 \\
0.0345 \\
0.0365 \\
0.0395 \\
0.0422 \\
0.0435 \\
0.0452 \\
0.0463 \\
0.0479 \\
0.0490 \\
0.0508 \\
0.0519 \\
0.0537 \\
0.0530 \\
0.0549 \\
0.0553 \\
0.0563 \\
0.0583 \\
0.0602 \\
0.0631 \\
0.0629 \\
0.0681 \\
0.0733 \\
0.0809 \\
0.0896 \\
0.0904 \\
0.0302\end{array}$ \\
\hline
\end{tabular}

High Pressure test

Pres 


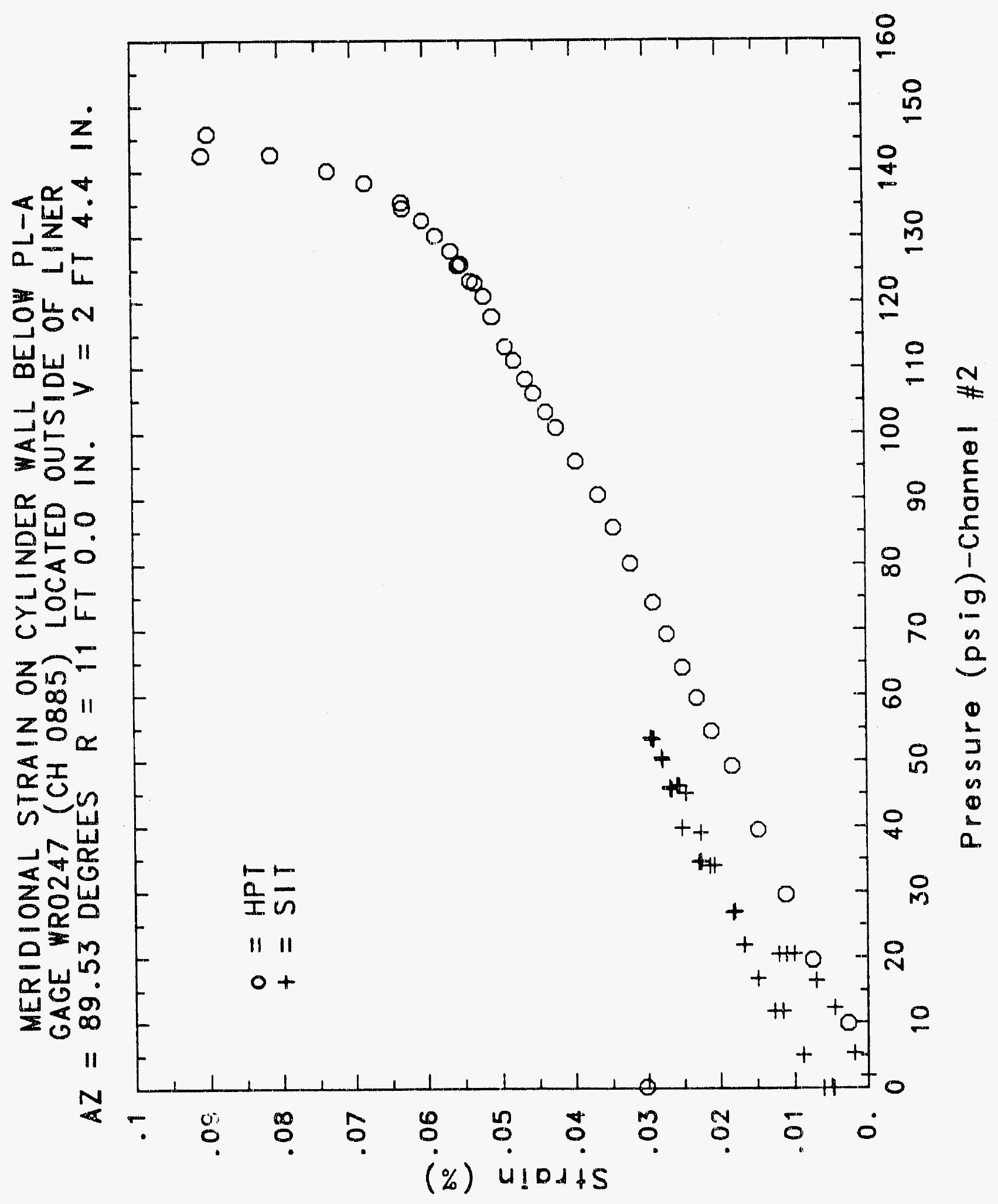




\section{Reinforced Concrete Test Data}

Weldable gage 248 Channel 886

Structural IntEgrity TEST

High Pressure test

\begin{tabular}{|c|c|c|c|}
\hline $\begin{array}{c}\text { Pressure } \\
\text { (psig) } \\
-0.05 \\
5.33 \\
12.31 \\
16.44 \\
20.51 \\
20.46 \\
20.47 \\
27.05 \\
34.69 \\
34.53 \\
34.53 \\
39.70 \\
45.90 \\
45.65 \\
45.62 \\
50.10 \\
53.47 \\
53.29 \\
53.21 \\
50.49 \\
46.14 \\
46.13 \\
44.97 \\
38.98 \\
33.99 \\
33.96 \\
26.83 \\
21.88 \\
21.90 \\
16.69 \\
11.74 \\
11.77 \\
5.05 \\
0.02 \\
-0.04 \\
-0.02 \\
-0.02 \\
0.02 \\
\end{array}$ & $\begin{array}{c}\text { \% Strain } \\
0.0004 \\
0.0002 \\
0.0004 \\
0.0003 \\
0.0012 \\
0.0010 \\
0.0019 \\
0.0009 \\
0.0008 \\
0.0004 \\
0.0002 \\
0.0001 \\
-0.0002 \\
-0.0009 \\
-0.0004 \\
-0.0006 \\
0.0003 \\
0.0006 \\
0.0008 \\
0.0010 \\
0.0011 \\
0.0013 \\
0.0028 \\
0.0024 \\
0.0020 \\
0.0023 \\
0.0011 \\
0.0011 \\
0.0000 \\
-0.0001 \\
-0.0007 \\
-0.0016 \\
-0.0010 \\
-0.0011 \\
0.0020 \\
0.0030 \\
0.0030 \\
0.0021\end{array}$ & $\begin{array}{c}\text { Pressure } \\
\text { (psig) } \\
9.89 \\
19.55 \\
29.57 \\
39.42 \\
49.16 \\
54.50 \\
59.57 \\
64.20 \\
69.32 \\
74.16 \\
80.16 \\
85.61 \\
90.58 \\
95.69 \\
100.92 \\
103.25 \\
106.11 \\
108.31 \\
111.08 \\
113.24 \\
117.83 \\
120.92 \\
123.28 \\
122.97 \\
125.82 \\
125.60 \\
127.84 \\
130.19 \\
132.53 \\
135.33 \\
134.42 \\
138.35 \\
140.16 \\
142.63 \\
145.78 \\
142.52 \\
0.22\end{array}$ & $\begin{array}{c}\text { \% Strain } \\
-0.0044 \\
-0.0051 \\
-0.0044 \\
-0.0037 \\
-0.0034 \\
-0.0030 \\
-0.0026 \\
-0.0016 \\
-0.0006 \\
-0.0010 \\
-0.0021 \\
-0.0017 \\
-0.0015 \\
-0.0007 \\
0.0005 \\
0.0012 \\
0.0016 \\
0.0019 \\
0.0033 \\
0.0040 \\
0.0058 \\
0.0068 \\
0.0083 \\
0.0088 \\
0.0098 \\
0.0097 \\
0.0109 \\
0.0126 \\
0.0153 \\
0.0185 \\
0.0201 \\
0.0263 \\
0.0308 \\
0.0381 \\
0.0447 \\
0.0465 \\
0.0411\end{array}$ \\
\hline
\end{tabular}




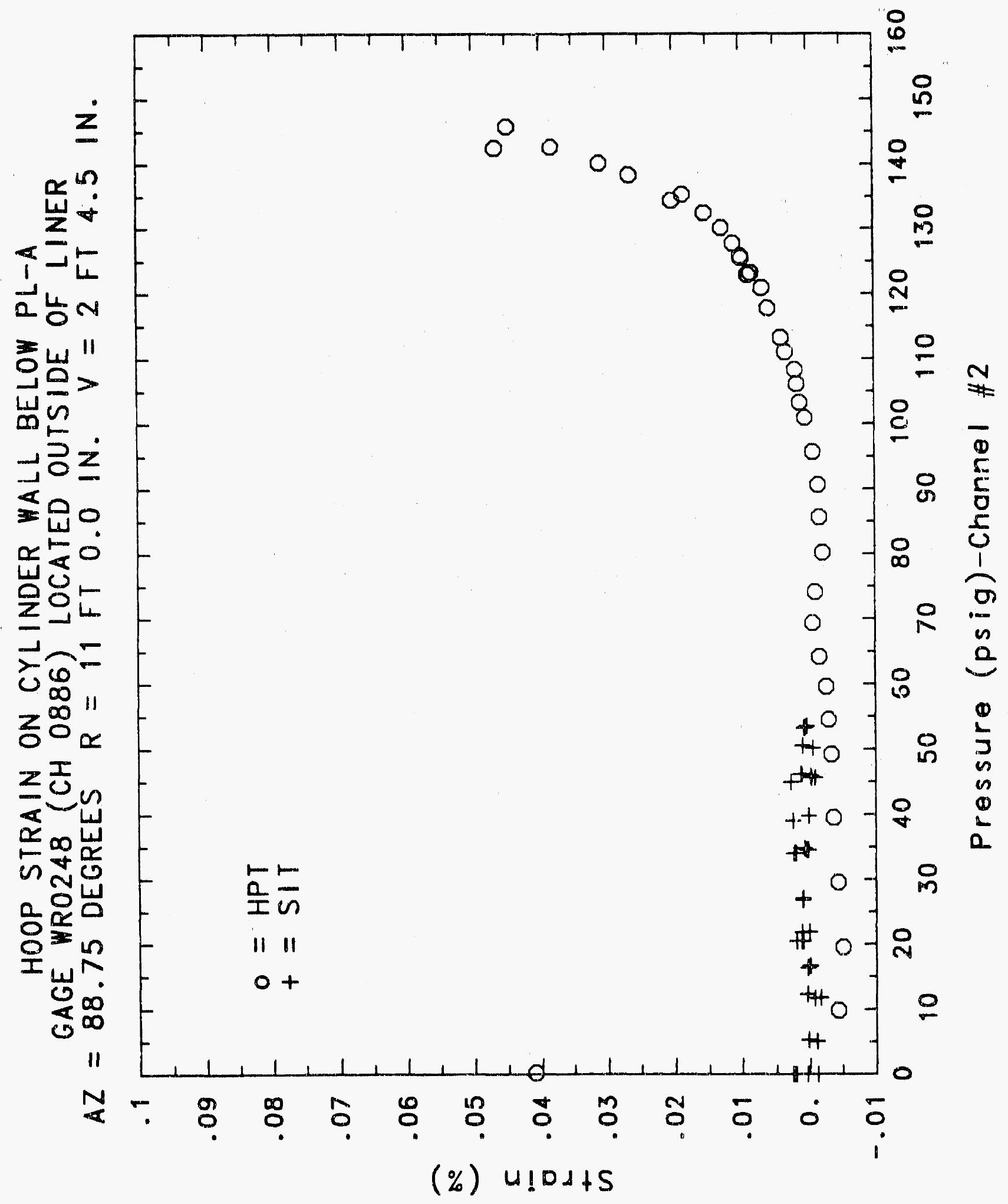




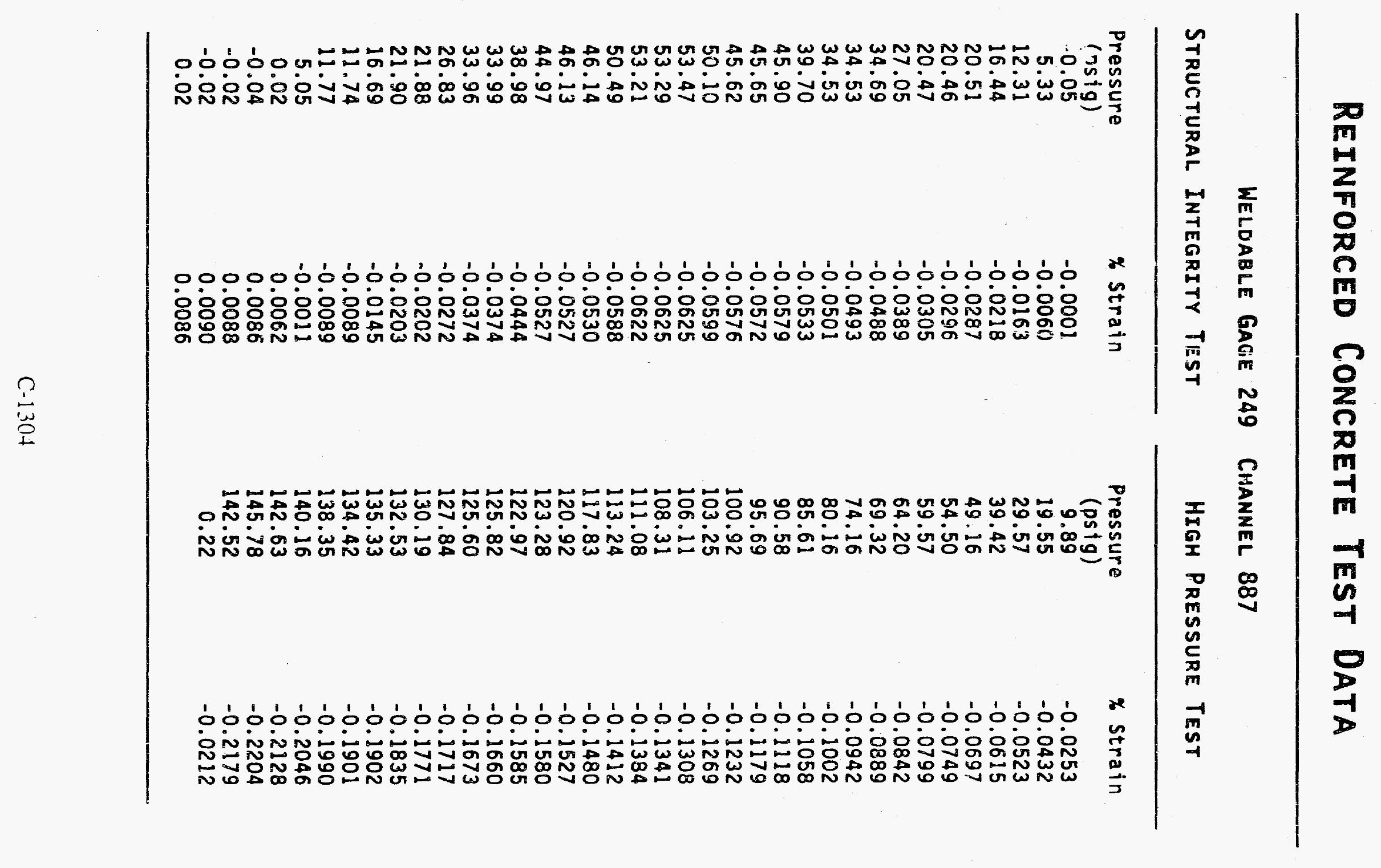




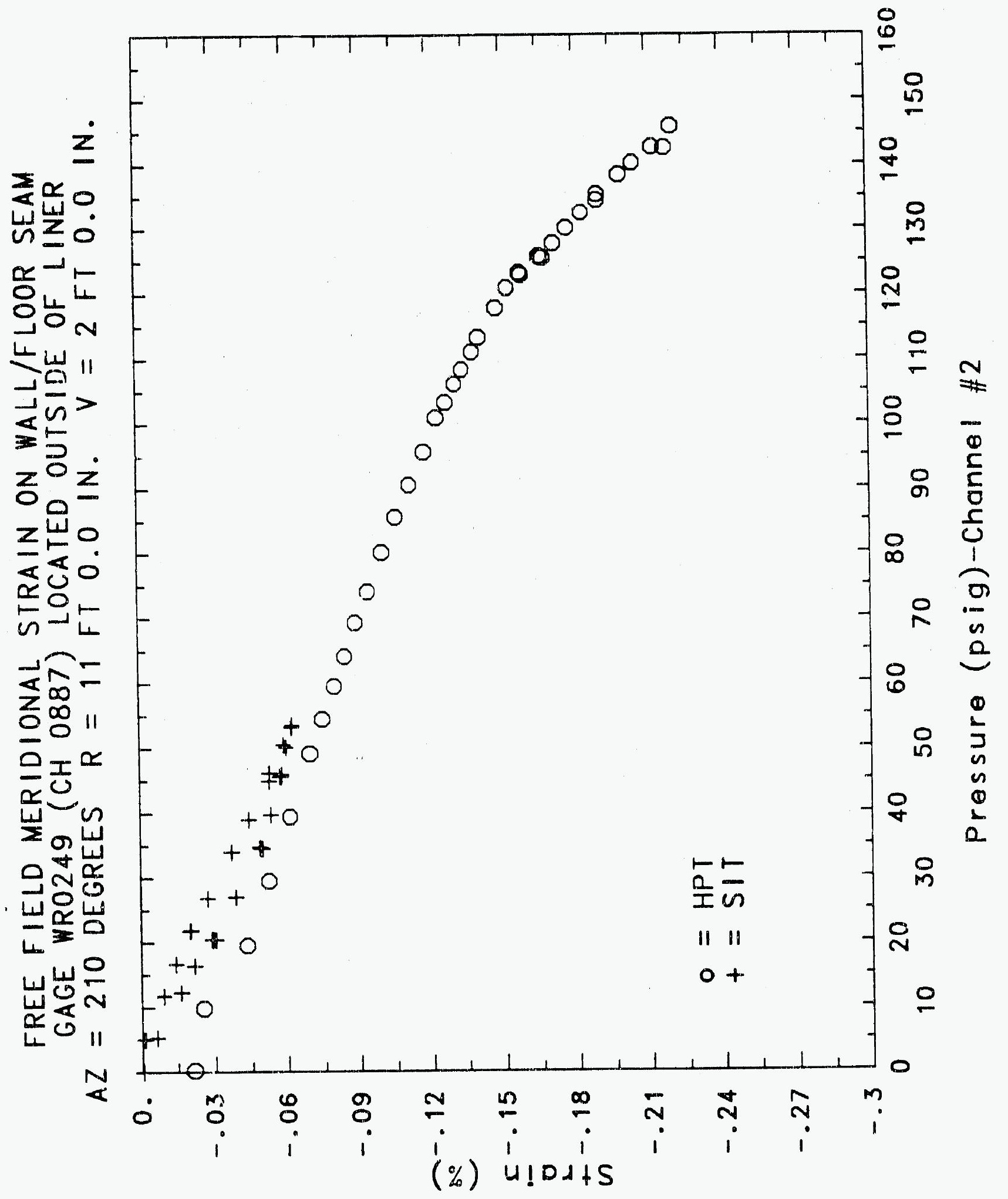

C- 1305 


\section{Reinforced Concrete Test Data}

We! Dable gage 250 Channel 888

Structural Integrity Test

High Pressure test

\begin{tabular}{|c|c|c|c|}
\hline $\begin{array}{l}\text { Pressure } \\
\text { (psig) } \\
-0.05 \\
55.33 \\
12.31 \\
16.44 \\
20.51 \\
20.46 \\
20.47 \\
27.05 \\
34.69 \\
34.53 \\
34.53 \\
39.70 \\
45.90 \\
45.65 \\
45.52 \\
50.10 \\
53.47 \\
53.29 \\
53.21 \\
50.49 \\
46.14 \\
46.13 \\
44.97 \\
38.9 \\
33 \\
33.99 \\
33.96 \\
26.83 \\
21.88 \\
21.90 \\
16.09 \\
11.74 \\
11.77 \\
5\end{array}$ & $\begin{array}{l}\text { * Strain } \\
-0.0003 \\
-0.0010 \\
-0.0020 \\
-0.0027 \\
-0.0033 \\
-0.0028 \\
-0.0032 \\
-0.0037 \\
-0.0040 \\
-0.0039 \\
-0.0043 \\
-0.0044 \\
-0.0052 \\
-0.0051 \\
-0.0053 \\
-0.0055 \\
-0.0060 \\
-0.0061 \\
-0.0057 \\
-0.0051 \\
-0.0039 \\
-0.0036 \\
-0.0027 \\
-0.0017 \\
-0.0010 \\
-0.0007 \\
-0.0003 \\
0.0004 \\
0.0004 \\
0.0000 \\
-0.0005 \\
-0.0011 \\
-0.0015 \\
-0.0018 \\
0.0018 \\
0.0012 \\
0.0010 \\
0.0000\end{array}$ & $\begin{array}{c}\text { Pressure } \\
(\text { psig) } \\
9.89 \\
19.55 \\
29.57 \\
39.42 \\
49.16 \\
54.50 \\
59.57 \\
64.20 \\
69.32 \\
74.16 \\
80.16 \\
85.61 \\
90.58 \\
95.69 \\
100.92 \\
103.25 \\
106.11 \\
108.31 \\
111.08 \\
113.24 \\
117.83 \\
120.92 \\
123.28 \\
122.97 \\
125.82 \\
125.60 \\
127.84 \\
130.19 \\
132.53 \\
135.33 \\
134.42 \\
138.35 \\
140.16 \\
142.63 \\
145.78 \\
142.52 \\
0.22\end{array}$ & $\begin{array}{l}\text { \% strain } \\
-0.0044 \\
-0.0065 \\
-0.0065 \\
-0.0076 \\
-0.0078 \\
-0.0085 \\
-0.0095 \\
-0.0089 \\
-0.0082 \\
-0.0074 \\
-0.0100 \\
-0.0108 \\
-0.0115 \\
-0.0123 \\
-0.0127 \\
-0.0132 \\
-0.0138 \\
-0.0145 \\
-0.0144 \\
-0.0153 \\
-0.0163 \\
-0.0174 \\
-0.0177 \\
-0.0174 \\
-0.0195 \\
-0.0200 \\
-0.0206 \\
-0.0215 \\
-0.0223 \\
-0.0240 \\
-0.0233 \\
-0.0224 \\
-0.0239 \\
-0.0267 \\
-0.0296 \\
-0.0298 \\
0.0164\end{array}$ \\
\hline
\end{tabular}




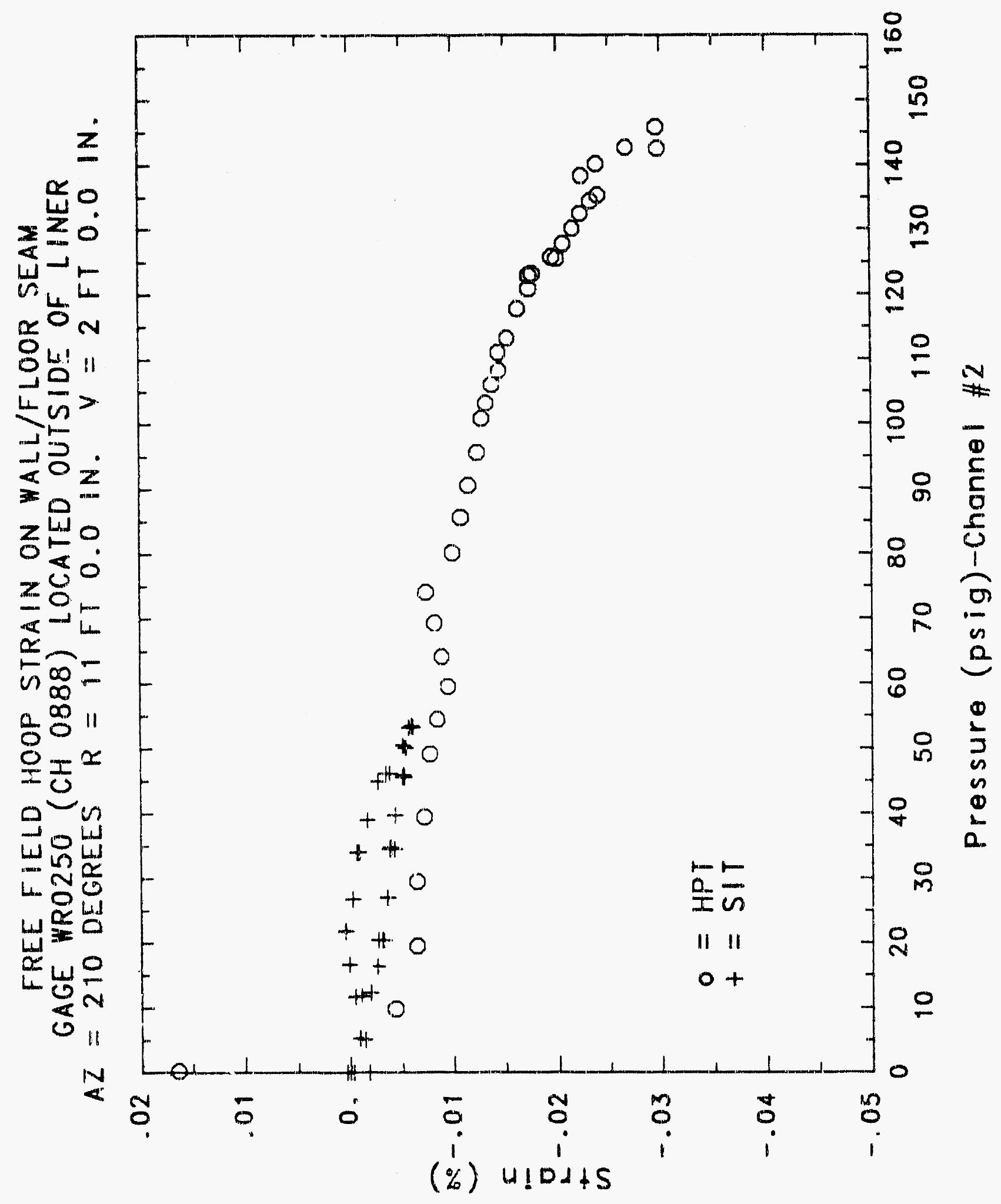




\section{Reinforced Concrete Test Data}

Weldable Gage 251 rhannel 889

Structural Integrity Test

High Pressure test

\begin{tabular}{|c|c|c|c|}
\hline $\begin{array}{c}\text { Pressure } \\
(\text { psig) } \\
-0.05 \\
5.33 \\
12.31 \\
16.44 \\
20.51 \\
20.46 \\
20.47 \\
27.05 \\
34.69 \\
34.53 \\
34.53 \\
39.70 \\
45.90 \\
45.65 \\
45.62 \\
50.10 \\
53.47 \\
53.29 \\
53.21 \\
50.49 \\
46.14 \\
46.13 \\
44.97 \\
38.98 \\
33.99 \\
33.96 \\
26.83 \\
21.88 \\
21.90 \\
16.69 \\
11.74 \\
11.77 \\
5.05 \\
0.02 \\
-0.04 \\
-0.02 \\
-0.02 \\
0.02\end{array}$ & $\begin{array}{l}\text { X Strain } \\
0.0005 \\
0.0049 \\
0.0111 \\
0.0152 \\
0.0201 \\
0.0217 \\
0.0222 \\
0.0304 \\
0.0428 \\
0.0441 \\
0.0444 \\
0.0488 \\
0.0548 \\
0.0554 \\
0.0557 \\
0.0586 \\
0.0616 \\
0.0616 \\
0.0618 \\
0.0593 \\
0.0545 \\
0.0540 \\
0.0539 \\
0.0470 \\
0.0410 \\
0.0411 \\
0.0327 \\
0.0271 \\
0.0275 \\
0.0225 \\
0.0180 \\
0.0175 \\
0.0118 \\
0.0067 \\
0.0039 \\
0.0035 \\
0.0039 \\
0.0036\end{array}$ & $\begin{array}{c}\text { Pressure } \\
\text { (ps1g) } \\
9.89 \\
19.55 \\
29.57 \\
39.42 \\
49.16 \\
54.50 \\
59.57 \\
54.20 \\
69.32 \\
74.16 \\
80.16 \\
85.61 \\
90.58 \\
95.69 \\
100.92 \\
103.25 \\
106.11 \\
108.31 \\
111.08 \\
113.24 \\
117.83 \\
120.92 \\
123.28 \\
122.97 \\
125.82 \\
125.60 \\
127.84 \\
130.19 \\
132.53 \\
135.33 \\
134.42 \\
138.35 \\
140.16 \\
142.63 \\
145.78 \\
142.52 \\
0.22 \\
\end{array}$ & $\begin{array}{l}\text { \% Strain } \\
0.0090 \\
0.0236 \\
0.0329 \\
0.0416 \\
0.0499 \\
0.0554 \\
0.0603 \\
0.0653 \\
0.0703 \\
0.0762 \\
0.0852 \\
0.0920 \\
0.0987 \\
0.1049 \\
0.1135 \\
0.1199 \\
0.1266 \\
0.1307 \\
0.1361 \\
0.1393 \\
0.1456 \\
0.1511 \\
0.1556 \\
0.1564 \\
0.1619 \\
0.1633 \\
0.1666 \\
0.1718 \\
0.1779 \\
0.1861 \\
0.1861 \\
0.1950 \\
0.2006 \\
0.2085 \\
0.2185 \\
0.2179 \\
0.0262\end{array}$ \\
\hline
\end{tabular}




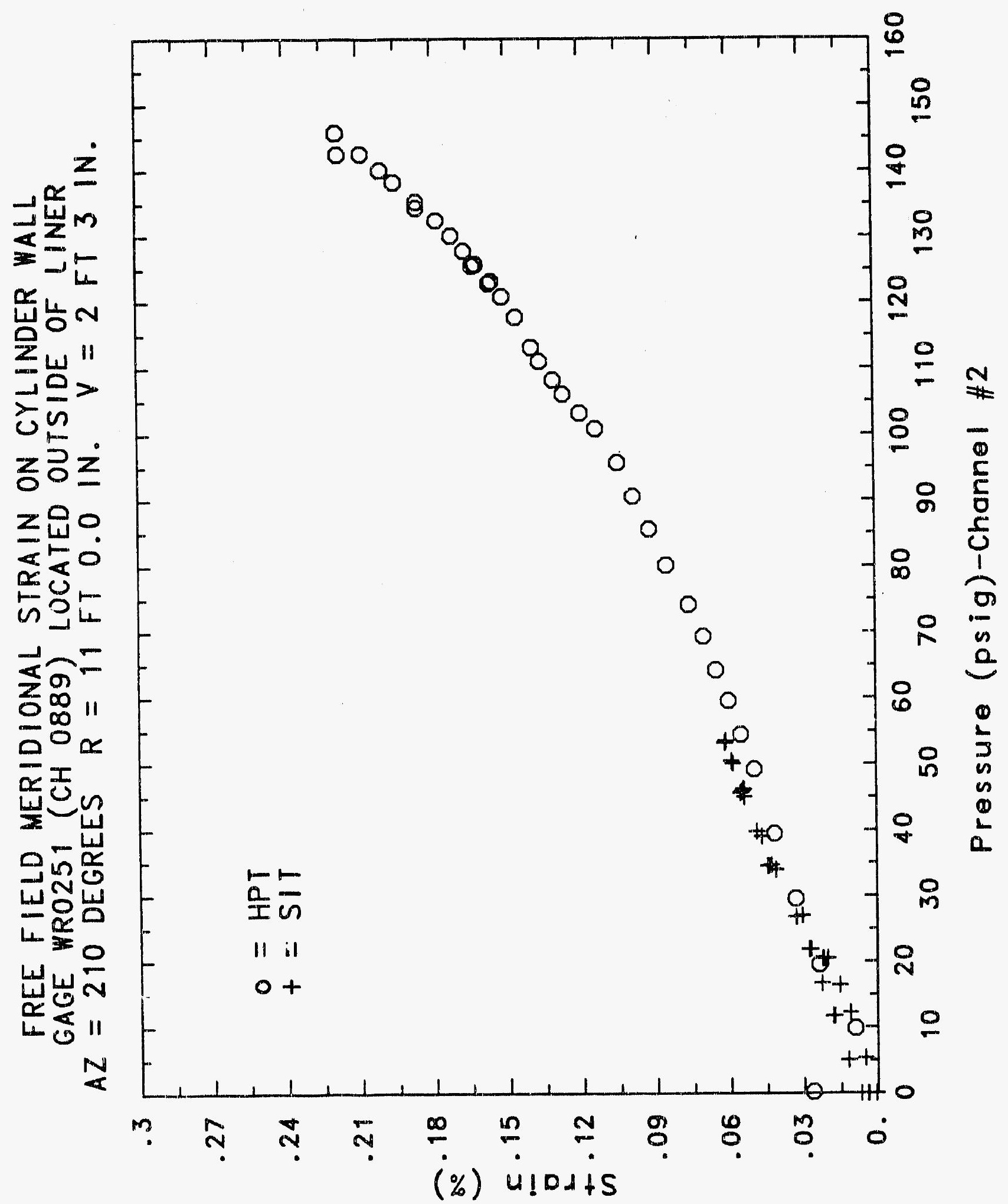


ㅇํㅇํ의

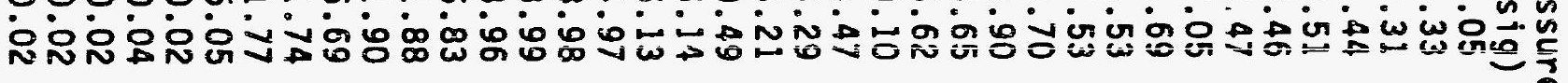

00000000000000000000000000000000000000

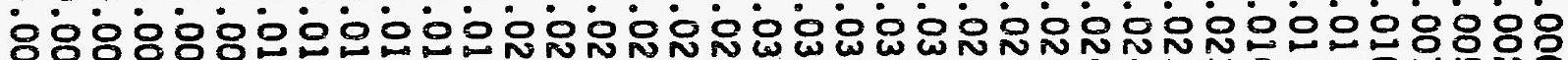

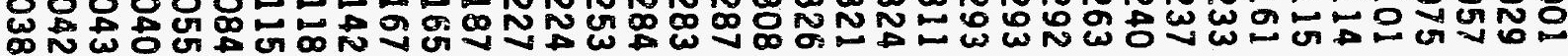

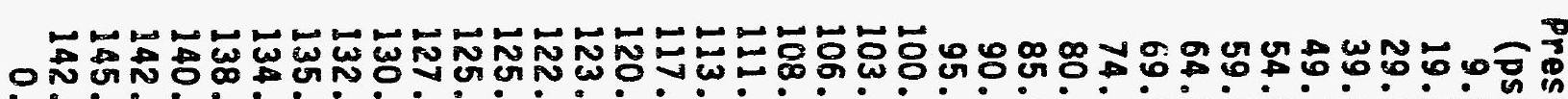

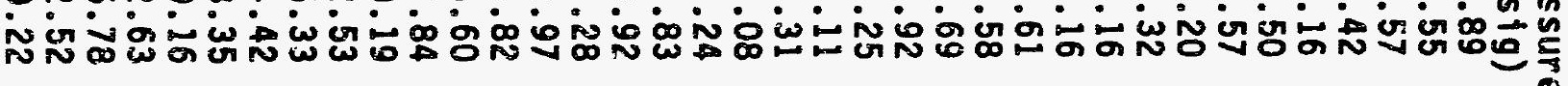




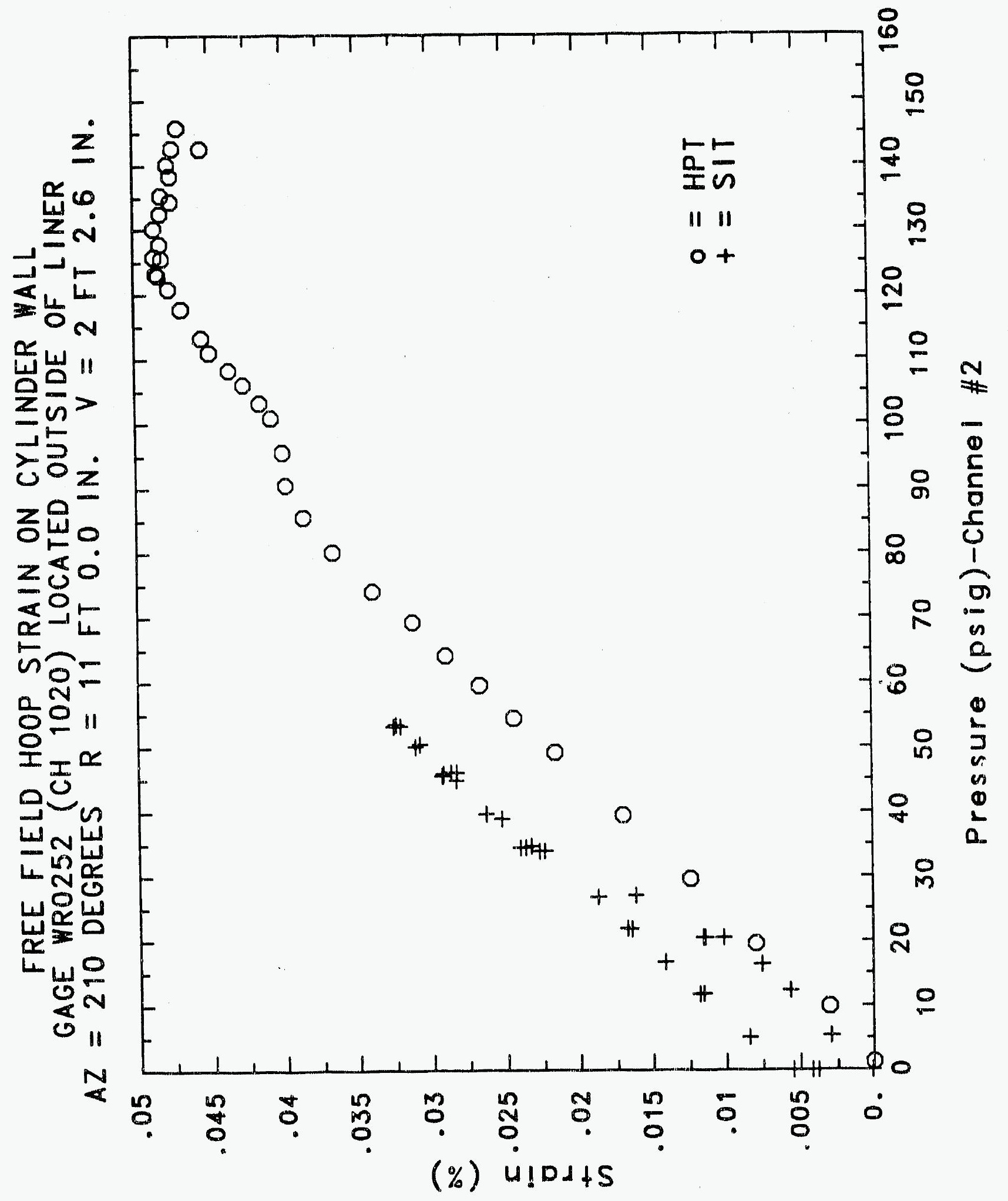




\section{Reinforced Concrete Test Data}

Weldable Gage 253 Chamel 1021

Structural InTEgrity Test

High Pressure test

\begin{tabular}{|c|c|c|c|}
\hline $\begin{array}{c}\text { Pressure } \\
\text { (psig) } \\
-0.05 \\
5.33 \\
12.31 \\
16.44 \\
20.51 \\
20.46 \\
20.47 \\
27.05 \\
34.69 \\
34.53 \\
34.53 \\
39.70 \\
45.90 \\
45.65 \\
45.62 \\
50.10 \\
53.47 \\
53.29 \\
53.21 \\
50.49 \\
46.14 \\
46.13 \\
44.97 \\
38.98 \\
33.99 \\
33.96 \\
26.83 \\
21.88 \\
21.90 \\
16.69 \\
11.74 \\
11.77 \\
5.05 \\
0.02 \\
-0.04 \\
-0.02 \\
-0.02 \\
0.02 \\
\end{array}$ & $\begin{array}{l}\text { \% Strain } \\
0.0003 \\
0.0002 \\
-0.0010 \\
-0.0016 \\
-0.0028 \\
-0.0019 \\
-0.0023 \\
-0.0030 \\
-0.0029 \\
-0.0020 \\
-0.0021 \\
-0.0029 \\
-0.0033 \\
-0.0028 \\
-0.0027 \\
-0.0028 \\
-0.0030 \\
-0.0031 \\
-0.0025 \\
-0.0020 \\
-0.0011 \\
-0.0011 \\
0.0008 \\
0.0009 \\
0.0007 \\
0.0010 \\
0.0009 \\
0.0012 \\
0.0011 \\
0.0014 \\
0.0012 \\
0.0008 \\
0.0012 \\
0.0018 \\
0.0040 \\
0.0034 \\
0.0030 \\
0.0020\end{array}$ & $\begin{array}{c}\text { Pressure } \\
(\text { psig) } \\
9.89 \\
19.55 \\
29.57 \\
39.42 \\
49.16 \\
54.50 \\
59.57 \\
64.20 \\
69.32 \\
74.16 \\
80.16 \\
85.61 \\
90.58 \\
95.69 \\
100.92 \\
103.25 \\
106.11 \\
108.31 \\
111.08 \\
113.24 \\
117.83 \\
120.92 \\
123.28 \\
122.97 \\
125.82 \\
125.60 \\
127.84 \\
130.19 \\
132.53 \\
135.33 \\
134.42 \\
138.35 \\
140.16 \\
142.63 \\
145.78 \\
142.52 \\
0.22\end{array}$ & $\begin{array}{l}\text { X Strain } \\
-0.0054 \\
-0.0061 \\
-0.0067 \\
-0.0075 \\
-0.0082 \\
-0.0082 \\
-0.0078 \\
-0.0077 \\
-0.0083 \\
-0.0091 \\
-0.0088 \\
-0.0089 \\
-0.0081 \\
-0.0073 \\
-0.0052 \\
-0.0040 \\
-0.0032 \\
-0.0027 \\
-0.0012 \\
-0.0005 \\
0.0003 \\
0.0023 \\
0.0029 \\
0.0034 \\
0.0019 \\
0.0022 \\
0.0011 \\
0.0007 \\
0.0004 \\
0.0013 \\
0.0015 \\
0.0034 \\
0.0050 \\
0.0071 \\
0.0094 \\
0.0111 \\
0.0234\end{array}$ \\
\hline
\end{tabular}




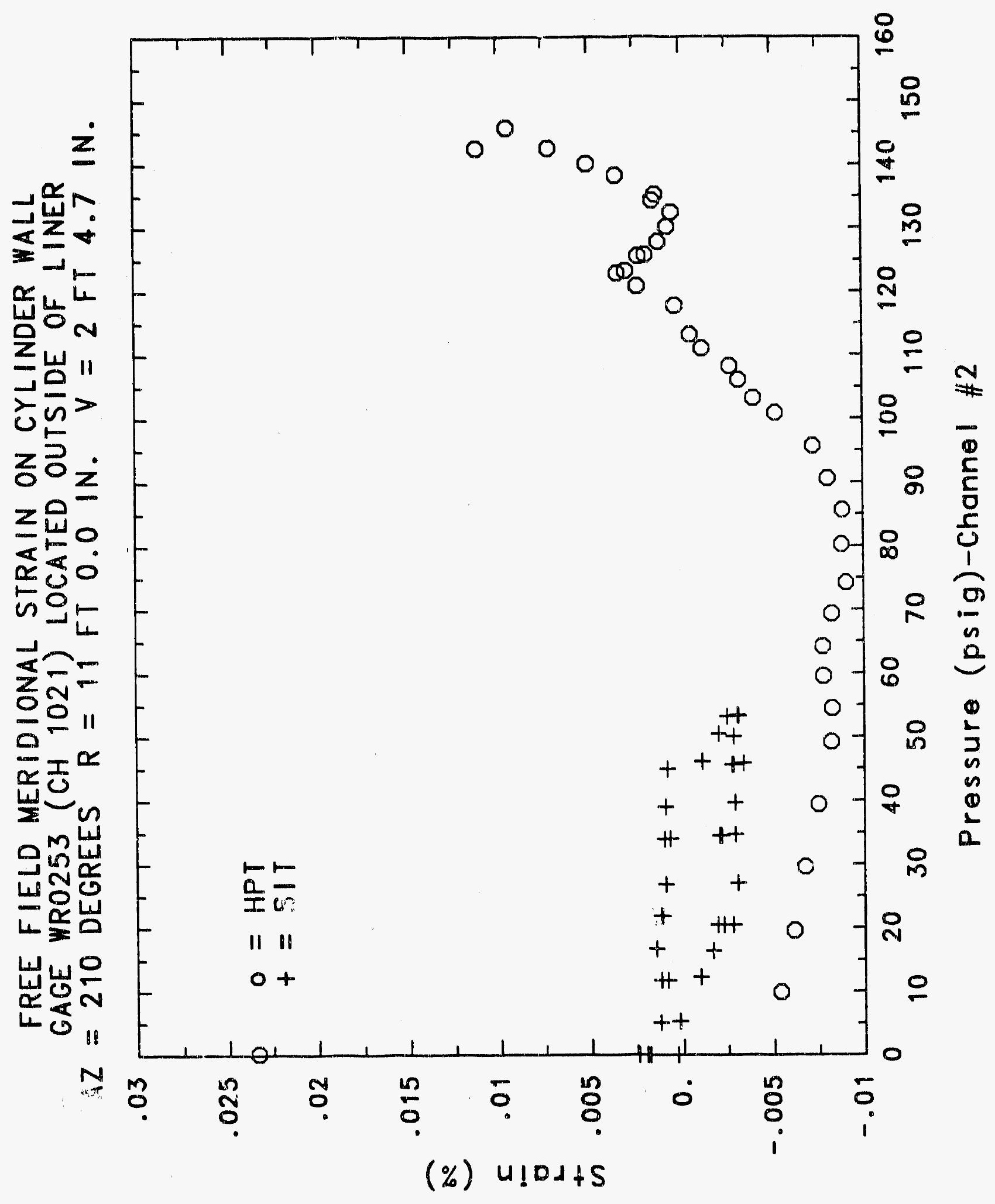




\section{Reinforced Concrete Test Data}

Weldable gage 254 Channel 1022

Structural Integrity Test

High Pressure test

\begin{tabular}{|c|c|c|c|}
\hline $\begin{array}{c}\text { Pressure } \\
\text { (psig) } \\
-0.05 \\
5.33 \\
12.31 \\
16.44 \\
20.51 \\
20.46 \\
20.47 \\
27.05 \\
34.69 \\
34.53 \\
34.53 \\
39.70 \\
45.90 \\
45.65 \\
45.62 \\
50.10 \\
53.47 \\
53.29 \\
53.21 \\
50.49 \\
46.14 \\
46.13 \\
44.97 \\
38.98 \\
33.99 \\
33.96 \\
26.83 \\
21.88 \\
21.90 \\
16.69 \\
11.74 \\
11.77 \\
5.05 \\
0.02 \\
-0.04 \\
-0.02 \\
-0.02 \\
0.02\end{array}$ & $\begin{array}{l}\text { \% Strain } \\
0.0010 \\
0.0012 \\
0.0016 \\
0.0020 \\
0.0024 \\
0.0029 \\
0.0027 \\
0.0037 \\
0.0034 \\
0.0038 \\
0.0038 \\
0.0035 \\
0.0036 \\
0.0038 \\
0.0038 \\
0.0041 \\
0.0044 \\
0.0048 \\
0.0047 \\
0.0048 \\
0.0050 \\
0.0051 \\
0.0059 \\
0.0055 \\
0.0049 \\
0.0048 \\
0.0041 \\
0.0039 \\
0.0041 \\
0.0037 \\
0.0032 \\
0.0030 \\
0.0021 \\
0.0016 \\
0.0036 \\
0.0032 \\
0.0027 \\
0.0016\end{array}$ & $\begin{array}{c}\text { Pressure } \\
(\text { psig) } \\
9.89 \\
19.55 \\
29.57 \\
39.42 \\
49.16 \\
54.50 \\
59.57 \\
64.20 \\
69.32 \\
74.16 \\
80.16 \\
85.61 \\
90.58 \\
95.69 \\
100.92 \\
103.25 \\
106.11 \\
108.31 \\
111.08 \\
113.24 \\
117.83 \\
120.92 \\
123.28 \\
122.97 \\
125.82 \\
125.60 \\
127.84 \\
130.19 \\
132.53 \\
135.33 \\
134.42 \\
138.35 \\
140.16 \\
142.63 \\
145.78 \\
142.52 \\
0.22\end{array}$ & $\begin{array}{c}\text { \% Strain } \\
-0.0027 \\
-0.0026 \\
-0.0021 \\
-0.0014 \\
-0.0007 \\
-0.0002 \\
0.0003 \\
0.0008 \\
0.0012 \\
0.0002 \\
0.0007 \\
0.0015 \\
0.0023 \\
0.0034 \\
0.0047 \\
0.0053 \\
0.0056 \\
0.0060 \\
0.0068 \\
0.0074 \\
0.0089 \\
0.0102 \\
0.0118 \\
0.0124 \\
0.0134 \\
0.0137 \\
0.0141 \\
0.0153 \\
0.0179 \\
0.0217 \\
0.0230 \\
0.0283 \\
0.0327 \\
0.0392 \\
0.0449 \\
0.0468 \\
0.0343\end{array}$ \\
\hline
\end{tabular}




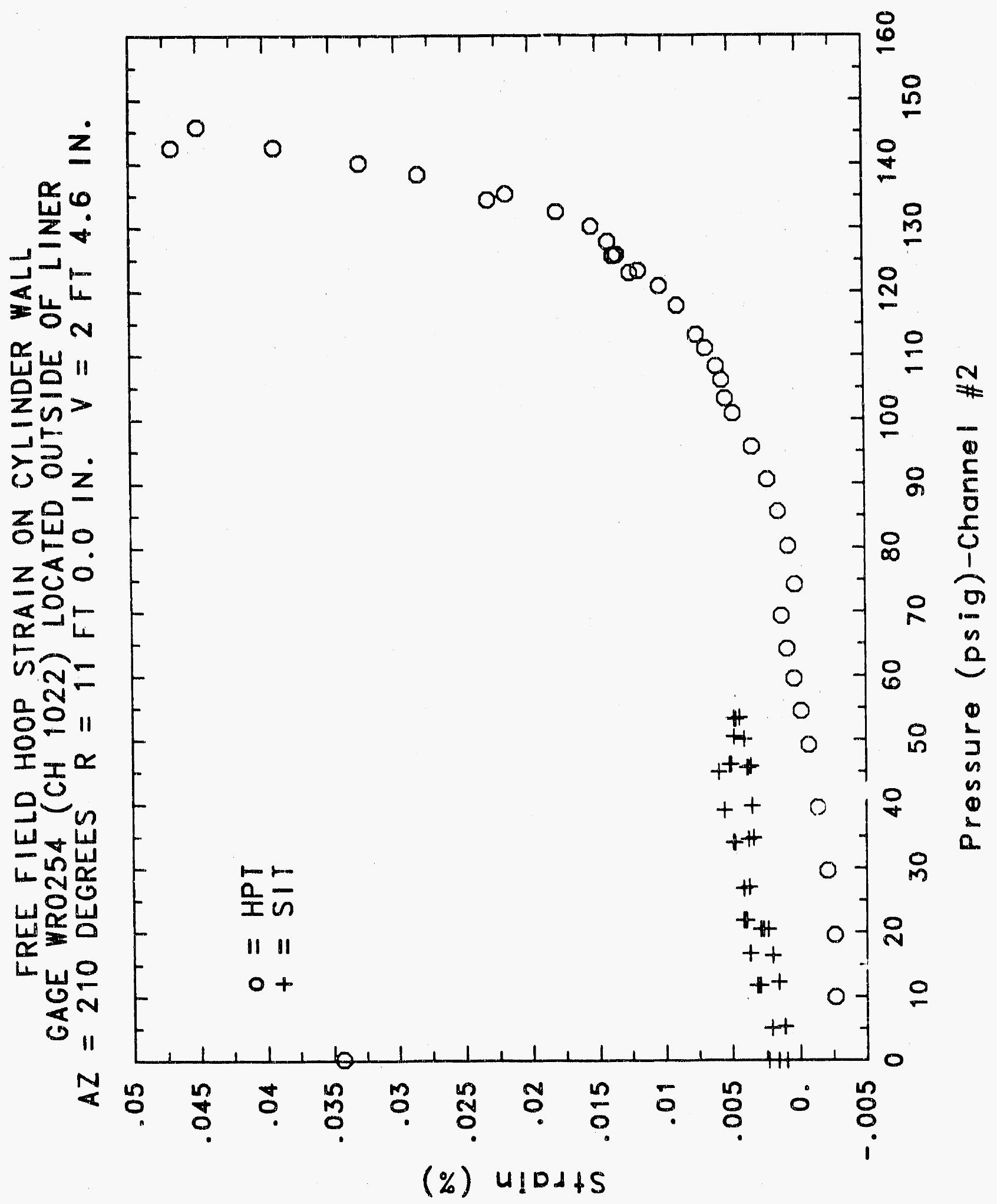




\section{Reinforced Concrete Test Data}

Weldable Gage 255 Chammel 1023

Structural Integrity Test

High Pressure test

\begin{tabular}{|c|c|c|c|}
\hline $\begin{array}{c}\text { Pressure } \\
\text { (psig) } \\
-0.05 \\
5.33 \\
12.31 \\
16.44 \\
20.51 \\
20.46 \\
20.47 \\
27.05 \\
34.69 \\
34.53 \\
34.53 \\
39.70 \\
45.90 \\
45.65 \\
45.62 \\
50.10 \\
53.47 \\
53.29 \\
53.21 \\
50.49 \\
46.14 \\
46.13 \\
44.97 \\
38.98 \\
33.99 \\
33.96 \\
26.83 \\
21.88 \\
21.90 \\
16.69 \\
11.74 \\
11.77 \\
5.05 \\
0.02 \\
-0.04 \\
-0.02 \\
-0.02 \\
0.02 \\
\end{array}$ & $\begin{array}{l}\text { \% Strain } \\
0.0002 \\
-0.0006 \\
-0.0030 \\
-0.0054 \\
-0.0093 \\
-0.0103 \\
-0.0111 \\
-0.0191 \\
-0.0313 \\
-0.0320 \\
-0.0322 \\
-0.0378 \\
-0.0451 \\
-0.0451 \\
-0.0454 \\
-0.0500 \\
-0.0538 \\
-0.0539 \\
-0.0539 \\
-0.0518 \\
-0.0473 \\
-0.0467 \\
-0.0476 \\
-0.0407 \\
-0.0358 \\
-0.0355 \\
-0.0280 \\
-0.0221 \\
-0.0220 \\
-0.0161 \\
-0.0101 \\
-0.0094 \\
-0.0019 \\
0.0012 \\
0.0030 \\
0.0004 \\
-0.0003 \\
0.0015\end{array}$ & $\begin{array}{c}\text { Pressure } \\
(\text { psig) } \\
9.89 \\
19.55 \\
29.57 \\
39.42 \\
49.16 \\
54.50 \\
59.57 \\
64.20 \\
69.32 \\
74.16 \\
80.16 \\
85.61 \\
90.58 \\
95.69 \\
100.92 \\
103.25 \\
106.11 \\
108.31 \\
111.08 \\
113.24 \\
117.83 \\
120.92 \\
123.28 \\
122.97 \\
125.82 \\
125.60 \\
127.84 \\
130.19 \\
132.53 \\
135.33 \\
134.42 \\
138.35 \\
140.16 \\
142.63 \\
145.78 \\
142.52 \\
0.22\end{array}$ & $\begin{array}{l}\text { \% Strain } \\
-0.0142 \\
-0.0248 \\
-0.0358 \\
-0.0464 \\
-0.0566 \\
-0.0625 \\
-0.0678 \\
-0.0741 \\
-0.0813 \\
-0.0953 \\
-0.1095 \\
-0.1186 \\
-0.1291 \\
-0.1402 \\
-0.1512 \\
-0.1245 \\
-0.1253 \\
-0.1299 \\
-0.1365 \\
-0.1416 \\
-0.1518 \\
-0.1591 \\
-0.1659 \\
-0.1671 \\
-0.1745 \\
-0.1766 \\
-0.1814 \\
-0.1889 \\
-0.1976 \\
-0.2097 \\
-0.2119 \\
-0.2288 \\
-0.2426 \\
-0.2641 \\
-0.2947 \\
-0.3017 \\
-0.0975\end{array}$ \\
\hline
\end{tabular}




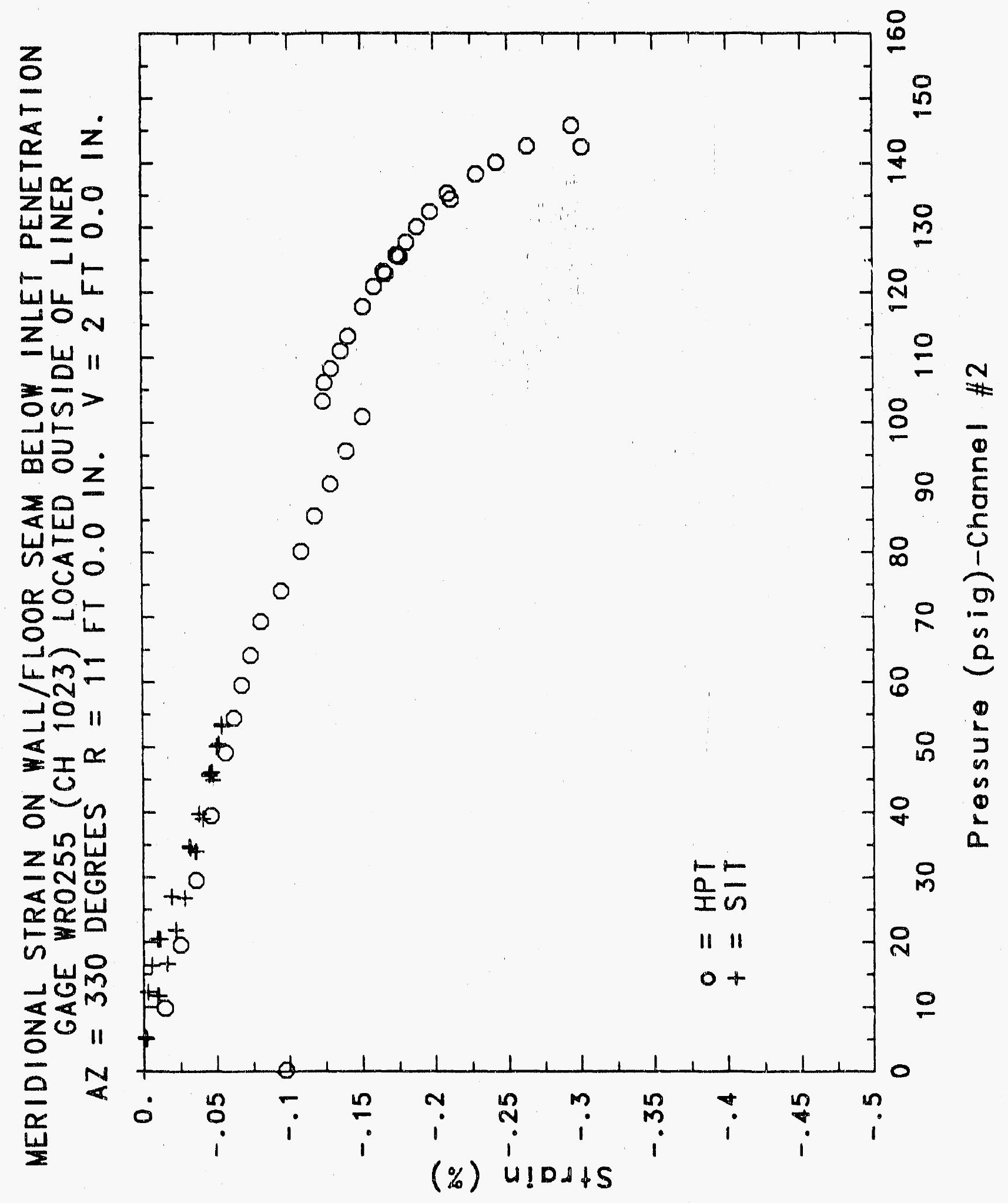


ㅇํㅇำ

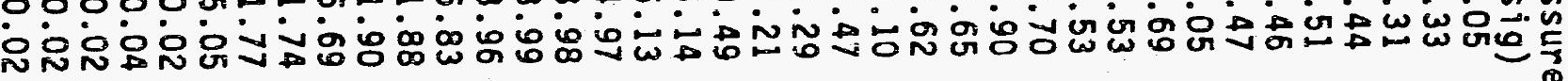

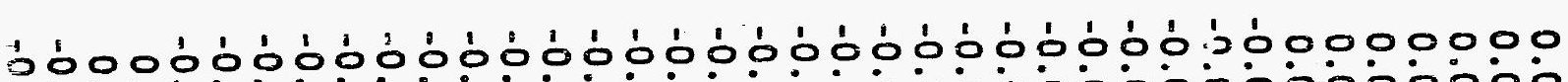
No

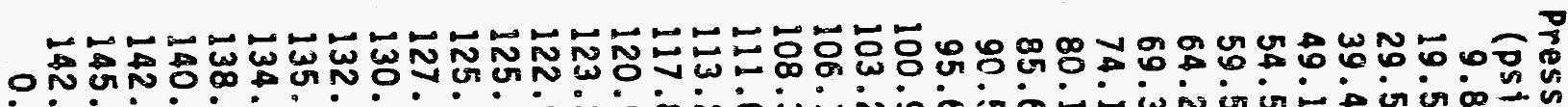
N

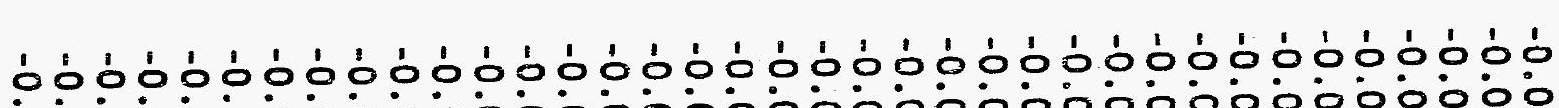
o

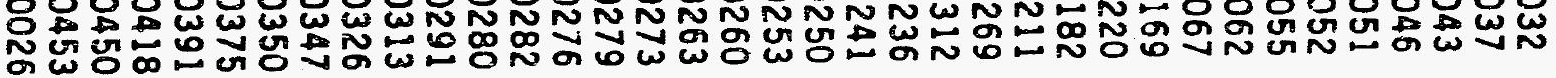




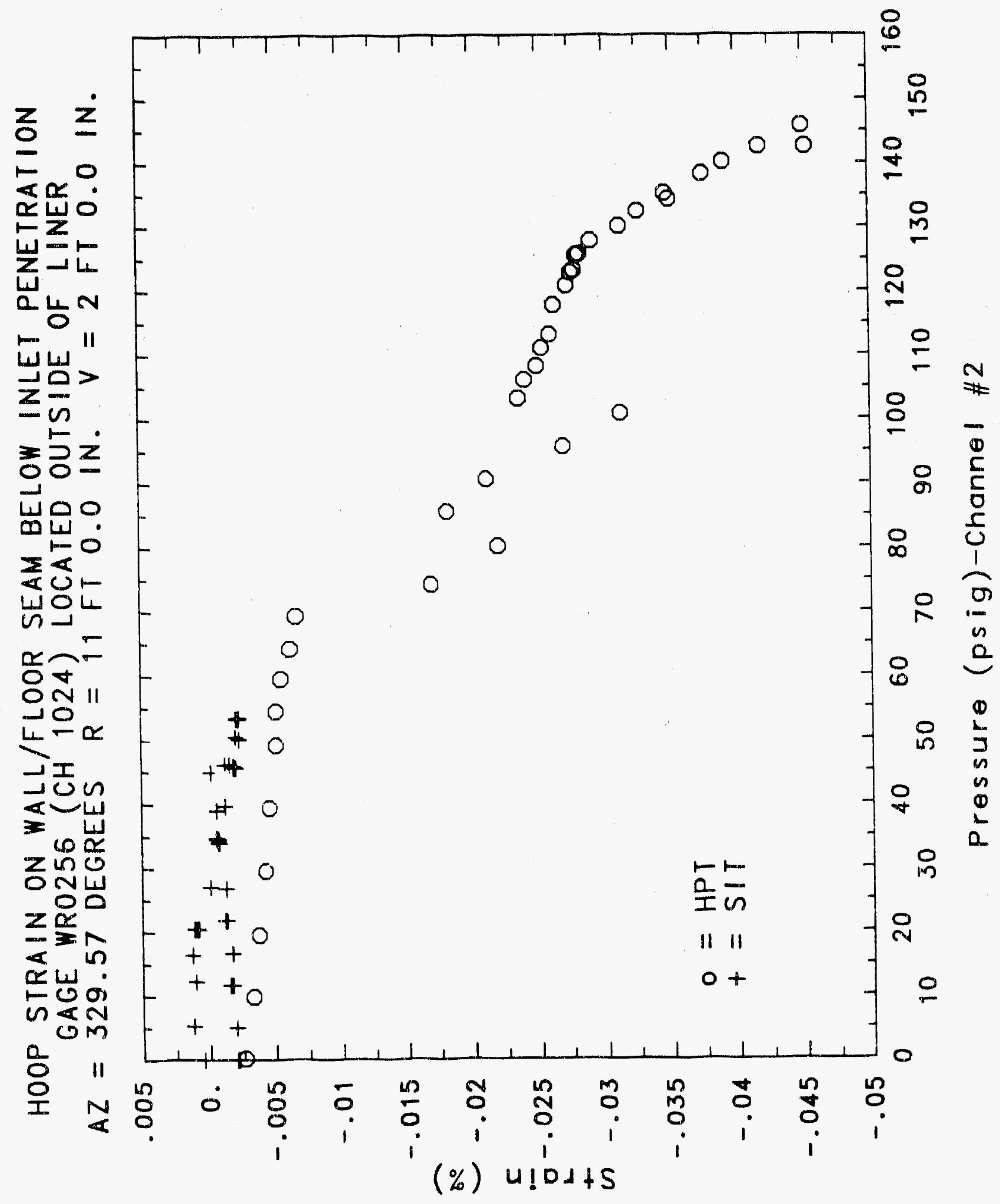




\section{Reinforced Concrete Test Data}

Weldable gage 257 Channel 1025

STRUCTURAL INTEGRITY TEST

high Pressure test

\begin{tabular}{|c|c|c|c|}
\hline $\begin{array}{c}\text { Pressure } \\
\text { (psig) } \\
-0.05 \\
5.33 \\
12.31 \\
16.44 \\
20.51 \\
20.46 \\
20.47 \\
27.05 \\
34.69 \\
34.53 \\
34.53 \\
39.70 \\
45.90 \\
45.65 \\
45.62 \\
50.10 \\
53.47 \\
53.29 \\
53.21 \\
50.49 \\
46.14 \\
46.13 \\
44.97 \\
38.98 \\
33.99 \\
33.96 \\
26.83 \\
21.88 \\
21.90 \\
16.69 \\
11.74 \\
11.77 \\
5.05 \\
0.02 \\
-0.04 \\
-0.02 \\
-0.02 \\
0.02 \\
\end{array}$ & $\begin{array}{l}\text { X Strain } \\
0.0008 \\
0.0028 \\
0.0052 \\
0.0071 \\
0.0095 \\
0.0119 \\
0.0115 \\
0.0176 \\
0.0286 \\
0.0278 \\
0.0280 \\
0.0321 \\
0.0388 \\
0.0390 \\
0.0392 \\
0.0418 \\
0.0438 \\
0.0441 \\
0.0440 \\
0.0427 \\
0.0402 \\
0.0398 \\
0.0414 \\
0.0379 \\
0.0347 \\
0.0340 \\
0.0288 \\
0.0249 \\
0.0248 \\
0.0208 \\
0.0171 \\
0.0168 \\
0.0126 \\
0.0097 \\
0.0078 \\
0.0087 \\
0.0090 \\
0.0087\end{array}$ & $\begin{array}{c}\text { Pressure } \\
\text { (psig) } \\
9.89 \\
19.55 \\
29.57 \\
39.42 \\
49.16 \\
54.50 \\
59.57 \\
64.20 \\
69.32 \\
74.16 \\
80.16 \\
85.61 \\
90.58 \\
95.69 \\
100.92 \\
103.25 \\
106.11 \\
108.31 \\
111.08 \\
113.24 \\
117.83 \\
120.92 \\
123.28 \\
122.97 \\
125.82 \\
125.60 \\
127.84 \\
130.19 \\
132.53 \\
135.33 \\
134.42 \\
138.35 \\
140.16 \\
142.63 \\
145.78 \\
142.52 \\
0.22\end{array}$ & $\begin{array}{l}\text { \% Strain } \\
0.0013 \\
0.0075 \\
0.0148 \\
0.0221 \\
0.0288 \\
0.0325 \\
0.0353 \\
0.0393 \\
0.0427 \\
0.0477 \\
0.0555 \\
0.0603 \\
0.0658 \\
0.0713 \\
0.0773 \\
0.0673 \\
0.0685 \\
0.0710 \\
0.0756 \\
0.0787 \\
0.0856 \\
0.0910 \\
0.0960 \\
0.0972 \\
0.1018 \\
0.1041 \\
0.1070 \\
0.1118 \\
0.1166 \\
0.1230 \\
0.1249 \\
0.1314 \\
0.1372 \\
0.1444 \\
0.1542 \\
0.1569 \\
0.0338\end{array}$ \\
\hline
\end{tabular}




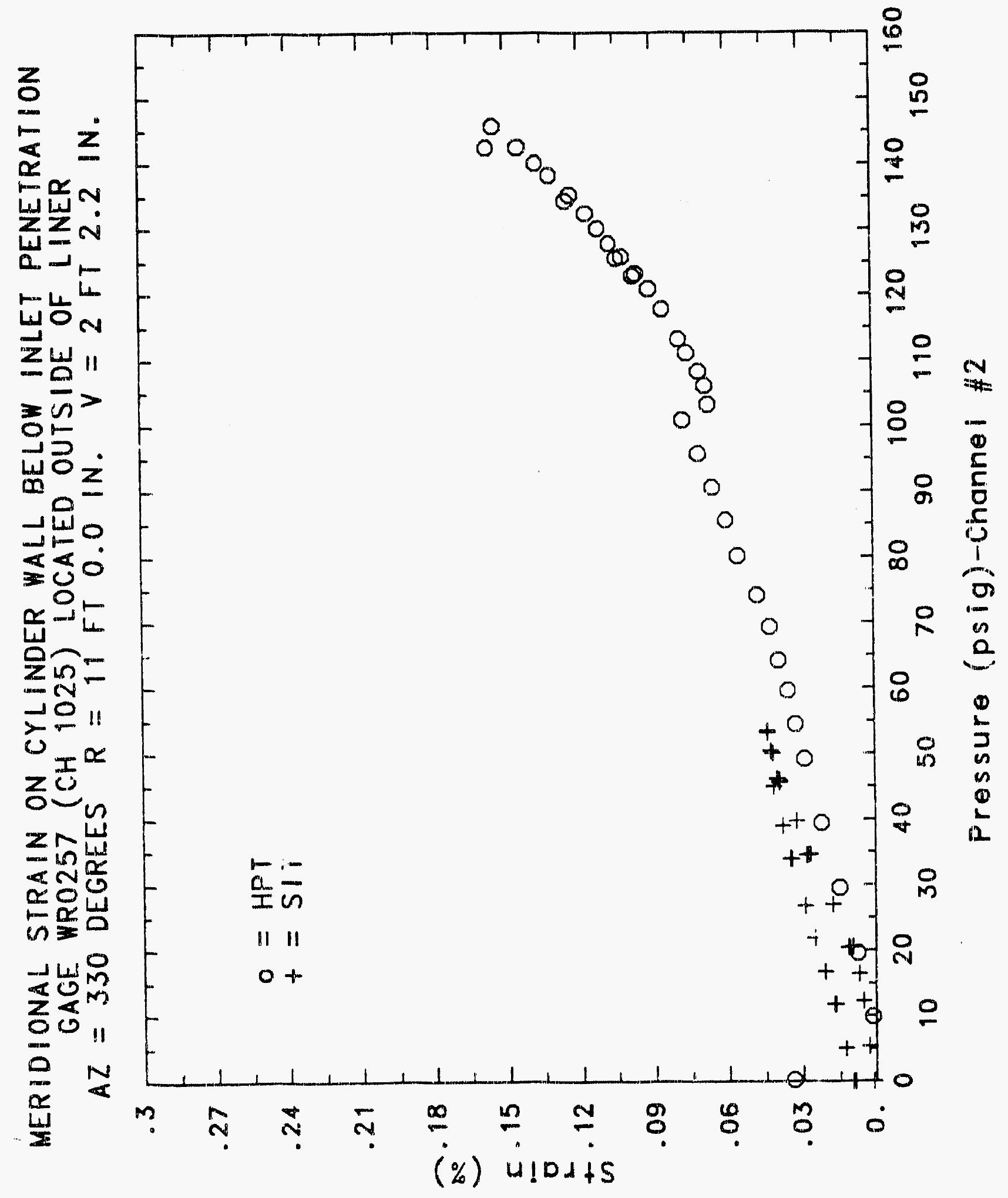




\section{Reinforged Concrete Test Data}

Weldable gage 258 Channel 1026

Structural Integrity Test

High Pressure test

\begin{tabular}{|c|c|c|c|}
\hline $\begin{array}{c}\text { Pressure } \\
\text { (psig) } \\
-0.05 \\
5.33 \\
12.31 \\
16.44 \\
20.51 \\
20.46 \\
20.47 \\
27.05 \\
34.69 \\
34.53 \\
34.53 \\
39.70 \\
45.90 \\
45.65 \\
45.62 \\
50.10 \\
53.47 \\
53.29 \\
53.21 \\
50.49 \\
46.14 \\
46.13 \\
44.97 \\
38.98 \\
33.99 \\
33.96 \\
26.83 \\
21.88 \\
21.90 \\
16.69 \\
11.74 \\
11.77 \\
5.05 \\
0.02 \\
-0.04 \\
-0.02 \\
-0.02 \\
0.02 \\
\end{array}$ & $\begin{array}{l}\text { \% Strain } \\
-0.0010 \\
-0.0003 \\
-0.0010 \\
-0.0014 \\
-0.0021 \\
-0.0021 \\
-0.0021 \\
-0.0035 \\
-0.0058 \\
-0.0052 \\
-0.0053 \\
-0.0061 \\
-0.0063 \\
-0.0063 \\
-0.0066 \\
-0.0064 \\
-0.0065 \\
-0.0059 \\
-0.0057 \\
-0.0053 \\
-0.0048 \\
-0.0048 \\
-0.0037 \\
-0.0041 \\
-0.0035 \\
-0.0037 \\
-0.0034 \\
-0.0030 \\
-0.0031 \\
-0.0028 \\
-0.0025 \\
-0.0024 \\
-0.0021 \\
-0.0020 \\
0.0008 \\
-0.0001 \\
-0.0004 \\
-0.0027\end{array}$ & $\begin{array}{c}\text { Pressure } \\
\text { (psig) } \\
9.89 \\
19.55 \\
29.57 \\
39.42 \\
19.16 \\
54.50 \\
59.57 \\
64.20 \\
69.32 \\
74.16 \\
80.16 \\
85.61 \\
90.58 \\
95.69 \\
100.92 \\
103.25 \\
106.11 \\
108.31 \\
111.08 \\
113.24 \\
117.83 \\
120.92 \\
123.28 \\
122.97 \\
125.82 \\
125.60 \\
127.84 \\
130.19 \\
132.53 \\
135.33 \\
134.42 \\
138.35 \\
140.16 \\
142.63 \\
145.78 \\
142.52 \\
0.22 \\
\end{array}$ & $\begin{array}{l}\text { \% Strain } \\
-0.0046 \\
-0.0060 \\
-0.0068 \\
-0.0074 \\
-0.0081 \\
-0.0083 \\
-0.0083 \\
-0.0087 \\
-0.0085 \\
-0.0087 \\
-0.0101 \\
-0.0110 \\
-0.0116 \\
-0.0127 \\
-0.0134 \\
-0.0146 \\
-0.0146 \\
-0.0148 \\
-0.0149 \\
-0.0150 \\
-0.0146 \\
-0.0146 \\
-0.0142 \\
-0.0135 \\
-0.0138 \\
-0.0135 \\
-0.0138 \\
-0.0149 \\
-0.0155 \\
-0.0165 \\
-0.0167 \\
-0.0174 \\
-0.0186 \\
-0.0191 \\
-0.0215 \\
-0.0215 \\
0.0054\end{array}$ \\
\hline
\end{tabular}




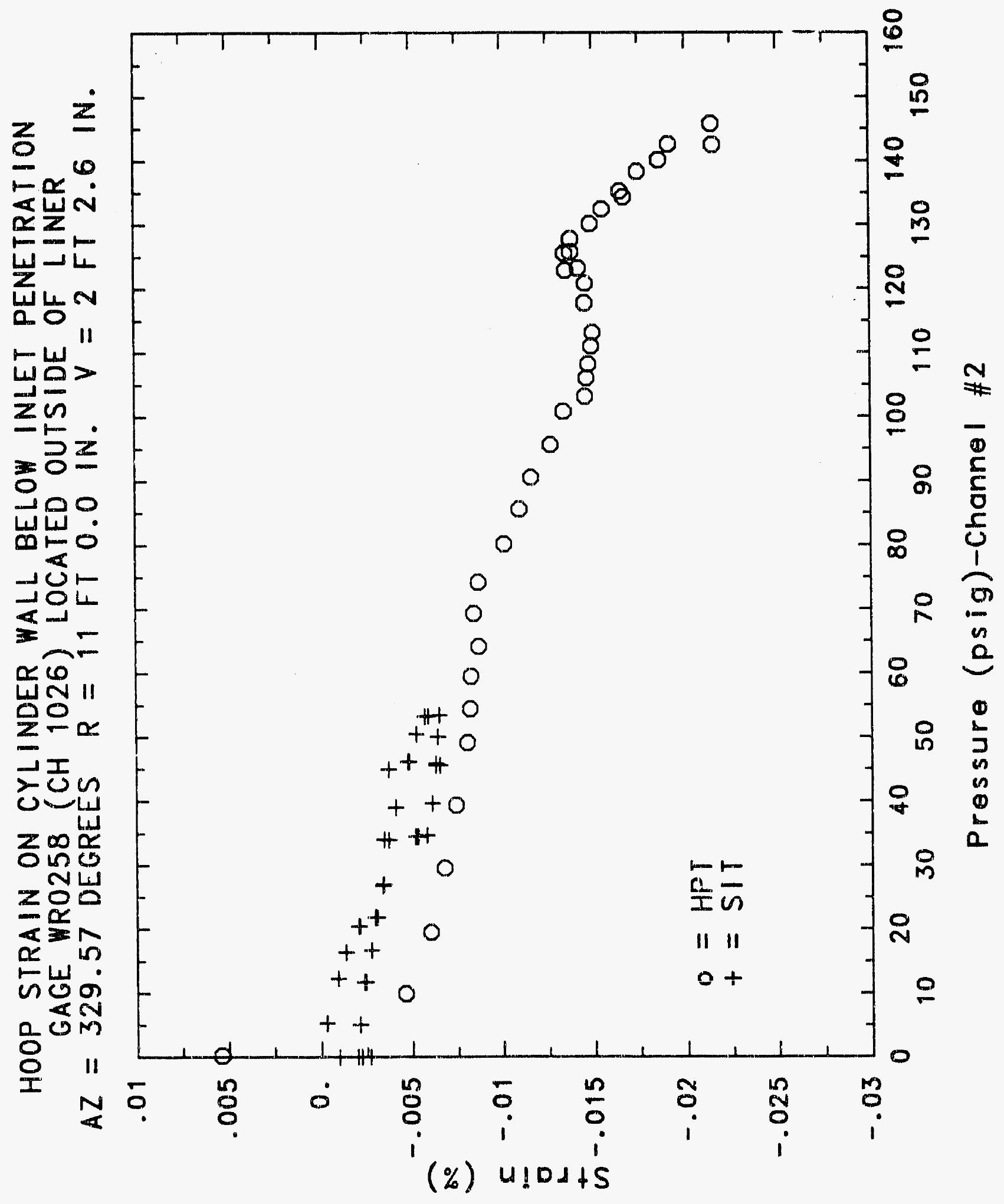




\section{Reinforced Concrete Test Data}

Weldable Gage 259 Channel 1027

StRUCTURAL INTEgRITY TEST

High Pressure Test

\begin{tabular}{|c|c|c|c|}
\hline $\begin{array}{l}\text { Pressure } \\
\text { (psig) } \\
-0.05 \\
5.33 \\
12.31 \\
16.44 \\
20.51 \\
20.46 \\
20.47 \\
27.05 \\
34.69 \\
34.53 \\
34.53 \\
39.70 \\
45.90 \\
45.65 \\
45.62 \\
50.10 \\
53.47 \\
53.29 \\
53.21 \\
50.49 \\
46.14 \\
46.13 \\
44.97 \\
38.98 \\
32.99 \\
33.96 \\
26.83 \\
21.88 \\
21.90 \\
16.69 \\
11.74 \\
11.77 \\
5.05 \\
0.02 \\
-0.04 \\
-0.02 \\
-0.02 \\
0.02\end{array}$ & $\begin{array}{l}\text { \% Strain } \\
-0.0002 \\
0.0023 \\
0.0054 \\
0.0072 \\
0.0104 \\
0.0110 \\
0.0116 \\
0.0171 \\
0.0219 \\
0.0222 \\
0.0223 \\
0.0245 \\
0.0262 \\
0.0264 \\
0.0263 \\
0.0275 \\
0.0281 \\
0.0281 \\
0.0280 \\
0.0268 \\
0.0247 \\
0.0243 \\
0.0258 \\
0.0227 \\
0.0204 \\
0.0204 \\
0.0171 \\
0.0148 \\
0.0149 \\
0.0125 \\
0.0105 \\
0.0103 \\
0.0077 \\
0.0056 \\
0.0057 \\
0.0062 \\
0.0065 \\
0.0056\end{array}$ & $\begin{array}{c}\text { Pressure } \\
\text { ipsig) } \\
9.89 \\
19.55 \\
29.57 \\
39.42 \\
49.16 \\
54.50 \\
59.57 \\
64.20 \\
69.32 \\
74.16 \\
80.16 \\
85.61 \\
90.58 \\
95.69 \\
100.92 \\
103.25 \\
106.11 \\
108.31 \\
111.08 \\
113.24 \\
117.83 \\
120.92 \\
123.28 \\
122.97 \\
125.82 \\
125.60 \\
127.84 \\
130.19 \\
132.53 \\
135.33 \\
134.42 \\
138.35 \\
140.16 \\
142.63 \\
145.78 \\
142.52 \\
0.22 \\
19\end{array}$ & $\begin{array}{l}\text { \% Strain } \\
0.0009 \\
0.0043 \\
0.0081 \\
0.0122 \\
0.0164 \\
0.0187 \\
0.0207 \\
0.0222 \\
0.0235 \\
0.0236 \\
0.0256 \\
0.0272 \\
0.0287 \\
0.0304 \\
0.0324 \\
0.0315 \\
0.0328 \\
0.0336 \\
0.0347 \\
0.0354 \\
0.0369 \\
0.0379 \\
0.0385 \\
0.0385 \\
0.0394 \\
0.0392 \\
0.0395 \\
0.0394 \\
0.0394 \\
0.0397 \\
0.0398 \\
0.0404 \\
0.0408 \\
0.0404 \\
0.0405 \\
0.0401 \\
0.0054\end{array}$ \\
\hline
\end{tabular}




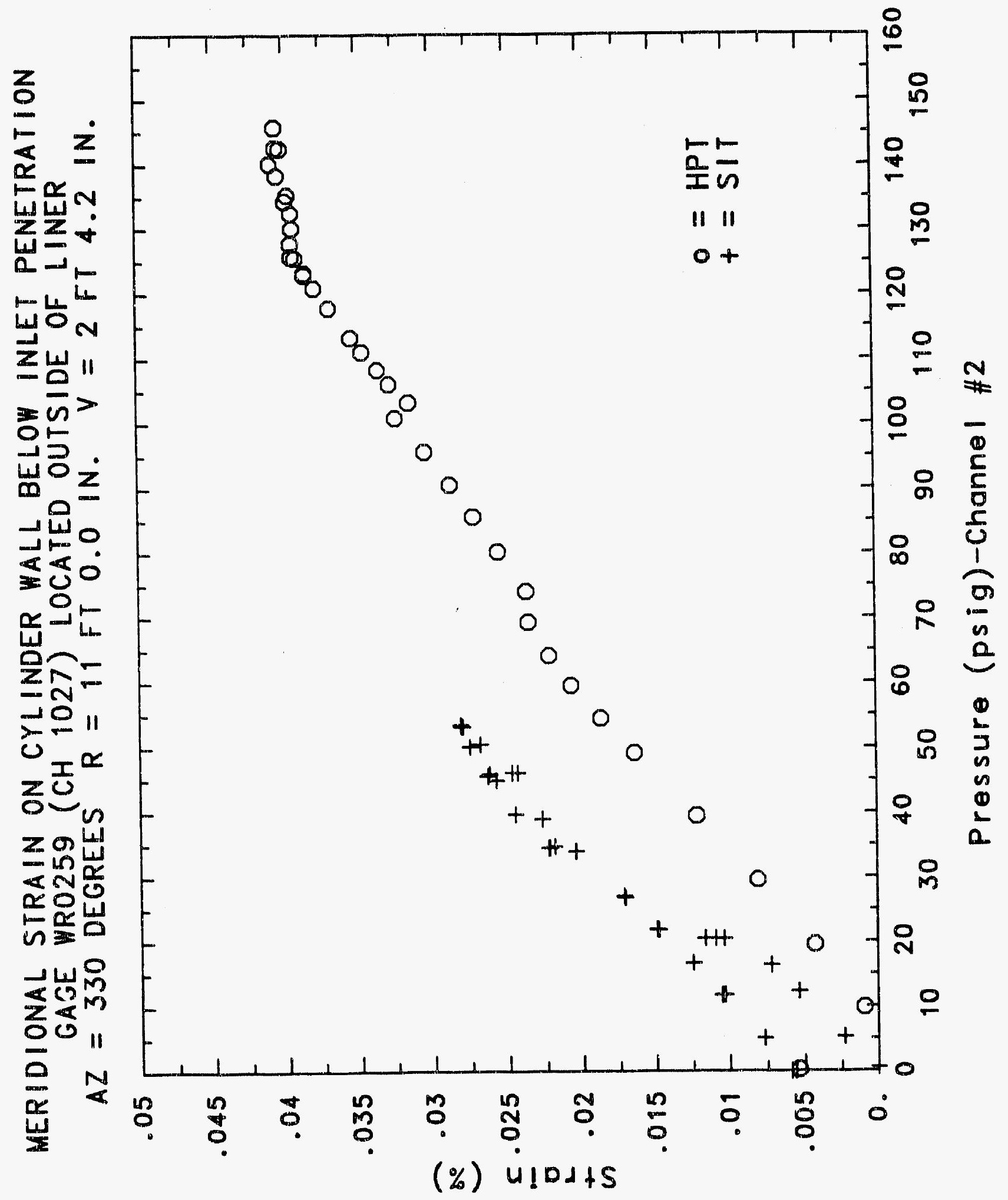




\section{Reinforced Concrete Test Data}

Weldable gage 260 Channel 1028

Structural Integrity Test

High Pressure test

\begin{tabular}{|c|c|c|c|}
\hline $\begin{array}{c}\text { Pressure } \\
\text { (psig) } \\
-0.05 \\
5.33 \\
12.31 \\
16.44 \\
20.51 \\
20.46 \\
20.47 \\
27.05 \\
34.69 \\
34.53 \\
34.53 \\
39.70 \\
45.90 \\
45.65 \\
45.62 \\
50.10 \\
53.47 \\
53.29 \\
53.21 \\
50.49 \\
46.14 \\
46.13 \\
44.97 \\
38.98 \\
33.99 \\
33.96 \\
26.83 \\
21.88 \\
21.90 \\
16.69 \\
11.74 \\
11.77 \\
5.05 \\
0.02 \\
-0.04 \\
-0.02 \\
-0.02 \\
0.02 \\
\end{array}$ & $\begin{array}{c}\text { \% Strain } \\
\\
-0.0003 \\
0.0000 \\
-0.0001 \\
-0.0001 \\
0.0003 \\
0.0003 \\
0.0007 \\
0.0010 \\
0.0017 \\
0.0018 \\
0.0019 \\
0.0023 \\
0.0025 \\
0.0032 \\
0.0033 \\
0.0036 \\
0.0039 \\
0.0042 \\
0.0046 \\
0.0050 \\
0.0049 \\
0.0050 \\
0.0058 \\
0.0048 \\
0.0047 \\
0.0047 \\
0.0043 \\
0.0039 \\
0.0038 \\
0.0034 \\
0.0031 \\
0.0029 \\
0.0023 \\
0.0013 \\
0.0046 \\
0.0038 \\
0.0031 \\
0.0011\end{array}$ & $\begin{array}{c}\text { Pressure } \\
\text { (psig) } \\
9.89 \\
19.55 \\
29.57 \\
39.42 \\
49.16 \\
54.50 \\
59.57 \\
64.20 \\
69.32 \\
74.16 \\
80.16 \\
85.61 \\
90.58 \\
95.69 \\
100.92 \\
103.25 \\
106.11 \\
108.31 \\
111.08 \\
113.24 \\
117.83 \\
120.92 \\
123.28 \\
122.97 \\
125.82 \\
125.60 \\
127.84 \\
130.19 \\
132.53 \\
135.33 \\
134.42 \\
138.35 \\
140.16 \\
142.63 \\
145.78 \\
142.52 \\
0.22\end{array}$ & $\begin{array}{l}\text { \% Strain } \\
-0.0032 \\
-0.0027 \\
-0.0026 \\
-0.0024 \\
-0.0022 \\
-0.0018 \\
-0.0016 \\
-0.0012 \\
-0.0001 \\
0.0007 \\
0.0013 \\
0.0022 \\
0.0029 \\
0.0038 \\
0.0047 \\
0.0054 \\
0.0059 \\
0.0061 \\
0.0070 \\
0.0076 \\
0.0086 \\
0.0096 \\
0.0108 \\
0.0118 \\
0.0125 \\
0.0134 \\
0.0134 \\
0.0136 \\
0.0147 \\
0.0167 \\
0.0174 \\
0.0206 \\
0.0236 \\
0.0279 \\
0.0308 \\
0.0322 \\
0.0199\end{array}$ \\
\hline
\end{tabular}




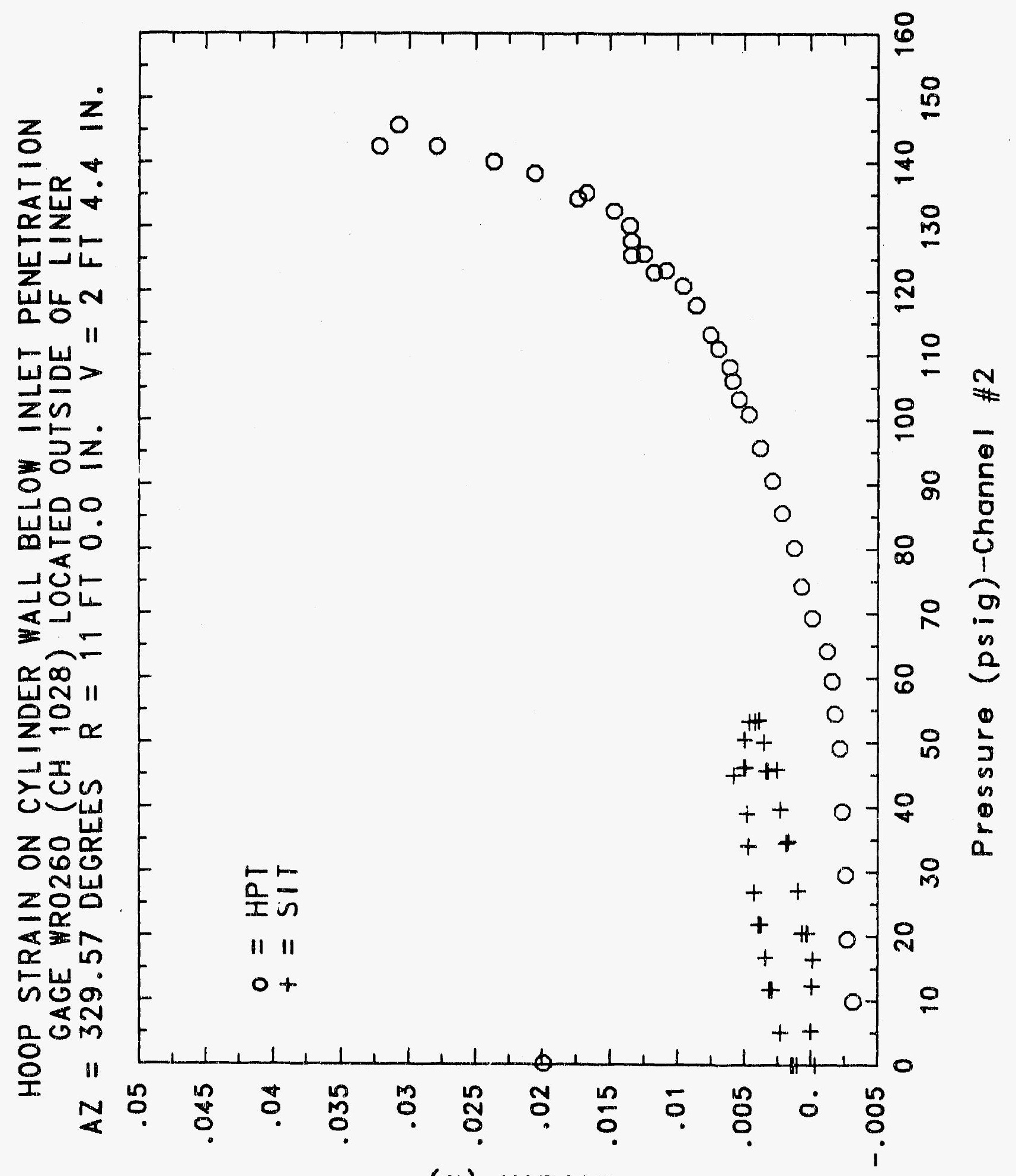

(\%) $4 ! 0 d+5$ 


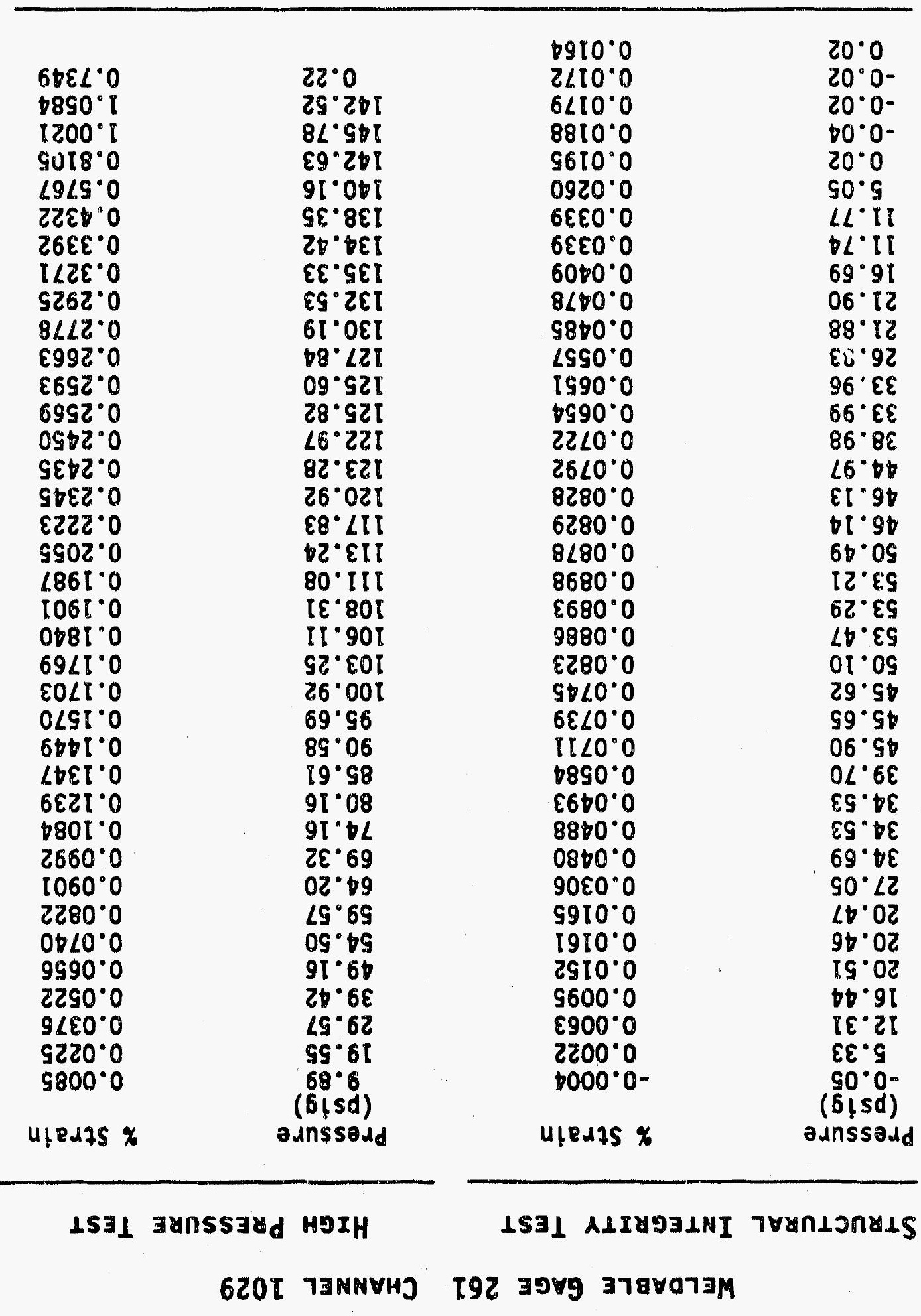

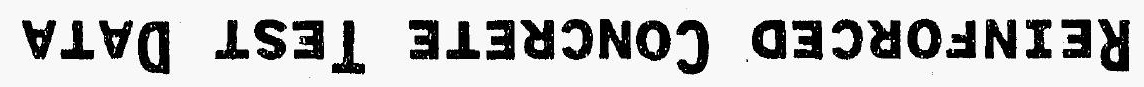




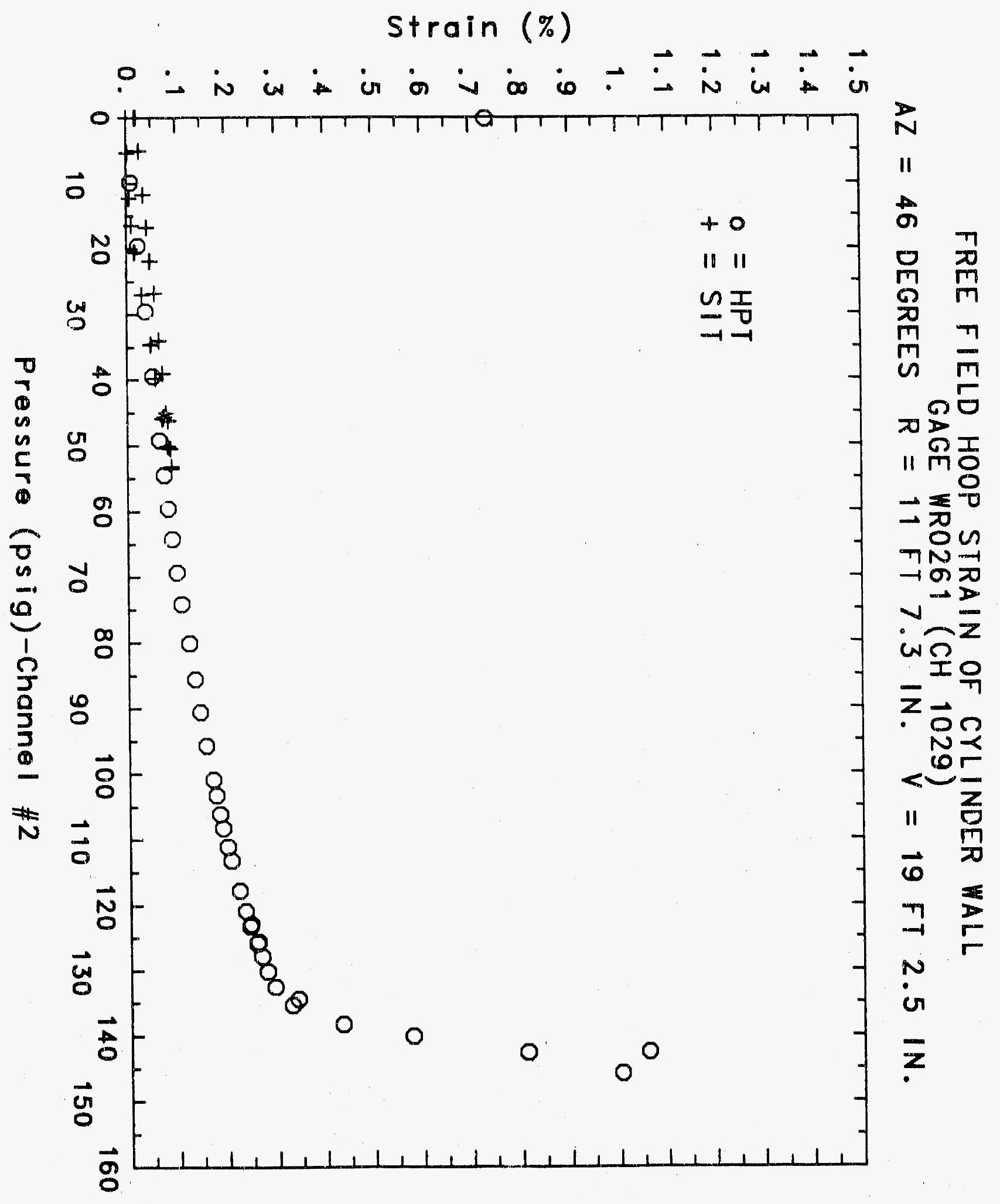




\section{Reinforced Concrete Test Data}

Weldable Gage 262 Channel 1040

STRUCTURAL INTEGRITY TEST

High Pressure test

\begin{tabular}{|c|c|c|c|}
\hline $\begin{array}{c}\text { Pressure } \\
\text { (psig) } \\
-0.05 \\
5.33 \\
12.31 \\
16.44 \\
20.51 \\
20.46 \\
20.47 \\
27.05 \\
34.69 \\
34.53 \\
34.53 \\
39.70 \\
45.90 \\
45.65 \\
45.62 \\
50.10 \\
53.47 \\
53.29 \\
53.21 \\
50.49 \\
46.14 \\
46.13 \\
44.97 \\
38.98 \\
33.99 \\
33.96 \\
26.83 \\
21.88 \\
21.90 \\
16.69 \\
11.74 \\
11.77 \\
5.05 \\
0.02 \\
-0.04 \\
-0.02 \\
-0.02 \\
0.02 \\
\end{array}$ & $\begin{array}{c}\text { \% Strain } \\
-0.0001 \\
0.0015 \\
0.0050 \\
0.0071 \\
0.0112 \\
0.0111 \\
0.0111 \\
0.0207 \\
0.0342 \\
0.0356 \\
0.0356 \\
0.0427 \\
0.0525 \\
0.0545 \\
0.0549 \\
0.0599 \\
0.0539 \\
0.0641 \\
0.0643 \\
0.0630 \\
0.0590 \\
0.0590 \\
0.0548 \\
0.0508 \\
0.0460 \\
0.0449 \\
0.0376 \\
0.0333 \\
0.0333 \\
0.0290 \\
0.0240 \\
0.0235 \\
0.0180 \\
0.0132 \\
0.0114 \\
0.0111 \\
0.0105 \\
0.0103\end{array}$ & $\begin{array}{c}\text { Pressure } \\
(\text { psig) } \\
9.89 \\
19.55 \\
29.57 \\
39.42 \\
49.16 \\
54.50 \\
59.57 \\
64.20 \\
69.32 \\
74.16 \\
80.16 \\
85.61 \\
90.58 \\
95.69 \\
100.92 \\
103.25 \\
106.11 \\
108.31 \\
111.08 \\
113.24 \\
117.83 \\
120.92 \\
123.28 \\
122.97 \\
125.82 \\
125.60 \\
127.84 \\
130.19 \\
132.53 \\
135.33 \\
134.42 \\
138.35 \\
140.16 \\
142.63 \\
145.78 \\
142.52 \\
0.22\end{array}$ & $\begin{array}{l}\text { \% Strain } \\
0.0058 \\
0.0159 \\
0.0262 \\
0.0359 \\
0.0459 \\
0.0510 \\
0.0555 \\
0.0610 \\
0.0666 \\
0.0738 \\
0.0837 \\
0.0907 \\
0.0969 \\
0.1038 \\
0.1118 \\
0.1159 \\
0.1206 \\
0.1241 \\
0.1290 \\
0.1332 \\
0.1427 \\
0.1500 \\
0.1564 \\
0.1570 \\
0.1633 \\
0.1648 \\
0.1689 \\
0.1761 \\
0.1843 \\
0.1967 \\
0.1990 \\
0.2134 \\
0.2249 \\
0.2435 \\
0.2667 \\
0.2641 \\
0.0746\end{array}$ \\
\hline
\end{tabular}




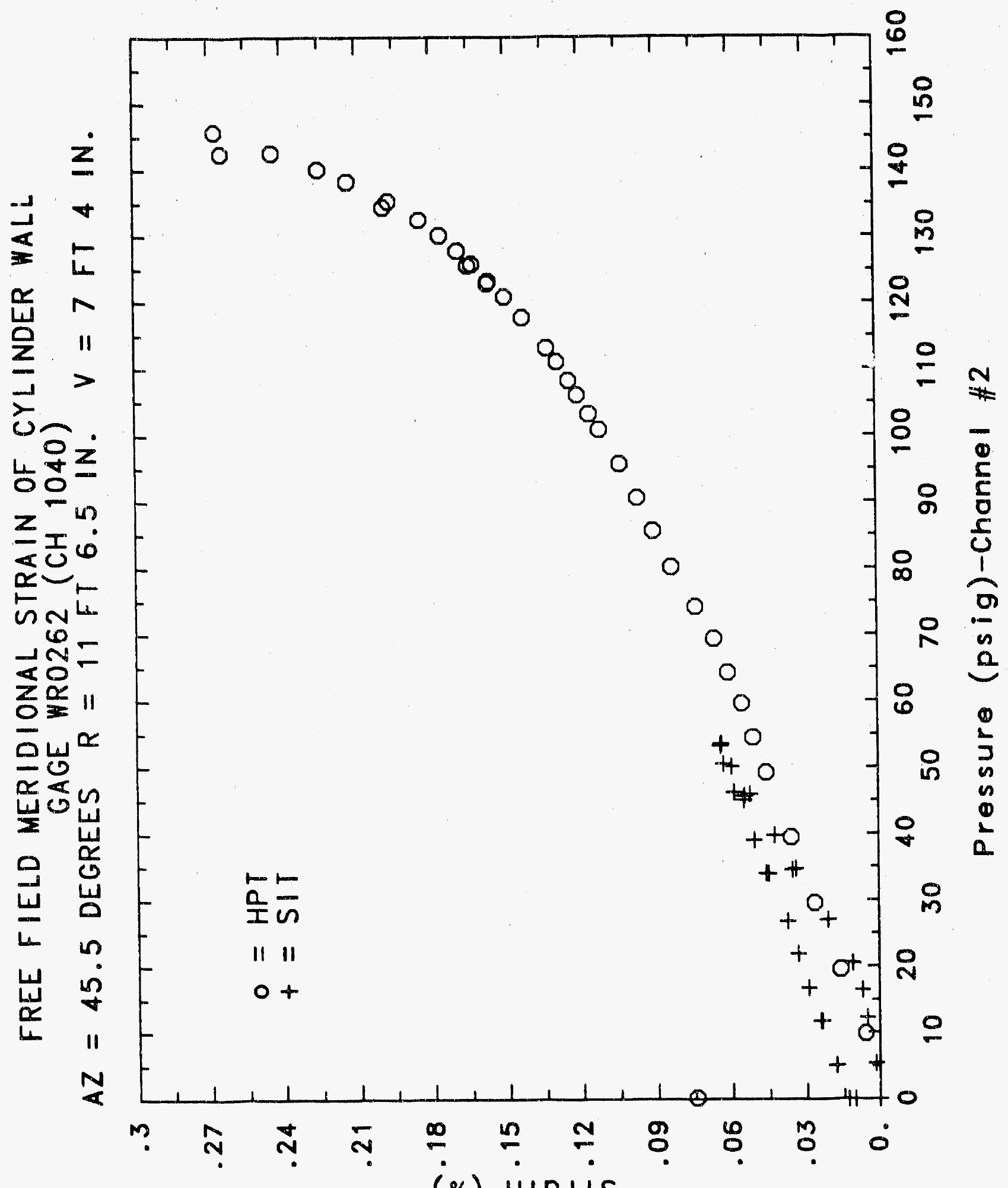

(\%) $u ! 0 d+S$ 


\section{Reinforced Concrete Test Data}

Weldable gage 263 Channel 1041

STRUCTURAL INTEGRITY TEST

\begin{tabular}{|c|c|c|c|}
\hline $\begin{array}{c}\text { Pressure } \\
\text { (psig) } \\
-0.05 \\
5.33 \\
12.31 \\
16.44 \\
20.51 \\
20.46 \\
20.47 \\
27.05 \\
34.69 \\
34.53 \\
34.53 \\
39.70 \\
45.90 \\
45.65 \\
45.62 \\
50.10 \\
53.47 \\
53.29 \\
53.21 \\
50.49 \\
46.14 \\
46.13 \\
44.97 \\
38.98 \\
33.99 \\
33.96 \\
26.83 \\
21.88 \\
21.90 \\
16.69 \\
11.74 \\
11.77 \\
5.05 \\
0.02 \\
-0.04 \\
-0.02 \\
-0.02 \\
0.02 \\
\end{array}$ & $\begin{array}{c}\text { \% Strain } \\
-0.0002 \\
0.0005 \\
0.0027 \\
0.0040 \\
0.0069 \\
0.0069 \\
0.0070 \\
0.0138 \\
0.0230 \\
0.0236 \\
0.0236 \\
0.0278 \\
0.0348 \\
0.0364 \\
0.0370 \\
0.0410 \\
0.0446 \\
0.0449 \\
0.0451 \\
0.0448 \\
0.0421 \\
0.0423 \\
0.0390 \\
0.0369 \\
0.0332 \\
0.0333 \\
0.0289 \\
0.0254 \\
0.0258 \\
0.0230 \\
0.0194 \\
0.0190 \\
0.0152 \\
0.0113 \\
0.0114 \\
0.0111 \\
0.0104 \\
0.0104\end{array}$ & $\begin{array}{c}\text { Pressure } \\
(\text { psig) } \\
9.89 \\
19.55 \\
29.57 \\
39.42 \\
49.16 \\
54.50 \\
59.57 \\
64.20 \\
69.32 \\
74.16 \\
80.16 \\
85.61 \\
90.58 \\
95.69 \\
100.92 \\
103.25 \\
106.11 \\
108.31 \\
111.08 \\
113.24 \\
117.83 \\
120.92 \\
123.28 \\
122.97 \\
125.82 \\
125.60 \\
127.84 \\
130.19 \\
132.53 \\
135.33 \\
134.42 \\
138.35 \\
140.16 \\
142.63 \\
145.78 \\
142.52 \\
0.22 \\
\end{array}$ & $\begin{array}{l}\text { \% Strain } \\
0.0029 \\
0.0101 \\
0.0174 \\
0.0242 \\
0.0310 \\
0.0349 \\
0.0390 \\
0.0431 \\
0.0474 \\
0.0523 \\
0.0611 \\
0.0679 \\
0.0741 \\
0.0815 \\
0.0894 \\
0.0931 \\
0.0971 \\
0.1005 \\
0.1051 \\
0.1087 \\
0.1168 \\
0.1229 \\
0.1278 \\
0.1281 \\
0.1341 \\
0.1346 \\
0.1391 \\
0.1458 \\
0.1556 \\
0.1647 \\
0.1657 \\
0.1748 \\
0.1792 \\
0.1859 \\
0.1924 \\
0.2037 \\
0.0668\end{array}$ \\
\hline
\end{tabular}

high Pressure test

Pressure

9.89

29.57

39.42

49.16

64.20

74.16

80.16

95.69

108.31

117.83

120.92

123.28

25.60

27.84

30.19

132.53

135.33

34.42

38.35

142.63

45.78

2.52

0.2037

0.0668 


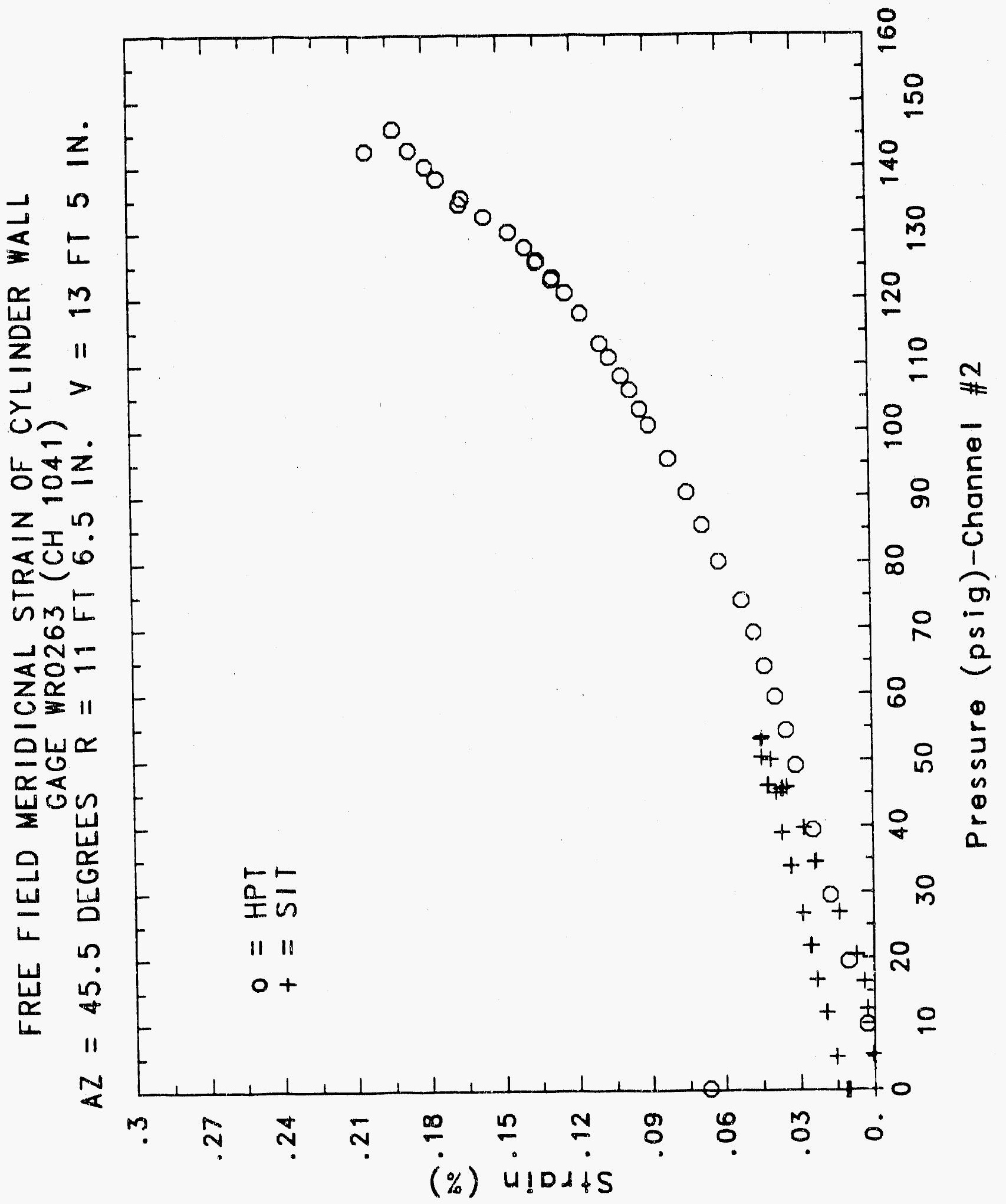




\section{Reinforced Concrete Test Data}

Weldable Gage 264 Channel 1042

Structural INTEgRITY TEST

High Pressure Test

\begin{tabular}{|c|c|c|c|}
\hline $\begin{array}{c}\text { Pressure } \\
\text { (psig) } \\
-0.05 \\
5.33 \\
12.31 \\
16.44 \\
20.51 \\
20.46 \\
20.47 \\
27.05 \\
34.69 \\
34.53 \\
34.53 \\
39.70 \\
45.90 \\
45.65 \\
45.62 \\
50.10 \\
53.47 \\
53.29 \\
53.21 \\
50.49 \\
46.14 \\
46.13 \\
44.97 \\
38.98 \\
33.99 \\
33.96 \\
26.83 \\
21.88 \\
21.90 \\
16.69 \\
11.74 \\
11.77 \\
5.05 \\
0.02 \\
-0.04 \\
-0.02 \\
-0.02 \\
0.02\end{array}$ & $\begin{array}{c}\text { x Strain } \\
-0.0004 \\
-0.0004 \\
0.0010 \\
0.0014 \\
0.0028 \\
0.0024 \\
0.0029 \\
0.0081 \\
0.0176 \\
0.0185 \\
0.0189 \\
0.0231 \\
0.0307 \\
0.0331 \\
0.0339 \\
0.0379 \\
0.0421 \\
0.0431 \\
0.0433 \\
0.0434 \\
0.0416 \\
0.0420 \\
0.0403 \\
0.0378 \\
0.0358 \\
0.0352 \\
0.0319 \\
0.0296 \\
0.0292 \\
0.0275 \\
0.0238 \\
0.0235 \\
0.0210 \\
0.0179 \\
0.0179 \\
0.0177 \\
0.0165 \\
0.0164\end{array}$ & $\begin{array}{c}\text { Pressure } \\
(\text { psig) } \\
9.89 \\
19.55 \\
29.57 \\
39.42 \\
49.16 \\
54.50 \\
59.57 \\
64.20 \\
69.32 \\
74.15 \\
80.16 \\
85.61 \\
90.58 \\
95.69 \\
100.92 \\
103.25 \\
106.11 \\
108.31 \\
111.08 \\
113.24 \\
117.83 \\
120.92 \\
123.28 \\
122.97 \\
125.82 \\
125.60 \\
127.84 \\
130.19 \\
132.53 \\
135.33 \\
134.42 \\
138.35 \\
140.16 \\
142.63 \\
145.78 \\
142.52 \\
0.22\end{array}$ & $\begin{array}{c}\text { * Strain } \\
0.0015 \\
0.0076 \\
0.0137 \\
0.0192 \\
0.0249 \\
0.0285 \\
0.0319 \\
0.0361 \\
0.0402 \\
0.0453 \\
0.0536 \\
0.0601 \\
0.0657 \\
0.0717 \\
0.0792 \\
0.0832 \\
0.0872 \\
0.0903 \\
0.0951 \\
0.0991 \\
0.1073 \\
0.1131 \\
0.1180 \\
0.1185 \\
0.1235 \\
0.1241 \\
0.1271 \\
0.1333 \\
0.1434 \\
0.1611 \\
0.1652 \\
0.1939 \\
0.2200 \\
0.2617 \\
0.2621 \\
0.2523 \\
0.0859\end{array}$ \\
\hline
\end{tabular}




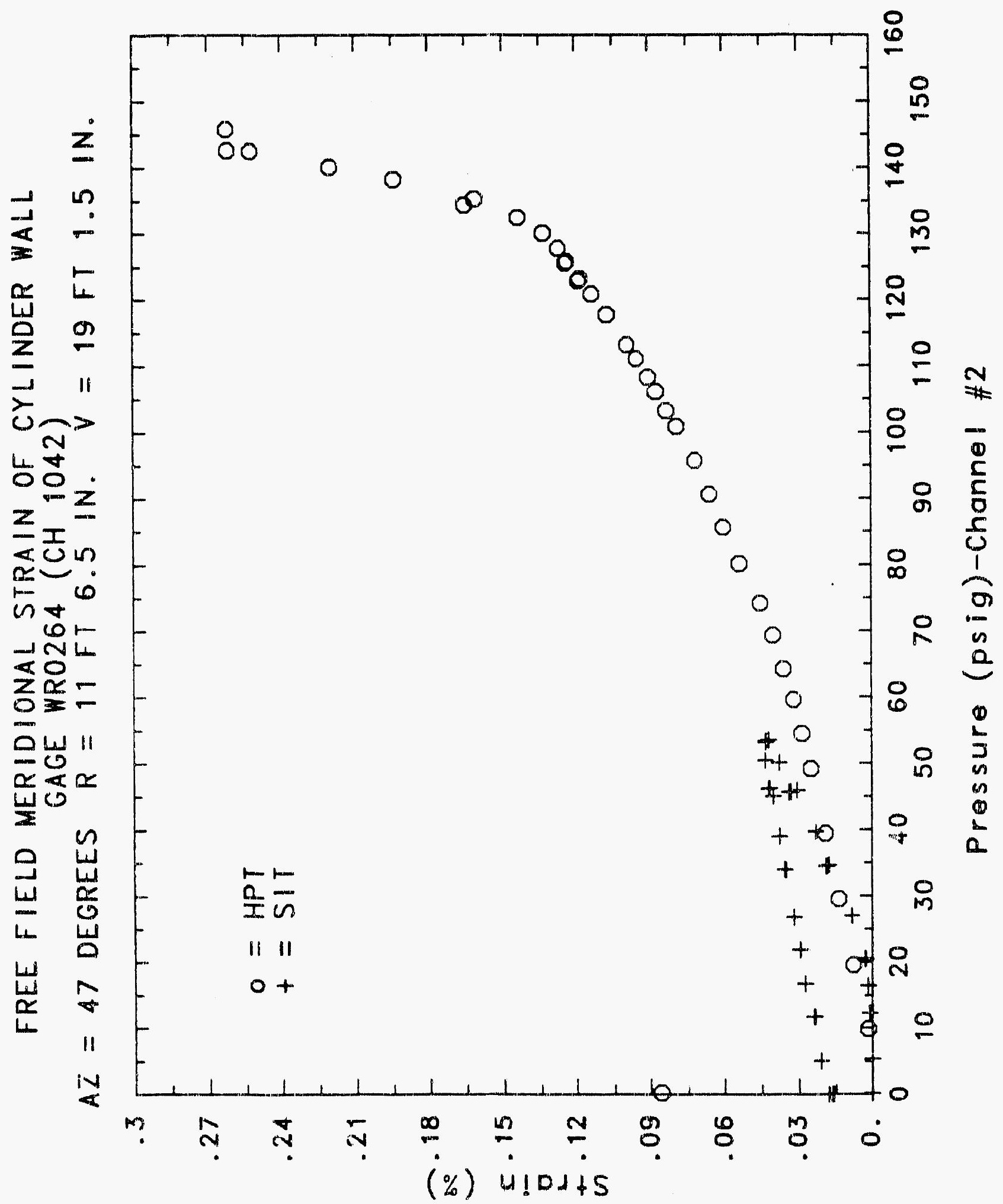




\section{Reinforced Concrete Test Data}

Weldable gage 265 Channel 1043

Structural InTEgRITY TEST

high Pressure test

\begin{tabular}{|c|c|c|c|}
\hline $\begin{array}{c}\text { Pressure } \\
\text { (psig) } \\
-0.05 \\
5.33 \\
12.31 \\
16.44 \\
20.51 \\
20.46 \\
20.47 \\
27.05 \\
34.69 \\
34.53 \\
34.53 \\
39.70 \\
45.90 \\
45.65 \\
45.62 \\
50.10 \\
53.47 \\
53.29 \\
53.21 \\
50.49 \\
46.14 \\
46.13 \\
44.97 \\
38.98 \\
33.99 \\
33.96 \\
26.83 \\
21.88 \\
21.90 \\
16.69 \\
11.74 \\
11.77 \\
5.05 \\
0.02 \\
-0.04 \\
-0.02 \\
-0.02 \\
0.02 \\
\end{array}$ & $\begin{array}{l}\text { \% Strain } \\
0.0003 \\
0.0029 \\
0.0070 \\
0.0102 \\
0.0163 \\
0.0189 \\
0.0195 \\
0.0347 \\
0.0530 \\
0.0545 \\
0.0550 \\
0.0635 \\
0.0761 \\
0.0766 \\
0.0768 \\
0.0842 \\
0.0910 \\
0.0916 \\
0.0908 \\
0.0894 \\
0.0846 \\
0.0846 \\
0.0845 \\
0.0770 \\
0.0706 \\
0.0700 \\
0.0603 \\
0.0531 \\
0.0523 \\
0.0448 \\
0.0374 \\
0.0362 \\
0.0268 \\
0.0192 \\
0.0171 \\
0.0177 \\
0.0176 \\
0.0178\end{array}$ & $\begin{array}{c}\text { Pressure } \\
\text { (psig) } \\
9.89 \\
19.55 \\
29.57 \\
39.42 \\
49.16 \\
54.50 \\
59.57 \\
64.20 \\
69.32 \\
74.16 \\
80.16 \\
85.61 \\
90.58 \\
95.69 \\
100.92 \\
103.25 \\
106.11 \\
108.31 \\
111.08 \\
113.24 \\
117.83 \\
120.92 \\
123.28 \\
122.97 \\
125.82 \\
125.60 \\
127.84 \\
130.19 \\
132.53 \\
135.33 \\
134.42 \\
138.35 \\
140.16 \\
142.63 \\
145.78 \\
142.52 \\
0.22\end{array}$ & $\begin{array}{l}\text { \% strain } \\
0.0110 \\
0.0269 \\
0.0428 \\
0.0576 \\
0.0717 \\
0.0796 \\
0.0880 \\
0.0970 \\
0.1059 \\
0.1156 \\
0.1272 \\
0.1385 \\
0.1492 \\
0.1612 \\
0.1733 \\
0.1787 \\
0.1852 \\
0.1904 \\
0.1983 \\
0.2043 \\
0.2168 \\
0.2224 \\
0.2262 \\
0.2267 \\
0.2301 \\
0.2308 \\
0.2331 \\
0.2335 \\
0.2364 \\
0.2421 \\
0.2430 \\
0.2492 \\
0.2441 \\
0.4045 \\
0.5924 \\
0.6151 \\
0.3236\end{array}$ \\
\hline
\end{tabular}




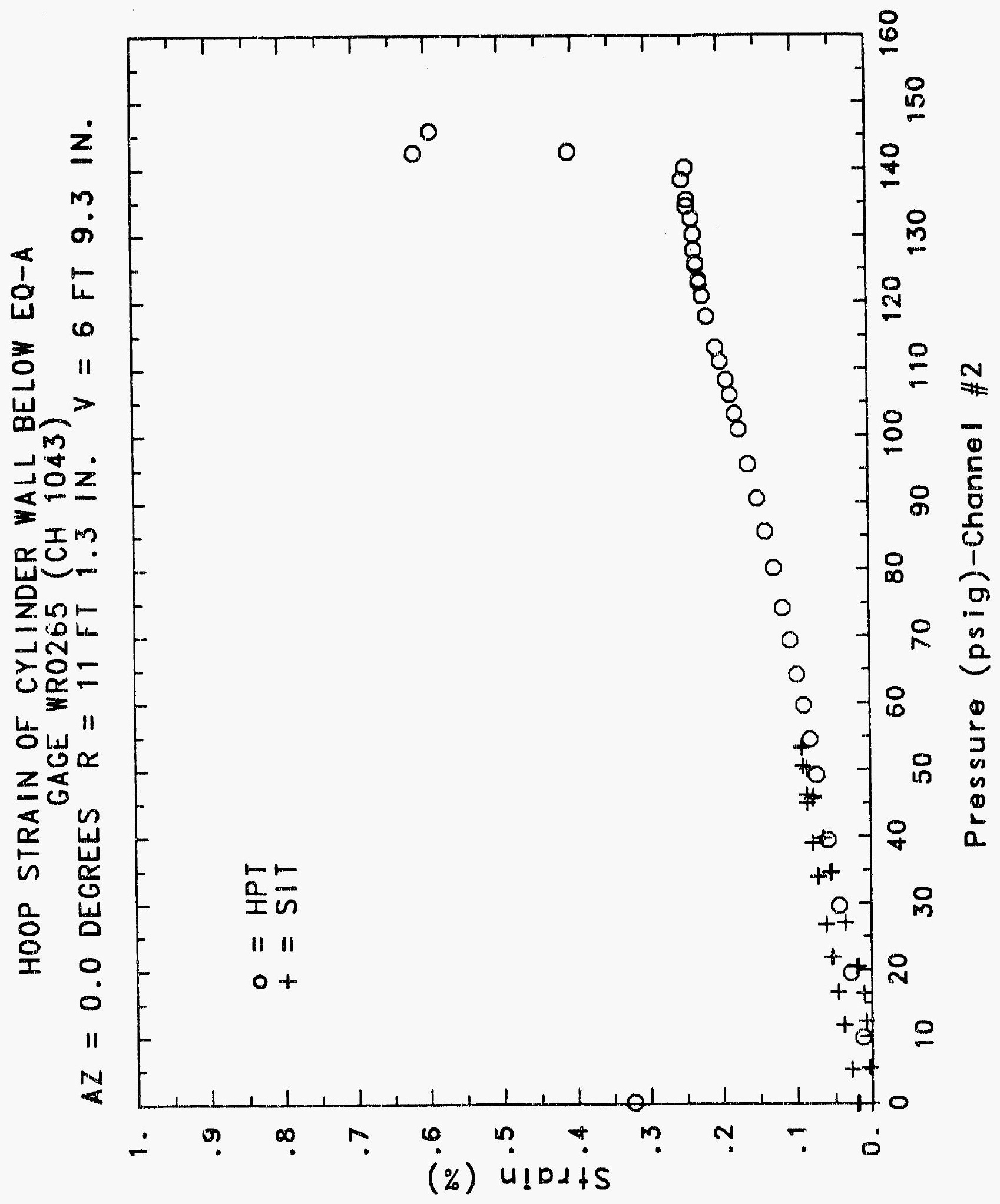




\section{Reinforced Concrete Test Data}

Weldable Gage 266 Chanmel 1044

Structural Integrity test

High Pressure test

\begin{tabular}{|c|c|c|c|}
\hline $\begin{array}{c}\text { Pressure } \\
(\text { psig) } \\
-0.05 \\
5.33 \\
12.31 \\
16.44 \\
20.51 \\
20.46 \\
20.47 \\
27.05 \\
34.69 \\
34.53 \\
34.53 \\
39.70 \\
45.90 \\
45.65 \\
45.62 \\
50.10 \\
53.47 \\
53.29 \\
53.21 \\
50.49 \\
46.14 \\
46.13 \\
44.97 \\
38.98 \\
33.99 \\
33.96 \\
26.83 \\
21.88 \\
21.90 \\
16.69 \\
11.74 \\
11.77 \\
5.05 \\
0.02 \\
-0.04 \\
-0.02 \\
-0.02 \\
0.02 \\
\end{array}$ & $\begin{array}{l}\text { Strain } \\
0.0006 \\
0.0031 \\
0.0072 \\
0.0099 \\
0.0142 \\
0.0152 \\
0.0155 \\
0.0252 \\
0.0391 \\
0.0397 \\
0.0408 \\
0.0480 \\
0.0589 \\
0.0602 \\
0.0602 \\
0.0669 \\
0.0729 \\
0.0740 \\
0.0745 \\
0.0729 \\
0.0685 \\
0.0685 \\
0.0686 \\
0.0624 \\
0.0572 \\
0.0565 \\
0.0483 \\
0.0422 \\
0.0417 \\
0.0357 \\
0.0290 \\
0.0284 \\
0.0201 \\
0.0143 \\
0.0115 \\
0.0112 \\
0.0117 \\
0.0119\end{array}$ & $\begin{array}{c}\text { Pressure } \\
\text { (psig) } \\
9.89 \\
19.55 \\
29.57 \\
39.42 \\
49.16 \\
54.50 \\
59.57 \\
64.20 \\
69.32 \\
74.16 \\
80.16 \\
85.61 \\
90.58 \\
95.69 \\
100.92 \\
103.25 \\
106.11 \\
108.31 \\
111.08 \\
113.24 \\
117.83 \\
120.92 \\
123.28 \\
122.97 \\
125.82 \\
125.60 \\
127.84 \\
130.19 \\
132.53 \\
135.33 \\
134.42 \\
138.35 \\
140.16 \\
142.63 \\
145.78 \\
142.52 \\
0.22 \\
\end{array}$ & $\begin{array}{l}\text { \% Strain } \\
0.0081 \\
0.0213 \\
0.0345 \\
0.0475 \\
0.0598 \\
0.0670 \\
0.0747 \\
0.0840 \\
0.0945 \\
0.1065 \\
0.1216 \\
0.1368 \\
0.1532 \\
0.1746 \\
0.2011 \\
0.2165 \\
0.2325 \\
0.2460 \\
0.2678 \\
0.2857 \\
0.3294 \\
0.3651 \\
0.3992 \\
0.4059 \\
0.4354 \\
0.4457 \\
0.4546 \\
0.4743 \\
0.5163 \\
0.6333 \\
0.6927 \\
0.7457 \\
0.7553 \\
0.7973 \\
0.8658 \\
0.8984 \\
0.5172\end{array}$ \\
\hline
\end{tabular}




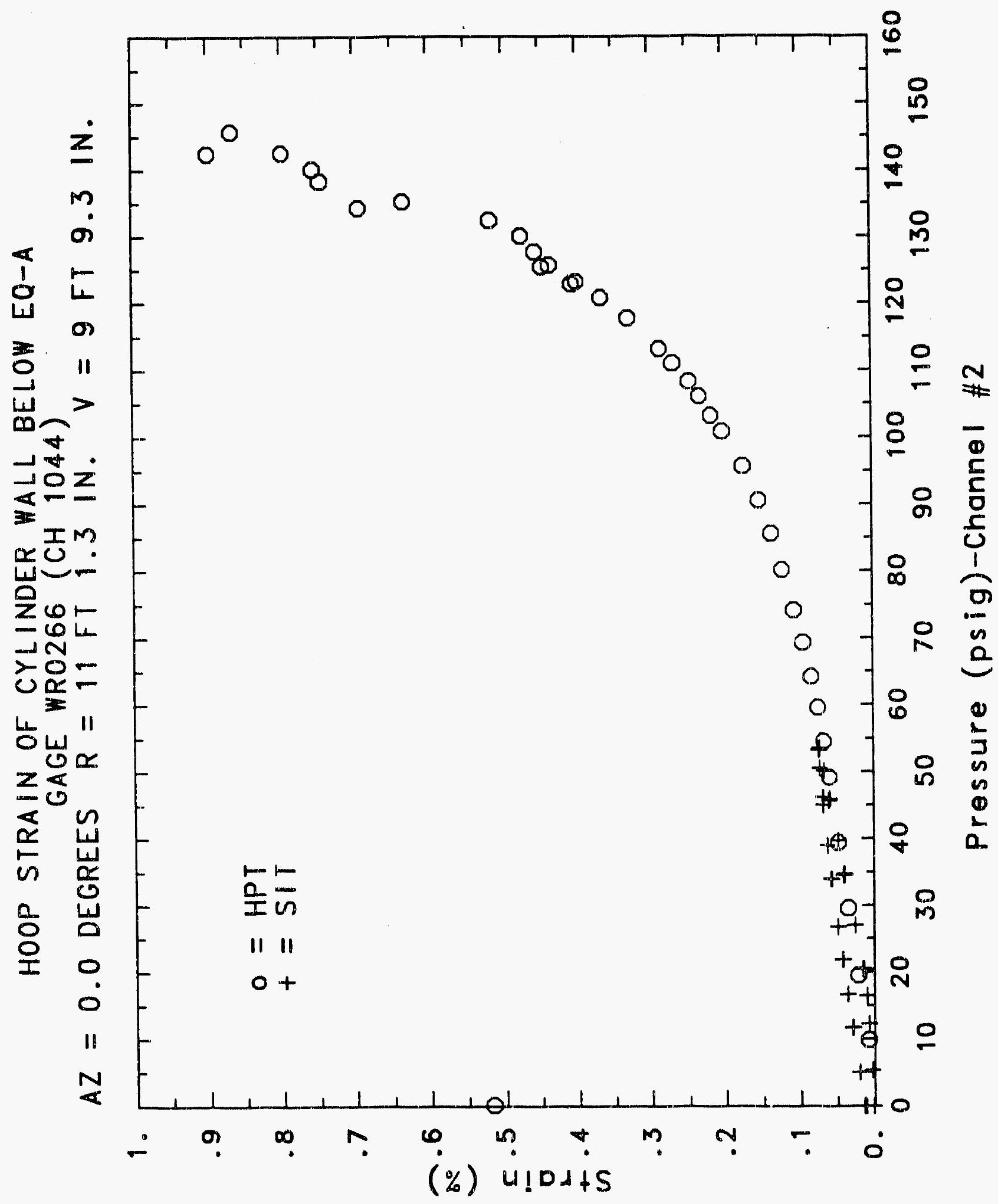




\section{Reinforced Concrete Test Data}

Weldable Gage 267 Chamnel 1045

Structural Integrity Test

High Pressure Test

\begin{tabular}{|c|c|c|c|}
\hline $\begin{array}{c}\text { Pressure } \\
\text { (psig) } \\
-0.05 \\
5.33 \\
12.31 \\
16.44 \\
20.51 \\
20.46 \\
20.47 \\
27.05 \\
34.69 \\
34.53 \\
34.53 \\
39.70 \\
45.90 \\
45.65 \\
45.62 \\
50.10 \\
53.47 \\
53.29 \\
53.21 \\
50.49 \\
46.14 \\
46.13 \\
44.97 \\
38.98 \\
33.99 \\
33.96 \\
26.83 \\
21.88 \\
21.90 \\
16.69 \\
11.74 \\
11.77 \\
5.05 \\
0.02 \\
-0.04 \\
-0.02 \\
-0.02 \\
0.02 \\
\end{array}$ & $\begin{array}{l}\text { \% Strain } \\
0.0000 \\
0.0048 \\
0.0116 \\
0.0160 \\
0.0205 \\
0.0222 \\
0.0224 \\
0.0364 \\
0.0530 \\
0.0548 \\
0.0555 \\
0.0643 \\
0.0764 \\
0.0761 \\
0.0761 \\
0.0834 \\
0.0899 \\
0.0904 \\
0.0905 \\
0.0877 \\
0.0814 \\
0.0818 \\
0.0820 \\
0.0731 \\
0.0665 \\
0.0660 \\
0.0555 \\
0.0478 \\
0.0474 \\
0.0396 \\
0.0316 \\
0.0302 \\
0.0191 \\
0.0110 \\
0.0084 \\
0.0085 \\
0.0082 \\
0.0089\end{array}$ & $\begin{array}{c}\text { Pressure } \\
(\text { psig) } \\
9.89 \\
19.55 \\
29.57 \\
39.42 \\
49.16 \\
54.50 \\
59.57 \\
64.20 \\
69.32 \\
74.16 \\
80.16 \\
85.61 \\
90.58 \\
95.69 \\
100.92 \\
103.25 \\
106.11 \\
108.31 \\
111.08 \\
113.24 \\
117.83 \\
120.92 \\
123.28 \\
122.97 \\
125.82 \\
125.60 \\
127.84 \\
130.19 \\
132.53 \\
135.33 \\
134.42 \\
138.35 \\
140.16 \\
142.63 \\
145.78 \\
142.52 \\
0.22\end{array}$ & $\begin{array}{l}\text { X Strain } \\
0.0128 \\
0.0298 \\
0.0467 \\
0.0633 \\
0.0780 \\
0.0866 \\
0.0965 \\
0.1060 \\
0.1169 \\
0.1271 \\
0.1417 \\
0.1549 \\
0.1681 \\
0.1819 \\
0.1964 \\
0.2024 \\
0.2097 \\
0.2162 \\
0.2252 \\
0.2345 \\
0.2619 \\
0.2833 \\
0.3076 \\
0.3139 \\
0.3511 \\
0.3604 \\
0.3809 \\
0.4362 \\
0.5611 \\
0.5984 \\
0.6027 \\
0.6308 \\
0.6478 \\
0.7057 \\
0.7476 \\
0.7545 \\
0.4598\end{array}$ \\
\hline
\end{tabular}




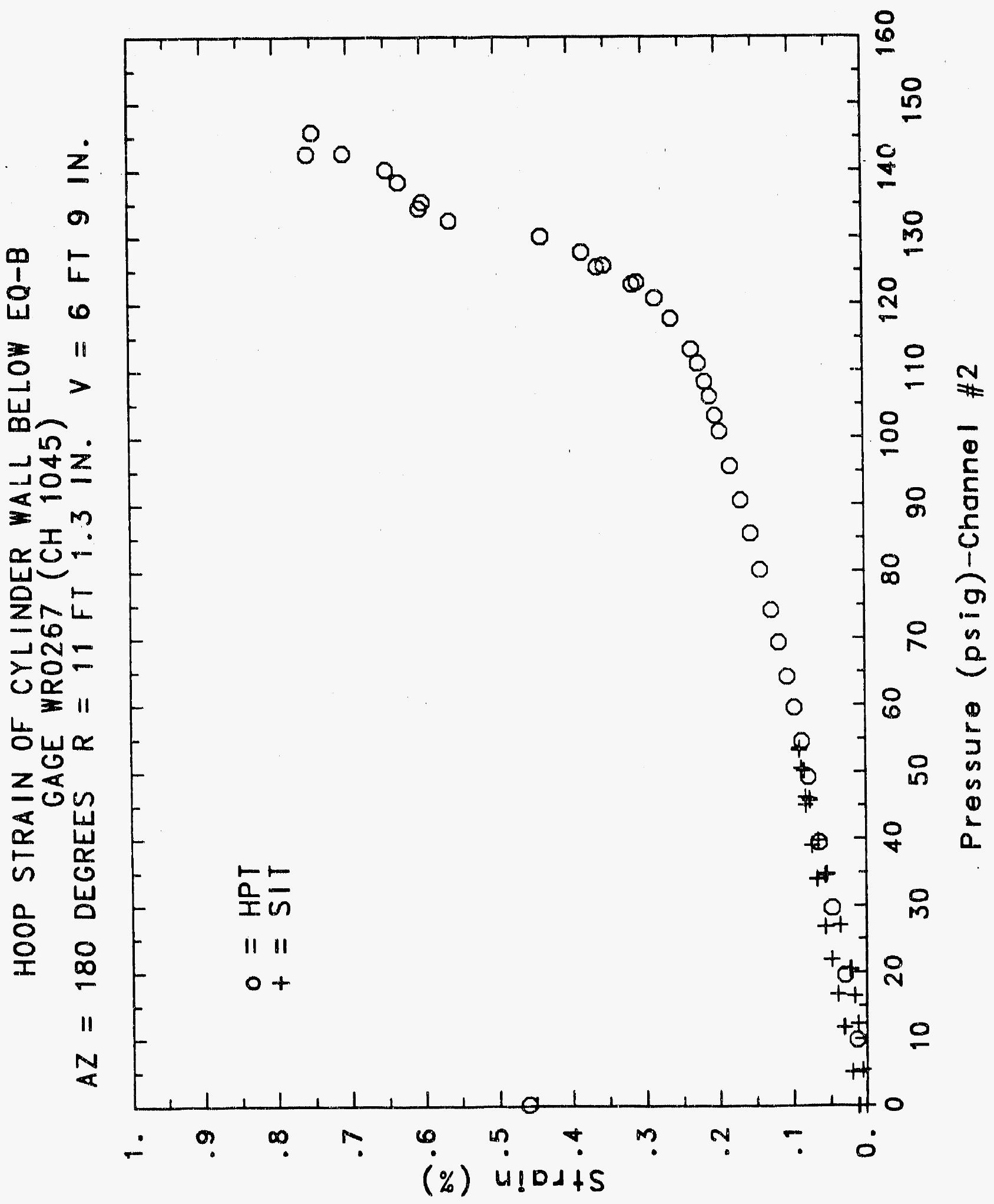




\section{Reinforced Concrete Test Data}

Weldable gage 268 Chammel 1046

STRUCTURAL INTEGRITY TEST

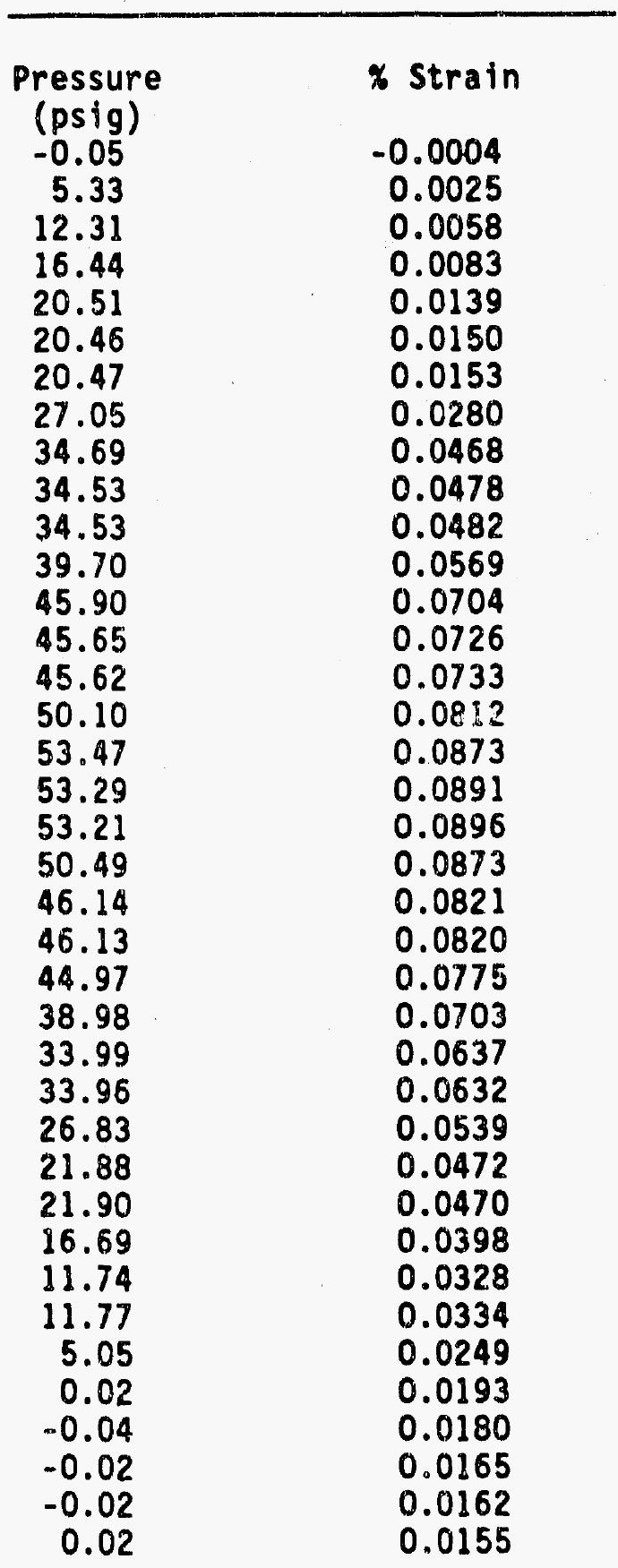

high Pressure test

Pressure
(psig)

9.89

19.55

29.57

39.42

49.16

54.50

59.57

64.20

69.32

74.16

80.16

85.61

90.58

95.69

100.92

103.25

106.11

108.31

111.08

113.24

117.83

120.92

123.28

122.97

125.82

125.60

127.84

130.19

132.53

135.33

134.42

138.35

140.16

142.63

145.78

142.52

0.22
* Strain

0.0085

0.0236

0.0384

0.0526

0.0667

0.0745

0.0828

0.0911

0.1000

0.1088

0.1227

0.1331

0.1421

0.1521

0.1629

0.1681

0.1749

0.1802

0.1873

0.1934

0.2091

0.2211

0.2314

0.2327

0.2454

0.2476

0.2551

0.2747

0.2989

0.3460

0.3813

0.4443

0.4661

0.7401

0.8490

0.9032

0.5886 


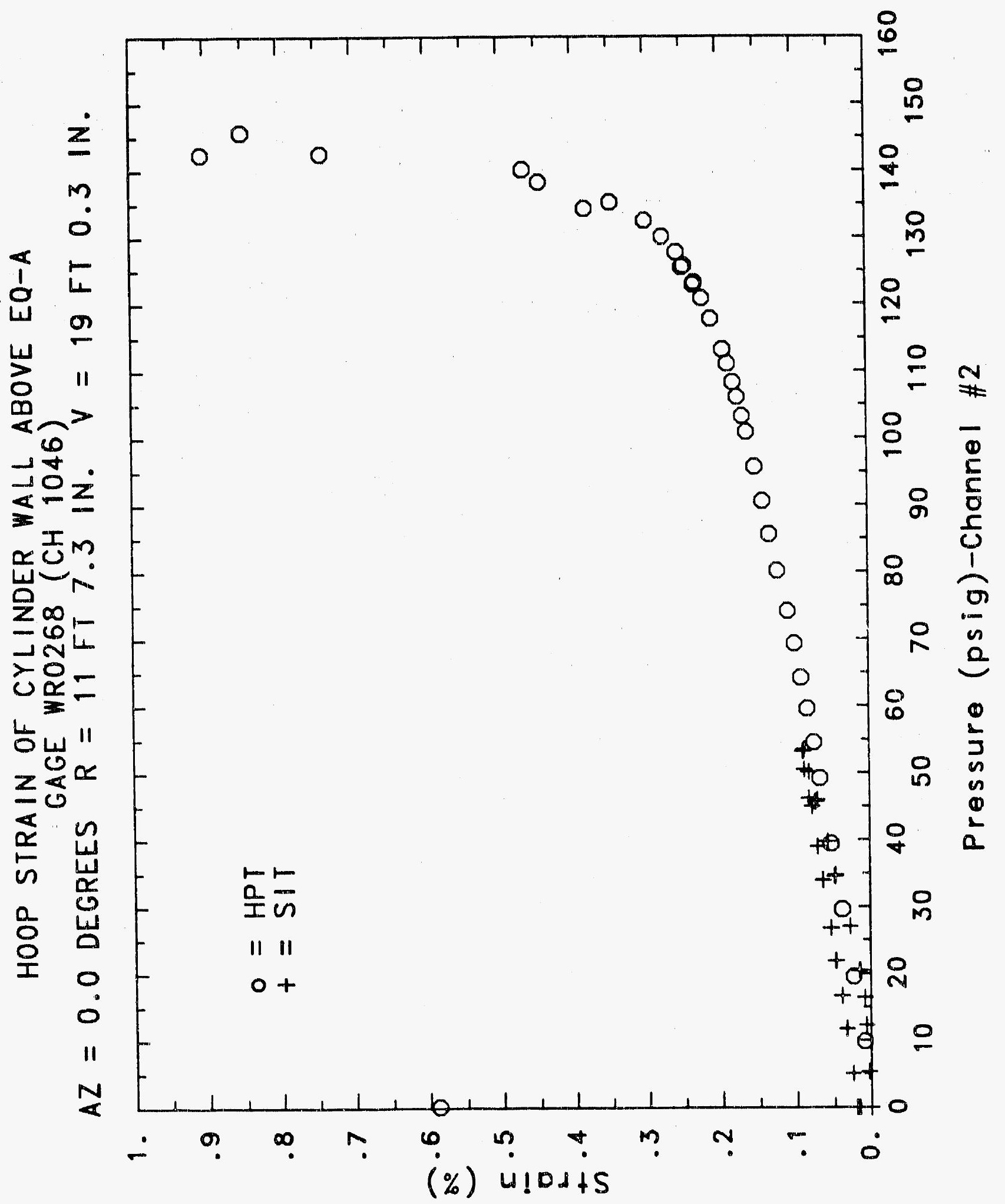




\section{Reinforced Concrete Test Data}

Weldable Gage 269 Channel 1047 ;

Structural IntEgrxty TEST

high Pressure test

\begin{tabular}{|c|c|c|c|}
\hline $\begin{array}{c}\text { Pressure } \\
\text { (psig) } \\
-0.05 \\
5.33 \\
12.31 \\
16.44 \\
20.51 \\
20.46 \\
20.47 \\
27.05 \\
34.69 \\
34.53 \\
34.53 \\
39.70 \\
45.90 \\
45.65 \\
45.62 \\
50.10 \\
53.47 \\
53.29 \\
53.21 \\
50.49 \\
46.14 \\
46.13 \\
44.97 \\
38.98 \\
33.99 \\
33.96 \\
26.83 \\
21.88 \\
21.90 \\
16.69 \\
11.74 \\
11.77 \\
5.05 \\
0.02 \\
-0.04 \\
-0.02 \\
-0.02 \\
0.02\end{array}$ & $\begin{array}{c}\text { x Strain } \\
-0.0005 \\
0.0029 \\
0.0069 \\
0.0097 \\
0.0163 \\
0.0176 \\
0.0178 \\
0.0342 \\
0.0556 \\
0.0545 \\
0.0541 \\
0.0631 \\
0.0768 \\
0.0797 \\
0.0807 \\
0.0889 \\
0.0959 \\
0.0967 \\
0.0968 \\
0.0941 \\
0.0881 \\
0.0877 \\
0.0831 \\
0.0742 \\
0.0662 \\
0.0665 \\
0.0556 \\
0.0471 \\
0.0457 \\
0.0374 \\
0.0293 \\
0.0310 \\
0.0221 \\
0.0161 \\
0.0153 \\
0.0145 \\
0.0141 \\
0.0132\end{array}$ & $\begin{array}{c}\text { Pressure } \\
\text { (psig) } \\
9.89 \\
19.55 \\
29.57 \\
39.42 \\
49.16 \\
54.50 \\
59.57 \\
64.20 \\
69.32 \\
74.16 \\
80.16 \\
85.61 \\
9058 \\
95.69 \\
100.92 \\
103.25 \\
106.11 \\
108.31 \\
111.08 \\
113.24 \\
117.83 \\
120.92 \\
123.28 \\
122.97 \\
125.82 \\
125.60 \\
127.84 \\
130.19 \\
132.53 \\
135.33 \\
134.42 \\
138.35 \\
140.16 \\
142.63 \\
145.78 \\
142.52 \\
0.22\end{array}$ & $\begin{array}{c}\text { * Strain } \\
0.0111 \\
0.0276 \\
0.0445 \\
0.0611 \\
0.0763 \\
0.0843 \\
0.0931 \\
0.1018 \\
0.1112 \\
0.1199 \\
0.1337 \\
0.1445 \\
0.1553 \\
0.1669 \\
0.1798 \\
0.1859 \\
0.1936 \\
0.2001 \\
0.2085 \\
0.2153 \\
0.2334 \\
0.2476 \\
0.2593 \\
0.2604 \\
0.2721 \\
0.2725 \\
0.2755 \\
0.2839 \\
0.3184 \\
0.5714 \\
0.6451 \\
0.9350 \\
1.0072 \\
1.0752 \\
1.1134 \\
1.1298 \\
0.8063\end{array}$ \\
\hline
\end{tabular}




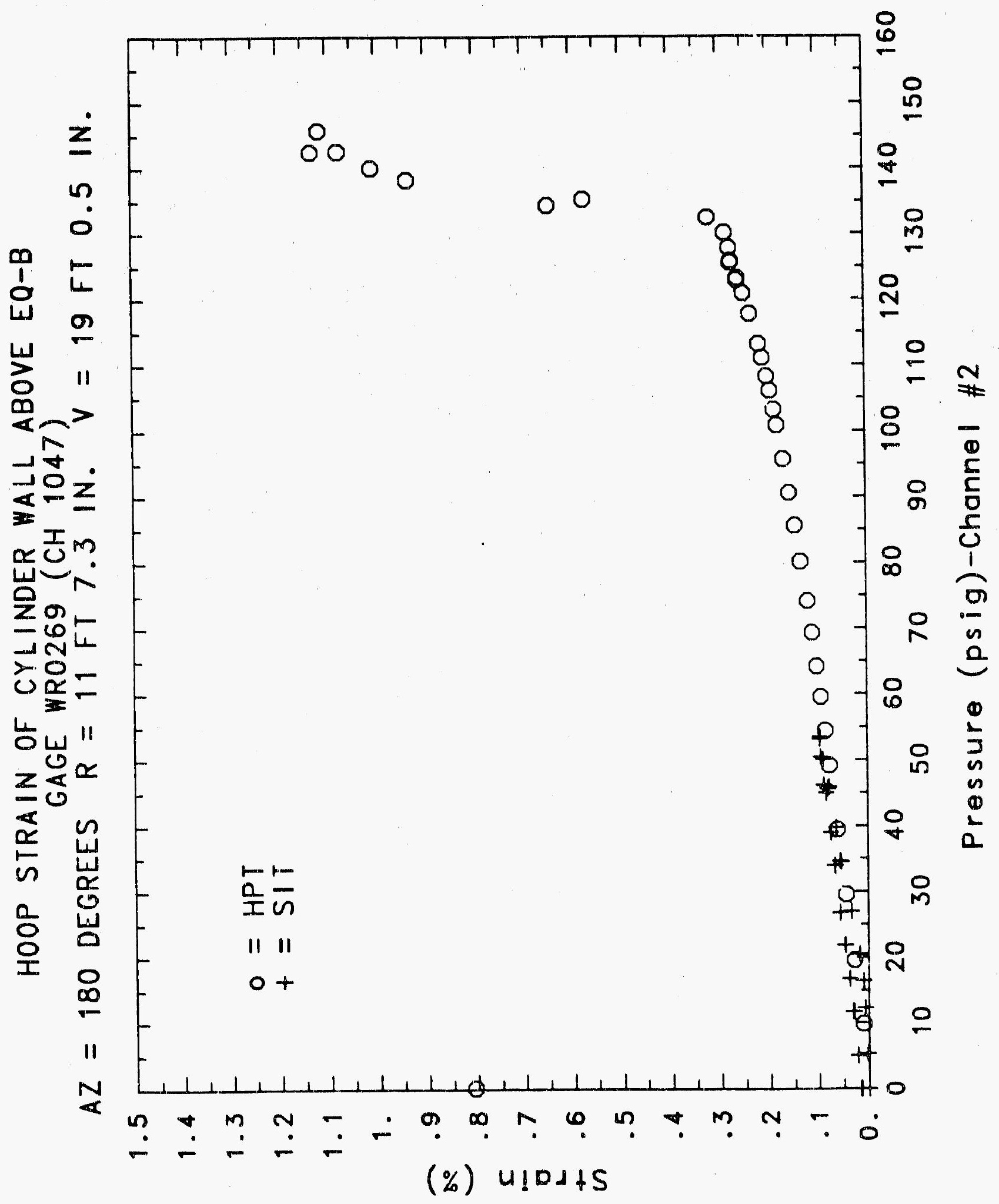




\section{Reinforced Concrete Test Data}

Weldable gage 270 Channel 1048

STRUCTURAL INTEGRITY TEST

High Pressure Test

\begin{tabular}{|c|c|c|c|}
\hline $\begin{array}{c}\text { Pressure } \\
\text { (psig) } \\
-0.05 \\
5.33 \\
12.31 \\
16.44 \\
20.51 \\
20.46 \\
20.47 \\
27.05 \\
34.69 \\
34.53 \\
34.53 \\
39.70 \\
45.90 \\
45.65 \\
45.62 \\
50.10 \\
53.47 \\
53.29 \\
53.21 \\
50.49 \\
46.14 \\
46.13 \\
44.97 \\
38.98 \\
33.99 \\
33.96 \\
26.83 \\
21.88 \\
21.90 \\
16.69 \\
11.74 \\
11.77 \\
5.05 \\
0.02 \\
-0.04 \\
-0.02 \\
-0.02 \\
0.02 \\
\end{array}$ & $\begin{array}{l}\text { X Strain } \\
0.0004 \\
0.0029 \\
0.0065 \\
0.0091 \\
0.0157 \\
0.0175 \\
0.0183 \\
0.0321 \\
0.0520 \\
0.0547 \\
0.0546 \\
0.0641 \\
0.0792 \\
0.0817 \\
0.0822 \\
0.0903 \\
0.0979 \\
0.0992 \\
0.0993 \\
0.0973 \\
0.0928 \\
0.0921 \\
0.0885 \\
0.0828 \\
0.0761 \\
0.0758 \\
0.0671 \\
0.0602 \\
0.0599 \\
0.0527 \\
0.0455 \\
0.0456 \\
0.0367 \\
0.0303 \\
0.0260 \\
0.0257 \\
0.0258 \\
0.0260\end{array}$ & $\begin{array}{c}\text { Pressure } \\
\text { (psig) } \\
9.89 \\
19.55 \\
29.57 \\
39.42 \\
49.16 \\
54.50 \\
59.57 \\
64.20 \\
69.32 \\
74.16 \\
80.16 \\
85.61 \\
90.58 \\
95.69 \\
100.92 \\
103.25 \\
106.11 \\
108.31 \\
111.08 \\
113.24 \\
117.83 \\
120.92 \\
123.28 \\
122.97 \\
125.82 \\
125.60 \\
127.84 \\
130.19 \\
132.53 \\
135.33 \\
134.42 \\
138.35 \\
140.16 \\
142.63 \\
145.78 \\
142.52 \\
0.22 \\
\end{array}$ & $\begin{array}{l}\text { \% Strain } \\
0.0106 \\
0.0265 \\
0.0417 \\
0.0563 \\
0.0703 \\
0.0786 \\
0.0874 \\
0.0962 \\
0.1065 \\
0.1174 \\
0.1327 \\
0.1457 \\
0.1574 \\
0.1718 \\
0.1880 \\
0.1957 \\
0.2047 \\
0.2118 \\
0.2218 \\
0.2289 \\
0.2459 \\
0.2544 \\
0.2619 \\
0.2618 \\
0.2727 \\
0.2771 \\
0.2872 \\
0.3234 \\
0.5454 \\
0.8297 \\
0.8544 \\
0.9807 \\
1.0627 \\
1.1725 \\
1.2912 \\
1.3195 \\
0.9668\end{array}$ \\
\hline
\end{tabular}




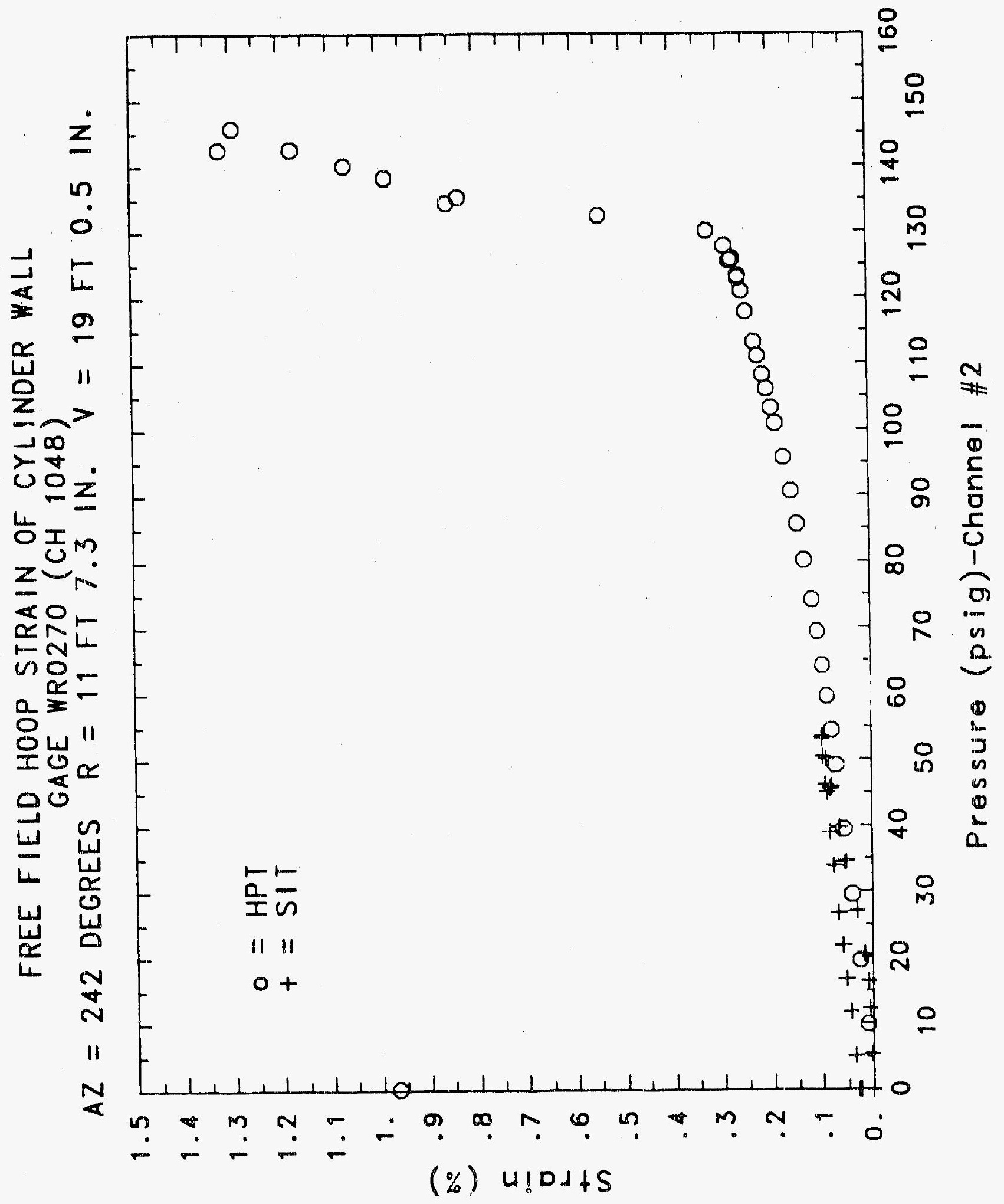




\section{Reinforced Concrete Test Data}

Weldable gage 271 Chanmel 1049

Structural. IntFgrity Test

High Pressure test

\begin{tabular}{|c|c|c|c|}
\hline $\begin{array}{c}\text { Pressure } \\
\text { (psig) } \\
-0.05 \\
5.33 \\
12.31 \\
16.44 \\
20.51 \\
20.46 \\
20.47 \\
27.05 \\
34.69 \\
34.53 \\
34.53 \\
39.70 \\
45.90 \\
45.65 \\
45.62 \\
50.10 \\
53.47 \\
53.29 \\
53.21 \\
50.49 \\
46.14 \\
46.13 \\
44.97 \\
38.98 \\
33.99 \\
33.96 \\
26.83 \\
21.88 \\
21.90 \\
16.69 \\
11.74 \\
11.77 \\
5.05 \\
0.02 \\
-0.04 \\
-0.02 \\
-0.02 \\
0.02 \\
\end{array}$ & $\begin{array}{l}\text { \% Strain } \\
0.0010 \\
0.0030 \\
0.0060 \\
0.0079 \\
0.0111 \\
0.0117 \\
0.0117 \\
0.0165 \\
0.0248 \\
0.0255 \\
0.0254 \\
0.0297 \\
0.0352 \\
0.0352 \\
0.0358 \\
0.0392 \\
0.0427 \\
0.0429 \\
0.0430 \\
0.0420 \\
0.0395 \\
0.0394 \\
0.0411 \\
0.0383 \\
0.0352 \\
0.0351 \\
0.0308 \\
0.0273 \\
0.0267 \\
0.0234 \\
0.0200 \\
0.0193 \\
0.0146 \\
0.0108 \\
0.0092 \\
0.0112 \\
0.0109 \\
0.0102\end{array}$ & $\begin{array}{c}\text { Pressure } \\
(\text { psig) } \\
9.89 \\
19.55 \\
29.57 \\
39.42 \\
49.16 \\
54.50 \\
59.57 \\
64.20 \\
69.32 \\
74.16 \\
80.16 \\
85.61 \\
90.58 \\
95.69 \\
100.92 \\
103.25 \\
106.11 \\
108.31 \\
111.08 \\
113.24 \\
117.83 \\
120.92 \\
123.28 \\
122.97 \\
125.82 \\
125.60 \\
127.84 \\
130.19 \\
132.53 \\
135.33 \\
134.42 \\
138.35 \\
140.16 \\
142.63 \\
145.78 \\
142.52 \\
0.22\end{array}$ & $\begin{array}{c}\text { * Strain } \\
0.0040 \\
0.0107 \\
0.0179 \\
0.0250 \\
0.0319 \\
0.0362 \\
0.0408 \\
0.0463 \\
0.0517 \\
0.0581 \\
0.0660 \\
0.0746 \\
0.0837 \\
0.0917 \\
0.0987 \\
0.1022 \\
0.1059 \\
0.1092 \\
0.1137 \\
0.1171 \\
0.1250 \\
0.1307 \\
0.1372 \\
0.1387 \\
0.1457 \\
0.1485 \\
0.1538 \\
0.1641 \\
0.1803 \\
0.2044 \\
0.2129 \\
0.2475 \\
0.2795 \\
0.3240 \\
0.3710 \\
0.3975 \\
0.2476\end{array}$ \\
\hline
\end{tabular}




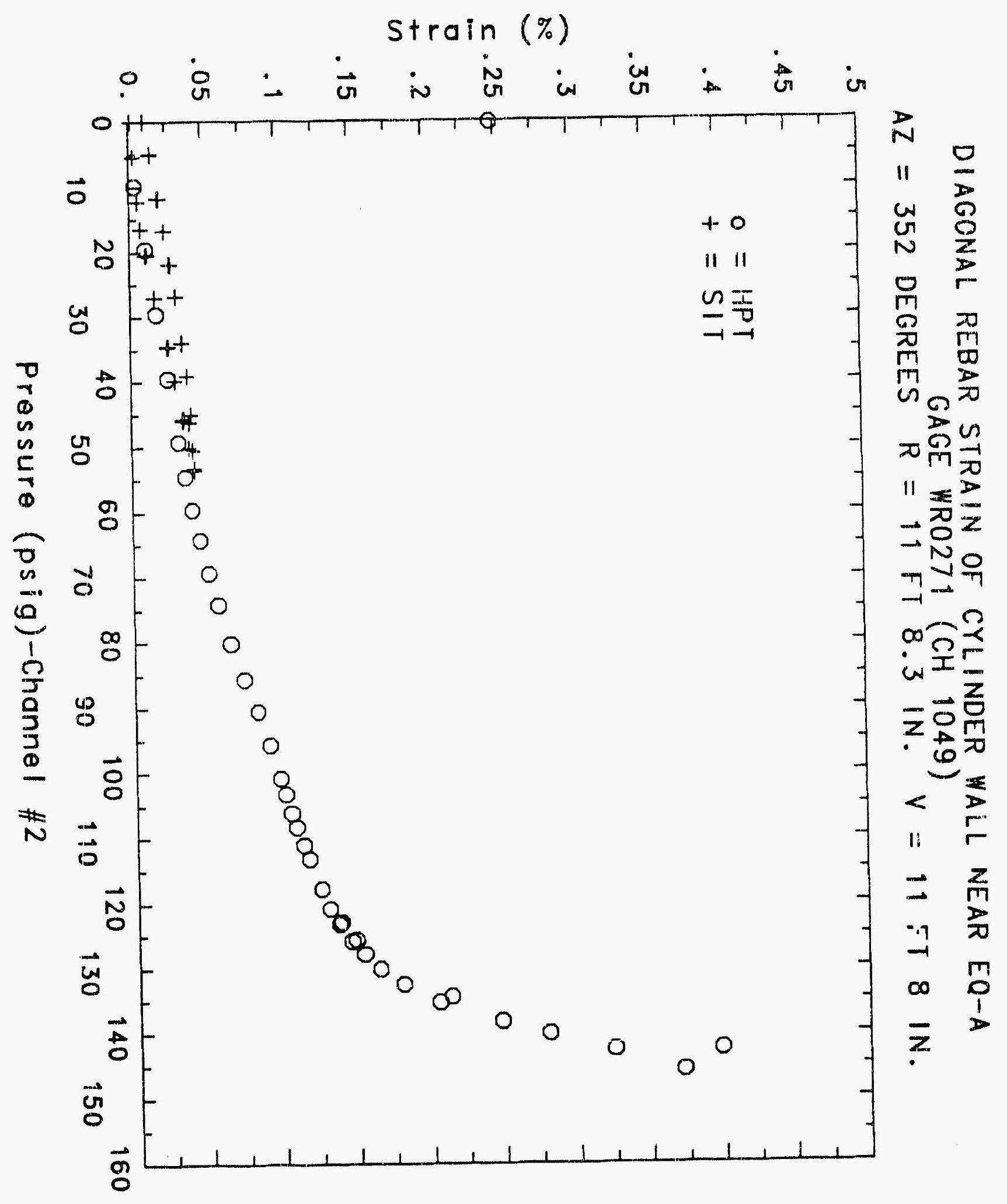




\section{Reinforced Concrete Test Data}

\begin{tabular}{|c|c|c|c|}
\hline STRUCTURAL & $\begin{array}{l}\text { WELDABLE GAGE } 272 \\
\text { INTEGRITY TEST }\end{array}$ & $\begin{aligned} & \text { Channel } 1060 \\
& \text { High Pressure }\end{aligned}$ & TEST \\
\hline $\begin{array}{c}\text { Pressure } \\
\text { (psig) } \\
-0.05 \\
5.33 \\
12.31 \\
16.44 \\
20.51 \\
20.46 \\
20.47 \\
27.05 \\
34.69 \\
34.53 \\
34.53 \\
39.70 \\
45.90 \\
45.65 \\
45.62 \\
50.10 \\
53.47 \\
53.29 \\
53.61 \\
50.49 \\
46.14 \\
46.13 \\
44.97 \\
38.98 \\
33.99 \\
33.96 \\
26.83 \\
21.88 \\
21.90 \\
16.69 \\
11.74 \\
11.77 \\
5.05 \\
0.02 \\
-0.04 \\
-0.02 \\
-0.02 \\
0.02 \\
\end{array}$ & $\begin{array}{l}\text { \% Strain } \\
0.0007 \\
0.0025 \\
0.0052 \\
0.0068 \\
0.0104 \\
0.0110 \\
0.0116 \\
0.0170 \\
0.0279 \\
0.0290 \\
0.0290 \\
0.0348 \\
0.0407 \\
0.0409 \\
0.0410 \\
0.0445 \\
0.0481 \\
0.0481 \\
0.0479 \\
0.0469 \\
0.0444 \\
0.0442 \\
0.0460 \\
0.0424 \\
0.0388 \\
0.0392 \\
0.0345 \\
0.0309 \\
0.0308 \\
0.0269 \\
0.0229 \\
0.0223 \\
0.0171 \\
0.0131 \\
0.0114 \\
0.0125 \\
0.0129 \\
0.0123\end{array}$ & $\begin{array}{c}\text { Pressure } \\
(\text { psig) } \\
9.89 \\
19.55 \\
29.57 \\
39.42 \\
49.16 \\
54.50 \\
59.57 \\
64.20 \\
69.32 \\
74.16 \\
80.16 \\
85.61 \\
90.58 \\
95.69 \\
100.92 \\
103.25 \\
106.11 \\
108.31 \\
111.08 \\
113.24 \\
117.83 \\
120.92 \\
123.28 \\
122.97 \\
125.82 \\
125.60 \\
127.84 \\
130.19 \\
132.53 \\
135.33 \\
134.42 \\
138.35 \\
140.16 \\
142.63 \\
145.78 \\
142.52 \\
0.22 \\
\end{array}$ & $\begin{array}{l}\text { \% Strain } \\
0.0040 \\
0.0116 \\
0.0194 \\
0.0259 \\
0.0326 \\
0.0365 \\
0.0400 \\
0.0441 \\
0.0488 \\
0.0544 \\
0.0605 \\
0.0678 \\
0.0751 \\
0.0844 \\
0.0980 \\
0.1039 \\
0.1102 \\
0.1153 \\
0.1228 \\
0.1286 \\
0.1411 \\
0.1510 \\
0.1608 \\
0.1636 \\
0.1739 \\
0.1784 \\
0.1845 \\
0.1963 \\
0.2092 \\
0.2325 \\
0.2400 \\
0.2672 \\
0.2945 \\
0.3362 \\
0.3831 \\
0.4032 \\
0.2437\end{array}$ \\
\hline
\end{tabular}




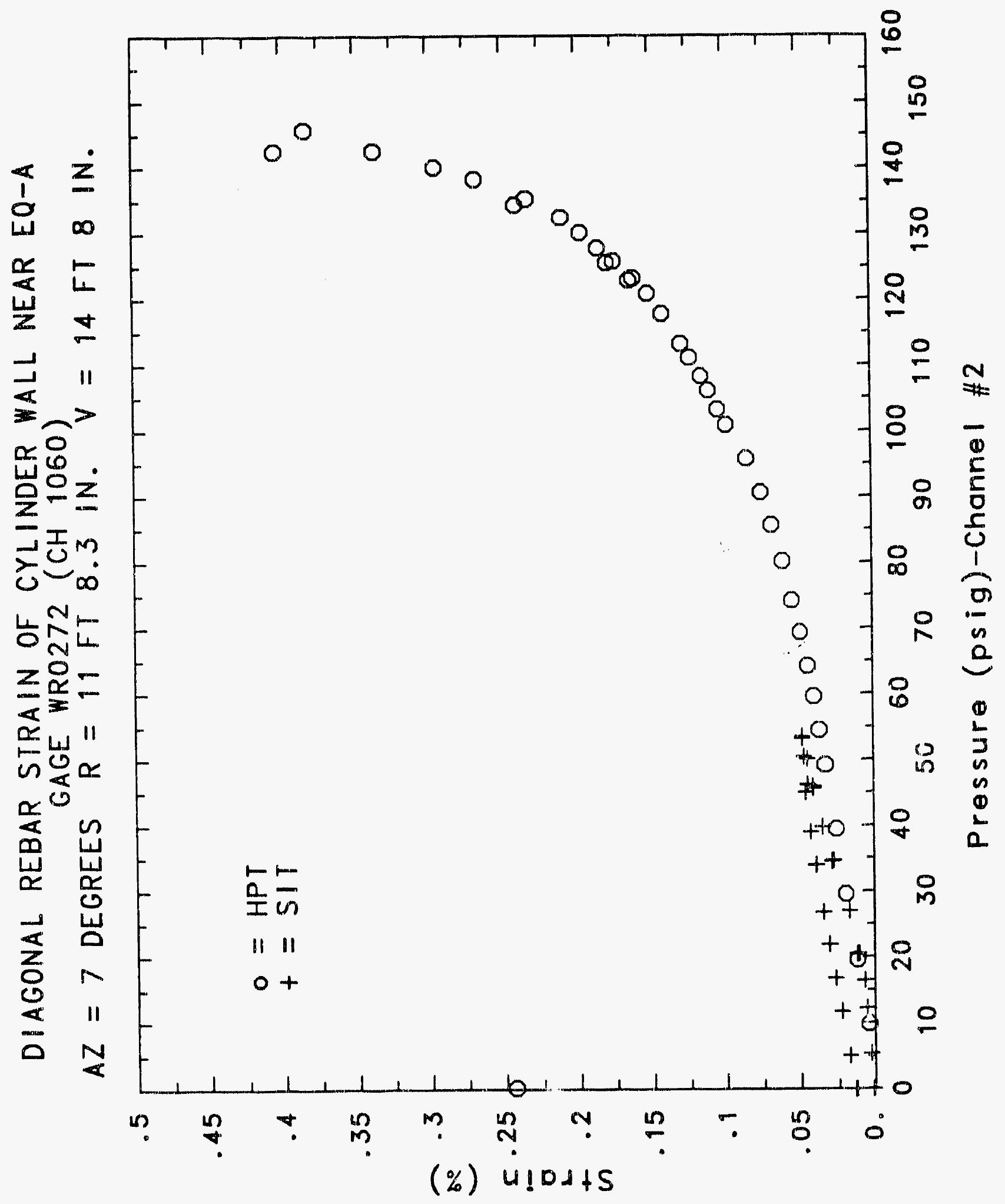




\section{Reinforced Concrete Test Data}

Weldable gage 273 Channel 1061

Structural Integrity Test

High Pressure Test

\begin{tabular}{cccc}
\hline Pressure & \% strain & $\begin{array}{c}\text { Pressure } \\
\text { (psig) }\end{array}$ & \% Strain \\
-0.05 & 0.0008 & 9.89 & 0.0046 \\
5.33 & 0.0024 & 19.55 & 0.0140 \\
12.31 & 0.0057 & 29.57 & 0.0241 \\
16.44 & 0.0079 & 39.42 & 0.0339 \\
20.51 & 0.0115 & 49.16 & 0.0436 \\
20.46 & 0.0124 & 54.50 & 0.0490 \\
20.47 & 0.0133 & 59.57 & 0.0547 \\
27.05 & 0.0222 & 64.20 & 0.0608 \\
34.69 & 0.0353 & 69.32 & 0.0680 \\
34.53 & 0.0372 & 74.16 & 0.0759 \\
34.53 & 0.0369 & 80.16 & 0.0869 \\
39.70 & 0.0427 & 85.61 & 0.0960 \\
45.90 & 0.0507 & 90.58 & 0.1041 \\
45.65 & 0.0515 & 95.69 & 0.1138 \\
45.62 & 0.0522 & 100.92 & 0.1255 \\
50.10 & 0.0569 & 103.25 & 0.1316 \\
53.47 & 0.0617 & 106.11 & 0.1380 \\
53.29 & 0.0623 & 108.31 & 0.1444 \\
53.21 & 0.0623 & 111.08 & 0.1550 \\
50.49 & 0.0611 & 113.24 & 0.1636 \\
46.14 & 0.0580 & 117.83 & 0.1845 \\
46.13 & 0.0581 & 120.92 & 0.1995 \\
44.97 & 0.0575 & 123.28 & 0.2109 \\
38.98 & 0.0530 & 122.97 & 0.2130 \\
33.99 & 0.0491 & 125.82 & 0.2238 \\
33.96 & 0.0488 & 125.60 & 0.2262 \\
26.83 & 0.0425 & 127.84 & 0.2328 \\
21.88 & 0.0379 & 130.19 & 0.2459 \\
21.90 & 0.0377 & 132.53 & 0.2569 \\
16.69 & 0.0327 & 135.33 & 0.2692 \\
11.74 & 0.0278 & 134.42 & 0.2708 \\
11.77 & 0.0266 & 138.35 & 0.2865 \\
5.05 & 0.0204 & 140.16 & 0.3132 \\
0.02 & 0.0153 & 142.63 & 0.3296 \\
-0.04 & 0.0139 & 145.78 & 0.1244 \\
-0.02 & 0.0135 & 142.52 & \\
-0.02 & 0.0137 & 0.22 & \\
0.02 & 0.0137 & & \\
\hline & & & \\
\hline
\end{tabular}




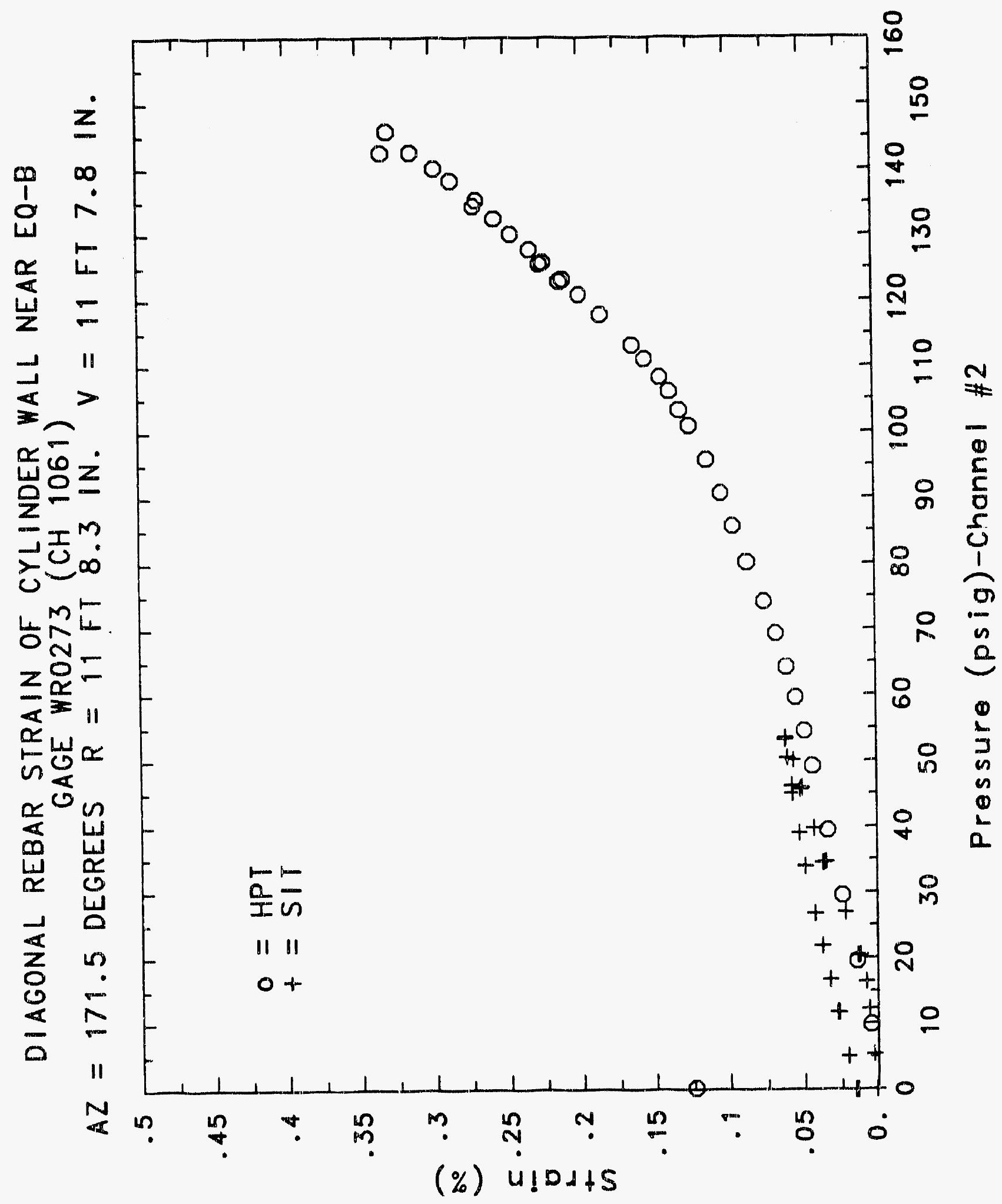




\section{Reinforced Concrete Test Data}

Weldable gage 274 Channel 1062

Structural. Integrity test

\begin{tabular}{|c|c|c|c|}
\hline $\begin{array}{c}\text { Pressure } \\
(\text { psig) } \\
-0.05 \\
5.33 \\
12.31 \\
16.44 \\
20.51 \\
20.46 \\
20.47 \\
27.05 \\
34.69 \\
34.53 \\
34.53 \\
39.70 \\
45.90 \\
45.65 \\
45.62 \\
50.10 \\
53.47 \\
53.29 \\
53.21 \\
50.49 \\
46.14 \\
46.13 \\
44.97 \\
38.98 \\
33.99 \\
33.96 \\
26.83 \\
21.88 \\
21.90 \\
16.69 \\
11.74 \\
11.77 \\
5.05 \\
0.02 \\
-0.04 \\
-0.02 \\
-0.02 \\
0.02\end{array}$ & $\begin{array}{l}\text { * Strain } \\
0.0005 \\
0.0015 \\
0.0033 \\
0.0047 \\
0.0073 \\
0.0084 \\
0.0092 \\
0.0218 \\
0.0383 \\
0.0402 \\
0.0405 \\
0.0468 \\
0.0573 \\
0.0587 \\
0.0590 \\
0.0652 \\
0.0708 \\
0.0717 \\
0.0723 \\
0.0711 \\
0.0681 \\
0.0680 \\
0.0684 \\
0.0638 \\
0.0600 \\
0.0594 \\
0.0532 \\
0.0481 \\
0.0481 \\
0.0430 \\
0.0379 \\
0.0363 \\
0.0301 \\
0.0248 \\
0.0227 \\
0.0220 \\
0.0221 \\
0.0223\end{array}$ & $\begin{array}{c}\text { Pressure } \\
(\text { psig) } \\
9.89 \\
19.55 \\
29.57 \\
39.42 \\
49.16 \\
54.50 \\
59.57 \\
64.20 \\
69.32 \\
74.16 \\
80.16 \\
85.61 \\
90.58 \\
95.69 \\
100.92 \\
103.25 \\
106.11 \\
108.31 \\
111.08 \\
113.24 \\
117.83 \\
120.92 \\
123.28 \\
122.97 \\
125.82 \\
125.60 \\
127.84 \\
130.19 \\
132.53 \\
135.33 \\
134.42 \\
138.35 \\
140.16 \\
142.63 \\
145.78 \\
142.52 \\
0.22 \\
\end{array}$ & $\begin{array}{l}\text { \% Strain } \\
0.0051 \\
0.0156 \\
0.0257 \\
0.0355 \\
0.0452 \\
0.0509 \\
0.0568 \\
0.0625 \\
0.0688 \\
0.0755 \\
0.0854 \\
0.0936 \\
0.1019 \\
0.1116 \\
0.1218 \\
0.1270 \\
0.1323 \\
0.1364 \\
0.1423 \\
0.1467 \\
0.1567 \\
0.1642 \\
0.1722 \\
0.1745 \\
0.1818 \\
0.1842 \\
0.1882 \\
0.1960 \\
0.2036 \\
0.2140 \\
0.2165 \\
0.2267 \\
0.2347 \\
0.2457 \\
0.2592 \\
0.2626 \\
0.0963\end{array}$ \\
\hline
\end{tabular}

High Pressure test

\% Strain

(psig)

19.55

49.16

54.50

69.32

74.16

85.61

90.58

103.25

113.24

117.83

122.97

125.82

132.53

135.33

134.42

145.78

2.52

0.2626

0.0221

0.0223 


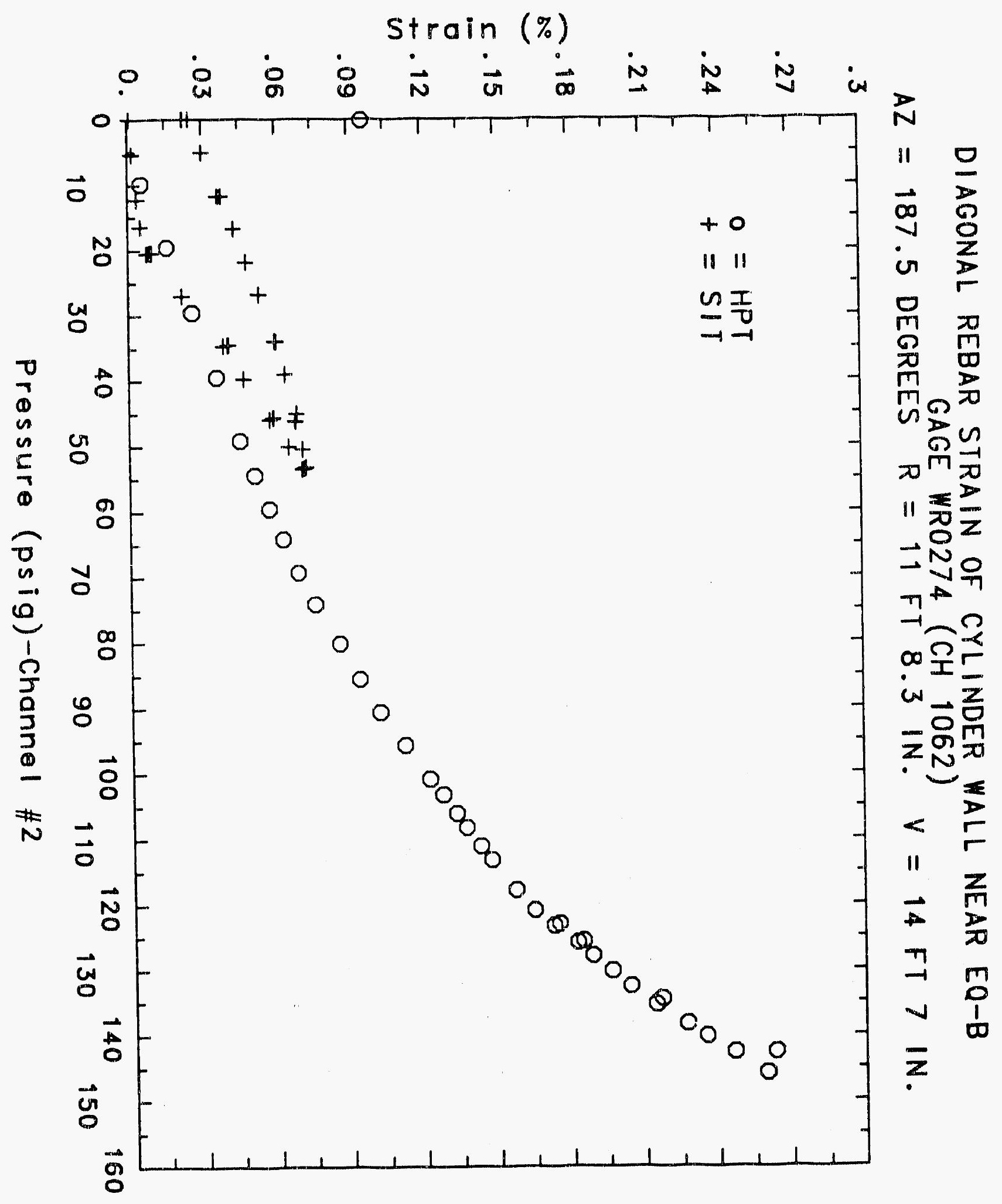




\section{Reinforced Concrete Test Data}

Weldable Gage 275 Channel 1063

StRUCTURAL InTEgrity TEST

\begin{tabular}{|c|c|c|c|}
\hline $\begin{array}{c}\text { Pressure } \\
\text { (psig) } \\
-0.05 \\
5.33 \\
12.31 \\
16.44 \\
20.51 \\
20.46 \\
20.47 \\
27.05 \\
34.69 \\
34.53 \\
34.53 \\
39.70 \\
45.90 \\
45.65 \\
45.62 \\
50.10 \\
53.47 \\
53.29 \\
53.21 \\
50.49 \\
46.14 \\
46.13 \\
44.97 \\
38.98 \\
33.99 \\
33.96 \\
26.83 \\
21.88 \\
21.90 \\
16.69 \\
11.74 \\
11.77 \\
5.05 \\
0.02 \\
-0.04 \\
-0.02 \\
-0.02 \\
0.02\end{array}$ & $\begin{array}{l}\text { x Strain } \\
0.0012 \\
0.0018 \\
0.0034 \\
0.0042 \\
0.0062 \\
0.0068 \\
0.0077 \\
0.0118 \\
0.0161 \\
0.0164 \\
0.0163 \\
0.0184 \\
0.0213 \\
0.0207 \\
0.0205 \\
0.0226 \\
0.0237 \\
0.0235 \\
0.0235 \\
0.0226 \\
0.0215 \\
0.0212 \\
0.0218 \\
0.0201 \\
0.0187 \\
0.0187 \\
0.0169 \\
0.0151 \\
0.0152 \\
0.0131 \\
0.0108 \\
0.0100 \\
0.0074 \\
0.0053 \\
0.0056 \\
0.0058 \\
0.0061 \\
0.0066\end{array}$ & $\begin{array}{c}\text { Pressure } \\
\text { (psig) } \\
9.89 \\
19.55 \\
29.57 \\
39.42 \\
49.16 \\
54.50 \\
59.57 \\
64.20 \\
69.32 \\
74.16 \\
80.16 \\
85.61 \\
90.58 \\
95.69 \\
100.92 \\
103.25 \\
106.11 \\
108.31 \\
111.08 \\
113.24 \\
117.83 \\
120.92 \\
123.28 \\
122.97 \\
125.82 \\
125.60 \\
127.84 \\
130.19 \\
132.53 \\
135.33 \\
134.42 \\
138.35 \\
140.16 \\
142.63 \\
145.78 \\
142.52 \\
0.22\end{array}$ & $\begin{array}{l}\text { X Strain } \\
0.0002 \\
0.0037 \\
0.0070 \\
0.0099 \\
0.0136 \\
0.0147 \\
0.0169 \\
0.0190 \\
0.0232 \\
0.0273 \\
0.0305 \\
0.0357 \\
0.0402 \\
0.0455 \\
0.0514 \\
0.0537 \\
0.0572 \\
0.0599 \\
0.0637 \\
0.0668 \\
0.0748 \\
0.0789 \\
0.0826 \\
0.0828 \\
0.0874 \\
0.0873 \\
0.0909 \\
0.0954 \\
0.1002 \\
0.1079 \\
0.1072 \\
0.1190 \\
0.1259 \\
0.1362 \\
0.1456 \\
0.1425 \\
0.0428\end{array}$ \\
\hline
\end{tabular}

High Pressure Test

ressure

9.89

29.57

39.42

49.16

64.20

74.16

80.16

85.61

100.92

106.11

108.31

117.83

122.97

125.82

0.0873

0.0909

0.1079

0.1072

0.1190

0.1456

.1425

0.0061

42.52 


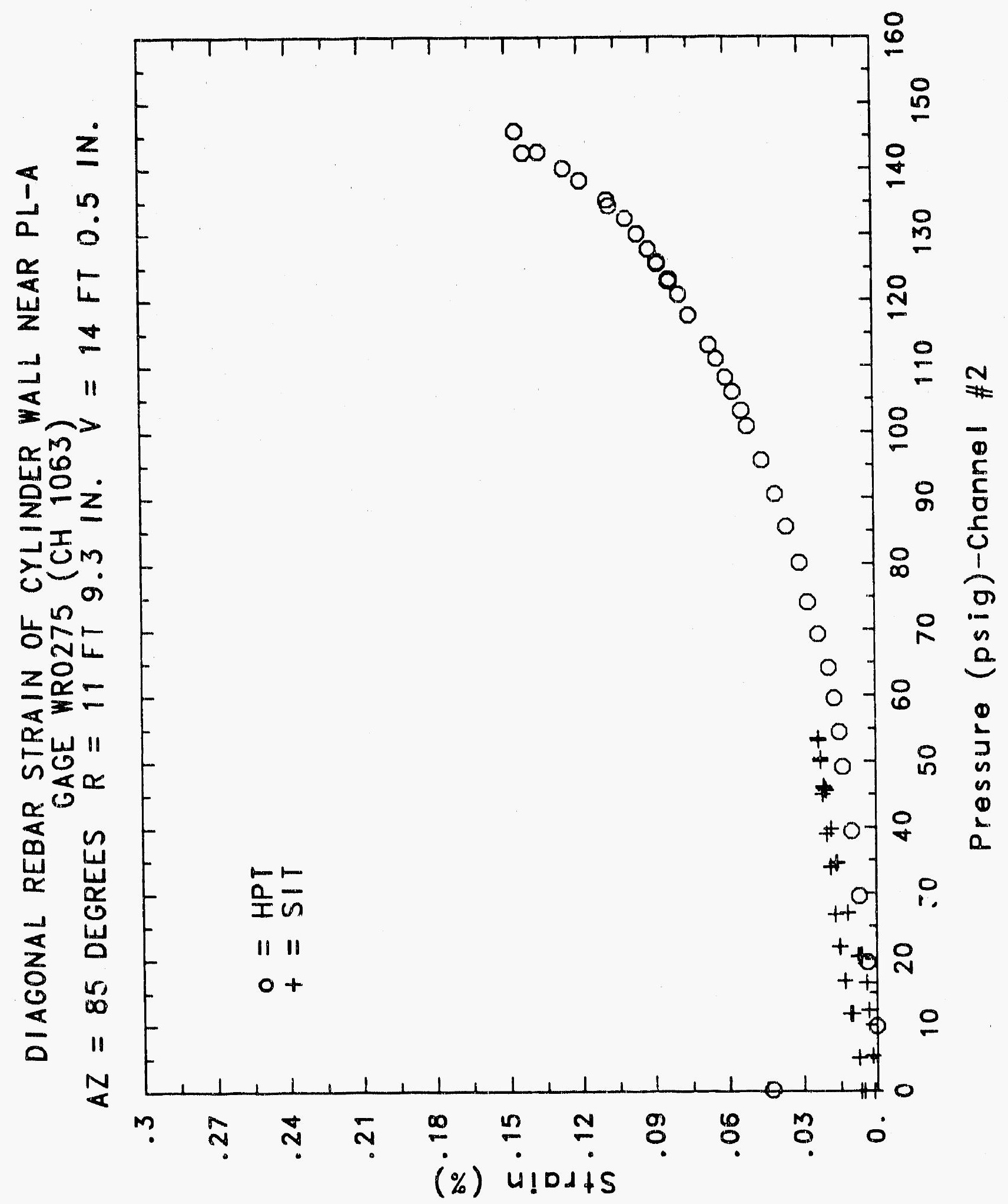




\section{Reinforced Concrete Test Data}

Weldable gage 276 Channel 1064

STRUCTURAL INTEGRITY TEST

\begin{tabular}{|c|c|c|c|}
\hline $\begin{array}{c}\text { Pressure } \\
\text { (psig) } \\
-0.05 \\
5.33 \\
12.31 \\
16.44 \\
20.51 \\
20.46 \\
20.47 \\
27.05 \\
34.69 \\
34.53 \\
34.53 \\
39.70 \\
45.90 \\
45.65 \\
45.62 \\
50.10 \\
53.47 \\
53.29 \\
53.21 \\
50.49 \\
46.14 \\
46.13 \\
44.97 \\
38.98 \\
33.99 \\
33.96 \\
26.83 \\
21.88 \\
21.90 \\
16.69 \\
11.74 \\
11.77 \\
5.05 \\
0.02 \\
-0.04 \\
-0.02 \\
-0.02 \\
0.02 \\
\end{array}$ & $\begin{array}{c}\text { \% Strain } \\
\text {-0.0008 } \\
0.0011 \\
0.0034 \\
0.0050 \\
0.0081 \\
0.0084 \\
0.0087 \\
0.0137 \\
0.0193 \\
0.0199 \\
0.0200 \\
0.0230 \\
0.0265 \\
0.0269 \\
0.0270 \\
0.0294 \\
0.0314 \\
0.0316 \\
0.0317 \\
0.0307 \\
0.0293 \\
0.0290 \\
0.0292 \\
0.0271 \\
0.0250 \\
0.0250 \\
0.0220 \\
0.0196 \\
0.0201 \\
0.0174 \\
0.0150 \\
0.0144 \\
0.0113 \\
0.0085 \\
0.0088 \\
0.0083 \\
0.0076 \\
0.0080\end{array}$ & $\begin{array}{c}\text { Pressure } \\
\text { (psig) } \\
9.89 \\
19.55 \\
29.57 \\
39.42 \\
49.16 \\
54.50 \\
59.57 \\
64.20 \\
69.32 \\
74.16 \\
80.16 \\
85.61 \\
90.58 \\
95.69 \\
100.92 \\
103.25 \\
106.11 \\
108.31 \\
111.08 \\
113.24 \\
117.83 \\
120.92 \\
123.28 \\
122.97 \\
125.82 \\
125.60 \\
127.84 \\
130.19 \\
132.53 \\
135.33 \\
134.42 \\
138.35 \\
140.16 \\
142.63 \\
145.78 \\
142.52 \\
0.22\end{array}$ & $\begin{array}{l}\text { X Strain } \\
0.0005 \\
0.0054 \\
0.0104 \\
0.0150 \\
0.0197 \\
0.0223 \\
0.0247 \\
0.0281 \\
0.0319 \\
0.0355 \\
0.0393 \\
0.0415 \\
0.0442 \\
0.0459 \\
0.0487 \\
0.0493 \\
0.0505 \\
0.0514 \\
0.0524 \\
0.0531 \\
0.0551 \\
0.0562 \\
0.0585 \\
0.0593 \\
0.0623 \\
0.0625 \\
0.0641 \\
0.0673 \\
0.0723 \\
0.0799 \\
0.0817 \\
0.0926 \\
0.1024 \\
0.1164 \\
0.1344 \\
0.1373 \\
0.0506\end{array}$ \\
\hline
\end{tabular}

High Pressure test

Pressure

0.0005

0.0104

0.0247

0.0281

0.0319

0.0415

0.0442

0.0493

0.0505

0.0524

0.0531

0.0551

0.0593

0.0673

0.0723

0.0817

0.0926

0.1024

0.1164

0.1344

0.0506 


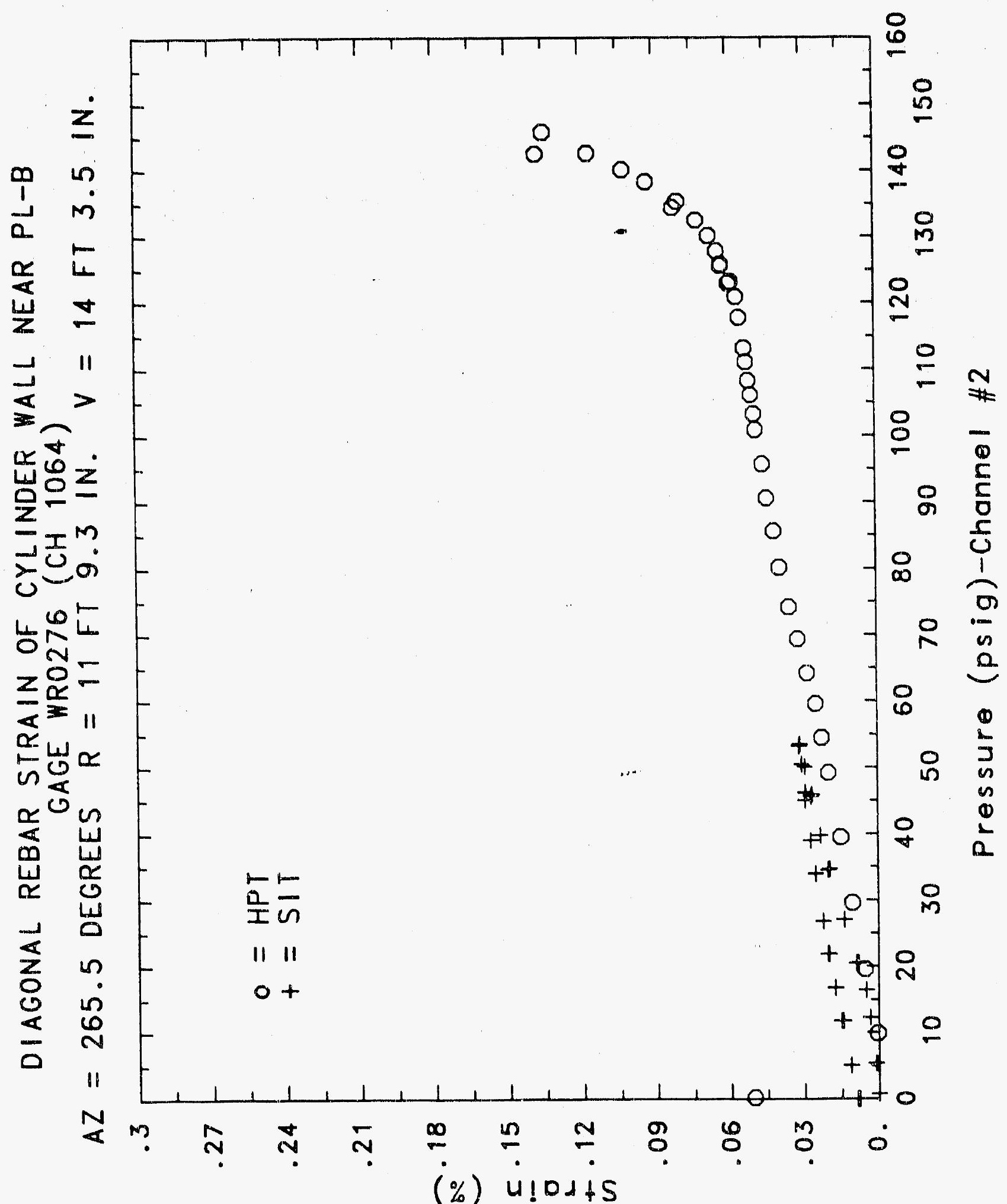




\section{Reinforced Concrete Test Data}

Weldable Gage 277 Chanmel 1065

Structural Integrity test

\begin{tabular}{|c|c|c|c|}
\hline $\begin{array}{c}\text { Pressure } \\
\text { (psig) } \\
-0.05 \\
5.33 \\
12.31 \\
16.44 \\
20.51 \\
20.46 \\
20.47 \\
27.05 \\
34.69 \\
34.53 \\
34.53 \\
39.70 \\
45.90 \\
45.65 \\
45.62 \\
50.10 \\
53.47 \\
53.29 \\
53.21 \\
50.49 \\
46.14 \\
46.13 \\
44.97 \\
38.98 \\
33.99 \\
33.96 \\
26.83 \\
21.88 \\
21.90 \\
16.69 \\
11.74 \\
11.77 \\
5.05 \\
0.02 \\
-0.04 \\
-0.02 \\
-0.02 \\
0.02 \\
\end{array}$ & $\begin{array}{c}\text { \% Strain } \\
-0.0001 \\
0.0012 \\
0.0035 \\
0.0049 \\
0.0074 \\
0.0074 \\
0.0071 \\
0.0156 \\
0.0272 \\
0.0282 \\
0.0286 \\
0.0348 \\
0.0453 \\
0.0477 \\
0.0490 \\
0.0549 \\
0.0603 \\
0.0614 \\
0.0623 \\
0.0610 \\
0.0586 \\
0.0582 \\
0.0551 \\
0.0511 \\
0.0468 \\
0.0458 \\
0.0394 \\
0.0351 \\
0.0351 \\
0.0309 \\
0.0264 \\
0.0265 \\
0.0221 \\
0.0179 \\
0.0172 \\
0.0164 \\
0.0155 \\
0.0150\end{array}$ & $\begin{array}{c}\text { Pressure } \\
(\text { psig) } \\
9.89 \\
19.55 \\
29.57 \\
39.42 \\
49.16 \\
54.50 \\
59.57 \\
64.20 \\
69.32 \\
74.16 \\
80.16 \\
85.61 \\
90.58 \\
95.69 \\
100.92 \\
103.25 \\
106.11 \\
108.31 \\
111.08 \\
113.24 \\
117.83 \\
120.92 \\
123.28 \\
122.97 \\
125.82 \\
125.60 \\
127.84 \\
130.19 \\
132.53 \\
135.33 \\
134.42 \\
138.35 \\
140.16 \\
142.63 \\
145.78 \\
142.52 \\
0.22 \\
\end{array}$ & $\begin{array}{l}\text { X Strain } \\
0.0043 \\
0.0133 \\
0.0234 \\
0.0327 \\
0.0421 \\
0.0476 \\
0.0535 \\
0.0596 \\
0.0670 \\
0.0754 \\
0.0920 \\
0.1037 \\
0.1149 \\
0.1271 \\
0.1404 \\
0.1473 \\
0.1553 \\
0.1612 \\
0.1704 \\
0.1774 \\
0.1959 \\
0.2130 \\
0.2292 \\
0.2324 \\
0.2556 \\
0.2616 \\
0.2790 \\
0.3600 \\
0.5015 \\
0.5681 \\
0.5916 \\
0.6699 \\
0.7205 \\
0.8152 \\
0.8855 \\
0.9510 \\
0.6889\end{array}$ \\
\hline
\end{tabular}

High Pressure test 


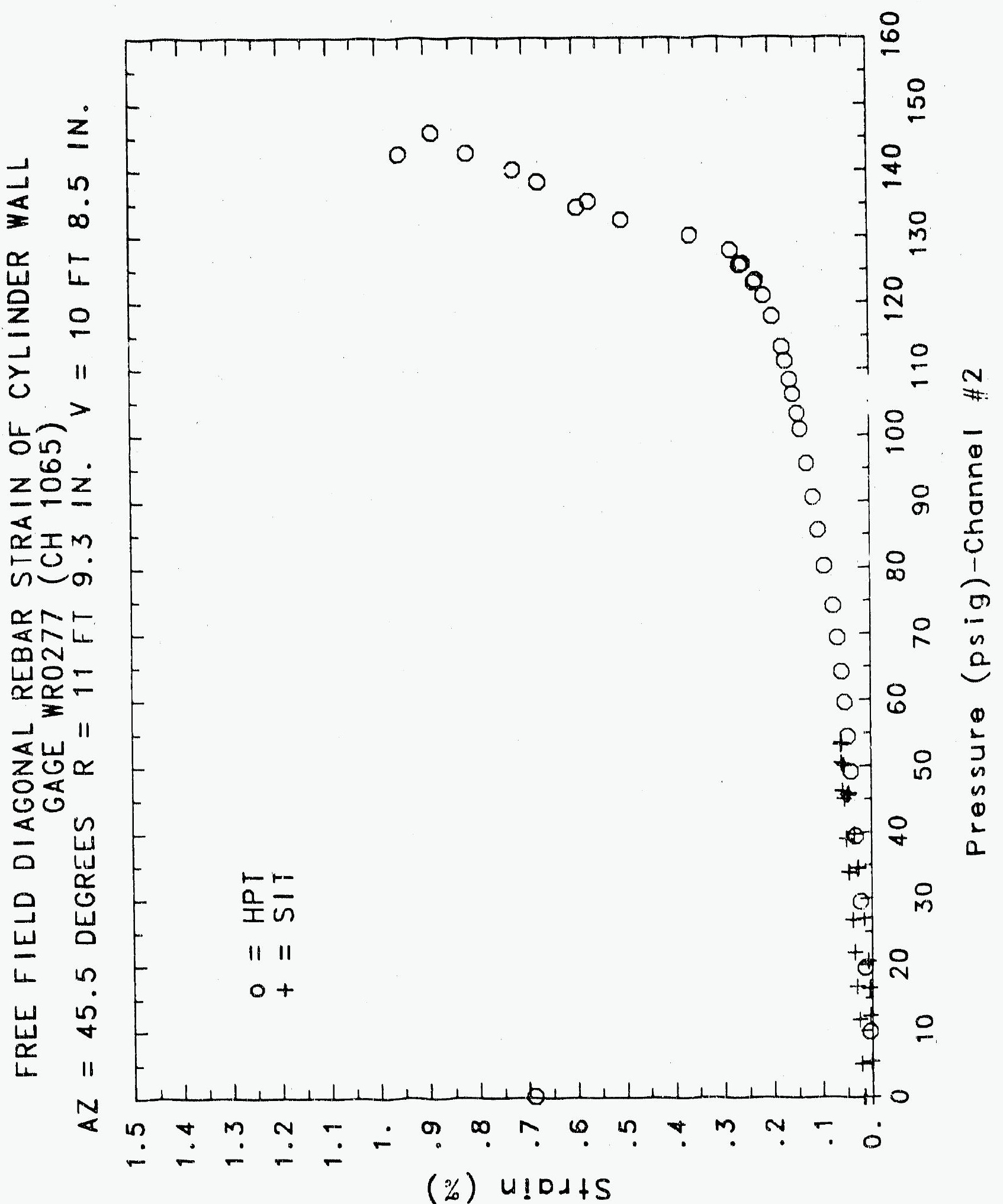

C -1.361 


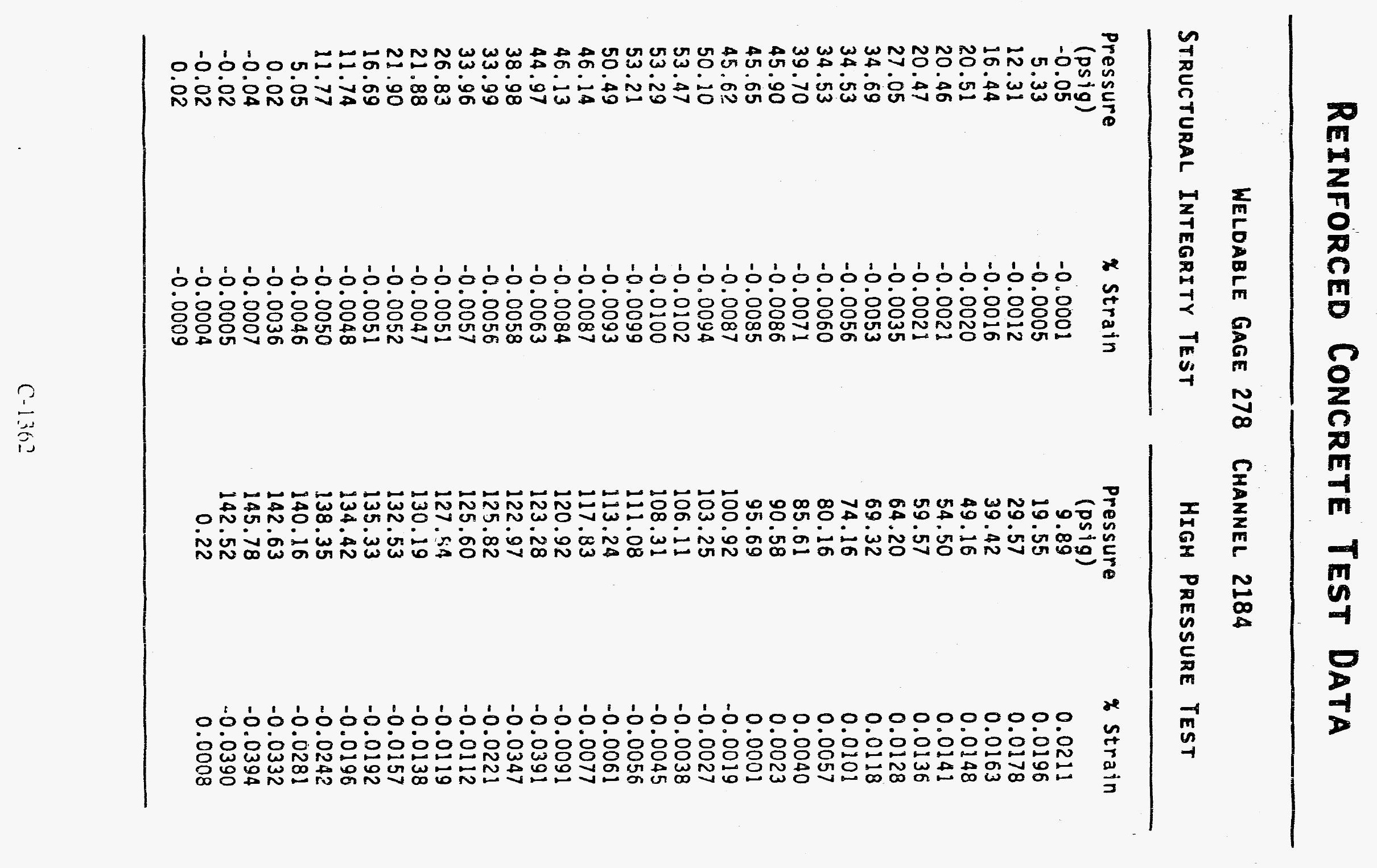




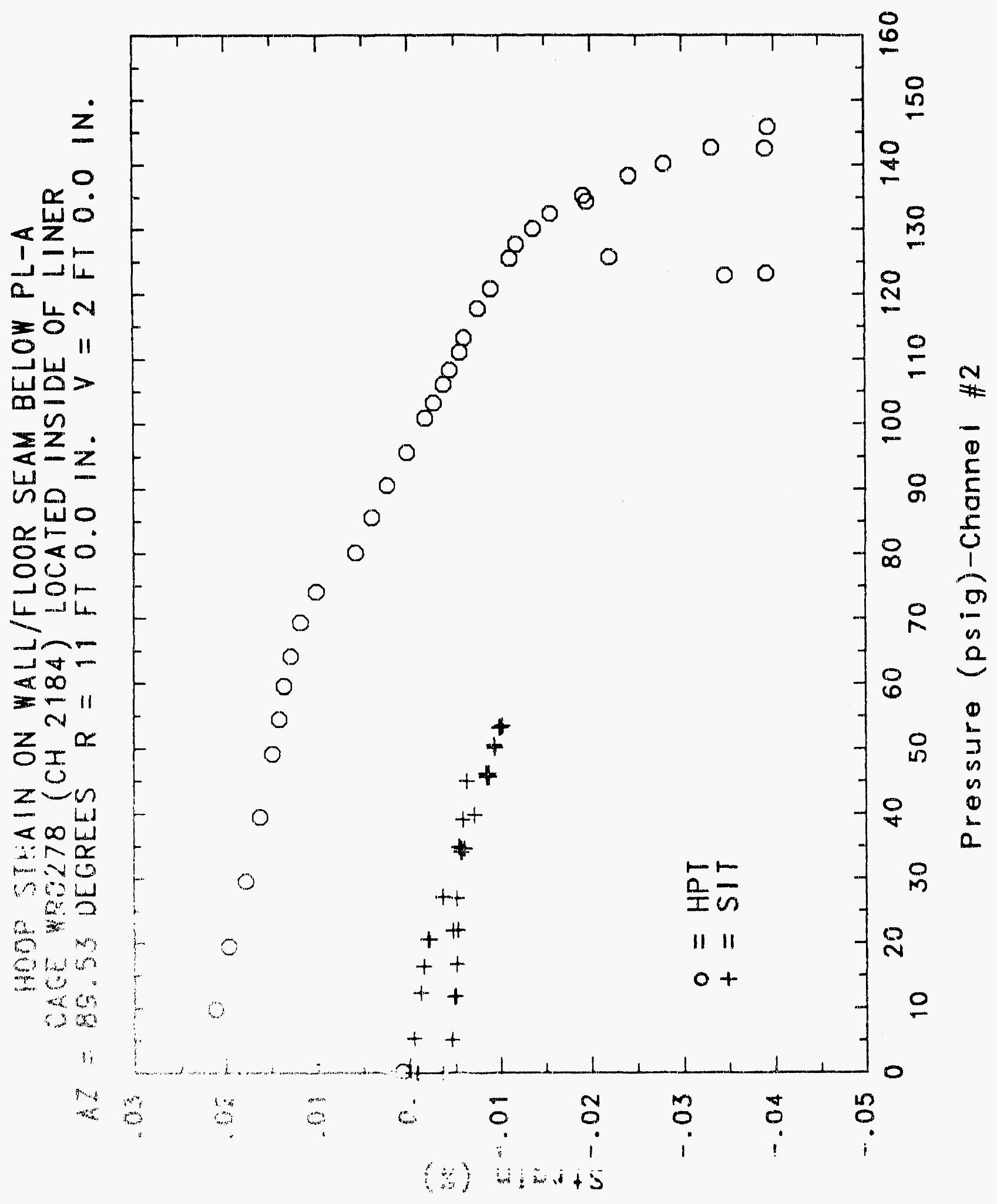




\section{ReInforced Concrete Test Data}

Weldable gage 279 Chamnel 2185

Structural Integrity Test

High Pressure test

\begin{tabular}{|c|c|c|c|}
\hline $\begin{array}{c}\text { Pressure } \\
\text { (psig) } \\
-0.05 \\
5.33 \\
12.31 \\
16.44 \\
20.51 \\
20.46 \\
20.47 \\
27.05 \\
34.69 \\
34.53 \\
34.53 \\
39.70 \\
45.90 \\
45.65 \\
45.62 \\
50.10 \\
53.47 \\
53.29 \\
53.21 \\
50.49 \\
46.14 \\
46.13 \\
44.97 \\
38.98 \\
33.99 \\
33.96 \\
26.83 \\
21.88 \\
21.90 \\
16.69 \\
11.74 \\
11.77 \\
5.05 \\
0.09 \\
-0.04 \\
-0.02 \\
-0.02 \\
0.02\end{array}$ & $\begin{array}{c}\text { \% Strain } \\
0.0003 \\
0.0010 \\
0.0017 \\
0.0017 \\
0.0011 \\
0.0007 \\
0.0001 \\
-0.0011 \\
0.0002 \\
-0.0001 \\
-0.0001 \\
0.0003 \\
0.0006 \\
0.0002 \\
0.0001 \\
0.0007 \\
0.0012 \\
0.0014 \\
0.0017 \\
0.0018 \\
0.0014 \\
0.0017 \\
0.0035 \\
0.0024 \\
0.0016 \\
0.0014 \\
0.0004 \\
-0.0005 \\
-0.0013 \\
-0.0020 \\
-0.0026 \\
-0.0029 \\
-0.0027 \\
-0.0021 \\
0.0017 \\
0.0018 \\
0.0019 \\
0.0014\end{array}$ & $\begin{array}{c}\text { Pressure } \\
(\text { psig) } \\
9.89 \\
19.55 \\
29.57 \\
39.42 \\
49.16 \\
54.50 \\
59.57 \\
64.20 \\
69.32 \\
74.16 \\
80.16 \\
85.61 \\
90.58 \\
95.69 \\
100.92 \\
103.25 \\
106.11 \\
108.31 \\
111.08 \\
113.24 \\
117.83 \\
120.92 \\
123.28 \\
122.97 \\
125.82 \\
125.60 \\
127.84 \\
130.19 \\
132.53 \\
135.33 \\
134.42 \\
138.35 \\
140.16 \\
142.63 \\
145.78 \\
142.52 \\
0.22\end{array}$ & $\begin{array}{l}\text { \% Strain } \\
-0.0047 \\
-0.0056 \\
-0.0051 \\
-0.0043 \\
-0.0033 \\
-0.0024 \\
-0.0020 \\
-0.0015 \\
-0.0012 \\
-0.0020 \\
-0.0030 \\
-0.0024 \\
-0.0016 \\
-0.0009 \\
-0.0018 \\
-0.0019 \\
-0.0017 \\
-0.0017 \\
-0.0012 \\
-0.0009 \\
-0.0007 \\
0.0005 \\
0.0006 \\
0.0009 \\
0.0008 \\
0.0009 \\
0.0006 \\
0.0003 \\
-0.0007 \\
-0.0011 \\
-0.0012 \\
0.0020 \\
-0.0038 \\
-0.0065 \\
-0.0107 \\
-0.0112 \\
-0.0018\end{array}$ \\
\hline
\end{tabular}




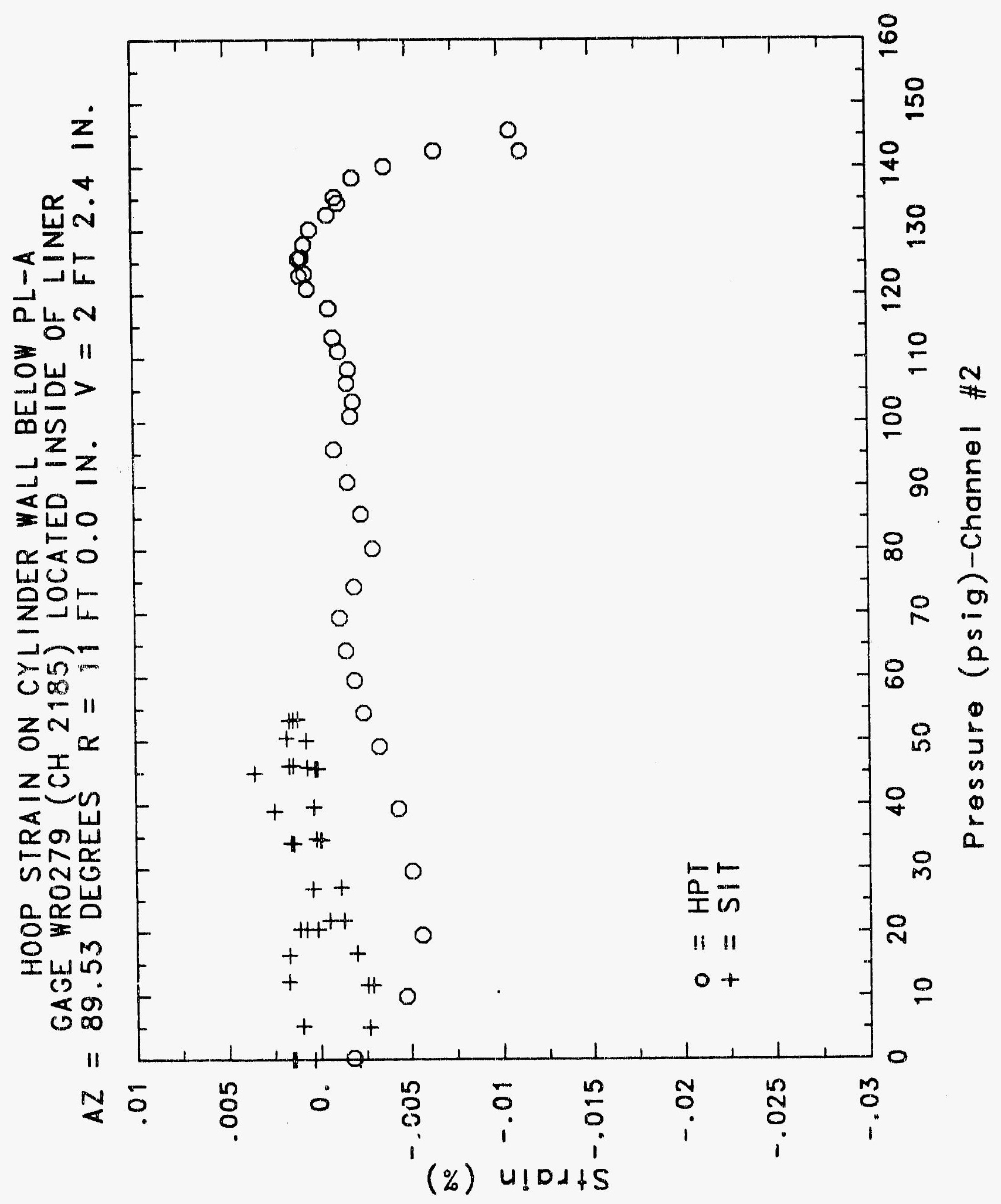




\section{Reinforced Concrete Test Data}

Weldable gage 280 Channel 2186

StRUCTURAL INTEGRITY TEST

\begin{tabular}{|c|c|c|c|}
\hline $\begin{array}{c}\text { Pressure } \\
\text { (psig) } \\
-0.05 \\
5.33 \\
12.31 \\
16.44 \\
20.51 \\
20.46 \\
20.47 \\
27.05 \\
34.69 \\
34.53 \\
34.53 \\
39.70 \\
45.90 \\
45.65 \\
45.62 \\
50.10 \\
53.47 \\
53.29 \\
53.21 \\
50.49 \\
46.14 \\
46.13 \\
44.97 \\
38.98 \\
33.99 \\
33.96 \\
26.83 \\
21.88 \\
21.90 \\
16.69 \\
11.74 \\
11.77 \\
5.05 \\
0.02 \\
-0.04 \\
-0.02 \\
-0.02 \\
0.02 \\
\end{array}$ & $\begin{array}{l}\text { \% Strain } \\
0.0004 \\
0.0005 \\
0.0014 \\
0.0017 \\
0.0023 \\
0.0020 \\
0.0021 \\
0.0015 \\
0.0019 \\
0.0015 \\
0.0013 \\
0.0013 \\
0.0013 \\
0.0010 \\
0.0012 \\
0.0019 \\
0.0027 \\
0.0031 \\
0.0032 \\
0.0035 \\
0.0032 \\
0.0036 \\
0.0057 \\
0.0046 \\
0.0038 \\
0.0037 \\
0.0023 \\
0.0015 \\
0.0004 \\
-0.0009 \\
-0.0016 \\
-0.0023 \\
-0.0027 \\
-0.0030 \\
0.0011 \\
0.0013 \\
0.0013 \\
0.0006\end{array}$ & $\begin{array}{c}\text { Pressure } \\
\text { (psig) } \\
9.89 \\
19.55 \\
29.57 \\
39.42 \\
49.16 \\
54.50 \\
59.57 \\
64.20 \\
69.32 \\
74.16 \\
80.16 \\
85.61 \\
90.58 \\
95.69 \\
100.92 \\
103.25 \\
106.11 \\
108.31 \\
111.08 \\
113.24 \\
117.83 \\
120.92 \\
123.28 \\
122.97 \\
125.82 \\
125.60 \\
127.84 \\
130.19 \\
132.53 \\
135.33 \\
134.42 \\
138.35 \\
140.16 \\
142.63 \\
145.78 \\
142.52 \\
0.22 \\
\end{array}$ & $\begin{array}{l}\text { \% Strain } \\
-0.0037 \\
-0.0034 \\
-0.0027 \\
-0.0017 \\
-0.0006 \\
0.0002 \\
0.0009 \\
0.0022 \\
0.0031 \\
0.0036 \\
0.0037 \\
0.0047 \\
0.0060 \\
0.0071 \\
0.0088 \\
0.0098 \\
0.0109 \\
0.0115 \\
0.0131 \\
0.0141 \\
0.0165 \\
0.0180 \\
0.0196 \\
0.0197 \\
0.0211 \\
0.0216 \\
0.0223 \\
0.0241 \\
0.0266 \\
0.0296 \\
0.0301 \\
0.0354 \\
0.0388 \\
0.0448 \\
0.0505 \\
0.0504 \\
-0.0028\end{array}$ \\
\hline
\end{tabular}

High Pressure test

Press

8.89

19.55

49.16

54.50

64.20

69.32

85.61

100.92

103.25

113.24

117.83

120.92

123.28

122.97

125.82

25.60

127.84

130.19

134.42

138.35

140.16

142.63

45.78

42.52

0.0504

0.0028 


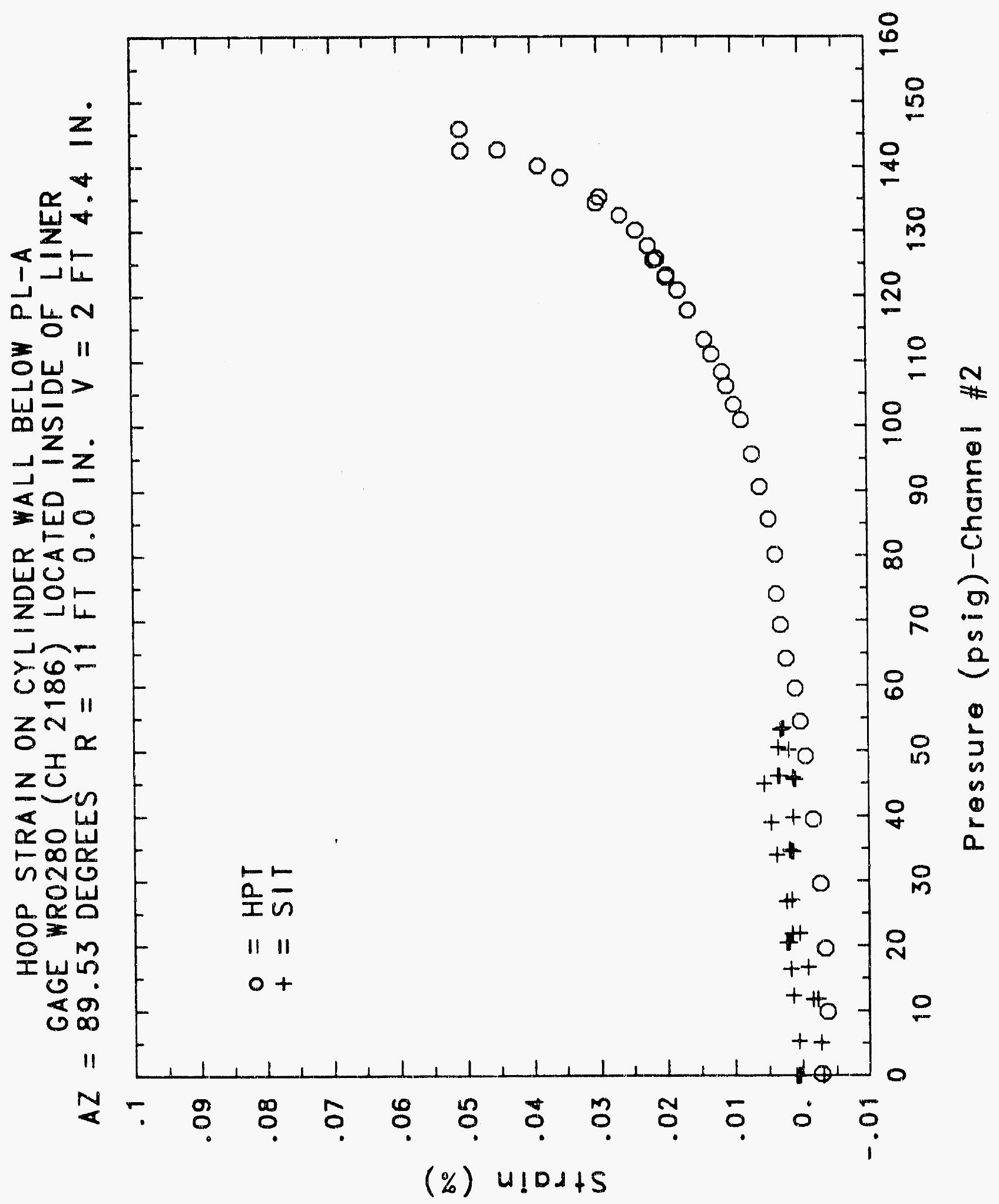


Reinforced Concrete Test Data

Weldable Gage 281 Channel 2187

Structural. Integrity Test

\begin{tabular}{|c|c|c|c|}
\hline $\begin{array}{c}\text { Pressure } \\
\text { (psig) } \\
-0.05 \\
5.33 \\
12.31 \\
16.44 \\
20.51 \\
20.46 \\
20.47 \\
27.05 \\
34.69 \\
34.53 \\
34.53 \\
39.70 \\
45.90 \\
45.65 \\
45.62 \\
50.10 \\
53.47 \\
53.29 \\
53.21 \\
50.49 \\
46.14 \\
46.13 \\
44.97 \\
38.98 \\
33.99 \\
33.96 \\
26.83 \\
21.88 \\
21.90 \\
16.69 \\
11.74 \\
11.77 \\
5.05 \\
0.02 \\
-0.04 \\
-0.02 \\
-0.02 \\
0.02 \\
\end{array}$ & $\begin{array}{c}\text { \% Strain } \\
0.0000 \\
0.0052 \\
0.0146 \\
0.0221 \\
0.0312 \\
0.0334 \\
0.0350 \\
0.0454 \\
0.0561 \\
0.0565 \\
0.0566 \\
0.0612 \\
0.0672 \\
0.0668 \\
0.0664 \\
0.0721 \\
0.0761 \\
0.0749 \\
0.0747 \\
0.0722 \\
0.0670 \\
0.0655 \\
0.0681 \\
0.0619 \\
0.0567 \\
0.0573 \\
0.0487 \\
0.0433 \\
0.0442 \\
0.0368 \\
0.0280 \\
0.0265 \\
0.0140 \\
0.0033 \\
-0.0027 \\
0.0000 \\
0.0013 \\
0.0046\end{array}$ & $\begin{array}{c}\text { Pressure } \\
(\text { psig) } \\
9.89 \\
19.55 \\
29.57 \\
39.42 \\
49.16 \\
54.50 \\
59.57 \\
64.20 \\
69.32 \\
74.16 \\
80.16 \\
85.61 \\
90.58 \\
95.69 \\
100.92 \\
103.25 \\
106.11 \\
108.31 \\
111.08 \\
113.24 \\
117.83 \\
120.92 \\
123.28 \\
122.97 \\
125.82 \\
125.60 \\
127.84 \\
130.19 \\
132.53 \\
135.33 \\
134.42 \\
138.35 \\
140.16 \\
142.63 \\
145.78 \\
142.52 \\
0.22\end{array}$ & $\begin{array}{l}\text { \% Strain } \\
0.0120 \\
0.0300 \\
0.0439 \\
0.0557 \\
0.0674 \\
0.0744 \\
0.0806 \\
0.0894 \\
0.0963 \\
0.1061 \\
0.1126 \\
0.1216 \\
0.1302 \\
0.1387 \\
0.1445 \\
0.1477 \\
0.1517 \\
0.1553 \\
0.1599 \\
0.1641 \\
0.1730 \\
0.1791 \\
0.1852 \\
0.1863 \\
0.1928 \\
0.1947 \\
0.2000 \\
0.2071 \\
0.2160 \\
0.12887 \\
0.2313 \\
0.2474 \\
0.2578 \\
0.2727 \\
0.2919 \\
0.2763 \\
0.0506\end{array}$ \\
\hline
\end{tabular}

High Pressure test 


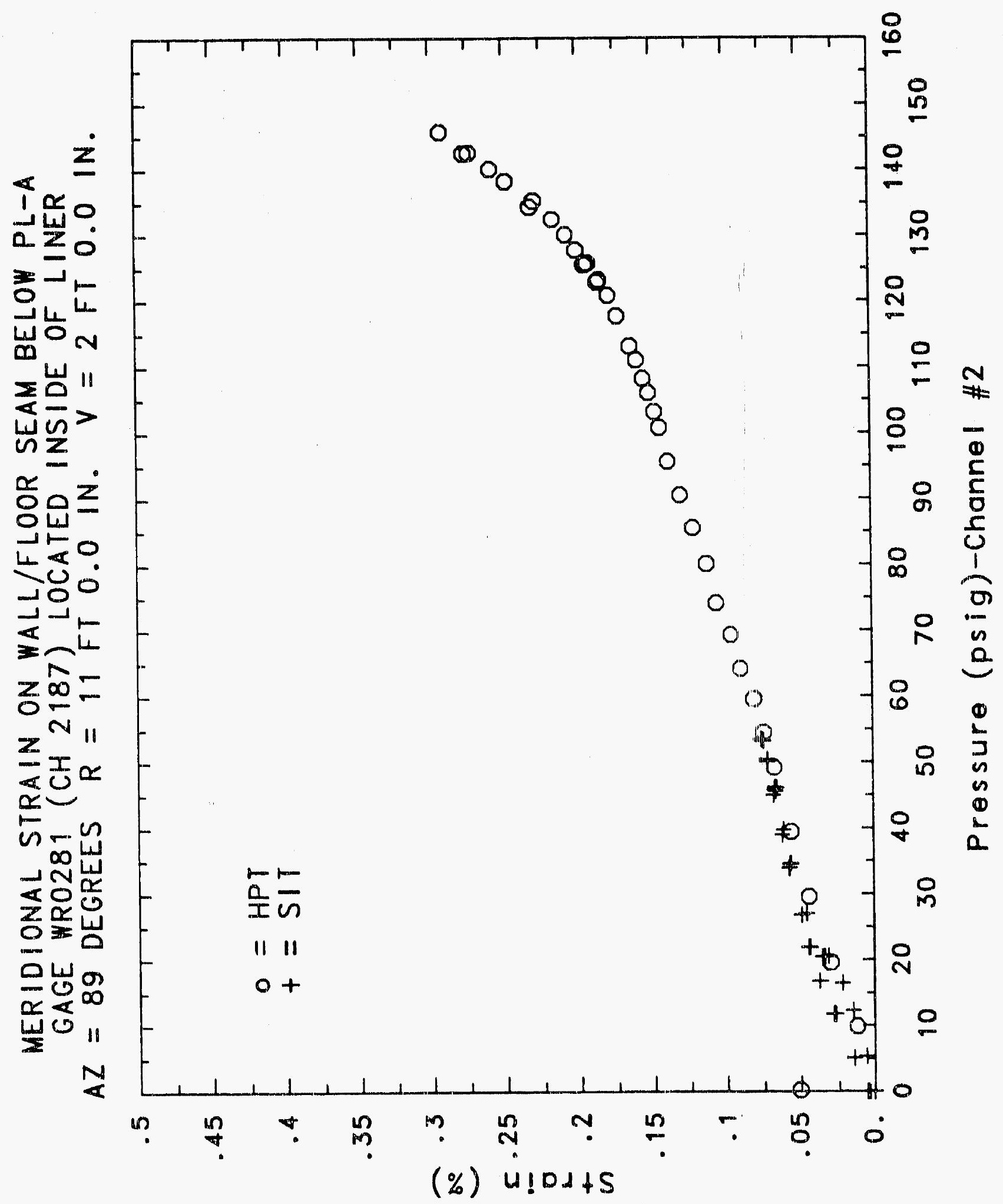




\section{Reinforced Concrete Test Data}

Weldable Gage 282 Channel 2188

STRUCTURAL INTEGRITY TEST

High Pressure test

\begin{tabular}{|c|c|c|c|}
\hline $\begin{array}{c}\text { Pressure } \\
(\text { psig) } \\
-0.05 \\
5.33 \\
12.31 \\
16.44 \\
20.51 \\
20.46 \\
20.47 \\
27.05 \\
34.69 \\
34.53 \\
34.53 \\
39.70 \\
45.90 \\
45.65 \\
45.62 \\
50.10 \\
53.47 \\
53.29 \\
53.21 \\
50.49 \\
46.14 \\
46.13 \\
44.97 \\
38.98 \\
33.99 \\
33.96 \\
26.83 \\
21.88 \\
21.90 \\
16.69 \\
11.74 \\
11.77 \\
5.05 \\
0.02 \\
-0.04 \\
-0.02 \\
-0.02 \\
0.02 \\
\end{array}$ & $\begin{array}{l}\text { \% Strain } \\
0.0002 \\
0.0027 \\
0.0088 \\
0.0114 \\
0.0124 \\
0.0127 \\
0.0130 \\
0.0147 \\
0.0170 \\
0.0178 \\
0.0180 \\
0.0191 \\
0.0212 \\
0.0211 \\
0.0208 \\
0.0208 \\
0.0208 \\
0.0204 \\
0.0203 \\
0.0195 \\
0.0188 \\
0.0186 \\
0.0181 \\
0.0177 \\
0.0173 \\
0.0176 \\
0.0170 \\
0.0166 \\
0.0166 \\
0.0164 \\
0.0162 \\
0.0160 \\
0.0154 \\
0.0134 \\
0.0078 \\
0.0077 \\
0.0079 \\
0.0082\end{array}$ & $\begin{array}{c}\text { Pressure } \\
(\text { psig) } \\
9.89 \\
19.55 \\
29.57 \\
39.42 \\
49.16 \\
54.50 \\
59.57 \\
64.20 \\
69.32 \\
74.16 \\
80.16 \\
85.61 \\
90.58 \\
95.69 \\
100.92 \\
103.25 \\
106.11 \\
108.31 \\
111.08 \\
113.24 \\
117.83 \\
120.92 \\
123.28 \\
122.97 \\
125.82 \\
125.60 \\
127.84 \\
130.19 \\
132.53 \\
135.33 \\
134.42 \\
138.35 \\
140.16 \\
142.63 \\
145.78 \\
142.52 \\
0.22 \\
10\end{array}$ & $\begin{array}{c}\text { \% Strain } \\
0.0057 \\
0.0062 \\
0.0063 \\
0.0071 \\
0.0084 \\
0.0094 \\
0.0099 \\
0.0102 \\
0.0111 \\
0.0112 \\
0.0116 \\
0.0122 \\
0.0145 \\
0.0193 \\
0.0260 \\
0.0283 \\
0.0303 \\
0.0316 \\
0.0330 \\
0.0335 \\
0.0350 \\
0.0365 \\
0.0376 \\
0.0372 \\
0.0389 \\
0.0390 \\
0.0403 \\
0.0424 \\
0.0461 \\
0.0509 \\
0.0510 \\
0.0597 \\
0.0654 \\
0.0747 \\
0.0846 \\
0.0761 \\
-0.0172\end{array}$ \\
\hline
\end{tabular}




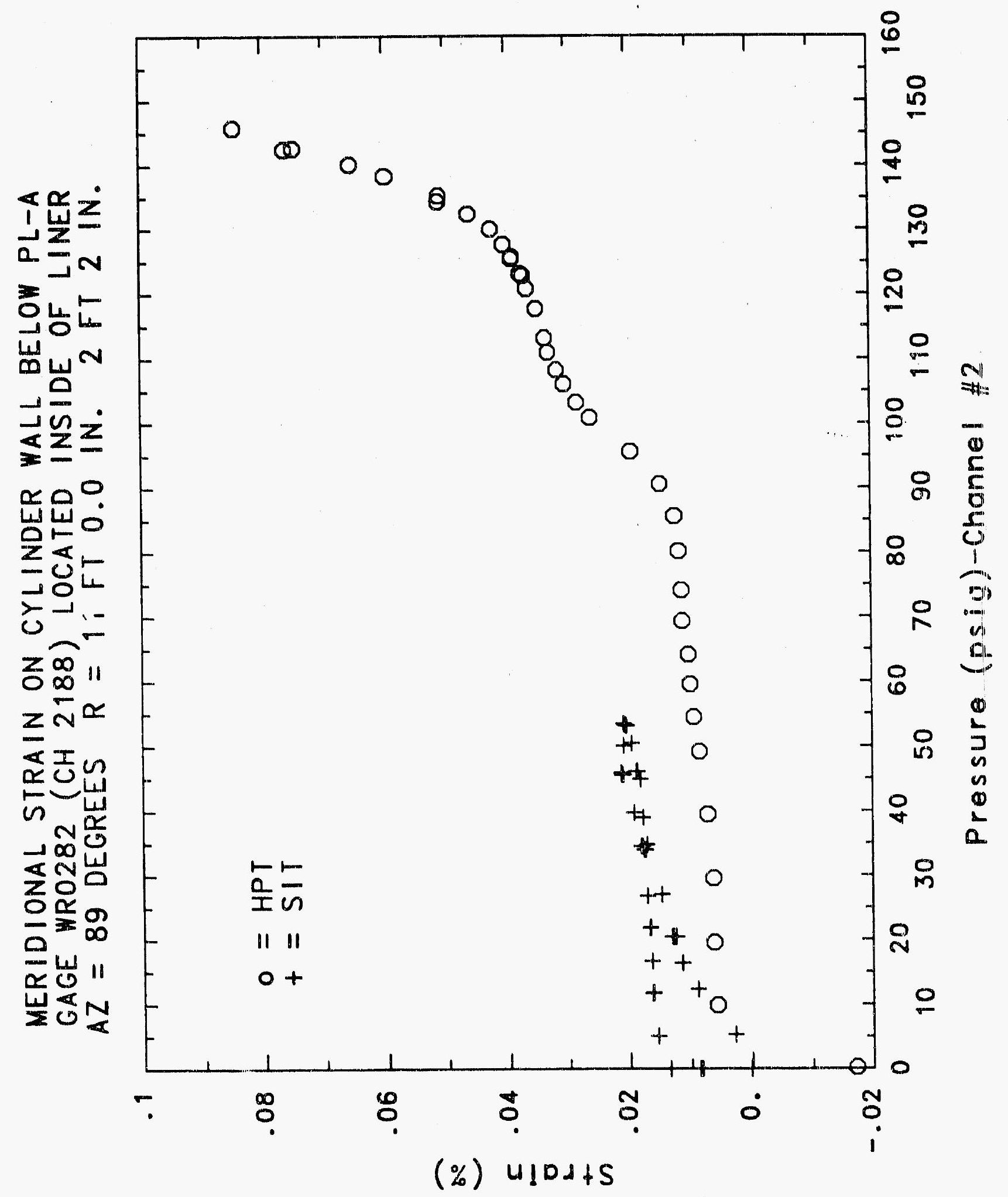




\section{Reinforced Concrete Test Data}

Weldable gage 283 Channel 2189

Structural Integrity Test

High Pressure test

\begin{tabular}{|c|c|c|c|}
\hline $\begin{array}{c}\text { Pressure } \\
\text { (psig) } \\
-0.05 \\
5.33 \\
12.31 \\
16.44 \\
20.51 \\
20.46 \\
20.47 \\
27.05 \\
34.69 \\
34.53 \\
34.53 \\
39.70 \\
45.90 \\
45.65 \\
45.62 \\
50.10 \\
53.47 \\
53.29 \\
53.21 \\
50.49 \\
46.14 \\
46.13 \\
44.97 \\
38.98 \\
33.99 \\
33.96 \\
26.83 \\
21.88 \\
21.90 \\
16.69 \\
11.74 \\
11.77 \\
5.05 \\
0.02 \\
-0.04 \\
-0.02 \\
-0.02 \\
0.02\end{array}$ & $\begin{array}{l}\text { X Strain } \\
0.0008 \\
0.0027 \\
0.0061 \\
0.0087 \\
0.0114 \\
0.0124 \\
0.0132 \\
0.0183 \\
0.0233 \\
0.0239 \\
0.0239 \\
0.0261 \\
0.0277 \\
0.0274 \\
0.0275 \\
0.0287 \\
0.0292 \\
0.0288 \\
0.0286 \\
0.0273 \\
0.0254 \\
0.0250 \\
0.0239 \\
0.0220 \\
0.0205 \\
0.0212 \\
0.0187 \\
0.0170 \\
0.0177 \\
0.0156 \\
0.0134 \\
0.0131 \\
0.0097 \\
0.0071 \\
0.0041 \\
0.0042 \\
0.0043 \\
0.00046\end{array}$ & $\begin{array}{c}\text { Pressure } \\
(\text { psig) } \\
9.89 \\
19.55 \\
29.57 \\
39.42 \\
49.16 \\
54.50 \\
59.57 \\
64.20 \\
69.32 \\
74.16 \\
80.16 \\
85.61 \\
90.58 \\
95.69 \\
100.92 \\
103.25 \\
106.11 \\
108.31 \\
111.08 \\
113.24 \\
117.83 \\
120.92 \\
123.28 \\
122.97 \\
125.82 \\
125.60 \\
127.84 \\
130.19 \\
132.53 \\
135.33 \\
134.42 \\
138.35 \\
140.16 \\
142.63 \\
145.78 \\
142.52 \\
0.22\end{array}$ & $\begin{array}{l}\text { \% Strain } \\
0.0031 \\
0.0081 \\
0.0118 \\
0.0153 \\
0.0187 \\
0.0207 \\
0.0221 \\
0.0229 \\
0.0238 \\
0.0256 \\
0.0271 \\
0.0279 \\
0.0284 \\
0.0294 \\
0.0297 \\
0.0299 \\
0.0301 \\
0.0305 \\
0.0305 \\
0.0305 \\
0.0308 \\
0.0307 \\
0.0306 \\
0.0304 \\
0.0306 \\
0.0300 \\
0.0303 \\
0.0302 \\
0.0300 \\
0.0296 \\
0.0282 \\
0.0257 \\
0.0224 \\
0.0756 \\
0.0103 \\
0.0048 \\
-0.0320\end{array}$ \\
\hline
\end{tabular}




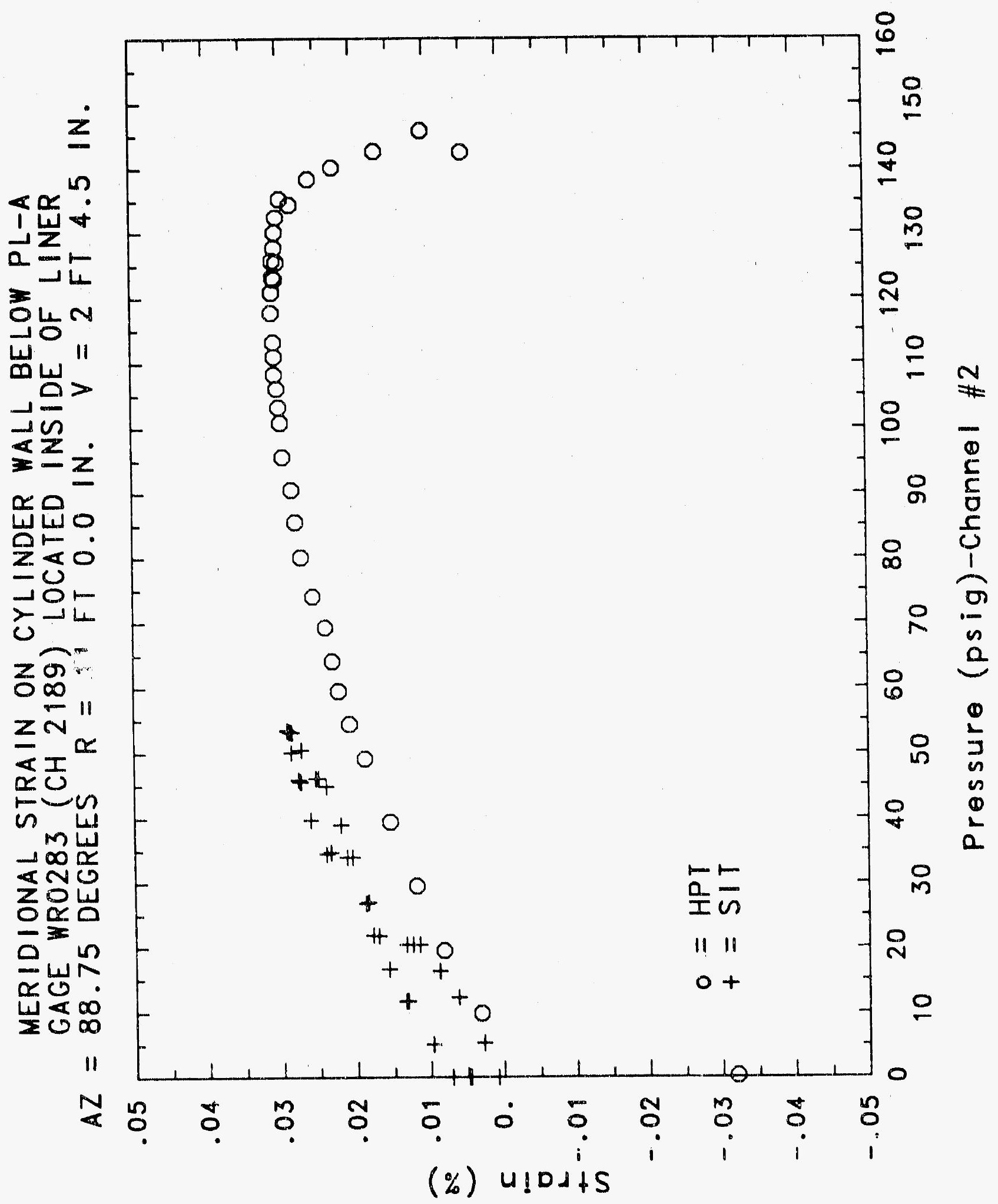




\section{Reinforced Concrete Test Data}

Weldable Gage 284 Channel 2200

Structural IntEgrity Test

High Pressure test

\begin{tabular}{|c|c|c|c|}
\hline $\begin{array}{c}\text { Pressure } \\
\text { (psig) } \\
-0.05 \\
5.33 \\
12.31 \\
16.44 \\
20.51 \\
20.46 \\
20.47 \\
27.05 \\
34.69 \\
34.53 \\
34.53 \\
39.70 \\
45.90 \\
45.65 \\
45.62 \\
50.10 \\
53.47 \\
53.29 \\
53.21 \\
50.49 \\
46.14 \\
46.13 \\
44.97 \\
38.98 \\
33.99 \\
33.96 \\
26.83 \\
21.88 \\
21.90 \\
16.69 \\
11.74 \\
11.77 \\
5.05 \\
0.02 \\
-0.04 \\
-0.02 \\
-0.02 \\
0.02\end{array}$ & $\begin{array}{r}\text { x Strain } \\
0.0001 \\
0.0015 \\
0.0007 \\
-0.0001 \\
-0.0010 \\
-0.0012 \\
-0.0015 \\
-0.0027 \\
-0.0036 \\
-0.0042 \\
-0.0042 \\
-0.0043 \\
-0.0068 \\
-0.0065 \\
-0.0065 \\
-0.0068 \\
-0.0071 \\
-0.0067 \\
-0.0069 \\
-0.0062 \\
-0.0050 \\
-0.0049 \\
-0.0038 \\
-0.0027 \\
-0.0020 \\
-0.0021 \\
-0.0008 \\
0.0001 \\
-0.0003 \\
-0.0003 \\
0.0003 \\
-0.0002 \\
0.0000 \\
-0.0009 \\
0.0016 \\
0.0013 \\
0.0008 \\
0.0001\end{array}$ & $\begin{array}{c}\text { Pressure } \\
(\text { psig) } \\
9.89 \\
19.55 \\
29.57 \\
39.42 \\
49.16 \\
54.50 \\
59.57 \\
64.20 \\
69.32 \\
74.16 \\
80.16 \\
85.61 \\
90.58 \\
95.69 \\
100.92 \\
103.25 \\
106.11 \\
108.31 \\
111.08 \\
113.24 \\
117.83 \\
120.92 \\
123.28 \\
122.97 \\
125.82 \\
125.60 \\
127.84 \\
130.19 \\
132.53 \\
135.33 \\
134.42 \\
138.35 \\
140.16 \\
142.63 \\
145.78 \\
142.52 \\
0.22\end{array}$ & $\begin{array}{l}\text { \% Strain } \\
-0.0041 \\
0.0069 \\
-0.0079 \\
-0.0088 \\
-0.0096 \\
-0.0103 \\
-0.0108 \\
-0.0112 \\
-0.0120 \\
-0.0134 \\
-0.0152 \\
-0.0165 \\
-0.0179 \\
-0.0195 \\
-0.0209 \\
-0.0219 \\
-0.0229 \\
-0.0235 \\
-0.0245 \\
-0.0258 \\
-0.0279 \\
-0.0296 \\
-0.0312 \\
-0.0313 \\
-0.0344 \\
-0.0349 \\
-0.0365 \\
-0.0388 \\
-0.0410 \\
-0.0436 \\
-0.0441 \\
-0.0470 \\
-0.0493 \\
-0.0532 \\
-0.0580 \\
-0.0558 \\
-0.0010\end{array}$ \\
\hline
\end{tabular}




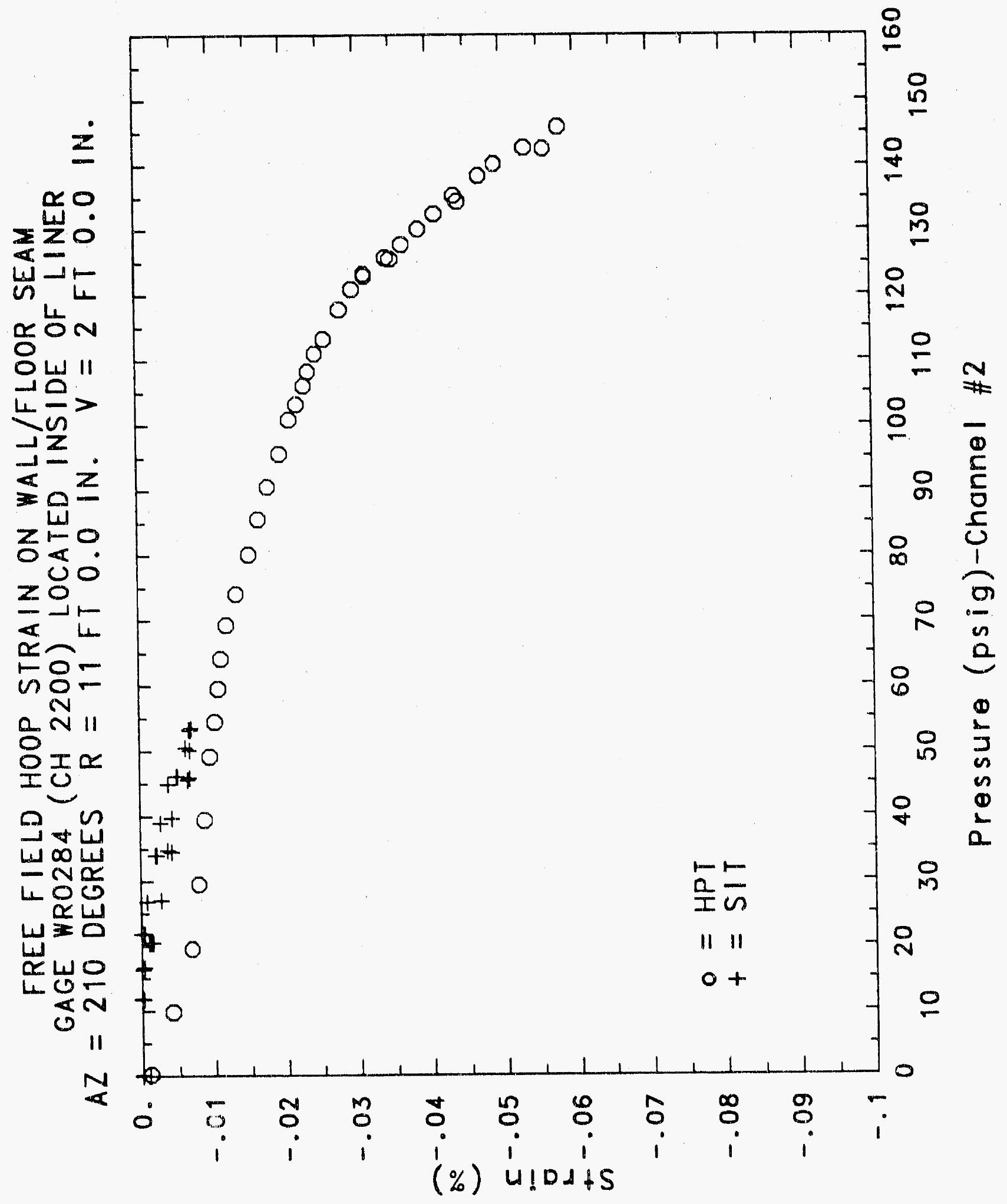




\section{Reinforced Concrete Test Data}

Weloable Gage 285 Channel 2201

STRUCTURAL INTEGRITY TEST

\begin{tabular}{|c|c|}
\hline $\begin{array}{c}\text { Pressure } \\
\text { (psig) } \\
-0.05 \\
5.33 \\
12.31 \\
16.44 \\
20.51 \\
20.46 \\
20.47 \\
27.05 \\
34.69 \\
34.53 \\
34.53 \\
39.70 \\
45.90 \\
45.65 \\
45.62 \\
51.10 \\
3.47 \\
53.29 \\
53.21 \\
50.49 \\
46.14 \\
46.13 \\
44.97 \\
38.98 \\
33.99 \\
33.96 \\
26.83 \\
21.88 \\
21.90 \\
16.69 \\
11.74 \\
11.77 \\
5.05 \\
0.02 \\
-0.04 \\
-0.02 \\
-0.02 \\
0.02\end{array}$ & $\begin{array}{l}\text { \% Strain } \\
\\
0.0004 \\
-0.0002 \\
-0.0014 \\
-0.0020 \\
-0.0026 \\
-0.0023 \\
-0.0022 \\
-0.0034 \\
-0.0046 \\
-0.0043 \\
-0.0043 \\
-0.0052 \\
-0.0058 \\
-0.0057 \\
-0.0056 \\
-0.0065 \\
-0.0064 \\
-0.0062 \\
-0.0060 \\
-0.0055 \\
-0.0049 \\
-0.0047 \\
-0.0029 \\
-0.0028 \\
-0.0024 \\
-0.0023 \\
-0.0017 \\
-0.0010 \\
-0.0008 \\
-0.0003 \\
0.0005 \\
0.0003 \\
0.0014 \\
0.0019 \\
0.0040 \\
0.0038 \\
0.0034 \\
0.0028\end{array}$ \\
\hline
\end{tabular}

High Pressure Test

\begin{tabular}{|c|c|}
\hline $\begin{array}{c}\text { Pressure } \\
\text { (psig) } \\
9.89 \\
19.55 \\
29.57 \\
39.42 \\
49.16 \\
54.50 \\
59.57 \\
64.20 \\
69.32 \\
74.16 \\
80.16 \\
85.61 \\
90.58 \\
95.69 \\
100.92 \\
103.25 \\
106.11 \\
108.31 \\
111.08 \\
113.24 \\
117.83 \\
120.92 \\
123.28 \\
122.97 \\
125.82 \\
125.60 \\
127.84 \\
130.19 \\
132.53 \\
135.33 \\
134.42 \\
138.35 \\
140.16 \\
142.63 \\
145.78 \\
142.52 \\
3.26\end{array}$ & 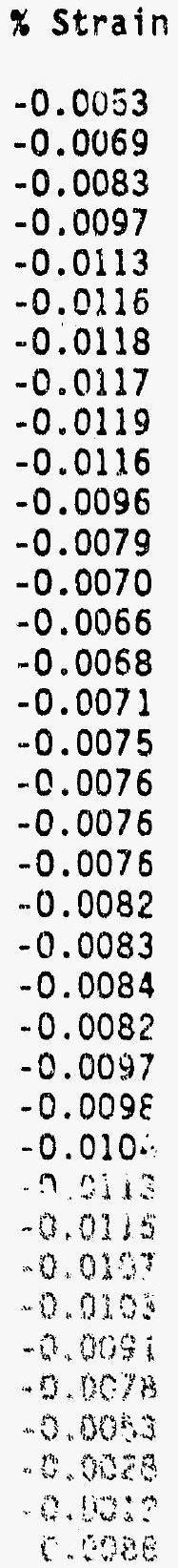 \\
\hline
\end{tabular}




\section{IMAGE EVALUATION TEST TARGET (MT-3)}
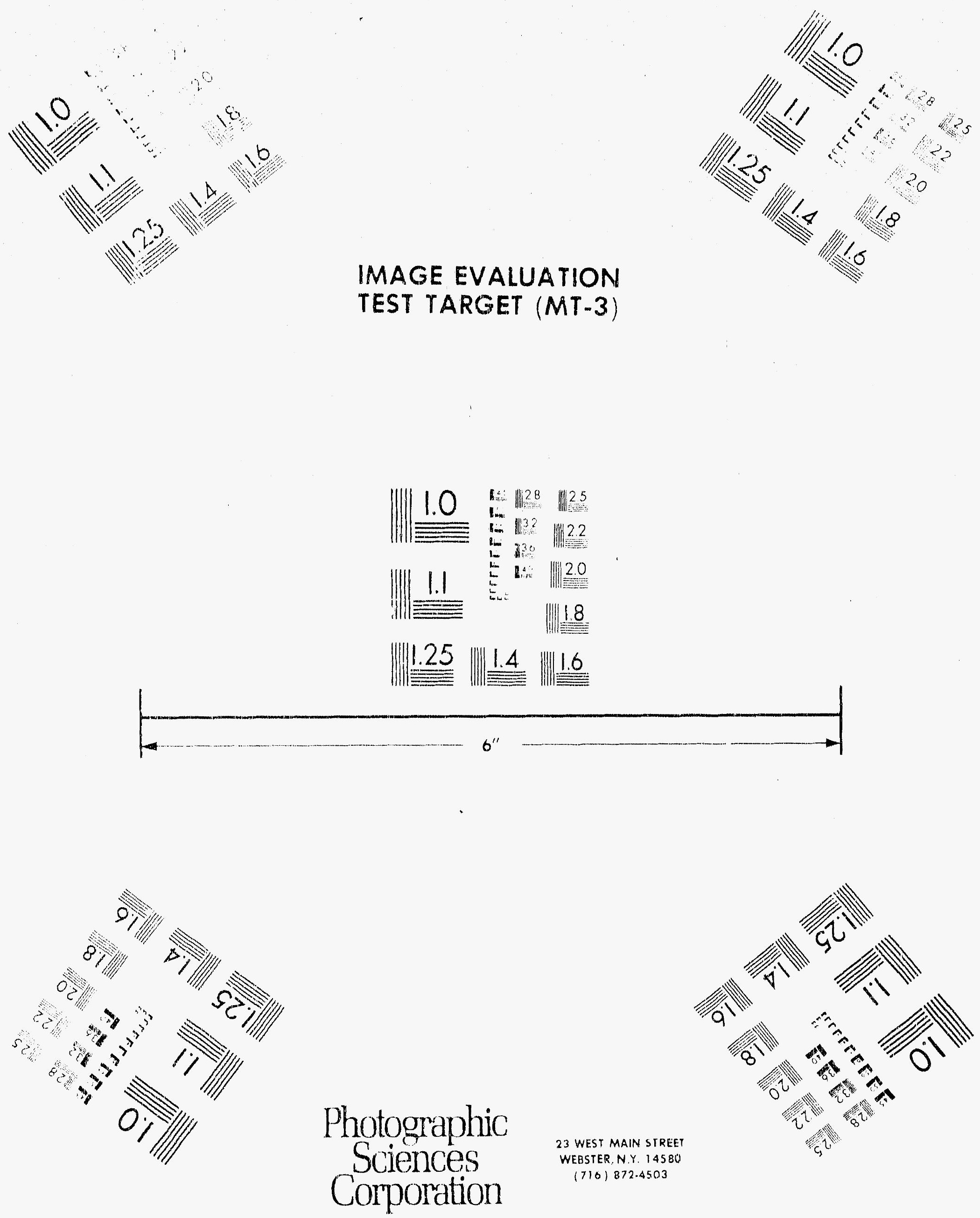

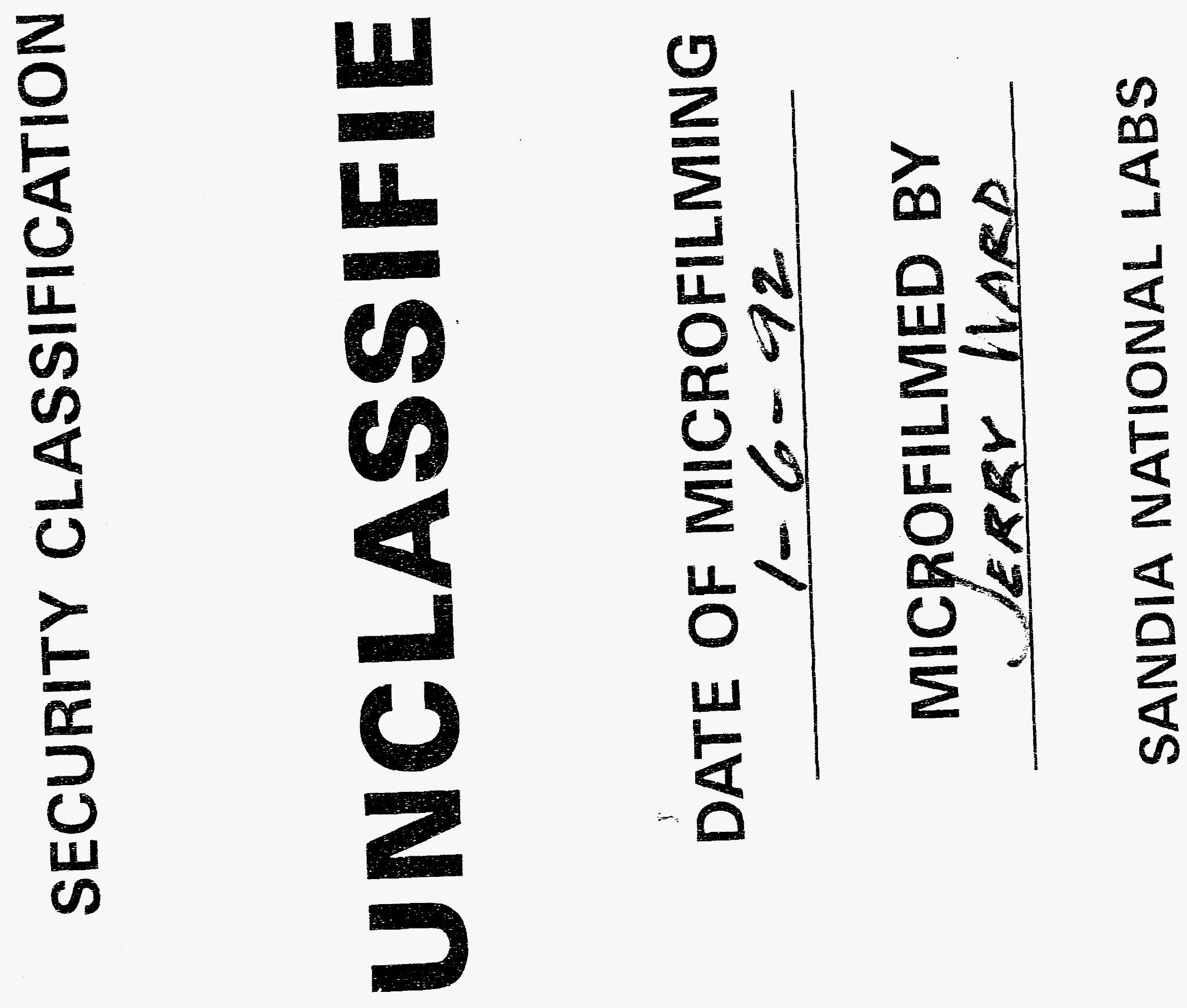


\section{THIS DOCUMENT FILMED}

AT

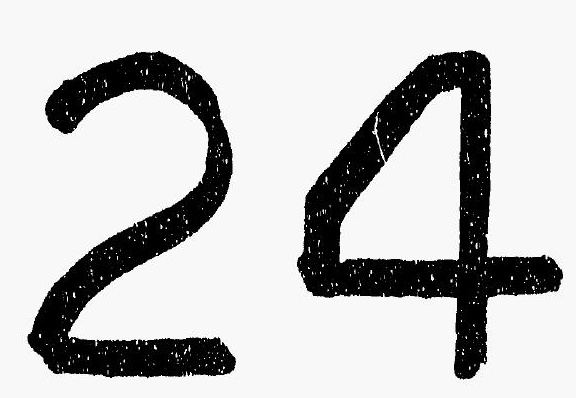

REDUCTION 


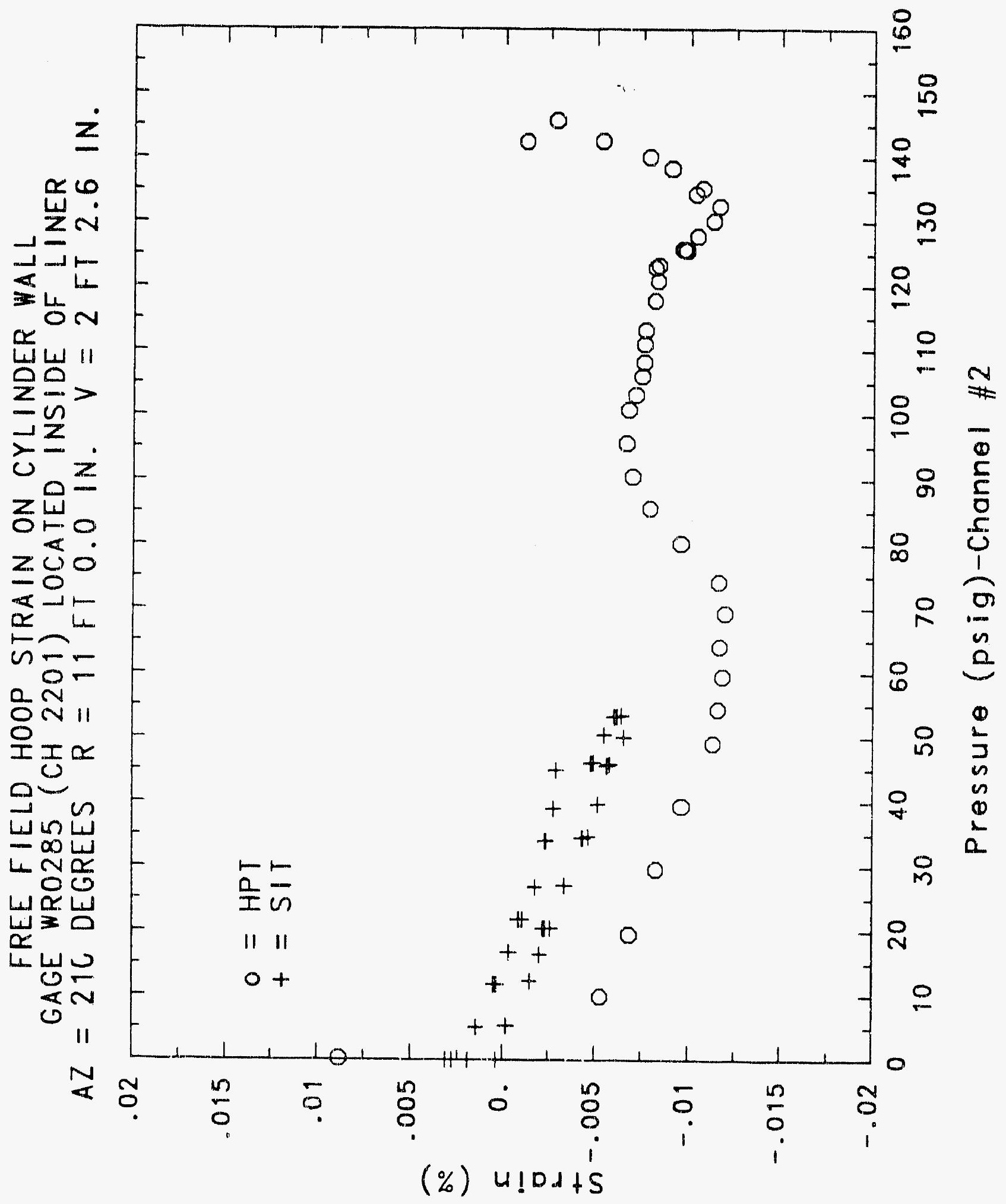




\section{Reinforced Concrete Test Data}

Weldabi.e gage 286 Channel 2202

Structural Integrity Test

High Pressure test

\begin{tabular}{|c|c|c|c|}
\hline $\begin{array}{c}\text { Pressure } \\
(p s i g) \\
-0.05 \\
5.33 \\
12.31 \\
16.44 \\
20.51 \\
20.46 \\
20.47 \\
27.05 \\
34.69 \\
34.53 \\
34.53 \\
39.70 \\
45.90 \\
45.65 \\
45.62 \\
50.10 \\
53.47 \\
53.29 \\
53.21 \\
50.49 \\
46.14 \\
46.13 \\
44.97 \\
38.98 \\
33.99 \\
33.96 \\
26.83 \\
21.88 \\
21.90 \\
16.69 \\
11.74 \\
11.77 \\
5.05 \\
0.02 \\
-0.04 \\
-0.02 \\
-0.02 \\
0.02 \\
\end{array}$ & $\begin{array}{l}\text { \% Strain } \\
\\
0.0004 \\
0.0008 \\
0.0007 \\
0.0005 \\
0.0006 \\
0.0010 \\
0.0010 \\
0.0013 \\
0.0005 \\
0.0008 \\
0.0009 \\
0.0003 \\
0.0003 \\
0.0004 \\
0.0004 \\
0.0003 \\
0.0002 \\
0.0006 \\
0.0007 \\
0.0012 \\
0.0015 \\
0.0015 \\
0.0029 \\
0.0026 \\
0.0027 \\
0.0023 \\
0.0027 \\
0.0027 \\
0.0025 \\
0.0026 \\
0.0026 \\
0.0020 \\
0.0023 \\
0.0018 \\
0.0041 \\
0.0036 \\
0.0030 \\
0.0021\end{array}$ & $\begin{array}{c}\text { Pressure } \\
\text { (psig) } \\
9.89 \\
19.55 \\
29.57 \\
39.42 \\
49.16 \\
54.50 \\
59.57 \\
64.20 \\
69.32 \\
74.16 \\
80.16 \\
85.61 \\
90.58 \\
95.69 \\
100.92 \\
103.25 \\
106.11 \\
108.31 \\
111.08 \\
113.24 \\
117.83 \\
120.92 \\
123.28 \\
122.97 \\
125.82 \\
125.60 \\
127.84 \\
130.19 \\
132.53 \\
135.33 \\
134.42 \\
138.35 \\
140.16 \\
142.63 \\
145.78 \\
142.52 \\
0.22 \\
\end{array}$ & $\begin{array}{l}\text { \% Strain } \\
-0.0035 \\
-0.0039 \\
-0.0046 \\
-0.0049 \\
-0.0051 \\
-0.0051 \\
-0.0047 \\
-0.0046 \\
-0.0051 \\
-0.0056 \\
-0.0065 \\
-0.0068 \\
-0.0063 \\
-0.0057 \\
-0.0051 \\
-0.0048 \\
-0.0051 \\
-0.0048 \\
-0.0045 \\
-0.0042 \\
-0.0032 \\
-0.0024 \\
-0.0014 \\
-0.0006 \\
-0.0005 \\
-0.0004 \\
-0.0004 \\
0.0006 \\
0.0033 \\
0.0068 \\
0.0077 \\
0.0130 \\
0.0176 \\
0.0241 \\
0.0301 \\
0.0319 \\
0.0270\end{array}$ \\
\hline
\end{tabular}




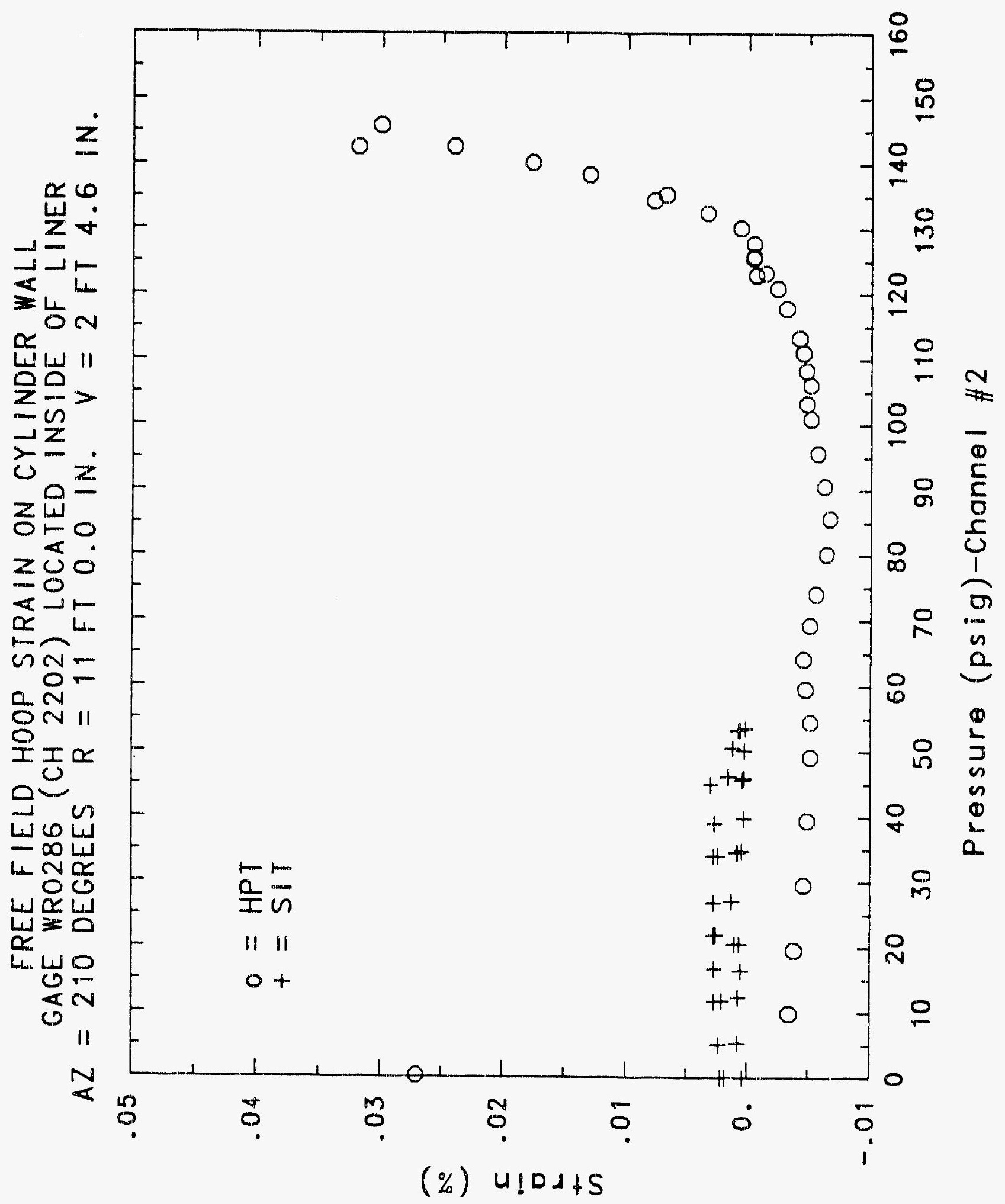




\section{Reinforced Concrete Test Data}

Weldable gage 287 Channel 2203

Structural IntEgrity test

\begin{tabular}{|c|c|c|c|}
\hline $\begin{array}{c}\text { Pressure } \\
(p s i g) \\
-0.05 \\
5.33 \\
12.31 \\
16.44 \\
20.51 \\
20.46 \\
20.47 \\
27.05 \\
34.69 \\
34.53 \\
34.53 \\
39.70 \\
45.90 \\
45.65 \\
45.62 \\
50.10 \\
53.47 \\
53.29 \\
53.21 \\
50.49 \\
46.14 \\
46.13 \\
44.97 \\
38.98 \\
33.99 \\
33.96 \\
26.83 \\
21.88 \\
21.90 \\
16.69 \\
11.74 \\
11.77 \\
5.05 \\
0.02 \\
-0.04 \\
-0.02 \\
-0.02 \\
0.02 \\
\end{array}$ & $\begin{array}{l}\text { \% Strain } \\
0.0000 \\
0.0097 \\
0.0226 \\
0.0306 \\
0.0403 \\
0.0416 \\
0.0425 \\
0.0564 \\
0.0688 \\
0.0693 \\
0.0695 \\
0.0748 \\
0.0804 \\
0.0805 \\
0.0805 \\
0.0844 \\
0.0879 \\
0.0880 \\
0.0880 \\
0.0830 \\
0.0751 \\
0.0746 \\
0.0736 \\
0.0621 \\
0.0526 \\
0.0530 \\
0.0390 \\
0.0301 \\
0.0305 \\
0.0228 \\
0.0152 \\
0.0149 \\
0.0031 \\
-0.0097 \\
-0.0160 \\
-0.0151 \\
-0.0155 \\
-0.0152\end{array}$ & $\begin{array}{c}\text { Pressure } \\
(\text { ps } 1 \text { g) } \\
9.89 \\
19.55 \\
29.57 \\
39.42 \\
49.16 \\
54.50 \\
59.57 \\
64.20 \\
69.32 \\
74.16 \\
80.16 \\
85.61 \\
90.58 \\
95.69 \\
100.92 \\
103.25 \\
106.11 \\
108.31 \\
111.08 \\
113.24 \\
117.83 \\
120.92 \\
123.28 \\
122.97 \\
125.82 \\
125.60 \\
127.84 \\
130.19 \\
132.53 \\
135.33 \\
134.42 \\
138.35 \\
140.16 \\
142.63 \\
145.78 \\
142.52 \\
0.22 \\
\end{array}$ & $\begin{array}{l}\text { \% Strain } \\
0.0304 \\
0.0554 \\
0.0691 \\
0.0814 \\
0.0932 \\
0.1012 \\
0.1078 \\
0.1148 \\
0.1230 \\
0.1321 \\
0.1446 \\
0.1570 \\
0.1691 \\
0.1805 \\
0.1869 \\
0.1901 \\
0.1952 \\
0.1994 \\
0.2054 \\
0.2115 \\
0.2240 \\
0.2334 \\
0.2438 \\
0.2453 \\
0.2637 \\
0.2671 \\
0.2766 \\
0.2907 \\
0.3046 \\
0.3207 \\
0.3218 \\
0.3413 \\
0.3541 \\
0.3721 \\
0.3926 \\
0.3758 \\
0.0809\end{array}$ \\
\hline
\end{tabular}

High Pressure test

Pressure \% Strain

(psig)

19.55

39.42

49.16

54.50

69.32

74.16

85.61

90.58

95.69

100.92

108.31

111.08

113.24

117.83

120.92

122.97

125.82

125.60

127.84

130.19

135.33

134.42

138.35

140.16

142.63

145.78

0.52

0.3758

0.0809 
18E

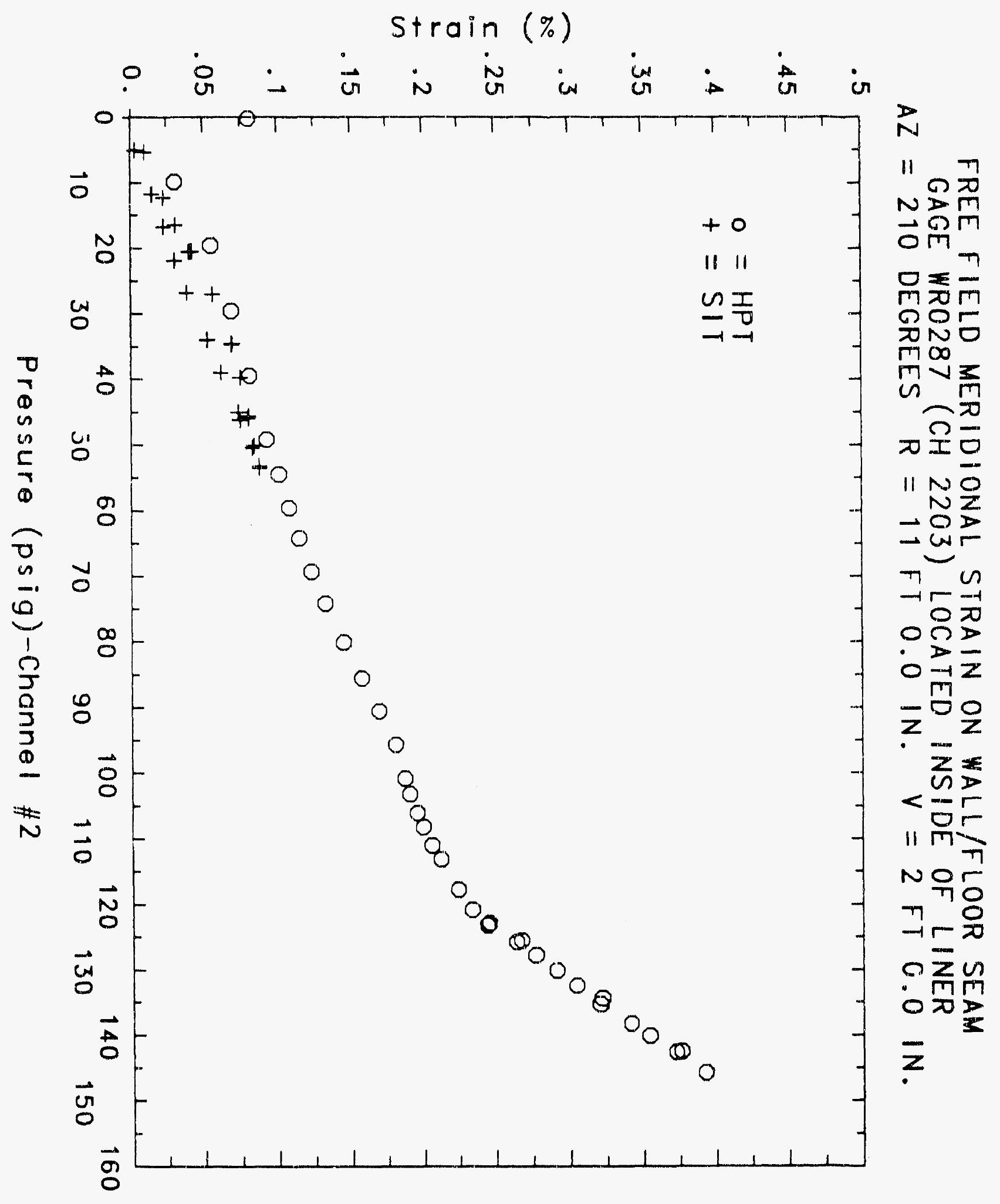




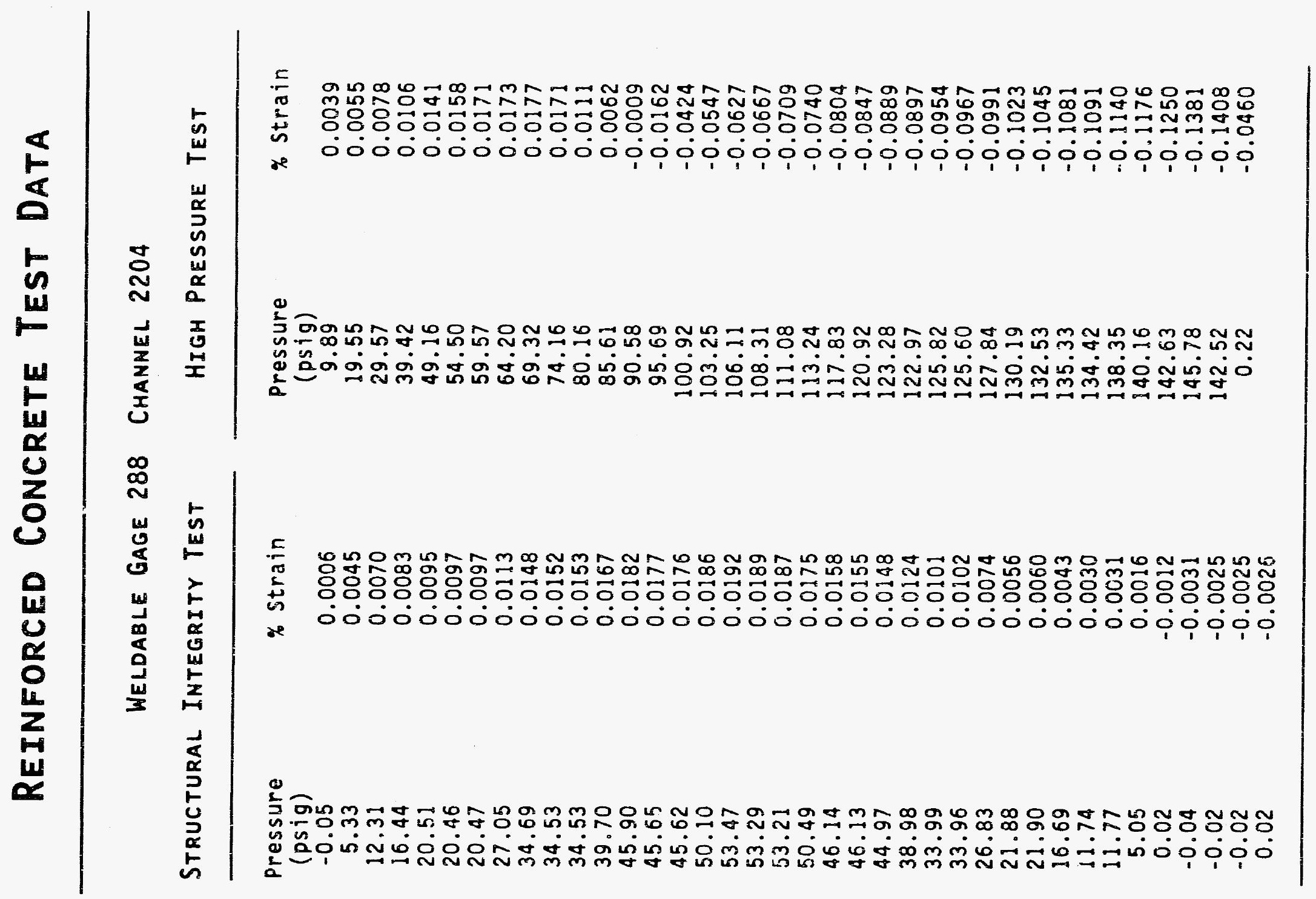




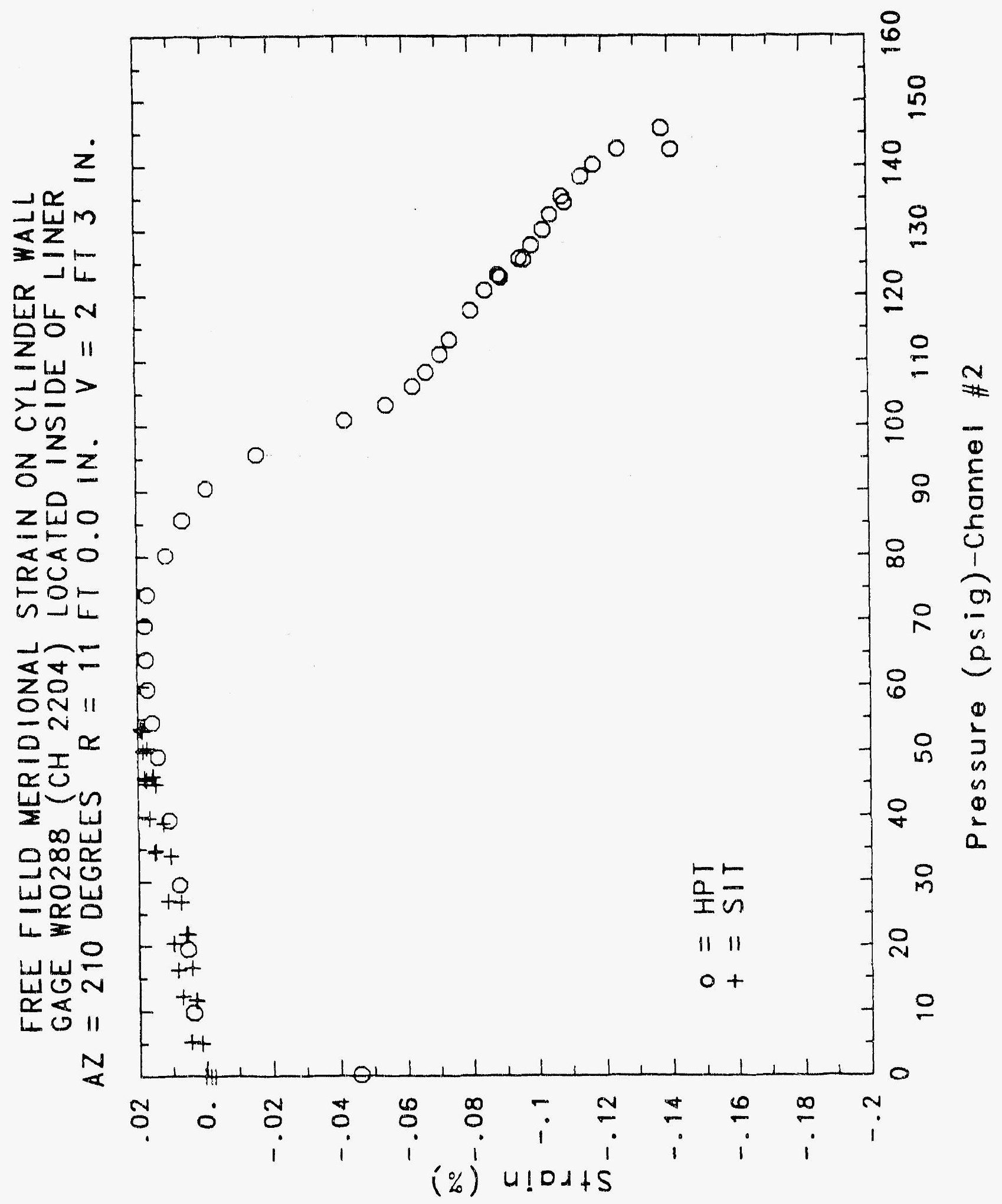




\section{Reinforced Concrete Test Data}

Weldable gage 289 Channel 2205

Structural INTEgRITY TEST

\begin{tabular}{|c|c|c|c|}
\hline $\begin{array}{c}\text { Pressure } \\
\text { (psig) } \\
-0.05 \\
5.33 \\
12.31 \\
16.44 \\
20.51 \\
20.46 \\
20.47 \\
27.05 \\
34.69 \\
34.53 \\
34.53 \\
39.70 \\
45.90 \\
45.65 \\
45.62 \\
50.10 \\
53.47 \\
53.29 \\
53.21 \\
50.49 \\
46.14 \\
46.13 \\
44.97 \\
38.98 \\
33.99 \\
33.96 \\
26.83 \\
21.88 \\
21.90 \\
16.69 \\
11.74 \\
11.77 \\
5.05 \\
0.02 \\
-0.04 \\
-0.02 \\
-0.02 \\
0.02\end{array}$ & $\begin{array}{l}\text { S Strain } \\
0.0000 \\
0.0031 \\
0.0060 \\
0.0080 \\
0.0107 \\
0.0114 \\
0.0118 \\
0.0175 \\
0.0252 \\
0.0255 \\
0.0258 \\
0.0287 \\
0.0319 \\
0.0322 \\
0.0322 \\
0.0343 \\
0.0357 \\
0.0357 \\
0.0356 \\
0.0339 \\
0.0313 \\
0.0309 \\
0.0305 \\
0.0268 \\
0.0238 \\
0.0239 \\
0.0196 \\
0.0167 \\
0.0167 \\
0.0141 \\
0.0116 \\
0.0116 \\
0.0086 \\
0.0050 \\
0.0024 \\
0.0032 \\
0.0032 \\
0.0031\end{array}$ & $\begin{array}{c}\text { Pressure } \\
(\text { psig) } \\
9.89 \\
19.55 \\
29.57 \\
39.42 \\
49.16 \\
54.50 \\
59.57 \\
64.20 \\
69.32 \\
74.16 \\
80.16 \\
85.61 \\
90.58 \\
95.69 \\
100.92 \\
103.25 \\
106.11 \\
108.31 \\
111.08 \\
113.24 \\
117.83 \\
120.92 \\
123.28 \\
122.97 \\
125.82 \\
125.60 \\
127.84 \\
130.19 \\
132.53 \\
135.33 \\
134.42 \\
138.35 \\
140.16 \\
142.63 \\
145.78 \\
142.52 \\
0.22\end{array}$ & $\begin{array}{l}\text { \% Strain } \\
0.0048 \\
0.0112 \\
0.0159 \\
0.0211 \\
0.0263 \\
0.0295 \\
0.0322 \\
0.0344 \\
0.0362 \\
0.0387 \\
0.0412 \\
0.0428 \\
0.0434 \\
0.0420 \\
0.0408 \\
0.0405 \\
0.0408 \\
0.0413 \\
0.0414 \\
0.0413 \\
0.0412 \\
0.0408 \\
0.0404 \\
0.0401 \\
0.0392 \\
0.0383 \\
0.0387 \\
0.0385 \\
0.0383 \\
0.0381 \\
0.0373 \\
0.0366 \\
0.0362 \\
0.0347 \\
0.0337 \\
0.0283 \\
-0.0183\end{array}$ \\
\hline
\end{tabular}




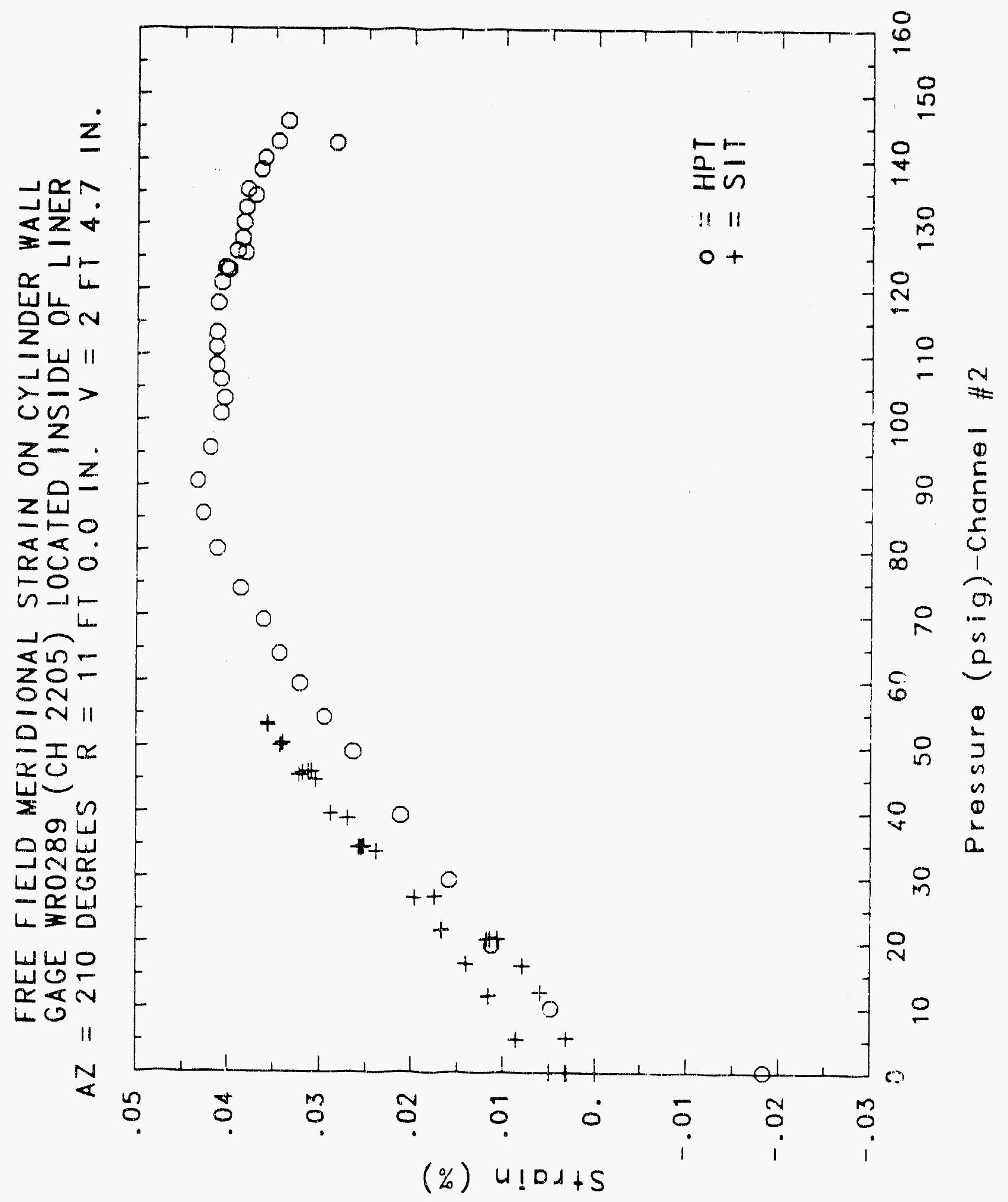




\section{Reinforced Concrete test Data}

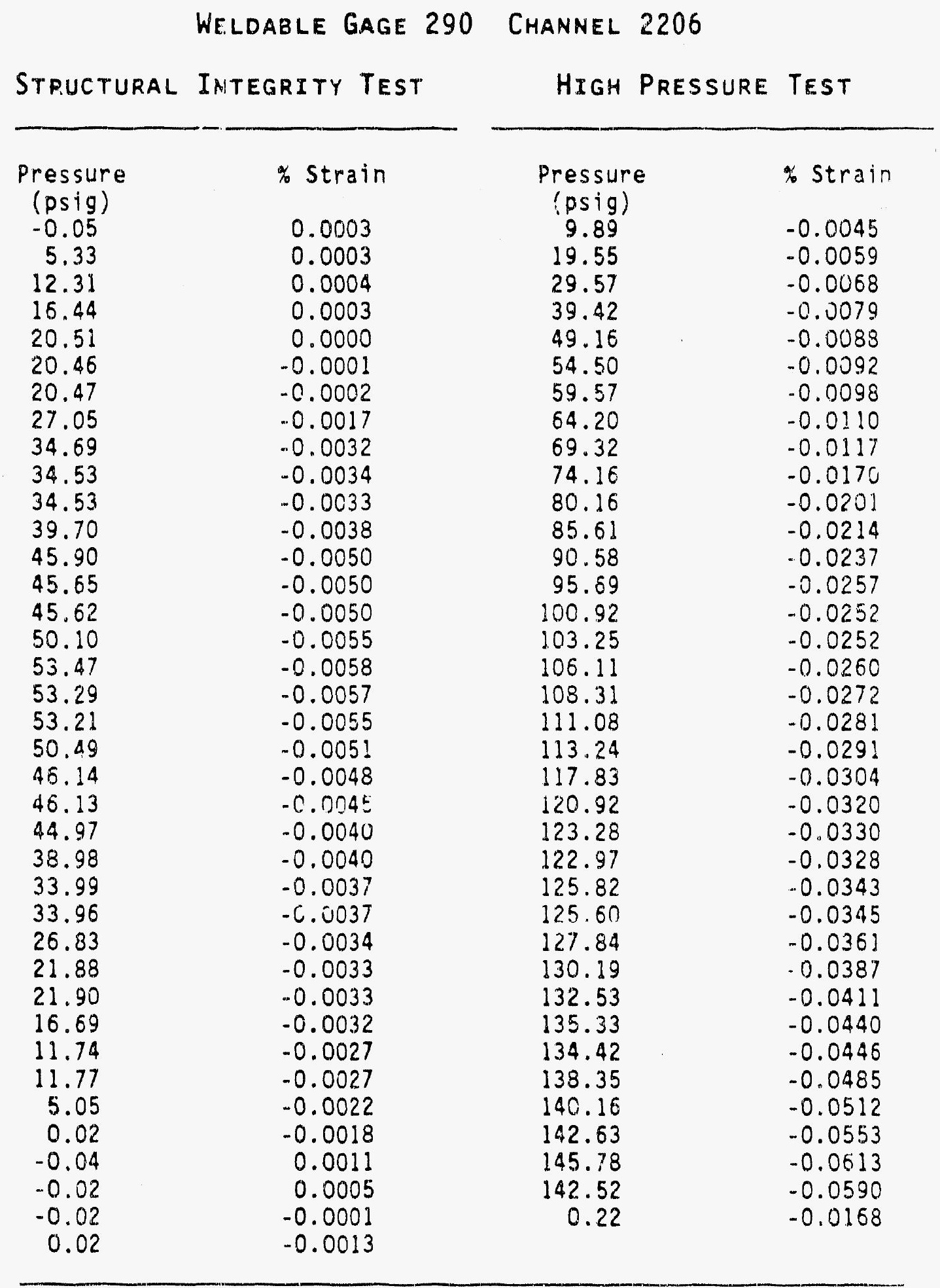




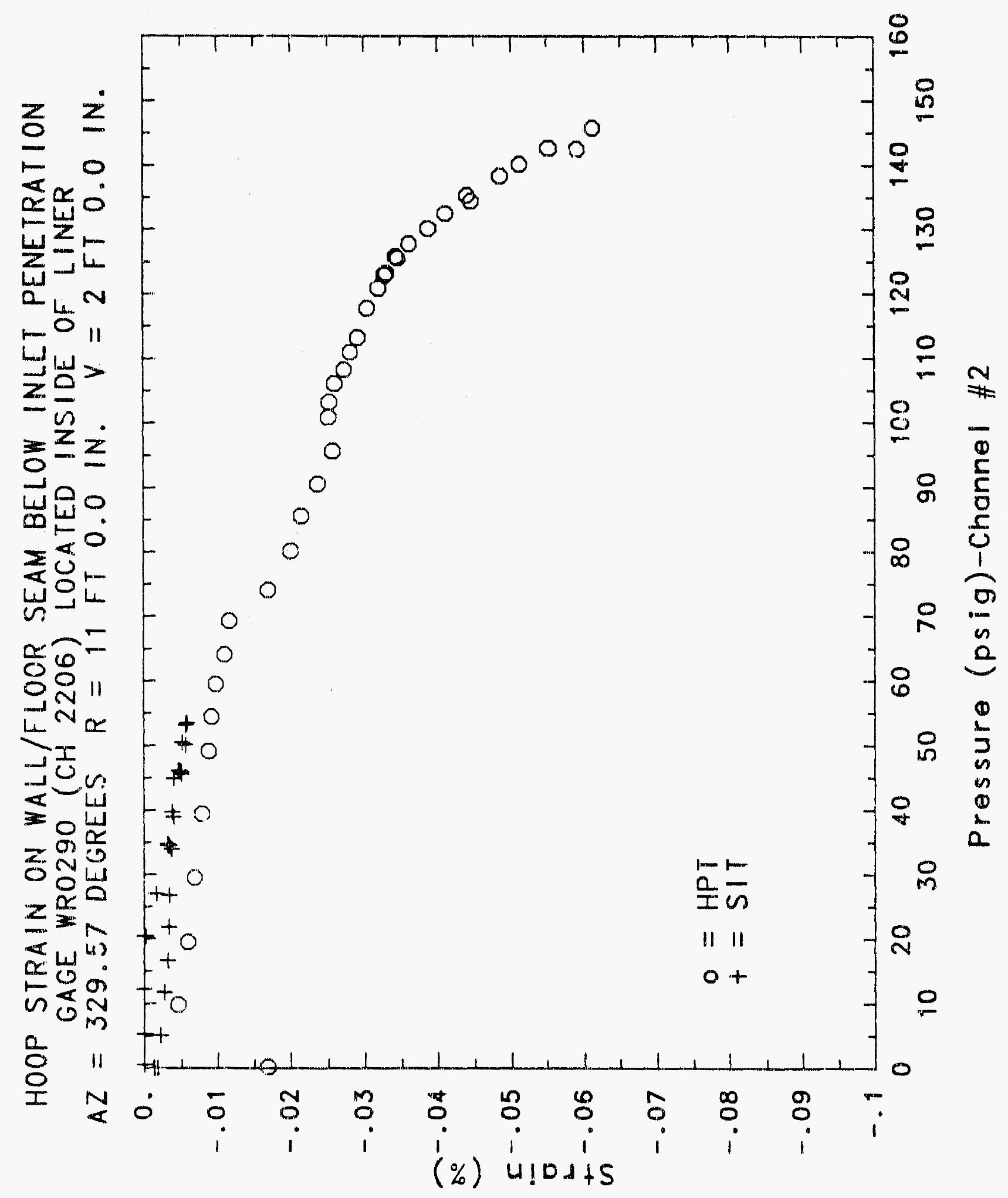




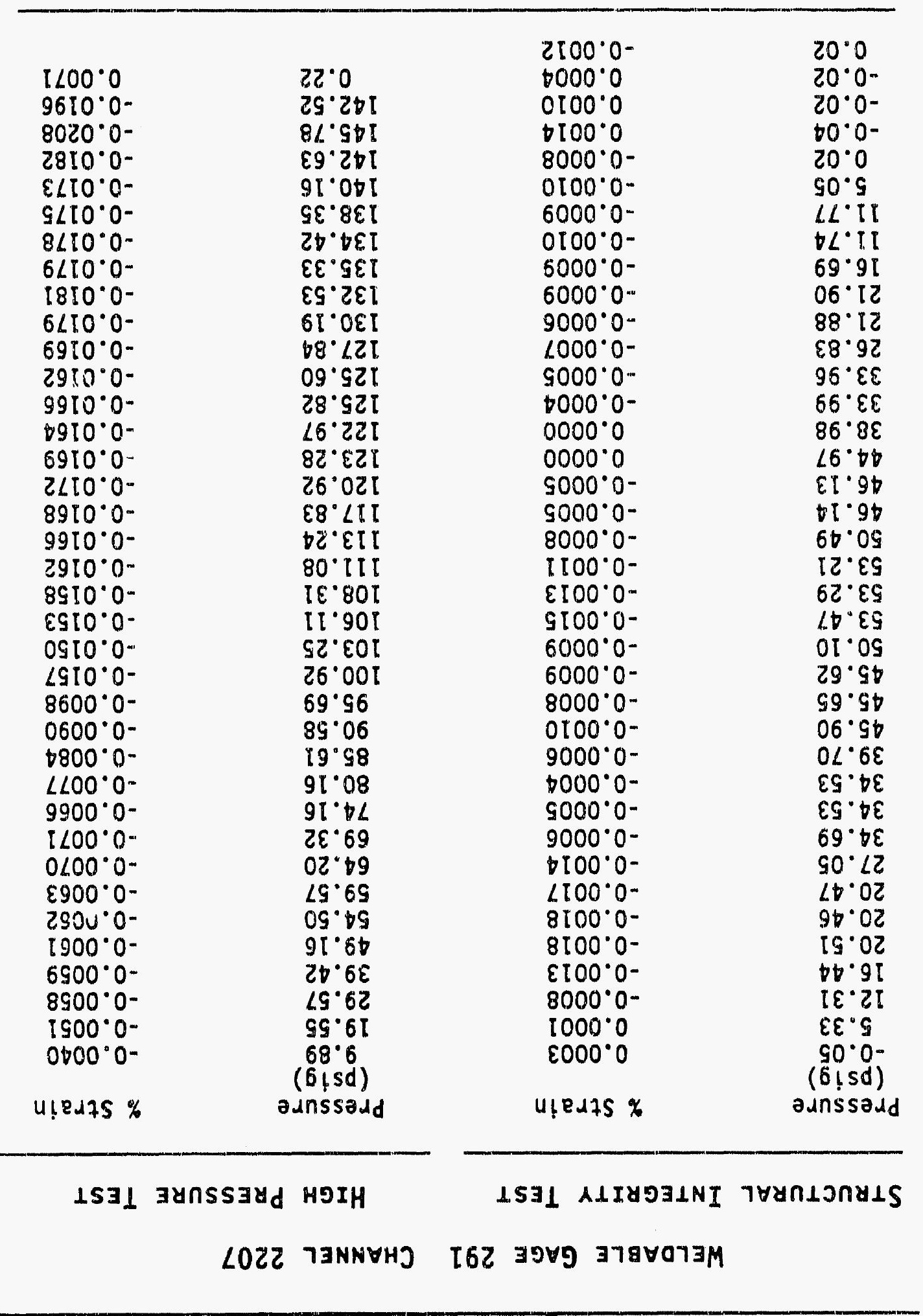

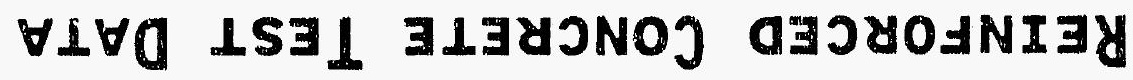




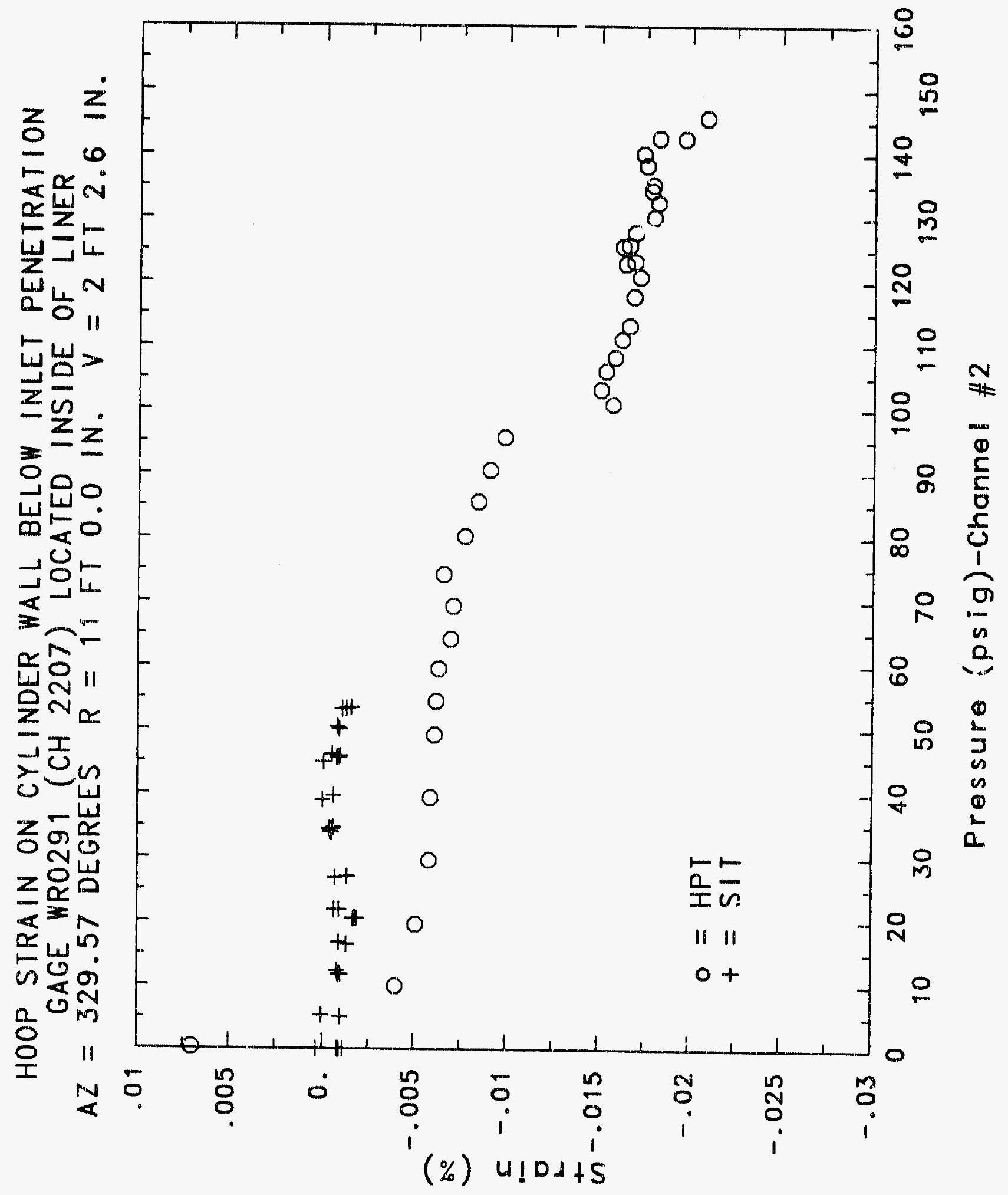




\section{Reinforced Concrete Test Data}

Weldable Gage 292 Channel 2208

StRUCTURAL INTEGRITY TEST

\begin{tabular}{|c|c|c|c|}
\hline $\begin{array}{c}\text { Pressure } \\
\text { (psig) } \\
-0.05 \\
5.33 \\
12.31 \\
16.44 \\
20.51 \\
20.46 \\
20.47 \\
27.05 \\
34.69 \\
34.53 \\
34.53 \\
39.70 \\
45.90 \\
45.65 \\
45.62 \\
50.10 \\
53.47 \\
53.29 \\
53.21 \\
56.49 \\
46.14 \\
46.13 \\
44.97 \\
38.98 \\
33.99 \\
33.96 \\
26.83 \\
21.88 \\
21.90 \\
16.69 \\
11.74 \\
11.77 \\
5.05 \\
0.02 \\
-0.04 \\
-0.02 \\
-0.02 \\
0.02 \\
\end{array}$ & $\begin{array}{c}\text { Strain } \\
\\
0.0003 \\
0.0003 \\
0.0002 \\
0.0006 \\
0.0006 \\
0.0000 \\
-0.0006 \\
-0.0004 \\
0.0005 \\
0.0003 \\
0.0004 \\
0.0008 \\
0.0027 \\
0.0040 \\
0.0044 \\
0.0048 \\
0.0056 \\
0.0068 \\
0.0070 \\
0.0075 \\
0.0077 \\
0.0078 \\
0.0077 \\
0.0071 \\
0.0059 \\
0.0058 \\
0.0044 \\
0.0037 \\
0.0039 \\
0.0031 \\
0.0031 \\
0.0035 \\
0.0028 \\
0.0019 \\
0.0058 \\
0.0050 \\
0.0040 \\
0.0025\end{array}$ & $\begin{array}{c}\text { Pressure } \\
\text { (psig) } \\
9.89 \\
19.55 \\
29.57 \\
39.42 \\
49.16 \\
54.50 \\
59.57 \\
64.20 \\
69.32 \\
74.16 \\
80.16 \\
35.61 \\
90.58 \\
95.69 \\
100.92 \\
103.25 \\
106.11 \\
108.31 \\
111.08 \\
113.21 \\
117.83 \\
120.92 \\
123.28 \\
122.97 \\
125.82 \\
125.60 \\
127.84 \\
130.19 \\
132.53 \\
135.33 \\
134.42 \\
138.35 \\
140.16 \\
142.63 \\
145.78 \\
142.52 \\
0.22 \\
\end{array}$ & $\begin{array}{c}\text { Xtrain } \\
-0.0022 \\
-0.0014 \\
-0.0007 \\
-0.0002 \\
0.0003 \\
0.0007 \\
0.0013 \\
0.0016 \\
0.0029 \\
0.0041 \\
0.0050 \\
0.0058 \\
0.0069 \\
0.0082 \\
0.0089 \\
0.0099 \\
0.0107 \\
0.0111 \\
0.0122 \\
0.0128 \\
0.0142 \\
0.0153 \\
0.0167 \\
0.0174 \\
0.0187 \\
0.0192 \\
0.0193 \\
0.0196 \\
0.0205 \\
0.0227 \\
0.0232 \\
0.0270 \\
0.0303 \\
0.0347 \\
0.0380 \\
0.0386 \\
0.0190\end{array}$ \\
\hline
\end{tabular}

High Pressure test

0.89

39.42

59.57

74.16

80.16

103.25

106.11

108.31

117.83

120.92

123.28

122.97

125.82

125.60

135.33

134.48

0.0270

0.0303

0.0347

0.0380

0386

0.0190 


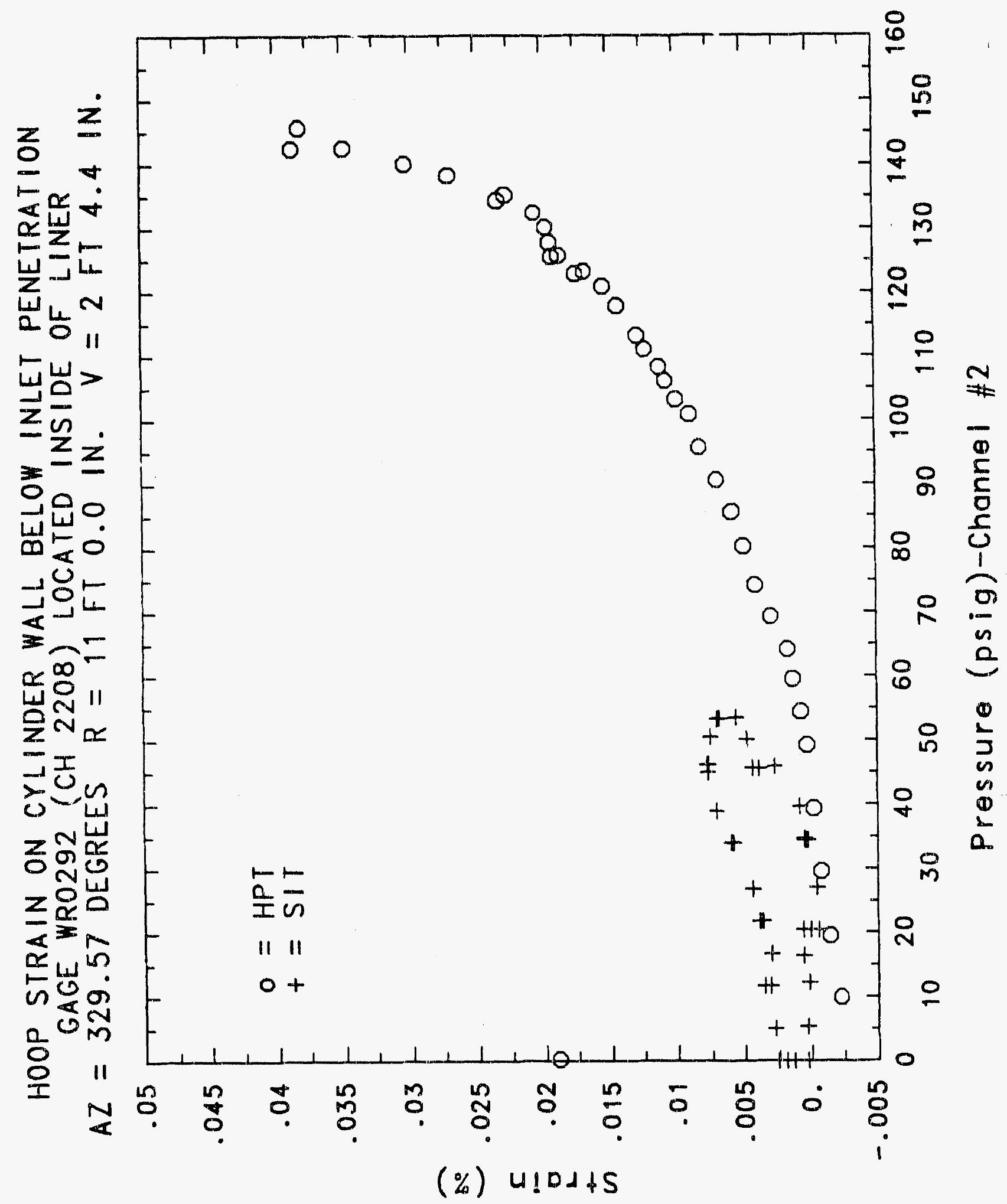




\section{Reinforced Concrete Test Data}

Weldable gage 293 Channel 2209

Structural IntEgrity TEST

High Pressure test

\begin{tabular}{rccc}
\hline Pressure & \% strain & $\begin{array}{c}\text { Pressure } \\
\text { (psig) }\end{array}$ & \% Strain \\
-0.05 & 0.0009 & 9.89 & 0.0103 \\
5.33 & 0.0028 & 19.55 & 0.0261 \\
12.31 & 0.0086 & 29.57 & 0.0424 \\
16.44 & 0.0128 & 39.42 & 0.0580 \\
20.51 & 0.0201 & 49.16 & 0.0737 \\
20.46 & 0.0220 & 54.50 & 0.0827 \\
20.47 & 0.0225 & 59.57 & 0.0903 \\
27.05 & 0.0347 & 64.20 & 0.0991 \\
34.69 & 0.0513 & 69.32 & 0.1086 \\
34.53 & 0.0513 & 74.16 & 0.1356 \\
34.53 & 0.0520 & 80.16 & 0.1563 \\
39.70 & 0.0596 & 85.61 & 0.1626 \\
45.90 & 0.0703 & 90.58 & 0.1793 \\
45.65 & 0.0715 & 95.69 & 0.1984 \\
45.62 & 0.0716 & 100.92 & 0.1651 \\
50.10 & 0.0783 & 103.25 & 0.1565 \\
53.47 & 0.0841 & 106.11 & 0.1598 \\
53.29 & 0.0840 & 108.31 & 0.1663 \\
53.21 & 0.0841 & 11.08 & 0.1750 \\
50.49 & 0.0803 & 113.24 & 0.1816 \\
46.14 & 0.0736 & 117.83 & 0.1937 \\
46.13 & 0.0729 & 120.92 & 0.2031 \\
44.97 & 0.0729 & 123.28 & 0.2105 \\
38.98 & 0.0633 & 122.97 & 0.2118 \\
33.99 & 0.0556 & 125.82 & 0.2200 \\
33.96 & 0.0558 & 125.60 & 0.2221 \\
26.83 & 0.0446 & 127.84 & 0.2282 \\
21.88 & 0.0365 & 130.19 & 0.2374 \\
21.90 & 0.0363 & 132.53 & 0.2479 \\
16.69 & 0.0276 & 135.33 & 0.2630 \\
11.74 & 0.0190 & 134.42 & 0.2650 \\
11.77 & 0.0189 & 138.35 & 0.2879 \\
5.05 & 0.0082 & 140.16 & 0.3069 \\
0.02 & 0.0038 & 142.63 & 0.3897 \\
-0.04 & 0.0004 & 145.78 & 0.3803 \\
-0.02 & 0.0043 & 142.52 & \\
-0.02 & 0.0050 & 0.22 & \\
0.02 & 0.0036 & & \\
\hline & & & \\
\hline
\end{tabular}




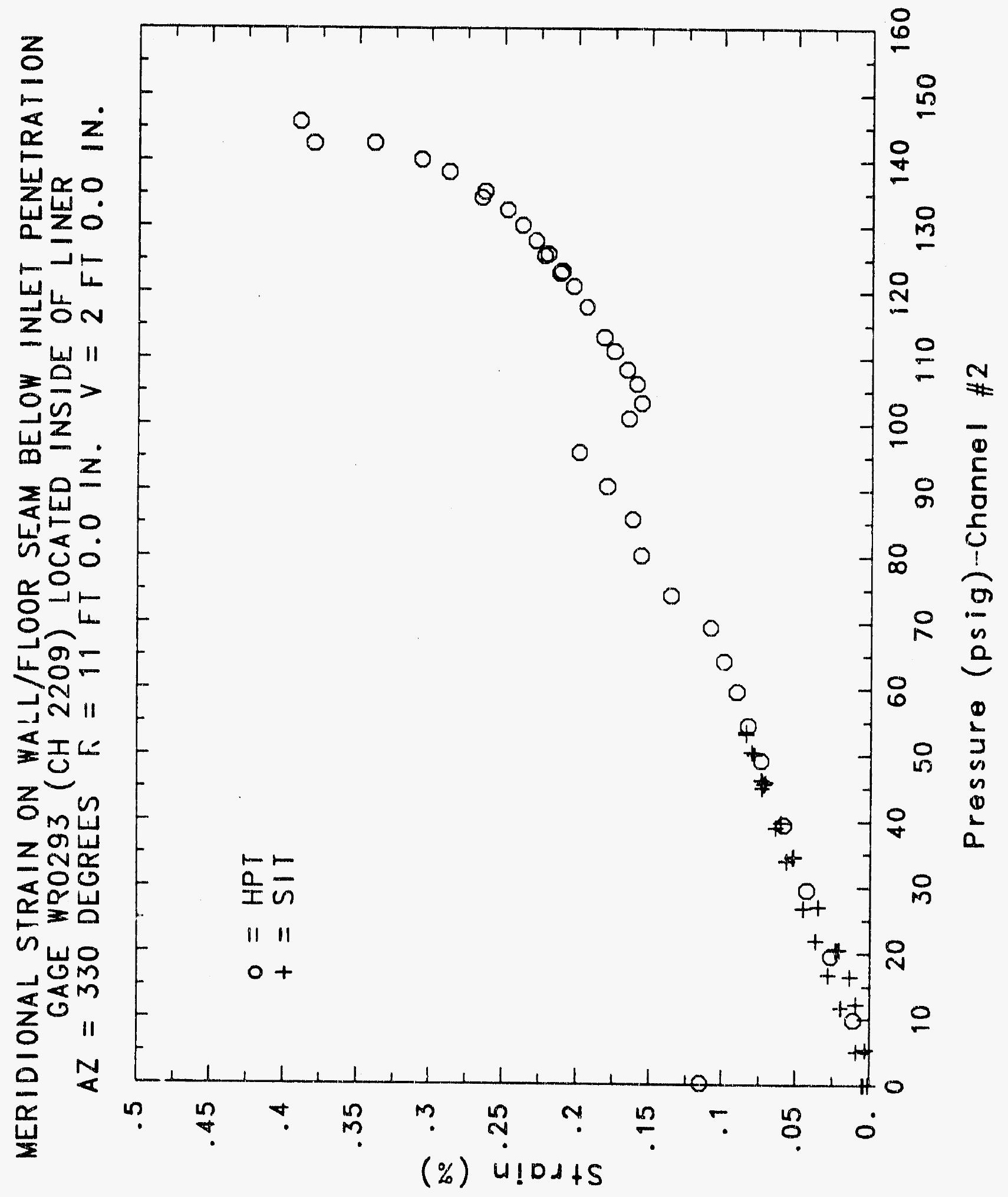




\section{Reinforced Concrete Test Data}

Weldable Gage 294 Channel 2220

Structural Integrity Test

\begin{tabular}{cccc}
\hline $\begin{array}{c}\text { Pressure } \\
\text { (psig) }\end{array}$ & \% Strain & $\begin{array}{c}\text { Pressure } \\
\text { (psig) }\end{array}$ & \% Strain \\
-0.05 & 0.0006 & 9.89 & -0.0029 \\
5.33 & 0.0026 & 19.55 & -0.0040 \\
12.31 & 0.0072 & 29.57 & -0.0054 \\
16.44 & 0.0079 & 39.42 & -0.0065 \\
20.51 & 0.0085 & 49.16 & -0.0065 \\
20.46 & 0.0077 & 54.50 & -0.0066 \\
20.47 & 0.0083 & 59.57 & -0.0076 \\
27.05 & 0.0110 & 64.20 & -0.0113 \\
34.69 & 0.0099 & 69.32 & -0.0161 \\
34.53 & 0.0124 & 74.16 & -0.0229 \\
34.53 & 0.0121 & 80.16 & -0.0281 \\
39.70 & 0.0112 & 85.61 & -0.0309 \\
45.90 & 0.0062 & 90.58 & -0.0340 \\
45.65 & 0.0044 & 95.69 & -0.0377 \\
45.62 & 0.0037 & 100.92 & -0.0419 \\
50.10 & 0.0027 & 103.25 & -0.0406 \\
53.47 & 0.0008 & 106.11 & -0.0413 \\
53.29 & -0.0005 & 108.31 & -0.0427 \\
53.21 & -0.0010 & 111.08 & -0.0445 \\
50.49 & -0.0016 & 113.24 & -0.0463 \\
46.14 & -0.0026 & 117.83 & -0.0493 \\
46.13 & -0.0029 & 120.92 & -0.0521 \\
44.97 & -0.0032 & 123.28 & -0.0544 \\
38.98 & -0.0046 & 122.97 & -0.0533 \\
33.99 & -0.0057 & 125.82 & -0.0522 \\
33.96 & -0.0062 & 125.60 & -0.0544 \\
26.83 & -0.0062 & 127.84 & -0.0567 \\
21.88 & -0.0056 & 130.19 & -0.0619 \\
21.90 & -0.0045 & 132.53 & -0.0641 \\
16.69 & -0.0038 & 135.33 & -0.0684 \\
11.74 & -0.0036 & 134.42 & -0.0695 \\
11.77 & -0.0046 & 138.35 & -0.0704 \\
5.05 & -0.0021 & 140.16 & -0.0723 \\
0.02 & -0.0028 & 142.63 & -0.0765 \\
-0.04 & -0.0024 & 145.78 & -0.0777 \\
-0.02 & -0.0016 & 142.52 & -0.0798 \\
-0.02 & -0.0013 & 0.22 & -0.0298 \\
0.02 & -0.0010 & & \\
\hline & & & \\
\hline
\end{tabular}

High Pressure test 


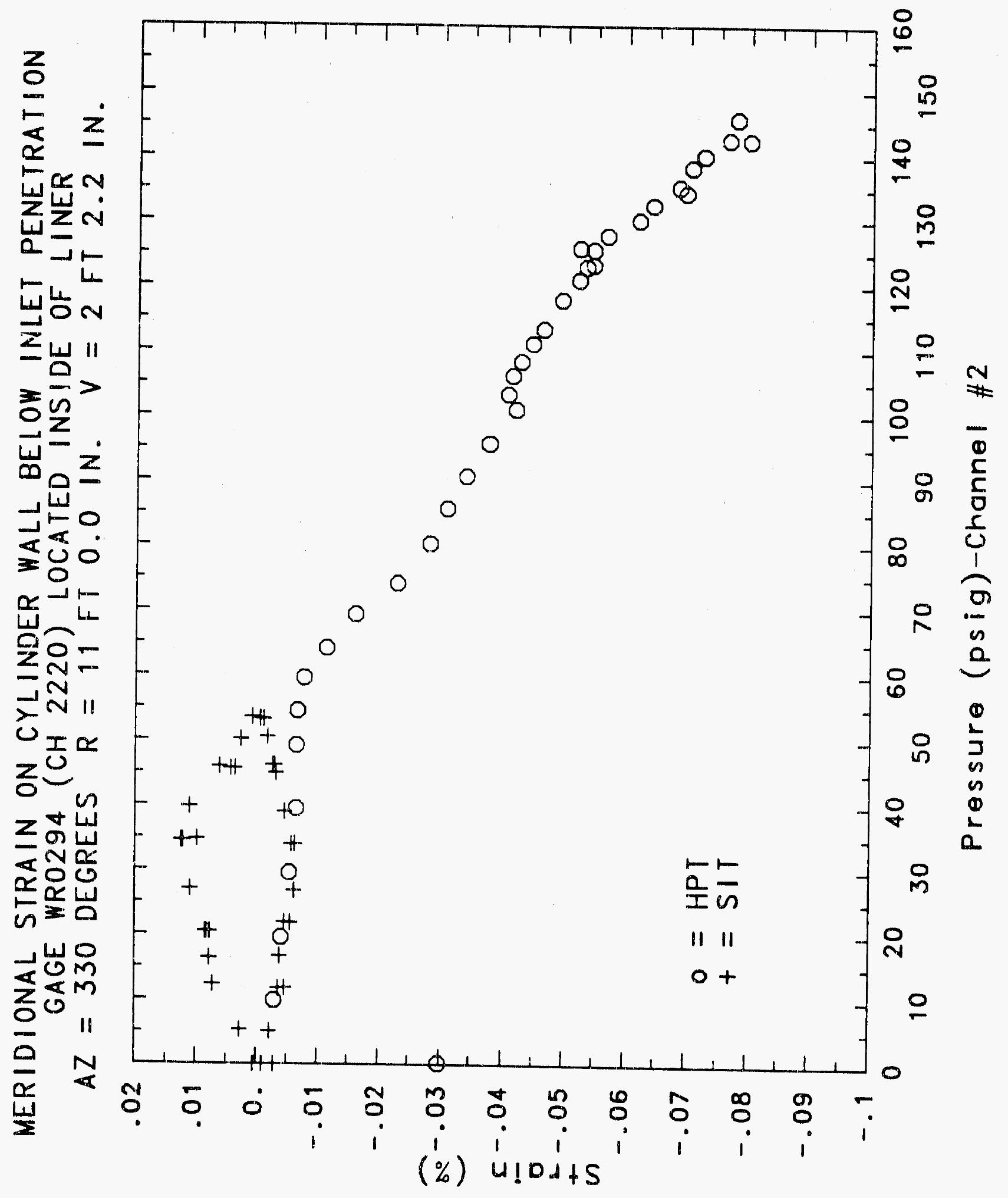




\section{Reinforced Concrete Test Data}

Weldable Gase 295 Channel 2221

Structural Integrity test

High Pressure test

\begin{tabular}{|c|c|c|c|}
\hline $\begin{array}{c}\text { Pressure } \\
\text { (psig) } \\
-0.05 \\
5.33 \\
12.31 \\
16.44 \\
20.51 \\
20.46 \\
20.47 \\
27.05 \\
34.69 \\
34.53 \\
34.53 \\
39.70 \\
45.90 \\
45.65 \\
45.62 \\
50.10 \\
53.47 \\
53.29 \\
53.21 \\
50.49 \\
16.14 \\
46.13 \\
44.97 \\
38.98 \\
33.99 \\
33.96 \\
26.83 \\
21.88 \\
21.90 \\
16.69 \\
11.74 \\
11.77 \\
5.05 \\
0.02 \\
-0.04 \\
-0.02 \\
-0.02 \\
0.02 \\
\end{array}$ & $\begin{array}{l}\text { \% Strain } \\
0.0006 \\
0.0021 \\
0.0044 \\
0.0060 \\
0.0085 \\
0.0088 \\
0.0091 \\
0.0135 \\
0.0169 \\
0.0170 \\
0.0170 \\
0.0184 \\
0.0195 \\
0.0188 \\
0.0191 \\
0.0196 \\
0.0199 \\
0.0194 \\
0.0192 \\
0.0182 \\
0.0167 \\
0.0165 \\
0.0173 \\
0.0155 \\
0.0140 \\
0.0141 \\
0.0119 \\
0.0104 \\
0.0103 \\
0.0088 \\
0.0075 \\
0.0075 \\
0.0058 \\
0.0047 \\
0.0026 \\
0.0042 \\
0.0045 \\
0.0047\end{array}$ & $\begin{array}{c}\text { Pressure } \\
(\text { psig) } \\
9.89 \\
19.55 \\
29.57 \\
39.42 \\
49.16 \\
54.50 \\
59.57 \\
64.20 \\
69.32 \\
74.16 \\
80.16 \\
85.61 \\
90.58 \\
95.69 \\
100.92 \\
103.25 \\
106.11 \\
108.31 \\
111.08 \\
113.24 \\
117.83 \\
120.92 \\
123.28 \\
122.97 \\
125.82 \\
125.60 \\
127.84 \\
130.19 \\
132.53 \\
135.33 \\
134.42 \\
138.35 \\
140.16 \\
142.63 \\
145.78 \\
142.52 \\
0.22\end{array}$ & $\begin{array}{c}\text { \% Strain } \\
\text {-0.0006 } \\
0.0017 \\
0.0045 \\
0.0070 \\
0.0096 \\
0.0111 \\
0.0123 \\
0.0132 \\
0.0134 \\
0.0124 \\
0.0115 \\
0.0118 \\
0.0116 \\
0.0111 \\
0.0101 \\
0.0099 \\
0.0100 \\
0.0100 \\
0.0096 \\
0.0096 \\
0.0096 \\
0.0093 \\
0.0089 \\
0.0084 \\
0.0084 \\
0.0079 \\
0.0081 \\
0.0080 \\
0.0076 \\
0.0070 \\
0.0069 \\
0.0057 \\
0.0050 \\
0.0035 \\
0.0017 \\
0.0000 \\
-0.0154\end{array}$ \\
\hline
\end{tabular}




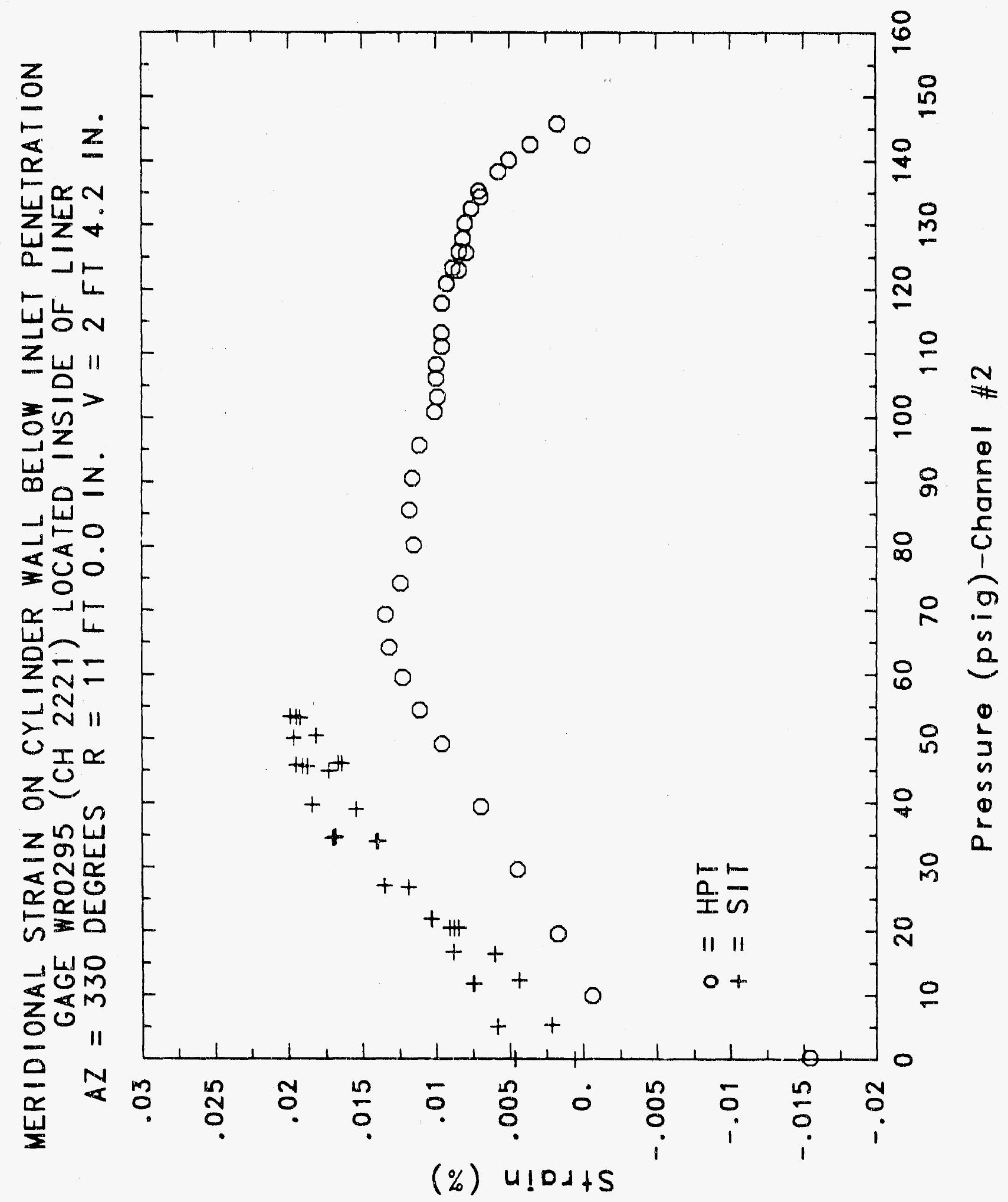



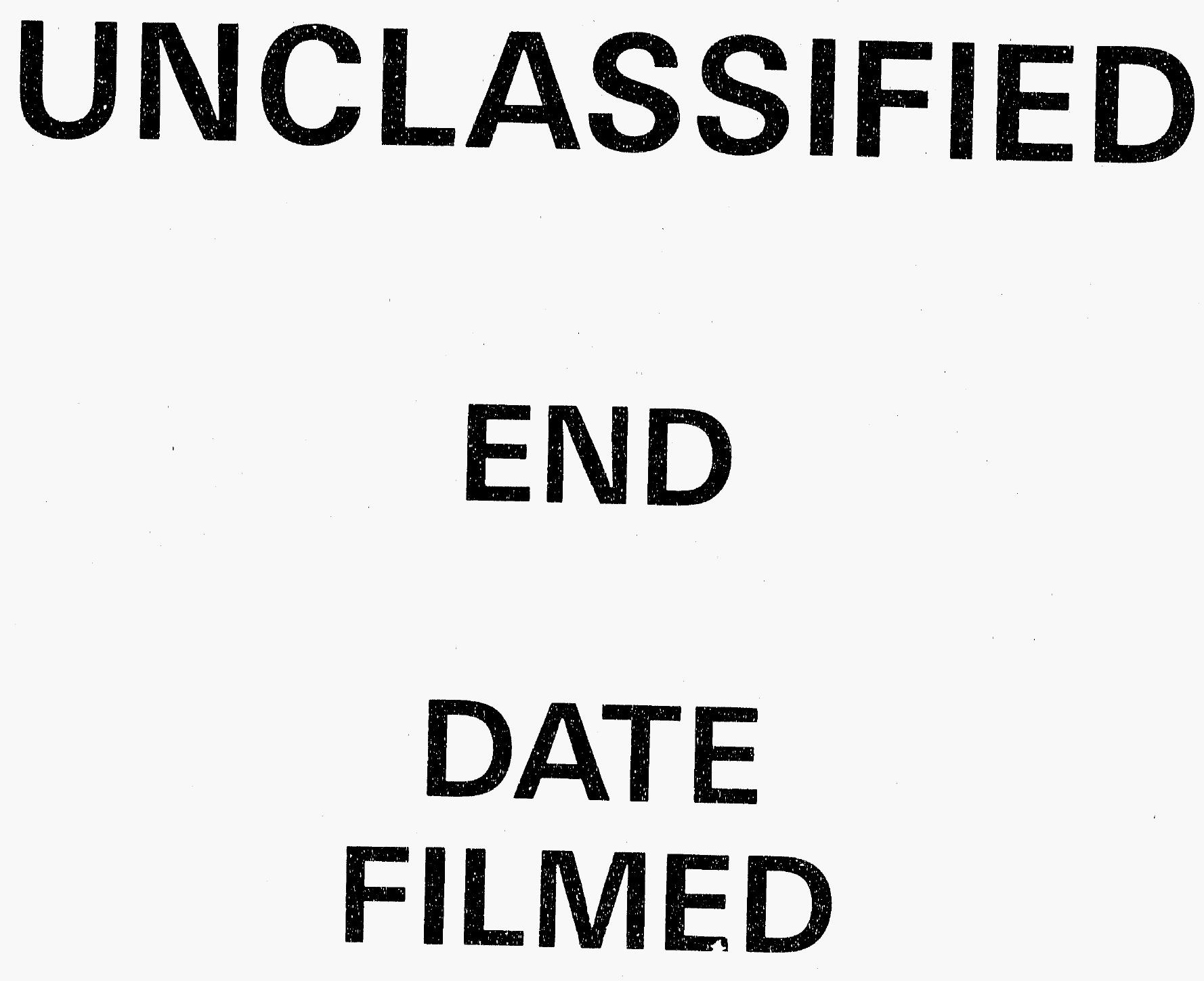

$1-6-92$

SANDIA NATIONAL LABS 
$2058-2 / 8(01 /)$

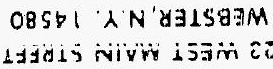

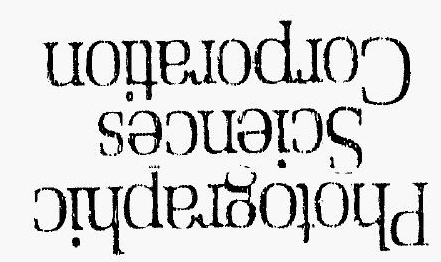
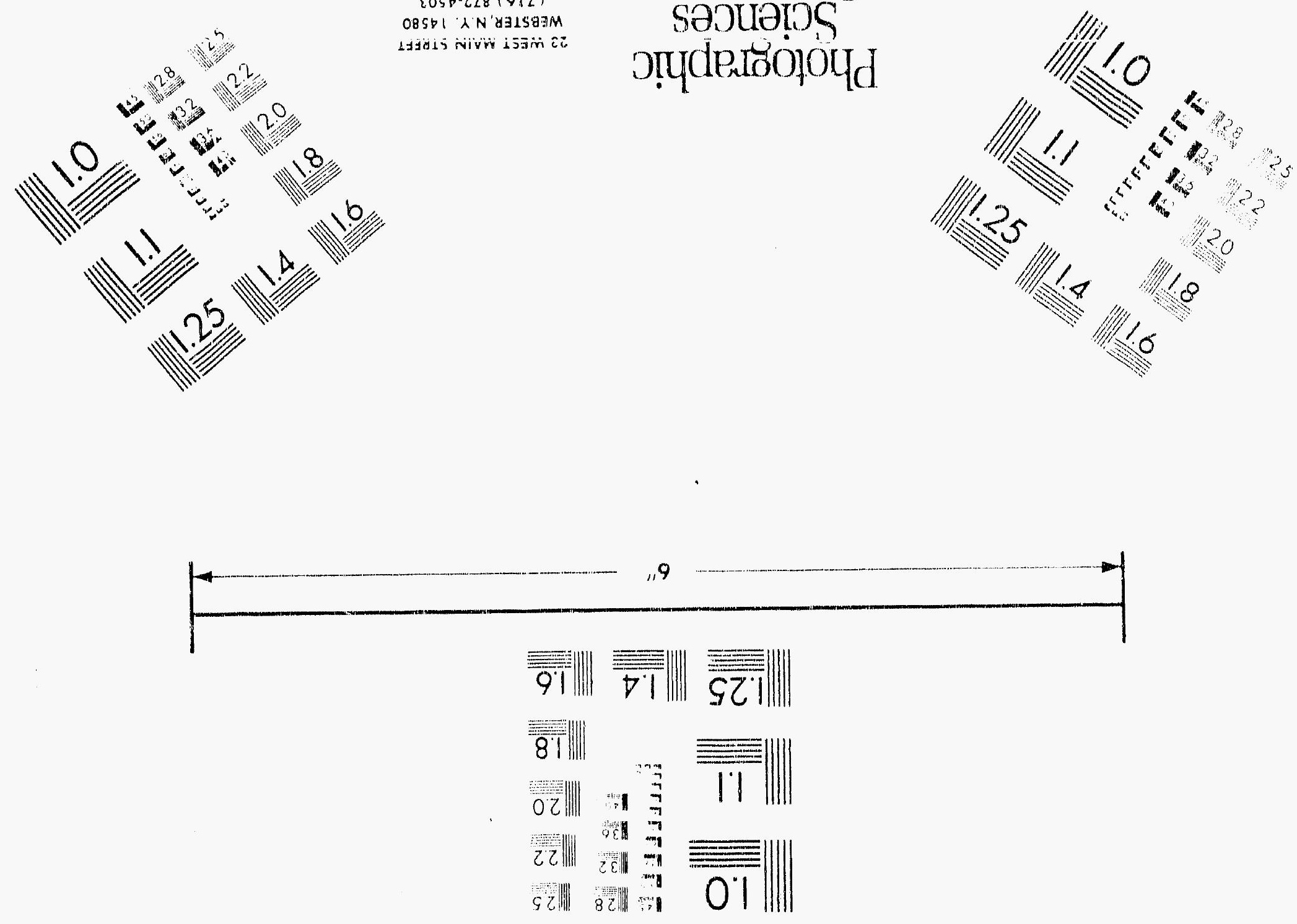

$(\varepsilon-1 W) \perp \exists S \forall \forall \perp 15 \exists \perp$

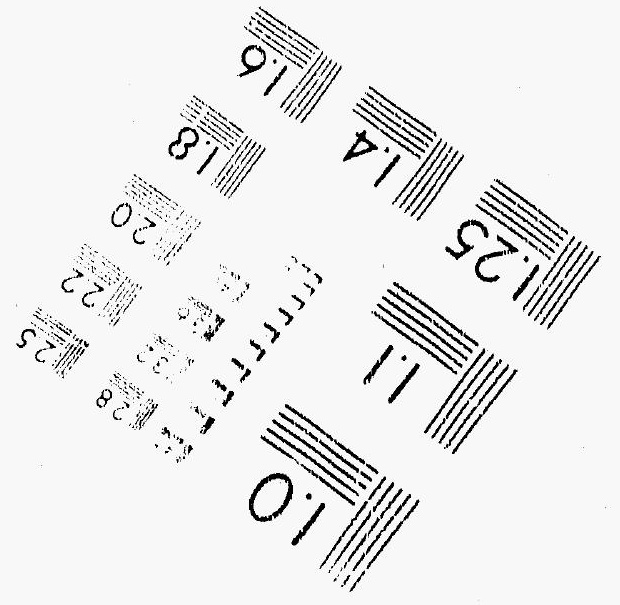

\section{NOII $\forall \cap 7 \forall \wedge \exists \exists$ 3 WI}

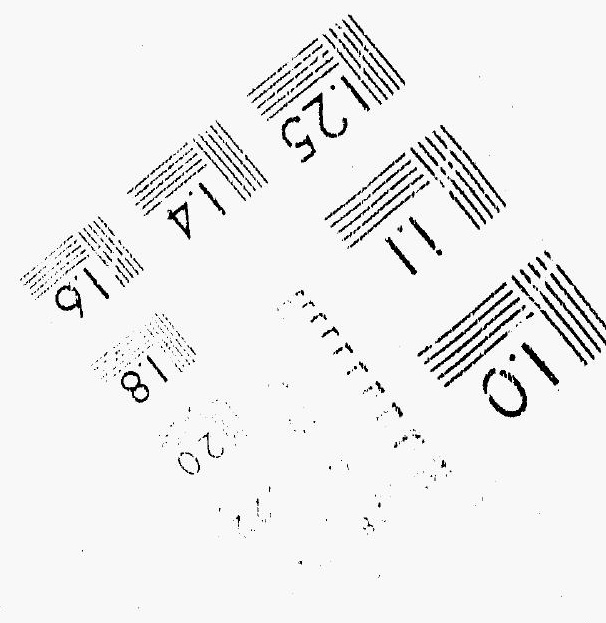




\section{SECURITY CLASSIFICATION}

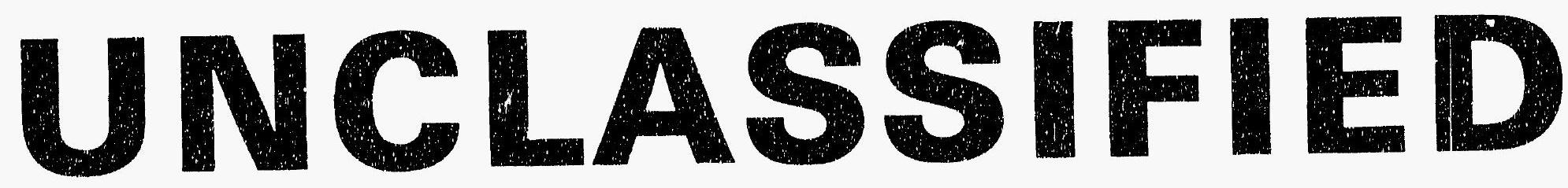

\section{DATE OF MICROFILMING $1-6-92$}

\section{MICROFILMED BY ERRY MARD}

SANDIA NATIONAL LABS 


\section{THIS DOCUMENT FILMED}

AT

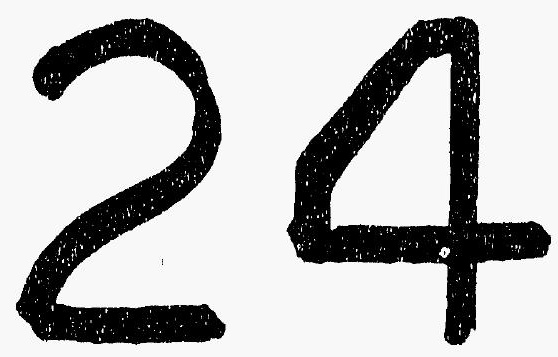

REDUCTION 


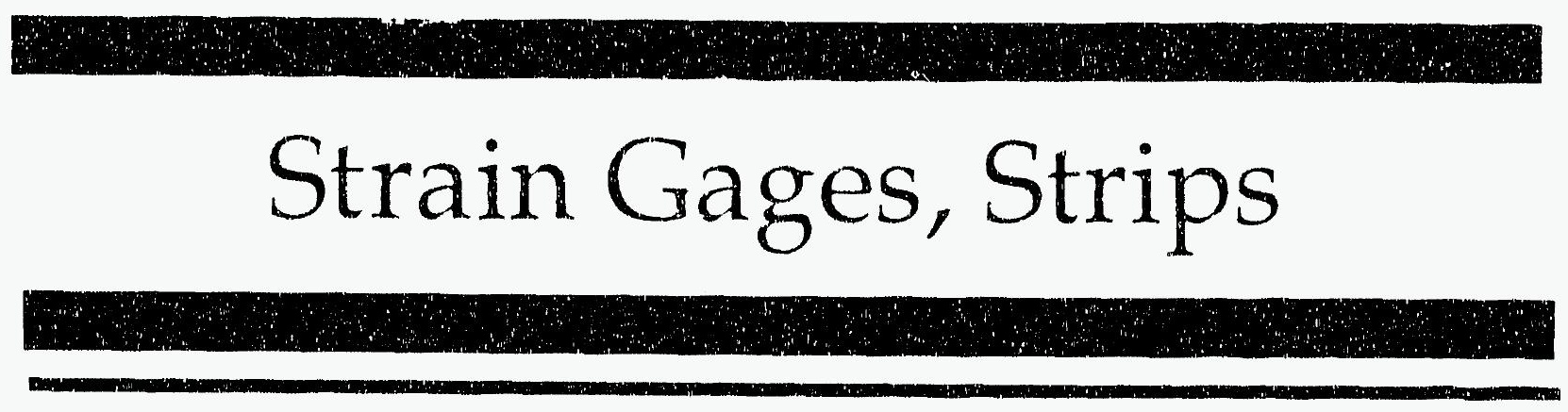


Strip 1 Channels $260,261,262,263,264$

Structural Integrity Test

\begin{tabular}{|c|c|c|c|c|c|}
\hline Pressure & Gage 1 & Gage 3 & Gage 5 & Gage 7 & Gage 3 \\
\hline Psig & \%strain & \%strain & Xstrain & zstrain & *strain \\
\hline-0.05 & 0.0020 & -0.0009 & -0.0015 & 0.0005 & -0.0007 \\
\hline 5.33 & 0.0166 & 0.0041 & -0.0053 & -0.0006 & 0.0032 \\
\hline 12.31 & 0.0350 & 0.0173 & -0.0016 & 0.0003 & 0.0092 \\
\hline 16.44 & 0.0400 & 0.0221 & 0.0021 & 0.0062 & 0.0149 \\
\hline 20.51 & 0.0431 & 0.0277 & $0.0: 03$ & 0.0149 & 0.0235 \\
\hline 20.46 & 0.0436 & 0.0295 & 0.0128 & 0.0165 & 0.025 .3 \\
\hline 20.47 & 0.0442 & 0.0297 & 0.0140 & 0.0175 & 0.0268 \\
\hline 27.05 & 0.0485 & 0.0382 & 0.0237 & 0.0298 & 0.0375 \\
\hline 34.69 & 0.0557 & 0.0477 & $0.035 !$ & 0.0430 & 0.0497 \\
\hline 3453 & 0.0550 & 0.0476 & 0.0360 & 0.0442 & 0.0508 \\
\hline 34.53 & 0.0554 & 0.0485 & 0.0366 & 0.0444 & 0.0514 \\
\hline 39.70 & 0.05 .88 & 0.0538 & 0.0434 & 0.0525 & 0.0576 \\
\hline 45.30 & 0.0644 & $0.061:$ & 0.0513 & 0.0621 & 0.0668 \\
\hline 45.65 & 0.0650 & 0.0614 & 0.0516 & 0.0615 & 0.0670 \\
\hline 4562 & 0.0658 & 0.0623 & 0.0521 & 0.06 .15 & 0.0661 \\
\hline 50.10 & 0.0693 & 0.0678 & 0.0574 & 0.0683 & 0.0731 \\
\hline 53.47 & 0.0726 & 0.0726 & 0.0534 & 0.0742 & 0.0782 \\
\hline 53.23 & 0.0732 & 0.0714 & 0.0644 & 0.0745 & 0.0773 \\
\hline 53.21 & 0.0732 & 0.0725 & 0.0630 & 0.0738 & 0.0775 \\
\hline 50.49 & 0.0727 & 0.0698 & 0.0603 & 0.06997 & 0.0732 \\
\hline 46.14 & 0.0705 & 0.0645 & 0.0545 & 00618 & 0.0657 \\
\hline 46.13 & 0.0706 & 0.0646 & 0.0537 & $0.06: 6$ & 0.0653 \\
\hline 44.97 & 0.0687 & 0.0622 & 0.0514 & 0.0588 & 0.0621 \\
\hline 38.98 & 0.0644 & 0.0553 & 0.0432 & 0.0496 & 0.0540 \\
\hline 33.99 & 0.0617 & 0.0500 & 0.0358 & 0.0411 & $0.04 E ?$ \\
\hline 33.36 & 0.0612 & 0.0513 & 0.0371 & 0.0424 & 0.0471 \\
\hline 26.83 & 0.0567 & 0.0432 & 0.0265 & 0.0307 & 0.0374 \\
\hline 21.88 & 0.0537 & 0.0386 & 0.0201 & 0.0237 & 0.0313 \\
\hline 21.90 & 0.0539 & 0.0381 & 0.0209 & 0.0253 & 0.0320 \\
\hline 16.69 & 0.0495 & 0.0322 & 0.0122 & 0.0157 & 0.0231 \\
\hline 11.74 & 0.0458 & 0.0261 & 0.0044 & 0.0074 & 0.0155 \\
\hline 11.77 & 0.0457 & 0.0251 & 0.0043 & 0.0065 & 0.0144 \\
\hline 5.05 & 0.0396 & 0.0171 & -0.0068 & -0.0037 & 0.0050 \\
\hline 0.02 & 0.0321 & 0.0094 & -0.0154 & -0.0134 & -0.0024 \\
\hline-0.04 & 0.0151 & .0 .0028 & -0.0177 & -0.0095 & -0.0020 \\
\hline .0 .02 & 0.0150 & -0.0037 & -0.0183 & -0.0095 & -0.0033 \\
\hline .002 & 0.0159 & -0.0034 & -0.0182 & -0.0097 & -0.0045 \\
\hline 0.02 & 0.0174 & -0.0005 & $\cdots 0.0181$ & -0.0104 & -0.0036 \\
\hline
\end{tabular}




\section{Reinforced Concrete Test Data}

Strip 1 Channels $260,261,262,263,264$

\section{High Pressure test}

\begin{tabular}{|c|c|c|c|c|c|}
\hline fressure & Gage 1 & Gage 3 & Gage 5 & Gage 7 & Gage 9 \\
\hline$P=10$ & zstrain & *Strain & XStrain & Kstrain & xstrain \\
\hline 383 & 0.0358 & 0.0236 & 0.0134 & 0.0109 & 0.0295 \\
\hline 19.55 & 0.0417 & 0.0391 & 0.0230 & 0.0218 & 0.0271 \\
\hline 2957 & 0.0502 & 0.0516 & 0.0391 & 0.0390 & 0.0443 \\
\hline $38.4 ?$ & 0.0548 & 0.0620 & 0.0516 & 0.0528 & $0.055 i$ \\
\hline 4616 & 00597 & 0.0721 & 0.0643 & 0.0670 & 0.0617 \\
\hline 54.50 & 0.0630 & 0.0776 & 0.0731 & 0.0750 & 0.0746 \\
\hline 59.57 & $0.066 ?$ & 0.0838 & 0.0794 & $0.08,30$ & 0.0815 \\
\hline 6.400 & 0.0717 & 0.0915 & $0.088 ?$ & 0.0925 & 0.0896 \\
\hline$f c_{1} \quad \because$ & 00755 & 0.0991 & 0.0971 & 0.1023 & 0.0980 \\
\hline $3 \therefore 16$ & 0.0828 & 0.1079 & 0.1072 & 0.1128 & 0.1072 \\
\hline$\Leftrightarrow 016$ & 0.0837 & 0.1199 & 0.1193 & 0.1268 & 0.1207 \\
\hline$\{: 1: 1$ & 0.0955 & 0.1284 & 0.1295 & 0.1385 & 0.1313 \\
\hline$\therefore \quad 5$ & 0.0977 & 0.1333 & 0.1368 & 01470 & $0.14 m$ \\
\hline 48 & 10980 & 0.1251 & 0.1416 & 0.1532 & 0.1484 \\
\hline 100 & 0.0500 & 0.1367 & 0.1472 & 0.1612 & 0.1575 \\
\hline$\therefore \therefore$ & $0096 ?$ & 0.1407 & 0.1498 & 0.1653 & 0.1611 \\
\hline lat 11 & 00,076 & 0.1439 & 0.1539 & 01701 & 0.166 .6 \\
\hline$\therefore \quad \therefore$ & 0.050 & 0.1463 & 0.1575 & 0.1744 & 0.1701 \\
\hline 111.06 & 0.1012 & 0.1495 & 0.1620 & 0.1801 & 0.1762 \\
\hline $3: 12.4$ & $0.106 \theta$ & 0.1533 & 0.1662 & 0.1850 & 0.1811 \\
\hline 117.43 & 0.1081 & 0.160 .3 & 0.1750 & 01954 & 0.1922 \\
\hline 120.82 & 01115 & 0.1649 & $0.181 ?$ & 0.2027 & 0.1987 \\
\hline 123.28 & 0.1138 & 0.1697 & 0.1858 & 0.2080 & 0.2045 \\
\hline 122.97 & 0.1139 & 0.1689 & D.: $: 86.4$ & 0.2076 & 0.2047 \\
\hline 12582 & C.:1EE & 0.1739 & 0.1919 & 02151 & 0.2110 \\
\hline $125 \mathrm{fr}^{\mathrm{n}}$ & 0.11 .1 & 0.1744 & $0.18: 3$ & $0.21 \%$ & 0.2126 \\
\hline 1278 & 0.1190 & $0.177 i$ & 0.1968 & $0: 2003$ & 0.2174 \\
\hline 130.19 & 0.12 .14 & 0.1820 & 0.2029 & 02079 & 0.226 .8 \\
\hline 1043 & 0.1232 & 0.1809 & 0.2025 & 0.2300 & 0.2371 \\
\hline 135.33 & 0.1259 & 0.1540 & 0.2203 & 0.2542 & $025 \%$ \\
\hline 13442 & 01253 & 0.1936 & 0.260. & $0 ? 545$ & $0.25 \%$ \\
\hline $1383 t$ & 0.1 .276 & 0.2016 & 0.2350 & 0.2770 & 0284 \\
\hline 14016 & 0.1278 & $0.205 i$ & 0.2444 & $0.64 \% 4$ & 0.3087 \\
\hline 14263 & 01267 & 0.2125 & 0.2568 & 0.3234 & 0.35 .39 \\
\hline 145.76 & 0.1275 & 0.2189 & 0.26 .97 & 0.3591 & 0.4139 \\
\hline $14: 8$ & 0.1269 & 0.2168 & 0.26 .74 & 03019 & $0.4: 54$ \\
\hline 022 & 0.0227 & 0.0353 & 0.0148 & O DGEA & $0.100 \mathrm{r}$ \\
\hline
\end{tabular}




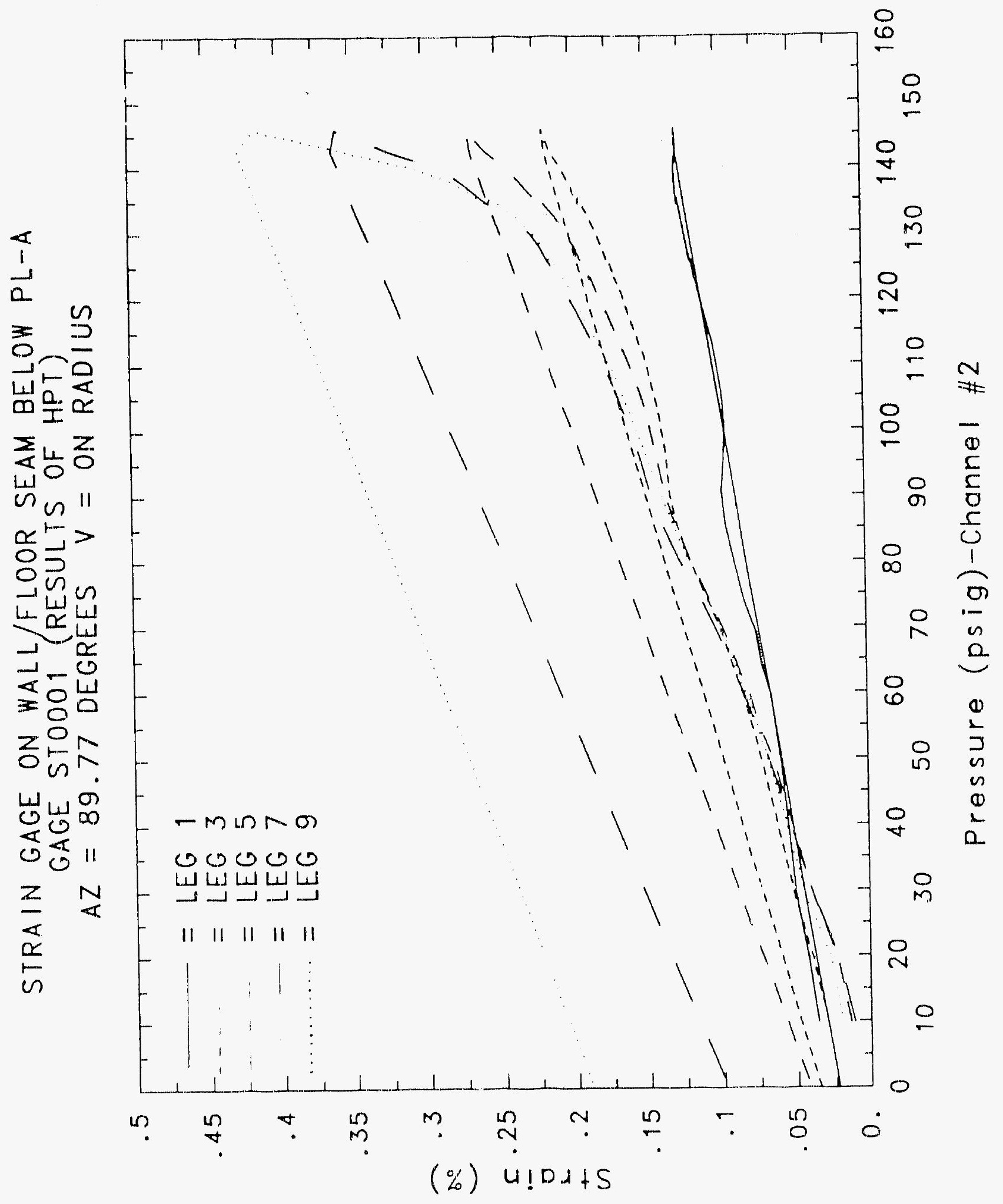

C 1400 


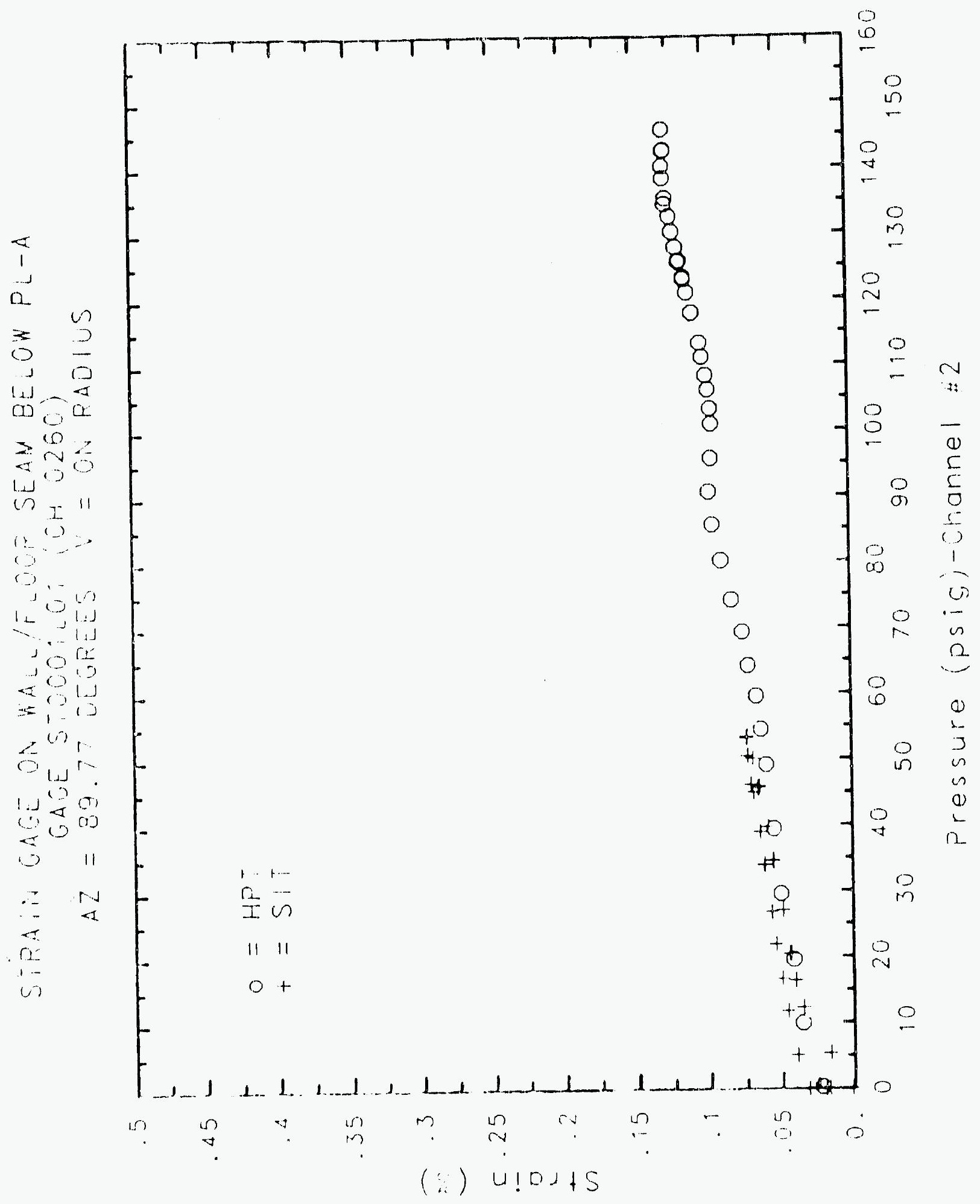


2()$+[-5$

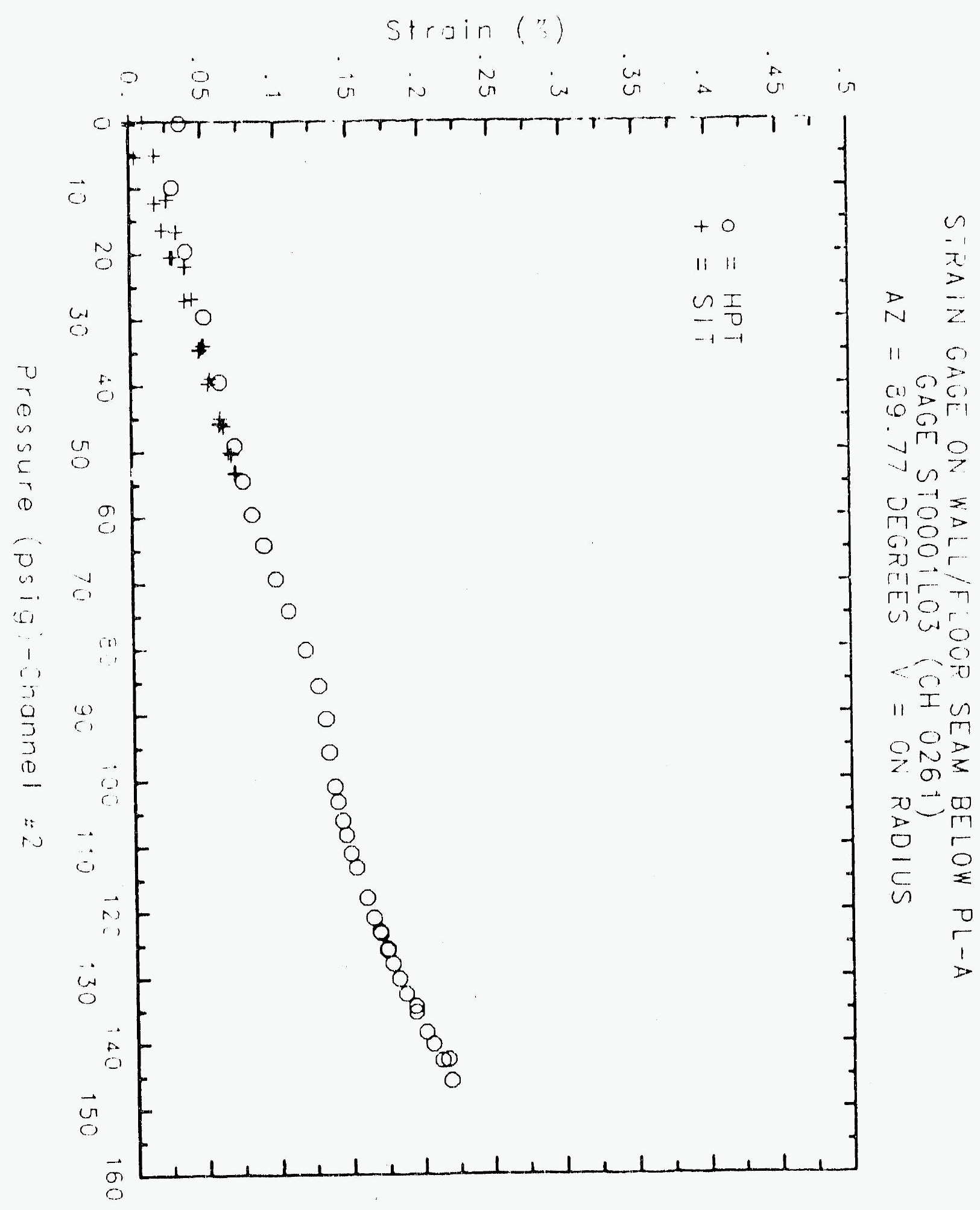


(c)t

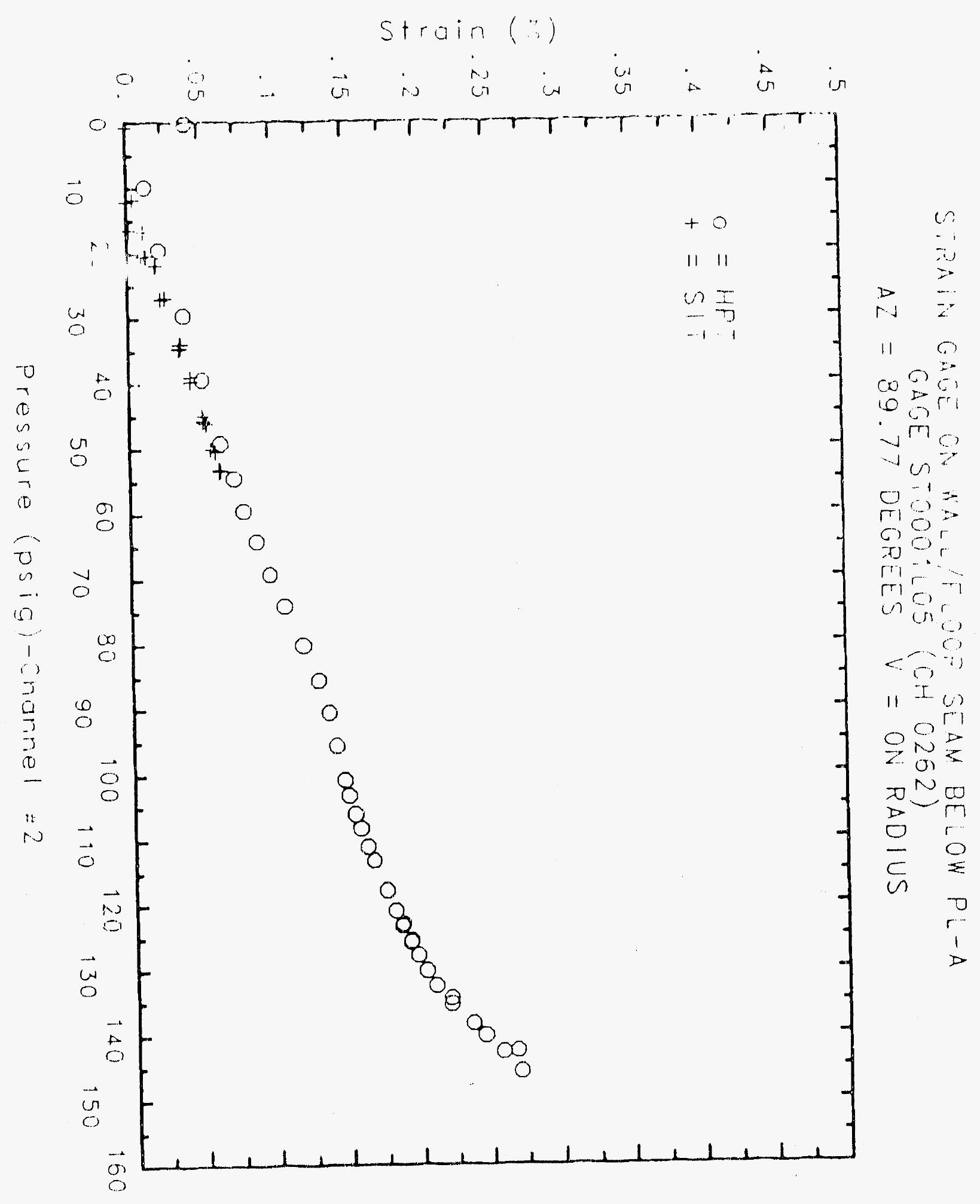




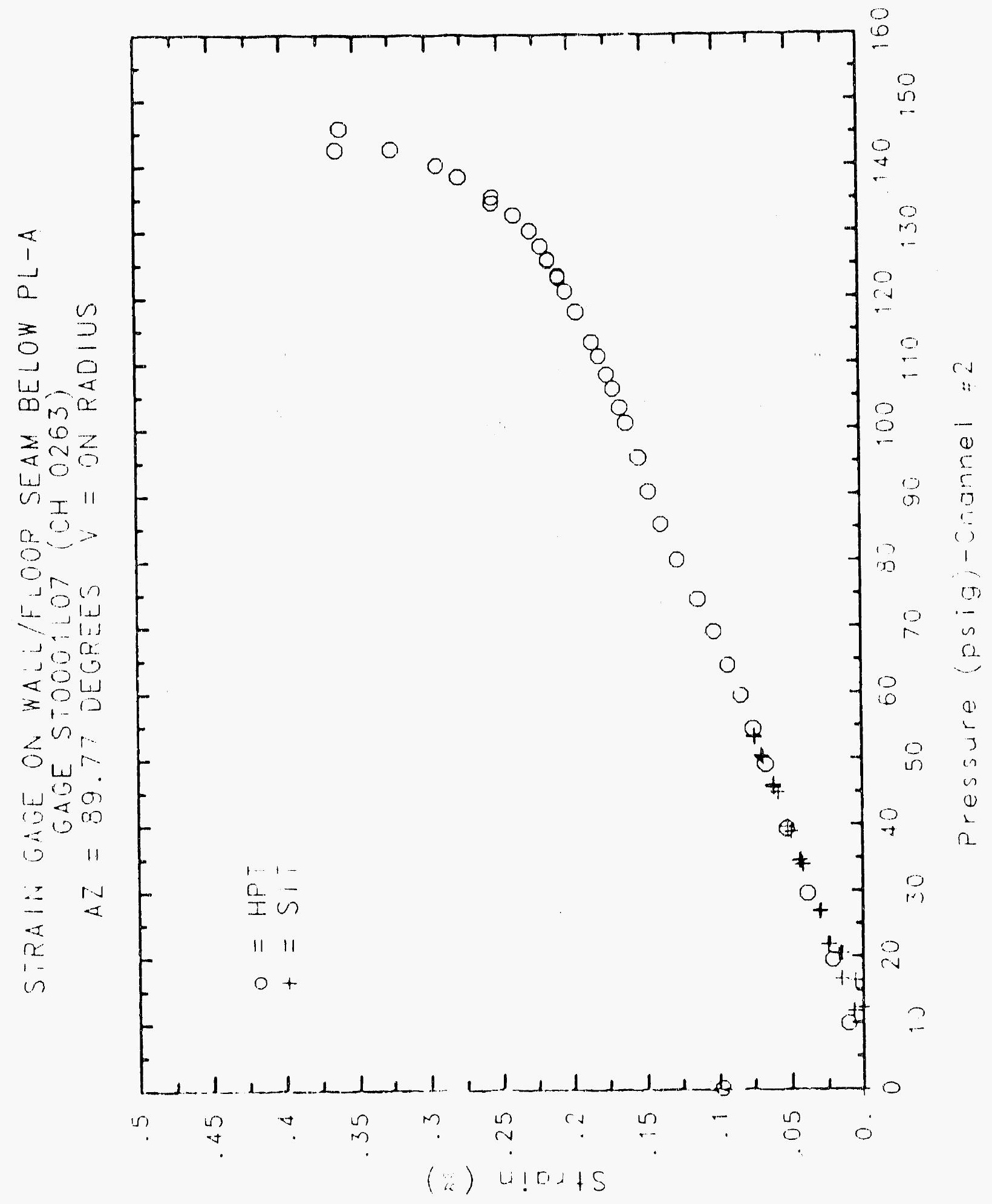

C. 1404 
S(1) $+1-0$

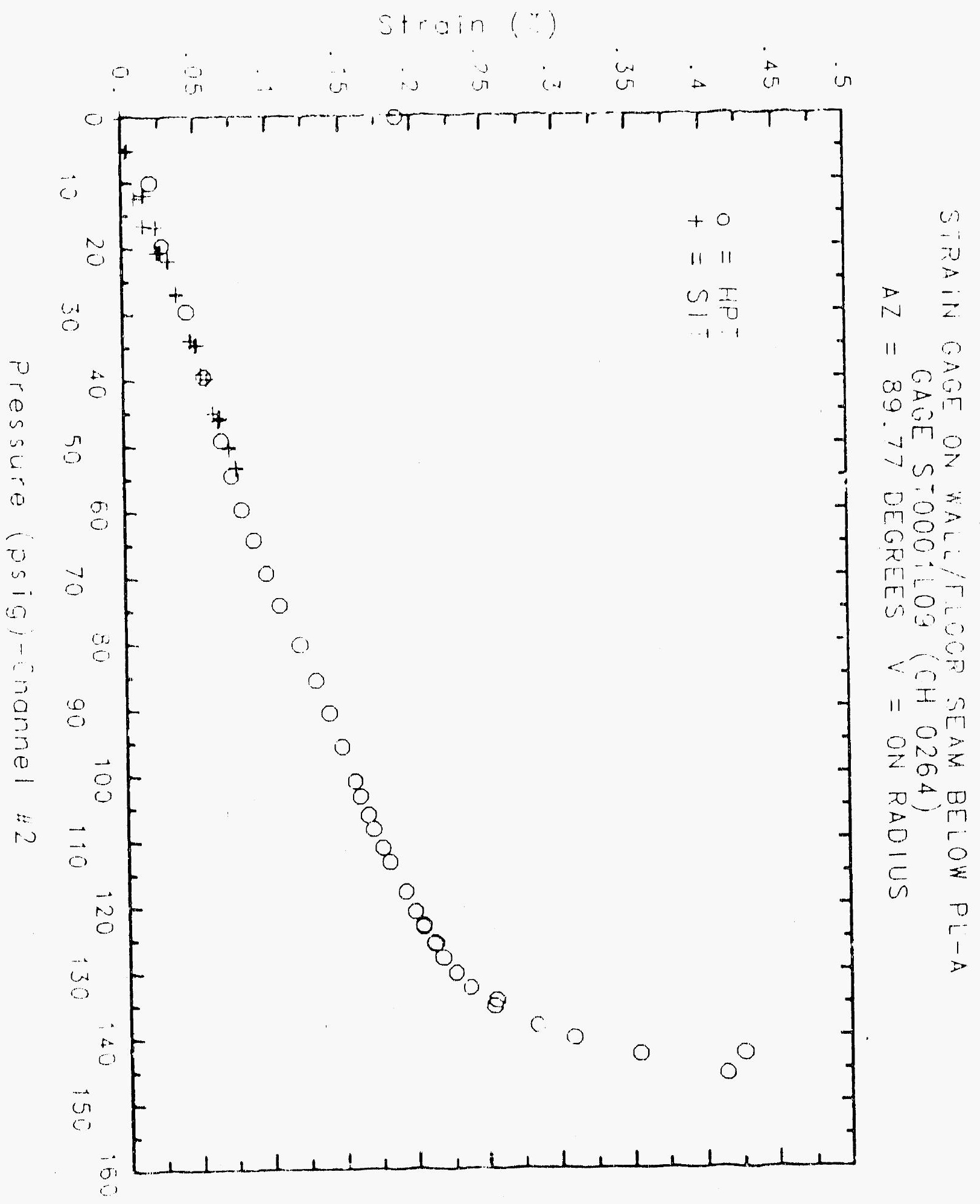




\section{Reinforced Concrete test Data}

Strip 2 Channels $265,266,267,268,269$

\section{STRUCTURAL INTEGRITY TEST}

\begin{tabular}{|c|c|c|c|c|c|}
\hline tresurn & Gage : & nge? & Gate 5 & Gage 7 & beze 9 \\
\hline$P E$ & $\begin{array}{l}\text { xsira:": } \\
0 \text { o } 0017\end{array}$ & $\begin{array}{l}\text { ketrain } \\
0 \text { grogs }\end{array}$ & $\begin{array}{l}x+5 a 1 \\
000\end{array}$ & $\begin{array}{l}\text { zitran } \\
\text { o } 000 \%\end{array}$ & $\begin{array}{l}\text { xstraw } \\
0.0000\end{array}$ \\
\hline 533 & 0.0073 & 0.0006 & 000 & 0.000 & $0.00 \%$ \\
\hline$\therefore: \vdots$ & 00.28 & 0.0131 & $00: i^{4}$ & 00150 & 0.016 \\
\hline 1E. 44 & 00100 & 0.0170 & 0.0152 & $00 ! 32$ & 0.0156 \\
\hline$\because \because \vdots$ & $0019 ?$ & $002:$ & 0.0 .12 & 000 & 0.016 \\
\hline & $0.20:$ & 0.0228 & 605 & $0.02: 1$ & 0 con \\
\hline ic 47 & 00209 & 0.02 .8 & o va? & 0.0265 & $C$ Cito \\
\hline$\therefore 05$ & $0.029 \%$ & $003 i 2$ & $c 0.4$ & 0.0381 & $003: 1$ \\
\hline 409 & 0045 & 0040 & 000 & $0.05,35$ & $0.04 \%$ \\
\hline$\because 13$ & 0.0 .55 & 0.0503 & 0.044 & 0.0547 & $0.04 \%$ \\
\hline$\therefore$ & 02444 & $0 . j \mathrm{ce}$ & $0 \cos$ & $0.056 ?$ & $0.04 \%$ \\
\hline$\therefore 0$ & 0057 & 000,3 & $0: \ldots$ & o oes: & 0.0524 \\
\hline$\therefore \quad \therefore$ & cosin 4 & 0014 & $00 \%$ & 0781 & Oor: \\
\hline$\because \vdots$ & $\therefore \therefore 6$ & 0005 & $i a$ & 0072 & $605:$ \\
\hline$\because \because$ & $\therefore$ & 0001 & $6 a$ & 0.0718 & con: 4 \\
\hline & C. & 0.0710 & $0 ?:$ & 0,0133 & cos \\
\hline$\therefore \quad \therefore$ & 6 & 0.6, & 067. & C 0242 & 0074 \\
\hline$\because \because$ & 0.20 & 0014 & $0: 4$ & c ne: & $0 \mathrm{CH}$ \\
\hline$\therefore \because:$ & 00 & o 034 & 0679 & 0.0435 & $0074:$ \\
\hline$\therefore<$ & 0.054 & 0.005 & $c 0,35$ & 0.0793 & $0.0 r s$ \\
\hline $46: 4$ & 0054 & 0.005 & 0 of & 0.0716 & 0.083 \\
\hline at & 00541 & 0.0846 & 0.0064 & C.07io & o cris \\
\hline $44 \div$ & $0.05: 5$ & 02.42 & 0 OEF 1 & 0.0719 & 0.0016 \\
\hline & 00453 & 0.0658 & 005,9 & 0062 & $0.05,41$ \\
\hline & 0402 & $0 \quad 345$ & 6060 & 00544 & $0.04 \pi$ \\
\hline$\therefore \quad \div$ & $\therefore 0409$ & 0.0409 & 0.0503 & 0.0557 & 0.0485 \\
\hline$\therefore-\therefore$ & 00205 & 0.344 & $0.030 i$ & 0.0443 & 0.031 \\
\hline$\therefore \varepsilon \alpha$ & $\therefore 020$ & 0.0 .54 & 0.0313 & 0035 & $0.03 \mathrm{i}^{\mathrm{r}}$ \\
\hline$\therefore 90$ & $0 \times 42$ & 0.298 & $0.0: 17$ & 0.0972 & $00 \%$ \\
\hline 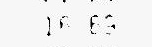 & 60173 & $0: 210$ & 0.0237 & 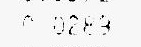 & 20054 \\
\hline$\because: 74$ & $00: 10$ & 00135 & 00 & 0020 & 0.0181 \\
\hline$\therefore 7$ & 00.21 & 0.0146 & 0014 & 0.0198 & 0.0176 \\
\hline$\vdots 05$ & 00047 & 70081 & $0 . \cos$ & 0.0103 & 0007 \\
\hline $0.0 ?$ & 0003 & -0.0019 & -00000 & 00004 & $0000 i$ \\
\hline$-6 c 4$ & 00032 & .0 .0039 & .0 .0067 & 0.002 & 0.0025 \\
\hline .32 & -00018 & -0.0016 & $-0.02: 1$ & con? & c.00\% \\
\hline 80 & 00016 & -0.0017 & 00023 & 00017 & 0 acs: \\
\hline$a$ & 0.018 & 0 DOOE & o cone & 00030 & 0.00 .6 \\
\hline
\end{tabular}




\section{Reinforced Concrete Test Data}

StRip 2 Channels $265,266,267,268,269$

High Pressure test

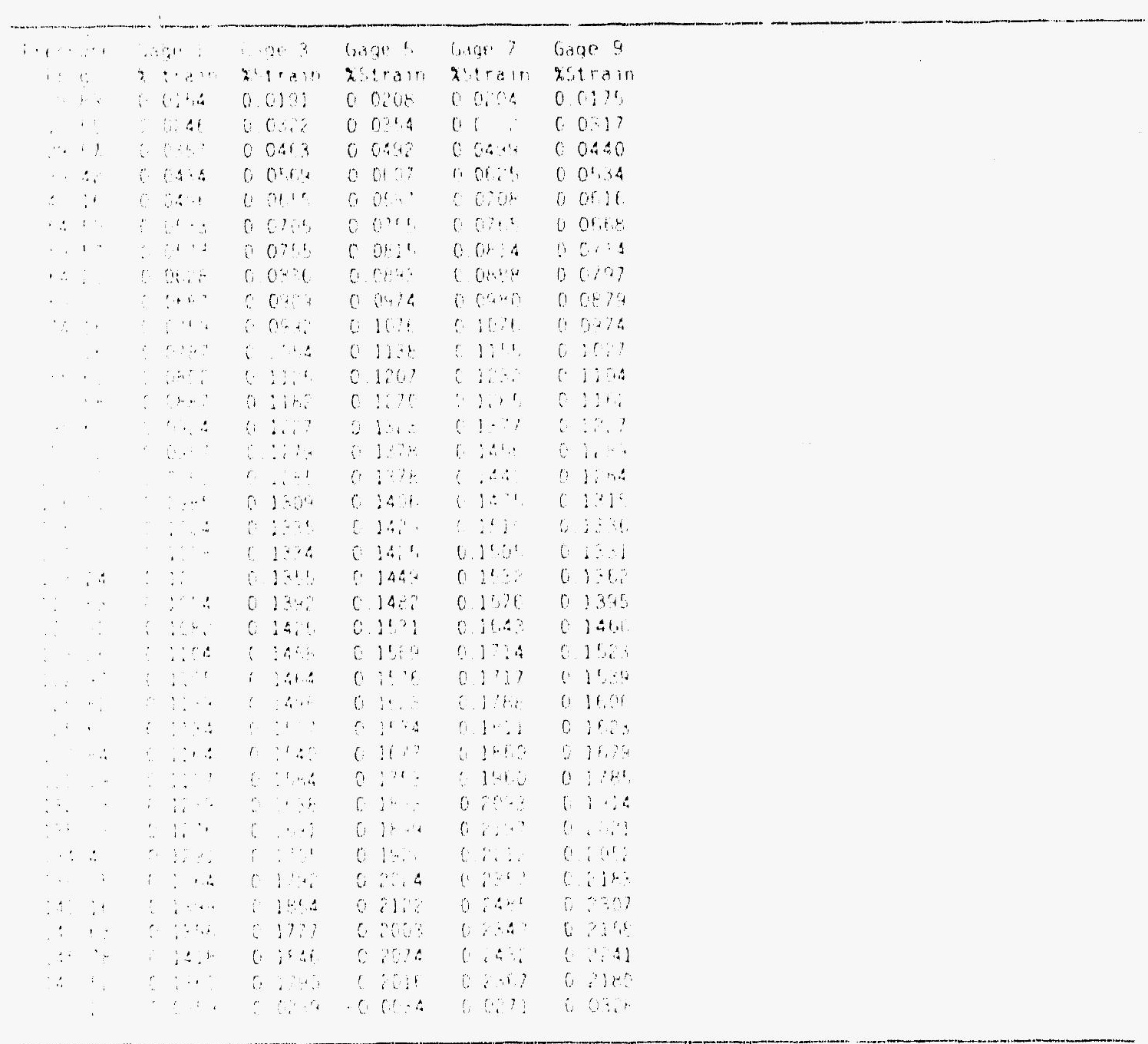




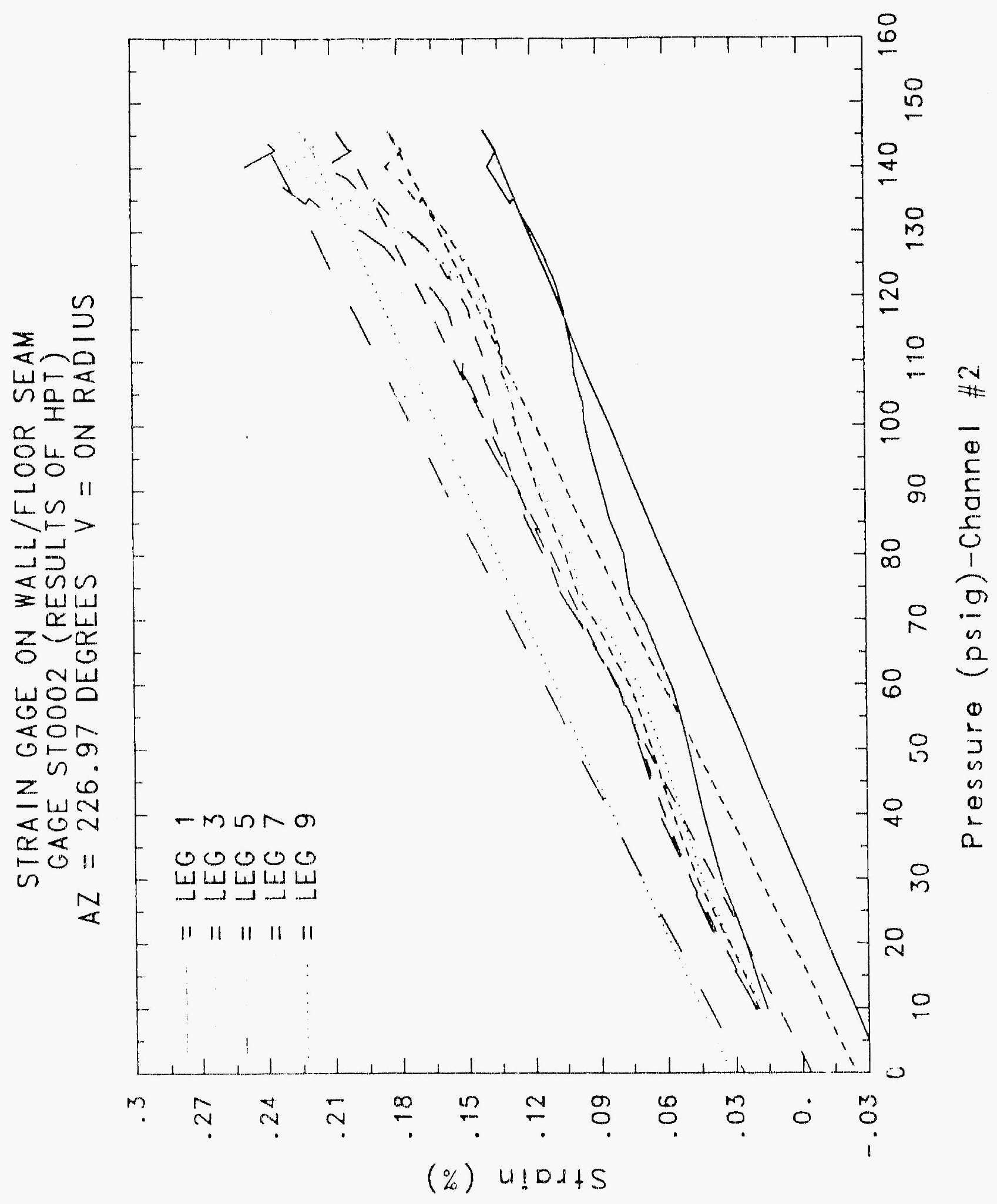




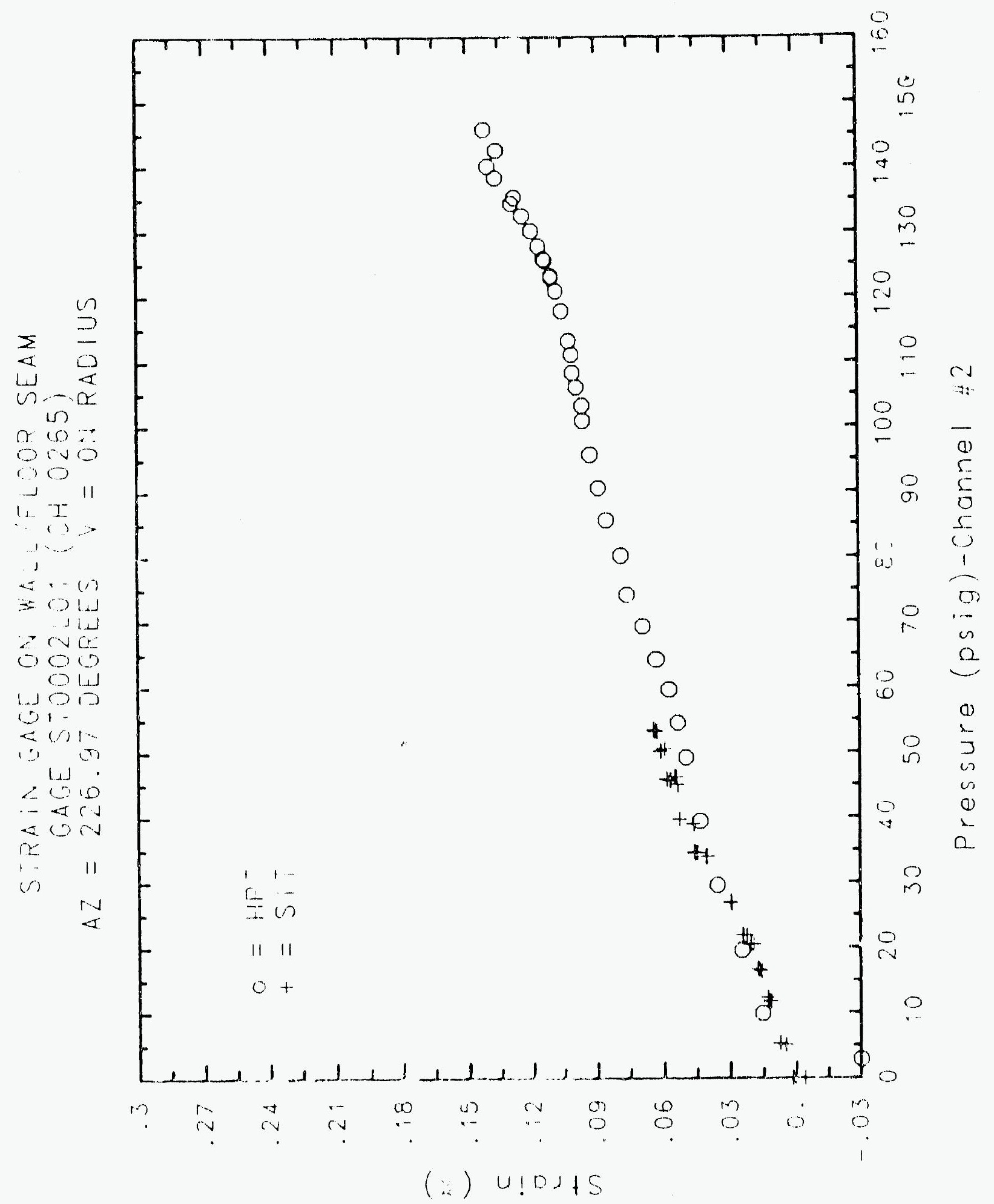

C. 1409 


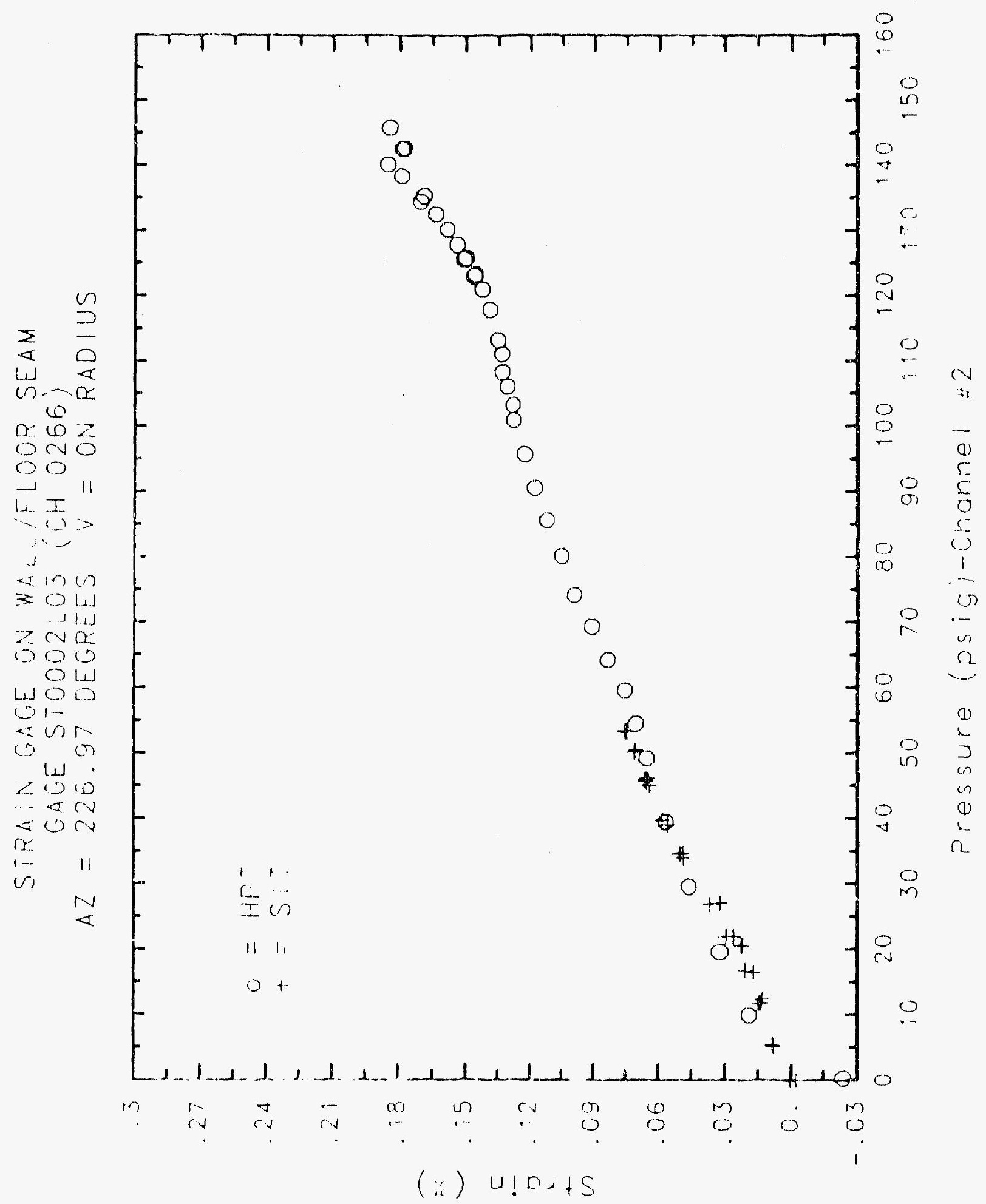

C-1410 


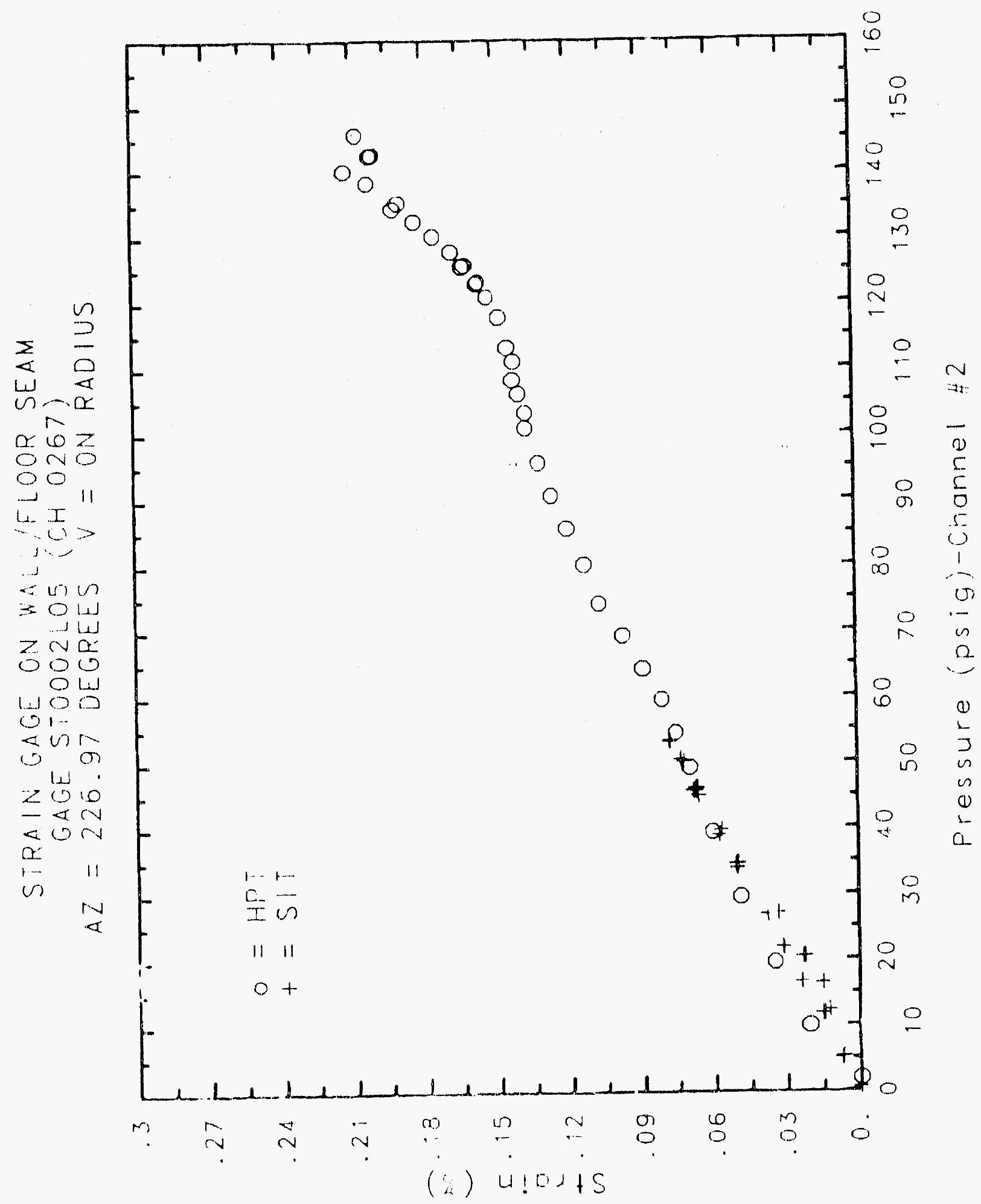




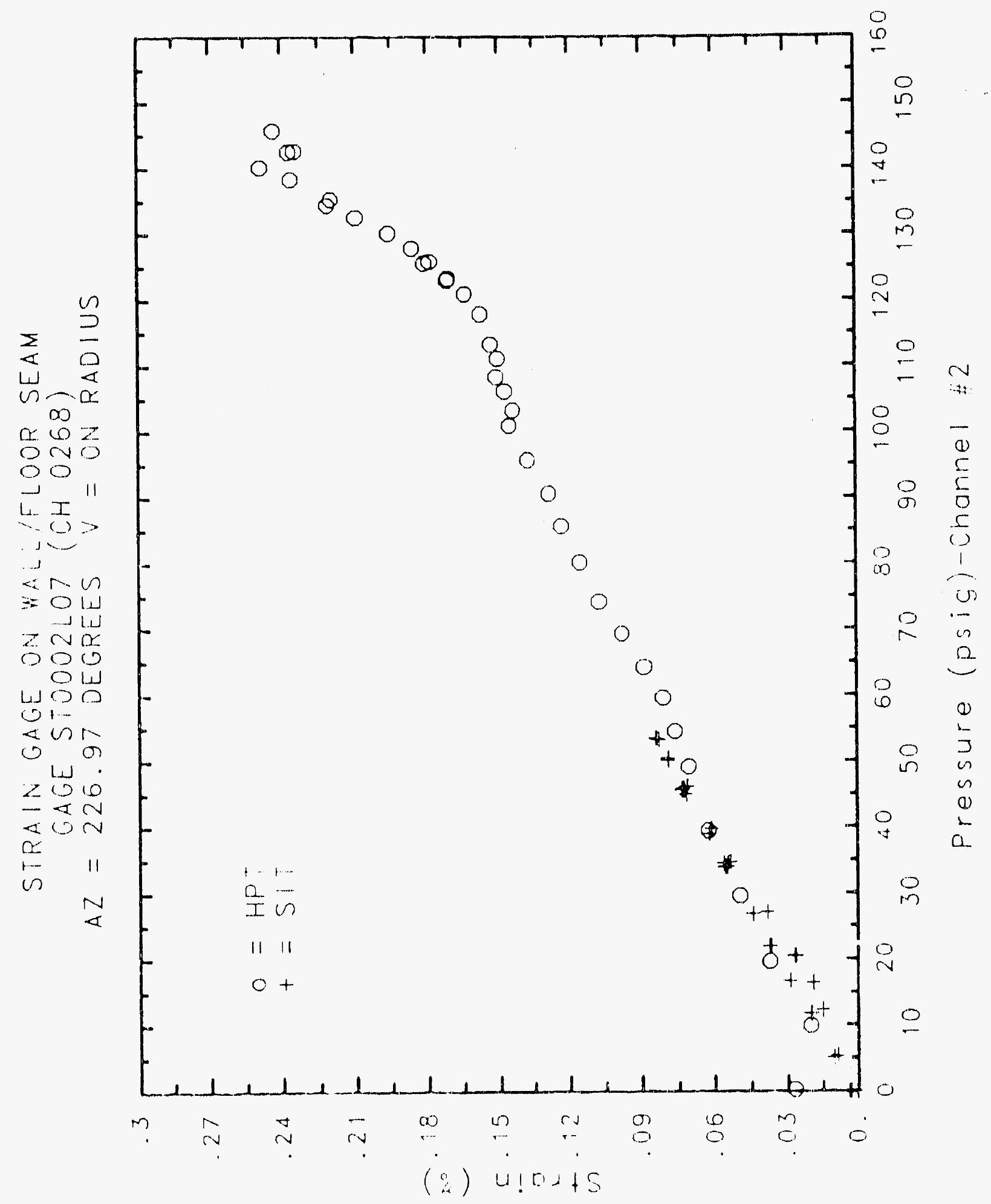

C. -1412 


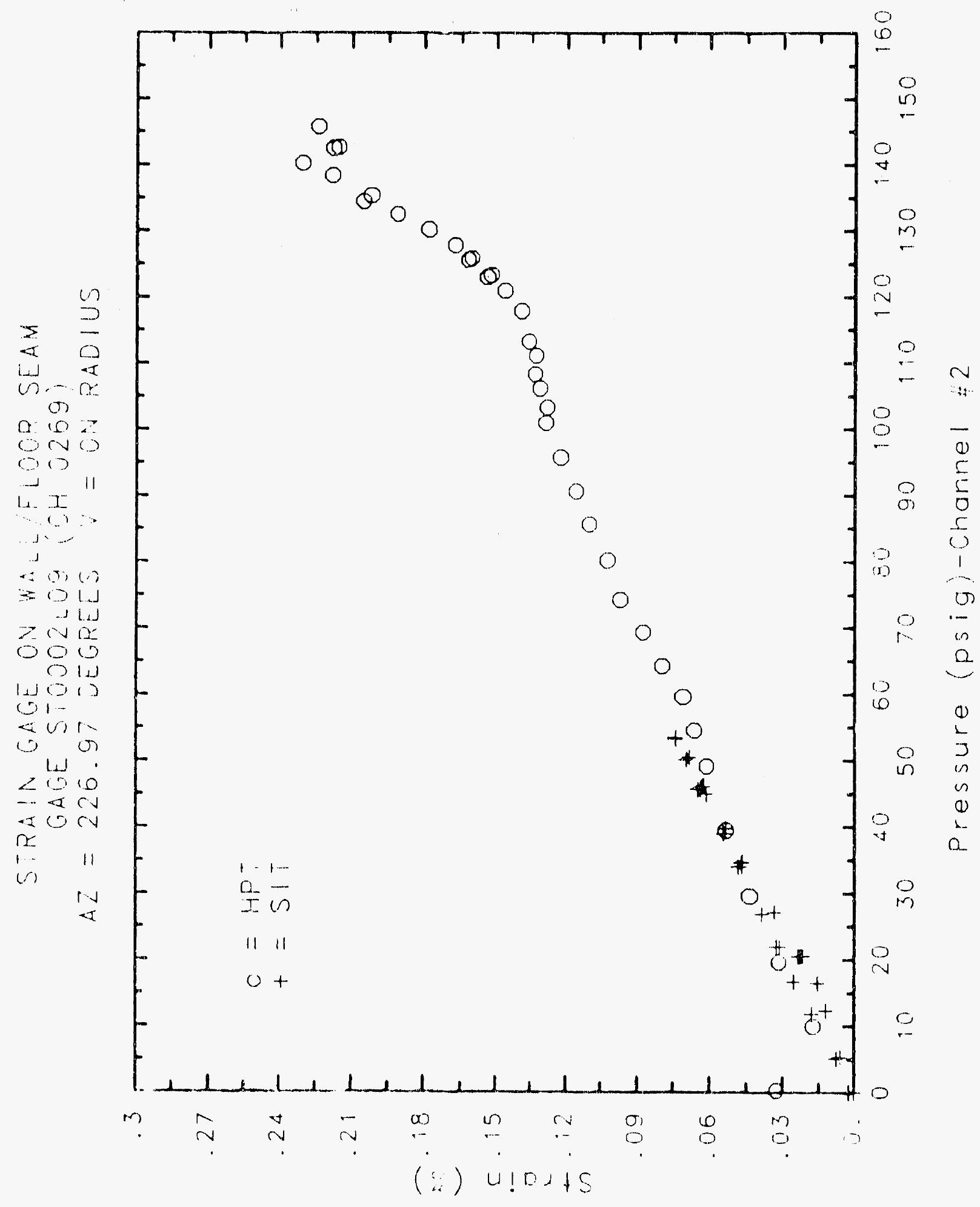

C -1413 


\section{Reinforced Concrete Test Data}

\section{Strip 3 Channels $280,281,282$}

\section{StRuCtural InTEgrity TEST}

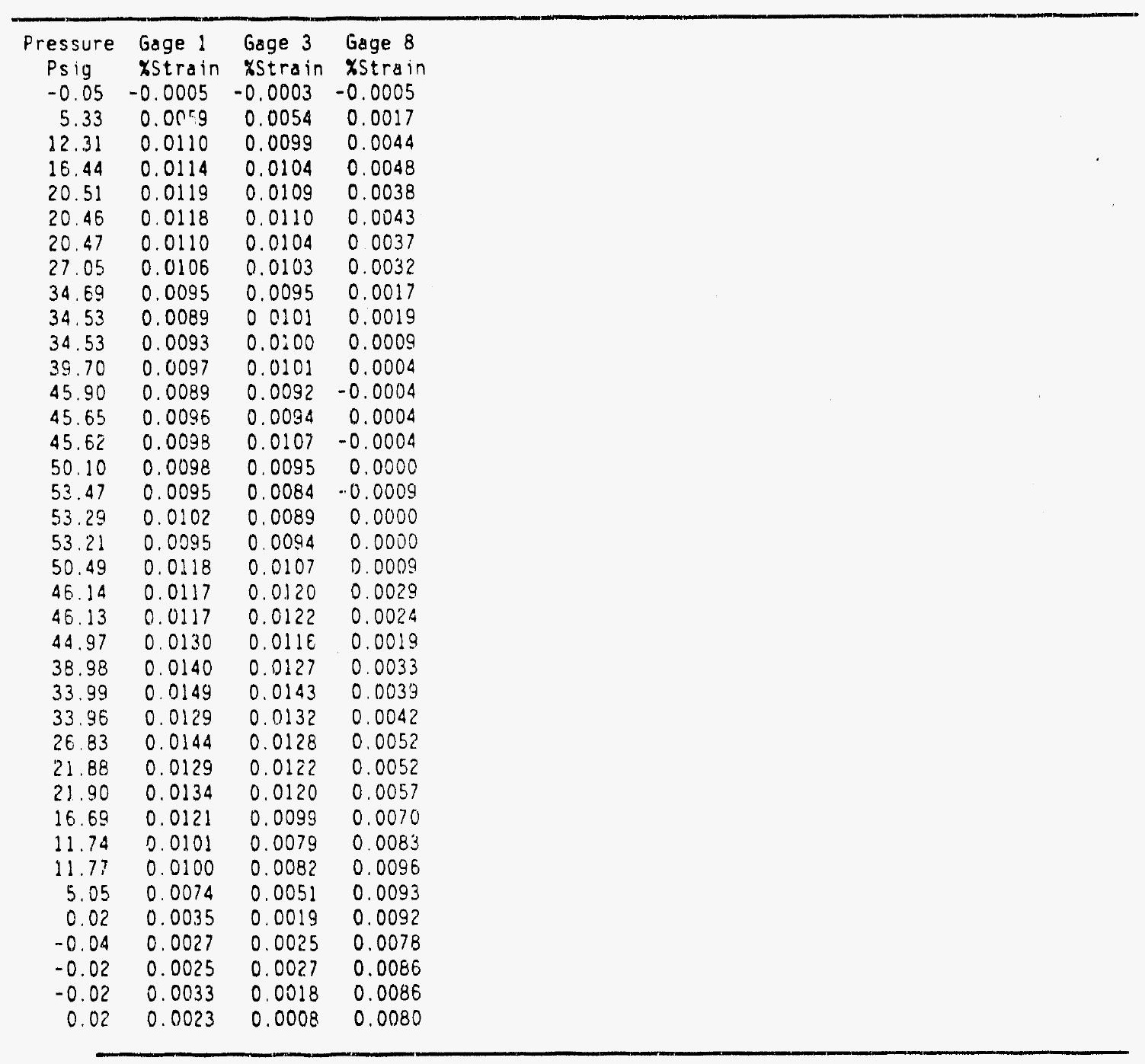




\section{Reinforced Concrete Test Data}

Strip 3 Channels $280,281,282$

High Pressure test

\begin{tabular}{|c|c|c|c|}
\hline $\begin{array}{c}\text { Pressure } \\
\text { Psig }\end{array}$ & Gage 1 & Gage 3 & $\begin{array}{l}\text { Gage } 8 \\
\text { Zstrain }\end{array}$ \\
\hline $\begin{array}{r}\text { P519 } \\
9.89\end{array}$ & $\begin{array}{l}\text { Xstrain } \\
0.0122\end{array}$ & $\begin{array}{l}\text { Xstrain } \\
0.0107\end{array}$ & $\begin{array}{l}\text { zstrain } \\
0.0056\end{array}$ \\
\hline 19.55 & 0.0175 & 0.0163 & 0.0055 \\
\hline 29.57 & 0.0212 & 0.0217 & 0.0068 \\
\hline 39.42 & 0.0207 & 0.0205 & 0.0048 \\
\hline 49.16 & 0.0198 & 0.0206 & 0.0025 \\
\hline 54.50 & 0.0190 & 0.0192 & 0.0008 \\
\hline 59.57 & 0.0191 & 0.0191 & -0.0012 \\
\hline 64.20 & 0.0197 & 0.0189 & -0.0026 \\
\hline 69.32 & 0.0195 & 0.0190 & -0.0045 \\
\hline 74.16 & 0.0187 & 0.0181 & -0.0072 \\
\hline 80.16 & 0.0184 & 0.0175 & -0.0059 \\
\hline 85.61 & 0.0178 & 0.0170 & -0.0071 \\
\hline 90.58 & 0.0177 & 0.0159 & -0.0088 \\
\hline 95.69 & 0.0170 & 0.0140 & -0.0095 \\
\hline 100.92 & 0.0153 & 0.0142 & -0.0107 \\
\hline 103.25 & 0.0153 & 0.0134 & -0.1114 \\
\hline 106.11 & 0.0150 & 0.0123 & -0.0116 \\
\hline 108.31 & 0.0142 & 0.0117 & -0.0127 \\
\hline 111.08 & 0.0147 & 0.0114 & -0.0136 \\
\hline 113.24 & 0.0140 & 0.0112 & -0.0142 \\
\hline 117.83 & 0.0136 & 0.0097 & -0.0155 \\
\hline 120.92 & 0.0128 & 0.0091 & -0.0172 \\
\hline 123.28 & 0.0127 & 0.0082 & -0.0179 \\
\hline 122.97 & 0.0128 & 0.0085 & -0.0181 \\
\hline 125.82 & 0.0120 & 0.0076 & -0.0190 \\
\hline 125.60 & 0.0122 & 0.0076 & -0.0194 \\
\hline 127.84 & 0.0112 & 0.0072 & -0.0199 \\
\hline 130.19 & 0.0103 & 0.0064 & -0.0220 \\
\hline 132.53 & 0.0092 & 0.0038 & -0.0247 \\
\hline 135.33 & 0.0076 & 0.0024 & -0.0274 \\
\hline 134.42 & 0.0073 & 0.0027 & -0.0276 \\
\hline 138.35 & 0.0049 & 0.0011 & -0.0303 \\
\hline 140.16 & & 0.0002 & -0.0326 \\
\hline 142.63 & 0.0029 & 0.0001 & -0.0331 \\
\hline 145.78 & 0.0014 & 0.0000 & $-0.034 !$ \\
\hline 142.52 & 0.0015 & 0.0007 & -0.0328 \\
\hline 0.22 & 0.0308 & 0.0313 & 0.0295 \\
\hline
\end{tabular}




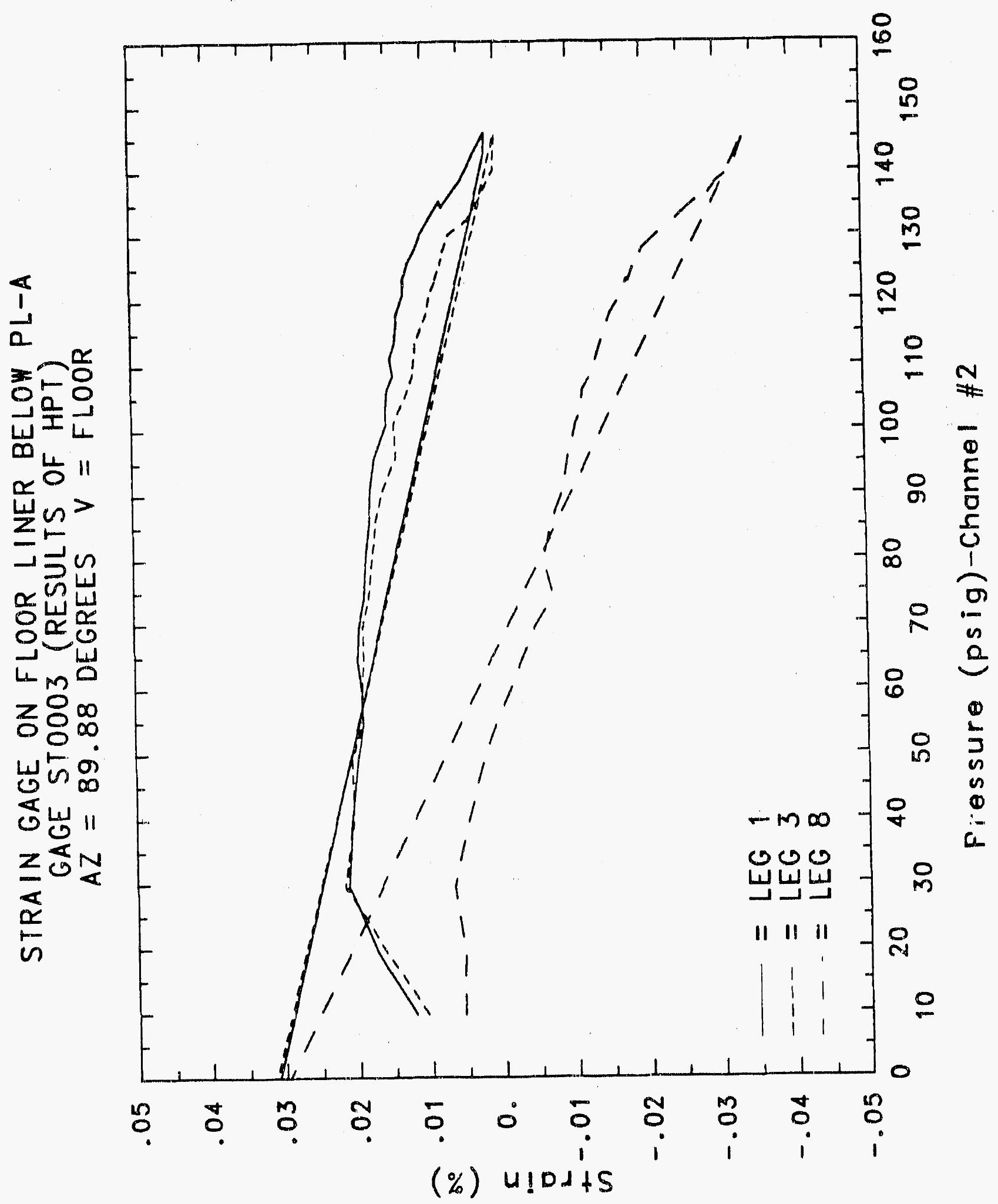




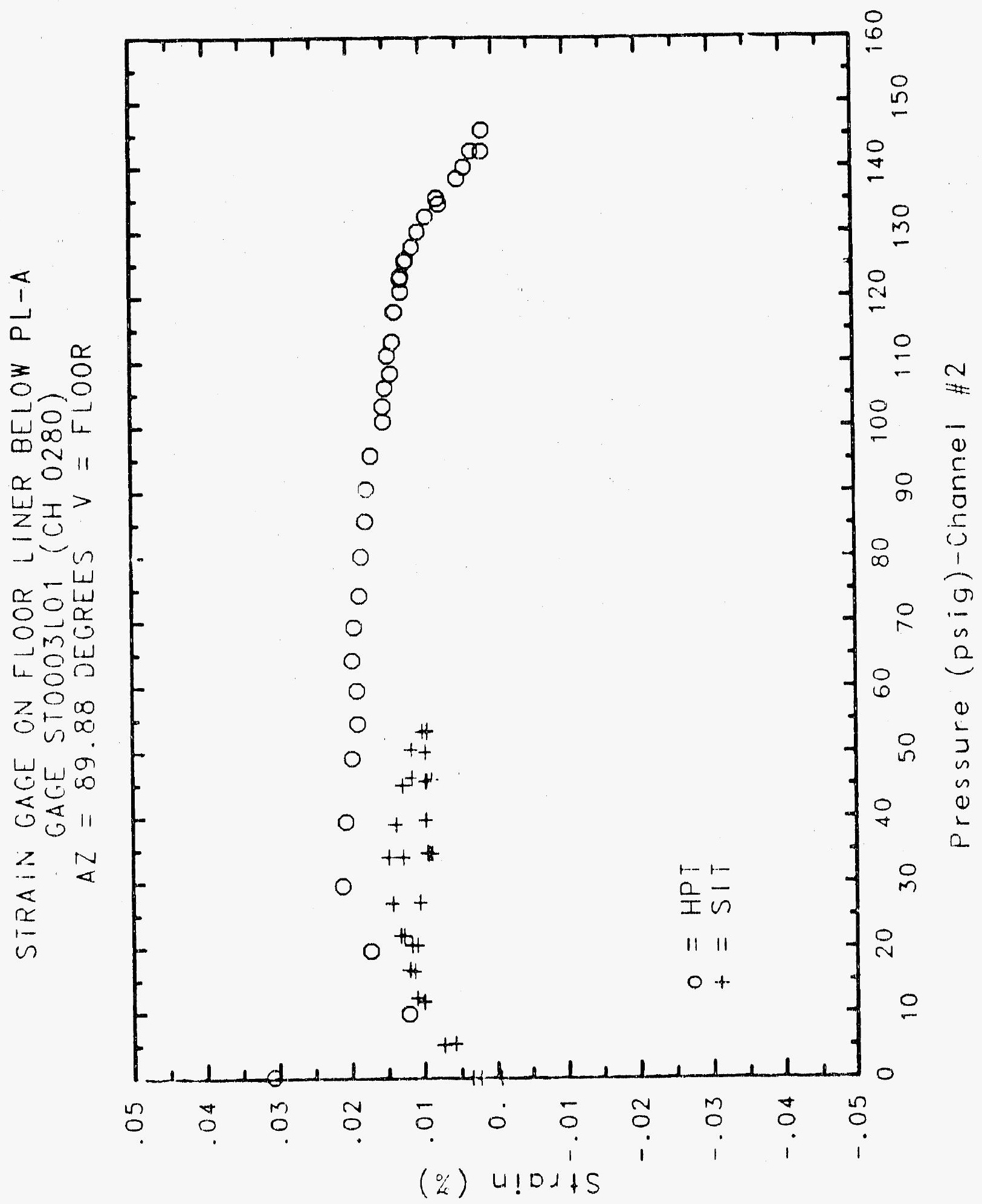

C. 1417 


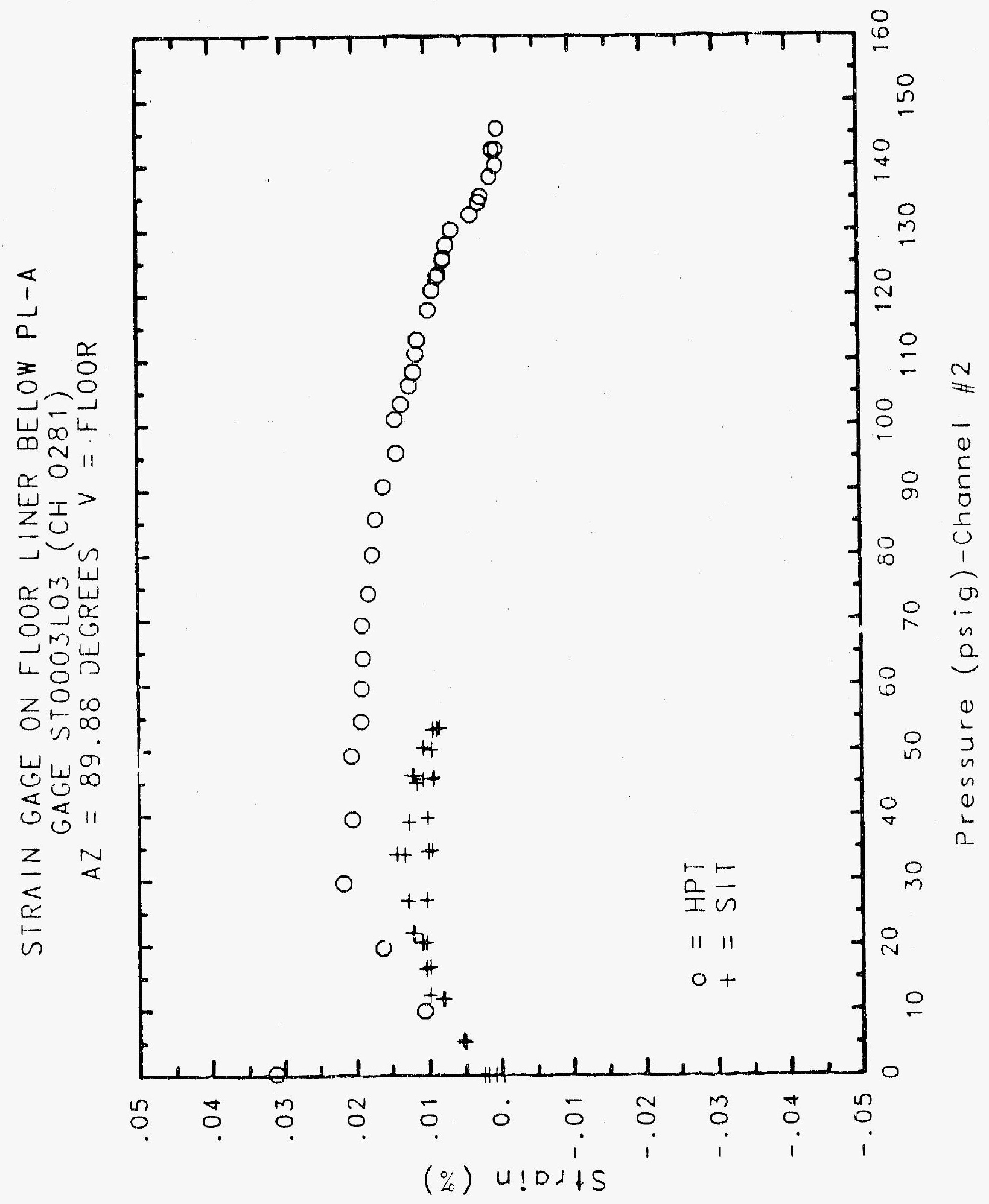

C. 1418 


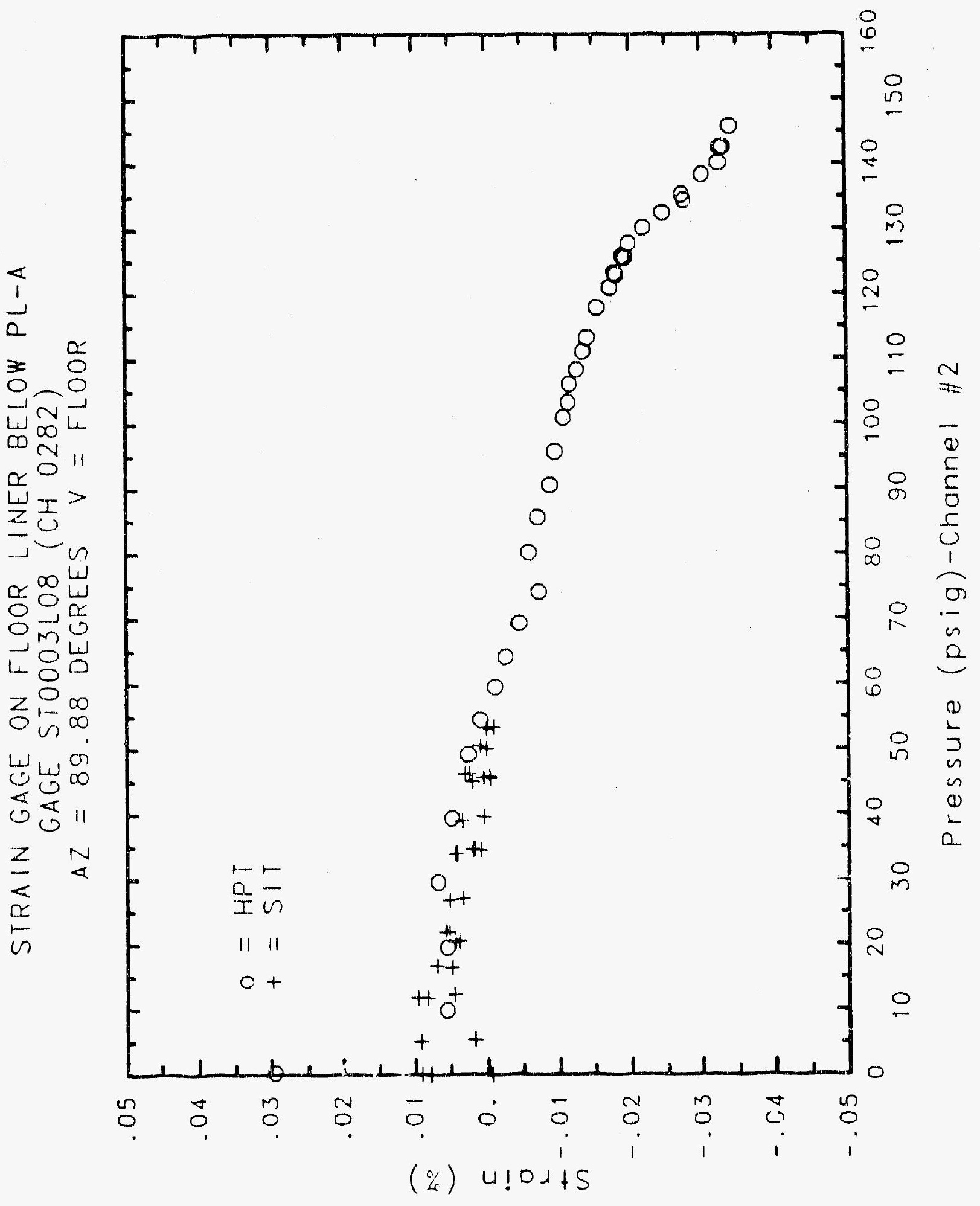




\section{Reinforced Concrete Test Data}

Strip 4 Channels $283,284,285,286,287$

Structural Integrity test

\begin{tabular}{|c|c|c|c|c|c|}
\hline Pressure & fiage 1 & Gage 3 & Gage 5 & Gage 7 & Gage 9 \\
\hline Ps:g & xstrain & xstrain & xstrain & xstrain & xst main \\
\hline-0.05 & 0.0006 & 0.0003 & -0.0027 & -0.0014 & 0.0020 \\
\hline 5.33 & 0.0038 & 0.0040 & 0.0002 & 0.0003 & 0.0020 \\
\hline 12.31 & 0.0048 & 0.0108 & 0.0049 & 0.0020 & 0.0048 \\
\hline 16.44 & 0.0059 & 0.0122 & 0.0059 & 0.0029 & 0.0069 \\
\hline 20.51 & 0.0031 & 0.0081 & 0.0054 & 0.0036 & $0.008 ?$ \\
\hline 20.46 & 0.0004 & 0.0065 & $0.006 !$ & 0.0040 & 0.0087 \\
\hline 20.47 & -0.0001 & 0.0054 & 0.0059 & 0.0045 & 0.0088 \\
\hline 27.05 & 0.0085 & -0.0019 & 0.0050 & 0.0053 & $0.010 \%$ \\
\hline 34.69 & $0.0: 93$ & -0.0178 & 0.0022 & 0.0102 & 0.015 \\
\hline 34.53 & 0.0217 & -0.0210 & 0.0016 & 0.0111 & 0.0176 \\
\hline 3453 & 0.0234 & -0.0218 & 0.0016 & 0.0115 & 0.0 .82 \\
\hline 39.70 & 0.0295 & -0.0301 & 0.0008 & $0.0: 35$ & 00393 \\
\hline 45.90 & $0.04 ! 7$ & -0.0462 & -0.0012 & 0.0183 & 0.0256 \\
\hline 45.65 & 0.0425 & -0.0154 & -0.0016 & 0.017 .3 & 0.0248 \\
\hline 45.62 & 0.0425 & -0.0463 & -0.0024 & 0.0168 & 00.240 \\
\hline 50.10 & 0.0454 & -0.0533 & -0.0022 & 0.0194 & 0.0674 \\
\hline 5347 & 0.0481 & -0.0813 & -0.0029 & 0.0201 & 0.0288 \\
\hline $5: 29$ & 0.0485 & -0.0813 & -0.0035 & 0.0200 & 0.0268 \\
\hline 5321 & 0.0480 & -0.0818 & -0.0037 & 0.0202 & 0.0275 \\
\hline 5043 & 0.0479 & -0.0585 & -00061 & $0.018 ?$ & 00200 \\
\hline $46: 4$ & 0.0483 & -0.0525 & -0.0002 & 0.0153 & 0.0235 \\
\hline$\Delta E .: 3$ & 0.3478 & -0.0516 & -0.0003 & 0.0155 & 0.02 .33 \\
\hline 44.97 & 0.0448 & -0.0492 & -00073 & 0.0125 & 0.0224 \\
\hline 3898 & 00434 & -0.0424 & -0.0080 & 0.0108 & $0.0: 98$ \\
\hline 3230 & 0.0386 & -0.0343 & $-0.007 \%$ & 0.0076 & 0.0174 \\
\hline 3390 & $\begin{array}{ll}0 & 0387\end{array}$ & -0.0366 & $-\approx .0075$ & 0.0083 & 0.0163 \\
\hline 26.83 & 0.0367 & -0.0264 & -0.0057 & 0.0051 & 0.0143 \\
\hline 21.88 & 0.0339 & -0.0207 & -0.0040 & 0.0042 & 0.6124 \\
\hline 2190 & 0.0356 & -0.0212 & -0.0038 & 0.0041 & 0.0134 \\
\hline 16.69 & 0.0328 & -0.0155 & -0.0021 & 0.0047 & 0.0118 \\
\hline 11.74 & 0.0309 & -0.0092 & 0.0012 & 0.0053 & 0.0114 \\
\hline 11.77 & 0.0330 & -0.0080 & 0.0010 & 0.0000 & 0.0124 \\
\hline 5.05 & 0.0307 & $-0.00,0$ & 0.0045 & 0.0085 & 0.0127 \\
\hline 0.02 & 0.0282 & 0.0013 & 0.0059 & 0.0094 & 0.0136 \\
\hline-0.04 & 0.0302 & 0.0029 & 0.0033 & 0.0057 & 0.0099 \\
\hline-0.02 & 0.0242 & 0.0026 & 0.0037 & 0.0062 & 0.0098 \\
\hline-0.02 & 0.0250 & 0.0028 & $0 . \operatorname{sn}_{46}$ & 0.0060 & 0.0092 \\
\hline 0.02 & 0.0245 & 0.0032 & 0.0052 & 0.0067 & 0.3118 \\
\hline
\end{tabular}




\section{Reinforced Concrete Test Data}

Strip 4 Channels $283,284,285,286,287$

HIgh Pressure TEST

\begin{tabular}{|c|c|c|c|c|c|}
\hline Fessiure & Guge ! & Gage 3 & Gage 5 & Gage 7 & Gaze 9 \\
\hline$P \leq 3$ & xstroir & Tram & $x \operatorname{sit} \sin$ & zstrain & zsirain \\
\hline 989 & 0.0063 & 0.0045 & 0.0022 & 0.0027 & 00022 \\
\hline 1955 & 0.0121 & -0.0054 & -0.0028 & $-0.001:$ & 00016 \\
\hline $295 ?$ & 00183 & -0.0193 & -0.0073 & 0.0002 & $0 \quad 0061$ \\
\hline 394 & 0.0240 & -00353 & -0.0096 & 0.0052 & 0.0108 \\
\hline $4: 26$ & 00263 & -00406 & $-0.009 ?$ & 0.0119 & 0.0169 \\
\hline$\therefore$ & $002 E$. & -0.0509 & -0.0084 & $0014 \%$ & 0.0398 \\
\hline 65 & 00500 & $\begin{array}{lll}-0 & 06,76\end{array}$ & $-c .007 t$ & 0.0175 & 0.0220 \\
\hline 142 & C C 314 & - 0203 & -0.0075 & 0.0198 & 0.0248 \\
\hline$\theta \vdots$ & 0.0245 & -00025 & -0.0058 & 0.0266 & 0.0257 \\
\hline$\therefore \therefore$ & 0.047 & $-0 \quad 060$ & $-0005:$ & 00038 & 0.0298 \\
\hline$\therefore:$ & $005:$ & - OLEA & -0.0006 & C. $02 \vdots 3$ & 0.0364 \\
\hline$\therefore \therefore$ & C OESO & $0134:$ & -0.0 cot & cosen & 00364 \\
\hline $4:$ is & (1) 074 & -01112 & - o ocot & $-0 \quad 0 c ?$ & 0.0 .41 \\
\hline or $\varepsilon$ & 0100 & $-0.07: 0$ & -0.0020 & - o cos? & .0000 \\
\hline$\therefore \quad 3$ & $0 \mathrm{ara}$ & 0054 & 000 & $603=7$ & -0.0220 \\
\hline$a z$ & C $: 396$ & $=04 \%$ & $-i \cos 34$ & $-0.62 \%$ & - O co5s \\
\hline$: 06 \quad 11$ & 01491 & 0040 & -0.0007 & - coost & -60.97 \\
\hline$\ldots$ & 01000 & cosos & $-c 000$ & - $024: 2$ & -0.021 \\
\hline$\because \therefore$ & $6 \quad 1642$ & 000 & 000 & $-c=246$ & octes \\
\hline 115 is & O DEE & 00024 & cotio & $c 045$ & $-0: 45$ \\
\hline$\because F$ & $\therefore: 14$ & $6 \quad \therefore \quad$ b & c ona & $\therefore$ os : & - 00 \\
\hline$\ldots$ & $0.25=0$ & 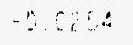 & Cona & $-0 \cos$ & - o dist \\
\hline 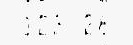 & $0.88 \%$ & - c ans & Cori & -605 & - C.83:1 \\
\hline$\ldots$ & C. $16 E:$ & - $\mathrm{CaI}^{\circ}$ & 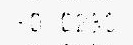 & $-c 005$ & .0005 \\
\hline 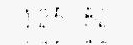 & $\therefore 16$ & $\because 664$ & $0 \quad \ldots \quad$ : & -0025 & -6006 \\
\hline$\therefore \quad \cdots$ & ( it) & 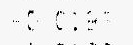 & 858 & $\cos 75$ & -60075 \\
\hline & 678 & $\because \quad \therefore \quad$ & $-c c i a$ & 60 & - O Dat \\
\hline$\ldots$ & b. $: 4$ & $c \& 4:$ & $-60 \mathrm{at}$ & -605 & -0006 \\
\hline$\therefore$ & $\because x$ & $\because 0$ & ocra & 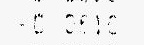 & -605 \\
\hline & $\therefore \therefore B$ & 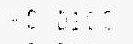 & $\because \because \because:$ & $\therefore: \because$ & $-0 \cos \theta$ \\
\hline 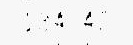 & r $10 . .6$ & $\cos x$ & 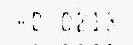 & $\therefore a d s$ & -005 \\
\hline$\therefore \quad \vdots$ & 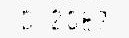 & $-6 c 0$ & - o:a: & -000.4 & 00 \\
\hline$\therefore \therefore \quad \therefore$ & $\therefore 2: 48$ & $=0.4$ & 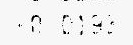 & $=0 \mathrm{cos}$ & $\therefore \cos$ \\
\hline$\therefore \quad \vdots$ & bay & $0005 t$ & $-00 \%=$ & $-000 \mathrm{~s}$ & $-\cos 3$ \\
\hline 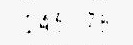 & 094 & 0002 & 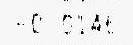 & - 0 ie: & ocotes \\
\hline$\therefore 1$ & c & o ont: & 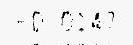 & -c ons & -0058 \\
\hline ! & $064 \%$ & $0 \mathrm{BA}=\mathrm{P}$ & $0 \quad 0: 1$ & $00: 0$ & 1 get \\
\hline
\end{tabular}




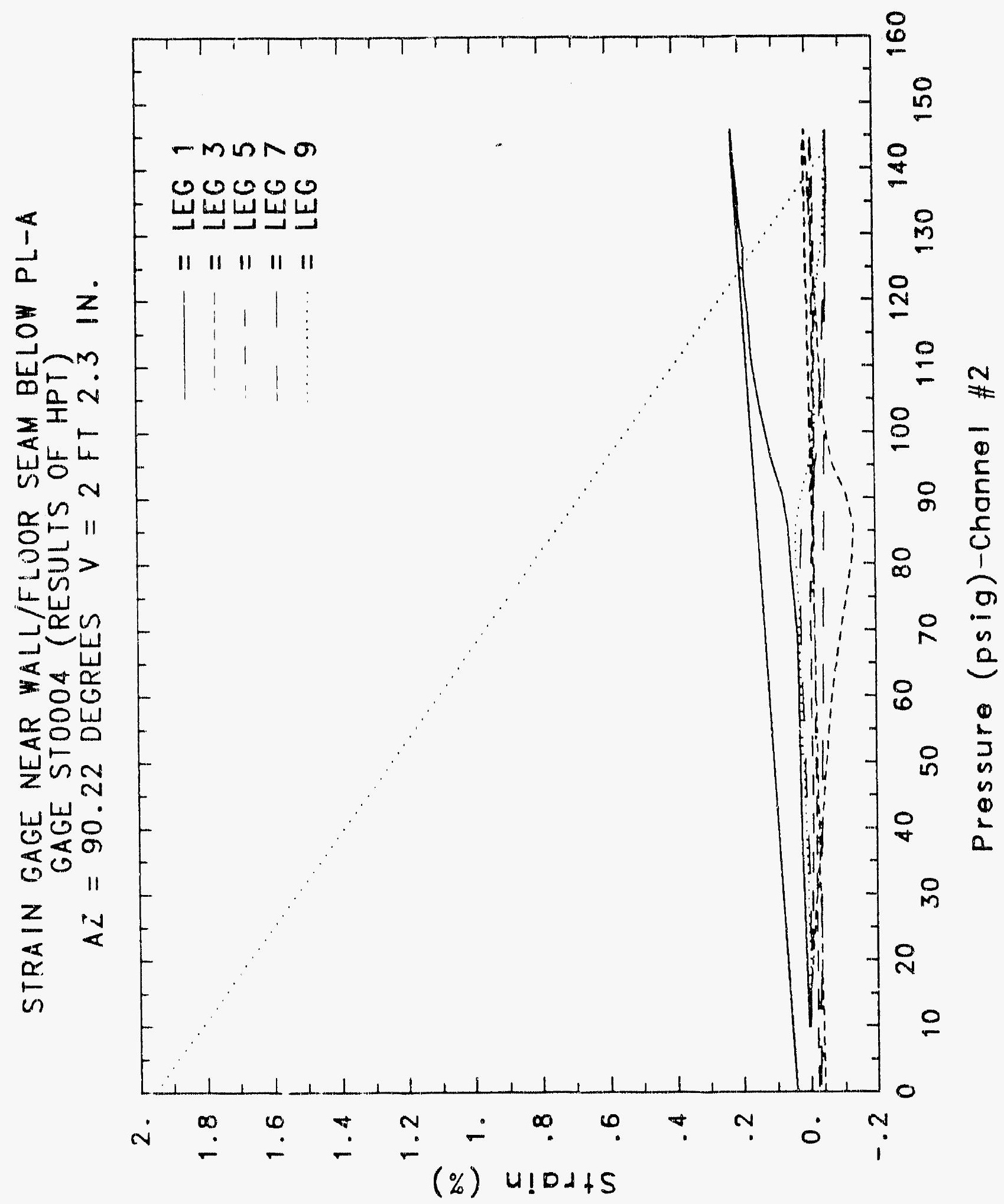




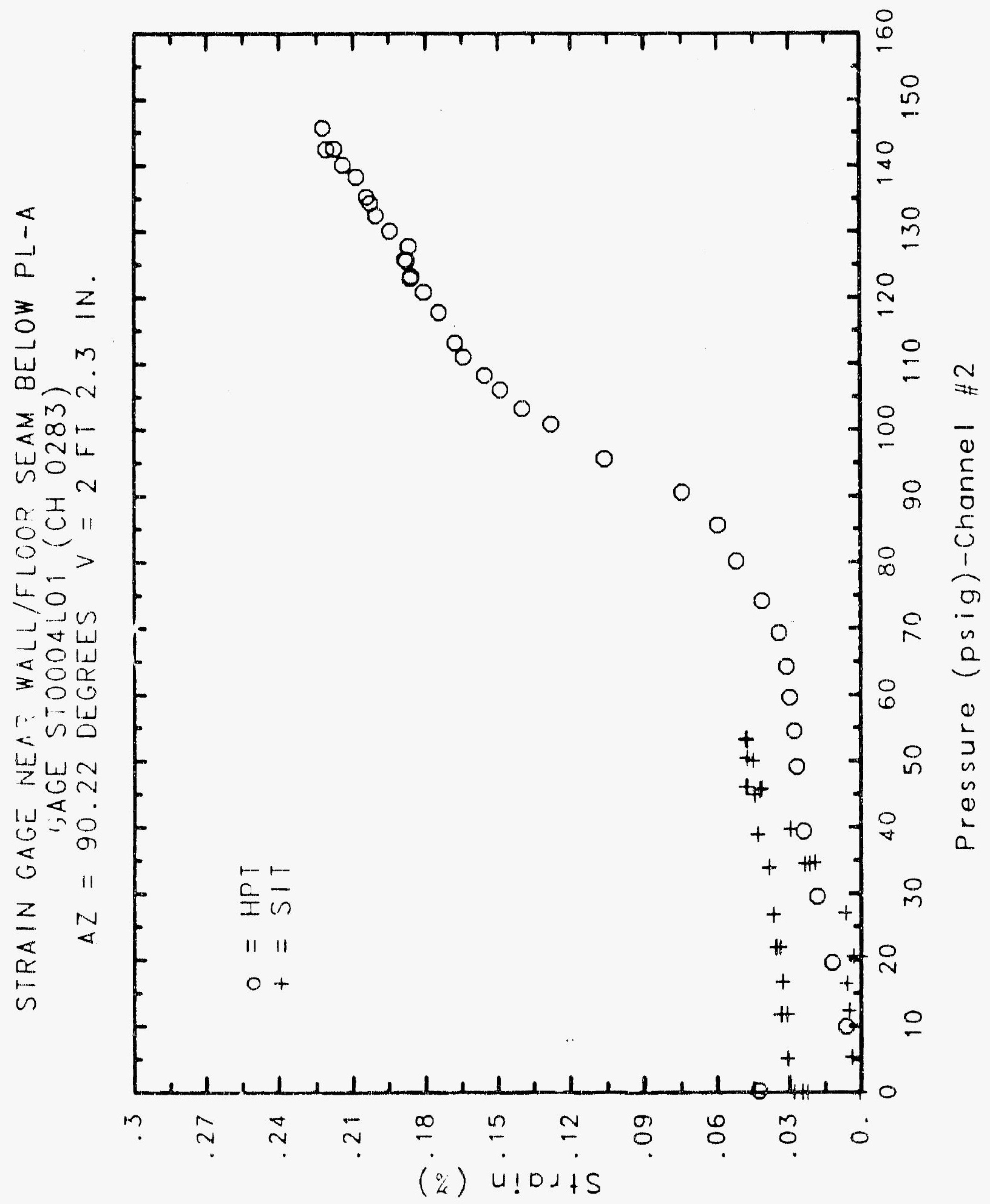




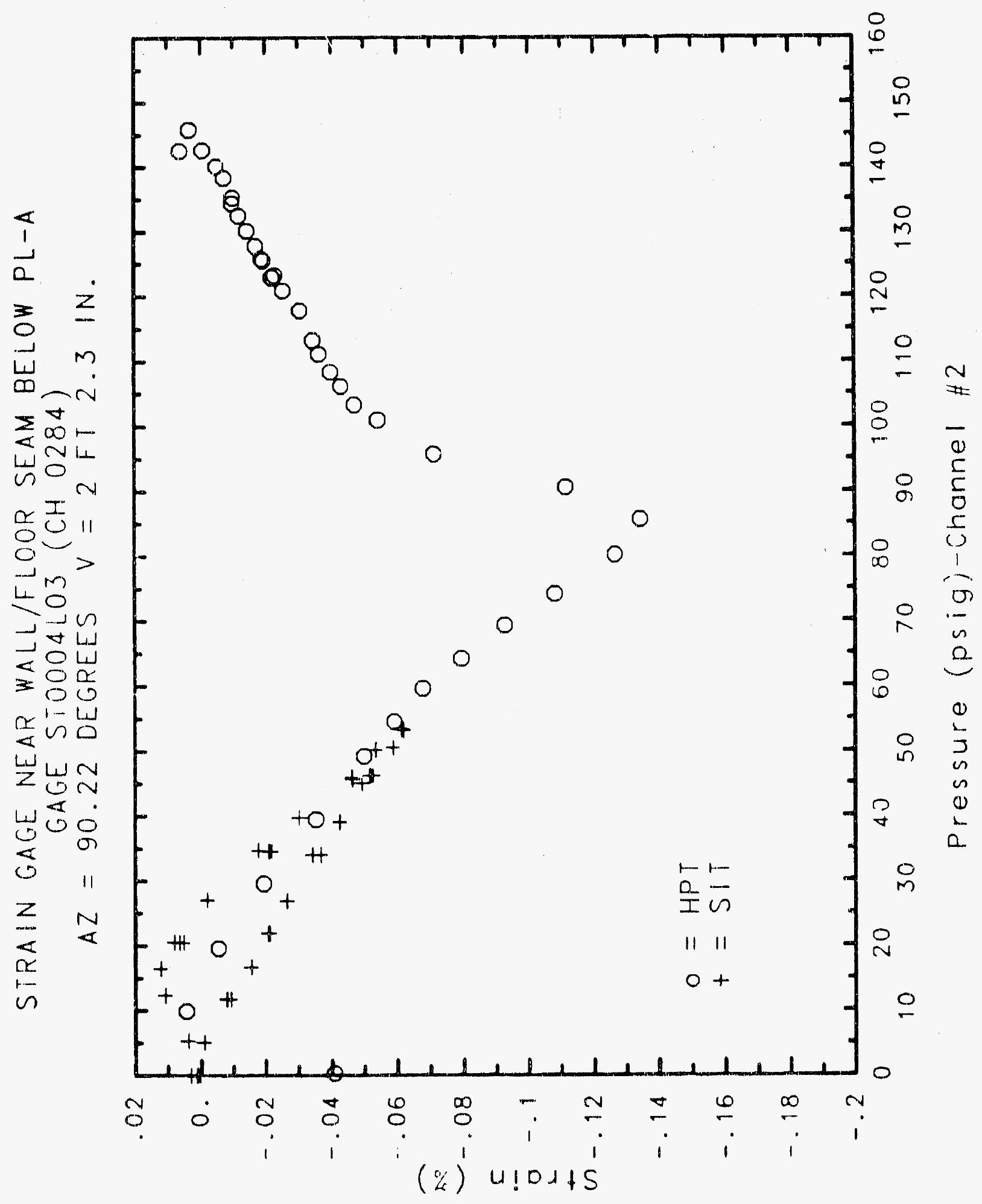

C- 1424 


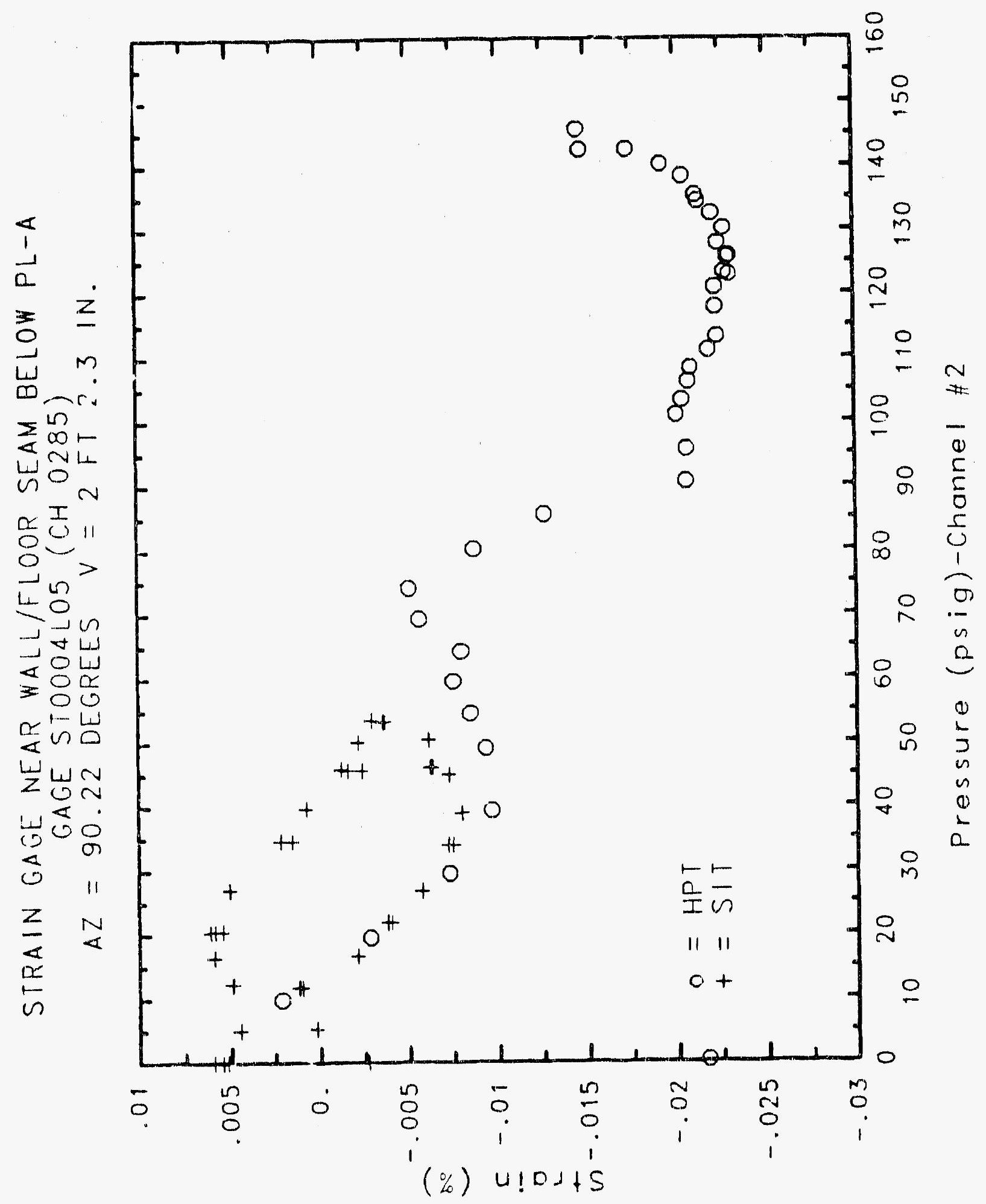




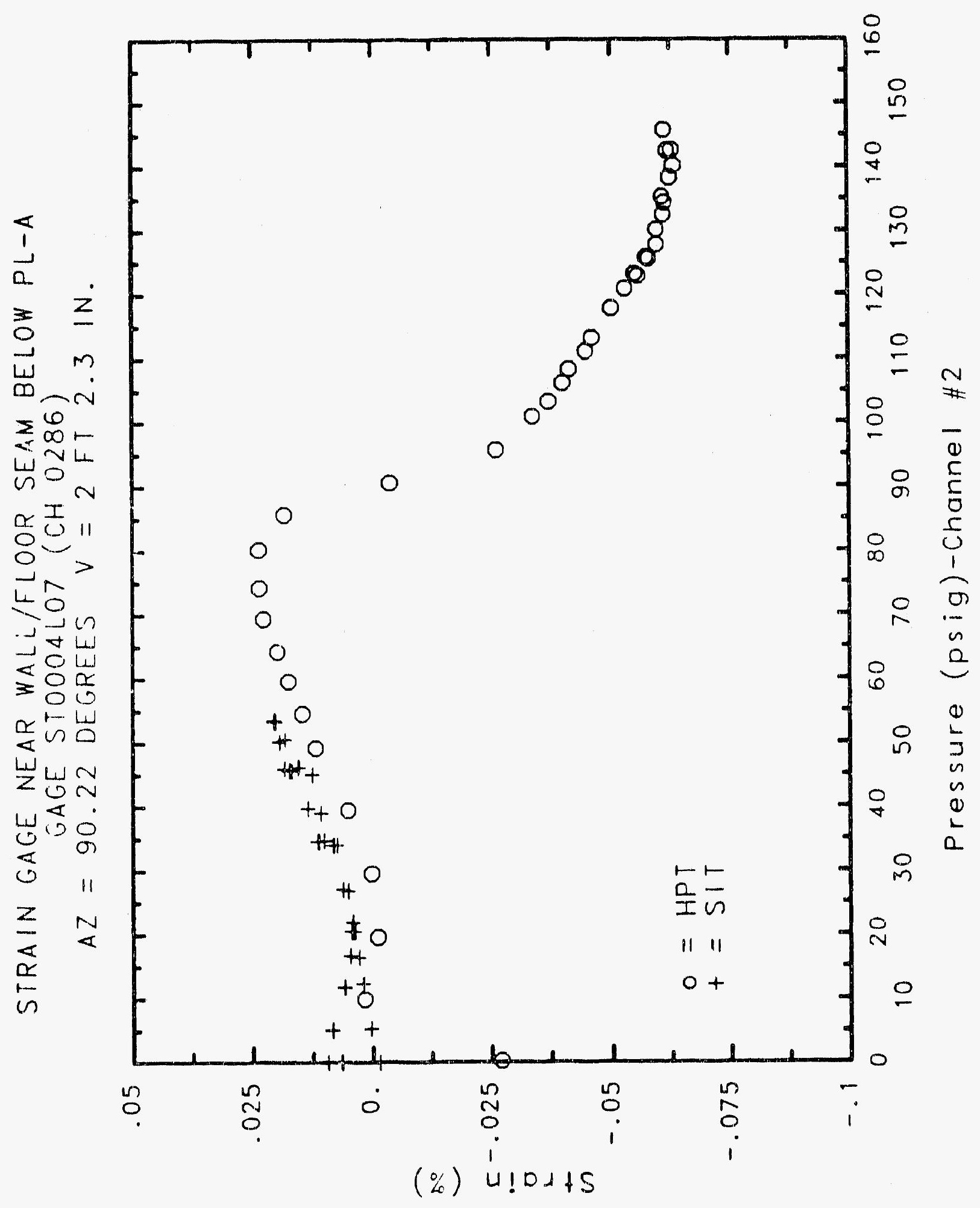




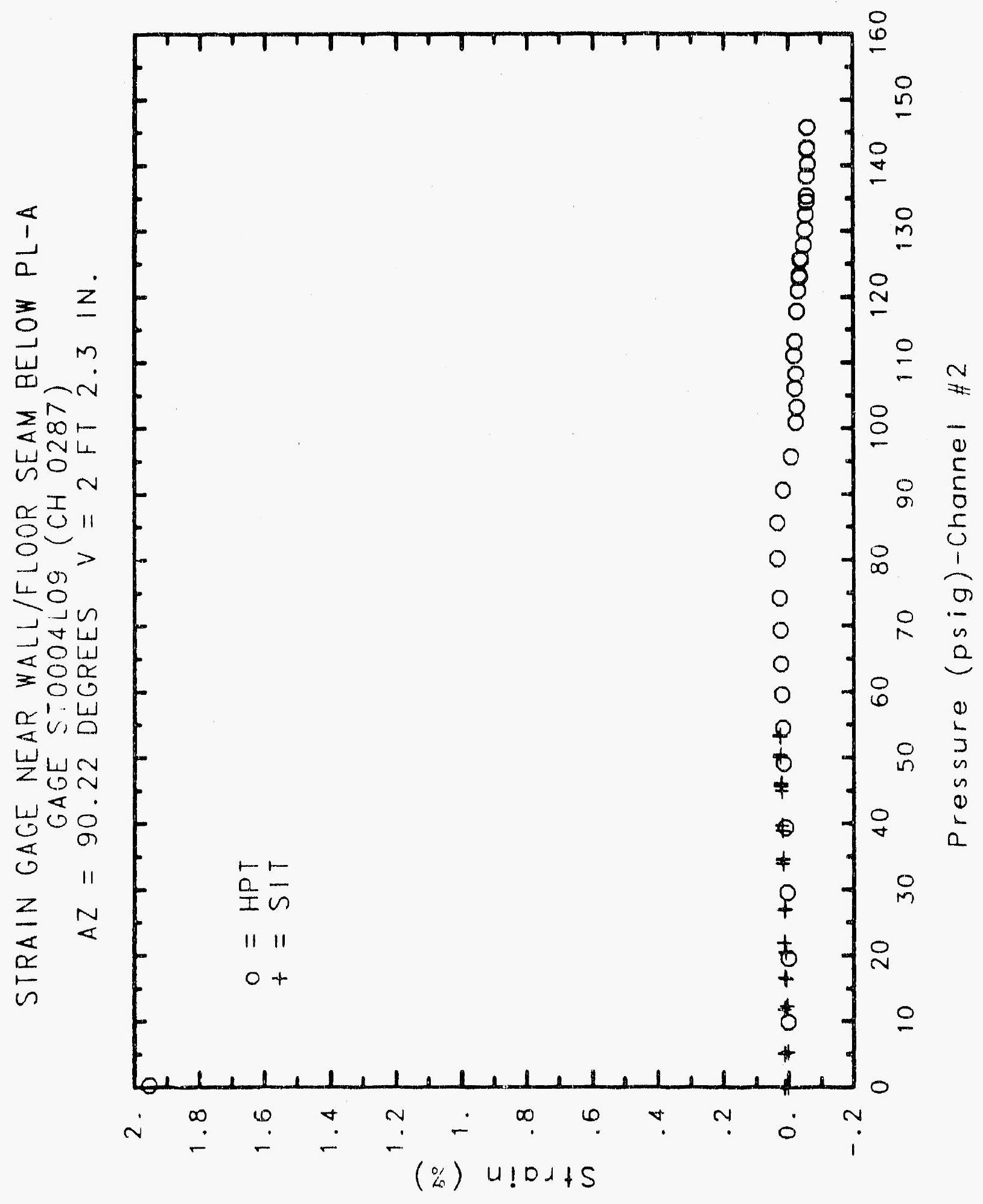




\section{Reinforced Concrete Test Data}

\section{Strip 5 Channels $288,289,300$}

\section{Structural InTEgRITY TEST}

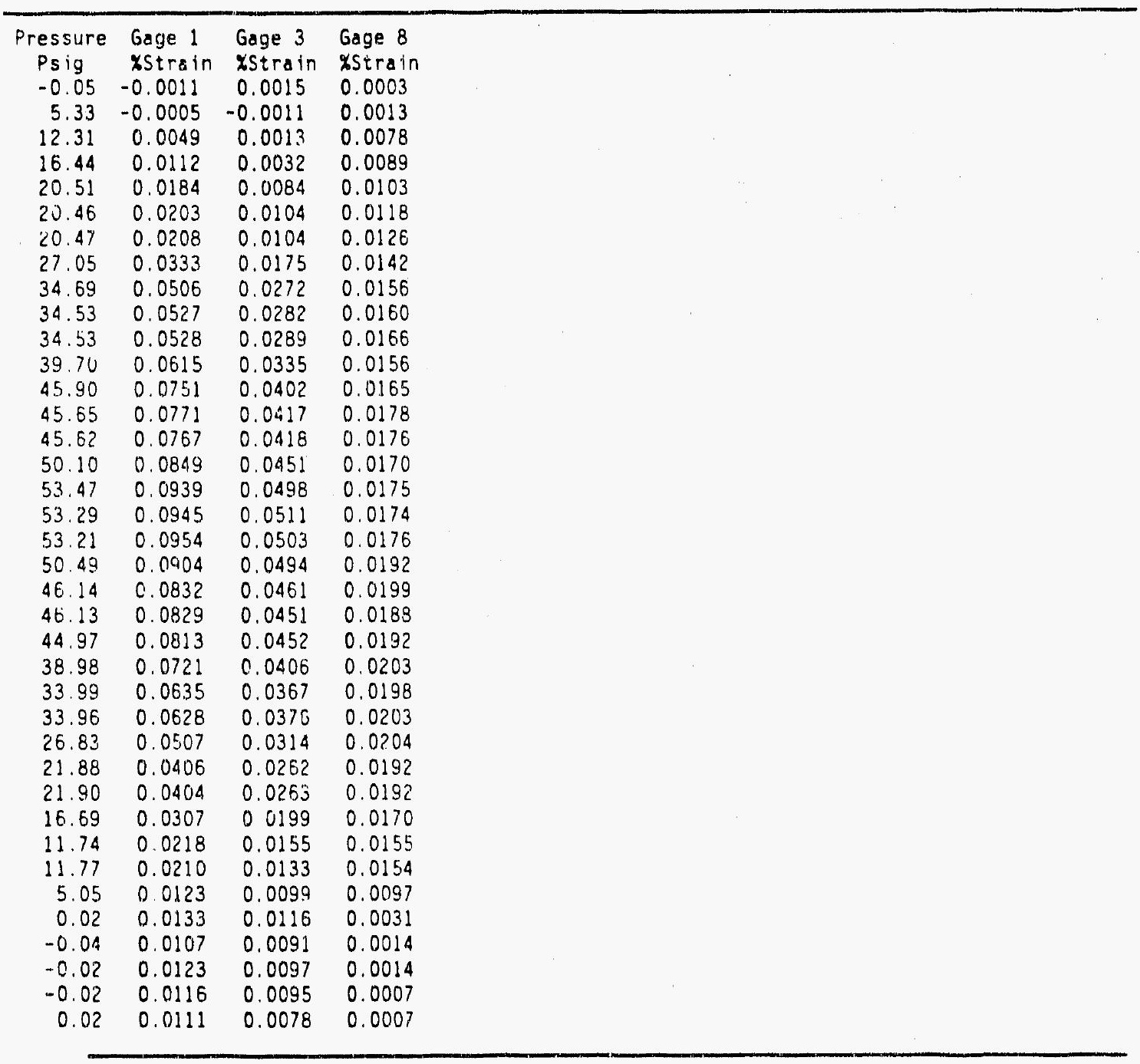




\section{Reinforced Concrete Test Data}

\section{Strip 5 Channels $288,289,300$}

\section{High Pressure Test}

\begin{tabular}{clll}
\hline Pressure & Gage 1 & Gage 3 & Gage 8 \\
Psig & Xstrain & Xstrain & XStrain \\
9.89 & 0.0074 & 0.0027 & 0.0135 \\
19.55 & 0.0263 & 0.0127 & 0.0178 \\
29.57 & 0.0457 & 0.0210 & 0.0188 \\
39.42 & 0.0642 & 0.0302 & 0.0176 \\
49.16 & 0.0809 & 0.0374 & 0.0171 \\
54.50 & 0.0912 & 0.0419 & 0.0162 \\
59.57 & 0.1009 & 0.0477 & 0.0151 \\
64.20 & 0.1114 & 0.0528 & 0.0147 \\
69.32 & 0.1266 & 0.0598 & 0.0133 \\
74.16 & 0.1413 & 0.0676 & 0.0110 \\
80.16 & 0.1570 & 0.0728 & 0.0063 \\
85.61 & 0.1713 & 0.0788 & 0.0024 \\
90.58 & 0.1846 & 0.0836 & -0.0013 \\
95.69 & 0.2005 & 0.0896 & -0.0062 \\
100.92 & 0.2152 & 0.0934 & -0.0110 \\
103.25 & 0.2224 & 0.0958 & -0.0130 \\
106.11 & 0.2294 & 0.0974 & -0.0161 \\
108.31 & 0.2358 & 0.0991 & -0.0181 \\
111.08 & 0.2440 & 0.1010 & -0.0211 \\
113.24 & 0.2500 & 0.1026 & -0.0230 \\
117.83 & 0.2607 & 0.1053 & -0.0285 \\
120.92 & 0.2673 & 0.1067 & -0.0315 \\
123.28 & 0.2739 & 0.1073 & -0.0339 \\
122.97 & 0.2747 & 0.1076 & -0.0350 \\
125.82 & 0.2799 & 0.1079 & -0.0375 \\
125.60 & 0.2818 & 0.1083 & -0.0385 \\
127.84 & 0.2847 & 0.1090 & -0.0124 \\
130.19 & 0.2899 & 0.1095 & -0.0464 \\
132.53 & 0.2945 & 0.1084 & -0.0517 \\
135.33 & 0.3001 & 0.1060 & -0.0584 \\
134.42 & 0.2993 & 0.1046 & -0.0578 \\
13835 & 0.3033 & 0.1019 & -0.0655 \\
140.16 & 0.3046 & 0.1006 & -0.0718 \\
142.63 & 0.3056 & 0.0946 & -0.0828 \\
145.78 & 0.3112 & 0.0938 & -0.0905 \\
142.52 & 0.3095 & 0.0935 & -0.0913 \\
0.22 & 0.0004 & -0.0794 & -0.0619 \\
& & & \\
110 & &
\end{tabular}




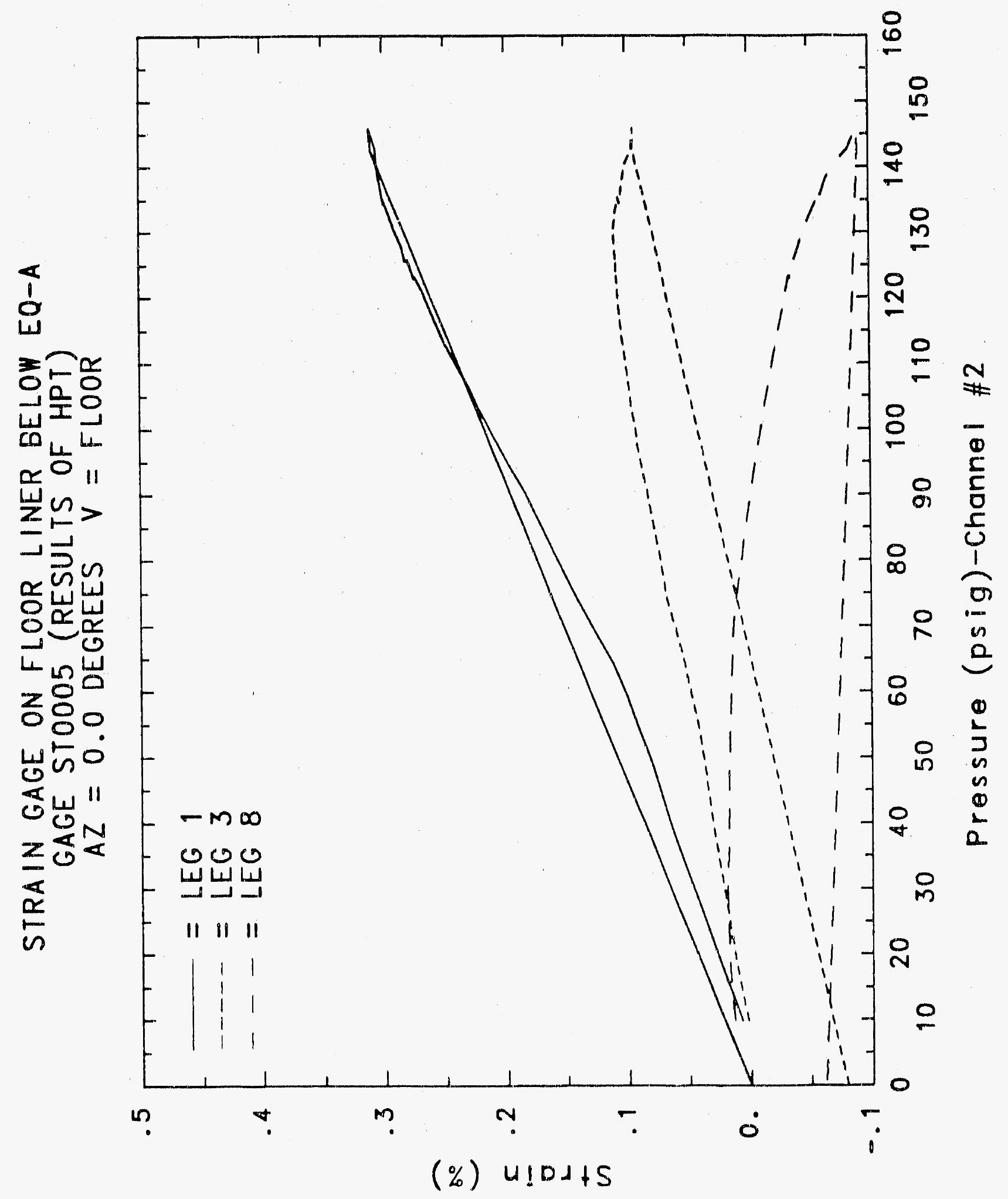




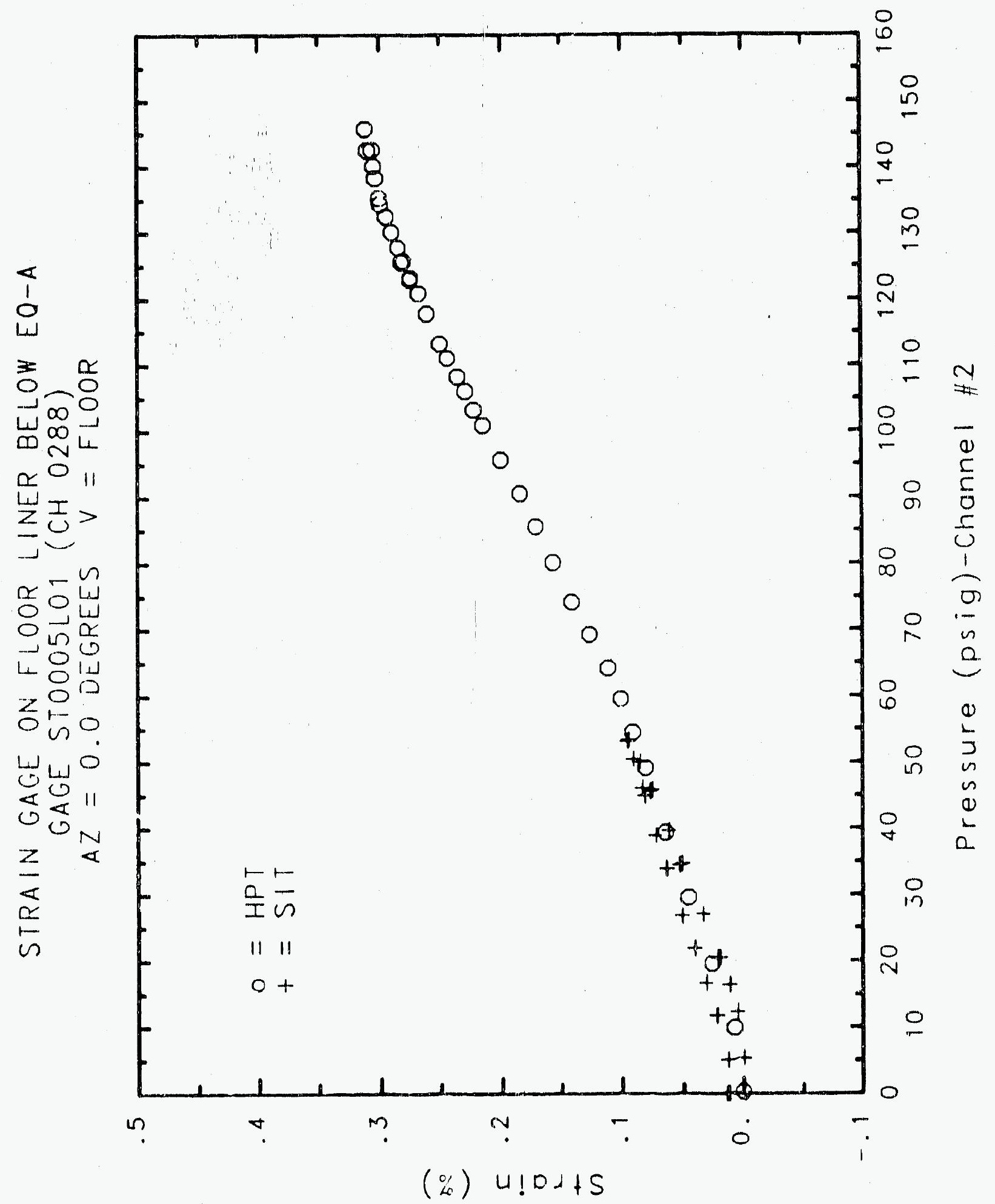




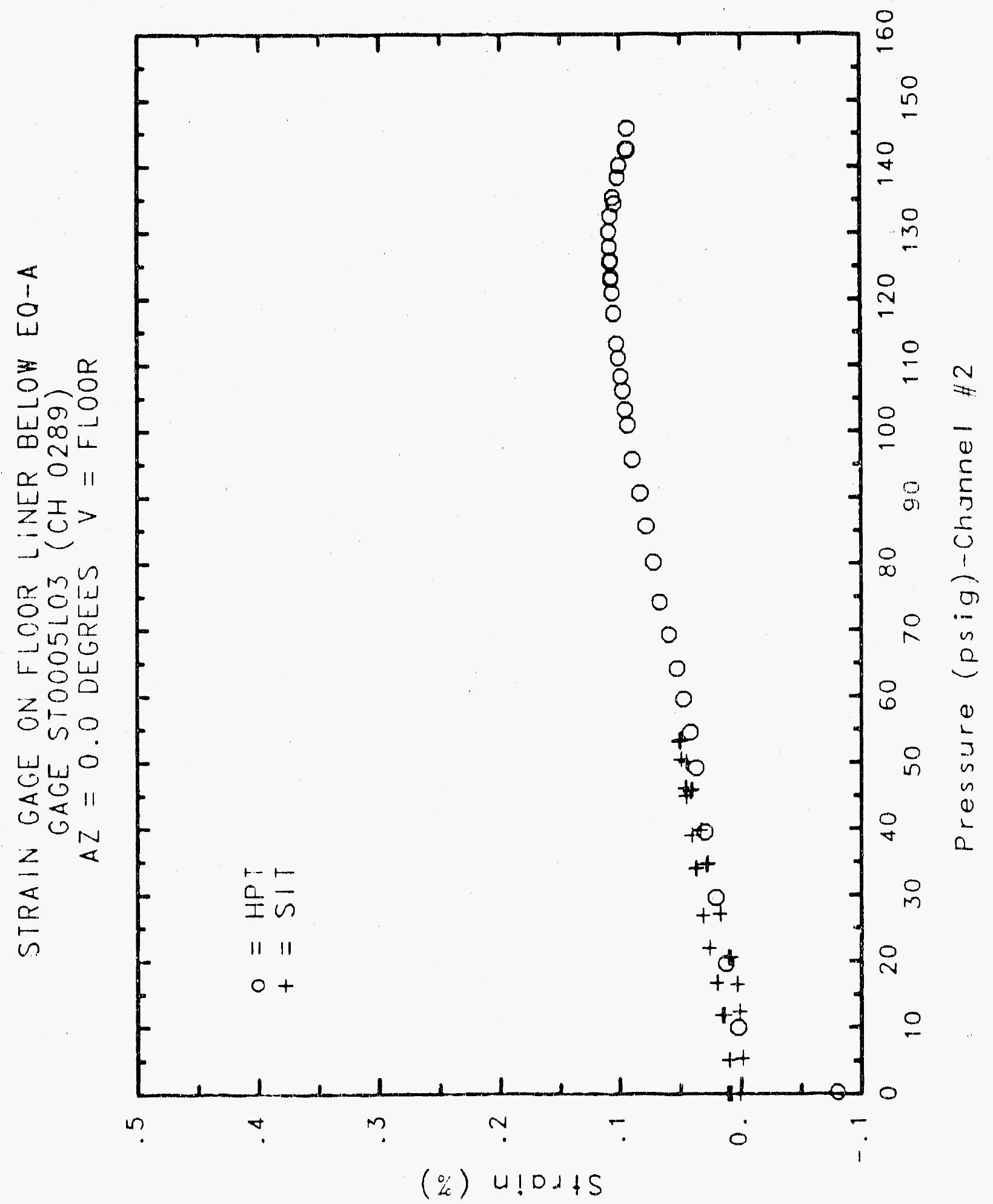




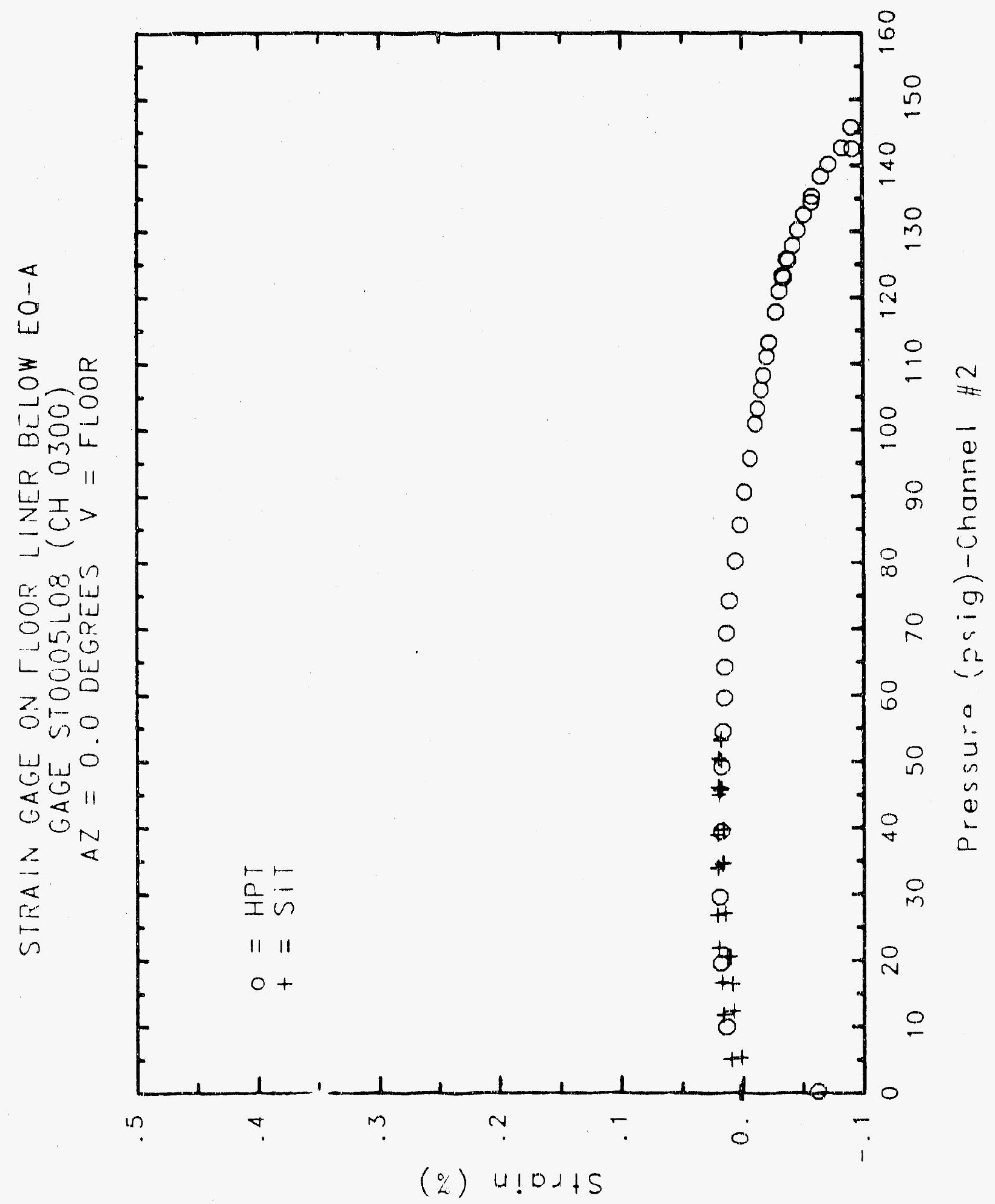




\section{Reinforced Concrete test Data}

Strip 6 Channels $304,305,306,308,309,320,321$

\section{STRUCTURAL INTEGRITY TEST}

\begin{tabular}{|c|c|c|c|c|c|c|c|}
\hline Pressure & Gage ' 1 & Gage 2 & Gage 3 & Gage 4 & Gage 6 & Gage 8 & Gagelo \\
\hline Psig & Xstrain & xstrain & Xstrain & zstrain & XStrain & Xstrain & xstrain \\
\hline-0.05 & 0.0000 & -0.0001 & -0.0003 & 0.0008 & 0.0008 & -0.0022 & -0.0015 \\
\hline 5.33 & 0.0047 & 0.0045 & 0.0018 & 0.0041 & 0.0012 & 0.0024 & 6.0053 \\
\hline 12.31 & 0.0091 & 0.0001 & 0.0064 & 0.0070 & 0.0018 & 0.0056 & $0.02 \div 2$ \\
\hline 16.44 & 10120 & 0.0030 & 0.0091 & 0.0095 & 0.0033 & 0.0066 & 0.0230 \\
\hline 20.51 & 0.0141 & 0.0044 & 0.0119 & 0.0112 & -0.4695 & 0.0047 & 0.0204 \\
\hline 20.46 & 0.0145 & 0.0046 & 0.0115 & 0.0108 & -0.4688 & 0.0026 & 0.0188 \\
\hline 20.47 & 0.0154 & 0.0055 & 0.0119 & $0.0: 25$ & -04700 & 0.0013 & 0.0171 \\
\hline 27.05 & 0.0174 & 0.0085 & 0.0145 & 0.0128 & -0.4668 & -0.0144 & 0.0028 \\
\hline 34.69 & 0.0246 & 0.0147 & 0.0213 & 0.0170 & -0.4627 & -0.0484 & -0.0332 \\
\hline 34.53 & 0.0253 & 0.0154 & 0.0215 & 0.0175 & -0.1530 & $-0.05 E 9$ & -0.0404 \\
\hline 34.53 & 0.0248 & 0.0160 & 0.0216 & 0.0179 & -0.4629 & -0.0601 & -0.0430 \\
\hline 39.70 & 0.0277 & 0.0180 & 0.0239 & 0.0195 & $-0.46 ! 3$ & -0.0829 & -0.0654 \\
\hline 45.90 & 0.0285 & 0.0198 & 0.0263 & 0.0202 & $-0.4 E, 03$ & -0.1130 & -0.0976 \\
\hline 45.65 & 0.0274 & 0.0190 & 0.0252 & 0.0196 & -0.4602 & $-6114 \vdots$ & -0.0984 \\
\hline 45.62 & 0.0277 & 0.0185 & 0.0251 & 0.0192 & $-0.40 C E$ & -1150 & $-0.098 !$ \\
\hline 50.10 & 00295 & 0.0195 & 0.0263 & 0.0211 & -0.4606 & -0.1284 & -0.1120 \\
\hline 53.47 & 00301 & 0.0204 & 0.0275 & 0.0214 & $-0.459 !$ & -0.1429 & $-0.125+1$ \\
\hline 53.29 & 0.0301 & 0.0209 & 0.0268 & 0.0208 & -0.4593 & -0.1433 & -0.1277 \\
\hline 5321 & 0.0292 & 0.0198 & 0.0271 & 0.0218 & -0.4599 & -0.1418 & -0.1273 \\
\hline 50.49 & 0.0287 & 0.0185 & 0.0257 & 0.0212 & -0.4502 & -0.1417 & -0.1250 \\
\hline 46.14 & 0.0262 & 0.0167 & 0.0240 & 0.0202 & -0.4606 & -0.1371 & -0.1213 \\
\hline 46.13 & 0.0258 & 0.0163 & 0.0242 & 0.0200 & -0.4612 & -0.1360 & -0.1198 \\
\hline 44.97 & 0.0270 & 0.0174 & 0.0244 & 0.0203 & -0.0304 & -0.1343 & -0.1182 \\
\hline 38.98 & 0.0233 & 0.0142 & 0.0220 & $0.018 !$ & -0.0297 & -0.1287 & -0.1116 \\
\hline 33.99 & 0.0212 & 0.0116 & 0.0190 & 0.0169 & -0.4645 & -0.1192 & -0.1033 \\
\hline 33.96 & C.0210 & 0.0125 & 0.0192 & 0.0158 & -0.4649 & -0.1194 & $-0103 !$ \\
\hline 26.83 & 0.0191 & 0.0101 & 0.0161 & 0.0128 & -0.4676 & -0.1013 & -0.0855 \\
\hline 2). 88 & 0.0179 & 0.0084 & $0.0: 44$ & 0.0103 & -0.4706 & -0.0859 & -0.0708 \\
\hline 21.30 & 0.0178 & 0.0030 & 0.0135 & 0.0391 & -0.4704 & -0.0888 & $-0.01: 9$ \\
\hline 16.09 & 0.0158 & 0.0060 & 0.0114 & 0.0084 & -0.473 & -0.0746 & -0.0591 \\
\hline 11.74 & 0.0134 & 0.0038 & 0.0087 & $0.006 ?$ & -0.149 & 0.0607 & -0.0450 \\
\hline 11.77 & 0.0123 & 0.0028 & 0.0078 & 0.0058 & -04741 & $-0 .\llcorner 576$ & -00417 \\
\hline 5.05 & 0.0066 & -0.0031 & 0.0044 & O. COE 1 & -0.4749 & -1.0472 & -0.0246 \\
\hline 002 & -0.0006 & -0.0106 & -0.0014 & 1.0021 & .0 .4786 & -0.0469 & -0.0219 \\
\hline-0.04 & 0.0016 & .0 .0071 & 0.0018 & 1.0053 & -0.4740 & -00321 & $=0.0155$ \\
\hline-0 v? & 0.0016 & -0.0068 & 0.0049 & $0.2=0 \mathrm{~s}$ & $-0.474 ?$ & -0.1324 & $=0.0158$ \\
\hline-01.02 & 0.0012 & -0.0067 & 0.0041 & $0.0 \div \div 5$ & -0.4737 & $-0.03 \%$ & $-0.016 !$ \\
\hline 0.02 & 0.0030 & -0.0080 & 0.0029 & 0.0053 & -0.4764 & -0.0333 & -00.175 \\
\hline
\end{tabular}




\section{Reinforced Concrete Test Data}

Strip 6 Channel.s $304,305,306,308,309,320,321$

\section{High Pressure test}

\begin{tabular}{|c|c|c|c|c|c|c|c|}
\hline Pressure & isage 1 & Gage $?$ & Gage 3 & Gage 4 & Gage 6 & Gage 8 & Sagelo \\
\hline$P:: 9$ & $x \sec$ in & xstroin & xstrain & xstrain & Xstrain & xstrain & Xstrain \\
\hline$\$ 9$ & 0.0105 & 0.0207 & 0.0287 & 0.0027 & 0.0024 & -0.0053 & -0.0055 \\
\hline 1955 & 0.0157 & 0.0154 & 0.0124 & 0.0014 & 0.0007 & -0.0286 & $-0.028 ?$ \\
\hline $29.5 ?$ & 00186 & 0.0190 & 0.0056 & 0.0023 & 0.0027 & $-0.05,58$ & -0.0566 \\
\hline 3942 & 0.0221 & 0.0221 & 0.0106 & 0.0071 & 0.0059 & -0.0829 & -0.0827 \\
\hline $4 \div .5$ & 0.035 & 0.025 .3 & 0.0136 & 0.0104 & 0.0084 & -0.1050 & -0.1060 \\
\hline $54: 0$ & $n: 023$ & 0.0271 & $0.016:$ & 0.0119 & 0.0114 & -0.1182 & -0.1183 \\
\hline 5.457 & 00260 & 00.76 & 0.0270 & 0.0136 & $0.012 \mathrm{E}$ & -0.1341 & -0.1345 \\
\hline $6: \cdots$ & 0.280 & $0028 ?$ & $0 . C: E, E$ & 0.0147 & 0.0150 & -0.1571 & -0.1573 \\
\hline 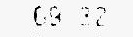 & 0,281 & 00283 & $0.0: 81$ & 0.0150 & 0.0133 & $-0.18 ! 1$ & -0.1807 \\
\hline $74: E$ & 6025 & $00: 77$ & 00190 & $0.0: 4 E$ & 00144 & -0.2059 & $-0.205 E$ \\
\hline $4: \quad: t$ & 6.680 & 0.0277 & 0.0180 & 0.0238 & $0013 \%$ & -0.2334 & -0.2133 \\
\hline$+5: 1$ & $\Leftrightarrow 6 R^{\circ}$ & 0.0278 & 0.0258 & 0.0148 & $002 \div$ & -0.2582 & -0.2585 \\
\hline $9:: 3$ & c 025: & 00275 & 0.0197 & 0.0153 & 00144 & -0.2614 & -0.2814 \\
\hline$\because 58$ & 0020 & $0.02 \leq:$ & 0.0205 & 0.0100 & 0.0150 & -0.3059 & -03056 \\
\hline$\because \therefore \quad$ & 0030 & $0<631$ & $000: 2$ & 0.0 .57 & $0: \vdots 5$ & $\because 03282$ & $-0.32 t 6$ \\
\hline$\therefore: \vdots$ & $0.032 i$ & 0.0254 & $002: 9$ & 0.0181 & $0 \quad 0: 73$ & -0.3 .391 & -0.3365 \\
\hline OF: : & $\therefore 030$ & 00302 & $0 \cos 3$ & 00191 & 00179 & -0.94 .94 & -03495 \\
\hline$\therefore 5 \quad \therefore$ & 0039 & 003.12 & 0.0220 & 0020 & $0.018 \%$ & -0.3560 & .0 .3572 \\
\hline$\therefore: \quad 8$ & 00.35 & $0.05 \%$ & 0.40 & 0.0 .25 & 0022 & - 03592 & $03 E A 5$ \\
\hline$\therefore \therefore a$ & C 0321 & 0.05 & 0.605 & 00225 & $002: 3$ & -0.3783 & -0.378 \\
\hline$\therefore 78$. & 0055 & 00315 & 0067 & 0254 & $0 . \cos 0$ & $-0: 405$ & $-0 \quad 40: ?$ \\
\hline$\ldots$ & $6237:$ & 00367 & 0.0 .90 & 00273 & 0.0264 & $.04: 95$ & -0.4193 \\
\hline 1326 & c 0374 & 0.0376 & $0.025 ?$ & 0.0273 & 0203 & -0.4357 & $-0.4 \% 5$ \\
\hline$\ldots$ & 60010 & 0030 & O ocis: & $0 \quad 0268$ & 0.0254 & -0.437 & $-0.4: 77$ \\
\hline 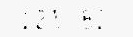 & 0070 & 0200 & 00275 & C.018? & $001 \%$ & $-0.44: 5$ & -0.4434 \\
\hline$\therefore=\therefore$ & $6.2 ? ?$ & $0.03 r ?$ & 00248 & c 005? & $0.000 ?$ & -0.4387 & -0438 \\
\hline$\therefore \quad 14$ & coss & 0.065 & 00272 & .00145 & $-[: 0: 55$ & .04368 & -040 \\
\hline$\therefore \therefore$ & C O4:C & c $04: 5$ & .00068 & $-[00544$ & -0.053 & -04567 & .0446 \\
\hline$\therefore \because 1$ & $0 \quad 4 \%$ & 00404 & $-0.07 t$ & $-00=54$ & 00610 & $-0.46 ? 8$ & - 04 4.: 4 \\
\hline$\therefore \quad 3:$ & 5031 & $0.6 \times 4$ & -008 & -0 lat & -01673 & -0.5257 & -0.535 \\
\hline$\therefore \therefore<$ & 0908 & o Cas & - o ot? & -01542 & $-0: \vdots B d$ & $=05407$ & .05400 \\
\hline$\therefore \quad \cdots$ & $\therefore 0: 5,0$ & .6015 & -01230 & -6.265 & 0.1674 & -0.5710 & $.057: 3$ \\
\hline $4:$ ir & 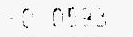 & Co 054 & .0143 & $-6: 17: 4$ & -0.1722 & $=0,575$ & -0.5710 \\
\hline $24: 13$ & $-C$ rete & $-0.088:$ & 01326 & $-0: 578$ & -0.1589 & -0.5748 & $-0575 !$ \\
\hline$\therefore A \div 78$ & 6092 & -0098 & .01215 & 0.1305 & -01303 & $-0.56 E E$ & $-0.567 \%$ \\
\hline $14: 18$ & 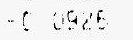 & $\therefore 040$ & .01170 & -01241 & -0.1250 & -0.5633 & -0.5630 \\
\hline 023 & $-C 0739$ & -0024 & 0.0325 & -01242 & -0.1249 & -0.6019 & -06022 \\
\hline
\end{tabular}




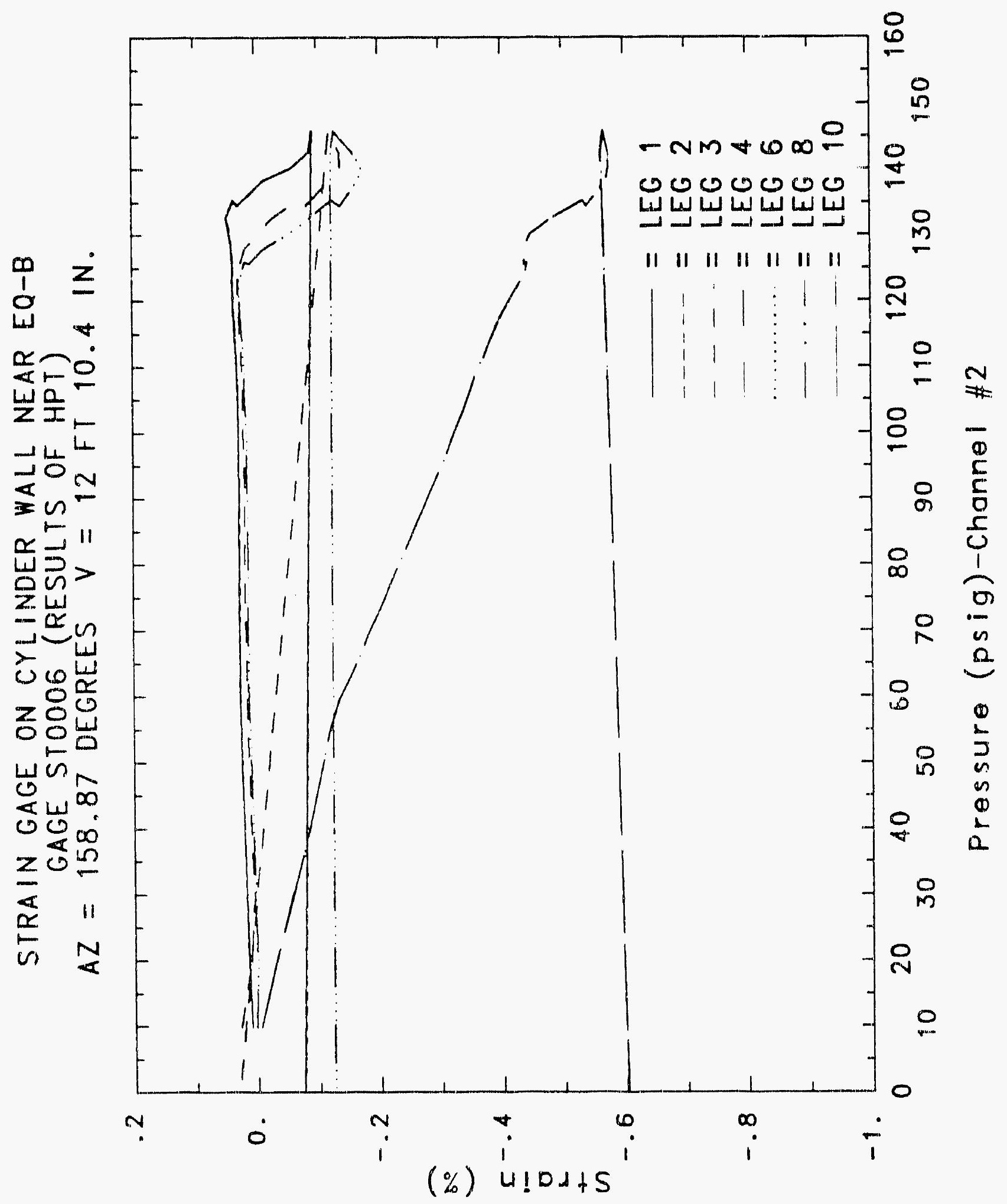




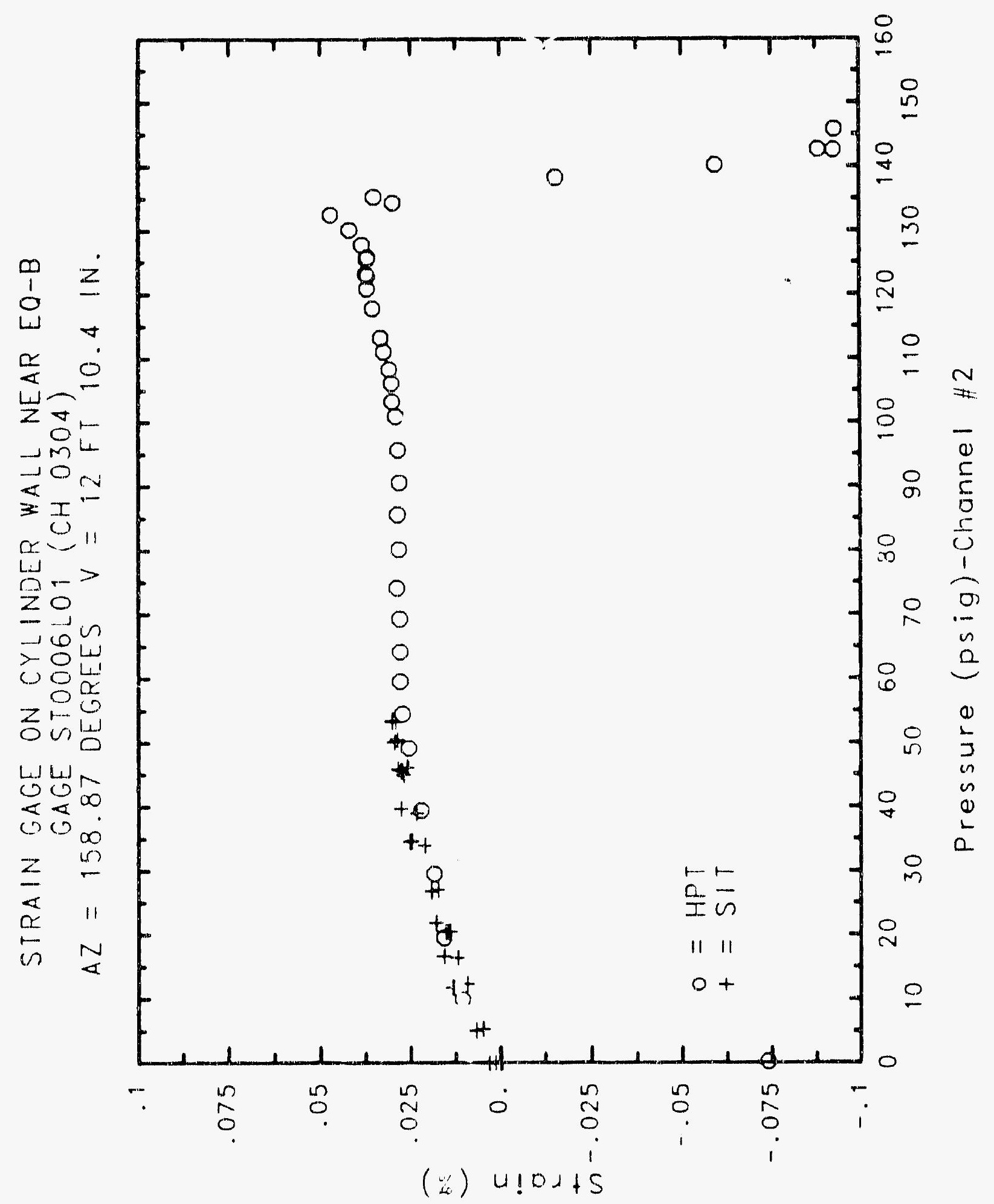




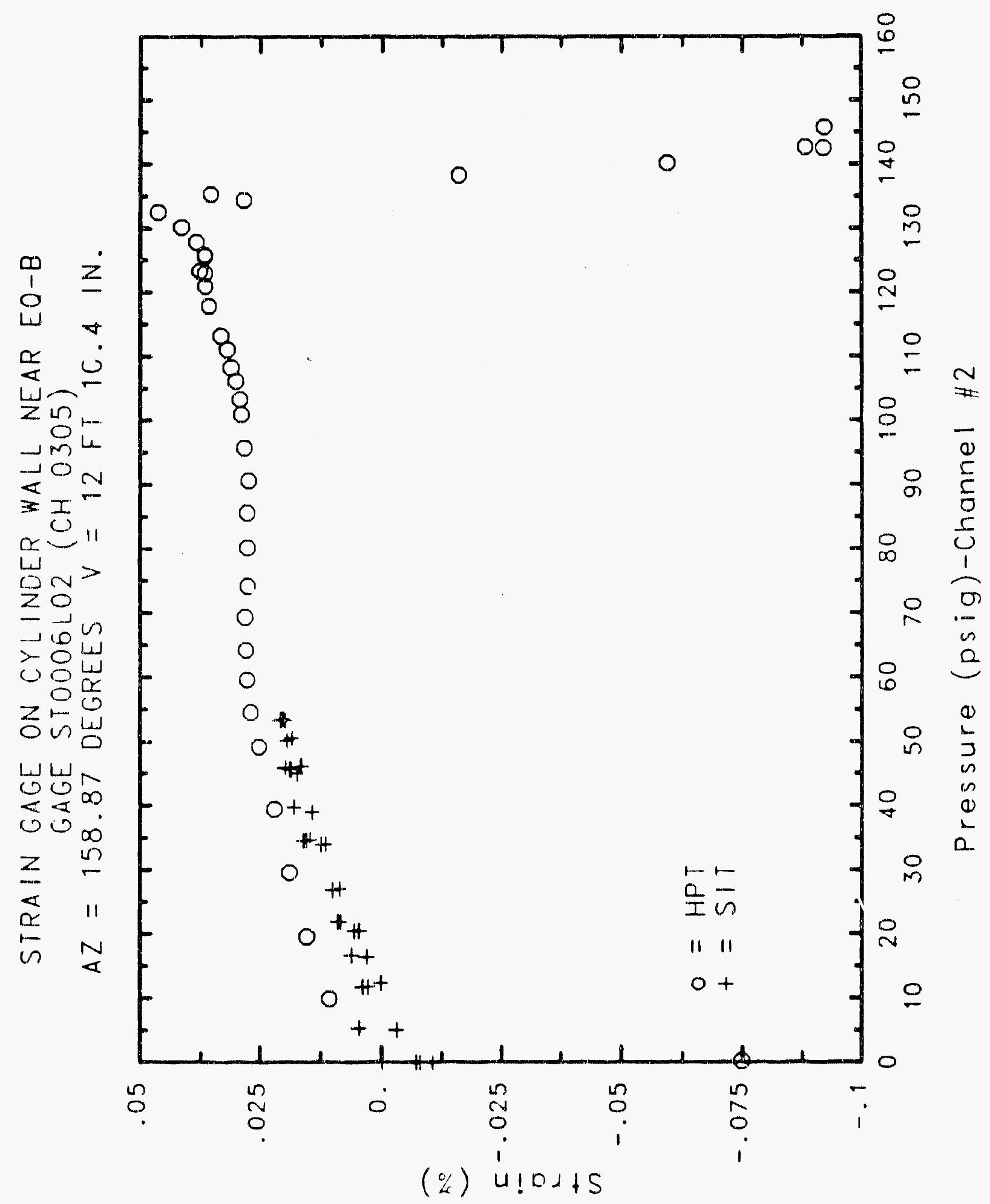




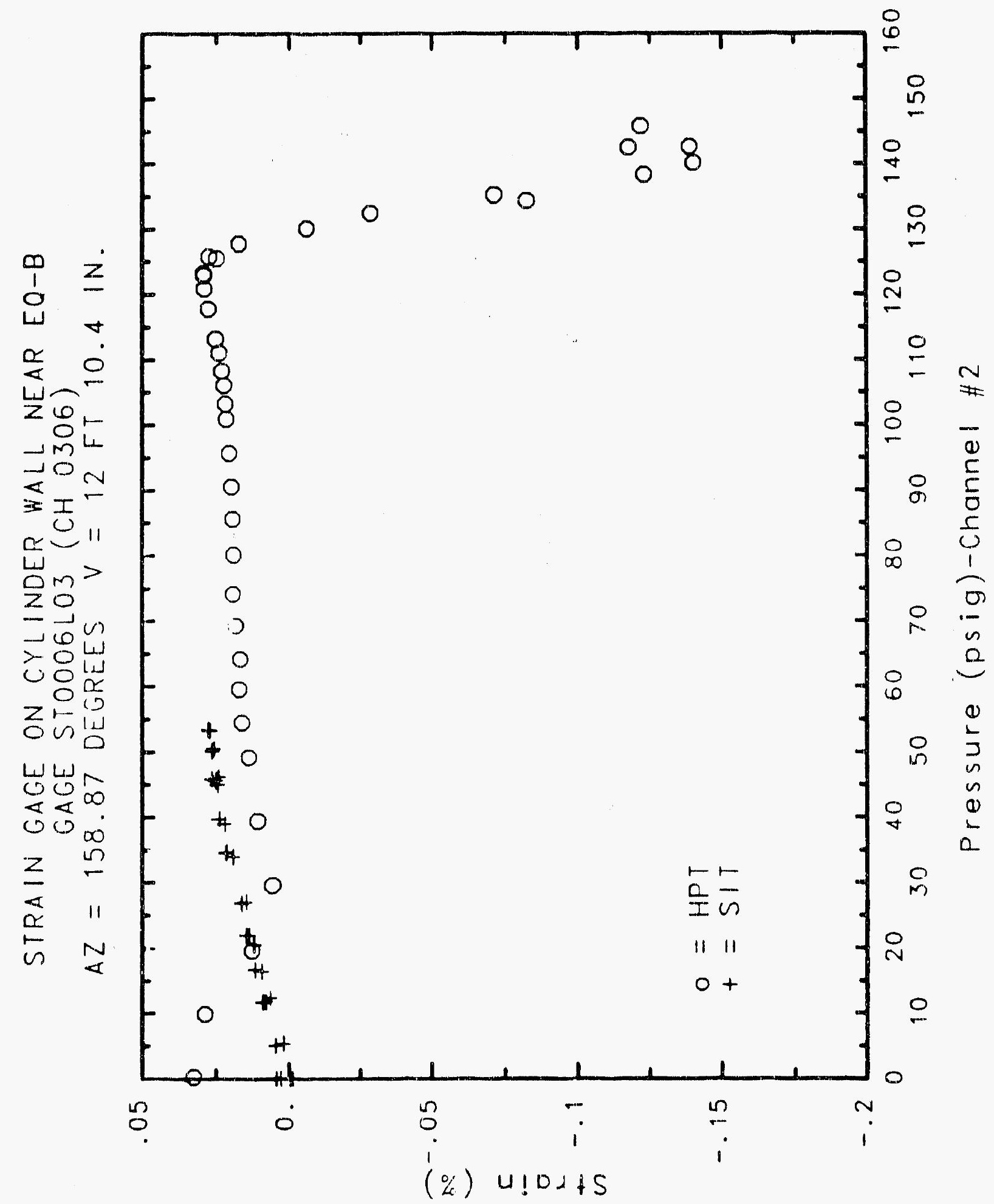

C-1439 


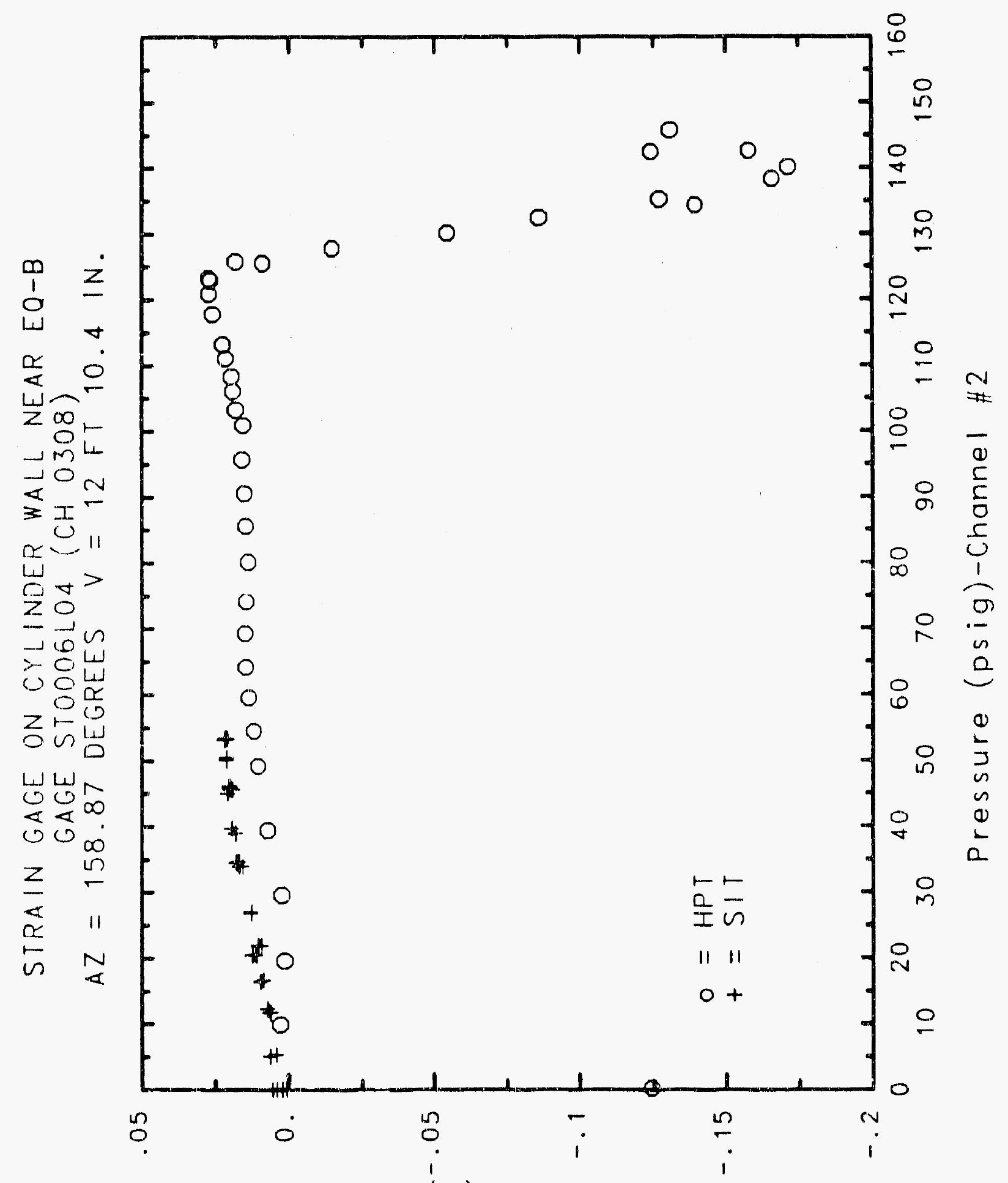

(\%) $4 ! 0 d+5$ 


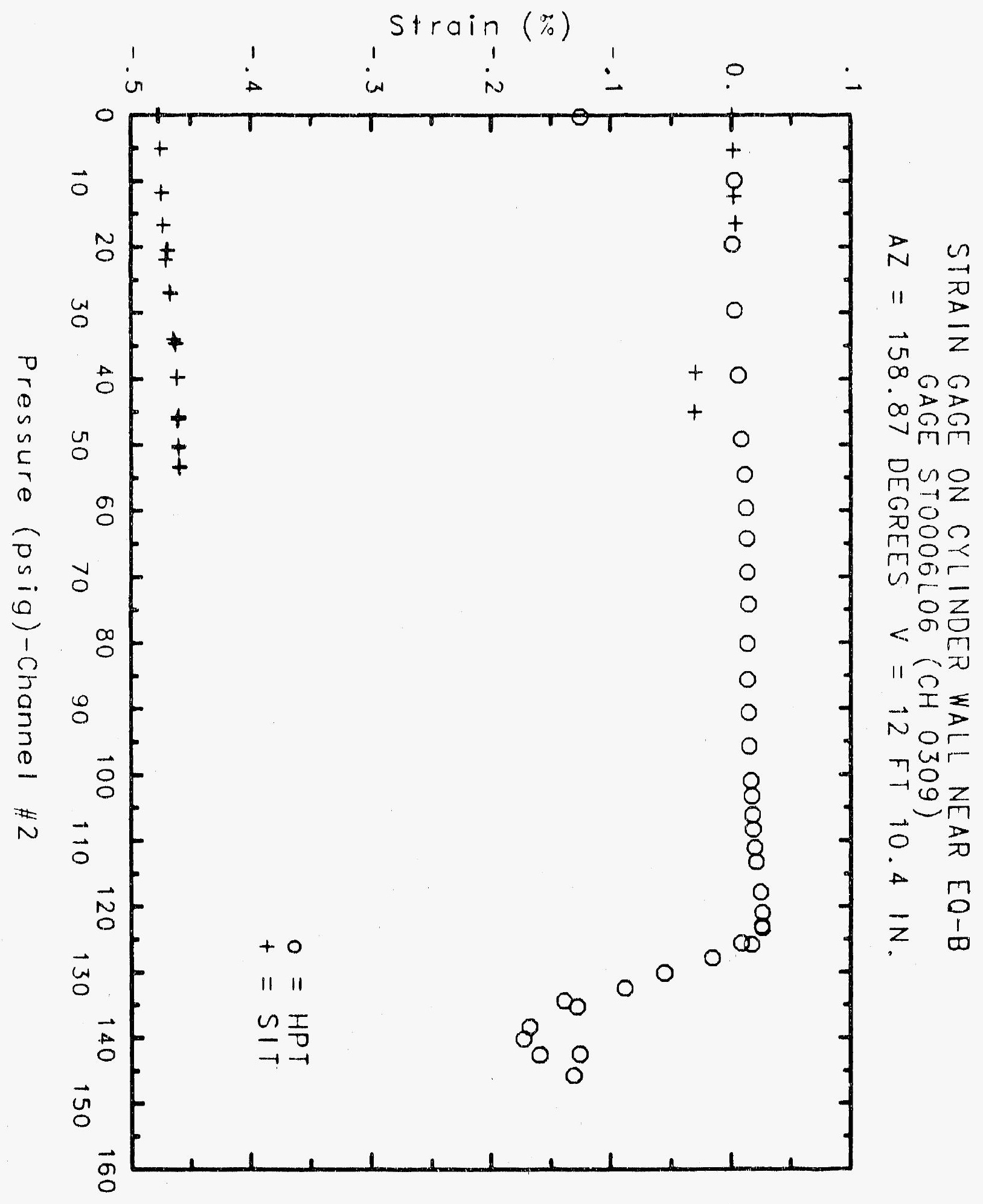




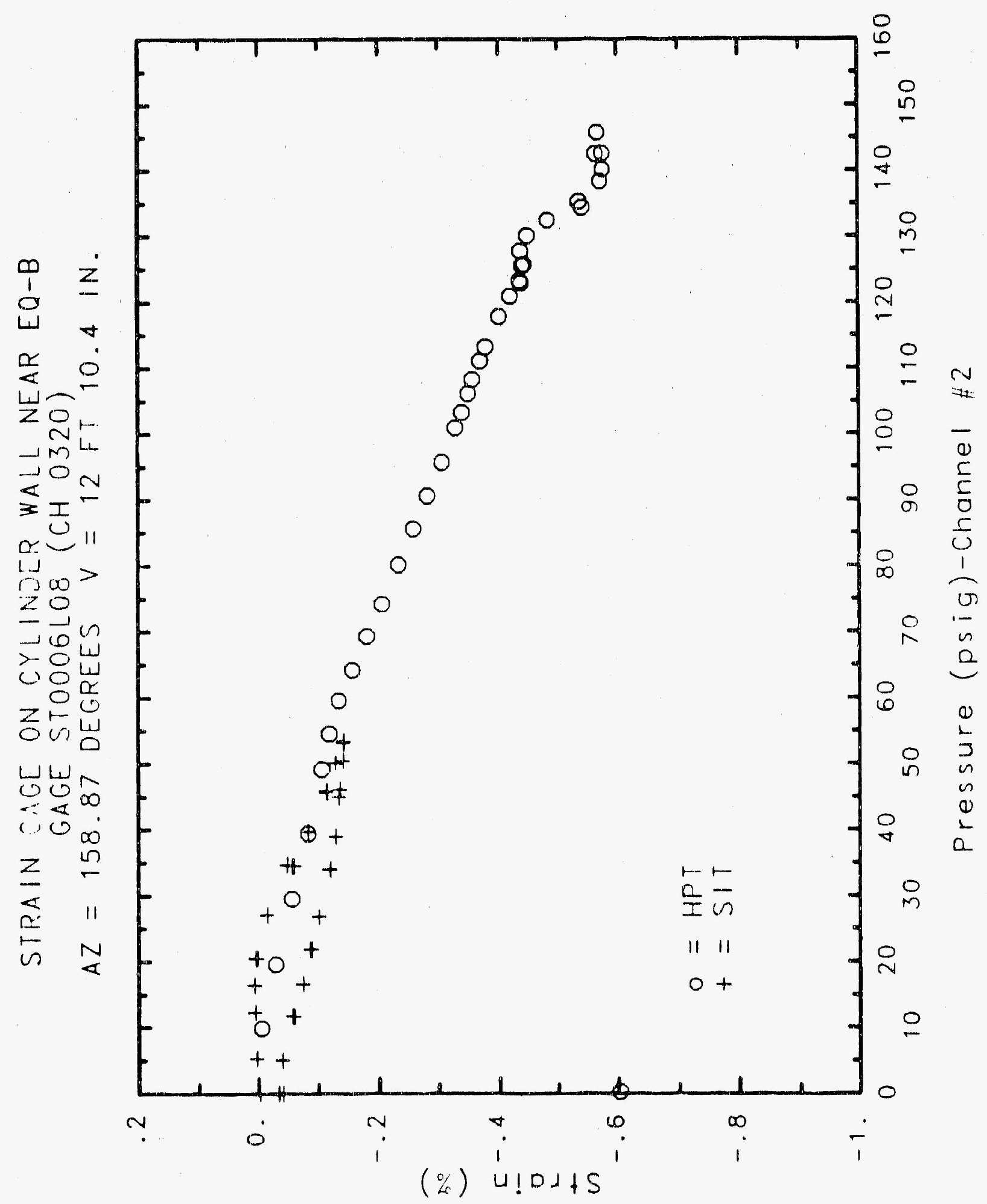




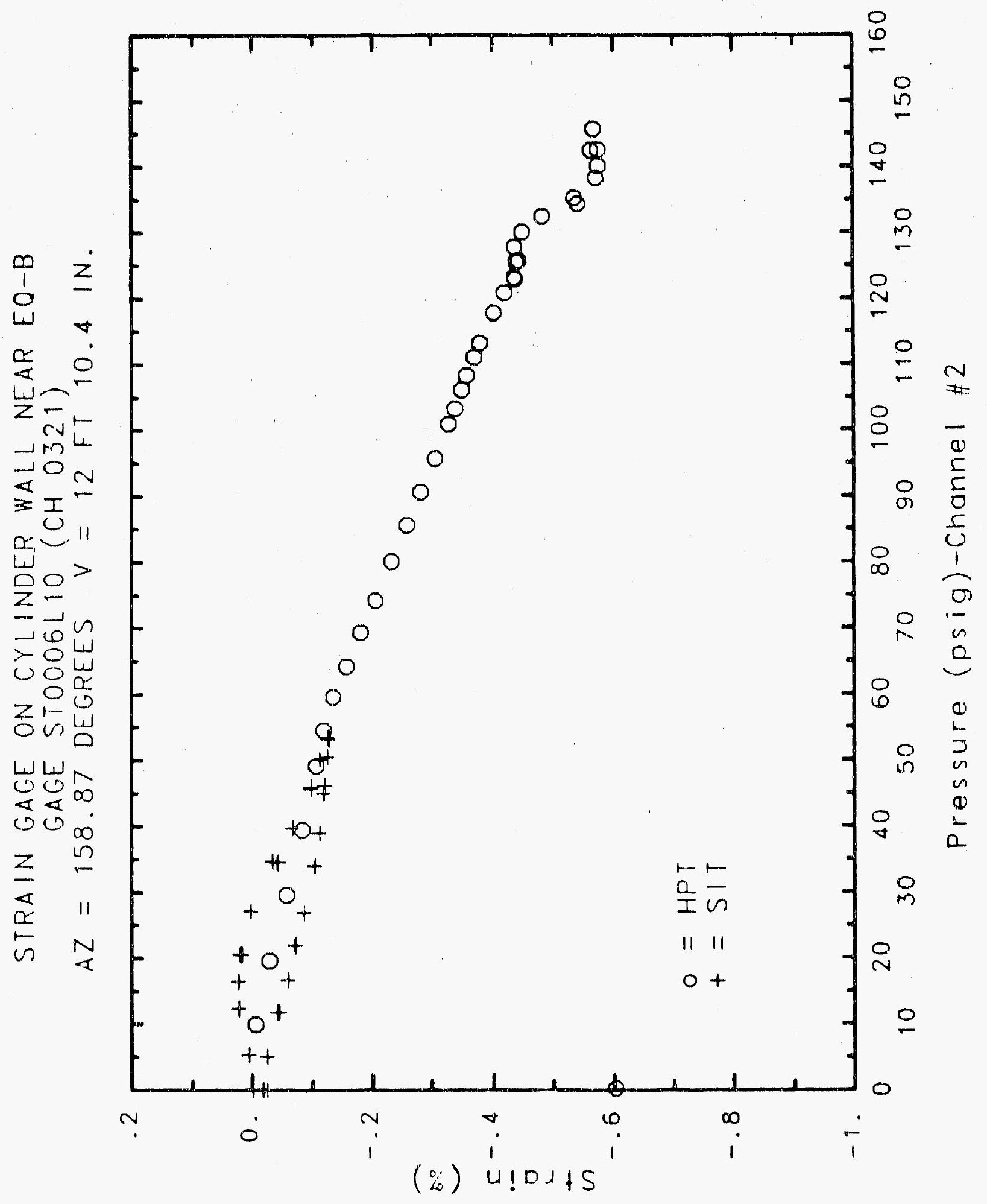




\section{Reinforced Concrete Test Data}

Strip 7 Channels $322,323,324,325,326,327,328$

\section{Structural Integrity Test}

\begin{tabular}{|c|c|c|c|c|c|c|c|}
\hline Pressure & Gage 1 & Gage 2 & Gage 3 & Gage 4 & Gage 6 & Gage 8 & Gage10 \\
\hline Psig & Xstrain & Xstrain & xstrain & Xstrain & xstrain & xstrain & xstrain \\
\hline-0.05 & 0.0009 & -0.0016 & -0.0023 & -0.0034 & -0.0016 & -0.0006 & -0.0007 \\
\hline 5.33 & 0.0369 & 0.0430 & 0.0436 & 0.0337 & 0.0175 & 0.0124 & 0.0099 \\
\hline 12.31 & 0.0824 & 0.0973 & 0.0990 & 0.0092 & 0.0412 & 0.0311 & 0.0247 \\
\hline 16.44 & 0.1087 & 0.1301 & 0.1315 & 0.0419 & 0.0550 & 0.0408 & 0.0341 \\
\hline 20.51 & 0.1399 & 0.1715 & 0.1757 & 0.0860 & 0.0697 & 0.0529 & 0.0443 \\
\hline 20.46 & 0.1426 & 0.1748 & 0.1797 & 0.0833 & 0.0713 & 0.0541 & 0.0462 \\
\hline 20.47 & 0.1440 & 0.1757 & 0.1815 & 0.0906 & 0.0724 & 0.0552 & 0.0467 \\
\hline 27.05 & 0.1866 & 0.2340 & 0.2464 & 0.1554 & 0.0985 & 0.0755 & 0.0659 \\
\hline 34.69 & 0.2369 & 0.3033 & 0.3182 & 0.2284 & 0.1204 & 0.0964 & 0.0876 \\
\hline 34.53 & 0.2427 & 0.3086 & 0.3247 & 0.2341 & 0.1212 & 0.0972 & 0.0882 \\
\hline 34.53 & 0.2436 & 0.3096 & 0.3262 & 0.2355 & 0.1213 & 0.0974 & 0.0894 \\
\hline 39.70 & 0.2737 & 0.3517 & 0.3705 & 02797 & 0.1316 & 0.1075 & 0.0996 \\
\hline 45.90 & 0.3219 & 0.4184 & 0.4449 & 0.3546 & 0.1473 & 0.1264 & 0.1172 \\
\hline 45.65 & 0.3280 & 0.4265 & 0.4540 & 0.3630 & 0.1492 & 0.1285 & 0.1187 \\
\hline 45.62 & 0.3291 & 0.4279 & 0.4558 & 0.3644 & 0.1492 & 0.1285 & 0.1186 \\
\hline 50.10 & 0.3582 & 0.4716 & 0.5082 & 0.4175 & 0.1636 & 0.1440 & 0.1303 \\
\hline 53.47 & 0.3873 & 0.5179 & 0.5678 & 0.4768 & 0.1830 & 0.1665 & 0.1456 \\
\hline 53.29 & 0.3921 & 0.5264 & 0.5789 & 0.4881 & 0.1886 & 0.1734 & 0.1488 \\
\hline 53.21 & 0.3938 & 0.5291 & 0.5824 & 0.4910 & 0.1896 & 01751 & 0.1502 \\
\hline 50.49 & 0.3886 & 0.5249 & 0.5783 & 0.4879 & 0.1866 & 0.1736 & 0.1482 \\
\hline 16.14 & 0.3796 & 0.5143 & 0.5674 & 0.4767 & 0.1794 & 0.1661 & 0.1398 \\
\hline 46.13 & 0.3799 & 0.5142 & 0.5675 & 0.4763 & 0.1784 & 0.1650 & 0.1393 \\
\hline 44.97 & $0.380 ?$ & 0.5140 & 0.5669 & 0.4760 & 0.1789 & 0.1647 & 0.1404 \\
\hline 38.98 & 0.3658 & 0.4978 & 0.5498 & 0.4593 & 0.1675 & 0.1538 & 0.1291 \\
\hline 33.99 & 0.3529 & 0.4827 & 0.5343 & 0.4434 & 0.1549 & 0.1433 & 0.1187 \\
\hline 33.96 & 03522 & 0.4816 & 0.5330 & 0.4424 & 0.1545 & 0.1421 & 0.1183 \\
\hline 26.83 & 0.3320 & 0.4589 & 0.5098 & 0.4194 & 0.1385 & 0.1256 & 0.1024 \\
\hline 21.88 & 0.3153 & 0.4410 & 0.4903 & 0.4006 & 0.1271 & 0.1144 & 0.0918 \\
\hline 21.90 & 0.3141 & 0.4399 & 0.4904 & 0.4003 & 0.1269 & 0.1135 & 0.0909 \\
\hline 16.69 & 0.2937 & 0.4170 & 0.4662 & 0.3768 & 0.1132 & 0.1011 & 0.0793 \\
\hline 11.74 & 0.2674 & 0.3845 & 0.4324 & 0.3425 & 0.0956 & 0.0872 & 0.0654 \\
\hline 11.77 & 0.2658 & 0.3833 & 0.4303 & 0.3415 & 0.0945 & 0,0868 & 0.0646 \\
\hline 5.05 & 0.2112 & 0.3178 & 0.3632 & 0.2730 & 0.0658 & 0.0655 & 0.0464 \\
\hline 0.02 & 0.1585 & 0.2453 & 0.2861 & 0.1948 & 0.0424 & 0.0507 & 0.0349 \\
\hline-0.04 & 0.1553 & 0.2429 & 0.2842 & 0.1928 & 0.0422 & 0.0509 & 0.0334 \\
\hline-0.02 & 0.1567 & 0.2451 & 0.2849 & 0.1955 & 0.0433 & 0.0498 & 0.0334 \\
\hline-0.02 & 0.1555 & 0.2438 & 0.2834 & 0.1941 & 0.0427 & 0.0496 & 0.0330 \\
\hline 0.02 & 0.1544 & 0.2426 & 0.2810 & 0.1918 & 0.0409 & 0.0479 & 0.0312 \\
\hline
\end{tabular}




\section{Reinforced Concrete Test Data}

Strip 7 Channels $322,323,324,325,326,327,328$

High Pressure test

\begin{tabular}{|c|c|c|c|c|c|c|c|}
\hline Pressure & Gage 1 & Gage 2 & Gage 3 & Gage 4 & Gage 6 & Gage 8 & Gage 10 \\
\hline Psig & Xstrain & xstrain & xstrain & xstrain & xstrain & xstrain & xstrain \\
\hline 9.89 & 0.0832 & 0.0981 & 0.0976 & 0.0980 & 0.0432 & 0.0271 & 0.0226 \\
\hline 19.55 & 0.1381 & 0.1615 & 0.1608 & 0.1615 & 0.0768 & 0.0554 & 0.0478 \\
\hline 29.57 & 0.1701 & 0.2014 & 0.2019 & 0.2021 & 0.1010 & 0.0780 & 0.0706 \\
\hline 39.42 & 0.1990 & 0.2372 & 0.2397 & 0.2407 & 0.1225 & 0.0997 & 0.0919 \\
\hline 49.16 & 0.2271 & 0.2736 & $0.2805^{\prime \prime}$ & 0.2810 & 0.1429 & 0.1212 & 0.1122 \\
\hline 54.50 & 0.2467 & 0.3044 & 0.3174 & 0.3176 & 0.1608 & 0.1408 & 0.1307 \\
\hline 59.57 & 0.2667 & 0.3603 & 0.3960 & 0.3962 & 0.2076 & 0.2331 & 0.2095 \\
\hline 64.20 & 0.2810 & 0.4094 & 0.4652 & 0.4652 & 0.2801 & 0.3637 & 0.3514 \\
\hline 69.32 & 0.3002 & 0.4567 & 0.5263 & 0.5261 & 0.3517 & 0.5011 & 0.4915 \\
\hline 74.16 & 0.3326 & 0.5208 & 0.6034 & 0.6038 & 0.4364 & 0.6525 & 0.6390 \\
\hline 80.16 & 0.4125 & 0.6232 & 0.7177 & 0.7178 & 0.6029 & 0.8735 & 0.8208 \\
\hline 85.61 & 0.5182 & 0.7304 & 0.8222 & 0.8225 & 0.7831 & 1.0616 & $0.995 \%$ \\
\hline 90.58 & 0.6551 & 0.8564 & 0.9355 & 0.9357 & 0.9382 & 1.1946 & 1.1508 \\
\hline 95.69 & 0.7847 & 1.0176 & 1.0694 & 1.0699 & 1.0683 & 1.2857 & 1. 3089 \\
\hline 100.92 & 0.9264 & 1.1756 & 1.1999 & 1. 1999 & 1.1593 & 1. 363 ? & 1.4280 \\
\hline 103.25 & 0.9891 & 1.2483 & 1. 2605 & 1.2609 & 1.1872 & 1.3973 & 1.4632 \\
\hline 106.11 & 1.0527 & 1.3243 & 1. 3237 & $1.3 \hat{\imath} 4 !$ & 1.2193 & 1.4344 & 1.5029 \\
\hline $108 \quad 31$ & 1.0964 & 1.3761 & 1.3706 & 1.3715 & 1.2454 & 1.4673 & 1.5309 \\
\hline 111.08 & 1.1520 & 1.4477 & 1.4371 & 1.4379 & 1.2837 & 1.5177 & 1.5734 \\
\hline 113.24 & 1.1889 & 1.4978 & 1.4876 & 1.4879 & 1. 3143 & 1. 5611 & 1.6087 \\
\hline 117.83 & 1.2710 & 1.6084 & 1.6011 & 1.6012 & 1. 3994 & 1.6709 & 1.7137 \\
\hline 120.92 & 1.3316 & 1.6898 & 1.6790 & 1.6795 & 1. 4552 & 1.7535 & 1.7887 \\
\hline 123.28 & 1.3819 & 1.7603 & 1.7456 & 1. 7458 & 1. 5033 & 1.8251 & 1.8509 \\
\hline 122.97 & 1.3941 & $1.778 ?$ & 1.7615 & 1.7622 & 1. 5150 & 1.8436 & 1.8632 \\
\hline 125.82 & 1.4514 & 1.8633 & 1.8453 & 1.8458 & 1. 5993 & 1.9372 & 1.9562 \\
\hline 125.60 & 1. 4667 & 1.8852 & 1.8640 & 1.8649 & 1.6137 & 1.9610 & 1.9746 \\
\hline 127.84 & 1.4956 & 1.9290 & 1.9055 & 1.9057 & 1.6537 & 2.0040 & 2.0241 \\
\hline 130.19 & 1.5480 & 2.0022 & 1.9747 & 1.9753 & 1.7403 & 2.0888 & 2.1170 \\
\hline 132.53 & 1. 6232 & 2.1061 & 2.0773 & 2.0773 & 1.8811 & 2.1983 & 2.2481 \\
\hline 135.33 & 1.7396 & 2.2556 & 2.2328 & 2.2332 & 2.0686 & 2.3410 & 2.4308 \\
\hline 134.42 & 1.7712 & 2.2896 & 2.2718 & 2.2720 & 2.1083 & 2.3750 & 2.4670 \\
\hline 138.35 & 2.0190 & 2.5306 & 2.5599 & 2.5608 & 2.3965 & 2.6414 & 2.7855 \\
\hline 140.16 & 2.2471 & 2.7359 & 2.8116 & 2.8121 & 2.6181 & 2.8797 & $3.035 ?$ \\
\hline 142.63 & 2. 5497 & 3.0297 & 3.2044 & 3.2050 & 2.9515 & 3.2493 & 3.3912 \\
\hline 145.78 & 2.8375 & 3.3556 & 3.6183 & 3.6189 & 3.2904 & 3.6323 & 3.7617 \\
\hline 142.52 & 2.9614 & 3.4848 & 3.1457 & 3.7464 & 3.4041 & 3.7759 & 3.8942 \\
\hline 0.22 & 2.5400 & 3.0404 & 3.2697 & 3.2700 & 2.9894 & 3.3844 & 3.5228 \\
\hline
\end{tabular}




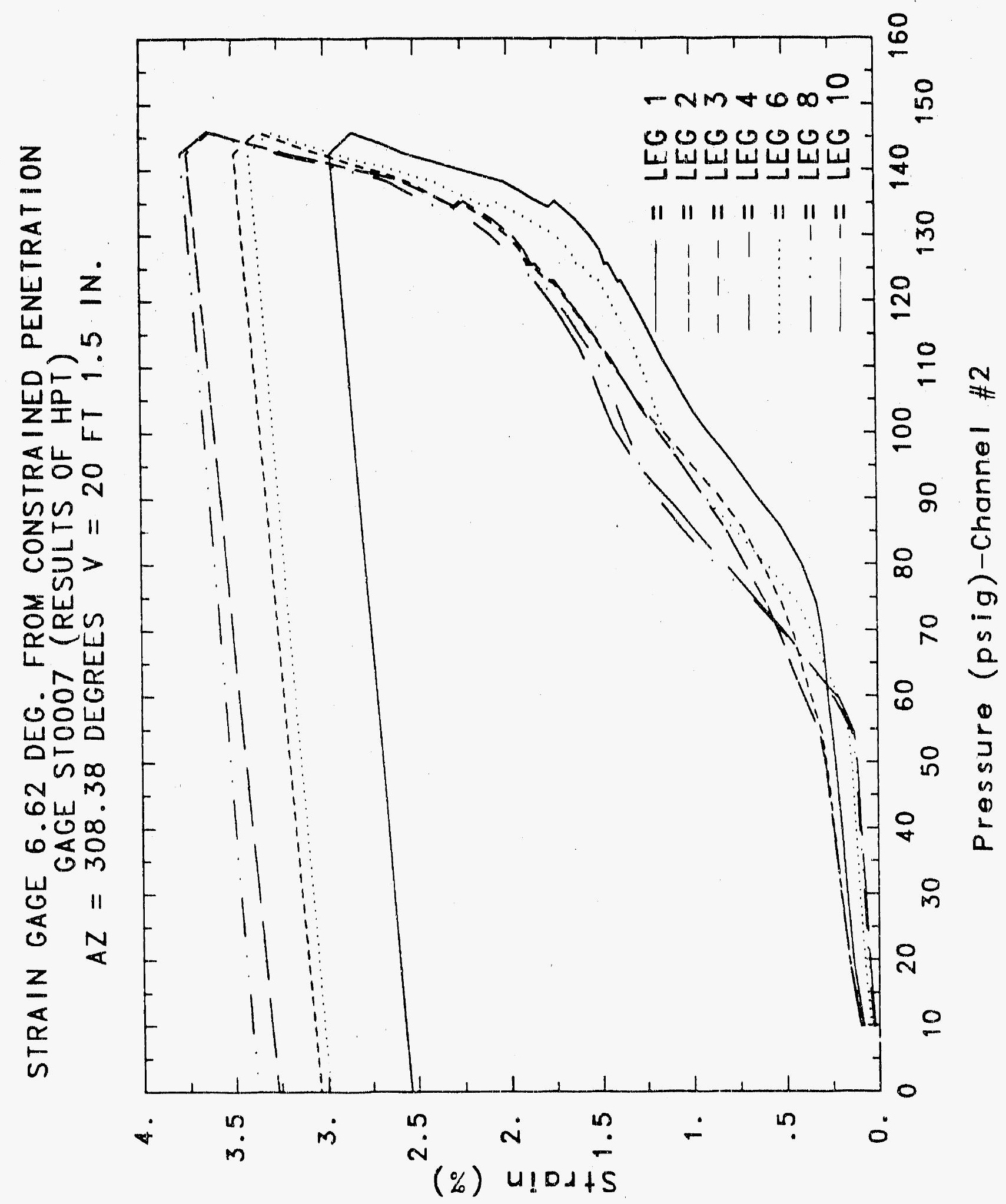




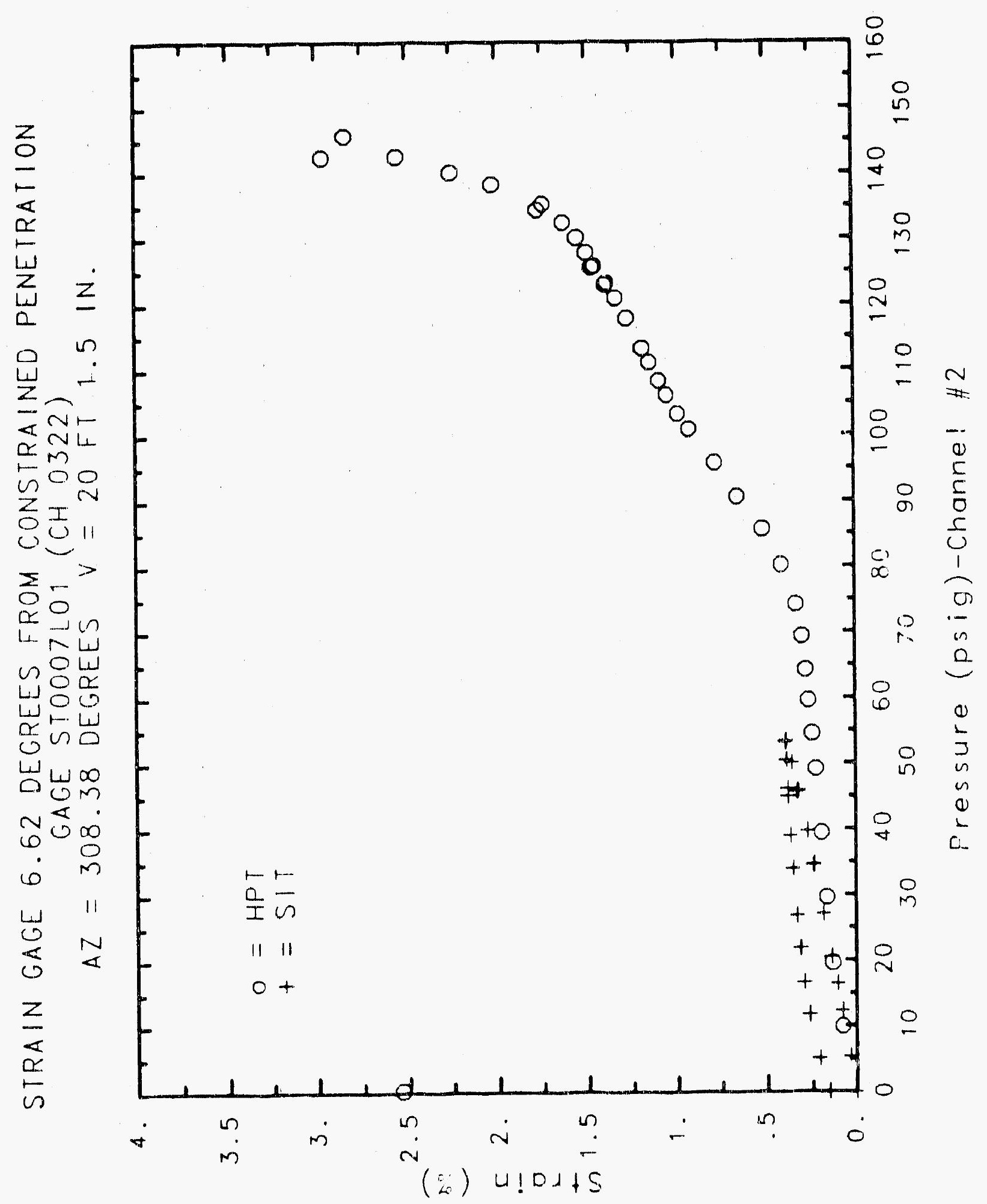




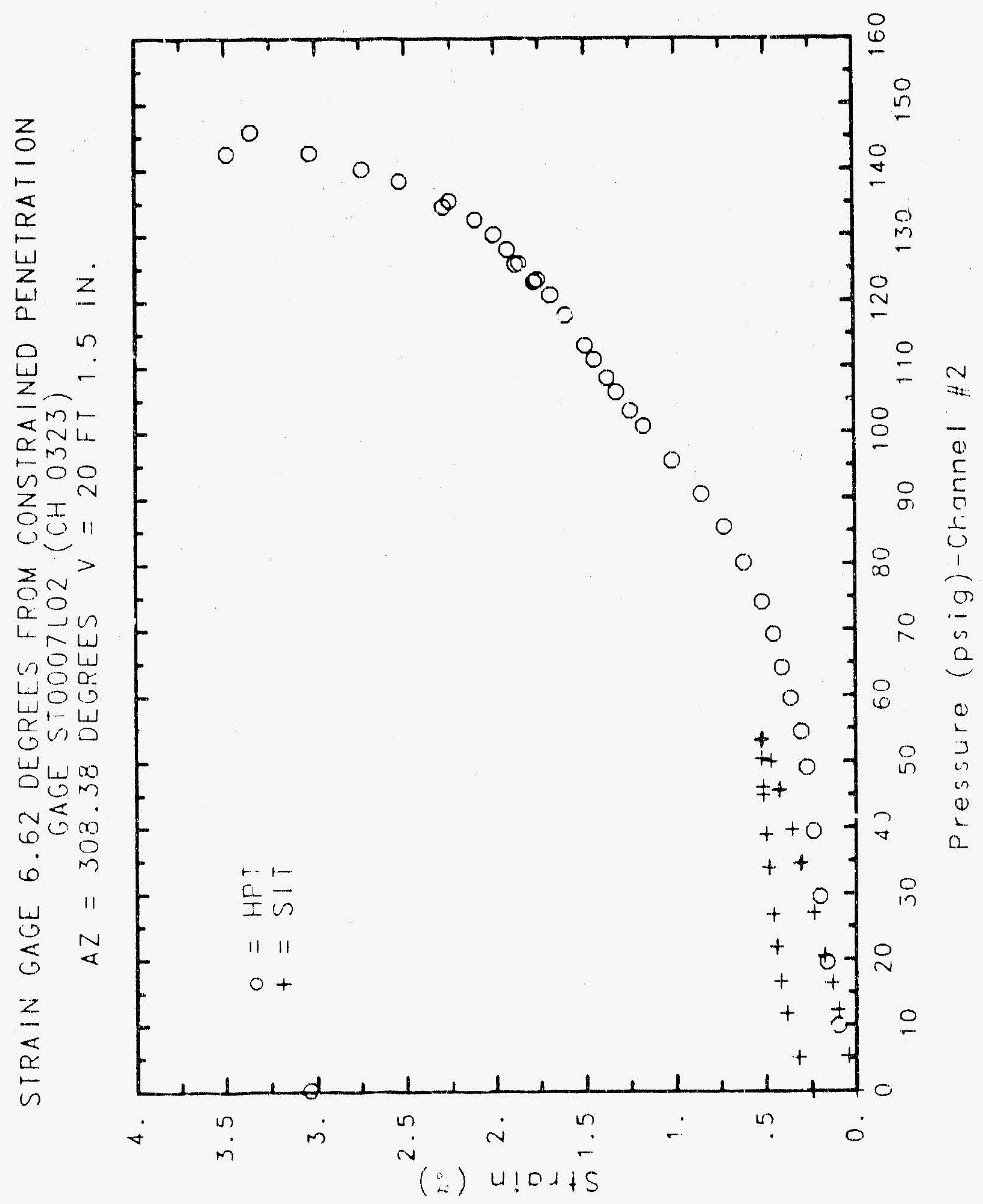




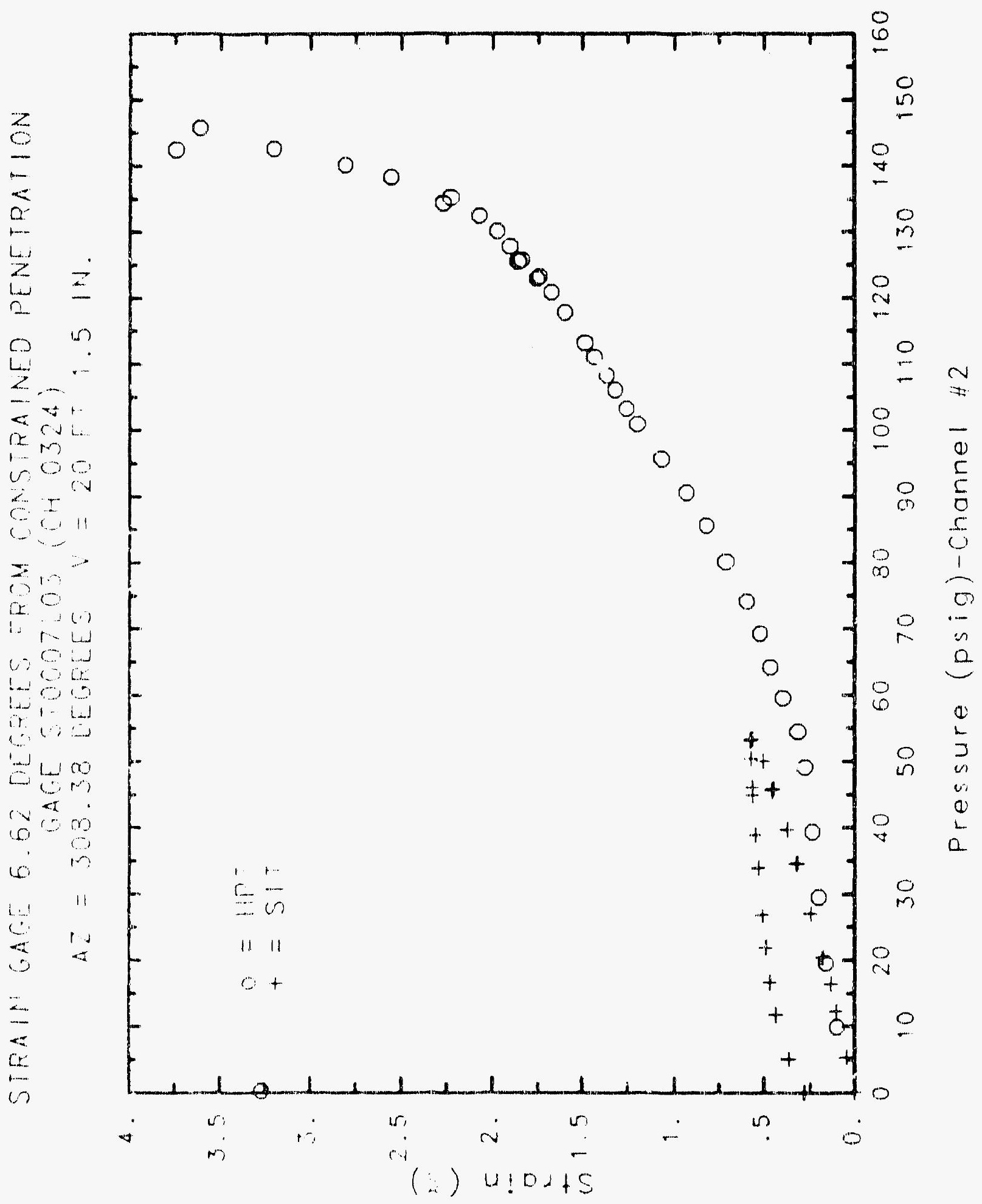

(..) $1+1)$ 


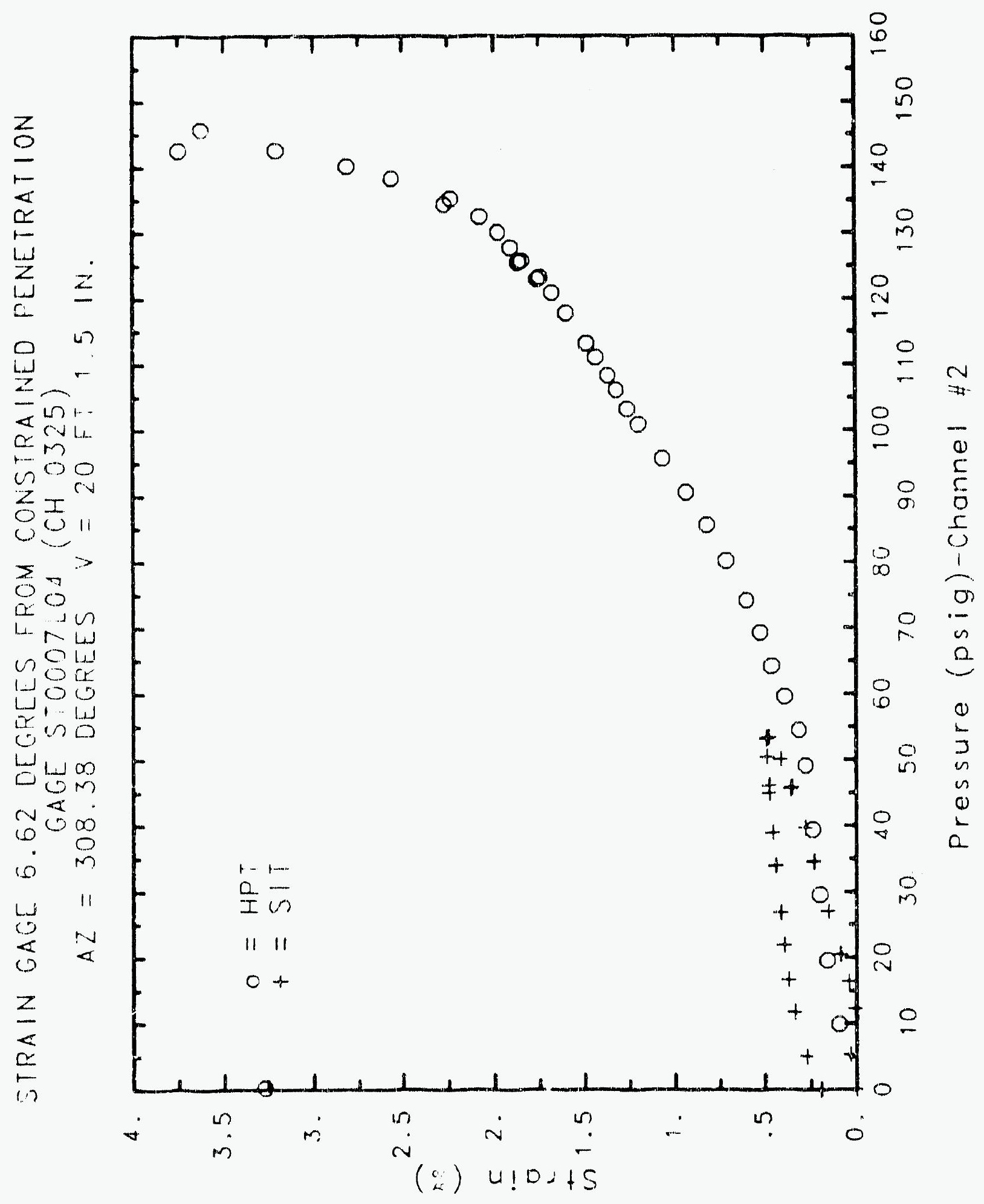




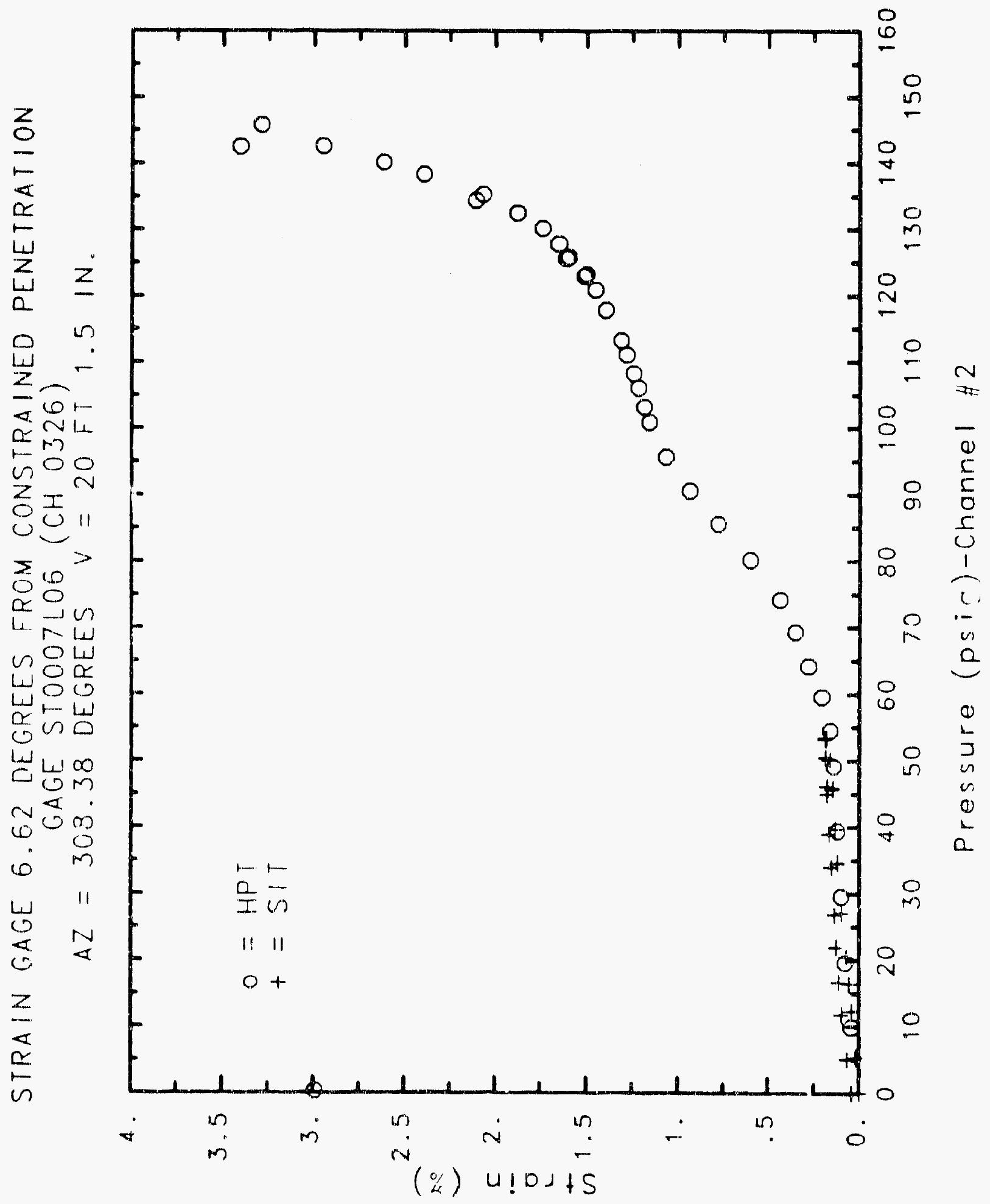

C- -1451 


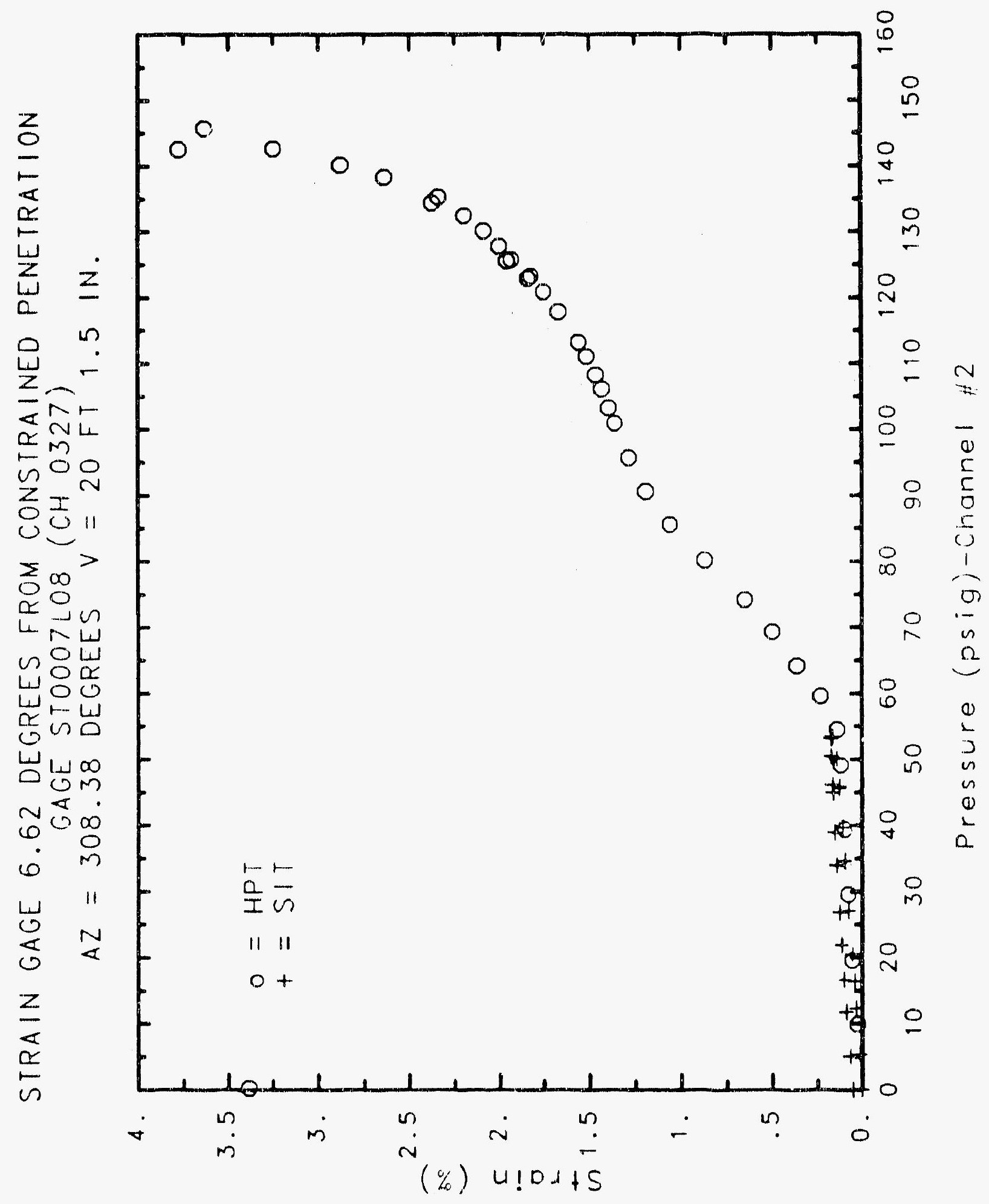




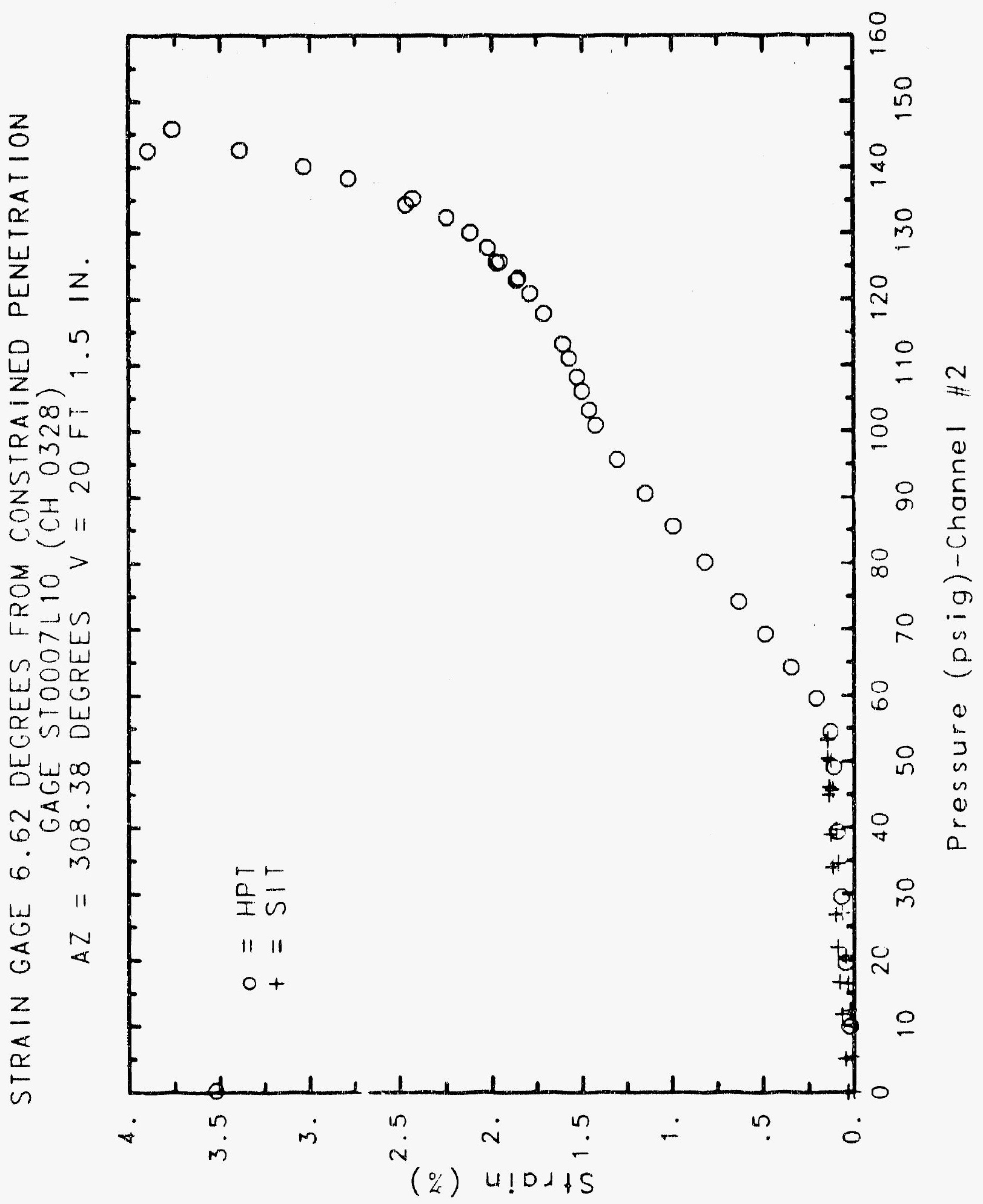




\section{Reinforced Concrete Test Data}

\section{Strip 8 Channels $386,387,388,389,2240,2241$}

\section{Structural Integrity TEST}

\begin{tabular}{|c|c|c|c|c|c|c|}
\hline $\begin{array}{c}\text { Pressure } \\
\text { Psig }\end{array}$ & $\begin{array}{l}\text { Gage } 1 \\
\text { Xstrain }\end{array}$ & $\begin{array}{l}\text { Gage ? } \\
\text { xStra in }\end{array}$ & $\begin{array}{l}\text { Gage } 3 \\
\text { xstrain }\end{array}$ & $\begin{array}{l}\text { Gage } 5 \\
\text { XStrain }\end{array}$ & $\begin{array}{l}\text { Gage } 7 \\
\text { XStrain }\end{array}$ & $\begin{array}{l}\text { Gage10 } \\
\text { xstrain }\end{array}$ \\
\hline-0.05 & 0.0006 & -0.0009 & -0.0013 & 0.0010 & -0.0024 & 0.0021 \\
\hline 5.33 & -0.0036 & -0.0052 & -0.0063 & -0.0028 & -0.0030 & -0.0011 \\
\hline 12.31 & -0.0083 & -0.0098 & -0.0109 & -0.0067 & -0.0067 & -0.0044 \\
\hline 16.44 & -0.0114 & -0.0136 & -0.0140 & -0.0100 & -0.0090 & -0.0064 \\
\hline 20.51 & -0.0162 & -0.0178 & -0.0183 & -0.0411 & -0.0116 & -0.0077 \\
\hline $20.4 E$ & -0.0174 & -0.0188 & -0.0193 & -0.0422 & -0.0111 & -0.0078 \\
\hline 20.47 & -0.0175 & -0.0181 & -0.0190 & -0.0426 & -0.0114 & -0.0075 \\
\hline 27.05 & -0.0403 & -0.0403 & -0.0386 & -0.0598 & -0.0279 & -0.0239 \\
\hline 34.69 & -0.0599 & -0.0571 & -0.0516 & -0.0740 & -0.0343 & -0.0290 \\
\hline 34.53 & -0.0604 & -0.0578 & -0.0520 & -0.0749 & -0.0346 & -0.0291 \\
\hline 34.53 & -0.0602 & -0.0577 & -0.0525 & -0.0755 & -0.0345 & -0.0295 \\
\hline 39.70 & -0.0708 & -0.0661 & -0.0500 & -0.0835 & -0.0404 & -0.0346 \\
\hline 45.90 & -0.0831 & -0.0773 & -0.0705 & -0.0926 & -0.0476 & -0.0421 \\
\hline 45.65 & -0.0829 & $-0.07 ? 1$ & -0.0696 & -0.0919 & -0.0483 & -0.0420 \\
\hline 45.62 & -0.0827 & -0.0770 & -0.0695 & -0.0927 & -0.0480 & -0.0430 \\
\hline 50.10 & -0.0910 & -0.0844 & -0.0763 & -0.0991 & -0.0528 & -0.0476 \\
\hline 53.47 & -0.0967 & -0.0905 & -0.0810 & -0.1040 & -0.0573 & -0.0508 \\
\hline 53.29 & -0.0961 & -0.0911 & -0.0816 & -0.1040 & -0.0579 & -0.0516 \\
\hline 53.21 & -0.0954 & -0.0897 & $-0.080 E$ & -0.1037 & -0.0583 & -0.0520 \\
\hline 50.49 & -0.0963 & -0.0890 & -0.0799 & -0.1021 & -0.0559 & -0.0504 \\
\hline 46.14 & -0.0907 & -0.0845 & -0.0754 & $\begin{array}{lll}-0 & 0983\end{array}$ & -0.0525 & -0.0470 \\
\hline$\Delta E .13$ & -0.0906 & -0.0846 & -0.0759 & -0.0980 & -0.0525 & -0.0471 \\
\hline 4.4 .97 & $-0 \quad 0885$ & -0.0821 & -0.0735 & -0.0570 & -0.0520 & -0.0449 \\
\hline 38.98 & .00823 & -0.0763 & -0.0679 & -0.0503 & -0.0458 & -0.0394 \\
\hline 33.99 & -0.0760 & -0.0705 & -0.0629 & -0.0847 & -0.0411 & -0.0357 \\
\hline 33.96 & -0.0770 & -0.0707 & -0.0621 & -0.0858 & -0.0407 & -0.0347 \\
\hline 26.83 & -0.0683 & .0 .0625 & -0.0556 & -0.0781 & -0.0342 & -0.0282 \\
\hline 21.88 & -0.0609 & -0.0562 & -0.0493 & -0.0714 & -0.0296 & -0.0230 \\
\hline $21: 0$ & -0.0612 & -0.0558 & -0.0495 & -0.0727 & -0.0293 & -0.0231 \\
\hline 16.69 & -0.0506 & -0.0468 & -0.0410 & -0.0637 & -0.0243 & -0.0181 \\
\hline 11.74 & -0.0399 & -0.0369 & -0.0327 & -0.0553 & $-0 \quad 0194$ & -0.0136 \\
\hline 11.77 & -0.0399 & -0.0360 & -0.0325 & -0.0551 & -0.0188 & -0.0145 \\
\hline 5.05 & -0.0244 & -0.0229 & -0.0205 & -0.0438 & -0.0112 & -0.0084 \\
\hline 0.02 & -0.0129 & -0.0135 & -0.0130 & -0.0358 & -0.0073 & -0.0037 \\
\hline-0.04 & -0.0113 & -0.0114 & -0.0111 & -0.0336 & -0.0070 & -0.0030 \\
\hline-0.02 & -0.0108 & -0.0101 & -0.0107 & -0.0338 & -0.0054 & $\because 0.0028$ \\
\hline-0.02 & -0.0105 & -0.0116 & -0.0110 & -0.0336 & -0.0057 & -0.0028 \\
\hline 0.02 & -0.0105 & -0.0111 & -0.0108 & -0.0341 & -0.0048 & -0.0009 \\
\hline
\end{tabular}




\section{Reinforced Concrete Test Data}

\section{Strip 8 Channels $386,387,388,389,2240,2241$}

\section{High Pressure test}

\begin{tabular}{|c|c|c|c|c|c|c|}
\hline Pressure & Gage 1 & Gage 2 & Gage 3 & Gage 5 & Gage 7 & Gage10 \\
\hline $\begin{array}{l}\text { Psig } \\
9.89\end{array}$ & $\begin{array}{l}\text { Xstrain } \\
-0.0112\end{array}$ & $\begin{array}{l}\text { XStrain } \\
-0.0110\end{array}$ & $\begin{array}{l}\text { Eotrain } \\
-0.0100\end{array}$ & $\begin{array}{l}\text { Xstrain } \\
-0.0101\end{array}$ & $\begin{array}{l}\text { xstrain } \\
-0.0077\end{array}$ & $\begin{array}{l}\text { Xstrain } \\
-0.0071\end{array}$ \\
\hline 19.55 & -0.0230 & -0.0222 & -0.0211 & -0.0209 & -0.0167 & .0 .0158 \\
\hline 29.57 & $-0: 0401$ & -0.0382 & -0.0355 & -0.0347 & -0.0265 & -0.0251 \\
\hline 39.42 & -0.0573 & -0.0531 & -0.0487 & -0.0489 & -0.0368 & -0.0343 \\
\hline 49.16 & -0.0723 & -0.0675 & -0.0612 & -0.0608 & -0.0464 & -0.0436 \\
\hline 54.50 & -0.0788 & -0.0748 & -0.0676 & -0.0678 & -0.0515 & -0.0484 \\
\hline 59.57 & -0.0891 & -0.0835 & -0.0755 & -0.0759 & -0.0573 & -0.0535 \\
\hline 64.20 & -0.0988 & -0.0920 & -0.0837 & -0.0829 & -0.0823 & 0.0577 \\
\hline 69.32 & -0.1082 & -0.1009 & -0.0899 & -0.0908 & -0.0672 & -0.0620 \\
\hline 74.16 & -0.1109 & -0.1039 & -0.0937 & -0.0936 & -0.0702 & -0.0650 \\
\hline 80.16 & -0.1167 & -0.1102 & -0.0986 & -0.0992 & -0.0748 & -0.0692 \\
\hline 85.61 & -0.1200 & -0.1135 & -0.1019 & -0.1017 & -0.0770 & -0.0710 \\
\hline 90.58 & -0.1223 & -0.1170 & -0.1042 & -0.1038 & -0.0793 & -0.0730 \\
\hline 95.69 & -0.1249 & -0.1181 & -0.1062 & -0.1066 & -0.0814 & $-0.075 !$ \\
\hline 100.92 & -0.1259 & -0.1199 & -0.1085 & -0.1081 & -0.0834 & -0.0771 \\
\hline 103.25 & -0.1318 & -0.1249 & -0.1129 & -0.1122 & -0.0854 & -0.0784 \\
\hline 106.11 & -0.1303 & -0.1243 & -0.1126 & -0.1126 & -0.0866 & -0.0798 \\
\hline 108.31 & -0.1303 & -0.1249 & -0.1132 & -0.1134 & -0.0875 & -0.0807 \\
\hline 111.08 & -0.1336 & -0.1278 & -0.1155 & -0.1159 & -0.0892 & -0.0821 \\
\hline 113.24 & -0.1334 & -0.1277 & -0.1160 & -0.1161 & -0.0902 & -0.0831 \\
\hline 117.83 & -0.1328 & -0.1285 & -0.1173 & -0.1169 & -0.0921 & -0.0851 \\
\hline 120.9 & 7.1414 & -0.1356 & -0.1234 & -01232 & -0.0355 & -0.0878 \\
\hline 123.28 & -0.1447 & -0.1388 & -0.1254 & -0.1253 & -0.0973 & -0.0890 \\
\hline 122.97 & -0.1425 & -0.1369 & -0.1248 & -0.1242 & -0.0967 & -0.0883 \\
\hline 125.82 & -0.1438 & -0.1377 & -0.1257 & -0.1256 & -0.0994 & -0.0912 \\
\hline 125.60 & -0.1416 & -0.1368 & -0.1247 & -0.1248 & -0.0987 & -0.0900 \\
\hline 127.84 & -0.1538 & -0.1461 & -0.1327 & -0.1329 & -0.1016 & -0.0920 \\
\hline 130.19 & -0.1505 & -0.1452 & -0.1322 & -0.1324 & -0.1027 & -0.0932 \\
\hline 132.53 & -0.1570 & -0.1507 & -0.1368 & -0.1370 & -0.1052 & -0.0950 \\
\hline 135.33 & -0.1574 & -0.2040 & -0.1348 & -0.1346 & -0.1085 & -0.0976 \\
\hline 134.42 & -0.1528 & -0.2009 & -0.1324 & -0.1327 & -0.1070 & -0.0959 \\
\hline 138.35 & -0.1627 & -0.2353 & -0.1416 & -0.1414 & -0.1137 & -0.1014 \\
\hline 140.16 & -0.1666 & -0.2507 & -0.1410 & -0.1409 & -0.1107 & -0.1019 \\
\hline 142.63 & -0.2399 & -0.2555 & -0.1461 & -0.1447 & -0.1126 & -0.1030 \\
\hline 145.78 & -0.3123 & -0.2955 & -0.1592 & -0.1597 & -0.1223 & -0.110 .5 \\
\hline 142.52 & -0.3158 & -0.2990 & -0.1595 & -0.1595 & -0.1144 & -0.1046 \\
\hline 0.22 & -0.0603 & -0.0643 & 0.0266 & 0.0274 & 0.0067 & 0.0000 \\
\hline
\end{tabular}




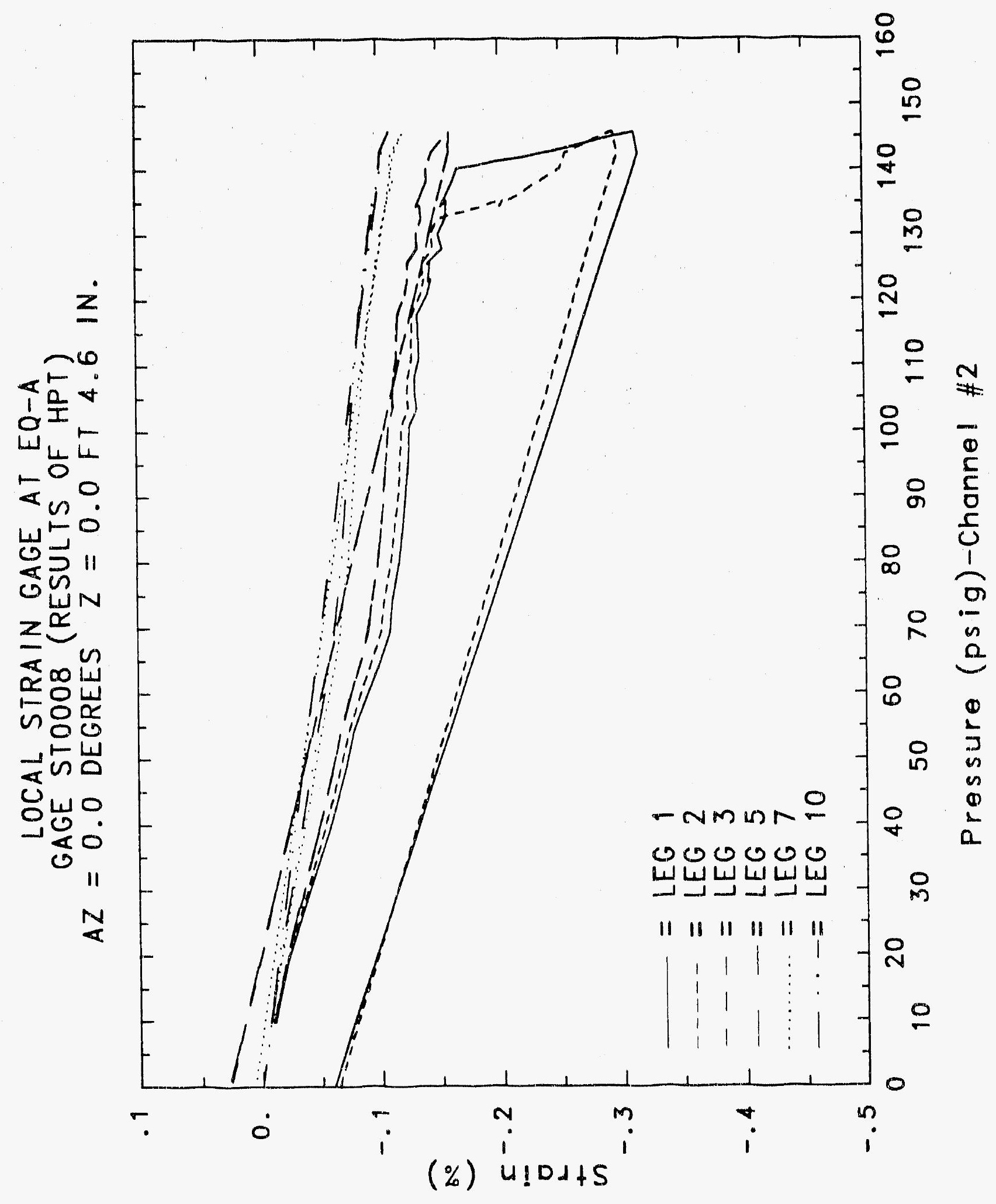




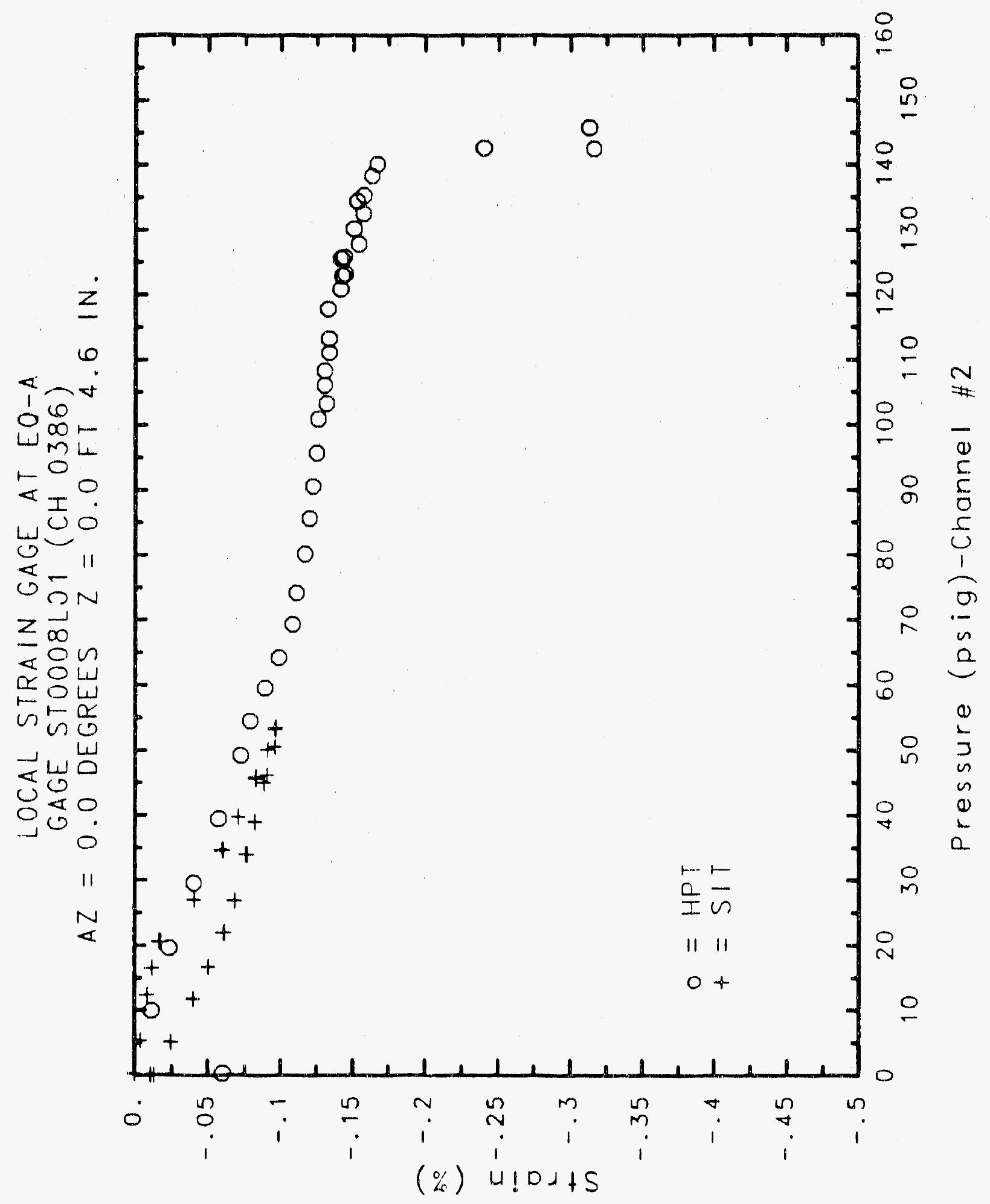




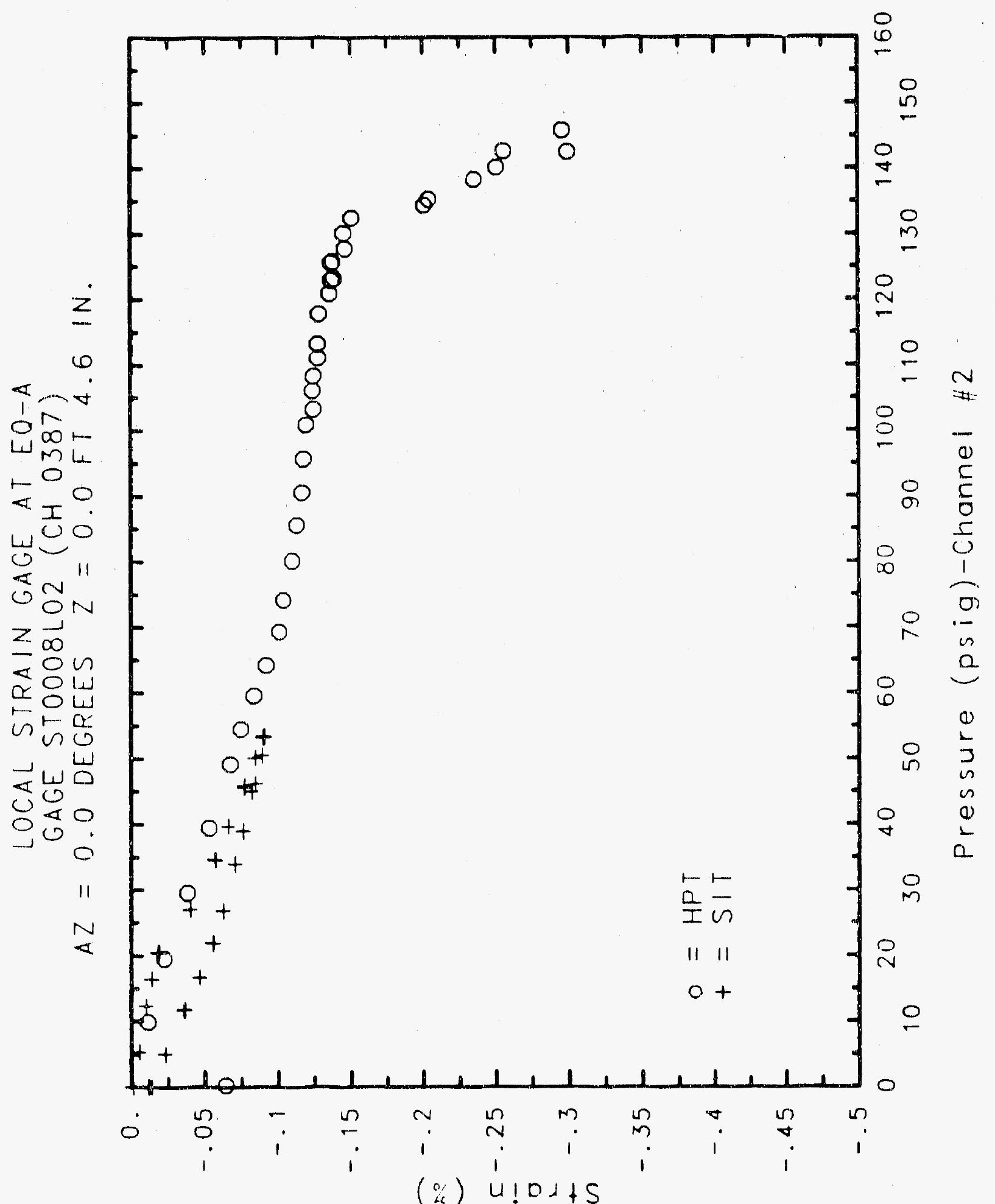




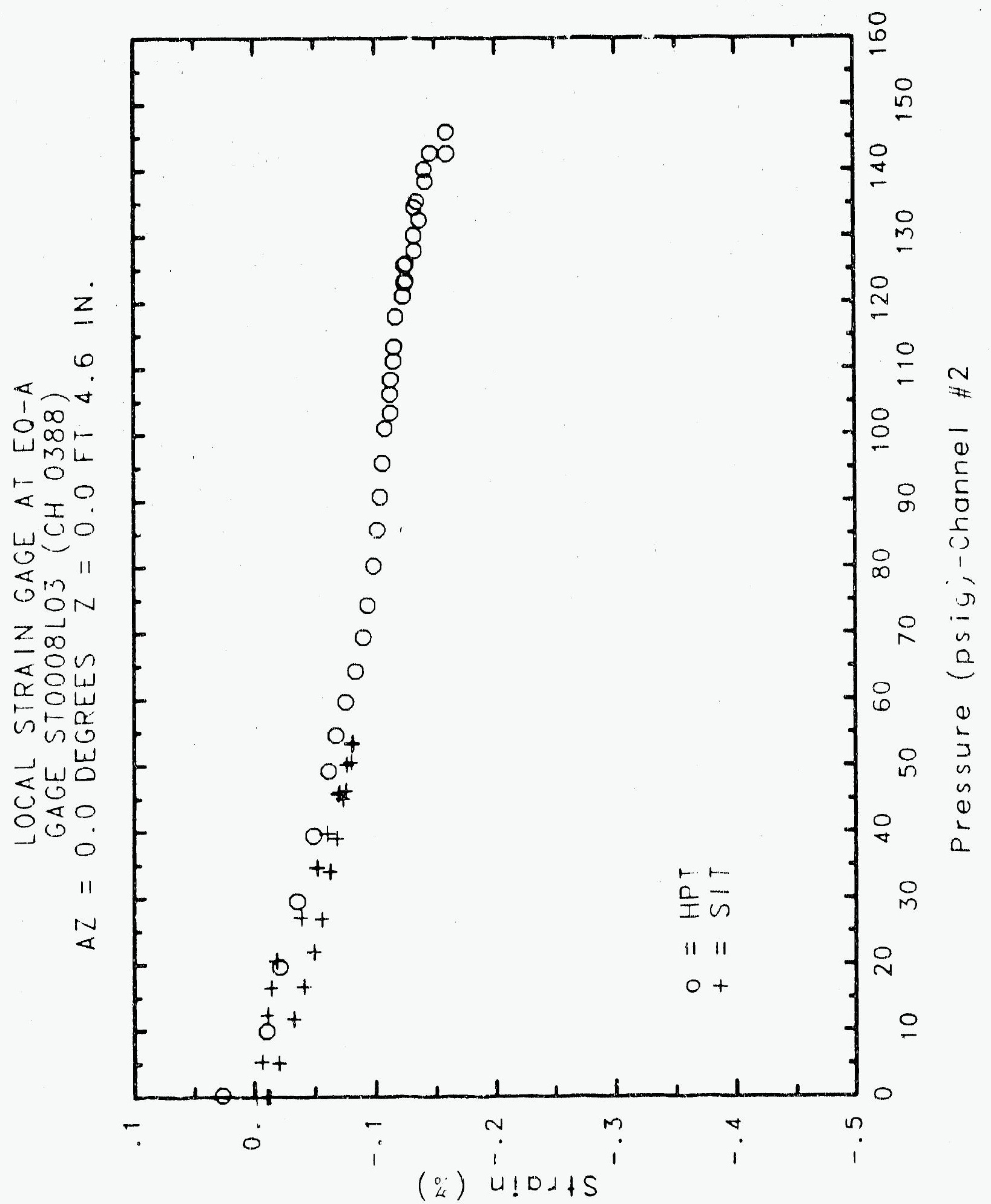




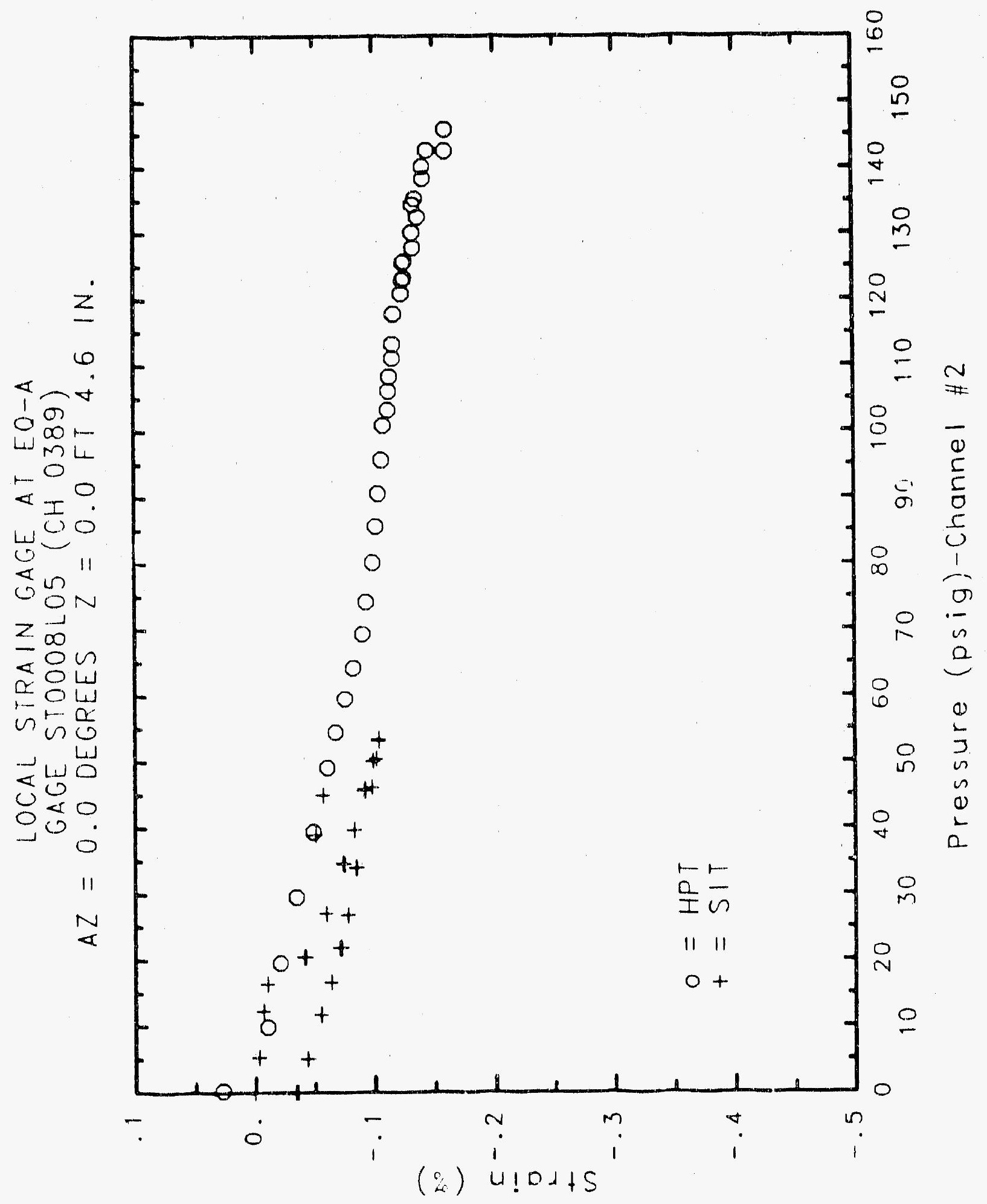




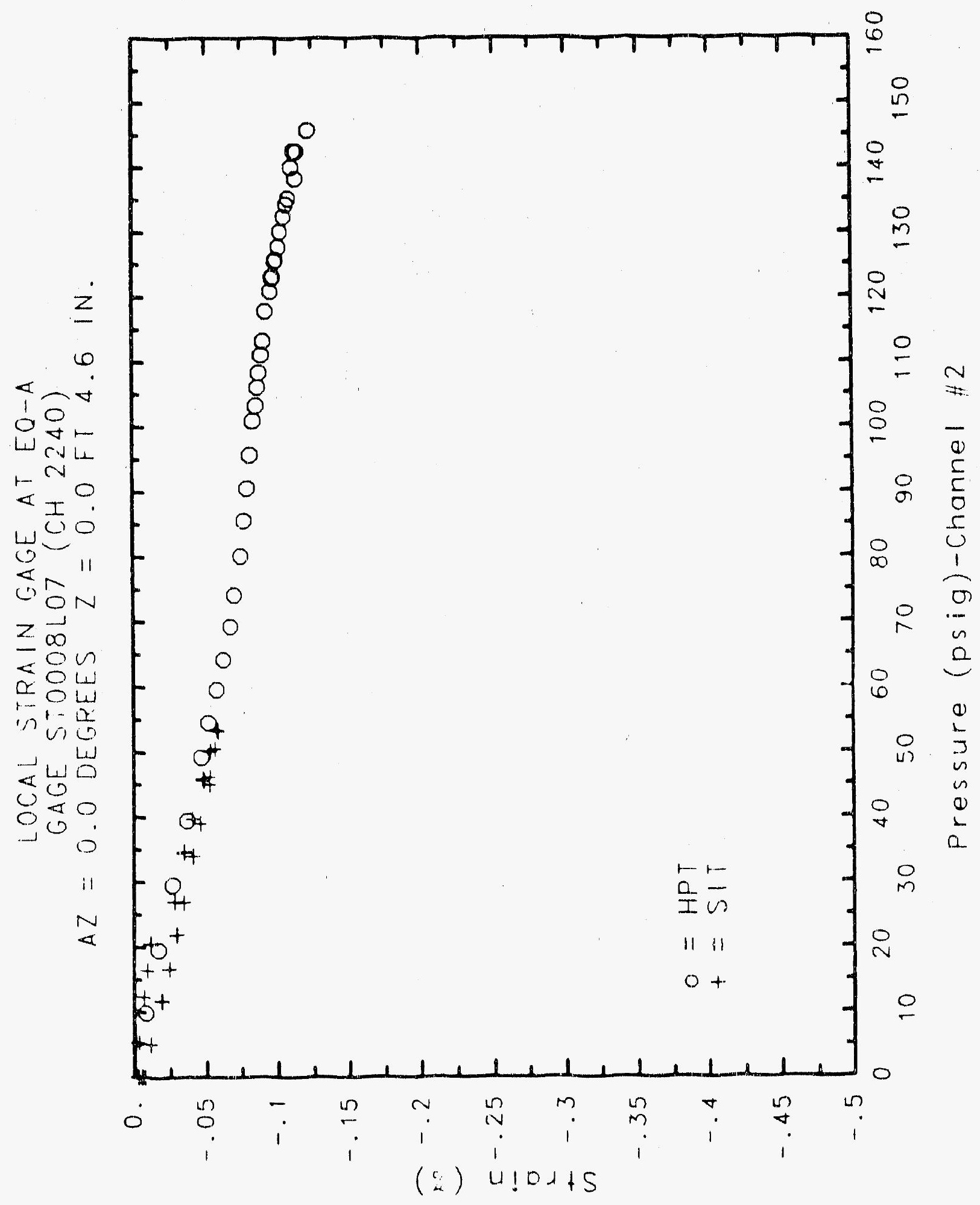




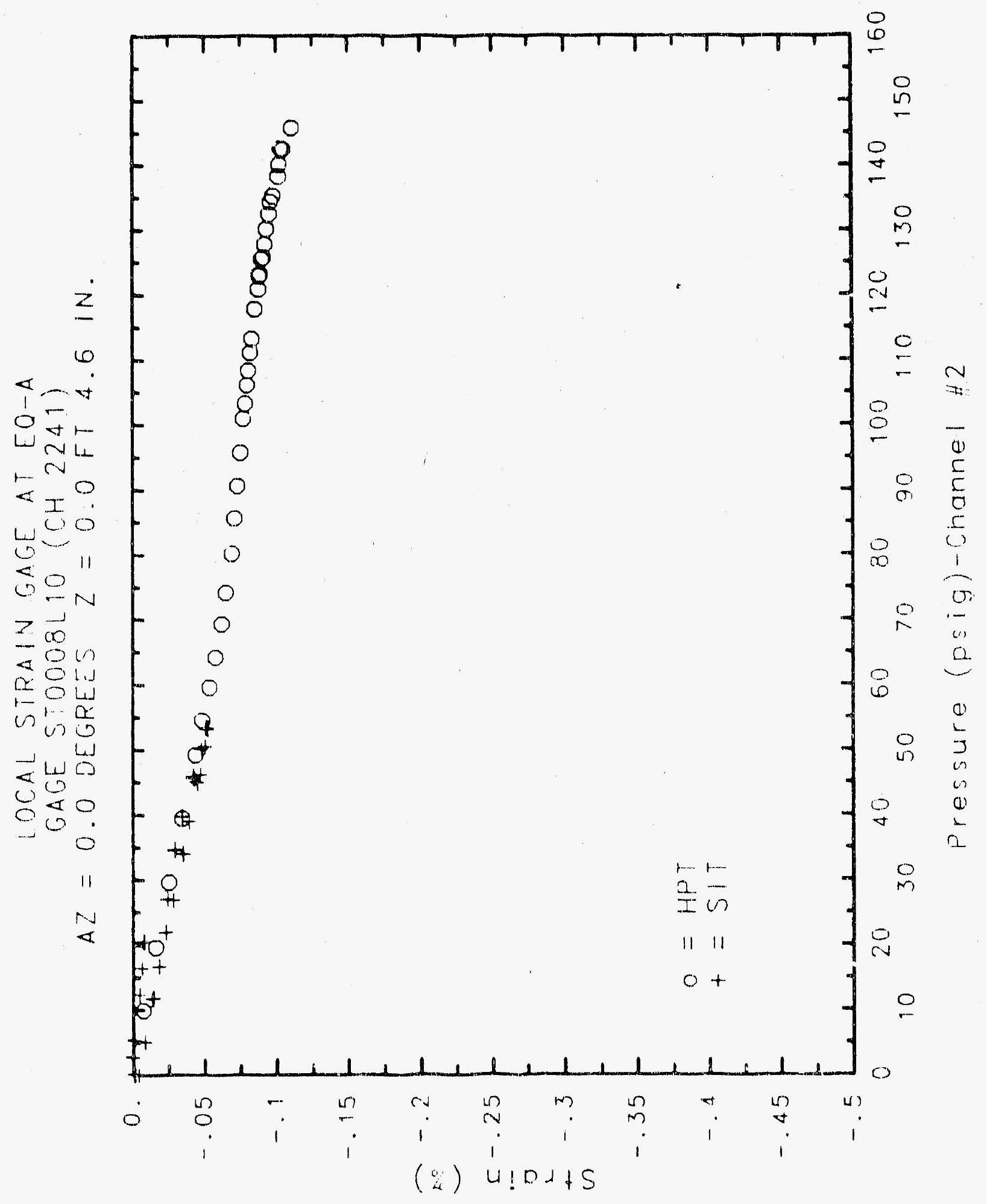




\section{Reinforced Concrete Test Data}

Strip 9 Channels $2242,2243,2244,2245,2246,2247$

\section{STRUCTURAL. INTEGRITY TEST}

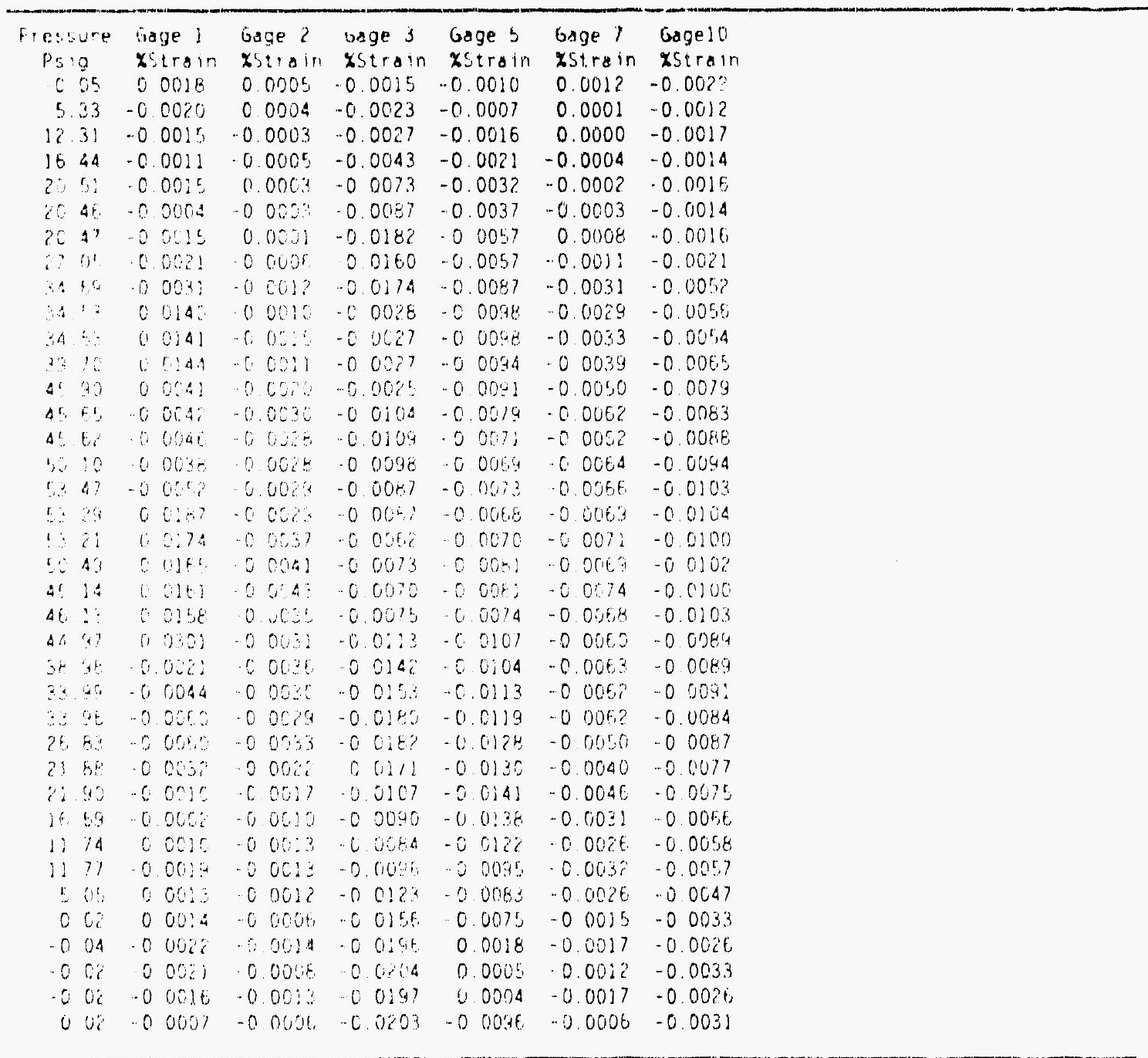




\section{Reinforced Concrete Test Data}

Strip 9 Channels $2242,2243,2244,2245,2246,2247$

High Pressure test

\begin{tabular}{|c|c|c|c|c|c|c|}
\hline Pressure & Gage 1 & Goje? & Gage 3 & Gage 5 & Gage 7 & Gage 10 \\
\hline$P_{5 i g}$ & xstrain & xstrain & xstrain & xStrain & xstrein & xstroin \\
\hline 9.89 & 0.0001 & 0.0004 & 0.0007 & 0.0002 & 0.0002 & 0.0023 \\
\hline 19.55 & $1 \quad 0017$ & 0.0019 & 0.0019 & 0.0004 & -0.0001 & $\therefore 0.0007$ \\
\hline 29.57 & 0.0031 & 0.0031 & 0.0029 & 0.0006 & -0.0007 & -0.0019 \\
\hline 39.42 & 0.0048 & 0.0046 & 0.0038 & 0.0011 & -0.0008 & -0.0025 \\
\hline 49.18 & 0.0073 & $0.6,65$ & 0.0055 & 0.0020 & -0.0003 & -0.0026 \\
\hline 54.50 & 0.0085 & 0.0078 & 0.0064 & 0.0025 & -0.0003 & -0.0023 \\
\hline $5 y .57$ & 0.0099 & 0.0091 & 0.0076 & 0.0125 & 0.0003 & -0.0025 \\
\hline 0.20 & 0.0108 & 0.0109 & 0.0091 & 0.0319 & 0.0010 & -0.0015 \\
\hline 69.32 & 0.0134 & 00123 & 0.0108 & 0.0354 & 0.0017 & -0.0014 \\
\hline 74.16 & $0.015,7$ & $0.015 ?$ & 0.0124 & 0.0476 & 0.0027 & -0.0008 \\
\hline 80.16 & 0.0170 & 0.0161 & 0.0138 & -0.0007 & 0.0024 & -0.0015 \\
\hline 85.61 & 0.0199 & 0.0194 & 0.0153 & 0.0008 & 0.0035 & -0.0011 \\
\hline 90.58 & 0.0223 & $0.0 \% 16$ & 0.0181 & 0.0020 & 0.0043 & -0.0004 \\
\hline 95,69 & 0.0258 & 0.0247 & 0.0204 & 0.0040 & 0.0053 & 0.0001 \\
\hline 100.92 & 0.0233 & 00275 & 0.0231 & $0.005 E$ & 0.0065 & 0.0005 \\
\hline $103: 5$ & 0.0308 & 0.0292 & 0.02 .43 & $0.006 y$ & 0.0069 & 0.0007 \\
\hline 106.11 & 0.0328 & 0.0310 & 0.0250 & 0.0079 & 0.0076 & 0.0010 \\
\hline 108.31 & 0.0343 & 0.03324 & 0.0269 & 0.0087 & 0.0060 & 0.0012 \\
\hline 111.08 & 0.0368 & 0.0345 & 0.0285 & 00099 & 0.0088 & 0.0016 \\
\hline 113.24 & 0.0385 & $003 E_{2}$ & 0.0238 & 0.0107 & 0.0093 & 0.0017 \\
\hline 117.83 & 0.0433 & 0.0401 & 0.0332 & 0.0130 & 0.0109 & 0.0021 \\
\hline $1 \hat{20} 0.92$ & 0.0467 & 0.0431 & 0.0355 & 0.0144 & 0.0119 & 0.0027 \\
\hline 123.28 & 0.0457 & 0.0457 & 0.0378 & 0.0157 & 0.0128 & 0.0031 \\
\hline$\lcm{22.97}$ & 0.0501 & 0.0462 & 0.0383 & 0.0178 & 0.0131 & 0.0032 \\
\hline 125.82 & 00531 & 0.0483 & 0.0403 & 0.0270 & 0.0142 & 0.0038 \\
\hline 12560 & 0.0535 & 0.0495 & 0.0411 & 0.0361 & 0.0144 & 0.0035 \\
\hline 127.84 & 0.0555 & 0.0514 & 0.0426 & 0.0440 & 0.0151 & 0.0038 \\
\hline 130.19 & 0.0580 & 0.0530 & 0.0444 & 0.0532 & 0.0155 & 0.0035 \\
\hline .28 .53 & $0.060 E$ & 0.0560 & 0 OAE3 & 0.0568 & 0.0160 & 0.0029 \\
\hline 135.33 & 0.0635 & 0.0586 & 0.0481 & 0.0595 & 0.0159 & 0.0016 \\
\hline $1342 \%$ & 00532 & 00587 & 0.0475 & 0.0573 & 0.0158 & 0.0012 \\
\hline 138.35 & $0.065 ?$ & $0.06,1 E$ & 0.0503 & 0.0531 & 0.0157 & .0 .0001 \\
\hline 140.16 & 0.0664 & $0 . C E 30$ & 0.0511 & 0.0537 & 0.0147 & -0.0021 \\
\hline 142.63 & 0.0694 & 0.0649 & 0.0524 & 0.0522 & 0.0134 & -0.0041 \\
\hline 145.78 & 00732 & 0.0677 & 0.0544 & 0.0514 & 0.0125 & -0.0063 \\
\hline 142.52 & 0.0735 & $0.067 E$ & 0.0540 & 0.0249 & 0.0114 & -0.0076 \\
\hline 0.22 & -0.0024 & 0.0030 & 0.0010 & -0.0213 & -0.0045 & -0.0044 \\
\hline
\end{tabular}




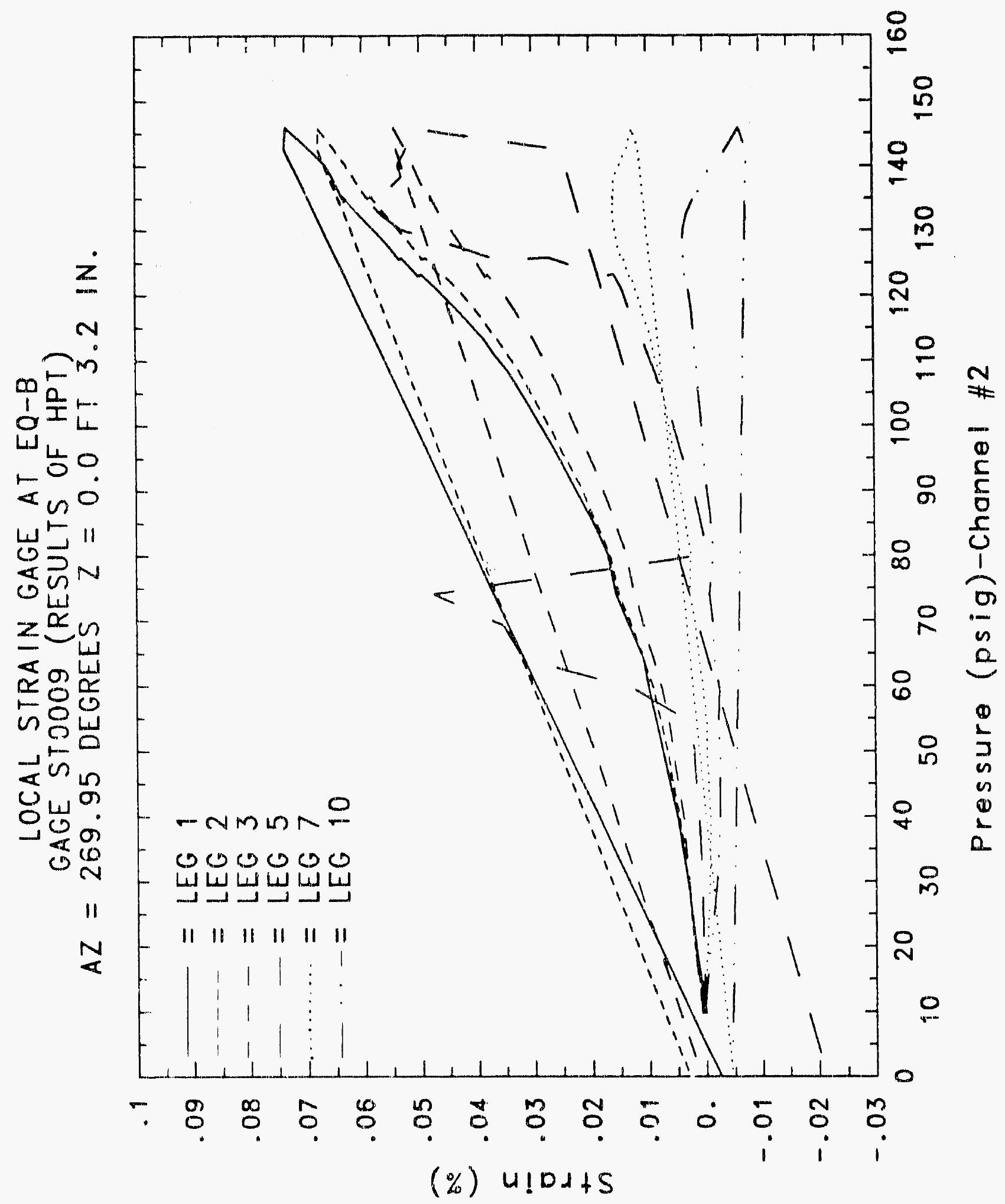




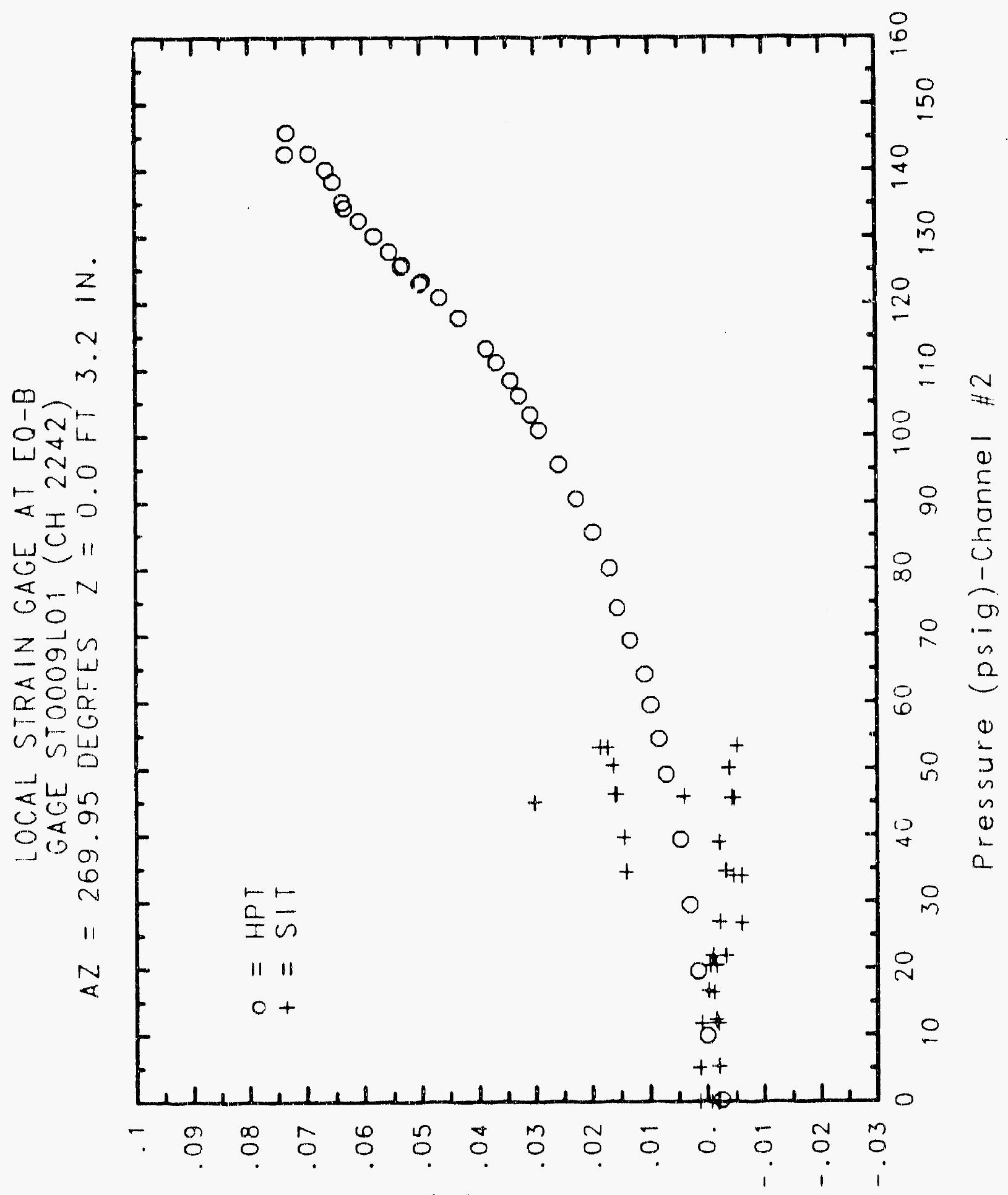

$\left(\begin{array}{c}c \\ 4\end{array}\right) U ! D+t S$ 


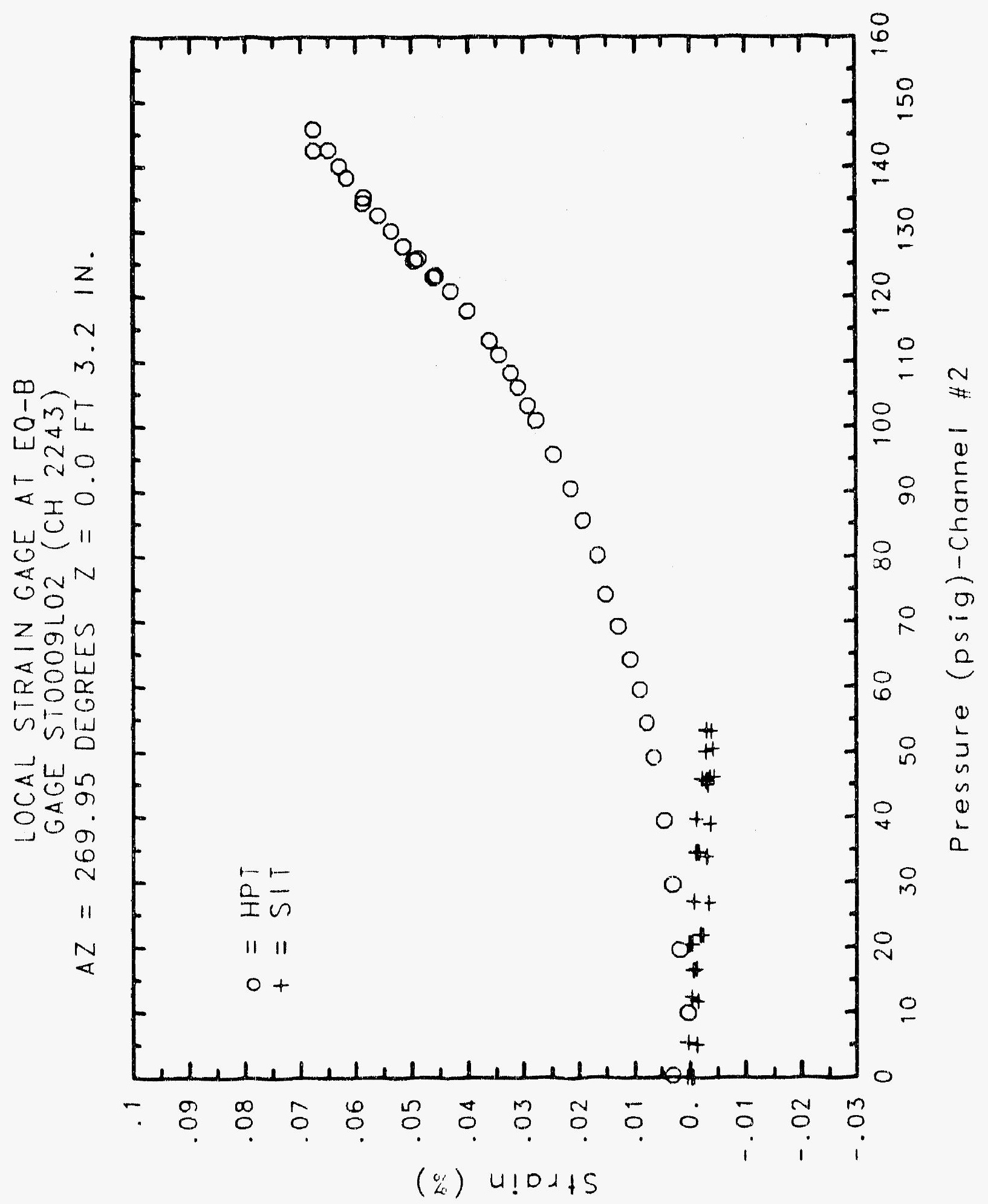

C. 1467 


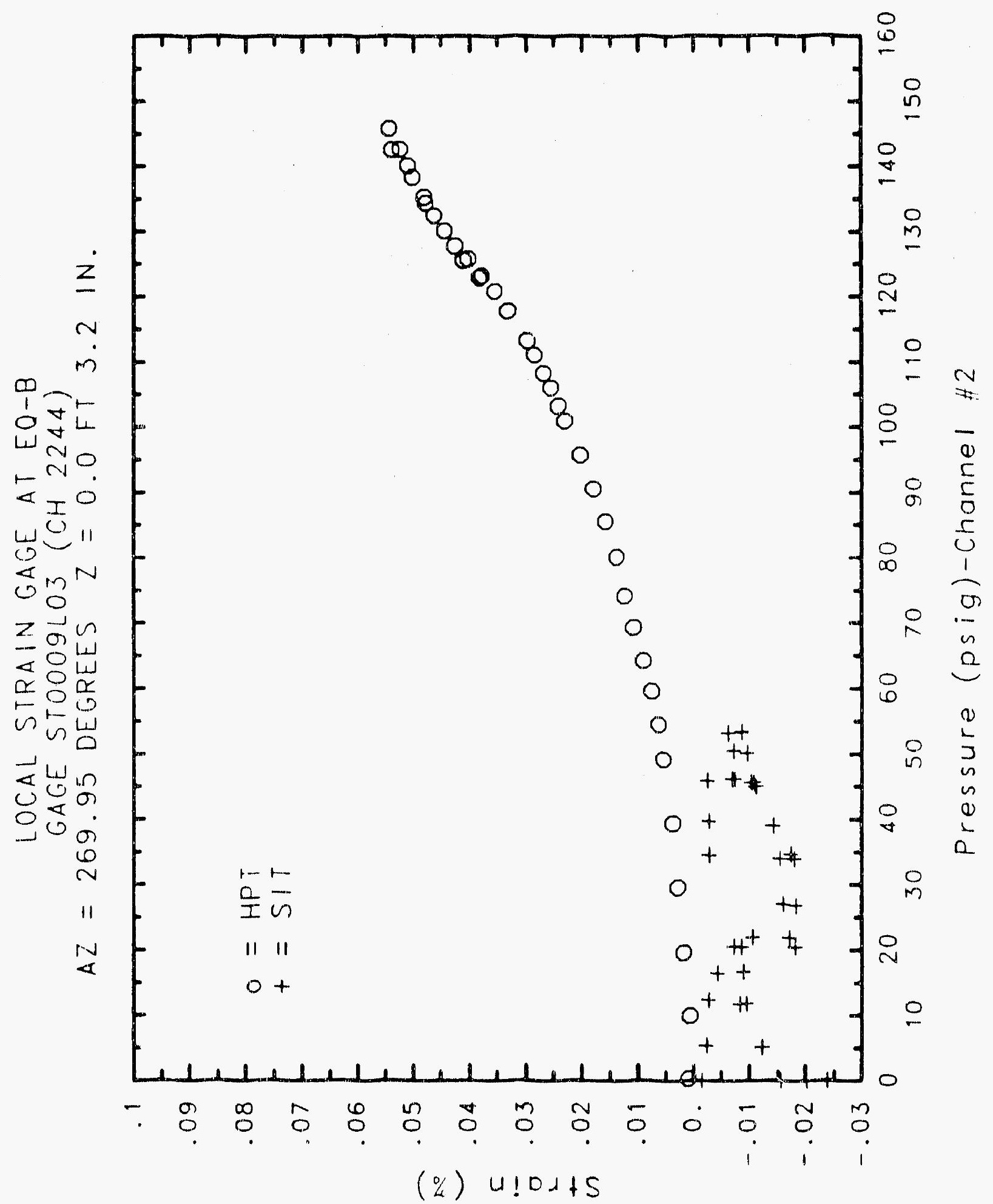




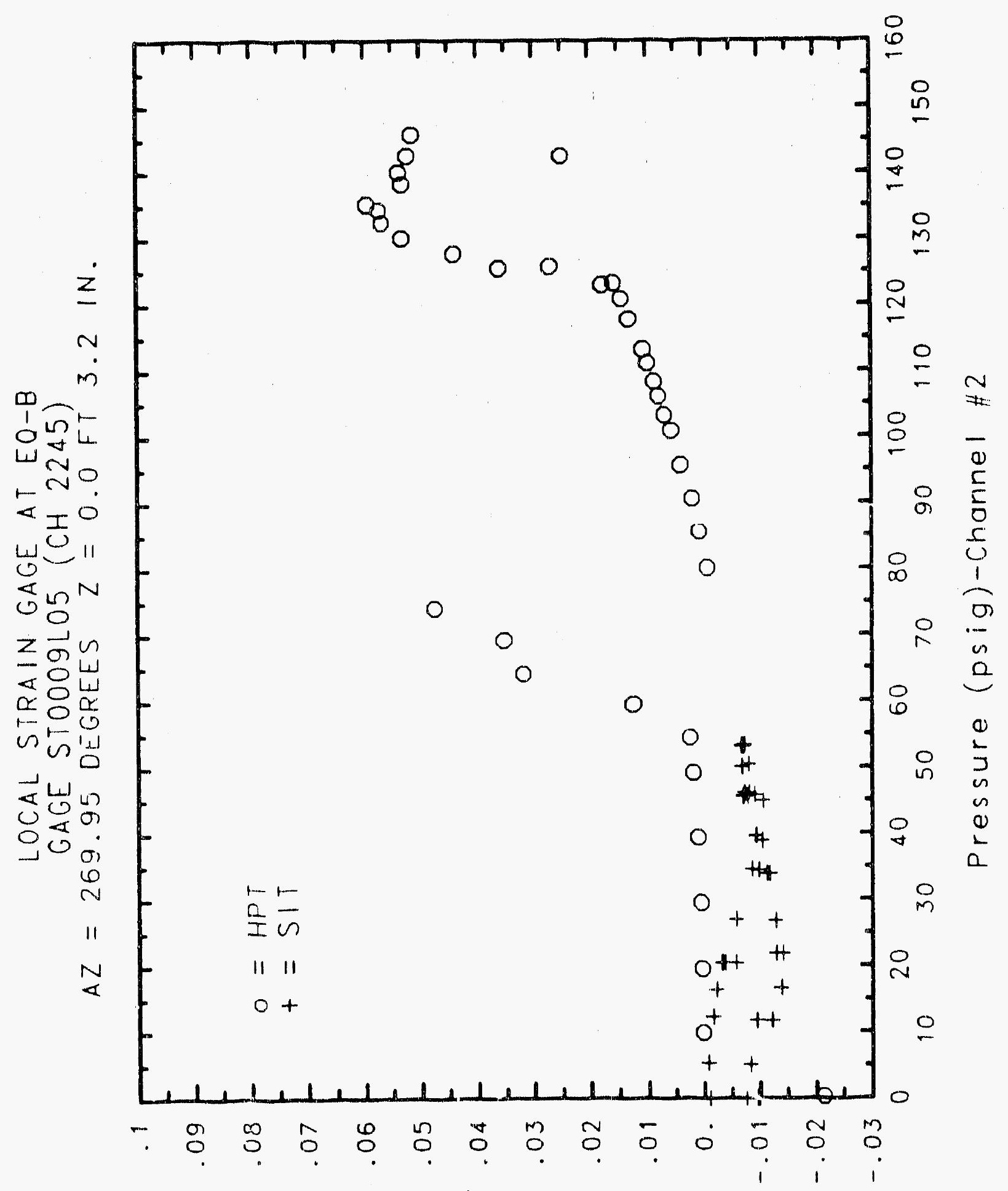

(\%) $4 ! 0+15$ 


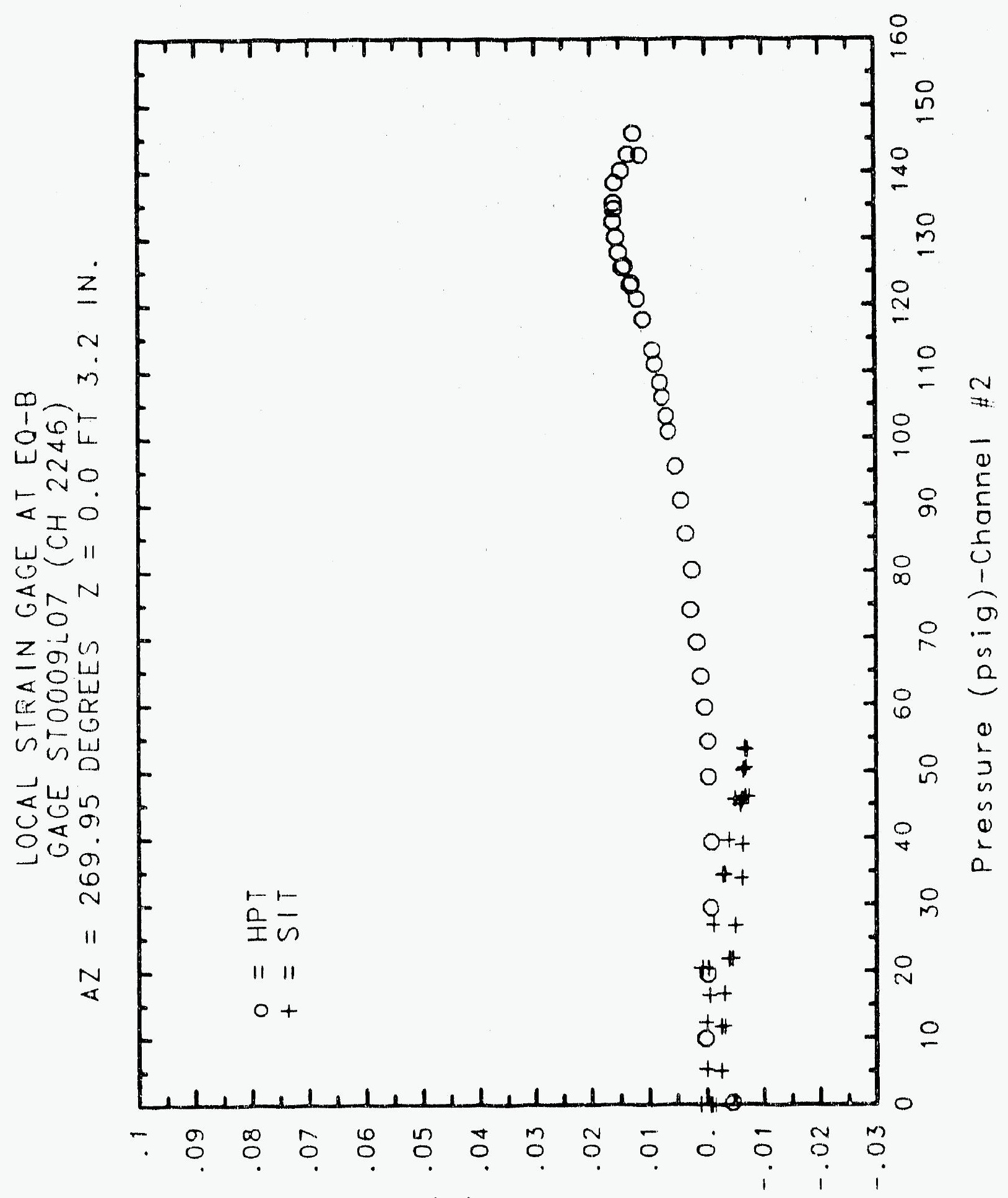

(\%) $u ! 0 d+5$ 


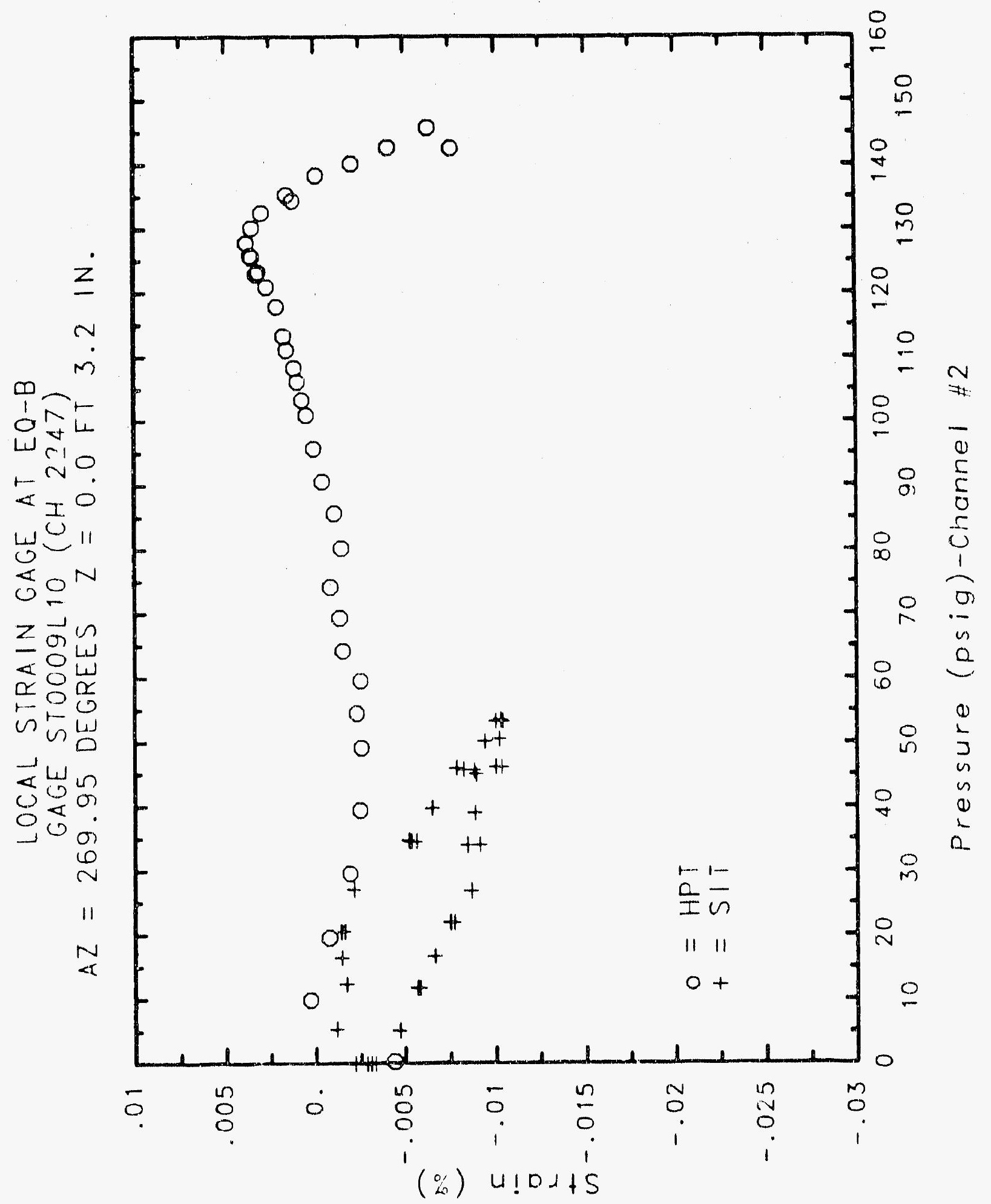




\section{Reinforced Concrete Test Data}

Strip 10 Channels $2248,2249,2260,2261,2262,2263$

\section{StRUCTURAL INTEgRITY TEST}

\begin{tabular}{|c|c|c|c|c|c|c|}
\hline $\begin{array}{c}\text { Pressure } \\
\text { Psig }\end{array}$ & $\begin{array}{l}\text { Gage } 1 \\
\text { xstrain }\end{array}$ & $\begin{array}{l}\text { Gage } 2 \\
\text { xstrain }\end{array}$ & $\begin{array}{l}\text { Gage } 3 \\
\text { xstrain }\end{array}$ & $\begin{array}{l}\text { Gage } 5 \\
\text { Xstrain }\end{array}$ & $\begin{array}{l}\text { Goge } 7 \\
\text { Xstra in }\end{array}$ & $\begin{array}{l}\text { Gagelo } \\
\text { Xstrain }\end{array}$ \\
\hline $\begin{array}{l}P .519 \\
-0.05\end{array}$ & 0.0020 & -0.0023 & -0.0008 & 0.0006 & -0.0018 & 0.0012 \\
\hline 5.33 & 0.0003 & -0.0023 & -0.0003 & -0.0014 & -0.0021 & -0.0004 \\
\hline 12.31 & 0.0005 & -0.0024 & 0.0002 & -0.0022 & -0.0024 & -0.0010 \\
\hline 16.44 & 0.0005 & -0.0014 & 0.0007 & 0.0007 & -0.0051 & 0.0004 \\
\hline 20.51 & 0.0009 & -0.0026 & 0.0012 & -0.0002 & -0.0129 & 0.0003 \\
\hline 20.46 & .0 .0005 & -0.0019 & 0.0004 & 0.0000 & -0.0141 & -0.0009 \\
\hline 20.47 & -0.0007 & -0.0025 & -0.0012 & -0.0006 & -0.0166 & -0.0004 \\
\hline 27.05 & -0.0006 & -0.0026 & 0.0007 & -0.0009 & -0.0165 & 0.0000 \\
\hline 34.69 & -0.0009 & -0.0036 & 0.0005 & 0.0000 & -0.0165 & 0.0000 \\
\hline 34.53 & -0.0040 & -0.0060 & -0.0027 & -0.0027 & -0.0222 & -0.0027 \\
\hline 34.53 & -0.0043 & -0.0056 & -0.0032 & -0.0023 & -0.0223 & -0.0038 \\
\hline 39.70 & .0 .0034 & -0.0059 & -0.0019 & -0.0032 & -0.0209 & -0.0037 \\
\hline 45.90 & -0.0019 & .0 .0041 & 0.0003 & -0.0005 & -0.0186 & -0.0013 \\
\hline 45.65 & 0.0000 & -0.0011 & 0.0020 & 0.0011 & -0.0159 & 0.0000 \\
\hline 45.62 & 0.0012 & .0 .0015 & 0.0036 & 0.0023 & -0.0161 & 0.0014 \\
\hline 50.10 & 0.0012 & -0.0011 & 0.0025 & 0.0027 & -0.0152 & 0.0018 \\
\hline 53.47 & 0.0011 & -0.0016 & 0.0026 & 0.0025 & -0.0151 & 0.0014 \\
\hline 33.29 & 0.0011 & -0.0005 & 0.0033 & 0.0033 & -0.0149 & 0.0016 \\
\hline $53.2 !$ & 0.0012 & -0.0006 & 0.0040 & 0.0023 & -0.0146 & 0.0024 \\
\hline 50.49 & 0.0018 & 0.0001 & 0.0030 & 0.0029 & -0.0142 & 0.0025 \\
\hline 46.14 & 0.0019 & -0.0003 & 0.0034 & 0.0037 & -0.0144 & 0.0027 \\
\hline 46.13 & 0.0027 & 0.0003 & 0.0037 & 0.0037 & -0.0139 & 0.0029 \\
\hline 44.97 & $0.000 i$ & -0.0007 & 0.0025 & $n .0016$ & -0.0161 & 0.0018 \\
\hline 38.98 & 0.0006 & -0.0016 & 0.0021 & 0.0012 & $-0,0162$ & 0.0015 \\
\hline 33.99 & 0.0017 & -0.0023 & 0.002 .4 & 0.0020 & -0.0171 & 0.0016 \\
\hline 33.96 & 0.0005 & -0.0012 & 0.0020 & 0.0014 & -0.0172 & 0.0007 \\
\hline 26.83 & -0.0003 & -00025 & 0.0007 & 0.0003 & -0.0188 & -0.0001 \\
\hline 21.88 & -0.0002 & -0.0029 & 0.0002 & 0.0005 & -0.0195 & -0.0003 \\
\hline 21.90 & -0.0026 & -0.0041 & $=0.0009$ & -0.0022 & -0.0213 & -0.0029 \\
\hline 16.69 & -0.0034 & -0.0053 & -0.0030 & -0.0018 & -0.0218 & -0.0023 \\
\hline 11.74 & -0.0025 & -0.0053 & -0.0012 & -0.0019 & -0.0213 & -0.0023 \\
\hline 11.77 & -0.0001 & -0.0034 & 0.0015 & 0.0000 & -0.0188 & -0.0009 \\
\hline 505 & 0.0008 & -0.0024 & 0.0018 & 0.0011 & -0.0183 & 0.0001 \\
\hline 0.02 & 0.0013 & -0.0018 & 0.0024 & 0.0016 & -0.0163 & 0.0001 \\
\hline-0.04 & 0.0020 & 00001 & 0.0042 & 0.0031 & -0.0135 & 0.0020 \\
\hline-0.02 & 0.0025 & -0.0003 & 0.0035 & 0.0034 & $=0.0149$ & 0.0015 \\
\hline-0.02 & 0.0017 & -0.0001 & 0.0033 & 0.0026 & -0.0150 & 0.0014 \\
\hline 0.02 & 0.0008 & -0.0008 & 0.0021 & 0.0026 & -0.0175 & 0.0013 \\
\hline
\end{tabular}




\section{Reinforced Concrete Test Data}

Strif 10 Channels $2248,2249,2260,2261,2262,2263$

High Pressure test

\begin{tabular}{|c|c|c|c|c|c|c|}
\hline Pressure & Gage 1 & Gage $?$ & Gage 3 & Gage 5 & Gage 7 & Gage 10 \\
\hline Psig & xstrain & xstrain & Xstrain & $x s t r a i n$ & xstrain & xstrain \\
\hline 9.89 & 0.0009 & 0.0012 & 0.0013 & 0.0011 & 0.0004 & 0.0007 \\
\hline 19.55 & 0.0009 & 0.0009 & 0.0013 & 0.0011 & 0.0006 & 0.0011 \\
\hline 29.57 & 0.0012 & 0.0013 & 0.0014 & 0.0019 & 0.0011 & 0.0017 \\
\hline 33.42 & 0.0020 & 0.0023 & 0.0023 & 0.0028 & 0.0020 & 0.0024 \\
\hline 49.16 & $0.003 ?$ & 0.0038 & 0.00 .35 & 0.0042 & 0.0033 & 0.0038 \\
\hline 54.50 & 0.0034 & 0.0042 & 0.0034 & 0.0046 & 0.0033 & 0.0045 \\
\hline 59.57 & 0.0036 & 0.0042 & 0.0035 & 0.0048 & -0.0026 & 0.0041 \\
\hline 64.20 & 0.0027 & 0.0034 & 0.0027 & 0.0041 & -0.0067 & 0.0042 \\
\hline 69.32 & 0.0016 & 0.0025 & 0.0017 & 0.0033 & -0.0078 & 0.0034 \\
\hline 74.16 & -0.0001 & $0 . n 009$ & 0.0006 & 0.0023 & -0.0104 & 0.0028 \\
\hline 80.16 & -0.0014 & -0.0005 & -0.0005 & 0.0015 & -0.0639 & 0.0009 \\
\hline 85.61 & -0.0028 & -0.0019 & -0.0018 & 0.0000 & -0.0256 & 0.0002 \\
\hline 90.58 & -0.0042 & -0.0032 & -0.0031 & -10.0011 & -0.0273 & -0.0007 \\
\hline 95.69 & -0.0056 & -0.0047 & -0.0049 & -0.0025 & -0.0288 & -0.0018 \\
\hline $100.9 ?$ & -0.0068 & -0.0059 & -0.0066 & -0.0041 & -0.0302 & -0.0029 \\
\hline 103.25 & -0.0076 & -0.0066 & -0.0073 & -0.0047 & -0.0312 & -0.0034 \\
\hline 106.11 & -0.0085 & -0.0077 & -0.0082 & -0.0054 & -0.0319 & -0.0040 \\
\hline 10831 & -0.0093 & -0.0005 & -0.0092 & -0.0064 & -0.0325 & -0.0044 \\
\hline 111.08 & -0.0103 & -0.0095 & -0.0101 & -0.0075 & -0.0334 & -0.0053 \\
\hline 113.24 & -0.0110 & -0.0102 & -0.0109 & -0.0083 & -0.0344 & -0.0058 \\
\hline 117.83 & -0.01 .70 & -0.0123 & -0.0134 & -0.0102 & -0.0361 & -0.0073 \\
\hline 120.92 & -0.0145 & -0.0137 & -0.0148 & -0.0120 & -0.0374 & -0.0084 \\
\hline 123.28 & .0 .0156 & -0.0153 & -0.0158 & -0.0127 & -0.0387 & -0.0089 \\
\hline 122.97 & -0.0150 & -0.0146 & -0.0160 & -0.0132 & -0.0392 & -0.0089 \\
\hline 125.82 & -0.0167 & -0.0164 & -0.0167 & -0.0137 & -0.0403 & -0.0095 \\
\hline 125.60 & -0.0161 & $=0.0161$ & -0.0171 & -0.0141 & -0.0413 & -0.0099 \\
\hline 127.84 & -0.0166 & -0.0165 & -0.0183 & -0.0154 & -0.0426 & -0.0107 \\
\hline 130.19 & -0.0177 & -0.0179 & -0.0195 & -0.0165 & -0.0442 & -0.0115 \\
\hline 132.53 & -0.0191 & -0.0197 & -0.0208 & -0.0180 & .0 .0456 & -0.0121 \\
\hline 135.33 & -0.0210 & -0.0219 & -0.0220 & -0.0193 & -0.0477 & -0.0133 \\
\hline 134.42 & -0.0215 & -0.0226 & -0.0218 & -0.0191 & -0.0497 & -0.0125 \\
\hline 138.35 & -0.0238 & -0.0255 & -0.0233 & -0.0206 & .0 .0519 & -0.0139 \\
\hline 140.16 & -0.0255 & -0.0278 & -0.0252 & -0.0228 & -0.0530 & -0.0145 \\
\hline 142.63 & -0.0259 & -0.0287 & -0.0271 & -0.0246 & -0.0548 & -0.0172 \\
\hline 145.78 & -0.0280 & -0.0313 & -0.0295 & -0.0271 & -0.0565 & -0.0192 \\
\hline 142.52 & $=0.0278$ & -0.0310 & -0.0294 & -0.0271 & -0.0561 & -0.0192 \\
\hline 0.22 & 0.0037 & 0.0021 & 0.0053 & 0.0030 & -0.0331 & 0.0024 \\
\hline
\end{tabular}




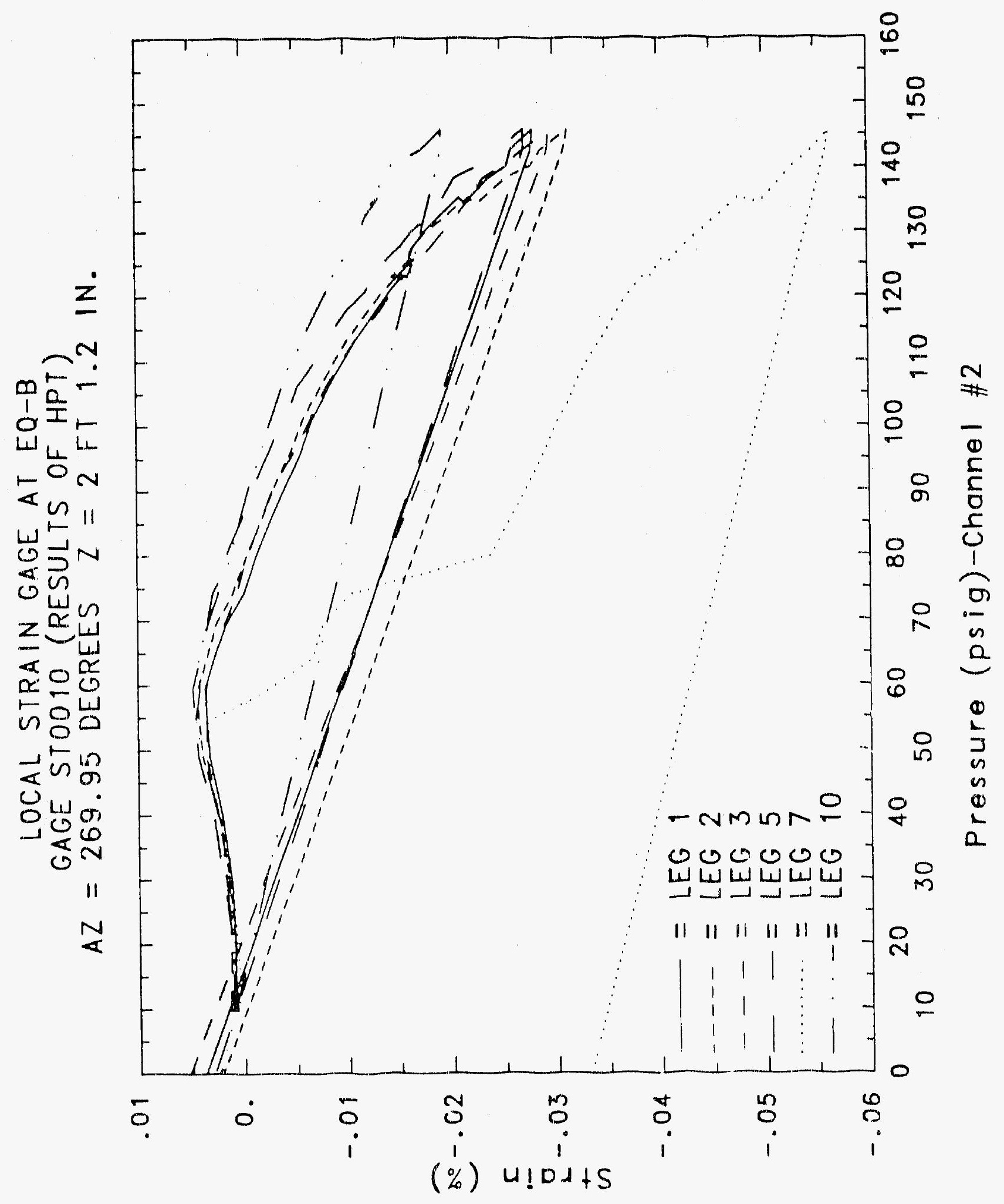




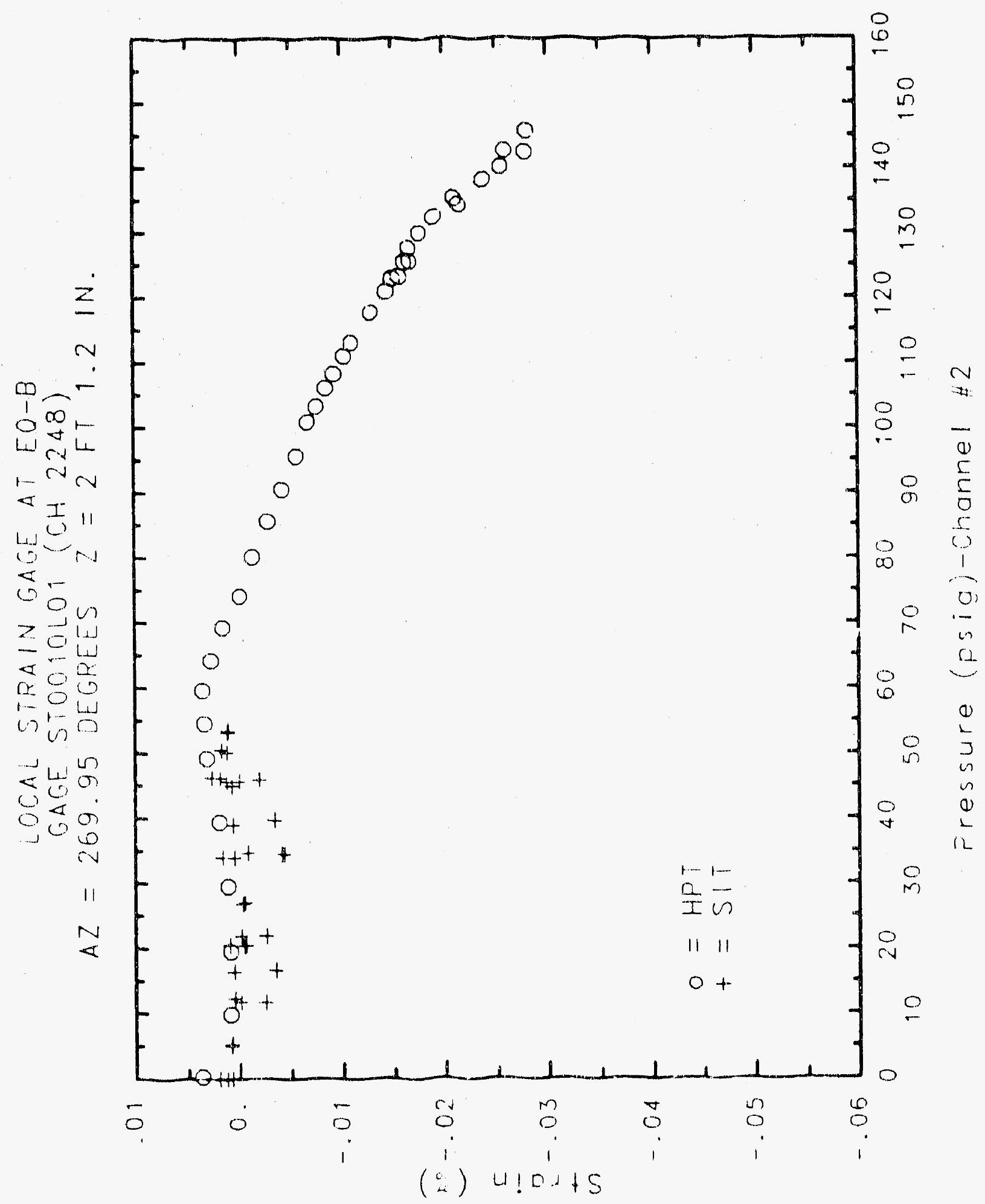




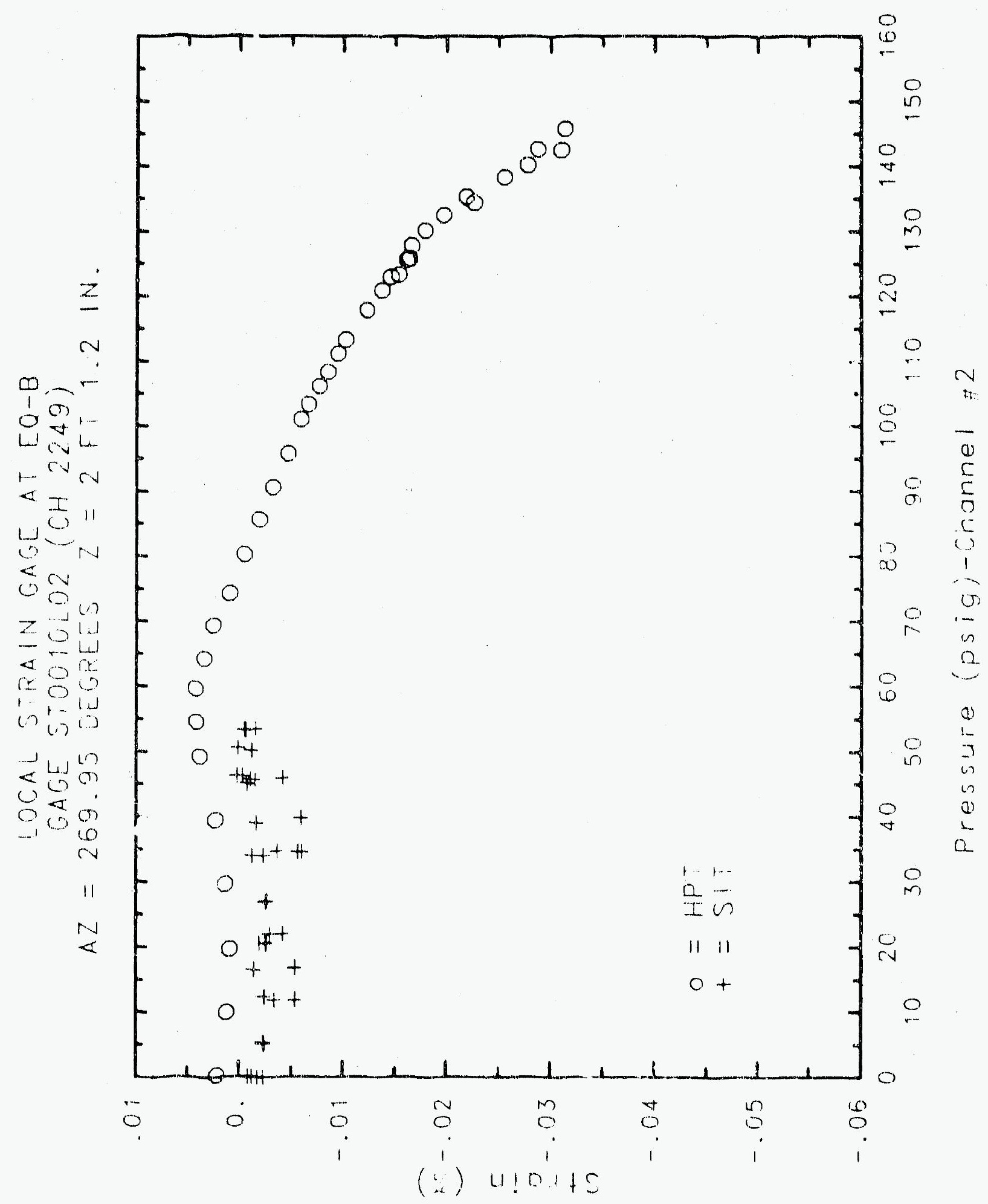




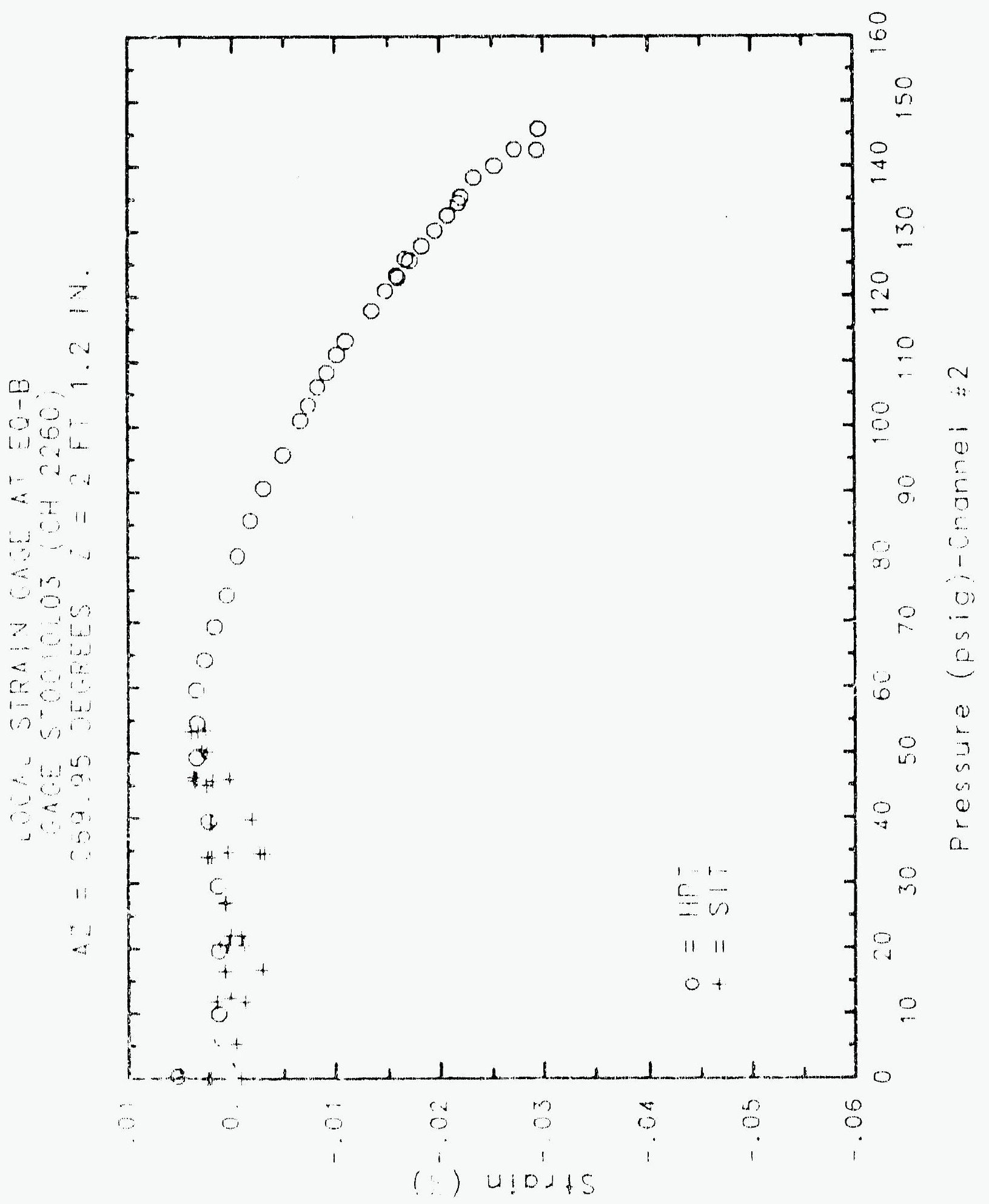




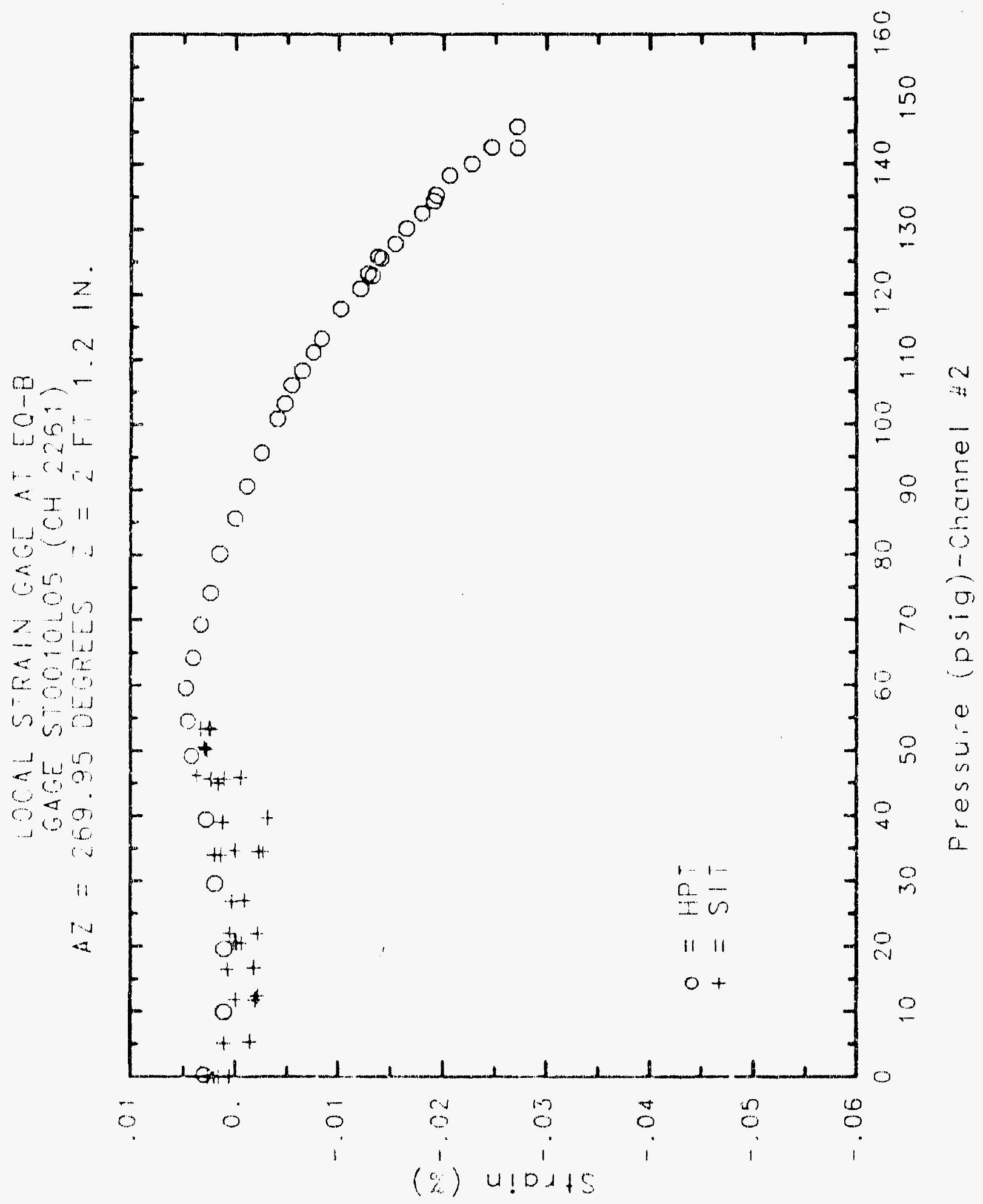




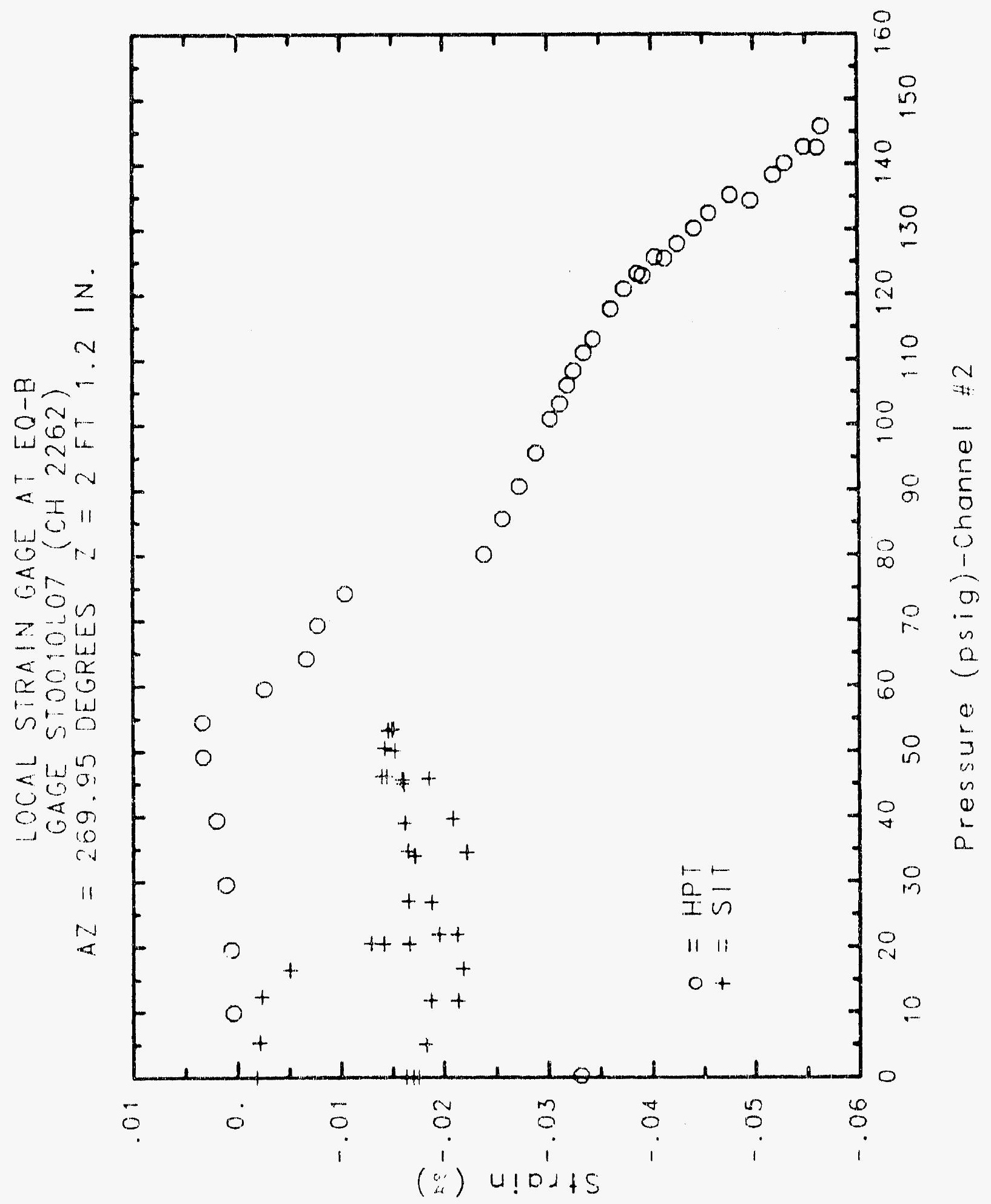




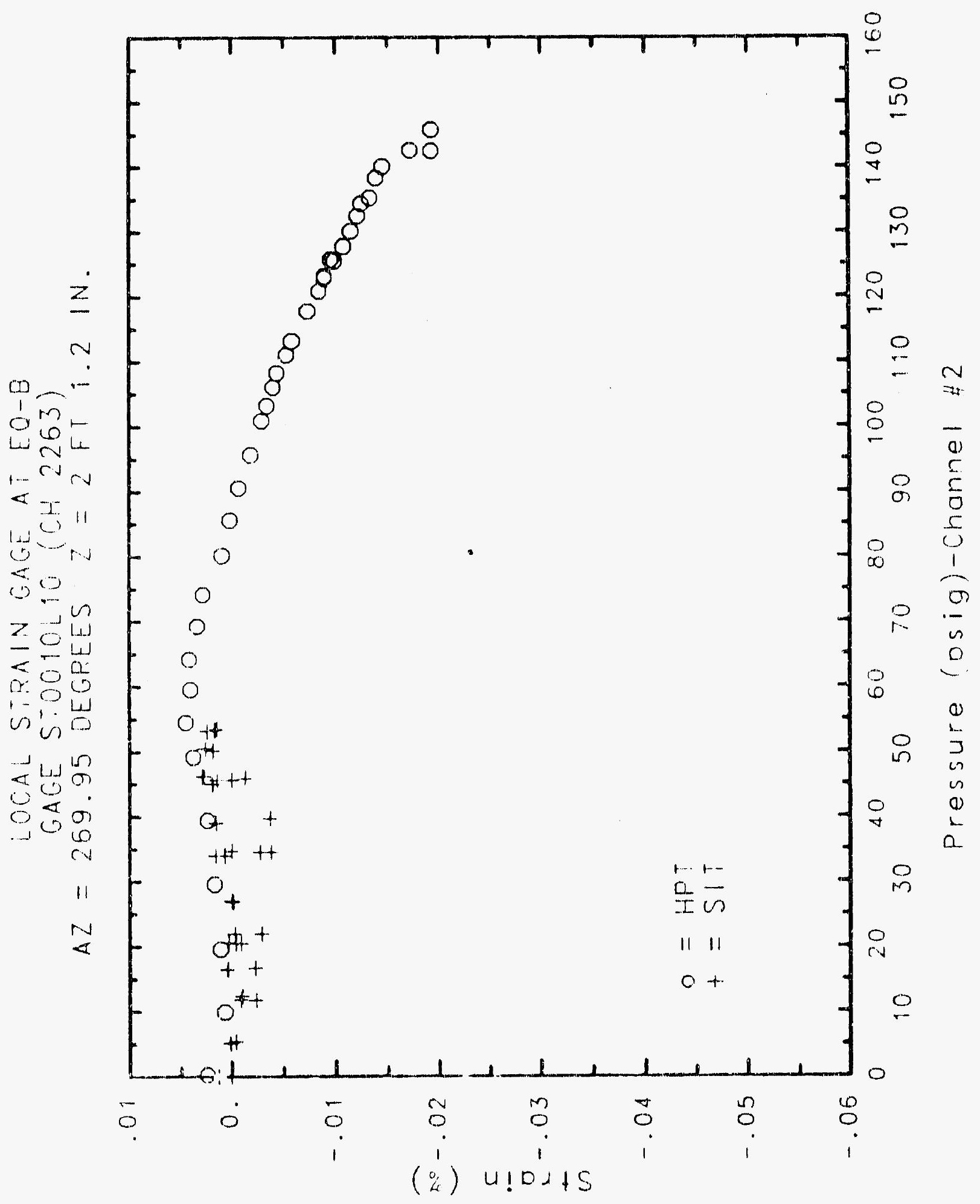




\section{Reinforced Concrete Test Data}

\section{Strip 11 Channels $2264,2265,2266,2267,2268,2269$}

\section{STRUCTURAL INTEGRITY TEST}

\begin{tabular}{|c|c|c|c|c|c|c|}
\hline Pressure & Gage 1 & Gage 2 & Gage 3 & Gage 5 & Gage 7 & Gage 10 \\
\hline Psig & xstrain & Xstroin & Xstrain & xstrain & xstrain & Xstrain \\
\hline-0.05 & 0.0005 & 0.0001 & 0.0016 & 0.0003 & -0.0003 & 0.0003 \\
\hline 5.33 & -0.0031 & -0.0018 & -0.0018 & -0.0008 & -0.0008 & -0.0017 \\
\hline 12.31 & -0.0050 & -0.0040 & -0.0036 & -0.0030 & -0.0032 & -0.0028 \\
\hline 16.44 & -0.0066 & -0.0059 & -0.0060 & -0.0055 & -0.0041 & -0.0044 \\
\hline 20.51 & -0.0093 & -0.0082 & -0.0084 & -0.0078 & -0.0072 & -0.0064 \\
\hline 20.46 & -0.0095 & -0.0098 & -0.0084 & -0.0030 & -0.0078 & -0.0068 \\
\hline 20.47 & -0.0101 & -0.0095 & -0.0093 & -0.0100 & $-0.00 \%$ & -0.0067 \\
\hline 27.05 & -0.0164 & -0.0149 & -0.0149 & -0.0147 & -0.0113 & -0.0095 \\
\hline 34.69 & -0.0204 & -0.0192 & -0.0185 & -0.0186 & -0.0128 & -0.0102 \\
\hline 34.53 & -0.0203 & -0.0190 & -0.0187 & -0.0517 & -0.0125 & -0.0103 \\
\hline 3453 & -0.0202 & -0.0199 & -0.0188 & -0.0533 & -0.0114 & -0.0106 \\
\hline 39.70 & $=0.0274$ & -0.0268 & -0.0256 & -0.0591 & -0.0187 & -0.0169 \\
\hline 45.90 & -0.0375 & $\therefore 0.0349$ & -0.0332 & -0.0640 & -0.0251 & -0.0229 \\
\hline 45.65 & -0.0383 & -0.0361 & -0.0336 & -0.0668 & -0.0255 & -0.0227 \\
\hline 45.62 & -0.0385 & -0.0352 & -0.0335 & -0.0679 & -0.0252 & -0.0235 \\
\hline 50.10 & -0.0435 & -0.0405 & -0.0371 & -0.0707 & $-0 \quad 0267$ & -0.0239 \\
\hline 53.47 & .0 .0470 & .0 .0438 & -0.0336 & -0.0733 & -0.0289 & -0.0252 \\
\hline 53.29 & -0.0474 & -0.0443 & -0.0399 & -0.0733 & $=0.0291$ & -0.0253 \\
\hline 5321 & -0.0485 & -0.0445 & -0.0401 & .0 .0730 & -0.0284 & -0.0256 \\
\hline 5049 & $-0.048 ?$ & $-0.043 ?$ & -0.0394 & -0.0720 & -0.0276 & -0.0249 \\
\hline 46.14 & .0 .0462 & 0.0410 & -0.0381 & -0.0700 & -0.0263 & -0.0232 \\
\hline 46.13 & -0.0463 & -0.0418 & -0.0371 & -0.0707 & -0.0259 & -0.0233 \\
\hline 44.97 & -0.0446 & -0.0392 & -0.0360 & -0.070 .3 & -0.0248 & -0.0223 \\
\hline 38.98 & -0.0427 & -0.0373 & -0.0342 & -0.0684 & -0.0225 & -0.0203 \\
\hline 33.99 & -0.0406 & -0.0359 & .0 .0311 & -0.0573 & -0.0203 & -0.0179 \\
\hline 33.96 & -0.0401 & -0.0360 & -0.0319 & -0.0669 & -0.0209 & -0.0178 \\
\hline 26.83 & -0.0377 & -0.0329 & -0.0285 & -0.0640 & -0.0179 & .0 .0154 \\
\hline 21.88 & -0.0359 & -0.0306 & -0.0265 & -0.0621 & $-0.015 E$ & -0.0139 \\
\hline 21.90 & -0.0358 & -0.0304 & -0.0268 & -0.0639 & -0.0157 & -0.0137 \\
\hline$i 6.69$ & -0.0333 & -0.0278 & $-0.023 E$ & -0.0625 & -0.0130 & -0.0128 \\
\hline 11.74 & -0.0308 & -0.0252 & -0.0218 & -0.0596 & 0.0122 & -0.0113 \\
\hline 1177 & -C 0310 & -0.0256 & -0.0219 & -0.0595 & -0.0120 & -0.0114 \\
\hline 5.05 & -00272 & -0.0225 & -0.0181 & -0.0575 & -0.0089 & -0.0093 \\
\hline 0.02 & -0.0236 & -0.0183 & -0.0158 & -0.0547 & -0.0081 & -0.0080 \\
\hline-0.04 & -0.0222 & -0.0169 & -0.0141 & -0.0552 & -0.0069 & -0.0077 \\
\hline-0.02 & -0.0220 & -0.0171 & -0.0143 & -0.0547 & -0.0070 & $=0.0069$ \\
\hline-0.02 & -0.0210 & -0.0175 & -0.0141 & -0.0550 & -0.0067 & -0.0063 \\
\hline 0.02 & -0.0213 & -0.0171 & -0.0132 & -0.0569 & 0.0062 & -0.0067 \\
\hline
\end{tabular}




\section{Reinforced Concrete Test Data}

Strip 11 Channels $2264,2265,2266,2267,2268,2269$

\section{High Pressume Test}

\begin{tabular}{|c|c|c|c|c|c|c|c|}
\hline Pressure & Gage 1 & Gage $?$ & Gage 3 & Gage 5 & Gage 7 & Gage 10 & \\
\hline $\begin{array}{l}\text { Psig } \\
9.89\end{array}$ & $\begin{array}{l}\text { xstrain } \\
-0.0031\end{array}$ & $\begin{array}{l}\text { XStrain } \\
-0.0033\end{array}$ & $\begin{array}{l}\text { Xstrain } \\
=0.0034\end{array}$ & $\begin{array}{l}\text { Xstrain } \\
-0.0032\end{array}$ & $\begin{array}{l}\text { xstrain } \\
-0.0028\end{array}$ & $\begin{array}{l}\text { Xstrain } \\
-0.0026\end{array}$ & \\
\hline 19.55 & -0.0069 & -0.0073 & -0.0072 & -0.0068 & -0.0058 & -0.0054 & \\
\hline 29.57 & -0.0114 & -0.0118 & -0.0111 & -0.0107 & -0.0091 & -0.0084 & \\
\hline 39.42 & -0.0179 & -0.0178 & -0.0170 & -0.0151 & -0.0127 & -0.0116 & \\
\hline 49.16 & -0.0242 & -0.0237 & -0.0225 & -0.0194 & -0.0171 & -0.0155 & \\
\hline 54.50 & -0.0280 & -0.0272 & -0.0255 & -0.0223 & -0.0199 & -0.0181 & \\
\hline 59.57 & -0.0326 & -0.0312 & -0.0290 & -0.0257 & -0.0223 & -0.0204 & \\
\hline 64.20 & -0.0390 & -0.0356 & -0.0323 & -0.0305 & -0.0261 & -0.0238 & \\
\hline 69.32 & -0.0451 & -0.0401 & -0.0357 & -0.0344 & -0.0290 & $-0.026 ?$ & \\
\hline 74.16 & -0.0507 & -0.0440 & -0.0384 & $-0.04 E 3$ & -0.0327 & -0.0297 & \\
\hline 80.16 & -0.0591 & -0.0506 & -0.0429 & -0.0507 & -0.0346 & -0.0328 & \\
\hline 85.61 & -0.0654 & -0.0553 & -0.0462 & -0.0538 & -0.0375 & -0.0353 & \\
\hline 90.58 & -0.0707 & -0.0593 & -0.0487 & -0.0564 & -0.0398 & -0.0376 & \\
\hline 95.69 & -0.0763 & -0.0634 & -0.0511 & -0.0588 & -0.0420 & -0.0396 & \\
\hline 100.92 & -0.0820 & -0.0676 & -0.0537 & -0.0614 & -0.0442 & -0.0418 & \\
\hline 103.25 & -0.0848 & $\cdots 0.0698$ & -0.0548 & -0.0626 & -0.0452 & -0.0427 & \\
\hline 10611 & -0.0875 & -0.0719 & -0.0559 & -0.0638 & -0.0463 & -0.0437 & \\
\hline 108.31 & .00894 & -0.0733 & -0.0566 & -0.0646 & -0.0470 & -0.0442 & \\
\hline 111.08 & -0.0919 & -0.0753 & -0.0576 & -0.0656 & -0.0481 & -0.0451 & \\
\hline 113.24 & -0.0938 & -0.0767 & -0.0583 & -0.0665 & -0.0488 & -0.0457 & \\
\hline 117.83 & -0.0975 & -0.0793 & .0 .0598 & -0.0680 & -0.0502 & -0.0463 & \\
\hline 120.92 & -0.1005 & -0.0816 & -0.0609 & -0.0692 & -0.0511 & -0.0476 & \\
\hline 123.28 & -0.1031 & -0.0834 & .0 .0615 & $-0.070 !$ & $-0.052 ?$ & .0 .0485 & \\
\hline 122.97 & -0.1027 & -0.0828 & -0.0608 & -0.0596 & -0.0524 & -0.0488 & \\
\hline $125.8 ?$ & -0.1058 & -0.0851 & $-0.062 ?$ & -0.0711 & -0.0534 & -0.0495 & \\
\hline 125.60 & -0.1060 & -0.0851 & -0.0618 & -0.0713 & -0.0531 & -0.0491 & \\
\hline $1 ? 7.84$ & -0.1090 & -0.0874 & -0.0636 & -0.0730 & .0 .0541 & -0.0500 & \\
\hline 130.19 & -0.1130 & -0.0903 & -0.0650 & -0.0747 & -0.0558 & -0.0513 & \\
\hline 132.53 & -0.1178 & -0.0939 & -0.0670 & -0.0767 & -0.0576 & -0.0526 & \\
\hline 135.33 & -0.1229 & -0.0980 & -0.0695 & -0.0792 & -0.0592 & -0.0539 & \\
\hline $1344 ?$ & -0.1223 & -0.0975 & -0.0688 & -0.0805 & -0.0594 & -0.0539 & \\
\hline 138.35 & -0.1298 & -0.1036 & -0.0726 & -0.0858 & .0 .0516 & .0 .2556 & \\
\hline 140.16 & -0.1355 & -0.1074 & $-0.0 / 42$ & -0.0866 & -0.0641 & -0.11577 & \\
\hline 142.63 & -0.1440 & -0.1125 & -00782 & -0.0900 & -0.06544 & -0.0581 & \\
\hline 145.78 & -0.1539 & -0.1208 & -0.0824 & -0.0934 & -0.0683 & -0.0505 & \\
\hline 142.52 & -0.1517 & -0.1176 & -0.0789 & -0.0390 & -0.0635 & $-0.036,6$ & " \\
\hline 0.22 & -0.0461 & -0.0209 & -0.0028 & -0.0190 & -0.0014 & -0.0055 & \\
\hline
\end{tabular}




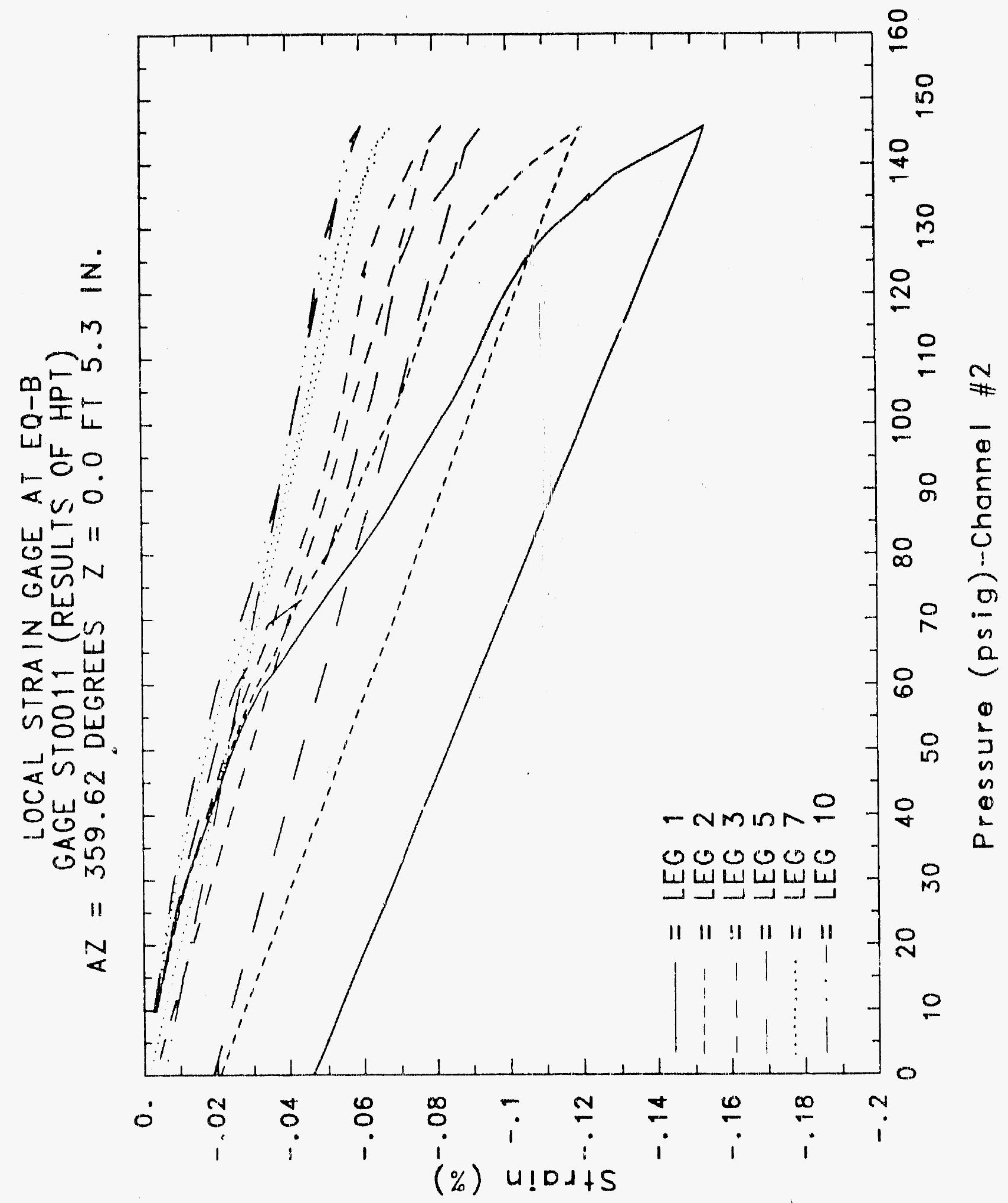




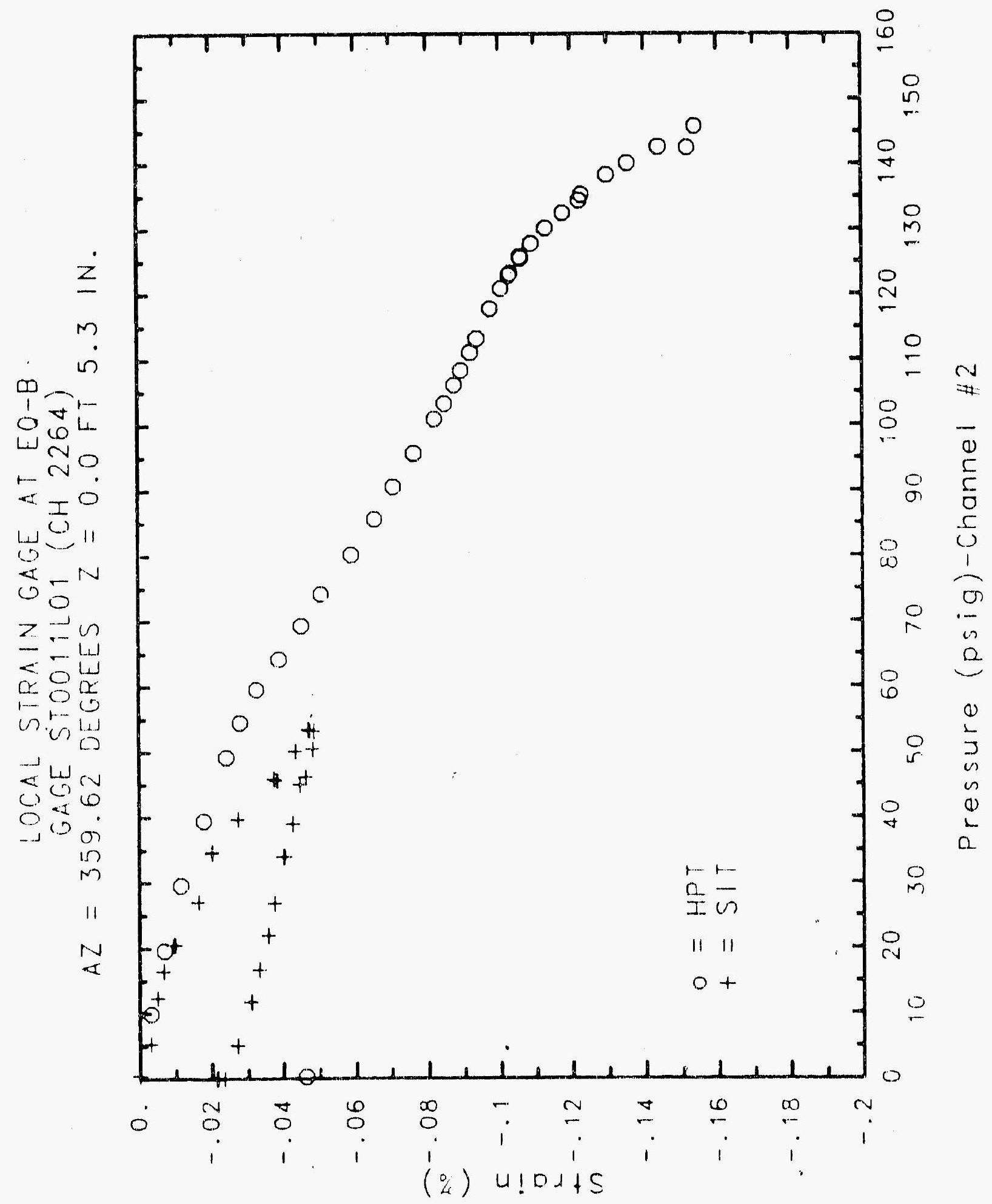




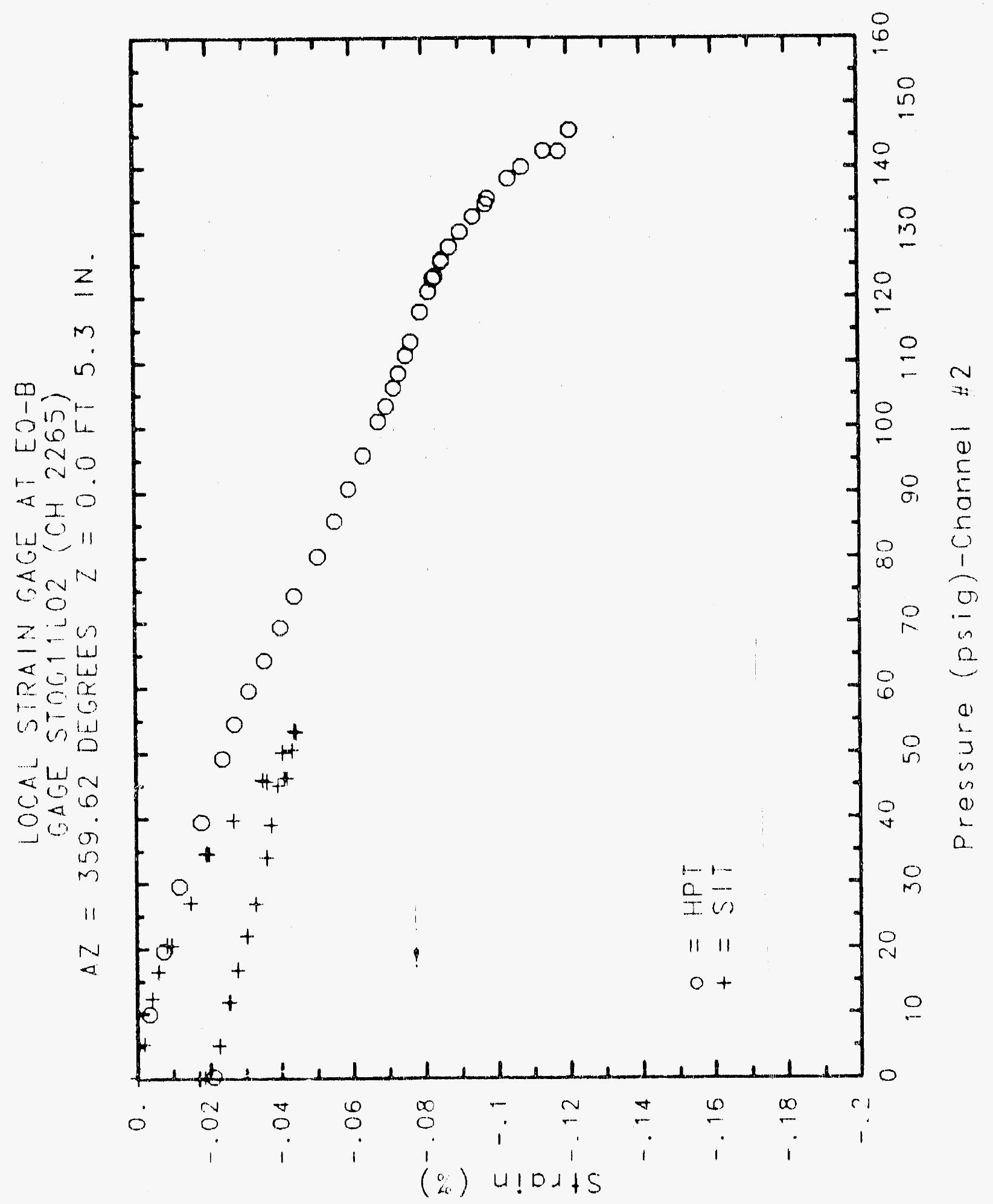




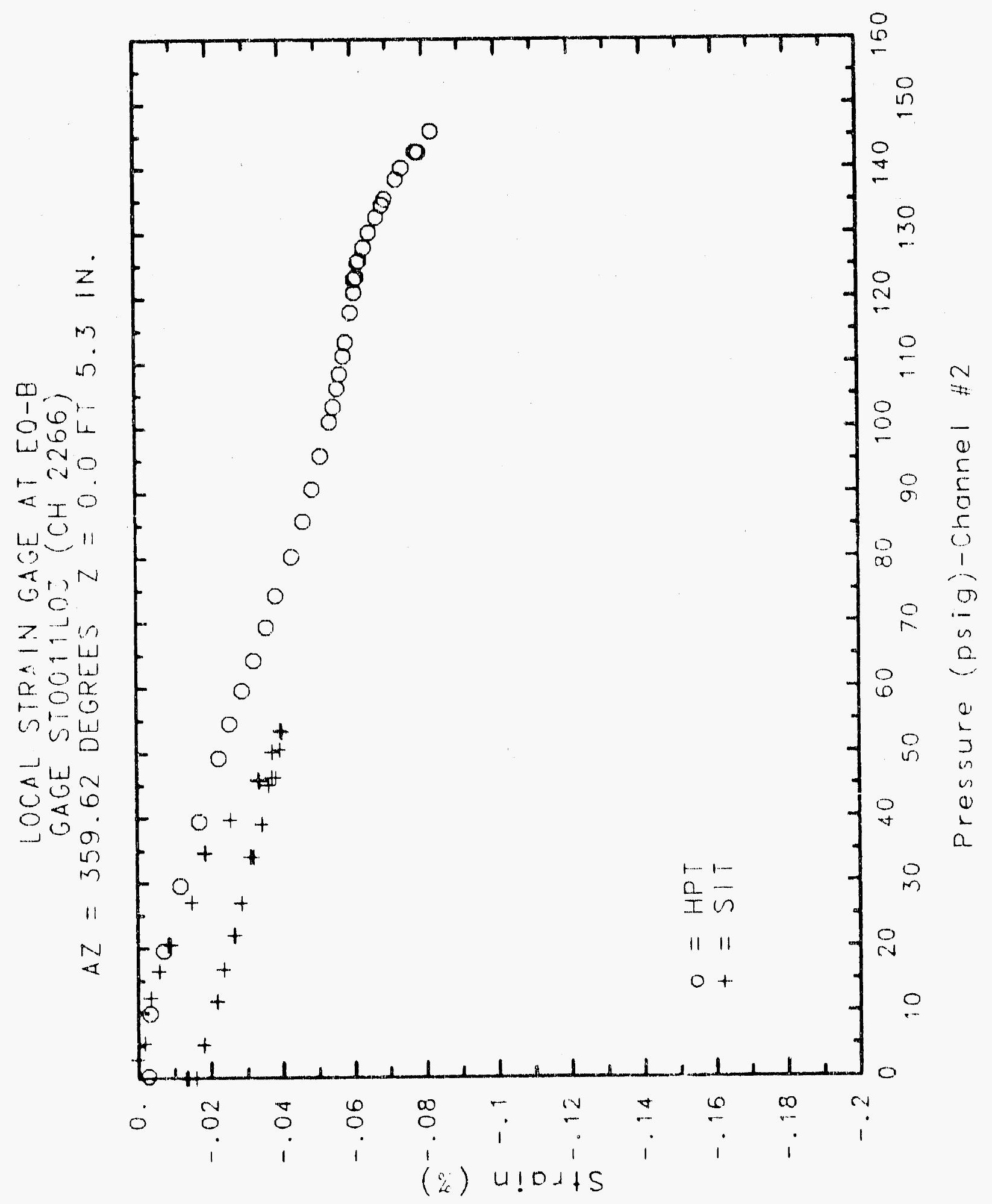




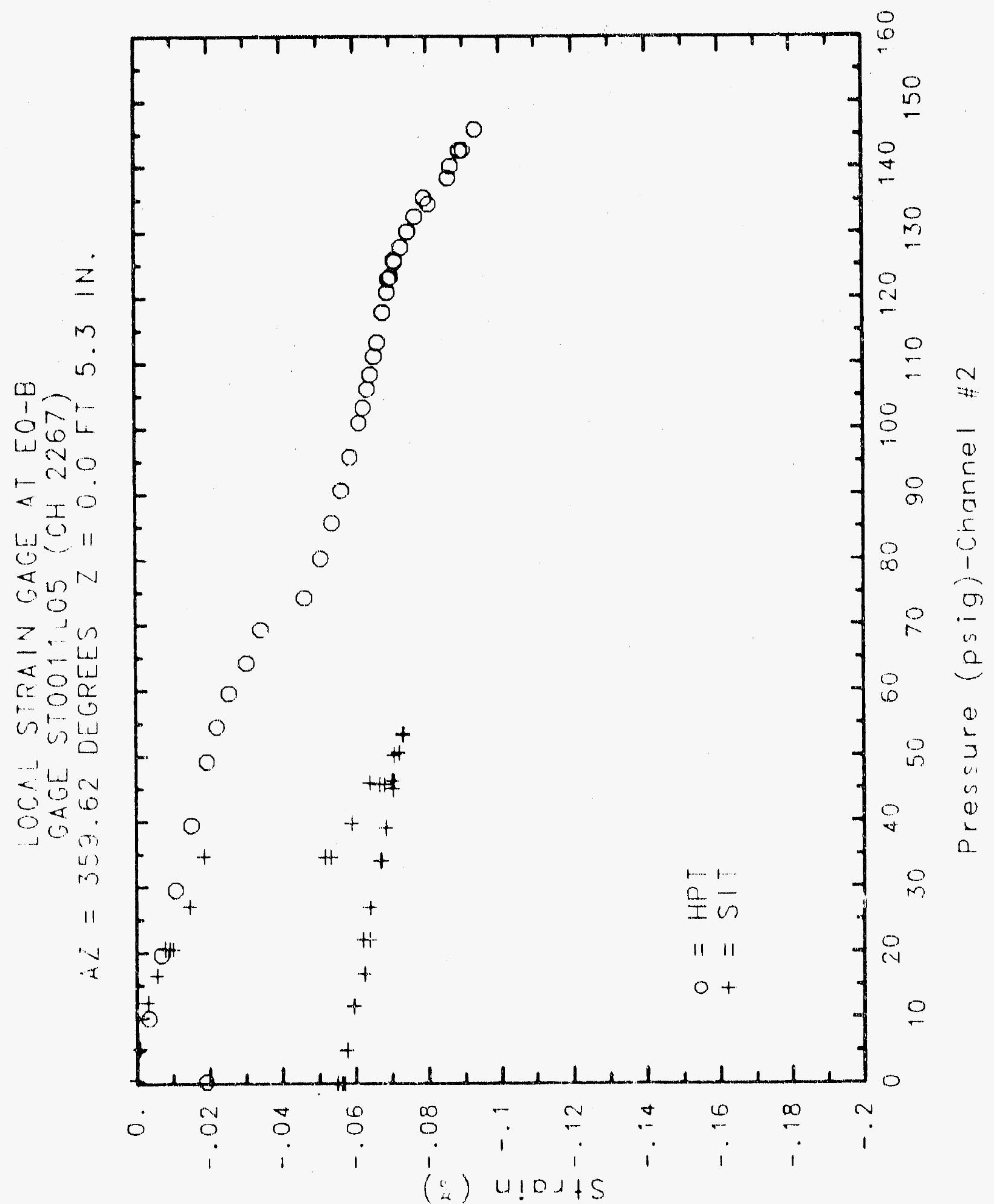




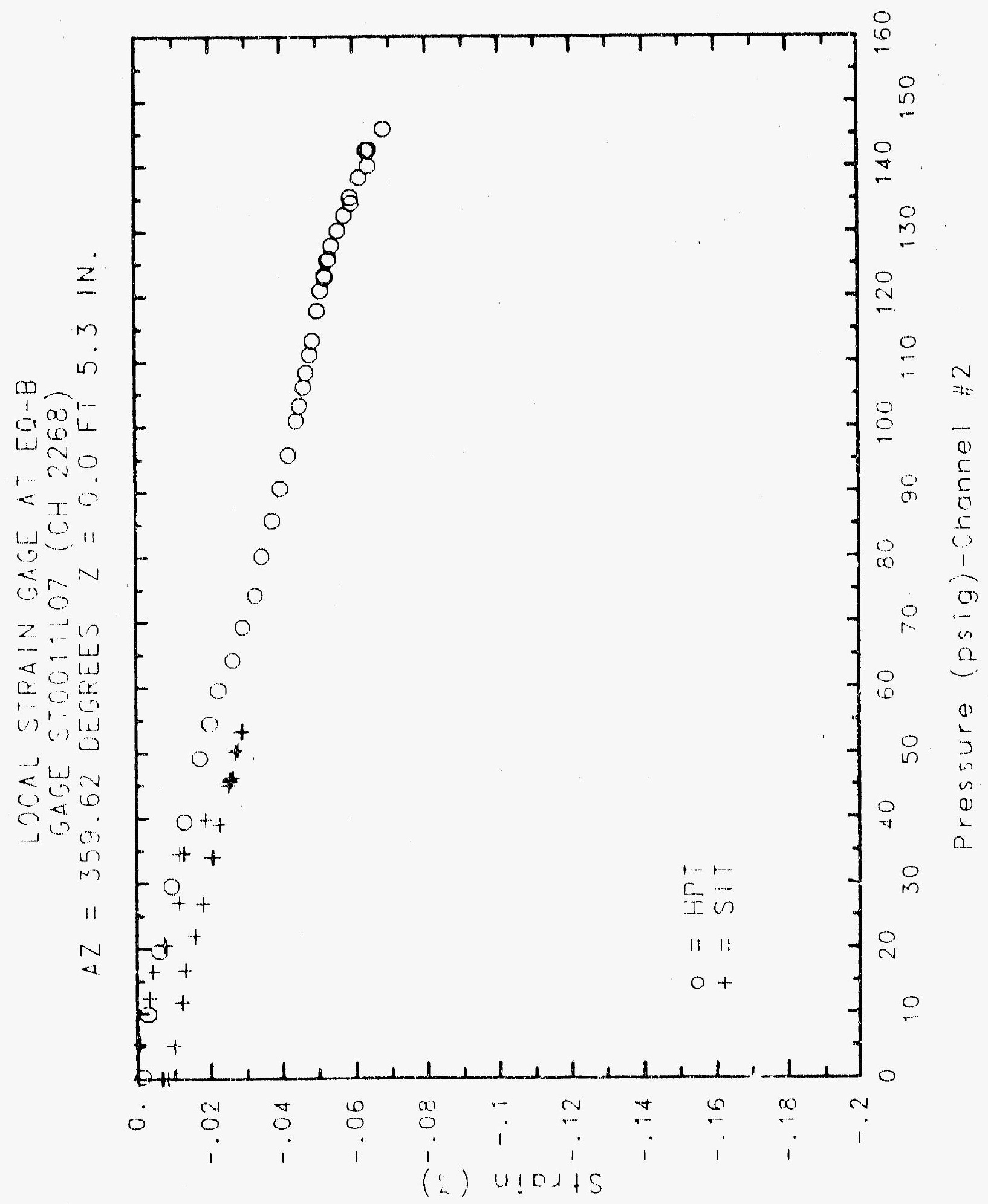




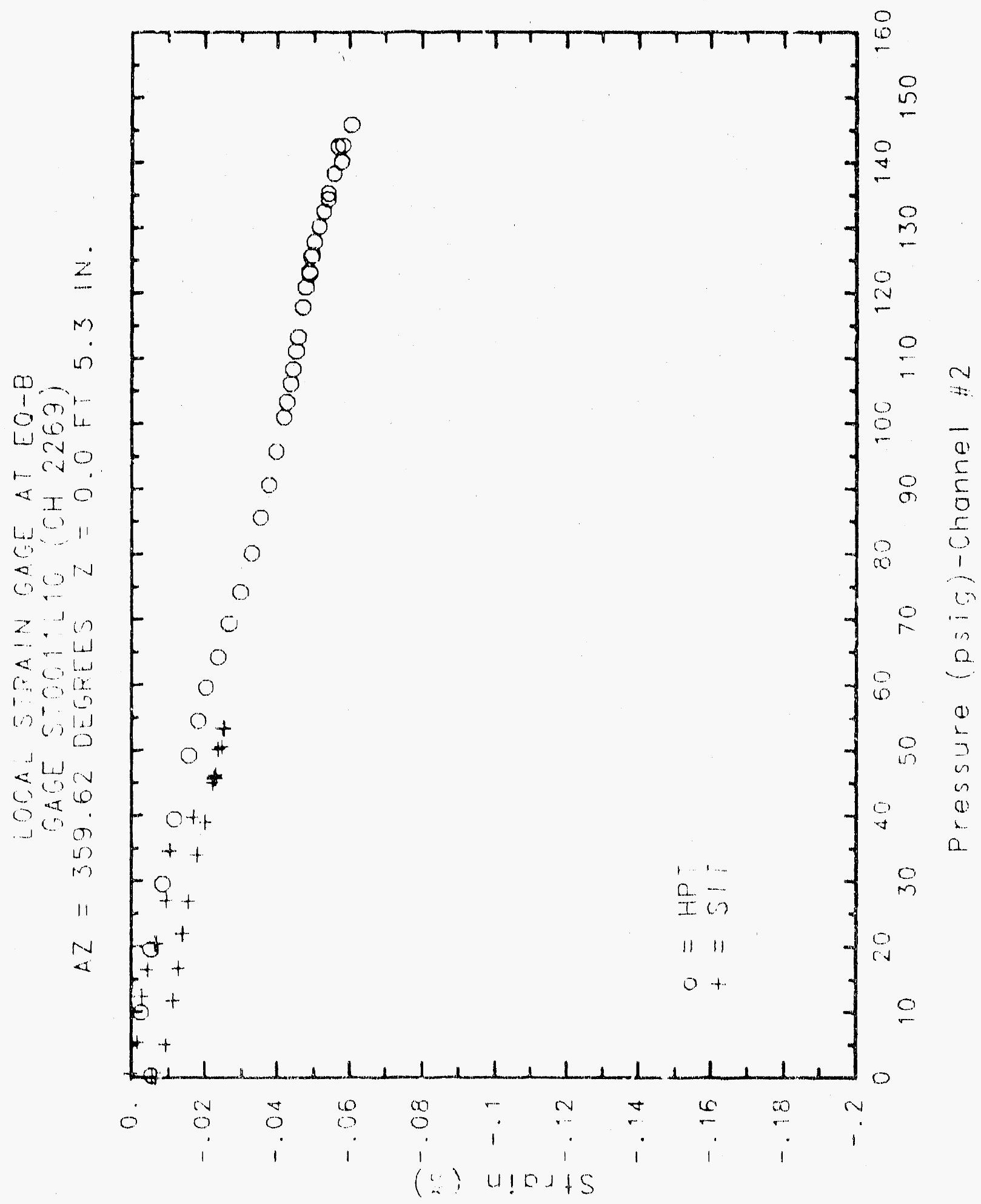




\section{Reinforced Concrete Test Data}

STRIP 12 ChANNELS $2280,2281,2282,2283,2284,2285$

\section{STRUCTURAL INTEGRITY TEST}

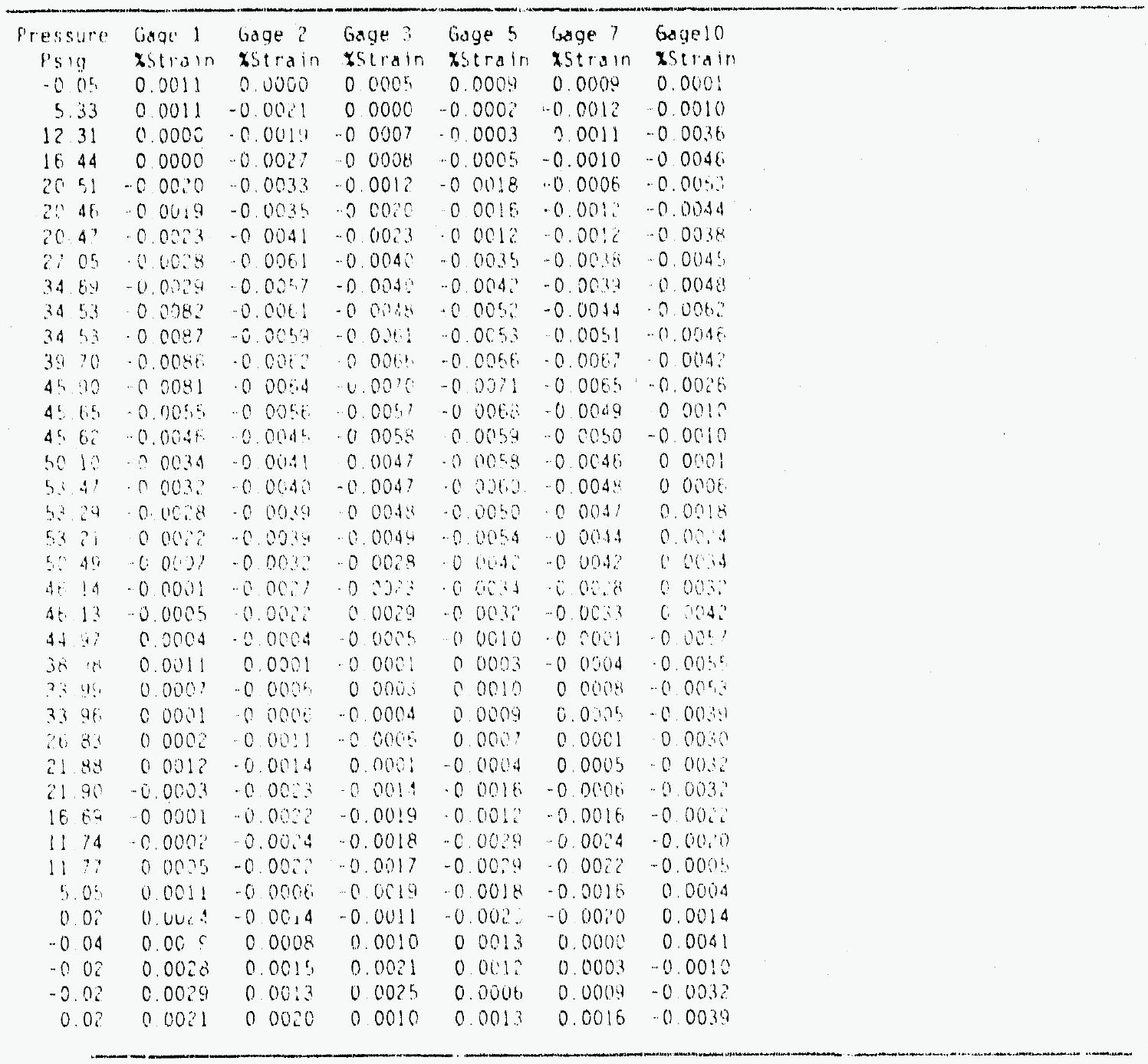



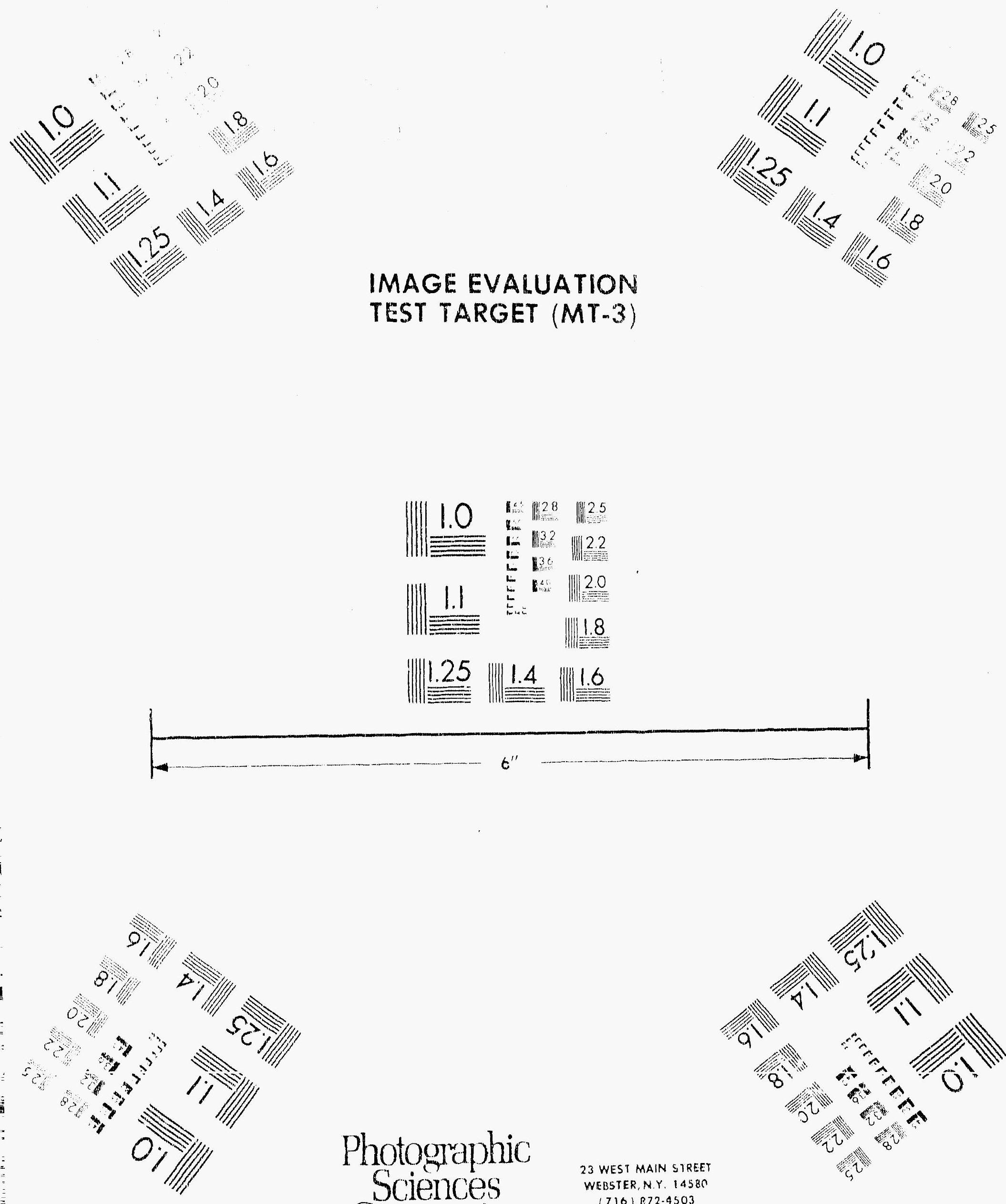

Photographic Sciences Corporation

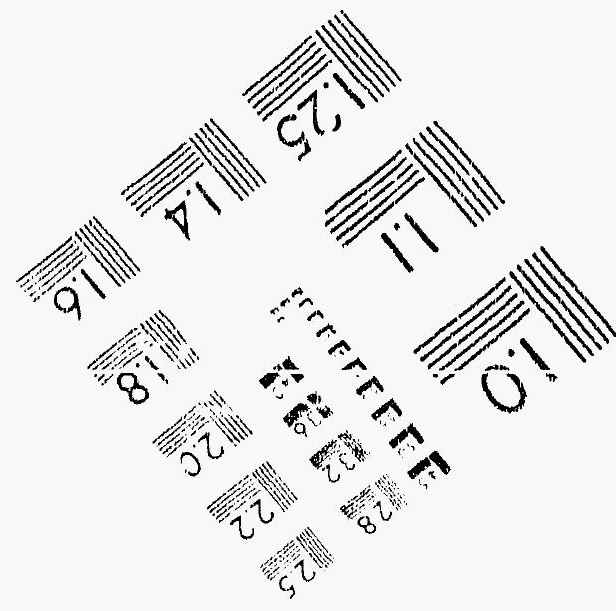




\section{SECURITY CLASSIFICATION}

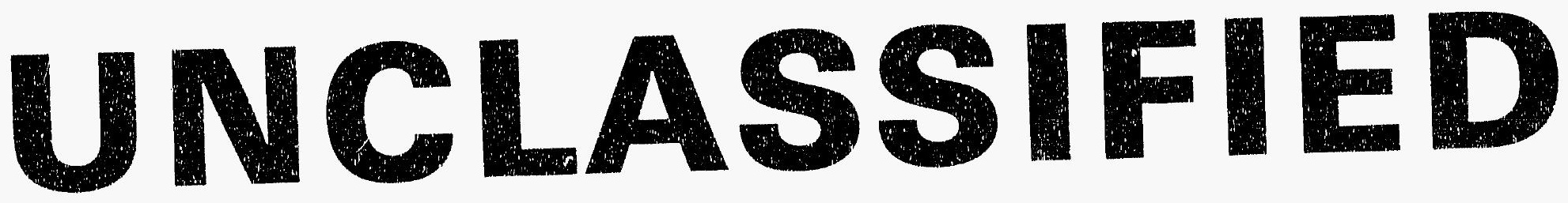

\section{DATE OF MICROFILMING $1-6-92$}

\section{MICROFILMED BY - SerEr hard}

SANDIA NATIONAL LABS 


\section{THIS DOCUMENT FILMED}

A T

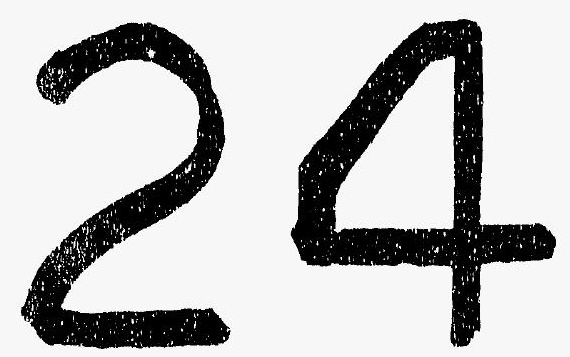

REDUCTION 


\section{Reinforced Concrete Test Data}

Strip 12 Channels $2280,2281,2282,2283,2284,2285$

\section{High Pressure test}

\begin{tabular}{|c|c|c|c|c|c|c|}
\hline Pressure & Gage 1 & Gasge 2 & Gage 3 & Gage 5 & Eage 7 & Gage10 \\
\hline Psig & Xstra in & xstra in & Xstrair. & XStraín & xstrain & xstratn \\
\hline 9.89 & 0.0002 & 0.0001 & -0.0001 & -0.0002 & 0.0000 & -0.0004 \\
\hline 19.55 & 0.0012 & 0.0009 & 0.0007 & 0.0007 & 0.0010 & 0.0004 \\
\hline 29.57 & 0.0020 & 0.0017 & 0.0018 & 0.0020 & 0.0023 & 0.0014 \\
\hline 39.42 & 0.0031 & 0.0031 & 0.0031 & 0.0035 & 0.0039 & 0.0024 \\
\hline 49.16 & 0.0042 & 0.0043 & $0.004 ?$ & 0.0047 & 0.0053 & 0.0034 \\
\hline 54.50 & 0.0046 & 0.0047 & 0.0046 & 0.0050 & 0.0054 & 0.0033 \\
\hline 59.57 & 0.0047 & 0.0047 & 0.0047 & 0.0047 & 0.0052 & 0.0031 \\
\hline 64.20 & 0.0042 & 0.0037 & 0.0037 & 0.0033 & 0.0034 & 0.0013 \\
\hline 69.32 & 0.0035 & 0.0031 & 0.0027 & 0.0020 & 0.0020 & -0.0002 \\
\hline 74.16 & 0.0027 & 0.0021 & 0.0018 & 0.0009 & 0.0007 & -0.0010 \\
\hline 80.16 & 0.024 & 0.0011 & 0.0005 & -0.0012 & -0.0016 & -0.0027 \\
\hline 85.61 & 0.0017 & 0.0002 & -0.0004 & -0.0024 & -0.0029 & -0.0037 \\
\hline 90.58 & 0.0010 & -0.0005 & -0.0014 & -0.0035 & -0.0037 & -0.0045 \\
\hline 95.69 & 0.0005 & 0.0014 & -0.0022 & -0.0046 & -0.0049 & -0.0053 \\
\hline 100.82 & -0.0001 & -0.0022 & -0.0031 & -0.0056 & -0.0059 & -0.0060 \\
\hline 10325 & -0.0003 & -0.0026 & -0.0035 & -0.0061 & -0.0065 & -0.0062 \\
\hline 106.11 & -0.0007 & -0.0030 & -0.0038 & -0.0065 & -0.0070 & -0.0066 \\
\hline 108.31 & -0.0009 & -0.0031 & -0.0042 & -0.0069 & $-0.007 ?$ & -0.0068 \\
\hline 111.08 & $-0.001 i$ & -0.0036 & -0.0045 & 0.0073 & -0.0077 & -0.0071 \\
\hline 113.24 & -0.0013 & -0.0039 & -0.0048 & -0.0076 & -0.0080 & -0.0073 \\
\hline 117.83 & -0.0013 & -0.0041 & .0 .0052 & -0.0079 & -0.008 .3 & -0.0076 \\
\hline 120.92 & -0.0017 & -0.0045 & -0.0055 & $-0 . c^{\prime} \cup 83$ & -0.0087 & -0.0077 \\
\hline 123.28 & -0.0014 & -0.0045 & -0.0055 & -0.0082 & -0.0085 & -0.0073 \\
\hline 122.97 & -0.0004 & -0.0041 & $=0.0050$ & -0.0076 & -0.0081 & -0.0063 \\
\hline 125.82 & -0.0002 & -0.0044 & -0.0055 & -0.0079 & -0.0082 & -0.0060 \\
\hline 125.60 & 0.0004 & -0.0045 & -0.0054 & -0.0080 & $-C .0082$ & -0.0056 \\
\hline 127.84 & 0.0004 & -0.0047 & -0.0056 & -0.0082 & -0.0084 & -0.0056 \\
\hline 130.19 & 0.0009 & -0.0047 & -0.0057 & -0.0083 & -0.0085 & -0.0053 \\
\hline 132.53 & 0.0012 & -0.0045 & -0.0057 & -0.0083 & -0.0085 & .0 .0052 \\
\hline 135.33 & 0.0021 & -0.0041 & -0.0055 & -0.0082 & -0.0081 & -0.0045 \\
\hline 134.42 & 0.0030 & -0.0034 & -0.0050 & -0.0075 & $-0.00 \% 4$ & -0.0039 \\
\hline 138.35 & 0.0042 & -0.0027 & -0.0046 & -0.0071 & -0.0069 & -0.0032 \\
\hline 140.16 & 0.0053 & -0.0017 & -0.0040 & -0.0007 & -0.0061 & -0.0024 \\
\hline 142.63 & 0.0067 & -0.0004 & -0.0028 & -0.0055 & -0.0048 & -0.0014 \\
\hline 145.78 & 0.0073 & 0.0005 & -0.0020 & -0.0047 & -0.0042 & -0.0007 \\
\hline 142.52 & 0.0100 & 0.0036 & 0.0010 & -0.0018 & -0.0016 & 0.0011 \\
\hline 0.22 & 0.0163 & 0.0119 & 0.0098 & 0.0090 & 0.0097 & 0.0115 \\
\hline
\end{tabular}




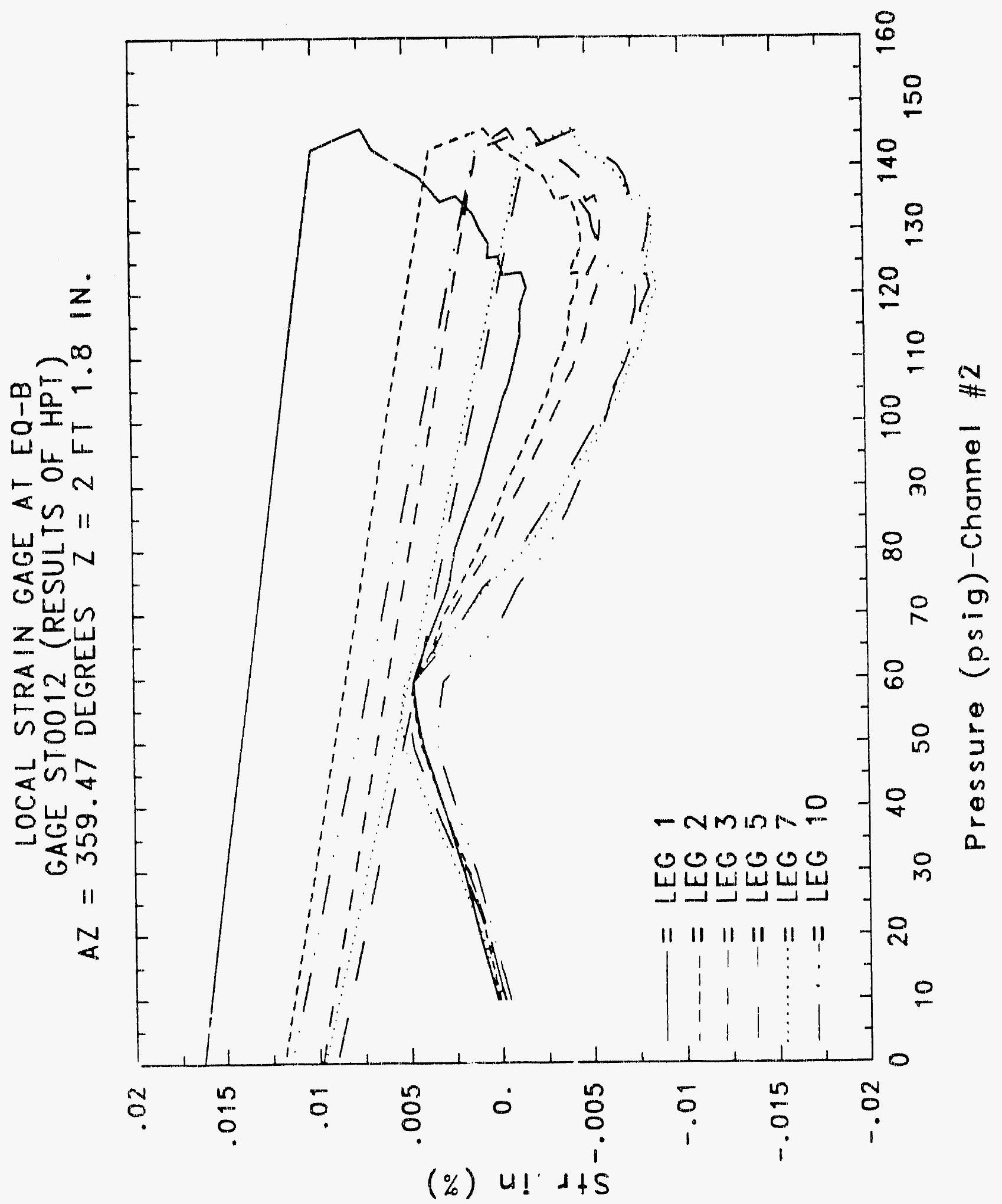




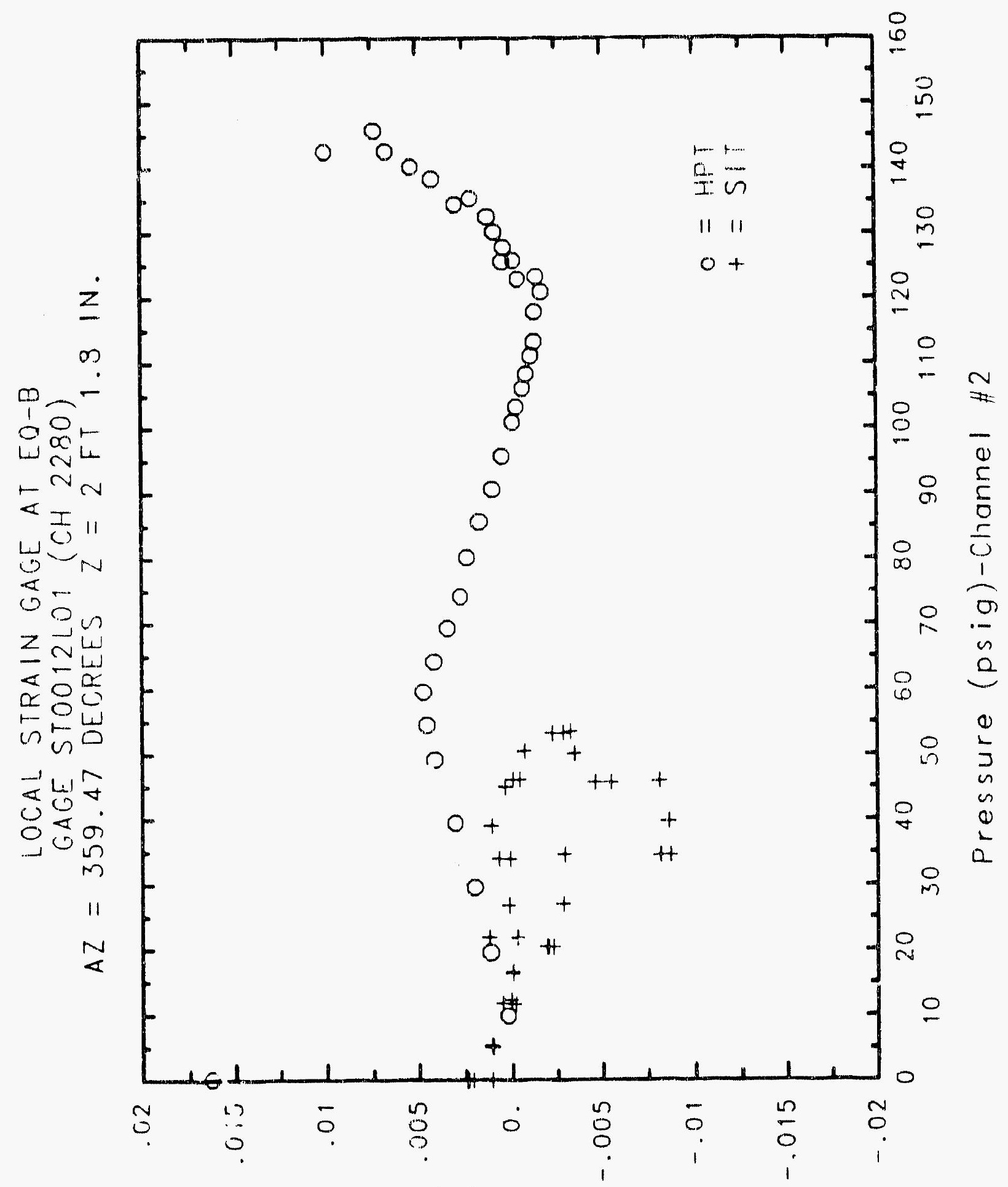

(i) $4 ! 0+5$ 


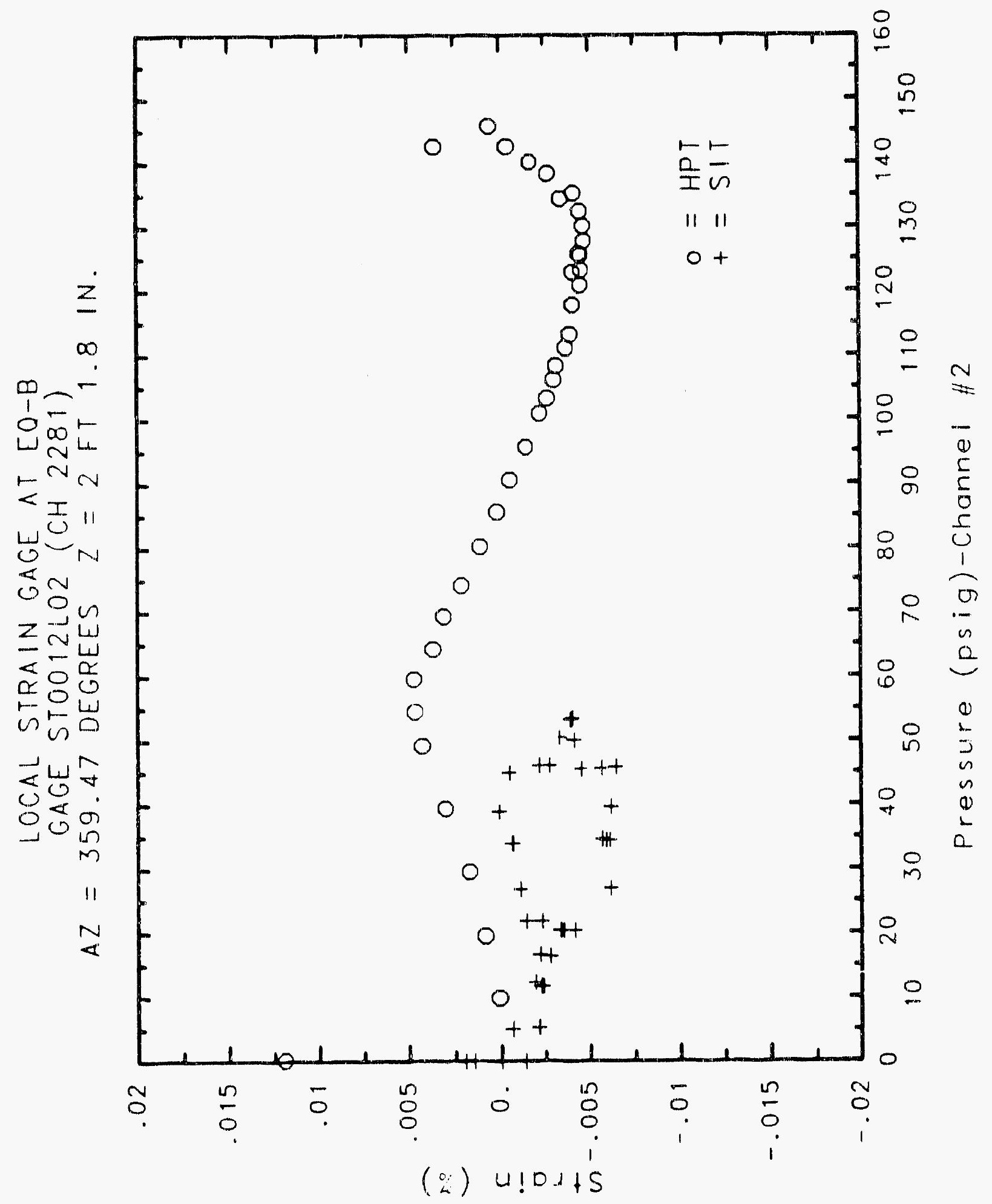

C. 1494 


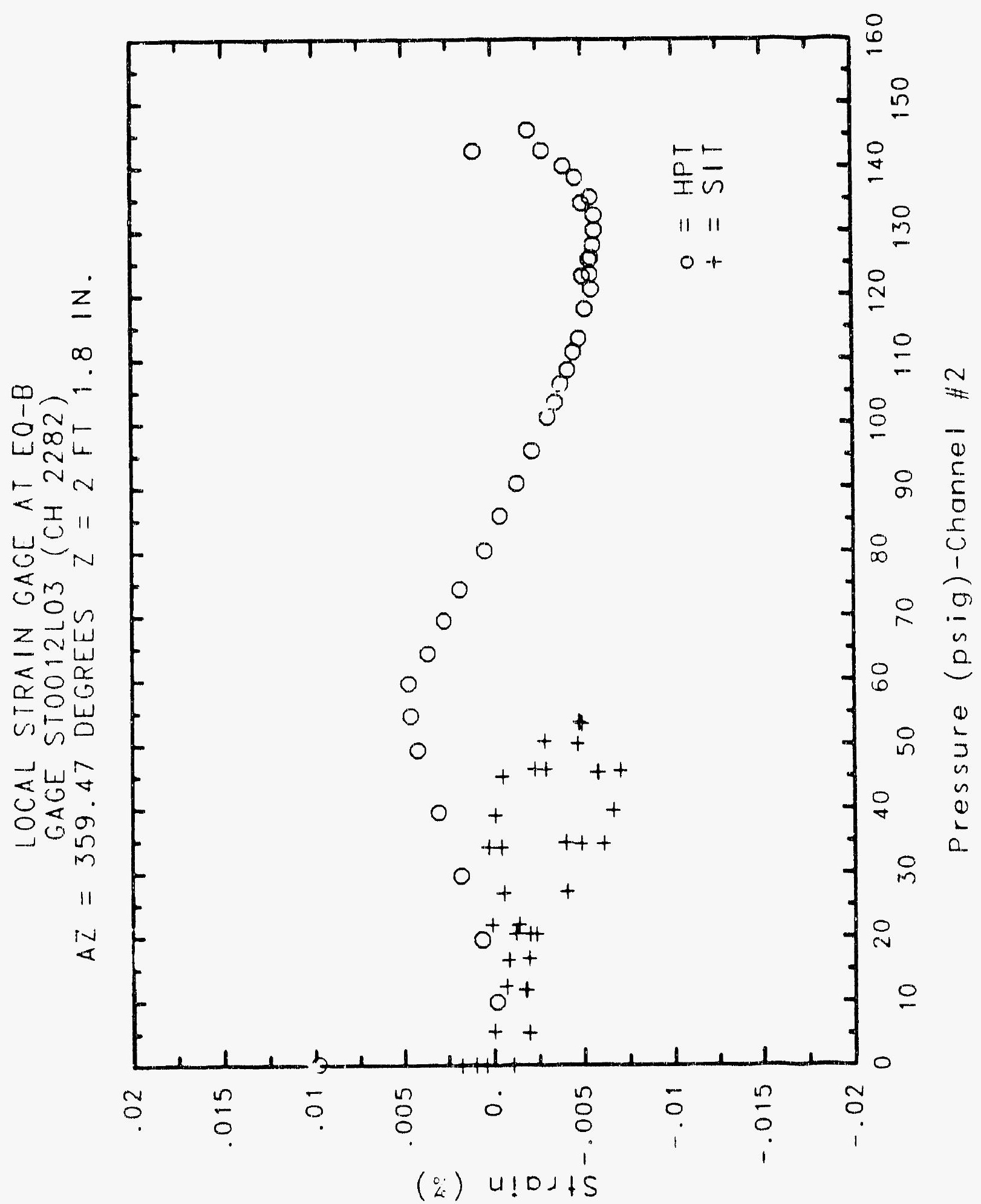




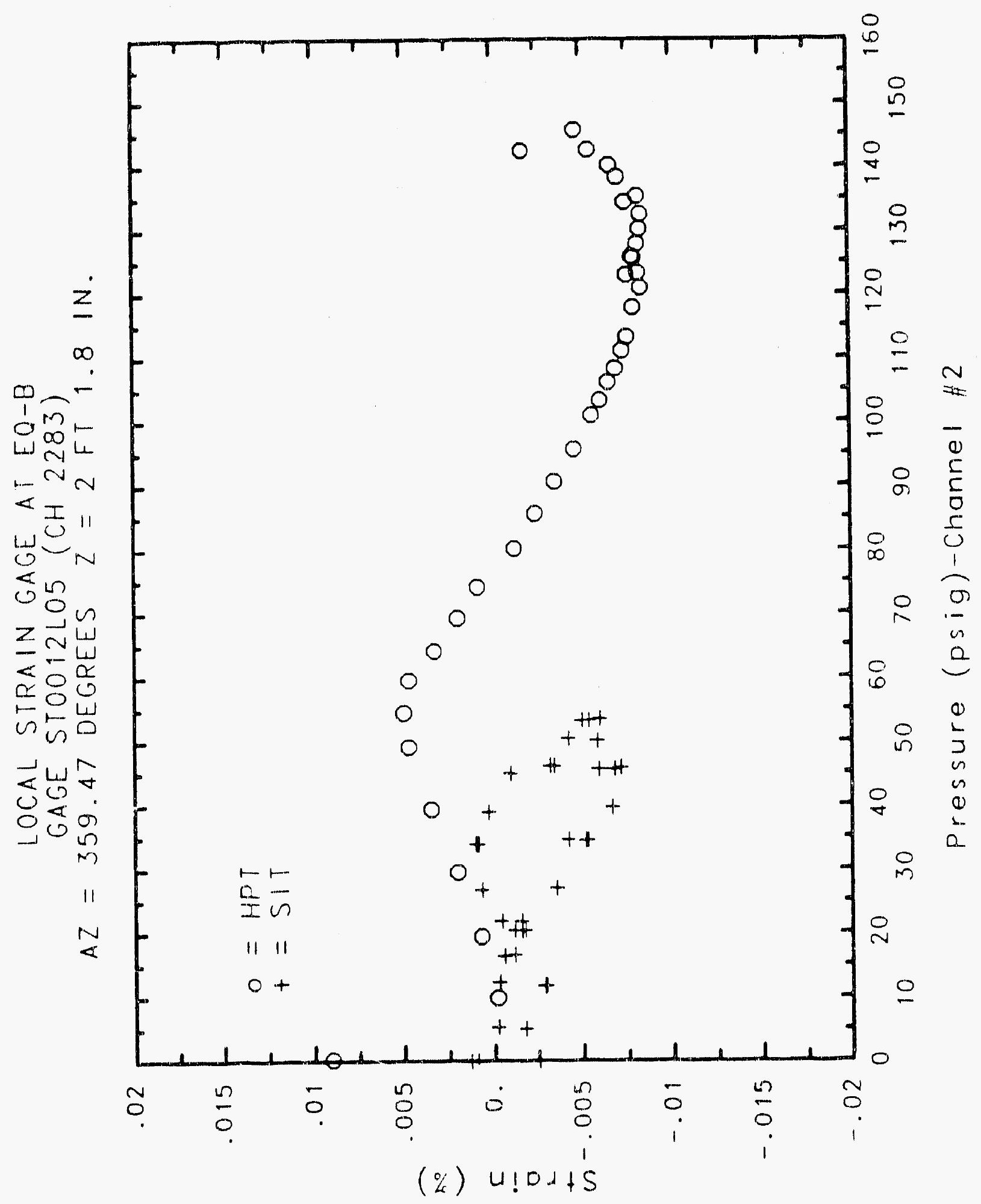




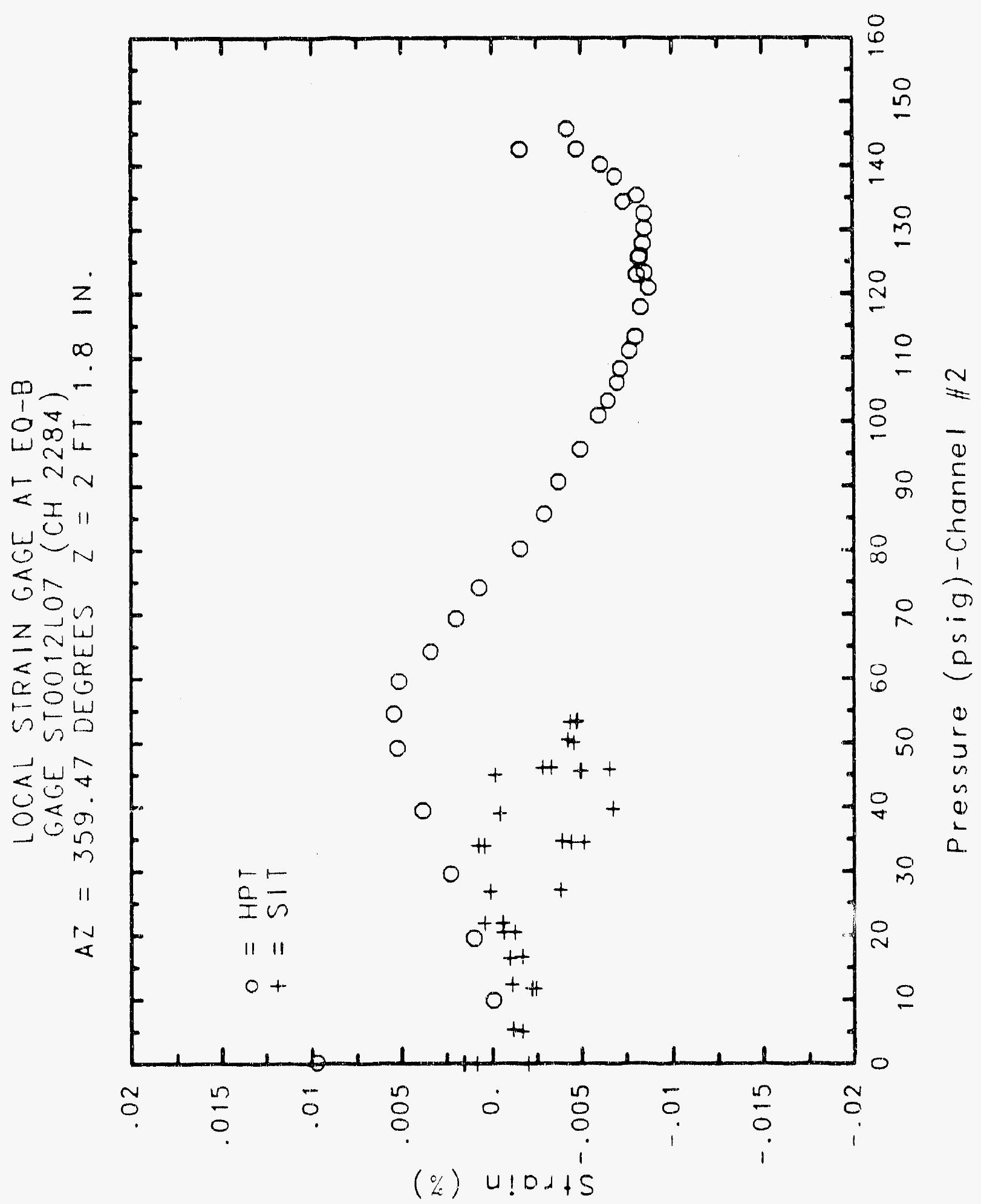




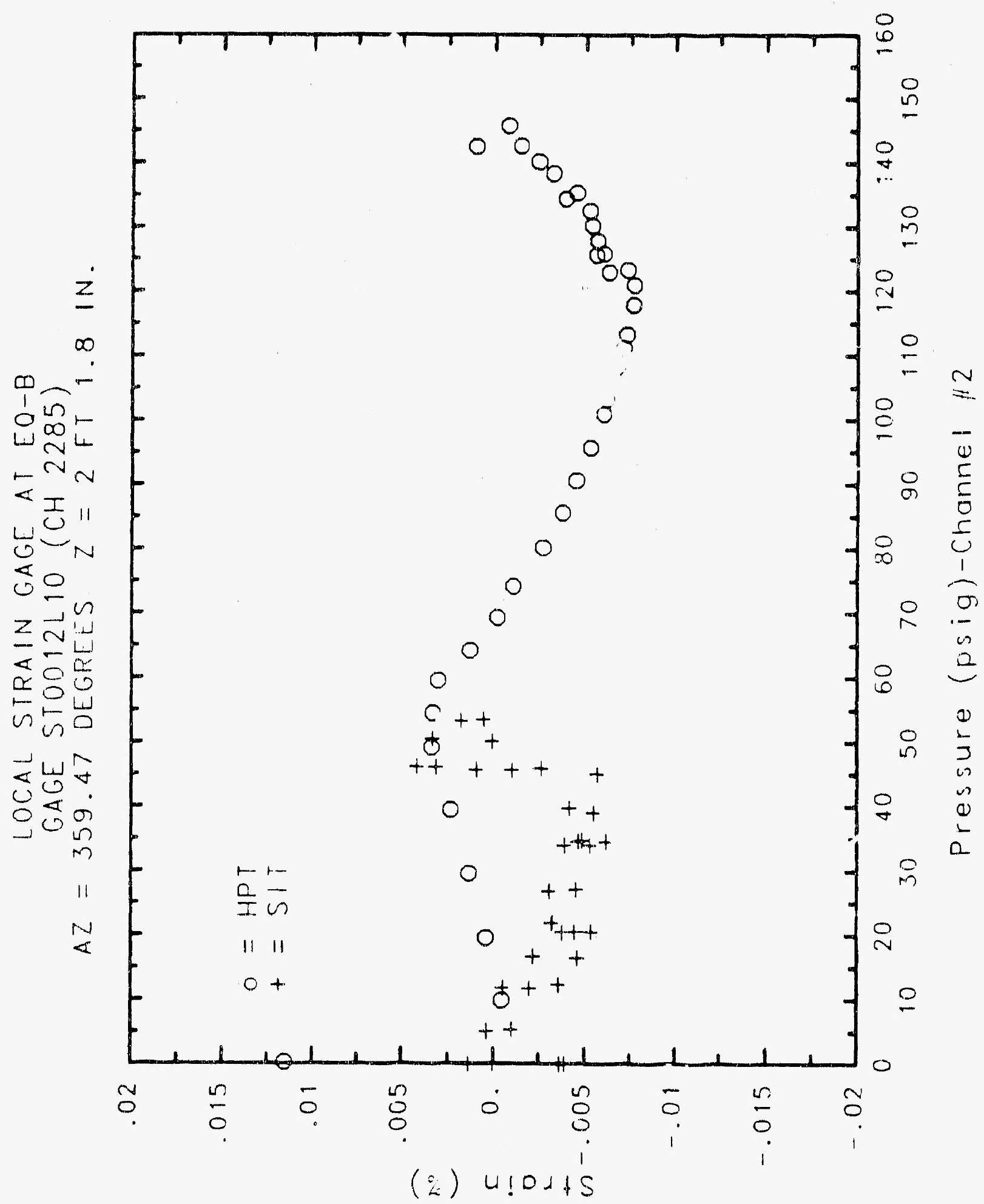




\section{Reinforced Concrete Test Data}

Strip 13 Channels $2286,2237,2288,2289,2300,2301$

STRUCTURAL INTEGRITY TEST

\begin{tabular}{|c|c|c|c|c|c|c|}
\hline Pressure & Gage 1 & bage 2 & Gage 3 & Gage 5 & Eage 7 & 6 oge 10 \\
\hline Psig & Xstrain & xstrain & xstrsin & xstrain & xstrain & Xstrain \\
\hline-0.05 & 0.0005 & 0.0008 & $-n .000$ & 0.0011 & -0.0005 & 0.0000 \\
\hline 5.33 & -00038 & -0.0008 & -0.0029 & -0.0018 & 0.0000 & -0.0019 \\
\hline 12.31 & $-0.008 !$ & $-0.005 \%$ & -0.0067 & -0.0039 & -0.0014 & -0.0027 \\
\hline 16.44 & -0.0121 & -0.0088 & $-0 \quad 3092$ & -0.0048 & -0.0019 & -0.0030 \\
\hline 20.51 & -0.0163 & -0.0136 & $\because 0134$ & $-0.008 ?$ & .0 .0045 & -0.0040 \\
\hline 20.46 & -0.0180 & -0.0149 & 0.0138 & -0.009 & -0.0042 & -0.0043 \\
\hline 20.47 & -0.0201 & -0.0163 & -0.0150 & -0.0098 & -0.0048 & -0.0049 \\
\hline 27.05 & $-0.029 E$ & .0 .0256 & -0.0228 & $-0.01 E:$ & -0.0100 & -0.0091 \\
\hline 34.59 & $-0.04 C 4$ & -0.0343 & -0.0313 & -0.0209 & $-0.0: 50$ & -0.0137 \\
\hline 34.53 & -0.0436 & -0.0381 & -0.0347 & -0.0238 & -0.0166 & -00140 \\
\hline 34.53 & -0.0444 & -0.8335 & -0.0344 & -0.0246 & -0.0155 & -0.0154 \\
\hline 39.70 & -0.0434 & $-0 \cup 434$ & $-0.039 ?$ & -0.0280 & -0.0190 & -0.0175 \\
\hline 45.90 & -0.0560 & $-1,050 i$ & -0.0447 & $-c .0330$ & -0.0231 & -0.0216 \\
\hline 45.65 & $-0.05 E 5$ & .00494 & -0.0445 & -0.0332 & -0.0235 & -0.0219 \\
\hline 45.52 & -00559 & -0.0495 & $-0.044 E$ & -0.0325 & -0.0231 & -0.0205 \\
\hline $5=10$ & -0.0598 & -0.0531 & -0.048: & -0.0355 & -0.0255 & -00237 \\
\hline 5347 & $-0.0 E 2 Z$ & $.0 .055 E$ & -0.0501 & -0.0365 & -0.0270 & $-0 . C 2 \Delta E$ \\
\hline 53.29 & -0.0018 & $-0.05 E 1$ & -0.0433 & $-003 E ?$ & -0.0273 & $-0 \quad 02.45$ \\
\hline 53.21 & $-\therefore . O E: ?$ & -0.0547 & -0.0492 & .0 .0354 & -0.0283 & -00254 \\
\hline 50.49 & $-0.058:$ & $-0.051:$ & -0.0453 & -0.0344 & -0.0257 & 0.0237 \\
\hline AE 14 & -0.0520 & $-0.046 \hat{3}$ & $-0.04: 7$ & -0.0322 & -0.0234 & $-0.02: 2$ \\
\hline $46 \vdots:$ & -0.0515 & -0.0464 & -0.0416 & $-0.03: 6$ & -0.0237 & -0.0213 \\
\hline 44.57 & -0.0454 & -0.0456 & -0.0405 & $-0.02 \geq 9$ & -0.0212 & -0.0189 \\
\hline 38.98 & -0.0426 & -0.0389 & -0.0354 & -0.0262 & -0.0175 & -0.0155 \\
\hline 33.99 & -3.0356 & -0.0331 & -0.0309 & -0.0224 & $-0.015 i$ & -0.0129 \\
\hline 33.96 & -0.0368 & -0.0331 & -0.0306 & -0.0232 & -0.0150 & -0.0132 \\
\hline 2683 & .0 .0282 & -0.0252 & -0.0249 & -0.0182 & -0.0110 & $-0.00=0$ \\
\hline 21.88 & $-0.024 i$ & -0.0216 & -0.0214 & $-02: 54$ & -0.0093 & -0.0070 \\
\hline 21.90 & $-0.024 E$ & -0.0221 & $-C .0217$ & -00.60 & $-\hat{i} \operatorname{cog} 5$ & -0.0075 \\
\hline $1 \in 69$ & -0.0225 & -0.0196 & .0 .0195 & -0.0148 & .00071 & -0.0053 \\
\hline 11.74 & -0.0200 & -0.0176 & -0.0178 & -0.0123 & $-0.005 F$ & -0.0045 \\
\hline 11.77 & -0.0202 & -0.0175 & -0.0180 & -0.0133 & -0.0050 & $-0.005:$ \\
\hline 5.05 & -0.0150 & -0.0139 & -0.0136 & -0.0109 & .00053 & -0.0038 \\
\hline 0.02 & -0.0124 & -0.0103 & -0.0116 & -0.0033 & -0.0043 & -0.0036 \\
\hline-0.04 & -0.0110 & -0.0087 & -0.0092 & -0.0086 & -0.0020 & -0.0033 \\
\hline-0.02 & -0.0111 & -0.0093 & -0.0098 & -0.0079 & -0.01022 & -0.0022 \\
\hline-0.02 & -0.0118 & -0.0093 & -0.0104 & -0.0083 & -0.0015 & -0.0028 \\
\hline 0.02 & -0.0112 & -0.0100 & -0.0097 & -0.0080 & -00016 & -0.0018 \\
\hline
\end{tabular}




\section{Reinforced Concrete Test Data}

STKIP 13 ChANnel.s $2286,2287,2288,2289,2300,2301$

High Pressure test

\begin{tabular}{|c|c|c|c|c|c|c|}
\hline Pressute & $\cos =1$ & Gage 2 & Goge 3 & Bage 5 & Goge ; & Gageio \\
\hline Ps:g & Xstain & $x 5: r \ln$ & xstroin & xstrain & Xstrain & xstrain \\
\hline 9.89 & -0.0372 & -0.0058 & -0.0058 & -0.0034 & -0.0022 & -00012 \\
\hline 19.55 & $-0.0: 50$ & -0.0149 & -0.0128 & -0.00185 & -0.0051 & -00530 \\
\hline 29.57 & -0.0 .52 & -0.0241 & -0.0209 & -0.0142 & -0.0109 & .0 .0579 \\
\hline 39.2 & -0.0357 & .00332 & -0.0283 & -0.0197 & -0.0158 & -0.0123 \\
\hline 49.16 & $-c \cos 19$ & $-0.04: 0$ & -0.0353 & -0.0252 & $-C .020 ?$ & -0.0160 \\
\hline 5450 & -0.0497 & -0 O4EE & -0.0339 & -2.0233 & -0.0223 & -0.0192 \\
\hline 59.57 & $-0.053:$ & $-0.050 !$ & $-0.04: 8$ & -0.0324 & -0.0259 & $-0.02: 3$ \\
\hline 6420 & -0.0552 & -0.0523 & -00449 & -0.0324 & -0.0255 & $-0.02: 8$ \\
\hline 63.32 & -0.0578 & -0.0547 & 0.0470 & 0.0331 & -0.0251 & $-c .0231$ \\
\hline $74: 6$ & -0.0585 & -0.0558 & -0.0473 & -0.0335 & $-0 \quad 0284$ & .00335 \\
\hline $8: 15$ & -00572 & $-0.055:$ & -00473 & -00350 & -0.0237 & -0.0253 \\
\hline 85.61 & -0.0553 & $-0.054:$ & $-0.046 i$ & $-0.034=$ & -00235 & -0.0252 \\
\hline 92.58 & $-0.054:$ & -0.0522 & -0.0446 & -0.0323 & $-0.02 ? 4$ & $-0 \quad 0>5=$ \\
\hline 9569 & $005: 8$ & -0.0498 & -0.0424 & $-6 \quad 0:: .:$ & -00255 & -0.0247 \\
\hline $10 \therefore 32$ & $-C .048:$ & $-0.24 E ?$ & -0.0393 & -0.025 & $=0253$ & -00242 \\
\hline 103.25 & $-0.045:$ & .0 .0447 & -0.0375 & $-6 \quad 0 z-5$ & -0.0245 & -0.0238 \\
\hline $1 \therefore \div 11$ & -0.0454 & $-i-2+3=$ & 0.0354 & -0.0268 & -0.2235 & -0.0238 \\
\hline $1083:$ & $.0 .044:$ & -00424 & -0.0355 & -0.0250 & -0 0:33 & -0.0234 \\
\hline$\because \therefore: 08$ & $-0.04: 9$ & -0.04 .54 & -00334 & -0.0242 & -0.5224 & -00632 \\
\hline$: 2324$ & $-0.040 \hat{\varepsilon}$ & -00333 & -0 cosid & .00235 & $-0.22: 5$ & .00225 \\
\hline iIi.83 & -0.0376 & -0.0051 & $-0.225 ?$ & -0.200 & -0.2198 & $-8.62: 7$ \\
\hline 12002 & -20345 & -0.0329 & $-00 \% 55$ & -0887 & -0.0179 & -0.0205 \\
\hline 12328 & $-0.03 \%:$ & -0.0308 & -0.0644 & $-00: 70$ & $-0.0: 58$ & -0.0336 \\
\hline 12297 & $-0.03: 0$ & -0030 & $-0.023 E$ & -0.150 & $-0.0: 58$ & $-0.0: 9 E$ \\
\hline$: 2582$ & $-0 \quad 2: 32$ & $-C 0274$ & -00216 & $=0.0: 43$ & $-60 ! 35$ & $-0.015 !$ \\
\hline 12560 & -0.0280 & $-0.02 E ?$ & -0.0205 & -0.0137 & $-00: 34$ & $-0.018:$ \\
\hline 227.84 & $-0 \quad 0257$ & $-0.02 \$ \hat{0}$ & $-0:: 83$ & $-001: 7$ & $-00: 25$ & U2:5 \\
\hline 13219 & -00225 & $-003 j 5$ & -0.0150 & -00065 & $-0.01: 2$ & -C.ODE: \\
\hline 13253 & -0 CIB: & -00172 & $-0 \div: 9$ & -3005 & -0.0053 & -0.014 \\
\hline 13533 & -0.0130 & -0.0121 & -3.0073 & - o OCLE & -0.0047 & 0.0123 \\
\hline$: 34.42$ & -0.0125 & $\because 0116$ & -0.0005 & -0.0021 & .0 .0032 & $-0.030 t$ \\
\hline$: 38 \quad 35$ & -00072 & -00050 & $-20: 57$ & 0.0018 & 00002 & .00086 \\
\hline $140: 0$ & -0.0025 & -0.0214 & 0.0025 & 00044 & 00020 & -2.0083 \\
\hline 14263 & 0.0021 & 0.0035 & 0.0071 & 0.0080 & 0.0032 & -0.0082 \\
\hline 14578 & 00057 & 0.0075 & 0.0111 & 0.0110 & 0.0043 & -0.0083 \\
\hline $142.5 ?$ & 0.0225 & 0.0219 & $0.0 ? 31$ & 0.0190 & 0.0111 & $-0.00=0$ \\
\hline 0.22 & $0.10: 4$ & $0.09: 1$ & 0.0822 & 0.0628 & $0.054 !$ & $0.040 \mathrm{~s}$ \\
\hline
\end{tabular}




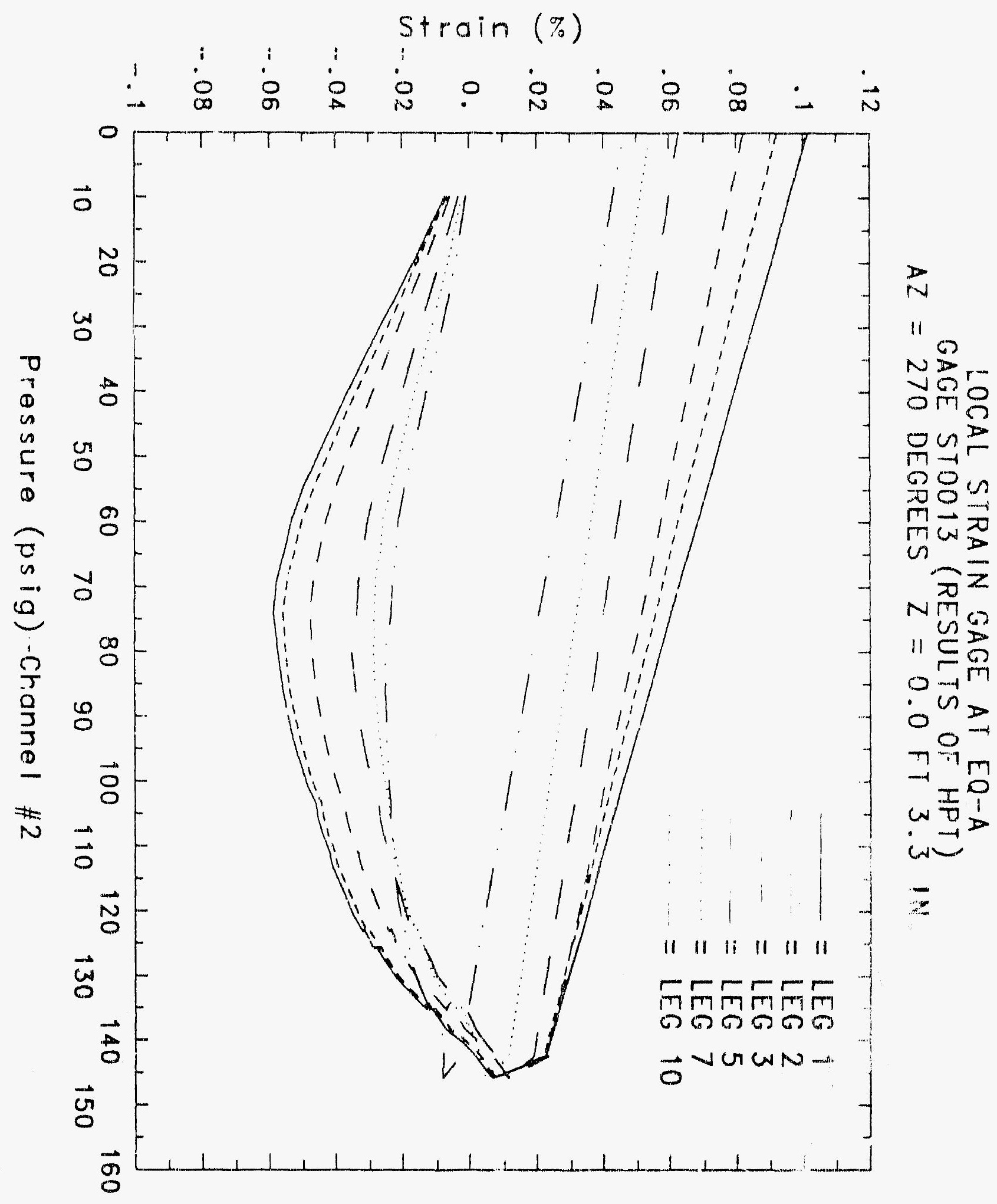




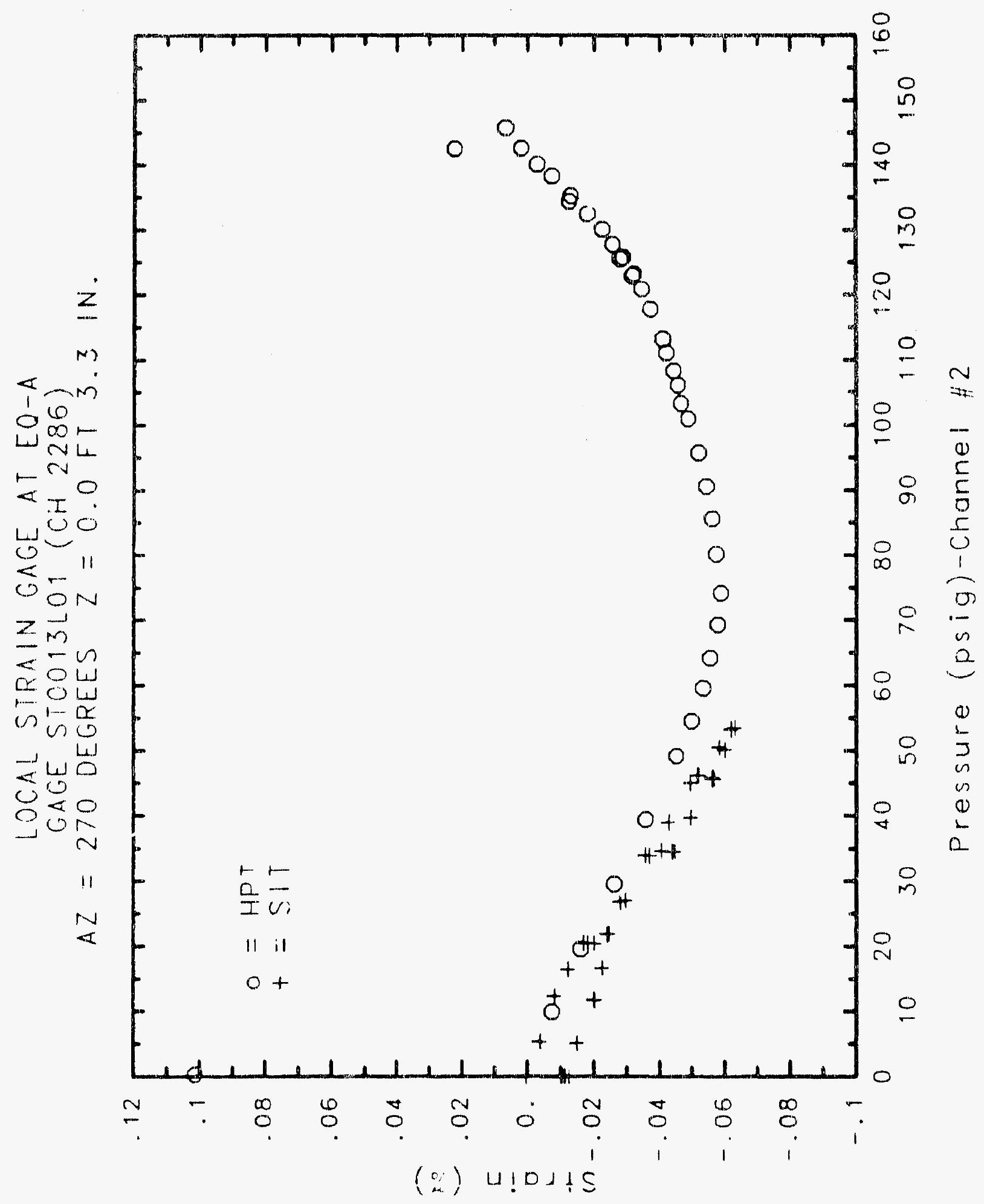




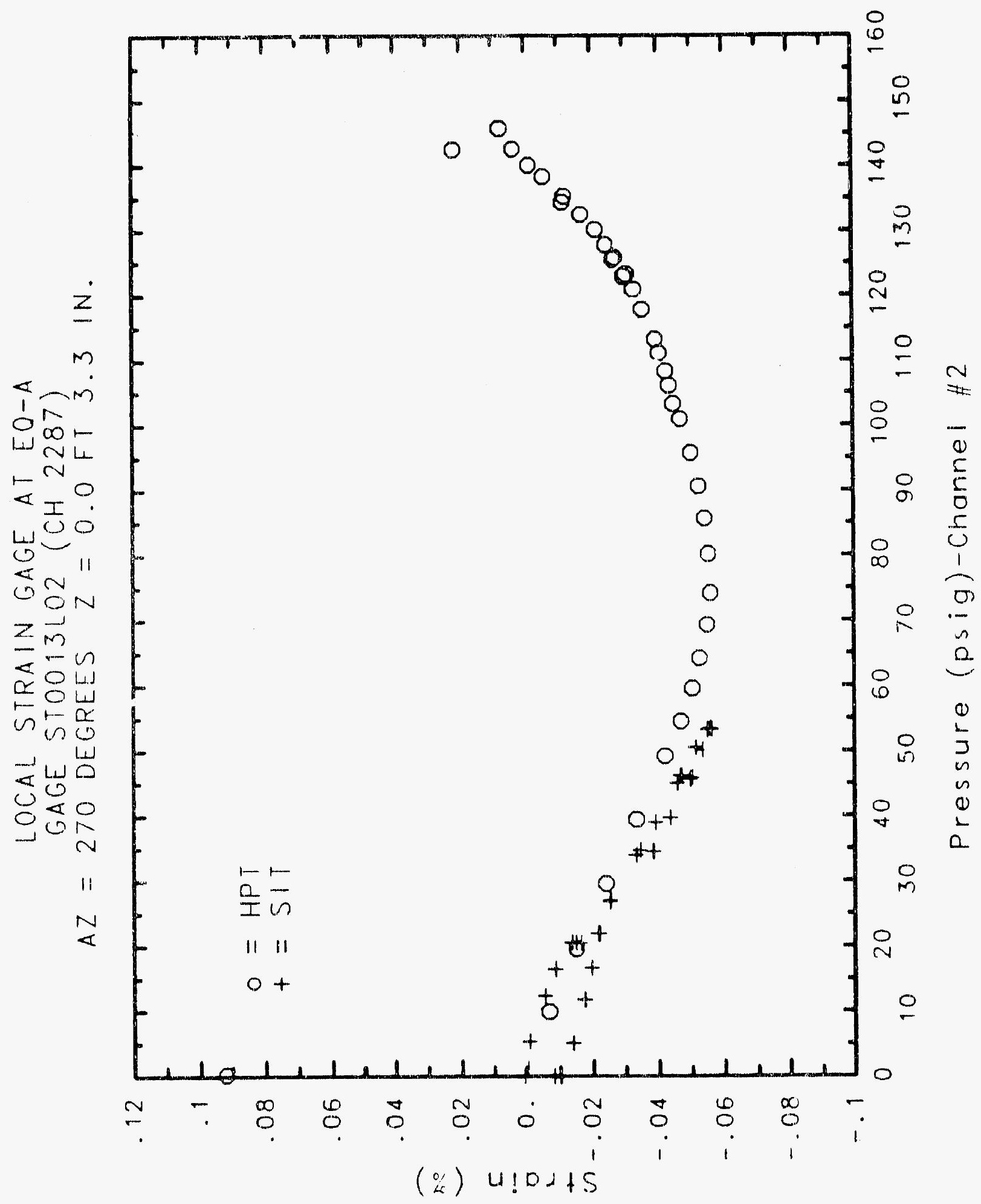

C. -1.503 


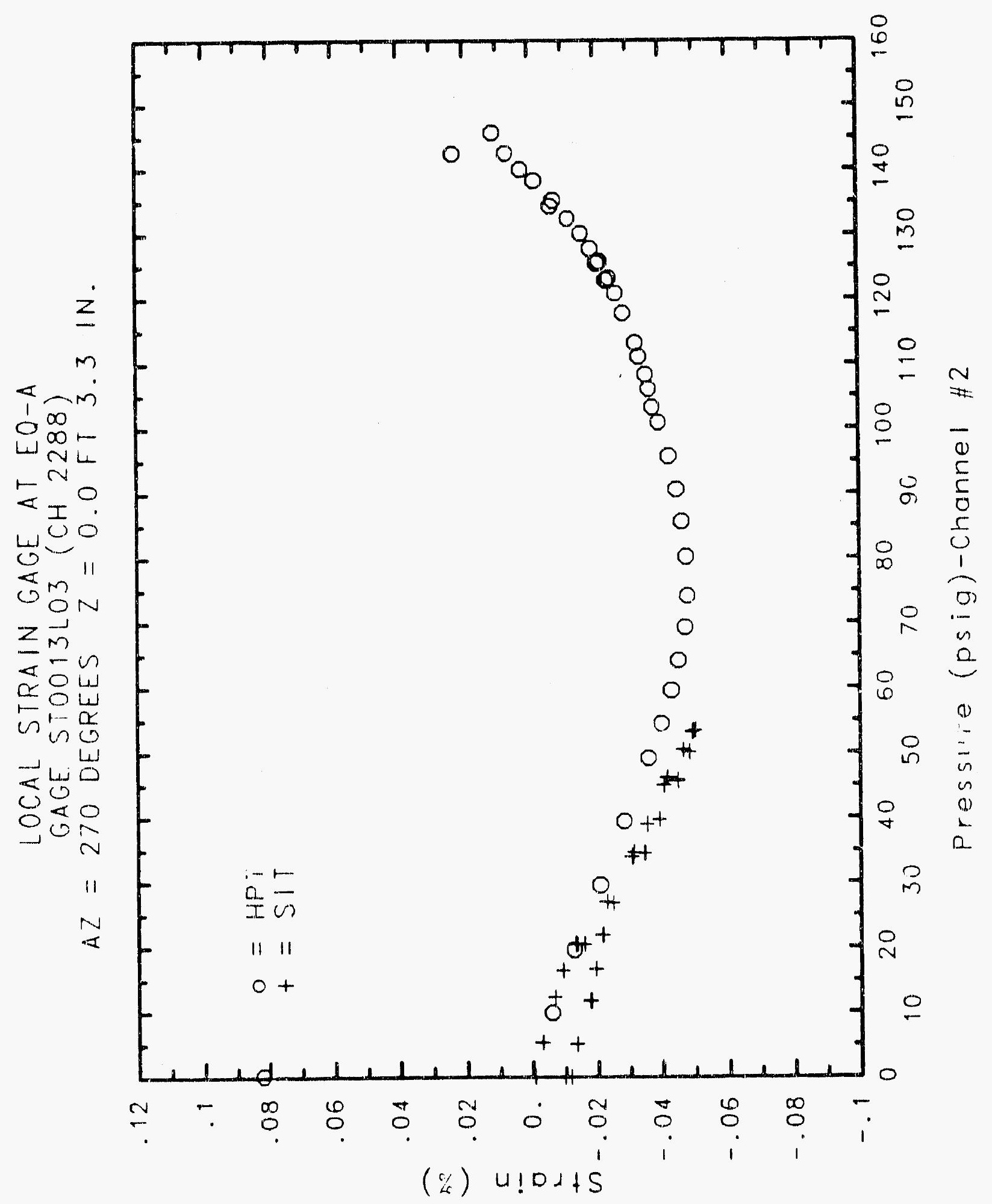

C- 1504 


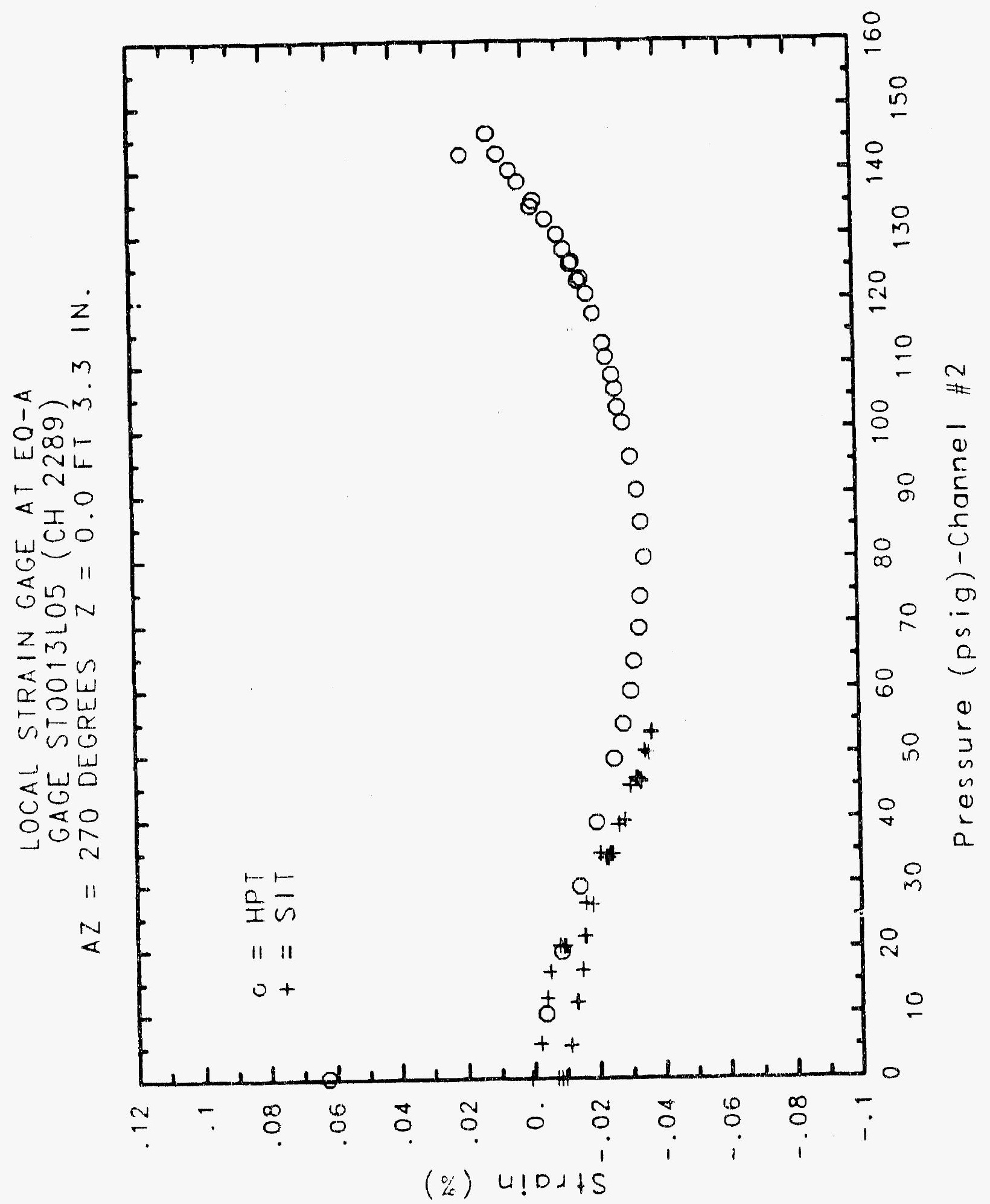

C. 1505 


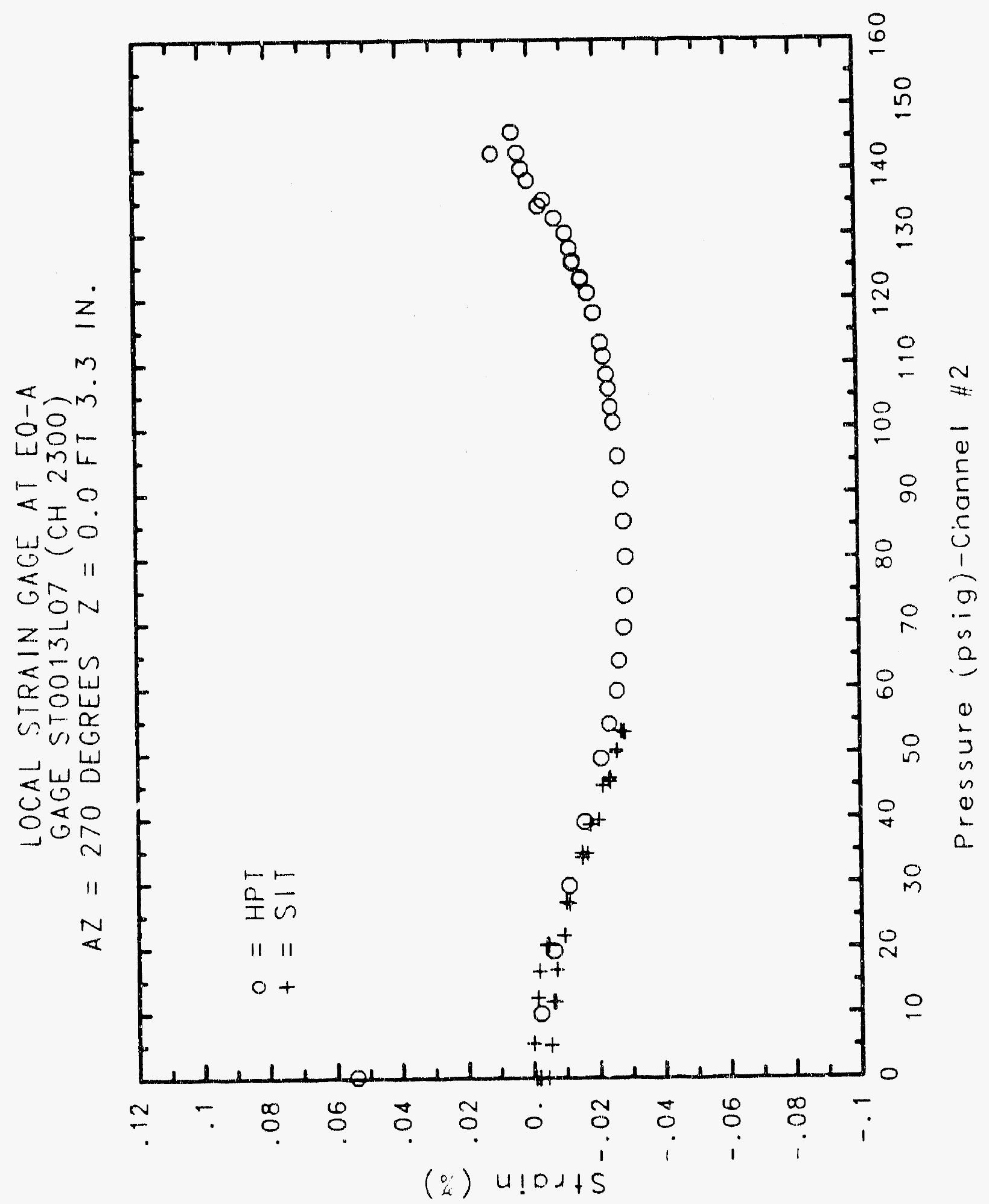




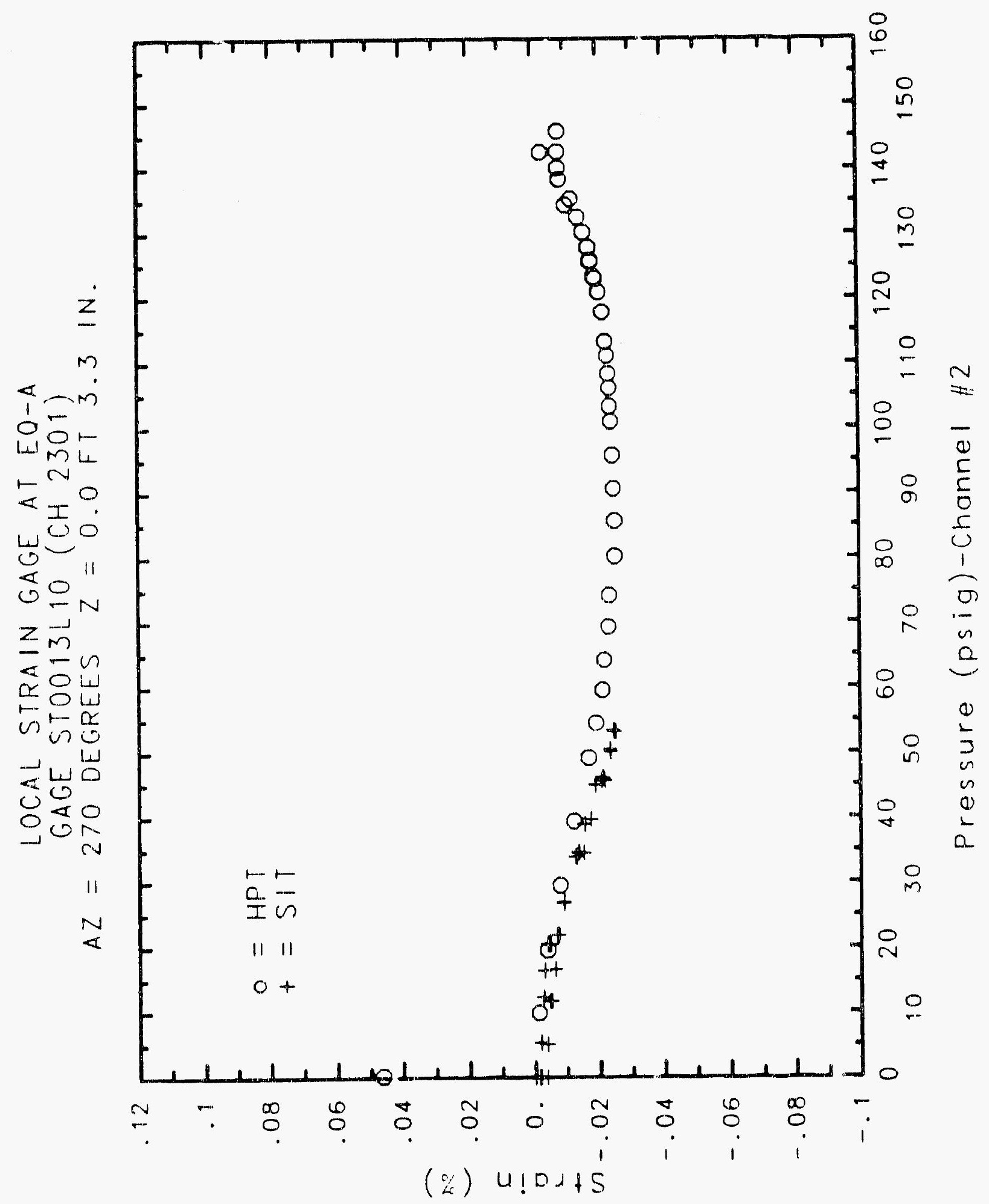




\section{Reinforced Concrete Test Data}

\section{Strip 14 Channels 2364,2365,2366, 2367,2368,}

$2369,2380,2381,2382,2383$

\section{High Pressure Test}

\begin{tabular}{|c|c|c|c|c|c|c|c|c|c|c|}
\hline Pressure & Gage 1 & Gage 2 & Gage 3 & Gage 4 & Goge 5 & Gage 6 & Gage 7 & Gage 8 & Gage 9 & Gagelo \\
\hline Psig & xstrain & XStroin & xstroin & xstrain & Xstrain & xstrain & XStre in & Xstra in & Strain & $\begin{array}{l}\text { xstrain } \\
0.0108\end{array}$ \\
\hline $\begin{array}{r}9.89 \\
19.55\end{array}$ & $\begin{array}{l}0.0188 \\
0.0301\end{array}$ & $\begin{array}{l}0.0167 \\
0.0295\end{array}$ & $\begin{array}{l}0.0155 \\
0.0276\end{array}$ & $\begin{array}{l}0.0531 \\
0.0899\end{array}$ & $\begin{array}{l}0.0133 \\
0.02 .44\end{array}$ & $\begin{array}{l}0.0123 \\
0.0229\end{array}$ & $\begin{array}{l}0.0113 \\
0.0213\end{array}$ & $\begin{array}{l}0.0110 \\
0.0205\end{array}$ & $\begin{array}{l}0.0109 \\
0.0213\end{array}$ & $\begin{array}{l}0.0108 \\
0.0208\end{array}$ \\
\hline 29.57 & 0.0411 & i.0419 & 0.0394 & 0.1109 & 0.0356 & 0.0338 & 0.0323 & 0.0316 & 0.0335 & 0.0308 \\
\hline 39.42 & & & & & & & .0427 & 0.0423 & & \\
\hline 49.16 & 0.0588 & 0.0642 & 0.0609 & 0.1567 & 0.0 .568 & .0549 & 0527 & 0.0521 & 574 & 0474 \\
\hline 54.50 & 0.0640 & 0.0709 & $0.06 \% 2$ & 0.2058 & 0.0635 & .0611 & .0586 & 0.0572 & .0645 & 0.05 .17 \\
\hline 59.57 & 0.0685 & 0.0768 & 0.0729 & 0.2283 & 0.0692 & 0.0669 & 0.0640 & 0.0630 & & 0.0580 \\
\hline 64.20 & 0.0713 & 0.0833 & 0.0789 & 0.2785 & $0.075 ?$ & 0.0733 & 0.0699 & 0.0658 & 787 & 0.0605 \\
\hline 69.32 & 0.0738 & 0.0897 & & & & & & & & \\
\hline 74.16 & 0.0764 & 0.0963 & 0.0913 & 0.45 & 0.0864 & 0.0856 & 0821 & & & 695 \\
\hline $8 C:: 6$ & 0.0776 & 0.0968 & 0.0914 & -2.7574 & 0.0871 & 0.0889 & 0.0863 & 0.0639 & 968 & $0.070 ?$ \\
\hline 8561 & 0.0830 & 0.1035 & 0.0979 & -2.7408 & & & & & & 0.0750 \\
\hline 90.58 & 0.0871 & & & -3.21 & & & & & & \\
\hline 9569 & 0.09 & & & & & & & & & 840 \\
\hline 102.92 & 0.0993 & 0.1249 & 0.1187 & -2.3605 & 0.1127 & 148 & 33 & & & 0905 \\
\hline 103.25 & 0.1021 & 0.1286 & 0.1223 & -2.3252 & 0.1159 & 0.1181 & 0.1170 & 0.0348 & 0.1285 & $0.09:=$ \\
\hline 16811 & 0.1049 & 0.1319 & 0.1253 & -3.07 & & & & & & 9950 \\
\hline $100.3 !$ & 0.1082 & 0.13 & & -2 . & & & & & & \\
\hline 111.08 & 0.1 & & & -2 & & & & & & 017 \\
\hline 113.24 & 0.1150 & 0.1448 & 0.1374 & -3.2167 & 0.1292 & 0.1323 & 0.1 & 0.1092 & & 037 \\
\hline 217.83 & 0.1243 & 0.155 .9 & 0.1483 & -1.8503 & 0.1389 & 0.1420 & 0.1411 & 0.1179 & 0.1536 & 0.1112 \\
\hline :20.92 & 0.1285 & 0.1618 & 0.1539 & -1.9952 & 0.1436 & 1469 & 0.1459 & 227 & 587 & 1146 \\
\hline 123.28 & 0.13 & & 0.1581 & -2.5 & & & & & & 174 \\
\hline 97 & & & & & & & & & & \\
\hline 125.82 & 0.1374 & 0.1727 & 0.1634 & -4.5083 & 0.1522 & 0.1 & 0.1 & 294 & & .1211 \\
\hline 125.60 & 0.1437 & 0.1781 & 0.1691 & -2.1107 & 0.1575 & 0.1599 & 0.1583 & 0.1313 & 0.1699 & 0.1234 \\
\hline 12784 & 0.1488 & 0.1859 & 0.1772 & -0.6156 & 0.1645 & 0.1667 & 0.1641 & 01362 & 0.1753 & 0.1278 \\
\hline 130.19 & 0.1583 & 0.1978 & 0.1883 & 0.0872 & & 0.1757 & 0.1729 & & & 0.1386 \\
\hline .53 & & & & & & & & & & \\
\hline 135.33 & 0.2019 & 0.2495 & 0.2303 & 0.3732 & & & & 794 & & 0.1649 \\
\hline 134.42 & 0.2458 & 0.2597 & 0.2451 & 0.4124 & 0.2223 & 0.2218 & 0.2166 & 0.1814 & 0.2255 & 0.1686 \\
\hline 138.35 & 0.3168 & 0.3434 & 0.3349 & 0.5345 & 0.3438 & 0.3561 & 0.3376 & 0.3733 & 0.3641 & 0.2877 \\
\hline 140.16 & 0.4304 & 0.5134 & 0.5240 & 0.7569 & 0.5904 & 0.6111 & 0.5896 & 0.6200 & 0.6062 & 0.4887 \\
\hline 142.63 & 0.6583 & 0.7689 & 0.7836 & & & & & & & 0.7237 \\
\hline & 0.8024 & 0.9293 & 0.9337 & 1.0355 & & & & 1.0337 & & 0.8477 \\
\hline 142.52 & 0.7858 & & & & & & & & & 0.8152 \\
\hline 0.22 & 0.4235 & 0.5133 & 0.4989 & & 0.4911 & 0.5268 & & 0.6541 & 0.6351 & 0.5466 \\
\hline
\end{tabular}




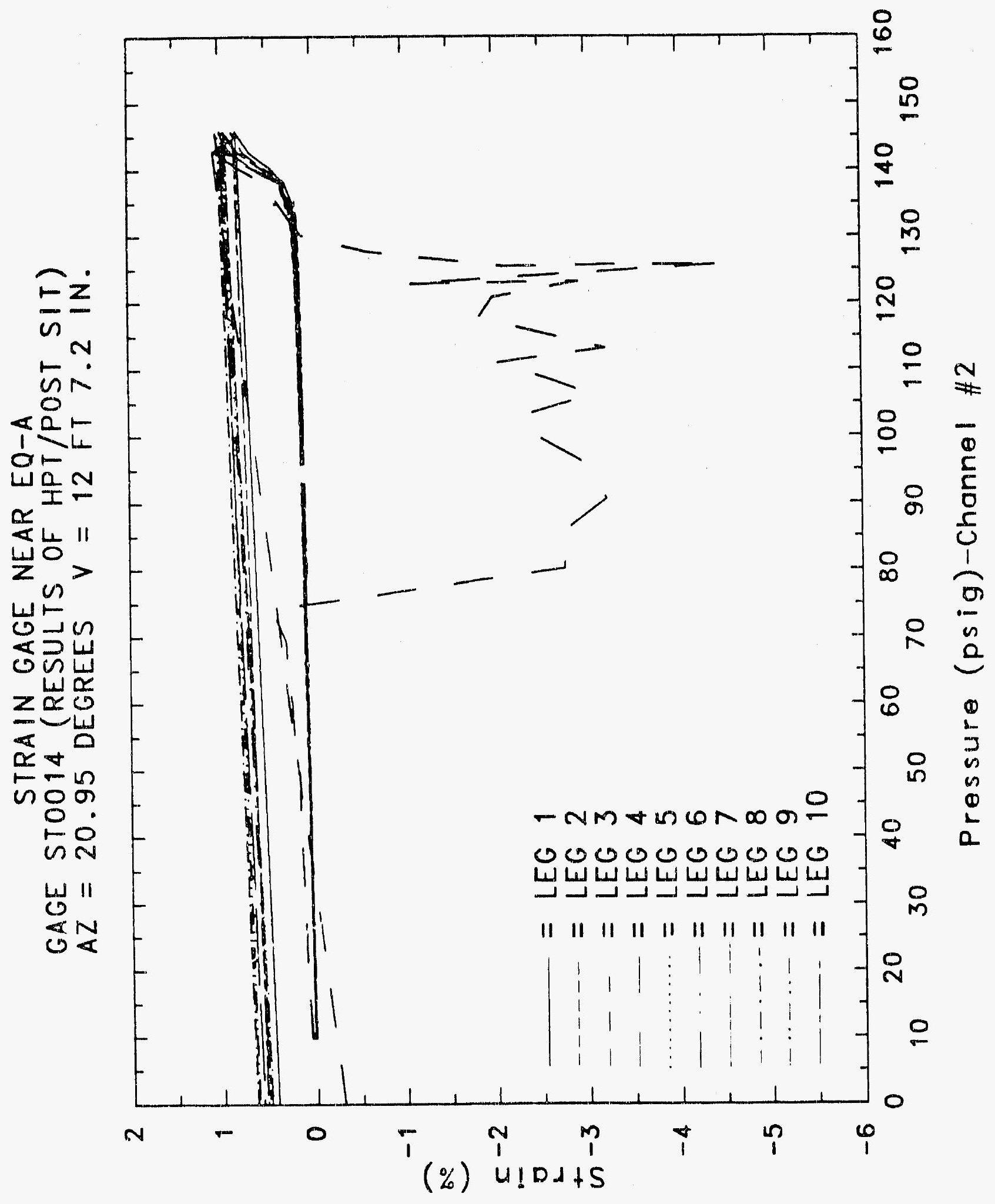

C. 1509 


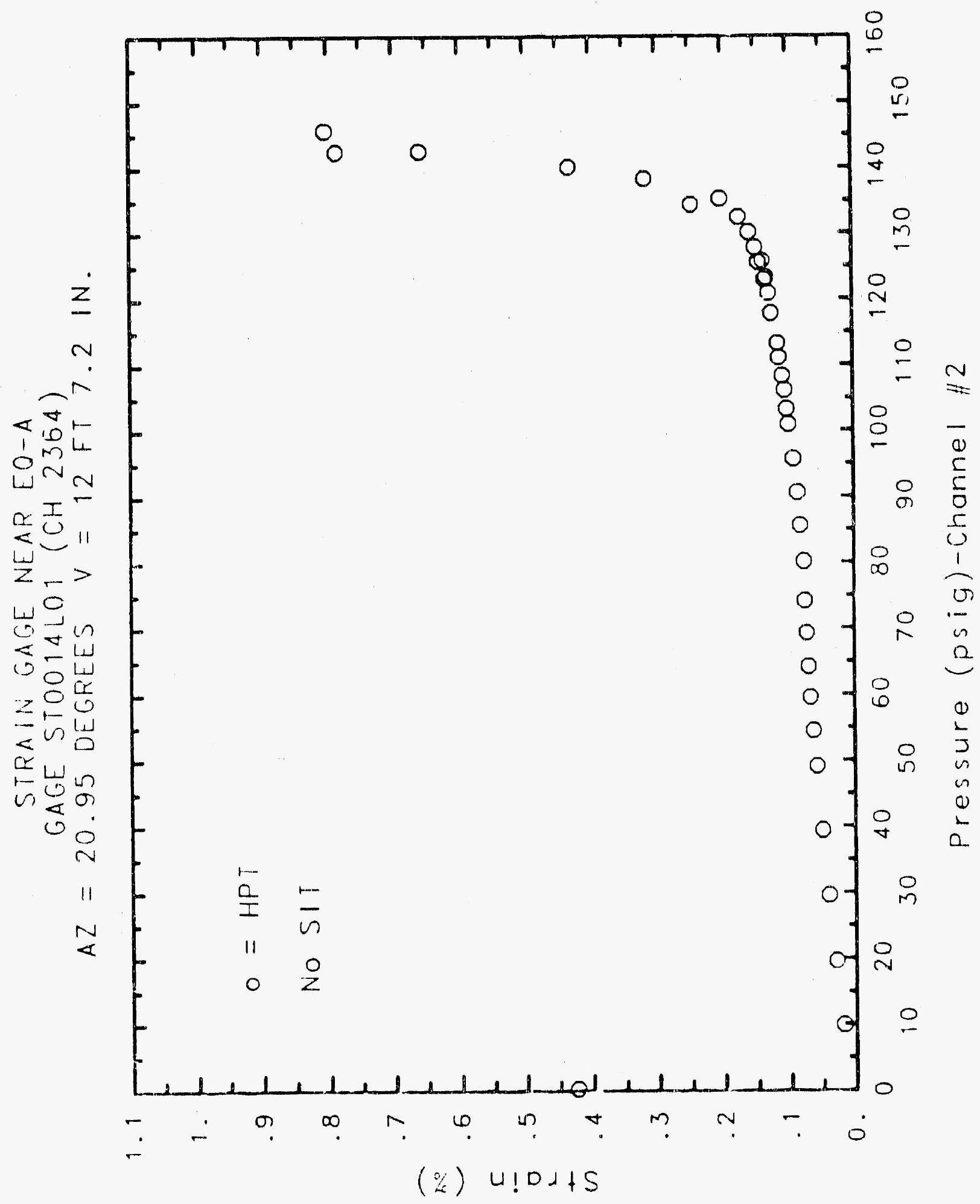




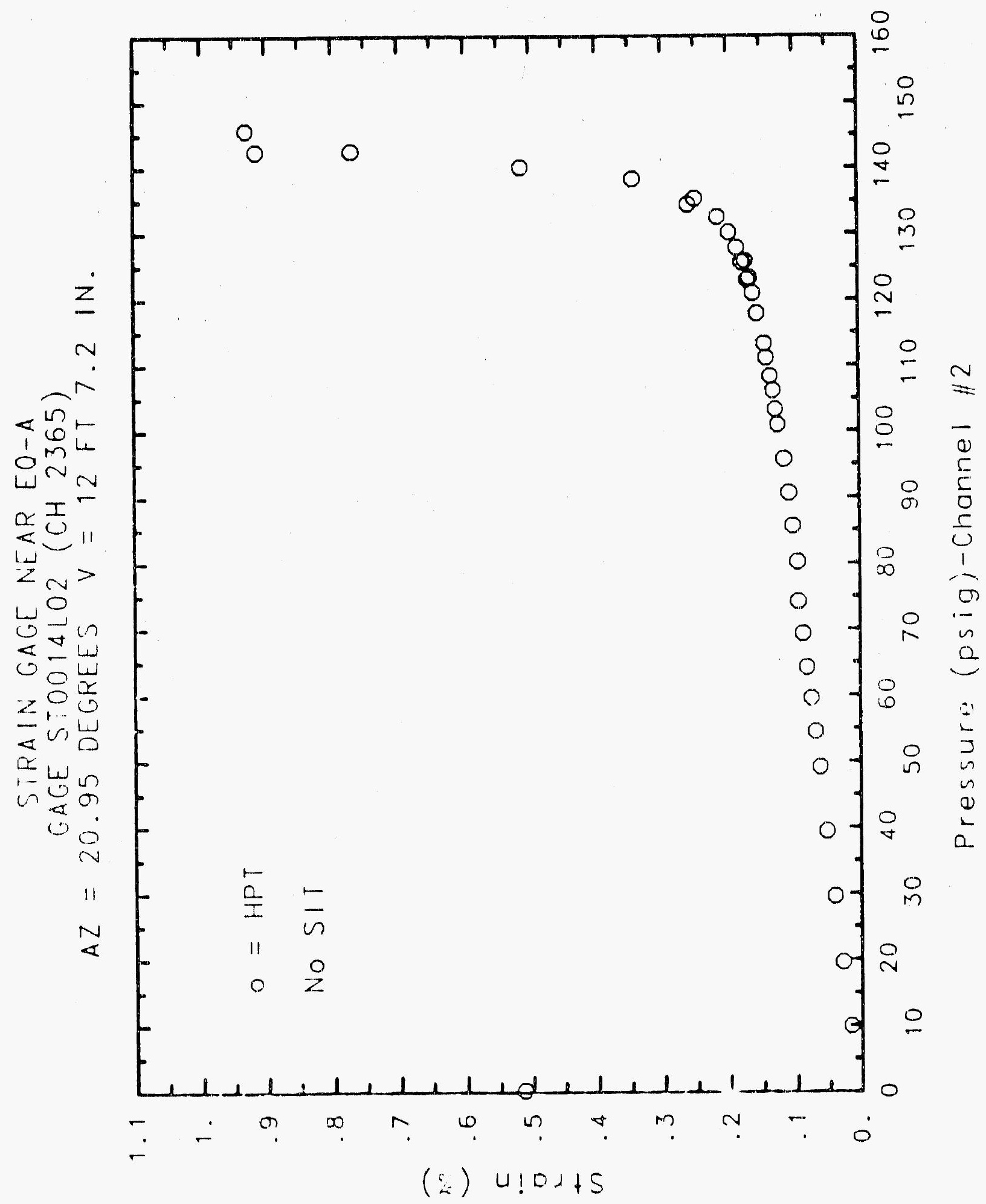

C. 1511 


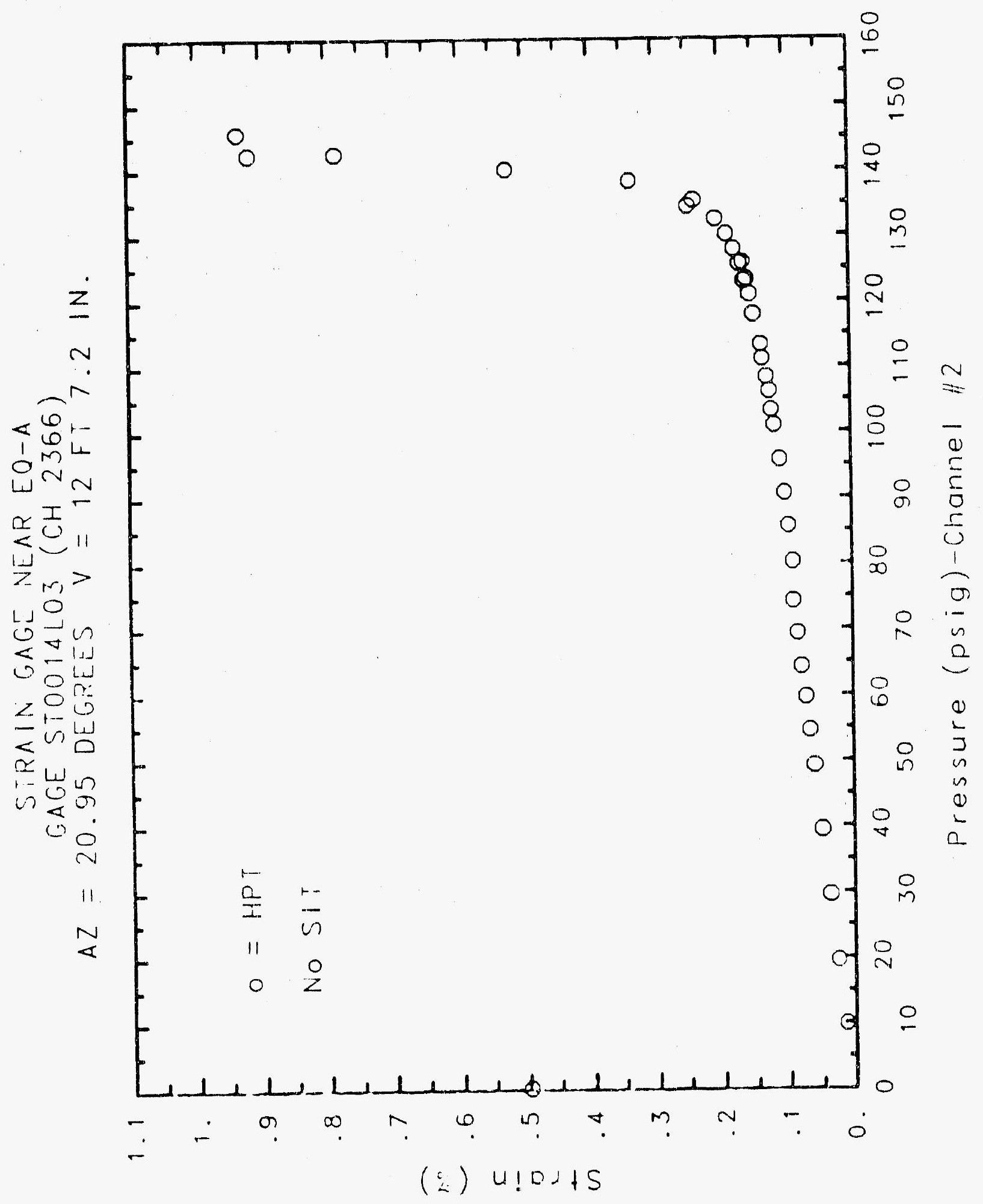




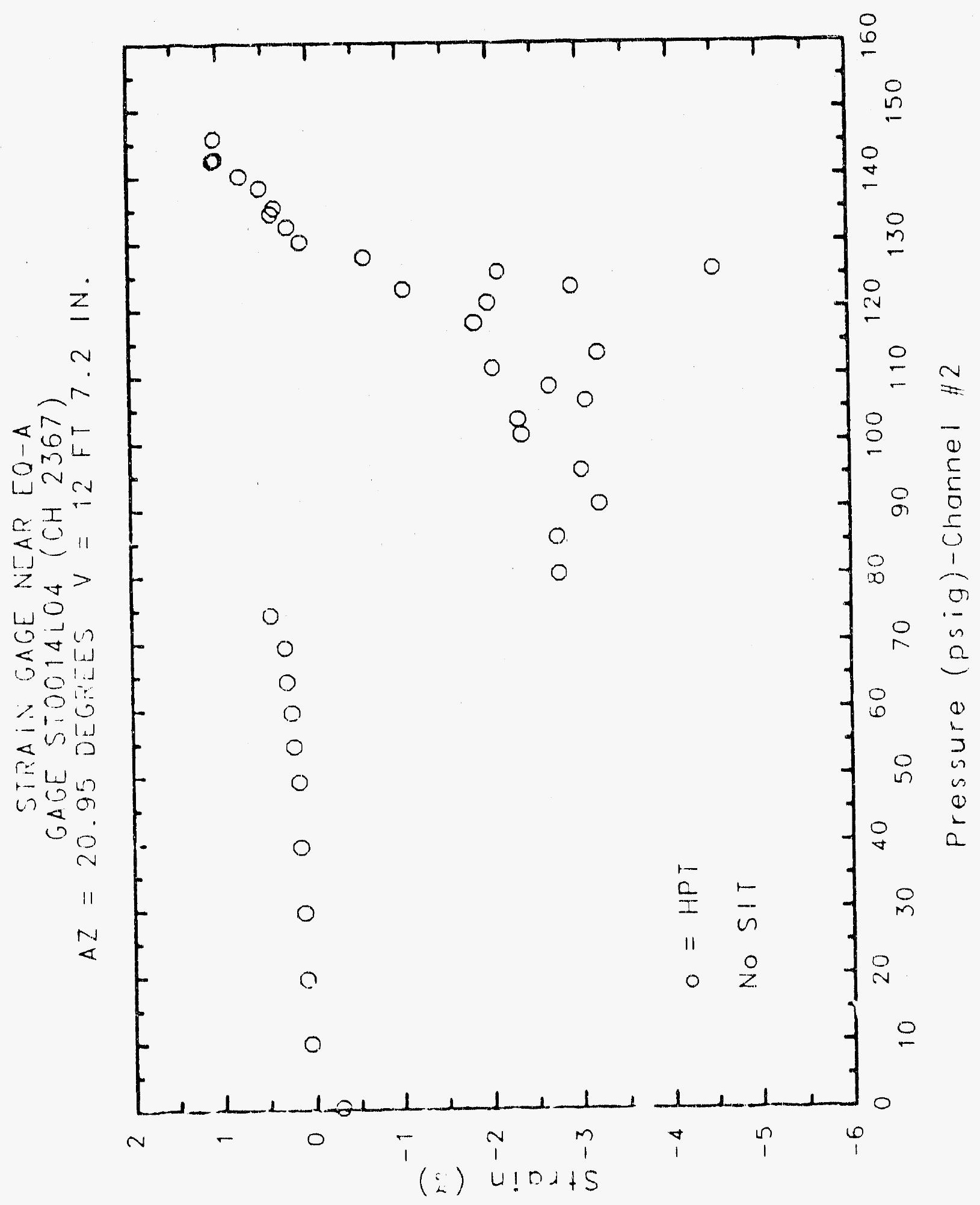

C-15.3 


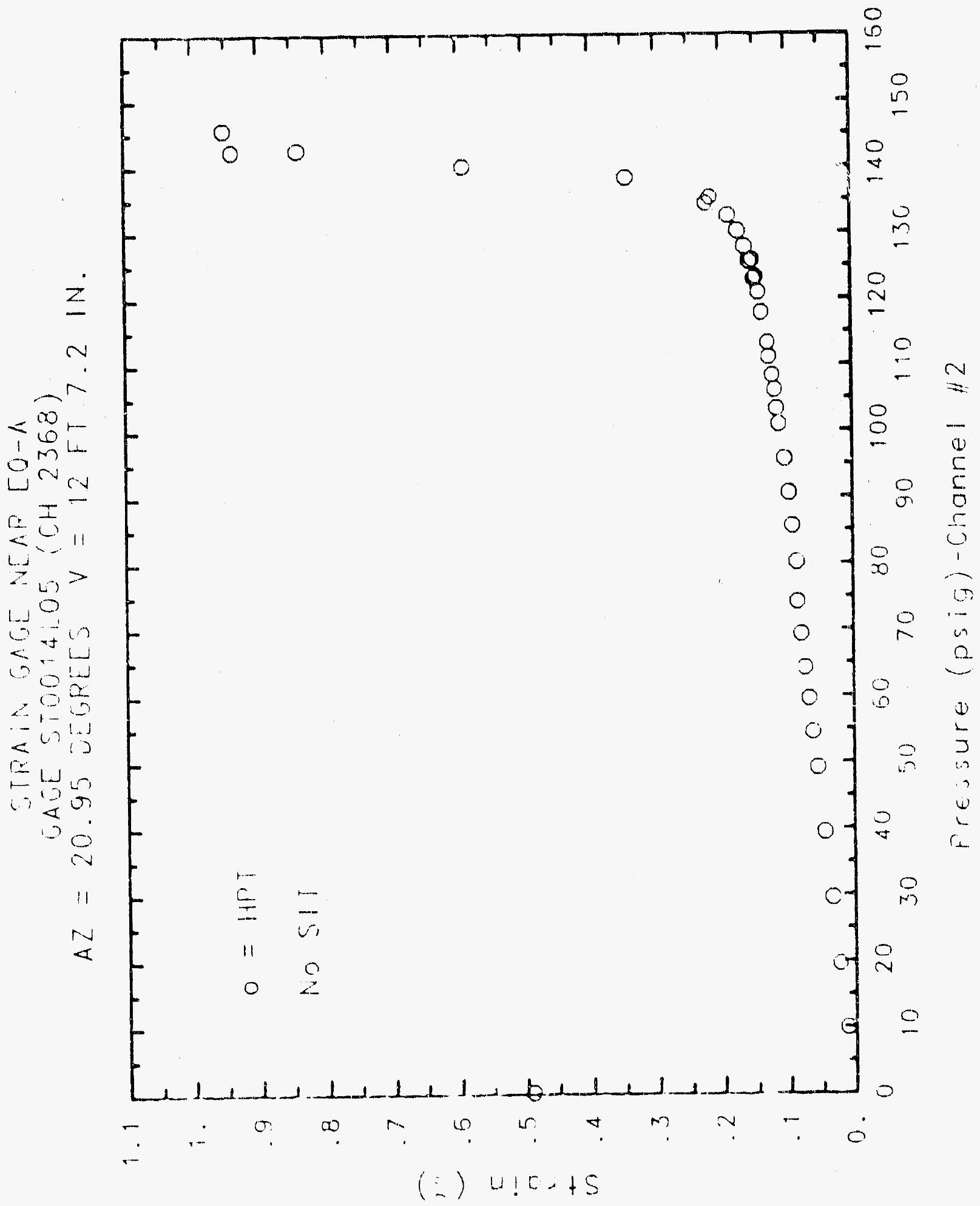

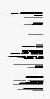

$C-151+$

$\bar{E}$ 


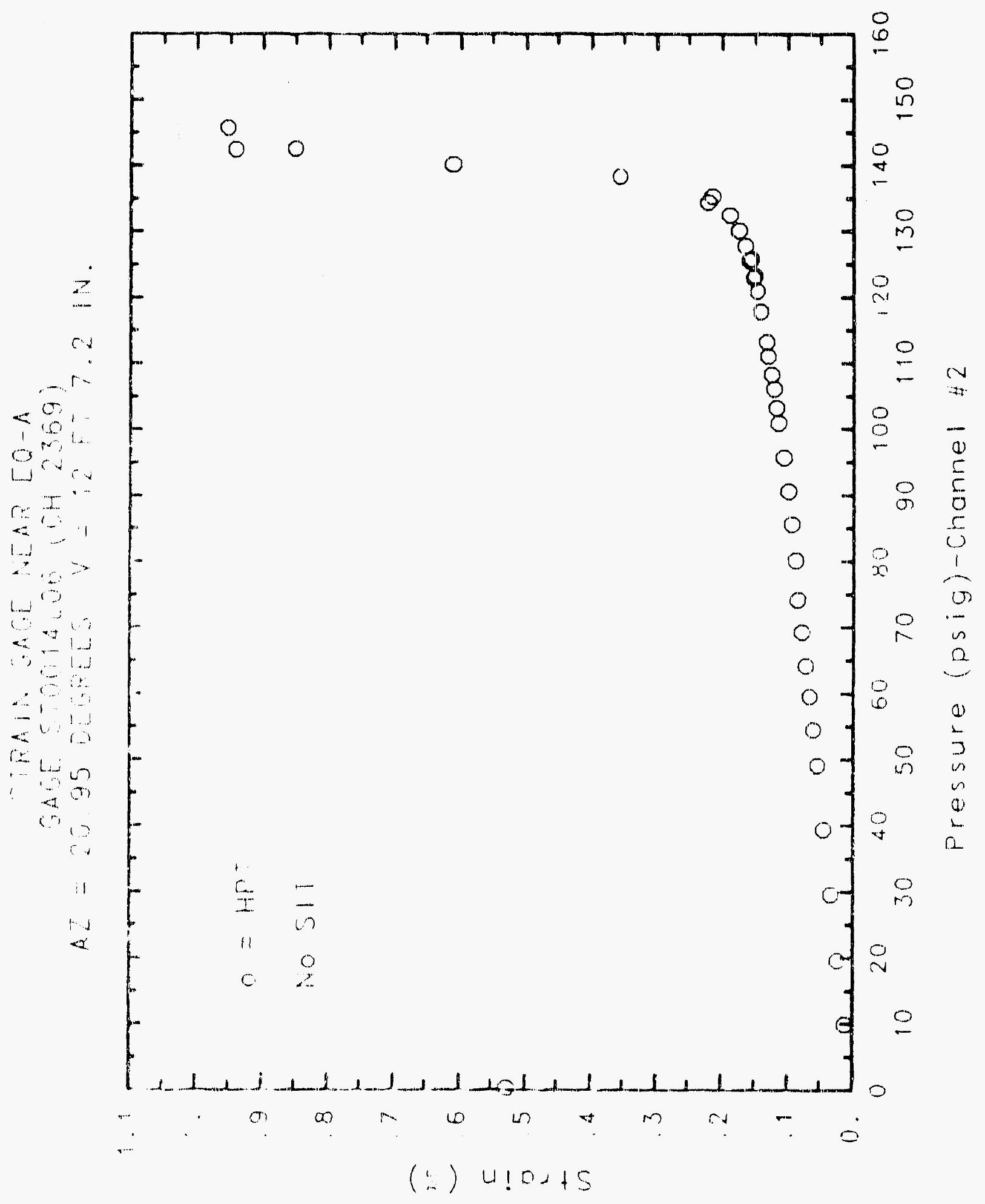

C.1515 


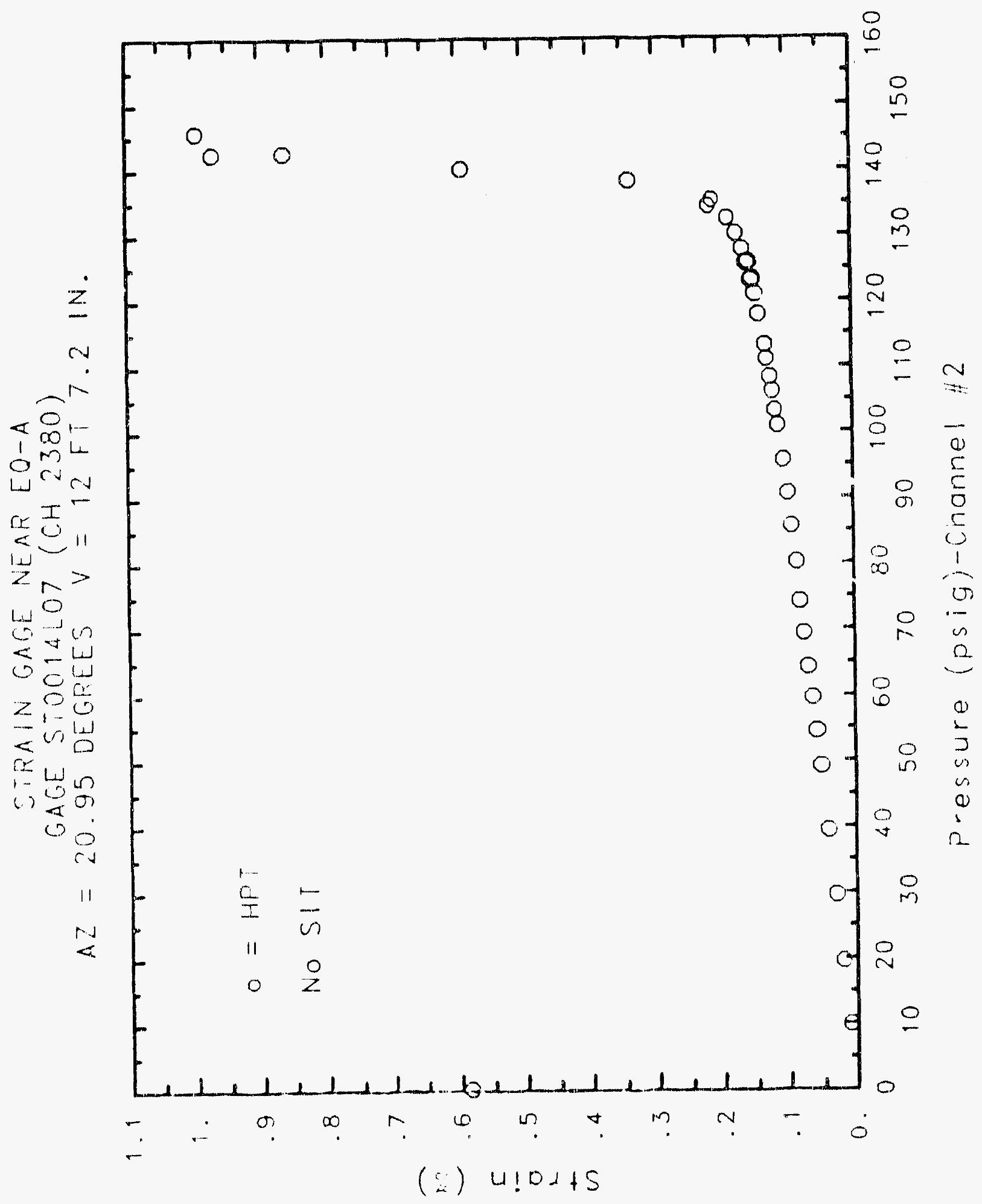

C. -1516 


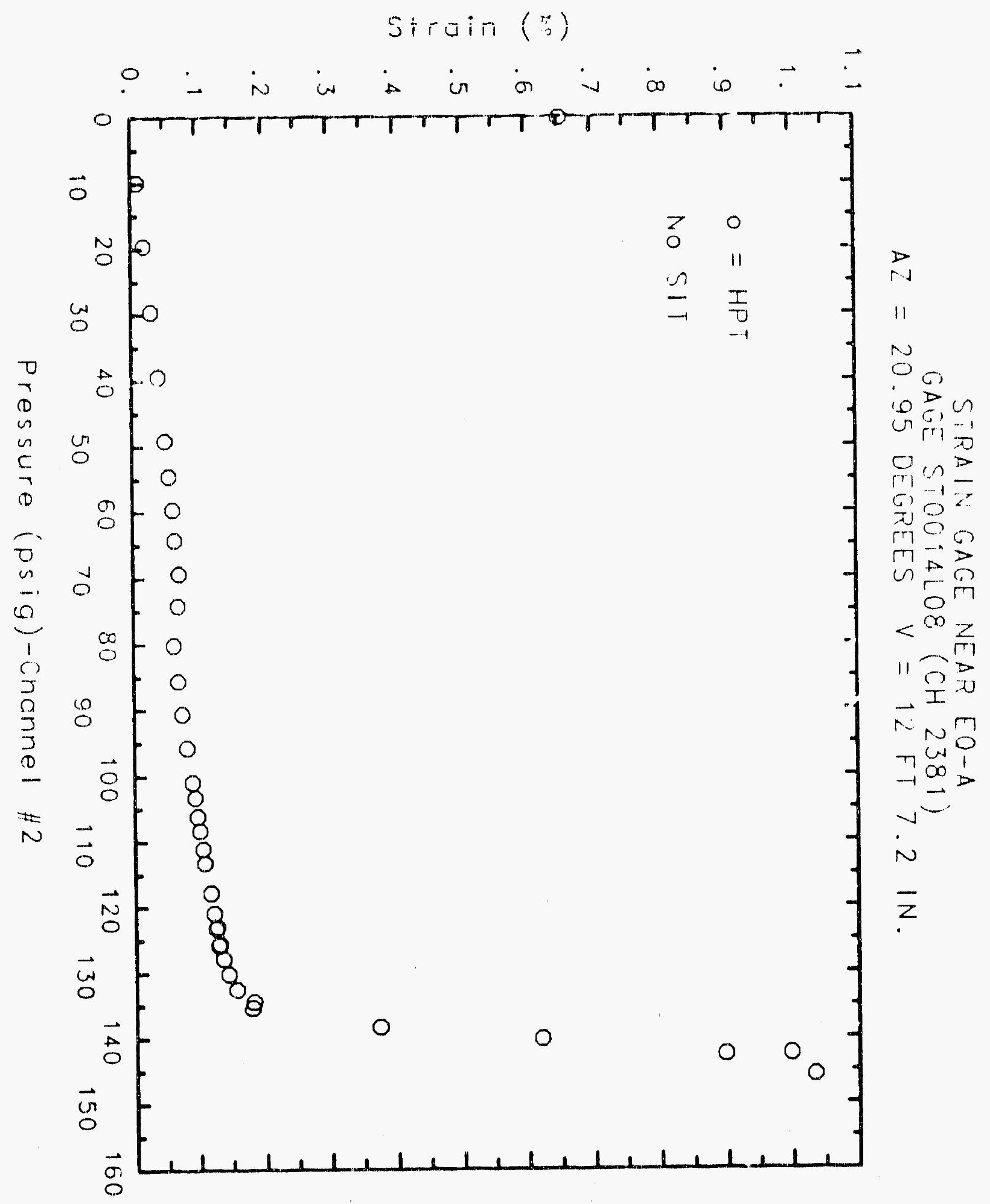




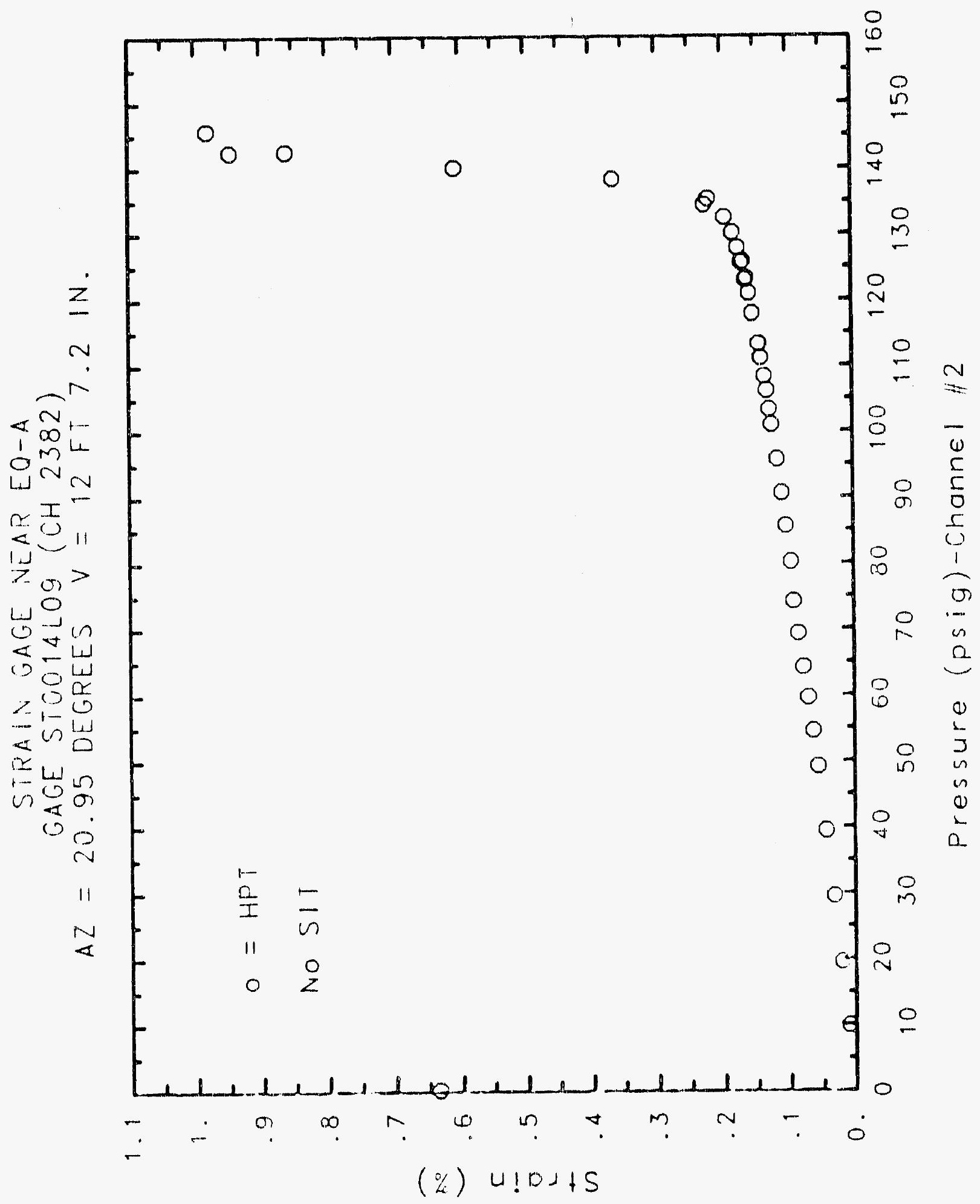

C. -1518 


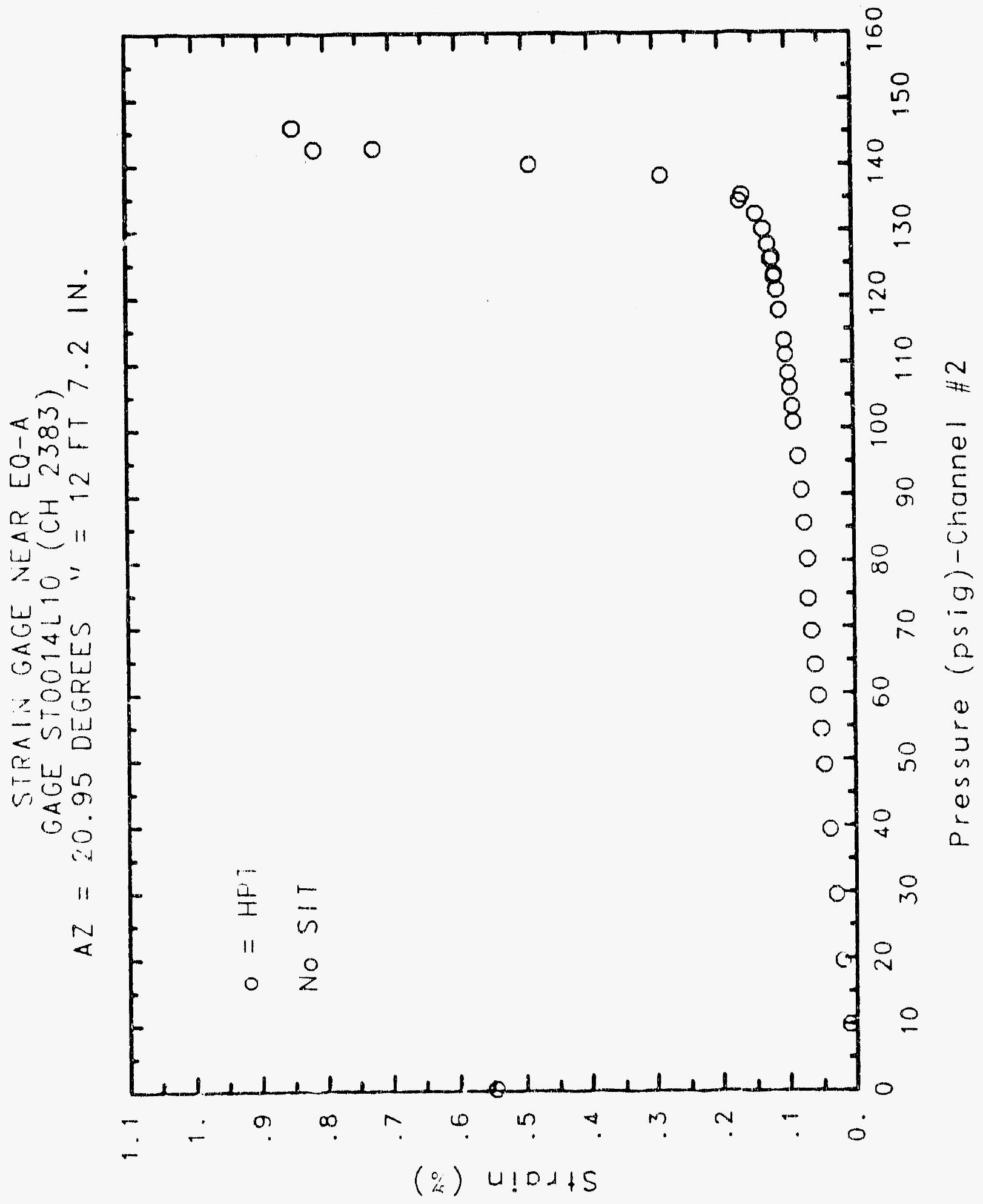

C. 1519 


\section{Reinforced Concrete Test Data}

Strip 15 Channels $\begin{array}{r}2384,2385,2386,2387,2388, \\ 2389,2400,2401,2402,2403\end{array}$

\section{High Pressure test}

\begin{tabular}{|c|c|c|c|c|c|c|c|c|c|c|}
\hline $\begin{array}{c}\text { Pressure } \\
\text { Psig }\end{array}$ & $\begin{array}{l}\text { Gage } 1 \\
\text { XStra in }\end{array}$ & $\begin{array}{l}\text { Gage } 2 \\
\text { xStrain }\end{array}$ & $\begin{array}{l}\text { Gage } 3 \\
\text { xstrain }\end{array}$ & $\begin{array}{l}\text { Gage } \\
\text { xstrain }\end{array}$ & $\begin{array}{l}\text { Gage } 5 \\
\text { Xstra in }\end{array}$ & $\begin{array}{l}\text { Gage } 6 \\
\text { xstrain }\end{array}$ & $\begin{array}{l}\text { Gage } 7 \\
\text { XStrain }\end{array}$ & $\begin{array}{l}\text { Gage } 8 \\
\text { xstrain }\end{array}$ & $\begin{array}{l}\text { Gage } 9 \\
\text { XStrain }\end{array}$ & $\begin{array}{l}\text { Gagelo } \\
\text { Xstrain }\end{array}$ \\
\hline 9.89 & 0.0109 & 0.0109 & 0.0097 & 0.0119 & 0.0105 & 0.0110 & 0.0120 & 0.0116 & 0.0101 & 0.0098 \\
\hline 19.55 & 0.0195 & 0.0222 & 0.0188 & 0.0235 & 0.0203 & $0.02 n 1$ & 0.0224 & 0.0215 & 0.0197 & 0.0184 \\
\hline $2 y .57$ & 0.0280 & 0.0292 & 0.0285 & 0.0336 & 0.0302 & 0.0310 & 0.0326 & 0.0315 & 0.0293 & 0.0261 \\
\hline 39.46 & 0.0357 & 0.0380 & 0.0377 & 0.0437 & 0.0402 & 0.0412 & 0.0417 & 0.0404 & 0.0373 & 0.0332 \\
\hline 49.16 & 0.0435 & 0.0466 & 0.0471 & 0.0536 & 0.0497 & 0.0514 & 0.0510 & 0.0500 & 0.0461 & 0.0407 \\
\hline 54.50 & $0.054 ?$ & 0.0526 & 0.0528 & 0.0613 & 0.0555 & 0.0571 & 0.0565 & 0.0552 & 0.0521 & 0.0451 \\
\hline 53.57 & 0.0519 & 0.0575 & 0.0582 & 0.0714 & 0.0614 & 0.0641 & 0.0632 & 0.0617 & 0.0569 & 0.0495 \\
\hline 64.20 & 0.0566 & 0.0633 & 0.0634 & 0.0730 & 0.0666 & 0.0701 & 0.0692 & 0.0686 & 0.0623 & 0.0544 \\
\hline 69.32 & 0.0602 & 0.0682 & 0.0703 & 0.0791 & 0.0727 & 0.0771 & 0.0773 & 0.0752 & 0.0701 & 0.0594 \\
\hline 74.16 & 0.0717 & 0.0809 & 0.0194 & 0.0763 & 0.0755 & 0.0840 & 0.0850 & 0.0819 & 0.0768 & 0.0656 \\
\hline 80.16 & 0.0750 & 0.0843 & 0.0885 & 0.0897 & 0.0842 & 0.0907 & 0.0934 & 0.0874 & 0.0811 & 0.0695 \\
\hline 85.61 & 0.0814 & 0.0916 & 0.0974 & 0.0968 & 0.0907 & 0.1004 & 0.1034 & 0.0856 & 0.0905 & 0.0763 \\
\hline 90.58 & 0.0877 & 0.0983 & 0.1061 & 0.103 & 0.0970 & 0.1082 & 0.1117 & 0.1032 & 0.0980 & 0.0821 \\
\hline 95.69 & 0.0929 & 0.1047 & 0.1162 & 0.1097 & 0.1037 & 0.1169 & 0.1187 & 0.1115 & 0.1065 & 0.0891 \\
\hline 100.92 & 0.0976 & 0.1104 & 0.1254 & 0.1148 & 0.1130 & 0.1327 & 0.1242 & 0.1193 & 0.1166 & 0.0945 \\
\hline 103.25 & 0.1001 & 0.1130 & 0.1293 & 0.1173 & 0.1188 & 0.1373 & 0.1270 & 0.1219 & 0.1216 & 0.0964 \\
\hline 106.11 & 0.1028 & 0.1170 & 0.1372 & 0.1189 & 0.1245 & 0.1448 & 0.1291 & 0.1246 & 0.1264 & 0.0985 \\
\hline 108.31 & 0.1049 & 0.1192 & 0.1409 & 0.1202 & 0.1310 & 0.1485 & 0.1314 & 0.1275 & 0.1300 & 0.1013 \\
\hline 111.08 & 0.1067 & 0.1227 & 0.1467 & 0.1223 & 0.1354 & 0.1534 & 0.1353 & 0.1307 & 0.1354 & 0.1080 \\
\hline 113.24 & 0.1079 & 0.1283 & 0.1509 & 0.1233 & 0.1395 & 0.1572 & 0.1373 & 0.1329 & 0.1382 & 0.1126 \\
\hline 117.83 & 0.1099 & 0.1332 & 0.1554 & 0.1222 & 0.1438 & 0.1644 & 0.1414 & 0.1364 & 0.1423 & 0.1136 \\
\hline 120.92 & 0.1115 & 0.1351 & 0.1580 & 0.1249 & 0.1465 & 0.1670 & 0.1434 & 0.1387 & 0.1452 & 0.1222 \\
\hline 123.28 & $0.117 j$ & 0.1382 & 0.1603 & 0.1259 & 0.1480 & 0.1690 & 0.1461 & 0.1402 & 0.1479 & 0.1234 \\
\hline 122.97 & 0.1157 & 0.1384 & 0.1604 & 0.1258 & 0.1481 & 0.1691 & 0.1467 & 0.1409 & 0.1478 & 0.1234 \\
\hline $125.8 ?$ & 0.1185 & 0.1410 & 0.1620 & 0.1255 & 0.1534 & 0.1707 & 0.1470 & 01412 & 0.1485 & 0.1229 \\
\hline 125.60 & 0.1197 & 0.1415 & 0.1616 & 0.1270 & 0.1519 & 0.1699 & 0.1482 & 0.1423 & 0.1490 & 0.1233 \\
\hline 127.84 & 0.1222 & 0.1 .437 & 0.1625 & 0.1290 & 0.1527 & 0.1708 & 0.1500 & 0.1449 & 0.1513 & 0.1243 \\
\hline 130.19 & 0.1251 & 0.1459 & 0.1659 & 0.1312 & 0.1600 & 0.1761 & 0.1573 & 0.1513 & 0.1592 & 0.1294 \\
\hline 132.53 & 0.1357 & 0.1530 & 0.1739 & 0.1362 & 0.1770 & 0.1894 & 0.1724 & 0.1734 & 0.1982 & 0.1573 \\
\hline 135.33 & 0.1802 & 0.1675 & 0.1840 & 0.1411 & 0.2129 & 0.2720 & 0.2535 & 0.4080 & 0.6729 & 0.4981 \\
\hline 134.42 & 0.1874 & 0.1721 & 0.1860 & 0.1312 & 0.2163 & 0.2800 & 0.2917 & 0.5116 & 0.7880 & 0.5896 \\
\hline 138.35 & 0.4068 & 0.2446 & 0.2094 & 0.1393 & 0.2425 & 0.3385 & 0.6274 & 0.9385 & 1.2605 & 0.9401 \\
\hline 140.16 & 1.0897 & 0.6107 & 0.2773 & 0.1633 & 0.3453 & 0.4942 & 0.8676 & 1.0887 & 1.3403 & 1.0388 \\
\hline 142.63 & j. 3125 & 1.3496 & 0.8429 & 0.4815 & 0.7665 & 0.8562 & 1.0780 & 1.1653 & 1.3937 & 1.1173 \\
\hline 145.78 & 1.3592 & 1.4402 & 1.2916 & 0.9824 & 1.0421 & 1.1876 & 1. 2258 & 1.2386 & 1.4535 & 1.2270 \\
\hline 142.52 & 1.3553 & 1.4314 & 1.3069 & 1.0199 & 1.0538 & 1.1958 & 1.2310 & 1.2361 & 1.4508 & 1.2327 \\
\hline 0.22 & 1.0891 & 1.1790 & 1.0932 & 0.8509 & 0.9137 & 1.0738 & 1.1401 & 1.1655 & 1.3837 & 1.1843 \\
\hline
\end{tabular}




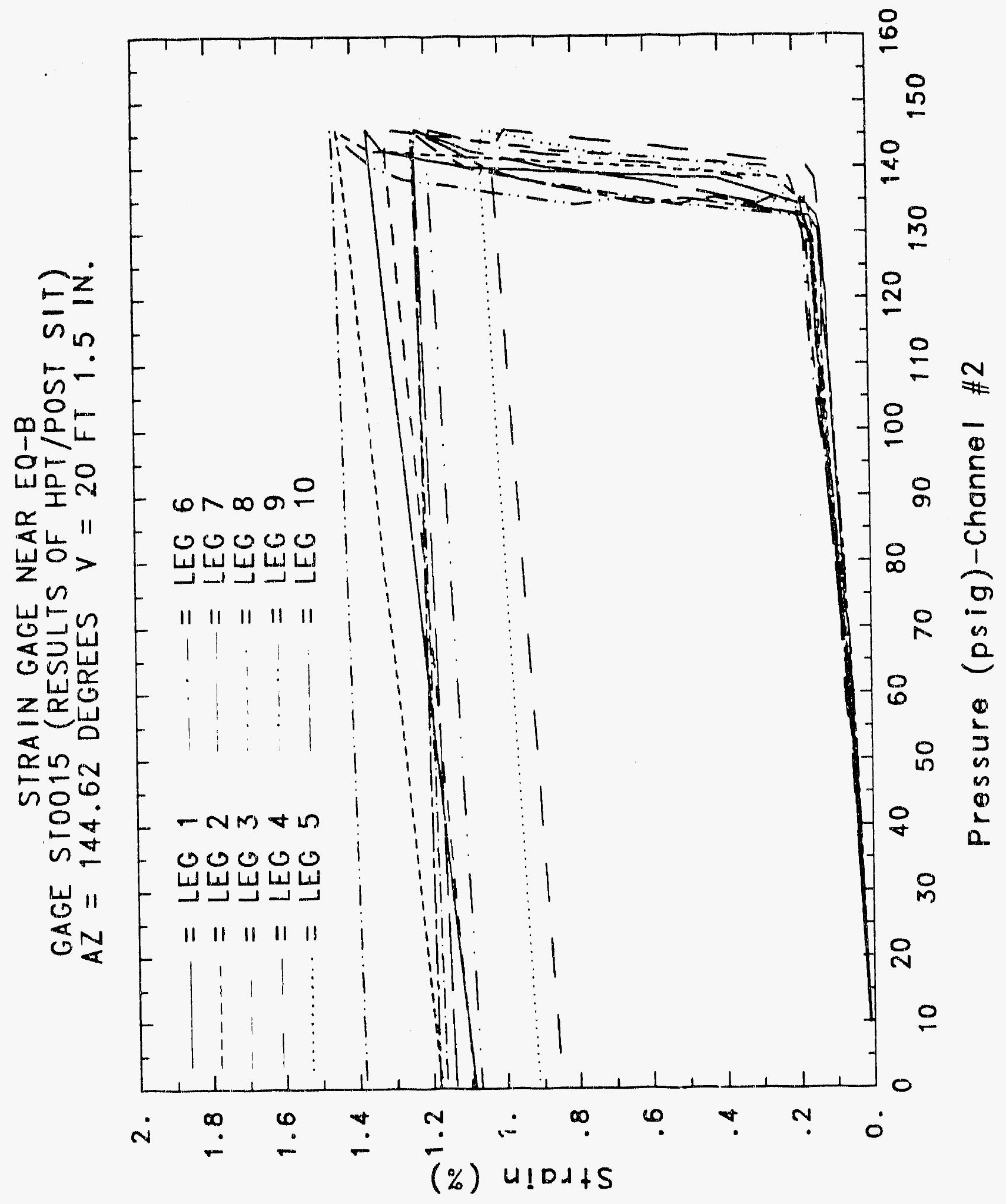




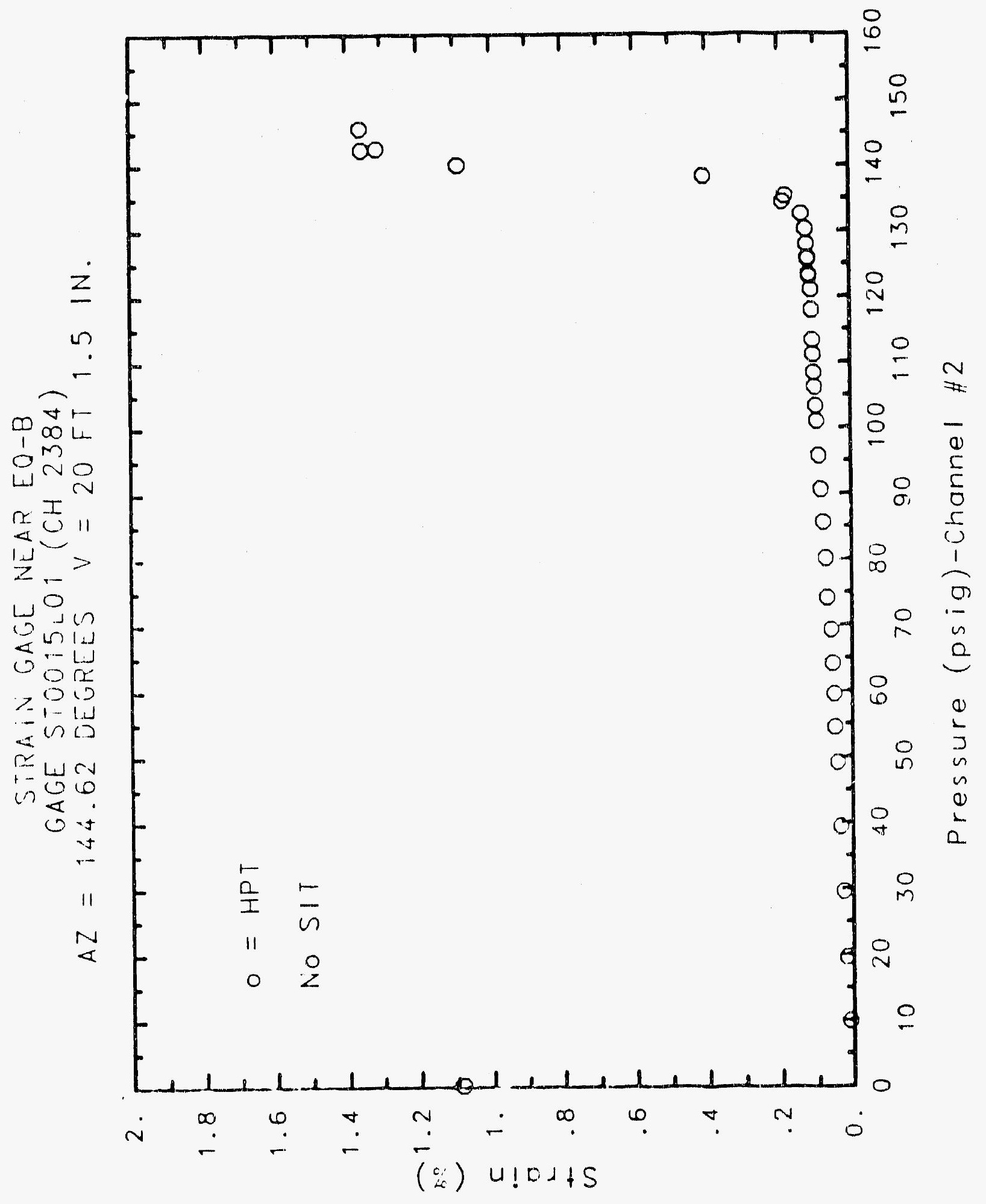

C-1522 


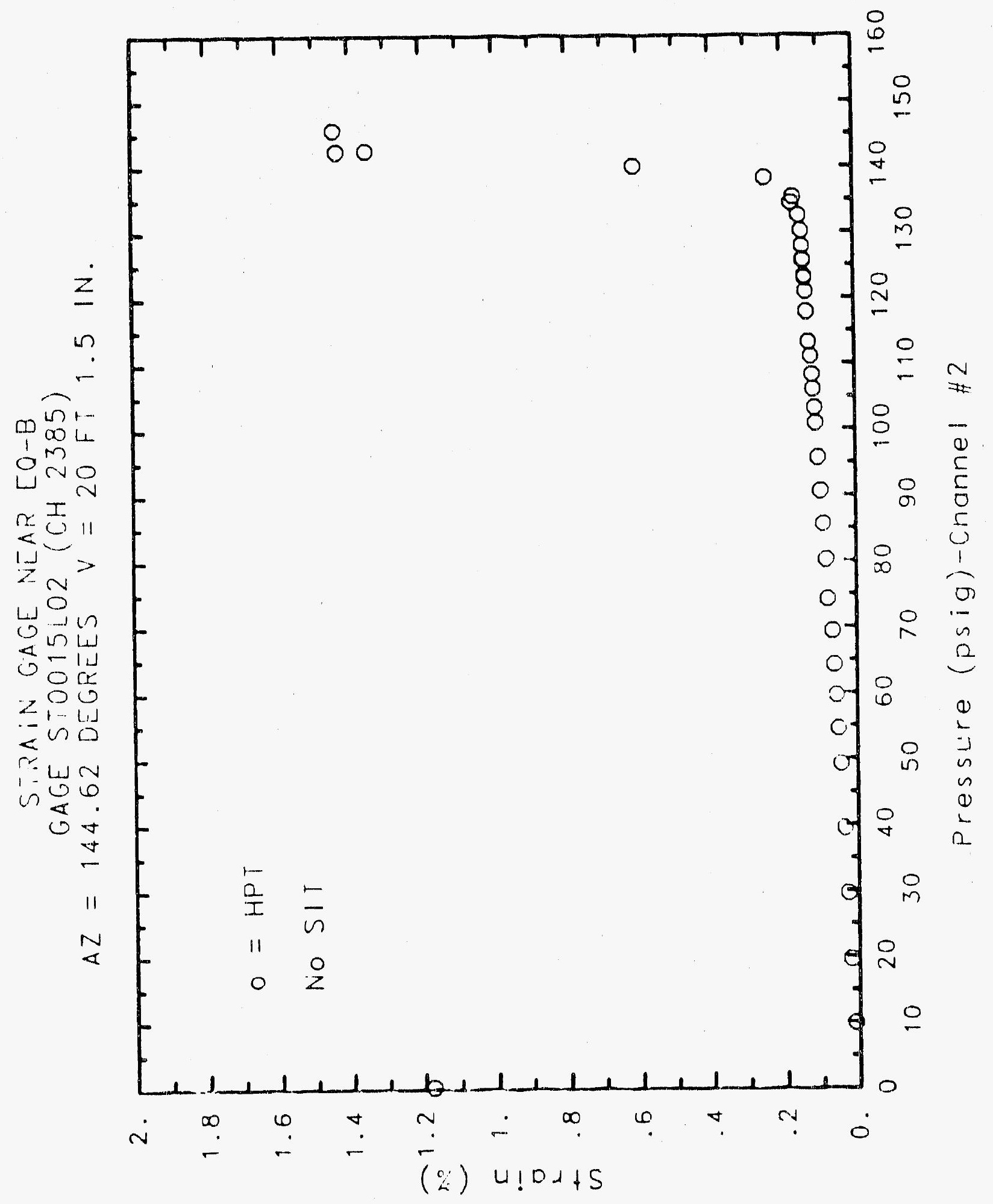

C- 1523 


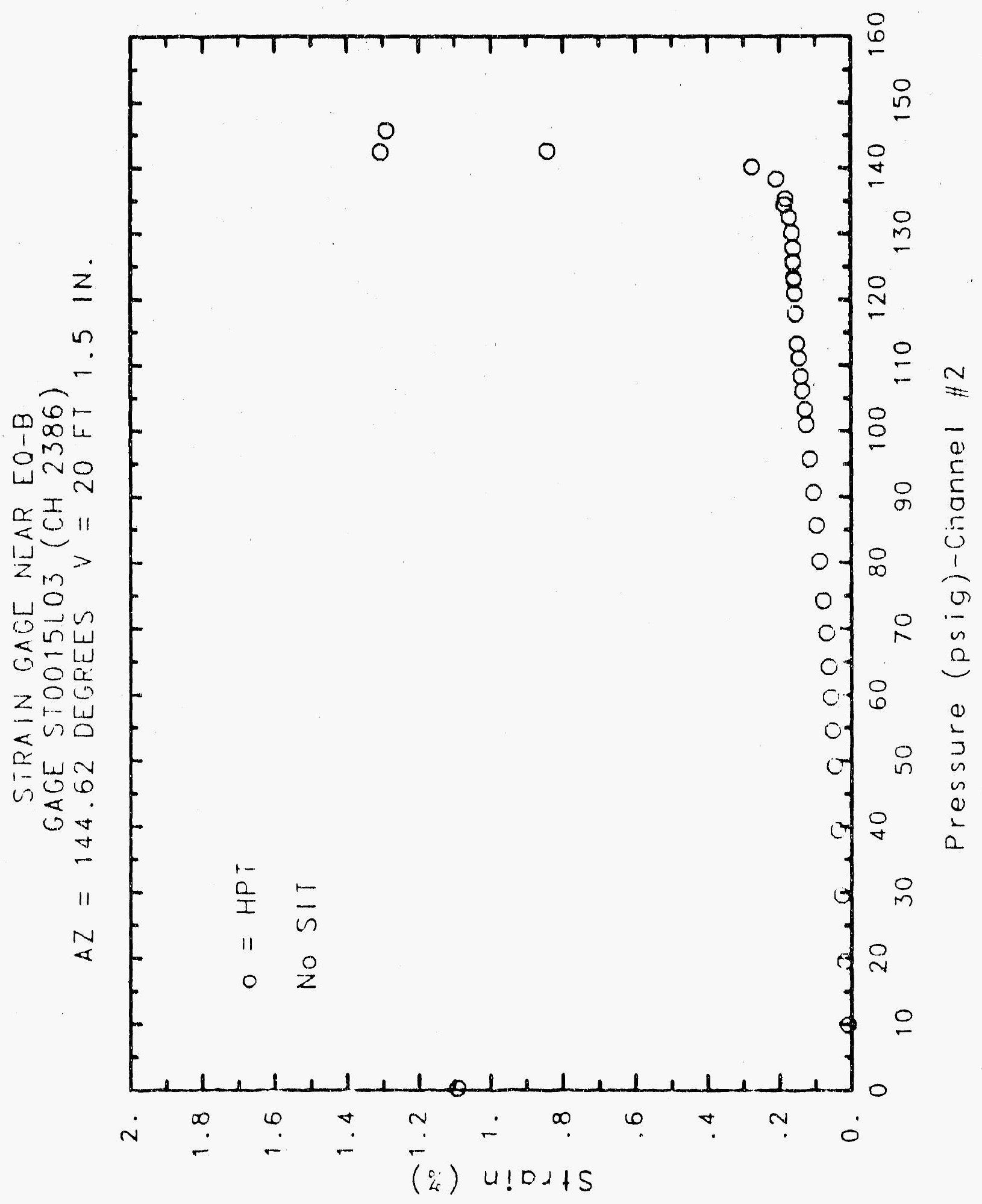

C-1524 


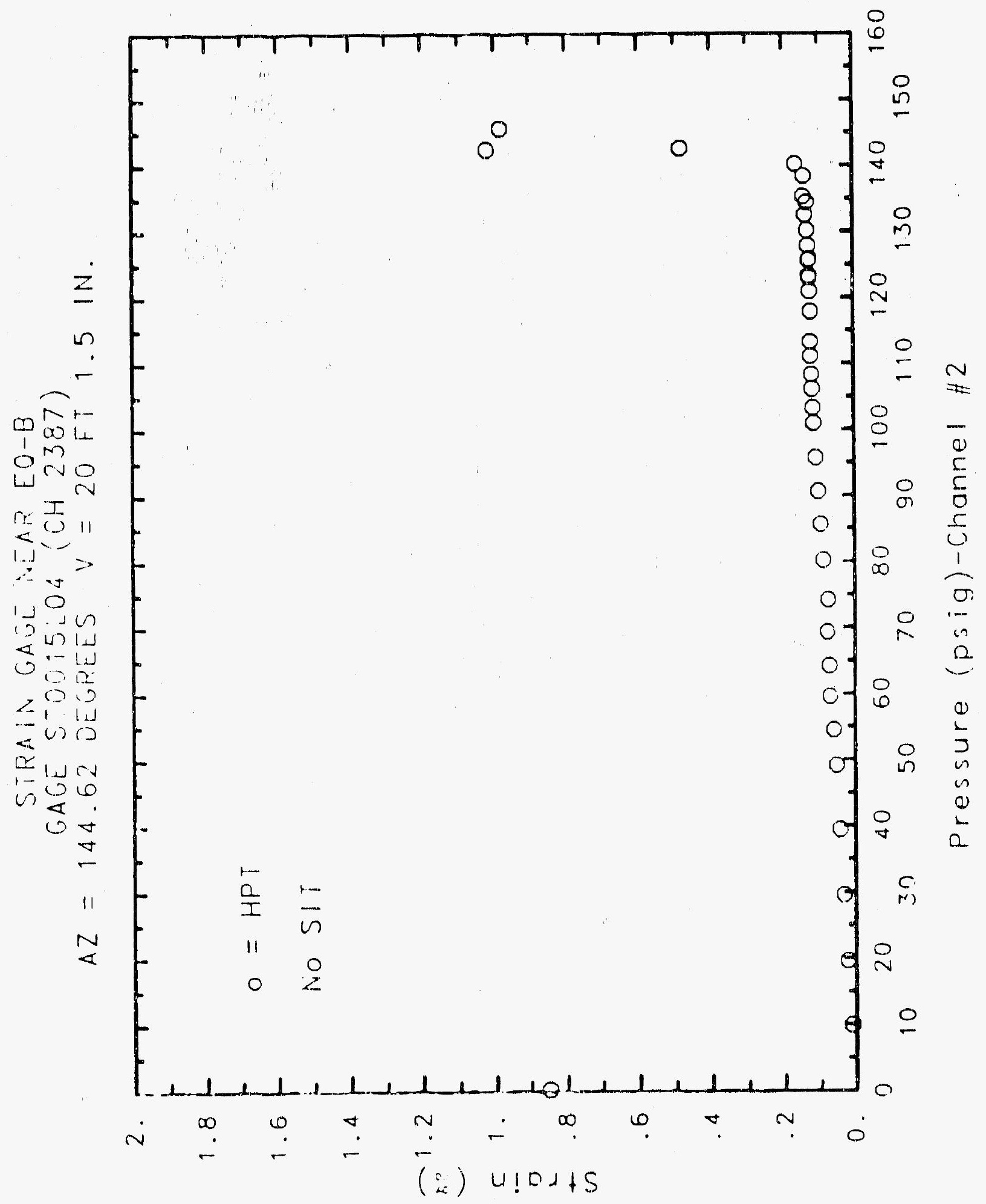

C- 1525 


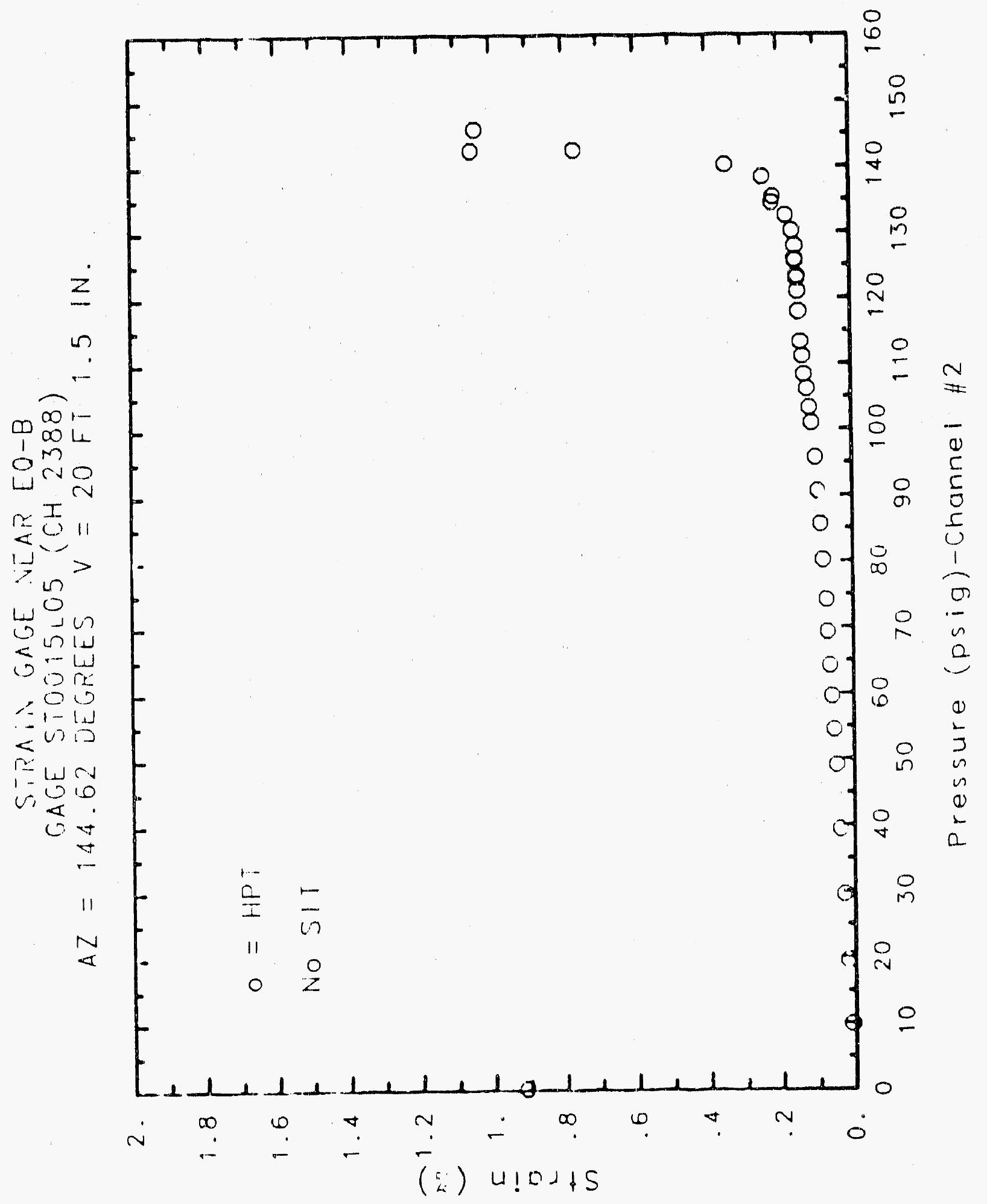

C- 1526 


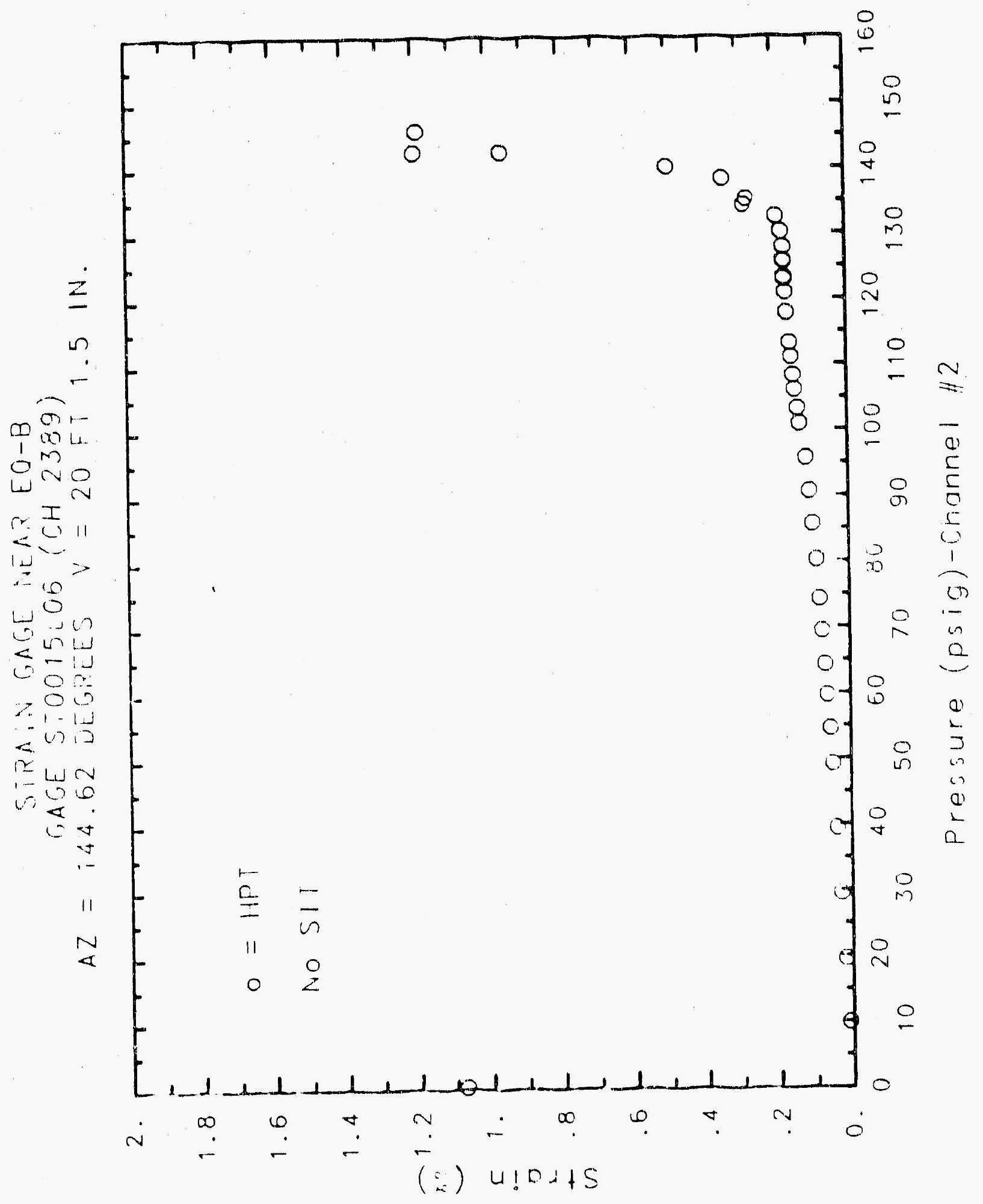

C- 1527 


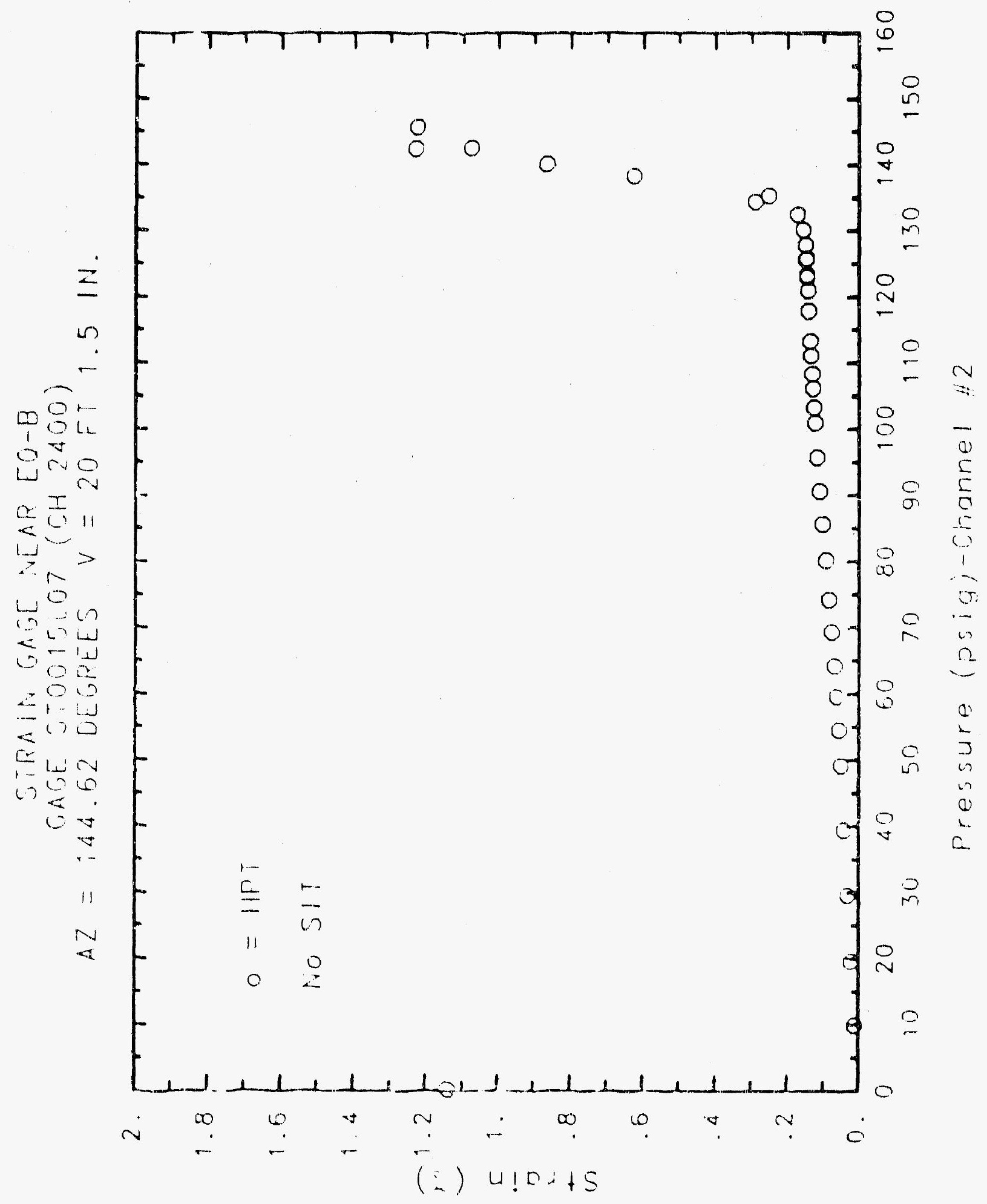

$C-15-1$ 


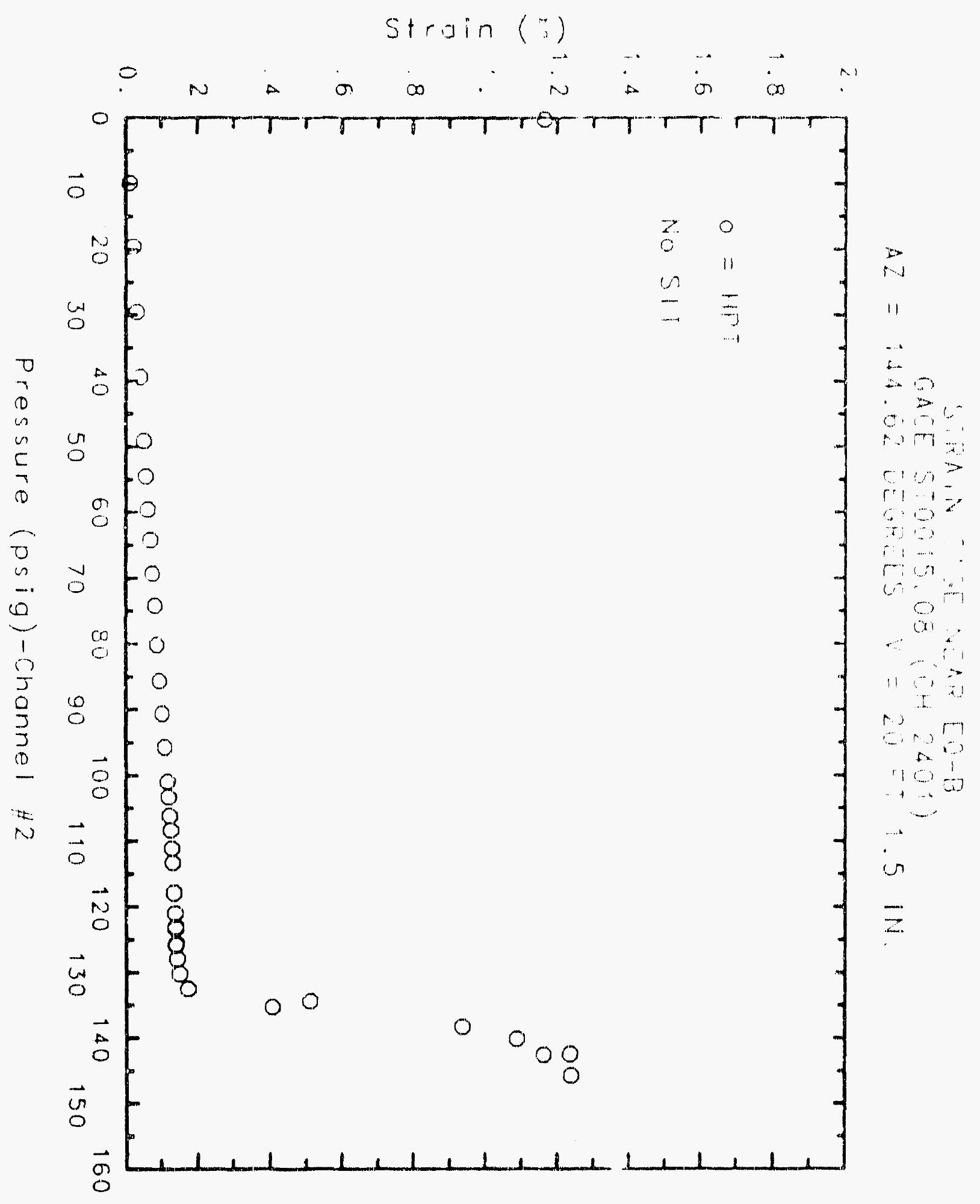




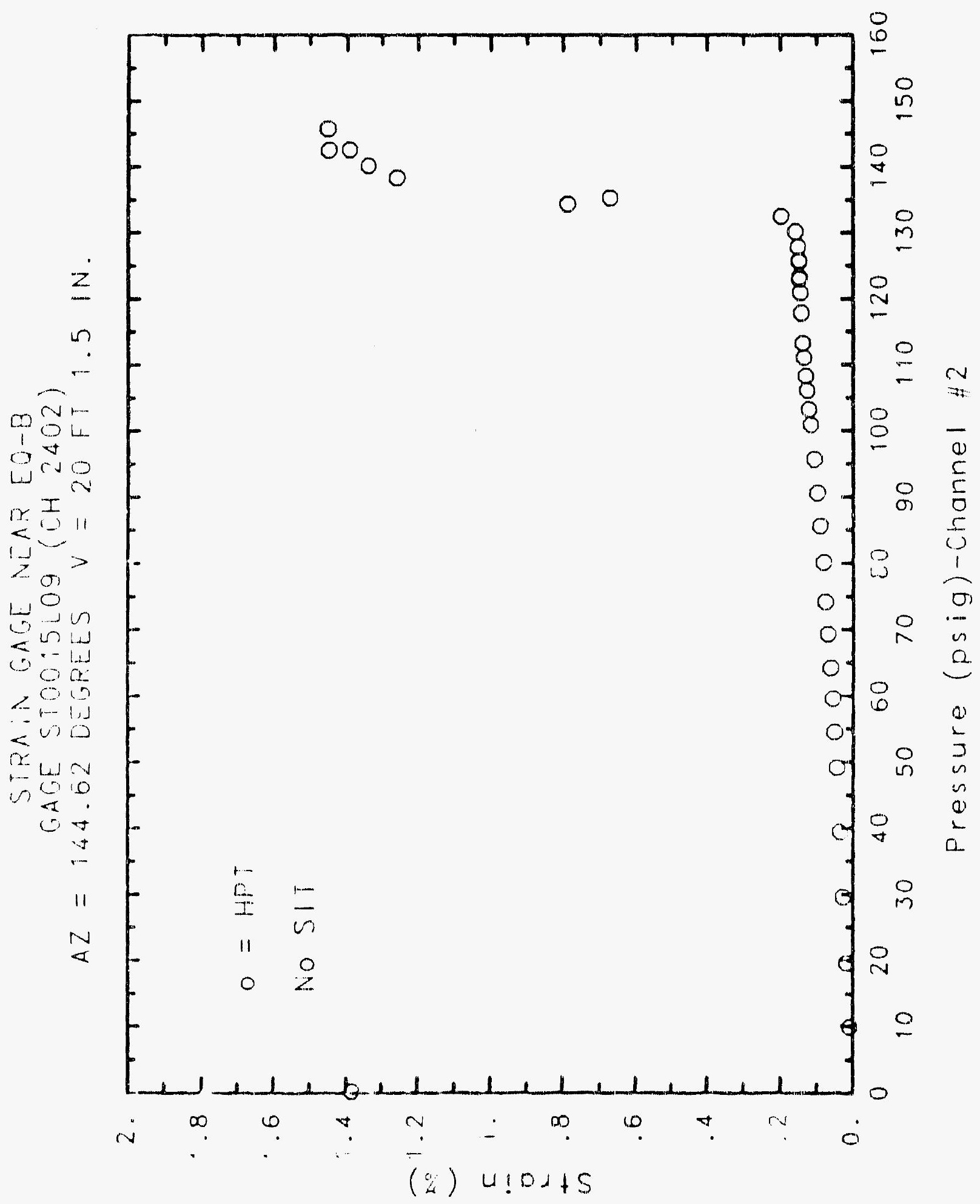

$C .15311$ 


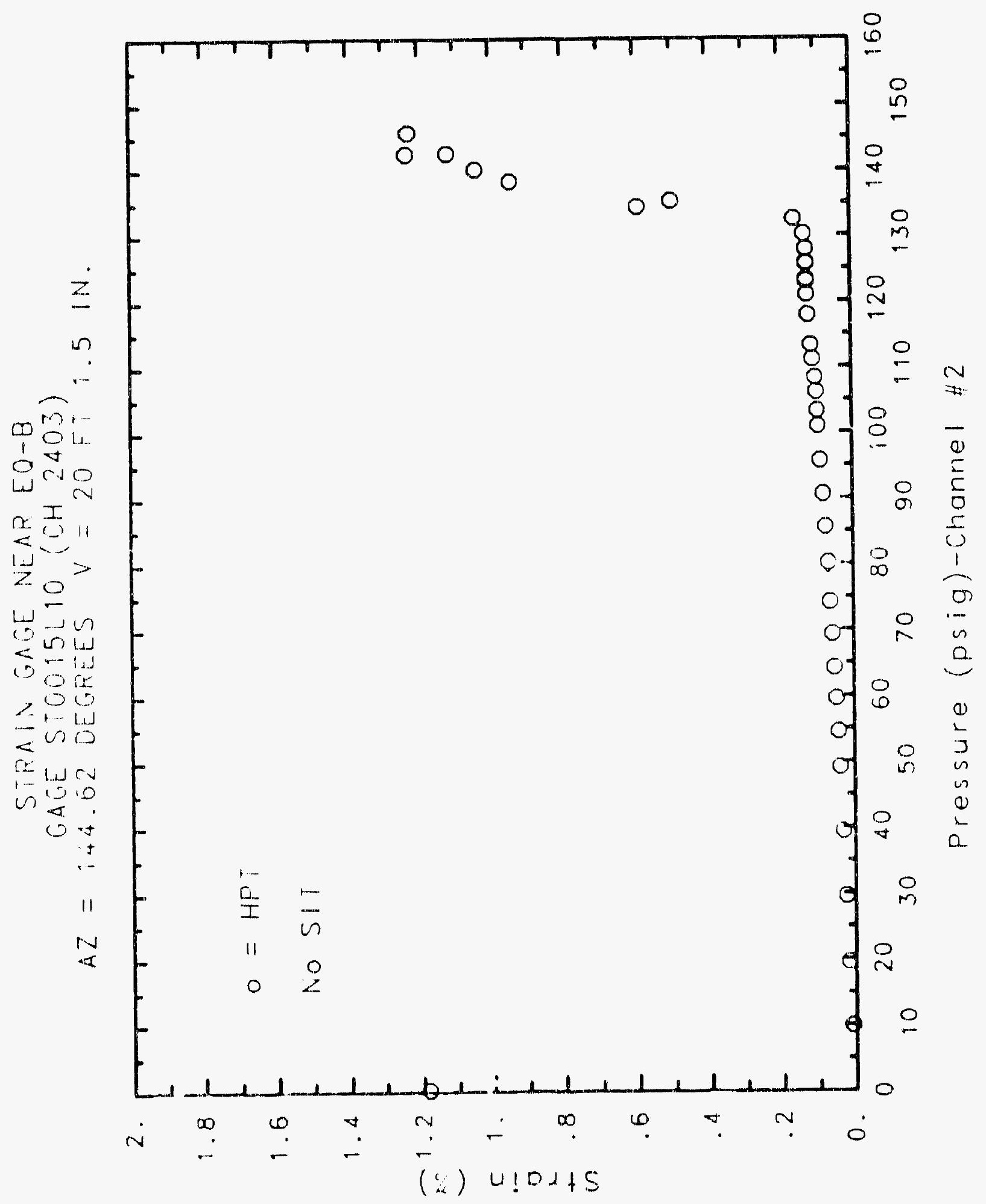

C. -1531 

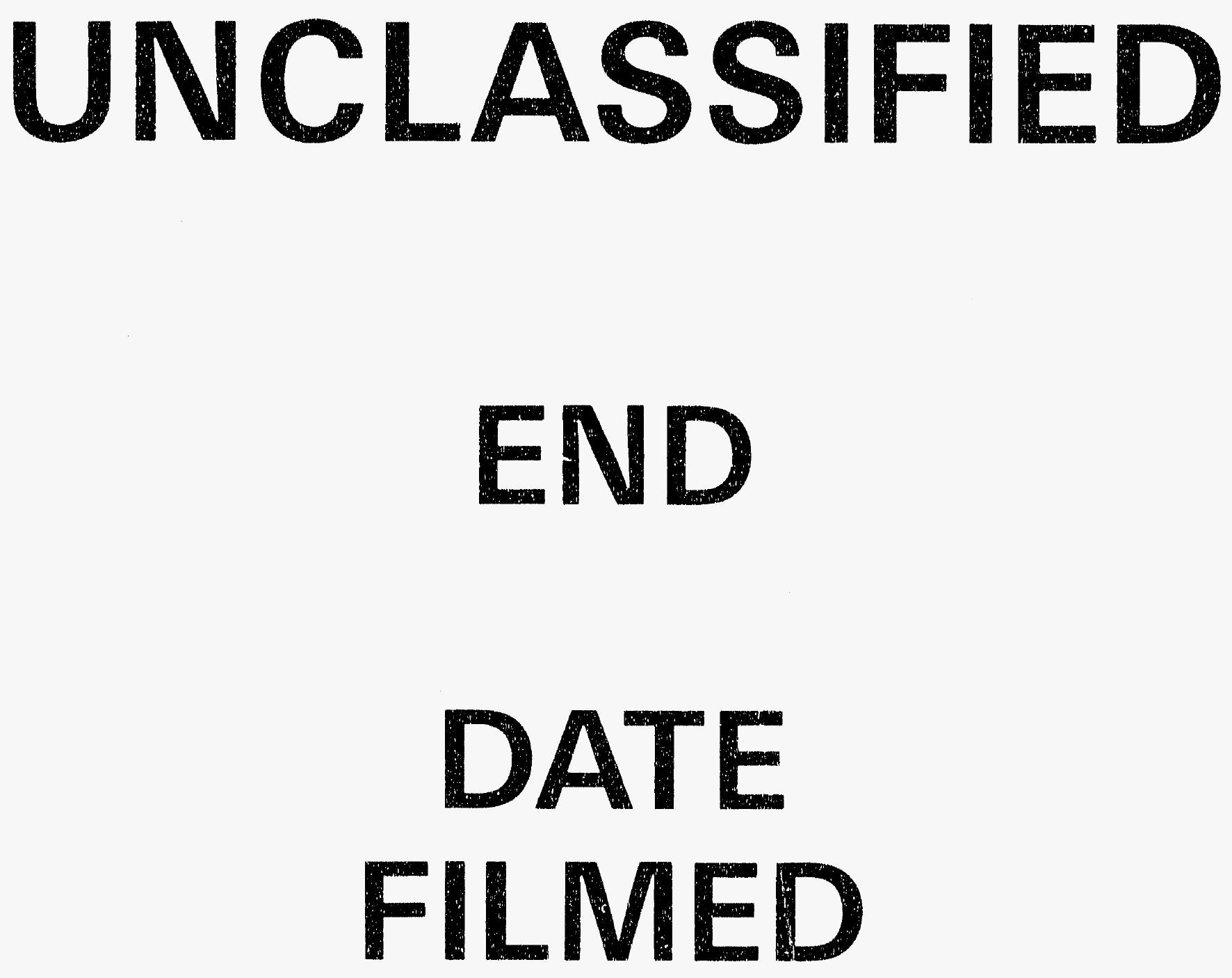

$$
1-6-92
$$

SANDIA NATIONAL LABS 

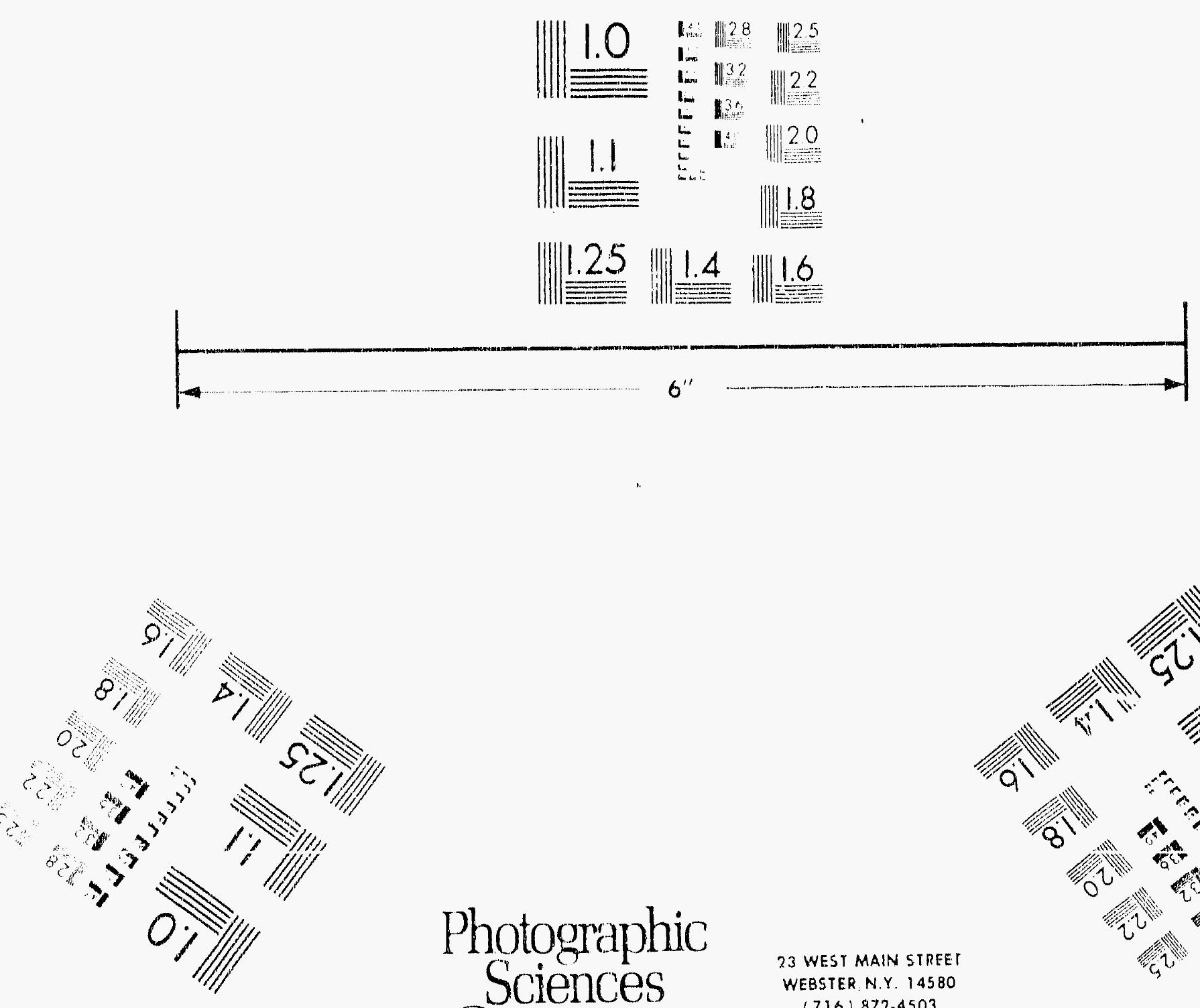

Photographic Sciences Corporation

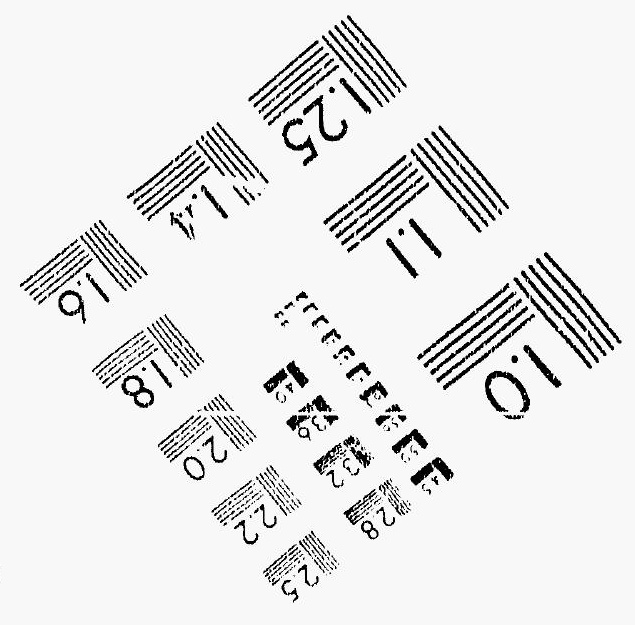




\section{SECURITY CLASSIFICATION}
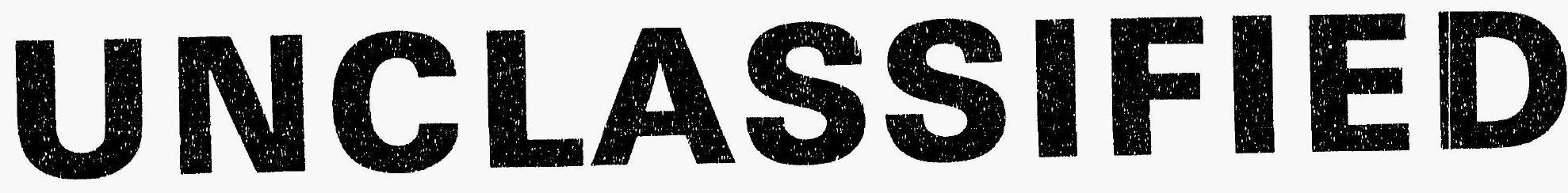

\section{DATE OF MICROFILMING $1-6-92$}

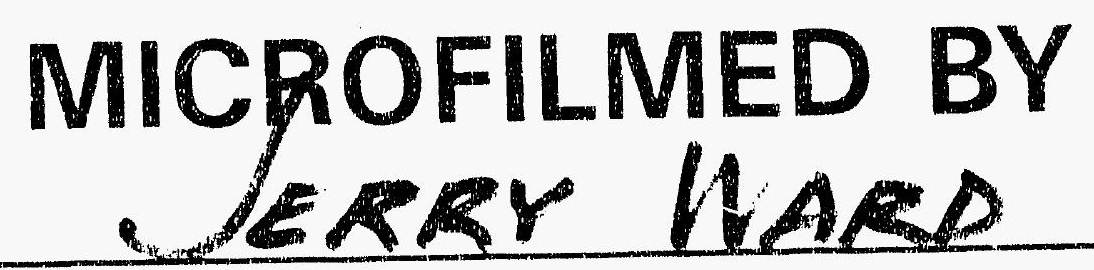

SANDIA NATIONAL LABS 


\section{THIS DOCUMENT FILMED}

AT

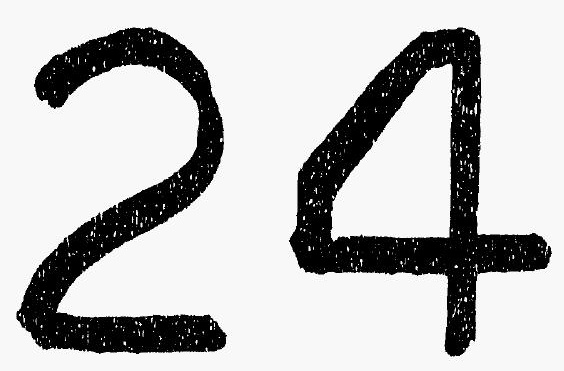

REDUCTION 


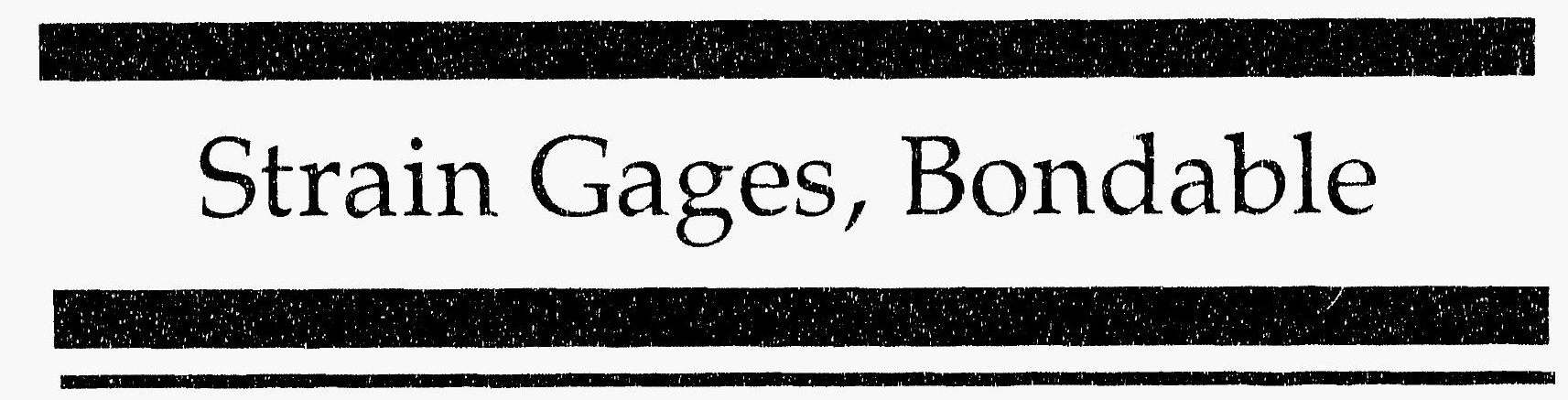




\section{Reinforced Concrete Test Data}

Bonded Gage 1 Channel 1066

Structural Integrity Test

High Pressure test

\begin{tabular}{|c|c|c|c|}
\hline $\begin{array}{c}\text { Pressure } \\
\text { (psig) } \\
-0.05 \\
5.33 \\
12.31 \\
16.44 \\
20.51 \\
20.46 \\
20.47 \\
27.05 \\
34.69 \\
34.53 \\
34.53 \\
39.70 \\
45.90 \\
45.65 \\
45.62 \\
50.10 \\
53.47 \\
53.29 \\
53.21 \\
50.49 \\
46.14 \\
46.13 \\
44.97 \\
38.98 \\
33.99 \\
33.96 \\
26.83 \\
21.88 \\
21.90 \\
16.69 \\
11.74 \\
11.77 \\
5.05 \\
0.02 \\
-0.04 \\
-0.02 \\
-0.02 \\
0.02 \\
\end{array}$ & $\begin{array}{c}\text { * Strain } \\
0.0006 \\
0.0043 \\
0.0115 \\
0.0162 \\
0.0228 \\
0.0251 \\
0.0270 \\
0.0408 \\
0.0576 \\
0.0596 \\
0.0604 \\
0.0696 \\
0.0820 \\
0.0815 \\
0.0814 \\
0.0892 \\
0.0958 \\
0.0953 \\
0.0950 \\
0.0912 \\
0.0852 \\
0.0839 \\
0.0801 \\
0.0727 \\
0.0655 \\
0.0677 \\
0.0577 \\
0.0511 \\
0.0516 \\
0.0436 \\
0.0354 \\
0.0339 \\
0.0229 \\
0.0145 \\
0.0073 \\
0.0078 \\
0.0077 \\
0.0090\end{array}$ & $\begin{array}{c}\text { Pressure } \\
(\text { ps 1g) } \\
9.89 \\
19.55 \\
29.57 \\
39.42 \\
49.10 \\
54.50 \\
59.57 \\
64.20 \\
69.32 \\
74.16 \\
80.16 \\
85.61 \\
90.58 \\
95.69 \\
100.92 \\
103.25 \\
106.11 \\
108.31 \\
111.08 \\
113.24 \\
117.83 \\
120.92 \\
123.28 \\
122.97 \\
125.82 \\
125.60 \\
127.84 \\
130.19 \\
132.53 \\
135.33 \\
134.42 \\
138.35 \\
140.16 \\
142.63 \\
145.78 \\
142.52 \\
0.22 \\
\end{array}$ & $\begin{array}{l}\text { \% Strain } \\
0.0146 \\
0.0316 \\
0.0495 \\
0.0658 \\
0.0818 \\
0.0904 \\
0.1000 \\
0.1096 \\
0.1205 \\
0.1328 \\
0.1482 \\
0.1618 \\
0.1732 \\
0.1847 \\
0.1961 \\
0.2025 \\
0.2096 \\
0.2181 \\
0.2305 \\
0.2462 \\
0.2763 \\
0.2910 \\
0.3037 \\
0.3054 \\
0.3193 \\
0.3275 \\
0.3496 \\
0.3851 \\
0.4354 \\
0.5986 \\
0.6326 \\
0.8865 \\
0.9373 \\
1.0438 \\
1.1597 \\
1.1764 \\
0.7968\end{array}$ \\
\hline
\end{tabular}


EESI

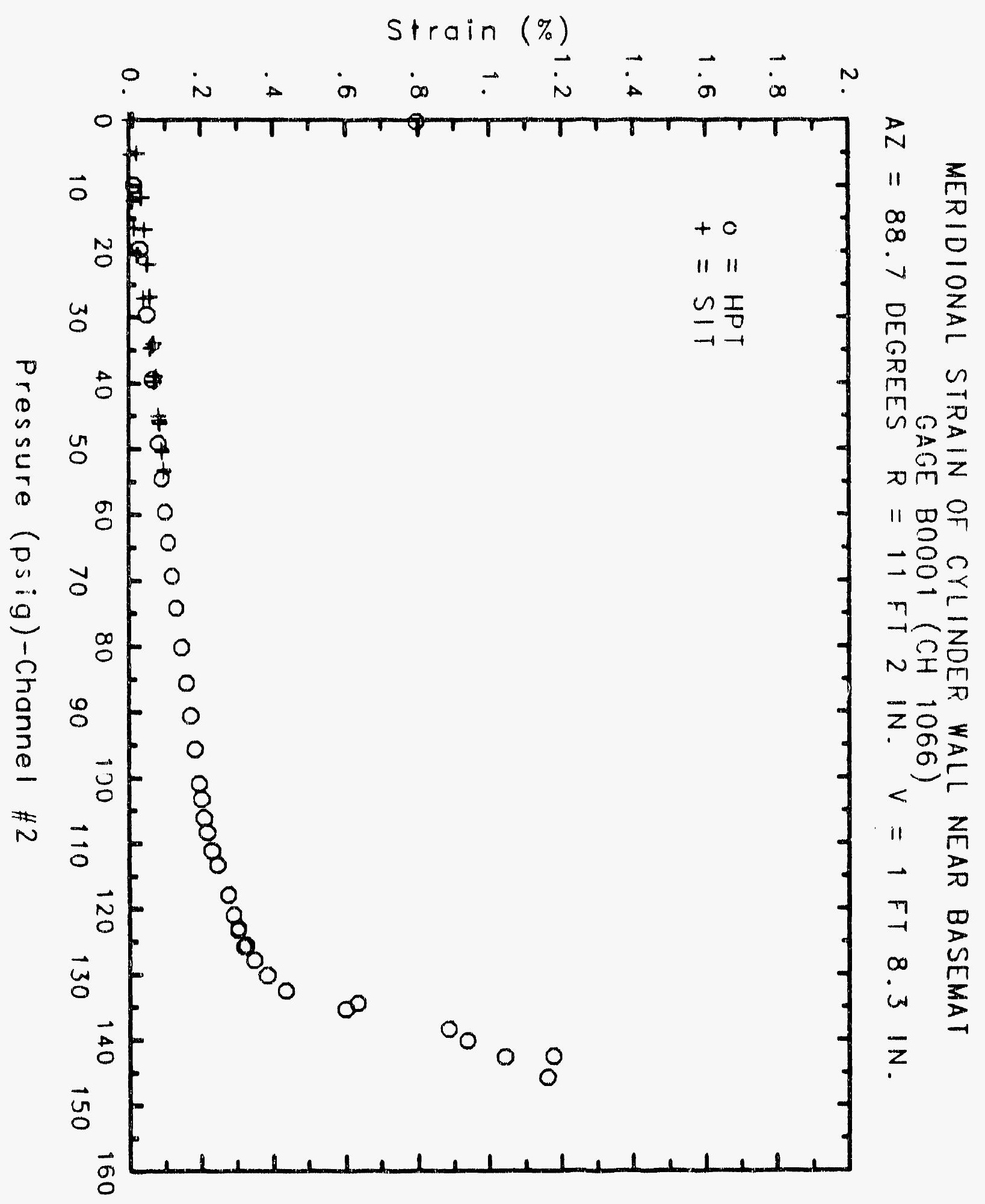




\section{Reinforced Concrete Test Data}

Bonded Gage 2 Channel 1067

Structural Integrity Test

high pressure test

\begin{tabular}{|c|c|c|c|}
\hline $\begin{array}{c}\text { Pressure } \\
(p s 19) \\
-0.05 \\
5.33 \\
12.31 \\
16.44 \\
20.51 \\
20.46 \\
20.47 \\
27.05 \\
34.69 \\
34.53 \\
34.53 \\
39.70 \\
45.90 \\
45.65 \\
45.62 \\
50.10 \\
53.47 \\
53.29 \\
53.21 \\
50.49 \\
46.14 \\
46.13 \\
44.97 \\
38.98 \\
33.99 \\
33.96 \\
26.83 \\
21.88 \\
21.90 \\
16.69 \\
11.74 \\
11.77 \\
5.05 \\
0.02 \\
-0.04 \\
-0.02 \\
-0.02 \\
0.02 \\
\end{array}$ & $\begin{array}{c}x \text { Strain } \\
\text {-0.0003 } \\
0.0048 \\
0.0126 \\
0.0173 \\
0.0242 \\
0.0268 \\
0.0290 \\
0.0429 \\
0.0597 \\
0.0624 \\
0.0632 \\
0.0727 \\
0.0854 \\
0.0847 \\
0.0841 \\
0.0914 \\
0.0980 \\
0.0973 \\
0.0967 \\
0.0926 \\
0.0862 \\
0.0847 \\
0.0806 \\
0.0726 \\
0.0558 \\
0.0684 \\
0.0584 \\
0.0520 \\
0.0535 \\
0.0456 \\
0.0374 \\
0.0359 \\
0.0248 \\
0.0168 \\
0.0087 \\
0.0085 \\
0.0086 \\
0.0097\end{array}$ & $\begin{array}{c}\text { Pressure } \\
\text { (psig) } \\
9.89 \\
19.55 \\
29.57 \\
39.42 \\
49.16 \\
54.50 \\
59.57 \\
64.20 \\
69.32 \\
74.16 \\
80.16 \\
85.61 \\
90.58 \\
95.69 \\
100.92 \\
103.25 \\
106.11 \\
108.31 \\
111.08 \\
113.24 \\
117.83 \\
120.92 \\
123.28 \\
122.97 \\
125.82 \\
125.60 \\
127.84 \\
130.19 \\
132.53 \\
135.33 \\
134.42 \\
138.35 \\
140.16 \\
142.63 \\
145.78 \\
142.52 \\
0.22 \\
\end{array}$ & $\begin{array}{l}\text { X Strain } \\
0.0150 \\
0.0317 \\
0.0493 \\
0.0662 \\
0.0827 \\
0.0919 \\
0.1017 \\
0.1117 \\
0.1234 \\
0.1360 \\
0.1518 \\
0.1667 \\
0.1791 \\
0.1915 \\
0.2055 \\
0.2128 \\
0.2219 \\
0.2300 \\
0.2443 \\
0.2604 \\
0.3918 \\
0.0473 \\
0.7416 \\
0.7422 \\
0.7523 \\
0.7568 \\
0.7796 \\
0.9371 \\
1.0062 \\
1.1303 \\
1.1613 \\
1.3163 \\
1.4142 \\
1.5499 \\
1.6518 \\
1.8601 \\
1.2578\end{array}$ \\
\hline
\end{tabular}




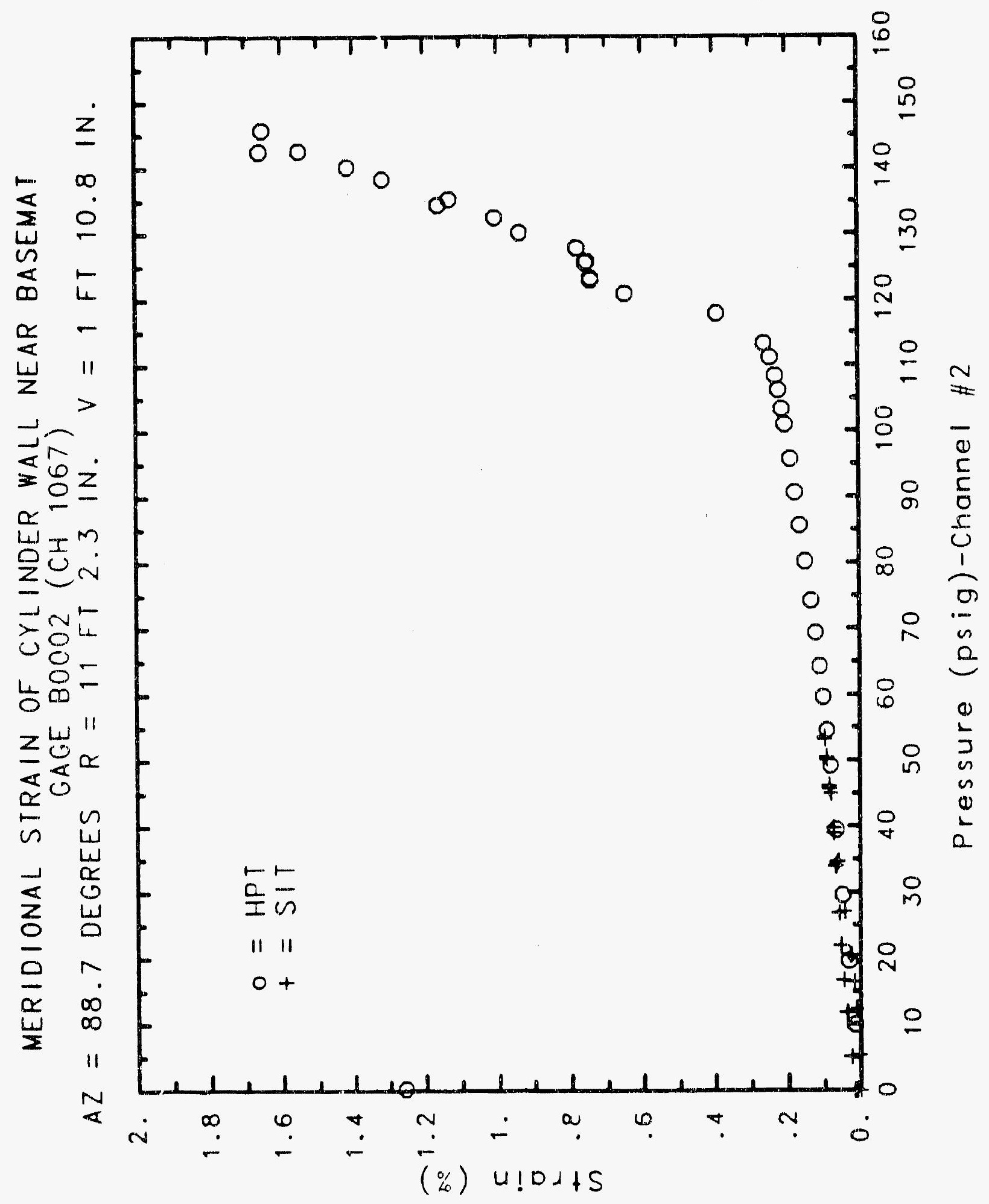




\section{Reinforced Concrete Test Data}

Bonded gage 4 Chanmel 1069

StRuCTURAL INTEGRITY TEST

\begin{tabular}{|c|c|}
\hline $\begin{array}{c}\text { Pressure } \\
\text { (psig) } \\
-0.05 \\
5.33 \\
12.31 \\
16.44 \\
20.51 \\
20.46 \\
20.47 \\
27.05 \\
34.69 \\
34.53 \\
34.53 \\
39.70 \\
45.90 \\
45.65 \\
45.62 \\
50.10 \\
53.47 \\
53.29 \\
53.21 \\
50.49 \\
46.14 \\
46.13 \\
44.97 \\
38.98 \\
33.99 \\
33.96 \\
26.83 \\
21.88 \\
2.1 .90 \\
16.69 \\
11.74 \\
11.77 \\
5.05 \\
0.02 \\
-0.04 \\
-0.02 \\
-0.02 \\
0.02 \\
\end{array}$ & $\begin{array}{c}\text { X Strain } \\
-0.0004 \\
0.0025 \\
0.0074 \\
0.0109 \\
0.0171 \\
0.0182 \\
0.0186 \\
0.0340 \\
0.0530 \\
0.0537 \\
0.0545 \\
0.0647 \\
0.0786 \\
0.0784 \\
0.0777 \\
0.0851 \\
0.0927 \\
0.0921 \\
0.0915 \\
0.0881 \\
0.0822 \\
0.0815 \\
0.0794 \\
0.0713 \\
0.0641 \\
0.0641 \\
0.0529 \\
0.0455 \\
0.0460 \\
0.0382 \\
0.0303 \\
0.0304 \\
0.0198 \\
0.0134 \\
0.0108 \\
0.0112 \\
0.0114 \\
0.0111\end{array}$ \\
\hline
\end{tabular}

High Pressure test

\begin{tabular}{|c|c|}
\hline $\begin{array}{c}\text { Pressure } \\
\text { (psig) } \\
9.89 \\
19.55 \\
29.57 \\
39.42 \\
49.16 \\
54.50 \\
59.57 \\
64.20 \\
69.32 \\
74.16 \\
80.16 \\
85.61 \\
90.58 \\
95.69 \\
100.92 \\
103.25 \\
106.11 \\
108.31 \\
111.08 \\
113.24 \\
117.83 \\
120.92 \\
123.28 \\
122.97 \\
125.82 \\
125.60 \\
127.84 \\
130.19 \\
132.53 \\
135.33 \\
134.42 \\
138.35 \\
140.16 \\
142.63 \\
145.78 \\
142.52 \\
0.22\end{array}$ & $\begin{array}{l}\text { \% Strain } \\
0.0143 \\
0.0311 \\
0.0478 \\
0.0633 \\
0.0784 \\
0.0867 \\
0.0961 \\
0.1050 \\
0.1142 \\
0.1261 \\
0.1469 \\
0.1609 \\
0.1734 \\
0.1855 \\
0.1990 \\
0.2055 \\
0.2151 \\
0.2229 \\
0.2354 \\
0.2467 \\
0.4067 \\
0.5705 \\
0.6442 \\
0.6576 \\
0.7176 \\
0.7429 \\
0.7842 \\
0.8303 \\
0.8889 \\
0.9675 \\
0.9765 \\
1.1053 \\
1.1788 \\
1.3024 \\
1.3840 \\
1.3657 \\
0.9942\end{array}$ \\
\hline
\end{tabular}




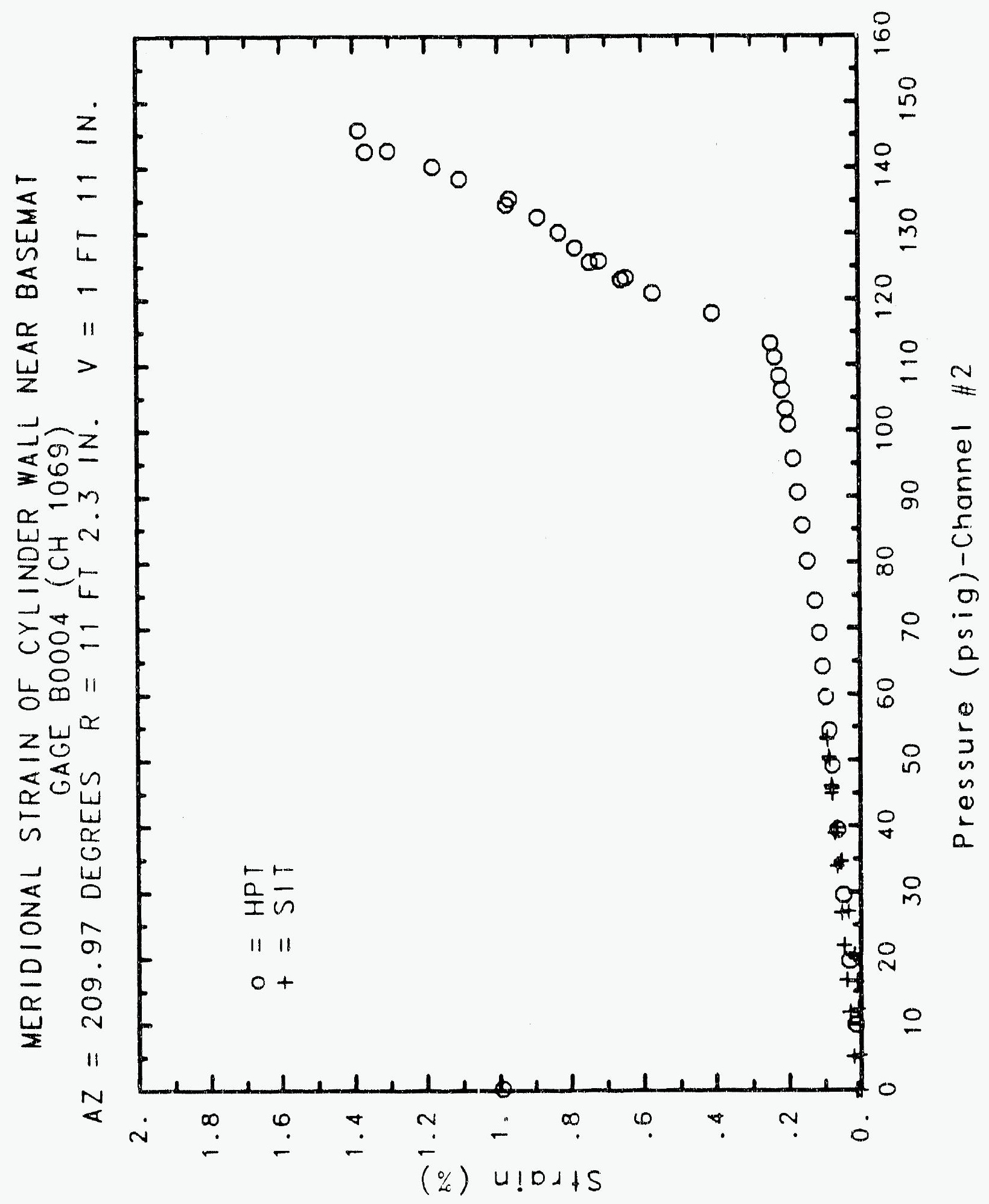




\section{Reinforced Concrete Test Data}

Bonded Gage 5 Channel 1080

StRuctural INTEgRity TEST

\begin{tabular}{|c|c|c|c|}
\hline $\begin{array}{c}\text { Pressure } \\
\text { (psig) } \\
-0.05 \\
5.33 \\
12.31 \\
16.44 \\
20.51 \\
20.46 \\
20.47 \\
27.05 \\
34.69 \\
34.53 \\
34.53 \\
39.70 \\
45.90 \\
45.65 \\
45.62 \\
50.10 \\
53.47 \\
53.29 \\
53.21 \\
50.49 \\
46.14 \\
46.13 \\
44.97 \\
38.98 \\
33.99 \\
33.96 \\
26.83 \\
21.88 \\
21.90 \\
16.69 \\
11.74 \\
11.77 \\
5.05 \\
0.02 \\
-0.04 \\
-0.02 \\
-0.02 \\
0.02 \\
\end{array}$ & $\begin{array}{l}\text { X Strain } \\
\\
0.0010 \\
0.0068 \\
0.0131 \\
0.0166 \\
0.0223 \\
0.0237 \\
0.0244 \\
0.0346 \\
0.0494 \\
0.0511 \\
0.0512 \\
0.0593 \\
0.0719 \\
0.0738 \\
0.0743 \\
0.080 ! \\
0.0859 \\
0.0867 \\
0.0866 \\
0.0844 \\
0.0799 \\
0.0794 \\
0.0809 \\
0.0742 \\
0.0681 \\
0.0678 \\
0.0581 \\
0.0515 \\
0.0507 \\
0.0439 \\
0.0370 \\
0.0361 \\
0.0263 \\
0.0193 \\
0.0107 \\
0.0139 \\
0.0163 \\
0.0161\end{array}$ & $\begin{array}{c}\text { Pressure } \\
\text { (psig) } \\
9.89 \\
19.55 \\
29.57 \\
39.42 \\
49.16 \\
54.50 \\
59.57 \\
64.20 \\
69.32 \\
74.16 \\
80.16 \\
85.61 \\
90.58 \\
95.69 \\
100.92 \\
103.25 \\
106.11 \\
108.31 \\
111.08 \\
113.24 \\
117.83 \\
120.92 \\
123.28 \\
122.97 \\
125.82 \\
125.60 \\
127.84 \\
130.19 \\
132.53 \\
135.33 \\
134.42 \\
138.35 \\
140.16 \\
142.53 \\
145.78 \\
142.52 \\
0.22\end{array}$ & $\begin{array}{l}\text { x Strain } \\
0.0152 \\
0.0297 \\
0.0441 \\
0.0585 \\
0.0718 \\
0.0780 \\
0.0864 \\
0.0961 \\
0.1059 \\
0.1208 \\
0.1389 \\
0.1532 \\
0.1648 \\
0.1782 \\
0.1937 \\
0.2001 \\
0.2096 \\
0.2164 \\
0.2262 \\
0.2343 \\
0.2514 \\
0.2519 \\
0.2534 \\
0.2546 \\
0.2518 \\
0.2545 \\
0.2537 \\
0.6070 \\
0.8403 \\
0.9107 \\
0.9182 \\
0.9632 \\
1.0442 \\
1.1504 \\
1.2560 \\
1.2651 \\
0.8935\end{array}$ \\
\hline
\end{tabular}




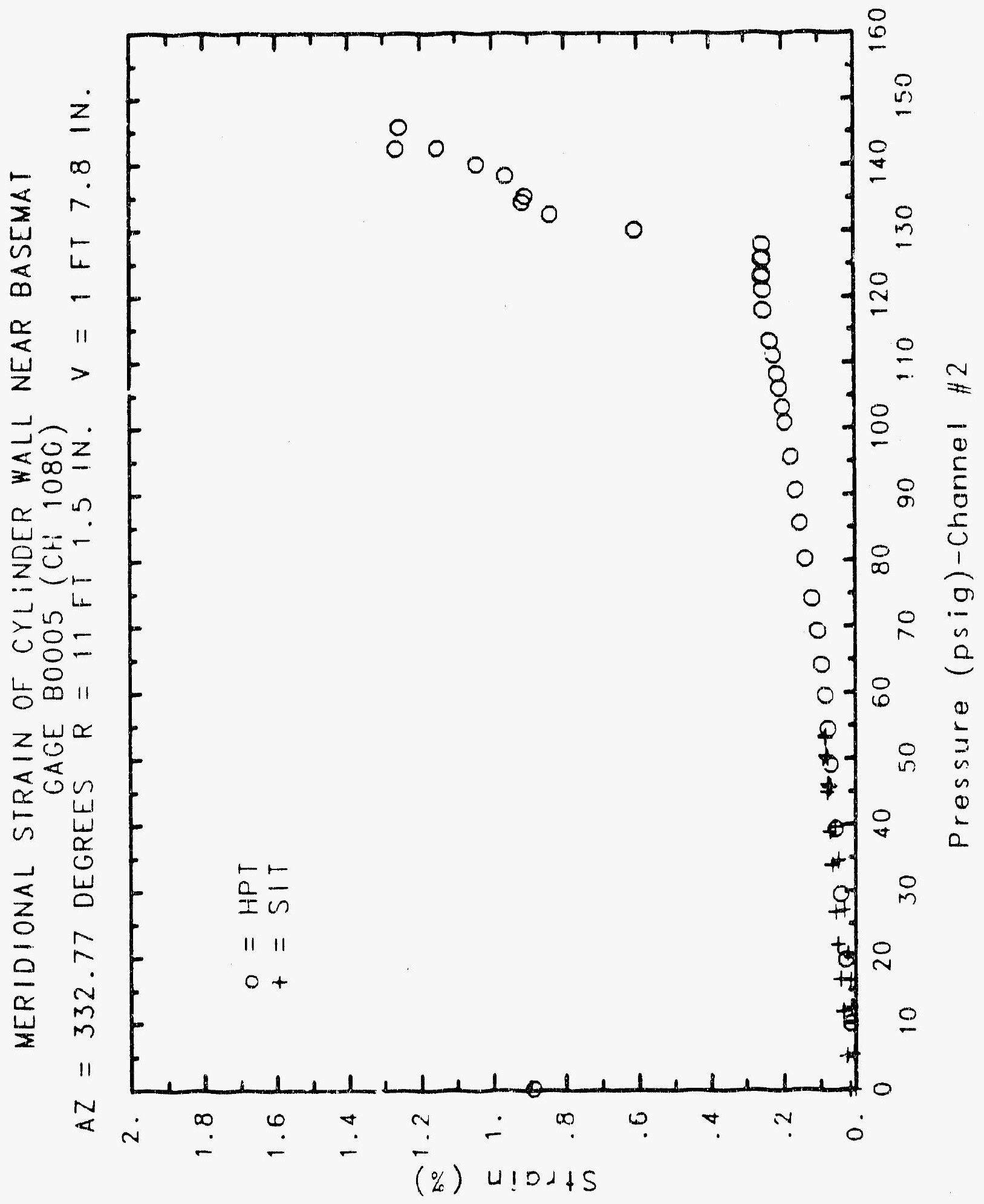




\section{Reinforced Concrete Test Data}

Bonded gage 7 Chanmel 1082

Structural InTEgrity test

\begin{tabular}{|c|c|c|c|}
\hline $\begin{array}{c}\text { Pressure } \\
\text { (psig) } \\
-0.05 \\
5.33 \\
12.31 \\
16.44 \\
20.51 \\
20.46 \\
20.47 \\
27.05 \\
34.69 \\
34.53 \\
34.53 \\
39.70 \\
45.90 \\
45.65 \\
45.62 \\
50.10 \\
53.47 \\
53.29 \\
53.21 \\
50.49 \\
46.14 \\
46.13 \\
44.97 \\
38.98 \\
33.99 \\
23.96 \\
26.83 \\
21.88 \\
21.30 \\
16.69 \\
11.74 \\
11.77 \\
5.05 \\
0.02 \\
-0.04 \\
-0.02 \\
-0.02 \\
0.02 \\
\end{array}$ & $\begin{array}{l}\text { X Strain } \\
\\
-0.0003 \\
-0.0017 \\
-0.0030 \\
-0.0043 \\
-0.0047 \\
-0.0055 \\
-0.0055 \\
-0.0034 \\
-0.0006 \\
0.0003 \\
0.0003 \\
0.0024 \\
0.0044 \\
0.0045 \\
0.0042 \\
0.0059 \\
0.0073 \\
0.0072 \\
0.0075 \\
0.0065 \\
0.0054 \\
0.0055 \\
0.0036 \\
0.0055 \\
0.0014 \\
0.0028 \\
0.0010 \\
0.0004 \\
0.0002 \\
-0.0002 \\
-0.0008 \\
-0.0003 \\
-0.0010 \\
0.0006 \\
0.0027 \\
0.0019 \\
0.0013 \\
-0.0001\end{array}$ & $\begin{array}{c}\text { Pressure } \\
(p s 1 g) \\
9.89 \\
19.55 \\
29.57 \\
39.42 \\
49.16 \\
54.50 \\
59.57 \\
64.20 \\
69.32 \\
74.16 \\
80.16 \\
85.51 \\
90.58 \\
95.69 \\
100.92 \\
103.25 \\
106.11 \\
108.31 \\
111.08 \\
133.24 \\
117.83 \\
120.92 \\
123.28 \\
122.97 \\
125.82 \\
125.60 \\
127.84 \\
130.19 \\
132.53 \\
135.33 \\
134.42 \\
138.35 \\
140.16 \\
142.63 \\
145.78 \\
142.52 \\
0.22\end{array}$ & $\begin{array}{l}\text { \% Strain } \\
\text {-0.0021 } \\
-0.0016 \\
0.0003 \\
0.0029 \\
0.0056 \\
0.0076 \\
0.0027 \\
0.0143 \\
0.0168 \\
0.0185 \\
0.0226 \\
0.0248 \\
0.0268 \\
0.0287 \\
0.0305 \\
0.0313 \\
0.0322 \\
0.0332 \\
0.0343 \\
0.0359 \\
0.0387 \\
0.0400 \\
0.0409 \\
0.0403 \\
0.0418 \\
0.0415 \\
0.0431 \\
0.0461 \\
0.0502 \\
0.0563 \\
0.0576 \\
0.0673 \\
0.0733 \\
0.0833 \\
0.0924 \\
0.0920 \\
0.0286\end{array}$ \\
\hline
\end{tabular}

High Pressure test

9.89

19.55

39.42

49.16

64.20

69.32

85.81

90.58

95.69

100.92

108.31

111.08

120.92

12328

122.97

125.82

130.19

132.53

135.33

140.16

142.63

145.78

42.52

0.0920

0.0286

$-0.0021$

0.0029

0.0027

0.0168

0.0248

0.0268

0.0305

0.0313

0.0332

0.0343

0.0400

0.0418

0.0415

0.0461

0.0502

0.0673

0.0733

0.0833
0.0019

0.0013 


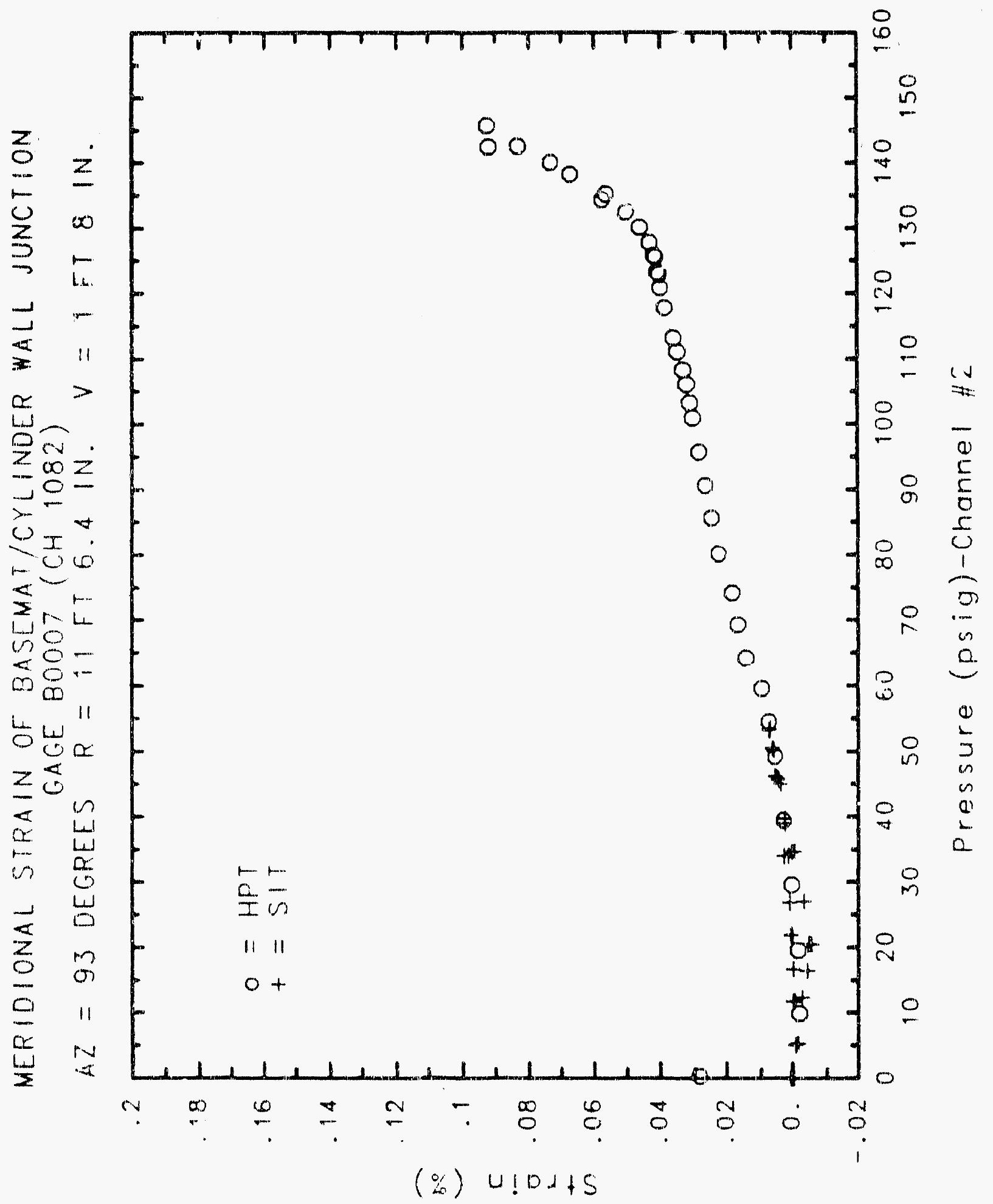




\section{Reinforced Concrete Test Data}

Bonded Gage 8 Channel 1083

STRUCTURAL INTEGRITY TEST

high Pressure test

\begin{tabular}{|c|c|c|c|}
\hline $\begin{array}{c}\text { Pressure } \\
(\text { psig) } \\
-0.05 \\
5.33 \\
12.31 \\
16.44 \\
20.51 \\
20.46 \\
20.47 \\
27.05 \\
3 . .19 \\
34.5 .3 \\
34.83 \\
39.70 \\
45.90 \\
45.65 \\
45.62 \\
50.10 \\
53.47 \\
53.29 \\
53.21 \\
50.49 \\
46.14 \\
46.13 \\
44.97 \\
38.98 \\
33.99 \\
33.96 \\
26.83 \\
21.88 \\
21.90 \\
16.69 \\
11.74 \\
11.77 \\
5.05 \\
0.02 \\
-0.04 \\
-0.02 \\
-0.02 \\
0.02 \\
\end{array}$ & $\begin{array}{l}\text { X Strain } \\
-0.0117 \\
-0.0256 \\
-0.0087 \\
-0.0109 \\
-0.0088 \\
-0.0131 \\
0.0210 \\
0.1968 \\
0.1056 \\
0.1433 \\
0.1631 \\
0.1880 \\
0.8812 \\
0.9097 \\
0.9338 \\
0.9816 \\
0.2520 \\
0.9527 \\
0.9538 \\
0.5482 \\
0.9379 \\
0.9193 \\
0.8927 \\
0.9060 \\
0.8717 \\
0.1924 \\
0.8816 \\
0.1662 \\
0.1373 \\
0.1688 \\
0.2318 \\
0.2427 \\
0.2420 \\
0.2423 \\
0.4102 \\
0.1718 \\
0.1417 \\
0.1227\end{array}$ & $\begin{array}{c}\text { Pressure } \\
(\text { psig) } \\
9.89 \\
19.55 \\
29.57 \\
39.42 \\
49.16 \\
54.50 \\
59.57 \\
64.20 \\
69.32 \\
74.16 \\
80.16 \\
85.61 \\
90.58 \\
95.69 \\
100.92 \\
103.25 \\
106.11 \\
108.31 \\
111.08 \\
113.24 \\
117.83 \\
120.92 \\
123.28 \\
122.97 \\
125.82 \\
125.60 \\
127.84 \\
130.19 \\
132.53 \\
135.33 \\
134.42 \\
138.35 \\
140.16 \\
142.63 \\
145.78 \\
142.52 \\
0.22 \\
\end{array}$ & $\begin{array}{l}\text { X Strain } \\
-0.0756 \\
-0.0853 \\
-0.0978 \\
-0.1145 \\
-0.0918 \\
-0.0774 \\
-0.0879 \\
-0.8369 \\
-0.8327 \\
-0.8063 \\
-0.8202 \\
-0.8355 \\
-0.8112 \\
-0.8460 \\
-0.8780 \\
-0.8810 \\
-0.8967 \\
-0.8950 \\
-0.9031 \\
-0.8847 \\
-0.8701 \\
-0.8830 \\
-0.8917 \\
-0.8942 \\
-0.8674 \\
-0.8603 \\
-0.8521 \\
-0.8192 \\
-0.7861 \\
-0.7407 \\
-0.7049 \\
-0.6541 \\
-0.6087 \\
-0.5586 \\
-0.5983 \\
-0.6167 \\
-0.5694\end{array}$ \\
\hline
\end{tabular}




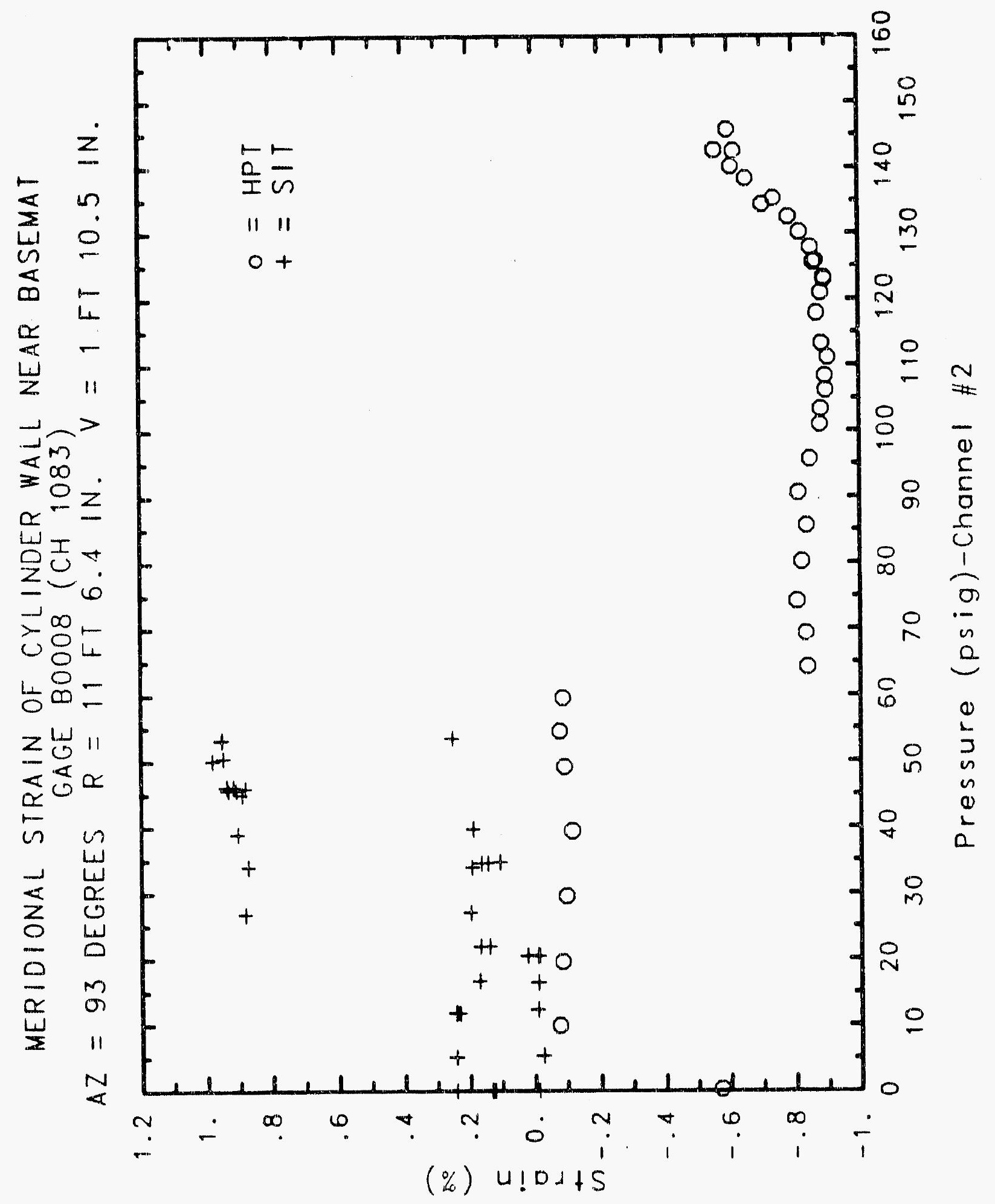




\section{Reinforced Concrete Test Data}

\begin{tabular}{|c|c|c|c|}
\hline STRUCTURAL & $\begin{array}{l}\text { BONDED Gage } 9 \\
\text { InTEgRITY TEST }\end{array}$ & $\begin{aligned} \text { Channel } 1084 \\
\text { High Pressure }\end{aligned}$ & TEST \\
\hline $\begin{array}{c}\text { Pressure } \\
\text { (psig) } \\
-0.05 \\
5.33 \\
12.31 \\
16.44 \\
20.51 \\
20.46 \\
20.47 \\
27.05 \\
34.69 \\
34.53 \\
34.53 \\
39.70 \\
45.90 \\
45.65 \\
45.62 \\
50.10 \\
53.47 \\
53.29 \\
53.21 \\
50.49 \\
46.14 \\
46.13 \\
44.97 \\
38.98 \\
33.99 \\
33.96 \\
26.83 \\
21.88 \\
21.90 \\
16.69 \\
11.74 \\
11.77 \\
5.05 \\
0.02 \\
-0.04 \\
-0.02 \\
-0.02 \\
0.02 \\
\end{array}$ & $\begin{array}{c}\text { strain } \\
\\
-0.0001 \\
-0.0010 \\
-0.0022 \\
-0.0026 \\
-0.0021 \\
-0.0020 \\
-0.0027 \\
0.0006 \\
0.0051 \\
0.0055 \\
0.0054 \\
0.0080 \\
0.0115 \\
0.0112 \\
0.0111 \\
0.0134 \\
0.0163 \\
0.0159 \\
0.0162 \\
0.0151 \\
0.0131 \\
0.0126 \\
0.0124 \\
0.0107 \\
0.0076 \\
0.0081 \\
0.0046 \\
0.0027 \\
0.0031 \\
0.0612 \\
-0.0005 \\
-0.0006 \\
-0.0009 \\
0.0000 \\
0.0010 \\
0.0002 \\
0.0000 \\
-0.0002\end{array}$ & $\begin{array}{c}\text { Pressure } \\
(\text { ps1g) } \\
9.89 \\
19.55 \\
29.57 \\
39.42 \\
49.16 \\
54.50 \\
59.57 \\
64.20 \\
69.32 \\
74.16 \\
80.16 \\
85.61 \\
90.58 \\
95.69 \\
100.92 \\
103.25 \\
106.11 \\
108.31 \\
111.08 \\
113.24 \\
117.83 \\
120.92 \\
123.28 \\
122.97 \\
125.82 \\
125.60 \\
127.84 \\
130.19 \\
132.53 \\
135.33 \\
134.42 \\
138.35 \\
140.16 \\
142.63 \\
145.78 \\
142.52 \\
0.22 \\
\end{array}$ & $\begin{array}{c}\text { \% Strain } \\
-0.0020 \\
-0.0006 \\
0.0032 \\
0.0080 \\
0.0132 \\
0.0163 \\
0.0200 \\
0.0233 \\
0.0265 \\
0.0278 \\
0.0390 \\
0.0445 \\
0.0492 \\
0.0537 \\
0.0589 \\
0.0613 \\
0.0651 \\
0.0679 \\
0.0721 \\
0.0755 \\
0.0835 \\
0.0888 \\
0.0940 \\
0.0946 \\
0.1015 \\
0.1016 \\
0.1067 \\
0.1153 \\
0.1270 \\
0.1398 \\
0.1400 \\
0.1565 \\
0.1677 \\
0.1859 \\
0.2041 \\
0.2044 \\
0.0994\end{array}$ \\
\hline
\end{tabular}




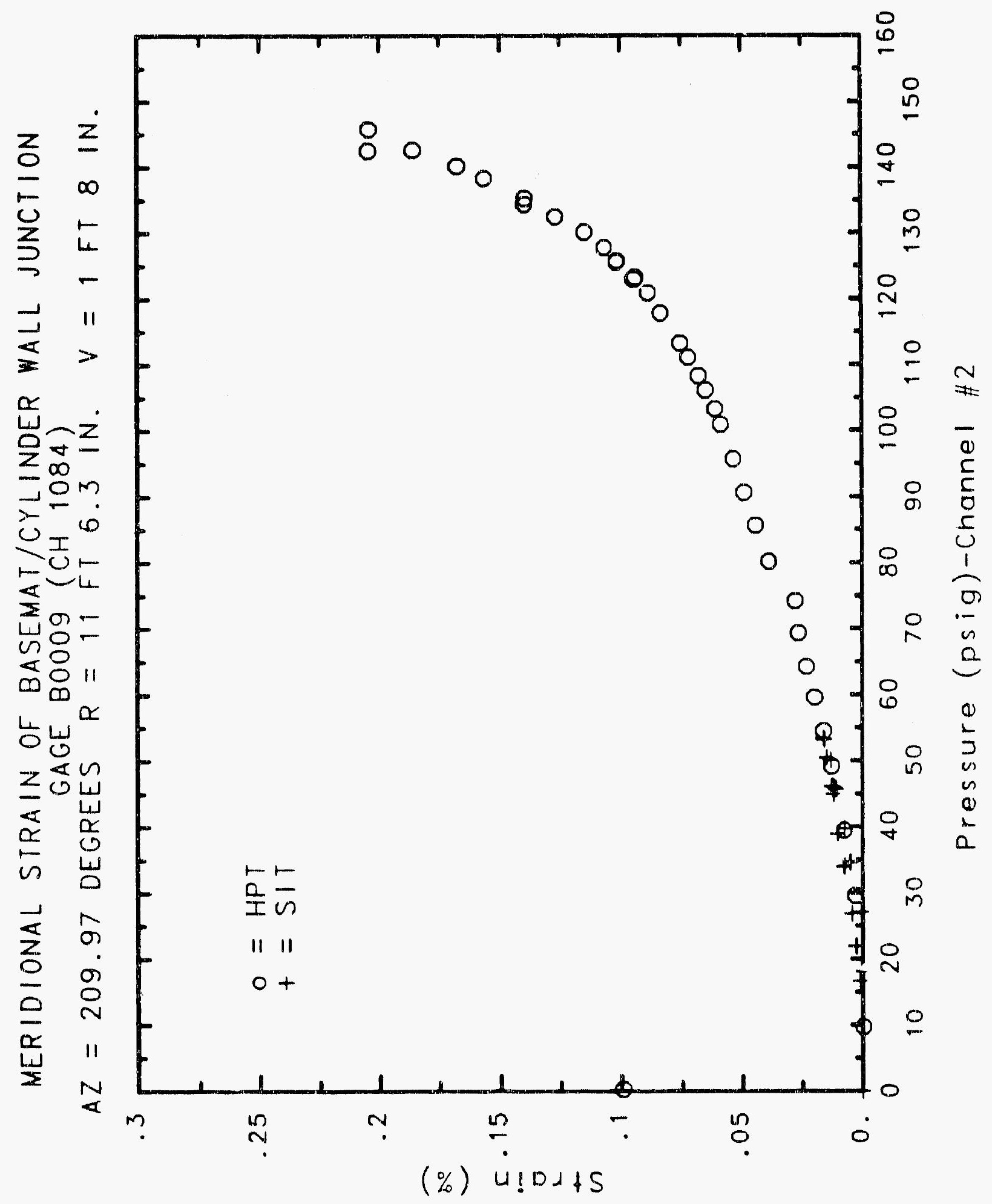




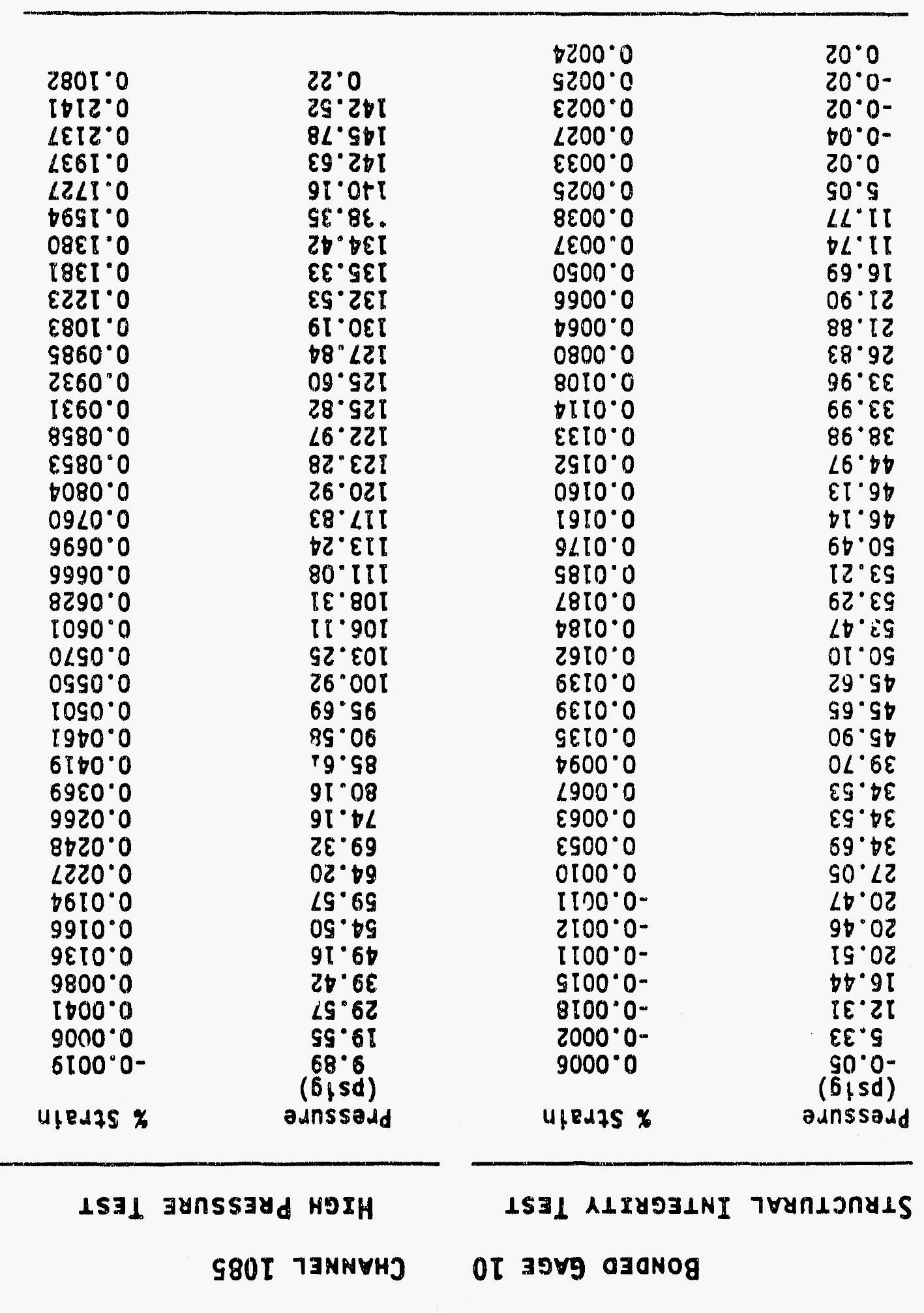

$\forall \perp \forall O$ LSI BLJUJNOS OBOUOANIJY 


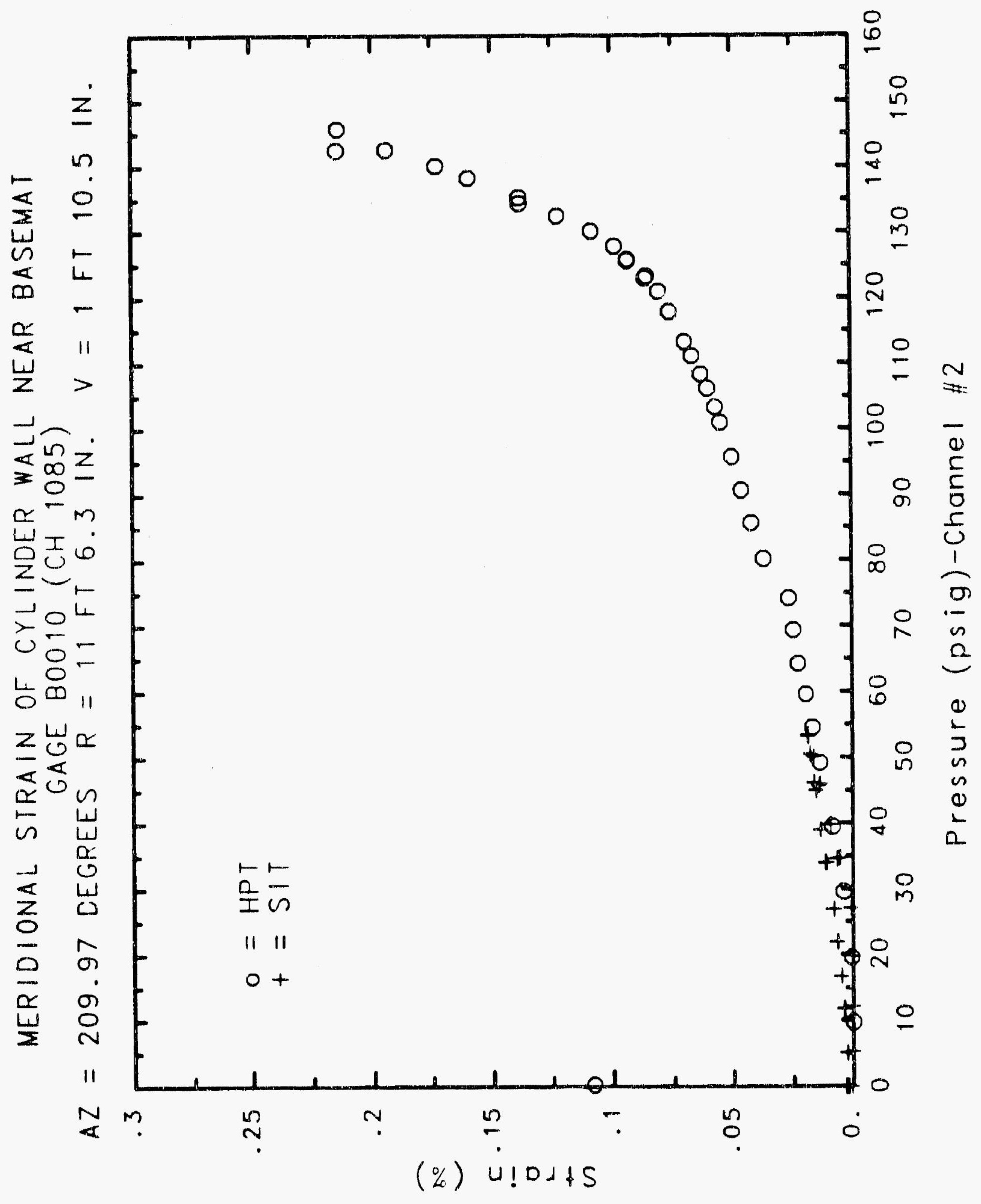




\section{Reinforced Concrete Test Data}

Bonded Gage 11 Channel 1086

Structural IntEgrity TEST

high Pressure test

\begin{tabular}{|c|c|c|c|}
\hline $\begin{array}{c}\text { Pressure } \\
\text { (psig) } \\
-0.05 \\
5.33 \\
12.31 \\
16.44 \\
20.51 \\
20.46 \\
20.47 \\
27.05 \\
34.69 \\
34.53 \\
34.53 \\
39.70 \\
45.90 \\
45.65 \\
45.62 \\
50.10 \\
53.47 \\
53.29 \\
53.21 \\
50.49 \\
46.14 \\
46.13 \\
44.97 \\
38.98 \\
33.99 \\
33.96 \\
26.83 \\
21.88 \\
21.90 \\
16.69 \\
11.74 \\
11.77 \\
5.05 \\
0.02 \\
-0.04 \\
-0.02 \\
-0.02 \\
0.02\end{array}$ & $\begin{array}{c}\text { X Strain } \\
-0.0004 \\
-0.0009 \\
-0.0016 \\
-0.0020 \\
-0.0018 \\
-0.0019 \\
-0.0021 \\
0.0001 \\
0.0036 \\
0.0043 \\
0.0035 \\
0.0059 \\
0.0110 \\
0.0107 \\
0.01112 \\
0.0137 \\
0.0174 \\
0.0175 \\
0.0172 \\
0.0168 \\
0.0149 \\
0.0141 \\
0.0165 \\
0.0132 \\
0.0111 \\
0.0109 \\
0.0074 \\
0.0050 \\
0.0052 \\
0.0029 \\
0.00111 \\
0.0010 \\
0.0005 \\
0.0012 \\
0.0047 \\
0.0027 \\
0.0018 \\
0.0015\end{array}$ & $\begin{array}{c}\text { Pressure } \\
(\text { ps } 1 \mathrm{~g}) \\
9.89 \\
19.55 \\
29.57 \\
39.42 \\
49.16 \\
54.50 \\
59.57 \\
64.20 \\
69.32 \\
74.16 \\
80.16 \\
85.61 \\
90.58 \\
95.69 \\
100.92 \\
103.25 \\
106.11 \\
108.31 \\
111.08 \\
113.24 \\
117.83 \\
120.92 \\
123.28 \\
122.97 \\
125.82 \\
125.60 \\
127.84 \\
130.19 \\
132.53 \\
135.33 \\
134.42 \\
138.35 \\
140.16 \\
142.63 \\
145.78 \\
142.52 \\
0.22\end{array}$ & $\begin{array}{l}\text { x Strain } \\
0.00 n 0 \\
0.0027 \\
0.0068 \\
0.0110 \\
0.0155 \\
0.0186 \\
0.0212 \\
0.0244 \\
0.0276 \\
0.0320 \\
0.0367 \\
0.0416 \\
0.0452 \\
0.0487 \\
0.0521 \\
0.0532 \\
0.0551 \\
0.0568 \\
0.0532 \\
0.0592 \\
0.0613 \\
0.0622 \\
0.0629 \\
0.0629 \\
0.0656 \\
0.0662 \\
0.0695 \\
0.0738 \\
0.0798 \\
0.0885 \\
0.0887 \\
0.1032 \\
0.1135 \\
0.1292 \\
0.1448 \\
0.1446 \\
0.0600\end{array}$ \\
\hline
\end{tabular}




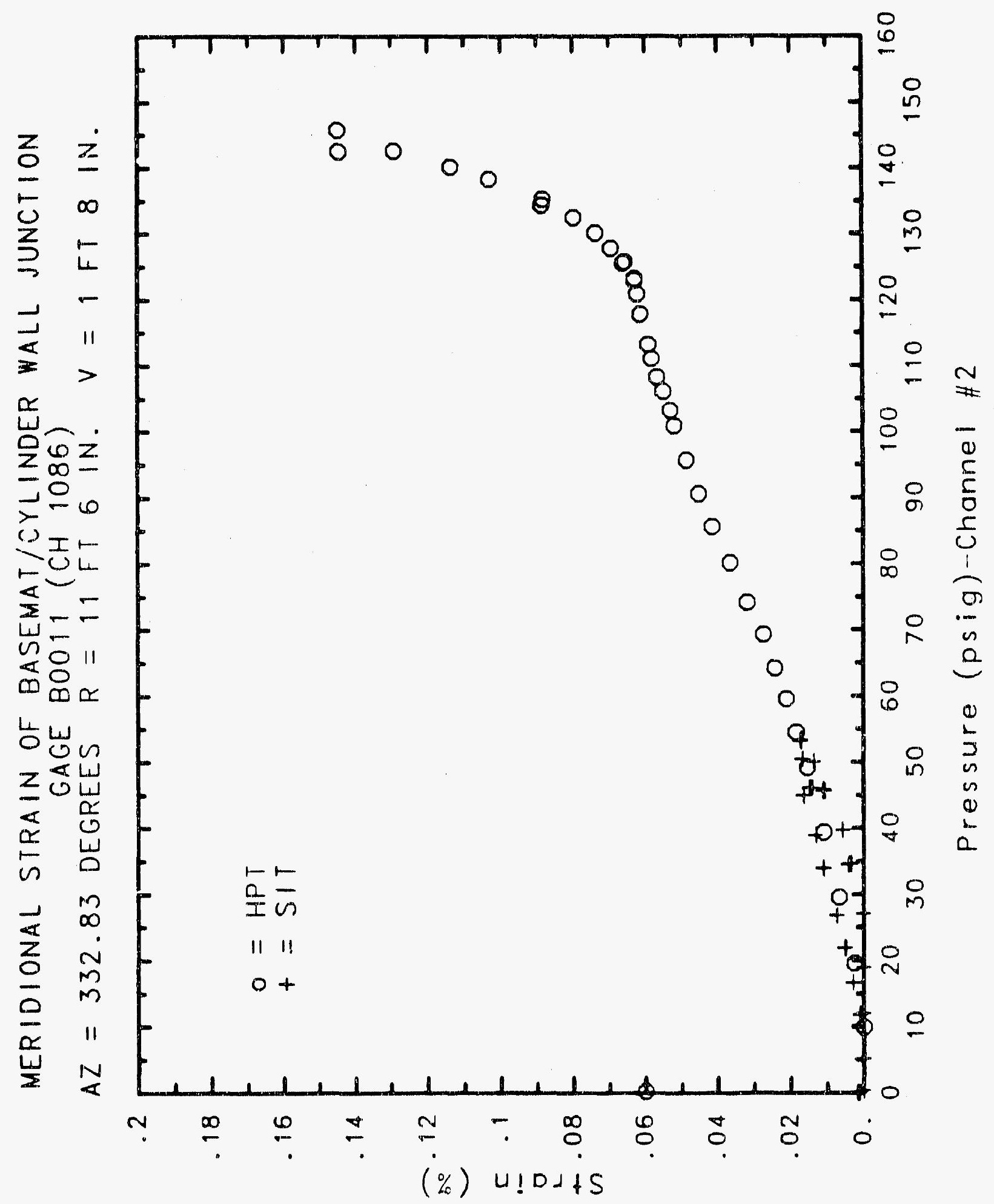




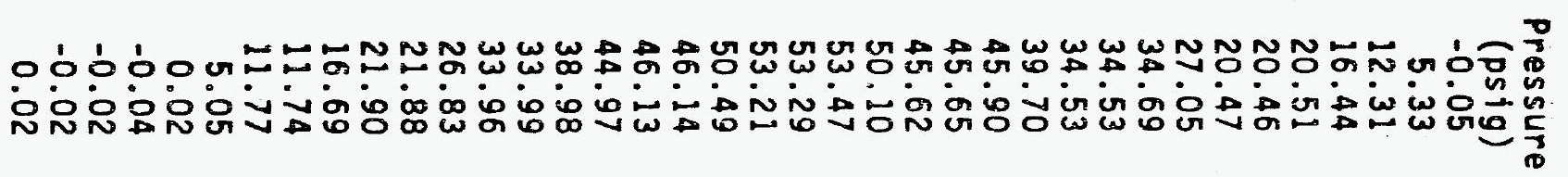

00000000000000000000000000000000000000

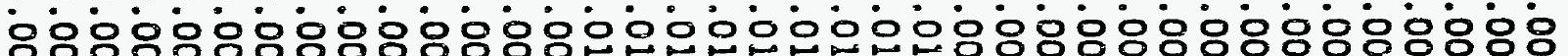

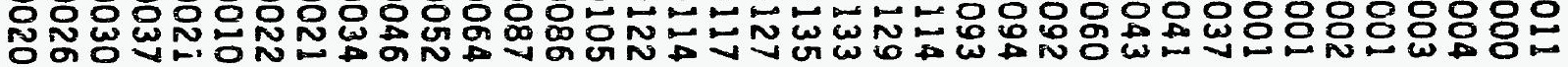

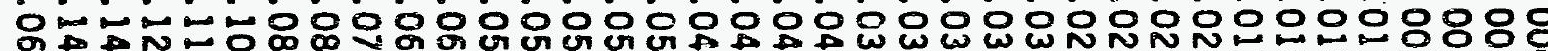

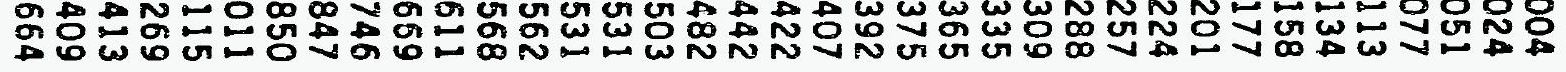

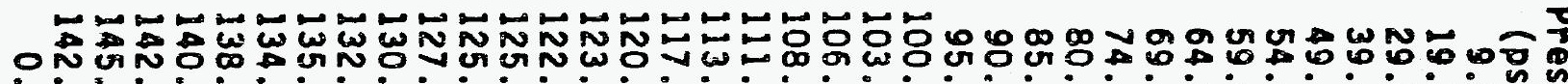
Nivis

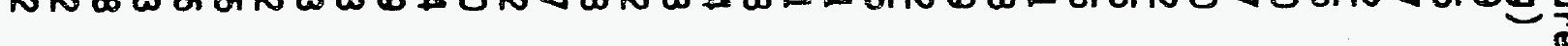

+
5
$\frac{3}{3}$
$\frac{3}{3}$




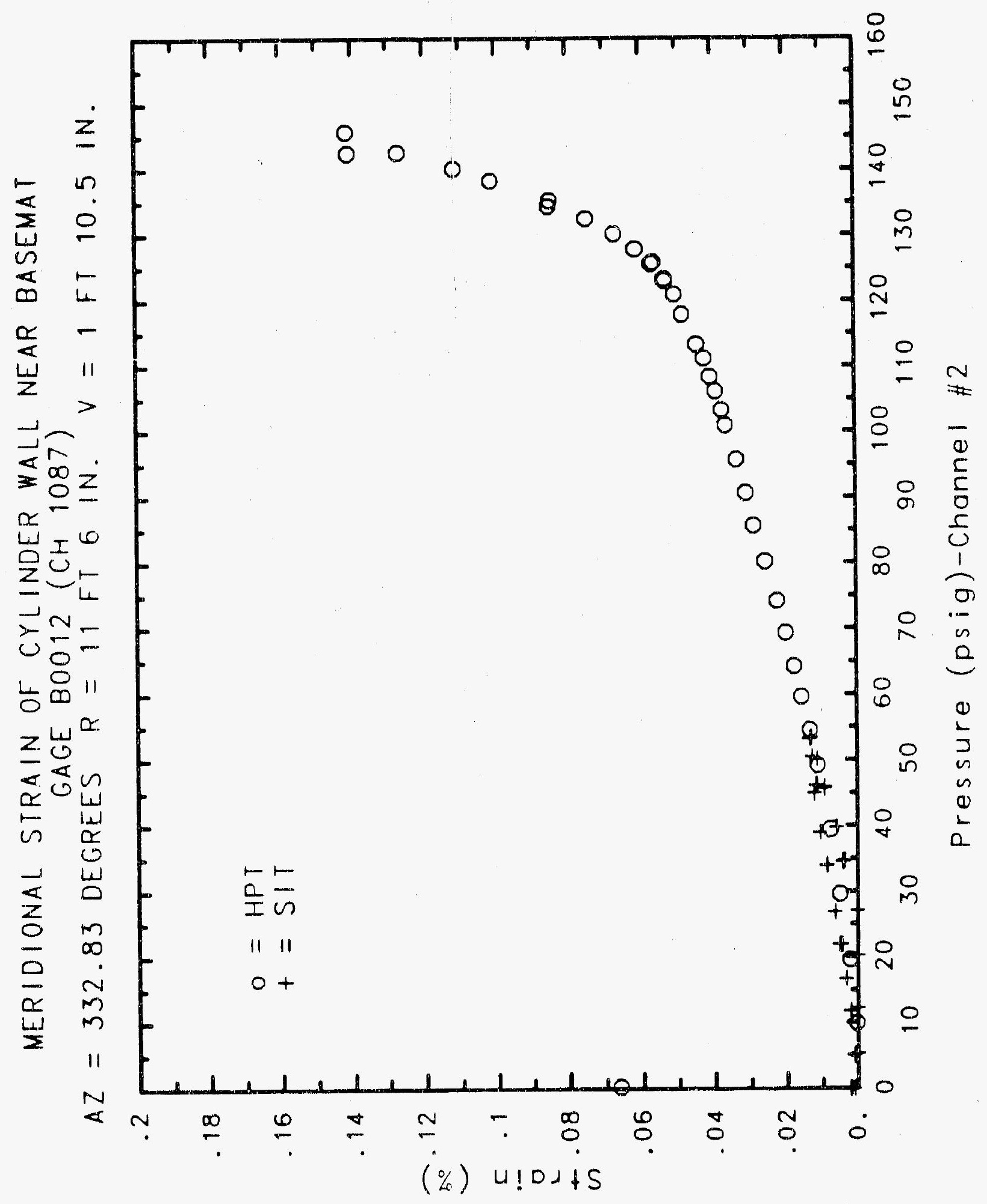




\section{Reinforced Concrete Test Data}

Bonded Gage 13 Channel 1088

StRUCTURAL INTEGRITY TEST

\begin{tabular}{|c|c|c|c|}
\hline $\begin{array}{c}\text { Pressure } \\
\text { (ps1g) } \\
-0.05 \\
5.33 \\
12.31 \\
16.44 \\
20.51 \\
20.46 \\
20.47 \\
27.05 \\
34.69 \\
34.53 \\
34.53 \\
39.70 \\
45.90 \\
45.65 \\
45.62 \\
50.10 \\
53.47 \\
53.29 \\
53.21 \\
50.49 \\
46.14 \\
46.13 \\
44.97 \\
38.98 \\
33.99 \\
33.96 \\
26.83 \\
21.88 \\
21.90 \\
16.69 \\
11.74 \\
11.77 \\
5.05 \\
0.02 \\
-0.04 \\
-0.02 \\
-0.02 \\
0.02 \\
\end{array}$ & $\begin{array}{l}\text { J Strain } \\
0.0008 \\
-0.0017 \\
-0.0057 \\
-0.0090 \\
-0.0140 \\
-0.0182 \\
-0.0230 \\
-0.0262 \\
-0.0279 \\
-0.0284 \\
-0.0282 \\
-0.0284 \\
-0.0286 \\
-0.0274 \\
-0.0263 \\
-0.0266 \\
-0.0254 \\
-0.0242 \\
-0.0242 \\
-0.0227 \\
-0.0223 \\
-0.0210 \\
-0.0198 \\
-0.0199 \\
-0.0209 \\
-0.0264 \\
-0.0281 \\
-0.0305 \\
-0.0304 \\
-0.0287 \\
-0.0266 \\
-0.0247 \\
-0.0198 \\
-0.0154 \\
-0.0019 \\
-0.0039 \\
-0.0043 \\
-0.0075\end{array}$ & $\begin{array}{c}\text { Pressure } \\
(\text { ps } 1 \text { g) } \\
9.89 \\
19.55 \\
29.57 \\
39.42 \\
49.16 \\
54.50 \\
59.57 \\
64.20 \\
69.32 \\
74.16 \\
80.16 \\
85.61 \\
90.58 \\
95.69 \\
100.92 \\
103.25 \\
106.11 \\
108.31 \\
111.08 \\
113.24 \\
117.83 \\
120.92 \\
123.28 \\
122.97 \\
125.82 \\
125.60 \\
127.84 \\
130.19 \\
132.53 \\
135.33 \\
134.42 \\
138.35 \\
140.16 \\
142.63 \\
145.78 \\
142.52 \\
0.22\end{array}$ & $\begin{array}{l}\text { X Strain } \\
-0.0059 \\
-0.0103 \\
-0.0135 \\
-0.0158 \\
-0.0178 \\
-0.0189 \\
-0.0201 \\
-0.0219 \\
-0.0238 \\
-0.0281 \\
-0.0225 \\
-0.0217 \\
-0.0209 \\
-0.0197 \\
-0.0199 \\
-0.0194 \\
-0.0194 \\
-0.0198 \\
-0.0197 \\
-0.0193 \\
-0.0178 \\
-0.0174 \\
-0.0174 \\
-0.0175 \\
-0.0178 \\
-0.0184 \\
-0.0187 \\
-0.0194 \\
-0.0189 \\
-0.0187 \\
-0.0205 \\
-0.0220 \\
-0.0207 \\
-0.0176 \\
-0.0136 \\
-0.0121 \\
-0.0177\end{array}$ \\
\hline
\end{tabular}

High Pressure test

Pressure

0.89

19.55

49.16

54.50

64.20

69.32

85.61

90.58

100.92

103.25

111.08

113.24

117.83

120.92

123.28

125.60

132.53

135.33

134.42

140.16

142.63

145.78

2.52

$-0.0136$

$-0.0121$

$-0.0177$ 


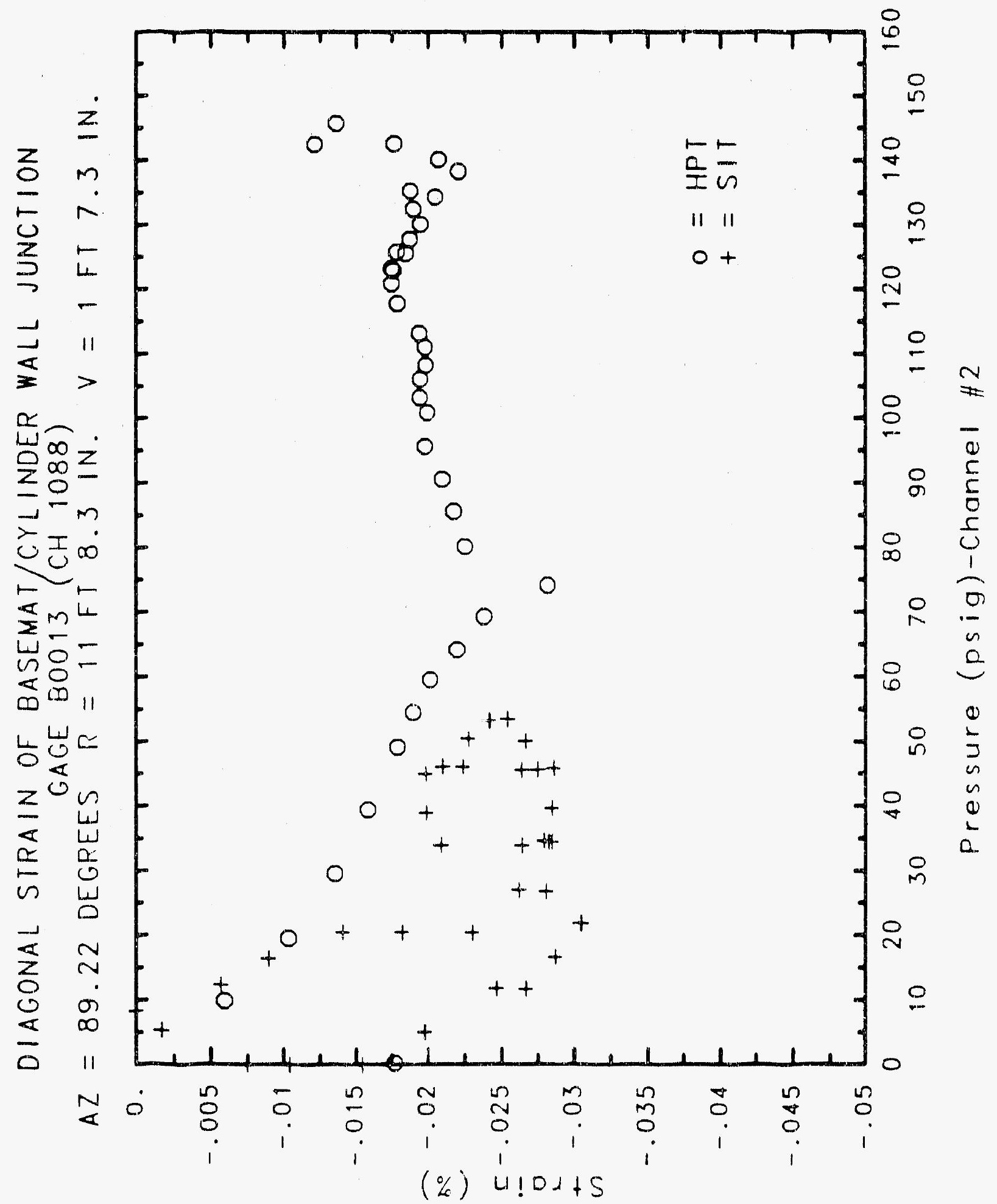




\section{Reinforced Concrete Test Data}

Bonded Gage 14 Channel 1089

STRUCTURAL INTEGRITY TEST

High Pressure test

\begin{tabular}{|c|c|c|c|c|}
\hline $\begin{array}{c}\text { Pressure } \\
\text { (psig) } \\
-0.05 \\
5.33 \\
12.31 \\
16.44 \\
20.51 \\
20.46 \\
20.47 \\
27.05 \\
34.69 \\
34.53 \\
34.53 \\
39.70 \\
45.90 \\
45.65 \\
45.62 \\
50.10 \\
53.47 \\
53.29 \\
53.21 \\
50.49 \\
46.14 \\
46.13 \\
44.97 \\
38.98 \\
33.99 \\
33.96 \\
26.83 \\
21.88 \\
21.90 \\
16.69 \\
11.74 \\
11.77 \\
5.05 \\
3.02 \\
-0.04 \\
-0.02 \\
-0.02 \\
0.02\end{array}$ & $\begin{array}{l}\text { \% strain } \\
-0.0003 \\
-0.0053 \\
-0.0114 \\
-0.0150 \\
-0.0194 \\
-0.0228 \\
-0.0261 \\
-0.0284 \\
-0.0296 \\
-0.0209 \\
-0.0303 \\
-0.0307 \\
-0.0313 \\
-0.0309 \\
-0.0302 \\
-0.0303 \\
-0.0302 \\
-0.0297 \\
-0.0291 \\
-0.0250 \\
-0.01281 \\
-0.0279 \\
-0.0254 \\
-0.0257 \\
-0.0258 \\
-0.0297 \\
-0.0295 \\
-0.0305 \\
-0.0303 \\
-0.0298 \\
-0.0292 \\
-0.0289 \\
-0.0256 \\
-0.0215 \\
-0.0029 \\
-0.0049 \\
-0.0061 \\
-0.0114\end{array}$ & 4 & $\begin{array}{c}\text { Pressure } \\
(\text { psig) } \\
9.89 \\
19.55 \\
29.57 \\
39.42 \\
49.16 \\
54.50 \\
59.57 \\
64.20 \\
59.32 \\
74.16 \\
80.16 \\
85.61 \\
90.58 \\
95.69 \\
120.92 \\
103.25 \\
106.11 \\
108.31 \\
111.08 \\
113.24 \\
117.83 \\
120.92 \\
123.28 \\
122.97 \\
125.82 \\
125.60 \\
127.84 \\
130.19 \\
132.53 \\
135.33 \\
134.42 \\
138.35 \\
140.16 \\
142.63 \\
145.78 \\
142.52 \\
0.22 \\
\end{array}$ & $\begin{array}{l}\text { J Strain } \\
-0.0111 \\
-0.0160 \\
-0.0189 \\
-0.0206 \\
-0.0219 \\
-0.0227 \\
-0.0232 \\
-0.0249 \\
-0.0262 \\
-0.0288 \\
-0.0276 \\
-0.0273 \\
-0.0206 \\
-0.0255 \\
-0.0248 \\
-0.0244 \\
-0.0247 \\
-0.0247 \\
-0.0245 \\
-0.0243 \\
-0.0237 \\
-0.0241 \\
-0.0238 \\
-0.0239 \\
-0.0242 \\
-0.0246 \\
-0.0245 \\
-0.0254 \\
-0.0266 \\
-0.0283 \\
-0.0307 \\
-0.0365 \\
-0.0392 \\
-0.0427 \\
-0.0428 \\
-0.0435 \\
-0.0379\end{array}$ \\
\hline
\end{tabular}




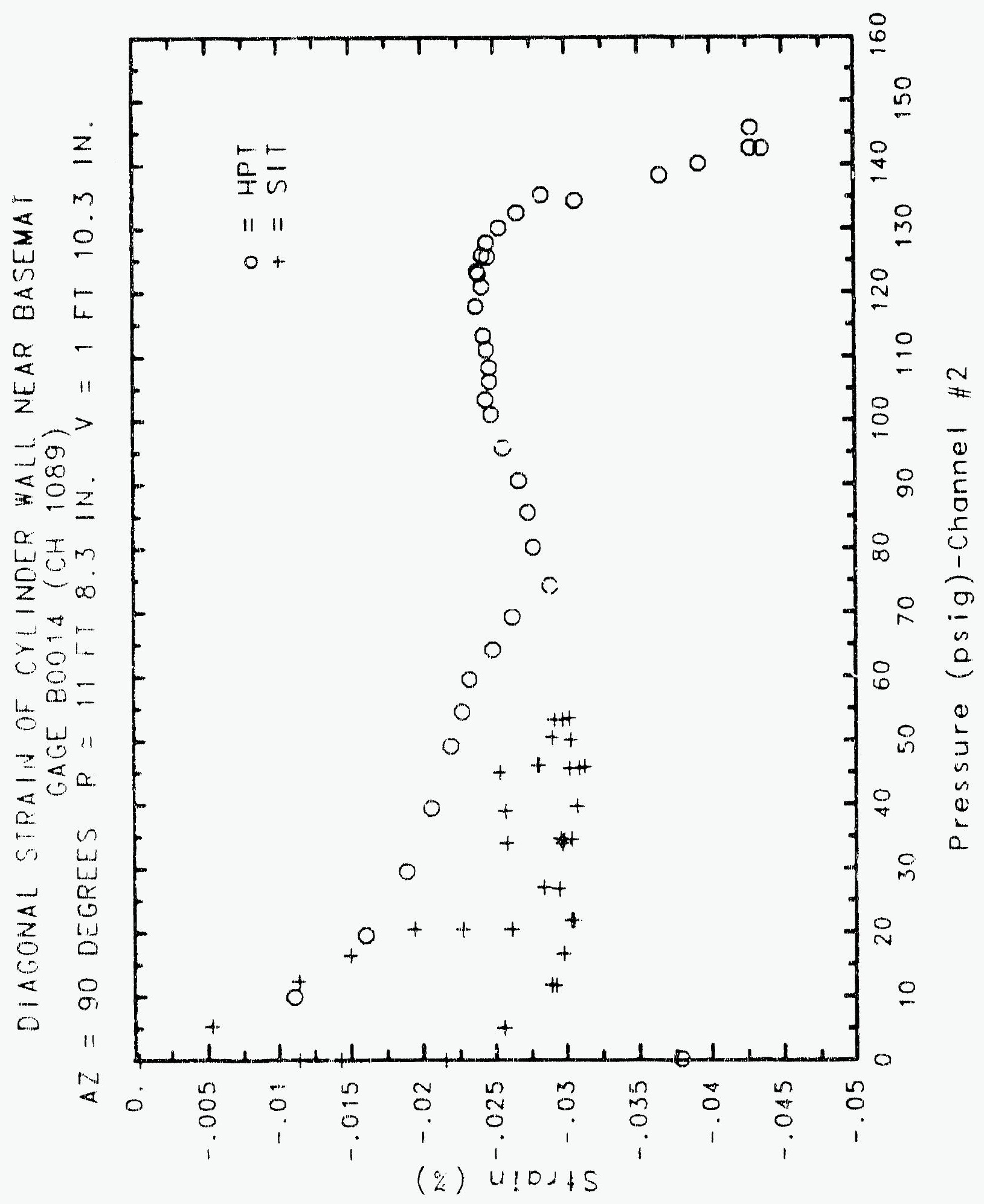




\section{Reinforced Concrete Test Data}

Bonded Gage 15 Channel 1100

Structural Integrity Test

High Pressure test

\begin{tabular}{|c|c|c|c|}
\hline $\begin{array}{c}\text { Pressure } \\
\text { (psig) } \\
-0.05 \\
5.33 \\
12.31 \\
16.44 \\
20.51 \\
20.46 \\
20.47 \\
27.05 \\
34.69 \\
34.53 \\
34.53 \\
39.70 \\
45.90 \\
45.65 \\
45.62 \\
50.10 \\
53.47 \\
53.29 \\
53.21 \\
50.49 \\
46.14 \\
46.13 \\
44.97 \\
38.98 \\
33.99 \\
33.96 \\
26.83 \\
21.88 \\
21.90 \\
16.69 \\
11.74 \\
11.77 \\
5.05 \\
0.02 \\
-0.04 \\
-0.02 \\
-0.02 \\
0.02 \\
\end{array}$ & $\begin{array}{l}\text { X Strain } \\
0.0002 \\
-0.0019 \\
-0.0046 \\
-0.0058 \\
-0.0069 \\
-0.0070 \\
-0.0071 \\
-0.0079 \\
-0.0088 \\
-0.0087 \\
-0.0085 \\
-0.0087 \\
-0.0085 \\
-0.0082 \\
-0.0084 \\
-0.0082 \\
-0.0081 \\
-0.0077 \\
-0.0078 \\
-0.0077 \\
-0.0080 \\
-0.0079 \\
-0.0092 \\
-0.0088 \\
-0.0093 \\
-0.0092 \\
-0.0091 \\
-0.0090 \\
-0.0091 \\
-0.0084 \\
-0.0076 \\
-0.0072 \\
-0.0054 \\
-0.0028 \\
-0.0027 \\
-0.0027 \\
-0.0025 \\
-0.0027\end{array}$ & $\begin{array}{c}\text { Pressure } \\
(\text { psig) } \\
9.89 \\
19.55 \\
29.57 \\
39.42 \\
49.16 \\
54.50 \\
59.57 \\
64.20 \\
69.32 \\
74.16 \\
80.16 \\
85.61 \\
90.58 \\
95.69 \\
100.92 \\
103.25 \\
106.11 \\
108.31 \\
111.08 \\
113.24 \\
117.83 \\
120.92 \\
123.28 \\
122.97 \\
125.82 \\
125.60 \\
127.84 \\
130.19 \\
132.53 \\
135.33 \\
134.42 \\
138.35 \\
140.16 \\
142.63 \\
145.78 \\
142.52 \\
0.22 \\
\end{array}$ & $\begin{array}{l}x \text { Strain } \\
-0.0056 \\
-0.0081 \\
-0.0090 \\
-0.0089 \\
-0.0093 \\
-0.0091 \\
-0.0088 \\
-0.0085 \\
-0.0084 \\
-0.0094 \\
-0.0068 \\
-0.0051 \\
-0.0035 \\
-0.0015 \\
0.0004 \\
0.0019 \\
0.0038 \\
0.0055 \\
0.0077 \\
0.0096 \\
0.0140 \\
0.0170 \\
0.0191 \\
0.0189 \\
0.0215 \\
0.0228 \\
0.0247 \\
0.0290 \\
0.0339 \\
0.0382 \\
0.0379 \\
0.0417 \\
0.0440 \\
0.0482 \\
0.0538 \\
0.0554 \\
0.0201\end{array}$ \\
\hline
\end{tabular}




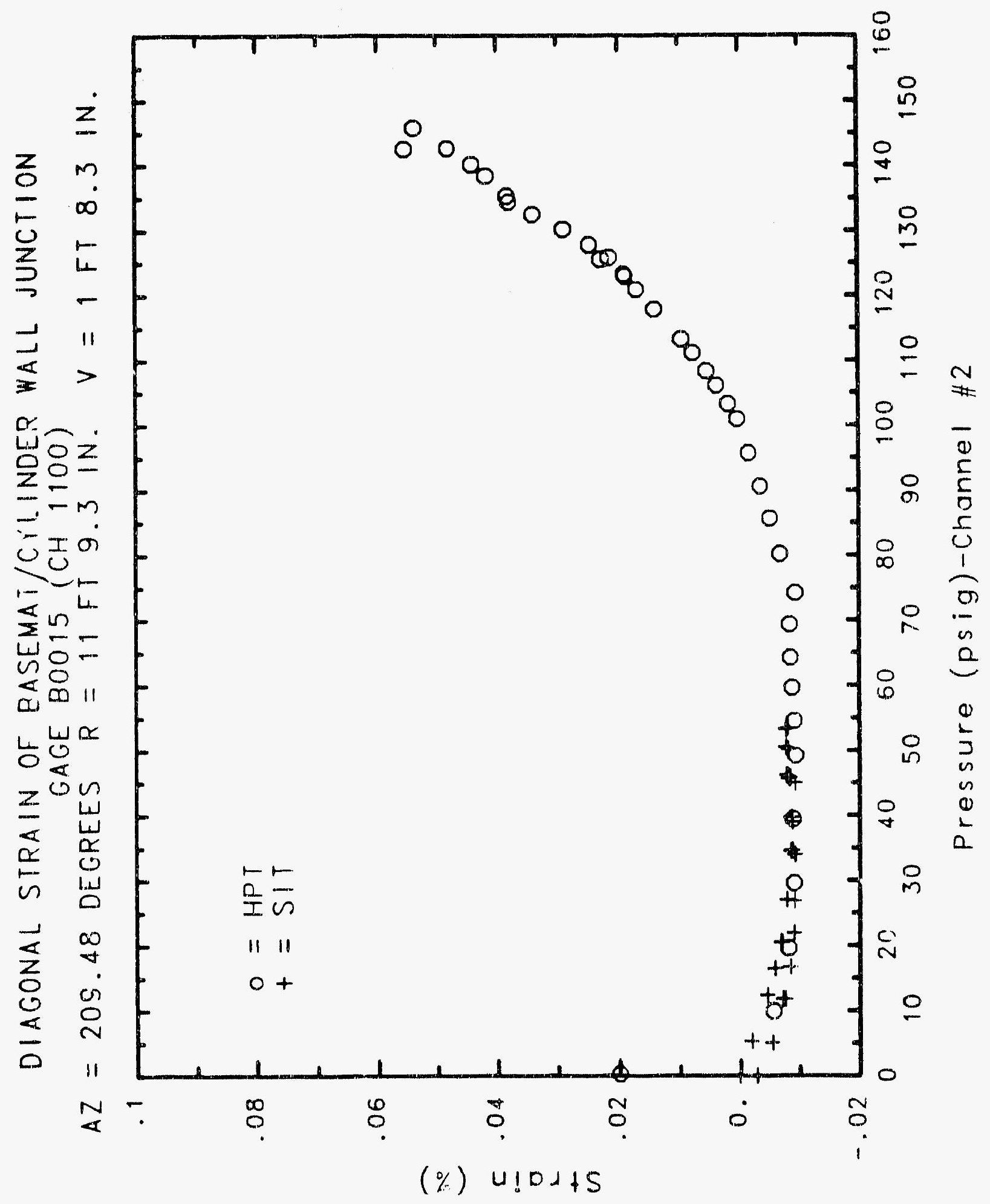




\section{Reinforced Concrete Test Data}

Bonded gage 16 Chammel 1101

STRUCTURAL INTEgRITY TEST

High Pressure test

\begin{tabular}{|c|c|c|c|}
\hline $\begin{array}{c}\text { Pressure } \\
\text { (psig) } \\
-0.05 \\
5.33 \\
12.31 \\
16.44 \\
20.51 \\
20.46 \\
20.47 \\
27.05 \\
34.59 \\
34.53 \\
34.53 \\
39.70 \\
45.40 \\
45.65 \\
45.62 \\
50.10 \\
53.47 \\
53.29 \\
53.21 \\
50.49 \\
46.14 \\
46.13 \\
44.97 \\
38.98 \\
33.99 \\
33.96 \\
26.83 \\
21.88 \\
21.90 \\
16.69 \\
11.74 \\
11.77 \\
5.05 \\
0.02 \\
-0.04 \\
-0.02 \\
-0.02 \\
0.02 \\
\end{array}$ & $\begin{array}{l}\text { X Strain } \\
-0.0006 \\
-0.0010 \\
-0.0026 \\
-0.0040 \\
-0.0057 \\
-0.0065 \\
-0.0071 \\
-0.0081 \\
-0.0095 \\
-0.0106 \\
-0.0113 \\
-0.0111 \\
-0.0112 \\
-0.0108 \\
-0.0104 \\
-0.0105 \\
-0.0099 \\
-0.0097 \\
-0.0093 \\
-0.0086 \\
-0.0086 \\
-0.0083 \\
-0.0099 \\
-0.0098 \\
-0.0099 \\
-0.0105 \\
-0.0106 \\
-0.0102 \\
-0.0111 \\
-0.0113 \\
-0.0105 \\
-0.0102 \\
-0.0081 \\
-0.0060 \\
-0.0056 \\
-0.0067 \\
-0.0056 \\
-0.00653\end{array}$ & $\begin{array}{c}\text { Pressure } \\
\text { (ps1g) } \\
9.89 \\
19.55 \\
29.57 \\
39.42 \\
49.16 \\
54.50 \\
59.57 \\
64.20 \\
69.32 \\
74.16 \\
80.16 \\
85.61 \\
90.58 \\
95.69 \\
100.92 \\
103.25 \\
106.11 \\
108.31 \\
111.08 \\
113.24 \\
117.83 \\
120.92 \\
123.28 \\
122.97 \\
125.82 \\
125.60 \\
127.84 \\
130.19 \\
132.53 \\
135.33 \\
134.42 \\
138.35 \\
140.16 \\
142.63 \\
145.78 \\
142.52 \\
0.22\end{array}$ & $\begin{array}{l}\text { X Strain } \\
-0.0035 \\
-0.0054 \\
-0.0066 \\
-0.0071 \\
-0.0076 \\
-0.0078 \\
-0.0072 \\
-0.0084 \\
-0.0088 \\
-0.0106 \\
-0.0064 \\
-0.0054 \\
-0.0045 \\
-0.0029 \\
-0.0019 \\
-0.0010 \\
0.0001 \\
0.0009 \\
0.0018 \\
0.0023 \\
0.0032 \\
0.0040 \\
0.0043 \\
0.0025 \\
0.0027 \\
0.0024 \\
0.0041 \\
0.0077 \\
0.0112 \\
0.0151 \\
0.0159 \\
0.0192 \\
0.0218 \\
0.0264 \\
0.0317 \\
0.0335 \\
0.0098\end{array}$ \\
\hline
\end{tabular}




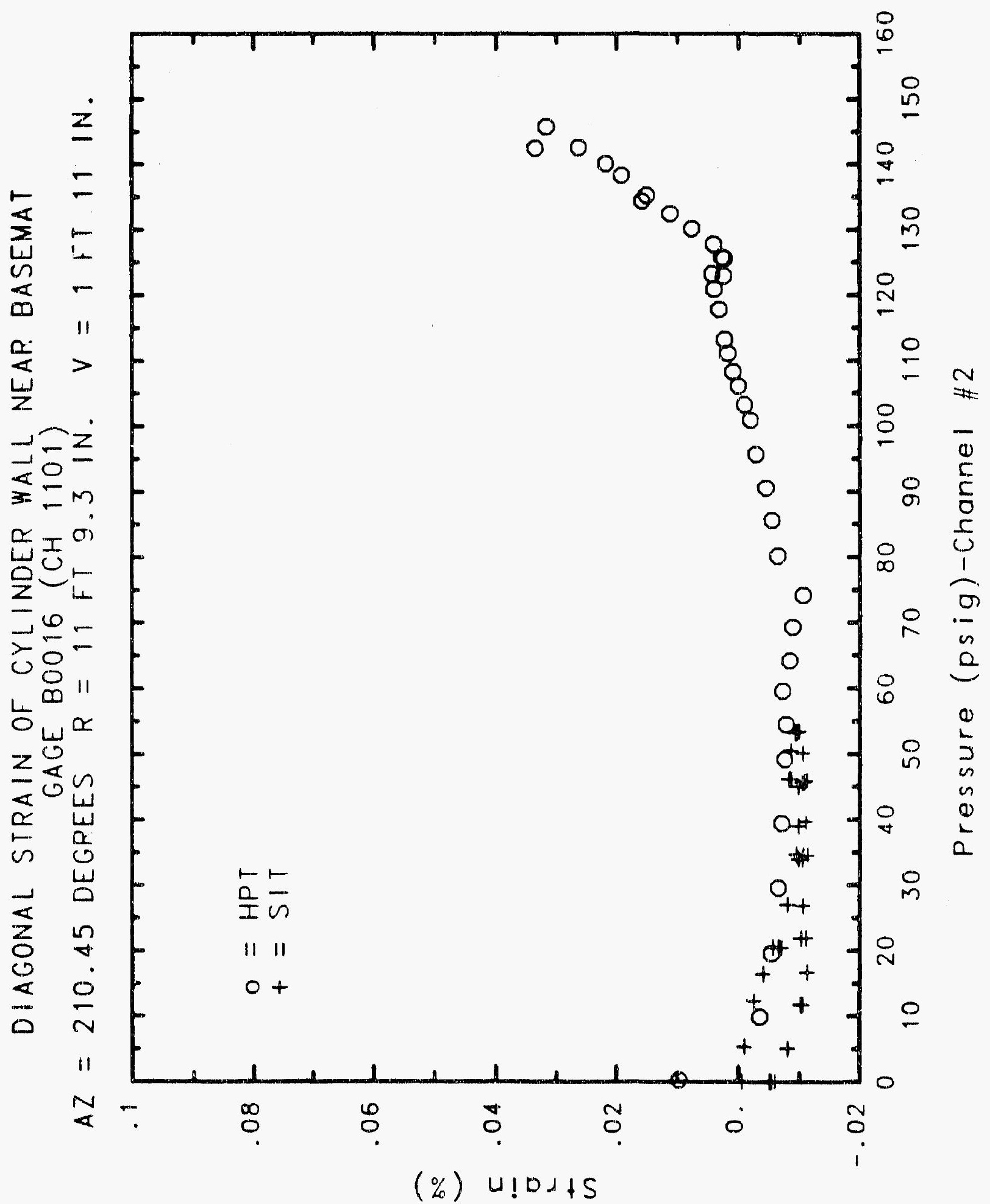




\section{Reinforced Concrete Test Data}

Bonded gage 17 Channel 1102

StRUCTURAL INTEgRITY TEST

\begin{tabular}{|c|c|c|c|}
\hline $\begin{array}{c}\text { Pressure } \\
(\text { psig) } \\
-0.05 \\
5.33 \\
12.31 \\
16.44 \\
20.51 \\
20.46 \\
20.47 \\
27.05 \\
34.69 \\
34.53 \\
34.53 \\
39.70 \\
45.90 \\
45.65 \\
45.62 \\
50.10 \\
53.47 \\
53.29 \\
53.21 \\
50.49 \\
46.14 \\
46.13 \\
44.97 \\
38.98 \\
33.99 \\
33.96 \\
26.83 \\
21.88 \\
21.90 \\
16.69 \\
11.74 \\
11.77 \\
5.05 \\
0.02 \\
-0.04 \\
-0.02 \\
-0.02 \\
0.02 \\
\end{array}$ & $\begin{array}{l}\text { S Strain } \\
-0.0009 \\
-0.0039 \\
-0.0053 \\
-0.0058 \\
-0.0071 \\
-0.0077 \\
-0.0079 \\
-0.0101 \\
-0.0113 \\
-0.0116 \\
-0.0116 \\
-0.0123 \\
-0.0126 \\
-0.0125 \\
-0.0125 \\
-0.0121 \\
-0.0127 \\
-0.0120 \\
-0.0118 \\
-0.0113 \\
-0.0113 \\
-0.0109 \\
-0.0110 \\
-0.0109 \\
-0.0106 \\
-0.0107 \\
-0.0104 \\
-0.0101 \\
-0.0106 \\
-0.0098 \\
-0.0092 \\
-0.0086 \\
-0.0063 \\
-0.0017 \\
0.0020 \\
-0.0048 \\
-0.0054 \\
-0.0034\end{array}$ & $\begin{array}{c}\text { Pressure } \\
(\text { psig) } \\
9.89 \\
19.55 \\
29.57 \\
39.42 \\
49.16 \\
54.50 \\
59.57 \\
64.20 \\
69.32 \\
74.16 \\
80.16 \\
85.61 \\
90.58 \\
95.69 \\
100.92 \\
103.25 \\
106.11 \\
108.31 \\
111.08 \\
113.24 \\
117.83 \\
120.92 \\
123.28 \\
122.97 \\
125.82 \\
125.60 \\
127.84 \\
130.19 \\
132.53 \\
135.33 \\
134.42 \\
138.35 \\
140.16 \\
142.63 \\
145.78 \\
142.52 \\
0.22\end{array}$ & $\begin{array}{c}\text { x Strain } \\
-0.0071 \\
-0.0094 \\
-0.0106 \\
-0.0113 \\
-0.0120 \\
-0.0121 \\
-0.0124 \\
-0.0124 \\
-0.0128 \\
-0.0112 \\
-0.0081 \\
-0.0061 \\
-0.0047 \\
-0.0031 \\
-0.0013 \\
0.0004 \\
0.0019 \\
0.0031 \\
0.0057 \\
0.0081 \\
0.0140 \\
0.0189 \\
0.0227 \\
0.0232 \\
0.0251 \\
0.0252 \\
0.0264 \\
0.0281 \\
0.0317 \\
0.0356 \\
0.0362 \\
0.0405 \\
0.0439 \\
0.0481 \\
0.0529 \\
0.0534 \\
0.0126\end{array}$ \\
\hline
\end{tabular}

\section{High Pressure test}

Pressure * Straín

9.89

19.55

39.42

49.16

54.50

59.57

64.20

69.32

74.16

80.16

85.61

90.58

06.11

108.31

111.08

113.24

122.97

125.82

125.60

132.53

135.33

134.42

138.35

140.16

2.52

0.0126 


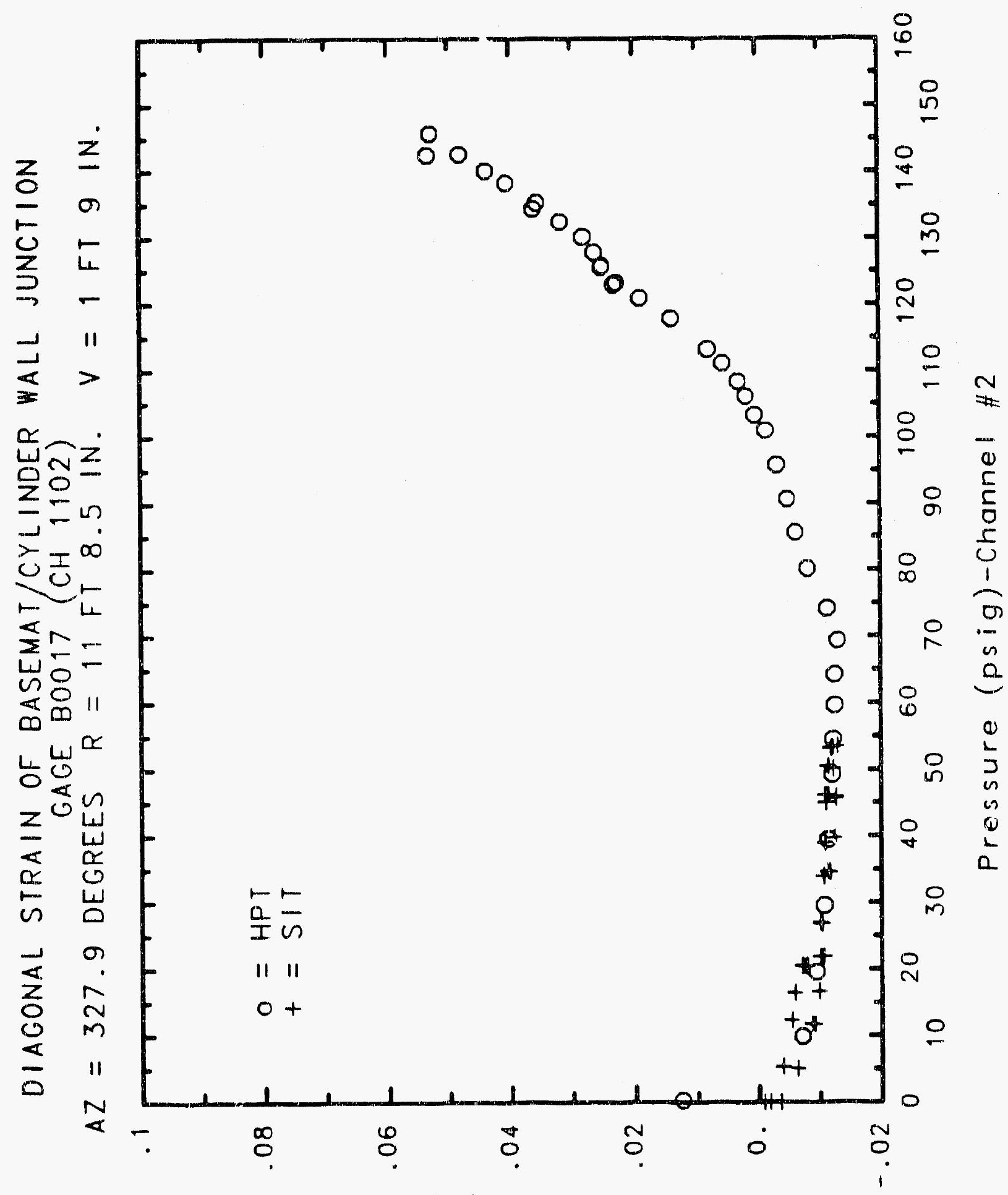

(\%) $u ! 0+t S$ 


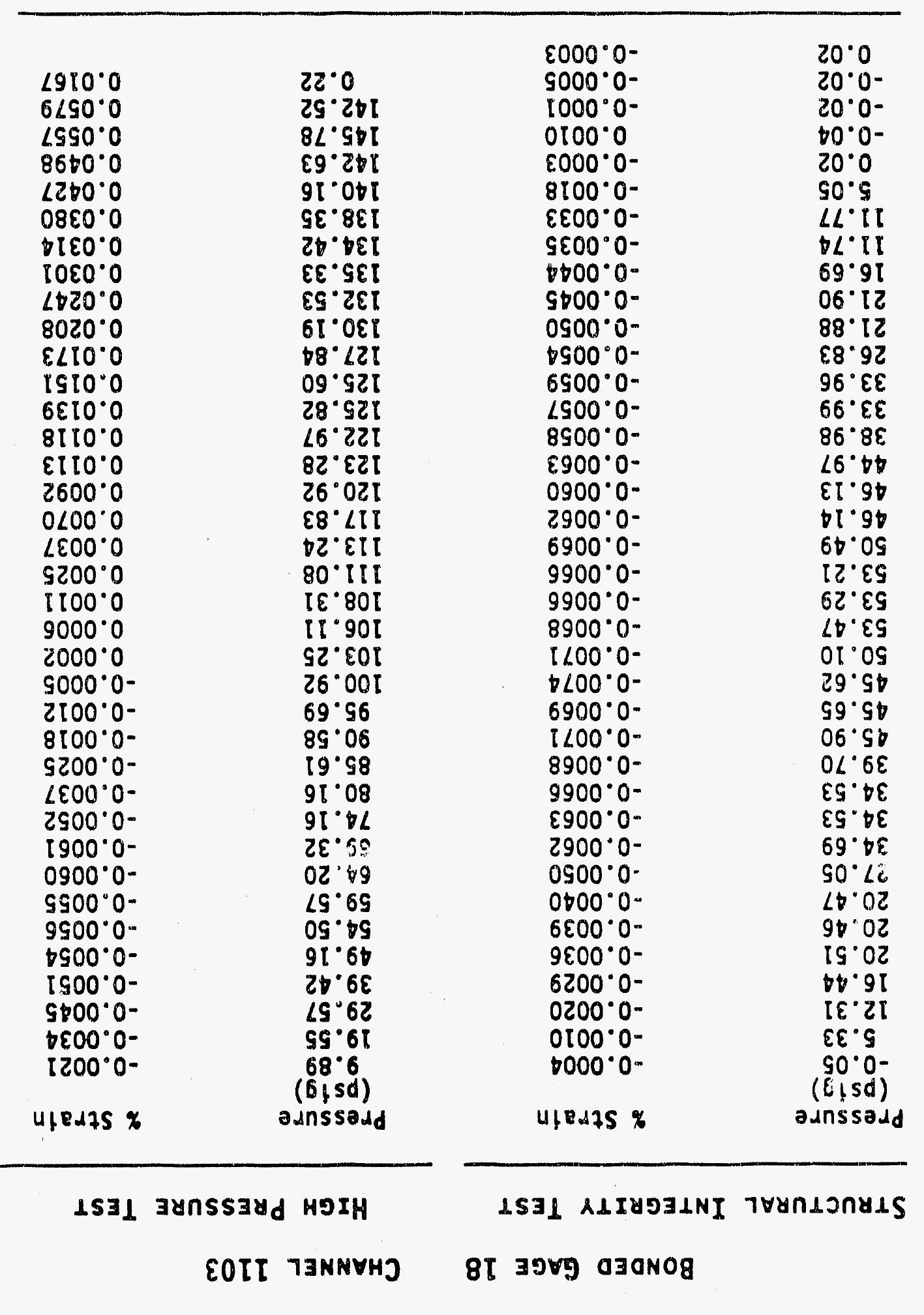

$\forall \perp \forall$ ISI JLJYJNOJ aJJUOANIJY 


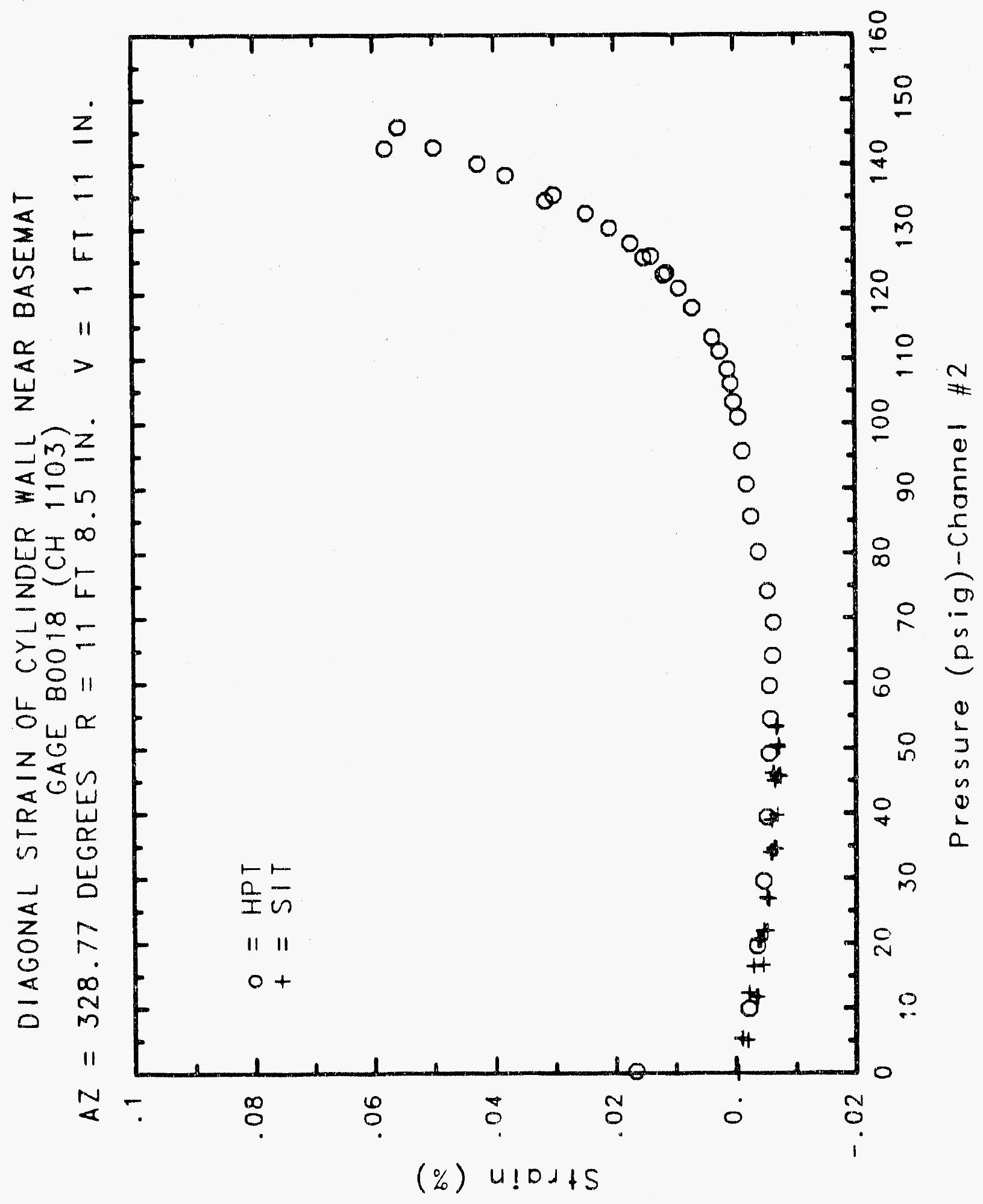




\section{Reinforced Concrete Test Data}

Bonded gage 19 Channel 1104

Structural Integrity test

High Pressure test

\begin{tabular}{|c|c|c|c|}
\hline $\begin{array}{c}\text { Pressure } \\
\text { (psig) } \\
-0.05 \\
5.33 \\
12.31 \\
16.44 \\
20.51 \\
20.46 \\
20.47 \\
27.05 \\
34.69 \\
34.53 \\
34.53 \\
39.70 \\
45.90 \\
45.65 \\
45.62 \\
50.10 \\
53.47 \\
53.29 \\
53.21 \\
50.49 \\
46.14 \\
46.13 \\
44.97 \\
38.98 \\
33.99 \\
33.96 \\
26.83 \\
21.88 \\
21.90 \\
16.69 \\
11.74 \\
11.77 \\
5.05 \\
0.02 \\
-0.04 \\
-0.02 \\
-0.02 \\
0.02 \\
\end{array}$ & $\begin{array}{l}\text { X Strain } \\
0.0000 \\
0.0035 \\
0.0087 \\
0.0125 \\
0.0177 \\
0.0183 \\
0.0186 \\
0.0286 \\
0.0428 \\
0.0445 \\
0.0454 \\
0.0528 \\
0.0650 \\
0.0649 \\
0.0650 \\
0.0708 \\
0.0768 \\
0.0768 \\
0.0770 \\
0.0748 \\
0.0697 \\
0.0694 \\
0.0686 \\
0.0619 \\
0.0559 \\
0.0559 \\
0.0466 \\
0.0408 \\
0.0411 \\
0.0349 \\
0.0287 \\
0.0279 \\
0.0199 \\
0.0136 \\
0.0092 \\
0.0094 \\
0.0098 \\
0.0099\end{array}$ & $\begin{array}{c}\text { Pressure } \\
\text { (psig) } \\
9.89 \\
19.55 \\
29.57 \\
39.42 \\
49.16 \\
54.50 \\
59.57 \\
64.20 \\
69.32 \\
74.16 \\
80.16 \\
85.61 \\
90.58 \\
95.69 \\
100.92 \\
103.25 \\
106.11 \\
108.31 \\
111.08 \\
113.24 \\
117.83 \\
120.92 \\
123.28 \\
122.97 \\
125.82 \\
125.60 \\
127.84 \\
130.19 \\
132.53 \\
135.33 \\
134.42 \\
138.35 \\
140.16 \\
142.63 \\
145.78 \\
142.52 \\
0.22\end{array}$ & $\begin{array}{l}\text { x Strain } \\
0.0126 \\
0.0265 \\
0.0406 \\
0.0542 \\
0.0673 \\
0.0748 \\
0.0824 \\
0.0902 \\
0.0990 \\
0.1078 \\
0.1184 \\
0.1290 \\
0.1392 \\
0.1507 \\
0.1630 \\
0.1690 \\
0.1759 \\
0.1814 \\
0.1898 \\
0.1966 \\
0.2122 \\
0.2229 \\
0.2323 \\
0.2338 \\
0.2471 \\
0.2564 \\
0.2684 \\
0.2781 \\
0.8371 \\
0.9816 \\
1.0371 \\
1.3404 \\
1.5195 \\
1.7260 \\
1.8837 \\
1.9140 \\
1.5445\end{array}$ \\
\hline
\end{tabular}




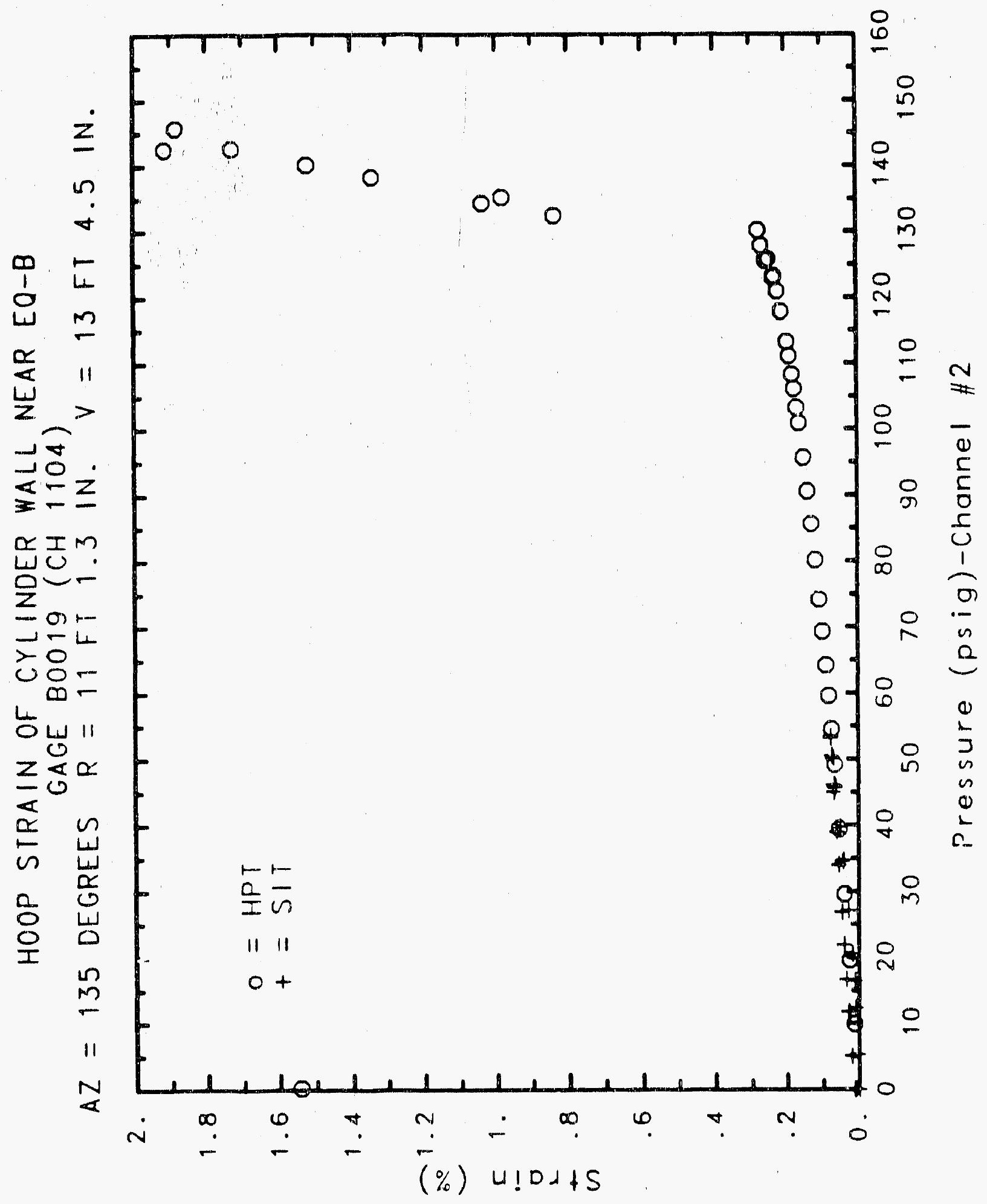




\section{Reinforced Concrete Test Data}

Bonded gage 20 Channel 1105

STRUCTURAL INTEGRITY TEST

High pressure test

\begin{tabular}{|c|c|c|c|}
\hline $\begin{array}{c}\text { Pressure } \\
\text { (psig) } \\
-0.05 \\
5.33 \\
12.31 \\
16.44 \\
20.51 \\
20.46 \\
20.47 \\
27.05 \\
34.69 \\
34.53 \\
34.53 \\
39.70 \\
45.90 \\
45.65 \\
45.62 \\
50.10 \\
53.47 \\
53.29 \\
53.21 \\
50.49 \\
46.14 \\
46.13 \\
44.97 \\
38.98 \\
33.99 \\
33.96 \\
26.83 \\
21.88 \\
21.90 \\
16.69 \\
11.74 \\
11.77 \\
5.05 \\
0.02 \\
-0.04 \\
-0.02 \\
-0.02 \\
0.02\end{array}$ & $\begin{array}{c}\text { Strain } \\
-0.0002 \\
0.0006 \\
0.0024 \\
0.0034 \\
0.0057 \\
0.0064 \\
0.0068 \\
0.0129 \\
0.0220 \\
0.0243 \\
0.0549 \\
0.0292 \\
0.0359 \\
0.0360 \\
0.0357 \\
0.0395 \\
0.0427 \\
0.0437 \\
0.0436 \\
0.0427 \\
0.0405 \\
0.0403 \\
0.0406 \\
0.0377 \\
0.0348 \\
0.0346 \\
0.0304 \\
0.0275 \\
0.0282 \\
0.0253 \\
0.0222 \\
0.0206 \\
0.0160 \\
0.0128 \\
0.0105 \\
0.0108 \\
0.0101 \\
0.0111\end{array}$ & $\begin{array}{c}\text { Pressure } \\
\text { (psig) } \\
9.89 \\
19.55 \\
29.57 \\
39.42 \\
49.16 \\
54.50 \\
59.57 \\
64.20 \\
69.32 \\
74.16 \\
80.16 \\
85.61 \\
90.58 \\
95.69 \\
100.92 \\
103.25 \\
106.11 \\
108.31 \\
111.08 \\
113.24 \\
117.83 \\
120.92 \\
123.98 \\
122.97 \\
125.82 \\
125.60 \\
127.84 \\
130.19 \\
132.53 \\
135.33 \\
134.42 \\
138.35 \\
140.16 \\
142.63 \\
145.78 \\
142.52 \\
0.22 \\
\end{array}$ & $\begin{array}{l}\text { \% Stra1li } \\
0.0059 \\
0.0129 \\
0.0198 \\
0.0265 \\
0.0331 \\
0.0369 \\
0.0412 \\
0.0457 \\
0.0510 \\
0.0562 \\
0.0626 \\
0.0686 \\
0.0739 \\
0.0804 \\
0.0869 \\
0.0901 \\
0.0936 \\
0.0964 \\
0.1006 \\
0.1035 \\
0.1108 \\
0.1150 \\
0.1182 \\
0.1180 \\
0.1196 \\
0.1195 \\
0.1206 \\
0.1219 \\
0.1226 \\
0.1313 \\
0.1318 \\
0.1398 \\
0.1529 \\
0.1949 \\
0.2261 \\
0.2260 \\
0.1170\end{array}$ \\
\hline
\end{tabular}




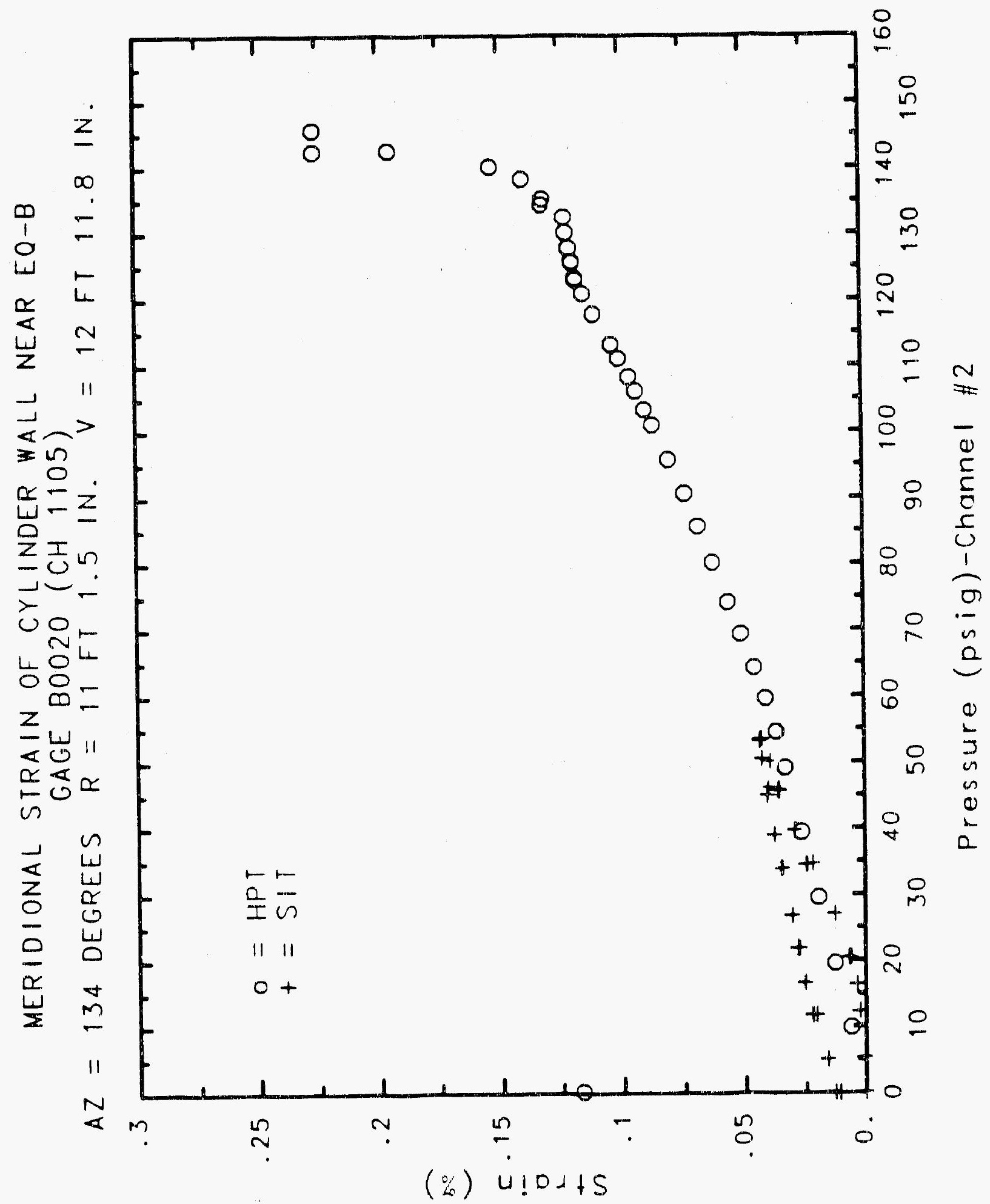




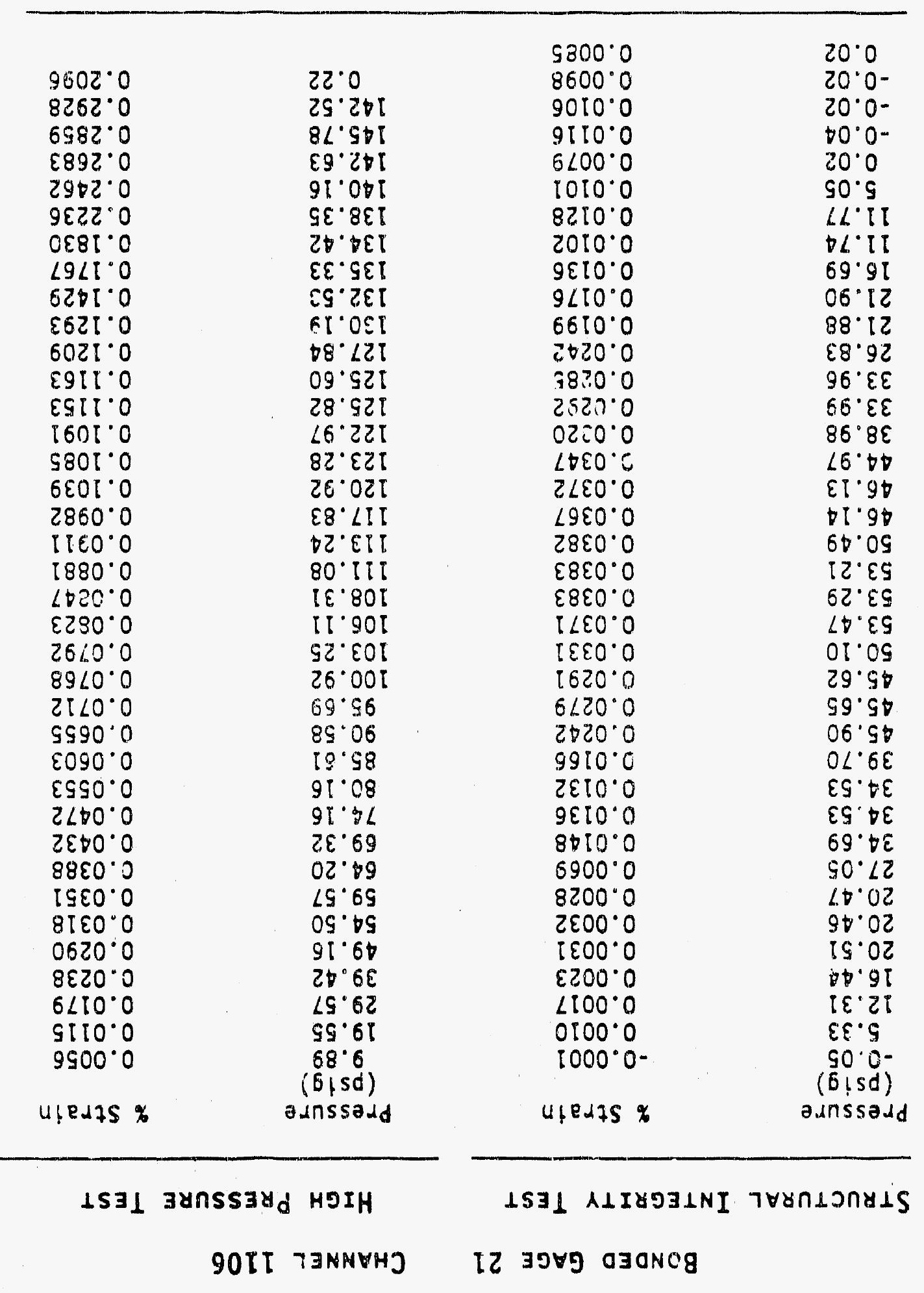

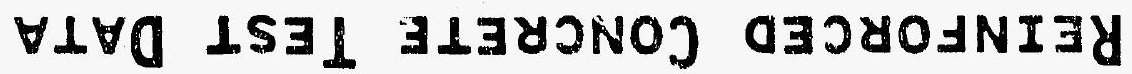




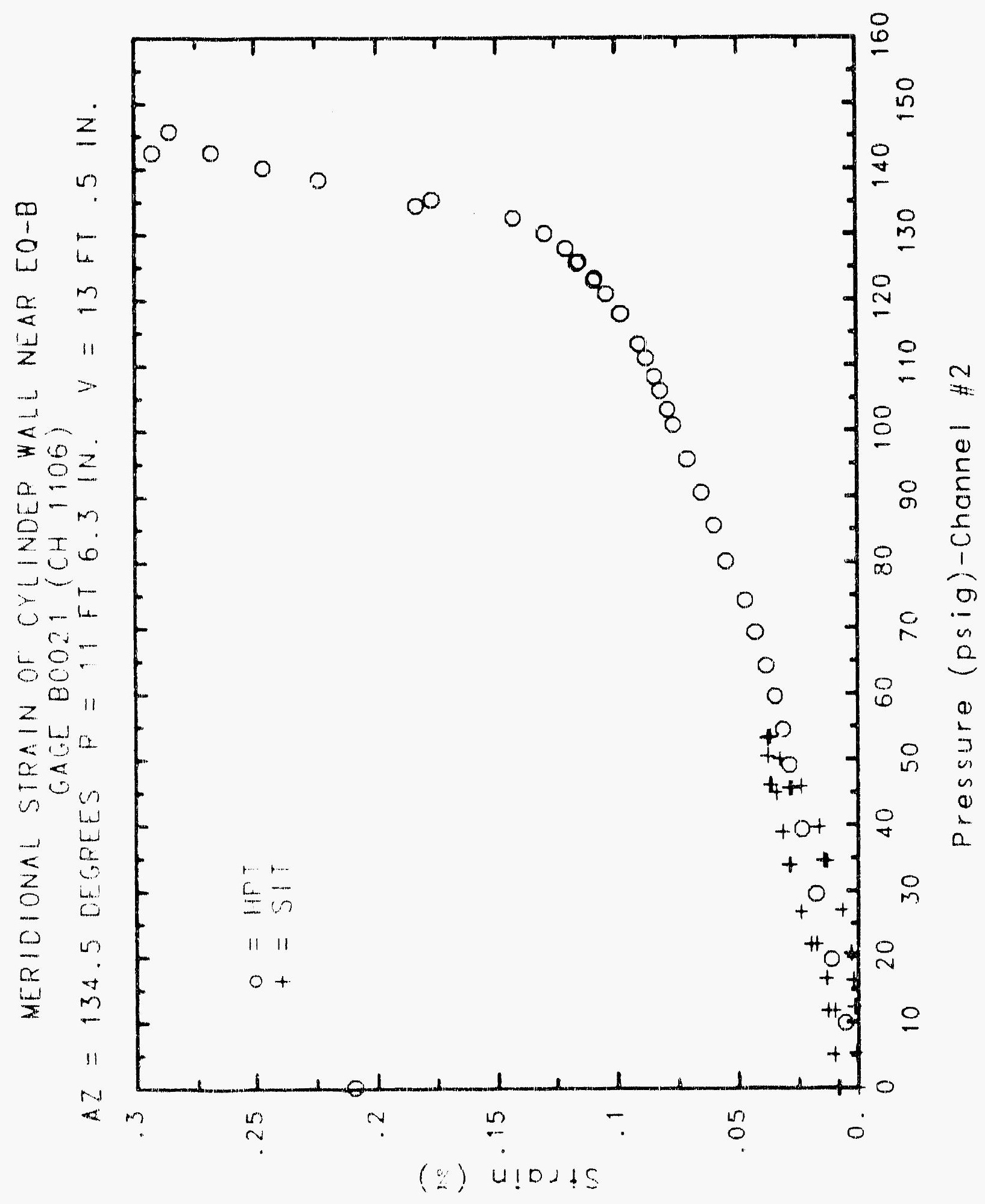




\section{Reinforced Concrete Test Data}

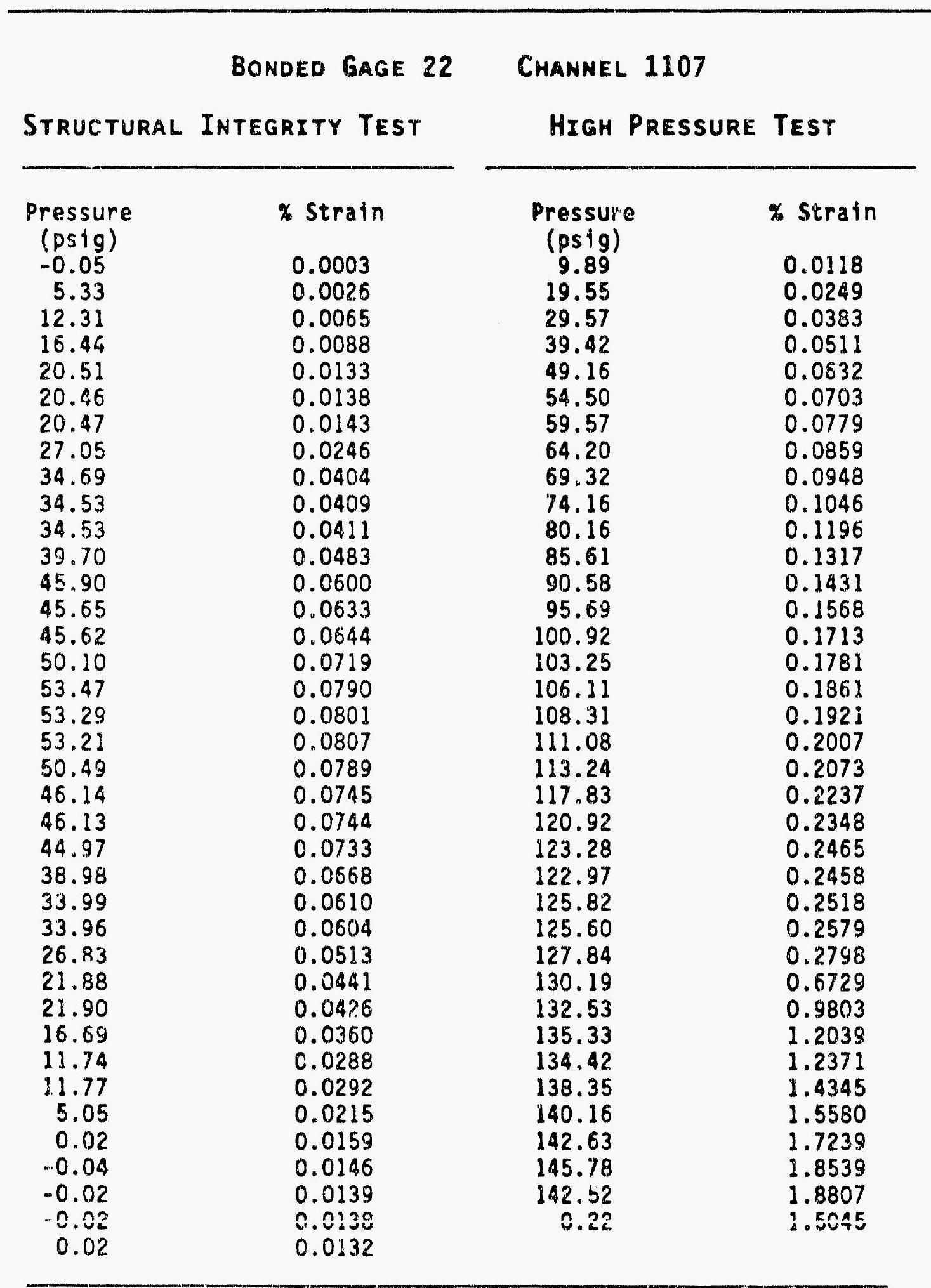




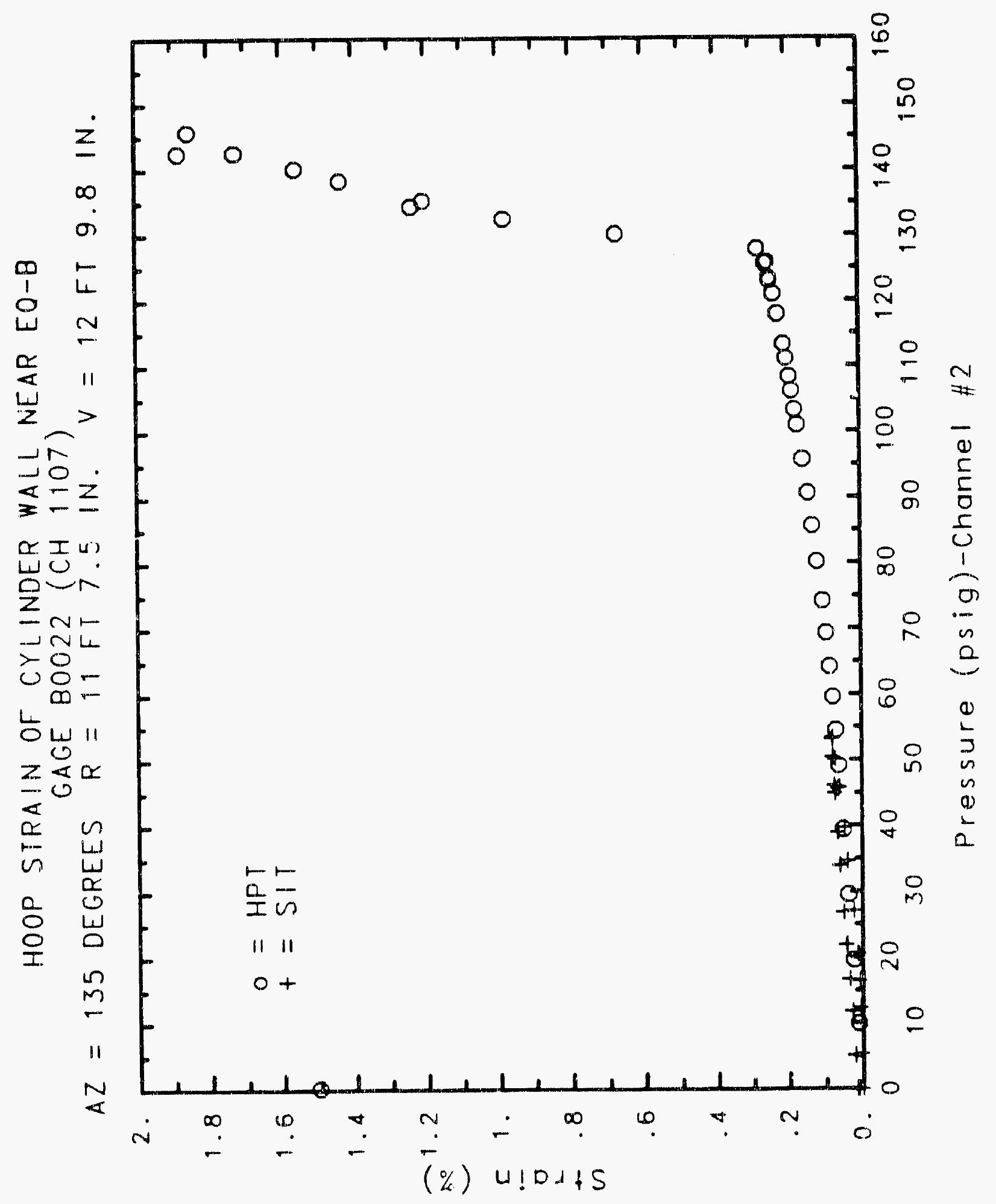




\section{Reinforced Concrete Test Data}

Bonded gage 23 Channel 1108

Structural Integrity Test

High Pressure test

\begin{tabular}{|c|c|c|c|}
\hline $\begin{array}{c}\text { Pressure } \\
\text { (psig) } \\
-0.05 \\
5.33 \\
12.31 \\
16.44 \\
20.51 \\
20.46 \\
20.47 \\
27.05 \\
34.69 \\
34.53 \\
34.53 \\
39.70 \\
45.90 \\
45.65 \\
45.62 \\
50.10 \\
53.47 \\
53.29 \\
53.21 \\
50.49 \\
46.14 \\
46.13 \\
44.97 \\
38.98 \\
33.90 \\
33.96 \\
26.83 \\
21.88 \\
21.90 \\
16.69 \\
11.74 \\
11.77 \\
5.05 \\
0.02 \\
-0.04 \\
-0.02 \\
-0.02 \\
0.02 \\
0.06\end{array}$ & $\begin{array}{l}\text { \% Strain } \\
-0.0008 \\
-0.0004 \\
-0.0002 \\
0.0007 \\
0.0018 \\
0.0022 \\
0.0021 \\
0.0065 \\
0.0103 \\
0.0102 \\
0.0100 \\
0.0126 \\
0.0153 \\
0.0152 \\
0.0148 \\
0.0167 \\
0.0182 \\
0.0184 \\
0.0180 \\
0.0164 \\
0.0148 \\
0.0144 \\
0.0121 \\
0.0099 \\
0.0074 \\
0.0074 \\
0.0046 \\
0.0028 \\
0.0033 \\
0.0014 \\
0.0001 \\
0.0012 \\
0.0007 \\
0.0005 \\
0.0019 \\
0.0019 \\
0.0013 \\
0.00112\end{array}$ & $\begin{array}{c}\text { Pressure } \\
(\text { psig) } \\
9.89 \\
19.55 \\
29.57 \\
39.42 \\
49.16 \\
54.50 \\
59.57 \\
64.20 \\
69.32 \\
74.16 \\
80.16 \\
85.61 \\
90.58 \\
95.69 \\
100.92 \\
103.25 \\
106.11 \\
108.31 \\
111.08 \\
113.24 \\
117.83 \\
120.92 \\
123.28 \\
122.97 \\
125.82 \\
125.60 \\
127.84 \\
130.19 \\
132.53 \\
135.33 \\
134.42 \\
138.35 \\
140.16 \\
142.63 \\
145.78 \\
142.52 \\
0.22\end{array}$ & $\begin{array}{l}\text { X Strain } \\
0.0004 \\
0.0026 \\
0.0057 \\
0.0094 \\
0.0144 \\
0.0162 \\
0.0185 \\
0.0196 \\
0.0216 \\
0.0231 \\
0.0255 \\
0.0270 \\
0.0279 \\
0.0275 \\
0.0275 \\
0.0268 \\
0.0268 \\
0.0273 \\
0.0267 \\
0.0263 \\
0.0267 \\
0.0292 \\
0.0319 \\
0.0323 \\
0.0359 \\
0.0362 \\
0.0394 \\
0.0443 \\
0.0475 \\
0.0507 \\
0.0493 \\
0.0522 \\
0.0531 \\
0.0508 \\
0.0527 \\
0.0512 \\
0.0223\end{array}$ \\
\hline
\end{tabular}




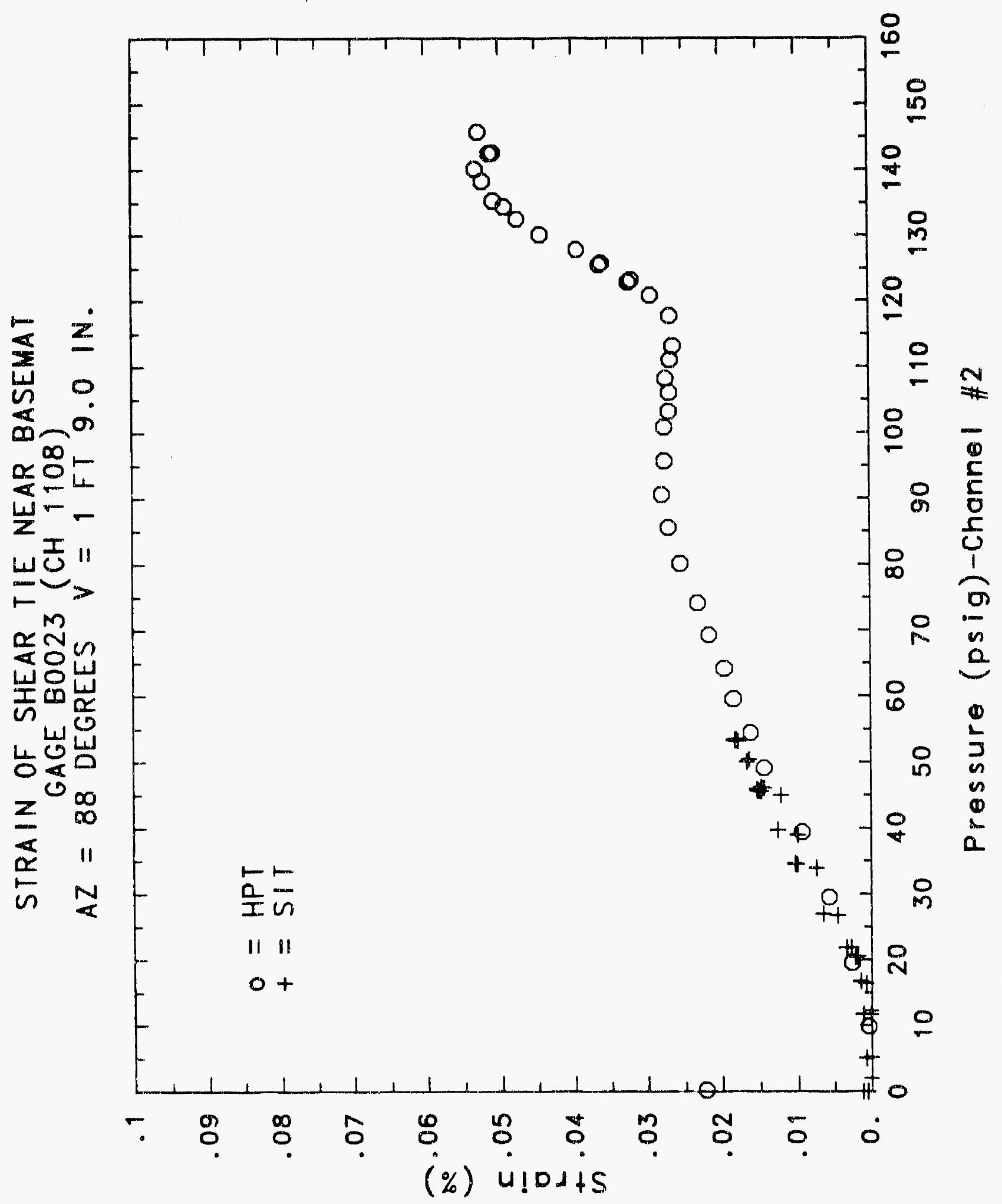




\section{Reinforced Concrete Test Data}

Bonded gage 24 Chamel 1109

Structural InTEgrity TEST

High Pressure test

\begin{tabular}{|c|c|c|c|}
\hline $\begin{array}{c}\text { Pressure } \\
\text { (psig) } \\
-0.05 \\
5.33 \\
12.31 \\
16.44 \\
20.51 \\
20.46 \\
20.47 \\
27.05 \\
34.69 \\
34.53 \\
34.53 \\
39.70 \\
45.90 \\
45.65 \\
45.62 \\
50.10 \\
53.47 \\
53.29 \\
53.21 \\
50.49 \\
46.14 \\
46.13 \\
44.97 \\
38.98 \\
33.99 \\
33.96 \\
26.83 \\
21.88 \\
21.90 \\
16.69 \\
11.74 \\
11.77 \\
5.05 \\
0.02 \\
-0.04 \\
-0.02 \\
-0.02 \\
0.02\end{array}$ & $\begin{array}{l}\text { \% Stra1n } \\
0.0001 \\
0.0032 \\
0.0082 \\
0.0117 \\
0.0176 \\
0.0185 \\
0.0184 \\
0.0358 \\
0.0514 \\
0.0514 \\
0.0521 \\
0.0606 \\
0.0720 \\
0.0733 \\
0.0741 \\
0.0807 \\
0.0869 \\
0.0870 \\
0.0869 \\
0.0847 \\
0.0790 \\
0.0792 \\
0.0750 \\
0.0674 \\
0.0600 \\
0.0596 \\
0.0489 \\
0.0413 \\
0.0409 \\
0.0335 \\
0.0261 \\
0.0270 \\
0.0173 \\
0.0111 \\
0.0101 \\
0.0090 \\
0.0084 \\
0.0078\end{array}$ & $\begin{array}{c}\text { Pressure } \\
(\text { psig) } \\
9.89 \\
19.55 \\
29.57 \\
39.42 \\
49.16 \\
54.50 \\
59.57 \\
64.20 \\
69.32 \\
74.16 \\
80.16 \\
85.61 \\
90.58 \\
95.69 \\
100.92 \\
103.25 \\
106.11 \\
108.31 \\
111.08 \\
113.24 \\
117.83 \\
120.92 \\
123.28 \\
122.97 \\
125.82 \\
125.60 \\
127.84 \\
130.19 \\
132.53 \\
135.33 \\
134.42 \\
138.35 \\
140.16 \\
142.63 \\
145.78 \\
142.52 \\
0.22\end{array}$ & $\begin{array}{l}\text { \% Strain } \\
0.0138 \\
0.0283 \\
0.0430 \\
0.0569 \\
0.0706 \\
0.0781 \\
0.0860 \\
0.0926 \\
0.1005 \\
0.1087 \\
0.1207 \\
0.1298 \\
0.1385 \\
0.1484 \\
0.1601 \\
0.1658 \\
0.1727 \\
0.1780 \\
0.1859 \\
0.1915 \\
0.2062 \\
0.2150 \\
0.2207 \\
0.2214 \\
0.2271 \\
0.2277 \\
0.2319 \\
0.2385 \\
0.2412 \\
0.2447 \\
0.2492 \\
0.4706 \\
0.7438 \\
0.8890 \\
1.0150 \\
1.2116 \\
0.9012\end{array}$ \\
\hline
\end{tabular}




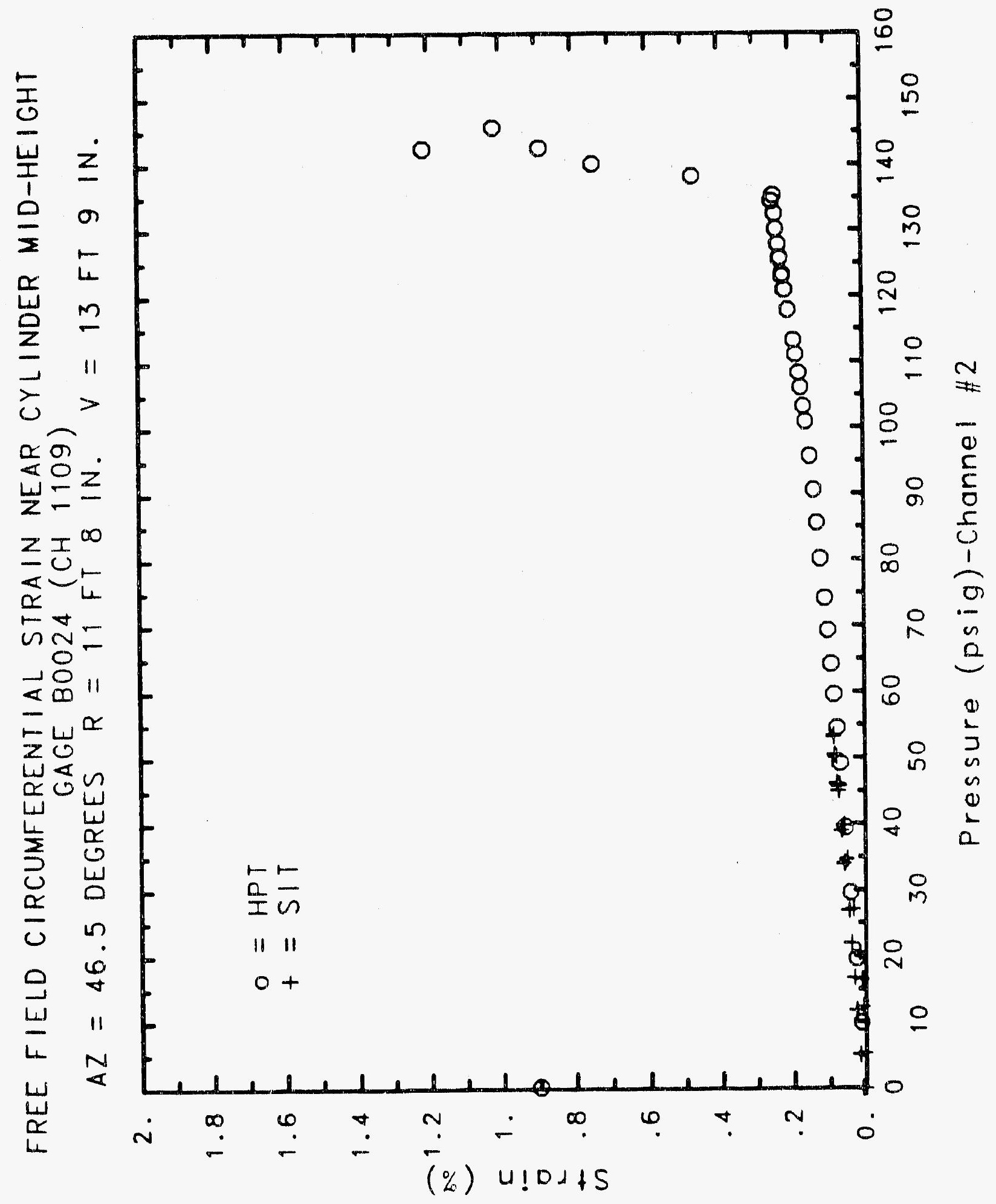




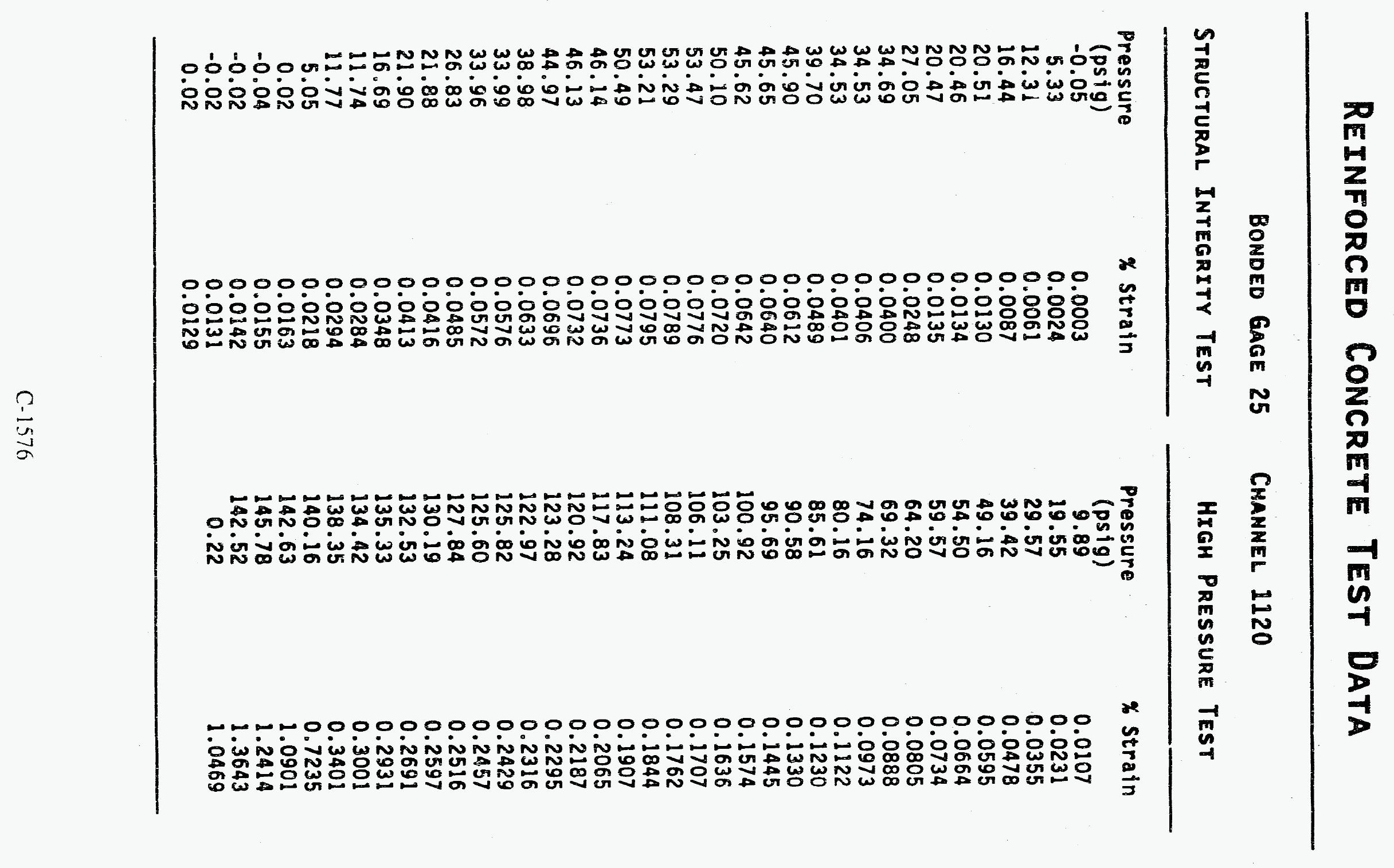




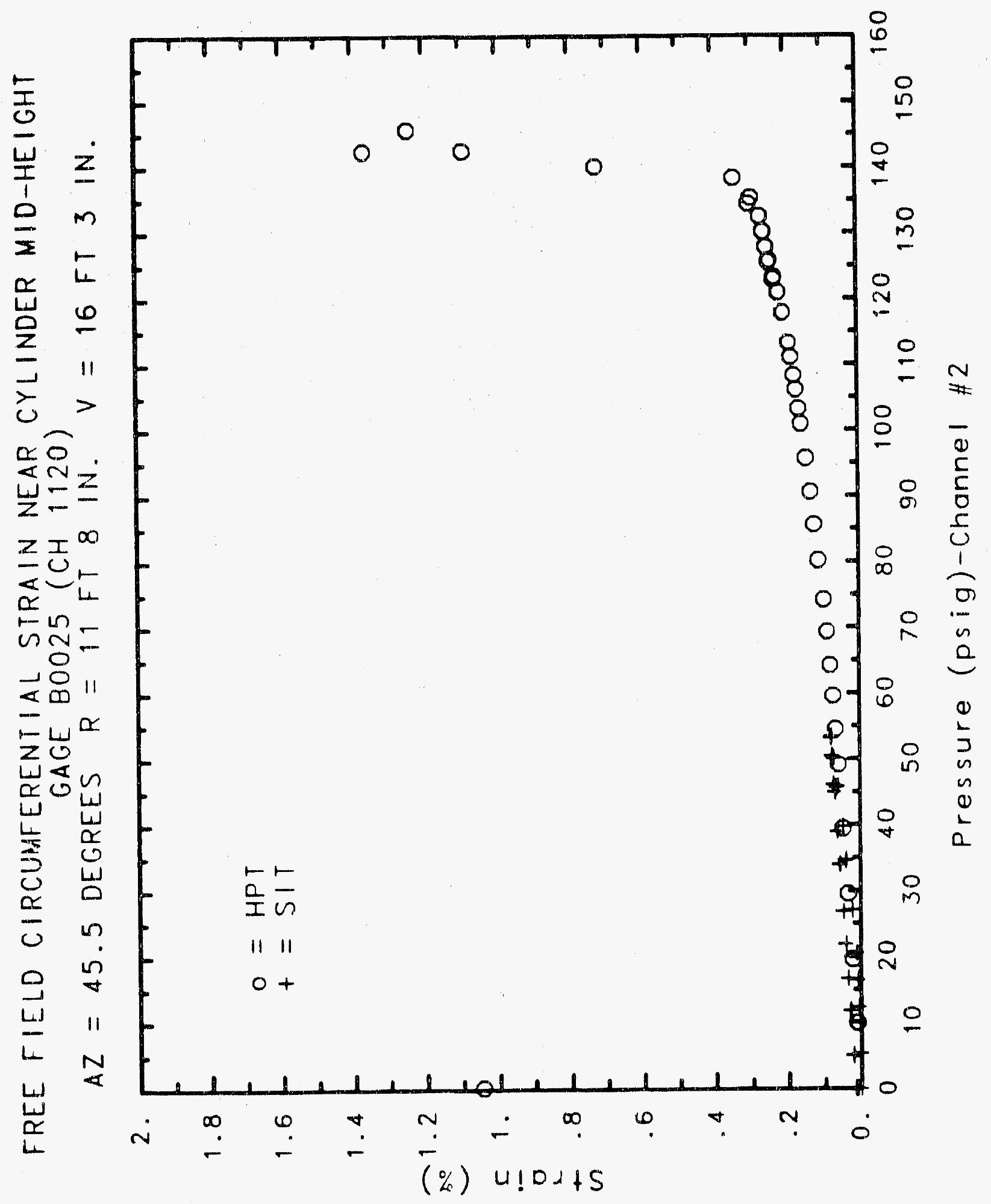




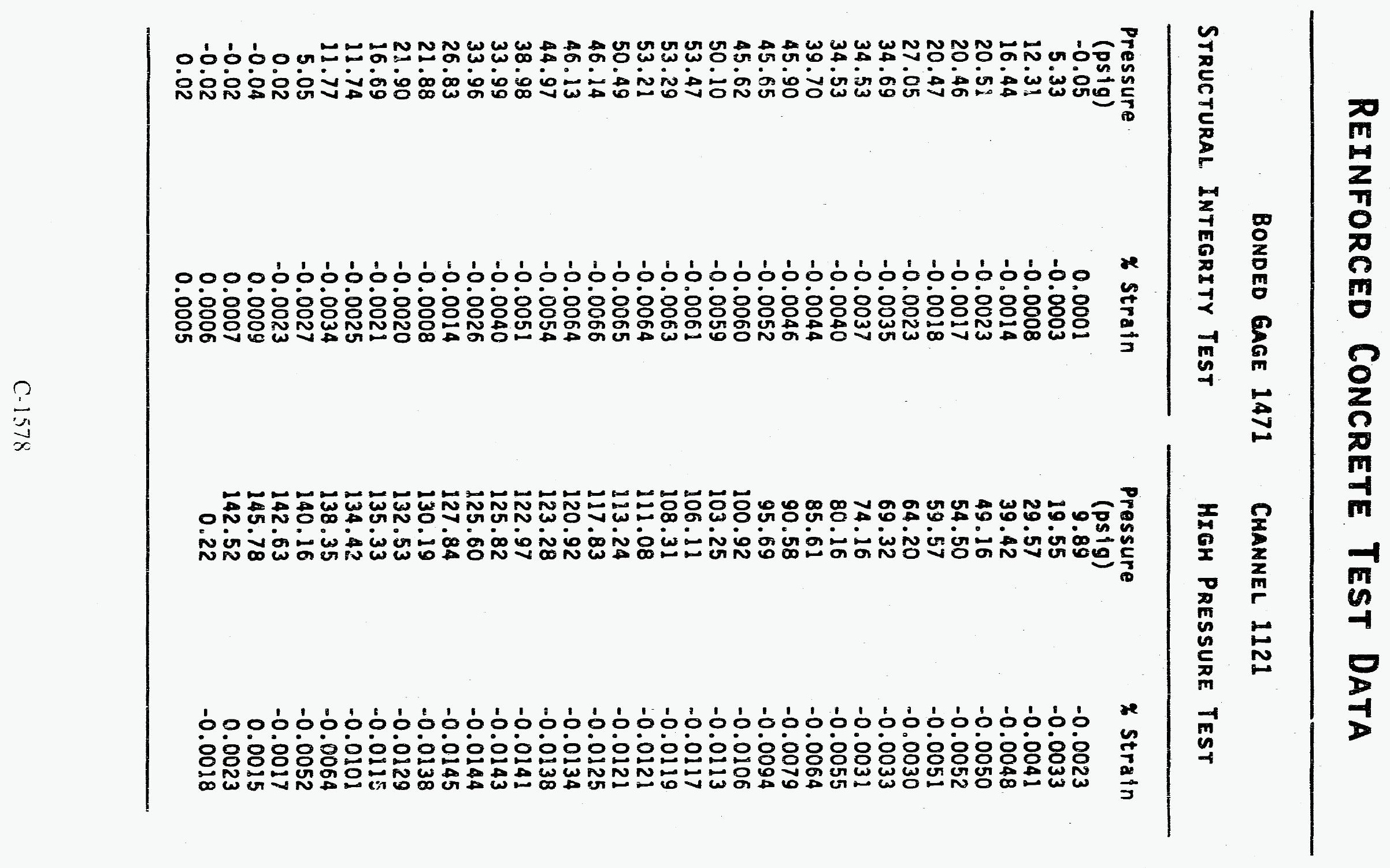




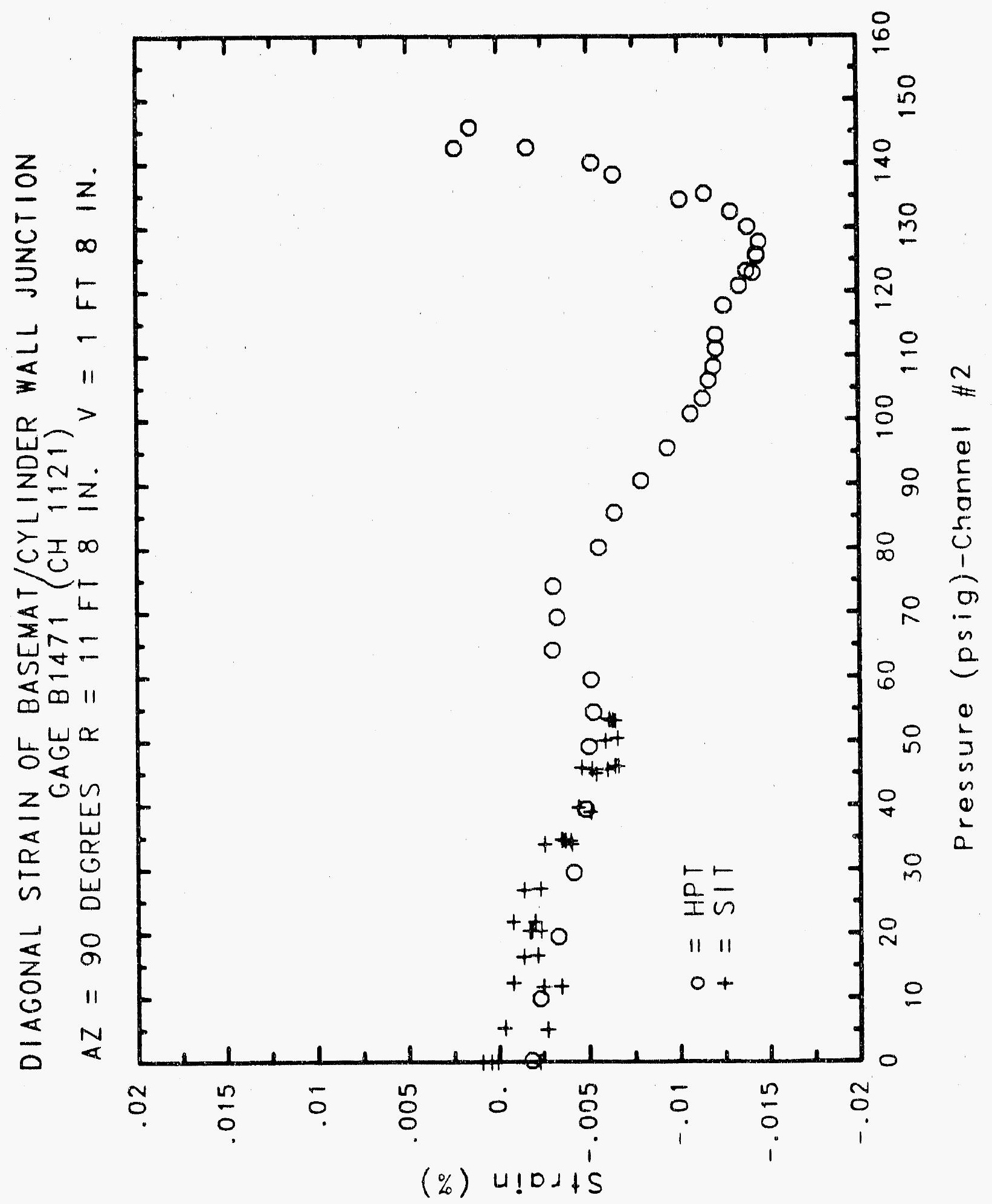




\section{Reinforced Concrete Test Data}

Bonded gage 1481 Cihannel 1122

Structural Integrity Test

\begin{tabular}{|c|c|c|c|}
\hline $\begin{array}{c}\text { Pressure } \\
\text { (psig) } \\
-0.05 \\
5.33 \\
12.31 \\
16.44 \\
20.51 \\
20.46 \\
20.47 \\
27.05 \\
34.69 \\
34.53 \\
34.53 \\
39.70 \\
45.90 \\
45.65 \\
45.62 \\
50.10 \\
53.47 \\
53.29 \\
53.21 \\
50.49 \\
46.14 \\
46.13 \\
44.97 \\
38.98 \\
33.99 \\
33.96 \\
26.83 \\
21.88 \\
21.90 \\
16.69 \\
11.74 \\
11.77 \\
5.05 \\
0.02 \\
-0.04 \\
-0.02 \\
-0.02 \\
0.02\end{array}$ & $\begin{array}{l}\text { Strain } \\
-0.0001 \\
-0.0023 \\
-0.0048 \\
-0.0064 \\
-0.0072 \\
-0.0071 \\
-0.0067 \\
-0.0077 \\
-0.0081 \\
-0.0078 \\
-0.0084 \\
-0.0089 \\
-0.0092 \\
-0.0096 \\
-0.0093 \\
-0.0099 \\
-0.0097 \\
-0.0100 \\
-0.0098 \\
-0.0099 \\
-0.0094 \\
-0.0095 \\
-0.0090 \\
-0.0087 \\
-0.0082 \\
-0.0073 \\
-0.0067 \\
-0.0066 \\
-0.0078 \\
-0.0073 \\
-0.0078 \\
-0.0080 \\
-0.0069 \\
-0.0052 \\
-0.0026 \\
-0.0022 \\
-0.0024 \\
-0.0028\end{array}$ & $\begin{array}{c}\text { Pressure } \\
(\text { psig) } \\
9.89 \\
19.55 \\
29.57 \\
39.42 \\
49.16 \\
54.50 \\
59.57 \\
64.20 \\
69.32 \\
74.16 \\
80.16 \\
85.61 \\
90.58 \\
95.69 \\
100.92 \\
103.25 \\
106.11 \\
108.31 \\
111.08 \\
113.24 \\
117.83 \\
120.92 \\
123.28 \\
122.97 \\
125.82 \\
125.60 \\
127.84 \\
130.19 \\
132.53 \\
135.33 \\
134.42 \\
138.35 \\
140.16 \\
142.63 \\
145.78 \\
142.52 \\
0.22 \\
\end{array}$ & $\begin{array}{l}\text { \% Strain } \\
-0.0047 \\
-0.0066 \\
-0.0078 \\
-0.0086 \\
-0.0089 \\
-0.0091 \\
-0.0089 \\
-0.0077 \\
-0.0074 \\
-0.0081 \\
-0.0094 \\
-0.0100 \\
-0.0101 \\
-0.0103 \\
-0.0106 \\
-0.0112 \\
-0.0115 \\
-0.0117 \\
-0.0126 \\
-0.0126 \\
-0.0125 \\
-0.0126 \\
-0.0125 \\
-0.0124 \\
-0.0123 \\
-0.0122 \\
-0.0117 \\
-0.0104 \\
-0.0081 \\
-0.0052 \\
-0.0049 \\
-0.0006 \\
0.0019 \\
0.0071 \\
0.0114 \\
0.0110 \\
-0.0029\end{array}$ \\
\hline
\end{tabular}

High Pressure test

Press

9.89

39.42

49.16

54.50

69.32

80.16

85.61

100.92

.

08.31

111.08

113.24

123.28

125.82

125.60

127.84

130.19

32.53

138.35

140.16

0.0071

0.0114

0110
0.0024 


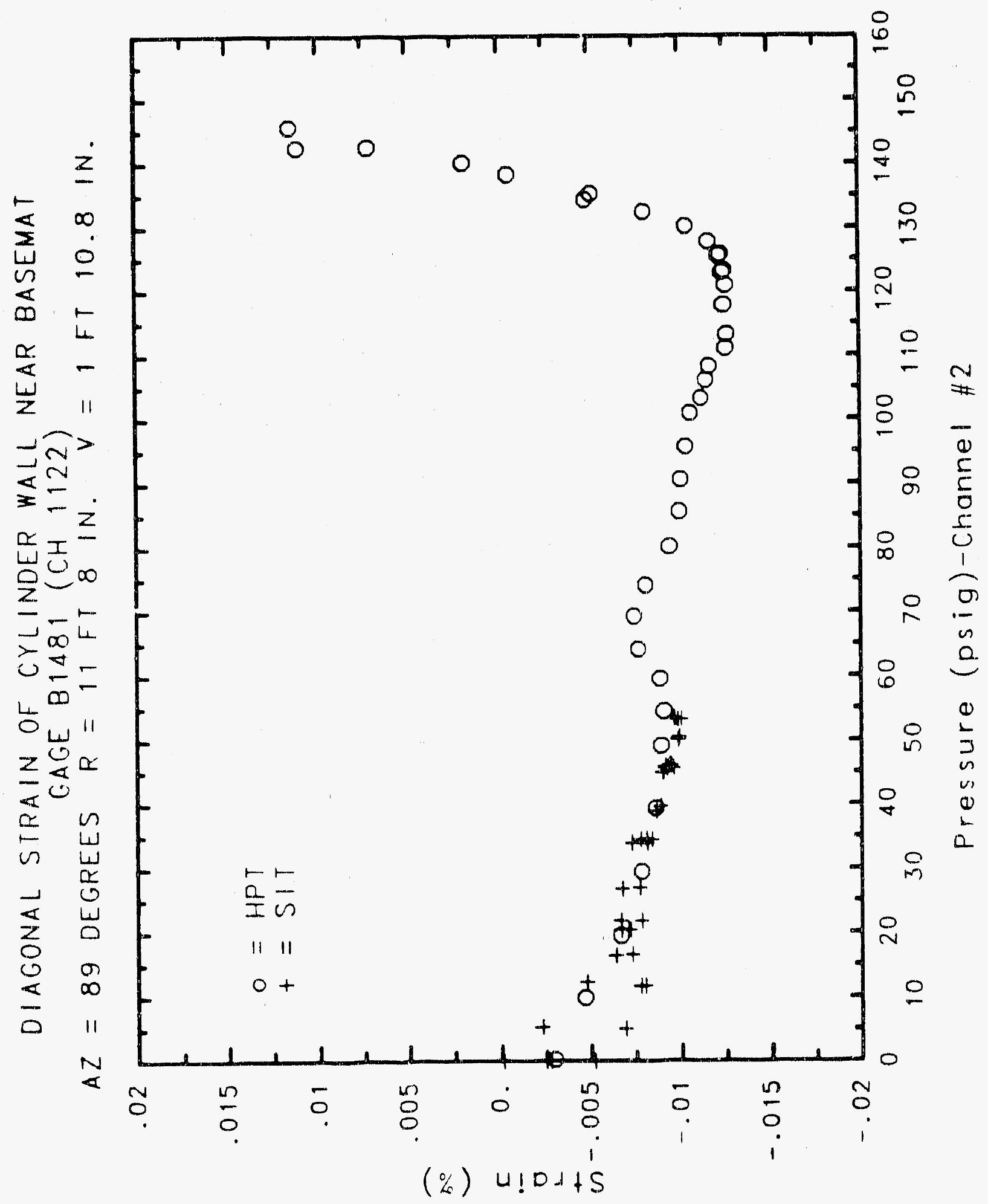




\section{Reinforced Concrete Test Data}

Bonded Gage 1801 Channel 1123

StruCtURAL INTEGRITY TEST

High Pressure test

\begin{tabular}{|c|c|c|c|}
\hline $\begin{array}{c}\text { Pressure } \\
\text { (psig) } \\
-0.05 \\
5.33 \\
12.31 \\
16.44 \\
20.51 \\
20.46 \\
20.47 \\
27.05 \\
34.69 \\
34.53 \\
34.53 \\
39.70 \\
45.90 \\
45.65 \\
45.62 \\
50.10 \\
53.47 \\
53.29 \\
53.21 \\
50.49 \\
46.14 \\
46.13 \\
44.97 \\
38.98 \\
33.99 \\
33.96 \\
26.83 \\
21.88 \\
21.90 \\
16.69 \\
11.74 \\
11.77 \\
5.05 \\
0.02 \\
-0.04 \\
-0.02 \\
-0.02 \\
0.02\end{array}$ & $\begin{array}{l}\text { X Strain } \\
0.0002 \\
-0.0007 \\
-0.0018 \\
-0.0027 \\
-0.0039 \\
-0.0034 \\
-0.0038 \\
-0.1603 \\
-0.8294 \\
-0.0069 \\
-0.0070 \\
-1.4581 \\
-0.3820 \\
-0.0087 \\
-0.0090 \\
-0.0090 \\
-0.0097 \\
-0.0093 \\
-0.0095 \\
-0.0096 \\
-0.0095 \\
-0.0094 \\
-0.0081 \\
-0.0074 \\
-0.0069 \\
-0.0069 \\
-0.0060 \\
-0.0055 \\
-0.0050 \\
-0.0044 \\
-0.1500 \\
-0.0029 \\
-2.6418 \\
0.0009 \\
0.0007 \\
-0.4497 \\
-0.0003 \\
0.0010\end{array}$ & $\begin{array}{c}\text { Pressure } \\
\text { (psig) } \\
9.89 \\
19.55 \\
29.57 \\
39.42 \\
49.16 \\
54.50 \\
59.57 \\
64.20 \\
69.32 \\
74.16 \\
80.16 \\
85.61 \\
90.58 \\
95.69 \\
100.92 \\
103.25 \\
106.11 \\
108.31 \\
111.08 \\
113.24 \\
117.83 \\
120.92 \\
123.28 \\
122.97 \\
125.82 \\
125.60 \\
127.84 \\
130.19 \\
132.53 \\
135.33 \\
134.42 \\
138.35 \\
140.16 \\
142.63 \\
145.78 \\
142.52 \\
0.22\end{array}$ & $\begin{array}{l}\text { Strain } \\
-0.0049 \\
-0.0072 \\
-0.2936 \\
1.1614 \\
-0.0110 \\
-0.0111 \\
-0.0115 \\
-0.0116 \\
-0.0114 \\
-0.0111 \\
-0.0111 \\
-0.0104 \\
-0.0095 \\
-0.0089 \\
-0.0084 \\
-0.0081 \\
-0.0077 \\
-0.0075 \\
-0.0069 \\
-0.0065 \\
-0.0051 \\
-0.0048 \\
-0.0049 \\
-0.0055 \\
-0.0051 \\
-0.0059 \\
-0.0050 \\
-0.0035 \\
-0.0029 \\
-0.0029 \\
-0.0035 \\
-0.0009 \\
0.0015 \\
0.0050 \\
0.0088 \\
0.0099 \\
-0.0091\end{array}$ \\
\hline
\end{tabular}




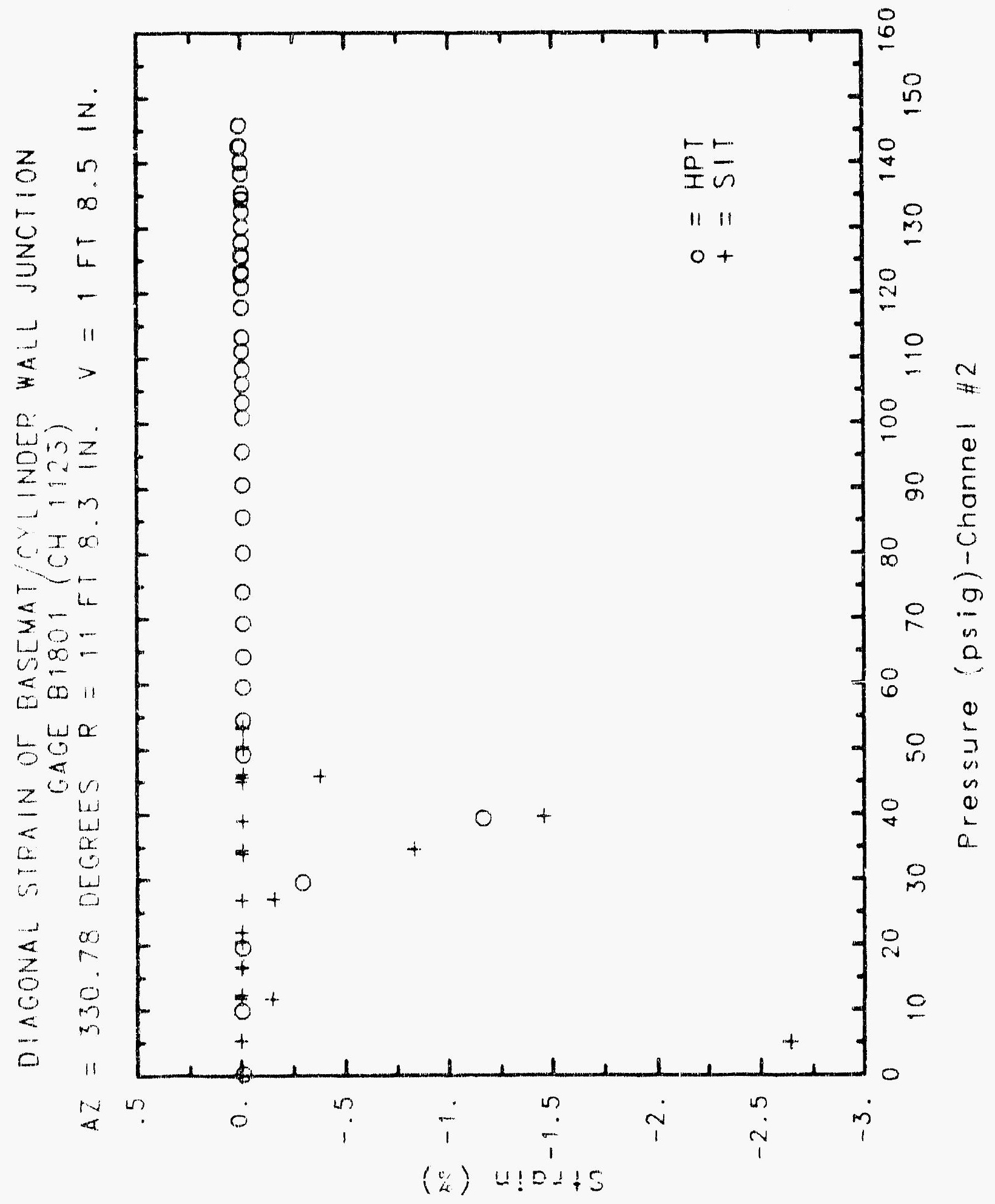




\section{Reinforced Concrete Test Data}

Bonded Gage 1811 Channel 1124

StRuCtURAL INTEgRITY TEST

High Pressure test

\begin{tabular}{|c|c|c|c|}
\hline $\begin{array}{c}\text { Pressure } \\
\text { (psig) } \\
-0.05 \\
5.33 \\
12.31 \\
16.44 \\
20.51 \\
20.46 \\
20.47 \\
27.05 \\
34.69 \\
34.53 \\
34.53 \\
39.70 \\
45.90 \\
45.65 \\
45.62 \\
50.10 \\
53.47 \\
53.29 \\
53.21 \\
50.49 \\
46.14 \\
46.13 \\
44.97 \\
38.98 \\
33.99 \\
33.96 \\
26.83 \\
21.80 \\
21.90 \\
16.69 \\
11.74 \\
11.77 \\
5.05 \\
0.02 \\
-0.04 \\
-0.02 \\
-0.02 \\
0.02\end{array}$ & $\begin{array}{l}\text { \% Strain } \\
-0.0003 \\
-0.0015 \\
-0.0014 \\
-0.0019 \\
-0.0025 \\
-0.0028 \\
-0.0029 \\
-0.0044 \\
-0.0056 \\
-0.0058 \\
-0.0057 \\
-0.0063 \\
-0.0065 \\
-0.0070 \\
-0.0063 \\
-0.0070 \\
-0.0063 \\
-0.0064 \\
-0.0063 \\
-0.0057 \\
-0.0054 \\
-0.0051 \\
-0.0071 \\
-0.0066 \\
-0.0062 \\
-0.0063 \\
-0.0057 \\
-0.0051 \\
-0.0050 \\
-0.0041 \\
-0.0033 \\
-0.0034 \\
-0.0020 \\
-0.0009 \\
0.0005 \\
-0.0026 \\
-0.0030 \\
-0.0017\end{array}$ & $\begin{array}{c}\text { Pressure } \\
\text { (psig) } \\
9.89 \\
19.55 \\
29.57 \\
39.42 \\
49.16 \\
54.50 \\
59.57 \\
64.20 \\
69.32 \\
74.16 \\
80.16 \\
85.61 \\
90.58 \\
95.69 \\
100.92 \\
103.25 \\
106.11 \\
108.31 \\
111.08 \\
113.24 \\
117.83 \\
120.92 \\
123.28 \\
122.97 \\
125.82 \\
125.60 \\
127.84 \\
130.19 \\
132.53 \\
135.33 \\
134.42 \\
138.35 \\
140.16 \\
142.83 \\
145.78 \\
142.52 \\
0.22 \\
\end{array}$ & $\begin{array}{l}\text { \% St.rain } \\
-0.0031 \\
-0.0048 \\
-0.0060 \\
-0.0065 \\
-0.0079 \\
-0.0077 \\
-0.0079 \\
-0.0079 \\
-0.0082 \\
-0.0088 \\
-0.0079 \\
-0.0076 \\
-0.0078 \\
-0.0075 \\
-0.0070 \\
-0.0064 \\
-0.0053 \\
-0.0061 \\
-0.0060 \\
-0.0058 \\
-0.0055 \\
-0.0059 \\
-0.0068 \\
-0.0076 \\
-0.0084 \\
-0.0101 \\
-0.0101 \\
-0.0116 \\
-0.0115 \\
-0.0127 \\
-0.0147 \\
-0.0137 \\
-0.0134 \\
-0.0126 \\
-0.0104 \\
-0.0105 \\
-0.0157\end{array}$ \\
\hline
\end{tabular}




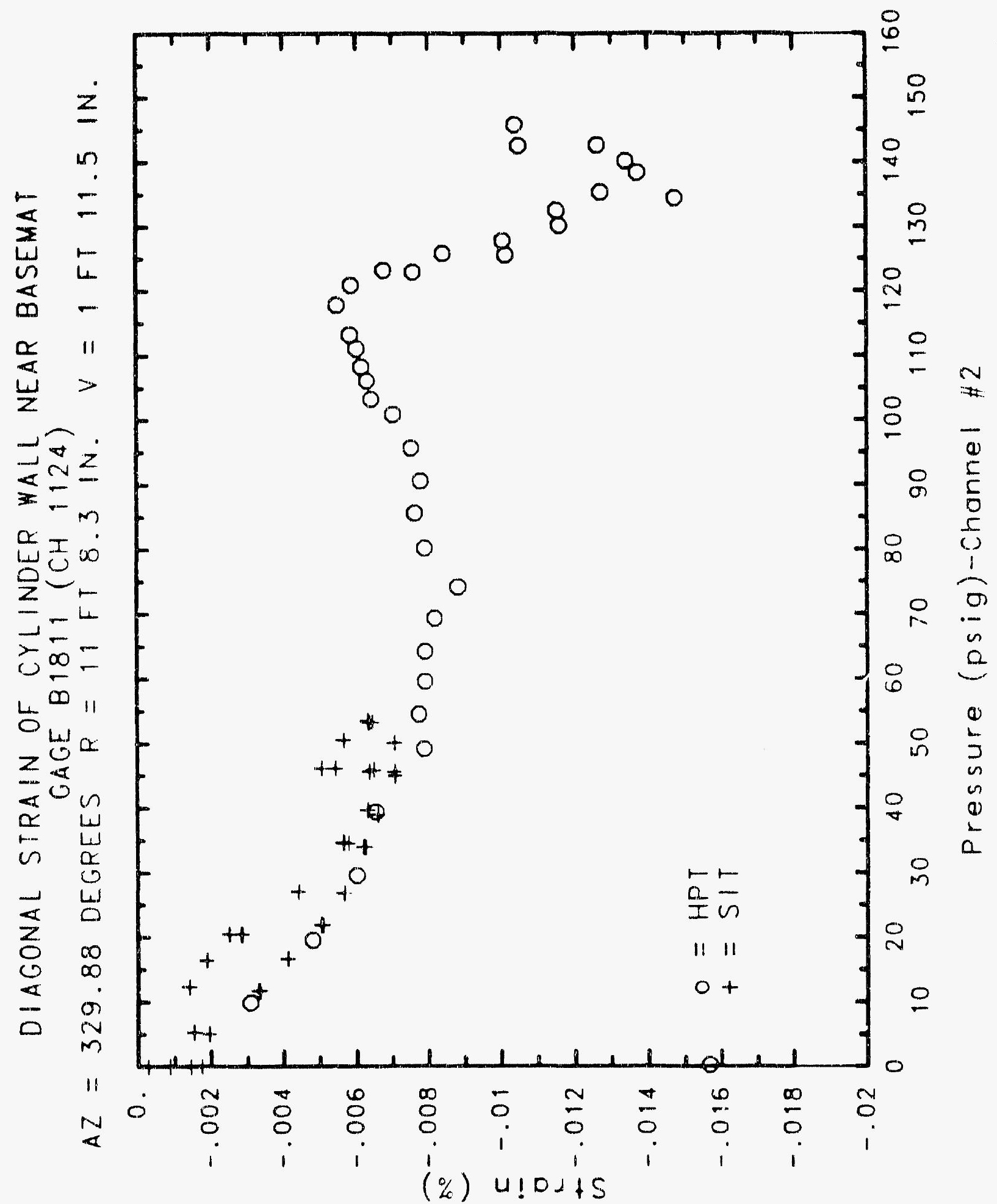



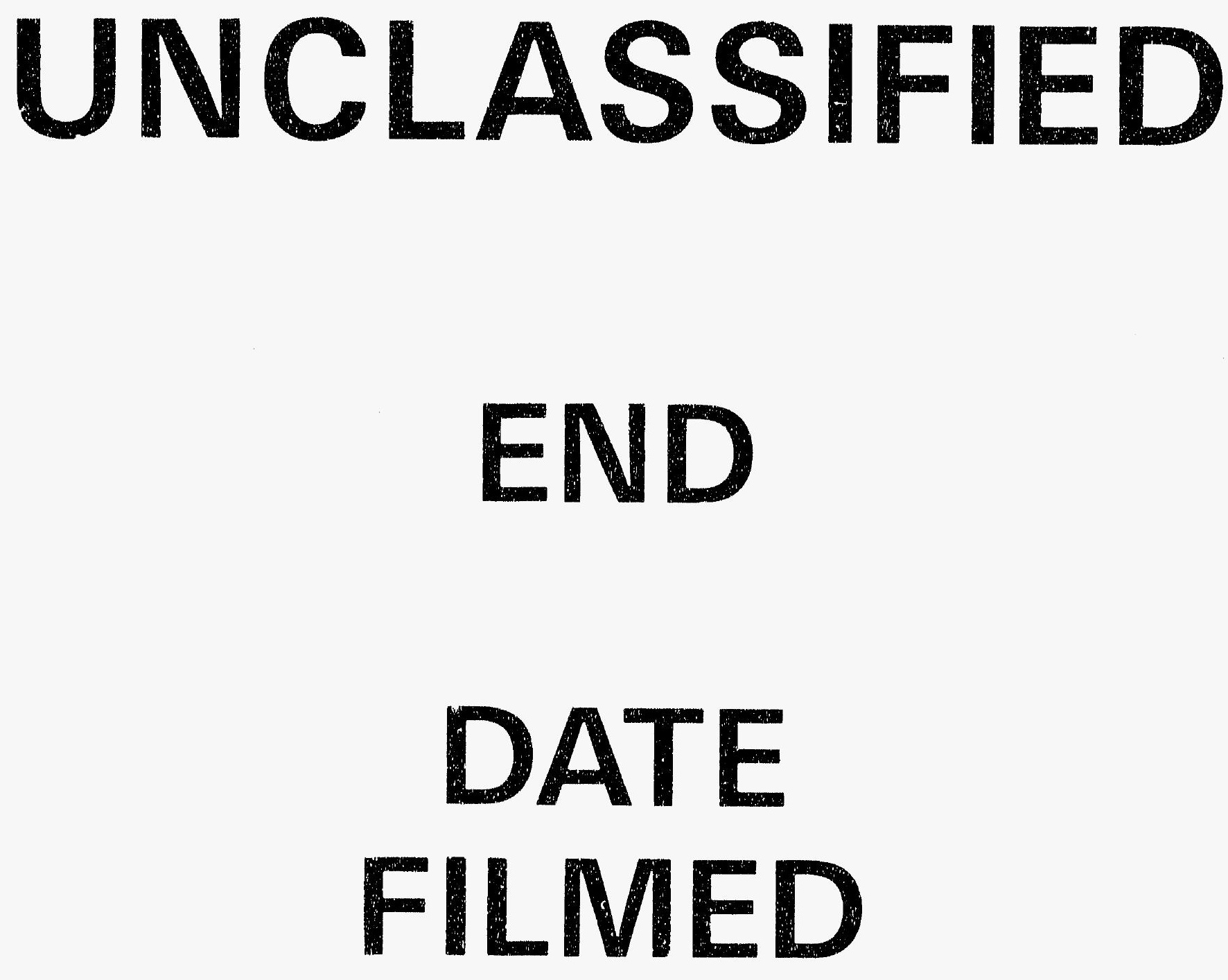

$$
1-6-92
$$

\section{SANDIA NATIONAL LABS}




\section{IMAGE EVALUATION TEST TARGET (MT-3)}
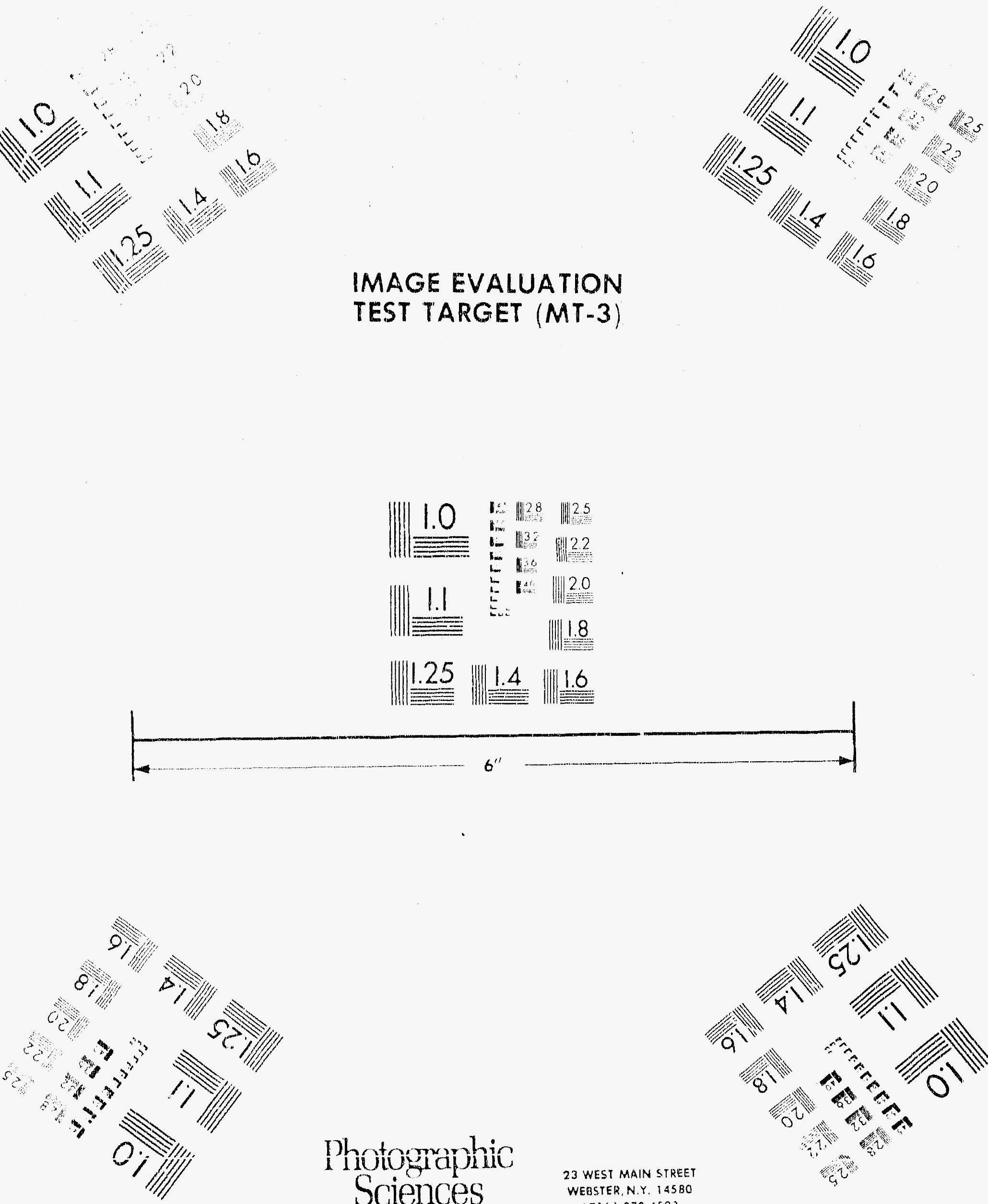

Photographic Sciences Corporation

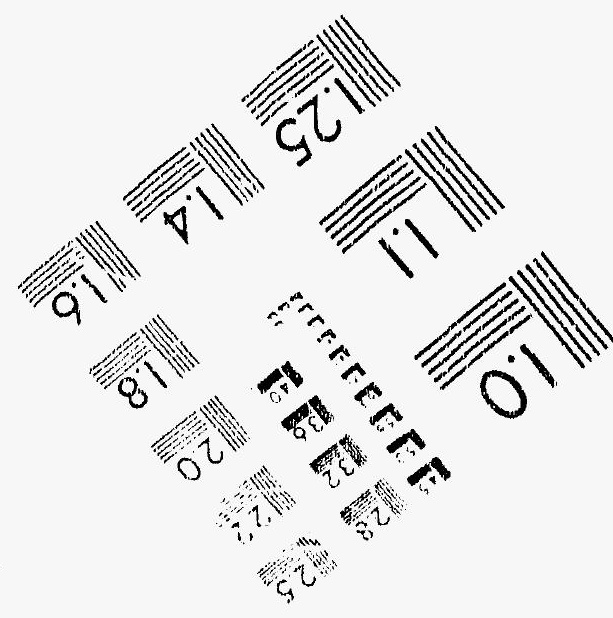




\section{SECURITY CLASSIFICATION}

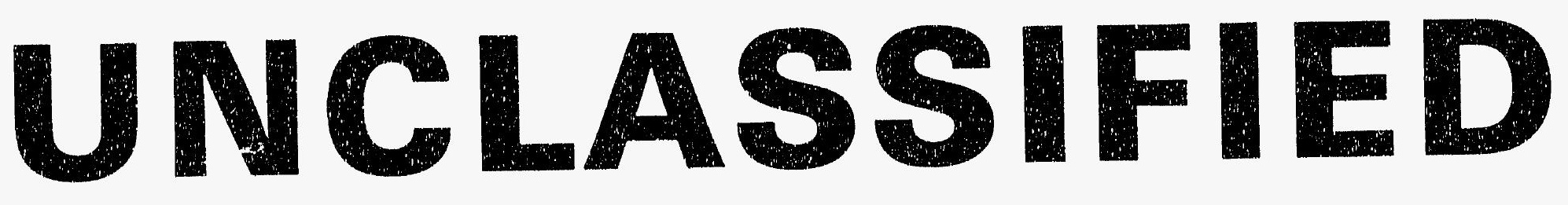

\section{DATE OF MICROFILMING $1-6-92$}

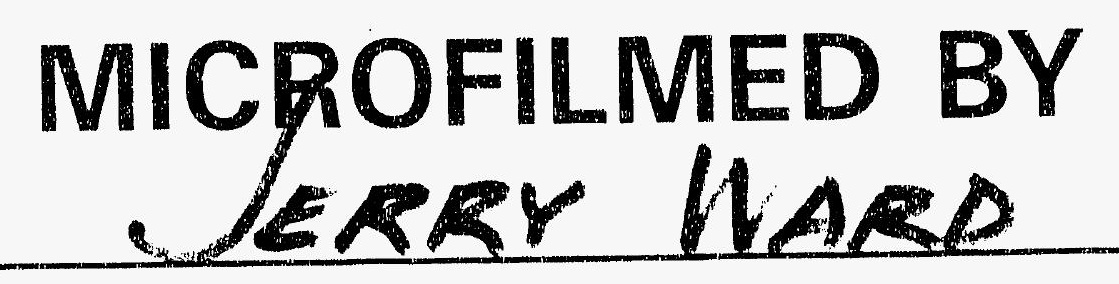

SANDIA NATIONAL LABS 


\section{THIS DOCUMENT FILMED}

AT

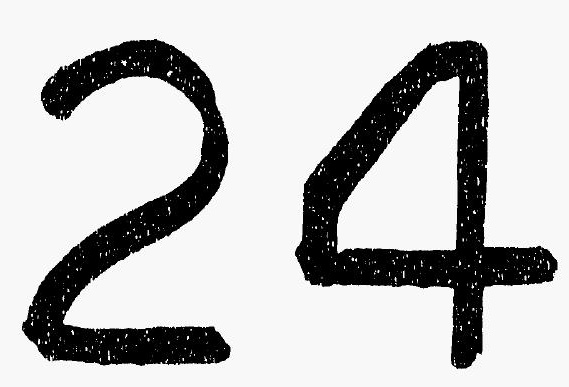

REDUCTION 


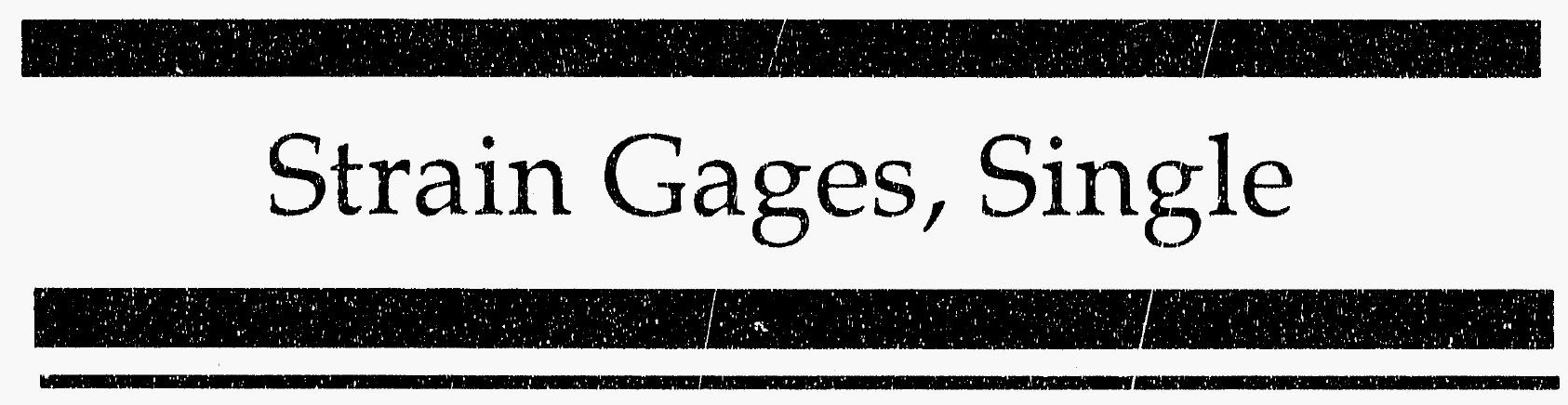




\section{Reinforcee Coiltrete Test Data}

Strain gage 0

STRUCTURAL INTEGRITY TEST

\begin{tabular}{|c|c|c|c|}
\hline $\begin{array}{c}\text { Pressure } \\
\text { (psig) } \\
-0.05 \\
5.33 \\
12.31 \\
16.44 \\
20.51 \\
20.46 \\
20.47 \\
27.05 \\
34.69 \\
34.53 \\
34.53 \\
39.70 \\
45.90 \\
45.65 \\
45.62 \\
50.10 \\
53.47 \\
53.29 \\
53.21 \\
50.19 \\
46.14 \\
46.13 \\
44.97 \\
38.98 \\
33.99 \\
33.96 \\
26.83 \\
21.88 \\
21.90 \\
16.53 \\
11.14 \\
11.77 \\
5.05 \\
11.02 \\
-0.04 \\
-0.02 \\
-0.02 \\
0.02 \\
\end{array}$ & $\begin{array}{c}\text { X Strain } \\
0.0024 \\
-0.0010 \\
0.0053 \\
0.0143 \\
0.0150 \\
0.0168 \\
0.0170 \\
0.0165 \\
0.0169 \\
0.0183 \\
0.0177 \\
0.0151 \\
0.0133 \\
0.0096 \\
0.0088 \\
0.0082 \\
0.0081 \\
0.0073 \\
0.0013 \\
0.0044 \\
0.0055 \\
0.0067 \\
0.0168 \\
0.0159 \\
0.0170 \\
0.0166 \\
0.0169 \\
0.0188 \\
0.0201 \\
0.0200 \\
0.0179 \\
0.0136 \\
0.0108 \\
0.0181 \\
0.0200 \\
0.0217 \\
0.0225 \\
0.0239\end{array}$ & $\begin{array}{c}\text { Pressure } \\
(\text { psig) } \\
9.89 \\
19.55 \\
29.57 \\
39.42 \\
49.16 \\
54.50 \\
59.57 \\
64.20 \\
69.32 \\
74.16 \\
80.16 \\
85.61 \\
90.58 \\
95.69 \\
100.92 \\
103.25 \\
106.11 \\
108.31 \\
111.08 \\
113.24 \\
117.83 \\
120.92 \\
123.28 \\
122.97 \\
125.82 \\
125.60 \\
127.84 \\
130.19 \\
132.53 \\
135.33 \\
134.42 \\
138.35 \\
140.16 \\
142.63 \\
145.78 \\
142.52 \\
0.22 \\
\end{array}$ & $\begin{array}{l}\text { \% Strain } \\
-0.0071 \\
-0.0085 \\
-0.0090 \\
-0.0089 \\
-0.0088 \\
-0.0088 \\
-0.0086 \\
-0.0089 \\
-0.0094 \\
-0.0099 \\
-0.0112 \\
-0.0112 \\
-0.0116 \\
-0.0117 \\
-0.0118 \\
-0.0114 \\
-0.0115 \\
-0.0117 \\
-0.0114 \\
-0.0108 \\
-0.0103 \\
-0.0096 \\
-0.0089 \\
-0.0086 \\
-0.0081 \\
-0.0077 \\
-0.0075 \\
-0.0075 \\
-0.0074 \\
-0.0086 \\
-0.0092 \\
-0.0119 \\
-0.0146 \\
-0.0190 \\
-0.0218 \\
-0.0211 \\
0.0073\end{array}$ \\
\hline
\end{tabular}

Channel 2302

High Pressure test

ressur

9.89

19.55

29.57

39.42

49.16

64.20

74.16

80.16

95.69

106.11

113.24

117.83

122.97

12.5 .82

127.84

130.19

132.53

135.33

134.42

138.35

140.16

2.52

0.22
$-0.007$

$-0.0085$

$-0.0090$

$-0.0089$

0.0088

$-0.0088$

$-0.0094$

$-0.0116$

$-0.0117$

0.0115

$-0.0103$

$-0.0096$

$-0.0089$

0.007

0.0086

0.0190

0.0073 


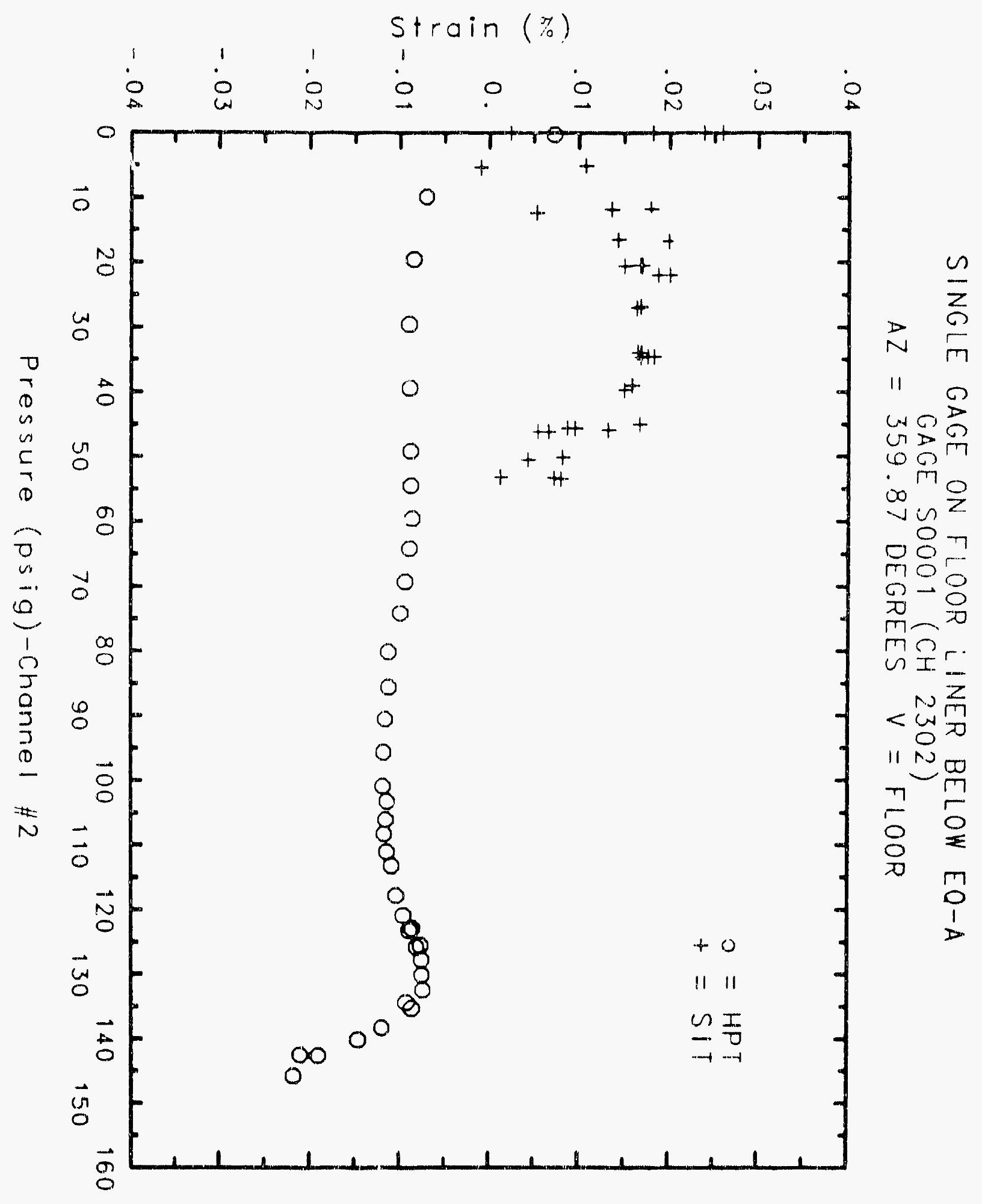




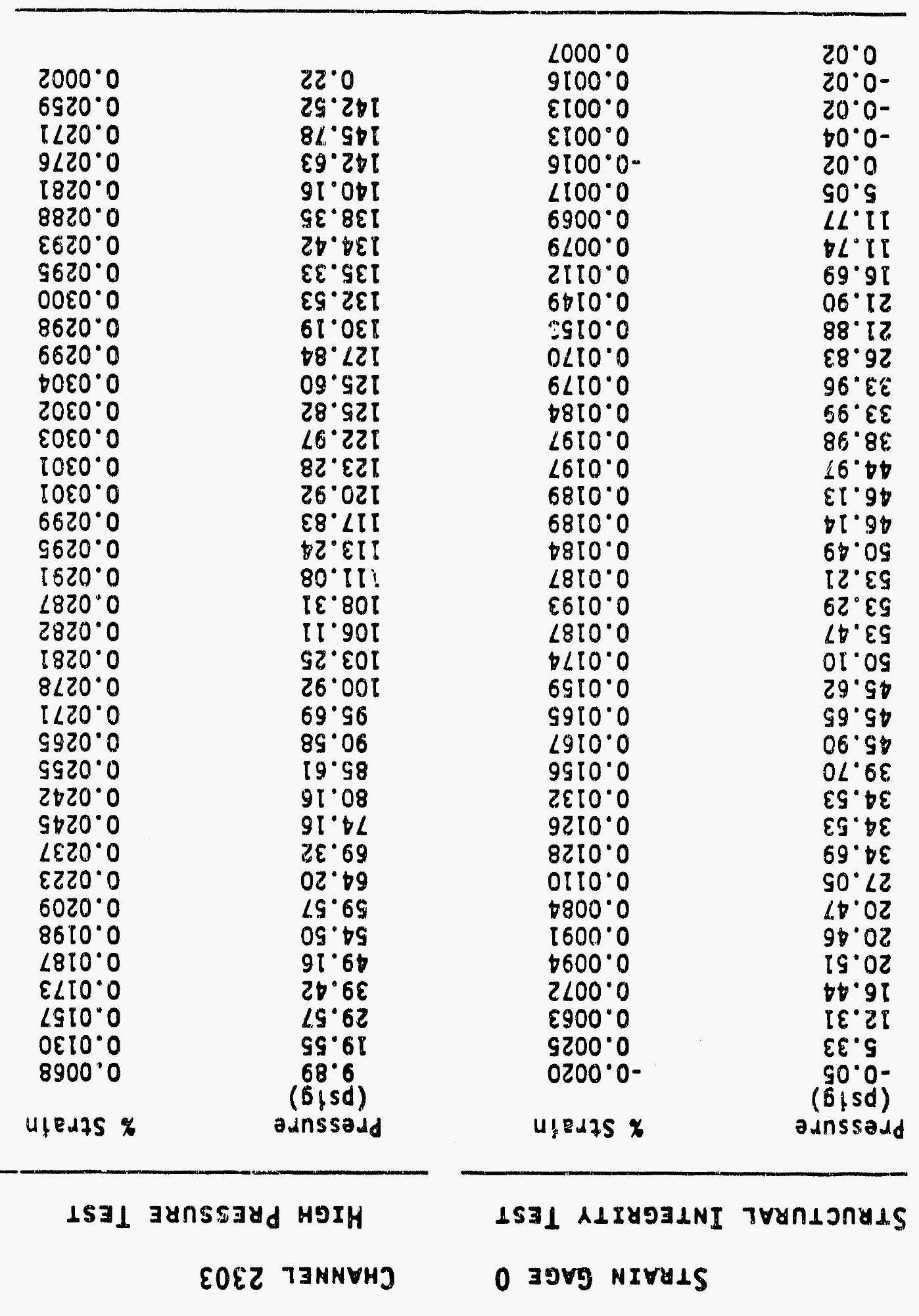




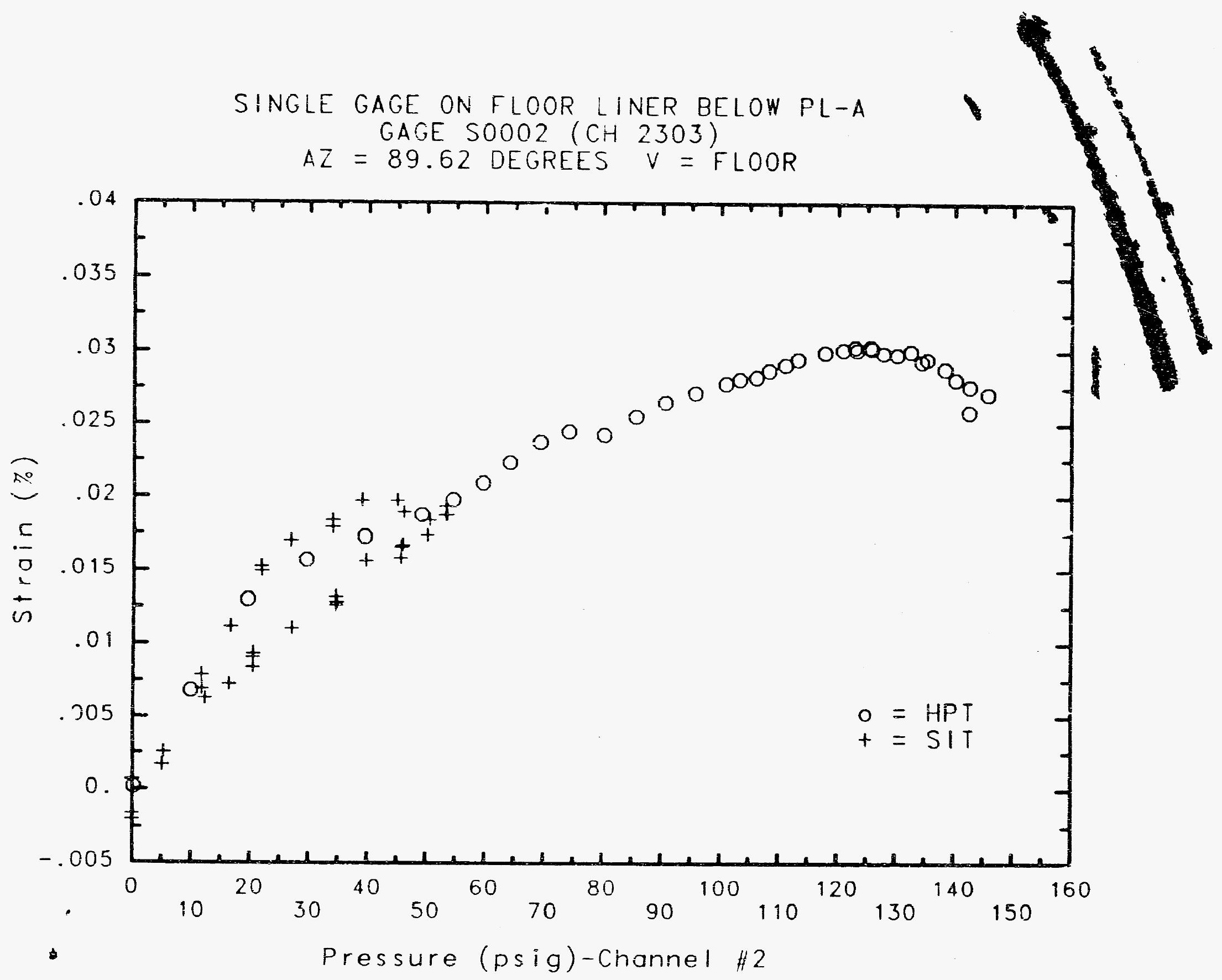




\section{Reinforced Concrete Test Data}

Strain Gage 0

Structural Integrity Test

\begin{tabular}{|c|c|c|c|}
\hline $\begin{array}{c}\text { Pressure } \\
(\text { psig) } \\
-0.05 \\
5.33 \\
12.31 \\
16.44 \\
20.51 \\
20.46 \\
20.47 \\
27.05 \\
34.69 \\
34.53 \\
34.53 \\
39.70 \\
45.90 \\
45.65 \\
45.62 \\
50.10 \\
53.47 \\
53.29 \\
53.21 \\
50.49 \\
46.14 \\
46.13 \\
44.97 \\
38.98 \\
33.99 \\
33.96 \\
26.83 \\
21.88 \\
21.90 \\
16.69 \\
11.74 \\
11.77 \\
5.05 \\
0.02 \\
-0.04 \\
-0.02 \\
-0.02 \\
0.02 \\
\end{array}$ & $\begin{array}{l}\text { \% Strain } \\
0.0018 \\
-0.0063 \\
-0.0139 \\
-0.0166 \\
-0.0175 \\
-0.0174 \\
-0.0175 \\
-0.0191 \\
-0.0211 \\
-0.0210 \\
-0.0212 \\
-0.0234 \\
-0.0141 \\
-0.0246 \\
-0.0244 \\
-0.0254 \\
-0.0263 \\
-0.0260 \\
-0.0269 \\
-10.0251 \\
-0.0257 \\
-0.0254 \\
-0.0220 \\
-0.0227 \\
-0.0211 \\
-0.0212 \\
-0.0218 \\
-0.0206 \\
-0.0217 \\
-0.02016 \\
-0.0206 \\
-0.0210 \\
-0.0204 \\
-0.0196 \\
-0.0137 \\
-0.0124 \\
-0.0133 \\
-0.0152\end{array}$ & $\begin{array}{c}\text { Pressure } \\
(\text { psig) } \\
9.89 \\
19.55 \\
29.57 \\
39.42 \\
49.16 \\
54.50 \\
59.57 \\
64.20 \\
69.32 \\
74.16 \\
80.16 \\
85.61 \\
90.58 \\
95.69 \\
100.92 \\
103.25 \\
106.11 \\
108.31 \\
111.08 \\
113.24 \\
117.83 \\
120.92 \\
123.28 \\
122.97 \\
125.82 \\
125.60 \\
127.84 \\
130.19 \\
132.53 \\
135.33 \\
134.42 \\
138.35 \\
140.16 \\
142.63 \\
145.78 \\
142.52 \\
0.22 \\
\end{array}$ & $\begin{array}{l}\text { \% Strain } \\
-0.0077 \\
-0.0088 \\
-0.0101 \\
-0.0117 \\
-0.0132 \\
-0.0142 \\
-0.0149 \\
-0.0157 \\
-0.0170 \\
-0.0189 \\
-0.0225 \\
-0.0243 \\
-0.0262 \\
-0.0289 \\
-0.0317 \\
-0.0327 \\
-0.0337 \\
-0.0348 \\
-0.0359 \\
-0.0367 \\
-0.0385 \\
-0.0401 \\
-0.0411 \\
-0.0411 \\
-0.0426 \\
-0.0427 \\
-0.0439 \\
-0.0460 \\
-0.0483 \\
-0.0520 \\
-0.0527 \\
-0.0582 \\
-0.0621 \\
-0.0673 \\
-0.0726 \\
-0.0710 \\
-0.0286\end{array}$ \\
\hline
\end{tabular}

Channel 2304

High Priessure Test

Pressur

9.89

39.42

59.57

69.32

74.16

90.58

103.25

106.11

113.24

117.83

122.97

125.82

130.19

132.53

135.33

134.42

138.35

140.16

142.63

145.78

42.52

0.22
$-0.0077$

$-0.0088$

$-0.0101$

$-0.0117$

$-0.0132$

$-0.0142$

$-0.0143$

$-0.0189$

$-0.0225$

$-0.0243$

$-0.0262$

$-0.0289$

$-0.0317$

$-0.0348$

$-0.0401$

$-0.0411$

$-0.0411$

$-0.0439$

$-0.0527$

$-0.0582$

0.0621

0.0710

0.0286 


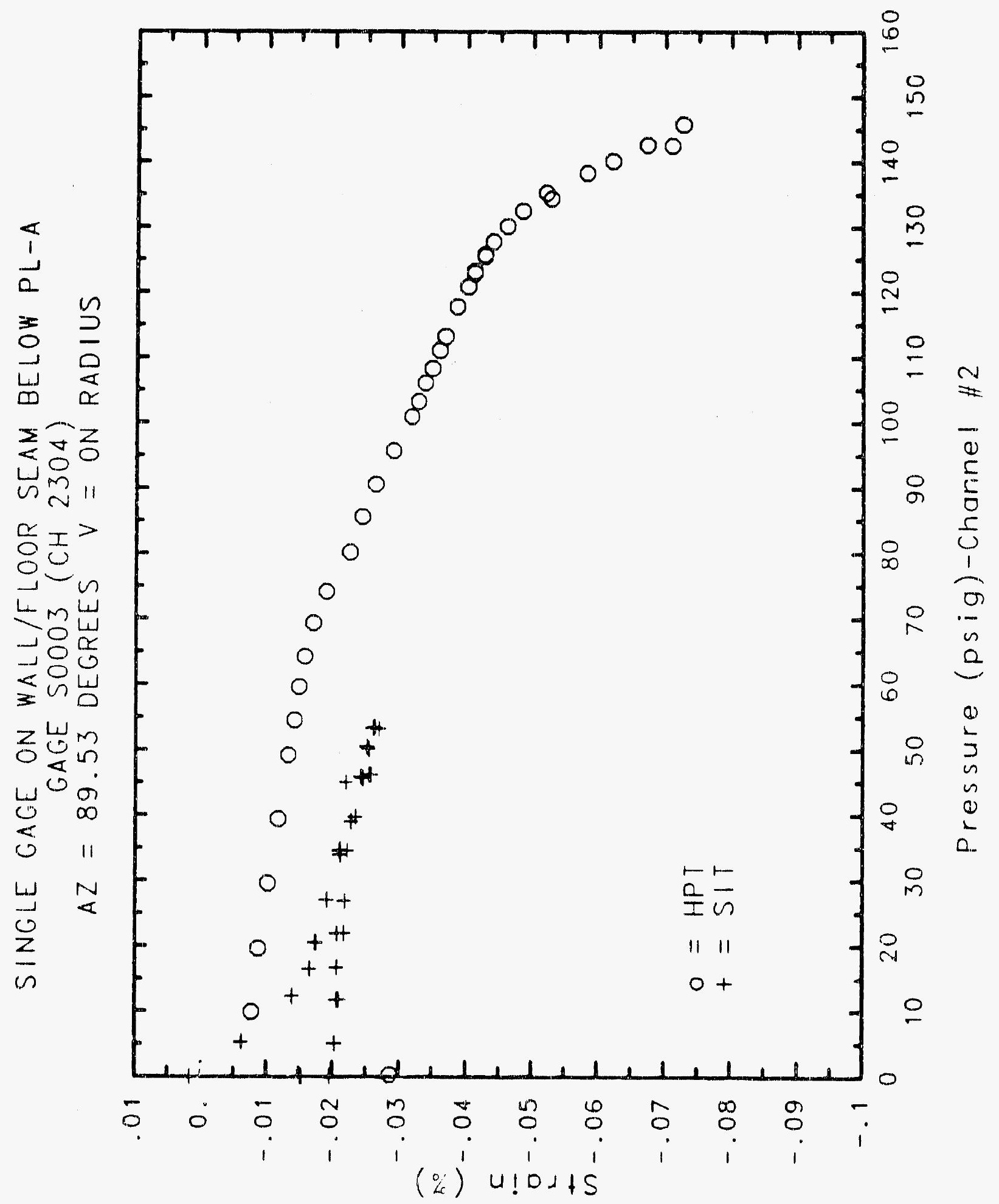




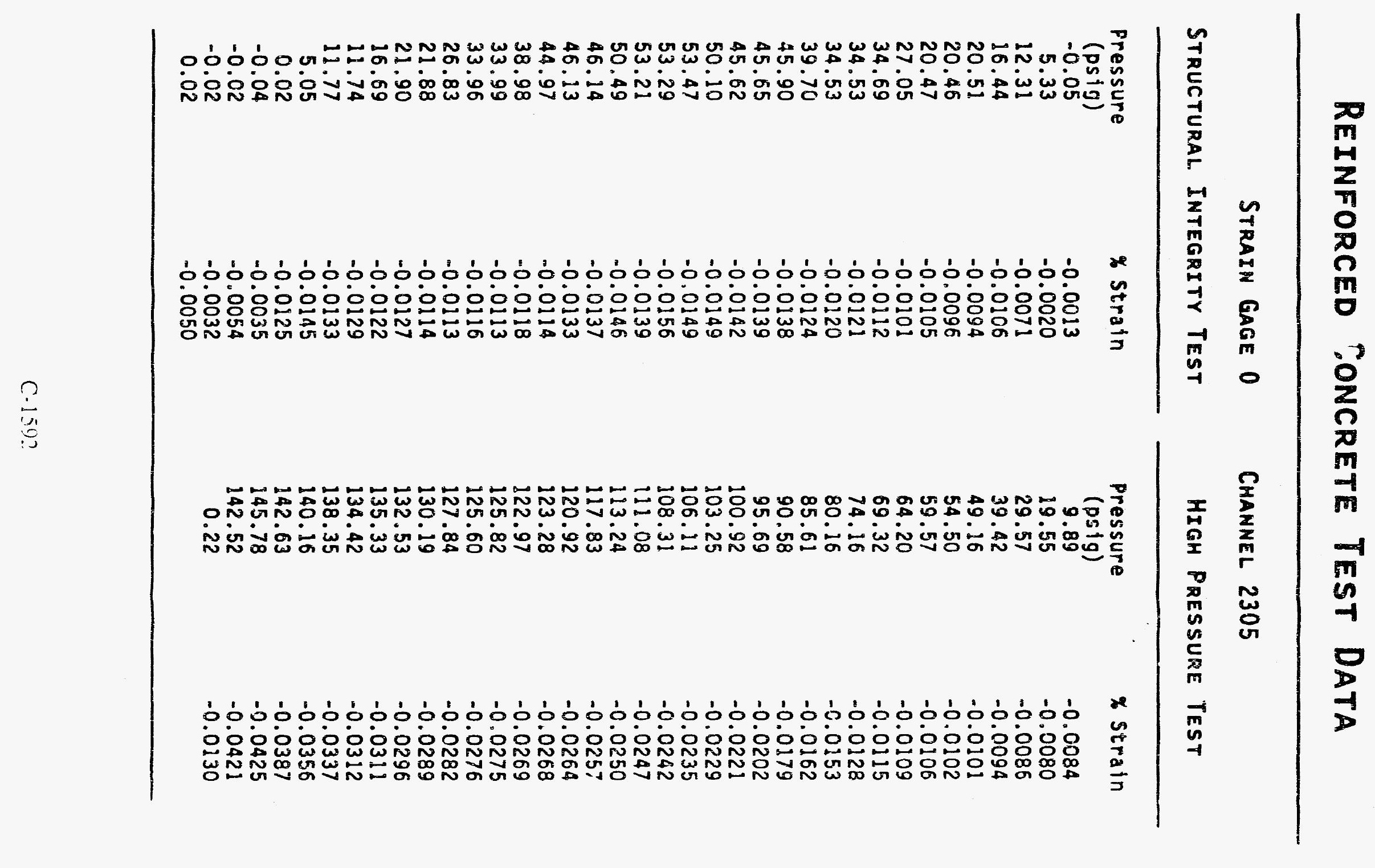




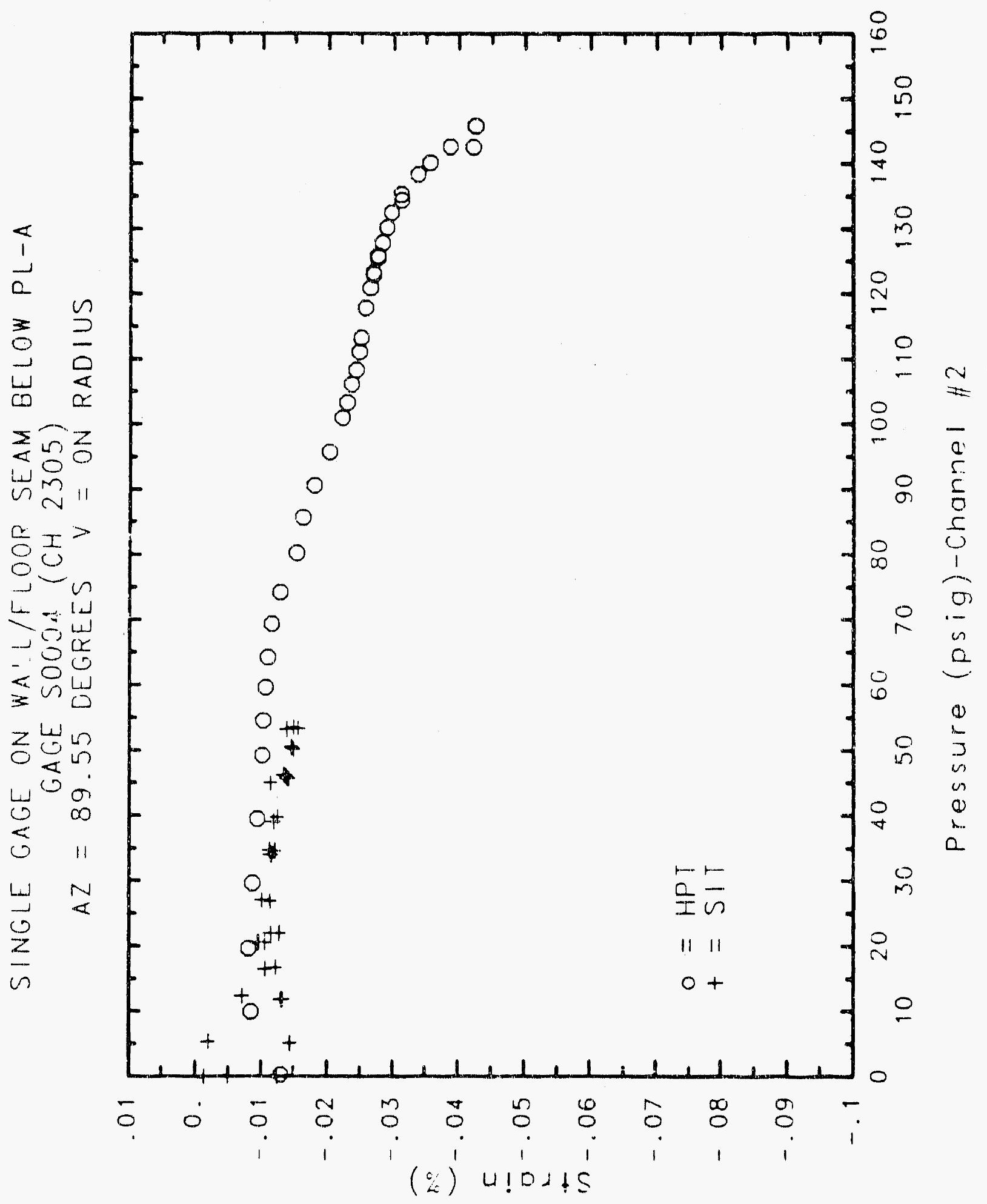




\section{Reinforced Concrete test Data}

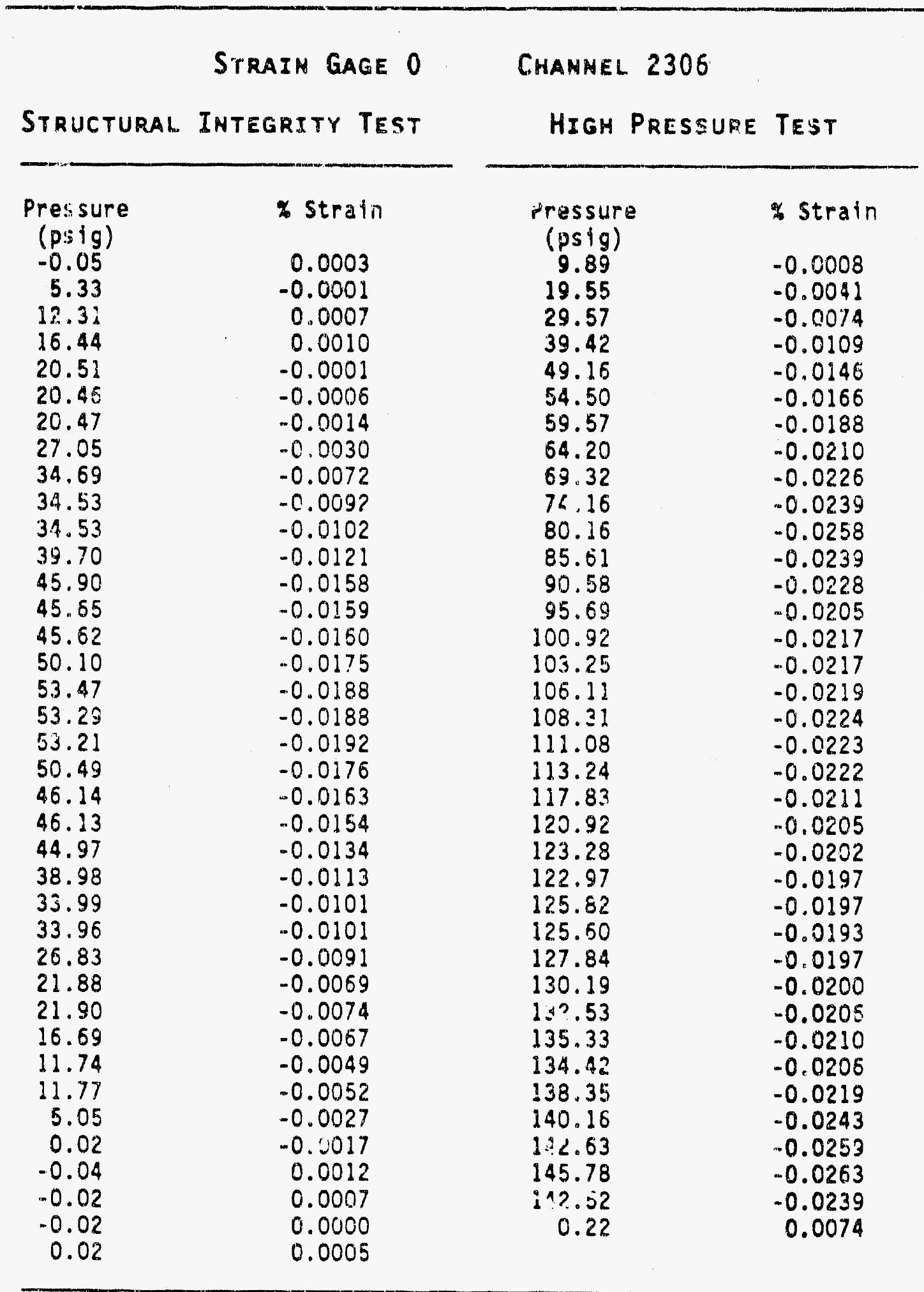




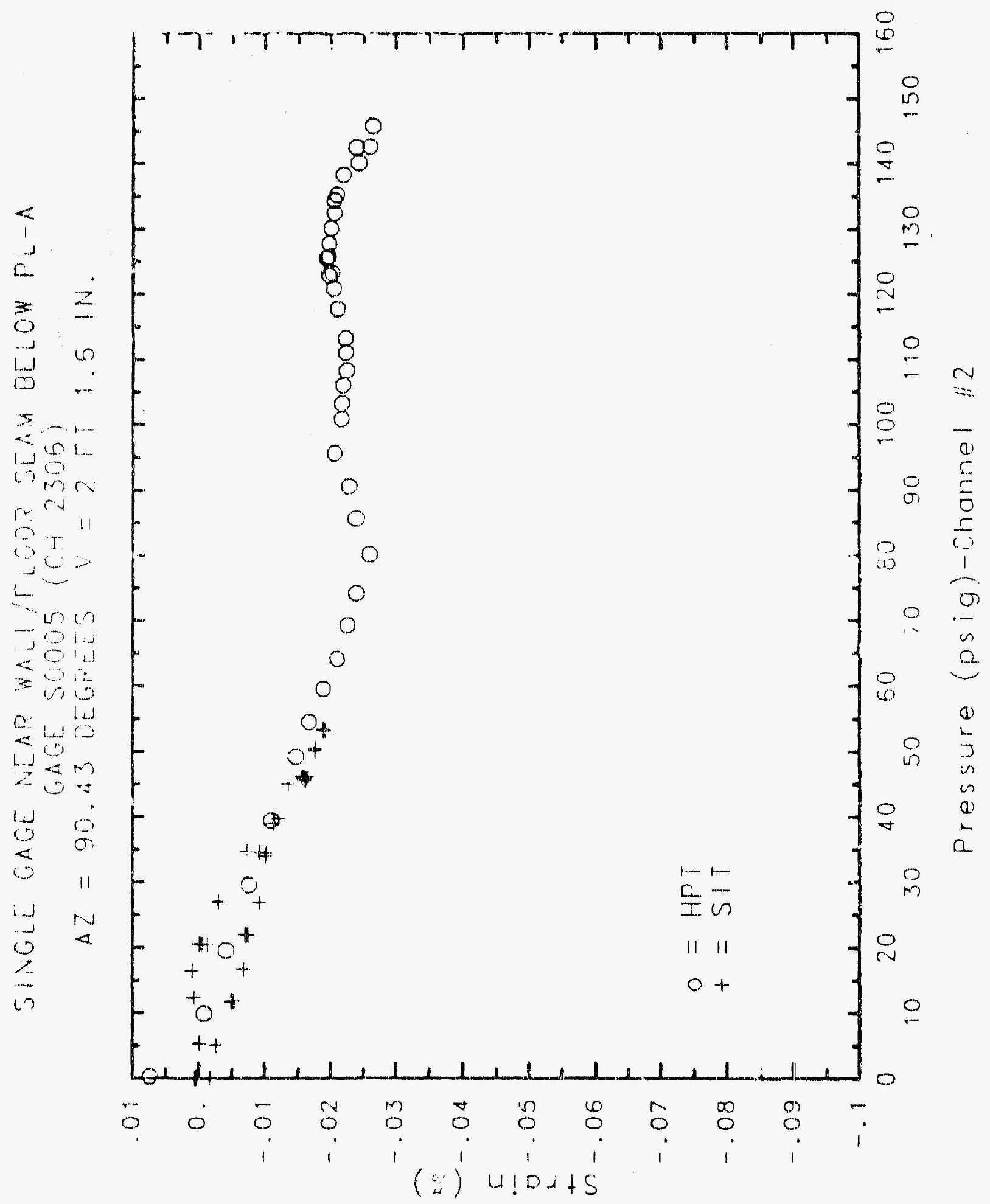

$C \cdot 1.595$ 


\section{Reinforced Concrete Test Data}

Strain Gage 0

STRUCTURAL INTEGRITY TEST

\begin{tabular}{|c|c|c|c|}
\hline $\begin{array}{c}\text { Pressure } \\
\text { (psig) } \\
-0.05 \\
5.33 \\
12.31 \\
16.44 \\
20.51 \\
20.46 \\
20.47 \\
27.05 \\
34.69 \\
34.53 \\
34.53 \\
39.70 \\
45.90 \\
45.65 \\
45.62 \\
50.10 \\
53.47 \\
53.29 \\
53.21 \\
50.49 \\
46.14 \\
46.13 \\
44.97 \\
38.98 \\
33.99 \\
33.96 \\
26.83 \\
21.88 \\
21.90 \\
16.69 \\
11.74 \\
11.77 \\
5.05 \\
0.02 \\
-0.04 \\
-0.02 \\
-0.02 \\
0.02 \\
\end{array}$ & $\begin{array}{l}\text { S Strain } \\
0.0012 \\
0.0005 \\
-0.0014 \\
-0.0027 \\
-0.0066 \\
-0.0071 \\
-0.0092 \\
-0.0152 \\
-0.0242 \\
-0.0277 \\
-0.0276 \\
-0.0317 \\
-0.0384 \\
-0.0385 \\
-0.0382 \\
-0.0403 \\
-0.0432 \\
-0.0428 \\
-0.0422 \\
-0.0414 \\
-0.0393 \\
-0.0393 \\
-0.0358 \\
-0.0334 \\
-0.0306 \\
-0.0314 \\
-0.0283 \\
-0.0259 \\
-0.0276 \\
-0.0226 \\
-0.0185 \\
-0.0174 \\
-0.0133 \\
-0.0086 \\
-0.0040 \\
-0.0039 \\
-0.0037 \\
-0.0052\end{array}$ & $\begin{array}{c}\text { Pressure } \\
\text { (psig) } \\
9.89 \\
19.55 \\
29.57 \\
39.42 \\
49.16 \\
54.50 \\
59.57 \\
64.20 \\
69.32 \\
74.16 \\
80.16 \\
85.61 \\
90.58 \\
95.69 \\
100.92 \\
103.25 \\
106.11 \\
108.31 \\
111.08 \\
113.24 \\
117.83 \\
120.92 \\
123.28 \\
122.97 \\
125.82 \\
125.60 \\
127.84 \\
130.19 \\
132.53 \\
135.33 \\
134.42 \\
138.35 \\
140.16 \\
142.63 \\
145.78 \\
142.52 \\
0.22 \\
\end{array}$ & $\begin{array}{l}\text { \% Strain } \\
-0.0057 \\
-0.0145 \\
-0.0227 \\
-0.0284 \\
-0.0339 \\
-0.0370 \\
-0.0400 \\
-0.0443 \\
-0.0489 \\
-0.0553 \\
-0.0645 \\
-0.0701 \\
-0.0710 \\
-0.0627 \\
-0.0616 \\
-0.0607 \\
-0.0605 \\
-0.0602 \\
-0.0599 \\
-0.0600 \\
-0.0597 \\
-0.0582 \\
-0.0568 \\
-0.0564 \\
-0.0555 \\
-0.0550 \\
-0.0549 \\
-0.0540 \\
-0.0537 \\
-0.0543 \\
-0.0549 \\
-0.0575 \\
-0.0591 \\
-0.0611 \\
-0.0621 \\
-0.0579 \\
-0.0098\end{array}$ \\
\hline
\end{tabular}

Channel 2307

high Pressure test

Pressure

9.89

29.57

69.32

85.61

95.69

106.11

108.31

113.24

117.83

120.92

123.28

125.60

127.84

130.19

32.53

135.33

140.16

2.52

$-0.0621$

$-0.0579$

$-0.0098$ 


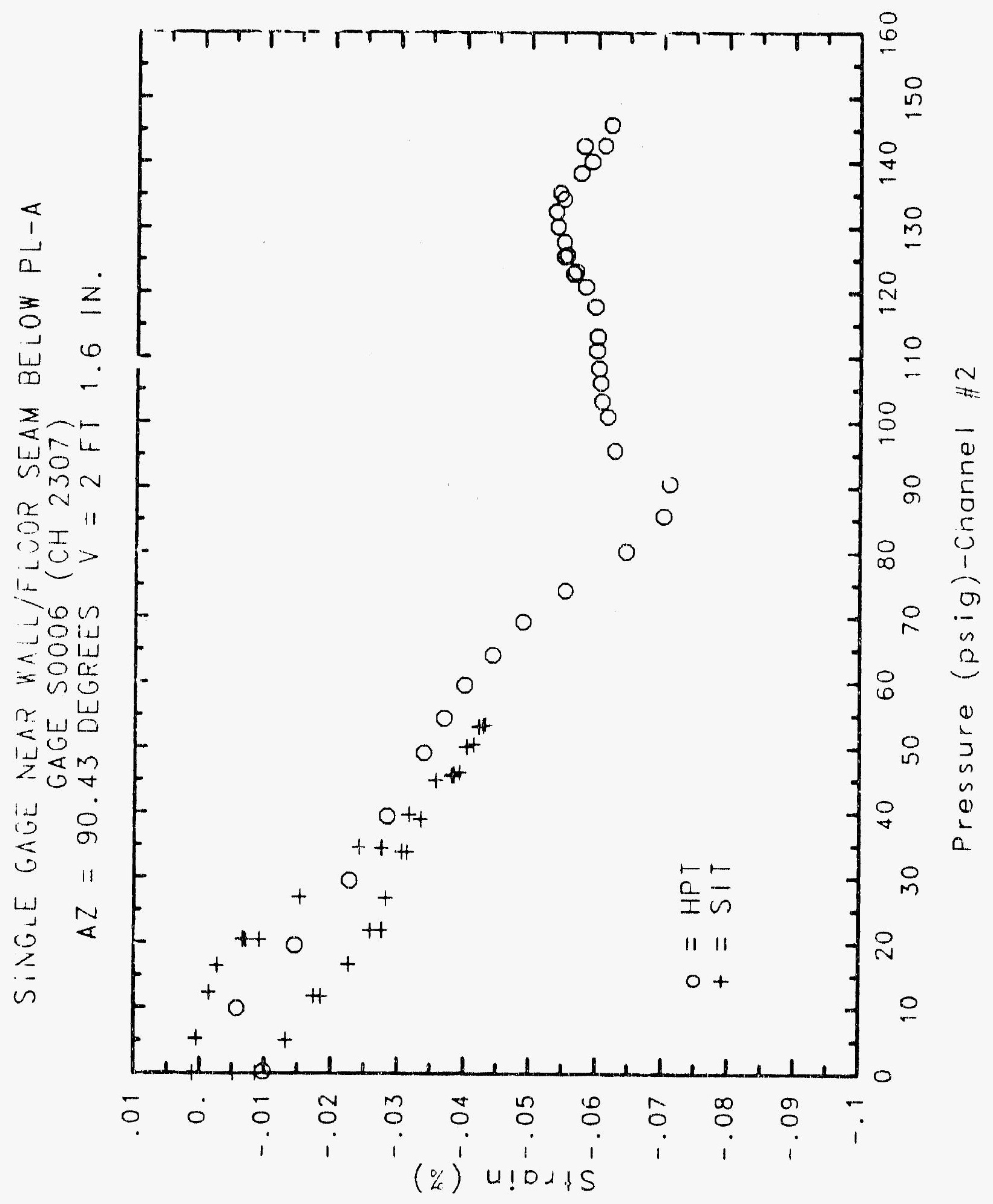




\section{Reinforced Concrete Test Data}

Strain gage 0

StRUCTURAL INTEGRITY TEST

\begin{tabular}{ll}
$\begin{array}{c}\text { Pressure } \\
\text { (psig) }\end{array}$ & \% strain \\
-0.05 & \\
5.33 & -0.0012 \\
12.31 & -0.0006 \\
16.44 & -0.0009 \\
20.51 & -0.0014 \\
20.46 & -0.0013 \\
20.47 & -0.0019 \\
27.05 & -0.0017 \\
34.69 & -0.0023 \\
34.53 & -0.0053 \\
34.53 & -0.0058 \\
39.70 & -0.0064 \\
45.90 & -0.0069 \\
45.55 & -0.0077 \\
45.62 & -0.0077 \\
50.10 & -0.0081 \\
53.47 & -0.0080 \\
53.29 & -0.0082 \\
53.21 & -0.0082 \\
50.49 & -0.0069 \\
46.14 & -0.0053 \\
46.13 & -0.0054 \\
44.97 & -0.0045 \\
38.98 & -0.0025 \\
33.99 & -0.0017 \\
33.96 & -0.0020 \\
26.83 & -0.0016 \\
21.88 & -0.0026 \\
21.90 & -0.0026 \\
16.69 & -0.0027 \\
11.74 & -0.0040 \\
11.77 & -0.0023 \\
5.05 & -0.0027 \\
0.02 & -0.0010 \\
-0.04 & 0.0010 \\
-0.02 & 0.0062 \\
-0.02 & 0.0062 \\
0.02 & 0.0053 \\
& 0.0057 \\
\hline
\end{tabular}

Chanhel 2308

High Pressure test

\begin{tabular}{|c|c|}
\hline $\begin{array}{c}\text { Pressure } \\
(\text { psig) } \\
9.89 \\
19.55 \\
29.57 \\
39.42 \\
49.16 \\
54.50 \\
59.57 \\
64.20 \\
69.32 \\
74.16 \\
80.16 \\
85.61 \\
90.58 \\
95.69 \\
100.92 \\
103.25 \\
106.11 \\
108.31 \\
111.08 \\
113.24 \\
117.83 \\
120.92 \\
123.28 \\
122.97 \\
125.82 \\
125.60 \\
127.84 \\
130.19 \\
132.53 \\
135.33 \\
134.42 \\
138.35 \\
140.16 \\
142.63 \\
145.78 \\
142.52 \\
0.22\end{array}$ & $\begin{array}{l}\text { \% Strain } \\
-0.0016 \\
-0.0051 \\
-0.0065 \\
-0.0070 \\
-0.0082 \\
-0.0088 \\
-0.0093 \\
-0.0099 \\
-0.0110 \\
-0.0130 \\
-0.0168 \\
-0.0182 \\
-0.0203 \\
-0.0282 \\
-0.0309 \\
-0.0316 \\
-0.0326 \\
-0.0332 \\
-0.0339 \\
-0.0347 \\
-0.0356 \\
-0.0356 \\
-0.0356 \\
-0.0352 \\
-0.0351 \\
-0.0347 \\
-0.0348 \\
-0.0341 \\
-0.0339 \\
-0.0343 \\
-0.0345 \\
-0.0370 \\
-0.0383 \\
-0.0399 \\
-0.0403 \\
-0.0371 \\
-0.0158\end{array}$ \\
\hline
\end{tabular}




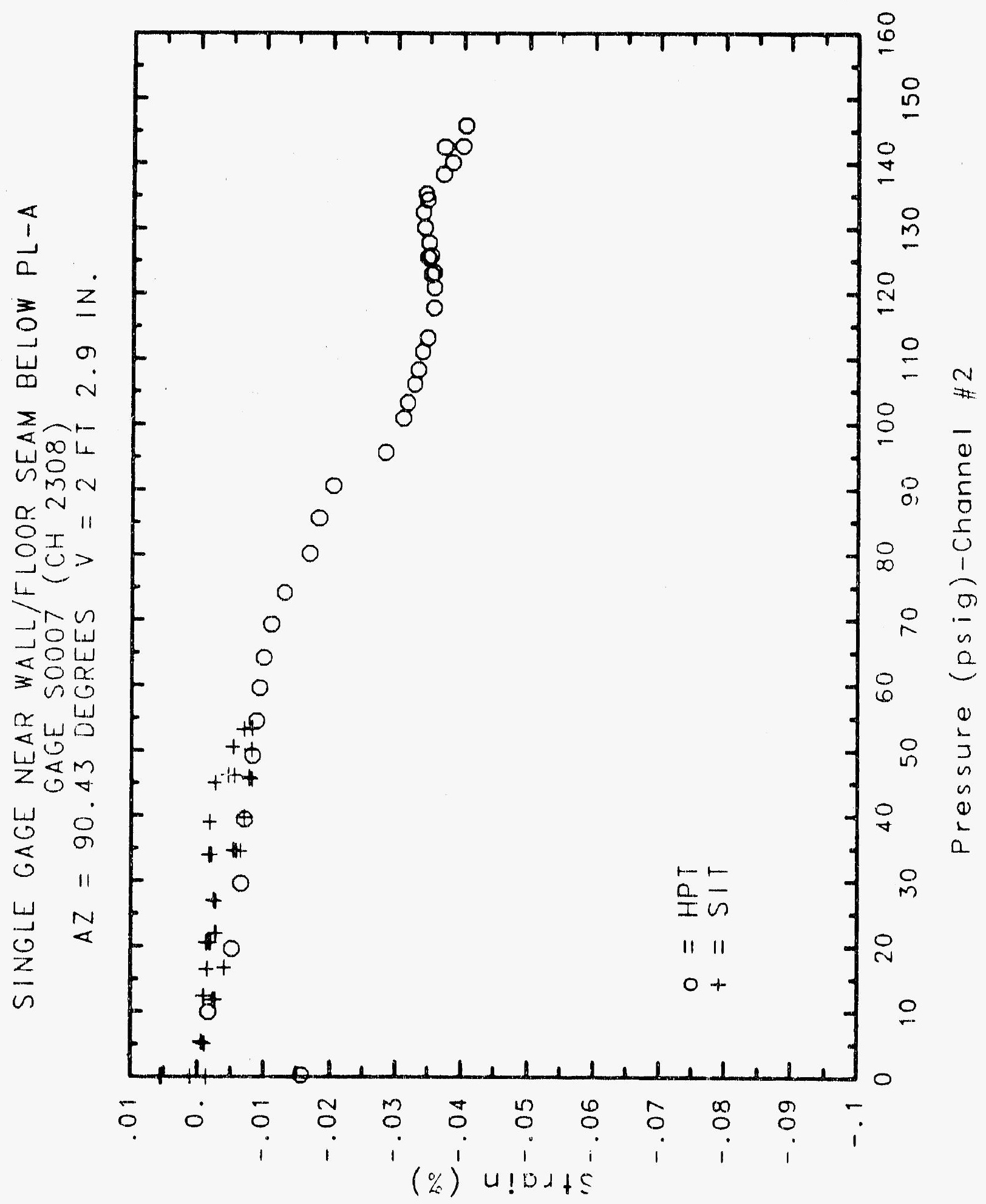

C-1509 


\section{Reinforced Concrete Test Data}

Strain Gage 0

StRUCTURAL INTEGRITY TEST

\begin{tabular}{|c|c|}
\hline $\begin{array}{c}\text { Pressure } \\
(\text { ps } 19) \\
-0.05 \\
5.33 \\
12.31 \\
16.44 \\
20.51 \\
20.46 \\
20.47 \\
27.05 \\
34.69 \\
34.53 \\
34.53 \\
39.70 \\
45.90 \\
45.65 \\
45.62 \\
50.10 \\
53.47 \\
53.29 \\
53.21 \\
50.49 \\
46.14 \\
46.13 \\
44.97 \\
38.98 \\
33.99 \\
33.96 \\
26.83 \\
21.88 \\
21.90 \\
16.69 \\
11.74 \\
11.77 \\
5.05 \\
0.02 \\
-0.04 \\
-0.02 \\
-0.02 \\
0.02 \\
\end{array}$ & $\begin{array}{l}\text { X Stra1n } \\
0.0018 \\
0.0028 \\
0.0026 \\
0.0031 \\
0.0013 \\
0.0023 \\
0.0024 \\
0.0004 \\
0.0004 \\
-0.0010 \\
-0.0011 \\
-0.0023 \\
-0.0031 \\
-0.0036 \\
-0.0040 \\
-0.0048 \\
-0.0045 \\
-0.0043 \\
-0.0051 \\
-0.0037 \\
-0.0031 \\
-0.0030 \\
-0.0019 \\
-0.0002 \\
0.0006 \\
0.0007 \\
0.0024 \\
0.0027 \\
0.0025 \\
0.0032 \\
0.0029 \\
0.0031 \\
0.0026 \\
0.0008 \\
0.0018 \\
0.0021 \\
0.0015 \\
0.0004\end{array}$ \\
\hline
\end{tabular}

Channel 2309

High Pressure test

Pressure $\quad$ strain

(psig)

9.89

19.55

29.57

39.42

49.16

54.50

59.57

64.20

69.32

74.16

80.16

85.61

90.58

95.69

100.92

103.25

106.11

108,31

111.08

113.24

117.83

120.92

123.28

122.97

125.82

125.60

127.84

130.19

132.53

135.33

134.42

138.35

140.16

142.63

145.78

142.52

0.22
0.0020

0.0010

0.0000

$-0.0009$

$-0.0023$

$-0.0030$

$-0.0039$

$-0.0055$

$-0.0070$

$-0.0096$

$-0.0126$

$-0.0147$

$-0.0164$

$-0.0186$

$-0.0204$

$-0.0210$

$-0.0225$

$-0.0238$

$-0.0246$

$-0.0257$

$-0.0286$

$-0.0310$

$-0.0331$

$-0.0331$

$-0.0359$

$-0.0367$

$-0.0387$

$-0.0429$

$-0.0473$

$-0.0520$

$-0.0529$

$-0.0603$

$-0.0656$

$-0.0681$

$-0.0751$

$-0.0699$

$-0.0320$ 


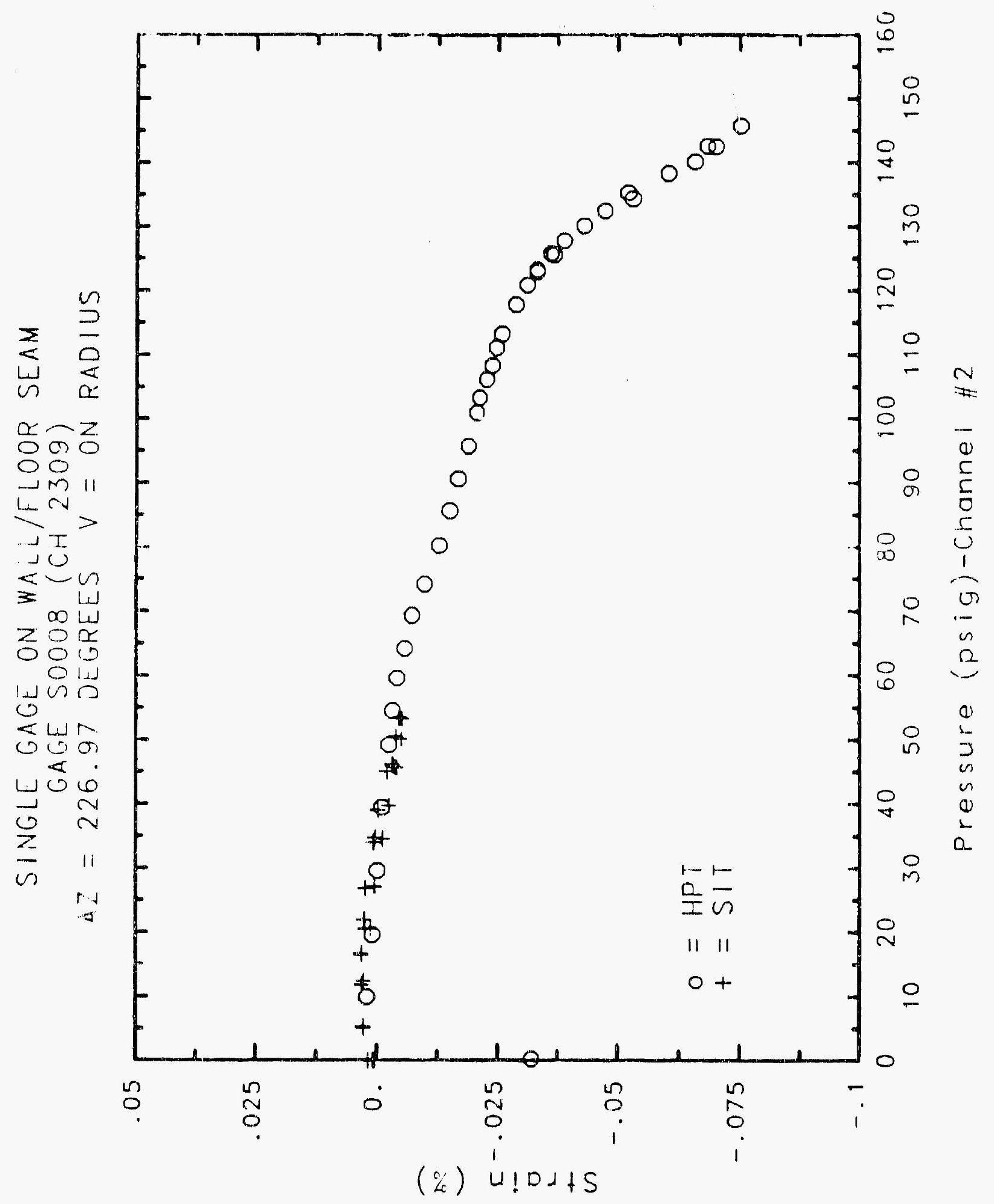

C-1601 


\section{Reinforced Concrete Test Data}

Strain gage 0

StruCtURAL Integrity Test

\begin{tabular}{|c|c|c|c|}
\hline $\begin{array}{c}\text { Pressure } \\
\text { (psig) } \\
-0.05 \\
5.33 \\
12.31 \\
16.44 \\
20.51 \\
20.46 \\
20.47 \\
27.05 \\
34.69 \\
34.53 \\
34.53 \\
39.70 \\
45.90 \\
45.65 \\
45.62 \\
50.10 \\
53.47 \\
53.29 \\
53.21 \\
50.49 \\
46.14 \\
46.13 \\
44.97 \\
38.98 \\
33.99 \\
33.96 \\
26.83 \\
21.88 \\
21.90 \\
16.69 \\
11.74 \\
11.77 \\
5.05 \\
0.02 \\
-0.04 \\
-0.02 \\
-0.02 \\
0.02 \\
\end{array}$ & $\begin{array}{c}\text { \% Strain } \\
\text {-0.0003 } \\
0.0077 \\
0.0124 \\
0.0217 \\
0.0235 \\
0.0240 \\
0.0276 \\
0.0257 \\
0.0370 \\
0.0307 \\
0.0295 \\
0.0142 \\
0.0052 \\
0.0071 \\
0.0055 \\
0.0047 \\
0.0045 \\
0.0039 \\
0.0051 \\
0.0063 \\
0.0069 \\
0.0074 \\
0.0175 \\
0.0191 \\
0.0201 \\
0.0185 \\
0.0175 \\
0.0334 \\
0.0421 \\
0.0397 \\
0.0211 \\
0.0082 \\
0.0077 \\
0.0084 \\
0.0071 \\
0.0129 \\
0.0146 \\
0.0148\end{array}$ & $\begin{array}{c}\text { Pressure } \\
(\text { ps } 19) \\
9.89 \\
19.55 \\
29.57 \\
39.42 \\
49.16 \\
54.50 \\
59.57 \\
64.20 \\
59.32 \\
74.16 \\
80.16 \\
85.61 \\
90.58 \\
95.69 \\
100.92 \\
103.25 \\
106.11 \\
108.31 \\
111.08 \\
113.24 \\
117.83 \\
120.92 \\
123.28 \\
122.97 \\
125.82 \\
125.60 \\
127.84 \\
130.19 \\
132.53 \\
135.33 \\
134.42 \\
138.35 \\
140.16 \\
142.63 \\
145.78 \\
142.52 \\
0.22 \\
\end{array}$ & $\begin{array}{l}\text { X Strain } \\
0.0082 \\
0.0081 \\
0.0063 \\
0.0049 \\
0.0042 \\
0.0039 \\
0.0036 \\
0.0023 \\
0.0016 \\
-0.0005 \\
-0.0026 \\
-0.0043 \\
-0.0056 \\
-0.0073 \\
-0.0091 \\
-0.0094 \\
-0.0104 \\
-0.0113 \\
-0.0116 \\
-0.0120 \\
-0.0133 \\
-0.0152 \\
-0.0165 \\
-0.0165 \\
-0.0182 \\
-0.0186 \\
-0.0204 \\
-0.0234 \\
-0.0267 \\
-0.0301 \\
-0.0308 \\
-0.0355 \\
-0.0393 \\
-0.0431 \\
-0.0492 \\
-0.0459 \\
-0.0171\end{array}$ \\
\hline
\end{tabular}

Channel 2320

High Pressure test

Pressure

9.89

19.55

29.57

39.42

74.16

30.16

95.69

103.25

106.11

113.24

123.28

122.97

125.60

127.84

130.19

132.53

138.35

140.16

145.78

2.52

$-0.0459$

$-0.0171$ 


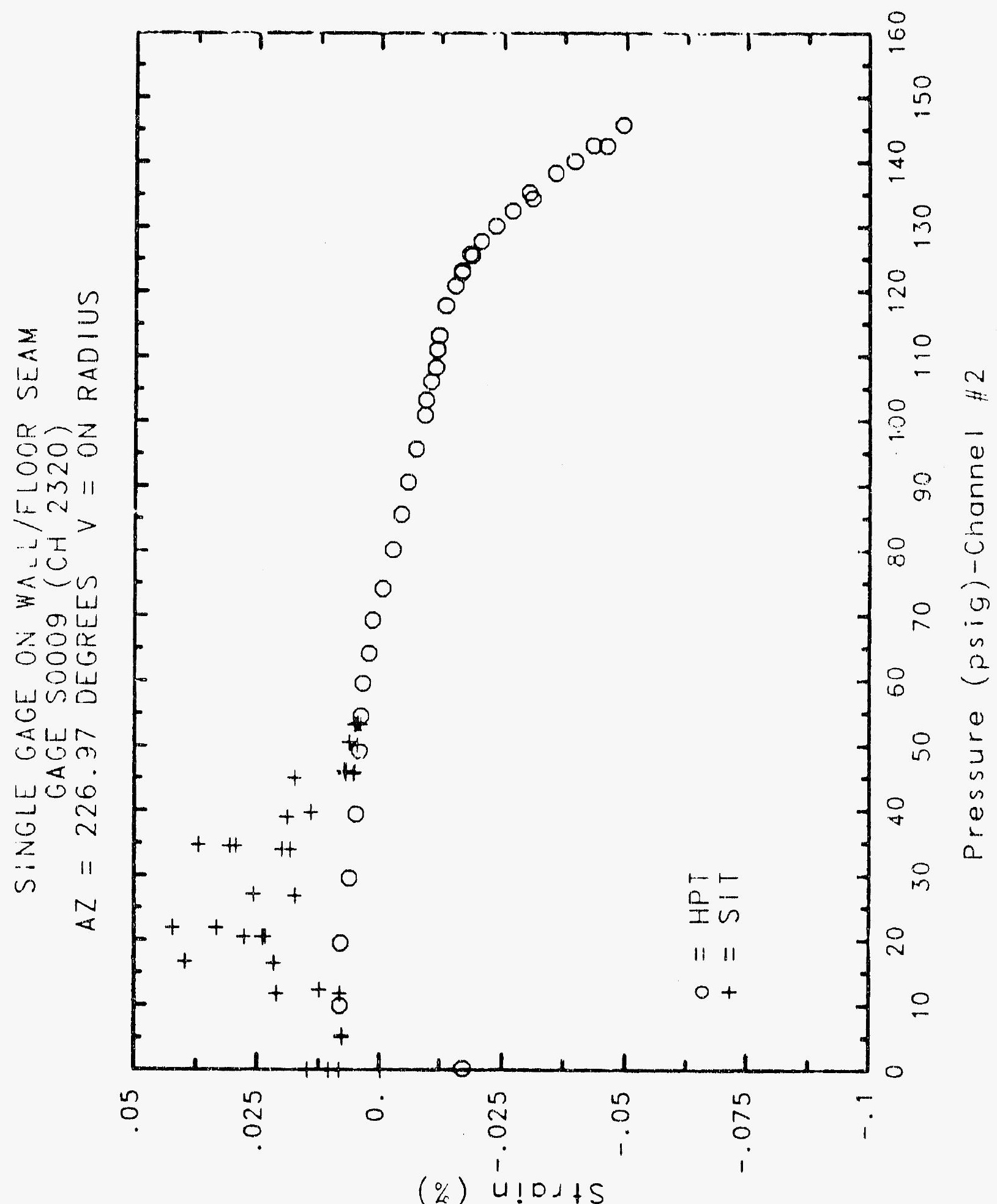




\section{Reinforced Concrete Test Data}

Strain Gage 0

StRUCTURAL INTEgRITY TEST

\begin{tabular}{|c|c|c|c|}
\hline $\begin{array}{c}\text { Pressure } \\
\text { (ps 1g) } \\
-0.05 \\
5.33 \\
12.31 \\
16.44 \\
20.51 \\
20.46 \\
20.47 \\
27.05 \\
34.69 \\
34.53 \\
34.53 \\
39.70 \\
45.90 \\
45.65 \\
45.62 \\
50.10 \\
53.47 \\
53.29 \\
53.21 \\
50.49 \\
46.14 \\
46.13 \\
44.97 \\
38.98 \\
33.99 \\
33.96 \\
26.83 \\
21.88 \\
21.90 \\
16.69 \\
11.74 \\
11.77 \\
5.05 \\
0.02 \\
-0.04 \\
-0.02 \\
-0.02 \\
0.02 \\
\end{array}$ & $\begin{array}{l}\text { X Straln } \\
0.0011 \\
0.0061 \\
0.0110 \\
0.0136 \\
0.0187 \\
0.0198 \\
0.0203 \\
0.0289 \\
0.0403 \\
0.0423 \\
0.0427 \\
0.0505 \\
0.0616 \\
0.0619 \\
0.0617 \\
0.0674 \\
0.0733 \\
0.0741 \\
0.0745 \\
0.0739 \\
0.0701 \\
0.0698 \\
0.0636 \\
0.0640 \\
0.0581 \\
0.0583 \\
0.0499 \\
0.0442 \\
0.0453 \\
0.0391 \\
0.0338 \\
0.0316 \\
0.0234 \\
0.0147 \\
0.0135 \\
0.0128 \\
0.0133 \\
0.0133\end{array}$ & $\begin{array}{c}\text { Pressure } \\
\text { (ps1g) } \\
9.89 \\
19.55 \\
29.57 \\
39.42 \\
49.16 \\
54.50 \\
59.57 \\
64.20 \\
69.32 \\
74.16 \\
80.16 \\
85.61 \\
90.58 \\
95.69 \\
100.92 \\
103.25 \\
106.11 \\
108.31 \\
111.08 \\
113.24 \\
117.83 \\
120.92 \\
123.28 \\
122.97 \\
125.82 \\
125.60 \\
127.84 \\
130.19 \\
132.53 \\
135.33 \\
134.42 \\
138.35 \\
140.16 \\
142.63 \\
145.78 \\
142.52 \\
0.22 \\
\end{array}$ & $\begin{array}{l}\text { \% Strain } \\
0.0132 \\
0.0255 \\
0.0374 \\
0.0485 \\
0.0589 \\
0.0654 \\
0.0722 \\
0.0805 \\
0.0891 \\
0.0989 \\
0.1078 \\
0.1183 \\
0.1288 \\
0.1404 \\
0.1532 \\
0.1593 \\
0.1663 \\
0.1716 \\
0.1792 \\
0.1852 \\
0.2005 \\
0.2138 \\
0.2252 \\
0.2267 \\
0.2382 \\
0.2404 \\
0.2501 \\
0.2751 \\
0.3126 \\
0.3751 \\
0.3885 \\
0.4740 \\
0.5436 \\
0.6569 \\
0.7609 \\
0.7667 \\
0.5389\end{array}$ \\
\hline
\end{tabular}

Channel 2321

High Pressure Test

Pressure

0.0255

0.0374

0.0485

0.0589

0.0654

0.0722

0.0989

0.1183

0.1288

0.1593

0.1663

0.1792

0.1852

0.2005

0.2138

0.2252

0.2267

0.2751

0.3126

0.3751

0.3885

0.4740

0.5436

0.6569

.7609

0.7667

0.5389 


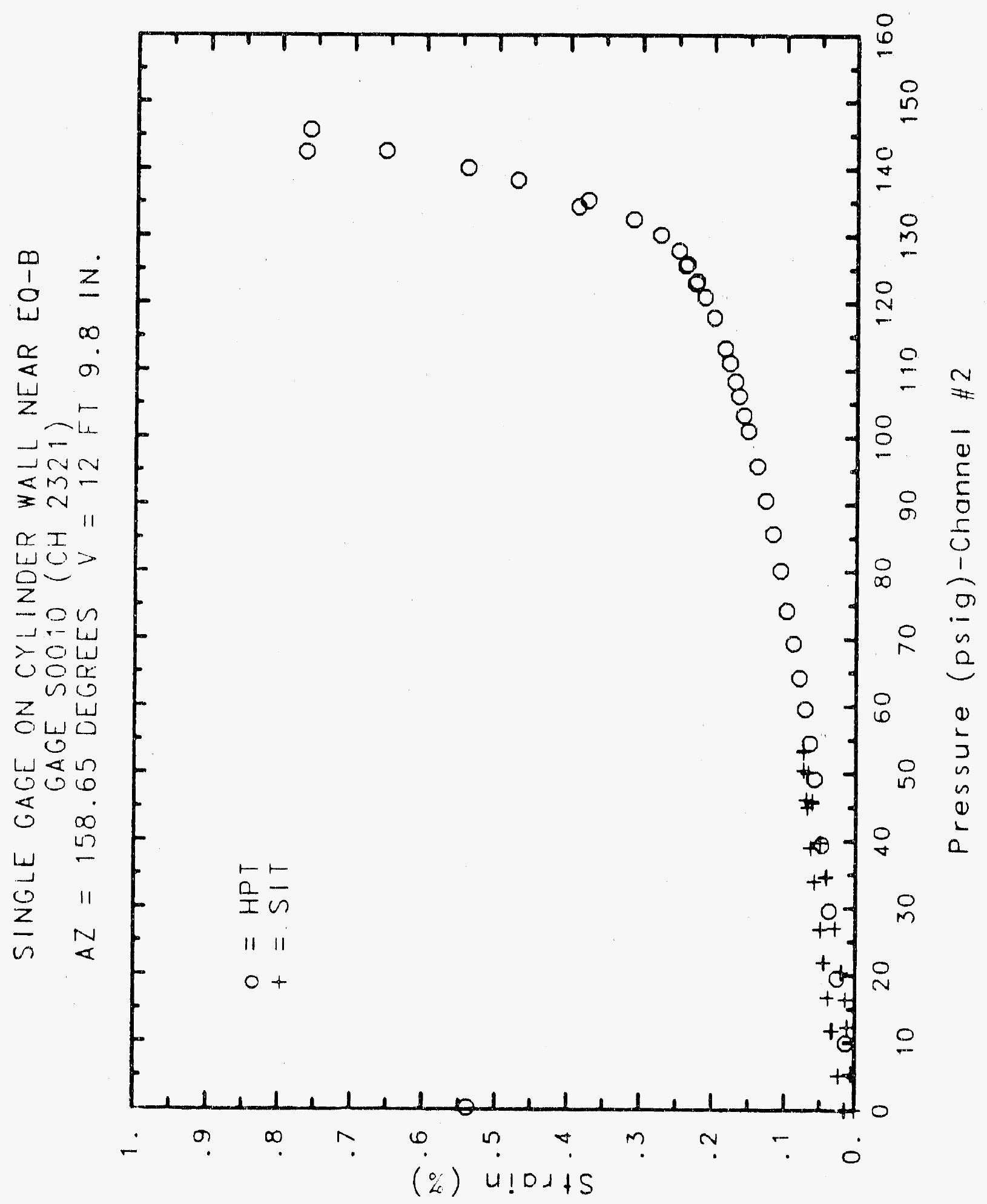




\section{Reinforced Concrete Test Data}

Strain Gage 0

STRUCTURAL INTEGRITY TEST

\begin{tabular}{|c|c|c|c|}
\hline $\begin{array}{c}\text { Pressure } \\
\text { (ps 1g) } \\
-0.05 \\
5.33 \\
12.31 \\
16.44 \\
20.51 \\
20.46 \\
20.47 \\
27.05 \\
34.69 \\
34.53 \\
34.53 \\
39.70 \\
45.90 \\
45.65 \\
45.62 \\
50.10 \\
53.47 \\
53.29 \\
53.21 \\
50.49 \\
46.14 \\
46.13 \\
44.97 \\
38.98 \\
33.99 \\
33.96 \\
26.83 \\
21.88 \\
21.90 \\
16.69 \\
11.74 \\
11.77 \\
5.05 \\
0.02 \\
-0.04 \\
-0.02 \\
-0.02 \\
0.02 \\
\end{array}$ & $\begin{array}{l}\text { Strain } \\
0.0005 \\
0.0035 \\
0.0062 \\
0.0095 \\
0.0132 \\
0.0150 \\
0.0153 \\
0.0249 \\
0.0377 \\
0.0408 \\
0.0422 \\
0.0495 \\
0.0606 \\
0.0607 \\
0.0614 \\
0.0670 \\
0.0725 \\
0.0729 \\
0.0724 \\
0.0714 \\
0.0687 \\
0.0685 \\
0.0675 \\
0.0629 \\
0.0579 \\
0.0573 \\
0.0495 \\
0.0440 \\
0.0440 \\
0.0389 \\
0.0325 \\
0.0312 \\
0.0225 \\
0.0141 \\
0.0123 \\
0.0135 \\
0.0138 \\
0.0138\end{array}$ & $\begin{array}{c}\text { Pressure } \\
\text { (ps1g) } \\
9.89 \\
19.55 \\
29.57 \\
39.42 \\
49.16 \\
54.50 \\
59.57 \\
54.20 \\
69.32 \\
74.16 \\
80.16 \\
85.61 \\
90.58 \\
95.69 \\
100.92 \\
103.25 \\
106.11 \\
108.31 \\
111.08 \\
113.24 \\
117.83 \\
120.92 \\
123.28 \\
122.97 \\
125.82 \\
125.60 \\
127.84 \\
130.19 \\
132.53 \\
135.33 \\
134.42 \\
138.35 \\
140.16 \\
142.63 \\
145.78 \\
142.52 \\
0.22 \\
103\end{array}$ & $\begin{array}{l}\text { \% Strain } \\
0.0114 \\
0.0232 \\
0.0354 \\
0.0465 \\
0.0570 \\
0.0632 \\
0.0703 \\
0.0780 \\
0.0867 \\
0.0963 \\
0.1061 \\
0.1167 \\
0.1268 \\
0.1376 \\
0.1493 \\
0.1546 \\
0.1605 \\
0.1657 \\
0.1729 \\
0.1788 \\
0.1946 \\
0.2093 \\
0.2216 \\
0.2229 \\
0.2332 \\
0.2348 \\
0.2423 \\
0.2636 \\
0.2987 \\
0.3550 \\
0.3652 \\
0.4477 \\
0.5255 \\
0.6550 \\
0.7826 \\
0.7903 \\
0.5389\end{array}$ \\
\hline
\end{tabular}

Chanmel 2.322

High Pressure test

ressure

9.89

19.55

29.57

54.50

64.20

69.32

74.16

90.58

103.25

111.08

113.24

123.28

122.97

125.82

127.84

130.19

132.53

135.33

140.16

142.63

45.78

42.52

0.7903

0.5389 


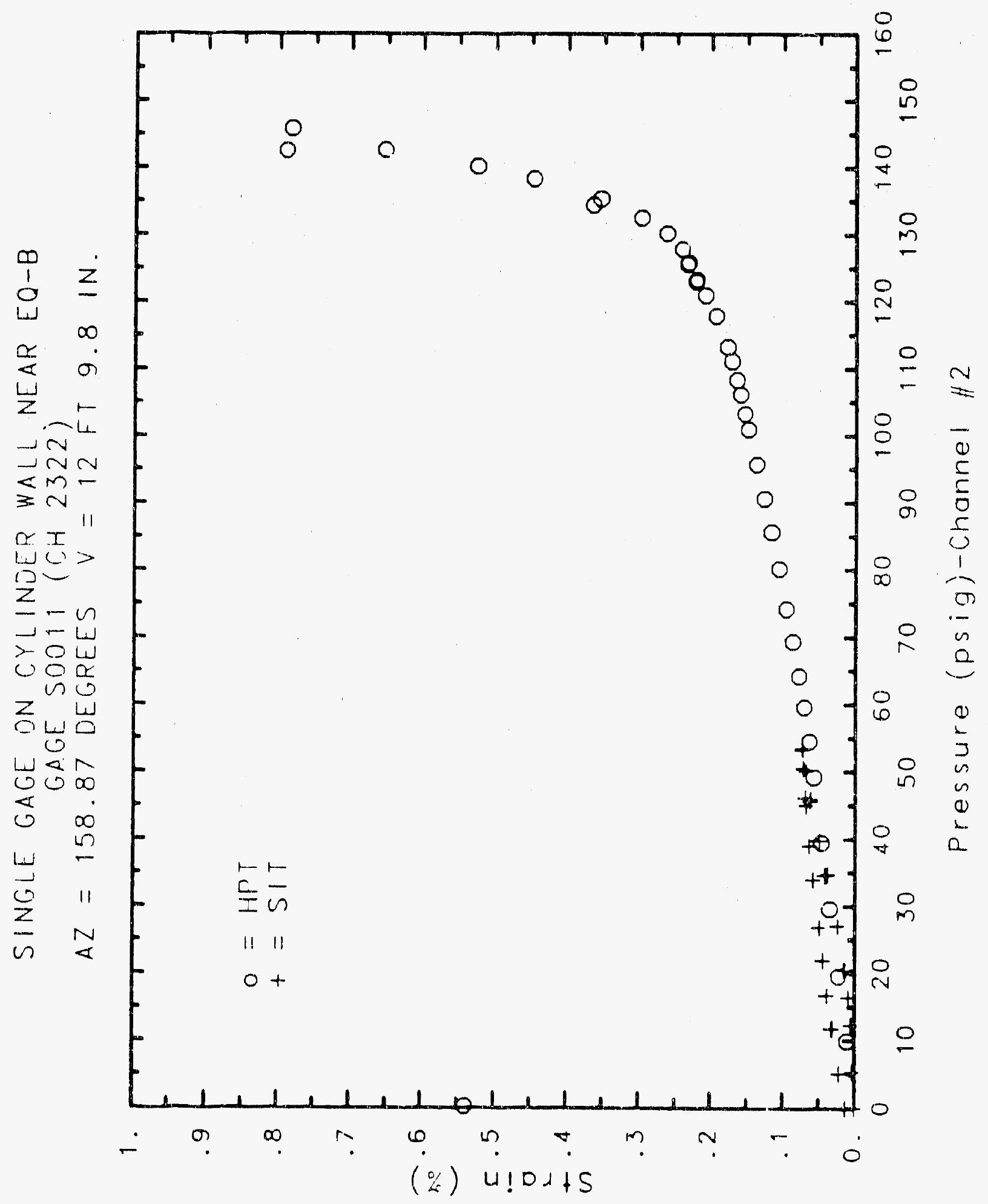




\section{Reinforced Concrete Test Data}

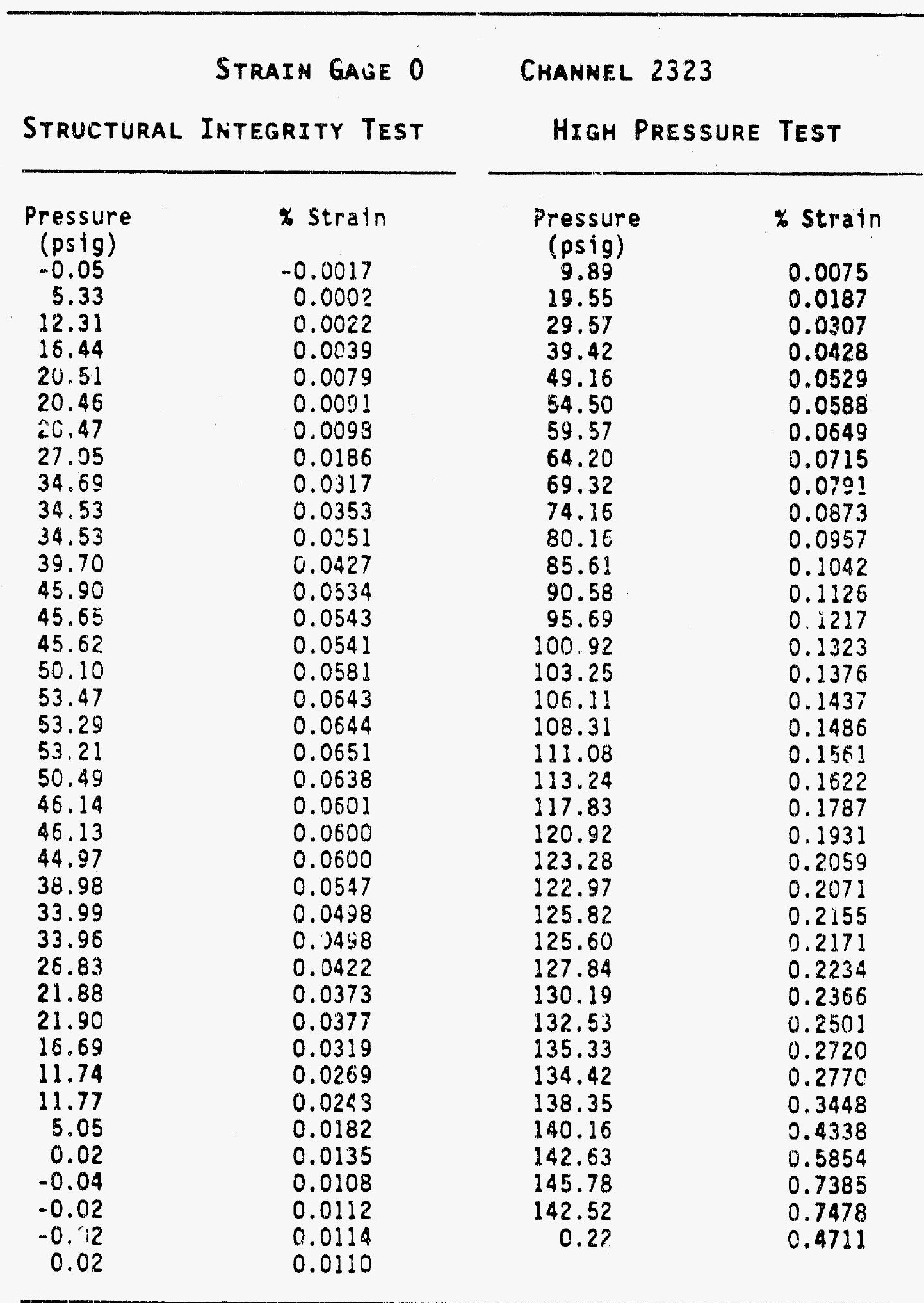




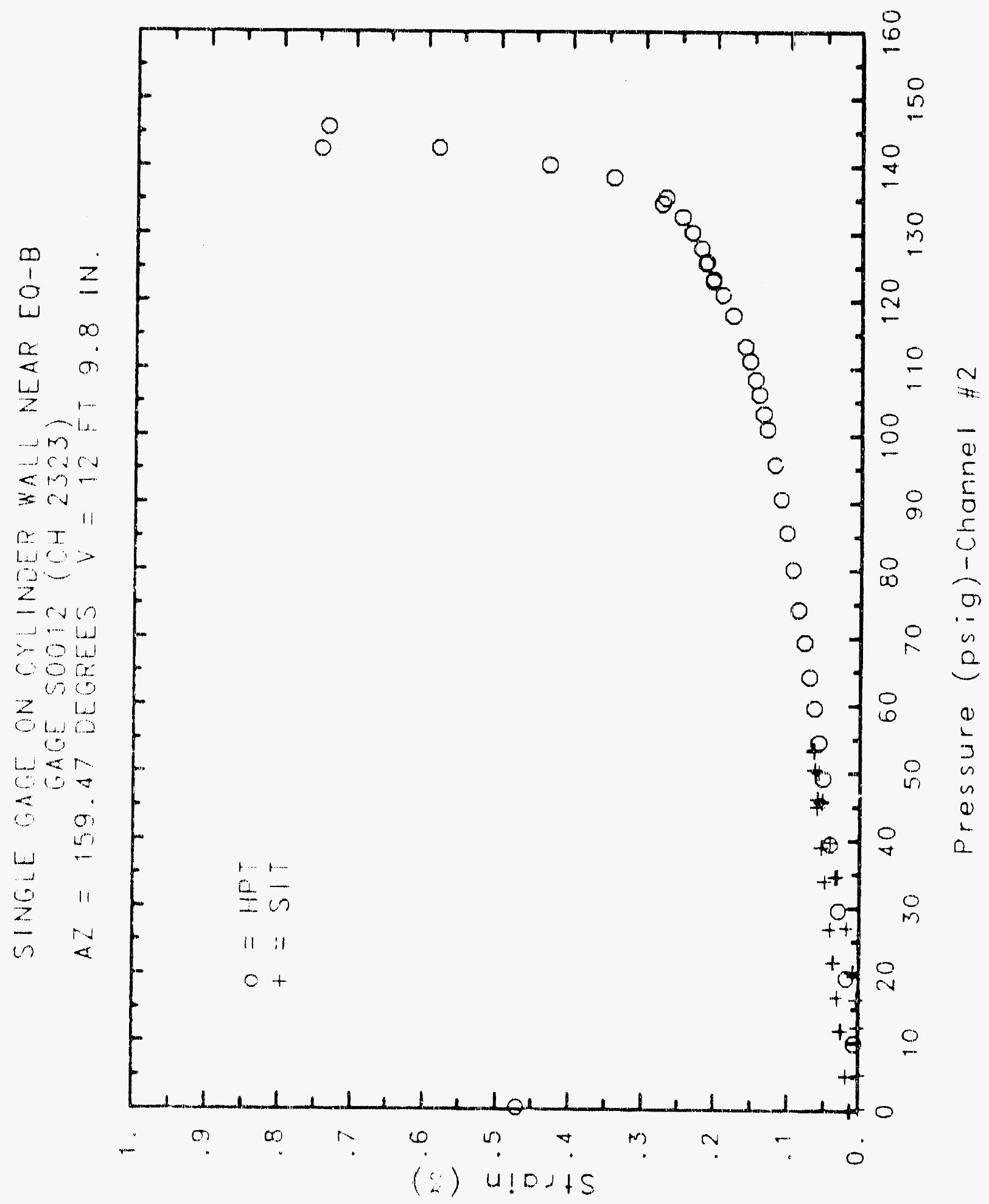

C. $16(1)$ 


\section{Reinforced Concrete Test Data}

Strain gage 0

Structural Integrity TEST

\begin{tabular}{|c|c|c|c|}
\hline $\begin{array}{c}\text { Pressure } \\
\text { (psig) } \\
-0.05 \\
5.33 \\
12.31 \\
16.44 \\
20.51 \\
20.46 \\
20.47 \\
27.05 \\
34.69 \\
34.53 \\
34.53 \\
39.70 \\
45.90 \\
45.65 \\
45.62 \\
50.10 \\
53.47 \\
53.29 \\
53.21 \\
50.49 \\
46.14 \\
46.13 \\
44.97 \\
38.98 \\
33.99 \\
33.96 \\
26.83 \\
21.88 \\
21.90 \\
16.69 \\
11.74 \\
11.77 \\
5.05 \\
0.02 \\
-0.04 \\
-0.02 \\
-0.02 \\
0.02 \\
\end{array}$ & $\begin{array}{l}\text { X Strain } \\
0.0015 \\
0.0113 \\
0.0212 \\
0.0254 \\
0.0281 \\
0.0288 \\
0.0288 \\
0.0313 \\
0.0354 \\
0.0356 \\
0.0355 \\
0.0375 \\
0.0422 \\
0.0422 \\
0.0425 \\
0.0448 \\
0.0468 \\
0.0469 \\
0.0473 \\
0.0465 \\
0.0462 \\
0.0461 \\
0.0457 \\
0.0446 \\
0.0432 \\
0.0424 \\
0.0401 \\
0.0373 \\
0.0375 \\
0.0345 \\
0.0298 \\
0.0307 \\
0.0206 \\
0.0092 \\
0.0118 \\
0.0122 \\
0.0115 \\
0.0108\end{array}$ & $\begin{array}{c}\text { Pressure } \\
(\text { psig) } \\
9.89 \\
19.55 \\
29.57 \\
39.42 \\
49.16 \\
54.50 \\
59.57 \\
64.20 \\
69.32 \\
74.16 \\
80.16 \\
85.61 \\
90.58 \\
95.69 \\
100.92 \\
103.25 \\
106.11 \\
108.31 \\
111.08 \\
113.24 \\
117.83 \\
120.92 \\
123.28 \\
122.97 \\
125.82 \\
125.60 \\
127.84 \\
130.19 \\
132.53 \\
135.33 \\
134.42 \\
138.35 \\
140.16 \\
142.63 \\
145.78 \\
142.52 \\
0.22 \\
\end{array}$ & $\begin{array}{l}\text { \% Strain } \\
0.0169 \\
0.0247 \\
0.0275 \\
0.0289 \\
0.0295 \\
0.0293 \\
0.0262 \\
0.0241 \\
0.0242 \\
0.0256 \\
0.0251 \\
0.0266 \\
0.0278 \\
0.0279 \\
0.0264 \\
0.0248 \\
0.0225 \\
0.0203 \\
0.0160 \\
0.0123 \\
0.0029 \\
-0.0031 \\
-0.0070 \\
-0.0070 \\
-0.0112 \\
-0.0104 \\
-0.0117 \\
-0.0130 \\
-0.0153 \\
-0.0163 \\
-0.0156 \\
-0.0229 \\
-0.0293 \\
-0.0408 \\
-0.0516 \\
-0.0521 \\
-0.0638\end{array}$ \\
\hline
\end{tabular}

Channel 2324

High Pressure test

(psig)

19.55

29.57

39.42

59.57

64.20

74.16

80.16

95.69

100.92

106.11

108.31

111.08

113.24

117.83

20.92

122.97

125.82

125.60

35.33

140.16

142.63

45.78

42.52

0.22
0.0247

0.0275

0.0289

0.0295

0.0293

0.0242

0.0256

0.0251

0.0266

0.0278

0.0279

0.0248

0.0225

0.0160

0.0123

0.0029

$-0.0070$

$-0.0112$

$-0.0104$

$-0.0117$

$-0.0130$

$-0.0156$

$-0.0229$

$-0.0293$

$-0.0408$

$-0.0516$

$-0.0521$
0.0203 


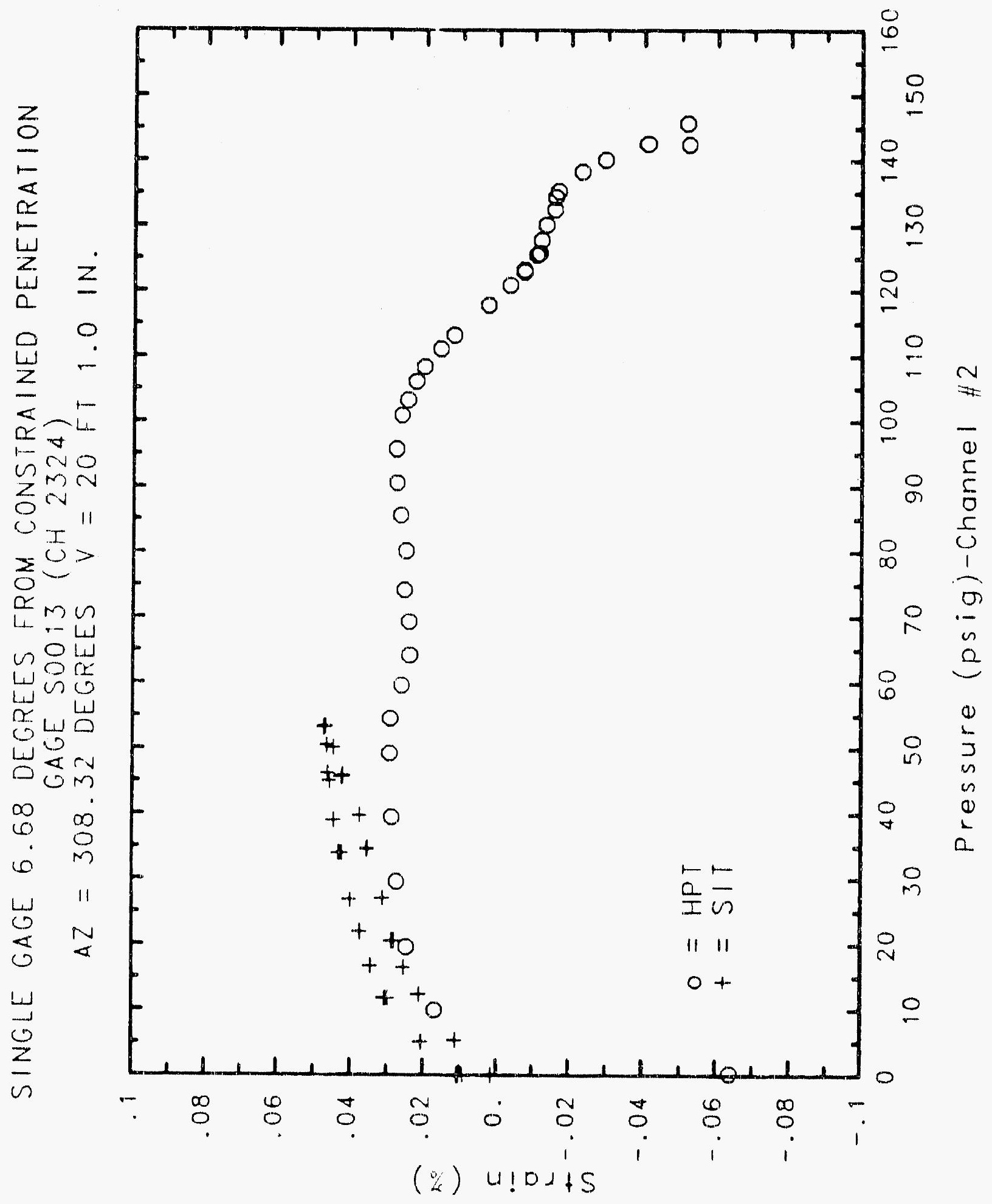

C. -1611 


\section{Reinforced Concrete Test Data}

Strain Gage 0

Structural Integrity Test

\begin{tabular}{|c|c|}
\hline $\begin{array}{c}\text { Pressure } \\
\text { (psig) } \\
-0.05 \\
5.33 \\
12.31 \\
16.44 \\
20.51 \\
20.46 \\
20.47 \\
27.05 \\
34.69 \\
34.53 \\
34.53 \\
39.70 \\
45.90 \\
45.65 \\
45.62 \\
50.10 \\
53.47 \\
53.29 \\
53.21 \\
50.49 \\
46.14 \\
46.13 \\
44.97 \\
38.98 \\
33.99 \\
33.96 \\
26.83 \\
21.88 \\
21.90 \\
16.69 \\
11.74 \\
11.77 \\
5.05 \\
0.02 \\
-0.04 \\
-0.02 \\
-0.02 \\
0.02 \\
\end{array}$ & $\begin{array}{c}\text { X Strain } \\
-0.0010 \\
0.0111 \\
0.0229 \\
0.0281 \\
0.536 \\
0.0343 \\
0.0345 \\
0.0395 \\
0.0433 \\
0.0432 \\
0.0427 \\
0.0447 \\
0.0494 \\
0.0497 \\
0.0497 \\
0.0525 \\
0.0553 \\
0.0558 \\
0.0556 \\
0.0552 \\
0.0537 \\
0.0534 \\
0.0555 \\
0.0526 \\
0.0502 \\
0.0505 \\
0.0463 \\
0.0438 \\
0.0431 \\
0.0385 \\
0.0332 \\
0.0324 \\
0.0193 \\
0.0057 \\
0.0095 \\
0.0107 \\
0.0098 \\
0.0083\end{array}$ \\
\hline
\end{tabular}

Chanmel 2325

Hygh Pressure test

Pressure \% Strain

(psig)

9.89

19.55

29.57

0.0203

0.0306

39.42

0.0353

49.16

54.50

59.57

64.20

69.32

74.16

80.16

0.0383

0.0402

0.0409

0.0387

0.0357

0.0363

0.0379

85.61

0.0358

90.58

95.69

0.0357

0.0356

0.0347

100.92

103.25

106.11

108.31

111.08

0.0348

0.0347

0.0343

0.0343

0.0340

113.24

0.0343

117.83

0.0350

120.92

123.28

122.97

125.82

125.60

127.84

130.19

132.53

0.0352

0.0353

0.0356

0.0356

0.0361

0.0362

0.0352

0.0341

135.33

134.42

0.0341

0.0338

0.0334

0.0321

0.0289

0.0254

0.0208

$-0.0128$ 


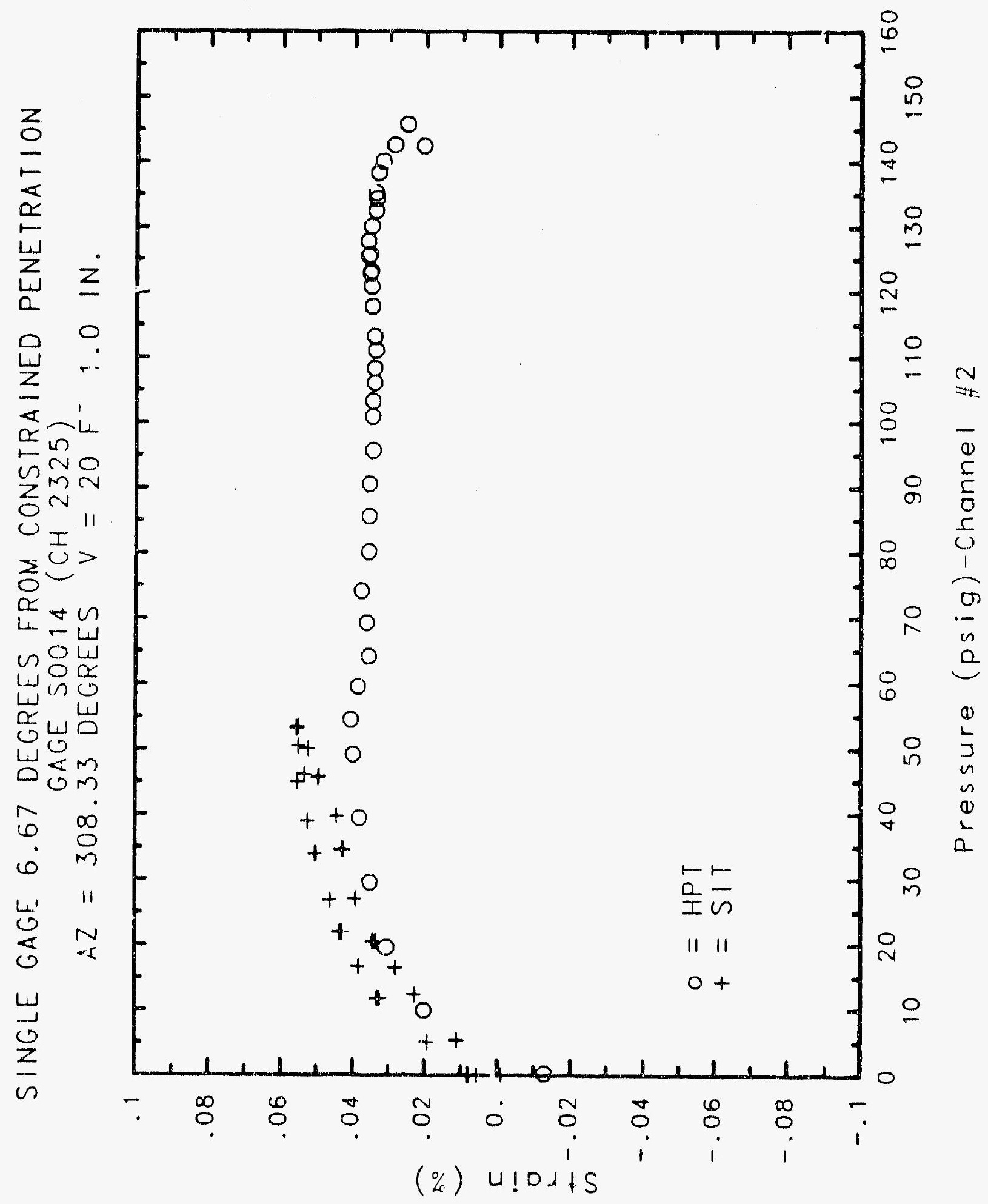




\section{Reinforced Concrete Test Data}

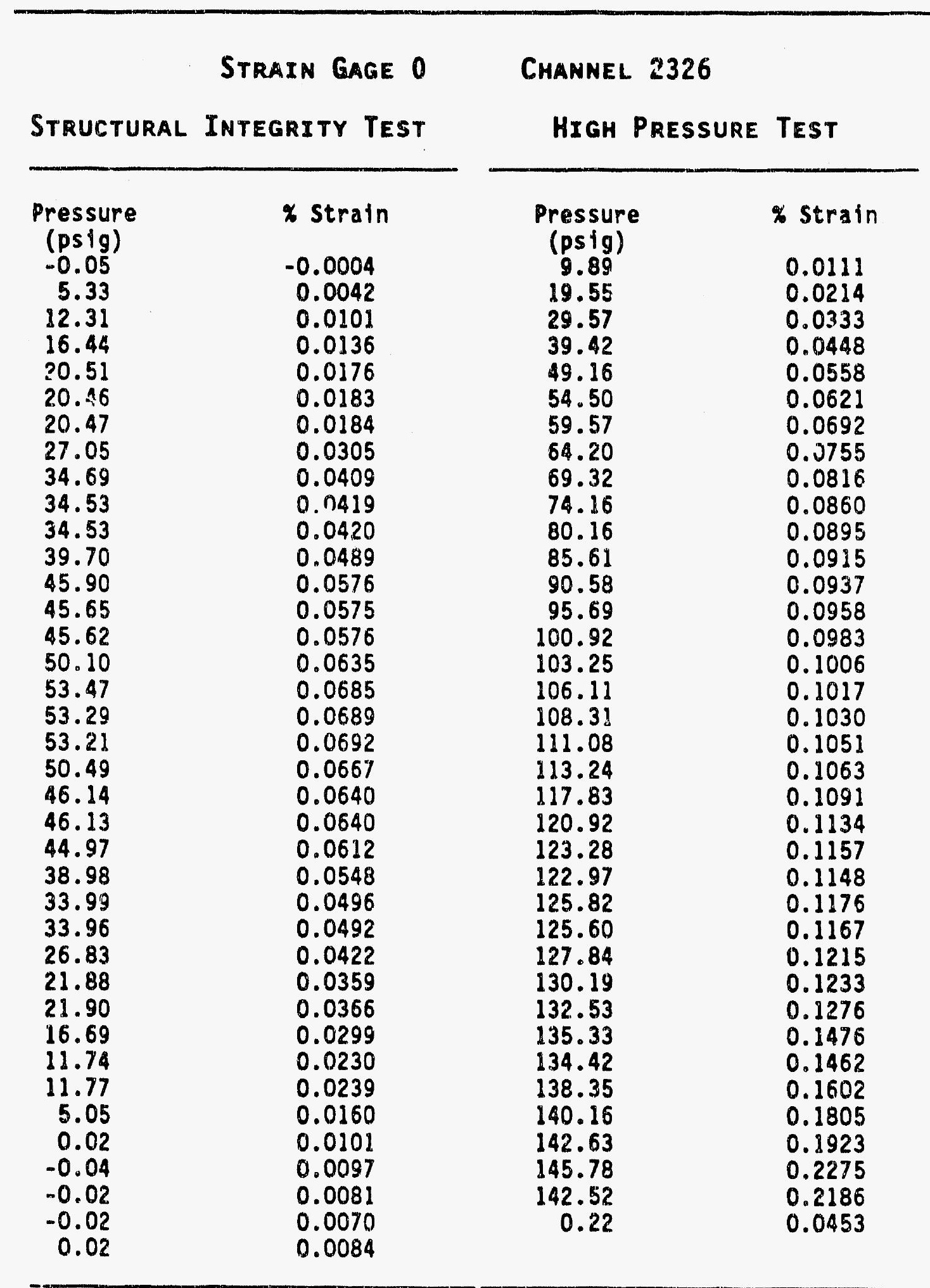




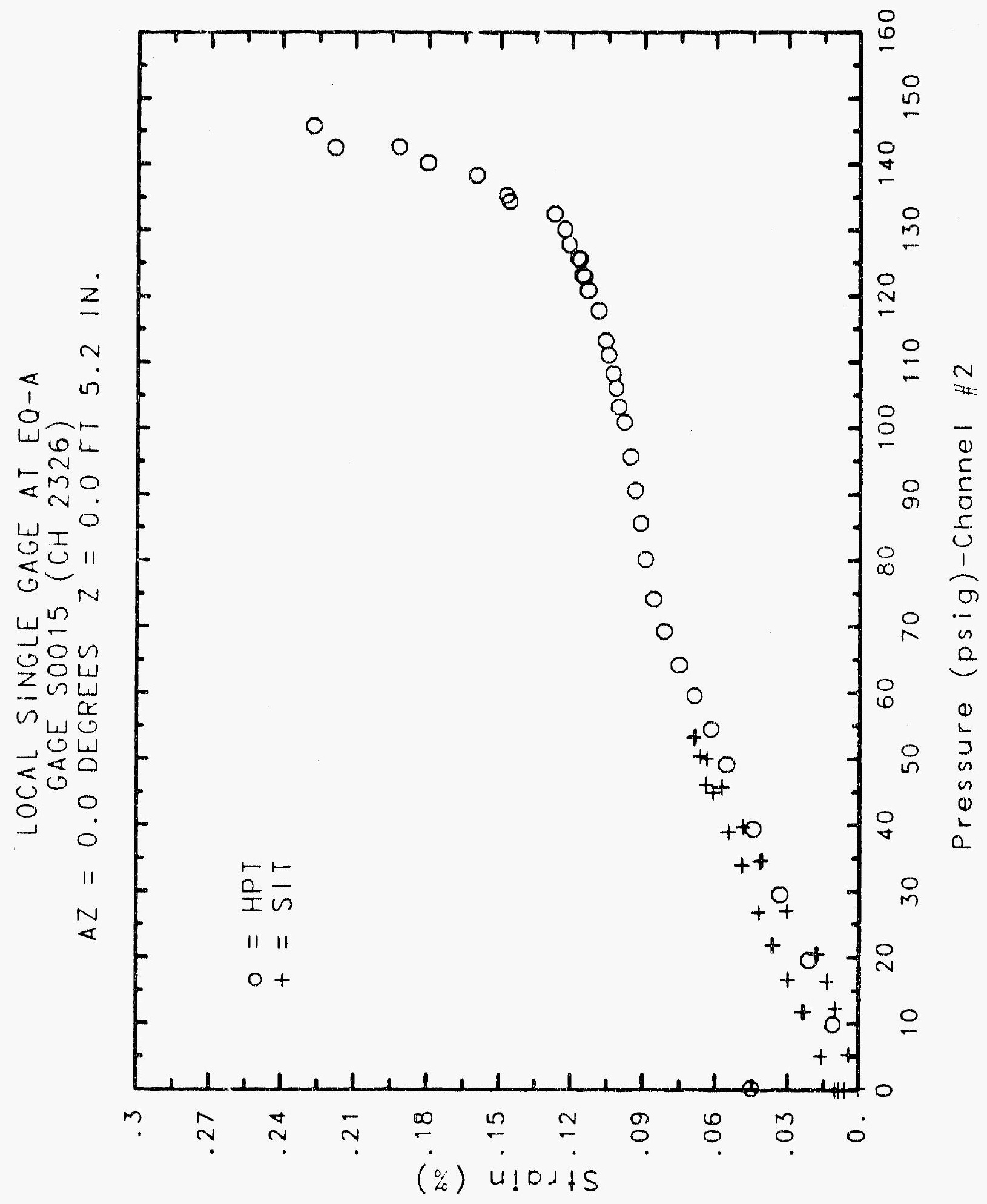

C- -1615 


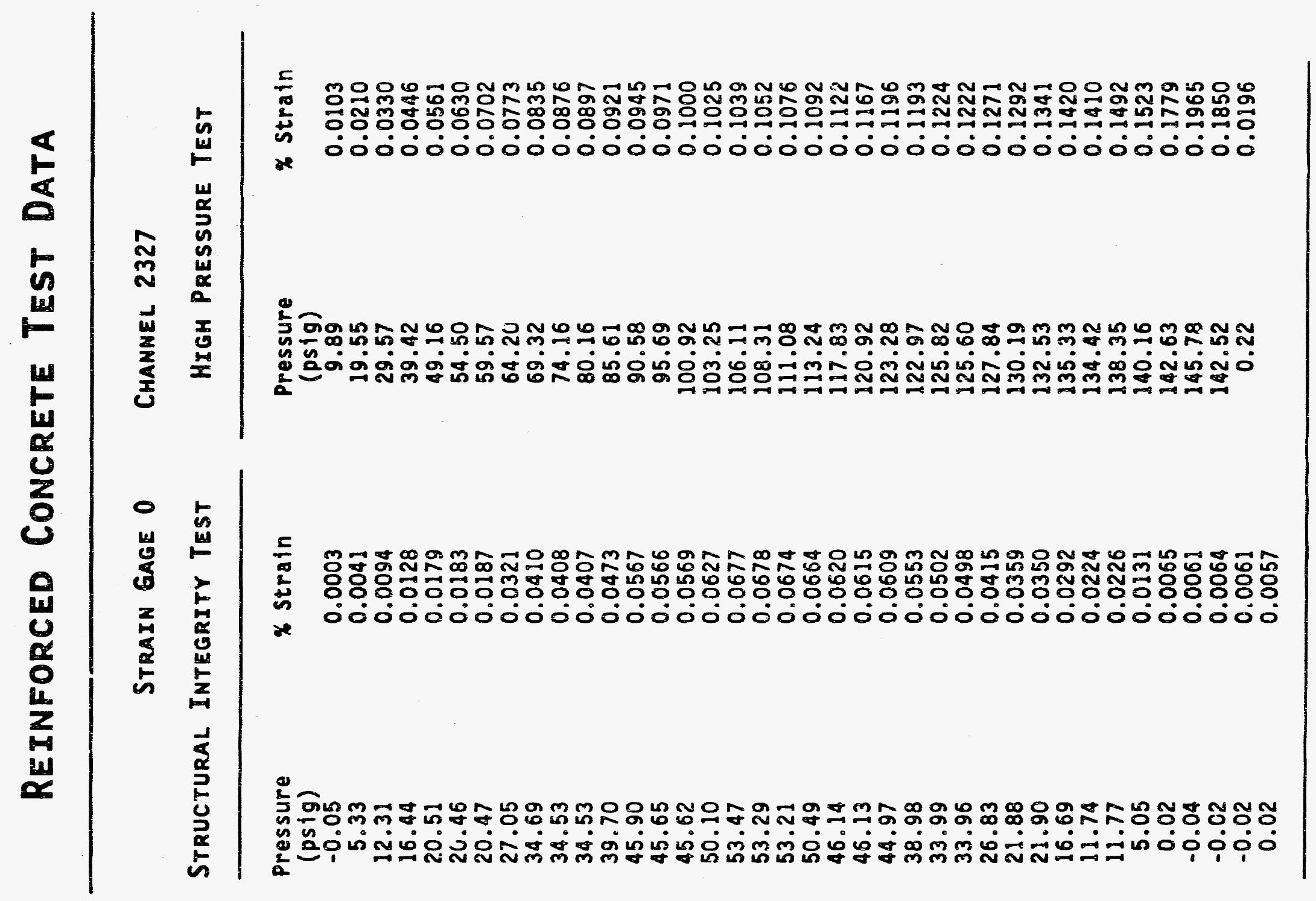




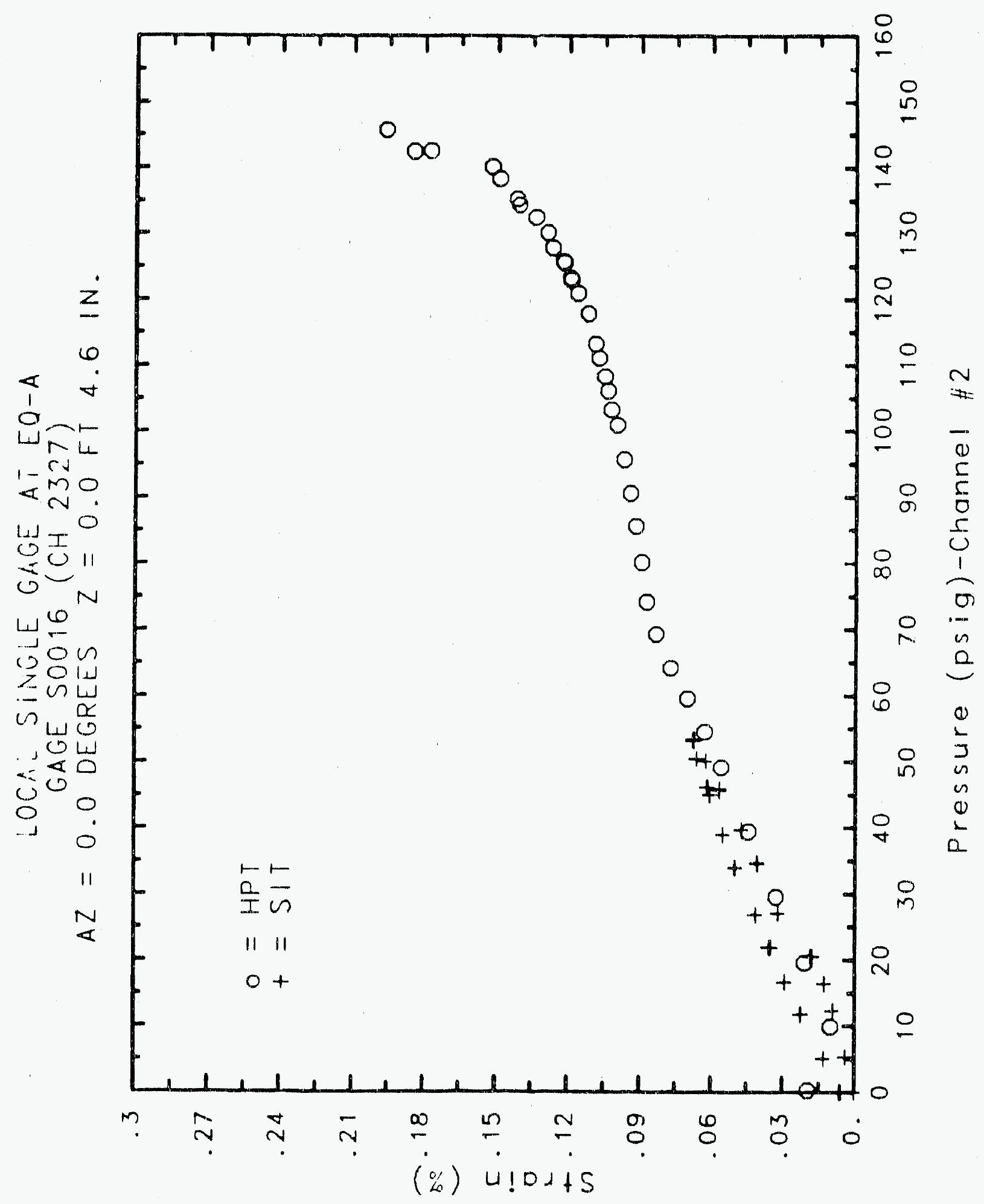




\section{Reinforced Concrete Test Data}

Strayn Gage 0

StRuctural Integrity TEst

\begin{tabular}{|c|c|c|c|}
\hline $\begin{array}{c}\text { Pressure } \\
\text { (psig) } \\
-0.05 \\
5.33 \\
12.31 \\
16.44 \\
20.51 \\
20.46 \\
20.47 \\
27.05 \\
34.69 \\
34.53 \\
34.53 \\
39.70 \\
45.90 \\
45.65 \\
45.62 \\
50.10 \\
53.47 \\
53.29 \\
53.21 \\
50.49 \\
46.14 \\
46.13 \\
44.97 \\
38.98 \\
33.99 \\
33.96 \\
26.83 \\
21.88 \\
21.90 \\
16.69 \\
11.74 \\
11.77 \\
5.05 \\
0.02 \\
-0.04 \\
-0.02 \\
-0.02 \\
0.02 \\
\end{array}$ & $\begin{array}{l}\text { X Strain } \\
\text {-0.0014 } \\
0.0033 \\
0.0098 \\
0.0136 \\
0.0181 \\
0.0185 \\
0.0185 \\
0.0317 \\
0.0380 \\
0.0380 \\
0.0381 \\
0.0441 \\
0.0518 \\
0.0528 \\
0.0526 \\
0.0575 \\
0.0624 \\
0.0621 \\
0.0626 \\
0.0609 \\
0.0574 \\
0.0572 \\
0.0551 \\
0.0492 \\
0.0440 \\
0.0440 \\
0.0371 \\
0.0314 \\
0.0309 \\
0.0249 \\
0.0195 \\
0.0191 \\
0.0114 \\
0.0058 \\
0.0048 \\
0.0047 \\
0.0044 \\
0.0038\end{array}$ & $\begin{array}{c}\text { Pressure } \\
(\text { ps1g) } \\
9.89 \\
19.55 \\
29.57 \\
39.42 \\
49.16 \\
54.50 \\
59.57 \\
64.20 \\
69.32 \\
74.16 \\
80.16 \\
85.61 \\
90.58 \\
95.69 \\
100.92 \\
103.25 \\
106.11 \\
108.31 \\
111.08 \\
113.24 \\
117.83 \\
120.92 \\
123.28 \\
122.97 \\
125.82 \\
125.60 \\
127.84 \\
130.19 \\
132.53 \\
135.33 \\
134.42 \\
138.35 \\
140.16 \\
142.63 \\
145.78 \\
142.52 \\
0.22\end{array}$ & $\begin{array}{l}\text { X strain } \\
0.0102 \\
0.0202 \\
0.0311 \\
0.0416 \\
0.0520 \\
0.0581 \\
0.0645 \\
0.0704 \\
0.0759 \\
0.0796 \\
0.0826 \\
0.0848 \\
0.0872 \\
0.0896 \\
0.0923 \\
0.0944 \\
0.0958 \\
0.0971 \\
0.0991 \\
0.1005 \\
0.1036 \\
0.1077 \\
0.1100 \\
0.1093 \\
0.1122 \\
0.1117 \\
0.1160 \\
0.1183 \\
0.1223 \\
0.1290 \\
0.1280 \\
0.1369 \\
0.1413 \\
0.1462 \\
0.1621 \\
0.1508 \\
0.0024\end{array}$ \\
\hline
\end{tabular}

Channel 2328

high Pressure test 


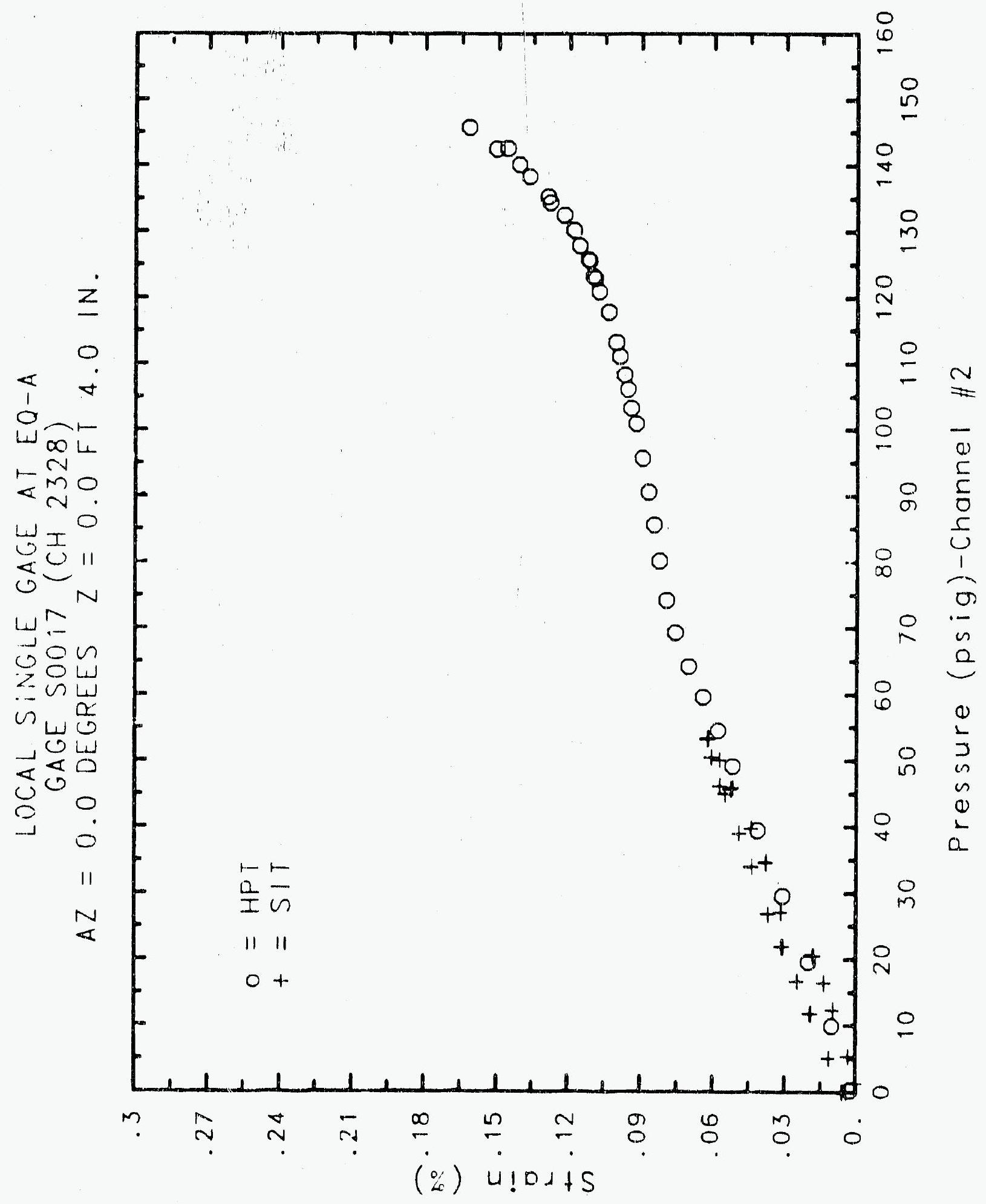




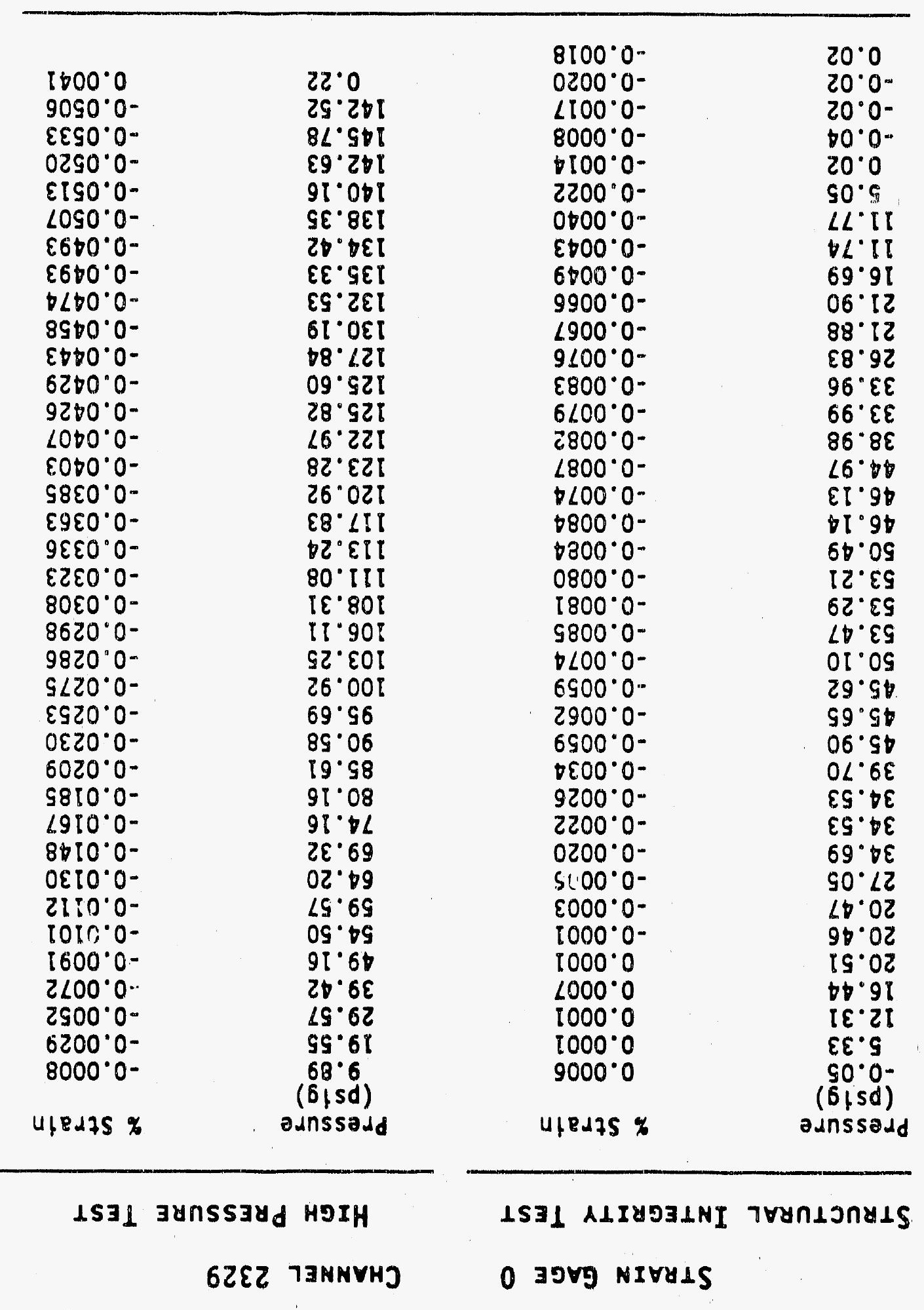

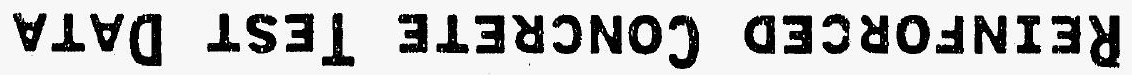




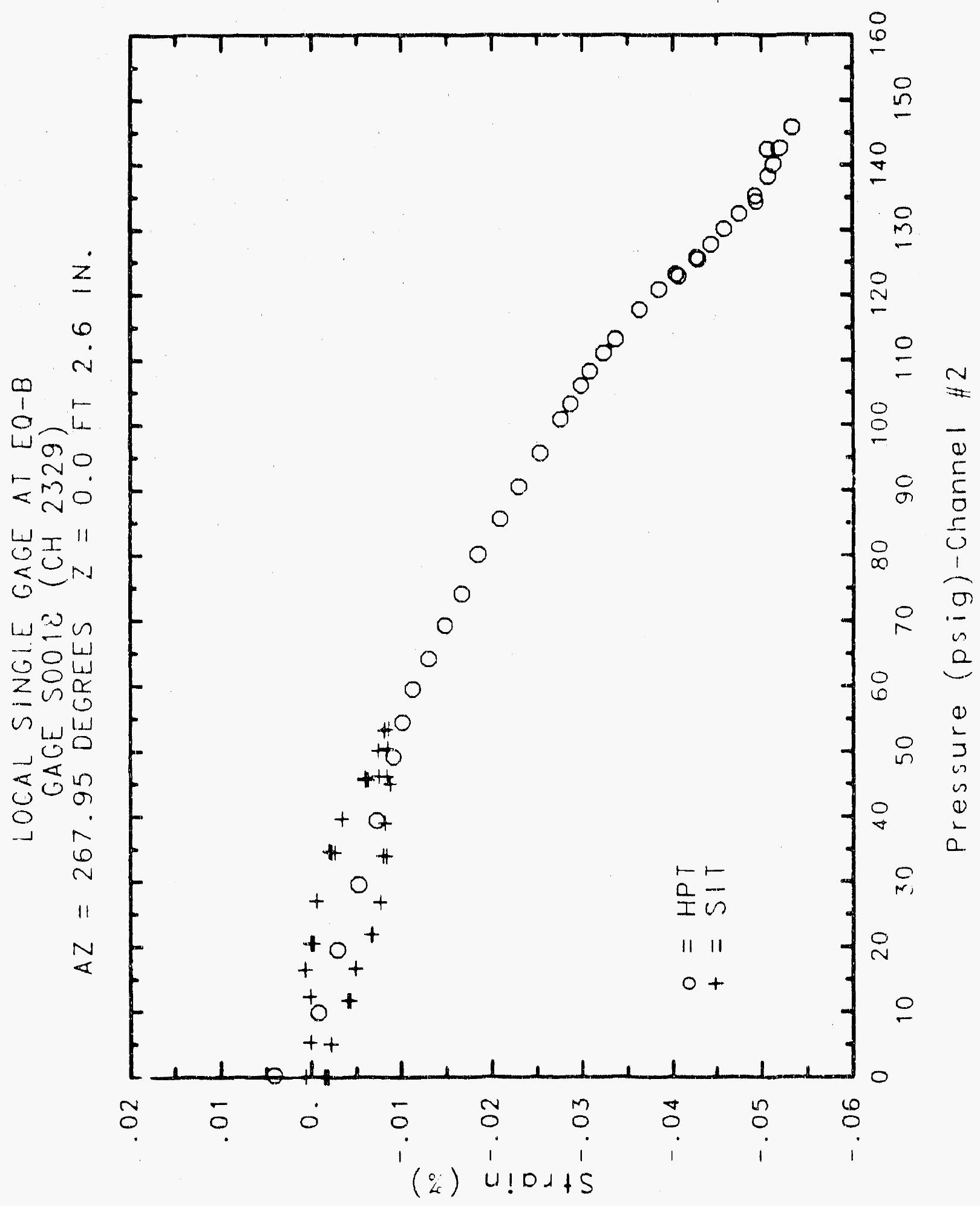




\section{Reinforced Concrete Test Data}

Strain Gage 0

STRUCTURAL INTEGRITY TEST

\begin{tabular}{|c|c|}
\hline $\begin{array}{c}\text { Pressure } \\
\text { (psig) } \\
-0.05 \\
5.33 \\
12.31 \\
16.44 \\
20.51 \\
20.46 \\
20.47 \\
27.05 \\
34.69 \\
34.53 \\
34.53 \\
39.70 \\
45.90 \\
45.65 \\
45.62 \\
50.10 \\
53.47 \\
53.29 \\
53.21 \\
50.49 \\
46.14 \\
46.13 \\
44.97 \\
38.98 \\
33.99 \\
33.96 \\
26.83 \\
21.88 \\
21.90 \\
16.69 \\
11.74 \\
11.77 \\
5.05 \\
0.02 \\
-0.04 \\
-0.02 \\
-0.02 \\
0.02 \\
\end{array}$ & $\begin{array}{r}\text { X Strain } \\
-0.0003 \\
0.0000 \\
0.0010 \\
0.0023 \\
0.0025 \\
0.0027 \\
0.0034 \\
0.0028 \\
0.0018 \\
0.0028 \\
0.0030 \\
0.0020 \\
-0.0001 \\
0.0005 \\
0.0011 \\
0.0001 \\
-0.0012 \\
-0.0009 \\
-0.0017 \\
-0.0016 \\
-0.0008 \\
0.0015 \\
-0.0013 \\
-0.0015 \\
-0.0013 \\
-0.0006 \\
-0.0006 \\
0.0007 \\
0.0005 \\
0.0001 \\
0.0017 \\
0.0039 \\
0.0018 \\
0.0037 \\
0.0017 \\
0.0085 \\
0.0092 \\
0.0087\end{array}$ \\
\hline
\end{tabular}

Channel 2340

\section{High pressure test}

Pressure

9.89

19.55

29.57

39.42

49.16

54.50

59.57

64.20

69.32

74.16

80.16

85.61

90.58

95.69

100.92

103.25

106.11

108.31

111.08

113.24

117.83

120.92

123.28

122.97

125.82

125.60

127.27

130.19

132.53

135.33

134.42

138.35

140.16

142.63

145.78

142.52

0.22
* Strain

0.0037

0.0025

0.0010

$-0.0004$

$-0.0019$

$-0.0026$

$-0.0038$

$-0.0052$

$-0.0068$

$-0.0085$

$-0.0098$

$-0.0116$

$-0.0132$

$-0.0150$

$-0.0168$

$-0.0177$

$-0.0187$

$-0.0195$

$-0.0207$

$-0.0215$

$-0.0236$

$-0.0251$

$-0.0265$

$-0.0267$

$-0.0283$

$-0.0284$

$-0.0294$

$-0.0306$

$-0.0313$

$-0.0318$

$-0.0313$

$-0.0316$

$-0.0315$

$-0.0310$

$-0.0313$

$-0.0292$

0.0091 


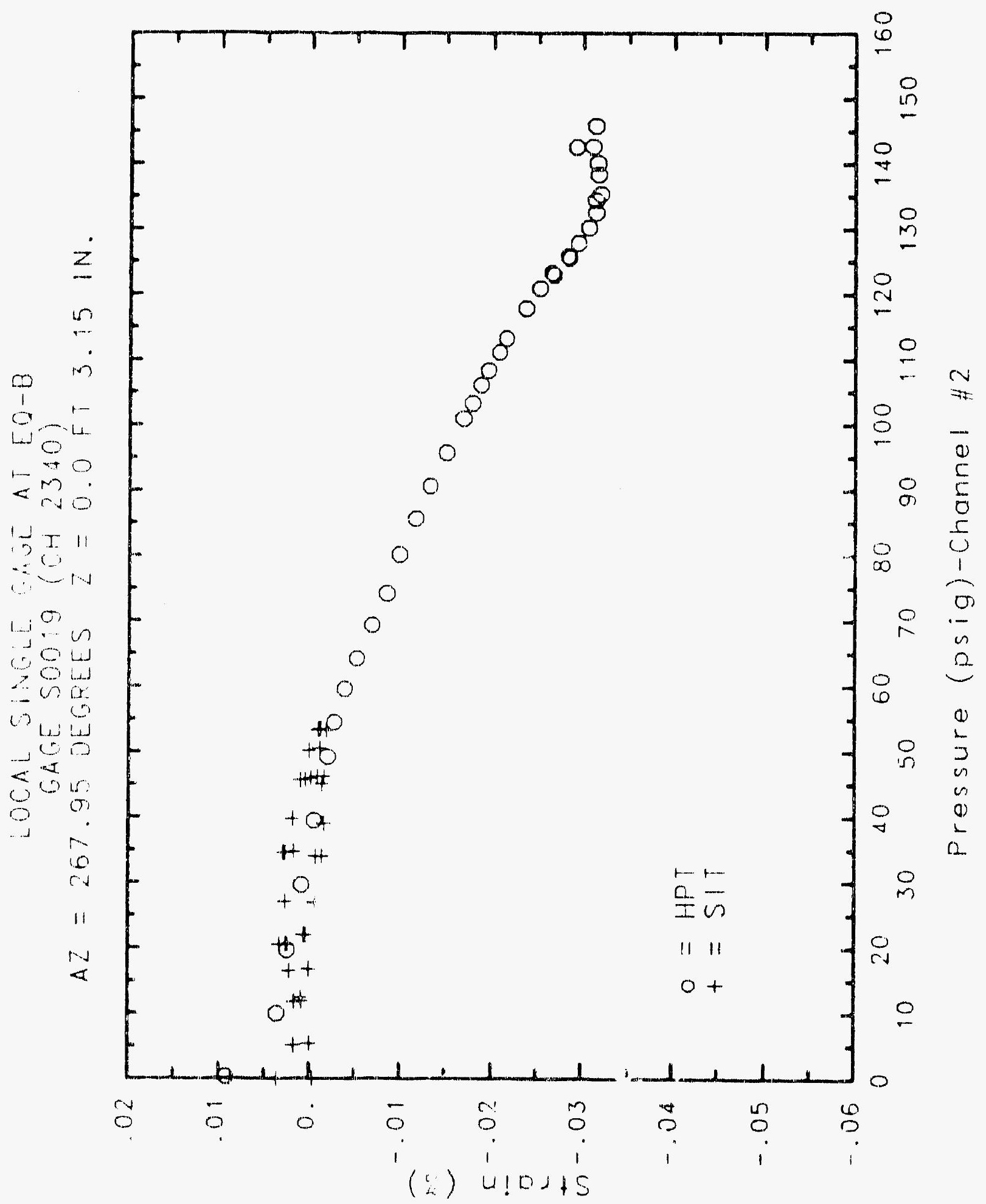

(.) 16$) .3$ 


\section{Reinforced Concrete Test Data}

Strain gage 0

STRUCTURAL INTEGRITY TEST

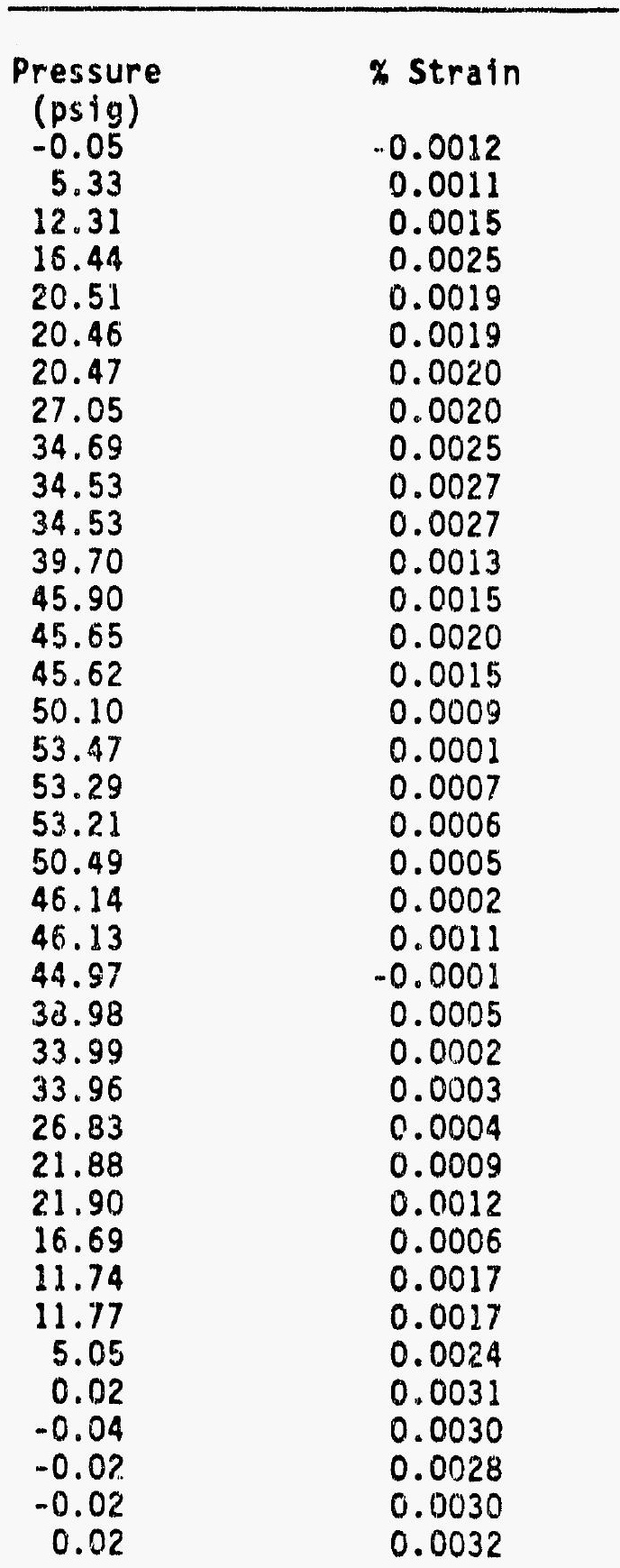

Channel 2341

High Pressure test

\begin{tabular}{|c|c|}
\hline $\begin{array}{c}\text { Pressure } \\
\text { (ps1g) } \\
9.89 \\
19.55 \\
29.57 \\
39.42 \\
49.16 \\
54.50 \\
59.57 \\
64.20 \\
69.32 \\
74.16 \\
80.16 \\
85.61 \\
90.58 \\
95.69 \\
100.92 \\
103.25 \\
106.11 \\
108.31 \\
111.08 \\
113.24 \\
117.83 \\
120.92 \\
123.28 \\
122.97 \\
125.82 \\
125.60 \\
12 \% .84 \\
130.19 \\
132.53 \\
135.33 \\
134.42 \\
138.35 \\
140.16 \\
142.63 \\
145.78 \\
142.52 \\
0.22 \\
\end{array}$ & $\begin{array}{l}x \text { Strain } \\
0.0004 \\
0.0001 \\
-0.0011 \\
-0.0019 \\
-0.0029 \\
-0.0034 \\
-0.0042 \\
-0.0053 \\
-0.0067 \\
-0.0078 \\
-0.0087 \\
-0.0097 \\
-0.0108 \\
-0.0119 \\
-0.0130 \\
-0.0135 \\
-0.0141 \\
-0.0147 \\
-0.0153 \\
-0.0159 \\
-0.0172 \\
-0.0181 \\
-0.0191 \\
-0.0193 \\
-0.0201 \\
-0.0203 \\
-0.0209 \\
-0.0214 \\
-0.0217 \\
-0.0217 \\
-0.0214 \\
-0.0209 \\
-0.0201 \\
-0.0191 \\
-0.0184 \\
-0.0173 \\
0.0048\end{array}$ \\
\hline
\end{tabular}




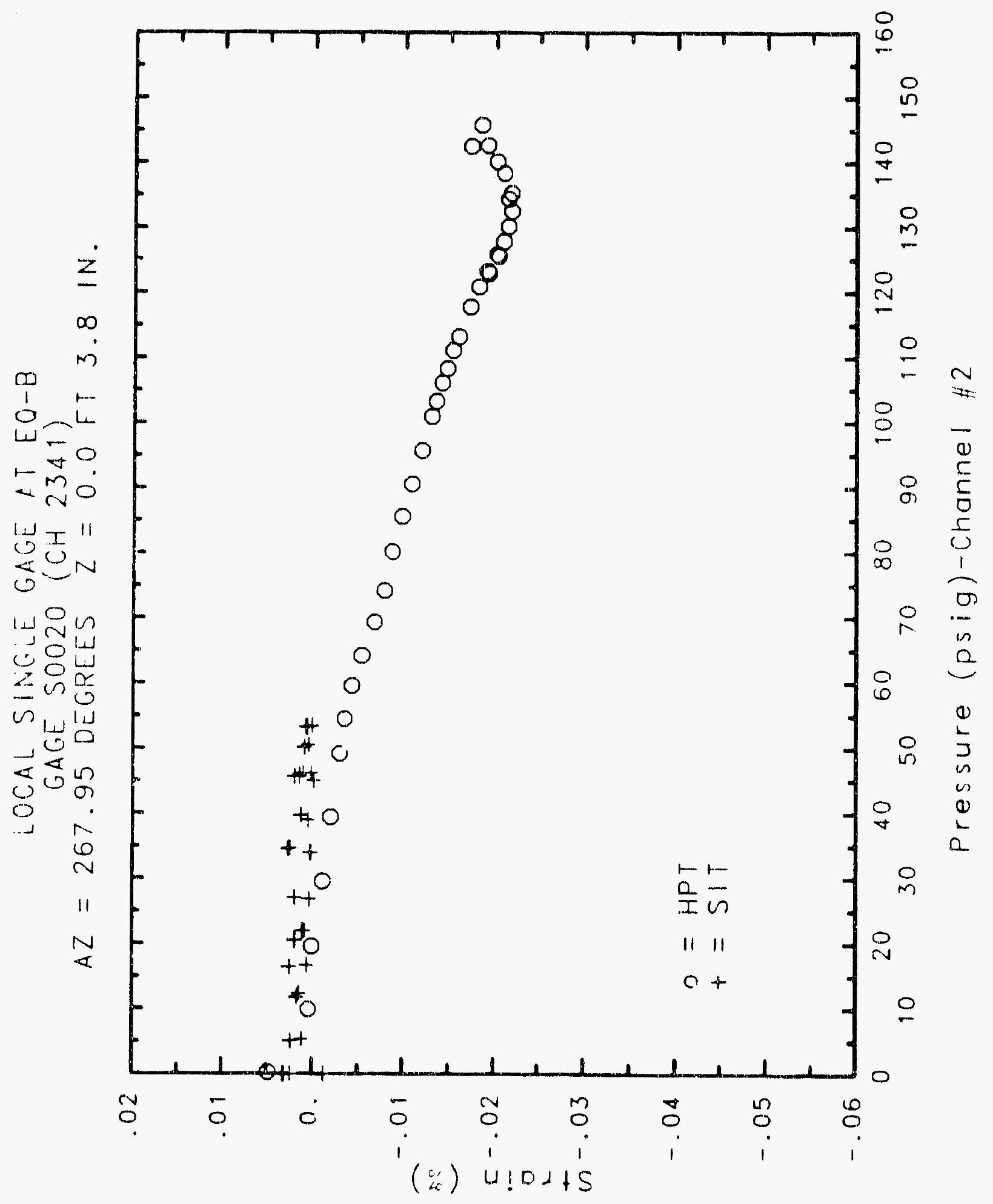

C. 16.25 


\section{Reinforced Concrete Test Data}

Strain gage 0

StRUCTURAL INTEgRITY TEST

\begin{tabular}{|c|c|c|c|}
\hline $\begin{array}{c}\text { Pressure } \\
\text { (psig) } \\
-0.05 \\
5.33 \\
12.31 \\
16.44 \\
20.51 \\
20.46 \\
20.47 \\
27.05 \\
34.69 \\
34.53 \\
34.53 \\
39.70 \\
45.90 \\
45.65 \\
45.62 \\
50.10 \\
53.47 \\
53.29 \\
53.21 \\
50.49 \\
46.14 \\
46.13 \\
44.97 \\
38.98 \\
33.99 \\
33.96 \\
26.83 \\
21.58 \\
21.90 \\
16.69 \\
11.74 \\
11.77 \\
5.05 \\
0.02 \\
-0.04 \\
-0.02 \\
-0.02 \\
0.02 \\
\end{array}$ & $\begin{array}{l}\text { X Straln } \\
-0.0022 \\
-0.0009 \\
-0.0007 \\
-0.0003 \\
0.0010 \\
0.0013 \\
0.0008 \\
-0.0003 \\
-0.0024 \\
-0.0035 \\
-0.0035 \\
-0.0035 \\
-0.0038 \\
-0.0034 \\
-0.0024 \\
-0.0030 \\
-0.0034 \\
-0.0032 \\
-0.0035 \\
-0.0035 \\
-0.0035 \\
-0.0043 \\
-0.0049 \\
-0.0038 \\
-0.0044 \\
-0.0035 \\
-0.0034 \\
-0.0027 \\
-0.0038 \\
-0.0031 \\
-0.0026 \\
-0.0024 \\
-0.0023 \\
-0.0012 \\
-0.0017 \\
-0.0014 \\
-0.0009 \\
-0.0016\end{array}$ & $\begin{array}{c}\text { Pressure } \\
\text { (psig) } \\
9.89 \\
19.55 \\
29.57 \\
39.42 \\
49.16 \\
54.50 \\
59.57 \\
64.20 \\
69.32 \\
74.16 \\
80.16 \\
85.61 \\
90.58 \\
95.69 \\
100.92 \\
103.25 \\
106.11 \\
108.31 \\
111.08 \\
113.24 \\
117.83 \\
120.92 \\
123.28 \\
122.97 \\
125.82 \\
125.60 \\
127.84 \\
130.19 \\
132.53 \\
135.33 \\
134.42 \\
138.35 \\
140.16 \\
142.63 \\
145.78 \\
142.52 \\
0.22 \\
\end{array}$ & $\begin{array}{c}x \text { Strain } \\
\text { O. } 00006 \\
-0.0002 \\
-0.0014 \\
-0.0030 \\
-0.0041 \\
-0.0045 \\
-0.0046 \\
-0.0043 \\
-0.0031 \\
-0.0023 \\
-0.0013 \\
0.0000 \\
0.0013 \\
0.0027 \\
0.0032 \\
0.0036 \\
0.0045 \\
0.0050 \\
0.0058 \\
0.0066 \\
0.0083 \\
0.0039 \\
0.0111 \\
0.0114 \\
0.0127 \\
0.0127 \\
0.0136 \\
0.0142 \\
0.0150 \\
0.0161 \\
0.0164 \\
0.0177 \\
0.0187 \\
0.0197 \\
0.0208 \\
0.0196 \\
-0.0104\end{array}$ \\
\hline
\end{tabular}

Chammel 2342

High Pressure test

Pressure

9.89

19.55

29.57

64.20

69.32

90.58

100.92

103.25

106.11

108.31

120.92

125.60

127.84

32.53

135.33

134.42

138.35

140.16

142.63

5.78

2.52

0.0104 


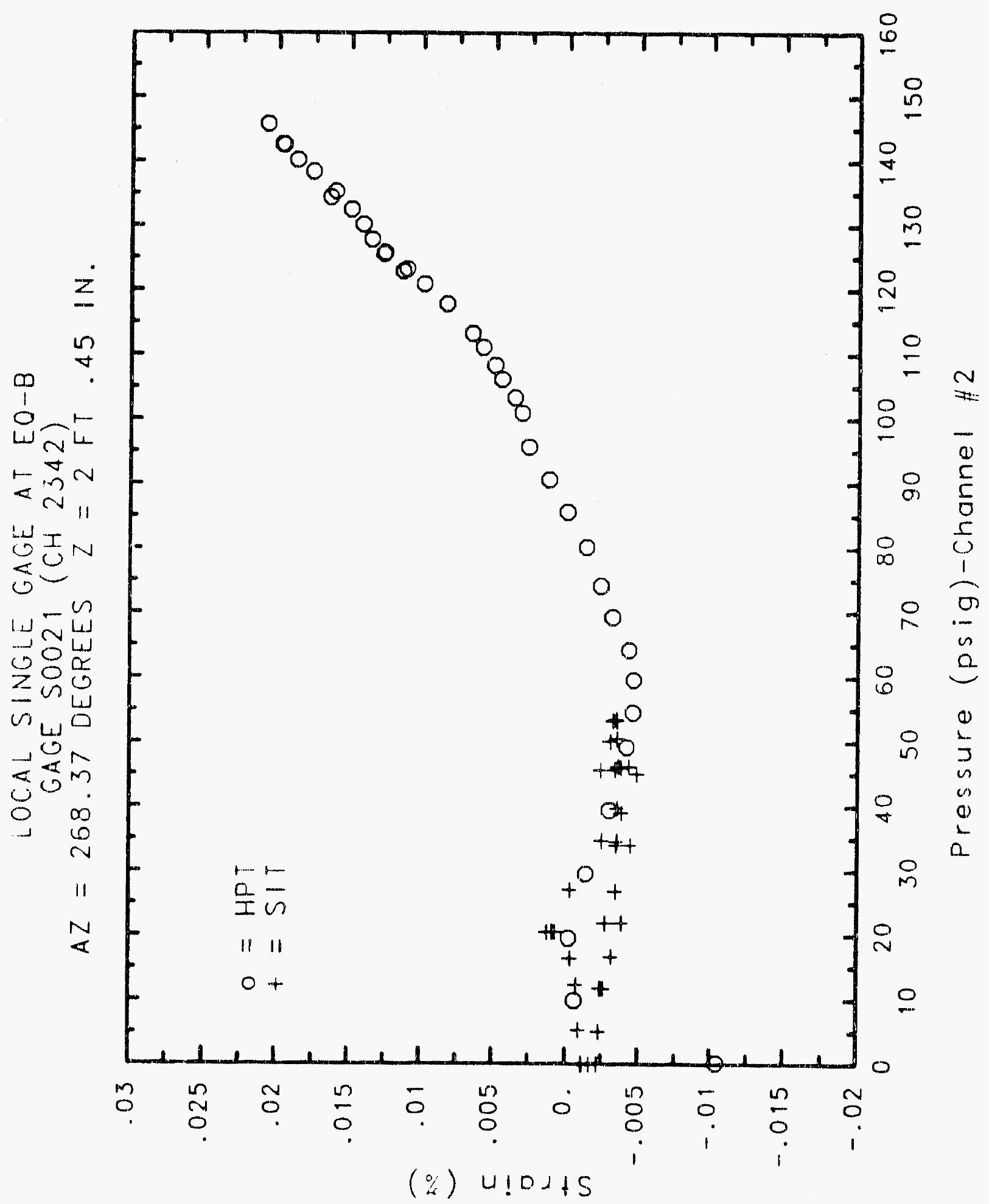

C. 1627 


\section{Reinforced Concrete Test Data}

Strain Gage 0

STRUCTURAL INTEGRITY TEST

\begin{tabular}{ll}
\hline Pressure & \\
(psig) & x Strain \\
-0.05 & \\
5.33 & 0.0014 \\
12.31 & 0.0022 \\
16.44 & 0.0029 \\
20.51 & 0.0035 \\
20.46 & 0.0039 \\
20.47 & 0.0032 \\
27.05 & 0.0024 \\
34.69 & 0.0021 \\
34.53 & -0.0001 \\
34.53 & -0.0007 \\
39.70 & -0.0009 \\
45.90 & -0.0010 \\
45.65 & -0.0008 \\
45.62 & 0.0002 \\
50.10 & -0.0002 \\
53.47 & -0.0003 \\
53.29 & -0.0005 \\
53.21 & -0.0010 \\
50.49 & -0.0010 \\
46.14 & -0.0013 \\
46.13 & -0.0006 \\
44.97 & -0.0009 \\
38.98 & -0.0017 \\
33.99 & -0.0015 \\
33.96 & -0.0013 \\
26.83 & -0.0014 \\
21.88 & -0.0013 \\
21.90 & -0.0012 \\
16.69 & -0.0006 \\
11.74 & -0.0016 \\
11.77 & -0.0004 \\
5.05 & -0.0004 \\
0.02 & 0.0008 \\
-0.04 & 0.0017 \\
-0.02 & 0.0018 \\
-0.02 & 0.0021 \\
0.02 & 0.0022 \\
& 0.0018 \\
\hline
\end{tabular}

Channel 2343

High Pressure Test

Pressure

( $p s \mid g$ )

9.89

19.55

29.57

39.42

49.16

54.50

59.57

64.20

69.32

74.16

80.16

85.61

90.58

95.69

100.92

103.25

106.11

108.31

111.08

113.24

117.83

120.92

123.28

122.97

125.82

125.60

127.84

130.19

132.53

135.33

134.42

138.35

140.16

142.63

145.78

142.52

0.22
* Strain

$-0.0013$

$-0.0013$

$-0.0030$

$-0.0048$

$-0.0060$

$-0.0062$

$-0.0064$

$-0.0058$

$-0.0047$

$-0.0038$

$-0.0018$

$-0.0002$

0.0017

0.0036

0.0043

0.0060

0.0071

0.0078

0.0089

0.0099

0.0119

0.0137

0.0156

0.0162

0.0176

0.0179

0.0183

0.0194

0.0198

0.0206

0.020 ?

0.0218

0.0222

0.0230

0.0233

0.0223

$-0.0021$ 


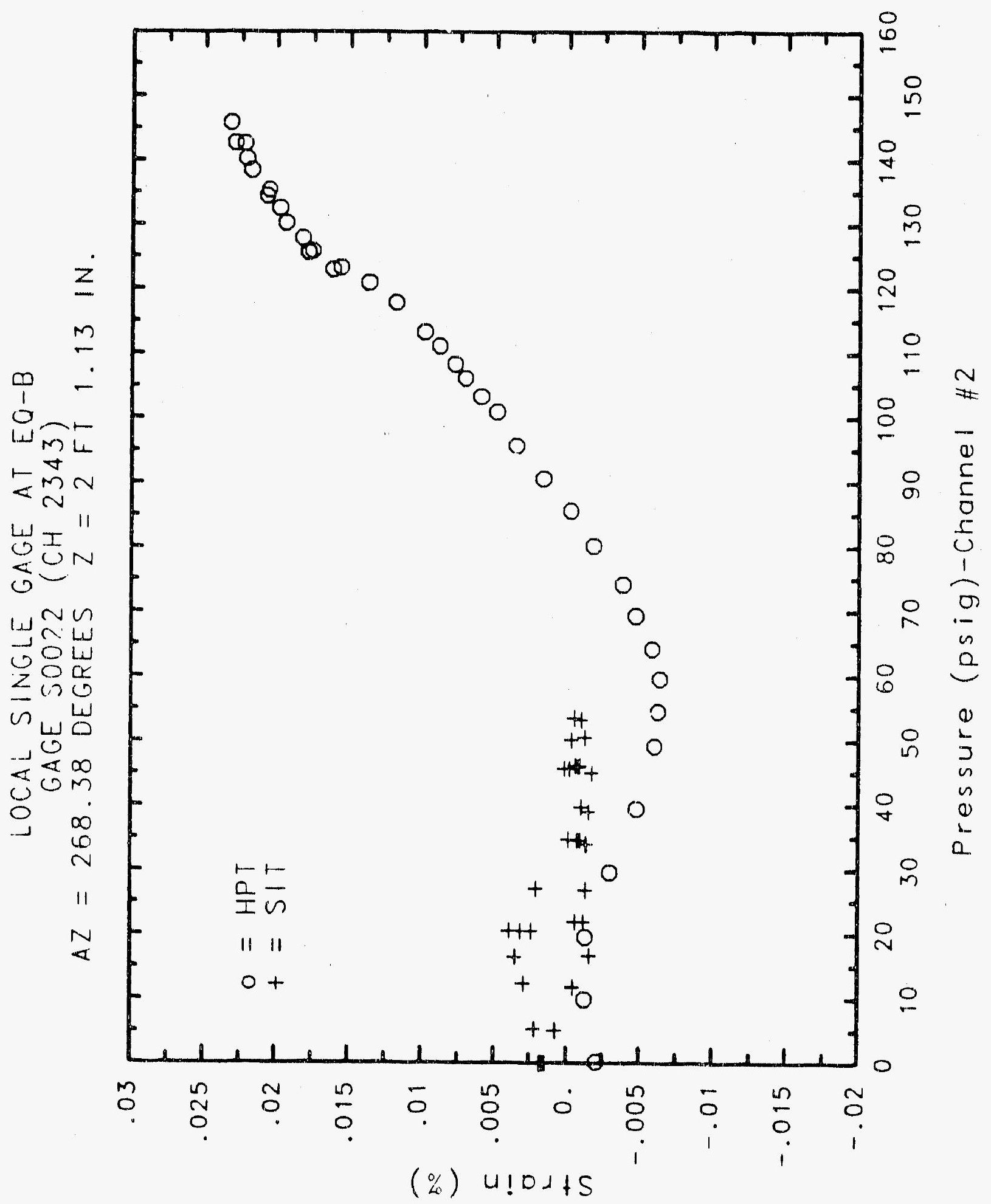

C. 1629 


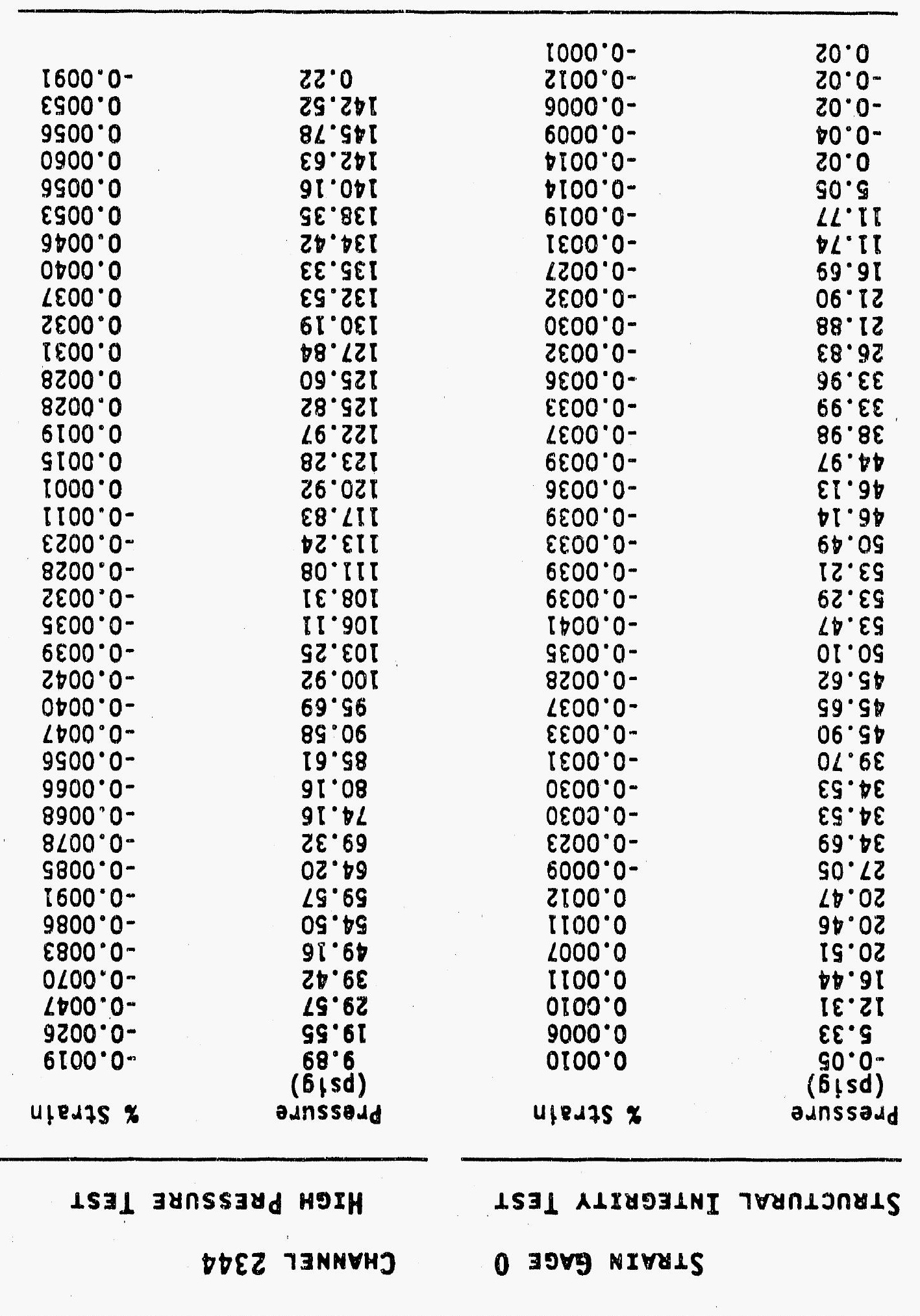

$\forall \perp \forall 0 \quad 153 \perp$ ILIVINOJ a3J4OJNIJY 


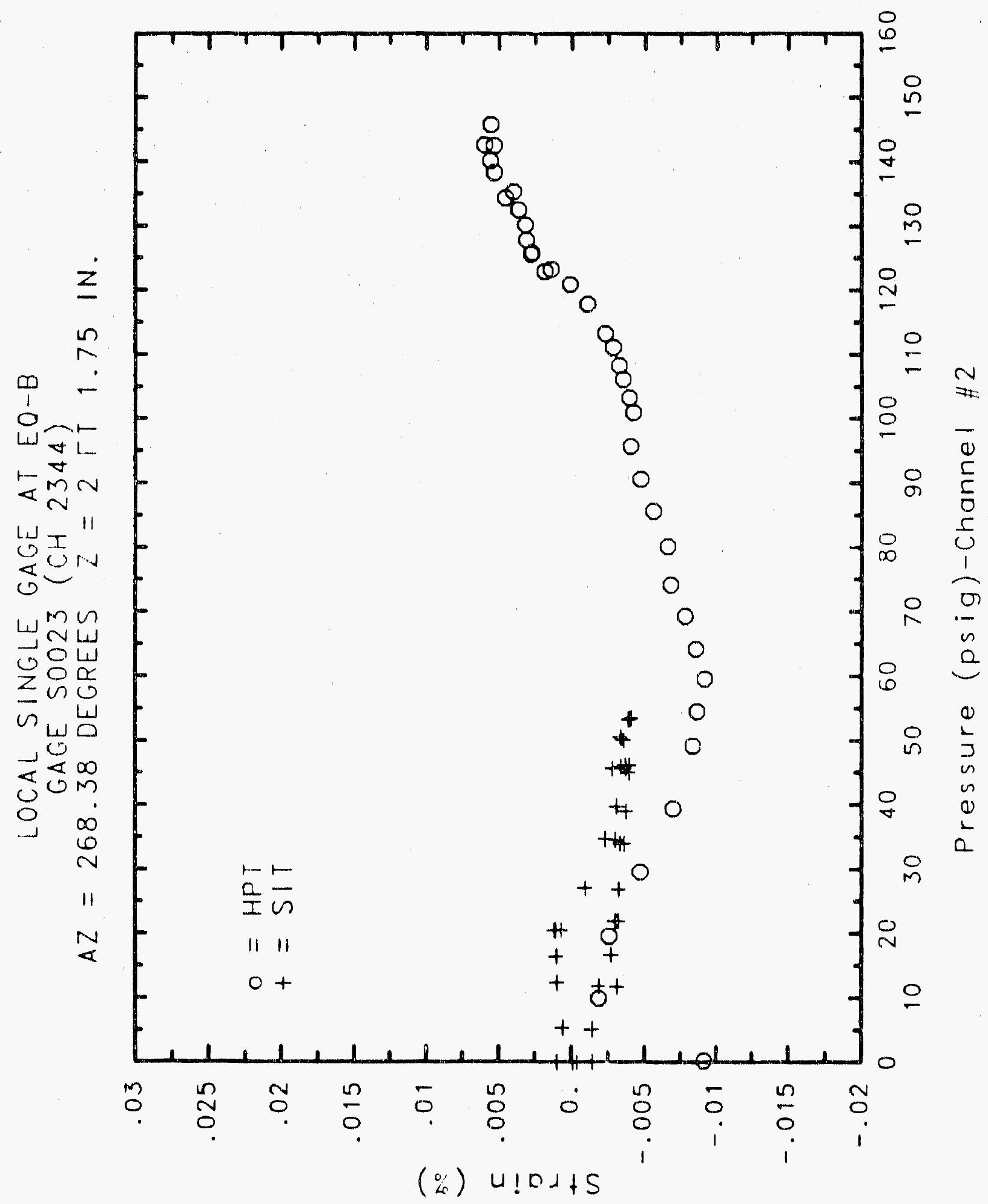




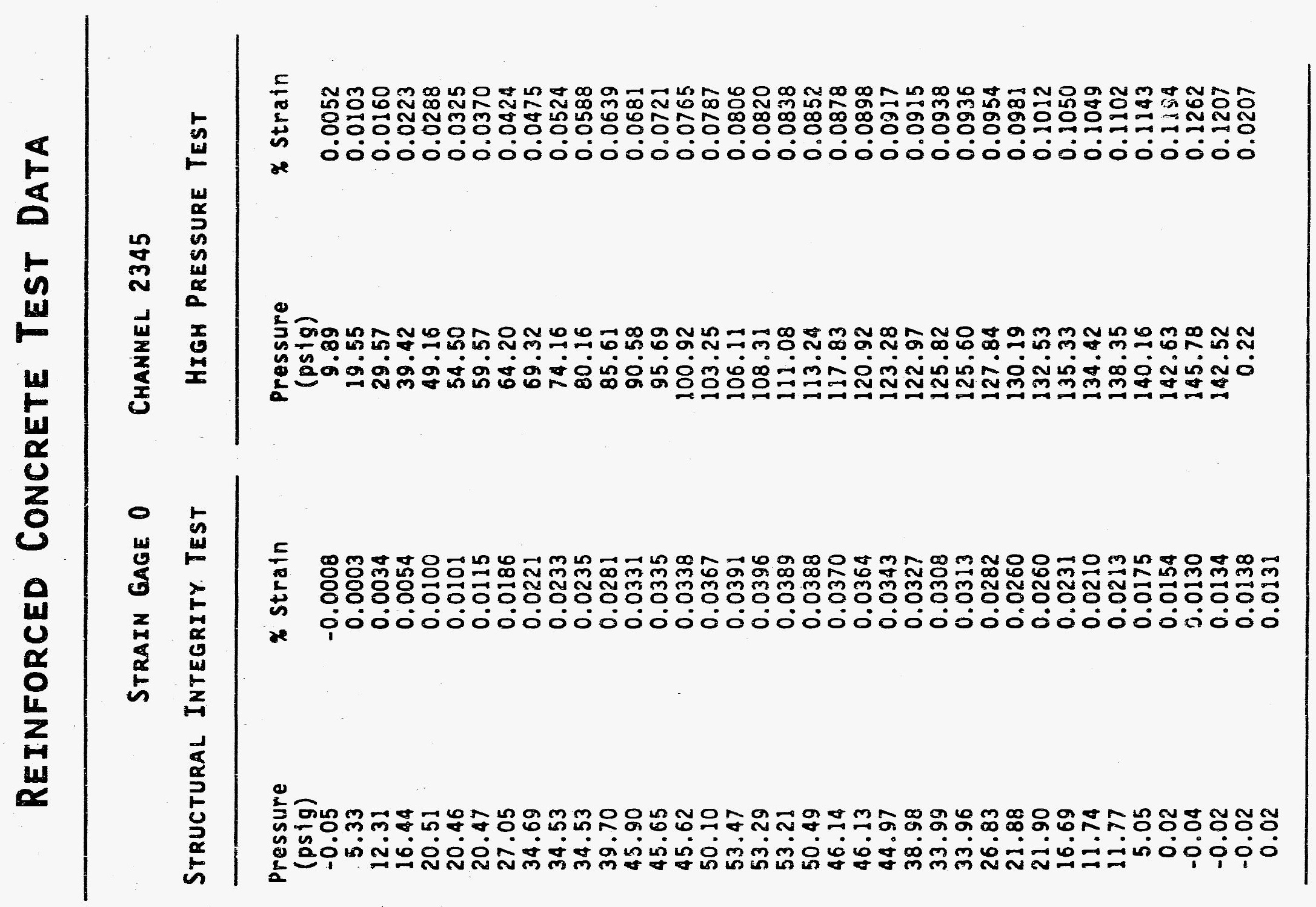




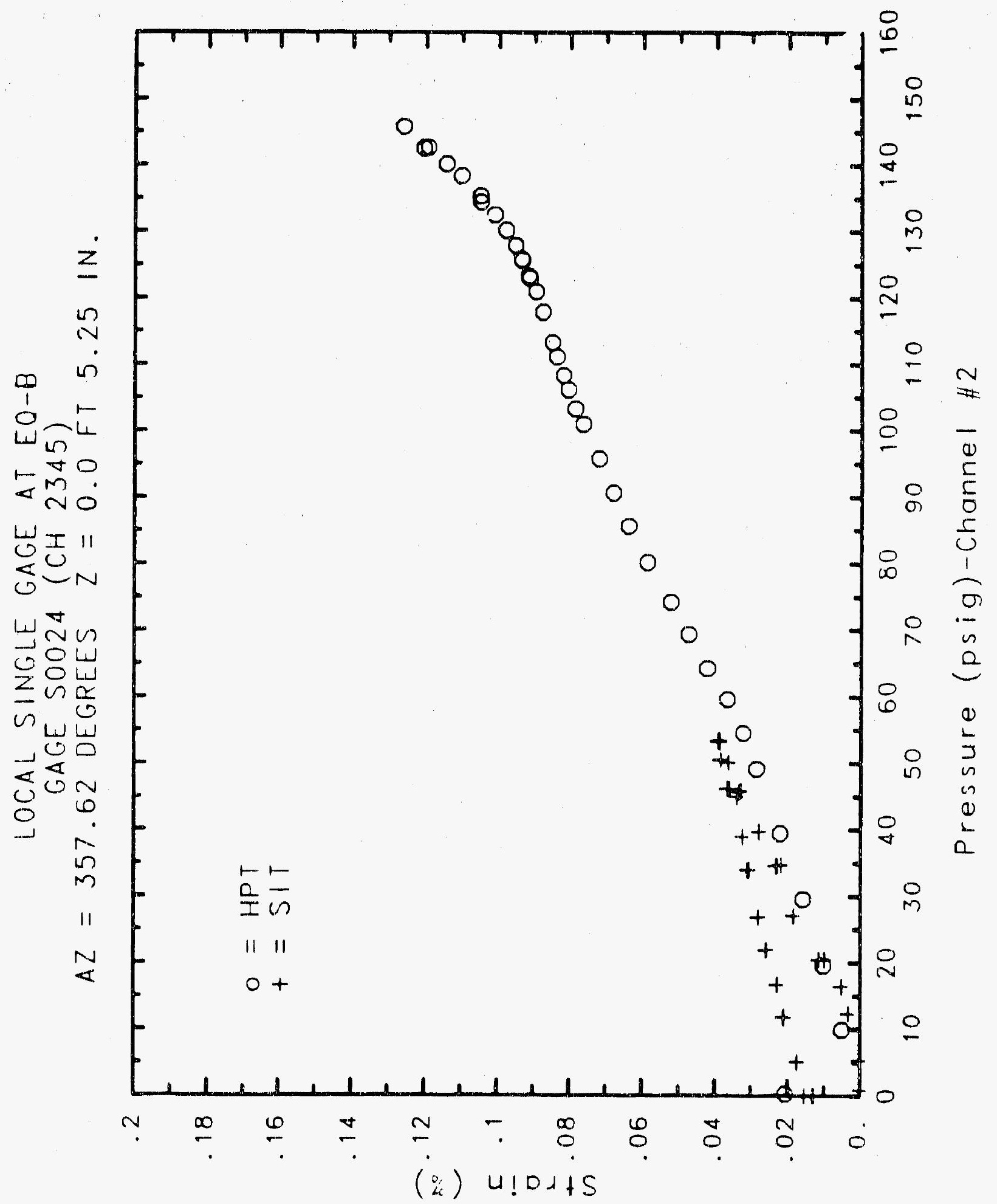

C. -16.3 .3$ 


\section{Reinforced Concrete Test Data}

Strain gage 0

StRUCTURAL INTEgRITY TEST

\begin{tabular}{|c|c|c|c|}
\hline $\begin{array}{c}\text { Pressure } \\
\text { (psig) } \\
-0.05 \\
5.33 \\
12.31 \\
16.44 \\
20.51 \\
20.46 \\
20.47 \\
27.05 \\
34.69 \\
34.53 \\
34.53 \\
39.70 \\
45.90 \\
45.65 \\
45.62 \\
50.10 \\
53.47 \\
53.29 \\
53.21 \\
50.49 \\
46.14 \\
46.13 \\
44.97 \\
38.98 \\
33.99 \\
33.96 \\
26.83 \\
21.88 \\
21.90 \\
16.69 \\
11.74 \\
11.77 \\
5.05 \\
0.02 \\
-0.04 \\
-0.02 \\
-0.02 \\
0.02 \\
\end{array}$ & $\begin{array}{l}\text { X Strain } \\
\text {-0.0008 } \\
0.0028 \\
0.0052 \\
0.0077 \\
0.0118 \\
0.0131 \\
0.0134 \\
0.0205 \\
0.0257 \\
0.0249 \\
0.0255 \\
0.0309 \\
0.0368 \\
0.0377 \\
0.0373 \\
0.0394 \\
0.0421 \\
0.0421 \\
0.0428 \\
0.0414 \\
0.0393 \\
0.0397 \\
0.0379 \\
0.0359 \\
0.0332 \\
0.0339 \\
0.0299 \\
0.0272 \\
0.0276 \\
0.0247 \\
0.0218 \\
0.0221 \\
0.0187 \\
0.0152 \\
0.0149 \\
0.0149 \\
0.0140 \\
0.0140\end{array}$ & $\begin{array}{c}\text { Pressure } \\
(\text { psig) } \\
9.89 \\
19.55 \\
29.57 \\
39.42 \\
49.16 \\
54.50 \\
59.57 \\
64.20 \\
69.32 \\
74.16 \\
80.16 \\
85.61 \\
90.58 \\
95.69 \\
100.92 \\
103.25 \\
106.11 \\
108.31 \\
111.08 \\
113.24 \\
117.83 \\
120.92 \\
123.28 \\
122.97 \\
125.82 \\
125.60 \\
127.84 \\
130.19 \\
132.53 \\
135.33 \\
134.42 \\
138.35 \\
140.16 \\
142.63 \\
145.78 \\
142.52 \\
0.22 \\
\end{array}$ & $\begin{array}{l}x \text { Strain } \\
0.0054 \\
0.0111 \\
0.0170 \\
0.0235 \\
0.0302 \\
0.0346 \\
0.0352 \\
0.0453 \\
0.0509 \\
0.0561 \\
0.0619 \\
0.0671 \\
0.0713 \\
0.0756 \\
0.0799 \\
0.0819 \\
0.0838 \\
0.0854 \\
0.0872 \\
0.0887 \\
0.0914 \\
0.0934 \\
0.0951 \\
0.0951 \\
0.0973 \\
0.0978 \\
0.0998 \\
0.1025 \\
0.1055 \\
0.1090 \\
0.1089 \\
0.1141 \\
0.1173 \\
0.1225 \\
0.1287 \\
0.1220 \\
0.0183\end{array}$ \\
\hline
\end{tabular}

Chammel 2346

high Pressure test

ressure

0.89

39.42

59.57

64.20

74.16

80.16

85.61

90.58

95.69

100.92

108.31

111.08

113.24

117.83

120.92

123.28

127.84

130.19

132.53

135.33

134.42

142.63

145.78

42.52

0.22
0.0054

0.0111

0.0170

0.0235

0.0302

0.0346

0.035 ?

0.0453

0.0561

0.0619

0.0671

0.0713

0.0756

0.0799

0.0854

0.0872

0.0887

0.0914

0.0934

0.0951

0.0951

0.0998

0.1025

0.1055

0.1090

0.1089

0.1141

0.1173

0.1225

0.1287

0.1220

0.0183 


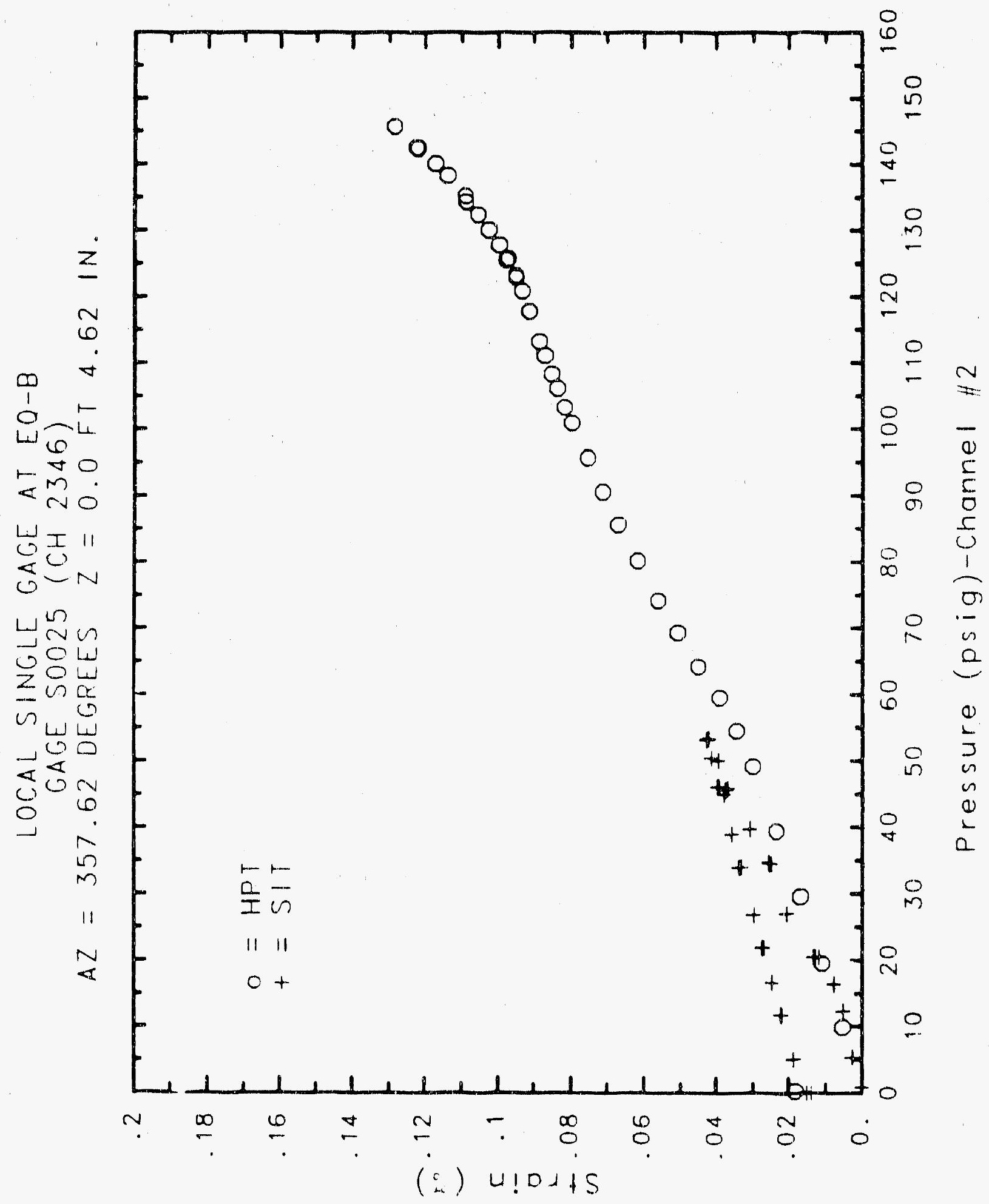




\section{Reinforced Concrete Test Data}

Strain gage 0

STRUCTURAL INTEGRITY TEST

\begin{tabular}{rccc}
\hline $\begin{array}{c}\text { Pressure } \\
\text { (psig) }\end{array}$ & \% strain & $\begin{array}{c}\text { Pressure } \\
\text { (psig) }\end{array}$ & \% Strain \\
-0.05 & 0.0003 & 9.89 & 0.0054 \\
5.33 & 0.0013 & 19.55 & 0.0109 \\
12.31 & 0.0041 & 29.57 & 0.0169 \\
16.44 & 0.0057 & 39.42 & 0.0230 \\
20.51 & 0.0108 & 49.16 & 0.0296 \\
20.46 & 0.0118 & 54.50 & 0.0336 \\
20.47 & 0.0118 & 59.57 & 0.0381 \\
27.05 & 0.0180 & 64.20 & 0.0437 \\
34.69 & 0.0220 & 69.32 & 0.0489 \\
34.53 & 0.0225 & 74.16 & 0.0537 \\
34.53 & 0.0234 & 80.16 & 0.0596 \\
39.70 & 0.0288 & 85.61 & 0.0643 \\
45.90 & 0.0333 & 90.58 & 0.0684 \\
45.65 & 0.0335 & 95.69 & 0.0721 \\
45.62 & 0.0339 & 100.92 & 0.0763 \\
50.10 & 0.0346 & 103.25 & 0.0780 \\
53.47 & 0.0372 & 106.11 & 0.0798 \\
53.29 & 0.0376 & 108.31 & 0.0813 \\
53.21 & 0.0365 & 111.08 & 0.0829 \\
50.49 & 0.0365 & 113.24 & 0.0844 \\
46.14 & 0.0347 & 117.83 & 0.0869 \\
46.13 & 0.0339 & 120.92 & 0.0887 \\
44.97 & 0.0335 & 123.28 & 0.0904 \\
38.98 & 0.0305 & 122.97 & 0.0904 \\
33.99 & 0.0283 & 125.82 & 0.0924 \\
33.96 & 0.0283 & 125.60 & 0.0328 \\
26.83 & 0.0250 & 127.84 & 0.0942 \\
21.88 & 0.0227 & 130.19 & 0.0966 \\
21.90 & 0.0222 & 132.53 & 0.0993 \\
16.69 & 0.0197 & 135.33 & 0.1025 \\
11.74 & 0.0171 & 134.42 & 0.1021 \\
11.77 & 0.0164 & 138.35 & 0.1067 \\
5.05 & 0.0126 & 140.16 & 0.1098 \\
0.02 & 0.0098 & 142.63 & 0.1144 \\
-0.04 & 0.0075 & 145.78 & 0.1127 \\
-0.02 & 0.0082 & 142.52 & 0.0121 \\
-0.02 & 0.0088 & 0.22 & \\
0.02 & 0.0086 & & \\
\hline & & & \\
\hline
\end{tabular}

Chanmel 2347

High Pressure test

Pressure

0.89

29.57

39.42

$\$ 9.16$

64.20

74.16

80.16

85.61

90.58

100.92

103.25

106.11

111.08

113.24

117.83

120.92

123.28

122.97

125.82

125.60

127.84

132.53

135.33

134.42

138.35

140.16

142.63

145.78

42.52

0.1127

0.0121 


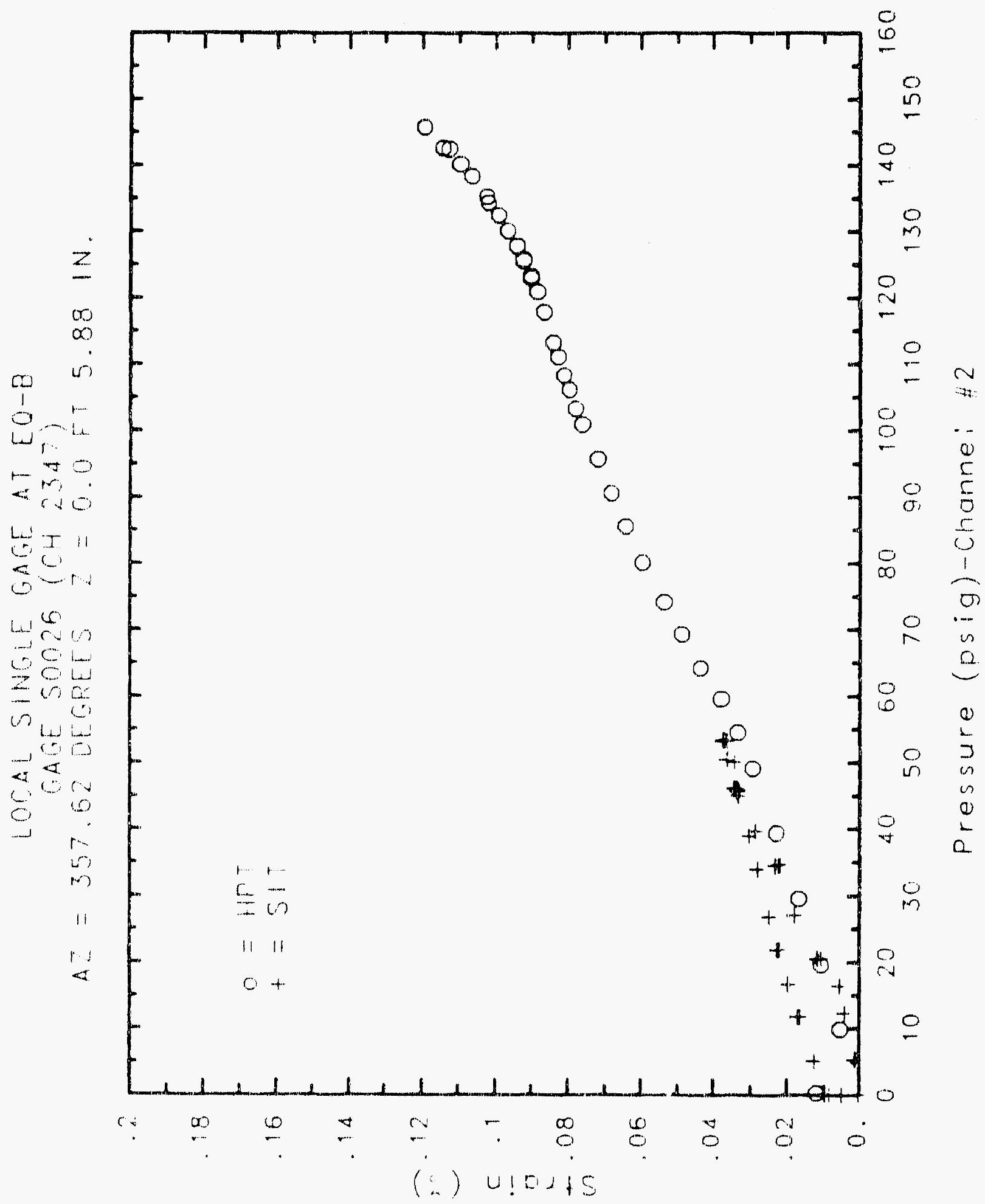




\section{Reinforced Concrete Test Data}

Strain gage 0

Structural Integrity Test

\begin{tabular}{|c|c|c|c|}
\hline $\begin{array}{c}\text { Pressure } \\
(\text { psig) } \\
-0.05 \\
5.33 \\
12.31 \\
16.44 \\
20.51 \\
20.46 \\
20.47 \\
27.05 \\
34.69 \\
34.53 \\
34.53 \\
39.70 \\
45.90 \\
45.65 \\
45.62 \\
50.10 \\
53.47 \\
53.29 \\
53.21 \\
50.49 \\
46.14 \\
46.13 \\
44.97 \\
38.98 \\
33.99 \\
33.96 \\
26.83 \\
21.88 \\
21.90 \\
16.69 \\
11.74 \\
11.77 \\
5.05 \\
3.02 \\
-0.04 \\
-0.02 \\
-0.02 \\
0.02 \\
\end{array}$ & $\begin{array}{l}\text { X Strain } \\
\\
0.0001 \\
0.0024 \\
0.0045 \\
0.0062 \\
0.0051 \\
0.0048 \\
0.0044 \\
0.0056 \\
0.0056 \\
0.0047 \\
0.0046 \\
0.0060 \\
0.0074 \\
0.0092 \\
0.0090 \\
0.0089 \\
0.0096 \\
0.0085 \\
0.0092 \\
0.0082 \\
0.0071 \\
0.0073 \\
0.0048 \\
0.0038 \\
0.0027 \\
0.0031 \\
0.0024 \\
0.0018 \\
0.0022 \\
0.0014 \\
0.0020 \\
0.0028 \\
0.0027 \\
0.0028 \\
0.0005 \\
0.0011 \\
0.0016 \\
0.0010\end{array}$ & $\begin{array}{c}\text { Prossure } \\
(\text { ps } 1 \text { g) } \\
9.89 \\
19.55 \\
29.57 \\
39.42 \\
49.16 \\
54.50 \\
59.57 \\
64.20 \\
69.32 \\
74.16 \\
80.16 \\
85.61 \\
90.58 \\
95.69 \\
100.92 \\
103.25 \\
106.11 \\
108.31 \\
111.08 \\
113.24 \\
117.83 \\
120.92 \\
123.28 \\
122.97 \\
125.82 \\
125.60 \\
127.84 \\
130.19 \\
132.53 \\
135.33 \\
134.42 \\
138.35 \\
140.16 \\
142.63 \\
145.78 \\
142.52 \\
0.22 \\
100\end{array}$ & $\begin{array}{l}x \text { Strain } \\
0.0037 \\
0.0060 \\
0.0070 \\
0.0076 \\
0.0091 \\
0.0102 \\
0.0117 \\
0.0149 \\
0.0184 \\
0.0209 \\
0.0239 \\
0.0263 \\
0.0286 \\
0.0308 \\
0.0330 \\
0.0339 \\
0.0350 \\
0.0358 \\
0.0369 \\
0.0375 \\
0.0391 \\
0.0404 \\
0.0407 \\
0.0400 \\
0.0412 \\
0.0411 \\
0.0417 \\
0.0427 \\
0.0434 \\
0.0443 \\
0.0442 \\
0.0454 \\
0.0459 \\
0.0466 \\
0.0474 \\
0.0432 \\
0.0080\end{array}$ \\
\hline
\end{tabular}

Channel 2348

High Pressure test

Pressure

0.89

49.16

64.20

80.16

106.11

108.31

117.83

120.92

125.60

127.84

i35.33

134.42

138.35

145.78

42.52

0.22
* Strain

0.0037

0.0070

0.0076

0.011

0.0184

0.0209

0.0239

0.02 .63

0.0286

0.0339

0.0350

0.0369

.0375

0.0404

0.0407

0.0412

$0.04 \mathrm{~J}$

0.0417

0.0443

0.0442

0.0454

0.0459

0.0466

0.047

0.0080 


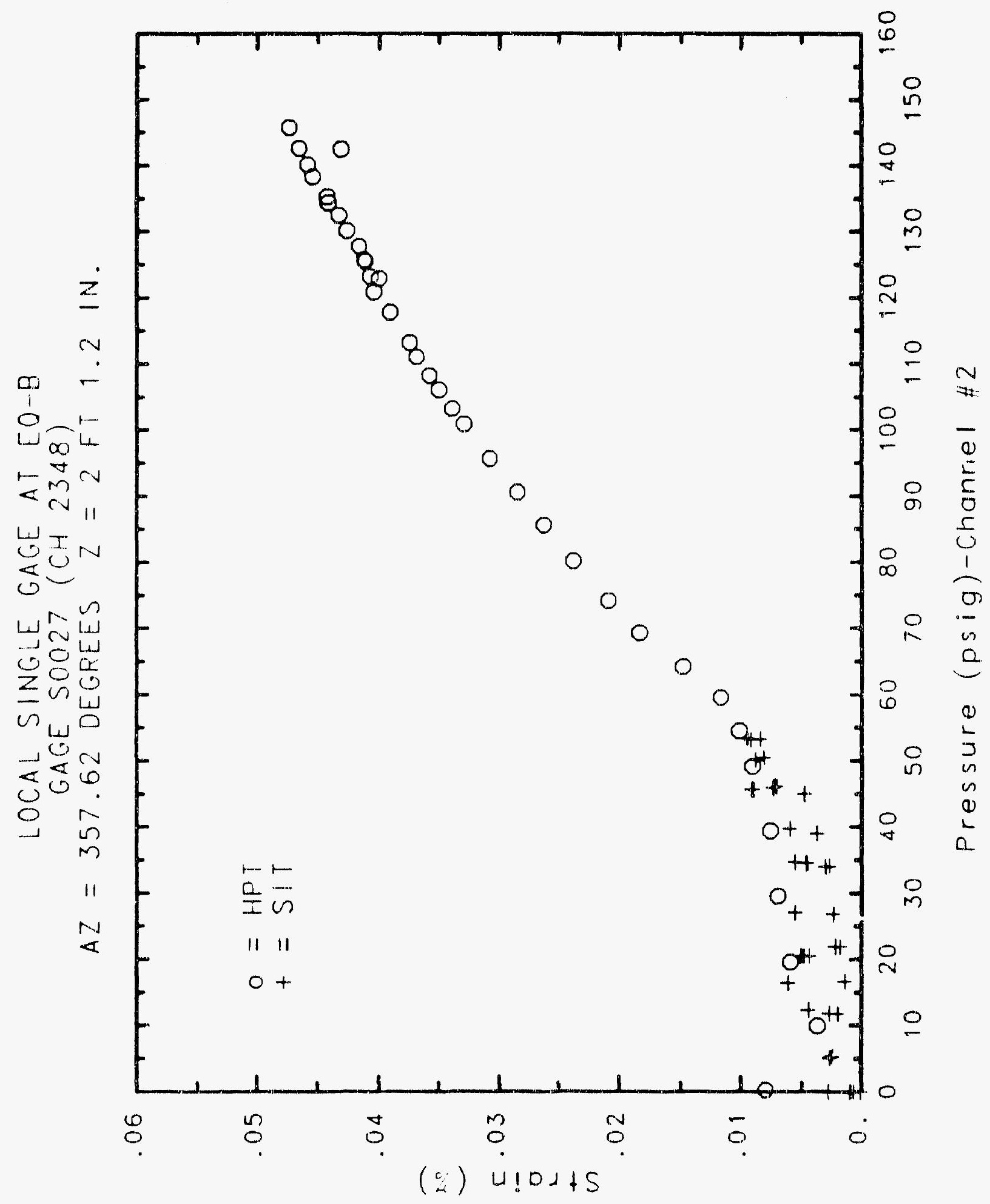




\section{Reinforced Concrete Test Data}

Strain Gage 0

STRUCTURAL INTEGRITY TEST

\begin{tabular}{|c|c|}
\hline $\begin{array}{c}\text { Pressure } \\
(\text { ps1g) } \\
-0.05 \\
5.33 \\
12.31 \\
16.44 \\
20.51 \\
20.46 \\
20.47 \\
27.05 \\
34.69 \\
34.53 \\
34.53 \\
39.70 \\
45.90 \\
45.65 \\
45.62 \\
50.10 \\
53.47 \\
53.29 \\
53.21 \\
50.49 \\
46.14 \\
46.13 \\
44.97 \\
38.98 \\
33.99 \\
33.96 \\
26.83 \\
21.88 \\
21.90 \\
16.69 \\
11.74 \\
11.77 \\
5.05 \\
0.02 \\
-0.04 \\
-0.02 \\
-0.02 \\
0.02\end{array}$ & $\begin{array}{l}\text { \% Strain } \\
\\
0.0010 \\
0.0025 \\
0.0048 \\
0.0061 \\
0.0066 \\
0.0075 \\
0.0077 \\
0.0083 \\
0.0081 \\
0.0080 \\
0.0085 \\
0.0093 \\
0.0106 \\
0.0107 \\
0.0105 \\
0.0103 \\
0.0106 \\
0.0105 \\
0.0111 \\
0.0097 \\
0.0085 \\
0.0087 \\
0.0072 \\
0.0072 \\
0.0065 \\
0.0069 \\
0.0061 \\
0.0061 \\
0.0055 \\
0.0061 \\
0.0056 \\
0.0067 \\
0.0060 \\
0.0046 \\
0.0038 \\
0.0045 \\
0.0040 \\
0.0054\end{array}$ \\
\hline
\end{tabular}

Channel 2349

High Pressure test

Pressure

* Strain

9.89

19.55

29.57

39.42

49.16

54.50

59.57

64.20

69.32

74.16

80.16

85.61

90.58

95.69

100.92

103.25

106.11

108.31

111.08

113.24

117.83

120.92

123.28

122.97

125.82

125.60

127.84

130.19

132.53

135.33

134.42

138.35

140.16

142.63

145.78

142.52

0.22
0.0042

0.0062

0.0075

0.0085

0.0103

0.0118

0.0133

0.0169

0.0207

0.0232

0.0262

0.0288

0.0312

0.0335

0.0358

0.0368

0.0380

0.0387

0.0399

0.0407

0.0424

0.0437

0.0443

0.0436

0.0445

0.0442

0.0450

0.0457

0.0464

0.0474

0.0467

0.0484

0.0490

0.0497

0.0508

0.0463

0.0111 


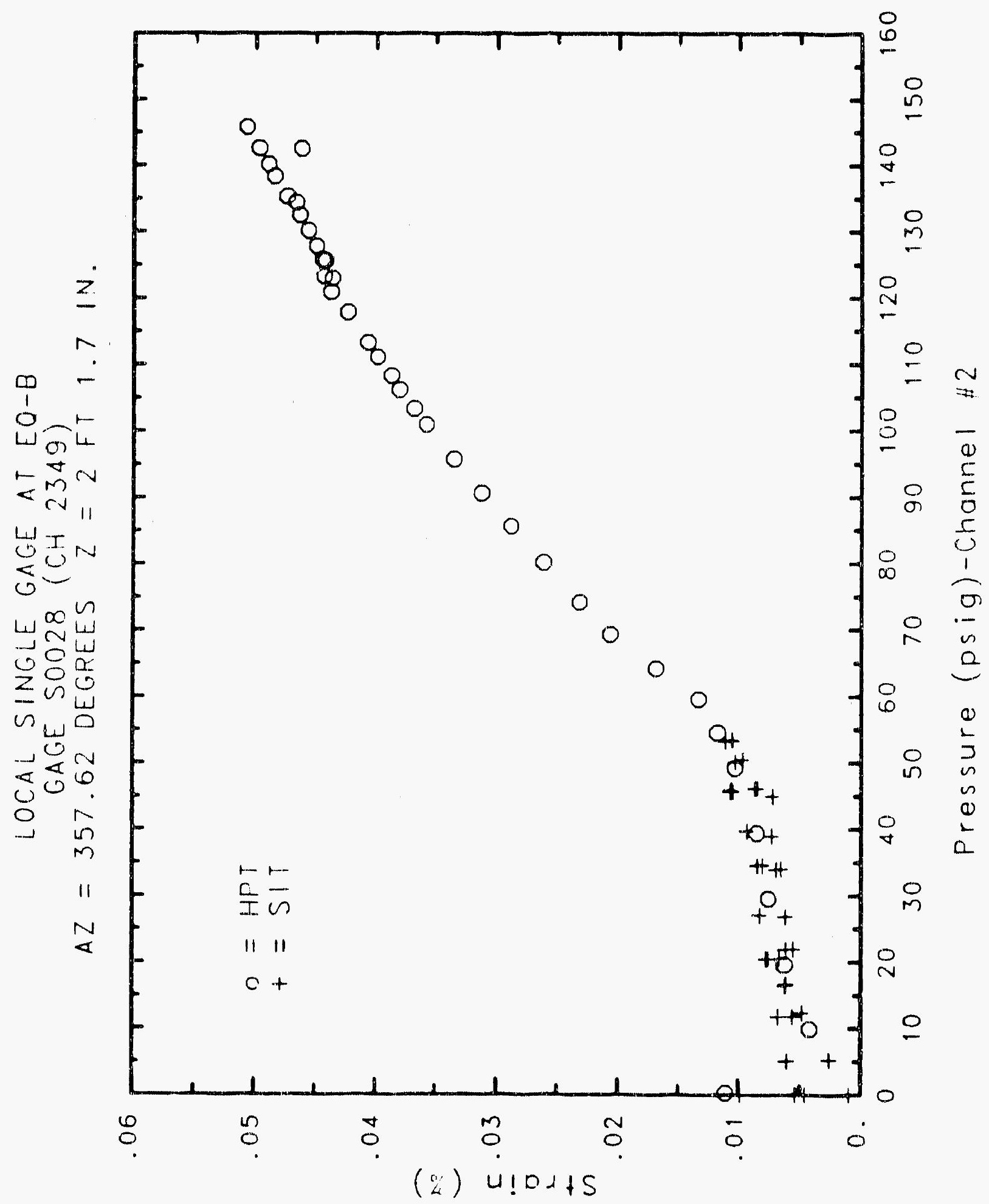

C. $-16+1$ 


\section{Reinforced Concrete Test Data}

Strain Gage 0

StRUCTURAL IMTEGRITY TEST

\begin{tabular}{|c|c|c|c|}
\hline $\begin{array}{c}\text { Pressure } \\
\text { (psig) } \\
-0.05 \\
5.33 \\
12.31 \\
16.44 \\
20.51 \\
20.46 \\
20.47 \\
27.05 \\
34.69 \\
34.53 \\
34.53 \\
39.70 \\
45.90 \\
45.65 \\
45.62 \\
50.10 \\
53.47 \\
53.29 \\
53.21 \\
50.49 \\
46.14 \\
46.13 \\
44.97 \\
38.98 \\
33.99 \\
33.96 \\
26.83 \\
21.88 \\
21.90 \\
16.69 \\
11.74 \\
11.77 \\
5.05 \\
0.02 \\
-0.04 \\
-0.02 \\
-0.02 \\
0.02 \\
\end{array}$ & $\begin{array}{l}\text { \% Strain } \\
\text {-0.0007 } \\
0.0007 \\
0.0029 \\
0.0040 \\
0.0039 \\
0.0038 \\
0.0042 \\
0.0054 \\
0.0041 \\
0.0082 \\
0.0082 \\
0.0088 \\
0.0100 \\
0.0096 \\
0.0102 \\
0.0103 \\
0.0109 \\
0.0106 \\
0.0096 \\
0.0096 \\
0.0081 \\
0.0078 \\
0.0078 \\
0.0068 \\
0.0059 \\
0.0058 \\
0.0051 \\
0.0056 \\
0.0057 \\
0.0047 \\
0.0055 \\
0.0059 \\
0.0050 \\
0.0050 \\
0.0034 \\
0.0037 \\
0.0049 \\
0.0046\end{array}$ & $\begin{array}{c}\text { Pressure } \\
\text { (psig) } \\
9.89 \\
19.55 \\
29.57 \\
39.42 \\
49.16 \\
54.50 \\
59.57 \\
64.20 \\
69.32 \\
74.16 \\
80.16 \\
85.61 \\
90.58 \\
95.69 \\
100.92 \\
103.25 \\
106.11 \\
108.31 \\
111.08 \\
113.24 \\
117.83 \\
120.92 \\
123.28 \\
122.97 \\
125.82 \\
125.60 \\
127.84 \\
130.19 \\
132.53 \\
135.33 \\
134.42 \\
138.35 \\
140.16 \\
142.63 \\
145.78 \\
142.52 \\
0.22\end{array}$ & $\begin{array}{l}\text { x Strain } \\
0.0012 \\
0.0033 \\
0.0046 \\
0.0054 \\
0.0073 \\
0.0086 \\
0.0102 \\
0.0140 \\
0.0176 \\
0.0200 \\
0.0222 \\
0.0245 \\
0.0267 \\
0.0288 \\
0.0310 \\
0.0321 \\
0.0330 \\
0.0338 \\
0.0350 \\
0.0357 \\
0.0375 \\
0.0388 \\
0.0398 \\
0.0396 \\
0.0407 \\
0.0408 \\
0.0418 \\
0.0425 \\
0.0436 \\
0.0449 \\
0.0450 \\
0.0463 \\
0.0468 \\
0.0478 \\
0.0491 \\
0.0454 \\
0.0138\end{array}$ \\
\hline
\end{tabular}

Chanmel 2360

High Pressure test

Pressure

0.89

19.55

49.16

64.20

74.16

80.16

85.61

100.92

106.11

108.31

111.08

113.24

117.83

120.92

123.28

125.60

127.84

130.19

132.53

135.33

134.42

138.35

140.16

42.63

75

2.52

0.22
0.0012

0.0033

0.0046

0.0054

0.0073

0.0140

0.0200

0.0222

0.0245

0.0267

0.0288

0.0330

0.0338

0.0350

0.0357

0.0375

0.0388

0.0398

0.0408

0.0418

0.0425

0.0436

0.0449

0.0450

0.0463

0.0468

0.0454

0.0138 


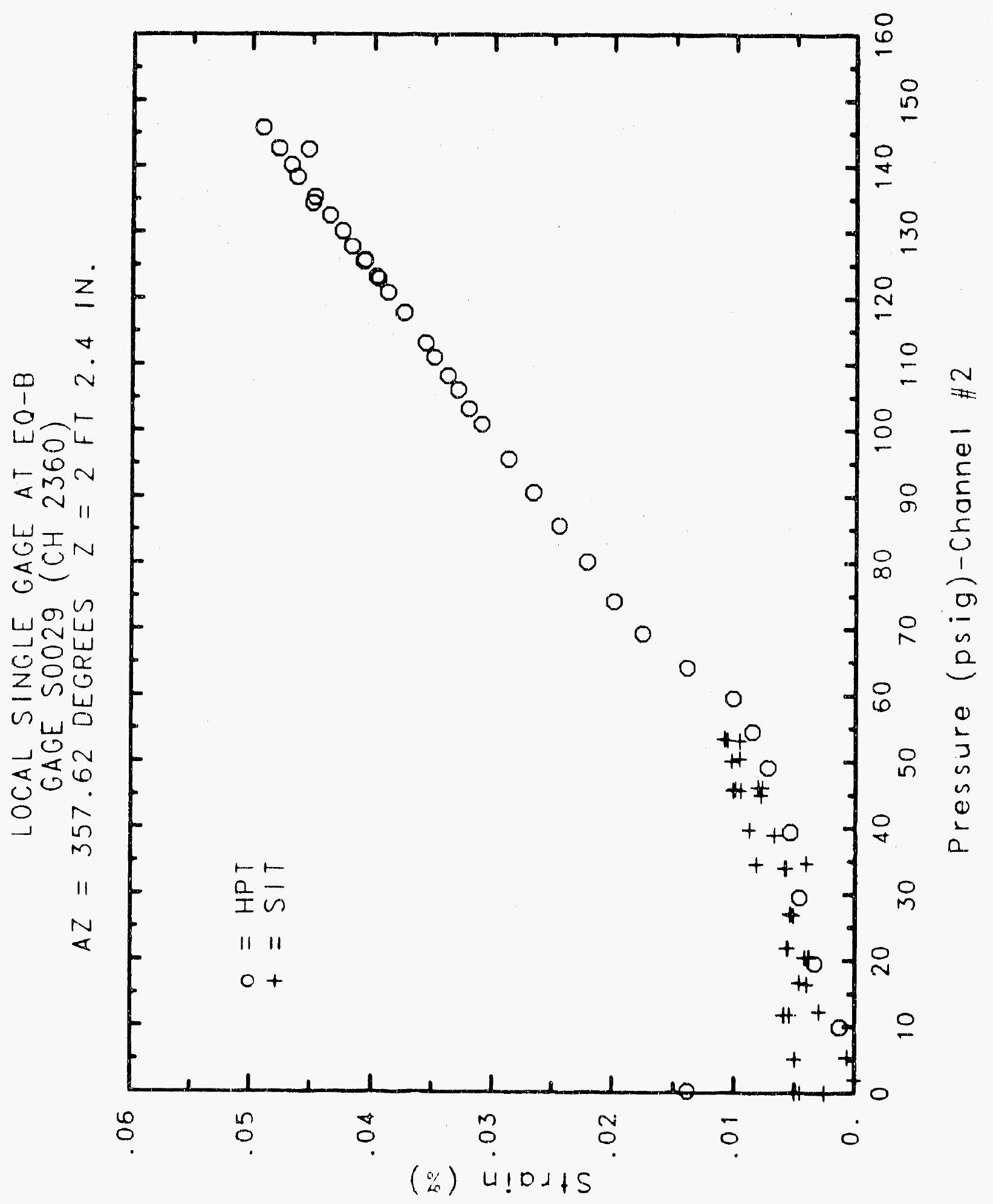

C- 1643 


\section{Reinforced Concrete Test Data}

Strain gage 0

Structural InTEgRITy TEST

\begin{tabular}{|c|c|c|c|}
\hline $\begin{array}{c}\text { Pressure } \\
\text { (psig) } \\
-0.05 \\
5.33 \\
12.31 \\
16.44 \\
20.51 \\
20.46 \\
20.47 \\
27.05 \\
34.69 \\
34.53 \\
34.53 \\
39.70 \\
45.90 \\
45.65 \\
45.62 \\
50.10 \\
53.47 \\
53.29 \\
53.21 \\
50.49 \\
46.14 \\
46.13 \\
44.97 \\
38.98 \\
33.99 \\
33.96 \\
26.83 \\
21.88 \\
21.90 \\
16.69 \\
11.74 \\
11.77 \\
5.05 \\
0.02 \\
-0.04 \\
-0.02 \\
-0.02 \\
0.02 \\
\end{array}$ & $\begin{array}{c}\text { X Strain } \\
-0.0001 \\
0.0015 \\
0.0036 \\
0.0047 \\
0.0064 \\
0.0078 \\
0.0076 \\
0.0110 \\
0.0148 \\
0.0142 \\
0.0145 \\
0.0172 \\
0.0199 \\
0.0207 \\
0.0203 \\
0.0216 \\
0.0229 \\
0.0222 \\
0.0223 \\
0.0205 \\
0.0185 \\
0.0189 \\
0.0159 \\
0.0137 \\
0.0118 \\
0.0119 \\
0.0087 \\
0.0071 \\
0.0073 \\
0.0060 \\
0.0043 \\
0.0044 \\
0.0027 \\
0.0000 \\
0.0019 \\
0.0012 \\
0.0000 \\
0.0004\end{array}$ & $\begin{array}{c}\text { Pressure } \\
(\text { psig) } \\
9.89 \\
19.55 \\
29.57 \\
39.42 \\
49.16 \\
54.50 \\
59.57 \\
64.20 \\
69.32 \\
74.16 \\
80.16 \\
85.61 \\
90.58 \\
95.69 \\
100.92 \\
103.25 \\
106.11 \\
108.31 \\
111.08 \\
113.24 \\
117.83 \\
120.92 \\
123.28 \\
122.97 \\
125.82 \\
125.60 \\
127.84 \\
130.19 \\
132.53 \\
135.33 \\
134.42 \\
138.35 \\
140.16 \\
142.63 \\
145.78 \\
142.52 \\
0.22 \\
19\end{array}$ & $\begin{array}{l}\text { \% Strain } \\
0.0038 \\
0.0071 \\
0.0111 \\
0.0149 \\
0.0187 \\
0.0207 \\
0.0216 \\
0.0221 \\
0.0218 \\
0.0207 \\
0.0173 \\
0.0140 \\
0.0102 \\
0.0059 \\
0.0007 \\
-0.0020 \\
-0.0044 \\
-0.0067 \\
-0.0100 \\
-0.0125 \\
-0.0187 \\
-0.0234 \\
-0.0268 \\
-0.0268 \\
-0.0315 \\
-0.0324 \\
-0.0353 \\
-0.0405 \\
-0.0468 \\
-0.0536 \\
-0.0543 \\
-0.0634 \\
-0.0695 \\
-0.0783 \\
-0.0883 \\
-0.0984 \\
-0.0980\end{array}$ \\
\hline
\end{tabular}

Chanhel 2361

High Pressure test

Pressure

0.89

19.55

29.57

54.50

59.57

64.20

80.16

90.58

95.69

100.92

103.25

113.24

123.28

122.97

127.84

130.19

134.42

138.35

42.52

0.22
0.0038

0.0071

0.0187

0.0207

0.0216

0.0221

0.0173

0.0102

0.0059

0.0007

$-0.0020$

$-0.0044$

$-0.0067$

$-0.0187$

0.0234

$-0.0268$

$-0.0268$

$-0.0315$

$-0.0405$

$-0.0468$

$-0.0536$

$-0.0543$

$-0.0634$

0.0695

0.0984 


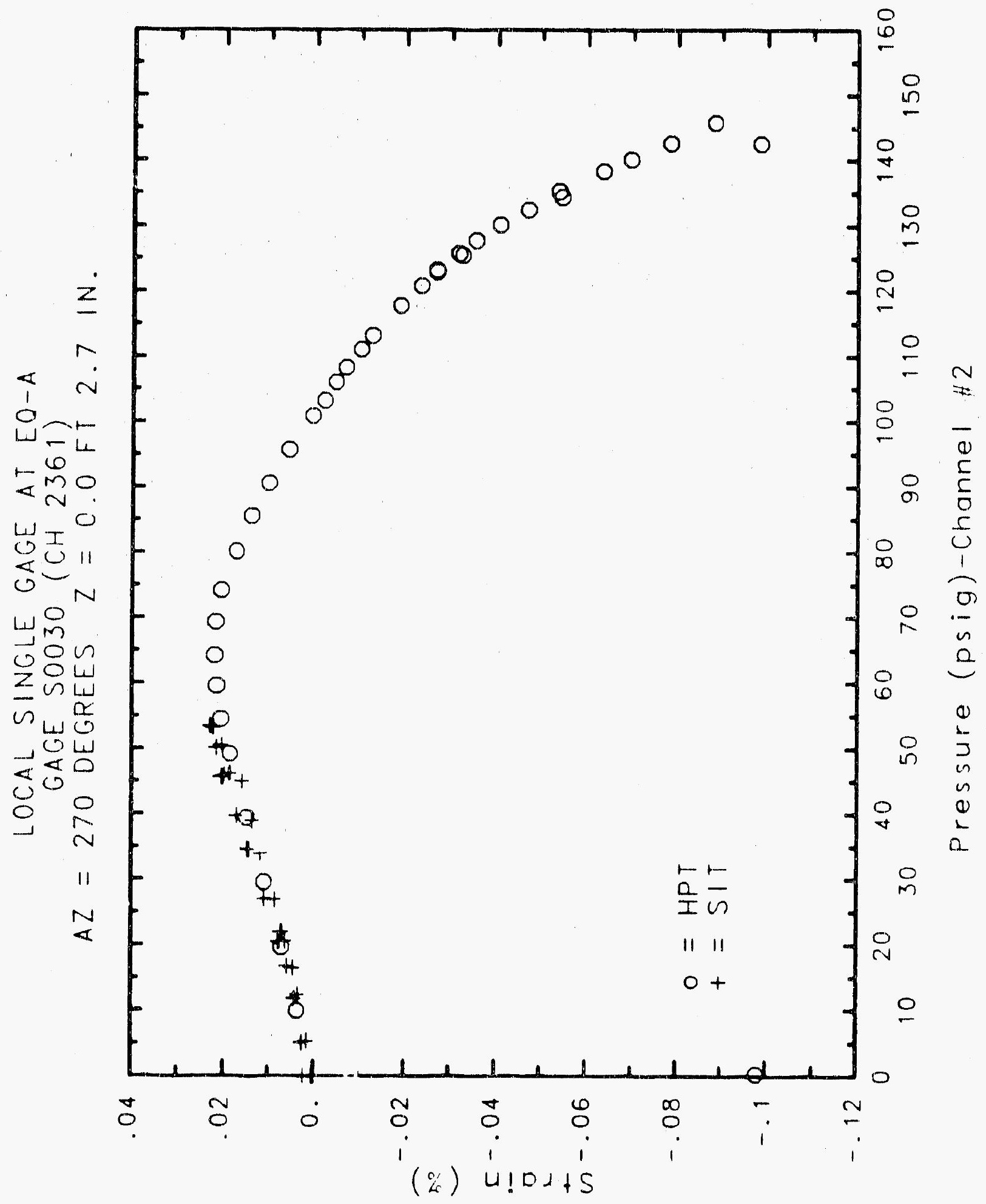




\section{Reinforced Concrete Test Data}

\begin{tabular}{|c|c|c|c|}
\hline STRUCTURAL & $\begin{array}{l}\text { StRAIN GAgE } 0 \\
\text { INTEGRITY TEST }\end{array}$ & $\begin{array}{l}\text { Channel } 2362 \\
\text { High Pressure }\end{array}$ & TEST \\
\hline $\begin{array}{c}\text { Pressure } \\
\text { (psig) } \\
-0.05 \\
5.33 \\
12.31 \\
16.44 \\
20.51 \\
20.46 \\
20.47 \\
27.05 \\
34.69 \\
34.53 \\
34.53 \\
39.70 \\
45.90 \\
45.65 \\
45.62 \\
50.10 \\
53.47 \\
53.29 \\
53.21 \\
50.49 \\
46.14 \\
46.13 \\
44.97 \\
38.98 \\
33.99 \\
33.96 \\
26.83 \\
21.88 \\
21.90 \\
16.69 \\
11.74 \\
11.77 \\
5.05 \\
0.02 \\
-0.04 \\
-0.02 \\
-0.02 \\
0.02 \\
\end{array}$ & $\begin{array}{l}\text { X Stra In } \\
\\
-0.0018 \\
0.0002 \\
0.0029 \\
0.0042 \\
0.0069 \\
0.0075 \\
0.0087 \\
0.0123 \\
0.0156 \\
0.0162 \\
0.0165 \\
0.0177 \\
0.0207 \\
0.0199 \\
0.0202 \\
0.0212 \\
0.0220 \\
0.0212 \\
0.0219 \\
0.0205 \\
0.0178 \\
0.0171 \\
0.0169 \\
0.0146 \\
0.0126 \\
0.0124 \\
0.0097 \\
0.0077 \\
0.0088 \\
0.0073 \\
0.0060 \\
0.0055 \\
0.0036 \\
0.0027 \\
0.0019 \\
0.0022 \\
0.0020 \\
0.0025\end{array}$ & $\begin{array}{c}\text { Pressure } \\
(\text { ps } 19) \\
9.89 \\
19.55 \\
29.57 \\
39.42 \\
49.16 \\
54.50 \\
59.57 \\
64.20 \\
69.32 \\
74.16 \\
80.16 \\
85.61 \\
90.58 \\
95.69 \\
100.92 \\
103.25 \\
106.11 \\
108.31 \\
111.08 \\
113.24 \\
117.83 \\
120.92 \\
123.28 \\
122.97 \\
125.82 \\
125.60 \\
127.84 \\
130.19 \\
132.53 \\
135.33 \\
134.42 \\
138.35 \\
140.16 \\
142.63 \\
145.78 \\
142.52 \\
0.22 \\
\end{array}$ & $\begin{array}{l}\text { X Strain } \\
0.0037 \\
0.0072 \\
0.0112 \\
0.0147 \\
0.0185 \\
0.0207 \\
0.0216 \\
0.0221 \\
0.0220 \\
0.0212 \\
0.0172 \\
0.0143 \\
0.0110 \\
0.0068 \\
0.0023 \\
-0.0003 \\
-0.0024 \\
-0.0042 \\
-0.0074 \\
-0.0095 \\
-0.0154 \\
-0.0197 \\
-0.0226 \\
-0.0227 \\
-0.0267 \\
-0.0271 \\
-0.0296 \\
-0.0338 \\
-0.0389 \\
-0.0439 \\
-0.0437 \\
-0.0511 \\
-0.0561 \\
-0.0644 \\
-0.0748 \\
-0.0855 \\
-0.0941\end{array}$ \\
\hline
\end{tabular}




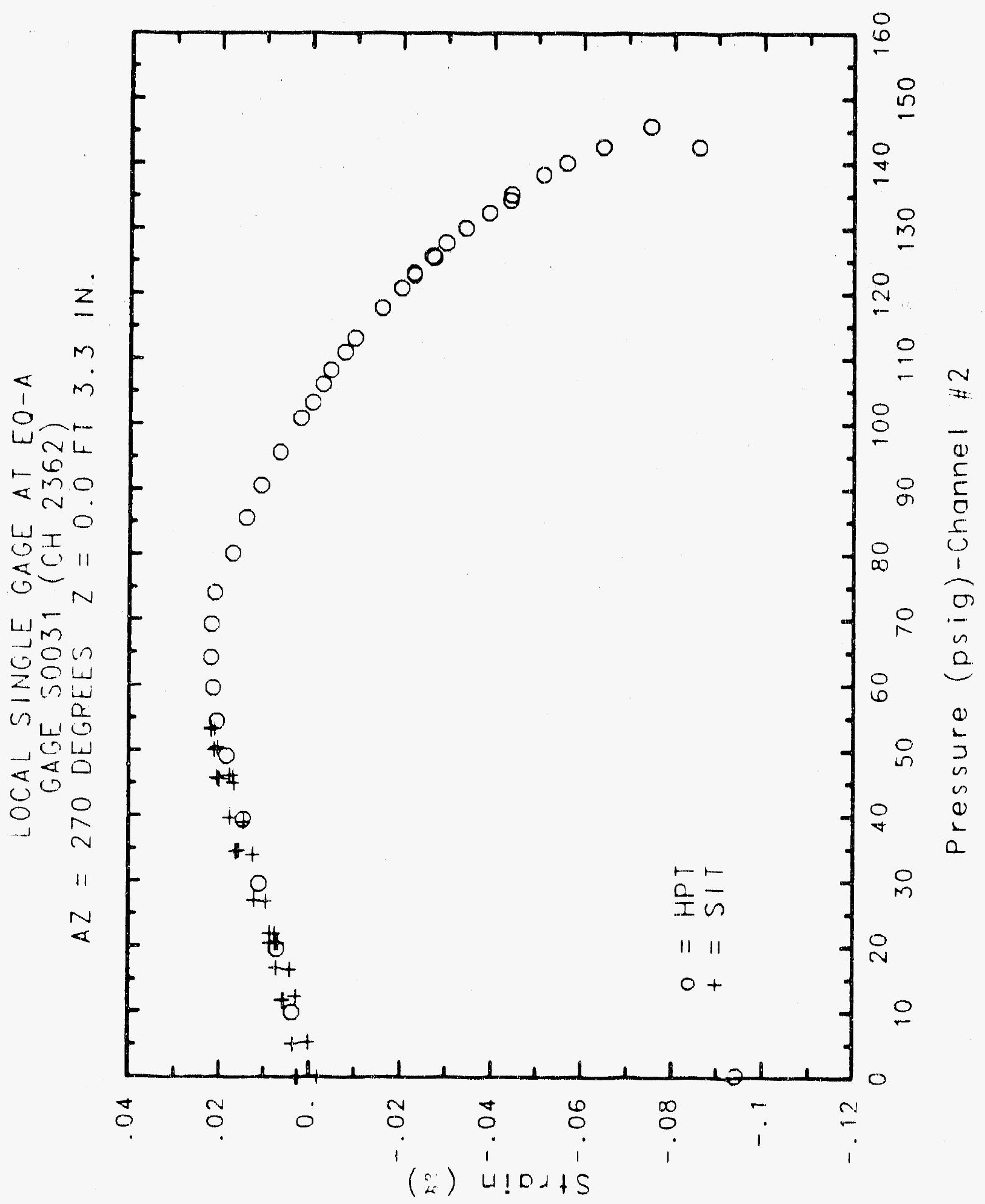

C $-16+7$ 


\section{Reinforced Concrete Test Data}

Strain gage 0

StRUCTURAL IntEgrity TEST

\begin{tabular}{|c|c|c|c|}
\hline $\begin{array}{c}\text { Pressure } \\
\text { (psig) } \\
-0.05 \\
5.33 \\
12.31 \\
16.44 \\
20.51 \\
20.46 \\
20.47 \\
27.05 \\
34.69 \\
34.53 \\
34.53 \\
39.70 \\
45.90 \\
45.65 \\
45.62 \\
50.10 \\
53.47 \\
53.29 \\
53.21 \\
50.49 \\
46.14 \\
46.13 \\
44.97 \\
38.98 \\
33.99 \\
33.96 \\
26.83 \\
21.88 \\
21.90 \\
16.69 \\
11.74 \\
11.77 \\
5.05 \\
0.02 \\
-0.04 \\
-0.02 \\
-0.02 \\
0.02 \\
\end{array}$ & $\begin{array}{l}\text { \% strain } \\
\\
0.0017 \\
0.0021 \\
0.0042 \\
0.0061 \\
0.0078 \\
0.0084 \\
0.0098 \\
0.0123 \\
0.0159 \\
0.0172 \\
0.0169 \\
0.0195 \\
0.0213 \\
0.0212 \\
0.0215 \\
0.0228 \\
0.0232 \\
0.0234 \\
0.0225 \\
0.0211 \\
0.0196 \\
0.0193 \\
0.0178 \\
0.0153 \\
0.0133 \\
0.0133 \\
0.0112 \\
0.0102 \\
0.0099 \\
0.0081 \\
0.0074 \\
0.0080 \\
0.0068 \\
0.0053 \\
0.0046 \\
0.0049 \\
0.0054 \\
0.0052\end{array}$ & $\begin{array}{c}\text { Pressure } \\
(\text { psig) } \\
9.89 \\
19.55 \\
69.57 \\
39.42 \\
49.16 \\
54.50 \\
59.57 \\
64.20 \\
69.32 \\
74.16 \\
80.16 \\
85.61 \\
90.58 \\
95.69 \\
100.92 \\
103.25 \\
106.11 \\
108.31 \\
111.08 \\
113.24 \\
117.83 \\
120.92 \\
123.28 \\
122.97 \\
125.82 \\
125.60 \\
127.84 \\
130.19 \\
132.53 \\
135.33 \\
134.42 \\
138.35 \\
140.16 \\
142.63 \\
145.78 \\
142.52 \\
0.22 \\
\end{array}$ & $\begin{array}{c}\text { \% Strain } \\
0.0030 \\
0.0064 \\
0.0099 \\
0.0134 \\
0.0170 \\
0.0189 \\
0.0200 \\
0.0204 \\
0.0208 \\
0.0205 \\
0.0186 \\
0.0160 \\
0.0131 \\
0.0097 \\
0.0056 \\
0.0036 \\
0.0018 \\
-0.0001 \\
-0.0027 \\
-0.0046 \\
-0.0100 \\
-0.0137 \\
-0.0171 \\
-0.0174 \\
-0.0212 \\
-0.0218 \\
-0.0243 \\
-0.0279 \\
-0.0328 \\
-0.0381 \\
-0.0387 \\
-0.0456 \\
-0.0504 \\
-0.0580 \\
-0.0677 \\
-0.0778 \\
-0.0906\end{array}$ \\
\hline
\end{tabular}

Channel 2363

High Pressure Test

(psig)

63.57

49.16

54.50

85.61

90.58

103.25

113.24

123.28

122.97

127.84

134.42

138.35

140.16

42.52

$-0.0677$

$-0.0778$

$-0.0906$ 


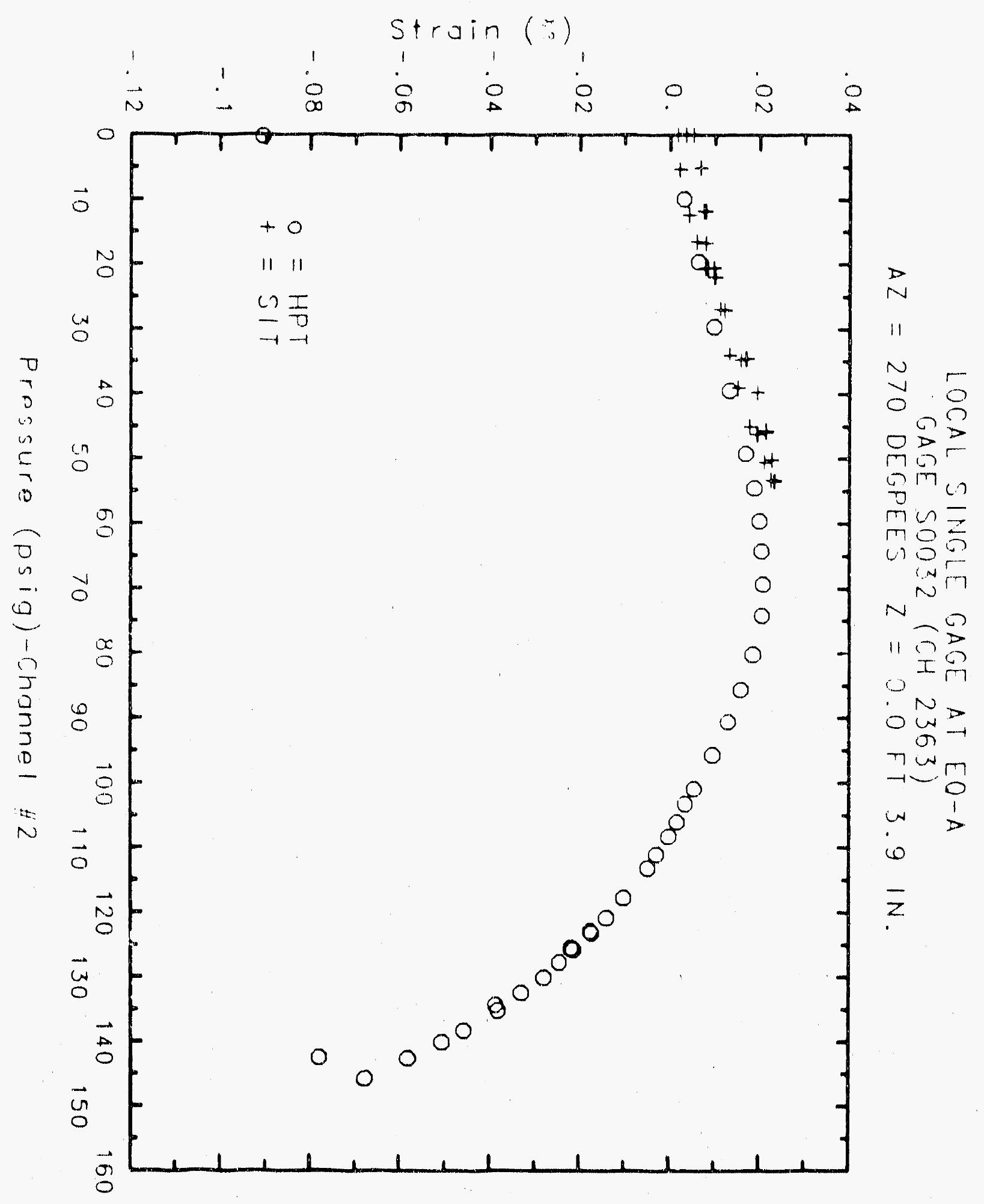




\section{Reinforced Concrete Test Data}

\begin{tabular}{|c|c|c|}
\hline $\begin{array}{r}\text { Strain Gage } 0 \\
\text { Structural Integrity Test }\end{array}$ & $\begin{array}{l}\text { Channel } 2424 \\
\text { High Pressure }\end{array}$ & TEST \\
\hline $\begin{array}{l}\text { Pressure } \quad \text { Strain } \\
\text { (psig) } \\
\text { No SIT data } \\
\text { gage was added after SIT }\end{array}$ & $\begin{array}{c}\text { Pressure } \\
(p s 1 g) \\
9.89 \\
19.55 \\
29.57 \\
39.42 \\
49.16 \\
54.50 \\
59.57 \\
64.20 \\
69.32 \\
74.16 \\
80.16 \\
85.61 \\
90.58 \\
95.69 \\
100.92 \\
103.25 \\
106.11 \\
108.31 \\
111.08 \\
113.24 \\
117.83 \\
120.92 \\
123.28 \\
122.97 \\
125.82 \\
125.60 \\
127.84 \\
130.19 \\
132.53 \\
135.33 \\
134.42 \\
138.35 \\
140.16 \\
142.63 \\
145.78 \\
142.52 \\
0.22 \\
\end{array}$ & $\begin{array}{l}\text { x Strain } \\
\text {-0.0074 } \\
-0.0015 \\
0.0035 \\
0.0084 \\
0.0141 \\
0.0143 \\
0.0122 \\
0.0214 \\
0.0262 \\
0.0072 \\
0.0232 \\
0.0285 \\
0.0333 \\
0.0384 \\
0.0479 \\
0.0532 \\
0.0566 \\
0.0592 \\
0.0629 \\
0.0657 \\
0.0716 \\
0.0755 \\
0.0787 \\
0.0792 \\
0.0847 \\
0.0875 \\
0.0917 \\
0.0958 \\
0.1018 \\
0.1037 \\
0.0810 \\
0.0633 \\
0.0786 \\
0.1011 \\
0.1411 \\
0.1525 \\
0.1064\end{array}$ \\
\hline
\end{tabular}




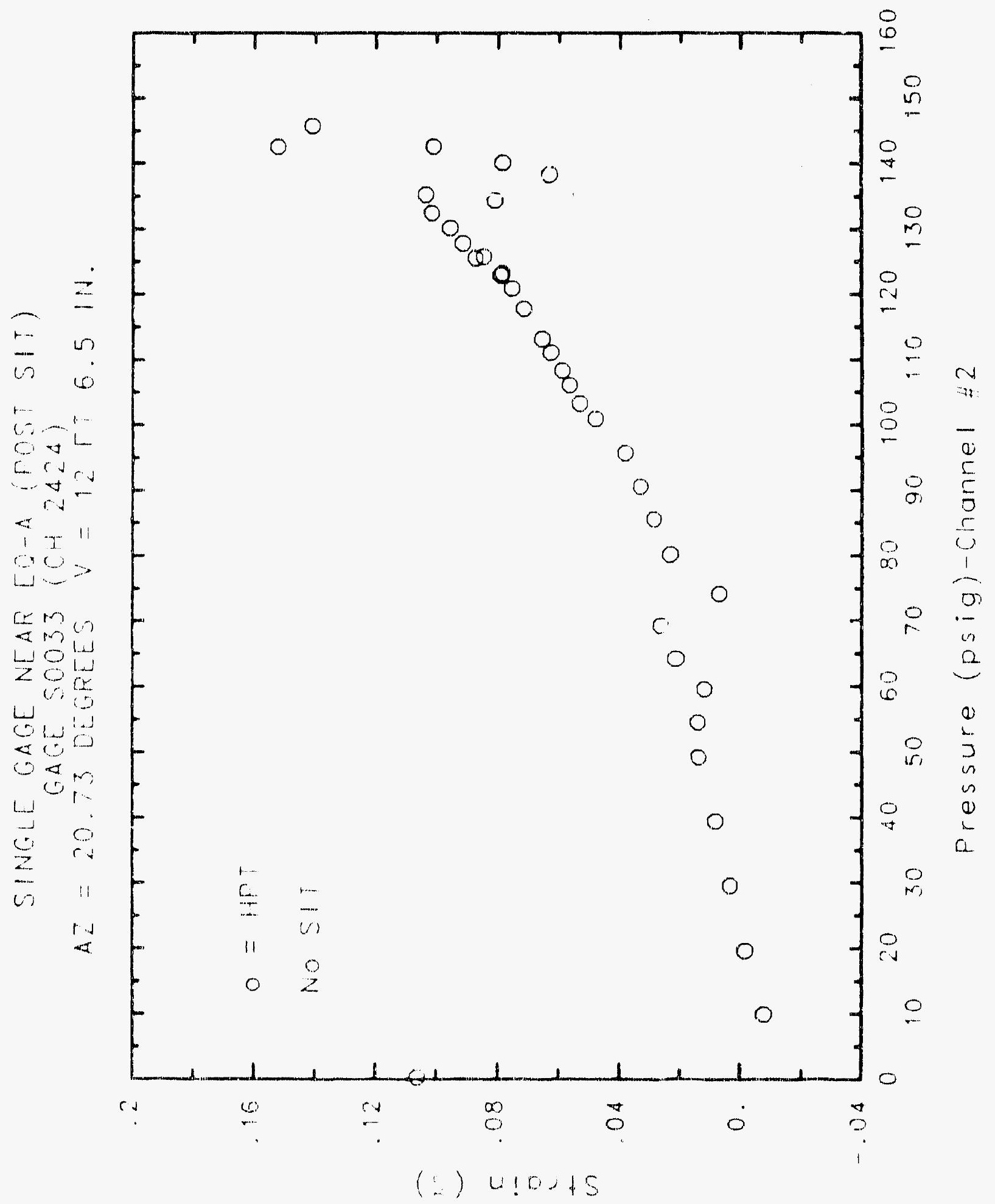

( . 10,51 


\section{Reinforced Concrete Test Data}

Strain Gage 0

Structural INTEgRity TEST

Pressure

( $\mu s i g$ )

No SIT data

This gage was added after SIT
Channel 2425

High Pressure Test

Pressure

(psig)

9.89

19.55

29.57

39.42

49.16

54.50

59.57

64.20

69.32

74.16

80.16

85.61

90.58

95.69

100.92

103.25

106.11

108.21

111.08

113.24

117.83

12C. 92

123.28

122.97

12.5 .82

125.60

127.84

130.19

132.53

135.33

134.42

138.35

140.16

142.63

145.78

142.52

0.22
* Strain

0.0135

0.6185

0.0248

0.0308

0.0373

0.0400

0.0452

0.0469

0.0526

0.0502

0.0183

0.0221

0.0277

0.0328

0.038 .

0.0391

0.0395

0.0412

0.0445

0.0469

0.0521

0.0562

0.0576

0.0596

0.0597

0.0588

0.0599

0.0617

0.0640

0.0831

0.0785

$-0.0290$

$-0.0285$

$-0.0057$

U. 0162

0.0341

$-0.0058$ 


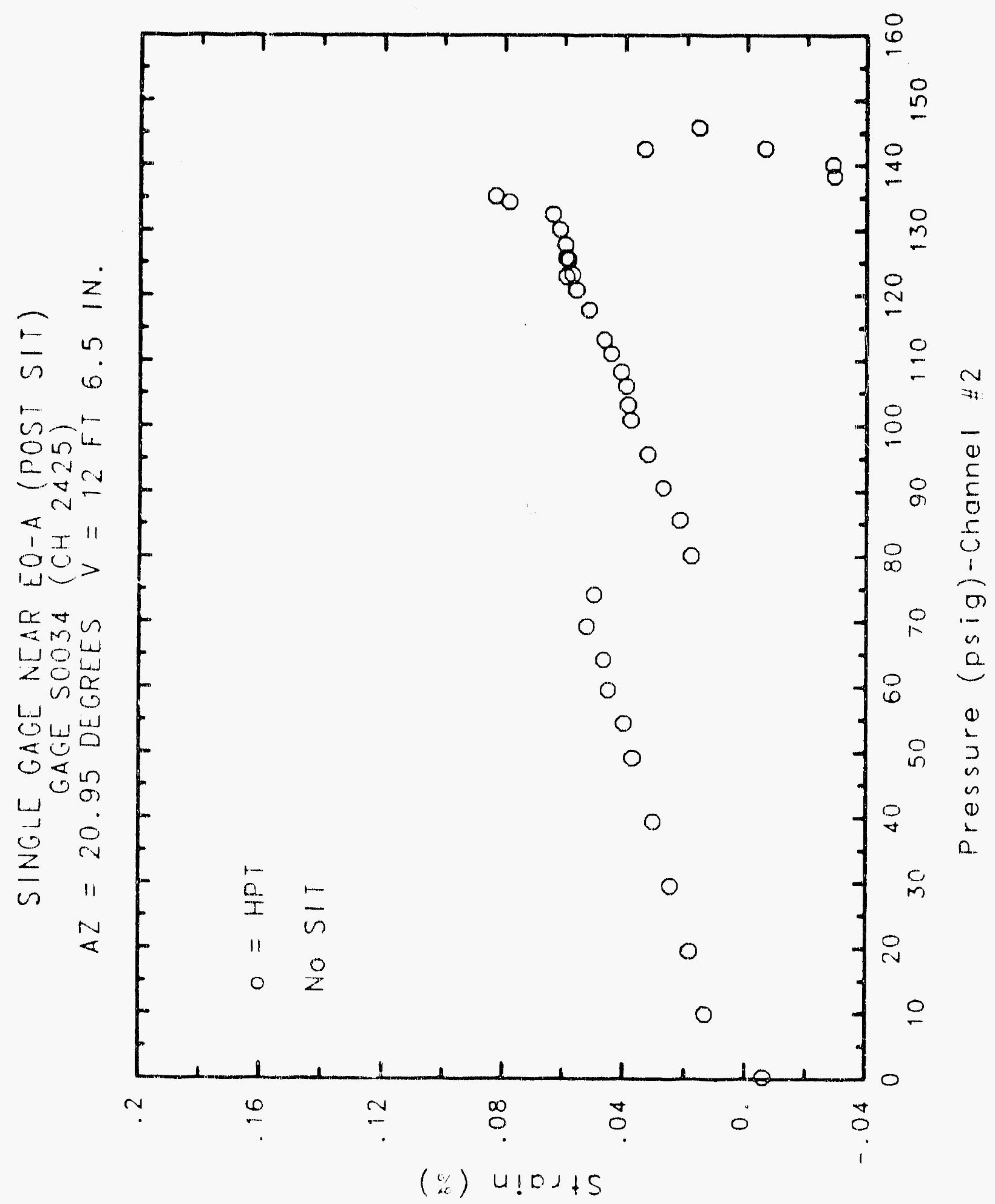

C. -16.53 
Strain Gage 0

Structural Integrity Test

Pressure
(psig)
Ho SIT data
s gage was added after SIT

This gage was added after SIT

Channel 2426

High Pressure test

Pr

Pressur

9.89

19.55

29.57

39.42

49.16

54.50

59.57

64.20

69.32

74.16

80.16

85.61

90.58

95.69

100.92

103.25

106.11

108.31

111.08

113.24

117.83

120.92

123.28

122.97

125.82

125.60

127.84

130.19

132.53

135.33

134.42

138.35

140.16

142.63

145.78

142.52

0.22

* Strain

$-0.0016$

0.0020

0.0072

0.0127

0.0179

0.0208

0.0240

0.0275

0.0308

0.0357

0.0386

0.0427

0.0466

0.0510

0.0559

0.0583

0.0611

0.0632

0.0663

0.0686

0.0736

0.0770

0.0802

0.0807

0.0842

0.0851

0.0876

0.0916

0.0958

0.1023

0.1042

0.1150

0.1231

0.1361

0.1514

0.1463

0.0941 


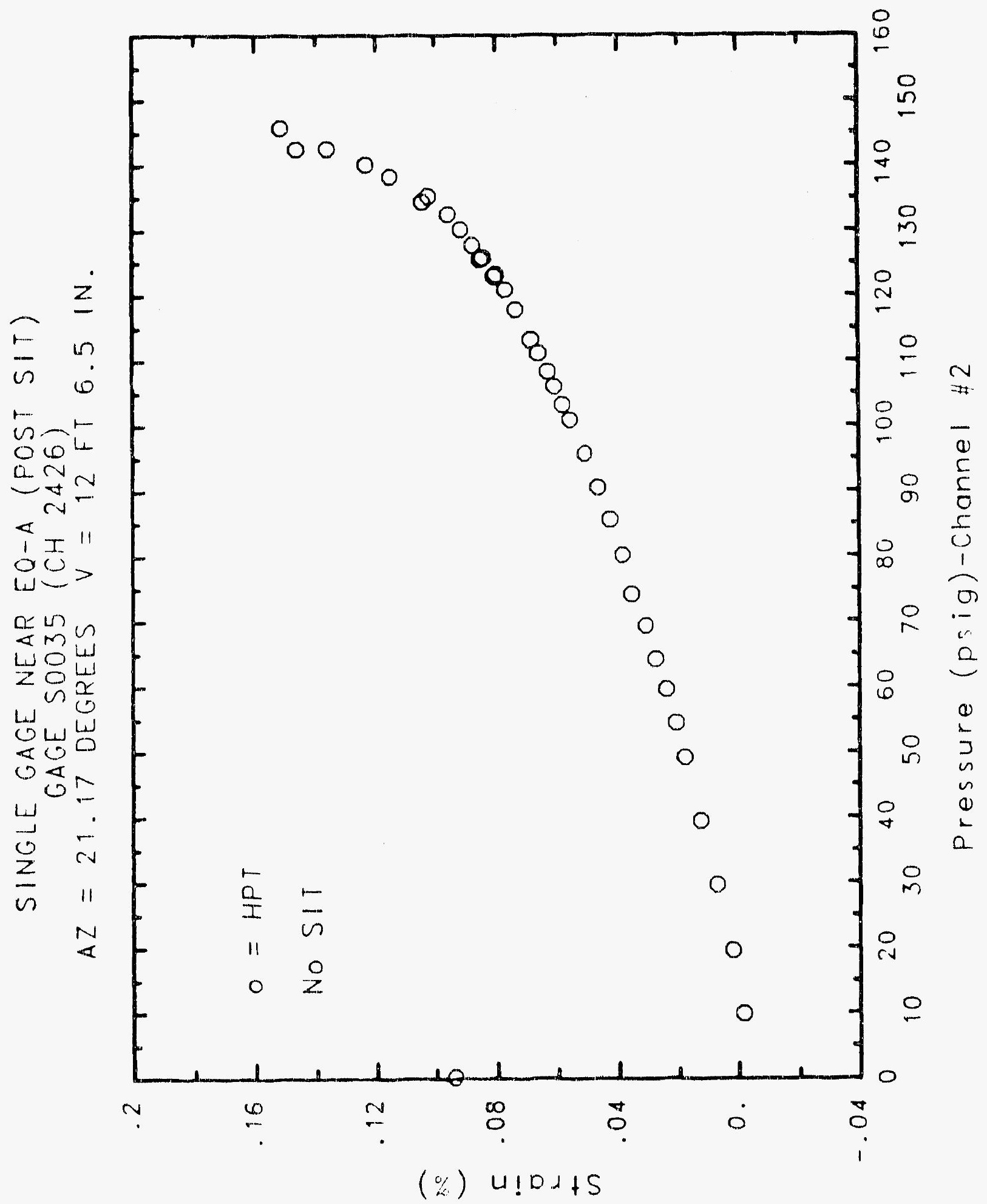

C. -1655 


\section{Reinforced Concrete Test Data}

Strain Gage 0

Structural Integrity Test

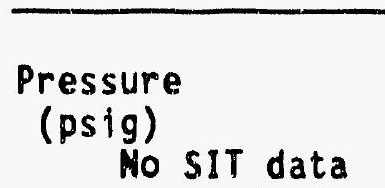

This gage was added after SIT

* Strain
Chammel 2427

High Pressure test

Pressure

(Fsig)

9.89

19.55

29.57

39.42

49.16

54.50

59.57

64.20

69.32

74.16

80.16

85.61

90.58

95.69

100.92

103.25

106.11

108.31

111.08

113.24

117.83

120.92

123.28

122.97

125.82

125.60

127.84

130.19

132.53

135.33

134.42

138.35

140.16

142.53

145.78

142.52

0.22
* Strain

0.0062

0.0118

0.0172

0.0224

0.0275

0.0306

0.0336

0.0370

0.0406

0.0421

0.0445

0.0474

0.0506

0.0538

0.0570

0.0584

0.0598

0.0610

0.0625

0.0638

0.0670

0.0690

0.0707

0.0707

0.0720

0.0719

0.0720

0.0693

0.0649

0.0667

$0.06 \%$

0.0713

0.0669

0.0585

0.0708

0.0662

$-0.0168$ 


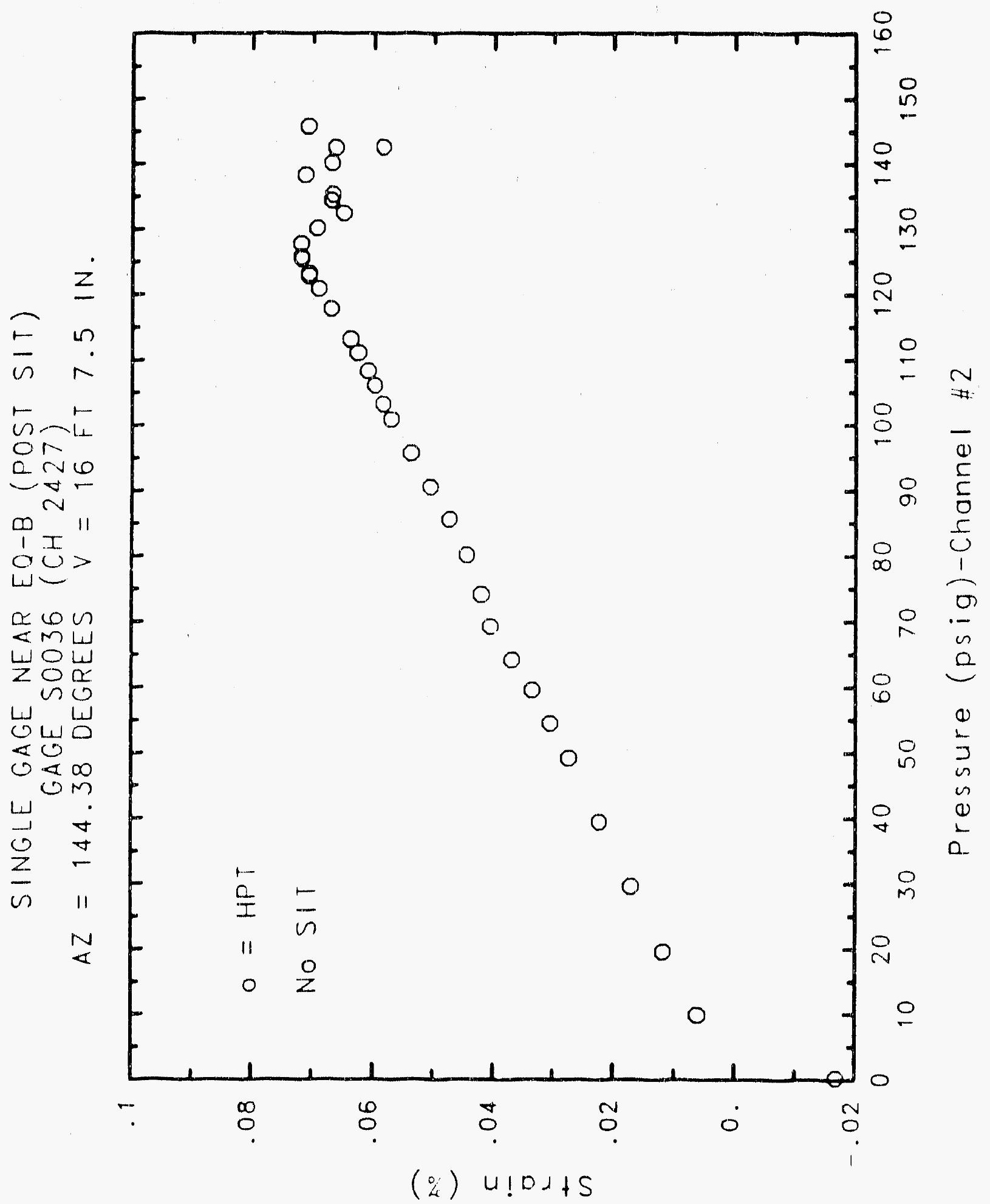

C. -1657 


\section{Reinforced Concrete Test Data}

Strain Gage 0

Structural Integrity Test

Pressure
(psig)
No sir data strain

This gage was added after SIT

Chanmel 2428

high Pressure test

\begin{tabular}{|c|c|}
\hline $\begin{array}{c}\text { Pressure } \\
\text { (psig) } \\
9.89 \\
19.55 \\
29.57 \\
39.42 \\
49.16 \\
54.50 \\
59.57 \\
64.20 \\
69.32 \\
74.16 \\
80.16 \\
85.61 \\
90.58 \\
95.69 \\
100.92 \\
103.25 \\
106.11 \\
108.31 \\
111.08 \\
113.24 \\
117.83 \\
120.92 \\
123.28 \\
122.97 \\
125.82 \\
125.60 \\
127.84 \\
130.19 \\
132.53 \\
135.33 \\
134.42 \\
138.35 \\
140.16 \\
142.63 \\
145.78 \\
142.52 \\
0.22\end{array}$ & $\begin{array}{l}\text { X Strin in } \\
0.0065 \\
0.0127 \\
0.0185 \\
0.0242 \\
0.0301 \\
0.0336 \\
0.0371 \\
0.0409 \\
0.0452 \\
0.0496 \\
0.0525 \\
0.0566 \\
0.0605 \\
0.0649 \\
0.0687 \\
0.0704 \\
0.0722 \\
0.0735 \\
0.0754 \\
0.0768 \\
0.0802 \\
0.0824 \\
0.0834 \\
0.0820 \\
0.0809 \\
0.0789 \\
0.0785 \\
0.0749 \\
0.0703 \\
0.0689 \\
0.0665 \\
0.0655 \\
0.0705 \\
0.0727 \\
0.0825 \\
0.0766 \\
-0.0014\end{array}$ \\
\hline
\end{tabular}




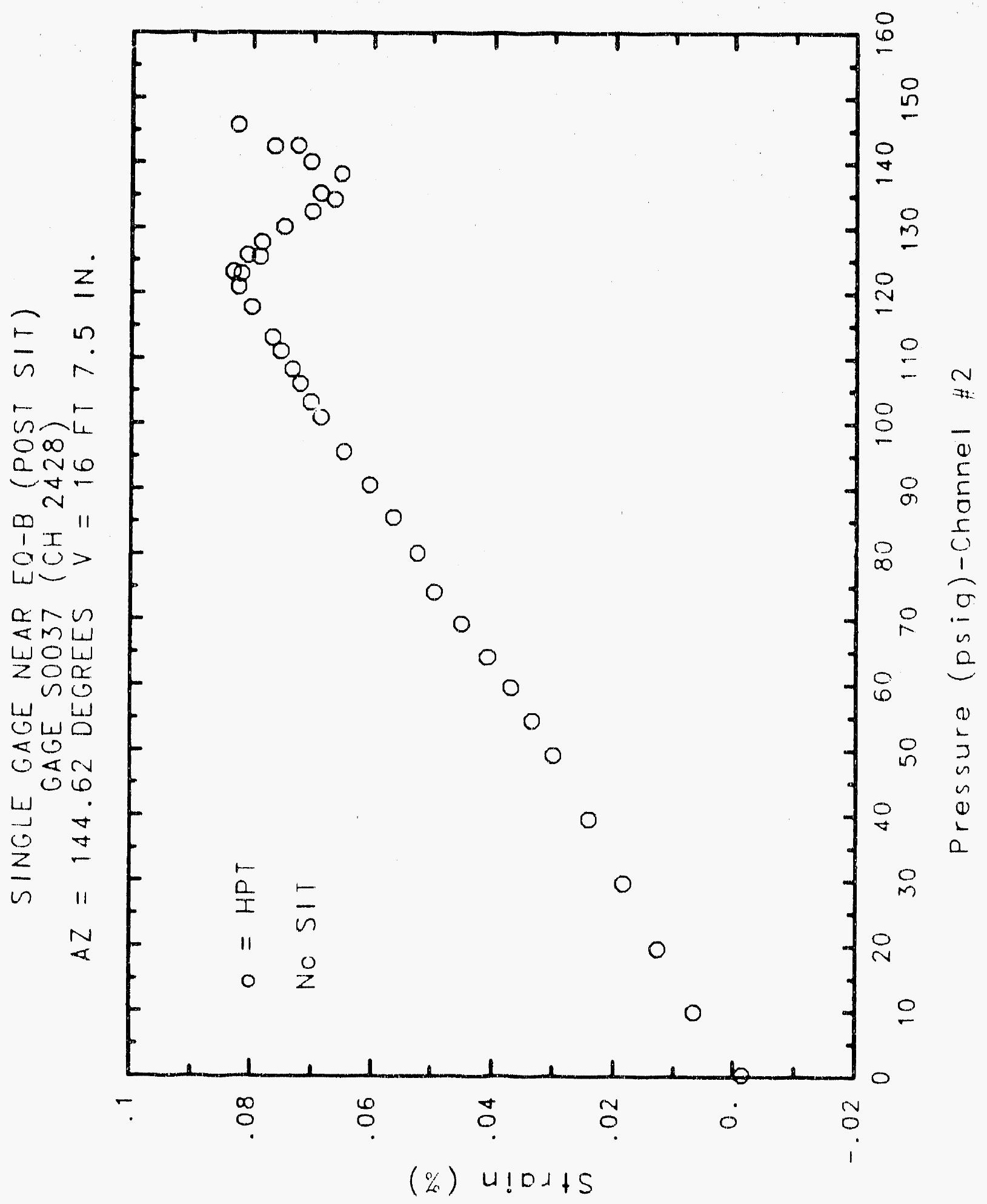

C. -16.59 


\section{Reinforced Concrete Test Data}

Strain Gage 0

STRUCTURAL InTEgRITY TEST

\begin{tabular}{|c|c|c|c|}
\hline $\begin{array}{c}\text { Pressure } \\
\text { (psig) } \\
-0.05 \\
5.33 \\
12.31 \\
16.44 \\
20.51 \\
20.46 \\
20.47 \\
27.05 \\
34.69 \\
34.53 \\
34.53 \\
39.70 \\
45.90 \\
45.65 \\
45.62 \\
50.10 \\
53.47 \\
53.29 \\
53.21 \\
50.49 \\
46.14 \\
46.13 \\
44.97 \\
38.98 \\
33.99 \\
33.96 \\
26.83 \\
21.88 \\
21.90 \\
16.69 \\
11.74 \\
11.77 \\
5.05 \\
0.02 \\
-0.04 \\
-0.02 \\
-0.02 \\
0.02 \\
\end{array}$ & $\begin{array}{l}x \text { Strain } \\
0.0000 \\
0.0000 \\
0.0000 \\
0.0000 \\
0.0000 \\
0.0000 \\
0.0000 \\
0.0000 \\
0.0000 \\
0.0000 \\
0.0000 \\
0.0000 \\
0.0000 \\
0.0000 \\
0.0000 \\
0.0000 \\
0.0000 \\
0.0000 \\
0.0000 \\
0.0000 \\
0.0000 \\
0.0000 \\
0.0000 \\
0.0000 \\
0.0000 \\
0.0000 \\
0.0000 \\
0.0000 \\
0.0000 \\
0.0000 \\
0.0000 \\
0.0000 \\
0.0000 \\
0.0000 \\
0.0000 \\
0.0000 \\
0.0000 \\
0.0000\end{array}$ & $\begin{array}{c}\text { Pressure } \\
(\text { psig) } \\
9.89 \\
19.55 \\
29.57 \\
39.42 \\
49.16 \\
54.50 \\
59.57 \\
64.20 \\
69.32 \\
74.16 \\
80.16 \\
85.61 \\
90.58 \\
95.69 \\
100.92 \\
103.25 \\
106.11 \\
108.31 \\
111.08 \\
113.24 \\
117.83 \\
120.92 \\
123.28 \\
122.97 \\
125.82 \\
125.60 \\
127.84 \\
130.19 \\
132.53 \\
135.33 \\
134.42 \\
138.35 \\
140.16 \\
142.63 \\
145.78 \\
142.52 \\
0.22 \\
\end{array}$ & $\begin{array}{l}\text { X Strain } \\
0.0054 \\
0.0111 \\
0.0167 \\
0.0222 \\
0.0278 \\
0.0308 \\
0.0342 \\
0.0371 \\
0.0408 \\
0.0437 \\
0.0533 \\
0.0574 \\
0.0612 \\
0.0658 \\
0.0697 \\
0.0714 \\
0.0733 \\
0.0746 \\
0.0764 \\
0.0777 \\
0.0811 \\
0.0831 \\
0.0843 \\
0.0836 \\
0.0845 \\
0.0832 \\
0.0828 \\
0.0797 \\
0.0732 \\
0.0705 \\
0.0624 \\
0.0599 \\
0.0652 \\
0.0756 \\
0.0934 \\
0.0906 \\
0.0357\end{array}$ \\
\hline
\end{tabular}

Chanmel 2429

high Pressure test

(psig)

9.89

29.57

39.42

59.57

69.32

74.16

80.16

85.61

90.58

103.25

106.11

111.08

113.24

117.83

120.92

23.28

125.60

27.84

30.19

32.53

35.33

134.42

38.35

145.78

2.52

0.0000
0.0906

Strain

0.0054

0.0167

0.0222

0.0278

0.0408

0.0437

0.0533

0.0574

0.0658

0.0733

0.0764

0.0777

0.0811

0.0831

0.0843

0.0828

0.0797

0.0732

0.0705

0.0624

0.0599

0.0652

0.0756

0.0357 


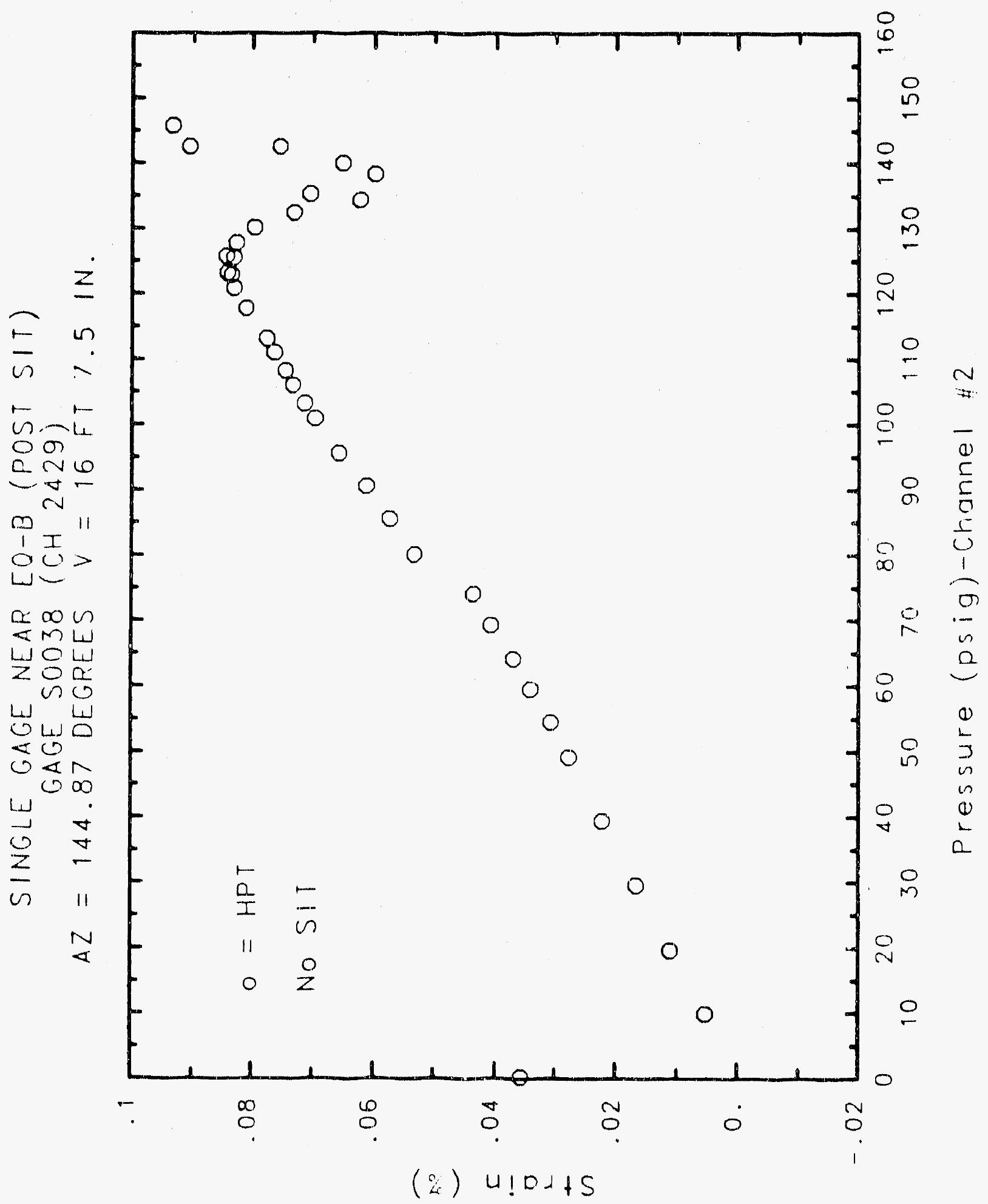

C. 1601 


\section{Reinforced Concrete Test Data}

Strain Gage 0

StRUC TURAL INTEgRITY TEST

\begin{tabular}{|c|c|}
\hline $\begin{array}{c}\text { Pressure } \\
\text { (psig) } \\
-0.05 \\
5.33 \\
12.31 \\
16.44 \\
20.51 \\
20.46 \\
20.47 \\
27.05 \\
34.69 \\
34.53 \\
34.53 \\
39.70 \\
45.90 \\
45.65 \\
45.62 \\
50.10 \\
53.47 \\
53.29 \\
53.21 \\
50.49 \\
46.14 \\
46.13 \\
44.91 \\
38.98 \\
33.99 \\
33.96 \\
26.83 \\
21.88 \\
21.90 \\
16.69 \\
11.74 \\
11.77 \\
5.05 \\
0.02 \\
-0.04 \\
-0.02 \\
-0.02 \\
0.02\end{array}$ & $\begin{array}{l}\text { X Strain } \\
0.0000 \\
0.0000 \\
0.0000 \\
0.0000 \\
0.0000 \\
0.0000 \\
0.0000 \\
0.0000 \\
0.0000 \\
0.0000 \\
0.0000 \\
0.0000 \\
0.0000 \\
0.0000 \\
0.0000 \\
0.0000 \\
0.0000 \\
0.0000 \\
0.0000 \\
0.0000 \\
0.0000 \\
0.0000 \\
0.0000 \\
0.0000 \\
0.0000 \\
0.0000 \\
0.0000 \\
0.0000 \\
0.0000 \\
0.0000 \\
0.0000 \\
0.0000 \\
0.0000 \\
0.0000 \\
0.0000 \\
0.0000 \\
0.0000 \\
0.0000\end{array}$ \\
\hline
\end{tabular}

Chammel 2465

High Pressure test

(psig)

9.89

19.55

29.57

39.42

49.16

54.50

59.57

64.20

69.32

74.16

80.16

85.61

90.58

95.69

100.92

103.25

106.11

108.31

111.08

113.24

117.83

120.92

123.28

122.97

125.82

125.60

127.84

130.19

132.53

135.33

134.42

138.35

140.16

142.63

145.78

142.52

0.22
* Strain

$-0.0017$

$-0.0047$

$-0.0081$

$-0.0118$

$-0.0162$

$-0.0181$

$-0.0198$

$-0.0214$

$-0.0190$

$-0.0131$

$-0.0067$

$-0.0002$

0.0062

0.0126

0.0197

0.0234

0.0265

0.0288

0.0328

0.0352

0.0386

0.0423

0.0437

0.0430

0.0463

0.0462

0.0469

0.0480

0.0494

0.0506

0.0494

0.0505

0.0502

0.0499

0.0492

0.0398

0.0024 


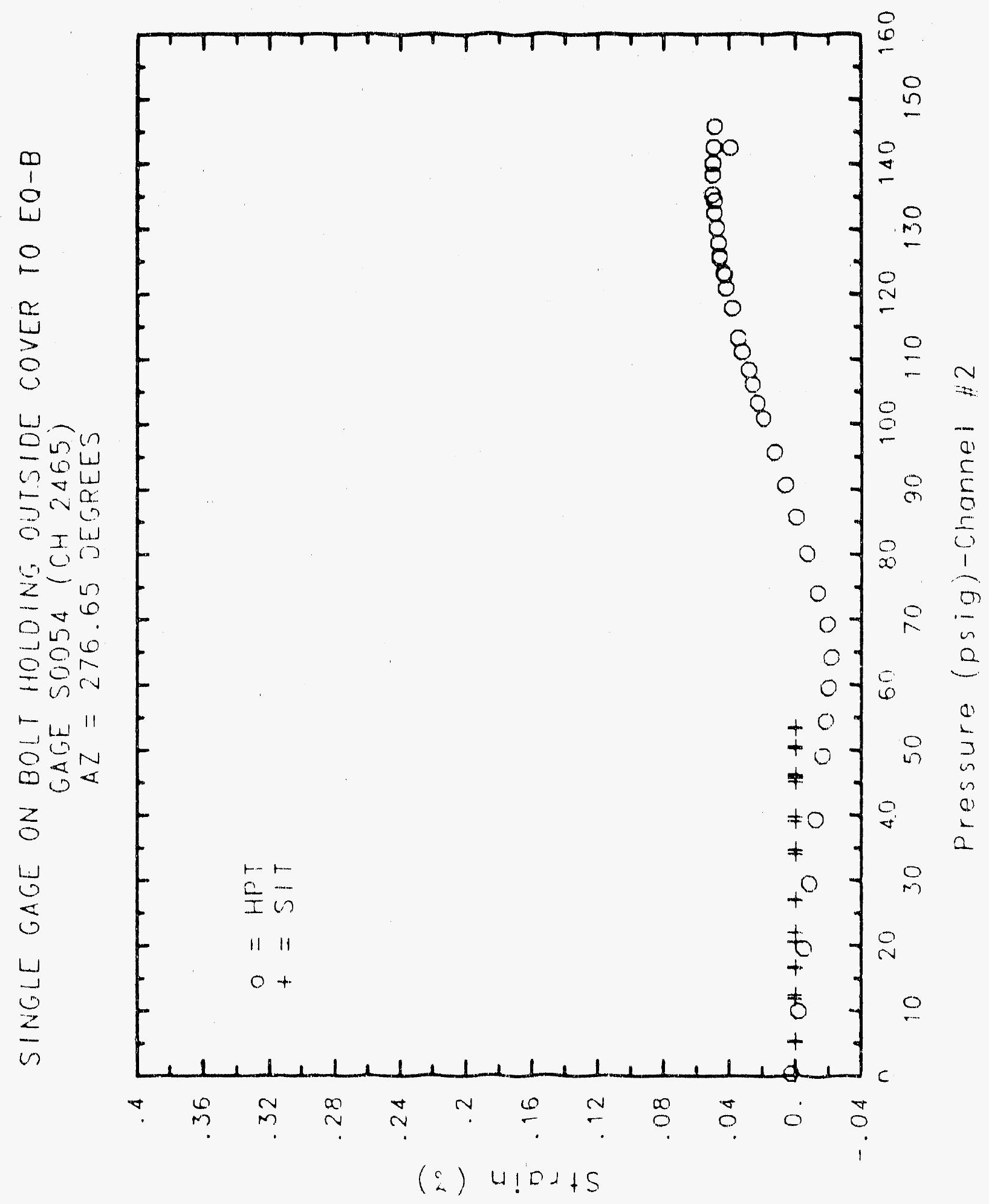




\section{Reinforced Concrete Test Data}

Strain gage 0

STRUCTURAL INTEGRITY TEST

\begin{tabular}{|c|c|c|c|}
\hline $\begin{array}{c}\text { Pressure } \\
(p s 1 g) \\
-0.05 \\
5.33 \\
12.31 \\
16.44 \\
20.51 \\
20.46 \\
20.47 \\
27.05 \\
34.69 \\
34.53 \\
34.53 \\
39.70 \\
45.90 \\
45.65 \\
45.52 \\
50.10 \\
53.47 \\
53.29 \\
53.21 \\
50.49 \\
46.14 \\
46.13 \\
44.97 \\
38.98 \\
33.99 \\
33.96 \\
26.83 \\
21.88 \\
21.90 \\
16.69 \\
11.74 \\
11.77 \\
5.05 \\
0.02 \\
-0.04 \\
-0.02 \\
-0.02 \\
0.02 \\
\end{array}$ & $\begin{array}{l}\text { X Strain } \\
\text { 0.0000 } \\
0.0000 \\
0.0000 \\
0.0000 \\
0.0000 \\
0.0000 \\
0.0000 \\
0.0000 \\
0.0000 \\
0.0000 \\
0.0000 \\
0.0000 \\
0.0000 \\
0.0000 \\
0.0000 \\
0.0000 \\
0.0000 \\
0.0000 \\
0.0000 \\
0.0000 \\
0.0000 \\
0.0000 \\
0.0000 \\
0.0000 \\
0.0000 \\
0.0000 \\
0.0000 \\
0.0000 \\
0.0000 \\
0.0000 \\
0.0000 \\
0.0000 \\
0.0000 \\
0.0000 \\
0.0000 \\
0.0000 \\
0.0000 \\
0.0000\end{array}$ & $\begin{array}{c}\text { Pressure } \\
(\text { pstg) } \\
9.89 \\
19.55 \\
29.57 \\
39.42 \\
49.16 \\
54.50 \\
59.57 \\
64.20 \\
69.32 \\
74.16 \\
80.16 \\
85.61 \\
90.58 \\
95.69 \\
100.92 \\
103.25 \\
106.11 \\
108.31 \\
111.08 \\
113.24 \\
117.83 \\
120.92 \\
123.28 \\
122.97 \\
125.82 \\
125.60 \\
127.84 \\
130.19 \\
132.53 \\
135.32 \\
134.42 \\
138.35 \\
140.16 \\
142.63 \\
145.78 \\
142.52 \\
0.22\end{array}$ & $\begin{array}{l}\text { X Strain } \\
0.0002 \\
0.0009 \\
0.0011 \\
0.0010 \\
0.0003 \\
0.0002 \\
0.0023 \\
0.0049 \\
0.0086 \\
0.0120 \\
0.0200 \\
0.0279 \\
0.0365 \\
0.0470 \\
0.0576 \\
0.0626 \\
0.0688 \\
0.0735 \\
0.0801 \\
0.0850 \\
0.0963 \\
0.1056 \\
0.1127 \\
0.1133 \\
0.1215 \\
0.1225 \\
0.1278 \\
0.1365 \\
0.1467 \\
0.1627 \\
0.1669 \\
0.1866 \\
0.2060 \\
0.2422 \\
0.2895 \\
0.3104 \\
1.1296\end{array}$ \\
\hline
\end{tabular}

Channel 2467

high Pressure test

Pressure

19.55

29.57

0.0009

0.0011

0.0002

0.0023

0.0049

0.0086

0.0279

0.0365

0.0576

0.0626

0.0688

0.0735

0.0801

0.0963

0.1056

0.1127

0.1133

0.1215

0.1225

1278

0.1627

0.1669

0.1866

0.2060

0.2422

0.3104

1. 1296 


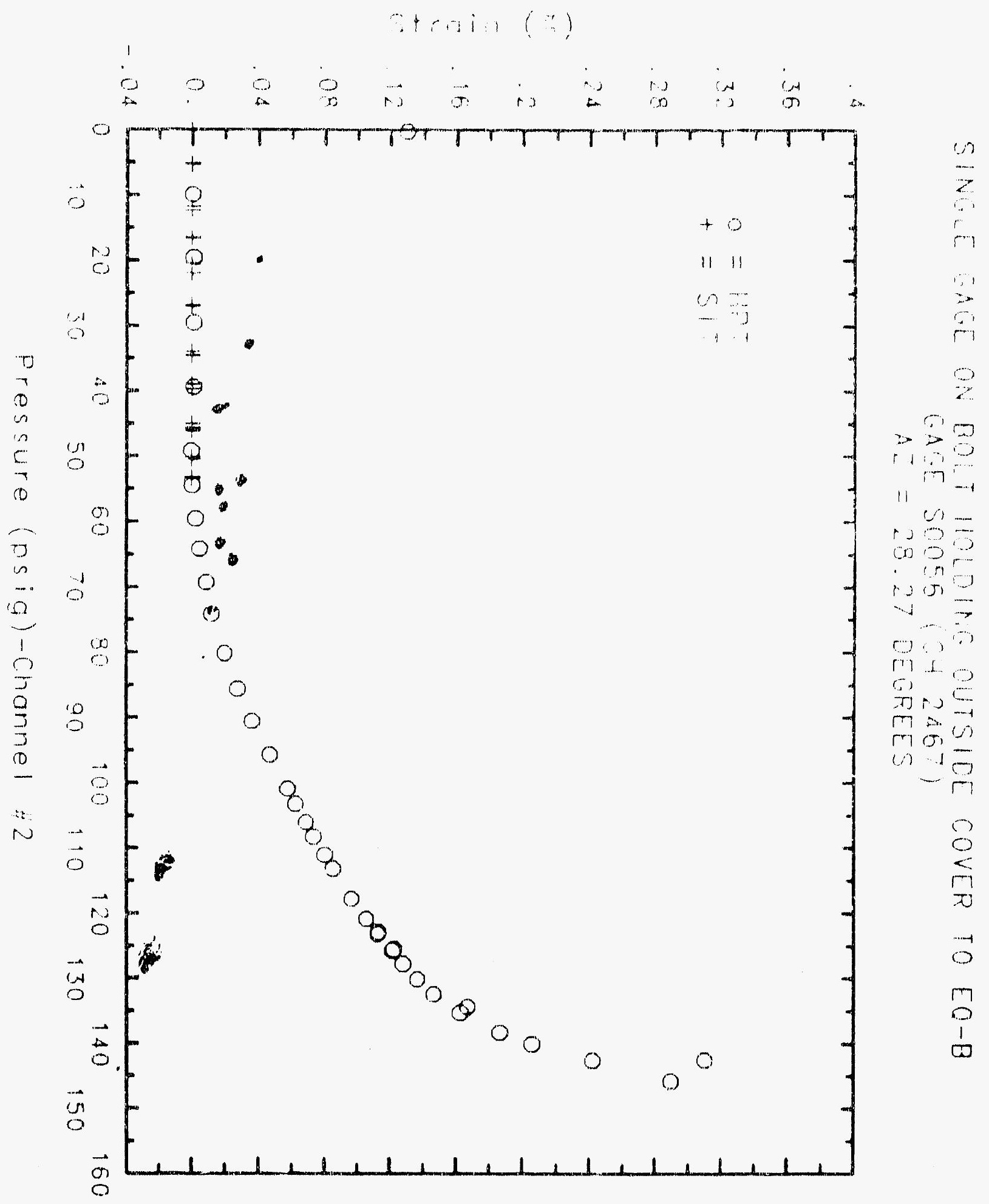




\section{Reinforced Concrete Test Data}

Strain Gage 0

STRUCTURAL INTEgRITY TEST

\begin{tabular}{cccc}
\hline $\begin{array}{c}\text { Pressure } \\
\text { (psig) }\end{array}$ & \% strain & $\begin{array}{c}\text { Pressure } \\
\text { (psig) }\end{array}$ & \% strain \\
-0.05 & 0.0000 & 9.89 & -0.0008 \\
5.33 & 0.0000 & 19.55 & -0.0021 \\
12.31 & 0.0000 & 29.57 & -0.0037 \\
16.44 & 0.0000 & 39.42 & -0.0054 \\
20.51 & 0.0000 & 49.16 & -0.0079 \\
20.46 & 0.0000 & 54.50 & -0.0096 \\
20.47 & 0.0000 & 59.57 & -0.0115 \\
27.05 & 0.0000 & 64.20 & -0.0146 \\
34.69 & 0.0000 & 69.32 & -0.0163 \\
34.53 & 0.0000 & 74.16 & -0.0128 \\
34.53 & 0.0000 & 80.16 & -0.0078 \\
39.70 & 0.0000 & 85.61 & -0.0022 \\
45.90 & 0.0000 & 90.58 & 0.0035 \\
45.65 & 0.0000 & 95.69 & 0.0094 \\
45.62 & 0.0000 & 100.92 & 0.0157 \\
50.10 & 0.0000 & 103.25 & 0.0184 \\
53.47 & 0.0000 & 106.11 & 0.0212 \\
53.29 & 0.0000 & 108.31 & 0.0237 \\
53.21 & 0.0000 & 111.08 & 0.0271 \\
50.49 & 0.0000 & 113.24 & 0.0296 \\
46.14 & 0.0000 & 117.83 & 0.0339 \\
46.13 & 0.0000 & 120.92 & 0.0377 \\
44.97 & 0.0000 & 123.28 & 0.0403 \\
38.98 & 0.0000 & 122.97 & 0.0407 \\
33.99 & 0.0000 & 125.82 & 0.0430 \\
33.95 & 0.0000 & 125.60 & 0.0427 \\
26.83 & 0.0000 & 127.84 & 0.0431 \\
21.88 & 0.0000 & 130.19 & 0.0447 \\
21.90 & 0.0000 & 132.53 & 0.0465 \\
16.69 & 0.0000 & 135.33 & 0.0485 \\
11.74 & 0.0000 & 134.42 & 0.0486 \\
11.77 & 0.0000 & 138.35 & 0.0498 \\
5.05 & 0.0000 & 140.16 & 0.0503 \\
0.02 & 0.0000 & 142.63 & 0.0517 \\
-0.04 & 0.0000 & 145.78 & 0.0523 \\
-0.02 & 0.0000 & 142.52 & 0.0493 \\
-0.02 & 0.0000 & 0.22 & 0.0345 \\
0.02 & 0.0000 & & \\
\hline & & & \\
\hline
\end{tabular}

Chanhel 2469

High Pressure test 


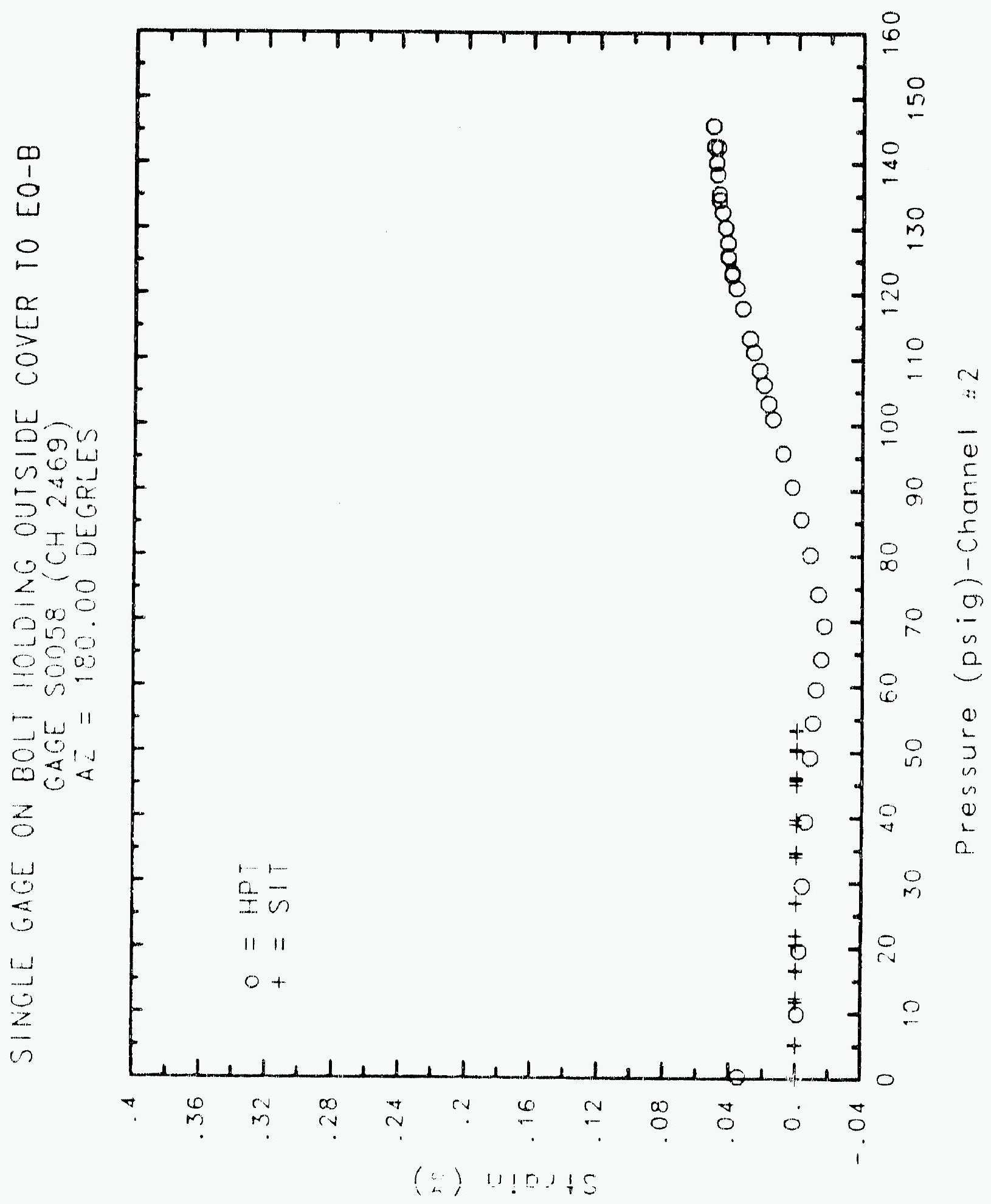



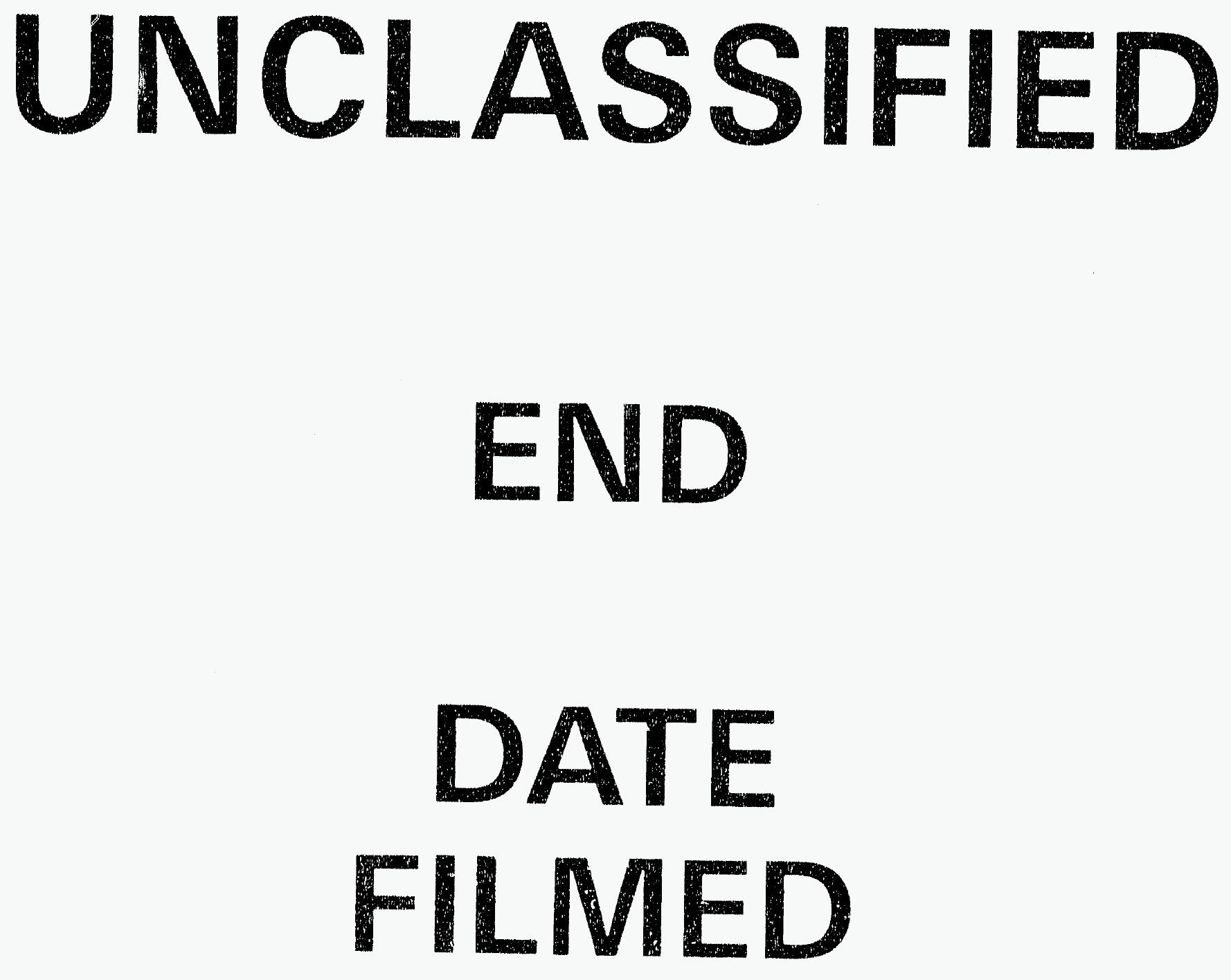

$$
1-6.9 \%
$$



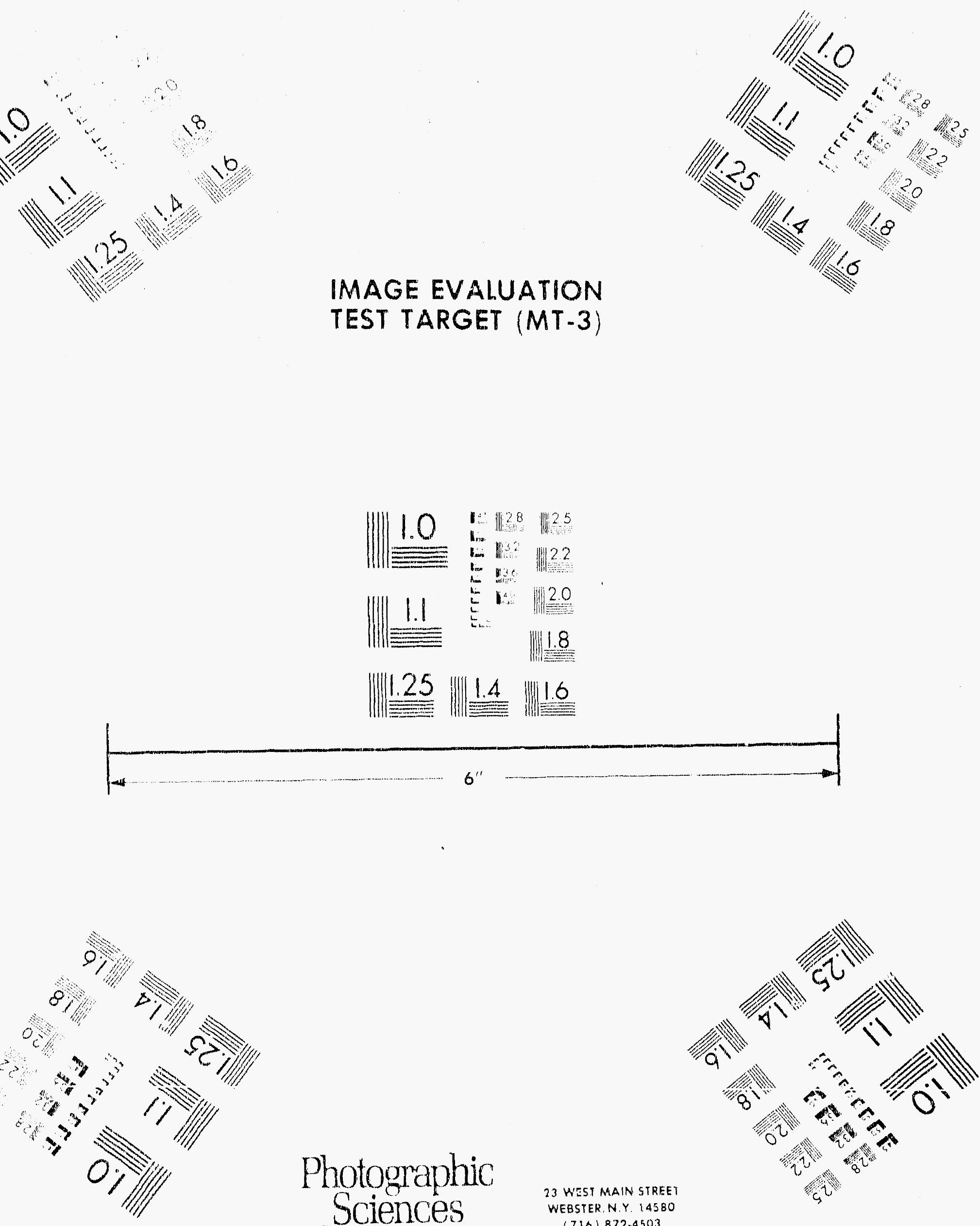

Photographic Sciences Corporation
23 WEST MAIN STREET WEBSTER. N.Y. 14580 (716) 872.4503

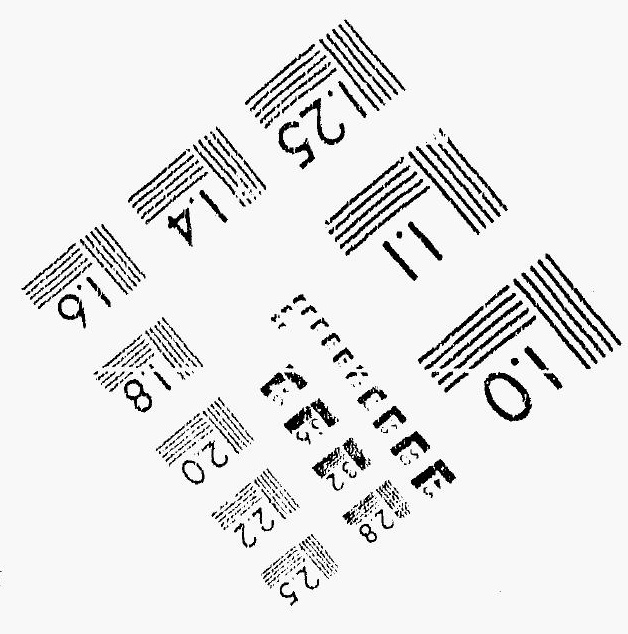




\section{SECURITY CLASSIFICATION}
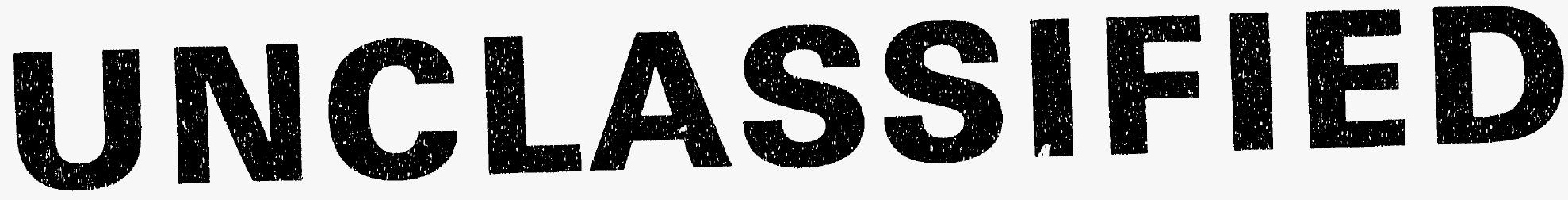

\section{DATE OF MICROFILIMING \\ $1-6-92$}
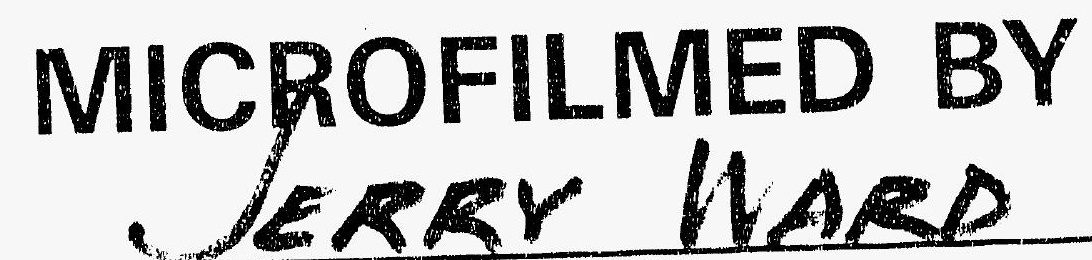

SANDIA NATIONAL LABS 
THIS DOCUMENT FILMED

AT

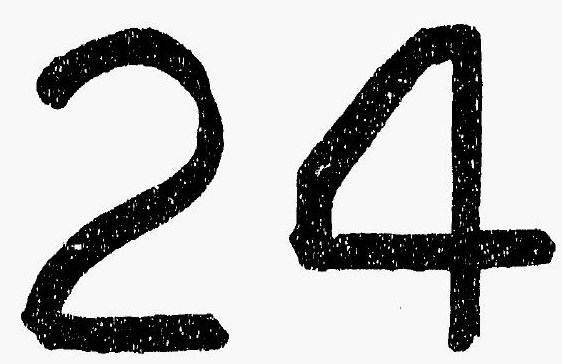

REDUCTION 


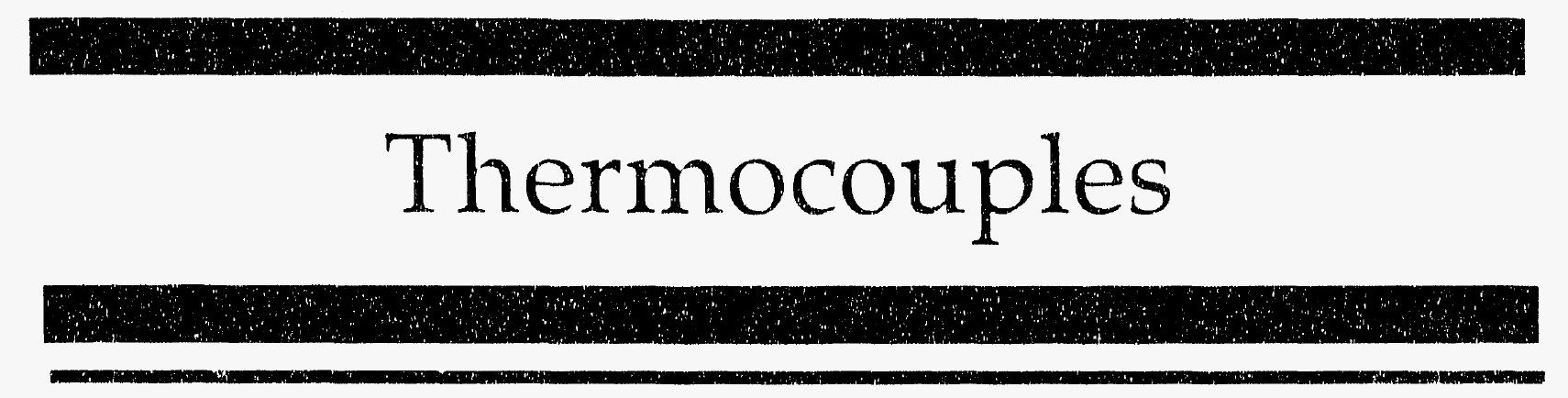




\section{Reinforced Concrete Test Data}

Thermocouple Channel 60

StRUCTURAL INTEGRITY TEST

\begin{tabular}{|c|c|c|c|}
\hline $\begin{array}{c}\text { Pressure } \\
\text { (psig) } \\
-0.05 \\
5.33 \\
12.31 \\
16.44 \\
20.51 \\
20.46 \\
20.47 \\
27.05 \\
34.69 \\
34.53 \\
34.53 \\
39.70 \\
45.90 \\
45.65 \\
45.62 \\
50.10 \\
53.47 \\
53.29 \\
53.21 \\
50.49 \\
46.14 \\
46.13 \\
44.97 \\
38.98 \\
33.99 \\
33.96 \\
26.83 \\
21.88 \\
21.90 \\
16.69 \\
11.74 \\
11.77 \\
5.05 \\
0.02 \\
-0.04 \\
-0.02 \\
-0.02 \\
0.02\end{array}$ & $\begin{array}{c}\text { Temperature } \\
\text { (Fahrenheit) } \\
75.4680 \\
75.5120 \\
75.6010 \\
75.8660 \\
75.3350 \\
76.5740 \\
76.8390 \\
76.8840 \\
76.6180 \\
75.4680 \\
75.4680 \\
76.3530 \\
75.1130 \\
74.9810 \\
74.9360 \\
75.0250 \\
75.7330 \\
75.5560 \\
75.5120 \\
75.6010 \\
75.6450 \\
75.6010 \\
76.4410 \\
76.7510 \\
77.0160 \\
77.2810 \\
77.2370 \\
77.2370 \\
77.2810 \\
77.0600 \\
76.9280 \\
76.5300 \\
76.3530 \\
76.0870 \\
75.0690 \\
74.8480 \\
74.9360 \\
75.0250 \\
750\end{array}$ & $\begin{array}{c}\text { Pressure } \\
\text { (psig) } \\
9.89 \\
19.55 \\
29.57 \\
39.42 \\
49.16 \\
54.50 \\
59.57 \\
64.20 \\
69.32 \\
74.16 \\
80.16 \\
85.61 \\
90.58 \\
95.69 \\
100.92 \\
103.25 \\
106.11 \\
108.31 \\
111.08 \\
113.24 \\
117.83 \\
120.92 \\
123.28 \\
122.97 \\
125.82 \\
125.60 \\
127.84 \\
130.19 \\
132.53 \\
135.33 \\
134.42 \\
138.35 \\
140.16 \\
142.63 \\
145.78 \\
142.52 \\
0.22\end{array}$ & $\begin{array}{c}\text { Temperature } \\
\text { (Fahrenheit) } \\
75.9550 \\
75.9550 \\
75.9550 \\
75.9990 \\
75.9100 \\
75.9100 \\
75.8660 \\
75.9100 \\
75.9990 \\
75.9550 \\
76.0430 \\
75.9990 \\
75.9550 \\
76.0870 \\
76.0430 \\
76.0870 \\
76.0870 \\
76.0430 \\
76.0870 \\
76.0870 \\
76.0870 \\
76.1320 \\
76.1320 \\
76.0870 \\
76.0430 \\
76.0430 \\
76.0870 \\
76.1320 \\
76.1320 \\
76.1320 \\
76.1760 \\
76.1320 \\
76.1320 \\
76.0870 \\
76.0870 \\
76.0870 \\
76.0870\end{array}$ \\
\hline
\end{tabular}

High Pressure test

pressure

Fahrenheit

75.9550

75.9550

75.9990

75.9100

75.9100

75.9990

75.9550

76.0430

75.9990

76.0430

76.0870

76.0870

76.0430

76.0870

76.0870

76.0430

76.1320

76.0870

76.0870 


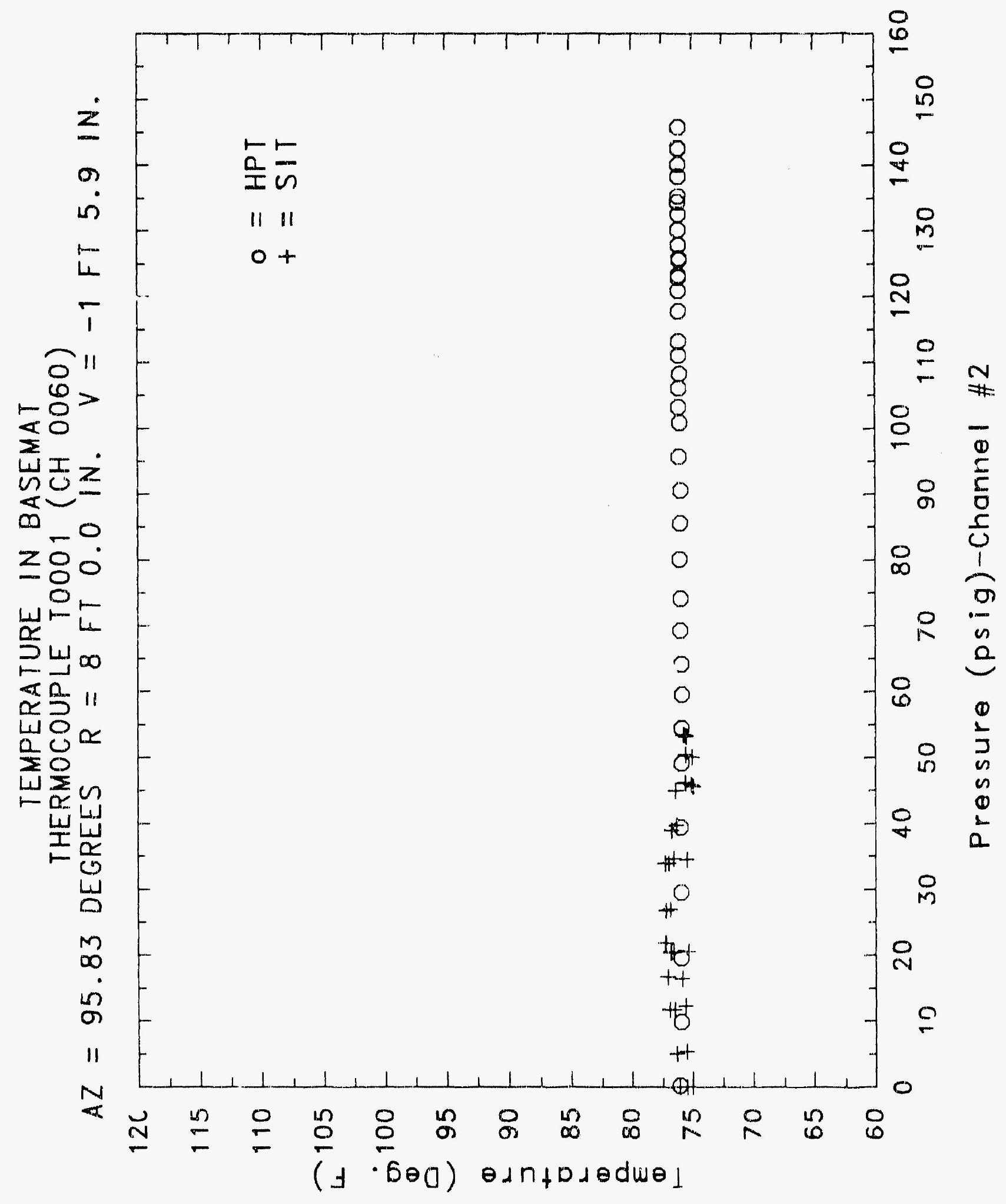




\section{Reinforced Concrete Test Data}

Thermocouple Channel 61

Structural Integrity Test

\begin{tabular}{|c|c|c|c|}
\hline $\begin{array}{l}\text { Pressure } \\
\text { (psig) } \\
-0.05 \\
5.33 \\
12.31 \\
16.44 \\
20.51 \\
20.46 \\
20.47 \\
27.05 \\
34.69 \\
34.53 \\
34.53 \\
39.70 \\
45.90 \\
45.65 \\
45.62 \\
50.10 \\
53.47 \\
53.29 \\
53.21 \\
50.49 \\
46.14 \\
46.13 \\
44.97 \\
38.98 \\
33.99 \\
33.96 \\
26.83 \\
21.88 \\
21.90 \\
16.69 \\
11.74 \\
11.77 \\
5.05 \\
0.02 \\
-0.04 \\
-0.02 \\
-0.02 \\
0.02\end{array}$ & $\begin{array}{c}\text { Temperature } \\
\text { (Fahrenheit) } \\
101.7400 \\
102.6000 \\
102.6400 \\
103.4600 \\
92.3140 \\
101.6600 \\
100.7900 \\
101.7000 \\
102.1300 \\
90.8350 \\
91.4440 \\
103.1600 \\
91.5310 \\
91.3140 \\
91.6180 \\
91.4440 \\
102.3000 \\
101.0500 \\
100.6200 \\
101.2700 \\
102.1700 \\
102.2100 \\
104.3600 \\
105.1300 \\
104.4500 \\
104.1100 \\
104.2800 \\
104.6200 \\
104.4100 \\
104.7900 \\
105.1800 \\
104.8800 \\
105.4800 \\
104.3200 \\
86.7330 \\
83.1400 \\
83.4470 \\
83.6660\end{array}$ & $\begin{array}{c}\text { Pressure } \\
(\text { psig) } \\
9.89 \\
19.55 \\
29.57 \\
39.42 \\
49.16 \\
54.50 \\
59.57 \\
64.20 \\
69.32 \\
74.16 \\
80.16 \\
85.61 \\
90.58 \\
95.69 \\
100.92 \\
103.25 \\
106.11 \\
108.31 \\
111.08 \\
113.24 \\
117.83 \\
120.32 \\
123.28 \\
122.97 \\
125.82 \\
125.60 \\
127.84 \\
130.19 \\
132.53 \\
135.33 \\
134.42 \\
138.35 \\
140.16 \\
142.63 \\
145.78 \\
142.52 \\
0.22\end{array}$ & $\begin{array}{c}\text { Temperature } \\
\text { (Fahrenheit) } \\
78.6060 \\
78.6940 \\
78.6500 \\
78.6940 \\
78.6940 \\
78.6060 \\
78.6500 \\
78.6060 \\
78.6940 \\
78.6060 \\
78.3410 \\
78.2970 \\
78.2970 \\
78.2530 \\
78.2090 \\
78.2090 \\
78.0320 \\
78.0760 \\
78.0320 \\
77.9880 \\
78.0320 \\
77.9880 \\
77.8560 \\
77.6790 \\
77.6790 \\
77.5460 \\
77.4140 \\
77.5910 \\
77.5460 \\
77.5460 \\
77.5460 \\
77.1490 \\
77.3260 \\
77.5460 \\
77.7230 \\
77.7670 \\
77.9880\end{array}$ \\
\hline
\end{tabular}

High Pressure test

78.6060

78.6940

78.6500

78.6940

78.6500

78.6060

78.6940

78.6060

78.2970

78.2530

78.2090

78.0760

78.0320

77.9880

78.0320

77.9880

77.6790

77.6790

77.5460

77.4140

7.5910

7.5460

77.5460

77.1490

77.3260

7.5460

77.7670

77.9880 emperature 


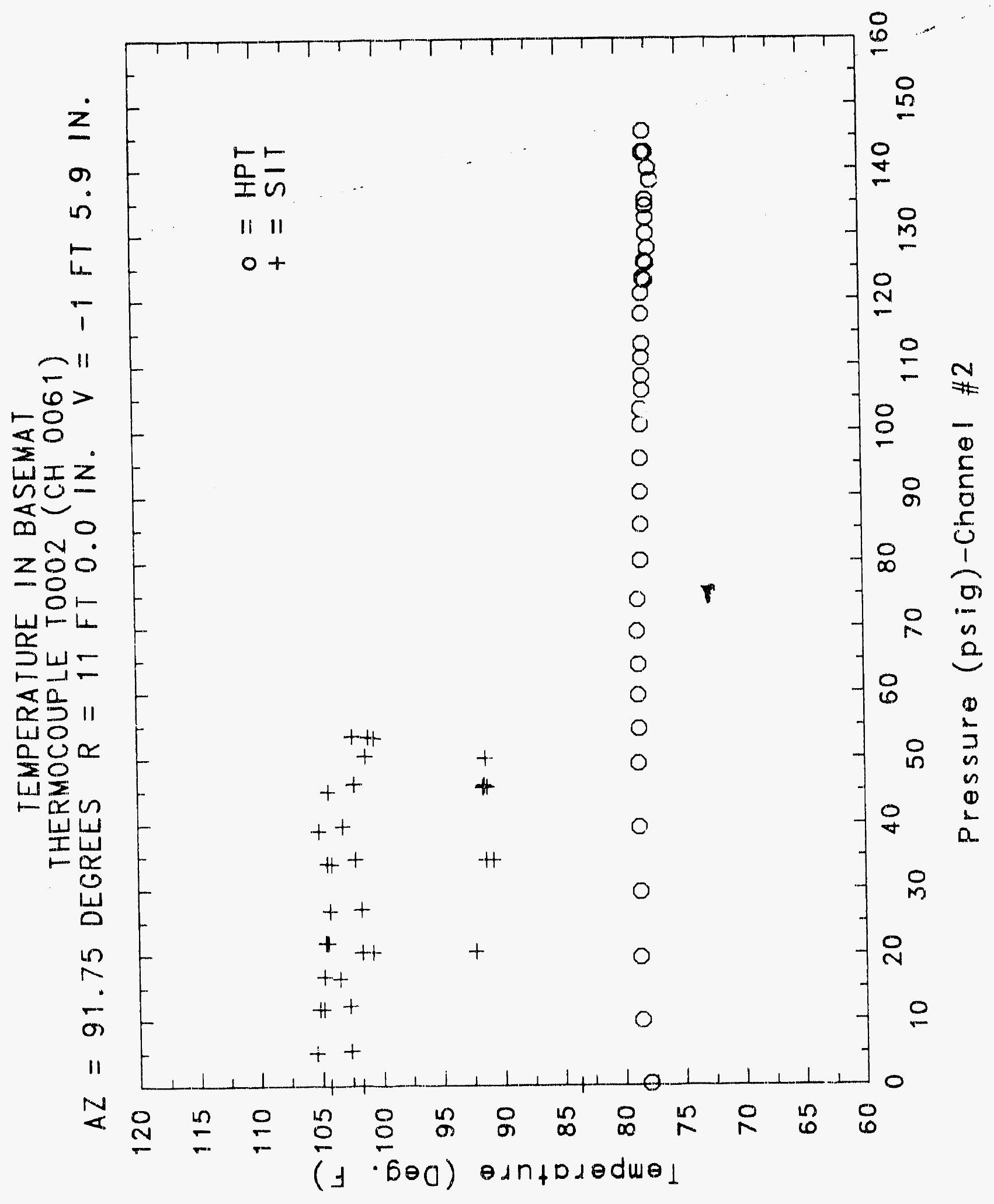

C- 1671 


\section{Reinforced Concrete Test Data}

Thermocouple Channel 62

Structural INTEgrity TEST

\begin{tabular}{|c|c|c|c|}
\hline $\begin{array}{c}\text { Pressure } \\
\text { (psig) } \\
-0.05 \\
5.33 \\
12.31 \\
16.44 \\
20.51 \\
20.46 \\
20.47 \\
27.05 \\
34.62 \\
34.53 \\
34.53 \\
39.70 \\
45.90 \\
45.65 \\
45.62 \\
50.10 \\
53.47 \\
53.29 \\
53.21 \\
50.49 \\
46.14 \\
46.13 \\
44.97 \\
38.98 \\
33.99 \\
33.96 \\
26.83 \\
21.88 \\
21.90 \\
16.69 \\
11.74 \\
11.77 \\
5.05 \\
0.02 \\
-0.04 \\
-0.02 \\
-0.02 \\
0.02\end{array}$ & $\begin{array}{c}\text { Temperature } \\
\text { (Fahrenheit) } \\
79.0470 \\
78.8270 \\
78.6500 \\
78.5620 \\
78.3410 \\
78.5620 \\
78.7380 \\
79.0910 \\
79.4000 \\
79.7970 \\
80.0610 \\
80.7660 \\
81.0300 \\
81.6900 \\
81.9970 \\
82.3930 \\
82.9640 \\
83.2270 \\
83.3590 \\
83.4470 \\
83.4470 \\
83.5790 \\
80.9860 \\
80.9420 \\
80.9420 \\
81.0740 \\
81.1620 \\
81.5140 \\
81.9970 \\
82.3490 \\
82.9640 \\
83.7100 \\
84.1490 \\
84.6310 \\
82.7440 \\
81.9090 \\
81.8460 \\
81.2940\end{array}$ & $\begin{array}{c}\text { Pressure } \\
(\text { psig) } \\
9.89 \\
19.55 \\
29.57 \\
39.42 \\
49.16 \\
54.50 \\
59.57 \\
64.20 \\
69.32 \\
74.16 \\
80.16 \\
85.61 \\
90.58 \\
95.69 \\
100.92 \\
103.25 \\
106.11 \\
108.31 \\
111.08 \\
113.24 \\
117.83 \\
120.92 \\
123.28 \\
122.97 \\
125.82 \\
125.60 \\
127.84 \\
130.19 \\
132.53 \\
135.33 \\
134.42 \\
138.35 \\
140.16 \\
142.63 \\
145.78 \\
142.52 \\
0.22\end{array}$ & $\begin{array}{c}\text { Temperature } \\
\text { (Fahrenheit) } \\
79.1790 \\
79.1790 \\
79.1350 \\
79.0910 \\
79.0910 \\
79.1790 \\
79.2240 \\
79.4000 \\
79.4440 \\
79.6640 \\
81.2940 \\
81.4260 \\
81.2940 \\
81.2500 \\
81.0300 \\
80.6780 \\
80.5010 \\
80.4130 \\
80.1050 \\
80.0170 \\
79.8410 \\
79.6200 \\
79.2240 \\
79.0030 \\
78.7380 \\
78.4740 \\
78.3850 \\
78.2530 \\
78.2090 \\
78.2090 \\
78.2530 \\
78.4300 \\
78.7830 \\
79.3120 \\
79.8850 \\
80.6340 \\
81.3820\end{array}$ \\
\hline
\end{tabular}

High Pressure test

Pressure

Fahrenhe it

79.1790

79.1790

79.0910

79.1790

79.2240

79.4000

81.2940

81.4260

81.2940

80.1050

80.0170

.

78.4740

78.2090

78.2090

78.2530

78.4300

78.7830

9.3120

2.52 


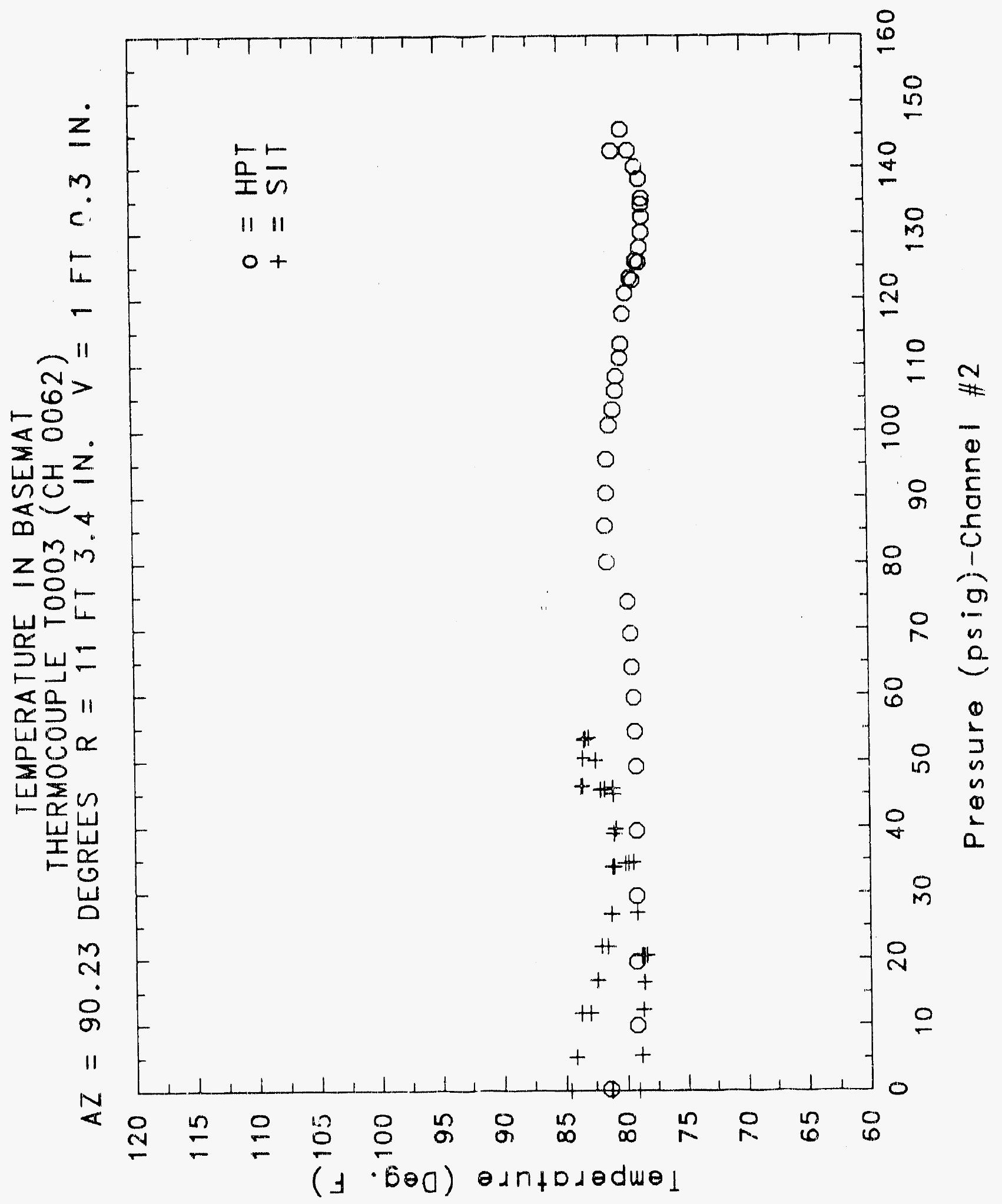




\section{Reinforced Concrete Test Data}

Thermocouple Chan..el 63

STRUCTURAL INTEGRITY TEST

\begin{tabular}{|c|c|c|c|}
\hline $\begin{array}{l}\text { Pressure } \\
\text { (psig) } \\
-0.05 \\
5.33 \\
12.31 \\
16.44 \\
20.51 \\
20.46 \\
20.47 \\
27.05 \\
34.69 \\
34.53 \\
34.53 \\
39.70 \\
45.90 \\
45.65 \\
45.62 \\
50.10 \\
53.47 \\
53.29 \\
53.21 \\
50.49 \\
46.14 \\
46.13 \\
44.97 \\
38.98 \\
33.99 \\
33.96 \\
26.83 \\
21.88 \\
21.90 \\
16.69 \\
11.74 \\
11.77 \\
5.05 \\
0.02 \\
-0.04 \\
-0.02 \\
-0.02 \\
0.02\end{array}$ & $\begin{array}{c}\text { Temperature } \\
\text { (Fahrenheit) } \\
77.9440 \\
77.8560 \\
77.8560 \\
77.8110 \\
77.7670 \\
77.8560 \\
77.9440 \\
77.9880 \\
78.0320 \\
77.9000 \\
77.9880 \\
78.1210 \\
78.0760 \\
78.1210 \\
78.1650 \\
78.1650 \\
78.3410 \\
78.4300 \\
78.4740 \\
78.4740 \\
78.5180 \\
78.7380 \\
79.5760 \\
79.5760 \\
79.6200 \\
79.6200 \\
79.7090 \\
79.6640 \\
79.6200 \\
79.6200 \\
79.7090 \\
79.6640 \\
79.7530 \\
79.7530 \\
80.1050 \\
80.1050 \\
80.1490 \\
80.0610\end{array}$ & $\begin{array}{c}\text { Pressure } \\
(\text { psig) } \\
9.89 \\
19.55 \\
29.57 \\
39.42 \\
49.16 \\
54.50 \\
59.57 \\
64.20 \\
69.32 \\
74.16 \\
80.16 \\
85.61 \\
90.58 \\
95.69 \\
100.92 \\
103.25 \\
106.11 \\
108.31 \\
111.08 \\
113.24 \\
117.83 \\
120.92 \\
123.28 \\
122.97 \\
125.82 \\
125.60 \\
127.84 \\
130.19 \\
132.53 \\
135.33 \\
134.42 \\
138.35 \\
140.16 \\
142.63 \\
145.78 \\
142.52 \\
0.22\end{array}$ & $\begin{array}{c}\text { Temperature } \\
\text { (Fahrenheit) } \\
78.8710 \\
78.8710 \\
78.7830 \\
78.7830 \\
78.6940 \\
78.7380 \\
78.6940 \\
78.7380 \\
78.7380 \\
78.8270 \\
79.0910 \\
79.2240 \\
79.2680 \\
79.4440 \\
79.5320 \\
79.6640 \\
79.7090 \\
79.7530 \\
79.7970 \\
79.8410 \\
79.8850 \\
79.9290 \\
79.9730 \\
79.9730 \\
80.0170 \\
79.9290 \\
79.8410 \\
80.1490 \\
80.1050 \\
80.1050 \\
80.1050 \\
80.1050 \\
80.1490 \\
80.0610 \\
80.0170 \\
79.9730 \\
80.0170\end{array}$ \\
\hline
\end{tabular}

High Pressure test

Temperature

78.8710

78.7830

78.7830

78.6940

78.7380

78.7380

79.2240

79.2680

79.6640

79.7090

79.7530

79.7970

79.9290

79.9730

79.9730

80.0170

80.1490

80.1050

80.1050

80.1050

80.1050

80.1490

80.0170

79.9730

80.0170 


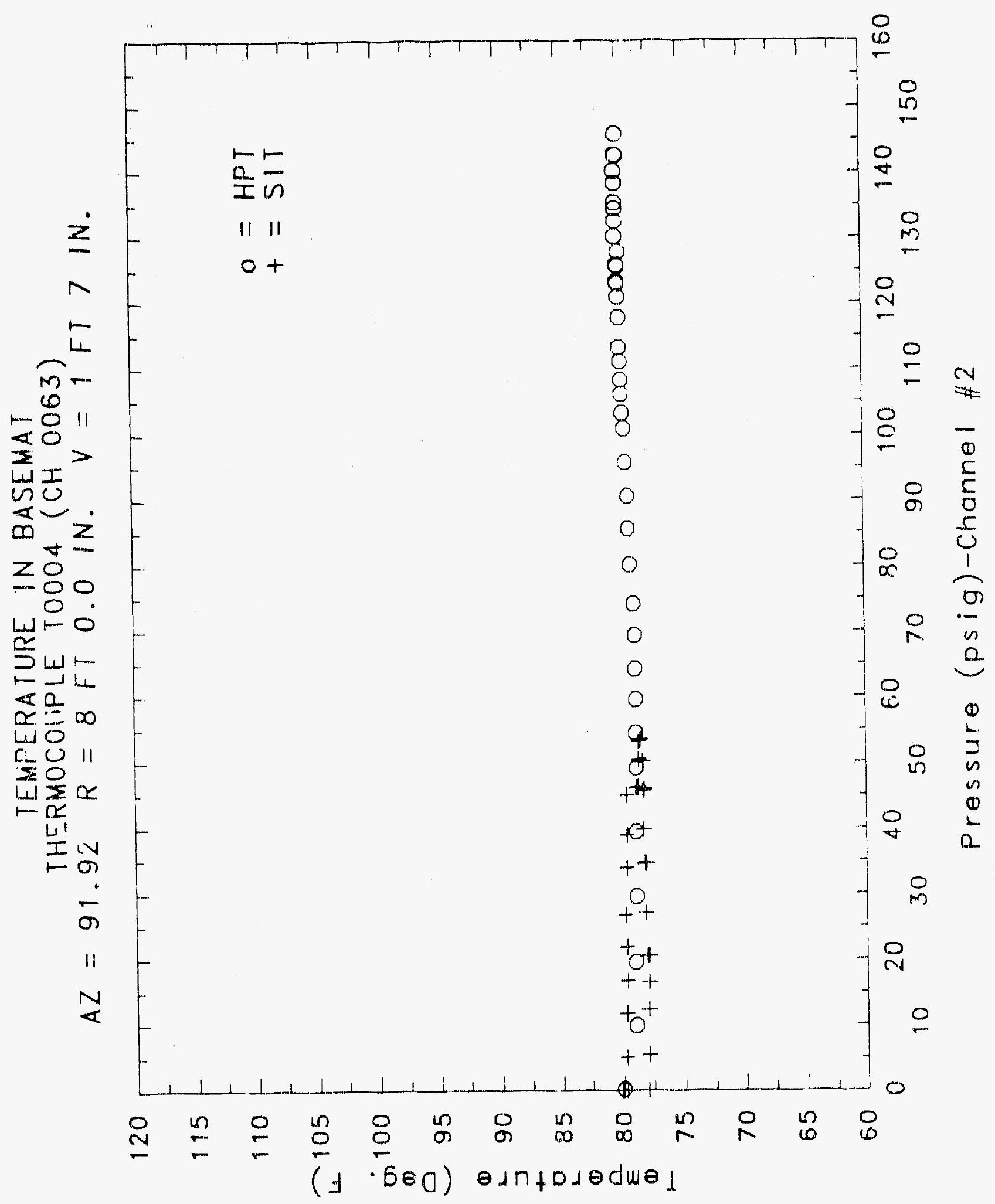




\section{Reinforced Concrete Test Data}

\begin{tabular}{|c|c|c|c|}
\hline \multirow[b]{2}{*}{ STRUCTURAL } & \multicolumn{3}{|c|}{ ChANNE: 64} \\
\hline & INTEGRITY TEST & $H I G H \quad P$ & RE TEST \\
\hline $\begin{array}{l}\text { Pressure } \\
\text { (psig) } \\
-0.05 \\
5.33 \\
12.31 \\
16.44 \\
20.51 \\
20.46 \\
20.47 \\
27.05 \\
34.69 \\
34.53 \\
34.53 \\
39.70 \\
45.90 \\
45.65 \\
45.62 \\
50.10 \\
53.47 \\
53.29 \\
53.21 \\
50.49 \\
46.14 \\
46.13 \\
44.97 \\
38.98 \\
33.99 \\
33.96 \\
66.83 \\
21.88 \\
21.90 \\
16.69 \\
11.74 \\
11.77 \\
5.05 \\
0.02 \\
-0.04 \\
-0.02 \\
-0.02 \\
0.02\end{array}$ & $\begin{array}{c}\text { Temperature } \\
\text { (Fahrenheit) } \\
78.8270 \\
78.9590 \\
79.1790 \\
79.3120 \\
79.5320 \\
80.1490 \\
80.7220 \\
81.5140 \\
82.1730 \\
83.0520 \\
83.4030 \\
83.9740 \\
84.2370 \\
84.6310 \\
84.7190 \\
84.7630 \\
85.0700 \\
85.0260 \\
84.9380 \\
84.8500 \\
84.8070 \\
84.5870 \\
81.7340 \\
81.9090 \\
82.0410 \\
83.6570 \\
83.1840 \\
83.9740 \\
85.2890 \\
85.8580 \\
86.4270 \\
86.8640 \\
87.0390 \\
87.1260 \\
82.5690 \\
82.0410 \\
81.8650 \\
81.9970\end{array}$ & $\begin{array}{c}\text { Pressure } \\
\text { (psig) } \\
9.89 \\
19.55 \\
29.57 \\
39.42 \\
49.16 \\
54.50 \\
59.57 \\
64.20 \\
69.32 \\
74.16 \\
80.16 \\
85.61 \\
90.59 \\
95.69 \\
100.92 \\
103.25 \\
106.11 \\
108.31 \\
111.08 \\
113.24 \\
117.83 \\
120.92 \\
123.28 \\
122.97 \\
125.82 \\
125.50 \\
167.84 \\
130.19 \\
132.53 \\
135.33 \\
134.42 \\
138.35 \\
140.16 \\
142.63 \\
115.78 \\
142.52 \\
0.22 \\
\end{array}$ & $\begin{array}{c}\text { Temparature } \\
\text { (Fahrenheit) } \\
79.4440 \\
79.5750 \\
79.7090 \\
79.8850 \\
80.1050 \\
80.2810 \\
80.5150 \\
80.8980 \\
81.2500 \\
81.8220 \\
82.8320 \\
82.5690 \\
82.4370 \\
82.1290 \\
81.8220 \\
81.3820 \\
81.1180 \\
80.9860 \\
80.7650 \\
80.5900 \\
80.3690 \\
80.1930 \\
79.7530 \\
79.5760 \\
79.4000 \\
79.2680 \\
79.2240 \\
79.2240 \\
79.3120 \\
79.5200 \\
79.9290 \\
80.5340 \\
81.4260 \\
82.3490 \\
83.0030 \\
83.4030 \\
83.5350\end{array}$ \\
\hline
\end{tabular}




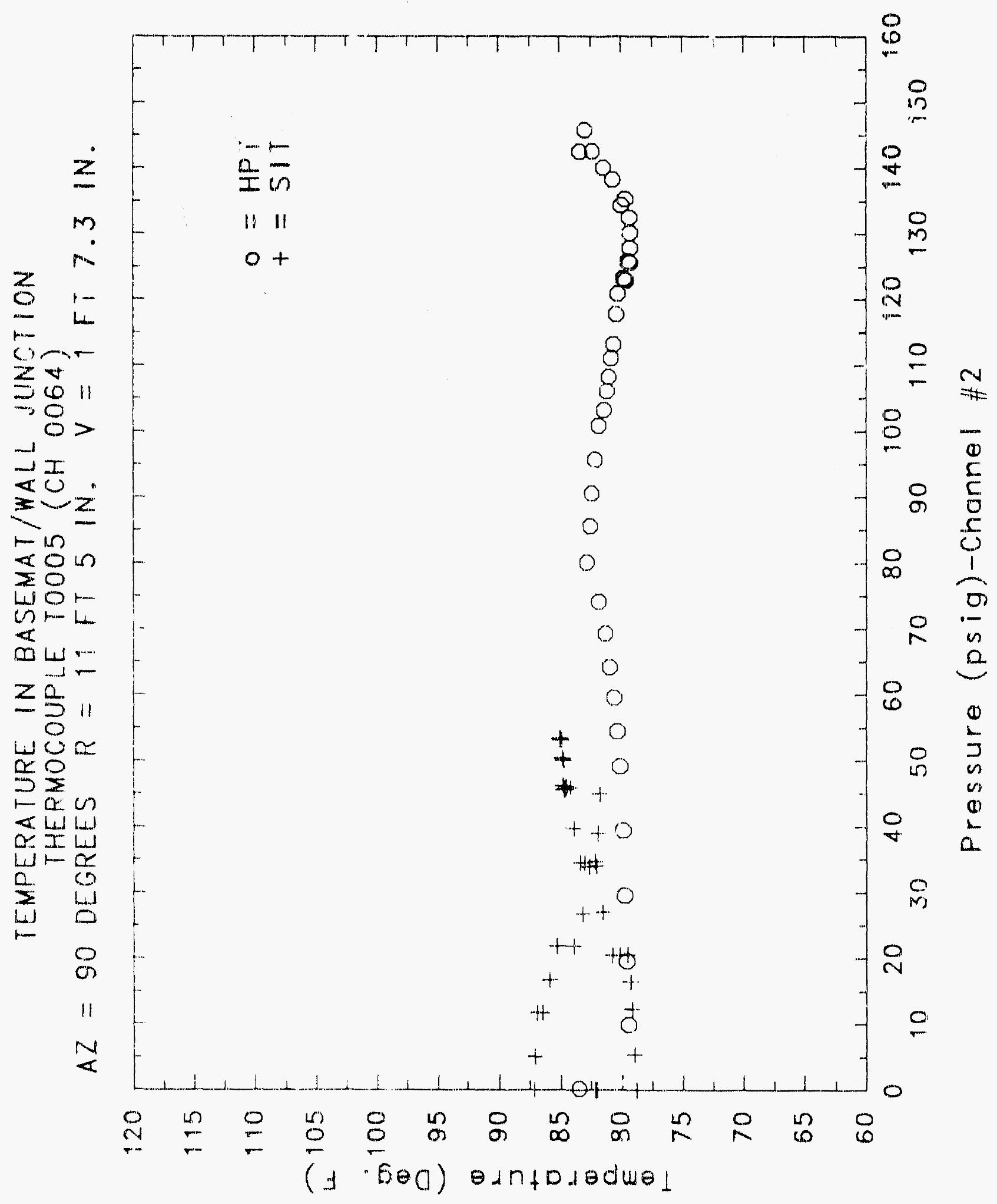




\section{Reinforced Concrete test Data}

\section{Thermocouple Channel 65}

Structural INTEGRITY TEST

\begin{tabular}{|c|c|c|c|}
\hline $\begin{array}{l}\text { Pressure } \\
\text { (psig) } \\
-0.05 \\
5.33 \\
12.31 \\
16.44 \\
20.51 \\
20.46 \\
20.47 \\
27.05 \\
34.69 \\
34.53 \\
34.53 \\
39.70 \\
45.90 \\
45.65 \\
45.62 \\
50.10 \\
53.47 \\
53.29 \\
53.21 \\
50.49 \\
46.14 \\
46.13 \\
44.97 \\
38.98 \\
33.99 \\
33.96 \\
26.83 \\
21.88 \\
21.90 \\
16.69 \\
11.74 \\
11.77 \\
5.05 \\
0.02 \\
.0 .04 \\
-0.02 \\
-0.02 \\
0.02\end{array}$ & $\begin{array}{c}\text { Temperature } \\
\text { (Fahrenheit; } \\
78.9590 \\
79.2680 \\
79.7530 \\
80.1050 \\
80.6340 \\
81.5580 \\
82.4370 \\
83.9300 \\
85.1130 \\
86.2080 \\
86.7330 \\
87.3450 \\
87.4760 \\
87.5640 \\
87.4760 \\
87.2580 \\
87.2140 \\
86.9080 \\
86.6890 \\
86.3830 \\
86.0770 \\
85.6390 \\
82.3050 \\
82.7000 \\
83.0080 \\
84.1930 \\
85.1570 \\
86.4700 \\
88.6990 \\
89.4840 \\
90.0940 \\
90.2690 \\
90.1380 \\
89.7890 \\
82.1290 \\
81.8650 \\
81.8220 \\
82.3930\end{array}$ & $\begin{array}{c}\text { Pressure } \\
(p s i g) \\
9.89 \\
19.55 \\
29.57 \\
39.42 \\
49.16 \\
54.50 \\
59.57 \\
64.20 \\
69.32 \\
74.16 \\
80.16 \\
85.61 \\
90.58 \\
95.69 \\
100.92 \\
103.25 \\
106.11 \\
108.31 \\
111.08 \\
113.24 \\
117.83 \\
120.92 \\
123.28 \\
122.97 \\
125.82 \\
125.60 \\
127.84 \\
130.19 \\
132.53 \\
135.33 \\
134.42 \\
138.35 \\
140.16 \\
142.63 \\
145.78 \\
142.52 \\
10.22\end{array}$ & $\begin{array}{c}\text { Temperature } \\
\text { (Fahrenheit) } \\
79.7090 \\
80.0610 \\
80.3250 \\
80.7660 \\
81.2500 \\
81.6460 \\
81.9530 \\
82.5250 \\
83.0520 \\
83.9300 \\
84.3680 \\
83.8860 \\
83.4030 \\
82.7440 \\
82.3050 \\
81.6460 \\
81.3380 \\
81.1620 \\
80.8100 \\
80.6780 \\
80.3250 \\
80.1430 \\
79.6200 \\
79.4440 \\
79.3120 \\
79.3120 \\
79.4000 \\
79.5760 \\
79.7090 \\
80.3250 \\
80.8980 \\
82.1290 \\
83.5790 \\
84.8070 \\
85.6830 \\
86.0330 \\
84.7030\end{array}$ \\
\hline
\end{tabular}

High Pressure test

emperature

79.7090

80.0610

80.3250

81.6460

81.9530

82.5250

83.0520

84.3680

83.8860

83.4030

81.6460

81.3380

81.1620

80.1490

79.6200

79.4440

79.4000

80

83.5790

84.8070

5.6830

.0330 


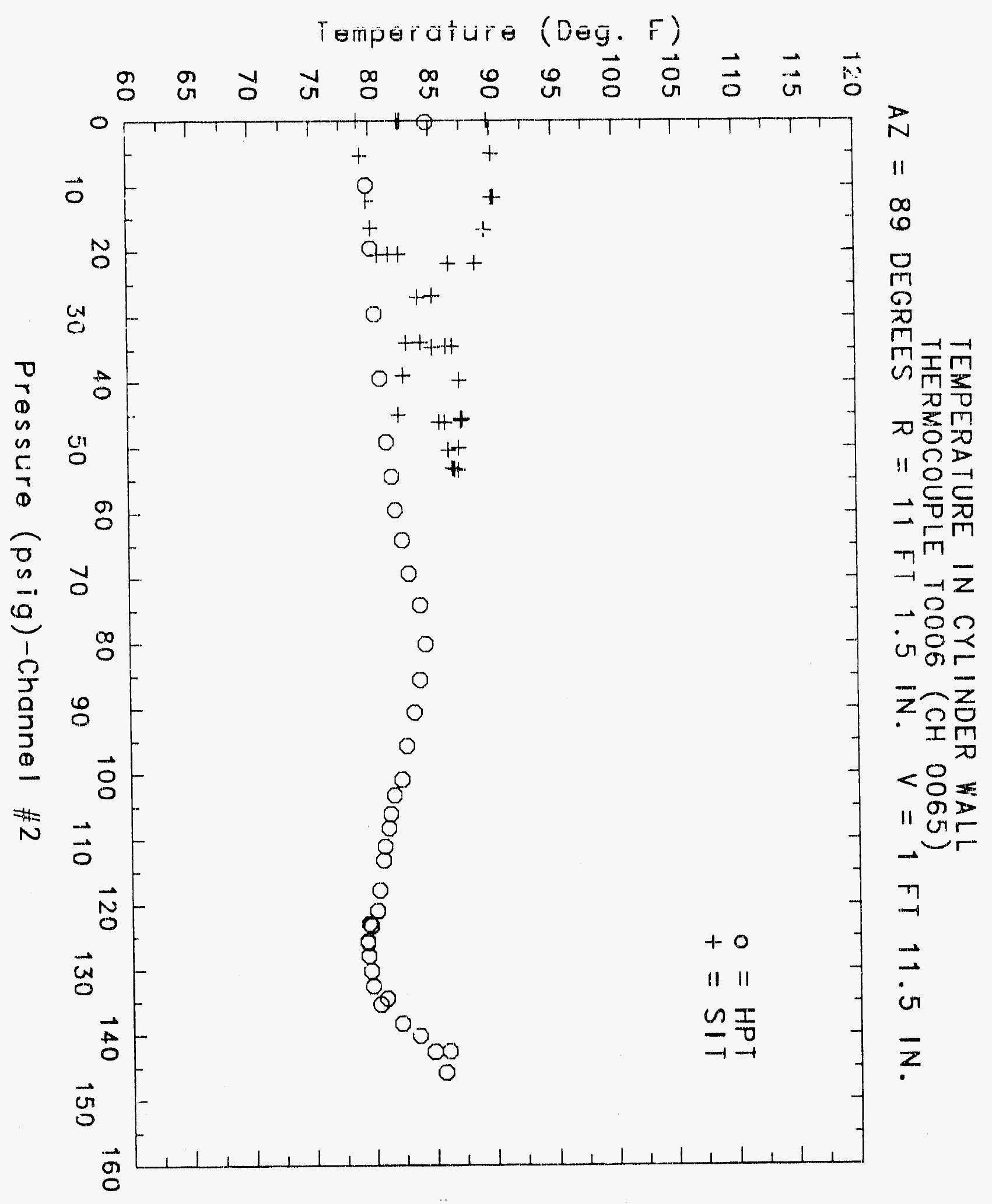




\section{Reinforced Concrete Test Data}

Thermocouple Chanmel 66

Structural Integrity Test

\begin{tabular}{|c|c|c|c|}
\hline $\begin{array}{c}\text { Pressure } \\
\text { (psig) } \\
-0.05 \\
5.33 \\
12.31 \\
16.44 \\
20.51 \\
20.46 \\
20.47 \\
27.05 \\
34.69 \\
34.53 \\
34.53 \\
39.70 \\
45.90 \\
45.65 \\
45.62 \\
50.10 \\
53.47 \\
53.29 \\
53.21 \\
50.49 \\
46.14 \\
46.13 \\
44.97 \\
38.98 \\
33.99 \\
33.96 \\
26.83 \\
21.88 \\
21.40 \\
16.69 \\
11.74 \\
11.77 \\
5.05 \\
0.02 \\
-0.04 \\
-0.02 \\
-0.02 \\
0.02\end{array}$ & $\begin{array}{c}\text { Temperature } \\
\text { (Fahrenheit) } \\
81.0300 \\
81.2500 \\
81.6900 \\
82.0410 \\
82.5690 \\
83.1400 \\
83.7540 \\
85.2450 \\
86.7330 \\
87.9570 \\
88.4370 \\
89.4400 \\
90.0940 \\
90.3560 \\
90.2250 \\
90.2250 \\
90.0940 \\
89.7460 \\
89.3970 \\
88.7430 \\
88.2190 \\
87.6950 \\
83.6230 \\
84.2810 \\
84.7190 \\
85.4200 \\
86.3390 \\
87.2580 \\
89.1790 \\
90.3990 \\
91.3140 \\
92.0530 \\
92.0530 \\
91.6180 \\
82.4370 \\
81.8650 \\
81.8220 \\
83.0080\end{array}$ & $\begin{array}{c}\text { Pressure } \\
\text { (psig) } \\
9.89 \\
19.55 \\
29.57 \\
39.42 \\
49.16 \\
54.50 \\
59.57 \\
64.20 \\
69.32 \\
74.16 \\
80.16 \\
85.61 \\
90.58 \\
95.69 \\
100.92 \\
103.25 \\
106.11 \\
108.31 \\
111.08 \\
113.24 \\
117.83 \\
120.92 \\
123.28 \\
122.97 \\
125.82 \\
125.60 \\
127.84 \\
130.19 \\
132.53 \\
105.33 \\
134.42 \\
138.35 \\
140.16 \\
142.63 \\
145.78 \\
142.52 \\
0.22\end{array}$ & $\begin{array}{c}\text { Temperature } \\
\text { (Fahrenheit) } \\
81.0300 \\
81.6900 \\
82.3930 \\
83.1400 \\
83.7540 \\
84.1930 \\
84.5870 \\
85.0260 \\
85.5080 \\
86.2080 \\
87.0390 \\
86.5140 \\
85.9890 \\
85.2450 \\
84.5440 \\
83.7100 \\
83.3150 \\
83.2270 \\
82.7440 \\
82.5690 \\
82.3050 \\
81.9970 \\
81.3380 \\
81.0300 \\
80.9420 \\
80.6780 \\
80.8540 \\
81.1620 \\
81.5140 \\
82.1290 \\
82.5690 \\
83.7100 \\
84.9820 \\
86.3830 \\
87.3450 \\
87.6950 \\
80.3690\end{array}$ \\
\hline
\end{tabular}

High Pressure test

emperature

81.0300

81.6900

82.3930

3.7540

84.1930

84.5870

35.0260

85.5080

85.9890

85.2450

83.7100

83.3150

83.2270

82.7440

82.3050

81.9970

81.3380

80.6780

80.8540

81.1620

82.5690

83.7100

84.9820

86.3830

30.3690 


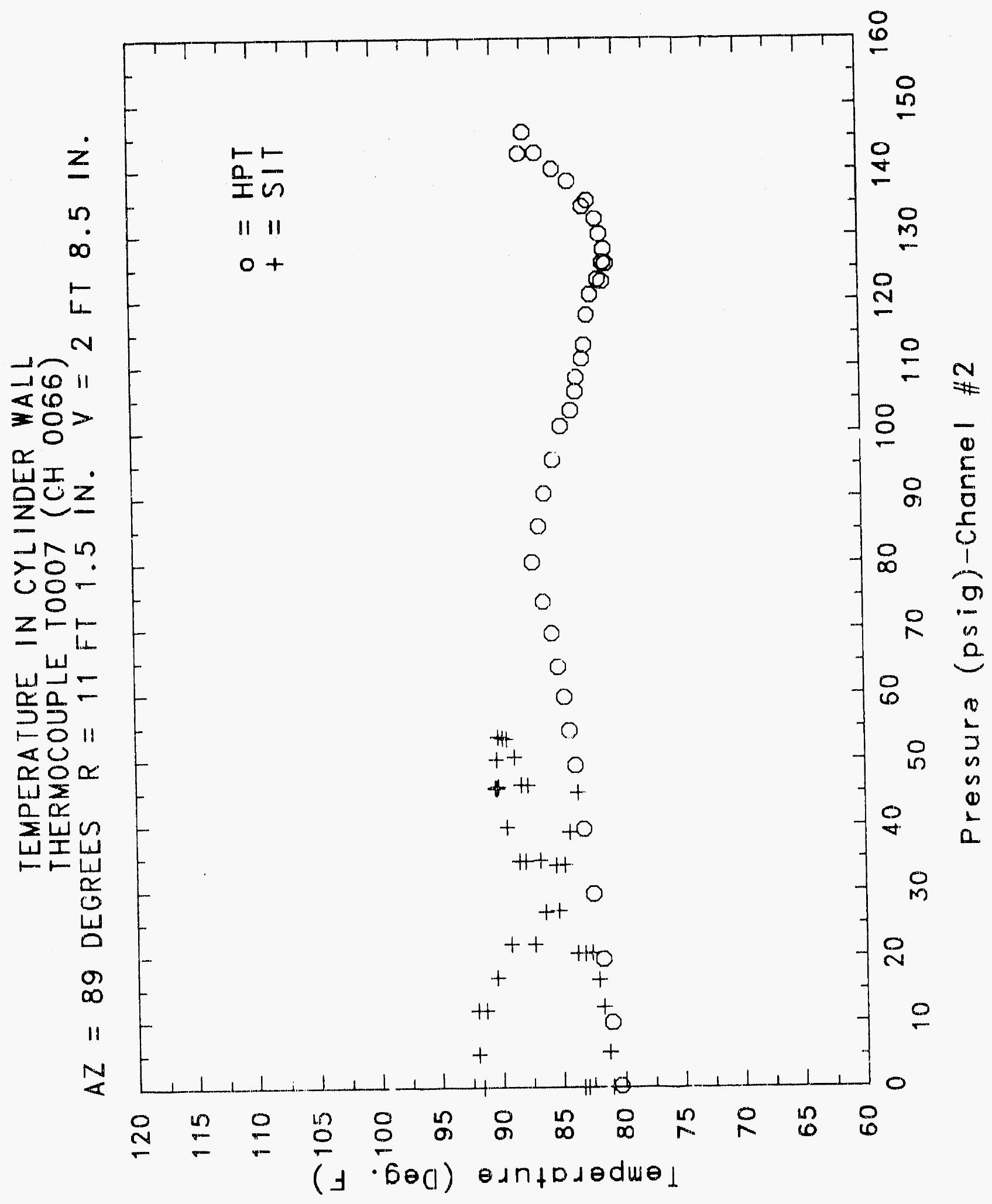

C-168: 


\section{Reinforced Concrete Test Data}

Thermocouple Channel 67

Structural INTEgRity TEST

\begin{tabular}{|c|c|c|c|}
\hline $\begin{array}{l}\text { Pressure } \\
\text { (psig) } \\
-0.05 \\
5.33 \\
12.31 \\
16.44 \\
20.51 \\
20.46 \\
20.47 \\
27.05 \\
34.69 \\
34.53 \\
34.53 \\
39.70 \\
45.90 \\
45.65 \\
45.62 \\
50.10 \\
53.47 \\
53.29 \\
53.21 \\
50.49 \\
46.14 \\
46.13 \\
44.97 \\
38.98 \\
33.99 \\
33.96 \\
20.83 \\
21.88 \\
21.90 \\
16.69 \\
11.74 \\
11.77 \\
5.05 \\
0.02 \\
-0.04 \\
-0.02 \\
-0.02 \\
0.02\end{array}$ & $\begin{array}{c}\text { Temperature } \\
\text { (Fahrenhe it) } \\
80.8540 \\
81.2500 \\
81.8650 \\
82.3050 \\
82.9640 \\
83.7980 \\
84.7630 \\
86.0330 \\
87.6510 \\
89.0040 \\
89.5710 \\
90.4430 \\
91.0960 \\
91.4440 \\
91.3570 \\
91.3140 \\
91.2270 \\
90.8350 \\
90.4430 \\
89.8760 \\
89.3100 \\
88.6550 \\
83.8860 \\
84.5870 \\
85.2450 \\
86.0770 \\
87.0390 \\
88.1310 \\
90.1380 \\
91.2700 \\
92.1400 \\
92.9220 \\
93.0530 \\
92.6620 \\
83.0960 \\
82.3930 \\
82.3490 \\
83.2710\end{array}$ & $\begin{array}{c}\text { Pressure } \\
\text { (psig) } \\
9.89 \\
19.55 \\
29.57 \\
39.42 \\
49.16 \\
54.50 \\
59.57 \\
64.20 \\
69.32 \\
74.16 \\
80.16 \\
85.61 \\
90.58 \\
95.69 \\
100.92 \\
103.25 \\
106.11 \\
108.31 \\
111.08 \\
113.24 \\
117.83 \\
120.92 \\
123.28 \\
122.91 \\
125.82 \\
125.60 \\
127.84 \\
130.19 \\
132.53 \\
135.33 \\
134.42 \\
138.35 \\
140.16 \\
142.63 \\
145.78 \\
142.52 \\
0.22\end{array}$ & $\begin{array}{c}\text { Temperature } \\
\text { (Fahrenheit) } \\
80.8100 \\
81.4260 \\
82.1290 \\
82.9640 \\
83.6230 \\
84.1490 \\
84.5870 \\
84.9820 \\
85.6390 \\
86.5140 \\
87.3890 \\
86.5200 \\
86.2960 \\
85.4640 \\
84.8500 \\
84.0170 \\
83.5350 \\
83.4470 \\
82.9640 \\
82.7440 \\
82.5250 \\
82.1730 \\
81.4700 \\
81.2500 \\
81.0740 \\
80.8540 \\
80.9420 \\
81.2060 \\
81.4700 \\
82.1730 \\
82.7880 \\
84.0170 \\
85.4200 \\
86.6890 \\
87.7380 \\
88.3050 \\
82.6570\end{array}$ \\
\hline
\end{tabular}

High Pressure test

emperature

80.8100

81.4260

84.1490

84.5870

85.5140

87.3890

86.5200

84.0170

83.5350

82.5250

82.1730

81.0740

80.8540

82.1730

82.7880

84.0170

4200

8.3060

82.3490

83.2710 


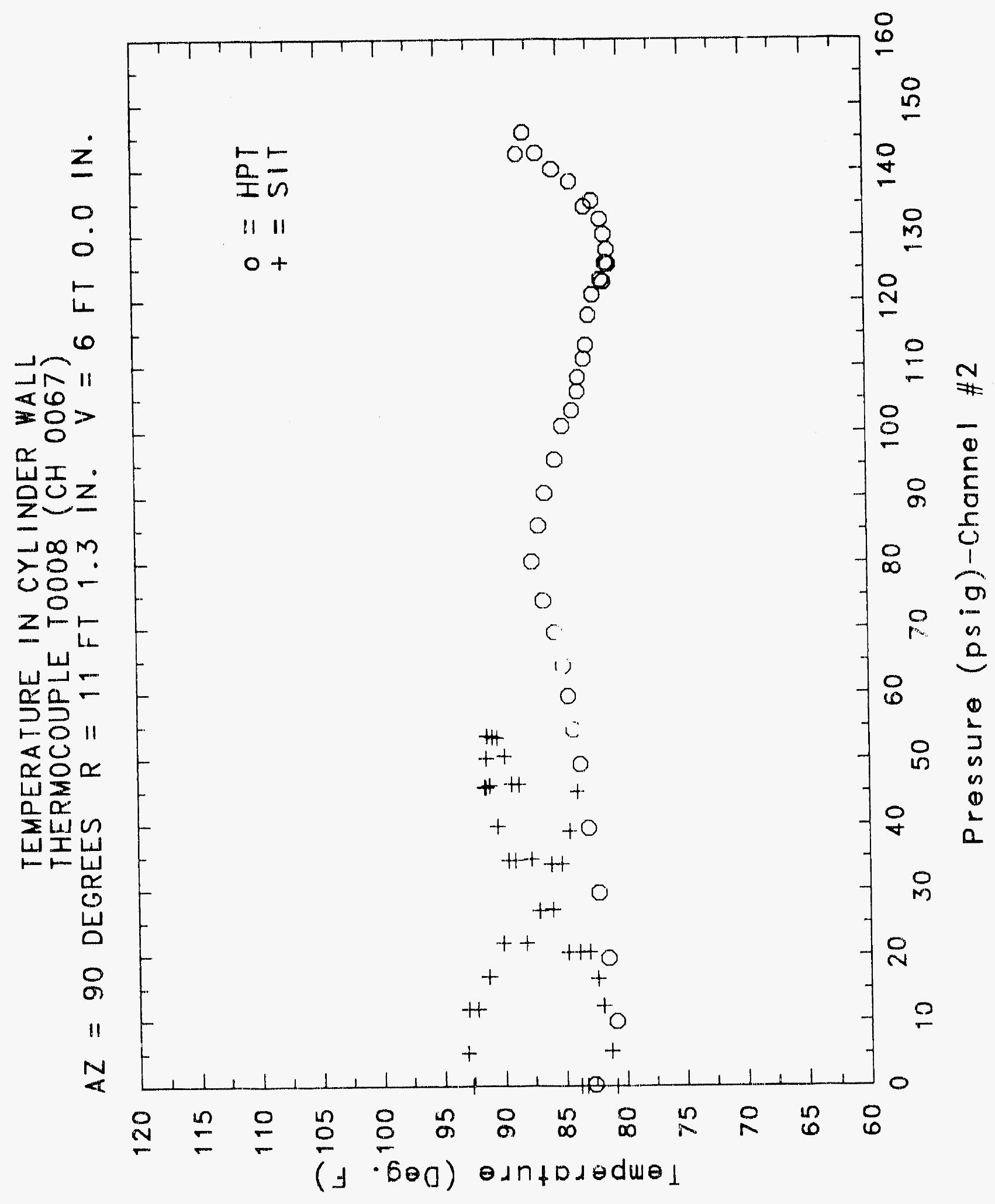

C. 1683 


\section{Reinforced Concrete Test Data}

Thermocouple Channel 68

\section{STRUCTURAL INTEGRITY TEST}

\begin{tabular}{|c|c|c|c|}
\hline $\begin{array}{c}\text { Pressure } \\
\text { (psig) } \\
-0.05 \\
5.33 \\
12.31 \\
16.44 \\
20.51 \\
20.46 \\
20.47 \\
27.05 \\
34.69 \\
34.53 \\
34.53 \\
39.70 \\
45.90 \\
45.05 \\
45.62 \\
50.10 \\
53.47 \\
53.29 \\
53.21 \\
50.49 \\
46.14 \\
46.13 \\
44.97 \\
38.98 \\
33.99 \\
33.96 \\
26.83 \\
21.88 \\
21.90 \\
16.69 \\
11.74 \\
11.77 \\
5.05 \\
0.02 \\
-0.04 \\
-0.02 \\
-0.02 \\
0.02\end{array}$ & $\begin{array}{c}\text { Temperature } \\
\text { (Fahrenheit) } \\
81.0300 \\
81.4260 \\
81.9970 \\
82.3490 \\
82.8320 \\
83.4910 \\
84.0170 \\
85.0700 \\
86.2080 \\
87.3890 \\
88.0000 \\
88.7860 \\
89.4840 \\
90.1380 \\
90.1380 \\
90.2690 \\
90.2690 \\
90.0940 \\
89.8760 \\
89.3970 \\
88.9610 \\
88.4810 \\
84.2370 \\
84.7630 \\
85.1570 \\
85.7700 \\
86.3390 \\
86.8640 \\
88.2620 \\
89.1350 \\
90.0510 \\
90.8780 \\
91.0960 \\
91.0960 \\
83.7100 \\
83.0520 \\
83.0080 \\
83.6230\end{array}$ & $\begin{array}{c}\text { Pressure } \\
(\text { psig) } \\
9.89 \\
19.55 \\
29.57 \\
39.42 \\
49.16 \\
54.50 \\
59.57 \\
64.20 \\
69.32 \\
74.16 \\
80.16 \\
85.61 \\
90.58 \\
95.69 \\
100.92 \\
103.25 \\
106.11 \\
108.31 \\
111.08 \\
113.24 \\
117.83 \\
120.92 \\
123.28 \\
122.97 \\
125.82 \\
125.60 \\
127.84 \\
130.19 \\
132.53 \\
135.33 \\
134.42 \\
138.35 \\
140.16 \\
142.63 \\
145.78 \\
142.52 \\
0.22\end{array}$ & $\begin{array}{c}\text { Temperature } \\
\text { (Fahrenheit) } \\
80.8980 \\
81.3820 \\
82.0410 \\
82.7440 \\
83.2710 \\
83.7980 \\
84.1490 \\
84.5440 \\
84.8940 \\
85.4640 \\
80.5580 \\
86.2520 \\
85.8140 \\
85.2010 \\
84.5870 \\
83.8860 \\
83.4470 \\
83.3150 \\
82.8760 \\
82.7880 \\
82.3930 \\
82.0850 \\
81.3820 \\
81.1180 \\
80.8980 \\
80.7660 \\
80.8100 \\
80.9420 \\
81.0740 \\
81.6900 \\
82.1290 \\
82.7440 \\
83.7540 \\
84.7630 \\
85.8140 \\
86.4270 \\
81.7780\end{array}$ \\
\hline
\end{tabular}

High Pressure Test

Pressure

9.89

19.55

29.57

39.42

59.57

64.20

85.61

100.92

117.83

122.97

130.19

134.42

38.35

145.78

2.52

86.4270

81.7780 


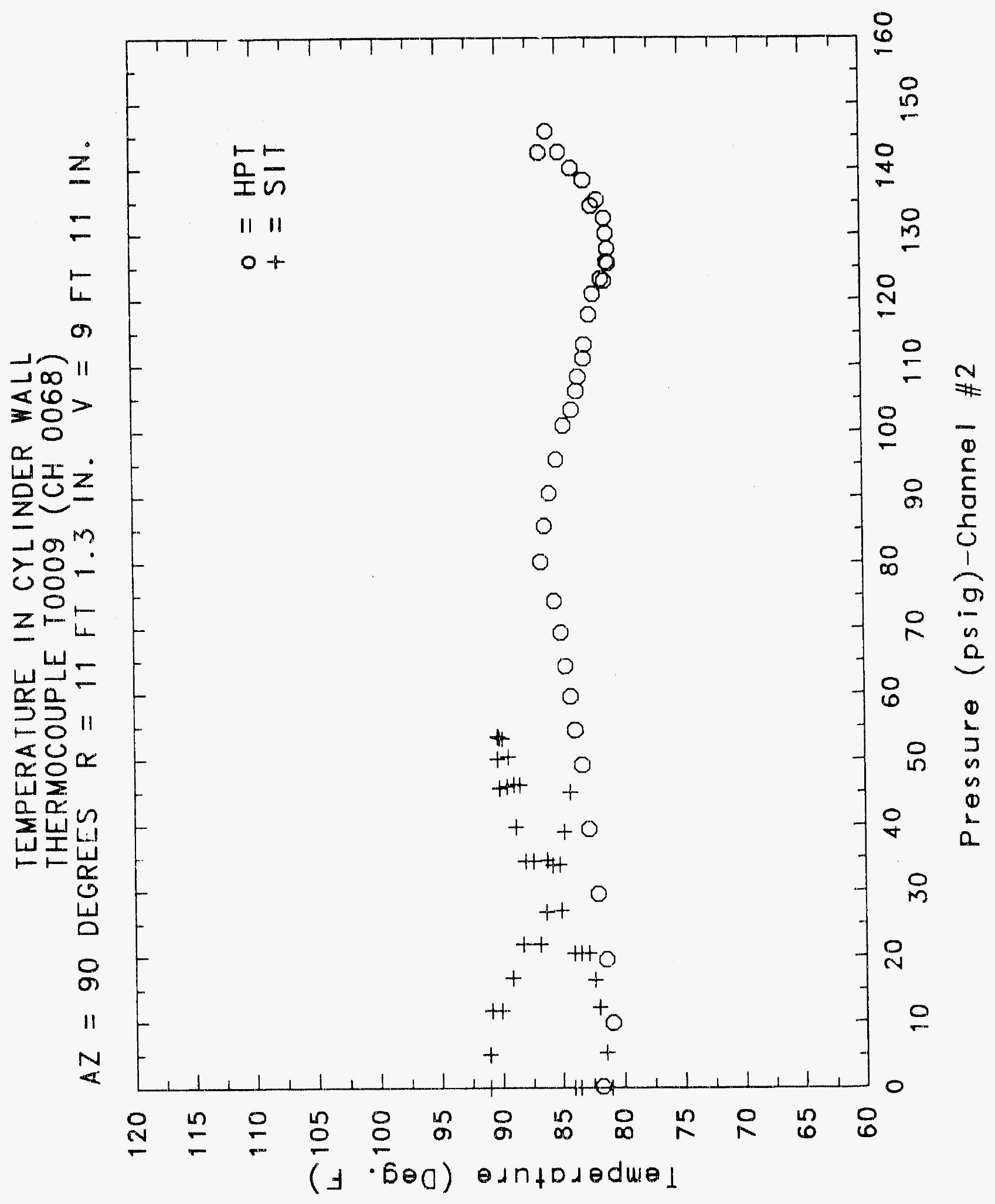




\section{Reinforced Concrete Test Data}

Thermocouple Channel 69

STRUCTURAL INTEGRITY TEST

\begin{tabular}{|c|c|c|c|}
\hline $\begin{array}{c}\text { Pressure } \\
\text { (psig) } \\
-0.05 \\
5.33 \\
12.31 \\
16.44 \\
20.51 \\
20.46 \\
20.47 \\
27.05 \\
34.69 \\
34.53 \\
34.53 \\
39.70 \\
45.90 \\
45.65 \\
45.62 \\
50.10 \\
53.47 \\
53.29 \\
53.21 \\
50.49 \\
46.14 \\
46.13 \\
44.97 \\
38.98 \\
33.99 \\
33.96 \\
26.83 \\
21.88 \\
21.90 \\
16.69 \\
11.74 \\
11.77 \\
5.05 \\
0.02 \\
-0.04 \\
-0.02 \\
-0.02 \\
0.02\end{array}$ & $\begin{array}{c}\text { Temperature } \\
\text { (Fahrenheit) } \\
81.0740 \\
81.4260 \\
81.7780 \\
82.0850 \\
82.3050 \\
82.7000 \\
83.0520 \\
83.4910 \\
84.1930 \\
84.8500 \\
85.1130 \\
85.7700 \\
86.2960 \\
87.0830 \\
87.3890 \\
87.8260 \\
88.3060 \\
88.4810 \\
88.5240 \\
88.5240 \\
88.3500 \\
88.1310 \\
83.7100 \\
84.0610 \\
84.4560 \\
84.9820 \\
85.2890 \\
85.7260 \\
86.2960 \\
86.6020 \\
87.0390 \\
87.6510 \\
87.9570 \\
88.1310 \\
84.1050 \\
83.4030 \\
83.0960 \\
83.4910\end{array}$ & $\begin{array}{c}\text { Pressure } \\
(\text { psig) } \\
9.89 \\
19.55 \\
29.57 \\
39.42 \\
49.16 \\
54.50 \\
59.57 \\
64.20 \\
69.32 \\
74.16 \\
80.16 \\
85.61 \\
90.58 \\
95.69 \\
100.92 \\
103.25 \\
106.11 \\
108.31 \\
111.08 \\
113.24 \\
117.83 \\
120.92 \\
123.28 \\
122.97 \\
125.82 \\
125.60 \\
127.84 \\
130.19 \\
132.53 \\
135.33 \\
134.42 \\
138.35 \\
140.16 \\
142.63 \\
145.78 \\
142.52 \\
0.22\end{array}$ & $\begin{array}{c}\text { Temperature } \\
\text { (Fahrenheit) } \\
80.3690 \\
80.7220 \\
81.1620 \\
81.7340 \\
82.0410 \\
82.4370 \\
82.7880 \\
83.0520 \\
83.4910 \\
83.7980 \\
84.5870 \\
84.0750 \\
84.5870 \\
84.2810 \\
83.7980 \\
83.2710 \\
82.8760 \\
82.7440 \\
82.3490 \\
82.1290 \\
81.8650 \\
81.5580 \\
80.8540 \\
80.6340 \\
80.3690 \\
80.1050 \\
80.0610 \\
80.0170 \\
80.2370 \\
80.5010 \\
80.8980 \\
81.3820 \\
81.9090 \\
82.5690 \\
83.0520 \\
83.5350 \\
79.9730\end{array}$ \\
\hline
\end{tabular}

High pressure test

Temperature

80.3690

80.7220

82.4370

82.7880

83.0520

84.5870

84.0750

84.5870

83.2710

82.8760

82.7440

82.3490

80.8540

80.1050

80.0610

80.0170

80.2370

81.3820

81.9090

82.5690

83.0520

83.5350 


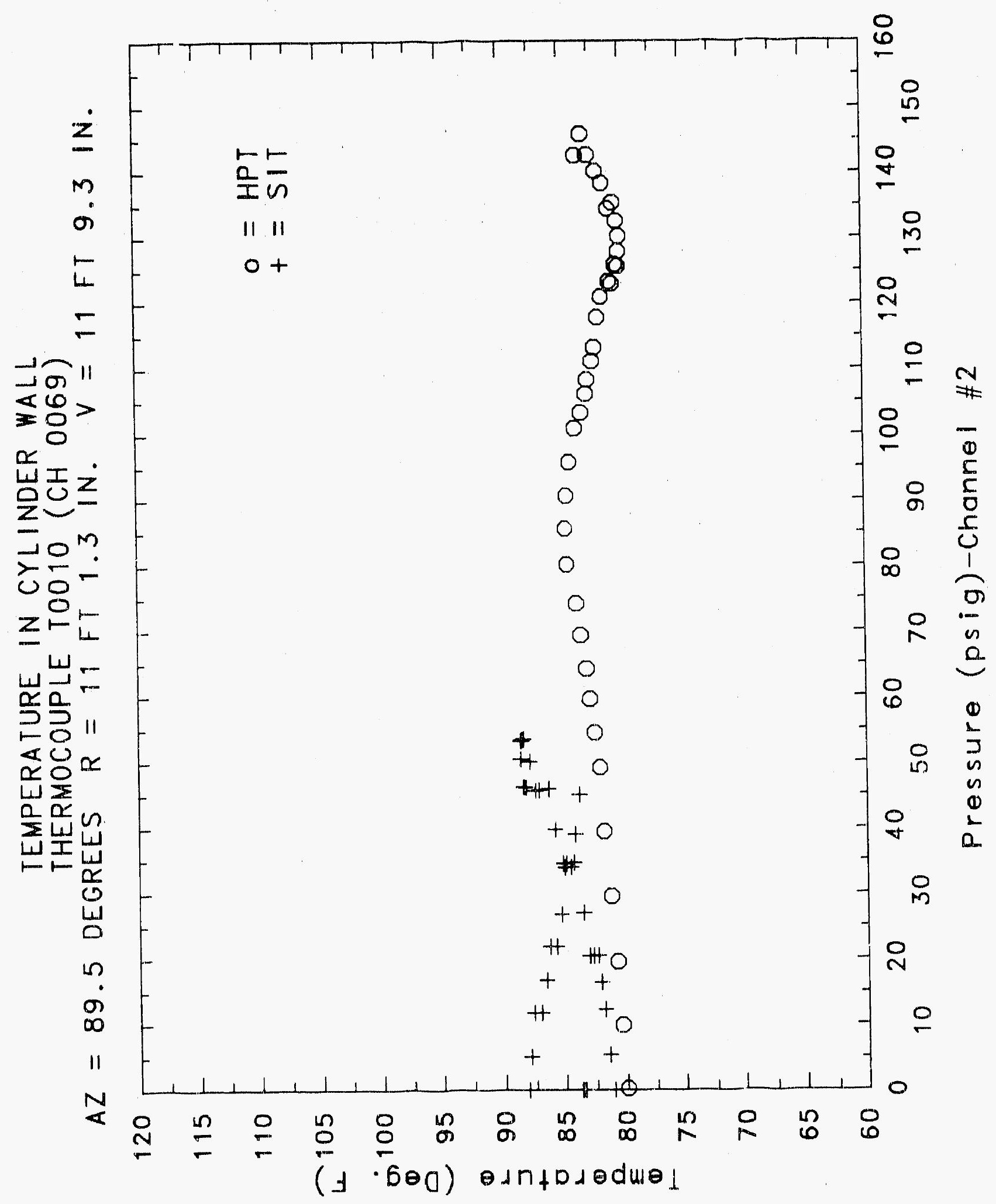




\section{Reinforced Concrete Test Data}

Thermocouple Channel 70

StRUCTURAL INTEgRITY TEST

\begin{tabular}{|c|c|c|c|}
\hline $\begin{array}{c}\text { Pressure } \\
\text { (psig) } \\
-0.05 \\
5.33 \\
12.31 \\
16.44 \\
20.51 \\
20.46 \\
20.47 \\
27.05 \\
34.69 \\
34.53 \\
34.53 \\
39.70 \\
45.90 \\
45.65 \\
45.02 \\
50.10 \\
53.47 \\
53.29 \\
53.21 \\
50.49 \\
46.14 \\
46.13 \\
44.97 \\
38.98 \\
33.99 \\
33.96 \\
26.83 \\
21.88 \\
21.90 \\
16.69 \\
11.74 \\
11.77 \\
5.05 \\
0.02 \\
-0.04 \\
-0.02 \\
-0.02 \\
0.02 \\
\end{array}$ & $\begin{array}{c}\text { Temperature } \\
\text { (Fahrenheit) } \\
79.8850 \\
80.1930 \\
80.6780 \\
81.1620 \\
81.7780 \\
82.5250 \\
83.1840 \\
84.0170 \\
84.8940 \\
86.0770 \\
86.6450 \\
87.4320 \\
88.0440 \\
88.8300 \\
89.0480 \\
89.1790 \\
89.2660 \\
89.0920 \\
88.9610 \\
88.6550 \\
88.3500 \\
87.9130 \\
83.9740 \\
84.5000 \\
84.8940 \\
85.5950 \\
86.0330 \\
86.4270 \\
87.6070 \\
88.3500 \\
89.1350 \\
90.0510 \\
90.3120 \\
90.3990 \\
83.3590 \\
82.8320 \\
82.8760 \\
83.9300\end{array}$ & $\begin{array}{l}\text { Pressure } \\
(\text { psig) } \\
9.89 \\
19.55 \\
29.57 \\
39.42 \\
49.16 \\
54.50 \\
59.57 \\
64.20 \\
69.32 \\
74.16 \\
80.16 \\
85.61 \\
90.58 \\
95.69 \\
100.92 \\
103.25 \\
106.11 \\
108.31 \\
111.08 \\
113.24 \\
117.83 \\
120.92 \\
123.28 \\
122.97 \\
125.82 \\
125.60 \\
127.84 \\
130.19 \\
132.53 \\
135.33 \\
134.42 \\
138.35 \\
140.16 \\
142.63 \\
145.78 \\
142.52 \\
0.22\end{array}$ & $\begin{array}{c}\text { Temperature } \\
\text { (Fahreni:ait) } \\
79.8850 \\
80.3690 \\
80.7220 \\
81.4260 \\
81.9970 \\
82.5250 \\
82.8760 \\
83.4030 \\
83.7980 \\
84.3680 \\
85.5080 \\
85.1130 \\
84.7190 \\
84.0170 \\
83.4030 \\
82.7000 \\
82.2610 \\
82.1190 \\
81.7340 \\
81.4700 \\
81.1180 \\
80.8100 \\
80.1490 \\
79.9290 \\
79.7530 \\
79.6200 \\
79.7090 \\
80.0170 \\
80.2370 \\
80.9860 \\
81.6020 \\
82.4370 \\
83.1400 \\
83.9740 \\
84.8500 \\
85.5510 \\
82.3050\end{array}$ \\
\hline
\end{tabular}

High Pressure test

Temperature

ahroni:ait

30.3690

80.7220

81.4260

81.9970

83.4030

83.7980

84.3680

85.5080

84.7190

84.0170

83.4030

$82.1<90$

81.7340

81.4700

80.1490

79.9290

79.7530

79.6200

80.2370

80.9860

81.6020

83.9740

84.8500

85.5510

82.3050 


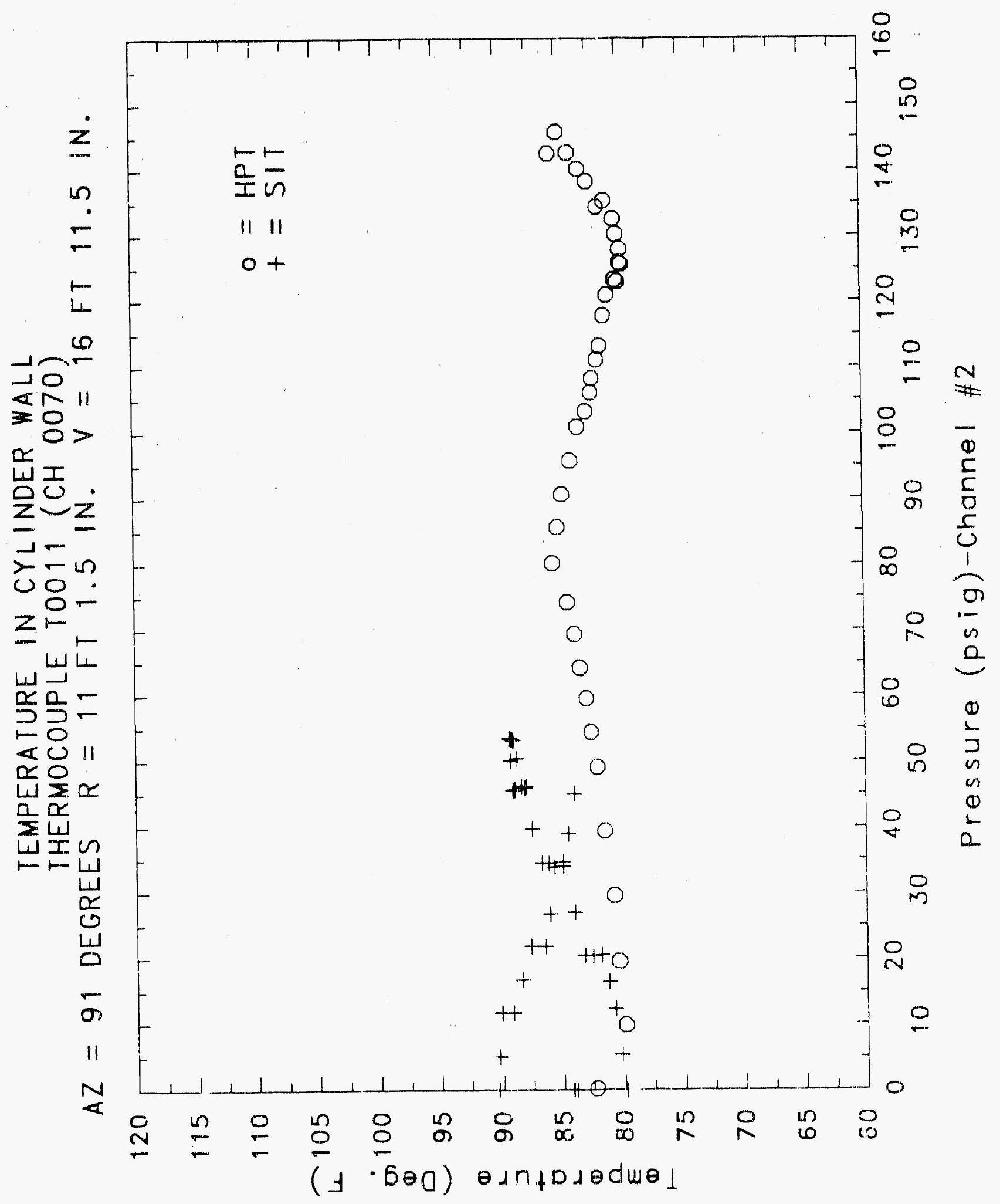




\section{Reinforced Concrete test Data}

Thermocouple Channel 71

Structural. IntEgrity TEST

\begin{tabular}{|c|c|c|c|}
\hline $\begin{array}{l}\text { Pressure } \\
(p s i g) \\
-0.05 \\
5.33 \\
12.31 \\
16.44 \\
20.51 \\
20.46 \\
20.47 \\
27.05 \\
34.69 \\
34.53 \\
34.53 \\
39.70 \\
45.90 \\
45.65 \\
45.62 \\
50.10 \\
53.47 \\
53.29 \\
53.21 \\
50.49 \\
46.14 \\
46.13 \\
64.97 \\
38.98 \\
33.99 \\
33.96 \\
26.83 \\
21.88 \\
21.90 \\
16.69 \\
11.74 \\
11.77 \\
5.05 \\
0.02 \\
-0.04 \\
-0.07 \\
-0.02 \\
0.02\end{array}$ & $\begin{array}{c}\text { Temperature } \\
\text { (Fahrenheit) } \\
80.3690 \\
80.5450 \\
81.1180 \\
31.5900 \\
8 \% .3490 \\
83.0520 \\
83.6230 \\
84.4560 \\
85.2890 \\
86.2080 \\
86.6450 \\
87.6510 \\
88.5680 \\
90.0940 \\
90.4430 \\
90.6610 \\
90.7480 \\
90.4860 \\
90.2690 \\
89.7460 \\
89.2660 \\
38.6990 \\
84.3240 \\
84.7630 \\
85.2450 \\
85.9890 \\
86.4270 \\
86.8640 \\
87.5640 \\
88.0880 \\
80.6120 \\
89.6150 \\
90.0070 \\
90.1810 \\
83.2710 \\
82.7480 \\
82.8320 \\
84.1930\end{array}$ & $\begin{array}{c}\text { Pressure } \\
(p 519) \\
9.89 \\
19.55 \\
29.57 \\
39.42 \\
49.16 \\
54.50 \\
59.57 \\
64.20 \\
69.32 \\
74.16 \\
80.16 \\
85.61 \\
96.58 \\
95.69 \\
100.92 \\
103.25 \\
106.11 \\
108.31 \\
111.08 \\
113.24 \\
117.83 \\
120.92 \\
123.28 \\
122.97 \\
125.82 \\
125.60 \\
127.84 \\
130.19 \\
132.53 \\
135.33 \\
134.42 \\
138.35 \\
140.16 \\
142.63 \\
145.78 \\
142.52 \\
0.22\end{array}$ & $\begin{array}{c}\text { Temperature } \\
\text { (Fahrenheit) } \\
79.8410 \\
80.2370 \\
80.7660 \\
81.5580 \\
82.0850 \\
82.5570 \\
83.0080 \\
83.5790 \\
83.9300 \\
84.5000 \\
85.1570 \\
84.8500 \\
8 . .1120 \\
83.7980 \\
83.2270 \\
82.5250 \\
82.0850 \\
81.9970 \\
81.4700 \\
81.3820 \\
81.0300 \\
80.6780 \\
79.9730 \\
79.8410 \\
79.6200 \\
79.5760 \\
79.6640 \\
80.0170 \\
80.3250 \\
80.8980 \\
81.6300 \\
82.4810 \\
83.1400 \\
83.9300 \\
84.5440 \\
85.1570 \\
31.2500\end{array}$ \\
\hline
\end{tabular}

High Pressure test

Temperature

ahrenheit)

80.7660

81.5580

83.0080

83.5790

85.1570

84.8500

84.0120

82.5250

82.0850

81.9970

9.9730

79.8410

79.6200

9.5760

9.6640

83.1400

83.9300

31.2500 


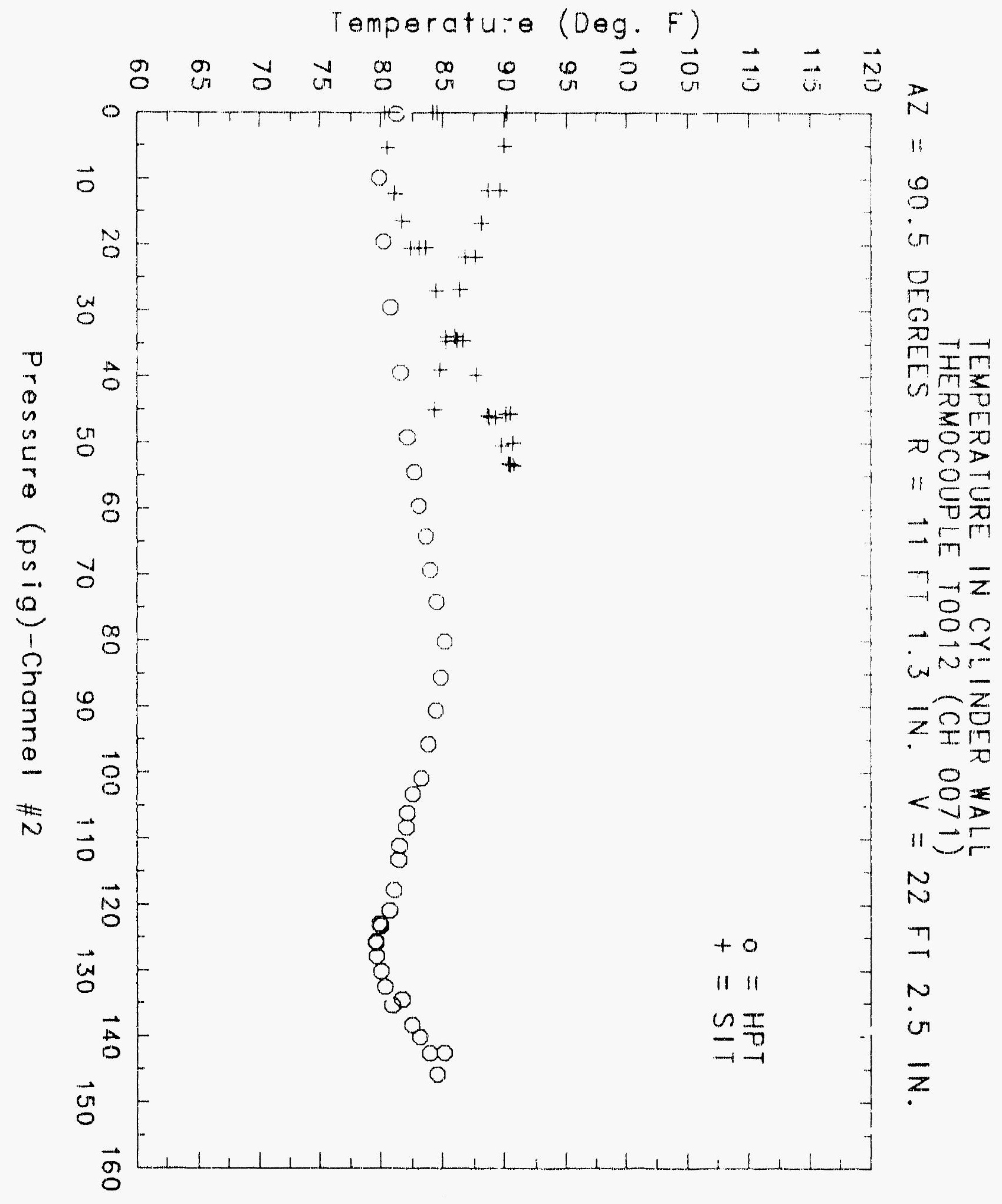




\title{
Reinforced Concrete T'est Data
}

\author{
Thermocouple Channel $; 2$
}

Structural Integrity Test

\begin{tabular}{|c|c|c|c|}
\hline $\begin{array}{l}\text { Pressure } \\
(p s i g) \\
-0.05 \\
5.33 \\
12.31 \\
16.44 \\
20.51 \\
20.46 \\
20.47 \\
27.05 \\
34.69 \\
34.53 \\
34.53 \\
39.70 \\
45.90 \\
45.65 \\
45.62 \\
50.10 \\
53.47 \\
53.29 \\
53.21 \\
50.49 \\
46.14 \\
46.13 \\
44.97 \\
38.98 \\
33.99 \\
33.96 \\
26.83 \\
21.88 \\
21.90 \\
16.69 \\
11.74 \\
31.77 \\
5.05 \\
0.02 \\
-0.04 \\
-0.02 \\
-0.02 \\
0.02 \\
\end{array}$ & $\begin{array}{c}\text { Temperature } \\
\text { (Fahrenheit) } \\
80.3250 \\
80.7220 \\
81.4260 \\
82.0850 \\
82.7000 \\
83.5790 \\
84.2370 \\
85.1130 \\
85.9020 \\
86.7330 \\
87.2580 \\
88.3500 \\
89.4400 \\
90.9650 \\
91.2270 \\
91.4880 \\
91.4880 \\
91.1830 \\
90.8780 \\
90.3120 \\
89.8330 \\
89.1350 \\
84.5870 \\
85.2450 \\
85.7700 \\
86.5580 \\
86.9520 \\
87.4320 \\
88.1750 \\
88.4810 \\
89.0480 \\
90.0510 \\
90.4430 \\
90.5740 \\
83.0960 \\
82.6570 \\
82.9200 \\
84.5000\end{array}$ & $\begin{array}{c}\text { Pressure } \\
(p s i g) \\
9.89 \\
19.55 \\
29.57 \\
39.42 \\
49.16 \\
54.50 \\
59.57 \\
64.20 \\
69.32 \\
74.16 \\
80.16 \\
85.61 \\
90.58 \\
95.69 \\
100.92 \\
103.25 \\
106.11 \\
108.31 \\
111.08 \\
113.24 \\
117.83 \\
120.92 \\
123.28 \\
122.97 \\
125.82 \\
125.60 \\
127.84 \\
130.19 \\
132.53 \\
135.33 \\
134.42 \\
138.35 \\
140.16 \\
142.63 \\
145.78 \\
142.52 \\
0.22\end{array}$ & $\begin{array}{c}\text { Temperature } \\
\text { (Fahrenheit) } \\
79.8850 \\
80.3250 \\
80.8980 \\
81.6900 \\
82.4810 \\
82.9640 \\
83.4470 \\
83.9300 \\
84.4120 \\
84.5020 \\
85.6390 \\
85.1130 \\
84.6310 \\
83.8860 \\
83.3590 \\
82.5250 \\
82.0850 \\
81.9090 \\
81.4260 \\
81.2060 \\
80.9420 \\
80.5450 \\
79.8410 \\
79.5760 \\
79.5760 \\
79.5760 \\
79.6640 \\
80.2370 \\
80.6780 \\
81.4260 \\
82.2170 \\
83.1400 \\
83.7980 \\
84.5870 \\
85.1130 \\
85.5080 \\
81.5140\end{array}$ \\
\hline
\end{tabular}

\section{High Pressure test}

ressure Temperature

9.89

79.8850

80.3250

80.8980

81.6900

82.4810

83.9470

83.9300

84.4120

84.3620

85.1130

84.6310

83.8860

83.3590

82.5250

81.9090

81.4260

81.2060

80.5450

79.8410

79.5760

79.5760

79.6640

80.2370

80.6780

81.4260

82.2170

83.1400

83.7980

84.5870

85.1130

85.5080 


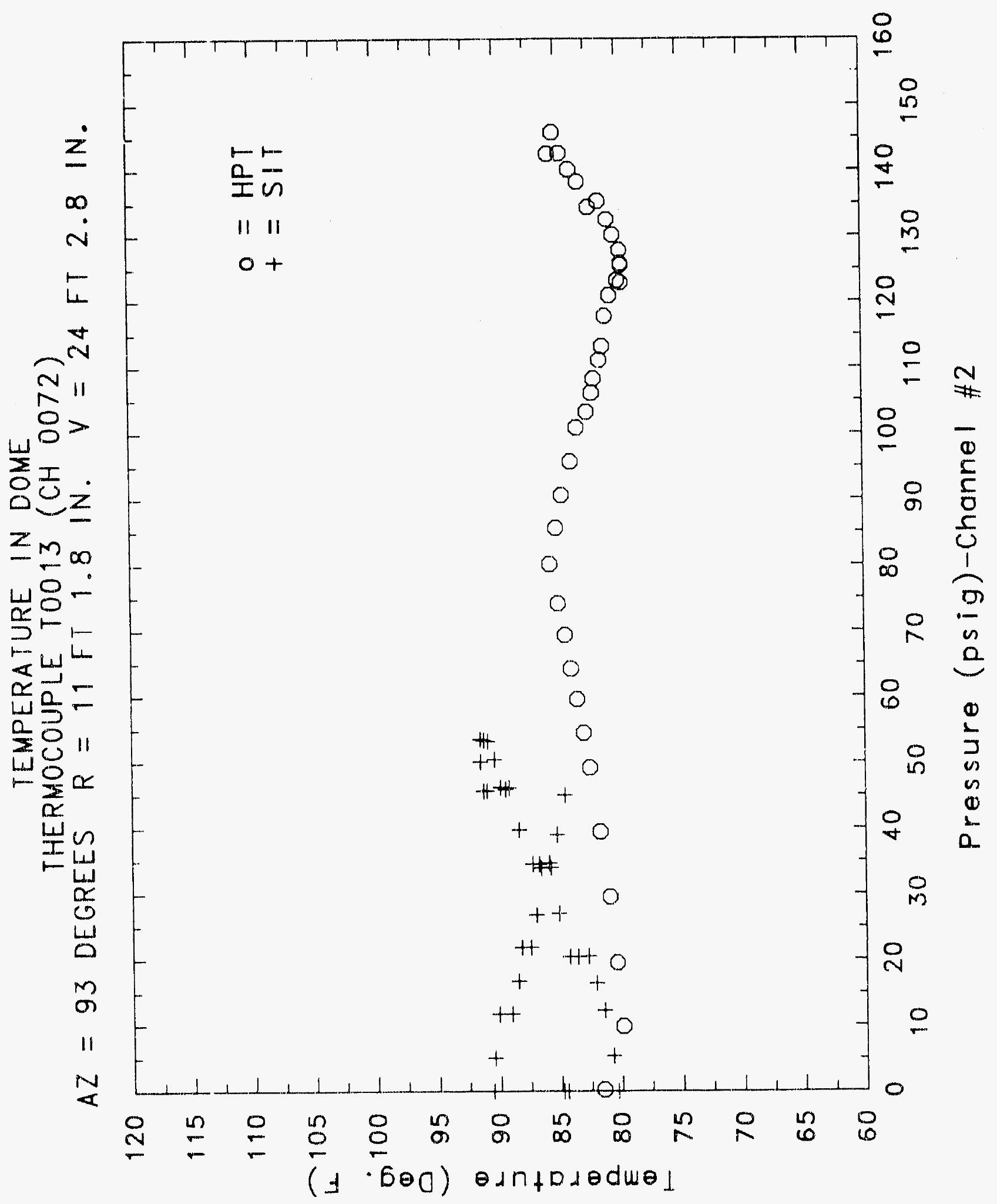

C. -1693 


\section{Reinforced Concrete Test Data}

Thermocouple Channel 73

Structural InTEgRITY TEST

\begin{tabular}{l}
\hline Pressure \\
(psig) \\
-0.05 \\
5.33 \\
12.31 \\
16.44 \\
20.51 \\
20.46 \\
20.47 \\
27.05 \\
34.69 \\
34.53 \\
34.53 \\
39.70 \\
45.90 \\
45.65 \\
45.62 \\
50.10 \\
53.47 \\
53.29 \\
53.21 \\
50.49 \\
46.14 \\
46.13 \\
44.97 \\
38.98 \\
33.99 \\
33.96 \\
26.83 \\
21.88 \\
21.90 \\
16.69 \\
11.74 \\
11.77 \\
5.05 \\
0.02 \\
-0.04 \\
-0.02 \\
-0.09 \\
0.02 \\
\\
\hline
\end{tabular}

Temperature

(Fahrenheit)

79.6200

$82.525 r$

85.0260

88.6990

92.0530

91.4010

96.5210

94.5280

99.1130

102.8200

112.8600

105.2200

91.2700

84.2810

83.9740

83.1400

82.4810

77.0600

77.9000

81.8650

81.6460

76.3090

89.5280

89.3970

90.2690

91.5750

96.5210

94.5720

104.3600

100.1500

91.2700

88.8730

86.1640

81.8220

76.3530

82.0850

84.3240

88.4810
High Pressure Test
Temperature

$\begin{array}{cc}\text { (psig) } & \text { (Fahrenheit) } \\ 9.89 & 82.1730\end{array}$

$19.55 \quad 82.0410$

$29.57 \quad 82.6570$

$39.42 \quad 81.9090$

$49.16 \quad 84.1930$

$54.50 \quad 84.7630$

$59.57 \quad 83.0960$

$64.20 \quad 87.0830$

$69.32 \quad 87.6070$

$74.16 \quad 91.0520$

$80.16 \quad 72.5850$

$85.61 \quad 70.8510$

$90.58 \quad 71.6520$

$95.69 \quad 68.9800$

$100.92 \quad 67.9090$

$103.25 \quad 68.0430$

$106.11 \quad 67.3730$

$108.31 \quad 67.0160$

$111.08 \quad 67.2390$

$113.24 \quad 65.8980$

$117.83 \quad 66.7030$

$120.92 \quad 67.5520$

$123.28 \quad 70.8070$

$122.97 \quad 72.7180$

$125.82 \quad 73.5620$

$125.60 \quad 75.9550$

$127.84 \quad 77.0600$

$130.19 \quad 77.6350$

$\begin{array}{ll}132.53 & 81.9970\end{array}$

$135.33 \quad 83.3150$

$134.42 \quad 85.9890$

$138.35 \quad 89.7020$

$140.16 \quad 93.5740$

$142.63 \quad 89.5710$

$\begin{array}{ll}145.78 & 86.2960\end{array}$

$142.52 \quad 83.3590$

$0.22 \quad 77.3700$ 


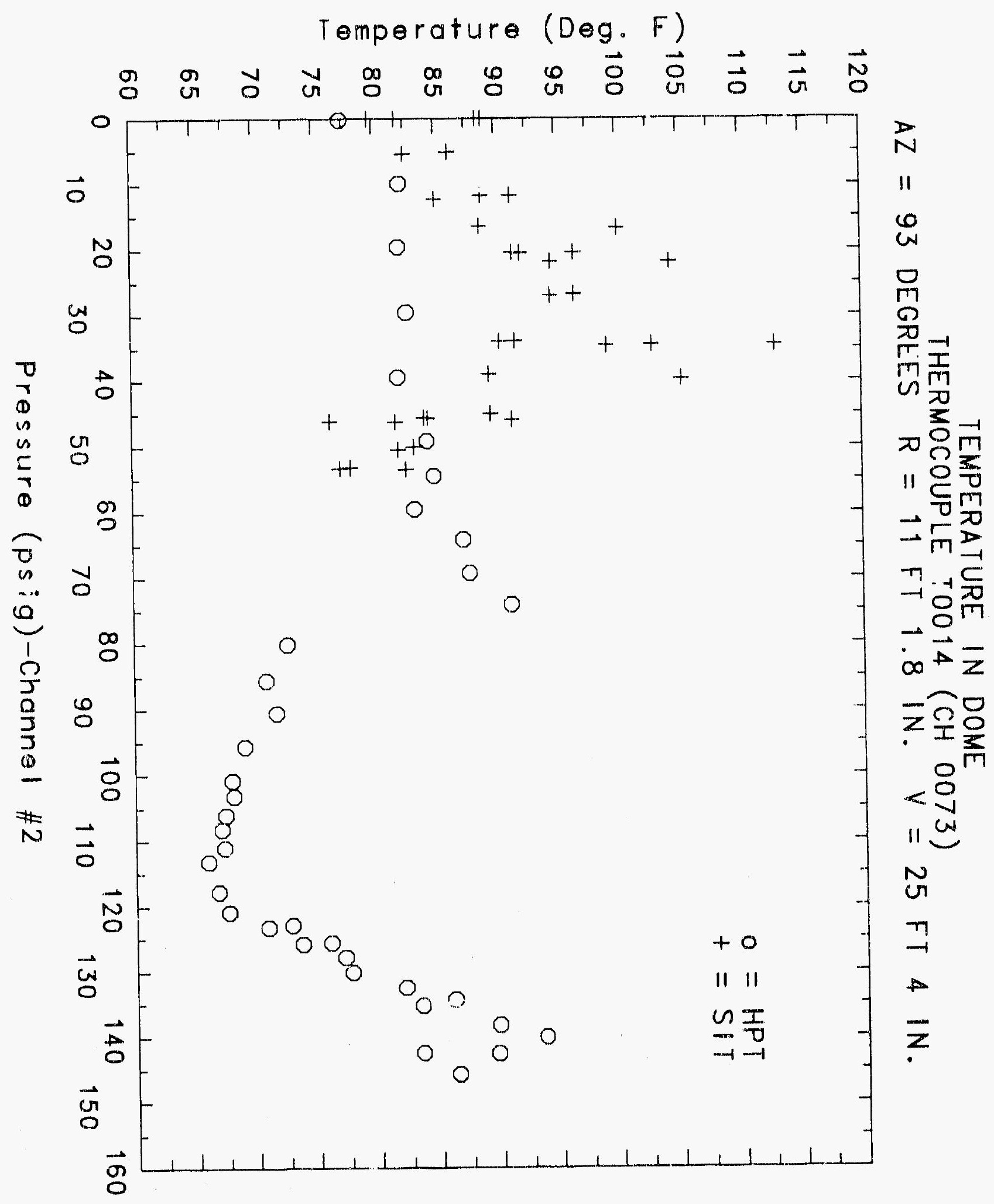




\section{Reinforced Concrete Test Data}

Thermocouple Channel 74

Structural INTEgRITY TEST

\begin{tabular}{|c|c|c|c|}
\hline $\begin{array}{c}\text { Pressure } \\
\text { (psig) } \\
-0.05 \\
5.33 \\
12.31 \\
16.44 \\
20.51 \\
20.46 \\
20.47 \\
27.05 \\
34.69 \\
34.53 \\
34.53 \\
39.70 \\
45.90 \\
45.65 \\
45.62 \\
50.10 \\
53.47 \\
53.29 \\
53.21 \\
50.49 \\
46.14 \\
46.13 \\
44.97 \\
38.98 \\
33.99 \\
33.96 \\
26.83 \\
21.88 \\
21.90 \\
16.69 \\
11.74 \\
11.77 \\
5.05 \\
0.02 \\
-0.04 \\
-0.02 \\
-0.02 \\
0.02\end{array}$ & $\begin{array}{c}\text { Temperature } \\
\text { (Fahrenheit) } \\
79.0470 \\
79.6200 \\
80.8100 \\
81.8220 \\
82.7440 \\
83.7980 \\
84.4120 \\
85.3760 \\
86.2080 \\
86.9520 \\
87.3890 \\
88.1750 \\
89.3100 \\
90.0510 \\
90.0510 \\
89.9200 \\
89.7890 \\
89.4840 \\
89.2660 \\
88.6990 \\
88.2620 \\
87.6950 \\
84.5870 \\
85.4200 \\
86.0770 \\
87.0830 \\
87.4760 \\
88.0000 \\
88.6120 \\
89.0040 \\
89.3970 \\
89.7460 \\
89.8760 \\
89.7890 \\
81.8650 \\
81.7780 \\
82.5690 \\
84.7630\end{array}$ & $\begin{array}{c}\text { Pressure } \\
(\text { psig) } \\
9.89 \\
19.55 \\
29.57 \\
39.42 \\
49.16 \\
54.50 \\
59.57 \\
64.20 \\
69.32 \\
74.16 \\
80.16 \\
85.61 \\
90.58 \\
95.69 \\
100.92 \\
103.25 \\
106.11 \\
108.31 \\
111.08 \\
113.24 \\
117.83 \\
120.92 \\
123.28 \\
122.97 \\
125.82 \\
125.60 \\
127.84 \\
130.19 \\
132.53 \\
135.33 \\
134.42 \\
138.35 \\
140.16 \\
142.63 \\
145.78 \\
142.52 \\
0.22\end{array}$ & $\begin{array}{c}\text { Temperature } \\
\text { (Fahrenheit) } \\
78.8710 \\
79.4000 \\
80.0610 \\
80.9860 \\
81.6020 \\
82.2610 \\
82.7000 \\
83.4030 \\
83.7980 \\
84.4120 \\
83.9300 \\
83.4030 \\
83.0520 \\
82.4810 \\
81.9090 \\
81.2940 \\
80.8540 \\
80.6780 \\
80.2810 \\
80.1050 \\
79.8850 \\
79.4880 \\
78.8710 \\
78.7830 \\
78.9150 \\
79.0470 \\
79.3120 \\
80.0170 \\
80.4130 \\
81.2500 \\
82.3490 \\
83.2710 \\
84.1490 \\
84.7190 \\
85.1570 \\
85.5510 \\
83.0080\end{array}$ \\
\hline
\end{tabular}

High Pressure. Test

remperature

78.8710

79.4000

80.9860

81.6020

82.2610

83.7980

84.4120

83.9300

83.4030

83.0520

81.9090

81.2940

80.8540

80.1050

79.8850

79.4880

78.8710

79.0470

79.3120

80.0170

80.4130

83.2710

84.1490

84.7190

$\begin{array}{rr}42.52 & 85.5510 \\ 0.22 & 83.0080\end{array}$ 


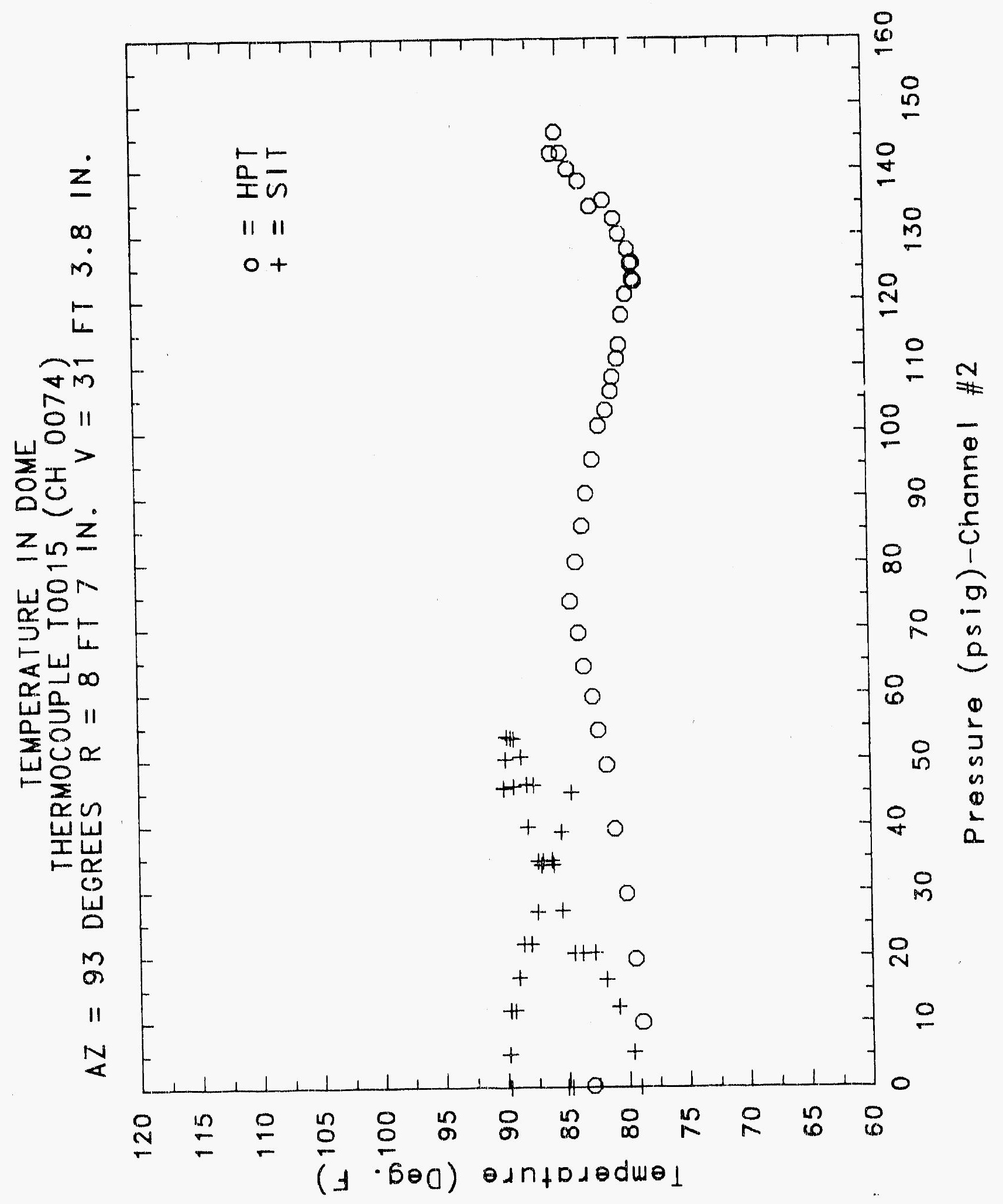




\section{Reinforced Concrete Test Data}

\section{Thermocouple Channel 75}

\section{StRUCTURAL INTEgRITY TEST}

\begin{tabular}{|c|c|}
\hline $\begin{array}{c}\text { Pressure } \\
\text { (psig) } \\
-0.05 \\
5.33 \\
12.31 \\
16.44 \\
20.51 \\
20.46 \\
20.47 \\
27.05 \\
34.69 \\
34.53 \\
34.53 \\
39.70 \\
45.90 \\
45.65 \\
45.62 \\
50.10 \\
53.47 \\
53.29 \\
53.21 \\
50.49 \\
46.14 \\
46.13 \\
44.97 \\
38.98 \\
33.99 \\
33.96 \\
26.83 \\
21.88 \\
21.90 \\
16.69 \\
11.74 \\
11.77 \\
5.05 \\
0.02 \\
-0.04 \\
-0.02 \\
-0.02 \\
0.02\end{array}$ & $\begin{array}{c}\text { Temperature } \\
\text { (Fahrenheit) } \\
84.7190 \\
85.5510 \\
86.0330 \\
86.0770 \\
86.2960 \\
86.5580 \\
86.7330 \\
87.2580 \\
87.6070 \\
87.8690 \\
88.0880 \\
88.4370 \\
88.7430 \\
89.0040 \\
89.0920 \\
89.0920 \\
89.1350 \\
89.0920 \\
88.9610 \\
88.6120 \\
88.2620 \\
88.0000 \\
86.9520 \\
87.3890 \\
87.6510 \\
87.9570 \\
88.0880 \\
88.3060 \\
88.4810 \\
88.6120 \\
88.7430 \\
89.0920 \\
89.1790 \\
89.0480 \\
84.1930 \\
87.3450 \\
88.9170 \\
89.5710\end{array}$ \\
\hline
\end{tabular}

\section{High Pressure test}

\begin{tabular}{|c|c|}
\hline $\begin{array}{c}\text { Pressure } \\
(\text { psig) } \\
9.89 \\
19.55 \\
29.57 \\
39.42 \\
49.16 \\
54.50 \\
59.57 \\
64.20 \\
69.32 \\
74.16 \\
80.16 \\
85.61 \\
90.58 \\
95.69 \\
100.92 \\
103.25 \\
106.11 \\
108.31 \\
111.08 \\
113.24 \\
117.83 \\
120.92 \\
123.28 \\
122.97 \\
125.82 \\
125.60 \\
127.84 \\
130.19 \\
132.53 \\
135.33 \\
134.42 \\
138.35 \\
140.16 \\
142.63 \\
145.78 \\
142.52 \\
0.22\end{array}$ & $\begin{array}{c}\text { Temperature } \\
\text { (Fahrenheit) } \\
84.1490 \\
84.6310 \\
85.3760 \\
86.0770 \\
86.5140 \\
86.8640 \\
87.1260 \\
87.3890 \\
87.6070 \\
87.9130 \\
87.5640 \\
87.1700 \\
86.6890 \\
86.0330 \\
85.4640 \\
84.7630 \\
84.2810 \\
84.1050 \\
83.6660 \\
83.4910 \\
83.2270 \\
82.7880 \\
81.9090 \\
81.8220 \\
82.4810 \\
83.8860 \\
84.6750 \\
85.8140 \\
86.2080 \\
86.4270 \\
86.5580 \\
86.7330 \\
87.0390 \\
87.1700 \\
87.2140 \\
87.3010 \\
82.4810\end{array}$ \\
\hline
\end{tabular}




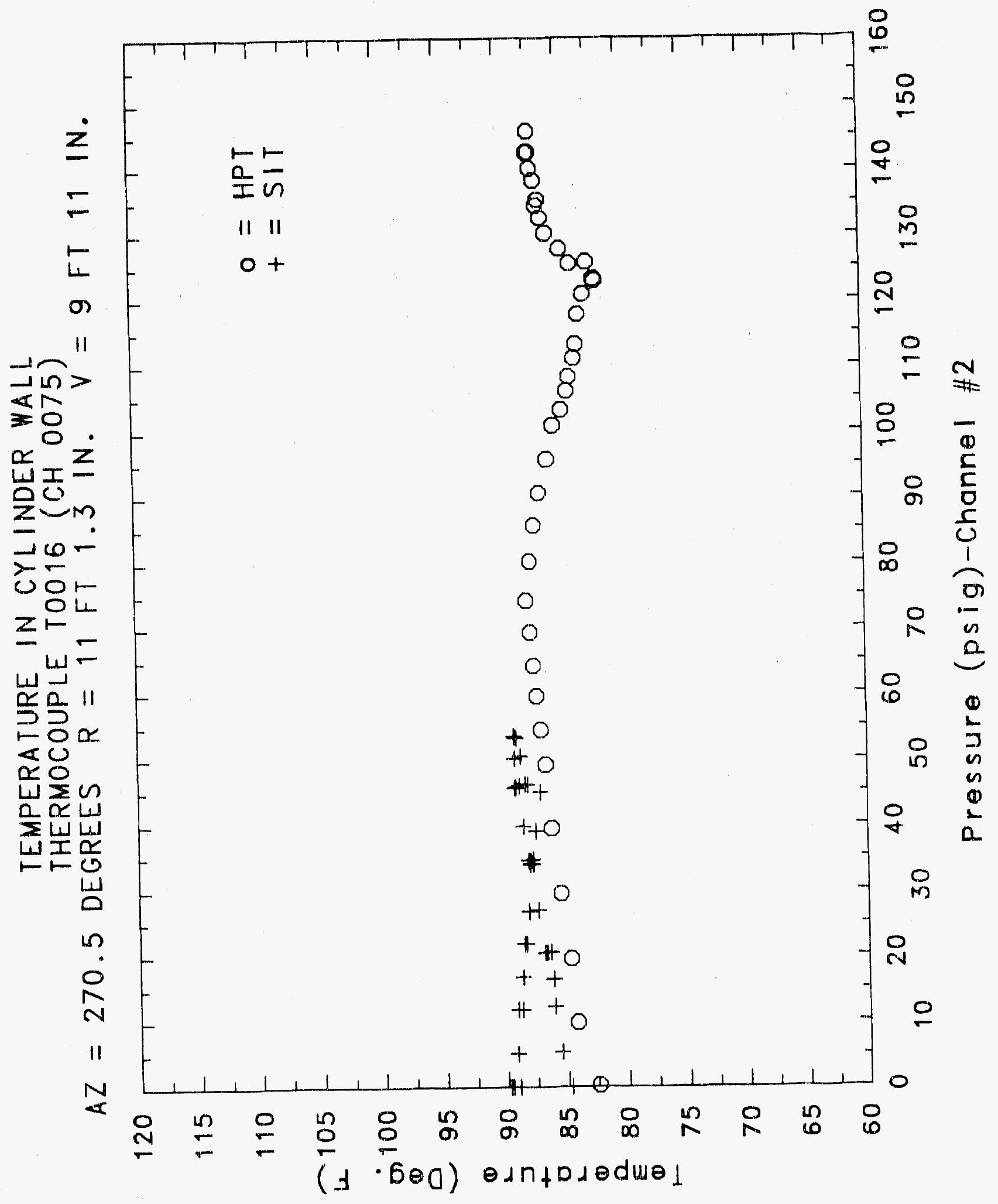




\section{Reinforced Concrete Test Data}

Thermocouple Channel 76

StRUCTURAL INTEgRITY TEST

\begin{tabular}{cccc} 
Pressure & Temperature & & \\
\cline { 4 - 4 }$($ psig) & Pressure & Temperature \\
-0.05 & $($ psig) & (Fahrenheit) \\
5.33 & 82.6130 & 9.89 & 81.0300 \\
12.31 & 83.0520 & 19.55 & 81.5580 \\
16.44 & 83.7100 & 29.57 & 82.1290 \\
20.51 & 84.1050 & 39.42 & 82.8760 \\
20.46 & 84.2370 & 49.16 & 83.5350 \\
20.47 & 81.7630 & 54.50 & 84.0610 \\
27.05 & 84.9380 & 59.57 & 84.4560 \\
34.69 & 85.4640 & 64.20 & 84.8940 \\
34.53 & 85.9890 & 69.32 & 85.2010 \\
34.53 & 86.2960 & 74.16 & 85.6390 \\
39.70 & 86.5140 & 80.16 & 86.3830 \\
45.90 & 87.0830 & 85.61 & 86.3830 \\
45.65 & 87.3450 & 90.58 & 86.3390 \\
45.62 & 87.7820 & 95.69 & 85.9450 \\
50.10 & 87.9570 & 100.92 & 85.5950 \\
53.47 & 88.3500 & 103.25 & 84.9820 \\
53.29 & 88.7860 & 106.11 & 84.5870 \\
53.21 & 88.9610 & 108.31 & 84.5870 \\
50.49 & 89.0040 & 111.08 & 84.1490 \\
46.14 & 88.9170 & 113.24 & 83.9740 \\
46.13 & 88.6990 & 117.83 & 83.6660 \\
44.97 & 88.4810 & 120.92 & 83.4030 \\
38.98 & 84.9820 & 123.28 & 82.5690 \\
33.99 & 85.5080 & 122.97 & 82.0850 \\
33.96 & 85.8580 & 125.82 & 81.9530 \\
26.83 & 86.4700 & 125.60 & 81.7340 \\
21.88 & 86.6890 & 127.84 & 81.6900 \\
21.90 & 87.0390 & 130.19 & 82.4810 \\
16.69 & 87.4760 & 132.53 & 82.7880 \\
11.74 & 87.6950 & 135.33 & 83.4470 \\
11.77 & 87.9130 & 134.42 & 83.9300 \\
5.05 & 88.2620 & 138.35 & 84.4120 \\
0.02 & 88.3500 & 140.16 & 84.8070 \\
-0.04 & 88.3500 & 142.63 & 85.0260 \\
-0.02 & 84.1490 & 145.78 & 85.4640 \\
-0.02 & 83.7980 & 142.52 & 85.6390 \\
0.02 & 84.5870 & 0.22 & 81.4260 \\
& 86.1200 & & \\
\hline & & & \\
\hline
\end{tabular}

High Pressure Test

Temperature

81.0300

81.5580

82.1290

82.8760

84.4560

84.8940

85.2010

86.3830

86.3390

85.9450

84.9820

84.5870

84.5870

84.1490

83.6660

83.4030

82.5690

82.0850

81.7340

81.6900

82.4810

82.7880

83.9300

84.4120

84.8070

85.0260

85.6390

81.4260 


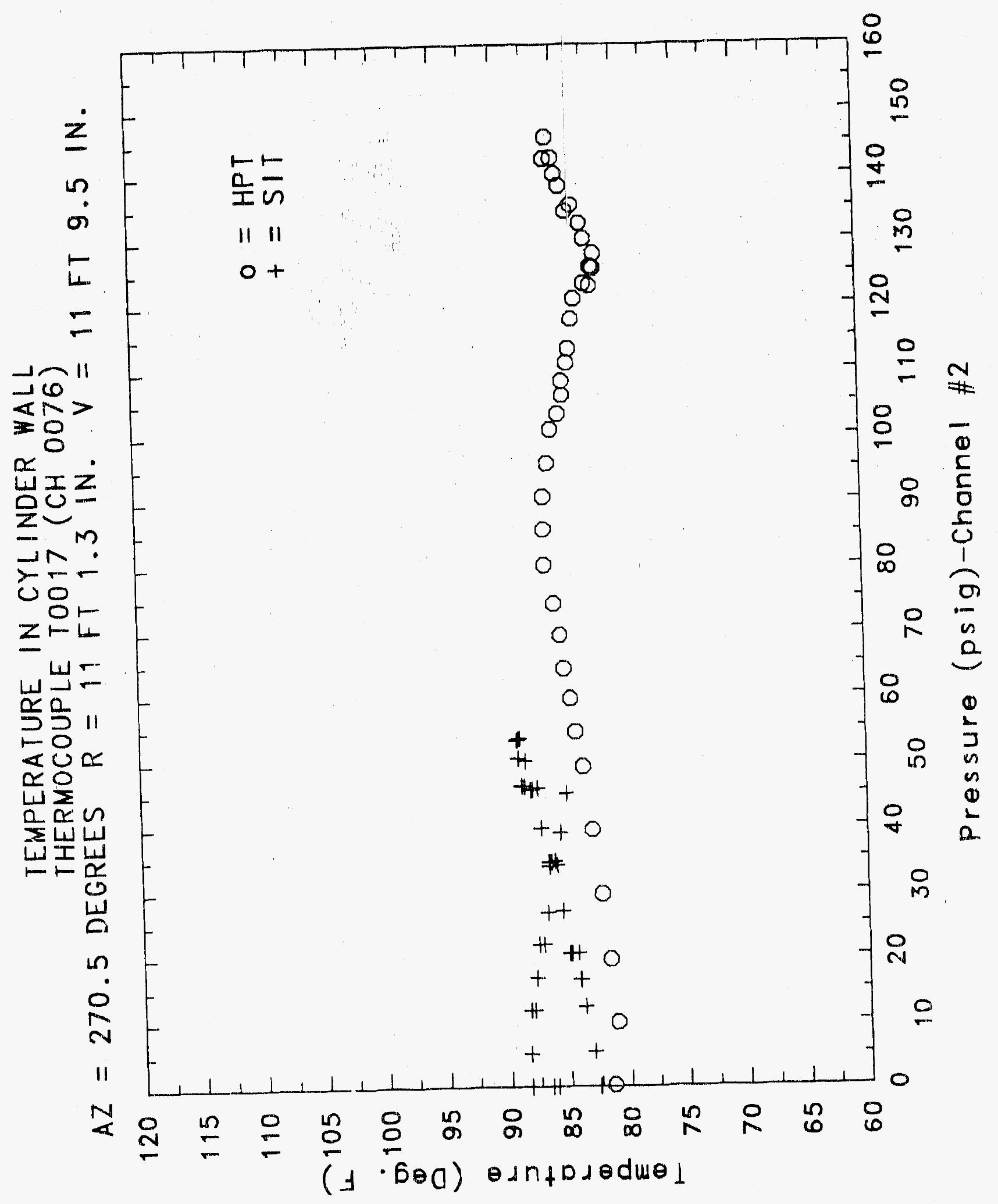




\section{Reinforced Concrete Test Data}

Thermocouple Channel 77

STRUCTURAL INTEgRITY TEST

\begin{tabular}{|c|c|c|c|}
\hline $\begin{array}{c}\text { Pressure } \\
\text { (psig) } \\
-0.05 \\
5.33 \\
12.31 \\
16.44 \\
20.51 \\
20.46 \\
20.47 \\
27.05 \\
34.69 \\
34.53 \\
34.53 \\
39.70 \\
45.90 \\
45.65 \\
45.62 \\
50.10 \\
53.47 \\
53.29 \\
53.21 \\
50.49 \\
46.14 \\
46.13 \\
44.97 \\
38.98 \\
33.99 \\
33.96 \\
26.83 \\
21.88 \\
21.90 \\
16.69 \\
11.74 \\
11.77 \\
5.05 \\
0.02 \\
-0.04 \\
-0.02 \\
-0.02 \\
0.02\end{array}$ & $\begin{array}{c}\text { Temperature } \\
\text { (Fahrenhe it) } \\
81.2060 \\
81.0740 \\
81.0740 \\
81.2500 \\
81.4260 \\
81.7780 \\
82.0850 \\
82.4370 \\
82.7440 \\
83.4470 \\
83.8420 \\
84.3240 \\
84.8940 \\
86.0770 \\
86.6450 \\
87.3890 \\
87.8690 \\
88.2190 \\
88.3930 \\
88.4810 \\
88.5240 \\
88.3930 \\
84.6310 \\
84.7190 \\
84.8940 \\
85.1130 \\
85.2890 \\
85.5080 \\
85.9450 \\
86.2960 \\
86.6890 \\
87.3450 \\
87.9130 \\
88.3930 \\
85.5510 \\
84.9820 \\
84.8070 \\
84.8070\end{array}$ & $\begin{array}{c}\text { Pressure } \\
\text { (psig) } \\
9.89 \\
19.55 \\
29.57 \\
39.42 \\
49.16 \\
54.50 \\
59.57 \\
64.20 \\
69.32 \\
74.16 \\
80.16 \\
85.61 \\
90.58 \\
95.69 \\
100.92 \\
103.25 \\
106.11 \\
108.31 \\
111.08 \\
113.24 \\
117.83 \\
120.92 \\
123.28 \\
122.97 \\
125.82 \\
125.60 \\
127.84 \\
130.19 \\
132.53 \\
135.33 \\
134.42 \\
138.35 \\
140.16 \\
142.63 \\
145.78 \\
142.52 \\
0.22\end{array}$ & $\begin{array}{c}\text { Temperature } \\
\text { (Fahrenheit) } \\
80.5450 \\
80.5900 \\
80.5900 \\
80.7660 \\
80.9420 \\
81.2500 \\
81.4260 \\
81.8220 \\
81.9970 \\
82.4370 \\
83.7100 \\
83.7540 \\
83.7100 \\
83.4910 \\
83.2270 \\
82.8320 \\
82.5690 \\
82.4810 \\
82.2170 \\
81.9970 \\
81.7340 \\
81.4260 \\
80.9420 \\
80.7660 \\
80.5450 \\
80.3690 \\
80.2810 \\
80.3250 \\
80.3250 \\
80.4570 \\
80.6780 \\
80.9860 \\
81.2060 \\
81.5140 \\
81.8220 \\
82.2610 \\
82.6570\end{array}$ \\
\hline
\end{tabular}

High Pressure Test

Temperature

80.5450

80.5900

80.5900

80.7660

81.4260

81.8220

81.9970

82.4370

83.7100

83.4910

83.2270

2.8320

82.5690

2.4810

81.9970

81.7340

81.4260

80.9420

80.3690

80.2810

80.3250

80.3250

80.0860

81.2060

81.5140

81.8220

82.2610

82.6570 


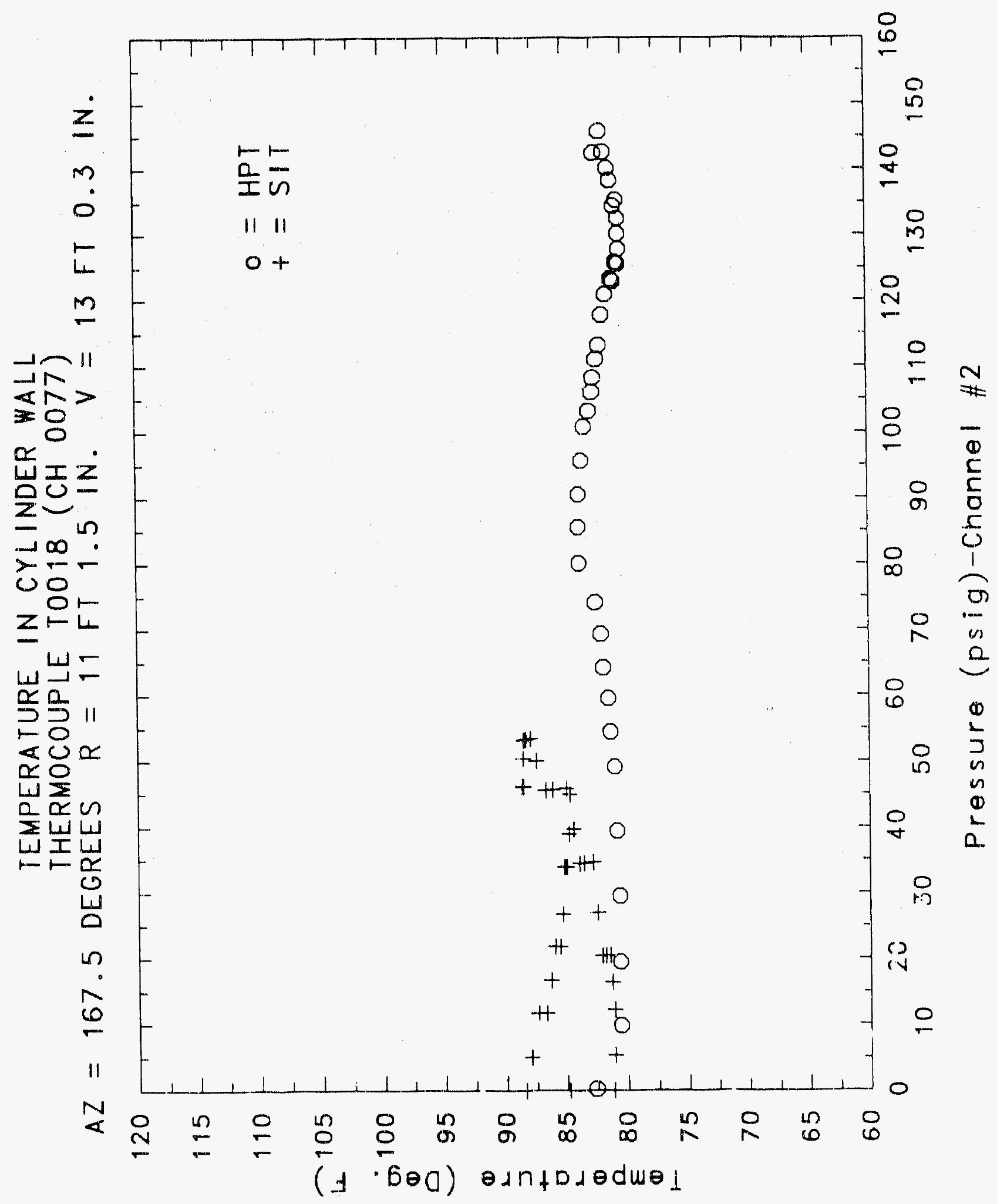

C. -1703 


\section{Reinforced Concrete Test Data}

Thermocouple Channel 78

StRUCTURAL INTEgRITY TEST

High Pressure Test

\begin{tabular}{|c|c|c|c|}
\hline $\begin{array}{c}\text { Pressure } \\
(\text { psig) } \\
-0.05 \\
5.33 \\
12.31 \\
16.44 \\
20.51 \\
20.46 \\
20.47 \\
27.05 \\
34.69 \\
34.53 \\
34.53 \\
39.70 \\
45.90 \\
45.65 \\
45.62 \\
50.10 \\
53.47 \\
53.29 \\
53.21 \\
50.49 \\
46.14 \\
46.13 \\
44.97 \\
38.98 \\
33.99 \\
33.96 \\
26.85 \\
21.88 \\
21.90 \\
16.69 \\
11.74 \\
11.77 \\
5.05 \\
0.02 \\
-0.04 \\
-0.02 \\
-0.02 \\
0.02\end{array}$ & $\begin{array}{c}\text { Temperature } \\
\text { (Fahrenheit) } \\
81.2060 \\
81.6020 \\
82.1200 \\
82.4370 \\
82.9200 \\
83.5790 \\
84.0170 \\
84.8500 \\
85.5080 \\
36.5580 \\
87.3010 \\
88.5240 \\
89.6580 \\
91.0960 \\
91.3140 \\
91.4010 \\
91.3570 \\
91.0960 \\
90.8350 \\
90.3560 \\
89.9200 \\
89.2660 \\
85.0700 \\
85.4640 \\
85.8140 \\
86.3030 \\
86.7770 \\
87.1700 \\
88.1750 \\
88.8300 \\
89.9200 \\
91.1830 \\
91.6620 \\
91.8790 \\
84.5000 \\
84.1490 \\
84.2370 \\
84.8070\end{array}$ & $\begin{array}{c}\text { Pressure } \\
(\text { psig) } \\
9.89 \\
19.55 \\
29.57 \\
39.42 \\
49.16 \\
54.50 \\
59.57 \\
64.20 \\
69.32 \\
74.16 \\
80.16 \\
85.61 \\
90.58 \\
95.69 \\
100.92 \\
103.25 \\
106.11 \\
108.31 \\
111.08 \\
113.24 \\
117.83 \\
120.92 \\
123.28 \\
122.97 \\
125.82 \\
125.60 \\
127.84 \\
130.19 \\
132.53 \\
135.33 \\
134.42 \\
138.35 \\
140.16 \\
142.63 \\
145.78 \\
142.52 \\
0.22\end{array}$ & $\begin{array}{c}\text { Temperature } \\
\text { (Fahrenheit) } \\
80.8980 \\
81.2500 \\
81.7780 \\
82.3930 \\
82.7440 \\
83.2710 \\
83.5790 \\
84.0170 \\
84.3680 \\
84.7190 \\
85.5950 \\
85.2890 \\
84.9380 \\
84.3240 \\
83.7100 \\
83.0520 \\
82.5570 \\
82.4370 \\
81.9530 \\
81.7780 \\
81.4700 \\
81.1180 \\
80.5010 \\
80.2370 \\
80.1050 \\
80.1050 \\
80.3250 \\
80.5450 \\
80.7660 \\
81.1620 \\
80.3690 \\
79.9290 \\
80.1050 \\
79.5320 \\
78.2530 \\
77.6790 \\
77.4140\end{array}$ \\
\hline
\end{tabular}




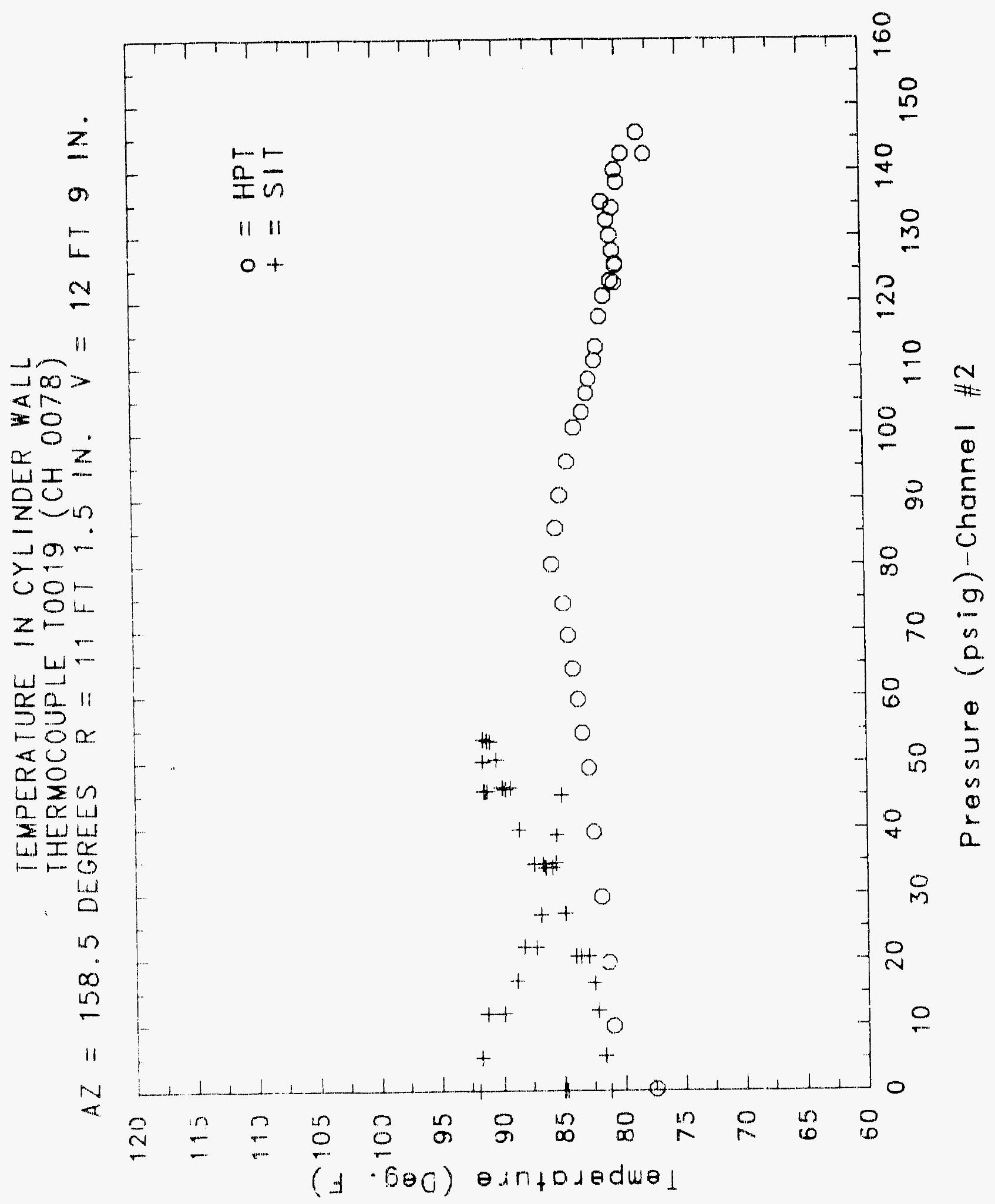




\section{Reinforced Concrete Test Data}

Thermocouple Channel 79

Structural INTEGRITY TEST

\begin{tabular}{|c|c|c|c|}
\hline $\begin{array}{c}\text { Pressure } \\
\text { (psig) } \\
-0.05 \\
5.33 \\
12.31 \\
16.44 \\
20.51 \\
20.46 \\
20.47 \\
27.05 \\
34.69 \\
34.53 \\
34.53 \\
39.70 \\
45.90 \\
45.65 \\
45.62 \\
50.10 \\
53.47 \\
53.29 \\
53.21 \\
50.49 \\
46.14 \\
46.13 \\
44.97 \\
38.98 \\
33.99 \\
33.96 \\
26.83 \\
21.88 \\
21.90 \\
16.69 \\
11.74 \\
11.77 \\
5.05 \\
0.02 \\
-0.06 \\
-0.02 \\
-0.02 \\
0.02\end{array}$ & $\begin{array}{c}\text { Temperature } \\
\text { (Fahrenhe it) } \\
80.7220 \\
81.0740 \\
81.6900 \\
82.2170 \\
82.7440 \\
83.3590 \\
83.7980 \\
84.2810 \\
84.8070 \\
85.5080 \\
85.7700 \\
86.1640 \\
86.5140 \\
87.0390 \\
87.2140 \\
87.5200 \\
87.6950 \\
87.9570 \\
88.0000 \\
87.9570 \\
87.8690 \\
87.6950 \\
84.6310 \\
85.1570 \\
85.5510 \\
86.2520 \\
86.5580 \\
86.9520 \\
87.5200 \\
87.7820 \\
88.0440 \\
88.4370 \\
88.6120 \\
88.6990 \\
64.4500 \\
84.0170 \\
84.3240 \\
85.7700\end{array}$ & $\begin{array}{c}\text { Pressure } \\
\text { (psig) } \\
9.89 \\
19.55 \\
29.57 \\
39.42 \\
49.16 \\
54.50 \\
59.57 \\
64.23 \\
59.32 \\
74.16 \\
80.16 \\
85.61 \\
90.58 \\
95.69 \\
100.92 \\
103.25 \\
106.11 \\
108.31 \\
111.08 \\
113.24 \\
117.83 \\
120.92 \\
123.28 \\
122.97 \\
125.82 \\
125.60 \\
127.84 \\
130.19 \\
132.53 \\
135.33 \\
134.42 \\
138.35 \\
140.16 \\
142.63 \\
145.78 \\
142.52 \\
0.22\end{array}$ & $\begin{array}{c}\text { Temperature } \\
\text { (Fahrenheit } \\
81.2500 \\
81.4700 \\
81.8650 \\
82.3050 \\
82.8760 \\
83.3150 \\
83.7540 \\
84.1490 \\
84.5000 \\
84.8500 \\
85.6390 \\
85.6830 \\
85.6390 \\
85.3760 \\
85.0700 \\
84.5440 \\
84.2370 \\
84.0610 \\
83.7540 \\
83.5350 \\
83.2270 \\
82.8760 \\
82.3490 \\
82.0410 \\
81.7340 \\
81.4700 \\
81.4260 \\
81.6900 \\
81.9530 \\
82.7440 \\
83.2710 \\
83.8420 \\
84.2370 \\
84.2810 \\
82.2610 \\
80.3250 \\
78.2530\end{array}$ \\
\hline
\end{tabular}

\section{High Pressure test}

Pressure

9.55

29.57

54.50

69.32

85.61

90.58

95.69

103.25

106.11

108.31

111.08

113.24

117.83

120.92

123.28

122.97

125.82

132.53

135.33

134.42

138.35

140.16

142.63

2.52

80.3250

78.2530 


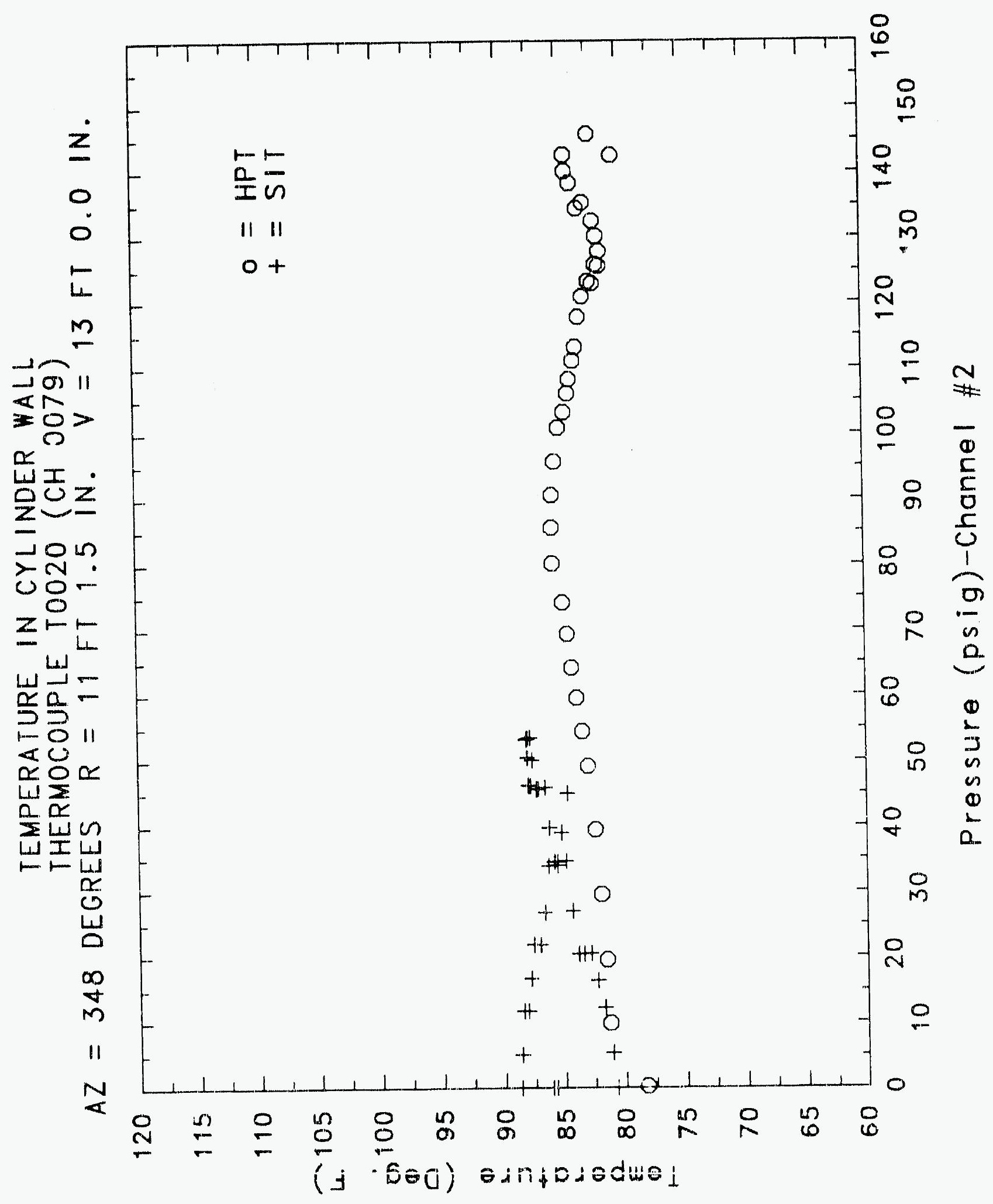




\section{Reinforced Concrete Test Data}

Thermocouple Channel 80

Structural IntEgrity TEST

\begin{tabular}{|c|c|c|c|}
\hline $\begin{array}{c}\text { Pressure } \\
\text { (psig) } \\
-0.05 \\
5.33 \\
12.31 \\
16.44 \\
20.51 \\
20.46 \\
20.47 \\
27.05 \\
34.69 \\
34.53 \\
34.53 \\
39.70 \\
45.90 \\
45.65 \\
45.62 \\
50.10 \\
53.47 \\
53.29 \\
53.21 \\
50.49 \\
46.14 \\
46.13 \\
44.97 \\
38.98 \\
33.99 \\
33.96 \\
26.83 \\
21.88 \\
21.90 \\
16.69 \\
11.74 \\
11.77 \\
5.05 \\
0.02 \\
-0.04 \\
-0.02 \\
-0.02 \\
0.02\end{array}$ & $\begin{array}{c}\text { Temperature } \\
\text { (Fahrenheit) } \\
77.3700 \\
78.3410 \\
79.3120 \\
79.8410 \\
80.2810 \\
80.8980 \\
81.2500 \\
81.8220 \\
82.3490 \\
82.7440 \\
82.8760 \\
83.3590 \\
83.6660 \\
83.9300 \\
83.9740 \\
83.9740 \\
84.1050 \\
84.0170 \\
83.8860 \\
83.5790 \\
83.3150 \\
83.0080 \\
82.0410 \\
82.6130 \\
83.0520 \\
83.4910 \\
83.7540 \\
83.9740 \\
84.3680 \\
84.5870 \\
84.8070 \\
85.1130 \\
85.2010 \\
85.1570 \\
79.1350 \\
79.9730 \\
81.4700 \\
83.4030\end{array}$ & $\begin{array}{c}\text { Pressure } \\
(\text { psig) } \\
9.89 \\
19.55 \\
29.5 i \\
39.42 \\
49.16 \\
54.50 \\
59.57 \\
64.20 \\
69.32 \\
74.16 \\
80.16 \\
85.61 \\
90.58 \\
95.69 \\
100.92 \\
103.25 \\
106.11 \\
108.31 \\
111.08 \\
113.24 \\
117.83 \\
120.92 \\
123.28 \\
122.97 \\
125.82 \\
125.60 \\
127.84 \\
130.19 \\
132.53 \\
135.33 \\
134.42 \\
138.35 \\
140.16 \\
142.63 \\
145.78 \\
142.52 \\
0.22\end{array}$ & $\begin{array}{c}\text { Temperature } \\
\text { (Fahrenheit) } \\
77.9000 \\
78.5180 \\
79.0910 \\
79.7970 \\
80.3250 \\
80.8540 \\
81.1180 \\
81.4700 \\
81.6900 \\
82.0850 \\
82.2170 \\
81.8220 \\
81.3380 \\
80.6340 \\
80.0170 \\
79.3120 \\
78.8710 \\
78.6060 \\
78.1650 \\
77.9880 \\
77.5910 \\
77.3260 \\
76.3090 \\
76.1760 \\
76.4860 \\
77.2810 \\
77.9440 \\
79.7090 \\
80.2370 \\
80.9420 \\
81.3820 \\
81.6020 \\
82.0410 \\
82.2610 \\
82.3930 \\
82.4370 \\
79.1350\end{array}$ \\
\hline
\end{tabular}

High Pressure Test

emperature

(Fahrenheit)

77.9000

78.5180

79.0910

80.3250

80.8540

81.1180

81.4700

82.0850

82.2170

81.8220

80.0170

79.3120

78.1650

77.9880

77.5910

77.3260

76.1760

76.4860

77.2810

79.7090

80.9420

81.3820

81.6020

82.0410

82.2610

82.3930

82.4370
77.9440 


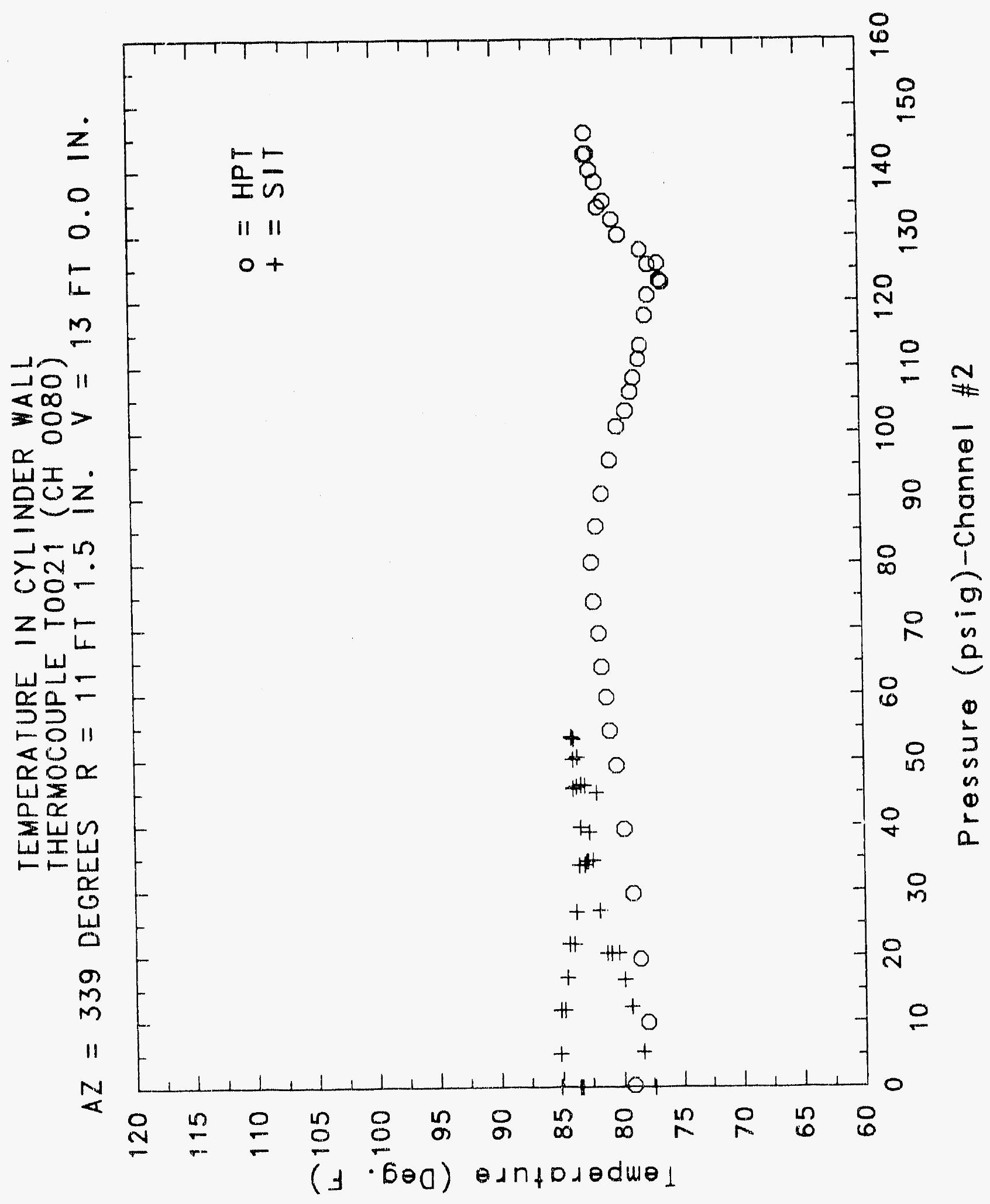

C. 1709 


\section{Reinforced Concrete Test Data}

Thermocouple Channel 81

Structural Integrity Test

\begin{tabular}{|c|c|c|c|}
\hline $\begin{array}{c}\text { Pressure } \\
\text { (psiy) } \\
-0.05 \\
5.33 \\
12.31 \\
16.44 \\
20.51 \\
20.46 \\
20.47 \\
27.05 \\
34.65 \\
34.53 \\
34.53 \\
39.70 \\
45.90 \\
45.65 \\
45.62 \\
50.10 \\
53.47 \\
53.29 \\
53.21 \\
50.49 \\
46.14 \\
46.13 \\
44.97 \\
38.98 \\
33.99 \\
33.96 \\
26.83 \\
21.88 \\
21.90 \\
16.69 \\
11.74 \\
11.77 \\
5.00 \\
0.02 \\
-0.04 \\
-0.02 \\
-0.02 \\
0.02\end{array}$ & $\begin{array}{c}\text { Temperature } \\
\text { (Fahrenheit) } \\
72.8520 \\
73.6510 \\
74.8480 \\
75.8660 \\
76.9280 \\
77.8110 \\
78.4740 \\
79.5760 \\
80.4130 \\
81.1620 \\
81.5140 \\
82.1730 \\
82.7000 \\
82.9200 \\
82.8760 \\
83.0080 \\
83.0520 \\
83.0080 \\
82.7880 \\
82.4370 \\
82.1290 \\
81.7780 \\
78.8270 \\
79.6640 \\
80.2370 \\
81.2060 \\
81.7340 \\
82.1290 \\
82.7880 \\
83.0520 \\
83.4470 \\
83.7100 \\
83.7540 \\
83.7100 \\
76.4410 \\
76.3090 \\
77.0160 \\
79.0910\end{array}$ & $\begin{array}{c}\text { Pressure } \\
(\text { psig) } \\
9.89 \\
19.55 \\
29.57 \\
39.42 \\
49.16 \\
54.50 \\
59.57 \\
64.20 \\
69.32 \\
74.16 \\
80.16 \\
85.61 \\
90.58 \\
95.69 \\
100.92 \\
103.25 \\
106.11 \\
108.31 \\
111.08 \\
113.24 \\
117.83 \\
120.92 \\
123.28 \\
122.97 \\
125.82 \\
125.60 \\
127.84 \\
130.19 \\
132.53 \\
135.33 \\
134.42 \\
138.35 \\
140.16 \\
142.63 \\
145.78 \\
142.52 \\
0.22\end{array}$ & $\begin{array}{c}\text { Temperature } \\
\text { (Fahrenheit) } \\
73.2510 \\
73.8720 \\
74.5820 \\
75.5120 \\
76.1320 \\
76.7510 \\
77.1930 \\
77.6350 \\
78.1210 \\
78.6940 \\
78.2970 \\
77.8560 \\
77.5910 \\
77.1490 \\
76.7510 \\
76.1320 \\
75.7330 \\
75.5560 \\
75.2020 \\
75.0690 \\
74.7590 \\
74.4490 \\
73.8720 \\
73.7390 \\
73.8280 \\
73.9170 \\
74.0940 \\
74.7150 \\
75.0690 \\
75.8220 \\
76.8840 \\
77.8560 \\
78.6500 \\
79.0910 \\
79.5320 \\
79.7530 \\
77.5460\end{array}$ \\
\hline
\end{tabular}

\section{High Pressure test}

emperature

Fahrenheit)

73.8720

74.5820

75.5120

76.1320

78.1210

78.6940

77.5910

77.1490

76.7510

75.5560

75.2020

5.0690

74.7590

73.7390

73.8280

73.9170

74.0940

75.8220

76.8840

77.8560

8.6500

79.7530

77.5460 


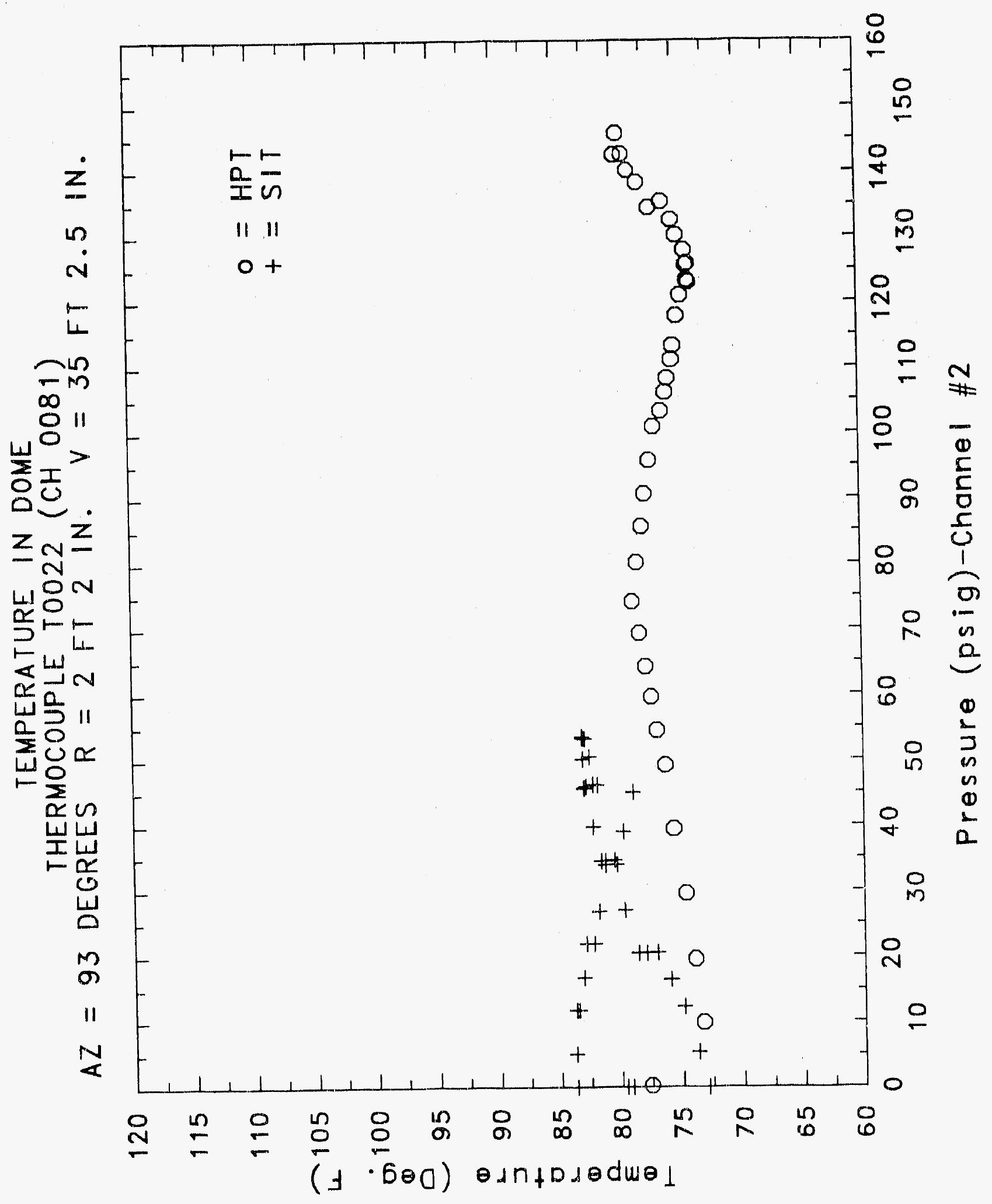

C. 1711 


\section{Reinforced Concrete Test Data}

Thermocouple Channel 82

Structural Integrity Test

\begin{tabular}{rccc}
\hline & & & \\
Pressure & Temperature & Pressure & Temperature \\
(psig) & $($ Fahrenheit) & $($ psig) & (Fahrenheit) \\
-0.05 & 72.2300 & 9.89 & 75.2910 \\
5.33 & 73.1180 & 19.55 & 75.9990 \\
12.31 & 74.4930 & 29.57 & 76.4860 \\
16.44 & 75.7330 & 39.42 & 76.9280 \\
20.51 & 78.0320 & 49.16 & 77.4140 \\
20.46 & 81.7340 & 54.50 & 78.0320 \\
20.47 & 85.6390 & 59.57 & 78.5620 \\
27.05 & 88.3060 & 64.20 & 81.6460 \\
34.69 & 88.1310 & 69.32 & 82.9640 \\
34.53 & 87.5640 & 74.16 & 86.6020 \\
34.53 & 87.2140 & 80.16 & 77.5020 \\
39.70 & 87.0390 & 85.61 & 76.7070 \\
45.90 & 85.7260 & 90.58 & 75.9990 \\
45.65 & 83.4030 & 95.69 & 74.7590 \\
45.62 & 82.8760 & 100.92 & 74.0500 \\
50.10 & 82.0410 & 103.25 & 73.3840 \\
53.47 & 81.4260 & 106.11 & 72.7630 \\
53.29 & 80.4130 & 108.31 & 72.7180 \\
53.21 & 79.9730 & 111.08 & 72.3190 \\
50.49 & 79.3120 & 113.24 & 71.9190 \\
46.14 & 79.2240 & 117.83 & 71.6960 \\
46.13 & 78.6500 & 120.92 & 71.6070 \\
44.97 & 78.1210 & 123.28 & 71.6520 \\
38.98 & 78.9590 & 122.91 & 72.0080 \\
33.99 & 80.4570 & 125.82 & 72.2740 \\
33.96 & 86.9520 & 125.60 & 72.7630 \\
26.83 & 89.6580 & 127.84 & 73.0290 \\
21.88 & 93.7470 & 130.19 & 74.3160 \\
21.90 & 94.7020 & 132.53 & 75.1580 \\
16.69 & 92.0530 & 135.33 & 76.0430 \\
11.74 & 90.4860 & 134.42 & 89.7530 \\
11.77 & 87.7820 & 138.35 & 86.6890 \\
5.05 & 86.4700 & 140.16 & 88.1200 \\
0.02 & 85.1570 & 142.63 & 85.5080 \\
-0.04 & 74.9810 & 145.78 & 83.2270 \\
-0.02 & 75.7330 & 142.52 & \\
-0.02 & 76.6180 & 0.22 & \\
0.02 & 79.3560 & & 8010 \\
& & & \\
\hline & & & \\
\hline
\end{tabular}

High Pressure test

29.57

39.42

64.20

74.16

80.16

95.69

106.11

113.24

125.82

125.60

127.84

135.33

134.42

138.35

140.16

42.52

42.52
0.22
75.2910

76.9280

77.4140

6460

86.6020

77.5020

74.7590

74.0500

73.3840

72.7630

71.9190

71.6960

71.6070

72.2740

72.7630

73.0290

76.0430

79.7530

86.6890

86.1200

83.2270

80.5010 


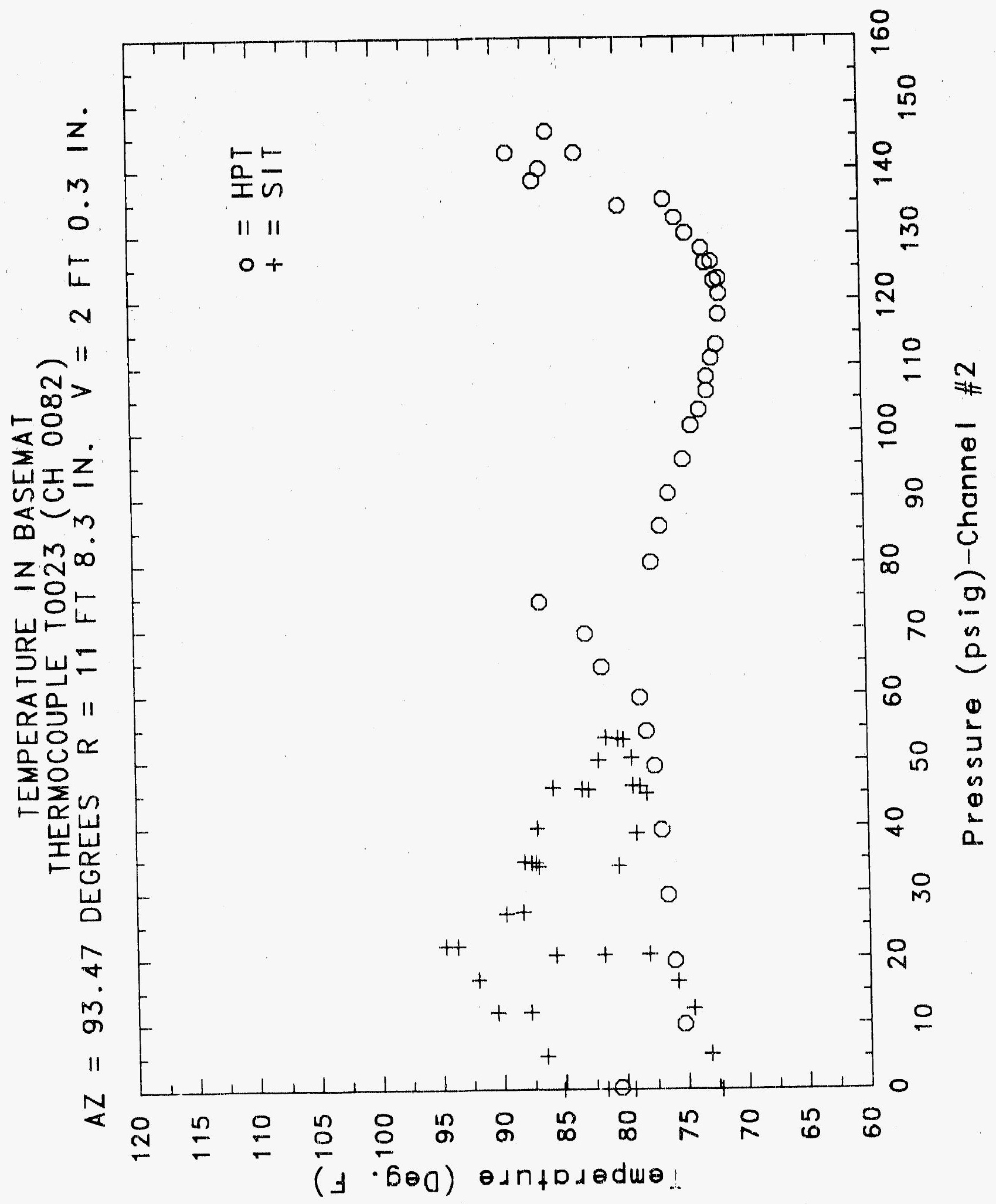




\section{Reinforced Concrete Test Data}

Thermocouple Channel 83

Structural INTEGRITY TEST

\begin{tabular}{|c|c|c|c|}
\hline $\begin{array}{c}\text { Pressire } \\
\text { (psig) } \\
-0.05 \\
5.33 \\
12.31 \\
16.44 \\
20.51 \\
20.46 \\
20.47 \\
27.05 \\
34.69 \\
34.53 \\
34.53 \\
39.70 \\
45.90 \\
45.65 \\
45.62 \\
50.10 \\
53.47 \\
53.29 \\
53.21 \\
50.49 \\
46.14 \\
46.13 \\
44.97 \\
38.98 \\
33.99 \\
33.96 \\
26.83 \\
21.88 \\
21.90 \\
16.69 \\
11.74 \\
11.77 \\
5.05 \\
0.02 \\
-0.04 \\
-0.02 \\
-0.02 \\
0.02\end{array}$ & $\begin{array}{c}\text { Temperature } \\
\text { (Fahrenheit) } \\
72.3630 \\
73.4730 \\
75.0690 \\
76.7070 \\
79.1790 \\
83.0520 \\
86.9520 \\
89.8760 \\
90.3120 \\
90.2690 \\
89.9640 \\
89.6150 \\
88.0880 \\
85.6390 \\
84.8500 \\
83.8860 \\
83.2710 \\
82.1730 \\
81.5580 \\
80.8100 \\
80.5010 \\
79.7090 \\
78.8710 \\
80.0170 \\
81.5580 \\
88.0880 \\
90.7040 \\
95.0920 \\
97.8180 \\
95.3080 \\
93.5300 \\
90.4430 \\
88.8300 \\
87.3450 \\
74.9360 \\
75.6890 \\
76.6180 \\
79.8410\end{array}$ & $\begin{array}{c}\text { Pressure } \\
\text { (psig) } \\
9.89 \\
19.55 \\
29.57 \\
39.42 \\
49.16 \\
54.50 \\
59.57 \\
64.20 \\
69.32 \\
74.16 \\
80.16 \\
85.61 \\
90.58 \\
95.69 \\
100.92 \\
103.25 \\
106.11 \\
108.31 \\
111.08 \\
113.24 \\
117.83 \\
120.92 \\
123.28 \\
122.97 \\
125.82 \\
125.60 \\
127.84 \\
130.19 \\
132.53 \\
135.33 \\
134.42 \\
138.35 \\
140.16 \\
142.63 \\
145.78 \\
142.52 \\
0.22\end{array}$ & $\begin{array}{c}\text { Temperature } \\
\text { (Fahrenheit) } \\
75.3790 \\
76.2200 \\
76.7510 \\
77.3260 \\
78.0320 \\
78.8270 \\
79.4440 \\
82.8320 \\
84.1050 \\
88.0000 \\
78.7830 \\
77.7230 \\
76.8840 \\
75.3350 \\
74.4930 \\
73.6510 \\
72.8960 \\
72.7180 \\
72.3630 \\
72.0080 \\
71.7850 \\
71.6520 \\
71.7850 \\
72.0960 \\
72.4960 \\
73.1180 \\
73.4290 \\
74.8480 \\
75.6890 \\
76.9720 \\
80.9420 \\
87.6950 \\
87.1700 \\
89.8760 \\
86.9520 \\
84.6750 \\
81.7340\end{array}$ \\
\hline
\end{tabular}

High Pressure test

Temperature

(Fhrenheit)

75.3790

77.3260

78.0320

78.8270

84.1050

88.0000

78.7830

77.7230

74.4930

73.6510

72.8960

72.0080

72.0960

73.1180

73.4290

75.6890

87.6950

87.1700

89.8760

81.7340
74.8480 


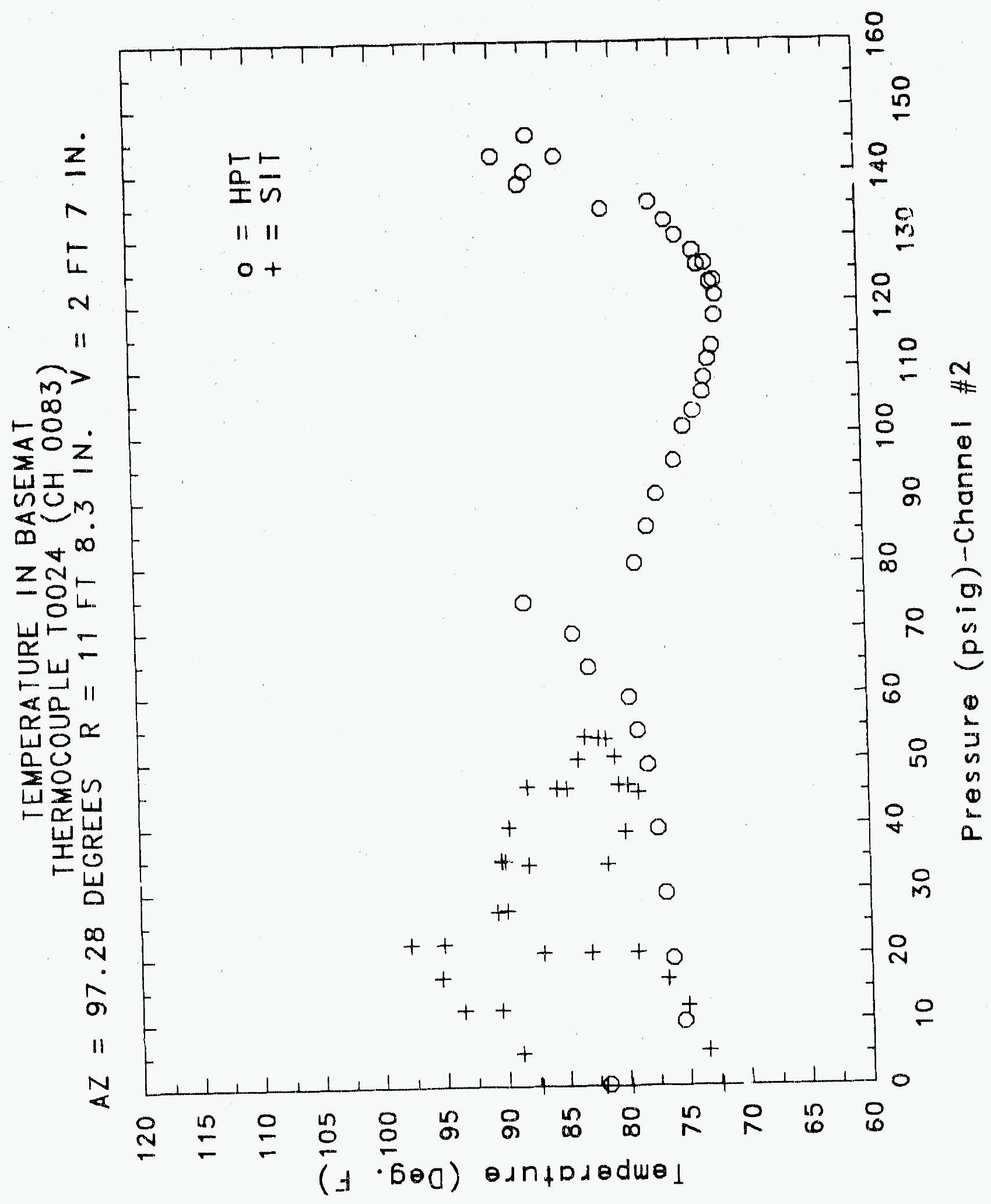

C. 1715 


\section{Reinforced Concrete Test Data}

Thermocouple Channel 84

StRUCTURAL INTEgRITY TEST

\begin{tabular}{|c|c|c|c|}
\hline $\begin{array}{c}\text { Pressure } \\
\text { (psig) } \\
-0.05 \\
5.33 \\
12.31 \\
16.44 \\
20.51 \\
20.46 \\
20.47 \\
27.05 \\
34.69 \\
34.53 \\
34.53 \\
39.70 \\
45.90 \\
45.65 \\
45.62 \\
50.10 \\
53.47 \\
53.29 \\
53.21 \\
50.49 \\
46.14 \\
46.13 \\
44.97 \\
38.98 \\
33.99 \\
33.96 \\
26.83 \\
21.88 \\
21.90 \\
16.69 \\
11.74 \\
11.77 \\
5.05 \\
0.02 \\
-0.04 \\
-0.02 \\
-0.02 \\
0.02\end{array}$ & $\begin{array}{c}\text { Temperature } \\
\text { (Fahrenheit) } \\
72.9400 \\
74.2270 \\
76.0430 \\
78.0320 \\
80.3690 \\
84.0610 \\
88.6120 \\
90.1810 \\
90.9220 \\
93.7910 \\
92.7050 \\
91.1830 \\
89.3530 \\
86.7770 \\
86.1200 \\
85.2890 \\
84.5000 \\
83.1400 \\
82.4370 \\
81.6460 \\
81.3380 \\
80.4130 \\
79.9730 \\
81.3380 \\
82.4370 \\
88.6990 \\
91.4880 \\
95.1780 \\
99.5020 \\
97.8610 \\
95.5680 \\
91.4440 \\
89.5280 \\
87.9130 \\
75.6010 \\
76.5740 \\
77.6790 \\
81.4260\end{array}$ & $\begin{array}{c}\text { Pressure } \\
\text { (psig) } \\
9.89 \\
19.55 \\
29.57 \\
39.42 \\
49.16 \\
54.50 \\
59.57 \\
64.20 \\
69.32 \\
74.16 \\
80.16 \\
85.61 \\
90.58 \\
95.69 \\
100.92 \\
103.25 \\
106.11 \\
108.31 \\
111.08 \\
113.24 \\
117.83 \\
120.92 \\
123.28 \\
122.97 \\
125.82 \\
125.60 \\
127.84 \\
130.19 \\
132.53 \\
135.33 \\
134.47 \\
138.30 \\
140.16 \\
142.63 \\
145.78 \\
142.52 \\
0.22\end{array}$ & $\begin{array}{c}\text { Temperature } \\
\text { (Fahrenheit) } \\
76.2640 \\
77.0600 \\
77.5460 \\
77.9880 \\
78.6940 \\
79.4440 \\
80.0610 \\
83.5790 \\
84.8070 \\
88.3060 \\
79.1790 \\
78.2970 \\
77.5020 \\
76.3090 \\
75.4680 \\
74.6260 \\
74.0500 \\
73.8720 \\
73.3400 \\
73.0290 \\
72.8070 \\
72.5850 \\
72.4080 \\
72.7630 \\
73.1180 \\
73.7390 \\
74.0940 \\
75.4680 \\
76.4860 \\
77.9880 \\
81.0740 \\
87.6070 \\
87.0830 \\
89.9200 \\
87.0390 \\
84.8940 \\
82.0850\end{array}$ \\
\hline
\end{tabular}

High Pressure test

Pressure

9.89

19.55

39.42

49.16

85.61

90.58

100.92

103.25

106.11

120.92

123.28

122.97

127.84

130.19

132.53

135.33

140.16

142.63

145.78

52

0.22
Temperature

76.2640

77.0600

77.9880

78.6940

83.5790

84.8070

88.3060

79.1790

76.3090

74.0500

73.0290

72.8070

72.5850

73.1180

73.7390

74.0940

75.4680

76.4860

81.0740

87.6070

87.0830

89.9200

87.0390

.8940

2.0850 


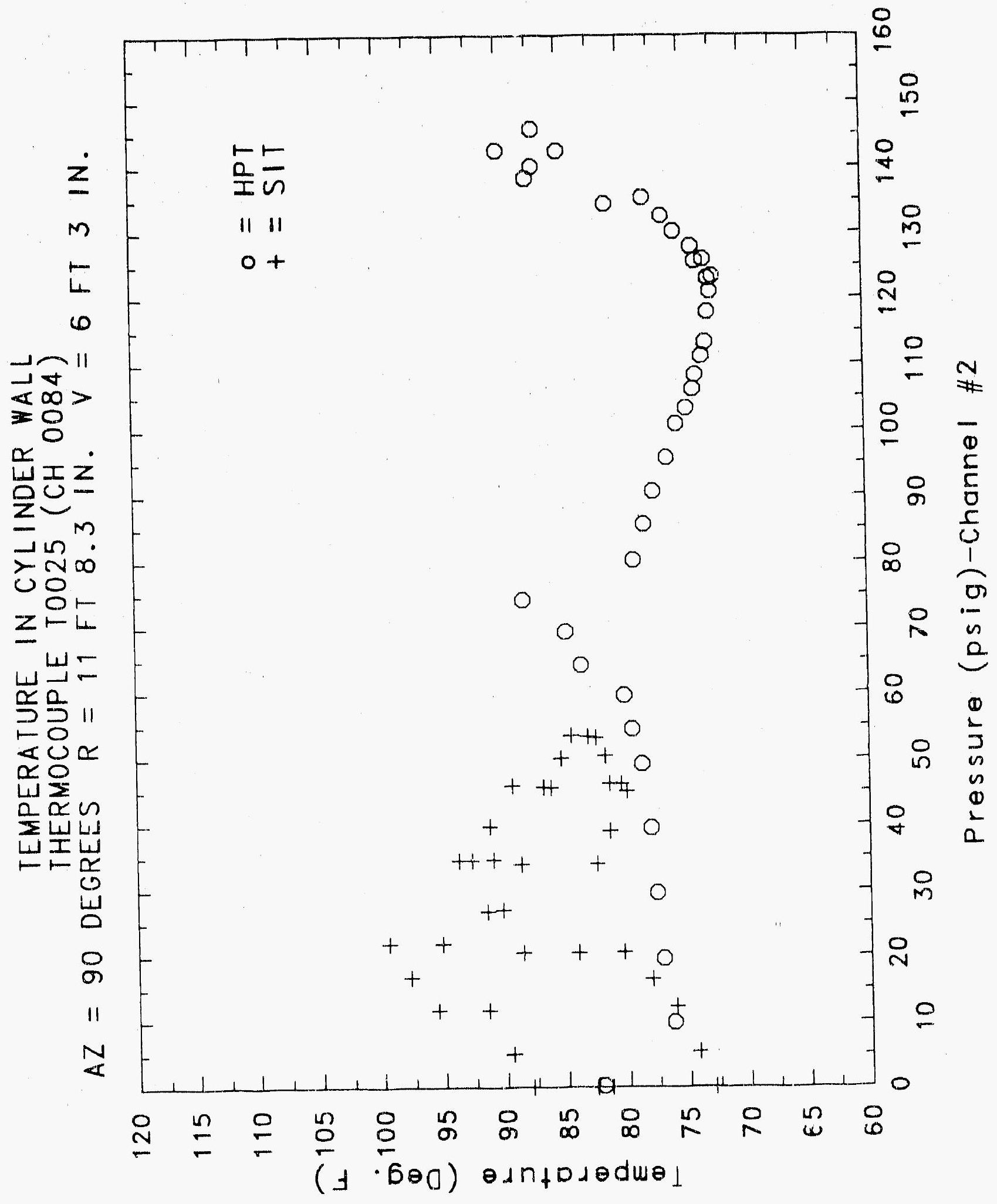




\section{Reinforced Concrete test Data}

Thermocouple "Channel 85

Structural IntEgrity TEST

\begin{tabular}{|c|c|c|c|}
\hline $\begin{array}{c}\text { Pressure } \\
(p \text { ig) } \\
-0.05 \\
5.33 \\
12.31 \\
16.44 \\
20.51 \\
20.46 \\
20.47 \\
27.05 \\
34.69 \\
34.53 \\
34.53 \\
39.70 \\
45.90 \\
45.65 \\
45.62 \\
50.10 \\
53.47 \\
53.29 \\
53.21 \\
50.49 \\
46.14 \\
46.13 \\
44.97 \\
38.98 \\
33.99 \\
33.96 \\
26.83 \\
21.88 \\
21.90 \\
16.69 \\
11.74 \\
11.77 \\
5.05 \\
0.02 \\
-0.04 \\
-0.02 \\
-0.02 \\
0.02\end{array}$ & $\begin{array}{c}\text { Temperature } \\
\text { (Fahrenheit) } \\
73.5170 \\
73.7840 \\
74.2270 \\
74.9360 \\
75.6450 \\
76.5300 \\
77.3370 \\
78.5620 \\
79.7090 \\
81.2940 \\
82.0850 \\
83.0960 \\
83.8860 \\
84.7630 \\
84.7190 \\
84.5000 \\
84.2370 \\
83.8420 \\
83.5790 \\
83.1400 \\
82.7440 \\
82.2170 \\
73.1210 \\
78.5180 \\
78.9590 \\
79.7090 \\
80.1930 \\
80.9420 \\
82.7000 \\
83.6660 \\
84.6310 \\
85.6830 \\
85.8580 \\
85.8580 \\
77.3700 \\
76.9720 \\
77.1490 \\
78.2530\end{array}$ & $\begin{array}{c}\text { Pressure } \\
\text { (psig) } \\
r .89 \\
19.55 \\
29.57 \\
39.42 \\
49.16 \\
49 \\
54.50 \\
59.57 \\
64.20 \\
69.32 \\
74.16 \\
80.16 \\
85.61 \\
90.58 \\
95.69 \\
100.92 \\
103.25 \\
106.11 \\
108.31 \\
111.08 \\
113.24 \\
117.83 \\
120.92 \\
123.28 \\
122.97 \\
125.82 \\
125.60 \\
127.84 \\
130.19 \\
132.53 \\
135.33 \\
134.42 \\
138.35 \\
140.16 \\
142.63 \\
145.78 \\
142.52 \\
0.22\end{array}$ & $\begin{array}{c}\text { Temperature } \\
\text { (Fahrenheit) } \\
73.9170 \\
74.2270 \\
74.4040 \\
74.8030 \\
75.3350 \\
75.8220 \\
76.0870 \\
76.7070 \\
77.3490 \\
77.8560 \\
79.0470 \\
78.3410 \\
77.8110 \\
77.0160 \\
76.3530 \\
75.6450 \\
75.1580 \\
74.8920 \\
74.4930 \\
74.2710 \\
73.8720 \\
73.5170 \\
72.9400 \\
72.6740 \\
72.5410 \\
72.5850 \\
72.7180 \\
73.0290 \\
73.2070 \\
73.9170 \\
74.6700 \\
75.5560 \\
76.7510 \\
78.0320 \\
79.0470 \\
75.0250 \\
75.1130\end{array}$ \\
\hline
\end{tabular}

High Pressure test

Temporature
Fahrenheit)

74.4040

74.8030

75.3350

76.7070

77.1490

77.8560

77.8110

77.0160

76.3530

74.8920

74.4930

74.2710

72.9400

72.6740

72.5410

.5850

73.0290

73.2070

73.9170

.6700

76.7510

78.0320

79.0470

75.0250

75.1130 


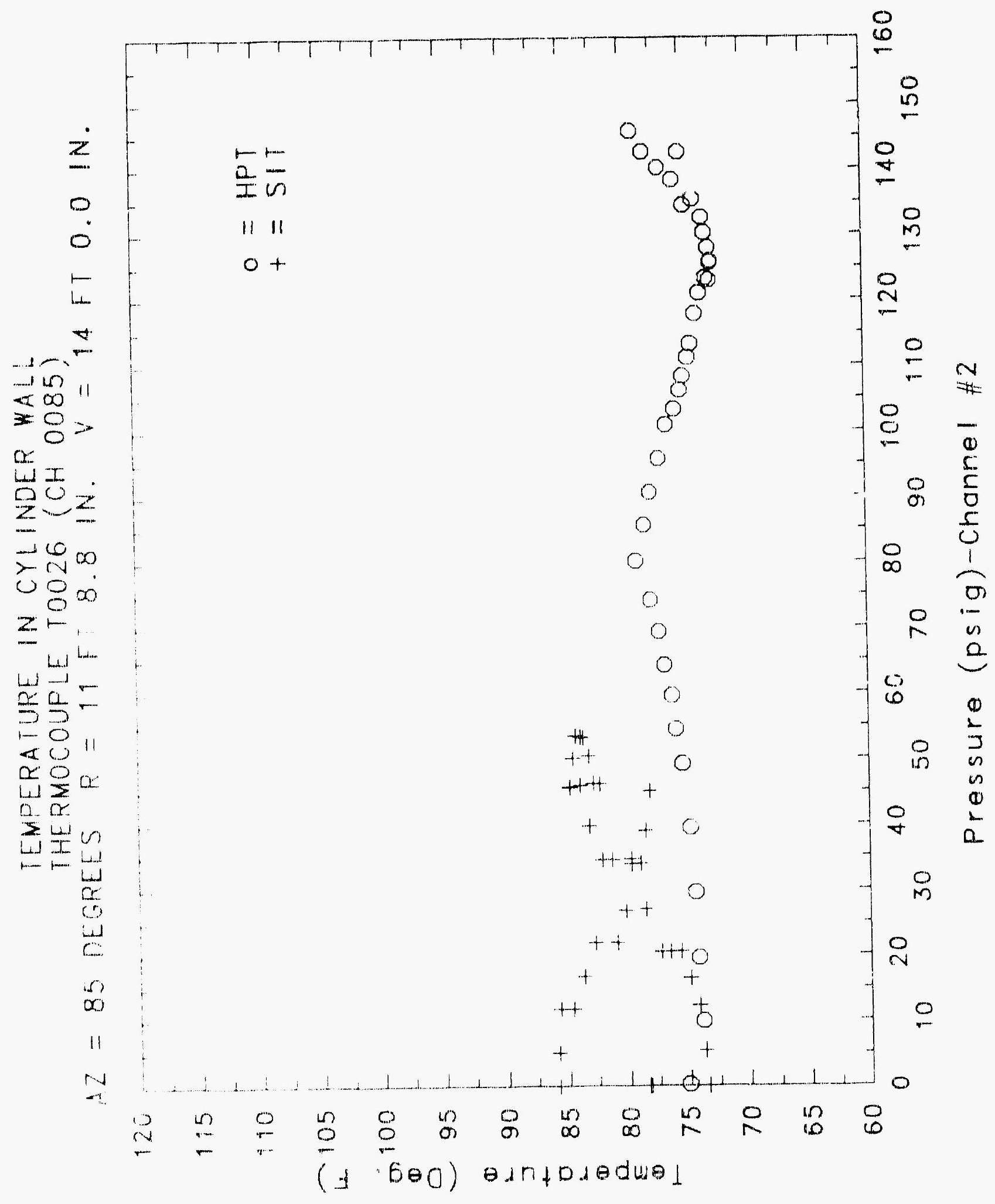




\section{Reinforced Concrete Test Data}

Thermocouple Channel 88

StRUCTURAL INTEGRITY TEST

\begin{tabular}{|c|c|c|c|}
\hline $\begin{array}{l}\text { Pressure } \\
\text { (psig) } \\
-0.05 \\
5.33 \\
12.31 \\
16.44 \\
20.51 \\
20.46 \\
20.47 \\
27.05 \\
34.69 \\
34.53 \\
34.53 \\
39.70 \\
45.90 \\
45.65 \\
45.62 \\
50.10 \\
53.41 \\
53.29 \\
53.21 \\
50.49 \\
46.14 \\
46.13 \\
44.97 \\
38.98 \\
33.99 \\
33.96 \\
26.83 \\
21.88 \\
21.90 \\
16.69 \\
11.74 \\
11.77 \\
5.05 \\
0.02 \\
-0.04 \\
-0.02 \\
-0.02 \\
0.02\end{array}$ & $\begin{array}{c}\text { Temperature } \\
\text { (Fahrenheit) } \\
73.7390 \\
74.8920 \\
76.4860 \\
77.9000 \\
79.2240 \\
80.3250 \\
81.0740 \\
82.0410 \\
82.5250 \\
83.5350 \\
83.9740 \\
86.6890 \\
86.7330 \\
85.2010 \\
84.6750 \\
84.1050 \\
83.7540 \\
83.2710 \\
82.7440 \\
82.1730 \\
81.8650 \\
81.0740 \\
80.8100 \\
81.9530 \\
82.6130 \\
83.6660 \\
84.1050 \\
84.4560 \\
85.1570 \\
85.4200 \\
85.6830 \\
85.2890 \\
85.0260 \\
84.5000 \\
75.7330 \\
76.9720 \\
78.3850 \\
81.0300\end{array}$ & $\begin{array}{c}\text { Pressure } \\
(p s 1 g) \\
9.89 \\
19.55 \\
29.57 \\
39.42 \\
49.16 \\
54.50 \\
59.57 \\
64.20 \\
69.32 \\
74.16 \\
80.16 \\
85.61 \\
90.58 \\
95.69 \\
100.92 \\
103.25 \\
108.11 \\
108.31 \\
111.08 \\
113.24 \\
117.83 \\
120.92 \\
123.28 \\
122.97 \\
125.82 \\
125.50 \\
127.84 \\
130.19 \\
132.53 \\
135.33 \\
134.42 \\
138.35 \\
140.16 \\
142.63 \\
145.78 \\
142.52 \\
0.22\end{array}$ & $\begin{array}{c}\text { Temperature } \\
\text { (Fahrenheit) } \\
74.1830 \\
74.8920 \\
75.2910 \\
75.7780 \\
76.2200 \\
76.8840 \\
77.4580 \\
78.1650 \\
78.8270 \\
79.7090 \\
76.6620 \\
76.1760 \\
75.7330 \\
75.0250 \\
74.40 .80 \\
73.8720 \\
73.3400 \\
73.0740 \\
72.7180 \\
72.4960 \\
72.1410 \\
71.9630 \\
71.7410 \\
72.0080 \\
72.4520 \\
73.2510 \\
73.7840 \\
74.7150 \\
75.0690 \\
76.8840 \\
78.3410 \\
79.4880 \\
79.9730 \\
80.4570 \\
80.5010 \\
80.4130 \\
78.8270\end{array}$ \\
\hline
\end{tabular}

High Pressure Test

\author{
Fahrenheit)
}

74.1830

74.8920

75.2910

76.8840

77.4580

78.1650

76.6520

76.1760

75.7330

75.0250

73.3400

73.0740

72.7180

71.9630

71.7410

72.0080

72.4520

74.7150

75.0690

76.8840

78.3410

80.4570

80.5010

80.4130

78.8270 


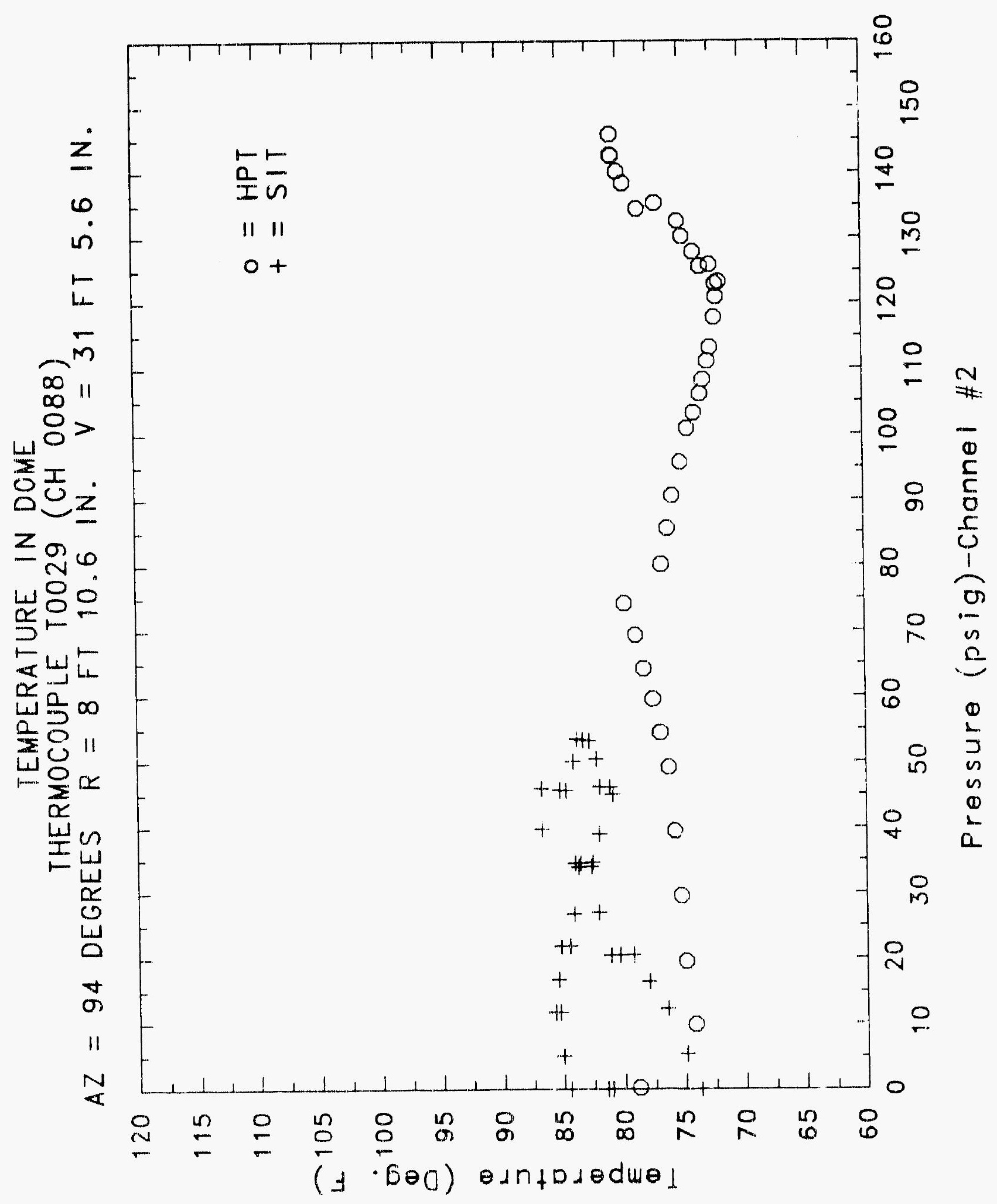




\section{Reinforced Concrete Test Data}

\section{Thermocouple Channel 89}

Structural INTEGRITY TEST

\begin{tabular}{|c|c|}
\hline $\begin{array}{l}\text { Pressure } \\
\text { (psig) } \\
-0.05 \\
5.33 \\
12.31 \\
16.44 \\
20.51 \\
20.46 \\
20.47 \\
27.05 \\
34.69 \\
34.53 \\
34.53 \\
39.70 \\
45.90 \\
45.65 \\
45.62 \\
50.10 \\
53.47 \\
53.29 \\
53.21 \\
50.49 \\
46.14 \\
46.13 \\
44.97 \\
38.98 \\
33.99 \\
33.96 \\
26.83 \\
21.88 \\
21.90 \\
16.69 \\
11.74 \\
11.77 \\
5.05 \\
0.02 \\
-0.04 \\
-0.02 \\
-0.02 \\
0.02\end{array}$ & $\begin{array}{c}\text { Temperature } \\
\text { (Fahrenheit) } \\
72.4080 \\
73.9170 \\
75.7780 \\
77.4140 \\
78.6940 \\
79.7970 \\
80.6340 \\
81.2500 \\
81.6460 \\
82.6130 \\
82.9200 \\
83.0520 \\
82.6130 \\
81.9090 \\
81.6900 \\
81.5140 \\
81.5140 \\
81.1620 \\
80.7660 \\
80.4570 \\
80.3250 \\
79.6640 \\
80.3690 \\
81.5580 \\
82.1730 \\
83.1400 \\
83.4910 \\
83.7980 \\
84.2810 \\
84.4560 \\
84.5440 \\
84.0170 \\
83.5350 \\
82.9640 \\
523.1600 \\
76.5740 \\
78.0320 \\
80.5010\end{array}$ \\
\hline
\end{tabular}

High Pressure test

\begin{tabular}{|c|c|}
\hline $\begin{array}{c}\text { Pressure } \\
\text { (psig) } \\
9.89 \\
19.55 \\
29.57 \\
39.42 \\
49.16 \\
54.50 \\
59.57 \\
64.20 \\
69.32 \\
74.16 \\
80.16 \\
85.61 \\
90.58 \\
95.69 \\
100.92 \\
103.25 \\
106.11 \\
108.31 \\
111.08 \\
113.24 \\
117.83 \\
120.92 \\
123.28 \\
122.97 \\
125.82 \\
125.60 \\
127.84 \\
130.19 \\
132.53 \\
135.33 \\
134.42 \\
138.35 \\
140.16 \\
142.63 \\
145.78 \\
142.52 \\
0.22\end{array}$ & $\begin{array}{c}\text { Temperature } \\
\text { (Fahrenheit) } \\
73.3840 \\
73.8720 \\
74.2270 \\
74.6700 \\
75.0250 \\
75.6010 \\
76.2640 \\
77.0600 \\
77.6790 \\
78.4300 \\
74.9360 \\
74.5370 \\
74.2710 \\
73.7390 \\
73.2950 \\
72.7180 \\
72.2740 \\
72.0520 \\
71.7410 \\
71.3850 \\
71.2520 \\
71.1630 \\
71.1180 \\
71.5190 \\
71.8300 \\
72.6740 \\
73.2510 \\
73.9170 \\
74.3160 \\
76.5740 \\
78.1650 \\
2109.4000 \\
78.9150 \\
79.5760 \\
79.4880 \\
79.1350 \\
77.7670\end{array}$ \\
\hline
\end{tabular}




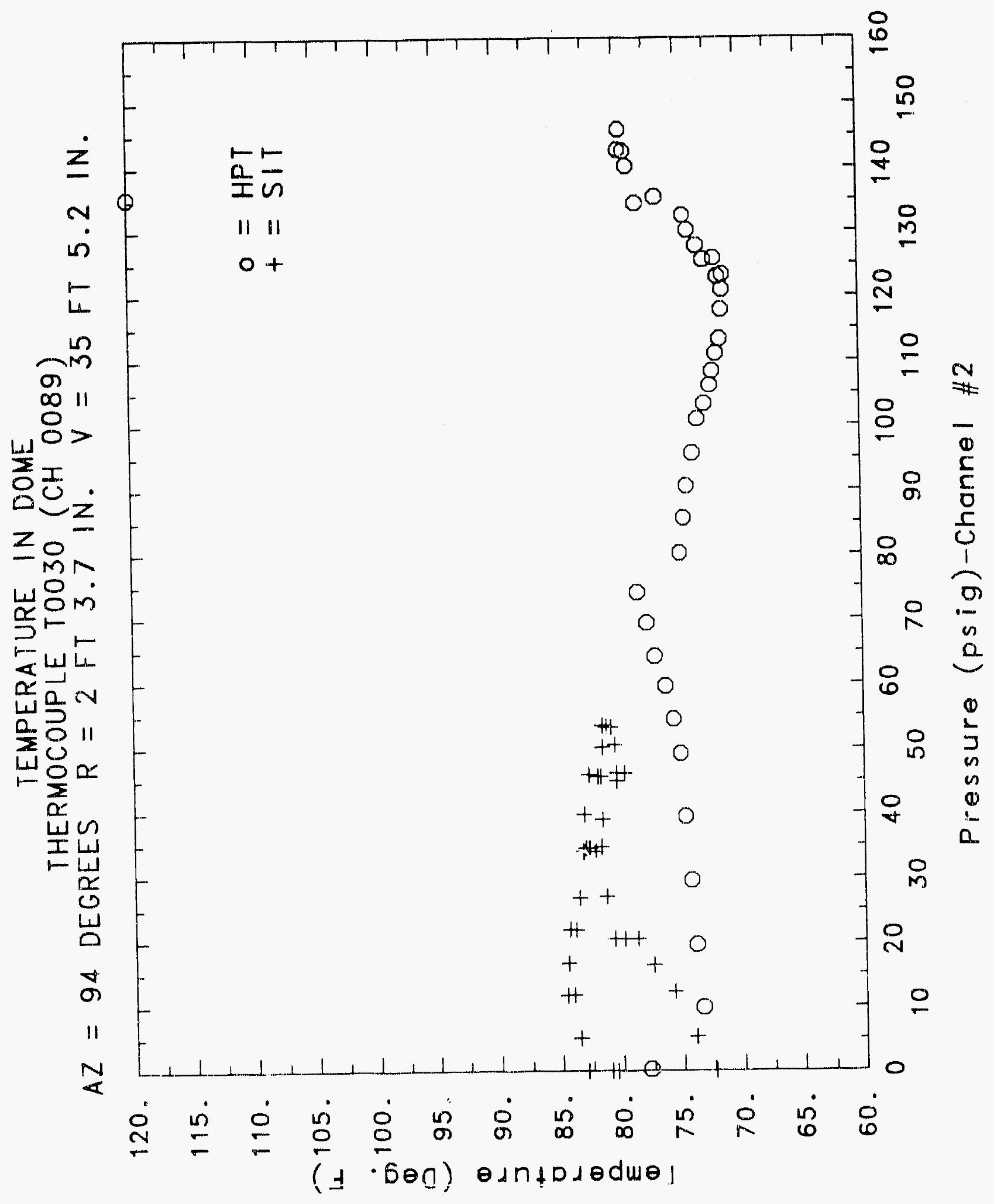

C- 1723 


\section{Reinforced Concrete Test Data}

Thermocouple Channel 90

\begin{tabular}{|c|c|c|c|}
\hline \multicolumn{2}{|c|}{ STRUCTURAL INTEGRITY TEST } & \multicolumn{2}{|c|}{ High Pressure Test } \\
\hline $\begin{array}{l}\text { Pressure } \\
\text { (psig) } \\
-0.05 \\
5.33 \\
12.31 \\
16.44 \\
20.51 \\
20.46 \\
20.47 \\
27.05 \\
34.69 \\
34.53 \\
34.53 \\
39.70 \\
45.90 \\
45.65 \\
45.62 \\
50.10 \\
53.47 \\
53.29 \\
53.21 \\
50.49 \\
46.14 \\
46.13 \\
44.97 \\
38.98 \\
33.99 \\
33.96 \\
26.83 \\
21.88 \\
21.90 \\
16.69 \\
11.74 \\
11.77 \\
5.05 \\
0.02 \\
-0.04 \\
-0.02 \\
-0.02 \\
0.02\end{array}$ & $\begin{array}{c}\text { Temperature } \\
\text { (Fahrenheit) } \\
74.3600 \\
74.6700 \\
75.2020 \\
75.7780 \\
76.3970 \\
77.3260 \\
78.0320 \\
78.9590 \\
79.7530 \\
81.1620 \\
82.1290 \\
83.5790 \\
84.8940 \\
85.8580 \\
85.8140 \\
85.3760 \\
84.8940 \\
84.3240 \\
83.8420 \\
83.1840 \\
82.7440 \\
82.0850 \\
79.4440 \\
79.8850 \\
80.2370 \\
80.8540 \\
81.2500 \\
81.7340 \\
83.0080 \\
83.7980 \\
84.9380 \\
86.3390 \\
86.5580 \\
86.4700 \\
77.9440 \\
78.2970 \\
78.6940 \\
79.7970\end{array}$ & $\begin{array}{c}\text { Pressure } \\
(\text { psig) } \\
9.89 \\
19.55 \\
29.57 \\
39.42 \\
49.16 \\
54.50 \\
59.57 \\
64.20 \\
69.32 \\
74.16 \\
80.16 \\
85.61 \\
90.58 \\
95.69 \\
100.92 \\
103.25 \\
106.11 \\
108.31 \\
111.08 \\
113.24 \\
117.83 \\
120.92 \\
123.28 \\
122.97 \\
125.82 \\
125.60 \\
127.84 \\
130.19 \\
132.53 \\
135.33 \\
134.42 \\
138.35 \\
140.16 \\
142.63 \\
145.78 \\
142.52 \\
0.22\end{array}$ & $\begin{array}{c}\text { Temperature } \\
\text { (Fahrenheit) } \\
75.0690 \\
75.4230 \\
75.6890 \\
76.0870 \\
76.4410 \\
76.7510 \\
77.0600 \\
77.5910 \\
77.8560 \\
78.2970 \\
78.2970 \\
77.7230 \\
77.2370 \\
76.5740 \\
75.9990 \\
75.3790 \\
75.1130 \\
74.9360 \\
74.5370 \\
74.3160 \\
74.0050 \\
73.6510 \\
73.2960 \\
73.2510 \\
73.3400 \\
73.7390 \\
73.9170 \\
74.3600 \\
74.5820 \\
75.2460 \\
75.9100 \\
76.5740 \\
77.0600 \\
77.6790 \\
78.2530 \\
78.5620 \\
78.5620\end{array}$ \\
\hline
\end{tabular}




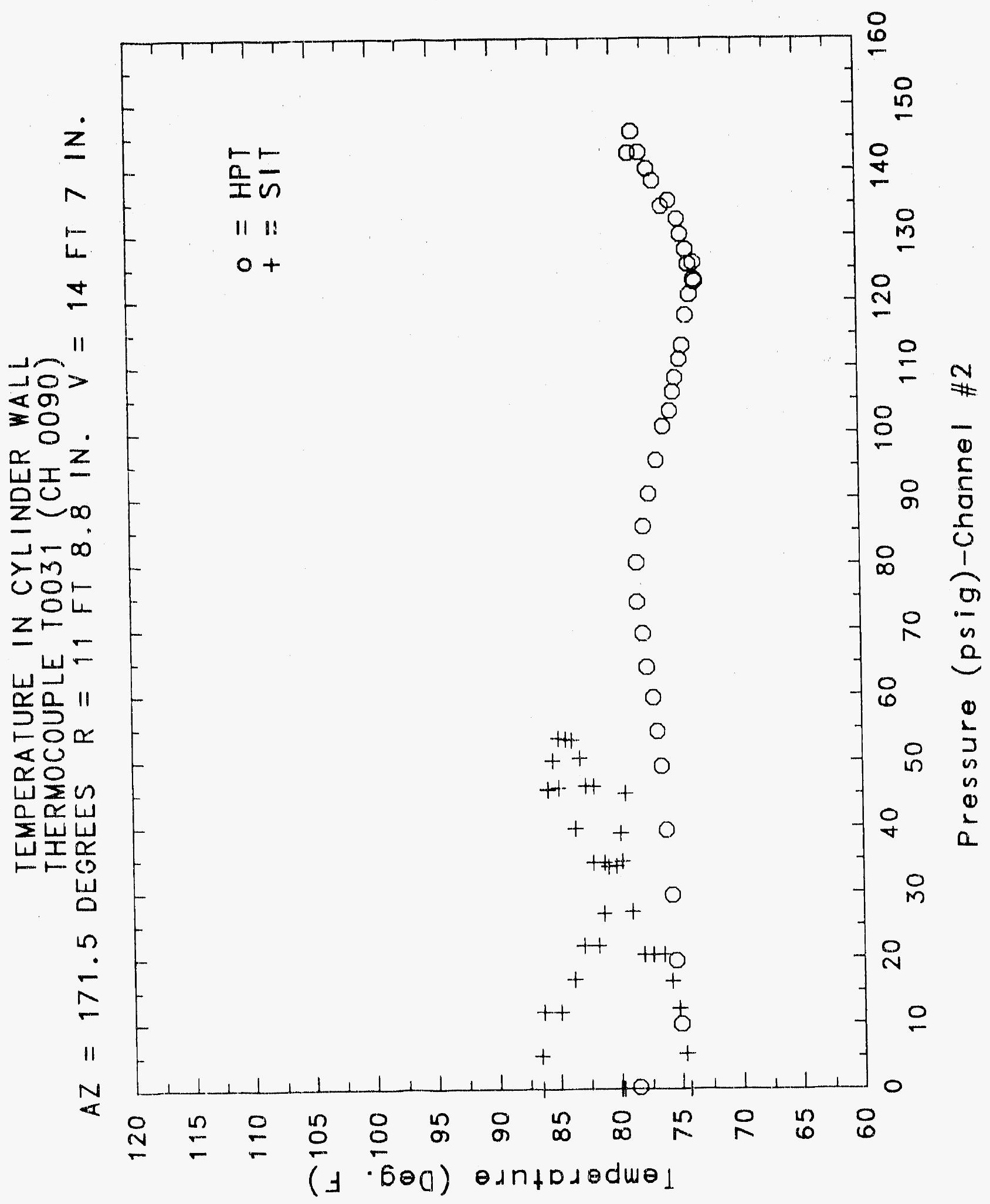




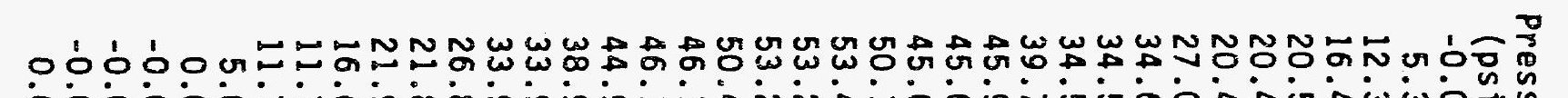

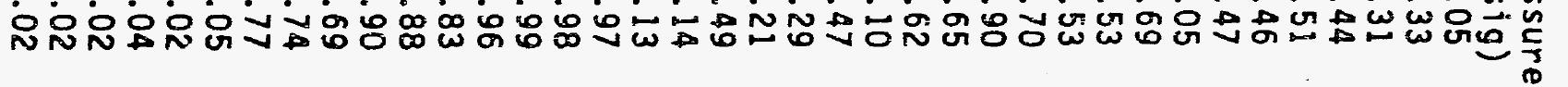

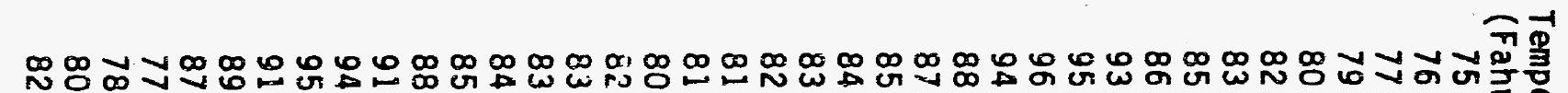

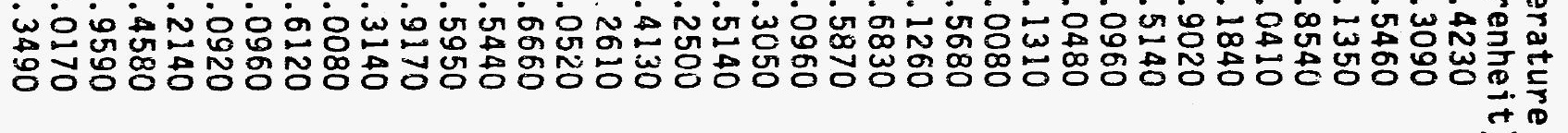

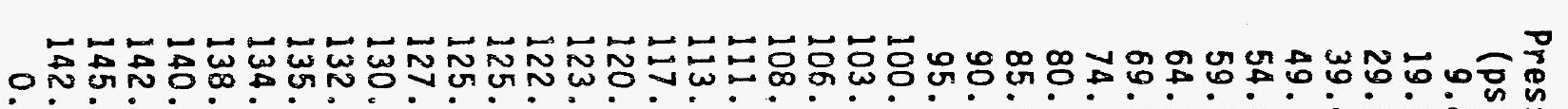

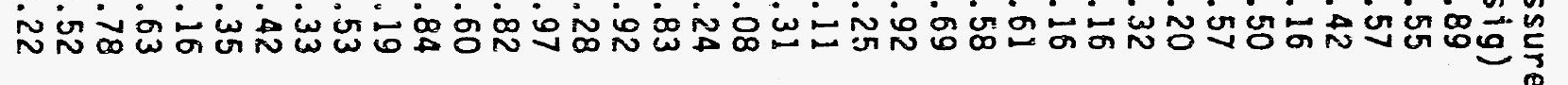

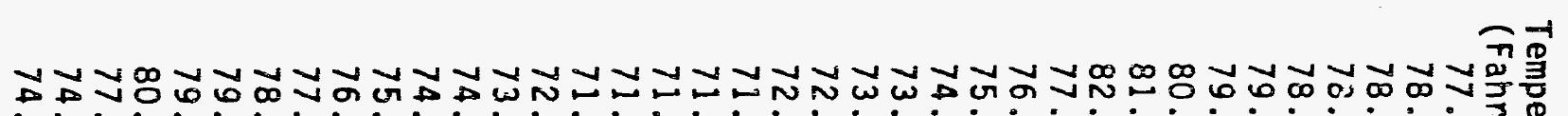

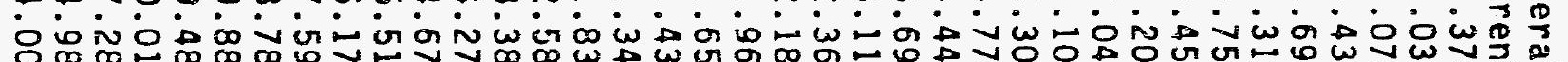

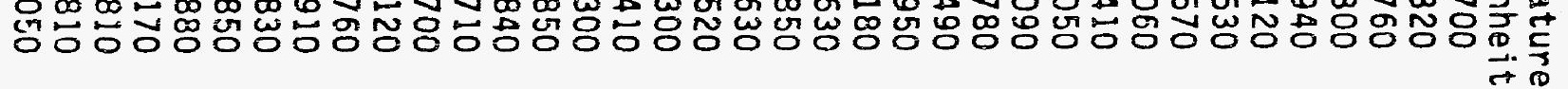




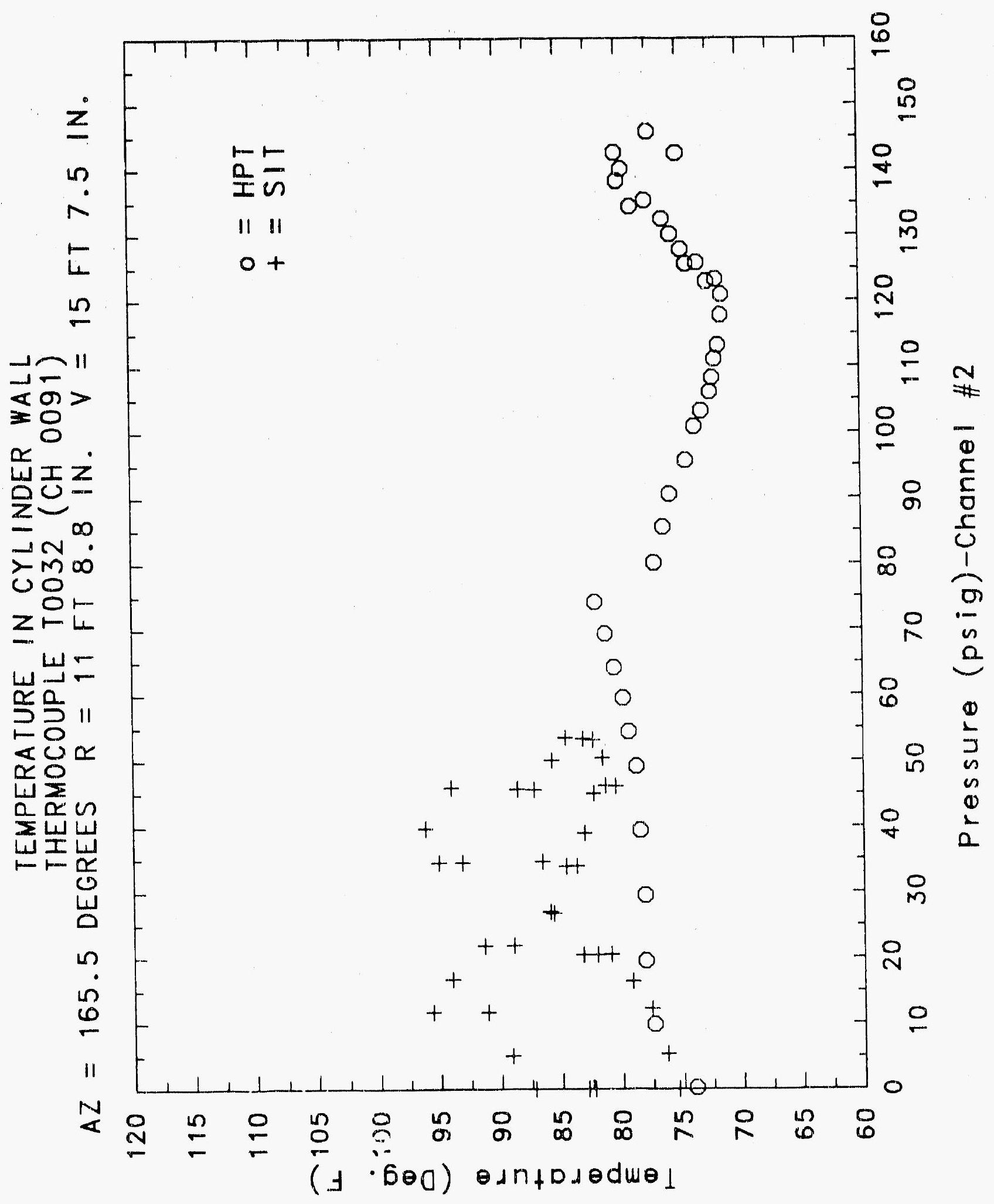




\section{Reinforced Concrete Test Data}

Thermocouple Channel 92

Structural Integrity Test

\begin{tabular}{|c|c|c|c|}
\hline $\begin{array}{c}\text { Pressure } \\
\text { (psig) } \\
-0.05 \\
5.33 \\
12.31 \\
16.44 \\
20.51 \\
20.46 \\
20.47 \\
27.05 \\
34.69 \\
34.53 \\
34.53 \\
39.70 \\
45.90 \\
45.65 \\
45.62 \\
50.10 \\
53.47 \\
53.29 \\
53.21 \\
50.49 \\
46.14 \\
46.13 \\
44.97 \\
38.98 \\
33.99 \\
33.96 \\
26.83 \\
21.88 \\
21.90 \\
16.69 \\
11.74 \\
11.77 \\
5.05 \\
0.02 \\
-0.04 \\
-0.02 \\
-0.02 \\
0.02\end{array}$ & $\begin{array}{c}\text { Temperature } \\
\text { (Fahrenheit) } \\
77.6790 \\
78.2090 \\
78.6940 \\
79.2240 \\
79.0910 \\
80.0170 \\
80.1490 \\
80.6780 \\
80.9860 \\
81.7340 \\
81.6460 \\
81.9970 \\
82.3930 \\
82.7000 \\
82.9640 \\
82.5250 \\
82.6130 \\
82.6570 \\
82.7880 \\
82.3050 \\
82.4370 \\
81.6900 \\
80.8100 \\
81.3380 \\
81.6020 \\
81.9090 \\
81.9970 \\
82.1290 \\
82.6130 \\
82.7000 \\
83.2270 \\
83.0960 \\
83.6660 \\
83.3590 \\
78.1210 \\
81.5580 \\
83.0520 \\
83.4910\end{array}$ & $\begin{array}{c}\text { Pressure } \\
(\text { psig) } \\
9.89 \\
19.55 \\
29.57 \\
39.42 \\
49.16 \\
54.50 \\
59.57 \\
64.20 \\
69.32 \\
74.16 \\
80.16 \\
85.61 \\
90.58 \\
95.69 \\
100.92 \\
103.25 \\
106.11 \\
108.31 \\
111.08 \\
113.24 \\
117.83 \\
120.92 \\
123.28 \\
122.97 \\
125.82 \\
125.60 \\
127.84 \\
130.19 \\
132.53 \\
135.33 \\
134.42 \\
138.35 \\
140.16 \\
142.63 \\
145.78 \\
142.52 \\
0.22\end{array}$ & $\begin{array}{c}\text { Temperature } \\
\text { (Fahrenheit) } \\
77.1490 \\
77.3700 \\
77.9880 \\
78.4300 \\
78.8710 \\
79.0470 \\
79.6200 \\
80.1930 \\
80.4570 \\
80.8100 \\
81.1180 \\
80.5010 \\
79.6640 \\
79.2680 \\
78.3850 \\
77.8110 \\
77.4140 \\
77.0600 \\
76.4410 \\
76.3090 \\
75.9990 \\
75.4230 \\
74.7590 \\
74.4930 \\
75.2460 \\
77.0160 \\
77.8110 \\
79.0910 \\
79.6640 \\
80.1930 \\
80.4570 \\
80.7220 \\
80.8540 \\
81.0740 \\
81.1620 \\
81.2500 \\
80.9860\end{array}$ \\
\hline
\end{tabular}

High Pressure test

emperature

77.1490

77.3700

77.9880

79.0470

79.6200

80.1930

80.4570

80.5010

79.6640

79.2680

78.3850

77.4140

77.0600

75.9990

75.4230

74.7590

77.8110

79.0910

79.6640

0.1930

80.7220

80.8540

81.0740

81.1620

81.2500

80.3860 


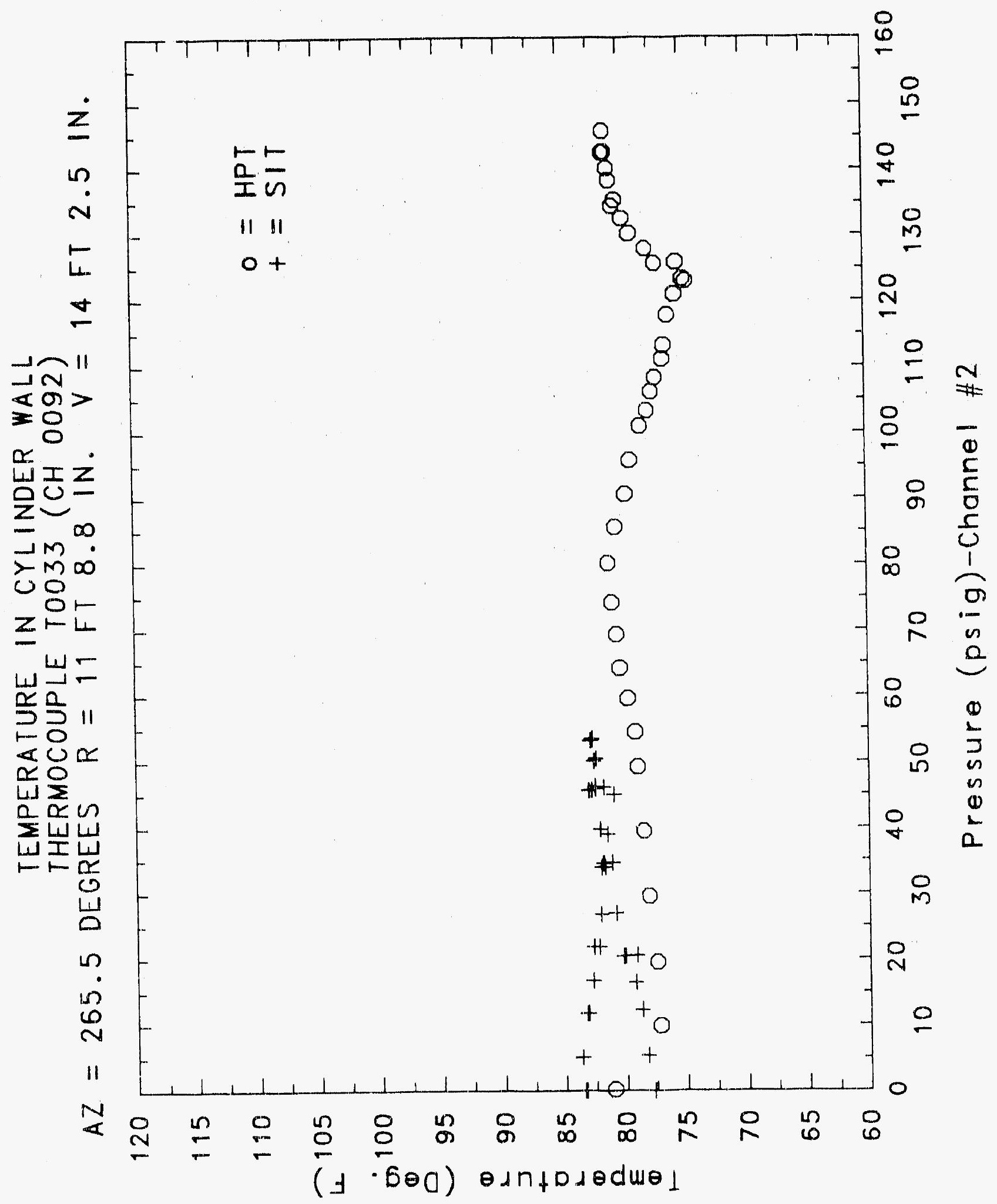

C-1729 


\section{Reinforced Concrete Test Data}

Thermocouple Channel 93

StruCtURAL INTEGRITY TEST

\begin{tabular}{|c|c|c|c|}
\hline $\begin{array}{l}\text { Pressure } \\
\text { (psig) } \\
-0.05 \\
5.33 \\
12.31 \\
16.44 \\
20.51 \\
20.46 \\
20.47 \\
27.05 \\
34.69 \\
34.53 \\
34.53 \\
39.70 \\
45.90 \\
45.65 \\
45.62 \\
50.10 \\
53.47 \\
53.29 \\
53.21 \\
50.49 \\
46.14 \\
46.13 \\
44.97 \\
38.98 \\
33.99 \\
33.96 \\
26.83 \\
21.88 \\
21.90 \\
16.69 \\
11.74 \\
11.77 \\
5.05 \\
0.02 \\
-0.04 \\
-0.02 \\
-0.02 \\
0.02\end{array}$ & $\begin{array}{c}\text { Temperature } \\
\text { (Fahrenheit) } \\
74.4490 \\
75.0250 \\
75.8660 \\
76.6180 \\
77.2810 \\
78.1650 \\
78.7830 \\
79.4000 \\
79.9290 \\
80.6340 \\
81.1180 \\
81.4260 \\
81.8650 \\
82.0410 \\
82.2170 \\
82.3490 \\
82.3050 \\
82.2610 \\
82.1730 \\
82.0850 \\
81.9970 \\
81.8650 \\
79.6200 \\
80.3250 \\
80.8540 \\
81.6460 \\
81.9970 \\
82.5690 \\
83.0960 \\
83.4470 \\
83.7540 \\
84.0610 \\
84.1930 \\
84.2370 \\
78.3850 \\
78.1650 \\
79.1790 \\
81.3500\end{array}$ & $\begin{array}{c}\text { Pressure } \\
(\text { psig) } \\
9.89 \\
19.55 \\
29.57 \\
39.42 \\
49.16 \\
54.50 \\
59.57 \\
64.20 \\
69.32 \\
74.16 \\
80.16 \\
85.61 \\
90.58 \\
95.69 \\
100.92 \\
103.25 \\
106.11 \\
108.31 \\
111.08 \\
113.24 \\
117.83 \\
120.92 \\
123.28 \\
122.97 \\
125.82 \\
125.00 \\
127.84 \\
130.19 \\
132.53 \\
135.33 \\
134.42 \\
138.35 \\
140.16 \\
142.63 \\
145.78 \\
142.52 \\
0.22\end{array}$ & $\begin{array}{c}\text { Temperature } \\
\text { (Fahrenheit) } \\
75.4680 \\
75.9100 \\
76.1320 \\
76.5300 \\
77.1050 \\
77.6350 \\
77.8110 \\
78.2970 \\
78.6060 \\
79.1790 \\
79.8850 \\
79.6640 \\
79.4440 \\
78.9590 \\
78.5180 \\
78.0320 \\
77.5910 \\
77.4580 \\
77.1490 \\
76.9720 \\
76.5740 \\
76.2200 \\
75.4680 \\
75.3790 \\
75.1130 \\
75.2020 \\
75.2460 \\
75.9100 \\
75.9100 \\
76.6180 \\
77.3700 \\
78.0320 \\
78.8270 \\
78.8710 \\
75.2910 \\
71.2960 \\
69.6040\end{array}$ \\
\hline
\end{tabular}

High Pressure test

$\operatorname{Pr}$

39.42

49.16

59.57

64.20

69.32

90.58

108.31

111.08

113.24

123.28

122.97

30.19

132.53

35.33

34.42

138.35

140.16

142.52

71.2960

69.6040 


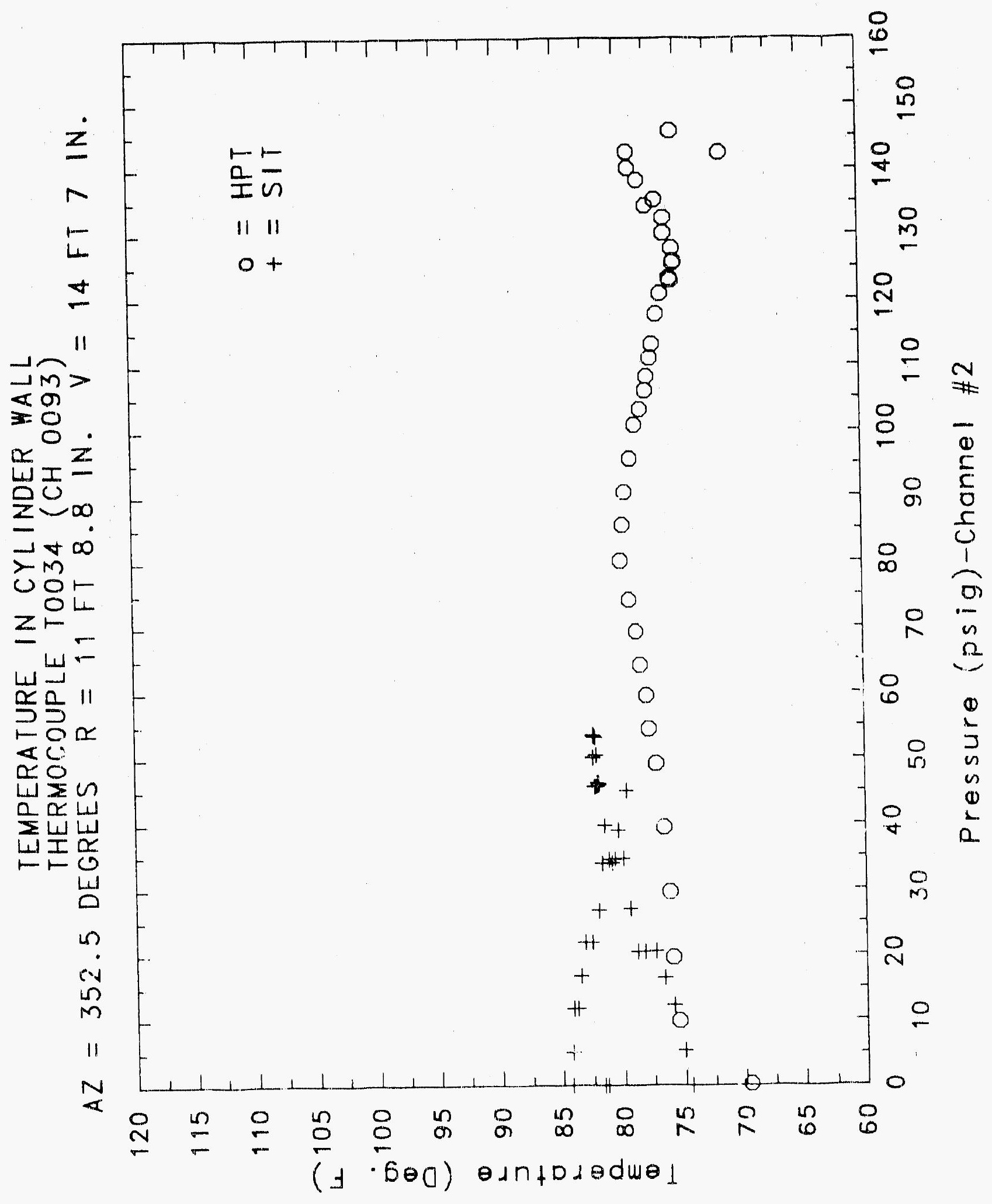




\section{Reinforced Concrete Test Data}

Thermocouple Channel 94

StRUCTURAL INTEgRITY TEST

\begin{tabular}{|c|c|c|c|}
\hline $\begin{array}{l}\text { Pressure } \\
\text { (psig) } \\
-0.05 \\
5.33 \\
12.31 \\
16.44 \\
20.51 \\
20.46 \\
20.47 \\
27.05 \\
34.69 \\
34.53 \\
34.53 \\
39.70 \\
45.90 \\
45.65 \\
45.62 \\
50.10 \\
53.47 \\
53.29 \\
53.21 \\
50.49 \\
46.14 \\
46.13 \\
44.97 \\
38.98 \\
33.99 \\
33.96 \\
26.83 \\
21.88 \\
21.90 \\
16.69 \\
11.74 \\
11.77 \\
5.05 \\
0.02 \\
-0.04 \\
-0.02 \\
-0.02 \\
0.02\end{array}$ & $\begin{array}{l}\text { Temperature } \\
\text { (Fahrenheit) } \\
79.7090 \\
79.2680 \\
79.4880 \\
79.9290 \\
80.5450 \\
81.4260 \\
81.9530 \\
62.2170 \\
82.4370 \\
83.0080 \\
83.1840 \\
83.3150 \\
83.1840 \\
82.5690 \\
82.1730 \\
81.8220 \\
81.5140 \\
81.2940 \\
81.0300 \\
80.5450 \\
80.4130 \\
80.1050 \\
83.5790 \\
83.8420 \\
83.9300 \\
84.1930 \\
84.3680 \\
84.5000 \\
84.8500 \\
84.9380 \\
85.0700 \\
84.7190 \\
84.4560 \\
84.1050 \\
80.5450 \\
85.2010 \\
85.1130 \\
85.0700\end{array}$ & $\begin{array}{l}\text { Pressure } \\
(p s i g) \\
9.89 \\
19.55 \\
29.57 \\
39.42 \\
49.16 \\
54.50 \\
59.57 \\
64.20 \\
69.32 \\
74.10 \\
80.16 \\
85.61 \\
90.58 \\
95.69 \\
100.92 \\
103.25 \\
106.11 \\
108.31 \\
111.08 \\
113.24 \\
117.83 \\
120.92 \\
123.28 \\
122.97 \\
125.82 \\
125.60 \\
127.84 \\
130.19 \\
132.53 \\
135.33 \\
134.42 \\
138.35 \\
140.16 \\
142.63 \\
145.78 \\
142.52 \\
0.22\end{array}$ & $\begin{array}{c}\text { Temperature } \\
\text { (Fahrenheit) } \\
80.1490 \\
80.4130 \\
80.4130 \\
80.5010 \\
80.5450 \\
80.6780 \\
80.8540 \\
81.1620 \\
81.5140 \\
81.9530 \\
79.5320 \\
78.7830 \\
78.2530 \\
77.3700 \\
76.6180 \\
75.9990 \\
75.5120 \\
75.2910 \\
74.8920 \\
74.5700 \\
74.2710 \\
74.0940 \\
74.2710 \\
76.1750 \\
73.2530 \\
78.9590 \\
79.5760 \\
80.4570 \\
80.1930 \\
80.5400 \\
80.9420 \\
81.2940 \\
81.5580 \\
81.5140 \\
81.5140 \\
81.2500 \\
80.2810\end{array}$ \\
\hline
\end{tabular}

High Pressure test

19.55

29.57

39.42

49.16

64.20

69.32

74.16

90.58

95.69

106.11

108.31

117.83

120.92

25.60

127.84

34.42

38.35

40.16

42.52
0.22
Temperature

ahrenheit)

80.1490

80.4130

80.5010

80.5450

80.6780

81.5140

81.9530

78.2530

77.3700

75.5120

75.2910

74.8920

74.0940

74.2710

76.1760

80.4570

80.4420

81.2940

81.5580

81.2500

80.2810 


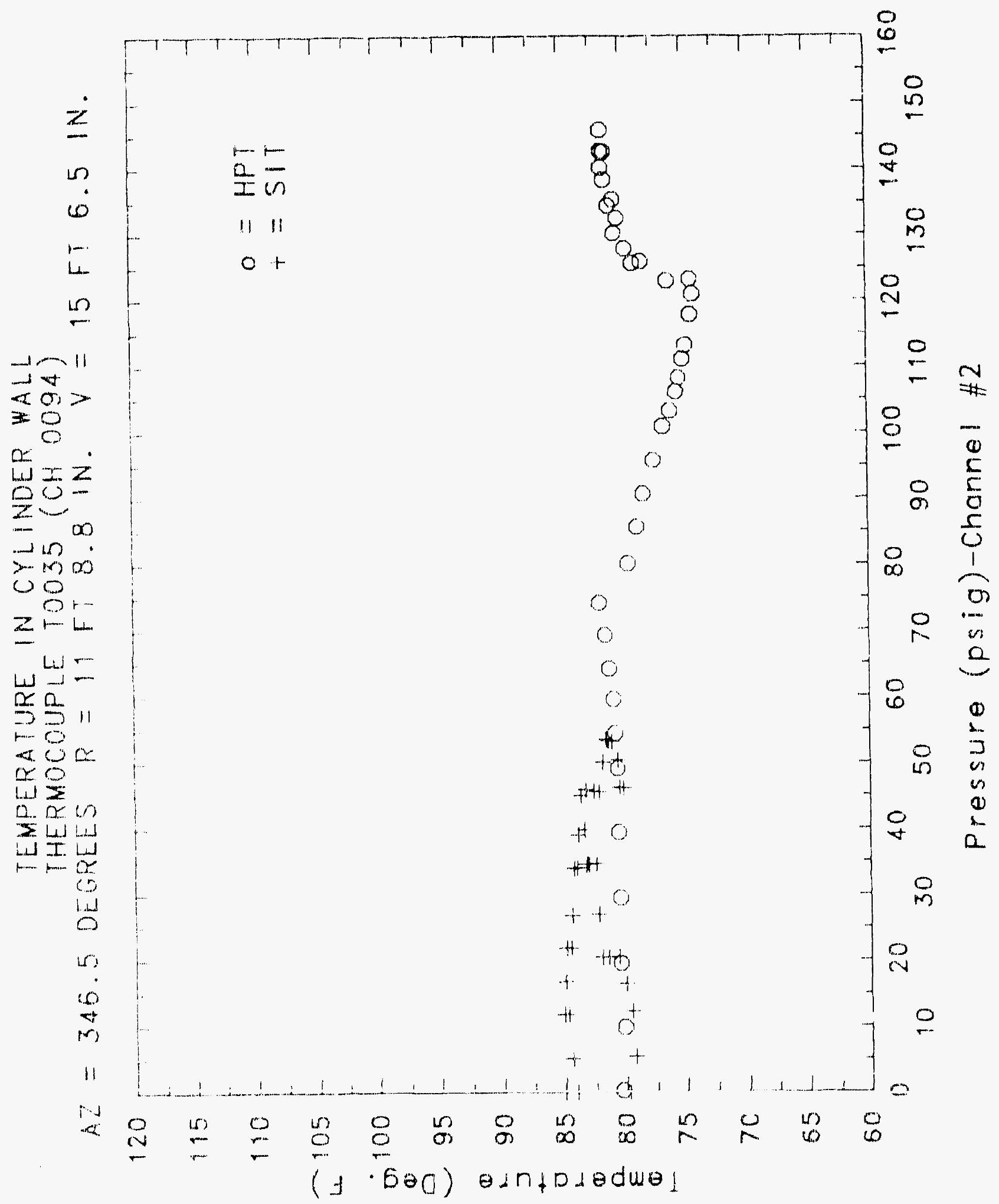




\section{Reinforced Concrete Test Data}

\section{Thermocouple Channel 95}

STRUCTURAL INTEGRITY TEST

\begin{tabular}{|c|c|c|c|}
\hline $\begin{array}{l}\text { Pressure } \\
\text { (psig) } \\
-0.05 \\
5.33 \\
12.31 \\
16.44 \\
20.51 \\
20.46 \\
20.47 \\
27.05 \\
34.69 \\
34.53 \\
34.53 \\
39.70 \\
45.90 \\
45.65 \\
45.82 \\
50.10 \\
53.47 \\
53.29 \\
53.21 \\
50.49 \\
46.14 \\
46.13 \\
44.97 \\
38.98 \\
33.99 \\
33.96 \\
26.83 \\
7.88 \\
21.90 \\
16.69 \\
11.74 \\
11.77 \\
5.05 \\
0.02 \\
-0.04 \\
-0.02 \\
-0.02 \\
0.02 \\
\end{array}$ & $\begin{array}{c}\text { Temperature } \\
\text { (Fahrenheit) } \\
77.9880 \\
77.9880 \\
77.9880 \\
77.9440 \\
78.0320 \\
78.0320 \\
78.0760 \\
78.4300 \\
78.7830 \\
79.0470 \\
79.2240 \\
79.7090 \\
80.1050 \\
80.5900 \\
80.8540 \\
81.3380 \\
81.9090 \\
82.2610 \\
82.4810 \\
82.6570 \\
82.6130 \\
82.7880 \\
80.0170 \\
80.1050 \\
80.2370 \\
80.3690 \\
80.5900 \\
80.6780 \\
80.9420 \\
81.1620 \\
81.1620 \\
81.6020 \\
81.6900 \\
81.7780 \\
81.1620 \\
80.5900 \\
80.2370 \\
80.3690\end{array}$ & $\begin{array}{c}\text { Pressure } \\
(\text { psig) } \\
9.89 \\
19.55 \\
29.57 \\
39.42 \\
49.16 \\
54.50 \\
59.57 \\
64.20 \\
69.32 \\
74.15 \\
80.16 \\
85.61 \\
90.58 \\
95.69 \\
100.92 \\
103.25 \\
106.11 \\
108.31 \\
111.08 \\
113.24 \\
117.83 \\
120.92 \\
123.28 \\
122.97 \\
125.82 \\
125.60 \\
127.84 \\
130.19 \\
132.53 \\
135.33 \\
134.42 \\
138.35 \\
140.16 \\
142.63 \\
145.78 \\
142.52 \\
0.22\end{array}$ & $\begin{array}{c}\text { Temperature } \\
\text { (Fahrenheit) } \\
76.7950 \\
77.1050 \\
77.5020 \\
77.7670 \\
78.0760 \\
78.2090 \\
78.5620 \\
78.7380 \\
79.0030 \\
79.210 \\
79.9290 \\
80.1930 \\
80.4130 \\
80.3690 \\
80.2810 \\
79.9290 \\
79.6640 \\
79.6200 \\
79.3560 \\
79.2680 \\
79.1350 \\
78.8770 \\
78.2530 \\
77.9000 \\
77.7230 \\
77.2810 \\
77.2810 \\
77.1490 \\
77.2370 \\
77.3260 \\
77.4140 \\
77.6350 \\
77.8560 \\
78.0760 \\
78.3410 \\
78.4740 \\
73.6510\end{array}$ \\
\hline
\end{tabular}

High Pressure test

Temperature

ahrenheit)

76.7950

77.1050

78.0760

78.2090

78.0030

$79.26: 0$

79.9290

80.2810

79.3290

79.6640

79.2680

79.1350

78.2770

77.7230

77.2810

77.2810

77.1490

77.4140

77.6350

77.8560

78.4740

73.6510 


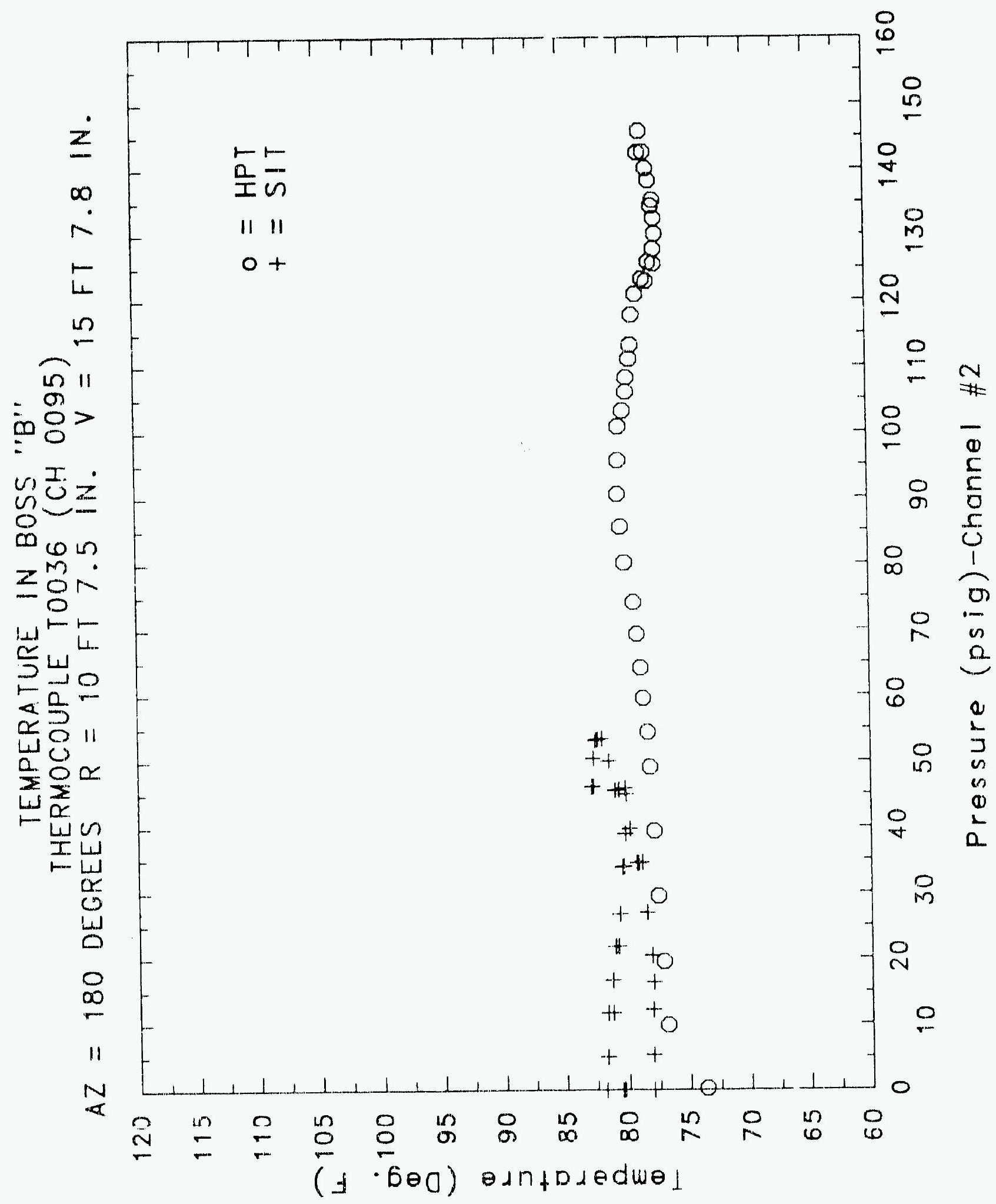




\section{Reinforced Concrete Test Data}

\section{Thermocouple Channel 96}

\section{Structural. Integrity TEST}

\begin{tabular}{|c|c|}
\hline $\begin{array}{l}\text { Pressure } \\
\text { (psig) } \\
-0.05 \\
5.33 \\
12.31 \\
16.44 \\
20.51 \\
20.46 \\
20.47 \\
27.05 \\
34.69 \\
34.53 \\
34.53 \\
39.70 \\
45.90 \\
45.65 \\
45.62 \\
50.10 \\
53.47 \\
53.29 \\
53.21 \\
50.49 \\
46.14 \\
46.13 \\
44.97 \\
38.98 \\
33.99 \\
33.96 \\
26.83 \\
21.88 \\
21.90 \\
16.69 \\
11.74 \\
11.77 \\
5.05 \\
0.02 \\
-0.04 \\
-0.02 \\
-0.02 \\
0.02\end{array}$ & $\begin{array}{c}\text { Temperature } \\
\text { (Fahrenheit) } \\
76.3530 \\
76.2200 \\
76.2200 \\
76.2200 \\
76.3090 \\
76.6620 \\
76.9280 \\
77.3260 \\
77.5460 \\
77.7670 \\
78.1210 \\
78.6500 \\
79.3560 \\
80.3690 \\
80.8540 \\
81.4700 \\
81.9970 \\
82.3050 \\
82.5250 \\
82.5250 \\
82.6570 \\
82.7440 \\
79.4440 \\
79.5760 \\
79.7530 \\
79.9730 \\
80.1930 \\
80.4570 \\
80.8100 \\
80.7220 \\
81.2500 \\
81.9970 \\
82.4370 \\
82.8760 \\
80.5010 \\
79.9730 \\
79.8410 \\
79.8850\end{array}$ \\
\hline
\end{tabular}

\section{High Pressure test}

\begin{tabular}{|c|c|}
\hline $\begin{array}{c}\text { Pressure } \\
(p s i g) \\
9.89 \\
19.55 \\
29.57 \\
39.42 \\
49.16 \\
54.50 \\
59.57 \\
64.20 \\
69.32 \\
74.16 \\
80.16 \\
85.61 \\
90.58 \\
95.69 \\
100.92 \\
103.25 \\
106.11 \\
108.31 \\
111.08 \\
113.24 \\
117.83 \\
120.92 \\
123.28 \\
122.97 \\
125.82 \\
125.60 \\
127.84 \\
130.19 \\
132.53 \\
135.33 \\
134.42 \\
138.35 \\
140.16 \\
142.63 \\
145.78 \\
142.52 \\
0.22\end{array}$ & $\begin{array}{c}\text { Temperature } \\
\text { (Fahrenheit) } \\
75.6450 \\
75.6890 \\
75.7330 \\
75.8220 \\
75.9990 \\
76.3530 \\
76.4860 \\
76.7950 \\
77.1050 \\
77.3260 \\
79.0910 \\
79.2240 \\
79.2680 \\
79.0910 \\
78.8270 \\
78.5620 \\
78.2530 \\
78.1210 \\
77.9440 \\
77.7670 \\
77.5020 \\
77.2370 \\
76.6620 \\
76.3530 \\
76.0430 \\
75.9550 \\
75.9550 \\
75.9100 \\
75.7780 \\
75.9990 \\
76.2640 \\
76.4860 \\
76.6620 \\
76.8390 \\
77.2810 \\
77.5020 \\
77.8110\end{array}$ \\
\hline
\end{tabular}




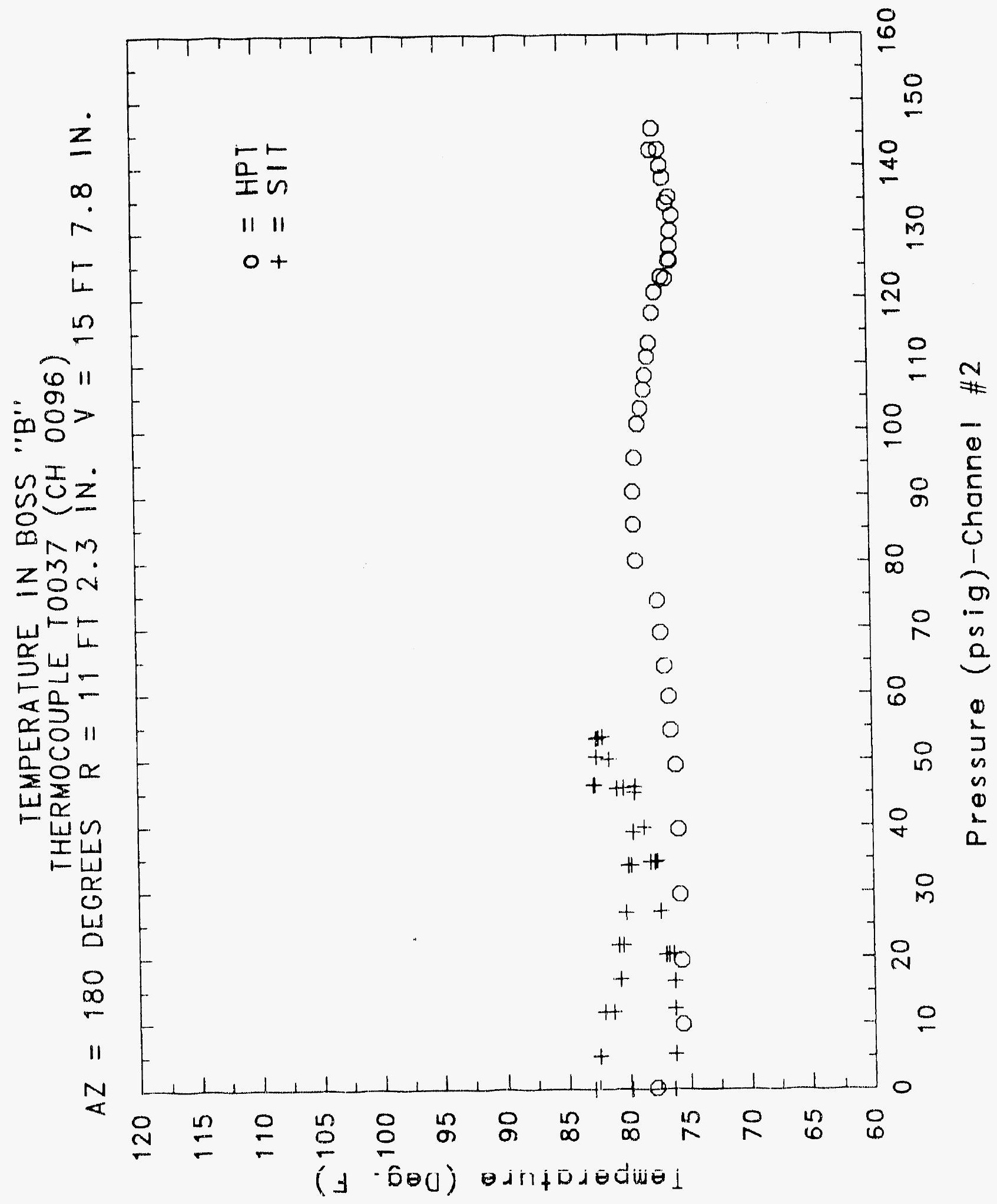




\section{Reinforced Concrete Test Data}

Thermocouple Channel 97

Structural Integrity Test

\begin{tabular}{|c|c|c|c|}
\hline $\begin{array}{c}\text { Pressure } \\
\text { (psig) } \\
-0.05 \\
5.33 \\
12.31 \\
16.44 \\
20.51 \\
20.76 \\
20.47 \\
27.05 \\
34.69 \\
34.53 \\
34.53 \\
39.70 \\
45.90 \\
45.65 \\
45.62 \\
50.10 \\
53.47 \\
53.29 \\
53.21 \\
50.49 \\
46.14 \\
46.13 \\
44.97 \\
38.98 \\
33.99 \\
33.96 \\
26.83 \\
21.88 \\
21.90 \\
16.69 \\
11.74 \\
11.77 \\
5.05 \\
0.02 \\
-0.04 \\
-0.02 \\
-0.02 \\
0.02 \\
\end{array}$ & $\begin{array}{c}\text { Temperature } \\
\text { (Fahrenheit) } \\
74.4490 \\
74.6700 \\
75.1130 \\
75.6450 \\
76.1320 \\
77.0160 \\
77.5910 \\
78.2530 \\
78.8710 \\
79.9290 \\
80.5900 \\
81.7780 \\
82.7440 \\
83.7540 \\
83.7980 \\
83.6660 \\
83.4030 \\
83.1400 \\
82.7880 \\
82.3490 \\
81.9970 \\
81.5580 \\
79.6640 \\
79.9290 \\
80.2810 \\
80.8980 \\
81.1180 \\
81.5580 \\
82.2170 \\
82.8760 \\
83.7100 \\
84.8070 \\
85.1130 \\
85.2890 \\
78.0320 \\
78.6060 \\
79.0910 \\
80.0610\end{array}$ & $\begin{array}{c}\text { Pressure } \\
(\text { psig) } \\
9.89 \\
19.55 \\
29.57 \\
39.42 \\
49.16 \\
54.50 \\
59.57 \\
64.20 \\
69.32 \\
74.16 \\
80.16 \\
85.61 \\
90.58 \\
95.69 \\
100.92 \\
103.25 \\
106.11 \\
108.31 \\
111.08 \\
113.24 \\
117.83 \\
120.92 \\
123.28 \\
122.97 \\
125.82 \\
125.60 \\
127.84 \\
130.19 \\
132.53 \\
135.33 \\
134.42 \\
138.35 \\
140.16 \\
142.63 \\
145.78 \\
142.52 \\
0.22\end{array}$ & $\begin{array}{c}\text { Temperature } \\
\text { (Fahrenteit) } \\
75.2020 \\
75.3790 \\
75.8220 \\
76.0430 \\
76.3090 \\
76.6180 \\
76.8840 \\
77.4140 \\
77.6790 \\
78.0760 \\
78.6060 \\
78.2090 \\
77.7230 \\
77.1490 \\
76.5300 \\
75.9550 \\
75.6010 \\
75.4680 \\
74.9810 \\
74.8030 \\
74.5370 \\
74.1830 \\
73.4730 \\
73.3840 \\
73.3840 \\
73.8720 \\
74.2270 \\
74.8480 \\
75.0690 \\
75.6890 \\
76.3090 \\
76.7950 \\
77.2370 \\
77.8110 \\
78.0760 \\
78.3410 \\
78.2970\end{array}$ \\
\hline
\end{tabular}

High Pressure test

(Fahrentieit)

75.2020

76.0430

76.3090

76.6180

76.8840

78.0760

78.6060

78.2090

76.5300

75.9550

75.6010

75.4680

74.5370

74.1830

73.4730

73.3840

73.8720

74.2270

74.8480

75.0690

76.7950

77.2370

77.8110

78.0760

78.3410

79.0910 


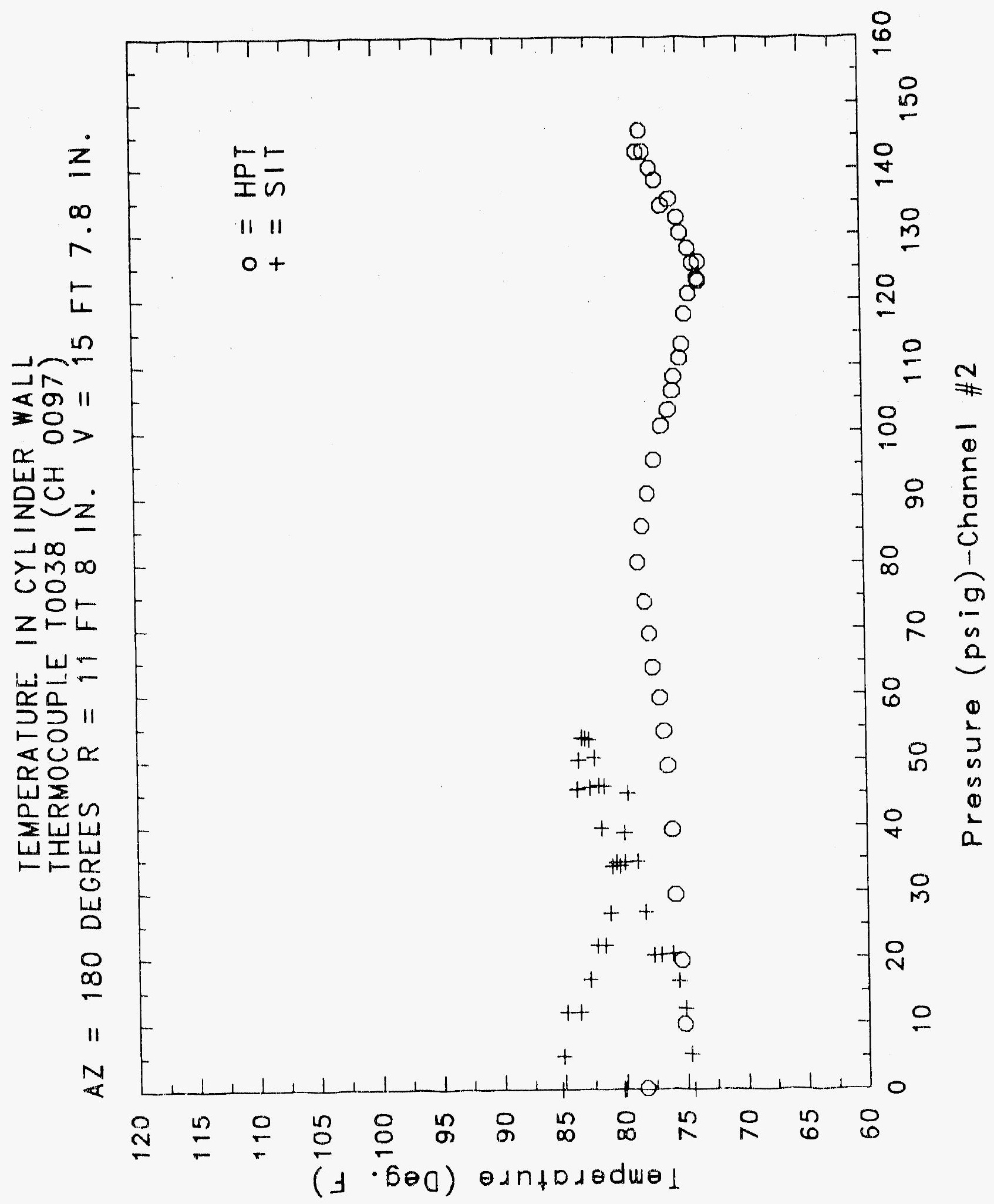




\section{Reinforced Concrete Test Data}

Thermocouple Channel 98

StRuCtURAL INTEgRITY TEST

\begin{tabular}{|c|c|c|c|}
\hline $\begin{array}{l}\text { Pressure } \\
\text { (psig) } \\
-0.05 \\
5.33 \\
12.31 \\
16.44 \\
20.51 \\
20.46 \\
20.47 \\
27.05 \\
34.69 \\
34.53 \\
34.53 \\
39.70 \\
45.90 \\
45.65 \\
45.62 \\
50.10 \\
53.47 \\
53.29 \\
53.21 \\
50.49 \\
46.14 \\
46.13 \\
44.97 \\
38.98 \\
33.99 \\
33.96 \\
26.83 \\
21.88 \\
21.90 \\
16.69 \\
11.74 \\
11.77 \\
5.05 \\
0.02 \\
-0.04 \\
-0.02 \\
-0.02 \\
0.02\end{array}$ & $\begin{array}{c}\text { Temperature } \\
\text { (Fahrenheit) } \\
73.9610 \\
74.7150 \\
75.6890 \\
76.8840 \\
78.2090 \\
79.4440 \\
80.1490 \\
81.2940 \\
82.0410 \\
84.8500 \\
86.2080 \\
87.7380 \\
88.1750 \\
86.2960 \\
85.3760 \\
84.2810 \\
83.4030 \\
82.4370 \\
81.6460 \\
80.8540 \\
80.4570 \\
79.8850 \\
80.8100 \\
81.5580 \\
82.0410 \\
82.8320 \\
83.2270 \\
84.0610 \\
85.8140 \\
87.2140 \\
88.4810 \\
87.9130 \\
87.1260 \\
86.2080 \\
76.7070 \\
78.3850 \\
79.3120 \\
81.3380\end{array}$ & $\begin{array}{c}\text { Pressure } \\
\text { (psig) } \\
9.89 \\
19.55 \\
29.57 \\
39.42 \\
49.16 \\
54.50 \\
59.57 \\
64.20 \\
69.32 \\
74.16 \\
80.16 \\
85.61 \\
90.58 \\
95.69 \\
100.92 \\
103.25 \\
106.11 \\
108.31 \\
111.08 \\
113.24 \\
117.83 \\
120.92 \\
123.28 \\
122.97 \\
125.82 \\
125.60 \\
127.84 \\
130.19 \\
132.53 \\
135.33 \\
134.42 \\
138.35 \\
140.16 \\
142.63 \\
145.78 \\
142.52 \\
0.22\end{array}$ & $\begin{array}{c}\text { Temperature } \\
\text { (Fahrenheit) } \\
76.3090 \\
76.7070 \\
77.1050 \\
77.4140 \\
77.6790 \\
78.1210 \\
78.6500 \\
79.0470 \\
79.5760 \\
80.0610 \\
77.4140 \\
76.5300 \\
75.7780 \\
74.8030 \\
74.0050 \\
73.2960 \\
72.7630 \\
72.5410 \\
72.2300 \\
72.0080 \\
71.6520 \\
71.4740 \\
71.3410 \\
71.8300 \\
72.6740 \\
73.7390 \\
74.2270 \\
75.0690 \\
75.6010 \\
76.8390 \\
77.7670 \\
78.8270 \\
79.3120 \\
79.8850 \\
79.8410 \\
79.5320 \\
78.7830\end{array}$ \\
\hline
\end{tabular}

High Pressure Test

Temperatur

76.3090

76.7070

77.4140

77.6790

78.1210

79.5760

80.0610

77.4140

74.8030

74.0050

73.2960

72.2300

72.0080

71.6520

71.4740

72.6740

73.7390

74.2270

75.0690

77.7670

78.8270

79.3120

79.8850

.8410

79.5320

78.7830 


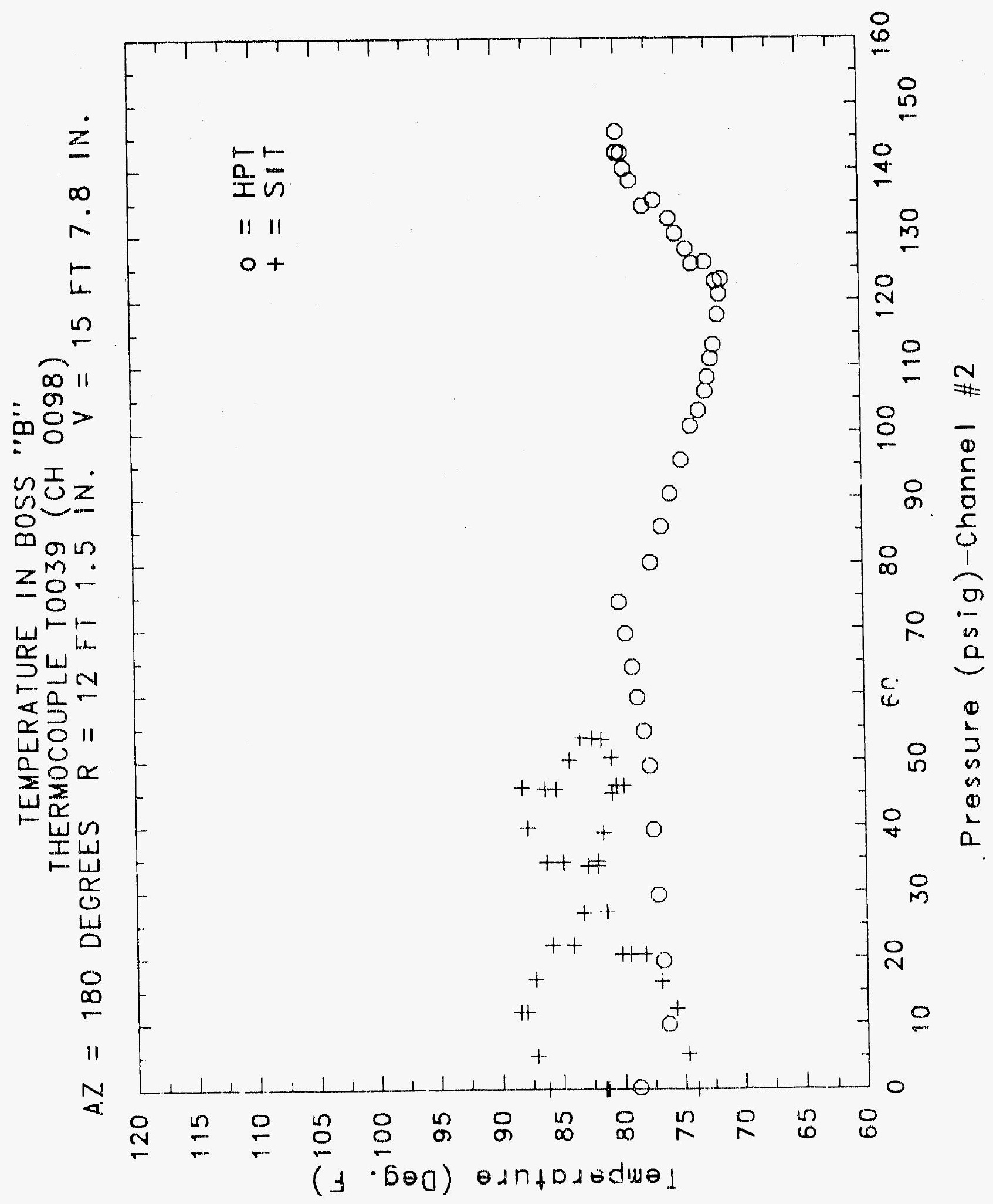




\section{Reinforced Concrete Test Data}

Thermocouple Channel 99

STRUCTURAL INTEGRITY TEST

\begin{tabular}{|c|c|}
\hline $\begin{array}{c}\text { Pressure } \\
\text { (psig) } \\
-0.05 \\
5.33 \\
12.31 \\
16.44 \\
20.51 \\
20.46 \\
20.47 \\
27.05 \\
34.69 \\
34.53 \\
34.53 \\
39.70 \\
45.90 \\
45.65 \\
45.62 \\
50.10 \\
53.47 \\
53.29 \\
53.21 \\
50.49 \\
46.14 \\
46.13 \\
44.97 \\
38.98 \\
33.99 \\
33.96 \\
26.83 \\
21.88 \\
21.90 \\
16.69 \\
11.74 \\
11.77 \\
5.05 \\
0.02 \\
-0.04 \\
-0.02 \\
-0.02 \\
0.02\end{array}$ & $\begin{array}{c}\text { Temperature } \\
\text { (Fahrenhei.t) } \\
74.5820 \\
75.1580 \\
76.0430 \\
76.7510 \\
77.5460 \\
78.8710 \\
79.9730 \\
81.9090 \\
83.3590 \\
85.1570 \\
85.7260 \\
86.4270 \\
86.8640 \\
86.8640 \\
86.6450 \\
86.2520 \\
85.9890 \\
85.5080 \\
85.0700 \\
84.5000 \\
83.9300 \\
83.2270 \\
78.7380 \\
79.4440 \\
80.0170 \\
81.3380 \\
82.5690 \\
84.1490 \\
86.9520 \\
88.1750 \\
88.9610 \\
89.0920 \\
88.8730 \\
88.3930 \\
77.6790 \\
17.2370 \\
77.3700 \\
78.5620\end{array}$ \\
\hline
\end{tabular}

High Pressure test

\begin{tabular}{|c|c|}
\hline $\begin{array}{c}\text { Pressure } \\
(\text { psig) } \\
9.89 \\
19.55 \\
29.57 \\
39.42 \\
49.16 \\
54.50 \\
59.57 \\
64.20 \\
69.32 \\
74.16 \\
80.16 \\
85.61 \\
90.58 \\
95.69 \\
100.92 \\
103.25 \\
106.11 \\
108.31 \\
111.08 \\
113.24 \\
117.83 \\
120.92 \\
123.28 \\
122.97 \\
125.82 \\
125.60 \\
127.84 \\
130.19 \\
132.53 \\
135.33 \\
134.42 \\
138.35 \\
140.16 \\
142.63 \\
145.78 \\
142.52 \\
0.22\end{array}$ & $\begin{array}{c}\text { Temperatıre } \\
\text { (Fahrenht }: t \text { ) } \\
75.1580 \\
75.7330 \\
76.2640 \\
77.1050 \\
77.8110 \\
78.5180 \\
78.9590 \\
79.7090 \\
80.5010 \\
81.6020 \\
81.8650 \\
81.2500 \\
80.5900 \\
79.6200 \\
78.9150 \\
78.12 .10 \\
77.5910 \\
77.3700 \\
76.9720 \\
76.7070 \\
76.3970 \\
76.0430 \\
75.3350 \\
75.2020 \\
75.2020 \\
75.2020 \\
75.2910 \\
75.5560 \\
75.9100 \\
76.7510 \\
77.5460 \\
79.3120 \\
81.2060 \\
82.8320 \\
83.8420 \\
84.0170 \\
81.1180\end{array}$ \\
\hline
\end{tabular}




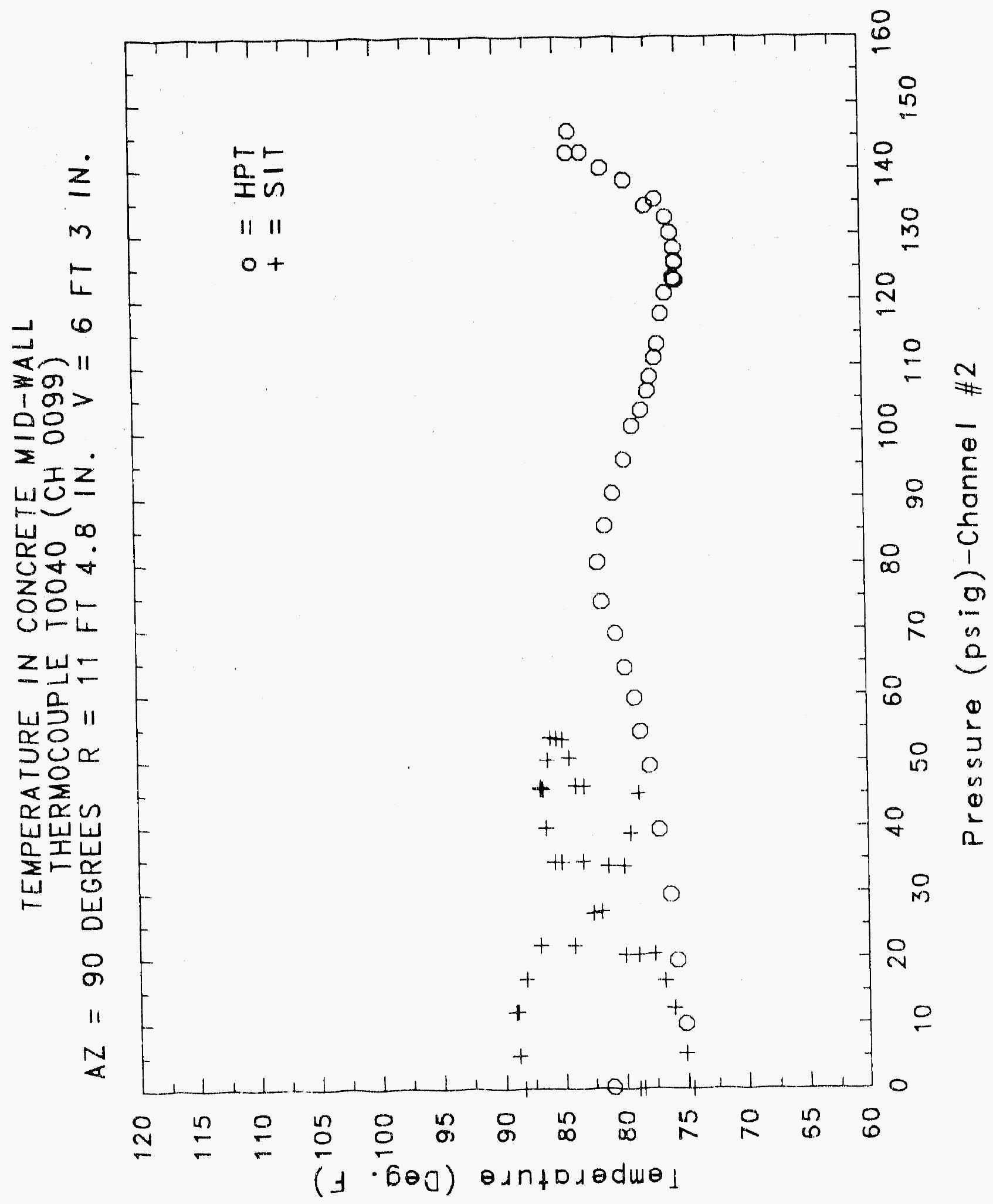

C -1743 


\section{Reinforced Concrete Test Data}

Thermocouple Channel 100

Structural Integrity Test

High Pressure test

\begin{tabular}{|c|c|c|c|}
\hline $\begin{array}{l}\text { Pressure } \\
\text { (psig) } \\
-0.05 \\
5.33 \\
12.31 \\
16.44 \\
20.51 \\
20.46 \\
20.47 \\
27.05 \\
34.69 \\
34.53 \\
34.53 \\
39.70 \\
45.90 \\
45.65 \\
45.62 \\
50.10 \\
53.47 \\
53.29 \\
53.21 \\
50.49 \\
46.14 \\
46.13 \\
44.97 \\
38.98 \\
33.99 \\
33.96 \\
26.83 \\
21.88 \\
21.90 \\
16.69 \\
11.74 \\
11.77 \\
5.05 \\
0.02 \\
-0.04 \\
-0.02 \\
-0.02 \\
0.02\end{array}$ & $\begin{array}{c}\text { Temperature } \\
\text { (Fahrenheit) } \\
82.6130 \\
82.6130 \\
82.5250 \\
83.2270 \\
83.1840 \\
83.7100 \\
83.3590 \\
84.5000 \\
85.2450 \\
86.0330 \\
86.2960 \\
87.1260 \\
87.6950 \\
88.3930 \\
88.1750 \\
88.8730 \\
88.7860 \\
88.8300 \\
89.0920 \\
88.2190 \\
88.1750 \\
87.5200 \\
84.1930 \\
85.2010 \\
85.5080 \\
85.4640 \\
85.9890 \\
86.0770 \\
87.4760 \\
87.9570 \\
88.3060 \\
89.0480 \\
88.9170 \\
88.7430 \\
83.7540 \\
83.5350 \\
82.8320 \\
84.5440\end{array}$ & $\begin{array}{c}\text { Pressure } \\
\text { (psig) } \\
9.89 \\
19.55 \\
29.57 \\
39.42 \\
49.16 \\
54.50 \\
59.57 \\
64.20 \\
69.32 \\
74.16 \\
80.16 \\
85.61 \\
90.58 \\
95.69 \\
100.92 \\
103.25 \\
106.11 \\
108.31 \\
111.08 \\
113.24 \\
117.83 \\
120.92 \\
123.28 \\
122.97 \\
125.82 \\
125.60 \\
127.84 \\
130.19 \\
132.53 \\
135.33 \\
134.42 \\
138.35 \\
140.16 \\
142.63 \\
145.78 \\
142.52 \\
0.22\end{array}$ & $\begin{array}{c}\text { Temperature } \\
\text { (Fahrenhe it) } \\
82.3490 \\
83.1840 \\
83.2710 \\
84.1490 \\
84.6310 \\
84.8940 \\
84.9820 \\
85.3320 \\
85.7700 \\
86.1640 \\
86.5580 \\
86.8640 \\
86.6890 \\
86.0770 \\
85.6830 \\
85.1130 \\
84.8500 \\
84.7630 \\
84.3680 \\
84.5440 \\
84.2810 \\
84.2810 \\
83.3150 \\
82.8320 \\
82.7000 \\
82.1290 \\
82.3050 \\
82.7440 \\
82.5690 \\
83.4030 \\
83.3590 \\
84.0170 \\
84.4560 \\
85.5950 \\
85.9890 \\
86.1200 \\
76.3970\end{array}$ \\
\hline
\end{tabular}




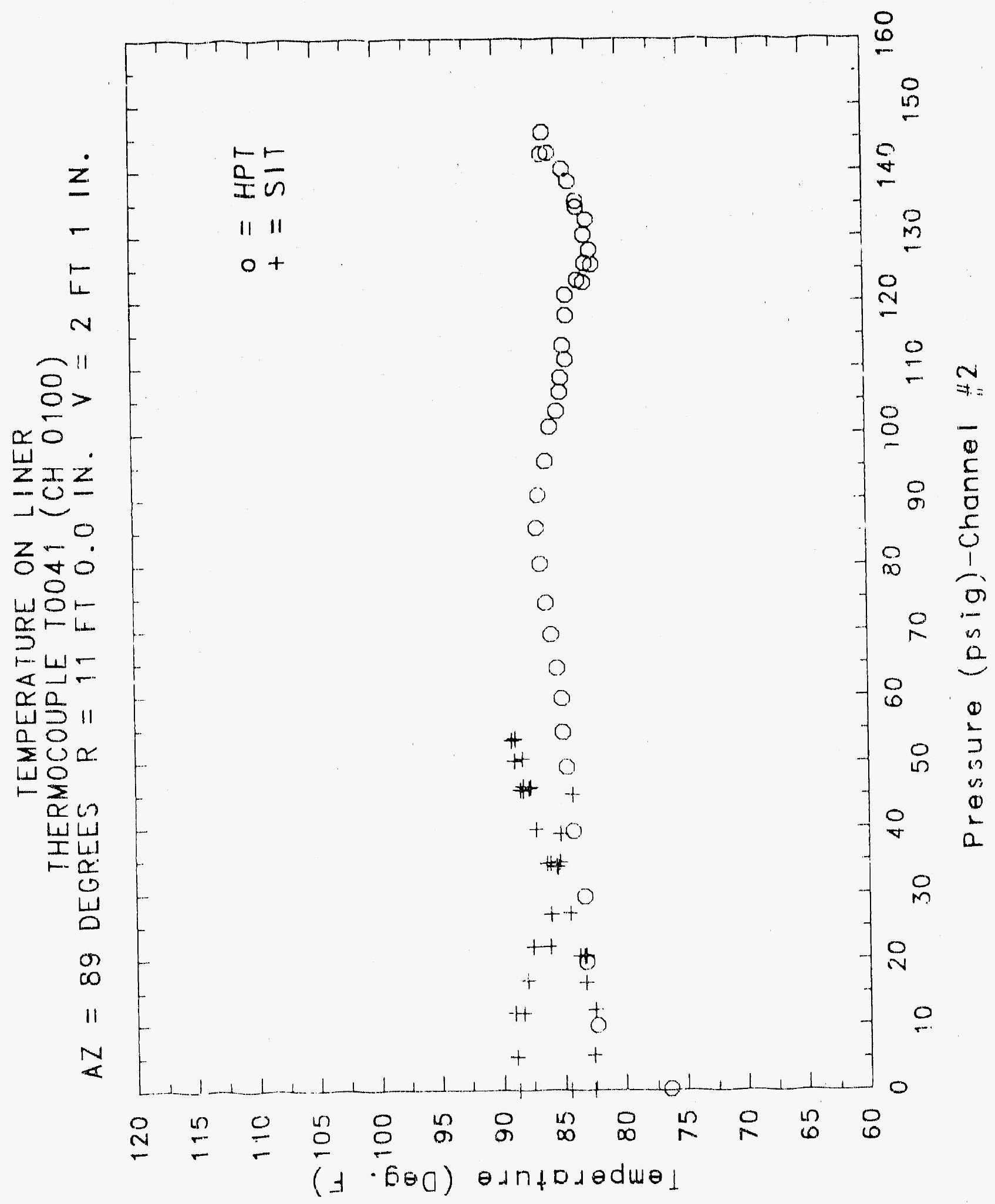




\section{Reinforced Concrete Test Data}

Thermocouple Channel 101

StRUCTURAL InTEGRITY TEST

\begin{tabular}{|c|c|}
\hline $\begin{array}{l}\text { Prejsure } \\
\text { (psig) } \\
-0.05 \\
5.33 \\
12.31 \\
16.44 \\
20.51 \\
20.46 \\
20.47 \\
27.05 \\
34.59 \\
34.53 \\
34.53 \\
39.70 \\
45.90 \\
45.65 \\
45.62 \\
50.10 \\
53.47 \\
53.29 \\
53.21 \\
50.49 \\
46.14 \\
46.13 \\
44.97 \\
38.98 \\
33.99 \\
33.96 \\
26.83 \\
21.88 \\
21.90 \\
16.69 \\
11.74 \\
11.77 \\
5.05 \\
0.02 \\
-0.04 \\
-0.02 \\
-0.02 \\
0.02\end{array}$ & $\begin{array}{c}\text { Temperature } \\
\text { (Fahrenheit) } \\
82.5690 \\
82.9200 \\
83.2710 \\
83.0960 \\
83.5790 \\
83.4910 \\
83.2270 \\
84.5000 \\
84.9820 \\
85.4640 \\
85.0260 \\
85.5950 \\
86.4270 \\
86.3390 \\
86.4700 \\
86.7770 \\
87.1260 \\
86.9080 \\
81.0830 \\
86.6450 \\
86.6450 \\
86.6450 \\
84.1930 \\
84.7630 \\
85.1130 \\
85.1570 \\
85.4200 \\
85.8580 \\
85.6830 \\
85.9890 \\
85.9450 \\
86.6890 \\
86.4700 \\
86.6890 \\
82.1290 \\
82.8320 \\
83.6660 \\
85.1570\end{array}$ \\
\hline
\end{tabular}

High Pressure test

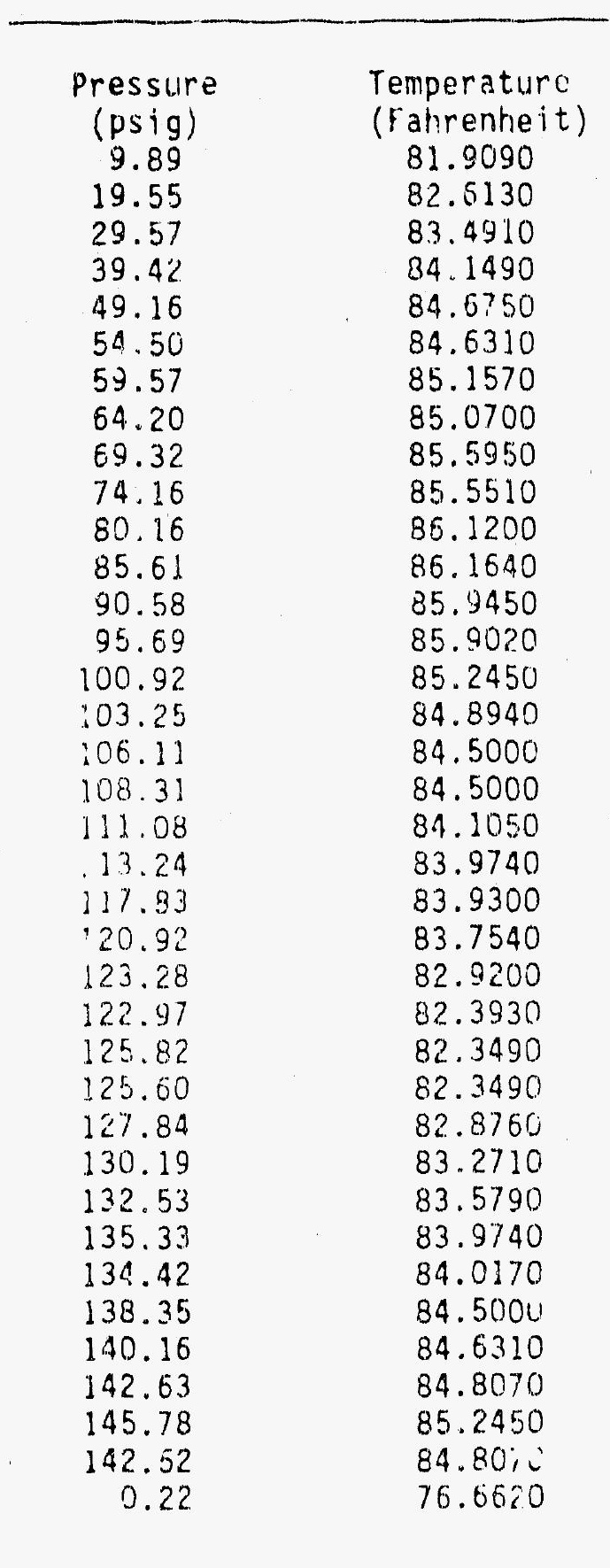




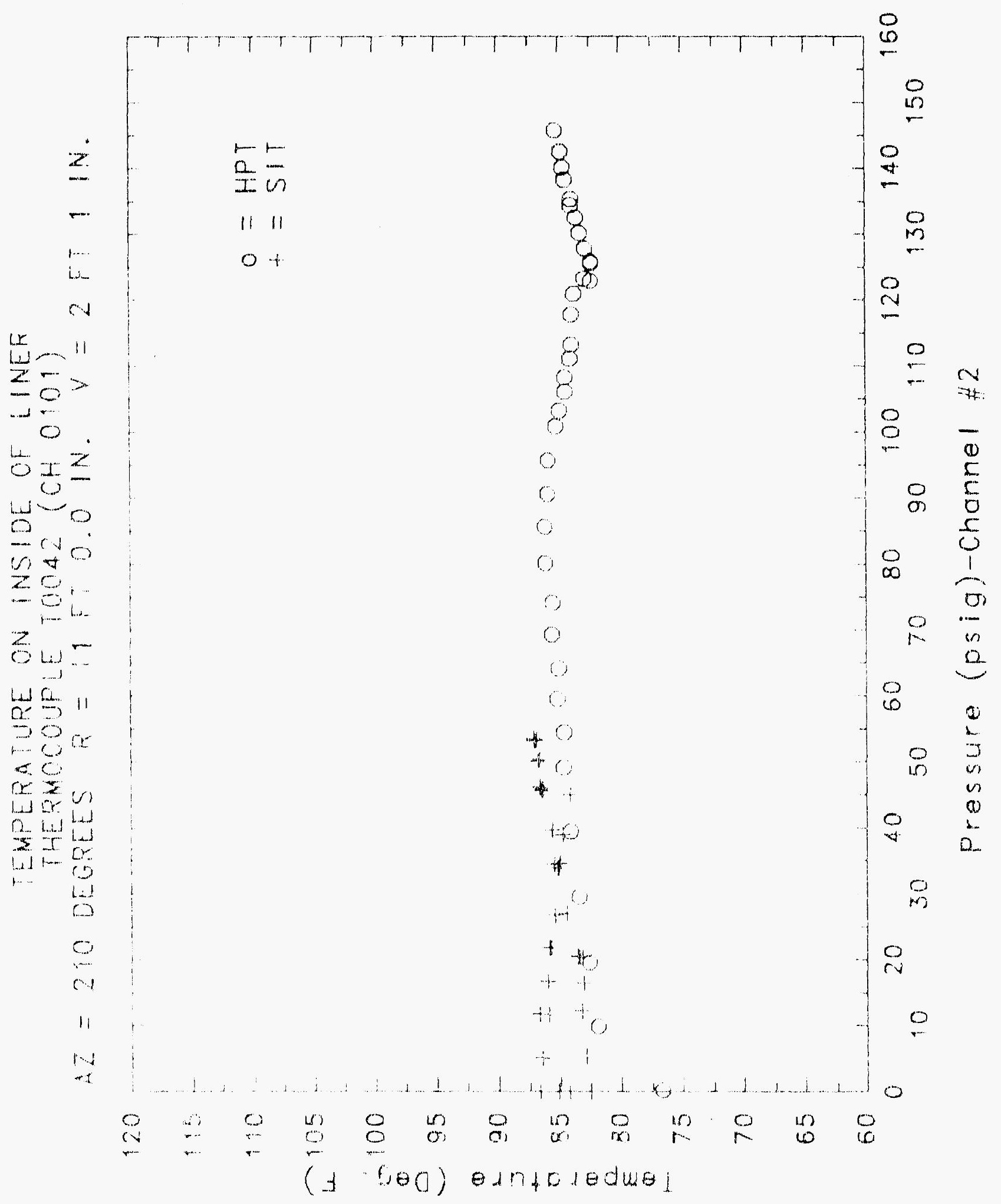




\section{Reinforced Concrete Test Data}

Thermocouple Channel 102

STRUCTURAL INTEGRITY TEST

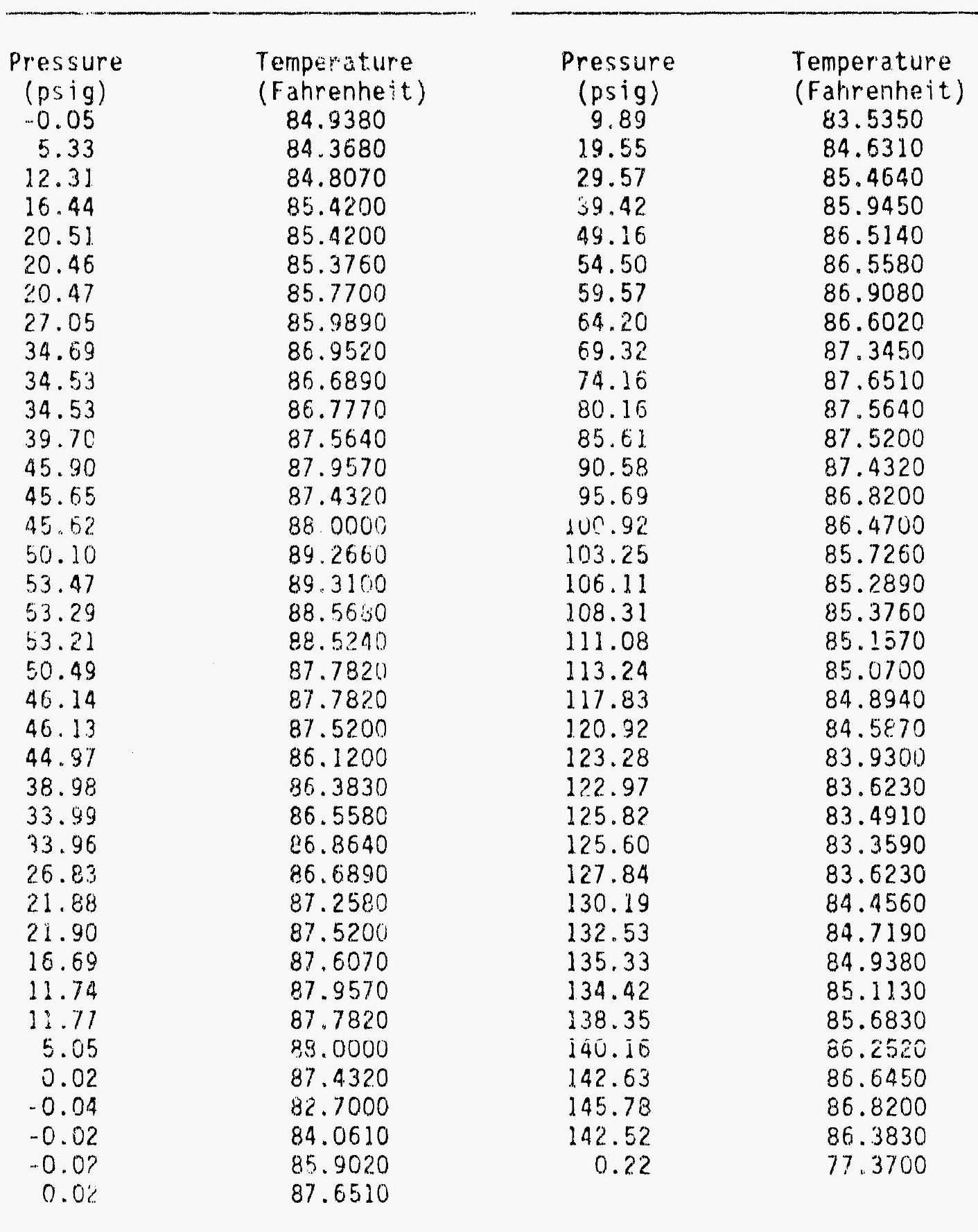

High Pressure Test

Pressure

9.89

Fahrenheit)

85.4640

85.9450

86.5140

86.6020

87.3450

87.5200

87.4320

86.8200

85.2890

85.3760

85.1570

84.5870

83.9300

83.6230

83.6230

84.4560

84.7190

84.9380

85.6830

86.2520

86.6450

$\begin{array}{rr}42.52 & 86.3830 \\ 0.22 & 77.3700\end{array}$ 


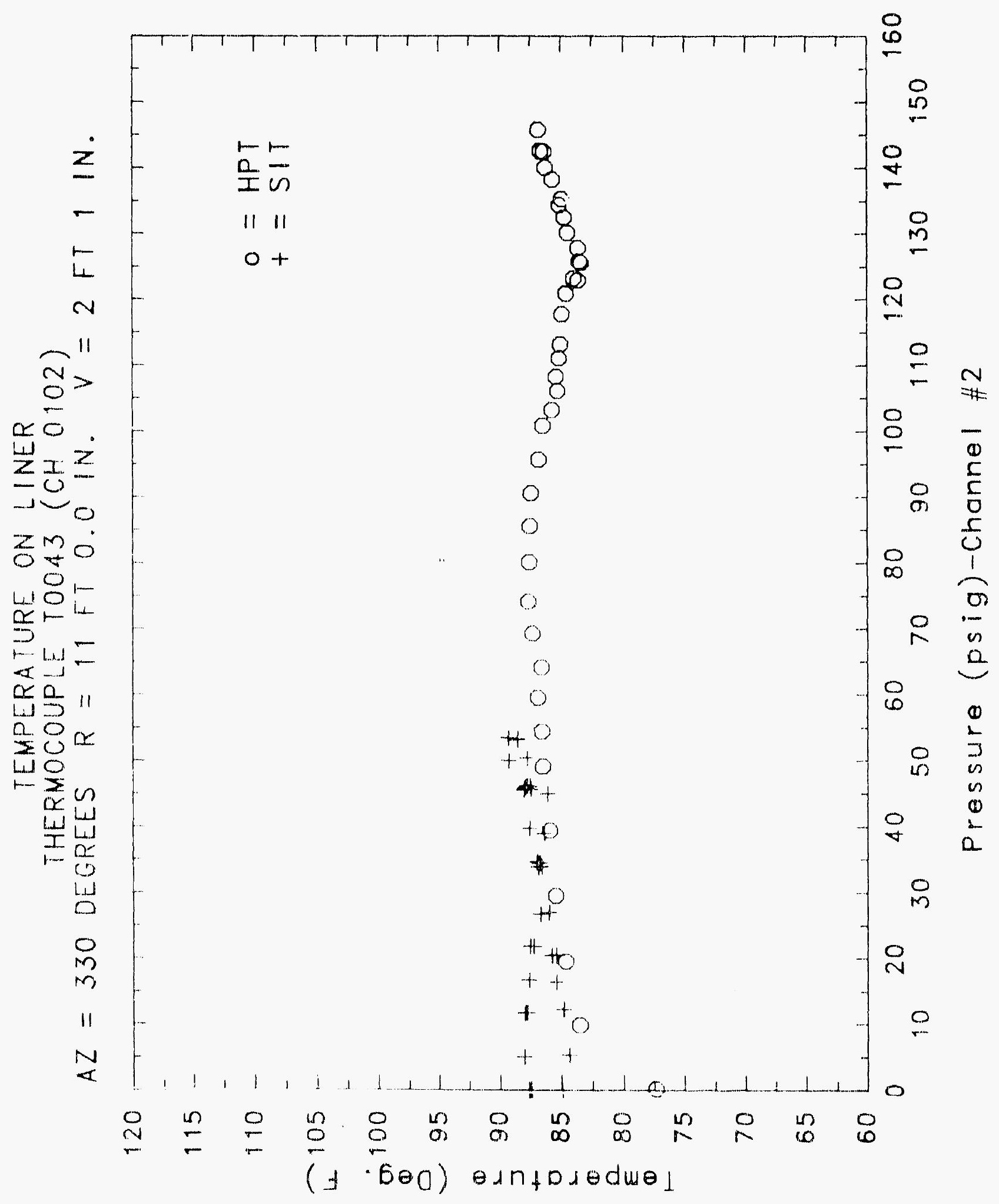




\section{Reinforced Concrete Test Data}

Thermocouple Channel 103

StRuctural INTEGRITY TEST

\begin{tabular}{|c|c|}
\hline $\begin{array}{l}\text { Pressure } \\
\text { (psig) } \\
-0.05 \\
5.33 \\
12.31 \\
16.44 \\
20.51 \\
20.46 \\
20.47 \\
27.05 \\
34.69 \\
34.53 \\
34.53 \\
39.70 \\
45.90 \\
45.65 \\
45.62 \\
50.10 \\
53.47 \\
53.29 \\
53.21 \\
50.49 \\
46.14 \\
46.13 \\
44.97 \\
38.98 \\
33.99 \\
33.96 \\
26.83 \\
21.88 \\
21.90 \\
16.69 \\
11.74 \\
11.77 \\
5.05 \\
0.02 \\
-0.04 \\
-0.02 \\
-0.02 \\
0.02\end{array}$ & $\begin{array}{c}\text { Temperature } \\
\text { (Fahrenheit) } \\
83.4910 \\
84.3680 \\
84.6750 \\
85.2010 \\
86.4700 \\
86.5140 \\
86.3830 \\
87.2580 \\
88.2190 \\
88.2190 \\
88.0440 \\
88.4810 \\
83.3060 \\
87.5200 \\
87.5640 \\
87.8260 \\
88.1310 \\
87.5640 \\
87.3450 \\
86.5580 \\
86.1640 \\
86.2080 \\
86.6450 \\
86.9950 \\
87.2140 \\
87.6070 \\
87.9130 \\
88.1310 \\
88.3930 \\
88.7430 \\
88.5240 \\
88.5680 \\
87.8260 \\
86.7330 \\
81.2500 \\
84.1930 \\
85.4200 \\
87.4760\end{array}$ \\
\hline
\end{tabular}

High Pressure test

\begin{tabular}{|c|c|}
\hline $\begin{array}{l}\text { iressure } \\
\text { (psig) } \\
9.89 \\
19.55 \\
29.57 \\
39.42 \\
49.16 \\
54.50 \\
59.57 \\
64.20 \\
69.32 \\
74.16 \\
80.16 \\
85.61 \\
90.58 \\
95.69 \\
100.92 \\
103.25 \\
106.11 \\
108.31 \\
111.08 \\
113.24 \\
117.83 \\
120.92 \\
123.28 \\
122.97 \\
125.82 \\
125.60 \\
127.84 \\
130.19 \\
132.53 \\
135.33 \\
134.42 \\
138.35 \\
140.16 \\
142.63 \\
145.78 \\
142.52 \\
0.22\end{array}$ & $\begin{array}{c}\text { Temperature } \\
\text { (Fahrenheit) } \\
84.9820 \\
85.6830 \\
86.6890 \\
87.0390 \\
87.1260 \\
87.0390 \\
87.2140 \\
87.7380 \\
88.1310 \\
88.1750 \\
84.7190 \\
84.8940 \\
84.5000 \\
84.0610 \\
83.4470 \\
82.7000 \\
82.2610 \\
82.2170 \\
81.7340 \\
81.3380 \\
81.7780 \\
81.2940 \\
81.0300 \\
80.9860 \\
81.1620 \\
81.6020 \\
82.2610 \\
83.0960 \\
84.4560 \\
84.3680 \\
84.5870 \\
85.9020 \\
85.1570 \\
86.0770 \\
85.6830 \\
83.6660 \\
68.7570\end{array}$ \\
\hline
\end{tabular}




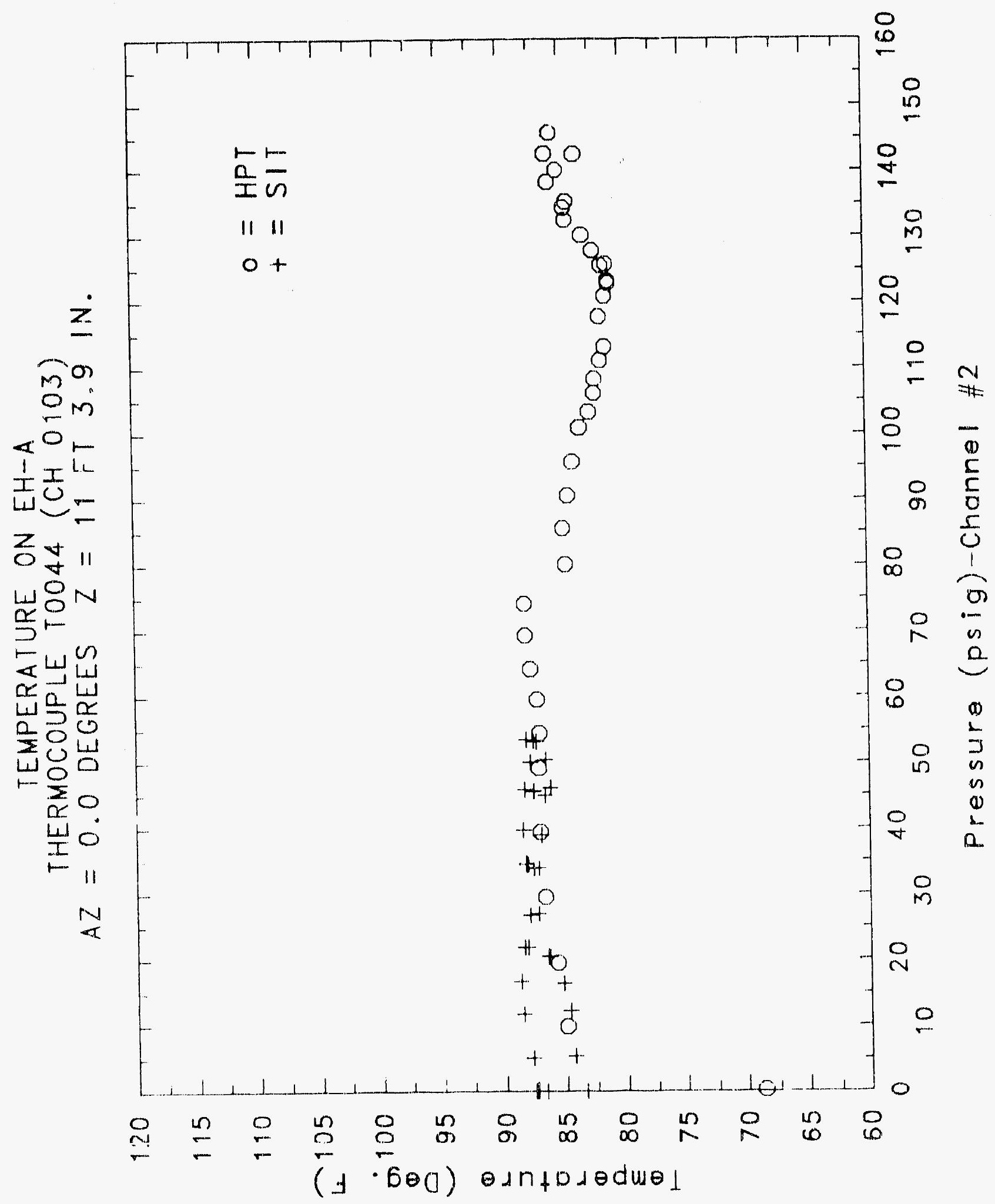




\section{Reinforced Concrete Test Data}

Thermocouple Channe!: 104

Structural InTEgrity TEST

\begin{tabular}{|c|c|c|c|}
\hline $\begin{array}{l}\text { Pressure } \\
\text { (psig) } \\
-0.05 \\
5.33 \\
12.31 \\
16.44 \\
20.51 \\
20.46 \\
20.47 \\
27.05 \\
34.69 \\
34.53 \\
34.53 \\
39.70 \\
45.90 \\
45.65 \\
45.62 \\
50.10 \\
53.47 \\
53.29 \\
53.21 \\
50.49 \\
46.14 \\
46.13 \\
44.97 \\
38.98 \\
33.99 \\
33.96 \\
26.83 \\
21.88 \\
21.90 \\
16.69 \\
11.74 \\
11.77 \\
5.05 \\
0.02 \\
-0.04 \\
0.02 \\
-0.02 \\
0.02 \\
\end{array}$ & $\begin{array}{c}\text { Temperature } \\
\text { (Fahrenheit) } \\
82.3930 \\
84.3240 \\
85.4640 \\
86.2960 \\
86.9520 \\
87.2580 \\
87.6950 \\
88.2190 \\
88.8300 \\
89.0920 \\
88.9610 \\
88.9610 \\
88.5240 \\
87.6070 \\
87.4760 \\
88.0000 \\
88.3500 \\
88.0000 \\
87.6510 \\
86.8200 \\
86.1640 \\
85.7700 \\
88.5680 \\
88.8730 \\
89.0480 \\
89.6150 \\
89.7020 \\
89.9200 \\
90.3120 \\
90.0940 \\
89.8330 \\
89.0920 \\
88.4810 \\
88.0440 \\
82.7880 \\
86.0330 \\
87.4320 \\
88.6550\end{array}$ & $\begin{array}{c}\text { Pressure } \\
(p s i g) \\
9.89 \\
19.55 \\
29.57 \\
39.42 \\
49.16 \\
54.50 \\
59.57 \\
64.20 \\
69.32 \\
74.16 \\
80.16 \\
85.61 \\
90.58 \\
95.69 \\
100.92 \\
103.25 \\
106.11 \\
108.31 \\
111.08 \\
113.24 \\
117.83 \\
120.92 \\
123.28 \\
122.97 \\
125.82 \\
125.61 \\
127.84 \\
130.19 \\
132.53 \\
135.33 \\
134.42 \\
138.35 \\
140.16 \\
142.63 \\
145.78 \\
142.52 \\
0.22 \\
\end{array}$ & $\begin{array}{c}\text { Temperature } \\
\text { (Fahrenheit) } \\
84.1490 \\
84.9380 \\
85.5510 \\
86.0770 \\
86.3390 \\
86.3830 \\
86.4700 \\
87.0390 \\
87.5200 \\
88.0440 \\
84.7630 \\
84.9380 \\
85.1570 \\
84.7630 \\
84.3240 \\
83.1840 \\
83.0080 \\
83.1840 \\
82.7000 \\
82.6570 \\
82.6130 \\
82.2170 \\
82.0850 \\
81.8650 \\
81.9530 \\
82.2610 \\
83.0960 \\
82.7880 \\
83.7100 \\
85.0260 \\
85.4200 \\
86.6450 \\
85.5510 \\
86.5140 \\
85.5080 \\
83.4030 \\
69.9160\end{array}$ \\
\hline
\end{tabular}

High Pressure Test 


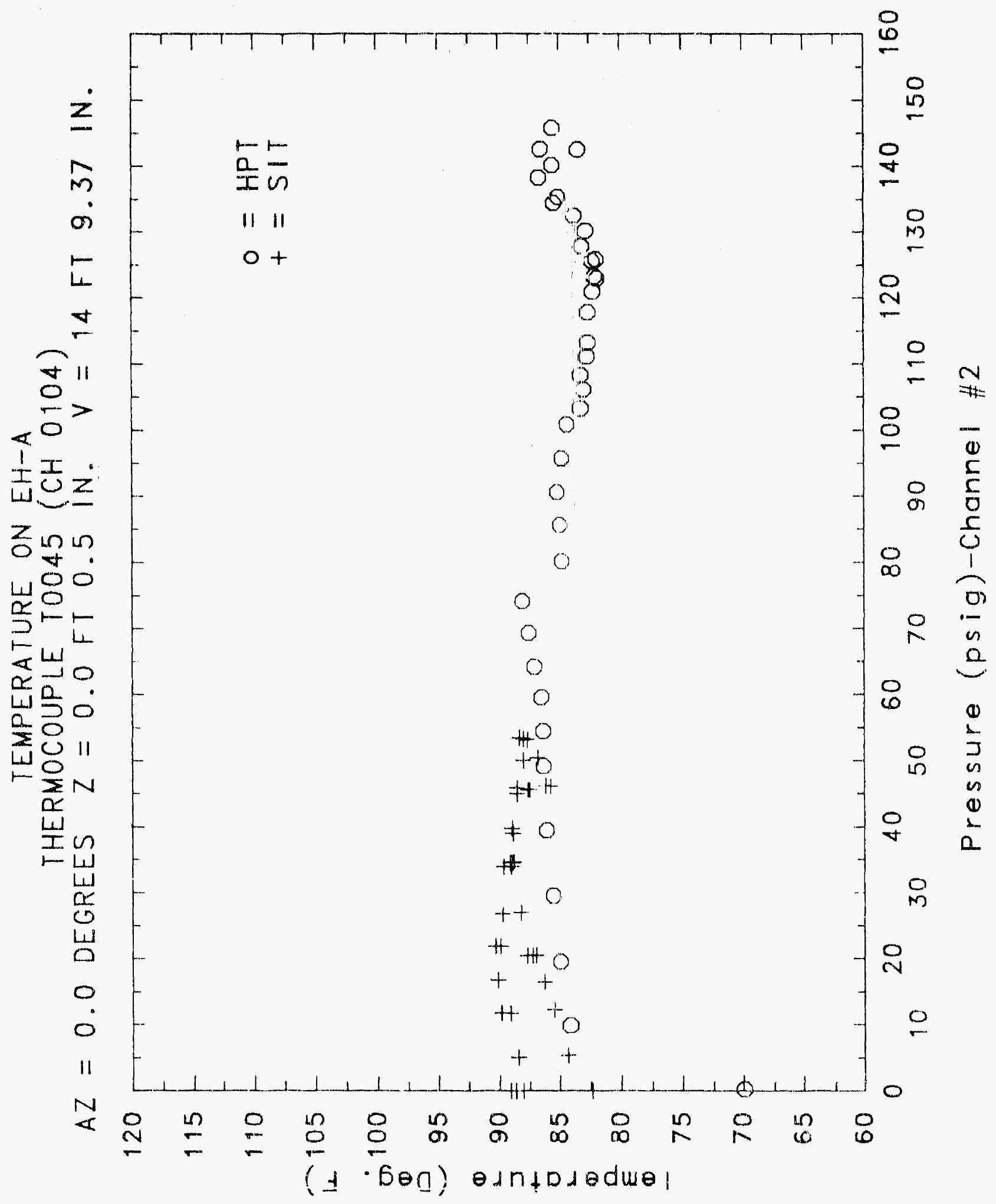

C. -1753 


\section{Reinforced Concrete Test Data}

Thermocouple ChanNel 105

Structural Integrity Test

\begin{tabular}{|c|c|}
\hline $\begin{array}{l}\text { Pressure } \\
(p s i g) \\
-0.05 \\
5.33 \\
12.31 \\
16.44 \\
20.51 \\
20.46 \\
20.47 \\
27.05 \\
34.69 \\
34.53 \\
34.53 \\
39.70 \\
45.90 \\
45.65 \\
45.62 \\
50.10 \\
53.47 \\
53.29 \\
53.21 \\
50.49 \\
46.14 \\
46.13 \\
44.97 \\
38.98 \\
33.99 \\
33.96 \\
26.83 \\
21.88 \\
21.90 \\
16.69 \\
11.74 \\
11.77 \\
5.05 \\
0.02 \\
-0.04 \\
-0.02 \\
-0.02 \\
0.02\end{array}$ & $\begin{array}{l}\text { Temperature } \\
\text { (Fahrenheit) } \\
81.7340 \\
81.9090 \\
82.1290 \\
82.4370 \\
82.7000 \\
82.9200 \\
83.1840 \\
83.8420 \\
84.5870 \\
84.8940 \\
85.0700 \\
85.7700 \\
86.3830 \\
86.7770 \\
86.9520 \\
87.5640 \\
87.9130 \\
87.9570 \\
88.0000 \\
87.8690 \\
87.6950 \\
87.6510 \\
84.9380 \\
85.2450 \\
85.4640 \\
85.8580 \\
86.1200 \\
86.3390 \\
86.8200 \\
86.9950 \\
87.2140 \\
87.7380 \\
87.8260 \\
87.8260 \\
85.1570 \\
84.5000 \\
84.5000 \\
85.6390\end{array}$ \\
\hline
\end{tabular}

High Pressure Test

\begin{tabular}{|c|c|}
\hline $\begin{array}{c}\text { Pressure } \\
\text { (psig) } \\
9.89 \\
19.55 \\
29.57 \\
39.42 \\
49.16 \\
54.50 \\
59.57 \\
64.20 \\
69.32 \\
74.16 \\
80.16 \\
85.61 \\
90.58 \\
95.69 \\
100.92 \\
103.25 \\
106.11 \\
108.31 \\
111.08 \\
113.24 \\
117.83 \\
120.92 \\
123.28 \\
122.97 \\
125.82 \\
125.60 \\
127.84 \\
130.19 \\
132.53 \\
135.33 \\
134.42 \\
138.35 \\
140.16 \\
142.63 \\
145.78 \\
142.52 \\
0.22\end{array}$ & $\begin{array}{c}\text { Temperature } \\
\text { (Fahrenheit) } \\
81.6460 \\
82.2610 \\
82.7880 \\
83.1840 \\
83.7540 \\
84.0610 \\
84.2810 \\
84.5870 \\
84.8940 \\
85.3320 \\
85.9890 \\
86.2080 \\
86.2520 \\
85.9020 \\
85.6390 \\
85.1130 \\
84.8070 \\
84.7190 \\
84.4120 \\
81.2810 \\
84.1930 \\
83.7980 \\
83.1840 \\
82.7440 \\
82.5690 \\
82.1290 \\
82.2170 \\
82.2170 \\
82.3930 \\
82.7000 \\
82.8320 \\
83.3590 \\
83.7100 \\
84.1930 \\
84.5870 \\
84.1490 \\
76.2640\end{array}$ \\
\hline
\end{tabular}




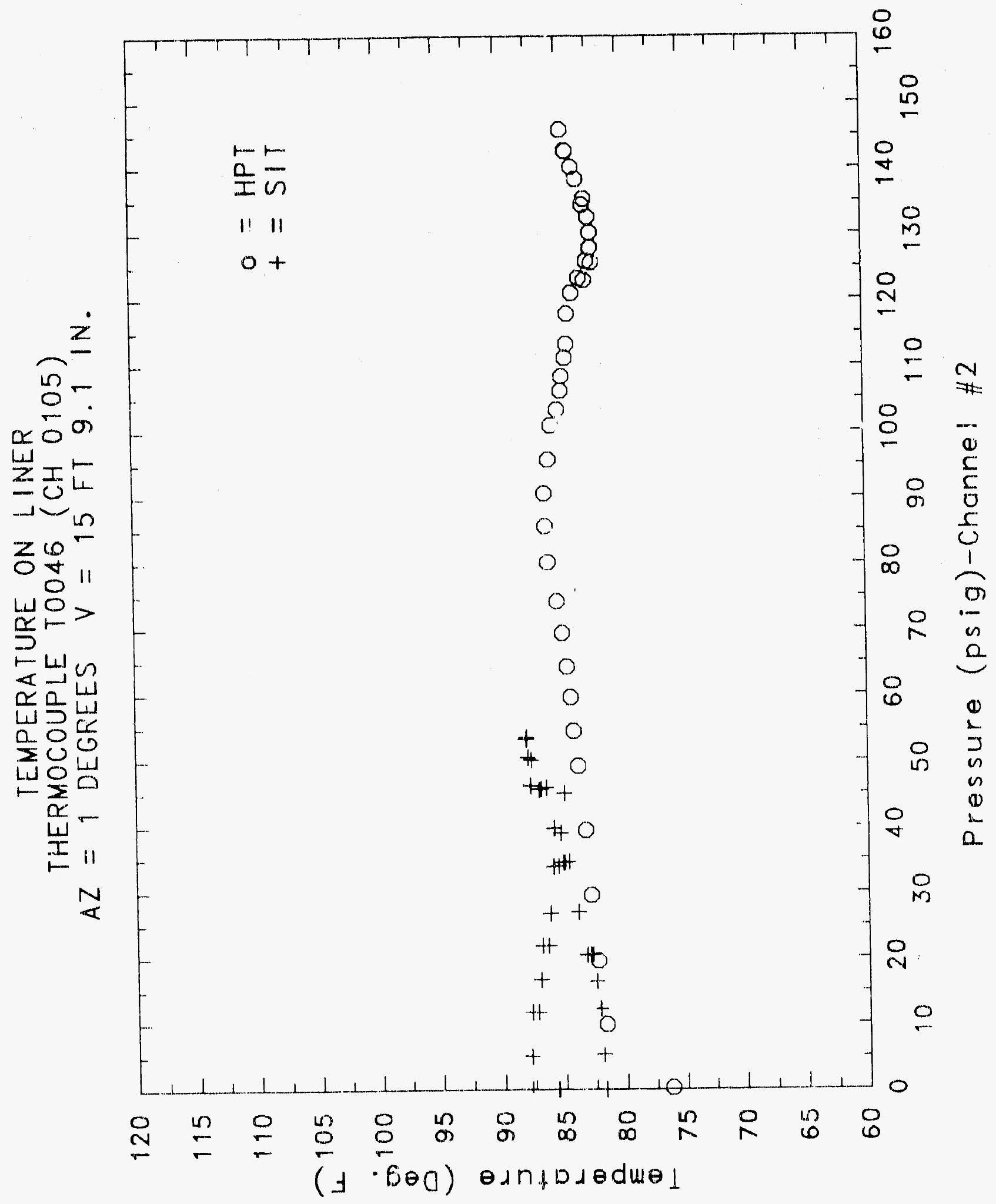




\section{Reinforced Concrete Test Data}

Thermocouple Channel 106

STRUCTURAL INTEGRITY TEST

\begin{tabular}{|c|c|}
\hline $\begin{array}{c}\text { Pressure } \\
\text { (psig) } \\
-0.05 \\
5.33 \\
12.31 \\
16.44 \\
20.51 \\
20.46 \\
20.47 \\
27.05 \\
34.69 \\
34.53 \\
34.53 \\
39.70 \\
45.90 \\
45.65 \\
45.52 \\
50.10 \\
53.47 \\
53.29 \\
53.21 \\
50.49 \\
46.14 \\
46.13 \\
44.97 \\
38.98 \\
33.99 \\
33.96 \\
26.83 \\
21.88 \\
21.90 \\
16.69 \\
11.74 \\
11.77 \\
5.05 \\
0.02 \\
-0.04 \\
-0.02 \\
-0.02 \\
0.02\end{array}$ & $\begin{array}{c}\text { Temperature } \\
\text { (Fahrenheit) } \\
81.5140 \\
81.9090 \\
82.4810 \\
82.8760 \\
83.4910 \\
83.9300 \\
84.3240 \\
85.4200 \\
86.2960 \\
86.9950 \\
87.3890 \\
88.2190 \\
88.9170 \\
89.3530 \\
89.5280 \\
89.8330 \\
89.9640 \\
89.6580 \\
89.4840 \\
88.9610 \\
88.6120 \\
88.2620 \\
84.7630 \\
85.3320 \\
85.7700 \\
86.3390 \\
86.7330 \\
87.0830 \\
87.8690 \\
88.3500 \\
88.6550 \\
89.4400 \\
89.6150 \\
89.4840 \\
83.6660 \\
83.2270 \\
83.4470 \\
84.8500\end{array}$ \\
\hline
\end{tabular}

High Pressure test

\begin{tabular}{|c|c|}
\hline $\begin{array}{c}\text { Pressure } \\
(p \text { ig) } \\
9.89 \\
19.55 \\
29.57 \\
39.42 \\
49.16 \\
54.50 \\
59.57 \\
64.20 \\
69.32 \\
74.16 \\
80.16 \\
85.61 \\
90.58 \\
95.69 \\
100.92 \\
103.25 \\
106.11 \\
108.31 \\
111.08 \\
113.24 \\
117.83 \\
120.92 \\
123.28 \\
122.97 \\
125.82 \\
125.60 \\
127.84 \\
130.19 \\
132.53 \\
135.33 \\
134.42 \\
138.35 \\
140.16 \\
142.53 \\
145.78 \\
142.52 \\
0.22\end{array}$ & $\begin{array}{c}\text { Temperature } \\
\text { (Fahrenheit) } \\
81.2060 \\
81.8650 \\
82.5250 \\
83.2270 \\
83.8420 \\
84.2370 \\
84.5870 \\
85.0700 \\
85.5080 \\
85.9890 \\
86.9080 \\
86.7330 \\
86.2960 \\
85.6390 \\
85.1130 \\
84.2810 \\
83.8860 \\
83.7980 \\
83.3150 \\
83.0520 \\
82.8320 \\
82.4370 \\
81.6900 \\
81.3820 \\
81.2060 \\
80.8980 \\
81.0300 \\
81.3380 \\
81.6460 \\
82.2170 \\
82.6570 \\
83.5350 \\
84.2370 \\
85.1130 \\
85.9020 \\
85.9890 \\
76.8390\end{array}$ \\
\hline
\end{tabular}




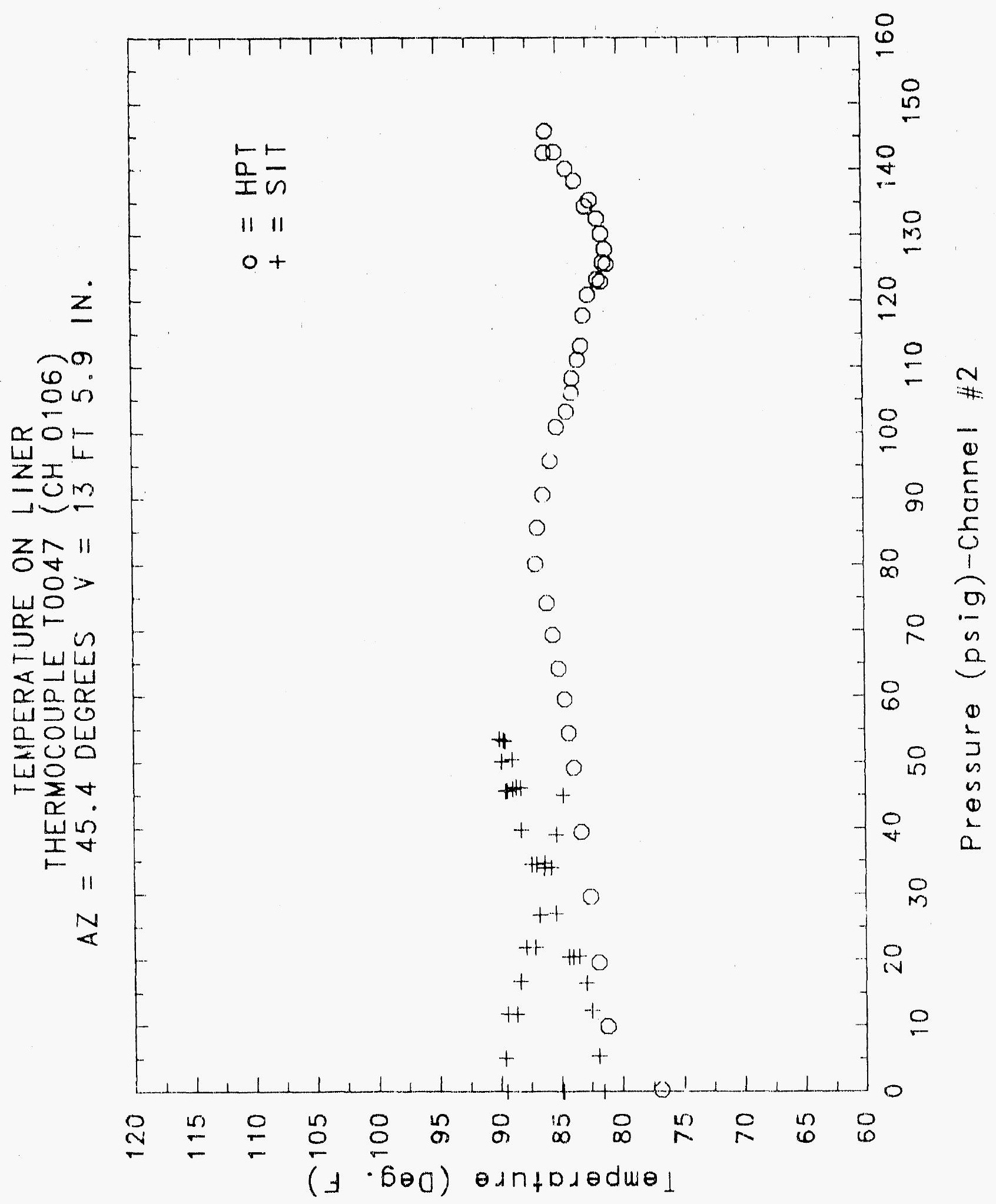




\section{Reinforced Concrete Test Data}

Thermocouple Channel 107

Structural Integrity test

\begin{tabular}{|c|c|c|c|}
\hline $\begin{array}{l}\text { Pressure } \\
(p \text { pig) } \\
-0.05 \\
5.33 \\
12.31 \\
16.44 \\
20.51 \\
20.46 \\
20.47 \\
27.05 \\
34.69 \\
34.53 \\
34.53 \\
39.70 \\
45.90 \\
45.65 \\
45.62 \\
50.10 \\
53.47 \\
53.29 \\
53.21 \\
50.49 \\
46.14 \\
46.13 \\
44.97 \\
38.98 \\
33.99 \\
.33 .96 \\
26.83 \\
21.88 \\
21.90 \\
16.69 \\
11.74 \\
11.77 \\
5.05 \\
0.02 \\
-0.04 \\
-0.02 \\
-0.02 \\
0.02\end{array}$ & $\begin{array}{l}\text { Temperature } \\
\text { (Fahrenheit) } \\
80.8980 \\
81.2060 \\
81.7340 \\
82.3050 \\
82.8760 \\
83.3590 \\
83.8860 \\
84.8940 \\
85.8580 \\
86.5140 \\
86.7770 \\
87.6070 \\
88.4370 \\
88.9610 \\
89.1350 \\
89.4900 \\
89.5710 \\
89.3970 \\
89.2220 \\
88.7860 \\
88.3930 \\
88.1310 \\
84.9380 \\
85.3320 \\
85.6830 \\
86.3830 \\
86.7770 \\
87.2140 \\
87.9130 \\
80.2520 \\
88.5680 \\
89.3100 \\
89.4840 \\
89.4840 \\
83.9300 \\
83.4030 \\
83.6230 \\
85.3320\end{array}$ & $\begin{array}{c}\text { Pressure } \\
(p s i g) \\
9.89 \\
19.55 \\
29.57 \\
39.42 \\
49.16 \\
54.50 \\
59.57 \\
64.20 \\
69.32 \\
74.16 \\
80.16 \\
85.61 \\
90.58 \\
95.69 \\
100.92 \\
103.25 \\
106.11 \\
108.31 \\
111.08 \\
113.24 \\
117.83 \\
120.92 \\
123.28 \\
122.97 \\
125.82 \\
125.60 \\
127.84 \\
130.19 \\
132.53 \\
135.33 \\
134.42 \\
138.35 \\
140.16 \\
142.63 \\
145.78 \\
142.52 \\
0.22 \\
\end{array}$ & $\begin{array}{c}\text { Temperature } \\
\text { (Fahrenheit } \\
80.8100 \\
81.6900 \\
82.4370 \\
83.1400 \\
83.9740 \\
84.3240 \\
84.6750 \\
85.0260 \\
85.4640 \\
85.9890 \\
86.6890 \\
86.5580 \\
86.2520 \\
85.4640 \\
84.9820 \\
84.1050 \\
83.7980 \\
83.6230 \\
83.2270 \\
83.0080 \\
82.8760 \\
82.4810 \\
81.6900 \\
81.2060 \\
81.1620 \\
80.9860 \\
81.2060 \\
81.5140 \\
81.9090 \\
82.4370 \\
82.7880 \\
83.6660 \\
84.3680 \\
84.9820 \\
85.72 .60 \\
85.8140 \\
78.3410\end{array}$ \\
\hline
\end{tabular}

High pressure test

ressure

( 8.89

19.55

29.57

54.50

59.57

64.20

69.32

85.6

100.92

103.25

111.08

120.92

125.82

125.60

132.53

135.33

134.42

138.35

140.16

42.52

85.8140

Temperature

80.8100

81.6900

82.4370

83.9740

84.3240

84.6750

85.0260

BE. 4640

85.9890

86.5580

86.2520

85.4640

84.9820

84.1050

83.6230

83.2270

83.0080

82.8760

81.2060

80.9860

81.2060

82.4370

83.6660

84.3680

84.9820

83.9300

83.6230 


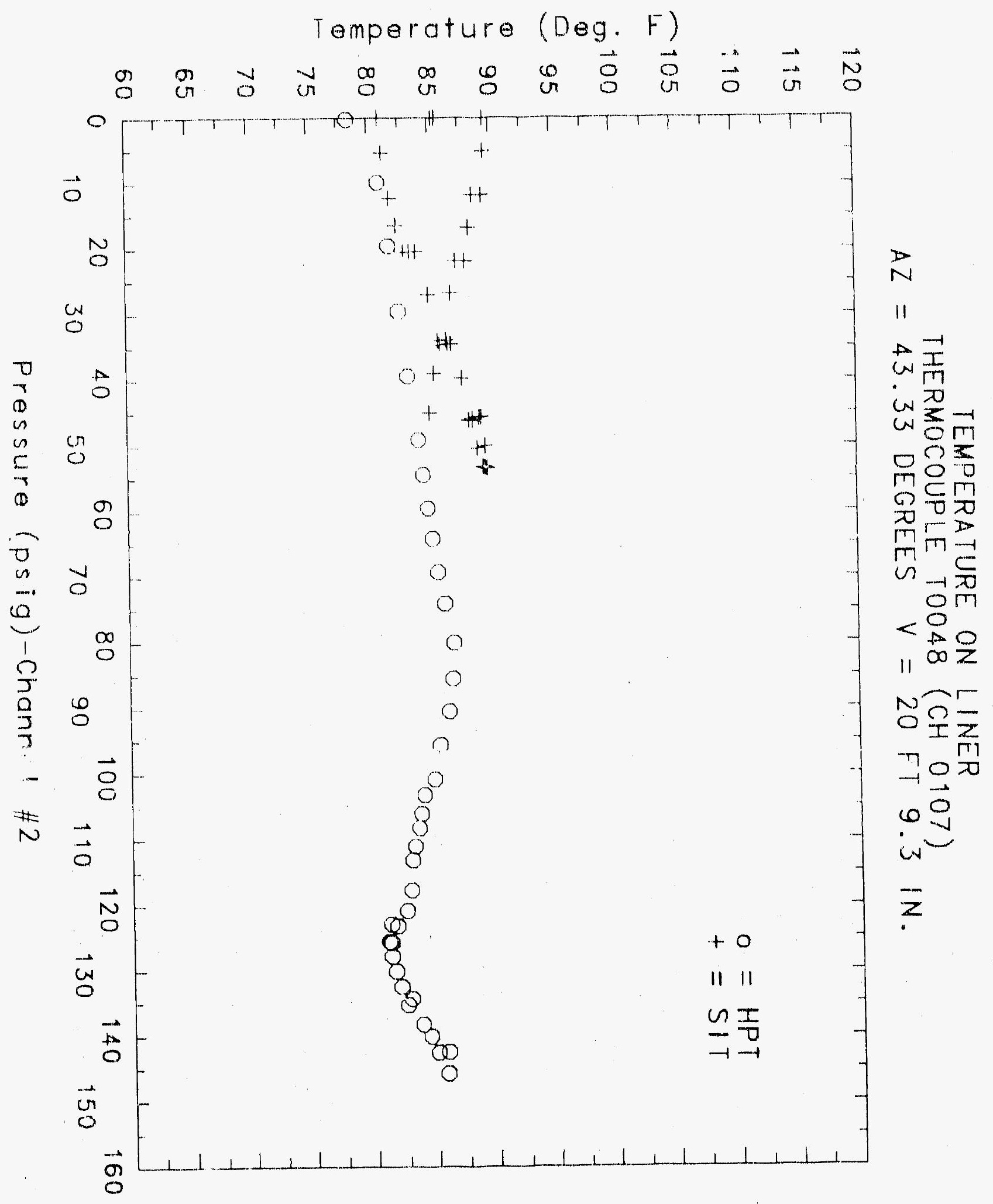




\section{- Reinforced Concrete test Data}

Thermocouple Channel 108

Structi,imal Integrity teg

Pressure

(psig)

$-0.05$

5.32

12.31

16.44

20.51

20.46

20.47

27.05

34.69

34.53

34.53

39.70

45.90

45.65

45,62

50.10

53.47

53.29

53.21

50.49

46.14

46.13

44.97

38.98

33.99

33.96

26. 8 ?

21.88

21.90

16.69

11.74

11.77

5.05

0.02

$-0.04$

$-0.02$

$-0.02$

0.02

\section{Temperature}

(Fahrenheit)

82.1730

82.3050

82.5250

82.7000

83.1400

83.3590

83.7100

85.1130

86.5140

$87: 1260$

87.4760

88.4370

89.1790

89.3970

89.3530

89.6580

83.7020

89.3530

83.1350

88.5240

88.0880

87.8690

84.2810

34.3070

85.1130

85,5510

86.2080

$86.860^{\circ}$

88.2620

89.1790

89.7020

90.5300

90.5740

90.1810

83.1840

82.7000

82.6570

84.2810
HXgh Pressure test

Fressure

(psig)

9.89

19.55

29.57

39.42

49.16

54.50

59.57

64.20

69.32

74.16

80.16

85.61

90.58

95.69

100.92

103.25

106.11

108.31

111.08

113.24

117.83

120.92

12328

12297

125.82

125.70

127.14

130.13

132.5 .

$135.3:$

134.42

138.35

340.16

142.63

145.78

142.52

0.22
Temperature

(Fahrenheit)

81.9970

82.9200

83.5350

84.1050

84.7190

84.9820

35.2450

85.5510

85.9450

86.5140

87.0390

86.8640

86.5580

85.9450

85.4200

84.7190

84.4120

$84.23 \%$

83.8860

83.6660

83.6230

83.3150

82.6570

82.1730

82.1730

81.73417

82.0410

82.2170

82.5250

82.9640

83.0960

83.9740

84.8070

85.8140

86.5580

8 6. 3390

78.12 .10 


\section{IMAGE EVAI.UATION \\ TEST TARGET (MT-3)}
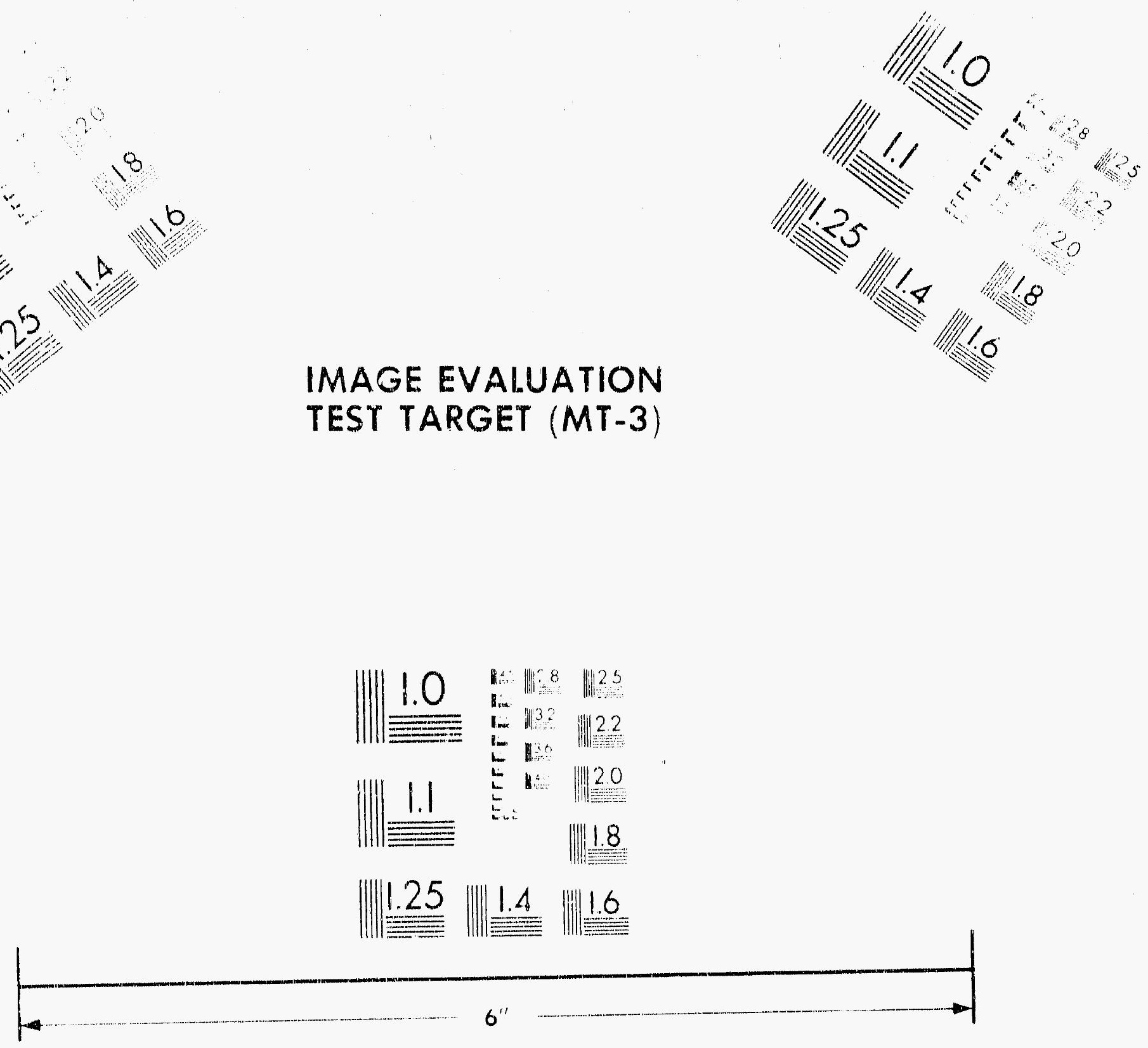

Photographic Sciences Co poration

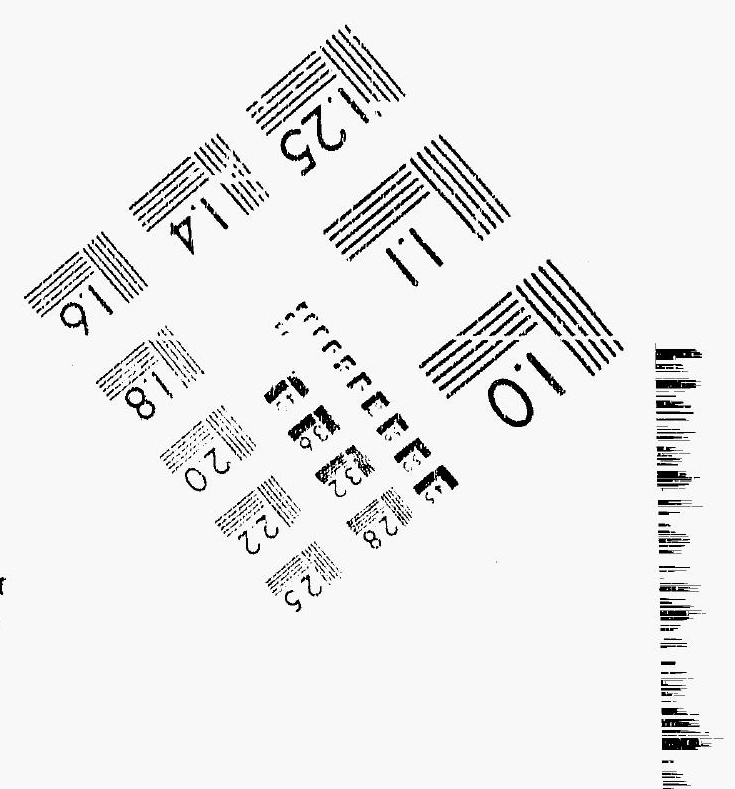




\section{SECURITY CLASSIFICATION}

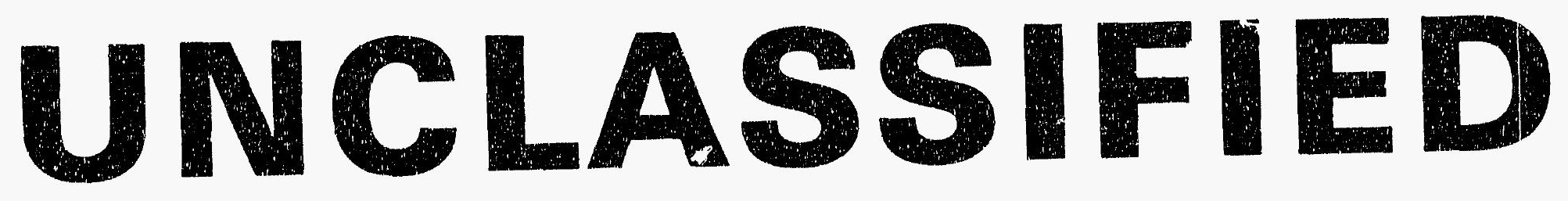

\section{DATE OF MICROFILMING $1-6-92$}

\section{MICROFILMED BY - Lerer hard}

SANDIA NATIONAL LABS 
THIS DOCUMENT FILMED AT

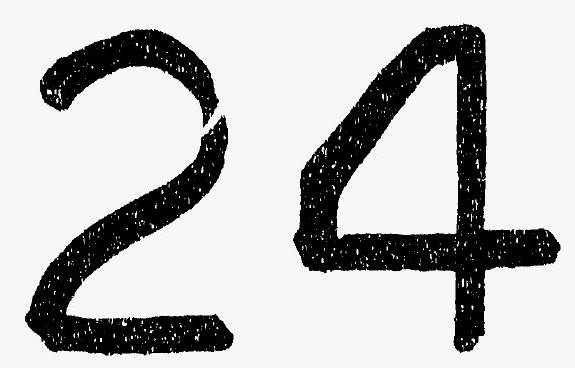

REDUCTION 


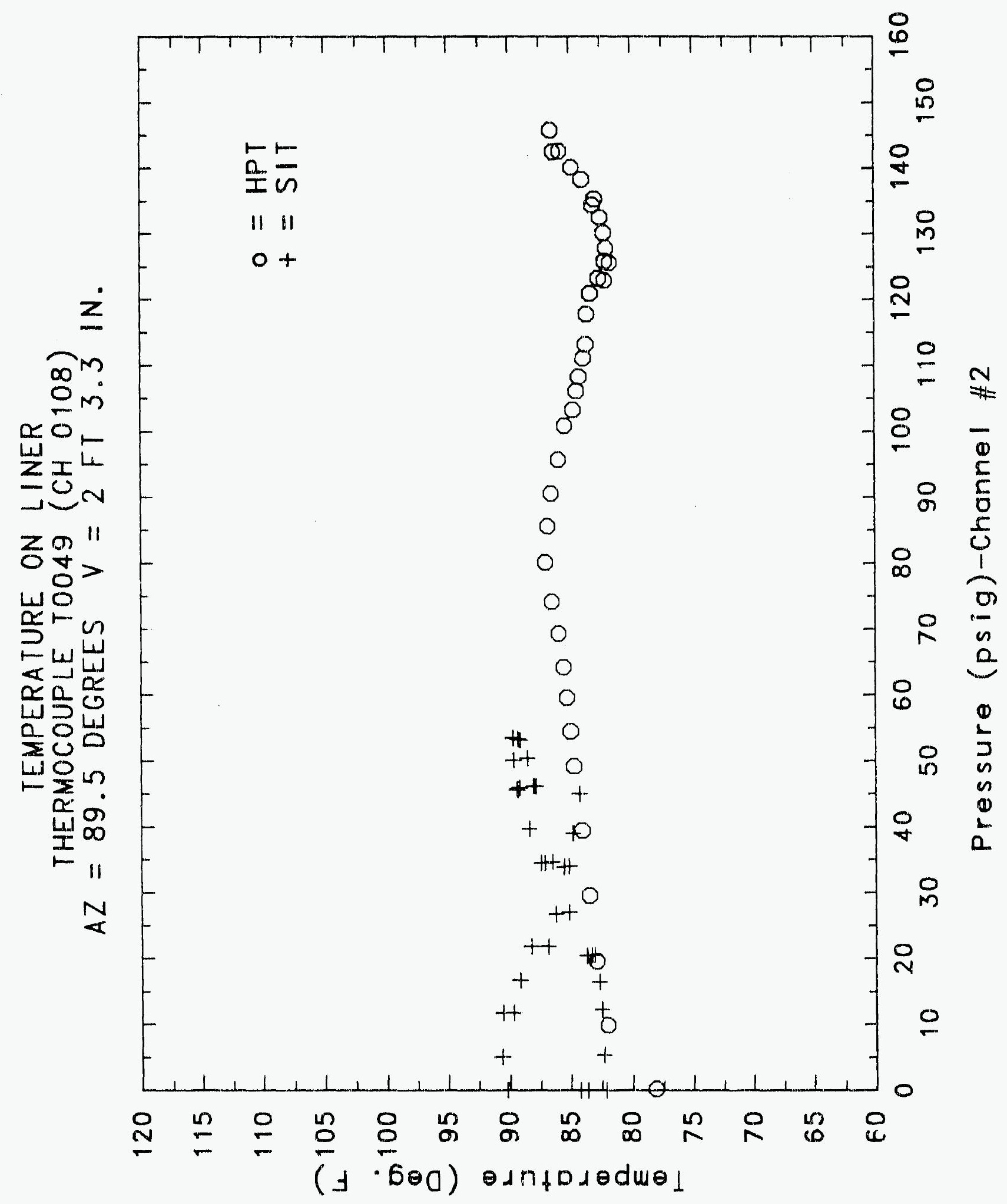

C- 1761 


\section{Reinforced Concrete Test Data}

Thermocouple Channel 109

StRUCTURAL INTEgRITY TEST

\begin{tabular}{|c|c|c|c|}
\hline $\begin{array}{c}\text { Pressure } \\
(\text { psig) } \\
-0.05 \\
5.33 \\
12.31 \\
16.44 \\
20.51 \\
20.46 \\
20.47 \\
27.05 \\
34.69 \\
34.53 \\
34.53 \\
39.70 \\
45.90 \\
45.65 \\
45.62 \\
50.10 \\
53.47 \\
53.29 \\
53.21 \\
50.49 \\
46.14 \\
46.13 \\
44.97 \\
38.98 \\
33.99 \\
33.96 \\
26.83 \\
21.88 \\
21.80 \\
16.69 \\
11.74 \\
1177 \\
5.05 \\
0.62 \\
-0.04 \\
-0.02 \\
-0.02 \\
0.02\end{array}$ & $\begin{array}{c}\text { Temperature } \\
\text { (Fahrenheit) } \\
84.1930 \\
84.8070 \\
84.6750 \\
85.1130 \\
85.5950 \\
85.5950 \\
86.4270 \\
87.6950 \\
88.6120 \\
88.4370 \\
88.6550 \\
89.4400 \\
89.7890 \\
88.9610 \\
88.8730 \\
89.7020 \\
90.0070 \\
89.1350 \\
88.9170 \\
88.0880 \\
87.6070 \\
87.7380 \\
86.1200 \\
86.9520 \\
87.1700 \\
87.8260 \\
88.4810 \\
88.6550 \\
89.2220 \\
89.2660 \\
89.2220 \\
89.0480 \\
88.6120 \\
87.7820 \\
83.1400 \\
83.6230 \\
84.1050 \\
86.2960\end{array}$ & $\begin{array}{c}\text { Pressure } \\
(\text { psig) } \\
9.89 \\
19.55 \\
29.57 \\
39.42 \\
49.16 \\
54.50 \\
59.57 \\
64.20 \\
69.32 \\
74.16 \\
80.16 \\
85.61 \\
90.58 \\
95.69 \\
100.92 \\
103.25 \\
106.11 \\
108.31 \\
111.08 \\
113.24 \\
117.83 \\
120.92 \\
123.28 \\
122.97 \\
125.82 \\
125.60 \\
127.84 \\
130.19 \\
132.53 \\
135.33 \\
134.42 \\
138.35 \\
140.16 \\
142.63 \\
145.78 \\
142.52 \\
0.22\end{array}$ & $\begin{array}{c}\text { Temperature } \\
\text { (Fahrenheit) } \\
84.1490 \\
85.2450 \\
86.2520 \\
86.5140 \\
86.9080 \\
86.7330 \\
86.8640 \\
87.0830 \\
87.6070 \\
88.3060 \\
86.0770 \\
86.1200 \\
85.8580 \\
85.1130 \\
84.6310 \\
83.5350 \\
83.1840 \\
83.2270 \\
82.7880 \\
82.6130 \\
82.7000 \\
82.3050 \\
81.8220 \\
81.3820 \\
81.5140 \\
81.2940 \\
81.7340 \\
82.0410 \\
82.6570 \\
83.4030 \\
83.6230 \\
85.5510 \\
85.2890 \\
86.2080 \\
86.0330 \\
84.9380 \\
66.5690\end{array}$ \\
\hline
\end{tabular}

High Pressute test

Fahrenheit)

84.1490

85.2450

86.2520

86.7330

86.8640

87.0830

86.0770

86.1200

85.8580

83.5350

83.1840

83.2270

82.7880

82.3050

81.8220

81.3820

81.7340

82.0410

82.6570

85.5510

85.2890

86.2080

86.0330

84.9380

66.5690 


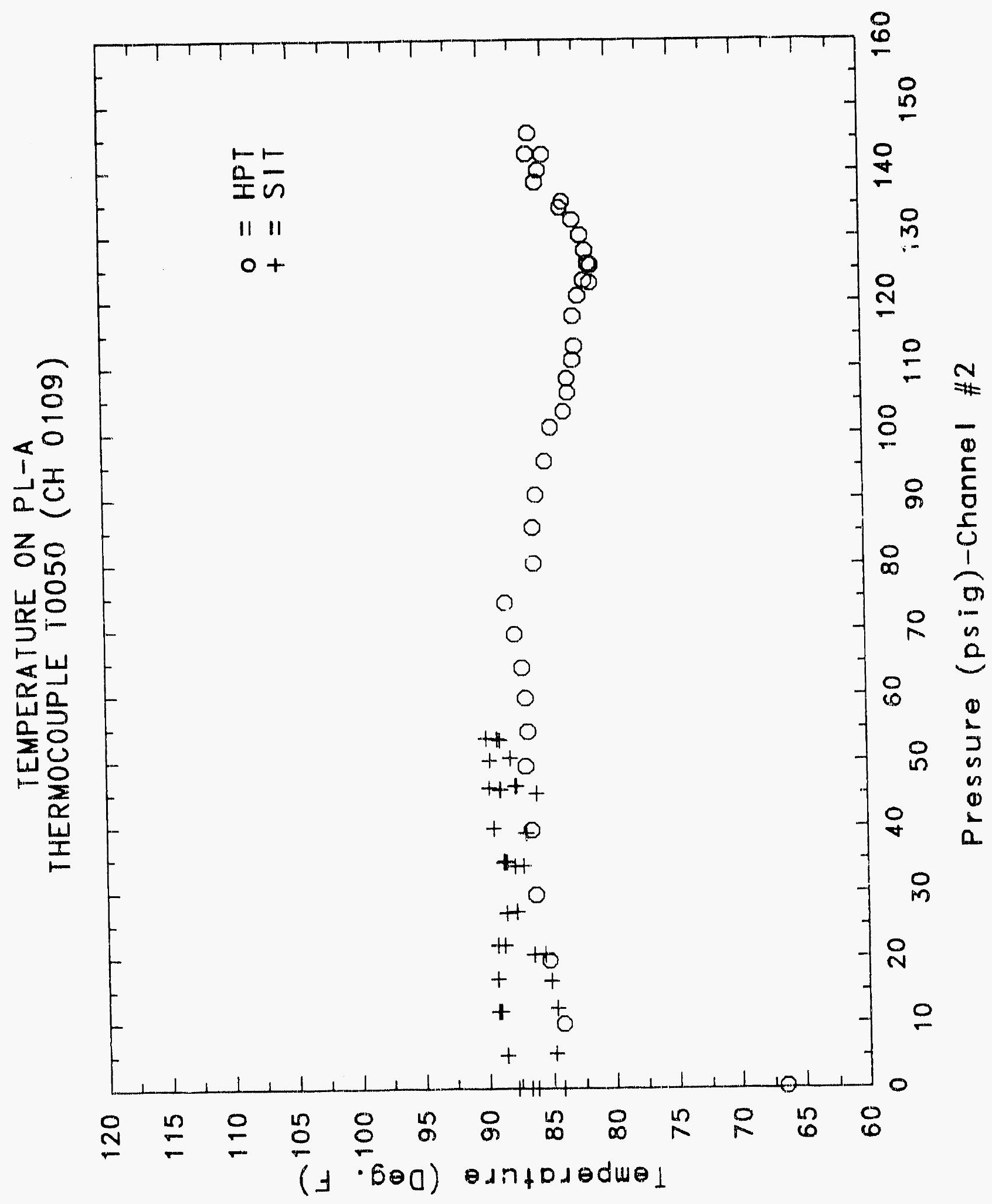




\section{Reinforced Concrete Test Data}

Thermocouple Channel 110

StRuctural IntEgrity TEST

\begin{tabular}{|c|c|c|c|}
\hline $\begin{array}{l}\text { Pressure } \\
\text { (psig) } \\
-0.05 \\
5.33 \\
12.31 \\
16.44 \\
20.51 \\
20.46 \\
20.47 \\
27.05 \\
34.69 \\
34.53 \\
34.53 \\
39.70 \\
45.90 \\
45.65 \\
45.62 \\
50.10 \\
53.47 \\
53.29 \\
53.21 \\
50.49 \\
46.14 \\
46.13 \\
44.97 \\
38.98 \\
33.99 \\
33.96 \\
26.83 \\
21.88 \\
21.90 \\
16.69 \\
11.74 \\
11.77 \\
5.05 \\
0.02 \\
-0.04 \\
-0.02 \\
-0.02 \\
0.02\end{array}$ & $\begin{array}{c}\text { Temperature } \\
\text { (Fahrenteit) } \\
81.9530 \\
82.2170 \\
82.3490 \\
82.5690 \\
82.8320 \\
83.0960 \\
83.3590 \\
84.0610 \\
84.8500 \\
85.5080 \\
85.7700 \\
86.6020 \\
87.3010 \\
88.1310 \\
88.3930 \\
88.9170 \\
89.3530 \\
89.5280 \\
89.3970 \\
89.0320 \\
88.6990 \\
88.6550 \\
84.4120 \\
84.8070 \\
85.1570 \\
85.5080 \\
85.8140 \\
86.0770 \\
86.8640 \\
87.1260 \\
87.4320 \\
88.2620 \\
88.4370 \\
88.4370 \\
84.4560 \\
83.7540 \\
83.4910 \\
84.4560\end{array}$ & $\begin{array}{c}\text { Pressure } \\
(\text { psig) } \\
9.89 \\
19.55 \\
29.57 \\
39.42 \\
49.16 \\
54.50 \\
59.57 \\
64.20 \\
69.32 \\
74.16 \\
80.16 \\
85.61 \\
90.58 \\
95.69 \\
100.92 \\
103.25 \\
106.11 \\
108.31 \\
111.08 \\
113.24 \\
117.83 \\
120.92 \\
123.28 \\
122.97 \\
125.82 \\
125.60 \\
127.84 \\
130.19 \\
132.53 \\
135.33 \\
134.42 \\
138.35 \\
140.16 \\
142.63 \\
145.78 \\
142.52 \\
0.22 \\
\end{array}$ & $\begin{array}{c}\text { Temperature } \\
\text { (Fahrenheit) } \\
81.3820 \\
82.0850 \\
82.6130 \\
82.9200 \\
83.5350 \\
83.7540 \\
83.9740 \\
84.1490 \\
84.5440 \\
84.9820 \\
85.5510 \\
85.8140 \\
85.8140 \\
85.2890 \\
84.8500 \\
84.1050 \\
83.7100 \\
83.6230 \\
83.3150 \\
83.0960 \\
82.9200 \\
82.5690 \\
81.7340 \\
81.4260 \\
81.2940 \\
80.8540 \\
80.9860 \\
81.0300 \\
81.1180 \\
81.4700 \\
81.6900 \\
82.3490 \\
82.8320 \\
83.4470 \\
84.1050 \\
84.1490 \\
75.1580\end{array}$ \\
\hline
\end{tabular}

\author{
High Pressure test
}

remperature

81.3820

82.0850

82.6130

82.9200

83.5350

83.7540

84.5440

84.9820

85.5510

85.8140

84.8500

84.1050

83.7100

83.6230

82.9200

81.7340

81.4260

81.2940

80.8540

81.1180

81.4700

81.6900

82.3490

82.8320

84.1050

84.1490

75.1580 


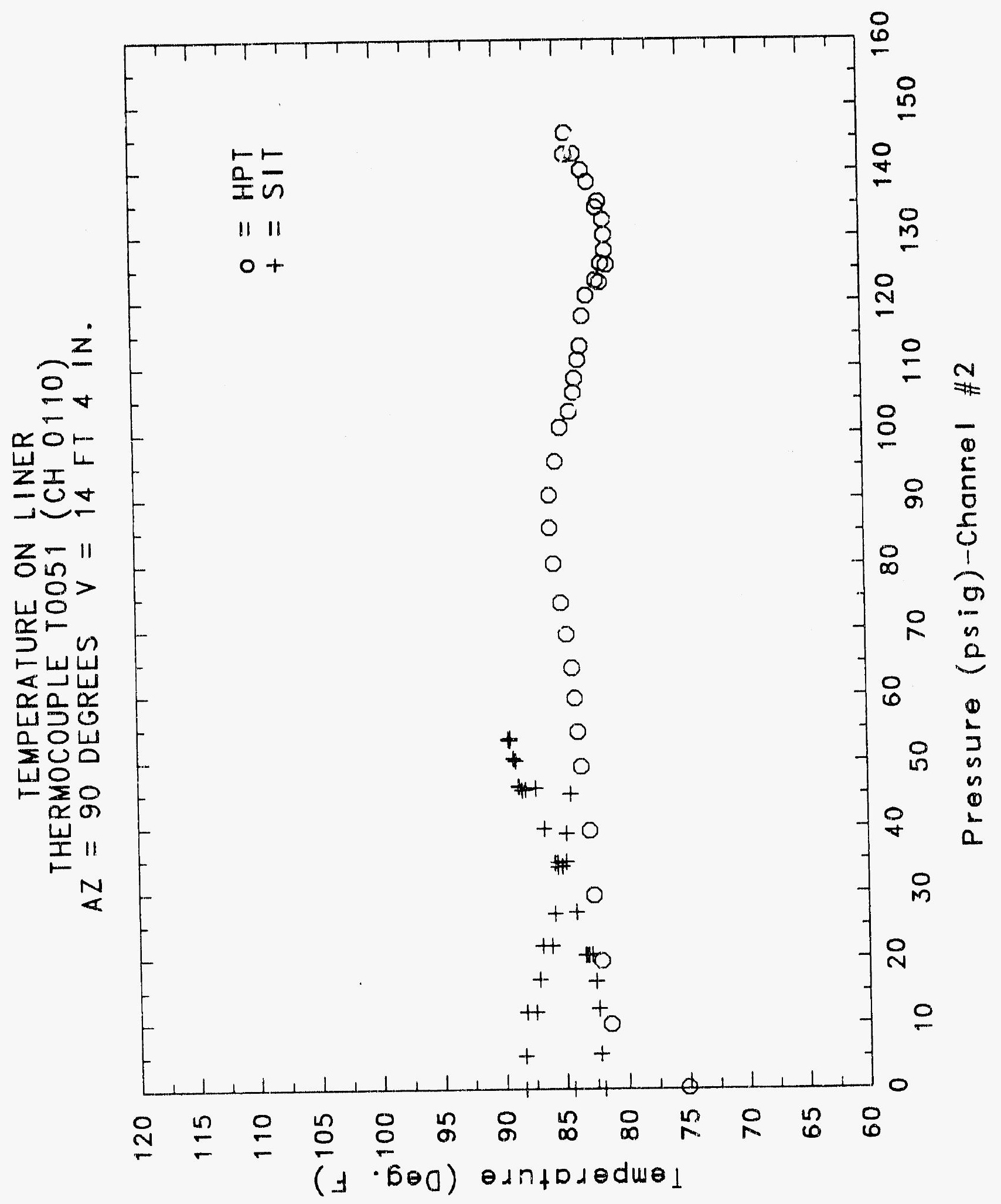




\section{Reinforced Concrete Test Data}

Thermocouple Channel 111

STRUCTURAL INTEGRITY TEST

\begin{tabular}{|c|c|c|c|}
\hline $\begin{array}{l}\text { Pressure } \\
\text { (psig) } \\
-0.05 \\
5.33 \\
12.31 \\
16.44 \\
20.51 \\
20.46 \\
20.47 \\
27.05 \\
34.69 \\
34.53 \\
34.53 \\
39.70 \\
45.90 \\
45.65 \\
45.62 \\
50.10 \\
53.47 \\
53.29 \\
53.21 \\
50.49 \\
46.14 \\
46.13 \\
44.97 \\
38.98 \\
33.99 \\
33.96 \\
26.83 \\
21.88 \\
21.90 \\
16.69 \\
11.74 \\
11.77 \\
5.05 \\
0.02 \\
-0.04 \\
-0.02 \\
-0.02 \\
0.02\end{array}$ & $\begin{array}{c}\text { Temperature } \\
\text { (Fahrenheit) } \\
81.0740 \\
81.5580 \\
82.1730 \\
82.9200 \\
83.6230 \\
84.2370 \\
84.8070 \\
85.9890 \\
87.0390 \\
87.5640 \\
88.0000 \\
89.3100 \\
90.5740 \\
91.4880 \\
91.6620 \\
91.8790 \\
91.7490 \\
91.1400 \\
90.8350 \\
90.0940 \\
89.7020 \\
88.9610 \\
85.1130 \\
85.8140 \\
86.3390 \\
87.0830 \\
87.5200 \\
87.9570 \\
88.6120 \\
89.0040 \\
89.3970 \\
90.5300 \\
90.7910 \\
90.5740 \\
82.9200 \\
82.5690 \\
83.2270 \\
85.7700\end{array}$ & $\begin{array}{c}\text { Pressure } \\
\text { (psig) } \\
9.89 \\
19.55 \\
29.57 \\
39.42 \\
49.16 \\
54.50 \\
59.57 \\
64.20 \\
69.32 \\
74.16 \\
80.16 \\
85.61 \\
90.58 \\
95.69 \\
100.92 \\
103.25 \\
106.11 \\
108.31 \\
111.08 \\
113.24 \\
117.83 \\
120.92 \\
123.28 \\
122.97 \\
125.82 \\
125.60 \\
127.84 \\
130.19 \\
132.53 \\
135.33 \\
134.42 \\
138.35 \\
140.16 \\
142.63 \\
145.78 \\
142.52 \\
0.22\end{array}$ & $\begin{array}{c}\text { Temperature } \\
\text { (Fahrenheit) } \\
80.5450 \\
81.4700 \\
82.2610 \\
83.0520 \\
83.8860 \\
84.4120 \\
84.8070 \\
85.2010 \\
85.7260 \\
86.2520 \\
86.3390 \\
85.9020 \\
85.4640 \\
84.6750 \\
84.0610 \\
83.1840 \\
82.7440 \\
82.6130 \\
82.1730 \\
81.9530 \\
81.8220 \\
81.4260 \\
80.6340 \\
80.3250 \\
80.2810 \\
80.3250 \\
80.5900 \\
81.2060 \\
81.6020 \\
82.3490 \\
83.0520 \\
83.9740 \\
84.5870 \\
85.4200 \\
85.9890 \\
86.1200 \\
79.6200\end{array}$ \\
\hline
\end{tabular}

High Pressure test 


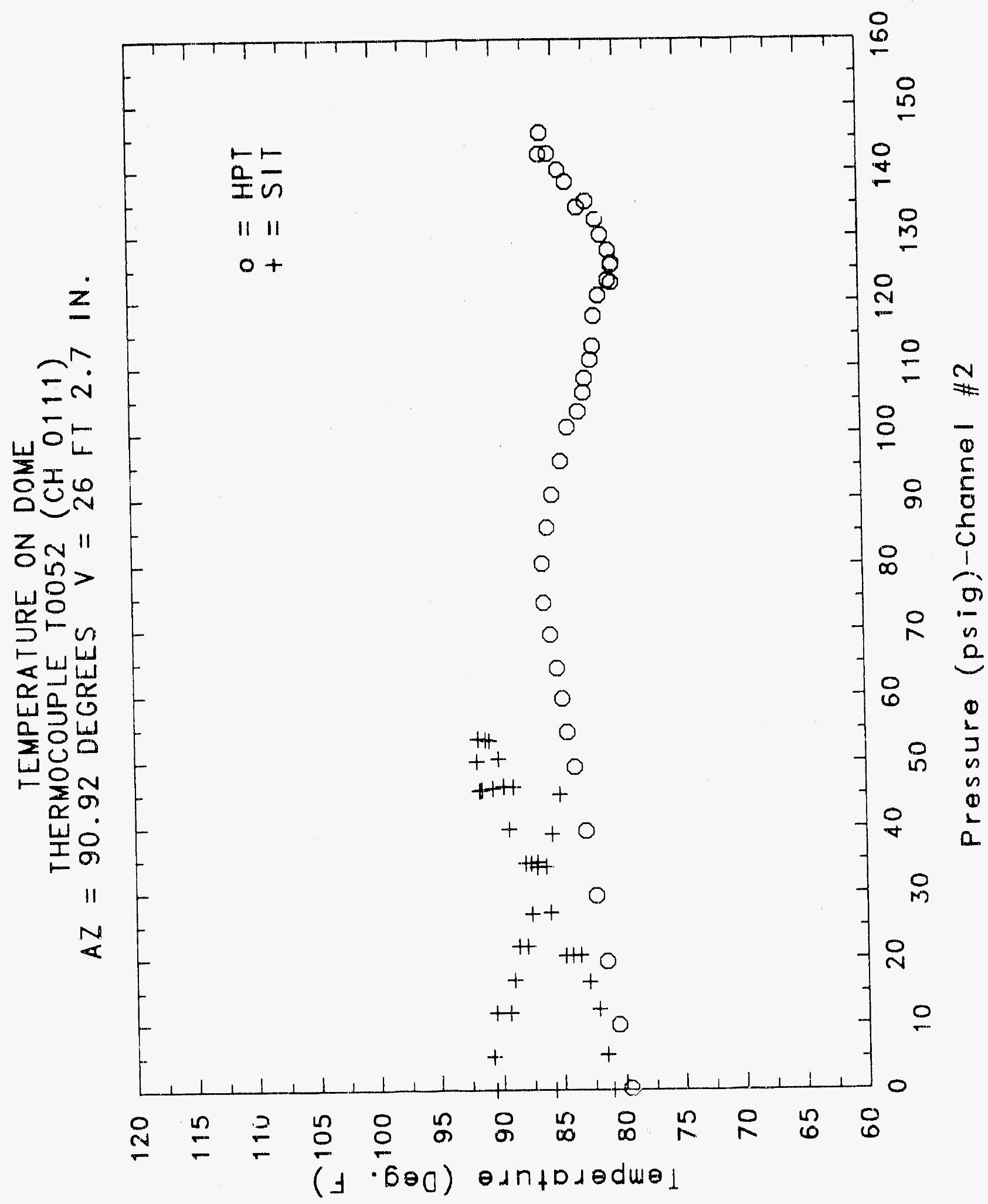




\section{Reinforced Concrete Test Data}

Thermocouple jhannel 112

Structural INTEgRity test

\begin{tabular}{|c|c|c|c|}
\hline $\begin{array}{l}\text { Pressure } \\
\text { (psig) } \\
-0.05 \\
5.33 \\
12.31 \\
16.44 \\
20.51 \\
20.46 \\
20.47 \\
27.05 \\
34.69 \\
34.53 \\
34.53 \\
39.70 \\
45.90 \\
45.65 \\
45.62 \\
50.10 \\
53.47 \\
53.29 \\
53.21 \\
50.49 \\
46.14 \\
46.13 \\
44.97 \\
38.98 \\
33.99 \\
33.96 \\
26.83 \\
21.88 \\
21.90 \\
16.69 \\
11.74 \\
11.77 \\
5.05 \\
0.02 \\
-0.04 \\
-0.02 \\
-0.02 \\
0.02\end{array}$ & $\begin{array}{c}\text { Temperature } \\
\text { (Fahrenheit) } \\
81.2500 \\
81.4700 \\
81.7340 \\
82.3050 \\
82.7440 \\
83.3150 \\
83.8420 \\
84.7190 \\
85.7260 \\
86.3830 \\
87.0390 \\
88.2620 \\
89.5710 \\
91.1830 \\
91.6180 \\
92.2270 \\
92.4010 \\
92.1840 \\
91.8790 \\
91.2700 \\
90.6610 \\
90.2250 \\
84.8500 \\
85.2890 \\
85.6390 \\
86.2960 \\
86.6450 \\
86.9520 \\
87.8260 \\
88.3060 \\
89.0920 \\
90.7910 \\
91.4010 \\
91.7490 \\
84.4560 \\
83.6660 \\
83.5350 \\
84.9820\end{array}$ & $\begin{array}{c}\text { Pressure } \\
(\text { psig) } \\
9.89 \\
19.55 \\
29.57 \\
39.42 \\
49.16 \\
54.50 \\
59.57 \\
64.20 \\
59.32 \\
74.16 \\
80.16 \\
85.61 \\
90.58 \\
95.69 \\
100.92 \\
103.25 \\
106.11 \\
108.31 \\
111.08 \\
113.24 \\
117.83 \\
120.92 \\
123.28 \\
122.97 \\
125.82 \\
125.60 \\
127.84 \\
130.19 \\
132.53 \\
135.33 \\
134.42 \\
138.35 \\
140.16 \\
142.63 \\
145.78 \\
142.52 \\
0.22\end{array}$ & $\begin{array}{c}\text { Temperature } \\
\text { (Fahrenheit } \\
80.6340 \\
81.4260 \\
82.2610 \\
82.9200 \\
83.6660 \\
83.8420 \\
84.3240 \\
84.6750 \\
85.1570 \\
85.5950 \\
86.1200 \\
85.9450 \\
85.6830 \\
84.9380 \\
84.4560 \\
83.5790 \\
83.1840 \\
83.1840 \\
82.6570 \\
82.3930 \\
82.2170 \\
81.8650 \\
81.1180 \\
80.6780 \\
80.7660 \\
80.4570 \\
80.7220 \\
81.0300 \\
81.2940 \\
81.8650 \\
82.2610 \\
83.1400 \\
83.5350 \\
84.2810 \\
85.0700 \\
84.9820 \\
78.3850\end{array}$ \\
\hline
\end{tabular}

High Pressure test

Temperature

ahrenheit

80.6340

81.4260

83.6660

83.8420

84.3240

85.5950

86.1200

85.9450

35.6830

83.5790

83.1840

83.1840

82.2170

81.8650

81.1180

80.4570

80.7220

81.0300

81.2940

81.8650

83.5350

84.2810

85.0700

84.9820

78.3850 
69LI-J

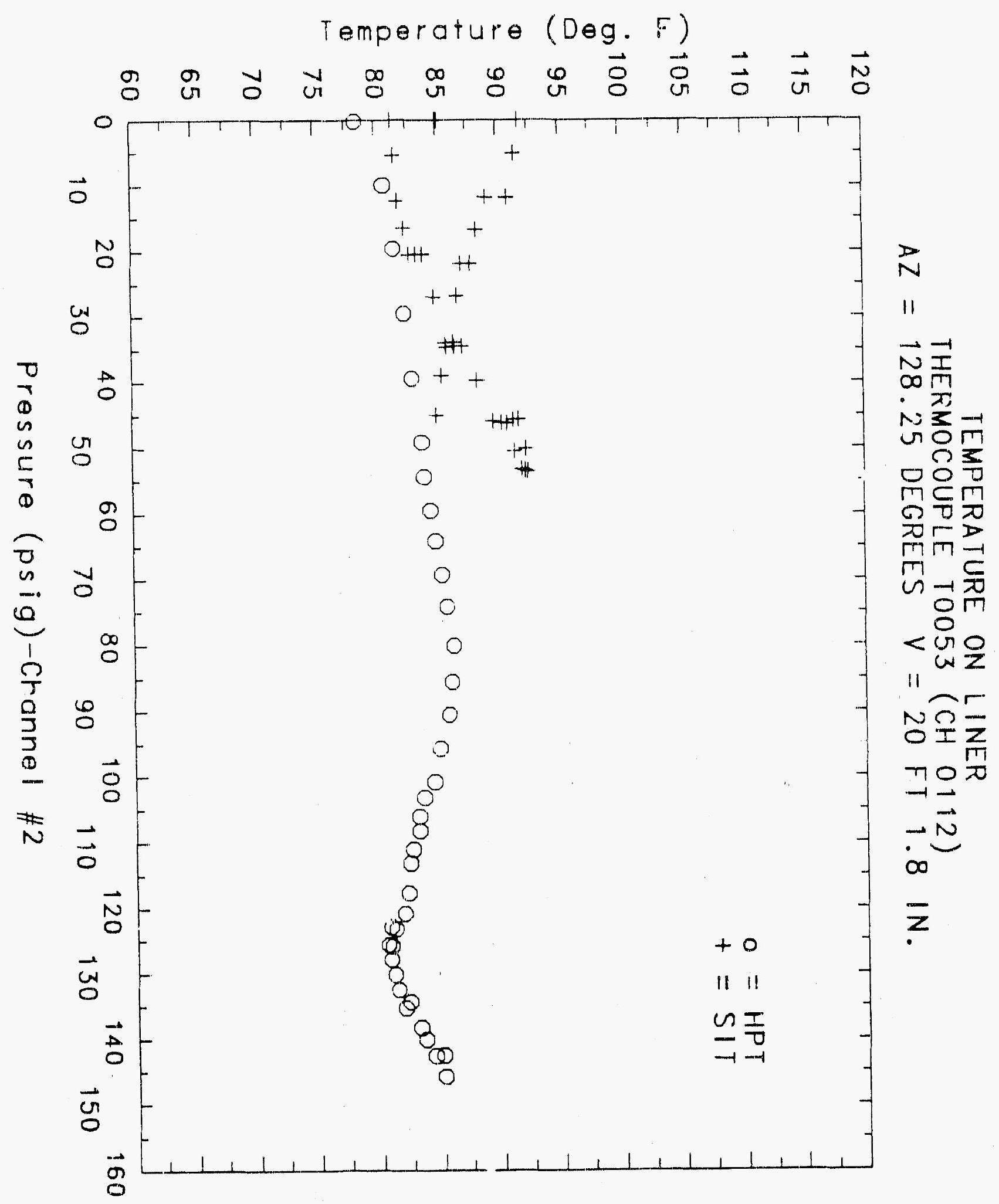




\section{Reinfcrced Concrete test Data}

\section{Thermocuuple Channel $\$ 13$}

Structural INTEGRITY TEST

\begin{tabular}{|c|c|c|c|}
\hline $\begin{array}{l}\text { Pressure } \\
(p s i g) \\
-0.05 \\
5.33 \\
12.31 \\
16.44 \\
20.51 \\
20.46 \\
20.47 \\
27.05 \\
34.59 \\
34.53 \\
34.53 \\
39.70 \\
45.90 \\
45.65 \\
45.62 \\
50.10 \\
53.47 \\
53.29 \\
53.21 \\
50.49 \\
46.14 \\
46.13 \\
44.97 \\
38.98 \\
33.99 \\
33.96 \\
26.833 \\
21.88 \\
21.90 \\
16.69 \\
11.74 \\
11.77 \\
5.05 \\
0.02 \\
-0.04 \\
-0.02 \\
-0.02 \\
0.02\end{array}$ & $\begin{array}{c}\text { Temperature } \\
\text { (Fahrenheit) } \\
85.7260 \\
85.6830 \\
85.1570 \\
85.4200 \\
85.6390 \\
85.0700 \\
85.1130 \\
86.6890 \\
87.7380 \\
87.4760 \\
87.6070 \\
88.7860 \\
89.4840 \\
888.7860 \\
88.7430 \\
89.3970 \\
89.6150 \\
89.1350 \\
88.9170 \\
88.1310 \\
87.7820 \\
88.0440 \\
86.2960 \\
86.8640 \\
87.0390 \\
86.9520 \\
87.4760 \\
87.4320 \\
87.9570 \\
88.4810 \\
88.3930 \\
88.7430 \\
88.4810 \\
87.4320 \\
84.7630 \\
84.9380 \\
85.2010 \\
87.9570\end{array}$ & $\begin{array}{l}\text { Prossure } \\
(\text { psig) } \\
9.89 \\
19.55 \\
29.57 \\
39.42 \\
49.16 \\
54.50 \\
59.57 \\
64.20 \\
69.32 \\
74.16 \\
80.16 \\
35.61 \\
90.58 \\
95.09 \\
100.12 \\
103.25 \\
106.11 \\
108.31 \\
111.08 \\
113.24 \\
117.83 \\
120.92 \\
123.29 \\
122.97 \\
125.82 \\
125.60 \\
127.84 \\
130.19 \\
132.53 \\
135.33 \\
134.42 \\
133.35 \\
140.16 \\
14.63 \\
145.78 \\
142.52 \\
0.22\end{array}$ & $\begin{array}{c}\text { Temperature } \\
\text { (Fahrentieit) } \\
85.0260 \\
86.5140 \\
87.3890 \\
87.3010 \\
87.6950 \\
87.3890 \\
87.3890 \\
87.2140 \\
87.6070 \\
87.8590 \\
36.3390 \\
86.3830 \\
86.1640 \\
85.4200 \\
84.9380 \\
84.0610 \\
83.6230 \\
83.4910 \\
83.2710 \\
83.0080 \\
83.0960 \\
82.7880 \\
82.6130 \\
82.5250 \\
83.0520 \\
82.6130 \\
83.1400 \\
83.4030 \\
83.6230 \\
84.0610 \\
83.9300 \\
84.9380 \\
85.2010 \\
85.6330 \\
86.1200 \\
85.1130 \\
65.4950\end{array}$ \\
\hline
\end{tabular}

\section{High PRESSURE TEST}

(Fahrentieit)

86.5140

87.3890

87.3890

87.3890

87.8690

36.3390

86.3830

84.9380

84.0610

83.2710

63.0080

83.0960

82.5250

83.0520

83.4030

83.6230

84.0610

85.2010

85.6330

86.1200

85.1130

65.4950 


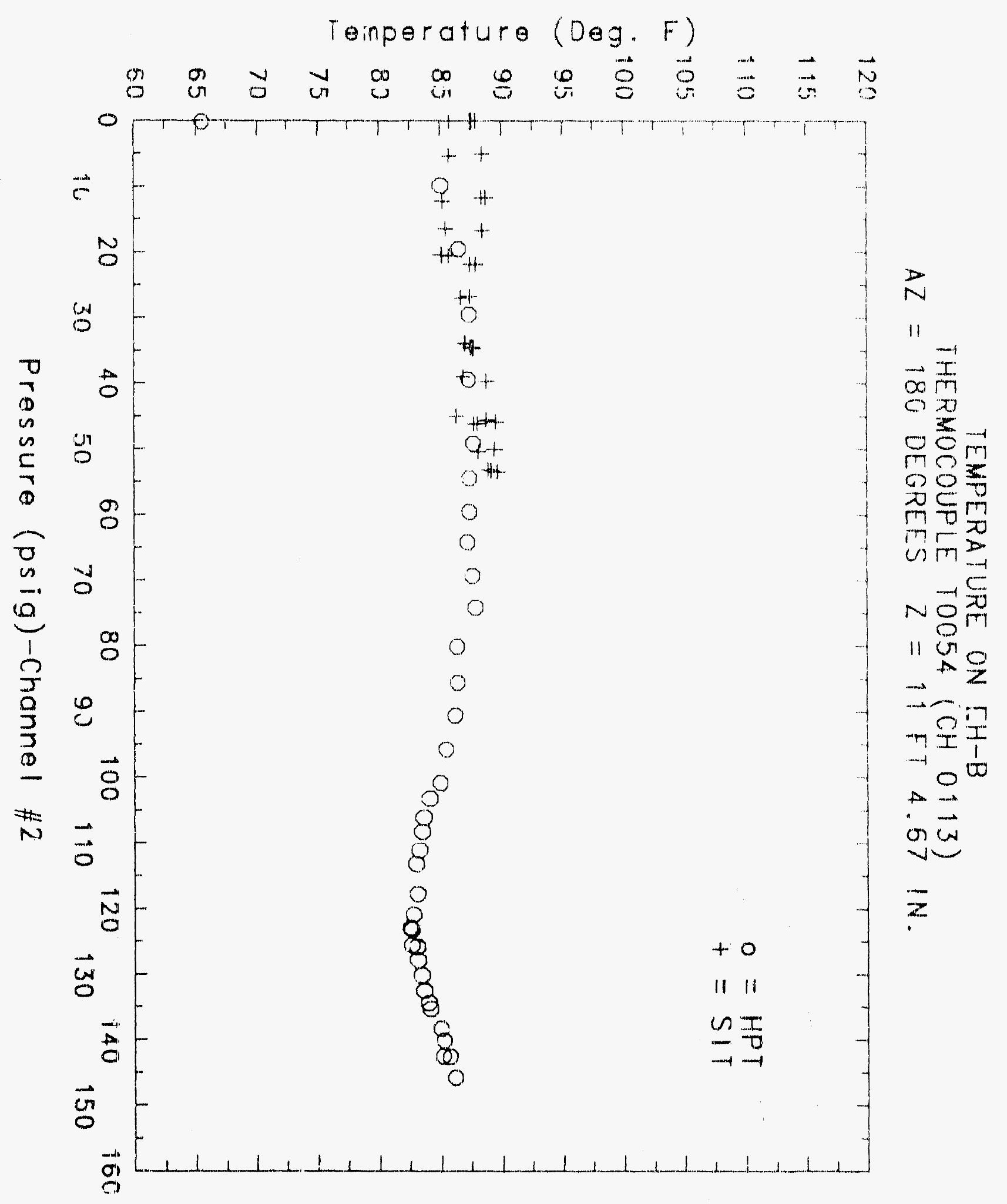




\section{Reinforced Concrete: Test Data}

Thermocouple Channel 114

STRUCTURAL INTEGRITY TEST

\begin{tabular}{|c|c|c|c|}
\hline $\begin{array}{l}\text { Pressure } \\
\text { (psig) } \\
-0.05 \\
5.33 \\
12.31 \\
16.44 \\
20.51 \\
20.46 \\
20.47 \\
27.05 \\
34.69 \\
34.53 \\
34.53 \\
39.70 \\
45.90 \\
45.65 \\
45.62 \\
50.10 \\
53.47 \\
53.29 \\
53.21 \\
50.49 \\
46.14 \\
46.13 \\
44.97 \\
38.98 \\
33.99 \\
33.96 \\
26.83 \\
21.88 \\
21.90 \\
16.69 \\
11.74 \\
11.77 \\
5.05 \\
0.02 \\
-0.04 \\
-0.02 \\
-0.02 \\
0.02\end{array}$ & $\begin{array}{c}\text { Temperature } \\
\text { (Fahrenheit) } \\
82.9200 \\
83.3150 \\
83.4910 \\
83.7100 \\
84.0610 \\
84.1930 \\
84.5440 \\
85.5080 \\
86.3830 \\
86.9520 \\
87.3010 \\
88.3060 \\
89.2660 \\
90.1380 \\
90.4860 \\
91.0960 \\
91.2700 \\
91.0090 \\
90.8350 \\
90.2250 \\
89.7890 \\
89.4840 \\
85.5080 \\
86.0330 \\
86.2960 \\
86.6890 \\
86.9080 \\
87.1250 \\
87.8690 \\
88.3500 \\
88.9170 \\
90.0510 \\
90.4860 \\
90.3990 \\
85.1130 \\
84.5440 \\
84.5870 \\
86.0330\end{array}$ & $\begin{array}{c}\text { Pressure } \\
(\text { psig) } \\
9.89 \\
19.55 \\
29.57 \\
39.42 \\
49.16 \\
54.50 \\
59.57 \\
64.20 \\
69.32 \\
74.16 \\
80.16 \\
85.61 \\
90.58 \\
95.69 \\
100.92 \\
103.25 \\
106.11 \\
108.31 \\
111.08 \\
113.24 \\
117.83 \\
120.92 \\
123.28 \\
122.97 \\
125.82 \\
125.60 \\
127.84 \\
130.19 \\
132.53 \\
135.33 \\
134.42 \\
138.35 \\
140.16 \\
142.63 \\
145.78 \\
142.52 \\
0.22\end{array}$ & $\begin{array}{c}\text { Temperature } \\
\text { (Fahrenheit) } \\
82.4370 \\
83.3590 \\
84.1050 \\
84.4560 \\
84.9380 \\
85.2010 \\
85.4640 \\
85.6390 \\
85.9450 \\
86.2960 \\
86.7770 \\
86.7770 \\
86.5140 \\
85.9020 \\
85.3760 \\
84.6310 \\
84.2370 \\
84.1490 \\
83.6660 \\
83.4910 \\
83.3590 \\
82.9640 \\
82.3050 \\
81.7780 \\
81.7340 \\
81.5140 \\
81.6900 \\
81.8650 \\
82.1730 \\
82.5690 \\
81.9530 \\
32.2610 \\
82.4810 \\
82.7880 \\
81.9530 \\
81.3380 \\
72.2300\end{array}$ \\
\hline
\end{tabular}

High Pressure test

ressure

emperature

82.4370

83.3590

84.1050

85.2010

85.4640

86.7770

86.7770

86.5140

84.6310

84.2370

84.1490

83.6660

83.3590

82.9640

82.3050

5140

81.6900

81.8650

82.1730

32.2610

82.4810

82.7880

81.9530

1.3380

72.2300 


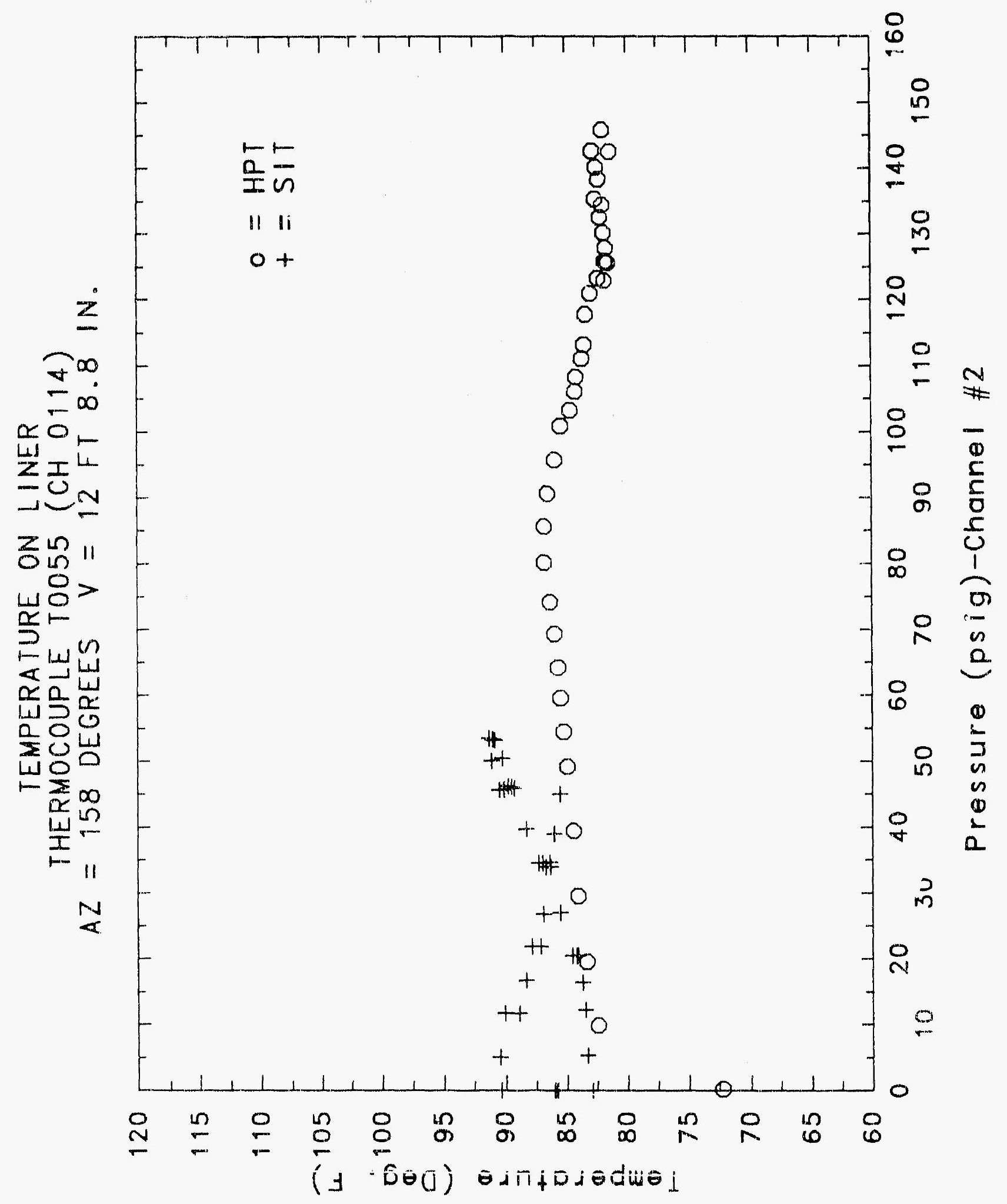

C. 1773 


\section{Reinforced Concrete Test Data}

Thermocouple Channel 115

Structural Integrity TEST

\begin{tabular}{|c|c|c|c|}
\hline $\begin{array}{c}\text { Pressure } \\
\text { (psig) } \\
-0.05 \\
5.33 \\
12.31 \\
16.44 \\
20.51 \\
20.46 \\
20.47 \\
27.05 \\
34.69 \\
34.53 \\
34.53 \\
39.70 \\
45.90 \\
45.65 \\
45.62 \\
50.10 \\
53.47 \\
53.29 \\
53.21 \\
50.49 \\
46.14 \\
46.13 \\
44.97 \\
38.98 \\
33.99 \\
33.96 \\
26.83 \\
? 1.88 \\
21.90 \\
16.69 \\
11.74 \\
11.77 \\
5.05 \\
0.02 \\
-0.04 \\
-0.02 \\
-0.02 \\
0.02\end{array}$ & $\begin{array}{c}\text { Temperature } \\
\text { (Fahrenheit) } \\
83.7540 \\
83.6230 \\
83.5790 \\
83.7100 \\
83.7540 \\
83.7100 \\
83.7980 \\
84.2370 \\
84.7630 \\
85.0700 \\
85.2450 \\
85.7260 \\
86.2960 \\
86.7770 \\
87.0390 \\
87.6510 \\
88.1310 \\
88.4370 \\
88.5240 \\
88.4810 \\
88.3500 \\
88.4370 \\
85.5080 \\
85.7260 \\
85.9020 \\
86.0770 \\
86.2080 \\
86.2960 \\
86.7330 \\
86.8640 \\
86.9080 \\
87.4320 \\
87.5640 \\
87.5640 \\
86.2080 \\
85.5950 \\
85.4200 \\
86.2080\end{array}$ & $\begin{array}{c}\text { Pressure } \\
(\text { psig) } \\
9.89 \\
19.55 \\
29.57 \\
39.42 \\
49.16 \\
54.50 \\
59.57 \\
64.20 \\
69.32 \\
74.16 \\
80.16 \\
85.61 \\
90.58 \\
95.69 \\
100.92 \\
103.25 \\
106.11 \\
108.31 \\
111.08 \\
113.24 \\
117.83 \\
120.92 \\
123.28 \\
122.97 \\
125.82 \\
125.60 \\
127.84 \\
130.19 \\
132.53 \\
135.33 \\
134.42 \\
138.35 \\
140.16 \\
142.63 \\
145.78 \\
142.52 \\
0.22\end{array}$ & $\begin{array}{c}\text { Temperature } \\
\text { (Fahrenheit) } \\
82.5250 \\
83.0960 \\
83.6230 \\
84.0610 \\
84.5030 \\
84.5870 \\
84.9380 \\
85.0700 \\
85.3760 \\
85.6830 \\
86.0770 \\
86.4270 \\
86.6020 \\
86.3390 \\
86.1640 \\
85.5080 \\
85.2890 \\
85.2450 \\
84.8940 \\
84.8070 \\
84.6750 \\
84.3580 \\
83.7100 \\
83.3590 \\
83.2710 \\
82.8760 \\
82.9640 \\
82.9640 \\
83.0960 \\
83.3150 \\
83.0080 \\
83.4030 \\
83.5350 \\
83.7540 \\
83.9740 \\
83.5790 \\
75.1580\end{array}$ \\
\hline
\end{tabular}

\section{High Pressure Test}

emperature

82.5250

83.0960

83.6230

84.0610

84.5070

9380

85.0700

85.3760

85.6830

$86.60<0$

86.3390

86.1640

84.8070

84.6750

83.3590

83.2710

82.8760

82.9640

83.4030

83.5350

. 7540

83.9740

83.5790

75.1580 


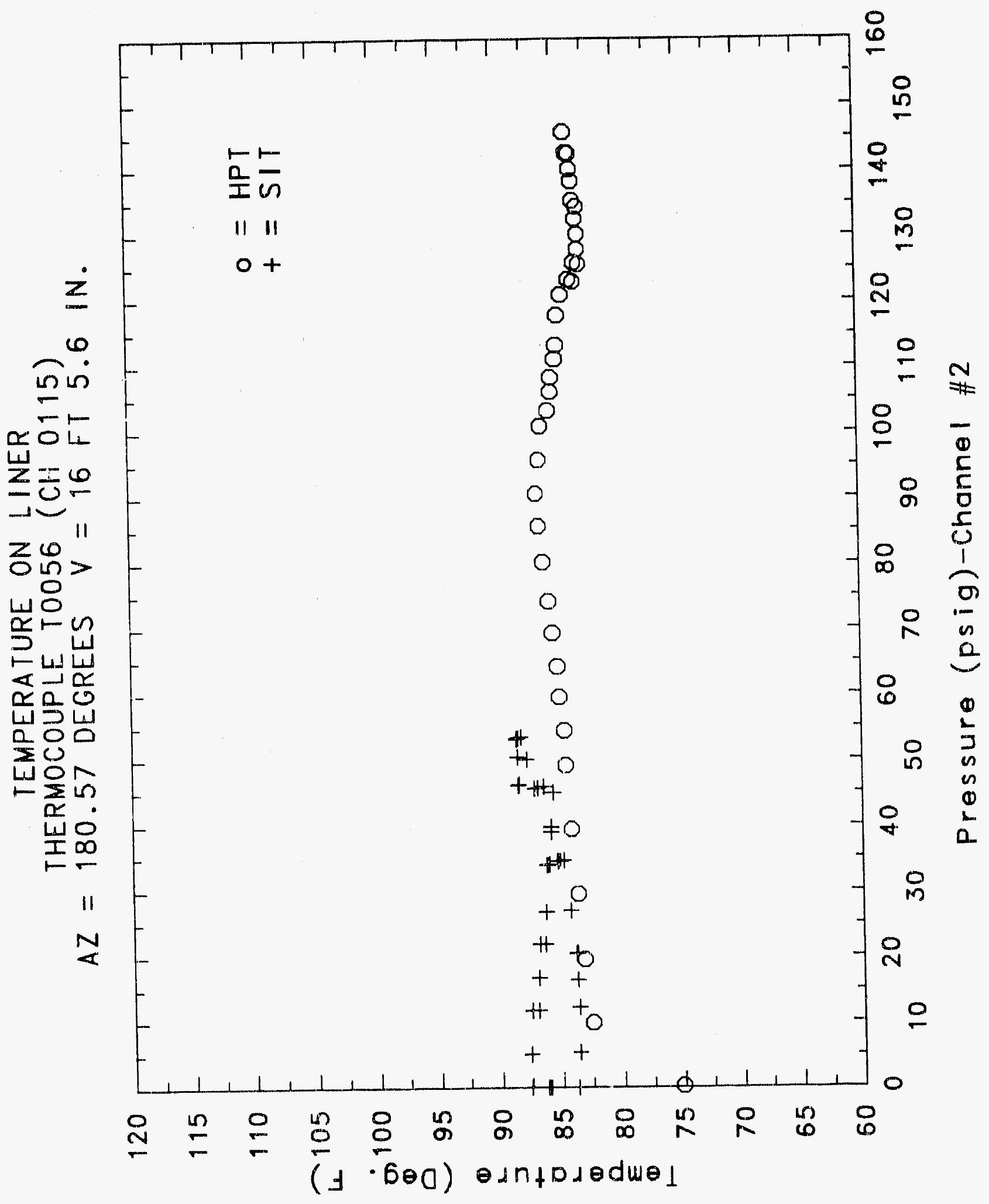

C- 1775 


\section{Reinforced Concrete Test Data}

Thermocouple Channel 116

STRUCTURAL INTEGRITY TEST

\begin{tabular}{|c|c|}
\hline $\begin{array}{c}\text { Pressure } \\
\text { (psig) } \\
-0.05 \\
5.33 \\
12.31 \\
16.44 \\
20.51 \\
20.46 \\
20.47 \\
27.05 \\
34.69 \\
34.53 \\
34.53 \\
39.70 \\
45.90 \\
45.65 \\
45.62 \\
50.10 \\
53.47 \\
53.29 \\
53.21 \\
50.49 \\
46.14 \\
46.13 \\
44.97 \\
38.98 \\
33.99 \\
33.96 \\
26.83 \\
21.88 \\
21.90 \\
16.69 \\
11.74 \\
11.77 \\
5.05 \\
0.02 \\
-0.04 \\
-0.02 \\
-0.02 \\
0.02\end{array}$ & $\begin{array}{c}\text { Temperature } \\
\text { (Fahrenheit) } \\
83.2270 \\
83.4030 \\
83.5790 \\
83.8420 \\
84.0610 \\
83.9740 \\
84.1050 \\
84.9820 \\
85.7260 \\
85.8580 \\
85.9450 \\
86.6450 \\
87.2140 \\
87.3010 \\
87.4320 \\
87.9130 \\
88.1750 \\
87.9130 \\
87.9570 \\
87.3890 \\
87.2140 \\
87.0830 \\
84.9380 \\
85.5510 \\
85.8140 \\
85.9020 \\
86.2080 \\
86.2960 \\
86.6020 \\
86.9080 \\
86.9520 \\
87.3890 \\
87.4760 \\
86.9080 \\
82.7000 \\
83.6230 \\
84.4120 \\
86.0770\end{array}$ \\
\hline
\end{tabular}

High Pressure Test

\begin{tabular}{|c|c|}
\hline $\begin{array}{c}\text { Pressure } \\
\text { (psig) } \\
9.89 \\
19.55 \\
29.57 \\
39.42 \\
49.16 \\
54.50 \\
59.57 \\
64.20 \\
69.32 \\
74.16 \\
80.16 \\
85.61 \\
90.58 \\
95.69 \\
100.92 \\
103.25 \\
106.11 \\
108.31 \\
111.08 \\
113.24 \\
117.83 \\
120.92 \\
123.28 \\
122.97 \\
125.82 \\
125.60 \\
127.84 \\
130.19 \\
132.53 \\
135.33 \\
134.42 \\
138.35 \\
140.16 \\
142.63 \\
145.78 \\
142.52 \\
0.22\end{array}$ & $\begin{array}{c}\text { Temperature } \\
\text { (Fahrenheit) } \\
82.2610 \\
83.3150 \\
84.1050 \\
84.5870 \\
85.2010 \\
85.3760 \\
85.5950 \\
85.7700 \\
86.1640 \\
86.5140 \\
86.4700 \\
86.6020 \\
86.4700 \\
85.9020 \\
85.5080 \\
84.7630 \\
84.5000 \\
84.4120 \\
84.1490 \\
83.9300 \\
83.8420 \\
83.4910 \\
82.8320 \\
82.4370 \\
82.6130 \\
82.6130 \\
83.0520 \\
83.5790 \\
83.7980 \\
84.1050 \\
84.1490 \\
84.7630 \\
85.0260 \\
85.3320 \\
85.7700 \\
85.1570 \\
77.1050\end{array}$ \\
\hline
\end{tabular}




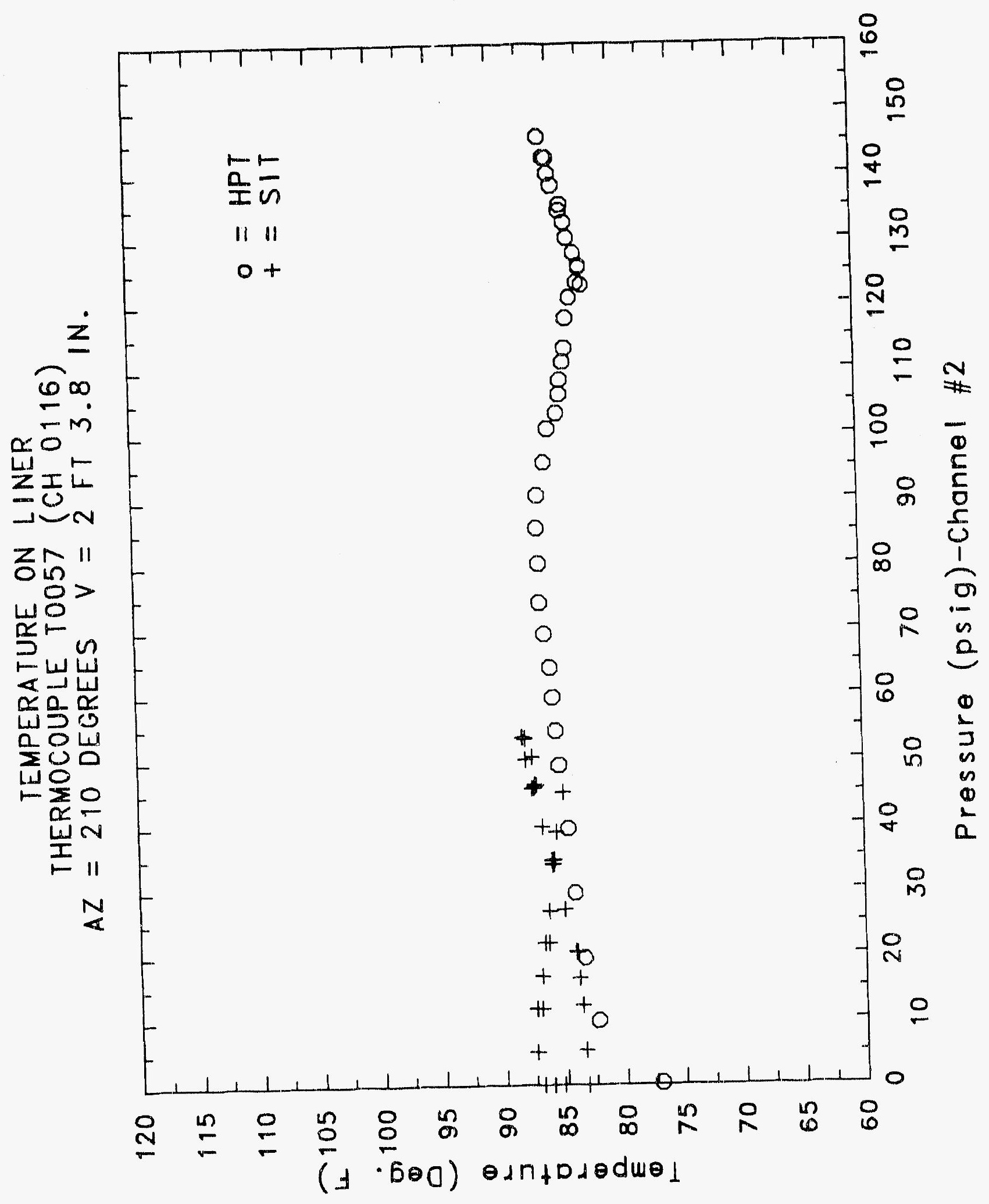




\section{Reinforced Concrete Test Data}

Thermocouple Channel 117

Structural Integrity TEST

\begin{tabular}{|c|c|c|c|}
\hline $\begin{array}{l}\text { Pressure } \\
\text { (psig) } \\
-0.05 \\
5.33 \\
12.31 \\
16.44 \\
20.51 \\
20.46 \\
20.47 \\
27.05 \\
34.69 \\
34.53 \\
34.53 \\
39.70 \\
45.90 \\
45.65 \\
45.62 \\
50.10 \\
53.47 \\
53.29 \\
53.21 \\
50.49 \\
46.14 \\
46.13 \\
44.97 \\
38.98 \\
33.99 \\
33.96 \\
26.83 \\
21.88 \\
21.90 \\
16.69 \\
11.74 \\
11.77 \\
5.05 \\
0.02 \\
-0.04 \\
-0.02 \\
-0.02 \\
0.02 \\
\end{array}$ & $\begin{array}{c}\text { Temperature } \\
\text { (Fahrenheit) } \\
83.4030 \\
83.9740 \\
84.4120 \\
84.6310 \\
85.0700 \\
85.1570 \\
85.3320 \\
86.3830 \\
87.2140 \\
87.3890 \\
87.5200 \\
88.2620 \\
88.9170 \\
88.9610 \\
89.0480 \\
89.5710 \\
89.6580 \\
89.4840 \\
89.2660 \\
88.7430 \\
88.2620 \\
88.1310 \\
85.9450 \\
86.5580 \\
86.9520 \\
87.1700 \\
87.4320 \\
87.5200 \\
88.0000 \\
88.1750 \\
88.35 n 0 \\
88.8730 \\
88.6990 \\
88.4810 \\
83.5790 \\
84.4560 \\
85.6390 \\
87.8690\end{array}$ & $\begin{array}{c}\text { Pressure } \\
(\text { psig) } \\
9.89 \\
19.55 \\
29.57 \\
39.42 \\
49.16 \\
54.50 \\
59.57 \\
64.20 \\
69.32 \\
74.16 \\
80.16 \\
85.61 \\
90.58 \\
95.69 \\
100.92 \\
103.25 \\
106.11 \\
108.31 \\
111.08 \\
113.24 \\
117.83 \\
120.92 \\
123.28 \\
122.97 \\
125.82 \\
125.60 \\
127.84 \\
130.19 \\
132.53 \\
135.33 \\
134.42 \\
138.35 \\
140.16 \\
142.63 \\
145.78 \\
142.52 \\
0.22\end{array}$ & $\begin{array}{c}\text { Temperature } \\
\text { (Fahrenheit) } \\
83.7100 \\
84.8070 \\
85.6830 \\
86.2960 \\
86.9080 \\
87.2140 \\
87.3450 \\
87.6070 \\
87.9130 \\
88.2190 \\
87.8690 \\
87.6950 \\
87.4370 \\
86.8200 \\
86.1640 \\
85.3320 \\
84.9380 \\
84.80 \% 0 \\
84.4120 \\
84.1930 \\
84.1050 \\
83.6660 \\
82.9640 \\
82.5690 \\
82.8760 \\
83.0520 \\
83.5790 \\
84.2370 \\
84.6750 \\
85.0260 \\
84.9820 \\
85.6390 \\
85.9450 \\
86.3390 \\
86.6890 \\
86.2520 \\
76.1760\end{array}$ \\
\hline
\end{tabular}

High Pressure Test

Fahrenheit)

83.7100

84.8070

85.6830

87.2140

87.3450

87.6070

87.8690

87.6950

$87.43 ? 0$

86.8200

84.9380

$84.80 \%$

84.4120

83.6660

82.9540

82.5690

$82.876 ?$

83.5790

84.2370

84.6750

85.0260

85.9450

86.3390

86.6890

86.2520

76.1760 


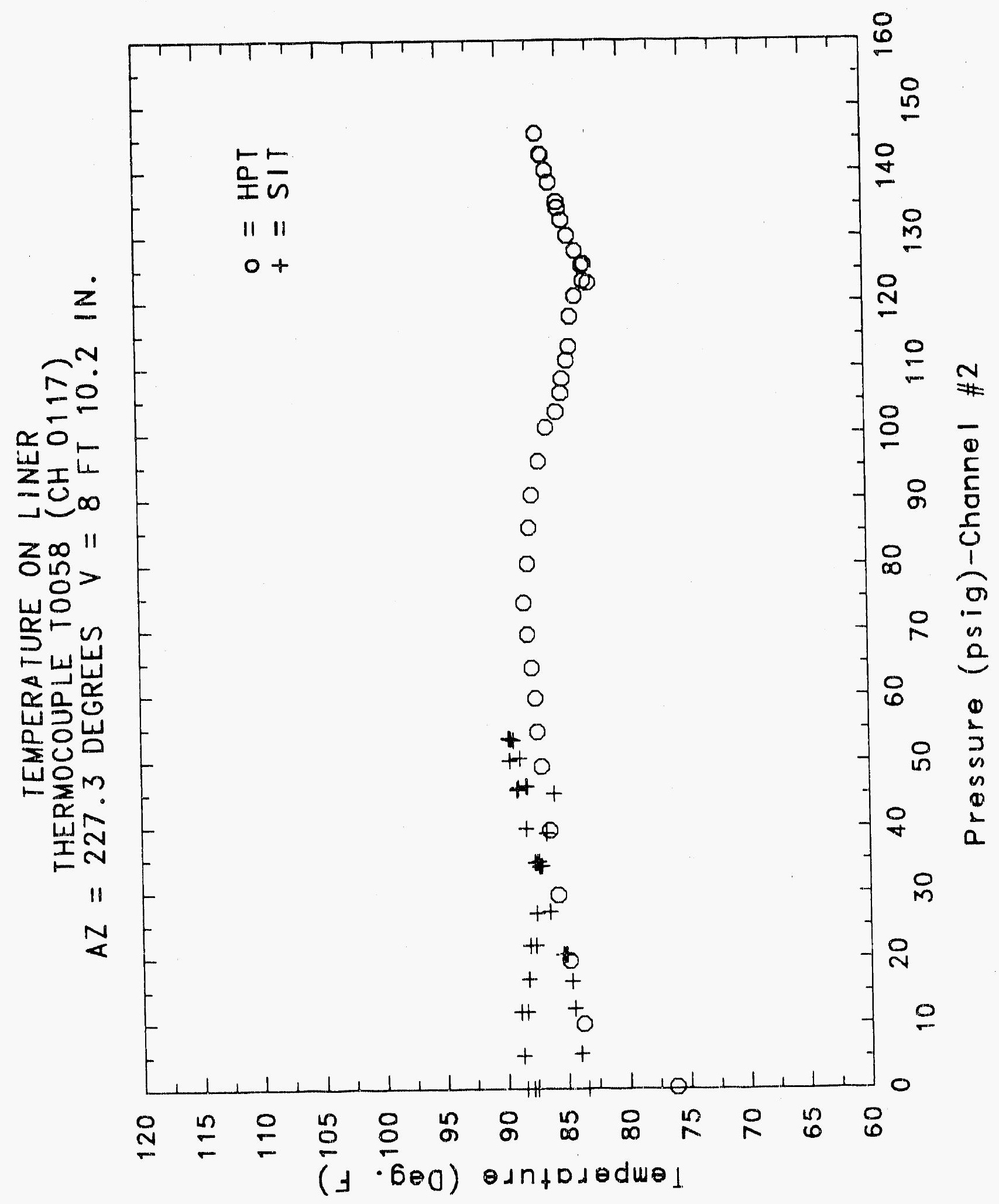




\section{Reinforced Concrete Test Data}

Thermocouple Channel 118

Structural IntEgrity Test

\begin{tabular}{|c|c|c|c|}
\hline $\begin{array}{c}\text { Pressure } \\
\text { (psig) } \\
-0.05 \\
5.33 \\
12.31 \\
16.44 \\
20.51 \\
20.46 \\
20.47 \\
27.05 \\
34.69 \\
34.53 \\
34.53 \\
39.70 \\
45.90 \\
45.65 \\
45.62 \\
50.10 \\
53.47 \\
53.29 \\
53.21 \\
50.49 \\
46.14 \\
46.13 \\
44.97 \\
38.98 \\
33.99 \\
33.96 \\
26.83 \\
21.88 \\
21.90 \\
16.69 \\
11.74 \\
11.77 \\
5.05 \\
0.02 \\
-0.04 \\
-0.02 \\
-0.02 \\
0.02\end{array}$ & $\begin{array}{l}\text { Temperature } \\
\text { (Fahrenheit) } \\
82.5250 \\
82.6130 \\
83.0520 \\
83.5790 \\
84.1490 \\
84.6310 \\
85.0260 \\
85.9020 \\
86.7770 \\
87.2140 \\
87.3890 \\
88.1310 \\
88.7860 \\
89.0040 \\
89.1350 \\
89.4400 \\
89.5710 \\
89.2660 \\
89.0480 \\
88.6550 \\
88.2620 \\
87.9570 \\
85.6830 \\
86.2520 \\
86.6890 \\
87.3010 \\
87.6510 \\
87.9570 \\
88.5680 \\
88.8300 \\
89.0920 \\
89.5710 \\
89.7020 \\
89.4840 \\
84.1930 \\
85.2010 \\
85.9020 \\
87.7380\end{array}$ & $\begin{array}{c}\text { Pressure } \\
(p s 1 g) \\
9.89 \\
19.55 \\
29.57 \\
39.42 \\
49.16 \\
54.50 \\
59.57 \\
64.20 \\
69.32 \\
74.16 \\
80.16 \\
85.61 \\
90.58 \\
95.69 \\
100.92 \\
103.25 \\
106.11 \\
108.31 \\
111.08 \\
113.24 \\
117.83 \\
120.92 \\
123.28 \\
122.97 \\
125.82 \\
125.60 \\
127.84 \\
130.19 \\
132.53 \\
135.33 \\
134.42 \\
138.35 \\
140.16 \\
142.63 \\
145.78 \\
142.52 \\
0.22\end{array}$ & $\begin{array}{c}\text { Temperature } \\
\text { (Fahrenheit) } \\
82.7440 \\
83.4470 \\
84.2810 \\
84.9380 \\
85.7700 \\
85.9020 \\
86.3390 \\
86.5580 \\
86.9950 \\
87.3010 \\
87.5640 \\
87.3450 \\
86.9080 \\
86.0330 \\
85.4640 \\
84.3680 \\
84.0170 \\
83.8860 \\
83.3590 \\
83.1400 \\
82.9200 \\
82.5250 \\
81.7730 \\
81.2940 \\
81.6460 \\
82.2170 \\
82.8320 \\
83.5790 \\
84.0170 \\
84.5870 \\
84.8500 \\
85.5950 \\
85.9890 \\
86.5140 \\
85.9520 \\
86.6890 \\
79.4440\end{array}$ \\
\hline
\end{tabular}

High Pressure test

Temperature

82.7440

83.4470

85.7700

85.9020

86.3390

87.3010

87.5640

87.3450

86.9080

86.0330

84.3680

84.0170

83.8860

83.3590

82.5250

81.7780

81.2940

82.8320

83.5790

84.0170

84.5870

84.8500

85.5950

85.9890

86.5140

85.9520

86.6890

79.4440 


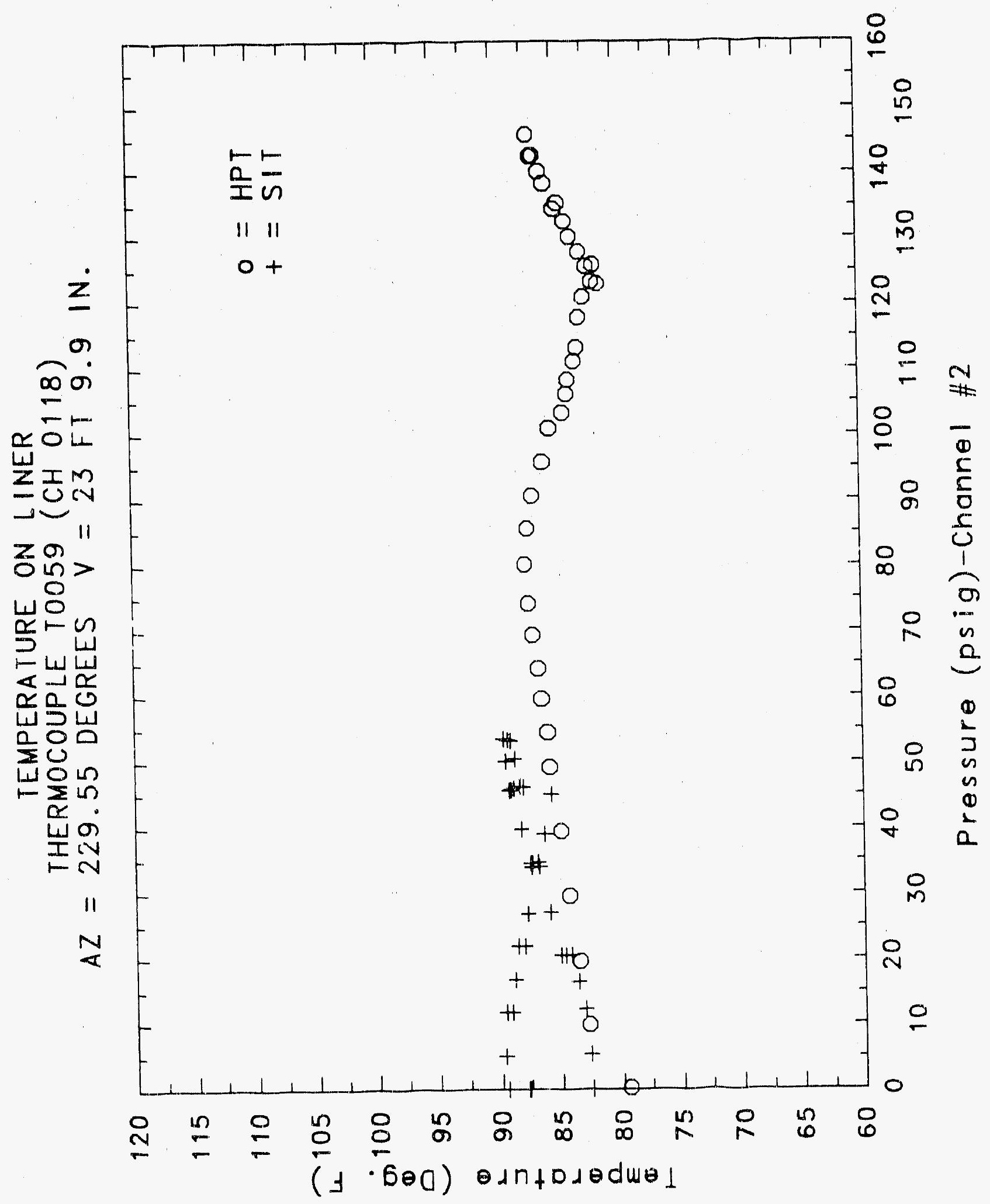

C. -1781 


\section{Reinforced Concrete Test Data}

Thermocouple Channel 119

StRUCTURAL INTEgRITY TEST

\begin{tabular}{|c|c|c|c|}
\hline $\begin{array}{c}\text { Pressure } \\
\text { (psig) } \\
-0.05 \\
5.33 \\
12.31 \\
16.44 \\
20.51 \\
20.46 \\
20.47 \\
27.05 \\
34.69 \\
34.53 \\
34.53 \\
39.70 \\
45.90 \\
45.65 \\
45.62 \\
50.10 \\
53.47 \\
53.29 \\
53.21 \\
50.49 \\
46.14 \\
46.13 \\
44.97 \\
38.98 \\
33.99 \\
33.96 \\
26.83 \\
21.88 \\
21.50 \\
16.69 \\
11.74 \\
11.77 \\
5.05 \\
0.02 \\
-0.04 \\
-0.02 \\
-0.02 \\
0.02\end{array}$ & $\begin{array}{c}\text { Temperature } \\
\text { (Fahrenheit) } \\
79.8850 \\
79.7530 \\
80.8100 \\
82.3930 \\
82.8760 \\
83.5790 \\
83.9300 \\
85.1570 \\
86.1640 \\
86.5140 \\
86.6890 \\
87.9130 \\
88.7430 \\
88.4370 \\
88.5680 \\
88.8730 \\
89.1350 \\
88.6550 \\
88.4370 \\
88.0000 \\
88.0880 \\
87.5200 \\
84.4560 \\
85.0700 \\
85.5080 \\
86.2520 \\
86.9520 \\
87.4760 \\
81.8690 \\
88.9610 \\
88.5680 \\
88.8730 \\
88.7860 \\
88.8300 \\
82.0410 \\
81.4260 \\
82.7000 \\
85.0260\end{array}$ & $\begin{array}{c}\text { Pressure } \\
(p s i g) \\
9.89 \\
19.55 \\
29.57 \\
39.42 \\
49.16 \\
54.50 \\
59.57 \\
64.20 \\
69.32 \\
74.16 \\
80.16 \\
85.61 \\
90.58 \\
95.69 \\
100.92 \\
103.25 \\
106.11 \\
108.31 \\
111.08 \\
113.24 \\
117.83 \\
120.92 \\
123.28 \\
122.97 \\
125.82 \\
125.60 \\
127.84 \\
130.19 \\
132.53 \\
135.33 \\
134.42 \\
138.35 \\
140.16 \\
142.63 \\
145.78 \\
142.52 \\
0.22\end{array}$ & $\begin{array}{c}\text { Temperature } \\
\text { (Fihrenheit) } \\
79.4440 \\
80.5010 \\
81.5140 \\
82.2170 \\
83.3590 \\
83.4470 \\
83.7100 \\
83.9740 \\
84.5870 \\
85.0260 \\
84.9380 \\
84.3680 \\
84.3240 \\
83.5350 \\
83.7980 \\
82.6570 \\
81.9090 \\
82.1290 \\
81.6900 \\
81.5580 \\
81.3380 \\
81.2500 \\
80.6340 \\
79.8410 \\
80.4130 \\
80.1490 \\
80.5450 \\
80.8100 \\
81.2940 \\
82.3930 \\
82.7880 \\
83.8420 \\
84.2370 \\
84.8940 \\
85.4640 \\
85.3320 \\
81.2500\end{array}$ \\
\hline
\end{tabular}

\section{High Pressure test}

Temperature

79.4440

81.5140

82.2170

83.3590

83.4470

83.7100

84.5870

85.0260

84.9380

84.3680

83.7980

82.6570

81.9090

81.5580

81.3380

81.2500

80.6340

80.1490

80.5450

80.8100

81.2940

82.3930

83.8420

84.2370

84.8940

85.4640

85.3320

81.2500 


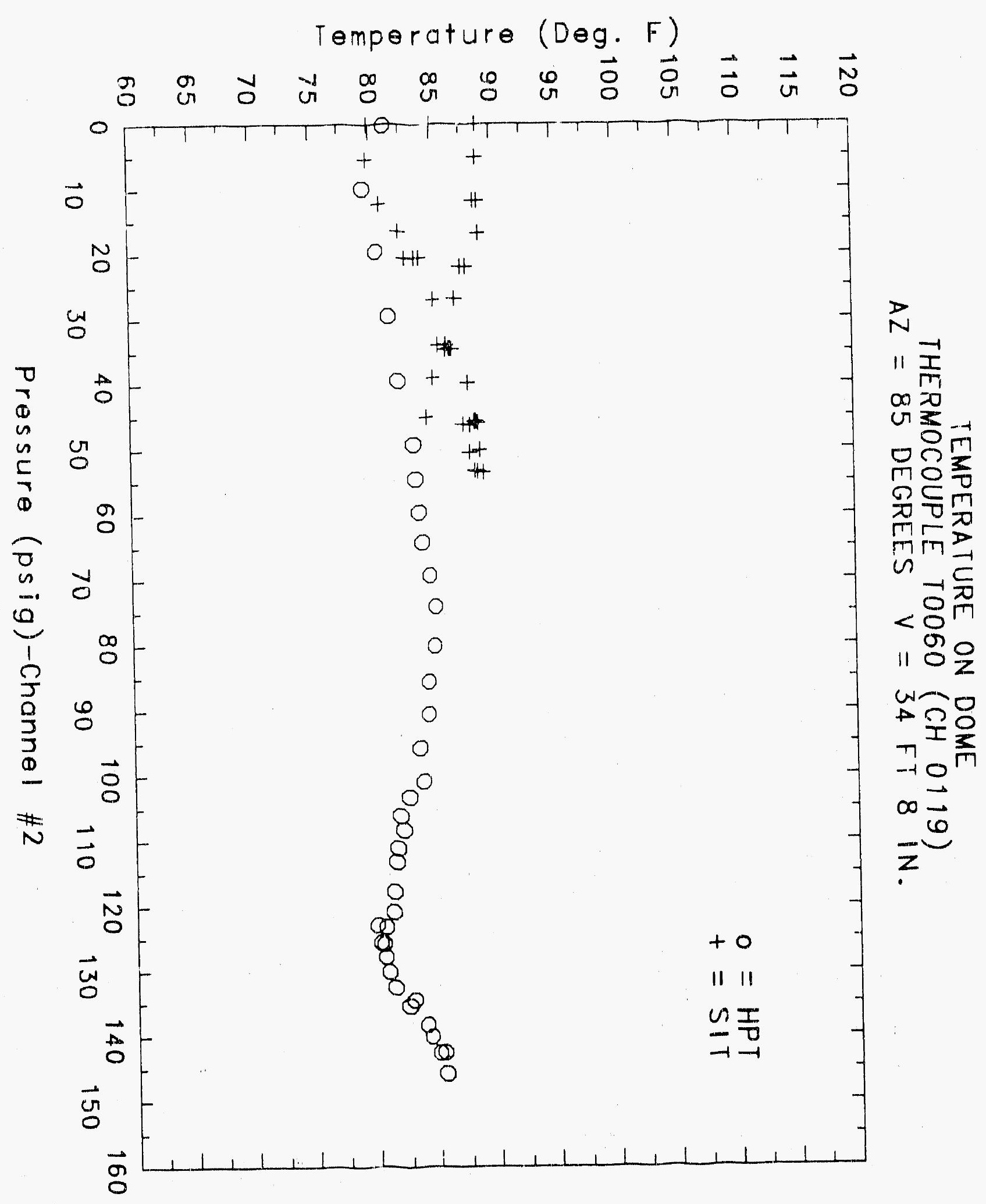




\section{Reinforced Concrete test Data}

Thermocouple Channel 2012

STRUCTURAL INTEGRITY TEST

\begin{tabular}{|c|c|c|c|}
\hline $\begin{array}{l}\text { Pressure } \\
\text { (psig) } \\
-0.05 \\
5.33 \\
12.31 \\
15.44 \\
20.51 \\
20.46 \\
20.47 \\
27.05 \\
34.69 \\
34.53 \\
34.53 \\
39.70 \\
45.90 \\
45.65 \\
45.62 \\
50.10 \\
53.47 \\
53.29 \\
53.21 \\
50.49 \\
46.14 \\
46.13 \\
44.97 \\
38.98 \\
33.99 \\
33.96 \\
26.83 \\
21.88 \\
21.90 \\
16.69 \\
11.74 \\
11.79 \\
5.05 \\
0.02 \\
-0.04 \\
-0.02 \\
-0.02 \\
0.02\end{array}$ & $\begin{array}{c}\text { Temperature } \\
\text { (Fahrenheit) } \\
80.5010 \\
81.1180 \\
81.3820 \\
81.6460 \\
81.8650 \\
81.7340 \\
81.8220 \\
82.6570 \\
83.1840 \\
83.3150 \\
83.3150 \\
84.0170 \\
84.5000 \\
84.5440 \\
84.4560 \\
85.0260 \\
83.1570 \\
84.8500 \\
84.7190 \\
84.1050 \\
83.6230 \\
83.5350 \\
82.0410 \\
82.6570 \\
82.8760 \\
83.1400 \\
83.3150 \\
83.3590 \\
83.7980 \\
83.8860 \\
84.0170 \\
84.3240 \\
84.2370 \\
84.0170 \\
78.8270 \\
81.4260 \\
83.4910 \\
85.5510\end{array}$ & $\begin{array}{c}\text { Pressure } \\
(\text { psig) } \\
9.89 \\
19.55 \\
29.57 \\
39.42 \\
49.16 \\
54.50 \\
59.57 \\
64.20 \\
69.32 \\
74.16 \\
80.16 \\
85.61 \\
90.58 \\
95.63 \\
100.92 \\
103.25 \\
106.11 \\
108.31 \\
111.08 \\
113.24 \\
117.83 \\
120.92 \\
123.28 \\
122.97 \\
125.82 \\
125.60 \\
127.84 \\
130.19 \\
132.53 \\
135.33 \\
134.42 \\
138.35 \\
140.16 \\
142.63 \\
145.78 \\
142.52 \\
0.22\end{array}$ & $\begin{array}{c}\text { Temperature } \\
\text { (Fahrenheit) } \\
79.5320 \\
80.6340 \\
81.2940 \\
81.9530 \\
82.5690 \\
82.8320 \\
33.0080 \\
83.1840 \\
83.5350 \\
83.7980 \\
83.4030 \\
83.1400 \\
82.8320 \\
81.9530 \\
81.3820 \\
80.5900 \\
80.1930 \\
80.0610 \\
79.5321 \\
79.3120 \\
79.2240 \\
78.8270 \\
77.9880 \\
77.5460 \\
78.0320 \\
78.6940 \\
79.4440 \\
80.4570 \\
80.8980 \\
81.2060 \\
81.2060 \\
81.7340 \\
81.9090 \\
32.3050 \\
82.5690 \\
82.0850 \\
73.9610\end{array}$ \\
\hline
\end{tabular}

High Presgure test

Pressure

(psig)

19.55

29.57

54.50

74.16

95.6 ?

106.11

108.31

113.24

117.83

122.97

125.82

125.60

132.53

135.33

134.42

138.35

142.63

145.78

42.52

0.22
Temperature

79.5320

80.6340

81.2940

82.8320

33.0080

83.1840

83.5350

83.4030

83.1400

82.8320

80.5900

80.1930

79.3120

79.2240

77.5460

78.0320

78.6940

80.8980

81.2060

81.2060

32.3050

82.5690

82.0850

73.9610 


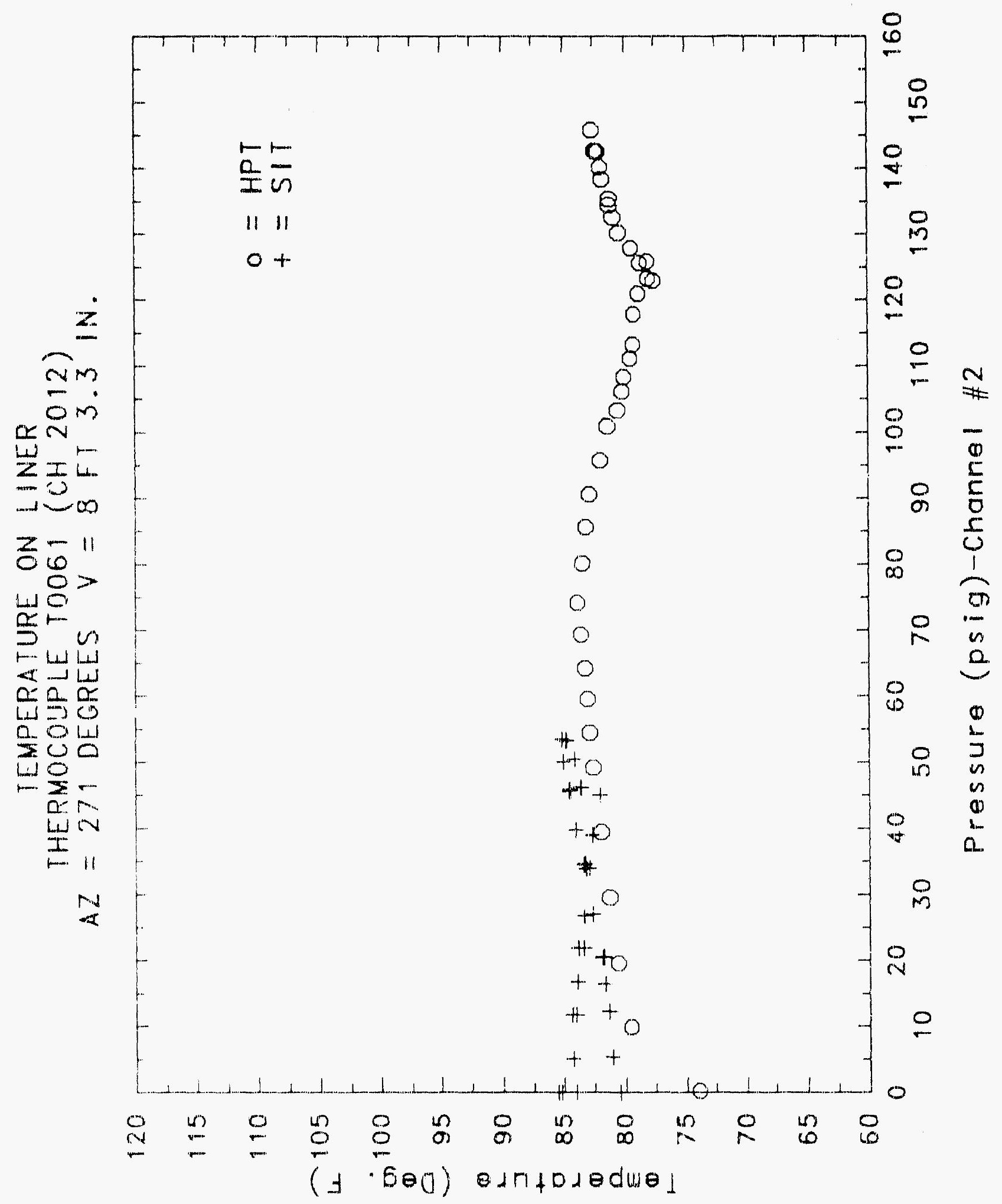




\section{Reinforced Concrete Test Data}

Thermocouple Channel 20001

StRUCTURAL INTEgRITY TEST

\begin{tabular}{|c|c|c|c|}
\hline $\begin{array}{l}\text { Pressure } \\
(p s i g) \\
-0.05 \\
5.33 \\
12.31 \\
16.44 \\
20.51 \\
20.46 \\
20.47 \\
27.05 \\
34.69 \\
34.53 \\
34.53 \\
33.70 \\
45.90 \\
45.65 \\
45.62 \\
50.90 \\
63.47 \\
53.79 \\
53.21 \\
50.49 \\
40.14 \\
46.13 \\
44.97 \\
38.98 \\
33.99 \\
33.96 \\
26.83 \\
21.88 \\
21.90 \\
16.60 \\
11.7 \\
11.77 \\
5.05 \\
0.02 \\
-0.04 \\
-0.02 \\
-0.02 \\
0.02\end{array}$ & $\begin{array}{c}\text { Temperature } \\
\text { (Fahrenhe it) } \\
79.8410 \\
80.1490 \\
80.5450 \\
80.8540 \\
81.0300 \\
81.1180 \\
81.2500 \\
81.9530 \\
82.7440 \\
82.7880 \\
82.9200 \\
83.6230 \\
84.1490 \\
84.4120 \\
34.5000 \\
84.9380 \\
85.7260 \\
85.6390 \\
85.5510 \\
85.0700 \\
84.7190 \\
84.7190 \\
81.1590 \\
82.4370 \\
82.7000 \\
83.0520 \\
83.3150 \\
83.4030 \\
83.9740 \\
84.1050 \\
84.1490 \\
84.6750 \\
84.5870 \\
84.3240 \\
79.6040 \\
79.8850 \\
80.9860 \\
83.4030\end{array}$ & $\begin{array}{c}\text { Pressure } \\
(p s i g) \\
9.89 \\
19.55 \\
29.57 \\
39.42 \\
49.16 \\
54.50 \\
59.57 \\
64.20 \\
69.32 \\
74.16 \\
80.16 \\
85.61 \\
90.58 \\
95.69 \\
100.92 \\
103.25 \\
106.11 \\
108.31 \\
111.08 \\
113.24 \\
117.83 \\
120.92 \\
123.28 \\
122.97 \\
125.82 \\
125.60 \\
127.84 \\
130.19 \\
132.53 \\
135.33 \\
134.42 \\
138.35 \\
140.16 \\
142.63 \\
145.78 \\
142.52 \\
0.22 \\
\end{array}$ & $\begin{array}{c}\text { Temperature } \\
\text { (Fahrenheit) } \\
78.3850 \\
79.4000 \\
80.2370 \\
80.8540 \\
81.5580 \\
81.7340 \\
81.9530 \\
82.1730 \\
82.5690 \\
82.9200 \\
83.0080 \\
83.0960 \\
82.9200 \\
82.3050 \\
81.8650 \\
81.0740 \\
80.7220 \\
80.7220 \\
80.1930 \\
80.0610 \\
79.8850 \\
79.5320 \\
78.7380 \\
78.2530 \\
78.3410 \\
78.0760 \\
78.3410 \\
78.9590 \\
79.3120 \\
79.9290 \\
80.1490 \\
80.7660 \\
81.0740 \\
81.5020 \\
82.0650 \\
81.3820 \\
72.7630\end{array}$ \\
\hline
\end{tabular}

High Pressure test

(Fahrenhe it)

78.3850

79.4000

81.5580

81.7340

81.9530

82.9200

83.0080

83.0960

82.9200

81.0740

80.7220

80.7220

79.8850

78.7380

78.2530

78.3410

78.9590

79.3120

79.9290

81.0740

81.6020

82.0550

81.3820

72.7630 


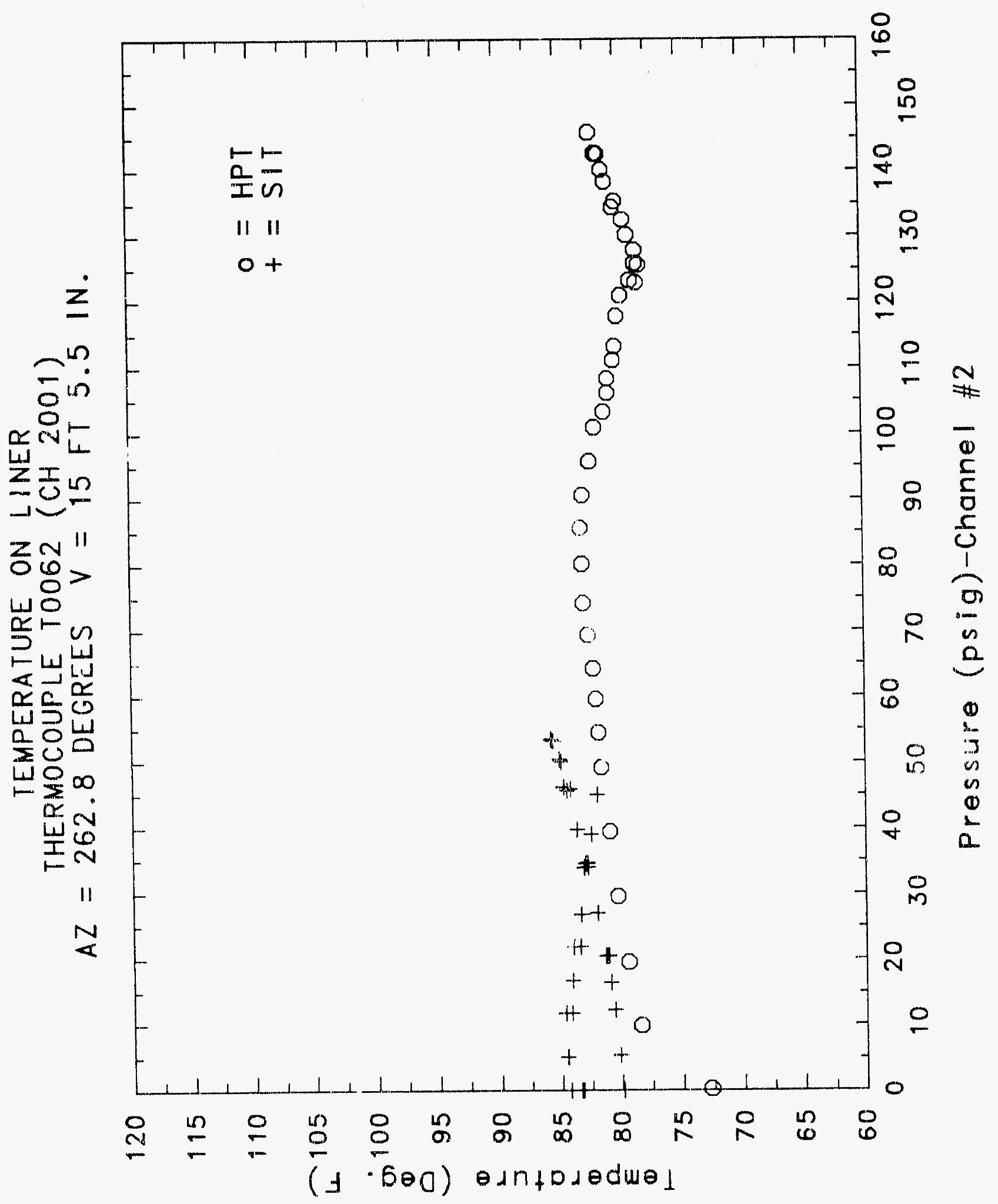




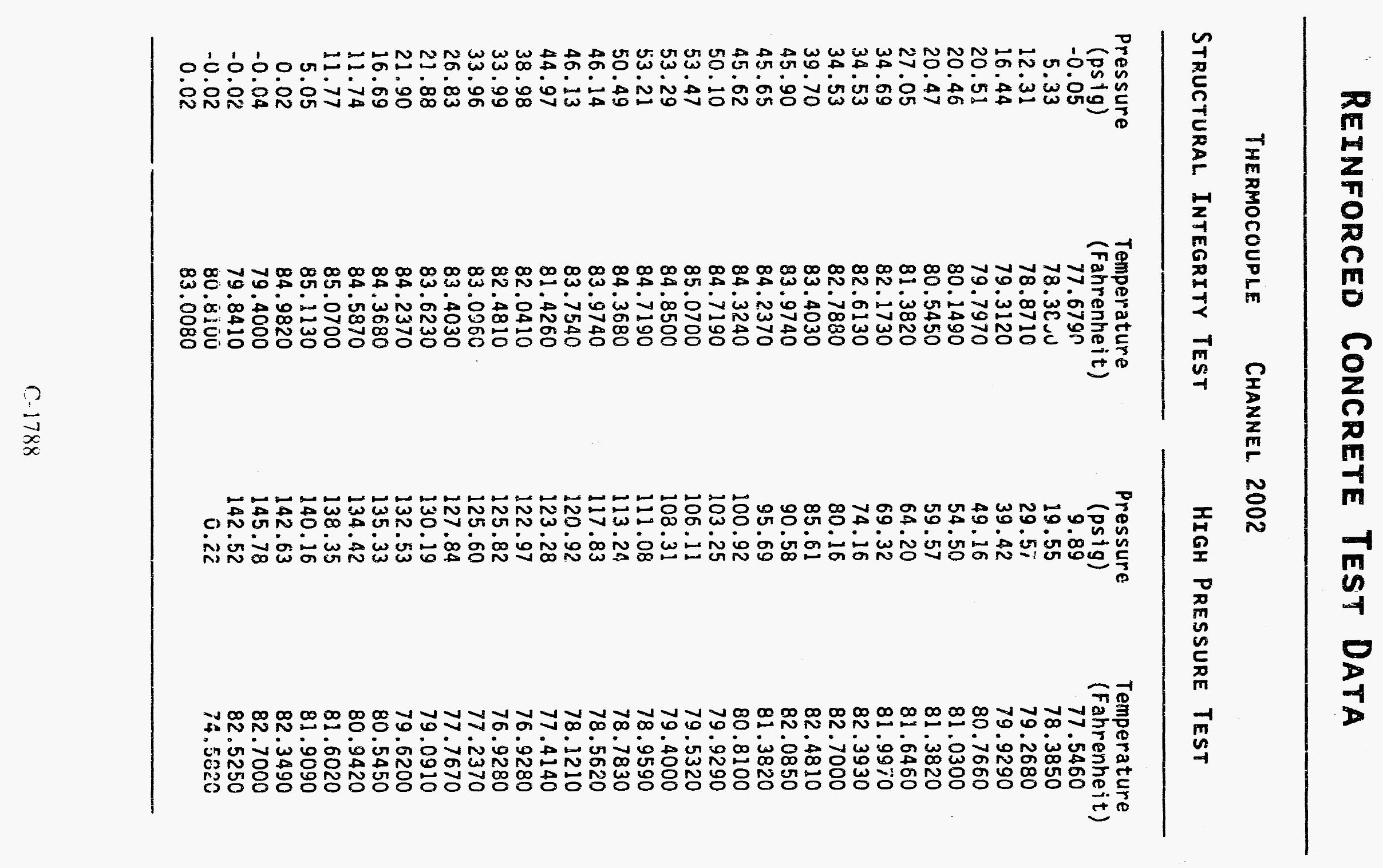




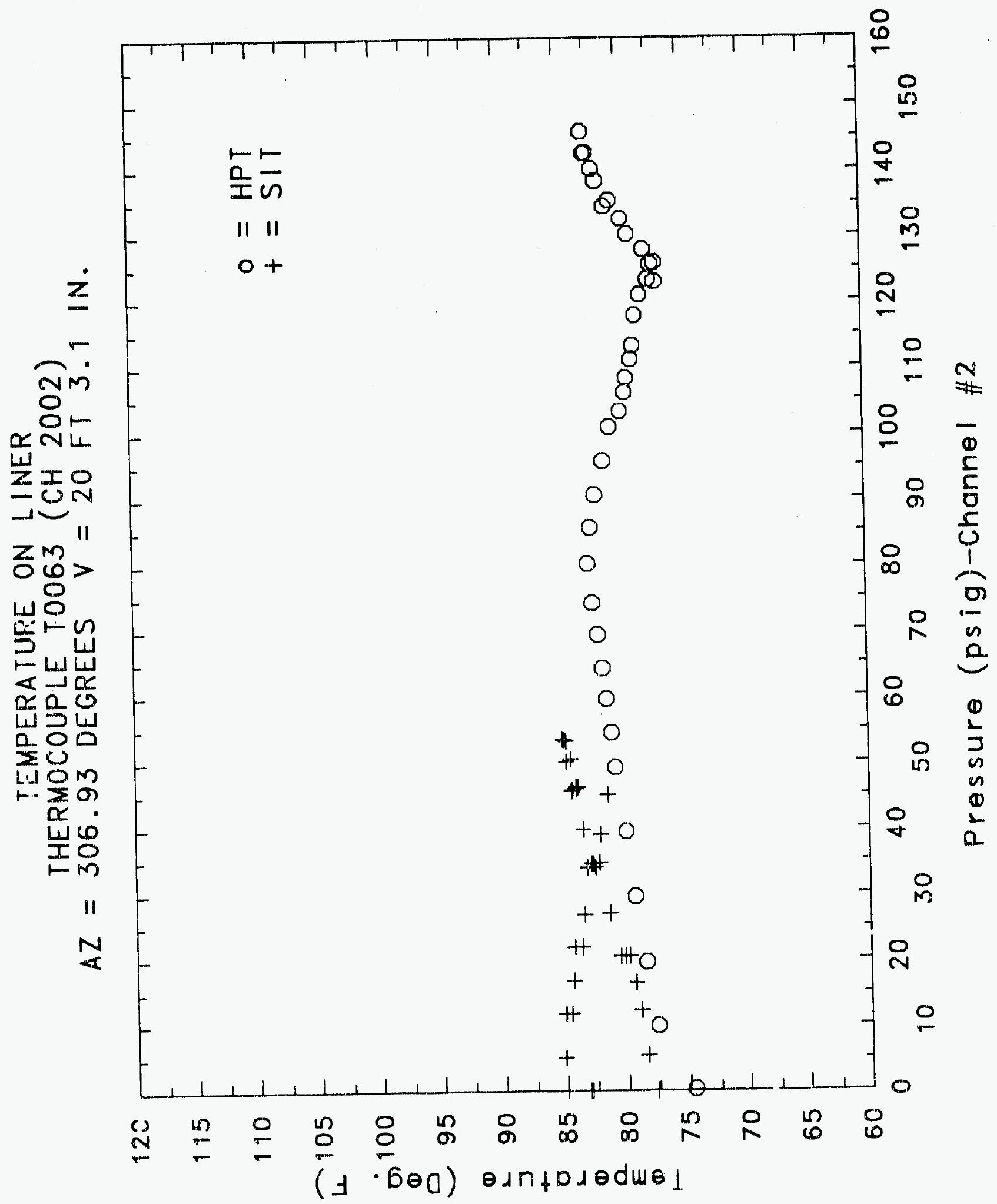

C. 1789 


\section{Reinforced Concrete Tesi Data}

Thermocouple Channel 2003

StRuctural IntEgrity TEST

\begin{tabular}{|c|c|c|c|}
\hline $\begin{array}{c}\text { Pressure } \\
\text { (psig) } \\
-0.05 \\
5.33 \\
12.31 \\
16.44 \\
20.51 \\
20.46 \\
20.47 \\
27.05 \\
34.69 \\
34.53 \\
34.53 \\
39.70 \\
45.90 \\
45.65 \\
45.62 \\
50.10 \\
53.47 \\
53.29 \\
53.21 \\
50.49 \\
46.14 \\
46.13 \\
44.97 \\
38.98 \\
33.99 \\
33.96 \\
26.83 \\
21.88 \\
21.90 \\
16.69 \\
11.74 \\
11.77 \\
5.05 \\
0.02 \\
-0.04 \\
-0.02 \\
-0.02 \\
0.02\end{array}$ & $\begin{array}{c}\text { Temperature } \\
\text { (Fahrenheit) } \\
79.9730 \\
80.5450 \\
81.0740 \\
81.4700 \\
81.6460 \\
81.4700 \\
81.5580 \\
82.2610 \\
82.9200 \\
82.8760 \\
82.9640 \\
83.5350 \\
84.1050 \\
84.0170 \\
84.1050 \\
84.4120 \\
84.7190 \\
84.4120 \\
84.2810 \\
83.6660 \\
83.4030 \\
83.2270 \\
82.0850 \\
82.7000 \\
82.9200 \\
83.0080 \\
83.3150 \\
83.3590 \\
83.6230 \\
83.8860 \\
83.7980 \\
84.0610 \\
84.0610 \\
83.5350 \\
78.6060 \\
79.7530 \\
81.3380 \\
84.1930\end{array}$ & $\begin{array}{c}\text { Pressure } \\
\text { (psig) } \\
9.89 \\
19.55 \\
29.57 \\
39.42 \\
49.16 \\
54.50 \\
59.57 \\
64.20 \\
69.32 \\
74.16 \\
80.16 \\
85.61 \\
90.58 \\
95.69 \\
100.92 \\
103.25 \\
106.11 \\
108.31 \\
111.08 \\
113.24 \\
117.83 \\
120.92 \\
123.28 \\
122.97 \\
125.82 \\
125.60 \\
127.84 \\
130.19 \\
132.53 \\
135.33 \\
134.42 \\
138.35 \\
140.16 \\
142.63 \\
145.78 \\
142.52 \\
0.22\end{array}$ & $\begin{array}{c}\text { Temperature } \\
\text { (Fahrenheit) } \\
79.4000 \\
80.5010 \\
81.3380 \\
81.9090 \\
82.5690 \\
82.7000 \\
82.8760 \\
82.9640 \\
83.2710 \\
83.5350 \\
83.0960 \\
82.9640 \\
82.7440 \\
82.0850 \\
81.6460 \\
80.8980 \\
80.5900 \\
80.4130 \\
80.1050 \\
79.8850 \\
79.8410 \\
79.5320 \\
78.8270 \\
78.2530 \\
78.5620 \\
78.5180 \\
79.0910 \\
79.9730 \\
80.5010 \\
80.9860 \\
80.9420 \\
81.5140 \\
81.7780 \\
82.0850 \\
82.4810 \\
81.7340 \\
73.1180\end{array}$ \\
\hline
\end{tabular}

High Pressure test 


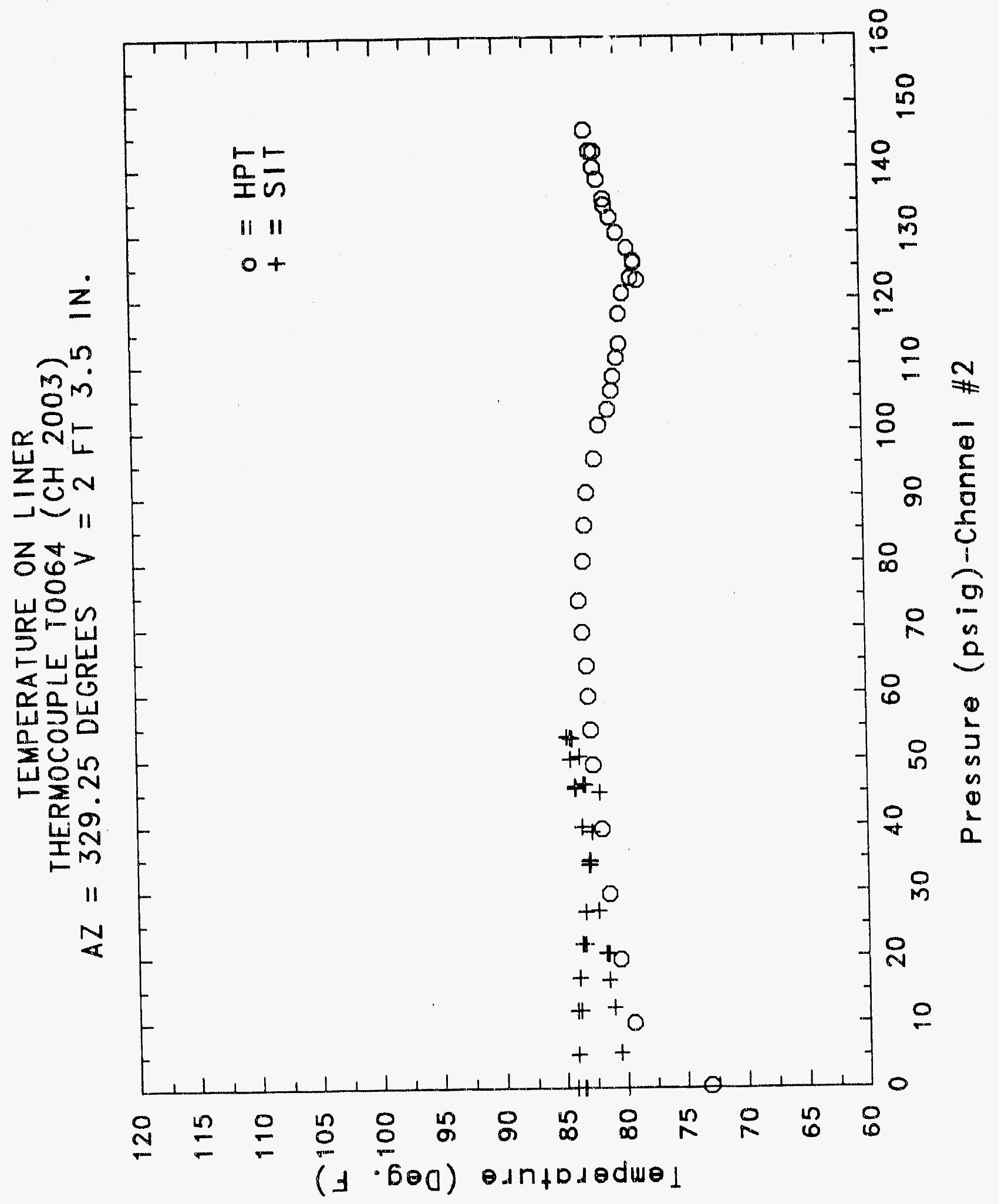




\title{
Reinforced Concrete Test Data
}

\author{
Thermocouple Channel 2004
}

\section{StRUCTURAL INTEgRITY TEST}

\begin{tabular}{c} 
Pressure \\
(psig) \\
-0.05 \\
5.33 \\
12.31 \\
16.44 \\
20.51 \\
20.46 \\
20.47 \\
27.05 \\
34.69 \\
34.53 \\
34.53 \\
39.70 \\
45.90 \\
45.65 \\
45.62 \\
50.10 \\
53.47 \\
53.29 \\
53.21 \\
50.49 \\
46.14 \\
46.13 \\
44.97 \\
38.98 \\
33.99 \\
33.96 \\
26.83 \\
21.88 \\
21.90 \\
16.69 \\
11.74 \\
11.77 \\
5.05 \\
0.02 \\
-0.04 \\
-0.02 \\
-0.02 \\
0.02 \\
7 \\
\hline
\end{tabular}

High Pressure test

\begin{tabular}{|c|c|}
\hline $\begin{array}{c}\text { Pressure } \\
\text { (psig) } \\
9.89 \\
19.55 \\
29.57 \\
39.42 \\
49.16 \\
54.50 \\
59.57 \\
64.20 \\
69.32 \\
74.16 \\
80.16 \\
85.61 \\
90.58 \\
95.69 \\
100.92 \\
103.25 \\
106.11 \\
108.31 \\
111.08 \\
113.24 \\
117.83 \\
120.92 \\
123.28 \\
122.97 \\
125.82 \\
125.60 \\
127.84 \\
130.19 \\
132.53 \\
135.33 \\
134.42 \\
138.35 \\
140.16 \\
142.63 \\
145.78 \\
142.52 \\
0.22\end{array}$ & $\begin{array}{c}\text { Temperature } \\
\text { (Fahrenheit) } \\
80.2370 \\
81.2060 \\
81.8220 \\
82.1730 \\
82.3930 \\
82.6130 \\
82.4810 \\
83.3150 \\
83.3590 \\
83.7100 \\
79.6640 \\
79.6200 \\
79.4880 \\
78.6060 \\
78.1210 \\
77.3700 \\
76.8390 \\
76.7950 \\
76.4410 \\
76.1320 \\
76.4410 \\
76.0870 \\
75.9550 \\
76.1760 \\
76.0870 \\
76.9720 \\
77.6790 \\
78.3850 \\
79.6200 \\
79.6640 \\
79.8410 \\
81.6020 \\
80.8100 \\
81.5140 \\
80.9420 \\
79.0470 \\
64.1960\end{array}$ \\
\hline
\end{tabular}




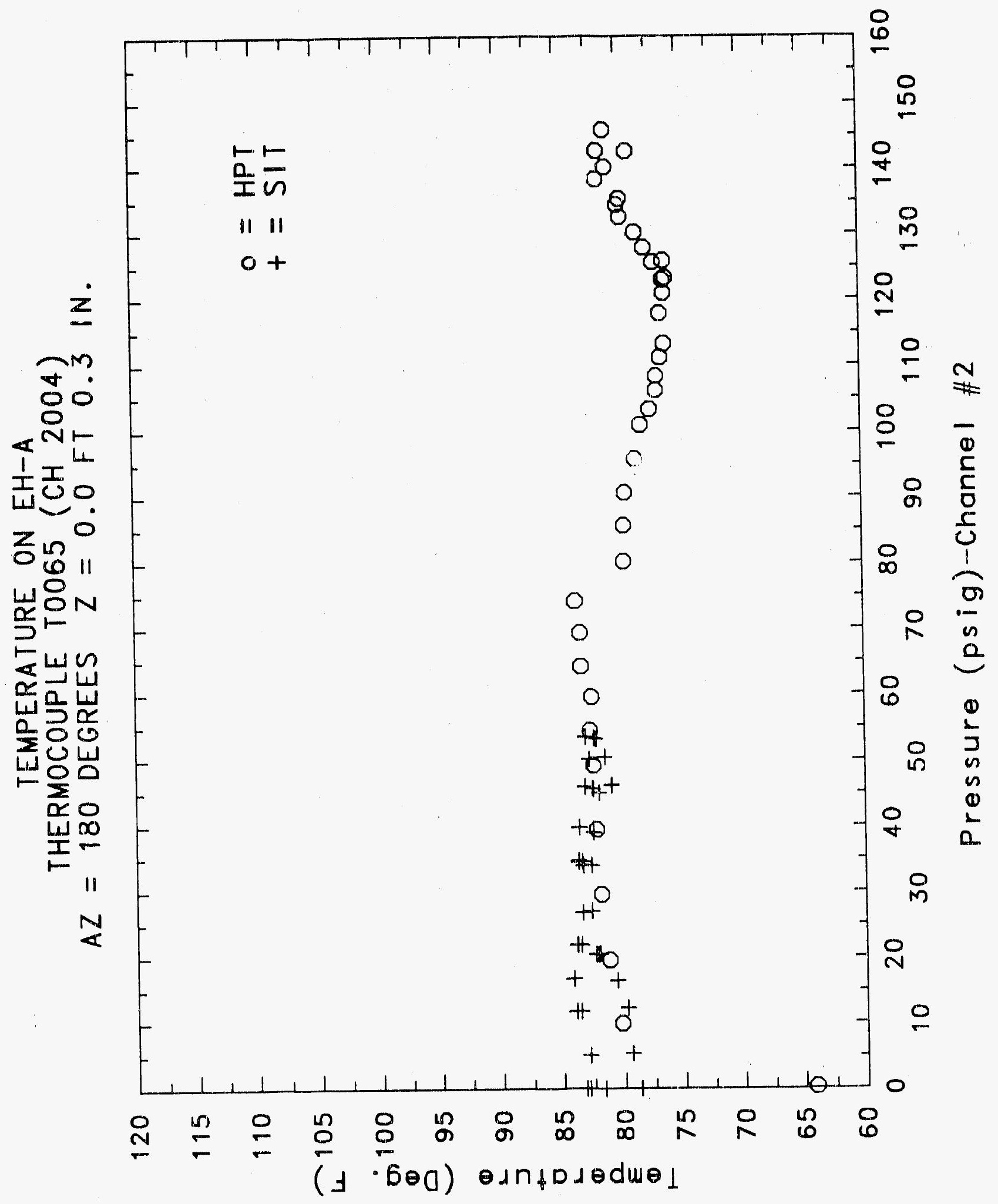




\section{Reinforced Concrete Test Data}

Thermocouple Channel 2005

Structural Integrity TEST

\begin{tabular}{|c|c|c|c|}
\hline $\begin{array}{c}\text { Pressure } \\
\text { (psig) } \\
-0.05 \\
5.33 \\
12.31 \\
16.44 \\
20.51 \\
20.46 \\
20.47 \\
27.05 \\
34.69 \\
34.53 \\
34.53 \\
39.70 \\
45.90 \\
45.65 \\
45.62 \\
50.10 \\
53.47 \\
53.29 \\
53.21 \\
50.49 \\
46.14 \\
46.13 \\
44.97 \\
38.98 \\
33.99 \\
33.96 \\
26.83 \\
21.38 \\
21.90 \\
16.69 \\
11.74 \\
11.77 \\
5.05 \\
0.02 \\
-0.04 \\
-0.02 \\
-0.02 \\
0.02\end{array}$ & $\begin{array}{c}\text { Temperature } \\
\text { (Fahrenheit) } \\
77.4580 \\
79.4440 \\
80.6780 \\
81.6020 \\
82.2170 \\
82.4810 \\
82.9640 \\
83.4470 \\
84.1490 \\
84.5440 \\
84.3240 \\
84.1490 \\
83.6230 \\
82.6570 \\
82.6130 \\
83.0520 \\
83.4910 \\
83.1400 \\
82.8760 \\
81.9090 \\
81.0740 \\
80.8540 \\
83.8420 \\
84.1930 \\
84.3680 \\
84.8940 \\
84.9380 \\
85.2450 \\
85.5950 \\
85.4640 \\
85.0260 \\
84.3240 \\
83.7100 \\
83.2270 \\
77.8110 \\
81.1620 \\
82.7000 \\
84.0170\end{array}$ & $\begin{array}{c}\text { Pressure } \\
(\text { psig) } \\
9.89 \\
19.55 \\
29.57 \\
39.42 \\
49.16 \\
54.50 \\
59.57 \\
64.20 \\
69.32 \\
74.16 \\
80.16 \\
85.61 \\
90.58 \\
95.69 \\
100.92 \\
103.25 \\
106.11 \\
108.31 \\
111.08 \\
113.24 \\
117.83 \\
120.92 \\
123.28 \\
122.97 \\
125.82 \\
125.60 \\
127.84 \\
130.19 \\
132.53 \\
135.33 \\
134.42 \\
138.35 \\
140.16 \\
142.63 \\
145.78 \\
142.52 \\
0.22 \\
\end{array}$ & $\begin{array}{c}\text { Temperature } \\
\text { (Fahrenheit) } \\
79.3120 \\
79.9290 \\
80.7220 \\
81.2500 \\
81.6020 \\
81.7340 \\
81.7780 \\
82.2170 \\
82.6570 \\
83.1400 \\
79.9290 \\
80.2370 \\
80.4570 \\
80.0170 \\
79.4440 \\
78.3850 \\
78.0760 \\
78.4300 \\
77.8560 \\
77.7230 \\
77.9440 \\
77.2810 \\
77.1490 \\
76.7510 \\
76.7950 \\
77.5910 \\
78.3850 \\
77.8560 \\
78.8710 \\
80.4130 \\
80.5900 \\
81.9090 \\
80.8540 \\
81.7340 \\
80.5900 \\
78.6060 \\
65.1820\end{array}$ \\
\hline
\end{tabular}

high Pressure test

Pressure

9.89

19.55

49.16

54.50

59.57

64.20

80.16

85.61

90.58

106.11

108.31

111.08

113.24

117.83

123.28

122.97

125.82

25.60

27.84

30.19

140.16

42.63

45.78

2.52

78.6060

65.1820 


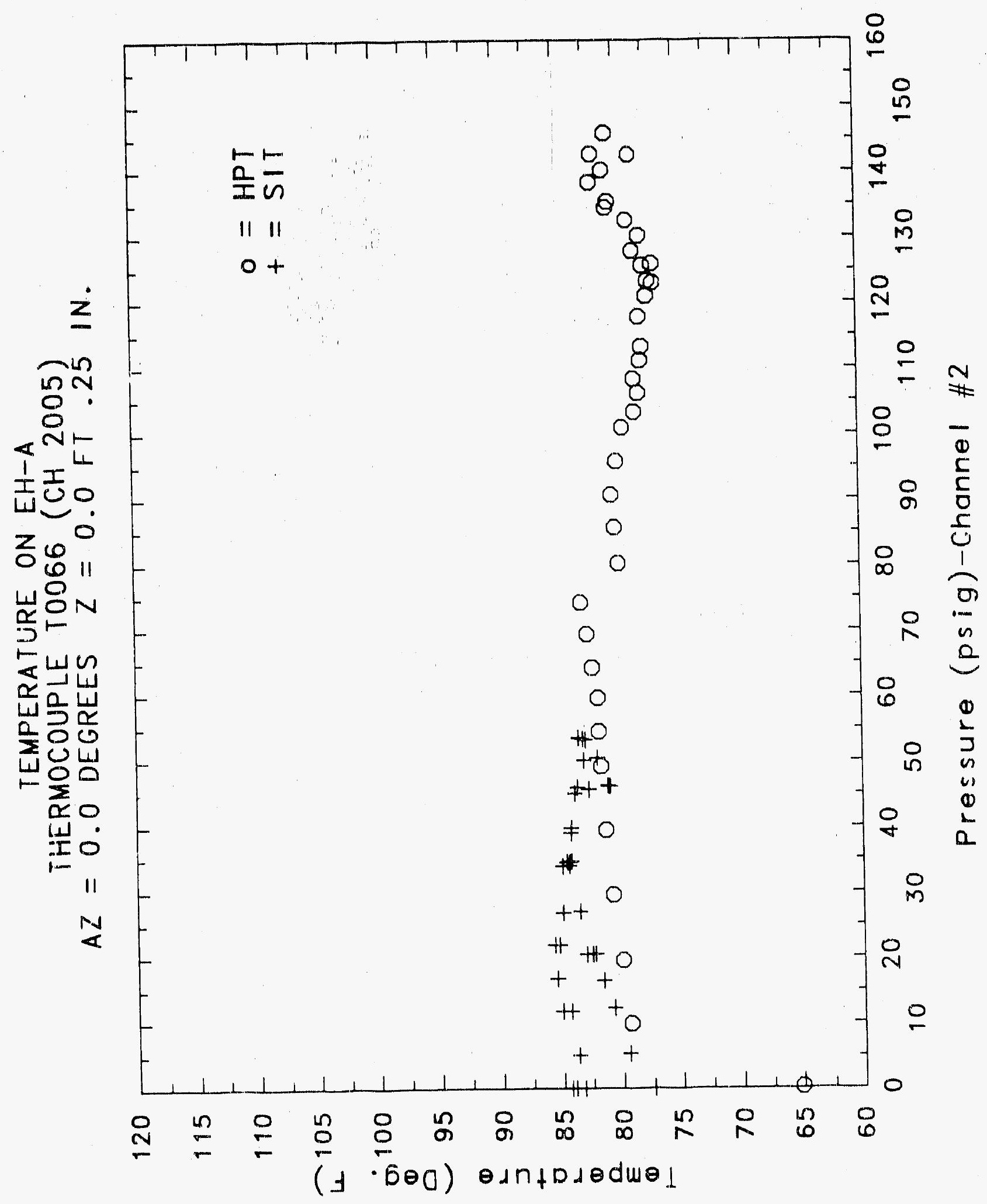

C. 1795 


\section{Reinforced Concrete Test Data}

Thermucouple Channel 2006

Structural InTEgRITY TEST

\begin{tabular}{|c|c|c|c|}
\hline $\begin{array}{l}\text { Pressure } \\
\text { (psig) } \\
-0.05 \\
5.33 \\
12.31 \\
16.44 \\
20.51 \\
20.46 \\
20.47 \\
27.05 \\
34.69 \\
34.53 \\
34.53 \\
39.70 \\
45.90 \\
45.65 \\
45.62 \\
50.10 \\
53.47 \\
53.29 \\
53.21 \\
50.49 \\
46.14 \\
46.13 \\
44.97 \\
38.98 \\
33.99 \\
33.96 \\
26.83 \\
21.88 \\
21.90 \\
16.69 \\
11.74 \\
11.77 \\
5.05 \\
0.02 \\
-0.04 \\
-0.02 \\
-0.02 \\
0.02\end{array}$ & $\begin{array}{c}\text { Temperature } \\
\text { (Fahrenheit) } \\
81.0740 \\
80.9860 \\
80.4570 \\
80.8100 \\
81.0300 \\
80.5900 \\
80.6340 \\
82.1730 \\
83.2270 \\
82.9640 \\
83.0960 \\
84.2810 \\
84.9820 \\
84.1490 \\
84.1050 \\
84.7630 \\
85.1570 \\
84.6310 \\
84.4120 \\
83.5790 \\
83.2710 \\
83.4910 \\
81.8650 \\
82.4810 \\
82.6570 \\
82.6130 \\
83.0960 \\
83.1400 \\
83.7100 \\
84.1930 \\
84.1050 \\
84.3240 \\
84.1490 \\
83.0520 \\
80.0610 \\
80.1050 \\
80.4130 \\
83.3590 \\
\end{array}$ & $\begin{array}{c}\text { Pressure } \\
(\text { psig) } \\
9.89 \\
19.55 \\
29.57 \\
39.42 \\
49.16 \\
54.50 \\
59.57 \\
64.20 \\
69.32 \\
74.16 \\
80.16 \\
85.61 \\
90.58 \\
95.69 \\
100.9 ? \\
103.25 \\
106.11 \\
108.31 \\
111.08 \\
113.24 \\
117.83 \\
120.92 \\
123.28 \\
122.97 \\
125.82 \\
125.60 \\
127.84 \\
130.19 \\
132.53 \\
135.33 \\
134.42 \\
138.35 \\
140.16 \\
142.63 \\
145.78 \\
142.52 \\
0.22 \\
\end{array}$ & $\begin{array}{c}\text { Temperature } \\
\text { (Fahrenheit) } \\
80.4130 \\
81.9530 \\
82.7440 \\
82.6130 \\
83.0520 \\
82.7000 \\
82.7000 \\
82.5250 \\
83.0080 \\
83.2710 \\
81.4260 \\
81.5140 \\
81.2500 \\
80.4130 \\
80.0170 \\
79.0910 \\
78.6500 \\
78.5180 \\
78.2530 \\
77.9440 \\
78.1210 \\
77.8110 \\
77.7670 \\
77.6350 \\
78.1650 \\
77.8110 \\
78.2970 \\
78.6060 \\
78.9150 \\
79.3120 \\
79.2680 \\
80.2810 \\
80.5900 \\
81.2060 \\
81.5140 \\
80.5450 \\
60.7380\end{array}$ \\
\hline
\end{tabular}




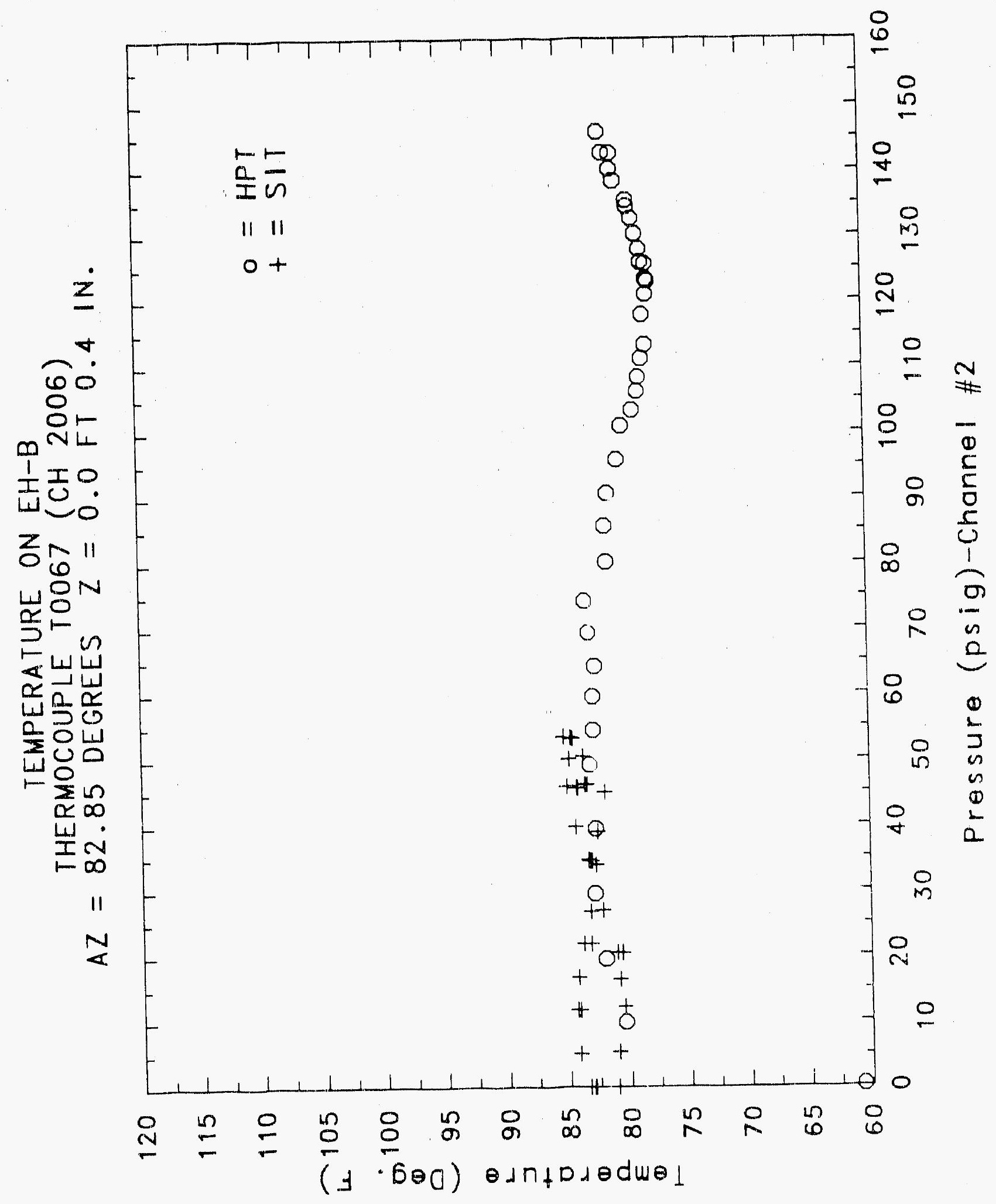

C. -1797 


\section{Reinforced Concrete Test Data}

Thermocouple Channel 2007

Structural INTEgRity TEST

\begin{tabular}{|c|c|}
\hline $\begin{array}{l}\text { Pressure } \\
(p s i g) \\
-0.05 \\
5.33 \\
12.31 \\
16.44 \\
20.51 \\
20.46 \\
60.47 \\
27.05 \\
34.69 \\
34.53 \\
34.53 \\
39.70 \\
45.90 \\
45.65 \\
45.62 \\
50.10 \\
53.47 \\
53.29 \\
53.21 \\
50.49 \\
46.14 \\
46.13 \\
44.97 \\
38.98 \\
33.99 \\
33.96 \\
26.83 \\
21.88 \\
21.90 \\
16.69 \\
11.74 \\
11.77 \\
5.01 \\
0.02 \\
-0.04 \\
-0.02 \\
-6.02 \\
000\end{array}$ & $\begin{array}{l}\text { Temperature } \\
\text { (Fahrenhei } \\
75.4230 \\
75.7780 \\
76.4410 \\
76.9720 \\
77.7230 \\
78.4300 \\
79.0030 \\
80.0610 \\
80.8980 \\
82.8760 \\
83.7540 \\
85.0700 \\
85.8140 \\
85.2450 \\
84.8070 \\
84.4120 \\
84.0610 \\
83.5790 \\
83.2270 \\
82.7000 \\
82.4810 \\
82.2610 \\
80.3590 \\
80.8100 \\
81.2050 \\
81.7780 \\
82.1290 \\
82.7880 \\
84.2370 \\
84.9380 \\
85.9020 \\
86.1640 \\
85.8140 \\
85.5950 \\
79.0030 \\
79.3120 \\
79.4880 \\
80.4130\end{array}$ \\
\hline
\end{tabular}

High pressure Test

\begin{tabular}{|c|c|}
\hline $\begin{array}{c}\text { Pressure } \\
\text { (psig) } \\
9.89 \\
19.55 \\
29.57 \\
39.42 \\
49.16 \\
54.50 \\
59.57 \\
64.20 \\
69.32 \\
74.16 \\
80.16 \\
85.61 \\
90.58 \\
95.69 \\
100.92 \\
103.25 \\
106.11 \\
108.31 \\
111.08 \\
113.24 \\
117.83 \\
120.92 \\
123.28 \\
122.97 \\
125.82 \\
125.60 \\
127.84 \\
130.19 \\
132.53 \\
135.33 \\
134.42 \\
138.35 \\
140.16 \\
142.63 \\
145.78 \\
142.52 \\
0.22\end{array}$ & $\begin{array}{c}\text { Temperature } \\
\text { (Fahrenhe it) } \\
76.1760 \\
76.5740 \\
77.1930 \\
77.6350 \\
77.9880 \\
78.4300 \\
78.8710 \\
79.2240 \\
79.5320 \\
79.9730 \\
78.5180 \\
78.2090 \\
77.7670 \\
77.1931 \\
76.7070 \\
76.0870 \\
75.6010 \\
75.4680 \\
75.1130 \\
74.8920 \\
74.6260 \\
74.450 \\
74.4040 \\
74.7590 \\
75.5120 \\
75.7780 \\
75.9550 \\
76.0430 \\
76.3090 \\
77.1490 \\
77.6790 \\
78.6940 \\
79.0910 \\
79.9290 \\
79.9290 \\
79.6640 \\
75.8220\end{array}$ \\
\hline
\end{tabular}




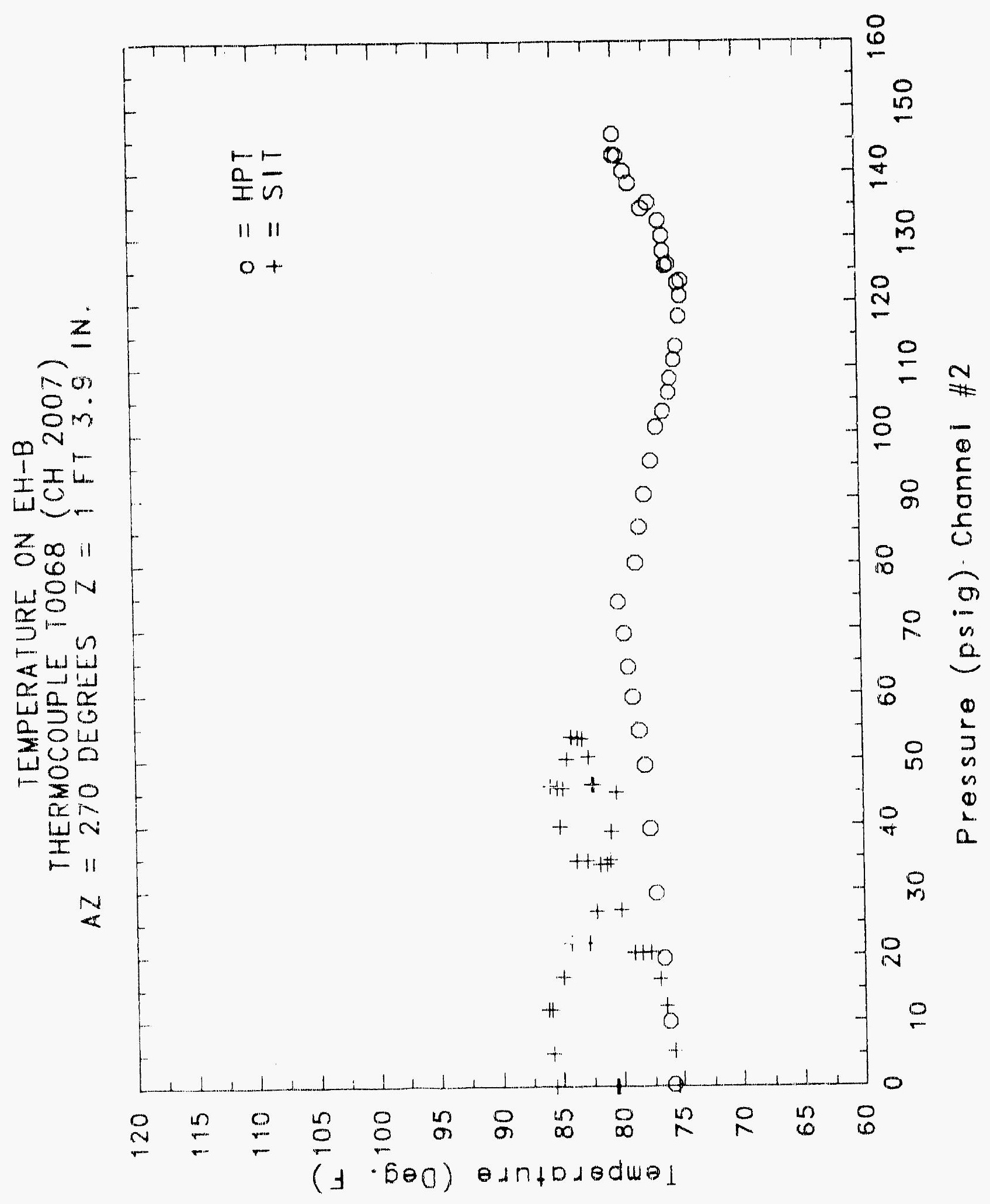




\section{Reinforced Concrete Test Data}

Thermocouple Channel 2008

Structural INTEgrity TEST

\begin{tabular}{|c|c|c|c|}
\hline $\begin{array}{l}\text { Pressure } \\
\text { (psig) } \\
-0.05 \\
5.33 \\
12.31 \\
16.44 \\
20.51 \\
20.46 \\
20.47 \\
27.05 \\
34.69 \\
34.53 \\
34.53 \\
39.70 \\
45.90 \\
45.65 \\
45.62 \\
50.10 \\
53.47 \\
53.29 \\
53.21 \\
50.49 \\
46.14 \\
46.13 \\
44.97 \\
38.98 \\
33.99 \\
33.96 \\
26.83 \\
21.88 \\
21.90 \\
16.69 \\
11.74 \\
11.77 \\
5.05 \\
0.02 \\
-0.04 \\
-0.02 \\
-0.02 \\
0.02\end{array}$ & $\begin{array}{c}\text { Temperature } \\
\text { (Fahrenheit) } \\
75.5560 \\
76.1320 \\
76.7950 \\
77.0600 \\
78.1650 \\
79.0470 \\
79.8410 \\
80.6340 \\
81.6460 \\
84.2370 \\
85.0260 \\
85.5080 \\
85.2450 \\
84.4120 \\
83.8850 \\
83.3590 \\
83.4030 \\
82.7440 \\
82.4810 \\
82.2610 \\
82.0850 \\
81.5580 \\
80.6780 \\
81.1620 \\
81.5140 \\
81.8220 \\
82.5250 \\
83.1840 \\
85.0250 \\
85.7260 \\
86.2080 \\
85.7700 \\
85.2890 \\
85.0260 \\
73.4000 \\
80.1490 \\
80.0170 \\
80.8540\end{array}$ & $\begin{array}{c}\text { Fressure } \\
(p s 1 g) \\
9.89 \\
19.55 \\
29.57 \\
39.42 \\
49.16 \\
54.50 \\
59.57 \\
64.20 \\
69.32 \\
74.16 \\
80.10 \\
85.61 \\
90.58 \\
95.69 \\
100.92 \\
103.25 \\
106.11 \\
108.31 \\
111.08 \\
113.24 \\
117.83 \\
120.92 \\
123.28 \\
122.97 \\
125.82 \\
125.60 \\
127.84 \\
130.19 \\
132.53 \\
135.33 \\
134.42 \\
138.35 \\
140.16 \\
142.63 \\
146.79 \\
142.52 \\
0.22\end{array}$ & $\begin{array}{c}\text { Temperature } \\
\text { (Fahrenheit) } \\
77.1490 \\
77.4140 \\
77.9440 \\
78.3410 \\
78.8270 \\
79.0030 \\
79.5320 \\
79.8410 \\
80.1050 \\
80.8100 \\
79.2680 \\
79.0030 \\
78.6060 \\
77.8560 \\
77.5460 \\
77.1050 \\
76.7510 \\
76.9720 \\
76.3970 \\
76.0870 \\
75.7330 \\
75.9100 \\
75.4230 \\
75.7780 \\
76.6180 \\
77.0160 \\
76.9280 \\
77.0160 \\
77.1050 \\
77.9000 \\
78.5620 \\
79.5760 \\
79.5320 \\
80.3750 \\
80.9370 \\
79.7090 \\
77.5910\end{array}$ \\
\hline
\end{tabular}

High Pressure test

Temperature

77.1490

77.4140

77.9440

78.3410

78.8270

79.8410

80.1050

79.0030

7.1050

75.7780

6.6180

77.1050

.9000

80.3250

77.5910 


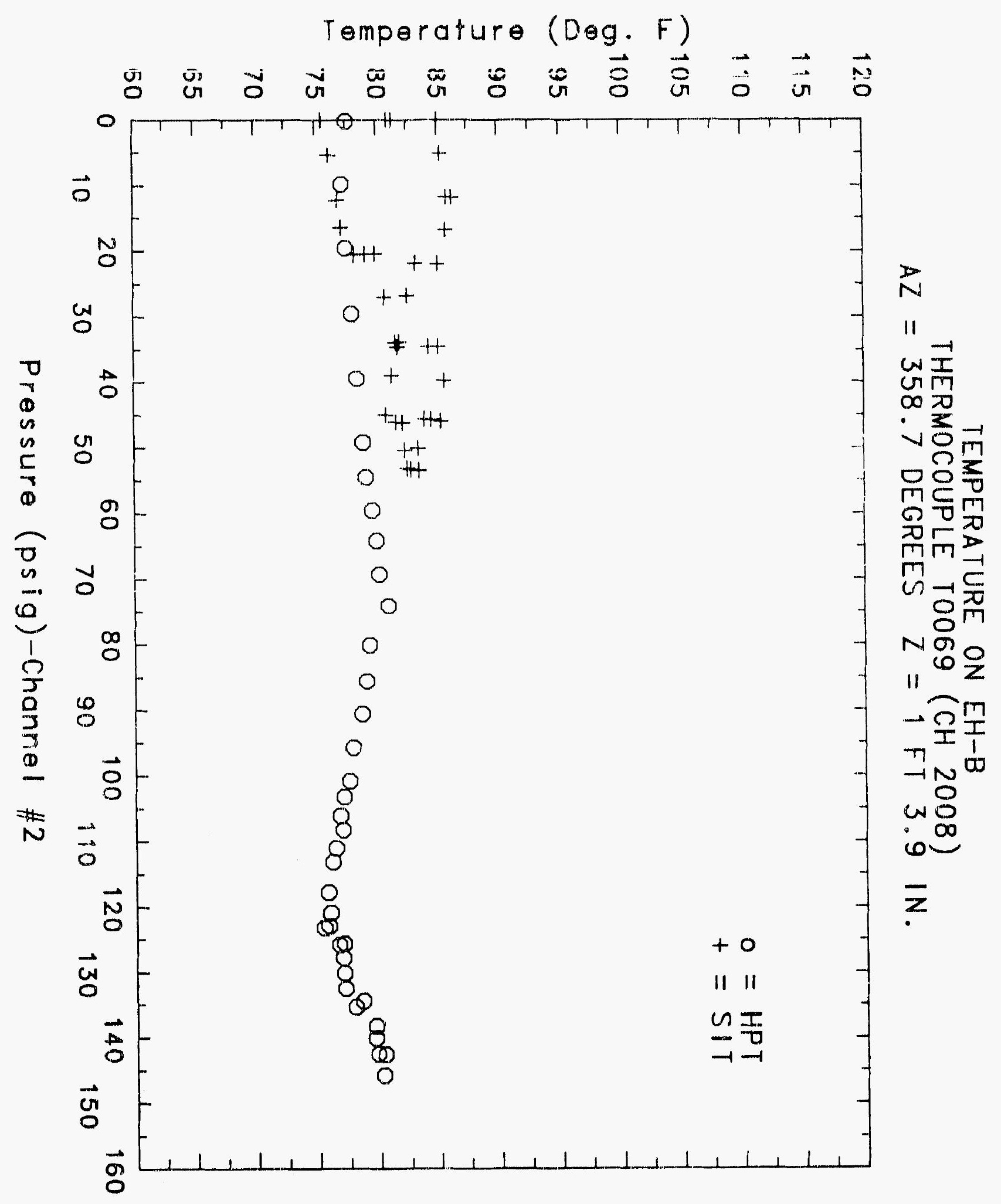




\section{Reinforced Concrete Test Data}

Thermocouple Channel 2009

Structural InTEgrity TEST

\begin{tabular}{|c|c|c|c|}
\hline $\begin{array}{c}\text { Pressure } \\
(\text { psig) } \\
-0.05 \\
5.33 \\
12.31 \\
16.44 \\
20.51 \\
20.46 \\
20.47 \\
27.05 \\
34.69 \\
34.53 \\
34.53 \\
39.70 \\
45.90 \\
45.65 \\
45.62 \\
50.10 \\
53.47 \\
53.29 \\
53.21 \\
50.49 \\
46.14 \\
46.13 \\
44.97 \\
38.98 \\
33.99 \\
33.96 \\
26.83 \\
21.88 \\
21.90 \\
16.69 \\
11.74 \\
11.77 \\
5.05 \\
0.02 \\
-0.04 \\
-0.02 \\
-0.02 \\
0.02 \\
\end{array}$ & $\begin{array}{c}\text { Temperature } \\
\text { (Fahrenheit) } \\
76.3090 \\
77.4140 \\
78.7830 \\
80.3250 \\
82.2170 \\
83.2710 \\
84.2810 \\
86.6020 \\
87.6510 \\
94.4410 \\
95.4380 \\
95.4380 \\
92.4440 \\
85.4200 \\
83.8420 \\
82.9200 \\
82.3490 \\
81.1620 \\
80.5010 \\
79.7530 \\
79.8850 \\
79.7090 \\
82.2170 \\
83.2270 \\
83.7980 \\
84.6310 \\
85.9450 \\
88.5240 \\
91.9230 \\
93.6170 \\
94.2680 \\
88.4810 \\
85.8580 \\
83.8860 \\
79.3120 \\
73.8410 \\
80.3250 \\
82.7440\end{array}$ & $\begin{array}{l}\text { Pressure } \\
(\text { psig) } \\
9.89 \\
19.55 \\
29.57 \\
39.42 \\
49.16 \\
54.50 \\
59.57 \\
64.20 \\
69.32 \\
74.16 \\
80.16 \\
85.61 \\
90.58 \\
95.69 \\
100.92 \\
103.25 \\
106.11 \\
108.31 \\
111.08 \\
113.24 \\
117.83 \\
120.92 \\
123.28 \\
122.97 \\
125.82 \\
125.60 \\
127.84 \\
130.19 \\
132.53 \\
135.33 \\
134.42 \\
138.35 \\
140.16 \\
142.63 \\
145.78 \\
142.59 \\
0.22 \\
\end{array}$ & $\begin{array}{c}\text { Temperature } \\
\text { (Fahrenheit) } \\
79.8850 \\
80.7660 \\
80.9860 \\
80.6780 \\
81.5140 \\
82.8320 \\
83.2710 \\
83.3150 \\
83.6230 \\
84.9820 \\
74.5820 \\
74.2270 \\
73.6510 \\
72.8520 \\
72.4080 \\
71.2960 \\
70.2730 \\
70.4060 \\
70.0500 \\
69.4710 \\
70.0940 \\
70.4950 \\
73.4290 \\
76.1320 \\
77.4580 \\
77.2370 \\
76.7950 \\
77.3260 \\
78.4740 \\
79.4000 \\
80.5010 \\
82.6130 \\
82.3050 \\
83.5790 \\
81.7340 \\
80.5900 \\
76.0430\end{array}$ \\
\hline
\end{tabular}

High Pressure test

Pressure

Fahrenheit)

79.8850

80.7660

80.9860

80.6780

83.2710

83.3150

83.6230

74.2270

73.6510

72.8520

70.2730

70.4060

70.0500

70.4950

73.4290

76.1320

76.7950

77.3260

78.4740

82.6130

82.3050

83.5790

80.5900 


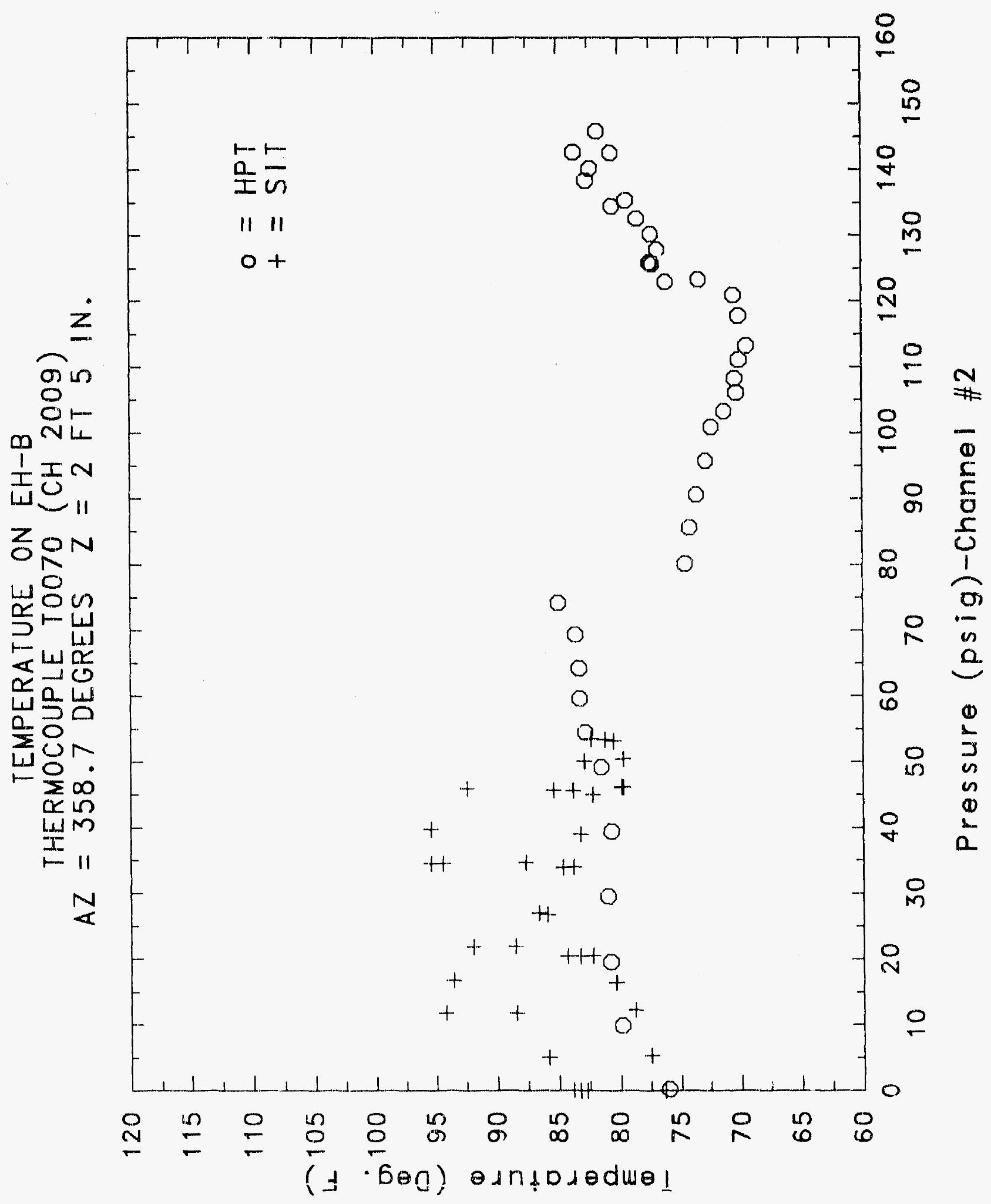




\title{
Reinforced Concrete Test Data
}

\author{
Thermocouple Channel 2010
}

StRUCTURAL INTEGRITY TEST

\begin{tabular}{|c|c|c|c|}
\hline $\begin{array}{c}\text { Pressure } \\
\text { (psig) } \\
-0.05 \\
5.33 \\
12.31 \\
16.44 \\
20.51 \\
20.46 \\
20.47 \\
27.05 \\
34.69 \\
34.53 \\
34.53 \\
39.70 \\
45.90 \\
45.65 \\
45.62 \\
50.10 \\
53.47 \\
53.29 \\
53.21 \\
50.49 \\
46.14 \\
46.13 \\
44.97 \\
38.98 \\
33.99 \\
33.96 \\
26.83 \\
21.88 \\
21.90 \\
16.69 \\
11.74 \\
11.77 \\
5.05 \\
0.02 \\
-0.04 \\
-0.02 \\
-0.02 \\
0.02\end{array}$ & $\begin{array}{c}\text { Temperature } \\
\text { (Fahrenheit) } \\
74.4930 \\
76.3090 \\
78.6500 \\
81.3380 \\
84.3680 \\
85.6390 \\
88.9170 \\
91.0520 \\
93.4870 \\
102.1300 \\
102.9500 \\
99.8470 \\
94.0510 \\
85.2010 \\
83.0960 \\
81.3820 \\
80.5450 \\
78.2090 \\
77.3700 \\
76.9720 \\
77.5020 \\
76.5300 \\
83.0520 \\
84.2810 \\
84.9380 \\
85.8580 \\
90.3560 \\
93.5300 \\
98.9410 \\
100.2300 \\
98.7250 \\
89.3100 \\
85.5510 \\
82.6570 \\
80.1930 \\
79.9730 \\
80.5010 \\
83.5350\end{array}$ & $\begin{array}{c}\text { Pressure } \\
\text { (psig) } \\
9.89 \\
19.55 \\
29.57 \\
39.42 \\
49.16 \\
54.50 \\
59.57 \\
64.20 \\
69.32 \\
74.16 \\
80.16 \\
85.61 \\
90.58 \\
95.69 \\
100.92 \\
103.25 \\
106.11 \\
108.31 \\
111.08 \\
113.24 \\
117.83 \\
120.92 \\
123.28 \\
122.97 \\
125.82 \\
125.60 \\
127.84 \\
130.19 \\
132.53 \\
135.33 \\
134.42 \\
138.35 \\
140.16 \\
142.63 \\
145.78 \\
142.52 \\
0.22\end{array}$ & $\begin{array}{c}\text { Temperature } \\
\text { (Fahrenheit) } \\
80.0170 \\
80.8980 \\
81.0300 \\
80.7220 \\
81.7340 \\
82.9200 \\
83.2710 \\
83.4470 \\
83.7540 \\
85.2450 \\
74.5370 \\
74.2270 \\
73.6950 \\
72.5850 \\
72.1410 \\
70.9400 \\
69.9610 \\
70.2280 \\
69.9160 \\
69.3370 \\
70.0500 \\
70.3170 \\
73.3400 \\
76.1760 \\
77.6350 \\
77.3700 \\
76.8840 \\
77.5910 \\
78.5180 \\
79.5760 \\
80.7220 \\
82.8760 \\
83.1400 \\
83.7980 \\
81.8220 \\
80.5900 \\
75.6010\end{array}$ \\
\hline
\end{tabular}

High Pressure Test

emperature

80.0170

80.8980

81.0300

80.7220

82.9200

83.2710

83.4470

83.7540

85.2450

74.2270

73.6950

2.5850

69.9610

70.2280

69.9160

69.3370

73.3400

76.1760

77.6350

77.3700

(

78.5180

79.5760

80.7220

83. 1400

83.7980

81.8220

80.5900

75.6010 


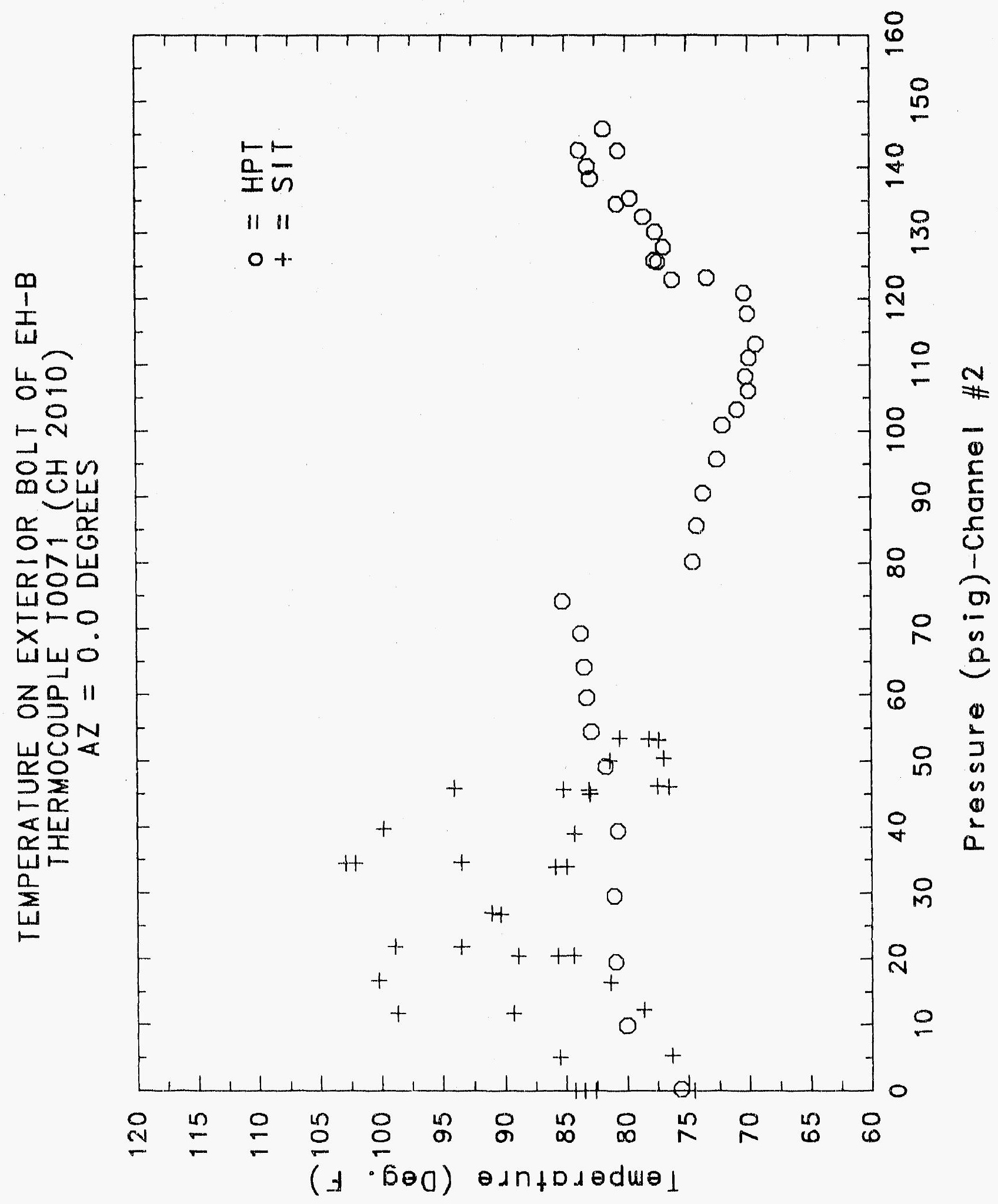




\section{Reinforced Concrete Test Data}

Thermocouple Channel 2011

Structural INTEGRITY TEST

\begin{tabular}{|c|c|c|c|}
\hline $\begin{array}{c}\text { Pressure } \\
\text { (psig) } \\
-0.05 \\
5.33 \\
12.31 \\
16.44 \\
20.51 \\
20.46 \\
20.47 \\
27.05 \\
34.69 \\
34.53 \\
34.53 \\
39.70 \\
45.90 \\
45.65 \\
45.62 \\
50.10 \\
53.47 \\
53.29 \\
53.21 \\
50.49 \\
46.14 \\
46.13 \\
44.97 \\
38.98 \\
33.99 \\
33.96 \\
26.83 \\
21.88 \\
21.90 \\
16.69 \\
11.74 \\
11.77 \\
5.05 \\
0.02 \\
-0.04 \\
-0.02 \\
-0.02 \\
0.02\end{array}$ & $\begin{array}{c}\text { Temperature } \\
\text { (Fahrenheit) } \\
74.4040 \\
76.3090 \\
78.8270 \\
81.2940 \\
83.6660 \\
85.2450 \\
87.5640 \\
88.0000 \\
89.7020 \\
96.9530 \\
99.5450 \\
98.0340 \\
92.4010 \\
84.7630 \\
82.8760 \\
81.2500 \\
80.5010 \\
77.5020 \\
76.3090 \\
76.5300 \\
77.1930 \\
75.1130 \\
83.0080 \\
84.5000 \\
85.3320 \\
87.4320 \\
89.3530 \\
90.6610 \\
92.3140 \\
95.4380 \\
94.6150 \\
86.6020 \\
84.9820 \\
82.5250 \\
76.9280 \\
78.9150 \\
79.7530 \\
83.9300\end{array}$ & $\begin{array}{c}\text { Pressure } \\
\text { (psig) } \\
9.89 \\
19.55 \\
29.57 \\
39.42 \\
49.16 \\
54.50 \\
59.57 \\
64.20 \\
69.32 \\
74.16 \\
80.16 \\
85.61 \\
90.58 \\
95.69 \\
100.92 \\
103.25 \\
106.11 \\
108.31 \\
111.08 \\
113.24 \\
117.83 \\
120.92 \\
123.28 \\
122.97 \\
125.82 \\
125.60 \\
127.84 \\
130.19 \\
132.53 \\
135.33 \\
134.42 \\
138.35 \\
140.16 \\
142.63 \\
145.78 \\
142.52 \\
0.22\end{array}$ & $\begin{array}{c}\text { Temperature } \\
\text { (Fahrenheit) } \\
78.4740 \\
78.7380 \\
78.4300 \\
78.2530 \\
79.7530 \\
80.7660 \\
80.8980 \\
81.5580 \\
81.1620 \\
83.8860 \\
72.5850 \\
71.7850 \\
71.4300 \\
70.0940 \\
68.9360 \\
67.8650 \\
67.3290 \\
67.4630 \\
66.8820 \\
66.0770 \\
67.0160 \\
67.0160 \\
69.6490 \\
72.0080 \\
74.0940 \\
74.8480 \\
75.1130 \\
75.6890 \\
76.5300 \\
78.5180 \\
80.8540 \\
83.2710 \\
81.2060 \\
82.8320 \\
81.0740 \\
79.7970 \\
73.5620\end{array}$ \\
\hline
\end{tabular}

High Pressure test

Pressure

9.89

29.57

59.57

64.20

69.32

90.58

95.69

100.92

103.25

111.08

113.24

117.83

120.92

125.82

$125.6 \mathrm{C}$

127.84

130.19

132.53

135.33

134.42

140.16

142.63

145.78

2.52

79.7970

73.5620 


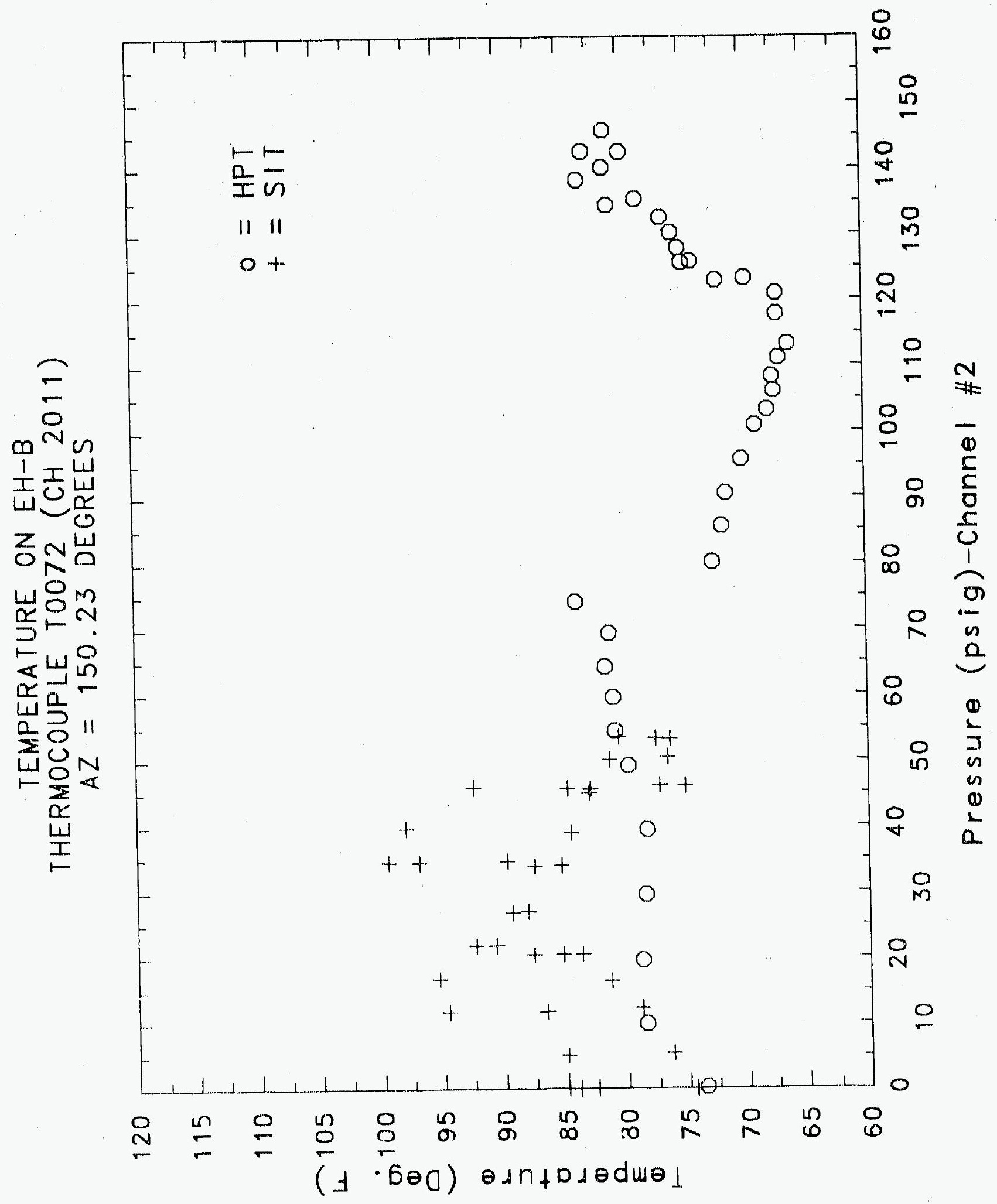

(c) $18(17$ 


\section{Reinforced Concrete Test Data}

Thermocouple Channel 2013

STRUCTURAL INTEGRITY TEST

\begin{tabular}{|c|c|c|c|}
\hline $\begin{array}{c}\text { Pressure } \\
\text { (psig) } \\
-0.05 \\
5.33 \\
12.31 \\
16.44 \\
20.51 \\
20.46 \\
20.47 \\
27.05 \\
34.69 \\
34.53 \\
34.53 \\
39.70 \\
45.90 \\
45.65 \\
45.62 \\
50.10 \\
53.47 \\
53.29 \\
53.21 \\
50.49 \\
46.14 \\
46.13 \\
44.97 \\
38.98 \\
33.99 \\
33.96 \\
26.83 \\
21.88 \\
21.90 \\
16.69 \\
11.74 \\
11.77 \\
5.05 \\
0.02 \\
-0.04 \\
-0.02 \\
-0.02 \\
0.02\end{array}$ & $\begin{array}{c}\text { Temperature } \\
\text { (Fahrenheit) } \\
74.9810 \\
76.1760 \\
80.0610 \\
81.5580 \\
84.5440 \\
87.0830 \\
88.5240 \\
87.3890 \\
88.1310 \\
89.8330 \\
87.2580 \\
83.4910 \\
80.8980 \\
78.8710 \\
78.2090 \\
78.2090 \\
77.7230 \\
70.5400 \\
69.7380 \\
76.2200 \\
76.1320 \\
71.1180 \\
83.3150 \\
85.1130 \\
85.7260 \\
87.4760 \\
88.7430 \\
88.7860 \\
90.2250 \\
87.8690 \\
85.7700 \\
82.0850 \\
79.9290 \\
76.3970 \\
71.5630 \\
76.2640 \\
78.5620 \\
86.1640 \\
80\end{array}$ & $\begin{array}{c}\text { Pressure } \\
\text { (psig) } \\
9.89 \\
19.55 \\
29.57 \\
39.42 \\
49.16 \\
54.50 \\
59.57 \\
64.20 \\
69.32 \\
74.16 \\
80.16 \\
85.61 \\
90.58 \\
95.69 \\
100.92 \\
103.25 \\
106.11 \\
108.31 \\
111.08 \\
113.24 \\
117.83 \\
120.92 \\
123.28 \\
122.97 \\
125.82 \\
125.60 \\
127.84 \\
130.19 \\
132.53 \\
135.33 \\
134.42 \\
138.35 \\
140.16 \\
142.63 \\
145.78 \\
142.52 \\
0.22\end{array}$ & $\begin{array}{c}\text { Temperature } \\
\text { (Fahrenheit) } \\
74.9810 \\
75.6450 \\
75.8220 \\
75.2020 \\
76.9280 \\
81.2940 \\
77.6350 \\
82.3930 \\
80.1050 \\
81.4700 \\
66.1220 \\
65.4500 \\
65.4950 \\
61.9970 \\
62.0870 \\
61.2330 \\
61.0080 \\
60.4230 \\
60.6030 \\
59.2970 \\
60.0620 \\
60.1930 \\
63.9270 \\
66.9710 \\
67.9990 \\
70.4510 \\
71.0290 \\
71.8740 \\
74.1830 \\
80.1050 \\
82.3930 \\
85.4640 \\
82.8760 \\
82.7440 \\
79.9730 \\
77.3260 \\
72.2300\end{array}$ \\
\hline
\end{tabular}

High Pressure Test

Pressure

9.89

19.55

39.42

49.16

64.20

69.32

85.61

90.58

$(100.92$

103.25

106.11

108.31

111.08

113.24

120.92

123.28

122.97

125.82

30.19

135.33

134.42

138.35

140.16

42.52

0.22
(Fahrenheit)

74.9810

75.6450

75.8220

75.2020

7.6350

80.1050

81.4700

66.1220

61.9970

62.0870

61.2330

63.9270

66.9710

71.0290

71.8740

74.1830

80.1050

82.8760

77.3260

72.2300 


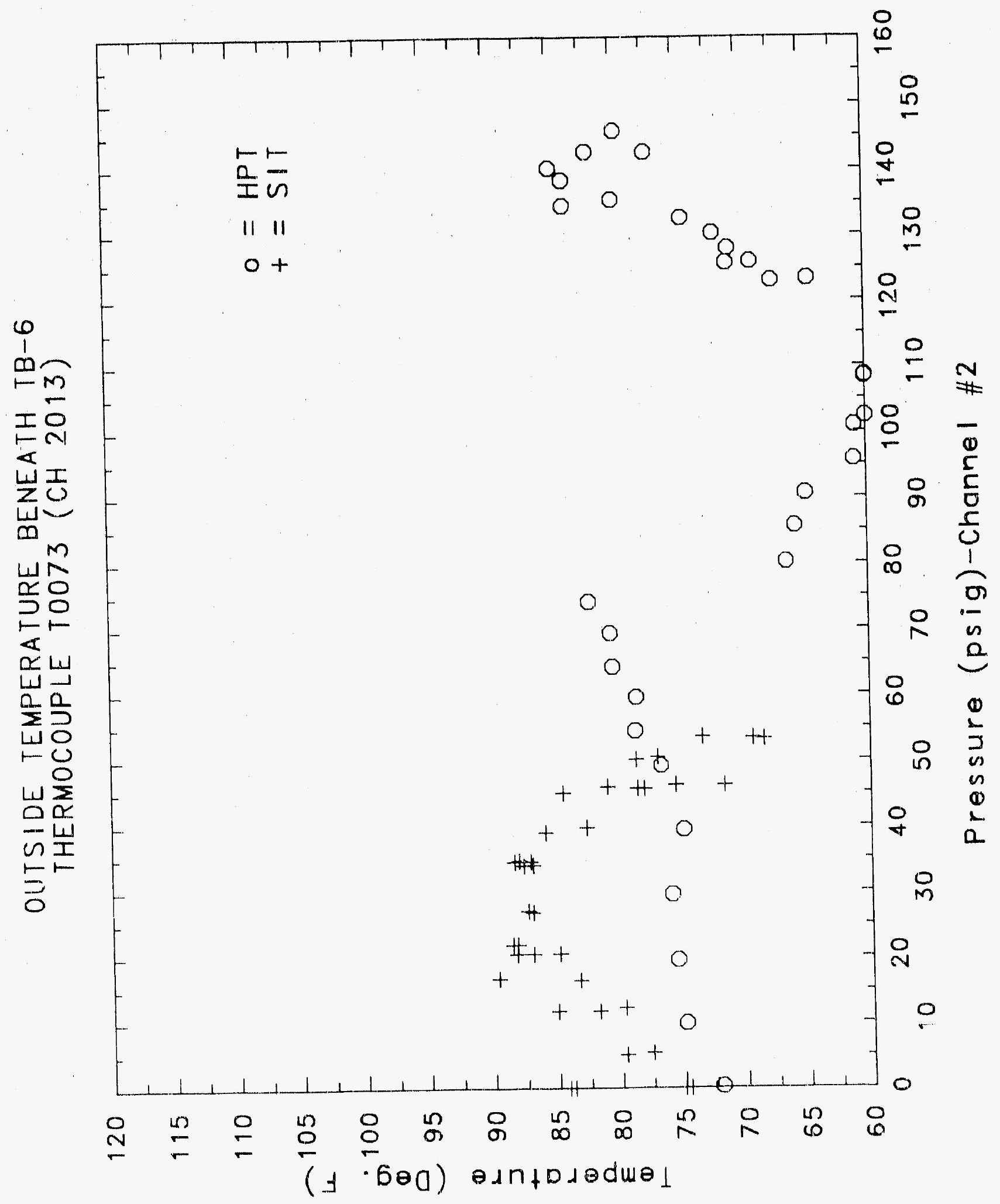

$(-180)$ 


\section{Reinforced Concrete Test Data}

Thermocouple ChanNel 2014

Structural Integrity test

\begin{tabular}{|c|c|}
\hline $\begin{array}{c}\text { Pressure } \\
\text { (psig) } \\
-0.05 \\
5.33 \\
12.31 \\
16.44 \\
20.51 \\
20.46 \\
20.47 \\
27.05 \\
34.69 \\
34.53 \\
34.53 \\
39.70 \\
45.90 \\
45.65 \\
45.62 \\
50.10 \\
53.47 \\
53.29 \\
53.21 \\
50.49 \\
46.14 \\
46.13 \\
44.97 \\
38.98 \\
33.99 \\
33.96 \\
26.83 \\
21.88 \\
21.90 \\
16.69 \\
11.74 \\
11.77 \\
5.05 \\
0.02 \\
-0.04 \\
-0.02 \\
-0.02 \\
0.02 \\
\end{array}$ & $\begin{array}{c}\text { Temperature } \\
\text { (Fahrenhe it) } \\
75.2460 \\
76.3530 \\
80.1930 \\
81.7340 \\
84.4120 \\
87.1260 \\
88.9170 \\
87.4320 \\
87.6510 \\
90.7040 \\
87.1260 \\
83.1840 \\
80.7660 \\
78.8710 \\
78.1210 \\
78.2970 \\
77.8110 \\
70.4510 \\
69.6040 \\
76.3090 \\
76.0430 \\
70.9400 \\
83.5790 \\
85.1130 \\
86.0770 \\
87.6510 \\
88.9170 \\
88.4810 \\
90.1380 \\
88.3500 \\
85.6390 \\
81.7340 \\
79.8410 \\
75.9990 \\
71.7410 \\
76.3530 \\
78.5180 \\
86.1200\end{array}$ \\
\hline
\end{tabular}

High Pressure test

\begin{tabular}{|c|c|}
\hline $\begin{array}{c}\text { Pressure } \\
\text { (psig) } \\
9.89 \\
19.55 \\
29.57 \\
39.42 \\
49.16 \\
54.50 \\
59.57 \\
64.20 \\
69.32 \\
74.16 \\
80.16 \\
85.61 \\
90.58 \\
95.69 \\
100.92 \\
103.25 \\
106.11 \\
108.31 \\
111.08 \\
113.24 \\
117.83 \\
120.92 \\
123.28 \\
122.97 \\
125.82 \\
125.60 \\
127.84 \\
130.19 \\
132.53 \\
135.33 \\
134.42 \\
138.35 \\
140.16 \\
142.63 \\
145.78 \\
142.52 \\
0.22\end{array}$ & $\begin{array}{c}\text { Temperature } \\
\text { (Fahrenheit) } \\
74.5820 \\
75.6010 \\
74.8480 \\
73.7390 \\
76.9280 \\
81.2060 \\
76.8390 \\
79.3120 \\
78.3410 \\
80.7660 \\
65.2710 \\
64.8680 \\
63.9270 \\
60.6030 \\
59.3420 \\
59.0260 \\
58.3500 \\
59.2510 \\
58.1240 \\
57.7180 \\
58.6650 \\
58.8010 \\
63.2540 \\
66.7480 \\
67.9990 \\
70.8960 \\
71.0290 \\
71.8740 \\
74.1830 \\
77.5910 \\
82.4810 \\
83.5790 \\
83.6660 \\
82.2170 \\
80.0170 \\
77.5020 \\
71.2960\end{array}$ \\
\hline
\end{tabular}




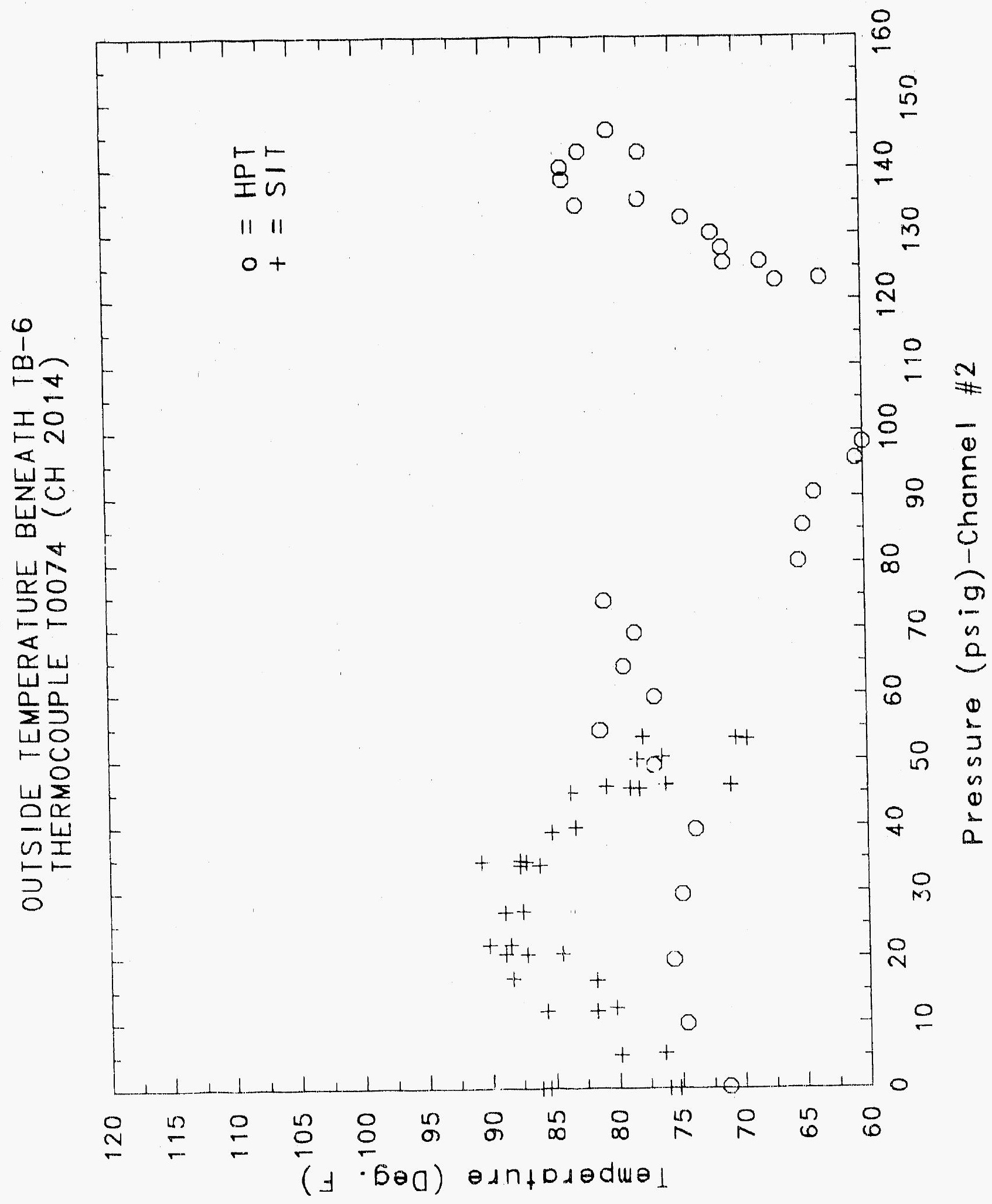

C. $\mid 811$ 


\section{Reinforced Concrete Test Data}

\section{Thermocouplf Channel 2015}

STRUCTURAL INTEgRITY TEST

\begin{tabular}{cc}
\hline & \\
Pressure & Temperature \\
(psig) & (Fahrenheit) \\
No SIT data &
\end{tabular}

This gage was added aiter SIT

\section{High pressure test}

\begin{tabular}{|c|c|}
\hline $\begin{array}{c}\text { Pressure } \\
(p s i g) \\
9.89 \\
19.55 \\
29.57 \\
39.42 \\
49.16 \\
54.50 \\
59.57 \\
64.20 \\
69.32 \\
74.16 \\
80.16 \\
85.61 \\
90.58 \\
95.69 \\
100.92 \\
103.25 \\
106.11 \\
108.31 \\
111.08 \\
113.24 \\
117.83 \\
120.92 \\
123.28 \\
122.97 \\
125.82 \\
125.60 \\
127.84 \\
130.19 \\
132.53 \\
135.33 \\
134.42 \\
139.35 \\
140.16 \\
142.63 \\
145.78 \\
142.52 \\
0.22\end{array}$ & $\begin{array}{c}\text { Temperature } \\
\text { (Fahrenheit) } \\
78.7830 \\
79.0030 \\
78.7830 \\
78.3850 \\
79.8850 \\
81.5140 \\
80.2810 \\
83.7540 \\
83.2710 \\
85.7700 \\
74.6260 \\
73.8280 \\
73.2960 \\
71.7410 \\
71.3410 \\
70.4950 \\
69.8720 \\
70.0500 \\
69.4260 \\
68.5790 \\
69.0250 \\
68.9800 \\
70.2280 \\
71.4740 \\
72.1410 \\
74.1830 \\
74.8480 \\
75.8660 \\
77.9440 \\
80.0170 \\
81.3380 \\
84.1050 \\
83.0080 \\
84.5870 \\
82.2170 \\
80.3250 \\
77.2370\end{array}$ \\
\hline
\end{tabular}




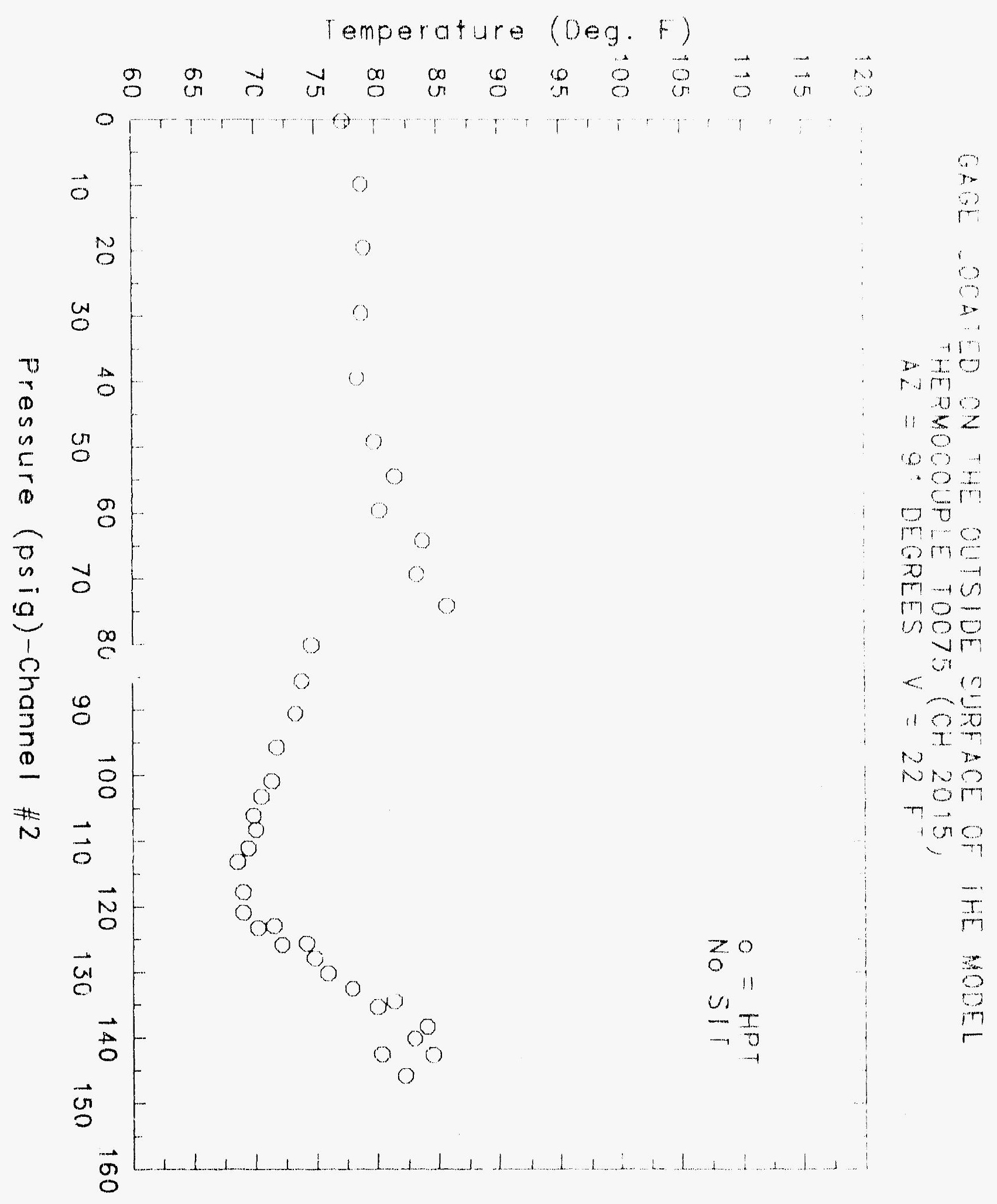



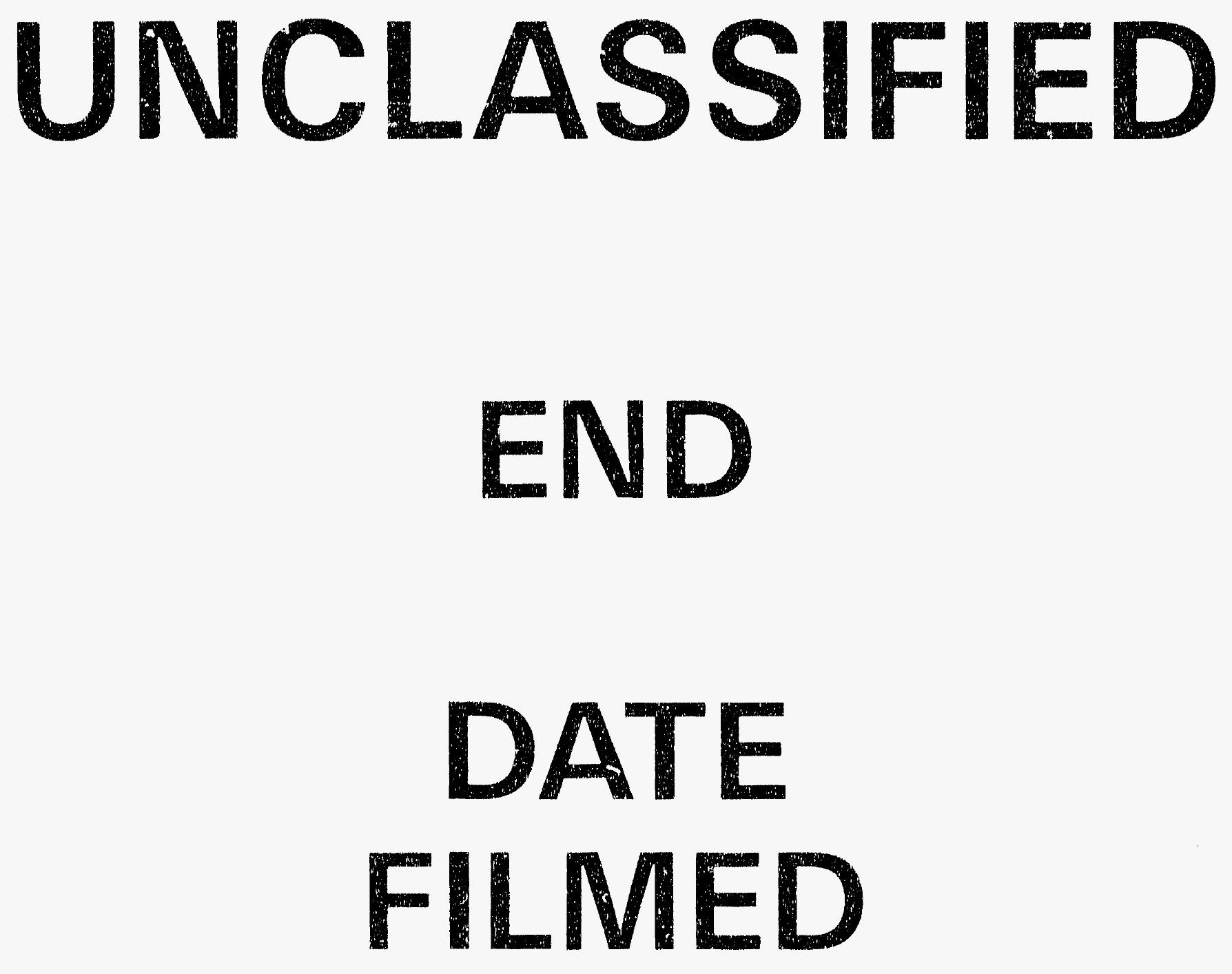

$$
1-6.92 .
$$

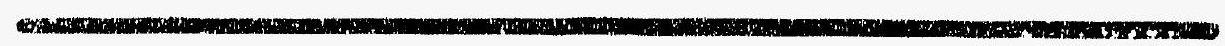

SANDIA NATIONAL LABS 


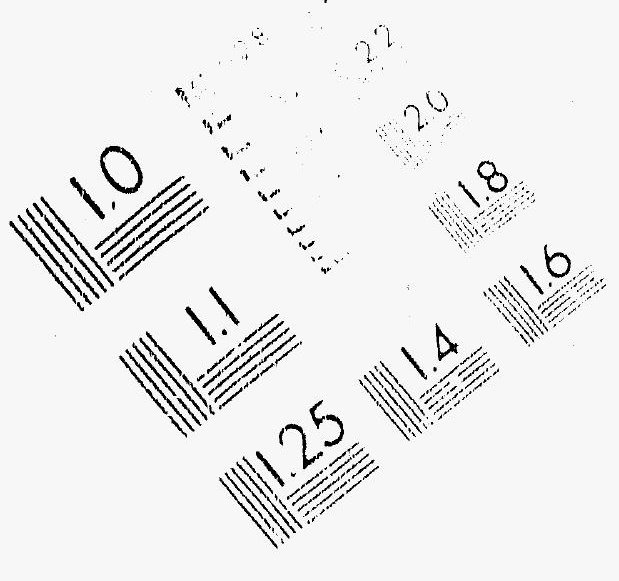

\section{IMAGE EVALUATION \\ TEST TARGET (MT-3)}
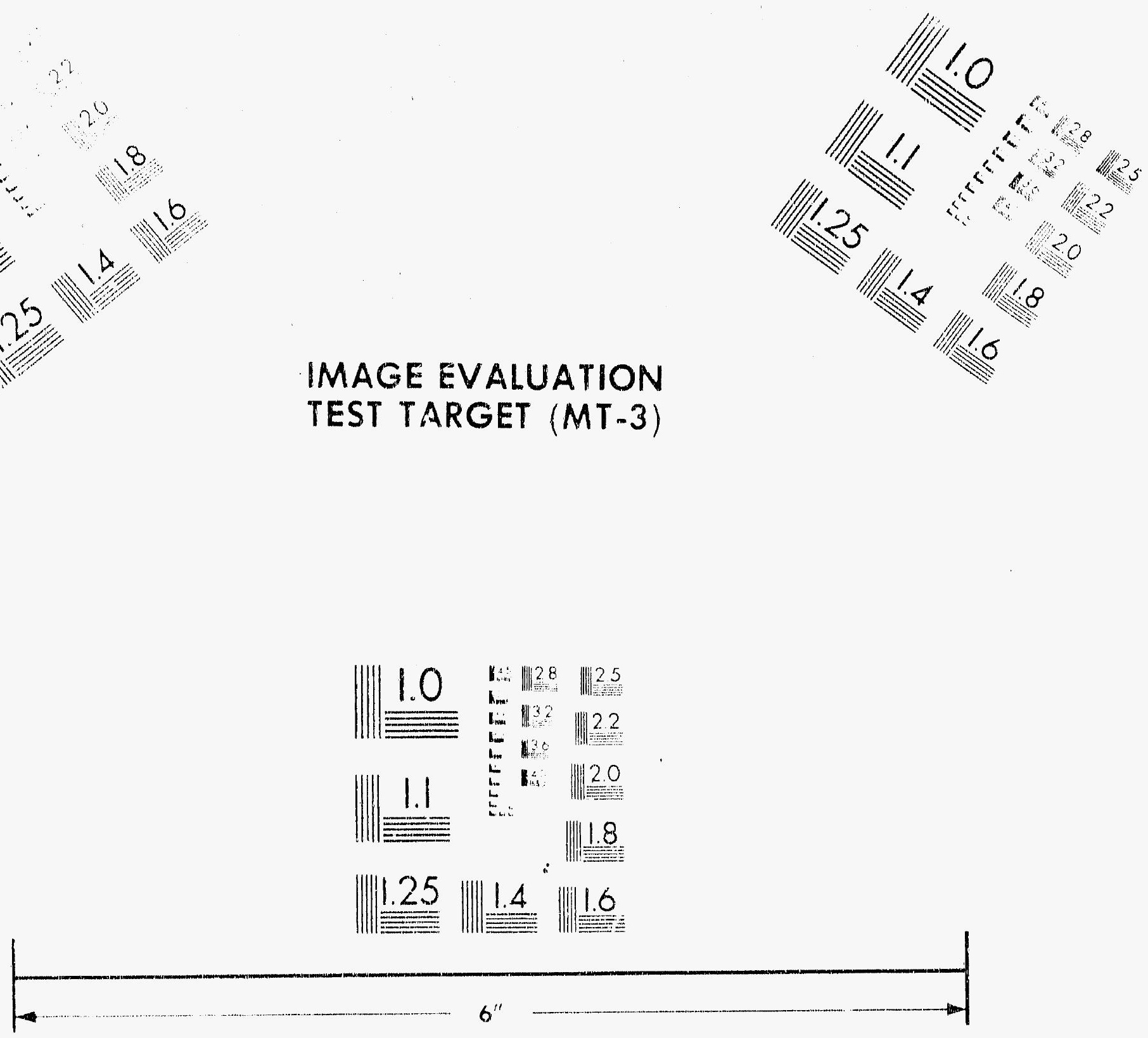

Photographic Sciences Corporation
23 WEST MAIN STREET

WEBSTER, N.Y. 14580 ( 716$) 872.4503$

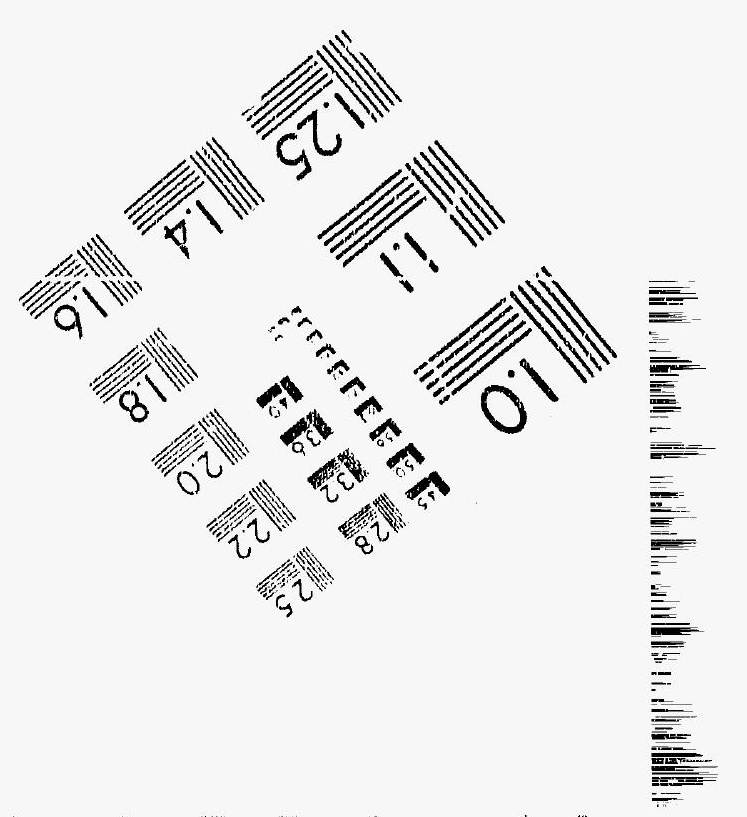




\section{SECURITY CLASSIFICATION}

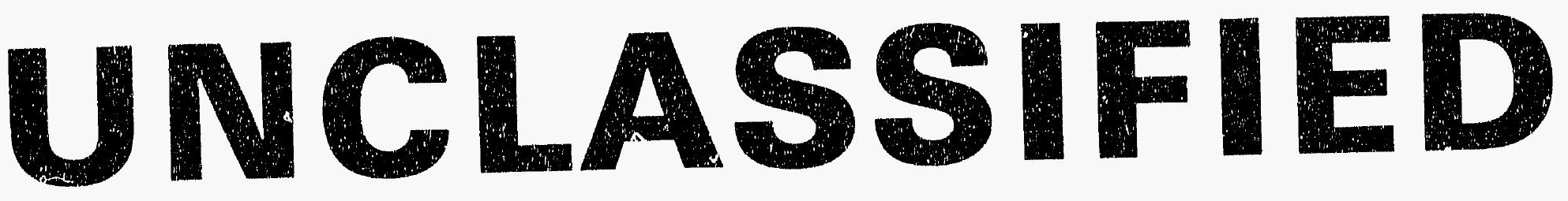

\section{DATE OF MICROFILMING $1-6-92$}




\section{THIS DOCUMENT FILMED}

AT

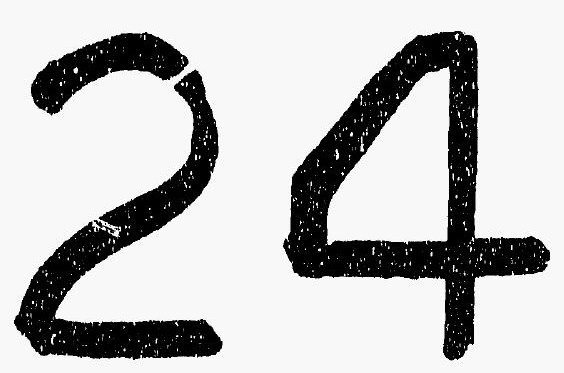

REDUCTION 


\section{Other Instrumentation}

Pressure Transducers, Load Links, Embedment Gages, and Inclinometers$$
\text { , }
$$ 


\section{Reinforced Concrete Test Data}

Pressure Transducer Channel 1

Structural INTEGRITY TEST

\begin{tabular}{|c|c|c|c|}
\hline $\begin{array}{c}\text { Pressure } \\
\text { (psig) } \\
-0.05 \\
5.33 \\
12.31 \\
16.44 \\
20.51 \\
20.46 \\
20.47 \\
27.05 \\
34.69 \\
34.53 \\
34.53 \\
39.70 \\
45.90 \\
45.65 \\
45.62 \\
50.10 \\
53.47 \\
53.29 \\
53.21 \\
50.49 \\
46.14 \\
46.13 \\
44.97 \\
38.98 \\
33.99 \\
33.96 \\
26.153 \\
21.88 \\
21.90 \\
16.69 \\
11.74 \\
11.77 \\
5.05 \\
0.02 \\
-0.04 \\
-0.02 \\
-0.02 \\
0.02 \\
\end{array}$ & $\begin{array}{l}\text { Pressure } \\
(\text { psi) } \\
11.9050 \\
17.2450 \\
24.1530 \\
28.2460 \\
32.2750 \\
32.2200 \\
32.2120 \\
38.7400 \\
46.3400 \\
46.1700 \\
46.1550 \\
51.2920 \\
57.4570 \\
57.2150 \\
57.1850 \\
61.6420 \\
65.0100 \\
64.8270 \\
64.7550 \\
62.0470 \\
57.7250 \\
57.7370 \\
56.6370 \\
50.6600 \\
45.6920 \\
45.6400 \\
38.5370 \\
33.5970 \\
33.6070 \\
28.4080 \\
23.4830 \\
23.5080 \\
16.8340 \\
11.8260 \\
11.8400 \\
11.8480 \\
11.8440 \\
11.8270\end{array}$ & $\begin{array}{c}\text { Pressure } \\
(\text { psig) } \\
9.89 \\
19.55 \\
29.57 \\
39.42 \\
49.16 \\
54.56 \\
59.57 \\
64.20 \\
69.32 \\
74.16 \\
80.16 \\
85.61 \\
90.58 \\
95.69 \\
100.92 \\
103.25 \\
106.11 \\
108.31 \\
111.08 \\
113.24 \\
117.83 \\
120.92 \\
123.28 \\
122.97 \\
125.82 \\
125.60 \\
127.84 \\
130.19 \\
132.53 \\
135.33 \\
134.42 \\
138.35 \\
140.16 \\
142.63 \\
145.78 \\
142.52 \\
0.22\end{array}$ & $\begin{array}{c}\text { Pressurt } \\
(F i) \\
21.7370 \\
31.3320 \\
41.2870 \\
51.0780 \\
60.7780 \\
66.1030 \\
71.1510 \\
75.7560 \\
80.8540 \\
85.6720 \\
91.5970 \\
97.0370 \\
102.0000 \\
107.1100 \\
112.3200 \\
114.6400 \\
117.4900 \\
119.6900 \\
122.4500 \\
124.6100 \\
129.2000 \\
132.2900 \\
134.6500 \\
134.3500 \\
137.2100 \\
136.9900 \\
139.2300 \\
141.5700 \\
143.8900 \\
146.6300 \\
145.7100 \\
149.6100 \\
151.3900 \\
153.8400 \\
156.9800 \\
153.7100 \\
11.8650\end{array}$ \\
\hline
\end{tabular}

High Pressure Test

31.3320

41.2870

51.0780

60.7780

75.7560

80.8540

85.6720

91.5970

107.1100

112.3200

114.6400

117.4900

119.6900

122.4500

132.2900

134.6500

134.3500

137.2100

136.9900

39.2300

146.6300

145.7100

149.6100

51.3900

53.8400

153.7100

11.8650 


\section{Reinforced Concrete Test Data}

Pressure transoucer Channel 2

STRUCTURAL INTEGRITY TEST

\begin{tabular}{|c|c|c|c|}
\hline $\begin{array}{c}\text { Pressure } \\
(\text { psig) } \\
-0.05 \\
5.33 \\
12.31 \\
16.44 \\
20.51 \\
20.46 \\
20.47 \\
27.05 \\
34.69 \\
34.53 \\
34.53 \\
39.70 \\
45.90 \\
45.65 \\
45.62 \\
50.10 \\
53.47 \\
53.29 \\
53.21 \\
50.49 \\
46.14 \\
46.13 \\
44.97 \\
38.98 \\
33.99 \\
33.96 \\
26.83 \\
21.88 \\
21.90 \\
16.69 \\
11.74 \\
11.77 \\
5.05 \\
0.02 \\
-0.04 \\
-0.02 \\
-0.02 \\
0.02 \\
\end{array}$ & $\begin{array}{c}\text { Pressure } \\
(p s i) \\
-0.0541 \\
5.3329 \\
12.3070 \\
16.4390 \\
20.5060 \\
20.4580 \\
20.4730 \\
27.0470 \\
34.6910 \\
34.5340 \\
34.5300 \\
39.7010 \\
45.8970 \\
45.6540 \\
45.6230 \\
50.0970 \\
53.4730 \\
53.2890 \\
53.2110 \\
50.4860 \\
46.1430 \\
46.1340 \\
44.9750 \\
38.9830 \\
33.9940 \\
33.9580 \\
26.8300 \\
21.8780 \\
21.9030 \\
16.6870 \\
11.7450 \\
11.7660 \\
5.0549 \\
0.0155 \\
-0.0363 \\
-0.0208 \\
-0.0229 \\
0.0167\end{array}$ & $\begin{array}{c}\text { Pressure } \\
(p s i g) \\
9.89 \\
19.55 \\
29.57 \\
39.42 \\
49.16 \\
54.50 \\
59.57 \\
64.20 \\
69.32 \\
74.16 \\
80.16 \\
85.61 \\
90.58 \\
95.69 \\
100.92 \\
103.25 \\
106.11 \\
108.31 \\
111.08 \\
113.24 \\
117.83 \\
120.92 \\
123.28 \\
123.97 \\
125.82 \\
125.60 \\
127.84 \\
130.19 \\
132.53 \\
135.33 \\
134.42 \\
138.35 \\
140.16 \\
142.63 \\
145.78 \\
142.52 \\
0.22 \\
\end{array}$ & $\begin{array}{c}\text { Pressure } \\
\text { (psi) } \\
9.8909 \\
19.5540 \\
29.5670 \\
39.4160 \\
49.1570 \\
54.5010 \\
59.5730 \\
64.1980 \\
69.3230 \\
74.1630 \\
80.1630 \\
85.6080 \\
90.5780 \\
95.6930 \\
100.9200 \\
103.2500 \\
106.1100 \\
108.3100 \\
111.0800 \\
113.2400 \\
117.8300 \\
120.9200 \\
123.2800 \\
122.9700 \\
125.8200 \\
125.6000 \\
127.8400 \\
130.1900 \\
132.5300 \\
135.3300 \\
134.4200 \\
138.3500 \\
140.1600 \\
142.6300 \\
145.7800 \\
142.5200 \\
0.2213\end{array}$ \\
\hline
\end{tabular}

High Pressure test

Pressure

(psi)

19.5540

29.5670

39.4160

49.1570

54.5010

64.1980

69.3230

74.1630

80.1630

8.5 .6080

90.5780

100.9200

103.2500

106.1100

108.3100

111.0800

113.2400

20.9200

123.2800

122.9700

125.8200

125.6000

127.8400

130.1900

135.3300

134.4200

138.3500

140.1600

142.6300

145.7800

0.2213 


\section{Reinforced Concrete Test Data}

Pressure Transducer Channel 3

STRUCTURAL INTEGRITY TEST

\begin{tabular}{|c|c|c|c|}
\hline $\begin{array}{c}\text { Pressure } \\
(\text { psig) } \\
-0.05 \\
5.33 \\
12.31 \\
16.44 \\
20.51 \\
20.46 \\
20.47 \\
27.05 \\
34.69 \\
34.53 \\
34.53 \\
39.70 \\
45.90 \\
45.65 \\
45.62 \\
50.10 \\
53.47 \\
53.29 \\
53.21 \\
50.49 \\
46.14 \\
46.13 \\
44.97 \\
38.98 \\
33.99 \\
33.96 \\
26.83 \\
21.88 \\
21.90 \\
16.69 \\
11.74 \\
11.77 \\
5.05 \\
0.02 \\
-0.04 \\
-0.02 \\
.0 .02 \\
0.02 \\
\end{array}$ & $\begin{array}{l}\text { Pressure } \\
(\text { ps } 1) \\
11.1110 \\
11.1340 \\
11.1610 \\
11.1670 \\
11.2630 \\
11.2230 \\
11.3110 \\
11.3230 \\
11.2890 \\
11.4560 \\
11.4460 \\
11.4200 \\
11.3510 \\
11.1900 \\
11.1640 \\
11.1710 \\
11.1810 \\
11.1180 \\
11.0460 \\
11.0470 \\
11.1560 \\
11.1780 \\
11.2130 \\
11.2330 \\
11.3210 \\
11.2390 \\
11.2850 \\
11.3940 \\
11.3430 \\
11.4200 \\
11.4140 \\
11.3420 \\
11.2220 \\
11.1970 \\
11.1780 \\
11.1540 \\
11.2200 \\
11.2450\end{array}$ & $\begin{array}{c}\text { Pressure } \\
(\text { psig) } \\
9.89 \\
19.55 \\
29.57 \\
39.42 \\
49.16 \\
54.50 \\
59.57 \\
64.20 \\
69.32 \\
74.16 \\
80.16 \\
85.61 \\
90.58 \\
95.69 \\
100.92 \\
103.25 \\
106.11 \\
108.31 \\
111.08 \\
113.24 \\
117.83 \\
120.92 \\
123.28 \\
122.97 \\
125.82 \\
125.60 \\
127.84 \\
130.19 \\
132.53 \\
135.33 \\
134.42 \\
138.35 \\
140.16 \\
142.63 \\
145.78 \\
142.52 \\
0.22\end{array}$ & $\begin{array}{c}\text { Pressure } \\
(\text { psi) } \\
0.0035 \\
30.4300 \\
40.4770 \\
50.3490 \\
60.0900 \\
65.4650 \\
70.6150 \\
75.1850 \\
80.3700 \\
85.2500 \\
91.0060 \\
96.4760 \\
101.4700 \\
106.5600 \\
111.8100 \\
114.1400 \\
116.9700 \\
119.1400 \\
121.9800 \\
124.1000 \\
128.7200 \\
131.8500 \\
134.2900 \\
134.0300 \\
136.9100 \\
136.6900 \\
138.9500 \\
141.2900 \\
143.6300 \\
146.4400 \\
145.5200 \\
149.4600 \\
151.1000 \\
153.8100 \\
156.9000 \\
153.5900 \\
10.8010\end{array}$ \\
\hline
\end{tabular}

\section{High Pressure test}

Pressure

0.0035

50.3490

65.4650

70.6150

75.1850

80.3700

95.4760

101.4700

106.5600

111.8100

114.1400

121.9800

124.1000

128.7200

$\$ 31.8500$

134.2900

134.0300

138.9500

1.2900

146.4400

145.5200

51.1000

100

42.52

10.8010
149.4600 


\section{Reinforced Concrete Test Data}

Pressure transducer Channel 6

Structural INTEgRITY TEST

\begin{tabular}{|c|c|c|c|}
\hline $\begin{array}{c}\text { Pressure } \\
(\text { psig) } \\
-0.05 \\
5.33 \\
12.31 \\
16.44 \\
20.51 \\
20.46 \\
20.47 \\
27.05 \\
34.69 \\
34.53 \\
34.53 \\
39.70 \\
45.90 \\
45.65 \\
45.62 \\
50.10 \\
53.47 \\
53.29 \\
53.21 \\
50.49 \\
46.14 \\
46.13 \\
44.97 \\
38.98 \\
33.99 \\
33.96 \\
26.83 \\
21.88 \\
21.90 \\
16.69 \\
11.74 \\
11.77 \\
5.05 \\
0.02 \\
-0.04 \\
-0.02 \\
-0.02 \\
0.02 \\
\end{array}$ & $\begin{array}{c}\text { Pressure } \\
\text { (psi) } \\
12.3290 \\
17.6780 \\
24.5950 \\
28.7090 \\
32.7770 \\
32.7550 \\
32.7850 \\
39.3570 \\
46.9600 \\
46.8000 \\
46.7900 \\
51.9250 \\
58.0570 \\
57.7350 \\
57.6750 \\
62.1070 \\
65.4600 \\
65.2720 \\
65.1920 \\
62.4720 \\
58.1500 \\
58.1670 \\
57.1220 \\
51.1750 \\
46.2300 \\
46.2370 \\
39.1620 \\
34.2500 \\
34.2950 \\
29.1020 \\
24.1680 \\
24.1130 \\
17.3910 \\
12.3410 \\
12.2540 \\
12.2900 \\
12.3000 \\
12.3310\end{array}$ & $\begin{array}{c}\text { Pressure } \\
\text { (psig) } \\
9.89 \\
19.55 \\
29.57 \\
39.42 \\
49.16 \\
54.50 \\
59.57 \\
64.20 \\
69.32 \\
74.16 \\
80.16 \\
85.61 \\
90.58 \\
95.69 \\
100.92 \\
103.25 \\
106.11 \\
108.31 \\
111.08 \\
113.24 \\
117.83 \\
120.92 \\
123.28 \\
122.97 \\
125.82 \\
125.60 \\
127.84 \\
130.19 \\
132.53 \\
135.33 \\
134.42 \\
138.35 \\
140.16 \\
142.63 \\
145.78 \\
142.52 \\
0.22 \\
\end{array}$ & $\begin{array}{c}\text { Pressure } \\
\text { (psi) } \\
22.1580 \\
31.7540 \\
41.7040 \\
51.5040 \\
61.2110 \\
66.5330 \\
71.5910 \\
76.2110 \\
81.3250 \\
86.1700 \\
92.0230 \\
97.4560 \\
102.4200 \\
107.5300 \\
112.7400 \\
115.0700 \\
117.9300 \\
120.1300 \\
122.8900 \\
125.0500 \\
129.6400 \\
132.7300 \\
125.0300 \\
134.7900 \\
137.6500 \\
137.4300 \\
139.6700 \\
142.0100 \\
144.3400 \\
147.1000 \\
146.2200 \\
150.1700 \\
151.9600 \\
154.4200 \\
157.5300 \\
154.2200 \\
12.3650\end{array}$ \\
\hline
\end{tabular}

high Pressure test

Pressur

31.7540

41.7040

51.5040

61.2110

66.5330

71.5910

76.2110

86.1700

92.0230

$97.45 \therefore 0$

102.4200

107.5300

117.9300

120.1300

122.8900

125.0500

129.6400

137.6500

137.4300

$\$ 39.6700$

142.0100

144.3400

150.1700

151.9600

154.4200

157.5300

54.2200

12.3650 


\section{Reinforced Concrete test Data}

Pressure Transoucer Channel 19

Structural Integrity Test

\begin{tabular}{|c|c|c|c|}
\hline $\begin{array}{c}\text { Pressure } \\
\text { (psig) } \\
-0.05 \\
5.33 \\
12.31 \\
16.44 \\
20.51 \\
20.46 \\
20.47 \\
27.05 \\
34.69 \\
34.53 \\
34.53 \\
39.70 \\
45.90 \\
45.65 \\
45.62 \\
50.10 \\
53.47 \\
53.29 \\
53.21 \\
50.49 \\
46.14 \\
46.13 \\
44.97 \\
38.98 \\
33.99 \\
33.96 \\
26.83 \\
21.88 \\
21.90 \\
16.69 \\
11.74 \\
11.77 \\
5.05 \\
0.02 \\
-0.04 \\
-0.02 \\
-0.02 \\
0.02 \\
\end{array}$ & $\begin{array}{l}\text { Pressure } \\
(\text { psi) } \\
0.0072 \\
0.0198 \\
0.0372 \\
0.0364 \\
0.0661 \\
0.0531 \\
0.0930 \\
0.0940 \\
0.0492 \\
-0.0308 \\
-0.0268 \\
0.0375 \\
0.0730 \\
0.0218 \\
0.0225 \\
0.02112 \\
0.0301 \\
-0.0125 \\
-0.0256 \\
-0.0132 \\
0.0281 \\
0.0195 \\
0.0589 \\
0.0724 \\
0.0880 \\
0.0660 \\
0.0832 \\
0.0872 \\
0.1007 \\
0.0494 \\
0.0578 \\
0.1173 \\
0.0789 \\
0.0498 \\
0.0738 \\
0.0685 \\
0.0948 \\
0.1167\end{array}$ & $\begin{array}{c}\text { Pressure } \\
(\text { ps } 1 \text { g) } \\
9.89 \\
19.55 \\
29.57 \\
39.42 \\
49.16 \\
54.50 \\
59.57 \\
64.20 \\
69.32 \\
74.16 \\
80.16 \\
85.61 \\
90.58 \\
95.69 \\
100.92 \\
103.25 \\
106.11 \\
108.31 \\
111.08 \\
113.24 \\
117.83 \\
120.92 \\
123.28 \\
122.97 \\
125.82 \\
125.60 \\
127.84 \\
130.19 \\
132.53 \\
135.33 \\
134.42 \\
138.35 \\
140.16 \\
142.63 \\
145.78 \\
142.52 \\
0.22\end{array}$ & $\begin{array}{c}\text { Pressure } \\
(\text { psi) } \\
-0.0040 \\
18.6950 \\
28.6170 \\
38.3960 \\
48.1040 \\
53.4270 \\
58.4960 \\
63.0850 \\
68.1990 \\
73.0450 \\
78.8030 \\
84.2440 \\
89.1990 \\
94.2580 \\
99.4760 \\
101.7800 \\
104.6200 \\
106.8100 \\
109.5800 \\
111.7300 \\
116.3300 \\
119.4100 \\
121.8600 \\
121.6100 \\
124.4600 \\
124.2000 \\
126.4600 \\
128.8100 \\
131.1400 \\
133.9200 \\
133.0200 \\
136.9500 \\
138.6600 \\
141.2300 \\
144.3200 \\
141.0700 \\
-0.7628\end{array}$ \\
\hline
\end{tabular}

High Pressure test

Pressure

$-0.0040$

18.6950

28.6170

38.3960

48.1040

53.4270

68.1990

73.0450

78.8030

84.2440

89.1990

94.2580

104.6200

106.8100

116.3300

119.4100

121.8600

124.2000

8600

$13 i .1400$

133.9200

133.0200

136.9500

138.6600

141.2300

44.3200

141.0700

$-0.7628$ 


\section{Reinforced Concrete Test Data}

Load Link Chaninel 18

Structural Integrity Test

\begin{tabular}{|c|c|c|c|}
\hline $\begin{array}{c}\text { Pressure } \\
\text { (psig) } \\
-0.05 \\
5.33 \\
12.31 \\
16.44 \\
20.51 \\
20.46 \\
20.47 \\
27.05 \\
34.69 \\
34.53 \\
34.53 \\
39.70 \\
45.90 \\
45.65 \\
45.62 \\
50.10 \\
53.47 \\
53.29 \\
53.21 \\
50.49 \\
46.14 \\
46.13 \\
44.97 \\
38.98 \\
33.99 \\
33.96 \\
26.83 \\
21.88 \\
21.90 \\
16.69 \\
11.74 \\
11.77 \\
5.05 \\
0.02 \\
-0.04 \\
-0.02 \\
-0.02 \\
0.02\end{array}$ & $\begin{array}{c}\text { Load } \\
\text { (pounds) } \\
-18.8726 \\
31.6980 \\
135.3001 \\
190.1445 \\
284.5916 \\
324.8096 \\
330.3268 \\
506.9651 \\
744.5326 \\
817.6155 \\
829.2055 \\
934.5779 \\
1097.7815 \\
1118.5206 \\
1116.0398 \\
1192.8438 \\
1274.4109 \\
1283.5401 \\
1277.4870 \\
1261.6102 \\
1189.1723 \\
1161.6856 \\
1140.0535 \\
1041.4188 \\
943.3400 \\
946.3962 \\
797.1741 \\
699.7203 \\
699.1249 \\
607.7540 \\
505.4280 \\
482.8928 \\
347.6622 \\
259.3376 \\
157.4482 \\
174.4563 \\
174.9623 \\
158.5398\end{array}$ & $\begin{array}{c}\text { Pressure } \\
(\text { psig) } \\
9.89 \\
19.55 \\
29.57 \\
39.42 \\
49.16 \\
54.50 \\
59.57 \\
64.20 \\
09.32 \\
74.16 \\
80.16 \\
85.61 \\
90.58 \\
95.69 \\
100.92 \\
103.25 \\
106.11 \\
108.31 \\
111.08 \\
113.24 \\
117.83 \\
120.92 \\
123.28 \\
122.97 \\
125.82 \\
125.60 \\
127.84 \\
130.19 \\
132.53 \\
135.33 \\
134.42 \\
138.35 \\
140.16 \\
142.63 \\
145.78 \\
142.52 \\
0.22\end{array}$ & $\begin{array}{c}\text { Load } \\
\text { (pounds) } \\
149.2915 \\
412.4693 \\
661.6259 \\
911.3482 \\
1028.2213 \\
1302.2945 \\
1358.9549 \\
1543.5226 \\
1652.5764 \\
1754.8826 \\
1823.1528 \\
1924.2682 \\
1965.0517 \\
1994.9199 \\
2053.9618 \\
2125.8043 \\
2134.3381 \\
2122.7282 \\
2170.8547 \\
2220.8666 \\
2212.9282 \\
2220.7674 \\
2321.2874 \\
2284.0761 \\
2280.4046 \\
2345.0034 \\
2373.6808 \\
2354.2318 \\
2443.73 .2 \\
2463.1863 \\
2456.3394 \\
2513.5951 \\
2622.0535 \\
2668.3939 \\
2691.5145 \\
2613.5197 \\
-751.4986\end{array}$ \\
\hline
\end{tabular}

High Pressure test 


\section{Reinforced Concrete Test Data}

Concrete Embedment Gage 2 Channel 1126

Structural INTEgRIty Test

\begin{tabular}{|c|c|c|c|}
\hline $\begin{array}{c}\text { Pressure } \\
(\text { psig) } \\
-0.05 \\
5.33 \\
12.31 \\
16.44 \\
20.51 \\
20.46 \\
20.47 \\
27.05 \\
34.69 \\
34.53 \\
34.53 \\
39.70 \\
45.90 \\
45.65 \\
45.62 \\
50.10 \\
53.47 \\
53.29 \\
53.21 \\
50.49 \\
46.14 \\
46.13 \\
44.97 \\
38.98 \\
33.99 \\
33.96 \\
26.83 \\
21.88 \\
21.90 \\
16.69 \\
11.74 \\
11.77 \\
5.05 \\
0.02 \\
-0.04 \\
-0.02 \\
-0.02 \\
0.02\end{array}$ & $\begin{array}{l}\text { X Strain } \\
-0.0009 \\
-0.0009 \\
-0.0004 \\
0.0005 \\
0.0029 \\
0.0027 \\
0.0019 \\
0.0074 \\
0.0141 \\
0.0166 \\
0.0180 \\
0.0260 \\
0.0357 \\
0.0486 \\
0.0468 \\
0.0491 \\
0.0542 \\
0.0496 \\
0.0497 \\
0.0485 \\
0.0446 \\
0.0440 \\
0.0370 \\
0.0328 \\
0.0271 \\
0.0255 \\
0.0166 \\
0.0116 \\
0.0152 \\
0.0105 \\
0.0130 \\
0.0136 \\
0.0100 \\
0.0066 \\
0.0001 \\
-0.0015 \\
-0.0028 \\
-0.0045\end{array}$ & $\begin{array}{c}\text { Pressure } \\
\text { (psig) } \\
9.89 \\
19.55 \\
29.57 \\
39.42 \\
49.16 \\
54.50 \\
59.57 \\
64.20 \\
69.32 \\
74.16 \\
80.16 \\
85.61 \\
90.58 \\
95.69 \\
100.92 \\
103.25 \\
106.11 \\
108.31 \\
111.08 \\
113.24 \\
117.83 \\
120.92 \\
123.28 \\
122.97 \\
125.82 \\
125.60 \\
127.84 \\
130.19 \\
132.53 \\
135.33 \\
134.42 \\
138.35 \\
140.16 \\
142.63 \\
145.78 \\
142.52 \\
0.22 \\
\end{array}$ & $\begin{array}{l}\text { * Strain } \\
-0.0027 \\
0.0048 \\
0.0137 \\
0.0255 \\
0.0356 \\
0.0445 \\
0.0622 \\
0.0737 \\
0.0823 \\
0.0769 \\
0.0452 \\
0.0512 \\
0.0484 \\
0.0349 \\
0.0509 \\
0.0577 \\
0.0419 \\
0.0442 \\
0.0464 \\
0.0428 \\
0.0348 \\
0.0310 \\
0.0232 \\
0.0162 \\
0.0273 \\
0.0196 \\
0.0216 \\
0.0195 \\
0.0114 \\
0.0132 \\
0.0133 \\
0.0135 \\
0.0157 \\
0.0257 \\
0.0391 \\
0.0458 \\
0.0258\end{array}$ \\
\hline
\end{tabular}

High Pressure Test

Pressure

8.89

19.55

49.16

64.20

80.16

95.69

106.11

108.31

111.08

13.24

123.28

122.97

25.82

25.60

32.53

135.33

140.16

42.63

45.78

2.52

0.0258 


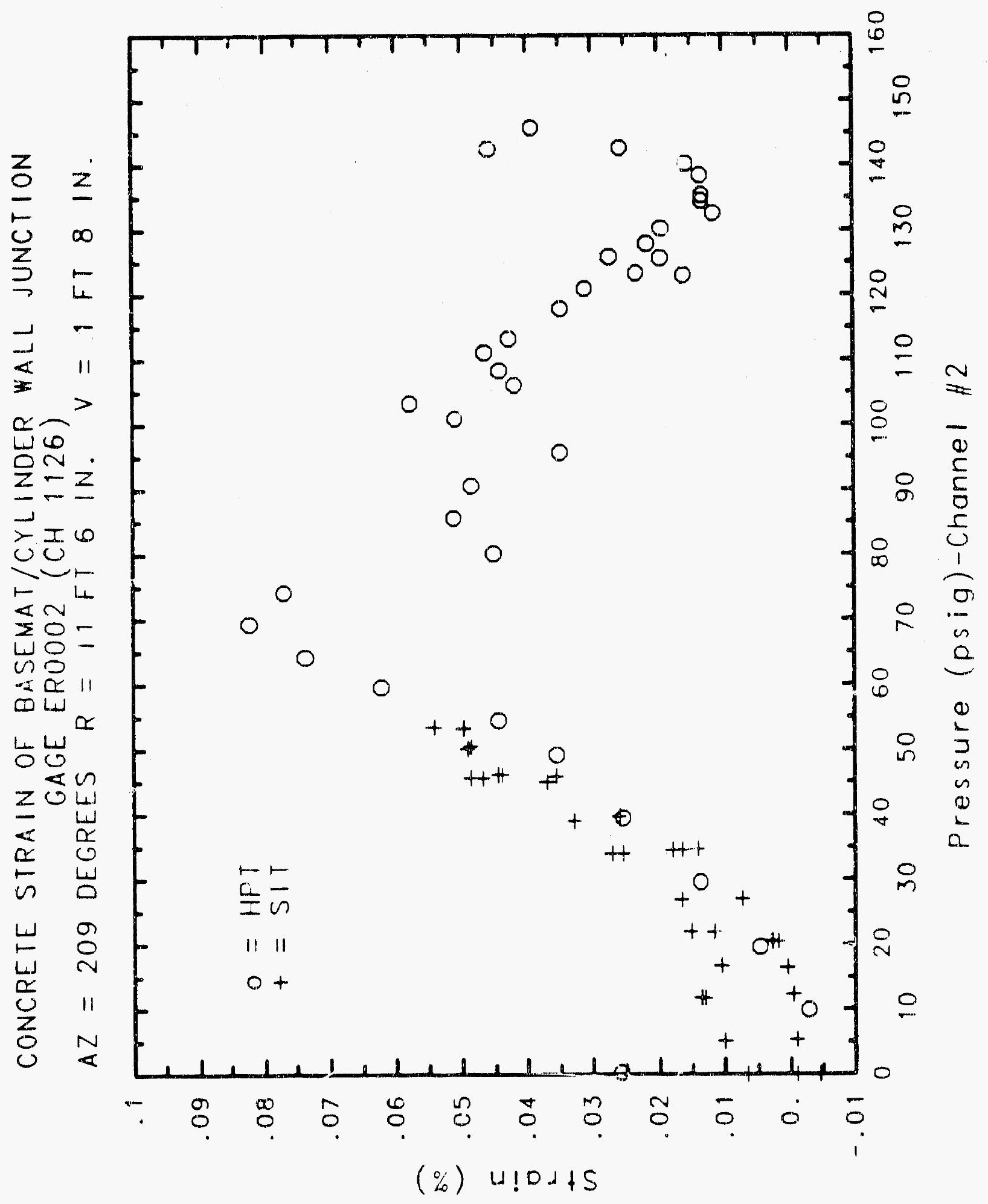

C-1821 


\section{Reinforced Concrete Test Data}

Concrete embedment gage 3 inannel 1127

StRuctural INTEgRITY TEST

High pressurf test

\begin{tabular}{|c|c|c|c|}
\hline $\begin{array}{c}\text { Pressure } \\
\text { (psig) } \\
-0.05 \\
5.33 \\
12.31 \\
16.44 \\
20.51 \\
20.46 \\
20.47 \\
27.05 \\
34.69 \\
34.53 \\
34.53 \\
39.70 \\
45.90 \\
45.05 \\
45.62 \\
50.10 \\
53.47 \\
53.29 \\
53.21 \\
50.49 \\
46.14 \\
46.13 \\
44.97 \\
38.98 \\
33.99 \\
33.96 \\
26.83 \\
21.88 \\
21.90 \\
16.69 \\
11.74 \\
11.77 \\
5.05 \\
0.02 \\
-0.04 \\
-0.02 \\
-0.02 \\
0.02 \\
\end{array}$ & $\begin{array}{c}\text { X Strain } \\
-0.0001 \\
-0.0003 \\
0.0016 \\
0.0031 \\
0.0058 \\
0.0059 \\
0.0062 \\
0.0118 \\
0.0205 \\
0.0205 \\
0.0207 \\
0.0242 \\
0.0305 \\
0.0302 \\
0.0298 \\
0.0327 \\
0.0349 \\
0.0342 \\
0.0338 \\
0.0323 \\
0.0293 \\
0.0291 \\
0.0293 \\
0.0248 \\
0.0209 \\
0.0205 \\
0.0148 \\
0.0109 \\
0.0107 \\
0.0069 \\
0.0033 \\
0.0031 \\
-0.000 \\
-0.0006 \\
0.0009 \\
-0.0018 \\
-0.0023 \\
-0.0015\end{array}$ & $\begin{array}{c}\text { Pressure } \\
(\text { ps1g) } \\
9.89 \\
19.55 \\
29.57 \\
39.42 \\
49.16 \\
54.50 \\
59.57 \\
64.20 \\
69.32 \\
74.16 \\
80.16 \\
85.61 \\
90.58 \\
95.69 \\
100.92 \\
103.25 \\
16.11 \\
108.31 \\
111.08 \\
113.24 \\
117.83 \\
120.92 \\
123.28 \\
122.97 \\
125.82 \\
125.60 \\
127.84 \\
130.19 \\
132.53 \\
135.33 \\
134.42 \\
138.35 \\
140.16 \\
142.63 \\
145.72 \\
142.52 \\
0.22 \\
\end{array}$ & $\begin{array}{c}\text { Strain } \\
\text { O. } 0.0013 \\
0.0047 \\
0.0119 \\
0.0134 \\
0.0272 \\
0.0309 \\
0.0347 \\
0.0384 \\
0.0408 \\
0.0444 \\
0.0476 \\
0.0497 \\
0.0495 \\
0.0504 \\
0.0519 \\
0.0496 \\
0.0500 \\
0.0503 \\
0.0475 \\
0.0426 \\
0.0305 \\
0.0248 \\
0.0222 \\
0.0219 \\
0.0258 \\
0.0274 \\
0.0354 \\
0.0380 \\
0.0382 \\
0.0385 \\
0.0369 \\
0.0396 \\
0.0427 \\
0.0491 \\
0.0583 \\
0.0572 \\
0.0494\end{array}$ \\
\hline
\end{tabular}




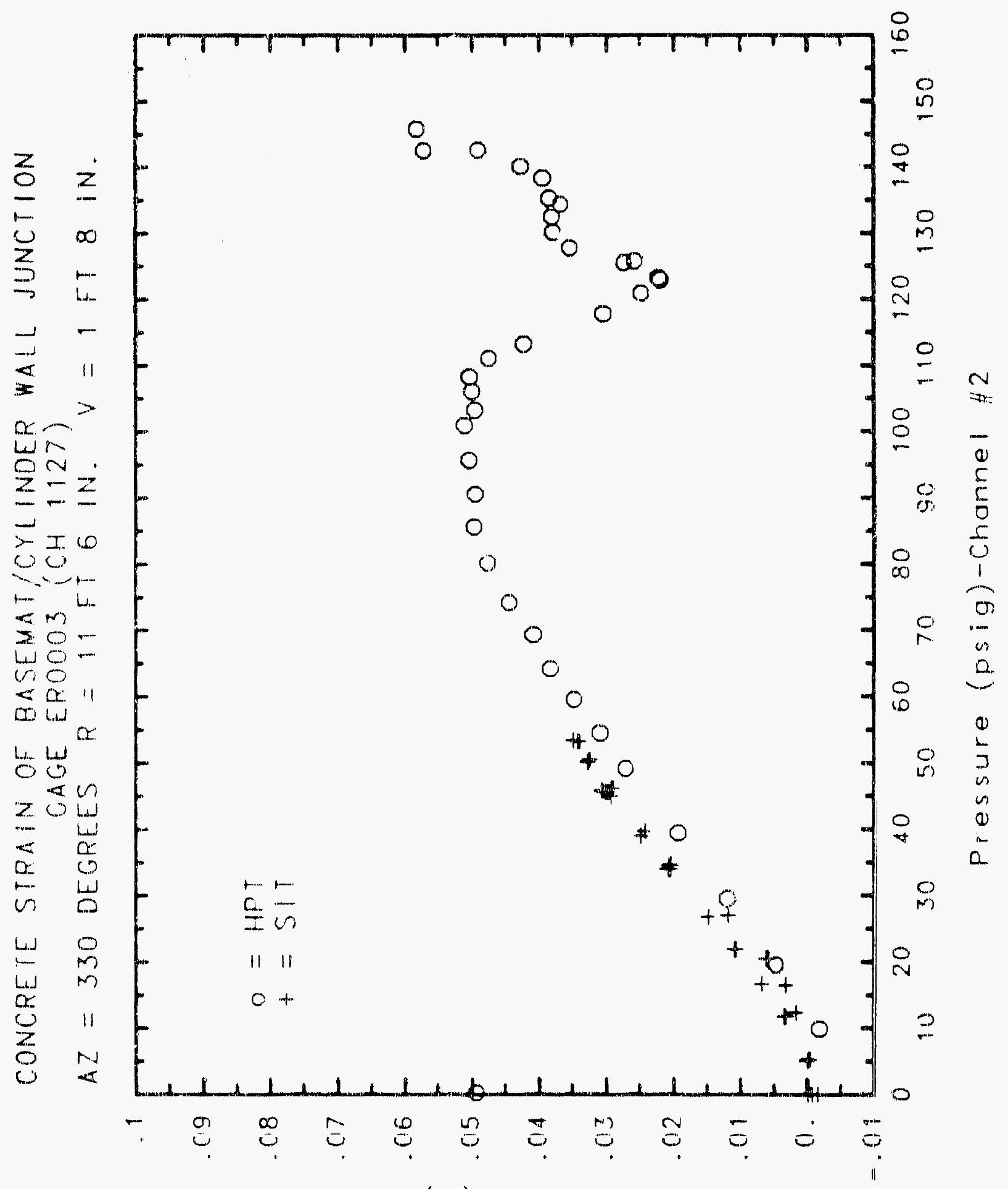

(c) $4 ! 0+15$ 


\section{Reinforced Concrete Test Data}

Ni

Concrete Embedment Gage 4 Channel 1128

Structural Integrity Test

\begin{tabular}{|c|c|c|c|}
\hline $\begin{array}{c}\text { Pressure } \\
(\text { psig) } \\
-0.05 \\
5.33 \\
12.31 \\
16.44 \\
20.51 \\
20.46 \\
20.47 \\
27.05 \\
34.69 \\
34.53 \\
34.53 \\
39.70 \\
45.90 \\
45.65 \\
45.62 \\
50.10 \\
53.47 \\
53.29 \\
53.21 \\
50.49 \\
46.14 \\
46.13 \\
44.97 \\
38.98 \\
33.99 \\
33.96 \\
26.83 \\
21.88 \\
21.90 \\
16.69 \\
11.74 \\
11.77 \\
5.05 \\
0.02 \\
-0.04 \\
-0.02 \\
-0.02 \\
0.02 \\
\end{array}$ & $\begin{array}{l}\text { \% Strain } \\
0.0003 \\
0.0013 \\
0.0027 \\
0.0034 \\
0.0042 \\
0.0048 \\
0.0048 \\
0.0078 \\
0.0132 \\
0.0149 \\
0.0156 \\
0.0188 \\
0.0248 \\
0.0252 \\
0.0256 \\
0.0281 \\
0.0303 \\
0.0308 \\
0.0312 \\
0.0303 \\
0.0287 \\
0.0284 \\
0.0284 \\
0.0263 \\
0.0245 \\
0.0249 \\
0.0218 \\
0.0192 \\
0.0189 \\
0.0166 \\
0.0146 \\
0.0141 \\
0.0113 \\
0.0086 \\
0.0075 \\
0.0072 \\
0.0071 \\
0.0072\end{array}$ & $\begin{array}{c}\text { Pressure } \\
(\text { ps } 19) \\
9.89 \\
19.55 \\
29.57 \\
39.42 \\
49.16 \\
54.50 \\
59.57 \\
64.20 \\
69.32 \\
74.16 \\
80.16 \\
85.61 \\
90.58 \\
95.69 \\
100.92 \\
103.25 \\
106.11 \\
108.31 \\
111.08 \\
113.24 \\
117.83 \\
120.92 \\
123.28 \\
122.97 \\
125.82 \\
125.60 \\
127.84 \\
130.19 \\
132.53 \\
135.33 \\
134.42 \\
138.35 \\
140.16 \\
142.63 \\
145.78 \\
142.52 \\
0.22\end{array}$ & $\begin{array}{l}\text { * Strain } \\
.0 .0002 \\
0.0040 \\
0.0098 \\
0.0150 \\
0.0198 \\
0.0228 \\
0.0258 \\
0.0294 \\
0.0337 \\
0.0363 \\
0.0438 \\
0.0508 \\
0.0576 \\
0.0644 \\
0.0716 \\
0.0758 \\
0.0795 \\
0.0827 \\
0.0880 \\
0.0919 \\
0.1014 \\
0.1081 \\
0.1136 \\
0.1147 \\
0.1206 \\
0.1219 \\
0.1259 \\
0.1326 \\
0.1376 \\
0.1413 \\
0.1418 \\
0.1591 \\
0.1866 \\
0.2250 \\
0.2601 \\
0.2688 \\
0.1050\end{array}$ \\
\hline
\end{tabular}

high Pressure test

Pres?

(psig)

19.55

39.42

49.16

54.50

59.57

74.16

90.58

103.25

106.1 .

113.24

117.83

122.97

125.82

125.60

127.84

130.19

135.33

134.42

138.35

140.16

142.63

42.52

0.22
$-0.0002$

0.0040

0.0150

0.0198

0.0228

0.0337

0.0438

0.0508

0.0716

0.0795

0.0827

0.0880

0.1081

0.1147

0.1206

0.1219

0.1376

0.1418

0.1591

0.1866

0.2250

0.2601

0.1050 


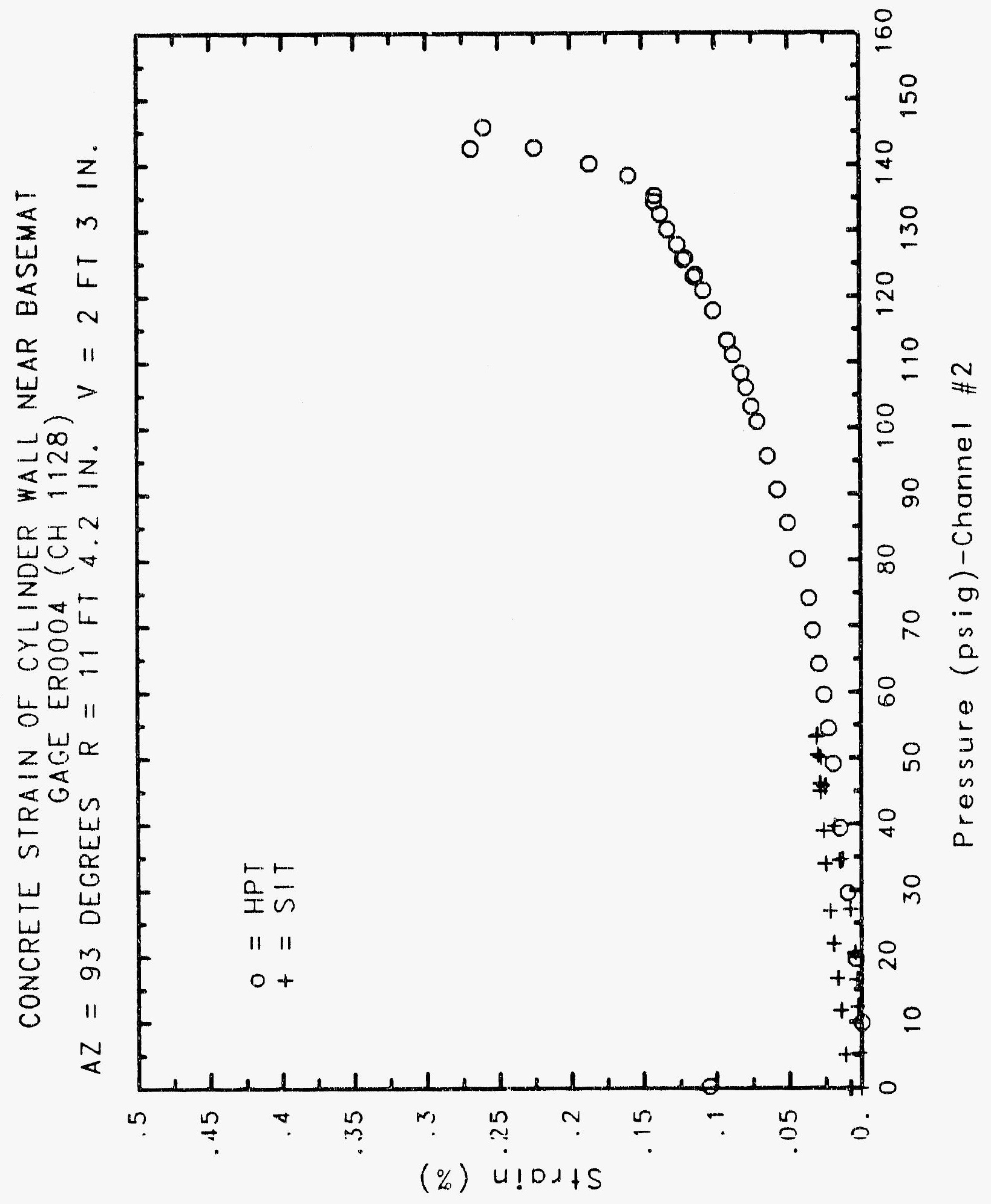

C- 1825 


\section{Reinforced Concrete Test Data}

Concrete Embedment Gage 5 Channel 1129

Structural INTEgrity TEST

\begin{tabular}{|c|c|c|c|}
\hline $\begin{array}{c}\text { Pressure } \\
\text { (psig) } \\
-0.05 \\
5.33 \\
12.31 \\
16.44 \\
20.51 \\
20.46 \\
20.47 \\
27.05 \\
34.69 \\
34.53 \\
34.53 \\
39.70 \\
45.90 \\
45.65 \\
45.62 \\
50.10 \\
53.47 \\
53.29 \\
53.21 \\
50.49 \\
46.14 \\
46.13 \\
44.97 \\
38.98 \\
33.99 \\
33.96 \\
26.83 \\
21.88 \\
21.90 \\
16.69 \\
11.74 \\
11.77 \\
5.05 \\
0.02 \\
-0.04 \\
-0.02 \\
-0.02 \\
0.02 \\
\end{array}$ & $\begin{array}{c}\text { X Strain } \\
\\
-0.0006 \\
-0.0003 \\
0.0007 \\
0.0013 \\
0.0028 \\
0.0026 \\
0.0029 \\
0.0059 \\
0.0122 \\
0.0133 \\
0.0137 \\
0.0176 \\
0.0226 \\
0.0230 \\
0.0227 \\
0.0251 \\
0.0275 \\
0.0282 \\
0.0276 \\
0.0272 \\
0.0259 \\
0.0259 \\
0.0255 \\
0.0239 \\
0.0218 \\
0.0219 \\
0.0190 \\
0.0167 \\
0.0169 \\
0.0150 \\
0.0126 \\
0.0122 \\
0.0091 \\
0.0070 \\
0.0060 \\
0.0061 \\
0.0064 \\
0.0060\end{array}$ & $\begin{array}{c}\text { Pressure } \\
(\text { psig) } \\
9.89 \\
19.55 \\
29.57 \\
39.42 \\
49.16 \\
54.50 \\
59.57 \\
64.20 \\
69.32 \\
74.16 \\
80.16 \\
85.61 \\
90.58 \\
95.69 \\
100.92 \\
103.25 \\
106.11 \\
108.31 \\
111.08 \\
113.24 \\
117.83 \\
120.92 \\
123.28 \\
122.97 \\
125.82 \\
125.60 \\
127.84 \\
130.19 \\
1=2.53 \\
135.33 \\
134.42 \\
138.35 \\
140.16 \\
142.63 \\
145.78 \\
142.52 \\
0.22\end{array}$ & $\begin{array}{c}\text { \% Strain } \\
-0.0005 \\
0.0041 \\
0.0090 \\
0.0135 \\
0.0180 \\
0.0205 \\
0.0236 \\
0.0283 \\
0.0333 \\
0.0404 \\
0.0462 \\
0.0515 \\
0.0553 \\
0.0601 \\
0.0652 \\
0.0672 \\
0.0701 \\
0.0725 \\
0.0754 \\
0.0781 \\
0.0838 \\
0.0885 \\
0.0928 \\
0.0937 \\
0.0986 \\
0.0998 \\
0.1035 \\
0.1092 \\
0.1158 \\
0.1208 \\
0.1207 \\
0.1296 \\
0.1412 \\
0.2172 \\
0.2929 \\
0.3051 \\
0.1742\end{array}$ \\
\hline
\end{tabular}

High Pressure Test

Pressure

0.89

19.55

29.57

39.42

49.16

54.50

59.57

74.16

80.16

85.61

90.58

95.69

100.92

106.11

108.31

111.08

120.92

123.28

122.97

125.82

125.60

127.84

$1=2.53$

135.33

134.42

138.35

140.16

45.78

42.52

0.22
Strain

0.0005

0.0135

0.0205

0.0236

0.0404

0.0553

0.0601

0.0701

0.0725

0.0754

0.0781

0.0928

0.0937

0.0986

0.0998

0.1035

0.1158

0.1208

0.1207

0.1296

0.1412

0.2929

0.3051

0.1742 


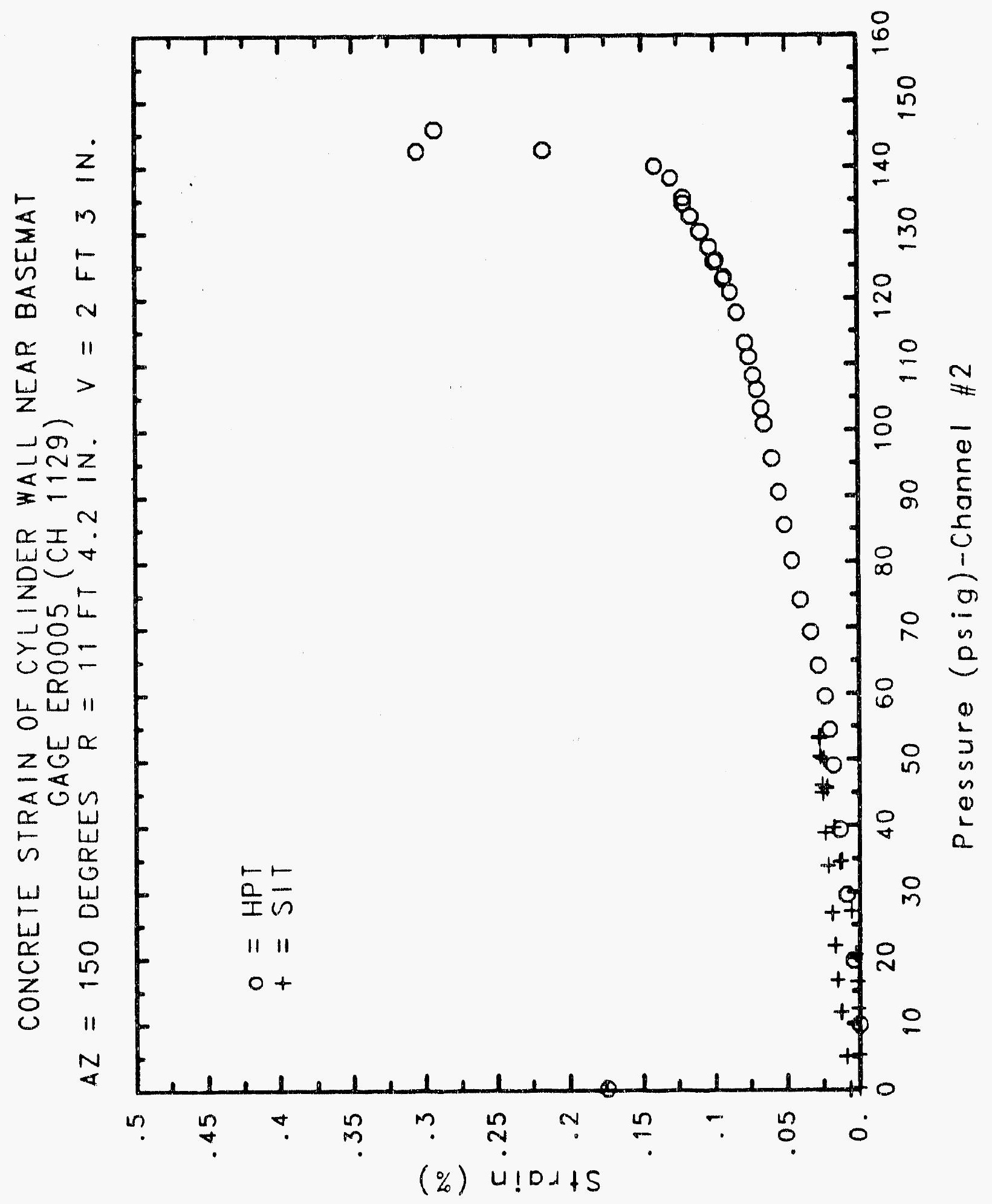




\section{Reinforced Concrete Test Data}

Concrete Embedment Gage 6 Channel 1140

STRUCTURAL. INTEGRITY TEST

High Pressure Test

\begin{tabular}{|c|c|c|c|}
\hline $\begin{array}{c}\text { Pressure } \\
\text { (psig) } \\
-0.05 \\
5.33 \\
12.31 \\
16.44 \\
20.51 \\
20.46 \\
20.47 \\
27.05 \\
34.69 \\
34.53 \\
34.53 \\
39.70 \\
45.90 \\
45.85 \\
45.62 \\
50.10 \\
53.47 \\
53.29 \\
53.21 \\
50.49 \\
46.14 \\
46.13 \\
44.97 \\
38.98 \\
33.99 \\
33.96 \\
26.83 \\
21.88 \\
21.90 \\
16.69 \\
11.74 \\
11.77 \\
5.05 \\
0.02 \\
-0.04 \\
-0.02 \\
-0.02 \\
0.02 \\
\end{array}$ & $\begin{array}{l}\text { X Strain } \\
0.0005 \\
0.0009 \\
0.0020 \\
0.0025 \\
0.0032 \\
0.0031 \\
0.0032 \\
0.0064 \\
0.0131 \\
0.0143 \\
0.0146 \\
0.0178 \\
0.0223 \\
0.0227 \\
0.0229 \\
0.0254 \\
0.0276 \\
0.0275 \\
0.0278 \\
0.0273 \\
0.0258 \\
0.0253 \\
0.0253 \\
0.0233 \\
0.0215 \\
0.0215 \\
0.0188 \\
0.0171 \\
0.017 \\
0.0151 \\
0.0134 \\
0.0134 \\
0.0110 \\
0.0093 \\
0.0086 \\
0.0082 \\
0.0078 \\
0.0081\end{array}$ & $\begin{array}{c}\text { Pressure } \\
(\text { psig) } \\
9.89 \\
19.55 \\
29.57 \\
39.42 \\
49.16 \\
54.50 \\
59.57 \\
64.20 \\
69.32 \\
74.16 \\
80.16 \\
85.61 \\
90.58 \\
95.69 \\
100.92 \\
103.25 \\
106.11 \\
108.31 \\
111.08 \\
113.24 \\
117.83 \\
120.92 \\
123.28 \\
122.97 \\
125.82 \\
125.60 \\
127.84 \\
130.19 \\
132.53 \\
135.23 \\
134.42 \\
138.35 \\
140.16 \\
142.63 \\
145.78 \\
142.52 \\
0.22 \\
\end{array}$ & $\begin{array}{c}\text { \% Strain } \\
-0.0022 \\
0.0016 \\
0.0058 \\
0.0100 \\
0.0142 \\
0.0167 \\
0.0195 \\
0.0221 \\
0.0248 \\
0.0266 \\
0.0376 \\
0.0447 \\
0.0508 \\
0.0568 \\
0.0634 \\
0.0673 \\
0.0720 \\
0.0754 \\
0.0810 \\
0.0855 \\
0.0975 \\
0.1065 \\
0.1156 \\
0.1177 \\
0.1271 \\
0.1281 \\
0.1322 \\
0.1361 \\
0.1380 \\
0.1417 \\
0.1404 \\
0.1569 \\
0.1719 \\
0.2062 \\
0.2323 \\
0.2381 \\
0.0959\end{array}$ \\
\hline
\end{tabular}




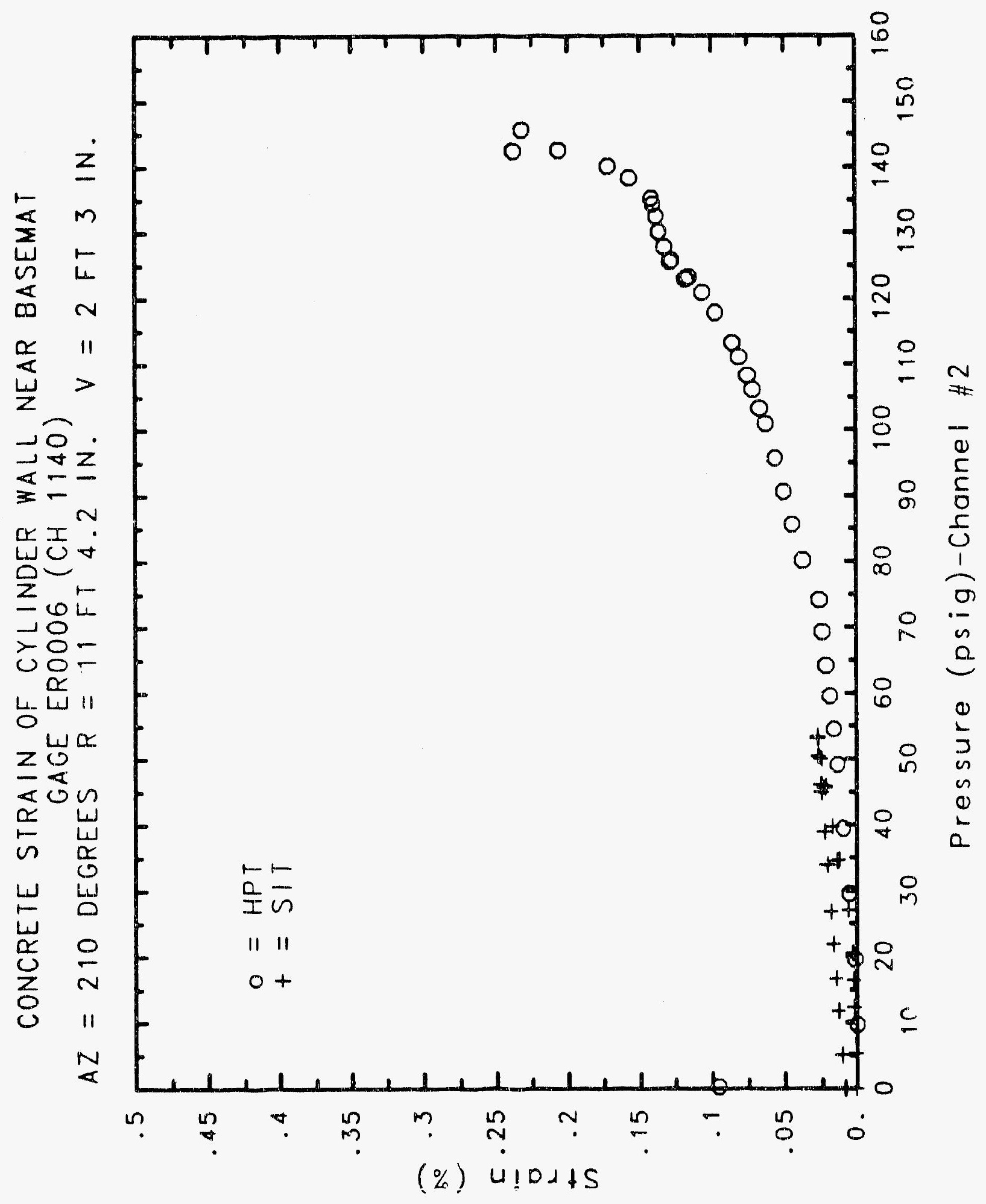

C- 1829 


\section{Reinforced Concrete Test Data}

Concrete Embedment gage 7 Chamnel 1141

StRUCTURAL InTEGRITY TEST

High Pressure test

\begin{tabular}{|c|c|c|c|}
\hline $\begin{array}{c}\text { Pressure } \\
\text { (psig) } \\
-0.05 \\
5.33 \\
12.31 \\
16.44 \\
20.51 \\
20.46 \\
20.47 \\
27.05 \\
34.69 \\
34.53 \\
34.53 \\
39.70 \\
45.90 \\
45.65 \\
45.62 \\
50.10 \\
53.47 \\
53.29 \\
53.21 \\
50.49 \\
46.14 \\
46.13 \\
44.97 \\
38.98 \\
33.99 \\
33.96 \\
26.83 \\
21.88 \\
21.90 \\
16.69 \\
11.74 \\
11.77 \\
5.05 \\
0.02 \\
-0.04 \\
-0.02 \\
-0.02 \\
0.02 \\
\end{array}$ & $\begin{array}{c}\text { Strain } \\
-0.0003 \\
0.0006 \\
0.0020 \\
0.0027 \\
0.0039 \\
0.0044 \\
0.0045 \\
0.0138 \\
0.0294 \\
0.0310 \\
0.0315 \\
0.0371 \\
0.0468 \\
0.0479 \\
0.0483 \\
0.0532 \\
0.0571 \\
0.0579 \\
0.0583 \\
0.0569 \\
0.0543 \\
0.0540 \\
0.0545 \\
0.0516 \\
0.0477 \\
0.0478 \\
0.0424 \\
0.0383 \\
0.0384 \\
0.0345 \\
0.0302 \\
0.0300 \\
0.0250 \\
0.0210 \\
0.0198 \\
0.0192 \\
0.0194 \\
0.0194\end{array}$ & $\begin{array}{c}\text { Pressure } \\
(\text { psig) } \\
9.89 \\
19.55 \\
29.57 \\
39.42 \\
49.16 \\
54.50 \\
59.57 \\
64.20 \\
69.32 \\
74.16 \\
80.16 \\
85.61 \\
90.58 \\
95.69 \\
100.92 \\
103.25 \\
106.11 \\
108.31 \\
111.08 \\
113.24 \\
117.83 \\
120.92 \\
123.28 \\
122.97 \\
125.82 \\
125.60 \\
127.84 \\
130.19 \\
132.53 \\
135.33 \\
134.42 \\
138.35 \\
140.16 \\
142.63 \\
145.78 \\
142.52 \\
0.22 \\
\end{array}$ & $\begin{array}{l}\text { * Strain } \\
0.0033 \\
0.0125 \\
0.0217 \\
0.0304 \\
0.0383 \\
0.0441 \\
0.0497 \\
0.0558 \\
0.0633 \\
0.0742 \\
0.0851 \\
0.0957 \\
0.1056 \\
0.1166 \\
0.1280 \\
0.1329 \\
0.1386 \\
0.1432 \\
0.1505 \\
0.1562 \\
0.1688 \\
0.1771 \\
0.1865 \\
0.1894 \\
0.1998 \\
0.1996 \\
0.2057 \\
0.2125 \\
0.2202 \\
0.3296 \\
0.2295 \\
0.2421 \\
0.2521 \\
0.3003 \\
0.3820 \\
0.4041 \\
0.2120\end{array}$ \\
\hline
\end{tabular}




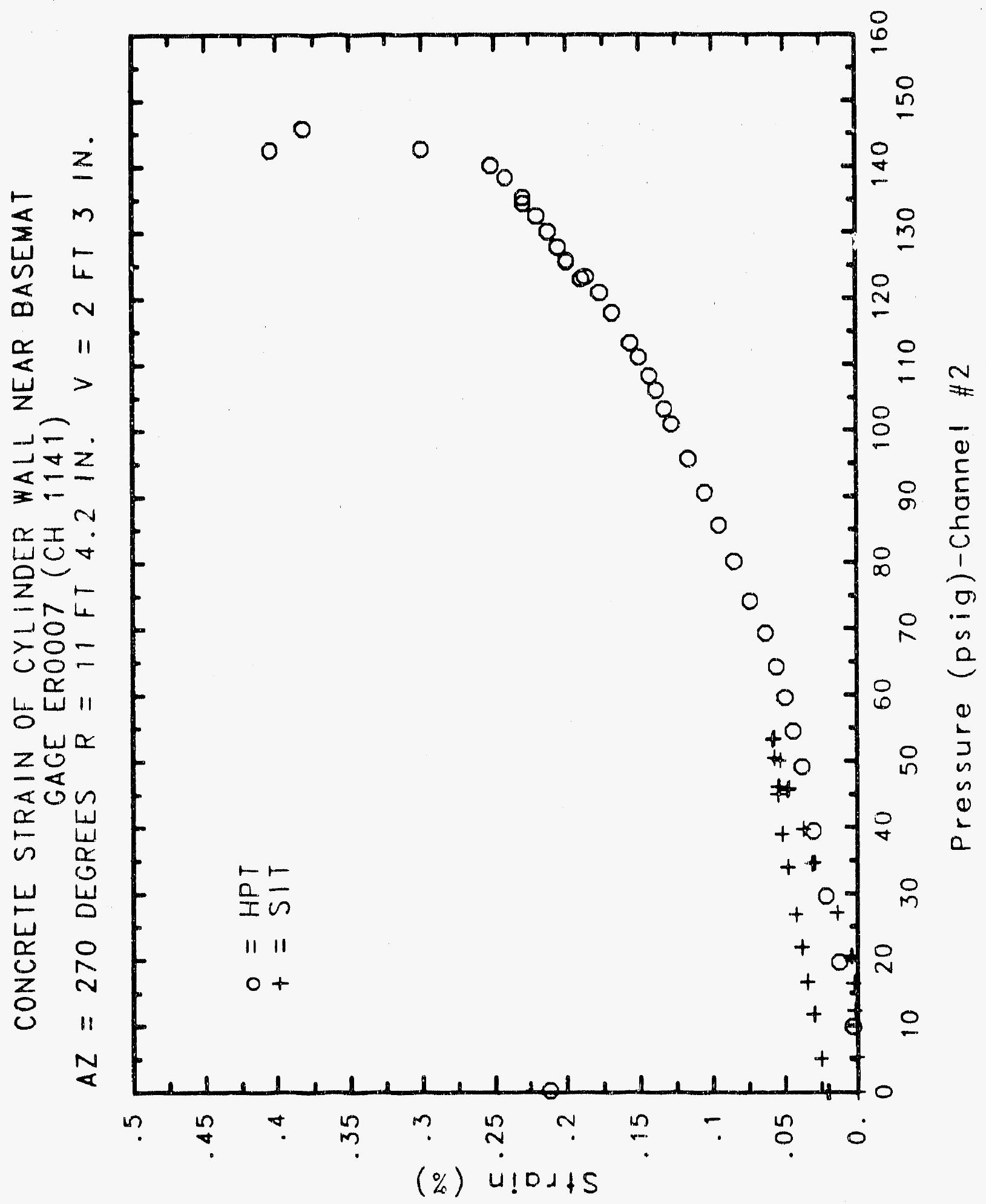

C-1831 


\section{Reinforced Concrete Test Data}

Concrete Embedment Gage 8 Channel 1142

Structural. Integrity TEST

\begin{tabular}{|c|c|c|c|}
\hline $\begin{array}{c}\text { Pressure } \\
\text { (psig) } \\
-0.05 \\
5.33 \\
12.31 \\
16.44 \\
20.51 \\
20.46 \\
20.47 \\
27.05 \\
34.69 \\
34.53 \\
34.53 \\
39.70 \\
45.90 \\
45.65 \\
45.62 \\
50.10 \\
53.47 \\
53.29 \\
53.21 \\
50.49 \\
46.14 \\
46.13 \\
44.97 \\
38.93 \\
33.99 \\
33.96 \\
26.83 \\
21.88 \\
21.90 \\
16.69 \\
11.74 \\
11.77 \\
5.05 \\
0.02 \\
-0.04 \\
-0.02 \\
-0.02 \\
0.02 \\
\end{array}$ & $\begin{array}{l}\text { X Strain } \\
0.0000 \\
0.0011 \\
0.0023 \\
0.0031 \\
0.0047 \\
0.0052 \\
0.0054 \\
0.0099 \\
0.0202 \\
0.0214 \\
0.0215 \\
0.0256 \\
0.0328 \\
0.0339 \\
0.0340 \\
0.0377 \\
0.0413 \\
0.0417 \\
0.0415 \\
0.0407 \\
0.0385 \\
0.0383 \\
0.0386 \\
0.0357 \\
0.0334 \\
0.0334 \\
0.0297 \\
0.0269 \\
0.0268 \\
0.0240 \\
0.0209 \\
0.02112 \\
0.0174 \\
0.0149 \\
0.0134 \\
0.0139 \\
0.0137 \\
0.0142\end{array}$ & $\begin{array}{c}\text { Pressure } \\
(\text { ps } 1 g \text { ) } \\
189 \\
19.55 \\
29.57 \\
39.42 \\
49.16 \\
54.50 \\
59.57 \\
64.20 \\
69.32 \\
71.16 \\
80.16 \\
85.61 \\
90.58 \\
95.69 \\
100.92 \\
103.25 \\
106.11 \\
108.31 \\
111.08 \\
113.24 \\
117.83 \\
120.92 \\
123.28 \\
122.97 \\
125.82 \\
125.60 \\
127.84 \\
130.19 \\
132.53 \\
135.33 \\
134.42 \\
138.35 \\
140.16 \\
142.63 \\
145.78 \\
142.52 \\
0.22\end{array}$ & $\begin{array}{l}\text { X Strain } \\
0.0010 \\
0.0078 \\
0.0144 \\
0.0207 \\
0.0269 \\
0.0308 \\
0.0345 \\
0.0389 \\
0.0435 \\
0.0498 \\
0.0581 \\
0.0655 \\
0.0708 \\
0.0776 \\
0.0839 \\
0.0874 \\
0.0908 \\
0.0935 \\
0.0970 \\
0.0998 \\
0.1059 \\
0.1105 \\
0.1151 \\
0.1163 \\
0.1216 \\
0.1233 \\
0.1257 \\
0.1282 \\
0.1317 \\
0.1364 \\
0.1361 \\
0.1426 \\
0.1458 \\
0.1518 \\
0.1805 \\
0.1883 \\
0.0392\end{array}$ \\
\hline
\end{tabular}

High Pressure test

Pressur

89

19.55

49.16

54.50

64.20

80.16

90.58

95.69

106.11

108.31

113.28

117.83

122.97

125.82

125.60

132.53

135.33

134.42

138.35

140.16

142.63

42.52

0.22
0.0010

0207

0.0308

0.0345

0.0389

0.0581

0.0708

0.0776

0.0839

0.0874

0.0970

0.1059

0.1105

0.1151

0.1233

0.1282

0.1317

0.1364

0.1361

0.1518

0.0392 


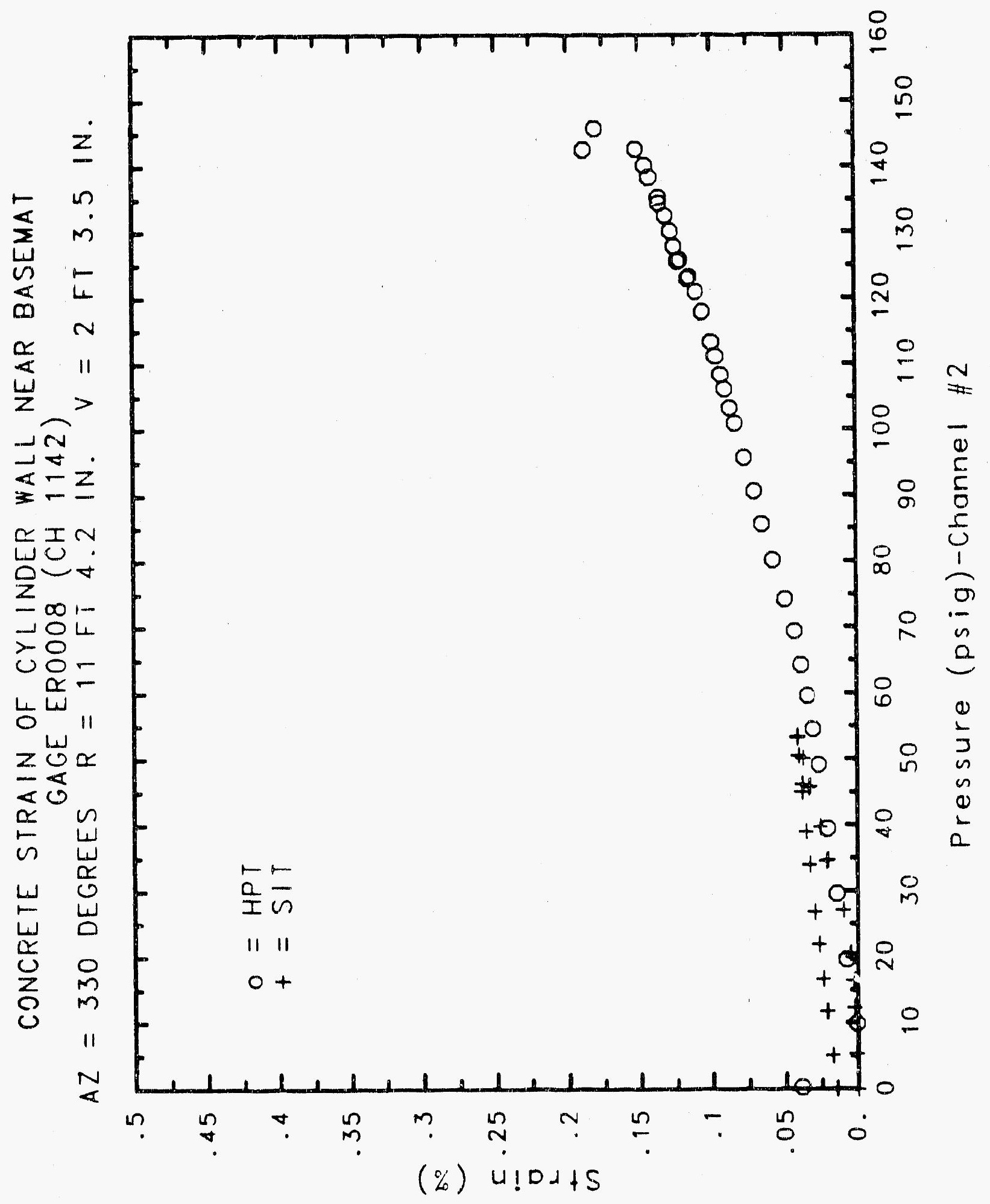




\section{Reinforced Concrete Test Data}

Concrete embedment Gage 9 Channel 1143

STRUCTURAL INTEGRITY TEST

\begin{tabular}{|c|c|c|c|}
\hline $\begin{array}{c}\text { Pressure } \\
\text { (psig) } \\
-0.05 \\
5.33 \\
12.31 \\
16.44 \\
20.51 \\
20.46 \\
20.47 \\
27.05 \\
34.69 \\
31.53 \\
34.53 \\
39.70 \\
45.90 \\
45.65 \\
45.62 \\
50.10 \\
53.47 \\
53.29 \\
53.21 \\
50.49 \\
46.14 \\
46.13 \\
44.97 \\
38.98 \\
33.99 \\
33.96 \\
26.83 \\
21.88 \\
21.90 \\
16.69 \\
11.74 \\
11.77 \\
5.05 \\
0.02 \\
-0.04 \\
-0.02 \\
-0.02 \\
0.02 \\
\end{array}$ & $\begin{array}{l}\text { X Strain } \\
0.0005 \\
0.0006 \\
0.0018 \\
0.0020 \\
0.0026 \\
0.0028 \\
0.0025 \\
0.0037 \\
0.0064 \\
0.0075 \\
0.0076 \\
0.0111 \\
0.0186 \\
0.0195 \\
0.0200 \\
0.0235 \\
0.0270 \\
0.0277 \\
0.0280 \\
0.0276 \\
0.0262 \\
0.0262 \\
0.0264 \\
0.0244 \\
0.0229 \\
0.0224 \\
0.0198 \\
0.0179 \\
0.0177 \\
0.0157 \\
0.0141 \\
0.0137 \\
0.0118 \\
0.0397 \\
0.0094 \\
0.0094 \\
0.0091 \\
0.0089\end{array}$ & $\begin{array}{c}\text { Pressure } \\
(\text { psig) } \\
9.89 \\
19.55 \\
29.57 \\
39.42 \\
49.16 \\
54.50 \\
59.57 \\
64.20 \\
69.32 \\
74.16 \\
80.16 \\
85.61 \\
90.58 \\
95.69 \\
100.92 \\
103.25 \\
106.11 \\
108.31 \\
111.08 \\
113.24 \\
117.83 \\
120.92 \\
123.28 \\
122.97 \\
125.82 \\
125.60 \\
127.84 \\
130.19 \\
132.53 \\
135.33 \\
134.42 \\
138.35 \\
140.16 \\
142.63 \\
145.78 \\
142.52 \\
0.22\end{array}$ & $\begin{array}{c}\text { \% Stra n } \\
-0.0012 \\
0.0030 \\
0.0077 \\
0.0123 \\
0.0169 \\
0.0205 \\
0.0236 \\
0.0285 \\
0.0336 \\
0.0463 \\
0.0560 \\
0.0652 \\
0.0747 \\
0.0850 \\
0.0943 \\
0.0991 \\
0.1045 \\
0.1083 \\
0.1144 \\
0.1195 \\
0.1316 \\
0.1414 \\
0.1521 \\
0.1545 \\
0.1659 \\
0.1698 \\
0.1773 \\
0.1910 \\
0.2005 \\
0.2096 \\
0.2105 \\
0.2203 \\
0.2265 \\
0.2291 \\
0.2293 \\
0.2244 \\
0.0688\end{array}$ \\
\hline
\end{tabular}

High Pressure test

Pressure

19.55

49.16

4.50

64.20

85.61

90.58

95.69

106.11

13.24

117.83

120.92

123.28

22.97

130.19

132.53

135.33

134.42

138.35

140.16

145.78

42.52

0.22
$-0.0012$

0.0030

0.0077

0.0205

0.0236

0.0285

0.0336

0.0652

0.0747

0.0850

0.0943

0.0991

0.1045

0.1083

0.1185

0.1316

0.1414

0.1521

0.1545

0.1659

0.1698

0.1910

0.2005

0.2096

0.2105

0.2203

0.2265

.2291

0.2244

0.0688 


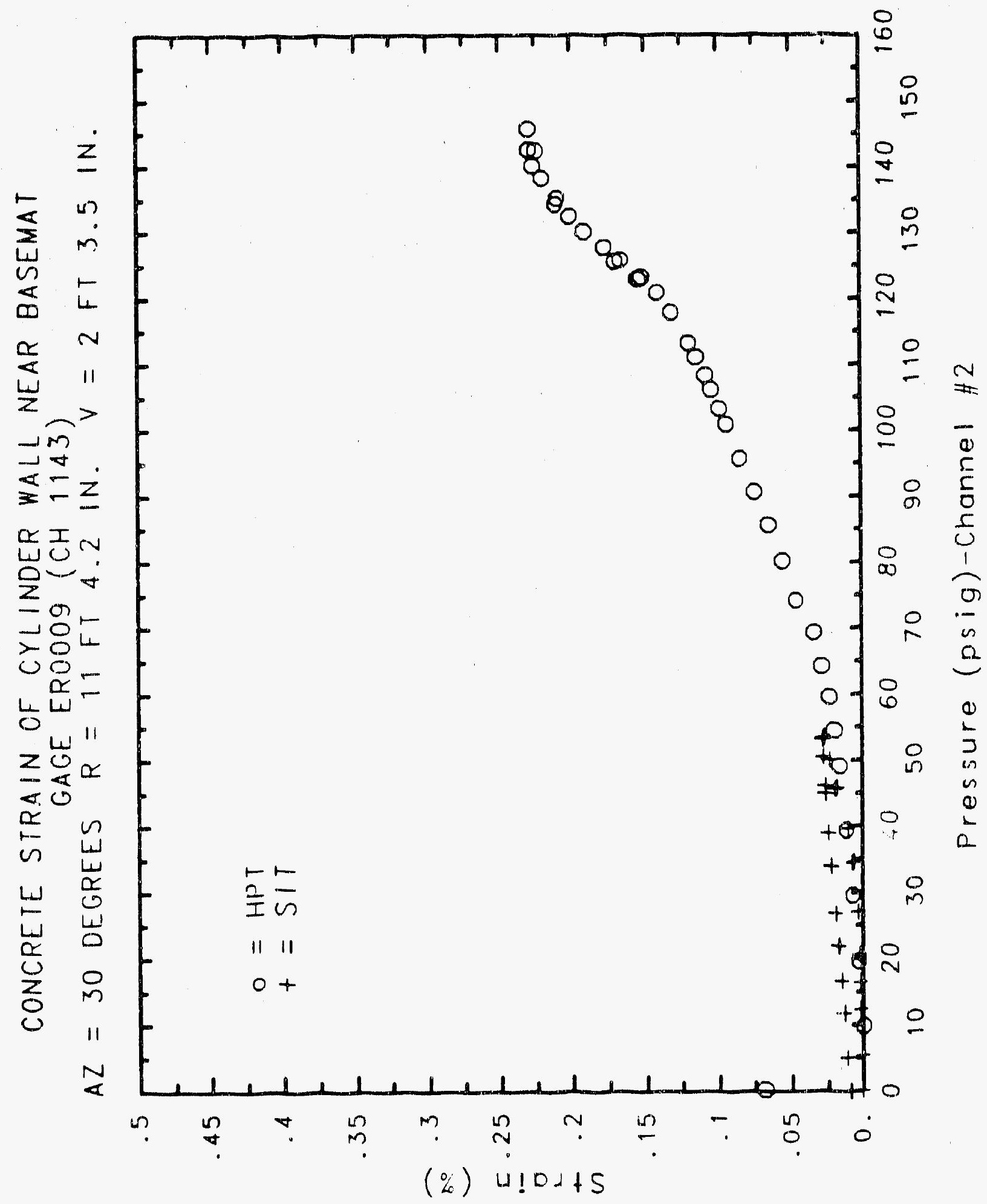

C. 18.35 


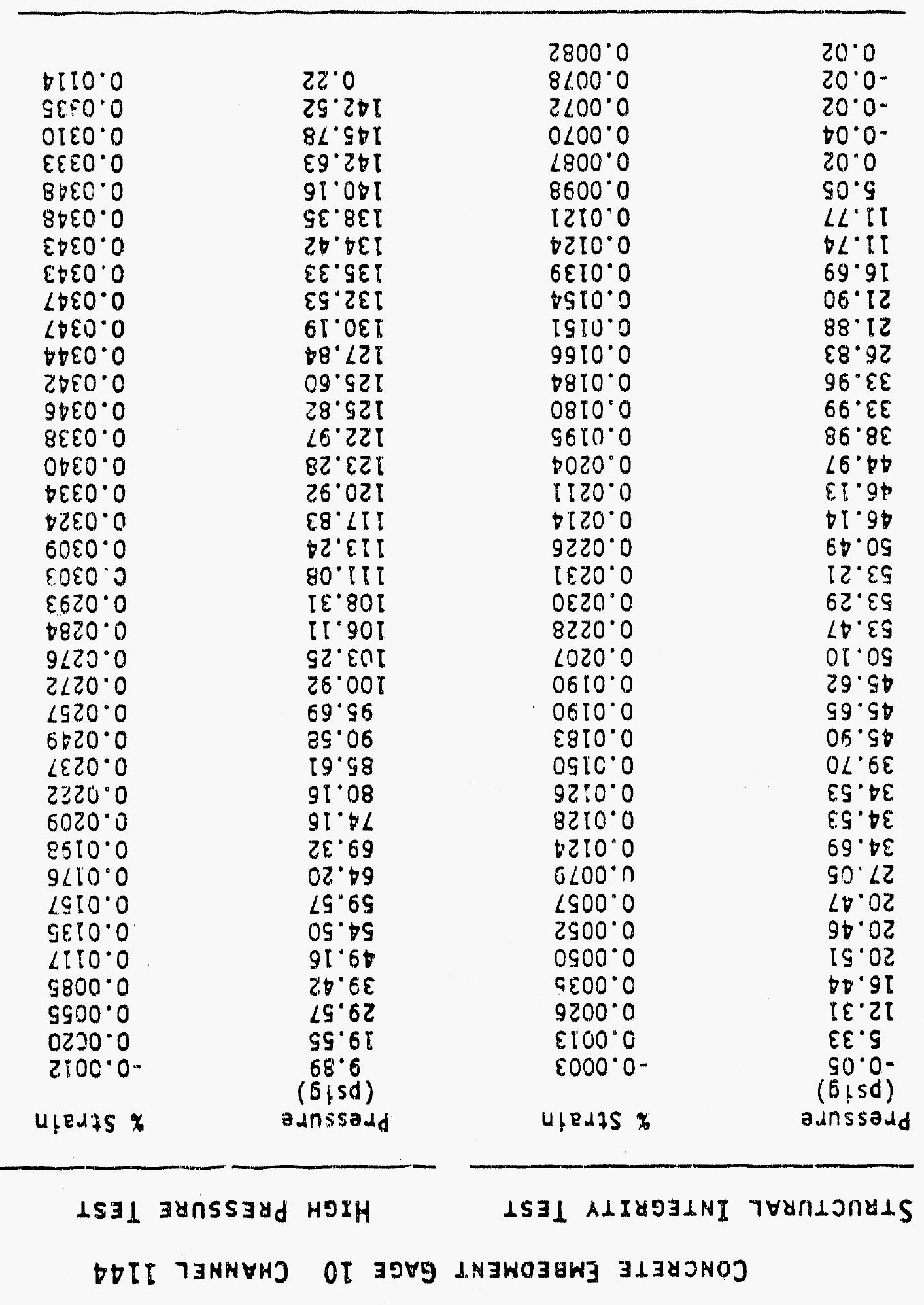

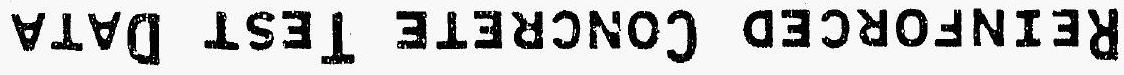




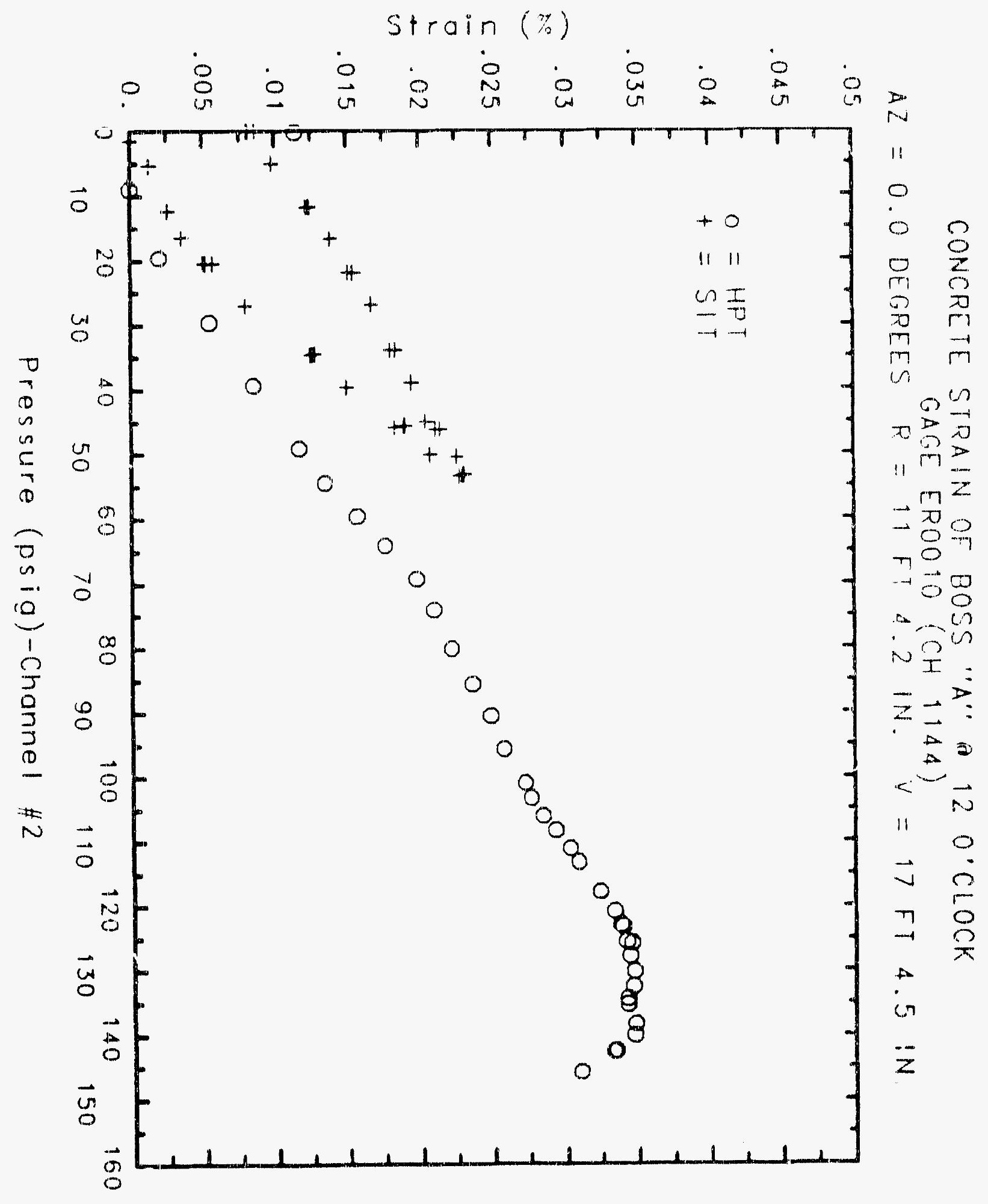




\section{Reinforced ConcretE Test Data}

\section{Concrete Embedment gage 11 Channel 1145}

Structural INTEgRITY TEst

\begin{tabular}{|c|c|c|c|}
\hline $\begin{array}{c}\text { Pressure } \\
(\text { psig) } \\
-0.05 \\
5.33 \\
12.31 \\
16.44 \\
20.51 \\
20.46 \\
20.47 \\
27.05 \\
34.69 \\
34.53 \\
34.53 \\
39.70 \\
45.90 \\
45.65 \\
45.62 \\
50.10 \\
53.47 \\
53.29 \\
53.21 \\
50.49 \\
46.14 \\
46.13 \\
44.97 \\
38.98 \\
33.99 \\
33.96 \\
26.83 \\
21.88 \\
21.90 \\
16.69 \\
11.74 \\
11.77 \\
5.05 \\
0.052 \\
-0.04 \\
-0.02 \\
-0.02 \\
0.02 \\
\end{array}$ & $\begin{array}{l}\text { z Strain } \\
0.0006 \\
0.0020 \\
0.0041 \\
0.0055 \\
0.0096 \\
0.0107 \\
0.0112 \\
0.0210 \\
0.0319 \\
0.0330 \\
0.0331 \\
0.0383 \\
0.0470 \\
0.0480 \\
0.0481 \\
0.0528 \\
0.0568 \\
0.0571 \\
0.0571 \\
0.0559 \\
0.0530 \\
0.0529 \\
0.0508 \\
0.0469 \\
0.0433 \\
0.0432 \\
0.0386 \\
0.0348 \\
0.0346 \\
0.0309 \\
0.0271 \\
0.0271 \\
0.0224 \\
0.01887 \\
0.0172 \\
0.0174 \\
0.0168 \\
0.0165\end{array}$ & $\begin{array}{c}\text { Pressure } \\
(\text { psig) } \\
9.89 \\
19.55 \\
29.57 \\
39.42 \\
49.16 \\
54.50 \\
59.57 \\
64.20 \\
69.32 \\
74.16 \\
80.16 \\
85.61 \\
90.58 \\
95.69 \\
100.92 \\
103.25 \\
106.11 \\
108.31 \\
111.08 \\
113.24 \\
117.83 \\
120.92 \\
123.28 \\
122.97 \\
125.82 \\
125.60 \\
127.84 \\
130.19 \\
132.53 \\
135.33 \\
134.42 \\
138.35 \\
140.16 \\
142.63 \\
145.78 \\
142.52 \\
0.22\end{array}$ & $\begin{array}{l}\text { X strain } \\
0.0031 \\
0.0118 \\
0.0200 \\
0.0268 \\
0.0346 \\
0.0389 \\
0.0429 \\
0.0465 \\
0.0499 \\
0.0529 \\
0.0583 \\
0.0633 \\
0.0683 \\
0.0744 \\
0.0809 \\
0.0840 \\
0.0879 \\
0.0911 \\
0.0957 \\
0.0999 \\
0.1098 \\
0.1166 \\
0.1227 \\
0.1236 \\
0.1318 \\
0.1327 \\
0.1388 \\
0.1522 \\
0.1718 \\
0.2000 \\
0.2073 \\
0.2688 \\
0.3429 \\
0.4763 \\
0.5937 \\
0.6199 \\
0.4374\end{array}$ \\
\hline
\end{tabular}

High Pressure Test

Pressure

19.55

54.50

59.57

69.32

74.16

80.16

95.69

111.08

113.24

11.83

123.28

122.97

125.82

125.60

132.53

135.33

134.42

138.35

40.16

42.52

0.22
* Strain

0.0031

0.0200

.0389

0.0465

0.0499

0.0529

0.0583

0.0744

0.0879

0.0911

0.1098

0.1227

0.1236

0.1318

0.1327

0.1388

1522

0.2000

0.2073

0.2688

0.3429

0.4763

0.5937

49

0.4374 


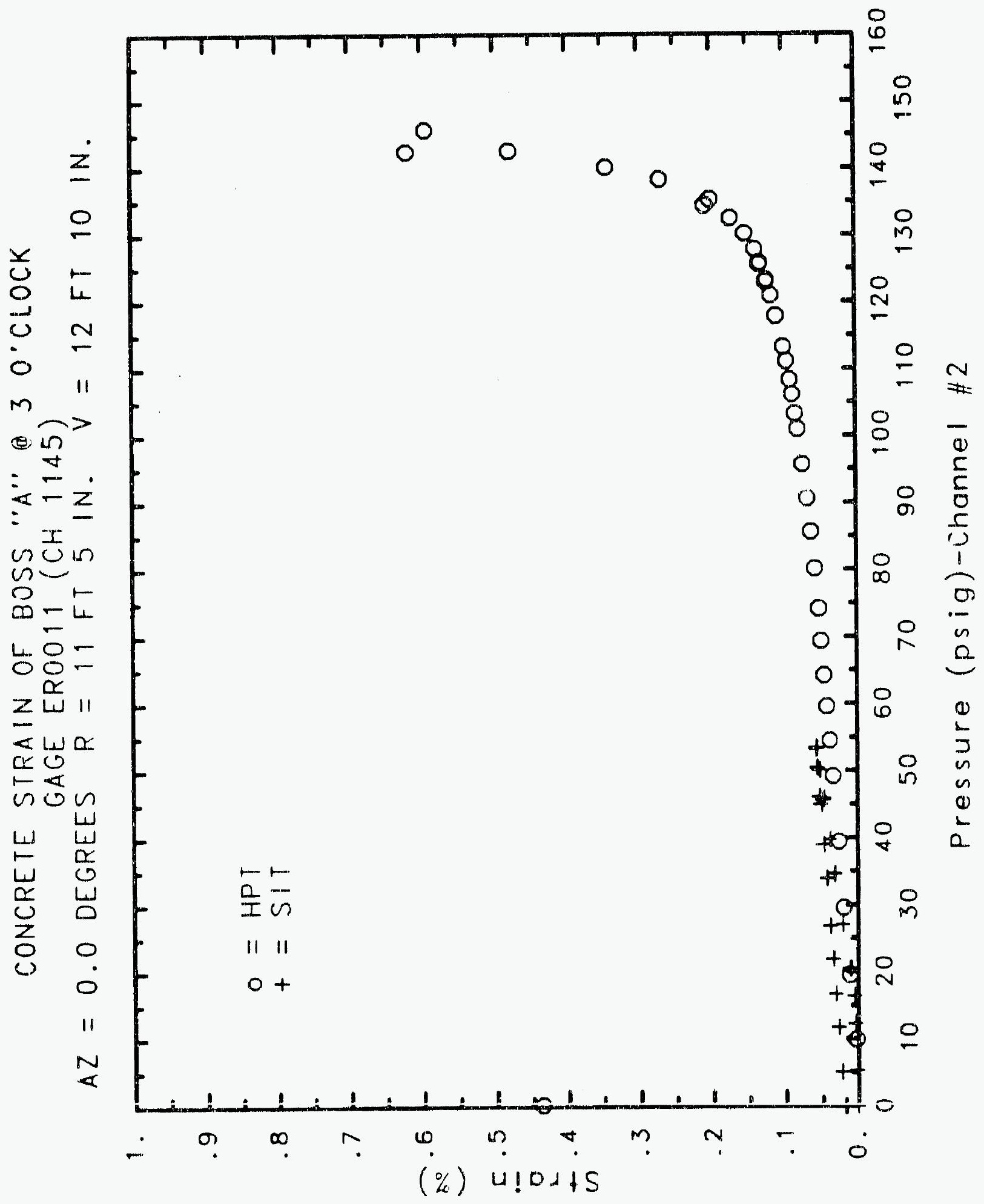




\section{Reinforced Concrete Test Data}

\section{Concrete Embedment gage 12 Channel 1146}

Structural InTEgRITY TEST

\begin{tabular}{|c|c|c|c|}
\hline $\begin{array}{c}\text { Pressure } \\
\text { (psig) } \\
-0.05 \\
5.33 \\
12.31 \\
16.44 \\
20.51 \\
20.46 \\
20.47 \\
27.05 \\
34.69 \\
34.53 \\
34.53 \\
39.70 \\
45.90 \\
45.65 \\
45.62 \\
50.10 \\
53.47 \\
53.29 \\
53.21 \\
50.49 \\
46.14 \\
46.13 \\
44.97 \\
38.98 \\
33.99 \\
33.96 \\
26.83 \\
21.88 \\
21.90 \\
16.69 \\
11.74 \\
11.77 \\
5.05 \\
0.02 \\
-0.04 \\
-0.02 \\
-0.02 \\
0.02\end{array}$ & $\begin{array}{c}\text { Strain } \\
-0.0003 \\
0.0032 \\
0.0081 \\
0.0121 \\
0.0190 \\
0.0207 \\
0.0220 \\
0.0383 \\
0.0582 \\
0.0599 \\
0.0602 \\
0.0687 \\
0.0822 \\
0.0826 \\
0.0834 \\
0.0912 \\
0.0976 \\
0.0979 \\
0.0983 \\
0.0955 \\
0.0900 \\
0.0899 \\
0.0885 \\
0.0813 \\
0.0743 \\
0.0745 \\
0.0642 \\
0.0565 \\
0.0564 \\
0.0486 \\
0.0404 \\
0.0384 \\
0.0287 \\
0.0214 \\
0.0191 \\
0.0190 \\
0.0192 \\
0.0193\end{array}$ & $\begin{array}{c}\text { Pressure } \\
\text { (psig) } \\
9.89 \\
19.55 \\
29.57 \\
39.42 \\
49.16 \\
54.50 \\
59.57 \\
64.20 \\
69.32 \\
74.16 \\
80.16 \\
15.61 \\
90.58 \\
95.69 \\
100.92 \\
103.25 \\
106.11 \\
108.31 \\
111.08 \\
113.24 \\
117.83 \\
120.92 \\
123.28 \\
122.97 \\
125.82 \\
125.60 \\
127.84 \\
130.19 \\
132.53 \\
135.33 \\
134.42 \\
138.35 \\
140.16 \\
142.63 \\
145.78 \\
142.54 \\
0.22\end{array}$ & $\begin{array}{l}\text { \% Strain } \\
0.0116 \\
0.0279 \\
0.0443 \\
0.0598 \\
0.0745 \\
0.0830 \\
0.0914 \\
0.1005 \\
0.1103 \\
0.1201 \\
0.1327 \\
0.1454 \\
0.1577 \\
0.1719 \\
0.1883 \\
0.1959 \\
0.2051 \\
0.2127 \\
0.2239 \\
0.2328 \\
0.2562 \\
0.2743 \\
0.2925 \\
0.2957 \\
0.3214 \\
0.3271 \\
0.3466 \\
0.4076 \\
0.5255 \\
0.7019 \\
0.7445 \\
0.9707 \\
1.1208 \\
1.3143 \\
1.4641 \\
1.4953 \\
1.1513\end{array}$ \\
\hline
\end{tabular}

High Pressure test

Pr

9.89

19.55

29.57

49.16

54.50

69.32

85.61

90.58

95.69

103.25

106.11

108.31

11.08

117.83

120.92

123.28

122.97

125.82

25.60

132.53

135.33

134.42

140.16

142.63

145.78

$42.5 \%$

0.22
0.0116

0.0279

0.0598

0.0745

0.0830

0.0914

0.1005

0.1103

0.1201

. 1327

0.1454

0.1577

0.1719

0.1883

0.1959

0.2051

0.2239

0.2328

0.2562

0.2743

0.2925

0.2957

0.3466

0.5255

0.7019

0.7445

0.9707

1.1208

1.3143

1.4641

1.4953

1.1513 


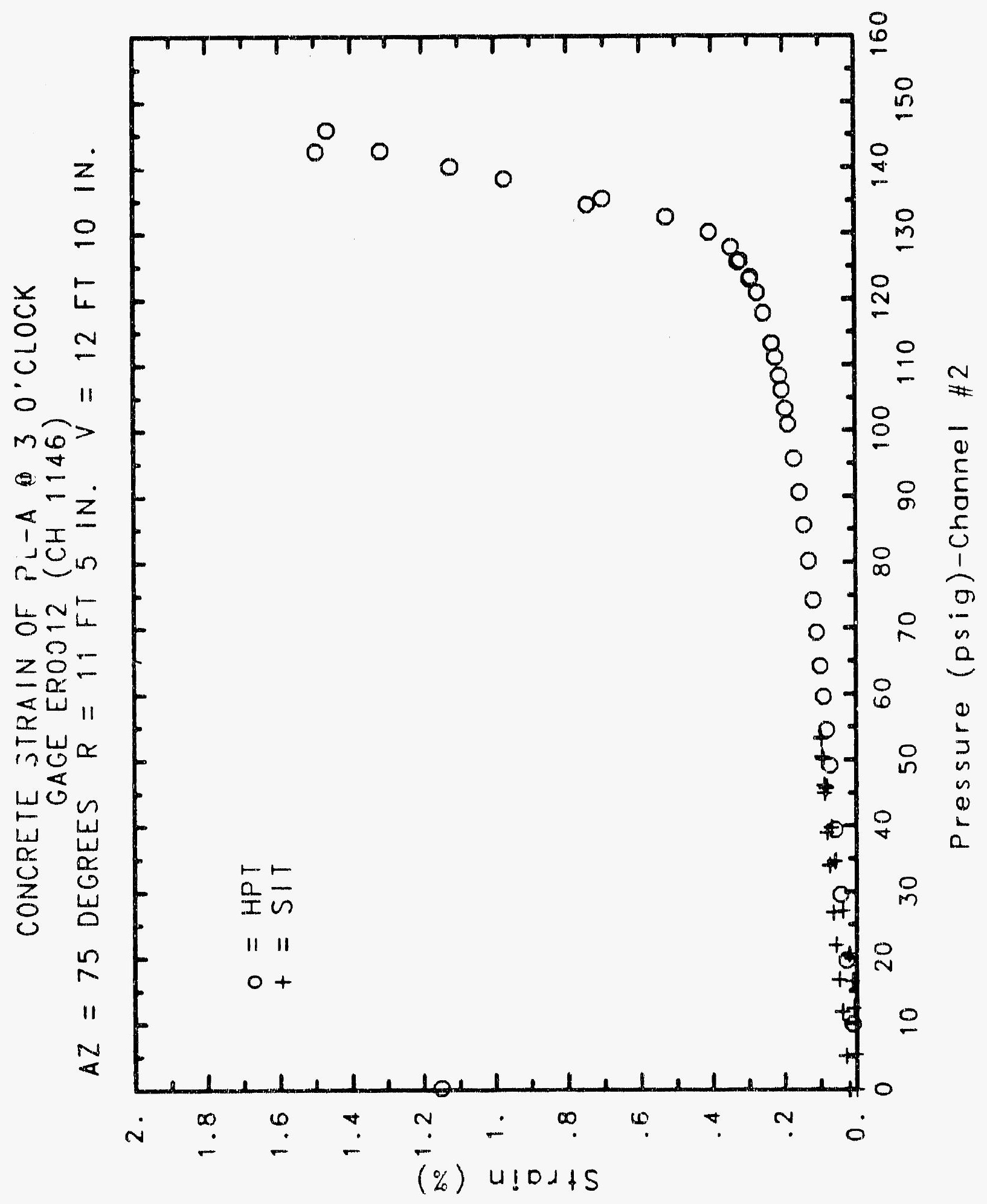




\section{Reinforced Concrete Test Data}

Concrete Embedment Gage 13 Channel 1147

STRUCTURAL INTEGRITY TEST

\begin{tabular}{|c|c|c|c|}
\hline $\begin{array}{c}\text { Pressure } \\
(\text { psig) } \\
-0.05 \\
5.33 \\
12.31 \\
16.44 \\
20.51 \\
20.46 \\
20.47 \\
27.05 \\
34.69 \\
34.53 \\
34.53 \\
39.70 \\
45.90 \\
45.65 \\
45.62 \\
50.10 \\
53.47 \\
53.29 \\
53.21 \\
50.49 \\
46.14 \\
46.13 \\
44.97 \\
38.98 \\
33.99 \\
33.96 \\
26.83 \\
21.88 \\
21.90 \\
16.69 \\
11.74 \\
11.77 \\
5.05 \\
0.02 \\
-0.04 \\
-0.02 \\
-0.02 \\
0.02 \\
\end{array}$ & $\begin{array}{l}\text { X Strain } \\
0.0008 \\
0.0024 \\
0.0042 \\
0.0053 \\
0.0065 \\
0.0067 \\
0.0067 \\
0.0129 \\
0.0204 \\
0.0209 \\
0.0209 \\
0.0249 \\
0.0309 \\
0.0313 \\
0.0312 \\
0.0345 \\
0.0372 \\
0.0377 \\
0.0377 \\
0.0371 \\
0.0356 \\
0.0352 \\
0.0339 \\
0.0322 \\
0.0301 \\
0.0301 \\
0.0273 \\
0.0252 \\
0.0253 \\
0.0228 \\
0.0208 \\
0.0210 \\
0.0180 \\
0.0158 \\
0.0149 \\
0.0146 \\
0.0151 \\
0.0152\end{array}$ & $\begin{array}{c}\text { Pressure } \\
(\text { psig) } \\
9.89 \\
19.55 \\
29.57 \\
39.42 \\
49.16 \\
54.50 \\
59.57 \\
64.20 \\
69.32 \\
74.16 \\
80.16 \\
85.61 \\
90.58 \\
95.69 \\
100.92 \\
103.25 \\
106.11 \\
108.31 \\
111.08 \\
113.24 \\
117.83 \\
120.92 \\
123.28 \\
122.97 \\
125.82 \\
125.60 \\
127.84 \\
130.19 \\
132.53 \\
135.33 \\
134.42 \\
138.35 \\
140.16 \\
142.63 \\
145.78 \\
142.52 \\
0.22 \\
\end{array}$ & $\begin{array}{l}\text { X strain } \\
0.0002 \\
0.0060 \\
0.0118 \\
0.0164 \\
0.0211 \\
0.0250 \\
0.0285 \\
0.0321 \\
0.0359 \\
0.0398 \\
0.0448 \\
0.0494 \\
0.0532 \\
0.0578 \\
0.0626 \\
0.0647 \\
0.0669 \\
0.0688 \\
0.0717 \\
0.0742 \\
0.0799 \\
0.0843 \\
0.0877 \\
0.0885 \\
0.0948 \\
0.0961 \\
0.1005 \\
0.1137 \\
0.1471 \\
0.2171 \\
0.2231 \\
0.3073 \\
0.3476 \\
0.4676 \\
0.5506 \\
0.5728 \\
0.4497\end{array}$ \\
\hline
\end{tabular}

High Pressure Test

Pressure

0.89

19.55

29.57

39.42

59.57

69.32

74.16

80.16

85.61

103.25

111.08

113.24

17.83

122.97

125.82

125.60

127.84

130.19

132.53

134.42

138.35

140.16

42.52

0.5506

0.5728

0.4497 


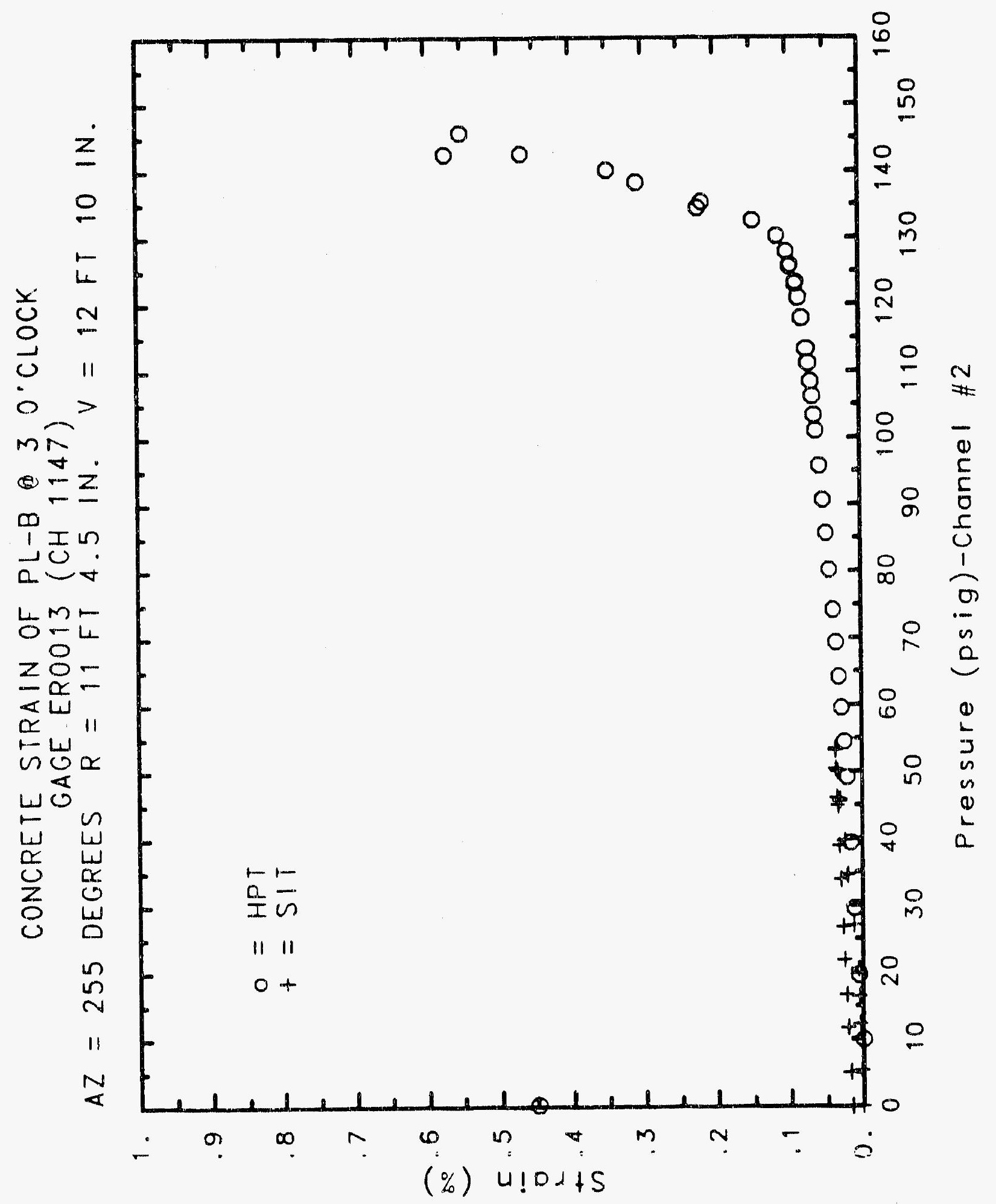

C- 1843 
Reinforced Concrete Test Data

Concrete Embedment gage 14 Channel 1148

STRUCTURAL INTEGRITY TEST

High Pressure test

\begin{tabular}{|c|c|c|c|}
\hline $\begin{array}{c}\text { Pressure } \\
\text { (psig) } \\
-0.05 \\
5.33 \\
12.31 \\
16.44 \\
20.51 \\
20.46 \\
20.47 \\
27.05 \\
34.69 \\
34.53 \\
34.53 \\
39.70 \\
45.90 \\
45.65 \\
45.62 \\
50.10 \\
53.47 \\
53.29 \\
53.21 \\
50.49 \\
46.14 \\
46.13 \\
44.97 \\
38.98 \\
33.99 \\
33.96 \\
26.83 \\
21.88 \\
21.90 \\
16.69 \\
11.74 \\
11.77 \\
5.05 \\
0.02 \\
-0.04 \\
-0.02 \\
-0.02 \\
0.02\end{array}$ & $\begin{array}{l}\text { \% Strain } \\
\text {-0.0003 } \\
0.0007 \\
0.0025 \\
0.0038 \\
0.0070 \\
0.0080 \\
0.0084 \\
0.0143 \\
0.0240 \\
0.0259 \\
0.0263 \\
0.0314 \\
0.0402 \\
0.0411 \\
0.0414 \\
0.0461 \\
0.0508 \\
0.0512 \\
0.0516 \\
0.0507 \\
0.0486 \\
0.0487 \\
0.0474 \\
0.0445 \\
0.0420 \\
0.0421 \\
0.0372 \\
0.0340 \\
0.0342 \\
0.0308 \\
0.0269 \\
0.0261 \\
0.0214 \\
0.0176 \\
0.0148 \\
0.01440 \\
0.0149 \\
0.0153\end{array}$ & $\begin{array}{c}\text { Pressure } \\
\text { (psig) } \\
9.89 \\
19.55 \\
29.57 \\
39.42 \\
49.16 \\
54.50 \\
59.57 \\
64.20 \\
69.32 \\
74.16 \\
80.16 \\
85.61 \\
90.58 \\
95.69 \\
100.92 \\
103.25 \\
106.11 \\
108.31 \\
111.08 \\
113.24 \\
117.83 \\
120.92 \\
123.28 \\
122.97 \\
125.82 \\
125.60 \\
127.84 \\
130.19 \\
132.53 \\
135.33 \\
134.42 \\
138.35 \\
140.16 \\
142.63 \\
145.78 \\
142.52 \\
0.22 \\
\end{array}$ & $\begin{array}{l}\text { \% strain } \\
0.0029 \\
0.0113 \\
0.0195 \\
0.0276 \\
0.0351 \\
0.0397 \\
0.0447 \\
0.0506 \\
0.0579 \\
0.0646 \\
0.0732 \\
0.0807 \\
0.0875 \\
0.0946 \\
0.1017 \\
0.1048 \\
0.1088 \\
0.1121 \\
0.1171 \\
0.1213 \\
0.1330 \\
0.1387 \\
0.1435 \\
0.1445 \\
0.1487 \\
0.1494 \\
0.1531 \\
0.1586 \\
0.1678 \\
0.2044 \\
0.2197 \\
0.3004 \\
0.3795 \\
0.4731 \\
0.5228 \\
0.5309 \\
0.3495\end{array}$ \\
\hline
\end{tabular}




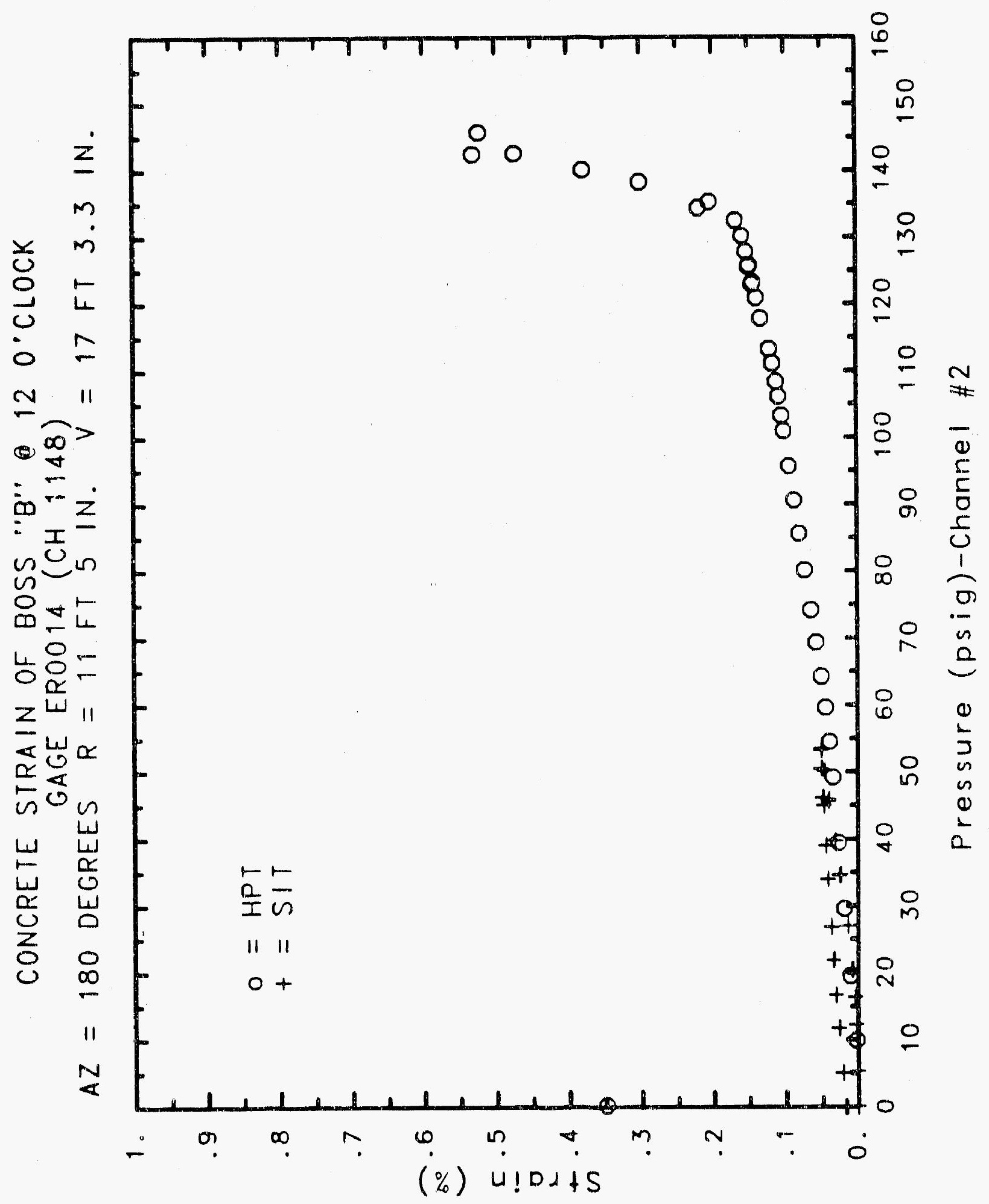




\section{Reinforced Concrete Test Data}

\section{Concrete Embedment Gage 15 Channel 1149}

Structural InTEgRITY TEST

\begin{tabular}{|c|c|c|c|}
\hline $\begin{array}{c}\text { Pressure } \\
\text { (psig) } \\
-0.05 \\
5.33 \\
12.31 \\
16.44 \\
20.51 \\
20.46 \\
20.47 \\
27.05 \\
34.69 \\
34.53 \\
34.53 \\
39.70 \\
45.90 \\
45.65 \\
45.62 \\
50.10 \\
53.47 \\
53.29 \\
53.21 \\
50.49 \\
46.14 \\
46.13 \\
44.97 \\
38.98 \\
33.99 \\
33.96 \\
26.83 \\
21.88 \\
21.90 \\
16.69 \\
11.74 \\
11.77 \\
5.05 \\
0.02 \\
-0.04 \\
-0.02 \\
-0.06 \\
0.02 \\
\end{array}$ & $\begin{array}{l}\text { x Strain } \\
-0.0005 \\
0.0044 \\
0.0102 \\
0.0139 \\
0.0194 \\
0.0204 \\
0.0211 \\
0.0350 \\
0.0511 \\
0.0531 \\
0.0537 \\
0.0622 \\
0.0735 \\
0.0735 \\
0.0734 \\
0.0807 \\
0.0872 \\
0.0878 \\
0.0875 \\
0.0844 \\
0.0786 \\
0.0775 \\
0.0766 \\
0.0688 \\
0.0611 \\
0.0613 \\
0.0507 \\
0.0440 \\
0.0438 \\
0.0366 \\
0.0290 \\
0.0283 \\
0.0195 \\
0.0130 \\
0.0101 \\
0.0103 \\
0.0103 \\
0.0107\end{array}$ & $\begin{array}{c}\text { Pressure } \\
(\text { ps } 19) \\
9.89 \\
19.55 \\
29.57 \\
39.42 \\
49.16 \\
54.50 \\
59.57 \\
64.20 \\
69.32 \\
74.16 \\
80.16 \\
85.61 \\
90.58 \\
95.69 \\
100.92 \\
103.25 \\
106.11 \\
108.31 \\
111.08 \\
113.24 \\
117.83 \\
120.92 \\
123.28 \\
122.97 \\
125.82 \\
125.60 \\
127.84 \\
130.19 \\
132.53 \\
135.33 \\
134.42 \\
138.35 \\
140.16 \\
142.63 \\
145.78 \\
142.52 \\
0.22 \\
\end{array}$ & $\begin{array}{l}\text { \% Strain } \\
0.0097 \\
0.0252 \\
0.0412 \\
0.0565 \\
0.0714 \\
0.0799 \\
0.0894 \\
0.0993 \\
0.1106 \\
0.1230 \\
0.1386 \\
0.1555 \\
0.1728 \\
0.1944 \\
0.2222 \\
0.2364 \\
0.2552 \\
0.2716 \\
0.2988 \\
0.3235 \\
0.3995 \\
0.4632 \\
0.5252 \\
0.5398 \\
0.6302 \\
0.6507 \\
0.7011 \\
0.8162 \\
0.9632 \\
1.1091 \\
1.1405 \\
1.3092 \\
1.4341 \\
1.6259 \\
1.7996 \\
1.8423 \\
1.4513\end{array}$ \\
\hline
\end{tabular}

High Pressure test

Pressure

0.0097

0.0252

0.0714

0.0799

0.0894

0.0993

0.1106

0.1555

0.1728

0.1944

0.2222

0.2364

0.2988

0.3235

0.3995

0.4632

0.5252

0.6507

0.7011

0.8162

0.9632

1.1091

1.1405

1.4341

1.6259

1.7996

1.8423

1.4513 


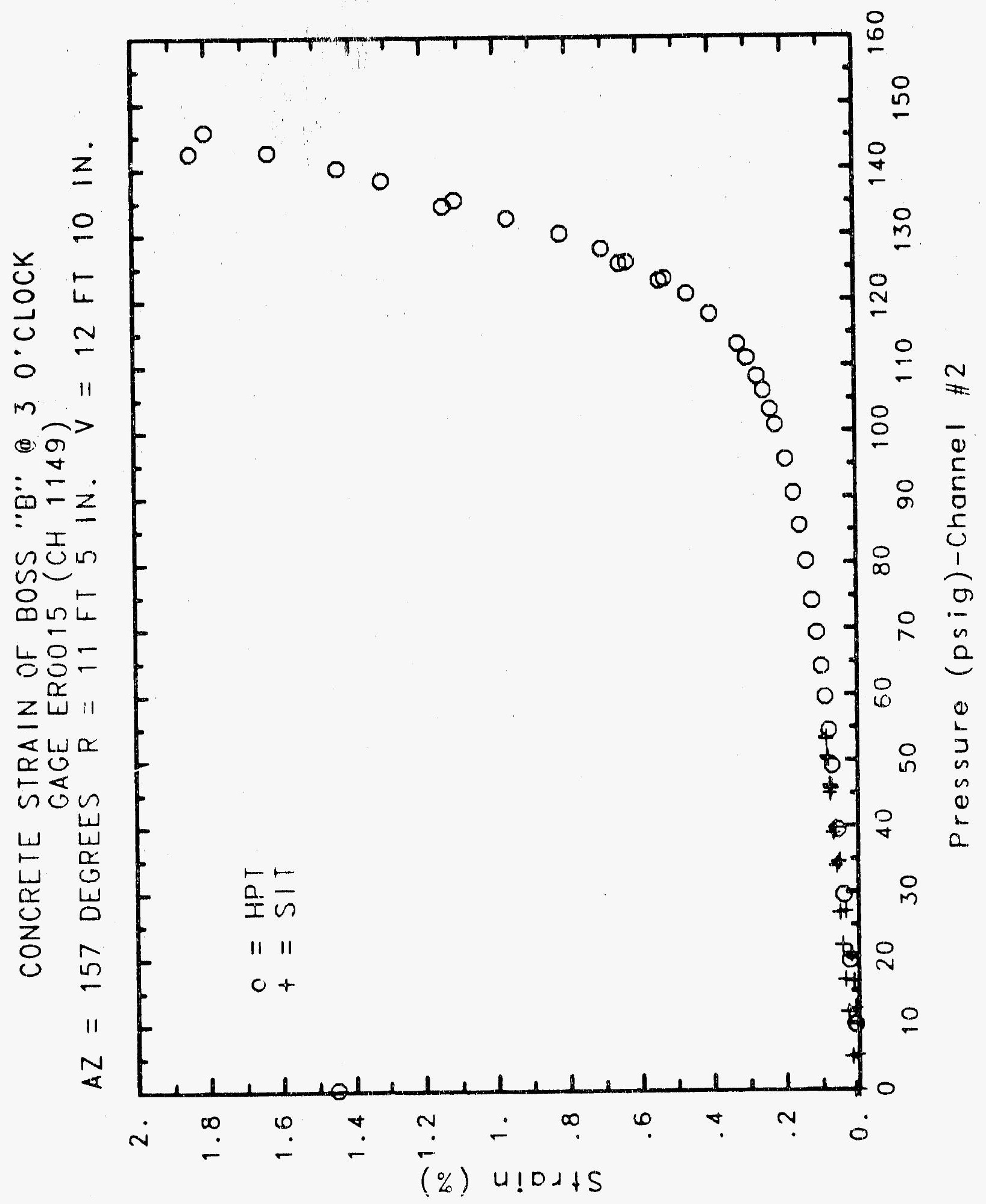




\section{Reinforced Concrete Test Data}

Concrete Embedment Gage 16 Channel 1160

Structural InTEGRITY TEST

\begin{tabular}{|c|c|c|c|}
\hline $\begin{array}{c}\text { Pressure } \\
\text { (psig) } \\
-0.05 \\
5.33 \\
12.31 \\
16.44 \\
20.51 \\
20.46 \\
20.47 \\
27.05 \\
34.69 \\
34.53 \\
34.53 \\
39.70 \\
45.90 \\
45.65 \\
45.62 \\
50.10 \\
53.47 \\
53.29 \\
53.21 \\
50.49 \\
46.14 \\
46.13 \\
44.97 \\
38.98 \\
33.99 \\
33.96 \\
26.83 \\
21.88 \\
21.90 \\
16.69 \\
11.74 \\
11.77 \\
5.05 \\
0.02 \\
-0.04 \\
-0.02 \\
-0.02 \\
0.02 \\
\end{array}$ & $\begin{array}{l}\text { X Stratn } \\
0.0003 \\
0.0007 \\
0.0025 \\
0.0028 \\
0.0049 \\
0.0055 \\
0.0357 \\
0.0102 \\
0.0180 \\
0.0198 \\
0.0204 \\
0.0235 \\
0.0295 \\
0.0305 \\
0.0305 \\
0.0332 \\
0.0357 \\
0.0359 \\
0.0353 \\
0.0353 \\
0.0337 \\
0.0339 \\
0.0328 \\
0.0314 \\
0.0293 \\
0.0294 \\
0.0263 \\
0.0241 \\
0.0229 \\
0.0213 \\
0.0195 \\
0.0194 \\
0.0159 \\
0.0142 \\
0.0125 \\
0.0118 \\
0.0123 \\
0.0123\end{array}$ & $\begin{array}{c}\text { Pressure } \\
(\text { psig) } \\
9.89 \\
19.55 \\
29.57 \\
39.42 \\
49.16 \\
54.50 \\
59.57 \\
64.20 \\
69.32 \\
74.16 \\
80.16 \\
85.61 \\
90.58 \\
95.69 \\
100.92 \\
103.25 \\
106.11 \\
108.31 \\
111.08 \\
113.24 \\
117.83 \\
120.92 \\
123.28 \\
122.97 \\
125.82 \\
125.60 \\
127.84 \\
130.19 \\
132.53 \\
135.33 \\
134.42 \\
138.35 \\
140.16 \\
142.63 \\
145.78 \\
142.52 \\
0.22\end{array}$ & $\begin{array}{c}\text { * Strain } \\
-0.0002 \\
0.0043 \\
0.0088 \\
0.0133 \\
0.0176 \\
0.0201 \\
0.0222 \\
0.0254 \\
0.0285 \\
0.0313 \\
0.0347 \\
0.0376 \\
0.0405 \\
0.0435 \\
0.0463 \\
0.0480 \\
0.0498 \\
0.0514 \\
0.0534 \\
0.0549 \\
0.0585 \\
0.0613 \\
0.0638 \\
0.0642 \\
0.0669 \\
0.0673 \\
0.0690 \\
0.0721 \\
0.0764 \\
0.0825 \\
0.0837 \\
0.0963 \\
0.1054 \\
0.1137 \\
0.1165 \\
0.1175 \\
0.0463\end{array}$ \\
\hline
\end{tabular}

High Pressure Test

Pressure

9.89

19.55

49.15

54.50

69.32

74.16

85.61

90.58

95.69

100.92

111.08

113.24

117.83

122.97

125.82

130.19

32.53

35.33

140.16

142.63

0.22
0.0043

0.0088

0.0176

0.0201

0.0222

0.0254

0.0285

0.0347

0.0376

0.0405

0.0435

0.0463

0.0514

0.0534

0.0549

0.0585

0.0613

0.0669

0.0764

0.0963

0.1054

0.1137

0.1165

0.1175

0.0463 


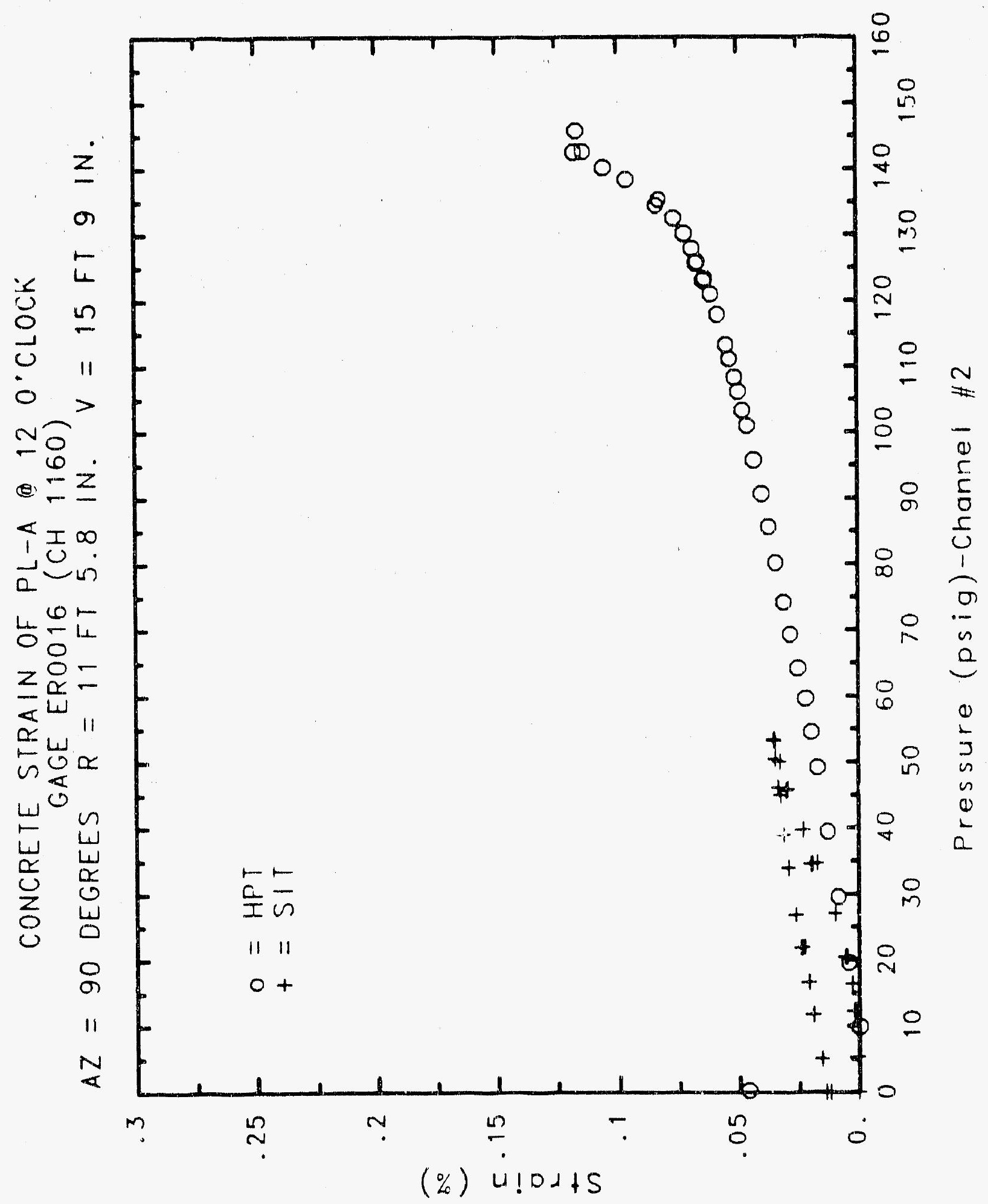




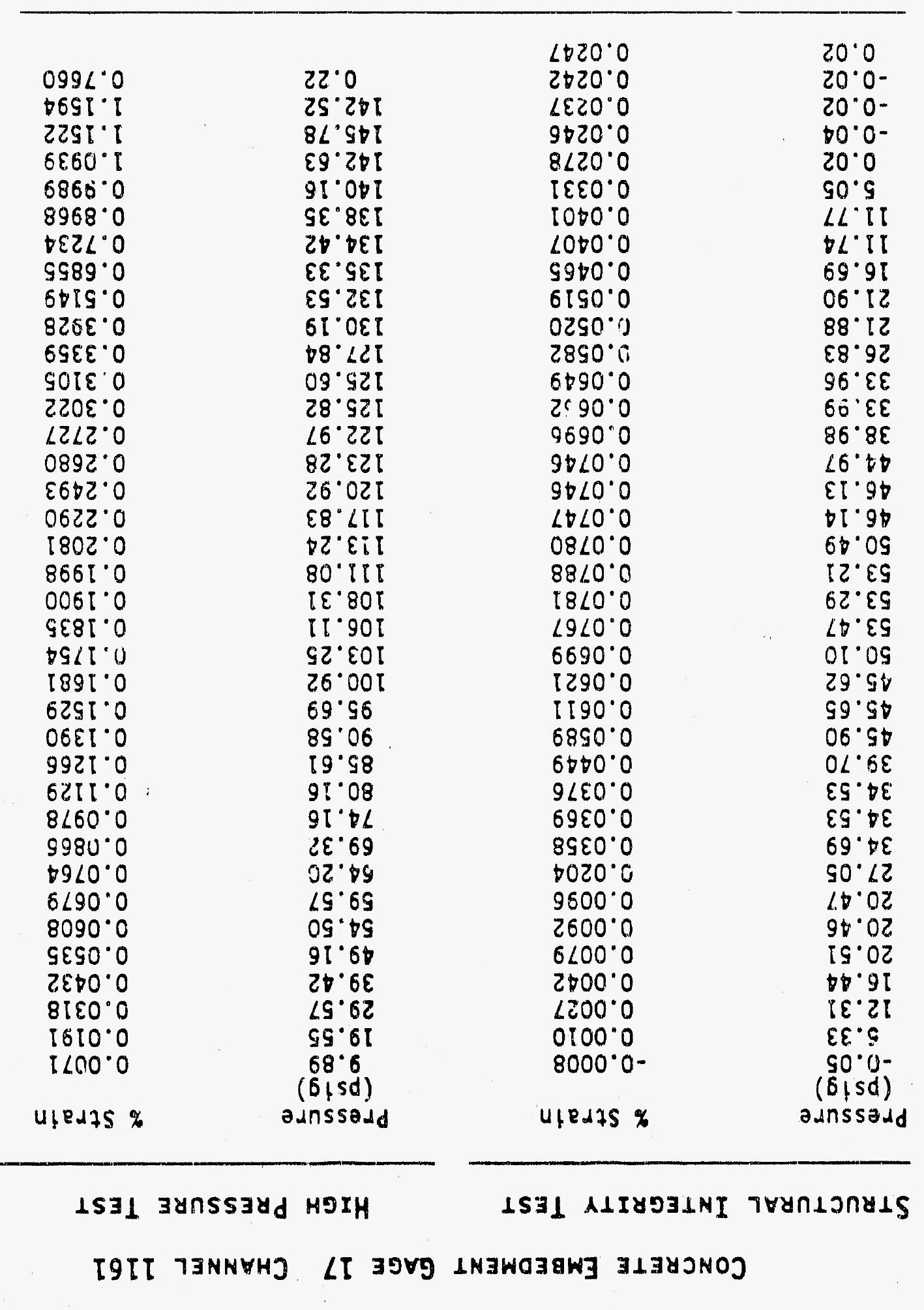

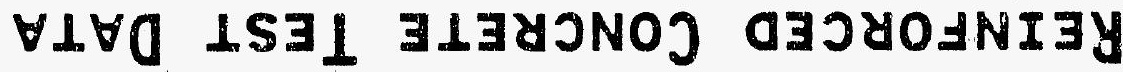




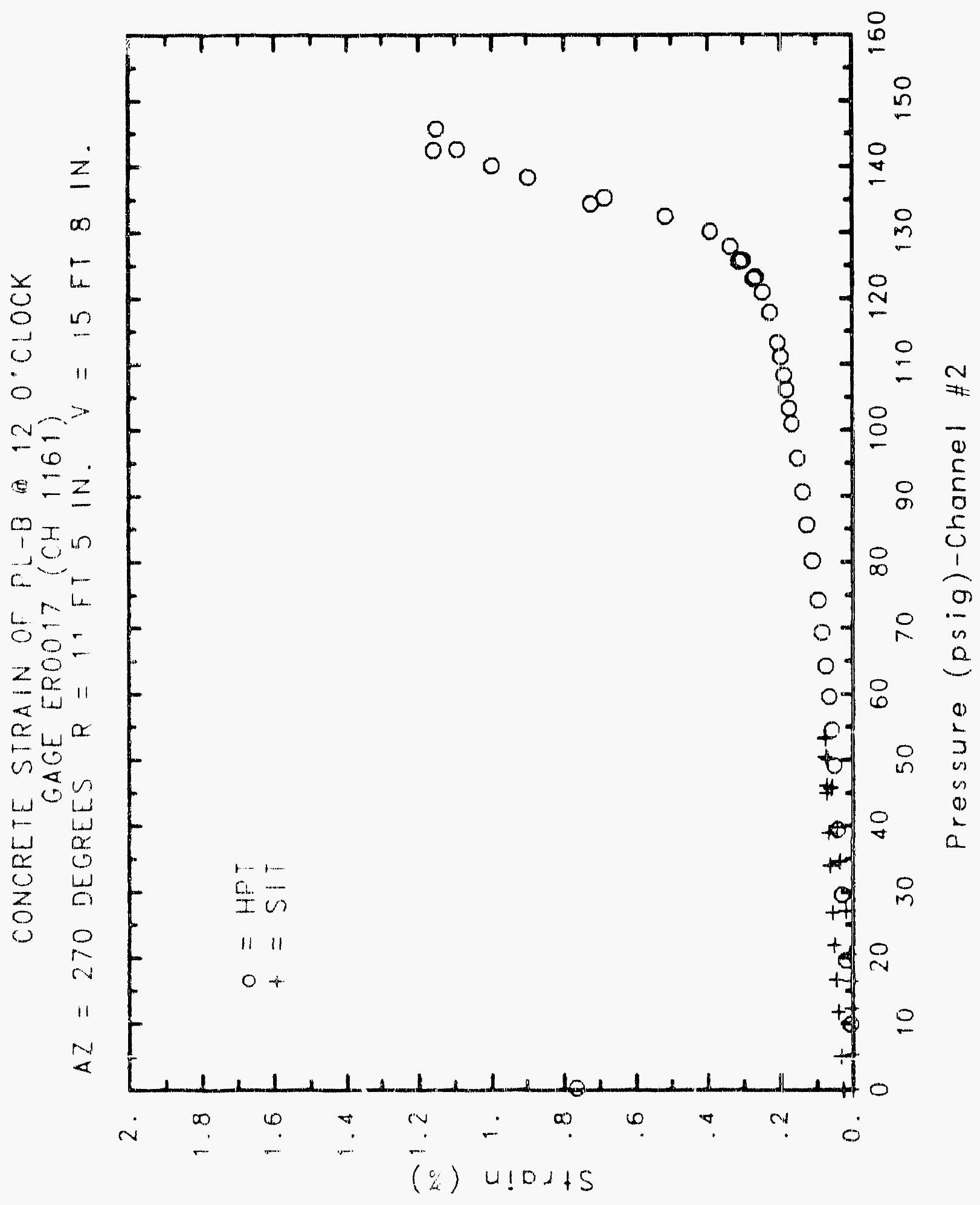




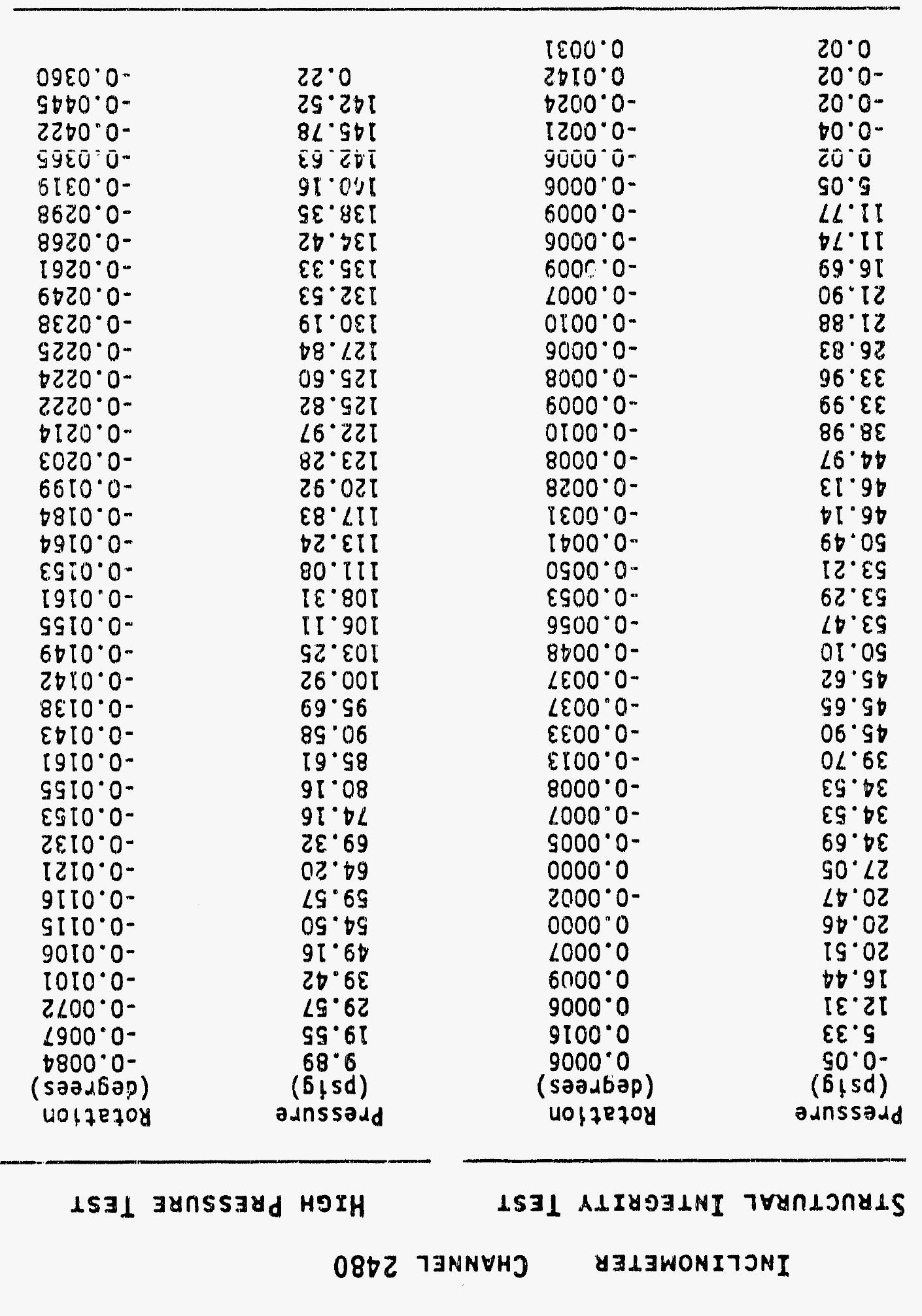

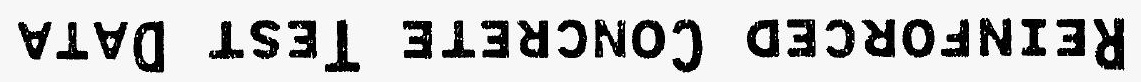




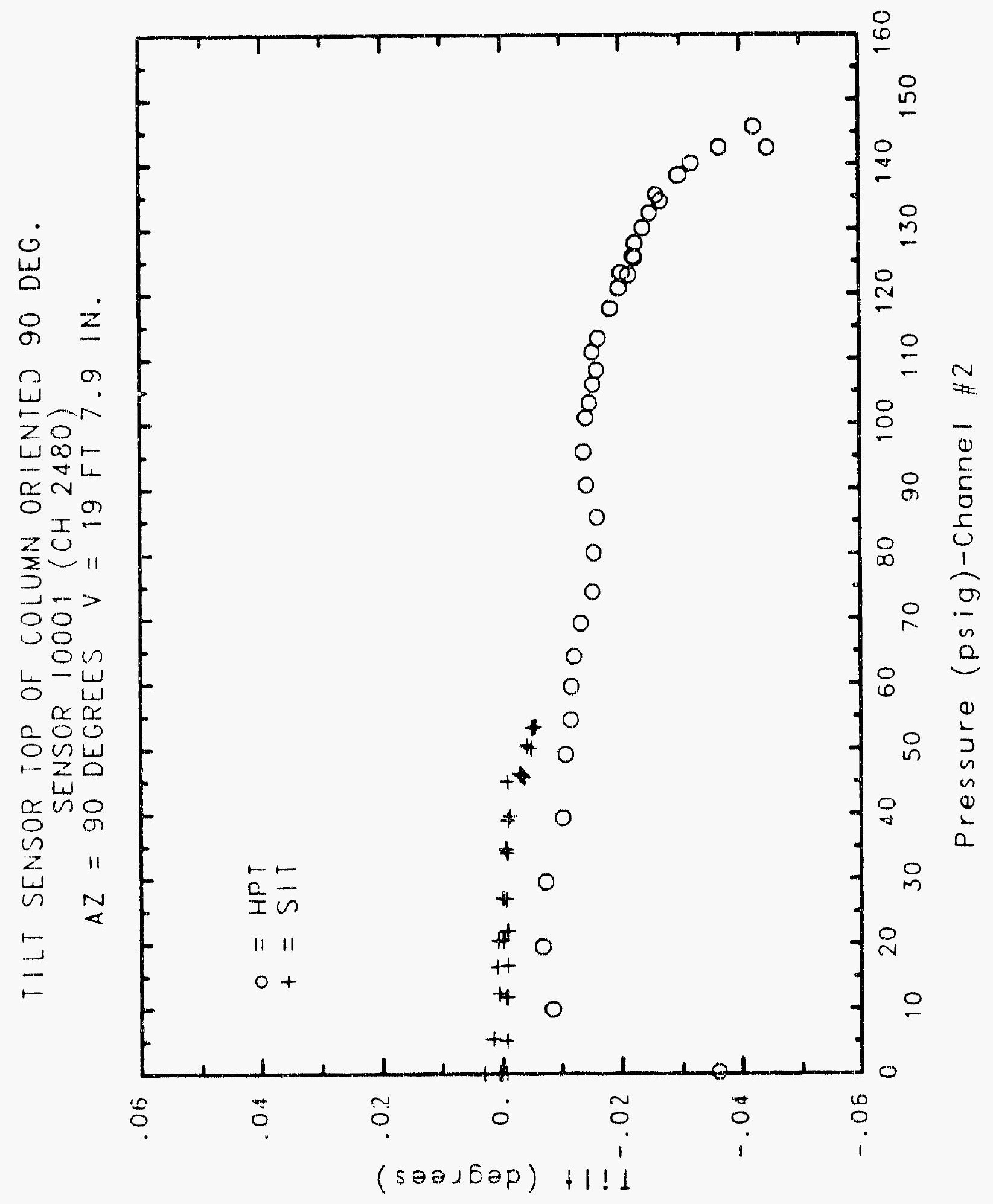




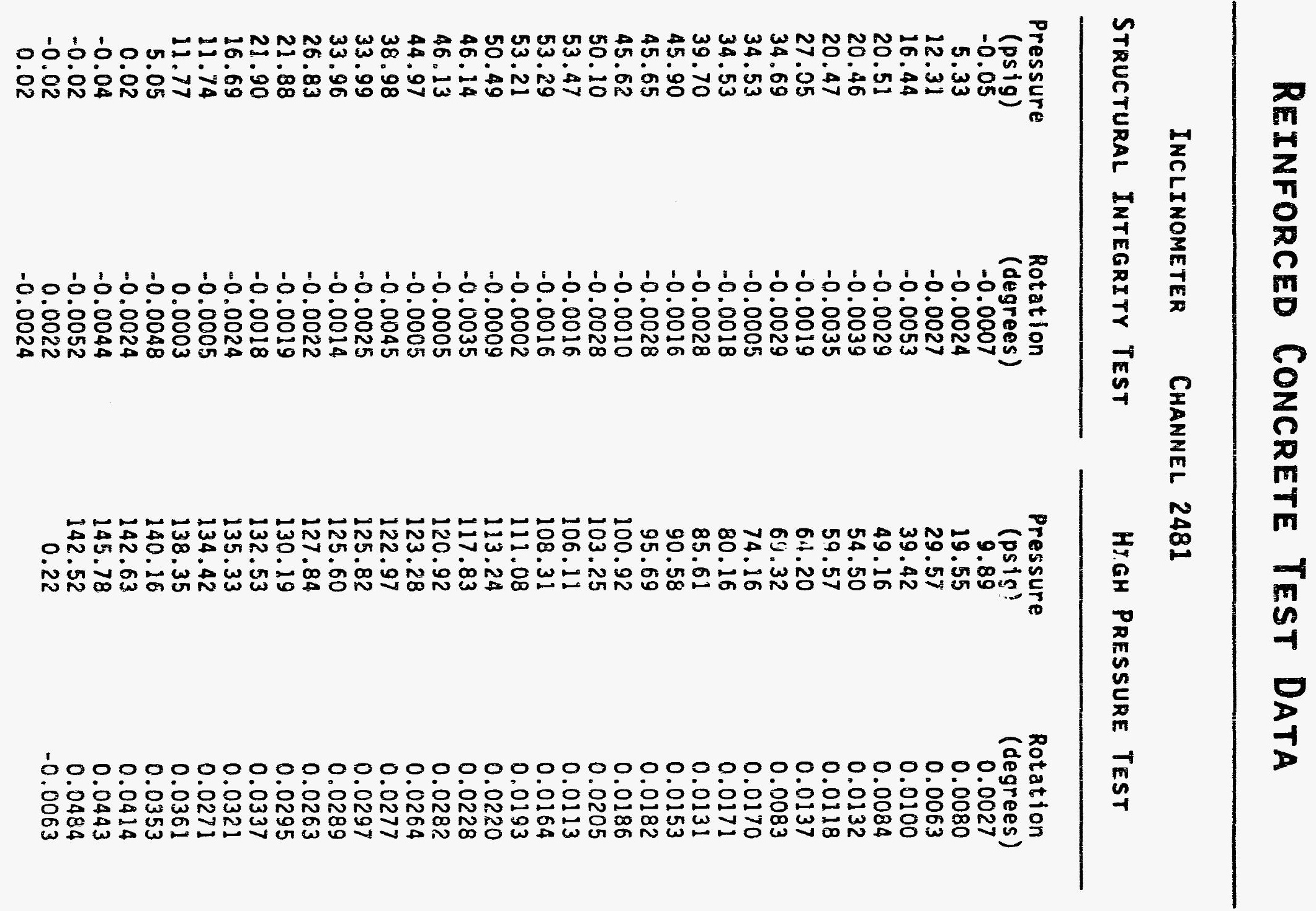




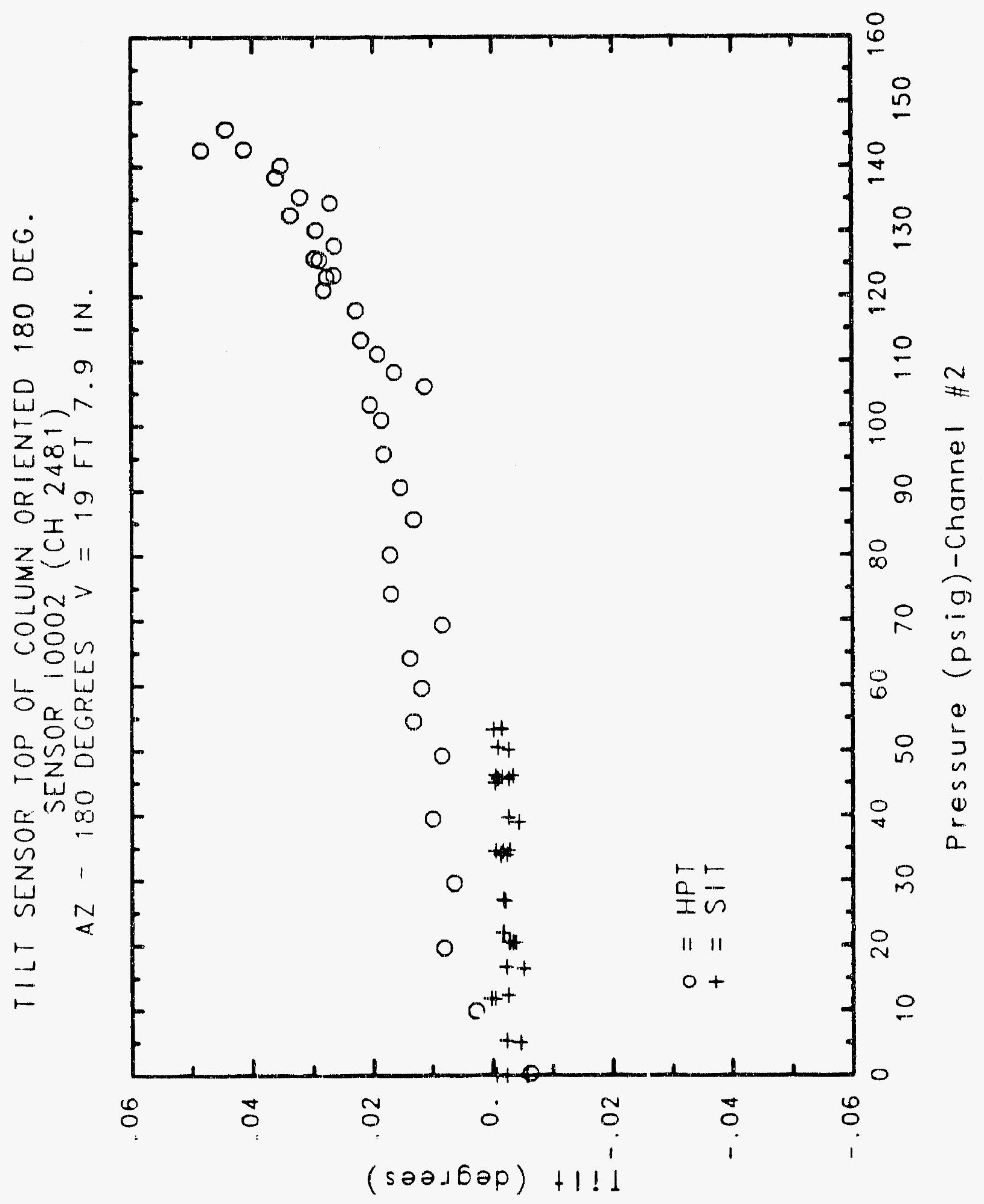




\section{Reinforced Concrete Test Data}

Inclinometer Channel 2482

STRUCTURAL InTEgRITY TEST

\begin{tabular}{|c|c|c|c|}
\hline $\begin{array}{c}\text { Pressure } \\
\text { (psig) } \\
-0.05 \\
5.33 \\
12.31 \\
16.44 \\
20.51 \\
20.46 \\
20.47 \\
27.05 \\
34.69 \\
34.53 \\
34.53 \\
39.70 \\
45.90 \\
45.65 \\
45.62 \\
50.10 \\
53.47 \\
53.29 \\
53.21 \\
50.49 \\
46.14 \\
46.13 \\
44.97 \\
38.98 \\
33.99 \\
33.96 \\
26.83 \\
21.88 \\
21.90 \\
16.69 \\
11.74 \\
11.77 \\
5.05 \\
0.02 \\
-0.04 \\
-0.02 \\
-0.02 \\
0.02\end{array}$ & $\begin{array}{c}\text { Rotation } \\
\text { (degrees) } \\
0.0169 \\
0.0118 \\
0.0069 \\
0.0131 \\
0.0217 \\
0.0224 \\
0.0500 \\
0.0441 \\
0.0398 \\
0.0398 \\
0.0418 \\
0.0386 \\
0.0406 \\
0.0447 \\
0.0459 \\
0.0473 \\
0.0441 \\
0.0454 \\
0.0386 \\
0.0438 \\
0.0338 \\
0.0376 \\
0.0412 \\
0.0516 \\
0.0438 \\
0.0562 \\
0.0643 \\
0.0426 \\
0.0563 \\
0.0424 \\
0.0436 \\
0.0418 \\
0.0393 \\
0.0432 \\
0.0483 \\
0.0388 \\
0.0424 \\
0.0501\end{array}$ & $\begin{array}{c}\text { Pressure } \\
\text { (psig) } \\
9.89 \\
19.55 \\
29.57 \\
39.42 \\
49.16 \\
54.50 \\
59.57 \\
64.20 \\
69.32 \\
74.16 \\
80.16 \\
85.61 \\
90.58 \\
95.69 \\
100.92 \\
103.25 \\
106.11 \\
108.31 \\
111.08 \\
113.24 \\
117.83 \\
120.92 \\
123.28 \\
122.97 \\
125.82 \\
125.60 \\
127.84 \\
130.19 \\
132.53 \\
135.33 \\
134.42 \\
138.35 \\
140.16 \\
142.63 \\
145.78 \\
142.52 \\
0.22\end{array}$ & $\begin{array}{c}\text { Rotation } \\
\text { (degrees) } \\
0.0193 \\
0.0220 \\
0.0276 \\
0.0273 \\
0.0271 \\
0.0266 \\
0.0244 \\
0.0338 \\
0.0354 \\
0.0383 \\
-0.0010 \\
0.0042 \\
0.0035 \\
-0.0003 \\
-0.0019 \\
0.0046 \\
-0.0045 \\
0.0001 \\
-0.0003 \\
0.0022 \\
0.0028 \\
-0.0009 \\
0.0340 \\
0.0481 \\
0.0218 \\
0.0271 \\
0.0306 \\
0.0227 \\
0.0264 \\
0.0345 \\
0.0355 \\
0.0423 \\
0.0373 \\
0.0444 \\
0.0390 \\
0.0304 \\
-0.0234\end{array}$ \\
\hline
\end{tabular}

High Pressure test

Rotation

0.0193

0.0273

0.0271

0.0266

0.0354

0.0042

0.0035

0.0046

$-0.0045$

0.0022

$-0.0009$

0.0340

0.0481

0.0306

0.0264

0.0345

0.0373

ก. $03 n a$ 


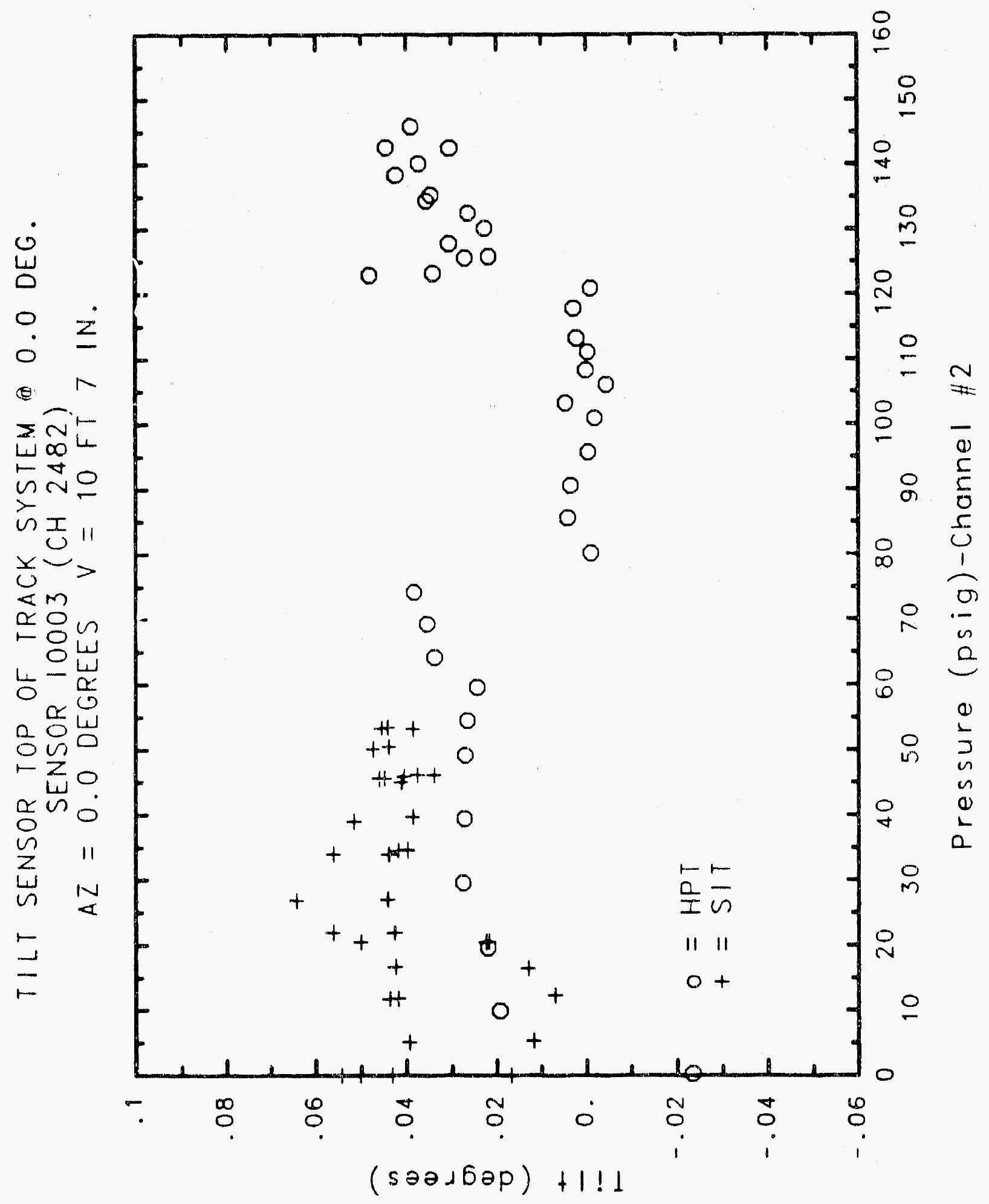




\section{Reinforced Concrete Test Data}

InClinometer Channel 2483

Structural Integrity Test

\begin{tabular}{|c|c|c|c|}
\hline $\begin{array}{c}\text { Pressure } \\
\text { (psig) } \\
-0.05 \\
5.33 \\
12.31 \\
16.44 \\
20.51 \\
20.46 \\
20.47 \\
27.05 \\
34.69 \\
34.53 \\
34.53 \\
39.70 \\
45.90 \\
45.65 \\
45.62 \\
50.10 \\
53.47 \\
53.29 \\
53.2 .1 \\
50.49 \\
46.14 \\
46.13 \\
44.97 \\
38.98 \\
33.99 \\
33.96 \\
26.83 \\
21.88 \\
21.90 \\
16.69 \\
11.74 \\
11.77 \\
5.05 \\
0.02 \\
-0.04 \\
-0.02 \\
-0.02 \\
0.02 \\
\end{array}$ & $\begin{array}{c}\text { Rotation } \\
\text { (degrees) } \\
-0.0204 \\
-0.0262 \\
-0.0195 \\
-0.0212 \\
-0.0224 \\
-0.0255 \\
-0.0418 \\
-0.0221 \\
-0.0189 \\
-0.0259 \\
-0.0247 \\
-0.0199 \\
-0.0152 \\
-0.0129 \\
-0.0129 \\
-0.0145 \\
-0.0127 \\
-0.0011 \\
-0.0057 \\
-0.0080 \\
-0.0068 \\
-0.0004 \\
-0.0067 \\
0.0143 \\
0.0026 \\
-0.0170 \\
-0.0047 \\
-0.0194 \\
-0.0177 \\
-0.0157 \\
-0.0147 \\
-0.0096 \\
-0.0105 \\
-0.0056 \\
0.0121 \\
-0.0012 \\
-0.0031 \\
-0.0030\end{array}$ & $\begin{array}{c}\text { Pressure } \\
\text { (psig) } \\
9.89 \\
19.55 \\
29.57 \\
39.42 \\
49.16 \\
54.50 \\
59.57 \\
64.20 \\
69.32 \\
74.16 \\
80.16 \\
85.51 \\
90.58 \\
95.69 \\
100.92 \\
103.25 \\
106.11 \\
108.31 \\
111.08 \\
113.24 \\
117.83 \\
120.92 \\
123.28 \\
122.97 \\
125.82 \\
125.60 \\
127.84 \\
130.19 \\
132.53 \\
135.33 \\
134.42 \\
138.35 \\
140.16 \\
142.63 \\
145.78 \\
142.52 \\
0.22\end{array}$ & $\begin{array}{c}\text { Rotation } \\
\text { (degrees) } \\
-0.0083 \\
-0.0063 \\
-0.0065 \\
-0.0075 \\
-0.0076 \\
-0.0052 \\
-0.0025 \\
-0.0034 \\
0.0010 \\
-0.0010 \\
0.0309 \\
0.0379 \\
0.0419 \\
0.0484 \\
0.0532 \\
0.0551 \\
0.0580 \\
0.0604 \\
0.0640 \\
0.0665 \\
0.0738 \\
0.0781 \\
0.0755 \\
0.0846 \\
0.0771 \\
0.0916 \\
0.1006 \\
0.0947 \\
0.0926 \\
0.1051 \\
0.1138 \\
0.1167 \\
0.1163 \\
0.1294 \\
0.1487 \\
0.1503 \\
0.0175\end{array}$ \\
\hline
\end{tabular}

\section{High Pressure test}

Pressure

degrees )

$-0.0083$

$-0.0063$

$-0.0075$

$-0.0076$

$-0.0052$

$-0.0025$

0.0010

0.0309

0.0379

0.0419

0.0484

0.0532

0.0580

0.0604

0.0640

0.0665

0.0738

0.0846

0.0771

0.0916

0.1006

0.1051

0.1138

0.1167

0.1163

0.1294

0.1503

0.0175 


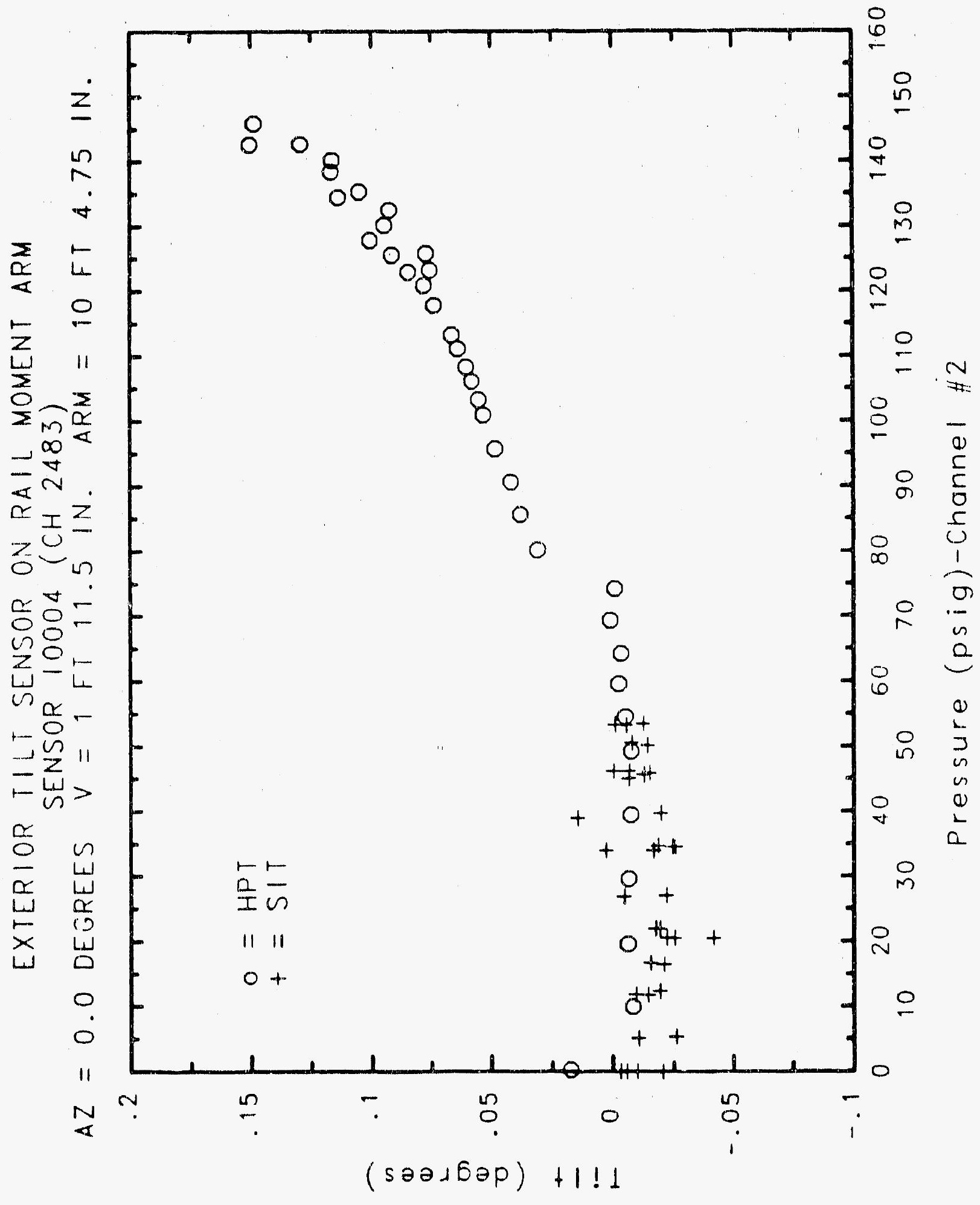

C- 1859 


\section{Reinforced Concrete Test Data}

Inclinometer Channel 2484

StRUCTURAL INTEGRITY TEST

\begin{tabular}{|c|c|c|c|}
\hline $\begin{array}{l}\text { Pressure } \\
\text { (psig) } \\
-0.05 \\
5.33 \\
12.31 \\
16.44 \\
20.51 \\
20.46 \\
20.47 \\
27.05 \\
34.69 \\
34.53 \\
34.53 \\
39.70 \\
45.90 \\
45.65 \\
45.62 \\
50.10 \\
53.47 \\
53.29 \\
53.21 \\
50.49 \\
46.14 \\
46.13 \\
44.97 \\
38.98 \\
33.99 \\
33.96 \\
26.83 \\
21.88 \\
21.90 \\
16.69 \\
11.74 \\
11.77 \\
5.05 \\
0.02 \\
-0.04 \\
-0.02 \\
-0.02 \\
0.02 \\
\end{array}$ & $\begin{array}{c}\text { Rotation } \\
\text { (degrees) } \\
0.0014 \\
0.0024 \\
0.0072 \\
0.0075 \\
0.0129 \\
0.0132 \\
0.0154 \\
0.0101 \\
0.0068 \\
0.0074 \\
0.0066 \\
0.0050 \\
0.0033 \\
-0.0003 \\
-0.0015 \\
-0.00134 \\
-0.0039 \\
-0.0083 \\
-0.0092 \\
-0.0073 \\
-0.0039 \\
-0.0094 \\
-0.0011 \\
0.0020 \\
0.0065 \\
0.0094 \\
0.0099 \\
0.0072 \\
0.0037 \\
0.0051 \\
0.0051 \\
0.0057 \\
0.0044 \\
0.0040 \\
-0.0045 \\
0.0017 \\
0.0043 \\
0.0089\end{array}$ & $\begin{array}{c}\text { Pressure } \\
(\text { psig) } \\
9.89 \\
19.55 \\
29.57 \\
39.42 \\
49.16 \\
54.50 \\
59.57 \\
64.20 \\
69.32 \\
74.16 \\
80.16 \\
85.61 \\
90.58 \\
95.69 \\
100.92 \\
103.25 \\
106.11 \\
108.31 \\
111.08 \\
113.24 \\
117.83 \\
120.92 \\
123.28 \\
122.97 \\
125.82 \\
125.60 \\
127.84 \\
130.19 \\
132.53 \\
135.33 \\
134.42 \\
138.35 \\
140.16 \\
142.63 \\
145.78 \\
142.52 \\
0.22\end{array}$ & $\begin{array}{c}\text { Rotation } \\
\text { (degrees) } \\
0.0013 \\
0.0012 \\
-0.0002 \\
-0.0045 \\
0.0008 \\
-0.0006 \\
-0.0048 \\
-0.0022 \\
-0.0094 \\
-0.0263 \\
-0.0602 \\
-0.0749 \\
-0.0902 \\
-0.1081 \\
-0.1287 \\
-0.1438 \\
-0.1556 \\
-0.1655 \\
-0.1795 \\
-0.1877 \\
-0.2112 \\
-0.2293 \\
-0.2483 \\
-0.2525 \\
-0.2676 \\
-0.2745 \\
-0.2840 \\
-0.3040 \\
-0.3269 \\
-0.3556 \\
-0.3910 \\
-0.4214 \\
-0.4417 \\
-0.4774 \\
-0.5031 \\
-0.5166 \\
-0.2590\end{array}$ \\
\hline
\end{tabular}

High Pressure test

Pres:

9.89

19.55

29.57

54.50

59.57

64.20

69.32

85.61

.58

100.92

111.08

123.28

122.97

132.53

134.42

138.35

140.16

142.52

$0 . \hat{2} 2$
Rolution

(degrees)

0.0013

0.0012

$-0.0002$

0.0008

$-0.0006$

$-0.0048$

$-0.0022$

$-0.0094$

.0749

$-0.108$

$-0.1287$

$-0.1438$

$-0.1556$

$-0.1877$

$-0.2112$

$-0.2293$

$-0.2483$

$-0.2525$

$-0.2676$

$-0.2745$

$-0.2840$

$-0.3269$

$-0.3556$

$-0.3910$

$-0.4214$

$-0.4417$

$-0.4774$

$-0.5031$

$-0.5166$

$-0.2590$ 


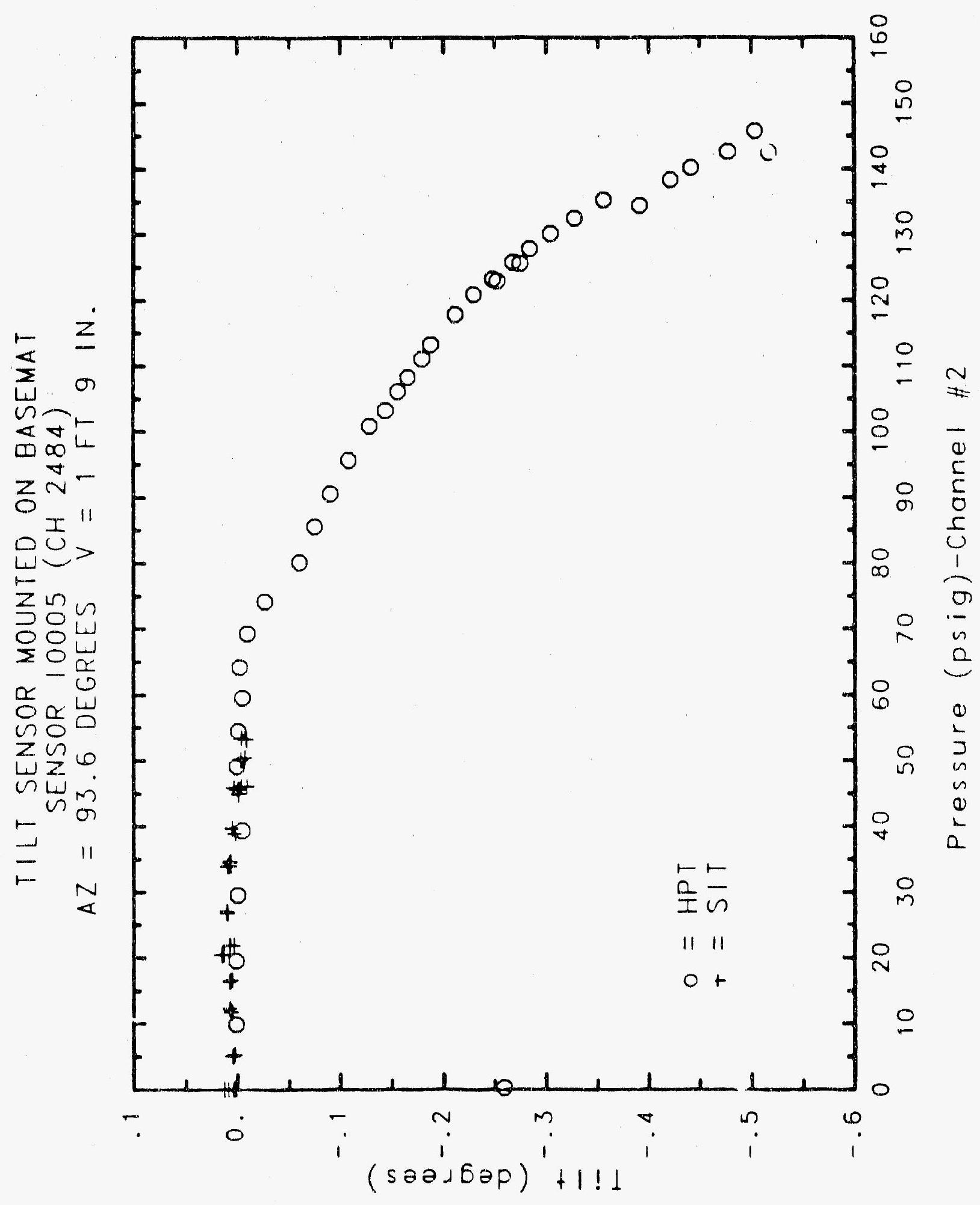




\section{Reinforced Concrete Test Data}

InClinometer Channel 2485

STRUCTURAL INTEGRITY TEST

\begin{tabular}{|c|c|c|c|}
\hline $\begin{array}{l}\text { Pressure } \\
\text { (psig) } \\
-0.05 \\
5.33 \\
12.31 \\
16.44 \\
20.51 \\
20.46 \\
20.47 \\
27.05 \\
34.69 \\
34.53 \\
34.53 \\
39.70 \\
45.90 \\
45.65 \\
45.62 \\
50.10 \\
53.47 \\
53.29 \\
53.21 \\
50.49 \\
46.14 \\
46.13 \\
44.97 \\
38.98 \\
33.99 \\
33.96 \\
26.83 \\
21.88 \\
21.90 \\
16.69 \\
11.74 \\
11.77 \\
5.05 \\
0.02 \\
-0.04 \\
-0.02 \\
-0.02 \\
0.02 \\
\end{array}$ & $\begin{array}{l}\text { Rotation } \\
\text { (degrees) } \\
-0.0011 \\
0.0025 \\
0.0159 \\
0.0087 \\
0.0063 \\
0.0149 \\
0.0074 \\
0.0126 \\
0.0152 \\
0.0145 \\
0.0120 \\
0.0139 \\
0.0145 \\
0.0115 \\
0.0244 \\
0.0223 \\
0.0250 \\
0.0218 \\
0.0167 \\
0.0136 \\
0.0332 \\
0.0210 \\
0.0139 \\
0.0442 \\
0.0293 \\
0.0389 \\
0.0571 \\
0.0360 \\
0.0190 \\
0.0250 \\
0.0246 \\
0.0247 \\
0.0134 \\
0.0166 \\
0.0114 \\
0.0079 \\
0.0104 \\
0.0714\end{array}$ & $\begin{array}{c}\text { Pressure } \\
\text { (psig) } \\
9.89 \\
19.55 \\
29.57 \\
39.42 \\
49.16 \\
54.50 \\
59.57 \\
64.20 \\
69.32 \\
74.16 \\
80.16 \\
85.61 \\
90.58 \\
95.69 \\
100.92 \\
103.25 \\
106.11 \\
108.31 \\
111.08 \\
113.24 \\
117.83 \\
120.92 \\
123.28 \\
122.97 \\
125.82 \\
125.60 \\
127.8 \\
130.19 \\
132.53 \\
135.33 \\
134.42 \\
138.35 \\
140.16 \\
142.63 \\
145.78 \\
142.52 \\
0.22\end{array}$ & $\begin{array}{c}\text { Rotation } \\
\text { (degrees) } \\
0.0054 \\
0.0040 \\
0.0051 \\
0.0056 \\
0.0049 \\
0.0057 \\
0.0089 \\
0.0115 \\
0.0146 \\
0.0233 \\
0.0241 \\
0.0289 \\
0.0353 \\
0.0404 \\
0.0472 \\
0.0505 \\
0.0547 \\
0.0603 \\
0.0651 \\
0.0692 \\
0.0826 \\
0.0880 \\
0.0941 \\
0.0946 \\
0.1020 \\
0.1030 \\
0.1056 \\
0.1188 \\
0.1237 \\
0.1345 \\
0.1498 \\
0.1534 \\
0.1558 \\
0.1824 \\
0.2005 \\
0.2109 \\
0.0342\end{array}$ \\
\hline
\end{tabular}

High Pressure test

Pressure degrees

0.0051

0.0056

0.0049

0.0057

0.0089

0.0115

0.0146

.

0.0353

0.0404

0.0472

0.0505

0.0651

0.0692

0.0826

0.0880

0.1020

0.1188

0.1237

0.1345

0.1498

0.1824

0.2109

0.0342 


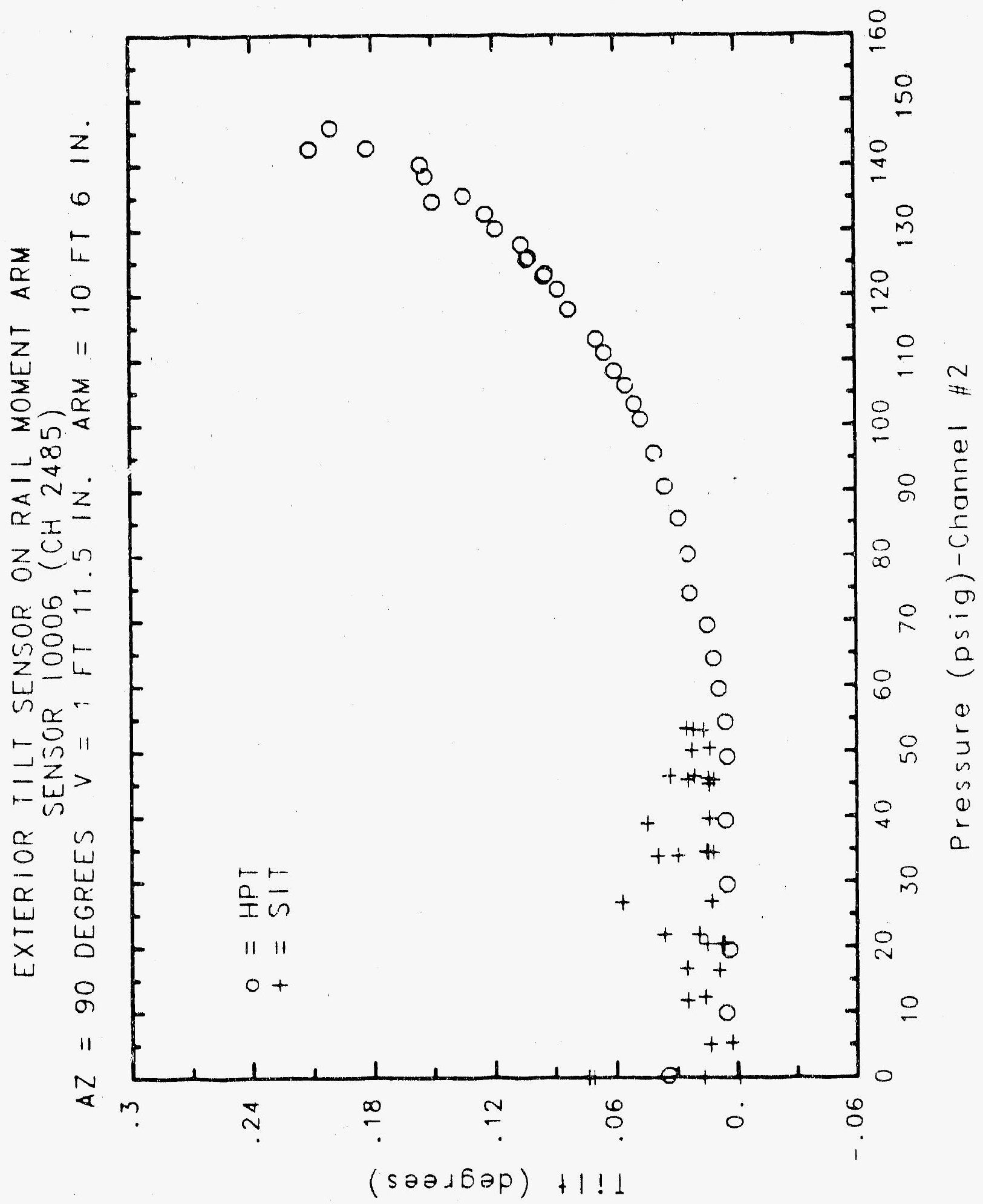

C. -186.3 
Reinforced Concrete Test Data

Inclinometer Channel 2486

Structural INTEgRITY TEST

\begin{tabular}{|c|c|c|c|}
\hline $\begin{array}{c}\text { Pressure } \\
(p s i g) \\
-0.05 \\
5.33 \\
12.31 \\
16.44 \\
20.51 \\
20.46 \\
20.47 \\
27.05 \\
34.69 \\
34.53 \\
34.53 \\
39.70 \\
45.90 \\
45.65 \\
45.62 \\
50.10 \\
53.47 \\
53.29 \\
53.21 \\
50.49 \\
46.14 \\
46.13 \\
44.97 \\
38.98 \\
33.99 \\
33.96 \\
26.83 \\
21.88 \\
21.90 \\
15.69 \\
11.74 \\
11.77 \\
5.05 \\
0.02 \\
-0.04 \\
-0.02 \\
-0.02 \\
0.02 \\
\end{array}$ & $\begin{array}{c}\text { Rotation } \\
\text { (degrees) } \\
0.0055 \\
0.0149 \\
0.0167 \\
0.0116 \\
.0 .0082 \\
-0.0063 \\
0.0008 \\
-0.0049 \\
-0.0239 \\
-0.0214 \\
-0.0195 \\
-0.0180 \\
0.0253 \\
0.0607 \\
0.0500 \\
0.0502 \\
0.0498 \\
0.0426 \\
0.0549 \\
0.0536 \\
0.0428 \\
0.0314 \\
0.0435 \\
0.0395 \\
0.0182 \\
0.0220 \\
0.0135 \\
0.0007 \\
0.0027 \\
-0.0024 \\
0.0021 \\
0.0234 \\
0.0386 \\
0.0365 \\
0.0676 \\
0.0385 \\
0.0288 \\
0.0478\end{array}$ & $\begin{array}{c}\text { Pressure } \\
\text { (psig) } \\
9.89 \\
19.55 \\
29.57 \\
39.42 \\
49.16 \\
54.50 \\
59.57 \\
64.20 \\
69.32 \\
74.16 \\
80.16 \\
85.61 \\
90.58 \\
95.69 \\
100.92 \\
103.25 \\
106.11 \\
108.31 \\
111.08 \\
113.24 \\
117.83 \\
120.92 \\
123.28 \\
122.97 \\
125.82 \\
125.60 \\
127.84 \\
130.19 \\
132.53 \\
135.33 \\
134.42 \\
138.35 \\
140.16 \\
142.63 \\
145.78 \\
142.52 \\
0.22 \\
\end{array}$ & $\begin{array}{l}\text { Rotation } \\
\text { (degrees) } \\
-0.0192 \\
-0.0275 \\
-0.0283 \\
-0.0309 \\
-0.0352 \\
-0.0355 \\
-0.0494 \\
-0.0434 \\
-0.0545 \\
-0.0783 \\
-0.0308 \\
-0.0401 \\
-0.0496 \\
-0.0497 \\
-0.0575 \\
-0.0565 \\
-0.0592 \\
-0.0547 \\
-0.0663 \\
-0.0678 \\
-0.0723 \\
-0.0850 \\
-0.1322 \\
-0.1344 \\
-0.1471 \\
-0.1167 \\
-0.1214 \\
-0.1384 \\
-0.1380 \\
-0.1581 \\
-0.1576 \\
-0.1785 \\
-0.1396 \\
-0.1878 \\
-0.1778 \\
-0.1818 \\
-0.0514\end{array}$ \\
\hline
\end{tabular}

High Pressure test

Rotation

$-0.0192$

$-0.0275$

$-0.0283$

$-0.0355$

$-0.0494$

$-0.0434$

$-0.0308$

$-0.0401$

$-0.0496$

$-0.0497$

$-0.0592$

$-0.0547$

$-0.0663$

$-0.0678$

$-0.1322$

$-0.1344$

$-0.1471$

$-0.1167$

$-0.1380$

$-0.1581$

$-0.1576$

$-0.1785$

$-0.1396$

$-0.1818$

$-0.0514$ 


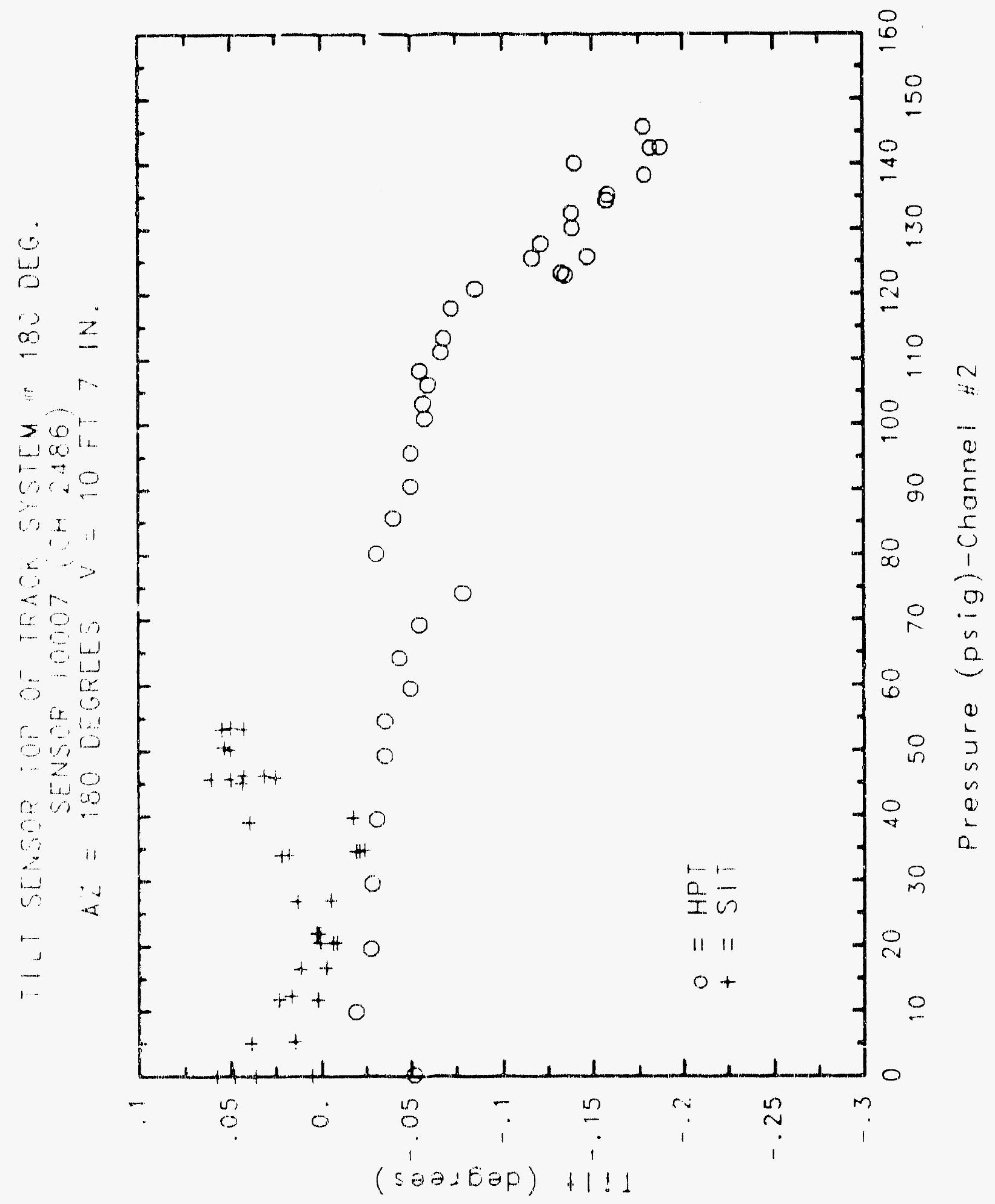




\section{Reinforced Concrete Test Data}

InClinometer Channel 2487

STRUCTURAL INTEGRITY TEST

High Pressure test

\begin{tabular}{|c|c|c|c|}
\hline $\begin{array}{c}\text { Pressure } \\
(\text { ps } 1 \text { g) } \\
-0.05 \\
5.33 \\
12.31 \\
16.44 \\
20.51 \\
20.46 \\
60.47 \\
27.05 \\
34.69 \\
34.53 \\
34.53 \\
39.70 \\
45.90 \\
45.65 \\
45.62 \\
50.10 \\
53.47 \\
53.29 \\
53.21 \\
50.49 \\
46.14 \\
46.13 \\
44.97 \\
38.98 \\
33.99 \\
33.96 \\
26.83 \\
21.88 \\
21.90 \\
16.69 \\
11.74 \\
11.77 \\
5.05 \\
0.02 \\
-0.04 \\
-0.02 \\
-0.02 \\
0.02 \\
\end{array}$ & $\begin{array}{l}\text { Rotation } \\
\text { (degrees) } \\
-0.0134 \\
-0.0223 \\
-0.0220 \\
-0.0285 \\
-0.0373 \\
-0.0359 \\
-0.0373 \\
-0.0387 \\
-0.0419 \\
-0.0450 \\
0.0437 \\
-0.0329 \\
-0.0201 \\
-0.0229 \\
-0.0251 \\
-0.0173 \\
-0.0221 \\
-0.0193 \\
-0.0213 \\
-0.0241 \\
-0.0246 \\
-0.0214 \\
-0.0325 \\
-0.0350 \\
-0.0359 \\
-0.0383 \\
-0.0421 \\
-0.0443 \\
-0.0493 \\
-0.0498 \\
-0.0539 \\
0.0361 \\
-0.0345 \\
-0.0294 \\
-0.0516 \\
-0.0378 \\
-0.0361 \\
-0.0368\end{array}$ & $\begin{array}{c}\text { Pressure } \\
(\text { psig) } \\
9.89 \\
19.55 \\
29.57 \\
39.42 \\
49.16 \\
54.50 \\
59.57 \\
64.20 \\
69.32 \\
74.16 \\
80.16 \\
85.61 \\
90.58 \\
95.69 \\
100.92 \\
103.25 \\
106.11 \\
108.31 \\
111.08 \\
113.24 \\
117.83 \\
120.92 \\
123.28 \\
122.97 \\
125.82 \\
125.60 \\
127.84 \\
130.19 \\
132.53 \\
135.33 \\
134.42 \\
138.35 \\
140.15 \\
142.63 \\
145.78 \\
142.52 \\
0.22 \\
\end{array}$ & $\begin{array}{c}\text { Rotation } \\
\text { (degrees) } \\
0.0085 \\
0.0012 \\
0.0066 \\
0.0046 \\
0.0006 \\
-0.0013 \\
0.0033 \\
-0.0018 \\
0.0016 \\
0.0009 \\
0.0301 \\
0.0339 \\
0.0368 \\
0.0450 \\
0.0493 \\
0.0523 \\
0.0551 \\
0.0573 \\
0.0598 \\
0.0628 \\
0.0688 \\
0.0741 \\
0.0648 \\
0.0513 \\
0.0526 \\
0.0649 \\
0.0647 \\
0.0749 \\
0.0755 \\
0.0873 \\
0.0821 \\
0.0873 \\
0.1081 \\
0.1088 \\
0.1263 \\
0.1436 \\
0.0103\end{array}$ \\
\hline
\end{tabular}




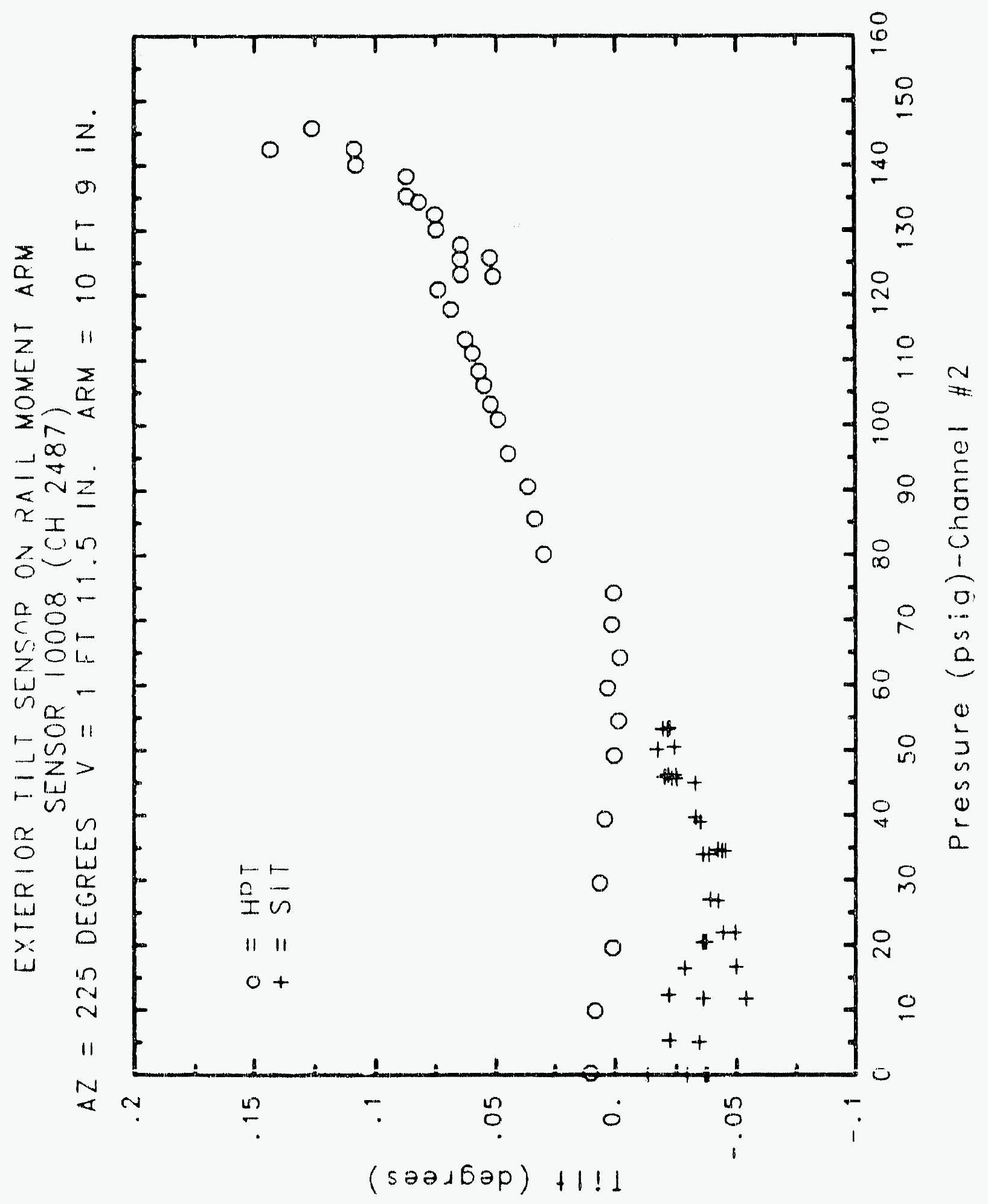

C. -1867 

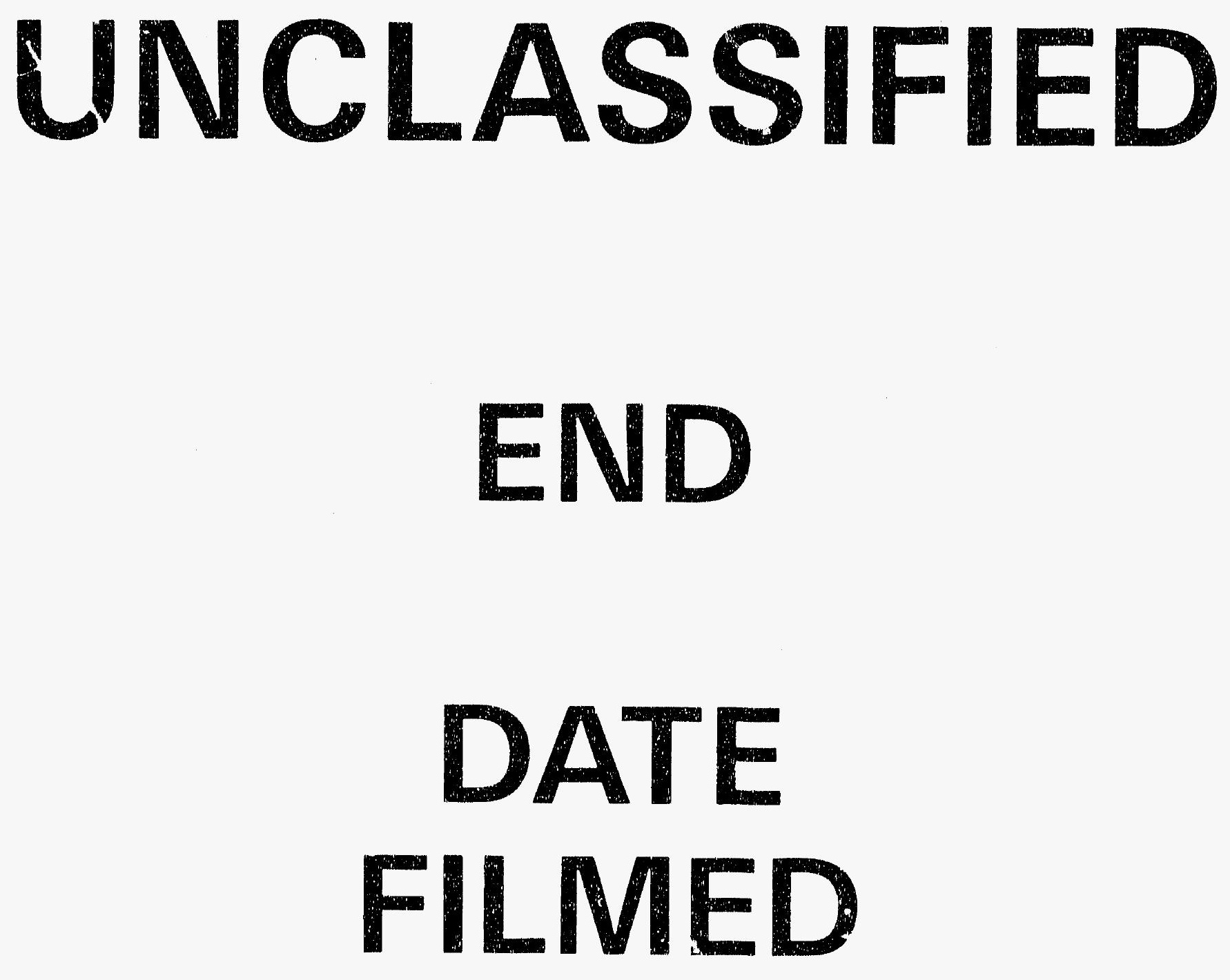

$$
1-6-92
$$

SANDIA NATIONAL LABS 